\section{Pacific Northwest} National Laboratory

Operated by Battelle for the

U.S. Department of Energy

\title{
River Data Package for the 2004 Composite Analysis
}

\author{
C. L. Rakowski \\ G. R. Guensch \\ G. W. Patton
}

August 2004

Prepared for the U.S. Department of Energy under Contract DE-AC06-76RL01830 


\title{
DISCLAIMER
}

This report was prepared as an account of work sponsored by an agency of the United States Government. Neither the United States Government nor any agency thereof, nor Battelle Memorial Institute, nor any of their employees, makes any warranty, express or implied, or assumes any legal liability or responsibility for the accuracy, completeness, or usefulness of any information, apparatus, product, or process disclosed, or represents that its use would not infringe privately owned rights. Reference herein to any specific commercial product, process, or service by trade name, trademark, manufacturer, or otherwise does not necessarily constitute or imply its endorsement, recommendation, or favoring by the United States Government or any agency thereof, or Battelle Memorial Institute. The views and opinions of authors expressed herein do not necessarily state or reflect those of the United States Government or any agency thereof.

\author{
PACIFIC NORTHWEST NATIONAL LABORATORY \\ operated by \\ BATTELLE \\ for the \\ UNITED STATES DEPARTMENT OF ENERGY \\ under Contract DE-AC06-76RL01830
}

Printed in the United States of America
Available to DOE and DOE contractors from the Office of Scientific and Technical Information,
P.O. Box 62, Oak Ridge, TN 37831-0062;
ph: (865) 576-8401
fax: (865) 576-5728
email: reports@adonis.osti.gov

\author{
Available to the public from the National Technical Information Service, \\ U.S. Department of Commerce, 5285 Port Royal Rd., Springfield, VA 22161 \\ ph: (800) 553-6847 \\ fax: (703) 605-6900 \\ email: orders@ntis.fedworld.gov \\ online ordering: http://www.ntis.gov/ordering.htm
}

This document was printed on recycled paper. 


\title{
River Data Package for the 2004 Composite Analysis
}

\author{
C. L. Rakowski \\ G. R. Guensch \\ G. W. Patton
}

August 2004

Prepared for

the U.S. Department of Energy

under Contract DE-AC06-76RL01830

Pacific Northwest National Laboratory

Richland, Washington 99352 


\section{Summary}

A composite analysis is required by U.S. Department of Energy (DOE) Order 435.1 to ensure public safety through the management of active and planned low-level radioactive waste disposal facilities associated with the Hanford Site. The original composite analysis in 1998 must be revised and submitted to DOE Headquarters in 2004 because of revisions to waste site information in the 100, 200, and 300 Areas; updated performance assessments and environmental impact statements; changes in inventory estimates for key sites and constituents; and a change in the definition of offsite receptors.

Beginning in fiscal year 2003, the DOE Richland Operations Office initiated activities, including the development of data packages, to support the 2004 Composite Analysis. The river data package provides calculations of flow and transport in the Columbia River system. The module is based on the legacy code for the Modular Aquatic Simulation System II (MASS2), which is a two-dimensional, depth-averaged model that provides the capability to simulate the lateral (bank-to-bank) variation of flow and contaminants. It simulates river hydrodynamics (water velocities and surface elevations), sediment transport, contaminant transport, biotic transport, and sediment-contaminant interaction, including both suspended sediments and bed sediments. MASS2 applies the general finite-volume principles to a multi-block curvilinear computational grid. Momentum and conservation of mass are coupled using a variation of the Patankar (1980) SIMPLE algorithm.

This document presents the data assembled to run the river module components for the section of the Columbia River from Vernita Bridge to the confluence with the Yakima River. MASS2 requires data on the river flow rate, downstream water surface elevation, groundwater influx, channel bathymetry, and the bed and suspended sediment properties. Stochastic variability for some input parameters such as partition coefficient $\left(\mathrm{k}_{\mathrm{d}}\right)$ values and radionuclide concentrations is generated by the Environmental Stochastic Preprocessor (Eslinger et al. 2002). River flow is randomized on a yearly basis. At this time, the conceptual model does not incorporate extreme flooding (for example, 50 to 100 years) or dam removal scenarios.

Flow data are adequate to the current task of running at a monthly time step. Daily and hourly flow data are available if the time step is reduced in the future. A very good bathymetric grid has been developed that provides increased resolution where necessary. Sediment data are the least available, but there is little evidence demonstrating an immediate need for additional resolution on this model component. Partition coefficient values have been broadly defined with carefully defined distributions based on the best available data and stochastically incorporated into the model. Groundwater inputs are defined by the groundwater model results. Substantial data on radionuclide background levels and river inputs exist, and additional information is continually becoming available through programs such as the Hanford Environmental Information System and Hanford Site Environmental Surveillance. 


\section{Acronyms}

2D

CD

CRCIA

CSTF

DOE

DOE-RL

EIS

ESP

FY

GIS

GWDROP

HEIS

LiDAR

MASS2

NASQAN

RM

SAC

SHOALS

STOMP

USGS

VZDROP two-dimensional

compact disc

Columbia River Comprehensive Impact Assessment

Containment Systems Test Facility

U.S. Department of Energy

U.S. Department of Energy, Richland Operations Office

environmental impact statement

Environmental Stochastic Preprocessor

fiscal year

geographic information system

groundwater data translator

Hanford Environmental Information System

light detection and ranging

Modular Aquatic Simulation System in Two Dimensions

National Stream Water Quality Network

river mile

System Assessment Capability

scanning hydrographic operational LiDAR survey

Surface Transport Over Multiple Phases

U.S. Geological Survey

VADose zone Environmental Release 


\section{Contents}

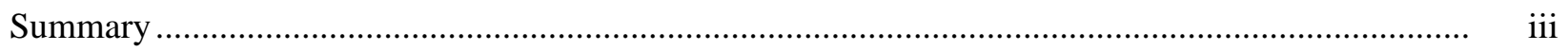

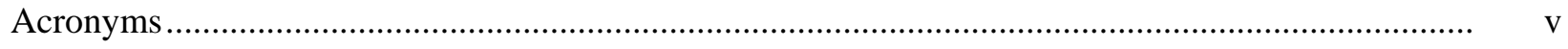

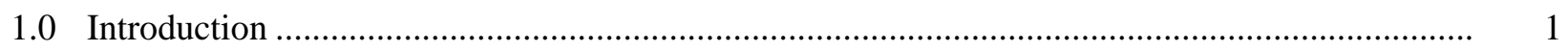

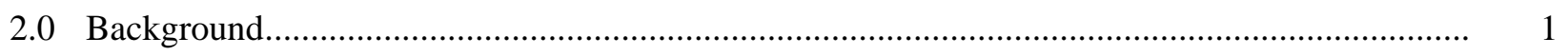

3.0 Data Gathering Methods and Data Limitations .............................................................. 2

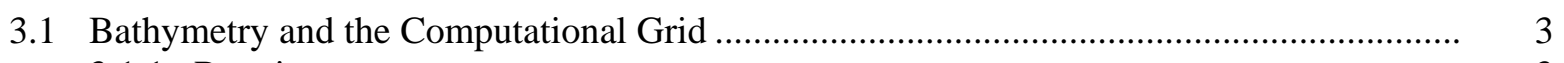

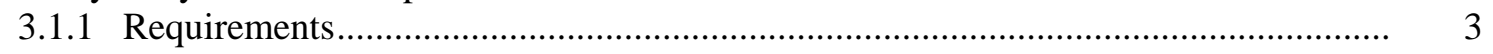

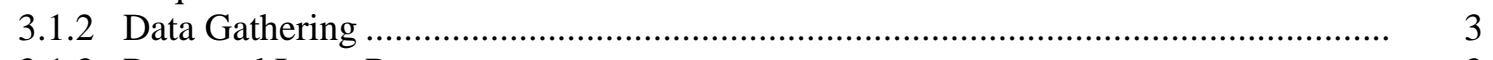

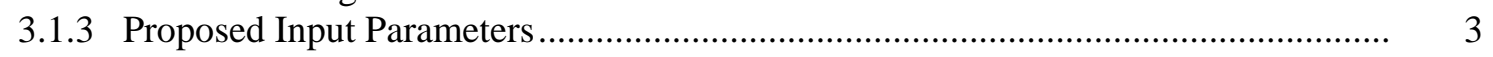

3.1.4 Data Issues, Uncertainties, and Recommendations ............................................... 8

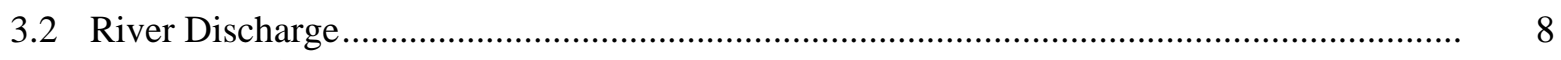

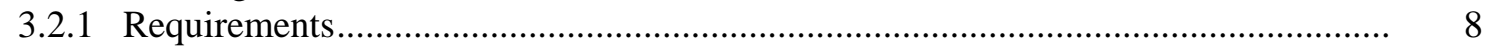

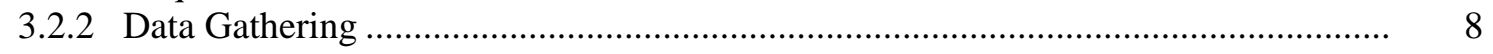

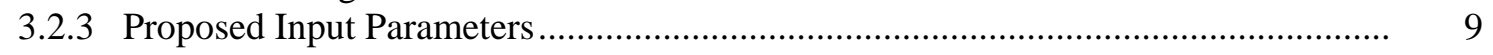

3.2.4 Data Issues, Uncertainties, and Recommendations ............................................... 10

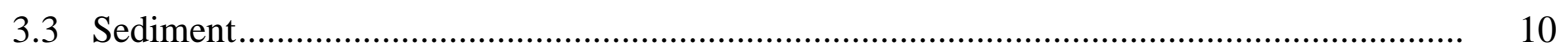

3.3.1 Requirements............................................................................................ 10

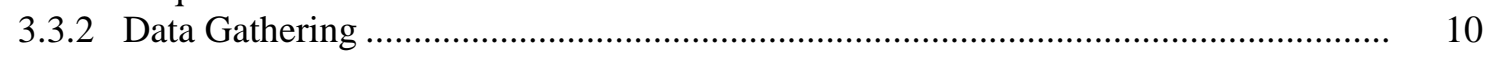

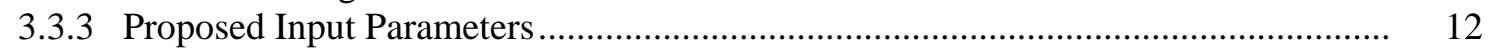

3.3.4 Data Issues, Uncertainties, and Recommendations ................................................ 12

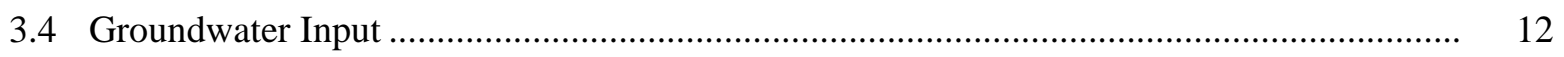

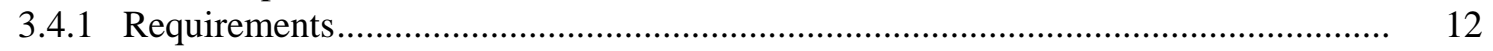

3.4.2 Data Issues, Uncertainties, and Recommendations ................................................ 13

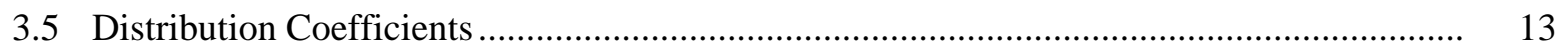

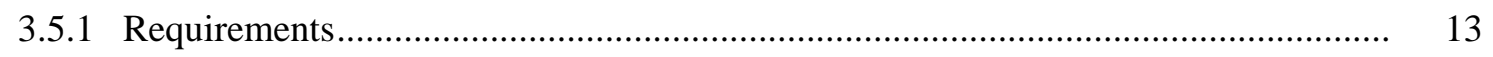

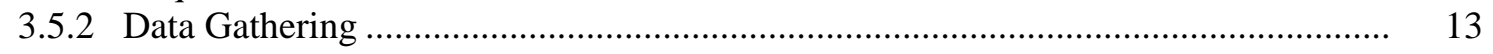

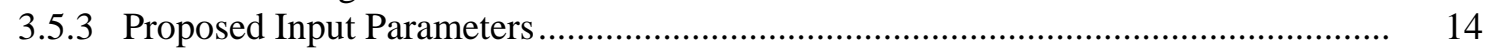

3.5.4 Data Issues, Uncertainties, and Recommendations .................................................. 14

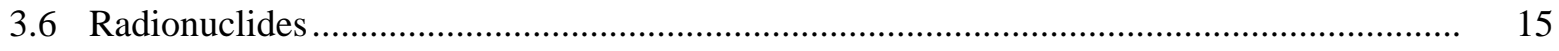

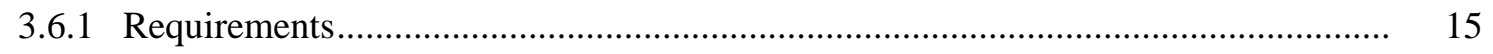

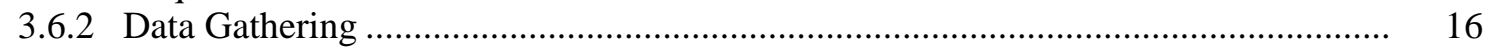

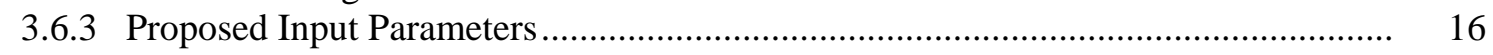

3.6.4 Data Issues, Uncertainties, and Recommendations .............................................. 16 
4.0 Conclusions and Recommendations......

\section{Figures}

$1 \quad$ Updated Bathymetry, River Mile 368 to 381 ..................................................................... 4

$2 \quad$ Updated Bathymetry, River Mile 359 to 367 ................................................................... 5

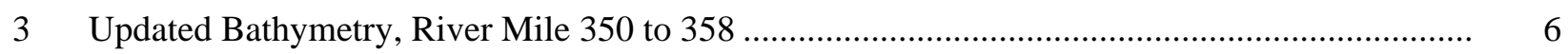

4 Computational Grid Example for the Upper Portion of the Study Area..................................... 7

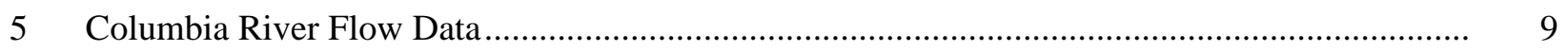

6 Probability Density Function of Priest Rapids Dam Flow Data for the Post-Dam Period ........... 9

$7 \quad$ Sample of Color Coded Bed Material Coverage .................................................................... 11

8 Cumulative Probability Distributions of $k_{d}$ Values for Bed Sediment from Which Model Input was Stochastically Derived ........................................................................................

9 Cumulative Probability Distributions of $k_{d}$ Values in Suspended River Sediment for Use in the Stochastic Determination of Model Input.

\section{Tables}

1 Examples of River Water and Sediment Data Available from the Hanford Environmental Information System .....

2 Examples of River Water Data for Samples Collected on Cross-River Transects in the Columbia River..

3 Examples of Radionuclide Data for Sediment Samples Collected from Columbia River Dams, 1996-1999, in pCi/g, dry wt 


\subsection{Introduction}

A composite analysis is required by U.S. Department of Energy (DOE) Order 435.1 to ensure public safety through the management of active and planned low-level waste disposal facilities associated with the Hanford Site (DOE M 435.1-1). The original composite analysis detailed in Kincaid et al. (1998) must be revised and submitted to DOE Headquarters because of revisions to waste site information in the 100, 200, and 300 Areas; updated performance assessments and environmental impact statements (EIS); changes in inventory estimates for key sites and constituents; and a change in the definition of offsite receptors.

Kincaid et al. (2004) describes the technical scope of the 2004 Composite Analysis for the Hanford Site and the approach to perform this analysis. It will be a site-wide analysis, considering final remedial action for the Columbia River corridor and the Central Plateau, and will be a companion site-wide assessment to waste-specific and site-specific assessments. The 2004 Composite Analysis also will provide supporting information on a regional or site-wide basis for use in important Hanford assessments and decisions such as the Comprehensive Environmental Response, Compensation, and Liability Act (CERCLA) 5-year review in 2005, tank closure decisions, decisions on final groundwater remedies for the 200 Areas, decisions of final groundwater remedies for the 100 Areas, and the Columbia River corridor final record of decision.

Beginning in fiscal year (FY) 2003, the DOE Richland Operations Office (DOE-RL) initiated activities, including the development of data packages, to support the 2004 Composite Analysis. This report describes the data compiled in FY 2003 to support Columbia River modeling for the 2004 Composite Analysis. This work was conducted as part of the Characterization of Systems Task of the Groundwater Remediation Project (formerly the Groundwater Protection Project) managed by Fluor Hanford, Inc., Richland, Washington.

The purpose of this data package is to summarize the underlying data used as input for numerical simulation as part of the 2004 Composite Analysis and to provide the input parameters needed for the simulations. The general approach for this work was to extract data and interpreted information from existing documents and databases. Every attempt was made to provide traceability back to the original source of the data or interpretations.

\subsection{Background}

The long-term impact of the Hanford Site on ecological and human health is of interest to environmental managers, engineers, and scientists responsible for the cleanup, as well as the public. The Systems Assessment Capability (SAC) is a system of linked modeling tools and data intended to provide a way to evaluate these impacts. Details of the SAC models and the initial assessments are presented in Kincaid et al. (2000) and background information on the development of SAC is presented in Bryce et al. (2002), 
which can be found at http://www.hanford.gov/docs/gpp/modeling/pnnl14027/river.pdf. SAC primarily consists of contaminant inventory estimates and models of contaminant release; vadose zone, groundwater, river, and shoreline models; and impact or risk assessment tools.

The River Flow and Transport Module provides the capability to calculate flow and transport in the Columbia River system. The module is based on the Modular Aquatic Simulation System in Two Dimensions (MASS2) is a two-dimensional (2D), depth-averaged hydrodynamics and transport model. The theoretical basis and use of MASS2 are presented in Perkins and Richmond (2004a) and Perkins and Richmond (2004b), respectively. The model simulates time varying distributions of depth-averaged velocities, water surface elevations, and water quality constituents. MASS2 uses a structured, multiblock, boundary-fitted, curvilinear computational mesh, which allows the simulation of very complex riverine or estuarine networks. The blocks may be of varying resolution, which allows high resolution to be used only where needed. MASS2 can simulate a wide variety of hydrodynamic conditions, including supercritical flow and hydraulic jumps. It can also simulate a wide variety of water quality conditions, including sediment, conservative or decaying contaminants, sediment-sorbed contaminants, water temperature, and total dissolved gas. Any number of these constituents may be simulated simultaneously. In addition, transport simulations may be performed using pre-calculated hydrodynamic conditions, allowing long-term transport simulations unencumbered by the more intensive hydrodynamic calculations, or repeated transport simulations without re-simulating hydrodynamics.

For the composite analysis, MASS2 is used to simulate river flows and contaminant transport through the Hanford Reach of the Columbia River. MASS2 uses groundwater and vadose zone inputs from other models in the Composite Analysis, and its output is used by the modules for impact assessment. This document presents the data assembled to run the river module for the section of the Columbia River from Vernita Bridge to the confluence with the Yakima River. The Environmental Stochastic Preprocessor (ESP) generates stochastic variability for some input parameters for many of the SAC modules (Eslinger et al. 2002). For the River Flow and Transport Module, ESP provides inputs such as the partition coefficient $\left(\mathrm{k}_{\mathrm{d}}\right)$ values and radionuclide concentrations. River flow is randomized on a yearly basis. At this time, the conceptual model does not incorporate scenarios for extreme flooding (50 to 100 years) or dam removal.

\subsection{Data Gathering Methods and Data Limitations}

MASS2 requires data on the river flow rate, downstream water surface elevation, groundwater influx, channel bathymetry, and the bed and suspended sediment properties. In the subsections below, the data requirements, data sources, model inputs, and data limitations are discussed for each required component for the River Flow and Transport Module. 


\subsection{Bathymetry and the Computational Grid}

River bathymetry describes the shape of the channel. Multiple data sources were integrated into a single bathymetric surface, then the bathymetric surface was used in the creation of the computational grid. The computational grid is the representation of the channel shape that is input into MASS2. Two distinct types of data are required for the creation of the computational grid: shoreline data defining the lateral extent of the river and bathymetric data to represent the shape of the river bottom.

\subsubsection{Requirements}

Accurate representation of the channel bathymetry is vital to realistically simulate the river depth and velocity characteristics. However, a coarse computational grid is necessary to maximize processing speed and ensure that the long time periods required by the composite analysis can be simulated in reasonable amounts of time. Therefore, the river channel features must be represented using as coarse of a grid as possible. To achieve this, a computational grid with variable grid cell densities was developed. The composite analysis computational grid has double the resolution in the cross-stream direction for complex regions of interest. These complex regions are represented by smaller grid cells while the less complex river segments are represented by larger, coarser grid cells (see Section 3.1.3).

\subsubsection{Data Gathering}

River bathymetry was based on data obtained in 1998 by the U.S. Geological Survey Biological Resource Division in Cook, Washington (Tiffan et al. 2002). A scanning hydrographic operational LiDAR survey (SHOALS) light detection and ranging (LIDAR) system (Irish et al. 2000) was used to measure bottom elevations in near shore and shallow areas. These point data and data from cross section surveys were used to create a continuous, three-dimensional bathymetric surface of the study area in ArcInfo (Figures 1 through 3).

The computational grid for the river simulations was based on this bathymetric surface and shorelines that included the large islands in the Hanford Reach. The shorelines were based on flows of $80 \mathrm{kcfs}$ and digitized from aerial photos. The ArcInfo bathymetric surface was used to determine the bed elevation at each point in the computational grid. Recent MASS2 code enhancements allow grids with grid density changes at matched boundaries to be used. These enhancements allow the increased resolution around the islands, where it is needed, while reducing the computational time relative to having the higher resolution in all areas of the river. The new grid contains 58 blocks with a total of 2,708 cells.

\subsubsection{Proposed Input Parameters}

The computational grid, created in Gridgen, had 7 cells across in areas without islands, 14 cells across in areas with islands (Figure 4), and a total of 2,708 cells partitioned into 58 blocks. The grid extended from the Yakima River confluence (Columbia River mile [RM] 335) upstream to RM 389 near Vernita Bridge. 


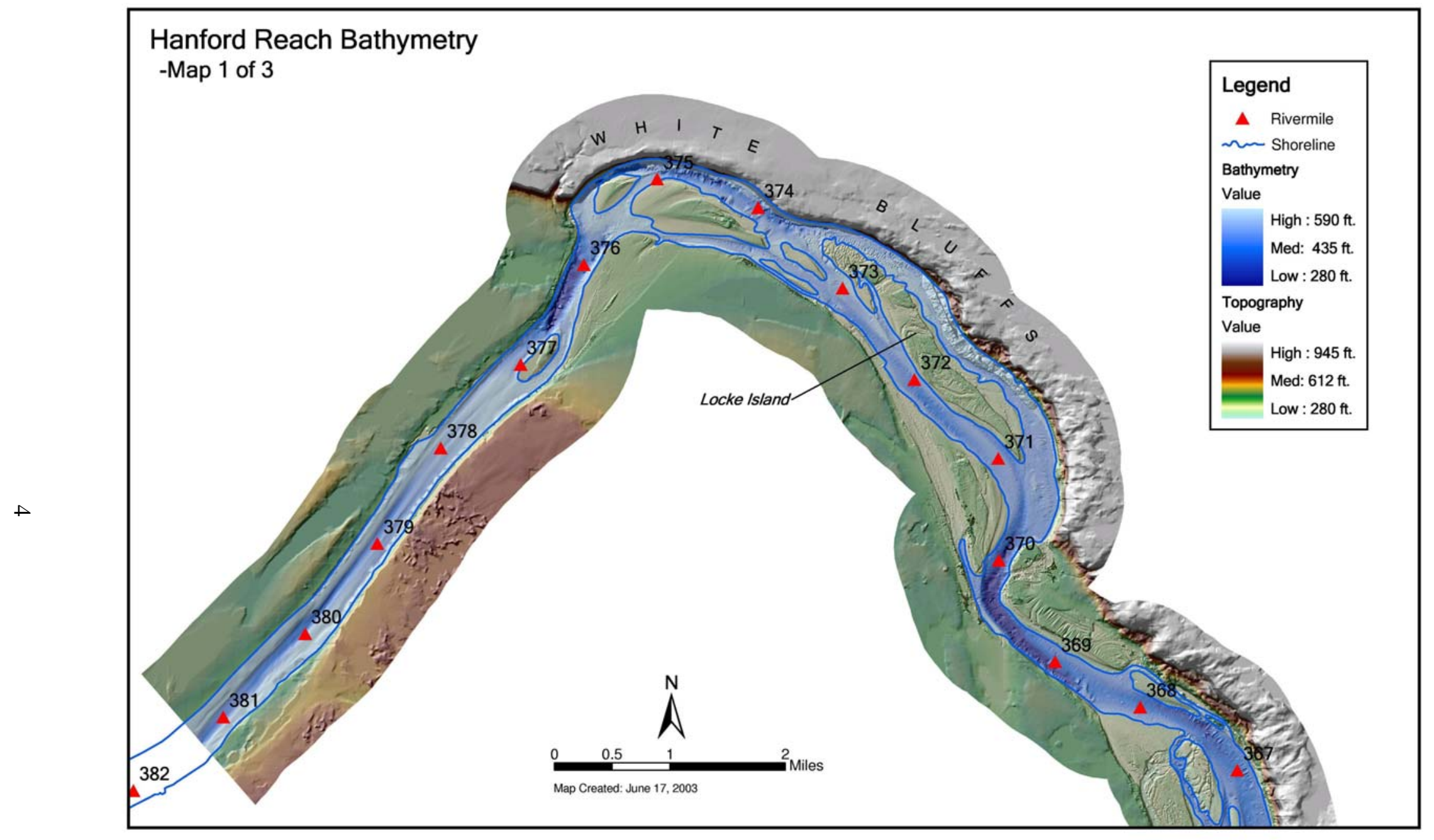

Figure 1. Updated Bathymetry, River Mile 368 to 381 


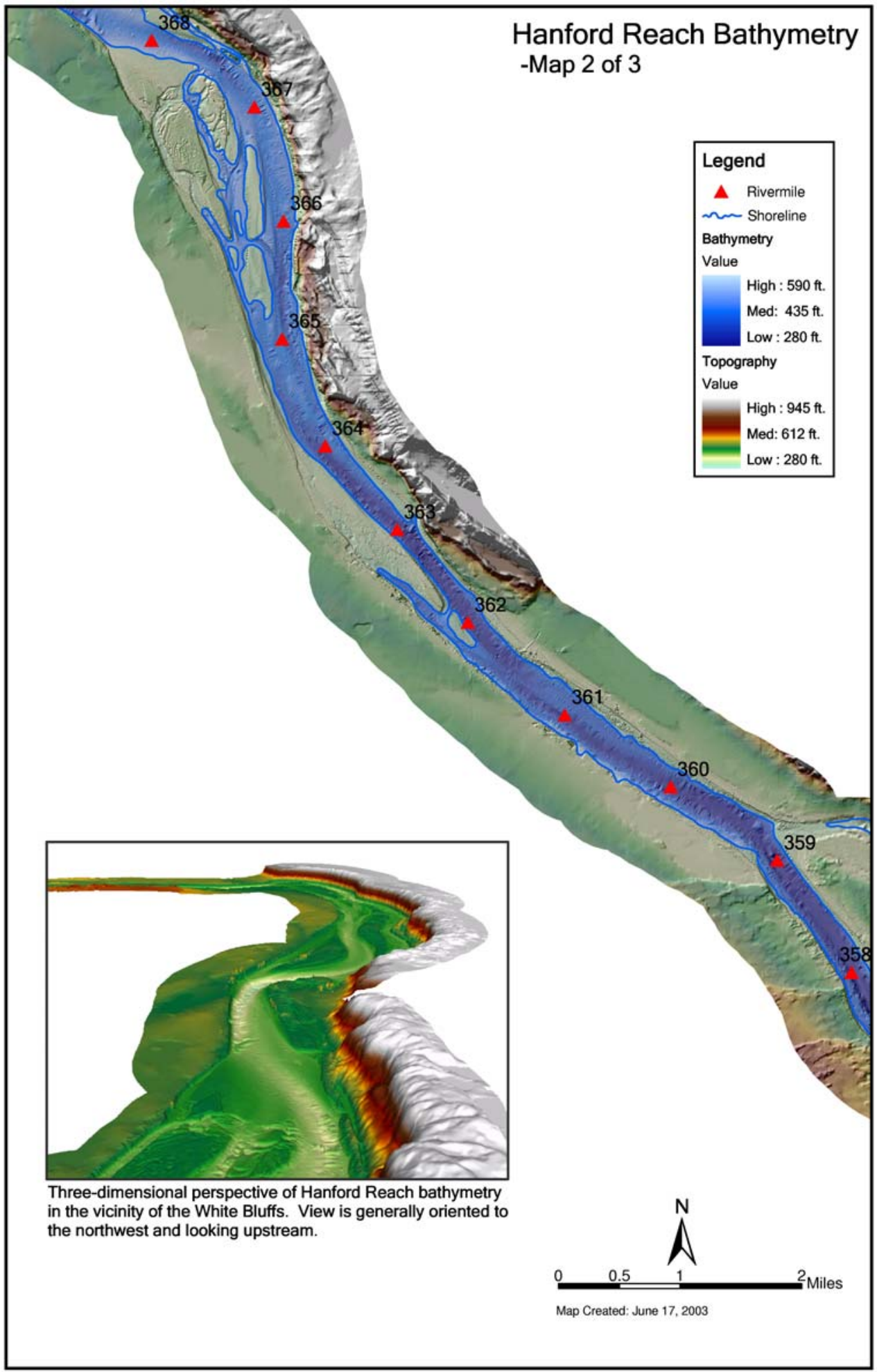

Figure 2. Updated Bathymetry, River Mile 359 to 367 


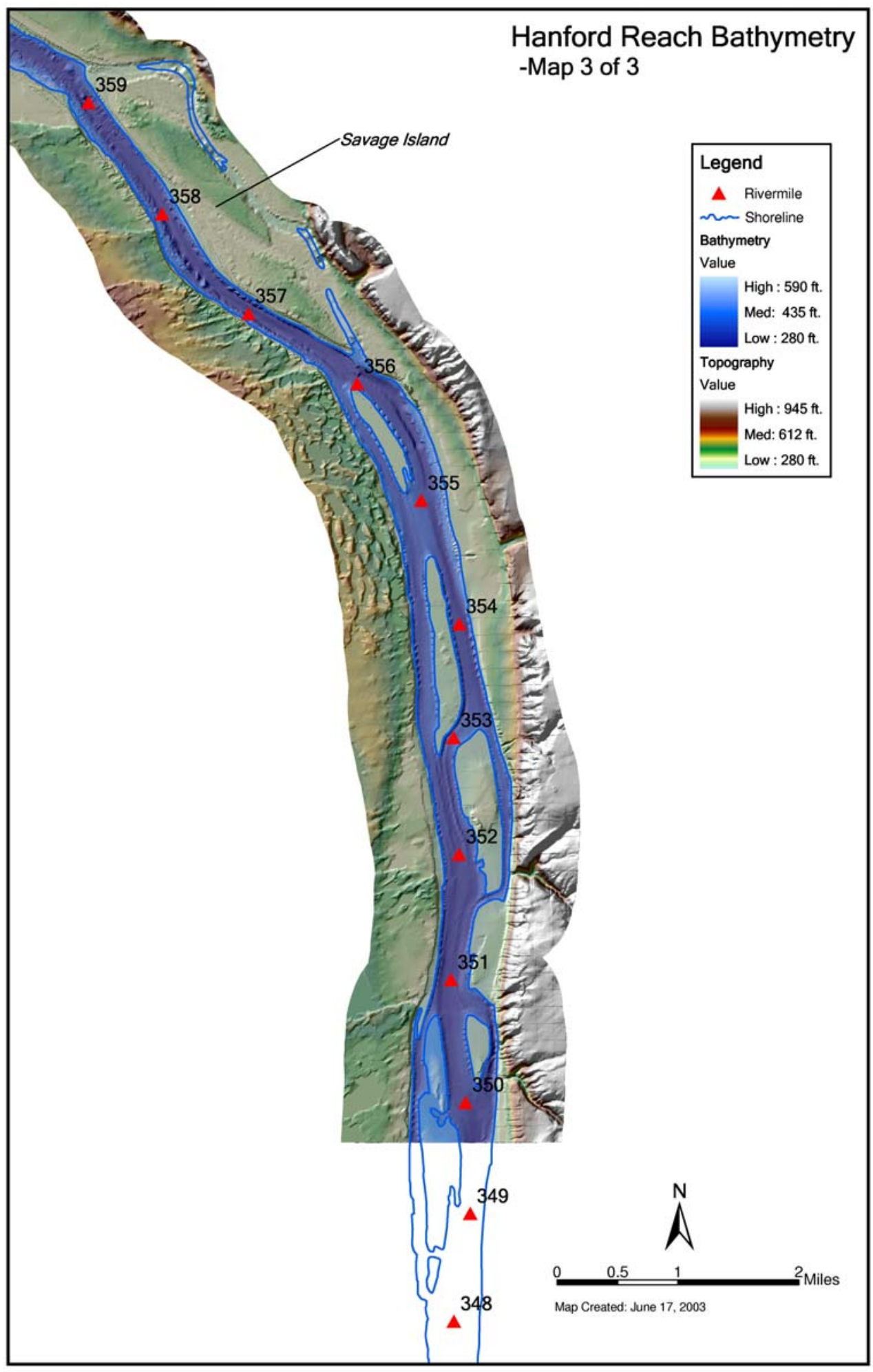

Figure 3. Updated Bathymetry, River Mile 350 to 358 


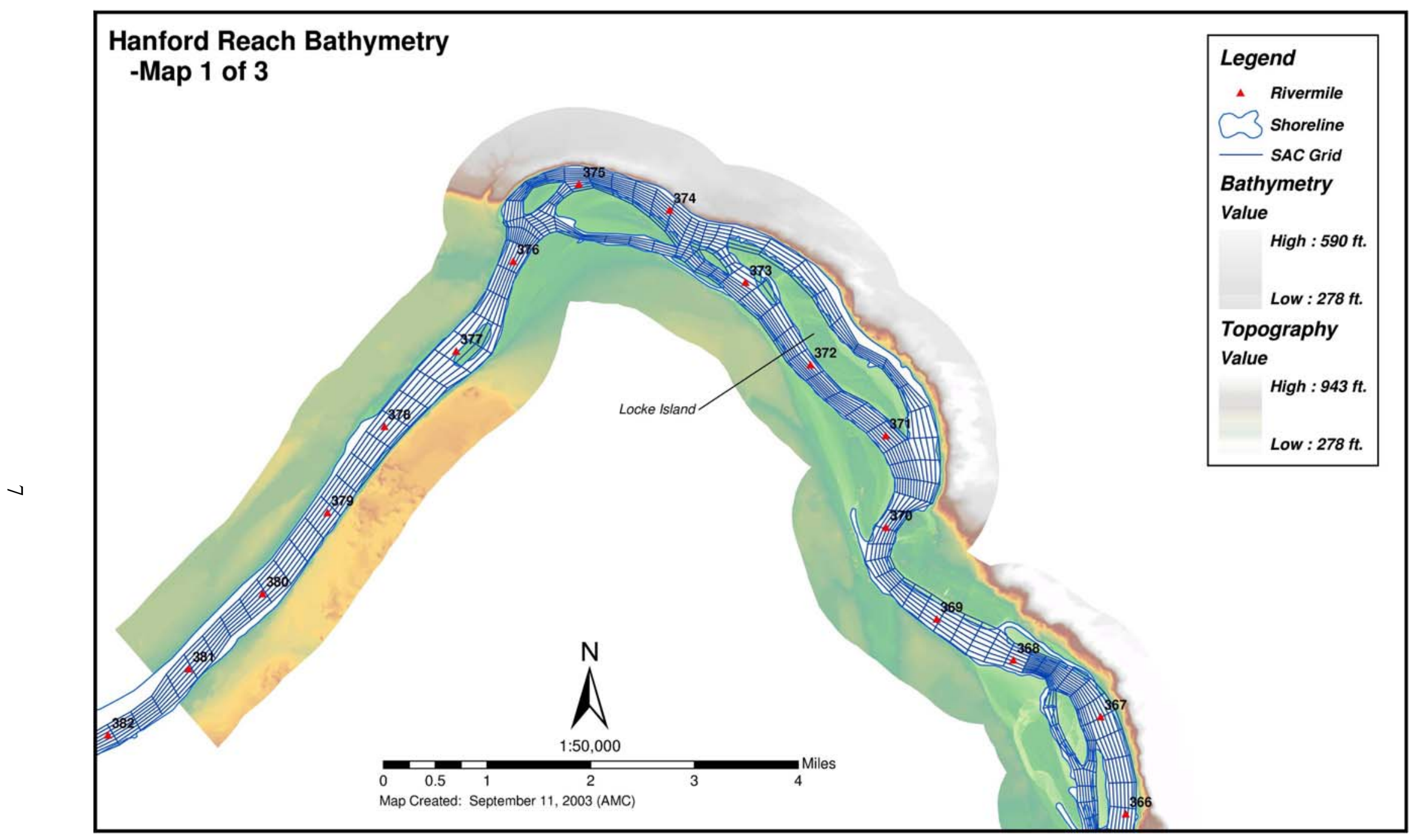

Figure 4. Computational Grid Example for the Upper Portion of the Study Area 


\subsubsection{Data Issues, Uncertainties, and Recommendations}

The most notable technical issue with the bathymetry is that the areal extent of the river is limited to that of the simulated lowest flow channel because the current implementation of the model does not allow any cells to go dry. As a result, the model will underestimate the channel width at higher or flood flows and, therefore, may overestimate the depth in extreme cases.

The other technical issues result from gaps in geographic coverage of the data and limitations to the data collection technique. The SHOALS LiDAR data were only collected for a portion of RM 355 to 377 and can only penetrate into 4.6 meters (15 feet) of water. Consequently, there were data gaps in: areas not covered by the SHOALS survey or in areas too deep for LiDAR, or areas not included in the Pacific Northwest National Laboratory (PNNL) hydrosurvey data. ${ }^{(a)}$ In these areas, a bathymetric data set was derived from the U.S. Army Corps of Engineers cross-section data. The cross-section data were interpolated in the stream-wise direction on an orthogonal grid system to fill the gaps between the cross sections. Only data from those areas not represented by any other source were used in the creation of a bathymetric surface.

\subsection{River Discharge}

A realistic representation of river discharge is important to simulate the hydrodynamic conditions within the river. Consequently, the choice of representative inflow conditions is crucial to obtaining the realistic transport of contaminants in the river.

\subsubsection{Requirements}

Two hydrodynamic inputs are required for MASS2: upstream discharge and the downstream water surface elevation. The downstream boundary of the model is the Columbia River near its confluence with the Yakima River. The water surface elevation is controlled by McNary Dam, so the only variable hydrologic data needed to run MASS2 are the flow of the Columbia River at the upstream end of the study area. This information is available through hydropower project operations records and U.S. Geological Survey (USGS) flow gaging data.

\subsubsection{Data Gathering}

Mean monthly flows were computed from the USGS Vernita Bridge gage data for use as model inflows. These monthly flows were not taken individually, but were grouped as flow years (i.e., January through December for each year), which preserved the character of the yearly hydrograph. The downstream stage was held at a constant 103.63 meters (340 feet) above sea level, the normal operating stage of McNary Dam.

(a) Unpublished data from Tim Hanrahan, Pacific Northwest National Laboratory, Richland, Washington. 


\subsubsection{Proposed Input Parameters}

The historic Columbia River flow data at Priest Rapids Dam are shown in Figure 5. For the post-dam period (1975 to 2001), these data were grouped into a series of flow years from which the model selected at random. The probability density plot of the flow data is positively skewed (Figure 6).

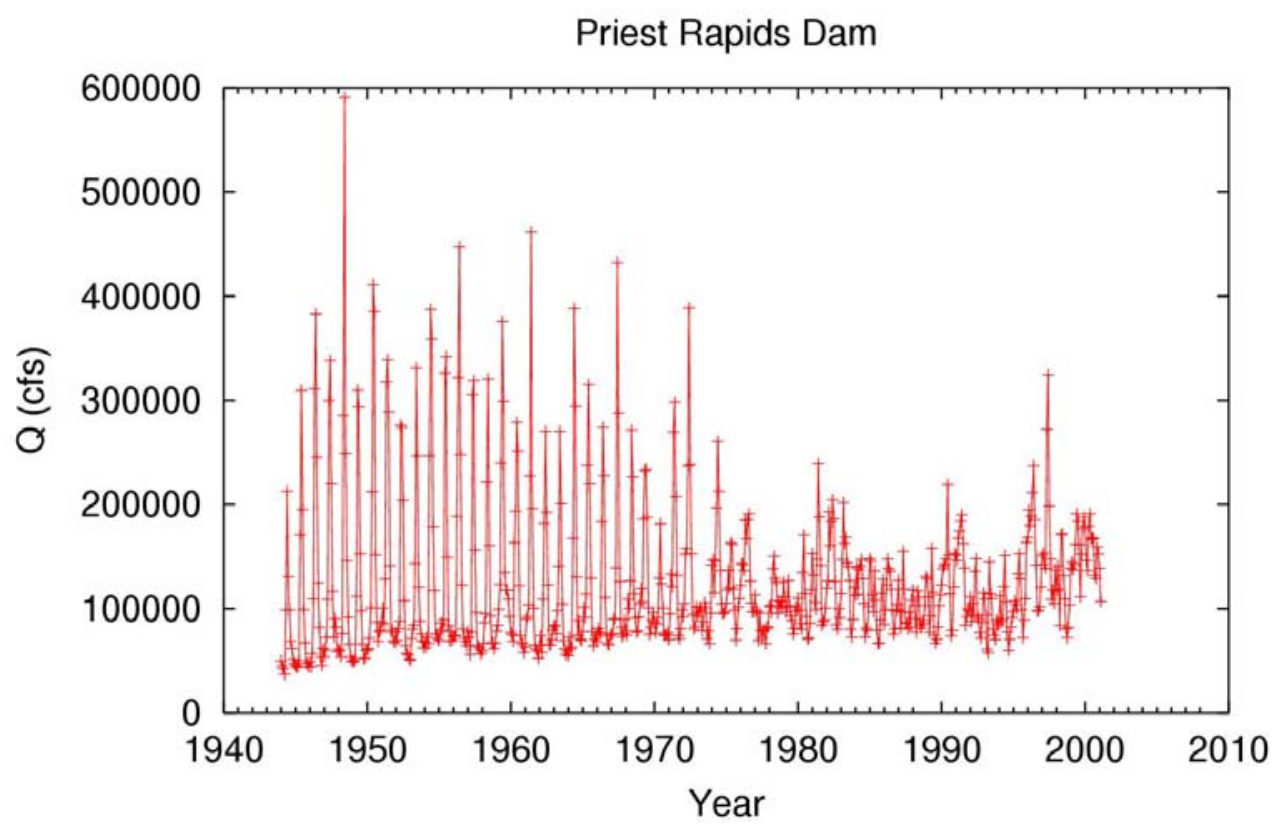

Figure 5. Columbia River Flow Data

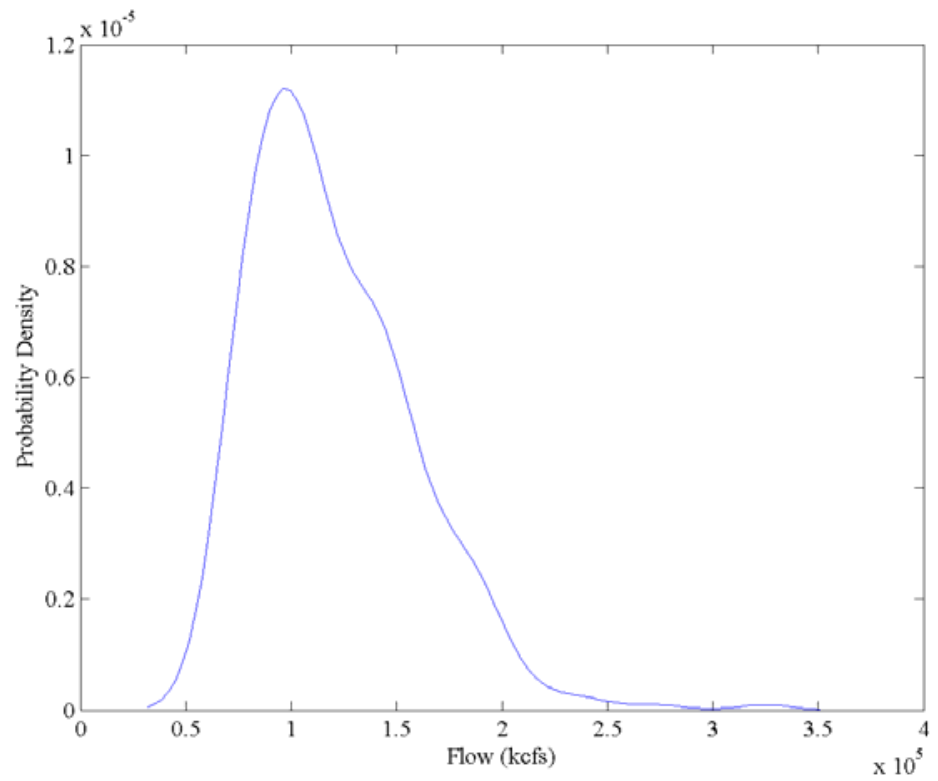

Figure 6. Probability Density Function of Priest Rapids Dam Flow Data for the Post-Dam Period (1975 to 2001) 


\subsubsection{Data Issues, Uncertainties, and Recommendations}

The flow data are fairly straightforward and complete, although it does not reflect the daily flow fluctuations. The flow rate of the Columbia River in the Hanford Reach fluctuates significantly and is controlled primarily by releases from upstream dams. There are both seasonal and daily fluctuations in flow, which also cause fluctuations in river stage. Seasonal flows typically peak from April through June, during spring runoff from snowmelt, and are lowest from September through October. The seasonal change in average water level is up to about 2 meters (6.6 feet). Daily fluctuations in discharge are caused by releases from dams based on demand for power production. Because of these changes in flow, the river stage varies significantly over a short time period. Vertical fluctuations of more than 1.5 meters (4.9 feet) during a 24-hour period are common along the Hanford Reach (Poston et al. 2003). These fluctuations are not significant at the monthly output interval currently employed in the model, however. The flow data also represent only current conditions and the current conceptual model does not include future climate or hydropower changes that may affect the hydrograph, nor does it include extreme flood events.

\subsection{Sediment}

The characteristics of suspended and bed sediment are important for estimating the adsorption and subsequent fate and transport of contaminants in the study area. Many parameters are needed to accurately characterize the bed and suspended sediments.

\subsubsection{Requirements}

The bed sediment can be characterized for each river cell by material type, depth, median particle size, density, settling velocity, erodibility, and erosional and depositional critical shear values. The suspended sediment is characterized by these same properties, except that concentration $\left(\mathrm{kg} / \mathrm{m}^{3}\right)$ is used to characterize the amount of suspended sediment, instead of the depth, as for bed sediment. These characteristics are estimated from available data or established relationships derived from empirical data.

\subsubsection{Data Gathering}

The background suspended sediment data were obtained from the USGS National Stream Water Quality Network (NASQAN) web site (http://water.usgs.gov/nasqan). Water quality parameters for the Columbia River water samples collected at Vernita Bridge were downloaded and used as model boundary conditions. Each dataset included additional information on numerous aspects of water quality including temperature, conductance, dissolved oxygen, $\mathrm{pH}$, alkalinity, dissolved constituents, and suspended sediment concentrations. These data consisted of several measurements per year (typically quarterly) starting in 1996. All of the suspended sediment concentration data were averaged for each location to estimate the background suspended sediment concentration.

In past analyses, multiple bed depths had been assigned based on observed sediment size. The material types were derived from a geographic information system (GIS) coverage of the approximate spatial distribution of bottom material types, created by Tim Hanrahan from USGS data (Figure 7). When the sediment size was determined for the computational grid, it was observed that only $1 \%$ of the nodes 
had a depth, based on past criteria, which would be different than 0.3 meter ( 1 foot) depth. Previous work had varied bed depths, in meters (feet), based on material types:

- Organic

- Organic-sand

- Organic-cobble

- Clay (soft)

- Silt

- Silt-sand

- Silt-gravel

- Silt-cobble

- Clay (hard)
$0.3(1.00)$

$0.3(1.00)$

$0.3(1.00)$

$0.9(3.00)$

$0.9(3.00)$

$0.6(2.00)$

$0.3(1.00)$

$0.3(1.00)$

$0.9(3.00)$

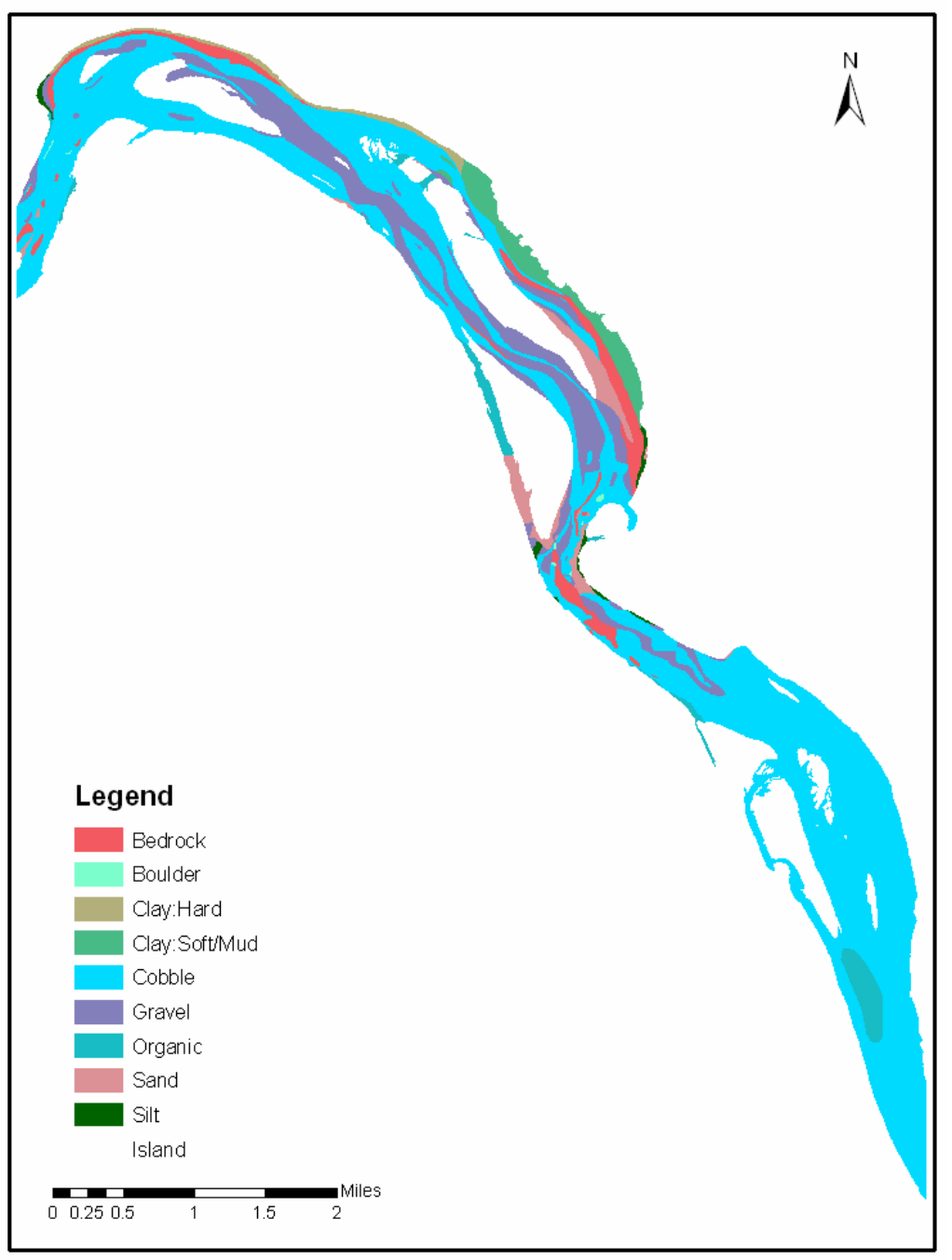

Figure 7. Sample of Color Coded Bed Material Coverage (Tim Hanrahan, Pacific Northwest National Laboratory, Richland, Washington) 
More resistant or organic types have $1 \mathrm{ft}$ depths. Consequently, all initial bed depths were set to 0.3 meter ( $1 \mathrm{foot}$ ). The presence of coarse or resistant sediments at the grid locations is reasonable as the river grid was restricted to locations that are inundated at all flows.

\subsubsection{Proposed Input Parameters}

Suspended sediment concentration in the Columbia River was set to a constant $3.75 \mathrm{mg} / \mathrm{L}$. One sediment class with the following properties was used:

- Median particle diameter (d50): $0.003 \mathrm{~cm}$ (0.0001 feet)

- Solids density: $2,650 \mathrm{~kg} / \mathrm{m}^{3}\left(75.04 \mathrm{~kg} / \mathrm{ft}^{3}\right)$

- Settling velocity: $0.000010 \mathrm{~m} / \mathrm{s}(0.000033 \mathrm{ft} / \mathrm{s})$

- Erodibility: 0.0

- Critical shear for erosion: $0.0073 \mathrm{kgf} / \mathrm{m}^{2}\left(0.0015 \mathrm{lbf} / \mathrm{ft}^{2}\right)$

- Critical shear for deposition: $0.0073 \mathrm{kgf} / \mathrm{m}^{2}\left(0.0015 \mathrm{lbf} / \mathrm{ft}^{2}\right)$

The river bed was represented as one sediment class that was 0.3 meter ( $1 \mathrm{foot}$ ) deep.

\subsubsection{Data Issues, Uncertainties, and Recommendations}

Almost all data gathered since the closure of the Hanford Site single pass reactors (early 1970s) have been for upper sediment (river/sediment interface). Only limited information is available for sediment grain size, total organic content, and sequestration by sulfide (Blanton et al. 1995; Patton and Crecelius 2001). Total organic content plays a large role in the variability of $k_{d}$ values, which are discussed in Section 3.5.

The relative importance of sediment transport in the fate of in-river contaminants in the Columbia River is uncertain. The low concentrations of suspended and bed sediment moving in the Columbia River suggests that sediment may not be a high priority factor at this time. Therefore, in effort to minimize processing time, continuing to use one sediment class is recommended until the need for additional refinement is demonstrated with reliable data and analysis.

\subsection{Groundwater Input (CFEST)}

A series of scripts have been developed to allow the use of model outputs for groundwater and vadose zone flows and contaminants into the Hanford Reach of the Columbia River. The output locations for both models are mapped onto the MASS2 grid then time series of flows and transported variables used as inputs to MASS2.

\subsubsection{Requirements}

MASS2 receives input from the coupled fluid energy and solute transport (CFEST) groundwater module, the vadose zone model (STOMP), and in the case of reactor discharges, directly from the inventory module. Contaminant and water influx from CFEST is input to the bed sediment layer in MASS2 using output from the groundwater data translator (GWDROP), which matched the cells from the 
CFEST model to the appropriate cells in the MASS2 computational grid. Vadose zone releases from STOMP are input into MASS2 with the VZDROP code. MASS2 then generates and outputs annual average concentrations of contaminants in the water column (dissolved and total sediment-sorbed) and in the bed sediment (pore water and total sediment-sorbed).

\subsubsection{Data Issues, Uncertainties, and Recommendations}

The data issues and uncertainties for the CFEST data are documented in Thorne (2004). The vadose zone model data issues and uncertainties are documented in Last et al. (2004).

\subsection{Distribution Coefficients ( $k_{d}$ Values)}

Partition coefficient $\left(\mathrm{k}_{\mathrm{d}}\right)$ values are used to describe the adsorption of dissolved contaminants to ground, bed, and suspended sediment particles. This process affects both the bio-availability, and the fate and transport of contaminants. The basic $\mathrm{k}_{\mathrm{d}}$ linear sorption isotherm model is

$$
k_{d}=S / C_{a q}
$$

where $S$ is the sorbed concentration (or the concentration of the contaminant on the solid, $\mathrm{Ci} / \mathrm{kg}$ ), and $C_{a q}$ is the concentration of the contaminant in the aqueous or dissolved phase $\left(\mathrm{Ci} / \mathrm{m}^{3}\right)$. Suspended and bed sediment $\mathrm{k}_{\mathrm{d}}$ distributions are estimated from available data for each contaminant. This linear model was deemed adequate for transport through the Hanford Reach (Cantrell et al. 2003).

A stochastic approach was taken to capture the wide range of values of $\mathrm{k}_{\mathrm{d}}$, reported in field and laboratory studies.

\subsubsection{Requirements}

MASS2 requires input of $\mathrm{k}_{\mathrm{d}}$ for each transported particulate phase for use in the sediment-contaminant interaction portion of the model.

\subsubsection{Data Gathering}

Distribution coefficient estimates for the linear sorption isotherm model are site-specific because they are affected by numerous site-specific characteristics including $\mathrm{pH}$, salinity, substrate size and composition, substrate cation exchange capacity, the presence of organics, the concentrations of competing ions, and redox potential. Although many partition coefficient studies exist, most pertain to groundwater or marine environments with relatively few applying to freshwater, aquatic systems. Of those, even fewer apply to the sections of the Columbia River affected by the Hanford Site. Available data for studies that involved fresh water, aquatic environments, and basalt substrates were examined to develop the most appropriate $\mathrm{k}_{\mathrm{d}}$ distributions for our application (Kaplan and Serne 2000; Cantrell et al. 2003;). In consultation with Pacific Northwest National Laboratory geochemists, site-specific ranges of values of $\mathrm{k}_{\mathrm{d}}$ for multiple contaminants for suspended sediments were developed (see Cantrell et al. 2003 for a discussion). The Environmental Stochastic Preprocessor (Eslinger et al. 2002) was developed to generate the needed variability in $\mathrm{k}_{\mathrm{d}}$ as an input for the suite of MASS2 simulations used for the composite analysis. 


\subsubsection{Proposed Input Parameters}

Partition coefficient values were represented with a number of basic distribution types based on the characteristics of each constituent. These distribution types include log normal, uniform, triangular, and constant. Partition coefficient distributions used for bed sediment, shown in Figure 8, consist of log normal distributions for selenium (Se), neptunium (Np), cesium (Cs), uranium (U), and strontium (Sr); a uniform distribution between 0 and 0.1 for carbon (C); and a triangular distribution for iodine (I). Also in Figure 8 , the median $\mathrm{k}_{\mathrm{d}}$ values occur at 0.5 cumulative probability for the lognormal distributions and the distribution used for technetium (Tc) was generated so that half of the values would be simulated as zero. Distributions for tritium and chlorine do not appear in Figure 8 because these constituents were assigned constant $\mathrm{k}_{\mathrm{d}}$ values of zero, indicating that they do not adsorb to sediment. For strontium, technetium, iodine, and uranium, suspended sediment $\mathrm{k}_{\mathrm{d}}$ distributions are believed to differ from those used for bed sediment. Distributions for these constituents, shown in Figure 9, were all log-normally distributed.

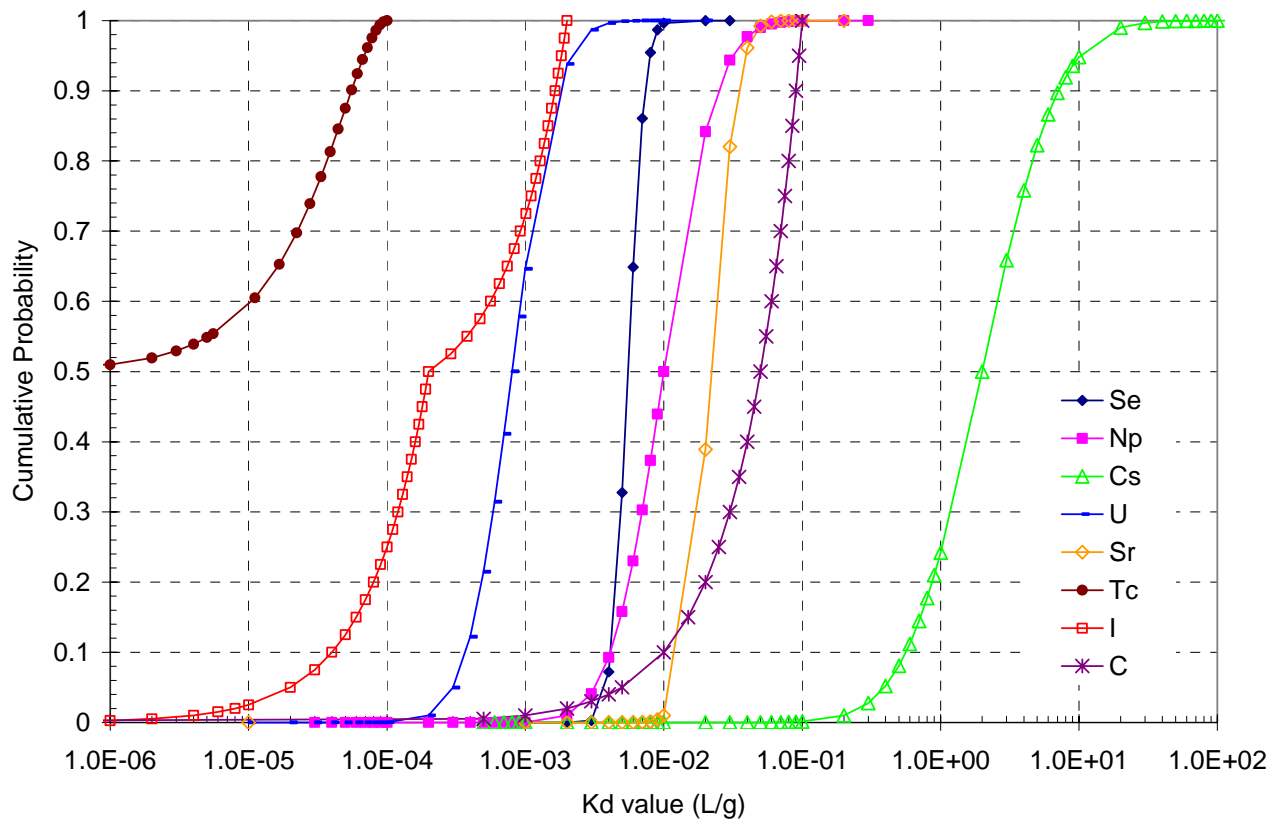

Figure 8. Cumulative Probability Distributions of $k_{d}$ Values for Bed Sediment from Which Model Input was Stochastically Derived. (Note that the lower $\mathrm{k}_{\mathrm{d}}$ limit for Tc, I, and C is zero, which cannot be plotted on a log scale.)

\subsubsection{Data Issues, Uncertainties, and Recommendations}

Uncertainties in the $k_{d}$ values were determined by using values derived stochastically from carefully designed distributions for each constituent that span the range of possible values. These distributions should be refined as new information becomes available. 


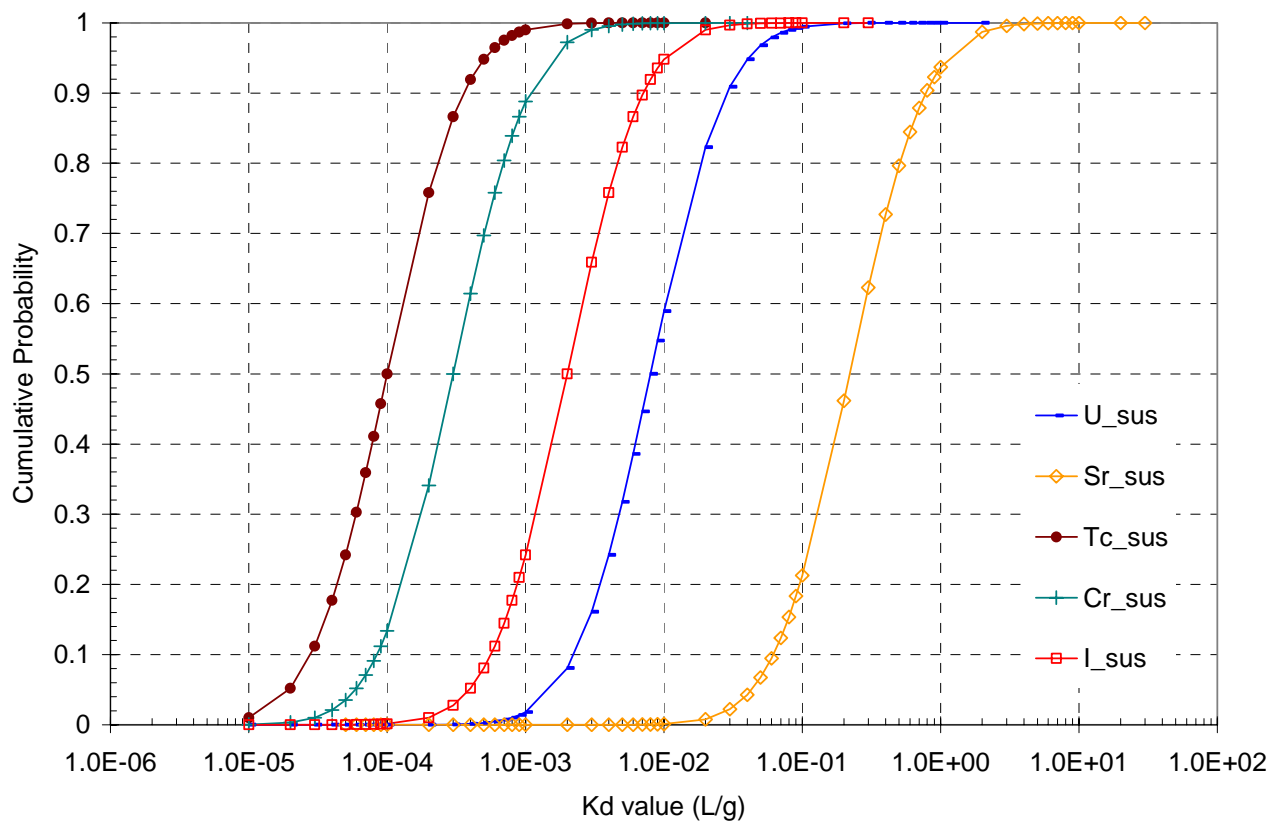

Figure 9. Cumulative Probability Distributions of $k_{d}$ Values in Suspended River Sediment for Use in the Stochastic Determination of Model Input. Constituents not shown are unchanged from bed $\mathrm{k}_{\mathrm{d}}$ distributions.

\subsection{Radionuclides}

The concentrations of radionuclides in Columbia River water and sediment samples collected (or modeled) at both background locations and locations potentially affected by Hanford contaminants are needed for the composite analysis. The requirements, data gathering methods, proposed model input parameters, and other data issues are discussed this section.

\subsubsection{Requirements}

Background values of radionuclide concentrations are necessary for MASS2 to simulate contaminant fate and transport. Background concentrations of dissolved radionuclides in the Columbia River were determined from available data for contaminants with measurable background levels. Background concentrations of radionuclides adsorbed to sediment were either estimated from measured data or computed from the dissolved concentrations assuming equilibrium partitioning.

Dissolved and sorbed radionuclide concentrations in sections of the Hanford Reach affected by Hanford Site contaminants are also necessary for MASS2 to simulate contaminant fate and transport. Concentrations of radionuclides in this section of the Columbia River were determined from available data with measurable levels. Concentrations of radionuclides in sediment were either estimated from measured data or computed from the dissolved concentrations assuming equilibrium partitioning. Radionuclide concentrations in reactor discharges during operational years (1944 to 1971) are not incorporated at this time. 


\subsubsection{Data Gathering}

Surface water and sediment background radionuclide data for the initial model runs were obtained from 5-year geometric means for 1990 to 1995 taken from the Columbia River Comprehensive Impact Assessment (CRCIA) Project (DOE 1998). This data set is based on a data compendium (Eslinger et al. 1994) and a data volume (Miley et al. 1997) that were produced for CRCIA. The data compendium provides a collection of references, data source, descriptions of data formats, and a discussion of the search process.) These data are discussed further and presented in the next section and are included in the companion compact disk (CD) issued with this report.

Additional radionuclide data from the Hanford Environmental Information System (HEIS) database has been summarized and are included in the companion CD issued with this report. The majority of the radionuclide data were collected for the Hanford Site's Surface Environmental Surveillance Project with the sampling locations, sampling and analytical methods, and results reported annually (e.g., Poston et al. 2003; Bisping et al. 2003).

\subsubsection{Proposed Input Parameters}

Radionuclide inputs were based on primarily on data from the HEIS database. This information been summarized in Tables 1 through 3 to provide examples of the data (see CD issued with this report).

Most of the available data for radionuclides in the water column is for total (unfiltered) water samples with limited information about dissolved and particle associated fractions. There was a reasonable amount of dissolved and particle associated data from the continuous filter/resin systems operated by the Hanford Site Surface Environmental Surveillance Project (e.g., Poston et al. 2003) at Priest Rapids Dam, the 300 Area drinking water intake, and the Richland pumphouse. Dissolved/particle data were available for iodine-129, plutonium isotopes, and gamma-emitting sorbed radionuclides; however, a significant portion of the data were reported as less than the detection limit for at least one fraction. Another potential source of information on sorption is available from metals data (filtered and unfiltered) collected from Columbia River transect water samples. The metals data are reported in the Hanford Site Environmental Surveillance Data Reports (e.g., Bisping 2003), with results for samples collected after 2002 available in the HEIS database.

\subsubsection{Data Issues, Uncertainties, and Recommendations}

Most of the available water data for radionuclides were for total (unfiltered) water samples with limited information on dissolved and particle associated fractions. No data were available for chorine-36 for sediment or water samples. Data analysis is complicated for some radionuclides because a significant portion of the results are reported as undetected values. 
Table 1. Examples of River Water and Sediment Data Available from the Hanford Environmental Information System (attached to companion CD)

\begin{tabular}{|c|c|c|c|c|}
\hline Media & Locations & Dates & Analytes & File Name \\
\hline \multirow[t]{6}{*}{ River water } & Multiple On/Off Site, VB, Regional & $1970-1989$ & Cr6+, Cs-137, I-129, Pu-iso, Sr-90, Tc-99, U-iso, Particle/Dissolved & 70-89 all water \\
\hline & VB, 100-F, 100-N, RPH, 300-A, HTS & $1990-1995$ & Pu-iso, U-235, Particle/Dissolved & 90-95 SW \\
\hline & VB, 100-F, 100-N, RPH, 300-A, HTS & $1990-2000$ & Cr, Cs-137, Sr-90, Tc-99, Tritium, U-iso & 90-00 Transect \\
\hline & Multiple Onsite, VB & $1999-2003$ & Cr, Cs-137, I-129, Pu-iso, Sr-90, Tc-99, Tritium, U-iso, Particle/Dissolved & 99-03 SW SAC \\
\hline & PRD, RPH, 100-K & 2003 & C-14 & 90_pres C-14 \\
\hline & Multiple Onsite, VB & $1996-1999$ & Cr, Cs-137, I-129, Pu-iso, Sr-90, Tc-99, Trit, U-iso, Particle/Dissolved & 96-99 SW_FNL \\
\hline \multirow[t]{5}{*}{ Sediment } & PRD, McNary, Hanford Reach & $1988-1990$ & Cs-137, Pu-iso, Sr-90, U-iso & 88-90 Sediment \\
\hline & PRD, McNary, Hanford Reach, IH & $1999-2003$ & Cs-137, Pu-iso, Sr-90, Tc-99, U-iso & 99-03 SD SAC \\
\hline & PRD, McNary, Hanford Reach & 1999 & Sediment Grain Size & 99 SED_SANDS \\
\hline & PRD, McNary, Hanford Reach & $1990-1995$ & $\mathrm{Pu}, \mathrm{U}-235$ & 90-95 SEDIMENT \\
\hline & PRD, McNary, Hanford Reach, IH & $1996-1999$ & Cr, Cs-137, Pu-iso, Sr-90, U-iso & 96 - 99 Sediment_FNL \\
\hline $\begin{array}{l}\mathrm{FNL}=\mathrm{F} \\
\mathrm{HTS}=\mathrm{H} \\
\mathrm{IH}=\mathrm{Ic} \\
\mathrm{RPH}=\mathrm{R} \\
\mathrm{SED}=\mathrm{S} \\
\mathrm{SW}=\mathrm{S} \\
\mathrm{VB}=\mathrm{V} \\
100 \mathrm{~F}=1 \\
100 \mathrm{~N}=10 \\
300 \mathrm{~A}=3\end{array}$ & $\begin{array}{l}\text { nal. } \\
\text { anford Town Site. } \\
\text { Harbor. } \\
\text { chland Pumphouse. } \\
\text { diment. } \\
\text { rface water. } \\
\text { arnita Bridge. } \\
\text { 0-F Area. } \\
\text { 0-N Area. } \\
0 \text { Area. }\end{array}$ & & & \\
\hline
\end{tabular}


Table 2. Examples of River Water Data for Samples Collected on Cross-River Transects in the Columbia River. (Data is available on the CD issued with this report.)

\begin{tabular}{|c|c|c|c|c|c|c|c|c|c|}
\hline Location & Data & $\mathrm{I}-129$ & $\mathrm{Pu}-239 / 240$ & Sr-90 & Tc-99 & Tritium & U-234 & U-235 & U-238 \\
\hline \multirow[t]{4}{*}{ 100-F Area } & Count & & & 41 & & 45 & 40 & 40 & 40 \\
\hline & Max & & & 1.12 & & 96 & 0.315 & 0.0287 & 0.279 \\
\hline & Average & & & 0.107 & & 34.3 & 0.226 & 0.00586 & 0.176 \\
\hline & Std Dev & & & 0.163 & & 18.5 & 0.0343 & 0.00693 & 0.0286 \\
\hline \multirow[t]{4}{*}{ 100-N Area } & Count & & & 38 & & 47 & 37 & 37 & 37 \\
\hline & Max & & & 109 & & 207 & 0.303 & 0.0327 & 0.275 \\
\hline & Average & & & 3.09 & & 32.1 & 0.220 & 0.00646 & 0.174 \\
\hline & Std Dev & & & 17.9 & & 65.9 & 0.036 & 0.00715 & 0.0304 \\
\hline \multirow[t]{4}{*}{ 300-A Area } & Count & & & 49 & 13 & 51 & 49 & 49 & 49 \\
\hline & Max & & & 0.417 & 0.663 & 2660 & 1.33 & 0.0561 & 1.29 \\
\hline & Average & & & 0.0867 & 0.164 & 176.3 & 0.356 & 0.0124 & 0.3049 \\
\hline & Std Dev & & & 0.0712 & 0.212 & 402.4 & 0.250 & 0.0151 & 0.2372 \\
\hline \multirow[t]{4}{*}{ Hanford Town Site } & Count & & & 40 & & 43 & 40 & 40 & 40 \\
\hline & Max & & & 0.125 & & 9000 & 0.355 & 0.0167 & 0.248 \\
\hline & Average & & & 0.0840 & & 449 & 0.224 & 0.00483 & 0.169 \\
\hline & Std Dev & & & 0.0188 & & 1534 & 0.044 & 0.00523 & 0.0288 \\
\hline \multirow[t]{4}{*}{ Priest Rapids Dam } & Count & 17 & 34 & 49 & 49 & 47 & 49 & 49 & 49 \\
\hline & Max & 0.000022 & 0.000282 & 0.127 & 1.61 & 205 & 0.418 & 0.0251 & 0.38 \\
\hline & Average & 0.000010 & 0.000038 & 0.0792 & 0.0446 & 36.6 & 0.252 & 0.00826 & 0.203 \\
\hline & Std Dev & 0.000007 & 0.000053 & 0.0191 & 0.272 & 26.5 & 0.052 & 0.00675 & 0.0500 \\
\hline \multirow[t]{4}{*}{ Richland Pumphouse } & Count & 16 & 32 & 196 & 49 & 194 & 196 & 196 & 196 \\
\hline & Max & 0.000163 & 0.00017 & 0.305 & 0.534 & 244 & 0.501 & 0.0237 & 0.364 \\
\hline & Average & 0.000105 & 0.00004 & 0.0825 & 0.0486 & 55.5 & 0.256 & 0.00747 & 0.207 \\
\hline & Std Dev & 0.000045 & 0.00004 & 0.0283 & 0.159 & 29.3 & 0.0552 & 0.00595 & 0.0420 \\
\hline \multirow[t]{4}{*}{ Vernita Bridge } & Count & & & 57 & & 58 & 56 & 56 & 56 \\
\hline & Max & & & 0.147 & & 85.4 & 0.33 & 0.0277 & 0.265 \\
\hline & Average & & & 0.0804 & & 32.9 & 0.239 & 0.0081 & 0.186 \\
\hline & Std Dev & & & 0.0189 & & 19.6 & 0.0356 & 0.0061 & 0.0306 \\
\hline
\end{tabular}


Table 3. Examples of Radionuclide Data for Sediment Samples Collected from Columbia River Dams, 1996-1999, in pCi/g, dry wt. (Data is available on the CD issued with this report.)

\begin{tabular}{|c|c|c|c|c|c|}
\hline Location & & Hanford Reach Dam & Ice Harbor Dam & McNary Dam & Priest Rapids Dam \\
\hline \multirow[t]{4}{*}{ Cs-137 } & Number of Samples & 16 & 6 & 20 & 19 \\
\hline & Maximum & 0.599 & 0.293 & 0.811 & 0.674 \\
\hline & Average & 0.323 & 0.195 & 0.389 & 0.357 \\
\hline & Standard Deviation & 0.163 & 0.116 & 0.137 & 0.143 \\
\hline \multirow[t]{4}{*}{$\mathrm{Pu}-239 / 240$} & Number of Samples & 16 & 6 & 20 & 19 \\
\hline & Maximum & 0.00758 & 0.00949 & 0.01270 & 0.01730 \\
\hline & Average & 0.00305 & 0.00621 & 0.00800 & 0.00927 \\
\hline & Standard Deviation & 0.00167 & 0.00369 & 0.00161 & 0.00385 \\
\hline \multirow[t]{4}{*}{ Sr-90 } & Number of Samples & 16 & 6 & 20 & 19 \\
\hline & Maximum & 0.0163 & 0.0236 & 0.0477 & 0.0194 \\
\hline & Average & 0.0060 & 0.0159 & 0.0232 & 0.0127 \\
\hline & Standard Deviation & 0.0037 & 0.0070 & 0.0090 & 0.0047 \\
\hline \multirow[t]{4}{*}{ U-234 } & Number of Samples & 8 & 6 & 12 & 12 \\
\hline & Maximum & 0.301 & 1.100 & 0.861 & 0.829 \\
\hline & Average & 0.203 & 0.759 & 0.690 & 0.512 \\
\hline & Standard Deviation & 0.066 & 0.306 & 0.134 & 0.213 \\
\hline \multirow[t]{4}{*}{ U-235 } & Number of Samples & 16 & 6 & 20 & 19 \\
\hline & Maximum & 0.16 & 0.0555 & 0.206 & 0.168 \\
\hline & Average & 0.0362 & 0.0268 & 0.0392 & 0.0386 \\
\hline & Standard Deviation & 0.0498 & 0.0157 & 0.0509 & 0.0525 \\
\hline \multirow[t]{4}{*}{ U-238 } & Number of Samples & 16 & 6 & 20 & 19 \\
\hline & Maximum & 2.09 & 0.732 & 2.32 & 1.54 \\
\hline & Average & 0.8012 & 0.5402 & 0.9679 & 0.6988 \\
\hline & Standard Deviation & 0.6987 & 0.1919 & 0.6050 & 0.3993 \\
\hline
\end{tabular}

\subsection{Conclusions and Recommendations}

River discharge estimates were adequate to the current task of running the simulation model at a monthly time interval. Daily and hourly flow data are available if the time is reduced in the future. A good bathymetric grid has been developed that provides increased resolution where necessary. Sediment data are the least available, but there is little evidence demonstrating an immediate need for additional resolution on this model component. Partition coefficient values have been broadly defined with carefully defined distributions based on the best available data, and stochastically incorporated into the model. Groundwater inputs are defined by the groundwater model results. Substantial data on radionuclide background levels and river inputs exist, and additional information is continually becoming available through programs such as the HEIS and Hanford Site Surface Environmental Surveillance Project. 


\subsection{References}

Bisping LE. 2003. Hanford Site Environmental Surveillance Data Report for Calendar Year 2002. PNNL-14295, APP. 1, Pacific Northwest National Laboratory, Richland, Washington.

Blanton ML, WW Gardiner, and RL Dirkes. 1995. Environmental Monitoring of Columbia River Sediments: Grain-Size Distributions and Contaminant Associations. PNL-10535, Pacific Northwest Laboratory, Richland, Washington.

Bryce RW, CT Kincaid, PW Eslinger, and LF Morasch (eds.). 2002. An Initial Assessment of Hanford Impact Performed with the System Assessment Capability. PNNL-14027, Pacific Northwest National Laboratory, Richland, Washington.

Cantrell KJ, RJ Serne, and GV Last. 2003. Hanford Contaminant Distribution Coefficient Database Coefficient Database and Users Guide. PNNL-13895, Rev. 1, Pacific Northwest National Laboratory, Richland, Washington.

Comprehensive Environmental Response, Compensation, and Liability Act (CERCLA). 1980. Public Law 96-150, as amended, 94 Stat. 2767, 42 USC 9601 et seq.

DOE. 1998. Screening Assessment and Requirements for a Comprehensive Assessment, Columbia River Comprehensive Impact Assessment. DOE/RL-96-16, Rev. 1, U.S. Department of Energy, Richland Operations Office, Richland, Washington.

DOE M 435.1-1. 1999. Radioactive Waste Management Manual. U.S. Department of Energy, Washington, D.C. Available on the Internet at http://www.directives.doe.gov/pdfs/doe/doetext/neword/435/m4351-1c1.html

DOE Order 435.1. 1999. Radioactive Waste Management. U.S. Department of Energy, Washington, D.C. Available on the Internet at http://www.hanford.gov/wastemgt/doe/psg/pdf/doeo435.1.pdf

Eslinger PW, LR Huesties, AD Maughan, TM Miley, and WH Walters. 1994. Data Compendium for the Columbia River Impact Assessment. PNL-9785, Pacific Northwest Laboratory, Richland, Washington.

Eslinger PW, DW Engel, LH Gerhardstein, CA Lopresti, WE Nichols, and DL Strenge. 2002. "User Instructions for the Systems Assessment Capability, Rev. 0, Computer Codes Volume 1: Inventory, Release, and Transport Modules.” PNNL-13932, Pacific Northwest National Laboratory, Richland, Washington.

Irish JL, JK McClung, and WJ Lillyrop. 2000. “Airborne Lidar Bathymetry: The SHOALS System.” PIANC Bulletin 103:43-53. 
Kaplan DI and RJ Serne. 2000. Geochemical Data Package for the Hanford Immobilized Low-Activity Tank Waste Performance Assessment (ILAW PA). PNNL-13037, Rev. 1, Pacific Northwest National Laboratory, Richland, Washington.

Kincaid CT, MP Bergeron, CR Cole, MD Freshley, NL Hassig, VG Johnson, DI Kaplan, RJ Serne, GP Streile, DL Strenge, PD Thorne, LW Vail, GA Whyatt, and SK Wurstner. 1998. Composite Analysis for Low-Level Waste Disposal in the 200 Area Plateau of the Hanford Site. PNNL-11800, Pacific Northwest National Laboratory, Richland, Washington.

Kincaid CT, PW Eslinger, WE Nichols, AL Bunn, RW Bryce, TB Miley, MC Richmond, SF Snyder, and RL Aaberg. 2000. System Assessment Capability (Revision 0); Assessment Description, Requirements, Software Design, and Test Plan. BHI-01365, Draft A, Bechtel Hanford, Inc., Richland, Washington.

Kincaid CT, RW Bryce, and JW Buck. 2004. Technical Scope and Approach for the 2004 Composite Analysis of Low-Level Waste Disposal at the Hanford Site. PNNL-14372, Pacific Northwest National Laboratory, Richland, Washington.

Last GV, EJ Freeman, KJ Cantrell, MJ Fayer, GW Gee, WE Nichols, BN Bjornstad, and DG Horton. 2004. Vadose Zone Hydrogeology Data Package for the 2004 Composite Analysis. PNNL-14702, Rev. 0, prepared for the Groundwater Remediation Project by Pacific Northwest National Laboratory, Richland, Washington.

Miley TB, RT O’Neil, RO Gilbert, LA Klevgard, and TB Walters. 1997. Data for the Screening Assessment, Volume II, - Appendixes. DOE/RL-96-16c, Rev. 1, Richland, Washington.

McMichael GA, WA Perkins, CJ McMurray, JY Chien, CL Rakowski, A Coleman, MC Richmond, J Vucelic, EV Arntzen, RP Mueller, CA Duberstein, and J Lukas. 2003. Subyearling Chinook Salmon Stranding in the Hanford Reach of the Columbia River. PNWD-3308, prepared for Grand County Public Utility District No. 2 by Battelle-Pacific Northwest Division, Richland, Washington.

Patankar S. 1980. Numerical Heat Transfer and Fluid Flow. Hemisphere, New York, New York.

Patton GW and EA Crecelius. 2001. Simultaneously Extracted Metals/Acid-Volatile Sulfide and Total Metals in Surface Sediment from the Hanford Reach of the Columbia River and the Lower Snake River. PNNL-13417, Pacific Northwest National Laboratory, Richland, Washington.

Perkins WA and MC Richmond. 2004a. MASS2 Modular Aquatic Simulation System in Two Dimensions: Theory and Numerical Methods. PNNL-14820-1, Pacific Northwest National Laboratory, Richland, Washington.

Perkins WA and MC Richmond. 2004b. MASS2 Modular Aquatic Simulation System in Two Dimensions. User Guide and Reference. PNNL-14820-1, Pacific Northwest National Laboratory, Richland, Washington.

Poston TM, RW Hanf, RL Dirkes, and LF Morasch (eds.). 2003. Hanford Site Environmental Report for Calendar Year 2002. PNNL-14295, Pacific Northwest National Laboratory, Richland, Washington. 
Thorne PD. 2004. Groundwater Data Package for the 2004 Composite Analysis. PNNL-14753, Rev. 0, Pacific Northwest National Laboratory, Richland, Washington.

Tiffan KF, RD Garland, and DW Rondorf. 2002. “Quantifying Flow-Dependent Changes in Subyearling Fall Chinook Salmon Rearing Habitat Using Two-Dimensional Spatially Explicit Modeling. North American Journal of Fisheries Management 22(3):713-726. 


\section{Distribution}

No. of

Copies

OFFSITE

Greg Guench

Balance Hydrologics, Inc.

841 Folger Avenue

Berkeley, CA 94710

\section{ONSITE}

2 DOE Office of River Protection
R. M. Yasek
H6-60
R. W. Lober
H6-60

9 DOE Richland Operations Office
B. L. Charboneau
B. L. Foley
A6-33
J. P. Hanson
R. D. Hildebrand
J. G. Morse
K. M. Thompson
S. H. Wisness
DOE Public Reading Room (2)

5 Bechtel Hanford Inc.

P. G. Doctor

H9-01

K. R. Fecht

H9-04

K A Gano

J. K. Linville

H6-60

$\mathrm{H} 0-23$

S. G. Weiss

$\mathrm{H} 0-23$

9 CH2M HILL Hanford Group, Inc.
F. J. Anderson
A. J. Knepp
M. N. Jarayssi

E6-35

H6-03

H6-03
No. of

Copies

F. M. Mann E6-35

W. J. McMahon E6-35

C. W. Miller H6-62

D. A. Myers E6-35

C. D. Wittreich H6-62

M. I. Wood H8-44

2 Fluor Federal Services

$\begin{array}{ll}\text { R. Khaleel } & \text { E6-17 } \\ \text { R. J. Puigh } & \text { E6-17 }\end{array}$

8 Fluor Hanford, Inc.

J. V. Borghese E6-35

F. M. Coony E6-35

B. H. Ford E6-35

T. W. Fogwell E6-35

R. Jackson E6-35

V. J. Rohay E6-35

L. C. Swanson E6-35

M. E. Todd-Robertson E6-35

Stoller

R. G. McCain

48 Pacific Northwest National Laboratory

R. L. Aaberg K3-54

C. Arimescu K6-04

M. P. Bergeron K9-36

B. N. Bjornstad K6-81

R. W. Bryce E6-35

A. L. Bunn K6-85

K. J. Cantrell K6-81

Y. J. Chien K6-81

R. L. Dirkes K6-75 
No. of

Copies

J. L. Downs

D. W. Engel

P. W. Eslinger

M. J. Fayer

E. J. Freeman

M. D. Freshley

G. W. Gee

T. J Gilmore

D. G. Horton

C. T. Kincaid

G. V. Last (5)

C. A. LoPresti

W. J. Martin

T. B. Miley

C. J. Murray

B. A. Napier

W. E. Nichols
No. of

Copies

K6-85

K6-04

K9-33

K9-36

K9-33

K9-33

K6-81

K6-81

K9-33

K6-81

K5-12

K6-81

K6-04

K6-81

K3-54

K9-33
G. W. Patton

C. L. Rakowski

J. V. Ramsdell, Jr.

S. P. Reidel

M. C. Richmond

R. G. Riley

M. L. Rockhold

R. J. Serne

D. L. Strenge

M. B. Triplett

P. D. Thorne

A. L. Ward

M. D. White

M. D. Williams

S. K. Wurstner

S. B. Yabusaki

Hanford Technical Library (2)
K6-75

K9-33

K3-54

K6-81

K9-33

K6-96

K9-36

P7-22

K3-54

K6-04

K9-33

K9-33

K9-36

K9-36

K9-36

K9-36

H2-53 


\section{Data Files from CD}


$70-89$ all water 


\begin{tabular}{|c|c|c|c|c|c|c|c|c|c|c|c|c|c|}
\hline & A & $\mathrm{B}$ & $\mathrm{C}$ & $\mathrm{D}$ & $E$ & $\mathrm{~F}$ & $\mathrm{G}$ & $\mathrm{H}$ & $\mathrm{I}$ & $\mathrm{J}$ & $\mathrm{K}$ & $\mathrm{L}$ & $\mathrm{M}$ \\
\hline 1 & SAMPLE_NUM_ASSIGNED_TO & SAMPLER & MEDIA & BIOTA_MEDIA & TAG_ID & SAMP_NUM & SAMP_DATE_TIME & SAMP_DATE_TIME_ON & CON_ID & CON_SHORT_NAME & METHOD_NAME & LAB_CODE & VALUE_RPTD \\
\hline 2 & PDMS & & SW & & & E034558 & 13-Jul-70 & & $10098-97-2$ & SR-90 & SRISO_SEP_PRECIP_GPC & USTEST & 1720 \\
\hline 3 & PDMS & & SW & & & E002411 & 04-Jan-71 & & $18540-29-9$ & $\mathrm{CR}+6$ & UNKNOWN_METALS & PNL-KI & 0.005 \\
\hline 4 & PDMS & & SW & & & E011010 & 05-Jan-71 & & $18540-29-9$ & $\mathrm{CR}+6$ & UNKNOWN_METALS & PNL-KI & 0 \\
\hline 5 & PDMS & & SW & & & E011295 & 05-Jan-71 & & $18540-29-9$ & $\mathrm{CR}+6$ & UNKNOWN_METALS & PNL-KI & 0.002 \\
\hline 6 & PDMS & & SW & & & E001675 & 05-Jan-71 & & 18540-29-9 & $\mathrm{CR}+6$ & UNKNOWN_METALS & PNL-KI & 0.001 \\
\hline 7 & PDMS & & SW & & & E032747 & 05-Jan-71 & & $10098-97-2$ & SR-90 & SRISO_SEP_PRECIP_GPC & USTEST & 1220 \\
\hline 8 & PDMS & & SW & & & E028082 & 06-Jan-71 & & $18540-29-9$ & $\mathrm{CR}+6$ & UNKNŌWN_METALS & PNL-KI & 2.7 \\
\hline 9 & PDMS & & SW & & & E028083 & 12-Jan-71 & & $18540-29-9$ & $\mathrm{CR}+6$ & UNKNOWN_METALS & PNL-KI & 2.7 \\
\hline 10 & PDMS & & SW & & & E001676 & 12-Jan-71 & & $18540-29-9$ & $\mathrm{CR}+6$ & UNKNOWN_METALS & PNL-KI & 0.003 \\
\hline 11 & PDMS & & SW & & & E002413 & 18-Jan-71 & & $18540-29-9$ & $\mathrm{CR}+6$ & UNKNOWN_METALS & PNL-KI & 0.004 \\
\hline 12 & PDMS & & SW & & & E011011 & 19-Jan-71 & & $18540-29-9$ & $\mathrm{CR}+6$ & UNKNOWN_METALS & PNL-KI & 0.007 \\
\hline 13 & PDMS & & SW & & & E004189 & 19-Jan-71 & & $10045-97-3$ & CS-137 & GAMMA_GS & USTEST & 13 \\
\hline 14 & PDMS & & SW & & & E011296 & 19-Jan-71 & & $18540-29-9$ & $\mathrm{CR}+6$ & UNKNOWN_METALS & PNL-KI & 0.002 \\
\hline 15 & PDMS & & SW & & & E027922 & 19-Jan-71 & & 18540-29-9 & $\mathrm{CR}+6$ & UNKNOWN_METALS & PNL-KI & 2.7 \\
\hline 16 & PDMS & & SW & & & E000364 & 22-Jan-71 & & $10045-97-3$ & CS-137 & GAMMA_GS & USTEST & 69.6 \\
\hline 17 & PDMS & & SW & & & E004103 & 22-Jan-71 & & $10045-97-3$ & CS-137 & GAMMA_GS & USTEST & 407 \\
\hline 18 & PDMS & & SW & & & E028594 & 22-Jan-71 & & $10045-97-3$ & CS-137 & GAMMA_GS & USTEST & -109 \\
\hline 19 & PDMS & & SW & & & E034518 & 22-Jan-71 & & $10045-97-3$ & CS-137 & GAMMA_GS & USTEST & 24.5 \\
\hline 20 & PDMS & & SW & & & E027480 & 22-Jan-71 & & $10045-97-3$ & CS-137 & GAMMA_GS & USTEST & 0 \\
\hline 21 & PDMS & & SW & & & E015839 & 22-Jan-71 & & $10045-97-3$ & CS-137 & GAMMA_GS & PNL-KI & 318 \\
\hline 22 & PDMS & & SW & & & E022355 & 22-Jan-71 & & $10045-97-3$ & CS-137 & GAMMA_GS & USTEST & 51.8 \\
\hline 23 & PDMS & & SW & & & E002414 & 25-Jan-71 & & $18540-29-9$ & $\mathrm{CR}+6$ & UNKNOWN_METALS & PNL-KI & 0.001 \\
\hline 24 & PDMS & & SW & & & E026334 & 25-Jan-71 & & $10045-97-3$ & CS-137 & GAMMA_GS & USTEST & 0 \\
\hline 25 & PDMS & & SW & & & E004190 & 26-Jan-71 & & $10028-17-8$ & TRITIUM & TRITIUM_DIST_LSC & USTEST & 645 \\
\hline 26 & PDMS & & SW & & & E004190 & 26-Jan-71 & & $10045-97-3$ & CS-137 & GAMMA_GS & USTEST & 1.95 \\
\hline 27 & PDMS & & SW & & & E004190 & 26-Jan-71 & & $10098-97-2$ & SR-90 & SRISO_SEP_PRECIP_GPC & USTEST & 0.414 \\
\hline 28 & PDMS & & SW & & & E028084 & 26-Jan-71 & & $10098-97-2$ & SR-90 & SRISO_SEP_PRECIP_GPC & USTEST & 0.382 \\
\hline 29 & PDMS & & SW & & & E028084 & 26-Jan-71 & & $18540-29-9$ & $\mathrm{CR}+6$ & UNKNOWN_METALS & PNL-KI & 2.7 \\
\hline 30 & PDMS & & SW & & & E028084 & 26-Jan-71 & & $10028-17-8$ & TRITIUM & TRITIUM_DIST_LSC & USTEST & 1170 \\
\hline 31 & PDMS & & SW & & & E001608 & 26-Jan-71 & & 18540-29-9 & $\mathrm{CR}+6$ & UNKNOWN_METALS & PNL-KI & 0.001 \\
\hline 32 & PDMS & & SW & & & E011012 & 02-Feb-71 & & $18540-29-9$ & $\mathrm{CR}+6$ & UNKNOWN_METALS & PNL-KI & 0.003 \\
\hline 33 & PDMS & & SW & & & E004191 & 02-Feb-71 & & $10045-97-3$ & CS-137 & GAMMA_GS & USTEST & -0.404 \\
\hline 34 & PDMS & & SW & & & E011297 & 02-Feb-71 & & 18540-29-9 & $\mathrm{CR}+6$ & UNKNOWN_METALS & PNL-KI & 0.003 \\
\hline 35 & PDMS & & SW & & & E028085 & 02-Feb-71 & & $18540-29-9$ & $\mathrm{CR}+6$ & UNKNOWN_METALS & PNL-KI & 2.7 \\
\hline 36 & PDMS & & SW & & & E021389 & 02-Feb-71 & & $18540-29-9$ & $\mathrm{CR}+6$ & UNKNOWN_METALS & PNL-KI & 0.001 \\
\hline 37 & PDMS & & SW & & & E020833 & 03-Feb-71 & & $18540-29-9$ & $\mathrm{CR}+6$ & UNKNOWN_METALS & PNL-KI & 0.002 \\
\hline 38 & PDMS & & SW & & & E002338 & 08-Feb-71 & & $18540-29-9$ & $\mathrm{CR}+6$ & UNKNOWN_METALS & PNL-KI & 0.001 \\
\hline 39 & PDMS & & SW & & & E028086 & 09-Feb-71 & & 18540-29-9 & $\mathrm{CR}+6$ & UNKNOWN_METALS & PNL-KI & 2.7 \\
\hline 40 & PDMS & & SW & & & E001610 & 09-Feb-71 & & $18540-29-9$ & $\mathrm{CR}+6$ & UNKNOWN_METALS & PNL-KI & 0.001 \\
\hline 41 & PDMS & & SW & & & E021390 & 15-Feb-71 & & $18540-29-9$ & $\mathrm{CR}+6$ & UNKNOWN_METALS & PNL-KI & 0.002 \\
\hline 42 & PDMS & & SW & & & E027923 & 16-Feb-71 & & $18540-29-9$ & $\mathrm{CR}+6$ & UNKNOWN_METALS & PNL-KI & 2.7 \\
\hline 43 & PDMS & & SW & & & E020834 & 16-Feb-71 & & $18540-29-9$ & $\mathrm{CR}+6$ & UNKNOWN_METALS & PNL-KI & 0.002 \\
\hline 44 & PDMS & & SW & & & E010967 & 17-Feb-71 & & $18540-29-9$ & $\mathrm{CR}+6$ & UNKNOWN_METALS & PNL-KI & 0.004 \\
\hline 45 & PDMS & & SW & & & E011298 & 17-Feb-71 & & $18540-29-9$ & $\mathrm{CR}+6$ & UNKNOWN_METALS & PNL-KI & 0.004 \\
\hline 46 & PDMS & & SW & & & E010576 & 23-Feb-71 & & $10028-17-8$ & TRITIUM & TRITIUM_DIST_LSC & USTEST & 1140 \\
\hline 47 & PDMS & & SW & & & E010576 & 23-Feb-71 & & $10098-97-2$ & SR-90 & SRISO_SEP_PRECIP_GPC & USTEST & 0.318 \\
\hline 48 & PDMS & & SW & & & E012255 & 23-Feb-71 & & $10098-97-2$ & SR-90 & SRISO_SEP_PRECIP_GPC & USTEST & 0.405 \\
\hline 49 & PDMS & & SW & & & E012255 & 23-Feb-71 & & $18540-29-9$ & $\mathrm{CR}+6$ & UNKNOWN_METALS & PNL-KI & 2.7 \\
\hline 50 & PDMS & & SW & & & E012255 & 23-Feb-71 & & $10028-17-8$ & TRITIUM & TRITIUM_DIST_LSC & USTEST & 1470 \\
\hline 51 & PDMS & & SW & & & E020835 & 23-Feb-71 & & $18540-29-9$ & $\mathrm{CR}+6$ & UNKNOWN_METALS & PNL-KI & 0.001 \\
\hline 52 & PDMS & & SW & & & E010968 & 02-Mar-71 & & $18540-29-9$ & $\mathrm{CR}+6$ & UNKNOWN_METALS & PNL-KI & 0.002 \\
\hline 53 & PDMS & & SW & & & E011299 & 02-Mar-71 & & 18540-29-9 & $\mathrm{CR}+6$ & UNKNOWN_METALS & PNL-KI & 0.002 \\
\hline
\end{tabular}




\begin{tabular}{|c|c|c|c|c|c|c|c|c|c|c|c|c|c|}
\hline & A & $\mathrm{B}$ & $\mathrm{C}$ & $\mathrm{D}$ & $E$ & $\mathrm{~F}$ & $\mathrm{G}$ & $\mathrm{H}$ & $\mathrm{I}$ & $\mathrm{J}$ & $\mathrm{K}$ & $\mathrm{L}$ & $\mathrm{M}$ \\
\hline 1 & SAMPLE_NUM_ASSIGNED_TO & SAMPLER & MEDIA & BIOTA_MEDIA & TAG_ID & SAMP_NUM & SAMP_DATE_TIME & SAMP_DATE_TIME_ON & CON_ID & CON_SHORT_NAME & METHOD_NAME & LAB_CODE & VALUE_RPTD \\
\hline 54 & PDMS & & SW & & & E028087 & 02-Mar-71 & & $18540-29-9$ & $\mathrm{CR}+6$ & UNKNOWN_METALS & PNL-KI & 2.7 \\
\hline 55 & PDMS & & SW & & & E047167 & 02-Mar-71 & & $18540-29-9$ & $\mathrm{CR}+6$ & UNKNOWN_METALS & PNL-KI & c \\
\hline 56 & PDMS & & SW & & & E002340 & 08-Mar-71 & & $18540-29-9$ & $\mathrm{CR}+6$ & UNKNOWN_METALS & PNL-KI & 0.001 \\
\hline 57 & PDMS & & SW & & & E028088 & 09-Mar-71 & & $18540-29-9$ & $\mathrm{CR}+6$ & UNKNOWN_METALS & PNL-KI & 2.7 \\
\hline 58 & PDMS & & SW & & & E020795 & 09-Mar-71 & & $18540-29-9$ & $\mathrm{CR}+6$ & UNKNOWN_METALS & PNL-KI & 0.002 \\
\hline 59 & PDMS & & SW & & & E021391 & 15-Mar-71 & & $18540-29-9$ & $\mathrm{CR}+6$ & UNKNOWN_METALS & PNL-KI & 0.002 \\
\hline 60 & PDMS & & SW & & & E010969 & 16-Mar-71 & & $18540-29-9$ & $\mathrm{CR}+6$ & UNKNOWN_METALS & PNL-KI & 0.003 \\
\hline 61 & PDMS & & SW & & & E011300 & 16-Mar-71 & & $18540-29-9$ & $\mathrm{CR}+6$ & UNKNOWN_METALS & PNL-KI & 0.003 \\
\hline 62 & PDMS & & SW & & & E027852 & 16-Mar-71 & & $18540-29-9$ & $\mathrm{CR}+6$ & UNKNOWN_METALS & PNL-KI & 2.7 \\
\hline 63 & PDMS & & SW & & & E047168 & 16-Mar-71 & & $18540-29-9$ & $\mathrm{CR}+6$ & UNKNOWN_METALS & PNL-KI & c \\
\hline 64 & PDMS & & SW & & & E032749 & 16-Mar-71 & & $10098-97-2$ & SR-90 & SRISO_SEP_PRECIP_GPC & USTEST & 938 \\
\hline 65 & PDMS & & SW & & & E021392 & 22-Mar-71 & & $18540-29-9$ & $\mathrm{CR}+6$ & UNKNŌWN_METALS & PNL-KI & 0.007 \\
\hline 66 & PDMS & & SW & & & E026292 & 22-Mar-71 & & $10045-97-3$ & CS-137 & GAMMA_GS & USTEST & 0.2 \\
\hline 67 & PDMS & & SW & & & E010532 & 23-Mar-71 & & $10028-17-8$ & TRITIUM & TRITIUM_DIST_LSC & USTEST & 277 \\
\hline 68 & PDMS & & SW & & & E010532 & 23-Mar-71 & & $10098-97-2$ & SR-90 & SRISO_SEP_PRECIP_GPC & USTEST & 3.78 \\
\hline 69 & PDMS & & SW & & & E012257 & 23-Mar-71 & & $10098-97-2$ & SR-90 & SRISO_SEP_PRECIP_GPC & USTEST & 0.309 \\
\hline 70 & PDMS & & SW & & & E012257 & 23-Mar-71 & & $18540-29-9$ & $\mathrm{CR}+6$ & UNKNOWN_METALS & PNL-KI & 2.7 \\
\hline 71 & PDMS & & SW & & & E012257 & 23-Mar-71 & & $10028-17-8$ & TRITIUM & TRITIUM_DIST_LSC & USTEST & 263 \\
\hline 72 & PDMS & & SW & & & E020796 & 23-Mar-71 & & $18540-29-9$ & $\mathrm{CR}+6$ & UNKNOWN_METALS & PNL-KI & 0.001 \\
\hline 73 & PDMS & & SW & & & E021393 & 29-Mar-71 & & $18540-29-9$ & $\mathrm{CR}+6$ & UNKNOWN_METALS & PNL-KI & 0.001 \\
\hline 74 & PDMS & & SW & & & E011301 & 30-Mar-71 & & $18540-29-9$ & $\mathrm{CR}+6$ & UNKNOWN_METALS & PNL-KI & 0.004 \\
\hline 75 & PDMS & & SW & & & E020797 & 30-Mar-71 & & $18540-29-9$ & $\mathrm{CR}+6$ & UNKNOWN_METALS & PNL-KI & 0.001 \\
\hline 76 & PDMS & & SW & & & E010970 & 31-Mar-71 & & $18540-29-9$ & $\mathrm{CR}+6$ & UNKNOWN_METALS & PNL-KI & 0.006 \\
\hline 77 & PDMS & & SW & & & E021394 & 05-Apr-71 & & $18540-29-9$ & $\mathrm{CR}+6$ & UNKNOWN_METALS & PNL-KI & 0.001 \\
\hline 78 & PDMS & & SW & & & E020798 & 06-Apr-71 & & 18540-29-9 & $\mathrm{CR}+6$ & UNKNOWN_METALS & PNL-KI & 0.001 \\
\hline 79 & PDMS & & SW & & & E021395 & 12-Apr-71 & & $18540-29-9$ & $\mathrm{CR}+6$ & UNKNOWN_METALS & PNL-KI & 0.001 \\
\hline 80 & PDMS & & SW & & & E010971 & 13-Apr-71 & & $18540-29-9$ & $\mathrm{CR}+6$ & UNKNOWN_METALS & PNL-KI & 0.06 \\
\hline 81 & PDMS & & SW & & & E011302 & 13-Apr-71 & & $18540-29-9$ & $\mathrm{CR}+6$ & UNKNOWN_METALS & PNL-KI & 0.04 \\
\hline 82 & PDMS & & SW & & & E003777 & 13-Apr-71 & & $18540-29-9$ & $\mathrm{CR}+6$ & UNKNOWN_METALS & PNL-KI & 0.001 \\
\hline 83 & PDMS & & SW & & & E032750 & 13-Apr-71 & & $10098-97-2$ & SR-90 & SRISO_SEP_PRECIP_GPC & USTEST & 333 \\
\hline 84 & PDMS & & SW & & & E035851 & 16-Apr-71 & & $10045-97-3$ & CS-137 & GAMMĀ_GS & USTEST & 24.4 \\
\hline 85 & PDMS & & SW & & & E028545 & 16-Apr-71 & & $10045-97-3$ & CS-137 & GAMMA_GS & USTEST & 31.3 \\
\hline 86 & PDMS & & SW & & & E024867 & 16-Apr-71 & & $10045-97-3$ & CS-137 & GAMMA_GS & USTEST & 39.5 \\
\hline 87 & PDMS & & SW & & & E027483 & 16-Apr-71 & & $10045-97-3$ & CS-137 & GAMMA_GS & USTEST & 16.7 \\
\hline 88 & PDMS & & SW & & & E015840 & 16-Apr-71 & & $10045-97-3$ & CS-137 & GAMMA_GS & PNL-KI & 101 \\
\hline 89 & PDMS & & SW & & & E069435 & 16-Apr-71 & & $10045-97-3$ & CS-137 & GAMMA_GS & USTEST & 48.8 \\
\hline 90 & PDMS & & SW & & & E002341 & 19-Apr-71 & & $18540-29-9$ & $\mathrm{CR}+6$ & UNKNOWN_METALS & PNL-KI & 0.001 \\
\hline 91 & PDMS & & SW & & & E020799 & 20-Apr-71 & & 18540-29-9 & $\mathrm{CR}+6$ & UNKNOWN_METALS & PNL-KI & 0.001 \\
\hline 92 & PDMS & & SW & & & E002342 & 26-Apr-71 & & $18540-29-9$ & $\mathrm{CR}+6$ & UNKNOWN_METALS & PNL-KI & 0.001 \\
\hline 93 & PDMS & & SW & & & E010495 & 27-Apr-71 & & $10028-17-8$ & TRITIUM & TRITIUM_DIST_LSC & USTEST & 0 \\
\hline 94 & PDMS & & SW & & & E010495 & 27-Apr-71 & & $10098-97-2$ & SR-90 & SRISO_SEP_PRECIP_GPC & USTEST & 1.07 \\
\hline 95 & PDMS & & SW & & & E012259 & 27-Apr-71 & & $10098-97-2$ & SR-90 & SRISO_SEP_PRECIP_GPC & USTEST & 0.42 \\
\hline 96 & PDMS & & SW & & & E012259 & 27-Apr-71 & & $10028-17-8$ & TRITIUM & TRITIUM_DIST_LSC & USTEST & 2.6 \\
\hline 97 & PDMS & & SW & & & E020800 & 27-Apr-71 & & 18540-29-9 & $\mathrm{CR}+6$ & UNKNOWN_METALS & PNL-KI & 0.001 \\
\hline 98 & PDMS & & SW & & & E002343 & 03-May-71 & & $18540-29-9$ & $\mathrm{CR}+6$ & UNKNOWN_METALS & PNL-KI & 0.001 \\
\hline 99 & PDMS & & SW & & & E010972 & 04-May-71 & & $18540-29-9$ & $\mathrm{CR}+6$ & UNKNOWN_METALS & PNL-KI & 0.011 \\
\hline 100 & PDMS & & SW & & & E011303 & 04-May-71 & & $18540-29-9$ & $\mathrm{CR}+6$ & UNKNOWN_METALS & PNL-KI & 0.011 \\
\hline 101 & PDMS & & SW & & & E020801 & 04-May-71 & & $18540-29-9$ & $\mathrm{CR}+6$ & UNKNOWN_METALS & PNL-KI & 0.001 \\
\hline 102 & PDMS & & SW & & & E002344 & 10-May-71 & & $18540-29-9$ & $\mathrm{CR}+6$ & UNKNOWN_METALS & PNL-KI & 0.001 \\
\hline 103 & PDMS & & SW & & & E020802 & 11-May-71 & & $18540-29-9$ & $\mathrm{CR}+6$ & UNKNOWN_METALS & PNL-KI & 0.001 \\
\hline 104 & PDMS & & SW & & & E002345 & 17-May-71 & & 18540-29-9 & $\mathrm{CR}+6$ & UNKNOWN_METALS & PNL-KI & 0.001 \\
\hline 105 & PDMS & & SW & & & E010973 & 18-May-71 & & $18540-29-9$ & $\mathrm{CR}+6$ & UNKNOWN_METALS & PNL-KI & 0.008 \\
\hline
\end{tabular}




\begin{tabular}{|c|c|c|c|c|c|c|c|c|c|c|c|c|c|}
\hline & $\bar{A}$ & $\mathrm{~B}$ & $\bar{C}$ & $\mathrm{D}$ & $\mathrm{E}$ & $\mathrm{F}$ & $\bar{G}$ & $\mathrm{H}$ & 1 & $\mathrm{~J}$ & $\bar{K}$ & $\bar{L}$ & $\mathrm{M}$ \\
\hline 1 & SAMPLE_NUM_ASSIGNED_TO & SAMPLER & MEDIA & BIOTA_MEDIA & TAG_ID & SAMP_NUM & SAMP_DATE_TIME & SAMP_DATE_TIME_ON & CON_ID & CON_SHORT_NAME & METHOD_NAME & LAB_CODE & VALUE_RPTD \\
\hline 106 & PDMS & & SW & & & E011304 & 18-May-71 & & $18540-29-9$ & $\mathrm{CR}+6$ & UNKNOWN_METALS & PNL-KI & 0.008 \\
\hline 107 & PDMS & & SW & & & E003778 & 18-May-71 & & $18540-29-9$ & $\mathrm{CR}+6$ & UNKNOWN_METALS & PNL-KI & 0.001 \\
\hline 108 & PDMS & & SW & & & E032751 & 18-May-71 & & $10098-97-2$ & SR-90 & SRISO_SEP_PRECIP_GPC & USTEST & 1630 \\
\hline 109 & PDMS & & SW & & & E039808 & 18-May-71 & & $10045-97-3$ & CS-137 & GAMMA_GS & USTEST & 14.1 \\
\hline 110 & PDMS & & SW & & & E021396 & 24-May-71 & & 18540-29-9 & $\mathrm{CR}+6$ & UNKNOW̄N_METALS & PNL-KI & 0.001 \\
\hline 111 & PDMS & & SW & & & E010523 & 25-Мау-71 & & $10028-17-8$ & TRITIUM & TRITIUM_DIST_LSC & USTEST & 1290 \\
\hline 112 & PDMS & & SW & & & E010523 & 25-Мау-71 & & $10098-97-2$ & SR-90 & SRISO_SEP_PRECIP_GPC & USTEST & 0.321 \\
\hline 113 & PDMS & & SW & & & E012260 & 25-May-71 & & $10098-97-2$ & SR-90 & SRISO_SEP_PRECIP_GPC & USTEST & 0.271 \\
\hline 114 & PDMS & & SW & & & E012260 & 25-May-71 & & $10028-17-8$ & TRITIUM & TRITIŪM_DIST_LSC & USTEST & 541 \\
\hline 115 & PDMS & & SW & & & E003779 & 25-May-71 & & $18540-29-9$ & $\mathrm{CR}+6$ & UNKNOW̄N_MĒTALS & PNL-KI & 0.001 \\
\hline 116 & PDMS & & SW & & & E010974 & 01-Jun-71 & & $18540-29-9$ & $\mathrm{CR}+6$ & UNKNOWN_METALS & PNL-KI & 0.008 \\
\hline 117 & PDMS & & SW & & & E011305 & 01-Jun-71 & & 18540-29-9 & $\mathrm{CR}+6$ & UNKNOWN_METALS & PNL-KI & 0.008 \\
\hline 118 & PDMS & & SW & & & E002346 & 01-Jun-71 & & 18540-29-9 & $\mathrm{CR}+6$ & UNKNOWN_METALS & PNL-KI & 0.001 \\
\hline 119 & PDMS & & SW & & & E020803 & 02-Jun-71 & & $18540-29-9$ & $\mathrm{CR}+6$ & UNKNOWN_METALS & PNL-KI & 0.001 \\
\hline 120 & PDMS & & SW & & & E002347 & 07-Jun-71 & & $18540-29-9$ & $\mathrm{CR}+6$ & UNKNOWN_METALS & PNL-KI & 0.001 \\
\hline 121 & PDMS & & SW & & & E003781 & 08-Jun-71 & & 18540-29-9 & $\mathrm{CR}+6$ & UNKNOWN_METALS & PNL-KI & 0.001 \\
\hline 122 & PDMS & & SW & & & E021397 & 14-Jun-71 & & $18540-29-9$ & $\mathrm{CR}+6$ & UNKNOWN_METALS & PNL-KI & 0.002 \\
\hline 123 & PDMS & & SW & & & E026304 & 14-Jun-71 & & $10045-97-3$ & CS-137 & GAMMA_GS & USTEST & 4.96 \\
\hline 124 & PDMS & & SW & & & E010975 & 15-Jun-71 & & 18540-29-9 & $\mathrm{CR}+6$ & UNKNOWNN_METALS & PNL-KI & 0.007 \\
\hline 125 & PDMS & & SW & & & E011306 & 15-Jun-71 & & 18540-29-9 & $\mathrm{CR}+6$ & UNKNOWN_METALS & PNL-KI & 0.008 \\
\hline 126 & PDMS & & SW & & & E003782 & 15-Jun-71 & & 18540-29-9 & $\mathrm{CR}+6$ & UNKNOWN_METALS & PNL-KI & 0.002 \\
\hline 127 & PDMS & & SW & & & E032752 & 15-Jun-71 & & $10098-97-2$ & SR-90 & SRISO_SEP_PRECIP_GPC & USTEST & 1570 \\
\hline 128 & PDMS & & SW & & & E068613 & 15-Jun-71 & & $10045-97-3$ & CS-137 & GAMMA_GS & USTEST & 13.2 \\
\hline 129 & PDMS & & SW & & & E021398 & 21-Jun-71 & & $18540-29-9$ & $\mathrm{CR}+6$ & UNKNOWN_METALS & PNL-KI & 0.001 \\
\hline 130 & PDMS & & SW & & & E010479 & 22-Jun-71 & & 10028-17-8 & TRITIUM & TRITIUM_DIST_LSC & USTEST & 1450 \\
\hline 131 & PDMS & & SW & & & E010479 & 22-Jun-71 & & $10098-97-2$ & SR-90 & SRISO_SEP_PRECIP_GPC & USTEST & 0.756 \\
\hline 132 & PDMS & & SW & & & E012261 & 22-Jun-71 & & $10098-97-2$ & SR-90 & SRISO_SEP_PRECIP_GPC & USTEST & 0.209 \\
\hline 133 & PDMS & & SW & & & E012261 & 22-Jun-71 & & $10028-17-8$ & TRITIUM & TRITIUM_DIST_LSC & USTEST & 2030 \\
\hline 134 & PDMS & & SW & & & E003783 & 22-Jun-71 & & $18540-29-9$ & $\mathrm{CR}+6$ & UNKNOWN_METALS & PNL-KI & 0.001 \\
\hline 135 & PDMS & & SW & & & E002348 & 28-Jun-71 & & 18540-29-9 & $\mathrm{CR}+6$ & UNKNOWN_METALS & PNL-KI & 0.001 \\
\hline 136 & PDMS & & SW & & & E010976 & 29-Jun-71 & & $18540-29-9$ & $\mathrm{CR}+6$ & UNKNOWN_METALS & PNL-KI & 0.007 \\
\hline 137 & PDMS & & SW & & & E011307 & 29-Jun-71 & & 18540-29-9 & $\mathrm{CR}+6$ & UNKNOWN_METALS & PNL-KI & 0.005 \\
\hline 138 & PDMS & & SW & & & E003784 & 29-Jun-71 & & 18540-29-9 & $\mathrm{CR}+6$ & UNKNOWN_METALS & PNL-KI & 0.001 \\
\hline 139 & PDMS & & SW & & & E021385 & 05-Jul-71 & & 18540-29-9 & $\mathrm{CR}+6$ & UNKNOWN_METALS & PNL-KI & 0.001 \\
\hline 140 & PDMS & & SW & & & E002350 & 12-Jul-71 & & $18540-29-9$ & $\mathrm{CR}+6$ & UNKNOWN_METALS & PNL-KI & 0.001 \\
\hline 141 & PDMS & & SW & & & E026308 & 12-Jul-71 & & $10045-97-3$ & CS-137 & GAMMA_GS & USTEST & 25.3 \\
\hline 142 & PDMS & & SW & & & E025587 & 16-Jul-71 & & $10045-97-3$ & CS-137 & GAMMA_GS & USTEST & 47.3 \\
\hline 143 & PDMS & & SW & & & E014930 & 16-Jul-71 & & $10045-97-3$ & CS-137 & GAMMA_GS & USTEST & 37.9 \\
\hline 144 & PDMS & & SW & & & E025951 & 16-Jul-71 & & $10045-97-3$ & CS-137 & GAMMA_GS & USTEST & 54.1 \\
\hline 145 & PDMS & & SW & & & E016387 & 16-Jul-71 & & $10045-97-3$ & CS-137 & GAMMA_GS & USTEST & 40.6 \\
\hline 146 & PDMS & & SW & & & E024868 & 16-Jul-71 & & $10045-97-3$ & CS-137 & GAMMA_GS & USTEST & 103 \\
\hline 147 & PDMS & & SW & & & E030839 & 16-Jul-71 & & $10045-97-3$ & CS-137 & GAMMA_GS & PNL-KI & 112 \\
\hline 148 & PDMS & & SW & & & E036894 & 16-Jul-71 & & $10045-97-3$ & CS-137 & GAMMA_GS & USTEST & 60.5 \\
\hline 149 & PDMS & & SW & & & E002351 & 19-Jul-71 & & $18540-29-9$ & $\mathrm{CR}+6$ & UNKNOWN_METALS & PNL-KI & 0.001 \\
\hline 150 & PDMS & & SW & & & E002352 & 26-Jul-71 & & 18540-29-9 & $\mathrm{CR}+6$ & UNKNOWN_METALS & PNL-KI & 0.001 \\
\hline 151 & PDMS & & SW & & & E010442 & 27-Jul-71 & & $10028-17-8$ & TRITIUM & TRITIUM_DIST_LSC & USTEST & 1090 \\
\hline 152 & PDMS & & SW & & & E010442 & 27-Jul-71 & & $10045-97-3$ & CS-137 & GAMMA_GS & USTEST & 1.14 \\
\hline 153 & PDMS & & SW & & & E010442 & 27-Jul-71 & & $10098-97-2$ & SR-90 & SRISO_SEP_PRECIP_GPC & USTEST & 0.629 \\
\hline 154 & PDMS & & SW & & & E012262 & 28-Jul-71 & & $10098-97-2$ & SR-90 & SRISO_SEP_PRECIP_GPC & USTEST & 0.386 \\
\hline 155 & PDMS & & SW & & & E012262 & 28-Jul-71 & & $10045-97-3$ & CS-137 & GAMMA_GS & USTEST & 1.2 \\
\hline 156 & PDMS & & SW & & & E012262 & 28-Jul-71 & & $10028-17-8$ & TRITIUM & TRITIUM_DIST_LSC & USTEST & 479 \\
\hline 157 & PDMS & & SW & & & E002353 & 02-Aug-71 & & 18540-29-9 & $\mathrm{CR}+6$ & UNKNOWN_METALS & PNL-KI & 0.001 \\
\hline
\end{tabular}




\begin{tabular}{|c|c|c|c|c|c|c|c|c|c|c|c|c|c|}
\hline & $\bar{A}$ & $\mathrm{~B}$ & $\bar{C}$ & $\mathrm{D}$ & $\mathrm{E}$ & $\mathrm{F}$ & $\bar{G}$ & $\mathrm{H}$ & 1 & $\mathrm{~J}$ & $\bar{K}$ & $\bar{L}$ & $\mathrm{M}$ \\
\hline 1 & SAMPLE_NUM_ASSIGNED_TO & SAMPLER & MEDIA & BIOTA_MEDIA & TAG_ID & SAMP_NUM & SAMP_DATE_TIME & SAMP_DATE_TIME_ON & CON_ID & CON_SHORT_NAME & METHOD_NAME & LAB_CODE & VALUE_RPTD \\
\hline 158 & PDMS & & SW & & & E002354 & 09-Aug-71 & & $18540-29-9$ & $\mathrm{CR}+6$ & UNKNOWN_METALS & PNL-KI & 0.001 \\
\hline 159 & PDMS & & SW & & & E002355 & 16-Aug-71 & & $18540-29-9$ & $\mathrm{CR}+6$ & UNKNOWN_METALS & PNL-KI & 0.001 \\
\hline 160 & PDMS & & SW & & & E032754 & 17-Aug-71 & & $10098-97-2$ & SR-90 & SRISO_SEP_PRECIP_GPC & USTEST & 25100 \\
\hline 161 & PDMS & & SW & & & E065384 & 18-Aug-71 & & $10028-17-8$ & TRITIUM & TRITIUM_DIST_LSC & USTEST & 7600 \\
\hline 162 & PDMS & & SW & & & E002356 & 23-Aug-71 & & 18540-29-9 & $\mathrm{CR}+6$ & UNKNOW̄N_MĒTALS & PNL-KI & 0.001 \\
\hline 163 & PDMS & & SW & & & E010398 & 24-Aug-71 & & $10028-17-8$ & TRITIUM & TRITIUM_DIST_LSC & USTEST & 1500 \\
\hline 164 & PDMS & & SW & & & E010398 & 24-Aug-71 & & $10045-97-3$ & CS-137 & GAMMA_GS & USTEST & 27.8 \\
\hline 165 & PDMS & & SW & & & E010398 & 24-Aug-71 & & $10098-97-2$ & SR-90 & SRISO_SEEP_PRECIP_GPC & USTEST & 0.937 \\
\hline 166 & PDMS & & SW & & & E012263 & 24-Aug-71 & & $10098-97-2$ & SR-90 & SRISO_SEP_PRECIP_GPC & USTEST & 0.544 \\
\hline 167 & PDMS & & SW & & & E012263 & 24-Aug-71 & & $10028-17-8$ & TRITIUM & TRITIUM_DIST'_LSC & USTEST & 1570 \\
\hline 168 & PDMS & & SW & & & E017117 & 26-Aug-71 & & $18540-29-9$ & $\mathrm{CR}+6$ & UNKNOWN_METALS & PNL-KI & 0.003 \\
\hline 169 & PDMS & & SW & & & E072232 & 30-Aug-71 & & 18540-29-9 & $\mathrm{CR}+6$ & UNKNOWN_METALS & PNL-KI & 0.4 \\
\hline 170 & PDMS & & SW & & & E002357 & 30-Aug-71 & & 18540-29-9 & $\mathrm{CR}+6$ & UNKNOWN_METALS & PNL-KI & 0.001 \\
\hline 171 & PDMS & & SW & & & E021386 & 06-Sep-71 & & $18540-29-9$ & $\mathrm{CR}+6$ & UNKNOWN_METALS & PNL-KI & 0.001 \\
\hline 172 & PDMS & & SW & & & E002359 & 13-Sep-71 & & $18540-29-9$ & $\mathrm{CR}+6$ & UNKNOWN_METALS & PNL-KI & 0.001 \\
\hline 173 & PDMS & & SW & & & E026316 & 13-Sep-71 & & $10045-97-3$ & CS-137 & GAMMA_GS & USTEST & 31.8 \\
\hline 174 & PDMS & & SW & & & E032755 & 14-Sep-71 & & $10098-97-2$ & SR-90 & SRISO_SEPP_PRECIP_GPC & USTEST & 2520 \\
\hline 175 & PDMS & & SW & & & E002360 & 20-Sep-71 & & $18540-29-9$ & $\mathrm{CR}+6$ & UNKNOWN_METALS & PNL-KI & 0.001 \\
\hline 176 & PDMS & & SW & & & E010354 & 21-Sep-71 & & $10028-17-8$ & TRITIUM & TRITIUM_DIST_LSC & USTEST & 1120 \\
\hline 177 & PDMS & & SW & & & E010354 & 21-Sep-71 & & $10045-97-3$ & CS-137 & GAMMA_GS & USTEST & 0.929 \\
\hline 178 & PDMS & & SW & & & E010354 & 21-Sep-71 & & $10098-97-2$ & SR-90 & SRISO_SEEP_PRECIP_GPC & USTEST & 0.604 \\
\hline 179 & PDMS & & SW & & & E012264 & 21-Sep-71 & & $10098-97-2$ & SR-90 & SRISO_SEP_PRECIP_GPC & USTEST & 0.157 \\
\hline 180 & PDMS & & SW & & & E012264 & 21-Sep-71 & & $10045-97-3$ & CS-137 & GAMMA_GS & USTEST & 0.0331 \\
\hline 181 & PDMS & & SW & & & E012264 & 21-Sep-71 & & $10028-17-8$ & TRITIUM & TRITIUM_DIST_LSC & USTEST & 4300 \\
\hline 182 & PDMS & & SW & & & E002361 & 27-Sep-71 & & 18540-29-9 & $\mathrm{CR}+6$ & UNKNOWNN_METALS & PNL-KI & 0.001 \\
\hline 183 & PDMS & & SW & & & E004106 & 27-Sep-71 & & $10045-97-3$ & CS-137 & GAMMA_GS & USTEST & 55.8 \\
\hline 184 & PDMS & & SW & & & E026319 & 11-Oct-71 & & $10045-97-3$ & CS-137 & GAMMA_GS & USTEST & 0.204 \\
\hline 185 & PDMS & & SW & & & E032756 & 12-Oct-71 & & $10098-97-2$ & SR-90 & SRISO_SEP_PRECIP_GPC & USTEST & 27.4 \\
\hline 186 & PDMS & & SW & & & E014931 & 15-Oct-71 & & $10045-97-3$ & CS-137 & GAMMA_GS & USTEST & 21.8 \\
\hline 187 & PDMS & & SW & & & E004104 & 15-Oct-71 & & $10045-97-3$ & CS-137 & GAMMA_GS & USTEST & 128 \\
\hline 188 & PDMS & & SW & & & E016388 & 15-Oct-71 & & $10045-97-3$ & CS-137 & GAMMA_GS & USTEST & 35.3 \\
\hline 189 & PDMS & & SW & & & E022466 & 15-Oct-71 & & $10045-97-3$ & CS-137 & GAMMA_GS & USTEST & 95.7 \\
\hline 190 & PDMS & & SW & & & E027489 & 15-Oct-71 & & $10045-97-3$ & CS-137 & GAMMA_GS & USTEST & 13.2 \\
\hline 191 & PDMS & & SW & & & E003548 & 15-Oct-71 & & $10045-97-3$ & CS-137 & GAMMA_GS & PNL-KI & 33.2 \\
\hline 192 & PDMS & & SW & & & E036895 & 15-Oct-71 & & $10045-97-3$ & CS-137 & GAMMA_GS & USTEST & 40.7 \\
\hline 193 & PDMS & & SW & & & E003785 & 18-Oct-71 & & $10045-97-3$ & CS-137 & GAMMA_GS & USTEST & 4.89 \\
\hline 194 & PDMS & & SW & & & E003786 & 25-Oct-71 & & $10045-97-3$ & CS-137 & GAMMA_GS & USTEST & 17.6 \\
\hline 195 & PDMS & & SW & & & E010318 & 26-Oct-71 & & $10028-17-8$ & TRITIUM & TRITIUM_DIST_LSC & USTEST & 346 \\
\hline 196 & PDMS & & SW & & & E010318 & 26-Oct-71 & & $10098-97-2$ & SR-90 & SRISO_SEP_PRECIP_GPC & USTEST & 0.459 \\
\hline 197 & PDMS & & SW & & & E012265 & 26-Oct-71 & & $10098-97-2$ & SR-90 & SRISO_SEP_PRECIP_GPC & USTEST & 0.439 \\
\hline 198 & PDMS & & SW & & & E012265 & 26-Oct-71 & & $10028-17-8$ & TRITIUM & TRITIUM_DIST_LSC & USTEST & 392 \\
\hline 199 & PDMS & & SW & & & E030271 & 28-Oct-71 & & $10028-17-8$ & TRITIUM & TRITIUM_DIST_LSC & PNL-KI & 820 \\
\hline 200 & PDMS & & SW & & & E003787 & 01-Nov-71 & & $10045-97-3$ & CS-137 & GAMMA_GS & USTEST & 0.154 \\
\hline 201 & PDMS & & SW & & & E003788 & 08-Nov-71 & & $10045-97-3$ & CS-137 & GAMMA_GS & USTEST & 8.46 \\
\hline 202 & PDMS & & SW & & & E010332 & 09-Nov-71 & & $10045-97-3$ & CS-137 & GAMMA_GS & USTEST & 25.8 \\
\hline 203 & PDMS & & SW & & & E030272 & 15-Nov-71 & & $10028-17-8$ & TRITIUM & TRITIUM_DIST_LSC & PNL-KI & 610 \\
\hline 204 & PDMS & & SW & & & E026323 & 15-Nov-71 & & $10045-97-3$ & CS-137 & GAMMA_GS & USTEST & -0.735 \\
\hline 205 & PDMS & & SW & & & E032757 & 16-Nov-71 & & $10098-97-2$ & SR-90 & SRISO_SEP_PRECIP_GPC & USTEST & 2240 \\
\hline 206 & PDMS & & SW & & & E030273 & 22-Nov-71 & & $10028-17-8$ & TRITIUM & TRITIUM_DIST_LSC & PNL-KI & 850 \\
\hline 207 & PDMS & & SW & & & E002369 & 22-Nov-71 & & $10098-97-2$ & SR-90 & SRISO_SEP_PRECIP_GPC & USTEST & 0.349 \\
\hline 208 & PDMS & & SW & & & E004192 & 23-Nov-71 & & $10028-17-8$ & TRITIUM & TRITIUM_DIST_LSC & USTEST & \\
\hline 209 & PDMS & & SW & & & E004192 & 23-Nov-71 & & $10045-97-3$ & CS-137 & GAMMA_GS & USTEST & 0.0557 \\
\hline
\end{tabular}




\begin{tabular}{|c|c|c|c|c|c|c|c|c|c|c|c|c|c|}
\hline & A & $\mathrm{B}$ & $\mathrm{C}$ & $\mathrm{D}$ & $E$ & $\mathrm{~F}$ & $\mathrm{G}$ & $\mathrm{H}$ & $\mathrm{I}$ & $\mathrm{J}$ & $\mathrm{K}$ & $\mathrm{L}$ & $\mathrm{M}$ \\
\hline 1 & SAMPLE_NUM_ASSIGNED_TO & SAMPLER & MEDIA & BIOTA_MEDIA & TAG_ID & SAMP_NUM & SAMP_DATE_TIME & SAMP_DATE_TIME_ON & CON_ID & CON_SHORT_NAME & METHOD_NAME & LAB_CODE & VALUE_RPTD \\
\hline 210 & PDMS & & SW & & & E004192 & $23-N o v-71$ & & $10098-97-2$ & SR-90 & SRISO_SEP_PRECIP_GPC & USTEST & 0.441 \\
\hline 211 & PDMS & & SW & & & E012266 & 23-Nov-71 & & $10098-97-2$ & SR-90 & SRISO_SEP_PRECIP_GPC & USTEST & 0.333 \\
\hline 212 & PDMS & & SW & & & E012266 & 23-Nov-71 & & $10045-97-3$ & CS-137 & GAMMA_GS & USTEST & -0.00713 \\
\hline 213 & PDMS & & SW & & & E012266 & 23-Nov-71 & & $10028-17-8$ & TRITIUM & TRITIUM_DIST_LSC & USTEST & 602 \\
\hline 214 & PDMS & & SW & & & E003792 & 13-Dec-71 & & $10045-97-3$ & CS-137 & GAMMA_GS & USTEST & 8.98 \\
\hline 215 & PDMS & & SW & & & E010303 & 21-Dec-71 & & $10045-97-3$ & CS-137 & GAMMA_GS & USTEST & 23.3 \\
\hline 216 & PDMS & & SW & & & E002374 & 27-Dec-71 & & $10098-97-2$ & SR-90 & SRISO_SEEP_PRECIP_GPC & USTEST & 0.404 \\
\hline 217 & PDMS & & SW & & & E004193 & 28-Dec-71 & & $10028-17-8$ & TRITIUM & TRITIUM_DIST_LSC & USTEST & 491 \\
\hline 218 & PDMS & & SW & & & E004193 & 28-Dec-71 & & $10098-97-2$ & SR-90 & SRISO_SEP_PRECIP_GPC & USTEST & 0.401 \\
\hline 219 & PDMS & & SW & & & E012267 & 28-Dec-71 & & $10098-97-2$ & SR-90 & SRISO_SEP_PRECIP_GPC & USTEST & 0.405 \\
\hline 220 & PDMS & & SW & & & E012267 & 28-Dec-71 & & $10028-17-8$ & TRITIUM & TRITIUM_DIST_LSC & USTEST & 0 \\
\hline 221 & PDMS & & SW & & & E030274 & 30-Dec-71 & & $10028-17-8$ & TRITIUM & TRITIUM_DIST_LSC & PNL-KI & 1000 \\
\hline 222 & PDMS & & SW & & & E026254 & 10-Jan-72 & & $10045-97-3$ & CS-137 & GAMMA_GS & USTEST & -3.52 \\
\hline 223 & PDMS & & SW & & & E025918 & 14-Jan-72 & & $10045-97-3$ & CS-137 & GAMMA_GS & USTEST & -0.0905 \\
\hline 224 & PDMS & & SW & & & E014839 & 14-Jan-72 & & $10045-97-3$ & CS-137 & GAMMA_GS & USTEST & 10.9 \\
\hline 225 & PDMS & & SW & & & E022467 & 14-Jan-72 & & $10045-97-3$ & CS-137 & GAMMA_GS & USTEST & 9.42 \\
\hline 226 & PDMS & & SW & & & E003549 & 14-Jan-72 & & $10045-97-3$ & CS-137 & GAMMA_GS & PNL-KI & 34.7 \\
\hline 227 & PDMS & & SW & & & E002378 & 24-Jan-72 & & $10098-97-2$ & SR-90 & SRISO_SEP_PRECIP_GPC & USTEST & 0.27 \\
\hline 228 & PDMS & & SW & & & E004194 & 25-Jan-72 & & $10028-17-8$ & TRITIUM & TRITIUM_DIST_LSC & USTEST & 1280 \\
\hline 229 & PDMS & & SW & & & E004194 & 25-Jan-72 & & $10098-97-2$ & SR-90 & SRISO_SEP_PRECIP_GPC & USTEST & 0.546 \\
\hline 230 & PDMS & & SW & & & E012268 & 25-Jan-72 & & $10098-97-2$ & SR-90 & SRISO_SEP_PRECIP_GPC & USTEST & 0.481 \\
\hline 231 & PDMS & & SW & & & E012268 & 25-Jan-72 & & $10028-17-8$ & TRITIUM & TRITIUM_DIST_LSC & USTEST & 67.9 \\
\hline 232 & PDMS & & SW & & & E002379 & 31-Jan-72 & & $10045-97-3$ & CS-137 & GAMMA_GS & USTEST & 10.4 \\
\hline 233 & PDMS & & SW & & & E010273 & 01-Feb-72 & & $10045-97-3$ & CS-137 & GAMMA_GS & USTEST & 14.3 \\
\hline 234 & PDMS & & SW & & & E032758 & $08-F e b-72$ & & $10098-97-2$ & SR-90 & SRISO_SEP_PRECIP_GPC & USTEST & 749 \\
\hline 235 & PDMS & & SW & & & E004195 & 22-Feb-72 & & $10028-17-8$ & TRITIUM & TRITIUM_DIST_LSC & USTEST & 215 \\
\hline 236 & PDMS & & SW & & & E004195 & $22-F e b-72$ & & $10045-97-3$ & CS-137 & GAMMA_GS & USTEST & -0.0184 \\
\hline 237 & PDMS & & SW & & & E004195 & 22-Feb-72 & & $10098-97-2$ & SR-90 & SRISO_SEP_PRECIP_GPC & USTEST & 0.536 \\
\hline 238 & PDMS & & SW & & & E012269 & $22-F e b-72$ & & $10098-97-2$ & SR-90 & SRISO_SEP_PRECIP_GPC & USTEST & 0.421 \\
\hline 239 & PDMS & & SW & & & E012269 & 22-Feb-72 & & $10045-97-3$ & CS-137 & GAMMA_GS & USTEST & 0.0332 \\
\hline 240 & PDMS & & SW & & & E012269 & 22-Feb-72 & & $10028-17-8$ & TRITIUM & TRITIUM_DIST_LSC & USTEST & 1390 \\
\hline 241 & PDMS & & SW & & & E002300 & 22-Feb-72 & & $10098-97-2$ & SR-90 & SRISO_SEP_PRECIP_GPC & USTEST & 0.423 \\
\hline 242 & PDMS & & SW & & & E026260 & 06-Mar-72 & & $10045-97-3$ & CS-137 & GAMMA_GS & USTEST & -0.129 \\
\hline 243 & PDMS & & SW & & & E032759 & 07-Mar-72 & & $10098-97-2$ & SR-90 & SRISO_SEP_PRECIP_GPC & USTEST & 411 \\
\hline 244 & PDMS & & SW & & & E003735 & 20-Mar-72 & & $10045-97-3$ & CS-137 & GAMMA_GS & USTEST & 12.5 \\
\hline 245 & PDMS & & SW & & & E002304 & 20-Mar-72 & & $10045-97-3$ & CS-137 & GAMMA_GS & USTEST & 13 \\
\hline 246 & PDMS & & SW & & & E004196 & 21-Mar-72 & & $10028-17-8$ & TRITIUM & TRITIUM_DIST_LSC & USTEST & -454 \\
\hline 247 & PDMS & & SW & & & E004196 & 21-Mar-72 & & $10098-97-2$ & SR-90 & SRISO_SEP_PRECIP_GPC & USTEST & 0.468 \\
\hline 248 & PDMS & & SW & & & E012270 & 21-Mar-72 & & $10098-97-2$ & SR-90 & SRISO_SEP_PRECIP_GPC & USTEST & 0.346 \\
\hline 249 & PDMS & & SW & & & E012270 & 21-Mar-72 & & $10028-17-8$ & TRITIUM & TRITIUM_DIST_LSC & USTEST & 363 \\
\hline 250 & PDMS & & SW & & & E002305 & 27-Mar-72 & & $10098-97-2$ & SR-90 & SRISO_SEP_PRECIP_GPC & USTEST & 0.226 \\
\hline 251 & PDMS & & SW & & & E064997 & 03-Apr-72 & & $10028-17-8$ & TRITIUM & TRITIUM_DIST_LSC & USTEST & 225 \\
\hline 252 & PDMS & & SW & & & E032760 & 04-Apr-72 & & $10098-97-2$ & SR-90 & SRISO_SEP_PRECIP_GPC & USTEST & 940 \\
\hline 253 & PDMS & & SW & & & E027460 & 04-Apr-72 & & $10045-97-3$ & CS-137 & GAMMA_GS & USTEST & -3.58 \\
\hline 254 & PDMS & & SW & & & E013043 & 07-Apr-72 & & $10045-97-3$ & CS-137 & GAMMA_GS & USTEST & -5.02 \\
\hline 255 & PDMS & & SW & & & E025955 & 07-Apr-72 & & $10045-97-3$ & CS-137 & GAMMA_GS & USTEST & 62.2 \\
\hline 256 & PDMS & & SW & & & E028041 & 07-Apr-72 & & $10045-97-3$ & CS-137 & GAMMA_GS & USTEST & -2.27 \\
\hline 257 & PDMS & & SW & & & E023910 & 07-Apr-72 & & $10045-97-3$ & CS-137 & GAMMA_GS & USTEST & 37 \\
\hline 258 & PDMS & & SW & & & E003550 & 07-Apr-72 & & $10045-97-3$ & CS-137 & GAMMA_GS & PNL-KI & 19.9 \\
\hline 259 & PDMS & & SW & & & E036896 & 07-Apr-72 & & $10045-97-3$ & CS-137 & GAMMA_GS & USTEST & 19.7 \\
\hline 260 & PDMS & & SW & & & E003738 & 10-Apr-72 & & $10045-97-3$ & CS-137 & GAMMA_GS & USTEST & -0.334 \\
\hline 261 & PDMS & & SW & & & E002307 & 10-Apr-72 & & $10045-97-3$ & CS-137 & GAMMA_GS & USTEST & 48.6 \\
\hline
\end{tabular}




\begin{tabular}{|c|c|c|c|c|c|c|c|c|c|c|c|c|c|}
\hline & $\bar{A}$ & $\mathrm{~B}$ & $\bar{C}$ & $\mathrm{D}$ & $\mathrm{E}$ & $\mathrm{F}$ & $\bar{G}$ & $\mathrm{H}$ & 1 & $\mathrm{~J}$ & $\bar{K}$ & $\bar{L}$ & $\mathrm{M}$ \\
\hline 1 & SAMPLE_NUM_ASSIGNED_TO & SAMPLER & MEDIA & BIOTA_MEDIA & TAG_ID & SAMP_NUM & SAMP_DATE_TIME & SAMP_DATE_TIME_ON & CON_ID & CON_SHORT_NAME & METHOD_NAME & LAB_CODE & VALUE_RPTD \\
\hline 262 & PDMS & & SW & & & E010200 & 11-Apr-72 & & $10045-97-3$ & CS-137 & GAMMA_GS & USTEST & 23.1 \\
\hline 263 & PDMS & & SW & & & E003740 & 24-Apr-72 & & $10045-97-3$ & CS-137 & GAMMA_GS & USTEST & 13.2 \\
\hline 264 & PDMS & & SW & & & E002309 & 24-Apr-72 & & $10098-97-2$ & SR-90 & SRISO_SEP_PRECIP_GPC & USTEST & 0.197 \\
\hline 265 & PDMS & & SW & & & E004197 & 25-Apr-72 & & $10028-17-8$ & TRITIUM & TRITIUM_DIST_LSC & USTEST & -190 \\
\hline 266 & PDMS & & SW & & & E004197 & 25-Apr-72 & & $10098-97-2$ & SR-90 & SRISO_SEP_PRECIP_GPC & USTEST & 0.192 \\
\hline 267 & PDMS & & SW & & & E012271 & 25-Apr-72 & & $10098-97-2$ & SR-90 & SRISO_SEP_PRECIP_GPC & USTEST & 0.243 \\
\hline 268 & PDMS & & SW & & & E012271 & 25-Apr-72 & & $10028-17-8$ & TRITIUM & TRITIUM_DISTT_LSC & USTEST & -306 \\
\hline 269 & PDMS & & SW & & & E026268 & 01-Мау-72 & & $10045-97-3$ & CS-137 & GAMMA_GS & USTEST & -1.19 \\
\hline 270 & PDMS & & SW & & & E010151 & 02-May-72 & & $10045-97-3$ & CS-137 & GAMMA_GS & USTEST & 25.4 \\
\hline 271 & PDMS & & SW & & & E032761 & 02-May-72 & & $10098-97-2$ & SR-90 & SRISO_SEEP_PRECIP_GPC & USTEST & 3.73 \\
\hline 272 & PDMS & & SW & & & E003743 & 15-May-72 & & $10045-97-3$ & CS-137 & GAMMA_GS & USTEST & -0.177 \\
\hline 273 & PDMS & & SW & & & E002312 & 15-Мау-72 & & $10045-97-3$ & CS-137 & GAMMA_GS & USTEST & -0.0678 \\
\hline 274 & PDMS & & SW & & & E010165 & 16-Мау-72 & & $10045-97-3$ & CS-137 & GAMMA_GS & USTEST & 18.6 \\
\hline 275 & PDMS & & SW & & & E002313 & 22-May-72 & & $10098-97-2$ & SR-90 & SRISO_SEP_PRECIP_GPC & USTEST & 0.191 \\
\hline 276 & PDMS & & SW & & & E004165 & 23-May-72 & & $10028-17-8$ & TRITIUM & TRITIUM_DIST_LSC & USTEST & 811 \\
\hline 277 & PDMS & & SW & & & E004198 & 23-May-72 & & $10028-17-8$ & TRITIUM & TRITIUM_DIST_LSC & USTEST & 16 \\
\hline 278 & PDMS & & SW & & & E004198 & 23-May-72 & & $10045-97-3$ & CS-137 & GAMMA_GS & USTEST & 1.06 \\
\hline 279 & PDMS & & SW & & & E004198 & 23-May-72 & & $10098-97-2$ & SR-90 & SRISO_SEPP_PRECIP_GPC & USTEST & 0.266 \\
\hline 280 & PDMS & & SW & & & E012272 & 23-May-72 & & $10098-97-2$ & SR-90 & SRISO_SEP_PRECIP_GPC & USTEST & 0.181 \\
\hline 281 & PDMS & & SW & & & E012272 & 23-Мау-72 & & $10045-97-3$ & CS-137 & GAMMA_GS & USTEST & 0.214 \\
\hline 282 & PDMS & & SW & & & E012272 & 23-Мау-72 & & $10028-17-8$ & TRITIUM & TRITIUM_DIST_LSC & USTEST & -198 \\
\hline 283 & PDMS & & SW & & & E010108 & 30-Mау-72 & & $10045-97-3$ & CS-137 & GAMMA_GS & USTEST & 2.57 \\
\hline 284 & PDMS & & SW & & & E003745 & 30-May-72 & & $10045-97-3$ & CS-137 & GAMMA_GS & USTEST & 2.47 \\
\hline 285 & PDMS & & SW & & & E002314 & 30-May-72 & & $10045-97-3$ & CS-137 & GAMMA_GS & USTEST & 3.62 \\
\hline 286 & PDMS & & SW & & & E026565 & 02-Jun-72 & & $10098-97-2$ & SR-90 & SRISO_SEPP_PRECIP_GPC & USTEST & 8.83 \\
\hline 287 & PDMS & & SW & & & E003746 & 05-Jun-72 & & $10045-97-3$ & CS-137 & GAMMA_GS & USTEST & 4.54 \\
\hline 288 & PDMS & & SW & & & E002315 & 05-Jun-72 & & $10045-97-3$ & CS-137 & GAMMA_GS & USTEST & 8 \\
\hline 289 & PDMS & & SW & & & E010115 & 06-Jun-72 & & $10045-97-3$ & CS-137 & GAMMA_GS & USTEST & 18 \\
\hline 290 & PDMS & & SW & & & E010122 & 13-Jun-72 & & $10045-97-3$ & CS-137 & GAMMA_GS & USTEST & 22.9 \\
\hline 291 & PDMS & & SW & & & E032762 & 13-Jun-72 & & $10098-97-2$ & SR-90 & SRISO_SEP_PRECIP_GPC & USTEST & 2540 \\
\hline 292 & PDMS & & SW & & & E003748 & 19-Jun-72 & & $10045-97-3$ & CS-137 & GAMMA_GS & USTEST & 14.4 \\
\hline 293 & PDMS & & SW & & & E002318 & 26-Jun-72 & & $10098-97-2$ & SR-90 & SRISO_SEPP_PRECIP_GPC & USTEST & 0.297 \\
\hline 294 & PDMS & & SW & & & E002318 & 26-Jun-72 & & $10045-97-3$ & CS-137 & GAMMA__GS & USTEST & 15.8 \\
\hline 295 & PDMS & & SW & & & E004199 & 27-Jun-72 & & 10028-17-8 & TRITIUM & TRITIUM_DIST_LSC & USTEST & -211 \\
\hline 296 & PDMS & & SW & & & E004199 & 27-Jun-72 & & $10045-97-3$ & CS-137 & GAMMA_GS & USTEST & 1.02 \\
\hline 297 & PDMS & & SW & & & E004199 & 27-Jun-72 & & $10098-97-2$ & SR-90 & SRISO_SEP_PRECIP_GPC & USTEST & 0.124 \\
\hline 298 & PDMS & & SW & & & E012273 & 27-Jun-72 & & $10098-97-2$ & SR-90 & SRISO_SEP_PRECIP_GPC & USTEST & 0.322 \\
\hline 299 & PDMS & & SW & & & E012273 & 27-Jun-72 & & $10028-17-8$ & TRITIUM & TRITIUM_DISTT_LSC & USTEST & -256 \\
\hline 300 & PDMS & & SW & & & E003750 & 03-Jul-72 & & $10045-97-3$ & CS-137 & GAMMA_GS & USTEST & 0.166 \\
\hline 301 & PDMS & & SW & & & E002319 & 03-Jul-72 & & $10045-97-3$ & CS-137 & GAMMA_GS & USTEST & -0.106 \\
\hline 302 & PDMS & & SW & & & E010073 & 05-Jul-72 & & $10045-97-3$ & CS-137 & GAMMA_GS & USTEST & 0.164 \\
\hline 303 & PDMS & & SW & & & E000365 & 07-Jul-72 & & $10045-97-3$ & CS-137 & GAMMA_GS & USTEST & 13.2 \\
\hline 304 & PDMS & & SW & & & E025919 & 07-Jul-72 & & $10045-97-3$ & CS-137 & GAMMA_GS & USTEST & 40.5 \\
\hline 305 & PDMS & & SW & & & E016389 & 07-Jul-72 & & $10045-97-3$ & CS-137 & GAMMA_GS & USTEST & 18.7 \\
\hline 306 & PDMS & & SW & & & E023911 & 07-Jul-72 & & $10045-97-3$ & CS-137 & GAMMA_GS & USTEST & 89.8 \\
\hline 307 & PDMS & & SW & & & E030840 & 07-Jul-72 & & $10045-97-3$ & CS-137 & GAMMA_GS & PNL-KI & 28.1 \\
\hline 308 & PDMS & & SW & & & E035515 & 07-Jul-72 & & $10045-97-3$ & CS-137 & GAMMA_GS & USTEST & 215 \\
\hline 309 & PDMS & & SW & & & E014261 & 07-Jul-72 & & $10045-97-3$ & CS-137 & GAMMA_GS & USTEST & 16.4 \\
\hline 310 & PDMS & & SW & & & E014261 & 07-Jul-72 & & $10098-97-2$ & SR-90 & SRISO_SEP_PRECIP_GPC & USTEST & 12.7 \\
\hline 311 & PDMS & & SW & & & E003751 & 10-Jul-72 & & $10045-97-3$ & CS-137 & GAMMA_GS & USTEST & 4.76 \\
\hline 312 & PDMS & & SW & & & E002320 & 10-Jul-72 & & $10045-97-3$ & CS-137 & GAMMA_GS & USTEST & 12.2 \\
\hline 313 & PDMS & & SW & & & E032763 & 11-Jul-72 & & $10098-97-2$ & SR-90 & SRISO_SEP_PRECIP_GPC & USTEST & 462 \\
\hline
\end{tabular}




\begin{tabular}{|c|c|c|c|c|c|c|c|c|c|c|c|c|c|}
\hline & $\bar{A}$ & $\mathrm{~B}$ & $\mathrm{C}$ & $\bar{D}$ & $\mathrm{E}$ & $\mathrm{F}$ & $\bar{G}$ & $\mathrm{H}$ & 1 & $\mathrm{~J}$ & $\mathrm{~K}$ & $\mathrm{~L}$ & $\bar{M}$ \\
\hline 1 & SAMPLE_NUM_ASSIGNED_TO & SAMPLER & MEDIA & BIOTA_MEDIA & TAG_ID & SAMP_NUM & SAMP_DATE_TIME & SAMP_DATE_TIME_ON & CON_ID & \begin{tabular}{|l|} 
CON_SHORT_NAME \\
\end{tabular} & METHOD_NAME & LAB_CODE & VALUE_RPTD \\
\hline 314 & PDMS & & SW & & & E039818 & 11-Jul-72 & & $10045-97-3$ & CS-137 & GAMMA_GS & USTEST & -2.04 \\
\hline 315 & PDMS & & sW & & & E010086 & 18-Jul-72 & & $10045-97-3$ & CS-137 & GAMMA_GS & USTEST & 6.62 \\
\hline 316 & PDMS & & SW & & & E003753 & 24-Jul-72 & & $10045-97-3$ & CS-137 & GAMMA_GS & USTEST & 2.66 \\
\hline 317 & PDMS & & SW & & & E002322 & 24-Jul-72 & & $10098-97-2$ & SR-90 & SRISO_SEP_PRECIP_GPC & USTEST & 0.286 \\
\hline 318 & PDMS & & SW & & & E002322 & 24-Jul-72 & & $10045-97-3$ & CS-137 & GAMMA_GS & USTEST & 1.51 \\
\hline 319 & PDMS & & SW & & & E004200 & 25-Jul-72 & & $10028-17-8$ & TRITIUM & TRITIUM_DIST_LSC & USTEST & -422 \\
\hline 320 & PDMS & & sW & & & E004200 & 25-Jul-72 & & $10045-97-3$ & CS-137 & GAMMA_GS & USTEST & 13.4 \\
\hline 321 & PDMS & & sW & & & E004200 & 25-Jul-72 & & $10098-97-2$ & SR-90 & SRISO_SEEP_PRECIP_GPC & USTEST & 0.295 \\
\hline 322 & PDMS & & sW & & & E012274 & 25-Jul-72 & & $10098-97-2$ & SR-90 & SRISO_SEP_PRECIP_GPC & USTEST & 0.241 \\
\hline 323 & PDMS & & sW & & & E012274 & 25-Jul-72 & & $10045-97-3$ & CS-137 & GAMMA_GS ${ }^{-}$ & USTEST & 0.00831 \\
\hline 324 & PDMS & & SW & & & E012274 & 25-Jul-72 & & $10028-17-8$ & TRITIUM & TRITIUM_DIST_LSC & USTEST & -0.000532 \\
\hline 325 & PDMS & & SW & & & E003754 & 31-Jul-72 & & $10045-97-3$ & CS-137 & GAMMA_GS & USTEST & 5.63 \\
\hline 326 & PDMS & & sW & & & E026278 & 31-Jul-72 & & $10045-97-3$ & CS-137 & GAMMA_GS & USTEST & 13.7 \\
\hline 327 & PDMS & & SW & & & E026566 & 04-Aug-72 & & $10098-97-2$ & SR-90 & SRISO_SEP_PRECIP_GPC & USTEST & 4.86 \\
\hline 328 & PDMS & & SW & & & E032764 & 08-Aug-72 & & $10098-97-2$ & SR-90 & SRISO_SEP_PRECIP_GPC & USTEST & 129 \\
\hline 329 & PDMS & & SW & & & E002326 & 21-Aug-72 & & $10098-97-2$ & SR-90 & SRISO_SEP_PRECIP_GPC & USTEST & 0.0238 \\
\hline 330 & PDMS & & SW & & & E026282 & 28-Aug-72 & & $10045-97-3$ & CS-137 & GAMMA_GS & USTEST & 0.161 \\
\hline 331 & PDMS & & sW & & & E004201 & 29-Aug-72 & & $10028-17-8$ & TRITIUM & TRITIUM_DIST_LSC & USTEST & 224 \\
\hline 332 & PDMS & & sW & & & E004201 & 29-Aug-72 & & $10045-97-3$ & CS-137 & GAMMA_GS & USTEST & 0.00831 \\
\hline 333 & PDMS & & sW & & & E004201 & 29-Aug-72 & & $10098-97-2$ & SR-90 & SRISO_SEEP_PRECIP_GPC & USTEST & 0.299 \\
\hline 334 & PDMS & & sW & & & E012275 & 29-Aug-72 & & $10098-97-2$ & SR-90 & SRISO_SEP_PRECIP_GPC & USTEST & 2.77 \\
\hline 335 & PDMS & & SW & & & E012275 & 29-Aug-72 & & $10045-97-3$ & CS-137 & GAMMA_GS & USTEST & 0.435 \\
\hline 336 & PDMS & & SW & & & E012275 & 29-Aug-72 & & $10028-17-8$ & TRITIUM & TRITIUM_DIST_LSC & USTEST & -213 \\
\hline 337 & PDMS & & SW & & & E032765 & 29-Aug-72 & & $10098-97-2$ & SR-90 & SRISO_SEP_PRECIP_GPC & USTEST & 588 \\
\hline 338 & PDMS & & SW & & & E026567 & 01-Sep-72 & & $10098-97-2$ & SR-90 & SRISO_SEP_PRECIP_GPC & USTEST & 2.25 \\
\hline 339 & PDMS & & sW & & & E003760 & 11-Sep-72 & & $10045-97-3$ & CS-137 & GAMMA_GS & USTEST & 3.61 \\
\hline 340 & PDMS & & SW & & & E002329 & 11-Sep-72 & & $10045-97-3$ & CS-137 & GAMMA_GS & USTEST & 10.7 \\
\hline 341 & PDMS & & SW & & & E010000 & 12-Sep-72 & & $10045-97-3$ & CS-137 & GAMMA_GS & USTEST & -0.643 \\
\hline 342 & PDMS & & SW & & & E004167 & 25-Sep-72 & & $10028-17-8$ & TRITIUM & TRITIUM_DIST_LSC & USTEST & 24800 \\
\hline 343 & PDMS & & SW & & & E002331 & 25-Sep-72 & & $10098-97-2$ & SR-90 & SRISO_SEP_PRECIP_GPC & USTEST & 0.0962 \\
\hline 344 & PDMS & & SW & & & E004157 & 26-Sep-72 & & $10028-17-8$ & TRITIUM & TRITIUM_DIST_LSC & USTEST & 0 \\
\hline 345 & PDMS & & SW & & & E004157 & 26-Sep-72 & & $10045-97-3$ & CS-137 & GAMMA_GS & USTEST & 0.64 \\
\hline 346 & PDMS & & SW & & & E004157 & $26-\operatorname{Sep}-72$ & & $10098-97-2$ & SR-90 & SRISO_SEEP_PRECIP_GPC & USTEST & 0.402 \\
\hline 347 & PDMS & & SW & & & E012276 & $26-\operatorname{Sep}-72$ & & $10098-97-2$ & SR-90 & SRISO_SEP_PRECIP_GPC & USTEST & 0.313 \\
\hline 348 & PDMS & & SW & & & E012276 & 26-Sep-72 & & $10045-97-3$ & CS-137 & GAMMA_GS & USTEST & 0.762 \\
\hline 349 & PDMS & & SW & & & E012276 & 26-Sep-72 & & $10028-17-8$ & TRITIUM & TRITIUM_DIST_LSC & USTEST & 0 \\
\hline 350 & PDMS & & SW & & & E002332 & $02-$ Oct-72 & & $10045-97-3$ & CS-137 & GAMMA_GS & USTEST & 0.166 \\
\hline 351 & PDMS & & sW & & & E032766 & 03-Oct-72 & & 10098-97-2 & SR-90 & SRISO_SEP_PRECIP_GPC & USTEST & 770 \\
\hline 352 & PDMS & & SW & & & E014933 & 13-Oct-72 & & $10045-97-3$ & CS-137 & GAMMA_GS & USTEST & 0.204 \\
\hline 353 & PDMS & & SW & & & E025920 & 13-Oct-72 & & $10045-97-3$ & CS-137 & GAMMA_GS & USTEST & 170 \\
\hline 354 & PDMS & & SW & & & E016390 & 13-Oct-72 & & $10045-97-3$ & CS-137 & GAMMA_GS & USTEST & 0.971 \\
\hline 355 & PDMS & & SW & & & E024869 & 13-Oct-72 & & $10045-97-3$ & CS-137 & GAMMA_GS & USTEST & 32.2 \\
\hline 356 & PDMS & & SW & & & E036897 & 13-Oct-72 & & $10045-97-3$ & CS-137 & GAMMA_GS & USTEST & 17.8 \\
\hline 357 & PDMS & & SW & & & E014262 & 13-Oct-72 & & $10045-97-3$ & CS-137 & GAMMA_GS & USTEST & 14 \\
\hline 358 & PDMS & & sW & & & E014262 & 13-Oct-72 & & $10098-97-2$ & SR-90 & SRISO_SEP_PRECIP_GPC & USTEST & 6.77 \\
\hline 359 & PDMS & & SW & & & E002335 & 23-Oct-72 & & 10098-97-2 & SR-90 & SRISO_SEP_PRECIP_GPC & USTEST & 0 \\
\hline 360 & PDMS & & SW & & & E026213 & $30-$ Oct-72 & & $10045-97-3$ & CS-137 & GAMMA_GS & USTEST & -1.41 \\
\hline 361 & PDMS & & sW & & & E014707 & 31-Oct-72 & & $10045-97-3$ & CS-137 & GAMMA_GS & USTEST & 11.5 \\
\hline 362 & PDMS & & SW & & & E004158 & 31-Oct-72 & & $10028-17-8$ & TRITIUM & TRITIUM_DIST_LSC & USTEST & 69.4 \\
\hline 363 & PDMS & & SW & & & E004158 & 31-Oct-72 & & $10098-97-2$ & SR-90 & SRISO_SEPP_PRECIP_GPC & USTEST & 0.391 \\
\hline 364 & PDMS & & SW & & & E004158 & 31-Oct-72 & & $10045-97-3$ & CS-137 & GAMMA_GS & USTEST & -0.0146 \\
\hline 365 & PDMS & & SW & & & E012231 & 31-Oct-72 & & $10098-97-2$ & SR-90 & SRISO_SEP_PRECIP_GPC & USTEST & 0.331 \\
\hline
\end{tabular}




\begin{tabular}{|c|c|c|c|c|c|c|c|c|c|c|c|c|c|}
\hline & $\bar{A}$ & $\mathrm{~B}$ & $\mathrm{C}$ & $\bar{D}$ & $\mathrm{E}$ & $\mathrm{F}$ & $\bar{G}$ & $\mathrm{H}$ & 1 & $\mathrm{~J}$ & $\mathrm{~K}$ & $\mathrm{~L}$ & $\bar{M}$ \\
\hline 1 & SAMPLE_NUM_ASSIGNED_TO & SAMPLER & MEDIA & BIOTA_MEDIA & TAG_ID & SAMP_NUM & SAMP_DATE_TIME| & SAMP_DATE_TIME_ON & CON_ID & CON_SHORT_NAME & METHOD_NAME & LAB_CODE & VALUE_RPTD \\
\hline 366 & PDMS & & SW & & & E012231 & 31-Oct-72 & & $10045-97-3$ & CS-137 & GAMMA_GS & USTEST & 0.743 \\
\hline 367 & PDMS & & sW & & & E012231 & 31-Oct-72 & & $10028-17-8$ & TRITIUM & TRITIUM_DIST_LSC & USTEST & 0 \\
\hline 368 & PDMS & & SW & & & E032767 & $31-$ Oct-72 & & $10098-97-2$ & SR-90 & SRISO_SEP_PRECIP_GPC & USTEST & 973 \\
\hline 369 & PDMS & & SW & & & E009984 & 07-Nov-72 & & $10045-97-3$ & CS-137 & GAMMA_GS & USTEST & 6.02 \\
\hline 370 & PDMS & & SW & & & E026568 & 10-Nov-72 & & $10098-97-2$ & SR-90 & SRISO_SEPP_PRECIP_GPC & USTEST & 5.07 \\
\hline 371 & PDMS & & SW & & & E002261 & 13-Nov-72 & & $10045-97-3$ & CS-137 & GAMMA_GS & USTEST & -1.64 \\
\hline 372 & PDMS & & sW & & & E009991 & 14-Nov-72 & & $10045-97-3$ & CS-137 & GAMMA_GS & USTEST & 4.08 \\
\hline 373 & PDMS & & sW & & & E004168 & 16-Nov-72 & & $10028-17-8$ & TRITIUM & TRITIUM_DIST_LSC & USTEST & 7970 \\
\hline 374 & PDMS & & sW & & & E021383 & 20-Nov-72 & & $10098-97-2$ & SR-90 & SRISO_SEP_PRECIP_GPC & USTEST & 0.38 \\
\hline 375 & PDMS & & sW & & & E021383 & 20-Nov-72 & & $10045-97-3$ & CS-137 & GAMMA_GS & USTEST & 0.166 \\
\hline 376 & PDMS & & SW & & & E003694 & 27-Nov-72 & & $10045-97-3$ & CS-137 & GAMMA_GS & USTEST & -0.648 \\
\hline 377 & PDMS & & SW & & & E002262 & 27-Nov-72 & & $10045-97-3$ & CS-137 & GAMMA_GS & USTEST & -0.634 \\
\hline 378 & PDMS & & sW & & & E026217 & 27-Nov-72 & & $10045-97-3$ & CS-137 & GAMMA_GS & USTEST & 14.5 \\
\hline 379 & PDMS & & SW & & & E004159 & 28-Nov-72 & & $10028-17-8$ & TRITIUM & TRITIUM_DIST_LSC & USTEST & 154 \\
\hline 380 & PDMS & & SW & & & E004159 & 28-Nov-72 & & $10098-97-2$ & SR-90 & SRISO_SEP_PRECIP_GPC & USTEST & 0.207 \\
\hline 381 & PDMS & & SW & & & E004159 & 28-Nov-72 & & $10045-97-3$ & CS-137 & GAMMA_GS & USTEST & 0.00767 \\
\hline 382 & PDMS & & SW & & & E012232 & 28-Nov-72 & & $10098-97-2$ & SR-90 & SRISO_SEP_PRECIP_GPC & USTEST & 0.179 \\
\hline 383 & PDMS & & sW & & & E012232 & 28-Nov-72 & & $10045-97-3$ & CS-137 & GAMMA_GS & USTEST & 0.00766 \\
\hline 384 & PDMS & & sW & & & E012232 & 28-Nov-72 & & $10028-17-8$ & TRITIUM & TRITIUM_DIST_LSC & USTEST & 0 \\
\hline 385 & PDMS & & sW & & & E032768 & 28-Nov-72 & & $10098-97-2$ & SR-90 & SRISO_SEP_PRECIP_GPC & USTEST & 996 \\
\hline 386 & PDMS & & sW & & & E002263 & 04-Dec-72 & & $10045-97-3$ & CS-137 & GAMMĀGS' & USTEST & -0.684 \\
\hline 387 & PDMS & & SW & & & E009948 & 12-Dec-72 & & $10098-97-2$ & SR-90 & SRISO_SEEP_PRECIP_GPC & USTEST & 0.495 \\
\hline 388 & PDMS & & SW & & & E009948 & 12-Dec-72 & & $10045-97-3$ & CS-137 & GAMMA_GS & USTEST & 7.76 \\
\hline 389 & PDMS & & SW & & & E004169 & 19-Dec-72 & & $10028-17-8$ & TRITIUM & TRITIUM_DIST_LSC & USTEST & 3940 \\
\hline 390 & PDMS & & SW & & & E003698 & 21-Dec-72 & & $10045-97-3$ & CS-137 & GAMMA_GS & USTEST & -0.0592 \\
\hline 391 & PDMS & & sW & & & E002266 & 21-Dec-72 & & 10098-97-2 & SR-90 & SRISO_SEEP_PRECIP_GPC & USTEST & 0.239 \\
\hline 392 & PDMS & & SW & & & E002266 & 21-Dec-72 & & $10045-97-3$ & CS-137 & GAMMA_GS & USTEST & 8.36 \\
\hline 393 & PDMS & & SW & & & E009957 & 22-Dec-72 & & $10028-17-8$ & TRITIUM & TRITIUM_DIST_LSC & USTEST & 0 \\
\hline 394 & PDMS & & SW & & & E009957 & 22-Dec-72 & & 10098-97-2 & SR-90 & SRISO_SEP_PRECIP_GPC & USTEST & 0.492 \\
\hline 395 & PDMS & & SW & & & E014708 & 22-Dec-72 & & $10045-97-3$ & CS-137 & GAMMA_GS & USTEST & 0.459 \\
\hline 396 & PDMS & & SW & & & E009957 & 22-Dec-72 & & $10045-97-3$ & CS-137 & GAMMA_GS & USTEST & -0.06 \\
\hline 397 & PDMS & & SW & & & E012233 & 22-Dec-72 & & $10098-97-2$ & SR-90 & SRISO_SEP_PRECIP_GPC & USTEST & 0.183 \\
\hline 398 & PDMS & & SW & & & E012233 & 22-Dec-72 & & $10045-97-3$ & CS-137 & GAMMA_GS & USTEST & -0.00705 \\
\hline 399 & PDMS & & SW & & & E012233 & 22-Dec-72 & & $10028-17-8$ & TRITIUM & TRITIUM_DIST_LSC & USTEST & 0 \\
\hline 400 & PDMS & & SW & & & E032769 & 22-Dec-72 & & 10098-97-2 & SR-90 & SRISO_SËP_PRECIP_GPC & USTEST & 1210 \\
\hline 401 & PDMS & & SW & & & E026569 & 22-Dec-72 & & $10098-97-2$ & SR-90 & SRISO_SEP_PRECIP_GPC & USTEST & 0.432 \\
\hline 402 & PDMS & & SW & & & E009897 & 02-Jan-73 & & $10045-97-3$ & CS-137 & GAMMA_GS & USTEST & -1.38 \\
\hline 403 & PDMS & & sW & & & E014028 & 02-Jan-73 & & $10028-17-8$ & TRITIUM & TRITIUM_DIST_LSC & USTEST & 941 \\
\hline 404 & PDMS & & SW & & & E003699 & 02-Jan-73 & & $10045-97-3$ & CS-137 & GAMMA_GS & USTEST & 0.153 \\
\hline 405 & PDMS & & SW & & & E002268 & 08-Jan-73 & & $10045-97-3$ & CS-137 & GAMMA_GS & USTEST & -0.121 \\
\hline 406 & PDMS & & SW & & & E026223 & 08-Jan-73 & & $10045-97-3$ & CS-137 & GAMMA_GS & USTEST & 15.4 \\
\hline 407 & PDMS & & SW & & & E027462 & 09-Jan-73 & & $10045-97-3$ & CS-137 & GAMMA_GS & USTEST & 13.9 \\
\hline 408 & PDMS & & SW & & & E014934 & 12-Jan-73 & & $10045-97-3$ & CS-137 & GAMMA_GS & USTEST & 12.6 \\
\hline 409 & PDMS & & SW & & & E004170 & 18-Jan-73 & & $10028-17-8$ & TRITIUM & TRITIUM_DIST_LSC & USTEST & 15000 \\
\hline 410 & PDMS & & sW & & & E004170 & 18-Jan-73 & & $10045-97-3$ & CS-137 & GAMMA_GS & USTEST & 5.83 \\
\hline 411 & PDMS & & SW & & & E004230 & 18-Jan-73 & & $10045-97-3$ & CS-137 & GAMMA_GS & USTEST & -4.57 \\
\hline 412 & PDMS & & SW & & & E004230 & 18-Jan-73 & & $10028-17-8$ & TRITIUM & TRITIUM_DIST_LSC & USTEST & 308000 \\
\hline 413 & PDMS & & SW & & & E009914 & 19-Jan-73 & & $10045-97-3$ & CS-137 & GAMMA_GS & USTEST & -0.417 \\
\hline 414 & PDMS & & SW & & & E016391 & 19-Jan-73 & & $10098-97-2$ & SR-90 & SRISO_SEP_PRECIP_GPC & USTEST & 0.00664 \\
\hline 415 & PDMS & & SW & & & E016391 & 19-Jan-73 & & $10045-97-3$ & CS-137 & GAMMA_GS & USTEST & 22.5 \\
\hline 416 & PDMS & & SW & & & E022468 & 19-Jan-73 & & $10045-97-3$ & CS-137 & GAMMA_GS & USTEST & 12.7 \\
\hline 417 & PDMS & & SW & & & E022468 & 19-Jan-73 & & $10098-97-2$ & SR-90 & SRISO_SEP_PRECIP_GPC & USTEST & 0.00222 \\
\hline
\end{tabular}




\begin{tabular}{|c|c|c|c|c|c|c|c|c|c|c|c|c|c|}
\hline & $\bar{A}$ & $\mathrm{~B}$ & $\bar{C}$ & $\mathrm{D}$ & $\mathrm{E}$ & $\mathrm{F}$ & $\bar{G}$ & $\mathrm{H}$ & 1 & $\mathrm{~J}$ & $\bar{K}$ & $\bar{L}$ & $\mathrm{M}$ \\
\hline 1 & SAMPLE_NUM_ASSIGNED_TO & \begin{tabular}{|l} 
SAMPLER \\
\end{tabular} & MEDIA & BIOTA_MEDIA & TAG_ID & SAMP_NUM & \begin{tabular}{|l|} 
SAMP_DATE_TIME \\
\end{tabular} & SAMP_DATE_TIME_ON & CON_ID & \begin{tabular}{|l} 
CON_SHORT_NAME \\
\end{tabular} & METHOD_NAME & LAB_CODE & VALUE_RPTD \\
\hline 418 & PDMS & & SW & & & E027632 & 19-Jan-73 & & $10045-97-3$ & CS-137 & GAMMA_GS & USTEST & 27.1 \\
\hline 419 & PDMS & & SW & & & E002271 & 22-Jan-73 & & $10098-97-2$ & SR-90 & SRISO_SEEP_PRECIP_GPC & USTEST & 0.0298 \\
\hline 420 & PDMS & & SW & & & E032770 & 26-Jan-73 & & $10098-97-2$ & SR-90 & SRISO_SEP_PRECIP_GPC & USTEST & 0.158 \\
\hline 421 & PDMS & & SW & & & E009924 & 29-Jan-73 & & $10028-17-8$ & TRITIUM & TRITIUM DIST_LSC & USTEST & 329 \\
\hline 422 & PDMS & & SW & & & E009924 & 29-Jan-73 & & $10098-97-2$ & SR-90 & SRISO_SEP_PRECIP_GPC & USTEST & 0.259 \\
\hline 423 & PDMS & & SW & & & E009924 & 29-Jan-73 & & $10045-97-3$ & CS-137 & GAMMA_GS & USTEST & -0.811 \\
\hline 424 & PDMS & & SW & & & E012234 & 29-Jan-73 & & $10098-97-2$ & SR-90 & SRISO_SEEP_PRECIP_GPC & USTEST & 0.271 \\
\hline 425 & PDMS & & SW & & & E012234 & 29-Jan-73 & & $10045-97-3$ & CS-137 & GAMMA_GS & USTEST & -0.385 \\
\hline 426 & PDMS & & SW & & & E012234 & 29-Jan-73 & & $10028-17-8$ & TRITIUM & TRITIUM_DIST_LSC & USTEST & 645 \\
\hline 427 & PDMS & & SW & & & E010876 & 29-Jan-73 & & $10045-97-3$ & CS-137 & GAMMA_GS & USTEST & -0.713 \\
\hline 428 & PDMS & & SW & & & E021384 & 29-Jan-73 & & $10045-97-3$ & CS-137 & GAMMA_GS & USTEST & -0.559 \\
\hline 429 & PDMS & & SW & & & E009925 & 30-Jan-73 & & $10045-97-3$ & CS-137 & GAMMA_GS & USTEST & 6.32 \\
\hline 430 & PDMS & & SW & & & E026227 & 05-Feb-73 & & $10045-97-3$ & CS-137 & GAMMA_GS & USTEST & -0.0369 \\
\hline 431 & PDMS & & SW & & & E009867 & 13-Feb-73 & & $10045-97-3$ & CS-137 & GAMMA_GS & USTEST & 0.373 \\
\hline 432 & PDMS & & SW & & & E026570 & 16-Feb-73 & & $10098-97-2$ & SR-90 & SRISO_SEP_PRECIP_GPC & USTEST & 2.43 \\
\hline 433 & PDMS & & SW & & & E003705 & 20-Feb-73 & & $10045-97-3$ & CS-137 & GAMMA_GS & USTEST & 0.405 \\
\hline 434 & PDMS & & SW & & & E002274 & 20-Feb-73 & & $10098-97-2$ & SR-90 & SRISO_SEP_PRECIP_GPC & USTEST & 0 \\
\hline 435 & PDMS & & SW & & & E032771 & 20-Feb-73 & & $10098-97-2$ & SR-90 & SRISO_SEP_PRECIP_GPC & USTEST & 274 \\
\hline 436 & PDMS & & SW & & & E004171 & 21-Feb-73 & & $10028-17-8$ & TRITIUM & TRITIUM_DIST_LSC & USTEST & 6640 \\
\hline 437 & PDMS & & SW & & & E009880 & 26-Feb-73 & & $10028-17-8$ & TRITIUM & TRITIUM_DIST_LSC & USTEST & 982 \\
\hline 438 & PDMS & & SW & & & E009880 & 26-Feb-73 & & $10098-97-2$ & SR-90 & SRISO_SEP_PRECIP_GPC & USTEST & 0.404 \\
\hline 439 & PDMS & & SW & & & E009880 & 26-Feb-73 & & $10045-97-3$ & CS-137 & GAMMĀGS & USTEST & -0.00195 \\
\hline 440 & PDMS & & SW & & & E012235 & 26-Feb-73 & & $10098-97-2$ & SR-90 & SRISO_SEP_PRECIP_GPC & USTEST & 1.41 \\
\hline 441 & PDMS & & SW & & & E012235 & 26-Feb-73 & & $10045-97-3$ & CS-137 & GAMMA_GS & USTEST & -0.0301 \\
\hline 442 & PDMS & & SW & & & E003706 & 26-Feb-73 & & $10045-97-3$ & CS-137 & GAMMA_GS & USTEST & -1.23 \\
\hline 443 & PDMS & & SW & & & E002275 & 26-Feb-73 & & $10045-97-3$ & CS-137 & GAMMA_GS & USTEST & 1.13 \\
\hline 444 & PDMS & & SW & & & E009881 & $27-F e b-73$ & & $10045-97-3$ & CS-137 & GAMMA_GS & USTEST & 0.00523 \\
\hline 445 & PDMS & & SW & & & E003707 & 05-Mar-73 & & $10045-97-3$ & CS-137 & GAMMA_GS & USTEST & -2.56 \\
\hline 446 & PDMS & & SW & & & E026231 & 05-Mar-73 & & $10045-97-3$ & CS-137 & GAMMA_GS & USTEST & -1.34 \\
\hline 447 & PDMS & & SW & & & E009888 & 06-Mar-73 & & $10045-97-3$ & CS-137 & GAMMA_GS & USTEST & -0.424 \\
\hline 448 & PDMS & & SW & & & E026571 & 09-Mar-73 & & $10098-97-2$ & SR-90 & SRISO_SEP_PRECIP_GPC & USTEST & 5.78 \\
\hline 449 & PDMS & & SW & & & E004172 & 16-Mar-73 & & $10028-17-8$ & TRITIUM & TRITIUM_DIST_LSC & USTEST & 10400 \\
\hline 450 & PDMS & & SW & & & E004172 & 16-Mar-73 & & $10045-97-3$ & CS-137 & GAMMA_GS & USTEST & 5.02 \\
\hline 451 & PDMS & & SW & & & E004594 & 19-Mar-73 & & $10045-97-3$ & CS-137 & GAMMA_GS & USTEST & 12.3 \\
\hline 452 & PDMS & & SW & & & E003709 & 19-Mar-73 & & $10045-97-3$ & CS-137 & GAMMA_GS & USTEST & 0.316 \\
\hline 453 & PDMS & & SW & & & E002278 & 19-Mar-73 & & $10045-97-3$ & CS-137 & GAMMA_GS & USTEST & -0.287 \\
\hline 454 & PDMS & & SW & & & E009830 & 20-Mar-73 & & $10045-97-3$ & CS-137 & GAMMA_GS & USTEST & 0.0682 \\
\hline 455 & PDMS & & SW & & & E032772 & 20-Mar-73 & & $10098-97-2$ & SR-90 & SRISO_SEPP_PRECIP_GPC & USTEST & 481 \\
\hline 456 & PDMS & & SW & & & E039723 & 26-Mar-73 & & $10045-97-3$ & CS-137 & GAMMA_GS & USTEST & 13 \\
\hline 457 & PDMS & & SW & & & E003710 & 26-Mar-73 & & $10045-97-3$ & CS-137 & GAMMA_GS & USTEST & 17.8 \\
\hline 458 & PDMS & & SW & & & E002279 & 26-Mar-73 & & $10098-97-2$ & SR-90 & SRISO_SEP_PRECIP_GPC & USTEST & 0.257 \\
\hline 459 & PDMS & & SW & & & E002279 & 26-Mar-73 & & $10045-97-3$ & CS-137 & GAMMA_GS & USTEST & 12.4 \\
\hline 460 & PDMS & & SW & & & E009837 & 27-Mar-73 & & $10028-17-8$ & TRITIUM & TRITIUM_DIST_LSC & USTEST & 792 \\
\hline 461 & PDMS & & SW & & & E009837 & 27-Mar-73 & & $10098-97-2$ & SR-90 & SRISO_SEP_PRECIP_GPC & USTEST & 0.345 \\
\hline 462 & PDMS & & SW & & & E009837 & 27-Mar-73 & & $10045-97-3$ & CS-137 & GAMMA_GS & USTEST & 0.00255 \\
\hline 463 & PDMS & & SW & & & E012236 & 27-Mar-73 & & $10098-97-2$ & SR-90 & SRISO_SEEP_PRECIP_GPC & USTEST & 0.306 \\
\hline 464 & PDMS & & SW & & & E012236 & 27-Mar-73 & & $10045-97-3$ & CS-137 & GAMMA_GS & USTEST & 0.594 \\
\hline 465 & PDMS & & SW & & & E012236 & 27-Mar-73 & & $10028-17-8$ & TRITIUM & TRITIUM_DIST_LSC & USTEST & 604 \\
\hline 466 & PDMS & & SW & & & E003711 & 02-Apr-73 & & $10045-97-3$ & CS-137 & GAMMA_GS & USTEST & -1.1 \\
\hline 467 & PDMS & & SW & & & E002280 & 02-Apr-73 & & $10045-97-3$ & CS-137 & GAMMA_GS & USTEST & 3.45 \\
\hline 468 & PDMS & & SW & & & E026235 & 02-Apr-73 & & $10045-97-3$ & CS-137 & GAMMA_GS & USTEST & 26.6 \\
\hline 469 & PDMS & & SW & & & E016392 & 06-Apr-73 & & $10098-97-2$ & SR-90 & SRISO_SEP_PRECIP_GPC & USTEST & 3.71 \\
\hline
\end{tabular}




\begin{tabular}{|c|c|c|c|c|c|c|c|c|c|c|c|c|c|}
\hline & A & $\mathrm{B}$ & $\mathrm{C}$ & $\mathrm{D}$ & $E$ & $\mathrm{~F}$ & G & $\mathrm{H}$ & $\mathrm{I}$ & $\mathrm{J}$ & $\mathrm{K}$ & L & $\mathrm{M}$ \\
\hline 1 & SAMPLE_NUM_ASSIGNED_TO & SAMPLER & MEDIA & BIOTA_MEDIA & TAG_ID & SAMP_NUM & SAMP_DATE_TIME & SAMP_DATE_TIME_ON & CON_ID & CON_SHORT_NAME & METHOD_NAME & LAB_CODE & VALUE_RPTD \\
\hline 470 & PDMS & & SW & & & E016392 & 06-Apr-73 & & $10045-97-3$ & CS-137 & GAMMA_GS & USTEST & 9.54 \\
\hline 471 & PDMS & & SW & & & E022469 & 06-Apr-73 & & $10045-97-3$ & CS-137 & GAMMA_GS & USTEST & 35 \\
\hline 472 & PDMS & & sw & & & E022469 & 06-Apr-73 & & $10098-97-2$ & SR-90 & SRISO_SEPP_PRECIP_GPC & USTEST & 1.21 \\
\hline 473 & PDMS & & SW & & & E027633 & 06-Apr-73 & & $10045-97-3$ & CS-137 & GAMMA_GS & USTEST & 35.7 \\
\hline 474 & PDMS & & sw & & & E014263 & 06-Apr-73 & & $10045-97-3$ & CS-137 & GAMMA_GS & USTEST & 11.8 \\
\hline 475 & PDMS & & sw & & & E014263 & 06-Apr-73 & & $10098-97-2$ & SR-90 & SRISO_SEP_PRECIP_GPC & USTEST & 8.32 \\
\hline 476 & PDMS & & sw & & & E004596 & 09-Apr-73 & & $10045-97-3$ & CS-137 & GAMMA_GS & USTEST & 9.58 \\
\hline 477 & PDMS & & sw & & & E004597 & 16-Apr-73 & & $10045-97-3$ & CS-137 & GAMMA_GS & USTEST & 13 \\
\hline 478 & PDMS & & sW & & & E003713 & 16-Apr-73 & & $10045-97-3$ & CS-137 & GAMMA_GS & USTEST & -0.182 \\
\hline 479 & PDMS & & SW & & & E002282 & 16-Apr-73 & & $10045-97-3$ & CS-137 & GAMMA_GS & USTEST & -0.0435 \\
\hline 480 & PDMS & & SW & & & E009775 & 17-Apr-73 & & $10045-97-3$ & CS-137 & GAMMA_GS & USTEST & 2.49 \\
\hline 481 & PDMS & & sw & & & E032773 & 17-Apr-73 & & $10098-97-2$ & SR-90 & SRISO_SEP_PRECIP_GPC & USTEST & 1920 \\
\hline 482 & PDMS & & sw & & & E026530 & 20-Apr-73 & & $10098-97-2$ & SR-90 & SRISO_SEP_PRECIP_GPC & USTEST & 6.84 \\
\hline 483 & PDMS & & sw & & & E004173 & 23-Apr-73 & & $10028-17-8$ & TRITIUM & TRITIUM_DIST_LSC & USTEST & 2390 \\
\hline 484 & PDMS & & sW & & & E004173 & 23-Apr-73 & & $10098-97-2$ & SR-90 & SRISO_SEP_PRECIP_GPC & USTEST & 0.49 \\
\hline 485 & PDMS & & sW & & & E004173 & 23-Apr-73 & & $10045-97-3$ & CS-137 & GAMMA_GS & USTEST & -0.0825 \\
\hline 486 & PDMS & & SW & & & E003714 & 23-Apr-73 & & $10045-97-3$ & CS-137 & GAMMA_GS & USTEST & 5.32 \\
\hline 487 & PDMS & & sw & & & E002283 & 23-Apr-73 & & $10098-97-2$ & SR-90 & SRISO_SEP_PRECIP_GPC & USTEST & 0.293 \\
\hline 488 & PDMS & & sw & & & E009782 & 24-Apr-73 & & $10028-17-8$ & TRITIUM & TRITIUM_DIST_LSC & USTEST & 1100 \\
\hline 489 & PDMS & & sw & & & E009782 & 24-Apr-73 & & $10098-97-2$ & SR-90 & SRISO_SEP_PRECIP_GPC & USTEST & 0.386 \\
\hline 490 & PDMS & & sw & & & E009782 & 24-Apr-73 & & $10045-97-3$ & CS-137 & GAMMA_GS & USTEST & 3.44 \\
\hline 491 & PDMS & & sw & & & E014700 & 24-Apr-73 & & $10045-97-3$ & CS-137 & GAMMA_GS & USTEST & 0.406 \\
\hline 492 & PDMS & & sW & & & E035056 & 24-Apr-73 & & $10028-17-8$ & TRITIUM & TRITIUM_DIST_LSC & USTEST & 1070 \\
\hline 493 & PDMS & & sW & & & E046079 & 25-Apr-73 & & $10028-17-8$ & TRITIUM & TRITIUM_DIST_LSC & USTEST & 13000 \\
\hline 494 & PDMS & & sw & & & E037553 & 30-Apr-73 & & $10098-97-2$ & SR-90 & SRISO_SEP_PRECIP_GPC & USTEST & 0.394 \\
\hline 495 & PDMS & & sw & & & E003715 & 30-Apr-73 & & $10045-97-3$ & CS-137 & GAMMA_GS & USTEST & -0.534 \\
\hline 496 & PDMS & & sw & & & E002284 & 30-Apr-73 & & $10045-97-3$ & CS-137 & GAMMA_GS & USTEST & -0.0951 \\
\hline 497 & PDMS & & sw & & & E026239 & 30-Apr-73 & & $10045-97-3$ & CS-137 & GAMMA_GS & USTEST & 2.87 \\
\hline 498 & PDMS & & sw & & & E004598 & 01-May-73 & & $10045-97-3$ & CS-137 & GAMMA_GS & USTEST & -0.000743 \\
\hline 499 & PDMS & & sW & & & E026531 & 04-May-73 & & $10098-97-2$ & SR-90 & SRISO_SEP_PRECIP_GPC & USTEST & 0.833 \\
\hline 500 & PDMS & & SW & & & E003716 & 07-May-73 & & $10045-97-3$ & CS-137 & GAMMA_GS & USTEST & 2.17 \\
\hline 501 & PDMS & & sw & & & E002285 & 07-May-73 & & $10045-97-3$ & CS-137 & GAMMA_GS & USTEST & 3.68 \\
\hline 502 & PDMS & & sw & & & E009796 & 08-May-73 & & $10045-97-3$ & CS-137 & GAMMA_GS & USTEST & 2.82 \\
\hline 503 & PDMS & & sw & & & E002286 & 14-May-73 & & $10045-97-3$ & CS-137 & GAMMA_GS & USTEST & -0.598 \\
\hline 504 & PDMS & & SW & & & E032708 & 15-May-73 & & $10098-97-2$ & SR-90 & SRISO_SEP_PRECIP_GPC & USTEST & 2040 \\
\hline 505 & PDMS & & sw & & & E004174 & 18-May-73 & & $10098-97-2$ & SR-90 & SRISO_SEP_PRECIP_GPC & USTEST & 0.699 \\
\hline 506 & PDMS & & sW & & & E004174 & 18-May-73 & & $10045-97-3$ & CS-137 & GAMMA_GS & USTEST & -0.488 \\
\hline 507 & PDMS & & sw & & & E004174 & 18-May-73 & & $10028-17-8$ & TRITIUM & TRITIUM_DIST_LSC & USTEST & 7770 \\
\hline 508 & PDMS & & sw & & & E003718 & 21-May-73 & & $10045-97-3$ & CS-137 & GAMMA_GS & USTEST & 4.44 \\
\hline 509 & PDMS & & sw & & & E002287 & 21-May-73 & & $10098-97-2$ & SR-90 & SRISO_SEP_PRECIP_GPC & USTEST & 0.242 \\
\hline 510 & PDMS & & sw & & & E002287 & 21-May-73 & & $10045-97-3$ & CS-137 & GAMMA_GS & USTEST & 20.4 \\
\hline 511 & PDMS & & sw & & & E009810 & 22-May-73 & & $10045-97-3$ & CS-137 & GAMMA_GS & USTEST & 11.4 \\
\hline 512 & PDMS & & sW & & & E004599 & 29-May-73 & & $10098-97-2$ & SR-90 & SRISO_SEP_PRECIP_GPC & USTEST & 0.261 \\
\hline 513 & PDMS & & SW & & & E009817 & 29-May-73 & & $10028-17-8$ & TRITIUM & TRITIUM_DIST_LSC & USTEST & 513 \\
\hline 514 & PDMS & & sw & & & E009817 & 29-May-73 & & $10098-97-2$ & SR-90 & SRISO_SEP_PRECIP_GPC & USTEST & 0.383 \\
\hline 515 & PDMS & & sw & & & E009817 & 29-May-73 & & $10045-97-3$ & CS-137 & GAMMA_GS & USTEST & 1.98 \\
\hline 516 & PDMS & & sw & & & E014701 & 29-May-73 & & $10045-97-3$ & CS-137 & GAMMA_GS & USTEST & 0.413 \\
\hline 517 & PDMS & & sw & & & E041047 & 29-May-73 & & $10028-17-8$ & TRITIUM & TRITIUM_DIST_LSC & USTEST & 503 \\
\hline 518 & PDMS & & sw & & & E003719 & 29-May-73 & & $10045-97-3$ & CS-137 & GAMMA_GS & USTEST & 16.9 \\
\hline 519 & PDMS & & SW & & & E002288 & 29-May-73 & & $10045-97-3$ & CS-137 & GAMMA_GS & USTEST & 14.4 \\
\hline 520 & PDMS & & sW & & & E026243 & 29-May-73 & & $10045-97-3$ & CS-137 & GAMMA_GS & USTEST & 8.46 \\
\hline 521 & PDMS & & SW & & & E026532 & 01-Jun-73 & & $10098-97-2$ & SR-90 & SRISO_SEP_PRECIP_GPC & USTEST & 12.9 \\
\hline
\end{tabular}




\begin{tabular}{|c|c|c|c|c|c|c|c|c|c|c|c|c|c|}
\hline & $\bar{A}$ & $\mathrm{~B}$ & $\mathrm{C}$ & $\mathrm{D}$ & $E$ & $\mathrm{~F}$ & G & $\mathrm{H}$ & $\mathrm{I}$ & $\mathrm{J}$ & $\mathrm{K}$ & $\bar{L}$ & $\mathrm{M}$ \\
\hline 1 & SAMPLE_NUM_ASSIGNED_TO & SAMPLER & MEDIA & BIOTA_MEDIA & TAG_ID & SAMP_NUM & SAMP_DATE_TIME & SAMP_DATE_TIME_ON & $\overline{C O N} \_I D$ & CON_SHORT_NAME & METHOD_NAME & LAB_CODE & VALUE_RPTD \\
\hline 522 & PDMS & & SW & & & E003720 & 04-Jun-73 & & $10045-97-3$ & CS-137 & GAMMA_GS & USTEST & -6.91 \\
\hline 523 & PDMS & & SW & & & E002289 & 04-Jun-73 & & $10045-97-3$ & CS-137 & GAMMA_GS & USTEST & -7.79 \\
\hline 524 & PDMS & & SW & & & E009730 & 05-Jun-73 & & $10045-97-3$ & CS-137 & GAMMA_GS & USTEST & -8.57 \\
\hline 525 & PDMS & & SW & & & E003721 & 11-Jun-73 & & $10045-97-3$ & CS-137 & GAMMA_GS & USTEST & 16.1 \\
\hline 526 & PDMS & & SW & & & E002290 & 11-Jun-73 & & $10045-97-3$ & CS-137 & GAMMA_GS & USTEST & 1.06 \\
\hline 527 & PDMS & & SW & & & E009737 & 12-Jun-73 & & $10045-97-3$ & CS-137 & GAMMA_GS & USTEST & 1.97 \\
\hline 528 & PDMS & & SW & & & E032709 & 12-Jun-73 & & $10098-97-2$ & SR-90 & SRISO_SEP_PRECIP_GPC & USTEST & 2950 \\
\hline 529 & PDMS & & SW & & & E004175 & 14-Jun-73 & & $10028-17-8$ & TRITIUM & TRITIUM_DIST_LSC & USTEST & 4270 \\
\hline 530 & PDMS & & SW & & & E004175 & 14-Jun-73 & & $10098-97-2$ & SR-90 & SRISO_SEP_PRECIP_GPC & USTEST & 0.845 \\
\hline 531 & PDMS & & SW & & & E004175 & 14-Jun-73 & & $10045-97-3$ & CS-137 & GAMMA_GS & USTEST & 13 \\
\hline 532 & PDMS & & SW & & & E003722 & 18-Jun-73 & & $10045-97-3$ & CS-137 & GAMMA_GS & USTEST & -0.0196 \\
\hline 533 & PDMS & & SW & & & E002291 & 18-Jun-73 & & $10045-97-3$ & CS-137 & GAMMA_GS & USTEST & 0.0436 \\
\hline 534 & PDMS & & SW & & & E009744 & 19-Jun-73 & & $10045-97-3$ & CS-137 & GAMMA_GS & USTEST & 0.409 \\
\hline 535 & PDMS & & SW & & & E004600 & 25-Jun-73 & & $10045-97-3$ & CS-137 & GAMMA_GS & USTEST & -0.0187 \\
\hline 536 & PDMS & & SW & & & E004600 & 25-Jun-73 & & $10098-97-2$ & SR-90 & SRISO_SEP_PRECIP_GPC & USTEST & 0.242 \\
\hline 537 & PDMS & & SW & & & E009750 & 25-Jun-73 & & $10028-17-8$ & TRITIUM & TRITIUM_DIST_LSC & USTEST & 414 \\
\hline 538 & PDMS & & SW & & & E041048 & 25-Jun-73 & & $10028-17-8$ & TRITIUM & TRITIUM_DIST_LSC & USTEST & 295 \\
\hline 539 & PDMS & & SW & & & E003723 & 25-Jun-73 & & $10045-97-3$ & CS-137 & GAMMA_GS & USTEST & 0.043 \\
\hline 540 & PDMS & & SW & & & E002292 & 25-Jun-73 & & $10098-97-2$ & SR-90 & SRISO_SEP_PRECIP_GPC & USTEST & 0.334 \\
\hline 541 & PDMS & & SW & & & E002292 & 25-Jun-73 & & $10045-97-3$ & CS-137 & GAMMA_GS & USTEST & 8.6 \\
\hline 542 & PDMS & & SW & & & E014702 & 26-Jun-73 & & $10045-97-3$ & CS-137 & GAMMA_GS & USTEST & 0.832 \\
\hline 543 & PDMS & & SW & & & E009751 & 26-Jun-73 & & $10098-97-2$ & SR-90 & SRISO_SEP_PRECIP_GPC & USTEST & 0.312 \\
\hline 544 & PDMS & & SW & & & E009751 & 26-Jun-73 & & $10045-97-3$ & CS-137 & GAMMA_GS & USTEST & 7.69 \\
\hline 545 & PDMS & & SW & & & E003724 & 02-Jul-73 & & $10045-97-3$ & CS-137 & GAMMA_GS & USTEST & 2.01 \\
\hline 546 & PDMS & & SW & & & E002293 & 02-Jul-73 & & $10045-97-3$ & CS-137 & GAMMA_GS & USTEST & -0.171 \\
\hline 547 & PDMS & & SW & & & E009758 & 03-Jul-73 & & $10045-97-3$ & CS-137 & GAMMA_GS & USTEST & -0.473 \\
\hline 548 & PDMS & & SW & & & E014936 & 06-Jul-73 & & $10045-97-3$ & CS-137 & GAMMA_GS & USTEST & -0.387 \\
\hline 549 & PDMS & & SW & & & E025956 & 06-Jul-73 & & $10045-97-3$ & CS-137 & GAMMA_GS & USTEST & -0.908 \\
\hline 550 & PDMS & & SW & & & E016393 & 06-Jul-73 & & $10098-97-2$ & SR-90 & SRISO_SEP_PRECIP_GPC & USTEST & 3.78 \\
\hline 551 & PDMS & & SW & & & E016393 & 06-Jul-73 & & $10045-97-3$ & CS-137 & GAMMA_GS & USTEST & -0.595 \\
\hline 552 & PDMS & & SW & & & E022414 & 06-Jul-73 & & $10045-97-3$ & CS-137 & GAMMA_GS & USTEST & 23.7 \\
\hline 553 & PDMS & & SW & & & E022414 & 06-Jul-73 & & $10098-97-2$ & SR-90 & SRISO_SEP_PRECIP_GPC & USTEST & 3.66 \\
\hline 554 & PDMS & & SW & & & E027634 & 06-Jul-73 & & $10045-97-3$ & CS-137 & GAMMA_GS & USTEST & 108 \\
\hline 555 & PDMS & & SW & & & E026533 & 06-Jul-73 & & $10045-97-3$ & CS-137 & GAMMA_GS & USTEST & 12.4 \\
\hline 556 & PDMS & & SW & & & E026533 & $06-J u l-73$ & & $10098-97-2$ & SR-90 & SRISO_SEP_PRECIP_GPC & USTEST & 12.7 \\
\hline 557 & PDMS & & SW & & & E003725 & 09-Jul-73 & & $10045-97-3$ & CS-137 & GAMMA_GS & USTEST & 2.41 \\
\hline 558 & PDMS & & SW & & & E002294 & 09-Jul-73 & & $10045-97-3$ & CS-137 & GAMMA_GS & USTEST & 4.92 \\
\hline 559 & PDMS & & SW & & & E009765 & 10-Jul-73 & & $10045-97-3$ & CS-137 & GAMMA_GS & USTEST & -0.372 \\
\hline 560 & PDMS & & SW & & & E032710 & 10-Jul-73 & & $10098-97-2$ & SR-90 & SRISO_SEP_PRECIP_GPC & USTEST & 2820 \\
\hline 561 & PDMS & & SW & & & E003726 & 16-Jul-73 & & $10045-97-3$ & CS-137 & GAMMA_GS & USTEST & -2.34 \\
\hline 562 & PDMS & & SW & & & E002295 & 16-Jul-73 & & $10045-97-3$ & CS-137 & GAMMA_GS & USTEST & -1.53 \\
\hline 563 & PDMS & & SW & & & E009772 & 17-Jul-73 & & $10045-97-3$ & CS-137 & GAMMA_GS & USTEST & 6.48 \\
\hline 564 & PDMS & & SW & & & E027464 & 17-Jul-73 & & $10045-97-3$ & CS-137 & GAMMA_GS & USTEST & -3.51 \\
\hline 565 & PDMS & & SW & & & E010870 & 23-Jul-73 & & $10045-97-3$ & CS-137 & GAMMA_GS & USTEST & -4.4 \\
\hline 566 & PDMS & & SW & & & E002296 & 23-Jul-73 & & $10098-97-2$ & SR-90 & SRISO_SEP_PRECIP_GPC & USTEST & 0.273 \\
\hline 567 & PDMS & & SW & & & E002296 & 23-Jul-73 & & $10045-97-3$ & CS-137 & GAMMA_GS & USTEST & -0.193 \\
\hline 568 & PDMS & & SW & & & E009685 & 24-Jul-73 & & $10028-17-8$ & TRITIUM & TRITIUM_DIST_LSC & USTEST & 533 \\
\hline 569 & PDMS & & SW & & & E009685 & 24-Jul-73 & & $10045-97-3$ & CS-137 & GAMMA_GS & USTEST & -4.58 \\
\hline 570 & PDMS & & SW & & & E014703 & 24-Jul-73 & & $10045-97-3$ & CS-137 & GAMMA_GS & USTEST & 0.838 \\
\hline 571 & PDMS & & SW & & & E035067 & 24-Jul-73 & & $10028-17-8$ & TRITIUM & TRITIUM_DIST_LSC & USTEST & 506 \\
\hline 572 & PDMS & & SW & & & E004176 & 26-Jul-73 & & $10098-97-2$ & SR-90 & SRISO_SEP_PRECIP_GPC & USTEST & 0.054 \\
\hline 573 & PDMS & & SW & & & E004176 & 26-Jul-73 & & $10045-97-3$ & CS-137 & GAMMA_GS & USTEST & -0.451 \\
\hline
\end{tabular}




\begin{tabular}{|c|c|c|c|c|c|c|c|c|c|c|c|c|c|}
\hline & $\bar{A}$ & $\mathrm{~B}$ & $\mathrm{C}$ & $\bar{D}$ & $\mathrm{E}$ & $\mathrm{F}$ & $\bar{G}$ & $\mathrm{H}$ & 1 & $\mathrm{~J}$ & $\mathrm{~K}$ & $\mathrm{~L}$ & $\bar{M}$ \\
\hline 1 & SAMPLE_NUM_ASSIGNED_TO & SAMPLER & MEDIA & BIOTA_MEDIA & TAG_ID & SAMP_NUM & SAMP_DATE_TIME| & SAMP_DATE_TIME_ON & CON_ID & CON_SHORT_NAME & METHOD_NAME & LAB_CODE & VALUE_RPTD \\
\hline 574 & PDMS & & SW & & & E004176 & 26-Jul-73 & & $10028-17-8$ & TRITIUM & TRITIUM_DIST_LSC & USTEST & 1450 \\
\hline 575 & PDMS & & sW & & & E046080 & 26-Jul-73 & & $10028-17-8$ & TRITIUM & TRITIUM_DIST_LSC & USTEST & 1900 \\
\hline 576 & PDMS & & SW & & & E080001 & 30-Jul-73 & & $10098-97-2$ & SR-90 & SRISO_SEP_PRECIP_GPC & USTEST & 0.37 \\
\hline 577 & PDMS & & SW & & & E010871 & 30-Jul-73 & & $10045-97-3$ & CS-137 & GAMMA_GS & USTEST & -6.27 \\
\hline 578 & PDMS & & SW & & & E002297 & 30-Jul-73 & & $10045-97-3$ & CS-137 & GAMMA_GS & USTEST & 1.75 \\
\hline 579 & PDMS & & SW & & & E026175 & 30-Jul-73 & & $10045-97-3$ & CS-137 & GAMMA_GS & USTEST & 6.68 \\
\hline 580 & PDMS & & sW & & & E009692 & 31-Jul-73 & & 10098-97-2 & SR-90 & SRISO_SEEP_PRECIP_GPC & USTEST & 0.343 \\
\hline 581 & PDMS & & sW & & & E009692 & 31-Jul-73 & & $10045-97-3$ & CS-137 & GAMMĀGS' & USTEST & 3.04 \\
\hline 582 & PDMS & & sW & & & E026534 & 03-Aug-73 & & $10098-97-2$ & SR-90 & SRISO_SEEP_PRECIP_GPC & USTEST & 10.9 \\
\hline 583 & PDMS & & sW & & & E003727 & 06-Aug-73 & & $10045-97-3$ & CS-137 & GAMMA_GS & USTEST & -0.0607 \\
\hline 584 & PDMS & & sW & & & E002222 & 06-Aug-73 & & $10045-97-3$ & CS-137 & GAMMA_GS & USTEST & 9.36 \\
\hline 585 & PDMS & & SW & & & E009699 & 07-Aug-73 & & $10045-97-3$ & CS-137 & GAMMA_GS & USTEST & 0.781 \\
\hline 586 & PDMS & & sW & & & E032711 & 07-Aug-73 & & $10098-97-2$ & SR-90 & SRISO_SEEP_PRECIP_GPC & USTEST & 217 \\
\hline 587 & PDMS & & SW & & & E004177 & 13-Aug-73 & & $10098-97-2$ & SR-90 & SRISO_SEP_PRECIP_GPC & USTEST & 0.779 \\
\hline 588 & PDMS & & SW & & & E004177 & 13-Aug-73 & & $10045-97-3$ & CS-137 & GAMMA_GS & USTEST & -0.777 \\
\hline 589 & PDMS & & SW & & & E004177 & 13-Aug-73 & & $10028-17-8$ & TRITIUM & TRITIUM_DIST_LSC & USTEST & 7770 \\
\hline 590 & PDMS & & SW & & & E003728 & 13-Aug-73 & & $10045-97-3$ & CS-137 & GAMMA_GS & USTEST & -1.19 \\
\hline 591 & PDMS & & sW & & & E002223 & 13-Aug-73 & & $10045-97-3$ & CS-137 & GAMMA_GS & USTEST & 6.02 \\
\hline 592 & PDMS & & sW & & & E002224 & 20-Aug-73 & & $10098-97-2$ & SR-90 & SRISO_SEP_PRECIP_GPC & USTEST & 0.22 \\
\hline 593 & PDMS & & sW & & & E004602 & 27-Aug-73 & & $10045-97-3$ & CS-137 & GAMMA_GS & USTEST & -0.0955 \\
\hline 594 & PDMS & & sW & & & E004602 & 27-Aug-73 & & $10098-97-2$ & SR-90 & SRISO_SEEP_PRECIP_GPC & USTEST & 0.35 \\
\hline 595 & PDMS & & SW & & & E026179 & 27-Aug-73 & & $10045-97-3$ & CS-137 & GAMMĀGS ${ }^{-}$ & USTEST & 11.7 \\
\hline 596 & PDMS & & SW & & & E009720 & 28-Aug-73 & & $10098-97-2$ & SR-90 & SRISO_SEP_PRECIP_GPC & USTEST & 0.43 \\
\hline 597 & PDMS & & SW & & & E009720 & 28-Aug-73 & & $10045-97-3$ & CS-137 & GAMMA_GS & USTEST & -0.903 \\
\hline 598 & PDMS & & SW & & & E014704 & 28-Aug-73 & & $10045-97-3$ & CS-137 & GAMMA_GS & USTEST & 0.361 \\
\hline 599 & PDMS & & sW & & & E035071 & 28-Aug-73 & & 10028-17-8 & TRITIUM & TRITIUM_DIST_LSC & USTEST & 367 \\
\hline 600 & PDMS & & SW & & & E026535 & 31-Aug-73 & & $10098-97-2$ & SR-90 & SRISO_SEP_PRECIP_GPC & USTEST & 10.3 \\
\hline 601 & PDMS & & SW & & & E009633 & 04-Sep-73 & & $10045-97-3$ & CS-137 & GAMMA_GS & USTEST & 10.9 \\
\hline 602 & PDMS & & SW & & & E003730 & 04-Sep-73 & & $10045-97-3$ & CS-137 & GAMMA_GS & USTEST & -6.23 \\
\hline 603 & PDMS & & SW & & & E032712 & 04-Sep-73 & & $10098-97-2$ & SR-90 & SRISO_SEP_PRECIP_GPC & USTEST & 2760 \\
\hline 604 & PDMS & & SW & & & E004178 & 07-Sep-73 & & $10028-17-8$ & TRITIUM & TRITIUM_DIST_LSC & USTEST & 9510 \\
\hline 605 & PDMS & & SW & & & E004178 & $07-S e p-73$ & & $10098-97-2$ & SR-90 & SRISO_SEP_PRECIP_GPC & USTEST & 0.854 \\
\hline 606 & PDMS & & SW & & & E004178 & 07-Sep-73 & & $10045-97-3$ & CS-137 & GAMMA_GS & USTEST & 5.54 \\
\hline 607 & PDMS & & SW & & & E003656 & 10-Sep-73 & & $10045-97-3$ & CS-137 & GAMMA_GS & USTEST & 3.08 \\
\hline 608 & PDMS & & SW & & & E002227 & 10-Sep-73 & & $10045-97-3$ & CS-137 & GAMMA_GS & USTEST & 0.0511 \\
\hline 609 & PDMS & & SW & & & E009640 & 11-Sep-73 & & $10045-97-3$ & CS-137 & GAMMA_GS & USTEST & 12.1 \\
\hline 610 & PDMS & & SW & & & E009647 & 18-Sep-73 & & $10045-97-3$ & CS-137 & GAMMA_GS & USTEST & 5.85 \\
\hline 611 & PDMS & & sW & & & E003657 & 24-Sep-73 & & $10045-97-3$ & CS-137 & GAMMA_GS & USTEST & 2.32 \\
\hline 612 & PDMS & & SW & & & E002229 & 24-Sep-73 & & $10098-97-2$ & SR-90 & SRISO_SEP_PRECIP_GPC & USTEST & 1.49 \\
\hline 613 & PDMS & & SW & & & E002229 & 24-Sep-73 & & $10045-97-3$ & CS-137 & GAMMA_GS & USTEST & 0.0402 \\
\hline 614 & PDMS & & SW & & & E037554 & $25-$ Sep-73 & & 10098-97-2 & SR-90 & SRISO_SEP_PRECIP_GPC & USTEST & 0.418 \\
\hline 615 & PDMS & & SW & & & E037554 & $25-\operatorname{Sep}-73$ & & $10045-97-3$ & CS-137 & GAMMA_GS & USTEST & -0.0626 \\
\hline 616 & PDMS & & SW & & & E014578 & 25-Sep-73 & & $10028-17-8$ & TRITIUM & TRITIUM_DIST_LSC & USTEST & 504 \\
\hline 617 & PDMS & & SW & & & E014578 & 25-Sep-73 & & $10098-97-2$ & SR-90 & SRISO_SEP_PRECIP_GPC & USTEST & 0.315 \\
\hline 618 & PDMS & & SW & & & E014578 & $25-$ Sep-73 & & $10045-97-3$ & CS-137 & GAMMA_GS & USTEST & 0.0536 \\
\hline 619 & PDMS & & SW & & & E035001 & 25-Sep-73 & & $10028-17-8$ & TRITIUM & TRITIUM_DIST_LSC & USTEST & 385 \\
\hline 620 & PDMS & & SW & & & E003658 & 01-Oct-73 & & $10045-97-3$ & CS-137 & GAMMA_GS & USTEST & -0.563 \\
\hline 621 & PDMS & & SW & & & E002230 & 01-Oct-73 & & $10045-97-3$ & CS-137 & GAMMA_GS & USTEST & 5.82 \\
\hline 622 & PDMS & & SW & & & E026184 & 01-Oct-73 & & $10045-97-3$ & CS-137 & GAMMA_GS & USTEST & 18.4 \\
\hline 623 & PDMS & & SW & & & E009660 & $02-$ Oct-73 & & $10045-97-3$ & CS-137 & GAMMA_GS & USTEST & 4.29 \\
\hline 624 & PDMS & & SW & & & E003659 & 08-Oct-73 & & $10045-97-3$ & CS-137 & GAMMA_GS & USTEST & 6.54 \\
\hline 625 & PDMS & & SW & & & E002231 & 08-Oct-73 & & $10045-97-3$ & CS-137 & GAMMA_GS & USTEST & 19.7 \\
\hline
\end{tabular}




\begin{tabular}{|c|c|c|c|c|c|c|c|c|c|c|c|c|c|}
\hline & A & $\mathrm{B}$ & $\mathrm{c}$ & $\mathrm{D}$ & $\mathrm{E}$ & $\mathrm{F}$ & G & $\mathrm{H}$ & $\mathrm{I}$ & $\mathrm{J}$ & $\mathrm{K}$ & $\mathrm{L}$ & $\mathrm{M}$ \\
\hline 1 & SAMPLE_NUM_ASSIGNED_TO & SAMPLER & MEDIA & BIOTA_MEDIA & TAG_ID & SAMP_NUM & SAMP_DATE_TIME & SAMP_DATE_TIME_ON & CON_ID & CON_SHORT_NAME & METHOD_NAME & LAB_CODE & \begin{tabular}{|l|} 
VALUE_RPTD \\
\end{tabular} \\
\hline 626 & PDMS & & SW & & & E009667 & 09-Oct-73 & & $10045-97-3$ & CS-137 & GAMMA_GS & USTEST & 7.53 \\
\hline \begin{tabular}{|c|}
627 \\
\end{tabular} & PDMS & & sW & & & E027465 & 09-Oct-73 & & $10045-97-3$ & CS-137 & GAMMA_GS & USTEST & 22.7 \\
\hline 628 & PDMS & & sW & & & E004179 & 11-Oct-73 & & $10098-97-2$ & SR-90 & SRISO_SEP_PRECIP_GPC & USTEST & 0.628 \\
\hline 629 & PDMS & & sW & & & E004179 & 11-Oct-73 & & $10045-97-3$ & CS-137 & GAMMA_GS & USTEST & 7.64 \\
\hline 630 & PDMS & & SW & & & E004179 & 11-Oct-73 & & $10028-17-8$ & TRITIUM & TRITIUM_DIST_LSC & USTEST & 2690 \\
\hline 631 & PDMS & & sW & & & E046081 & 11-Oct-73 & & $10028-17-8$ & TRITIUM & TRITIUM_DIST_LSC & USTEST & 2590 \\
\hline 632 & PDMS & & SW & & & E014937 & 12-Oct-73 & & $10045-97-3$ & CS-137 & GAMMA_GS & USTEST & 0.177 \\
\hline 633 & PDMS & & SW & & & E025921 & 12-Oct-73 & & $10045-97-3$ & CS-137 & GAMMA_GS & USTEST & 12.9 \\
\hline 634 & PDMS & & sW & & & E016394 & 12-Oct-73 & & $10098-97-2$ & SR-90 & SRISO_SEP_PRECIP_GPC & USTEST & 0.387 \\
\hline 635 & PDMS & & SW & & & E016394 & 12-Oct-73 & & $10045-97-3$ & CS-137 & GAMMA_GS & USTEST & -0.814 \\
\hline 636 & PDMS & & sW & & & E022415 & 12-Oct-73 & & $10045-97-3$ & CS-137 & GAMMA_GS & USTEST & 75.9 \\
\hline 637 & PDMS & & SW & & & E022415 & 12-Oct-73 & & 10098-97-2 & SR-90 & SRISO_SEEP_PRECIP_GPC & USTEST & 0.148 \\
\hline 638 & PDMS & & SW & & & E027502 & 12-Oct-73 & & $10045-97-3$ & CS-137 & GAMMA_GS & USTEST & 0.0743 \\
\hline 639 & PDMS & & SW & & & E003551 & 12-Oct-73 & & $10045-97-3$ & CS-137 & GAMMA_GS & PNL-KI & 30.4 \\
\hline 640 & PDMS & & SW & & & E027635 & 12-Oct-73 & & $10045-97-3$ & CS-137 & GAMMA_GS & USTEST & 19.2 \\
\hline 641 & PDMS & & SW & & & E003660 & 15-Oct-73 & & $10045-97-3$ & CS-137 & GAMMA_GS & USTEST & 4.26 \\
\hline 642 & PDMS & & sW & & & E002232 & 15-Oct-73 & & $10045-97-3$ & CS-137 & GAMMA_GS & USTEST & -5.12 \\
\hline 643 & PDMS & & sW & & & E003661 & 22-Oct-73 & & $10045-97-3$ & CS-137 & GAMMA_GS & USTEST & -0.348 \\
\hline 644 & PDMS & & SW & & & E002233 & 22-Oct-73 & & $10098-97-2$ & SR-90 & SRISO_SEEP_PRECIP_GPC & USTEST & 0.356 \\
\hline 645 & PDMS & & SW & & & E002233 & 22-Oct-73 & & $10045-97-3$ & CS-137 & GAMMĀ_GS & USTEST & -0.0705 \\
\hline 646 & PDMS & & SW & & & E009587 & 23-Oct-73 & & $10045-97-3$ & CS-137 & GAMMA_GS & USTEST & 0.0481 \\
\hline 647 & PDMS & & SW & & & E004603 & 29-Oct-73 & & $10045-97-3$ & CS-137 & GAMMA_GS & USTEST & 0.00256 \\
\hline 648 & PDMS & & SW & & & E004603 & 29-Oct-73 & & 10098-97-2 & SR-90 & SRISO_SEPP_PRECIP_GPC & USTEST & 0.378 \\
\hline 649 & PDMS & & sW & & & E009593 & 29-Oct-73 & & $10028-17-8$ & TRITIUM & TRITIUM_DIST_LSC & USTEST & 738 \\
\hline 650 & PDMS & & SW & & & E009593 & 29-Oct-73 & & $10098-97-2$ & SR-90 & SRISO_SEP_PRECIP_GPC & USTEST & 0.351 \\
\hline 651 & PDMS & & SW & & & E009593 & 29-Oct-73 & & $10045-97-3$ & CS-137 & GAMMĀ_GS & USTEST & 0.176 \\
\hline 652 & PDMS & & SW & & & E041049 & 29-Oct-73 & & $10028-17-8$ & TRITIUM & TRITIUM_DIST_LSC & USTEST & 308 \\
\hline 653 & PDMS & & SW & & & E003662 & 29-Oct-73 & & $10045-97-3$ & CS-137 & GAMMA_GS & USTEST & -0.517 \\
\hline 654 & PDMS & & SW & & & E002234 & 29-Oct-73 & & $10045-97-3$ & CS-137 & GAMMA_GS & USTEST & -0.608 \\
\hline 655 & PDMS & & SW & & & E000842 & 29-Oct-73 & & $10045-97-3$ & CS-137 & GAMMA_GS & USTEST & -0.658 \\
\hline 656 & PDMS & & sW & & & E009594 & 30-Oct-73 & & $10045-97-3$ & CS-137 & GAMMA_GS & USTEST & 0.218 \\
\hline 657 & PDMS & & SW & & & E032713 & 30-Oct-73 & & $10098-97-2$ & SR-90 & SRISO_SEEP_PRECIP_GPC & USTEST & 869 \\
\hline 658 & PDMS & & SW & & & E003663 & 05-Nov-73 & & $10045-97-3$ & CS-137 & GAMMĀ_GS & USTEST & 4.8 \\
\hline 659 & PDMS & & SW & & & E002235 & 05-Nov-73 & & $10045-97-3$ & CS-137 & GAMMA_GS & USTEST & -0.184 \\
\hline 660 & PDMS & & SW & & & E009601 & 06-Nov-73 & & $10045-97-3$ & CS-137 & GAMMA_GS & USTEST & -0.0546 \\
\hline 661 & PDMS & & sW & & & E004180 & 08-Nov-73 & & $10028-17-8$ & TRITIUM & TRITIUM_DIST_LSC & USTEST & 1890 \\
\hline 662 & PDMS & & SW & & & E004180 & 08-Nov-73 & & $10098-97-2$ & SR-90 & SRISO_SEP_PRECIP_GPC & USTEST & 0.782 \\
\hline 663 & PDMS & & SW & & & E004180 & 08-Nov-73 & & $10045-97-3$ & CS-137 & GAMMA_GS & USTEST & 3.69 \\
\hline 664 & PDMS & & SW & & & E035534 & 09-Nov-73 & & $10098-97-2$ & SR-90 & SRISO_SEEP_PRECIP_GPC & USTEST & 6.18 \\
\hline 665 & PDMS & & SW & & & E010874 & 12-Nov-73 & & $10045-97-3$ & CS-137 & GAMMĀ_GS & USTEST & 0.625 \\
\hline 666 & PDMS & & SW & & & E002236 & 12-Nov-73 & & $10045-97-3$ & CS-137 & GAMMA_GS & USTEST & -7.49 \\
\hline 667 & PDMS & & SW & & & E009608 & 13-Nov-73 & & $10045-97-3$ & CS-137 & GAMMA_GS & USTEST & 7.13 \\
\hline 668 & PDMS & & SW & & & E003664 & 19-Nov-73 & & $10045-97-3$ & CS-137 & GAMMA_GS & USTEST & -0.669 \\
\hline 669 & PDMS & & sW & & & E002237 & 19-Nov-73 & & $10098-97-2$ & SR-90 & SRISO_SEP_PRECIP_GPC & USTEST & 0.389 \\
\hline 670 & PDMS & & SW & & & E002237 & 19-Nov-73 & & $10045-97-3$ & CS-137 & GAMMA__GS & USTEST & 0.181 \\
\hline 671 & PDMS & & SW & & & E009615 & 20-Nov-73 & & $10045-97-3$ & CS-137 & GAMMA_GS & USTEST & 1.97 \\
\hline 672 & PDMS & & SW & & & E004604 & 26-Nov-73 & & $10045-97-3$ & CS-137 & GAMMA_GS & USTEST & 0.576 \\
\hline 673 & PDMS & & SW & & & E004604 & 26-Nov-73 & & $10098-97-2$ & SR-90 & SRISO_SEEP_PRECIP_GPC & USTEST & 0.437 \\
\hline 674 & PDMS & & SW & & & E003665 & 26-Nov-73 & & $10045-97-3$ & CS-137 & GAMMA_G_G & USTEST & -2.66 \\
\hline 675 & PDMS & & SW & & & E002238 & 26-Nov-73 & & $10045-97-3$ & CS-137 & GAMMA_GS & USTEST & -2.34 \\
\hline 676 & PDMS & & sW & & & E026188 & 26-Nov-73 & & $10045-97-3$ & CS-137 & GAMMA_GS & USTEST & 0.865 \\
\hline 677 & PDMS & & SW & & & E009622 & 27-Nov-73 & & $10028-17-8$ & TRITIUM & TRITIUM_DIST_LSC & USTEST & 199 \\
\hline
\end{tabular}




\begin{tabular}{|c|c|c|c|c|c|c|c|c|c|c|c|c|c|}
\hline & A & $\mathrm{B}$ & $\mathrm{C}$ & $\mathrm{D}$ & $E$ & $\mathrm{~F}$ & $\mathrm{G}$ & $\mathrm{H}$ & $\mathrm{I}$ & $\mathrm{J}$ & $\mathrm{K}$ & $\mathrm{L}$ & $\mathrm{M}$ \\
\hline 1 & SAMPLE_NUM_ASSIGNED_TO & SAMPLER & MEDIA & BIOTA_MEDIA & TAG_ID & SAMP_NUM & SAMP_DATE_TIME & SAMP_DATE_TIME_ON & CON_ID & CON_SHORT_NAME & METHOD_NAME & LAB_CODE & VALUE_RPTD \\
\hline 678 & PDMS & & SW & & & E009622 & 27-Nov-73 & & $10098-97-2$ & SR-90 & SRISO_SEP_PRECIP_GPC & USTEST & 0.324 \\
\hline 679 & PDMS & & SW & & & E009622 & 27-Nov-73 & & $10045-97-3$ & CS-137 & GAMMA_GS & USTEST & -4.23 \\
\hline 680 & PDMS & & SW & & & E014705 & 27-Nov-73 & & $10045-97-3$ & CS-137 & GAMMA_GS & USTEST & 0.944 \\
\hline 681 & PDMS & & SW & & & E027885 & 27-Nov-73 & & $10028-17-8$ & TRITIUM & TRITIUM_DIST_LSC & USTEST & 284 \\
\hline 682 & PDMS & & SW & & & E032714 & 27-Nov-73 & & $10098-97-2$ & SR-90 & SRISO_SEP_PRECIP_GPC & USTEST & 215 \\
\hline 683 & PDMS & & SW & & & E003666 & 03-Dec-73 & & $10045-97-3$ & CS-137 & GAMMA__GS & USTEST & 6.58 \\
\hline 684 & PDMS & & SW & & & E002239 & 03-Dec-73 & & $10045-97-3$ & CS-137 & GAMMA_GS & USTEST & 14.8 \\
\hline 685 & PDMS & & SW & & & E009629 & 04-Dec-73 & & $10045-97-3$ & CS-137 & GAMMA_GS & USTEST & 5.33 \\
\hline 686 & PDMS & & SW & & & E004181 & 07-Dec-73 & & $10028-17-8$ & TRITIUM & TRITIUM_DIST_LSC & USTEST & 2440 \\
\hline 687 & PDMS & & SW & & & E004181 & 07-Dec-73 & & $10098-97-2$ & SR-90 & SRISO_SEP_PRECIP_GPC & USTEST & 0.77 \\
\hline 688 & PDMS & & SW & & & E004181 & 07-Dec-73 & & $10045-97-3$ & CS-137 & GAMMA_GS & USTEST & 0.631 \\
\hline 689 & PDMS & & SW & & & E026536 & 07-Dec-73 & & $10098-97-2$ & SR-90 & SRISO_SEP_PRECIP_GPC & USTEST & 0.045 \\
\hline 690 & PDMS & & SW & & & E001611 & 10-Dec-73 & & $10045-97-3$ & CS-137 & GAMMA_GS & USTEST & -1.18 \\
\hline 691 & PDMS & & SW & & & E002240 & 10-Dec-73 & & $10045-97-3$ & CS-137 & GAMMA_GS & USTEST & -0.762 \\
\hline 692 & PDMS & & SW & & & E009542 & 11-Dec-73 & & $10045-97-3$ & CS-137 & GAMMA_GS & USTEST & 3.61 \\
\hline 693 & PDMS & & SW & & & E003667 & 17-Dec-73 & & $10045-97-3$ & CS-137 & GAMMA_GS & USTEST & 2.04 \\
\hline 694 & PDMS & & SW & & & E002241 & 17-Dec-73 & & $10045-97-3$ & CS-137 & GAMMA_GS & USTEST & 1.52 \\
\hline 695 & PDMS & & SW & & & E009549 & 18-Dec-73 & & $10045-97-3$ & CS-137 & GAMMA_GS & USTEST & 18.9 \\
\hline 696 & PDMS & & SW & & & E003668 & 21-Dec-73 & & $10045-97-3$ & CS-137 & GAMMA_GS & USTEST & 4.46 \\
\hline 697 & PDMS & & SW & & & E002242 & 21-Dec-73 & & $10098-97-2$ & SR-90 & SRISO_SEP_PRECIP_GPC & USTEST & 0.261 \\
\hline 698 & PDMS & & SW & & & E002242 & 21-Dec-73 & & $10045-97-3$ & CS-137 & GAMMA_GS & USTEST & -0.0278 \\
\hline 699 & PDMS & & SW & & & E004605 & 26-Dec-73 & & $10045-97-3$ & CS-137 & GAMMA_GS & USTEST & 0.00255 \\
\hline 700 & PDMS & & SW & & & E004605 & 26-Dec-73 & & $10098-97-2$ & SR-90 & SRISO_SEP_PRECIP_GPC & USTEST & 0.243 \\
\hline 701 & PDMS & & SW & & & E009557 & 26-Dec-73 & & $10028-17-8$ & TRITIUM & TRITIUM̄DISTT_LSC & USTEST & 749 \\
\hline 702 & PDMS & & SW & & & E009557 & 26-Dec-73 & & $10098-97-2$ & SR-90 & SRISO_SEP_PRECIP_GPC & USTEST & 0.161 \\
\hline 703 & PDMS & & SW & & & E014706 & 26-Dec-73 & & $10045-97-3$ & CS-137 & GAMMA_GS & USTEST & -0.0743 \\
\hline 704 & PDMS & & SW & & & E035011 & 26-Dec-73 & & $10028-17-8$ & TRITIUM & TRITIUM_DIST_LSC & USTEST & 228 \\
\hline 705 & PDMS & & SW & & & E032715 & 26-Dec-73 & & $10098-97-2$ & SR-90 & SRISO_SEP_PRECIP_GPC & USTEST & 175 \\
\hline 706 & PDMS & & SW & & & E003669 & 31-Dec-73 & & $10045-97-3$ & CS-137 & GAMMA_GS & USTEST & 0.963 \\
\hline 707 & PDMS & & SW & & & E002243 & 31-Dec-73 & & $10045-97-3$ & CS-137 & GAMMA_GS & USTEST & -0.0756 \\
\hline 708 & PDMS & & SW & & & E009564 & 02-Jan-74 & & $10045-97-3$ & CS-137 & GAMMA_GS & USTEST & 1.71 \\
\hline 709 & PDMS & & SW & & & E027466 & 02-Jan-74 & & $10045-97-3$ & CS-137 & GAMMA_GS & USTEST & -2.54 \\
\hline 710 & PDMS & & SW & & & E003670 & 07-Jan-74 & & $10045-97-3$ & CS-137 & GAMMA_GS & USTEST & -0.00652 \\
\hline 711 & PDMS & & SW & & & E002244 & 07-Jan-74 & & $10045-97-3$ & CS-137 & GAMMA_GS & USTEST & -0.0142 \\
\hline 712 & PDMS & & SW & & & E009570 & 08-Jan-74 & & $10045-97-3$ & CS-137 & GAMMA_GS & USTEST & 4.19 \\
\hline 713 & PDMS & & SW & & & E037678 & 11-Jan-74 & & $10045-97-3$ & CS-137 & GAMMA_GS & USTEST & 6.1 \\
\hline 714 & PDMS & & SW & & & E025922 & 11-Jan-74 & & $10045-97-3$ & CS-137 & GAMMA_GS & USTEST & 17.3 \\
\hline 715 & PDMS & & SW & & & E003552 & 11-Jan-74 & & $10045-97-3$ & CS-137 & GAMMA_GS & PNL-KI & -17.2 \\
\hline 716 & PDMS & & SW & & & E003671 & 14-Jan-74 & & $10045-97-3$ & CS-137 & GAMMA_GS & USTEST & -1.15 \\
\hline 717 & PDMS & & SW & & & E002245 & 14-Jan-74 & & $10045-97-3$ & CS-137 & GAMMA_GS & USTEST & 3.07 \\
\hline 718 & PDMS & & SW & & & E009577 & 15-Jan-74 & & $10045-97-3$ & CS-137 & GAMMA_GS & USTEST & -0.749 \\
\hline 719 & PDMS & & SW & & & E016395 & 18-Jan-74 & & $10098-97-2$ & SR-90 & SRISO_SEP_PRECIP_GPC & USTEST & 2.67 \\
\hline 720 & PDMS & & SW & & & E016395 & 18-Jan-74 & & $10045-97-3$ & CS-137 & GAMMA_GS & USTEST & 16.5 \\
\hline 721 & PDMS & & SW & & & E022416 & 18-Jan-74 & & $10045-97-3$ & CS-137 & GAMMA_GS & USTEST & 3.74 \\
\hline 722 & PDMS & & SW & & & E022416 & 18-Jan-74 & & $10098-97-2$ & SR-90 & SRISO_SEP_PRECIP_GPC & USTEST & 0.203 \\
\hline 723 & PDMS & & SW & & & E027636 & 18-Jan-74 & & $10045-97-3$ & CS-137 & GAMMA_GS & USTEST & -0.442 \\
\hline 724 & PDMS & & SW & & & E014264 & 18-Jan-74 & & $10045-97-3$ & CS-137 & GAMMA_GS & USTEST & -0.487 \\
\hline 725 & PDMS & & SW & & & E014264 & 18-Jan-74 & & $10098-97-2$ & SR-90 & SRISO_SEP_PRECIP_GPC & USTEST & 3.41 \\
\hline 726 & PDMS & & SW & & & E028036 & 21-Jan-74 & & $10098-97-2$ & SR-90 & SRISO_SEP_PRECIP_GPC & USTEST & 0.118 \\
\hline 727 & PDMS & & SW & & & E003672 & 21-Jan-74 & & $10045-97-3$ & CS-137 & GAMMA_GS & USTEST & -0.345 \\
\hline 728 & PDMS & & SW & & & E002246 & 21-Jan-74 & & $10098-97-2$ & SR-90 & SRISO_SEP_PRECIP_GPC & USTEST & 0.263 \\
\hline 729 & PDMS & & SW & & & E002246 & 21-Jan-74 & & $10045-97-3$ & CS-137 & GAMMA_GS & USTEST & 0.0511 \\
\hline
\end{tabular}




\begin{tabular}{|c|c|c|c|c|c|c|c|c|c|c|c|c|c|}
\hline & A & $\mathrm{B}$ & $C$ & $\mathrm{D}$ & $E$ & $\mathrm{~F}$ & G & $\mathrm{H}$ & $\mathrm{I}$ & $\mathrm{J}$ & $\mathrm{K}$ & $\mathrm{L}$ & $\mathrm{M}$ \\
\hline 1 & SAMPLE_NUM_ASSIGNED_TO & SAMPLER & MEDIA & BIOTA_MEDIA & TAG_ID & SAMP_NUM & SAMP_DATE_TIME & SAMP_DATE_TIME_ON & CON_ID & CON_SHORT_NAME & METHOD_NAME & LAB_CODE & VALUE_RPTD \\
\hline 730 & PDMS & & SW & & & E009584 & 22-Jan-74 & & $10045-97-3$ & CS-137 & GAMMA_GS & USTEST & -0.752 \\
\hline 731 & PDMS & & SW & & & E032716 & 22-Jan-74 & & $10098-97-2$ & SR-90 & SRISO_SEP_PRECIP_GPC & USTEST & 178 \\
\hline 732 & PDMS & & SW & & & E004606 & 28-Jan-74 & & $10045-97-3$ & CS-137 & GAMMA_GS & USTEST & 0.00255 \\
\hline 733 & PDMS & & SW & & & E004606 & 28-Jan-74 & & $10098-97-2$ & SR-90 & SRISO_SEP_PRECIP_GPC & USTEST & 0.279 \\
\hline 734 & PDMS & & SW & & & E003673 & 28-Jan-74 & & $10045-97-3$ & CS-137 & GAMMA_GS & USTEST & 0.626 \\
\hline 735 & PDMS & & SW & & & E084936 & 28-Jan-74 & & $10028-17-8$ & TRITIUM & TRITIUM_DIST_LSC & USTEST & 642 \\
\hline 736 & PDMS & & SW & & & E002247 & 28-Jan-74 & & $10045-97-3$ & CS-137 & GAMMA_GS & USTEST & -0.0904 \\
\hline 737 & PDMS & & SW & & & E009497 & 29-Jan-74 & & $10028-17-8$ & TRITIUM & TRITIUM_DIST_LSC & USTEST & 231 \\
\hline 738 & PDMS & & SW & & & E009497 & 29-Jan-74 & & $10098-97-2$ & SR-90 & SRISO_SEP_PRECIP_GPC & USTEST & 0.275 \\
\hline 739 & PDMS & & SW & & & E009497 & 29-Jan-74 & & $10045-97-3$ & CS-137 & GAMMA_GS & USTEST & -0.931 \\
\hline 740 & PDMS & & SW & & & E014691 & 29-Jan-74 & & $10045-97-3$ & CS-137 & GAMMA_GS & USTEST & -0.00342 \\
\hline 741 & PDMS & & sW & & & E035015 & 29-Jan-74 & & $10028-17-8$ & TRITIUM & TRITIUM_DIST_LSC & USTEST & 334 \\
\hline 742 & PDMS & & SW & & & E003674 & 04-Feb-74 & & $10045-97-3$ & CS-137 & GAMMA_GS & USTEST & 0.0508 \\
\hline 743 & PDMS & & SW & & & E009504 & 05-Feb-74 & & $10045-97-3$ & CS-137 & GAMMA_GS & USTEST & 0.0511 \\
\hline 744 & PDMS & & SW & & & E037704 & 08-Feb-74 & & $10045-97-3$ & CS-137 & GAMMA_GS & USTEST & 9.78 \\
\hline 745 & PDMS & & SW & & & E037704 & 08-Feb-74 & & $10098-97-2$ & SR-90 & SRISO_SEP_PRECIP_GPC & USTEST & 0.243 \\
\hline 746 & PDMS & & SW & & & E026537 & 08-Feb-74 & & $10098-97-2$ & SR-90 & SRISO_SEP_PRECIP_GPC & USTEST & 6.59 \\
\hline 747 & PDMS & & SW & & & E003675 & 11-Feb-74 & & $10045-97-3$ & CS-137 & GAMMA_GS & USTEST & 10.7 \\
\hline 748 & PDMS & & SW & & & E084937 & 11-Feb-74 & & $10028-17-8$ & TRITIUM & TRITIUM_DIST_LSC & USTEST & 269 \\
\hline 749 & PDMS & & SW & & & E002249 & 11-Feb-74 & & $10045-97-3$ & CS-137 & GAMMA_GS & USTEST & 14.1 \\
\hline 750 & PDMS & & SW & & & E009511 & 12-Feb-74 & & $10045-97-3$ & CS-137 & GAMMA_GS & USTEST & -0.22 \\
\hline 751 & PDMS & & SW & & & E009518 & 19-Feb-74 & & $10045-97-3$ & CS-137 & GAMMA_GS & USTEST & 0.0511 \\
\hline 752 & PDMS & & SW & & & E003676 & 19-Feb-74 & & $10045-97-3$ & CS-137 & GAMMA_GS & USTEST & -0.325 \\
\hline 753 & PDMS & & SW & & & E002250 & 19-Feb-74 & & $10098-97-2$ & SR-90 & SRISO_SEP_PRECIP_GPC & USTEST & 0.0725 \\
\hline 754 & PDMS & & SW & & & E002250 & 19-Feb-74 & & $10045-97-3$ & CS-137 & GAMMA_GS & USTEST & -0.279 \\
\hline 755 & PDMS & & SW & & & E032717 & 19-Feb-74 & & $10098-97-2$ & SR-90 & SRISO_SEP_PRECIP_GPC & USTEST & 11.6 \\
\hline 756 & PDMS & & SW & & & E004607 & 25-Feb-74 & & $10098-97-2$ & SR-90 & SRISO_SEP_PRECIP_GPC & USTEST & 0.347 \\
\hline 757 & PDMS & & SW & & & E004607 & 25-Feb-74 & & $10045-97-3$ & CS-137 & GAMMA_GS & USTEST & 1.45 \\
\hline 758 & PDMS & & SW & & & E003677 & 25-Feb-74 & & $10045-97-3$ & CS-137 & GAMMA_GS & USTEST & 12.8 \\
\hline 759 & PDMS & & SW & & & E002251 & 25-Feb-74 & & $10045-97-3$ & CS-137 & GAMMA_GS & USTEST & 1.52 \\
\hline 760 & PDMS & & SW & & & E009525 & 26-Feb-74 & & $10028-17-8$ & TRITIUM & TRITIUM_DIST_LSC & USTEST & 466 \\
\hline 761 & PDMS & & SW & & & E009525 & $26-F e b-74$ & & $10098-97-2$ & SR-90 & SRISO_SEP_PRECIP_GPC & USTEST & 0.207 \\
\hline 762 & PDMS & & SW & & & E009525 & 26-Feb-74 & & $10045-97-3$ & CS-137 & GAMMA_GS & USTEST & -0.664 \\
\hline 763 & PDMS & & SW & & & E014692 & 26-Feb-74 & & $10045-97-3$ & CS-137 & GAMMA_GS & USTEST & -0.0215 \\
\hline 764 & PDMS & & SW & & & E035020 & 26-Feb-74 & & $10028-17-8$ & TRITIUM & TRITIUM_DIST_LSC & USTEST & 321 \\
\hline 765 & PDMS & & SW & & & E003678 & 04-Mar-74 & & $10045-97-3$ & CS-137 & GAMMA_GS & USTEST & -0.228 \\
\hline 766 & PDMS & & SW & & & E002252 & 04-Mar-74 & & $10045-97-3$ & CS-137 & GAMMA_GS & USTEST & 0.0536 \\
\hline 767 & PDMS & & SW & & & E026197 & 04-Mar-74 & & $10045-97-3$ & CS-137 & GAMMA_GS & USTEST & -5.47 \\
\hline 768 & PDMS & & sW & & & E009532 & 05-Mar-74 & & $10045-97-3$ & CS-137 & GAMMA_GS & USTEST & 0.0511 \\
\hline 769 & PDMS & & SW & & & E026538 & 08-Mar-74 & & $10098-97-2$ & SR-90 & SRISO_SEP_PRECIP_GPC & USTEST & 6.94 \\
\hline 770 & PDMS & & SW & & & E003679 & 11-Mar-74 & & $10045-97-3$ & CS-137 & GAMMA_GS & USTEST & 0.894 \\
\hline 771 & PDMS & & SW & & & E002253 & 11-Mar-74 & & $10045-97-3$ & CS-137 & GAMMA_GS & USTEST & -0.0564 \\
\hline 772 & PDMS & & SW & & & E009445 & 12-Mar-74 & & $10045-97-3$ & CS-137 & GAMMA_GS & USTEST & -0.227 \\
\hline 773 & PDMS & & SW & & & E028026 & 15-Mar-74 & & $10045-97-3$ & CS-137 & GAMMA_GS & USTEST & 0.0478 \\
\hline 774 & PDMS & & SW & & & E028026 & 15-Mar-74 & & $10028-17-8$ & TRITIUM & TRITIUM_DIST_LSC & USTEST & 628 \\
\hline 775 & PDMS & & SW & & & E028026 & 15-Mar-74 & & $10098-97-2$ & SR-90 & SRISO_SEP_PRECIP_GPC & USTEST & 0.212 \\
\hline 776 & PDMS & & SW & & & E003680 & 18-Mar-74 & & $10045-97-3$ & CS-137 & GAMMA_GS & USTEST & 0.0113 \\
\hline 777 & PDMS & & SW & & & E002254 & 18-Mar-74 & & $10045-97-3$ & CS-137 & GAMMA_GS & USTEST & 0.639 \\
\hline 778 & PDMS & & SW & & & E009452 & 19-Mar-74 & & $10045-97-3$ & CS-137 & GAMMA_GS & USTEST & 1.08 \\
\hline 779 & PDMS & & SW & & & E032718 & 19-Mar-74 & & $10098-97-2$ & SR-90 & SRISO_SEP_PRECIP_GPC & USTEST & 288 \\
\hline 780 & PDMS & & SW & & & E004608 & 25-Mar-74 & & $10045-97-3$ & CS-137 & GAMMA_GS & USTEST & -0.472 \\
\hline 781 & PDMS & & SW & & & E009458 & 25-Mar-74 & & 10028-17-8 & TRITIUM & TRITIUM_DIST_LSC & USTEST & 1990 \\
\hline
\end{tabular}




\begin{tabular}{|c|c|c|c|c|c|c|c|c|c|c|c|c|c|}
\hline & $\bar{A}$ & $\mathrm{~B}$ & $\mathrm{C}$ & $\bar{D}$ & $\mathrm{E}$ & $\mathrm{F}$ & $\bar{G}$ & $\mathrm{H}$ & 1 & $\mathrm{~J}$ & $\mathrm{~K}$ & $\mathrm{~L}$ & $\bar{M}$ \\
\hline 1 & SAMPLE_NUM_ASSIGNED_TO & SAMPLER & MEDIA & BIOTA_MEDIA & TAG_ID & SAMP_NUM & SAMP_DATE_TIME & SAMP_DATE_TIME_ON & CON_ID & \begin{tabular}{|l|} 
CON_SHORT_NAME \\
\end{tabular} & METHOD_NAME & LAB_CODE & VALUE_RPTD \\
\hline 782 & PDMS & & SW & & & E009458 & 25-Mar-74 & & $10098-97-2$ & SR-90 & SRISO_SEP_PRECIP_GPC & USTEST & 0.239 \\
\hline 783 & PDMS & & sW & & & E014693 & 25-Mar-74 & & $10045-97-3$ & CS-137 & GAMMĀGS ${ }^{-}$ & USTEST & 0.00333 \\
\hline 784 & PDMS & & SW & & & E003681 & 25-Mar-74 & & $10045-97-3$ & CS-137 & GAMMA_GS & USTEST & -0.712 \\
\hline 785 & PDMS & & SW & & & E002255 & 25-Mar-74 & & $10098-97-2$ & SR-90 & SRISO_SEP_PRECIP_GPC & USTEST & 0.568 \\
\hline 786 & PDMS & & SW & & & E002255 & 25-Mar-74 & & $10045-97-3$ & CS-137 & GAMMA_GS & USTEST & -0.607 \\
\hline 787 & PDMS & & SW & & & E009459 & 26-Mar-74 & & $10045-97-3$ & CS-137 & GAMMA_GS & USTEST & 3.72 \\
\hline 788 & PDMS & & sW & & & E041050 & 27-Mar-74 & & $10028-17-8$ & TRITIUM & TRITIUM_DIST_LSC & USTEST & 286 \\
\hline 789 & PDMS & & sW & & & E003682 & 01-Apr-74 & & $10045-97-3$ & CS-137 & GAMMA_GS & USTEST & 0.0382 \\
\hline 790 & PDMS & & sW & & & E002256 & 01-Apr-74 & & $10045-97-3$ & CS-137 & GAMMA_GS & USTEST & 0.0167 \\
\hline 791 & PDMS & & sW & & & E009466 & 02-Apr-74 & & $10045-97-3$ & CS-137 & GAMMA_GS & USTEST & 0.0516 \\
\hline 792 & PDMS & & SW & & & E014938 & 05-Apr-74 & & $10045-97-3$ & CS-137 & GAMMA_GS & USTEST & 3.93 \\
\hline 793 & PDMS & & SW & & & E016396 & 05-Apr-74 & & $10045-97-3$ & CS-137 & GAMMA_GS & USTEST & -0.0667 \\
\hline 794 & PDMS & & sW & & & E016396 & 05-Apr-74 & & $10098-97-2$ & SR-90 & SRISO_SEEP_PRECIP_GPC & USTEST & 3.47 \\
\hline 795 & PDMS & & SW & & & E022417 & 05-Apr-74 & & $10045-97-3$ & CS-137 & GAMMA_GS & USTEST & 34.4 \\
\hline 796 & PDMS & & SW & & & E022417 & 05-Apr-74 & & $10098-97-2$ & SR-90 & SRISO_SEP_PRECIP_GPC & USTEST & 0.839 \\
\hline 797 & PDMS & & SW & & & E027637 & 05-Apr-74 & & $10045-97-3$ & CS-137 & GAMMA_GS & USTEST & 9.9 \\
\hline 798 & PDMS & & SW & & & E014265 & 05-Apr-74 & & $10045-97-3$ & CS-137 & GAMMA_GS & USTEST & 1.64 \\
\hline 799 & PDMS & & sW & & & E014265 & 05-Apr-74 & & 10098-97-2 & SR-90 & SRISO_SEEP_PRECIP_GPC & USTEST & 8.99 \\
\hline 800 & PDMS & & sW & & & E003683 & 08-Apr-74 & & $10045-97-3$ & CS-137 & GAMMA_GS & USTEST & 8.06 \\
\hline 801 & PDMS & & sW & & & E002257 & 08-Apr-74 & & $10045-97-3$ & CS-137 & GAMMA_GS & USTEST & 5.44 \\
\hline 802 & PDMS & & sW & & & E026200 & 08-Apr-74 & & $10045-97-3$ & CS-137 & GAMMA_GS & USTEST & -3.42 \\
\hline 803 & PDMS & & SW & & & E009473 & 09-Apr-74 & & $10045-97-3$ & CS-137 & GAMMA_GS & USTEST & 15.8 \\
\hline 804 & PDMS & & SW & & & E046083 & 15-Apr-74 & & $10028-17-8$ & TRITIUM & TRITIUM_DIST_LSC & USTEST & 845 \\
\hline 805 & PDMS & & SW & & & E028027 & 15-Apr-74 & & $10045-97-3$ & CS-137 & GAMMA_GS & USTEST & 5.92 \\
\hline 806 & PDMS & & SW & & & E028027 & 15-Apr-74 & & $10098-97-2$ & SR-90 & SRISO_SEP_PRECIP_GPC & USTEST & 0.248 \\
\hline 807 & PDMS & & sW & & & E003684 & 15-Apr-74 & & $10045-97-3$ & CS-137 & GAMMA_GS & USTEST & -0.806 \\
\hline 808 & PDMS & & SW & & & E002258 & 15-Apr-74 & & $10045-97-3$ & CS-137 & GAMMA_GS & USTEST & 3.9 \\
\hline 809 & PDMS & & SW & & & E009480 & 16-Apr-74 & & $10045-97-3$ & CS-137 & GAMMA_GS & USTEST & 0.0511 \\
\hline 810 & PDMS & & SW & & & E032719 & 16-Apr-74 & & 10098-97-2 & SR-90 & SRISO_SEP_PRECIP_GPC & USTEST & 165 \\
\hline 811 & PDMS & & SW & & & E003685 & 22-Apr-74 & & $10045-97-3$ & CS-137 & GAMMA_GS & USTEST & 0.0511 \\
\hline 812 & PDMS & & SW & & & E002259 & 22-Apr-74 & & $10098-97-2$ & SR-90 & SRISO_SEP_PRECIP_GPC & USTEST & 0 \\
\hline 813 & PDMS & & SW & & & E002259 & 22-Apr-74 & & $10045-97-3$ & CS-137 & GAMMA_GS & USTEST & 0.0511 \\
\hline 814 & PDMS & & SW & & & E009487 & 23-Apr-74 & & $10028-17-8$ & TRITIUM & TRITIUM_DIST_LSC & USTEST & 469 \\
\hline 815 & PDMS & & SW & & & E009487 & 23-Apr-74 & & $10098-97-2$ & SR-90 & SRISO_SEP_PRECIP_GPC & USTEST & 0.23 \\
\hline 816 & PDMS & & SW & & & E009487 & 23-Apr-74 & & $10045-97-3$ & CS-137 & GAMMA_GS & USTEST & 0.051 \\
\hline 817 & PDMS & & SW & & & E014694 & 23-Apr-74 & & $10045-97-3$ & CS-137 & GAMMA_GS & USTEST & 0.646 \\
\hline 818 & PDMS & & SW & & & E004609 & 29-Apr-74 & & $10098-97-2$ & SR-90 & SRISO_SEP_PRECIP_GPC & USTEST & 0.284 \\
\hline 819 & PDMS & & sW & & & E004609 & 29-Apr-74 & & $10045-97-3$ & CS-137 & GAMMA_GS & USTEST & 5.1 \\
\hline 820 & PDMS & & SW & & & E003686 & 29-Apr-74 & & $10045-97-3$ & CS-137 & GAMMA_GS & USTEST & -0.417 \\
\hline 821 & PDMS & & SW & & & E002184 & 29-Apr-74 & & $10045-97-3$ & CS-137 & GAMMA_GS & USTEST & -0.644 \\
\hline 822 & PDMS & & SW & & & E009400 & 30-Apr-74 & & $10045-97-3$ & CS-137 & GAMMA_GS & USTEST & 5.38 \\
\hline 823 & PDMS & & SW & & & E026539 & 03-May-74 & & $10098-97-2$ & SR-90 & SRISO_SEP_PRECIP_GPC & USTEST & 6.68 \\
\hline 824 & PDMS & & SW & & & E003687 & 06-May-74 & & $10045-97-3$ & CS-137 & GAMMA_GS & USTEST & 15.6 \\
\hline 825 & PDMS & & SW & & & E002185 & 06-May-74 & & $10045-97-3$ & CS-137 & GAMMA_GS & USTEST & 18.4 \\
\hline 826 & PDMS & & SW & & & E009407 & 07-May-74 & & $10045-97-3$ & CS-137 & GAMMA_GS & USTEST & -0.56 \\
\hline 827 & PDMS & & SW & & & E028028 & 10-May-74 & & $10045-97-3$ & CS-137 & GAMMA_GS & USTEST & 2.2 \\
\hline 828 & PDMS & & SW & & & E028028 & 10-May-74 & & $10028-17-8$ & TRITIUM & TRITIUM_DIST_LSC & USTEST & 718 \\
\hline 829 & PDMS & & SW & & & E028028 & 10-May-74 & & $10098-97-2$ & SR-90 & SRISO_SEP_PRECIP_GPC & USTEST & 0.185 \\
\hline 830 & PDMS & & SW & & & E003688 & 13-May-74 & & $10045-97-3$ & CS-137 & GAMMA_GS & USTEST & 0.0179 \\
\hline 831 & PDMS & & SW & & & E002186 & 13-May-74 & & $10045-97-3$ & CS-137 & GAMMA_GS & USTEST & -0.16 \\
\hline 832 & PDMS & & SW & & & E009414 & 14-May-74 & & $10045-97-3$ & CS-137 & GAMMA_GS & USTEST & 0.0495 \\
\hline 833 & PDMS & & SW & & & E032720 & 14-May-74 & & $10098-97-2$ & SR-90 & SRISO_SEP_PRECIP_GPC & USTEST & 122 \\
\hline
\end{tabular}




\begin{tabular}{|c|c|c|c|c|c|c|c|c|c|c|c|c|c|}
\hline & A & $\mathrm{B}$ & $\mathrm{c}$ & $\mathrm{D}$ & $\mathrm{E}$ & $\mathrm{F}$ & G & $\mathrm{H}$ & $\mathrm{I}$ & $\mathrm{J}$ & $\mathrm{K}$ & $\mathrm{L}$ & $\mathrm{M}$ \\
\hline 1 & SAMPLE_NUM_ASSIGNED_TO & SAMPLER & MEDIA & BIOTA_MEDIA & TAG_ID & SAMP_NUM & SAMP_DATE_TIME & SAMP_DATE_TIME_ON & CON_ID & CON_SHORT_NAME & METHOD_NAME & LAB_CODE & \begin{tabular}{|l|} 
VALUE_RPTD \\
\end{tabular} \\
\hline 834 & PDMS & & SW & & & E003689 & 20-May-74 & & $10045-97-3$ & CS-137 & GAMMA GS & USTEST & 8.13 \\
\hline 835 & PDMS & & sW & & & E002187 & 20-May-74 & & $10045-97-3$ & CS-137 & GAMMA_GS & USTEST & 10.2 \\
\hline 836 & PDMS & & sW & & & E004610 & 21-May-74 & & $10098-97-2$ & SR-90 & SRISO_SEP_PRECIP_GPC & USTEST & 0.356 \\
\hline 837 & PDMS & & sW & & & E004610 & 21-May-74 & & $10045-97-3$ & CS-137 & GAMMA_GS & USTEST & 5.15 \\
\hline 838 & PDMS & & sW & & & E009421 & 21-May-74 & & $10098-97-2$ & SR-90 & SRISO_SEP_PRECIP_GPC & USTEST & 0.207 \\
\hline 839 & PDMS & & sW & & & E014695 & 21-May-74 & & $10045-97-3$ & CS-137 & GAMMA_GS & USTEST & 0.0256 \\
\hline 840 & PDMS & & SW & & & E009428 & 28-May-74 & & $10045-97-3$ & CS-137 & GAMMA_GS & USTEST & 3.95 \\
\hline 841 & PDMS & & SW & & & E003690 & 28-May-74 & & $10045-97-3$ & CS-137 & GAMMA_GS & USTEST & -0.214 \\
\hline 842 & PDMS & & sW & & & E002188 & 28-May-74 & & $10045-97-3$ & CS-137 & GAMMA GS & USTEST & -0.11 \\
\hline 843 & PDMS & & SW & & & E026540 & 31-May-74 & & $10098-97-2$ & SR-90 & SRISO_S̄EP_PRECIP_GPC & USTEST & 7.23 \\
\hline 844 & PDMS & & sW & & & E003691 & 03-Jun-74 & & $10045-97-3$ & CS-137 & GAMMA_GS & USTEST & 5.01 \\
\hline 845 & PDMS & & SW & & & E002189 & 03-Jun-74 & & $10045-97-3$ & CS-137 & GAMMA_GS & USTEST & 6.35 \\
\hline 846 & PDMS & & SW & & & E009435 & 04-Jun-74 & & $10045-97-3$ & CS-137 & GAMMA_GS & USTEST & -0.0869 \\
\hline 847 & PDMS & & SW & & & E028029 & 07-Jun-74 & & $10045-97-3$ & CS-137 & GAMMA GS & USTEST & 5.28 \\
\hline 848 & PDMS & & SW & & & E028029 & 07-Jun-74 & & $10098-97-2$ & SR-90 & SRISO_SEP_PRECIP_GPC & USTEST & 0.266 \\
\hline 849 & PDMS & & SW & & & E003692 & 10-Jun-74 & & $10045-97-3$ & CS-137 & GAMMA_GS & USTEST & -0.28 \\
\hline 850 & PDMS & & sW & & & E002190 & 10-Jun-74 & & $10045-97-3$ & CS-137 & GAMMA_GS & USTEST & -0.559 \\
\hline 851 & PDMS & & sW & & & E009442 & 11-Jun-74 & & $10045-97-3$ & CS-137 & GAMMA_GS & USTEST & -0.112 \\
\hline 852 & PDMS & & SW & & & E034555 & 11-Jun-74 & & $10098-97-2$ & SR-90 & SRISO_SEEP_PRECIP_GPC & USTEST & 10.2 \\
\hline 853 & PDMS & & SW & & & E009355 & 18-Jun-74 & & $10045-97-3$ & CS-137 & GAMMĀ_GS & USTEST & 7.85 \\
\hline 854 & PDMS & & SW & & & E004611 & 24-Jun-74 & & $10045-97-3$ & CS-137 & GAMMA_GS & USTEST & -0.124 \\
\hline 855 & PDMS & & SW & & & E004611 & 24-Jun-74 & & $10098-97-2$ & SR-90 & SRISO_SEP_PRECIP_GPC & USTEST & 0.198 \\
\hline 856 & PDMS & & SW & & & E016123 & 24-Jun-74 & & $10045-97-3$ & CS-137 & GAMMA_GS & USTEST & 0.0089 \\
\hline 857 & PDMS & & sW & & & E003618 & 24-Jun-74 & & $10045-97-3$ & CS-137 & GAMMA_GS & USTEST & 6.84 \\
\hline 858 & PDMS & & SW & & & E002191 & 24-Jun-74 & & $10098-97-2$ & SR-90 & SRISO_SEEP_PRECIP_GPC & USTEST & 0.24 \\
\hline 859 & PDMS & & SW & & & E002191 & 24-Jun-74 & & $10045-97-3$ & CS-137 & GAMMA_GS & USTEST & -0.904 \\
\hline 860 & PDMS & & SW & & & E009362 & 25-Jun-74 & & $10028-17-8$ & TRITIUM & TRITIUM_DIST_LSC & USTEST & 213 \\
\hline 861 & PDMS & & SW & & & E009362 & 25-Jun-74 & & $10098-97-2$ & SR-90 & SRISO_SEP_PRECIP_GPC & USTEST & 0.302 \\
\hline 862 & PDMS & & SW & & & E009362 & 25-Jun-74 & & $10045-97-3$ & CS-137 & GAMMĀGS & USTEST & -0.573 \\
\hline 863 & PDMS & & SW & & & E014696 & 25-Jun-74 & & $10045-97-3$ & CS-137 & GAMMA_GS & USTEST & -0.105 \\
\hline 864 & PDMS & & sW & & & E041051 & 27-Jun-74 & & $10028-17-8$ & TRITIUM & TRITIUM_DIST_LSC & USTEST & 431 \\
\hline 865 & PDMS & & SW & & & E003619 & 01-Jul-74 & & $10045-97-3$ & CS-137 & GAMMA_GS & USTEST & 8.14 \\
\hline 866 & PDMS & & SW & & & E002192 & 01-Jul-74 & & $10045-97-3$ & CS-137 & GAMMA_GS & USTEST & 9.11 \\
\hline 867 & PDMS & & SW & & & E026206 & 01-Jul-74 & & $10045-97-3$ & CS-137 & GAMMA_GS & USTEST & -1.19 \\
\hline 868 & PDMS & & SW & & & E021379 & 08-Jul-74 & & $10045-97-3$ & CS-137 & GAMMA_GS & USTEST & 0.0516 \\
\hline 869 & PDMS & & sW & & & E009376 & 09-Jul-74 & & $10045-97-3$ & CS-137 & GAMMA_GS & USTEST & 10.2 \\
\hline 870 & PDMS & & SW & & & E032721 & 09-Jul-74 & & $10098-97-2$ & SR-90 & SRISO_SEP_PRECIP_GPC & USTEST & 76.4 \\
\hline 871 & PDMS & & SW & & & E028030 & 12-Jul-74 & & $10045-97-3$ & CS-137 & GAMMA_GS & USTEST & -0.0296 \\
\hline 872 & PDMS & & SW & & & E028030 & 12-Jul-74 & & $10028-17-8$ & TRITIUM & TRITIUM_DIST_LSC & USTEST & 1120 \\
\hline 873 & PDMS & & SW & & & E028030 & 12-Jul-74 & & $10098-97-2$ & SR-90 & SRISO_SEPP_PRECIP_GPC & USTEST & 0.194 \\
\hline 874 & PDMS & & SW & & & E014939 & 12-Jul-74 & & $10045-97-3$ & CS-137 & GAMMĀ_GS & USTEST & -0.713 \\
\hline 875 & PDMS & & SW & & & E016397 & 12-Jul-74 & & $10098-97-2$ & SR-90 & SRISO_S̄EP_PRECIP_GPC & USTEST & 1.68 \\
\hline 876 & PDMS & & SW & & & E016397 & 12-Jul-74 & & $10045-97-3$ & CS-137 & GAMMĀGS & USTEST & 6.67 \\
\hline 877 & PDMS & & sW & & & E022418 & 12-Jul-74 & & $10045-97-3$ & CS-137 & GAMMA_GS & USTEST & 14.9 \\
\hline 878 & PDMS & & SW & & & E022418 & 12-Jul-74 & & $10098-97-2$ & SR-90 & SRISO_SEEP_PRECIP_GPC & USTEST & 1.14 \\
\hline 879 & PDMS & & SW & & & E003553 & 12-Jul-74 & & $10045-97-3$ & CS-137 & GAMMĀ_GS & USTEST & -1.06 \\
\hline 880 & PDMS & & SW & & & E027638 & 12-Jul-74 & & $10045-97-3$ & CS-137 & GAMMA_GS & USTEST & 11.2 \\
\hline 881 & PDMS & & SW & & & E014266 & 12-Jul-74 & & $10045-97-3$ & CS-137 & GAMMA_GS & USTEST & -0.166 \\
\hline 882 & PDMS & & SW & & & E014266 & 12-Jul-74 & & $10098-97-2$ & SR-90 & SRISO_SEEP_PRECIP_GPC & USTEST & 9.1 \\
\hline 883 & PDMS & & sW & & & E003620 & 15-Jul-74 & & $10045-97-3$ & CS-137 & GAMMĀGS & USTEST & -0.444 \\
\hline 884 & PDMS & & sW & & & E021380 & 15-Jul-74 & & $10045-97-3$ & CS-137 & GAMMA_GS & USTEST & -0.0722 \\
\hline 885 & PDMS & & SW & & & E009383 & 16-Jul-74 & & $10045-97-3$ & CS-137 & GAMMA_GS & USTEST & 0.051 \\
\hline
\end{tabular}




\begin{tabular}{|c|c|c|c|c|c|c|c|c|c|c|c|c|c|}
\hline & $\bar{A}$ & $\mathrm{~B}$ & $\bar{C}$ & $\mathrm{D}$ & $\mathrm{E}$ & $\mathrm{F}$ & $\bar{G}$ & $\mathrm{H}$ & 1 & $\mathrm{~J}$ & $\bar{K}$ & $\bar{L}$ & $\mathrm{M}$ \\
\hline 1 & SAMPLE_NUM_ASSIGNED_TO & SAMPLER & MEDIA & BIOTA_MEDIA & TAG_ID & SAMP_NUM & SAMP_DATE_TIME & SAMP_DATE_TIME_ON & CON_ID & CON_SHORT_NAME & METHOD_NAME & LAB_CODE & VALUE_RPTD \\
\hline 886 & PDMS & & SW & & & E010868 & 22-Jul-74 & & $10045-97-3$ & CS-137 & GAMMA_GS & USTEST & 2.48 \\
\hline 887 & PDMS & & SW & & & E002193 & 22-Jul-74 & & $10098-97-2$ & SR-90 & SRISO_S̄EP_PRECIP_GPC & USTEST & 0.396 \\
\hline 888 & PDMS & & SW & & & E002193 & 22-Jul-74 & & $10045-97-3$ & CS-137 & GAMMA_GS & USTEST & -0.433 \\
\hline 889 & PDMS & & SW & & & E009390 & 23-Jul-74 & & $10045-97-3$ & CS-137 & GAMMA_GS & USTEST & -0.291 \\
\hline 890 & PDMS & & SW & & & E004612 & 29-Jul-74 & & $10098-97-2$ & SR-90 & SRISO_SEEP_PRECIP_GPC & USTEST & 0.288 \\
\hline 891 & PDMS & & SW & & & E004612 & 29-Jul-74 & & $10045-97-3$ & CS-137 & GAMMA_GS & USTEST & 4.87 \\
\hline 892 & PDMS & & SW & & & E010869 & 29-Jul-74 & & $10045-97-3$ & CS-137 & GAMMA_GS & USTEST & 1.72 \\
\hline 893 & PDMS & & SW & & & E002194 & 29-Jul-74 & & $10045-97-3$ & CS-137 & GAMMA_GS & USTEST & 0.437 \\
\hline 894 & PDMS & & SW & & & E026133 & 29-Jul-74 & & $10045-97-3$ & CS-137 & GAMMA_GS & USTEST & -2.24 \\
\hline 895 & PDMS & & SW & & & E009303 & 30-Jul-74 & & $10028-17-8$ & TRITIUM & TRITIUM_DIST_LSC & USTEST & 253 \\
\hline 896 & PDMS & & SW & & & E009303 & 30-Jul-74 & & $10098-97-2$ & SR-90 & SRISO_SEP_PRECIP_GPC & USTEST & 0.374 \\
\hline 897 & PDMS & & SW & & & E009303 & 30-Jul-74 & & $10045-97-3$ & CS-137 & GAMMA_GS & USTEST & -0.174 \\
\hline 898 & PDMS & & SW & & & E014697 & 30-Jul-74 & & $10045-97-3$ & CS-137 & GAMMA_GS & USTEST & 3.68 \\
\hline 899 & PDMS & & SW & & & E035039 & 30-Jul-74 & & $10028-17-8$ & TRITIUM & TRITIUM_DIST_LSC & USTEST & 0 \\
\hline 900 & PDMS & & SW & & & E026541 & 02-Aug-74 & & $10098-97-2$ & SR-90 & SRISO_SEP_PRECIP_GPC & USTEST & 10.7 \\
\hline 901 & PDMS & & SW & & & E003621 & 05-Aug-74 & & $10045-97-3$ & CS-137 & GAMMA_GS & USTEST & 6.39 \\
\hline 902 & PDMS & & SW & & & E002195 & 05-Aug-74 & & $10045-97-3$ & CS-137 & GAMMA_GS & USTEST & -0.112 \\
\hline 903 & PDMS & & SW & & & E009310 & 06-Aug-74 & & $10045-97-3$ & CS-137 & GAMMA_GS & USTEST & 5.94 \\
\hline 904 & PDMS & & SW & & & E032722 & 06-Aug-74 & & $10098-97-2$ & SR-90 & SRISO_SEP_PRECIP_GPC & USTEST & 269 \\
\hline 905 & PDMS & & SW & & & E028031 & 08-Aug-74 & & $10045-97-3$ & CS-137 & GAMMA_GS & USTEST & 2.1 \\
\hline 906 & PDMS & & SW & & & E028031 & 08-Aug-74 & & $10028-17-8$ & TRITIUM & TRITIUM_DIST_LSC & USTEST & 504 \\
\hline 907 & PDMS & & SW & & & E028031 & 08-Aug-74 & & $10098-97-2$ & SR-90 & SRISO_SEPP_PRECIP_GPC & USTEST & 3.54 \\
\hline 908 & PDMS & & SW & & & E003622 & 12-Aug-74 & & $10045-97-3$ & CS-137 & GAMMA_GS & USTEST & 1.75 \\
\hline 909 & PDMS & & SW & & & E002196 & 12-Aug-74 & & $10045-97-3$ & CS-137 & GAMMA_GS & USTEST & 8.84 \\
\hline 910 & PDMS & & SW & & & E009317 & 13-Aug-74 & & $10045-97-3$ & CS-137 & GAMMA_GS & USTEST & 0.0511 \\
\hline 911 & PDMS & & SW & & & E003623 & 19-Aug-74 & & $10045-97-3$ & CS-137 & GAMMA_GS & USTEST & -0.545 \\
\hline 912 & PDMS & & SW & & & E002197 & 19-Aug-74 & & $10045-97-3$ & CS-137 & GAMMA_GS & USTEST & -0.401 \\
\hline 913 & PDMS & & SW & & & E009324 & 20-Aug-74 & & $10045-97-3$ & CS-137 & GAMMA_GS & USTEST & 1.26 \\
\hline 914 & PDMS & & SW & & & E004232 & 23-Aug-74 & & $10098-97-2$ & SR-90 & SRISO_SEP_PRECIP_GPC & USTEST & 0 \\
\hline 915 & PDMS & & SW & & & E004613 & 26-Aug-74 & & $10098-97-2$ & SR-90 & SRISO_SEP_PRECIP_GPC & USTEST & 0.883 \\
\hline 916 & PDMS & & SW & & & E004613 & 26-Aug-74 & & $10045-97-3$ & CS-137 & GAMMA_GS & USTEST & -0.207 \\
\hline 917 & PDMS & & SW & & & E003624 & 26-Aug-74 & & $10045-97-3$ & CS-137 & GAMMA_GS & USTEST & 1.88 \\
\hline 918 & PDMS & & SW & & & E002198 & 26-Aug-74 & & $10045-97-3$ & CS-137 & GAMMA_GS & USTEST & -0.0976 \\
\hline 919 & PDMS & & SW & & & E026137 & 26-Aug-74 & & $10045-97-3$ & CS-137 & GAMMA_GS & USTEST & 10 \\
\hline 920 & PDMS & & SW & & & E009331 & 27-Aug-74 & & $10028-17-8$ & TRITIUM & TRITIUM_DIST_LSC & USTEST & 232 \\
\hline 921 & PDMS & & SW & & & E009331 & 27-Aug-74 & & $10045-97-3$ & CS-137 & GAMMA_GS & USTEST & 0.0511 \\
\hline 922 & PDMS & & SW & & & E009331 & 27-Aug-74 & & $10098-97-2$ & SR-90 & SRISO_SEP_PRECIP_GPC & USTEST & 0.36 \\
\hline 923 & PDMS & & SW & & & E014698 & 27-Aug-74 & & $10045-97-3$ & CS-137 & GAMMA_GS & USTEST & -0.27 \\
\hline 924 & PDMS & & SW & & & E034959 & 27-Aug-74 & & $10028-17-8$ & TRITIUM & TRITIUM_DIST_LSC & USTEST & 712 \\
\hline 925 & PDMS & & SW & & & E026542 & 30-Aug-74 & & $10098-97-2$ & SR-90 & SRISO_SEP_PRECIP_GPC & USTEST & 11.4 \\
\hline 926 & PDMS & & SW & & & E009338 & 03-Sep-74 & & $10045-97-3$ & CS-137 & GAMMA_GS & USTEST & -0.11 \\
\hline 927 & PDMS & & SW & & & E003625 & 03-Sep-74 & & $10045-97-3$ & CS-137 & GAMMA_GS & USTEST & 0.708 \\
\hline 928 & PDMS & & SW & & & E002199 & 03-Sep-74 & & $10045-97-3$ & CS-137 & GAMMA_GS & USTEST & 0.17 \\
\hline 929 & PDMS & & SW & & & E032723 & 03-Sep-74 & & $10098-97-2$ & SR-90 & SRISO_SEP_PRECIP_GPC & USTEST & 946 \\
\hline 930 & PDMS & & SW & & & E003626 & 09-Sep-74 & & $10045-97-3$ & CS-137 & GAMMA_GS & USTEST & 0.61 \\
\hline 931 & PDMS & & SW & & & E002200 & 09-Sep-74 & & $10045-97-3$ & CS-137 & GAMMA_GS & USTEST & -0.0614 \\
\hline 932 & PDMS & & SW & & & E009345 & 10-Sep-74 & & $10045-97-3$ & CS-137 & GAMMA_GS & USTEST & -1.43 \\
\hline 933 & PDMS & & SW & & & E037682 & 12-Sep-74 & & $10045-97-3$ & CS-137 & GAMMA_GS & USTEST & 0.0258 \\
\hline 934 & PDMS & & SW & & & E037682 & 12-Sep-74 & & $10098-97-2$ & SR-90 & SRISO_SEP_PRECIP_GPC & USTEST & 5.02 \\
\hline 935 & PDMS & & SW & & & E003627 & 16-Sep-74 & & $10045-97-3$ & CS-137 & GAMMA_GS & USTEST & -0.155 \\
\hline 936 & PDMS & & SW & & & E002201 & 16-Sep-74 & & $10045-97-3$ & CS-137 & GAMMA_GS & USTEST & 2.85 \\
\hline 937 & PDMS & & SW & & & E009258 & 17-Sep-74 & & $10045-97-3$ & CS-137 & GAMMA_GS & USTEST & 0.748 \\
\hline
\end{tabular}




\begin{tabular}{|c|c|c|c|c|c|c|c|c|c|c|c|c|c|}
\hline & $\bar{A}$ & $\mathrm{~B}$ & $\bar{C}$ & $\mathrm{D}$ & $\mathrm{E}$ & $\mathrm{F}$ & $\bar{G}$ & $\mathrm{H}$ & 1 & $\mathrm{~J}$ & $\bar{K}$ & $\bar{L}$ & $\mathrm{M}$ \\
\hline 1 & SAMPLE_NUM_ASSIGNED_TO & \begin{tabular}{|l} 
SAMPLER \\
\end{tabular} & MEDIA & BIOTA_MEDIA & TAG_ID & SAMP_NUM & \begin{tabular}{|l|} 
SAMP_DATE_TIME \\
\end{tabular} & SAMP_DATE_TIME_ON & CON_ID & \begin{tabular}{|l} 
CON_SHORT_NAME \\
\end{tabular} & METHOD_NAME & LAB_CODE & VALUE_RPTD \\
\hline 938 & PDMS & & SW & & & E034961 & 17-Sep-74 & & $10028-17-8$ & TRITIUM & TRITIUM_DIST_LSC & USTEST & 490 \\
\hline 939 & PDMS & & SW & & & E003628 & 23-Sep-74 & & $10045-97-3$ & CS-137 & GAMMA_GS & USTEST & 0.0511 \\
\hline 940 & PDMS & & SW & & & E002202 & 23-Sep-74 & & $10098-97-2$ & SR-90 & SRISO_SEP_PRECIP_GPC & USTEST & 0.541 \\
\hline 941 & PDMS & & SW & & & E002202 & 23-Sep-74 & & $10045-97-3$ & CS-137 & GAMMA_GS & USTEST & -0.14 \\
\hline 942 & PDMS & & SW & & & E009265 & 24-Sep-74 & & $10028-17-8$ & TRITIUM & TRITIUM_DIST_LSC & USTEST & 353 \\
\hline 943 & PDMS & & SW & & & E009265 & 24-Sep-74 & & $10045-97-3$ & CS-137 & GAMMA_GS & USTEST & 0.0491 \\
\hline 944 & PDMS & & SW & & & E009265 & 24-Sep-74 & & $10098-97-2$ & SR-90 & SRISO_SEEP_PRECIP_GPC & USTEST & 0.338 \\
\hline 945 & PDMS & & SW & & & E014684 & 24-Sep-74 & & $10045-97-3$ & CS-137 & GAMMA_GS & USTEST & -0.0154 \\
\hline 946 & PDMS & & SW & & & E004614 & 30-Sep-74 & & $10045-97-3$ & CS-137 & GAMMA_GS & USTEST & 0.85 \\
\hline 947 & PDMS & & SW & & & E004614 & 30-Sep-74 & & $10098-97-2$ & SR-90 & SRISO_SEP_PRECIP_GPC & USTEST & 2.54 \\
\hline 948 & PDMS & & SW & & & E004233 & 30-Sep-74 & & $10045-97-3$ & CS-137 & GAMMA_GS & USTEST & 6.67 \\
\hline 949 & PDMS & & SW & & & E003629 & 30-Sep-74 & & $10045-97-3$ & CS-137 & GAMMA_GS & USTEST & 3.91 \\
\hline 950 & PDMS & & SW & & & E002203 & $30-S e p-74$ & & $10045-97-3$ & CS-137 & GAMMA_GS & USTEST & -0.376 \\
\hline 951 & PDMS & & SW & & & E026142 & 30-Sep-74 & & $10045-97-3$ & CS-137 & GAMMA_GS & USTEST & -0.478 \\
\hline 952 & PDMS & & SW & & & E009272 & 01-Oct-74 & & $10045-97-3$ & CS-137 & GAMMA_GS & USTEST & 0.0511 \\
\hline 953 & PDMS & & SW & & & E032724 & 01-Oct-74 & & $10098-97-2$ & SR-90 & SRISO_SEP_PRECIP_GPC & USTEST & 88.6 \\
\hline 954 & PDMS & & SW & & & E002204 & $07-$ Oct-74 & & $10045-97-3$ & CS-137 & GAMMA_GS & USTEST & 0.0511 \\
\hline 955 & PDMS & & SW & & & E009279 & $08-O c t-74$ & & $10045-97-3$ & CS-137 & GAMMA_GS & USTEST & 7.54 \\
\hline 956 & PDMS & & SW & & & E064998 & 10-Oct-74 & & $10028-17-8$ & TRITIUM & TRITIUM_DIST_LSC & USTEST & 12300 \\
\hline 957 & PDMS & & SW & & & E028032 & 10-Oct-74 & & $10045-97-3$ & CS-137 & GAMMA_GS & USTEST & 0.0212 \\
\hline 958 & PDMS & & SW & & & E028032 & 10-Oct-74 & & $10028-17-8$ & TRITIUM & TRITIUM_DIST_LSC & USTEST & 288 \\
\hline 959 & PDMS & & SW & & & E028032 & 10-Oct-74 & & $10098-97-2$ & SR-90 & SRISO_SEPP_PRECIP_GPC & USTEST & 0.239 \\
\hline 960 & PDMS & & SW & & & E014940 & 11-Oct-74 & & $10045-97-3$ & CS-137 & GAMMA_GS & USTEST & 0.307 \\
\hline 961 & PDMS & & SW & & & E016398 & 11-Oct-74 & & $10098-97-2$ & SR-90 & SRISO_SEP_PRECIP_GPC & USTEST & 3.18 \\
\hline 962 & PDMS & & SW & & & E016398 & 11-Oct-74 & & $10045-97-3$ & CS-137 & GAMMA_GS & USTEST & 0.371 \\
\hline 963 & PDMS & & SW & & & E022419 & 11-Oct-74 & & $10045-97-3$ & CS-137 & GAMMA_GS & USTEST & 43.2 \\
\hline 964 & PDMS & & SW & & & E022419 & 11-Oct-74 & & $10098-97-2$ & SR-90 & SRISO_SEP_PRECIP_GPC & USTEST & 6.35 \\
\hline 965 & PDMS & & SW & & & E003554 & 11-Oct-74 & & $10045-97-3$ & CS-137 & GAMMA_GS & USTEST & 0.0511 \\
\hline 966 & PDMS & & SW & & & E027639 & 11-Oct-74 & & $10045-97-3$ & CS-137 & GAMMA_GS & USTEST & 10 \\
\hline 967 & PDMS & & SW & & & E014267 & 11-Oct-74 & & $10045-97-3$ & CS-137 & GAMMA_GS & USTEST & 1.01 \\
\hline 968 & PDMS & & SW & & & E003630 & 14-Oct-74 & & $10045-97-3$ & CS-137 & GAMMA_GS & USTEST & 0.051 \\
\hline 969 & PDMS & & SW & & & E002205 & 14-Oct-74 & & $10045-97-3$ & CS-137 & GAMMA_GS & USTEST & 4.79 \\
\hline 970 & PDMS & & SW & & & E009286 & 15-Oct-74 & & $10045-97-3$ & CS-137 & GAMMA_GS & USTEST & -0.422 \\
\hline 971 & PDMS & & SW & & & E010861 & 21-Oct-74 & & $10045-97-3$ & CS-137 & GAMMA_GS & USTEST & 0.0536 \\
\hline 972 & PDMS & & SW & & & E002206 & 21-Oct-74 & & $10045-97-3$ & CS-137 & GAMMA_GS & USTEST & -0.385 \\
\hline 973 & PDMS & & SW & & & E009293 & 22-Oct-74 & & $10045-97-3$ & CS-137 & GAMMA_GS & USTEST & 9.38 \\
\hline 974 & PDMS & & SW & & & E034966 & 22-Oct-74 & & 10028-17-8 & TRITIUM & TRITIUM_DIST_LSC & USTEST & 149 \\
\hline 975 & PDMS & & SW & & & E016124 & 28-Oct-74 & & $10045-97-3$ & CS-137 & GAMMA_GS & USTEST & 17 \\
\hline 976 & PDMS & & SW & & & E016459 & 28-Oct-74 & & $10045-97-3$ & CS-137 & GAMMA_GS & USTEST & 7.22 \\
\hline 977 & PDMS & & SW & & & E016459 & 28-Oct-74 & & $10028-17-8$ & TRITIUM & TRITIUM_DIST_LSC & USTEST & 441 \\
\hline 978 & PDMS & & SW & & & E010862 & 28-Oct-74 & & $10045-97-3$ & CS-137 & GAMMA_GS & USTEST & -0.179 \\
\hline 979 & PDMS & & SW & & & E002207 & 28-Oct-74 & & $10098-97-2$ & SR-90 & SRISO_SEP_PRECIP_GPC & USTEST & 0.285 \\
\hline 980 & PDMS & & SW & & & E002207 & 28-Oct-74 & & $10045-97-3$ & CS-137 & GAMMA_GS & USTEST & 5.67 \\
\hline 981 & PDMS & & SW & & & E069707 & 29-Oct-74 & & $10098-97-2$ & SR-90 & SRISO_SEP_PRECIP_GPC & USTEST & 0.167 \\
\hline 982 & PDMS & & SW & & & E009300 & 29-Oct-74 & & $10028-17-8$ & TRITIUM & TRITIUM_DIST_LSC & USTEST & 209 \\
\hline 983 & PDMS & & SW & & & E009300 & 29-Oct-74 & & $10045-97-3$ & CS-137 & GAMMA_GS & USTEST & 0.0536 \\
\hline 984 & PDMS & & SW & & & E009300 & 29-Oct-74 & & $10098-97-2$ & SR-90 & SRISO_SEP_PRECIP_GPC & USTEST & 0.329 \\
\hline 985 & PDMS & & SW & & & E032725 & 29-Oct-74 & & $10098-97-2$ & SR-90 & SRISO_SEP_PRECIP_GPC & USTEST & 126 \\
\hline 986 & PDMS & & SW & & & E026146 & 29-Oct-74 & & $10045-97-3$ & CS-137 & GAMMA_GS & USTEST & -0.232 \\
\hline 987 & PDMS & & SW & & & E003631 & 04-Nov-74 & & $10045-97-3$ & CS-137 & GAMMA_GS & USTEST & -0.0822 \\
\hline 988 & PDMS & & SW & & & E009213 & 05-Nov-74 & & $10045-97-3$ & CS-137 & GAMMA_GS & USTEST & -0.51 \\
\hline 989 & PDMS & & SW & & & E003632 & 11-Nov-74 & & $10045-97-3$ & CS-137 & GAMMA_GS & USTEST & 7.72 \\
\hline
\end{tabular}




\begin{tabular}{|c|c|c|c|c|c|c|c|c|c|c|c|c|c|}
\hline & A & $\mathrm{B}$ & $\mathrm{c}$ & $\mathrm{D}$ & $\mathrm{E}$ & $\mathrm{F}$ & G & $\mathrm{H}$ & $\mathrm{I}$ & $\mathrm{J}$ & $\mathrm{K}$ & $\mathrm{L}$ & $\mathrm{M}$ \\
\hline 1 & SAMPLE_NUM_ASSIGNED_TO & SAMPLER & MEDIA & BIOTA_MEDIA & TAG_ID & SAMP_NUM & SAMP_DATE_TIME & SAMP_DATE_TIME_ON & CON_ID & CON_SHORT_NAME & METHOD_NAME & LAB_CODE & \begin{tabular}{|l|} 
VALUE_RPTD \\
\end{tabular} \\
\hline 990 & PDMS & & SW & & & E023893 & 14-Nov-74 & & $10045-97-3$ & CS-137 & GAMMA_GS & USTEST & -0.778 \\
\hline \begin{tabular}{|l|}
991 \\
\end{tabular} & PDMS & & sW & & & E016129 & 14-Nov-74 & & $10045-97-3$ & CS-137 & GAMMA_GS & USTEST & 6.49 \\
\hline \begin{tabular}{|l|}
992 \\
\end{tabular} & PDMS & & sW & & & E016129 & 14-Nov-74 & & $10028-17-8$ & TRITIUM & TRITIUM_DIST_LSC & USTEST & 186 \\
\hline \begin{tabular}{|l|}
993 \\
\end{tabular} & PDMS & & sW & & & E016129 & 14-Nov-74 & & $10098-97-2$ & SR-90 & SRISO_SEP_PRECIP_GPC & USTEST & 0.423 \\
\hline \begin{tabular}{|l|l|}
994 \\
\end{tabular} & PDMS & & sW & & & E010863 & 18-Nov-74 & & $10045-97-3$ & CS-137 & GAMMA_GS & USTEST & -0.285 \\
\hline \begin{tabular}{|l|}
995 \\
\end{tabular} & PDMS & & sW & & & E004615 & 25-Nov-74 & & $10098-97-2$ & SR-90 & SRISO_SEP_PRECIP_GPC & USTEST & 0.0897 \\
\hline 996 & PDMS & & SW & & & E004615 & 25-Nov-74 & & $10045-97-3$ & CS-137 & GAMMA_GS & USTEST & 0.00234 \\
\hline 997 & PDMS & & SW & & & E016125 & 25-Nov-74 & & $10045-97-3$ & CS-137 & GAMMA_GS & USTEST & 8.12 \\
\hline \begin{tabular}{|l|}
998 \\
\end{tabular} & PDMS & & sW & & & E016460 & 25-Nov-74 & & $10045-97-3$ & CS-137 & GAMMA GS & USTEST & 6.06 \\
\hline \begin{tabular}{|l|}
999 \\
\end{tabular} & PDMS & & SW & & & E016460 & 25-Nov-74 & & 10028-17-8 & TRITIUM & TRITIUM_DIST_LSC & USTEST & 316 \\
\hline 1000 & PDMS & & SW & & & E003633 & 25-Nov-74 & & $10045-97-3$ & CS-137 & GAMMA_GS & USTEST & 0.0511 \\
\hline 1001 & PDMS & & SW & & & E002208 & 25-Nov-74 & & $10098-97-2$ & SR-90 & SRISO_SEPP_PRECIP_GPC & USTEST & 0.345 \\
\hline 1002 & PDMS & & SW & & & E002208 & 25-Nov-74 & & $10045-97-3$ & CS-137 & GAMMA_GS & USTEST & 2.19 \\
\hline 1003 & PDMS & & SW & & & E026150 & 25-Nov-74 & & $10045-97-3$ & CS-137 & GAMMA GS & USTEST & -0.344 \\
\hline 1004 & PDMS & & SW & & & E009234 & 26-Nov-74 & & $10028-17-8$ & TRITIUM & TRITIUM_DIST_LSC & USTEST & 306 \\
\hline 1005 & PDMS & & SW & & & E009234 & 26-Nov-74 & & $10045-97-3$ & CS-137 & GAMMA_GS & USTEST & 0.0296 \\
\hline 1006 & PDMS & & sW & & & E009234 & 26-Nov-74 & & $10098-97-2$ & SR-90 & SRISO_SEP_PRECIP_GPC & USTEST & 0.297 \\
\hline \begin{tabular}{|c|}
1007 \\
\end{tabular} & PDMS & & sW & & & E014685 & 26-Nov-74 & & $10045-97-3$ & CS-137 & GAMMA_GS & USTEST & 0.003 \\
\hline 1008 & PDMS & & SW & & & E034970 & 26-Nov-74 & & $10028-17-8$ & TRITIUM & TRITIUM_DIST_LSC & USTEST & 358 \\
\hline 1009 & PDMS & & SW & & & E032726 & 26-Nov-74 & & $10098-97-2$ & SR-90 & SRISO_SEP_PRECIP_GPC & USTEST & 423 \\
\hline 1010 & PDMS & & SW & & & E003634 & 02-Dec-74 & & $10045-97-3$ & CS-137 & GAMMA_GS & USTEST & -0.298 \\
\hline 1011 & PDMS & & SW & & & E009241 & 03-Dec-74 & & $10045-97-3$ & CS-137 & GAMMA_GS & USTEST & -0.168 \\
\hline 1012 & PDMS & & SW & & & E004234 & 05-Dec-74 & & $10045-97-3$ & CS-137 & GAMMA_GS & USTEST & 10.3 \\
\hline 1013 & PDMS & & SW & & & E016130 & 05-Dec-74 & & $10045-97-3$ & CS-137 & GAMMA_GS & USTEST & 0.0255 \\
\hline 1014 & PDMS & & SW & & & E016130 & 05-Dec-74 & & $10098-97-2$ & SR-90 & SRISO_SEP_PRECIP_GPC & USTEST & 0.016 \\
\hline 1015 & PDMS & & SW & & & E003635 & 09-Dec-74 & & $10045-97-3$ & CS-137 & GAMMA_GS & USTEST & -0.322 \\
\hline 1016 & PDMS & & SW & & & E002209 & 09-Dec-74 & & $10045-97-3$ & CS-137 & GAMMA_GS & USTEST & 7.36 \\
\hline $\mid 1017$ & PDMS & & SW & & & E009248 & 10-Dec-74 & & $10045-97-3$ & CS-137 & GAMMA_GS & USTEST & 4.29 \\
\hline 1018 & PDMS & & SW & & & E043984 & 15-Dec-74 & & $10028-17-8$ & TRITIUM & TRITIUM_DIST_LSC & USTEST & 291 \\
\hline 1019 & PDMS & & SW & & & E003636 & 16-Dec-74 & & $10045-97-3$ & CS-137 & GAMMA_GS & USTEST & 3.39 \\
\hline 1020 & PDMS & & sW & & & E009255 & 17-Dec-74 & & $10045-97-3$ & CS-137 & GAMMA_GS & USTEST & 5.17 \\
\hline 1021 & PDMS & & SW & & & E009167 & 23-Dec-74 & & $10045-97-3$ & CS-137 & GAMMA_GS & USTEST & 6.81 \\
\hline 1022 & PDMS & & SW & & & E003637 & 23-Dec-74 & & $10045-97-3$ & CS-137 & GAMMA_GS & USTEST & 15.8 \\
\hline 1023 & PDMS & & SW & & & E032727 & 23-Dec-74 & & $10098-97-2$ & SR-90 & SRISO_SEEP_PRECIP_GPC & USTEST & 375 \\
\hline 1024 & PDMS & & SW & & & E004616 & 30-Dec-74 & & $10098-97-2$ & SR-90 & SRISO_SEP_PRECIP_GPC & USTEST & 0.131 \\
\hline 1025 & PDMS & & sW & & & E004616 & 30-Dec-74 & & $10045-97-3$ & CS-137 & GAMMA_GS & USTEST & -0.0318 \\
\hline 1026 & PDMS & & SW & & & E016461 & 30-Dec-74 & & $10045-97-3$ & CS-137 & GAMMA_GS & USTEST & -0.336 \\
\hline 1027 & PDMS & & SW & & & E003638 & 30-Dec-74 & & $10045-97-3$ & CS-137 & GAMMA_GS & USTEST & -0.213 \\
\hline 1028 & PDMS & & SW & & & E002210 & 30-Dec-74 & & $10098-97-2$ & SR-90 & SRISO_SEPP_PRECIP_GPC & USTEST & 0.375 \\
\hline 1029 & PDMS & & SW & & & E002210 & 30-Dec-74 & & $10045-97-3$ & CS-137 & GAMMĀGS & USTEST & -0.676 \\
\hline 1030 & PDMS & & SW & & & E026155 & 30-Dec-74 & & $10045-97-3$ & CS-137 & GAMMA_GS & USTEST & -0.751 \\
\hline 1031 & PDMS & & SW & & & E009175 & 31-Dec-74 & & $10028-17-8$ & TRITIUM & TRITIUM_DIST_LSC & USTEST & 864 \\
\hline 1032 & PDMS & & SW & & & E009175 & 31-Dec-74 & & $10045-97-3$ & CS-137 & GAMMA_GS & USTEST & 0.0468 \\
\hline 1033 & PDMS & & SW & & & E009175 & 31-Dec-74 & & $10098-97-2$ & SR-90 & SRISO_SEP_PRECIP_GPC & USTEST & 0.167 \\
\hline 1034 & PDMS & & SW & & & E014686 & 31-Dec-74 & & $10045-97-3$ & CS-137 & GAMMA_GS & USTEST & 0.198 \\
\hline 1035 & PDMS & & SW & & & E034973 & 31-Dec-74 & & $10028-17-8$ & TRITIUM & TRITIUM_DIST_LSC & USTEST & 620 \\
\hline 1036 & PDMS & & SW & & & E016126 & 06-Jan-75 & & $10045-97-3$ & CS-137 & GAMMA_GS & USTEST & -4.66 \\
\hline \begin{tabular}{|l|l|}
1037 \\
\end{tabular} & PDMS & & SW & & & E003639 & 06-Jan-75 & & $10045-97-3$ & CS-137 & GAMMA_GS & USTEST & 2.24 \\
\hline 1038 & PDMS & & SW & & & E009182 & 07-Jan-75 & & $10045-97-3$ & CS-137 & GAMMA_GS & USTEST & 11.5 \\
\hline 1039 & PDMS & & SW & & & E016131 & 09-Jan-75 & & $10045-97-3$ & CS-137 & GAMMA_GS & USTEST & -0.0118 \\
\hline 1040 & PDMS & & SW & & & E016131 & 09-Jan-75 & & 10028-17-8 & TRITIUM & TRITIUM_DIST_LSC & USTEST & 1250 \\
\hline 1041 & PDMS & & SW & & & E016131 & 09-Jan-75 & & $10098-97-2$ & SR-90 & SRISO_SEP_PRECIP_GPC & USTEST & 0.374 \\
\hline
\end{tabular}




\begin{tabular}{|c|c|c|c|c|c|c|c|c|c|c|c|c|c|}
\hline & $\bar{A}$ & $\mathrm{~B}$ & $\bar{C}$ & $\mathrm{D}$ & $\mathrm{E}$ & $\mathrm{F}$ & $\bar{G}$ & $\mathrm{H}$ & 1 & $\mathrm{~J}$ & $\bar{K}$ & $\bar{L}$ & $\mathrm{M}$ \\
\hline 1 & SAMPLE_NUM_ASSIGNED_TO & \begin{tabular}{|l} 
SAMPLER \\
\end{tabular} & MEDIA & BIOTA_MEDIA & TAG_ID & SAMP_NUM & SAMP_DATE_TIME & SAMP_DATE_TIME_ON & CON_ID & CON_SHORT_NAME & METHOD_NAME & LAB_CODE & VALUE_RPTD \\
\hline 1042 & PDMS & & SW & & & E012096 & 09-Jan-75 & & $10045-97-3$ & CS-137 & GAMMA_GS & USTEST & -0.358 \\
\hline 1043 & PDMS & & SW & & & E037679 & 10-Jan-75 & & $10045-97-3$ & CS-137 & GAMMA_GS & USTEST & 0.0465 \\
\hline 1044 & PDMS & & SW & & & E016399 & 10-Jan-75 & & $10098-97-2$ & SR-90 & SRISO_SEP_PRECIP_GPC & USTEST & 12.4 \\
\hline 1045 & PDMS & & SW & & & E016399 & 10-Jan-75 & & $10045-97-3$ & CS-137 & GAMMA_GS & USTEST & 0.0562 \\
\hline 1046 & PDMS & & SW & & & E027640 & 10-Jan-75 & & $10045-97-3$ & CS-137 & GAMMA_GS & USTEST & 41.8 \\
\hline 1047 & PDMS & & SW & & & E014268 & 10-Jan-75 & & $10045-97-3$ & CS-137 & GAMMA_GS & USTEST & 2.51 \\
\hline 1048 & PDMS & & SW & & & E014268 & 10-Jan-75 & & $10098-97-2$ & SR-90 & SRISO_SEEP_PRECIP_GPC & USTEST & 4.23 \\
\hline 1049 & PDMS & & SW & & & E003640 & 13-Jan-75 & & $10045-97-3$ & CS-137 & GAMMA_GS & USTEST & -0.227 \\
\hline 1050 & PDMS & & SW & & & E009189 & 14-Jan-75 & & $10045-97-3$ & CS-137 & GAMMA_GS & USTEST & -0.409 \\
\hline 1051 & PDMS & & SW & & & E003641 & 20-Jan-75 & & $10045-97-3$ & CS-137 & GAMMA_GS & USTEST & 0.84 \\
\hline 1052 & PDMS & & SW & & & E009196 & 21-Jan-75 & & $10045-97-3$ & CS-137 & GAMMA_GS & USTEST & 4.62 \\
\hline 1053 & PDMS & & SW & & & E032728 & 21-Jan-75 & & $10098-97-2$ & SR-90 & SRISO_SEP_PRECIP_GPC & USTEST & 128 \\
\hline 1054 & PDMS & & SW & & & E004617 & 27-Jan-75 & & $10098-97-2$ & SR-90 & SRISO_SEP_PRECIP_GPC & USTEST & 0.302 \\
\hline 1055 & PDMS & & SW & & & E004617 & 27-Jan-75 & & $10045-97-3$ & CS-137 & GAMMA_GS & USTEST & 0.418 \\
\hline 1056 & PDMS & & SW & & & E037115 & 27-Jan-75 & & $10028-17-8$ & TRITIUM & TRITIUM_DIST_LSC & USTEST & 855 \\
\hline 1057 & PDMS & & SW & & & E003642 & 27-Jan-75 & & $10045-97-3$ & CS-137 & GAMMA_GS & USTEST & -0.156 \\
\hline 1058 & PDMS & & SW & & & E002211 & 27-Jan-75 & & $10098-97-2$ & SR-90 & SRISO_SEP_PRECIP_GPC & USTEST & 0.27 \\
\hline 1059 & PDMS & & SW & & & E002211 & 27-Jan-75 & & $10045-97-3$ & CS-137 & GAMMA_GS & USTEST & -0.0622 \\
\hline 1060 & PDMS & & SW & & & E026159 & 27-Jan-75 & & $10045-97-3$ & CS-137 & GAMMA_GS & USTEST & -0.324 \\
\hline 1061 & PDMS & & SW & & & E009203 & 28-Jan-75 & & $10028-17-8$ & TRITIUM & TRITIUM_DIST_LSC & USTEST & 937 \\
\hline 1062 & PDMS & & SW & & & E009203 & 28-Jan-75 & & $10045-97-3$ & CS-137 & GAMMA_GS & USTEST & -0.196 \\
\hline 1063 & PDMS & & SW & & & E009203 & 28-Jan-75 & & $10098-97-2$ & SR-90 & SRISO_SEEP_PRECIP_GPC & USTEST & 0.365 \\
\hline 1064 & PDMS & & SW & & & E014687 & 28-Jan-75 & & $10045-97-3$ & CS-137 & GAMMA_GS & USTEST & 0.767 \\
\hline 1065 & PDMS & & SW & & & E034976 & 28-Jan-75 & & $10028-17-8$ & TRITIUM & TRITIUM_DIST_LSC & USTEST & 401 \\
\hline 1066 & PDMS & & SW & & & E010864 & 03-Feb-75 & & $10045-97-3$ & CS-137 & GAMMA_GS & USTEST & -5.31 \\
\hline 1067 & PDMS & & SW & & & E009116 & 04-Feb-75 & & $10045-97-3$ & CS-137 & GAMMA_GS & USTEST & 9.03 \\
\hline 1068 & PDMS & & SW & & & E003643 & $10-F e b-75$ & & $10045-97-3$ & CS-137 & GAMMA_GS & USTEST & 0.0511 \\
\hline 1069 & PDMS & & SW & & & E009123 & 11-Feb-75 & & $10045-97-3$ & CS-137 & GAMMA_GS & USTEST & 3.21 \\
\hline 1070 & PDMS & & SW & & & E016132 & 14-Feb-75 & & $10045-97-3$ & CS-137 & GAMMA_GS & USTEST & -0.0558 \\
\hline 1071 & PDMS & & SW & & & E016132 & 14-Feb-75 & & $10028-17-8$ & TRITIUM & TRITIUM_DIST_LSC & USTEST & 129 \\
\hline 1072 & PDMS & & SW & & & E016132 & 14-Feb-75 & & $10098-97-2$ & SR-90 & SRISO_SEP_PRECIP_GPC & USTEST & 0.27 \\
\hline 1073 & PDMS & & SW & & & E012097 & 14-Feb-75 & & $10045-97-3$ & CS-137 & GAMMA_GS & USTEST & 0.461 \\
\hline 1074 & PDMS & & SW & & & E003644 & 18-Feb-75 & & $10045-97-3$ & CS-137 & GAMMA_GS & USTEST & 7.48 \\
\hline 1075 & PDMS & & SW & & & E032729 & 18-Feb-75 & & $10098-97-2$ & SR-90 & SRISO_SEEP_PRECIP_GPC & USTEST & 138 \\
\hline 1076 & PDMS & & SW & & & E004618 & 24-Feb-75 & & $10098-97-2$ & SR-90 & SRISO_SEP_PRECIP_GPC & USTEST & 0.649 \\
\hline 1077 & PDMS & & SW & & & E004618 & 24-Feb-75 & & $10045-97-3$ & CS-137 & GAMMA_GS & USTEST & 0.0461 \\
\hline 1078 & PDMS & & SW & & & E016462 & 24-Feb-75 & & $10045-97-3$ & CS-137 & GAMMA_GS & USTEST & 0.0467 \\
\hline 1079 & PDMS & & SW & & & E016462 & 24-Feb-75 & & $10028-17-8$ & TRITIUM & TRITIUM_DIST_LSC & USTEST & 477 \\
\hline 1080 & PDMS & & SW & & & E003645 & 24-Feb-75 & & $10045-97-3$ & CS-137 & GAMMA_GS & USTEST & -0.662 \\
\hline 1081 & PDMS & & SW & & & E021359 & 24-Feb-75 & & $10098-97-2$ & SR-90 & SRISO_SEP_PRECIP_GPC & USTEST & 0.55 \\
\hline 1082 & PDMS & & SW & & & E026163 & 24-Feb-75 & & $10045-97-3$ & CS-137 & GAMMA_GS & USTEST & 1.87 \\
\hline 1083 & PDMS & & SW & & & E009137 & $25-F e b-75$ & & $10028-17-8$ & TRITIUM & TRITIUM_DIST_LSC & USTEST & 221 \\
\hline 1084 & PDMS & & SW & & & E009137 & $25-F e b-75$ & & $10045-97-3$ & CS-137 & GAMMA_GS & USTEST & 7.13 \\
\hline 1085 & PDMS & & SW & & & E009137 & $25-F e b-75$ & & $10098-97-2$ & SR-90 & SRISO_SEP_PRECIP_GPC & USTEST & 0.563 \\
\hline 1086 & PDMS & & SW & & & E014688 & 25 -Feb-75 & & $10045-97-3$ & CS-137 & GAMMA_GS & USTEST & 0.0911 \\
\hline 1087 & PDMS & & SW & & & E034978 & 25-Feb-75 & & $10028-17-8$ & TRITIUM & TRITIUM_DIST_LSC & USTEST & 93 \\
\hline 1088 & PDMS & & SW & & & E016133 & 06-Mar-75 & & $10045-97-3$ & CS-137 & GAMMA_GS & USTEST & -0.0139 \\
\hline 1089 & PDMS & & SW & & & E016133 & 06-Mar-75 & & $10028-17-8$ & TRITIUM & TRITIUM_DIST_LSC & USTEST & 359 \\
\hline 1090 & PDMS & & SW & & & E016133 & 06-Mar-75 & & $10098-97-2$ & SR-90 & SRISO_SEP_PRECIP_GPC & USTEST & 0.509 \\
\hline 1091 & PDMS & & SW & & & E012098 & 06-Mar-75 & & $10045-97-3$ & CS-137 & GAMMA_GS & USTEST & 5.29 \\
\hline 1092 & PDMS & & SW & & & E003646 & 10-Mar-75 & & $10045-97-3$ & CS-137 & GAMMA_GS & USTEST & 4.46 \\
\hline 1093 & PDMS & & SW & & & E009151 & 11-Mar-75 & & 10045-97-3 & CS-137 & GAMMA_GS & USTEST & 4.43 \\
\hline
\end{tabular}




\begin{tabular}{|c|c|c|c|c|c|c|c|c|c|c|c|c|c|}
\hline & $\bar{A}$ & $\mathrm{~B}$ & $\mathrm{C}$ & $\mathrm{D}$ & $E$ & $\mathrm{~F}$ & G & $\mathrm{H}$ & $\mathrm{I}$ & $\mathrm{J}$ & $\mathrm{K}$ & $\mathrm{L}$ & $\mathrm{M}$ \\
\hline 1 & SAMPLE_NUM_ASSIGNED_TO & SAMPLER & MEDIA & BIOTA_MEDIA & TAG_ID & SAMP_NUM & SAMP_DATE_TIME & SAMP_DATE_TIME_ON & CON_ID & CON_SHORT_NAME & METHOD_NAME & LAB_CODE & VALUE_RPTD \\
\hline 1094 & PDMS & & SW & & & E003647 & 17-Mar-75 & & $10045-97-3$ & CS-137 & GAMMA_GS & USTEST & 13.3 \\
\hline 1095 & PDMS & & SW & & & E009158 & 18-Mar-75 & & $10045-97-3$ & CS-137 & GAMMA_GS & USTEST & 6.77 \\
\hline 1096 & PDMS & & SW & & & E032730 & 18-Mar-75 & & $10098-97-2$ & SR-90 & SRISO_SEPPPRECIP_GPC & USTEST & 203 \\
\hline 1097 & PDMS & & SW & & & E016463 & 24-Mar-75 & & $10045-97-3$ & CS-137 & GAMMA_GS & USTEST & -1.06 \\
\hline 1098 & PDMS & & SW & & & E016463 & 24-Mar-75 & & $10028-17-8$ & TRITIUM & TRITIUM_DIST_LSC & USTEST & 559 \\
\hline 1099 & PDMS & & SW & & & E003648 & 24-Mar-75 & & $10045-97-3$ & CS-137 & GAMMA_GS & USTEST & -0.447 \\
\hline 1100 & PDMS & & SW & & & E002212 & 24-Mar-75 & & $10098-97-2$ & SR-90 & SRISO_SEP_PRECIP_GPC & USTEST & 0.387 \\
\hline 1101 & PDMS & & SW & & & E002212 & 24-Mar-75 & & $10045-97-3$ & CS-137 & GAMMA_GS & USTEST & -0.00143 \\
\hline 1102 & PDMS & & SW & & & E026167 & 24-Mar-75 & & $10045-97-3$ & CS-137 & GAMMA_GS & USTEST & -0.81 \\
\hline 1103 & PDMS & & SW & & & E009071 & 25-Mar-75 & & 10028-17-8 & TRITIUM & TRITIUM_DIST_LSC & USTEST & 405 \\
\hline 1104 & PDMS & & SW & & & E009071 & 25-Mar-75 & & $10045-97-3$ & CS-137 & GAMMA_GS & USTEST & 12.6 \\
\hline 1105 & PDMS & & SW & & & E009071 & 25-Mar-75 & & $10098-97-2$ & SR-90 & SRISO_SEPP_PRECIP_GPC & USTEST & 0.0901 \\
\hline 1106 & PDMS & & SW & & & E014689 & 25-Mar-75 & & $10045-97-3$ & CS-137 & GAMMA_GS & USTEST & 0.00255 \\
\hline 1107 & PDMS & & SW & & & E034982 & 25-Mar-75 & & $10028-17-8$ & TRITIUM & TRITIUM_DIST_LSC & USTEST & 466 \\
\hline 1108 & PDMS & & SW & & & E004619 & 31-Mar-75 & & $10045-97-3$ & CS-137 & GAMMA_GS & USTEST & -0.572 \\
\hline 1109 & PDMS & & SW & & & E004619 & 31-Mar-75 & & $10098-97-2$ & SR-90 & SRISO_SEP_PRECIP_GPC & USTEST & 0.239 \\
\hline 1110 & PDMS & & SW & & & E003649 & 31-Mar-75 & & $10045-97-3$ & CS-137 & GAMMA_GS & USTEST & 3.18 \\
\hline 1111 & PDMS & & SW & & & E009078 & 01-Apr-75 & & $10045-97-3$ & CS-137 & GAMMA_GS & USTEST & 5.66 \\
\hline 1112 & PDMS & & SW & & & E014941 & 04-Apr-75 & & $10045-97-3$ & CS-137 & GAMMA_GS & USTEST & -0.679 \\
\hline 1113 & PDMS & & SW & & & E016400 & 04-Apr-75 & & $10098-97-2$ & SR-90 & SRISO_SEP_PRECIP_GPC & USTEST & 5.44 \\
\hline 1114 & PDMS & & SW & & & E016400 & 04-Apr-75 & & $10045-97-3$ & CS-137 & GAMMA_GS & USTEST & 8.09 \\
\hline 1115 & PDMS & & SW & & & E022420 & 04-Apr-75 & & $10045-97-3$ & CS-137 & GAMMA_GS & USTEST & 63.3 \\
\hline 1116 & PDMS & & SW & & & E022420 & 04-Apr-75 & & $10098-97-2$ & SR-90 & SRISO_SEP_PRECIP_GPC & USTEST & 0.414 \\
\hline 1117 & PDMS & & SW & & & E027641 & 04-Apr-75 & & $10045-97-3$ & CS-137 & GAMMA_GS & USTEST & 5.55 \\
\hline 1118 & PDMS & & SW & & & E014269 & 04-Apr-75 & & $10045-97-3$ & CS-137 & GAMMA_GS & USTEST & 0.944 \\
\hline 1119 & PDMS & & SW & & & E014269 & 04-Apr-75 & & $10098-97-2$ & SR-90 & SRISO_SEP_PRECIP_GPC & USTEST & 0.18 \\
\hline 1120 & PDMS & & SW & & & E003650 & 07-Apr-75 & & $10045-97-3$ & CS-137 & GAMMA_GS & USTEST & 4.89 \\
\hline 1121 & PDMS & & SW & & & E009085 & 08-Apr-75 & & $10045-97-3$ & CS-137 & GAMMA_GS & USTEST & -0.0693 \\
\hline 1122 & PDMS & & SW & & & E016134 & 10-Apr-75 & & $10045-97-3$ & CS-137 & GAMMA_GS & USTEST & 0.0122 \\
\hline 1123 & PDMS & & SW & & & E016134 & 10-Apr-75 & & $10028-17-8$ & TRITIUM & TRITIUM_DIST_LSC & USTEST & 511 \\
\hline 1124 & PDMS & & SW & & & E016134 & 10-Apr-75 & & $10098-97-2$ & SR-90 & SRISO_SEP_PRECIP_GPC & USTEST & 0.225 \\
\hline 1125 & PDMS & & SW & & & E012099 & 10-Apr-75 & & $10045-97-3$ & CS-137 & GAMMA_GS & USTEST & -1 \\
\hline 1126 & PDMS & & SW & & & E003651 & 14-Apr-75 & & $10045-97-3$ & CS-137 & GAMMA_GS & USTEST & 12.6 \\
\hline 1127 & PDMS & & SW & & & E009092 & 15-Apr-75 & & $10045-97-3$ & CS-137 & GAMMA_GS & USTEST & 11.6 \\
\hline 1128 & PDMS & & SW & & & E032731 & 15-Apr-75 & & $10098-97-2$ & SR-90 & SRISO_SEP_PRECIP_GPC & USTEST & 98.9 \\
\hline 1129 & PDMS & & SW & & & E003652 & 21-Apr-75 & & $10045-97-3$ & CS-137 & GAMMA_GS & USTEST & -0.012 \\
\hline 1130 & PDMS & & SW & & & E009099 & 22-Apr-75 & & $10045-97-3$ & CS-137 & GAMMA_GS & USTEST & 0.0511 \\
\hline 1131 & PDMS & & SW & & & E039724 & 28-Apr-75 & & $10098-97-2$ & SR-90 & SRISO_SEP_PRECIP_GPC & USTEST & 0.76 \\
\hline 1132 & PDMS & & SW & & & E039724 & 28-Apr-75 & & $10045-97-3$ & CS-137 & GAMMA_GS & USTEST & 0.33 \\
\hline 1133 & PDMS & & SW & & & E016464 & 28-Apr-75 & & $10045-97-3$ & CS-137 & GAMMA_GS & USTEST & 5.78 \\
\hline 1134 & PDMS & & SW & & & E016464 & 28-Apr-75 & & $10028-17-8$ & TRITIUM & TRITIUM_DIST_LSC & USTEST & 493 \\
\hline 1135 & PDMS & & SW & & & E003653 & 28-Apr-75 & & $10045-97-3$ & CS-137 & GAMMA_GS & USTEST & 3 \\
\hline 1136 & PDMS & & SW & & & E002213 & 28-Apr-75 & & $10098-97-2$ & SR-90 & SRISO_SEP_PRECIP_GPC & USTEST & 0.0719 \\
\hline 1137 & PDMS & & SW & & & E002213 & 28-Apr-75 & & $10045-97-3$ & CS-137 & GAMMA_GS & USTEST & 0.275 \\
\hline 1138 & PDMS & & SW & & & E026094 & 28-Apr-75 & & $10045-97-3$ & CS-137 & GAMMA_GS & USTEST & 9.36 \\
\hline 1139 & PDMS & & SW & & & E009106 & 29-Apr-75 & & $10028-17-8$ & TRITIUM & TRITIUM_DIST_LSC & USTEST & 636 \\
\hline 1140 & PDMS & & SW & & & E009106 & 29-Apr-75 & & $10045-97-3$ & CS-137 & GAMMA_GS & USTEST & -0.563 \\
\hline 1141 & PDMS & & SW & & & E009106 & 29-Apr-75 & & $10098-97-2$ & SR-90 & SRISO_SEP_PRECIP_GPC & USTEST & 0.568 \\
\hline 1142 & PDMS & & SW & & & E014690 & 29-Apr-75 & & $10045-97-3$ & CS-137 & GAMMA_GS & USTEST & 0.193 \\
\hline 1143 & PDMS & & SW & & & E027809 & 29-Apr-75 & & $10028-17-8$ & TRITIUM & TRITIUM_DIST_LSC & USTEST & 666 \\
\hline 1144 & PDMS & & SW & & & E003654 & 05-May-75 & & $10045-97-3$ & CS-137 & GAMMA_GS & USTEST & 11.3 \\
\hline 1145 & PDMS & & SW & & & E009113 & 06-Мау-75 & & $10045-97-3$ & CS-137 & GAMMA_GS & USTEST & 0.969 \\
\hline
\end{tabular}




\begin{tabular}{|c|c|c|c|c|c|c|c|c|c|c|c|c|c|}
\hline & $\bar{A}$ & $\mathrm{~B}$ & $\bar{C}$ & $\mathrm{D}$ & $\mathrm{E}$ & $\mathrm{F}$ & $\bar{G}$ & $\mathrm{H}$ & 1 & $\mathrm{~J}$ & $\bar{K}$ & $\bar{L}$ & $\mathrm{M}$ \\
\hline 1 & SAMPLE_NUM_ASSIGNED_TO & SAMPLER & MEDIA & BIOTA_MEDIA & TAG_ID & SAMP_NUM & SAMP_DATE_TIME & SAMP_DATE_TIME_ON & CON_ID & CON_SHORT_NAME & METHOD_NAME & LAB_CODE & VALUE_RPTD \\
\hline 1146 & PDMS & & SW & & & E016135 & 08-May-75 & & $10045-97-3$ & CS-137 & GAMMA_GS & USTEST & 0.0331 \\
\hline 1147 & PDMS & & SW & & & E016135 & 08-May-75 & & $10028-17-8$ & TRITIUM & TRITIUM_DIST_LSC & USTEST & 501 \\
\hline 1148 & PDMS & & SW & & & E016135 & 08-May-75 & & $10098-97-2$ & SR-90 & SRISO_SEP_PRECIP_GPC & USTEST & 0.572 \\
\hline 1149 & PDMS & & SW & & & E012100 & 08-May-75 & & $10045-97-3$ & CS-137 & GAMMA_GS & USTEST & 2.73 \\
\hline 1150 & PDMS & & SW & & & E010866 & 12-May-75 & & $10045-97-3$ & CS-137 & GAMMA_GS & USTEST & -0.412 \\
\hline 1151 & PDMS & & SW & & & E009026 & 13-Мау-75 & & $10045-97-3$ & CS-137 & GAMMA_GS & USTEST & -0.479 \\
\hline 1152 & PDMS & & SW & & & E032732 & 13-Мау-75 & & $10098-97-2$ & SR-90 & SRISO_SEEP_PRECIP_GPC & USTEST & 113 \\
\hline 1153 & PDMS & & SW & & & E009032 & 19-May-75 & & $10045-97-3$ & CS-137 & GAMMA_GS & USTEST & 1.16 \\
\hline 1154 & PDMS & & SW & & & E003655 & 19-May-75 & & $10045-97-3$ & CS-137 & GAMMA_GS & USTEST & 1.67 \\
\hline 1155 & PDMS & & SW & & & E004620 & 27-May-75 & & $10045-97-3$ & CS-137 & GAMMA_GS & USTEST & 0.0835 \\
\hline 1156 & PDMS & & SW & & & E004620 & 27-May-75 & & $10098-97-2$ & SR-90 & SRISO_SEP_PRECIP_GPC & USTEST & 0.387 \\
\hline 1157 & PDMS & & SW & & & E009040 & 27-May-75 & & $10028-17-8$ & TRITIUM & TRITIUM_DIST_LSC & USTEST & 546 \\
\hline 1158 & PDMS & & SW & & & E009040 & 27-May-75 & & $10045-97-3$ & CS-137 & GAMMA_GS & USTEST & 0.347 \\
\hline 1159 & PDMS & & SW & & & E009040 & 27-May-75 & & $10098-97-2$ & SR-90 & SRISO_SEP_PRECIP_GPC & USTEST & 0.91 \\
\hline 1160 & PDMS & & SW & & & E014677 & 27-May-75 & & $10045-97-3$ & CS-137 & GAMMA_GS & USTEST & 6.89 \\
\hline 1161 & PDMS & & SW & & & E041052 & 27-May-75 & & $10028-17-8$ & TRITIUM & TRITIUM_DIST_LSC & USTEST & 102 \\
\hline 1162 & PDMS & & SW & & & E016465 & 27-May-75 & & $10045-97-3$ & CS-137 & GAMMA_GS & USTEST & 0.0511 \\
\hline 1163 & PDMS & & SW & & & E003584 & 27-May-75 & & $10045-97-3$ & CS-137 & GAMMA_GS & USTEST & -0.37 \\
\hline 1164 & PDMS & & SW & & & E002214 & 27-May-75 & & $10098-97-2$ & SR-90 & SRISO_SEP_PRECIP_GPC & USTEST & 0.338 \\
\hline 1165 & PDMS & & SW & & & E002214 & 27-May-75 & & $10045-97-3$ & CS-137 & GAMMA_GS & USTEST & 0.282 \\
\hline 1166 & PDMS & & SW & & & E026098 & 27-May-75 & & $10045-97-3$ & CS-137 & GAMMA_GS & USTEST & 20 \\
\hline 1167 & PDMS & & SW & & & E003585 & 02-Jun-75 & & $10045-97-3$ & CS-137 & GAMMA_GS & USTEST & 0.0511 \\
\hline 1168 & PDMS & & SW & & & E009047 & 03-Jun-75 & & $10045-97-3$ & CS-137 & GAMMA_GS & USTEST & 1.2 \\
\hline 1169 & PDMS & & SW & & & E016136 & 05-Jun-75 & & $10045-97-3$ & CS-137 & GAMMA_GS & USTEST & 1.84 \\
\hline 1170 & PDMS & & SW & & & E016136 & 05-Jun-75 & & 10028-17-8 & TRITIUM & TRITIUM_DIST_LSC & USTEST & -254 \\
\hline 1171 & PDMS & & SW & & & E016136 & 05-Jun-75 & & $10098-97-2$ & SR-90 & SRISO_SEP_PRECIP_GPC & USTEST & 1.48 \\
\hline 1172 & PDMS & & SW & & & E012101 & 05-Jun-75 & & $10045-97-3$ & CS-137 & GAMMA_GS & USTEST & 3.11 \\
\hline 1173 & PDMS & & SW & & & E003586 & 09-Jun-75 & & $10045-97-3$ & CS-137 & GAMMA_GS & USTEST & 1.01 \\
\hline 1174 & PDMS & & SW & & & E009054 & 10-Jun-75 & & $10045-97-3$ & CS-137 & GAMMA_GS & USTEST & 7.58 \\
\hline 1175 & PDMS & & SW & & & E032733 & 10-Jun-75 & & $10098-97-2$ & SR-90 & SRISO_SEP_PRECIP_GPC & USTEST & 317 \\
\hline 1176 & PDMS & & SW & & & E003587 & 16-Jun-75 & & $10045-97-3$ & CS-137 & GAMMA_GS & USTEST & -0.125 \\
\hline 1177 & PDMS & & SW & & & E009061 & 17-Jun-75 & & $10045-97-3$ & CS-137 & GAMMA_GS & USTEST & 4.62 \\
\hline 1178 & PDMS & & SW & & & E016466 & 23-Jun-75 & & $10045-97-3$ & CS-137 & GAMMA_GS & USTEST & 0.0511 \\
\hline 1179 & PDMS & & SW & & & E016466 & 23-Jun-75 & & 10028-17-8 & TRITIUM & TRITIUM_DIST_LSC & USTEST & 465 \\
\hline 1180 & PDMS & & SW & & & E003588 & 23-Jun-75 & & $10045-97-3$ & CS-137 & GAMMA_GS & USTEST & 3.91 \\
\hline 1181 & PDMS & & SW & & & E002215 & 23-Jun-75 & & $10098-97-2$ & SR-90 & SRISO_SEP_PRECIP_GPC & USTEST & 0.833 \\
\hline 1182 & PDMS & & SW & & & E002215 & 23-Jun-75 & & $10045-97-3$ & CS-137 & GAMMA_GS & USTEST & 0.774 \\
\hline 1183 & PDMS & & SW & & & E026102 & 23-Jun-75 & & $10045-97-3$ & CS-137 & GAMMA_GS & USTEST & 0.128 \\
\hline 1184 & PDMS & & SW & & & E014678 & 24-Jun-75 & & $10045-97-3$ & CS-137 & GAMMA_GS & USTEST & 0.00267 \\
\hline 1185 & PDMS & & SW & & & E008974 & 24-Jun-75 & & $10028-17-8$ & TRITIUM & TRITIUM_DIST_LSC & USTEST & 129 \\
\hline 1186 & PDMS & & SW & & & E008974 & 24-Jun-75 & & $10045-97-3$ & CS-137 & GAMMA_GS & USTEST & -0.345 \\
\hline 1187 & PDMS & & SW & & & E008974 & 24-Jun-75 & & $10098-97-2$ & SR-90 & SRISO_SEP_PRECIP_GPC & USTEST & 0.734 \\
\hline 1188 & PDMS & & SW & & & E034993 & 24-Jun-75 & & $10028-17-8$ & TRITIUM & TRITIUM_DIST_LSC & USTEST & 404 \\
\hline 1189 & PDMS & & SW & & & E075058 & 27-Jun-75 & & $10028-17-8$ & TRITIUM & TRITIUM_DIST_LSC & USTEST & 381 \\
\hline 1190 & PDMS & & SW & & & E070701 & 30-Jun-75 & & $10045-97-3$ & CS-137 & GAMMA_GS & USTEST & 0.00252 \\
\hline 1191 & PDMS & & SW & & & E004621 & 30-Jun-75 & & $10098-97-2$ & SR-90 & SRISO_SEP_PRECIP_GPC & USTEST & 0.295 \\
\hline 1192 & PDMS & & SW & & & E004621 & 30-Jun-75 & & $10045-97-3$ & CS-137 & GAMMA_GS & USTEST & -0.0464 \\
\hline 1193 & PDMS & & SW & & & E003589 & 30-Jun-75 & & $10045-97-3$ & CS-137 & GAMMA_GS & USTEST & -0.101 \\
\hline 1194 & PDMS & & SW & & & E008981 & 01-Jul-75 & & $10045-97-3$ & CS-137 & GAMMA_GS & USTEST & 3.58 \\
\hline 1195 & PDMS & & SW & & & E003590 & 07-Jul-75 & & $10045-97-3$ & CS-137 & GAMMA_GS & USTEST & 0.0511 \\
\hline 1196 & PDMS & & SW & & & E008988 & 08-Jul-75 & & $10045-97-3$ & CS-137 & GAMMA_GS & USTEST & -0.325 \\
\hline 1197 & PDMS & & SW & & & E032734 & 08-Jul-75 & & $10098-97-2$ & SR-90 & SRISO_SEP_PRECIP_GPC & USTEST & 2770 \\
\hline
\end{tabular}




\begin{tabular}{|c|c|c|c|c|c|c|c|c|c|c|c|c|c|}
\hline & A & $\mathrm{B}$ & $\mathrm{c}$ & $\mathrm{D}$ & $\mathrm{E}$ & $\mathrm{F}$ & G & $\mathrm{H}$ & $\mathrm{I}$ & $\mathrm{J}$ & $\mathrm{K}$ & $\mathrm{L}$ & $\mathrm{M}$ \\
\hline 1 & SAMPLE_NUM_ASSIGNED_TO & SAMPLER & MEDIA & BIOTA_MEDIA & TAG_ID & SAMP_NUM & SAMP_DATE_TIME & SAMP_DATE_TIME_ON & CON_ID & CON_SHORT_NAME & METHOD_NAME & LAB_CODE & \begin{tabular}{|l|} 
VALUE_RPTD \\
\end{tabular} \\
\hline 1198 & PDMS & & SW & & & E016137 & 10-Jul-75 & & $10045-97-3$ & CS-137 & GAMMA GS & USTEST & 0.177 \\
\hline 1199 & PDMS & & sW & & & E016137 & 10-Jul-75 & & $10028-17-8$ & TRITIUM & TRITIUM_DIST_LSC & USTEST & 284 \\
\hline 1200 & PDMS & & sW & & & E016137 & 10-Jul-75 & & $10098-97-2$ & SR-90 & SRISO_SEP_PRECIP_GPC & USTEST & 4.82 \\
\hline 1201 & PDMS & & sW & & & E012102 & 10-Jul-75 & & $10028-17-8$ & TRITIUM & TRITIUM_DIST_LSC & USTEST & 25000 \\
\hline 1202 & PDMS & & sW & & & E012102 & 10-Jul-75 & & $10045-97-3$ & CS-137 & GAMMA_GS & USTEST & 113 \\
\hline 1203 & PDMS & & sW & & & E010851 & 14-Jul-75 & & $10045-97-3$ & CS-137 & GAMMA_GS & USTEST & 0.0522 \\
\hline 1204 & PDMS & & SW & & & E008995 & 15-Jul-75 & & $10045-97-3$ & CS-137 & GAMMA_GS & USTEST & 0.0512 \\
\hline 1205 & PDMS & & SW & & & E009002 & 22-Jul-75 & & $10045-97-3$ & CS-137 & GAMMA_GS & USTEST & -0.000221 \\
\hline 1206 & PDMS & & sW & & & E004622 & 28-Jul-75 & & $10098-97-2$ & SR-90 & SRISO_SEP_PRECIP_GPC & USTEST & 0.139 \\
\hline 1207 & PDMS & & SW & & & E004622 & 28-Jul-75 & & $10045-97-3$ & CS-137 & GAMMA_GS & USTEST & 0.0016 \\
\hline 1208 & PDMS & & SW & & & E037055 & 28-Jul-75 & & $10028-17-8$ & TRITIUM & TRITIUM_DIST_LSC & USTEST & 554 \\
\hline 1209 & PDMS & & SW & & & E003592 & 28-Jul-75 & & $10045-97-3$ & CS-137 & GAMMA_GS & USTEST & -0.121 \\
\hline 1210 & PDMS & & SW & & & E002216 & 28-Jul-75 & & $10098-97-2$ & SR-90 & SRISO_SEPPPRECIP_GPC & USTEST & 0.171 \\
\hline 1211 & PDMS & & SW & & & E002216 & 28-Jul-75 & & $10045-97-3$ & CS-137 & GAMMĀGS & USTEST & -0.0261 \\
\hline 1212 & PDMS & & SW & & & E026107 & 28-Jul-75 & & $10045-97-3$ & CS-137 & GAMMA_GS & USTEST & 0.0887 \\
\hline 1213 & PDMS & & SW & & & E009009 & 29-Jul-75 & & $10045-97-3$ & CS-137 & GAMMA_GS & USTEST & 20.3 \\
\hline 1214 & PDMS & & sW & & & E009009 & 29-Jul-75 & & $10098-97-2$ & SR-90 & SRISO_SEP_PRECIP_GPC & USTEST & 0.213 \\
\hline 1215 & PDMS & & sW & & & E014679 & 29-Jul-75 & & $10045-97-3$ & CS-137 & GAMMA_GS & USTEST & 0.342 \\
\hline 1216 & PDMS & & SW & & & E041425 & 29-Jul-75 & & $10028-17-8$ & TRITIUM & TRITIUM_DIST_LSC & USTEST & 480 \\
\hline \begin{tabular}{|l|l|}
1217 \\
\end{tabular} & PDMS & & SW & & & E034998 & 29-Jul-75 & & $10028-17-8$ & TRITIUM & TRITIUM_DIST_LSC & USTEST & 389 \\
\hline 1218 & PDMS & & SW & & & E014885 & 01-Aug-75 & & $10045-97-3$ & CS-137 & GAMMA_GS & USTEST & 19.8 \\
\hline 1219 & PDMS & & SW & & & E016401 & 01-Aug-75 & & $10098-97-2$ & SR-90 & SRISO_SEPPPRECIP_GPC & USTEST & 1.44 \\
\hline 1220 & PDMS & & SW & & & E016401 & 01-Aug-75 & & $10045-97-3$ & CS-137 & GAMMA_GS & USTEST & 22.2 \\
\hline 1221 & PDMS & & SW & & & E022421 & 01-Aug-75 & & $10045-97-3$ & CS-137 & GAMMA_GS & USTEST & 34.5 \\
\hline 1222 & PDMS & & SW & & & E022421 & 01-Aug-75 & & $10098-97-2$ & SR-90 & SRISO_SEP_PRECIP_GPC & USTEST & 0.641 \\
\hline 1223 & PDMS & & SW & & & E003555 & 01-Aug-75 & & $10045-97-3$ & CS-137 & GAMMA_GS & USTEST & 20.8 \\
\hline 1224 & PDMS & & SW & & & E027642 & 01-Aug-75 & & $10045-97-3$ & CS-137 & GAMMA_GS & USTEST & 6.74 \\
\hline 1225 & PDMS & & SW & & & E014270 & 01-Aug-75 & & $10045-97-3$ & CS-137 & GAMMA_GS & USTEST & -0.156 \\
\hline 1226 & PDMS & & SW & & & E014270 & 01-Aug-75 & & $10098-97-2$ & SR-90 & SRISO_SEP_PRECIP_GPC & USTEST & 13.3 \\
\hline \begin{tabular}{|l|l|}
1227 \\
\end{tabular} & PDMS & & SW & & & E009015 & 04-Aug-75 & & $10045-97-3$ & CS-137 & GAMMA_GS & USTEST & 7.59 \\
\hline 1228 & PDMS & & SW & & & E003593 & 04-Aug-75 & & $10045-97-3$ & CS-137 & GAMMA_GS & USTEST & -0.182 \\
\hline 1229 & PDMS & & SW & & & E032735 & 05-Aug-75 & & $10098-97-2$ & SR-90 & SRISO_SEPP_PRECIP_GPC & USTEST & 1410 \\
\hline 1230 & PDMS & & SW & & & E016138 & 08-Aug-75 & & $10045-97-3$ & CS-137 & GAMMA_GS & USTEST & 0.713 \\
\hline 1231 & PDMS & & SW & & & E016138 & 08-Aug-75 & & $10028-17-8$ & TRITIUM & TRITIUM_DIST_LSC & USTEST & 148 \\
\hline 1232 & PDMS & & SW & & & E016138 & 08-Aug-75 & & $10098-97-2$ & SR-90 & SRISO_SEPP_PRECIP_GPC & USTEST & 0.172 \\
\hline 1233 & PDMS & & SW & & & E012103 & 08-Aug-75 & & $10028-17-8$ & TRITIUM & TRITIUM_DIST_LSC & USTEST & 31900 \\
\hline 1234 & PDMS & & SW & & & E012103 & 08-Aug-75 & & $10045-97-3$ & CS-137 & GAMMA_GS & USTEST & 2.08 \\
\hline 1235 & PDMS & & SW & & & E003594 & 11-Aug-75 & & $10045-97-3$ & CS-137 & GAMMA_GS & USTEST & 1.14 \\
\hline 1236 & PDMS & & SW & & & E008929 & 12-Aug-75 & & $10045-97-3$ & CS-137 & GAMMA_GS & USTEST & -0.616 \\
\hline 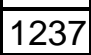 & PDMS & & SW & & & E003595 & 18-Aug-75 & & $10045-97-3$ & CS-137 & GAMMA_GS & USTEST & -0.916 \\
\hline 1238 & PDMS & & SW & & & E008936 & 19-Aug-75 & & $10045-97-3$ & CS-137 & GAMMA_GS & USTEST & 0.0511 \\
\hline 1239 & PDMS & & SW & & & E004623 & 25-Aug-75 & & $10098-97-2$ & SR-90 & SRISO_SEEP_PRECIP_GPC & USTEST & 0.356 \\
\hline 1240 & PDMS & & SW & & & E004623 & 25-Aug-75 & & $10045-97-3$ & CS-137 & GAMMA_GS & USTEST & 0.392 \\
\hline 1241 & PDMS & & SW & & & E037059 & 25-Aug-75 & & $10028-17-8$ & TRITIUM & TRITIUM_DIST_LSC & USTEST & 347 \\
\hline 1242 & PDMS & & SW & & & E003596 & 25-Aug-75 & & $10045-97-3$ & CS-137 & GAMMA_GS & USTEST & 1.08 \\
\hline 1243 & PDMS & & SW & & & E002217 & 25-Aug-75 & & $10045-97-3$ & CS-137 & GAMMA_GS & USTEST & 2.61 \\
\hline 1244 & PDMS & & SW & & & E026111 & 25-Aug-75 & & $10045-97-3$ & CS-137 & GAMMA_GS & USTEST & 22.9 \\
\hline 1245 & PDMS & & SW & & & E014680 & 26-Aug-75 & & $10045-97-3$ & CS-137 & GAMMA_GS & USTEST & 1.12 \\
\hline 1246 & PDMS & & SW & & & E008943 & 26-Aug-75 & & $10028-17-8$ & TRITIUM & TRITIUM_DIST_LSC & USTEST & 565 \\
\hline \begin{tabular}{|l|l|}
1247 & \\
\end{tabular} & PDMS & & sW & & & E008943 & 26-Aug-75 & & $10045-97-3$ & CS-137 & GAMMA_GS & USTEST & 3.7 \\
\hline 1248 & PDMS & & SW & & & E008943 & 26-Aug-75 & & $10098-97-2$ & SR-90 & SRISO_SEPPPRECIP_GPC & USTEST & 0.31 \\
\hline 1249 & PDMS & & SW & & & E041429 & 26-Aug-75 & & $10028-17-8$ & TRITIUM & TRITIUM_DISTT_LSC & USTEST & 202 \\
\hline
\end{tabular}




\begin{tabular}{|c|c|c|c|c|c|c|c|c|c|c|c|c|c|}
\hline & A & $\mathrm{B}$ & $\mathrm{c}$ & $\mathrm{D}$ & $\mathrm{E}$ & $\mathrm{F}$ & G & $\mathrm{H}$ & $\mathrm{I}$ & $\mathrm{J}$ & $\mathrm{K}$ & $\mathrm{L}$ & $\mathrm{M}$ \\
\hline 1 & SAMPLE_NUM_ASSIGNED_TO & SAMPLER & MEDIA & BIOTA_MEDIA & TAG_ID & SAMP_NUM & SAMP_DATE_TIME & SAMP_DATE_TIME_ON & CON_ID & CON_SHORT_NAME & METHOD_NAME & LAB_CODE & \begin{tabular}{|l|} 
VALUE_RPTD \\
\end{tabular} \\
\hline 1250 & PDMS & & SW & & & E041053 & 26-Aug-75 & & $10028-17-8$ & TRITIUM & TRITIUM_DIST_LSC & USTEST & 260 \\
\hline 1251 & PDMS & & sW & & & E008950 & 02-Sep-75 & & $10045-97-3$ & CS-137 & GAMMA_GS & USTEST & -0.335 \\
\hline 1252 & PDMS & & sW & & & E003597 & 02-Sep-75 & & $10045-97-3$ & CS-137 & GAMMA_GS & USTEST & -0.635 \\
\hline 1253 & PDMS & & sW & & & E032736 & 02-Sep-75 & & $10098-97-2$ & SR-90 & SRISO_SEP_PRECIP_GPC & USTEST & 116 \\
\hline 1254 & PDMS & & SW & & & E016139 & 04-Sep-75 & & $10045-97-3$ & CS-137 & GAMMA_GS & USTEST & 0.591 \\
\hline 1255 & PDMS & & SW & & & E016139 & 04-Sep-75 & & $10028-17-8$ & TRITIUM & TRITIUM_DIST_LSC & USTEST & 146 \\
\hline 1256 & PDMS & & SW & & & E016139 & 04-Sep-75 & & $10098-97-2$ & SR-90 & SRISO_SEP_PRECIP_GPC & USTEST & 0.405 \\
\hline 1257 & PDMS & & SW & & & E012104 & 04-Sep-75 & & $10028-17-8$ & TRITIUM & TRITIUM_DIST_LSC & USTEST & 14000 \\
\hline 1258 & PDMS & & SW & & & E012104 & 04-Sep-75 & & $10045-97-3$ & CS-137 & GAMMA_GS & USTEST & 69.9 \\
\hline 1259 & PDMS & & SW & & & E003598 & 08-Sep-75 & & $10045-97-3$ & CS-137 & GAMMA_GS & USTEST & -0.0836 \\
\hline 1260 & PDMS & & SW & & & E008957 & 09-Sep-75 & & $10045-97-3$ & CS-137 & GAMMA_GS & USTEST & -0.0835 \\
\hline 1261 & PDMS & & SW & & & E003599 & 15-Sep-75 & & $10045-97-3$ & CS-137 & GAMMA_GS & USTEST & 0.0115 \\
\hline 1262 & PDMS & & SW & & & E008964 & $16-$ Sep-75 & & $10045-97-3$ & CS-137 & GAMMA_GS & USTEST & 4.79 \\
\hline 1263 & PDMS & & SW & & & E003600 & 22-Sep-75 & & $10045-97-3$ & CS-137 & GAMMA GS & USTEST & -0.284 \\
\hline 1264 & PDMS & & SW & & & E008971 & 23-Sep-75 & & $10045-97-3$ & CS-137 & GAMMA_GS & USTEST & -0.568 \\
\hline 1265 & PDMS & & SW & & & E004624 & 29-Sep-75 & & $10098-97-2$ & SR-90 & SRISO_SEP_PRECIP_GPC & USTEST & 0.279 \\
\hline 1266 & PDMS & & sW & & & E004624 & 29-Sep-75 & & $10045-97-3$ & CS-137 & GAMMA_GS & USTEST & 6.96 \\
\hline \begin{tabular}{|c|}
1267 \\
\end{tabular} & PDMS & & sW & & & E070702 & 29-Sep-75 & & $10045-97-3$ & CS-137 & GAMMA_GS & USTEST & -0.016 \\
\hline 1268 & PDMS & & SW & & & E003601 & 29-Sep-75 & & $10045-97-3$ & CS-137 & GAMMA_GS & USTEST & -0.272 \\
\hline 1269 & PDMS & & SW & & & E021315 & 29-Sep-75 & & $10098-97-2$ & SR-90 & SRISO_SEEP_PRECIP_GPC & USTEST & 0.446 \\
\hline 1270 & PDMS & & SW & & & E021315 & 29-Sep-75 & & $10045-97-3$ & CS-137 & GAMMA_GS & USTEST & 0.521 \\
\hline 1271 & PDMS & & SW & & & E026116 & 29-Sep-75 & & $10045-97-3$ & CS-137 & GAMMA_GS & USTEST & 18.6 \\
\hline 1272 & PDMS & & SW & & & E014681 & $30-S e p-75$ & & $10045-97-3$ & CS-137 & GAMMA_GS & USTEST & -0.00571 \\
\hline 1273 & PDMS & & SW & & & E041433 & $30-S e p-75$ & & $10028-17-8$ & TRITIUM & TRITIUM_DIST_LSC & USTEST & 359 \\
\hline 1274 & PDMS & & SW & & & E008884 & $30-$ Sep-75 & & $10028-17-8$ & TRITIUM & TRITIUM_DIST_LSC & USTEST & 631 \\
\hline 1275 & PDMS & & SW & & & E008884 & $30-$ Sep-75 & & $10045-97-3$ & CS-137 & GAMMA_GS & USTEST & -0.769 \\
\hline 1276 & PDMS & & SW & & & E008884 & 30-Sep-75 & & $10098-97-2$ & SR-90 & SRISO_SEP_PRECIP_GPC & USTEST & 0.387 \\
\hline 1277 & PDMS & & SW & & & E034929 & 30-Sep-75 & & $10028-17-8$ & TRITIUM & TRITIUM_DISTT_LSC & USTEST & 161 \\
\hline 1278 & PDMS & & SW & & & E021224 & 30-Sep-75 & & $10045-97-3$ & CS-137 & GAMMA_GS & USTEST & -0.542 \\
\hline 1279 & PDMS & & SW & & & E021224 & 30-Sep-75 & & $10028-17-8$ & TRITIUM & TRITIUM_DIST_LSC & USTEST & -72.1 \\
\hline 1280 & PDMS & & SW & & & E021224 & $30-$ Sep-75 & & $10098-97-2$ & SR-90 & SRISO_SEP_PRECIP_GPC & USTEST & 1.85 \\
\hline 1281 & PDMS & & SW & & & E032737 & $30-$ Sep-75 & & $10098-97-2$ & SR-90 & SRISO_SEP_PRECIP_GPC & USTEST & 103 \\
\hline 1282 & PDMS & & SW & & & E003602 & $06-$ Oct-75 & & $10045-97-3$ & CS-137 & GAMMA_GS & USTEST & 0.0511 \\
\hline 1283 & PDMS & & SW & & & E008891 & 07-Oct-75 & & $10045-97-3$ & CS-137 & GAMMA_GS & USTEST & 0.0511 \\
\hline 1284 & PDMS & & SW & & & E016140 & 09-Oct-75 & & $10045-97-3$ & CS-137 & GAMMA_GS & USTEST & 0.933 \\
\hline 1285 & PDMS & & sW & & & E016140 & 09-Oct-75 & & $10028-17-8$ & TRITIUM & TRITIUM_DIST_LSC & USTEST & 124 \\
\hline 1286 & PDMS & & SW & & & E016140 & 09-Oct-75 & & $10098-97-2$ & SR-90 & SRISO_SEP_PRECIP_GPC & USTEST & 0.264 \\
\hline 1287 & PDMS & & SW & & & E012105 & $09-$ Oct-75 & & $10028-17-8$ & TRITIUM & TRITIUM_DIST_LSC & USTEST & 46900 \\
\hline 1288 & PDMS & & SW & & & E012105 & $09-$ Oct-75 & & $10045-97-3$ & CS-137 & GAMMA_GS & USTEST & 7.79 \\
\hline 1289 & PDMS & & SW & & & E003603 & 13-Oct-75 & & $10045-97-3$ & CS-137 & GAMMA_GS & USTEST & 3.68 \\
\hline 1290 & PDMS & & SW & & & E008898 & 14-Oct-75 & & $10045-97-3$ & CS-137 & GAMMA_GS & USTEST & 3.46 \\
\hline 1291 & PDMS & & SW & & & E003604 & 20-Oct-75 & & $10045-97-3$ & CS-137 & GAMMA_GS & USTEST & 8.31 \\
\hline 1292 & PDMS & & SW & & & E008905 & 21-Oct-75 & & $10045-97-3$ & CS-137 & GAMMA_GS & USTEST & 5.94 \\
\hline 1293 & PDMS & & SW & & & E039725 & 27-Oct-75 & & $10098-97-2$ & SR-90 & SRISO_SEP_PRECIP_GPC & USTEST & 0.167 \\
\hline 1294 & PDMS & & SW & & & E039725 & 27-Oct-75 & & $10045-97-3$ & CS-137 & GAMMA_GS & USTEST & 0.00268 \\
\hline 1295 & PDMS & & SW & & & E037068 & $27-$ Oct-75 & & $10028-17-8$ & TRITIUM & TRITIUM_DIST_LSC & USTEST & 712 \\
\hline 1296 & PDMS & & SW & & & E010852 & 27-Oct-75 & & $10045-97-3$ & CS-137 & GAMMA_GS & USTEST & 1.73 \\
\hline 1297 & PDMS & & SW & & & E002218 & 27-Oct-75 & & $10098-97-2$ & SR-90 & SRISO_SEEP_PRECIP_GPC & USTEST & 0.464 \\
\hline 1298 & PDMS & & SW & & & E002218 & 27-Oct-75 & & $10045-97-3$ & CS-137 & GAMMA_GS & USTEST & -0.0232 \\
\hline 1299 & PDMS & & SW & & & E026120 & 27-Oct-75 & & $10045-97-3$ & CS-137 & GAMMA_GS & USTEST & 12.5 \\
\hline 1300 & PDMS & & SW & & & E008912 & $28-O c t-75$ & & $10028-17-8$ & TRITIUM & TRITIUM_DIST_LSC & USTEST & 190 \\
\hline 1301 & PDMS & & SW & & & E014682 & 28-Oct-75 & & $10045-97-3$ & CS-137 & GAMMA_GS & USTEST & -0.0612 \\
\hline
\end{tabular}




\begin{tabular}{|c|c|c|c|c|c|c|c|c|c|c|c|c|c|}
\hline & A & $\mathrm{B}$ & $C$ & $\mathrm{D}$ & $E$ & $\mathrm{~F}$ & G & $\mathrm{H}$ & $\mathrm{I}$ & $\mathrm{J}$ & $\mathrm{K}$ & $\mathrm{L}$ & $\mathrm{M}$ \\
\hline 1 & SAMPLE_NUM_ASSIGNED_TO & SAMPLER & MEDIA & BIOTA_MEDIA & TAG_ID & SAMP_NUM & SAMP_DATE_TIME & SAMP_DATE_TIME_ON & CON_ID & CON_SHORT_NAME & METHOD_NAME & LAB_CODE & \begin{tabular}{|l|} 
VALUE_RPTD \\
\end{tabular} \\
\hline 1302 & PDMS & & SW & & & E008912 & 28-Oct-75 & & $10045-97-3$ & CS-137 & GAMMA_GS & USTEST & -0.29 \\
\hline 1303 & PDMS & & SW & & & E008912 & 28-Oct-75 & & $10098-97-2$ & SR-90 & SRISO_SEP_PRECIP_GPC & USTEST & 0.135 \\
\hline 1304 & PDMS & & SW & & & E041437 & 28-Oct-75 & & 10028-17-8 & TRITIUM & TRITIUM_DIST_LSC & USTEST & 823 \\
\hline 1305 & PDMS & & SW & & & E034933 & 28-Oct-75 & & $10028-17-8$ & TRITIUM & TRITIUM_DIST_LSC & USTEST & 863 \\
\hline 1306 & PDMS & & SW & & & E032738 & 28-Oct-75 & & $10098-97-2$ & SR-90 & SRISO_SEP_PRECIP_GPC & USTEST & 49.2 \\
\hline 1307 & PDMS & & SW & & & E003605 & 03-Nov-75 & & $10045-97-3$ & CS-137 & GAMMA_GS & USTEST & -0.371 \\
\hline 1308 & PDMS & & SW & & & E008919 & 04-Nov-75 & & $10045-97-3$ & CS-137 & GAMMA_GS & USTEST & 6.69 \\
\hline 1309 & PDMS & & SW & & & E016141 & 06-Nov-75 & & $10045-97-3$ & CS-137 & GAMMA_GS & USTEST & 0.0316 \\
\hline 1310 & PDMS & & SW & & & E016141 & 06-Nov-75 & & $10028-17-8$ & TRITIUM & TRITIUM_DIST_LSC & USTEST & 904 \\
\hline 1311 & PDMS & & SW & & & E016141 & 06-Nov-75 & & $10098-97-2$ & SR-90 & SRISO_SEP_PRECIP_GPC & USTEST & 0.281 \\
\hline 1312 & PDMS & & SW & & & E012106 & 06-Nov-75 & & 10028-17-8 & TRITIUM & TRITIUM_DIST_LSC & USTEST & 33600 \\
\hline 1313 & PDMS & & SW & & & E012106 & 06-Nov-75 & & $10045-97-3$ & CS-137 & GAMMA_GS & USTEST & 74.8 \\
\hline 1314 & PDMS & & SW & & & E037680 & 07-Nov-75 & & $10045-97-3$ & CS-137 & GAMMA_GS & USTEST & 2.26 \\
\hline 1315 & PDMS & & SW & & & E016402 & 07-Nov-75 & & $10098-97-2$ & SR-90 & SRISO_SEP_PRECIP_GPC & USTEST & 4.86 \\
\hline 1316 & PDMS & & SW & & & E016402 & 07-Nov-75 & & $10045-97-3$ & CS-137 & GAMMA_GS & USTEST & 3.75 \\
\hline 1317 & PDMS & & SW & & & E022422 & 07-Nov-75 & & $10045-97-3$ & CS-137 & GAMMA_GS & USTEST & 59.1 \\
\hline 1318 & PDMS & & SW & & & E022422 & 07-Nov-75 & & $10098-97-2$ & SR-90 & SRISO_SEP_PRECIP_GPC & USTEST & 0.455 \\
\hline 1319 & PDMS & & SW & & & E027643 & 07-Nov-75 & & $10045-97-3$ & CS-137 & GAMMA_GS & USTEST & 2.72 \\
\hline 1320 & PDMS & & SW & & & E021775 & 07-Nov-75 & & $10045-97-3$ & CS-137 & GAMMA_GS & USTEST & 4.36 \\
\hline 1321 & PDMS & & SW & & & E021775 & 07-Nov-75 & & $10098-97-2$ & SR-90 & SRISO_SEP_PRECIP_GPC & USTEST & 0.86 \\
\hline 1322 & PDMS & & SW & & & E010853 & 10-Nov-75 & & $10045-97-3$ & CS-137 & GAMMA_GS & USTEST & -0.161 \\
\hline 1323 & PDMS & & SW & & & E008926 & 11-Nov-75 & & $10045-97-3$ & CS-137 & GAMMA_GS & USTEST & -0.547 \\
\hline 1324 & PDMS & & SW & & & E003606 & 17-Nov-75 & & $10045-97-3$ & CS-137 & GAMMA_GS & USTEST & 11.3 \\
\hline 1325 & PDMS & & SW & & & E008839 & 18-Nov-75 & & $10045-97-3$ & CS-137 & GAMMA_GS & USTEST & 6.7 \\
\hline 1326 & PDMS & & SW & & & E004625 & 24-Nov-75 & & $10098-97-2$ & SR-90 & SRISO_SEP_PRECIP_GPC & USTEST & 0.536 \\
\hline \begin{tabular}{|c|}
1327 \\
\end{tabular} & PDMS & & SW & & & E004625 & 24-Nov-75 & & $10045-97-3$ & CS-137 & GAMMA_GS & USTEST & 9.51 \\
\hline 1328 & PDMS & & SW & & & E037072 & 24-Nov-75 & & $10028-17-8$ & TRITIUM & TRITIUM_DIST_LSC & USTEST & 420 \\
\hline 1329 & PDMS & & SW & & & E003607 & 24-Nov-75 & & $10045-97-3$ & CS-137 & GAMMA_GS & USTEST & -0.24 \\
\hline 1330 & PDMS & & SW & & & E002219 & 24-Nov-75 & & $10098-97-2$ & SR-90 & SRISO_SEP_PRECIP_GPC & USTEST & 0.217 \\
\hline 1331 & PDMS & & SW & & & E002219 & 24-Nov-75 & & $10045-97-3$ & CS-137 & GAMMA_GS & USTEST & 8.58 \\
\hline 1332 & PDMS & & SW & & & E026124 & 24-Nov-75 & & $10045-97-3$ & CS-137 & GAMMA_GS & USTEST & 0.0591 \\
\hline 1333 & PDMS & & SW & & & E008846 & 25-Nov-75 & & $10028-17-8$ & TRITIUM & TRITIUM_DIST_LSC & USTEST & 473 \\
\hline 1334 & PDMS & & SW & & & E008846 & 25-Nov-75 & & $10045-97-3$ & CS-137 & GAMMA_GS & USTEST & 3.45 \\
\hline 1335 & PDMS & & SW & & & E008846 & 25-Nov-75 & & $10098-97-2$ & SR-90 & SRISO_SEP_PRECIP_GPC & USTEST & 1 \\
\hline 1336 & PDMS & & SW & & & E014683 & 25-Nov-75 & & $10045-97-3$ & CS-137 & GAMMA_GS & USTEST & 0.591 \\
\hline 1337 & PDMS & & SW & & & E041441 & 25-Nov-75 & & $10028-17-8$ & TRITIUM & TRITIUM_DIST_LSC & USTEST & 386 \\
\hline 1338 & PDMS & & SW & & & E034937 & 25-Nov-75 & & $10028-17-8$ & TRITIUM & TRITIUM_DIST_LSC & USTEST & 546 \\
\hline 1339 & PDMS & & SW & & & E032739 & 25-Nov-75 & & $10098-97-2$ & SR-90 & SRISO_SEP_PRECIP_GPC & USTEST & 12.2 \\
\hline 1340 & PDMS & & SW & & & E003608 & 01-Dec-75 & & $10045-97-3$ & CS-137 & GAMMA_GS & USTEST & 0.0511 \\
\hline 1341 & PDMS & & SW & & & E008853 & 02-Dec-75 & & $10045-97-3$ & CS-137 & GAMMA_GS & USTEST & -0.318 \\
\hline 1342 & PDMS & & SW & & & E016142 & 05-Dec-75 & & $10045-97-3$ & CS-137 & GAMMA_GS & USTEST & 0.0481 \\
\hline 1343 & PDMS & & SW & & & E016142 & 05-Dec-75 & & $10028-17-8$ & TRITIUM & TRITIUM_DIST_LSC & USTEST & 209 \\
\hline 1344 & PDMS & & SW & & & E016142 & 05-Dec-75 & & $10098-97-2$ & SR-90 & SRISO_SEP_PRECIP_GPC & USTEST & 0.286 \\
\hline 1345 & PDMS & & SW & & & E012107 & 05-Dec-75 & & $10028-17-8$ & TRITIUM & TRITIUM_DIST_LSC & USTEST & 6100 \\
\hline 1346 & PDMS & & SW & & & E012107 & 05-Dec-75 & & $10045-97-3$ & CS-137 & GAMMA_GS & USTEST & -0.127 \\
\hline 1347 & PDMS & & SW & & & E003609 & 08-Dec-75 & & $10045-97-3$ & CS-137 & GAMMA_GS & USTEST & 0.0511 \\
\hline 1348 & PDMS & & SW & & & E008860 & 09-Dec-75 & & $10045-97-3$ & CS-137 & GAMMA_GS & USTEST & -0.355 \\
\hline 1349 & PDMS & & SW & & & E003610 & 15-Dec-75 & & $10045-97-3$ & CS-137 & GAMMA_GS & USTEST & -0.0291 \\
\hline 1350 & PDMS & & SW & & & E008867 & 16-Dec-75 & & $10045-97-3$ & CS-137 & GAMMA_GS & USTEST & -0.267 \\
\hline 1351 & PDMS & & SW & & & E003611 & 22-Dec-75 & & $10045-97-3$ & CS-137 & GAMMA_GS & USTEST & 0.0511 \\
\hline 1352 & PDMS & & SW & & & E008874 & 23-Dec-75 & & $10045-97-3$ & CS-137 & GAMMA_GS & USTEST & 6.8 \\
\hline 1353 & PDMS & & SW & & & E032740 & 23-Dec-75 & & $10098-97-2$ & SR-90 & SRISO_SEP_PRECIP_GPC & USTEST & 10.2 \\
\hline
\end{tabular}




\begin{tabular}{|c|c|c|c|c|c|c|c|c|c|c|c|c|c|}
\hline & $\bar{A}$ & $\mathrm{~B}$ & $\bar{C}$ & $\mathrm{D}$ & $\mathrm{E}$ & $\mathrm{F}$ & $\bar{G}$ & $\mathrm{H}$ & 1 & $\mathrm{~J}$ & $\bar{K}$ & $\bar{L}$ & $\mathrm{M}$ \\
\hline 1 & SAMPLE_NUM_ASSIGNED_TO & SAMPLER & MEDIA & BIOTA_MEDIA & TAG_ID & SAMP_NUM & SAMP_DATE_TIME & SAMP_DATE_TIME_ON & CON_ID & CON_SHORT_NAME & METHOD_NAME & LAB_CODE & VALUE_RPTD \\
\hline 1354 & PDMS & & SW & & & E004626 & 29-Dec-75 & & $10098-97-2$ & SR-90 & SRISO_SEP_PRECIP_GPC & USTEST & 0.135 \\
\hline 1355 & PDMS & & SW & & & E004626 & 29-Dec-75 & & $10028-17-8$ & TRITIUM & TRITIUM_DISTT_LSC & USTEST & 1280 \\
\hline 1356 & PDMS & & SW & & & E004626 & 29-Dec-75 & & $10045-97-3$ & CS-137 & GAMMA_GS & USTEST & -0.781 \\
\hline 1357 & PDMS & & SW & & & E016467 & 29-Dec-75 & & $10045-97-3$ & CS-137 & GAMMA_GS & USTEST & -0.299 \\
\hline 1358 & PDMS & & SW & & & E029661 & 29-Dec-75 & & $10045-97-3$ & CS-137 & GAMMA_GS & USTEST & 9.5 \\
\hline 1359 & PDMS & & SW & & & E002220 & 29-Dec-75 & & $10045-97-3$ & CS-137 & GAMMA_GS & USTEST & -12.3 \\
\hline 1360 & PDMS & & SW & & & E026129 & 29-Dec-75 & & $10045-97-3$ & CS-137 & GAMMA_GS & USTEST & -1.8 \\
\hline 1361 & PDMS & & SW & & & E012077 & 30-Dec-75 & & 15046-84-1 & I-129 & 1129_SEP_LEPS_GS & PNL-KI & 0.000035 \\
\hline 1362 & PDMS & & SW & & & E008787 & 30-Dec-75 & & $10028-17-8$ & TRITIUM & TRITIUM_DIST_L̄SC & USTEST & -625 \\
\hline 1363 & PDMS & & SW & & & E008787 & 30-Dec-75 & & $10045-97-3$ & CS-137 & GAMMA_GS & USTEST & 1.15 \\
\hline 1364 & PDMS & & SW & & & E008787 & 30-Dec-75 & & $10098-97-2$ & SR-90 & SRISO_SEEP_PRECIP_GPC & USTEST & 0.277 \\
\hline 1365 & PDMS & & SW & & & E075059 & 30-Dec-75 & & 10028-17-8 & TRITIUM & TRITIUM_DIST_LSC & USTEST & 674 \\
\hline 1366 & PDMS & & SW & & & E034941 & 30-Dec-75 & & 10028-17-8 & TRITIUM & TRITIUM_DIST_LSC & USTEST & 113 \\
\hline 1367 & PDMS & & SW & & & E001824 & 30-Dec-75 & & $10045-97-3$ & CS-137 & GAMMA_GS & USTEST & -0.354 \\
\hline 1368 & PDMS & & SW & & & E000843 & 31-Dec-75 & & $10045-97-3$ & CS-137 & 901.1_GAMMA_GS & USTEST & -1.05 \\
\hline 1369 & PDMS & & SW & & & E000843 & 31-Dec-75 & & $10028-17-8$ & TRITIUM & 906.0_H3_LSC & USTEST & -806 \\
\hline 1370 & PDMS & & SW & & & E074211 & 31-Dec-75 & & 15046-84-1 & I-129 & I129_SEP_LEPS_GS & PNL-KI & 0.00001 \\
\hline 1371 & PDMS & & SW & & & E012108 & 08-Jan-76 & & $10028-17-8$ & TRITIUM & TRITIUM_DIST_LSC & USTEST & 56300 \\
\hline 1372 & PDMS & & SW & & & E012108 & 08-Jan-76 & & $10045-97-3$ & CS-137 & GAMMA_GS & USTEST & -8.59 \\
\hline 1373 & PDMS & & SW & & & E014886 & 09-Jan-76 & & $10045-97-3$ & CS-137 & GAMMA_GS & USTEST & 0.0511 \\
\hline 1374 & PDMS & & SW & & & E066731 & 09-Jan-76 & & $10045-97-3$ & CS-137 & GAMMA_GS & USTEST & \\
\hline 1375 & PDMS & & SW & & & E066731 & 09-Jan-76 & & $10098-97-2$ & SR-90 & SRISO_SEEP_PRECIP_GPC & USTEST & \\
\hline 1376 & PDMS & & SW & & & E027644 & 09-Jan-76 & & $10045-97-3$ & CS-137 & GAMMA_GS & USTEST & 1.31 \\
\hline 1377 & PDMS & & SW & & & E027644 & 09-Jan-76 & & $10098-97-2$ & SR-90 & SRISO_SEP_PRECIP_GPC & USTEST & 5.95 \\
\hline 1378 & PDMS & & SW & & & E013528 & 19-Jan-76 & & $10045-97-3$ & CS-137 & GAMMA_GS & USTEST & 0.0026 \\
\hline 1379 & PDMS & & SW & & & E013528 & 19-Jan-76 & & $10028-17-8$ & TRITIUM & TRITIUM_DIST_LSC & USTEST & 902 \\
\hline 1380 & PDMS & & SW & & & E022423 & 19-Jan-76 & & $10045-97-3$ & CS-137 & GAMMA_GS & USTEST & 0.288 \\
\hline 1381 & PDMS & & SW & & & E004552 & 26-Jan-76 & & $10098-97-2$ & SR-90 & SRISO_SEP_PRECIP_GPC & USTEST & 0.423 \\
\hline 1382 & PDMS & & SW & & & E004552 & 26-Jan-76 & & $10028-17-8$ & TRITIUM & TRITIUM_DIST_LSC & USTEST & 81.8 \\
\hline 1383 & PDMS & & SW & & & E004552 & 26-Jan-76 & & $10045-97-3$ & CS-137 & GAMMA_GS & USTEST & -0.351 \\
\hline 1384 & PDMS & & SW & & & E016468 & 26-Jan-76 & & $10045-97-3$ & CS-137 & GAMMA_GS & USTEST & 0.0444 \\
\hline 1385 & PDMS & & SW & & & E001825 & 26-Jan-76 & & 10028-17-8 & TRITIUM & TRITIUM_DIST_LSC & USTEST & 55900 \\
\hline \begin{tabular}{|l|l|}
1386 \\
\end{tabular} & PDMS & & SW & & & E001825 & 26-Jan-76 & & $10045-97-3$ & CS-137 & GAMMA_GS & USTEST & 3.02 \\
\hline \begin{tabular}{|l|l|}
1387 \\
\end{tabular} & PDMS & & SW & & & E029662 & 26-Jan-76 & & $10045-97-3$ & CS-137 & GAMMA_GS & USTEST & -0.327 \\
\hline 1388 & PDMS & & SW & & & E002221 & 26-Jan-76 & & $10045-97-3$ & CS-137 & GAMMA_GS & USTEST & 0.0467 \\
\hline 1389 & PDMS & & SW & & & E024887 & 26-Jan-76 & & $10028-17-8$ & TRITIUM & TRITIUM_DIST_LSC & USTEST & 18100 \\
\hline 1390 & PDMS & & SW & & & E024887 & 26-Jan-76 & & $10045-97-3$ & CS-137 & GAMMA_GS & USTEST & -0.13 \\
\hline 1391 & PDMS & & SW & & & E026065 & 26-Jan-76 & & $10045-97-3$ & CS-137 & GAMMA_GS & USTEST & -0.314 \\
\hline 1392 & PDMS & & SW & & & E008815 & 27-Jan-76 & & $10028-17-8$ & TRITIUM & TRITIUM_DIST_LSC & USTEST & 616 \\
\hline 1393 & PDMS & & SW & & & E008815 & 27-Jan-76 & & $10045-97-3$ & CS-137 & GAMMA_GS & USTEST & 0.239 \\
\hline 1394 & PDMS & & SW & & & E008815 & 27-Jan-76 & & $10098-97-2$ & SR-90 & SRISO_SEP_PRECIP_GPC & USTEST & 0.212 \\
\hline 1395 & PDMS & & SW & & & E041446 & 27-Jan-76 & & $10028-17-8$ & TRITIUM & TRITIUM_DIST_LSC & USTEST & -383 \\
\hline 1396 & PDMS & & SW & & & E034945 & 27-Jan-76 & & $10028-17-8$ & TRITIUM & TRITIUM_DIST_LSC & USTEST & 86.2 \\
\hline 1397 & PDMS & & SW & & & E000844 & 28-Jan-76 & & $10045-97-3$ & CS-137 & 901.1_GAMMA_GS & USTEST & -0.304 \\
\hline 1398 & PDMS & & SW & & & E000844 & 28-Jan-76 & & $10028-17-8$ & TRITIUM & 906.0_H3_LSC & USTEST & 467 \\
\hline 1399 & PDMS & & SW & & & E012109 & 07-Feb-76 & & $10028-17-8$ & TRITIUM & TRITIUMM_DIST_LSC & USTEST & 23900 \\
\hline 1400 & PDMS & & SW & & & E012109 & 07-Feb-76 & & $10045-97-3$ & CS-137 & GAMMA_GS & USTEST & -3.68 \\
\hline 1401 & PDMS & & SW & & & E012078 & 17-Feb-76 & & 15046-84-1 & I-129 & 1129_SEP_LEPS_GS & PNL-KI & 0.000039 \\
\hline 1402 & PDMS & & SW & & & E074212 & $17-F e b-76$ & & 15046-84-1 & I-129 & 1129_SEP_LEPS_GS & PNL-KI & 0.00009 \\
\hline 1403 & PDMS & & SW & & & E004553 & 23-Feb-76 & & $10098-97-2$ & SR-90 & SRISO_SEP_PRECIP_GPC & USTEST & 0.248 \\
\hline 1404 & PDMS & & SW & & & E004553 & 23-Feb-76 & & $10028-17-8$ & TRITIUM & TRITIUM_DIST_LSC & USTEST & 852 \\
\hline 1405 & PDMS & & SW & & & E004553 & $23-F e b-76$ & & 10045-97-3 & CS-137 & GAMMA_GS & USTEST & -0.749 \\
\hline
\end{tabular}




\begin{tabular}{|c|c|c|c|c|c|c|c|c|c|c|c|c|c|}
\hline & A & $\mathrm{B}$ & $\mathrm{c}$ & $\mathrm{D}$ & $\mathrm{E}$ & $\mathrm{F}$ & G & $\mathrm{H}$ & $\mathrm{I}$ & $\mathrm{J}$ & $\mathrm{K}$ & $\mathrm{L}$ & $\mathrm{M}$ \\
\hline 1 & SAMPLE_NUM_ASSIGNED_TO & SAMPLER & MEDIA & BIOTA_MEDIA & TAG_ID & SAMP_NUM & SAMP_DATE_TIME & SAMP_DATE_TIME_ON & CON_ID & CON_SHORT_NAME & METHOD_NAME & LAB_CODE & \begin{tabular}{|l|} 
VALUE_RPTD \\
\end{tabular} \\
\hline 1406 & PDMS & & SW & & & E016469 & 23-Feb-76 & & $10045-97-3$ & CS-137 & GAMMA GS & USTEST & 1.64 \\
\hline 1407 & PDMS & & sW & & & E001826 & 23-Feb-76 & & $10028-17-8$ & TRITIUM & TRITIUM_DIST_LSC & USTEST & 57300 \\
\hline 1408 & PDMS & & sW & & & E001826 & 23-Feb-76 & & $10045-97-3$ & CS-137 & GAMMA_GS & USTEST & 7.03 \\
\hline 1409 & PDMS & & sW & & & E029663 & 23-Feb-76 & & $10045-97-3$ & CS-137 & GAMMA_GS & USTEST & 4.46 \\
\hline 1410 & PDMS & & sW & & & E002147 & 23-Feb-76 & & $10045-97-3$ & CS-137 & GAMMA_GS & USTEST & -0.441 \\
\hline 1411 & PDMS & & SW & & & E024888 & 23-Feb-76 & & $10028-17-8$ & TRITIUM & TRITIUM_DIST_LSC & USTEST & 85700 \\
\hline 1412 & PDMS & & SW & & & E024888 & 23-Feb-76 & & $10045-97-3$ & CS-137 & GAMMA_GS & USTEST & 4.75 \\
\hline 1413 & PDMS & & SW & & & E025537 & 23-Feb-76 & & $10045-97-3$ & CS-137 & GAMMA_GS & USTEST & -0.361 \\
\hline 1414 & PDMS & & sW & & & E008749 & 24-Feb-76 & & $10028-17-8$ & TRITIUM & TRITIUM_DIST_LSC & USTEST & 239 \\
\hline 1415 & PDMS & & SW & & & E008749 & 24-Feb-76 & & $10045-97-3$ & CS-137 & GAMMA_GS & USTEST & 3.24 \\
\hline 1416 & PDMS & & SW & & & E008749 & 24-Feb-76 & & $10098-97-2$ & SR-90 & SRISO_SEP_PRECIP_GPC & USTEST & 0.187 \\
\hline 1417 & PDMS & & SW & & & E041449 & 24-Feb-76 & & $10028-17-8$ & TRITIUM & TRITIUM_DISTT_LSC & USTEST & 1920 \\
\hline 1418 & PDMS & & SW & & & E034947 & 24-Feb-76 & & $10028-17-8$ & TRITIUM & TRITIUM_DIST_LSC & USTEST & 848 \\
\hline 1419 & PDMS & & SW & & & E000845 & 03-Mar-76 & & $10045-97-3$ & CS-137 & 901.1 GAMMA GS & USTEST & 2.35 \\
\hline 1420 & PDMS & & SW & & & E000845 & 03-Mar-76 & & $10028-17-8$ & TRITIUM & 906.0_H3_LSC & USTEST & -23.5 \\
\hline 1421 & PDMS & & SW & & & E012110 & 11-Mar-76 & & $10028-17-8$ & TRITIUM & TRITIUM_DIST_LSC & USTEST & 45400 \\
\hline 1422 & PDMS & & sW & & & E012110 & 11-Mar-76 & & $10045-97-3$ & CS-137 & GAMMA_GS & USTEST & -37.6 \\
\hline 1423 & PDMS & & sW & & & E029664 & 22-Mar-76 & & $10045-97-3$ & CS-137 & GAMMA_GS & USTEST & -0.169 \\
\hline 1424 & PDMS & & SW & & & E025541 & 22-Mar-76 & & $10045-97-3$ & CS-137 & GAMMA_GS & USTEST & 0.0487 \\
\hline 1425 & PDMS & & SW & & & E012079 & 26-Mar-76 & & 15046-84-1 & I-129 & 1129_SEP_LEPS_GS & PNL-KI & 0.000038 \\
\hline 1426 & PDMS & & SW & & & E008780 & 26-Mar-76 & & $10045-97-3$ & CS-137 & GAMMA_GS & USTEST & -0.858 \\
\hline \begin{tabular}{|l|l|}
1427 \\
\end{tabular} & PDMS & & SW & & & E004554 & 29-Mar-76 & & $10045-97-3$ & CS-137 & GAMMA_GS & USTEST & -0.45 \\
\hline 1428 & PDMS & & SW & & & E004554 & 29-Mar-76 & & 10028-17-8 & TRITIUM & TRITIUM_DIST_LSC & USTEST & 796 \\
\hline 1429 & PDMS & & SW & & & E004554 & 29-Mar-76 & & $10098-97-2$ & SR-90 & SRISO_SEP_PRECIP_GPC & USTEST & 0.26 \\
\hline 1430 & PDMS & & SW & & & E075060 & 29-Mar-76 & & 10028-17-8 & TRITIUM & TRITIUM_DISTT_LSC & USTEST & 755 \\
\hline 1431 & PDMS & & SW & & & E008783 & 29-Mar-76 & & $10028-17-8$ & TRITIUM & TRITIUM_DIST_LSC & USTEST & 139 \\
\hline 1432 & PDMS & & SW & & & E008783 & 29-Mar-76 & & $10045-97-3$ & CS-137 & GAMMA_GS & USTEST & 2.58 \\
\hline 1433 & PDMS & & SW & & & E008783 & 29-Mar-76 & & $10098-97-2$ & SR-90 & SRISO_SEPP_PRECIP_GPC & USTEST & 0.249 \\
\hline 1434 & PDMS & & SW & & & E041042 & 29-Mar-76 & & $10028-17-8$ & TRITIUM & TRITIUM_DIST_LSC & USTEST & 272 \\
\hline 1435 & PDMS & & SW & & & E016470 & 29-Mar-76 & & $10045-97-3$ & CS-137 & GAMMA_GS & USTEST & -0.361 \\
\hline 1436 & PDMS & & SW & & & E001827 & 29-Mar-76 & & $10028-17-8$ & TRITIUM & TRITIUM_DIST_LSC & USTEST & 38300 \\
\hline 1437 & PDMS & & SW & & & E001827 & 29-Mar-76 & & $10045-97-3$ & CS-137 & GAMMA_GS & USTEST & -0.481 \\
\hline 1438 & PDMS & & SW & & & E002148 & 29-Mar-76 & & $10045-97-3$ & CS-137 & GAMMA_GS & USTEST & -0.111 \\
\hline 1439 & PDMS & & SW & & & E024889 & 29-Mar-76 & & $10028-17-8$ & TRITIUM & TRITIUM_DIST_LSC & USTEST & 117000 \\
\hline 1440 & PDMS & & SW & & & E024889 & 29-Mar-76 & & $10045-97-3$ & CS-137 & GAMMA_GS & USTEST & -0.619 \\
\hline 1441 & PDMS & & sW & & & E014887 & 02-Apr-76 & & $10045-97-3$ & CS-137 & GAMMA_GS & USTEST & -0.246 \\
\hline 1442 & PDMS & & SW & & & E016403 & 02-Apr-76 & & $10098-97-2$ & SR-90 & SRISO_SEP_PRECIP_GPC & USTEST & 5.19 \\
\hline 1443 & PDMS & & SW & & & E016403 & 02-Apr-76 & & $10045-97-3$ & CS-137 & GAMMA_GS & USTEST & 1.62 \\
\hline 1444 & PDMS & & SW & & & E022424 & 02-Apr-76 & & $10045-97-3$ & CS-137 & GAMMA_GS & USTEST & 34.2 \\
\hline 1445 & PDMS & & SW & & & E022424 & 02-Apr-76 & & $10098-97-2$ & SR-90 & SRISO_SEEP_PRECIP_GPC & USTEST & 0.577 \\
\hline 1446 & PDMS & & SW & & & E003472 & 02-Apr-76 & & $10045-97-3$ & CS-137 & GAMMĀGS & USTEST & 10.7 \\
\hline \begin{tabular}{|l|l|}
1447 \\
\end{tabular} & PDMS & & SW & & & E003472 & 02-Apr-76 & & $10098-97-2$ & SR-90 & SRISO_SEEP_PRECIP_GPC & USTEST & 7.57 \\
\hline 1448 & PDMS & & SW & & & E027645 & 02-Apr-76 & & $10045-97-3$ & CS-137 & GAMMA_GS & USTEST & 12.1 \\
\hline 1449 & PDMS & & SW & & & E027645 & 02-Apr-76 & & $10098-97-2$ & SR-90 & SRISO_SEP_PRECIP_GPC & USTEST & 8.44 \\
\hline 1450 & PDMS & & SW & & & E014271 & 02-Apr-76 & & $10045-97-3$ & CS-137 & GAMMA_GS & USTEST & 2.14 \\
\hline 1451 & PDMS & & SW & & & E014271 & 02-Apr-76 & & $10098-97-2$ & SR-90 & SRISO_SEEP_PRECIP_GPC & USTEST & 1.46 \\
\hline 1452 & PDMS & & SW & & & E074213 & 05-Apr-76 & & 15046-84-1 & I-129 & 1129_SEP_LËPS_GS & PNL-KI & 0.0000092 \\
\hline 1453 & PDMS & & SW & & & E012111 & 09-Apr-76 & & $10028-17-8$ & TRITIUM & TRITIUM_DIST_LESC & USTEST & 35700 \\
\hline 1454 & PDMS & & SW & & & E012111 & 09-Apr-76 & & $10045-97-3$ & CS-137 & GAMMA_GS & USTEST & 20.2 \\
\hline 1455 & PDMS & & SW & & & E004555 & 12-Apr-76 & & $10098-97-2$ & SR-90 & SRISO_SEP_PRECIP_GPC & USTEST & 0.402 \\
\hline 1456 & PDMS & & SW & & & E004555 & 12-Apr-76 & & 10028-17-8 & TRITIUM & TRITIUM_DIST_LSC & USTEST & 130 \\
\hline 1457. & PDMS & & SW & & & E004555 & 12-Apr-76 & & $10045-97-3$ & CS-137 & GAMMA_GS & USTEST & 4.55 \\
\hline
\end{tabular}




\begin{tabular}{|c|c|c|c|c|c|c|c|c|c|c|c|c|c|}
\hline & A & $\mathrm{B}$ & $\mathrm{c}$ & $\mathrm{D}$ & $\mathrm{E}$ & $\mathrm{F}$ & G & $\mathrm{H}$ & $\mathrm{I}$ & $\mathrm{J}$ & $\mathrm{K}$ & $\mathrm{L}$ & $\mathrm{M}$ \\
\hline 1 & SAMPLE_NUM_ASSIGNED_TO & SAMPLER & MEDIA & BIOTA_MEDIA & TAG_ID & SAMP_NUM & SAMP_DATE_TIME & SAMP_DATE_TIME_ON & CON_ID & CON_SHORT_NAME & METHOD_NAME & LAB_CODE & \begin{tabular}{|l|} 
VALUE_RPTD \\
\end{tabular} \\
\hline 1458 & PDMS & & SW & & & E029665 & 19-Apr-76 & & $10045-97-3$ & CS-137 & GAMMA_GS & USTEST & 0.0511 \\
\hline 1459 & PDMS & & sW & & & E026739 & 22-Apr-76 & & $10045-97-3$ & CS-137 & GAMMA_GS & USTEST & -0.39 \\
\hline 1460 & PDMS & & sW & & & E016471 & 26-Apr-76 & & $10045-97-3$ & CS-137 & GAMMA_GS & USTEST & 0.048 \\
\hline 1461 & PDMS & & sW & & & E001828 & 26-Apr-76 & & $10028-17-8$ & TRITIUM & TRITIUM_DIST_LSC & USTEST & 81500 \\
\hline 1462 & PDMS & & sW & & & E001828 & 26-Apr-76 & & $10045-97-3$ & CS-137 & GAMMA_GS & USTEST & 0.0201 \\
\hline 1463 & PDMS & & sW & & & E002149 & 26-Apr-76 & & $10045-97-3$ & CS-137 & GAMMA_GS & USTEST & -0.603 \\
\hline 1464 & PDMS & & SW & & & E024890 & 26-Apr-76 & & $10028-17-8$ & TRITIUM & TRITIUM_DIST_LSC & USTEST & 126000 \\
\hline 1465 & PDMS & & SW & & & E024890 & 26-Apr-76 & & $10045-97-3$ & CS-137 & GAMMA_GS & USTEST & 1.11 \\
\hline 1466 & PDMS & & sW & & & E025545 & 26-Apr-76 & & $10045-97-3$ & CS-137 & GAMMA GS & USTEST & -0.381 \\
\hline 1467. & PDMS & & SW & & & E008718 & 27-Apr-76 & & $10028-17-8$ & TRITIUM & TRITIUM_DIST_LSC & USTEST & -355 \\
\hline 1468 & PDMS & & SW & & & E008718 & 27-Apr-76 & & $10045-97-3$ & CS-137 & GAMMA_GS & USTEST & 1.69 \\
\hline 1469 & PDMS & & SW & & & E008718 & 27-Apr-76 & & 10098-97-2 & SR-90 & SRISO_SEPP_PRECIP_GPC & USTEST & 0.234 \\
\hline 1470 & PDMS & & SW & & & E027398 & 27-Apr-76 & & $10028-17-8$ & TRITIUM & TRITIUM_DISTT_LSC & USTEST & 506 \\
\hline 1471 & PDMS & & SW & & & E012227 & 27-Apr-76 & & $10028-17-8$ & TRITIUM & TRITIUM_DIST_LSC & USTEST & 528 \\
\hline 1472 & PDMS & & SW & & & E000846 & 28-Apr-76 & & $10045-97-3$ & CS-137 & 901.1_GAMMA_GS & USTEST & 0.895 \\
\hline 1473 & PDMS & & SW & & & E000846 & 28-Apr-76 & & $10028-17-8$ & TRITIUM & 906.0_H3_LSC & USTEST & -443 \\
\hline 1474 & PDMS & & sW & & & E074214 & 04-May-76 & & 15046-84-1 & $1-129$ & I129_SEP_LEPS_GS & PNL-KI & 0.000013 \\
\hline 1475 & PDMS & & sW & & & E047394 & 06-May-76 & & $10028-17-8$ & TRITIUM & TRITIUM_DIST_LSC & USTEST & 930 \\
\hline 1476 & PDMS & & SW & & & E012080 & 09-May-76 & & 15046-84-1 & I-129 & 1129_SEP_LEPS_GS & PNL-KI & 0.0000285 \\
\hline 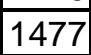 & PDMS & & SW & & & E012112 & 13-May-76 & & $10028-17-8$ & TRITIUM & TRITIUM_DIST_LESC & USTEST & 45100 \\
\hline 1478 & PDMS & & SW & & & E012112 & 13-May-76 & & $10045-97-3$ & CS-137 & GAMMA_GS & USTEST & 150 \\
\hline 1479 & PDMS & & SW & & & E000847 & 17-May-76 & & $10045-97-3$ & CS-137 & 901.1_GAMMA_GS & USTEST & -0.543 \\
\hline 1480 & PDMS & & SW & & & E000847 & 17-May-76 & & 10028-17-8 & TRITIUM & 906.0_H3_LSC & USTEST & 846 \\
\hline 1481 & PDMS & & SW & & & E029666 & 17-May-76 & & $10045-97-3$ & CS-137 & GAMMA_ĒGS & USTEST & 1.07 \\
\hline 1482 & PDMS & & SW & & & E004556 & 24-May-76 & & $10098-97-2$ & SR-90 & SRISO_SEP_PRECIP_GPC & USTEST & 0.251 \\
\hline 1483 & PDMS & & SW & & & E004556 & 24-May-76 & & $10028-17-8$ & TRITIUM & TRITIUM_DISTT_LSC & USTEST & 62.4 \\
\hline 1484 & PDMS & & SW & & & E004556 & 24-May-76 & & $10045-97-3$ & CS-137 & GAMMA_GS & USTEST & 0.00365 \\
\hline 1485 & PDMS & & SW & & & E016472 & 24-May-76 & & $10045-97-3$ & CS-137 & GAMMA_GS & USTEST & 0.0511 \\
\hline 1486 & PDMS & & SW & & & E001829 & 24-May-76 & & $10028-17-8$ & TRITIUM & TRITIUM_DIST_LSC & USTEST & 57300 \\
\hline \begin{tabular}{|l|l|}
1487 \\
\end{tabular} & PDMS & & SW & & & E001829 & 24-May-76 & & $10045-97-3$ & CS-137 & GAMMA_GS & USTEST & -0.0572 \\
\hline 1488 & PDMS & & SW & & & E002150 & 24-May-76 & & $10045-97-3$ & CS-137 & GAMMA_GS & USTEST & 0.0511 \\
\hline 1489 & PDMS & & SW & & & E024891 & 24-May-76 & & $10028-17-8$ & TRITIUM & TRITIUM_DIST_LSC & USTEST & 96700 \\
\hline 1490 & PDMS & & SW & & & E024891 & 24-May-76 & & $10045-97-3$ & CS-137 & GAMMA_GS & USTEST & -0.0142 \\
\hline 1491 & PDMS & & SW & & & E008652 & 25-May-76 & & $10028-17-8$ & TRITIUM & TRITIUM_DIST_LSC & USTEST & 819 \\
\hline 1492 & PDMS & & SW & & & E008652 & 25-May-76 & & $10045-97-3$ & CS-137 & GAMMA_GS & USTEST & 0.00256 \\
\hline 1493 & PDMS & & sW & & & E008652 & 25-May-76 & & $10098-97-2$ & SR-90 & SRISO_SEP_PRECIP_GPC & USTEST & 0.281 \\
\hline 1494 & PDMS & & SW & & & E041459 & 25-May-76 & & 10028-17-8 & TRITIUM & TRITIUM_DIST_LSC & USTEST & 297 \\
\hline 1495 & PDMS & & SW & & & E012228 & 25-May-76 & & 10028-17-8 & TRITIUM & TRITIUM_DIST_LSC & USTEST & 842 \\
\hline 1496 & PDMS & & SW & & & E025549 & 25-May-76 & & $10045-97-3$ & CS-137 & GAMMA_GS & USTEST & -0.0152 \\
\hline 1497 & PDMS & & SW & & & E023854 & 27-May-76 & & $10028-17-8$ & TRITIUM & TRITIUM_DIST_LSC & USTEST & 29.9 \\
\hline 1498 & PDMS & & SW & & & E023854 & 27-May-76 & & $10045-97-3$ & CS-137 & GAMMA_GS & USTEST & -0.0263 \\
\hline 1499 & PDMS & & SW & & & E012081 & 01-Jun-76 & & $15046-84-1$ & I-129 & 1129_SEP_LEPS_GS & PNL-KI & 0.00077 \\
\hline 1500 & PDMS & & SW & & & E074215 & 01-Jun-76 & & 15046-84-1 & I-129 & I129_SEP_LEPS_GS & PNL-KI & 0.000035 \\
\hline 1501 & PDMS & & SW & & & E029667 & 14-Jun-76 & & $10045-97-3$ & CS-137 & GAMMA_ESS & USTEST & 0.0492 \\
\hline 1502 & PDMS & & SW & & & E000848 & 16-Jun-76 & & $10045-97-3$ & CS-137 & 901.1_GAMMA_GS & USTEST & 0.0511 \\
\hline 1503 & PDMS & & SW & & & E000848 & 16-Jun-76 & & $10028-17-8$ & TRITIUM & 906.0_H3_LSC & USTEST & 965 \\
\hline 1504 & PDMS & & SW & & & E004557 & 28-Jun-76 & & $10045-97-3$ & CS-137 & GAMMĀĒSS & USTEST & -0.0157 \\
\hline 1505 & PDMS & & SW & & & E004557 & 28-Jun-76 & & $10028-17-8$ & TRITIUM & TRITIUM_DIST_LSC & USTEST & 259 \\
\hline 1506 & PDMS & & SW & & & E004557 & 28-Jun-76 & & $10098-97-2$ & SR-90 & SRISO_SEP_PRECIP_GPC & USTEST & 0.216 \\
\hline 1507 & PDMS & & sW & & & E016419 & 28-Jun-76 & & $10045-97-3$ & CS-137 & GAMMA_GS & USTEST & 0.428 \\
\hline 1508 & PDMS & & SW & & & E023855 & 28-Jun-76 & & $10028-17-8$ & TRITIUM & TRITIUM_DIST_LSC & USTEST & 69.9 \\
\hline 1509 & PDMS & & SW & & & E023855 & 28-Jun-76 & & $10045-97-3$ & CS-137 & GAMMA_GS & USTEST & -0.503 \\
\hline
\end{tabular}




\begin{tabular}{|c|c|c|c|c|c|c|c|c|c|c|c|c|c|}
\hline & A & $\mathrm{B}$ & $\mathrm{c}$ & $\mathrm{D}$ & $\mathrm{E}$ & $\mathrm{F}$ & G & $\mathrm{H}$ & $\mathrm{I}$ & $\mathrm{J}$ & $\mathrm{K}$ & $\mathrm{L}$ & $\mathrm{M}$ \\
\hline 1 & SAMPLE_NUM_ASSIGNED_TO & SAMPLER & MEDIA & BIOTA_MEDIA & TAG_ID & SAMP_NUM & SAMP_DATE_TIME & SAMP_DATE_TIME_ON & CON_ID & CON_SHORT_NAME & METHOD_NAME & LAB_CODE & \begin{tabular}{|l|} 
VALUE_RPTD \\
\end{tabular} \\
\hline 1510 & PDMS & & SW & & & E001830 & 28-Jun-76 & & $10028-17-8$ & TRITIUM & TRITIUM_DIST_LSC & USTEST & 31200 \\
\hline 1511 & PDMS & & sW & & & E001830 & 28-Jun-76 & & $10045-97-3$ & CS-137 & GAMMA_GS & USTEST & -0.286 \\
\hline 1512 & PDMS & & sW & & & E002151 & 28-Jun-76 & & $10045-97-3$ & CS-137 & GAMMA_GS & USTEST & 8.17 \\
\hline 1513 & PDMS & & sW & & & E024892 & 28-Jun-76 & & $10028-17-8$ & TRITIUM & TRITIUM_DIST_LSC & USTEST & 81100 \\
\hline 1514 & PDMS & & sW & & & E024892 & 28-Jun-76 & & $10045-97-3$ & CS-137 & GAMMA_GS & USTEST & -0.759 \\
\hline 1515 & PDMS & & sW & & & E012082 & 29-Jun-76 & & $15046-84-1$ & $1-129$ & I129_SEP_LEPS_GS & PNL-KI & 0.000015 \\
\hline 1516 & PDMS & & SW & & & E074216 & 29-Jun-76 & & 15046-84-1 & I-129 & I129_SEP_LEPS_GS & PNL-KI & 0.000011 \\
\hline 1517 & PDMS & & SW & & & E008687 & 29-Jun-76 & & $10028-17-8$ & TRITIUM & TRITIUM_DIST_LSC & USTEST & -351 \\
\hline 1518 & PDMS & & sW & & & E008687 & 29-Jun-76 & & $10098-97-2$ & SR-90 & SRISO_SEP_PRECIP_GPC & USTEST & 0.23 \\
\hline 1519 & PDMS & & SW & & & E008687 & 29-Jun-76 & & $10045-97-3$ & CS-137 & GAMMA_GS & USTEST & -0.00961 \\
\hline 1520 & PDMS & & sW & & & E041464 & 29-Jun-76 & & $10028-17-8$ & TRITIUM & TRITIUM_DIST_LSC & USTEST & -202 \\
\hline 1521 & PDMS & & SW & & & E012229 & 29-Jun-76 & & $10028-17-8$ & TRITIUM & TRITIUM_DIST_LSC & USTEST & -133 \\
\hline 1522 & PDMS & & SW & & & E025554 & 29-Jun-76 & & $10045-97-3$ & CS-137 & GAMMA_GS & USTEST & -0.0311 \\
\hline 1523 & PDMS & & SW & & & E029668 & 12-Jul-76 & & $10045-97-3$ & CS-137 & GAMMA_GS & USTEST & 0.0511 \\
\hline 1524 & PDMS & & SW & & & E000849 & 14-Jul-76 & & $10045-97-3$ & CS-137 & 901.1_GAMMA_GS & USTEST & 0.0536 \\
\hline 1525 & PDMS & & SW & & & E000849 & 14-Jul-76 & & $10028-17-8$ & TRITIUM & 906.0_H3_LSC & USTEST & 800 \\
\hline 1526 & PDMS & & sW & & & E023856 & 19-Jul-76 & & $10028-17-8$ & TRITIUM & TRITIUM_DIST_LSC & USTEST & 1080 \\
\hline \begin{tabular}{|c|}
1527 \\
\end{tabular} & PDMS & & sW & & & E023856 & 19-Jul-76 & & $10045-97-3$ & CS-137 & GAMMA_GS & USTEST & -0.244 \\
\hline 1528 & PDMS & & SW & & & E014888 & 23-Jul-76 & & $10045-97-3$ & CS-137 & GAMMA_GS & USTEST & 1.65 \\
\hline 1529 & PDMS & & SW & & & E016404 & 23-Jul-76 & & $10098-97-2$ & SR-90 & SRISO_SEEP_PRECIP_GPC & USTEST & 1.09 \\
\hline 1530 & PDMS & & SW & & & E016404 & 23-Jul-76 & & $10045-97-3$ & CS-137 & GAMMA_GS & USTEST & -0.000653 \\
\hline 1531 & PDMS & & SW & & & E022425 & 23-Jul-76 & & $10045-97-3$ & CS-137 & GAMMA_GS & USTEST & 0.00227 \\
\hline 1532 & PDMS & & SW & & & E022425 & 23-Jul-76 & & $10098-97-2$ & SR-90 & SRISO_SEP_PRECIP_GPC & USTEST & 0.696 \\
\hline 1533 & PDMS & & SW & & & E027646 & 23-Jul-76 & & $10045-97-3$ & CS-137 & GAMMA_GS & USTEST & 4.01 \\
\hline 1534 & PDMS & & SW & & & E027646 & 23-Jul-76 & & $10098-97-2$ & SR-90 & SRISO_SEP_PRECIP_GPC & USTEST & 1.24 \\
\hline 1535 & PDMS & & SW & & & E014272 & 23-Jul-76 & & $10045-97-3$ & CS-137 & GAMMA_GS & USTEST & -0.512 \\
\hline 1536 & PDMS & & SW & & & E014272 & 23-Jul-76 & & $10098-97-2$ & SR-90 & SRISO_SEP_PRECIP_GPC & USTEST & 10.4 \\
\hline 1537 & PDMS & & SW & & & E004558 & 26-Jul-76 & & $10098-97-2$ & SR-90 & SRISO_SEP_PRECIP_GPC & USTEST & 0.27 \\
\hline 1538 & PDMS & & SW & & & E004558 & 26-Jul-76 & & $10045-97-3$ & CS-137 & GAMMA_GS & USTEST & 0.136 \\
\hline 1539 & PDMS & & SW & & & E016420 & 26-Jul-76 & & $10045-97-3$ & CS-137 & GAMMA_GS & USTEST & 0.0511 \\
\hline 1540 & PDMS & & SW & & & E001831 & 26-Jul-76 & & $10028-17-8$ & TRITIUM & TRITIUM_DIST_LSC & USTEST & 46400 \\
\hline 1541 & PDMS & & SW & & & E001831 & 26-Jul-76 & & $10045-97-3$ & CS-137 & GAMMA_GS & USTEST & 0.0511 \\
\hline 1542 & PDMS & & SW & & & E002152 & 26-Jul-76 & & $10045-97-3$ & CS-137 & GAMMA_GS & USTEST & 4.02 \\
\hline 1543 & PDMS & & SW & & & E024893 & 26-Jul-76 & & $10028-17-8$ & TRITIUM & TRITIUM_DIST_LSC & USTEST & 130000 \\
\hline 1544 & PDMS & & SW & & & E024893 & 26-Jul-76 & & $10045-97-3$ & CS-137 & GAMMA_GS & USTEST & 0.0511 \\
\hline 1545 & PDMS & & sW & & & E025496 & 26-Jul-76 & & $10045-97-3$ & CS-137 & GAMMA_GS & USTEST & -0.21 \\
\hline 1546 & PDMS & & SW & & & E041388 & 27-Jul-76 & & 10028-17-8 & TRITIUM & TRITIUM_DIST_LSC & USTEST & 367 \\
\hline 1547 & PDMS & & SW & & & E008621 & 27-Jul-76 & & 10028-17-8 & TRITIUM & TRITIUM_DIST_LSC & USTEST & 432 \\
\hline 1548 & PDMS & & SW & & & E008621 & $27-J u l-76$ & & $10098-97-2$ & SR-90 & SRISO_SEP_PRECIP_GPC & USTEST & 0.194 \\
\hline 1549 & PDMS & & SW & & & E008621 & $27-J u l-76$ & & $10045-97-3$ & CS-137 & GAMMĀGS & USTEST & 0.734 \\
\hline 1550 & PDMS & & SW & & & E012230 & $27-J u l-76$ & & $10028-17-8$ & TRITIUM & TRITIUM_DIST_LSC & USTEST & 777 \\
\hline 1551 & PDMS & & SW & & & E012083 & 03-Aug-76 & & 15046-84-1 & I-129 & 1129_SEP_LEPS_GS & PNL-KI & 0.00003 \\
\hline 1552 & PDMS & & SW & & & E074217 & 03-Aug-76 & & 15046-84-1 & I-129 & I129_SEP_LEPS_GS & PNL-KI & 0.0000071 \\
\hline 1553 & PDMS & & SW & & & E000850 & 09-Aug-76 & & $10045-97-3$ & CS-137 & 901.1_GAMMA_ḠS & USTEST & 0.0511 \\
\hline 1554 & PDMS & & SW & & & E000850 & 09-Aug-76 & & 10028-17-8 & TRITIUM & 906.0_H3_LSC & USTEST & 87.6 \\
\hline 1555 & PDMS & & SW & & & E029669 & 09-Aug-76 & & $10045-97-3$ & CS-137 & GAMMĀE-GS & USTEST & 0.0502 \\
\hline 1556 & PDMS & & SW & & & E012185 & 24-Aug-76 & & $10028-17-8$ & TRITIUM & TRITIUM_DIST_LSC & USTEST & 858 \\
\hline 1557 & PDMS & & SW & & & E004559 & 31-Aug-76 & & $10045-97-3$ & CS-137 & GAMMA_GS & USTEST & 0.00256 \\
\hline 1558 & PDMS & & SW & & & E004559 & 31-Aug-76 & & $10028-17-8$ & TRITIUM & TRITIUM_DIST_LSC & USTEST & 726 \\
\hline 1559 & PDMS & & sW & & & E004559 & 31-Aug-76 & & $10098-97-2$ & SR-90 & SRISO_SEP_PRECIP_GPC & USTEST & 0.234 \\
\hline 1560 & PDMS & & SW & & & E012084 & 31-Aug-76 & & 15046-84-1 & I-129 & I129_SEP_LËPS_GS & PNL-KI & 0.000018 \\
\hline 1561 & PDMS & & SW & & & E074218 & 31-Aug-76 & & 15046-84-1 & I-129 & 1129_SEP_LEPS_GS & PNL-KI & 0.0000038 \\
\hline
\end{tabular}




\begin{tabular}{|c|c|c|c|c|c|c|c|c|c|c|c|c|c|}
\hline & A & $\mathrm{B}$ & $C$ & $\mathrm{D}$ & $E$ & $\mathrm{~F}$ & G & $\mathrm{H}$ & $\mathrm{I}$ & $\mathrm{J}$ & $\mathrm{K}$ & $\mathrm{L}$ & $\mathrm{M}$ \\
\hline 1 & SAMPLE_NUM_ASSIGNED_TO & SAMPLER & MEDIA & BIOTA_MEDIA & TAG_ID & SAMP_NUM & SAMP_DATE_TIME & SAMP_DATE_TIME_ON & CON_ID & CON_SHORT_NAME & METHOD_NAME & LAB_CODE & \begin{tabular}{|l|} 
VALUE_RPTD \\
\end{tabular} \\
\hline $\mid 1562$ & PDMS & & SW & & & E075061 & 31-Aug-76 & & $10028-17-8$ & TRITIUM & TRITIUM_DIST_LSC & USTEST & 794 \\
\hline 1563 & PDMS & & SW & & & E016421 & 31-Aug-76 & & $10045-97-3$ & CS-137 & GAMMA_GS & USTEST & -0.192 \\
\hline 1564 & PDMS & & SW & & & E023857 & 31-Aug-76 & & 10028-17-8 & TRITIUM & TRITIUM_DIST_LSC & USTEST & 1380 \\
\hline 1565 & PDMS & & SW & & & E023857 & 31-Aug-76 & & $10045-97-3$ & CS-137 & GAMMA_GS & USTEST & 0.0429 \\
\hline 1566 & PDMS & & SW & & & E001832 & 31-Aug-76 & & $10028-17-8$ & TRITIUM & TRITIUM_DIST_LSC & USTEST & 47000 \\
\hline 1567 & PDMS & & SW & & & E001832 & 31-Aug-76 & & $10045-97-3$ & CS-137 & GAMMA_GS & USTEST & -0.0579 \\
\hline 1568 & PDMS & & SW & & & E002153 & 31-Aug-76 & & $10045-97-3$ & CS-137 & GAMMA_GS & USTEST & 0.0511 \\
\hline 1569 & PDMS & & SW & & & E024894 & 31-Aug-76 & & $10028-17-8$ & TRITIUM & TRITIUM_DIST_LSC & USTEST & 237000 \\
\hline 1570 & PDMS & & SW & & & E024894 & 31-Aug-76 & & $10045-97-3$ & CS-137 & GAMMA_GS & USTEST & 0.0472 \\
\hline 1571 & PDMS & & SW & & & E008563 & 01-Sep-76 & & $10028-17-8$ & TRITIUM & TRITIUM_DIST_LSC & USTEST & 827 \\
\hline 1572 & PDMS & & SW & & & E008563 & 01-Sep-76 & & $10098-97-2$ & SR-90 & SRISO_SEP_PRECIP_GPC & USTEST & 0.277 \\
\hline 1573 & PDMS & & SW & & & E000851 & 07-Sep-76 & & $10028-17-8$ & TRITIUM & 906.0_H̄ 3 LLSC & USTEST & 981 \\
\hline 1574 & PDMS & & SW & & & E029670 & 07-Sep-76 & & $10045-97-3$ & CS-137 & GAMMA_ESS & USTEST & 1.51 \\
\hline 1575 & PDMS & & SW & & & E004560 & 27-Sep-76 & & $10098-97-2$ & SR-90 & SRISO_SEPPPRECIP_GPC & USTEST & 0.203 \\
\hline 1576 & PDMS & & SW & & & E004560 & 27-Sep-76 & & $10028-17-8$ & TRITIUM & TRITIUM_DIST_LSC & USTEST & 828 \\
\hline 1577 & PDMS & & SW & & & E004560 & 27-Sep-76 & & $10045-97-3$ & CS-137 & GAMMA_GS & USTEST & 0.027 \\
\hline 1578 & PDMS & & SW & & & E016422 & 27-Sep-76 & & $10045-97-3$ & CS-137 & GAMMA_GS & USTEST & 6.8 \\
\hline 1579 & PDMS & & SW & & & E023858 & 27-Sep-76 & & $10028-17-8$ & TRITIUM & TRITIUM_DIST_LSC & USTEST & 759 \\
\hline 1580 & PDMS & & SW & & & E023858 & 27-Sep-76 & & $10045-97-3$ & CS-137 & GAMMA_GS & USTEST & 0.604 \\
\hline 1581 & PDMS & & SW & & & E001833 & 27-Sep-76 & & $10028-17-8$ & TRITIUM & TRITIUM_DIST_LSC & USTEST & 52300 \\
\hline 1582 & PDMS & & SW & & & E001833 & 27-Sep-76 & & $10045-97-3$ & CS-137 & GAMMA_GS & USTEST & 0.641 \\
\hline 1583 & PDMS & & SW & & & E002154 & 27-Sep-76 & & $10045-97-3$ & CS-137 & GAMMA_GS & USTEST & 0.35 \\
\hline 1584 & PDMS & & SW & & & E024895 & 27-Sep-76 & & $10028-17-8$ & TRITIUM & TRITIUM_DIST_LSC & USTEST & 313000 \\
\hline 1585 & PDMS & & SW & & & E024895 & 27-Sep-76 & & $10045-97-3$ & CS-137 & GAMMA_GS & USTEST & 0.706 \\
\hline 1586 & PDMS & & SW & & & E012085 & 28-Sep-76 & & 15046-84-1 & I-129 & 1129_SEP_LEPS_GS & PNL-KI & 0.000058 \\
\hline 1587 & PDMS & & SW & & & E074219 & 28-Sep-76 & & 15046-84-1 & I-129 & 1129_SEP_LEPS_GS & PNL-KI & 0.0000071 \\
\hline 1588 & PDMS & & SW & & & E041395 & 29-Sep-76 & & $10028-17-8$ & TRITIUM & TRITIUM_DIST_LSC & USTEST & 1720 \\
\hline 1589 & PDMS & & SW & & & E008591 & 29-Sep-76 & & $10028-17-8$ & TRITIUM & TRITIUM_DIST_LSC & USTEST & 882 \\
\hline 1590 & PDMS & & SW & & & E008591 & 29-Sep-76 & & $10098-97-2$ & SR-90 & SRISO_SEP_PRECIP_GPC & USTEST & 0.194 \\
\hline 1591 & PDMS & & SW & & & E008591 & 29-Sep-76 & & $10045-97-3$ & CS-137 & GAMMA_GS & USTEST & 0.334 \\
\hline 1592 & PDMS & & SW & & & E012186 & 29-Sep-76 & & $10028-17-8$ & TRITIUM & TRITIUM_DIST_LSC & USTEST & 428 \\
\hline 1593 & PDMS & & SW & & & E029671 & $04-O c t-76$ & & $10045-97-3$ & CS-137 & GAMMA_GS & USTEST & 0.0511 \\
\hline 1594 & PDMS & & SW & & & E000852 & $05-$ Oct-76 & & $10045-97-3$ & CS-137 & 901.1_GAMMA_GS & USTEST & -0.00268 \\
\hline 1595 & PDMS & & SW & & & E000852 & $05-$ Oct-76 & & $10028-17-8$ & TRITIUM & 906.0_H3_LSC & USTEST & -108 \\
\hline 1596 & PDMS & & SW & & & E023859 & 11-Oct-76 & & $10028-17-8$ & TRITIUM & TRITIUM_DIST_LSC & USTEST & 36.9 \\
\hline 1597 & PDMS & & SW & & & E023859 & 11-Oct-76 & & $10045-97-3$ & CS-137 & GAMMA_GS & USTEST & 4.57 \\
\hline 1598 & PDMS & & SW & & & E004561 & 25-Oct-76 & & $10098-97-2$ & SR-90 & SRISO_SEP_PRECIP_GPC & USTEST & 0.257 \\
\hline 1599 & PDMS & & SW & & & E004561 & 25-Oct-76 & & 10028-17-8 & TRITIUM & TRITIUM_DIST_LSC & USTEST & 1010 \\
\hline 1600 & PDMS & & SW & & & E004561 & 25-Oct-76 & & $10045-97-3$ & CS-137 & GAMMA_GS & USTEST & -0.0167 \\
\hline 1601 & PDMS & & SW & & & E016423 & 25-Oct-76 & & $10045-97-3$ & CS-137 & GAMMA_GS & USTEST & -0.541 \\
\hline 1602 & PDMS & & SW & & & E001834 & $25-$ Oct-76 & & $10028-17-8$ & TRITIUM & TRITIUM_DIST_LSC & USTEST & 31500 \\
\hline 1603 & PDMS & & SW & & & E001834 & 25-Oct-76 & & $10045-97-3$ & CS-137 & GAMMA_GS & USTEST & -0.233 \\
\hline 1604 & PDMS & & SW & & & E002155 & 25-Oct-76 & & $10045-97-3$ & CS-137 & GAMMA_GS & USTEST & -0.0342 \\
\hline 1605 & PDMS & & SW & & & E024876 & 25-Oct-76 & & $10028-17-8$ & TRITIUM & TRITIUM_DIST_LSC & USTEST & 134000 \\
\hline 1606 & PDMS & & SW & & & E024876 & 25-Oct-76 & & $10045-97-3$ & CS-137 & GAMMA_GS & USTEST & 0.0382 \\
\hline 1607 & PDMS & & SW & & & E014889 & 25-Oct-76 & & $10045-97-3$ & CS-137 & GAMMA_GS & USTEST & 1.63 \\
\hline 1608 & PDMS & & SW & & & E016405 & 25-Oct-76 & & $10098-97-2$ & SR-90 & SRISO_SEP_PRECIP_GPC & USTEST & 3.19 \\
\hline 1609 & PDMS & & SW & & & E016405 & $25-$ Oct-76 & & $10045-97-3$ & CS-137 & GAMMA_GS & USTEST & 2.58 \\
\hline 1610 & PDMS & & SW & & & E022426 & 25-Oct-76 & & $10045-97-3$ & CS-137 & GAMMA_GS & USTEST & 27.8 \\
\hline 1611 & PDMS & & SW & & & E022426 & 25-Oct-76 & & $10098-97-2$ & SR-90 & SRISO_SEP_PRECIP_GPC & USTEST & $\exists$ \\
\hline 1612 & PDMS & & SW & & & E027647 & 25-Oct-76 & & $10045-97-3$ & CS-137 & GAMMA_GS & USTEST & 11.8 \\
\hline 1613 & PDMS & & SW & & & E027647 & 25-Oct-76 & & $10098-97-2$ & SR-90 & SRISO_SEP_PRECIP_GPC & USTEST & 0.0048 \\
\hline
\end{tabular}




\begin{tabular}{|c|c|c|c|c|c|c|c|c|c|c|c|c|c|}
\hline & $\bar{A}$ & $\mathrm{~B}$ & $\bar{C}$ & $\mathrm{D}$ & $\mathrm{E}$ & $\mathrm{F}$ & $\bar{G}$ & $\mathrm{H}$ & 1 & $\mathrm{~J}$ & $\bar{K}$ & $\bar{L}$ & $\mathrm{M}$ \\
\hline 1 & SAMPLE_NUM_ASSIGNED_TO & SAMPLER & MEDIA & BIOTA_MEDIA & TAG_ID & SAMP_NUM & SAMP_DATE_TIME & SAMP_DATE_TIME_ON & CON_ID & CON_SHORT_NAME & METHOD_NAME & LAB_CODE & VALUE_RPTD \\
\hline 1614 & PDMS & & SW & & & E014273 & 25 -Oct-76 & & $10045-97-3$ & CS-137 & GAMMA_GS & USTEST & -0.247 \\
\hline 1615 & PDMS & & SW & & & E014273 & 25-Oct-76 & & $10098-97-2$ & SR-90 & SRISO_SEP_PRECIP_GPC & USTEST & 0.0214 \\
\hline 1616 & PDMS & & SW & & & E012086 & 26-Oct-76 & & 15046-84-1 & I-129 & 1129 SEP_LEPS GS & PNL-KI & 0.000059 \\
\hline \begin{tabular}{|l|l|}
1617 \\
\end{tabular} & PDMS & & SW & & & E074220 & 26-Oct-76 & & $15046-84-1$ & I-129 & I129_SEP_LEPS_GS & PNL-KI & 0.0000103 \\
\hline 1618 & PDMS & & SW & & & E008525 & 27-Oct-76 & & $10028-17-8$ & TRITIUM & TRITIUM_DIST_LSC & USTEST & 49.7 \\
\hline 1619 & PDMS & & sW & & & E008525 & 27-Oct-76 & & $10098-97-2$ & SR-90 & SRISO SEP PRECIP GPC & USTEST & 0.243 \\
\hline 1620 & PDMS & & SW & & & E008525 & 27-Oct-76 & & $10045-97-3$ & CS-137 & GAMMA_GS & USTEST & -0.000536 \\
\hline 1621 & PDMS & & SW & & & E041398 & 27-Oct-76 & & $10028-17-8$ & TRITIUM & TRITIUM_DIST_LSC & USTEST & -90 \\
\hline 1622 & PDMS & & SW & & & E012187 & 27-Oct-76 & & $10028-17-8$ & TRITIUM & TRITIUM_DIST_LSC & USTEST & 288 \\
\hline 1623 & PDMS & & SW & & & E000853 & 01-Nov-76 & & $10045-97-3$ & CS-137 & 901.1_GAMMA_GS & USTEST & 4.8 \\
\hline 1624 & PDMS & & SW & & & E000853 & 01-Nov-76 & & 10028-17-8 & TRITIUM & 906.0 H3 LSC & USTEST & 45.7 \\
\hline 1625 & PDMS & & sW & & & E029672 & 01-Nov-76 & & $10045-97-3$ & CS-137 & GAMMA_GS & USTEST & 0.499 \\
\hline \begin{tabular}{|l|l|}
1626 \\
\end{tabular} & PDMS & & sW & & & E041401 & 24-Nov-76 & & $10028-17-8$ & TRITIUM & TRITIUM_DIST_LSC & USTEST & 105 \\
\hline \begin{tabular}{|l|l|}
1627 \\
162
\end{tabular} & PDMS & & sW & & & E008459 & 24-Nov-76 & & $10028-17-8$ & TRITIUM & TRITIUM_DIST_LSC & USTEST & -204 \\
\hline \begin{tabular}{|c|}
1628 \\
\end{tabular} & PDMS & & sW & & & E008459 & 24-Nov-76 & & $10098-97-2$ & SR-90 & SRISO_SEP_PRECIP_GPC & USTEST & 0.257 \\
\hline \begin{tabular}{|l|}
1629 \\
\end{tabular} & PDMS & & sW & & & E008459 & 24-Nov-76 & & $10045-97-3$ & CS-137 & GAMMA_GS & USTEST & 0.00256 \\
\hline 1630 & PDMS & & SW & & & E012188 & 24-Nov-76 & & $10028-17-8$ & TRITIUM & TRITIUM DIST_LSC & USTEST & 342 \\
\hline 1631 & PDMS & & SW & & & E000854 & 29-Nov-76 & & $10045-97-3$ & CS-137 & 901.1_GAMMA_GS & USTEST & 6.17 \\
\hline 1632 & PDMS & & SW & & & E000854 & 29-Nov-76 & & $10028-17-8$ & TRITIUM & 906.0_H3_LSC & USTEST & 314 \\
\hline 1633 & PDMS & & SW & & & E004562 & 29-Nov-76 & & $10098-97-2$ & SR-90 & SRISO_SĒP_PRECIP_GPC & USTEST & 0.207 \\
\hline 1634 & PDMS & & SW & & & E004562 & 29-Nov-76 & & $10028-17-8$ & TRITIUM & TRITIUM_DISTT_LSC & USTEST & 348 \\
\hline 1635 & PDMS & & SW & & & E004562 & 29-Nov-76 & & $10045-97-3$ & CS-137 & GAMMA_GS & USTEST & 0.483 \\
\hline 1636 & PDMS & & SW & & & E012087 & 29-Nov-76 & & 15046-84-1 & I-129 & 1129_SEP_LEPS_GS & PNL-KI & 0.000037 \\
\hline \begin{tabular}{|l|l|}
1637 \\
\end{tabular} & PDMS & & SW & & & E074221 & 29-Nov-76 & & 15046-84-1 & I-129 & 1129_SEP_LEPS_GS & PNL-KI & 0.000015 \\
\hline 1638 & PDMS & & sW & & & E016424 & 29-Nov-76 & & $10045-97-3$ & CS-137 & GAMMA_GSS & USTEST & 12 \\
\hline 1639 & PDMS & & sW & & & E001835 & 29-Nov-76 & & $10028-17-8$ & TRITIUM & TRITIUM_DIST_LSC & USTEST & 46400 \\
\hline 1640 & PDMS & & sW & & & E001835 & 29-Nov-76 & & $10045-97-3$ & CS-137 & GAMMA_GS & USTEST & 0.045 \\
\hline \begin{tabular}{|l|}
1641 \\
\end{tabular} & PDMS & & sW & & & E029673 & 29-Nov-76 & & $10045-97-3$ & CS-137 & GAMMA_GS & USTEST & -0.0303 \\
\hline \begin{tabular}{|l|}
1642 \\
\end{tabular} & PDMS & & sW & & & E002156 & 29-Nov-76 & & $10045-97-3$ & CS-137 & GAMMA_GS & USTEST & 1.65 \\
\hline 1643 & PDMS & & SW & & & E024877 & 29-Nov-76 & & $10028-17-8$ & TRITIUM & TRITIUM_DIST_LSC & USTEST & 160000 \\
\hline 1644 & PDMS & & sW & & & E024877 & 29-Nov-76 & & $10045-97-3$ & CS-137 & GAMMA_GS & USTEST & 1.1 \\
\hline 1645 & PDMS & & sW & & & E000855 & 27-Dec-76 & & $10045-97-3$ & CS-137 & 901.1_GAMMA_GS & USTEST & -0.6 \\
\hline 1646 & PDMS & & sW & & & E000855 & 27-Dec-76 & & $10028-17-8$ & TRITIUM & 906.0_H3_LSC & USTEST & 118 \\
\hline \begin{tabular}{|l|l|}
1647 \\
\end{tabular} & PDMS & & sW & & & E004563 & 27-Dec-76 & & $10098-97-2$ & SR-90 & SRISO_SEP_PRECIP_GPC & USTEST & 0.297 \\
\hline 1648 & PDMS & & SW & & & E004563 & 27-Dec-76 & & $10028-17-8$ & TRITIUM & TRITIUM_DISTT_LSC & USTEST & -128 \\
\hline 1649 & PDMS & & SW & & & E004563 & 27-Dec-76 & & $10045-97-3$ & CS-137 & GAMMA_GS & USTEST & 0.682 \\
\hline 1650 & PDMS & & SW & & & E016425 & 27-Dec-76 & & $10045-97-3$ & CS-137 & GAMMA_GS & USTEST & -0.272 \\
\hline 1651 & PDMS & & SW & & & E001836 & 27-Dec-76 & & $10028-17-8$ & TRITIUM & TRITIUM_DIST_LSC & USTEST & 45900 \\
\hline 1652 & PDMS & & sW & & & E001836 & 27-Dec-76 & & $10045-97-3$ & CS-137 & GAMMA_GS & USTEST & -0.108 \\
\hline 1653 & PDMS & & sW & & & E029674 & 27-Dec-76 & & $10045-97-3$ & CS-137 & GAMMA_GS & USTEST & -0.134 \\
\hline 1654 & PDMS & & sW & & & E002157 & 27-Dec-76 & & $10045-97-3$ & CS-137 & GAMMA_GS & USTEST & -0.331 \\
\hline 1655 & PDMS & & sW & & & E040731 & 27-Dec-76 & & $10028-17-8$ & TRITIUM & TRITIUM_DIST_LSC & USTEST & 176000 \\
\hline 1656 & PDMS & & SW & & & E012088 & 28-Dec-76 & & 15046-84-1 & I-129 & 1129_SEP_LEPS_GS & PNL-KI & 0.000056 \\
\hline 1657 & PDMS & & SW & & & E074222 & 28-Dec-76 & & 15046-84-1 & I-129 & 1129_SEP_LEPS_GS & PNL-KI & 0.0000089 \\
\hline 1658 & PDMS & & SW & & & E008494 & 29-Dec-76 & & $10028-17-8$ & TRITIUM & TRITIUM_DIST_LSC & USTEST & 36.5 \\
\hline 1659 & PDMS & & SW & & & E008494 & 29-Dec-76 & & $10098-97-2$ & SR-90 & SRISO_SEP_PRECIP_GPC & USTEST & 0.315 \\
\hline 1660 & PDMS & & sW & & & E008494 & 29-Dec-76 & & $10045-97-3$ & CS-137 & GAMMA_GS & USTEST & -0.0185 \\
\hline 1661 & PDMS & & sW & & & E041405 & 29-Dec-76 & & $10028-17-8$ & TRITIUM & TRITIUM_DIST_LSC & USTEST & -79.2 \\
\hline \begin{tabular}{|l|}
1662 \\
\end{tabular} & PDMS & & sW & & & E012189 & 29-Dec-76 & & $10028-17-8$ & TRITIUM & TRITIUM_DIST_LSC & USTEST & -97.4 \\
\hline 1663 & PDMS & & sW & & & E024878 & 29-Dec-76 & & $10045-97-3$ & CS-137 & GAMMA GS & USTEST & -0.48 \\
\hline 1664 & PDMS & & sW & & & E066732 & 03-Jan-77 & & $10045-97-3$ & CS-137 & GAMMA_GS & USTEST & \\
\hline 1665 & PDMS & & sW & & & E066732 & 03-Jan-77 & & $10098-97-2$ & SR-90 & SRISO SEP PRECIP GPC & USTEST & \\
\hline
\end{tabular}




\begin{tabular}{|c|c|c|c|c|c|c|c|c|c|c|c|c|c|}
\hline & A & $\mathrm{B}$ & $\mathrm{c}$ & $\mathrm{D}$ & $\mathrm{E}$ & $\mathrm{F}$ & G & $\mathrm{H}$ & $\mathrm{I}$ & $\mathrm{J}$ & $\mathrm{K}$ & $\mathrm{L}$ & $\mathrm{M}$ \\
\hline 1 & SAMPLE_NUM_ASSIGNED_TO & SAMPLER & MEDIA & BIOTA_MEDIA & TAG_ID & SAMP_NUM & SAMP_DATE_TIME & SAMP_DATE_TIME_ON & CON_ID & CON_SHORT_NAME & METHOD_NAME & LAB_CODE & VALUE_RPTD \\
\hline 1666 & PDMS & & SW & & & E011329 & 11-Jan-77 & & $10045-97-3$ & CS-137 & GAMMA GS & PNL-KI & 0.006 \\
\hline \begin{tabular}{|l|l|}
1667 & \\
\end{tabular} & PDMS & & sW & & & E002044 & 11-Jan-77 & & $10045-97-3$ & CS-137 & GAMMA_GS & PNL-KI & 0.02 \\
\hline 1668 & PDMS & & sW & & & E001760 & 11-Jan-77 & & $10045-97-3$ & CS-137 & GAMMA_GS & PNL-KI & 0.02 \\
\hline 1669 & PDMS & & sW & & & E014147 & 11-Jan-77 & & $10045-97-3$ & CS-137 & GAMMA_GS & PNL-KI & 0.004 \\
\hline 1670 & PDMS & & SW & & & E000856 & 24-Jan-77 & & $10045-97-3$ & CS-137 & 901.1_GAMMA_GS & USTEST & 0.0511 \\
\hline 1671 & PDMS & & SW & & & E000856 & 24-Jan-77 & & $10028-17-8$ & TRITIUM & 906.0_H3_LSC & USTEST & 159 \\
\hline 1672 & PDMS & & SW & & & E001837 & 24-Jan-77 & & $10028-17-8$ & TRITIUM & TRITIUM_DIST_LSC & USTEST & 43200 \\
\hline 1673 & PDMS & & SW & & & E001837 & 24-Jan-77 & & $10045-97-3$ & CS-137 & GAMMA_GS & USTEST & -0.219 \\
\hline 1674 & PDMS & & sW & & & E024879 & 24-Jan-77 & & $10028-17-8$ & TRITIUM & TRITIUM_DIST_LSC & USTEST & 198000 \\
\hline 1675 & PDMS & & SW & & & E024879 & 24-Jan-77 & & $10045-97-3$ & CS-137 & GAMMA_GS & USTEST & 0.0511 \\
\hline 1676 & PDMS & & SW & & & E011330 & 25-Jan-77 & & $10045-97-3$ & CS-137 & GAMMA_GS & PNL-KI & 0.002 \\
\hline \begin{tabular}{|l|l|}
1677 \\
\end{tabular} & PDMS & & SW & & & E002045 & 25-Jan-77 & & $10045-97-3$ & CS-137 & GAMMA_GS & PNL-KI & 0.02 \\
\hline 1678 & PDMS & & SW & & & E002045 & 25-Jan-77 & & 15046-84-1 & $1-129$ & 1129_SEP_LEPS_GS & PNL-KI & 0.00005 \\
\hline 1679 & PDMS & & SW & & & E001761 & 25-Jan-77 & & 15046-84-1 & $\mid-129$ & 1129_SEP_LEPS_GS & PNL-KI & 0.0000076 \\
\hline 1680 & PDMS & & SW & & & E001761 & 25-Jan-77 & & $10045-97-3$ & CS-137 & GAMMA_GS & PNL-KI & 0.01 \\
\hline \begin{tabular}{|l|}
1681 \\
\end{tabular} & PDMS & & SW & & & E014148 & 25-Jan-77 & & $10045-97-3$ & CS-137 & GAMMA_GS & PNL-KI & 0.002 \\
\hline 1682 & PDMS & & SW & & & E008428 & 26-Jan-77 & & $10028-17-8$ & TRITIUM & TRITIUM_DIST_LSC & USTEST & 100 \\
\hline \begin{tabular}{|l|}
1683 \\
\end{tabular} & PDMS & & sW & & & E008428 & 26-Jan-77 & & $10045-97-3$ & CS-137 & GAMMA_GS & USTEST & 0.0464 \\
\hline 1684 & PDMS & & SW & & & E041408 & 26-Jan-77 & & 10028-17-8 & TRITIUM & TRITIUM_DIST_LSC & USTEST & 254 \\
\hline 1685 & PDMS & & SW & & & E034897 & 26-Jan-77 & & 10028-17-8 & TRITIUM & TRITIUM_DIST_LSC & USTEST & 222 \\
\hline 1686 & PDMS & & SW & & & E004564 & 31-Jan-77 & & $10045-97-3$ & CS-137 & GAMMA_GS & USTEST & 6.78 \\
\hline \begin{tabular}{|l|l|}
1687 \\
\end{tabular} & PDMS & & SW & & & E004564 & 31-Jan-77 & & $10028-17-8$ & TRITIUM & TRITIUM_DIST_LSC & USTEST & 253 \\
\hline 1688 & PDMS & & SW & & & E016426 & 31-Jan-77 & & $10045-97-3$ & CS-137 & GAMMA_GS & USTEST & 11.3 \\
\hline 1689 & PDMS & & SW & & & E002158 & 31-Jan-77 & & $10045-97-3$ & CS-137 & GAMMA_GS & USTEST & 8.65 \\
\hline 1690 & PDMS & & SW & & & E002159 & 07-Feb-77 & & $10045-97-3$ & CS-137 & GAMMA_GS & USTEST & -0.954 \\
\hline 1691 & PDMS & & SW & & & E002046 & 08-Feb-77 & & $10045-97-3$ & CS-137 & GAMMA_GS & PNL-KI & 0.02 \\
\hline 1692 & PDMS & & SW & & & E002046 & 08-Feb-77 & & 15046-84-1 & I-129 & 1129_SEP_LEPS_GS & PNL-KI & 0.000069 \\
\hline 1693 & PDMS & & SW & & & E011331 & 08-Feb-77 & & $10045-97-3$ & CS-137 & GAMMA_GS & PNL-KI & 0.005 \\
\hline 1694 & PDMS & & SW & & & E001762 & 08-Feb-77 & & $10045-97-3$ & CS-137 & GAMMA_GS & PNL-KI & 0.09 \\
\hline 1695 & PDMS & & SW & & & E014149 & 08-Feb-77 & & $10045-97-3$ & CS-137 & GAMMA_GS & PNL-KI & 0.003 \\
\hline 1696 & PDMS & & SW & & & E000857 & 14-Feb-77 & & $10045-97-3$ & CS-137 & 901.1_GAMMA_GS & USTEST & -0.165 \\
\hline \begin{tabular}{|l|l|}
1697 \\
\end{tabular} & PDMS & & SW & & & E000857 & 14-Feb-77 & & $10028-17-8$ & TRITIUM & 906.0_H3_LSC & USTEST & -283 \\
\hline 1698 & PDMS & & SW & & & E001763 & 14-Feb-77 & & 15046-84-1 & $1-129$ & I129_SEP_LEPS_GS & PNL-KI & 0.000008 \\
\hline 1699 & PDMS & & SW & & & E001763 & 14-Feb-77 & & $10045-97-3$ & CS-137 & GAMMA_ĒS & PNL-KI & 0.03 \\
\hline 1700 & PDMS & & SW & & & E014150 & 14-Feb-77 & & $10045-97-3$ & CS-137 & GAMMA_GS & PNL-KI & 0.006 \\
\hline 1701 & PDMS & & SW & & & E001838 & 14-Feb-77 & & $10028-17-8$ & TRITIUM & TRITIUM_DIST_LSC & USTEST & 53900 \\
\hline 1702 & PDMS & & SW & & & E001838 & 14-Feb-77 & & $10045-97-3$ & CS-137 & GAMMA_GS & USTEST & -0.26 \\
\hline 1703 & PDMS & & SW & & & E024880 & 14-Feb-77 & & 10028-17-8 & TRITIUM & TRITIUM_DIST_LSC & USTEST & 186000 \\
\hline 1704 & PDMS & & SW & & & E024880 & 14-Feb-77 & & $10045-97-3$ & CS-137 & GAMMA_GS & USTEST & 0.0511 \\
\hline 1705 & PDMS & & SW & & & E014890 & 16-Feb-77 & & $10045-97-3$ & CS-137 & GAMMA_GS & USTEST & 2.19 \\
\hline 1706 & PDMS & & SW & & & E016406 & 16-Feb-77 & & $10098-97-2$ & SR-90 & SRISO_SEEP_PRECIP_GPC & USTEST & 1.61 \\
\hline $\mid 1707$ & PDMS & & SW & & & E016406 & 16-Feb-77 & & $10045-97-3$ & CS-137 & GAMMĀGS & USTEST & 2.52 \\
\hline 1708 & PDMS & & SW & & & E022427 & 16-Feb-77 & & $10045-97-3$ & CS-137 & GAMMA_GS & USTEST & 1.85 \\
\hline 1709 & PDMS & & SW & & & E022427 & 16-Feb-77 & & $10098-97-2$ & SR-90 & SRISO_SEP_PRECIP_GPC & USTEST & 1.25 \\
\hline 1710 & PDMS & & SW & & & E027648 & 16-Feb-77 & & $10045-97-3$ & CS-137 & GAMMA_GS & USTEST & 13.7 \\
\hline 1711 & PDMS & & SW & & & E027648 & 16-Feb-77 & & $10098-97-2$ & SR-90 & SRISO_SEEP_PRECIP_GPC & USTEST & 4.94 \\
\hline 1712 & PDMS & & SW & & & E014274 & 16-Feb-77 & & $10045-97-3$ & CS-137 & GAMMA_GS & USTEST & 1.7 \\
\hline 1713 & PDMS & & SW & & & E014274 & 16-Feb-77 & & $10098-97-2$ & SR-90 & SRISO_SEEP_PRECIP_GPC & USTEST & 3.32 \\
\hline 1714 & PDMS & & SW & & & E002047 & 22-Feb-77 & & $10045-97-3$ & CS-137 & GAMMA_GS & PNL-KI & 0.02 \\
\hline 1715 & PDMS & & SW & & & E011332 & 22-Feb-77 & & $10045-97-3$ & CS-137 & GAMMA_GS & PNL-KI & 0.002 \\
\hline 1716 & PDMS & & SW & & & E008456 & 23-Feb-77 & & $10028-17-8$ & TRITIUM & TRITIUM_DIST_LSC & USTEST & 521 \\
\hline 1717 & PDMS & & SW & & & E008456 & 23-Feb-77 & & $10045-97-3$ & CS-137 & GAMMA_GS & USTEST & -0.0416 \\
\hline
\end{tabular}




\begin{tabular}{|c|c|c|c|c|c|c|c|c|c|c|c|c|c|}
\hline & 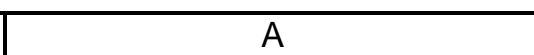 & $\mathrm{B}$ & $\mathrm{C}$ & $\mathrm{D}$ & $\mathrm{E}$ & $\mathrm{F}$ & $\bar{G}$ & $\mathrm{H}$ & $\mathrm{T}$ & $\mathrm{J}$ & $\mathrm{K}$ & $\bar{L}$ & $\mathrm{M}$ \\
\hline 1 & SAMPLE_NUM_ASSIGNED_TO & SAMPLER & MEDIA & BIOTA_MEDIA & TAG_ID & SAMP_NUM & SAMP_DATE_TIME & SAMP_DATE_TIME_ON & CON_ID & CON_SHORT_NAME & METHOD_NAME & LAB_CODE & VALUE_RPTD \\
\hline 1718 & PDMS & & SW & & & E041411 & $23-F e b-77$ & & $10028-17-8$ & TRITIUM & TRITIUM_DIST_LSC & USTEST & 446 \\
\hline 1719 & PDMS & & SW & & & E034900 & 23-Feb-77 & & $10028-17-8$ & TRITIUM & TRITIUM_DIST_LSC & USTEST & 468 \\
\hline 1720 & PDMS & & SW & & & E004565 & 28-Feb-77 & & $10028-17-8$ & TRITIUM & TRITIUM_DIST_LSC & USTEST & 385 \\
\hline 1721 & PDMS & & sw & & & E004565 & 28-Feb-77 & & $10045-97-3$ & CS-137 & GAMMA_GS & USTEST & -0.448 \\
\hline 1722 & PDMS & & SW & & & E016427 & 28-Feb-77 & & $10045-97-3$ & CS-137 & GAMMA_GS & USTEST & -0.452 \\
\hline 1723 & PDMS & & sW & & & E002160 & 28-Feb-77 & & $10045-97-3$ & CS-137 & GAMMA_GS & USTEST & 0.0511 \\
\hline 1724 & PDMS & & sW & & & E002048 & 08-Mar-77 & & $10045-97-3$ & CS-137 & GAMMA_GS & PNL-KI & 0.02 \\
\hline 1725 & PDMS & & sW & & & E011333 & 08-Mar-77 & & $10045-97-3$ & CS-137 & GAMMA_GS & PNL-KI & 0.004 \\
\hline 1726 & PDMS & & sW & & & E000858 & 14-Mar-77 & & $10098-97-2$ & SR-90 & 905.0_SR_GPC & USTEST & 0.0255 \\
\hline \begin{tabular}{|l|l|}
1727 \\
\end{tabular} & PDMS & & sW & & & E000858 & 14-Mar-77 & & $10045-97-3$ & CS-137 & 901.1_GAMMA_GS & USTEST & -0.267 \\
\hline 1728 & PDMS & & SW & & & E000858 & 14-Mar-77 & & $10028-17-8$ & TRITIUM & 906.0_H3_LSC & USTEST & 121 \\
\hline 1729 & PDMS & & sw & & & E001839 & 14-Mar-77 & & $10028-17-8$ & TRITIUM & TRITIUM_DIST_LSC & USTEST & 44200 \\
\hline 1730 & PDMS & & sW & & & E001839 & 14-Mar-77 & & $10098-97-2$ & SR-90 & SRISO_SEP_PRECIP_GPC & USTEST & 0.183 \\
\hline 1731 & PDMS & & SW & & & E001839 & 14-Mar-77 & & $10045-97-3$ & CS-137 & GAMMĀ_GS & USTEST & 0.0511 \\
\hline 1732 & PDMS & & sw & & & E024881 & 14-Mar-77 & & $10028-17-8$ & TRITIUM & TRITIUM_DIST_LSC & USTEST & 242000 \\
\hline 1733 & PDMS & & SW & & & E024881 & 14-Mar-77 & & $10045-97-3$ & CS-137 & GAMMA_GS & USTEST & -0.0564 \\
\hline 1734 & PDMS & & SW & & & E002049 & 22-Mar-77 & & $10045-97-3$ & CS-137 & GAMMA_GS & PNL-KI & 0.02 \\
\hline 1735 & PDMS & & sW & & & E011334 & 22-Mar-77 & & $10045-97-3$ & CS-137 & GAMMA_GS & PNL-KI & 0.003 \\
\hline 1736 & PDMS & & sW & & & E001764 & 22-Mar-77 & & $10045-97-3$ & CS-137 & GAMMA_GS & PNL-KI & 0.01 \\
\hline 1737 & PDMS & & SW & & & E014151 & 22-Mar-77 & & $10045-97-3$ & CS-137 & GAMMA_GS & PNL-KI & 0.004 \\
\hline 1738 & PDMS & & sW & & & E004566 & 28-Mar-77 & & $10045-97-3$ & CS-137 & GAMMA_GS & USTEST & 0.0434 \\
\hline 1739 & PDMS & & SW & & & E004566 & 28-Mar-77 & & $10028-17-8$ & TRITIUM & TRITIUM_DIST_LSC & USTEST & 224 \\
\hline 1740 & PDMS & & sW & & & E004566 & 28-Mar-77 & & $10098-97-2$ & SR-90 & SRISO_SEPPPERECIP_GPC & USTEST & 0.243 \\
\hline 1741 & PDMS & & sW & & & E016428 & 28-Mar-77 & & $10045-97-3$ & CS-137 & GAMMA__GS & USTEST & -0.155 \\
\hline 1742 & PDMS & & sW & & & E002161 & 28-Mar-77 & & $10045-97-3$ & CS-137 & GAMMA_GS & USTEST & -0.0241 \\
\hline 1743 & PDMS & & sW & & & E041415 & 30-Mar-77 & & $10028-17-8$ & TRITIUM & TRITIUM_DIST_LSC & USTEST & 539 \\
\hline 1744 & PDMS & & sW & & & E008397 & 30-Mar-77 & & $10028-17-8$ & TRITIUM & TRITIUM_DIST_LSC & USTEST & 556 \\
\hline 1745 & PDMS & & sW & & & E008397 & 30-Mar-77 & & $10098-97-2$ & SR-90 & SRISO_SEP_PRECIP_GPC & USTEST & 0.219 \\
\hline 1746 & PDMS & & SW & & & E008397 & 30-Mar-77 & & $10045-97-3$ & CS-137 & GAMMĀ_GS & USTEST & -0.614 \\
\hline \begin{tabular}{|l|l|}
1747 \\
\end{tabular} & PDMS & & sW & & & E034904 & 30-Mar-77 & & $10028-17-8$ & TRITIUM & TRITIUM_DIST_LSC & USTEST & 243 \\
\hline 1748 & PDMS & & sW & & & E002050 & 05-Apr-77 & & $10045-97-3$ & CS-137 & GAMMA_GS & PNL-KI & 0.02 \\
\hline 1749 & PDMS & & sW & & & E002050 & 05-Apr-77 & & 15046-84-1 & $\mid-129$ & I129_SEP_LEPS_GS & PNL-KI & 0.000055 \\
\hline 1750 & PDMS & & sW & & & E011335 & 05-Apr-77 & & $10045-97-3$ & CS-137 & GAMMA_GS & PNL-KI & 0.003 \\
\hline 1751 & PDMS & & sW & & & E001765 & 05-Apr-77 & & 15046-84-1 & $\mid-129$ & I129_SEP_LEPS_GS & PNL-KI & 0.00001 \\
\hline 1752 & PDMS & & SW & & & E001765 & 05-Apr-77 & & $10045-97-3$ & CS-137 & GAMMA_ĒS & PNL-KI & 0.02 \\
\hline 1753 & PDMS & & SW & & & E014152 & 05-Apr-77 & & $10045-97-3$ & CS-137 & GAMMA_GS & PNL-KI & 0.006 \\
\hline 1754 & PDMS & & sW & & & E000859 & 11-Apr-77 & & $10045-97-3$ & CS-137 & 901.1_GAMMA_GS & USTEST & 5.97 \\
\hline 1755 & PDMS & & sW & & & E000859 & 11-Apr-77 & & $10028-17-8$ & TRITIUM & 906.0_H3_LSC & USTEST & -318 \\
\hline 1756 & PDMS & & sW & & & E001840 & 11-Apr-77 & & $10028-17-8$ & TRITIUM & TRITIUM_DIST_LSC & USTEST & 75900 \\
\hline \begin{tabular}{|l|l|}
1757 \\
\end{tabular} & PDMS & & sW & & & E001840 & 11-Apr-77 & & $10045-97-3$ & CS-137 & GAMMA_GS & USTEST & -0.459 \\
\hline 1758 & PDMS & & sW & & & E024882 & 11-Apr-77 & & $10028-17-8$ & TRITIUM & TRITIUM_DIST_LSC & USTEST & 338000 \\
\hline 1759 & PDMS & & SW & & & E024882 & 11-Apr-77 & & $10045-97-3$ & CS-137 & GAMMA_GS & USTEST & 2.02 \\
\hline 1760 & PDMS & & sW & & & E002051 & 21-Apr-77 & & $10045-97-3$ & CS-137 & GAMMA_GS & PNL-KI & 0.03 \\
\hline 1761 & PDMS & & sW & & & E011336 & 21-Apr-77 & & $10045-97-3$ & CS-137 & GAMMA_GS & PNL-KI & 0.004 \\
\hline 1762 & PDMS & & sW & & & E001766 & 21-Apr-77 & & $10045-97-3$ & CS-137 & GAMMA_GS & PNL-KI & 0.05 \\
\hline 1763 & PDMS & & sW & & & E014153 & 21-Apr-77 & & $10045-97-3$ & CS-137 & GAMMA_GS & PNL-KI & 0.01 \\
\hline 1764 & PDMS & & sW & & & E004567 & 25-Apr-77 & & $10045-97-3$ & CS-137 & GAMMA_GS & USTEST & 0.0472 \\
\hline 1765 & PDMS & & sW & & & E004567 & 25-Apr-77 & & $10028-17-8$ & TRITIUM & TRITIUM_DIST_LSC & USTEST & -25.2 \\
\hline 1766 & PDMS & & sW & & & E016429 & 25-Apr-77 & & $10045-97-3$ & CS-137 & GAMMA_GS & USTEST & 8.57 \\
\hline \begin{tabular}{|l|l|}
1767 \\
\end{tabular} & PDMS & & sW & & & E002162 & 25-Apr-77 & & $10045-97-3$ & CS-137 & GAMMA_GS & USTEST & 16.8 \\
\hline 1768 & PDMS & & SW & & & E014891 & 25-Apr-77 & & $10045-97-3$ & CS-137 & GAMMA_GS & USTEST & 3.47 \\
\hline 1769 & PDMS & & sW & & & E014840 & 25-Apr-77 & & $10098-97-2$ & SR-90 & SRISO SEP PRECIP GPC & USTEST & 3.42 \\
\hline
\end{tabular}




\begin{tabular}{|c|c|c|c|c|c|c|c|c|c|c|c|c|c|}
\hline & $\bar{A}$ & $\mathrm{~B}$ & $\bar{C}$ & $\mathrm{D}$ & $\mathrm{E}$ & $\mathrm{F}$ & $\bar{G}$ & $\mathrm{H}$ & 1 & $\mathrm{~J}$ & $\bar{K}$ & $\mathrm{~L}$ & $\mathrm{M}$ \\
\hline 1 & SAMPLE_NUM_ASSIGNED_TO & \begin{tabular}{|l} 
SAMPLER \\
\end{tabular} & MEDIA & BIOTA_MEDIA & TAG_ID & SAMP_NUM & SAMP_DATE_TIME & SAMP_DATE_TIME_ON & CON_ID & \begin{tabular}{|l|} 
CON_SHORT_NAME \\
\end{tabular} & METHOD_NAME & LAB_CODE & VALUE_RPTD \\
\hline 1770 & PDMS & & SW & & & E014840 & $25-A p r-77$ & & $10045-97-3$ & CS-137 & GAMMA_GS & USTEST & 2.3 \\
\hline 1771 & PDMS & & SW & & & E022428 & 25-Apr-77 & & $10045-97-3$ & CS-137 & GAMMA_GS & USTEST & 19.6 \\
\hline 1772 & PDMS & & SW & & & E022428 & 25-Apr-77 & & $10098-97-2$ & SR-90 & SRISO_SEP_PRECIP_GPC & USTEST & 0.456 \\
\hline 1773 & PDMS & & SW & & & E022356 & 25-Apr-77 & & $10045-97-3$ & CS-137 & GAMMA_GS & USTEST & 15 \\
\hline 1774 & PDMS & & SW & & & E022356 & 25-Apr-77 & & $10098-97-2$ & SR-90 & SRISO_SEEP_PRECIP_GPC & USTEST & 4.4 \\
\hline 1775 & PDMS & & SW & & & E014275 & 25-Apr-77 & & $10045-97-3$ & CS-137 & GAMMA_GS & USTEST & 2.13 \\
\hline 1776 & PDMS & & SW & & & E014275 & 25-Apr-77 & & $10098-97-2$ & SR-90 & SRISO_SEEP_PRECIP_GPC & USTEST & 6.08 \\
\hline 1777 & PDMS & & SW & & & E041419 & 27-Apr-77 & & $10028-17-8$ & TRITIUM & TRITIUM_DISTT_LSC & USTEST & 1300 \\
\hline 1778 & PDMS & & SW & & & E008331 & 27-Apr-77 & & $10028-17-8$ & TRITIUM & TRITIUM_DIST_LEC & USTEST & 1350 \\
\hline 1779 & PDMS & & SW & & & E008331 & 27-Apr-77 & & $10045-97-3$ & CS-137 & GAMMA_GS & USTEST & -0.0263 \\
\hline 1780 & PDMS & & SW & & & E034908 & 27-Apr-77 & & $10028-17-8$ & TRITIUM & TRITIUM_DIST_LSC & USTEST & 891 \\
\hline 1781 & PDMS & & SW & & & E002052 & 03-May-77 & & $10045-97-3$ & CS-137 & GAMMA_GS & PNL-KI & 0.02 \\
\hline 1782 & PDMS & & SW & & & E002052 & 03-Мау-77 & & 15046-84-1 & $1-129$ & I129_SEP_LEPS_GS & PNL-KI & 0.0000695 \\
\hline 1783 & PDMS & & SW & & & E011337 & 03-May-77 & & $10045-97-3$ & CS-137 & GAMMA_GS & PNL-KI & 0.004 \\
\hline 1784 & PDMS & & SW & & & E014154 & 03-May-77 & & $10045-97-3$ & CS-137 & GAMMA_GS & PNL-KI & 0.008 \\
\hline 1785 & PDMS & & SW & & & E001767 & 03-May-77 & & 15046-84-1 & $1-129$ & I129_SEP_LEPS_GS & PNL-KI & 0.000012 \\
\hline 1786 & PDMS & & SW & & & E001767 & 03-May-77 & & $10045-97-3$ & CS-137 & GAMMA_GS & PNL-KI & 0.02 \\
\hline 1787 & PDMS & & SW & & & E000860 & 09-May-77 & & $10045-97-3$ & CS-137 & 901.1_GAMMA_GS & USTEST & 2.87 \\
\hline 1788 & PDMS & & SW & & & E000860 & 09-Мау-77 & & $10028-17-8$ & TRITIUM & 906.0_H3_LSC & USTEST & 24.3 \\
\hline 1789 & PDMS & & SW & & & E001841 & 09-Мау-77 & & $10028-17-8$ & TRITIUM & TRITIŪM_DIST_LSC & USTEST & 53400 \\
\hline 1790 & PDMS & & SW & & & E001841 & 09-Мау-77 & & $10045-97-3$ & CS-137 & GAMMA_GS & USTEST & -0.00103 \\
\hline 1791 & PDMS & & SW & & & E024883 & 09-Мау-77 & & 10028-17-8 & TRITIUM & TRITIUM_DIST_LSC & USTEST & 295000 \\
\hline 1792 & PDMS & & SW & & & E024883 & 09-May-77 & & $10045-97-3$ & CS-137 & GAMMA_GS & USTEST & -0.11 \\
\hline 1793 & PDMS & & SW & & & E002053 & 17-May-77 & & $10045-97-3$ & CS-137 & GAMMA_GS & PNL-KI & 0.02 \\
\hline 1794 & PDMS & & SW & & & E011338 & 17-Мау-77 & & $10045-97-3$ & CS-137 & GAMMA_GS & PNL-KI & 0.0009 \\
\hline 1795 & PDMS & & SW & & & E001768 & 17-Мау-77 & & $10045-97-3$ & CS-137 & GAMMA_GS & PNL-KI & 0.008 \\
\hline 1796 & PDMS & & SW & & & E014155 & 17-May-77 & & $10045-97-3$ & CS-137 & GAMMA_GS & PNL-KI & 0.0005 \\
\hline 1797 & PDMS & & SW & & & E008359 & 25-May-77 & & $10028-17-8$ & TRITIUM & TRITIUM_DIST_LSC & USTEST & 675 \\
\hline 1798 & PDMS & & SW & & & E008359 & 25-May-77 & & $10045-97-3$ & CS-137 & GAMMA_GS & USTEST & 0.0511 \\
\hline 1799 & PDMS & & SW & & & E041422 & 25-May-77 & & $10028-17-8$ & TRITIUM & TRITIUM_DIST_LSC & USTEST & 724 \\
\hline 1800 & PDMS & & SW & & & E034911 & 25-May-77 & & 10028-17-8 & TRITIUM & TRITIUM_DIST_LSC & USTEST & 889 \\
\hline 1801 & PDMS & & SW & & & E004568 & 31-May-77 & & $10028-17-8$ & TRITIUM & TRITIUM_DIST_LSC & USTEST & -514 \\
\hline 1802 & PDMS & & SW & & & E004568 & 31-Мау-77 & & $10045-97-3$ & CS-137 & GAMMA_GS & USTEST & 3.34 \\
\hline 1803 & PDMS & & SW & & & E002054 & 31-Мау-77 & & $10045-97-3$ & CS-137 & GAMMA_GS & PNL-KI & 0.02 \\
\hline 1804 & PDMS & & SW & & & E002054 & 31-May-77 & & 15046-84-1 & I-129 & I129_SEP_LEPS_GS & PNL-KI & 0.000043 \\
\hline 1805 & PDMS & & SW & & & E011339 & 31-May-77 & & $10045-97-3$ & CS-137 & GAMMA_GS & PNL-KI & 0.0009 \\
\hline 1806 & PDMS & & SW & & & E001769 & 31-May-77 & & 15046-84-1 & I-129 & I129_SEP_LEPS_GS & PNL-KI & 0.000012 \\
\hline 1807 & PDMS & & SW & & & E001769 & 31-May-77 & & $10045-97-3$ & CS-137 & GAMMA_GS & PNL-KI & 0.01 \\
\hline 1808 & PDMS & & SW & & & E014156 & 31-May-77 & & $10045-97-3$ & CS-137 & GAMMA_GS & PNL-KI & 0.0005 \\
\hline 1809 & PDMS & & SW & & & E016430 & 31-May-77 & & $10045-97-3$ & CS-137 & GAMMA_GS & USTEST & 7.03 \\
\hline 1810 & PDMS & & SW & & & E002163 & 31-May-77 & & $10045-97-3$ & CS-137 & GAMMA_GS & USTEST & 2.29 \\
\hline 1811 & PDMS & & SW & & & E000861 & 06-Jun-77 & & $10098-97-2$ & SR-90 & 905.0_SR_GPC & USTEST & 0.0631 \\
\hline 1812 & PDMS & & SW & & & E000861 & 06-Jun-77 & & $10045-97-3$ & CS-137 & 901.1_GAMMA_GS & USTEST & 0.62 \\
\hline 1813 & PDMS & & SW & & & E000861 & 06-Jun-77 & & $10028-17-8$ & TRITIUM & 906.0_H3_LSC & USTEST & 31.3 \\
\hline 1814 & PDMS & & SW & & & E001842 & 06-Jun-77 & & $10028-17-8$ & TRITIUM & TRITIUM_DIST_LSC & USTEST & 30400 \\
\hline 1815 & PDMS & & SW & & & E001842 & 06-Jun-77 & & $10098-97-2$ & SR-90 & SRISO_SEP_PRECIP_GPC & USTEST & 0.094 \\
\hline 1816 & PDMS & & SW & & & E001842 & 06-Jun-77 & & $10045-97-3$ & CS-137 & GAMMA_GS & USTEST & -0.0908 \\
\hline 1817 & PDMS & & SW & & & E020876 & 06-Jun-77 & & $10028-17-8$ & TRITIUM & TRITIUM_DIST_LSC & USTEST & 81200 \\
\hline 1818 & PDMS & & SW & & & E020876 & 06-Jun-77 & & $10045-97-3$ & CS-137 & GAMMA_GS & USTEST & 1.58 \\
\hline 1819 & PDMS & & SW & & & E020876 & 06-Jun-77 & & $10098-97-2$ & SR-90 & SRISO_SEP_PRECIP_GPC & USTEST & 0.0377 \\
\hline 1820 & PDMS & & SW & & & E002055 & 14-Jun-77 & & $10045-97-3$ & CS-137 & GAMMA_GS & PNL-KI & 0.02 \\
\hline 1821 & PDMS & & SW & & & E011340 & 14-Jun-77 & & $10045-97-3$ & CS-137 & GAMMA_GS & PNL-KI & 0.0009 \\
\hline
\end{tabular}




\begin{tabular}{|c|c|c|c|c|c|c|c|c|c|c|c|c|c|}
\hline & A & $\mathrm{B}$ & $\mathrm{c}$ & $\mathrm{D}$ & $\mathrm{E}$ & $\mathrm{F}$ & G & $\mathrm{H}$ & $\mathrm{I}$ & $\mathrm{J}$ & $\mathrm{K}$ & $\mathrm{L}$ & $\mathrm{M}$ \\
\hline 1 & SAMPLE_NUM_ASSIGNED_TO & SAMPLER & MEDIA & BIOTA_MEDIA & TAG_ID & SAMP_NUM & SAMP_DATE_TIME & SAMP_DATE_TIME_ON & CON_ID & CON_SHORT_NAME & METHOD_NAME & LAB_CODE & VALUE_RPTD \\
\hline 1822 & PDMS & & SW & & & E026984 & 14-Jun-77 & & $10045-97-3$ & CS-137 & GAMMA_GS & PNL-KI & 0.01 \\
\hline 1823 & PDMS & & sW & & & E030846 & 14-Jun-77 & & $10045-97-3$ & CS-137 & GAMMA_GS & PNL-KI & 0.0005 \\
\hline 1824 & PDMS & & sW & & & E004569 & 27-Jun-77 & & $10045-97-3$ & CS-137 & GAMMA_GS & USTEST & 1.16 \\
\hline 1825 & PDMS & & sW & & & E004569 & 27-Jun-77 & & $10028-17-8$ & TRITIUM & TRITIUM_DIST_LSC & USTEST & 672 \\
\hline 1826 & PDMS & & sW & & & E004569 & 27-Jun-77 & & $10098-97-2$ & SR-90 & SRISO_SEP_PRECIP_GPC & USTEST & 0.284 \\
\hline \begin{tabular}{|l|l|}
1827 \\
182
\end{tabular} & PDMS & & sW & & & E016431 & 27-Jun-77 & & $10045-97-3$ & CS-137 & GAMMA_GS & USTEST & 5.81 \\
\hline 1828 & PDMS & & SW & & & E002164 & 27-Jun-77 & & $10045-97-3$ & CS-137 & GAMMA_GS & USTEST & 15.1 \\
\hline 1829 & PDMS & & SW & & & E002056 & 28-Jun-77 & & $10045-97-3$ & CS-137 & GAMMA_GS & PNL-KI & 0.02 \\
\hline 1830 & PDMS & & sW & & & E002056 & 28-Jun-77 & & $15046-84-1$ & $1-129$ & 1129_SEP_LEPS_GS & PNL-KI & 0.00008 \\
\hline 1831 & PDMS & & SW & & & E011341 & 28-Jun-77 & & $10045-97-3$ & CS-137 & GAMMA_ḠS & PNL-KI & 0.0009 \\
\hline 1832 & PDMS & & SW & & & E001771 & 28-Jun-77 & & 15046-84-1 & $1-129$ & 1129_SEP_LEPS_GS & PNL-KI & 0.000011 \\
\hline 1833 & PDMS & & SW & & & E001771 & 28-Jun-77 & & $10045-97-3$ & CS-137 & GAMMA_ḠS & PNL-KI & 0.01 \\
\hline 1834 & PDMS & & SW & & & E014158 & 28-Jun-77 & & $10045-97-3$ & CS-137 & GAMMA_GS & PNL-KI & 0.0005 \\
\hline 1835 & PDMS & & SW & & & E008300 & 29-Jun-77 & & $10028-17-8$ & TRITIUM & TRITIUM_DIST_LSC & USTEST & 425 \\
\hline 1836 & PDMS & & SW & & & E008300 & 29-Jun-77 & & $10098-97-2$ & SR-90 & SRISO_SEP_PRECIP_GPC & USTEST & 0.356 \\
\hline \begin{tabular}{|l|l|}
1837 \\
\end{tabular} & PDMS & & SW & & & E008300 & 29-Jun-77 & & $10045-97-3$ & CS-137 & GAMMA_GS & USTEST & 0.0405 \\
\hline 1838 & PDMS & & sW & & & E041352 & 29-Jun-77 & & $10028-17-8$ & TRITIUM & TRITIUM_DIST_LSC & USTEST & 336 \\
\hline 1839 & PDMS & & sW & & & E034915 & 29-Jun-77 & & $10028-17-8$ & TRITIUM & TRITIUM_DIST_LSC & USTEST & -67.2 \\
\hline 1840 & PDMS & & SW & & & E000862 & 05-Jul-77 & & $10045-97-3$ & CS-137 & 901.1_GAMMMA_GS & USTEST & 0.126 \\
\hline 1841 & PDMS & & SW & & & E000862 & 05-Jul-77 & & $10028-17-8$ & TRITIUM & 906.0_H3_LSC & USTEST & 72 \\
\hline 1842 & PDMS & & SW & & & E023860 & 05-Jul-77 & & $10028-17-8$ & TRITIUM & TRITIUM_DIST_LSC & USTEST & 263 \\
\hline 1843 & PDMS & & SW & & & E023860 & 05-Jul-77 & & $10045-97-3$ & CS-137 & GAMMA_GS & USTEST & -0.168 \\
\hline 1844 & PDMS & & SW & & & E001843 & 05-Jul-77 & & 10028-17-8 & TRITIUM & TRITIUM_DIST_LSC & USTEST & 69500 \\
\hline 1845 & PDMS & & SW & & & E001843 & 05-Jul-77 & & $10045-97-3$ & CS-137 & GAMMA_GS & USTEST & -0.597 \\
\hline 1846 & PDMS & & SW & & & E024884 & 05-Jul-77 & & 10028-17-8 & TRITIUM & TRITIUM_DIST_LSC & USTEST & 184000 \\
\hline \begin{tabular}{|l|l|}
1847 \\
\end{tabular} & PDMS & & SW & & & E024884 & 05-Jul-77 & & $10045-97-3$ & CS-137 & GAMMA_GS & USTEST & -0.108 \\
\hline 1848 & PDMS & & SW & & & E002057 & 12-Jul-77 & & $10045-97-3$ & CS-137 & GAMMA_GS & PNL-KI & 0.02 \\
\hline 1849 & PDMS & & SW & & & E011342 & 12-Jul-77 & & $10045-97-3$ & CS-137 & GAMMA_GS & PNL-KI & 0.0009 \\
\hline 1850 & PDMS & & SW & & & E001772 & 12-Jul-77 & & 15046-84-1 & $\mid-129$ & 1129_SEP_LEPS_GS & PNL-KI & 0.0000067 \\
\hline \begin{tabular}{|l|}
1851 \\
\end{tabular} & PDMS & & SW & & & E001772 & 12-Jul-77 & & $10045-97-3$ & CS-137 & GAMMA_GS & PNL-KI & 0.01 \\
\hline 1852 & PDMS & & SW & & & E014159 & 12-Jul-77 & & $10045-97-3$ & CS-137 & GAMMA_GS & PNL-KI & 0.0005 \\
\hline 1853 & PDMS & & SW & & & E075062 & 18-Jul-77 & & $10028-17-8$ & TRITIUM & TRITIUM_DIST_LSC & USTEST & 83.8 \\
\hline 1854 & PDMS & & SW & & & E014892 & 18-Jul-77 & & $10045-97-3$ & CS-137 & GAMMA_GS & USTEST & 3.28 \\
\hline 1855 & PDMS & & SW & & & E016407 & 18-Jul-77 & & $10098-97-2$ & SR-90 & SRISO_SEEP_PRECIP_GPC & USTEST & 3.42 \\
\hline 1856 & PDMS & & SW & & & E016407 & 18-Jul-77 & & $10045-97-3$ & CS-137 & GAMMĀGS & USTEST & 6.73 \\
\hline \begin{tabular}{|l|l|}
1857 \\
\end{tabular} & PDMS & & sW & & & E022429 & 18-Jul-77 & & $10045-97-3$ & CS-137 & GAMMA_GS & USTEST & 7.18 \\
\hline 1858 & PDMS & & SW & & & E022429 & 18-Jul-77 & & $10098-97-2$ & SR-90 & SRISO_SEP_PRECIP_GPC & USTEST & 3.66 \\
\hline 1859 & PDMS & & SW & & & E027649 & 18-Jul-77 & & $10045-97-3$ & CS-137 & GAMMA_GS & USTEST & 6.37 \\
\hline 1860 & PDMS & & SW & & & E027649 & 18-Jul-77 & & $10098-97-2$ & SR-90 & SRISO_SEPP_PRECIP_GPC & USTEST & 0.708 \\
\hline 1861 & PDMS & & SW & & & E014276 & 18-Jul-77 & & $10045-97-3$ & CS-137 & GAMMĀGS & USTEST & 5.7 \\
\hline 1862 & PDMS & & SW & & & E014276 & 18-Jul-77 & & $10098-97-2$ & SR-90 & SRISO_SEEP_PRECIP_GPC & USTEST & 7.25 \\
\hline 1863 & PDMS & & SW & & & E004570 & 25-Jul-77 & & $10028-17-8$ & TRITIUM & TRITIUM_DISTT_LSC & USTEST & 671 \\
\hline 1864 & PDMS & & SW & & & E004570 & 25-Jul-77 & & $10045-97-3$ & CS-137 & GAMMA_GS & USTEST & 5.08 \\
\hline 1865 & PDMS & & SW & & & E016432 & 25-Jul-77 & & $10045-97-3$ & CS-137 & GAMMA_GS & USTEST & 0.785 \\
\hline 1866 & PDMS & & sW & & & E023861 & 25-Jul-77 & & 10028-17-8 & TRITIUM & TRITIUM_DIST_LSC & USTEST & -88 \\
\hline \begin{tabular}{|l|l|}
1867 \\
\end{tabular} & PDMS & & SW & & & E023861 & 25-Jul-77 & & $10045-97-3$ & CS-137 & GAMMA_GS & USTEST & 7.09 \\
\hline 1868 & PDMS & & SW & & & E002165 & 25-Jul-77 & & $10045-97-3$ & CS-137 & GAMMA_GS & USTEST & 3.96 \\
\hline 1869 & PDMS & & SW & & & E002058 & 26-Jul-77 & & $10045-97-3$ & CS-137 & GAMMA_GS & PNL-KI & 0.02 \\
\hline 1870 & PDMS & & SW & & & E002058 & 26-Jul-77 & & $15046-84-1$ & I-129 & 1129_SEP_LEPS_GS & PNL-KI & 0.000054 \\
\hline 1871 & PDMS & & SW & & & E011343 & 26-Jul-77 & & $10045-97-3$ & CS-137 & GAMMA_GS & PNL-KI & 0.0009 \\
\hline 1872 & PDMS & & SW & & & E008234 & 27-Jul-77 & & $10028-17-8$ & TRITIUM & TRITIUM_DIST_LSC & USTEST & 2010 \\
\hline 1873 & PDMS & & SW & & & E008234 & 27-Jul-77 & & $10045-97-3$ & CS-137 & GAMMA_GS & USTEST & -0.88 \\
\hline
\end{tabular}




\begin{tabular}{|c|c|c|c|c|c|c|c|c|c|c|c|c|c|}
\hline & 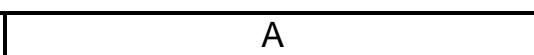 & $\mathrm{B}$ & $\mathrm{C}$ & $\mathrm{D}$ & $\mathrm{E}$ & $\mathrm{F}$ & $\mathrm{G}$ & $\mathrm{H}$ & $\mathrm{T}$ & $\mathrm{J}$ & $\mathrm{K}$ & $\mathrm{L}$ & $\mathrm{M}$ \\
\hline 1 & SAMPLE_NUM_ASSIGNED_TO & \begin{tabular}{|l} 
SAMPLER \\
\end{tabular} & MEDIA & BIOTA_MEDIA & TAG_ID & SAMP_NUM & \begin{tabular}{|l|} 
SAMP_DATE_TIME \\
\end{tabular} & SAMP_DATE_TIME_ON & CON_ID & \begin{tabular}{|l} 
CON_SHORT_NAME \\
\end{tabular} & METHOD_NAME & LAB_CODE & VALUE_RPTD \\
\hline 1874 & PDMS & & SW & & & E027832 & 27-Jul-77 & & $10028-17-8$ & TRITIUM & TRITIUM_DIST_LSC & USTEST & 806 \\
\hline 1875 & PDMS & & SW & & & E011344 & 09-Aug-77 & & $10045-97-3$ & CS-137 & GAMMA_GS & PNL-KI & 0.002 \\
\hline 1876 & PDMS & & SW & & & E002059 & 09-Aug-77 & & $10045-97-3$ & CS-137 & GAMMA_GS & PNL-KI & 0.02 \\
\hline 1877 & PDMS & & SW & & & E001773 & 09-Aug-77 & & $10045-97-3$ & CS-137 & GAMMA_GS & PNL-KI & 0.01 \\
\hline 1878 & PDMS & & SW & & & E014089 & 09-Aug-77 & & $10045-97-3$ & CS-137 & GAMMA_GS & PNL-KI & 0.001 \\
\hline 1879 & PDMS & & SW & & & E014843 & 22-Aug-77 & & $10098-97-2$ & SR-90 & SRISO_SEEP_PRECIP_GPC & USTEST & 0.0196 \\
\hline 1880 & PDMS & & sW & & & E011345 & 23-Aug-77 & & $10045-97-3$ & CS-137 & GAMMĀ_GS & PNL-KI & 0.0009 \\
\hline 1881 & PDMS & & sW & & & E002060 & 23-Aug-77 & & $10045-97-3$ & CS-137 & GAMMA_GS & PNL-KI & 0.02 \\
\hline 1882 & PDMS & & sW & & & E001774 & 23-Aug-77 & & $10045-97-3$ & CS-137 & GAMMA_GS & PNL-KI & 0.005 \\
\hline 1883 & PDMS & & SW & & & E014090 & 23-Aug-77 & & $10045-97-3$ & CS-137 & GAMMA_GS & PNL-KI & 0.005 \\
\hline 1884 & PDMS & & SW & & & E004964 & 26-Aug-77 & & $10098-97-2$ & SR-90 & 905.0_SR_GPC & USTEST & 0.0726 \\
\hline 1885 & PDMS & & SW & & & E004964 & 26-Aug-77 & & $10028-17-8$ & TRITIUM & 906.0_H3_LSC & USTEST & 380 \\
\hline 1886 & PDMS & & SW & & & E010725 & 26-Aug-77 & & $10098-97-2$ & SR-90 & 905.0_SR_GPC & USTEST & 0.0382 \\
\hline 1887 & PDMS & & SW & & & E010725 & 26-Aug-77 & & $10028-17-8$ & TRITIUM & 906.0_H3_LSC & USTEST & 488 \\
\hline 1888 & PDMS & & SW & & & E004571 & 29-Aug-77 & & $10028-17-8$ & TRITIUM & TRITIUM_DIST_LSC & USTEST & 651 \\
\hline 1889 & PDMS & & SW & & & E004571 & 29-Aug-77 & & $10045-97-3$ & CS-137 & GAMMA_GS & USTEST & 11.9 \\
\hline 1890 & PDMS & & SW & & & E001612 & 29-Aug-77 & & $10098-97-2$ & SR-90 & SRISO_SEP_PRECIP_GPC & USTEST & 0.152 \\
\hline 1891 & PDMS & & SW & & & E001612 & 29-Aug-77 & & $10028-17-8$ & TRITIUM & TRITIUM_DIST_LSC & USTEST & 574 \\
\hline 1892 & PDMS & & SW & & & E001612 & 29-Aug-77 & & $10045-97-3$ & CS-137 & GAMMA_GS & USTEST & -0.294 \\
\hline 1893 & PDMS & & SW & & & E044471 & 29-Aug-77 & & $10045-97-3$ & CS-137 & GAMMA_GS & USTEST & 5.48 \\
\hline 1894 & PDMS & & SW & & & E044471 & 29-Aug-77 & & $10028-17-8$ & TRITIUM & TRITIUM_DIST_LSC & USTEST & 47500 \\
\hline 1895 & PDMS & & SW & & & E002166 & 29-Aug-77 & & $10045-97-3$ & CS-137 & GAMMA_GS & USTEST & 13.5 \\
\hline 1896 & PDMS & & SW & & & E041358 & 31-Aug-77 & & $10028-17-8$ & TRITIUM & TRITIUM_DIST_LSC & USTEST & 325 \\
\hline 1897 & PDMS & & SW & & & E008175 & 31-Aug-77 & & $10045-97-3$ & CS-137 & GAMMA_GS & USTEST & 3.16 \\
\hline 1898 & PDMS & & SW & & & E034921 & 31-Aug-77 & & $10028-17-8$ & TRITIUM & TRITIUM_DIST_LSC & USTEST & 703 \\
\hline 1899 & PDMS & & SW & & & E002061 & 08-Sep-77 & & $10045-97-3$ & CS-137 & GAMMA_GS & PNL-KI & 0.02 \\
\hline 1900 & PDMS & & SW & & & E002061 & 08-Sep-77 & & 15046-84-1 & I-129 & I129_SEP_LEPS_GS & PNL-KI & 0.000039 \\
\hline 1901 & PDMS & & SW & & & E011346 & 08-Sep-77 & & $10045-97-3$ & CS-137 & GAMMA_GS & PNL-KI & 0.003 \\
\hline 1902 & PDMS & & SW & & & E001775 & 08-Sep-77 & & $15046-84-1$ & I-129 & 1129_SEP_LEPS_GS & PNL-KI & 0.0000042 \\
\hline 1903 & PDMS & & SW & & & E001775 & 08-Sep-77 & & $10045-97-3$ & CS-137 & GAMMA_GS & PNL-KI & 0.02 \\
\hline 1904 & PDMS & & SW & & & E014091 & 08-Sep-77 & & $10045-97-3$ & CS-137 & GAMMA_GS & PNL-KI & 0.002 \\
\hline 1905 & PDMS & & SW & & & E011347 & 20-Sep-77 & & $10045-97-3$ & CS-137 & GAMMA_GS & PNL-KI & 0.0009 \\
\hline 1906 & PDMS & & SW & & & E002062 & 20-Sep-77 & & $10045-97-3$ & CS-137 & GAMMA_GS & PNL-KI & 0.02 \\
\hline 1907 & PDMS & & SW & & & E001776 & $20-$ Sep-77 & & $10045-97-3$ & CS-137 & GAMMA_GS & PNL-KI & 0.02 \\
\hline 1908 & PDMS & & SW & & & E014092 & 20-Sep-77 & & $10045-97-3$ & CS-137 & GAMMA_GS & PNL-KI & 0.0005 \\
\hline 1909 & PDMS & & SW & & & E004572 & 26-Sep-77 & & $10045-97-3$ & CS-137 & GAMMA_GS & USTEST & 3.92 \\
\hline 1910 & PDMS & & SW & & & E004572 & 26-Sep-77 & & $10028-17-8$ & TRITIUM & TRITIUM_DIST_LSC & USTEST & 216 \\
\hline 1911 & PDMS & & SW & & & E004572 & 26-Sep-77 & & $10098-97-2$ & SR-90 & SRISO_SEP_PRECIP_GPC & USTEST & 0.586 \\
\hline 1912 & PDMS & & SW & & & E003612 & 26-Sep-77 & & $10045-97-3$ & CS-137 & GAMMA_GS & USTEST & -0.0768 \\
\hline 1913 & PDMS & & SW & & & E044472 & 26-Sep-77 & & $10045-97-3$ & CS-137 & GAMMA_GS & USTEST & 0.049 \\
\hline 1914 & PDMS & & SW & & & E044472 & 26-Sep-77 & & $10028-17-8$ & TRITIUM & TRITIUM_DIST_LSC & USTEST & 53700 \\
\hline 1915 & PDMS & & SW & & & E002167 & 26-Sep-77 & & $10045-97-3$ & CS-137 & GAMMA_GS & USTEST & 0.0511 \\
\hline 1916 & PDMS & & SW & & & E008202 & 28-Sep-77 & & $10028-17-8$ & TRITIUM & TRITIUM_DIST_LSC & USTEST & -158 \\
\hline 1917 & PDMS & & SW & & & E008202 & 28-Sep-77 & & $10098-97-2$ & SR-90 & SRISO_SEP_PRECIP_GPC & USTEST & 0.428 \\
\hline 1918 & PDMS & & SW & & & E008202 & 28-Sep-77 & & $10045-97-3$ & CS-137 & GAMMA_GS & USTEST & 4.9 \\
\hline 1919 & PDMS & & SW & & & E041361 & 28-Sep-77 & & $10028-17-8$ & TRITIUM & TRITIUM_DIST_LSC & USTEST & 44.1 \\
\hline 1920 & PDMS & & SW & & & E034856 & 28-Sep-77 & & $10028-17-8$ & TRITIUM & TRITIUM_DIST_LSC & USTEST & -227 \\
\hline 1921 & PDMS & & SW & & & E002063 & 04-Oct-77 & & $10045-97-3$ & CS-137 & GAMMA_GS & PNL-KI & 0.04 \\
\hline 1922 & PDMS & & SW & & & E011348 & 04-Oct-77 & & $10045-97-3$ & CS-137 & GAMMA_GS & PNL-KI & 0.003 \\
\hline 1923 & PDMS & & SW & & & E001777 & 04-Oct-77 & & 15046-84-1 & $1-129$ & I129_SEP_LEPS_GS & PNL-KI & 0.000005 \\
\hline 1924 & PDMS & & SW & & & E001777 & 04-Oct-77 & & $10045-97-3$ & CS-137 & GAMMA_GS & PNL-KI & 0.03 \\
\hline 1925 & PDMS & & SW & & & E014093 & 04-Oct-77 & & $10045-97-3$ & CS-137 & GAMMA_GS & PNL-KI & 0.002 \\
\hline
\end{tabular}




\begin{tabular}{|c|c|c|c|c|c|c|c|c|c|c|c|c|c|}
\hline & 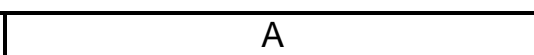 & $\mathrm{B}$ & $\mathrm{C}$ & $\mathrm{D}$ & $\mathrm{E}$ & $\mathrm{F}$ & $\bar{G}$ & $\mathrm{H}$ & $\mathrm{T}$ & $\mathrm{J}$ & $\mathrm{K}$ & $\bar{L}$ & $\mathrm{M}$ \\
\hline 1 & SAMPLE_NUM_ASSIGNED_TO & SAMPLER & MEDIA & BIOTA_MEDIA & TAG_ID & SAMP_NUM & SAMP_DATE_TIME & SAMP_DATE_TIME_ON & CON_ID & CON_SHORT_NAME & METHOD_NAME & LAB_CODE & VALUE_RPTD \\
\hline 1926 & PDMS & & SW & & & E012089 & 09-Oct-77 & & $15046-84-1$ & $1-129$ & 1129_SEP_LEPS_GS & PNL-KI & 0.00006 \\
\hline 1927 & PDMS & & SW & & & E008214 & 10-Oct-77 & & $10045-97-3$ & CS-137 & GAMMA GS & USTEST & 0.642 \\
\hline 1928 & PDMS & & SW & & & E000366 & 10-Oct-77 & & $10045-97-3$ & CS-137 & GAMMA_GS & USTEST & 1.34 \\
\hline 1929 & PDMS & & sW & & & E014841 & 10-Oct-77 & & $10098-97-2$ & SR-90 & SRISO SEP PRECIP GPC & USTEST & 0.534 \\
\hline 1930 & PDMS & & SW & & & E014841 & 10-Oct-77 & & $10045-97-3$ & CS-137 & GAMMA_GS & USTEST & 2.42 \\
\hline 1931 & PDMS & & sW & & & E022430 & 10-Oct-77 & & $10045-97-3$ & CS-137 & GAMMA_GS & USTEST & 0.209 \\
\hline 1932 & PDMS & & sW & & & E022430 & 10-Oct-77 & & $10098-97-2$ & SR-90 & SRISO_SEEP_PRECIP_GPC & USTEST & 0.302 \\
\hline 1933 & PDMS & & sW & & & E022357 & 10-Oct-77 & & $10045-97-3$ & CS-137 & GAMMĀ_GS & USTEST & 16.1 \\
\hline 1934 & PDMS & & sW & & & E022357 & 10-Oct-77 & & $10098-97-2$ & SR-90 & SRISO SE SEP PRECIP GPC & USTEST & 5.39 \\
\hline 1935 & PDMS & & sW & & & E014277 & 10-Oct-77 & & $10045-97-3$ & CS-137 & GAMMA_GS & USTEST & 7.79 \\
\hline 1936 & PDMS & & SW & & & E014277 & 10-Oct-77 & & $10098-97-2$ & SR-90 & SRISO_SEPP_PRECIP_GPC & USTEST & 5.45 \\
\hline \begin{tabular}{|l|l|}
1937 \\
\end{tabular} & PDMS & & sw & & & E002064 & 18-Oct-77 & & $10045-97-3$ & CS-137 & GAMMA_GS & PNL-KI & 0.03 \\
\hline 1938 & PDMS & & sW & & & E011349 & 18-Oct-77 & & $10045-97-3$ & CS-137 & GAMMA_GS & PNL-KI & 0.004 \\
\hline 1939 & PDMS & & sW & & & E001778 & 18-Oct-77 & & $10045-97-3$ & CS-137 & GAMMA_GS & PNL-KI & 0.06 \\
\hline 1940 & PDMS & & sw & & & E014094 & $18-$ Oct- 77 & & $10045-97-3$ & CS-137 & GAMMA_GS & PNL-KI & 0.002 \\
\hline 1941 & PDMS & & SW & & & E008136 & $26-$ Oct- 77 & & $10028-17-8$ & TRITIUM & TRITIUM_DIST_LSC & USTEST & 57.9 \\
\hline 1942 & PDMS & & SW & & & E008136 & 26-Oct-77 & & $10045-97-3$ & CS-137 & GAMMA_GS & USTEST & -0.342 \\
\hline 1943 & PDMS & & sW & & & E041364 & 26-Oct-77 & & $10028-17-8$ & TRITIUM & TRITIUM_DIST_LSC & USTEST & -97.9 \\
\hline 1944 & PDMS & & sW & & & E034859 & 26-Oct-77 & & $10028-17-8$ & TRITIUM & TRITIUM_DIST_LSC & USTEST & 311 \\
\hline 1945 & PDMS & & SW & & & E004573 & 31-Oct-77 & & $10045-97-3$ & CS-137 & GAMMA_GS & USTEST & -0.137 \\
\hline 1946 & PDMS & & SW & & & E004573 & 31-Oct-77 & & $10028-17-8$ & TRITIUM & TRITIUM_DIST_LSC & USTEST & -117 \\
\hline \begin{tabular}{|l|l|}
1947 \\
\end{tabular} & PDMS & & SW & & & E003613 & 31-Oct-77 & & $10045-97-3$ & CS-137 & GAMMA_GS & USTEST & 2.46 \\
\hline 1948 & PDMS & & sW & & & E044473 & 31-Oct-77 & & $10045-97-3$ & CS-137 & GAMMA_GS & USTEST & 0.0957 \\
\hline 1949 & PDMS & & sW & & & E044473 & 31-Oct-77 & & $10028-17-8$ & TRITIUM & TRITIUM_DIST_LSC & USTEST & 55300 \\
\hline 1950 & PDMS & & sW & & & E002168 & 31-Oct-77 & & $10045-97-3$ & CS-137 & GAMMA_GS & USTEST & 2.46 \\
\hline 1951 & PDMS & & sW & & & E002065 & 01-Nov-77 & & $10045-97-3$ & CS-137 & GAMMA_GS & PNL-KI & 0.03 \\
\hline \begin{tabular}{|l|l|}
1952 \\
\end{tabular} & PDMS & & sW & & & E002065 & 01-Nov-77 & & 15046-84-1 & $\mathrm{I}-129$ & I129_SEP_LEPS_GS & PNL-KI & 0.000043 \\
\hline \begin{tabular}{|l|l|}
1953 \\
\end{tabular} & PDMS & & sW & & & E011350 & 01-Nov-77 & & $10045-97-3$ & CS-137 & GAMMA_GS & PNL-KI & 0.0005 \\
\hline 1954 & PDMS & & SW & & & E001779 & 01-Nov-77 & & 15046-84-1 & $1-129$ & I129_SEP_LEPS_GS & PNL-KI & 0.0000047 \\
\hline 1955 & PDMS & & sw & & & E001779 & 01-Nov-77 & & $10045-97-3$ & CS-137 & GAMMA_GS & PNL-KI & 0.02 \\
\hline 1956 & PDMS & & sW & & & E014095 & 01-Nov-77 & & $10045-97-3$ & CS-137 & GAMMA_GS & PNL-KI & 0.002 \\
\hline \begin{tabular}{|l|l|}
1957 \\
\end{tabular} & PDMS & & sW & & & E068770 & 07-Nov-77 & & $10098-97-2$ & SR-90 & SRISO_SEP_PRECIP_GPC & USTEST & 0.78 \\
\hline 1958 & PDMS & & sW & & & E070791 & 07-Nov-77 & & $10098-97-2$ & SR-90 & SRISO_SEP_PRECIP_GPC & USTEST & 0.26 \\
\hline 1959 & PDMS & & sW & & & E035533 & 07-Nov-77 & & $10098-97-2$ & SR-90 & SRISO_SEP_PRECIP_GPC & USTEST & 9 \\
\hline 1960 & PDMS & & sW & & & E002066 & 15-Nov-77 & & $10045-97-3$ & CS-137 & GAMMA_GS & PNL-KI & 0.04 \\
\hline 1961 & PDMS & & SW & & & E011351 & 15-Nov-77 & & $10045-97-3$ & CS-137 & GAMMA_GS & PNL-KI & 0.003 \\
\hline 1962 & PDMS & & sW & & & E001780 & 15-Nov-77 & & $10045-97-3$ & CS-137 & GAMMA_GS & PNL-KI & 0.02 \\
\hline 1963 & PDMS & & sW & & & E014096 & 15-Nov-77 & & $10045-97-3$ & CS-137 & GAMMA_GS & PNL-KI & 0.003 \\
\hline 1964 & PDMS & & sw & & & E004574 & 28-Nov-77 & & $10028-17-8$ & TRITIUM & TRITIUM_DIST_LSC & USTEST & 256 \\
\hline 1965 & PDMS & & sW & & & E004574 & 28-Nov-77 & & $10045-97-3$ & CS-137 & GAMMA_GS & USTEST & 0.0511 \\
\hline 1966 & PDMS & & sW & & & E001613 & 28-Nov-77 & & $10098-97-2$ & SR-90 & SRISO_SEP_PRECIP_GPC & USTEST & 0.258 \\
\hline 1967. & PDMS & & sW & & & E001613 & 28-Nov-77 & & $10028-17-8$ & TRITIUM & TRITIUM_DISTT_LSC & USTEST & 514 \\
\hline 1968 & PDMS & & sW & & & E001613 & 28-Nov-77 & & $10045-97-3$ & CS-137 & GAMMA_GS & USTEST & 2.68 \\
\hline 1969 & PDMS & & sW & & & E014844 & 28-Nov-77 & & $10098-97-2$ & SR-90 & SRISO_SEPP_PRECIP_GPC & USTEST & 0.0922 \\
\hline 1970 & PDMS & & sW & & & E014844 & 28-Nov-77 & & $10045-97-3$ & CS-137 & GAMMA_GS & USTEST & -0.13 \\
\hline 1971 & PDMS & & sW & & & E014844 & 28-Nov-77 & & $10028-17-8$ & TRITIUM & TRITIUM_DIST_LSC & USTEST & 39700 \\
\hline 1972 & PDMS & & sW & & & E002169 & 28-Nov-77 & & $10045-97-3$ & CS-137 & GAMMA_GS & USTEST & 4.61 \\
\hline 1973 & PDMS & & sW & & & E002067 & 29-Nov-77 & & $10045-97-3$ & CS-137 & GAMMA_GS & PNL-KI & 0.04 \\
\hline 1974 & PDMS & & sW & & & E002067 & 29-Nov-77 & & 15046-84-1 & I-129 & I129_SEP_LEPS_GS & PNL-KI & 0.000047 \\
\hline 1975 & PDMS & & sW & & & E011352 & 29-Nov-77 & & $10045-97-3$ & CS-137 & GAMMA_ĒS & PNL-KI & 0.0005 \\
\hline 1976 & PDMS & & SW & & & E014097 & 29-Nov-77 & & $10045-97-3$ & CS-137 & GAMMA_GS & PNL-KI & 0.02 \\
\hline \begin{tabular}{|l|l|}
1977 \\
\end{tabular} & PDMS & & sW & & & E001781 & 29-Nov-77 & & $15046-84-1$ & I-129 & I129 SEP LEPS GS & PNL-KI & 0.0000038 \\
\hline
\end{tabular}




\begin{tabular}{|c|c|c|c|c|c|c|c|c|c|c|c|c|c|}
\hline & $\bar{A}$ & $\mathrm{~B}$ & $\bar{C}$ & $\mathrm{D}$ & $\mathrm{E}$ & $\mathrm{F}$ & $\bar{G}$ & $\mathrm{H}$ & 1 & $\mathrm{~J}$ & $\bar{K}$ & $\bar{L}$ & $\mathrm{M}$ \\
\hline 1 & SAMPLE_NUM_ASSIGNED_TO & \begin{tabular}{|l} 
SAMPLER \\
\end{tabular} & MEDIA & BIOTA_MEDIA & TAG_ID & SAMP_NUM & SAMP_DATE_TIME & SAMP_DATE_TIME_ON & CON_ID & \begin{tabular}{|l|} 
CON_SHORT_NAME \\
\end{tabular} & METHOD_NAME & LAB_CODE & VALUE_RPTD \\
\hline 1978 & PDMS & & SW & & & E001781 & 29-Nov-77 & & $10045-97-3$ & CS-137 & GAMMA_GS & PNL-KI & 0.02 \\
\hline 1979 & PDMS & & SW & & & E008171 & 30-Nov-77 & & $10028-17-8$ & TRITIUM & TRITIUM_DIST_LSC & USTEST & 573 \\
\hline 1980 & PDMS & & SW & & & E008171 & 30-Nov-77 & & $10045-97-3$ & CS-137 & GAMMA_GS & USTEST & 0.0511 \\
\hline 1981 & PDMS & & SW & & & E041368 & 30-Nov-77 & & $10028-17-8$ & TRITIUM & TRITIUM_DIST_LSC & USTEST & 504 \\
\hline 1982 & PDMS & & SW & & & E041043 & 30-Nov-77 & & $10028-17-8$ & TRITIUM & TRITIUM_DIST_LSC & USTEST & 433 \\
\hline 1983 & PDMS & & SW & & & E002068 & 13-Dec-77 & & $10045-97-3$ & CS-137 & GAMMA_GS & PNL-KI & 0.04 \\
\hline 1984 & PDMS & & SW & & & E011353 & 13-Dec-77 & & $10045-97-3$ & CS-137 & GAMMA_GS & PNL-KI & 0.003 \\
\hline 1985 & PDMS & & SW & & & E001782 & 13-Dec-77 & & $10045-97-3$ & CS-137 & GAMMA_GS & PNL-KI & 0.03 \\
\hline 1986 & PDMS & & SW & & & E014098 & 13-Dec-77 & & $10045-97-3$ & CS-137 & GAMMA_GS & PNL-KI & 0.005 \\
\hline 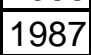 & PDMS & & SW & & & E020721 & 18-Dec-77 & & $18540-29-9$ & $\mathrm{CR}+6$ & UNKNOW̄N_METALS & PNL-KI & 0.6 \\
\hline 1988 & PDMS & & SW & & & E021272 & 26-Dec-77 & & $18540-29-9$ & $\mathrm{CR}+6$ & UNKNOWN_METALS & PNL-KI & 12.2 \\
\hline 1989 & PDMS & & SW & & & E004575 & 27-Dec-77 & & $10045-97-3$ & CS-137 & GAMMA_GS & USTEST & 8.95 \\
\hline 1990 & PDMS & & SW & & & E004575 & 27-Dec-77 & & 10028-17-8 & TRITIUM & TRITIUM_DIST_LSC & USTEST & 275 \\
\hline 1991 & PDMS & & SW & & & E004575 & 27-Dec-77 & & $10098-97-2$ & SR-90 & SRISO_SEP_PRECIP_GPC & USTEST & 0.258 \\
\hline 1992 & PDMS & & SW & & & E002069 & 27-Dec-77 & & $10045-97-3$ & CS-137 & GAMMA_GS & PNL-KI & 0.03 \\
\hline 1993 & PDMS & & SW & & & E002069 & 27-Dec-77 & & 15046-84-1 & 1-129 & I129_SEP_LEPS_GS & PNL-KI & 0.000039 \\
\hline 1994 & PDMS & & SW & & & E011354 & 27-Dec-77 & & $10045-97-3$ & CS-137 & GAMMA_GS & PNL-KI & 0.004 \\
\hline 1995 & PDMS & & SW & & & E001783 & 27-Dec-77 & & 15046-84-1 & I-129 & I129_SEP_LEPS_GS & PNL-KI & 0.0000058 \\
\hline 1996 & PDMS & & SW & & & E001783 & 27-Dec-77 & & $10045-97-3$ & CS-137 & GAMMA_ĒS & PNL-KI & 0.03 \\
\hline 1997 & PDMS & & SW & & & E014099 & 27-Dec-77 & & $10045-97-3$ & CS-137 & GAMMA_GS & PNL-KI & 0.005 \\
\hline 1998 & PDMS & & SW & & & E003614 & 27-Dec-77 & & $10045-97-3$ & CS-137 & GAMMA_GS & USTEST & 3.17 \\
\hline 1999 & PDMS & & SW & & & E044474 & 27-Dec-77 & & $10045-97-3$ & CS-137 & GAMMA_GS & USTEST & 8.5 \\
\hline 2000 & PDMS & & SW & & & E044474 & 27-Dec-77 & & $10028-17-8$ & TRITIUM & TRITIUM_DIST_LSC & USTEST & 48100 \\
\hline 2001 & PDMS & & SW & & & E002170 & 27-Dec-77 & & $10045-97-3$ & CS-137 & GAMMA_GS & USTEST & 6.62 \\
\hline 2002 & PDMS & & SW & & & E008105 & 28-Dec-77 & & 10028-17-8 & TRITIUM & TRITIUM_DIST_LSC & USTEST & 326 \\
\hline 2003 & PDMS & & SW & & & E008105 & 28-Dec-77 & & $10098-97-2$ & SR-90 & SRISO_SEP_PRECIP_GPC & USTEST & 0.282 \\
\hline 2004 & PDMS & & SW & & & E008105 & 28-Dec-77 & & $10045-97-3$ & CS-137 & GAMMA_GS & USTEST & 3.19 \\
\hline 2005 & PDMS & & SW & & & E075063 & 28-Dec-77 & & $10028-17-8$ & TRITIUM & TRITIUM_DIST_LSC & USTEST & 438 \\
\hline 2006 & PDMS & & SW & & & E041044 & 28-Dec-77 & & $10028-17-8$ & TRITIUM & TRITIUM_DIST_LSC & USTEST & 274 \\
\hline 2007 & PDMS & & SW & & & E001917 & 10-Jan-78 & & $10045-97-3$ & CS-137 & GAMMA_GS & PNL-KI & 0.03 \\
\hline 2008 & PDMS & & SW & & & E014100 & 10-Jan-78 & & $10045-97-3$ & CS-137 & GAMMA_GS & PNL-KI & 0.0064 \\
\hline 2009 & PDMS & & SW & & & E001784 & 10-Jan-78 & & $10045-97-3$ & CS-137 & GAMMA_GS & PNL-KI & 0.01 \\
\hline 2010 & PDMS & & SW & & & E001918 & 13-Jan-78 & & $10045-97-3$ & CS-137 & GAMMA_GS & PNL-KI & 0.07 \\
\hline 2011 & PDMS & & SW & & & E001919 & 17-Jan-78 & & $10045-97-3$ & CS-137 & GAMMA_GS & PNL-KI & 0.03 \\
\hline 2012 & PDMS & & SW & & & E001920 & 24-Jan-78 & & $10045-97-3$ & CS-137 & GAMMA_GS & PNL-KI & 0.05 \\
\hline 2013 & PDMS & & SW & & & E001920 & 24-Jan-78 & & 15046-84-1 & I-129 & I129_SEP_LEPS_GS & PNL-KI & 0.000035 \\
\hline 2014 & PDMS & & SW & & & E014101 & 24-Jan-78 & & $10045-97-3$ & CS-137 & GAMMA_GS & PNL-KI & 0.005 \\
\hline 2015 & PDMS & & SW & & & E001785 & 24-Jan-78 & & 15046-84-1 & I-129 & I129_SEP_LEPS_GS & PNL-KI & 0.0000085 \\
\hline 2016 & PDMS & & SW & & & E001785 & 24-Jan-78 & & $10045-97-3$ & CS-137 & GAMMA_GS & PNL-KI & 0.02 \\
\hline 2017 & PDMS & & SW & & & E041375 & 25-Jan-78 & & $10028-17-8$ & TRITIUM & TRITIUM_DIST_LSC & USTEST & 243 \\
\hline 2018 & PDMS & & SW & & & E041045 & 25-Jan-78 & & $10028-17-8$ & TRITIUM & TRITIUM_DIST_LSC & USTEST & 175 \\
\hline 2019 & PDMS & & SW & & & E004576 & 30-Jan-78 & & $10045-97-3$ & CS-137 & GAMMA_GS & USTEST & 1.13 \\
\hline 2020 & PDMS & & SW & & & E004576 & 30-Jan-78 & & $10028-17-8$ & TRITIUM & TRITIUM_DIST_LSC & USTEST & 257 \\
\hline 2021 & PDMS & & SW & & & E003615 & 30-Jan-78 & & $10045-97-3$ & CS-137 & GAMMA_GS & USTEST & 3.79 \\
\hline 2022 & PDMS & & SW & & & E044475 & 30-Jan-78 & & $10045-97-3$ & CS-137 & GAMMA_GS & USTEST & 6.43 \\
\hline 2023 & PDMS & & SW & & & E044475 & 30-Jan-78 & & $10028-17-8$ & TRITIUM & TRITIUM_DIST_LSC & USTEST & 48600 \\
\hline 2024 & PDMS & & SW & & & E002171 & 30-Jan-78 & & $10045-97-3$ & CS-137 & GAMMA_GS & USTEST & 4.36 \\
\hline 2025 & PDMS & & SW & & & E001921 & 07-Feb-78 & & $10045-97-3$ & CS-137 & GAMMA_GS & PNL-KI & 0.02 \\
\hline 2026 & PDMS & & SW & & & E003955 & 07-Feb-78 & & $10045-97-3$ & CS-137 & GAMMA_GS & PNL-KI & 0.01 \\
\hline 2027 & PDMS & & SW & & & E001362 & 07-Feb-78 & & $10045-97-3$ & CS-137 & GAMMA_GS & PNL-KI & 0.02 \\
\hline 2028 & PDMS & & SW & & & E013993 & 07-Feb-78 & & $10045-97-3$ & CS-137 & GAMMA_GS & PNL-KI & 0.02 \\
\hline 2029 & PDMS & & SW & & & E000367 & 13-Feb-78 & & $10045-97-3$ & CS-137 & GAMMA_GS & USTEST & 1.26 \\
\hline
\end{tabular}




\begin{tabular}{|c|c|c|c|c|c|c|c|c|c|c|c|c|c|}
\hline & A & $\mathrm{B}$ & $\mathrm{C}$ & $\mathrm{D}$ & $\mathrm{E}$ & $\mathrm{F}$ & $\mathrm{G}$ & $\mathrm{H}$ & $\mathrm{I}$ & $\mathrm{J}$ & $\mathrm{K}$ & $\mathrm{L}$ & $\mathrm{M}$ \\
\hline 1 & SAMPLE_NUM_ASSIGNED_TO & SAMPLER & MEDIA & BIOTA_MEDIA & TAG_ID & SAMP_NUM & SAMP_DATE_TIME & SAMP_DATE_TIME_ON & CON_ID & CON_SHORT_NAME & METHOD_NAME & LAB_CODE & VALUE_RPTD \\
\hline 2030 & PDMS & & SW & & & E014842 & 13-Feb-78 & & $10098-97-2$ & SR-90 & SRISO_SEP_PRECIP_GPC & USTEST & 1.45 \\
\hline 2031 & PDMS & & SW & & & E014842 & 13-Feb-78 & & $10045-97-3$ & CS-137 & GAMMA_GS & USTEST & 9.41 \\
\hline 2032 & PDMS & & SW & & & E022431 & 13-Feb-78 & & $10045-97-3$ & CS-137 & GAMMA_GS & USTEST & 22 \\
\hline 2033 & PDMS & & SW & & & E022431 & 13-Feb-78 & & $10098-97-2$ & SR-90 & SRISO_SEP_PRECIP_GPC & USTEST & 4.37 \\
\hline 2034 & PDMS & & SW & & & E022358 & 13-Feb-78 & & $10045-97-3$ & CS-137 & GAMMA_GS & USTEST & 11.2 \\
\hline 2035 & PDMS & & SW & & & E022358 & 13-Feb-78 & & $10098-97-2$ & SR-90 & SRISO_SEP_PRECIP_GPC & USTEST & 8.8 \\
\hline 2036 & PDMS & & SW & & & E021776 & 13-Feb-78 & & $10045-97-3$ & CS-137 & GAMMA_GS & USTEST & 11 \\
\hline 2037 & PDMS & & SW & & & E021776 & 13-Feb-78 & & $10098-97-2$ & SR-90 & SRISO_SEP_PRECIP_GPC & USTEST & 3.13 \\
\hline 2038 & PDMS & & SW & & & E002070 & 21-Feb-78 & & $10045-97-3$ & CS-137 & GAMMA_GS & PNL-KI & 0.02 \\
\hline 2039 & PDMS & & SW & & & E002070 & 21-Feb-78 & & 15046-84-1 & I-129 & I129_SEP_LEPS_GS & PNL-KI & 0.000029 \\
\hline 2040 & PDMS & & SW & & & E003956 & 21-Feb-78 & & $10045-97-3$ & CS-137 & GAMMA_GS & PNL-KI & 0.009 \\
\hline 2041 & PDMS & & SW & & & E001363 & 21-Feb-78 & & 15046-84-1 & I-129 & I129_SEP_LEPS_GS & PNL-KI & 0.0000087 \\
\hline 2042 & PDMS & & SW & & & E001363 & 21-Feb-78 & & $10045-97-3$ & CS-137 & GAMMA_ḠS & PNL-KI & 0.01 \\
\hline 2043 & PDMS & & SW & & & E013994 & 21-Feb-78 & & $10045-97-3$ & CS-137 & GAMMA_GS & PNL-KI & 0.01 \\
\hline 2044 & PDMS & & SW & & & E008067 & 22-Feb-78 & & 10028-17-8 & TRITIUM & TRITIUM_DIST_LSC & USTEST & 218 \\
\hline 2045 & PDMS & & SW & & & E008067 & 22-Feb-78 & & $10045-97-3$ & CS-137 & GAMMA_GS & USTEST & 8.56 \\
\hline 2046 & PDMS & & SW & & & E041378 & 22-Feb-78 & & 10028-17-8 & TRITIUM & TRITIUM_DIST_LSC & USTEST & 206 \\
\hline 2047 & PDMS & & SW & & & E034864 & 22-Feb-78 & & $10028-17-8$ & TRITIUM & TRITIUM_DIST_LSC & USTEST & 172 \\
\hline 2048 & PDMS & & SW & & & E004577 & 27-Feb-78 & & $10045-97-3$ & CS-137 & GAMMA_GS & USTEST & 1.58 \\
\hline 2049 & PDMS & & SW & & & E004577 & 27-Feb-78 & & $10028-17-8$ & TRITIUM & TRITIUM_DIST_LSC & USTEST & 210 \\
\hline 2050 & PDMS & & SW & & & E004577 & 27-Feb-78 & & $10098-97-2$ & SR-90 & SRISO_SEP_PRECIP_GPC & USTEST & 0.324 \\
\hline 2051 & PDMS & & SW & & & E001614 & 27-Feb-78 & & $10098-97-2$ & SR-90 & SRISO_SEP_PRECIP_GPC & USTEST & 0.685 \\
\hline 2052 & PDMS & & SW & & & E001614 & 27-Feb-78 & & $10028-17-8$ & TRITIUM & TRITIUM_DIST_LSC & USTEST & 163 \\
\hline 2053 & PDMS & & SW & & & E001614 & 27-Feb-78 & & $10045-97-3$ & CS-137 & GAMMA_GS & USTEST & -0.0118 \\
\hline 2054 & PDMS & & SW & & & E014845 & 27-Feb-78 & & 10098-97-2 & SR-90 & SRISO_SEP_PRECIP_GPC & USTEST & 2.13 \\
\hline 2055 & PDMS & & SW & & & E014845 & 27-Feb-78 & & $10045-97-3$ & CS-137 & GAMMA_GS & USTEST & -2.57 \\
\hline 2056 & PDMS & & sW & & & E014845 & 27-Feb-78 & & $10028-17-8$ & TRITIUM & TRITIUM_DIST_LSC & USTEST & 53600 \\
\hline 2057 & PDMS & & SW & & & E002172 & 27-Feb-78 & & $10045-97-3$ & CS-137 & GAMMA_GS & USTEST & -0.885 \\
\hline 2058 & PDMS & & SW & & & E002071 & 07-Mar-78 & & $10045-97-3$ & CS-137 & GAMMA_GS & PNL-KI & 0.02 \\
\hline 2059 & PDMS & & SW & & & E003957 & 07-Mar-78 & & $10045-97-3$ & CS-137 & GAMMA_GS & PNL-KI & 0.01 \\
\hline 2060 & PDMS & & SW & & & E013995 & 07-Mar-78 & & $10045-97-3$ & CS-137 & GAMMA_GS & PNL-KI & 0.009 \\
\hline 2061 & PDMS & & SW & & & E001364 & 07-Mar-78 & & $10045-97-3$ & CS-137 & GAMMA_GS & PNL-KI & 0.01 \\
\hline 2062 & PDMS & & SW & & & E002072 & 21-Mar-78 & & $10045-97-3$ & CS-137 & GAMMA_GS & PNL-KI & 0.02 \\
\hline 2063 & PDMS & & SW & & & E003958 & 21-Mar-78 & & $10045-97-3$ & CS-137 & GAMMA_GS & PNL-KI & 0.01 \\
\hline 2064 & PDMS & & SW & & & E004578 & 27-Mar-78 & & $10028-17-8$ & TRITIUM & TRITIUM_DIST_LSC & USTEST & 488 \\
\hline 2065 & PDMS & & SW & & & E004578 & 27-Mar-78 & & $10045-97-3$ & CS-137 & GAMMA_GS & USTEST & 0.621 \\
\hline 2066 & PDMS & & SW & & & E003616 & 27-Mar-78 & & $10045-97-3$ & CS-137 & GAMMA_GS & USTEST & -0.722 \\
\hline 2067 & PDMS & & SW & & & E044476 & 27-Mar-78 & & $10045-97-3$ & CS-137 & GAMMA_GS & USTEST & -0.0503 \\
\hline 2068 & PDMS & & SW & & & E044476 & 27-Mar-78 & & 10028-17-8 & TRITIUM & TRITIUM_DIST_LSC & USTEST & 55800 \\
\hline 2069 & PDMS & & SW & & & E002173 & 27-Mar-78 & & $10045-97-3$ & CS-137 & GAMMA_GS & USTEST & -1.82 \\
\hline 2070 & PDMS & & SW & & & E008008 & 29-Mar-78 & & $10028-17-8$ & TRITIUM & TRITIUM_DIST_LSC & USTEST & 521 \\
\hline 2071 & PDMS & & SW & & & E008008 & 29-Mar-78 & & $10098-97-2$ & SR-90 & SRISO_SEP_PRECIP_GPC & USTEST & 0.258 \\
\hline 2072 & PDMS & & SW & & & E008008 & 29-Mar-78 & & $10045-97-3$ & CS-137 & GAMMA_GS & USTEST & -0.671 \\
\hline 2073 & PDMS & & SW & & & E041382 & 29-Mar-78 & & 10028-17-8 & TRITIUM & TRITIUM_DIST_LSC & USTEST & 598 \\
\hline 2074 & PDMS & & SW & & & E034868 & 29-Mar-78 & & $10028-17-8$ & TRITIUM & TRITIUM_DIST_LSC & USTEST & 320 \\
\hline 2075 & PDMS & & SW & & & E002000 & 04-Apr-78 & & PU-239/240 & PU-239/240 & PUISO_IE_AEĀ & PNL-KI & 0.000085 \\
\hline 2076 & PDMS & & SW & & & E002000 & 04-Apr-78 & & $10045-97-3$ & CS-137 & GAMMA_GS & PNL-KI & 0.01 \\
\hline 2077 r r l & PDMS & & SW & & & E002000 & 04-Apr-78 & & 15046-84-1 & $\mid-129$ & I129_SEP_LEPS_GS & PNL-KI & 0.000043 \\
\hline 2078 & PDMS & & SW & & & E003959 & 04-Apr-78 & & PU-239/240 & PU-239/240 & PUIS̄O_IE_AEA & PNL-KI & 0.000033 \\
\hline 2079 & PDMS & & sW & & & E003959 & 04-Apr-78 & & $10045-97-3$ & CS-137 & GAMMA_GS & PNL-KI & 0.02 \\
\hline 2080 & PDMS & & SW & & & E013996 & 04-Apr-78 & & PU-239/240 & PU-239/240 & PUISO_IE_AEA & PNL-KI & 0.000027 \\
\hline 2081 & PDMS & & SW & & & E013996 & 04-Apr-78 & & $10045-97-3$ & CS-137 & GAMMA_GS & PNL-KI & 0.01 \\
\hline
\end{tabular}




\begin{tabular}{|c|c|c|c|c|c|c|c|c|c|c|c|c|c|}
\hline & A & $\mathrm{B}$ & $\mathrm{c}$ & $\mathrm{D}$ & $\mathrm{E}$ & $\mathrm{F}$ & G & $\mathrm{H}$ & $\mathrm{I}$ & $\mathrm{J}$ & $\mathrm{K}$ & $\mathrm{L}$ & $\mathrm{M}$ \\
\hline 1 & SAMPLE_NUM_ASSIGNED_TO & SAMPLER & MEDIA & BIOTA_MEDIA & TAG_ID & SAMP_NUM & SAMP_DATE_TIME & SAMP_DATE_TIME_ON & CON_ID & CON_SHORT_NAME & METHOD_NAME & LAB_CODE & VALUE_RPTD \\
\hline 2082 & PDMS & & SW & & & E001365 & 04-Apr-78 & & $15046-84-1$ & $1-129$ & 1129 SEP_LEPS GS & PNL-KI & 0.0000051 \\
\hline 2083 & PDMS & & sW & & & E001365 & 04-Apr-78 & & PU-239/240 & PU-239/240 & PUISOO_IE_AEA & PNL-KI & 0.000093 \\
\hline 2084 & PDMS & & sW & & & E001365 & 04-Apr-78 & & $10045-97-3$ & CS-137 & GAMMA_GS & PNL-KI & 0.007 \\
\hline 2085 & PDMS & & sW & & & E075064 & 06-Apr-78 & & $10028-17-8$ & TRITIUM & TRITIUM_DIST_LSC & USTEST & 437 \\
\hline 2086 & PDMS & & sW & & & E002001 & 18-Apr-78 & & $10045-97-3$ & CS-137 & GAMMA_GS & PNL-KI & 0.02 \\
\hline 2087 & PDMS & & SW & & & E003960 & 18-Apr-78 & & $10045-97-3$ & CS-137 & GAMMA_GS & PNL-KI & 0.01 \\
\hline 2088 & PDMS & & SW & & & E001366 & 18-Apr-78 & & 15046-84-1 & I-129 & I129_SEP_LEPS_GS & PNL-KI & 0.000014 \\
\hline 2089 & PDMS & & SW & & & E001366 & 18-Apr-78 & & $10045-97-3$ & CS-137 & GAMMA_GS & PNL-KI & 0.04 \\
\hline 2090 & PDMS & & SW & & & E013997 & 18-Apr-78 & & $10045-97-3$ & CS-137 & GAMMA_GS & PNL-KI & 0.009 \\
\hline 2091 & PDMS & & SW & & & E004579 & 24-Apr-78 & & $10045-97-3$ & CS-137 & GAMMA_GS & USTEST & -0.00094 \\
\hline 2092 & PDMS & & SW & & & E004579 & 24-Apr-78 & & $10028-17-8$ & TRITIUM & TRITIUM_DIST_LSC & USTEST & 285 \\
\hline 2093 & PDMS & & sW & & & E003617 & 24-Apr-78 & & $10045-97-3$ & CS-137 & GAMMA GS & USTEST & 5.78 \\
\hline \begin{tabular}{|l|}
2094 \\
\end{tabular} & PDMS & & SW & & & E044477 & 24-Apr-78 & & $10045-97-3$ & CS-137 & GAMMA_GS & USTEST & -0.232 \\
\hline \begin{tabular}{|l|}
2095 \\
\end{tabular} & PDMS & & SW & & & E044477 & 24-Apr-78 & & $10028-17-8$ & TRITIUM & TRITIUM DIST_LSC & USTEST & 46200 \\
\hline 2096 & PDMS & & SW & & & E002174 & 24-Apr-78 & & $10045-97-3$ & CS-137 & GAMMA_GS & USTEST & -0.596 \\
\hline \begin{tabular}{|l|}
2097 \\
\end{tabular} & PDMS & & SW & & & E007942 & 26-Apr-78 & & $10028-17-8$ & TRITIUM & TRITIUM_DIST_LSC & USTEST & 366 \\
\hline \begin{tabular}{|l|}
2098 \\
\end{tabular} & PDMS & & SW & & & E007942 & 26-Apr-78 & & $10045-97-3$ & CS-137 & GAMMA_GS & USTEST & 0.0508 \\
\hline \begin{tabular}{|l|}
2099 \\
\end{tabular} & PDMS & & sW & & & E034871 & 26-Apr-78 & & $10028-17-8$ & TRITIUM & TRITIUM_DIST_LSC & USTEST & 430 \\
\hline 2100 & PDMS & & SW & & & E003961 & 02-May-78 & & $10045-97-3$ & CS-137 & GAMMA_GS & PNL-KI & 0.01 \\
\hline 2101 & PDMS & & SW & & & E002002 & 02-May-78 & & $10045-97-3$ & CS-137 & GAMMA_GS & PNL-KI & 0.01 \\
\hline 2102 & PDMS & & SW & & & E002002 & 02-May-78 & & 15046-84-1 & $\mid-129$ & 1129_SEP_LEPS_GS & PNL-KI & 0.000012 \\
\hline 2103 & PDMS & & SW & & & E013998 & 02-May-78 & & $10045-97-3$ & CS-137 & GAMMA_GS & PNL-KI & 0.009 \\
\hline 2104 & PDMS & & SW & & & E011818 & 08-May-78 & & $10045-97-3$ & CS-137 & GAMMA_GS & USTEST & 12.6 \\
\hline \begin{tabular}{|l|}
2105 \\
\end{tabular} & PDMS & & SW & & & E016408 & 08-May-78 & & $10098-97-2$ & SR-90 & SRISO_SEP_PRECIP_GPC & USTEST & 1.41 \\
\hline \begin{tabular}{|l|l|l|l|l|}
2106 \\
\end{tabular} & PDMS & & SW & & & E016408 & 08-May-78 & & $10045-97-3$ & CS-137 & GAMMA_GS & USTEST & 2.41 \\
\hline \begin{tabular}{|l|l|}
2107 \\
\end{tabular} & PDMS & & SW & & & E022432 & 08-May-78 & & $10045-97-3$ & CS-137 & GAMMA_GS & USTEST & 53.2 \\
\hline 2108 & PDMS & & SW & & & E022432 & 08-May-78 & & $10098-97-2$ & SR-90 & SRISO_SEP_PRECIP_GPC & USTEST & 0.547 \\
\hline 2109 & PDMS & & SW & & & E022359 & 08-May-78 & & $10045-97-3$ & CS-137 & GAMMA_GS & USTEST & 13.3 \\
\hline 2110 & PDMS & & SW & & & E022359 & 08-May-78 & & $10098-97-2$ & SR-90 & SRISO_SEP_PRECIP_GPC & USTEST & 5.69 \\
\hline 2111 & PDMS & & SW & & & E014278 & 08-May-78 & & $10045-97-3$ & CS-137 & GAMMA_GS & USTEST & 2.91 \\
\hline 2112 & PDMS & & SW & & & E014278 & 08-May-78 & & $10098-97-2$ & SR-90 & SRISO_SEP_PRECIP_GPC & USTEST & 3.57 \\
\hline 2113 & PDMS & & SW & & & E002003 & 16-May-78 & & $10045-97-3$ & CS-137 & GAMMA_GS & PNL-KI & 0.02 \\
\hline 2114 & PDMS & & SW & & & E003962 & 16-May-78 & & $10045-97-3$ & CS-137 & GAMMA_GS & PNL-KI & 0.009 \\
\hline 2115 & PDMS & & SW & & & E013999 & 22-May-78 & & $10045-97-3$ & CS-137 & GAMMA_GS & PNL-KI & 0.007 \\
\hline 2116 & PDMS & & SW & & & E001367 & 22-May-78 & & $10045-97-3$ & CS-137 & GAMMA_GS & PNL-KI & 0.007 \\
\hline 2117 & PDMS & & sW & & & E001615 & 26-May-78 & & $10098-97-2$ & SR-90 & SRISO_SEP_PRECIP_GPC & USTEST & 0.258 \\
\hline 2118 & PDMS & & SW & & & E001615 & 26-May-78 & & 10028-17-8 & TRITIUM & TRITIUM_DIST_LSC & USTEST & 404 \\
\hline 2119 & PDMS & & SW & & & E001615 & 26-May-78 & & $10045-97-3$ & CS-137 & GAMMA_GS & USTEST & 0.0511 \\
\hline 2120 & PDMS & & SW & & & E014846 & 26-May-78 & & $10098-97-2$ & SR-90 & SRISO_SEPP_PRECIP_GPC & USTEST & 0.0589 \\
\hline 2121 & PDMS & & SW & & & E014846 & 26-May-78 & & $10045-97-3$ & CS-137 & GAMMĀGS & USTEST & -0.128 \\
\hline 2122 & PDMS & & SW & & & E014846 & 26-May-78 & & $10028-17-8$ & TRITIUM & TRITIUM_DIST_LSC & USTEST & 50000 \\
\hline 2123 & PDMS & & SW & & & E002175 & 26-May-78 & & $10045-97-3$ & CS-137 & GAMMA_GS & USTEST & 2.07 \\
\hline 2124 & PDMS & & SW & & & E004580 & 30-May-78 & & $10045-97-3$ & CS-137 & GAMMA_GS & USTEST & 0.423 \\
\hline 2125 & PDMS & & SW & & & E004580 & 30-May-78 & & $10028-17-8$ & TRITIUM & TRITIUM_DIST_LSC & USTEST & 291 \\
\hline 2126 & PDMS & & SW & & & E004580 & 30-May-78 & & $10098-97-2$ & SR-90 & SRISO_SEP_PRECIP_GPC & USTEST & 0.444 \\
\hline 2127 & PDMS & & SW & & & E002004 & 30-May-78 & & $10045-97-3$ & CS-137 & GAMMA_GS & PNL-KI & 0.02 \\
\hline 2128 & PDMS & & SW & & & E002004 & 30-May-78 & & 15046-84-1 & I-129 & 1129_SEP_LEPS_GS & PNL-KI & 0.000025 \\
\hline 2129 & PDMS & & SW & & & E003963 & 30-May-78 & & $10045-97-3$ & CS-137 & GAMMA_ĒS & PNL-KI & 0.007 \\
\hline 2130 & PDMS & & SW & & & E014000 & 30-May-78 & & $10045-97-3$ & CS-137 & GAMMA_GS & PNL-KI & 0.08 \\
\hline 2131 & PDMS & & SW & & & E001368 & 30-May-78 & & 15046-84-1 & I-129 & 1129_SEP_LEPS_GS & PNL-KI & 0.000011 \\
\hline 2132 & PDMS & & SW & & & E001368 & 30-May-78 & & $10045-97-3$ & CS-137 & GAMMA_GS & PNL-KI & 0.09 \\
\hline 2133 & PDMS & & SW & & & E075065 & 30-May-78 & & $10028-17-8$ & TRITIUM & TRITIUM_DIST_LSC & USTEST & -470 \\
\hline
\end{tabular}




\begin{tabular}{|c|c|c|c|c|c|c|c|c|c|c|c|c|c|}
\hline & 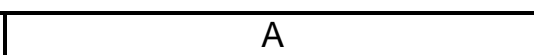 & $\mathrm{B}$ & $\mathrm{C}$ & $\mathrm{D}$ & $\mathrm{E}$ & $\mathrm{F}$ & $\bar{G}$ & $\mathrm{H}$ & $\mathrm{T}$ & $\mathrm{J}$ & $\mathrm{K}$ & $\bar{L}$ & $\mathrm{M}$ \\
\hline 1 & SAMPLE_NUM_ASSIGNED_TO & SAMPLER & MEDIA & BIOTA_MEDIA & TAG_ID & SAMP_NUM & SAMP_DATE_TIME & SAMP_DATE_TIME_ON & CON_ID & CON_SHORT_NAME & METHOD_NAME & LAB_CODE & VALUE_RPTD \\
\hline 2134 & PDMS & & SW & & & E041046 & 30-May-78 & & $10028-17-8$ & TRITIUM & TRITIUM_DIST_LSC & USTEST & 11.3 \\
\hline 2135 & PDMS & & sW & & & E007977 & 31-May-78 & & $10028-17-8$ & TRITIUM & TRITIUM_DIST_LSC & USTEST & 357 \\
\hline 2136 & PDMS & & SW & & & E007977 & 31-May-78 & & $10045-97-3$ & CS-137 & GAMMA_GS & USTEST & -0.343 \\
\hline 2137 & PDMS & & sW & & & E011358 & 13-Jun-78 & & $10045-97-3$ & CS-137 & GAMMA GS & PNL-KI & 0.01 \\
\hline 2138 & PDMS & & SW & & & E002005 & 13-Jun-78 & & $10045-97-3$ & CS-137 & GAMMA_GS & PNL-KI & 0.02 \\
\hline 2139 & PDMS & & sW & & & E014001 & 13-Jun-78 & & $10045-97-3$ & CS-137 & GAMMA_GS & PNL-KI & 0.007 \\
\hline 2140 & PDMS & & sW & & & E001369 & 13-Jun-78 & & $10045-97-3$ & CS-137 & GAMMA_GS & PNL-KI & 0.01 \\
\hline 2141 & PDMS & & sW & & & E004581 & 19-Jun-78 & & $10028-17-8$ & TRITIUM & TRITIUM_DIST_LSC & USTEST & 340 \\
\hline 2142 & PDMS & & sW & & & E004581 & 19-Jun-78 & & $10045-97-3$ & CS-137 & GAMMA_GS & USTEST & -0.0592 \\
\hline 2143 & PDMS & & sW & & & E007909 & 26-Jun-78 & & $10028-17-8$ & TRITIUM & TRITIUM_DIST_LSC & USTEST & 823 \\
\hline 2144 & PDMS & & SW & & & E007909 & 26-Jun-78 & & $10098-97-2$ & SR-90 & SRISO SEP PRECIP GPC & USTEST & 0.216 \\
\hline 2145 & PDMS & & sw & & & E007909 & 26-Jun-78 & & $10045-97-3$ & CS-137 & GAMMA_GS & USTEST & 10.7 \\
\hline \begin{tabular}{|l|l|}
2146 & \\
\end{tabular} & PDMS & & sW & & & E003556 & 26-Jun-78 & & $10045-97-3$ & CS-137 & GAMMA_GS & USTEST & -0.0573 \\
\hline \begin{tabular}{|l|l|}
2147 & \\
\end{tabular} & PDMS & & sW & & & E044478 & 26-Jun-78 & & $10045-97-3$ & CS-137 & GAMMA_GS & USTEST & 1.3 \\
\hline 2148 & PDMS & & sw & & & E044478 & 26-Jun-78 & & $10028-17-8$ & TRITIUM & TRITIUM_DIST_LSC & USTEST & 41400 \\
\hline 2149 & PDMS & & SW & & & E002176 & 26-Jun-78 & & $10045-97-3$ & CS-137 & GAMMA_GS & USTEST & 0.0473 \\
\hline 2150 & PDMS & & SW & & & E002006 & 27-Jun-78 & & PU-239/240 & PU-239/240 & PUISO_IE_AEA & PNL-KI & 0.00021 \\
\hline 2151 & PDMS & & sW & & & E002006 & 27-Jun-78 & & $10045-97-3$ & CS-137 & GAMMA_GS & PNL-KI & 0.02 \\
\hline 2152 & PDMS & & sW & & & E002006 & 27-Jun-78 & & $15046-84-1$ & I-129 & I129_SEP_LEPS_GS & PNL-KI & 0.000062 \\
\hline 2153 & PDMS & & SW & & & E005455 & 27-Jun-78 & & $10045-97-3$ & CS-137 & GAMMA_ĒS & PNL-KI & 0.03 \\
\hline 2154 & PDMS & & SW & & & E005455 & 27-Jun-78 & & PU-239/240 & PU-239/240 & PUISO_IE_AEA & PNL-KI & 0.000095 \\
\hline 2155 & PDMS & & SW & & & E001291 & 27-Jun-78 & & 15046-84-1 & $1-129$ & 1129_SEP_LEPS_GS & PNL-KI & 0.0000091 \\
\hline \begin{tabular}{|l|l|}
2156 \\
\end{tabular} & PDMS & & sW & & & E001291 & 27-Jun-78 & & PU-239/240 & PU-239/240 & PUISOO_IE_AEA & PNL-KI & 0.000055 \\
\hline \begin{tabular}{|l|l|}
2157 \\
\end{tabular} & PDMS & & sW & & & E001291 & 27-Jun-78 & & $10045-97-3$ & CS-137 & GAMMA_GS & PNL-KI & 0.02 \\
\hline 2158 & PDMS & & SW & & & E014002 & 27-Jun-78 & & PU-239/240 & PU-239/240 & PUISO_IE_AEA & PNL-KI & 0.000045 \\
\hline 2159 & PDMS & & sW & & & E014002 & 27-Jun-78 & & $10045-97-3$ & CS-137 & GAMMA_GS & PNL-KI & 0.002 \\
\hline 2160 & PDMS & & sW & & & E042417 & 28-Jun-78 & & $10028-17-8$ & TRITIUM & TRITIUM_DIST_LSC & USTEST & 611 \\
\hline \begin{tabular}{|l|}
2161 \\
\end{tabular} & PDMS & & sW & & & E034878 & 28-Jun-78 & & $10028-17-8$ & TRITIUM & TRITIUM_DIST_LSC & USTEST & 217 \\
\hline \begin{tabular}{|l|}
2162 \\
\end{tabular} & PDMS & & SW & & & E001292 & 11-Jul-78 & & 15046-84-1 & $1-129$ & 1129_SEP_LEPS_GS & PNL-KI & 0.000013 \\
\hline 2163 & PDMS & & sW & & & E001292 & 11-Jul-78 & & $10045-97-3$ & CS-137 & GAMMA_ĒS & PNL-KI & 0.02 \\
\hline \begin{tabular}{|l|l|}
2164 & \\
\end{tabular} & PDMS & & SW & & & E014003 & 11-Jul-78 & & $10045-97-3$ & CS-137 & GAMMA_GS & PNL-KI & 0.01 \\
\hline 2165 & PDMS & & sW & & & E002007 & 16-Jul-78 & & $10045-97-3$ & CS-137 & GAMMA_GS & PNL-KI & 0.03 \\
\hline 2166 & PDMS & & sW & & & E014461 & 16-Jul-78 & & $10045-97-3$ & CS-137 & GAMMA_GS & PNL-KI & 0.03 \\
\hline \begin{tabular}{|l|l|}
2167 \\
\end{tabular} & PDMS & & sW & & & E002008 & 25-Jul-78 & & $10045-97-3$ & CS-137 & GAMMA_GS & PNL-KI & 0.03 \\
\hline \begin{tabular}{|l|}
2168 \\
\end{tabular} & PDMS & & sW & & & E002008 & 25-Jul-78 & & $15046-84-1$ & $1-129$ & I129_SEP_LEPS_GS & PNL-KI & 0.000044 \\
\hline 2169 & PDMS & & SW & & & E012070 & 25-Jul-78 & & $10045-97-3$ & CS-137 & GAMMA_ḠS & PNL-KI & 0.01 \\
\hline 2170 & PDMS & & sW & & & E007939 & 26-Jul-78 & & $10028-17-8$ & TRITIUM & TRITIUM_DIST_LSC & USTEST & 182 \\
\hline \begin{tabular}{|l|l|}
2171 & \\
\end{tabular} & PDMS & & SW & & & E007939 & 26-Jul-78 & & $10045-97-3$ & CS-137 & GAMMA_GS & USTEST & 0.0165 \\
\hline \begin{tabular}{|l|}
2172 \\
\end{tabular} & PDMS & & sW & & & E027333 & 26-Jul-78 & & $10028-17-8$ & TRITIUM & TRITIUM_DIST_LSC & USTEST & 240 \\
\hline 2173 & PDMS & & sW & & & E027845 & 26-Jul-78 & & $10028-17-8$ & TRITIUM & TRITIUM_DIST_LSC & USTEST & 463 \\
\hline \begin{tabular}{|l|}
2174 \\
\end{tabular} & PDMS & & sW & & & E004582 & 31-Jul-78 & & $10028-17-8$ & TRITIUM & TRITIUM_DIST_LSC & USTEST & 431 \\
\hline 2175 & PDMS & & SW & & & E004582 & 31-Jul-78 & & $10045-97-3$ & CS-137 & GAMMA_GS & USTEST & 2.47 \\
\hline 2176 & PDMS & & sW & & & E003557 & 31-Jul-78 & & $10045-97-3$ & CS-137 & GAMMA_GS & USTEST & 17.3 \\
\hline \begin{tabular}{|l|l|}
2177 \\
\end{tabular} & PDMS & & sW & & & E044479 & 31-Jul-78 & & $10045-97-3$ & CS-137 & GAMMA_GS & USTEST & 3.02 \\
\hline 2178 & PDMS & & SW & & & E044479 & 31-Jul-78 & & $10028-17-8$ & TRITIUM & TRITIUM_DIST_LSC & USTEST & 38200 \\
\hline 2179 & PDMS & & sW & & & E002177 & 31-Jul-78 & & $10045-97-3$ & CS-137 & GAMMA_GS & USTEST & 8.65 \\
\hline 2180 & PDMS & & sW & & & E002009 & 08-Aug-78 & & $10045-97-3$ & CS-137 & GAMMA_GS & PNL-KI & 0.05 \\
\hline \begin{tabular}{|l|}
2181 \\
\end{tabular} & PDMS & & sW & & & E014893 & 15-Aug-78 & & $10045-97-3$ & CS-137 & GAMMA_GS & USTEST & 20.5 \\
\hline 2182 & PDMS & & SW & & & E016409 & 15-Aug-78 & & $10098-97-2$ & SR-90 & SRISO_SEPP_PRECIP_GPC & USTEST & 0.991 \\
\hline 2183 & PDMS & & sW & & & E016409 & 15-Aug-78 & & $10045-97-3$ & CS-137 & GAMMĀ_GS & USTEST & 6.94 \\
\hline 2184 & PDMS & & SW & & & E022433 & 15-Aug-78 & & $10045-97-3$ & CS-137 & GAMMA_GS & USTEST & 28.5 \\
\hline 2185 & PDMS & & sW & & & E022433 & 15-Aug-78 & & $10098-97-2$ & SR-90 & SRISO SEP PRECIP GPC & USTEST & 0.811 \\
\hline
\end{tabular}




\begin{tabular}{|c|c|c|c|c|c|c|c|c|c|c|c|c|c|}
\hline & A & $\mathrm{B}$ & $\mathrm{C}$ & $\mathrm{D}$ & $E$ & $\mathrm{~F}$ & $\mathrm{G}$ & $\mathrm{H}$ & $\mathrm{I}$ & $\mathrm{J}$ & $\mathrm{K}$ & $\mathrm{L}$ & $\mathrm{M}$ \\
\hline 1 & SAMPLE_NUM_ASSIGNED_TO & SAMPLER & MEDIA & BIOTA_MEDIA & TAG_ID & SAMP_NUM & SAMP_DATE_TIME & SAMP_DATE_TIME_ON & CON_ID & CON_SHORT_NAME & METHOD_NAME & LAB_CODE & VALUE_RPTD \\
\hline 2186 & PDMS & & SW & & & E027650 & 15-Aug-78 & & $10045-97-3$ & CS-137 & GAMMA_GS & USTEST & 17.9 \\
\hline 2187 & PDMS & & SW & & & E027650 & 15-Aug-78 & & $10098-97-2$ & SR-90 & SRISO_SEP_PRECIP_GPC & USTEST & 4.85 \\
\hline 2188 & PDMS & & SW & & & E014279 & 15-Aug-78 & & $10045-97-3$ & CS-137 & GAMMA_GS & USTEST & 10.1 \\
\hline 2189 & PDMS & & SW & & & E014279 & 15-Aug-78 & & $10098-97-2$ & SR-90 & SRISO_SEP_PRECIP_GPC & USTEST & 7.45 \\
\hline 2190 & PDMS & & SW & & & E002010 & 22-Aug-78 & & $10045-97-3$ & CS-137 & GAMMA_GS & PNL-KI & 0.03 \\
\hline 2191 & PDMS & & SW & & & E002010 & 22-Aug-78 & & 15046-84-1 & I-129 & 1129_SEP_LEPS_GS & PNL-KI & 0.000099 \\
\hline 2192 & PDMS & & SW & & & E005420 & 22-Aug-78 & & $10045-97-3$ & CS-137 & GAMMA_ḠS & PNL-KI & 0.02 \\
\hline 2193 & PDMS & & SW & & & E004583 & 28-Aug-78 & & $10045-97-3$ & CS-137 & GAMMA_GS & USTEST & -0.0668 \\
\hline 2194 & PDMS & & SW & & & E004583 & 28-Aug-78 & & $10028-17-8$ & TRITIUM & TRITIUM_DIST_LSC & USTEST & 247 \\
\hline 2195 & PDMS & & SW & & & E004583 & 28-Aug-78 & & $10098-97-2$ & SR-90 & SRISO_SEP_PRECIP_GPC & USTEST & 0.187 \\
\hline 2196 & PDMS & & SW & & & E001616 & 28-Aug-78 & & $10098-97-2$ & SR-90 & SRISO_SEP_PRECIP_GPC & USTEST & 0.38 \\
\hline 2197 & PDMS & & SW & & & E001616 & 28-Aug-78 & & $10028-17-8$ & TRITIUM & TRITIUM_DIST_LSC & USTEST & 407 \\
\hline 2198 & PDMS & & SW & & & E001616 & 28-Aug-78 & & $10045-97-3$ & CS-137 & GAMMA_GS & USTEST & 0.0281 \\
\hline 2199 & PDMS & & SW & & & E014818 & 28-Aug-78 & & $10098-97-2$ & SR-90 & SRISO_SEP_PRECIP_GPC & USTEST & 0.109 \\
\hline 2200 & PDMS & & SW & & & E014818 & 28-Aug-78 & & $10045-97-3$ & CS-137 & GAMMA_GS & USTEST & 1.75 \\
\hline 2201 & PDMS & & SW & & & E014818 & 28-Aug-78 & & $10028-17-8$ & TRITIUM & TRITIUM_DIST_LSC & USTEST & 40200 \\
\hline 2202 & PDMS & & SW & & & E002178 & 28-Aug-78 & & $10045-97-3$ & CS-137 & GAMMA_GS & USTEST & 2.56 \\
\hline 2203 & PDMS & & SW & & & E007880 & 30-Aug-78 & & $10028-17-8$ & TRITIUM & TRITIUM_DIST_LSC & USTEST & 330 \\
\hline 2204 & PDMS & & SW & & & E007880 & 30-Aug-78 & & $10045-97-3$ & CS-137 & GAMMA_GS & USTEST & 0.0511 \\
\hline 2205 & PDMS & & SW & & & E042423 & 30-Aug-78 & & $10028-17-8$ & TRITIUM & TRITIUM_DIST_LSC & USTEST & 321 \\
\hline 2206 & PDMS & & SW & & & E034884 & 30-Aug-78 & & $10028-17-8$ & TRITIUM & TRITIUM_DIST_LSC & USTEST & 1250 \\
\hline 2207 & PDMS & & SW & & & E002011 & 08-Sep-78 & & $10045-97-3$ & CS-137 & GAMMA_GS & PNL-KI & 0.02 \\
\hline 2208 & PDMS & & SW & & & E003964 & 08-Sep-78 & & $10045-97-3$ & CS-137 & GAMMA_GS & PNL-KI & 0.009 \\
\hline 2209 & PDMS & & SW & & & E001293 & 08-Sep-78 & & $10045-97-3$ & CS-137 & GAMMA_GS & PNL-KI & 0.01 \\
\hline 2210 & PDMS & & SW & & & E014004 & 08-Sep-78 & & $10045-97-3$ & CS-137 & GAMMA_GS & PNL-KI & 0.006 \\
\hline 2211 & PDMS & & SW & & & E002012 & 19-Sep-78 & & $10045-97-3$ & CS-137 & GAMMA_GS & PNL-KI & 0.04 \\
\hline 2212 & PDMS & & SW & & & E003965 & 19-Sep-78 & & $10045-97-3$ & CS-137 & GAMMA_GS & PNL-KI & 0.02 \\
\hline 2213 & PDMS & & SW & & & E001294 & 19-Sep-78 & & $10045-97-3$ & CS-137 & GAMMA_GS & PNL-KI & 0.01 \\
\hline 2214 & PDMS & & SW & & & E014005 & 19-Sep-78 & & $10045-97-3$ & CS-137 & GAMMA_GS & PNL-KI & 0.01 \\
\hline 2215 & PDMS & & SW & & & E039722 & 25-Sep-78 & & $10028-17-8$ & TRITIUM & TRITIUM_DIST_LSC & USTEST & 561 \\
\hline 2216 & PDMS & & SW & & & E039722 & 25-Sep-78 & & $10045-97-3$ & CS-137 & GAMMA_GS & USTEST & -0.117 \\
\hline 2217 & PDMS & & SW & & & E034631 & 25-Sep-78 & & $10045-97-3$ & CS-137 & GAMMA_GS & USTEST & -0.563 \\
\hline 2218 & PDMS & & SW & & & E044480 & 25-Sep-78 & & $10045-97-3$ & CS-137 & GAMMA_GS & USTEST & -0.0635 \\
\hline 2219 & PDMS & & SW & & & E044480 & 25-Sep-78 & & $10028-17-8$ & TRITIUM & TRITIUM_DIST_LSC & USTEST & 52300 \\
\hline 2220 & PDMS & & SW & & & E002179 & 25-Sep-78 & & $10045-97-3$ & CS-137 & GAMMA_GS & USTEST & -0.505 \\
\hline 2221 & PDMS & & SW & & & E007814 & 27-Sep-78 & & $10028-17-8$ & TRITIUM & TRITIUM_DIST_LSC & USTEST & 946 \\
\hline 2222 & PDMS & & SW & & & E007814 & 27-Sep-78 & & $10098-97-2$ & SR-90 & SRISO_SEP_PRECIP_GPC & USTEST & 0.283 \\
\hline 2223 & PDMS & & SW & & & E007814 & 27-Sep-78 & & $10045-97-3$ & CS-137 & GAMMA_GS & USTEST & 7.55 \\
\hline 2224 & PDMS & & SW & & & E042426 & 27-Sep-78 & & $10028-17-8$ & TRITIUM & TRITIUM_DIST_LSC & USTEST & 345 \\
\hline 2225 & PDMS & & SW & & & E034887 & 27-Sep-78 & & $10028-17-8$ & TRITIUM & TRITIUM_DIST_LSC & USTEST & 560 \\
\hline 2226 & PDMS & & SW & & & E003966 & 03-Oct-78 & & PU-239/240 & PU-239/240 & PUISO_IE_AEA & PNL-KI & 0.000076 \\
\hline 2227 & PDMS & & SW & & & E003966 & 03-Oct-78 & & $10045-97-3$ & CS-137 & GAMMA_GS & PNL-KI & 0.01 \\
\hline 2228 & PDMS & & SW & & & E002013 & 03-Oct-78 & & PU-239/240 & PU-239/240 & PUISO_IE_AEA & PNL-KI & 0.000087 \\
\hline 2229 & PDMS & & SW & & & E002013 & 03-Oct-78 & & $10045-97-3$ & CS-137 & GAMMA_GS & PNL-KI & 0.03 \\
\hline 2230 & PDMS & & SW & & & E002013 & 03-Oct-78 & & 15046-84-1 & I-129 & I129_SEP_LEPS_GS & PNL-KI & 0.0001 \\
\hline 2231 & PDMS & & SW & & & E001295 & 03-Oct-78 & & 15046-84-1 & $\mid-129$ & 1129_SEP_LEPS_GS & PNL-KI & 0.000016 \\
\hline 2232 & PDMS & & SW & & & E001295 & 03-Oct-78 & & PU-239/240 & PU-239/240 & PUISO_IE_AEA & PNL-KI & 0.00019 \\
\hline 2233 & PDMS & & SW & & & E001295 & 03-Oct-78 & & $10045-97-3$ & CS-137 & GAMMA_GS & PNL-KI & 0.03 \\
\hline 2234 & PDMS & & SW & & & E014006 & 03-Oct-78 & & PU-239/240 & PU-239/240 & PUISO_IE_AEA & PNL-KI & 0.000083 \\
\hline 2235 & PDMS & & SW & & & E014006 & 03-Oct-78 & & $10045-97-3$ & CS-137 & GAMMA_GS & PNL-KI & 0.03 \\
\hline 2236 & PDMS & & SW & & & E002014 & 17-Oct-78 & & $10045-97-3$ & CS-137 & GAMMA_GS & PNL-KI & 0.06 \\
\hline 2237 & PDMS & & SW & & & E003967 & 17-Oct-78 & & $10045-97-3$ & CS-137 & GAMMA_GS & PNL-KI & 0.04 \\
\hline
\end{tabular}




\begin{tabular}{|c|c|c|c|c|c|c|c|c|c|c|c|c|c|}
\hline & $\bar{A}$ & $\mathrm{~B}$ & $\bar{C}$ & $\mathrm{D}$ & $\mathrm{E}$ & $\mathrm{F}$ & $\bar{G}$ & $\mathrm{H}$ & 1 & $\mathrm{~J}$ & $\bar{K}$ & $\bar{L}$ & $\mathrm{M}$ \\
\hline 1 & SAMPLE_NUM_ASSIGNED_TO & \begin{tabular}{|l} 
SAMPLER \\
\end{tabular} & MEDIA & BIOTA_MEDIA & TAG_ID & SAMP_NUM & SAMP_DATE_TIME & SAMP_DATE_TIME_ON & CON_ID & \begin{tabular}{|l} 
CON_SHORT_NAME \\
\end{tabular} & METHOD_NAME & LAB_CODE & VALUE_RPTD \\
\hline 2238 & PDMS & & SW & & & E001296 & $17-$ Oct-78 & & $10045-97-3$ & CS-137 & GAMMA_GS & PNL-KI & 0.02 \\
\hline 2239 & PDMS & & SW & & & E014007 & 17-Oct-78 & & $10045-97-3$ & CS-137 & GAMMA_GS & PNL-KI & 0.02 \\
\hline 2240 & PDMS & & SW & & & E007842 & $25-$ Oct-78 & & $10028-17-8$ & TRITIUM & TRITIUM_DIST_LSC & USTEST & 544 \\
\hline 2241 & PDMS & & SW & & & E007842 & $25-$ Oct-78 & & $10045-97-3$ & CS-137 & GAMMA_GS & USTEST & -0.325 \\
\hline 2242 & PDMS & & SW & & & E042429 & 25-Oct-78 & & $10028-17-8$ & TRITIUM & TRITIUM_DIST_LSC & USTEST & 871 \\
\hline 2243 & PDMS & & SW & & & E034819 & $25-$ Oct-78 & & $10028-17-8$ & TRITIUM & TRITIUM_DIST_LSC & USTEST & 746 \\
\hline 2244 & PDMS & & SW & & & E004584 & $30-$ Oct-78 & & $10028-17-8$ & TRITIUM & TRITIUM_DIST_LSC & USTEST & 353 \\
\hline 2245 & PDMS & & SW & & & E004584 & $30-$ Oct-78 & & $10045-97-3$ & CS-137 & GAMMA_GS & USTEST & -0.0864 \\
\hline 2246 & PDMS & & SW & & & E003558 & $30-$ Oct-78 & & $10045-97-3$ & CS-137 & GAMMA_GS & USTEST & 5.24 \\
\hline 2247 & PDMS & & SW & & & E044481 & $30-$ Oct-78 & & $10045-97-3$ & CS-137 & GAMMA_GS & USTEST & 0.562 \\
\hline 2248 & PDMS & & SW & & & E044481 & $30-$ Oct-78 & & $10028-17-8$ & TRITIUM & TRITIUM_DIST_LSC & USTEST & 34700 \\
\hline 2249 & PDMS & & SW & & & E002180 & $30-$ Oct-78 & & $10045-97-3$ & CS-137 & GAMMA_GS & USTEST & -0.519 \\
\hline 2250 & PDMS & & SW & & & E002015 & 02-Nov-78 & & $10045-97-3$ & CS-137 & GAMMA_GS & PNL-KI & 0.005 \\
\hline 2251 & PDMS & & SW & & & E002015 & 02-Nov-78 & & 15046-84-1 & $1-129$ & I129_SEP_LEPS_GS & PNL-KI & 0.000095 \\
\hline 2252 & PDMS & & SW & & & E003968 & 02-Nov-78 & & $10045-97-3$ & CS-137 & GAMMA_GS & PNL-KI & 0.009 \\
\hline 2253 & PDMS & & SW & & & E001297 & 02-Nov-78 & & $10045-97-3$ & CS-137 & GAMMA_GS & PNL-KI & 0.05 \\
\hline 2254 & PDMS & & SW & & & E001297 & 02-Nov-78 & & 15046-84-1 & I-129 & I129_SEP_LEPS_GS & PNL-KI & 0.000014 \\
\hline 2255 & PDMS & & SW & & & E014008 & 02-Nov-78 & & $10045-97-3$ & CS-137 & GAMMA_GS & PNL-KI & 0.02 \\
\hline 2256 & PDMS & & SW & & & E044482 & 06-Nov-78 & & $10028-17-8$ & TRITIUM & TRITIUM_DIST_LSC & USTEST & 16600 \\
\hline 2257 & PDMS & & SW & & & E014894 & 07-Nov-78 & & $10045-97-3$ & CS-137 & GAMMA_GS & USTEST & 3.19 \\
\hline 2258 & PDMS & & SW & & & E016410 & 07-Nov-78 & & $10098-97-2$ & SR-90 & SRISO_SEEP_PRECIP_GPC & USTEST & 0.781 \\
\hline 2259 & PDMS & & SW & & & E016410 & 07-Nov-78 & & $10045-97-3$ & CS-137 & GAMMA_GS & USTEST & 3.55 \\
\hline 2260 & PDMS & & SW & & & E022434 & 07-Nov-78 & & $10045-97-3$ & CS-137 & GAMMA_GS & USTEST & 1.45 \\
\hline 2261 & PDMS & & SW & & & E022434 & 07-Nov-78 & & $10098-97-2$ & SR-90 & SRISO_SEP_PRECIP_GPC & USTEST & 0.258 \\
\hline 2262 & PDMS & & SW & & & E027651 & 07-Nov-78 & & $10045-97-3$ & CS-137 & GAMMA_GS & USTEST & 9.69 \\
\hline 2263 & PDMS & & SW & & & E027651 & 07-Nov-78 & & $10098-97-2$ & SR-90 & SRISO_SEEP_PRECIP_GPC & USTEST & 3.96 \\
\hline 2264 & PDMS & & SW & & & E014280 & 07-Nov-78 & & $10045-97-3$ & CS-137 & GAMMA_GS & USTEST & 6.11 \\
\hline 2265 & PDMS & & SW & & & E014280 & 07-Nov-78 & & $10098-97-2$ & SR-90 & SRISO_SEP_PRECIP_GPC & USTEST & \\
\hline 2266 & PDMS & & SW & & & E044483 & 13-Nov-78 & & $10028-17-8$ & TRITIUM & TRITIUM_DIST_LSC & USTEST & 13900 \\
\hline 2267 & PDMS & & SW & & & E003969 & 14-Nov-78 & & $10045-97-3$ & CS-137 & GAMMA_GS & PNL-KI & 0.007 \\
\hline 2268 & PDMS & & SW & & & E002016 & 14-Nov-78 & & $10045-97-3$ & CS-137 & GAMMA_GS & PNL-KI & 0.01 \\
\hline 2269 & PDMS & & SW & & & E044484 & 21-Nov-78 & & $10028-17-8$ & TRITIUM & TRITIUM_DIST_LSC & USTEST & 54000 \\
\hline 2270 & PDMS & & SW & & & E004585 & 27-Nov-78 & & $10098-97-2$ & SR-90 & SRISO_SEP_PRECIP_GPC & USTEST & 0.354 \\
\hline 2271 & PDMS & & SW & & & E004585 & $27-N o v-78$ & & $10028-17-8$ & TRITIUM & TRITIUM_DIST_LSC & USTEST & 439 \\
\hline 2272 & PDMS & & SW & & & E004585 & $27-N o v-78$ & & $10045-97-3$ & CS-137 & GAMMA_GS & USTEST & -0.0736 \\
\hline 2273 & PDMS & & SW & & & E001617 & 27-Nov-78 & & $10098-97-2$ & SR-90 & SRISO_SEP_PRECIP_GPC & USTEST & 0.252 \\
\hline 2274 & PDMS & & SW & & & E001617 & 27-Nov-78 & & $10028-17-8$ & TRITIUM & TRITIUM_DIST_LSC & USTEST & 212 \\
\hline 2275 & PDMS & & SW & & & E001617 & 27-Nov-78 & & $10045-97-3$ & CS-137 & GAMMA_GS & USTEST & -0.181 \\
\hline 2276 & PDMS & & SW & & & E014819 & $27-N o v-78$ & & $10098-97-2$ & SR-90 & SRISO_SEP_PRECIP_GPC & USTEST & 0.0574 \\
\hline 2277 & PDMS & & SW & & & E014819 & $27-N o v-78$ & & $10045-97-3$ & CS-137 & GAMMA_GS & USTEST & -0.0553 \\
\hline 2278 & PDMS & & SW & & & E014819 & 27-Nov-78 & & $10028-17-8$ & TRITIUM & TRITIUM_DIST_LSC & USTEST & 36600 \\
\hline 2279 & PDMS & & SW & & & E002181 & $27-N o v-78$ & & $10045-97-3$ & CS-137 & GAMMA_GS & USTEST & 0.851 \\
\hline 2280 & PDMS & & SW & & & E002017 & 28-Nov-78 & & $10045-97-3$ & CS-137 & GAMMA_GS & PNL-KI & 0.008 \\
\hline 2281 & PDMS & & SW & & & E002017 & 28-Nov-78 & & 15046-84-1 & I-129 & I129_SEP_LEPS_GS & PNL-KI & 0.000067 \\
\hline 2282 & PDMS & & SW & & & E003970 & 28-Nov-78 & & $10045-97-3$ & CS-137 & GAMMA_GS & PNL-KI & 0.006 \\
\hline 2283 & PDMS & & SW & & & E001298 & 28-Nov-78 & & $10045-97-3$ & CS-137 & GAMMA_GS & PNL-KI & 0.01 \\
\hline 2284 & PDMS & & SW & & & E001298 & 28-Nov-78 & & 15046-84-1 & I-129 & I129_SEP_LEPS_GS & PNL-KI & 0.000022 \\
\hline 2285 & PDMS & & SW & & & E014009 & 28-Nov-78 & & $10045-97-3$ & CS-137 & GAMMA_GS & PNL-KI & 0.02 \\
\hline 2286 & PDMS & & SW & & & E007783 & 29-Nov-78 & & $10028-17-8$ & TRITIUM & TRITIUM_DIST_LSC & USTEST & 174 \\
\hline 2287 & PDMS & & SW & & & E007783 & 29-Nov-78 & & $10045-97-3$ & CS-137 & GAMMA_GS & USTEST & -0.0816 \\
\hline 2288 & PDMS & & SW & & & E042434 & 29-Nov-78 & & $10028-17-8$ & TRITIUM & TRITIUM_DIST_LSC & USTEST & 395 \\
\hline 2289 & PDMS & & SW & & & E034824 & 29-Nov-78 & & $10028-17-8$ & TRITIUM & TRITIUM_DIST_LSC & USTEST & 287 \\
\hline
\end{tabular}




\begin{tabular}{|c|c|c|c|c|c|c|c|c|c|c|c|c|c|}
\hline & $\bar{A}$ & $\mathrm{~B}$ & $\mathrm{C}$ & $\mathrm{D}$ & $E$ & $\mathrm{~F}$ & G & $\mathrm{H}$ & $\mathrm{I}$ & $\mathrm{J}$ & $\mathrm{K}$ & $\mathrm{L}$ & $\mathrm{M}$ \\
\hline 1 & SAMPLE_NUM_ASSIGNED_TO & SAMPLER & MEDIA & BIOTA_MEDIA & TAG_ID & SAMP_NUM & SAMP_DATE_TIME & SAMP_DATE_TIME_ON & CON_ID & CON_SHORT_NAME & METHOD_NAME & LAB_CODE & \begin{tabular}{|l|} 
VALUE_RPTD \\
\end{tabular} \\
\hline 2290 & PDMS & & SW & & & E044485 & 04-Dec-78 & & $10028-17-8$ & TRITIUM & TRITIUM_DIST_LSC & USTEST & 78000 \\
\hline 2291 & PDMS & & SW & & & E044486 & 11-Dec-78 & & $10028-17-8$ & TRITIUM & TRITIUM_DIST_LSC & USTEST & 69000 \\
\hline 2292 & PDMS & & SW & & & E002018 & 12-Dec-78 & & $10045-97-3$ & CS-137 & GAMMA_GS & PNL-KI & 0.01 \\
\hline 2293 & PDMS & & SW & & & E003971 & 12-Dec-78 & & $10045-97-3$ & CS-137 & GAMMA_GS & PNL-KI & 0.004 \\
\hline 2294 & PDMS & & SW & & & E001299 & 12-Dec-78 & & $10045-97-3$ & CS-137 & GAMMA_GS & PNL-KI & 0.02 \\
\hline 2295 & PDMS & & SW & & & E014010 & 12-Dec-78 & & $10045-97-3$ & CS-137 & GAMMA_GS & PNL-KI & 0.004 \\
\hline 2296 & PDMS & & SW & & & E044487 & 18-Dec-78 & & $10028-17-8$ & TRITIUM & TRITIUM_DIST_LSC & USTEST & 89600 \\
\hline 2297 & PDMS & & SW & & & E042436 & 20-Dec-78 & & $10028-17-8$ & TRITIUM & TRITIUM_DIST_LSC & USTEST & 301 \\
\hline 2298 & PDMS & & SW & & & E034826 & 20-Dec-78 & & 10028-17-8 & TRITIUM & TRITIUM_DIST_LSC & USTEST & 169 \\
\hline 2299 & PDMS & & SW & & & E004586 & 22-Dec-78 & & 10028-17-8 & TRITIUM & TRITIUM_DIST_LSC & USTEST & 114 \\
\hline 2300 & PDMS & & SW & & & E004586 & 22-Dec-78 & & $10045-97-3$ & CS-137 & GAMMA_GS & USTEST & -0.0173 \\
\hline 2301 & PDMS & & SW & & & E003559 & 22-Dec-78 & & $10045-97-3$ & CS-137 & GAMMA_GS & USTEST & -1.16 \\
\hline \begin{tabular}{|l|}
2302 \\
\end{tabular} & PDMS & & SW & & & E044488 & 22-Dec-78 & & $10045-97-3$ & CS-137 & GAMMA_GS & USTEST & -0.305 \\
\hline 2303 & PDMS & & SW & & & E044488 & 22-Dec-78 & & $10028-17-8$ & TRITIUM & TRITIUM_DIST_LSC & USTEST & 75400 \\
\hline 2304 & PDMS & & SW & & & E002182 & 22-Dec-78 & & $10045-97-3$ & CS-137 & GAMMA_GS & USTEST & -0.589 \\
\hline 2305 & PDMS & & SW & & & E002019 & 27-Dec-78 & & PU-239/240 & PU-239/240 & PUISO_IE_AEA & PNL-KI & 0.000068 \\
\hline \begin{tabular}{|l|}
2306 \\
\end{tabular} & PDMS & & SW & & & E002019 & 27-Dec-78 & & $10045-97-3$ & CS-137 & GAMMA_GS & PNL-KI & 0.007 \\
\hline \begin{tabular}{|l|l|}
2307 \\
\end{tabular} & PDMS & & SW & & & E002019 & 27-Dec-78 & & 15046-84-1 & I-129 & 1129_SEP_LEPS_GS & PNL-KI & 0.000079 \\
\hline 2308 & PDMS & & SW & & & E003972 & 27-Dec-78 & & $10045-97-3$ & CS-137 & GAMMA_GS & PNL-KI & 0.005 \\
\hline 2309 & PDMS & & SW & & & E003972 & 27-Dec-78 & & PU-239/240 & PU-239/240 & PUISO_IE_AEA & PNL-KI & 0.000031 \\
\hline 2310 & PDMS & & SW & & & E014011 & 27-Dec-78 & & PU-239/240 & PU-239/240 & PUISO_IE_AEA & PNL-KI & 0.000058 \\
\hline 2311 & PDMS & & SW & & & E014011 & 27-Dec-78 & & $10045-97-3$ & CS-137 & GAMMA_GS & PNL-KI & 0.003 \\
\hline \begin{tabular}{|l|}
2312 \\
\end{tabular} & PDMS & & SW & & & E001300 & 27-Dec-78 & & PU-239/240 & PU-239/240 & PUISO_IE_AEA & PNL-KI & 0.00017 \\
\hline 2313 & PDMS & & SW & & & E001300 & 27-Dec-78 & & $10045-97-3$ & CS-137 & GAMMA_GS & PNL-KI & 0.008 \\
\hline \begin{tabular}{|l|}
2314 \\
\end{tabular} & PDMS & & SW & & & E001300 & 27-Dec-78 & & 15046-84-1 & I-129 & I129_SEP_LEPS_GS & PNL-KI & 0.000012 \\
\hline \begin{tabular}{|l|l|}
2315 \\
\end{tabular} & PDMS & & SW & & & E003543 & 27-Dec-78 & & $10028-17-8$ & TRITIUM & TRITIUM_DIST_L̄ESC & USTEST & 255 \\
\hline \begin{tabular}{|l|l}
2316 \\
\end{tabular} & PDMS & & SW & & & E003543 & 27-Dec-78 & & $10098-97-2$ & SR-90 & SRISO_SEP_PRECIP_GPC & USTEST & 1.14 \\
\hline \begin{tabular}{|l|l|}
2317 \\
\end{tabular} & PDMS & & SW & & & E003543 & 27-Dec-78 & & $10045-97-3$ & CS-137 & GAMMA_GS & USTEST & -0.785 \\
\hline 2318 & PDMS & & SW & & & E007723 & 02-Jan-79 & & $10028-17-8$ & TRITIUM & TRITIUM_DIST_LSC & HISTOR & 424 \\
\hline 2319 & PDMS & & SW & & & E007723 & 02-Jan-79 & & $10045-97-3$ & CS-137 & GAMMA_GS & USTEST & 0.0615 \\
\hline 2320 & PDMS & & SW & & & E002020 & 09-Jan-79 & & $10045-97-3$ & CS-137 & GAMMA_GS & HISTOR & 0.026 \\
\hline 2321 & PDMS & & SW & & & E003973 & 09-Jan-79 & & $10045-97-3$ & CS-137 & GAMMA_GS & HISTOR & 0.017 \\
\hline 2322 & PDMS & & SW & & & E014012 & 09-Jan-79 & & $10045-97-3$ & CS-137 & GAMMA_GS & HISTOR & 0.005 \\
\hline 2323 & PDMS & & SW & & & E001301 & 09-Jan-79 & & $10045-97-3$ & CS-137 & GAMMA_GS & HISTOR & 0.004 \\
\hline 2324 & PDMS & & SW & & & E004587 & 22-Jan-79 & & $10098-97-2$ & SR-90 & SRISO_SEP_PRECIP_GPC & USTEST & 0.311 \\
\hline 2325 & PDMS & & SW & & & E004587 & 22-Jan-79 & & 10028-17-8 & TRITIUM & TRITIUM_DIST_LSC & HISTOR & 335 \\
\hline \begin{tabular}{|l|}
2326 \\
\end{tabular} & PDMS & & SW & & & E004587 & 22-Jan-79 & & $10045-97-3$ & CS-137 & GAMMA_GS & USTEST & 0.775 \\
\hline \begin{tabular}{|l|}
2327 \\
\end{tabular} & PDMS & & SW & & & E003560 & 22-Jan-79 & & $10045-97-3$ & CS-137 & GAMMA_GS & USTEST & 0.00971 \\
\hline \begin{tabular}{|l|}
2328 \\
\end{tabular} & PDMS & & SW & & & E044489 & 22-Jan-79 & & $10045-97-3$ & CS-137 & GAMMA_GS & USTEST & 5.03 \\
\hline 2329 & PDMS & & SW & & & E044489 & 22-Jan-79 & & 10028-17-8 & TRITIUM & TRITIUM_DIST_LSC & USTEST & 22300 \\
\hline 2330 & PDMS & & SW & & & E002183 & 22-Jan-79 & & $10045-97-3$ & CS-137 & GAMMA_GS & USTEST & 7.52 \\
\hline 2331 & PDMS & & SW & & & E002021 & 23-Jan-79 & & $10045-97-3$ & CS-137 & GAMMA_GS & HISTOR & 0.01 \\
\hline 2332 & PDMS & & SW & & & E003974 & 23-Jan-79 & & $10045-97-3$ & CS-137 & GAMMA_GS & HISTOR & 0.003 \\
\hline 2333 & PDMS & & SW & & & E001302 & 23-Jan-79 & & $10045-97-3$ & CS-137 & GAMMA_GS & HISTOR & 0.03 \\
\hline 2334 & PDMS & & SW & & & E014013 & 23-Jan-79 & & $10045-97-3$ & CS-137 & GAMMA_GS & HISTOR & 0.005 \\
\hline 2335 & PDMS & & SW & & & E003975 & 06-Feb-79 & & $10045-97-3$ & CS-137 & GAMMA_GS & HISTOR & 0.002 \\
\hline 2336 & PDMS & & SW & & & E002022 & 06-Feb-79 & & $10045-97-3$ & CS-137 & GAMMA_GS & HISTOR & 0.007 \\
\hline 2337 & PDMS & & SW & & & E001303 & 06-Feb-79 & & $10045-97-3$ & CS-137 & GAMMA_GS & HISTOR & 0.02 \\
\hline 2338 & PDMS & & SW & & & E014014 & 06-Feb-79 & & $10045-97-3$ & CS-137 & GAMMA_GS & HISTOR & 0.004 \\
\hline 2339 & PDMS & & SW & & & E016411 & 13-Feb-79 & & 10098-97-2 & SR-90 & SRISO_SEP_PRECIP_GPC & USTEST & 0.763 \\
\hline 2340 & PDMS & & SW & & & E016411 & 13-Feb-79 & & $10045-97-3$ & CS-137 & GAMMA_GS & USTEST & 15.7 \\
\hline 2341 & PDMS & & SW & & & E022435 & 13-Feb-79 & & $10045-97-3$ & CS-137 & GAMMA_GS & USTEST & 0.166 \\
\hline
\end{tabular}




\begin{tabular}{|c|c|c|c|c|c|c|c|c|c|c|c|c|c|}
\hline & A & $\mathrm{B}$ & $\mathrm{C}$ & $\mathrm{D}$ & $E$ & $\mathrm{~F}$ & $\mathrm{G}$ & $\mathrm{H}$ & $\mathrm{I}$ & $\mathrm{J}$ & $\mathrm{K}$ & $\mathrm{L}$ & $\mathrm{M}$ \\
\hline 1 & SAMPLE_NUM_ASSIGNED_TO & SAMPLER & MEDIA & BIOTA_MEDIA & TAG_ID & SAMP_NUM & SAMP_DATE_TIME & SAMP_DATE_TIME_ON & CON_ID & CON_SHORT_NAME & METHOD_NAME & LAB_CODE & VALUE_RPTD \\
\hline 2342 & PDMS & & SW & & & E022435 & $13-F e b-79$ & & $10098-97-2$ & SR-90 & SRISO_SEP_PRECIP_GPC & USTEST & 0.517 \\
\hline 2343 & PDMS & & SW & & & E014281 & 13-Feb-79 & & $10045-97-3$ & CS-137 & GAMMA_GS & USTEST & 3.79 \\
\hline 2344 & PDMS & & SW & & & E014281 & 13-Feb-79 & & $10098-97-2$ & SR-90 & SRISO_SEP_PRECIP_GPC & USTEST & 0.901 \\
\hline 2345 & PDMS & & SW & & & E004588 & 20-Feb-79 & & $10045-97-3$ & CS-137 & GAMMA_GS & USTEST & 1.56 \\
\hline 2346 & PDMS & & SW & & & E004588 & 20-Feb-79 & & $10028-17-8$ & TRITIUM & TRITIUM_DIST_LSC & HISTOR & 708 \\
\hline 2347 & PDMS & & SW & & & E003976 & 20-Feb-79 & & $10045-97-3$ & CS-137 & GAMMA_GS & HISTOR & 0.005 \\
\hline 2348 & PDMS & & SW & & & E002023 & 20-Feb-79 & & $10045-97-3$ & CS-137 & GAMMA_GS & HISTOR & 0.009 \\
\hline 2349 & PDMS & & SW & & & E014015 & 20-Feb-79 & & $10045-97-3$ & CS-137 & GAMMA_GS & HISTOR & 0.005 \\
\hline 2350 & PDMS & & SW & & & E001304 & 20-Feb-79 & & $10045-97-3$ & CS-137 & GAMMA_GS & HISTOR & 0.02 \\
\hline 2351 & PDMS & & SW & & & E007680 & 21-Feb-79 & & $10028-17-8$ & TRITIUM & TRITIUM_DIST_LSC & HISTOR & 317 \\
\hline 2352 & PDMS & & SW & & & E007680 & 21-Feb-79 & & $10098-97-2$ & SR-90 & SRISO_SEP_PRECIP_GPC & USTEST & 0.33 \\
\hline 2353 & PDMS & & SW & & & E007680 & 21-Feb-79 & & $10045-97-3$ & CS-137 & GAMMA_GS & USTEST & 8.37 \\
\hline 2354 & PDMS & & SW & & & E001618 & 26-Feb-79 & & $10098-97-2$ & SR-90 & SRISO_SEP_PRECIP_GPC & USTEST & 0.198 \\
\hline 2355 & PDMS & & SW & & & E001618 & 26-Feb-79 & & $10028-17-8$ & TRITIUM & TRITIUM_DIST_LSC & HISTOR & 239 \\
\hline 2356 & PDMS & & SW & & & E001618 & 26-Feb-79 & & $10045-97-3$ & CS-137 & GAMMA_GS & USTEST & -0.683 \\
\hline 2357 & PDMS & & SW & & & E014820 & 26-Feb-79 & & $10098-97-2$ & SR-90 & SRISO_SEP_PRECIP_GPC & USTEST & 0.0348 \\
\hline 2358 & PDMS & & SW & & & E014820 & 26-Feb-79 & & $10045-97-3$ & CS-137 & GAMMA_GS & USTEST & 1.93 \\
\hline 2359 & PDMS & & SW & & & E014820 & 26-Feb-79 & & $10028-17-8$ & TRITIUM & TRITIUM_DIST_LSC & USTEST & 17200 \\
\hline 2360 & PDMS & & SW & & & E002110 & 26-Feb-79 & & $10045-97-3$ & CS-137 & GAMMA_GS & USTEST & 0.0615 \\
\hline 2361 & PDMS & & SW & & & E002024 & 06-Mar-79 & & $10045-97-3$ & CS-137 & GAMMA_GS & HISTOR & 0.006 \\
\hline 2362 & PDMS & & SW & & & E003977 & 06-Mar-79 & & $10045-97-3$ & CS-137 & GAMMA_GS & HISTOR & 0.004 \\
\hline 2363 & PDMS & & SW & & & E014016 & 06-Mar-79 & & $10045-97-3$ & CS-137 & GAMMA_GS & HISTOR & 0.2 \\
\hline 2364 & PDMS & & SW & & & E001305 & 06-Mar-79 & & $10045-97-3$ & CS-137 & GAMMA_GS & HISTOR & 1.2 \\
\hline 2365 & PDMS & & SW & & & E002025 & 20-Mar-79 & & PU-239/240 & PU-239/240 & PUISO_IE_AEA & HISTOR & 0.00044 \\
\hline 2366 & PDMS & & SW & & & E002025 & 20-Mar-79 & & $10045-97-3$ & CS-137 & GAMMA_GS & HISTOR & 0.01 \\
\hline 2367 & PDMS & & SW & & & E002025 & 20-Mar-79 & & 15046-84-1 & $1-129$ & I129_SEP_TEMS & 320 & 0.00015 \\
\hline 2368 & PDMS & & SW & & & E003978 & 20-Mar-79 & & PU-239/240 & PU-239/240 & PUISO_IE_AEA & HISTOR & 0.0000316 \\
\hline 2369 & PDMS & & SW & & & E003978 & 20-Mar-79 & & $10045-97-3$ & CS-137 & GAMMA_GS & HISTOR & 0.005 \\
\hline 2370 & PDMS & & SW & & & E014017 & 20-Mar-79 & & PU-239/240 & PU-239/240 & PUISO_IE_AEA & HISTOR & 0.0000301 \\
\hline 2371 & PDMS & & SW & & & E014017 & 20-Mar-79 & & $10045-97-3$ & CS-137 & GAMMA_GS & HISTOR & 0.003 \\
\hline 2372 & PDMS & & SW & & & E001306 & 20-Mar-79 & & PU-239/240 & PU-239/240 & PUISO_IE_AEA & HISTOR & 0.00028 \\
\hline 2373 & PDMS & & SW & & & E001306 & 20-Mar-79 & & $10045-97-3$ & CS-137 & GAMMA_GS & HISTOR & 0.01 \\
\hline 2374 & PDMS & & SW & & & E001306 & 20-Mar-79 & & 15046-84-1 & $1-129$ & I129_SEP_TEMS & 320 & 0.0004 \\
\hline 2375 & PDMS & & SW & & & E004516 & 26-Mar-79 & & $10045-97-3$ & CS-137 & GAMMA_GS & USTEST & 0.948 \\
\hline 2376 & PDMS & & SW & & & E004516 & 26-Mar-79 & & $10028-17-8$ & TRITIUM & TRITIUM_DIST_LSC & HISTOR & 65.5 \\
\hline 2377 & PDMS & & SW & & & E003561 & 26-Mar-79 & & $10045-97-3$ & CS-137 & GAMMA_GS & USTEST & -0.141 \\
\hline 2378 & PDMS & & SW & & & E044490 & 26-Mar-79 & & $10045-97-3$ & CS-137 & GAMMA_GS & USTEST & -0.0417 \\
\hline 2379 & PDMS & & SW & & & E044490 & 26-Mar-79 & & $10028-17-8$ & TRITIUM & TRITIUM_DIST_LSC & USTEST & 28200 \\
\hline 2380 & PDMS & & SW & & & E002111 & 26-Mar-79 & & $10045-97-3$ & CS-137 & GAMMA_GS & USTEST & 0.0613 \\
\hline 2381 & PDMS & & SW & & & E007621 & 28-Mar-79 & & $10028-17-8$ & TRITIUM & TRITIUM_DIST_LSC & HISTOR & 319 \\
\hline 2382 & PDMS & & SW & & & E007621 & 28-Mar-79 & & $10045-97-3$ & CS-137 & GAMMA_GS & USTEST & 8.26 \\
\hline 2383 & PDMS & & SW & & & E002026 & 03-Apr-79 & & $10045-97-3$ & CS-137 & GAMMA_GS & HISTOR & 0.009 \\
\hline 2384 & PDMS & & SW & & & E003979 & 03-Apr-79 & & $10045-97-3$ & CS-137 & GAMMA_GS & HISTOR & 0.002 \\
\hline 2385 & PDMS & & SW & & & E001307 & 03-Apr-79 & & $10045-97-3$ & CS-137 & GAMMA_GS & HISTOR & 0.005 \\
\hline 2386 & PDMS & & SW & & & E014018 & 03-Apr-79 & & $10045-97-3$ & CS-137 & GAMMA_GS & HISTOR & 0.03 \\
\hline 2387 & PDMS & & SW & & & E003980 & 17-Apr-79 & & $10045-97-3$ & CS-137 & GAMMA_GS & HISTOR & 0.007 \\
\hline 2388 & PDMS & & SW & & & E002027 & 17-Apr-79 & & $10045-97-3$ & CS-137 & GAMMA_GS & HISTOR & 0.01 \\
\hline 2389 & PDMS & & SW & & & E001308 & 17-Apr-79 & & $10045-97-3$ & CS-137 & GAMMA_GS & HISTOR & 0.01 \\
\hline 2390 & PDMS & & SW & & & E014019 & 17-Apr-79 & & $10045-97-3$ & CS-137 & GAMMA_GS & HISTOR & 0.006 \\
\hline 2391 & PDMS & & SW & & & E003562 & 23-Apr-79 & & $10045-97-3$ & CS-137 & GAMMA_GS & USTEST & -0.645 \\
\hline 2392 & PDMS & & SW & & & E044491 & 23-Apr-79 & & $10045-97-3$ & CS-137 & GAMMA_GS & USTEST & 0.529 \\
\hline 2393 & PDMS & & SW & & & E044491 & 23-Apr-79 & & $10028-17-8$ & TRITIUM & TRITIUM_DIST_LSC & USTEST & 17800 \\
\hline
\end{tabular}




\begin{tabular}{|c|c|c|c|c|c|c|c|c|c|c|c|c|c|}
\hline & 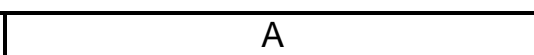 & $\mathrm{B}$ & $\mathrm{C}$ & $\mathrm{D}$ & $\mathrm{E}$ & $\mathrm{F}$ & $\mathrm{G}$ & $\mathrm{H}$ & $\mathrm{T}$ & $\mathrm{J}$ & $\mathrm{K}$ & $\mathrm{L}$ & $\mathrm{M}$ \\
\hline 1 & SAMPLE_NUM_ASSIGNED_TO & \begin{tabular}{|l} 
SAMPLER \\
\end{tabular} & MEDIA & BIOTA_MEDIA & TAG_ID & SAMP_NUM & \begin{tabular}{|l|} 
SAMP_DATE_TIME \\
\end{tabular} & SAMP_DATE_TIME_ON & CON_ID & \begin{tabular}{|l|} 
CON_SHORT_NAME \\
\end{tabular} & METHOD_NAME & LAB_CODE & VALUE_RPTD \\
\hline 2394 & PDMS & & SW & & & E002112 & 23-Apr-79 & & $10045-97-3$ & CS-137 & GAMMA_GS & USTEST & -0.0468 \\
\hline 2395 & PDMS & & SW & & & E007649 & 25-Apr-79 & & $10028-17-8$ & TRITIUM & TRITIUM_DIST_LSC & HISTOR & 803 \\
\hline 2396 & PDMS & & SW & & & E007649 & 25-Apr-79 & & $10098-97-2$ & SR-90 & SRISO_SEP_PRECIP_GPC & USTEST & 0.522 \\
\hline 2397 & PDMS & & SW & & & E007649 & 25-Apr-79 & & $10045-97-3$ & CS-137 & GAMMA_GS & USTEST & -0.104 \\
\hline 2398 & PDMS & & SW & & & E004517 & 30-Apr-79 & & $10045-97-3$ & CS-137 & GAMMA_GS & USTEST & 2.31 \\
\hline 2399 & PDMS & & SW & & & E004517 & 30-Apr-79 & & $10028-17-8$ & TRITIUM & TRITIUM_DIST_LSC & HISTOR & 173 \\
\hline 2400 & PDMS & & sW & & & E004517 & 30-Apr-79 & & $10098-97-2$ & SR-90 & SRISO_SEP_PRECIP_GPC & USTEST & 1.77 \\
\hline 2401 & PDMS & & sW & & & E002028 & 01-May-79 & & $10045-97-3$ & CS-137 & GAMMĀGS' & HISTOR & 0.007 \\
\hline 2402 & PDMS & & sW & & & E003981 & 01-May-79 & & $10045-97-3$ & CS-137 & GAMMA_GS & HISTOR & 0.008 \\
\hline 2403 & PDMS & & SW & & & E001309 & 01-May-79 & & $10045-97-3$ & CS-137 & GAMMA_GS & HISTOR & 0.14 \\
\hline 2404 & PDMS & & SW & & & E014020 & 01-May-79 & & $10045-97-3$ & CS-137 & GAMMA_GS & HISTOR & 0.14 \\
\hline 2405 & PDMS & & SW & & & E016412 & 08-May-79 & & $10098-97-2$ & SR-90 & SRISO_SEP_PRECIP_GPC & USTEST & 0.946 \\
\hline 2406 & PDMS & & SW & & & E016412 & 08-May-79 & & $10045-97-3$ & CS-137 & GAMMA_GS & USTEST & 5.79 \\
\hline 2407 & PDMS & & SW & & & E022436 & 08-May-79 & & $10045-97-3$ & CS-137 & GAMMA_GS & USTEST & 40.5 \\
\hline 2408 & PDMS & & SW & & & E022436 & 08-May-79 & & $10098-97-2$ & SR-90 & SRISO_SEP_PRECIP_GPC & USTEST & 0.444 \\
\hline 2409 & PDMS & & SW & & & E014282 & 08-May-79 & & $10045-97-3$ & CS-137 & GAMMA_GS & USTEST & 30.9 \\
\hline 2410 & PDMS & & SW & & & E014282 & 08-May-79 & & $10098-97-2$ & SR-90 & SRISO_SEP_PRECIP_GPC & USTEST & 0.435 \\
\hline 2411 & PDMS & & SW & & & E007569 & 09-May-79 & & $10028-17-8$ & TRITIUM & TRITIUM_DIST_LSC & HISTOR & 94.5 \\
\hline 2412 & PDMS & & SW & & & E007569 & 09-May-79 & & $10045-97-3$ & CS-137 & GAMMA_GS & USTEST & -0.563 \\
\hline 2413 & PDMS & & SW & & & E002029 & 15-May-79 & & $10045-97-3$ & CS-137 & GAMMA_GS & HISTOR & 0.01 \\
\hline \begin{tabular}{|l|}
2414 \\
\end{tabular} & PDMS & & SW & & & E003982 & 15-May-79 & & $10045-97-3$ & CS-137 & GAMMA_GS & HISTOR & 0.005 \\
\hline 2415 & PDMS & & SW & & & E001310 & 15-May-79 & & $10045-97-3$ & CS-137 & GAMMA_GS & HISTOR & 0.13 \\
\hline 2416 & PDMS & & SW & & & E014021 & 15-May-79 & & $10045-97-3$ & CS-137 & GAMMA_GS & HISTOR & 0.05 \\
\hline 2417 & PDMS & & SW & & & E004518 & 29-May-79 & & $10028-17-8$ & TRITIUM & TRITIUM_DIST_LSC & HISTOR & 220 \\
\hline 2418 & PDMS & & SW & & & E004518 & 29-May-79 & & $10045-97-3$ & CS-137 & GAMMA_GS & USTEST & 1.01 \\
\hline 2419 & PDMS & & SW & & & E002030 & 29-May-79 & & $10045-97-3$ & CS-137 & GAMMA_GS & HISTOR & 0.007 \\
\hline 2420 & PDMS & & SW & & & E003983 & 29-May-79 & & $10045-97-3$ & CS-137 & GAMMA_GS & HISTOR & 0.005 \\
\hline 2421 & PDMS & & SW & & & E001311 & 29-May-79 & & $10045-97-3$ & CS-137 & GAMMA_GS & HISTOR & 0.01 \\
\hline \begin{tabular}{|l|}
2422 \\
\end{tabular} & PDMS & & SW & & & E013944 & 29-May-79 & & $10045-97-3$ & CS-137 & GAMMA_GS & HISTOR & 0.02 \\
\hline 2423 & PDMS & & SW & & & E001619 & 29-May-79 & & $10098-97-2$ & SR-90 & SRISO_SEP_PRECIP_GPC & USTEST & 0.312 \\
\hline 2424 & PDMS & & SW & & & E001619 & 29-May-79 & & $10028-17-8$ & TRITIUM & TRITIUM_DIST_LSC & HISTOR & 280 \\
\hline 2425 & PDMS & & SW & & & E001619 & 29-May-79 & & $10045-97-3$ & CS-137 & GAMMA_GS & USTEST & 3.76 \\
\hline 2426 & PDMS & & SW & & & E014821 & 29-May-79 & & $10098-97-2$ & SR-90 & SRISO_SEPP_PRECIP_GPC & USTEST & 0.12 \\
\hline 2427 & PDMS & & SW & & & E014821 & 29-May-79 & & $10045-97-3$ & CS-137 & GAMMA_GS & USTEST & 2.27 \\
\hline 2428 & PDMS & & SW & & & E014821 & 29-May-79 & & $10028-17-8$ & TRITIUM & TRITIUM_DIST_LSC & USTEST & 15600 \\
\hline 2429 & PDMS & & SW & & & E014821 & 29-May-79 & & 14133-76-7 & TC-99 & TC99_SEP_LSC & HISTOR & 1.48 \\
\hline 2430 & PDMS & & SW & & & E002113 & 29-May-79 & & $10045-97-3$ & CS-137 & GAMMA_GS & USTEST & 0.0614 \\
\hline 2431 & PDMS & & SW & & & E003984 & 12-Jun-79 & & $10045-97-3$ & CS-137 & GAMMA_GS & HISTOR & 0.009 \\
\hline 2432 & PDMS & & SW & & & E002031 & 12-Jun-79 & & $10045-97-3$ & CS-137 & GAMMA_GS & HISTOR & 0.01 \\
\hline 2433 & PDMS & & SW & & & E001312 & 12-Jun-79 & & $10045-97-3$ & CS-137 & GAMMA_GS & HISTOR & 0.01 \\
\hline 2434 & PDMS & & SW & & & E013945 & 12-Jun-79 & & $10045-97-3$ & CS-137 & GAMMA_GS & HISTOR & 0.02 \\
\hline 2435 & PDMS & & SW & & & E004519 & 25-Jun-79 & & $10045-97-3$ & CS-137 & GAMMA_GS & USTEST & 7.93 \\
\hline 2436 & PDMS & & SW & & & E004519 & 25-Jun-79 & & $10028-17-8$ & TRITIUM & TRITIUM_DIST_LSC & HISTOR & 187 \\
\hline 2437 & PDMS & & SW & & & E003563 & 25-Jun-79 & & $10045-97-3$ & CS-137 & GAMMA_GS & USTEST & 3.97 \\
\hline 2438 & PDMS & & SW & & & E044492 & 25-Jun-79 & & $10045-97-3$ & CS-137 & GAMMA_GS & USTEST & -0.0971 \\
\hline 2439 & PDMS & & SW & & & E044492 & 25-Jun-79 & & $10028-17-8$ & TRITIUM & TRITIUM_DIST_LSC & USTEST & 19100 \\
\hline 2440 & PDMS & & SW & & & E002114 & 25-Jun-79 & & $10045-97-3$ & CS-137 & GAMMA_GS & USTEST & 12.7 \\
\hline 2441 & PDMS & & SW & & & E002032 & 26-Jun-79 & & PU-239/240 & PU-239/240 & PUISO_IE_AEA & HISTOR & 0.000038 \\
\hline 2442 & PDMS & & SW & & & E002032 & 26-Jun-79 & & $10045-97-3$ & CS-137 & GAMMA_GS & HISTOR & 0.07 \\
\hline 2443 & PDMS & & SW & & & E002032 & 26-Jun-79 & & 15046-84-1 & I-129 & I129_SEP_TEMS & 320 & 0.000095 \\
\hline 2444 & PDMS & & SW & & & E003985 & 26-Jun-79 & & $10045-97-3$ & CS-137 & GAMMA_GS & HISTOR & 0.01 \\
\hline 2445 & PDMS & & SW & & & E003985 & 26-Jun-79 & & PU-239/240 & PU-239/240 & PUISO_IE_AEA & HISTOR & 0.0000618 \\
\hline
\end{tabular}




\begin{tabular}{|c|c|c|c|c|c|c|c|c|c|c|c|c|c|}
\hline & $\bar{A}$ & $\mathrm{~B}$ & $\bar{C}$ & $\mathrm{D}$ & $\mathrm{E}$ & $\mathrm{F}$ & $\bar{G}$ & $\mathrm{H}$ & 1 & $\mathrm{~J}$ & $\bar{K}$ & $\bar{L}$ & $\mathrm{M}$ \\
\hline 1 & SAMPLE_NUM_ASSIGNED_TO & SAMPLER & MEDIA & BIOTA_MEDIA & TAG_ID & SAMP_NUM & SAMP_DATE_TIME & SAMP_DATE_TIME_ON & CON_ID & CON_SHORT_NAME & METHOD_NAME & LAB_CODE & VALUE_RPTD \\
\hline 2446 & PDMS & & SW & & & E013946 & 26-Jun-79 & & PU-239/240 & PU-239/240 & PUISO_IE_AEA & HISTOR & 0.0000393 \\
\hline 2447 & PDMS & & SW & & & E013946 & 26-Jun-79 & & $10045-97-3$ & CS-137 & GAMMA_ĒS & HISTOR & 0.02 \\
\hline 2448 & PDMS & & SW & & & E001313 & 26-Jun-79 & & PU-239/240 & PU-239/240 & PUISO_IE_AEA & HISTOR & 0.00045 \\
\hline 2449 & PDMS & & SW & & & E001313 & 26-Jun-79 & & $10045-97-3$ & CS-137 & GAMMA_GS & HISTOR & 0.07 \\
\hline 2450 & PDMS & & SW & & & E001313 & 26-Jun-79 & & 15046-84-1 & I-129 & I129_SEP_TEMS & 320 & 0.000028 \\
\hline 2451 & PDMS & & SW & & & E004648 & 30-Jun-79 & & $10045-97-3$ & CS-137 & GAMMA_ĒS & USTEST & 0.0749 \\
\hline 2452 & PDMS & & SW & & & E044683 & 30-Jun-79 & & $10098-97-2$ & SR-90 & SRISO_S̄EP_PRECIP_GPC & USTEST & 0.653 \\
\hline 2453 & PDMS & & SW & & & E002033 & 04-Jul-79 & & $10045-97-3$ & CS-137 & GAMMA_GS & HISTOR & 0.006 \\
\hline 2454 & PDMS & & SW & & & E003986 & 04-Jul-79 & & $10045-97-3$ & CS-137 & GAMMA_GS & HISTOR & 0.005 \\
\hline 2455 & PDMS & & SW & & & E001314 & 04-Jul-79 & & $10045-97-3$ & CS-137 & GAMMA_GS & HISTOR & 0.01 \\
\hline 2456 & PDMS & & SW & & & E013947 & 04-Jul-79 & & $10045-97-3$ & CS-137 & GAMMA_GS & HISTOR & 0.009 \\
\hline 2457 & PDMS & & SW & & & E002034 & 18-Jul-79 & & $10045-97-3$ & CS-137 & GAMMA_GS & HISTOR & 0.01 \\
\hline 2458 & PDMS & & SW & & & E003987 & 18-Jul-79 & & $10045-97-3$ & CS-137 & GAMMA_GS & HISTOR & 0.01 \\
\hline 2459 & PDMS & & SW & & & E001315 & 18-Jul-79 & & $10045-97-3$ & CS-137 & GAMMA_GS & HISTOR & 0.007 \\
\hline 2460 & PDMS & & SW & & & E013948 & 18-Jul-79 & & $10045-97-3$ & CS-137 & GAMMA_GS & HISTOR & 0.005 \\
\hline 2461 & PDMS & & SW & & & E004520 & 30-Jul-79 & & $10028-17-8$ & TRITIUM & TRITIUM_DIST_LSC & HISTOR & 348 \\
\hline 2462 & PDMS & & SW & & & E004520 & 30-Jul-79 & & $10045-97-3$ & CS-137 & GAMMA_GS & USTEST & 0.0102 \\
\hline 2463 & PDMS & & SW & & & E004520 & 30-Jul-79 & & $10098-97-2$ & SR-90 & SRISO_SEPP_PRECIP_GPC & USTEST & 0.426 \\
\hline 2464 & PDMS & & SW & & & E003564 & 30-Jul-79 & & $10045-97-3$ & CS-137 & GAMMA_GS & USTEST & -0.23 \\
\hline 2465 & PDMS & & SW & & & E044459 & 30-Jul-79 & & $10045-97-3$ & CS-137 & GAMMA_GS & USTEST & -0.089 \\
\hline \begin{tabular}{|l|l|}
2466 \\
\end{tabular} & PDMS & & SW & & & E044459 & 30-Jul-79 & & $10028-17-8$ & TRITIUM & TRITIUM_DIST_LSC & USTEST & 40900 \\
\hline \begin{tabular}{|l|l|}
2467 & -1 \\
\end{tabular} & PDMS & & SW & & & E002115 & $30-J u l-79$ & & $10045-97-3$ & CS-137 & GAMMA_GS & USTEST & 0.0582 \\
\hline 2468 & PDMS & & SW & & & E002035 & 01-Aug-79 & & $10045-97-3$ & CS-137 & GAMMA_GS & HISTOR & 0.01 \\
\hline 2469 & PDMS & & SW & & & E003988 & 01-Aug-79 & & $10045-97-3$ & CS-137 & GAMMA_GS & HISTOR & 0.004 \\
\hline 2470 & PDMS & & SW & & & E001316 & 01-Aug-79 & & $10045-97-3$ & CS-137 & GAMMA_GS & HISTOR & 0.02 \\
\hline 2471 & PDMS & & SW & & & E013949 & 01-Aug-79 & & $10045-97-3$ & CS-137 & GAMMA_GS & HISTOR & 0.005 \\
\hline 2472 & PDMS & & SW & & & E016413 & 14-Aug-79 & & $10098-97-2$ & SR-90 & SRISO_SEP_PRECIP_GPC & HISTOR & 1.03 \\
\hline 2473 & PDMS & & SW & & & E016413 & 14-Aug-79 & & $10045-97-3$ & CS-137 & GAMMA_GS & USTEST & 4.43 \\
\hline 2474 & PDMS & & SW & & & E022437 & 14-Aug-79 & & $10045-97-3$ & CS-137 & GAMMA_GS & USTEST & 27.3 \\
\hline 2475 & PDMS & & SW & & & E022437 & 14-Aug-79 & & $10098-97-2$ & SR-90 & SRISO_SEP_PRECIP_GPC & HISTOR & 0.384 \\
\hline 2476 & PDMS & & SW & & & E014283 & 14-Aug-79 & & $10045-97-3$ & CS-137 & GAMMA_GS & USTEST & 9.8 \\
\hline 2477 & PDMS & & SW & & & E014283 & 14-Aug-79 & & $10098-97-2$ & SR-90 & SRISO_SEP_PRECIP_GPC & HISTOR & 0.946 \\
\hline 2478 & PDMS & & SW & & & E003911 & 15-Aug-79 & & $10045-97-3$ & CS-137 & GAMMA__GS & HISTOR & 0.03 \\
\hline 2479 & PDMS & & SW & & & E002036 & 15-Aug-79 & & $10045-97-3$ & CS-137 & GAMMA_GS & HISTOR & 0.01 \\
\hline 2480 & PDMS & & SW & & & E013950 & 15-Aug-79 & & $10045-97-3$ & CS-137 & GAMMA_GS & HISTOR & 0.008 \\
\hline 2481 & PDMS & & SW & & & E001317 & 15-Aug-79 & & $10045-97-3$ & CS-137 & GAMMA_GS & HISTOR & 0.008 \\
\hline 2482 & PDMS & & SW & & & E004521 & 27-Aug-79 & & $10045-97-3$ & CS-137 & GAMMA_GS & USTEST & 0.285 \\
\hline 2483 & PDMS & & SW & & & E004521 & 27-Aug-79 & & $10028-17-8$ & TRITIUM & TRITIUM_DIST_LSC & HISTOR & 164 \\
\hline 2484 & PDMS & & SW & & & E001620 & 27-Aug-79 & & $10098-97-2$ & SR-90 & SRISO_SEP_PRECIP_GPC & HISTOR & 0.198 \\
\hline 2485 & PDMS & & SW & & & E001620 & 27-Aug-79 & & $10028-17-8$ & TRITIUM & TRITIUM_DIST_LSC & HISTOR & 324 \\
\hline 2486 & PDMS & & SW & & & E001620 & 27-Aug-79 & & $10045-97-3$ & CS-137 & GAMMA_GS & USTEST & 0.6 \\
\hline 2487 & PDMS & & SW & & & E014822 & 27-Aug-79 & & $10098-97-2$ & SR-90 & SRISO_SEP_PRECIP_GPC & HISTOR & 0.0901 \\
\hline 2488 & PDMS & & SW & & & E014822 & 27-Aug-79 & & $10045-97-3$ & CS-137 & GAMMA_GS & USTEST & -0.132 \\
\hline 2489 & PDMS & & SW & & & E014822 & 27-Aug-79 & & $10028-17-8$ & TRITIUM & TRITIUM_DIST_LSC & USTEST & 39900 \\
\hline 2490 & PDMS & & SW & & & E014822 & 27-Aug-79 & & $14133-76-7$ & TC-99 & TC99_SEP_LSC & HISTOR & 3.1 \\
\hline 2491 & PDMS & & SW & & & E002116 & 27-Aug-79 & & $10045-97-3$ & CS-137 & GAMM̄A_GS & USTEST & 3.6 \\
\hline 2492 & PDMS & & SW & & & E002037 & 29-Aug-79 & & $10045-97-3$ & CS-137 & GAMMA_GS & HISTOR & 0.02 \\
\hline 2493 & PDMS & & SW & & & E003912 & 29-Aug-79 & & $10045-97-3$ & CS-137 & GAMMA_GS & HISTOR & 0.02 \\
\hline 2494 & PDMS & & SW & & & E001318 & 29-Aug-79 & & $10045-97-3$ & CS-137 & GAMMA_GS & HISTOR & 0.01 \\
\hline 2495 & PDMS & & SW & & & E013951 & 29-Aug-79 & & $10045-97-3$ & CS-137 & GAMMA_GS & HISTOR & 0.003 \\
\hline 2496 & PDMS & & SW & & & E007493 & 29-Aug-79 & & $10028-17-8$ & TRITIUM & TRITIUM_DIST_LSC & HISTOR & 414 \\
\hline 2497 & PDMS & & SW & & & E007493 & 29-Aug-79 & & $10098-97-2$ & SR-90 & SRISO_SEP_PRECIP_GPC & USTEST & 0.21 \\
\hline
\end{tabular}




\begin{tabular}{|c|c|c|c|c|c|c|c|c|c|c|c|c|c|}
\hline & A & $\mathrm{B}$ & $\mathrm{C}$ & $\mathrm{D}$ & $\mathrm{E}$ & $\mathrm{F}$ & G & $\mathrm{H}$ & $\mathrm{I}$ & $\mathrm{J}$ & $\mathrm{K}$ & $\mathrm{L}$ & $\mathrm{M}$ \\
\hline 1 & SAMPLE_NUM_ASSIGNED_TO & SAMPLER & MEDIA & BIOTA_MEDIA & TAG_ID & SAMP_NUM & SAMP_DATE_TIME & SAMP_DATE_TIME_ON & CON_ID & CON_SHORT_NAME & METHOD_NAME & LAB_CODE & VALUE_RPTD \\
\hline 2498 & PDMS & & SW & & & E007493 & 29-Aug-79 & & $10045-97-3$ & CS-137 & GAMMA_GS & USTEST & 2.68 \\
\hline 2499 & PDMS & & SW & & & E003913 & 12-Sep-79 & & $10045-97-3$ & CS-137 & GAMMA_GS & HISTOR & 0.009 \\
\hline 2500 & PDMS & & SW & & & E002038 & 12-Sep-79 & & $10045-97-3$ & CS-137 & GAMMA_GS & HISTOR & 0.03 \\
\hline 2501 & PDMS & & SW & & & E001319 & 12-Sep-79 & & $10045-97-3$ & CS-137 & GAMMA_GS & HISTOR & 0.007 \\
\hline 2502 & PDMS & & SW & & & E013952 & 12-Sep-79 & & $10045-97-3$ & CS-137 & GAMMA_GS & HISTOR & 0.006 \\
\hline 2503 & PDMS & & SW & & & E002117 & 17-Sep-79 & & $10045-97-3$ & CS-137 & GAMMA_GS & HISTOR & 4.22 \\
\hline 2504 & PDMS & & SW & & & E004522 & 24-Sep-79 & & $10045-97-3$ & CS-137 & GAMMA_GS & HISTOR & 0.0102 \\
\hline 2505 & PDMS & & SW & & & E004522 & 24-Sep-79 & & $10028-17-8$ & TRITIUM & TRITIUM_DIST_LSC & HISTOR & 4.4 \\
\hline 2506 & PDMS & & SW & & & E003565 & 24-Sep-79 & & $10045-97-3$ & CS-137 & GAMMA_GS & HISTOR & 0.725 \\
\hline 2507 & PDMS & & SW & & & E044460 & 24-Sep-79 & & $10045-97-3$ & CS-137 & GAMMA_GS & HISTOR & 0.639 \\
\hline 2508 & PDMS & & SW & & & E044460 & 24-Sep-79 & & 10028-17-8 & TRITIUM & TRITIUM_DIST_LSC & HISTOR & 40100 \\
\hline 2509 & PDMS & & SW & & & E001975 & 26-Sep-79 & & $10045-97-3$ & CS-137 & GAMMA_GS & HISTOR & 0.006 \\
\hline 2510 & PDMS & & SW & & & E003914 & 26-Sep-79 & & $10045-97-3$ & CS-137 & GAMMA_GS & HISTOR & 0.006 \\
\hline 2511 & PDMS & & SW & & & E013953 & 26-Sep-79 & & $10045-97-3$ & CS-137 & GAMMA_GS & HISTOR & 0.007 \\
\hline 2512 & PDMS & & SW & & & E001320 & 26-Sep-79 & & $10045-97-3$ & CS-137 & GAMMA_GS & HISTOR & 0.01 \\
\hline 2513 & PDMS & & SW & & & E007427 & 26-Sep-79 & & $10028-17-8$ & TRITIUM & TRITIUM_DIST_LSC & HISTOR & 101 \\
\hline 2514 & PDMS & & SW & & & E007427 & 26-Sep-79 & & $10045-97-3$ & CS-137 & GAMMA_GS & HISTOR & -0.252 \\
\hline 2515 & PDMS & & SW & & & E012071 & $02-$ Oct-79 & & PU-239/240 & PU-239/240 & PUISO_IE_AEA & HISTOR & 0.000024 \\
\hline 2516 & PDMS & & SW & & & E012071 & $02-$ Oct-79 & & 15046-84-1 & I-129 & 1129_SEP_TEMS & 320 & 0.0001 \\
\hline 2517 & PDMS & & SW & & & E073581 & $02-$ Oct-79 & & PU-239/240 & PU-239/240 & PUISO_IE_AEA & HISTOR & 0.0000592 \\
\hline 2518 & PDMS & & SW & & & E030585 & $02-$ Oct-79 & & PU-239/240 & PU-239/240 & PUISO_IE_AEA & HISTOR & 0.000085 \\
\hline 2519 & PDMS & & SW & & & E030585 & $02-$ Oct-79 & & 15046-84-1 & I-129 & 1129_SEP_TEMS & 320 & 0.000014 \\
\hline 2520 & PDMS & & SW & & & E040618 & $02-$ Oct-79 & & PU-239/240 & PU-239/240 & PUISO_IE_AEA & HISTOR & 0.0000331 \\
\hline 2521 & PDMS & & SW & & & E003915 & $10-$ Oct-79 & & $10045-97-3$ & CS-137 & GAMMA_GS & HISTOR & 0.003 \\
\hline 2522 & PDMS & & SW & & & E001976 & $10-$ Oct-79 & & $10045-97-3$ & CS-137 & GAMMA_GS & HISTOR & 0.02 \\
\hline 2523 & PDMS & & SW & & & E001977 & 24-Oct-79 & & $10045-97-3$ & CS-137 & GAMMA_GS & HISTOR & 0.02 \\
\hline 2524 & PDMS & & SW & & & E003916 & 24-Oct-79 & & $10045-97-3$ & CS-137 & GAMMA_GS & HISTOR & 0.005 \\
\hline 2525 & PDMS & & SW & & & E013954 & 24-Oct-79 & & $10045-97-3$ & CS-137 & GAMMA_GS & HISTOR & 0.02 \\
\hline 2526 & PDMS & & SW & & & E001321 & 24-Oct-79 & & $10045-97-3$ & CS-137 & GAMMA_GS & HISTOR & 0.03 \\
\hline 2527 & PDMS & & SW & & & E007455 & 24-Oct-79 & & $10028-17-8$ & TRITIUM & TRITIUM_DIST_LSC & HISTOR & 287 \\
\hline 2528 & PDMS & & SW & & & E007455 & 24-Oct-79 & & $10045-97-3$ & CS-137 & GAMMA_GS & HISTOR & -0.233 \\
\hline 2529 & PDMS & & SW & & & E004523 & 29-Oct-79 & & $10045-97-3$ & CS-137 & GAMMA_GS & HISTOR & -0.211 \\
\hline 2530 & PDMS & & SW & & & E004523 & 29-Oct-79 & & $10098-97-2$ & SR-90 & SRISO_SEP_PRECIP_GPC & HISTOR & 0.335 \\
\hline 2531 & PDMS & & SW & & & E004523 & 29-Oct-79 & & $10028-17-8$ & TRITIUM & TRITIUM_DIST_LSC & HISTOR & 315 \\
\hline 2532 & PDMS & & SW & & & E003566 & 29-Oct-79 & & $10045-97-3$ & CS-137 & GAMMA_GS & HISTOR & -0.147 \\
\hline 2533 & PDMS & & SW & & & E044461 & 29-Oct-79 & & $10045-97-3$ & CS-137 & GAMMA_GS & HISTOR & -0.0335 \\
\hline $2534 \mathrm{~F}$ & PDMS & & SW & & & E044461 & 29-Oct-79 & & $10028-17-8$ & TRITIUM & TRITIUM_DIST_LSC & HISTOR & 36500 \\
\hline 2535 & PDMS & & SW & & & E002118 & 29-Oct-79 & & $10045-97-3$ & CS-137 & GAMMA_GS & HISTOR & -0.726 \\
\hline 2536 & PDMS & & SW & & & E016414 & 06-Nov-79 & & $10098-97-2$ & SR-90 & SRISO_SEEP_PRECIP_GPC & HISTOR & 0.835 \\
\hline 2537 & PDMS & & SW & & & E016414 & 06-Nov-79 & & $10045-97-3$ & CS-137 & GAMMA_GS & HISTOR & 11.1 \\
\hline 2538 & PDMS & & SW & & & E022438 & 06-Nov-79 & & $10045-97-3$ & CS-137 & GAMMA_GS & HISTOR & 30.8 \\
\hline 2539 & PDMS & & SW & & & E022438 & 06-Nov-79 & & $10098-97-2$ & SR-90 & SRISO_SEP_PRECIP_GPC & HISTOR & 0.444 \\
\hline 2540 & PDMS & & SW & & & E014284 & 06-Nov-79 & & $10045-97-3$ & CS-137 & GAMMA_GS & HISTOR & 0.0307 \\
\hline $2541 \mathrm{~F}$ & PDMS & & SW & & & E014284 & 06-Nov-79 & & $10098-97-2$ & SR-90 & SRISO_SEP_PRECIP_GPC & HISTOR & 3.15 \\
\hline 2542 & PDMS & & SW & & & E001978 & 13-Nov-79 & & $10045-97-3$ & CS-137 & GAMMA_GS & HISTOR & 0.009 \\
\hline 2543 & PDMS & & SW & & & E003917 & 13-Nov-79 & & $10045-97-3$ & CS-137 & GAMMA_GS & HISTOR & 0.006 \\
\hline 2544 & PDMS & & SW & & & E004524 & $26-N o v-79$ & & 10028-17-8 & TRITIUM & TRITIUM_DIST_LSC & HISTOR & 729 \\
\hline 2545 & PDMS & & SW & & & E004524 & $26-N o v-79$ & & $10045-97-3$ & CS-137 & GAMMA_GS & HISTOR & 0.665 \\
\hline 2546 & PDMS & & SW & & & E001621 & 26-Nov-79 & & $10098-97-2$ & SR-90 & SRISO_SEP_PRECIP_GPC & HISTOR & 0.264 \\
\hline 2547 & PDMS & & SW & & & E001621 & 26-Nov-79 & & $10028-17-8$ & TRITIUM & TRITIUM_DIST_LSC & HISTOR & 280 \\
\hline 2548 & PDMS & & SW & & & E001621 & 26-Nov-79 & & $10045-97-3$ & CS-137 & GAMMA_GS & HISTOR & 10.2 \\
\hline 2549 & PDMS & & SW & & & E014823 & 26-Nov-79 & & $10098-97-2$ & SR-90 & SRISO_SEPP_PRECIP_GPC & HISTOR & 0 \\
\hline
\end{tabular}




\begin{tabular}{|c|c|c|c|c|c|c|c|c|c|c|c|c|c|}
\hline & A & $\mathrm{B}$ & $\mathrm{C}$ & $\mathrm{D}$ & $\mathrm{E}$ & $\mathrm{F}$ & $\mathrm{G}$ & $\mathrm{H}$ & $\mathrm{I}$ & $\mathrm{J}$ & $\mathrm{K}$ & $\mathrm{L}$ & $\mathrm{M}$ \\
\hline 1 & SAMPLE_NUM_ASSIGNED_TO & \begin{tabular}{|l|} 
SAMPLER \\
\end{tabular} & MEDIA & BIOTA_MEDIA & TAG_ID & SAMP_NUM & SAMP_DATE_TIME & SAMP_DATE_TIME_ON & CON_ID & CON_SHORT_NAME & METHOD_NAME & LAB_CODE & VALUE_RPTD \\
\hline 2550 & PDMS & & SW & & & E014823 & $26-$ Nov-79 & & $10045-97-3$ & CS-137 & GAMMA GS & HISTOR & -0.175 \\
\hline 2551 & PDMS & & sw & & & E014823 & 26-Nov-79 & & $10028-17-8$ & TRITIUM & TRITIUM_DIST_LSC & HISTOR & 25400 \\
\hline 2552 & PDMS & & sw & & & E014823 & 26-Nov-79 & & $14133-76-7$ & TC-99 & TC99_SEP_LSC & HISTOR & -3.18 \\
\hline 2553 & PDMS & & sw & & & E002119 & 26-Nov-79 & & $10045-97-3$ & CS-137 & GAMMA_GS & HISTOR & 0.0614 \\
\hline 2554 & PDMS & & sw & & & E003918 & $27-N o v-79$ & & $10045-97-3$ & CS-137 & GAMMA_GS & HISTOR & 0.01 \\
\hline \begin{tabular}{|l|}
2555 \\
\end{tabular} & PDMS & & sw & & & E001979 & $27-$ Nov-79 & & $10045-97-3$ & CS-137 & GAMMA_GS & HISTOR & 0.02 \\
\hline 2556 & PDMS & & sW & & & E013955 & 27-Nov-79 & & $10045-97-3$ & CS-137 & GAMMA_GS & HISTOR & 0.02 \\
\hline 2557 & PDMS & & SW & & & E001322 & 27-Nov-79 & & $10045-97-3$ & CS-137 & GAMMA_GS & HISTOR & 0.04 \\
\hline 2558 & PDMS & & sw & & & E007397 & 29-Nov-79 & & $10028-17-8$ & TRITIUM & TRITIUM DIST_LSC & HISTOR & 584 \\
\hline 2559 & PDMS & & SW & & & E007397 & 29-Nov-79 & & $10098-97-2$ & SR-90 & SRISO_SEP_PRECIP_GPC & HISTOR & 0.282 \\
\hline 2560 & PDMS & & sw & & & E007397 & 29-Nov-79 & & $10045-97-3$ & CS-137 & GAMMA_GS & HISTOR & 0.0615 \\
\hline 2561 & PDMS & & sW & & & E003919 & 11-Dec-79 & & $10045-97-3$ & CS-137 & GAMMA_GS & HISTOR & 0.02 \\
\hline 2562 & PDMS & & sW & & & E001980 & 11-Dec-79 & & $10045-97-3$ & CS-137 & GAMMA_GS & HISTOR & 0.01 \\
\hline 2563 & PDMS & & sW & & & E001323 & 11-Dec-79 & & $10045-97-3$ & CS-137 & GAMMA GS & HISTOR & 0.008 \\
\hline 2564 & PDMS & & sw & & & E013956 & 11-Dec-79 & & $10045-97-3$ & CS-137 & GAMMA_GS & HISTOR & 0.004 \\
\hline 2565 & PDMS & & sw & & & E001981 & 20-Dec-79 & & $10045-97-3$ & CS-137 & GAMMA_GS & HISTOR & 0.006 \\
\hline 2566 & PDMS & & sw & & & E003920 & 20-Dec-79 & & $10045-97-3$ & CS-137 & GAMMA_GS & HISTOR & 0.002 \\
\hline 2567 & PDMS & & sw & & & E001324 & 20-Dec-79 & & $10045-97-3$ & CS-137 & GAMMA_GS & HISTOR & 0.03 \\
\hline 2568 & PDMS & & sW & & & E013957 & 20-Dec-79 & & $10045-97-3$ & CS-137 & GAMMA_GS & HISTOR & 0.006 \\
\hline 2569 & PDMS & & sW & & & E007330 & 26-Dec-79 & & $10028-17-8$ & TRITIUM & TRITIUM_DIST_LSC & HISTOR & 211 \\
\hline 2570 & PDMS & & sw & & & E007330 & 26-Dec-79 & & $10045-97-3$ & CS-137 & GAMMA_GS & HISTOR & 2.74 \\
\hline 2571 & PDMS & & sW & & & E004525 & 31-Dec-79 & & $10045-97-3$ & CS-137 & GAMMA_GS & HISTOR & 0.386 \\
\hline 2572 & PDMS & & sW & & & E004525 & 31-Dec-79 & & 10028-17-8 & TRITIUM & TRITIUM_DIST_LSC & HISTOR & 249 \\
\hline 2573 & PDMS & & sW & & & E003567 & 31-Dec-79 & & $10045-97-3$ & CS-137 & GAMMA_GS & HISTOR & 15.8 \\
\hline 2574 & PDMS & & sW & & & E044462 & 31-Dec-79 & & $10045-97-3$ & CS-137 & GAMMA_GS & HISTOR & 0.0102 \\
\hline 2575 & PDMS & & sW & & & E044462 & 31-Dec-79 & & $10028-17-8$ & TRITIUM & TRITIUM_DIST_LSC & HISTOR & 24300 \\
\hline 2576 & PDMS & & sW & & & E002120 & 31-Dec-79 & & $10045-97-3$ & CS-137 & GAMMA_GS & HISTOR & 0.0614 \\
\hline 2577 & PDMS & & sW & & & E001922 & 08-Jan-80 & & PU-239/240 & PU-239/240 & PUISO_IE_AEA & HISTOR & 0.00026 \\
\hline 2578 & PDMS & & sw & & & E001922 & 08-Jan-80 & & $10045-97-3$ & CS-137 & GAMMA_GS & HISTOR & 0.006 \\
\hline 2579 & PDMS & & sW & & & E001922 & 08-Jan-80 & & 15046-84-1 & I-129 & I129_SEP_TEMS & 320 & 0.000096 \\
\hline 2580 & PDMS & & sW & & & E003921 & 08-Jan-80 & & $10045-97-3$ & CS-137 & GAMMA_GS & HISTOR & 0.008 \\
\hline 2581 & PDMS & & sW & & & E003921 & 08-Jan-80 & & PU-239/240 & PU-239/240 & PUISO_IE_AEA & HISTOR & 0.0000357 \\
\hline 2582 & PDMS & & sW & & & E001325 & 08-Jan-80 & & $10045-97-3$ & CS-137 & GAMMA_GSS & HISTOR & 0.008 \\
\hline 2583 & PDMS & & SW & & & E001325 & 08-Jan-80 & & PU-239/240 & PU-239/240 & PUISO_IE_AEA & HISTOR & -0.000039 \\
\hline 2584 & PDMS & & sW & & & E001325 & 08-Jan-80 & & 15046-84-1 & I-129 & 1129_SEP_TEMS & 320 & 0.0000079 \\
\hline 2585 & PDMS & & sw & & & E013958 & 08-Jan-80 & & PU-239/240 & PU-239/240 & PUISO_IE_AEA & HISTOR & 0.0000321 \\
\hline 2586 & PDMS & & SW & & & E013958 & 08-Jan-80 & & $10045-97-3$ & CS-137 & GAMMA_GS & HISTOR & 0.002 \\
\hline 2587 & PDMS & & sW & & & E003568 & 14-Jan-80 & & $10045-97-3$ & CS-137 & GAMMA_GS & HISTOR & 7.07 \\
\hline 2588 & PDMS & & sW & & & E001923 & 22-Jan-80 & & $10045-97-3$ & CS-137 & GAMMA_GS & HISTOR & 0.15 \\
\hline 2589 & PDMS & & SW & & & E003922 & 22-Jan-80 & & $10045-97-3$ & CS-137 & GAMMA_GS & HISTOR & 0.04 \\
\hline 2590 & PDMS & & sW & & & E004526 & 28-Jan-80 & & $10098-97-2$ & SR-90 & SRISO_SEEP_PRECIP_GPC & HISTOR & 0.135 \\
\hline 2591 & PDMS & & sW & & & E004526 & 28-Jan-80 & & $10028-17-8$ & TRITIUM & TRITIUM_DISTT_LSC & HISTOR & 148 \\
\hline 2592 & PDMS & & SW & & & E004526 & 28-Jan-80 & & $10045-97-3$ & CS-137 & GAMMA_GS & HISTOR & 0.0617 \\
\hline 2593 & PDMS & & sW & & & E003569 & 28-Jan-80 & & $10045-97-3$ & CS-137 & GAMMA_GS & HISTOR & 3.02 \\
\hline 2594 & PDMS & & SW & & & E044463 & 28-Jan-80 & & $10045-97-3$ & CS-137 & GAMMA_GS & HISTOR & 0.809 \\
\hline 2595 & PDMS & & SW & & & E044463 & 28-Jan-80 & & $10028-17-8$ & TRITIUM & TRITIUM_DIST_LSC & HISTOR & 17400 \\
\hline 2596 & PDMS & & sW & & & E002121 & 28-Jan-80 & & $10045-97-3$ & CS-137 & GAMMA_GS & HISTOR & -0.487 \\
\hline $2597 \mathrm{~F}$ & PDMS & & sW & & & E007365 & 30-Jan-80 & & $10028-17-8$ & TRITIUM & TRITIUM_DIST_LSC & HISTOR & 492 \\
\hline 2598 & PDMS & & sw & & & E007365 & 30-Jan-80 & & $10045-97-3$ & CS-137 & GAMMA_GS & HISTOR & 0.0614 \\
\hline 2599 & PDMS & & SW & & & E003923 & 05-Feb-80 & & $10045-97-3$ & CS-137 & GAMMA_GS & HISTOR & 0.005 \\
\hline 2600 & PDMS & & SW & & & E001924 & 05-Feb-80 & & $10045-97-3$ & CS-137 & GAMMA_GS & HISTOR & 0.01 \\
\hline 2601 & PDMS & & SW & & & E016415 & 12-Feb-80 & & $10098-97-2$ & SR-90 & SRISO_SEPP_PRECIP_GPC & HISTOR & 1.7 \\
\hline
\end{tabular}




\begin{tabular}{|c|c|c|c|c|c|c|c|c|c|c|c|c|c|}
\hline & A & $\mathrm{B}$ & $\mathrm{C}$ & $\mathrm{D}$ & $E$ & $\mathrm{~F}$ & $\mathrm{G}$ & $\mathrm{H}$ & $\mathrm{I}$ & $\mathrm{J}$ & $\mathrm{K}$ & $\mathrm{L}$ & $\mathrm{M}$ \\
\hline 1 & SAMPLE_NUM_ASSIGNED_TO & SAMPLER & MEDIA & BIOTA_MEDIA & TAG_ID & SAMP_NUM & SAMP_DATE_TIME & SAMP_DATE_TIME_ON & CON_ID & CON_SHORT_NAME & METHOD_NAME & LAB_CODE & VALUE_RPTD \\
\hline 2602 & PDMS & & SW & & & E016415 & 12-Feb-80 & & $10045-97-3$ & CS-137 & GAMMA_GS & HISTOR & 12 \\
\hline 2603 & PDMS & & SW & & & E022439 & $12-F e b-80$ & & $10045-97-3$ & CS-137 & GAMMA_GS & HISTOR & 110 \\
\hline 2604 & PDMS & & SW & & & E022439 & 12-Feb-80 & & $10098-97-2$ & SR-90 & SRISO_SEP_PRECIP_GPC & HISTOR & 0.411 \\
\hline 2605 & PDMS & & SW & & & E014285 & 12-Feb-80 & & $10045-97-3$ & CS-137 & GAMMA_GS & HISTOR & 22.7 \\
\hline 2606 & PDMS & & SW & & & E014285 & $12-F e b-80$ & & 10098-97-2 & SR-90 & SRISO_SEP_PRECIP_GPC & HISTOR & 0.09 \\
\hline 2607 & PDMS & & SW & & & E001925 & 19-Feb-80 & & $10045-97-3$ & CS-137 & GAMMA_GS & HISTOR & 0.12 \\
\hline 2608 & PDMS & & SW & & & E003924 & $19-F e b-80$ & & $10045-97-3$ & CS-137 & GAMMA_GS & HISTOR & 0.04 \\
\hline 2609 & PDMS & & SW & & & E001326 & $19-F e b-80$ & & $10045-97-3$ & CS-137 & GAMMA_GS & HISTOR & 0.06 \\
\hline 2610 & PDMS & & SW & & & E013959 & $19-F e b-80$ & & $10045-97-3$ & CS-137 & GAMMA_GS & HISTOR & 0.03 \\
\hline 2611 & PDMS & & SW & & & E004527 & $25-F e b-80$ & & $10045-97-3$ & CS-137 & GAMMA_GS & HISTOR & 0.199 \\
\hline 2612 & PDMS & & SW & & & E004527 & $25-F e b-80$ & & $10028-17-8$ & TRITIUM & TRITIUM_DIST_LSC & HISTOR & 27.4 \\
\hline 2613 & PDMS & & SW & & & E001622 & $25-F e b-80$ & & $10098-97-2$ & SR-90 & SRISO_SEP_PRECIP_GPC & HISTOR & 0.453 \\
\hline 2614 & PDMS & & SW & & & E001622 & $25-F e b-80$ & & $10028-17-8$ & TRITIUM & TRITIUM_DIST_LSC & HISTOR & 217 \\
\hline 2615 & PDMS & & SW & & & E001622 & $25-F e b-80$ & & $10045-97-3$ & CS-137 & GAMMA_GS & HISTOR & 0.0614 \\
\hline 2616 & PDMS & & SW & & & E014824 & $25-F e b-80$ & & $10098-97-2$ & SR-90 & SRISO_SEP_PRECIP_GPC & HISTOR & 0.105 \\
\hline 2617 & PDMS & & SW & & & E014824 & 25-Feb-80 & & $10045-97-3$ & CS-137 & GAMMA_GS & HISTOR & -0.0535 \\
\hline 2618 & PDMS & & SW & & & E014824 & 25-Feb-80 & & $10028-17-8$ & TRITIUM & TRITIUM_DIST_LSC & HISTOR & 24100 \\
\hline 2619 & PDMS & & SW & & & E014824 & 25 -Feb-80 & & 14133-76-7 & TC-99 & TC99_SEP_LSC & HISTOR & 5.42 \\
\hline 2620 & PDMS & & SW & & & E002122 & $25-F e b-80$ & & $10045-97-3$ & CS-137 & GAMMA_GS & HISTOR & 0.0614 \\
\hline 2621 & PDMS & & SW & & & E007299 & $27-F e b-80$ & & $10028-17-8$ & TRITIUM & TRITIUM_DIST_LSC & HISTOR & 174 \\
\hline 2622 & PDMS & & SW & & & E007299 & 27-Feb-80 & & $10098-97-2$ & SR-90 & SRISO_SEP_PRECIP_GPC & HISTOR & 0.198 \\
\hline 2623 & PDMS & & SW & & & E007299 & $27-F e b-80$ & & $10045-97-3$ & CS-137 & GAMMA_GS & HISTOR & 2.35 \\
\hline 2624 & PDMS & & SW & & & E003925 & 04-Mar-80 & & $10045-97-3$ & CS-137 & GAMMA_GS & HISTOR & 0.008 \\
\hline 2625 & PDMS & & SW & & & E001926 & 04-Mar-80 & & $10045-97-3$ & CS-137 & GAMMA_GS & HISTOR & 0.01 \\
\hline 2626 & PDMS & & SW & & & E001327 & 04-Mar-80 & & $10045-97-3$ & CS-137 & GAMMA_GS & HISTOR & 0.02 \\
\hline 2627 & PDMS & & SW & & & E013960 & 04-Mar-80 & & $10045-97-3$ & CS-137 & GAMMA_GS & HISTOR & 0.007 \\
\hline 2628 & PDMS & & SW & & & E001927 & 18-Mar-80 & & $10045-97-3$ & CS-137 & GAMMA_GS & HISTOR & 0.005 \\
\hline 2629 & PDMS & & SW & & & E003926 & 18-Mar-80 & & $10045-97-3$ & CS-137 & GAMMA_GS & HISTOR & 0.007 \\
\hline 2630 & PDMS & & SW & & & E013961 & 18-Mar-80 & & $10045-97-3$ & CS-137 & GAMMA_GS & HISTOR & 0.007 \\
\hline 2631 & PDMS & & SW & & & E001328 & 18-Mar-80 & & $10045-97-3$ & CS-137 & GAMMA_GS & HISTOR & 0.004 \\
\hline 2632 & PDMS & & SW & & & E007327 & 26-Mar-80 & & $10028-17-8$ & TRITIUM & TRITIUM_DIST_LSC & HISTOR & 206 \\
\hline 2633 & PDMS & & SW & & & E007327 & 26-Mar-80 & & $10045-97-3$ & CS-137 & GAMMA_GS & HISTOR & -0.77 \\
\hline 2634 & PDMS & & SW & & & E004528 & 31-Mar-80 & & $10028-17-8$ & TRITIUM & TRITIUM_DIST_LSC & HISTOR & 222 \\
\hline 2635 & PDMS & & SW & & & E004528 & 31-Mar-80 & & $10045-97-3$ & CS-137 & GAMMA_GS & HISTOR & 0.428 \\
\hline 2636 & PDMS & & SW & & & E003570 & 31-Mar-80 & & $10045-97-3$ & CS-137 & GAMMA_GS & HISTOR & 0.00615 \\
\hline 2637 & PDMS & & SW & & & E044464 & 31-Mar-80 & & $10045-97-3$ & CS-137 & GAMMA_GS & HISTOR & -0.109 \\
\hline 2638 & PDMS & & SW & & & E044464 & 31-Mar-80 & & $10028-17-8$ & TRITIUM & TRITIUM_DIST_LSC & HISTOR & 23700 \\
\hline 2639 & PDMS & & SW & & & E002123 & 31-Mar-80 & & $10045-97-3$ & CS-137 & GAMMA_GS & HISTOR & 0.861 \\
\hline 2640 & PDMS & & SW & & & E003927 & 01-Apr-80 & & PU-239/240 & PU-239/240 & PUISO_IE_AEA & PNL-RO & 0.000119 \\
\hline 2641 & PDMS & & SW & & & E003927 & 01-Apr-80 & & $10045-97-3$ & CS-137 & GAMMA_GS & HISTOR & 0.003 \\
\hline 2642 & PDMS & & SW & & & E001928 & 01-Apr-80 & & PU-239/240 & PU-239/240 & PUISO_IE_AEA & PNL-RO & 0.0000799 \\
\hline 2643 & PDMS & & SW & & & E001928 & 01-Apr-80 & & $10045-97-3$ & CS-137 & GAMMA_GS & HISTOR & 0.007 \\
\hline 2644 & PDMS & & SW & & & E001928 & 01-Apr-80 & & 15046-84-1 & I-129 & I129_SEP_TEMS & 320 & 0.000094 \\
\hline 2645 & PDMS & & SW & & & E001329 & 01-Apr-80 & & $10045-97-3$ & CS-137 & GAMMA_GS & HISTOR & 0.004 \\
\hline 2646 & PDMS & & SW & & & E001329 & 01-Apr-80 & & PU-239/240 & PU-239/240 & PUISO_IE_AEA & PNL-RO & 0.0000421 \\
\hline 2647 & PDMS & & SW & & & E001329 & 01-Apr-80 & & 15046-84-1 & $\mid-129$ & I129_SEP_TEMS & 320 & 0.0000057 \\
\hline 2648 & PDMS & & SW & & & E013962 & 01-Apr-80 & & PU-239/240 & PU-239/240 & PUISO_IE_AEA & PNL-RO & 0.0000557 \\
\hline 2649 & PDMS & & SW & & & E013962 & 01-Apr-80 & & $10045-97-3$ & CS-137 & GAMMA_GS & HISTOR & 0.01 \\
\hline 2650 & PDMS & & SW & & & E001929 & 15-Apr-80 & & $10045-97-3$ & CS-137 & GAMMA_GS & HISTOR & 0.03 \\
\hline 2651 & PDMS & & SW & & & E003928 & 15-Apr-80 & & $10045-97-3$ & CS-137 & GAMMA_GS & HISTOR & 0.02 \\
\hline 2652 & PDMS & & SW & & & E001253 & 15-Apr-80 & & $10045-97-3$ & CS-137 & GAMMA_GS & HISTOR & 0.003 \\
\hline 2653 & PDMS & & SW & & & E013963 & 15-Apr-80 & & $10045-97-3$ & CS-137 & GAMMA_GS & HISTOR & 0.005 \\
\hline
\end{tabular}




\begin{tabular}{|c|c|c|c|c|c|c|c|c|c|c|c|c|c|}
\hline & A & $\mathrm{B}$ & $\mathrm{C}$ & $\mathrm{D}$ & $E$ & $\mathrm{~F}$ & $\mathrm{G}$ & $\mathrm{H}$ & $\mathrm{I}$ & $\mathrm{J}$ & $\mathrm{K}$ & $\mathrm{L}$ & $\mathrm{M}$ \\
\hline 1 & SAMPLE_NUM_ASSIGNED_TO & SAMPLER & MEDIA & BIOTA_MEDIA & TAG_ID & SAMP_NUM & SAMP_DATE_TIME & SAMP_DATE_TIME_ON & CON_ID & CON_SHORT_NAME & METHOD_NAME & LAB_CODE & VALUE_RPTD \\
\hline 2654 & PDMS & & SW & & & E004529 & 28-Apr-80 & & $10045-97-3$ & CS-137 & GAMMA_GS & HISTOR & -0.106 \\
\hline 2655 & PDMS & & SW & & & E004529 & 28-Apr-80 & & $10098-97-2$ & SR-90 & SRISO_SEP_PRECIP_GPC & HISTOR & 0.228 \\
\hline 2656 & PDMS & & SW & & & E004529 & 28-Apr-80 & & $10028-17-8$ & TRITIUM & TRITIUM_DIST_LSC & HISTOR & 183 \\
\hline 2657 & PDMS & & SW & & & E003571 & 28-Apr-80 & & $10045-97-3$ & CS-137 & GAMMA_GS & HISTOR & 0.422 \\
\hline 2658 & PDMS & & SW & & & E044465 & 28-Apr-80 & & $10045-97-3$ & CS-137 & GAMMA_GS & HISTOR & 0.319 \\
\hline 2659 & PDMS & & SW & & & E044465 & 28-Apr-80 & & $10028-17-8$ & TRITIUM & TRITIUM_DIST_LSC & HISTOR & 41400 \\
\hline 2660 & PDMS & & SW & & & E002124 & 28-Apr-80 & & $10045-97-3$ & CS-137 & GAMMA_GS & HISTOR & -0.0228 \\
\hline 2661 & PDMS & & SW & & & E001930 & 29-Apr-80 & & $10045-97-3$ & CS-137 & GAMMA_GS & HISTOR & 0.005 \\
\hline 2662 & PDMS & & SW & & & E003929 & 29-Apr-80 & & $10045-97-3$ & CS-137 & GAMMA_GS & HISTOR & 0.008 \\
\hline 2663 & PDMS & & SW & & & E001254 & 29-Apr-80 & & $10045-97-3$ & CS-137 & GAMMA_GS & HISTOR & 0.01 \\
\hline 2664 & PDMS & & SW & & & E013964 & 29-Apr-80 & & $10045-97-3$ & CS-137 & GAMMA_GS & HISTOR & 0.004 \\
\hline 2665 & PDMS & & SW & & & E007267 & 29-Apr-80 & & $10028-17-8$ & TRITIUM & TRITIUM_DIST_LSC & HISTOR & 145 \\
\hline 2666 & PDMS & & SW & & & E007267 & 29-Apr-80 & & $10098-97-2$ & SR-90 & SRISO_SEP_PRECIP_GPC & HISTOR & 0.176 \\
\hline 2667 & PDMS & & SW & & & E007267 & 29-Apr-80 & & $10045-97-3$ & CS-137 & GAMMA_GS & HISTOR & 0.675 \\
\hline 2668 & PDMS & & SW & & & E016416 & 06-May-80 & & $10098-97-2$ & SR-90 & SRISO_SEP_PRECIP_GPC & HISTOR & 6.64 \\
\hline 2669 & PDMS & & SW & & & E016416 & 06-May-80 & & $10045-97-3$ & CS-137 & GAMMA_GS & HISTOR & 7.57 \\
\hline 2670 & PDMS & & SW & & & E012569 & 06-May-80 & & $10045-97-3$ & CS-137 & GAMMA_GS & HISTOR & 1.19 \\
\hline 2671 & PDMS & & SW & & & E022440 & 06-May-80 & & $10045-97-3$ & CS-137 & GAMMA_GS & HISTOR & 60.6 \\
\hline 2672 & PDMS & & SW & & & E022440 & 06-May-80 & & $10098-97-2$ & SR-90 & SRISO_SEP_PRECIP_GPC & HISTOR & 0.597 \\
\hline 2673 & PDMS & & SW & & & E014286 & 06-May-80 & & $10045-97-3$ & CS-137 & GAMMĀ_GS & HISTOR & 0.827 \\
\hline 2674 & PDMS & & SW & & & E014286 & 06-May-80 & & $10098-97-2$ & SR-90 & SRISO_SEP_PRECIP_GPC & HISTOR & 1.95 \\
\hline 2675 & PDMS & & SW & & & E001931 & 13-May-80 & & $10045-97-3$ & CS-137 & GAMMA_GS & HISTOR & 0.005 \\
\hline 2676 & PDMS & & SW & & & E003930 & 13-May-80 & & $10045-97-3$ & CS-137 & GAMMA_GS & HISTOR & 0.002 \\
\hline 2677 & PDMS & & SW & & & E013965 & 13-May-80 & & $10045-97-3$ & CS-137 & GAMMA_GS & HISTOR & 0.003 \\
\hline 2678 & PDMS & & SW & & & E001255 & 13-May-80 & & $10045-97-3$ & CS-137 & GAMMA_GS & HISTOR & 0.004 \\
\hline 2679 & PDMS & & SW & & & E004530 & 27-May-80 & & $10028-17-8$ & TRITIUM & TRITIUM_DIST_LSC & HISTOR & 417 \\
\hline 2680 & PDMS & & SW & & & E004530 & 27-May-80 & & $10045-97-3$ & CS-137 & GAMMA_GS & HISTOR & 0.299 \\
\hline 2681 & PDMS & & SW & & & E003931 & 27-May-80 & & $10045-97-3$ & CS-137 & GAMMA_GS & HISTOR & 0.004 \\
\hline 2682 & PDMS & & SW & & & E001932 & 27-May-80 & & $10045-97-3$ & CS-137 & GAMMA_GS & HISTOR & 0.003 \\
\hline 2683 & PDMS & & SW & & & E013966 & 27-May-80 & & $10045-97-3$ & CS-137 & GAMMA_GS & HISTOR & 0.004 \\
\hline 2684 & PDMS & & SW & & & E001256 & 27-May-80 & & $10045-97-3$ & CS-137 & GAMMA_GS & HISTOR & 0.004 \\
\hline 2685 & PDMS & & SW & & & E003572 & 27-May-80 & & $10045-97-3$ & CS-137 & GAMMA_GS & HISTOR & 0.783 \\
\hline 2686 & PDMS & & SW & & & E003572 & 27-May-80 & & $10028-17-8$ & TRITIUM & TRITIUM_DIST_LSC & HISTOR & 199 \\
\hline 2687 & PDMS & & SW & & & E003572 & 27-May-80 & & $10098-97-2$ & SR-90 & SRISO_SEP_PRECIP_GPC & HISTOR & 0.268 \\
\hline 2688 & PDMS & & SW & & & E014825 & 27-May-80 & & $10098-97-2$ & SR-90 & SRISO_SEP_PRECIP_GPC & HISTOR & 0.045 \\
\hline 2689 & PDMS & & SW & & & E014825 & 27-May-80 & & $10045-97-3$ & CS-137 & GAMMA_GS & HISTOR & -0.0589 \\
\hline 2690 & PDMS & & SW & & & E014825 & 27-May-80 & & $10028-17-8$ & TRITIUM & TRITIUM_DIST_LSC & HISTOR & 47300 \\
\hline 2691 & PDMS & & SW & & & E014825 & 27-May-80 & & 14133-76-7 & TC-99 & TC99_SEP_LSC & HISTOR & 5.77 \\
\hline 2692 & PDMS & & SW & & & E002125 & 27-May-80 & & $10045-97-3$ & CS-137 & GAMMA_GS & HISTOR & -0.0105 \\
\hline 2693 & PDMS & & SW & & & E007202 & 28-May-80 & & $10028-17-8$ & TRITIUM & TRITIUM_DIST_LSC & HISTOR & 220 \\
\hline 2694 & PDMS & & SW & & & E007202 & 28-May-80 & & $10045-97-3$ & CS-137 & GAMMA_GS & HISTOR & -0.00373 \\
\hline 2695 & PDMS & & SW & & & E001982 & 10-Jun-80 & & $10045-97-3$ & CS-137 & GAMMA_GS & HISTOR & 0.007 \\
\hline 2696 & PDMS & & SW & & & E003932 & 10-Jun-80 & & $10045-97-3$ & CS-137 & GAMMA_GS & HISTOR & 0.003 \\
\hline 2697 & PDMS & & SW & & & E013967 & 10-Jun-80 & & $10045-97-3$ & CS-137 & GAMMA_GS & HISTOR & 0.004 \\
\hline 2698 & PDMS & & SW & & & E001257 & 10-Jun-80 & & $10045-97-3$ & CS-137 & GAMMA_GS & HISTOR & 0.01 \\
\hline 2699 & PDMS & & SW & & & E001933 & 24-Jun-80 & & $10045-97-3$ & CS-137 & GAMMA_GS & HISTOR & 0.01 \\
\hline 2700 & PDMS & & SW & & & E003933 & 24-Jun-80 & & $10045-97-3$ & CS-137 & GAMMA_GS & HISTOR & 0.006 \\
\hline 2701 & PDMS & & SW & & & E001258 & 24-Jun-80 & & $10045-97-3$ & CS-137 & GAMMA_GS & HISTOR & 0.004 \\
\hline 2702 & PDMS & & SW & & & E015257 & 24-Jun-80 & & $10045-97-3$ & CS-137 & GAMMA_GS & HISTOR & 0.004 \\
\hline 2703 & PDMS & & SW & & & E004531 & 30-Jun-80 & & $10045-97-3$ & CS-137 & GAMMA_GS & HISTOR & -0.00162 \\
\hline 2704 & PDMS & & SW & & & E004531 & 30-Jun-80 & & $10028-17-8$ & TRITIUM & TRITIUM_DIST_LSC & HISTOR & 201 \\
\hline 2705 & PDMS & & SW & & & E003573 & 30-Jun-80 & & $10045-97-3$ & CS-137 & GAMMA_GS & HISTOR & -0.00886 \\
\hline
\end{tabular}




\begin{tabular}{|c|c|c|c|c|c|c|c|c|c|c|c|c|c|}
\hline & A & $\mathrm{B}$ & $\mathrm{C}$ & $\mathrm{D}$ & $E$ & $\mathrm{~F}$ & $\mathrm{G}$ & $\mathrm{H}$ & $\mathrm{I}$ & $\mathrm{J}$ & $\mathrm{K}$ & $\mathrm{L}$ & $\mathrm{M}$ \\
\hline 1 & SAMPLE_NUM_ASSIGNED_TO & SAMPLER & MEDIA & BIOTA_MEDIA & TAG_ID & SAMP_NUM & SAMP_DATE_TIME & SAMP_DATE_TIME_ON & CON_ID & CON_SHORT_NAME & METHOD_NAME & LAB_CODE & VALUE_RPTD \\
\hline 2706 & PDMS & & SW & & & E044466 & 30-Jun-80 & & $10045-97-3$ & CS-137 & GAMMA_GS & HISTOR & 1.61 \\
\hline 2707 & PDMS & & SW & & & E044466 & 30-Jun-80 & & $10028-17-8$ & TRITIUM & TRITIUM_DIST_LSC & HISTOR & 41800 \\
\hline 2708 & PDMS & & SW & & & E002126 & 30-Jun-80 & & $10045-97-3$ & CS-137 & GAMMA_GS & HISTOR & 0.64 \\
\hline 2709 & PDMS & & SW & & & E007143 & 02-Jul-80 & & $10028-17-8$ & TRITIUM & TRITIUM_DIST_LSC & HISTOR & 178 \\
\hline 2710 & PDMS & & SW & & & E007143 & 02-Jul-80 & & $10045-97-3$ & CS-137 & GAMMA_GS & HISTOR & 0.625 \\
\hline 2711 & PDMS & & SW & & & E001934 & 08-Jul-80 & & PU-239/240 & PU-239/240 & PUISO_IE_AEA & PNL-RO & 0.00017 \\
\hline 2712 & PDMS & & SW & & & E001934 & 08-Jul-80 & & $10045-97-3$ & CS-137 & GAMMA_GES & HISTOR & 0.007 \\
\hline 2713 & PDMS & & SW & & & E001934 & 08-Jul-80 & & 15046-84-1 & I-129 & I129_SEP_TEMS & 320 & 0.000029 \\
\hline 2714 & PDMS & & SW & & & E003934 & 08-Jul-80 & & $10045-97-3$ & CS-137 & GAMMA_GS & HISTOR & 0.003 \\
\hline 2715 & PDMS & & SW & & & E003934 & 08-Jul-80 & & PU-239/240 & PU-239/240 & PUISO_IE_AEA & PNL-RO & 0.000311 \\
\hline 2716 & PDMS & & SW & & & E015258 & 08-Jul-80 & & PU-239/240 & PU-239/240 & PUISO_IE_AEA & PNL-RO & 0.000169 \\
\hline 2717 & PDMS & & SW & & & E015258 & 08-Jul-80 & & $10045-97-3$ & CS-137 & GAMMA_GS & HISTOR & 0.004 \\
\hline 2718 & PDMS & & SW & & & E001259 & 08-Jul-80 & & $10045-97-3$ & CS-137 & GAMMA_GS & HISTOR & 0.003 \\
\hline 2719 & PDMS & & SW & & & E001259 & 08-Jul-80 & & PU-239/240 & PU-239/240 & PUISO_IE_AEA & PNL-RO & 0.000159 \\
\hline 2720 & PDMS & & SW & & & E001259 & 08-Jul-80 & & 15046-84-1 & $1-129$ & I129_SEP_TEMS & 320 & 0.0000074 \\
\hline 2721 & PDMS & & SW & & & E001983 & 22-Jul-80 & & $10045-97-3$ & CS-137 & GAMMA_GS & HISTOR & 0.005 \\
\hline \begin{tabular}{|l|}
2722 \\
\end{tabular} & PDMS & & SW & & & E003935 & 22-Jul-80 & & $10045-97-3$ & CS-137 & GAMMA_GS & HISTOR & 0.008 \\
\hline \begin{tabular}{|l|}
2723 \\
\end{tabular} & PDMS & & SW & & & E001260 & 22-Jul-80 & & $10045-97-3$ & CS-137 & GAMMA_GS & HISTOR & 0.006 \\
\hline 2724 & PDMS & & SW & & & E013968 & 22-Jul-80 & & $10045-97-3$ & CS-137 & GAMMA_GS & HISTOR & 0.005 \\
\hline 2725 & PDMS & & SW & & & E004532 & 28-Jul-80 & & $10098-97-2$ & SR-90 & SRISO_SEP_PRECIP_GPC & HISTOR & 0.261 \\
\hline 2726 & PDMS & & SW & & & E004532 & 28-Jul-80 & & $10045-97-3$ & CS-137 & GAMMA_GS & HISTOR & 0.00614 \\
\hline 2727 & PDMS & & SW & & & E004532 & 28-Jul-80 & & $10028-17-8$ & TRITIUM & TRITIUM_DIST_LSC & HISTOR & 142 \\
\hline 2728 & PDMS & & SW & & & E003574 & 28-Jul-80 & & $10045-97-3$ & CS-137 & GAMMA_GS & HISTOR & 0.00614 \\
\hline 2729 & PDMS & & SW & & & E044467 & 28-Jul-80 & & $10045-97-3$ & CS-137 & GAMMA_GS & HISTOR & 0.111 \\
\hline 2730 & PDMS & & SW & & & E044467 & 28-Jul-80 & & $10028-17-8$ & TRITIUM & TRITIUM_DIST_LSC & HISTOR & 47100 \\
\hline 2731 & PDMS & & SW & & & E002127 & 28-Jul-80 & & $10045-97-3$ & CS-137 & GAMMA_GS & HISTOR & -0.0264 \\
\hline 2732 & PDMS & & SW & & & E007171 & 30-Jul-80 & & $10028-17-8$ & TRITIUM & TRITIUM_DIST_LSC & HISTOR & 356 \\
\hline 2733 & PDMS & & SW & & & E007171 & 30-Jul-80 & & $10098-97-2$ & SR-90 & SRISO_SEP_PRECIP_GPC & HISTOR & 0.185 \\
\hline 2734 & PDMS & & SW & & & E007171 & 30-Jul-80 & & $10045-97-3$ & CS-137 & GAMMA_GS & HISTOR & 0.799 \\
\hline 2735 & PDMS & & SW & & & E003936 & 05-Aug-80 & & $10045-97-3$ & CS-137 & GAMMA_GS & HISTOR & 0.004 \\
\hline 2736 & PDMS & & SW & & & E001261 & 05-Aug-80 & & $10045-97-3$ & CS-137 & GAMMA_GS & HISTOR & 0.003 \\
\hline 2737 & PDMS & & SW & & & E013969 & 05-Aug-80 & & $10045-97-3$ & CS-137 & GAMMA_GS & HISTOR & 0.005 \\
\hline 2738 & PDMS & & SW & & & E016417 & 12-Aug-80 & & $10098-97-2$ & SR-90 & SRISO_SEP_PRECIP_GPC & HISTOR & 39.6 \\
\hline 2739 & PDMS & & SW & & & E016417 & 12-Aug-80 & & $10045-97-3$ & CS-137 & GAMMA_GS & HISTOR & 31.8 \\
\hline 2740 & PDMS & & SW & & & E012570 & 12-Aug-80 & & $10045-97-3$ & CS-137 & GAMMA_GS & HISTOR & 0.382 \\
\hline 2741 & PDMS & & SW & & & E022441 & 12-Aug-80 & & $10045-97-3$ & CS-137 & GAMMA_GS & HISTOR & 46.8 \\
\hline 2742 & PDMS & & SW & & & E022441 & 12-Aug-80 & & $10098-97-2$ & SR-90 & SRISO_SEP_PRECIP_GPC & HISTOR & 0.523 \\
\hline 2743 & PDMS & & SW & & & E014287 & 12-Aug-80 & & $10045-97-3$ & CS-137 & GAMMA_GS & HISTOR & 10.1 \\
\hline 2744 & PDMS & & SW & & & E014287 & 12-Aug-80 & & $10098-97-2$ & SR-90 & SRISO_SEP_PRECIP_GPC & HISTOR & 6.43 \\
\hline 2745 & PDMS & & SW & & & E003937 & 19-Aug-80 & & $10045-97-3$ & CS-137 & GAMMA_GS & HISTOR & 0.004 \\
\hline 2746 & PDMS & & SW & & & E001984 & 19-Aug-80 & & $10045-97-3$ & CS-137 & GAMMA_GS & HISTOR & 0.007 \\
\hline 2747 & PDMS & & SW & & & E013970 & 19-Aug-80 & & $10045-97-3$ & CS-137 & GAMMA_GS & HISTOR & 0.004 \\
\hline 2748 & PDMS & & SW & & & E001262 & 19-Aug-80 & & $10045-97-3$ & CS-137 & GAMMA_GS & HISTOR & 0.005 \\
\hline 2749 & PDMS & & SW & & & E004533 & 25-Aug-80 & & $10045-97-3$ & CS-137 & GAMMA_GS & HISTOR & 0.0832 \\
\hline 2750 & PDMS & & SW & & & E004533 & 25-Aug-80 & & $10028-17-8$ & TRITIUM & TRITIUM_DIST_LSC & HISTOR & 58.4 \\
\hline 2751 & PDMS & & SW & & & E001624 & 25-Aug-80 & & $10098-97-2$ & SR-90 & SRISO_SEP_PRECIP_GPC & HISTOR & 0.176 \\
\hline 2752 & PDMS & & SW & & & E001624 & 25-Aug-80 & & $10028-17-8$ & TRITIUM & TRITIUM_DIST_LSC & HISTOR & 237 \\
\hline 2753 & PDMS & & SW & & & E001624 & 25-Aug-80 & & $10045-97-3$ & CS-137 & GAMMA_GS & HISTOR & 0.593 \\
\hline 2754 & PDMS & & SW & & & E014826 & 25-Aug-80 & & $10098-97-2$ & SR-90 & SRISO_SEP_PRECIP_GPC & HISTOR & 0 \\
\hline 2755 & PDMS & & SW & & & E014826 & 25-Aug-80 & & $10045-97-3$ & CS-137 & GAMMA_GS & HISTOR & 0.00789 \\
\hline 2756 & PDMS & & SW & & & E014826 & 25-Aug-80 & & $10028-17-8$ & TRITIUM & TRITIUM_DIST_LSC & HISTOR & 39200 \\
\hline 2757 & PDMS & & SW & & & E014826 & 25-Aug-80 & & $14133-76-7$ & TC-99 & TC99_SEP_LSC & HISTOR & 10.5 \\
\hline
\end{tabular}




\begin{tabular}{|c|c|c|c|c|c|c|c|c|c|c|c|c|c|}
\hline & A & $\mathrm{B}$ & $\mathrm{C}$ & $\mathrm{D}$ & $E$ & $\mathrm{~F}$ & $\mathrm{G}$ & $\mathrm{H}$ & $\mathrm{I}$ & $\mathrm{J}$ & $\mathrm{K}$ & $\mathrm{L}$ & $\mathrm{M}$ \\
\hline 1 & SAMPLE_NUM_ASSIGNED_TO & SAMPLER & MEDIA & BIOTA_MEDIA & TAG_ID & SAMP_NUM & SAMP_DATE_TIME & SAMP_DATE_TIME_ON & CON_ID & CON_SHORT_NAME & METHOD_NAME & LAB_CODE & VALUE_RPTD \\
\hline 2758 & PDMS & & SW & & & E002129 & 25-Aug-80 & & $10045-97-3$ & CS-137 & GAMMA_GS & HISTOR & 0.00307 \\
\hline 2759 & PDMS & & SW & & & E007105 & 27-Aug-80 & & $10028-17-8$ & TRITIUM & TRITIUM_DIST_LSC & HISTOR & 276 \\
\hline 2760 & PDMS & & SW & & & E007105 & 27-Aug-80 & & $10045-97-3$ & CS-137 & GAMMA_GS & HISTOR & 0.0165 \\
\hline 2761 & PDMS & & SW & & & E001985 & 02-Sep-80 & & $10045-97-3$ & CS-137 & GAMMA_GS & HISTOR & 0.005 \\
\hline 2762 & PDMS & & SW & & & E003938 & 02-Sep-80 & & $10045-97-3$ & CS-137 & GAMMA_GS & HISTOR & 0.004 \\
\hline 2763 & PDMS & & SW & & & E001263 & $02-\operatorname{Sep}-80$ & & $10045-97-3$ & CS-137 & GAMMA_GS & HISTOR & 0.004 \\
\hline 2764 & PDMS & & SW & & & E013971 & 02-Sep-80 & & $10045-97-3$ & CS-137 & GAMMA_GS & HISTOR & 0.003 \\
\hline 2765 & PDMS & & SW & & & E001986 & $16-S e p-80$ & & PU-239/240 & PU-239/240 & PUISO_IE_AEA & PNL-RO & 0.000182 \\
\hline 2766 & PDMS & & SW & & & E001986 & $16-$ Sep-80 & & $10045-97-3$ & CS-137 & GAMMA_GS & HISTOR & 0.003 \\
\hline 2767 & PDMS & & SW & & & E003939 & 16-Sep-80 & & PU-239/240 & PU-239/240 & PUISO_IE_AEA & PNL-RO & 0.000242 \\
\hline 2768 & PDMS & & SW & & & E003939 & $16-$ Sep-80 & & $10045-97-3$ & CS-137 & GAMMA_GS & HISTOR & 0.002 \\
\hline 2769 & PDMS & & SW & & & E001264 & 16-Sep-80 & & $10045-97-3$ & CS-137 & GAMMA_GS & HISTOR & 0.006 \\
\hline 2770 & PDMS & & SW & & & E001264 & 16-Sep-80 & & PU-239/240 & PU-239/240 & PUISO_IE_AEA & PNL-RO & 0.000107 \\
\hline 2771 & PDMS & & SW & & & E013972 & 16-Sep-80 & & PU-239/240 & PU-239/240 & PUISO_IE_AEA & PNL-RO & 0.000262 \\
\hline 2772 & PDMS & & SW & & & E013972 & $16-S e p-80$ & & $10045-97-3$ & CS-137 & GAMMA_GS & HISTOR & 0.002 \\
\hline 2773 & PDMS & & SW & & & E016352 & 23-Sep-80 & & $10098-97-2$ & SR-90 & SRISO_SEP_PRECIP_GPC & HISTOR & 18.8 \\
\hline 2774 & PDMS & & SW & & & E016352 & 23-Sep-80 & & $10045-97-3$ & CS-137 & GAMMA_GS & HISTOR & 7.04 \\
\hline 2775 & PDMS & & SW & & & E004534 & 29-Sep-80 & & $10045-97-3$ & CS-137 & GAMMA_GS & HISTOR & -0.0167 \\
\hline 2776 & PDMS & & SW & & & E004534 & 29-Sep-80 & & $10028-17-8$ & TRITIUM & TRITIUM_DIST_LSC & HISTOR & 175 \\
\hline 2777 & PDMS & & SW & & & E003575 & 29-Sep-80 & & $10045-97-3$ & CS-137 & GAMMA_GS & HISTOR & -0.0109 \\
\hline 2778 & PDMS & & SW & & & E044468 & 29-Sep-80 & & $10045-97-3$ & CS-137 & GAMMA_GS & HISTOR & -0.095 \\
\hline 2779 & PDMS & & SW & & & E044468 & 29-Sep-80 & & $10028-17-8$ & TRITIUM & TRITIUM_DIST_LSC & HISTOR & 41800 \\
\hline 2780 & PDMS & & SW & & & E002130 & 29-Sep-80 & & $10045-97-3$ & CS-137 & GAMMA_GS & HISTOR & -0.0353 \\
\hline 2781 & PDMS & & SW & & & E001987 & 30-Sep-80 & & $10045-97-3$ & CS-137 & GAMMA_GS & HISTOR & 0.005 \\
\hline 2782 & PDMS & & SW & & & E001987 & 30-Sep-80 & & 15046-84-1 & I-129 & I129_SEP_TEMS & 320 & 0.000046 \\
\hline 2783 & PDMS & & SW & & & E003940 & 30-Sep-80 & & $10045-97-3$ & CS-137 & GAMMA_GS & HISTOR & 0.005 \\
\hline 2784 & PDMS & & SW & & & E001265 & $30-$ Sep-80 & & $10045-97-3$ & CS-137 & GAMMA_GS & HISTOR & 0.007 \\
\hline 2785 & PDMS & & SW & & & E001265 & $30-$ Sep-80 & & 15046-84-1 & I-129 & I129_SEP_TEMS & 320 & 0.0000042 \\
\hline 2786 & PDMS & & SW & & & E013973 & $30-$ Sep-80 & & $10045-97-3$ & CS-137 & GAMMA_GS & HISTOR & 0.004 \\
\hline 2787 & PDMS & & SW & & & E007140 & 01-Oct-80 & & $10028-17-8$ & TRITIUM & TRITIUM_DIST_LSC & HISTOR & 161 \\
\hline 2788 & PDMS & & SW & & & E007140 & 01-Oct-80 & & $10045-97-3$ & CS-137 & GAMMA_GS & HISTOR & 0.194 \\
\hline 2789 & PDMS & & SW & & & E001988 & 14-Oct-80 & & $10045-97-3$ & CS-137 & GAMMA_GS & HISTOR & 0.005 \\
\hline 2790 & PDMS & & SW & & & E003941 & 14-Oct-80 & & $10045-97-3$ & CS-137 & GAMMA_GS & HISTOR & 0.001 \\
\hline 2791 & PDMS & & SW & & & E001266 & 14-Oct-80 & & $10045-97-3$ & CS-137 & GAMMA_GS & HISTOR & 0.004 \\
\hline 2792 & PDMS & & SW & & & E013974 & 14-Oct-80 & & $10045-97-3$ & CS-137 & GAMMA_GS & HISTOR & 0.002 \\
\hline 2793 & PDMS & & SW & & & E004535 & $20-$ Oct-80 & & $10098-97-2$ & SR-90 & SRISO_SEP_PRECIP_GPC & HISTOR & 0.315 \\
\hline 2794 & PDMS & & SW & & & E004535 & 20-Oct-80 & & $10045-97-3$ & CS-137 & GAMMA_GS & HISTOR & 2.46 \\
\hline 2795 & PDMS & & SW & & & E004535 & 20-Oct-80 & & $10028-17-8$ & TRITIUM & TRITIUM_DIST_LSC & HISTOR & 402 \\
\hline 2796 & PDMS & & SW & & & E003576 & $27-$ Oct-80 & & $10045-97-3$ & CS-137 & GAMMA_GS & HISTOR & 1.86 \\
\hline 2797 & PDMS & & SW & & & E044469 & $27-$ Oct-80 & & $10045-97-3$ & CS-137 & GAMMA_GS & HISTOR & 1.5 \\
\hline 2798 & PDMS & & SW & & & E044469 & $27-$ Oct-80 & & $10028-17-8$ & TRITIUM & TRITIUM_DIST_LSC & HISTOR & 39200 \\
\hline 2799 & PDMS & & SW & & & E002131 & $27-$ Oct-80 & & $10045-97-3$ & CS-137 & GAMMA_GS & HISTOR & -0.0021 \\
\hline 2800 & PDMS & & SW & & & E001989 & 28-Oct-80 & & $10045-97-3$ & CS-137 & GAMMA_GS & HISTOR & 0.005 \\
\hline 2801 & PDMS & & SW & & & E003942 & 28-Oct-80 & & $10045-97-3$ & CS-137 & GAMMA_GS & HISTOR & 0.001 \\
\hline 2802 & PDMS & & SW & & & E013975 & 28-Oct-80 & & $10045-97-3$ & CS-137 & GAMMA_GS & HISTOR & 0.001 \\
\hline 2803 & PDMS & & SW & & & E001267 & 28-Oct-80 & & $10045-97-3$ & CS-137 & GAMMA_GS & HISTOR & 0.006 \\
\hline 2804 & PDMS & & SW & & & E007074 & 29-Oct-80 & & $10028-17-8$ & TRITIUM & TRITIUM_DIST_LSC & HISTOR & 459 \\
\hline 2805 & PDMS & & SW & & & E007074 & $29-$ Oct-80 & & $10098-97-2$ & SR-90 & SRISO_SEP_PRECIP_GPC & HISTOR & 0.221 \\
\hline 2806 & PDMS & & SW & & & E007074 & 29-Oct-80 & & $10045-97-3$ & CS-137 & GAMMA_GS & HISTOR & -0.0608 \\
\hline 2807 & PDMS & & SW & & & E003943 & $11-N o v-80$ & & $10045-97-3$ & CS-137 & GAMMA_GS & HISTOR & 0.003 \\
\hline 2808 & PDMS & & SW & & & E001935 & 11-Nov-80 & & $10045-97-3$ & CS-137 & GAMMA_GS & HISTOR & 0.005 \\
\hline 2809 & PDMS & & SW & & & E013976 & 11-Nov-80 & & $10045-97-3$ & CS-137 & GAMMA_GS & HISTOR & 0.001 \\
\hline
\end{tabular}




\begin{tabular}{|c|c|c|c|c|c|c|c|c|c|c|c|c|c|}
\hline & A & $\mathrm{B}$ & $\mathrm{C}$ & $\mathrm{D}$ & $E$ & $\mathrm{~F}$ & $\mathrm{G}$ & $\mathrm{H}$ & $\mathrm{I}$ & $\mathrm{J}$ & $\mathrm{K}$ & $\mathrm{L}$ & $\mathrm{M}$ \\
\hline 1 & SAMPLE_NUM_ASSIGNED_TO & SAMPLER & MEDIA & BIOTA_MEDIA & TAG_ID & SAMP_NUM & SAMP_DATE_TIME & SAMP_DATE_TIME_ON & CON_ID & CON_SHORT_NAME & METHOD_NAME & LAB_CODE & VALUE_RPTD \\
\hline 2810 & PDMS & & SW & & & E001268 & $11-$ Nov-80 & & $10045-97-3$ & CS-137 & GAMMA_GS & HISTOR & 0.003 \\
\hline 2811 & PDMS & & SW & & & E016353 & 18-Nov-80 & & $10098-97-2$ & SR-90 & SRISO_SEP_PRECIP_GPC & HISTOR & 2.63 \\
\hline 2812 & PDMS & & SW & & & E016353 & 18-Nov-80 & & $10045-97-3$ & CS-137 & GAMMA_GS & HISTOR & 29.7 \\
\hline 2813 & PDMS & & SW & & & E012571 & 18-Nov-80 & & $10045-97-3$ & CS-137 & GAMMA_GS & HISTOR & -0.139 \\
\hline 2814 & PDMS & & SW & & & E022442 & 18-Nov-80 & & $10045-97-3$ & CS-137 & GAMMA_GS & HISTOR & 23 \\
\hline 2815 & PDMS & & SW & & & E022442 & 18-Nov-80 & & $10098-97-2$ & SR-90 & SRISO_SEP_PRECIP_GPC & HISTOR & 0.369 \\
\hline 2816 & PDMS & & SW & & & E014288 & 18-Nov-80 & & $10045-97-3$ & CS-137 & GAMMĀ_GS & HISTOR & 0.832 \\
\hline 2817 & PDMS & & SW & & & E014288 & 18-Nov-80 & & $10098-97-2$ & SR-90 & SRISO_SEP_PRECIP_GPC & HISTOR & 3.09 \\
\hline 2818 & PDMS & & SW & & & E001625 & 24-Nov-80 & & $10098-97-2$ & SR-90 & SRISO_SEP_PRECIP_GPC & HISTOR & 0.275 \\
\hline 2819 & PDMS & & SW & & & E001625 & 24-Nov-80 & & $10028-17-8$ & TRITIUM & TRITIUM_DIST_LSC & HISTOR & 484 \\
\hline 2820 & PDMS & & SW & & & E001625 & 24-Nov-80 & & $10045-97-3$ & CS-137 & GAMMA_GS & HISTOR & -0.0039 \\
\hline 2821 & PDMS & & SW & & & E014827 & 24-Nov-80 & & $10098-97-2$ & SR-90 & SRISO_SEP_PRECIP_GPC & HISTOR & 0 \\
\hline 2822 & PDMS & & SW & & & E014827 & 24-Nov-80 & & $10045-97-3$ & CS-137 & GAMMA_GS & HISTOR & 0.032 \\
\hline 2823 & PDMS & & SW & & & E014827 & 24-Nov-80 & & $10028-17-8$ & TRITIUM & TRITIUM_DIST_LSC & HISTOR & 29300 \\
\hline 2824 & PDMS & & SW & & & E014827 & 24-Nov-80 & & 14133-76-7 & TC-99 & TC99_SEP_LSC & HISTOR & 7.76 \\
\hline 2825 & PDMS & & SW & & & E002132 & 24-Nov-80 & & $10045-97-3$ & CS-137 & GAMMA_GS & HISTOR & -0.0554 \\
\hline 2826 & PDMS & & SW & & & E001990 & $25-N o v-80$ & & PU-239/240 & PU-239/240 & PUISO_IE_AEA & PNL-RO & 0.0000976 \\
\hline 2827 & PDMS & & SW & & & E001990 & $25-N o v-80$ & & $10045-97-3$ & CS-137 & GAMMA_GS & HISTOR & 0.002 \\
\hline 2828 & PDMS & & SW & & & E001990 & $25-N o v-80$ & & 15046-84-1 & 1-129 & I129_SEP_TEMS & 320 & 0.00005 \\
\hline 2829 & PDMS & & SW & & & E003944 & 25-Nov-80 & & $10045-97-3$ & CS-137 & GAMMA_GS & HISTOR & 0.006 \\
\hline 2830 & PDMS & & SW & & & E003944 & $25-N o v-80$ & & PU-239/240 & PU-239/240 & PUISO_IE_AEA & PNL-RO & 0.0000738 \\
\hline 2831 & PDMS & & SW & & & E001269 & 25-Nov-80 & & $10045-97-3$ & CS-137 & GAMMA_GS & HISTOR & 0.003 \\
\hline 2832 & PDMS & & SW & & & E001269 & 25-Nov-80 & & PU-239/240 & PU-239/240 & PUISO_IE_AEA & PNL-RO & 0.000202 \\
\hline 2833 & PDMS & & SW & & & E001269 & 25-Nov-80 & & 15046-84-1 & I-129 & I129_SEP_TEMS & 320 & 0.0000063 \\
\hline 2834 & PDMS & & SW & & & E013977 & 25-Nov-80 & & PU-239/240 & PU-239/240 & PUISO_IE_AEA & PNL-RO & 0.000339 \\
\hline 2835 & PDMS & & SW & & & E013977 & 25-Nov-80 & & $10045-97-3$ & CS-137 & GAMMA_GS & HISTOR & 0.003 \\
\hline 2836 & PDMS & & SW & & & E007008 & 26-Nov-80 & & $10028-17-8$ & TRITIUM & TRITIUM_DIST_LSC & HISTOR & 235 \\
\hline 2837 & PDMS & & SW & & & E007008 & 26-Nov-80 & & $10045-97-3$ & CS-137 & GAMMA_GS & HISTOR & 0.312 \\
\hline 2838 & PDMS & & SW & & & E004536 & 01-Dec-80 & & $10045-97-3$ & CS-137 & GAMMA_GS & HISTOR & 0.809 \\
\hline 2839 & PDMS & & SW & & & E004536 & 01-Dec-80 & & $10028-17-8$ & TRITIUM & TRITIUM_DIST_LSC & HISTOR & 330 \\
\hline 2840 & PDMS & & SW & & & E001991 & 09-Dec-80 & & $10045-97-3$ & CS-137 & GAMMA_GS & PNL-RO & 0.004 \\
\hline 2841 & PDMS & & SW & & & E003945 & 09-Dec-80 & & $10045-97-3$ & CS-137 & GAMMA_GS & PNL-RO & 0.002 \\
\hline 2842 & PDMS & & SW & & & E001270 & 09-Dec-80 & & $10045-97-3$ & CS-137 & GAMMA_GS & PNL-RO & 0.003 \\
\hline 2843 & PDMS & & SW & & & E013978 & 09-Dec-80 & & $10045-97-3$ & CS-137 & GAMMA_GS & PNL-RO & 0.001 \\
\hline 2844 & PDMS & & SW & & & E016354 & 17-Dec-80 & & $10098-97-2$ & SR-90 & SRISO_SEP_PRECIP_GPC & HISTOR & 2.63 \\
\hline 2845 & PDMS & & SW & & & E016354 & 17-Dec-80 & & $10045-97-3$ & CS-137 & GAMMA_GS & HISTOR & 107 \\
\hline 2846 & PDMS & & SW & & & E005416 & 23-Dec-80 & & $10045-97-3$ & CS-137 & GAMMA_GS & PNL-RO & 0.003 \\
\hline 2847 & PDMS & & SW & & & E001992 & 23-Dec-80 & & $10045-97-3$ & CS-137 & GAMMA_GS & PNL-RO & 0.003 \\
\hline 2848 & PDMS & & SW & & & E001271 & 23-Dec-80 & & $10045-97-3$ & CS-137 & GAMMA_GS & PNL-RO & 0.01 \\
\hline 2849 & PDMS & & SW & & & E013979 & 23-Dec-80 & & $10045-97-3$ & CS-137 & GAMMA_GS & PNL-RO & 0.002 \\
\hline 2850 & PDMS & & SW & & & E003577 & 29-Dec-80 & & $10045-97-3$ & CS-137 & GAMMA_GS & HISTOR & -0.00627 \\
\hline 2851 & PDMS & & SW & & & E037542 & 29-Dec-80 & & $10045-97-3$ & CS-137 & GAMMA_GS & HISTOR & 0.0138 \\
\hline 2852 & PDMS & & SW & & & E037542 & 29-Dec-80 & & $10028-17-8$ & TRITIUM & TRITIUM_DIST_LSC & HISTOR & 32200 \\
\hline 2853 & PDMS & & SW & & & E002133 & 29-Dec-80 & & $10045-97-3$ & CS-137 & GAMMA_GS & HISTOR & 0.315 \\
\hline 2854 & PDMS & & SW & & & E004537 & 30-Dec-80 & & $10028-17-8$ & TRITIUM & TRITIUM_DIST_LSC & HISTOR & 407 \\
\hline 2855 & PDMS & & SW & & & E004537 & 30-Dec-80 & & $10045-97-3$ & CS-137 & GAMMA_GS & HISTOR & -0.0324 \\
\hline 2856 & PDMS & & SW & & & E007042 & 30-Dec-80 & & $10028-17-8$ & TRITIUM & TRITIUM_DIST_LSC & HISTOR & 276 \\
\hline 2857 & PDMS & & SW & & & E007042 & 30-Dec-80 & & $10045-97-3$ & CS-137 & GAMMA_GS & HISTOR & 0.758 \\
\hline 2858 & PDMS & & SW & & & E001993 & 06-Jan-81 & & $10045-97-3$ & CS-137 & GAMMA_GS & HISTOR & 0.004 \\
\hline 2859 & PDMS & & SW & & & E001993 & 06-Jan-81 & & 15046-84-1 & I-129 & I129_SEP_TEMS & 320 & 0.000031 \\
\hline 2860 & PDMS & & SW & & & E003946 & 06-Jan-81 & & $10045-97-3$ & CS-137 & GAMMA_GS & HISTOR & 0.004 \\
\hline 2861 & PDMS & & SW & & & E013980 & 06-Jan-81 & & $10045-97-3$ & CS-137 & GAMMA_GS & HISTOR & 0.003 \\
\hline
\end{tabular}




\begin{tabular}{|c|c|c|c|c|c|c|c|c|c|c|c|c|c|}
\hline & A & $\mathrm{B}$ & $\mathrm{c}$ & $\mathrm{D}$ & $\mathrm{E}$ & $\mathrm{F}$ & G & $\mathrm{H}$ & $\mathrm{I}$ & $\mathrm{J}$ & $\mathrm{K}$ & $\mathrm{L}$ & $\mathrm{M}$ \\
\hline 1 & SAMPLE_NUM_ASSIGNED_TO & SAMPLER & MEDIA & BIOTA_MEDIA & TAG_ID & SAMP_NUM & SAMP_DATE_TIME & SAMP_DATE_TIME_ON & CON_ID & CON_SHORT_NAME & METHOD_NAME & LAB_CODE & \begin{tabular}{|l|} 
VALUE_RPTD \\
\end{tabular} \\
\hline 2862 & PDMS & & SW & & & E001272 & 06-Jan-81 & & $10045-97-3$ & CS-137 & GAMMA_GS & HISTOR & 0.005 \\
\hline 2863 & PDMS & & sW & & & E001272 & 06-Jan-81 & & 15046-84-1 & I-129 & I129_SEP_TEMS & 320 & 0.0000082 \\
\hline 2864 & PDMS & & sW & & & E016355 & 13-Jan-81 & & $10098-97-2$ & SR-90 & SRISO_SËP_PRECIP_GPC & HISTOR & 2.32 \\
\hline 2865 & PDMS & & sW & & & E016355 & 13-Jan-81 & & $10045-97-3$ & CS-137 & GAMMA_GS & HISTOR & 93.7 \\
\hline 2866 & PDMS & & sW & & & E002134 & 19-Jan-81 & & $10045-97-3$ & CS-137 & GAMMA_GS & HISTOR & 0.469 \\
\hline \begin{tabular}{|l|l|}
2867 & \\
\end{tabular} & PDMS & & sW & & & E001994 & 20-Jan-81 & & $10045-97-3$ & CS-137 & GAMMA_GS & HISTOR & 0.002 \\
\hline 2868 & PDMS & & SW & & & E003947 & 20-Jan-81 & & $10045-97-3$ & CS-137 & GAMMA_GS & HISTOR & 0.002 \\
\hline 2869 & PDMS & & SW & & & E001273 & 20-Jan-81 & & $10045-97-3$ & CS-137 & GAMMA_GS & HISTOR & 0.003 \\
\hline 2870 & PDMS & & sW & & & E013981 & 20-Jan-81 & & $10045-97-3$ & CS-137 & GAMMA GS & HISTOR & 0.003 \\
\hline 2871 & PDMS & & SW & & & E021411 & 26-Jan-81 & & $10098-97-2$ & SR-90 & SRISO_SEEP_PRECIP_GPC & HISTOR & 0.279 \\
\hline 2872 & PDMS & & SW & & & E021411 & 26-Jan-81 & & $10028-17-8$ & TRITIUM & TRITIUM_DIST_LSC & HISTOR & 341 \\
\hline 2873 & PDMS & & SW & & & E014828 & 26-Jan-81 & & 10098-97-2 & SR-90 & SRISO_SEP_PRECIP_GPC & HISTOR & 0.036 \\
\hline 2874 & PDMS & & SW & & & E014828 & 26-Jan-81 & & $10045-97-3$ & CS-137 & GAMMA_GS & HISTOR & -0.0772 \\
\hline 2875 & PDMS & & SW & & & E014828 & 26-Jan-81 & & $10028-17-8$ & TRITIUM & TRITIUM_DIST_LSC & HISTOR & 39900 \\
\hline 2876 & PDMS & & SW & & & E024493 & 26-Jan-81 & & $10098-97-2$ & SR-90 & SRISO_SEP_PRECIP_GPC & HISTOR & 0.198 \\
\hline \begin{tabular}{|l|l|}
2877 & \\
\end{tabular} & PDMS & & SW & & & E001995 & 03-Feb-81 & & $10045-97-3$ & CS-137 & GAMMA_GS & HISTOR & 0.003 \\
\hline 2878 & PDMS & & sW & & & E001995 & 03-Feb-81 & & $15046-84-1$ & $1-129$ & I129_SEP_TEMS & 320 & 0.0000088 \\
\hline \begin{tabular}{|l|}
2879 \\
\end{tabular} & PDMS & & sW & & & E005417 & 03-Feb-81 & & $10045-97-3$ & CS-137 & GAMMA_GS & HISTOR & 0.004 \\
\hline 2880 & PDMS & & SW & & & E001357 & 03-Feb-81 & & $10028-17-8$ & TRITIUM & TRITIUM_ELELECT_LSC & HISTOR & 224 \\
\hline 2881 & PDMS & & SW & & & E001357 & 03-Feb-81 & & $10098-97-2$ & SR-90 & SRISO_SEP_PRECIP_GPC & HISTOR & 0.176 \\
\hline 2882 & PDMS & & SW & & & E001357 & 03-Feb-81 & & $10045-97-3$ & CS-137 & GAMMA_GS & HISTOR & 0.65 \\
\hline 2883 & PDMS & & SW & & & E013982 & 03-Feb-81 & & $10045-97-3$ & CS-137 & GAMMA_GS & HISTOR & 0.002 \\
\hline 2884 & PDMS & & SW & & & E001274 & 03-Feb-81 & & $10045-97-3$ & CS-137 & GAMMA_GS & HISTOR & 0.005 \\
\hline 2885 & PDMS & & SW & & & E001274 & 03-Feb-81 & & 15046-84-1 & I-129 & I129_SEP_TEMS & 320 & 0.0000059 \\
\hline 2886 & PDMS & & SW & & & E004356 & 03-Feb-81 & & $10028-17-8$ & TRITIUM & TRITIUM_ELECT_LSC & HISTOR & 246 \\
\hline \begin{tabular}{|l|l|}
2887 & \\
\end{tabular} & PDMS & & SW & & & E004356 & 03-Feb-81 & & $10098-97-2$ & SR-90 & SRISO_SEPP_PRËCIP_GPC & HISTOR & 0.162 \\
\hline 2888 & PDMS & & SW & & & E004356 & 03-Feb-81 & & $10045-97-3$ & CS-137 & GAMMA_GS & HISTOR & 1.13 \\
\hline 2889 & PDMS & & SW & & & E016356 & 10-Feb-81 & & $10098-97-2$ & SR-90 & SRISO_SEPP_PRECIP_GPC & HISTOR & 1.19 \\
\hline 2890 & PDMS & & SW & & & E016356 & 10-Feb-81 & & $10045-97-3$ & CS-137 & GAMMA_GS & HISTOR & 23.7 \\
\hline 2891 & PDMS & & SW & & & E012572 & 10-Feb-81 & & $10045-97-3$ & CS-137 & GAMMA_GS & HISTOR & 1.99 \\
\hline 2892 & PDMS & & sW & & & E022443 & 10-Feb-81 & & $10045-97-3$ & CS-137 & GAMMA_GS & HISTOR & 40.2 \\
\hline 2893 & PDMS & & SW & & & E022443 & 10-Feb-81 & & $10098-97-2$ & SR-90 & SRISO_SEPP_PRECIP_GPC & HISTOR & 0.568 \\
\hline 2894 & PDMS & & SW & & & E014289 & 10-Feb-81 & & $10045-97-3$ & CS-137 & GAMMA_GS & HISTOR & 6.4 \\
\hline 2895 & PDMS & & SW & & & E014289 & 10-Feb-81 & & $10098-97-2$ & SR-90 & SRISO_SEEP_PRECIP_GPC & HISTOR & 3.61 \\
\hline 2896 & PDMS & & SW & & & E001996 & 17-Feb-81 & & $10045-97-3$ & CS-137 & GAMMĀGS & HISTOR & 0.02 \\
\hline \begin{tabular}{|l|l|}
2897 & \\
\end{tabular} & PDMS & & sW & & & E005418 & 17-Feb-81 & & $10045-97-3$ & CS-137 & GAMMA_GS & HISTOR & 0.004 \\
\hline 2898 & PDMS & & SW & & & E001275 & 17-Feb-81 & & $10045-97-3$ & CS-137 & GAMMA_GS & HISTOR & 0.005 \\
\hline 2899 & PDMS & & SW & & & E013906 & 17-Feb-81 & & $10045-97-3$ & CS-137 & GAMMA_GS & HISTOR & 0.002 \\
\hline 2900 & PDMS & & SW & & & E014829 & 23-Feb-81 & & $10098-97-2$ & SR-90 & SRISO_SEPP_PRECIP_GPC & HISTOR & 0.195 \\
\hline 2901 & PDMS & & SW & & & E014829 & 23-Feb-81 & & $10028-17-8$ & TRITIUM & TRITIUM_DISTT_LSC & HISTOR & 34000 \\
\hline 2902 & PDMS & & SW & & & E002135 & 23-Feb-81 & & $10045-97-3$ & CS-137 & GAMMA_GS & HISTOR & 0.00223 \\
\hline 2903 & PDMS & & SW & & & E010746 & 02-Mar-81 & & $10028-17-8$ & TRITIUM & TRITIUM_ELELCT_LSC & HISTOR & 181 \\
\hline 2904 & PDMS & & SW & & & E010746 & 02-Mar-81 & & $10045-97-3$ & CS-137 & GAMMA_GS & HISTOR & -0.0599 \\
\hline 2905 & PDMS & & SW & & & E001997 & 03-Mar-81 & & $10045-97-3$ & CS-137 & GAMMA_GS & HISTOR & 0.002 \\
\hline 2906 & PDMS & & SW & & & E001997 & 03-Mar-81 & & 15046-84-1 & I-129 & I129_SEP_TEMS & 320 & 0.00008 \\
\hline 2907 & PDMS & & SW & & & E003948 & 03-Mar-81 & & $10045-97-3$ & CS-137 & GAMMA_ĒS & HISTOR & 0.006 \\
\hline 2908 & PDMS & & SW & & & E001276 & 03-Mar-81 & & $10045-97-3$ & CS-137 & GAMMA_GS & HISTOR & 0.003 \\
\hline 2909 & PDMS & & SW & & & E001276 & 03-Mar-81 & & 15046-84-1 & I-129 & I129_SEP_TEMS & 320 & 0.0000059 \\
\hline 2910 & PDMS & & SW & & & E013907 & 03-Mar-81 & & $10045-97-3$ & CS-137 & GAMMA_ḠS & HISTOR & 0.007 \\
\hline 2911 & PDMS & & SW & & & E004357 & 03-Mar-81 & & $10028-17-8$ & TRITIUM & TRITIUM_ELECT_LSC & HISTOR & 283 \\
\hline 2912 & PDMS & & sW & & & E004357 & 03-Mar-81 & & $10045-97-3$ & CS-137 & GAMMA_GS & HISTOR & 0.334 \\
\hline 2913 & PDMS & & SW & & & E016357 & 10-Mar-81 & & $10098-97-2$ & SR-90 & SRISO_SEP_PRECIP_GPC & HISTOR & 3.84 \\
\hline
\end{tabular}




\begin{tabular}{|c|c|c|c|c|c|c|c|c|c|c|c|c|c|}
\hline & $\bar{A}$ & $\mathrm{~B}$ & $\bar{C}$ & $\mathrm{D}$ & $\mathrm{E}$ & $\mathrm{F}$ & $\bar{G}$ & $\mathrm{H}$ & 1 & $\mathrm{~J}$ & $\bar{K}$ & $\bar{L}$ & $\mathrm{M}$ \\
\hline 1 & \begin{tabular}{|l} 
SAMPLE_NUM_ASSIGNED_TO \\
\end{tabular} & SAMPLER & MEDIA & BIOTA_MEDIA & TAG_ID & SAMP_NUM & SAMP_DATE_TIME & SAMP_DATE_TIME_ON & CON_ID & CON_SHORT_NAME & METHOD_NAME & LAB_CODE & VALUE_RPTD \\
\hline 2914 & PDMS & & SW & & & E016357 & 10-Mar-81 & & $10045-97-3$ & CS-137 & GAMMA_GS & HISTOR & 17.3 \\
\hline 2915 & PDMS & & SW & & & E010726 & 12-Mar-81 & & $10098-97-2$ & SR-90 & 905.0_SR_GPC & HISTOR & 0.0257 \\
\hline 2916 & PDMS & & SW & & & E010726 & 12-Mar-81 & & $10028-17-8$ & TRITIUM & 906.0_H3_LSC & HISTOR & 98.6 \\
\hline 2917 & PDMS & & SW & & & E001341 & 16-Mar-81 & & $10045-97-3$ & CS-137 & 901.1_GAMMA_GS & HISTOR & 1.03 \\
\hline 2918 & PDMS & & SW & & & E001341 & 16-Mar-81 & & $10098-97-2$ & SR-90 & 905.0_SR_GPC & HISTOR & 0.225 \\
\hline 2919 & PDMS & & SW & & & E001341 & 16-Mar-81 & & $10028-17-8$ & TRITIUM & TRITIŪM_ELECT_LSC & HISTOR & 75.5 \\
\hline 2920 & PDMS & & SW & & & E001998 & 17-Mar-81 & & $10045-97-3$ & CS-137 & GAMMA_GS & HISTOR & 0.003 \\
\hline 2921 & PDMS & & SW & & & E003949 & 17-Mar-81 & & $10045-97-3$ & CS-137 & GAMMA_GS & HISTOR & 0.001 \\
\hline 2922 & PDMS & & SW & & & E001277 & 17-Mar-81 & & $10045-97-3$ & CS-137 & GAMMA_GS & HISTOR & 0.004 \\
\hline 2923 & PDMS & & SW & & & E013908 & 17-Mar-81 & & $10045-97-3$ & CS-137 & GAMMA_GS & HISTOR & 0.005 \\
\hline 2924 & PDMS & & SW & & & E014830 & 23-Mar-81 & & $10098-97-2$ & SR-90 & SRISO_SEP_PRECIP_GPC & HISTOR & 0.045 \\
\hline 2925 & PDMS & & SW & & & E014830 & 23-Mar-81 & & 10028-17-8 & TRITIUM & TRITIUM_DIST_LSC & HISTOR & 35800 \\
\hline 2926 & PDMS & & SW & & & E002136 & 23-Mar-81 & & $10045-97-3$ & CS-137 & GAMMA_GS & HISTOR & 0.0847 \\
\hline 2927 & PDMS & & SW & & & E001999 & 31-Mar-81 & & $10045-97-3$ & CS-137 & GAMMA_GS & PNL-KI & 0.01 \\
\hline 2928 & PDMS & & SW & & & E001999 & 31-Mar-81 & & 15046-84-1 & $1-129$ & I129_SEP_TEMS & 320 & 0.000057 \\
\hline 2929 & PDMS & & SW & & & E003874 & 31-Mar-81 & & $10045-97-3$ & CS-137 & GAMMA_GS & HISTOR & 0.001 \\
\hline 2930 & PDMS & & SW & & & E010747 & 31-Mar-81 & & $10028-17-8$ & TRITIUM & TRITIUM_ELECT_LSC & HISTOR & 240 \\
\hline 2931 & PDMS & & SW & & & E013909 & 31-Mar-81 & & $10045-97-3$ & CS-137 & GAMMA_GS & HISTOR & 0.003 \\
\hline 2932 & PDMS & & SW & & & E010747 & 31-Mar-81 & & $10045-97-3$ & CS-137 & GAMMA_GS & HISTOR & -0.0261 \\
\hline 2933 & PDMS & & SW & & & E001278 & 31-Mar-81 & & $10045-97-3$ & CS-137 & GAMMA_GS & PNL-KI & 0.007 \\
\hline 2934 & PDMS & & SW & & & E001278 & 31-Mar-81 & & 15046-84-1 & $1-129$ & I129_SEP_TEMS & 320 & 0.0000067 \\
\hline 2935 & PDMS & & SW & & & E004358 & 31-Mar-81 & & $10028-17-8$ & TRITIUM & TRITIUMM_ELECT_LSC & HISTOR & 270 \\
\hline 2936 & PDMS & & SW & & & E004358 & 31-Mar-81 & & $10045-97-3$ & CS-137 & GAMMA_GS & HISTOR & -0.0738 \\
\hline 2937 & PDMS & & SW & & & E016358 & 06-Apr-81 & & $10098-97-2$ & SR-90 & SRISO_SEP_PRECIP_GPC & HISTOR & 1.94 \\
\hline 2938 & PDMS & & SW & & & E016358 & 06-Apr-81 & & $10045-97-3$ & CS-137 & GAMMA_GS & HISTOR & 12 \\
\hline 2939 & PDMS & & SW & & & E001956 & 14-Apr-81 & & PU-239/240 & PU-239/240 & PUISO_IE_AEA & PNL-RO & 0.00011 \\
\hline 2940 & PDMS & & SW & & & E001956 & 14-Apr-81 & & $10045-97-3$ & CS-137 & GAMMA_GS & PNL-KI & 0.006 \\
\hline 2941 & PDMS & & SW & & & E001956 & 14-Apr-81 & & 15046-84-1 & I-129 & I129_SEP_TEMS & 320 & 0.000035 \\
\hline 2942 & PDMS & & SW & & & E005419 & 14-Apr-81 & & $10045-97-3$ & CS-137 & GAMMA_GS & PNL-KI & 0.006 \\
\hline 2943 & PDMS & & SW & & & E005419 & 14-Apr-81 & & PU-239/240 & PU-239/240 & PUISO_IE_AEA & PNL-RO & 0.000025 \\
\hline 2944 & PDMS & & SW & & & E030586 & 14-Apr-81 & & PU-239/240 & PU-239/240 & PUISO_IE_AEA & PNL-RO & 0.00034 \\
\hline 2945 & PDMS & & SW & & & E040616 & 14-Apr-81 & & PU-239/240 & PU-239/240 & PUISO_IE_AEA & PNL-RO & 0.000029 \\
\hline 2946 & PDMS & & SW & & & E014831 & 27-Apr-81 & & $10098-97-2$ & SR-90 & SRISO_SEP_PRECIP_GPC & HISTOR & 0.0441 \\
\hline 2947 & PDMS & & SW & & & E014831 & 27-Apr-81 & & $10045-97-3$ & CS-137 & GAMMA_GS & HISTOR & -0.0487 \\
\hline 2948 & PDMS & & SW & & & E014831 & 27-Apr-81 & & $10028-17-8$ & TRITIUM & TRITIUM_DIST_LSC & HISTOR & 33100 \\
\hline 2949 & PDMS & & SW & & & E002137 & 27-Apr-81 & & $10098-97-2$ & SR-90 & SRISO_SEP_PRECIP_GPC & HISTOR & 0.214 \\
\hline 2950 & PDMS & & SW & & & E002137 & 27-Apr-81 & & $10045-97-3$ & CS-137 & GAMMA_GS & HISTOR & 0.413 \\
\hline 2951 & PDMS & & SW & & & E001358 & 28-Apr-81 & & $10028-17-8$ & TRITIUM & TRITIUM_ELECT_LSC & HISTOR & 190 \\
\hline 2952 & PDMS & & SW & & & E001358 & 28-Apr-81 & & $10098-97-2$ & SR-90 & SRISO_SEP_PRECIP_GPC & HISTOR & 0.153 \\
\hline 2953 & PDMS & & SW & & & E001358 & 28-Apr-81 & & $10045-97-3$ & CS-137 & GAMMA_GS & HISTOR & 0.445 \\
\hline 2954 & PDMS & & SW & & & E001279 & 28-Apr-81 & & $10045-97-3$ & CS-137 & GAMMA_GS & PNL-KI & 0.008 \\
\hline 2955 & PDMS & & SW & & & E001279 & 28-Apr-81 & & 15046-84-1 & I-129 & I129_SEP_TEMS & 320 & 0.0000058 \\
\hline 2956 & PDMS & & SW & & & E013910 & 28-Apr-81 & & $10045-97-3$ & CS-137 & GAMMA_GS & PNL-KI & 0.008 \\
\hline 2957 & PDMS & & SW & & & E004359 & 28-Apr-81 & & $10028-17-8$ & TRITIUM & TRITIUM_ELECT_LSC & HISTOR & 260 \\
\hline 2958 & PDMS & & SW & & & E004359 & 28-Apr-81 & & $10098-97-2$ & SR-90 & SRISO_SEP_PRECIP_GPC & HISTOR & 0.23 \\
\hline 2959 & PDMS & & SW & & & E004359 & 28-Apr-81 & & $10045-97-3$ & CS-137 & GAMMA_GS & HISTOR & 0.751 \\
\hline 2960 & PDMS & & SW & & & E016359 & 05-May-81 & & $10098-97-2$ & SR-90 & SRISO_SEP_PRECIP_GPC & HISTOR & 1.76 \\
\hline 2961 & PDMS & & SW & & & E016359 & 05-May-81 & & $10045-97-3$ & CS-137 & GAMMA_GS & HISTOR & 35.5 \\
\hline 2962 & PDMS & & SW & & & E012573 & 05-May-81 & & $10045-97-3$ & CS-137 & GAMMA_GS & HISTOR & -0.151 \\
\hline 2963 & PDMS & & SW & & & E022444 & 05-May-81 & & $10045-97-3$ & CS-137 & GAMMA_GS & HISTOR & 41.1 \\
\hline 2964 & PDMS & & SW & & & E022444 & 05-May-81 & & $10098-97-2$ & SR-90 & SRISO_SEP_PRECIP_GPC & HISTOR & 0.405 \\
\hline 2965 & PDMS & & SW & & & E014290 & 05-Мау-81 & & 10045-97-3 & CS-137 & GAMMA_GS & HISTOR & 4.59 \\
\hline
\end{tabular}




\begin{tabular}{|c|c|c|c|c|c|c|c|c|c|c|c|c|c|}
\hline & A & $\mathrm{B}$ & $\mathrm{C}$ & $\mathrm{D}$ & $E$ & $\mathrm{~F}$ & $\mathrm{G}$ & $\mathrm{H}$ & $\mathrm{I}$ & $\mathrm{J}$ & $\mathrm{K}$ & $\mathrm{L}$ & $\mathrm{M}$ \\
\hline 1 & SAMPLE_NUM_ASSIGNED_TO & SAMPLER & MEDIA & BIOTA_MEDIA & TAG_ID & SAMP_NUM & SAMP_DATE_TIME & SAMP_DATE_TIME_ON & CON_ID & CON_SHORT_NAME & METHOD_NAME & LAB_CODE & VALUE_RPTD \\
\hline 2966 & PDMS & & SW & & & E014290 & 05-May-81 & & $10098-97-2$ & SR-90 & SRISO_SEP_PRECIP_GPC & HISTOR & 2.97 \\
\hline 2967 & PDMS & & SW & & & E044684 & 06-May-81 & & $10098-97-2$ & SR-90 & SRISO_SEP_PRECIP_GPC & HISTOR & 0.0045 \\
\hline 2968 & PDMS & & SW & & & E001957 & 12-May-81 & & $10045-97-3$ & CS-137 & GAMMA_GS & PNL-KI & 0.004 \\
\hline 2969 & PDMS & & SW & & & E003875 & 12-May-81 & & $10045-97-3$ & CS-137 & GAMMA_GS & PNL-KI & 0.009 \\
\hline 2970 & PDMS & & SW & & & E001280 & 12-Мау-81 & & $10045-97-3$ & CS-137 & GAMMA_GS & PNL-KI & 0.005 \\
\hline 2971 & PDMS & & SW & & & E013911 & 12-May-81 & & $10045-97-3$ & CS-137 & GAMMA_GS & PNL-KI & 0.006 \\
\hline 2972 & PDMS & & SW & & & E001958 & 26-May-81 & & $10045-97-3$ & CS-137 & GAMMA_GS & PNL-KI & 0.004 \\
\hline 2973 & PDMS & & SW & & & E001958 & 26-Мay-81 & & 15046-84-1 & I-129 & I129_SEP_TEMS & 320 & 0.000024 \\
\hline 2974 & PDMS & & SW & & & E003876 & 26-May-81 & & $10045-97-3$ & CS-137 & GAMMA_GS & PNL-KI & 0.01 \\
\hline 2975 & PDMS & & SW & & & E001281 & 26-May-81 & & $10045-97-3$ & CS-137 & GAMMA_GS & PNL-KI & 0.004 \\
\hline 2976 & PDMS & & SW & & & E001281 & 26-May-81 & & $15046-84-1$ & I-129 & I129_SEP_TEMS & 320 & 0.0000061 \\
\hline 2977 & PDMS & & SW & & & E013912 & 26-May-81 & & $10045-97-3$ & CS-137 & GAMMA_GS & PNL-KI & 0.008 \\
\hline 2978 & PDMS & & SW & & & E021412 & 26-May-81 & & $10098-97-2$ & SR-90 & SRISO_SEP_PRECIP_GPC & HISTOR & 0.212 \\
\hline 2979 & PDMS & & SW & & & E021412 & 26-May-81 & & $10028-17-8$ & TRITIUM & TRITIUM_DIST_LSC & HISTOR & 189 \\
\hline 2980 & PDMS & & SW & & & E014832 & 01-Jun-81 & & $10098-97-2$ & SR-90 & SRISO_SEP_PRECIP_GPC & HISTOR & 0.018 \\
\hline 2981 & PDMS & & SW & & & E014832 & 01-Jun-81 & & $10028-17-8$ & TRITIUM & TRITIUM_DIST_LSC & HISTOR & 32200 \\
\hline 2982 & PDMS & & SW & & & E002138 & 01-Jun-81 & & $10045-97-3$ & CS-137 & GAMMA_GS & HISTOR & 0.00165 \\
\hline 2983 & PDMS & & SW & & & E010693 & 02-Jun-81 & & $10028-17-8$ & TRITIUM & TRITIUM_ELECT_LSC & HISTOR & 186 \\
\hline 2984 & PDMS & & SW & & & E010693 & 02-Jun-81 & & $10045-97-3$ & CS-137 & GAMMA_GS & HISTOR & 0.00614 \\
\hline 2985 & PDMS & & SW & & & E004360 & 02-Jun-81 & & $10028-17-8$ & TRITIUM & TRITIUM_ELECT_LSC & HISTOR & 161 \\
\hline 2986 & PDMS & & SW & & & E004360 & 02-Jun-81 & & $10045-97-3$ & CS-137 & GAMMA_GS & HISTOR & 0.00106 \\
\hline 2987 & PDMS & & SW & & & E010727 & 03-Jun-81 & & $10098-97-2$ & SR-90 & 905.0_SR_GPC & HISTOR & 0.0901 \\
\hline 2988 & PDMS & & SW & & & E010727 & 03-Jun-81 & & $10028-17-8$ & TRITIUM & 906.0_H3_LSC & HISTOR & 122 \\
\hline 2989 & PDMS & & SW & & & E001959 & 09-Jun-81 & & $10045-97-3$ & CS-137 & GAMMA_GS & PNL-KI & 0.007 \\
\hline 2990 & PDMS & & SW & & & E003877 & 09-Jun-81 & & $10045-97-3$ & CS-137 & GAMMA_GS & PNL-KI & 0.008 \\
\hline 2991 & PDMS & & SW & & & E001282 & 09-Jun-81 & & $10045-97-3$ & CS-137 & GAMMA_GS & PNL-KI & 0.01 \\
\hline 2992 & PDMS & & SW & & & E013913 & 09-Jun-81 & & $10045-97-3$ & CS-137 & GAMMA_GS & PNL-KI & 0.01 \\
\hline 2993 & PDMS & & SW & & & E001342 & 15-Jun-81 & & $10045-97-3$ & CS-137 & 901.1_GAMMA_GS & HISTOR & 0.708 \\
\hline 2994 & PDMS & & SW & & & E001342 & 15-Jun-81 & & $10098-97-2$ & SR-90 & 905.0_SR_GPC & HISTOR & 0.234 \\
\hline 2995 & PDMS & & SW & & & E001342 & 15-Jun-81 & & $10028-17-8$ & TRITIUM & TRITIUM_ELECT_LSC & USTEST & 183 \\
\hline 2996 & PDMS & & SW & & & E016360 & 16-Jun-81 & & $10098-97-2$ & SR-90 & SRISO_SEP_PRECIP_GPC & HISTOR & 1.2 \\
\hline 2997 & PDMS & & SW & & & E016360 & 16-Jun-81 & & $10045-97-3$ & CS-137 & GAMMA_GS & HISTOR & 24.1 \\
\hline 2998 & PDMS & & SW & & & E001960 & 23-Jun-81 & & PU-239/240 & PU-239/240 & PUISO_IE_AEA & PNL-RO & 0.00012 \\
\hline 2999 & PDMS & & SW & & & E001960 & 23-Jun-81 & & $10045-97-3$ & CS-137 & GAMMA_GS & PNL-KI & 0.004 \\
\hline 3000 & PDMS & & SW & & & E001960 & 23-Jun-81 & & 15046-84-1 & 1-129 & I129_SEP_TEMS & 320 & 0.0000096 \\
\hline 3001 & PDMS & & SW & & & E003878 & 23-Jun-81 & & $10045-97-3$ & CS-137 & GAMMA_GS & PNL-KI & 0.003 \\
\hline 3002 & PDMS & & SW & & & E003878 & 23-Jun-81 & & PU-239/240 & PU-239/240 & PUISO_IE_AEA & PNL-RO & 0.000057 \\
\hline 3003 & PDMS & & SW & & & E013914 & 23-Jun-81 & & PU-239/240 & PU-239/240 & PUISO_IE_AEA & PNL-RO & 0.000025 \\
\hline 3004 & PDMS & & SW & & & E013914 & 23-Jun-81 & & $10045-97-3$ & CS-137 & GAMMA_GS & PNL-KI & 0.01 \\
\hline 3005 & PDMS & & SW & & & E001283 & 23-Jun-81 & & $10045-97-3$ & CS-137 & GAMMA_GS & PNL-KI & 0.03 \\
\hline 3006 & PDMS & & SW & & & E001283 & 23-Jun-81 & & PU-239/240 & PU-239/240 & PUISO_IE_AEA & PNL-RO & 0.00015 \\
\hline 3007 & PDMS & & SW & & & E001283 & 23-Jun-81 & & 15046-84-1 & I-129 & I129_SEP_TEMS & 320 & 0.000011 \\
\hline 3008 & PDMS & & SW & & & E014833 & 29-Jun-81 & & $10098-97-2$ & SR-90 & SRISO_SEP_PRECIP_GPC & HISTOR & 0.0315 \\
\hline 3009 & PDMS & & SW & & & E014833 & 29-Jun-81 & & $10028-17-8$ & TRITIUM & TRITIUM_DIST_LSC & HISTOR & 63200 \\
\hline 3010 & PDMS & & SW & & & E002139 & 29-Jun-81 & & $10045-97-3$ & CS-137 & GAMMA_GS & HISTOR & 0.661 \\
\hline 3011 & PDMS & & SW & & & E010694 & 30-Jun-81 & & $10028-17-8$ & TRITIUM & TRITIUM_ELECT_LSC & USTEST & 171 \\
\hline 3012 & PDMS & & SW & & & E010694 & 30-Jun-81 & & $10045-97-3$ & CS-137 & GAMMA_GS & HISTOR & 0.61 \\
\hline 3013 & PDMS & & SW & & & E004362 & 30-Jun-81 & & $10028-17-8$ & TRITIUM & TRITIUM_ELECT_LSC & USTEST & 162 \\
\hline 3014 & PDMS & & SW & & & E004362 & 30-Jun-81 & & $10045-97-3$ & CS-137 & GAMMA_GS & HISTOR & 0.00372 \\
\hline 3015 & PDMS & & SW & & & E001961 & 07-Jul-81 & & $10045-97-3$ & CS-137 & GAMMA_GS & PNL-KI & 0.008 \\
\hline 3016 & PDMS & & SW & & & E003879 & 07-Jul-81 & & $10045-97-3$ & CS-137 & GAMMA_GS & PNL-KI & 0.004 \\
\hline 3017 & PDMS & & SW & & & E013915 & 07-Jul-81 & & $10045-97-3$ & CS-137 & GAMMA_GS & PNL-KI & 0.004 \\
\hline
\end{tabular}




\begin{tabular}{|c|c|c|c|c|c|c|c|c|c|c|c|c|c|}
\hline & A & $\mathrm{B}$ & $C$ & $\mathrm{D}$ & $E$ & $\mathrm{~F}$ & $\mathrm{G}$ & $\mathrm{H}$ & $\mathrm{I}$ & $\mathrm{J}$ & $\mathrm{K}$ & $\mathrm{L}$ & $\mathrm{M}$ \\
\hline 1 & SAMPLE_NUM_ASSIGNED_TO & SAMPLER & MEDIA & BIOTA_MEDIA & TAG_ID & SAMP_NUM & SAMP_DATE_TIME & SAMP_DATE_TIME_ON & CON_ID & CON_SHORT_NAME & METHOD_NAME & LAB_CODE & VALUE_RPTD \\
\hline 3018 & PDMS & & SW & & & E001284 & 07-Jul-81 & & $10045-97-3$ & CS-137 & GAMMA_GS & PNL-KI & 0.008 \\
\hline 3019 & PDMS & & SW & & & E016361 & 16-Jul-81 & & $10098-97-2$ & SR-90 & SRISO_SEP_PRECIP_GPC & HISTOR & 5.78 \\
\hline 3020 & PDMS & & SW & & & E016361 & 16-Jul-81 & & $10045-97-3$ & CS-137 & GAMMA_GS & HISTOR & 19.8 \\
\hline 3021 & PDMS & & SW & & & E001962 & 21-Jul-81 & & $10045-97-3$ & CS-137 & GAMMA_GS & PNL-KI & 0.01 \\
\hline 3022 & PDMS & & SW & & & E003880 & 21-Jul-81 & & $10045-97-3$ & CS-137 & GAMMA_GS & PNL-KI & 0.004 \\
\hline 3023 & PDMS & & SW & & & E013916 & 21-Jul-81 & & $10045-97-3$ & CS-137 & GAMMA_GS & PNL-KI & 0.005 \\
\hline 3024 & PDMS & & sW & & & E001285 & 21-Jul-81 & & $10045-97-3$ & CS-137 & GAMMA_GS & PNL-KI & 0.006 \\
\hline 3025 & PDMS & & SW & & & E014834 & 27-Jul-81 & & $10098-97-2$ & SR-90 & SRISO_SEP_PRECIP_GPC & HISTOR & 0.378 \\
\hline 3026 & PDMS & & SW & & & E014834 & 27-Jul-81 & & $10045-97-3$ & CS-137 & GAMMA_GS & HISTOR & -0.0569 \\
\hline 3027 & PDMS & & SW & & & E014834 & 27-Jul-81 & & $10028-17-8$ & TRITIUM & TRITIUM_DIST_LSC & HISTOR & 48000 \\
\hline 3028 & PDMS & & SW & & & E002140 & 27-Jul-81 & & $10098-97-2$ & SR-90 & SRISO_SEP_PRECIP_GPC & HISTOR & 0.203 \\
\hline 3029 & PDMS & & SW & & & E002140 & 27-Jul-81 & & $10045-97-3$ & CS-137 & GAMMA_GS & HISTOR & 0.226 \\
\hline 3030 & PDMS & & SW & & & E001359 & 28-Jul-81 & & $10028-17-8$ & TRITIUM & TRITIUM_ELECT_LSC & USTEST & 191 \\
\hline 3031 & PDMS & & SW & & & E001359 & 28-Jul-81 & & $10098-97-2$ & SR-90 & SRISO_SEP_PRECIP_GPC & HISTOR & 0.113 \\
\hline 3032 & PDMS & & SW & & & E001359 & 28-Jul-81 & & $10045-97-3$ & CS-137 & GAMMA_GS & HISTOR & -0.0651 \\
\hline 3033 & PDMS & & SW & & & E004361 & 28-Jul-81 & & $10028-17-8$ & TRITIUM & TRITIUM_ELECT_LSC & USTEST & 208 \\
\hline 3034 & PDMS & & SW & & & E004361 & 28-Jul-81 & & $10098-97-2$ & SR-90 & SRISO_SEP_PRECIP_GPC & HISTOR & 0.243 \\
\hline 3035 & PDMS & & SW & & & E004361 & 28-Jul-81 & & $10045-97-3$ & CS-137 & GAMMA_GS & HISTOR & 0.206 \\
\hline 3036 & PDMS & & SW & & & E021413 & 03-Aug-81 & & $10098-97-2$ & SR-90 & SRISO_SEP_PRECIP_GPC & HISTOR & 0.261 \\
\hline 3037 & PDMS & & SW & & & E021413 & 03-Aug-81 & & $10028-17-8$ & TRITIUM & TRITIUM_DISTT_LSC & HISTOR & 169 \\
\hline 3038 & PDMS & & SW & & & E003881 & 04-Aug-81 & & $10045-97-3$ & CS-137 & GAMMA_GS & PNL-KI & 0.004 \\
\hline 3039 & PDMS & & SW & & & E001963 & 04-Aug-81 & & $10045-97-3$ & CS-137 & GAMMA_GS & PNL-KI & 0.003 \\
\hline 3040 & PDMS & & SW & & & E001963 & 04-Aug-81 & & 15046-84-1 & I-129 & I129_SEP_TEMS & 320 & 0.000014 \\
\hline 3041 & PDMS & & SW & & & E013917 & 04-Aug-81 & & $10045-97-3$ & CS-137 & GAMMA_GS & PNL-KI & 0.005 \\
\hline 3042 & PDMS & & SW & & & E001286 & 04-Aug-81 & & $10045-97-3$ & CS-137 & GAMMA_GS & PNL-KI & 0.004 \\
\hline 3043 & PDMS & & SW & & & E001286 & 04-Aug-81 & & 15046-84-1 & I-129 & I129_SEP_TEMS & 320 & 0.0000057 \\
\hline 3044 & PDMS & & SW & & & E016362 & 11-Aug-81 & & $10098-97-2$ & SR-90 & SRISO_SEP_PRECIP_GPC & USTEST & 0.0746 \\
\hline 3045 & PDMS & & SW & & & E016362 & 11-Aug-81 & & $10045-97-3$ & CS-137 & GAMMA_GS & HISTOR & 5.76 \\
\hline 3046 & PDMS & & SW & & & E012574 & 11-Aug-81 & & $10045-97-3$ & CS-137 & GAMMA_GS & HISTOR & -0.118 \\
\hline 3047 & PDMS & & SW & & & E022445 & 11-Aug-81 & & $10045-97-3$ & CS-137 & GAMMA_GS & HISTOR & 1.92 \\
\hline 3048 & PDMS & & SW & & & E022445 & 11-Aug-81 & & $10098-97-2$ & SR-90 & SRISO_SEP_PRECIP_GPC & USTEST & 0.349 \\
\hline 3049 & PDMS & & SW & & & E044685 & 17-Aug-81 & & 10098-97-2 & SR-90 & SRISO_SEP_PRECIP_GPC & USTEST & 0.0856 \\
\hline 3050 & PDMS & & SW & & & E001964 & 18-Aug-81 & & $10045-97-3$ & CS-137 & GAMMA_GS & PNL-KI & 0.00 \\
\hline 3051 & PDMS & & SW & & & E003882 & 18-Aug-81 & & $10045-97-3$ & CS-137 & GAMMA_GS & PNL-KI & 0.002 \\
\hline 3052 & PDMS & & SW & & & E001287 & 18-Aug-81 & & $10045-97-3$ & CS-137 & GAMMA_GS & PNL-KI & 0.01 \\
\hline 3053 & PDMS & & SW & & & E013918 & 18-Aug-81 & & $10045-97-3$ & CS-137 & GAMMA_GS & PNL-KI & 0.003 \\
\hline 3054 & PDMS & & SW & & & E010728 & 26-Aug-81 & & $10098-97-2$ & SR-90 & 905.0_SR_GPC & USTEST & \\
\hline 3055 & PDMS & & SW & & & E010728 & 26-Aug-81 & & $10028-17-8$ & TRITIUM & 906.0_H3_LSC & HISTOR & 206 \\
\hline 3056 & PDMS & & SW & & & E014835 & 31-Aug-81 & & $10098-97-2$ & SR-90 & SRISO_SEP_PRECIP_GPC & USTEST & 0.018 \\
\hline 3057 & PDMS & & SW & & & E014835 & 31-Aug-81 & & $10028-17-8$ & TRITIUM & TRITIUM_DIST_LSC & HISTOR & 33800 \\
\hline 3058 & PDMS & & SW & & & E002141 & 31-Aug-81 & & $10045-97-3$ & CS-137 & GAMMA_GS & HISTOR & -0.0295 \\
\hline 3059 & PDMS & & SW & & & E001965 & 01-Sep-81 & & $10045-97-3$ & CS-137 & GAMMA_GS & PNL-KI & 0.004 \\
\hline 3060 & PDMS & & SW & & & E001965 & 01-Sep-81 & & 15046-84-1 & I-129 & I129_SEP_TEMS & 320 & 0.000057 \\
\hline 3061 & PDMS & & SW & & & E003883 & 01-Sep-81 & & $10045-97-3$ & CS-137 & GAMMA_GS & PNL-KI & 0.007 \\
\hline 3062 & PDMS & & SW & & & E013919 & 01-Sep-81 & & $10045-97-3$ & CS-137 & GAMMA_GS & PNL-KI & 0.003 \\
\hline 3063 & PDMS & & SW & & & E001288 & 01-Sep-81 & & $10045-97-3$ & CS-137 & GAMMA_GS & PNL-KI & 0.007 \\
\hline 3064 & PDMS & & SW & & & E001288 & 01-Sep-81 & & 15046-84-1 & I-129 & 1129_SEP_TEMS & 320 & 0.0000072 \\
\hline 3065 & PDMS & & SW & & & E010695 & 01-Sep-81 & & $10028-17-8$ & TRITIUM & TRITIUM_ELECT_LSC & USTEST & 89 \\
\hline 3066 & PDMS & & SW & & & E010695 & 01-Sep-81 & & $10045-97-3$ & CS-137 & GAMMA_GS & HISTOR & -0.0562 \\
\hline 3067 & PDMS & & SW & & & E004363 & 01-Sep-81 & & $10028-17-8$ & TRITIUM & TRITIUM_ELECT_LSC & USTEST & 120 \\
\hline 3068 & PDMS & & SW & & & E004363 & 01-Sep-81 & & $10045-97-3$ & CS-137 & GAMMA_GS & HISTOR & -0.0668 \\
\hline 3069 & PDMS & & SW & & & E016363 & 08-Sep-81 & & 10098-97-2 & SR-90 & SRISO_SEP_PRECIP_GPC & USTEST & 1.6 \\
\hline
\end{tabular}




\begin{tabular}{|c|c|c|c|c|c|c|c|c|c|c|c|c|c|}
\hline & A & $B$ & $\mathrm{C}$ & $\mathrm{D}$ & $\mathrm{E}$ & $\mathrm{F}$ & G & $\mathrm{H}$ & 1 & $\mathrm{~J}$ & $\mathrm{~K}$ & $\mathrm{~L}$ & $\mathrm{M}$ \\
\hline 1 & SAMPLE_NUM_ASSIGNED_TO & SAMPLER & MEDIA & BIOTA_MEDIA & TAG_ID & SAMP_NUM & SAMP_DATE_TIME & SAMP_DATE_TIME_ON & CON_ID & CON_SHORT_NAME & METHOD_NAME & LAB_CODE & VALUE_RPTD \\
\hline 3070 & PDMS & & SW & & & E016363 & 08-Sep-81 & & $10045-97-3$ & CS-137 & GAMMA_GS & HISTOR & 7.84 \\
\hline 3071 & PDMS & & SW & & & E003884 & 15-Sep-81 & & $10045-97-3$ & CS-137 & GAMMA_GS & PNL-KI & 0.005 \\
\hline 3072 & PDMS & & SW & & & E001966 & 15-Sep-81 & & $10045-97-3$ & CS-137 & GAMMA_GS & PNL-KI & 0.12 \\
\hline 3073 & PDMS & & SW & & & E001289 & 15-Sep-81 & & $10045-97-3$ & CS-137 & GAMMA_GS & PNL-KI & 0.008 \\
\hline 3074 & PDMS & & SW & & & E013920 & 15-Sep-81 & & $10045-97-3$ & CS-137 & GAMMA_GS & PNL-KI & 0.003 \\
\hline 3075 & PDMS & & SW & & & E001343 & 21-Sep-81 & & $10098-97-2$ & SR-90 & 905.0_SR_GPC & USTEST & 0.252 \\
\hline 3076 & PDMS & & SW & & & E001343 & 21-Sep-81 & & $10028-17-8$ & TRITIUM & TRITIUM_ELECT_LSC & USTEST & 78 \\
\hline 3077 & PDMS & & SW & & & E014836 & 28-Sep-81 & & $10098-97-2$ & SR-90 & SRISO_SEP_PRECIP_GPC & USTEST & 0.027 \\
\hline 3078 & PDMS & & sW & & & E014836 & 28-Sep-81 & & $10028-17-8$ & TRITIUM & TRITIUM_DIST_LSC & USTEST & 34400 \\
\hline 3079 & PDMS & & SW & & & E002142 & 28-Sep-81 & & $10045-97-3$ & CS-137 & GAMMA_GS & USTEST & 0.201 \\
\hline 3080 & PDMS & & SW & & & E001967 & 29-Sep-81 & & PU-239/240 & PU-239/240 & PUISO_IE_AEA & PNL-RO & 0.000064 \\
\hline 3081 & PDMS & & SW & & & E001967 & 29-Sep-81 & & $10045-97-3$ & CS-137 & GAMMA_GS & PNL-KI & 0.1 \\
\hline 3082 & PDMS & & SW & & & E001967 & 29-Sep-81 & & 15046-84-1 & I-129 & 1129_SEP_TEMS & 320 & 0.00013 \\
\hline 3083 & PDMS & & SW & & & E003885 & 29-Sep-81 & & $10045-97-3$ & CS-137 & GAMMA_GS & PNL-KI & 0.04 \\
\hline 3084 & PDMS & & SW & & & E003885 & 29-Sep-81 & & PU-239/240 & PU-239/240 & PUISO_IE_AEA & PNL-RO & 0.000055 \\
\hline 3085 & PDMS & & SW & & & E013921 & 29-Sep-81 & & PU-239/240 & PU-239/240 & PUISO_IE_AEA & PNL-RO & 0.000066 \\
\hline 3086 & PDMS & & SW & & & E013921 & 29-Sep-81 & & $10045-97-3$ & CS-137 & GAMMA_GS & PNL-KI & 0.04 \\
\hline 3087 & PDMS & & SW & & & E001290 & 29-Sep-81 & & $10045-97-3$ & CS-137 & GAMMA_GS & PNL-KI & 0.1 \\
\hline 3088 & PDMS & & SW & & & E001290 & 29-Sep-81 & & PU-239/240 & PU-239/240 & PUISO_IE_AEA & PNL-RO & 0.00015 \\
\hline 3089 & PDMS & & SW & & & E001290 & 29-Sep-81 & & 15046-84-1 & I-129 & 1129_SEP_TEMS & 320 & 0.0000074 \\
\hline 3090 & PDMS & & SW & & & E010696 & 29-Sep-81 & & $10028-17-8$ & TRITIUM & TRITIUM_ELECT_LSC & USTEST & 77 \\
\hline 3091 & PDMS & & SW & & & E010696 & 29-Sep-81 & & $10045-97-3$ & CS-137 & GAMMA_GS & USTEST & -0.0897 \\
\hline 3092 & PDMS & & SW & & & E004364 & 29-Sep-81 & & $10028-17-8$ & TRITIUM & TRITIUM_ELECT_LSC & USTEST & 192 \\
\hline 3093 & PDMS & & SW & & & E004364 & 29-Sep-81 & & $10045-97-3$ & CS-137 & GAMMA_GS & USTEST & -0.0131 \\
\hline 3094 & PDMS & & SW & & & E001968 & 13-Oct-81 & & $10045-97-3$ & CS-137 & GAMMA_GS & PNL-RO & 0.047 \\
\hline 3095 & PDMS & & SW & & & E005415 & 13-Oct-81 & & $10045-97-3$ & CS-137 & GAMMA_GS & PNL-RO & 0.017 \\
\hline 3096 & PDMS & & SW & & & E013922 & 13-Oct-81 & & $10045-97-3$ & CS-137 & GAMMA_GS & PNL-RO & 0.02 \\
\hline 3097 & PDMS & & SW & & & E001226 & 13-Oct-81 & & $10045-97-3$ & CS-137 & GAMMA_GS & PNL-RO & 0.05 \\
\hline 3098 & PDMS & & SW & & & E016364 & 20-Oct-81 & & $10098-97-2$ & SR-90 & SRISO_SEP_PRECIP_GPC & USTEST & 1.03 \\
\hline 3099 & PDMS & & SW & & & E016364 & 20-Oct-81 & & $10045-97-3$ & CS-137 & GAMMA_GS & USTEST & 19.2 \\
\hline 3100 & PDMS & & SW & & & E012592 & 21-Oct-81 & & $10098-97-2$ & SR-90 & 905.0_SR_GPC & USTEST & 0.0045 \\
\hline 3101 & PDMS & & SW & & & E012592 & 21-Oct-81 & & $10045-97-3$ & CS-137 & 901.1_GAMMA_GS & USTEST & 3.93 \\
\hline 3102 & PDMS & & SW & & & E012592 & 21-Oct-81 & & $10028-17-8$ & TRITIUM & 906.0_H3_LSC & USTEST & 189 \\
\hline 3103 & PDMS & & SW & & & E014837 & 26-Oct-81 & & $10098-97-2$ & SR-90 & SRISO_SEP_PRECIP_GPC & USTEST & 0 \\
\hline 3104 & PDMS & & SW & & & E014837 & 26-Oct-81 & & $10045-97-3$ & CS-137 & GAMMA_GS & USTEST & -0.0979 \\
\hline 3105 & PDMS & & SW & & & E014837 & 26-Oct-81 & & 10028-17-8 & TRITIUM & TRITIUM_DIST_LSC & USTEST & 42200 \\
\hline 3106 & PDMS & & SW & & & E001969 & $27-$ Oct-81 & & $10045-97-3$ & CS-137 & GAMMA_GS & PNL-RO & 0.099 \\
\hline 3107 & PDMS & & SW & & & E001969 & $27-$ Oct-81 & & 15046-84-1 & I-129 & I129_SEP_TEMS & 320 & 0.000078 \\
\hline 3108 & PDMS & & SW & & & E003886 & $27-$ Oct-81 & & $10045-97-3$ & CS-137 & GAMMA_GS & PNL-RO & 0.042 \\
\hline 3109 & PDMS & & SW & & & E032579 & $27-$ Oct-81 & & 15046-84-1 & I-129 & I129_SEP_TEMS & 320 & 0.0000044 \\
\hline 3110 & PDMS & & SW & & & E001227 & 27-Oct-81 & & $10045-97-3$ & CS-137 & GAMMA_GS & PNL-RO & 0.05 \\
\hline $3111 \mathrm{~F}$ & PDMS & & SW & & & E001227 & 27-Oct-81 & & 15046-84-1 & I-129 & 1129_SEP_TEMS & 320 & 0.0000063 \\
\hline 3112 & PDMS & & SW & & & E013923 & 27-Oct-81 & & $10045-97-3$ & CS-137 & GAMMA_GS & PNL-RO & 0.02 \\
\hline 3113 & PDMS & & SW & & & E006695 & $27-$ Oct-81 & & $10045-97-3$ & CS-137 & GAMMA_GS & USTEST & -0.0422 \\
\hline 3114 & PDMS & & SW & & & E021414 & 02-Nov-81 & & $10098-97-2$ & SR-90 & SRISO_SEP_PRECIP_GPC & USTEST & 0.212 \\
\hline 3115 & PDMS & & SW & & & E021414 & 02-Nov-81 & & $10028-17-8$ & TRITIUM & TRITIUM_DIST_LSC & USTEST & 483 \\
\hline 3116 & PDMS & & SW & & & E002143 & 02-Nov-81 & & $10098-97-2$ & SR-90 & SRISO_SEP_PRECIP_GPC & USTEST & 0.221 \\
\hline $3117 \mathrm{~F}$ & PDMS & & SW & & & E002143 & 02-Nov-81 & & $10045-97-3$ & CS-137 & GAMMA_GS & USTEST & 0.309 \\
\hline 3118 & PDMS & & SW & & & E038893 & 03-Nov-81 & & $10028-17-8$ & TRITIUM & TRITIUM_ELECT_LSC & USTEST & 142 \\
\hline 3119 & PDMS & & SW & & & E001360 & 03-Nov-81 & & 10028-17-8 & TRITIUM & TRITIUM_ELECT_LSC & USTEST & 208 \\
\hline 3120 & PDMS & & SW & & & E001360 & 03-Nov-81 & & $10098-97-2$ & SR-90 & SRISO_SEP_PRECIP_GPC & USTEST & 0.171 \\
\hline 3121 & PDMS & & SW & & & E001360 & 03-Nov-81 & & $10045-97-3$ & CS-137 & GAMMA_GS & USTEST & 0.00614 \\
\hline
\end{tabular}




\begin{tabular}{|c|c|c|c|c|c|c|c|c|c|c|c|c|c|}
\hline & $\bar{A}$ & $\mathrm{~B}$ & $\bar{C}$ & $\mathrm{D}$ & $\mathrm{E}$ & $\mathrm{F}$ & $\bar{G}$ & $\mathrm{H}$ & 1 & $\mathrm{~J}$ & $\bar{K}$ & $\bar{L}$ & $\mathrm{M}$ \\
\hline 1 & SAMPLE_NUM_ASSIGNED_TO & \begin{tabular}{|l} 
SAMPLER \\
\end{tabular} & MEDIA & BIOTA_MEDIA & TAG_ID & SAMP_NUM & SAMP_DATE_TIME & SAMP_DATE_TIME_ON & CON_ID & \begin{tabular}{|l|} 
CON_SHORT_NAME \\
\end{tabular} & METHOD_NAME & LAB_CODE & VALUE_RPTD \\
\hline 3122 & PDMS & & SW & & & E004365 & 03-Nov-81 & & $10028-17-8$ & TRITIUM & TRITIUM_ELECT_LSC & USTEST & 162 \\
\hline 3123 & PDMS & & SW & & & E004365 & 03-Nov-81 & & $10098-97-2$ & SR-90 & SRISO_SËP_PRËCIP_GPC & USTEST & 0.275 \\
\hline 3124 & PDMS & & SW & & & E004365 & 03-Nov-81 & & $10045-97-3$ & CS-137 & GAMMA_GS & USTEST & -0.0422 \\
\hline 3125 & PDMS & & SW & & & E010729 & 04-Nov-81 & & $10098-97-2$ & SR-90 & 905.0_SR_GPC & USTEST & 0.221 \\
\hline 3126 & PDMS & & SW & & & E010729 & 04-Nov-81 & & $10028-17-8$ & TRITIUM & 906.0_H3_LSC & USTEST & 261 \\
\hline 3127 & PDMS & & SW & & & E001970 & 10-Nov-81 & & $10045-97-3$ & CS-137 & GAMMA_ĒGS & PNL-RO & 0.046 \\
\hline 3128 & PDMS & & SW & & & E003887 & 10-Nov-81 & & $10045-97-3$ & CS-137 & GAMMA_GS & PNL-RO & 0.018 \\
\hline 3129 & PDMS & & SW & & & E013924 & 10-Nov-81 & & $10045-97-3$ & CS-137 & GAMMA_GS & PNL-RO & 0.03 \\
\hline 3130 & PDMS & & SW & & & E001228 & 10-Nov-81 & & $10045-97-3$ & CS-137 & GAMMA_GS & PNL-RO & 0.07 \\
\hline 3131 & PDMS & & SW & & & E016365 & 17-Nov-81 & & $10098-97-2$ & SR-90 & SRISO_SEP_PRECIP_GPC & USTEST & 0.495 \\
\hline 3132 & PDMS & & SW & & & E016365 & 17-Nov-81 & & $10045-97-3$ & CS-137 & GAMMA_GS & USTEST & 22.5 \\
\hline 3133 & PDMS & & SW & & & E012575 & 17-Nov-81 & & $10045-97-3$ & CS-137 & GAMMA_GS & USTEST & 19.2 \\
\hline 3134 & PDMS & & SW & & & E022446 & 17-Nov-81 & & $10045-97-3$ & CS-137 & GAMMA_GS & USTEST & 6.36 \\
\hline 3135 & PDMS & & SW & & & E022446 & 17-Nov-81 & & $10098-97-2$ & SR-90 & SRISO_SEP_PRECIP_GPC & USTEST & 0.212 \\
\hline 3136 & PDMS & & SW & & & E014291 & 17-Nov-81 & & $10045-97-3$ & CS-137 & GAMMA_GS & USTEST & -0.0365 \\
\hline 3137 & PDMS & & SW & & & E014291 & 17-Nov-81 & & $10098-97-2$ & SR-90 & SRISO_SEP_PRECIP_GPC & USTEST & 1.98 \\
\hline 3138 & PDMS & & SW & & & E001971 & 24-Nov-81 & & PU-239/240 & PU-239/240 & PUISO_IE_AEA & PNL-RO & 0.00015 \\
\hline 3139 & PDMS & & SW & & & E001971 & 24-Nov-81 & & $10045-97-3$ & CS-137 & GAMMA_GS & PNL-RO & 0.039 \\
\hline 3140 & PDMS & & SW & & & E001971 & 24-Nov-81 & & 15046-84-1 & I-129 & I129_SEP_TEMS & 320 & 0.000067 \\
\hline 3141 & PDMS & & SW & & & E003888 & 24-Nov-81 & & $10045-97-3$ & CS-137 & GAMMA_ĒS & PNL-RO & 0.021 \\
\hline 3142 & PDMS & & SW & & & E003888 & 24-Nov-81 & & PU-239/240 & PU-239/240 & PUISO_IE_AEA & PNL-RO & 0.00008 \\
\hline 3143 & PDMS & & SW & & & E032580 & 24-Nov-81 & & 15046-84-1 & $1-129$ & I129_SEP_TEMS & 320 & 0.0000042 \\
\hline 3144 & PDMS & & SW & & & E001229 & 24-Nov-81 & & $10045-97-3$ & CS-137 & GAMMA_GS & PNL-RO & 0.07 \\
\hline 3145 & PDMS & & SW & & & E001229 & 24-Nov-81 & & PU-239/240 & PU-239/240 & PUISO_IE_AEA & PNL-RO & 0.00009 \\
\hline 3146 & PDMS & & SW & & & E001229 & 24-Nov-81 & & 15046-84-1 & I-129 & I129_SEP_TEMS & 320 & 0.0000061 \\
\hline 3147 & PDMS & & SW & & & E013925 & 24-Nov-81 & & PU-239/240 & PU-239/240 & PUISO_IE_AEA & PNL-RO & 0.00002 \\
\hline 3148 & PDMS & & SW & & & E013925 & 24-Nov-81 & & $10045-97-3$ & CS-137 & GAMMA_GS & PNL-RO & 0.03 \\
\hline 3149 & PDMS & & SW & & & E014838 & 30-Nov-81 & & $10098-97-2$ & SR-90 & SRISO_SEP_PRECIP_GPC & USTEST & 0.0676 \\
\hline 3150 & PDMS & & SW & & & E014838 & 30-Nov-81 & & $10028-17-8$ & TRITIUM & TRITIUM_DIST_LSC & USTEST & 41800 \\
\hline 3151 & PDMS & & SW & & & E002144 & 30-Nov-81 & & $10098-97-2$ & SR-90 & SRISO_SEP_PRECIP_GPC & USTEST & 0.117 \\
\hline 3152 & PDMS & & SW & & & E002144 & 30-Nov-81 & & $10045-97-3$ & CS-137 & GAMMA_GS & USTEST & -0.0275 \\
\hline 3153 & PDMS & & SW & & & E038892 & 01-Dec-81 & & $10028-17-8$ & TRITIUM & TRITIUM_ELECT_LSC & USTEST & 239 \\
\hline 3154 & PDMS & & SW & & & E010697 & 01-Dec-81 & & 10028-17-8 & TRITIUM & TRITIUM_ELECT__LSC & USTEST & 150 \\
\hline 3155 & PDMS & & SW & & & E010697 & 01-Dec-81 & & $10045-97-3$ & CS-137 & GAMMA_GS & USTEST & 0.00614 \\
\hline 3156 & PDMS & & SW & & & E004366 & 01-Dec-81 & & $10028-17-8$ & TRITIUM & TRITIUM_ELECT_LSC & USTEST & 144 \\
\hline 3157 & PDMS & & SW & & & E004366 & 01-Dec-81 & & $10045-97-3$ & CS-137 & GAMMA_GS & USTEST & 0.137 \\
\hline 3158 & PDMS & & SW & & & E001972 & 08-Dec-81 & & $10045-97-3$ & CS-137 & GAMMA_GS & PNL-RO & 0.053 \\
\hline 3159 & PDMS & & SW & & & E003889 & 08-Dec-81 & & $10045-97-3$ & CS-137 & GAMMA_GS & PNL-RO & 0.021 \\
\hline 3160 & PDMS & & SW & & & E001230 & 08-Dec-81 & & $10045-97-3$ & CS-137 & GAMMA_GS & PNL-RO & 0.07 \\
\hline 3161 & PDMS & & SW & & & E013926 & 08-Dec-81 & & $10045-97-3$ & CS-137 & GAMMA_GS & PNL-RO & 0.03 \\
\hline 3162 & PDMS & & SW & & & E001344 & 14-Dec-81 & & $10045-97-3$ & CS-137 & 901.1_GAMMA_GS & USTEST & -0.00201 \\
\hline 3163 & PDMS & & SW & & & E001344 & 14-Dec-81 & & $10098-97-2$ & SR-90 & 905.0_SR_GPC & USTEST & 0.27 \\
\hline 3164 & PDMS & & SW & & & E001344 & 14-Dec-81 & & $10028-17-8$ & TRITIUM & 906.0_H3_LSC & USTEST & 221 \\
\hline 3165 & PDMS & & SW & & & E038894 & 21-Dec-81 & & $10028-17-8$ & TRITIUM & TRITIUMM_ELECT_LSC & USTEST & 135 \\
\hline 3166 & PDMS & & SW & & & E001361 & 21-Dec-81 & & $10028-17-8$ & TRITIUM & TRITIUM_ELECT_LSC & USTEST & 91.3 \\
\hline 3167 & PDMS & & SW & & & E001361 & 21-Dec-81 & & $10098-97-2$ & SR-90 & SRISO_SEP_PRËCIP_GPC & USTEST & 0.153 \\
\hline 3168 & PDMS & & SW & & & E001361 & 21-Dec-81 & & $10045-97-3$ & CS-137 & GAMMA_GS & USTEST & 0.376 \\
\hline 3169 & PDMS & & SW & & & E004367 & 21-Dec-81 & & $10028-17-8$ & TRITIUM & TRITIUM_ELECT_LSC & USTEST & 180 \\
\hline 3170 & PDMS & & SW & & & E004367 & 21-Dec-81 & & $10098-97-2$ & SR-90 & SRISO_SEP_PRECIP_GPC & USTEST & 0.239 \\
\hline 3171 & PDMS & & SW & & & E004367 & 21-Dec-81 & & $10045-97-3$ & CS-137 & GAMMA_GS & USTEST & -0.0401 \\
\hline 3172 & PDMS & & SW & & & E002145 & 21-Dec-81 & & $10098-97-2$ & SR-90 & SRISO_SEP_PRECIP_GPC & USTEST & 0.36 \\
\hline 3173 & PDMS & & SW & & & E002145 & 21-Dec-81 & & $10045-97-3$ & CS-137 & GAMMA_GS & USTEST & 0.612 \\
\hline
\end{tabular}




\begin{tabular}{|c|c|c|c|c|c|c|c|c|c|c|c|c|c|}
\hline & A & $\mathrm{B}$ & $\mathrm{C}$ & $\mathrm{D}$ & $E$ & $\mathrm{~F}$ & $\mathrm{G}$ & $\mathrm{H}$ & $\mathrm{I}$ & $\mathrm{J}$ & $\mathrm{K}$ & $\mathrm{L}$ & $\mathrm{M}$ \\
\hline 1 & SAMPLE_NUM_ASSIGNED_TO & SAMPLER & MEDIA & BIOTA_MEDIA & TAG_ID & SAMP_NUM & SAMP_DATE_TIME & SAMP_DATE_TIME_ON & CON_ID & CON_SHORT_NAME & METHOD_NAME & LAB_CODE & VALUE_RPTD \\
\hline 3174 & PDMS & & SW & & & E001973 & 22-Dec-81 & & $10045-97-3$ & CS-137 & GAMMA_GS & PNL-RO & 0.057 \\
\hline 3175 & PDMS & & SW & & & E003890 & 22-Dec-81 & & $10045-97-3$ & CS-137 & GAMMA_GS & PNL-RO & 0.025 \\
\hline 3176 & PDMS & & SW & & & E001231 & 22-Dec-81 & & $10045-97-3$ & CS-137 & GAMMA_GS & PNL-RO & 0.06 \\
\hline 3177 & PDMS & & SW & & & E013927 & 22-Dec-81 & & $10045-97-3$ & CS-137 & GAMMA_GS & PNL-RO & 0.03 \\
\hline 3178 & PDMS & & SW & & & E038895 & 28-Dec-81 & & $10028-17-8$ & TRITIUM & TRITIUM_ELECT_LSC & USTEST & 163 \\
\hline 3179 & PDMS & & SW & & & E021415 & 28-Dec-81 & & $10098-97-2$ & SR-90 & SRISO_SEP_PRECIP_GPC & USTEST & 0.293 \\
\hline 3180 & PDMS & & SW & & & E021415 & 28-Dec-81 & & $10028-17-8$ & TRITIUM & TRITIUM_DISTT_LSC & USTEST & 466 \\
\hline 3181 & PDMS & & SW & & & E001936 & 05-Jan-82 & & $10045-97-3$ & CS-137 & GAMMA_GS & PNL-RO & 0.0439 \\
\hline 3182 & PDMS & & SW & & & E001936 & 05-Jan-82 & & 15046-84-1 & I-129 & I129_SEP_TEMS & 320 & 0.000041 \\
\hline 3183 & PDMS & & SW & & & E003891 & 05-Jan-82 & & $10045-97-3$ & CS-137 & GAMMA_GS & PNL-RO & 0.0176 \\
\hline 3184 & PDMS & & SW & & & E001232 & 05-Jan-82 & & $10045-97-3$ & CS-137 & GAMMA_GS & PNL-RO & 0.0311 \\
\hline 3185 & PDMS & & SW & & & E001232 & 05-Jan-82 & & 15046-84-1 & $1-129$ & I129_SEP_TEMS & 320 & 0.0000035 \\
\hline 3186 & PDMS & & SW & & & E013928 & 05-Jan-82 & & $10045-97-3$ & CS-137 & GAMMA_GS & PNL-RO & 0.0142 \\
\hline 3187 & PDMS & & SW & & & E075507 & 09-Jan-82 & & $10028-17-8$ & TRITIUM & TRITIUM_ELECT_LSC & USTEST & 159 \\
\hline 3188 & PDMS & & SW & & & E004368 & 09-Jan-82 & & $10028-17-8$ & TRITIUM & TRITIUM_ELECT_LSC & USTEST & 206 \\
\hline 3189 & PDMS & & SW & & & E001937 & 19-Jan-82 & & $10045-97-3$ & CS-137 & GAMMA_GS & PNL-RO & 0.0554 \\
\hline 3190 & PDMS & & SW & & & E003892 & 19-Jan-82 & & $10045-97-3$ & CS-137 & GAMMA_GS & PNL-RO & 0.0288 \\
\hline 3191 & PDMS & & SW & & & E024668 & 19-Jan-82 & & $10045-97-3$ & CS-137 & GAMMA_GS & HISTOR & \\
\hline 3192 & PDMS & & SW & & & E014022 & 19-Jan-82 & & $10045-97-3$ & CS-137 & GAMMA_GS & HISTOR & \\
\hline 3193 & PDMS & & SW & & & E024668 & 19-Jan-82 & & 15046-84-1 & I-129 & 1129_SEP_LEPS_GS & HISTOR & \\
\hline 3194 & PDMS & & SW & & & E010901 & 19-Jan-82 & & $10098-97-2$ & SR-90 & SRISO_SEP_PRECIP_GPC & USTEST & 0.158 \\
\hline 3195 & PDMS & & SW & & & E006598 & 19-Jan-82 & & $10098-97-2$ & SR-90 & SRISO_SEP_PRECIP_GPC & USTEST & 0.345 \\
\hline 3196 & PDMS & & SW & & & E004649 & 22-Jan-82 & & $10028-17-8$ & TRITIUM & TRITIUM_ELECT_LSC & USTEST & 332 \\
\hline 3197 & PDMS & & SW & & & E004649 & 22-Jan-82 & & $10098-97-2$ & SR-90 & SRISO_SEP_PRECIP_GPC & USTEST & 0.946 \\
\hline 3198 & PDMS & & SW & & & E004649 & 22-Jan-82 & & $10045-97-3$ & CS-137 & GAMMA_GS & USTEST & 3.71 \\
\hline 3199 & PDMS & & SW & & & E011819 & 22-Jan-82 & & $10028-17-8$ & TRITIUM & TRITIUM_ELECT_LSC & USTEST & 2.61 \\
\hline 3200 & PDMS & & SW & & & E011819 & 22-Jan-82 & & $10098-97-2$ & SR-90 & SRISO_SEP_PRECIP_GPC & USTEST & 0.149 \\
\hline 3201 & PDMS & & SW & & & E011819 & 22-Jan-82 & & $10045-97-3$ & CS-137 & GAMMA_GS & USTEST & -0.108 \\
\hline 3202 & PDMS & & SW & & & E075508 & 22-Jan-82 & & $10028-17-8$ & TRITIUM & TRITIUM_ELECT_LSC & USTEST & 241 \\
\hline 3203 & PDMS & & SW & & & E004369 & 22-Jan-82 & & $10028-17-8$ & TRITIUM & TRITIUM_ELECT_LSC & USTEST & 219 \\
\hline 3204 & PDMS & & SW & & & E083350 & 22-Jan-82 & & $10098-97-2$ & SR-90 & SRISO_SEP_PRECIP_GPC & USTEST & 0.175 \\
\hline 3205 & PDMS & & SW & & & E010698 & 23-Jan-82 & & $10045-97-3$ & CS-137 & GAMMA_GS & USTEST & -0.00296 \\
\hline 3206 & PDMS & & SW & & & E006601 & 23-Jan-82 & & $10045-97-3$ & CS-137 & GAMMA_GS & USTEST & -0.0591 \\
\hline 3207 & PDMS & & SW & & & E044470 & 23-Jan-82 & & $10028-17-8$ & TRITIUM & TRITIUM_DIST_LSC & USTEST & 19700 \\
\hline 3208 & PDMS & & SW & & & E002146 & 28-Jan-82 & & $10045-97-3$ & CS-137 & GAMMA_GS & USTEST & 0.0134 \\
\hline 3209 & PDMS & & SW & & & E001938 & 02-Feb-82 & & $10045-97-3$ & CS-137 & GAMMA_GS & PNL-RO & 0.055 \\
\hline 3210 & PDMS & & SW & & & E001938 & 02-Feb-82 & & 15046-84-1 & I-129 & I129_SEP_TEMS & 320 & 0.000042 \\
\hline 3211 & PDMS & & SW & & & E003893 & 02-Feb-82 & & $10045-97-3$ & CS-137 & GAMMA_GS & PNL-RO & 0.0247 \\
\hline 3212 & PDMS & & SW & & & E074210 & 02-Feb-82 & & 15046-84-1 & I-129 & I129_SEP_TEMS & 320 & \\
\hline 3213 & PDMS & & SW & & & E004370 & $05-F e b-82$ & & $10028-17-8$ & TRITIUM & TRITIUM_ELECT_LSC & USTEST & 357 \\
\hline 3214 & PDMS & & SW & & & E015389 & $09-F e b-82$ & & $10045-97-3$ & CS-137 & GAMMA_GS & USTEST & 49.7 \\
\hline 3215 & PDMS & & SW & & & E015389 & 09-Feb-82 & & $10098-97-2$ & SR-90 & SRISO_SEP_PRECIP_GPC & USTEST & 0.82 \\
\hline 3216 & PDMS & & SW & & & E000908 & 09-Feb-82 & & $10045-97-3$ & CS-137 & GAMMA_GS & USTEST & 42.7 \\
\hline 3217 & PDMS & & SW & & & E000908 & 09-Feb-82 & & $10098-97-2$ & SR-90 & SRISO_SEP_PRECIP_GPC & USTEST & 0.0135 \\
\hline 3218 & PDMS & & SW & & & E016366 & 09-Feb-82 & & $10098-97-2$ & SR-90 & SRISO_SEP_PRECIP_GPC & USTEST & 0.991 \\
\hline 3219 & PDMS & & SW & & & E016366 & 09-Feb-82 & & $10045-97-3$ & CS-137 & GAMMA_GS & USTEST & 6.54 \\
\hline 3220 & PDMS & & SW & & & E012576 & 09-Feb-82 & & $10045-97-3$ & CS-137 & GAMMA_GS & USTEST & -0.00569 \\
\hline 3221 & PDMS & & SW & & & E022447 & 09-Feb-82 & & $10045-97-3$ & CS-137 & GAMMA_GS & USTEST & 24.8 \\
\hline 3222 & PDMS & & SW & & & E022447 & 09-Feb-82 & & $10098-97-2$ & SR-90 & SRISO_SEP_PRECIP_GPC & USTEST & 0.788 \\
\hline 3223 & PDMS & & SW & & & E014258 & 09-Feb-82 & & $10045-97-3$ & CS-137 & GAMMA_GS & USTEST & -0.282 \\
\hline 3224 & PDMS & & SW & & & E014258 & 09-Feb-82 & & $10098-97-2$ & SR-90 & SRISO_SEP_PRECIP_GPC & USTEST & 3.84 \\
\hline 3225 & PDMS & & SW & & & E001940 & 16-Feb-82 & & $10045-97-3$ & CS-137 & GAMMA_GS & PNL-RO & 0.058 \\
\hline
\end{tabular}




\begin{tabular}{|c|c|c|c|c|c|c|c|c|c|c|c|c|c|}
\hline & $\bar{A}$ & $\mathrm{~B}$ & $\mathrm{C}$ & $\bar{D}$ & $\mathrm{E}$ & $\mathrm{F}$ & $\bar{G}$ & $\mathrm{H}$ & 1 & $\mathrm{~J}$ & $\mathrm{~K}$ & $\mathrm{~L}$ & $\bar{M}$ \\
\hline 1 & SAMPLE_NUM_ASSIGNED_TO & SAMPLER & MEDIA & BIOTA_MEDIA & TAG_ID & SAMP_NUM & SAMP_DATE_TIME| & SAMP_DATE_TIME_ON & CON_ID & CON_SHORT_NAME & METHOD_NAME & LAB_CODE & VALUE_RPTD \\
\hline 3226 & PDMS & & SW & & & E003894 & 16-Feb-82 & & $10045-97-3$ & CS-137 & GAMMA_GS & PNL-RO & 0.0204 \\
\hline 3227 & PDMS & & sW & & & E032546 & 16-Feb-82 & & 15046-84-1 & I-129 & I129_SEP_TEMS & 320 & 0.0000027 \\
\hline 3228 & PDMS & & SW & & & E001233 & 16-Feb-82 & & $10045-97-3$ & CS-137 & GAMMA_GS & PNL-RO & 0.0511 \\
\hline 3229 & PDMS & & SW & & & E013929 & 16-Feb-82 & & $10045-97-3$ & CS-137 & GAMMA_GS & PNL-RO & 0.0233 \\
\hline 3230 & PDMS & & SW & & & E002073 & 16-Feb-82 & & $10045-97-3$ & CS-137 & GAMMA_GS & USTEST & 0.00614 \\
\hline 3231 & PDMS & & SW & & & E038896 & 19-Feb-82 & & $10028-17-8$ & TRITIUM & TRITIUM_ELECT_LSC & USTEST & 298 \\
\hline 3232 & PDMS & & sW & & & E010699 & 19-Feb-82 & & $10028-17-8$ & TRITIUM & TRITIUM_ELECT__LSC & USTEST & 325 \\
\hline 3233 & PDMS & & sW & & & E010699 & 19-Feb-82 & & $10045-97-3$ & CS-137 & GAMMA_GS & USTEST & -0.0686 \\
\hline 3234 & PDMS & & sW & & & E004371 & 19-Feb-82 & & $10028-17-8$ & TRITIUM & TRITIUM_ELELECT_LSC & USTEST & 668 \\
\hline 3235 & PDMS & & SW & & & E004371 & 19-Feb-82 & & $10045-97-3$ & CS-137 & GAMMA_GS & USTEST & 0.00614 \\
\hline 3236 & PDMS & & SW & & & E010730 & 02-Mar-82 & & $10098-97-2$ & SR-90 & 905.0_SR_GPC & USTEST & 2.76 \\
\hline 3237 & PDMS & & sW & & & E010730 & 02-Mar-82 & & $10028-17-8$ & TRITIUM & 906.0_H3_LSC & USTEST & 191 \\
\hline 3238 & PDMS & & sW & & & E001941 & 02-Mar-82 & & $10045-97-3$ & CS-137 & GAMMA_ĒS & PNL-RO & 0.0601 \\
\hline 3239 & PDMS & & SW & & & E001941 & 02-Mar-82 & & 15046-84-1 & I-129 & I129_SEP_TEMS & 320 & 0.000045 \\
\hline 3240 & PDMS & & SW & & & E003895 & 02-Mar-82 & & $10045-97-3$ & CS-137 & GAMMA_GS & PNL-RO & 0.0312 \\
\hline 3241 & PDMS & & SW & & & E038897 & 02-Mar-82 & & $10028-17-8$ & TRITIUM & TRITIUM_ELECT_LSC & USTEST & 332 \\
\hline 3242 & PDMS & & SW & & & E001234 & 02-Mar-82 & & $10045-97-3$ & CS-137 & GAMMA_GS & PNL-RO & 0.0579 \\
\hline 3243 & PDMS & & sW & & & E001234 & 02-Mar-82 & & 15046-84-1 & I-129 & I129_SEP_TEMS & 320 & 0.0000046 \\
\hline 3244 & PDMS & & sW & & & E013930 & 02-Mar-82 & & $10045-97-3$ & CS-137 & GAMMA_GE & PNL-RO & 0.0316 \\
\hline 3245 & PDMS & & sW & & & E075509 & 02-Mar-82 & & $10028-17-8$ & TRITIUM & TRITIUM_ELECT_LSC & USTEST & 155 \\
\hline 3246 & PDMS & & sW & & & E004372 & 02-Mar-82 & & $10028-17-8$ & TRITIUM & TRITIUM_ELECT__LSC & USTEST & 280 \\
\hline 3247 & PDMS & & SW & & & E013826 & 08-Mar-82 & & $10098-97-2$ & SR-90 & 905.0_SR_GPC & USTEST & 0 \\
\hline 3248 & PDMS & & SW & & & E013826 & 08-Mar-82 & & $10028-17-8$ & TRITIUM & 906.0_H3_LSC & USTEST & 21400 \\
\hline 3249 & PDMS & & SW & & & E013826 & 08-Mar-82 & & $10045-97-3$ & CS-137 & 901.1_GAMMA_GS & USTEST & 0.039 \\
\hline 3250 & PDMS & & SW & & & E021416 & 08-Mar-82 & & $10098-97-2$ & SR-90 & SRISO_SEP_PRECIP_GPC & USTEST & 0.153 \\
\hline 3251 & PDMS & & sW & & & E021416 & 08-Mar-82 & & 10028-17-8 & TRITIUM & TRITIUM_DIST_LSC & USTEST & 320 \\
\hline 3252 & PDMS & & SW & & & E010902 & 09-Mar-82 & & $10098-97-2$ & SR-90 & SRISO_SEP_PRECIP_GPC & USTEST & 0.167 \\
\hline 3253 & PDMS & & SW & & & E006556 & 09-Mar-82 & & $10098-97-2$ & SR-90 & SRISO_SEP_PRECIP_GPC & USTEST & 0.00901 \\
\hline 3254 & PDMS & & SW & & & E002074 & 15-Mar-82 & & $10045-97-3$ & CS-137 & GAMMA_GS & USTEST & 0.0117 \\
\hline 3255 & PDMS & & SW & & & E004538 & 16-Mar-82 & & $10045-97-3$ & CS-137 & GAMMA_GS & USTEST & 0.0099 \\
\hline 3256 & PDMS & & SW & & & E004538 & 16-Mar-82 & & $10028-17-8$ & TRITIUM & TRITIUM_DIST_LSC & USTEST & 295 \\
\hline 3257 & PDMS & & SW & & & E004538 & 16-Mar-82 & & $10098-97-2$ & SR-90 & SRISO_SEP_PRECIP_GPC & USTEST & 0.198 \\
\hline 3258 & PDMS & & SW & & & E003896 & 16-Mar-82 & & $10045-97-3$ & CS-137 & GAMMA_GS & PNL-RO & 0.0287 \\
\hline 3259 & PDMS & & SW & & & E001942 & 16-Mar-82 & & $10045-97-3$ & CS-137 & GAMMA_GS & PNL-RO & 0.0463 \\
\hline 3260 & PDMS & & SW & & & E038898 & 16-Mar-82 & & $10028-17-8$ & TRITIUM & TRITIUM_ELECT_LSC & USTEST & 292 \\
\hline 3261 & PDMS & & SW & & & E001235 & 16-Mar-82 & & $10045-97-3$ & CS-137 & GAMMA_GS & PNL-RO & 0.0397 \\
\hline 3262 & PDMS & & SW & & & E010700 & 16-Mar-82 & & $10028-17-8$ & TRITIUM & TRITIUM_ELECT_LSC & USTEST & 306 \\
\hline 3263 & PDMS & & sW & & & E010700 & 16-Mar-82 & & 10098-97-2 & SR-90 & SRISO_SEP_PRECIP_GPC & USTEST & 0.0496 \\
\hline 3264 & PDMS & & SW & & & E010700 & 16-Mar-82 & & $10045-97-3$ & CS-137 & GAMMA_GS & USTEST & 0.00172 \\
\hline 3265 & PDMS & & SW & & & E013931 & 16-Mar-82 & & $10045-97-3$ & CS-137 & GAMMA_GS & PNL-RO & 0.02 \\
\hline 3266 & PDMS & & SW & & & E004373 & 16-Mar-82 & & $10028-17-8$ & TRITIUM & TRITIUM_ELECT_LSC & USTEST & 330 \\
\hline 3267 & PDMS & & SW & & & E004373 & 16-Mar-82 & & $10098-97-2$ & SR-90 & SRISO_SEP_PRECIP_GPC & USTEST & 0.395 \\
\hline 3268 & PDMS & & SW & & & E004373 & 16-Mar-82 & & $10045-97-3$ & CS-137 & GAMMA_GS & USTEST & 0.0111 \\
\hline 3269 & PDMS & & SW & & & E017994 & 22-Mar-82 & & $10045-97-3$ & CS-137 & 901.1_GAMMA_GS & USTEST & -0.000672 \\
\hline 3270 & PDMS & & SW & & & E017994 & 22-Mar-82 & & $10028-17-8$ & TRITIUM & 906.0_H3_LSC & USTEST & 197 \\
\hline 3271 & PDMS & & SW & & & E038899 & 25-Mar-82 & & $10028-17-8$ & TRITIUM & TRITIUM_ELECT_LSC & USTEST & 302 \\
\hline 3272 & PDMS & & SW & & & E003897 & 30-Mar-82 & & $10045-97-3$ & CS-137 & GAMMA_GS & PNL-RO & 0.0422 \\
\hline 3273 & PDMS & & SW & & & E003897 & 30-Mar-82 & & PU-239/240 & PU-239/240 & PUISO_IE_AEA & PNL-RO & 0.000017 \\
\hline 3274 & PDMS & & SW & & & E001943 & 30-Mar-82 & & PU-239/240 & PU-239/240 & PUISO_IE_AEA & PNL-RO & 0.0000033 \\
\hline 3275 & PDMS & & SW & & & E001943 & 30-Mar-82 & & $10045-97-3$ & CS-137 & GAMMA_GS & PNL-RO & 0.0751 \\
\hline 3276 & PDMS & & SW & & & E001943 & 30-Mar-82 & & 15046-84-1 & I-129 & 1129_SEP_TEMS & 320 & 0.0000075 \\
\hline 3277 & PDMS & & SW & & & E032547 & 30-Mar-82 & & 15046-84-1 & I-129 & 1129_SEP_TEMS & 320 & 0.0000051 \\
\hline
\end{tabular}




\begin{tabular}{|c|c|c|c|c|c|c|c|c|c|c|c|c|c|}
\hline & $\bar{A}$ & $\mathrm{~B}$ & $\mathrm{C}$ & $\mathrm{D}$ & $E$ & $\mathrm{~F}$ & G & $\mathrm{H}$ & $\mathrm{I}$ & $\mathrm{J}$ & $\mathrm{K}$ & $\mathrm{L}$ & $\mathrm{M}$ \\
\hline 1 & SAMPLE_NUM_ASSIGNED_TO & SAMPLER & MEDIA & BIOTA_MEDIA & TAG_ID & SAMP_NUM & SAMP_DATE_TIME & SAMP_DATE_TIME_ON & CON_ID & CON_SHORT_NAME & METHOD_NAME & LAB_CODE & \begin{tabular}{|l|} 
VALUE_RPTD \\
\end{tabular} \\
\hline 3278 & PDMS & & SW & & & E001236 & 30-Mar-82 & & $10045-97-3$ & CS-137 & GAMMA_GS & PNL-RO & 0.0705 \\
\hline 3279 & PDMS & & sW & & & E001236 & 30-Mar-82 & & PU-239/240 & PU-239/240 & PUISO_IE_AEA & PNL-RO & 0 \\
\hline 3280 & PDMS & & SW & & & E001236 & 30-Mar-82 & & 15046-84-1 & I-129 & 1129_SEP_TEMS & 320 & 0.0000051 \\
\hline 3281 & PDMS & & SW & & & E013932 & 30-Mar-82 & & PU-239/240 & PU-239/240 & PUISO_IE_AEA & PNL-RO & 0.000026 \\
\hline 3282 & PDMS & & SW & & & E013932 & 30-Mar-82 & & $10045-97-3$ & CS-137 & GAMMA_GSS & PNL-RO & 0.0336 \\
\hline 3283 & PDMS & & SW & & & E075510 & 30-Mar-82 & & $10028-17-8$ & TRITIUM & TRITIUM_ELECT_LSC & USTEST & 304 \\
\hline 3284 & PDMS & & SW & & & E004374 & 30-Mar-82 & & $10028-17-8$ & TRITIUM & TRITIUM_ELECT__LSC & USTEST & 286 \\
\hline 3285 & PDMS & & SW & & & E002075 & 12-Apr-82 & & $10045-97-3$ & CS-137 & GAMMA_GS & USTEST & 0.00279 \\
\hline 3286 & PDMS & & SW & & & E003898 & 13-Apr-82 & & $10045-97-3$ & CS-137 & GAMMA_GS & PNL-RO & 0.0258 \\
\hline 3287 & PDMS & & SW & & & E001944 & 13-Apr-82 & & $10045-97-3$ & CS-137 & GAMMA_GS & PNL-RO & 0.0527 \\
\hline 3288 & PDMS & & SW & & & E010701 & 13-Apr-82 & & $10028-17-8$ & TRITIUM & TRITIUM_ELECT_LSC & USTEST & 89 \\
\hline 3289 & PDMS & & SW & & & E010701 & 13-Apr-82 & & $10098-97-2$ & SR-90 & SRISO_SEPP_PRËCIP_GPC & USTEST & 0.36 \\
\hline 3290 & PDMS & & SW & & & E010701 & 13-Apr-82 & & $10045-97-3$ & CS-137 & GAMMA_GS & USTEST & 0.0403 \\
\hline 3291 & PDMS & & SW & & & E001237 & 13-Apr-82 & & $10045-97-3$ & CS-137 & GAMMA_GS & PNL-RO & 0.054 \\
\hline 3292 & PDMS & & SW & & & E013933 & 13-Apr-82 & & $10045-97-3$ & CS-137 & GAMMA_GS & PNL-RO & 0.0278 \\
\hline 3293 & PDMS & & SW & & & E004375 & 13-Apr-82 & & $10028-17-8$ & TRITIUM & TRITIUM_ELECT_LSC & USTEST & 178 \\
\hline 3294 & PDMS & & SW & & & E004375 & 13-Apr-82 & & $10098-97-2$ & SR-90 & SRISO_SEP_PRECIP_GPC & USTEST & 0.216 \\
\hline 3295 & PDMS & & SW & & & E004375 & 13-Apr-82 & & $10045-97-3$ & CS-137 & GAMMA_GS & USTEST & 0.236 \\
\hline 3296 & PDMS & & SW & & & E004650 & 16-Apr-82 & & $10028-17-8$ & TRITIUM & TRITIUM_ELEECT_LSC & USTEST & 332 \\
\hline 3297 & PDMS & & SW & & & E004650 & 16-Apr-82 & & $10098-97-2$ & SR-90 & SRISO_SEP_PRECIP_GPC & USTEST & 0.559 \\
\hline 3298 & PDMS & & SW & & & E004650 & 16-Apr-82 & & $10045-97-3$ & CS-137 & GAMMA_GS & USTEST & 2.92 \\
\hline 3299 & PDMS & & SW & & & E011820 & 16-Apr-82 & & $10028-17-8$ & TRITIUM & TRITIUM_ELECT_LSC & USTEST & -69 \\
\hline 3300 & PDMS & & SW & & & E011820 & 16-Apr-82 & & $10098-97-2$ & SR-90 & SRISO_SEP_PRECIP_GPC & USTEST & 0.0495 \\
\hline 3301 & PDMS & & SW & & & E011820 & 16-Apr-82 & & $10045-97-3$ & CS-137 & GAMMA_GS & USTEST & 0.00205 \\
\hline 3302 & PDMS & & SW & & & E038900 & 20-Apr-82 & & 10028-17-8 & TRITIUM & TRITIUM_ELECT_LSC & USTEST & 124 \\
\hline 3303 & PDMS & & SW & & & E001945 & 27-Apr-82 & & $10045-97-3$ & CS-137 & GAMMA_GS & PNL-RO & 0.0615 \\
\hline 3304 & PDMS & & SW & & & E001945 & 27-Apr-82 & & 15046-84-1 & I-129 & I129_SEP_TEMS & 320 & 0.000026 \\
\hline 3305 & PDMS & & SW & & & E003899 & 27-Apr-82 & & $10045-97-3$ & CS-137 & GAMMA_ḠS & PNL-RO & 0.0308 \\
\hline 3306 & PDMS & & SW & & & E032548 & 27-Apr-82 & & 15046-84-1 & I-129 & I129_SEP_TEMS & 320 & 0.0000048 \\
\hline 3307 & PDMS & & SW & & & E001238 & 27-Apr-82 & & $10045-97-3$ & CS-137 & GAMMA_ĒS & PNL-RO & 0.0636 \\
\hline 3308 & PDMS & & SW & & & E001238 & 27-Apr-82 & & 15046-84-1 & I-129 & I129_SEP_TEMS & 320 & 0.0000063 \\
\hline 3309 & PDMS & & SW & & & E013934 & 27-Apr-82 & & $10045-97-3$ & CS-137 & GAMMA_ĒS & PNL-RO & 0.0279 \\
\hline 3310 & PDMS & & SW & & & E075511 & 27-Apr-82 & & $10028-17-8$ & TRITIUM & TRITIUM_ELECT_LSC & USTEST & 106 \\
\hline 3311 & PDMS & & SW & & & E004376 & 27-Apr-82 & & $10028-17-8$ & TRITIUM & TRITIUM_ELECT_LSC & USTEST & 157 \\
\hline 3312 & PDMS & & SW & & & E017876 & 04-May-82 & & $10045-97-3$ & CS-137 & GAMMA_GS & USTEST & 3.33 \\
\hline 3313 & PDMS & & SW & & & E017876 & 04-May-82 & & $10098-97-2$ & SR-90 & SRISO_SEP_PRECIP_GPC & USTEST & 6.06 \\
\hline 3314 & PDMS & & SW & & & E011850 & 04-May-82 & & $10098-97-2$ & SR-90 & SRISO_SEP_PRECIP_GPC & USTEST & 5.32 \\
\hline 3315 & PDMS & & SW & & & E011850 & 04-Мау-82 & & $10045-97-3$ & CS-137 & GAMMA_GS & USTEST & 3.09 \\
\hline 3316 & PDMS & & SW & & & E014540 & 04-Мау-82 & & $10098-97-2$ & SR-90 & SRISO_SEEP_PRECIP_GPC & USTEST & 3.13 \\
\hline 3317 & PDMS & & SW & & & E014540 & 04-Мау-82 & & $10045-97-3$ & CS-137 & GAMMĀGS & USTEST & 3.66 \\
\hline 3318 & PDMS & & SW & & & E024166 & 04-May-82 & & $10098-97-2$ & SR-90 & SRISO_SEP_PRECIP_GPC & USTEST & 0.0811 \\
\hline 3319 & PDMS & & SW & & & E024166 & 04-May-82 & & $10045-97-3$ & CS-137 & GAMMA_GS & USTEST & -0.151 \\
\hline 3320 & PDMS & & SW & & & E016367 & 04-May-82 & & $10098-97-2$ & SR-90 & SRISO_SEP_PRECIP_GPC & USTEST & 4.64 \\
\hline 3321 & PDMS & & SW & & & E016367 & 04-Мау-82 & & $10045-97-3$ & CS-137 & GAMMA_GS & USTEST & 2.99 \\
\hline 3322 & PDMS & & SW & & & E012577 & 04-Мау-82 & & $10045-97-3$ & CS-137 & GAMMA_GS & USTEST & 0.131 \\
\hline 3323 & PDMS & & SW & & & E022448 & 04-Мау-82 & & $10045-97-3$ & CS-137 & GAMMA_GS & USTEST & 45 \\
\hline 3324 & PDMS & & SW & & & E022448 & 04-Mау-82 & & $10098-97-2$ & SR-90 & SRISO_SEEP_PRECIP_GPC & USTEST & 3.71 \\
\hline 3325 & PDMS & & SW & & & E014259 & 04-May-82 & & $10045-97-3$ & CS-137 & GAMMA_GS & USTEST & -0.152 \\
\hline 3326 & PDMS & & SW & & & E014259 & 04-May-82 & & $10098-97-2$ & SR-90 & SRISO_SEP_PRECIP_GPC & USTEST & 2.73 \\
\hline 3327 & PDMS & & SW & & & E002076 & 10-Мау-82 & & $10045-97-3$ & CS-137 & GAMMA_GS & USTEST & 1.25 \\
\hline 3328 & PDMS & & SW & & & E001946 & 11-Мау-82 & & $10045-97-3$ & CS-137 & GAMMA_GS & PNL-RO & 0.0642 \\
\hline 3329 & PDMS & & SW & & & E003900 & 11-May-82 & & $10045-97-3$ & CS-137 & GAMMA_GS & PNL-RO & 0.026 \\
\hline
\end{tabular}




\begin{tabular}{|c|c|c|c|c|c|c|c|c|c|c|c|c|c|}
\hline & A & $B$ & $\mathrm{C}$ & $\mathrm{D}$ & $\mathrm{E}$ & $\mathrm{F}$ & G & $\mathrm{H}$ & 1 & $\mathrm{~J}$ & $\mathrm{~K}$ & $\mathrm{~L}$ & $\mathrm{M}$ \\
\hline 1 & SAMPLE_NUM_ASSIGNED_TO & SAMPLER & MEDIA & BIOTA_MEDIA & TAG_ID & SAMP_NUM & SAMP_DATE_TIME & SAMP_DATE_TIME_ON & CON_ID & CON_SHORT_NAME & METHOD_NAME & LAB_CODE & VALUE_RPTD \\
\hline 3330 & PDMS & & SW & & & E038901 & 11-Мау-82 & & $10028-17-8$ & TRITIUM & TRITIUM_ELECT_LSC & USTEST & 162 \\
\hline 3331 & PDMS & & SW & & & E010702 & 11-May-82 & & 10028-17-8 & TRITIUM & TRITIUM_ELECT_LSC & USTEST & 136 \\
\hline 3332 & PDMS & & SW & & & E010702 & 11-May-82 & & $10098-97-2$ & SR-90 & SRISO_SEP_PRECIP_GPC & USTEST & 0.153 \\
\hline 3333 & PDMS & & SW & & & E010702 & 11-May-82 & & $10045-97-3$ & CS-137 & GAMMA_GS & USTEST & -0.155 \\
\hline 3334 & PDMS & & SW & & & E013935 & 11-May-82 & & $10045-97-3$ & CS-137 & GAMMA_GS & PNL-RO & 0.0326 \\
\hline 3335 & PDMS & & SW & & & E001239 & 11-May-82 & & $10045-97-3$ & CS-137 & GAMMA_GS & PNL-RO & 0.0671 \\
\hline 3336 & PDMS & & SW & & & E004377 & 12-May-82 & & $10028-17-8$ & TRITIUM & TRITIUM_ELECT_LSC & USTEST & 134 \\
\hline 3337 | & PDMS & & SW & & & E004377 & 12-May-82 & & $10098-97-2$ & SR-90 & SRISO_SEP_PRECIP_GPC & USTEST & 0.198 \\
\hline 3338 & PDMS & & sW & & & E004377 & 12-Мay-82 & & $10045-97-3$ & CS-137 & GAMMA_GS & USTEST & 0.105 \\
\hline 3339 & PDMS & & SW & & & E038902 & 18-Мay-82 & & 10028-17-8 & TRITIUM & TRITIUM_ELECT_LSC & USTEST & 125 \\
\hline 3340 & PDMS & & SW & & & E075512 & 18-May-82 & & $10028-17-8$ & TRITIUM & TRITIUM_ELECT_LSC & USTEST & 71 \\
\hline 3341 & PDMS & & SW & & & E004378 & 18-May-82 & & $10028-17-8$ & TRITIUM & TRITIUM_ELECT_LSC & USTEST & 112 \\
\hline 3342 & PDMS & & sW & & & E010731 & 25-May-82 & & $10098-97-2$ & SR-90 & 905.0_SR_GPC & USTEST & 0.0135 \\
\hline 3343 & PDMS & & SW & & & E010731 & 25-May-82 & & $10028-17-8$ & TRITIUM & 906.0_H3_LSC & USTEST & 32.2 \\
\hline 3344 & PDMS & & SW & & & E001947 & 25-May-82 & & $10045-97-3$ & CS-137 & GAMMA_GS & PNL-RO & 0.0576 \\
\hline 3345 & PDMS & & SW & & & E001947 & 25-May-82 & & 15046-84-1 & I-129 & 1129_SEP_TEMS & 320 & 0.000025 \\
\hline 3346 & PDMS & & SW & & & E003901 & 25-May-82 & & $10045-97-3$ & CS-137 & GAMMA_GS & PNL-RO & 0.0237 \\
\hline 3347 & PDMS & & SW & & & E032549 & 25-May-82 & & 15046-84-1 & I-129 & I129_SEP_TEMS & 320 & 0.0000048 \\
\hline 3348 & PDMS & & SW & & & E015254 & 25-May-82 & & $10045-97-3$ & CS-137 & GAMMA_GS & HISTOR & \\
\hline 3349 & PDMS & & SW & & & E026977 & 25-May-82 & & $10045-97-3$ & CS-137 & GAMMA_GS & HISTOR & \\
\hline 3350 & PDMS & & SW & & & E026977 & 25-May-82 & & 15046-84-1 & I-129 & 1129_SEP_TEMS & 320 & 0.0000016 \\
\hline 3351 & PDMS & & SW & & & E042261 & 25-May-82 & & $18540-29-9$ & $\mathrm{CR}+6$ & UNKNOWN_METALS & PNL-KI & 0.1 \\
\hline 3352 & PDMS & & SW & & & E028081 & 25-May-82 & & $18540-29-9$ & $\mathrm{CR}+6$ & UNKNOWN_METALS & PNL-KI & 0.1 \\
\hline 3353 & PDMS & & SW & & & E013805 & 01-Jun-82 & & $10098-97-2$ & SR-90 & 905.0_SR_GPC & USTEST & 0.0765 \\
\hline 3354 & PDMS & & SW & & & E013805 & 01-Jun-82 & & $10028-17-8$ & TRITIUM & 906.0_H3_LSC & USTEST & 16900 \\
\hline 3355 & PDMS & & SW & & & E013805 & 01-Jun-82 & & $10045-97-3$ & CS-137 & 901.1_GAMMA_GS & USTEST & 1.98 \\
\hline 3356 & PDMS & & SW & & & E022922 & 07-Jun-82 & & PU-239/240 & PU-239/240 & PUISO_IE_AEA & PNL-RO & 0.000011 \\
\hline 3357 & PDMS & & SW & & & E073582 & 07-Jun-82 & & PU-239/240 & PU-239/240 & PUISO_IE_AEA & PNL-RO & 0.000027 \\
\hline 3358 & PDMS & & SW & & & E030587 & 07-Jun-82 & & PU-239/240 & PU-239/240 & PUISO_IE_AEA & PNL-RO & 0.000015 \\
\hline 3359 & PDMS & & SW & & & E040617 & 07-Jun-82 & & PU-239/240 & PU-239/240 & PUISO_IE_AEA & PNL-RO & 0.000022 \\
\hline 3360 & PDMS & & SW & & & E021417 & 07-Jun-82 & & $10098-97-2$ & SR-90 & SRISO_SEP_PRECIP_GPC & USTEST & 0.191 \\
\hline 3361 & PDMS & & SW & & & E021417 & 07-Jun-82 & & $10028-17-8$ & TRITIUM & TRITIUM_DIST_LSC & USTEST & 403 \\
\hline 3362 & PDMS & & SW & & & E002077 & 07-Jun-82 & & $10045-97-3$ & CS-137 & GAMMA_GS & USTEST & 0.637 \\
\hline 3363 & PDMS & & SW & & & E032599 & 08-Jun-82 & & $10098-97-2$ & SR-90 & SRISO_SEP_PRECIP_GPC & USTEST & 0.26 \\
\hline 3364 & PDMS & & SW & & & E032599 & 08-Jun-82 & & $10028-17-8$ & TRITIUM & TRITIUM_DIST_LSC & USTEST & 396 \\
\hline 3365 & PDMS & & SW & & & E032599 & 08-Jun-82 & & $10045-97-3$ & CS-137 & GAMMA_GS & USTEST & 0.341 \\
\hline 3366 & PDMS & & SW & & & E001948 & 08-Jun-82 & & $10045-97-3$ & CS-137 & GAMMA_GS & PNL-RO & 0.0539 \\
\hline 3367 & PDMS & & SW & & & E003902 & 08-Jun-82 & & $10045-97-3$ & CS-137 & GAMMA_GS & PNL-RO & 0.0247 \\
\hline 3368 & PDMS & & SW & & & E038903 & 08-Jun-82 & & $10028-17-8$ & TRITIUM & TRITIUM_ELECT_LSC & USTEST & 108 \\
\hline 3369 & PDMS & & SW & & & E010703 & 08-Jun-82 & & $10028-17-8$ & TRITIUM & TRITIUM_ELECT_LSC & USTEST & 125 \\
\hline 3370 & PDMS & & SW & & & E010703 & 08-Jun-82 & & $10098-97-2$ & SR-90 & SRISO_SEP_PRECIP_GPC & USTEST & 0.131 \\
\hline 3371 & PDMS & & SW & & & E010703 & 08-Jun-82 & & $10045-97-3$ & CS-137 & GAMMA_GS & USTEST & -0.174 \\
\hline $3372 \mathrm{~F}$ & PDMS & & SW & & & E015255 & 08-Jun-82 & & $10045-97-3$ & CS-137 & GAMMA_GS & HISTOR & \\
\hline 3373 & PDMS & & SW & & & E026978 & 08-Jun-82 & & $10045-97-3$ & CS-137 & GAMMA_GS & HISTOR & \\
\hline 3374 & PDMS & & SW & & & E004379 & 08-Jun-82 & & $10028-17-8$ & TRITIUM & TRITIUM_ELECT_LSC & USTEST & 153 \\
\hline 3375 & PDMS & & SW & & & E004379 & 08-Jun-82 & & $10098-97-2$ & SR-90 & SRISO_SEP_PRECIP_GPC & USTEST & 0.162 \\
\hline 3376 & PDMS & & SW & & & E004379 & 08-Jun-82 & & $10045-97-3$ & CS-137 & GAMMA_GS & USTEST & 0.661 \\
\hline $3377 \mathrm{~F}$ & PDMS & & SW & & & E017995 & 14-Jun-82 & & $10045-97-3$ & CS-137 & 901.1_GAMMA_GS & USTEST & 1.03 \\
\hline 3378 & PDMS & & SW & & & E017995 & 14-Jun-82 & & $10028-17-8$ & TRITIUM & 906.0_H3_LSC & USTEST & 185 \\
\hline 3379 & PDMS & & SW & & & E001949 & 22-Jun-82 & & $10045-97-3$ & CS-137 & GAMMA_GS & PNL-RO & 0.0881 \\
\hline 3380 & PDMS & & SW & & & E001949 & 22-Jun-82 & & 15046-84-1 & 1-129 & I129_SEP_TEMS & 320 & 0.000014 \\
\hline 3381 & PDMS & & SW & & & E003903 & 22-Jun-82 & & $10045-97-3$ & CS-137 & GAMMA_GS & PNL-RO & 0.0518 \\
\hline
\end{tabular}




\begin{tabular}{|c|c|c|c|c|c|c|c|c|c|c|c|c|c|}
\hline & $\bar{A}$ & $\mathrm{~B}$ & C & $\mathrm{D}$ & $E$ & $\mathrm{~F}$ & G & $\mathrm{H}$ & $\mathrm{I}$ & $\mathrm{J}$ & $\mathrm{K}$ & $\mathrm{L}$ & $\mathrm{M}$ \\
\hline 1 & SAMPLE_NUM_ASSIGNED_TO & SAMPLER & MEDIA & BIOTA_MEDIA & TAG_ID & SAMP_NUM & SAMP_DATE_TIME & SAMP_DATE_TIME_ON & CON_ID & CON_SHORT_NAME & METHOD_NAME & LAB_CODE & \begin{tabular}{|l|} 
VALUE_RPTD \\
\end{tabular} \\
\hline 3382 & PDMS & & SW & & & E032550 & 22-Jun-82 & & $15046-84-1$ & $1-129$ & I129_SEP_TEMS & 320 & 0.0000033 \\
\hline 3383 & PDMS & & sW & & & E001240 & 22-Jun-82 & & $10045-97-3$ & CS-137 & GAMMA_ḠS & PNL-RO & 0.0706 \\
\hline 3384 & PDMS & & SW & & & E001240 & 22-Jun-82 & & 15046-84-1 & I-129 & 1129_SEP_TEMS & 320 & 0.0000045 \\
\hline 3385 & PDMS & & SW & & & E013936 & 22-Jun-82 & & $10045-97-3$ & CS-137 & GAMMA_GS & PNL-RO & 0.0376 \\
\hline 3386 & PDMS & & SW & & & E004380 & 29-Jun-82 & & $10028-17-8$ & TRITIUM & TRITIUM_ELECET_LSC & USTEST & 100 \\
\hline 3387 & PDMS & & SW & & & E004380 & 29-Jun-82 & & $10098-97-2$ & SR-90 & SRISO_SEP_PRECIP_GPC & USTEST & 0.0631 \\
\hline 3388 & PDMS & & SW & & & E004380 & 29-Jun-82 & & $10045-97-3$ & CS-137 & GAMMA_GS & USTEST & 0.163 \\
\hline 3389 & PDMS & & SW & & & E000905 & 06-Jul-82 & & $10028-17-8$ & TRITIUM & TRITIUM_DIST_LSC & USTEST & 78 \\
\hline 3390 & PDMS & & SW & & & E000905 & 06-Jul-82 & & $10028-17-8$ & TRITIUM & TRITIUM_ELECT_LSC & USTEST & 292 \\
\hline 3391 & PDMS & & SW & & & E000905 & 06-Jul-82 & & $10098-97-2$ & SR-90 & SRISO_SEP_PRECIP_GPC & USTEST & 0.315 \\
\hline 3392 & PDMS & & SW & & & E000905 & 06-Jul-82 & & $10045-97-3$ & CS-137 & GAMMA_GS & USTEST & 6.67 \\
\hline 3393 & PDMS & & SW & & & E011821 & 06-Jul-82 & & $10028-17-8$ & TRITIUM & TRITIUM_DIST_LSC & USTEST & 64 \\
\hline 3394 & PDMS & & SW & & & E011821 & 06-Jul-82 & & $10028-17-8$ & TRITIUM & TRITIUM_ELECT_LSC & USTEST & 64 \\
\hline 3395 & PDMS & & SW & & & E011821 & 06-Jul-82 & & $10098-97-2$ & SR-90 & SRISO_SËP_PRËCIP_GPC & USTEST & 0.144 \\
\hline 3396 & PDMS & & SW & & & E011821 & 06-Jul-82 & & $10045-97-3$ & CS-137 & GAMMA_GS & USTEST & 0.765 \\
\hline 3397 & PDMS & & SW & & & E003904 & 06-Jul-82 & & $10045-97-3$ & CS-137 & GAMMA_GS & PNL-RO & 0.0276 \\
\hline 3398 & PDMS & & SW & & & E001950 & 06-Jul-82 & & $10045-97-3$ & CS-137 & GAMMA_GS & PNL-RO & 0.0522 \\
\hline 3399 & PDMS & & SW & & & E001241 & 06-Jul-82 & & $10045-97-3$ & CS-137 & GAMMA_GS & PNL-RO & 0.0363 \\
\hline 3400 & PDMS & & SW & & & E010704 & 06-Jul-82 & & $10028-17-8$ & TRITIUM & TRITIUM_ELEECT_LSC & USTEST & 117 \\
\hline 3401 & PDMS & & SW & & & E010704 & 06-Jul-82 & & $10098-97-2$ & SR-90 & SRISO_SEP_PRECIP_GPC & USTEST & 0.126 \\
\hline 3402 & PDMS & & SW & & & E013937 & 06-Jul-82 & & $10045-97-3$ & CS-137 & GAMMA_GS & PNL-RO & 0.0254 \\
\hline 3403 & PDMS & & SW & & & E010704 & 06-Jul-82 & & $10045-97-3$ & CS-137 & GAMMA_GS & USTEST & -0.277 \\
\hline 3404 & PDMS & & SW & & & E002078 & 06-Jul-82 & & $10045-97-3$ & CS-137 & GAMMA_GS & USTEST & 10.6 \\
\hline 3405 & PDMS & & SW & & & E038904 & 13-Jul-82 & & $10028-17-8$ & TRITIUM & TRITIUM_ELECT_LSC & HISTOR & \\
\hline 3406 & PDMS & & SW & & & E001974 & 20-Jul-82 & & $10045-97-3$ & CS-137 & GAMMA_GS & HISTOR & \\
\hline 3407 & PDMS & & SW & & & E014817 & 20-Jul-82 & & $10045-97-3$ & CS-137 & GAMMA_GS & HISTOR & \\
\hline 3408 & PDMS & & SW & & & E015256 & 20-Jul-82 & & $10045-97-3$ & CS-137 & GAMMA_GS & HISTOR & \\
\hline 3409 & PDMS & & SW & & & E026979 & 20-Jul-82 & & $10045-97-3$ & CS-137 & GAMMA_GS & HISTOR & \\
\hline 3410 & PDMS & & SW & & & E011288 & 27-Jul-82 & & $10098-97-2$ & SR-90 & SRISO_SEP_PRECIP_GPC & USTEST & 0.0631 \\
\hline 3411 & PDMS & & SW & & & E011288 & 27-Jul-82 & & $10045-97-3$ & CS-137 & GAMMA_GS & USTEST & -0.225 \\
\hline 3412 & PDMS & & SW & & & E047198 & 27-Jul-82 & & $10098-97-2$ & SR-90 & SRISO_SEEP_PRECIP_GPC & USTEST & 0.0631 \\
\hline 3413 & PDMS & & SW & & & E047198 & 27-Jul-82 & & $10045-97-3$ & CS-137 & GAMMĀGS & USTEST & 0.576 \\
\hline 3414 & PDMS & & SW & & & E015872 & 27-Jul-82 & & $10098-97-2$ & SR-90 & SRISO_SEP_PRECIP_GPC & USTEST & 3.21 \\
\hline 3415 & PDMS & & SW & & & E015872 & 27-Jul-82 & & $10045-97-3$ & CS-137 & GAMMA_GS & USTEST & 11 \\
\hline 3416 & PDMS & & SW & & & E012578 & 27-Jul-82 & & $10045-97-3$ & CS-137 & GAMMA_GS & USTEST & 0.328 \\
\hline 3417 & PDMS & & SW & & & E022449 & 27-Jul-82 & & $10045-97-3$ & CS-137 & GAMMA_GS & USTEST & 17.8 \\
\hline 3418 & PDMS & & SW & & & E022449 & 27-Jul-82 & & $10098-97-2$ & SR-90 & SRISO_SEP_PRECIP_GPC & USTEST & 1.28 \\
\hline 3419 & PDMS & & SW & & & E014322 & 27-Jul-82 & & $10045-97-3$ & CS-137 & GAMMA_GS & HISTOR & \\
\hline 3420 & PDMS & & SW & & & E014322 & 27-Jul-82 & & $10098-97-2$ & SR-90 & SRISO_SEEP_PRECIP_GPC & HISTOR & \\
\hline 3421 & PDMS & & SW & & & E027668 & 02-Aug-82 & & $10045-97-3$ & CS-137 & GAMMĀGS & USTEST & 0.132 \\
\hline 3422 & PDMS & & SW & & & E001951 & 03-Aug-82 & & $10045-97-3$ & CS-137 & GAMMA_GS & PNL-RO & 0.097 \\
\hline 3423 & PDMS & & SW & & & E001951 & 03-Aug-82 & & 15046-84-1 & I-129 & I129_SEP_TEMS & 320 & 0.000028 \\
\hline 3424 & PDMS & & SW & & & E003905 & 03-Aug-82 & & $10045-97-3$ & CS-137 & GAMMA_GS & PNL-RO & 0.0538 \\
\hline 3425 & PDMS & & SW & & & E032551 & 03-Aug-82 & & 15046-84-1 & I-129 & I129_SEP_TEMS & 320 & 0.0000025 \\
\hline 3426 & PDMS & & SW & & & E038905 & 03-Aug-82 & & 10028-17-8 & TRITIUM & TRITIUM_ELECT_LSC & USTEST & 137 \\
\hline 3427. & PDMS & & SW & & & E001242 & 03-Aug-82 & & $10045-97-3$ & CS-137 & GAMMA_GS & PNL-RO & 0.0554 \\
\hline 3428 & PDMS & & SW & & & E001242 & 03-Aug-82 & & 15046-84-1 & I-129 & I129_SEP__TEMS & 320 & 0.0000092 \\
\hline 3429 & PDMS & & SW & & & E013938 & 03-Aug-82 & & $10045-97-3$ & CS-137 & GAMMA_GS & PNL-RO & 0.028 \\
\hline 3430 & PDMS & & SW & & & E010705 & 03-Aug-82 & & $10028-17-8$ & TRITIUM & TRITIUM_ELECT_LSC & USTEST & 145 \\
\hline 3431 & PDMS & & SW & & & E010705 & 03-Aug-82 & & $10098-97-2$ & SR-90 & SRISO_SEP_PRECIP_GPC & USTEST & 0.185 \\
\hline 3432 & PDMS & & SW & & & E010705 & 03-Aug-82 & & $10045-97-3$ & CS-137 & GAMMA_GS & USTEST & -0.0651 \\
\hline 3433 & PDMS & & SW & & & E004381 & 03-Aug-82 & & $10028-17-8$ & TRITIUM & TRITIUM_ELECT_LSC & USTEST & 154 \\
\hline
\end{tabular}




\begin{tabular}{|c|c|c|c|c|c|c|c|c|c|c|c|c|c|}
\hline & $\bar{A}$ & $\mathrm{~B}$ & $\bar{C}$ & $\mathrm{D}$ & $\mathrm{E}$ & $\mathrm{F}$ & $\bar{G}$ & $\mathrm{H}$ & 1 & $\mathrm{~J}$ & $\bar{K}$ & $\bar{L}$ & $\mathrm{M}$ \\
\hline 1 & SAMPLE_NUM_ASSIGNED_TO & SAMPLER & MEDIA & BIOTA_MEDIA & TAG_ID & SAMP_NUM & SAMP_DATE_TIME & SAMP_DATE_TIME_ON & CON_ID & CON_SHORT_NAME & METHOD_NAME & LAB_CODE & VALUE_RPTD \\
\hline 3434 & PDMS & & SW & & & E004381 & 03-Aug-82 & & $10098-97-2$ & SR-90 & SRISO_SEP_PRECIP_GPC & USTEST & 0.027 \\
\hline 3435 & PDMS & & SW & & & E004381 & 03-Aug-82 & & $10045-97-3$ & CS-137 & GAMMĀGS & USTEST & 0.479 \\
\hline 3436 & PDMS & & SW & & & E038906 & 10-Aug-82 & & $10028-17-8$ & TRITIUM & TRITIUM_ELECT_LSC & HISTOR & \\
\hline 3437 & PDMS & & SW & & & E010732 & 17-Aug-82 & & $10098-97-2$ & SR-90 & 905.0_SR_GPC & USTEST & 0.0045 \\
\hline 3438 & PDMS & & SW & & & E010732 & 17-Aug-82 & & $10028-17-8$ & TRITIUM & 906.0_H3_LSC & USTEST & 89 \\
\hline 3439 & PDMS & & SW & & & E001881 & 17-Aug-82 & & $10045-97-3$ & CS-137 & GAMMA_ESS & PNL-RO & 0.078 \\
\hline 3440 & PDMS & & SW & & & E003906 & 17-Aug-82 & & $10045-97-3$ & CS-137 & GAMMA_GS & PNL-RO & 0.0353 \\
\hline 3441 & PDMS & & SW & & & E013939 & 17-Aug-82 & & $10045-97-3$ & CS-137 & GAMMA_GS & PNL-RO & 0.0263 \\
\hline 3442 & PDMS & & SW & & & E001243 & 17-Aug-82 & & $10045-97-3$ & CS-137 & GAMMA_GS & PNL-RO & 0.0511 \\
\hline 3443 & PDMS & & SW & & & E013806 & 30-Aug-82 & & $10098-97-2$ & SR-90 & 905.0_SR_GPC & USTEST & 0.102 \\
\hline 3444 & PDMS & & SW & & & E013806 & 30-Aug-82 & & $10028-17-8$ & TRITIUM & 906.0_H3_LSC & USTEST & 18900 \\
\hline 3445 & PDMS & & SW & & & E013806 & 30-Aug-82 & & $10045-97-3$ & CS-137 & 901.1_GAMMA_GS & USTEST & 1.14 \\
\hline 3446 & PDMS & & SW & & & E021418 & 30-Aug-82 & & $10098-97-2$ & SR-90 & SRISŌSEP_PRECIP_GPC & USTEST & 0.151 \\
\hline 3447 & PDMS & & SW & & & E021418 & 30-Aug-82 & & $10028-17-8$ & TRITIUM & TRITIUM_DIST_LSC & USTEST & 244 \\
\hline 3448 & PDMS & & SW & & & E037627 & 30-Aug-82 & & $10098-97-2$ & SR-90 & SRISO_SEP_PRECIP_GPC & USTEST & 0.159 \\
\hline 3449 & PDMS & & SW & & & E037627 & 30-Aug-82 & & $10045-97-3$ & CS-137 & GAMMA_GS & USTEST & 1.58 \\
\hline 3450 & PDMS & & SW & & & E032600 & 31-Aug-82 & & $10098-97-2$ & SR-90 & SRISO_SEP_PRECIP_GPC & USTEST & 0.151 \\
\hline 3451 & PDMS & & SW & & & E032600 & 31-Aug-82 & & $10028-17-8$ & TRITIUM & TRITIUM_DIST_LSC & USTEST & 234 \\
\hline 3452 & PDMS & & SW & & & E032600 & 31-Aug-82 & & $10045-97-3$ & CS-137 & GAMMA_GS & USTEST & -0.0304 \\
\hline 3453 & PDMS & & SW & & & E001882 & 31-Aug-82 & & PU-239/240 & PU-239/240 & PUISO_IE_AEA & PNL-RO & 0.000016 \\
\hline 3454 & PDMS & & SW & & & E001882 & 31-Aug-82 & & $10045-97-3$ & CS-137 & GAMMA_ḠS & PNL-RO & 0.0445 \\
\hline 3455 & PDMS & & SW & & & E001882 & 31-Aug-82 & & 15046-84-1 & $\mid-129$ & I129_SEP__TEMS & 320 & 0.00015 \\
\hline 3456 & PDMS & & SW & & & E003907 & 31-Aug-82 & & $10045-97-3$ & CS-137 & GAMMA_GS & PNL-RO & 0.0249 \\
\hline 3457 & PDMS & & SW & & & E003907 & 31-Aug-82 & & PU-239/240 & PU-239/240 & PUISO_IE_AEA & PNL-RO & 0.000054 \\
\hline 3458 & PDMS & & SW & & & E001244 & 31-Aug-82 & & $10045-97-3$ & CS-137 & GAMMA_GS & PNL-RO & 0.0446 \\
\hline 3459 & PDMS & & SW & & & E001244 & 31-Aug-82 & & PU-239/240 & PU-239/240 & PUISO_IE_AEA & PNL-RO & 0.0000096 \\
\hline 3460 & PDMS & & SW & & & E001244 & 31-Aug-82 & & 15046-84-1 & $1-129$ & I129_SEP_TEMS & 320 & 0.0000049 \\
\hline 3461 & PDMS & & SW & & & E010706 & 31-Aug-82 & & $10028-17-8$ & TRITIUM & TRITIUM_ELECT_LSC & USTEST & 86 \\
\hline 3462 & PDMS & & SW & & & E013940 & 31-Aug-82 & & PU-239/240 & PU-239/240 & PUISO_IE_AEA & PNL-RO & 0.000047 \\
\hline 3463 & PDMS & & SW & & & E010706 & 31-Aug-82 & & $10098-97-2$ & SR-90 & SRISO_SEP_PRECIP_GPC & USTEST & 0.146 \\
\hline 3464 & PDMS & & SW & & & E013940 & 31-Aug-82 & & $10045-97-3$ & CS-137 & GAMMA_GS & PNL-RO & 0.0203 \\
\hline 3465 & PDMS & & SW & & & E010706 & 31-Aug-82 & & $10045-97-3$ & CS-137 & GAMMA_GS & USTEST & -0.0696 \\
\hline 3466 & PDMS & & SW & & & E004382 & 31-Aug-82 & & 10028-17-8 & TRITIUM & TRITIUM_ELECCT_LSC & USTEST & 149 \\
\hline 3467. & PDMS & & SW & & & E004382 & 31-Aug-82 & & $10098-97-2$ & SR-90 & SRISO_SËP_PRËCIP_GPC & USTEST & 0.172 \\
\hline 3468 & PDMS & & SW & & & E004382 & 31-Aug-82 & & $10045-97-3$ & CS-137 & GAMMA_GS & USTEST & 0.294 \\
\hline 3469 & PDMS & & SW & & & E011953 & 07-Sep-82 & & $10045-97-3$ & CS-137 & 901.1_GAMMA_GS & USTEST & -0.177 \\
\hline 3470 & PDMS & & SW & & & E011953 & 07-Sep-82 & & $10028-17-8$ & TRITIUM & 906.0_H3_LSC & USTEST & 164 \\
\hline 3471 & PDMS & & SW & & & E038907 & 07-Sep-82 & & $10028-17-8$ & TRITIUM & TRITIUMM_ELECT_LSC & HISTOR & \\
\hline 3472 & PDMS & & SW & & & E001883 & 14-Sep-82 & & $10045-97-3$ & CS-137 & GAMMA_GS & PNL-RO & 0.0397 \\
\hline 3473 & PDMS & & SW & & & E003908 & 14-Sep-82 & & $10045-97-3$ & CS-137 & GAMMA_GS & PNL-RO & 0.0208 \\
\hline 3474 & PDMS & & SW & & & E015252 & 14-Sep-82 & & $10045-97-3$ & CS-137 & GAMMA_GS & HISTOR & \\
\hline 3475 & PDMS & & SW & & & E026980 & 14-Sep-82 & & $10045-97-3$ & CS-137 & GAMMA_GS & HISTOR & \\
\hline 3476 & PDMS & & SW & & & E004308 & 21-Sep-82 & & $10028-17-8$ & TRITIUM & TRITIUM_ELECT_LSC & USTEST & 120 \\
\hline 3477 & PDMS & & SW & & & E004308 & 21-Sep-82 & & $10098-97-2$ & SR-90 & SRISO_SEP_PRECIP_GPC & USTEST & 0.0993 \\
\hline 3478 & PDMS & & SW & & & E004308 & 21-Sep-82 & & $10045-97-3$ & CS-137 & GAMMA_GS & USTEST & 0.693 \\
\hline 3479 & PDMS & & SW & & & E005202 & 27-Sep-82 & & $10098-97-2$ & SR-90 & SRISO_SEEP_PRECIP_GPC & USTEST & 0.253 \\
\hline 3480 & PDMS & & SW & & & E005202 & 27-Sep-82 & & $10045-97-3$ & CS-137 & GAMMA_GS & USTEST & -0.0304 \\
\hline 3481 & PDMS & & SW & & & E001884 & 28-Sep-82 & & $10045-97-3$ & CS-137 & GAMMA_GS & PNL-RO & 0.0364 \\
\hline 3482 & PDMS & & SW & & & E001884 & 28-Sep-82 & & 15046-84-1 & I-129 & I129_SEP_TEMS & 320 & 0.00017 \\
\hline 3483 & PDMS & & SW & & & E003909 & 28-Sep-82 & & $10045-97-3$ & CS-137 & GAMMA_GS & PNL-RO & 0.023 \\
\hline 3484 & PDMS & & SW & & & E032552 & 28-Sep-82 & & 15046-84-1 & I-129 & 1129_SEP_TEMS & 320 & 0.0000074 \\
\hline 3485 & PDMS & & SW & & & E038908 & 28-Sep-82 & & $10028-17-8$ & TRITIUM & TRITIUM_ELECT_LSC & USTEST & 181 \\
\hline
\end{tabular}




\begin{tabular}{|c|c|c|c|c|c|c|c|c|c|c|c|c|c|}
\hline & A & $\mathrm{B}$ & $\mathrm{c}$ & $\mathrm{D}$ & $\mathrm{E}$ & $\mathrm{F}$ & G & $\mathrm{H}$ & $\mathrm{I}$ & $\mathrm{J}$ & $\mathrm{K}$ & $\mathrm{L}$ & $\mathrm{M}$ \\
\hline 1 & SAMPLE_NUM_ASSIGNED_TO & SAMPLER & MEDIA & BIOTA_MEDIA & TAG_ID & SAMP_NUM & SAMP_DATE_TIME & SAMP_DATE_TIME_ON & CON_ID & CON_SHORT_NAME & METHOD_NAME & LAB_CODE & \begin{tabular}{|l|} 
VALUE_RPTD \\
\end{tabular} \\
\hline 3486 & PDMS & & SW & & & E001245 & $28-\mathrm{Sep}-82$ & & $15046-84-1$ & $1-129$ & I129_SEP_TEMS & 320 & \\
\hline \begin{tabular}{|l|l|}
3487 & \\
\end{tabular} & PDMS & & sW & & & E001245 & 28-Sep-82 & & $10045-97-3$ & CS-137 & GAMMA_GSS & PNL-RO & 0.11 \\
\hline 3488 & PDMS & & sW & & & E010707 & 28-Sep-82 & & $10028-17-8$ & TRITIUM & TRITIUM_ELECT_LSC & USTEST & 77 \\
\hline 3489 & PDMS & & sW & & & E010707 & 28-Sep-82 & & $10098-97-2$ & SR-90 & SRISO_SEP_PRECIP_GPC & USTEST & 0.2 \\
\hline 3490 & PDMS & & sW & & & E010707 & 28-Sep-82 & & $10045-97-3$ & CS-137 & GAMMA_GS & USTEST & 0.306 \\
\hline 3491 & PDMS & & SW & & & E013941 & 28-Sep-82 & & $10045-97-3$ & CS-137 & GAMMA_GS & PNL-RO & 0.052 \\
\hline 3492 & PDMS & & SW & & & E001885 & 12-Oct-82 & & $10045-97-3$ & CS-137 & GAMMA_GS & PNL-RO & 0.0414 \\
\hline 3493 & PDMS & & SW & & & E003910 & $12-$ Oct-82 & & $10045-97-3$ & CS-137 & GAMMA_GS & PNL-RO & 0.0221 \\
\hline 3494 & PDMS & & sW & & & E001246 & $12-$ Oct-82 & & $10045-97-3$ & CS-137 & GAMMA GS & PNL-RO & 0.19 \\
\hline 3495 & PDMS & & SW & & & E013942 & $12-$ Oct-82 & & $10045-97-3$ & CS-137 & GAMMA_GS & PNL-RO & 0.0807 \\
\hline 3496 & PDMS & & sW & & & E017877 & $19-$ Oct-82 & & $10045-97-3$ & CS-137 & GAMMA_GS & USTEST & 4.16 \\
\hline 3497 & PDMS & & SW & & & E017877 & $19-$ Oct-82 & & 10098-97-2 & SR-90 & SRISO_SEEP_PRECIP_GPC & USTEST & 1.08 \\
\hline 3498 & PDMS & & SW & & & E011851 & $19-$ Oct-82 & & $10098-97-2$ & SR-90 & SRISO_SEP_PRECIP_GPC & USTEST & 0.874 \\
\hline 3499 & PDMS & & SW & & & E011851 & 19-Oct-82 & & $10045-97-3$ & CS-137 & GAMMA GS & USTEST & 6.77 \\
\hline 3500 & PDMS & & SW & & & E016368 & $19-$ Oct-82 & & $10098-97-2$ & SR-90 & SRISO_SEPP_PRECIP_GPC & USTEST & 0.983 \\
\hline 3501 & PDMS & & SW & & & E016368 & $19-$ Oct-82 & & $10045-97-3$ & CS-137 & GAMMA_GS & USTEST & 4.05 \\
\hline 3502 & PDMS & & sW & & & E012579 & $19-$ Oct-82 & & $10045-97-3$ & CS-137 & GAMMA_GS & USTEST & -0.182 \\
\hline 3503 & PDMS & & sW & & & E022450 & $19-$ Oct-82 & & $10045-97-3$ & CS-137 & GAMMA_GS & USTEST & 18.6 \\
\hline 3504 & PDMS & & SW & & & E022450 & $19-$ Oct-82 & & $10098-97-2$ & SR-90 & SRISO_SEEP_PRECIP_GPC & USTEST & 0.845 \\
\hline 3505 & PDMS & & SW & & & E021777 & 19-Oct-82 & & $10045-97-3$ & CS-137 & GAMMĀ_GS & USTEST & 0.662 \\
\hline 3506 & PDMS & & SW & & & E021777 & $19-$ Oct-82 & & $10098-97-2$ & SR-90 & SRISO_SEP_PRECIP_GPC & USTEST & 2.06 \\
\hline \begin{tabular}{|l|l|}
3507 & \\
\end{tabular} & PDMS & & SW & & & E001886 & 26-Oct-82 & & $10045-97-3$ & CS-137 & GAMMA_GS & PNL-RO & 0.0428 \\
\hline 3508 & PDMS & & SW & & & E001886 & $26-$ Oct-82 & & 15046-84-1 & I-129 & I129_SEP_TEMS & 320 & 0.00012 \\
\hline 3509 & PDMS & & SW & & & E003838 & $26-$ Oct-82 & & $10045-97-3$ & CS-137 & GAMMA_ĒS & PNL-RO & 0.0211 \\
\hline 3510 & PDMS & & SW & & & E032553 & $26-$ Oct-82 & & 15046-84-1 & I-129 & I129_SEP_TEMS & 320 & 0.0000065 \\
\hline 3511 & PDMS & & SW & & & E038909 & $26-$ Oct-82 & & $10028-17-8$ & TRITIUM & TRITIUM_ELEECT_LSC & USTEST & 109 \\
\hline 3512 & PDMS & & SW & & & E001247 & 26-Oct-82 & & 15046-84-1 & I-129 & I129_SEP_TEMS & 320 & 0.000013 \\
\hline 3513 & PDMS & & SW & & & E001247 & 26-Oct-82 & & $10045-97-3$ & CS-137 & GAMMA_GSS & PNL-RO & 0.0578 \\
\hline 3514 & PDMS & & SW & & & E013943 & $26-$ Oct-82 & & $10045-97-3$ & CS-137 & GAMMA_GS & PNL-RO & 0.0246 \\
\hline 3515 & PDMS & & SW & & & E010708 & $26-$ Oct-82 & & $10028-17-8$ & TRITIUM & TRITIUM_ELECT_LSC & USTEST & 128 \\
\hline 3516 & PDMS & & SW & & & E010708 & $26-$ Oct-82 & & $10098-97-2$ & SR-90 & SRISO_SEP_PRËCIP_GPC & USTEST & 0.166 \\
\hline 3517 & PDMS & & SW & & & E010708 & $26-$ Oct-82 & & $10045-97-3$ & CS-137 & GAMMĀ_GS & USTEST & -0.664 \\
\hline 3518 & PDMS & & SW & & & E004309 & $26-$ Oct-82 & & $10028-17-8$ & TRITIUM & TRITIUM_ELECT_LSC & USTEST & 151 \\
\hline 3519 & PDMS & & SW & & & E004309 & $26-$ Oct-82 & & $10098-97-2$ & SR-90 & SRISO_SEPP_PRĒCIP_GPC & USTEST & 0.159 \\
\hline 3520 & PDMS & & SW & & & E004309 & 26-Oct-82 & & $10045-97-3$ & CS-137 & GAMMĀ_GS & USTEST & -0.108 \\
\hline 3521 & PDMS & & sW & & & E037628 & $26-$ Oct-82 & & $10098-97-2$ & SR-90 & SRISO_SETEPPRECIP_GPC & USTEST & 0.267 \\
\hline 3522 & PDMS & & SW & & & E037628 & $26-$ Oct-82 & & $10045-97-3$ & CS-137 & GAMMA_GS & USTEST & 0.0209 \\
\hline 3523 & PDMS & & SW & & & E010733 & 09-Nov-82 & & $10098-97-2$ & SR-90 & 905.0_SR_GPC & USTEST & 0.0131 \\
\hline 3524 & PDMS & & SW & & & E010733 & 09-Nov-82 & & $10028-17-8$ & TRITIUM & 906.0_H3_LSC & USTEST & -115 \\
\hline 3525 & PDMS & & SW & & & E003839 & 09-Nov-82 & & $10045-97-3$ & CS-137 & GAMM̄A_GS & PNL-RO & 0.0186 \\
\hline 3526 & PDMS & & SW & & & E001887 & 09-Nov-82 & & $10045-97-3$ & CS-137 & GAMMA_GS & PNL-RO & 0.0412 \\
\hline \begin{tabular}{|l|l|}
3527 & \\
\end{tabular} & PDMS & & SW & & & E013870 & 09-Nov-82 & & $10045-97-3$ & CS-137 & GAMMA_GS & PNL-RO & 0.0557 \\
\hline 3528 & PDMS & & SW & & & E001248 & 09-Nov-82 & & $10045-97-3$ & CS-137 & GAMMA_GS & PNL-RO & 0.12 \\
\hline 3529 & PDMS & & SW & & & E013807 & 17-Nov-82 & & $10098-97-2$ & SR-90 & 905.0_SR_GPC & USTEST & 0.449 \\
\hline 3530 & PDMS & & SW & & & E013807 & 17-Nov-82 & & $10028-17-8$ & TRITIUM & 906.0_H3_LSC & USTEST & 14700 \\
\hline 3531 & PDMS & & SW & & & E013807 & 17-Nov-82 & & $10045-97-3$ & CS-137 & 901.1_GAMMA_GS & USTEST & -0.114 \\
\hline 3532 & PDMS & & SW & & & E021419 & 22-Nov-82 & & $10098-97-2$ & SR-90 & SRISŌSEP_PRECIP_GPC & USTEST & 0.25 \\
\hline 3533 & PDMS & & SW & & & E021419 & 22-Nov-82 & & $10028-17-8$ & TRITIUM & TRITIUM̄_DISTT_LSC & USTEST & -90 \\
\hline 3534 & PDMS & & SW & & & E005210 & 22-Nov-82 & & $10098-97-2$ & SR-90 & SRISO_SEP_PRECIP_GPC & USTEST & 0.0735 \\
\hline 3535 & PDMS & & sW & & & E005210 & 22-Nov-82 & & $10045-97-3$ & CS-137 & GAMMĀGS & USTEST & 0.413 \\
\hline 3536 & PDMS & & sW & & & E032601 & 23-Nov-82 & & $10098-97-2$ & SR-90 & SRISO SEP PRECIP GPC & USTEST & 0.184 \\
\hline 3537. & PDMS & & SW & & & E032601 & 23-Nov-82 & & $10028-17-8$ & TRITIUM & TRITIUM_DIS̄T_LSC & USTEST & -240 \\
\hline
\end{tabular}




\begin{tabular}{|c|c|c|c|c|c|c|c|c|c|c|c|c|c|}
\hline & $\bar{A}$ & $\mathrm{~B}$ & $\bar{C}$ & $\mathrm{D}$ & $\mathrm{E}$ & $\mathrm{F}$ & $\bar{G}$ & $\mathrm{H}$ & 1 & $\mathrm{~J}$ & $\bar{K}$ & $\bar{L}$ & $\mathrm{M}$ \\
\hline 1 & SAMPLE_NUM_ASSIGNED_TO & \begin{tabular}{|l} 
SAMPLER \\
\end{tabular} & MEDIA & BIOTA_MEDIA & TAG_ID & SAMP_NUM & SAMP_DATE_TIME & SAMP_DATE_TIME_ON & CON_ID & CON_SHORT_NAME & METHOD_NAME & LAB_CODE & VALUE_RPTD \\
\hline 3538 & PDMS & & SW & & & E032601 & $23-N o v-82$ & & $10045-97-3$ & CS-137 & GAMMA_GS & USTEST & 0.684 \\
\hline 3539 & PDMS & & SW & & & E001888 & 23-Nov-82 & & PU-239/240 & PU-239/240 & PUISO_IE_AEA & PNL-RO & 0.000011 \\
\hline 3540 & PDMS & & SW & & & E001888 & 23-Nov-82 & & $10045-97-3$ & CS-137 & GAMMA_GS & PNL-RO & 0.0488 \\
\hline 3541 & PDMS & & SW & & & E001888 & 23-Nov-82 & & 15046-84-1 & I-129 & I129_SEP_TEMS & 320 & 0.000086 \\
\hline 3542 & PDMS & & SW & & & E003840 & 23-Nov-82 & & $10045-97-3$ & CS-137 & GAMMA_ESS & PNL-RO & 0.0301 \\
\hline 3543 & PDMS & & SW & & & E003840 & 23-Nov-82 & & PU-239/240 & PU-239/240 & PUISO_IE_AEA & PNL-RO & 0.000022 \\
\hline 3544 & PDMS & & SW & & & E032554 & 23-Nov-82 & & 15046-84-1 & I-129 & I129_SEP_TEMS & 320 & 0.0000063 \\
\hline 3545 & PDMS & & SW & & & E038910 & 23-Nov-82 & & $10028-17-8$ & TRITIUM & TRITIUM_ELEECT_LSC & USTEST & 138 \\
\hline 3546 & PDMS & & SW & & & E001249 & 23-Nov-82 & & 15046-84-1 & I-129 & 1129_SEP_TEMS ${ }^{-}$ & 320 & 0.0000074 \\
\hline \begin{tabular}{|l|l|}
3547 & \\
\end{tabular} & PDMS & & SW & & & E001249 & 23-Nov-82 & & $10045-97-3$ & CS-137 & GAMMA_ḠS & PNL-RO & 0.0464 \\
\hline 3548 & PDMS & & SW & & & E001249 & 23-Nov-82 & & PU-239/240 & PU-239/240 & PUISO_IE_AEA & PNL-RO & 0.000011 \\
\hline 3549 & PDMS & & SW & & & E010709 & 23-Nov-82 & & 10028-17-8 & TRITIUM & TRITIUM_ELECT_LSC & USTEST & 121 \\
\hline 3550 & PDMS & & SW & & & E013871 & 23-Nov-82 & & PU-239/240 & PU-239/240 & PUISO_IE_AEA & PNL-RO & 0.000026 \\
\hline 3551 & PDMS & & SW & & & E013871 & 23-Nov-82 & & $10045-97-3$ & CS-137 & GAMMA_GS & PNL-RO & 0.0225 \\
\hline 3552 & PDMS & & SW & & & E010709 & 23-Nov-82 & & $10098-97-2$ & SR-90 & SRISO_SEP_PRECIP_GPC & USTEST & 0.206 \\
\hline 3553 & PDMS & & SW & & & E010709 & 23-Nov-82 & & $10045-97-3$ & CS-137 & GAMMA_GS & USTEST & 0.407 \\
\hline 3554 & PDMS & & SW & & & E004310 & 23-Nov-82 & & $10028-17-8$ & TRITIUM & TRITIUM_ELECT_LSC & USTEST & 217 \\
\hline 3555 & PDMS & & SW & & & E004310 & 23-Nov-82 & & $10098-97-2$ & SR-90 & SRISO_SEP_PRECIP_GPC & USTEST & 0.192 \\
\hline 3556 & PDMS & & SW & & & E004310 & 23-Nov-82 & & $10045-97-3$ & CS-137 & GAMMA_GS & USTEST & 0.00344 \\
\hline 3557. & PDMS & & SW & & & E036171 & 29-Nov-82 & & $10045-97-3$ & CS-137 & 901.1_GAMMA_GS & USTEST & 0.222 \\
\hline 3558 & PDMS & & SW & & & E036171 & 29-Nov-82 & & $10028-17-8$ & TRITIUM & 906.0_H3_LSC & USTEST & 135 \\
\hline 3559 & PDMS & & SW & & & E001889 & 07-Dec-82 & & $10045-97-3$ & CS-137 & GAMMĀE_GS & PNL-RO & 0.0472 \\
\hline 3560 & PDMS & & SW & & & E003841 & 07-Dec-82 & & $10045-97-3$ & CS-137 & GAMMA_GS & PNL-RO & 0.0242 \\
\hline 3561 & PDMS & & SW & & & E013872 & 07-Dec-82 & & $10045-97-3$ & CS-137 & GAMMA_GS & PNL-RO & 0.0275 \\
\hline 3562 & PDMS & & SW & & & E001250 & 07-Dec-82 & & $10045-97-3$ & CS-137 & GAMMA_GS & PNL-RO & 0.0527 \\
\hline 3563 & PDMS & & SW & & & E004311 & 07-Dec-82 & & $10028-17-8$ & TRITIUM & TRITIUM_ELECT_LSC & USTEST & 142 \\
\hline 3564 & PDMS & & SW & & & E004311 & 07-Dec-82 & & $10098-97-2$ & SR-90 & SRISO_SEPP_PRËCIP_GPC & USTEST & 0.18 \\
\hline 3565 & PDMS & & SW & & & E004311 & 07-Dec-82 & & $10045-97-3$ & CS-137 & GAMMA_GS & USTEST & 0.263 \\
\hline 3566 & PDMS & & SW & & & E003842 & 21-Dec-82 & & $10045-97-3$ & CS-137 & GAMMA_GS & PNL-RO & 0.0234 \\
\hline 3567 & PDMS & & SW & & & E001890 & 21-Dec-82 & & $10045-97-3$ & CS-137 & GAMMA_GS & PNL-RO & 0.0451 \\
\hline 3568 & PDMS & & SW & & & E001890 & 21-Dec-82 & & 15046-84-1 & I-129 & I129_SEP_TEMS & 320 & 0.00011 \\
\hline 3569 & PDMS & & SW & & & E032555 & 21-Dec-82 & & 15046-84-1 & I-129 & I129_SEP_TEMS & 320 & 0.0000083 \\
\hline 3570 & PDMS & & SW & & & E038911 & 21-Dec-82 & & 10028-17-8 & TRITIUM & TRITIUM_ELECT_LSC & USTEST & 111 \\
\hline 3571 & PDMS & & SW & & & E001251 & 21-Dec-82 & & 15046-84-1 & I-129 & I129_SEP_TEMS & 320 & 0.0000052 \\
\hline 3572 & PDMS & & SW & & & E001251 & 21-Dec-82 & & $10045-97-3$ & CS-137 & GAMMA_GS & PNL-RO & 0.11 \\
\hline 3573 & PDMS & & SW & & & E013873 & 21-Dec-82 & & $10045-97-3$ & CS-137 & GAMMA_GS & PNL-RO & 0.0556 \\
\hline 3574 & PDMS & & SW & & & E010710 & 21-Dec-82 & & $10028-17-8$ & TRITIUM & TRITIUM_ELECT_LSC & USTEST & 168 \\
\hline 3575 & PDMS & & SW & & & E010710 & 21-Dec-82 & & $10098-97-2$ & SR-90 & SRISO_SEP_PRECIP_GPC & USTEST & 0.337 \\
\hline 3576 & PDMS & & SW & & & E010710 & 21-Dec-82 & & $10045-97-3$ & CS-137 & GAMMA_GS & USTEST & 0.494 \\
\hline 3577 & PDMS & & SW & & & E002079 & 27-Dec-82 & & $10098-97-2$ & SR-90 & SRISO_SEP_PRECIP_GPC & USTEST & 0.183 \\
\hline 3578 & PDMS & & SW & & & E002079 & 27-Dec-82 & & $10045-97-3$ & CS-137 & GAMMA_GS & USTEST & 0.0464 \\
\hline 3579 & PDMS & & SW & & & E000906 & 28-Dec-82 & & $10028-17-8$ & TRITIUM & TRITIUM_DIST_LSC & USTEST & 289 \\
\hline 3580 & PDMS & & SW & & & E000906 & 28-Dec-82 & & $10098-97-2$ & SR-90 & SRISO_SEP_PRECIP_GPC & USTEST & 0.504 \\
\hline 3581 & PDMS & & SW & & & E000906 & 28-Dec-82 & & $10045-97-3$ & CS-137 & GAMMA_GS & USTEST & 6.21 \\
\hline 3582 & PDMS & & SW & & & E024522 & 28-Dec-82 & & $10028-17-8$ & TRITIUM & TRITIUM_DIST_LSC & USTEST & 376 \\
\hline 3583 & PDMS & & SW & & & E024522 & 28-Dec-82 & & $10098-97-2$ & SR-90 & SRISO_SEP_PRECIP_GPC & USTEST & 0.00682 \\
\hline 3584 & PDMS & & SW & & & E024522 & 28-Dec-82 & & $10045-97-3$ & CS-137 & GAMMA_GS & USTEST & -0.0618 \\
\hline 3585 & PDMS & & SW & & & E001891 & 04-Jan-83 & & $10045-97-3$ & CS-137 & GAMMA_GS & PNL-RO & 0.046 \\
\hline 3586 & PDMS & & SW & & & E003843 & 04-Jan-83 & & $10045-97-3$ & CS-137 & GAMMA_GS & PNL-RO & 0.0244 \\
\hline 3587 & PDMS & & SW & & & E038912 & 04-Jan-83 & & $10028-17-8$ & TRITIUM & TRITIUM_ELECT_LSC & USTEST & 109 \\
\hline 3588 & PDMS & & SW & & & E001252 & 04-Jan-83 & & $10045-97-3$ & CS-137 & GAMMA_GS & PNL-RO & 0.0549 \\
\hline 3589 & PDMS & & SW & & & E013874 & 04-Jan-83 & & $10045-97-3$ & CS-137 & GAMMA_GS & PNL-RO & 0.025 \\
\hline
\end{tabular}




\begin{tabular}{|c|c|c|c|c|c|c|c|c|c|c|c|c|c|}
\hline & $\bar{A}$ & $\mathrm{~B}$ & $\mathrm{C}$ & $\bar{D}$ & $\mathrm{E}$ & $\mathrm{F}$ & $\bar{G}$ & $\mathrm{H}$ & 1 & $\mathrm{~J}$ & $\mathrm{~K}$ & $\mathrm{~L}$ & $\bar{M}$ \\
\hline 1 & SAMPLE_NUM_ASSIGNED_TO & SAMPLER & MEDIA & BIOTA_MEDIA & TAG_ID & SAMP_NUM & SAMP_DATE_TIME| & SAMP_DATE_TIME_ON & CON_ID & CON_SHORT_NAME & METHOD_NAME & LAB_CODE & VALUE_RPTD \\
\hline 3590 & PDMS & & SW & & & E005142 & 17-Jan-83 & & $10098-97-2$ & SR-90 & SRISO_SEP_PRECIP_GPC & USTEST & 0.259 \\
\hline 3591 & PDMS & & SW & & & E005142 & 17-Jan-83 & & $10045-97-3$ & CS-137 & GAMMĀGS' & USTEST & 0.152 \\
\hline 3592 & PDMS & & SW & & & E001892 & 18-Jan-83 & & $10045-97-3$ & CS-137 & GAMMA_GS & PNL-RO & 0.0458 \\
\hline 3593 & PDMS & & SW & & & E003844 & 18-Jan-83 & & $10045-97-3$ & CS-137 & GAMMA_GS & PNL-RO & 0.0213 \\
\hline 3594 & PDMS & & SW & & & E001190 & 18-Jan-83 & & $10045-97-3$ & CS-137 & GAMMA_GS & PNL-RO & 0.0388 \\
\hline 3595 & PDMS & & SW & & & E010711 & 18-Jan-83 & & $10028-17-8$ & TRITIUM & TRITIUM_ELECT_LSC & USTEST & 120 \\
\hline 3596 & PDMS & & sW & & & E013875 & 18-Jan-83 & & $10045-97-3$ & CS-137 & GAMMA_GS & PNL-RO & 0.02168 \\
\hline 3597 & PDMS & & sW & & & E010711 & 18-Jan-83 & & $10098-97-2$ & SR-90 & SRISO_SEEP_PRECIP_GPC & USTEST & 0.189 \\
\hline 3598 & PDMS & & sW & & & E010711 & 18-Jan-83 & & $10045-97-3$ & CS-137 & GAMMĀ_GS & USTEST & -0.674 \\
\hline 3599 & PDMS & & sW & & & E004312 & 18-Jan-83 & & $10028-17-8$ & TRITIUM & TRITIUM_ELECT_LSC & USTEST & 90.7 \\
\hline 3600 & PDMS & & SW & & & E001893 & 01-Feb-83 & & $10045-97-3$ & CS-137 & GAMMA_GS & PNL-RO & 0.0362 \\
\hline 3601 & PDMS & & SW & & & E003845 & 01-Feb-83 & & $10045-97-3$ & CS-137 & GAMMA_GS & PNL-RO & 0.00187 \\
\hline 3602 & PDMS & & sW & & & E013876 & 01-Feb-83 & & $10045-97-3$ & CS-137 & GAMMA_GS & PNL-RO & 0.0216 \\
\hline 3603 & PDMS & & SW & & & E001191 & 01-Feb-83 & & $10045-97-3$ & CS-137 & GAMMA_GS & PNL-RO & 0.0397 \\
\hline 3604 & PDMS & & SW & & & E017787 & 08-Feb-83 & & $10045-97-3$ & CS-137 & GAMMA_GS & USTEST & 0.355 \\
\hline 3605 & PDMS & & SW & & & E017787 & 08-Feb-83 & & $10098-97-2$ & SR-90 & SRISO_SEP_PRECIP_GPC & USTEST & 11.5 \\
\hline 3606 & PDMS & & SW & & & E017787 & 08-Feb-83 & & $10028-17-8$ & TRITIUM & TRITIUM_DIST_LSC & USTEST & 50 \\
\hline 3607 & PDMS & & sW & & & E021420 & 14-Feb-83 & & 10098-97-2 & SR-90 & SRISO_SEP_PRECIP_GPC & USTEST & 0.205 \\
\hline 3608 & PDMS & & sW & & & E021420 & 14-Feb-83 & & $10028-17-8$ & TRITIUM & TRITIUM_DIST_LSC & USTEST & 170 \\
\hline 3609 & PDMS & & sW & & & E005146 & 14-Feb-83 & & $10098-97-2$ & SR-90 & SRISO_SEP_PRECIP_GPC & USTEST & 0.2 \\
\hline 3610 & PDMS & & sW & & & E005146 & 14-Feb-83 & & $10045-97-3$ & CS-137 & GAMMĀGS' & USTEST & 0.201 \\
\hline 3611 & PDMS & & SW & & & E032602 & $15-F e b-83$ & & $10098-97-2$ & SR-90 & SRISO_SEP_PRECIP_GPC & USTEST & 0.305 \\
\hline 3612 & PDMS & & SW & & & E032602 & 15-Feb-83 & & $10028-17-8$ & TRITIUM & TRITIUM_DIST_LSC & USTEST & 120 \\
\hline 3613 & PDMS & & SW & & & E032602 & 15-Feb-83 & & $10045-97-3$ & CS-137 & GAMMA_GS & USTEST & 0.341 \\
\hline 3614 & PDMS & & SW & & & E001894 & 15-Feb-83 & & PU-239/240 & PU-239/240 & PUISO_IE_AEA & PNL-RO & 0.000054 \\
\hline 3615 & PDMS & & SW & & & E001894 & 15-Feb-83 & & $10045-97-3$ & CS-137 & GAMMA_GS & PNL-RO & 0.0445 \\
\hline 3616 & PDMS & & SW & & & E001894 & $15-F e b-83$ & & 15046-84-1 & I-129 & I129_SEP_TEMS & 320 & 0.000068 \\
\hline 3617 & PDMS & & SW & & & E003846 & $15-F e b-83$ & & $10045-97-3$ & CS-137 & GAMMA_GS & PNL-RO & 0.019 \\
\hline 3618 & PDMS & & SW & & & E003846 & 15-Feb-83 & & PU-239/240 & PU-239/240 & PUISO_IE_AEA & PNL-RO & 0.000014 \\
\hline 3619 & PDMS & & SW & & & E010712 & 15-Feb-83 & & $10028-17-8$ & TRITIUM & TRITIUM_ELECT_LSC & USTEST & 135 \\
\hline 3620 & PDMS & & SW & & & E010712 & 15-Feb-83 & & $10098-97-2$ & SR-90 & SRISO_SEP_PRECIP_GPC & USTEST & 0.191 \\
\hline 3621 & PDMS & & SW & & & E010712 & $15-F e b-83$ & & $10045-97-3$ & CS-137 & GAMMA_GS & USTEST & 0.139 \\
\hline 3622 & PDMS & & SW & & & E001192 & 15-Feb-83 & & 15046-84-1 & I-129 & I129_SEP_TEMS & 320 & 0.000028 \\
\hline 3623 & PDMS & & SW & & & E001192 & $15-F e b-83$ & & $10045-97-3$ & CS-137 & GAMMA_GS & PNL-RO & 0.0555 \\
\hline 3624 & PDMS & & SW & & & E001192 & $15-F e b-83$ & & PU-239/240 & PU-239/240 & PUISO_IE_AEA & PNL-RO & 0.000093 \\
\hline 3625 & PDMS & & SW & & & E013877 & 15-Feb-83 & & PU-239/240 & PU-239/240 & PUISO_IE_AEA & PNL-RO & 0.000018 \\
\hline 3626 & PDMS & & SW & & & E013877 & 15-Feb-83 & & $10045-97-3$ & CS-137 & GAMMA_GS & PNL-RO & 0.00199 \\
\hline 3627 & PDMS & & sW & & & E004313 & 15-Feb-83 & & $10028-17-8$ & TRITIUM & TRITIUM_ELECT_LSC & USTEST & 118 \\
\hline 3628 & PDMS & & SW & & & E004313 & $15-F e b-83$ & & $10045-97-3$ & CS-137 & GAMMA_GS & USTEST & 0.304 \\
\hline 3629 & PDMS & & SW & & & E004313 & $15-F e b-83$ & & $10098-97-2$ & SR-90 & SRISO_SEP_PRECIP_GPC & USTEST & 0.996 \\
\hline 3630 & PDMS & & SW & & & E015390 & 22-Feb-83 & & $10045-97-3$ & CS-137 & GAMMA_GS & HISTOR & \\
\hline 3631 & PDMS & & SW & & & E015390 & $22-F e b-83$ & & $10098-97-2$ & SR-90 & SRISO_SEP_PRECIP_GPC & HISTOR & \\
\hline 3632 & PDMS & & SW & & & E037316 & 22-Feb-83 & & $10098-97-2$ & SR-90 & SRISO_SEP_PRECIP_GPC & HISTOR & \\
\hline 3633 & PDMS & & SW & & & E037316 & 22-Feb-83 & & $10028-17-8$ & TRITIUM & TRITIUM_DIST_LSC & HISTOR & \\
\hline $3634 \mathrm{~F}$ & PDMS & & SW & & & E037316 & 22-Feb-83 & & $10045-97-3$ & CS-137 & GAMMA_GS & HISTOR & \\
\hline 3635 & PDMS & & SW & & & E013529 & 22-Feb-83 & & $10098-97-2$ & SR-90 & SRISO_SEP_PRECIP_GPC & USTEST & 4.36 \\
\hline 3636 & PDMS & & SW & & & E013529 & $22-F e b-83$ & & $10045-97-3$ & CS-137 & GAMMA_GS & USTEST & 1.96 \\
\hline 3637 & PDMS & & SW & & & E013529 & $22-F e b-83$ & & $10028-17-8$ & TRITIUM & TRITIUM_DIST_LSC & USTEST & 468 \\
\hline 3638 & PDMS & & SW & & & E012580 & 22-Feb-83 & & $10028-17-8$ & TRITIUM & TRITIUM_DIST_LSC & USTEST & 19100 \\
\hline 3639 & PDMS & & SW & & & E012580 & 22-Feb-83 & & $10045-97-3$ & CS-137 & GAMMA_GS & USTEST & -0.922 \\
\hline 3640 & PDMS & & SW & & & E017788 & 22-Feb-83 & & $10045-97-3$ & CS-137 & GAMMA_GS & USTEST & 7.82 \\
\hline $3641 \mathrm{~F}$ & PDMS & & SW & & & E017788 & $22-F e b-83$ & & $10098-97-2$ & SR-90 & SRISO_SEP_PRECIP_GPC & USTEST & 7.28 \\
\hline
\end{tabular}




\begin{tabular}{|c|c|c|c|c|c|c|c|c|c|c|c|c|c|}
\hline & A & $\mathrm{B}$ & $\mathrm{c}$ & $\mathrm{D}$ & $\mathrm{E}$ & $\mathrm{F}$ & G & $\mathrm{H}$ & $\mathrm{I}$ & $\mathrm{J}$ & $\mathrm{K}$ & $\mathrm{L}$ & $\mathrm{M}$ \\
\hline 1 & SAMPLE_NUM_ASSIGNED_TO & SAMPLER & MEDIA & BIOTA_MEDIA & TAG_ID & SAMP_NUM & SAMP_DATE_TIME & SAMP_DATE_TIME_ON & CON_ID & CON_SHORT_NAME & METHOD_NAME & LAB_CODE & \begin{tabular}{|l|} 
VALUE_RPTD \\
\end{tabular} \\
\hline 3642 & PDMS & & SW & & & E017788 & $22-F e b-83$ & & $10028-17-8$ & TRITIUM & TRITIUM DIST_LSC & USTEST & 342 \\
\hline 3643 & PDMS & & sW & & & E011121 & 22-Feb-83 & & $10028-17-8$ & TRITIUM & TRITIUM_DIST_LSC & USTEST & 2950 \\
\hline 3644 & PDMS & & sW & & & E011121 & 22-Feb-83 & & $10045-97-3$ & CS-137 & GAMMA_GS & USTEST & 0.968 \\
\hline 3645 & PDMS & & sW & & & E011121 & 22-Feb-83 & & $10098-97-2$ & SR-90 & SRISO_SEP_PRECIP_GPC & USTEST & 0.122 \\
\hline \begin{tabular}{|l|l|}
3646 & \\
\end{tabular} & PDMS & & sW & & & E010734 & 01-Mar-83 & & $10098-97-2$ & SR-90 & 905.0_SR_GPC & USTEST & 0.321 \\
\hline \begin{tabular}{|l|l|}
3647 \\
\end{tabular} & PDMS & & sW & & & E010734 & 01-Mar-83 & & $10028-17-8$ & TRITIUM & 906.0_H3_LSC & USTEST & -97 \\
\hline 3648 & PDMS & & SW & & & E001895 & 01-Mar-83 & & $10045-97-3$ & CS-137 & GAMMA_GS & PNL-RO & 0.0406 \\
\hline 3649 & PDMS & & SW & & & E003847 & 01-Mar-83 & & $10045-97-3$ & CS-137 & GAMMA_GS & PNL-RO & 0.0179 \\
\hline 3650 & PDMS & & sW & & & E013878 & 01-Mar-83 & & $10045-97-3$ & CS-137 & GAMMA GS & PNL-RO & 0.0154 \\
\hline 3651 & PDMS & & SW & & & E001193 & 01-Mar-83 & & $10045-97-3$ & CS-137 & GAMMA_GS & PNL-RO & 0.0298 \\
\hline 3652 & PDMS & & sW & & & E013808 & 07-Mar-83 & & $10098-97-2$ & SR-90 & 905.0_SR_GPC & USTEST & 0.0802 \\
\hline 3653 & PDMS & & SW & & & E013808 & 07-Mar-83 & & $10028-17-8$ & TRITIUM & 906.0_H3_LSC & USTEST & 17900 \\
\hline 3654 & PDMS & & SW & & & E013808 & 07-Mar-83 & & $10045-97-3$ & CS-137 & 901.1_GAMMA_GS & USTEST & -0.315 \\
\hline 3655 & PDMS & & SW & & & E037629 & 14-Mar-83 & & $10098-97-2$ & SR-90 & SRISO SEP PRECIP GPC & USTEST & 0.19 \\
\hline 3656 & PDMS & & SW & & & E037629 & 14-Mar-83 & & $10045-97-3$ & CS-137 & GAMMA_GS & USTEST & 1.14 \\
\hline \begin{tabular}{|l|l|}
3657 & \\
\end{tabular} & PDMS & & SW & & & E003848 & 15-Mar-83 & & $10045-97-3$ & CS-137 & GAMMA_GS & PNL-RO & 0.0202 \\
\hline 3658 & PDMS & & sW & & & E001896 & 15-Mar-83 & & $10045-97-3$ & CS-137 & GAMMA_GS & PNL-RO & 0.0383 \\
\hline 3659 & PDMS & & sW & & & E001194 & 15-Mar-83 & & $10045-97-3$ & CS-137 & GAMMA_GS & PNL-RO & 0.0842 \\
\hline 3660 & PDMS & & SW & & & E013879 & 15-Mar-83 & & $10045-97-3$ & CS-137 & GAMMA_GS & PNL-RO & 0.0292 \\
\hline 3661 & PDMS & & SW & & & E010713 & 15-Mar-83 & & $10028-17-8$ & TRITIUM & TRITIUM_ELELECT_LSC & USTEST & 128 \\
\hline 3662 & PDMS & & SW & & & E010713 & 15-Mar-83 & & $10098-97-2$ & SR-90 & SRISO_SEP_PRËCIP_GPC & USTEST & 0.257 \\
\hline 3663 & PDMS & & SW & & & E010713 & 15-Mar-83 & & $10045-97-3$ & CS-137 & GAMMA_GS & USTEST & 0.171 \\
\hline 3664 & PDMS & & SW & & & E004314 & 15-Mar-83 & & $10028-17-8$ & TRITIUM & TRITIUM_ELECT_LSC & USTEST & 134 \\
\hline 3665 & PDMS & & sW & & & E004314 & 15-Mar-83 & & $10098-97-2$ & SR-90 & SRISO_SEP_PRECIP_GPC & USTEST & 0.104 \\
\hline 3666 & PDMS & & SW & & & E004314 & 15-Mar-83 & & $10045-97-3$ & CS-137 & GAMMA_GS & USTEST & -0.0965 \\
\hline 3667. & PDMS & & SW & & & E012593 & 21-Mar-83 & & $10098-97-2$ & SR-90 & 905.0_SR_GPC & USTEST & 0.0752 \\
\hline 3668 & PDMS & & SW & & & E012593 & 21-Mar-83 & & $10045-97-3$ & CS-137 & 901.1_GAMMA_GS & USTEST & 0.131 \\
\hline 3669 & PDMS & & SW & & & E012593 & 21-Mar-83 & & $10028-17-8$ & TRITIUM & 906.0_H3_LSC & USTEST & -11.8 \\
\hline 3670 & PDMS & & SW & & & E013110 & 25-Mar-83 & & $10045-97-3$ & CS-137 & 901.1_GAMMA_GS & USTEST & 0.0693 \\
\hline 3671 & PDMS & & SW & & & E013110 & 25-Mar-83 & & $10028-17-8$ & TRITIUM & 906.0_H3_LSC & USTEST & \\
\hline 3672 & PDMS & & sW & & & E013110 & 25-Mar-83 & & $10098-97-2$ & SR-90 & 905.0_SR_GPC & USTEST & 0.337 \\
\hline 3673 & PDMS & & SW & & & E003849 & 29-Mar-83 & & $10045-97-3$ & CS-137 & GAMMA_ĒGS & PNL-RO & 0.0239 \\
\hline 3674 & PDMS & & SW & & & E001897 & 29-Mar-83 & & $10045-97-3$ & CS-137 & GAMMA_GS & PNL-RO & 0.0467 \\
\hline 3675 & PDMS & & SW & & & E001195 & 29-Mar-83 & & $10045-97-3$ & CS-137 & GAMMA_GS & PNL-RO & 0.042 \\
\hline 3676 & PDMS & & SW & & & E013880 & 29-Mar-83 & & $10045-97-3$ & CS-137 & GAMMA_GS & PNL-RO & 0.0244 \\
\hline 3677 & PDMS & & SW & & & E032035 & 11-Apr-83 & & $10028-17-8$ & TRITIUM & 906.0_H3_LSC & USTEST & 104 \\
\hline 3678 & PDMS & & SW & & & E032035 & 11-Apr-83 & & $10098-97-2$ & SR-90 & 905.0_SR_GPC & USTEST & 0.204 \\
\hline 3679 & PDMS & & SW & & & E037630 & 11-Apr-83 & & $10098-97-2$ & SR-90 & SRISO_SĒP_PRECIP_GPC & USTEST & 0.206 \\
\hline 3680 & PDMS & & SW & & & E037630 & 11-Apr-83 & & $10045-97-3$ & CS-137 & GAMMA_GS & USTEST & 0.571 \\
\hline 3681 & PDMS & & SW & & & E001898 & 12-Apr-83 & & $10045-97-3$ & CS-137 & GAMMA_GS & PNL-RO & 0.0375 \\
\hline 3682 & PDMS & & SW & & & E003850 & 12-Apr-83 & & $10045-97-3$ & CS-137 & GAMMA_GS & PNL-RO & 0.0167 \\
\hline 3683 & PDMS & & SW & & & E001196 & 12-Apr-83 & & $10045-97-3$ & CS-137 & GAMMA_GS & PNL-RO & 0.0627 \\
\hline 3684 & PDMS & & SW & & & E010714 & 12-Apr-83 & & $10028-17-8$ & TRITIUM & TRITIUM_ELECT_LSC & USTEST & 123 \\
\hline 3685 & PDMS & & SW & & & E013881 & 12-Apr-83 & & $10045-97-3$ & CS-137 & GAMMA_GS & PNL-RO & 0.0297 \\
\hline 3686 & PDMS & & SW & & & E010714 & 12-Apr-83 & & $10098-97-2$ & SR-90 & SRISO_SEPPPRECIP_GPC & USTEST & 0.197 \\
\hline 3687 & PDMS & & SW & & & E010714 & 12-Apr-83 & & $10045-97-3$ & CS-137 & GAMMĀGS & USTEST & 0.0343 \\
\hline 3688 & PDMS & & SW & & & E004315 & 12-Apr-83 & & $10028-17-8$ & TRITIUM & TRITIUM_ELELECT_LSC & USTEST & 194 \\
\hline 3689 & PDMS & & SW & & & E004315 & 12-Apr-83 & & $10098-97-2$ & SR-90 & SRISO_SEPP_PRËCIP_GPC & USTEST & 0.191 \\
\hline 3690 & PDMS & & SW & & & E004315 & 12-Apr-83 & & $10045-97-3$ & CS-137 & GAMMA_GS & USTEST & -0.00792 \\
\hline 3691 & PDMS & & sW & & & E017379 & 19-Apr-83 & & $10098-97-2$ & SR-90 & SRISO_SEP_PRECIP_GPC & USTEST & 0.141 \\
\hline 3692 & PDMS & & sW & & & E017379 & 19-Apr-83 & & $10045-97-3$ & CS-137 & GAMMA GS & USTEST & 0.0465 \\
\hline 3693 & PDMS & & SW & & & E017379 & 19-Apr-83 & & $10028-17-8$ & TRITIUM & TRITIUM_ELEECT_LSC & USTEST & 201 \\
\hline
\end{tabular}




\begin{tabular}{|c|c|c|c|c|c|c|c|c|c|c|c|c|c|}
\hline & $\bar{A}$ & $\mathrm{~B}$ & $\mathrm{C}$ & $\bar{D}$ & $\mathrm{E}$ & $\mathrm{F}$ & $\bar{G}$ & $\mathrm{H}$ & 1 & $\mathrm{~J}$ & $\mathrm{~K}$ & $\mathrm{~L}$ & $\bar{M}$ \\
\hline 1 & SAMPLE_NUM_ASSIGNED_TO & SAMPLER & MEDIA & BIOTA_MEDIA & TAG_ID & SAMP_NUM & SAMP_DATE_TIME & SAMP_DATE_TIME_ON & CON_ID & \begin{tabular}{|l|} 
CON_SHORT_NAME \\
\end{tabular} & METHOD_NAME & LAB_CODE & VALUE_RPTD \\
\hline 3694 & PDMS & & SW & & & E028043 & 19-Apr-83 & & $10045-97-3$ & CS-137 & GAMMA_GS & USTEST & 0.187 \\
\hline 3695 & PDMS & & sW & & & E028043 & 19-Apr-83 & & $10098-97-2$ & SR-90 & SRISO_SEEP_PRECIP_GPC & USTEST & 0.124 \\
\hline 3696 & PDMS & & SW & & & E028043 & 19-Apr-83 & & $10028-17-8$ & TRITIUM & TRITIUM_ELECT_LSC & USTEST & 182 \\
\hline 3697 & PDMS & & SW & & & E003851 & 26-Apr-83 & & $10045-97-3$ & CS-137 & GAMMA_GS & PNL-RO & 0.0213 \\
\hline 3698 & PDMS & & sW & & & E001899 & 26-Apr-83 & & $10045-97-3$ & CS-137 & GAMMA_GS & PNL-RO & 0.0341 \\
\hline 3699 & PDMS & & SW & & & E001197 & 26-Apr-83 & & $10045-97-3$ & CS-137 & GAMMA_GS & PNL-RO & 0.03 \\
\hline 3700 & PDMS & & sW & & & E013882 & 26-Apr-83 & & $10045-97-3$ & CS-137 & GAMMA_GS & PNL-RO & 0.0203 \\
\hline 3701 & PDMS & & sW & & & E034553 & 09-Мау-83 & & $10098-97-2$ & SR-90 & SRISO_SEEP_PRECIP_GPC & USTEST & 0.234 \\
\hline 3702 & PDMS & & sW & & & E034553 & 09-Мау-83 & & $10045-97-3$ & CS-137 & GAMMĀGS' & USTEST & 0 \\
\hline 3703 & PDMS & & SW & & & E001900 & 10-May-83 & & PU-239/240 & PU-239/240 & PUISO_IE_AEA & PNL-RO & 0.00014 \\
\hline 3704 & PDMS & & SW & & & E001900 & 10-May-83 & & $10045-97-3$ & CS-137 & GAMMA_GS & PNL-RO & 0.0254 \\
\hline 3705 & PDMS & & sW & & & E001900 & 10-May-83 & & 15046-84-1 & I-129 & I129_SEP_TEMS & 320 & 0.0000391 \\
\hline 3706 & PDMS & & sW & & & E003852 & 10-Мау-83 & & $10045-97-3$ & CS-137 & GAMMA_GS & PNL-RO & 0.0194 \\
\hline 3707 & PDMS & & SW & & & E003852 & 10-May-83 & & PU-239/240 & PU-239/240 & PUISO_IE_AEA & PNL-RO & 0.000026 \\
\hline 3708 & PDMS & & SW & & & E001198 & 10-May-83 & & $10045-97-3$ & CS-137 & GAMMA_GS & PNL-RO & 0.03646 \\
\hline 3709 & PDMS & & SW & & & E001198 & 10-May-83 & & PU-239/240 & PU-239/240 & PUISO_IE_AEA & PNL-RO & -0.000013 \\
\hline 3710 & PDMS & & SW & & & E001198 & 10-May-83 & & 15046-84-1 & I-129 & 1129_SEP_TEMS & 320 & 0.0000379 \\
\hline 3711 & PDMS & & sW & & & E013883 & 10-May-83 & & PU-239/240 & PU-239/240 & PUISO_IE_AEA & PNL-RO & 0.000028 \\
\hline 3712 & PDMS & & sW & & & E013883 & 10-May-83 & & $10045-97-3$ & CS-137 & GAMMA_GS & PNL-RO & 0.0173 \\
\hline 3713 & PDMS & & SW & & & E010715 & 10-May-83 & & $10028-17-8$ & TRITIUM & TRITIUM_ELECT_LSC & USTEST & 75 \\
\hline 3714 & PDMS & & sW & & & E010715 & 10-Мау-83 & & $10098-97-2$ & SR-90 & SRISO_SËP_PRËCIP_GPC & USTEST & 0.153 \\
\hline 3715 & PDMS & & SW & & & E010715 & 10-May-83 & & $10045-97-3$ & CS-137 & GAMMA_GS & USTEST & 0.127 \\
\hline 3716 & PDMS & & SW & & & E004316 & 10-May-83 & & $10028-17-8$ & TRITIUM & TRITIUM_ELECT_LSC & USTEST & 149 \\
\hline 3717 & PDMS & & SW & & & E004316 & 10-May-83 & & $10098-97-2$ & SR-90 & SRISO_SEP_PRECIP_GPC & USTEST & 0.193 \\
\hline 3718 & PDMS & & SW & & & E004316 & 10-May-83 & & $10045-97-3$ & CS-137 & GAMMA_GS & USTEST & 0.41 \\
\hline 3719 & PDMS & & SW & & & E032603 & 17-May-83 & & 10098-97-2 & SR-90 & SRISO_SEEP_PRECIP_GPC & USTEST & 0.167 \\
\hline 3720 & PDMS & & SW & & & E032603 & 17-May-83 & & $10028-17-8$ & TRITIUM & TRITIUM_DIST_LSC & USTEST & 67 \\
\hline 3721 & PDMS & & SW & & & E032603 & 17-May-83 & & $10045-97-3$ & CS-137 & GAMMA_GS & USTEST & -0.564 \\
\hline 3722 & PDMS & & SW & & & E013530 & 17-May-83 & & 10098-97-2 & SR-90 & SRISO_SEP_PRECIP_GPC & USTEST & 2.85 \\
\hline 3723 & PDMS & & SW & & & E013530 & 17-May-83 & & $10045-97-3$ & CS-137 & GAMMA_GS & USTEST & 4.03 \\
\hline 3724 & PDMS & & SW & & & E013530 & 17-May-83 & & $10028-17-8$ & TRITIUM & TRITIUM_DIST_LSC & USTEST & 67 \\
\hline 3725 & PDMS & & SW & & & E012581 & 17-May-83 & & $10028-17-8$ & TRITIUM & TRITIUM_DIST_LSC & USTEST & 21500 \\
\hline 3726 & PDMS & & SW & & & E012581 & 17-May-83 & & $10045-97-3$ & CS-137 & GAMMA_GS & USTEST & 1 \\
\hline 3727 & PDMS & & SW & & & E017789 & 17-May-83 & & $10045-97-3$ & CS-137 & GAMMA_GS & USTEST & 31.3 \\
\hline 3728 & PDMS & & SW & & & E017789 & 17-May-83 & & $10098-97-2$ & SR-90 & SRISO_SEP_PRECIP_GPC & USTEST & 6.87 \\
\hline 3729 & PDMS & & SW & & & E017789 & 17-May-83 & & $10028-17-8$ & TRITIUM & TRITIUM_DIST_LSC & USTEST & 243 \\
\hline 3730 & PDMS & & SW & & & E021778 & 17-May-83 & & $10028-17-8$ & TRITIUM & TRITIUM_DIST_LSC & USTEST & 810 \\
\hline 3731 & PDMS & & sW & & & E021778 & 17-May-83 & & $10045-97-3$ & CS-137 & GAMMA_GS & USTEST & 1.55 \\
\hline 3732 & PDMS & & SW & & & E021778 & 17-May-83 & & $10098-97-2$ & SR-90 & SRISO_SEP_PRECIP_GPC & USTEST & 1.05 \\
\hline 3733 & PDMS & & SW & & & E014489 & 24-May-83 & & $10098-97-2$ & SR-90 & 905.0_SR_GPC & USTEST & -0.0156 \\
\hline 3734 & PDMS & & SW & & & E014489 & 24-May-83 & & $10028-17-8$ & TRITIUM & 906.0_H3_LSC & USTEST & 28 \\
\hline 3735 & PDMS & & SW & & & E014489 & 24-May-83 & & $10045-97-3$ & CS-137 & 901.1_GAMMA_GS & USTEST & -0.429 \\
\hline 3736 & PDMS & & SW & & & E010735 & 24-May-83 & & $10045-97-3$ & CS-137 & 901.1_GAMMA_GS & USTEST & -0.0948 \\
\hline 3737 & PDMS & & SW & & & E010735 & 24-May-83 & & $10028-17-8$ & TRITIUM & 906.0_H3_LSC & USTEST & -100 \\
\hline 3738 & PDMS & & SW & & & E001901 & 24-May-83 & & $10045-97-3$ & CS-137 & GAMMA_GS & PNL-RO & 0.028 \\
\hline 3739 & PDMS & & SW & & & E003853 & 24-May-83 & & $10045-97-3$ & CS-137 & GAMMA_GS & PNL-RO & 0.0128 \\
\hline 3740 & PDMS & & SW & & & E001199 & 24-May-83 & & $10045-97-3$ & CS-137 & GAMMA_GS & PNL-RO & 0.0308 \\
\hline 3741 & PDMS & & SW & & & E013884 & 24-May-83 & & $10045-97-3$ & CS-137 & GAMMA_GS & PNL-RO & 0.0123 \\
\hline 3742 & PDMS & & SW & & & E013809 & 27-May-83 & & $10098-97-2$ & SR-90 & 905.0_SR_GPC & USTEST & -0.017 \\
\hline 3743 & PDMS & & SW & & & E013809 & 27-May-83 & & $10028-17-8$ & TRITIUM & 906.0_H3_LSC & USTEST & 19300 \\
\hline 3744 & PDMS & & SW & & & E013809 & 27-May-83 & & $10045-97-3$ & CS-137 & 901.1_GAMMA_GS & USTEST & 1.84 \\
\hline 3745 & PDMS & & SW & & & E037631 & 06-Jun-83 & & $10098-97-2$ & SR-90 & SRISO_SEP_PRECIP_GPC & USTEST & 0.0988 \\
\hline
\end{tabular}




\begin{tabular}{|c|c|c|c|c|c|c|c|c|c|c|c|c|c|}
\hline & A & $\mathrm{B}$ & C & $\mathrm{D}$ & $\mathrm{E}$ & $\mathrm{F}$ & G & $\mathrm{H}$ & 1 & $\mathrm{~J}$ & $\mathrm{~K}$ & $\mathrm{~L}$ & $\mathrm{M}$ \\
\hline 1 & SAMPLE_NUM_ASSIGNED_TO & SAMPLER & MEDIA & BIOTA_MEDIA & TAG_ID & SAMP_NUM & SAMP_DATE_TIME & SAMP_DATE_TIME_ON & CON_ID & CON_SHORT_NAME & METHOD_NAME & LAB_CODE & VALUE_RPTD \\
\hline 3746 & PDMS & & SW & & & E037631 & 06-Jun-83 & & $10045-97-3$ & CS-137 & GAMMA_GS & USTEST & 0.649 \\
\hline 3747 & PDMS & & sW & & & E001902 & 07-Jun-83 & & $10045-97-3$ & CS-137 & GAMMA_GS & PNL-RO & 0.0322 \\
\hline 3748 & PDMS & & sW & & & E003854 & 07-Jun-83 & & $10045-97-3$ & CS-137 & GAMMA_GS & PNL-RO & 0.0158 \\
\hline 3749 & PDMS & & sW & & & E001200 & 07-Jun-83 & & $10045-97-3$ & CS-137 & GAMMA_GS & PNL-RO & 0.0428 \\
\hline 3750 & PDMS & & sW & & & E010716 & 07-Jun-83 & & $10028-17-8$ & TRITIUM & TRITIUM_ELECT_LSC & USTEST & 120 \\
\hline 3751 & PDMS & & SW & & & E010716 & 07-Jun-83 & & $10098-97-2$ & SR-90 & SRISO_SEPP_PRËCIP_GPC & USTEST & 0.12 \\
\hline 3752 & PDMS & & SW & & & E010716 & 07-Jun-83 & & $10045-97-3$ & CS-137 & GAMMA_GS & USTEST & 0.673 \\
\hline 3753 & PDMS & & SW & & & E013885 & 07-Jun-83 & & $10045-97-3$ & CS-137 & GAMMA_GS & PNL-RO & 0.0184 \\
\hline 3754 & PDMS & & sW & & & E004317 & 07-Jun-83 & & $10028-17-8$ & TRITIUM & TRITIUM ELECT LSC & USTEST & 163 \\
\hline 3755 & PDMS & & SW & & & E004317 & 07-Jun-83 & & $10098-97-2$ & SR-90 & SRISO_SEP_PRËCIP_GPC & USTEST & 0.226 \\
\hline 3756 & PDMS & & SW & & & E004317 & 07-Jun-83 & & $10045-97-3$ & CS-137 & GAMMA_GS & USTEST & 0.829 \\
\hline 3757 & PDMS & & SW & & & E084796 & 13-Jun-83 & & $10028-17-8$ & TRITIUM & 906.0_H3_LSC & USTEST & 229 \\
\hline 3758 & PDMS & & SW & & & E012562 & 15-Jun-83 & & $10098-97-2$ & SR-90 & 905.0_SR_GPC & USTEST & -0.0055 \\
\hline 3759 & PDMS & & SW & & & E012562 & 15-Jun-83 & & $10045-97-3$ & CS-137 & 901.1 GAMMA GS & USTEST & 0.119 \\
\hline 3760 & PDMS & & SW & & & E012562 & 15-Jun-83 & & $10028-17-8$ & TRITIUM & 906.0_H3_LSC & USTEST & 213 \\
\hline 3761 & PDMS & & SW & & & E013111 & 17-Jun-83 & & $10045-97-3$ & CS-137 & 901.1_GAMMA_GS & USTEST & -0.0949 \\
\hline 3762 & PDMS & & sW & & & E013111 & 17-Jun-83 & & $10098-97-2$ & SR-90 & 905.0_SR_GPC & USTEST & 0.501 \\
\hline \begin{tabular}{|c|}
3763 \\
\end{tabular} & PDMS & & sW & & & E001903 & 21-Jun-83 & & $10045-97-3$ & CS-137 & GAMMA_GS & PNL-RO & 0.0346 \\
\hline 3764 & PDMS & & SW & & & E003855 & 21-Jun-83 & & $10045-97-3$ & CS-137 & GAMMA_GS & PNL-RO & 0.000131 \\
\hline 3765 & PDMS & & SW & & & E001201 & 21-Jun-83 & & $10045-97-3$ & CS-137 & GAMMA_GS & PNL-RO & 0.0452 \\
\hline 3766 & PDMS & & SW & & & E013886 & 21-Jun-83 & & $10045-97-3$ & CS-137 & GAMMA_GS & PNL-RO & 0.0213 \\
\hline \begin{tabular}{|c|}
3767 \\
\end{tabular} & PDMS & & SW & & & E032036 & 05-Jul-83 & & $10028-17-8$ & TRITIUM & 906.0_H3_LSC & USTEST & 269 \\
\hline 3768 & PDMS & & SW & & & E032036 & 05-Jul-83 & & $10098-97-2$ & SR-90 & 905.0_SR_GPC & USTEST & 0.255 \\
\hline 3769 & PDMS & & SW & & & E032036 & 05-Jul-83 & & $10045-97-3$ & CS-137 & 901.1_GAMMA_GS & USTEST & -0.697 \\
\hline 3770 & PDMS & & SW & & & E003856 & 05-Jul-83 & & $10045-97-3$ & CS-137 & GAMMA_AS & PNL-RO & 0.0126 \\
\hline 3771 & PDMS & & SW & & & E001904 & 05-Jul-83 & & $10045-97-3$ & CS-137 & GAMMA_GS & PNL-RO & 0.0267 \\
\hline 3772 & PDMS & & SW & & & E010717 & 05-Jul-83 & & $10028-17-8$ & TRITIUM & TRITIUM_ELECT_LSC & USTEST & 65.3 \\
\hline 3773 & PDMS & & SW & & & E013887 & 05-Jul-83 & & $10045-97-3$ & CS-137 & GAMMA_GS & PNL-RO & 0.0161 \\
\hline 3774 & PDMS & & SW & & & E010717 & 05-Jul-83 & & $10098-97-2$ & SR-90 & SRISO_SEP_PRECIP_GPC & USTEST & 0.461 \\
\hline 3775 & PDMS & & SW & & & E010717 & 05-Jul-83 & & $10045-97-3$ & CS-137 & GAMMA_GS & USTEST & 0.0655 \\
\hline 3776 & PDMS & & sW & & & E001202 & 05-Jul-83 & & $10045-97-3$ & CS-137 & GAMMA_GS & PNL-RO & 0.0378 \\
\hline 3777 & PDMS & & SW & & & E004318 & 05-Jul-83 & & $10028-17-8$ & TRITIUM & TRITIUM_ELEECT_LSC & USTEST & 109 \\
\hline \begin{tabular}{|l|l|}
3778 \\
\end{tabular} & PDMS & & SW & & & E004318 & 05-Jul-83 & & $10098-97-2$ & SR-90 & SRISO_SEPP_PRËCIP_GPC & USTEST & 0.208 \\
\hline 3779 & PDMS & & SW & & & E004318 & 05-Jul-83 & & $10045-97-3$ & CS-137 & GAMMĀGS & USTEST & 0 \\
\hline 3780 & PDMS & & SW & & & E037632 & 05-Jul-83 & & $10098-97-2$ & SR-90 & SRISO_SEEP_PRECIP_GPC & USTEST & 0.163 \\
\hline 3781 & PDMS & & sW & & & E037632 & 05-Jul-83 & & $10045-97-3$ & CS-137 & GAMMA_GS & USTEST & 0.114 \\
\hline 3782 & PDMS & & SW & & & E001905 & 19-Jul-83 & & $10045-97-3$ & CS-137 & GAMMA_GS & PNL-RO & 0.0338 \\
\hline 3783 & PDMS & & SW & & & E003857 & 19-Jul-83 & & $10045-97-3$ & CS-137 & GAMMA_GS & PNL-RO & 0.0149 \\
\hline 3784 & PDMS & & SW & & & E001204 & 19-Jul-83 & & $10045-97-3$ & CS-137 & GAMMA_GS & PNL-RO & 0.0372 \\
\hline 3785 & PDMS & & SW & & & E013888 & 19-Jul-83 & & $10045-97-3$ & CS-137 & GAMMA_GS & PNL-RO & 0.0143 \\
\hline 3786 & PDMS & & SW & & & E004182 & 26-Jul-83 & & $10098-97-2$ & SR-90 & SRISO_SEEP_PRECIP_GPC & USTEST & 0.669 \\
\hline 3787 & PDMS & & SW & & & E004182 & 26-Jul-83 & & $10045-97-3$ & CS-137 & GAMMĀGS & USTEST & 0.811 \\
\hline 3788 & PDMS & & SW & & & E004182 & 26-Jul-83 & & $10028-17-8$ & TRITIUM & TRITIUM_DIST_LSC & USTEST & 217 \\
\hline 3789 & PDMS & & SW & & & E012072 & 29-Jul-83 & & $15046-84-1$ & I-129 & I129_SEP_TEMS & 320 & 0.00007474 \\
\hline 3790 & PDMS & & SW & & & E074198 & 29-Jul-83 & & 15046-84-1 & I-129 & 1129_SEP_TEMS & 320 & 0.00001619 \\
\hline 3791 & PDMS & & SW & & & E037633 & 01-Aug-83 & & $10098-97-2$ & SR-90 & SRIS̄O_SĒP_PRECIP_GPC & USTEST & 0.232 \\
\hline 3792 & PDMS & & SW & & & E037633 & 01-Aug-83 & & $10045-97-3$ & CS-137 & GAMMA_GS & USTEST & 0.246 \\
\hline 3793 & PDMS & & SW & & & E001906 & 02-Aug-83 & & PU-239/240 & PU-239/240 & PUISO_IE_AEA & PNL-RO & 0.000033 \\
\hline 3794 & PDMS & & SW & & & E001906 & 02-Aug-83 & & $10045-97-3$ & CS-137 & GAMMA_GS & PNL-RO & 0.0367 \\
\hline 3795 & PDMS & & SW & & & E003858 & 02-Aug-83 & & PU-239/240 & PU-239/240 & PUISO_IE_AEA & PNL-RO & 0.000033 \\
\hline 3796 & PDMS & & SW & & & E003858 & 02-Aug-83 & & $10045-97-3$ & CS-137 & GAMMA_GS & PNL-RO & 0.0177 \\
\hline 3797 & PDMS & & SW & & & E010718 & 02-Aug-83 & & 10028-17-8 & TRITIUM & TRITIUM_ELEECT_LSC & USTEST & 202 \\
\hline
\end{tabular}




\begin{tabular}{|c|c|c|c|c|c|c|c|c|c|c|c|c|c|}
\hline & A & $\mathrm{B}$ & $\mathrm{C}$ & $\mathrm{D}$ & $E$ & $\mathrm{~F}$ & $\mathrm{G}$ & $\mathrm{H}$ & 1 & $\mathrm{~J}$ & $\mathrm{~K}$ & L & $\mathrm{M}$ \\
\hline 1 & SAMPLE_NUM_ASSIGNED_TO & SAMPLER & MEDIA & BIOTA_MEDIA & TAG_ID & SAMP_NUM & SAMP_DATE_TIME| & SAMP_DATE_TIME_ON & CON_ID & CON_SHORT_NAME & METHOD_NAME & LAB_CODE & VALUE_RPTD \\
\hline 3798 & PDMS & & SW & & & E013889 & 02-Aug-83 & & PU-239/240 & PU-239/240 & PUISO_IE_AEA & PNL-RO & 0.000024 \\
\hline 3799 & PDMS & & SW & & & E013889 & 02-Aug-83 & & $10045-97-3$ & CS-137 & GAMMA_GS & PNL-RO & 0.0176 \\
\hline 3800 & PDMS & & SW & & & E010718 & 02-Aug-83 & & $10098-97-2$ & SR-90 & SRISO_SEP_PRECIP_GPC & USTEST & 0.0185 \\
\hline 3801 & PDMS & & SW & & & E010718 & 02-Aug-83 & & $10045-97-3$ & CS-137 & GAMMA_GS & USTEST & 0.232 \\
\hline 3802 & PDMS & & SW & & & E001205 & 02-Aug-83 & & $10045-97-3$ & CS-137 & GAMMA_GS & PNL-RO & 0.0245 \\
\hline 3803 & PDMS & & SW & & & E001205 & 02-Aug-83 & & PU-239/240 & PU-239/240 & PUISO_IE_AEA & PNL-RO & -0.00005 \\
\hline 3804 & PDMS & & SW & & & E004319 & 02-Aug-83 & & $10028-17-8$ & TRITIUM & TRITIUM_E_ELECT_LSC & USTEST & 236 \\
\hline 3805 & PDMS & & SW & & & E004319 & 02-Aug-83 & & $10098-97-2$ & SR-90 & SRISO_SEP_PRECIP_GPC & USTEST & 0.294 \\
\hline 3806 & PDMS & & SW & & & E004319 & 02-Aug-83 & & $10045-97-3$ & CS-137 & GAMMA_GS & USTEST & 0.0873 \\
\hline 3807 & PDMS & & SW & & & E013531 & 09-Aug-83 & & $10098-97-2$ & SR-90 & SRISO_SEP_PRECIP_GPC & USTEST & 4.12 \\
\hline 3808 & PDMS & & SW & & & E013531 & 09-Aug-83 & & $10045-97-3$ & CS-137 & GAMMA_GS & USTEST & 1.42 \\
\hline 3809 & PDMS & & SW & & & E013531 & 09-Aug-83 & & $10028-17-8$ & TRITIUM & TRITIUM_DIST_LSC & USTEST & 182 \\
\hline 3810 & PDMS & & SW & & & E012582 & 09-Aug-83 & & $10028-17-8$ & TRITIUM & TRITIUM_DIST_LSC & USTEST & 18300 \\
\hline 3811 & PDMS & & SW & & & E012582 & 09-Aug-83 & & $10045-97-3$ & CS-137 & GAMMA_GS & USTEST & -0.218 \\
\hline 3812 & PDMS & & SW & & & E017790 & 09-Aug-83 & & $10045-97-3$ & CS-137 & GAMMA_GS & USTEST & 64.6 \\
\hline 3813 & PDMS & & SW & & & E017790 & 09-Aug-83 & & $10098-97-2$ & SR-90 & SRISO_SEP_PRECIP_GPC & USTEST & 4.67 \\
\hline 3814 & PDMS & & SW & & & E017790 & 09-Aug-83 & & $10028-17-8$ & TRITIUM & TRITIUM_DIST_LSC & USTEST & -7 \\
\hline 3815 & PDMS & & SW & & & E021779 & 09-Aug-83 & & $10028-17-8$ & TRITIUM & TRITIUM_DIST_LSC & USTEST & 442 \\
\hline 3816 & PDMS & & SW & & & E021779 & 09-Aug-83 & & $10045-97-3$ & CS-137 & GAMMA_GS & USTEST & 3.47 \\
\hline 3817 & PDMS & & SW & & & E021779 & 09-Aug-83 & & $10098-97-2$ & SR-90 & SRISO_SEP_PRECIP_GPC & USTEST & 3.8 \\
\hline 3818 & PDMS & & SW & & & E014490 & 16-Aug-83 & & $10098-97-2$ & SR-90 & 905.0_ĒR_GPC & USTEST & 0.108 \\
\hline 3819 & PDMS & & SW & & & E014490 & 16-Aug-83 & & $10028-17-8$ & TRITIUM & 906.0_H3_LSC & USTEST & 62 \\
\hline 3820 & PDMS & & SW & & & E014490 & 16-Aug-83 & & $10045-97-3$ & CS-137 & 901.1_GAMMA_GS & USTEST & -0.0579 \\
\hline 3821 & PDMS & & SW & & & E010736 & 16-Aug-83 & & $10045-97-3$ & CS-137 & 901.1_GAMMA_GS & USTEST & 0.458 \\
\hline 3822 & PDMS & & SW & & & E010736 & 16-Aug-83 & & 10028-17-8 & TRITIUM & 906.0_H3_LSC & USTEST & 57 \\
\hline 3823 & PDMS & & SW & & & E001907 & 16-Aug-83 & & $10045-97-3$ & CS-137 & GAMMA_GS & PNL-RO & 0.0459 \\
\hline 3824 & PDMS & & SW & & & E003859 & 16-Aug-83 & & $10045-97-3$ & CS-137 & GAMMA_GS & PNL-RO & 0.021 \\
\hline 3825 & PDMS & & SW & & & E001206 & 16-Aug-83 & & $10045-97-3$ & CS-137 & GAMMA_GS & PNL-RO & 0.0228 \\
\hline 3826 & PDMS & & SW & & & E013890 & 16-Aug-83 & & $10045-97-3$ & CS-137 & GAMMA_GS & PNL-RO & 0.0136 \\
\hline 3827 & PDMS & & SW & & & E013810 & 22-Aug-83 & & $10098-97-2$ & SR-90 & 905.0_SR_GPC & USTEST & 0.185 \\
\hline 3828 & PDMS & & SW & & & E013810 & 22-Aug-83 & & $10028-17-8$ & TRITIUM & 906.0_H3_LSC & USTEST & 25500 \\
\hline 3829 & PDMS & & SW & & & E013810 & 22-Aug-83 & & $10045-97-3$ & CS-137 & 901.1_GAMMA_GS & USTEST & -0.828 \\
\hline 3830 & PDMS & & SW & & & E037634 & 29-Aug-83 & & $10098-97-2$ & SR-90 & SRISŌSEP_PRECIP_GPC & USTEST & 0.24 \\
\hline 3831 & PDMS & & SW & & & E037634 & 29-Aug-83 & & $10045-97-3$ & CS-137 & GAMMA_GS & USTEST & 0.265 \\
\hline 3832 & PDMS & & SW & & & E001908 & 30-Aug-83 & & $10045-97-3$ & CS-137 & GAMMA_GS & PNL-RO & 0.0633 \\
\hline 3833 & PDMS & & SW & & & E003860 & 30-Aug-83 & & $10045-97-3$ & CS-137 & GAMMA_GS & PNL-RO & 0.0269 \\
\hline 3834 & PDMS & & SW & & & E013891 & 30-Aug-83 & & $10045-97-3$ & CS-137 & GAMMA_GS & PNL-RO & 0.014 \\
\hline 3835 & PDMS & & SW & & & E001207 & 30-Aug-83 & & $10045-97-3$ & CS-137 & GAMMA_GS & PNL-RO & 0.0264 \\
\hline 3836 & PDMS & & SW & & & E010719 & 30-Aug-83 & & $10028-17-8$ & TRITIUM & TRITIUM_ELECT_LSC & USTEST & 144 \\
\hline 3837 & PDMS & & SW & & & E010719 & 30-Aug-83 & & $10098-97-2$ & SR-90 & SRISO_SEP_PRECIP_GPC & USTEST & 0.137 \\
\hline 3838 & PDMS & & SW & & & E010719 & 30-Aug-83 & & $10045-97-3$ & CS-137 & GAMMA_GS & USTEST & -0.917 \\
\hline 3839 & PDMS & & SW & & & E004320 & 30-Aug-83 & & $10028-17-8$ & TRITIUM & TRITIUM_ELECT_LSC & USTEST & 151 \\
\hline 3840 & PDMS & & SW & & & E004320 & 30-Aug-83 & & $10098-97-2$ & SR-90 & SRISO_SEP_PRECIP_GPC & USTEST & 0.24 \\
\hline 3841 & PDMS & & SW & & & E004320 & 30-Aug-83 & & $10045-97-3$ & CS-137 & GAMMA_GS & USTEST & -0.271 \\
\hline 3842 & PDMS & & SW & & & E012563 & 05-Sep-83 & & $10098-97-2$ & SR-90 & 905.0_SR_GPC & USTEST & 0.0233 \\
\hline 3843 & PDMS & & SW & & & E012563 & 05-Sep-83 & & $10045-97-3$ & CS-137 & 901.1_GAMMA_GS & USTEST & 0.776 \\
\hline 3844 & PDMS & & SW & & & E012563 & 05-Sep-83 & & 10028-17-8 & TRITIUM & 906.0_H3_LSC & USTEST & 176 \\
\hline 3845 & PDMS & & SW & & & E001909 & 13-Sep-83 & & $10045-97-3$ & CS-137 & GAMMA_GS & PNL-RO & 0.0348 \\
\hline 3846 & PDMS & & SW & & & E003861 & 13-Sep-83 & & $10045-97-3$ & CS-137 & GAMMA_GS & PNL-RO & 0.0154 \\
\hline 3847 & PDMS & & SW & & & E015253 & 13-Sep-83 & & $10045-97-3$ & CS-137 & GAMMA_GS & HISTOR & \\
\hline 3848 & PDMS & & SW & & & E026983 & 13-Sep-83 & & $10045-97-3$ & CS-137 & GAMMA_GS & HISTOR & \\
\hline 3849 & PDMS & & SW & & & E013112 & 23-Sep-83 & & $10045-97-3$ & CS-137 & 901.1_GAMMA_GS & USTEST & 0.306 \\
\hline
\end{tabular}




\begin{tabular}{|c|c|c|c|c|c|c|c|c|c|c|c|c|c|}
\hline & A & $\mathrm{B}$ & C & $\mathrm{D}$ & $E$ & $\mathrm{~F}$ & G & $\mathrm{H}$ & $\mathrm{I}$ & $\mathrm{J}$ & $\mathrm{K}$ & $\mathrm{L}$ & $\mathrm{M}$ \\
\hline 1 & SAMPLE_NUM_ASSIGNED_TO & SAMPLER & MEDIA & BIOTA_MEDIA & TAG_ID & SAMP_NUM & SAMP_DATE_TIME & SAMP_DATE_TIME_ON & CON_ID & CON_SHORT_NAME & METHOD_NAME & LAB_CODE & \begin{tabular}{|l|} 
VALUE_RPTD \\
\end{tabular} \\
\hline 3850 & PDMS & & SW & & & E013112 & 23-Sep-83 & & $10028-17-8$ & TRITIUM & 906.0_H3_LSC & USTEST & -2.61 \\
\hline 3851 & PDMS & & SW & & & E013112 & 23-Sep-83 & & $10098-97-2$ & SR-90 & 905.0_SR_GPC & USTEST & 1.1 \\
\hline 3852 & PDMS & & SW & & & E032037 & 27-Sep-83 & & 10028-17-8 & TRITIUM & 906.0_H3_LSC & USTEST & 1570 \\
\hline 3853 & PDMS & & SW & & & E032037 & 27-Sep-83 & & $10098-97-2$ & SR-90 & 905.0_SR_GPC & USTEST & 0.265 \\
\hline 3854 & PDMS & & SW & & & E032037 & 27-Sep-83 & & $10045-97-3$ & CS-137 & 901.1_GAMMA_GS & USTEST & -0.356 \\
\hline 3855 & PDMS & & SW & & & E017380 & 27-Sep-83 & & $10098-97-2$ & SR-90 & SRISO_SEP_PRECIP_GPC & USTEST & 0.221 \\
\hline 3856 & PDMS & & SW & & & E017380 & 27-Sep-83 & & $10045-97-3$ & CS-137 & GAMMA_GS & USTEST & -0.296 \\
\hline 3857 & PDMS & & SW & & & E017380 & 27-Sep-83 & & $10028-17-8$ & TRITIUM & TRITIUM_ELECT_LSC & USTEST & 136 \\
\hline 3858 & PDMS & & SW & & & E028044 & 27-Sep-83 & & $10028-17-8$ & TRITIUM & TRITIUM_ELECT_LSC & USTEST & 106 \\
\hline 3859 & PDMS & & SW & & & E028044 & 27-Sep-83 & & $10045-97-3$ & CS-137 & GAMMA_GS & USTEST & 0.946 \\
\hline 3860 & PDMS & & SW & & & E028044 & 27-Sep-83 & & $10098-97-2$ & SR-90 & SRISO_SEEP_PRECIP_GPC & USTEST & 0.258 \\
\hline 3861 & PDMS & & SW & & & E001910 & $27-$ Sep-83 & & $10045-97-3$ & CS-137 & GAMMA_GS & PNL-RO & 0.0289 \\
\hline 3862 & PDMS & & SW & & & E003862 & $27-$ Sep-83 & & $10045-97-3$ & CS-137 & GAMMA_GS & PNL-RO & 0.014 \\
\hline 3863 & PDMS & & SW & & & E001208 & 27-Sep-83 & & $10045-97-3$ & CS-137 & GAMMA_GS & PNL-RO & 0.0352 \\
\hline 3864 & PDMS & & SW & & & E010720 & 27-Sep-83 & & $10028-17-8$ & TRITIUM & TRITIUM_ELECT_LSC & USTEST & 75.4 \\
\hline 3865 & PDMS & & SW & & & E013892 & 27-Sep-83 & & $10045-97-3$ & CS-137 & GAMMA_GS & PNL-RO & 0.0172 \\
\hline 3866 & PDMS & & SW & & & E010720 & 27-Sep-83 & & $10098-97-2$ & SR-90 & SRISO_SEPPPRECIP_GPC & USTEST & 0.194 \\
\hline 3867. & PDMS & & SW & & & E010720 & 27-Sep-83 & & $10045-97-3$ & CS-137 & GAMMA_GS & USTEST & -0.533 \\
\hline 3868 & PDMS & & SW & & & E004321 & 27-Sep-83 & & $10028-17-8$ & TRITIUM & TRITIUM_ELECT_LSC & USTEST & 155 \\
\hline 3869 & PDMS & & SW & & & E004321 & 27-Sep-83 & & $10098-97-2$ & SR-90 & SRISO_SEP_PRECIP_GPC & USTEST & 0.35 \\
\hline 3870 & PDMS & & SW & & & E004321 & 27-Sep-83 & & $10045-97-3$ & CS-137 & GAMMA_GS & USTEST & 0.907 \\
\hline 3871 & PDMS & & SW & & & E002080 & 27-Sep-83 & & $10098-97-2$ & SR-90 & SRISO_SEP_PRECIP_GPC & USTEST & 0.682 \\
\hline 3872 & PDMS & & SW & & & E002080 & 27-Sep-83 & & $10045-97-3$ & CS-137 & GAMMA_GS & USTEST & -0.918 \\
\hline 3873 & PDMS & & SW & & & E001911 & 11-Oct-83 & & $10045-97-3$ & CS-137 & GAMMA_GS & PNL-RO & 0.0325 \\
\hline 3874 & PDMS & & SW & & & E003863 & 11-Oct-83 & & $10045-97-3$ & CS-137 & GAMMA_GS & PNL-RO & 0.0175 \\
\hline 3875 & PDMS & & SW & & & E013893 & 11-Oct-83 & & $10045-97-3$ & CS-137 & GAMMA_GS & PNL-RO & 0.014 \\
\hline 3876 & PDMS & & SW & & & E001209 & 11-Oct-83 & & $10045-97-3$ & CS-137 & GAMMA_GS & PNL-RO & 0.0337 \\
\hline \begin{tabular}{|l|l|}
3877 & \\
\end{tabular} & PDMS & & SW & & & E003864 & 25-Oct-83 & & PU-239/240 & PU-239/240 & PUISO_IE_AEA & PNL-RO & 0.000019 \\
\hline 3878 & PDMS & & SW & & & E003864 & 25-Oct-83 & & $10045-97-3$ & CS-137 & GAMMA_GS & PNL-RO & 0.00996 \\
\hline 3879 & PDMS & & SW & & & E001912 & 25-Oct-83 & & PU-239/240 & PU-239/240 & PUISO_IE_AEA & PNL-RO & 0.000019 \\
\hline 3880 & PDMS & & SW & & & E001912 & 25-Oct-83 & & $10045-97-3$ & CS-137 & GAMMA_GS & PNL-RO & 0.0294 \\
\hline 3881 & PDMS & & SW & & & E001912 & 25-Oct-83 & & 15046-84-1 & I-129 & I129_SEP_TEMS & 320 & 0.0001197 \\
\hline 3882 & PDMS & & SW & & & E001210 & $25-$ Oct-83 & & $10045-97-3$ & CS-137 & GAMMA_GS & PNL-RO & 0.0281 \\
\hline 3883 & PDMS & & SW & & & E001210 & $25-$ Oct-83 & & PU-239/240 & PU-239/240 & PUISO_IE_AEA & PNL-RO & -0.000054 \\
\hline 3884 & PDMS & & SW & & & E001210 & 25-Oct-83 & & 15046-84-1 & I-129 & I129_SEP_TEMS & 320 & 0.0000137 \\
\hline 3885 & PDMS & & SW & & & E010721 & 25-Oct-83 & & $10028-17-8$ & TRITIUM & TRITIUM_ELECT_LSC & USTEST & 57.3 \\
\hline 3886 & PDMS & & SW & & & E010721 & 25-Oct-83 & & $10098-97-2$ & SR-90 & SRISO_SEP_PRECIP_GPC & USTEST & 0.0591 \\
\hline 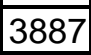 & PDMS & & SW & & & E010721 & 25-Oct-83 & & $10045-97-3$ & CS-137 & GAMMA_GS & USTEST & 0.083 \\
\hline 3888 & PDMS & & SW & & & E013894 & 25-Oct-83 & & PU-239/240 & PU-239/240 & PUISO_IE_AEA & PNL-RO & 0.000021 \\
\hline 3889 & PDMS & & SW & & & E013894 & 25-Oct-83 & & $10045-97-3$ & CS-137 & GAMMA_ĒGS & PNL-RO & 0.0178 \\
\hline 3890 & PDMS & & SW & & & E004322 & $25-$ Oct-83 & & $10028-17-8$ & TRITIUM & TRITIUM_ELECT_LSC & USTEST & 133 \\
\hline 3891 & PDMS & & SW & & & E004322 & 25-Oct-83 & & $10045-97-3$ & CS-137 & GAMMA_GS & USTEST & -0.152 \\
\hline 3892 & PDMS & & SW & & & E004322 & 25-Oct-83 & & $10098-97-2$ & SR-90 & SRISO_SEP_PRECIP_GPC & USTEST & 0.334 \\
\hline 3893 & PDMS & & SW & & & E037635 & 25-Oct-83 & & $10098-97-2$ & SR-90 & SRISO_SEP_PRECIP_GPC & USTEST & 0.072 \\
\hline 3894 & PDMS & & SW & & & E037635 & 25-Oct-83 & & $10045-97-3$ & CS-137 & GAMMA_GS & USTEST & 0.116 \\
\hline 3895 & PDMS & & SW & & & E032604 & 01-Nov-83 & & $10098-97-2$ & SR-90 & SRISO_SEEP_PRECIP_GPC & USTEST & 0.385 \\
\hline 3896 & PDMS & & SW & & & E032604 & 01-Nov-83 & & $10028-17-8$ & TRITIUM & TRITIUM_DIST_LSC & USTEST & 348 \\
\hline 3897 & PDMS & & SW & & & E032604 & 01-Nov-83 & & $10045-97-3$ & CS-137 & GAMMA_GS & USTEST & 1.04 \\
\hline 3898 & PDMS & & SW & & & E013532 & 01-Nov-83 & & $10098-97-2$ & SR-90 & SRISO_SEP_PRECIP_GPC & USTEST & 0.674 \\
\hline 3899 & PDMS & & SW & & & E013532 & 01-Nov-83 & & $10045-97-3$ & CS-137 & GAMMA_GS & USTEST & 2.35 \\
\hline 3900 & PDMS & & SW & & & E013532 & 01-Nov-83 & & 10028-17-8 & TRITIUM & TRITIUM_DIST_LSC & USTEST & 191 \\
\hline 3901 & PDMS & & SW & & & E012583 & 01-Nov-83 & & $10028-17-8$ & TRITIUM & TRITIUM_DIST_LEC & USTEST & 28600 \\
\hline
\end{tabular}




\begin{tabular}{|c|c|c|c|c|c|c|c|c|c|c|c|c|c|}
\hline & A & $\mathrm{B}$ & $\mathrm{c}$ & $\mathrm{D}$ & $\mathrm{E}$ & $\mathrm{F}$ & G & $\mathrm{H}$ & $\mathrm{I}$ & $\mathrm{J}$ & $\mathrm{K}$ & $\mathrm{L}$ & $\mathrm{M}$ \\
\hline 1 & SAMPLE_NUM_ASSIGNED_TO & SAMPLER & MEDIA & BIOTA_MEDIA & TAG_ID & SAMP_NUM & SAMP_DATE_TIME & SAMP_DATE_TIME_ON & CON_ID & CON_SHORT_NAME & METHOD_NAME & LAB_CODE & VALUE_RPTD \\
\hline 3902 & PDMS & & SW & & & E012583 & 01-Nov-83 & & $10045-97-3$ & CS-137 & GAMMA GS & USTEST & 0.154 \\
\hline 3903 & PDMS & & sW & & & E017791 & 01-Nov-83 & & $10045-97-3$ & CS-137 & GAMMA_GS & USTEST & 30.2 \\
\hline 3904 & PDMS & & sW & & & E017791 & 01-Nov-83 & & $10098-97-2$ & SR-90 & SRISO_SEP_PRECIP_GPC & USTEST & 1.2 \\
\hline 3905 & PDMS & & sW & & & E017791 & 01-Nov-83 & & $10028-17-8$ & TRITIUM & TRITIUM_DIST_LSC & USTEST & 324 \\
\hline 3906 & PDMS & & SW & & & E021780 & 01-Nov-83 & & $10028-17-8$ & TRITIUM & TRITIUM_DIST_LSC & USTEST & 871 \\
\hline \begin{tabular}{|l|l|}
3907 \\
\end{tabular} & PDMS & & sW & & & E021780 & 01-Nov-83 & & $10045-97-3$ & CS-137 & GAMMA_GS & USTEST & 1.01 \\
\hline 3908 & PDMS & & SW & & & E021780 & 01-Nov-83 & & $10098-97-2$ & SR-90 & SRISO_SEP_PRECIP_GPC & USTEST & 3.24 \\
\hline 3909 & PDMS & & SW & & & E014491 & 08-Nov-83 & & $10098-97-2$ & SR-90 & 905.0_SR_GPC & USTEST & 0.0914 \\
\hline 3910 & PDMS & & sW & & & E014491 & 08-Nov-83 & & $10028-17-8$ & TRITIUM & 906.0_H3_LSC & USTEST & 283 \\
\hline 3911 & PDMS & & SW & & & E014491 & 08-Nov-83 & & $10045-97-3$ & CS-137 & 901.1_GAMMA_GS & USTEST & 0.183 \\
\hline 3912 & PDMS & & SW & & & E010737 & 08-Nov-83 & & $10045-97-3$ & CS-137 & 901.1_GAMMA_GS & USTEST & 0.259 \\
\hline 3913 & PDMS & & SW & & & E010737 & 08-Nov-83 & & 10028-17-8 & TRITIUM & 906.0_H3_LSC & USTEST & 48.6 \\
\hline 3914 & PDMS & & SW & & & E003865 & 08-Nov-83 & & $10045-97-3$ & CS-137 & GAMMĀE-GS & PNL-RO & 0.0148 \\
\hline 3915 & PDMS & & SW & & & E001913 & 08-Nov-83 & & $10045-97-3$ & CS-137 & GAMMA_GS & PNL-RO & 0.0222 \\
\hline \begin{tabular}{|l|}
3916 \\
\end{tabular} & PDMS & & SW & & & E001211 & 08-Nov-83 & & $10045-97-3$ & CS-137 & GAMMA_GS & PNL-RO & 0.0321 \\
\hline \begin{tabular}{|l|}
3917 \\
\end{tabular} & PDMS & & SW & & & E013895 & 08-Nov-83 & & $10045-97-3$ & CS-137 & GAMMA_GS & PNL-RO & 0.0175 \\
\hline \begin{tabular}{|l|}
3918 \\
\end{tabular} & PDMS & & SW & & & E013811 & 14-Nov-83 & & $10098-97-2$ & SR-90 & 905.0_SR_GPC & USTEST & 0.0213 \\
\hline \begin{tabular}{|l|}
3919 \\
\end{tabular} & PDMS & & sW & & & E013811 & 14-Nov-83 & & $10028-17-8$ & TRITIUM & 906.0_H3_LSC & USTEST & 37600 \\
\hline 3920 & PDMS & & SW & & & E013811 & 14-Nov-83 & & $10045-97-3$ & CS-137 & 901.1_GAMMA_GS & USTEST & 0.0388 \\
\hline 3921 & PDMS & & SW & & & E001914 & 22-Nov-83 & & $10045-97-3$ & CS-137 & GAMM̄A_GS & PNL-RO & 0.0396 \\
\hline 3922 & PDMS & & SW & & & E003866 & 22-Nov-83 & & $10045-97-3$ & CS-137 & GAMMA_GS & PNL-RO & 0.027 \\
\hline 3923 & PDMS & & SW & & & E001212 & 22-Nov-83 & & $10045-97-3$ & CS-137 & GAMMA_GS & PNL-RO & 0.0435 \\
\hline 3924 & PDMS & & SW & & & E013896 & 22-Nov-83 & & $10045-97-3$ & CS-137 & GAMMA_GS & PNL-RO & 0.0154 \\
\hline 3925 & PDMS & & SW & & & E010722 & 22-Nov-83 & & $10028-17-8$ & TRITIUM & TRITIUM_ELEECT_LSC & USTEST & 39.5 \\
\hline 3926 & PDMS & & SW & & & E010722 & 22-Nov-83 & & $10098-97-2$ & SR-90 & SRISO_SEPPPRËCIP_GPC & USTEST & 0.231 \\
\hline 3927. & PDMS & & SW & & & E010722 & 22-Nov-83 & & $10045-97-3$ & CS-137 & GAMMA_GS & USTEST & 0.0489 \\
\hline 3928 & PDMS & & SW & & & E004323 & 22-Nov-83 & & $10028-17-8$ & TRITIUM & TRITIUM_ELECT_LSC & USTEST & 39.3 \\
\hline 3929 & PDMS & & SW & & & E004323 & 22-Nov-83 & & $10045-97-3$ & CS-137 & GAMMA_GS & USTEST & -0.13 \\
\hline 3930 & PDMS & & SW & & & E004323 & 22-Nov-83 & & $10098-97-2$ & SR-90 & SRISO_SEP_PRECIP_GPC & USTEST & 0.129 \\
\hline 3931 & PDMS & & SW & & & E037636 & 22-Nov-83 & & $10098-97-2$ & SR-90 & SRISO_SEP_PRECIP_GPC & USTEST & 0.352 \\
\hline 3932 & PDMS & & sW & & & E037636 & 22-Nov-83 & & $10045-97-3$ & CS-137 & GAMMA_GS & USTEST & -0.0259 \\
\hline 3933 & PDMS & & SW & & & E036172 & 06-Dec-83 & & $10098-97-2$ & SR-90 & 905.0_SR_GPC & USTEST & 0.0635 \\
\hline 3934 & PDMS & & SW & & & E036172 & 06-Dec-83 & & $10045-97-3$ & CS-137 & 901.1_GAMMA_GS & USTEST & 0.0379 \\
\hline 3935 & PDMS & & SW & & & E036172 & 06-Dec-83 & & $10028-17-8$ & TRITIUM & 906.0_H3_LSC & USTEST & 188 \\
\hline 3936 & PDMS & & SW & & & E001915 & 06-Dec-83 & & $10045-97-3$ & CS-137 & GAMMĀE_GS & PNL-RO & 0.0257 \\
\hline \begin{tabular}{|l|l|}
3937 \\
\end{tabular} & PDMS & & SW & & & E003867 & 06-Dec-83 & & $10045-97-3$ & CS-137 & GAMMA_GS & PNL-RO & 0.0134 \\
\hline 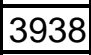 & PDMS & & SW & & & E001213 & 06-Dec-83 & & $10045-97-3$ & CS-137 & GAMMA_GS & PNL-RO & 0.0256 \\
\hline 3939 & PDMS & & SW & & & E013897 & 06-Dec-83 & & $10045-97-3$ & CS-137 & GAMMA_GS & PNL-RO & 0.00946 \\
\hline 3940 & PDMS & & SW & & & E013113 & 09-Dec-83 & & $10045-97-3$ & CS-137 & 901.1_GAMMMA_GS & USTEST & -0.118 \\
\hline 3941 & PDMS & & SW & & & E013113 & 09-Dec-83 & & $10028-17-8$ & TRITIUM & 906.0_H3_LSC & USTEST & 188 \\
\hline 3942 & PDMS & & SW & & & E013113 & 09-Dec-83 & & $10098-97-2$ & SR-90 & 905.0_SR_GPC & USTEST & 1.27 \\
\hline 3943 & PDMS & & SW & & & E032038 & 20-Dec-83 & & $10028-17-8$ & TRITIUM & 906.0_H3_LSC & USTEST & 255 \\
\hline 3944 & PDMS & & SW & & & E032038 & 20-Dec-83 & & $10098-97-2$ & SR-90 & 905.0_SR_GPC & USTEST & 0.199 \\
\hline 3945 & PDMS & & SW & & & E032038 & 20-Dec-83 & & $10045-97-3$ & CS-137 & 901.1_GAMMA_GS & USTEST & 0.219 \\
\hline 3946 & PDMS & & SW & & & E001845 & 20-Dec-83 & & $10045-97-3$ & CS-137 & GAMMA_AS & PNL-RO & 0.0272 \\
\hline 3947 & PDMS & & SW & & & E003868 & 20-Dec-83 & & $10045-97-3$ & CS-137 & GAMMA_GS & PNL-RO & 0.013 \\
\hline 3948 & PDMS & & SW & & & E001214 & 20-Dec-83 & & $10045-97-3$ & CS-137 & GAMMA_GS & PNL-RO & 0.0265 \\
\hline 3949 & PDMS & & SW & & & E010723 & 20-Dec-83 & & $10028-17-8$ & TRITIUM & TRITIUM_ELELECT_LSC & USTEST & 58.8 \\
\hline 3950 & PDMS & & SW & & & E013898 & 20-Dec-83 & & $10045-97-3$ & CS-137 & GAMMA_GS & PNL-RO & 0.0153 \\
\hline 3951 & PDMS & & sW & & & E010723 & 20-Dec-83 & & $10098-97-2$ & SR-90 & SRISO_SEP_PRECIP_GPC & USTEST & 0.112 \\
\hline 3952 & PDMS & & sW & & & E010723 & 20-Dec-83 & & $10045-97-3$ & CS-137 & GAMMA_GS & USTEST & 0.695 \\
\hline 3953 & PDMS & & SW & & & E004324 & 20-Dec-83 & & $10028-17-8$ & TRITIUM & TRITIUM_ĒELECT_LSC & USTEST & 78.1 \\
\hline
\end{tabular}




\begin{tabular}{|c|c|c|c|c|c|c|c|c|c|c|c|c|c|}
\hline & A & $\mathrm{B}$ & $\mathrm{C}$ & $\mathrm{D}$ & $\mathrm{E}$ & $\mathrm{F}$ & $\mathrm{G}$ & $\mathrm{H}$ & $\mathrm{I}$ & $\mathrm{J}$ & $\mathrm{K}$ & $\mathrm{L}$ & $\mathrm{M}$ \\
\hline 1 & SAMPLE_NUM_ASSIGNED_TO & \begin{tabular}{|l|} 
SAMPLER \\
\end{tabular} & MEDIA & BIOTA_MEDIA & TAG_ID & SAMP_NUM & SAMP_DATE_TIME & SAMP_DATE_TIME_ON & CON_ID & CON_SHORT_NAME & METHOD_NAME & LAB_CODE & VALUE_RPTD \\
\hline 3954 & PDMS & & SW & & & E004324 & $20-$ Dec-83 & & $10045-97-3$ & CS-137 & GAMMA_GS & USTEST & -0.0437 \\
\hline 3955 & PDMS & & sw & & & E004324 & 20-Dec-83 & & $10098-97-2$ & SR-90 & SRISO_SEPP_PRECIP_GPC & USTEST & 0.213 \\
\hline 3956 & PDMS & & sw & & & E037637 & 20-Dec-83 & & $10098-97-2$ & SR-90 & SRISO_SEP_PRECIP_GPC & USTEST & 0.18 \\
\hline \begin{tabular}{|c|}
3957 \\
\end{tabular} & PDMS & & sw & & & E037637 & 20-Dec-83 & & $10045-97-3$ & CS-137 & GAMMA_GS & USTEST & -0.796 \\
\hline \begin{tabular}{|c|}
3958 \\
\end{tabular} & PDMS & & sw & & & E001846 & 03-Jan-84 & & $10045-97-3$ & CS-137 & GAMMA_GS & PNL-RO & 0.0234 \\
\hline \begin{tabular}{|c|}
3959 \\
\end{tabular} & PDMS & & sw & & & E003869 & 03-Jan-84 & & $10045-97-3$ & CS-137 & GAMMA_GS & PNL-RO & 0.0134 \\
\hline 3960 & PDMS & & sW & & & E001215 & 03-Jan-84 & & $10045-97-3$ & CS-137 & GAMMA_GS & PNL-RO & 0.0331 \\
\hline 3961 & PDMS & & SW & & & E013899 & 03-Jan-84 & & $10045-97-3$ & CS-137 & GAMMA_GS & PNL-RO & 0.0163 \\
\hline 3962 & PDMS & & sw & & & E001847 & 17-Jan-84 & & PU-239/240 & PU-239/240 & PUISO_IE_AEA & PNL-RO & 0.00012 \\
\hline 3963 & PDMS & & SW & & & E001847 & 17-Jan-84 & & $10045-97-3$ & CS-137 & GAMMA_GSS & PNL-RO & 0.0241 \\
\hline 3964 & PDMS & & sw & & & E001847 & 17-Jan-84 & & 15046-84-1 & I-129 & I129_SEP_TEMS & 320 & 0.0000613 \\
\hline 3965 & PDMS & & sW & & & E003870 & 17-Jan-84 & & $10045-97-3$ & CS-137 & GAMMA_ḠS & PNL-RO & 0.0155 \\
\hline 3966 & PDMS & & sW & & & E003870 & 17-Jan-84 & & PU-239/240 & PU-239/240 & PUISO_IE_AEA & PNL-RO & 0.000018 \\
\hline 3967 & PDMS & & sW & & & E001216 & 17-Jan-84 & & $10045-97-3$ & CS-137 & GAMMA GS & PNL-RO & 0.0309 \\
\hline 3968 & PDMS & & sw & & & E001216 & 17-Jan-84 & & PU-239/240 & PU-239/240 & PUISO_IE_AEA & PNL-RO & 0.0005 \\
\hline 3969 & PDMS & & sw & & & E001216 & 17-Jan-84 & & 15046-84-1 & I-129 & I129_SEP_TEMS & 320 & 0.000011 \\
\hline 3970 & PDMS & & sw & & & E010724 & 17-Jan-84 & & $10028-17-8$ & TRITIUM & TRITIUM_ELECT_LSC & USTEST & 103 \\
\hline 3971 & PDMS & & sW & & & E010724 & 17-Jan-84 & & $10098-97-2$ & SR-90 & SRISO_SEPP_PRËCIP_GPC & USTEST & 0.134 \\
\hline 3972 & PDMS & & sW & & & E010724 & 17-Jan-84 & & $10045-97-3$ & CS-137 & GAMMA_GS & USTEST & 0.189 \\
\hline 3973 & PDMS & & sW & & & E013900 & 17-Jan-84 & & PU-239/240 & PU-239/240 & PUISO_IE_AEA & PNL-RO & 0.000009 \\
\hline 3974 & PDMS & & sw & & & E013900 & 17-Jan-84 & & $10045-97-3$ & CS-137 & GAMMA_GS & PNL-RO & 0.0158 \\
\hline 3975 & PDMS & & sW & & & E005959 & 17-Jan-84 & & $10028-17-8$ & TRITIUM & TRITIUM_DIST_LSC & USTEST & \\
\hline 3976 & PDMS & & sw & & & E005959 & 17-Jan-84 & & $10045-97-3$ & CS-137 & GAMMA_GS & USTEST & \\
\hline 3977 & PDMS & & sW & & & E005959 & 17-Jan-84 & & $10098-97-2$ & SR-90 & SRISO_SEEP_PRECIP_GPC & USTEST & \\
\hline 3978 & PDMS & & SW & & & E037317 & 24-Jan-84 & & $10098-97-2$ & SR-90 & SRISO_SEP_PRECIP_GPC & USTEST & 0.384 \\
\hline 3979 & PDMS & & sW & & & E037317 & 24-Jan-84 & & $10028-17-8$ & TRITIUM & TRITIUM_DISTT_LSC & USTEST & 254 \\
\hline 3980 & PDMS & & sW & & & E037317 & 24-Jan-84 & & $10045-97-3$ & CS-137 & GAMMA_GS & USTEST & -0.539 \\
\hline 3981 & PDMS & & sW & & & E003871 & 31-Jan-84 & & $10045-97-3$ & CS-137 & GAMMA_GS & PNL-RO & 0.0113 \\
\hline 3982 & PDMS & & sw & & & E001848 & 31-Jan-84 & & $10045-97-3$ & CS-137 & GAMMA_GS & PNL-RO & 0.0321 \\
\hline 3983 & PDMS & & sw & & & E015244 & 31-Jan-84 & & $10045-97-3$ & CS-137 & GAMMA_GS & HISTOR & \\
\hline 3984 & PDMS & & sw & & & E026972 & 31-Jan-84 & & $10045-97-3$ & CS-137 & GAMMA_GS & HISTOR & \\
\hline 3985 & PDMS & & sW & & & E014492 & 14-Feb-84 & & $10098-97-2$ & SR-90 & 905.0_SR_GPC & USTEST & 0.104 \\
\hline 3986 & PDMS & & sW & & & E014492 & 14-Feb-84 & & $10028-17-8$ & TRITIUM & 906.0_H3_LSC & USTEST & 136 \\
\hline 3987 & PDMS & & SW & & & E014492 & 14-Feb-84 & & $10045-97-3$ & CS-137 & 901.1_GAMMA_GS & USTEST & -0.582 \\
\hline 3988 & PDMS & & sW & & & E010738 & 14-Feb-84 & & $10045-97-3$ & CS-137 & 901.1_GAMMA_GS & USTEST & -0.244 \\
\hline 3989 & PDMS & & sw & & & E010738 & 14-Feb-84 & & $10028-17-8$ & TRITIUM & 906.0_H3_LSC & USTEST & 138 \\
\hline 3990 & PDMS & & SW & & & E014813 & 14-Feb-84 & & $10045-97-3$ & CS-137 & GAMMA_GS & HISTOR & \\
\hline 3991 & PDMS & & sW & & & E001955 & 14-Feb-84 & & $10045-97-3$ & CS-137 & GAMMA_GS & HISTOR & \\
\hline 3992 & PDMS & & sW & & & E001217 & 14-Feb-84 & & $10045-97-3$ & CS-137 & GAMMA_GS & PNL-RO & 0.0333 \\
\hline 3993 & PDMS & & SW & & & E010659 & 14-Feb-84 & & $10028-17-8$ & TRITIUM & TRITIUM_ELEECT_LSC & USTEST & 111 \\
\hline $3994 \mathrm{~F}$ & PDMS & & sW & & & E013901 & 14-Feb-84 & & $10045-97-3$ & CS-137 & GAMMA_GS & PNL-RO & 0.0126 \\
\hline 3995 & PDMS & & sW & & & E010659 & 14-Feb-84 & & $10098-97-2$ & SR-90 & SRISO_SEEP_PRECIP_GPC & USTEST & 0.167 \\
\hline 3996 & PDMS & & SW & & & E010659 & 14-Feb-84 & & $10045-97-3$ & CS-137 & GAMMA_GS & USTEST & -0.118 \\
\hline 3997 & PDMS & & sW & & & E004325 & 14-Feb-84 & & $10028-17-8$ & TRITIUM & TRITIUM_ELECT_LSC & USTEST & 137 \\
\hline 3998 & PDMS & & SW & & & E004325 & 14-Feb-84 & & $10045-97-3$ & CS-137 & GAMMA_GS & USTEST & 1.29 \\
\hline 3999 & PDMS & & SW & & & E004325 & 14-Feb-84 & & $10098-97-2$ & SR-90 & SRISO_SEEP_PRECIP_GPC & USTEST & 0.179 \\
\hline 4000 & PDMS & & sW & & & E013533 & 21-Feb-84 & & $10098-97-2$ & SR-90 & SRISO_SEP_PRECIP_GPC & USTEST & 1.76 \\
\hline $4001 \mathrm{~F}$ & PDMS & & sW & & & E013533 & 21-Feb-84 & & $10045-97-3$ & CS-137 & GAMMA_GS & USTEST & 10.6 \\
\hline $4002 \mathrm{~F}$ & PDMS & & sw & & & E013533 & 21-Feb-84 & & 10028-17-8 & TRITIUM & TRITIUM_DIST_LSC & USTEST & 5210 \\
\hline 4003 & PDMS & & SW & & & E012584 & 21-Feb-84 & & $10028-17-8$ & TRITIUM & TRITIUM_DIST_LSC & USTEST & 40500 \\
\hline 4004 & PDMS & & SW & & & E012584 & 21-Feb-84 & & $10045-97-3$ & CS-137 & GAMMA_GS & USTEST & 0.388 \\
\hline 4005 & PDMS & & SW & & & E017792 & 21-Feb-84 & & $10045-97-3$ & CS-137 & GAMMA_GS & USTEST & 11.5 \\
\hline
\end{tabular}




\begin{tabular}{|c|c|c|c|c|c|c|c|c|c|c|c|c|c|}
\hline & $\bar{A}$ & $\mathrm{~B}$ & C & $\mathrm{D}$ & $E$ & $\mathrm{~F}$ & G & $\mathrm{H}$ & $\mathrm{I}$ & $\mathrm{J}$ & $\mathrm{K}$ & $\mathrm{L}$ & $\mathrm{M}$ \\
\hline 1 & SAMPLE_NUM_ASSIGNED_TO & SAMPLER & MEDIA & BIOTA_MEDIA & TAG_ID & SAMP_NUM & SAMP_DATE_TIME & SAMP_DATE_TIME_ON & CON_ID & CON_SHORT_NAME & METHOD_NAME & LAB_CODE & VALUE_RPTD \\
\hline 4006 & PDMS & & SW & & & E017792 & 21-Feb-84 & & $10098-97-2$ & SR-90 & SRISO_SEP_PRECIP_GPC & USTEST & 0.833 \\
\hline 4007 & PDMS & & SW & & & E017792 & 21-Feb-84 & & $10028-17-8$ & TRITIUM & TRITIUM_DIST_LSC & USTEST & 195 \\
\hline 4008 & PDMS & & SW & & & E021781 & 21-Feb-84 & & $10028-17-8$ & TRITIUM & TRITIUM_DIST_LSC & USTEST & 1080 \\
\hline 4009 & PDMS & & SW & & & E021781 & 21-Feb-84 & & $10045-97-3$ & CS-137 & GAMMA_GS & USTEST & 1.1 \\
\hline 4010 & PDMS & & SW & & & E021781 & 21-Feb-84 & & $10098-97-2$ & SR-90 & SRISO_SEP_PRECIP_GPC & USTEST & 1.45 \\
\hline 4011 & PDMS & & SW & & & E001849 & 28-Feb-84 & & $10045-97-3$ & CS-137 & GAMMA_GS & PNL-RO & 0.0252 \\
\hline 4012 & PDMS & & SW & & & E003872 & 28-Feb-84 & & $10045-97-3$ & CS-137 & GAMMA_GS & PNL-RO & 0.0105 \\
\hline 4013 & PDMS & & SW & & & E013902 & 28-Feb-84 & & $10045-97-3$ & CS-137 & GAMMA_GS & PNL-RO & 0.0222 \\
\hline 4014 & PDMS & & SW & & & E001218 & 28-Feb-84 & & $10045-97-3$ & CS-137 & GAMMA_GS & PNL-RO & 0.0471 \\
\hline 4015 & PDMS & & SW & & & E013812 & 05-Mar-84 & & $10028-17-8$ & TRITIUM & 906.0_H3_LSC & USTEST & 35400 \\
\hline 4016 & PDMS & & SW & & & E013812 & 05-Mar-84 & & $10045-97-3$ & CS-137 & 901.1_GAMMA_GS & USTEST & -0.97 \\
\hline \begin{tabular}{|l|l|}
4017 \\
\end{tabular} & PDMS & & SW & & & E013812 & 05-Mar-84 & & $10098-97-2$ & SR-90 & 905.0_SR_GPC & USTEST & 0.0606 \\
\hline \begin{tabular}{|c|}
4018 \\
\end{tabular} & PDMS & & SW & & & E003873 & 13-Mar-84 & & $10045-97-3$ & CS-137 & GAMMĀĒSS & PNL-RO & 0.01 \\
\hline \begin{tabular}{|l|}
4019 \\
\end{tabular} & PDMS & & SW & & & E001850 & 13-Mar-84 & & $10045-97-3$ & CS-137 & GAMMA_GS & PNL-RO & 0.0259 \\
\hline 4020 & PDMS & & SW & & & E010660 & 13-Mar-84 & & $10028-17-8$ & TRITIUM & TRITIUM_ELECT_LSC & USTEST & 129 \\
\hline 4021 & PDMS & & SW & & & E010660 & 13-Mar-84 & & $10098-97-2$ & SR-90 & SRISO_SEP_PRECIP_GPC & USTEST & 0.177 \\
\hline 4022 & PDMS & & SW & & & E010660 & 13-Mar-84 & & $10045-97-3$ & CS-137 & GAMMA_GS & USTEST & -1.16 \\
\hline 4023 & PDMS & & SW & & & E001219 & 13-Mar-84 & & $10045-97-3$ & CS-137 & GAMMA_GS & PNL-RO & 0.0266 \\
\hline 4024 & PDMS & & SW & & & E013903 & 13-Mar-84 & & $10045-97-3$ & CS-137 & GAMMA_GS & PNL-RO & 0.0145 \\
\hline \begin{tabular}{|c|}
4025 \\
\end{tabular} & PDMS & & SW & & & E004326 & 13-Mar-84 & & $10028-17-8$ & TRITIUM & TRITIUM_ELELECT_LSC & USTEST & 150 \\
\hline 4026 & PDMS & & SW & & & E004326 & 13-Mar-84 & & $10045-97-3$ & CS-137 & GAMMA_GS & USTEST & -0.154 \\
\hline \begin{tabular}{|l|l|}
4027 \\
\end{tabular} & PDMS & & SW & & & E004326 & 13-Mar-84 & & $10098-97-2$ & SR-90 & SRISO_SEP_PRECIP_GPC & USTEST & 0.146 \\
\hline 4028 & PDMS & & SW & & & E036173 & 19-Mar-84 & & $10098-97-2$ & SR-90 & 905.0_SR_GPC & USTEST & 0.834 \\
\hline 4029 & PDMS & & SW & & & E036173 & 19-Mar-84 & & $10045-97-3$ & CS-137 & 901.1_GAMMA_GS & USTEST & -1.55 \\
\hline 4030 & PDMS & & SW & & & E036173 & 19-Mar-84 & & 10028-17-8 & TRITIUM & 906.0_H3_LSC & USTEST & 245 \\
\hline 4031 & PDMS & & SW & & & E032039 & 20-Mar-84 & & $10028-17-8$ & TRITIUM & 906.0_H3_LSC & USTEST & 490 \\
\hline 4032 & PDMS & & SW & & & E032039 & 20-Mar-84 & & $10098-97-2$ & SR-90 & 905.0_SR_GPC & USTEST & 0.364 \\
\hline 4033 & PDMS & & SW & & & E032039 & 20-Mar-84 & & $10045-97-3$ & CS-137 & 901.1_GAMMA_GS & USTEST & -0.389 \\
\hline 4034 & PDMS & & SW & & & E013114 & 23-Mar-84 & & $10045-97-3$ & CS-137 & 901.1_GAMMA_GS & USTEST & 0.388 \\
\hline 4035 & PDMS & & SW & & & E013114 & 23-Mar-84 & & $10028-17-8$ & TRITIUM & 906.0_H3_LSC & USTEST & 145 \\
\hline \begin{tabular}{|l|}
4036 \\
\end{tabular} & PDMS & & SW & & & E013114 & 23-Mar-84 & & $10098-97-2$ & SR-90 & 905.0_SR_GPC & USTEST & 0.807 \\
\hline \begin{tabular}{|c|}
4037 \\
\end{tabular} & PDMS & & SW & & & E003802 & 27-Mar-84 & & $10045-97-3$ & CS-137 & GAMMA_ĒGS & PNL-RO & 0.00905 \\
\hline 4038 & PDMS & & SW & & & E001851 & 27-Mar-84 & & $10045-97-3$ & CS-137 & GAMMA_GS & PNL-RO & 0.0168 \\
\hline 4039 & PDMS & & SW & & & E001221 & 27-Mar-84 & & $10045-97-3$ & CS-137 & GAMMA_GS & PNL-RO & 0.0385 \\
\hline 4040 & PDMS & & SW & & & E013904 & 27-Mar-84 & & $10045-97-3$ & CS-137 & GAMMA_GS & PNL-RO & 0.0146 \\
\hline 4041 & PDMS & & SW & & & E017381 & 10-Apr-84 & & $10098-97-2$ & SR-90 & SRISO_SEP_PRECIP_GPC & USTEST & 0.217 \\
\hline 4042 & PDMS & & SW & & & E017381 & 10-Apr-84 & & $10045-97-3$ & CS-137 & GAMMA_GS & USTEST & -0.341 \\
\hline 4043 & PDMS & & SW & & & E017381 & 10-Apr-84 & & 10028-17-8 & TRITIUM & TRITIUM_ELEECT_LSC & USTEST & 118 \\
\hline 4044 & PDMS & & SW & & & E028045 & 10-Apr-84 & & $10028-17-8$ & TRITIUM & TRITIUM_ELEECT_LESC & USTEST & 183 \\
\hline 4045 & PDMS & & SW & & & E028045 & 10-Apr-84 & & $10045-97-3$ & CS-137 & GAMMA_GS & USTEST & 0.183 \\
\hline 4046 & PDMS & & SW & & & E028045 & 10-Apr-84 & & $10098-97-2$ & SR-90 & SRISO_SEEP_PRECIP_GPC & USTEST & 0.184 \\
\hline 4047 & PDMS & & SW & & & E003803 & 10-Apr-84 & & $10045-97-3$ & CS-137 & GAMMA_GS & PNL-RO & 0.00843 \\
\hline 4048 & PDMS & & SW & & & E003803 & 10-Apr-84 & & PU-239/240 & PU-239/240 & PUISO_IE_AEA & PNL-RO & 0.000023 \\
\hline 4049 & PDMS & & SW & & & E001852 & 10-Apr-84 & & $10098-97-2$ & SR-90 & SRISO_SEP_PRECIP_GPC & PNL-RO & 0.26 \\
\hline 4050 & PDMS & & SW & & & E001852 & 10-Apr-84 & & PU-239/240 & PU-239/240 & PUISO_IE_AEAA & PNL-RO & 0.000087 \\
\hline 4051 & PDMS & & SW & & & E001852 & 10-Apr-84 & & $10045-97-3$ & CS-137 & GAMMA_GES & PNL-RO & 0.0167 \\
\hline 4052 & PDMS & & SW & & & E001852 & 10-Apr-84 & & 15046-84-1 & I-129 & I129_SEP_TEMS & 320 & 0.0000896 \\
\hline 4053 & PDMS & & SW & & & E026973 & 10-Apr-84 & & $10045-97-3$ & CS-137 & GAMMA_ḠS & HISTOR & \\
\hline 4054 & PDMS & & SW & & & E026973 & 10-Apr-84 & & $10098-97-2$ & SR-90 & SRISO_SEP_PRECIP_GPC & PNL-RO & 0.2 \\
\hline 4055 & PDMS & & SW & & & E026973 & 10-Apr-84 & & PU-239/240 & PU-239/240 & PUISO_IE_AEA & PNL-RO & 0.00032 \\
\hline 4056 & PDMS & & SW & & & E026973 & 10-Apr-84 & & 15046-84-1 & I-129 & I129_SEP_TEMS & 320 & 0.000011 \\
\hline \begin{tabular}{|c|c|}
4057 \\
\end{tabular} & PDMS & & SW & & & E010661 & 10-Apr-84 & & $10028-17-8$ & TRITIUM & TRITIUM_ELECT_LSC & USTEST & 166 \\
\hline
\end{tabular}




\begin{tabular}{|c|c|c|c|c|c|c|c|c|c|c|c|c|c|}
\hline & $\bar{A}$ & $\mathrm{~B}$ & $\mathrm{C}$ & $\mathrm{D}$ & $E$ & $\mathrm{~F}$ & G & $\mathrm{H}$ & $\mathrm{I}$ & $\mathrm{J}$ & $\mathrm{K}$ & $\mathrm{L}$ & $\mathrm{M}$ \\
\hline 1 & SAMPLE_NUM_ASSIGNED_TO & SAMPLER & MEDIA & BIOTA_MEDIA & TAG_ID & SAMP_NUM & SAMP_DATE_TIME & SAMP_DATE_TIME_ON & CON_ID & CON_SHORT_NAME & METHOD_NAME & LAB_CODE & VALUE_RPTD \\
\hline 4058 & PDMS & & SW & & & E010661 & 10-Apr-84 & & $10098-97-2$ & SR-90 & SRISO_SEP_PRECIP_GPC & USTEST & 0.152 \\
\hline 4059 & PDMS & & SW & & & E010661 & 10-Apr-84 & & $10045-97-3$ & CS-137 & GAMMA_GS & USTEST & 0.428 \\
\hline 4060 & PDMS & & SW & & & E015245 & 10-Apr-84 & & PU-239/240 & PU-239/240 & PUISO_IE_AEA & USTEST & 0.000028 \\
\hline 4061 & PDMS & & SW & & & E015245 & 10-Apr-84 & & $10045-97-3$ & CS-137 & GAMMA_GS & HISTOR & \\
\hline 4062 & PDMS & & SW & & & E004327 & 10-Apr-84 & & $10028-17-8$ & TRITIUM & TRITIUM_ELECT_LSC & USTEST & 200 \\
\hline 4063 & PDMS & & SW & & & E004327 & 10-Apr-84 & & $10045-97-3$ & CS-137 & GAMMA_GS & USTEST & 0.177 \\
\hline 4064 & PDMS & & SW & & & E004327 & 10-Apr-84 & & $10098-97-2$ & SR-90 & SRISO_SEP_PRECIP_GPC & USTEST & 0.0233 \\
\hline 4065 & PDMS & & SW & & & E037318 & 17-Apr-84 & & $10098-97-2$ & SR-90 & SRISO_SEP_PRECIP_GPC & USTEST & 0.251 \\
\hline 4066 & PDMS & & SW & & & E037318 & 17-Apr-84 & & 10028-17-8 & TRITIUM & TRITIUM_DIST_LSC & USTEST & 209 \\
\hline \begin{tabular}{|l|l|}
4067 \\
\end{tabular} & PDMS & & SW & & & E037318 & 17-Apr-84 & & $10045-97-3$ & CS-137 & GAMMA_GS & USTEST & -0.829 \\
\hline 4068 & PDMS & & SW & & & E001853 & 24-Apr-84 & & $10045-97-3$ & CS-137 & GAMMA_GS & PNL-RO & 0.0291 \\
\hline \begin{tabular}{|l|l|}
4069 \\
\end{tabular} & PDMS & & SW & & & E003804 & 24-Apr-84 & & $10045-97-3$ & CS-137 & GAMMA_GS & PNL-RO & 0.0122 \\
\hline 4070 & PDMS & & SW & & & E001222 & 24-Apr-84 & & $10045-97-3$ & CS-137 & GAMMA_GS & PNL-RO & 0.0288 \\
\hline \begin{tabular}{|l|l|}
4071 & \\
\end{tabular} & PDMS & & SW & & & E013905 & 24-Apr-84 & & $10045-97-3$ & CS-137 & GAMMA_GS & PNL-RO & 0.0154 \\
\hline \begin{tabular}{|l|l|}
4072 \\
\end{tabular} & PDMS & & SW & & & E014493 & 08-May-84 & & $10098-97-2$ & SR-90 & 905.0_SR_GPC & USTEST & 0.0358 \\
\hline 4073 & PDMS & & SW & & & E014493 & 08-May-84 & & 10028-17-8 & TRITIUM & 906.0_H3_LSC & USTEST & -26.5 \\
\hline 4074 & PDMS & & SW & & & E014493 & 08-May-84 & & $10045-97-3$ & CS-137 & 901.1_GAMMA_GS & USTEST & 0.358 \\
\hline 4075 & PDMS & & SW & & & E010739 & 08-May-84 & & $10045-97-3$ & CS-137 & 901.1_GAMMA_GS & USTEST & 0.678 \\
\hline 4076 & PDMS & & SW & & & E010739 & 08-May-84 & & $10028-17-8$ & TRITIUM & 906.0_H3_LSC & USTEST & -7.16 \\
\hline \begin{tabular}{|l|l|}
4077 \\
\end{tabular} & PDMS & & SW & & & E003805 & 08-May-84 & & $10045-97-3$ & CS-137 & GAMMA_GS & PNL-RO & 0.0128 \\
\hline 4078 & PDMS & & SW & & & E001854 & 08-May-84 & & $10045-97-3$ & CS-137 & GAMMA_GS & PNL-RO & 0.0272 \\
\hline \begin{tabular}{|l|l|}
4079 \\
\end{tabular} & PDMS & & SW & & & E001223 & 08-May-84 & & $10045-97-3$ & CS-137 & GAMMA_GS & PNL-RO & 0.0345 \\
\hline 4080 & PDMS & & SW & & & E010662 & 08-May-84 & & 10028-17-8 & TRITIUM & TRITIUM_ELECT_LSC & USTEST & 119 \\
\hline 4081 & PDMS & & SW & & & E013834 & 08-May-84 & & $10045-97-3$ & CS-137 & GAMMA_GS & PNL-RO & 0.011 \\
\hline 4082 & PDMS & & SW & & & E010662 & 08-May-84 & & $10098-97-2$ & SR-90 & SRISO_SEP_PRECIP_GPC & USTEST & 0.102 \\
\hline 4083 & PDMS & & SW & & & E010662 & 08-May-84 & & $10045-97-3$ & CS-137 & GAMMA_GS & USTEST & 0.153 \\
\hline 4084 & PDMS & & SW & & & E004328 & 08-May-84 & & $10028-17-8$ & TRITIUM & TRITIUM_ELECT_LSC & USTEST & 156 \\
\hline 4085 & PDMS & & SW & & & E004328 & 08-May-84 & & $10045-97-3$ & CS-137 & GAMMA_GS & USTEST & -0.242 \\
\hline 4086 & PDMS & & SW & & & E004328 & 08-May-84 & & $10098-97-2$ & SR-90 & SRISO_SEP_PRECIP_GPC & USTEST & 0.265 \\
\hline 4087 & PDMS & & SW & & & E013534 & 15-May-84 & & $10098-97-2$ & SR-90 & SRISO_SEP_PRECIP_GPC & USTEST & 33.1 \\
\hline 4088 & PDMS & & SW & & & E013534 & 15-May-84 & & $10045-97-3$ & CS-137 & GAMMA_GS & USTEST & 3.68 \\
\hline 4089 & PDMS & & SW & & & E013534 & 15-May-84 & & $10028-17-8$ & TRITIUM & TRITIUM_DIST_LSC & USTEST & 10100 \\
\hline 4090 & PDMS & & SW & & & E012585 & 15-May-84 & & $10028-17-8$ & TRITIUM & TRITIUM_DIST_LSC & USTEST & 26200 \\
\hline 4091 & PDMS & & SW & & & E012585 & 15-May-84 & & $10045-97-3$ & CS-137 & GAMMA_GS & USTEST & -0.622 \\
\hline \begin{tabular}{|l|}
4092 \\
\end{tabular} & PDMS & & SW & & & E017793 & 15-May-84 & & $10045-97-3$ & CS-137 & GAMMA_GS & USTEST & 23 \\
\hline 4093 & PDMS & & SW & & & E017793 & 15-May-84 & & $10098-97-2$ & SR-90 & SRISO_SEP_PRECIP_GPC & USTEST & 4.79 \\
\hline 4094 & PDMS & & SW & & & E017793 & 15-May-84 & & 10028-17-8 & TRITIUM & TRITIUM_DIST_LSC & USTEST & 197 \\
\hline 4095 & PDMS & & SW & & & E021782 & 15-May-84 & & $10028-17-8$ & TRITIUM & TRITIUM_DIST_LSC & USTEST & 1010 \\
\hline 4096 & PDMS & & SW & & & E021782 & 15-May-84 & & $10045-97-3$ & CS-137 & GAMMA_GS & USTEST & 2.5 \\
\hline 4097 & PDMS & & SW & & & E021782 & 15-May-84 & & $10098-97-2$ & SR-90 & SRISO_SEP_PRECIP_GPC & USTEST & 0.944 \\
\hline 4098 & PDMS & & SW & & & E000863 & 22-May-84 & & $10098-97-2$ & SR-90 & 905.0_SR_GPC & USTEST & 0.0417 \\
\hline 4099 & PDMS & & SW & & & E000863 & 22-May-84 & & $10045-97-3$ & CS-137 & 901.1_GAMMA_GS & USTEST & -0.678 \\
\hline 4100 & PDMS & & SW & & & E000863 & 22-May-84 & & $10028-17-8$ & TRITIUM & 906.0_H3_LSC & USTEST & 23.6 \\
\hline 4101 & PDMS & & SW & & & E001855 & 22-May-84 & & $10045-97-3$ & CS-137 & GAMMA_GS & PNL-RO & 0.0271 \\
\hline 4102 & PDMS & & SW & & & E003806 & 22-May-84 & & $10045-97-3$ & CS-137 & GAMMA_GS & PNL-RO & 0.00766 \\
\hline 4103 & PDMS & & SW & & & E015246 & 22-May-84 & & $10045-97-3$ & CS-137 & GAMMA_GS & HISTOR & \\
\hline 4104 & PDMS & & SW & & & E026974 & 22-May-84 & & $10045-97-3$ & CS-137 & GAMMA_GS & HISTOR & \\
\hline 4105 & PDMS & & SW & & & E013813 & 01-Jun-84 & & $10098-97-2$ & SR-90 & 905.0_SR_GPC & USTEST & 0.0991 \\
\hline 4106 & PDMS & & SW & & & E013813 & 01-Jun-84 & & 10028-17-8 & TRITIUM & 906.0_H3_LSC & USTEST & 26400 \\
\hline 4107 & PDMS & & SW & & & E013813 & 01-Jun-84 & & $10045-97-3$ & CS-137 & 901.1_GAMMA_GS & USTEST & -0.484 \\
\hline 4108 & PDMS & & SW & & & E003807 & 05-Jun-84 & & $10045-97-3$ & CS-137 & GAMMA_GS & PNL-RO & 0.0108 \\
\hline 4109 & PDMS & & SW & & & E001856 & 05-Jun-84 & & $10045-97-3$ & CS-137 & GAMMA_GS & PNL-RO & 0.0162 \\
\hline
\end{tabular}




\begin{tabular}{|c|c|c|c|c|c|c|c|c|c|c|c|c|c|}
\hline & $\bar{A}$ & $\mathrm{~B}$ & $\mathrm{C}$ & $\mathrm{D}$ & $E$ & $\mathrm{~F}$ & G & $\mathrm{H}$ & $\mathrm{I}$ & $\mathrm{J}$ & $\mathrm{K}$ & $\mathrm{L}$ & $\mathrm{M}$ \\
\hline 1 & SAMPLE_NUM_ASSIGNED_TO & SAMPLER & MEDIA & BIOTA_MEDIA & TAG_ID & SAMP_NUM & SAMP_DATE_TIME & SAMP_DATE_TIME_ON & CON_ID & CON_SHORT_NAME & METHOD_NAME & LAB_CODE & \begin{tabular}{|l|} 
VALUE_RPTD \\
\end{tabular} \\
\hline 4110 & PDMS & & SW & & & E010663 & 05-Jun-84 & & $10028-17-8$ & TRITIUM & TRITIUM_ELECT_LSC & USTEST & 137 \\
\hline 4111 & PDMS & & SW & & & E013835 & 05-Jun-84 & & $10045-97-3$ & CS-137 & GAMMA_GS & PNL-RO & 0.0168 \\
\hline 4112 & PDMS & & SW & & & E010663 & 05-Jun-84 & & $10098-97-2$ & SR-90 & SRISO_SEP_PRECIP_GPC & USTEST & 0.0731 \\
\hline 4113 & PDMS & & SW & & & E010663 & 05-Jun-84 & & $10045-97-3$ & CS-137 & GAMMA_GS & USTEST & -0.153 \\
\hline 4114 & PDMS & & SW & & & E001224 & 05-Jun-84 & & $10045-97-3$ & CS-137 & GAMMA_GS & PNL-RO & 0.0446 \\
\hline 4115 & PDMS & & SW & & & E004329 & 05-Jun-84 & & $10028-17-8$ & TRITIUM & TRITIUM_ELECT_LSC & USTEST & 179 \\
\hline 4116 & PDMS & & SW & & & E004329 & 05-Jun-84 & & $10098-97-2$ & SR-90 & SRISO_SËP_PRËCIP_GPC & USTEST & 0.17 \\
\hline 4117 & PDMS & & SW & & & E004329 & 05-Jun-84 & & $10045-97-3$ & CS-137 & GAMMA_GS & USTEST & -0.275 \\
\hline 4118 & PDMS & & SW & & & E036174 & 11-Jun-84 & & 10098-97-2 & SR-90 & 905.0_SR_GPC & USTEST & 0.038 \\
\hline 4119 & PDMS & & SW & & & E036174 & 11-Jun-84 & & $10045-97-3$ & CS-137 & 901.1_GAMMA_GS & USTEST & 0.172 \\
\hline 4120 & PDMS & & SW & & & E036174 & 11-Jun-84 & & 10028-17-8 & TRITIUM & 906.0_H3_LSC & USTEST & -90.8 \\
\hline 4121 & PDMS & & SW & & & E032040 & 12-Jun-84 & & $10028-17-8$ & TRITIUM & 906.0_H3_LSC & USTEST & 142 \\
\hline \begin{tabular}{|l|}
4122 \\
\end{tabular} & PDMS & & SW & & & E032040 & 12-Jun-84 & & 10098-97-2 & SR-90 & 905.0_SR_GPC & USTEST & 0.193 \\
\hline \begin{tabular}{|l|}
4123 \\
\end{tabular} & PDMS & & SW & & & E032040 & 12-Jun-84 & & $10045-97-3$ & CS-137 & 901.1_GAMMA_GS & USTEST & -0.935 \\
\hline \begin{tabular}{|l|l|}
4124 \\
\end{tabular} & PDMS & & SW & & & E013115 & 15-Jun-84 & & $10045-97-3$ & CS-137 & 901.1_GAMMA_GS & USTEST & -0.153 \\
\hline 4125 & PDMS & & SW & & & E013115 & 15-Jun-84 & & 10028-17-8 & TRITIUM & 906.0_H3_LSC & USTEST & 309 \\
\hline \begin{tabular}{|l|}
4126 \\
\end{tabular} & PDMS & & SW & & & E013115 & 15-Jun-84 & & 10098-97-2 & SR-90 & 905.0_SR_GPC & USTEST & 0.401 \\
\hline \begin{tabular}{|l|}
4127 \\
\end{tabular} & PDMS & & SW & & & E001857 & 19-Jun-84 & & $10045-97-3$ & CS-137 & GAMMA_ESS & PNL-RO & 0.0239 \\
\hline \begin{tabular}{|l|}
4128 \\
\end{tabular} & PDMS & & SW & & & E014814 & 19-Jun-84 & & $10045-97-3$ & CS-137 & GAMMA_GS & HISTOR & \\
\hline \begin{tabular}{|l|}
4129 \\
\end{tabular} & PDMS & & SW & & & E013836 & 19-Jun-84 & & $10045-97-3$ & CS-137 & GAMMA_GS & PNL-RO & 0.00899 \\
\hline \begin{tabular}{|l|}
4130 \\
\end{tabular} & PDMS & & SW & & & E001158 & 20-Jun-84 & & $10045-97-3$ & CS-137 & GAMMA_GS & PNL-RO & 0.0284 \\
\hline \begin{tabular}{|l|}
4131 \\
\end{tabular} & PDMS & & SW & & & E001858 & 03-Jul-84 & & $10098-97-2$ & SR-90 & SRISO_SEP_PRECIP_GPC & PNL-RO & \\
\hline \begin{tabular}{|l|}
4132 \\
\end{tabular} & PDMS & & SW & & & E001858 & 03-Jul-84 & & PU-239/240 & PU-239/240 & PUISO_IE_AEA & PNL-RO & 0.000051 \\
\hline 4133 & PDMS & & SW & & & E001858 & 03-Jul-84 & & $10045-97-3$ & CS-137 & GAMMA_GS & PNL-RO & 0.0211 \\
\hline \begin{tabular}{|l|}
4134 \\
\end{tabular} & PDMS & & SW & & & E001858 & 03-Jul-84 & & 15046-84-1 & I-129 & I129_SEP_TEMS & 320 & 0.000044 \\
\hline \begin{tabular}{|l|l}
4135 \\
\end{tabular} & PDMS & & SW & & & E003808 & 03-Jul-84 & & $10045-97-3$ & CS-137 & GAMMA_ḠS & PNL-RO & 0.0113 \\
\hline \begin{tabular}{|l|l|l}
4136 \\
\end{tabular} & PDMS & & SW & & & E003808 & 03-Jul-84 & & PU-239/240 & PU-239/240 & PUISO_IE_AEA & PNL-RO & 0.000025 \\
\hline 4137 & PDMS & & SW & & & E013837 & 03-Jul-84 & & PU-239/240 & PU-239/240 & PUISO_IE_AEA & USTEST & 0.00005 \\
\hline \begin{tabular}{|l|}
4138 \\
\end{tabular} & PDMS & & SW & & & E013837 & 03-Jul-84 & & $10045-97-3$ & CS-137 & GAMMA_GS & PNL-RO & 0.0162 \\
\hline \begin{tabular}{|l|}
4139 \\
\end{tabular} & PDMS & & SW & & & E001159 & 03-Jul-84 & & $10045-97-3$ & CS-137 & GAMMA_GS & PNL-RO & 0.0295 \\
\hline 4140 & PDMS & & SW & & & E001159 & 03-Jul-84 & & $10098-97-2$ & SR-90 & SRISO_SEP_PRECIP_GPC & PNL-RO & 0.2 \\
\hline \begin{tabular}{|l|}
4141 \\
\end{tabular} & PDMS & & SW & & & E001159 & 03-Jul-84 & & PU-239/240 & PU-239/240 & PUISO_IE_AEAA & PNL-RO & 0.00034 \\
\hline \begin{tabular}{|l|}
4142 \\
\end{tabular} & PDMS & & SW & & & E001159 & 03-Jul-84 & & 15046-84-1 & $1-129$ & I129_SEP_TEMS & 320 & 0.0000173 \\
\hline \begin{tabular}{|l|}
4143 \\
\end{tabular} & PDMS & & SW & & & E010664 & 03-Jul-84 & & $10028-17-8$ & TRITIUM & TRITIUM_ELECT_LSC & USTEST & 105 \\
\hline \begin{tabular}{|l|}
4144 \\
\end{tabular} & PDMS & & SW & & & E010664 & 03-Jul-84 & & $10098-97-2$ & SR-90 & SRISO_SEP_PRECIP_GPC & USTEST & 0.122 \\
\hline \begin{tabular}{|l|l|l|l|}
4145 \\
\end{tabular} & PDMS & & SW & & & E010664 & 03-Jul-84 & & $10045-97-3$ & CS-137 & GAMMA_GS & USTEST & -0.436 \\
\hline \begin{tabular}{|l|l|l|l|l|}
4146 \\
\end{tabular} & PDMS & & SW & & & E004330 & 03-Jul-84 & & 10028-17-8 & TRITIUM & TRITIUM_ELECT_LSC & USTEST & 159 \\
\hline \begin{tabular}{|l|l|}
4147 \\
\end{tabular} & PDMS & & SW & & & E004330 & 03-Jul-84 & & $10098-97-2$ & SR-90 & SRISO_SEP_PRËCIP_GPC & USTEST & 0.192 \\
\hline \begin{tabular}{|l|}
4148 \\
\end{tabular} & PDMS & & SW & & & E004330 & 03-Jul-84 & & $10045-97-3$ & CS-137 & GAMMA_GS & USTEST & 0.776 \\
\hline 4149 & PDMS & & SW & & & E037319 & 10-Jul-84 & & $10098-97-2$ & SR-90 & SRISO_SEEP_PRECIP_GPC & USTEST & 0.227 \\
\hline 4150 & PDMS & & SW & & & E037319 & 10-Jul-84 & & $10028-17-8$ & TRITIUM & TRITIUM_DIST_LSC & USTEST & 8.64 \\
\hline 4151 & PDMS & & SW & & & E037319 & 10-Jul-84 & & $10045-97-3$ & CS-137 & GAMMA_GS & USTEST & 0.819 \\
\hline \begin{tabular}{|l|l|}
4152 \\
\end{tabular} & PDMS & & SW & & & E001859 & 17-Jul-84 & & $10045-97-3$ & CS-137 & GAMMA_GS & PNL-RO & 0.0132 \\
\hline 4153 & PDMS & & SW & & & E003809 & 17-Jul-84 & & $10045-97-3$ & CS-137 & GAMMA_GS & PNL-RO & 0.00889 \\
\hline 4154 & PDMS & & SW & & & E001160 & 17-Jul-84 & & $10045-97-3$ & CS-137 & GAMMA_GS & PNL-RO & 0.0137 \\
\hline 4155 & PDMS & & SW & & & E013838 & 17-Jul-84 & & $10045-97-3$ & CS-137 & GAMMA_GS & PNL-RO & 0.0143 \\
\hline \begin{tabular}{|c|}
4156 \\
\end{tabular} & PDMS & & SW & & & E013535 & 19-Jul-84 & & $10098-97-2$ & SR-90 & SRISO_SEPPPRECIP_GPC & USTEST & 2.61 \\
\hline 4157 & PDMS & & SW & & & E013535 & 19-Jul-84 & & $10045-97-3$ & CS-137 & GAMMA_GS & USTEST & -0.0511 \\
\hline 4158 & PDMS & & SW & & & E013535 & 19-Jul-84 & & $10028-17-8$ & TRITIUM & TRITIUM_DIST_LSC & USTEST & 163 \\
\hline 4159 & PDMS & & SW & & & E010740 & 31-Jul-84 & & $10045-97-3$ & CS-137 & 901.1_GAMMA_GS & USTEST & 0.255 \\
\hline 4160 & PDMS & & SW & & & E010740 & 31-Jul-84 & & 10028-17-8 & TRITIUM & 906.0_H3_LSC & USTEST & -61.3 \\
\hline 4161 & PDMS & & SW & & & E001860 & 31-Jul-84 & & $10045-97-3$ & CS-137 & GAMMA_ĒSS & PNL-RO & 0.0247 \\
\hline
\end{tabular}




\begin{tabular}{|c|c|c|c|c|c|c|c|c|c|c|c|c|c|}
\hline & A & $\mathrm{B}$ & $\mathrm{c}$ & $\mathrm{D}$ & $\mathrm{E}$ & $\mathrm{F}$ & G & $\mathrm{H}$ & $\mathrm{I}$ & $\mathrm{J}$ & $\mathrm{K}$ & $\mathrm{L}$ & $\mathrm{M}$ \\
\hline 1 & SAMPLE_NUM_ASSIGNED_TO & SAMPLER & MEDIA & BIOTA_MEDIA & TAG_ID & SAMP_NUM & SAMP_DATE_TIME & SAMP_DATE_TIME_ON & CON_ID & CON_SHORT_NAME & METHOD_NAME & LAB_CODE & \begin{tabular}{|l|} 
VALUE_RPTD \\
\end{tabular} \\
\hline 4162 & PDMS & & SW & & & E003810 & 31-Jul-84 & & $10045-97-3$ & CS-137 & GAMMA_GS & PNL-RO & 0.00935 \\
\hline 4163 & PDMS & & sW & & & E015247 & 31-Jul-84 & & $10045-97-3$ & CS-137 & GAMMA_GS & HISTOR & \\
\hline 4164 & PDMS & & sW & & & E026975 & 31-Jul-84 & & $10045-97-3$ & CS-137 & GAMMA_GS & HISTOR & \\
\hline 4165 & PDMS & & sW & & & E010665 & 31-Jul-84 & & $10028-17-8$ & TRITIUM & TRITIUM_ELECT_LSC & USTEST & 132 \\
\hline \begin{tabular}{|l|l|}
4166 \\
\end{tabular} & PDMS & & sW & & & E010665 & 31-Jul-84 & & $10098-97-2$ & SR-90 & SRISO_SEP_PRECIP_GPC & USTEST & 0.14 \\
\hline \begin{tabular}{|l|l|}
4167 & \\
\end{tabular} & PDMS & & SW & & & E010665 & 31-Jul-84 & & $10045-97-3$ & CS-137 & GAMMA_GS & USTEST & -0.207 \\
\hline 4168 & PDMS & & SW & & & E004331 & 31-Jul-84 & & $10028-17-8$ & TRITIUM & TRITIUM_ELECT_LSC & USTEST & 134 \\
\hline 4169 & PDMS & & SW & & & E004331 & 31-Jul-84 & & $10098-97-2$ & SR-90 & SRISO_SEP_PRËCIP_GPC & USTEST & 0.201 \\
\hline 4170 & PDMS & & sW & & & E004331 & 31-Jul-84 & & $10045-97-3$ & CS-137 & GAMMA_GS & USTEST & -0.367 \\
\hline 4171 & PDMS & & SW & & & E000864 & 01-Aug-84 & & $10098-97-2$ & SR-90 & 905.0_SR_GPC & USTEST & 0.0258 \\
\hline 4172 & PDMS & & SW & & & E000864 & 01-Aug-84 & & $10045-97-3$ & CS-137 & 901.1_GAMMA_GS & USTEST & 0.194 \\
\hline 4173 & PDMS & & SW & & & E000864 & 01-Aug-84 & & $10028-17-8$ & TRITIUM & 906.0_H3_LSC & USTEST & 141 \\
\hline 4174 & PDMS & & SW & & & E014494 & 01-Aug-84 & & $10098-97-2$ & SR-90 & 905.0_SR_GPC & USTEST & 0.281 \\
\hline 4175 & PDMS & & SW & & & E014494 & 01-Aug-84 & & $10028-17-8$ & TRITIUM & 906.0_H3_LSC & USTEST & 28.9 \\
\hline 4176 & PDMS & & SW & & & E014494 & 01-Aug-84 & & $10045-97-3$ & CS-137 & 901.1_GAMMA_GS & USTEST & -0.415 \\
\hline \begin{tabular}{|l|l|}
4177 & \\
\end{tabular} & PDMS & & SW & & & E013536 & 07-Aug-84 & & $10098-97-2$ & SR-90 & SRISO_SEP_PRECIP_GPC & USTEST & 3.36 \\
\hline 4178 & PDMS & & sW & & & E013536 & 07-Aug-84 & & $10045-97-3$ & CS-137 & GAMMA_GS & USTEST & -0.818 \\
\hline \begin{tabular}{|l|}
4179 \\
\end{tabular} & PDMS & & sW & & & E013536 & 07-Aug-84 & & $10028-17-8$ & TRITIUM & TRITIUM_DIST_LSC & USTEST & 11900 \\
\hline 4180 & PDMS & & SW & & & E012586 & 07-Aug-84 & & $10028-17-8$ & TRITIUM & TRITIUM_DIST_LSC & USTEST & 30500 \\
\hline 4181 & PDMS & & SW & & & E012586 & 07-Aug-84 & & $10045-97-3$ & CS-137 & GAMMA_GS & USTEST & 1.33 \\
\hline 4182 & PDMS & & SW & & & E017794 & 07-Aug-84 & & $10045-97-3$ & CS-137 & GAMMA_GS & USTEST & 27.6 \\
\hline 4183 & PDMS & & SW & & & E017794 & 07-Aug-84 & & $10098-97-2$ & SR-90 & SRISO_SEPPPRECIP_GPC & USTEST & 3.41 \\
\hline 4184 & PDMS & & SW & & & E017794 & 07-Aug-84 & & $10028-17-8$ & TRITIUM & TRITIUM_DISTT_LSC & USTEST & 311 \\
\hline 4185 & PDMS & & SW & & & E021783 & 07-Aug-84 & & $10028-17-8$ & TRITIUM & TRITIUM_DIST_LSC & USTEST & 867 \\
\hline 4186 & PDMS & & SW & & & E021783 & 07-Aug-84 & & $10045-97-3$ & CS-137 & GAMMA_GS & USTEST & -0.894 \\
\hline \begin{tabular}{|l|l|}
4187 & \\
\end{tabular} & PDMS & & SW & & & E021783 & 07-Aug-84 & & $10098-97-2$ & SR-90 & SRISO_SEEP_PRECIP_GPC & USTEST & 4.97 \\
\hline 4188 & PDMS & & SW & & & E001952 & 14-Aug-84 & & $10045-97-3$ & CS-137 & GAMMĀGS & PNL-RO & 0.0264 \\
\hline 4189 & PDMS & & SW & & & E003811 & 14-Aug-84 & & $10045-97-3$ & CS-137 & GAMMA_GS & PNL-RO & 0.0141 \\
\hline 4190 & PDMS & & SW & & & E001161 & 14-Aug-84 & & $10045-97-3$ & CS-137 & GAMMA_GS & PNL-RO & 0.034 \\
\hline 4191 & PDMS & & SW & & & E013839 & 14-Aug-84 & & $10045-97-3$ & CS-137 & GAMMA_GS & PNL-RO & 0.012 \\
\hline 4192 & PDMS & & SW & & & E013814 & 20-Aug-84 & & $10098-97-2$ & SR-90 & 905.0_SR_GPC & USTEST & 0.0146 \\
\hline 4193 & PDMS & & SW & & & E013814 & 20-Aug-84 & & $10028-17-8$ & TRITIUM & 906.0_H3_LSC & USTEST & 29600 \\
\hline 4194 & PDMS & & SW & & & E013814 & 20-Aug-84 & & $10045-97-3$ & CS-137 & 901.1_GAMMA_GS & USTEST & 0.259 \\
\hline 4195 & PDMS & & SW & & & E001862 & 28-Aug-84 & & $10045-97-3$ & CS-137 & GAMM̄A_GS & PNL-RO & 0.0219 \\
\hline 4196 & PDMS & & SW & & & E003812 & 28-Aug-84 & & $10045-97-3$ & CS-137 & GAMMA_GS & PNL-RO & 0.0116 \\
\hline \begin{tabular}{|l|l|}
4197 & \\
\end{tabular} & PDMS & & sW & & & E001162 & 28-Aug-84 & & $10045-97-3$ & CS-137 & GAMMA_GS & PNL-RO & 0.0221 \\
\hline 4198 & PDMS & & SW & & & E010666 & 28-Aug-84 & & $10028-17-8$ & TRITIUM & TRITIUM_ELECT_LSC & USTEST & 94 \\
\hline 4199 & PDMS & & SW & & & E013840 & 28-Aug-84 & & $10045-97-3$ & CS-137 & GAMMA_GS & PNL-RO & 0.0127 \\
\hline 4200 & PDMS & & SW & & & E010666 & 28-Aug-84 & & $10098-97-2$ & SR-90 & SRISO_SEPP_PRECIP_GPC & USTEST & 0.134 \\
\hline 4201 & PDMS & & SW & & & E010666 & 28-Aug-84 & & $10045-97-3$ & CS-137 & GAMMĀGS & USTEST & 0.103 \\
\hline 4202 & PDMS & & SW & & & E004332 & 28-Aug-84 & & $10028-17-8$ & TRITIUM & TRITIUM_ELECT_LSC & USTEST & 144 \\
\hline 4203 & PDMS & & SW & & & E004332 & 28-Aug-84 & & $10098-97-2$ & SR-90 & SRISO_SËP_PRËCIP_GPC & USTEST & 0.0953 \\
\hline 4204 & PDMS & & SW & & & E004332 & 28-Aug-84 & & $10045-97-3$ & CS-137 & GAMMA_GS & USTEST & \\
\hline 4205 & PDMS & & SW & & & E036175 & 04-Sep-84 & & $10098-97-2$ & SR-90 & 905.0_SR_GPC & USTEST & 0.0328 \\
\hline 4206 & PDMS & & SW & & & E036175 & 04-Sep-84 & & $10045-97-3$ & CS-137 & 901.1_GAMMA_GS & USTEST & -0.229 \\
\hline 4207 & PDMS & & SW & & & E036175 & 04-Sep-84 & & $10028-17-8$ & TRITIUM & 906.0_H3_LSC & USTEST & 75.8 \\
\hline 4208 & PDMS & & SW & & & E032041 & 05-Sep-84 & & $10028-17-8$ & TRITIUM & 906.0_H3_LSC & USTEST & 119 \\
\hline 4209 & PDMS & & SW & & & E032041 & 05-Sep-84 & & $10098-97-2$ & SR-90 & 905.0_SR_GPC & USTEST & 0.224 \\
\hline 4210 & PDMS & & SW & & & E032041 & 05-Sep-84 & & $10045-97-3$ & CS-137 & 901.1_GAMMA_GS & USTEST & 0.0345 \\
\hline 4211 & PDMS & & SW & & & E013116 & 07-Sep-84 & & $10045-97-3$ & CS-137 & 901.1_GAMMA_GS & USTEST & -0.204 \\
\hline 4212 & PDMS & & SW & & & E013116 & 07-Sep-84 & & $10028-17-8$ & TRITIUM & 906.0_H3_LSC & USTEST & \\
\hline 4213 & PDMS & & SW & & & E013116 & 07-Sep-84 & & $10098-97-2$ & SR-90 & 905.0_SR_GPC & USTEST & 1.19 \\
\hline
\end{tabular}




\begin{tabular}{|c|c|c|c|c|c|c|c|c|c|c|c|c|c|}
\hline & A & $\mathrm{B}$ & $\mathrm{c}$ & $\mathrm{D}$ & $\mathrm{E}$ & $\mathrm{F}$ & G & $\mathrm{H}$ & $\mathrm{I}$ & $\mathrm{J}$ & $\mathrm{K}$ & $\mathrm{L}$ & $\mathrm{M}$ \\
\hline 1 & SAMPLE_NUM_ASSIGNED_TO & SAMPLER & MEDIA & BIOTA_MEDIA & TAG_ID & SAMP_NUM & SAMP_DATE_TIME & SAMP_DATE_TIME_ON & CON_ID & CON_SHORT_NAME & METHOD_NAME & LAB_CODE & \begin{tabular}{|l|} 
VALUE_RPTD \\
\end{tabular} \\
\hline 4214 & PDMS & & SW & & & E001863 & 11-Sep-84 & & $10045-97-3$ & CS-137 & GAMMA GS & PNL-RO & 0.0211 \\
\hline 4215 & PDMS & & sW & & & E003813 & 11-Sep-84 & & $10045-97-3$ & CS-137 & GAMMA_GS & PNL-RO & 0.0103 \\
\hline 4216 & PDMS & & sW & & & E015248 & 11-Sep-84 & & $10045-97-3$ & CS-137 & GAMMA_GS & HISTOR & \\
\hline \begin{tabular}{|l|l|}
4217 \\
\end{tabular} & PDMS & & sW & & & E026976 & 11-Sep-84 & & $10045-97-3$ & CS-137 & GAMMA_GS & HISTOR & \\
\hline 4218 & PDMS & & sW & & & E017382 & 25-Sep-84 & & $10098-97-2$ & SR-90 & SRISO_SEP_PRECIP_GPC & USTEST & 0.203 \\
\hline 4219 & PDMS & & SW & & & E017382 & 25-Sep-84 & & $10045-97-3$ & CS-137 & GAMMA_GS & USTEST & 0.383 \\
\hline 4220 & PDMS & & SW & & & E017382 & 25-Sep-84 & & $10028-17-8$ & TRITIUM & TRITIUM_ELECT_LSC & USTEST & 263 \\
\hline 4221 & PDMS & & SW & & & E028046 & 25-Sep-84 & & $10028-17-8$ & TRITIUM & TRITIUM_ELECT_LSC & USTEST & 221 \\
\hline 4222 & PDMS & & sW & & & E028046 & 25-Sep-84 & & $10045-97-3$ & CS-137 & GAMMA_GS & USTEST & 1.24 \\
\hline 4223 & PDMS & & SW & & & E028046 & 25-Sep-84 & & $10098-97-2$ & SR-90 & SRISO_S̄EP_PRECIP_GPC & USTEST & 0.205 \\
\hline 4224 & PDMS & & sW & & & E001864 & 25-Sep-84 & & $10098-97-2$ & SR-90 & SRISO_SEP_PRECIP_GPC & PNL-RO & 0.23 \\
\hline 4225 & PDMS & & SW & & & E001864 & 25-Sep-84 & & PU-239/240 & PU-239/240 & PUISO_IE_AEAA & PNL-RO & 0.00049 \\
\hline 4226 & PDMS & & SW & & & E001864 & 25-Sep-84 & & $10045-97-3$ & CS-137 & GAMMA_ESS & PNL-RO & 0.0234 \\
\hline 4227 & PDMS & & SW & & & E001864 & 25-Sep-84 & & 15046-84-1 & I-129 & 1129 SEP TEMS & 320 & 0.000119 \\
\hline 4228 & PDMS & & SW & & & E003814 & 25-Sep-84 & & $10045-97-3$ & CS-137 & GAMMA_GSS & PNL-RO & 0.00804 \\
\hline 4229 & PDMS & & SW & & & E003814 & 25-Sep-84 & & PU-239/240 & PU-239/240 & PUISO_IE_AEA & PNL-RO & 0.000004 \\
\hline 4230 & PDMS & & sW & & & E010667 & 25-Sep-84 & & $10028-17-8$ & TRITIUM & TRITIUM_ELECT_LSC & USTEST & 119 \\
\hline 4231 & PDMS & & sW & & & E013841 & 25-Sep-84 & & PU-239/240 & PU-239/240 & PUISO_IE_AEA & USTEST & 0.000037 \\
\hline 4232 & PDMS & & SW & & & E013841 & 25-Sep-84 & & $10045-97-3$ & CS-137 & GAMMA_ESS & PNL-RO & 0.00868 \\
\hline 4233 & PDMS & & SW & & & E010667 & 25-Sep-84 & & $10098-97-2$ & SR-90 & SRISO_SEEP_PRECIP_GPC & USTEST & 0.137 \\
\hline 4234 & PDMS & & SW & & & E010667 & 25-Sep-84 & & $10045-97-3$ & CS-137 & GAMMĀGS & USTEST & -0.306 \\
\hline 4235 & PDMS & & SW & & & E001163 & 25-Sep-84 & & $10045-97-3$ & CS-137 & GAMMA_GS & PNL-RO & 0.0183 \\
\hline 4236 & PDMS & & SW & & & E001163 & 25-Sep-84 & & $10098-97-2$ & SR-90 & SRISO_SEPP_PRECIP_GPC & PNL-RO & 0.26 \\
\hline 4237 & PDMS & & SW & & & E001163 & 25-Sep-84 & & PU-239/240 & PU-239/240 & PUISO_IE_AEA & PNL-RO & 0.00033 \\
\hline 4238 & PDMS & & SW & & & E001163 & 25-Sep-84 & & 15046-84-1 & I-129 & I129_SEP_TEMS & 320 & 0.0000128 \\
\hline 4239 & PDMS & & SW & & & E004333 & 25-Sep-84 & & $10028-17-8$ & TRITIUM & TRITIUUM_ELECT_LSC & USTEST & 270 \\
\hline 4240 & PDMS & & SW & & & E004333 & 25-Sep-84 & & $10098-97-2$ & SR-90 & SRISO_SEP_PRËCIP_GPC & USTEST & 0.18 \\
\hline 4241 & PDMS & & SW & & & E004333 & 25-Sep-84 & & $10045-97-3$ & CS-137 & GAMMA_GS & USTEST & -0.311 \\
\hline 4242 & PDMS & & SW & & & E037320 & 02-Oct-84 & & $10098-97-2$ & SR-90 & SRISO_SEEP_PRECIP_GPC & USTEST & 0.188 \\
\hline 4243 & PDMS & & SW & & & E037320 & 02-Oct-84 & & $10028-17-8$ & TRITIUM & TRITIUM_DISTT_LSC & USTEST & 13.9 \\
\hline 4244 & PDMS & & SW & & & E037320 & 02-Oct-84 & & $10045-97-3$ & CS-137 & GAMMA_GS & USTEST & 0.597 \\
\hline 4245 & PDMS & & SW & & & E001865 & 09-Oct-84 & & $10045-97-3$ & CS-137 & GAMMA_GS & PNL-RO & 0.0227 \\
\hline 4246 & PDMS & & SW & & & E003815 & 09-Oct-84 & & $10045-97-3$ & CS-137 & GAMMA_GS & PNL-RO & 0.0106 \\
\hline \begin{tabular}{|l|l|}
4247 \\
\end{tabular} & PDMS & & SW & & & E001164 & 09-Oct-84 & & $10045-97-3$ & CS-137 & GAMMA_GS & PNL-RO & 0.0347 \\
\hline 4248 & PDMS & & SW & & & E013842 & 09-Oct-84 & & $10045-97-3$ & CS-137 & GAMMA_GS & PNL-RO & 0.0128 \\
\hline 4249 & PDMS & & SW & & & E000865 & 23-Oct-84 & & $10098-97-2$ & SR-90 & 905.0_SR_GPC & USTEST & 0.249 \\
\hline 4250 & PDMS & & SW & & & E000865 & 23-Oct-84 & & $10045-97-3$ & CS-137 & 901.1_GAMMA_GS & USTEST & -0.919 \\
\hline 4251 & PDMS & & SW & & & E000865 & 23-Oct-84 & & 10028-17-8 & TRITIUM & 906.0_H3_LSC & USTEST & 82.5 \\
\hline 4252 & PDMS & & SW & & & E014495 & 23-Oct-84 & & $10098-97-2$ & SR-90 & 905.0_SR_GPC & USTEST & 0.0318 \\
\hline 4253 & PDMS & & SW & & & E014495 & 23-Oct-84 & & $10028-17-8$ & TRITIUM & 906.0_H3_LSC & USTEST & 23.8 \\
\hline 4254 & PDMS & & SW & & & E014495 & 23-Oct-84 & & $10045-97-3$ & CS-137 & 901.1_GAMMA_GS & USTEST & 0.506 \\
\hline 4255 & PDMS & & SW & & & E010741 & 23-Oct-84 & & $10045-97-3$ & CS-137 & 901.1_GAMMA_GS & USTEST & 0.505 \\
\hline 4256 & PDMS & & SW & & & E010741 & 23-Oct-84 & & $10028-17-8$ & TRITIUM & 906.0_H3_LSC & USTEST & -8.35 \\
\hline \begin{tabular}{|l|l|}
4257 & - \\
\end{tabular} & PDMS & & SW & & & E001866 & 23-Oct-84 & & $10045-97-3$ & CS-137 & GAMMA_ESS & PNL-RO & 0.0177 \\
\hline 4258 & PDMS & & SW & & & E003816 & 23-Oct-84 & & $10045-97-3$ & CS-137 & GAMMA_GS & PNL-RO & 0.0112 \\
\hline 4259 & PDMS & & SW & & & E010668 & 23-Oct-84 & & 10028-17-8 & TRITIUM & TRITIUM_ELECT_LSC & USTEST & 129 \\
\hline 4260 & PDMS & & SW & & & E010668 & 23-Oct-84 & & $10098-97-2$ & SR-90 & SRISO_SEP_PRËCIP_GPC & USTEST & 0.161 \\
\hline 4261 & PDMS & & SW & & & E010668 & 23-Oct-84 & & $10045-97-3$ & CS-137 & GAMMĀ_GS & USTEST & -0.732 \\
\hline 4262 & PDMS & & SW & & & E001165 & 23-Oct-84 & & $10045-97-3$ & CS-137 & GAMMA_GS & PNL-RO & 0.0154 \\
\hline 4263 & PDMS & & SW & & & E013843 & 23-Oct-84 & & $10045-97-3$ & CS-137 & GAMMA_GS & PNL-RO & 0.00892 \\
\hline 4264 & PDMS & & SW & & & E004334 & 23-Oct-84 & & $10028-17-8$ & TRITIUM & TRITIUM_ELECT_LSC & USTEST & 176 \\
\hline 4265 & PDMS & & SW & & & E004334 & 23-Oct-84 & & $10098-97-2$ & SR-90 & SRISO_SEP_PRËCIP_GPC & USTEST & 0.253 \\
\hline
\end{tabular}




\begin{tabular}{|c|c|c|c|c|c|c|c|c|c|c|c|c|c|}
\hline & $\bar{A}$ & $\mathrm{~B}$ & $\mathrm{C}$ & $\mathrm{D}$ & $E$ & $\mathrm{~F}$ & G & $\mathrm{H}$ & $\mathrm{I}$ & $\mathrm{J}$ & $\mathrm{K}$ & $\mathrm{L}$ & $\mathrm{M}$ \\
\hline 1 & SAMPLE_NUM_ASSIGNED_TO & SAMPLER & MEDIA & BIOTA_MEDIA & TAG_ID & SAMP_NUM & SAMP_DATE_TIME & SAMP_DATE_TIME_ON & CON_ID & CON_SHORT_NAME & METHOD_NAME & LAB_CODE & VALUE_RPTD \\
\hline 4266 & PDMS & & SW & & & E004334 & $23-$ Oct-84 & & $10045-97-3$ & CS-137 & GAMMA_GS & USTEST & 0.796 \\
\hline 4267 & PDMS & & SW & & & E068611 & 30-Oct-84 & & $10028-17-8$ & TRITIUM & TRITIUM_DIST_LSC & USTEST & 146 \\
\hline 4268 & PDMS & & SW & & & E013537 & 30-Oct-84 & & $10098-97-2$ & SR-90 & SRISO_SEP_PRECIP_GPC & USTEST & 2.23 \\
\hline 4269 & PDMS & & SW & & & E013537 & 30-Oct-84 & & $10045-97-3$ & CS-137 & GAMMA_GS & USTEST & -0.829 \\
\hline 4270 & PDMS & & SW & & & E013537 & 30-Oct-84 & & $10028-17-8$ & TRITIUM & TRITIUM_DIST_LSC & USTEST & 549 \\
\hline 4271 & PDMS & & SW & & & E012587 & $30-O c t-84$ & & $10028-17-8$ & TRITIUM & TRITIUM_DIST_LSC & USTEST & 20300 \\
\hline 4272 & PDMS & & SW & & & E012587 & 30-Oct-84 & & $10045-97-3$ & CS-137 & GAMMA_GS & USTEST & -1 \\
\hline 4273 & PDMS & & SW & & & E017795 & 30-Oct-84 & & $10045-97-3$ & CS-137 & GAMMA_GS & USTEST & 5.28 \\
\hline 4274 & PDMS & & SW & & & E017795 & $30-O c t-84$ & & $10098-97-2$ & SR-90 & SRISO_SEP_PRECIP_GPC & USTEST & 1.67 \\
\hline 4275 & PDMS & & SW & & & E017795 & $30-O c t-84$ & & $10028-17-8$ & TRITIUM & TRITIUM_DIST_LSC & USTEST & 160 \\
\hline 4276 & PDMS & & SW & & & E021784 & $30-0 c t-84$ & & $10028-17-8$ & TRITIUM & TRITIUM_DIST_LSC & USTEST & 805 \\
\hline 4277 & PDMS & & SW & & & E021784 & 30-Oct-84 & & $10045-97-3$ & CS-137 & GAMMA_GS & USTEST & 4.36 \\
\hline 4278 & PDMS & & SW & & & E021784 & 30-Oct-84 & & $10098-97-2$ & SR-90 & SRISO_SEP_PRECIP_GPC & USTEST & 2.03 \\
\hline 4279 & PDMS & & SW & & & E001867 & 06-Nov-84 & & $10045-97-3$ & CS-137 & GAMMA_GS & PNL-RO & 0.0247 \\
\hline 4280 & PDMS & & SW & & & E003817 & 06-Nov-84 & & $10045-97-3$ & CS-137 & GAMMA_GS & PNL-RO & 0.0126 \\
\hline 4281 & PDMS & & SW & & & E013844 & 06-Nov-84 & & $10045-97-3$ & CS-137 & GAMMA_GS & PNL-RO & 0.00999 \\
\hline 4282 & PDMS & & SW & & & E001166 & 06-Nov-84 & & $10045-97-3$ & CS-137 & GAMMA_GS & PNL-RO & 0.0204 \\
\hline 4283 & PDMS & & SW & & & E013815 & 19-Nov-84 & & $10098-97-2$ & SR-90 & 905.0_SR_GPC & USTEST & 0.0781 \\
\hline 4284 & PDMS & & SW & & & E013815 & 19-Nov-84 & & $10028-17-8$ & TRITIUM & 906.0_H3_LSC & USTEST & 23800 \\
\hline \begin{tabular}{|l|}
4285 \\
\end{tabular} & PDMS & & SW & & & E013815 & 19-Nov-84 & & $10045-97-3$ & CS-137 & 901.1_GAMMA_GS & USTEST & -1.3 \\
\hline \begin{tabular}{|l|}
4286 \\
\end{tabular} & PDMS & & SW & & & E001868 & 20-Nov-84 & & $10045-97-3$ & CS-137 & GAMMA_GS & PNL-RO & 0.0184 \\
\hline \begin{tabular}{|l|l|}
4287 \\
\end{tabular} & PDMS & & SW & & & E003818 & 20-Nov-84 & & $10045-97-3$ & CS-137 & GAMMA_GS & PNL-RO & 0.00951 \\
\hline \begin{tabular}{|l|}
4288 \\
\end{tabular} & PDMS & & SW & & & E001167 & 20-Nov-84 & & $10045-97-3$ & CS-137 & GAMMA_GS & PNL-RO & 0.0165 \\
\hline 4289 & PDMS & & SW & & & E010669 & 20-Nov-84 & & $10028-17-8$ & TRITIUM & TRITIUM_ELECT_LSC & USTEST & 113 \\
\hline 4290 & PDMS & & SW & & & E010669 & 20-Nov-84 & & $10098-97-2$ & SR-90 & SRISO_SEP_PRECIP_GPC & USTEST & 0.148 \\
\hline 4291 & PDMS & & SW & & & E010669 & 20-Nov-84 & & $10045-97-3$ & CS-137 & GAMMA_GS & USTEST & -0.0485 \\
\hline 4292 & PDMS & & SW & & & E013845 & 20-Nov-84 & & $10045-97-3$ & CS-137 & GAMMA_GS & PNL-RO & 0.0104 \\
\hline 4293 & PDMS & & SW & & & E004335 & 20-Nov-84 & & $10028-17-8$ & TRITIUM & TRITIUM_ELECT_LSC & USTEST & 132 \\
\hline 4294 & PDMS & & SW & & & E004335 & 20-Nov-84 & & $10098-97-2$ & SR-90 & SRISO_SEP_PRECIP_GPC & USTEST & 0.168 \\
\hline 4295 & PDMS & & SW & & & E004335 & 20-Nov-84 & & $10045-97-3$ & CS-137 & GAMMA_GS & USTEST & 0.322 \\
\hline \begin{tabular}{|l|}
4296 \\
\end{tabular} & PDMS & & SW & & & E036176 & 26-Nov-84 & & $10098-97-2$ & SR-90 & 905.0_SR_GPC & USTEST & 0.114 \\
\hline \begin{tabular}{|l|}
4297 \\
\end{tabular} & PDMS & & SW & & & E036176 & 26-Nov-84 & & $10045-97-3$ & CS-137 & 901.1_GAMMA_GS & USTEST & 0.765 \\
\hline \begin{tabular}{|l|}
4298 \\
\end{tabular} & PDMS & & SW & & & E036176 & 26-Nov-84 & & $10028-17-8$ & TRITIUM & 906.0_H3_LSC & USTEST & 114 \\
\hline \begin{tabular}{|l|}
4299 \\
\end{tabular} & PDMS & & SW & & & E032042 & 27-Nov-84 & & $10028-17-8$ & TRITIUM & 906.0_H3_LSC & USTEST & 208 \\
\hline \begin{tabular}{|l|}
4300 \\
\end{tabular} & PDMS & & SW & & & E032042 & 27-Nov-84 & & $10098-97-2$ & SR-90 & 905.0_SR_GPC & USTEST & 0.261 \\
\hline \begin{tabular}{|l|}
4301 \\
\end{tabular} & PDMS & & SW & & & E032042 & 27-Nov-84 & & $10045-97-3$ & CS-137 & 901.1_GAMMA_GS & USTEST & 0.922 \\
\hline \begin{tabular}{|l|}
4302 \\
\end{tabular} & PDMS & & SW & & & E013117 & 30-Nov-84 & & $10045-97-3$ & CS-137 & 901.1_GAMMA_GS & USTEST & -0.417 \\
\hline 4303 & PDMS & & SW & & & E013117 & 30-Nov-84 & & $10028-17-8$ & TRITIUM & 906.0_H3_LSC & USTEST & 81.9 \\
\hline \begin{tabular}{|l|l|}
4304 \\
\end{tabular} & PDMS & & SW & & & E013117 & 30-Nov-84 & & $10098-97-2$ & SR-90 & 905.0_SR_GPC & USTEST & 1.55 \\
\hline 4305 & PDMS & & SW & & & E001953 & 04-Dec-84 & & $10045-97-3$ & CS-137 & GAMMA_GS & HISTOR & \\
\hline 4306 & PDMS & & SW & & & E014815 & 04-Dec-84 & & $10045-97-3$ & CS-137 & GAMMA_GS & HISTOR & \\
\hline \begin{tabular}{|l|l|}
4307 \\
\end{tabular} & PDMS & & SW & & & E013846 & 04-Dec-84 & & $10045-97-3$ & CS-137 & GAMMA_GS & PNL-RO & 0.0173 \\
\hline 4308 & PDMS & & SW & & & E001168 & 04-Dec-84 & & $10045-97-3$ & CS-137 & GAMMA_GS & PNL-RO & 0.0281 \\
\hline 4309 & PDMS & & SW & & & E003819 & 18-Dec-84 & & $10045-97-3$ & CS-137 & GAMMA_GS & PNL-RO & 0.0124 \\
\hline 4310 & PDMS & & SW & & & E003819 & 18-Dec-84 & & PU-239/240 & PU-239/240 & PUISO_IE_AEA & PNL-RO & 0.0000188 \\
\hline \begin{tabular}{|l|l|}
4311 \\
\end{tabular} & PDMS & & SW & & & E001869 & 18-Dec-84 & & PU-239/240 & PU-239/240 & PUISO_IE_AEA & PNL-RO & 0.0000258 \\
\hline 4312 & PDMS & & SW & & & E001869 & 18-Dec-84 & & $10045-97-3$ & CS-137 & GAMMA_GS & PNL-RO & 0.0222 \\
\hline 4313 & PDMS & & SW & & & E001869 & 18-Dec-84 & & 15046-84-1 & I-129 & I129_SEP_TEMS & 320 & 0.000054 \\
\hline 4314 & PDMS & & SW & & & E001169 & 18-Dec-84 & & $10045-97-3$ & CS-137 & GAMMA_GS & PNL-RO & 0.0248 \\
\hline 4315 & PDMS & & SW & & & E001169 & 18-Dec-84 & & PU-239/240 & PU-239/240 & PUISO_IE_AEA & PNL-RO & 0.0000175 \\
\hline 4316 & PDMS & & SW & & & E001169 & 18-Dec-84 & & 15046-84-1 & $1-129$ & I129_SEP_TEMS & 320 & 0.00000748 \\
\hline \begin{tabular}{|c|}
4317 \\
\end{tabular} & PDMS & & SW & & & E010670 & 18-Dec-84 & & $10028-17-8$ & TRITIUM & TRITIUM_ELECT_LSC & USTEST & 190 \\
\hline
\end{tabular}




\begin{tabular}{|c|c|c|c|c|c|c|c|c|c|c|c|c|c|}
\hline & A & $\mathrm{B}$ & $\mathrm{c}$ & $\mathrm{D}$ & $\mathrm{E}$ & $\mathrm{F}$ & G & $\mathrm{H}$ & $\mathrm{I}$ & $\mathrm{J}$ & $\mathrm{K}$ & $\mathrm{L}$ & $\mathrm{M}$ \\
\hline 1 & SAMPLE_NUM_ASSIGNED_TO & SAMPLER & MEDIA & BIOTA_MEDIA & TAG_ID & SAMP_NUM & SAMP_DATE_TIME & SAMP_DATE_TIME_ON & CON_ID & CON_SHORT_NAME & METHOD_NAME & LAB_CODE & \begin{tabular}{|l|} 
VALUE_RPTD \\
\end{tabular} \\
\hline 4318 & PDMS & & SW & & & E013847 & 18-Dec-84 & & PU-239/240 & PU-239/240 & PUISO_IE_AEA & PNL-RO & 0.0000102 \\
\hline 4319 & PDMS & & sW & & & E013847 & 18-Dec-84 & & $10045-97-3$ & CS-137 & GAMMA_GSS & PNL-RO & 0.0152 \\
\hline 4320 & PDMS & & sW & & & E010670 & 18-Dec-84 & & $10098-97-2$ & SR-90 & SRISO_SEP_PRECIP_GPC & USTEST & 0.136 \\
\hline 4321 & PDMS & & sW & & & E010670 & 18-Dec-84 & & $10045-97-3$ & CS-137 & GAMMA_GS & USTEST & -0.696 \\
\hline 4322 & PDMS & & sW & & & E004336 & 18-Dec-84 & & $10028-17-8$ & TRITIUM & TRITIUM_ELECT_LSC & USTEST & 185 \\
\hline 4323 & PDMS & & SW & & & E004336 & 18-Dec-84 & & $10098-97-2$ & SR-90 & SRISO_SËP_PRËCIP_GPC & USTEST & 0.159 \\
\hline 4324 & PDMS & & SW & & & E004336 & 18-Dec-84 & & $10045-97-3$ & CS-137 & GAMMA_GS & USTEST & -0.89 \\
\hline 4325 & PDMS & & SW & & & E032043 & 26-Dec-84 & & $10028-17-8$ & TRITIUM & 906.0_H3_LSC & USTEST & 191 \\
\hline 4326 & PDMS & & sW & & & E032043 & 26-Dec-84 & & $10098-97-2$ & SR-90 & 905.0_SR_GPC & USTEST & 0.75 \\
\hline 4327 & PDMS & & SW & & & E032043 & 26-Dec-84 & & $10045-97-3$ & CS-137 & 901.1_GAMMA_GS & USTEST & -1.41 \\
\hline 4328 & PDMS & & SW & & & E001870 & 02-Jan-85 & & $10045-97-3$ & CS-137 & GAMMA_GS & PNL-RO & 0.0196 \\
\hline 4329 & PDMS & & SW & & & E003820 & 02-Jan-85 & & $10045-97-3$ & CS-137 & GAMMA_GS & PNL-RO & 0.00628 \\
\hline 4330 & PDMS & & SW & & & E001170 & 02-Jan-85 & & $10045-97-3$ & CS-137 & GAMMA_GS & PNL-RO & 0.0199 \\
\hline 4331 & PDMS & & SW & & & E013848 & 02-Jan-85 & & $10045-97-3$ & CS-137 & GAMMA_GS & PNL-RO & 0.0101 \\
\hline 4332 & PDMS & & SW & & & E001871 & 15-Jan-85 & & $10045-97-3$ & CS-137 & GAMMA_GS & PNL-RO & 0.0229 \\
\hline 4333 & PDMS & & SW & & & E003821 & 15-Jan-85 & & $10045-97-3$ & CS-137 & GAMMA_GS & PNL-RO & 0.0125 \\
\hline \begin{tabular}{|l|}
4334 \\
\end{tabular} & PDMS & & sW & & & E013849 & 15-Jan-85 & & $10045-97-3$ & CS-137 & GAMMA_GS & PNL-RO & 0.0129 \\
\hline \begin{tabular}{|l|}
4335 \\
\end{tabular} & PDMS & & sW & & & E001171 & 15-Jan-85 & & $10045-97-3$ & CS-137 & GAMMA_GS & PNL-RO & 0.0181 \\
\hline 4336 & PDMS & & SW & & & E001872 & 29-Jan-85 & & $10045-97-3$ & CS-137 & GAMMA_GS & PNL-RO & 0.0179 \\
\hline \begin{tabular}{|l|l|}
4337 & $-2 \cdot{ }^{2}$ \\
\end{tabular} & PDMS & & SW & & & E003822 & 29-Jan-85 & & $10045-97-3$ & CS-137 & GAMMA_GS & PNL-RO & 0.00926 \\
\hline 4338 & PDMS & & SW & & & E010671 & 29-Jan-85 & & $10028-17-8$ & TRITIUM & TRITIUM_ELECT_LSC & USTEST & 155 \\
\hline 4339 & PDMS & & SW & & & E013850 & 29-Jan-85 & & $10045-97-3$ & CS-137 & GAMMA_GS & PNL-RO & 0.0134 \\
\hline 4340 & PDMS & & SW & & & E010671 & 29-Jan-85 & & $10098-97-2$ & SR-90 & SRISO_SEPP_PRECIP_GPC & USTEST & 0.144 \\
\hline 4341 & PDMS & & SW & & & E010671 & 29-Jan-85 & & $10045-97-3$ & CS-137 & GAMMA GS & USTEST & 0.582 \\
\hline 4342 & PDMS & & SW & & & E001172 & 29-Jan-85 & & $10045-97-3$ & CS-137 & GAMMA_GS & PNL-RO & 0.0293 \\
\hline 4343 & PDMS & & SW & & & E004337 & 29-Jan-85 & & $10028-17-8$ & TRITIUM & TRITIUM_ELECT_LSC & USTEST & 162 \\
\hline 4344 & PDMS & & SW & & & E004337 & 29-Jan-85 & & $10045-97-3$ & CS-137 & GAMMA_GS & USTEST & -0.459 \\
\hline 4345 & PDMS & & SW & & & E004337 & 29-Jan-85 & & $10098-97-2$ & SR-90 & SRISO_SĒEP_PRECIP_GPC & USTEST & 0.171 \\
\hline 4346 & PDMS & & SW & & & E001873 & 11-Feb-85 & & $10045-97-3$ & CS-137 & GAMMĀGS & PNL-RO & 0.0289 \\
\hline 4347 & PDMS & & SW & & & E003823 & 11-Feb-85 & & $10045-97-3$ & CS-137 & GAMMA_GS & PNL-RO & 0.0106 \\
\hline 4348 & PDMS & & SW & & & E013851 & 11-Feb-85 & & $10045-97-3$ & CS-137 & GAMMA_GS & PNL-RO & 0.013 \\
\hline 4349 & PDMS & & SW & & & E001173 & 11-Feb-85 & & $10045-97-3$ & CS-137 & GAMMA_GS & PNL-RO & 0.0376 \\
\hline 4350 & PDMS & & SW & & & E004338 & 19-Feb-85 & & $10028-17-8$ & TRITIUM & TRITIUM_ELECT_LSC & USTEST & 147 \\
\hline 4351 & PDMS & & SW & & & E000866 & 26-Feb-85 & & $10098-97-2$ & SR-90 & 905.0_SR_GPC & USTEST & 0.298 \\
\hline 4352 & PDMS & & SW & & & E000866 & 26-Feb-85 & & $10045-97-3$ & CS-137 & 901.1_GAMMA_GS & USTEST & 0.346 \\
\hline 4353 & PDMS & & SW & & & E000866 & 26-Feb-85 & & $10028-17-8$ & TRITIUM & 906.0_H3_LSC & USTEST & -35.6 \\
\hline 4354 & PDMS & & SW & & & E014496 & 26-Feb-85 & & $10098-97-2$ & SR-90 & 905.0_SR_GPC & USTEST & 0.0658 \\
\hline 4355 & PDMS & & SW & & & E014496 & 26-Feb-85 & & $10028-17-8$ & TRITIUM & 906.0_H3_LSC & USTEST & -7.64 \\
\hline 4356 & PDMS & & SW & & & E014496 & 26-Feb-85 & & $10045-97-3$ & CS-137 & 901.1_GAMMA_GS & USTEST & 0.103 \\
\hline \begin{tabular}{|l|l|}
4357 & -1 \\
\end{tabular} & PDMS & & SW & & & E010742 & 26-Feb-85 & & $10045-97-3$ & CS-137 & 901.1_GAMMA_GS & USTEST & -1.1 \\
\hline 4358 & PDMS & & SW & & & E010742 & 26-Feb-85 & & $10028-17-8$ & TRITIUM & 906.0_H3_LSC & USTEST & -60.9 \\
\hline 4359 & PDMS & & SW & & & E001954 & 26-Feb-85 & & $10045-97-3$ & CS-137 & GAMM̄A_ESS & HISTOR & \\
\hline 4360 & PDMS & & SW & & & E014816 & 26-Feb-85 & & $10045-97-3$ & CS-137 & GAMMA_GS & HISTOR & \\
\hline 4361 & PDMS & & SW & & & E001174 & 26-Feb-85 & & $10045-97-3$ & CS-137 & GAMMA_GS & PNL-RO & 0.0331 \\
\hline 4362 & PDMS & & SW & & & E010672 & 26-Feb-85 & & $10028-17-8$ & TRITIUM & TRITIUM_ELECT_LSC & USTEST & 148 \\
\hline 4363 & PDMS & & SW & & & E010672 & 26-Feb-85 & & $10098-97-2$ & SR-90 & SRISO_SEP_PRËCIP_GPC & USTEST & 0.188 \\
\hline 4364 & PDMS & & SW & & & E010672 & 26-Feb-85 & & $10045-97-3$ & CS-137 & GAMMĀ_GS & USTEST & -0.92 \\
\hline 4365 & PDMS & & SW & & & E013852 & 26-Feb-85 & & $10045-97-3$ & CS-137 & GAMMA_GS & PNL-RO & 0.0221 \\
\hline 4366 & PDMS & & SW & & & E014572 & 26-Feb-85 & & $10045-97-3$ & CS-137 & GAMMA_GS & USTEST & 0.473 \\
\hline \begin{tabular}{|l|l|}
4367 & \\
\end{tabular} & PDMS & & sW & & & E014572 & 26-Feb-85 & & $10098-97-2$ & SR-90 & SRISO_SETEPPRECIP_GPC & USTEST & 0.14 \\
\hline 4368 & PDMS & & SW & & & E013538 & 26-Feb-85 & & $10098-97-2$ & SR-90 & SRISO_SEP_PRECIP_GPC & USTEST & 4.1 \\
\hline 4369 & PDMS & & SW & & & E013538 & 26-Feb-85 & & $10045-97-3$ & CS-137 & GAMMĀ_GS & USTEST & 1.73 \\
\hline
\end{tabular}




\begin{tabular}{|c|c|c|c|c|c|c|c|c|c|c|c|c|c|}
\hline & $\bar{A}$ & $\mathrm{~B}$ & $\mathrm{C}$ & $\mathrm{D}$ & $E$ & $\mathrm{~F}$ & G & $\mathrm{H}$ & $\mathrm{I}$ & $\mathrm{J}$ & $\mathrm{K}$ & $\bar{L}$ & $\mathrm{M}$ \\
\hline 1 & SAMPLE_NUM_ASSIGNED_TO & SAMPLER & MEDIA & BIOTA_MEDIA & TAG_ID & SAMP_NUM & SAMP_DATE_TIME & SAMP_DATE_TIME_ON & CON_ID & CON_SHORT_NAME & METHOD_NAME & LAB_CODE & VALUE_RPTD \\
\hline 4370 & PDMS & & SW & & & E013538 & $26-F e b-85$ & & $10028-17-8$ & TRITIUM & TRITIUM_DIST_LSC & USTEST & 1130 \\
\hline 4371 & PDMS & & SW & & & E012588 & 26-Feb-85 & & $10028-17-8$ & TRITIUM & TRITIUM_DIST_LSC & USTEST & 22700 \\
\hline 4372 & PDMS & & SW & & & E012588 & 26-Feb-85 & & $10045-97-3$ & CS-137 & GAMMA_GS & USTEST & 0.606 \\
\hline 4373 & PDMS & & SW & & & E017796 & 26-Feb-85 & & $10045-97-3$ & CS-137 & GAMMA_GS & USTEST & 6.45 \\
\hline 4374 & PDMS & & SW & & & E017796 & 26-Feb-85 & & $10098-97-2$ & SR-90 & SRISO_SEP_PRECIP_GPC & USTEST & 4.59 \\
\hline 4375 & PDMS & & SW & & & E017796 & 26-Feb-85 & & $10028-17-8$ & TRITIUM & TRITIUM_DIST_LSC & USTEST & 133 \\
\hline 4376 & PDMS & & SW & & & E021785 & 26-Feb-85 & & $10028-17-8$ & TRITIUM & TRITIUM_DIST_LSC & USTEST & 915 \\
\hline 4377 & PDMS & & SW & & & E021785 & 26-Feb-85 & & $10045-97-3$ & CS-137 & GAMMA_GS & USTEST & -1.52 \\
\hline 4378 & PDMS & & SW & & & E021785 & 26-Feb-85 & & $10098-97-2$ & SR-90 & SRISO_SEP_PRECIP_GPC & USTEST & 1.17 \\
\hline 4379 & PDMS & & SW & & & E013816 & 27-Feb-85 & & $10098-97-2$ & SR-90 & 905.0_E_R_GPC & USTEST & 0.238 \\
\hline 4380 & PDMS & & SW & & & E013816 & 27-Feb-85 & & $10028-17-8$ & TRITIUM & 906.0_H3_LSC & USTEST & 19900 \\
\hline 4381 & PDMS & & SW & & & E013816 & $27-F e b-85$ & & $10045-97-3$ & CS-137 & 901.1_GAMMA_GS & USTEST & 0.484 \\
\hline 4382 & PDMS & & SW & & & E036177 & 05-Mar-85 & & $10098-97-2$ & SR-90 & 905.0_SR_GPC & USTEST & 0.131 \\
\hline 4383 & PDMS & & SW & & & E036177 & 05-Mar-85 & & $10045-97-3$ & CS-137 & 901.1_GAMMA_GS & USTEST & 0.0485 \\
\hline 4384 & PDMS & & SW & & & E036177 & 05-Mar-85 & & $10028-17-8$ & TRITIUM & 906.0_H3_LSC & USTEST & -214 \\
\hline 4385 & PDMS & & SW & & & E013118 & 11-Mar-85 & & $10045-97-3$ & CS-137 & 901.1_GAMMA_GS & USTEST & 0.734 \\
\hline 4386 & PDMS & & SW & & & E013118 & 11-Mar-85 & & $10028-17-8$ & TRITIUM & 906.0_H3_LSC & USTEST & 53.2 \\
\hline 4387 & PDMS & & SW & & & E013118 & 11-Mar-85 & & $10098-97-2$ & SR-90 & 905.0_SR_GPC & USTEST & 1.07 \\
\hline 4388 & PDMS & & SW & & & E001874 & 12-Mar-85 & & $10045-97-3$ & CS-137 & GAMMA_GS & PNL-RO & 0.0153 \\
\hline 4389 & PDMS & & SW & & & E003824 & 12-Mar-85 & & $10045-97-3$ & CS-137 & GAMMA_GS & PNL-RO & 0.00912 \\
\hline 4390 & PDMS & & SW & & & E001175 & 12-Mar-85 & & PU-239/240 & PU-239/240 & PUISO_IE_AEA & PNL-RO & 0.000148 \\
\hline 4391 & PDMS & & SW & & & E001175 & 12-Mar-85 & & $10098-97-2$ & SR-90 & SRISO_SEP_PRECIP_GPC & PNL-RO & 0.0361 \\
\hline 4392 & PDMS & & SW & & & E001175 & 12-Mar-85 & & $10045-97-3$ & CS-137 & GAMMA_GS & PNL-RO & 0.0172 \\
\hline 4393 & PDMS & & SW & & & E013853 & 12-Mar-85 & & PU-239/240 & PU-239/240 & PUISO_IE_AEA & PNL-RO & 0.0000256 \\
\hline 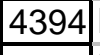 & PDMS & & SW & & & E013853 & 12-Mar-85 & & $10045-97-3$ & CS-137 & GAMMA_GS & PNL-RO & 0.0137 \\
\hline 4395 & PDMS & & SW & & & E032044 & 19-Mar-85 & & $10028-17-8$ & TRITIUM & 906.0_H3_LSC & USTEST & 67.6 \\
\hline 4396 & PDMS & & SW & & & E032044 & 19-Mar-85 & & $10098-97-2$ & SR-90 & 905.0_SR_GPC & USTEST & 0.217 \\
\hline 4397 & PDMS & & SW & & & E032044 & 19-Mar-85 & & $10045-97-3$ & CS-137 & 901.1_GAMMA_GS & USTEST & -0.689 \\
\hline 4398 & PDMS & & SW & & & E003825 & 26-Mar-85 & & $10045-97-3$ & CS-137 & GAMMA_GS & PNL-RO & 0.00712 \\
\hline 4399 & PDMS & & SW & & & E003825 & 26-Mar-85 & & PU-239/240 & PU-239/240 & PUISO_IE_AEA & PNL-RO & 0.0000134 \\
\hline 4400 & PDMS & & SW & & & E001875 & 26-Mar-85 & & 15046-84-1 & I-129 & I129_SEP_TEMS & 320 & 0.0000493 \\
\hline 4401 & PDMS & & SW & & & E001875 & 26-Mar-85 & & PU-239/240 & PU-239/240 & PUISO_IE_AEA & PNL-RO & 0.000422 \\
\hline 4402 & PDMS & & SW & & & E001875 & 26-Mar-85 & & $10098-97-2$ & SR-90 & SRISO_SEP_PRECIP_GPC & PNL-RO & 0.0278 \\
\hline 4403 & PDMS & & SW & & & E001875 & 26-Mar-85 & & $10045-97-3$ & CS-137 & GAMMA_GS & PNL-RO & 0.0204 \\
\hline 4404 & PDMS & & SW & & & E015249 & 26-Mar-85 & & $10045-97-3$ & CS-137 & GAMMA_GS & HISTOR & \\
\hline 4405 & PDMS & & SW & & & E026951 & 26-Mar-85 & & 15046-84-1 & I-129 & 1129_SEP_TEMS & 320 & 0.0000089 \\
\hline 4406 & PDMS & & SW & & & E026951 & 26-Mar-85 & & $10045-97-3$ & CS-137 & GAMMA_ḠS & HISTOR & \\
\hline 4407 & PDMS & & SW & & & E034556 & 01-Apr-85 & & $10045-97-3$ & CS-137 & 901.1_GAMMA_GS & USTEST & 0.138 \\
\hline 4408 & PDMS & & SW & & & E034556 & 01-Apr-85 & & $10098-97-2$ & SR-90 & 905.0_SR_GPC & USTEST & 0.269 \\
\hline 4409 & PDMS & & SW & & & E034556 & 01-Apr-85 & & $10028-17-8$ & TRITIUM & 906.0_H3_LSC & USTEST & 318 \\
\hline 4410 & PDMS & & SW & & & E010673 & 02-Apr-85 & & $10028-17-8$ & TRITIUM & TRITIUM_ELECT_LSC & USTEST & 105 \\
\hline 4411 & PDMS & & SW & & & E010673 & 02-Apr-85 & & $10098-97-2$ & SR-90 & SRISO_SEP_PRECIP_GPC & USTEST & 0.15 \\
\hline 4412 & PDMS & & SW & & & E010673 & 02-Apr-85 & & $10045-97-3$ & CS-137 & GAMMA_GS & USTEST & -0.62 \\
\hline 4413 & PDMS & & SW & & & E004339 & $02-A p r-85$ & & $10028-17-8$ & TRITIUM & TRITIUM_ELECT_LSC & USTEST & 164 \\
\hline 4414 & PDMS & & SW & & & E004339 & 02-Apr-85 & & $10045-97-3$ & CS-137 & GAMMA_GS & USTEST & 0.314 \\
\hline 4415 & PDMS & & SW & & & E004339 & 02-Apr-85 & & $10098-97-2$ & SR-90 & SRISO_SEP_PRECIP_GPC & USTEST & 0.207 \\
\hline 4416 & PDMS & & SW & & & E017383 & 10-Apr-85 & & $10098-97-2$ & SR-90 & SRISO_SEP_PRECIP_GPC & USTEST & 0.0599 \\
\hline \begin{tabular}{|l|l|l|}
4417 \\
\end{tabular} & PDMS & & SW & & & E017383 & 10-Apr-85 & & $10045-97-3$ & CS-137 & GAMMA_GS & USTEST & 0.487 \\
\hline 4418 & PDMS & & SW & & & E017383 & 10-Apr-85 & & $10028-17-8$ & TRITIUM & TRITIUM_ELECT_LSC & USTEST & 264 \\
\hline 4419 & PDMS & & SW & & & E028047 & 10-Apr-85 & & $10028-17-8$ & TRITIUM & TRITIUM_ELECT_LSC & USTEST & 94 \\
\hline 4420 & PDMS & & SW & & & E028047 & 10-Apr-85 & & $10045-97-3$ & CS-137 & GAMMA_GS & USTEST & -0.678 \\
\hline 4421 & PDMS & & SW & & & E028047 & 10-Apr-85 & & $10098-97-2$ & SR-90 & SRISO_SEP_PRECIP_GPC & USTEST & 0.22 \\
\hline
\end{tabular}




\begin{tabular}{|c|c|c|c|c|c|c|c|c|c|c|c|c|c|}
\hline & $\bar{A}$ & $\mathrm{~B}$ & C & $\mathrm{D}$ & $E$ & $\mathrm{~F}$ & G & $\mathrm{H}$ & $\mathrm{I}$ & $\mathrm{J}$ & $\mathrm{K}$ & $\mathrm{L}$ & $\mathrm{M}$ \\
\hline 1 & SAMPLE_NUM_ASSIGNED_TO & SAMPLER & MEDIA & BIOTA_MEDIA & TAG_ID & SAMP_NUM & SAMP_DATE_TIME & SAMP_DATE_TIME_ON & CON_ID & CON_SHORT_NAME & METHOD_NAME & LAB_CODE & \begin{tabular}{|l|} 
VALUE_RPTD \\
\end{tabular} \\
\hline 4422 & PDMS & & SW & & & E001876 & 10-Apr-85 & & $10045-97-3$ & CS-137 & GAMMA_GS & PNL-RO & 0.0215 \\
\hline 4423 & PDMS & & sW & & & E003826 & 10-Apr-85 & & $10045-97-3$ & CS-137 & GAMMA_GS & PNL-RO & 0.00834 \\
\hline 4424 & PDMS & & SW & & & E013854 & 10-Apr-85 & & $10045-97-3$ & CS-137 & GAMMA_GS & PNL-RO & 0.0108 \\
\hline 4425 & PDMS & & SW & & & E001176 & 10-Apr-85 & & $10045-97-3$ & CS-137 & GAMMA_GS & PNL-RO & 0.0275 \\
\hline 4426 & PDMS & & SW & & & E001877 & 22-Apr-85 & & $10045-97-3$ & CS-137 & GAMMA_GS & PNL-RO & 0.0147 \\
\hline 4427 & PDMS & & SW & & & E003827 & 22-Apr-85 & & $10045-97-3$ & CS-137 & GAMMA_GS & PNL-RO & 0.006 \\
\hline 4428 & PDMS & & SW & & & E015250 & 23-Apr-85 & & $10045-97-3$ & CS-137 & GAMMA_GS & HISTOR & \\
\hline 4429 & PDMS & & SW & & & E026952 & 23-Apr-85 & & $10045-97-3$ & CS-137 & GAMMA_GS & HISTOR & \\
\hline 4430 & PDMS & & SW & & & E010674 & 30-Apr-85 & & $10028-17-8$ & TRITIUM & TRITIUM_ELECT_LSC & USTEST & 63.7 \\
\hline 4431 & PDMS & & SW & & & E010674 & 30-Apr-85 & & $10098-97-2$ & SR-90 & SRISO_SEP_PRËCIP_GPC & USTEST & 0.211 \\
\hline 4432 & PDMS & & SW & & & E010674 & 30-Apr-85 & & $10045-97-3$ & CS-137 & GAMMA_GS & USTEST & -0.97 \\
\hline 4433 & PDMS & & SW & & & E004340 & 30-Apr-85 & & $10028-17-8$ & TRITIUM & TRITIUM_ELECCT_LSC & USTEST & 180 \\
\hline \begin{tabular}{|l|}
4434 \\
\end{tabular} & PDMS & & SW & & & E004340 & 30-Apr-85 & & $10045-97-3$ & CS-137 & GAMMA_GS & USTEST & 0.358 \\
\hline \begin{tabular}{|l|l|l|l}
4435 \\
\end{tabular} & PDMS & & SW & & & E004340 & 30-Apr-85 & & $10098-97-2$ & SR-90 & SRISO_S̄EP_PRECIP_GPC & USTEST & 0.206 \\
\hline \begin{tabular}{|l|l|}
4436 \\
\end{tabular} & PDMS & & SW & & & E001878 & 07-May-85 & & $10045-97-3$ & CS-137 & GAMMA_GS & PNL-RO & 0.0197 \\
\hline \begin{tabular}{|l|l|}
4437 \\
\end{tabular} & PDMS & & SW & & & E003828 & 07-May-85 & & $10045-97-3$ & CS-137 & GAMMA_GS & PNL-RO & 0.0106 \\
\hline \begin{tabular}{|l|}
4438 \\
\end{tabular} & PDMS & & SW & & & E026953 & 07-May-85 & & $10045-97-3$ & CS-137 & GAMMA_GS & HISTOR & \\
\hline \begin{tabular}{|l|}
4439 \\
\end{tabular} & PDMS & & SW & & & E015251 & 07-May-85 & & $10045-97-3$ & CS-137 & GAMMA_GS & HISTOR & \\
\hline \begin{tabular}{|c|}
4440 \\
\end{tabular} & PDMS & & SW & & & E000867 & 21-May-85 & & $10098-97-2$ & SR-90 & 905.0_SR_GPC & USTEST & 0.0326 \\
\hline \begin{tabular}{|l|l}
4441 \\
\end{tabular} & PDMS & & SW & & & E000867 & 21-May-85 & & $10045-97-3$ & CS-137 & 901.1_GAMMA_GS & USTEST & -0.367 \\
\hline \begin{tabular}{|l|}
4442 \\
\end{tabular} & PDMS & & SW & & & E000867 & 21-May-85 & & 10028-17-8 & TRITIUM & 906.0_H3_LSC & USTEST & 83.6 \\
\hline \begin{tabular}{|l|}
4443 \\
\end{tabular} & PDMS & & SW & & & E014434 & 21-May-85 & & 10098-97-2 & SR-90 & 905.0_SR_GPC & USTEST & 0.0408 \\
\hline \begin{tabular}{|l|}
4444 \\
\end{tabular} & PDMS & & SW & & & E014434 & 21-May-85 & & 10028-17-8 & TRITIUM & 906.0_H3_LSC & USTEST & -138 \\
\hline \begin{tabular}{|l|}
4445 \\
\end{tabular} & PDMS & & SW & & & E014434 & 21-May-85 & & $10045-97-3$ & CS-137 & 901.1_GAMMA_GS & USTEST & -0.0969 \\
\hline \begin{tabular}{|l|}
4446 \\
\end{tabular} & PDMS & & SW & & & E010743 & 21-May-85 & & $10045-97-3$ & CS-137 & 901.1_GAMMA_GS & USTEST & 0.817 \\
\hline \begin{tabular}{|l|}
4447 \\
\end{tabular} & PDMS & & SW & & & E010743 & 21-May-85 & & 10098-97-2 & SR-90 & 905.0_SR_GPC & USTEST & 0.0383 \\
\hline \begin{tabular}{|l|}
4448 \\
\end{tabular} & PDMS & & SW & & & E010743 & 21-May-85 & & 10028-17-8 & TRITIUM & 906.0_H3_LSC & USTEST & -1.08 \\
\hline \begin{tabular}{|l|}
4449 \\
\end{tabular} & PDMS & & SW & & & E003829 & 21-May-85 & & $10045-97-3$ & CS-137 & GAMMA_GS & PNL-RO & 0.0132 \\
\hline \begin{tabular}{|l|}
4450 \\
\end{tabular} & PDMS & & SW & & & E001879 & 21-May-85 & & $10045-97-3$ & CS-137 & GAMMA_GS & PNL-RO & 0.0271 \\
\hline \begin{tabular}{|l|}
4451 \\
\end{tabular} & PDMS & & SW & & & E013855 & 21-May-85 & & $10045-97-3$ & CS-137 & GAMMA_GS & PNL-RO & 0.014 \\
\hline \begin{tabular}{|l|}
4452 \\
\end{tabular} & PDMS & & SW & & & E001177 & 21-May-85 & & $10045-97-3$ & CS-137 & GAMMA_GS & PNL-RO & 0.0303 \\
\hline \begin{tabular}{|l|}
4453 \\
\end{tabular} & PDMS & & SW & & & E013539 & 21-May-85 & & $10098-97-2$ & SR-90 & SRISO_SEEP_PRECIP_GPC & USTEST & 0.657 \\
\hline \begin{tabular}{|c|}
4454 \\
\end{tabular} & PDMS & & SW & & & E013539 & 21-May-85 & & $10045-97-3$ & CS-137 & GAMMA_GS & USTEST & 1.28 \\
\hline 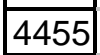 & PDMS & & SW & & & E013539 & 21-May-85 & & $10028-17-8$ & TRITIUM & TRITIUM_DIST_LSC & USTEST & 349 \\
\hline 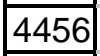 & PDMS & & SW & & & E012589 & 21-May-85 & & $10028-17-8$ & TRITIUM & TRITIUM_DIST_LSC & USTEST & 24800 \\
\hline \begin{tabular}{|l|l|l|l|l|l|}
4457 \\
\end{tabular} & PDMS & & SW & & & E012589 & 21-May-85 & & $10045-97-3$ & CS-137 & GAMMA_GS & USTEST & 0.817 \\
\hline \begin{tabular}{|l|}
4458 \\
\end{tabular} & PDMS & & SW & & & E017797 & 21-May-85 & & $10045-97-3$ & CS-137 & GAMMA_GS & USTEST & 16.8 \\
\hline \begin{tabular}{|l|l|}
4459 \\
\end{tabular} & PDMS & & SW & & & E017797 & 21-May-85 & & $10098-97-2$ & SR-90 & SRISO_SEEP_PRECIP_GPC & USTEST & 0.581 \\
\hline 4460 & PDMS & & SW & & & E017797 & 21-May-85 & & $10028-17-8$ & TRITIUM & TRITIUM_DIST_LLSC & USTEST & 375 \\
\hline \begin{tabular}{|l|l|l|}
4461 & -1 \\
\end{tabular} & PDMS & & SW & & & E021786 & 21-May-85 & & $10028-17-8$ & TRITIUM & TRITIUM_DIST_LEC & USTEST & 709 \\
\hline 4462 & PDMS & & SW & & & E021786 & 21-May-85 & & $10045-97-3$ & CS-137 & GAMMA_GS & USTEST & -0.2 \\
\hline 4463 & PDMS & & SW & & & E021786 & 21-May-85 & & $10098-97-2$ & SR-90 & SRISO_SEP_PRECIP_GPC & USTEST & 1.72 \\
\hline 4464 & PDMS & & SW & & & E013817 & 22-May-85 & & $10098-97-2$ & SR-90 & 905.0_SR_GPC & USTEST & 0.0608 \\
\hline 4465 & PDMS & & SW & & & E013817 & 22-May-85 & & 10028-17-8 & TRITIUM & 906.0_H3_LSC & USTEST & 22200 \\
\hline \begin{tabular}{|l|l|}
4466 & -2 \\
\end{tabular} & PDMS & & SW & & & E013817 & 22-May-85 & & $10045-97-3$ & CS-137 & 901.1_GAMMA_GS & USTEST & 0.0459 \\
\hline \begin{tabular}{|l|l|}
4467 \\
\end{tabular} & PDMS & & SW & & & E034557 & 28-May-85 & & $10045-97-3$ & CS-137 & 901.1_GAMMA_GS & USTEST & 0.46 \\
\hline \begin{tabular}{|l|l|}
4468 & -20 \\
\end{tabular} & PDMS & & SW & & & E034557 & 28-May-85 & & 10098-97-2 & SR-90 & 905.0_SR_GPC & USTEST & 0.127 \\
\hline \begin{tabular}{|l|l|}
4469 \\
\end{tabular} & PDMS & & SW & & & E034557 & 28-May-85 & & $10028-17-8$ & TRITIUM & 906.0_H3_LSC & USTEST & -59.9 \\
\hline 4470 & PDMS & & SW & & & E084797 & 28-May-85 & & 10028-17-8 & TRITIUM & 906.0_H3_LSC & USTEST & 192 \\
\hline \begin{tabular}{|l|l|}
4471 & \\
\end{tabular} & PDMS & & SW & & & E036178 & 28-May-85 & & 10098-97-2 & SR-90 & 905.0_SR_GPC & USTEST & 0.0231 \\
\hline \begin{tabular}{|l|}
4472 \\
\end{tabular} & PDMS & & SW & & & E036178 & 28-May-85 & & $10045-97-3$ & CS-137 & 901.1_GAMMA_GS & USTEST & 0.242 \\
\hline 4473 & PDMS & & SW & & & E036178 & 28-May-85 & & $10028-17-8$ & TRITIUM & 906.0_H3_LSC & USTEST & -82.1 \\
\hline
\end{tabular}




\begin{tabular}{|c|c|c|c|c|c|c|c|c|c|c|c|c|c|}
\hline & $\bar{A}$ & $\mathrm{~B}$ & $\bar{C}$ & $\mathrm{D}$ & $\mathrm{E}$ & $\mathrm{F}$ & $\bar{G}$ & $\mathrm{H}$ & 1 & $\mathrm{~J}$ & $\bar{K}$ & $\bar{L}$ & $\mathrm{M}$ \\
\hline 1 & SAMPLE_NUM_ASSIGNED_TO & SAMPLER & MEDIA & BIOTA_MEDIA & TAG_ID & SAMP_NUM & SAMP_DATE_TIME & SAMP_DATE_TIME_ON & CON_ID & CON_SHORT_NAME & METHOD_NAME & LAB_CODE & VALUE_RPTD \\
\hline 4474 & PDMS & & SW & & & E010675 & 28-May-85 & & $10028-17-8$ & TRITIUM & TRITIUM_ELECT_LSC & USTEST & 84.8 \\
\hline 4475 & PDMS & & SW & & & E010675 & 28-May-85 & & $10098-97-2$ & SR-90 & SRISO_SËP_PRËCIP_GPC & USTEST & 0.0851 \\
\hline 4476 & PDMS & & SW & & & E010675 & 28-May-85 & & $10045-97-3$ & CS-137 & GAMMA_GS & USTEST & -0.582 \\
\hline 4477 & PDMS & & SW & & & E004341 & 28-May-85 & & $10028-17-8$ & TRITIUM & TRITIUM_ELECT_LSC & USTEST & 94.2 \\
\hline 4478 & PDMS & & SW & & & E004341 & 28-May-85 & & $10045-97-3$ & CS-137 & GAMMA_GS & USTEST & 0.358 \\
\hline 4479 & PDMS & & SW & & & E004341 & 28-Мау-85 & & $10098-97-2$ & SR-90 & SRISO_SEEP_PRECIP_GPC & USTEST & 0.211 \\
\hline 4480 & PDMS & & SW & & & E013119 & 03-Jun-85 & & $10098-97-2$ & SR-90 & 905.0_SR_GPC & USTEST & 0.792 \\
\hline 4481 & PDMS & & SW & & & E001880 & 03-Jun-85 & & $10045-97-3$ & CS-137 & GAMMĀĒSS & PNL-RO & 0.0266 \\
\hline 4482 & PDMS & & SW & & & E003830 & 03-Jun-85 & & $10045-97-3$ & CS-137 & GAMMA_GS & PNL-RO & 0.0141 \\
\hline 4483 & PDMS & & SW & & & E001178 & 03-Jun-85 & & $10045-97-3$ & CS-137 & GAMMA_GS & PNL-RO & 0.0297 \\
\hline 4484 & PDMS & & SW & & & E013856 & 03-Jun-85 & & $10045-97-3$ & CS-137 & GAMMA_GS & PNL-RO & 0.0116 \\
\hline 4485 & PDMS & & SW & & & E001808 & 18-Jun-85 & & $10045-97-3$ & CS-137 & GAMMA_GS & PNL-RO & 0.0157 \\
\hline \begin{tabular}{|l|}
4486 \\
\end{tabular} & PDMS & & SW & & & E003831 & 18-Jun-85 & & $10045-97-3$ & CS-137 & GAMMA_GS & PNL-RO & 0.00839 \\
\hline 4487 & PDMS & & SW & & & E013857 & 18-Jun-85 & & $10045-97-3$ & CS-137 & GAMMA_GS & PNL-RO & 0.0189 \\
\hline 4488 & PDMS & & SW & & & E001179 & 18-Jun-85 & & $10045-97-3$ & CS-137 & GAMMA_GS & PNL-RO & 0.0242 \\
\hline 4489 & PDMS & & SW & & & E001809 & 02-Jul-85 & & 15046-84-1 & I-129 & I129_SEP_TEMS & 320 & 0.0000772 \\
\hline \begin{tabular}{|l|}
4490 \\
\end{tabular} & PDMS & & SW & & & E001809 & 02-Jul-85 & & PU-239/240 & PU-239/240 & PUISO_IE_AEA & PNL-RO & 0.000147 \\
\hline \begin{tabular}{|l|}
4491 \\
\end{tabular} & PDMS & & SW & & & E001809 & 02-Jul-85 & & $10098-97-2$ & SR-90 & SRISO_SEP_PRECIP_GPC & PNL-RO & 0.0203 \\
\hline \begin{tabular}{|l|}
4492 \\
\end{tabular} & PDMS & & SW & & & E001809 & 02-Jul-85 & & $10045-97-3$ & CS-137 & GAMMA_GS & PNL-RO & 0.00996 \\
\hline \begin{tabular}{|l|}
4493 \\
\end{tabular} & PDMS & & SW & & & E003832 & 02-Jul-85 & & $10045-97-3$ & CS-137 & GAMMA_GS & PNL-RO & 0.00773 \\
\hline 4494 & PDMS & & SW & & & E003832 & 02-Jul-85 & & PU-239/240 & PU-239/240 & PUISO_IE_AEA & PNL-RO & 0.0000779 \\
\hline \begin{tabular}{|l|l|l|l|}
4495 \\
\end{tabular} & PDMS & & SW & & & E001180 & 02-Jul-85 & & PU-239/240 & PU-239/240 & PUISO_IE_AEA & PNL-RO & 0.000506 \\
\hline \begin{tabular}{|l|}
4496 \\
\end{tabular} & PDMS & & SW & & & E001180 & 02-Jul-85 & & $10098-97-2$ & SR-90 & SRISO_SEP_PRECIP_GPC & PNL-RO & 0.0338 \\
\hline \begin{tabular}{|l|l|}
4497 \\
\end{tabular} & PDMS & & SW & & & E001180 & 02-Jul-85 & & $10045-97-3$ & CS-137 & GAMMA_GS & PNL-RO & 0.0207 \\
\hline \begin{tabular}{|l|}
4498 \\
\end{tabular} & PDMS & & SW & & & E010676 & 02-Jul-85 & & 10028-17-8 & TRITIUM & TRITIUM_ELECT_LSC & USTEST & 87.5 \\
\hline \begin{tabular}{|l|l|}
4499 \\
\end{tabular} & PDMS & & SW & & & E013858 & 02-Jul-85 & & PU-239/240 & PU-239/240 & PUISO_IE_AEA & PNL-RO & 0.0000184 \\
\hline 4500 & PDMS & & SW & & & E013858 & 02-Jul-85 & & $10045-97-3$ & CS-137 & GAMMA_GS & PNL-RO & 0.0106 \\
\hline 4501 & PDMS & & SW & & & E010676 & 02-Jul-85 & & $10098-97-2$ & SR-90 & SRISO_SEP_PRECIP_GPC & USTEST & 0.191 \\
\hline 4502 & PDMS & & SW & & & E010676 & 02-Jul-85 & & $10045-97-3$ & CS-137 & GAMMA_GS & USTEST & -0.704 \\
\hline 4503 & PDMS & & SW & & & E004342 & 02-Jul-85 & & $10028-17-8$ & TRITIUM & TRITIUM_ELECT_LSC & USTEST & 151 \\
\hline 4504 & PDMS & & SW & & & E004342 & 02-Jul-85 & & $10045-97-3$ & CS-137 & GAMMA_GS & USTEST & 0.31 \\
\hline \begin{tabular}{|l|}
4505 \\
\end{tabular} & PDMS & & SW & & & E004342 & 02-Jul-85 & & $10098-97-2$ & SR-90 & SRISO_SEP_PRECIP_GPC & USTEST & 0.14 \\
\hline \begin{tabular}{|l|}
4506 \\
\end{tabular} & PDMS & & SW & & & E032045 & 09-Jul-85 & & 10028-17-8 & TRITIUM & 906.0_H3_LSC & USTEST & 289 \\
\hline 4507 & PDMS & & SW & & & E032045 & 09-Jul-85 & & $10098-97-2$ & SR-90 & 905.0_SR_GPC & USTEST & 0.211 \\
\hline 4508 & PDMS & & SW & & & E032045 & 09-Jul-85 & & $10045-97-3$ & CS-137 & 901.1_GAMMA_GS & USTEST & -0.146 \\
\hline 4509 & PDMS & & SW & & & E003833 & 16-Jul-85 & & $10045-97-3$ & CS-137 & GAMMA_GS & PNL-RO & 0.0118 \\
\hline 4510 & PDMS & & SW & & & E001810 & 16-Jul-85 & & $10045-97-3$ & CS-137 & GAMMA_GS & PNL-RO & 0.0282 \\
\hline \begin{tabular}{|l|}
4511 \\
\end{tabular} & PDMS & & SW & & & E001181 & 17-Jul-85 & & $10045-97-3$ & CS-137 & GAMMA_GS & PNL-RO & 0.0183 \\
\hline 4512 & PDMS & & SW & & & E013859 & 17-Jul-85 & & $10045-97-3$ & CS-137 & GAMMA_GS & PNL-RO & 0.00904 \\
\hline 4513 & PDMS & & SW & & & E087936 & 26-Jul-85 & & 15046-84-1 & $\mathrm{I}-129$ & I129_SEP_TEMS & 320 & 0.0000082 \\
\hline 4514 & PDMS & & SW & & & E001811 & 30-Jul-85 & & $10045-97-3$ & CS-137 & GAMMA_GS & PNL-RO & 0.0291 \\
\hline 4515 & PDMS & & SW & & & E003834 & 30-Jul-85 & & $10045-97-3$ & CS-137 & GAMMA_GS & PNL-RO & 0.0129 \\
\hline 4516 & PDMS & & SW & & & E001182 & 30-Jul-85 & & $10045-97-3$ & CS-137 & GAMMA_GS & PNL-RO & 0.0191 \\
\hline 4517 & PDMS & & SW & & & E013860 & 30-Jul-85 & & $10045-97-3$ & CS-137 & GAMMA_GS & PNL-RO & 0.00901 \\
\hline 4518 & PDMS & & SW & & & E010677 & 30-Jul-85 & & $10028-17-8$ & TRITIUM & TRITIUM_ELECT_LSC & USTEST & 85.8 \\
\hline 4519 & PDMS & & SW & & & E010677 & 30-Jul-85 & & $10045-97-3$ & CS-137 & GAMMA_GS & USTEST & 1.53 \\
\hline 4520 & PDMS & & SW & & & E010677 & 30-Jul-85 & & $10098-97-2$ & SR-90 & SRISO_SEP_PRECIP_GPC & USTEST & 0.139 \\
\hline 4521 & PDMS & & SW & & & E014675 & 30-Jul-85 & & $10028-17-8$ & TRITIUM & TRITIUM_ELECT_LSC & USTEST & 172 \\
\hline \begin{tabular}{|l|}
4522 \\
\end{tabular} & PDMS & & SW & & & E014675 & 30-Jul-85 & & $10098-97-2$ & SR-90 & SRISO_SEP_PRECIP_GPC & USTEST & 0.157 \\
\hline 4523 & PDMS & & SW & & & E014675 & 30-Jul-85 & & $10045-97-3$ & CS-137 & GAMMA_GS & USTEST & -1.07 \\
\hline 4524 & PDMS & & SW & & & E000868 & 13-Aug-85 & & $10045-97-3$ & CS-137 & 901.1_GAMMA_GS & USTEST & 1.07 \\
\hline 4525 & PDMS & & SW & & & E000868 & 13-Aug-85 & & $10028-17-8$ & TRITIUM & 906.0_H3_LSC & USTEST & 106 \\
\hline
\end{tabular}




\begin{tabular}{|c|c|c|c|c|c|c|c|c|c|c|c|c|c|}
\hline & A & $\mathrm{B}$ & $\mathrm{c}$ & $\mathrm{D}$ & $\mathrm{E}$ & $\mathrm{F}$ & G & $\mathrm{H}$ & $\mathrm{I}$ & $\mathrm{J}$ & $\mathrm{K}$ & $\mathrm{L}$ & $\mathrm{M}$ \\
\hline 1 & SAMPLE_NUM_ASSIGNED_TO & SAMPLER & MEDIA & BIOTA_MEDIA & TAG_ID & SAMP_NUM & SAMP_DATE_TIME & SAMP_DATE_TIME_ON & CON_ID & CON_SHORT_NAME & METHOD_NAME & LAB_CODE & \begin{tabular}{|l|} 
VALUE_RPTD \\
\end{tabular} \\
\hline 4526 & PDMS & & SW & & & E000868 & 13-Aug-85 & & $10098-97-2$ & SR-90 & 905.0_SR_GPC & USTEST & 0.173 \\
\hline \begin{tabular}{|l|l|}
4527 & $-2 \cdot r$ \\
\end{tabular} & PDMS & & sW & & & E004161 & 13-Aug-85 & & $10028-17-8$ & TRITIUM & 906.0_H3_LSC & USTEST & 155 \\
\hline 4528 & PDMS & & sW & & & E004161 & 13-Aug-85 & & $10098-97-2$ & SR-90 & 905.0_SR_GPC & USTEST & 0.0563 \\
\hline 4529 & PDMS & & sW & & & E004161 & 13-Aug-85 & & $10045-97-3$ & CS-137 & 901.1_GAMMA_GS & USTEST & 1.63 \\
\hline 4530 & PDMS & & sW & & & E001812 & 13-Aug-85 & & $10045-97-3$ & CS-137 & GAMMA_GS & PNL-RO & 0.0205 \\
\hline \begin{tabular}{|l|}
4531 \\
\end{tabular} & PDMS & & sW & & & E003835 & 13-Aug-85 & & $10045-97-3$ & CS-137 & GAMMA_GS & PNL-RO & 0.00752 \\
\hline 4532 & PDMS & & SW & & & E001183 & 13-Aug-85 & & $10045-97-3$ & CS-137 & GAMMA_GS & PNL-RO & 0.0265 \\
\hline 4533 & PDMS & & SW & & & E013861 & 13-Aug-85 & & $10045-97-3$ & CS-137 & GAMMA_GS & PNL-RO & 0.0128 \\
\hline 4534 & PDMS & & sW & & & E013540 & 13-Aug-85 & & $10045-97-3$ & CS-137 & GAMMA GS & USTEST & -1.07 \\
\hline 4535 & PDMS & & SW & & & E013540 & 13-Aug-85 & & $10098-97-2$ & SR-90 & SRISO_SEEP_PRECIP_GPC & USTEST & 0.449 \\
\hline 4536 & PDMS & & sW & & & E013540 & 13-Aug-85 & & $10028-17-8$ & TRITIUM & TRITIUM_DIST_LSC & USTEST & 222 \\
\hline 4537 & PDMS & & SW & & & E012590 & 13-Aug-85 & & $10028-17-8$ & TRITIUM & TRITIUM_DIST_LSC & USTEST & 29400 \\
\hline 4538 & PDMS & & SW & & & E012590 & 13-Aug-85 & & $10045-97-3$ & CS-137 & GAMMA_GS & USTEST & 0.919 \\
\hline 4539 & PDMS & & SW & & & E017732 & 13-Aug-85 & & $10045-97-3$ & CS-137 & GAMMA_GS & USTEST & 8.06 \\
\hline 4540 & PDMS & & SW & & & E017732 & 13-Aug-85 & & $10028-17-8$ & TRITIUM & TRITIUM_DIST_LSC & USTEST & 190 \\
\hline 4541 & PDMS & & SW & & & E017732 & 13-Aug-85 & & $10098-97-2$ & SR-90 & SRISO_SEP_PRECIP_GPC & USTEST & 2.42 \\
\hline 4542 & PDMS & & sW & & & E021787 & 13-Aug-85 & & $10028-17-8$ & TRITIUM & TRITIUM_DIST_LSC & USTEST & 761 \\
\hline 4543 & PDMS & & sW & & & E021787 & 13-Aug-85 & & $10045-97-3$ & CS-137 & GAMMA_GS & USTEST & 3.41 \\
\hline 4544 & PDMS & & SW & & & E021787 & 13-Aug-85 & & $10098-97-2$ & SR-90 & SRISO_SEEP_PRECIP_GPC & USTEST & 2.66 \\
\hline 4545 & PDMS & & SW & & & E013818 & 14-Aug-85 & & 10028-17-8 & TRITIUM & 906.0_H3_LSC & USTEST & 23600 \\
\hline 4546 & PDMS & & SW & & & E013818 & 14-Aug-85 & & $10098-97-2$ & SR-90 & 905.0_SR_GPC & USTEST & 0.133 \\
\hline \begin{tabular}{|l|l|}
4547 \\
\end{tabular} & PDMS & & SW & & & E013818 & 14-Aug-85 & & $10045-97-3$ & CS-137 & 901.1_GAMMA_GS & USTEST & 0.339 \\
\hline 4548 & PDMS & & SW & & & E011192 & 19-Aug-85 & & $10045-97-3$ & CS-137 & 901.1_GAMMA_GS & USTEST & 0.306 \\
\hline 4549 & PDMS & & SW & & & E011192 & 19-Aug-85 & & $10028-17-8$ & TRITIUM & 906.0_H3_LSC & USTEST & 348 \\
\hline 4550 & PDMS & & SW & & & E011192 & 19-Aug-85 & & $10098-97-2$ & SR-90 & 905.0_SR_GPC & USTEST & 0.175 \\
\hline 4551 & PDMS & & SW & & & E036179 & 19-Aug-85 & & $10045-97-3$ & CS-137 & 901.1_GAMMA_GS & USTEST & 0.436 \\
\hline 4552 & PDMS & & SW & & & E036179 & 19-Aug-85 & & $10028-17-8$ & TRITIUM & 906.0 H3 LSC & USTEST & 21.5 \\
\hline 4553 & PDMS & & SW & & & E036179 & 19-Aug-85 & & $10098-97-2$ & SR-90 & 905.0_SR_GPC & USTEST & 0.0825 \\
\hline \begin{tabular}{|l|}
4554 \\
\end{tabular} & PDMS & & SW & & & E012474 & 21-Aug-85 & & $10045-97-3$ & CS-137 & 901.1_GAMMA_GS & USTEST & 0.34 \\
\hline 4555 & PDMS & & SW & & & E012474 & 21-Aug-85 & & $10028-17-8$ & TRITIUM & 906.0_H3_LSC & USTEST & 113 \\
\hline 4556 & PDMS & & SW & & & E012474 & 21-Aug-85 & & $10098-97-2$ & SR-90 & 905.0_SR_GPC & USTEST & 0.0269 \\
\hline \begin{tabular}{|l|}
4557 \\
\end{tabular} & PDMS & & SW & & & E001813 & 27-Aug-85 & & $10045-97-3$ & CS-137 & GAMMĀĒGS & PNL-RO & 0.0207 \\
\hline 4558 & PDMS & & SW & & & E003836 & 27-Aug-85 & & $10045-97-3$ & CS-137 & GAMMA_GS & PNL-RO & 0.0121 \\
\hline 4559 & PDMS & & SW & & & E001184 & 27-Aug-85 & & $10045-97-3$ & CS-137 & GAMMA_GS & PNL-RO & 0.0179 \\
\hline 4560 & PDMS & & SW & & & E013862 & 27-Aug-85 & & $10045-97-3$ & CS-137 & GAMMA_GS & PNL-RO & 0.00764 \\
\hline \begin{tabular}{|l|}
4561 \\
\end{tabular} & PDMS & & sW & & & E013120 & 30-Aug-85 & & $10028-17-8$ & TRITIUM & 906.0_H3_LSC & USTEST & 125 \\
\hline 4562 & PDMS & & SW & & & E013120 & 30-Aug-85 & & $10098-97-2$ & SR-90 & 905.0_SR_GPC & USTEST & 0.785 \\
\hline 4563 & PDMS & & SW & & & E013120 & 30-Aug-85 & & $10045-97-3$ & CS-137 & 901.1_GAMMA_GS & USTEST & 1.63 \\
\hline 4564 & PDMS & & SW & & & E086190 & 03-Sep-85 & & $10045-97-3$ & CS-137 & GAMM̄A_GS & USTEST & -0.388 \\
\hline 4565 & PDMS & & SW & & & E086190 & 03-Sep-85 & & $10098-97-2$ & SR-90 & SRISO_SEPE_PRECIP_GPC & USTEST & 0.16 \\
\hline 4566 & PDMS & & SW & & & E086190 & 03-Sep-85 & & $10028-17-8$ & TRITIUM & TRITIŪM_ELEECT_LSC̄ & USTEST & 88.8 \\
\hline \begin{tabular}{|l|l|}
4567 & -1 \\
\end{tabular} & PDMS & & SW & & & E086189 & 03-Sep-85 & & $10098-97-2$ & SR-90 & SRISO_SEPP_PRËCIP_GPC & USTEST & 0.117 \\
\hline 4568 & PDMS & & SW & & & E086189 & 03-Sep-85 & & $10045-97-3$ & CS-137 & GAMMĀGS & USTEST & -0.512 \\
\hline 4569 & PDMS & & SW & & & E086189 & 03-Sep-85 & & $10028-17-8$ & TRITIUM & TRITIUM_ELECT_LSC & USTEST & 164 \\
\hline 4570 & PDMS & & SW & & & E086262 & $10-$ Sep-85 & & PU-239/240 & PU-239/240 & PUISO_IE_AEA & PNL-RO & 0.000185 \\
\hline 4571 & PDMS & & SW & & & E086263 & $10-$ Sep-85 & & PU-239/240 & PU-239/240 & PUISO_IE_AEA & PNL-RO & 0.0000204 \\
\hline 4572 & PDMS & & SW & & & E086262 & $10-\operatorname{Sep}-85$ & & $10098-97-2$ & SR-90 & SRISO_SEPP_PRECIP_GPC & PNL-RO & 0.0507 \\
\hline 4573 & PDMS & & SW & & & E086262 & 10-Sep-85 & & $10045-97-3$ & CS-137 & GAMMĀ_GS & PNL-RO & 0.0304 \\
\hline 4574 & PDMS & & SW & & & E086263 & 10-Sep-85 & & $10045-97-3$ & CS-137 & GAMMA_GS & PNL-RO & 0.0115 \\
\hline 4575 & PDMS & & SW & & & E086266 & 10-Sep-85 & & $10045-97-3$ & CS-137 & GAMMA_GS & PNL-RO & 0.00534 \\
\hline 4576 & PDMS & & SW & & & E086264 & 10-Sep-85 & & $10045-97-3$ & CS-137 & GAMMA_GS & PNL-RO & 0.0158 \\
\hline 4577 & PDMS & & SW & & & E034199 & 24-Sep-85 & & 15046-84-1 & $\mathrm{I}-129$ & I129_SEP_TEMS & 320 & 0.0001493 \\
\hline
\end{tabular}




\begin{tabular}{|c|c|c|c|c|c|c|c|c|c|c|c|c|c|}
\hline & $\bar{A}$ & $\mathrm{~B}$ & C & $\mathrm{D}$ & $E$ & $\mathrm{~F}$ & G & $\mathrm{H}$ & $\mathrm{I}$ & $\mathrm{J}$ & $\mathrm{K}$ & $\mathrm{L}$ & $\mathrm{M}$ \\
\hline 1 & SAMPLE_NUM_ASSIGNED_TO & SAMPLER & MEDIA & BIOTA_MEDIA & TAG_ID & SAMP_NUM & SAMP_DATE_TIME & SAMP_DATE_TIME_ON & CON_ID & CON_SHORT_NAME & METHOD_NAME & LAB_CODE & \begin{tabular}{|l|} 
VALUE_RPTD \\
\end{tabular} \\
\hline 4578 & PDMS & & SW & & & E034199 & 24-Sep-85 & & $10045-97-3$ & CS-137 & GAMMA_GS & PNL-RO & \\
\hline 4579 & PDMS & & sW & & & E041096 & 24-Sep-85 & & $10045-97-3$ & CS-137 & GAMMA_GS & PNL-RO & \\
\hline 4580 & PDMS & & SW & & & E086265 & 24-Sep-85 & & 15046-84-1 & I-129 & 1129_SEP_TEMS & 320 & 0.000009 \\
\hline 4581 & PDMS & & SW & & & E086265 & 24-Sep-85 & & PU-239/240 & PU-239/240 & PUISO_IE_AEA & PNL-RO & 0.000221 \\
\hline 4582 & PDMS & & SW & & & E086267 & 24-Sep-85 & & PU-239/240 & PU-239/240 & PUISO_IE_AEA & PNL-RO & 0.0000228 \\
\hline 4583 & PDMS & & SW & & & E086265 & 24-Sep-85 & & $10098-97-2$ & SR-90 & SRISO_SEP_PRECIP_GPC & PNL-RO & 0.0478 \\
\hline 4584 & PDMS & & SW & & & E086265 & 24-Sep-85 & & $10045-97-3$ & CS-137 & GAMMA_GS & PNL-RO & 0.0233 \\
\hline 4585 & PDMS & & SW & & & E086267 & 24-Sep-85 & & $10045-97-3$ & CS-137 & GAMMA_GS & PNL-RO & 0.012 \\
\hline 4586 & PDMS & & SW & & & E086087 & 26-Sep-85 & & $10045-97-3$ & CS-137 & GAMMA_GS & USTEST & -0.894 \\
\hline 4587 & PDMS & & SW & & & E086087 & 26-Sep-85 & & $10098-97-2$ & SR-90 & SRISO_SEEP_PRECIP_GPC & USTEST & 0.102 \\
\hline 4588 & PDMS & & SW & & & E086377 & 01-Oct-85 & & 10028-17-8 & TRITIUM & 906.0_H3_LSC & USTEST & 174 \\
\hline 4589 & PDMS & & SW & & & E086377 & 01-Oct-85 & & $10045-97-3$ & CS-137 & 901.1_GAMMA_GS & USTEST & -0.0972 \\
\hline 4590 & PDMS & & SW & & & E086377 & 01-Oct-85 & & $10098-97-2$ & SR-90 & 905.0_SR_GPC & USTEST & 0.239 \\
\hline 4591 & PDMS & & SW & & & E086085 & $01-O c t-85$ & & $\mathrm{U}-238$ & U-238 & UISO_AEĀA & USTEST & 0.238 \\
\hline 4592 & PDMS & & SW & & & E086085 & $01-O c t-85$ & & 15117-96-1 & U-235 & UISO_AEA & USTEST & 0.0186 \\
\hline 4593 & PDMS & & SW & & & E086085 & 01-Oct-85 & & $13966-29-5$ & U-234 & UISO_AEA & USTEST & 0.183 \\
\hline 4594 & PDMS & & SW & & & E086085 & $01-O c t-85$ & & $10045-97-3$ & CS-137 & GAMMA_GS & USTEST & -0.242 \\
\hline 4595 & PDMS & & SW & & & E086085 & $01-O c t-85$ & & 10028-17-8 & TRITIUM & TRITIUM_ELECT_LSC & USTEST & 117 \\
\hline 4596 & PDMS & & SW & & & E086085 & 01-Oct-85 & & $10098-97-2$ & SR-90 & SRISO_SEPP_PRËCIP_GPC & USTEST & 0.0862 \\
\hline \begin{tabular}{|l|l|}
4597 & -1 \\
\end{tabular} & PDMS & & SW & & & E086086 & $01-O c t-85$ & & $10045-97-3$ & CS-137 & GAMMA_GS & USTEST & -0.275 \\
\hline 4598 & PDMS & & SW & & & E086086 & 01-Oct-85 & & $\mathrm{U}-238$ & $\mathrm{U}-238$ & UISO_AEA & USTEST & 0.153 \\
\hline 4599 & PDMS & & SW & & & E086086 & 01-Oct-85 & & 15117-96-1 & U-235 & UISO_AEA & USTEST & 0.0358 \\
\hline 4600 & PDMS & & SW & & & E086086 & $01-O c t-85$ & & $13966-29-5$ & U-234 & UISO_AEA & USTEST & 0.22 \\
\hline 4601 & PDMS & & SW & & & E086086 & $01-O c t-85$ & & $10028-17-8$ & TRITIUM & TRITIÜM_ELECT_LSC & USTEST & 99.8 \\
\hline 4602 & PDMS & & SW & & & E086086 & $01-O c t-85$ & & $10098-97-2$ & SR-90 & SRISO_SEP_PRËCIP_GPC & USTEST & 0.128 \\
\hline 4603 & PDMS & & SW & & & E086379 & $08-O c t-85$ & & $10098-97-2$ & SR-90 & SRISO_SEP_PRECIP_GPC & USTEST & 0.104 \\
\hline 4604 & PDMS & & SW & & & E086379 & $08-O c t-85$ & & $10045-97-3$ & CS-137 & GAMMĀGS & USTEST & 0.0605 \\
\hline 4605 & PDMS & & SW & & & E086379 & 08-Oct-85 & & $10028-17-8$ & TRITIUM & TRITIUM_ELECT_LSC & USTEST & 101 \\
\hline 4606 & PDMS & & SW & & & E086378 & $08-O c t-85$ & & $10098-97-2$ & SR-90 & SRISO_SEP_PRECIP_GPC & USTEST & 0.059 \\
\hline 4607 & PDMS & & SW & & & E086378 & $08-O c t-85$ & & $10045-97-3$ & CS-137 & GAMMA_GS & USTEST & -0.511 \\
\hline 4608 & PDMS & & SW & & & E086378 & $08-O c t-85$ & & $10028-17-8$ & TRITIUM & TRITIUM_ELECT_LSC & USTEST & 107 \\
\hline 4609 & PDMS & & SW & & & E086611 & 08-Oct-85 & & $10045-97-3$ & CS-137 & GAMMA_GS & PNL-RO & 0.00188 \\
\hline 4610 & PDMS & & SW & & & E086614 & 08-Oct-85 & & $10045-97-3$ & CS-137 & GAMMA_GS & PNL-RO & -0.00036 \\
\hline 4611 & PDMS & & SW & & & E086617 & 08-Oct-85 & & $10045-97-3$ & CS-137 & GAMMA_GS & PNL-RO & 0.00317 \\
\hline 4612 & PDMS & & SW & & & E086619 & $08-O c t-85$ & & $10045-97-3$ & CS-137 & GAMMA_GS & PNL-RO & 0.0008 \\
\hline 4613 & PDMS & & SW & & & E086615 & 22-Oct-85 & & $10045-97-3$ & CS-137 & GAMMA_GS & PNL-RO & 0.00187 \\
\hline 4614 & PDMS & & SW & & & E086612 & 22-Oct-85 & & $10045-97-3$ & CS-137 & GAMMA_GS & PNL-RO & -0.00208 \\
\hline 4615 & PDMS & & SW & & & E086620 & 22-Oct-85 & & $10045-97-3$ & CS-137 & GAMMA_GS & PNL-RO & -0.0000561 \\
\hline 4616 & PDMS & & SW & & & E086618 & 22-Oct-85 & & $10045-97-3$ & CS-137 & GAMMA_GS & PNL-RO & 0.00654 \\
\hline \begin{tabular}{|l|l|}
4617 \\
\end{tabular} & PDMS & & SW & & & E086533 & 29-Oct-85 & & $10028-17-8$ & TRITIUM & TRITIUM_ELECCT_LSC & USTEST & 129 \\
\hline 4618 & PDMS & & SW & & & E086533 & 29-Oct-85 & & $10045-97-3$ & CS-137 & GAMMA_GS & USTEST & -0.291 \\
\hline 4619 & PDMS & & SW & & & E086533 & 29-Oct-85 & & $10098-97-2$ & SR-90 & SRISO_SEP_PRECIP_GPC & USTEST & 0.145 \\
\hline 4620 & PDMS & & SW & & & E086533 & 29-Oct-85 & & $\mathrm{U}-238$ & $\mathrm{U}-238$ & UISO_AEA & USTEST & 0.193 \\
\hline 4621 & PDMS & & SW & & & E086533 & 29-Oct-85 & & 15117-96-1 & $\mathrm{U}-235$ & UISO_AEA & USTEST & 0.0142 \\
\hline 4622 & PDMS & & SW & & & E086533 & $29-O c t-85$ & & $13966-29-5$ & $\mathrm{U}-234$ & UISO_AEA & USTEST & 0.195 \\
\hline 4623 & PDMS & & SW & & & E086532 & $29-O c t-85$ & & $10028-17-8$ & TRITIUM & TRITIŪM_ELECT_LSC & USTEST & 107 \\
\hline 4624 & PDMS & & SW & & & E086532 & 29-Oct-85 & & $10045-97-3$ & CS-137 & GAMMA_GS & USTEST & 0.826 \\
\hline 4625 & PDMS & & SW & & & E086532 & $29-$ Oct-85 & & $10098-97-2$ & SR-90 & SRISO_S̄EP_PRECIP_GPC & USTEST & 0.181 \\
\hline 4626 & PDMS & & SW & & & E086532 & 29-Oct-85 & & $\mathrm{U}-238$ & $\mathrm{U}-238$ & UISO_AEA & USTEST & 0.256 \\
\hline 4627 & PDMS & & SW & & & E086532 & 29-Oct-85 & & 15117-96-1 & $\mathrm{U}-235$ & UISO_AEA & USTEST & 0.00528 \\
\hline 4628 & PDMS & & SW & & & E086532 & $29-O c t-85$ & & $13966-29-5$ & $\mathrm{U}-234$ & UISO_AEA & USTEST & 0.299 \\
\hline 4629 & PDMS & & SW & & & E086698 & 05-Nov-85 & & $10098-97-2$ & SR-90 & 905.0_SR_GPC & USTEST & 0.0278 \\
\hline
\end{tabular}




\begin{tabular}{|c|c|c|c|c|c|c|c|c|c|c|c|c|c|}
\hline & $\bar{A}$ & $\mathrm{~B}$ & $\bar{C}$ & $\mathrm{D}$ & $\mathrm{E}$ & $\mathrm{F}$ & $\bar{G}$ & $\mathrm{H}$ & 1 & $\mathrm{~J}$ & $\bar{K}$ & $\bar{L}$ & $\mathrm{M}$ \\
\hline 1 & SAMPLE_NUM_ASSIGNED_TO & SAMPLER & MEDIA & BIOTA_MEDIA & TAG_ID & SAMP_NUM & SAMP_DATE_TIME & SAMP_DATE_TIME_ON & CON_ID & CON_SHORT_NAME & METHOD_NAME & LAB_CODE & VALUE_RPTD \\
\hline 4630 & PDMS & & SW & & & E086698 & $05-N o v-85$ & & $10028-17-8$ & TRITIUM & 906.0_H3_LSC & USTEST & -34.8 \\
\hline 4631 & PDMS & & SW & & & E086698 & 05-Nov-85 & & $10045-97-3$ & CS-137 & 901.1_GAMMA_GS & USTEST & -0.255 \\
\hline 4632 & PDMS & & SW & & & E086697 & 05-Nov-85 & & $10098-97-2$ & SR-90 & 905.0_SR_GPC & USTEST & 0.14 \\
\hline 4633 & PDMS & & SW & & & E086697 & 05-Nov-85 & & $10028-17-8$ & TRITIUM & 906.0_H3_LSC & USTEST & 15.4 \\
\hline 4634 & PDMS & & SW & & & E086697 & 05-Nov-85 & & $10045-97-3$ & CS-137 & 901.1_GAMMA_GS & USTEST & 0.179 \\
\hline 4635 & PDMS & & SW & & & E086699 & 05-Nov-85 & & $10098-97-2$ & SR-90 & 905.0_SR_GPC & USTEST & 0.0301 \\
\hline 4636 & PDMS & & SW & & & E086699 & 05-Nov-85 & & $10028-17-8$ & TRITIUM & 906.0_H3_LSC & USTEST & -49.9 \\
\hline 4637 & PDMS & & SW & & & E086699 & 05-Nov-85 & & $10045-97-3$ & CS-137 & 901.1_GAMMA_GS & USTEST & 0.184 \\
\hline 4638 & PDMS & & SW & & & E086613 & 05-Nov-85 & & $10045-97-3$ & CS-137 & GAMM̄A_GS & PNL-RO & -0.0054 \\
\hline 4639 & PDMS & & SW & & & E086616 & 05-Nov-85 & & $10045-97-3$ & CS-137 & GAMMA_GS & PNL-RO & -0.00402 \\
\hline 4640 & PDMS & & SW & & & E086523 & 05-Nov-85 & & $10045-97-3$ & CS-137 & GAMMA_GS & PNL-RO & \\
\hline 4641 & PDMS & & SW & & & E086082 & 05-Nov-85 & & $10045-97-3$ & CS-137 & GAMMA_GS & PNL-RO & \\
\hline \begin{tabular}{|c|}
4642 \\
\end{tabular} & PDMS & & SW & & & E086692 & 05-Nov-85 & & $10098-97-2$ & SR-90 & SRISO_SEEP_PRECIP_GPC & USTEST & 0.632 \\
\hline 4643 & PDMS & & SW & & & E086692 & 05-Nov-85 & & $10045-97-3$ & CS-137 & GAMMA_GS & USTEST & 2.45 \\
\hline 4644 & PDMS & & SW & & & E086692 & 05-Nov-85 & & $10028-17-8$ & TRITIUM & TRITIUM_DIST_LSC & USTEST & 582 \\
\hline \begin{tabular}{|l|}
4645 \\
\end{tabular} & PDMS & & SW & & & E086696 & 05-Nov-85 & & $10045-97-3$ & CS-137 & GAMMA_GS & USTEST & 0.568 \\
\hline \begin{tabular}{|l|}
4646 \\
\end{tabular} & PDMS & & SW & & & E086696 & 05-Nov-85 & & $10028-17-8$ & TRITIUM & TRITIUM_DIST_LSC & USTEST & 21400 \\
\hline \begin{tabular}{|l|}
4647 \\
\end{tabular} & PDMS & & SW & & & E086693 & 05-Nov-85 & & $10098-97-2$ & SR-90 & SRISO_SEP_PRECIP_GPC & USTEST & 0.602 \\
\hline \begin{tabular}{|l|}
4648 \\
\end{tabular} & PDMS & & SW & & & E086693 & 05-Nov-85 & & $10045-97-3$ & CS-137 & GAMMA_GS & USTEST & 5.08 \\
\hline \begin{tabular}{|l|}
4649 \\
\end{tabular} & PDMS & & SW & & & E086693 & 05-Nov-85 & & $10028-17-8$ & TRITIUM & TRITIUM_DIST_LSC & USTEST & 66.5 \\
\hline 4650 & PDMS & & SW & & & E086695 & 05-Nov-85 & & $10098-97-2$ & SR-90 & SRISO_SEP_PRECIP_GPC & USTEST & 1.77 \\
\hline \begin{tabular}{|l|}
4651 \\
\end{tabular} & PDMS & & SW & & & E086695 & 05-Nov-85 & & $10045-97-3$ & CS-137 & GAMMA_GS & USTEST & -1.51 \\
\hline 4652 & PDMS & & SW & & & E086695 & 05-Nov-85 & & $10028-17-8$ & TRITIUM & TRITIUM_DIST_LSC & USTEST & 720 \\
\hline 4653 & PDMS & & SW & & & E086700 & $06-N o v-85$ & & $10098-97-2$ & SR-90 & 905.0_SR_GPC & USTEST & 0.0356 \\
\hline \begin{tabular}{|c|}
4654 \\
\end{tabular} & PDMS & & SW & & & E086700 & 06-Nov-85 & & 10028-17-8 & TRITIUM & 906.0_H3_LSC & USTEST & 21900 \\
\hline \begin{tabular}{|l|}
4655 \\
\end{tabular} & PDMS & & SW & & & E086700 & 06-Nov-85 & & $10045-97-3$ & CS-137 & 901.1_GAMMA_GS & USTEST & -0.404 \\
\hline 4656 & PDMS & & SW & & & E086691 & 12-Nov-85 & & $10098-97-2$ & SR-90 & 905.0_SR_GPC & USTEST & 0.206 \\
\hline 4657 & PDMS & & SW & & & E086691 & 12-Nov-85 & & $10045-97-3$ & CS-137 & 901.1_GAMMA_GS & USTEST & -0.7 \\
\hline 4658 & PDMS & & SW & & & E086691 & 12-Nov-85 & & $10028-17-8$ & TRITIUM & 906.0_H3_LSC & USTEST & 147 \\
\hline 4659 & PDMS & & SW & & & E086701 & 12-Nov-85 & & $10098-97-2$ & SR-90 & 905.0_SR_GPC & USTEST & 0.0159 \\
\hline 4660 & PDMS & & SW & & & E086701 & 12-Nov-85 & & $10028-17-8$ & TRITIUM & 906.0_H3_LSC & USTEST & -88.6 \\
\hline 4661 & PDMS & & SW & & & E086701 & 12-Nov-85 & & $10045-97-3$ & CS-137 & 901.1_GAMMA_GS & USTEST & 1 \\
\hline \begin{tabular}{|l|}
4662 \\
\end{tabular} & PDMS & & SW & & & E086871 & 18-Nov-85 & & 10028-17-8 & TRITIUM & 906.0_H3_LSC & USTEST & 92.2 \\
\hline \begin{tabular}{|l|}
4663 \\
\end{tabular} & PDMS & & SW & & & E086871 & 18-Nov-85 & & $10098-97-2$ & SR-90 & 905.0_SR_GPC & USTEST & 1.26 \\
\hline \begin{tabular}{|l|}
4664 \\
\end{tabular} & PDMS & & SW & & & E086871 & 18-Nov-85 & & $10045-97-3$ & CS-137 & 901.1_GAMMA_GS & USTEST & -0.242 \\
\hline \begin{tabular}{|l|}
4665 \\
\end{tabular} & PDMS & & SW & & & E087145 & 19-Nov-85 & & $10045-97-3$ & CS-137 & GAMMA_GS & PNL-RO & -0.00211 \\
\hline \begin{tabular}{|l|}
4666 \\
\end{tabular} & PDMS & & SW & & & E087142 & 19-Nov-85 & & $10045-97-3$ & CS-137 & GAMMA_GS & PNL-RO & 0.00167 \\
\hline \begin{tabular}{|l|l|}
4667 \\
\end{tabular} & PDMS & & SW & & & E086684 & 19-Nov-85 & & $10045-97-3$ & CS-137 & GAMMA_GS & PNL-RO & \\
\hline 4668 & PDMS & & SW & & & E086081 & 19-Nov-85 & & $10045-97-3$ & CS-137 & GAMMA_GS & PNL-RO & \\
\hline 4669 & PDMS & & SW & & & E087146 & 03-Dec-85 & & $10045-97-3$ & CS-137 & GAMMA_GS & PNL-RO & -0.00104 \\
\hline 4670 & PDMS & & SW & & & E087143 & 03-Dec-85 & & $10045-97-3$ & CS-137 & GAMMA_GS & PNL-RO & 0.00628 \\
\hline \begin{tabular}{|l|l|}
4671 & \\
\end{tabular} & PDMS & & SW & & & E087156 & 03-Dec-85 & & $10045-97-3$ & CS-137 & GAMMA_GS & PNL-RO & 0.0035 \\
\hline 4672 & PDMS & & SW & & & E086989 & 03-Dec-85 & & $10028-17-8$ & TRITIUM & TRITIUM_ELECT_LSC & USTEST & 132 \\
\hline 4673 & PDMS & & SW & & & E086989 & 03-Dec-85 & & $\mathrm{U}-238$ & $\mathrm{U}-238$ & UISO_AEA & USTEST & 0.199 \\
\hline 4674 & PDMS & & SW & & & E086989 & 03-Dec-85 & & $15117-96-1$ & U-235 & UISO_AEA & USTEST & 0.00518 \\
\hline 4675 & PDMS & & SW & & & E086989 & 03-Dec-85 & & $13966-29-5$ & $\mathrm{U}-234$ & UISO_AEA & USTEST & 0.243 \\
\hline 4676 & PDMS & & SW & & & E086989 & 03-Dec-85 & & $10098-97-2$ & SR-90 & SRISO_SEP_PRECIP_GPC & USTEST & 0.134 \\
\hline 4677 & PDMS & & SW & & & E087149 & 03-Dec-85 & & $10045-97-3$ & CS-137 & GAMMA_GS & PNL-RO & -0.00699 \\
\hline 4678 & PDMS & & SW & & & E086989 & 03-Dec-85 & & $10045-97-3$ & CS-137 & GAMMA_GS & USTEST & 0.256 \\
\hline 4679 & PDMS & & SW & & & E086988 & 03-Dec-85 & & $10045-97-3$ & CS-137 & GAMMA_GS & USTEST & 0.307 \\
\hline 4680 & PDMS & & SW & & & E086988 & 03-Dec-85 & & $10028-17-8$ & TRITIUM & TRITIUM_ELECT_LSC & USTEST & 167 \\
\hline 4681 & PDMS & & SW & & & E086988 & 03-Dec-85 & & $\mathrm{U}-238$ & $\mathrm{U}-238$ & UISO_AEA & USTEST & 0.229 \\
\hline
\end{tabular}




\begin{tabular}{|c|c|c|c|c|c|c|c|c|c|c|c|c|c|}
\hline & A & $\mathrm{B}$ & $\mathrm{c}$ & $\mathrm{D}$ & $\mathrm{E}$ & $\mathrm{F}$ & G & $\mathrm{H}$ & $\mathrm{I}$ & $\mathrm{J}$ & $\mathrm{K}$ & $\mathrm{L}$ & $\mathrm{M}$ \\
\hline 1 & SAMPLE_NUM_ASSIGNED_TO & SAMPLER & MEDIA & BIOTA_MEDIA & TAG_ID & SAMP_NUM & SAMP_DATE_TIME & SAMP_DATE_TIME_ON & CON_ID & CON_SHORT_NAME & METHOD_NAME & LAB_CODE & \begin{tabular}{|l|} 
VALUE_RPTD \\
\end{tabular} \\
\hline 4682 & PDMS & & SW & & & E086988 & 03-Dec-85 & & $10098-97-2$ & SR-90 & SRISO_SEP_PRECIP_GPC & USTEST & 0.052 \\
\hline 4683 & PDMS & & sW & & & E086988 & 03-Dec-85 & & 15117-96-1 & U-235 & UISO_AEA & USTEST & 0.0123 \\
\hline 4684 & PDMS & & sW & & & E086988 & 03-Dec-85 & & $13966-29-5$ & U-234 & UISO_AEA & USTEST & 0.35 \\
\hline 4685 & PDMS & & sW & & & E087157 & 17-Dec-85 & & $10045-97-3$ & CS-137 & GAMMA_GS & PNL-RO & -0.0039 \\
\hline \begin{tabular}{|l|l|}
4686 \\
\end{tabular} & PDMS & & sW & & & E087151 & 17-Dec-85 & & $10045-97-3$ & CS-137 & GAMMA_GS & PNL-RO & -0.000718 \\
\hline \begin{tabular}{|l|}
4687 \\
\end{tabular} & PDMS & & sW & & & E086987 & 18-Dec-85 & & $10045-97-3$ & CS-137 & GAMMA_GS & PNL-RO & \\
\hline 4688 & PDMS & & SW & & & E086986 & 18-Dec-85 & & $10045-97-3$ & CS-137 & GAMMA_GS & PNL-RO & \\
\hline 4689 & PDMS & & SW & & & E085910 & 24-Dec-85 & & $10098-97-2$ & SR-90 & 905.0_SR_GPC & USTEST & 0.189 \\
\hline 4690 & PDMS & & sW & & & E085910 & 24-Dec-85 & & $10045-97-3$ & CS-137 & 901.1 GAMMA GS & USTEST & -0.102 \\
\hline 4691 & PDMS & & SW & & & E085910 & 24-Dec-85 & & 10028-17-8 & TRITIUM & 906.0_H3_LSC & USTEST & 76.6 \\
\hline 4692 & PDMS & & sW & & & E087141 & 31-Dec-85 & & 15046-84-1 & $1-129$ & I129_SEP_TEMS & 320 & 0.0000757 \\
\hline 4693 & PDMS & & SW & & & E087141 & 31-Dec-85 & & $10045-97-3$ & CS-137 & GAMMA_ĒS & PNL-RO & 0.000473 \\
\hline 4694 & PDMS & & SW & & & E087141 & 31-Dec-85 & & PU-239/240 & PU-239/240 & PUISO_IE_AEA & PNL-RO & 0.000362 \\
\hline 4695 & PDMS & & SW & & & E087141 & 31-Dec-85 & & $10098-97-2$ & SR-90 & SRISO SEP PRECIP GPC & PNL-RO & 0.0434 \\
\hline 4696 & PDMS & & SW & & & E087144 & 31-Dec-85 & & $10045-97-3$ & CS-137 & GAMMA_GS & PNL-RO & -0.00149 \\
\hline \begin{tabular}{|l|l|}
4697 & -1 \\
\end{tabular} & PDMS & & SW & & & E087144 & 31-Dec-85 & & PU-239/240 & PU-239/240 & PUISO_IE_AEA & PNL-RO & 0.0000163 \\
\hline 4698 & PDMS & & sW & & & E087153 & 31-Dec-85 & & $10045-97-3$ & CS-137 & GAMMA_GS & PNL-RO & -0.00564 \\
\hline \begin{tabular}{|l|}
4699 \\
\end{tabular} & PDMS & & sW & & & E087147 & 31-Dec-85 & & $15046-84-1$ & $1-129$ & I129_SEP_TEMS & 320 & 0.0000094 \\
\hline 4700 & PDMS & & SW & & & E085909 & 31-Dec-85 & & $10028-17-8$ & TRITIUM & TRITIUM_ELEECT_LSC & USTEST & 143 \\
\hline 4701 & PDMS & & SW & & & E085909 & 31-Dec-85 & & U-238 & $\mathrm{U}-238$ & UISO_AEA & USTEST & 0.24 \\
\hline 4702 & PDMS & & SW & & & E085909 & 31-Dec-85 & & 15117-96-1 & U-235 & UISO_AEA & USTEST & 0.0111 \\
\hline 4703 & PDMS & & SW & & & E085909 & 31-Dec-85 & & $13966-29-5$ & $\mathrm{U}-234$ & UISO_AEA & USTEST & 0.336 \\
\hline 4704 & PDMS & & SW & & & E085909 & 31-Dec-85 & & $10098-97-2$ & SR-90 & SRISO_SEP_PRECIP_GPC & USTEST & 0.146 \\
\hline 4705 & PDMS & & SW & & & E087147 & 31-Dec-85 & & $10045-97-3$ & CS-137 & GAMMA_GS & PNL-RO & -0.00512 \\
\hline 4706 & PDMS & & SW & & & E087147 & 31-Dec-85 & & PU-239/240 & PU-239/240 & PUISO_IE_AEA & PNL-RO & 0.000503 \\
\hline 4707 & PDMS & & SW & & & E087153 & 31-Dec-85 & & PU-239/240 & PU-239/240 & PUISO_IE_AEA & PNL-RO & 0.0000186 \\
\hline 4708 & PDMS & & SW & & & E087147 & 31-Dec-85 & & $10098-97-2$ & SR-90 & SRISO_SEP_PRECIP_GPC & PNL-RO & 0.113 \\
\hline 4709 & PDMS & & SW & & & E085909 & 31-Dec-85 & & $10045-97-3$ & CS-137 & GAMMA_GS & USTEST & 0.613 \\
\hline 4710 & PDMS & & SW & & & E085911 & 31-Dec-85 & & $10098-97-2$ & SR-90 & SRISO_SEEP_PRECIP_GPC & USTEST & 0.19 \\
\hline 4711 & PDMS & & SW & & & E085911 & 31-Dec-85 & & $10028-17-8$ & TRITIUM & TRITIUM_ELEECT_LSC & USTEST & 217 \\
\hline 4712 & PDMS & & SW & & & E085911 & 31-Dec-85 & & $\mathrm{U}-238$ & $\mathrm{U}-238$ & UISO_AEA & USTEST & 0.321 \\
\hline 4713 & PDMS & & SW & & & E085911 & 31-Dec-85 & & 15117-96-1 & $\mathrm{U}-235$ & UISO_AEA & USTEST & 0.0126 \\
\hline 4714 & PDMS & & SW & & & E085911 & 31-Dec-85 & & $13966-29-5$ & $\mathrm{U}-234$ & UISO_AEA & USTEST & 0.392 \\
\hline \begin{tabular}{|l|l|}
4715 \\
\end{tabular} & PDMS & & SW & & & E085911 & 31-Dec-85 & & $10045-97-3$ & CS-137 & GAMM̄A_GS & USTEST & -1.89 \\
\hline \begin{tabular}{|l|}
4716 \\
\end{tabular} & PDMS & & SW & & & E086013 & 14-Jan-86 & & $10045-97-3$ & CS-137 & GAMMA_GS & PNL-RO & \\
\hline \begin{tabular}{|l|l|}
4717 \\
\end{tabular} & PDMS & & SW & & & E086012 & 14-Jan-86 & & $10045-97-3$ & CS-137 & GAMMA_GS & PNL-RO & \\
\hline \begin{tabular}{|l|l|}
4718 \\
\end{tabular} & PDMS & & SW & & & E087940 & 14-Jan-86 & & $10045-97-3$ & CS-137 & GAMMA_GS & PNL-RO & 0.00186 \\
\hline 4719 & PDMS & & SW & & & E087946 & 14-Jan-86 & & $10045-97-3$ & CS-137 & GAMMA_GS & PNL-RO & -0.00196 \\
\hline 4720 & PDMS & & SW & & & E086084 & 28-Jan-86 & & $10045-97-3$ & CS-137 & GAMMA_GS & PNL-RO & -0.00589 \\
\hline \begin{tabular}{|l|l|}
4721 \\
\end{tabular} & PDMS & & SW & & & E087938 & 28-Jan-86 & & $10045-97-3$ & CS-137 & GAMMA_GS & PNL-RO & -0.000686 \\
\hline 4722 & PDMS & & SW & & & E087942 & 28-Jan-86 & & $10045-97-3$ & CS-137 & GAMMA_GS & PNL-RO & 0.00111 \\
\hline \begin{tabular}{|l|}
4723 \\
\end{tabular} & PDMS & & SW & & & E087947 & 28-Jan-86 & & $10045-97-3$ & CS-137 & GAMMA_GS & PNL-RO & -0.0000751 \\
\hline \begin{tabular}{|l|}
4724 \\
\end{tabular} & PDMS & & SW & & & E087750 & 28-Jan-86 & & U-238 & $\mathrm{U}-238$ & UISO_AEA & USTEST & 0.269 \\
\hline 4725 & PDMS & & SW & & & E087750 & 28-Jan-86 & & 15117-96-1 & U-235 & UISO_AEA & USTEST & 0.0218 \\
\hline 4726 & PDMS & & SW & & & E087750 & 28-Jan-86 & & $13966-29-5$ & $\mathrm{U}-234$ & UISO_AEA & USTEST & 0.308 \\
\hline \begin{tabular}{|l|l|}
4727 \\
\end{tabular} & PDMS & & SW & & & E087750 & 28-Jan-86 & & 10098-97-2 & SR-90 & SRISŌ_SEP_PRECIP_GPC & USTEST & 0.128 \\
\hline 4728 & PDMS & & SW & & & E087750 & 28-Jan-86 & & $10045-97-3$ & CS-137 & GAMMĀ_GS & USTEST & -0.689 \\
\hline 4729 & PDMS & & SW & & & E087750 & 28-Jan-86 & & $10028-17-8$ & TRITIUM & TRITIUM_ELECT_LSC & USTEST & 102 \\
\hline 4730 & PDMS & & SW & & & E085953 & 28-Jan-86 & & $10045-97-3$ & CS-137 & GAMMA_GS & USTEST & 0.678 \\
\hline 4731 & PDMS & & SW & & & E085953 & 28-Jan-86 & & $\mathrm{U}-238$ & $\mathrm{U}-238$ & UISO_AĒA & USTEST & 0.342 \\
\hline 4732 & PDMS & & sW & & & E085953 & 28-Jan-86 & & 15117-96-1 & U-235 & UISO AEA & USTEST & 0.00427 \\
\hline 4733 & PDMS & & SW & & & E085953 & 28-Jan-86 & & $13966-29-5$ & $\mathrm{U}-234$ & UISO_AEA & USTEST & 0.384 \\
\hline
\end{tabular}




\begin{tabular}{|c|c|c|c|c|c|c|c|c|c|c|c|c|c|}
\hline & A & $\mathrm{B}$ & $\mathrm{C}$ & $\mathrm{D}$ & $\mathrm{E}$ & $\mathrm{F}$ & $\mathrm{G}$ & $\mathrm{H}$ & $\mathrm{I}$ & $\mathrm{J}$ & $\mathrm{K}$ & $\mathrm{L}$ & $\mathrm{M}$ \\
\hline 1 & SAMPLE_NUM_ASSIGNED_TO & SAMPLER & MEDIA & BIOTA_MEDIA & TAG_ID & SAMP_NUM & SAMP_DATE_TIME & SAMP_DATE_TIME_ON & CON_ID & CON_SHORT_NAME & METHOD_NAME & LAB_CODE & \begin{tabular}{|l|} 
VALUE_RPTD \\
\end{tabular} \\
\hline 4734 & PDMS & & SW & & & E085953 & 28-Jan-86 & & $10098-97-2$ & SR-90 & SRISO SEP PRECIP_GPC & USTEST & 0.163 \\
\hline 4735 & PDMS & & sW & & & E085953 & 28-Jan-86 & & $10028-17-8$ & TRITIUM & TRITIUMM_ELEECT_LSC̄ & USTEST & 209 \\
\hline 4736 & PDMS & & sW & & & E087753 & 03-Feb-86 & & $10028-17-8$ & TRITIUM & 906.0_H3_LSC & USTEST & -220 \\
\hline 4737 & PDMS & & sW & & & E087753 & 03-Feb-86 & & $10045-97-3$ & CS-137 & 901.1_GAMMA_GS & USTEST & 0.0484 \\
\hline 4738 & PDMS & & sW & & & E087753 & 03-Feb-86 & & $10098-97-2$ & SR-90 & 905.0_SR_GPC & USTEST & 0.0308 \\
\hline 4739 & PDMS & & sw & & & E087752 & 03-Feb-86 & & $10028-17-8$ & TRITIUM & 906.0_H3_LSC & USTEST & 26200 \\
\hline 4740 & PDMS & & SW & & & E087752 & 03-Feb-86 & & $10045-97-3$ & CS-137 & 901.1_GAMMA_GS & USTEST & 0.292 \\
\hline 4741 & PDMS & & SW & & & E087752 & 03-Feb-86 & & $10098-97-2$ & SR-90 & 905.0_SR_GPC & USTEST & 0.035 \\
\hline 4742 & PDMS & & sW & & & E087751 & 03-Feb-86 & & $10028-17-8$ & TRITIUM & 906.0_H3_LSC & USTEST & -161 \\
\hline \begin{tabular}{|l|}
4743 \\
\end{tabular} & PDMS & & SW & & & E087751 & 03-Feb-86 & & $10045-97-3$ & CS-137 & 901.1_GAMMA_GS & USTEST & -0.153 \\
\hline 4744 & PDMS & & sW & & & E087751 & 03-Feb-86 & & $10098-97-2$ & SR-90 & 905.0_SR_GPC & USTEST & 0.0156 \\
\hline \begin{tabular}{|l|l|}
4745 \\
\end{tabular} & PDMS & & SW & & & E087754 & 03-Feb-86 & & 10028-17-8 & TRITIUM & 906.0_H3_LSC & USTEST & -205 \\
\hline \begin{tabular}{|l|}
4746 \\
\end{tabular} & PDMS & & SW & & & E087754 & 03-Feb-86 & & $10045-97-3$ & CS-137 & 901.1_GAMMA_GS & USTEST & 0.562 \\
\hline \begin{tabular}{|l|}
4747 \\
\end{tabular} & PDMS & & sW & & & E087754 & 03-Feb-86 & & $10098-97-2$ & SR-90 & $905.0^{-} \mathrm{SR}$ GPC & USTEST & 0.0304 \\
\hline \begin{tabular}{|l|}
4748 \\
\end{tabular} & PDMS & & SW & & & E087893 & 11-Feb-86 & & $10045-97-3$ & CS-137 & 901.1_GAMMA_GS & USTEST & -0.306 \\
\hline \begin{tabular}{|l|l|}
4749 \\
\end{tabular} & PDMS & & sW & & & E087893 & 11-Feb-86 & & $10028-17-8$ & TRITIUM & 906.0_H3_LSC & USTEST & 5.37 \\
\hline \begin{tabular}{|l|}
4750 \\
\end{tabular} & PDMS & & sW & & & E087893 & 11-Feb-86 & & $10098-97-2$ & SR-90 & 905.0_SR_GPC & USTEST & 0.168 \\
\hline \begin{tabular}{|l|}
4751 \\
\end{tabular} & PDMS & & sW & & & E087937 & 11-Feb-86 & & $10045-97-3$ & CS-137 & GAMMA_GS & PNL-RO & -0.00552 \\
\hline \begin{tabular}{|l|}
4752 \\
\end{tabular} & PDMS & & SW & & & E087939 & 11-Feb-86 & & $10045-97-3$ & CS-137 & GAMMA_GS & PNL-RO & 0.00189 \\
\hline \begin{tabular}{|l|}
4753 \\
\end{tabular} & PDMS & & sW & & & E087949 & 11-Feb-86 & & $10045-97-3$ & CS-137 & GAMMA_GS & PNL-RO & -0.000831 \\
\hline \begin{tabular}{|l|}
4754 \\
\end{tabular} & PDMS & & sW & & & E087944 & 11-Feb-86 & & $10045-97-3$ & CS-137 & GAMMA_GS & PNL-RO & -0.00338 \\
\hline \begin{tabular}{|l|}
4755 \\
\end{tabular} & PDMS & & SW & & & E088246 & 25-Feb-86 & & $10045-97-3$ & CS-137 & GAMMA_GS & PNL-RO & -0.00589 \\
\hline \begin{tabular}{|l|}
4756 \\
\end{tabular} & PDMS & & SW & & & E088251 & 25-Feb-86 & & $10045-97-3$ & CS-137 & GAMMA_GS & PNL-RO & 0.000545 \\
\hline \begin{tabular}{|l|}
4757 \\
\end{tabular} & PDMS & & SW & & & E088019 & 25-Feb-86 & & $10045-97-3$ & CS-137 & GAMMA_GS & USTEST & -0.0511 \\
\hline \begin{tabular}{|l|}
4758 \\
\end{tabular} & PDMS & & SW & & & E088019 & 25-Feb-86 & & $13966-29-5$ & $\mathrm{U}-234$ & UISO_AEA & USTEST & 0.338 \\
\hline \begin{tabular}{|l|}
4759 \\
\end{tabular} & PDMS & & SW & & & E088019 & 25-Feb-86 & & 15117-96-1 & $\mathrm{U}-235$ & UISO_AEA & USTEST & 0.00606 \\
\hline 4760 & PDMS & & sW & & & E088019 & 25-Feb-86 & & U-238 & $\mathrm{U}-238$ & UISO_AEA & USTEST & 0.283 \\
\hline \begin{tabular}{|l|}
4761 \\
\end{tabular} & PDMS & & SW & & & E088255 & 25-Feb-86 & & $10045-97-3$ & CS-137 & GAMM̄A_GS & PNL-RO & -0.0036 \\
\hline \begin{tabular}{|l|l|}
4762 \\
\end{tabular} & PDMS & & sW & & & E088019 & $25-F e b-86$ & & $10028-17-8$ & TRITIUM & TRITIUM_ELECT_LSC & USTEST & 130 \\
\hline \begin{tabular}{|l|l|}
4763 \\
\end{tabular} & PDMS & & sW & & & E088019 & $25-$ Feb-86 & & $10098-97-2$ & SR-90 & SRISO_SEP_PRËCIP_GPC & USTEST & 0.159 \\
\hline \begin{tabular}{|l|}
4764 \\
\end{tabular} & PDMS & & SW & & & E088259 & 25-Feb-86 & & $10045-97-3$ & CS-137 & GAMMA_GS & PNL-RO & -0.0000879 \\
\hline \begin{tabular}{|l|l|}
4765 \\
\end{tabular} & PDMS & & sW & & & E088018 & 25-Feb-86 & & $10045-97-3$ & CS-137 & GAMMA_GS & USTEST & 0.097 \\
\hline \begin{tabular}{|l|l|}
4766 \\
\end{tabular} & PDMS & & sW & & & E088018 & 25-Feb-86 & & $13966-29-5$ & $\mathrm{U}-234$ & 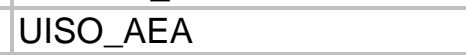 & USTEST & 0.319 \\
\hline \begin{tabular}{|l|l|}
4767 & -20 \\
\end{tabular} & PDMS & & SW & & & E088018 & 25-Feb-86 & & 15117-96-1 & $\mathrm{U}-235$ & UISO_AEA & USTEST & 0.00626 \\
\hline \begin{tabular}{|l|l|}
4768 \\
\end{tabular} & PDMS & & sW & & & E088018 & 25-Feb-86 & & U-238 & $\mathrm{U}-238$ & UISO_AEA & USTEST & 0.307 \\
\hline \begin{tabular}{|l|l|}
4769 \\
\end{tabular} & PDMS & & sW & & & E088018 & $25-$ Feb-86 & & 10028-17-8 & TRITIUM & TRITIŪM_ELECT_LSC & USTEST & 206 \\
\hline 4770 & PDMS & & SW & & & E088018 & 25-Feb-86 & & $10098-97-2$ & SR-90 & SRISO_SEP_PRECIP_GPC & USTEST & 0.166 \\
\hline \begin{tabular}{|l|}
4771 \\
\end{tabular} & PDMS & & SW & & & E088078 & 04-Mar-86 & & 10028-17-8 & TRITIUM & TRITIUM_DIST_LSC & USTEST & 187 \\
\hline \begin{tabular}{|l|}
4772 \\
\end{tabular} & PDMS & & sW & & & E088078 & 04-Mar-86 & & $10045-97-3$ & CS-137 & GAMMA_GS & USTEST & 0.275 \\
\hline 4773 & PDMS & & SW & & & E088078 & 04-Mar-86 & & $10098-97-2$ & SR-90 & SRISO_SEEP_PRECIP_GPC & USTEST & 0.536 \\
\hline 4774 & PDMS & & sW & & & E088081 & 04-Mar-86 & & $10045-97-3$ & CS-137 & GAMMĀ_GS & USTEST & -0.189 \\
\hline 4775 & PDMS & & sW & & & E088081 & 04-Mar-86 & & $10028-17-8$ & TRITIUM & TRITIUM_DIST_LSC & USTEST & 2530 \\
\hline 4776 & PDMS & & SW & & & E088079 & 04-Mar-86 & & $10028-17-8$ & TRITIUM & TRITIUM_DIST_LSC & USTEST & 150 \\
\hline 4777 & PDMS & & SW & & & E088079 & 04-Mar-86 & & $10045-97-3$ & CS-137 & GAMMA_GS & USTEST & 6.34 \\
\hline 4778 & PDMS & & SW & & & E088079 & 04-Mar-86 & & $10098-97-2$ & SR-90 & SRISO_SEPP_PRECIP_GPC & USTEST & 1.88 \\
\hline 4779 & PDMS & & SW & & & E088080 & 04-Mar-86 & & $10028-17-8$ & TRITIUM & TRITIUM_DIS̄T_LSC & USTEST & 719 \\
\hline 4780 & PDMS & & SW & & & E088080 & 04-Mar-86 & & $10045-97-3$ & CS-137 & GAMMA_GS & USTEST & -0.536 \\
\hline 4781 & PDMS & & sW & & & E088080 & 04-Mar-86 & & $10098-97-2$ & SR-90 & SRISO_SEEP_PRECIP_GPC & USTEST & 2.29 \\
\hline 4782 & PDMS & & sW & & & E088130 & 11-Mar-86 & & $10045-97-3$ & CS-137 & 901.1_GAMMA_GS & USTEST & -0.275 \\
\hline 4783 & PDMS & & SW & & & E088130 & 11-Mar-86 & & $10028-17-8$ & TRITIUM & 906.0_H3_LSC & USTEST & -27.9 \\
\hline 4784 & PDMS & & sW & & & E088130 & 11-Mar-86 & & $10098-97-2$ & SR-90 & 905.0 SR GPC & USTEST & 0.0393 \\
\hline 4785 & PDMS & & SW & & & E088247 & 11-Mar-86 & & $10045-97-3$ & CS-137 & GAMMĀA_GS & PNL-RO & -0.00263 \\
\hline
\end{tabular}




\begin{tabular}{|c|c|c|c|c|c|c|c|c|c|c|c|c|c|}
\hline & $\bar{A}$ & $\mathrm{~B}$ & C & $\mathrm{D}$ & $E$ & $\mathrm{~F}$ & G & $\mathrm{H}$ & $\mathrm{I}$ & $\mathrm{J}$ & $\mathrm{K}$ & $\mathrm{L}$ & $\mathrm{M}$ \\
\hline 1 & SAMPLE_NUM_ASSIGNED_TO & SAMPLER & MEDIA & BIOTA_MEDIA & TAG_ID & SAMP_NUM & SAMP_DATE_TIME & SAMP_DATE_TIME_ON & CON_ID & CON_SHORT_NAME & METHOD_NAME & LAB_CODE & \begin{tabular}{|l|} 
VALUE_RPTD \\
\end{tabular} \\
\hline \begin{tabular}{|l|l|}
4786 \\
\end{tabular} & PDMS & & SW & & & E088253 & 11-Mar-86 & & $10045-97-3$ & CS-137 & GAMMA_GS & PNL-RO & -0.00119 \\
\hline 4787 & PDMS & & SW & & & E088256 & 11-Mar-86 & & $10045-97-3$ & CS-137 & GAMMA_GS & PNL-RO & -0.00225 \\
\hline 4788 & PDMS & & SW & & & E088260 & 11-Mar-86 & & $10045-97-3$ & CS-137 & GAMMA_GS & PNL-RO & 0.00253 \\
\hline 4789 & PDMS & & SW & & & E088077 & 12-Mar-86 & & $10045-97-3$ & CS-137 & GAMMA_GS & USTEST & \\
\hline 4790 & PDMS & & SW & & & E088077 & 12-Mar-86 & & $10098-97-2$ & SR-90 & SRISO_SEEP_PRECIP_GPC & USTEST & \\
\hline 4791 & PDMS & & SW & & & E088129 & 12-Mar-86 & & $10028-17-8$ & TRITIUM & TRITIUM_DIST_LSC & USTEST & -12.9 \\
\hline 4792 & PDMS & & SW & & & E088127 & 12-Mar-86 & & $10028-17-8$ & TRITIUM & TRITIUM_DIST_LEC & USTEST & 94.5 \\
\hline 4793 & PDMS & & SW & & & E088126 & 12-Mar-86 & & $10028-17-8$ & TRITIUM & TRITIUM_DIST_LSC & USTEST & -169 \\
\hline 4794 & PDMS & & SW & & & E088128 & 12-Mar-86 & & $10028-17-8$ & TRITIUM & TRITIUM_DIST_LSC & USTEST & 172 \\
\hline 4795 & PDMS & & SW & & & E088125 & 18-Mar-86 & & $10045-97-3$ & CS-137 & 901.1_GAMMA_GS & USTEST & -0.106 \\
\hline 4796 & PDMS & & SW & & & E088125 & 18-Mar-86 & & $10098-97-2$ & SR-90 & 905.0_SR_GPC & USTEST & 0.174 \\
\hline \begin{tabular}{|l|l|}
4797 \\
\end{tabular} & PDMS & & SW & & & E088125 & 18-Mar-86 & & $13966-29-5$ & U-234 & UISO_IE_PLATE_AEA & USTEST & 0.315 \\
\hline 4798 & PDMS & & SW & & & E088125 & 18-Mar-86 & & 15117-96-1 & U-235 & UISO_IE_PLATE_AEA & USTEST & 0.00601 \\
\hline 4799 & PDMS & & SW & & & E088125 & 18-Mar-86 & & $\mathrm{U}-238$ & U-238 & UISO_IE_PLATE_AEA & USTEST & 0.297 \\
\hline 4800 & PDMS & & SW & & & E088125 & 18-Mar-86 & & 10028-17-8 & TRITIUM & TRITIŪM_ELECT_LSC & USTEST & 219 \\
\hline \begin{tabular}{|l|}
4801 \\
\end{tabular} & PDMS & & SW & & & E088254 & 25-Mar-86 & & $10045-97-3$ & CS-137 & GAMMA_GS & PNL-RO & 0.00291 \\
\hline 4802 & PDMS & & SW & & & E088254 & 25-Mar-86 & & PU-239/240 & PU-239/240 & PUISO_IE_AEA & PNL-RO & 0.0000224 \\
\hline 4803 & PDMS & & SW & & & E088250 & 25-Mar-86 & & $10045-97-3$ & CS-137 & GAMMA_GS & PNL-RO & -0.0031 \\
\hline 4804 & PDMS & & SW & & & E088250 & 25-Mar-86 & & 15046-84-1 & I-129 & I129_SEP__TEMS & 320 & 0.0000793 \\
\hline 4805 & PDMS & & SW & & & E088250 & 25-Mar-86 & & $10098-97-2$ & SR-90 & SRIS̄O_SËP_PRECIP_GPC & PNL-RO & 0.00393 \\
\hline 4806 & PDMS & & SW & & & E088250 & 25-Mar-86 & & PU-239/240 & PU-239/240 & PUISO_IE_AEA & PNL-RO & 0.0000337 \\
\hline 4807 & PDMS & & SW & & & E092071 & 25-Mar-86 & & 15046-84-1 & I-129 & I129_SEP_TEMS & 320 & 0.0000081 \\
\hline 4808 & PDMS & & SW & & & E092071 & 25-Mar-86 & & $10098-97-2$ & SR-90 & SRISO_SEP_PRECIP_GPC & PNL-RO & 0.00842 \\
\hline 4809 & PDMS & & SW & & & E092071 & 25-Mar-86 & & PU-239/240 & PU-239/240 & PUISO_IE_AEAA & PNL-RO & 0.0000208 \\
\hline 4810 & PDMS & & SW & & & E092345 & 25-Mar-86 & & PU-239/240 & PU-239/240 & PUISO_IE_AEA & PNL-RO & 0.00000679 \\
\hline 4811 & PDMS & & SW & & & E088261 & 26-Mar-86 & & $10098-97-2$ & SR-90 & SRISO_SEP_PRECIP_GPC & USTEST & 0.195 \\
\hline 4812 & PDMS & & SW & & & E088261 & 26-Mar-86 & & $10045-97-3$ & CS-137 & GAMMĀGS & USTEST & 0.387 \\
\hline 4813 & PDMS & & SW & & & E088449 & 01-Apr-86 & & $\mathrm{U}-238$ & U-238 & UISO_AĒAA & USTEST & 0.0238 \\
\hline 4814 & PDMS & & SW & & & E088449 & 01-Apr-86 & & 15117-96-1 & U-235 & UISO_AEA & USTEST & 0.00119 \\
\hline 4815 & PDMS & & SW & & & E088449 & 01-Apr-86 & & $13966-29-5$ & U-234 & UISO_AEA & USTEST & 0.0268 \\
\hline 4816 & PDMS & & SW & & & E088449 & 01-Apr-86 & & $10045-97-3$ & CS-137 & GAMMA_GS & USTEST & 0 \\
\hline 4817 & PDMS & & SW & & & E088449 & 01-Apr-86 & & $10098-97-2$ & SR-90 & SRISO_SEEP_PRECIP_GPC & USTEST & 0.191 \\
\hline 4818 & PDMS & & SW & & & E088449 & 01-Apr-86 & & $10028-17-8$ & TRITIUM & TRITIUM̄E_ELECT_LSC̄ & USTEST & 105 \\
\hline 4819 & PDMS & & SW & & & E088397 & 01-Apr-86 & & $\mathrm{U}-238$ & U-238 & UISO_AËA & USTEST & 0.0252 \\
\hline 4820 & PDMS & & SW & & & E088397 & 01-Apr-86 & & $15117-96-1$ & U-235 & UISO_AEA & USTEST & 0.00142 \\
\hline 4821 & PDMS & & SW & & & E088397 & 01-Apr-86 & & $13966-29-5$ & U-234 & UISO_AEA & USTEST & 0.0248 \\
\hline 4822 & PDMS & & SW & & & E088397 & 01-Apr-86 & & $10045-97-3$ & CS-137 & GAMMA_GS & USTEST & 0.367 \\
\hline 4823 & PDMS & & SW & & & E088397 & 01-Apr-86 & & $10098-97-2$ & SR-90 & SRISO_SEEP_PRECIP_GPC & USTEST & 0.185 \\
\hline 4824 & PDMS & & SW & & & E088397 & 01-Apr-86 & & $10028-17-8$ & TRITIUM & TRITIUM̄E_ELECT_LSC̄ & USTEST & 110 \\
\hline 4825 & PDMS & & SW & & & E088450 & 04-Apr-86 & & $10045-97-3$ & CS-137 & 901.1_GAMMMA_ḠS & USTEST & -0.459 \\
\hline 4826 & PDMS & & SW & & & E088450 & 04-Apr-86 & & $10028-17-8$ & TRITIUM & TRITIŪM_ELEC̄T_LSC & USTEST & 162 \\
\hline 4827 & PDMS & & SW & & & E088450 & 04-Apr-86 & & $10098-97-2$ & SR-90 & 905.0_SR_GPC & USTEST & 0.782 \\
\hline 4828 & PDMS & & SW & & & E088258 & 08-Apr-86 & & $10045-97-3$ & CS-137 & GAMMA_ḠS & PNL-RO & \\
\hline 4829 & PDMS & & SW & & & E088307 & 08-Apr-86 & & $10045-97-3$ & CS-137 & GAMMA_GS & PNL-RO & \\
\hline 4830 & PDMS & & SW & & & E088612 & 08-Apr-86 & & $10045-97-3$ & CS-137 & GAMMA_GS & PNL-RO & 0.00122 \\
\hline 4831 & PDMS & & SW & & & E088609 & 08-Apr-86 & & $10045-97-3$ & CS-137 & GAMMA_GS & PNL-RO & -0.00879 \\
\hline 4832 & PDMS & & SW & & & E088549 & 15-Apr-86 & & $10045-97-3$ & CS-137 & GAMMA_GS & USTEST & -1.15 \\
\hline 4833 & PDMS & & SW & & & E088549 & 15-Apr-86 & & $10098-97-2$ & SR-90 & SRISO_S̄EP_PRECIP_GPC & USTEST & 0.153 \\
\hline 4834 & PDMS & & SW & & & E088549 & 15-Apr-86 & & $10028-17-8$ & TRITIUM & TRITIUM_ELECT_LSC & USTEST & 106 \\
\hline 4835 & PDMS & & SW & & & E088549 & 15-Apr-86 & & $13966-29-5$ & U-234 & UISO_AEA & USTEST & 0.24 \\
\hline 4836 & PDMS & & SW & & & E088549 & 15-Apr-86 & & 15117-96-1 & U-235 & UISO_AEA & USTEST & 0.00694 \\
\hline 4837. & PDMS & & SW & & & E088549 & 15-Apr-86 & & $\mathrm{U}-238$ & $\mathrm{U}-238$ & UISO_AEA & USTEST & 0.222 \\
\hline
\end{tabular}




\begin{tabular}{|c|c|c|c|c|c|c|c|c|c|c|c|c|c|}
\hline & $\bar{A}$ & $\mathrm{~B}$ & $\bar{C}$ & $\mathrm{D}$ & $\mathrm{E}$ & $\mathrm{F}$ & $\bar{G}$ & $\mathrm{H}$ & 1 & $\mathrm{~J}$ & $\bar{K}$ & $\bar{L}$ & $\mathrm{M}$ \\
\hline 1 & SAMPLE_NUM_ASSIGNED_TO & SAMPLER & MEDIA & BIOTA_MEDIA & TAG_ID & SAMP_NUM & SAMP_DATE_TIME & SAMP_DATE_TIME_ON & CON_ID & CON_SHORT_NAME & METHOD_NAME & LAB_CODE & VALUE_RPTD \\
\hline 4838 & PDMS & & SW & & & E088607 & $22-A p r-86$ & & $10045-97-3$ & CS-137 & GAMMA_GS & PNL-RO & -0.000102 \\
\hline 4839 & PDMS & & SW & & & E088606 & 22-Apr-86 & & $10045-97-3$ & CS-137 & GAMMA_GS & PNL-RO & 0.00156 \\
\hline 4840 & PDMS & & SW & & & E088611 & 22-Apr-86 & & $10045-97-3$ & CS-137 & GAMMA_GS & PNL-RO & -0.00325 \\
\hline 4841 & PDMS & & SW & & & E088615 & 22-Apr-86 & & $10045-97-3$ & CS-137 & GAMMA_GS & PNL-RO & 0.00153 \\
\hline 4842 & PDMS & & SW & & & E088792 & 28-Apr-86 & & $10028-17-8$ & TRITIUM & 906.0_H3_LSC & USTEST & 46.1 \\
\hline 4843 & PDMS & & SW & & & E088792 & 28-Apr-86 & & $10098-97-2$ & SR-90 & 905.0_SR_GPC & USTEST & 0.0224 \\
\hline 4844 & PDMS & & SW & & & E088792 & 28-Apr-86 & & $10045-97-3$ & CS-137 & 901.1_GAMMA_GS & USTEST & -0.367 \\
\hline 4845 & PDMS & & SW & & & E088791 & 28-Apr-86 & & $10045-97-3$ & CS-137 & 901.1_GAMMA_GS & USTEST & 0.581 \\
\hline 4846 & PDMS & & SW & & & E088791 & 28-Apr-86 & & $10028-17-8$ & TRITIUM & 906.0_H3_LSC & USTEST & 130 \\
\hline 4847 & PDMS & & SW & & & E088791 & 28-Apr-86 & & $10098-97-2$ & SR-90 & 905.0_SR_GPC & USTEST & 0.108 \\
\hline 4848 & PDMS & & SW & & & E088790 & 28-Apr-86 & & $10045-97-3$ & CS-137 & 901.1_GAMMA_GS & USTEST & -0.413 \\
\hline 4849 & PDMS & & SW & & & E088790 & 28-Apr-86 & & $10098-97-2$ & SR-90 & 905.0_SR_GPC & USTEST & 0.0763 \\
\hline \begin{tabular}{|l|}
4850 \\
\end{tabular} & PDMS & & SW & & & E088790 & 28-Apr-86 & & 10028-17-8 & TRITIUM & 906.0_H3_LSC & USTEST & -40.8 \\
\hline \begin{tabular}{|l|l|}
4851 \\
\end{tabular} & PDMS & & SW & & & E088793 & 28-Apr-86 & & $10098-97-2$ & SR-90 & 905.0_SR_GPC & USTEST & 0.0355 \\
\hline \begin{tabular}{|l|}
4852 \\
\end{tabular} & PDMS & & SW & & & E088793 & 28-Apr-86 & & $10045-97-3$ & CS-137 & 901.1_GAMMA_GS & USTEST & 0.484 \\
\hline \begin{tabular}{|l|l|}
4853 \\
\end{tabular} & PDMS & & SW & & & E088793 & 28-Apr-86 & & $10028-17-8$ & TRITIUM & 906.0_H3_LSC & USTEST & 78.3 \\
\hline \begin{tabular}{|l|}
4854 \\
\end{tabular} & PDMS & & SW & & & E088788 & 29-Apr-86 & & $10045-97-3$ & CS-137 & GAMMA_GS & USTEST & 0.916 \\
\hline \begin{tabular}{|l|}
4855 \\
\end{tabular} & PDMS & & SW & & & E088788 & 29-Apr-86 & & $\mathrm{U}-238$ & $\mathrm{U}-238$ & UISO_AĒA & USTEST & 0.179 \\
\hline \begin{tabular}{|l|}
4856 \\
\end{tabular} & PDMS & & SW & & & E088788 & 29-Apr-86 & & 15117-96-1 & $\mathrm{U}-235$ & UISO_AEA & USTEST & 0.00957 \\
\hline \begin{tabular}{|l|l|}
4857 \\
\end{tabular} & PDMS & & SW & & & E088788 & 29-Apr-86 & & $13966-29-5$ & $\mathrm{U}-234$ & UISO_AEA & USTEST & 0.255 \\
\hline \begin{tabular}{|l|}
4858 \\
\end{tabular} & PDMS & & SW & & & E088788 & 29-Apr-86 & & $10028-17-8$ & TRITIUM & TRITIŪM_ELECT_LSC & USTEST & 119 \\
\hline \begin{tabular}{|l|}
4859 \\
\end{tabular} & PDMS & & SW & & & E088788 & 29-Apr-86 & & $10098-97-2$ & SR-90 & SRISO_SËP_PRËCIP_GPC & USTEST & 0.154 \\
\hline 4860 & PDMS & & SW & & & E088786 & 29-Apr-86 & & $10045-97-3$ & CS-137 & GAMMA_GS & USTEST & 1.37 \\
\hline \begin{tabular}{|l|l|}
4861 \\
\end{tabular} & PDMS & & SW & & & E088786 & 29-Apr-86 & & $\mathrm{U}-238$ & $\mathrm{U}-238$ & UISO_AEA & USTEST & 0.262 \\
\hline \begin{tabular}{|l|}
4862 \\
\end{tabular} & PDMS & & SW & & & E088786 & 29-Apr-86 & & 15117-96-1 & U-235 & UISO_AEA & USTEST & 0.00969 \\
\hline 4863 & PDMS & & SW & & & E088786 & 29-Apr-86 & & $13966-29-5$ & $\mathrm{U}-234$ & UISO_AEA & USTEST & 0.33 \\
\hline 4864 & PDMS & & SW & & & E088786 & 29-Apr-86 & & $10028-17-8$ & TRITIUM & TRITIUM_ELECT_LSC & USTEST & 128 \\
\hline 4865 & PDMS & & SW & & & E088786 & 29-Apr-86 & & $10098-97-2$ & SR-90 & SRISO_SEP_PRECIP_GPC & USTEST & 0.155 \\
\hline \begin{tabular}{|l|l|}
4866 \\
\end{tabular} & PDMS & & SW & & & E088931 & 06-May-86 & & $10045-97-3$ & CS-137 & 901.1_GAMMA_GS & USTEST & 0.381 \\
\hline \begin{tabular}{|l|l|}
4867 \\
\end{tabular} & PDMS & & SW & & & E088931 & 06-May-86 & & $10028-17-8$ & TRITIUM & TRITIUM_ELECT_LSC & USTEST & 145 \\
\hline \begin{tabular}{|l|}
4868 \\
\end{tabular} & PDMS & & SW & & & E088931 & 06-May-86 & & $10098-97-2$ & SR-90 & 905.0_SR_GPC & USTEST & 0.177 \\
\hline 4869 & PDMS & & SW & & & E034464 & 06-May-86 & & $10045-97-3$ & CS-137 & GAMMA_GS & PNL-RO & -0.00186 \\
\hline 4870 & PDMS & & SW & & & E033872 & 06-Мау-86 & & $10045-97-3$ & CS-137 & GAMMA_GS & PNL-RO & -0.00211 \\
\hline 4871 & PDMS & & SW & & & E034454 & 06-Мау-86 & & $10045-97-3$ & CS-137 & GAMMA_GS & PNL-RO & 0.000718 \\
\hline 4872 & PDMS & & SW & & & E034459 & 06-May-86 & & $10045-97-3$ & CS-137 & GAMMA_GS & PNL-RO & -0.00493 \\
\hline 4873 & PDMS & & SW & & & E088970 & 20-May-86 & & $10045-97-3$ & CS-137 & GAMMA_GS & PNL-RO & \\
\hline 4874 & PDMS & & SW & & & E088969 & 20-May-86 & & $10045-97-3$ & CS-137 & GAMMA_GS & PNL-RO & \\
\hline \begin{tabular}{|l|}
4875 \\
\end{tabular} & PDMS & & SW & & & E034452 & 20-May-86 & & $10045-97-3$ & CS-137 & GAMMA_GS & PNL-RO & 0.00706 \\
\hline 4876 & PDMS & & SW & & & E034461 & 20-May-86 & & $10045-97-3$ & CS-137 & GAMMA_GS & PNL-RO & 0.00255 \\
\hline 4877 & PDMS & & SW & & & E033967 & 27-May-86 & & $10098-97-2$ & SR-90 & SRISO_SEP_PRECIP_GPC & USTEST & 1.48 \\
\hline 4878 & PDMS & & SW & & & E033967 & 27-May-86 & & $10028-17-8$ & TRITIUM & TRITIUM_DIST_LSC & USTEST & 6070 \\
\hline 4879 & PDMS & & SW & & & E033967 & 27-May-86 & & $10045-97-3$ & CS-137 & GAMMA_GS & USTEST & 1.19 \\
\hline 4880 & PDMS & & SW & & & E089160 & 27-May-86 & & $10045-97-3$ & CS-137 & GAMMA_GS & USTEST & -1.38 \\
\hline 4881 & PDMS & & SW & & & E089160 & 27-May-86 & & $10028-17-8$ & TRITIUM & TRITIUM_DIST_LSC & USTEST & 232 \\
\hline 4882 & PDMS & & SW & & & E033968 & 27-May-86 & & $10098-97-2$ & SR-90 & SRISO_SEP_PRECIP_GPC & USTEST & 2.09 \\
\hline 4883 & PDMS & & SW & & & E033968 & 27-May-86 & & $10028-17-8$ & TRITIUM & TRITIUM_DISTT_LSC & USTEST & 67.8 \\
\hline 4884 & PDMS & & SW & & & E033968 & 27-May-86 & & $10045-97-3$ & CS-137 & GAMMA_GS & USTEST & 5.97 \\
\hline 4885 & PDMS & & SW & & & E033969 & 27-May-86 & & $10098-97-2$ & SR-90 & SRISO_SEP_PRECIP_GPC & USTEST & 1.99 \\
\hline 4886 & PDMS & & SW & & & E033969 & 27-May-86 & & $10045-97-3$ & CS-137 & GAMMA_GS & USTEST & 3.1 \\
\hline \begin{tabular}{|l|l|}
4887 \\
\end{tabular} & PDMS & & SW & & & E033969 & 27-May-86 & & $10028-17-8$ & TRITIUM & TRITIUM_DIST_LSC & USTEST & 706 \\
\hline 4888 & PDMS & & SW & & & E020899 & 03-Jun-86 & & $10045-97-3$ & CS-137 & 901.1_GAMMA_GS & USTEST & -0.183 \\
\hline 4889 & PDMS & & SW & & & E033942 & 03-Jun-86 & & $10098-97-2$ & SR-90 & 905.0_SR_GPC & USTEST & 0.049 \\
\hline
\end{tabular}




\begin{tabular}{|c|c|c|c|c|c|c|c|c|c|c|c|c|c|}
\hline & A & $\mathrm{B}$ & $\mathrm{c}$ & $\mathrm{D}$ & $\mathrm{E}$ & $\mathrm{F}$ & G & $\mathrm{H}$ & $\mathrm{I}$ & $\mathrm{J}$ & $\mathrm{K}$ & $\mathrm{L}$ & $\mathrm{M}$ \\
\hline 1 & SAMPLE_NUM_ASSIGNED_TO & SAMPLER & MEDIA & BIOTA_MEDIA & TAG_ID & SAMP_NUM & SAMP_DATE_TIME & SAMP_DATE_TIME_ON & CON_ID & CON_SHORT_NAME & METHOD_NAME & LAB_CODE & \begin{tabular}{|l|} 
VALUE_RPTD \\
\end{tabular} \\
\hline 4890 & PDMS & & SW & & & E033942 & 03-Jun-86 & & $10028-17-8$ & TRITIUM & 906.0_H3_LSC & USTEST & -167 \\
\hline 4891 & PDMS & & sW & & & E033942 & 03-Jun-86 & & $10045-97-3$ & CS-137 & 901.1_GAMMA_GS & USTEST & 0.0969 \\
\hline 4892 & PDMS & & sW & & & E089136 & 03-Jun-86 & & $10045-97-3$ & CS-137 & GAMMA_GS & PNL-RO & \\
\hline 4893 & PDMS & & sW & & & E089135 & 03-Jun-86 & & $10045-97-3$ & CS-137 & GAMMA_GS & PNL-RO & \\
\hline 4894 & PDMS & & sW & & & E034457 & 03-Jun-86 & & $10045-97-3$ & CS-137 & GAMMA_GS & PNL-RO & 0.0134 \\
\hline 4895 & PDMS & & SW & & & E034450 & 03-Jun-86 & & $10045-97-3$ & CS-137 & GAMMA_GS & PNL-RO & 0.00664 \\
\hline 4896 & PDMS & & SW & & & E033966 & 03-Jun-86 & & $10098-97-2$ & SR-90 & SRISO_SEP_PRECIP_GPC & USTEST & 0.13 \\
\hline 4897 & PDMS & & SW & & & E033966 & 03-Jun-86 & & $10045-97-3$ & CS-137 & GAMMA_GS & USTEST & -0.92 \\
\hline 4898 & PDMS & & sW & & & E033966 & 03-Jun-86 & & U-238 & U-238 & UISO AEA & USTEST & 0.216 \\
\hline 4899 & PDMS & & SW & & & E033966 & 03-Jun-86 & & 15117-96-1 & U-235 & UISO_AEA & USTEST & 0.00955 \\
\hline 4900 & PDMS & & sW & & & E033966 & 03-Jun-86 & & $13966-29-5$ & $\mathrm{U}-234$ & UISO_AEA & USTEST & 0.293 \\
\hline 4901 & PDMS & & SW & & & E033966 & 03-Jun-86 & & $10028-17-8$ & TRITIUM & TRITIÜM_ELECT_LSC & USTEST & 98.7 \\
\hline 4902 & PDMS & & SW & & & E033965 & 03-Jun-86 & & $10098-97-2$ & SR-90 & SRISO_SEPP_PRËCIP_GPC & USTEST & 0.0985 \\
\hline 4903 & PDMS & & SW & & & E033965 & 03-Jun-86 & & $10028-17-8$ & TRITIUM & TRITIUMM_ELEECT_LSC̄ & USTEST & 125 \\
\hline 4904 & PDMS & & SW & & & E033965 & 03-Jun-86 & & $\mathrm{U}-238$ & $\mathrm{U}-238$ & UISO_AEA & USTEST & 0.205 \\
\hline 4905 & PDMS & & SW & & & E033965 & 03-Jun-86 & & 15117-96-1 & U-235 & UISO_AEA & USTEST & 0.00692 \\
\hline 4906 & PDMS & & sW & & & E033965 & 03-Jun-86 & & $13966-29-5$ & $\mathrm{U}-234$ & UISO_AEA & USTEST & 0.251 \\
\hline \begin{tabular}{|l|l|}
4907 \\
\end{tabular} & PDMS & & sW & & & E033965 & 03-Jun-86 & & $10045-97-3$ & CS-137 & GAMMA_GS & USTEST & -0.145 \\
\hline 4908 & PDMS & & SW & & & E089265 & 04-Jun-86 & & $10098-97-2$ & SR-90 & SRISO_SEEP_PRECIP_GPC & USTEST & 0.222 \\
\hline 4909 & PDMS & & SW & & & E089265 & 04-Jun-86 & & $10045-97-3$ & CS-137 & GAMMĀ_GS & USTEST & -1.31 \\
\hline 4910 & PDMS & & SW & & & E088425 & 06-Jun-86 & & $10098-97-2$ & SR-90 & 905.0_SR_GPC & USTEST & 0.597 \\
\hline 4911 & PDMS & & SW & & & E088425 & 06-Jun-86 & & $10028-17-8$ & TRITIUM & TRITIŪM_ELECT_LSC & USTEST & 156 \\
\hline 4912 & PDMS & & SW & & & E088425 & 06-Jun-86 & & $10045-97-3$ & CS-137 & 901.1_GAMMA_GS & USTEST & 0.0484 \\
\hline 4913 & PDMS & & SW & & & E034463 & 17-Jun-86 & & $10045-97-3$ & CS-137 & GAMMĀASS & PNL-RO & 0.0101 \\
\hline 4914 & PDMS & & SW & & & E033868 & 17-Jun-86 & & $10045-97-3$ & CS-137 & GAMMA_GS & PNL-RO & 0.00983 \\
\hline 4915 & PDMS & & SW & & & E034451 & 17-Jun-86 & & $10045-97-3$ & CS-137 & GAMMA_GS & PNL-RO & 0.00549 \\
\hline 4916 & PDMS & & SW & & & E034455 & 17-Jun-86 & & $10045-97-3$ & CS-137 & GAMMA_GS & PNL-RO & 0.00434 \\
\hline \begin{tabular}{|l|l|}
4917 \\
\end{tabular} & PDMS & & SW & & & E033870 & 01-Jul-86 & & $10045-97-3$ & CS-137 & GAMMA_GS & PNL-RO & 0.00778 \\
\hline 4918 & PDMS & & SW & & & E033870 & 01-Jul-86 & & $15046-84-1$ & I-129 & I129_SEP_TEMS & 320 & 0.0000898 \\
\hline 4919 & PDMS & & SW & & & E033870 & 01-Jul-86 & & $10098-97-2$ & SR-90 & SRISO_SEEP_PRECIP_GPC & PNL-RO & 0.00192 \\
\hline 4920 & PDMS & & SW & & & E033870 & 01-Jul-86 & & PU-239/240 & PU-239/240 & PUISO_IE_AEA & PNL-RO & 0.000079 \\
\hline 4921 & PDMS & & SW & & & E034462 & 01-Jul-86 & & $10045-97-3$ & CS-137 & GAMMA_ḠS & PNL-RO & 0.00329 \\
\hline 4922 & PDMS & & SW & & & E034462 & 01-Jul-86 & & PU-239/240 & PU-239/240 & PUISO_IE_AEA & PNL-RO & 0.0000203 \\
\hline 4923 & PDMS & & SW & & & E034444 & 01-Jul-86 & & $10045-97-3$ & CS-137 & GAMMA_ḠS & PNL-RO & 0.00283 \\
\hline 4924 & PDMS & & SW & & & E034444 & 01-Jul-86 & & PU-239/240 & PU-239/240 & PUISO_IE_AEA & PNL-RO & 0.0000161 \\
\hline 4925 & PDMS & & sW & & & E089485 & 01-Jul-86 & & $10045-97-3$ & CS-137 & GAMMA_GS & USTEST & 0.643 \\
\hline 4926 & PDMS & & SW & & & E089485 & 01-Jul-86 & & $10098-97-2$ & SR-90 & SRISO_SEP_PRECIP_GPC & USTEST & 0.101 \\
\hline 4927 & PDMS & & SW & & & E089485 & 01-Jul-86 & & $\mathrm{U}-238$ & $\mathrm{U}-238$ & UISO_AEAA & USTEST & 0.0818 \\
\hline 4928 & PDMS & & SW & & & E089485 & 01-Jul-86 & & 15117-96-1 & U-235 & UISO_AEA & USTEST & 0.00454 \\
\hline 4929 & PDMS & & SW & & & E089485 & 01-Jul-86 & & $13966-29-5$ & $\mathrm{U}-234$ & UISO_AEA & USTEST & 0.109 \\
\hline 4930 & PDMS & & SW & & & E089485 & 01-Jul-86 & & $10028-17-8$ & TRITIUM & TRITIUM_ELECT_LSC & USTEST & 61.2 \\
\hline 4931 & PDMS & & SW & & & E034456 & 01-Jul-86 & & $10045-97-3$ & CS-137 & GAMMA_GS & PNL-RO & 0.00392 \\
\hline 4932 & PDMS & & SW & & & E034456 & 01-Jul-86 & & 15046-84-1 & I-129 & I129_SEP_TEMS & 320 & 0.0000082 \\
\hline 4933 & PDMS & & SW & & & E034456 & 01-Jul-86 & & $10098-97-2$ & SR-90 & SRISO_SEEP_PRECIP_GPC & PNL-RO & 0.00832 \\
\hline 4934 & PDMS & & SW & & & E034456 & 01-Jul-86 & & PU-239/240 & PU-239/240 & PUISO_IE_AEAA & PNL-RO & 0.000106 \\
\hline 4935 & PDMS & & SW & & & E089484 & 01-Jul-86 & & $10045-97-3$ & CS-137 & GAMMA_GES & USTEST & 0 \\
\hline 4936 & PDMS & & SW & & & E089484 & 01-Jul-86 & & $10098-97-2$ & SR-90 & SRISO_SEPPPRECIP_GPC & USTEST & 0.107 \\
\hline \begin{tabular}{|l|l|}
4937 & $-2 \cdot r$ \\
\end{tabular} & PDMS & & SW & & & E089484 & 01-Jul-86 & & $\mathrm{U}-238$ & $\mathrm{U}-238$ & UISO_AEA & USTEST & 0.149 \\
\hline 4938 & PDMS & & SW & & & E089484 & 01-Jul-86 & & 15117-96-1 & U-235 & UISO_AEA & USTEST & 0.0106 \\
\hline 4939 & PDMS & & sW & & & E089484 & 01-Jul-86 & & $13966-29-5$ & U-234 & UISO_AEA & USTEST & 0.212 \\
\hline 4940 & PDMS & & sW & & & E089484 & 01-Jul-86 & & $10028-17-8$ & TRITIUM & TRITIUMM_ELECT_LSC & USTEST & 138 \\
\hline 4941 & PDMS & & SW & & & E089486 & 08-Jul-86 & & $10045-97-3$ & CS-137 & 901.1_GAMMA_GS & USTEST & -0.136 \\
\hline
\end{tabular}




\begin{tabular}{|c|c|c|c|c|c|c|c|c|c|c|c|c|c|}
\hline & $\bar{A}$ & $\mathrm{~B}$ & $\mathrm{C}$ & $\mathrm{D}$ & $E$ & $\mathrm{~F}$ & G & $\mathrm{H}$ & $\mathrm{I}$ & $\mathrm{J}$ & $\mathrm{K}$ & $\mathrm{L}$ & $\mathrm{M}$ \\
\hline 1 & SAMPLE_NUM_ASSIGNED_TO & SAMPLER & MEDIA & BIOTA_MEDIA & TAG_ID & SAMP_NUM & SAMP_DATE_TIME & SAMP_DATE_TIME_ON & CON_ID & CON_SHORT_NAME & METHOD_NAME & LAB_CODE & VALUE_RPTD \\
\hline 4942 & PDMS & & SW & & & E089486 & 08-Jul-86 & & $10098-97-2$ & SR-90 & 905.0_SR_GPC & USTEST & 0.0824 \\
\hline 4943 & PDMS & & SW & & & E089486 & 08-Jul-86 & & $\mathrm{U}-238$ & $\mathrm{U}-238$ & UISO_IE_PLATE_AEA & USTEST & 0.263 \\
\hline 4944 & PDMS & & SW & & & E089486 & 08-Jul-86 & & 15117-96-1 & U-235 & UISO_IE_PLATE_AEA & USTEST & 0.0056 \\
\hline 4945 & PDMS & & SW & & & E089486 & 08-Jul-86 & & $13966-29-5$ & $\mathrm{U}-234$ & UISO_IE_PLATE_AEA & USTEST & 0.334 \\
\hline 4946 & PDMS & & SW & & & E089486 & 08-Jul-86 & & $10028-17-8$ & TRITIUM & TRITIŪM_ELECT__LSC & USTEST & 162 \\
\hline 4947 & PDMS & & SW & & & E089839 & 15-Jul-86 & & $10045-97-3$ & CS-137 & GAMMA_GS & PNL-RO & 0.000187 \\
\hline 4948 & PDMS & & SW & & & E089844 & 15-Jul-86 & & $10045-97-3$ & CS-137 & GAMMA_GS & PNL-RO & 0.00411 \\
\hline 4949 & PDMS & & SW & & & E089848 & 15-Jul-86 & & $10045-97-3$ & CS-137 & GAMMA_GS & PNL-RO & 0.00234 \\
\hline 4950 & PDMS & & SW & & & E089853 & 15-Jul-86 & & $10045-97-3$ & CS-137 & GAMMA_GS & PNL-RO & 0.000561 \\
\hline 4951 & PDMS & & SW & & & E089655 & 21-Jul-86 & & $10045-97-3$ & CS-137 & 901.1_GAMMA_GS & USTEST & 0.401 \\
\hline 4952 & PDMS & & SW & & & E089655 & 21-Jul-86 & & 10028-17-8 & TRITIUM & 906.0_H3_LSC & USTEST & -52.7 \\
\hline 4953 & PDMS & & SW & & & E089655 & 21-Jul-86 & & $10098-97-2$ & SR-90 & 905.0_SR_GPC & USTEST & 0.0976 \\
\hline \begin{tabular}{|l|}
4954 \\
\end{tabular} & PDMS & & SW & & & E089654 & 21-Jul-86 & & $10045-97-3$ & CS-137 & 901.1_GAMMA_GS & USTEST & -0.114 \\
\hline \begin{tabular}{|l|}
4955 \\
\end{tabular} & PDMS & & SW & & & E089654 & 21-Jul-86 & & 10028-17-8 & TRITIUM & 906.0_H3_LSC & USTEST & 1740 \\
\hline \begin{tabular}{|l|}
4956 \\
\end{tabular} & PDMS & & SW & & & E089654 & 21-Jul-86 & & $10098-97-2$ & SR-90 & 905.0_SR_GPC & USTEST & 0.0923 \\
\hline \begin{tabular}{|l|}
4957 \\
\end{tabular} & PDMS & & SW & & & E089653 & 21-Jul-86 & & $10045-97-3$ & CS-137 & 901.1_GAMMA_GS & USTEST & 0.401 \\
\hline 4958 & PDMS & & SW & & & E089653 & 21-Jul-86 & & $10028-17-8$ & TRITIUM & 906.0_H3_LSC & USTEST & 28 \\
\hline 4959 & PDMS & & SW & & & E089653 & 21-Jul-86 & & $10098-97-2$ & SR-90 & 905.0_SR_GPC & USTEST & 0.032 \\
\hline 4960 & PDMS & & SW & & & E089656 & 22-Jul-86 & & $10045-97-3$ & CS-137 & 901.1_GAMMA_GS & USTEST & -1.55 \\
\hline \begin{tabular}{|l|}
4961 \\
\end{tabular} & PDMS & & SW & & & E089656 & 22-Jul-86 & & $10028-17-8$ & TRITIUM & 906.0_H3_LSC & USTEST & 10.8 \\
\hline \begin{tabular}{|l|}
4962 \\
\end{tabular} & PDMS & & SW & & & E089656 & 22-Jul-86 & & 10098-97-2 & SR-90 & 905.0_SR_GPC & USTEST & 0.0251 \\
\hline \begin{tabular}{|l|}
4963 \\
\end{tabular} & PDMS & & SW & & & E089724 & 29-Jul-86 & & 10098-97-2 & SR-90 & 905.0_SR_GPC & USTEST & 0.103 \\
\hline \begin{tabular}{|l|}
4964 \\
\end{tabular} & PDMS & & SW & & & E089724 & 29-Jul-86 & & $10045-97-3$ & CS-137 & 901.1_GAMMA_GS & USTEST & 0.316 \\
\hline \begin{tabular}{|l|}
4965 \\
\end{tabular} & PDMS & & SW & & & E089724 & 29-Jul-86 & & 10028-17-8 & TRITIUM & TRITIÜM_ELECT_LSC & USTEST & 90.1 \\
\hline \begin{tabular}{|l|}
4966 \\
\end{tabular} & PDMS & & SW & & & E089841 & 29-Jul-86 & & $10045-97-3$ & CS-137 & GAMMA_GS & PNL-RO & 0.00335 \\
\hline 4967 & PDMS & & SW & & & E089846 & 29-Jul-86 & & $10045-97-3$ & CS-137 & GAMMA_GS & PNL-RO & 0.00306 \\
\hline 4968 & PDMS & & SW & & & E089726 & 29-Jul-86 & & U-238 & U-238 & UISO_AEA & USTEST & 0.207 \\
\hline 4969 & PDMS & & SW & & & E089726 & 29-Jul-86 & & 15117-96-1 & U-235 & UISO_AEA & USTEST & 0.00655 \\
\hline 4970 & PDMS & & SW & & & E089726 & 29-Jul-86 & & $13966-29-5$ & U-234 & UISO_AEA & USTEST & 0.271 \\
\hline 4971 & PDMS & & SW & & & E089726 & 29-Jul-86 & & $10098-97-2$ & SR-90 & SRISO_SEP_PRECIP_GPC & USTEST & 0.132 \\
\hline 4972 & PDMS & & SW & & & E089726 & 29-Jul-86 & & $10045-97-3$ & CS-137 & GAMMA_GS & USTEST & 0.242 \\
\hline 4973 & PDMS & & SW & & & E089726 & 29-Jul-86 & & $10028-17-8$ & TRITIUM & TRITIUM_ELECT_LSC & USTEST & 61.2 \\
\hline 4974 & PDMS & & SW & & & E089850 & 29-Jul-86 & & $10045-97-3$ & CS-137 & GAMMA_GS & PNL-RO & 0.00934 \\
\hline 4975 & PDMS & & SW & & & E089854 & 29-Jul-86 & & $10045-97-3$ & CS-137 & GAMMA_GS & PNL-RO & 0.00316 \\
\hline \begin{tabular}{|l|}
4976 \\
\end{tabular} & PDMS & & SW & & & E089725 & 29-Jul-86 & & $\mathrm{U}-238$ & U-238 & UISO_AEA & USTEST & 0.223 \\
\hline 4977 & PDMS & & SW & & & E089725 & 29-Jul-86 & & 15117-96-1 & U-235 & UISO_AEA & USTEST & 0.00841 \\
\hline \begin{tabular}{|l|}
4978 \\
\end{tabular} & PDMS & & SW & & & E089725 & 29-Jul-86 & & $13966-29-5$ & U-234 & UISO_AEA & USTEST & 0.282 \\
\hline 4979 & PDMS & & SW & & & E089725 & 29-Jul-86 & & $10098-97-2$ & SR-90 & SRISO_SEP_PRECIP_GPC & USTEST & 0.14 \\
\hline 4980 & PDMS & & SW & & & E089725 & 29-Jul-86 & & $10045-97-3$ & CS-137 & GAMMA_GS & USTEST & 0.551 \\
\hline 4981 & PDMS & & SW & & & E089725 & 29-Jul-86 & & $10028-17-8$ & TRITIUM & TRITIUM_ELECT_LSC & USTEST & 153 \\
\hline 4982 & PDMS & & SW & & & E089847 & 12-Aug-86 & & $10045-97-3$ & CS-137 & GAMMA_GS & PNL-RO & 0.00297 \\
\hline 4983 & PDMS & & SW & & & E089842 & 12-Aug-86 & & $10045-97-3$ & CS-137 & GAMMA_GS & PNL-RO & 0.00199 \\
\hline 4984 & PDMS & & SW & & & E089852 & 12-Aug-86 & & $10045-97-3$ & CS-137 & GAMMA_GS & PNL-RO & 0.0133 \\
\hline 4985 & PDMS & & SW & & & E089856 & 12-Aug-86 & & $10045-97-3$ & CS-137 & GAMMA_GS & PNL-RO & 0.0025 \\
\hline 4986 & PDMS & & SW & & & E089857 & 19-Aug-86 & & $10045-97-3$ & CS-137 & GAMMA_GS & USTEST & 1.9 \\
\hline 4987 & PDMS & & SW & & & E089857 & 19-Aug-86 & & $10098-97-2$ & SR-90 & SRISO_SEP_PRECIP_GPC & USTEST & -0.568 \\
\hline 4988 & PDMS & & SW & & & E089857 & 19-Aug-86 & & $10028-17-8$ & TRITIUM & TRITIUM_DIST_LSC & USTEST & 12.9 \\
\hline 4989 & PDMS & & SW & & & E089861 & 19-Aug-86 & & $10045-97-3$ & CS-137 & GAMMA_GS & USTEST & 2.5 \\
\hline 4990 & PDMS & & SW & & & E089861 & 19-Aug-86 & & $10028-17-8$ & TRITIUM & TRITIUM_DIST_LSC & USTEST & 659 \\
\hline 4991 & PDMS & & SW & & & E089858 & 19-Aug-86 & & $10045-97-3$ & CS-137 & GAMMA_GS & USTEST & 12 \\
\hline 4992 & PDMS & & SW & & & E089858 & 19-Aug-86 & & $10098-97-2$ & SR-90 & SRISO_SEP_PRECIP_GPC & USTEST & 4.35 \\
\hline 4993 & PDMS & & SW & & & E089858 & 19-Aug-86 & & $10028-17-8$ & TRITIUM & TRITIUM_DISTT_LSC & USTEST & 203 \\
\hline
\end{tabular}




\begin{tabular}{|c|c|c|c|c|c|c|c|c|c|c|c|c|c|}
\hline & $\bar{A}$ & $\mathrm{~B}$ & $\mathrm{C}$ & $\bar{D}$ & $\mathrm{E}$ & $\mathrm{F}$ & $\bar{G}$ & $\mathrm{H}$ & 1 & $\mathrm{~J}$ & $\mathrm{~K}$ & $\mathrm{~L}$ & $\bar{M}$ \\
\hline 1 & SAMPLE_NUM_ASSIGNED_TO & SAMPLER & MEDIA & BIOTA_MEDIA & TAG_ID & SAMP_NUM & SAMP_DATE_TIME & SAMP_DATE_TIME_ON & CON_ID & \begin{tabular}{|l|} 
CON_SHORT_NAME \\
\end{tabular} & METHOD_NAME & LAB_CODE & VALUE_RPTD \\
\hline 4994 & PDMS & & SW & & & E089859 & 19-Aug-86 & & $10045-97-3$ & CS-137 & GAMMA_GS & USTEST & 2.08 \\
\hline 4995 & PDMS & & sW & & & E089859 & 19-Aug-86 & & $10098-97-2$ & SR-90 & SRISO_SEEP_PRECIP_GPC & USTEST & 2.4 \\
\hline \begin{tabular}{|l|l|}
4996 \\
\end{tabular} & PDMS & & SW & & & E089859 & 19-Aug-86 & & $10028-17-8$ & TRITIUM & TRITIUM_DIST_LSC & USTEST & 526 \\
\hline \begin{tabular}{|l|l|}
4997 \\
\end{tabular} & PDMS & & SW & & & E090225 & 26-Aug-86 & & $10098-97-2$ & SR-90 & 905.0_SR_GPC & USTEST & 0.0986 \\
\hline 4998 & PDMS & & SW & & & E090225 & 26-Aug-86 & & $10045-97-3$ & CS-137 & 901.1_GAMMA_GS & USTEST & 0.4 \\
\hline \begin{tabular}{|l|l|}
4999 \\
\end{tabular} & PDMS & & SW & & & E090225 & 26-Aug-86 & & $10028-17-8$ & TRITIUM & 906.0_H3_LSC & USTEST & -76.2 \\
\hline 5000 & PDMS & & sW & & & E090910 & 26-Aug-86 & & $10045-97-3$ & CS-137 & GAMM̄A_GS & PNL-RO & 0.000972 \\
\hline 5001 & PDMS & & sW & & & E090905 & 26-Aug-86 & & $10045-97-3$ & CS-137 & GAMMA_GS & PNL-RO & 0.00267 \\
\hline 5002 & PDMS & & sW & & & E090915 & 26-Aug-86 & & $10045-97-3$ & CS-137 & GAMMA_GS & PNL-RO & -0.000261 \\
\hline 5003 & PDMS & & SW & & & E090920 & 26-Aug-86 & & $10045-97-3$ & CS-137 & GAMMA_GS & PNL-RO & 0.0011 \\
\hline 5004 & PDMS & & SW & & & E090224 & 27-Aug-86 & & $10098-97-2$ & SR-90 & SRISO_SEPP_PRECIP_GPC & USTEST & 0.189 \\
\hline 5005 & PDMS & & SW & & & E090224 & 27-Aug-86 & & $10045-97-3$ & CS-137 & GAMMA_GS & USTEST & 0.229 \\
\hline 5006 & PDMS & & SW & & & E090226 & 29-Aug-86 & & $10098-97-2$ & SR-90 & 905.0_SR_GPC & USTEST & 0.634 \\
\hline 5007 & PDMS & & SW & & & E090226 & 29-Aug-86 & & $10045-97-3$ & CS-137 & 901.1_GAMMA_GS & USTEST & 0.723 \\
\hline 5008 & PDMS & & SW & & & E090226 & 29-Aug-86 & & $10028-17-8$ & TRITIUM & TRITIUM_ELECT_LSC & USTEST & 162 \\
\hline 5009 & PDMS & & SW & & & E090280 & 02-Sep-86 & & $10098-97-2$ & SR-90 & SRISO_SEP_PRECIP_GPC & USTEST & 0.154 \\
\hline 5010 & PDMS & & SW & & & E090280 & 02-Sep-86 & & U-238 & U-238 & UISO_AEA & USTEST & 0.174 \\
\hline 5011 & PDMS & & sW & & & E090280 & 02-Sep-86 & & 15117-96-1 & U-235 & UISO_AEA & USTEST & 0.00392 \\
\hline 5012 & PDMS & & sW & & & E090280 & 02-Sep-86 & & $13966-29-5$ & U-234 & UISO_AEA & USTEST & 0.221 \\
\hline 5013 & PDMS & & sW & & & E090280 & 02-Sep-86 & & $10045-97-3$ & CS-137 & GAMMA_GS & USTEST & 0.138 \\
\hline 5014 & PDMS & & sW & & & E090280 & 02-Sep-86 & & $10028-17-8$ & TRITIUM & TRITIUM_ELEECT_LSC & USTEST & 106 \\
\hline 5015 & PDMS & & SW & & & E090279 & 02-Sep-86 & & $10098-97-2$ & SR-90 & SRISO_SËP_PRËCIP_GPC & USTEST & 0.176 \\
\hline 5016 & PDMS & & SW & & & E090279 & 02-Sep-86 & & U-238 & U-238 & UISO_AEA & USTEST & 0.218 \\
\hline 5017 & PDMS & & SW & & & E090279 & 02-Sep-86 & & $15117-96-1$ & U-235 & UISO_AEA & USTEST & 0 \\
\hline 5018 & PDMS & & SW & & & E090279 & 02-Sep-86 & & $13966-29-5$ & $\mathrm{U}-234$ & UISO_AEA & USTEST & 0.257 \\
\hline 5019 & PDMS & & SW & & & E090279 & 02-Sep-86 & & $10045-97-3$ & CS-137 & GAMMA_GS & USTEST & 0.631 \\
\hline 5020 & PDMS & & SW & & & E090279 & 02-Sep-86 & & $10028-17-8$ & TRITIUM & TRITIUM_ELECT_LSC & USTEST & 159 \\
\hline 5021 & PDMS & & SW & & & E090906 & 09-Sep-86 & & $10045-97-3$ & CS-137 & GAMMA_GS & PNL-RO & -0.00527 \\
\hline 5022 & PDMS & & SW & & & E090911 & 09-Sep-86 & & $10045-97-3$ & CS-137 & GAMMA_GS & PNL-RO & -0.000579 \\
\hline 5023 & PDMS & & SW & & & E090916 & 09-Sep-86 & & $10045-97-3$ & CS-137 & GAMMA_GS & PNL-RO & -0.00821 \\
\hline 5024 & PDMS & & SW & & & E090921 & 09-Sep-86 & & $10045-97-3$ & CS-137 & GAMMA_GS & PNL-RO & 0.00247 \\
\hline 5025 & PDMS & & SW & & & E090907 & 23-Sep-86 & & $10045-97-3$ & CS-137 & GAMMA_GS & PNL-RO & -0.00639 \\
\hline 5026 & PDMS & & sW & & & E090912 & 23-Sep-86 & & $10045-97-3$ & CS-137 & GAMMA_GS & PNL-RO & 0.000343 \\
\hline 5027 & PDMS & & SW & & & E090917 & 23-Sep-86 & & $10045-97-3$ & CS-137 & GAMMA_GS & PNL-RO & -0.000728 \\
\hline 5028 & PDMS & & SW & & & E090922 & 23-Sep-86 & & $10045-97-3$ & CS-137 & GAMMA_GS & PNL-RO & 0.000392 \\
\hline 5029 & PDMS & & SW & & & E090553 & 30-Sep-86 & & $10045-97-3$ & CS-137 & 901.1_GAMMA_GS & USTEST & 0.402 \\
\hline 5030 & PDMS & & SW & & & E090553 & 30-Sep-86 & & U-238 & $\mathrm{U}-238$ & UISO_IE_PLATE_AEA & USTEST & 0.157 \\
\hline 5031 & PDMS & & sW & & & E090553 & $30-S e p-86$ & & 15117-96-1 & U-235 & UISO_IE_PLATE_AEA & USTEST & 0.0216 \\
\hline 5032 & PDMS & & SW & & & E090553 & 30-Sep-86 & & 13966-29-5 & $\mathrm{U}-234$ & UISO_IE_PLATE_AEA & USTEST & 0.225 \\
\hline 5033 & PDMS & & SW & & & E090553 & 30-Sep-86 & & $10028-17-8$ & TRITIUM & TRITIUM_ELECT_LSC & USTEST & 251 \\
\hline 5034 & PDMS & & SW & & & E090553 & $30-S e p-86$ & & 10098-97-2 & SR-90 & 905.0_SR_GPC & USTEST & 0.134 \\
\hline 5035 & PDMS & & SW & & & E090557 & 30-Sep-86 & & $10045-97-3$ & CS-137 & GAMMA_GS & USTEST & -0.781 \\
\hline 5036 & PDMS & & SW & & & E090557 & 30-Sep-86 & & U-238 & $\mathrm{U}-238$ & UISO_AEA & USTEST & 0.264 \\
\hline 5037 & PDMS & & SW & & & E090557 & 30-Sep-86 & & $15117-96-1$ & U-235 & UISO_AEA & USTEST & 0.0114 \\
\hline 5038 & PDMS & & SW & & & E090557 & 30-Sep-86 & & 13966-29-5 & $\mathrm{U}-234$ & UISO_AEA & USTEST & 0.395 \\
\hline 5039 & PDMS & & SW & & & E090557 & 30-Sep-86 & & $10098-97-2$ & SR-90 & SRISO_SEP_PRECIP_GPC & USTEST & 0.143 \\
\hline 5040 & PDMS & & SW & & & E090557 & $30-$ Sep-86 & & $10028-17-8$ & TRITIUM & TRITIUM_ELECT_LSC & USTEST & 89.8 \\
\hline 5041 & PDMS & & SW & & & E090556 & 30-Sep-86 & & $10045-97-3$ & CS-137 & GAMMA_GS & USTEST & 0.158 \\
\hline 5042 & PDMS & & SW & & & E090556 & 30-Sep-86 & & U-238 & $\mathrm{U}-238$ & UISO_AEA & USTEST & 0.246 \\
\hline 5043 & PDMS & & SW & & & E090556 & 30-Sep-86 & & 15117-96-1 & U-235 & UISO_AEA & USTEST & 0.01 \\
\hline 5044 & PDMS & & SW & & & E090556 & 30-Sep-86 & & $13966-29-5$ & $\mathrm{U}-234$ & UISO_AEA & USTEST & 0.324 \\
\hline $5045 \mid \mathrm{F}$ & PDMS & & SW & & & E090556 & 30-Sep-86 & & $10098-97-2$ & SR-90 & SRISO_SEP_PRECIP_GPC & USTEST & 0.154 \\
\hline
\end{tabular}




\begin{tabular}{|c|c|c|c|c|c|c|c|c|c|c|c|c|c|}
\hline & A & $\mathrm{B}$ & $\mathrm{C}$ & $\mathrm{D}$ & $E$ & $\mathrm{~F}$ & $\mathrm{G}$ & $\mathrm{H}$ & $\mathrm{I}$ & $\mathrm{J}$ & $\mathrm{K}$ & $\mathrm{L}$ & $\mathrm{M}$ \\
\hline 1 & SAMPLE_NUM_ASSIGNED_TO & SAMPLER & MEDIA & BIOTA_MEDIA & TAG_ID & SAMP_NUM & SAMP_DATE_TIME & SAMP_DATE_TIME_ON & CON_ID & CON_SHORT_NAME & METHOD_NAME & LAB_CODE & VALUE_RPTD \\
\hline 5046 & PDMS & & SW & & & E090556 & 30-Sep-86 & & $10028-17-8$ & TRITIUM & TRITIUM_ELECT_LSC & USTEST & 154 \\
\hline 5047 & PDMS & & SW & & & E090908 & $07-$ Oct-86 & & $10045-97-3$ & CS-137 & GAMMA_GS & PNL-RO & 0.000403 \\
\hline 5048 & PDMS & & SW & & & E090913 & $07-$ Oct-86 & & $10045-97-3$ & CS-137 & GAMMA_GS & PNL-RO & 0.00144 \\
\hline 5049 & PDMS & & SW & & & E090908 & $07-$ Oct-86 & & 15046-84-1 & I-129 & I129_SEP_TEMS & 320 & 0.000161 \\
\hline 5050 & PDMS & & SW & & & E090908 & $07-O c t-86$ & & $10098-97-2$ & SR-90 & SRISO_SEP_PRECIP_GPC & PNL-RO & 0.0027 \\
\hline 5051 & PDMS & & SW & & & E090908 & $07-$ Oct-86 & & PU-239/240 & PU-239/240 & PUISO_IE_AEA & PNL-RO & 0.000277 \\
\hline 5052 & PDMS & & SW & & & E090913 & $07-$ Oct-86 & & PU-239/240 & PU-239/240 & PUISO_IE_AEA & PNL-RO & 0.0000129 \\
\hline 5053 & PDMS & & SW & & & E090918 & $07-$ Oct-86 & & $10045-97-3$ & CS-137 & GAMMA_GS & PNL-RO & -0.00211 \\
\hline 5054 & PDMS & & SW & & & E090923 & 07-Oct-86 & & $10045-97-3$ & CS-137 & GAMMA_GS & PNL-RO & -0.002 \\
\hline 5055 & PDMS & & SW & & & E090918 & 07-Oct-86 & & 15046-84-1 & I-129 & I129_SEP_TEMS & 320 & 0.0000104 \\
\hline 5056 & PDMS & & SW & & & E090918 & $07-$ Oct-86 & & $10098-97-2$ & SR-90 & SRISO_SEP_PRECIP_GPC & PNL-RO & 0.00557 \\
\hline 5057 & PDMS & & SW & & & E090918 & $07-$ Oct-86 & & PU-239/240 & PU-239/240 & PUISO_IE_AEA & PNL-RO & 0.0000159 \\
\hline 5058 & PDMS & & SW & & & E090923 & $07-$ Oct-86 & & PU-239/240 & PU-239/240 & PUISO_IE_AEA & PNL-RO & 0.0000244 \\
\hline 5059 & PDMS & & SW & & & E090753 & 13-Oct-86 & & $10045-97-3$ & CS-137 & 901.1_GAMMA_GS & USTEST & 0.242 \\
\hline 5060 & PDMS & & SW & & & E090753 & 13-Oct-86 & & $10028-17-8$ & TRITIUM & 906.0_H3_LSC & USTEST & -30 \\
\hline 5061 & PDMS & & SW & & & E090753 & 13-Oct-86 & & $10098-97-2$ & SR-90 & 905.0_SR_GPC & USTEST & 0.071 \\
\hline 5062 & PDMS & & SW & & & E090752 & 13-Oct-86 & & $10045-97-3$ & CS-137 & 901.1_GAMMA_GS & USTEST & -0.505 \\
\hline 5063 & PDMS & & SW & & & E090752 & 13-Oct-86 & & $10028-17-8$ & TRITIUM & 906.0_H3_LSC & USTEST & 6080 \\
\hline 5064 & PDMS & & SW & & & E090752 & 13-Oct-86 & & $10098-97-2$ & SR-90 & 905.0_SR_GPC & USTEST & 0.0925 \\
\hline 5065 & PDMS & & SW & & & E090751 & 13-Oct-86 & & $10045-97-3$ & CS-137 & 901.1_GAMMA_GS & USTEST & 0.172 \\
\hline 5066 & PDMS & & SW & & & E090751 & 13-Oct-86 & & $10028-17-8$ & TRITIUM & 906.0_H3_LSC & USTEST & -101 \\
\hline 5067 & PDMS & & SW & & & E090751 & 13-Oct-86 & & $10098-97-2$ & SR-90 & 905.0_SR_GPC & USTEST & 0.0635 \\
\hline 5068 & PDMS & & SW & & & E090754 & 13-Oct-86 & & $10045-97-3$ & CS-137 & 901.1_GAMMA_GS & USTEST & 0.734 \\
\hline 5069 & PDMS & & SW & & & E090754 & 13-Oct-86 & & $10028-17-8$ & TRITIUM & 906.0_H3_LSC & USTEST & -162 \\
\hline 5070 & PDMS & & SW & & & E090754 & 13-Oct-86 & & $10098-97-2$ & SR-90 & 905.0_SR_GPC & USTEST & 0.0239 \\
\hline 5071 & PDMS & & SW & & & E090860 & 21-Oct-86 & & $10045-97-3$ & CS-137 & 901.1_GAMMA_GS & USTEST & 0.286 \\
\hline 5072 & PDMS & & SW & & & E090860 & 21-Oct-86 & & $10098-97-2$ & SR-90 & 905.0_SR_GPC & USTEST & 0.148 \\
\hline 5073 & PDMS & & SW & & & E090860 & 21-Oct-86 & & $10028-17-8$ & TRITIUM & TRITIUM_ELECT_LSC & USTEST & 103 \\
\hline 5074 & PDMS & & SW & & & E090862 & 21-Oct-86 & & $10028-17-8$ & TRITIUM & TRITIUM_ELECT_LSC & USTEST & 120 \\
\hline 5075 & PDMS & & SW & & & E090862 & 21-Oct-86 & & $10045-97-3$ & CS-137 & GAMMA_GS & USTEST & 0.0452 \\
\hline 5076 & PDMS & & SW & & & E090862 & 21-Oct-86 & & $13966-29-5$ & $\mathrm{U}-234$ & UISO_AEA & USTEST & 0.305 \\
\hline 5077 & PDMS & & SW & & & E090862 & 21-Oct-86 & & $15117-96-1$ & U-235 & UISO_AEA & USTEST & 0.017 \\
\hline 5078 & PDMS & & SW & & & E090862 & 21-Oct-86 & & $\mathrm{U}-238$ & $\mathrm{U}-238$ & UISO_AEA & USTEST & 0.25 \\
\hline 5079 & PDMS & & SW & & & E090862 & 21-Oct-86 & & $10098-97-2$ & SR-90 & SRISO_SEP_PRECIP_GPC & USTEST & 0.138 \\
\hline 5080 & PDMS & & SW & & & E090861 & 21-Oct-86 & & $10028-17-8$ & TRITIUM & TRITIUM_ELECT_LSC & USTEST & 120 \\
\hline 5081 & PDMS & & SW & & & E090861 & 21-Oct-86 & & $10045-97-3$ & CS-137 & GAMMA_GS & USTEST & -0.688 \\
\hline 5082 & PDMS & & SW & & & E090861 & 21-Oct-86 & & $13966-29-5$ & $\mathrm{U}-234$ & UISO_AEA & USTEST & 0.332 \\
\hline 5083 & PDMS & & SW & & & E090861 & 21-Oct-86 & & $15117-96-1$ & U-235 & UISO_AEA & USTEST & 0.0138 \\
\hline 5084 & PDMS & & SW & & & E090861 & 21-Oct-86 & & $\mathrm{U}-238$ & U-238 & UISO_AEA & USTEST & 0.236 \\
\hline 5085 & PDMS & & SW & & & E090861 & 21-Oct-86 & & $10098-97-2$ & SR-90 & SRISO_SEP_PRECIP_GPC & USTEST & 0.139 \\
\hline 5086 & PDMS & & SW & & & E090909 & 21-Oct-86 & & $10045-97-3$ & CS-137 & GAMMA_GS & PNL-RO & -0.00327 \\
\hline 5087 & PDMS & & SW & & & E090914 & 21-Oct-86 & & $10045-97-3$ & CS-137 & GAMMA_GS & PNL-RO & -0.0028 \\
\hline 5088 & PDMS & & SW & & & E090924 & 21-Oct-86 & & $10045-97-3$ & CS-137 & GAMMA_GS & PNL-RO & -0.00337 \\
\hline 5089 & PDMS & & SW & & & E090919 & 21-Oct-86 & & $10045-97-3$ & CS-137 & GAMMA_GS & PNL-RO & -0.00681 \\
\hline 5090 & PDMS & & SW & & & E091020 & 28-Oct-86 & & $10098-97-2$ & SR-90 & SRISO_SEP_PRECIP_GPC & USTEST & 0.137 \\
\hline 5091 & PDMS & & SW & & & E091020 & 28-Oct-86 & & $\mathrm{U}-238$ & U-238 & UISO_AEA & USTEST & 0.237 \\
\hline 5092 & PDMS & & SW & & & E091020 & $28-O c t-86$ & & $15117-96-1$ & U-235 & UISO_AEA & USTEST & -0.00676 \\
\hline 5093 & PDMS & & SW & & & E091020 & $28-O c t-86$ & & $13966-29-5$ & U-234 & UISO_AEA & USTEST & 0.21 \\
\hline 5094 & PDMS & & SW & & & E091020 & $28-O c t-86$ & & $10045-97-3$ & CS-137 & GAMMA_GS & USTEST & 0.505 \\
\hline 5095 & PDMS & & SW & & & E091020 & 28-Oct-86 & & $10028-17-8$ & TRITIUM & TRITIUM_ELECT_LSC & USTEST & 116 \\
\hline 5096 & PDMS & & SW & & & E091013 & 28-Oct-86 & & $10098-97-2$ & SR-90 & SRISO_SEP_PRECIP_GPC & USTEST & 0.149 \\
\hline 5097 & PDMS & & SW & & & E091013 & 28-Oct-86 & & $\mathrm{U}-238$ & $\mathrm{U}-238$ & UISO_AEA & USTEST & 0.246 \\
\hline
\end{tabular}




\begin{tabular}{|c|c|c|c|c|c|c|c|c|c|c|c|c|c|}
\hline & A & $\mathrm{B}$ & $\mathrm{C}$ & $\mathrm{D}$ & $\mathrm{E}$ & $\mathrm{F}$ & $\mathrm{G}$ & $\mathrm{H}$ & $\mathrm{I}$ & $\mathrm{J}$ & $\mathrm{K}$ & $\mathrm{L}$ & $\mathrm{M}$ \\
\hline 1 & SAMPLE_NUM_ASSIGNED_TO & \begin{tabular}{|l|} 
SAMPLER \\
\end{tabular} & MEDIA & BIOTA_MEDIA & TAG_ID & SAMP_NUM & SAMP_DATE_TIME & SAMP_DATE_TIME_ON & CON_ID & CON_SHORT_NAME & METHOD_NAME & LAB_CODE & VALUE_RPTD \\
\hline \begin{tabular}{|c|}
5098 \\
\end{tabular} & PDMS & & SW & & & E091013 & $28-\mathrm{Oct}-86$ & & $15117-96-1$ & $\mathrm{U}-235$ & UISO AEA & USTEST & 0.00517 \\
\hline 5099 & PDMS & & sw & & & E091013 & 28-Oct-86 & & 13966-29-5 & U-234 & UISO_AEA & USTEST & 0.255 \\
\hline 5100 & PDMS & & sw & & & E091013 & 28-Oct-86 & & $10045-97-3$ & CS-137 & GAMMA_GS & USTEST & 0.0485 \\
\hline 5101 & PDMS & & sw & & & E091013 & 28-Oct-86 & & $10028-17-8$ & TRITIUM & TRITIUM_ELECT_LSC & USTEST & 148 \\
\hline \begin{tabular}{|l|l|}
5102 \\
\end{tabular} & PDMS & & sw & & & E090144 & 04-Nov-86 & & $10045-97-3$ & CS-137 & GAMMA_GS & PNL-RO & -0.000361 \\
\hline 5103 & PDMS & & SW & & & E090147 & 04-Nov-86 & & $10045-97-3$ & CS-137 & GAMMA_GS & PNL-RO & -0.00292 \\
\hline 5104 & PDMS & & sW & & & E090149 & 04-Nov-86 & & $10045-97-3$ & CS-137 & GAMMA_GS & PNL-RO & 0.00193 \\
\hline \begin{tabular}{|l|}
5105 \\
\end{tabular} & PDMS & & SW & & & E090153 & 04-Nov-86 & & $10045-97-3$ & CS-137 & GAMMA_GS & PNL-RO & -0.00235 \\
\hline 5106 & PDMS & & sw & & & E091110 & 11-Nov-86 & & $10028-17-8$ & TRITIUM & TRITIUM_DIST_LSC & USTEST & 49.9 \\
\hline \begin{tabular}{|l|l|}
5107 \\
\end{tabular} & PDMS & & SW & & & E091110 & 11-Nov-86 & & 10098-97-2 & SR-90 & SRISO_SEP_PRECIP_GPC & USTEST & 0.458 \\
\hline 5108 & PDMS & & sw & & & E091110 & 11-Nov-86 & & $10045-97-3$ & CS-137 & GAMMA_GS & USTEST & 1.07 \\
\hline 5109 & PDMS & & sW & & & E091115 & 11-Nov-86 & & $10028-17-8$ & TRITIUM & TRITIUM_DIST_LSC & USTEST & 1790 \\
\hline 5110 & PDMS & & sW & & & E091115 & 11-Nov-86 & & $10045-97-3$ & CS-137 & GAMMA_GS & USTEST & 1.03 \\
\hline 5111 & PDMS & & sW & & & E091113 & 11-Nov-86 & & $10028-17-8$ & TRITIUM & TRITIUM_DIST_LSC & USTEST & 11.3 \\
\hline 5112 & PDMS & & sw & & & E091113 & 11-Nov-86 & & $10098-97-2$ & SR-90 & SRISO_SEP_PRECIP_GPC & USTEST & 2.59 \\
\hline 5113 & PDMS & & sw & & & E091113 & 11-Nov-86 & & $10045-97-3$ & CS-137 & GAMMA_GS & USTEST & 2.48 \\
\hline 5114 & PDMS & & sw & & & E091114 & 11-Nov-86 & & U-238 & U-238 & UISO_AEA & USTEST & 97.8 \\
\hline 5115 & PDMS & & sW & & & E091114 & 11-Nov-86 & & 15117-96-1 & U-235 & UISO_AEA & USTEST & 3.7 \\
\hline 5116 & PDMS & & sW & & & E091114 & 11-Nov-86 & & $13966-29-5$ & U-234 & UISO_AEA & USTEST & 105 \\
\hline 5117 & PDMS & & sW & & & E091114 & 11-Nov-86 & & $10028-17-8$ & TRITIUM & TRITIŪM_DIST_LSC & USTEST & 612 \\
\hline 5118 & PDMS & & sw & & & E091114 & 11-Nov-86 & & $10098-97-2$ & SR-90 & SRISO_SEP_PRECIP_GPC & USTEST & 2.06 \\
\hline 5119 & PDMS & & sW & & & E091114 & 11-Nov-86 & & $10045-97-3$ & CS-137 & GAMMA_GS & USTEST & 0.755 \\
\hline 5120 & PDMS & & SW & & & E091118 & 18-Nov-86 & & $10028-17-8$ & TRITIUM & 906.0_H3_LSC & USTEST & -15.6 \\
\hline 5121 & PDMS & & sW & & & E091118 & 18-Nov-86 & & $10045-97-3$ & CS-137 & 901.1_GAMMA_GS & USTEST & 0.0452 \\
\hline 5122 & PDMS & & SW & & & E091118 & 18-Nov-86 & & $10098-97-2$ & SR-90 & 905.0_SR_GPC & USTEST & 0.0319 \\
\hline 5123 & PDMS & & sW & & & E090145 & 18-Nov-86 & & $10045-97-3$ & CS-137 & GAMM̄A_GSS & PNL-RO & -0.0156 \\
\hline 5124 & PDMS & & sW & & & E090148 & 18-Nov-86 & & $10045-97-3$ & CS-137 & GAMMA_GS & PNL-RO & -0.00826 \\
\hline 5125 & PDMS & & sW & & & E090150 & 18-Nov-86 & & $10045-97-3$ & CS-137 & GAMMA_GS & PNL-RO & 0.00805 \\
\hline 5126 & PDMS & & sw & & & E091116 & 18-Nov-86 & & $10045-97-3$ & CS-137 & GAMMA_GS & PNL-RO & -0.0103 \\
\hline 5127 & PDMS & & sw & & & E091112 & 19-Nov-86 & & $10098-97-2$ & SR-90 & SRISO_SEP_PRECIP_GPC & USTEST & \\
\hline 5128 & PDMS & & sW & & & E091112 & 19-Nov-86 & & $10045-97-3$ & CS-137 & GAMMA_GS & USTEST & \\
\hline 5129 & PDMS & & sW & & & E091119 & 21-Nov-86 & & $10045-97-3$ & CS-137 & 901.1_GAMMMA_GS & USTEST & -0.893 \\
\hline 5130 & PDMS & & sW & & & E091119 & 21-Nov-86 & & $10098-97-2$ & SR-90 & 905.0_SR_GPC & USTEST & 0.859 \\
\hline 5131 & PDMS & & SW & & & E091119 & 21-Nov-86 & & $10028-17-8$ & TRITIUM & TRITIŪM_ELEECT_LSC & USTEST & 166 \\
\hline 5132 & PDMS & & sW & & & E091437 & 02-Dec-86 & & $10045-97-3$ & CS-137 & GAMMA_GS & PNL-RO & -0.000321 \\
\hline 5133 & PDMS & & sw & & & E091434 & 02-Dec-86 & & $10045-97-3$ & CS-137 & GAMMA_GS & PNL-RO & -0.00112 \\
\hline 5134 & PDMS & & SW & & & E091441 & 02-Dec-86 & & $10045-97-3$ & CS-137 & GAMMA_GS & PNL-RO & -0.00431 \\
\hline 5135 & PDMS & & sW & & & E091446 & 02-Dec-86 & & $10045-97-3$ & CS-137 & GAMMA_GS & PNL-RO & -0.000413 \\
\hline 5136 & PDMS & & sW & & & E091232 & 02-Dec-86 & & $10045-97-3$ & CS-137 & GAMMA_GS & USTEST & 0.172 \\
\hline $5137 \mathrm{~F}$ & PDMS & & SW & & & E091232 & 02-Dec-86 & & $10098-97-2$ & SR-90 & SRISO_SEEP_PRECIP_GPC & USTEST & 0.145 \\
\hline 5138 & PDMS & & sW & & & E091232 & 02-Dec-86 & & $\mathrm{U}-238$ & $\mathrm{U}-238$ & UISO_AEAA & USTEST & 0.187 \\
\hline 5139 & PDMS & & sW & & & E091232 & 02-Dec-86 & & 15117-96-1 & U-235 & UISO_AEA & USTEST & 0.00585 \\
\hline 5140 & PDMS & & SW & & & E091232 & 02-Dec-86 & & $13966-29-5$ & $\mathrm{U}-234$ & UISO_AEA & USTEST & 0.259 \\
\hline 5141 & PDMS & & sW & & & E091232 & 02-Dec-86 & & $10028-17-8$ & TRITIUM & TRITIÜM_ELECT_LSC & USTEST & 104 \\
\hline 5142 & PDMS & & SW & & & E091231 & 02-Dec-86 & & $10045-97-3$ & CS-137 & GAMMA_GS & USTEST & 0.172 \\
\hline 5143 & PDMS & & SW & & & E091231 & 02-Dec-86 & & $\mathrm{U}-238$ & $\mathrm{U}-238$ & 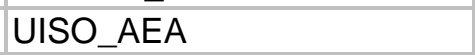 & USTEST & 0.2 \\
\hline $5144 \mathrm{~F}$ & PDMS & & sW & & & E091231 & 02-Dec-86 & & 15117-96-1 & U-235 & UISO_AEA & USTEST & 0.00196 \\
\hline 5145 & PDMS & & sW & & & E091231 & 02-Dec-86 & & $13966-29-5$ & $\mathrm{U}-234$ & UISO_AEA & USTEST & 0.269 \\
\hline 5146 & PDMS & & sw & & & E091231 & 02-Dec-86 & & $10098-97-2$ & SR-90 & SRISO_SEP_PRECIP_GPC & USTEST & 0.188 \\
\hline 5147 & PDMS & & SW & & & E091231 & 02-Dec-86 & & $10028-17-8$ & TRITIUM & TRITIUM_ELECT_LSC & USTEST & 134 \\
\hline 5148 & PDMS & & SW & & & E091435 & 16-Dec-86 & & $10045-97-3$ & CS-137 & GAMMA_GS & PNL-RO & 0.00139 \\
\hline 5149 & PDMS & & SW & & & E091438 & 16-Dec-86 & & $10045-97-3$ & CS-137 & GAMMA_GS & PNL-RO & -0.00189 \\
\hline
\end{tabular}




\begin{tabular}{|c|c|c|c|c|c|c|c|c|c|c|c|c|c|}
\hline & $\bar{A}$ & $\mathrm{~B}$ & $\mathrm{C}$ & $\bar{D}$ & $\mathrm{E}$ & $\mathrm{F}$ & $\bar{G}$ & $\mathrm{H}$ & 1 & $\mathrm{~J}$ & $\mathrm{~K}$ & $\mathrm{~L}$ & $\bar{M}$ \\
\hline 1 & SAMPLE_NUM_ASSIGNED_TO & SAMPLER & MEDIA & BIOTA_MEDIA & TAG_ID & SAMP_NUM & SAMP_DATE_TIME| & SAMP_DATE_TIME_ON & CON_ID & CON_SHORT_NAME & METHOD_NAME & LAB_CODE & VALUE_RPTD \\
\hline 5150 & PDMS & & SW & & & E091442 & 16-Dec-86 & & $10045-97-3$ & CS-137 & GAMMA_GS & PNL-RO & -0.00406 \\
\hline 5151 & PDMS & & sW & & & E091448 & 16-Dec-86 & & $10045-97-3$ & CS-137 & GAMMA_GS & PNL-RO & -0.00107 \\
\hline 5152 & PDMS & & SW & & & E091451 & 23-Dec-86 & & $10045-97-3$ & CS-137 & 901.1_GAMMA_GS & USTEST & 0.734 \\
\hline 5153 & PDMS & & SW & & & E091451 & 23-Dec-86 & & $10098-97-2$ & SR-90 & 905.0_SR_GPC & USTEST & 0.267 \\
\hline 5154 & PDMS & & sW & & & E091451 & 23-Dec-86 & & $13966-29-5$ & U-234 & UISO_IE_PLATE_AEA & USTEST & 0.343 \\
\hline 5155 & PDMS & & SW & & & E091451 & 23-Dec-86 & & 15117-96-1 & U-235 & UISO_IE_PLATE_AEA & USTEST & 0.0155 \\
\hline 5156 & PDMS & & sW & & & E091451 & 23-Dec-86 & & U-238 & $\mathrm{U}-238$ & UISO_IE_PLATE_AEA & USTEST & 0.225 \\
\hline 5157 & PDMS & & sW & & & E091451 & 23-Dec-86 & & $10028-17-8$ & TRITIUM & TRITIŪM_ELECT__LSC & USTEST & 159 \\
\hline 5158 & PDMS & & sW & & & E091436 & 30-Dec-86 & & $10045-97-3$ & CS-137 & GAMMA_GS & PNL-RO & -0.00234 \\
\hline 5159 & PDMS & & SW & & & E091439 & 30-Dec-86 & & $10045-97-3$ & CS-137 & GAMMA_GS & PNL-RO & 0.000272 \\
\hline 5160 & PDMS & & SW & & & E091436 & 30-Dec-86 & & 15046-84-1 & I-129 & 1129_SEP_TEMS & 320 & 0.0000787 \\
\hline 5161 & PDMS & & SW & & & E091436 & 30-Dec-86 & & $10098-97-2$ & SR-90 & SRISO_SEP_PRECIP_GPC & PNL-RO & 0.00229 \\
\hline 5162 & PDMS & & sW & & & E091436 & 30-Dec-86 & & PU-239/240 & PU-239/240 & PUISO_IE_AEA & PNL-RO & 0.0000978 \\
\hline 5163 & PDMS & & SW & & & E091439 & 30-Dec-86 & & PU-239/240 & PU-239/240 & PUISO_IE_AEA & PNL-RO & 0.0000317 \\
\hline 5164 & PDMS & & SW & & & E091444 & 30-Dec-86 & & $10045-97-3$ & CS-137 & GAMMA_GS & PNL-RO & -0.00161 \\
\hline 5165 & PDMS & & SW & & & E091444 & 30-Dec-86 & & 15046-84-1 & I-129 & 1129_SEP_TEMS & 320 & 0.0000076 \\
\hline 5166 & PDMS & & SW & & & E091444 & 30-Dec-86 & & $10098-97-2$ & SR-90 & SRISO_SEP_PRECIP_GPC & PNL-RO & 0.00723 \\
\hline 5167 & PDMS & & sW & & & E091444 & 30-Dec-86 & & PU-239/240 & PU-239/240 & PUISO_IE_AEA & PNL-RO & 0.0000465 \\
\hline 5168 & PDMS & & sW & & & E091454 & 30-Dec-86 & & $10045-97-3$ & CS-137 & GAMMA_GS & USTEST & -1.47 \\
\hline 5169 & PDMS & & SW & & & E091449 & 30-Dec-86 & & $10045-97-3$ & CS-137 & GAMMA_GS & PNL-RO & -0.00486 \\
\hline 5170 & PDMS & & sW & & & E091454 & 30-Dec-86 & & $10098-97-2$ & SR-90 & SRISO_SEEP_PRECIP_GPC & USTEST & 0.192 \\
\hline 5171 & PDMS & & SW & & & E091454 & 30-Dec-86 & & 13966-29-5 & U-234 & UISO_AEA & USTEST & 0.299 \\
\hline 5172 & PDMS & & SW & & & E091454 & 30-Dec-86 & & $15117-96-1$ & U-235 & UISO_AEA & USTEST & 0.00792 \\
\hline 5173 & PDMS & & SW & & & E091454 & 30-Dec-86 & & U-238 & U-238 & UISO_AEA & USTEST & 0.22 \\
\hline 5174 & PDMS & & SW & & & E091449 & 30-Dec-86 & & PU-239/240 & PU-239/240 & PUISO_IE_AEA & PNL-RO & 0.0000194 \\
\hline 5175 & PDMS & & SW & & & E091454 & 30-Dec-86 & & $10028-17-8$ & TRITIUM & TRITIUM_ELECT_LSC & USTEST & 88.3 \\
\hline 5176 & PDMS & & SW & & & E091453 & 30-Dec-86 & & $10045-97-3$ & CS-137 & GAMMA_GS & USTEST & 0.242 \\
\hline 5177 & PDMS & & SW & & & E091453 & 30-Dec-86 & & $10098-97-2$ & SR-90 & SRISO_SEP_PRECIP_GPC & USTEST & 0.242 \\
\hline 5178 & PDMS & & SW & & & E091453 & 30-Dec-86 & & $13966-29-5$ & $\mathrm{U}-234$ & UISO_AEA & USTEST & 0.297 \\
\hline 5179 & PDMS & & SW & & & E091453 & 30-Dec-86 & & $15117-96-1$ & U-235 & UISO_AEA & USTEST & 0.0189 \\
\hline 5180 & PDMS & & SW & & & E091453 & 30-Dec-86 & & U-238 & $\mathrm{U}-238$ & UISO_AEA & USTEST & 0.252 \\
\hline 5181 & PDMS & & SW & & & E091453 & 30-Dec-86 & & $10028-17-8$ & TRITIUM & TRITIUM_ELECT_LSC & USTEST & 126 \\
\hline 5182 & PDMS & & sW & & & E091457 & 05-Jan-87 & & $10028-17-8$ & TRITIUM & 906.0_H3_LSC & USTEST & -34.9 \\
\hline 5183 & PDMS & & SW & & & E091457 & 05-Jan-87 & & $10045-97-3$ & CS-137 & 901.1_GAMMA_GS & USTEST & -1.21 \\
\hline 5184 & PDMS & & SW & & & E091457 & 05-Jan-87 & & $10098-97-2$ & SR-90 & 905.0_SR_GPC & USTEST & 0.0155 \\
\hline 5185 & PDMS & & SW & & & E091456 & 05-Jan-87 & & $10028-17-8$ & TRITIUM & 906.0_H3_LSC & USTEST & 1440 \\
\hline 5186 & PDMS & & SW & & & E091456 & 05-Jan-87 & & $10045-97-3$ & CS-137 & 901.1_GAMMA_GS & USTEST & 0.92 \\
\hline 5187 & PDMS & & sW & & & E091456 & 05-Jan-87 & & 10098-97-2 & SR-90 & 905.0_SR_GPC & USTEST & 0.00562 \\
\hline 5188 & PDMS & & SW & & & E091455 & 05-Jan-87 & & $10045-97-3$ & CS-137 & 901.1_GAMMA_GS & USTEST & -0.497 \\
\hline 5189 & PDMS & & SW & & & E091455 & 05-Jan-87 & & $10028-17-8$ & TRITIUM & 906.0_H3_LSC & USTEST & 22 \\
\hline 5190 & PDMS & & SW & & & E091455 & 05-Jan-87 & & 10098-97-2 & SR-90 & 905.0_SR_GPC & USTEST & 0.046 \\
\hline 5191 & PDMS & & SW & & & E091458 & 05-Jan-87 & & $10028-17-8$ & TRITIUM & 906.0_H3_LSC & USTEST & -66 \\
\hline 5192 & PDMS & & SW & & & E091458 & 05-Jan-87 & & $10045-97-3$ & CS-137 & 901.1_GAMMA_GS & USTEST & -0.105 \\
\hline 5193 & PDMS & & SW & & & E091458 & 05-Jan-87 & & $10098-97-2$ & SR-90 & 905.0_SR_GPC & USTEST & 0.00623 \\
\hline 5194 & PDMS & & SW & & & E091692 & 13-Jan-87 & & $10045-97-3$ & CS-137 & 901.1_GAMMA_GS & USTEST & 0 \\
\hline 5195 & PDMS & & SW & & & E091692 & 13-Jan-87 & & $10028-17-8$ & TRITIUM & TRITIUM_ELECT_LSC & USTEST & 117 \\
\hline 5196 & PDMS & & SW & & & E091692 & 13-Jan-87 & & $10098-97-2$ & SR-90 & 905.0_SR_GPC & USTEST & 0.135 \\
\hline 5197 & PDMS & & SW & & & E027405 & 13-Jan-87 & & $10045-97-3$ & CS-137 & GAMMA_GS & PNL-RO & -0.000675 \\
\hline 5198 & PDMS & & SW & & & E092204 & 13-Jan-87 & & $10045-97-3$ & CS-137 & GAMMA_GS & PNL-RO & -0.00579 \\
\hline 5199 & PDMS & & SW & & & E092208 & 13-Jan-87 & & $10045-97-3$ & CS-137 & GAMMA_GS & PNL-RO & -0.00334 \\
\hline 5200 & PDMS & & SW & & & E092212 & 13-Jan-87 & & $10045-97-3$ & CS-137 & GAMMA_GS & PNL-RO & -0.00285 \\
\hline 5201 & PDMS & & SW & & & E092216 & 13-Jan-87 & & $10045-97-3$ & CS-137 & GAMMA_GS & PNL-RO & -0.00173 \\
\hline
\end{tabular}




\begin{tabular}{|c|c|c|c|c|c|c|c|c|c|c|c|c|c|}
\hline & $\bar{A}$ & $\mathrm{~B}$ & $\bar{C}$ & $\mathrm{D}$ & $\mathrm{E}$ & $\mathrm{F}$ & $\bar{G}$ & $\mathrm{H}$ & 1 & $\mathrm{~J}$ & $\bar{K}$ & $\bar{L}$ & $\mathrm{M}$ \\
\hline 1 & SAMPLE_NUM_ASSIGNED_TO & \begin{tabular}{|l} 
SAMPLER \\
\end{tabular} & MEDIA & BIOTA_MEDIA & TAG_ID & SAMP_NUM & SAMP_DATE_TIME & SAMP_DATE_TIME_ON & CON_ID & \begin{tabular}{|l|} 
CON_SHORT_NAME \\
\end{tabular} & METHOD_NAME & LAB_CODE & VALUE_RPTD \\
\hline 5202 & PDMS & & SW & & & E092220 & 13-Jan-87 & & $10045-97-3$ & CS-137 & GAMMA_GS & PNL-RO & -0.00193 \\
\hline 5203 & PDMS & & SW & & & E092201 & 27-Jan-87 & & $10045-97-3$ & CS-137 & GAMMA_GS & PNL-RO & -0.00155 \\
\hline 5204 & PDMS & & SW & & & E092205 & 27-Jan-87 & & $10045-97-3$ & CS-137 & GAMMA_GS & PNL-RO & -0.000588 \\
\hline 5205 & PDMS & & SW & & & E092209 & 27-Jan-87 & & $10045-97-3$ & CS-137 & GAMMA_GS & PNL-RO & 0.0000958 \\
\hline 5206 & PDMS & & SW & & & E092213 & 27-Jan-87 & & $10045-97-3$ & CS-137 & GAMMA_GS & PNL-RO & 0.000778 \\
\hline 5207 & PDMS & & SW & & & E092221 & 27-Jan-87 & & $10045-97-3$ & CS-137 & GAMMA_GS & PNL-RO & -0.0047 \\
\hline 5208 & PDMS & & SW & & & E092217 & 27-Jan-87 & & $10045-97-3$ & CS-137 & GAMMA_GS & PNL-RO & -0.00526 \\
\hline 5209 & PDMS & & SW & & & E091986 & 03-Feb-87 & & $10045-97-3$ & CS-137 & GAMMA_GS & USTEST & 0 \\
\hline 5210 & PDMS & & SW & & & E091986 & 03-Feb-87 & & $13966-29-5$ & $\mathrm{U}-234$ & UISO_AĒAA & USTEST & 0.273 \\
\hline 5211 & PDMS & & SW & & & E091986 & 03-Feb-87 & & 15117-96-1 & $\mathrm{U}-235$ & UISO_AEA & USTEST & 0.0149 \\
\hline 5212 & PDMS & & SW & & & E091986 & 03-Feb-87 & & U-238 & $\mathrm{U}-238$ & UISO_AEA & USTEST & 0.207 \\
\hline 5213 & PDMS & & SW & & & E091986 & 03-Feb-87 & & $10028-17-8$ & TRITIUM & TRITIÜM_ELECT_LSC & USTEST & 76.2 \\
\hline 5214 & PDMS & & SW & & & E091986 & 03-Feb-87 & & $10098-97-2$ & SR-90 & SRISO_SEP_PRËCIP_GPC & USTEST & 0.127 \\
\hline 5215 & PDMS & & SW & & & E091934 & 03-Feb-87 & & $10045-97-3$ & CS-137 & GAMMA_GS & USTEST & -0.473 \\
\hline 5216 & PDMS & & SW & & & E091934 & 03-Feb-87 & & $13966-29-5$ & $\mathrm{U}-234$ & UISO_AEA & USTEST & 0.302 \\
\hline 5217 & PDMS & & SW & & & E091934 & 03-Feb-87 & & 15117-96-1 & $\mathrm{U}-235$ & UISO_AEA & USTEST & 0.0148 \\
\hline 5218 & PDMS & & SW & & & E091934 & 03-Feb-87 & & $\mathrm{U}-238$ & $\mathrm{U}-238$ & UISO_AEA & USTEST & 0.282 \\
\hline 5219 & PDMS & & SW & & & E091934 & 03-Feb-87 & & $10028-17-8$ & TRITIUM & TRITIUMM_ELECT_LSC & USTEST & 143 \\
\hline 5220 & PDMS & & SW & & & E091934 & 03-Feb-87 & & $10098-97-2$ & SR-90 & SRISO_SEP_PRECIP_GPC & USTEST & 0.14 \\
\hline 5221 & PDMS & & SW & & & E092072 & 10-Feb-87 & & $10045-97-3$ & CS-137 & 901.1_GAMMA_GS & USTEST & 0.138 \\
\hline 5222 & PDMS & & SW & & & E092072 & 10-Feb-87 & & $10028-17-8$ & TRITIUM & 906.0_H3_LSC & USTEST & 41.9 \\
\hline 5223 & PDMS & & SW & & & E092072 & 10-Feb-87 & & $10098-97-2$ & SR-90 & 905.0_SR_GPC & USTEST & 0.0399 \\
\hline 5224 & PDMS & & SW & & & E092202 & 10-Feb-87 & & $10045-97-3$ & CS-137 & GAMMA_GS & PNL-RO & 0.00186 \\
\hline 5225 & PDMS & & SW & & & E092206 & 10-Feb-87 & & $10045-97-3$ & CS-137 & GAMMA_GS & PNL-RO & -0.00218 \\
\hline 5226 & PDMS & & SW & & & E092210 & 10-Feb-87 & & $10045-97-3$ & CS-137 & GAMMA_GS & PNL-RO & -0.00227 \\
\hline 5227 & PDMS & & SW & & & E092214 & 10-Feb-87 & & $10045-97-3$ & CS-137 & GAMMA_GS & PNL-RO & -0.00106 \\
\hline 5228 & PDMS & & SW & & & E092222 & 10-Feb-87 & & $10045-97-3$ & CS-137 & GAMMA_GS & PNL-RO & -0.00561 \\
\hline 5229 & PDMS & & SW & & & E092218 & 10-Feb-87 & & $10045-97-3$ & CS-137 & GAMMA_GS & PNL-RO & 0.0000333 \\
\hline 5230 & PDMS & & SW & & & E092073 & 13-Feb-87 & & $10045-97-3$ & CS-137 & 901.1_GAMMA_GS & USTEST & -0.229 \\
\hline 5231 & PDMS & & SW & & & E092073 & 13-Feb-87 & & $10098-97-2$ & SR-90 & 905.0_SR_GPC & USTEST & 0.679 \\
\hline 5232 & PDMS & & SW & & & E092073 & 13-Feb-87 & & $10028-17-8$ & TRITIUM & TRITIUM_ELECT_LSC & USTEST & 159 \\
\hline 5233 & PDMS & & SW & & & E092207 & 24-Feb-87 & & $10045-97-3$ & CS-137 & GAMMA_GS & PNL-RO & -0.00232 \\
\hline 5234 & PDMS & & SW & & & E092203 & 24-Feb-87 & & $10045-97-3$ & CS-137 & GAMMA_GS & PNL-RO & 0.00212 \\
\hline 5235 & PDMS & & SW & & & E092211 & 24-Feb-87 & & $10045-97-3$ & CS-137 & GAMMA_GS & PNL-RO & -0.00239 \\
\hline 5236 & PDMS & & SW & & & E092215 & 24-Feb-87 & & $10045-97-3$ & CS-137 & GAMMA_GS & PNL-RO & -0.00129 \\
\hline 5237 & PDMS & & SW & & & E092219 & 24-Feb-87 & & $10045-97-3$ & CS-137 & GAMMA_GS & PNL-RO & -0.00298 \\
\hline 5238 & PDMS & & SW & & & E092223 & 24-Feb-87 & & $10045-97-3$ & CS-137 & GAMMA_GS & PNL-RO & -0.00386 \\
\hline 5239 & PDMS & & SW & & & E092285 & 03-Mar-87 & & $10028-17-8$ & TRITIUM & TRITIUM_ELECT_LSC & USTEST & 67.6 \\
\hline 5240 & PDMS & & SW & & & E092285 & 03-Mar-87 & & $10098-97-2$ & SR-90 & SRISO_SEP_PRECIP_GPC & USTEST & 0.144 \\
\hline 5241 & PDMS & & SW & & & E092285 & 03-Mar-87 & & U-238 & U-238 & UISO_AEA & USTEST & 0.213 \\
\hline 5242 & PDMS & & SW & & & E092285 & 03-Mar-87 & & $15117-96-1$ & U-235 & UISO_AEA & USTEST & 0.00422 \\
\hline 5243 & PDMS & & SW & & & E092285 & 03-Mar-87 & & $13966-29-5$ & U-234 & UISO_AEA & USTEST & 0.293 \\
\hline 5244 & PDMS & & SW & & & E092285 & 03-Mar-87 & & $10045-97-3$ & CS-137 & GAMMA_GS & USTEST & 0.21 \\
\hline 5245 & PDMS & & SW & & & E092282 & 03-Mar-87 & & $10028-17-8$ & TRITIUM & TRITIUM_ELECT_LSC & USTEST & 129 \\
\hline 5246 & PDMS & & SW & & & E092282 & 03-Mar-87 & & $10098-97-2$ & SR-90 & SRISO_SEP_PRECIP_GPC & USTEST & 0.164 \\
\hline 5247 & PDMS & & SW & & & E092282 & 03-Mar-87 & & $\mathrm{U}-238$ & $\mathrm{U}-238$ & UISO_AEEA & USTEST & 0.355 \\
\hline 5248 & PDMS & & SW & & & E092282 & 03-Mar-87 & & $15117-96-1$ & U-235 & UISO_AEA & USTEST & 0.0367 \\
\hline 5249 & PDMS & & SW & & & E092282 & 03-Mar-87 & & $13966-29-5$ & U-234 & UISO_AEA & USTEST & 0.451 \\
\hline 5250 & PDMS & & SW & & & E092282 & 03-Mar-87 & & $10045-97-3$ & CS-137 & GAMMA_GS & USTEST & -0.736 \\
\hline 5251 & PDMS & & SW & & & E092286 & 03-Mar-87 & & $10028-17-8$ & TRITIUM & TRITIUM_DIST_LSC & USTEST & 163 \\
\hline 5252 & PDMS & & SW & & & E092286 & 03-Mar-87 & & $10098-97-2$ & SR-90 & SRISO_SEP_PRECIP_GPC & USTEST & 0.327 \\
\hline 5253 & PDMS & & SW & & & E092286 & 03-Mar-87 & & $10045-97-3$ & CS-137 & GAMMA_GS & USTEST & 0.687 \\
\hline
\end{tabular}




\begin{tabular}{|c|c|c|c|c|c|c|c|c|c|c|c|c|c|}
\hline & $\bar{A}$ & $\mathrm{~B}$ & $\bar{C}$ & $\mathrm{D}$ & $E$ & $\mathrm{~F}$ & $\mathrm{G}$ & $\mathrm{H}$ & 1 & $\mathrm{~J}$ & $\mathrm{~K}$ & $\mathrm{~L}$ & $\mathrm{M}$ \\
\hline 1 & SAMPLE_NUM_ASSIGNED_TO & SAMPLER & MEDIA & BIOTA_MEDIA & TAG_ID & SAMP_NUM & SAMP_DATE_TIME & SAMP_DATE_TIME_ON & CON_ID & CON_SHORT_NAME & METHOD_NAME & LAB_CODE & VALUE_RPTD \\
\hline 5254 & PDMS & & SW & & & E092306 & 03-Mar-87 & & $10045-97-3$ & CS-137 & GAMMA_GS & USTEST & 1.14 \\
\hline 5255 & PDMS & & sW & & & E092306 & 03-Mar-87 & & $10028-17-8$ & TRITIUM & TRITIUM_DIST_LSC & USTEST & 2410 \\
\hline 5256 & PDMS & & SW & & & E092287 & 03-Mar-87 & & $10098-97-2$ & SR-90 & SRISO_SEP_PRECIP_GPC & USTEST & 0.705 \\
\hline 5257 & PDMS & & sw & & & E092287 & 03-Mar-87 & & $10028-17-8$ & TRITIUM & TRITIUM_DIST_LSC & USTEST & 231 \\
\hline 5258 & PDMS & & SW & & & E092287 & 03-Mar-87 & & $10045-97-3$ & CS-137 & GAMMA_GS & USTEST & 13.1 \\
\hline 5259 & PDMS & & sw & & & E092288 & 03-Mar-87 & & $\mathrm{U}-238$ & U-238 & UISO AEA & USTEST & 75.5 \\
\hline 5260 & PDMS & & sW & & & E092288 & 03-Mar-87 & & 15117-96-1 & $\mathrm{U}-235$ & UISO_AEA & USTEST & 3.11 \\
\hline 5261 & PDMS & & sw & & & E092288 & 03-Mar-87 & & $13966-29-5$ & $\mathrm{U}-234$ & UISO_AEA & USTEST & 80.1 \\
\hline 5262 & PDMS & & sW & & & E092288 & 03-Mar-87 & & $10045-97-3$ & CS-137 & GAMM̄A_GS & USTEST & 1.27 \\
\hline 5263 & PDMS & & sw & & & E092288 & 03-Mar-87 & & $10098-97-2$ & SR-90 & SRISO_SEP_PRECIP_GPC & USTEST & 1.81 \\
\hline 5264 & PDMS & & sW & & & E092288 & 03-Mar-87 & & $10028-17-8$ & TRITIUM & TRITIUM DIST_LSC & USTEST & 553 \\
\hline 5265 & PDMS & & sw & & & E092267 & 10-Mar-87 & & $10045-97-3$ & CS-137 & GAMMA_GS & PNL-RO & \\
\hline 5266 & PDMS & & SW & & & E092266 & 10-Mar-87 & & $10045-97-3$ & CS-137 & GAMMA_GS & PNL-RO & \\
\hline 5267 & PDMS & & sw & & & E092659 & 10-Mar-87 & & $10045-97-3$ & CS-137 & GAMMA_GS & PNL-RO & -0.00926 \\
\hline 5268 & PDMS & & sW & & & E092661 & 10-Mar-87 & & $10045-97-3$ & CS-137 & GAMMA_GS & PNL-RO & -0.00544 \\
\hline 5269 & PDMS & & SW & & & E092663 & 10-Mar-87 & & $10045-97-3$ & CS-137 & GAMMA_GS & PNL-RO & -0.00437 \\
\hline 5270 & PDMS & & SW & & & E092665 & 10-Mar-87 & & $10045-97-3$ & CS-137 & GAMMA_GS & PNL-RO & -0.00475 \\
\hline 5271 & PDMS & & sW & & & E092658 & 24-Mar-87 & & $10045-97-3$ & CS-137 & GAMMA_GS & PNL-RO & -0.00123 \\
\hline 5272 & PDMS & & sw & & & E092537 & 24-Mar-87 & & $10045-97-3$ & CS-137 & GAMMA_GS & PNL-RO & -0.000962 \\
\hline 5273 & PDMS & & SW & & & E092660 & 24-Mar-87 & & $10045-97-3$ & CS-137 & GAMMA_GS & PNL-RO & -0.000672 \\
\hline 5274 & PDMS & & sW & & & E092662 & 24-Mar-87 & & $10045-97-3$ & CS-137 & GAMMA_GS & PNL-RO & -0.00334 \\
\hline 5275 & PDMS & & SW & & & E092664 & 24-Mar-87 & & $10045-97-3$ & CS-137 & GAMMA_GS & PNL-RO & -0.00473 \\
\hline 5276 & PDMS & & sW & & & E092666 & 24-Mar-87 & & $10045-97-3$ & CS-137 & GAMMA_GS & PNL-RO & -0.0137 \\
\hline 5277 & PDMS & & sW & & & E092484 & 30-Mar-87 & & $10098-97-2$ & SR-90 & 905.0_SR_GPC & USTEST & 0.024 \\
\hline 5278 & PDMS & & SW & & & E092484 & 30-Mar-87 & & 10028-17-8 & TRITIUM & 906.0_H3_LSC & USTEST & -41.9 \\
\hline 5279 & PDMS & & sW & & & E092484 & 30-Mar-87 & & $10045-97-3$ & CS-137 & 901.1_GAMMA_GS & USTEST & 0.136 \\
\hline 5280 & PDMS & & SW & & & E092483 & 30-Mar-87 & & 10098-97-2 & SR-90 & 905.0_SR_GPC & USTEST & 0.00286 \\
\hline 5281 & PDMS & & SW & & & E092483 & 30-Mar-87 & & $10028-17-8$ & TRITIUM & 906.0_H3_LSC & USTEST & 3860 \\
\hline 5282 & PDMS & & SW & & & E092483 & 30-Mar-87 & & $10045-97-3$ & CS-137 & 901.1_GAMMA_GS & USTEST & 0.63 \\
\hline 5283 & PDMS & & SW & & & E092482 & 30-Mar-87 & & $10098-97-2$ & SR-90 & 905.0_SR_GPC & USTEST & 0.024 \\
\hline 5284 & PDMS & & SW & & & E092482 & 30-Mar-87 & & 10028-17-8 & TRITIUM & 906.0_H3_LSC & USTEST & 35.4 \\
\hline 5285 & PDMS & & sw & & & E092482 & 30-Mar-87 & & $10045-97-3$ & CS-137 & 901.1 GAMMA GS & USTEST & 0.407 \\
\hline 5286 & PDMS & & sW & & & E092485 & 30-Mar-87 & & $10098-97-2$ & SR-90 & 905.0_SR_GPC & USTEST & 0.0149 \\
\hline 5287 & PDMS & & sW & & & E092485 & 30-Mar-87 & & $10028-17-8$ & TRITIUM & 906.0 H3 LSC & USTEST & -60.2 \\
\hline 5288 & PDMS & & sw & & & E092485 & 30-Mar-87 & & $10045-97-3$ & CS-137 & 901.1_GAMMA_GS & USTEST & -0.688 \\
\hline 5289 & PDMS & & sw & & & E092481 & 31-Mar-87 & & $10098-97-2$ & SR-90 & SRISO_SEP_PRECIP_GPC & USTEST & 0.175 \\
\hline 5290 & PDMS & & sW & & & E092481 & 31-Mar-87 & & $\mathrm{U}-238$ & U-238 & UISO_AEA & USTEST & 0.163 \\
\hline 5291 & PDMS & & sW & & & E092481 & 31-Mar-87 & & 15117-96-1 & U-235 & UISO_AEA & USTEST & 0.0155 \\
\hline 5292 & PDMS & & sw & & & E092481 & 31-Mar-87 & & 13966-29-5 & U-234 & UISO AEA & USTEST & 0.267 \\
\hline 5293 & PDMS & & SW & & & E092481 & 31-Mar-87 & & $10028-17-8$ & TRITIUM & TRITIÜM_ELECT_LSC & USTEST & 53.7 \\
\hline 5294 & PDMS & & SW & & & E092481 & 31-Mar-87 & & $10045-97-3$ & CS-137 & GAMMA_GS & USTEST & -0.683 \\
\hline 5295 & PDMS & & SW & & & E092480 & 31-Mar-87 & & $10098-97-2$ & SR-90 & SRISO_SEEP_PRECIP_GPC & USTEST & 0.183 \\
\hline 5296 & PDMS & & sw & & & E092480 & 31-Mar-87 & & $\mathrm{U}-238$ & U-238 & UISO_AEA & USTEST & 0.233 \\
\hline 5297 & PDMS & & sW & & & E092480 & 31-Mar-87 & & 15117-96-1 & U-235 & UISO_AEA & USTEST & 0.00978 \\
\hline 5298 & PDMS & & sw & & & E092480 & 31-Mar-87 & & $13966-29-5$ & U-234 & UISO AEA & USTEST & 0.307 \\
\hline 5299 & PDMS & & SW & & & E092480 & 31-Mar-87 & & $10028-17-8$ & TRITIUM & TRITIÜM_ELECT_LSC & USTEST & 127 \\
\hline 5300 & PDMS & & sw & & & E092480 & 31-Mar-87 & & $10045-97-3$ & CS-137 & GAMMA GS & USTEST & 0.413 \\
\hline 5301 & PDMS & & sw & & & E092667 & 07-Apr-87 & & $10098-97-2$ & SR-90 & 905.0_SR_GPC & USTEST & 0.15 \\
\hline 5302 & PDMS & & sw & & & E092667 & 07-Apr-87 & & $10045-97-3$ & CS-137 & 901.1_GAMMA_GS & USTEST & -0.2 \\
\hline 5303 & PDMS & & SW & & & E092667 & 07-Apr-87 & & $10028-17-8$ & TRITIUM & TRITIUMM_ELECT_LSC & USTEST & 73.4 \\
\hline 5304 & PDMS & & sw & & & E033674 & 07-Apr-87 & & PU-239/240 & PU-239/240 & PUISO_IE_AEA & PNL-RO & 0.00000781 \\
\hline 5305 & PDMS & & sw & & & E033642 & 07-Apr-87 & & PU-239/240 & PU-239/240 & PUISO IE AEA & PNL-RO & 0.0000593 \\
\hline
\end{tabular}




\begin{tabular}{|c|c|c|c|c|c|c|c|c|c|c|c|c|c|}
\hline & $\bar{A}$ & $\mathrm{~B}$ & $\mathrm{C}$ & $\bar{D}$ & $\mathrm{E}$ & $\mathrm{F}$ & $\bar{G}$ & $\mathrm{H}$ & 1 & $\mathrm{~J}$ & $\mathrm{~K}$ & $\mathrm{~L}$ & $\bar{M}$ \\
\hline 1 & SAMPLE_NUM_ASSIGNED_TO & SAMPLER & MEDIA & BIOTA_MEDIA & TAG_ID & SAMP_NUM & SAMP_DATE_TIME & SAMP_DATE_TIME_ON & CON_ID & CON_SHORT_NAME & METHOD_NAME & LAB_CODE & VALUE_RPTD \\
\hline 5306 & PDMS & & SW & & & E033642 & $07-A p r-87$ & & $15046-84-1$ & $1-129$ & 1129_SEP_TEMS & 320 & 0.0001176 \\
\hline 5307 & PDMS & & sW & & & E033642 & 07-Apr-87 & & $10045-97-3$ & CS-137 & GAMMA_GS & PNL-RO & -0.00897 \\
\hline 5308 & PDMS & & SW & & & E033674 & 07-Apr-87 & & $10045-97-3$ & CS-137 & GAMMA_GS & PNL-RO & -0.00351 \\
\hline 5309 & PDMS & & sw & & & E033671 & 07-Apr-87 & & PU-239/240 & PU-239/240 & PUISO_IE_AEA & PNL-RO & 0.000141 \\
\hline 5310 & PDMS & & SW & & & E033671 & 07-Apr-87 & & 15046-84-1 & I-129 & 1129_SEP_TEMS & 320 & 0.000004 \\
\hline 5311 & PDMS & & sw & & & E033671 & 07-Apr-87 & & $10045-97-3$ & CS-137 & GAMMA_GS & PNL-RO & -0.00633 \\
\hline 5312 & PDMS & & sW & & & E033667 & 07-Apr-87 & & PU-239/240 & PU-239/240 & PUISO_IE_AEA & PNL-RO & 0.00000382 \\
\hline 5313 & PDMS & & sW & & & E033667 & 07-Apr-87 & & $10045-97-3$ & CS-137 & GAMMA_GS & PNL-RO & -0.00234 \\
\hline 5314 & PDMS & & sW & & & E033646 & 07-Apr-87 & & PU-239/240 & PU-239/240 & PUISO_IE_AEA & PNL-RO & 0.0000168 \\
\hline 5315 & PDMS & & sW & & & E033646 & 07-Apr-87 & & $10045-97-3$ & CS-137 & GAMMA_GS & PNL-RO & -0.000759 \\
\hline 5316 & PDMS & & sW & & & E033650 & 07-Apr-87 & & PU-239/240 & PU-239/240 & PUISO_IE_AEA & PNL-RO & 0.0000976 \\
\hline 5317 & PDMS & & sw & & & E033650 & 07-Apr-87 & & $15046-84-1$ & I-129 & I129_SEP_TEMS & 320 & 0.000119 \\
\hline 5318 & PDMS & & sw & & & E033650 & 07-Apr-87 & & $10045-97-3$ & CS-137 & GAMMA_GS & PNL-RO & -0.0193 \\
\hline 5319 & PDMS & & sw & & & E092668 & 14-Apr-87 & & U-238 & U-238 & UISO_IE_PLATE_AEA & USTEST & 0.255 \\
\hline 5320 & PDMS & & sw & & & E092668 & 14-Apr-87 & & $15117-96-1$ & U-235 & UISO_IE_PLATE_AEA & USTEST & 0.0213 \\
\hline 5321 & PDMS & & sw & & & E092668 & 14-Apr-87 & & $13966-29-5$ & U-234 & UISO_IE_PLATE_AEA & USTEST & 0.331 \\
\hline 5322 & PDMS & & sW & & & E092668 & 14-Apr-87 & & $10045-97-3$ & CS-137 & 901.1_GAMMA_GS & USTEST & -0.58 \\
\hline 5323 & PDMS & & sw & & & E092668 & 14-Apr-87 & & $10028-17-8$ & TRITIUM & TRITIŪM_ELECT_LSC & USTEST & 170 \\
\hline 5324 & PDMS & & sw & & & E092668 & 14-Apr-87 & & $10098-97-2$ & SR-90 & 905.0_SR_GPC & USTEST & 0.154 \\
\hline 5325 & PDMS & & sW & & & E092875 & 21-Apr-87 & & $10045-97-3$ & CS-137 & GAMMA_ĒS & USTEST & 0.181 \\
\hline 5326 & PDMS & & sW & & & E092875 & 21-Apr-87 & & $10028-17-8$ & TRITIUM & TRITIUM_ELECCT_LSC & USTEST & 181 \\
\hline 5327 & PDMS & & SW & & & E092875 & 21-Apr-87 & & $10098-97-2$ & SR-90 & SRISO_SËP_PRËCIP_GPC & USTEST & 0.0965 \\
\hline 5328 & PDMS & & sW & & & E092875 & 21-Apr-87 & & $\mathrm{U}-238$ & U-238 & UISO_AEEA & USTEST & 0.471 \\
\hline 5329 & PDMS & & sw & & & E092875 & 21-Apr-87 & & 15117-96-1 & U-235 & UISO_AEA & USTEST & 0.037 \\
\hline 5330 & PDMS & & sw & & & E092875 & 21-Apr-87 & & $13966-29-5$ & U-234 & UISO_AEA & USTEST & 0.597 \\
\hline 5331 & PDMS & & sw & & & E092874 & 21-Apr-87 & & $10028-17-8$ & TRITIUM & TRITIÜM_ELECT_LSC & USTEST & 155 \\
\hline 5332 & PDMS & & sw & & & E092874 & 21-Apr-87 & & $10098-97-2$ & SR-90 & SRISO_SEP_PRECIP_GPC & USTEST & 0.106 \\
\hline 5333 & PDMS & & sw & & & E092874 & 21-Apr-87 & & U-238 & $\mathrm{U}-238$ & UISO_AEA & USTEST & 0.44 \\
\hline 5334 & PDMS & & sw & & & E092874 & 21-Apr-87 & & 15117-96-1 & $\mathrm{U}-235$ & UISO_AEA & USTEST & 0.0174 \\
\hline 5335 & PDMS & & sW & & & E092874 & 21-Apr-87 & & $13966-29-5$ & $\mathrm{U}-234$ & UISO_AEA & USTEST & 0.62 \\
\hline 5336 & PDMS & & sw & & & E092874 & 21-Apr-87 & & $10045-97-3$ & CS-137 & GAMMA_AS & USTEST & -0.0525 \\
\hline 5337 & PDMS & & sw & & & E093250 & 21-Apr-87 & & $10045-97-3$ & CS-137 & GAMMA_GS & PNL-RO & -0.000809 \\
\hline 5338 & PDMS & & sw & & & E093254 & 21-Apr-87 & & $10045-97-3$ & CS-137 & GAMMA_GS & PNL-RO & -0.00224 \\
\hline 5339 & PDMS & & sw & & & E093257 & 21-Apr-87 & & $10045-97-3$ & CS-137 & GAMMA_GS & PNL-RO & -0.00479 \\
\hline 5340 & PDMS & & SW & & & E093259 & 21-Apr-87 & & $10045-97-3$ & CS-137 & GAMMA_GS & PNL-RO & -0.00315 \\
\hline 5341 & PDMS & & sW & & & E093261 & 21-Apr-87 & & $10045-97-3$ & CS-137 & GAMMA_GS & PNL-RO & -0.00217 \\
\hline 5342 & PDMS & & sW & & & E093264 & 21-Apr-87 & & $10045-97-3$ & CS-137 & GAMMA_GS & PNL-RO & -0.0136 \\
\hline 5343 & PDMS & & sw & & & E092930 & 28-Apr-87 & & $10045-97-3$ & CS-137 & GAMMA_GS & USTEST & -0.194 \\
\hline 5344 & PDMS & & sw & & & E092930 & 28-Apr-87 & & $10098-97-2$ & SR-90 & SRISO_SEP_PRECIP_GPC & USTEST & 0.124 \\
\hline 5345 & PDMS & & sw & & & E092930 & 28-Apr-87 & & U-238 & $\mathrm{U}-238$ & UISO_AEA & USTEST & 0.223 \\
\hline 5346 & PDMS & & sw & & & E092930 & 28-Apr-87 & & 15117-96-1 & $\mathrm{U}-235$ & UISO_AEA & USTEST & 0.00592 \\
\hline 5347 & PDMS & & sW & & & E092930 & 28-Apr-87 & & 13966-29-5 & $\mathrm{U}-234$ & UISO_AEA & USTEST & 0.264 \\
\hline 5348 & PDMS & & sW & & & E092930 & 28-Apr-87 & & $10028-17-8$ & TRITIUM & TRITIŪM_ELECT_LSC & USTEST & 107 \\
\hline 5349 & PDMS & & sw & & & E092929 & 28-Apr-87 & & $10045-97-3$ & CS-137 & GAMMA_GS & USTEST & 0.588 \\
\hline 5350 & PDMS & & sw & & & E092929 & 28-Apr-87 & & $10098-97-2$ & SR-90 & SRISO_SEPP_PRECIP_GPC & USTEST & 0.161 \\
\hline 5351 & PDMS & & sw & & & E092929 & 28-Apr-87 & & U-238 & $\mathrm{U}-238$ & UISO_AEA & USTEST & 0.202 \\
\hline 5352 & PDMS & & sw & & & E092929 & 28-Apr-87 & & 15117-96-1 & U-235 & UISO_AEA & USTEST & 0.0143 \\
\hline 5353 & PDMS & & sw & & & E092929 & 28-Apr-87 & & $13966-29-5$ & $\mathrm{U}-234$ & UISO_AEA & USTEST & 0.304 \\
\hline 5354 & PDMS & & sW & & & E092929 & 28-Apr-87 & & $10028-17-8$ & TRITIUM & TRITIUM_ELECT_LSC & USTEST & 126 \\
\hline 5355 & PDMS & & sw & & & E093059 & 05-May-87 & & $10045-97-3$ & CS-137 & 901.1 GAMMA GS & USTEST & -0.105 \\
\hline 5356 & PDMS & & sw & & & E093059 & 05-May-87 & & $10028-17-8$ & TRITIUM & 906.0_H3_LSC & USTEST & 126 \\
\hline 5357 & PDMS & & sW & & & E093059 & 05-May-87 & & $10098-97-2$ & SR-90 & 905.0 SR GPC & USTEST & 0.0227 \\
\hline
\end{tabular}




\begin{tabular}{|c|c|c|c|c|c|c|c|c|c|c|c|c|c|}
\hline & A & $\mathrm{B}$ & $\mathrm{C}$ & $\mathrm{D}$ & $\mathrm{E}$ & $\mathrm{F}$ & $\mathrm{G}$ & $\mathrm{H}$ & $\mathrm{I}$ & $\mathrm{J}$ & $\mathrm{K}$ & $\mathrm{L}$ & $\mathrm{M}$ \\
\hline 1 & \begin{tabular}{|l|} 
SAMPLE_NUM_ASSIGNED_TO \\
\end{tabular} & \begin{tabular}{|l|} 
SAMPLER \\
\end{tabular} & MEDIA & BIOTA_MEDIA & TAG_ID & SAMP_NUM & SAMP_DATE_TIME & SAMP_DATE_TIME_ON & CON_ID & CON_SHORT_NAME & METHOD_NAME & LAB_CODE & VALUE_RPTD \\
\hline 5358 & PDMS & & SW & & & E093252 & 05-May-87 & & $10045-97-3$ & CS-137 & GAMMA_GS & PNL-RO & -0.00367 \\
\hline 5359 & PDMS & & sw & & & E093255 & 05-May-87 & & $10045-97-3$ & CS-137 & GAMMA_GS & PNL-RO & -0.00305 \\
\hline 5360 & PDMS & & sw & & & E093258 & 05-May-87 & & $10045-97-3$ & CS-137 & GAMMA_GS & PNL-RO & -0.0047 \\
\hline 5361 & PDMS & & sw & & & E093260 & 05-May-87 & & $10045-97-3$ & CS-137 & GAMMA_GS & PNL-RO & -0.003 \\
\hline \begin{tabular}{|l|l|}
5362 \\
\end{tabular} & PDMS & & sw & & & E093262 & 05-May-87 & & $10045-97-3$ & CS-137 & GAMMA_GS & PNL-RO & -0.00124 \\
\hline \begin{tabular}{|c|}
5363 \\
\end{tabular} & PDMS & & sw & & & E093265 & 05-May-87 & & $10045-97-3$ & CS-137 & GAMMA_GS & PNL-RO & -0.00855 \\
\hline 5364 & PDMS & & sW & & & E093060 & 08-May-87 & & $10045-97-3$ & CS-137 & 901.1_GAMMMA_GS & USTEST & -0.368 \\
\hline 5365 & PDMS & & SW & & & E093060 & 08-May-87 & & $10098-97-2$ & SR-90 & 905.0_SR_GPC & USTEST & 0.57 \\
\hline 5366 & PDMS & & sw & & & E093060 & 08-May-87 & & $10028-17-8$ & TRITIUM & TRITIUM_ELECT_LSC & USTEST & 135 \\
\hline \begin{tabular}{|l|l|}
5367 \\
\end{tabular} & PDMS & & SW & & & E093253 & 19-Мay-87 & & $10045-97-3$ & CS-137 & GAMMA_GS & PNL-RO & -0.00362 \\
\hline 5368 & PDMS & & sw & & & E093256 & 19-May-87 & & $10045-97-3$ & CS-137 & GAMMA_GS & PNL-RO & -0.0000857 \\
\hline 5369 & PDMS & & sW & & & E034048 & 19-Мау-87 & & $10045-97-3$ & CS-137 & GAMMA_GS & USTEST & \\
\hline 5370 & PDMS & & SW & & & E093263 & 19-Мay-87 & & $10045-97-3$ & CS-137 & GAMMA_GS & PNL-RO & 0.00375 \\
\hline 5371 & PDMS & & sW & & & E093266 & 19-May-87 & & $10045-97-3$ & CS-137 & GAMMA_GS & PNL-RO & -0.00952 \\
\hline 5372 & PDMS & & sw & & & E093202 & 20-May-87 & & $10045-97-3$ & CS-137 & GAMMA_GS & USTEST & -0.723 \\
\hline 5373 & PDMS & & sw & & & E093202 & 20-May-87 & & $10098-97-2$ & SR-90 & SRISO_SEP_PRECIP_GPC & USTEST & 0.148 \\
\hline 5374 & PDMS & & sw & & & E093267 & 26-May-87 & & $10045-97-3$ & CS-137 & GAMMA_GS & USTEST & 0.826 \\
\hline 5375 & PDMS & & sw & & & E093267 & 26-May-87 & & $10098-97-2$ & SR-90 & SRISO_SEP_PRECIP_GPC & USTEST & 0.12 \\
\hline 5376 & PDMS & & sW & & & E093267 & 26-Мау-87 & & $10028-17-8$ & TRITIUM & TRITIUM_DISTT_LSC & USTEST & -58 \\
\hline 5377 & PDMS & & sW & & & E093270 & 26-May-87 & & $10045-97-3$ & CS-137 & GAMMA_GS & USTEST & 0.362 \\
\hline 5378 & PDMS & & SW & & & E093270 & 26-May-87 & & $10028-17-8$ & TRITIUM & TRITIUM_DIST_LSC & USTEST & 9510 \\
\hline 5379 & PDMS & & sW & & & E093268 & 26-May-87 & & $10045-97-3$ & CS-137 & GAMMA_GS & USTEST & 0.92 \\
\hline 5380 & PDMS & & SW & & & E093268 & 26-May-87 & & $10098-97-2$ & SR-90 & SRISO_SEP_PRECIP_GPC & USTEST & 0.502 \\
\hline 5381 & PDMS & & sW & & & E093268 & 26-Мау-87 & & $10028-17-8$ & TRITIUM & TRITIUM_DIST_LSC & USTEST & 11.8 \\
\hline 5382 & PDMS & & SW & & & E093269 & 26-Мау-87 & & $10045-97-3$ & CS-137 & GAMMA_GS & USTEST & 0 \\
\hline 5383 & PDMS & & sW & & & E093269 & 26-Мау-87 & & $10098-97-2$ & SR-90 & SRISO_SEEP_PRECIP_GPC & USTEST & 1.77 \\
\hline 5384 & PDMS & & sW & & & E093269 & 26-May-87 & & $10028-17-8$ & TRITIUM & TRITIUM_DIST_LSC & USTEST & 455 \\
\hline 5385 & PDMS & & sW & & & E093269 & 26-May-87 & & U-238 & U-238 & UISO_AËA & USTEST & 92.7 \\
\hline 5386 & PDMS & & sw & & & E093269 & 26-May-87 & & 15117-96-1 & U-235 & UISO_AEA & USTEST & 3.74 \\
\hline 5387 & PDMS & & sw & & & E093269 & 26-May-87 & & $13966-29-5$ & U-234 & UISO_AEA & USTEST & 101 \\
\hline 5388 & PDMS & & sW & & & E093726 & 02-Jun-87 & & $10045-97-3$ & CS-137 & GAMMA_GS & PNL-RO & -0.00562 \\
\hline 5389 & PDMS & & sW & & & E093728 & 02-Jun-87 & & $10045-97-3$ & CS-137 & GAMMA_GS & PNL-RO & 0.000831 \\
\hline 5390 & PDMS & & sW & & & E093732 & 02-Jun-87 & & $10045-97-3$ & CS-137 & GAMMA_GS & PNL-RO & 0.00116 \\
\hline 5391 & PDMS & & SW & & & E093438 & 02-Jun-87 & & U-238 & U-238 & UISO_AĒAA & USTEST & 0.175 \\
\hline 5392 & PDMS & & sW & & & E093438 & 02-Jun-87 & & 15117-96-1 & U-235 & UISO_AEA & USTEST & 0.0127 \\
\hline 5393 & PDMS & & SW & & & E093438 & 02-Jun-87 & & $13966-29-5$ & U-234 & UISO_AEA & USTEST & 0.22 \\
\hline 5394 & PDMS & & SW & & & E093438 & 02-Jun-87 & & $10045-97-3$ & CS-137 & GAMMA_GS & USTEST & -1.1 \\
\hline 5395 & PDMS & & sW & & & E093730 & 02-Jun-87 & & $10045-97-3$ & CS-137 & GAMMA_GS & PNL-RO & -0.00332 \\
\hline 5396 & PDMS & & sW & & & E093438 & 02-Jun-87 & & $10098-97-2$ & SR-90 & SRISO_SEEP_PRECIP_GPC & USTEST & 0.161 \\
\hline $5397 \mathrm{~F}$ & PDMS & & SW & & & E093438 & 02-Jun-87 & & $10028-17-8$ & TRITIUM & TRITIUM̄E_ELËET_LSC̄ & USTEST & 94 \\
\hline 5398 & PDMS & & sW & & & E093736 & 02-Jun-87 & & $10045-97-3$ & CS-137 & GAMMA_GS & PNL-RO & -0.00643 \\
\hline 5399 & PDMS & & sW & & & E093437 & 02-Jun-87 & & U-238 & $\mathrm{U}-238$ & UISO_AĒAA & USTEST & 0.176 \\
\hline 5400 & PDMS & & SW & & & E093437 & 02-Jun-87 & & $15117-96-1$ & $\mathrm{U}-235$ & UISO_AEA & USTEST & 0.0103 \\
\hline 5401 & PDMS & & sW & & & E093437 & 02-Jun-87 & & $13966-29-5$ & $\mathrm{U}-234$ & UISO_AEA & USTEST & 0.139 \\
\hline 5402 & PDMS & & SW & & & E093437 & 02-Jun-87 & & $10045-97-3$ & CS-137 & GAMMA_GS & USTEST & 0.498 \\
\hline 5403 & PDMS & & SW & & & E093734 & 02-Jun-87 & & $10045-97-3$ & CS-137 & GAMMA_GS & PNL-RO & -0.00033 \\
\hline 5404 & PDMS & & sW & & & E093437 & 02-Jun-87 & & $10098-97-2$ & SR-90 & SRISO_SEEP_PRECIP_GPC & USTEST & 0.112 \\
\hline 5405 & PDMS & & sW & & & E093437 & 02-Jun-87 & & $10028-17-8$ & TRITIUM & TRITIUM̄E_ELËET_LSC̄ & USTEST & 67.8 \\
\hline 5406 & PDMS & & sw & & & E093729 & 16-Jun-87 & & $10045-97-3$ & CS-137 & GAMMA_GS & PNL-RO & -0.000502 \\
\hline 5407 & PDMS & & SW & & & E093727 & 16-Jun-87 & & $10045-97-3$ & CS-137 & GAMMA_GS & PNL-RO & -0.00838 \\
\hline 5408 & PDMS & & SW & & & E093731 & 16-Jun-87 & & $10045-97-3$ & CS-137 & GAMMA_GS & PNL-RO & -0.0121 \\
\hline 5409 & PDMS & & SW & & & E093733 & 16-Jun-87 & & $10045-97-3$ & CS-137 & GAMMA_GS & PNL-RO & -0.0082 \\
\hline
\end{tabular}




\begin{tabular}{|c|c|c|c|c|c|c|c|c|c|c|c|c|c|}
\hline & A & $\mathrm{B}$ & $\mathrm{C}$ & $\mathrm{D}$ & $\mathrm{E}$ & $\mathrm{F}$ & $\mathrm{G}$ & $\mathrm{H}$ & $\mathrm{I}$ & $\mathrm{J}$ & $\mathrm{K}$ & $\mathrm{L}$ & $\mathrm{M}$ \\
\hline 1 & SAMPLE_NUM_ASSIGNED_TO & SAMPLER & MEDIA & BIOTA_MEDIA & TAG_ID & SAMP_NUM & SAMP_DATE_TIME & SAMP_DATE_TIME_ON & CON_ID & CON_SHORT_NAME & METHOD_NAME & LAB_CODE & VALUE_RPTD \\
\hline 5410 & PDMS & & SW & & & E093737 & 16-Jun-87 & & $10045-97-3$ & CS-137 & GAMMA_GS & PNL-RO & -0.000239 \\
\hline 5411 & PDMS & & SW & & & E093735 & 16-Jun-87 & & $10045-97-3$ & CS-137 & GAMMA_GS & PNL-RO & 0.00136 \\
\hline 5412 & PDMS & & SW & & & E093675 & 22-Jun-87 & & $10045-97-3$ & CS-137 & 901.1_GAMMA_GS & USTEST & -0.55 \\
\hline 5413 & PDMS & & SW & & & E093675 & 22-Jun-87 & & $10098-97-2$ & SR-90 & 905.0_SR_GPC & USTEST & 0.0481 \\
\hline 5414 & PDMS & & SW & & & E093675 & 22-Jun-87 & & $10028-17-8$ & TRITIUM & 906.0_H3_LSC & USTEST & -117 \\
\hline 5415 & PDMS & & SW & & & E093674 & 22-Jun-87 & & $10045-97-3$ & CS-137 & 901.1_GAMMA_GS & USTEST & -0.158 \\
\hline 5416 & PDMS & & SW & & & E093674 & 22-Jun-87 & & $10098-97-2$ & SR-90 & 905.0_SR_GPC & USTEST & 0.0161 \\
\hline 5417 & PDMS & & SW & & & E093674 & 22-Jun-87 & & $10028-17-8$ & TRITIUM & 906.0_H3_LSC & USTEST & 2970 \\
\hline 5418 & PDMS & & SW & & & E093673 & 22-Jun-87 & & $10045-97-3$ & CS-137 & 901.1_GAMMA GS & USTEST & 0.0484 \\
\hline 5419 & PDMS & & SW & & & E093673 & 22-Jun-87 & & $10098-97-2$ & SR-90 & 905.0_SR_GPC & USTEST & 0.0147 \\
\hline 5420 & PDMS & & SW & & & E093673 & 22-Jun-87 & & $10028-17-8$ & TRITIUM & 906.0_H3_LSC & USTEST & -47.4 \\
\hline 5421 & PDMS & & SW & & & E093676 & 22-Jun-87 & & $10045-97-3$ & CS-137 & 901.1_GAMMA_GS & USTEST & 0.949 \\
\hline 5422 & PDMS & & SW & & & E093676 & 22-Jun-87 & & $10098-97-2$ & SR-90 & 905.0_SR_GPC & USTEST & -0.00509 \\
\hline 5423 & PDMS & & SW & & & E093676 & 22-Jun-87 & & $10028-17-8$ & TRITIUM & 906.0_H3_LSC & USTEST & -12.9 \\
\hline 5424 & PDMS & & SW & & & E093748 & 30-Jun-87 & & $10045-97-3$ & CS-137 & 901.1_GAMMA_GS & USTEST & 1.37 \\
\hline 5425 & PDMS & & SW & & & E093748 & 30-Jun-87 & & $10098-97-2$ & SR-90 & 905.0_SR_GPC & USTEST & 0.113 \\
\hline 5426 & PDMS & & SW & & & E093748 & 30-Jun-87 & & $10028-17-8$ & TRITIUM & TRITIŪM_ELECT_LSC & USTEST & 103 \\
\hline 5427 & PDMS & & SW & & & E033675 & 30-Jun-87 & & PU-239/240 & PU-239/240 & PUISO_IE_AEA & PNL-RO & 0.0000208 \\
\hline 5428 & PDMS & & SW & & & E033675 & 30-Jun-87 & & $10045-97-3$ & CS-137 & GAMMA_GS & PNL-RO & -0.00128 \\
\hline 5429 & PDMS & & SW & & & E033643 & 30-Jun-87 & & PU-239/240 & PU-239/240 & PUISO_IE_AEA & PNL-RO & 0.0000537 \\
\hline 5430 & PDMS & & SW & & & E033643 & 30-Jun-87 & & 15046-84-1 & $\mid-129$ & I129_SEP_TEMS & 320 & 0.0000789 \\
\hline 5431 & PDMS & & SW & & & E033643 & 30-Jun-87 & & $10045-97-3$ & CS-137 & GAMMA_ḠS & PNL-RO & -0.00477 \\
\hline 5432 & PDMS & & SW & & & E033672 & 30-Jun-87 & & PU-239/240 & PU-239/240 & PUISO_IE_AEA & PNL-RO & 0.000103 \\
\hline 5433 & PDMS & & SW & & & E033668 & 30-Jun-87 & & PU-239/240 & PU-239/240 & PUISO_IE_AEA & PNL-RO & 0.0000236 \\
\hline 5434 & PDMS & & SW & & & E033672 & 30-Jun-87 & & 15046-84-1 & I-129 & 1129_SEP_TEMS & 320 & 0.0000063 \\
\hline 5435 & PDMS & & SW & & & E033672 & 30-Jun-87 & & $10045-97-3$ & CS-137 & GAMMA_ḠS & PNL-RO & -0.00376 \\
\hline 5436 & PDMS & & sW & & & E033668 & 30-Jun-87 & & $10045-97-3$ & CS-137 & GAMMA_GS & PNL-RO & -0.0036 \\
\hline 5437 & PDMS & & SW & & & E093750 & 30-Jun-87 & & $13966-29-5$ & $\mathrm{U}-234$ & UISO_AĒA & USTEST & 0.232 \\
\hline 5438 & PDMS & & SW & & & E093750 & 30-Jun-87 & & $10045-97-3$ & CS-137 & GAMM̄A_GS & USTEST & 0.684 \\
\hline 5439 & PDMS & & SW & & & E093750 & 30-Jun-87 & & $10098-97-2$ & SR-90 & SRISO_SEP_PRECIP_GPC & USTEST & 0.123 \\
\hline 5440 & PDMS & & SW & & & E093750 & 30-Jun-87 & & $\mathrm{U}-238$ & $\mathrm{U}-238$ & UISO_AEA & USTEST & 0.167 \\
\hline 5441 & PDMS & & SW & & & E093750 & 30-Jun-87 & & 15117-96-1 & $\mathrm{U}-235$ & UISO_AEA & USTEST & 0.0124 \\
\hline 5442 & PDMS & & SW & & & E093750 & 30-Jun-87 & & $10028-17-8$ & TRITIUM & TRITIŪM_ELECT_LSC & USTEST & 71.3 \\
\hline 5443 & PDMS & & SW & & & E033651 & 30-Jun-87 & & PU-239/240 & PU-239/240 & PUISO_IE_AEA & PNL-RO & 0.0000977 \\
\hline 5444 & PDMS & & SW & & & E033647 & 30-Jun-87 & & PU-239/240 & PU-239/240 & PUISO_IE_AEA & PNL-RO & 0.0000451 \\
\hline 5445 & PDMS & & SW & & & E033651 & 30-Jun-87 & & 15046-84-1 & I-129 & I129_SEP_TEMS & 320 & 0.0000803 \\
\hline 5446 & PDMS & & SW & & & E033647 & 30-Jun-87 & & $10045-97-3$ & CS-137 & GAMMA_GS & PNL-RO & -0.00414 \\
\hline 5447 & PDMS & & SW & & & E033651 & 30-Jun-87 & & $10045-97-3$ & CS-137 & GAMMA_GS & PNL-RO & -0.00904 \\
\hline 5448 & PDMS & & SW & & & E093749 & 30-Jun-87 & & $\mathrm{U}-238$ & $\mathrm{U}-238$ & UISO_AĒA & USTEST & 0.193 \\
\hline 5449 & PDMS & & SW & & & E093749 & 30-Jun-87 & & 15117-96-1 & $\mathrm{U}-235$ & UISO_AEA & USTEST & 0.00354 \\
\hline 5450 & PDMS & & SW & & & E093749 & 30-Jun-87 & & $13966-29-5$ & $\mathrm{U}-234$ & UISO_AEA & USTEST & 0.284 \\
\hline 5451 & PDMS & & SW & & & E093749 & 30-Jun-87 & & $10045-97-3$ & CS-137 & GAMM̄A_GS & USTEST & -0.136 \\
\hline 5452 & PDMS & & SW & & & E093749 & 30-Jun-87 & & $10098-97-2$ & SR-90 & SRISO_SEP_PRECIP_GPC & USTEST & 0.109 \\
\hline 5453 & PDMS & & SW & & & E093749 & 30-Jun-87 & & $10028-17-8$ & TRITIUM & TRITIUM_ELECT_LSC & USTEST & 128 \\
\hline 5454 & PDMS & & SW & & & E093751 & 07-Jul-87 & & $10045-97-3$ & CS-137 & 901.1_GAMMA_GS & USTEST & 0.276 \\
\hline 5455 & PDMS & & SW & & & E093751 & 07-Jul-87 & & $10098-97-2$ & SR-90 & 905.0_SR_GPC & USTEST & 0.0917 \\
\hline 5456 & PDMS & & SW & & & E093751 & 07-Jul-87 & & $\mathrm{U}-238$ & $\mathrm{U}-238$ & UISO_IE_PLATE_AEA & USTEST & 0.236 \\
\hline 5457 & PDMS & & SW & & & E093751 & 07-Jul-87 & & 15117-96-1 & $\mathrm{U}-235$ & UISO_IE_PLATE_AEA & USTEST & 0.00727 \\
\hline 5458 & PDMS & & SW & & & E093751 & 07-Jul-87 & & $13966-29-5$ & $\mathrm{U}-234$ & UISO_IE_PLATE_AEA & USTEST & 0.247 \\
\hline 5459 & PDMS & & SW & & & E093751 & 07-Jul-87 & & $10028-17-8$ & TRITIUM & TRITIŪM_ELECT__LSC & USTEST & 130 \\
\hline 5460 & PDMS & & SW & & & E094351 & 14-Jul-87 & & $10045-97-3$ & CS-137 & GAMMA_GS & PNL-RO & -0.00369 \\
\hline 5461 & PDMS & & SW & & & E094348 & 14-Jul-87 & & $10045-97-3$ & CS-137 & GAMMA_GS & PNL-RO & -0.0000593 \\
\hline
\end{tabular}




\begin{tabular}{|c|c|c|c|c|c|c|c|c|c|c|c|c|c|}
\hline & A & $\mathrm{B}$ & $\mathrm{C}$ & $\mathrm{D}$ & $\mathrm{E}$ & $\mathrm{F}$ & G & $\mathrm{H}$ & $\mathrm{I}$ & $\mathrm{J}$ & $\mathrm{K}$ & $\mathrm{L}$ & $\mathrm{M}$ \\
\hline 1 & SAMPLE_NUM_ASSIGNED_TO & \begin{tabular}{|l|} 
SAMPLER \\
\end{tabular} & MEDIA & BIOTA_MEDIA & TAG_ID & SAMP_NUM & SAMP_DATE_TIME & SAMP_DATE_TIME_ON & CON_ID & CON_SHORT_NAME & METHOD_NAME & LAB_CODE & VALUE_RPTD \\
\hline 5462 & PDMS & & SW & & & E094354 & 14-Jul-87 & & $10045-97-3$ & CS-137 & GAMMA_GS & PNL-RO & -0.00375 \\
\hline 5463 & PDMS & & sw & & & E094357 & 14-Jul-87 & & $10045-97-3$ & CS-137 & GAMMA_GS & PNL-RO & -0.000785 \\
\hline 5464 & PDMS & & sw & & & E094360 & 14-Jul-87 & & $10045-97-3$ & CS-137 & GAMMA_GS & PNL-RO & -0.00759 \\
\hline 5465 & PDMS & & sw & & & E094363 & 14-Jul-87 & & $10045-97-3$ & CS-137 & GAMMA_GS & PNL-RO & -0.0123 \\
\hline \begin{tabular}{|l|l|}
5466 \\
\end{tabular} & PDMS & & sw & & & E093802 & 15-Jul-87 & & $10045-97-3$ & CS-137 & GAMMA_GS & USTEST & 0.362 \\
\hline \begin{tabular}{|l|l|}
5467 \\
\end{tabular} & PDMS & & sw & & & E093802 & 15-Jul-87 & & $10098-97-2$ & SR-90 & SRISO_SEP_PRECIP_GPC & USTEST & 0.124 \\
\hline \begin{tabular}{|l|l|}
5468 \\
\end{tabular} & PDMS & & sW & & & E093978 & 28-Jul-87 & & $10045-97-3$ & CS-137 & 901.1 GAMMA_GS & USTEST & -0.275 \\
\hline 5469 & PDMS & & SW & & & E093978 & 28-Jul-87 & & $10028-17-8$ & TRITIUM & 906.0_H3_LSC & USTEST & 8.6 \\
\hline 5470 & PDMS & & sw & & & E093978 & 28-Jul-87 & & $10098-97-2$ & SR-90 & 905.0_SR_GPC & USTEST & -0.0181 \\
\hline \begin{tabular}{|l|}
5471 \\
\end{tabular} & PDMS & & SW & & & E094349 & 28-Jul-87 & & $10045-97-3$ & CS-137 & GAMMĀA_GS & PNL-RO & -0.00129 \\
\hline 5472 & PDMS & & sw & & & E094352 & 28-Jul-87 & & $10045-97-3$ & CS-137 & GAMMA_GS & PNL-RO & -0.000806 \\
\hline 5473 & PDMS & & sW & & & E093977 & 28-Jul-87 & & U-238 & U-238 & UISO_AĒA & USTEST & 0.194 \\
\hline 5474 & PDMS & & sW & & & E093977 & 28-Jul-87 & & 15117-96-1 & U-235 & UISO_AEA & USTEST & 0.00632 \\
\hline 5475 & PDMS & & sW & & & E093977 & 28-Jul-87 & & $13966-29-5$ & U-234 & UISO_AEA & USTEST & 0.229 \\
\hline 5476 & PDMS & & sw & & & E093977 & 28-Jul-87 & & $10045-97-3$ & CS-137 & GAMMĀA_GS & USTEST & 0.242 \\
\hline 5477 & PDMS & & sw & & & E094358 & 28-Jul-87 & & $10045-97-3$ & CS-137 & GAMMA_GS & PNL-RO & -0.00503 \\
\hline 5478 & PDMS & & sw & & & E093977 & 28-Jul-87 & & $10098-97-2$ & SR-90 & SRISO_SEP_PRECIP_GPC & USTEST & 0.0963 \\
\hline 5479 & PDMS & & sw & & & E093977 & 28-Jul-87 & & $10028-17-8$ & TRITIUM & TRITIUM_ELECT_LSC & USTEST & 70.9 \\
\hline 5480 & PDMS & & sW & & & E094355 & 28-Jul-87 & & $10045-97-3$ & CS-137 & GAMMA_GS & PNL-RO & -0.00245 \\
\hline 5481 & PDMS & & sW & & & E094364 & 28-Jul-87 & & $10045-97-3$ & CS-137 & GAMMA_GS & PNL-RO & -0.0034 \\
\hline 5482 & PDMS & & sw & & & E093976 & 28-Jul-87 & & U-238 & U-238 & UISO_AĒA & USTEST & 0.265 \\
\hline 5483 & PDMS & & sW & & & E093976 & 28-Jul-87 & & 15117-96-1 & U-235 & UISO_AEA & USTEST & 0.00461 \\
\hline 5484 & PDMS & & SW & & & E093976 & 28-Jul-87 & & $13966-29-5$ & U-234 & UISO_AEA & USTEST & 0.298 \\
\hline 5485 & PDMS & & sW & & & E093976 & 28-Jul-87 & & $10045-97-3$ & CS-137 & GAMM̄A_GS & USTEST & 0.459 \\
\hline 5486 & PDMS & & SW & & & E094361 & 28-Jul-87 & & $10045-97-3$ & CS-137 & GAMMA_GS & PNL-RO & 0.0002 \\
\hline 5487 & PDMS & & sW & & & E093976 & 28-Jul-87 & & $10098-97-2$ & SR-90 & SRISO_SEEP_PRECIP_GPC & USTEST & 0.0986 \\
\hline 5488 & PDMS & & sW & & & E093976 & 28-Jul-87 & & $10028-17-8$ & TRITIUM & TRITIUM̄E_ELËCT_LSC̄ & USTEST & 131 \\
\hline 5489 & PDMS & & sW & & & E093979 & 31-Jul-87 & & $10045-97-3$ & CS-137 & 901.1_GAMMA_GS & USTEST & -0.339 \\
\hline 5490 & PDMS & & sw & & & E093979 & 31-Jul-87 & & $10098-97-2$ & SR-90 & 905.0_SR_GPC & USTEST & 0.436 \\
\hline 5491 & PDMS & & sW & & & E093979 & 31-Jul-87 & & $10028-17-8$ & TRITIUM & TRITIÜM_ELECT_LSC & USTEST & 105 \\
\hline 5492 & PDMS & & sW & & & E094350 & 11-Aug-87 & & $10045-97-3$ & CS-137 & GAMMA_GS & PNL-RO & 0.0000495 \\
\hline 5493 & PDMS & & sW & & & E094353 & 11-Aug-87 & & $10045-97-3$ & CS-137 & GAMMA_GS & PNL-RO & -0.0028 \\
\hline 5494 & PDMS & & sW & & & E094356 & 11-Aug-87 & & $10045-97-3$ & CS-137 & GAMMA_GS & PNL-RO & -0.00575 \\
\hline 5495 & PDMS & & SW & & & E094359 & 11-Aug-87 & & $10045-97-3$ & CS-137 & GAMMA_GS & PNL-RO & -0.0103 \\
\hline 5496 & PDMS & & sW & & & E094362 & 11-Aug-87 & & $10045-97-3$ & CS-137 & GAMMA_GS & PNL-RO & -0.00339 \\
\hline 5497 & PDMS & & sw & & & E094365 & 11-Aug-87 & & $10045-97-3$ & CS-137 & GAMMA_GS & PNL-RO & -0.0115 \\
\hline 5498 & PDMS & & SW & & & E094597 & 18-Aug-87 & & $10045-97-3$ & CS-137 & GAMMA_GS & USTEST & 1.79 \\
\hline 5499 & PDMS & & sW & & & E094597 & 18-Aug-87 & & $10028-17-8$ & TRITIUM & TRITIUM_DIST_LSC & USTEST & 125 \\
\hline 5500 & PDMS & & sW & & & E094597 & 18-Aug-87 & & $10098-97-2$ & SR-90 & SRISO_SEP_PRECIP_GPC & USTEST & 0.416 \\
\hline 5501 & PDMS & & SW & & & E094366 & 18-Aug-87 & & $10045-97-3$ & CS-137 & GAMMA_GS & USTEST & \\
\hline 5502 & PDMS & & sW & & & E094366 & 18-Aug-87 & & 10028-17-8 & TRITIUM & TRITIUM_DIST_LSC & USTEST & \\
\hline 5503 & PDMS & & sW & & & E094598 & 18-Aug-87 & & $10045-97-3$ & CS-137 & GAMMA_GS & USTEST & 50.2 \\
\hline 5504 & PDMS & & SW & & & E094598 & 18-Aug-87 & & $10028-17-8$ & TRITIUM & TRITIUM_DIST_LSC & USTEST & 222 \\
\hline 5505 & PDMS & & sW & & & E094598 & 18-Aug-87 & & $10098-97-2$ & SR-90 & SRISO_SEP_PRECIP_GPC & USTEST & 1.41 \\
\hline 5506 & PDMS & & SW & & & E094648 & 18-Aug-87 & & U-238 & $\mathrm{U}-238$ & UISO_AEA & USTEST & 166 \\
\hline 5507 & PDMS & & SW & & & E094648 & 18-Aug-87 & & 15117-96-1 & $\mathrm{U}-235$ & UISO_AEA & USTEST & 5.76 \\
\hline 5508 & PDMS & & sW & & & E094648 & 18-Aug-87 & & $13966-29-5$ & $\mathrm{U}-234$ & UISO_AEA & USTEST & 176 \\
\hline 5509 & PDMS & & sW & & & E094648 & 18-Aug-87 & & $10028-17-8$ & TRITIUM & TRITIUMM_DIST_LSC & USTEST & 571 \\
\hline 5510 & PDMS & & sw & & & E094648 & 18-Aug-87 & & $10045-97-3$ & CS-137 & GAMMA_GS & USTEST & 1.58 \\
\hline 5511 & PDMS & & SW & & & E094648 & 18-Aug-87 & & $10098-97-2$ & SR-90 & SRISO_SEP_PRECIP_GPC & USTEST & 2.76 \\
\hline 5512 & PDMS & & SW & & & E094968 & 25-Aug-87 & & $10045-97-3$ & CS-137 & GAMMA_GS & PNL-RO & -0.0139 \\
\hline 5513 & PDMS & & SW & & & E094970 & 25-Aug-87 & & $10045-97-3$ & CS-137 & GAMMA_GS & PNL-RO & -0.00293 \\
\hline
\end{tabular}




\begin{tabular}{|c|c|c|c|c|c|c|c|c|c|c|c|c|c|}
\hline & $\bar{A}$ & $\mathrm{~B}$ & $\mathrm{C}$ & $\bar{D}$ & $\mathrm{E}$ & $\mathrm{F}$ & $\bar{G}$ & $\mathrm{H}$ & 1 & $\mathrm{~J}$ & $\mathrm{~K}$ & $\bar{L}$ & $\bar{M}$ \\
\hline 1 & SAMPLE_NUM_ASSIGNED_TO & SAMPLER & MEDIA & BIOTA_MEDIA & TAG_ID & SAMP_NUM & SAMP_DATE_TIME & SAMP_DATE_TIME_ON & CON_ID & CON_SHORT_NAME & METHOD_NAME & LAB_CODE & VALUE_RPTD \\
\hline 5514 & PDMS & & SW & & & E034049 & 25-Aug-87 & & $10045-97-3$ & CS-137 & GAMMA_GS & USTEST & \\
\hline 5515 & PDMS & & sw & & & E094976 & 25-Aug-87 & & $10045-97-3$ & CS-137 & GAMMA_GS & PNL-RO & -0.00523 \\
\hline 5516 & PDMS & & SW & & & E094979 & 25-Aug-87 & & $10045-97-3$ & CS-137 & GAMMA_GS & PNL-RO & -0.00909 \\
\hline 5517 & PDMS & & sW & & & E094596 & 01-Sep-87 & & $10028-17-8$ & TRITIUM & TRITIUM ELECT_LSC & USTEST & 88.4 \\
\hline 5518 & PDMS & & SW & & & E094596 & 01-Sep-87 & & $10045-97-3$ & CS-137 & GAMMA_GS & USTEST & 0.436 \\
\hline 5519 & PDMS & & sw & & & E094596 & 01-Sep-87 & & $\mathrm{U}-238$ & U-238 & UISO_AEA & USTEST & 0.151 \\
\hline 5520 & PDMS & & sW & & & E094596 & 01-Sep-87 & & 15117-96-1 & U-235 & UISO_AEA & USTEST & 0.0114 \\
\hline 5521 & PDMS & & sW & & & E094596 & 01-Sep-87 & & $13966-29-5$ & U-234 & UISO_AEA & USTEST & 0.171 \\
\hline 5522 & PDMS & & sW & & & E094596 & 01-Sep-87 & & $10098-97-2$ & SR-90 & SRISŌSSEP_PRECIP_GPC & USTEST & 0.128 \\
\hline 5523 & PDMS & & SW & & & E094594 & 01-Sep-87 & & $10028-17-8$ & TRITIUM & TRITIUM_ELECT_LSC & USTEST & 130 \\
\hline 5524 & PDMS & & SW & & & E094594 & 01-Sep-87 & & $10045-97-3$ & CS-137 & GAMMA GS & USTEST & -0.745 \\
\hline 5525 & PDMS & & sw & & & E094594 & 01-Sep-87 & & $\mathrm{U}-238$ & U-238 & UISO_AEA & USTEST & 0.183 \\
\hline 5526 & PDMS & & sw & & & E094594 & 01-Sep-87 & & 15117-96-1 & U-235 & UISO_AEA & USTEST & 0.015 \\
\hline 5527 & PDMS & & sw & & & E094594 & 01-Sep-87 & & $13966-29-5$ & U-234 & UISO_AEA & USTEST & 0.214 \\
\hline 5528 & PDMS & & sw & & & E094594 & 01-Sep-87 & & $10098-97-2$ & SR-90 & SRISO_SEP_PRECIP_GPC & USTEST & 0.116 \\
\hline 5529 & PDMS & & sw & & & E094488 & 08-Sep-87 & & $10045-97-3$ & CS-137 & GAMMA GS & PNL-RO & \\
\hline 5530 & PDMS & & sW & & & E094489 & 08-Sep-87 & & $10045-97-3$ & CS-137 & GAMMA_GS & PNL-RO & \\
\hline 5531 & PDMS & & sw & & & E094974 & 08-Sep-87 & & $10045-97-3$ & CS-137 & GAMMA_GS & PNL-RO & 0.00000845 \\
\hline 5532 & PDMS & & sw & & & E094972 & 08-Sep-87 & & $10045-97-3$ & CS-137 & GAMMA_GS & PNL-RO & -0.00139 \\
\hline 5533 & PDMS & & sW & & & E094980 & 08-Sep-87 & & $10045-97-3$ & CS-137 & GAMMA_GS & PNL-RO & -0.000123 \\
\hline 5534 & PDMS & & sW & & & E094977 & 08-Sep-87 & & $10045-97-3$ & CS-137 & GAMMA_GS & PNL-RO & 0.0014 \\
\hline 5535 & PDMS & & SW & & & E094600 & 09-Sep-87 & & $10045-97-3$ & CS-137 & GAMMA_GS & USTEST & 0.138 \\
\hline 5536 & PDMS & & sW & & & E094600 & 09-Sep-87 & & $10098-97-2$ & SR-90 & SRISO_SEEP_PRECIP_GPC & USTEST & 0.16 \\
\hline 5537 & PDMS & & sw & & & E094651 & 14-Sep-87 & & $10028-17-8$ & TRITIUM & 906.0_H3_LSC & USTEST & 156 \\
\hline 5538 & PDMS & & sw & & & E094651 & 14-Sep-87 & & $10045-97-3$ & CS-137 & 901.1_GAMMA_GS & USTEST & 1.31 \\
\hline 5539 & PDMS & & sw & & & E094651 & 14-Sep-87 & & $10098-97-2$ & SR-90 & 905.0_SR_GPC & USTEST & 0.016 \\
\hline 5540 & PDMS & & sw & & & E094650 & 14-Sep-87 & & $10028-17-8$ & TRITIUM & 906.0_H3_LSC & USTEST & 4300 \\
\hline 5541 & PDMS & & sw & & & E094650 & 14-Sep-87 & & $10045-97-3$ & CS-137 & 901.1_GAMMA_GS & USTEST & -0.316 \\
\hline 5542 & PDMS & & sw & & & E094650 & 14-Sep-87 & & $10098-97-2$ & SR-90 & 905.0_SR_GPC & USTEST & -0.00593 \\
\hline 5543 & PDMS & & sW & & & E094649 & 14-Sep-87 & & $10028-17-8$ & TRITIUM & 906.0_H3_LSC & USTEST & 43 \\
\hline 5544 & PDMS & & sw & & & E094649 & 14-Sep-87 & & $10045-97-3$ & CS-137 & 901.1_GAMMA_GS & USTEST & 0.362 \\
\hline 5545 & PDMS & & sw & & & E094649 & 14-Sep-87 & & $10098-97-2$ & SR-90 & 905.0_SR_GPC & USTEST & 0.0238 \\
\hline 5546 & PDMS & & sw & & & E094652 & 14-Sep-87 & & $10028-17-8$ & TRITIUM & 906.0_H3_LSC & USTEST & 54.9 \\
\hline 5547 & PDMS & & sW & & & E094652 & 14-Sep-87 & & $10045-97-3$ & CS-137 & 901.1_GAMMA_GS & USTEST & -0.744 \\
\hline 5548 & PDMS & & SW & & & E094652 & 14-Sep-87 & & $10098-97-2$ & SR-90 & 905.0_SR_GPC & USTEST & -0.00696 \\
\hline 5549 & PDMS & & sW & & & E094810 & 22-Sep-87 & & $10028-17-8$ & TRITIUM & TRITIŪM_ELECT_LSC & USTEST & 111 \\
\hline 5550 & PDMS & & sW & & & E094810 & 22-Sep-87 & & $10045-97-3$ & CS-137 & 901.1_GAMMA_Ḡ & USTEST & 0.593 \\
\hline 5551 & PDMS & & sW & & & E094810 & 22-Sep-87 & & $10098-97-2$ & SR-90 & 905.0_SR_GPC & USTEST & 0.146 \\
\hline 5552 & PDMS & & sW & & & E094969 & 22-Sep-87 & & $10045-97-3$ & CS-137 & GAMMA GS & PNL-RO & -0.00307 \\
\hline 5553 & PDMS & & sw & & & E094971 & $22-\mathrm{Sep}-87$ & & $10045-97-3$ & CS-137 & GAMMA_GS & PNL-RO & -0.00427 \\
\hline 5554 & PDMS & & sW & & & E094973 & 22-Sep-87 & & $10045-97-3$ & CS-137 & GAMMA_GS & PNL-RO & 0.00189 \\
\hline 5555 & PDMS & & sW & & & E094975 & 22-Sep-87 & & $10045-97-3$ & CS-137 & GAMMA_GS & PNL-RO & 0.00264 \\
\hline 5556 & PDMS & & sW & & & E094978 & 22-Sep-87 & & $10045-97-3$ & CS-137 & GAMMA_GS & PNL-RO & 0.00102 \\
\hline 5557 & PDMS & & sw & & & E094981 & 22-Sep-87 & & $10045-97-3$ & CS-137 & GAMMA_GS & PNL-RO & 0.00436 \\
\hline 5558 & PDMS & & sw & & & E094813 & 29-Sep-87 & & U-238 & U-238 & UISO_IE_PLATE_AEA & USTEST & 0.257 \\
\hline 5559 & PDMS & & sw & & & E094813 & 29-Sep-87 & & 15117-96-1 & U-235 & UISO_IE_PLATE_AEA & USTEST & 0.00378 \\
\hline 5560 & PDMS & & sw & & & E094813 & 29-Sep-87 & & 13966-29-5 & $\mathrm{U}-234$ & UISO_IE_PLATE_AEA & USTEST & 0.328 \\
\hline 5561 & PDMS & & sw & & & E094813 & 29-Sep-87 & & $10028-17-8$ & TRITIUM & TRITIUM_ELECT_LSC & USTEST & 204 \\
\hline 5562 & PDMS & & sW & & & E094813 & 29-Sep-87 & & $10045-97-3$ & CS-137 & 901.1_GAMMA_GS & USTEST & -0.272 \\
\hline 5563 & PDMS & & sW & & & E094813 & 29-Sep-87 & & $10098-97-2$ & SR-90 & 905.0_SR_GPC & USTEST & 0.133 \\
\hline 5564 & PDMS & & SW & & & E094812 & 29-Sep-87 & & U-238 & U-238 & UISO_AEA & USTEST & 0.191 \\
\hline 5565 & PDMS & & sW & & & E094812 & 29-Sep-87 & & 15117-96-1 & $\mathrm{U}-235$ & UISO AEA & USTEST & 0.0278 \\
\hline
\end{tabular}




\begin{tabular}{|c|c|c|c|c|c|c|c|c|c|c|c|c|c|}
\hline & A & $B$ & $\mathrm{C}$ & $\mathrm{D}$ & $\mathrm{E}$ & $\mathrm{F}$ & G & $\mathrm{H}$ & 1 & $\mathrm{~J}$ & $\mathrm{~K}$ & $\mathrm{~L}$ & $\mathrm{M}$ \\
\hline 1 & SAMPLE_NUM_ASSIGNED_TO & SAMPLER & MEDIA & BIOTA_MEDIA & TAG_ID & SAMP_NUM & SAMP_DATE_TIME & SAMP_DATE_TIME_ON & CON_ID & CON_SHORT_NAME & METHOD_NAME & LAB_CODE & VALUE_RPTD \\
\hline 5566 & PDMS & & SW & & & E094812 & 29-Sep-87 & & $13966-29-5$ & $\mathrm{U}-234$ & UISO_AEA & USTEST & 0.273 \\
\hline 5567 & PDMS & & SW & & & E094812 & 29-Sep-87 & & 10028-17-8 & TRITIUM & TRITIUM_ELECT_LSC & USTEST & 65.1 \\
\hline 5568 & PDMS & & SW & & & E094812 & 29-Sep-87 & & $10045-97-3$ & CS-137 & GAMMA_GS & USTEST & -0.789 \\
\hline 5569 & PDMS & & SW & & & E094812 & 29-Sep-87 & & $10098-97-2$ & SR-90 & SRISO_SEP_PRECIP_GPC & USTEST & 0.167 \\
\hline 5570 & PDMS & & SW & & & E094811 & 29-Sep-87 & & U-238 & U-238 & UISO_AEA & USTEST & 0.175 \\
\hline 5571 & PDMS & & SW & & & E094811 & 29-Sep-87 & & 15117-96-1 & U-235 & UISO_AEA & USTEST & 0.00775 \\
\hline 5572 & PDMS & & SW & & & E094811 & 29-Sep-87 & & $13966-29-5$ & U-234 & UISO_AEA & USTEST & 0.241 \\
\hline 5573 & PDMS & & SW & & & E094811 & 29-Sep-87 & & $10028-17-8$ & TRITIUM & TRITIUM_ELECT_LSC & USTEST & 178 \\
\hline 5574 & PDMS & & SW & & & E094811 & 29-Sep-87 & & $10045-97-3$ & CS-137 & GAMMA_GS & USTEST & 0.643 \\
\hline 5575 & PDMS & & SW & & & E094811 & 29-Sep-87 & & $10098-97-2$ & SR-90 & SRISO_S̄EP_PRECIP_GPC & USTEST & 0.135 \\
\hline 5576 & PDMS & & SW & & & E033644 & 06-Oct-87 & & 15046-84-1 & I-129 & I129_SEP_TEMS & 320 & 0.0001301 \\
\hline 5577 & PDMS & & SW & & & E033676 & 06-Oct-87 & & PU-239/240 & PU-239/240 & PUISO_IE_AEA & PNL-RO & 0.0000326 \\
\hline 5578 & PDMS & & SW & & & E033644 & 06-Oct-87 & & PU-239/240 & PU-239/240 & PUISO_IE_AEA & PNL-RO & 0.0000556 \\
\hline 5579 & PDMS & & SW & & & E033644 & 06-Oct-87 & & $10045-97-3$ & CS-137 & GAMMA_GS & PNL-RO & 0.00228 \\
\hline 5580 & PDMS & & SW & & & E033676 & 06-Oct-87 & & $10045-97-3$ & CS-137 & GAMMA_GS & PNL-RO & -0.000233 \\
\hline 5581 & PDMS & & SW & & & E033664 & 06-Oct-87 & & PU-239/240 & PU-239/240 & PUISO_IE_AEA & PNL-RO & 0.0000675 \\
\hline 5582 & PDMS & & SW & & & E033664 & 06-Oct-87 & & 15046-84-1 & I-129 & 1129_SEP_TEMS & 320 & 0.0000052 \\
\hline 5583 & PDMS & & SW & & & E033664 & $06-$ Oct-87 & & $10045-97-3$ & CS-137 & GAMMA_GS & PNL-RO & 0.000947 \\
\hline 5584 & PDMS & & SW & & & E033669 & 06-Oct-87 & & PU-239/240 & PU-239/240 & PUISO_IE_AEA & PNL-RO & 0.0000221 \\
\hline 5585 & PDMS & & SW & & & E033669 & 06-Oct-87 & & $10045-97-3$ & CS-137 & GAMMA_GS & PNL-RO & 0.00116 \\
\hline 5586 & PDMS & & SW & & & E033652 & 06-Oct-87 & & 15046-84-1 & I-129 & 1129_SEP_TEMS & 320 & 0.000125 \\
\hline 5587 & PDMS & & SW & & & E033652 & 06-Oct-87 & & PU-239/240 & PU-239/240 & PUISO_IE_AEA & PNL-RO & 0.0000681 \\
\hline 5588 & PDMS & & sW & & & E033648 & 06-Oct-87 & & PU-239/240 & PU-239/240 & PUISO_IE_AEA & PNL-RO & 0.0000634 \\
\hline 5589 & PDMS & & SW & & & E033648 & 06-Oct-87 & & $10045-97-3$ & CS-137 & GAMMA_GS & PNL-RO & 0.00209 \\
\hline 5590 & PDMS & & SW & & & E033652 & 06-Oct-87 & & $10045-97-3$ & CS-137 & GAMMA_GS & PNL-RO & -0.00417 \\
\hline 5591 & PDMS & & SW & & & E095210 & 20-Oct-87 & & $10098-97-2$ & SR-90 & 905.0_SR_GPC & USTEST & 0.0022 \\
\hline 5592 & PDMS & & SW & & & E095210 & 20-Oct-87 & & $10028-17-8$ & TRITIUM & 906.0_H3_LSC & USTEST & 73 \\
\hline 5593 & PDMS & & SW & & & E095210 & 20-Oct-87 & & $10045-97-3$ & CS-137 & 901.1_GAMMA_GS & USTEST & 0.542 \\
\hline 5594 & PDMS & & sW & & & E033715 & 20-Oct-87 & & $10045-97-3$ & CS-137 & GAMMA_GS & USTEST & 0.63 \\
\hline 5595 & PDMS & & SW & & & E033715 & 20-Oct-87 & & U-238 & U-238 & UISO_AEA & USTEST & 0.236 \\
\hline 5596 & PDMS & & SW & & & E033715 & 20-Oct-87 & & 15117-96-1 & U-235 & UISO_AEA & USTEST & 0.0105 \\
\hline 5597 & PDMS & & SW & & & E033715 & 20-Oct-87 & & 13966-29-5 & U-234 & UISO_AEA & USTEST & 0.216 \\
\hline 5598 & PDMS & & SW & & & E033715 & 20-Oct-87 & & $10098-97-2$ & SR-90 & SRISO_SEP_PRECIP_GPC & USTEST & 0.127 \\
\hline 5599 & PDMS & & SW & & & E033715 & 20-Oct-87 & & $10028-17-8$ & TRITIUM & TRITIUM_ELECT_LSC & USTEST & 100 \\
\hline 5600 & PDMS & & SW & & & E033714 & 20-Oct-87 & & $10045-97-3$ & CS-137 & GAMMA_GS & USTEST & 0 \\
\hline 5601 & PDMS & & SW & & & E033714 & 20-Oct-87 & & 15117-96-1 & U-235 & UISO_AEAA & USTEST & 0.0106 \\
\hline 5602 & PDMS & & SW & & & E033714 & 20-Oct-87 & & $13966-29-5$ & U-234 & UISO_AEA & USTEST & 0.24 \\
\hline 5603 & PDMS & & SW & & & E033714 & $20-$ Oct-87 & & U-238 & $\mathrm{U}-238$ & UISO_AEA & USTEST & 0.182 \\
\hline 5604 & PDMS & & SW & & & E033714 & 20-Oct-87 & & $10098-97-2$ & SR-90 & SRISO_SEP_PRECIP_GPC & USTEST & 0.127 \\
\hline 5605 & PDMS & & SW & & & E033714 & 20-Oct-87 & & $10028-17-8$ & TRITIUM & TRITIUM_ELECT_LSC & USTEST & 97.4 \\
\hline 5606 & PDMS & & SW & & & E095681 & 20-Oct-87 & & $10045-97-3$ & CS-137 & GAMMA_GS & PNL-RO & 0.000927 \\
\hline 5607 & PDMS & & SW & & & E095294 & 20-Oct-87 & & $10045-97-3$ & CS-137 & GAMMA_GS & PNL-RO & 0.0000817 \\
\hline 5608 & PDMS & & SW & & & E095685 & 20-Oct-87 & & $10045-97-3$ & CS-137 & GAMMA_GS & PNL-RO & 0.00854 \\
\hline 5609 & PDMS & & SW & & & E095689 & $20-$ Oct-87 & & $10045-97-3$ & CS-137 & GAMMA_GS & PNL-RO & 0.000668 \\
\hline 5610 & PDMS & & SW & & & E095693 & 20-Oct-87 & & $10045-97-3$ & CS-137 & GAMMA_GS & PNL-RO & 0.00147 \\
\hline 5611 & PDMS & & SW & & & E095697 & $20-$ Oct-87 & & $10045-97-3$ & CS-137 & GAMMA_GS & PNL-RO & 0.00442 \\
\hline 5612 & PDMS & & SW & & & E033450 & 23-Oct-87 & & $10028-17-8$ & TRITIUM & TRITIUM_ELECT_LSC & USTEST & 209 \\
\hline 5613 & PDMS & & SW & & & E033450 & 23-Oct-87 & & $10098-97-2$ & SR-90 & 905.0_SR_GPC & USTEST & 0.409 \\
\hline 5614 & PDMS & & SW & & & E033450 & 23-Oct-87 & & $10045-97-3$ & CS-137 & 901.1_GAMMA_GS & USTEST & -0.242 \\
\hline 5615 & PDMS & & SW & & & E095295 & 03-Nov-87 & & $10045-97-3$ & CS-137 & GAMMA_GS & PNL-RO & 0.00296 \\
\hline 5616 & PDMS & & SW & & & E095682 & 03-Nov-87 & & $10045-97-3$ & CS-137 & GAMMA_GS & PNL-RO & -0.00146 \\
\hline $5617 \mid$ & PDMS & & SW & & & E033475 & 03-Nov-87 & & $10028-17-8$ & TRITIUM & TRITIUM_ELECT_LSC & USTEST & 52.3 \\
\hline
\end{tabular}




\begin{tabular}{|c|c|c|c|c|c|c|c|c|c|c|c|c|c|}
\hline & A & $\mathrm{B}$ & $\mathrm{C}$ & $\mathrm{D}$ & $\mathrm{E}$ & $\mathrm{F}$ & $\mathrm{G}$ & $\mathrm{H}$ & $\mathrm{I}$ & $\mathrm{J}$ & $\mathrm{K}$ & $\mathrm{L}$ & $\mathrm{M}$ \\
\hline 1 & SAMPLE_NUM_ASSIGNED_TO & \begin{tabular}{|l|} 
SAMPLER \\
\end{tabular} & MEDIA & BIOTA_MEDIA & TAG_ID & SAMP_NUM & SAMP_DATE_TIME & SAMP_DATE_TIME_ON & CON_ID & CON_SHORT_NAME & METHOD_NAME & LAB_CODE & VALUE_RPTD \\
\hline $5618 \mathrm{~F}$ & PDMS & & SW & & & E033475 & 03-Nov-87 & & U-238 & $U-238$ & UISO_AEA & USTEST & 0.366 \\
\hline 5619 & PDMS & & sw & & & E033475 & 03-Nov-87 & & 15117-96-1 & U-235 & UISO_AEA & USTEST & 0.00461 \\
\hline 5620 & PDMS & & sw & & & E033475 & 03-Nov-87 & & $13966-29-5$ & U-234 & UISO_AEA & USTEST & 0.197 \\
\hline $5621 \mathrm{~F}$ & PDMS & & sw & & & E033475 & 03-Nov-87 & & $10098-97-2$ & SR-90 & SRISO_SEP_PRECIP_GPC & USTEST & 0.127 \\
\hline 5622 & PDMS & & sw & & & E033475 & 03-Nov-87 & & $10045-97-3$ & CS-137 & GAMMA_GS & USTEST & 0.639 \\
\hline $5623 \mid$ & PDMS & & sw & & & E095690 & 03-Nov-87 & & $10045-97-3$ & CS-137 & GAMMA_GS & PNL-RO & 0.0000424 \\
\hline 5624 & PDMS & & sW & & & E095686 & 03-Nov-87 & & $10045-97-3$ & CS-137 & GAMMA_GS & PNL-RO & 0.00761 \\
\hline 5625 & PDMS & & SW & & & E095694 & 03-Nov-87 & & $10045-97-3$ & CS-137 & GAMMA_GS & PNL-RO & -0.00166 \\
\hline 5626 & PDMS & & sw & & & E095698 & 03-Nov-87 & & $10045-97-3$ & CS-137 & GAMMA_GS & PNL-RO & 0.00596 \\
\hline 5627 & PDMS & & SW & & & E033474 & 03-Nov-87 & & $10028-17-8$ & TRITIUM & TRITIUM_ELECT_LSC & USTEST & 112 \\
\hline $5628 \mid \mathrm{F}$ & PDMS & & sW & & & E033474 & 03-Nov-87 & & $10045-97-3$ & CS-137 & GAMMA_GS & USTEST & -0.776 \\
\hline 5629 & PDMS & & sW & & & E033474 & 03-Nov-87 & & $\mathrm{U}-238$ & U-238 & UISO_AĒA & USTEST & 0.187 \\
\hline 5630 & PDMS & & sW & & & E033474 & 03-Nov-87 & & 15117-96-1 & U-235 & UISO_AEA & USTEST & 0.00795 \\
\hline 5631 & PDMS & & sW & & & E033474 & 03-Nov-87 & & $13966-29-5$ & U-234 & UISO_AEA & USTEST & 0.264 \\
\hline $5632 \mathrm{~F}$ & PDMS & & sw & & & E033474 & 03-Nov-87 & & $10098-97-2$ & SR-90 & SRISO_SEP_PRECIP_GPC & USTEST & 0.123 \\
\hline 5633 & PDMS & & sw & & & E095296 & 10-Nov-87 & & $10045-97-3$ & CS-137 & GAMMA_GS & USTEST & 0.27 \\
\hline $5634 \mid \mathrm{F}$ & PDMS & & sw & & & E095296 & 10-Nov-87 & & $10098-97-2$ & SR-90 & SRISO_SEP_PRECIP_GPC & USTEST & 0.271 \\
\hline $5635 \mathrm{~F}$ & PDMS & & sw & & & E095296 & 10-Nov-87 & & $10028-17-8$ & TRITIUM & TRITIUM_DIST_LSC & USTEST & -10.7 \\
\hline 5636 & PDMS & & sW & & & E095298 & 10-Nov-87 & & $10045-97-3$ & CS-137 & GAMMA_GS & USTEST & -0.27 \\
\hline 5637 & PDMS & & sW & & & E095298 & 10-Nov-87 & & $10028-17-8$ & TRITIUM & TRITIUM_DIST_LSC & USTEST & 4750 \\
\hline 5638 & PDMS & & sw & & & E095349 & 10-Nov-87 & & $10098-97-2$ & SR-90 & SRISO_SEP_PRECIP_GPC & USTEST & \\
\hline 5639 & PDMS & & sW & & & E095349 & 10-Nov-87 & & $10028-17-8$ & TRITIUM & TRITIUM_DISTT_LSC & USTEST & \\
\hline 5640 & PDMS & & SW & & & E095349 & 10-Nov-87 & & $10045-97-3$ & CS-137 & GAMMA_GS & USTEST & \\
\hline 5641 & PDMS & & sW & & & E095297 & 10-Nov-87 & & $10045-97-3$ & CS-137 & GAMMA_GS & USTEST & 0.629 \\
\hline 5642 & PDMS & & SW & & & E095297 & 10-Nov-87 & & $\mathrm{U}-238$ & $\mathrm{U}-238$ & UISO_AĒA & USTEST & 128 \\
\hline 5643 & PDMS & & sW & & & E095297 & 10-Nov-87 & & 15117-96-1 & U-235 & UISO_AEA & USTEST & 7.32 \\
\hline $5644 \mid \mathrm{F}$ & PDMS & & sW & & & E095297 & 10-Nov-87 & & $13966-29-5$ & U-234 & UISO_AEA & USTEST & 132 \\
\hline 5645 & PDMS & & sW & & & E095297 & 10-Nov-87 & & $10098-97-2$ & SR-90 & SRISŌ_SEP_PRECIP_GPC & USTEST & 2.32 \\
\hline 5646 & PDMS & & sw & & & E095297 & 10-Nov-87 & & $10028-17-8$ & TRITIUM & TRITIUM_DIST_LSC & USTEST & 432 \\
\hline $5647 \mathrm{~F}$ & PDMS & & sw & & & E095282 & 13-Nov-87 & & $10045-97-3$ & CS-137 & GAMMA_GS & USTEST & 1.17 \\
\hline 5648 & PDMS & & sw & & & E095282 & 13-Nov-87 & & $10028-17-8$ & TRITIUM & TRITIUM_DIST_LSC & USTEST & -106 \\
\hline 5649 & PDMS & & sW & & & E095282 & 13-Nov-87 & & $\mathrm{U}-238$ & $\mathrm{U}-238$ & UISO_AËA & USTEST & 19.2 \\
\hline 5650 & PDMS & & sW & & & E095282 & 13-Nov-87 & & 15117-96-1 & $\mathrm{U}-235$ & UISO_AEA & USTEST & 1.15 \\
\hline 5651 & PDMS & & SW & & & E095282 & 13-Nov-87 & & $13966-29-5$ & $\mathrm{U}-234$ & UISO_AEA & USTEST & 28.9 \\
\hline 5652 & PDMS & & sW & & & E095280 & 13-Nov-87 & & $10045-97-3$ & CS-137 & GAMM̄A_GS & USTEST & 1.74 \\
\hline 5653 & PDMS & & SW & & & E095280 & 13-Nov-87 & & 10028-17-8 & TRITIUM & TRITIUM_DIST_LSC & USTEST & 14 \\
\hline 5654 & PDMS & & SW & & & E095280 & 13-Nov-87 & & $\mathrm{U}-238$ & $\mathrm{U}-238$ & UISO_AEA & USTEST & 3.26 \\
\hline 5655 & PDMS & & sW & & & E095280 & 13-Nov-87 & & 15117-96-1 & U-235 & UISO_AEA & USTEST & 0.124 \\
\hline 5656 & PDMS & & sW & & & E095280 & 13-Nov-87 & & $13966-29-5$ & $\mathrm{U}-234$ & UISO_AEA & USTEST & 3.84 \\
\hline 5657 & PDMS & & SW & & & E095281 & 13-Nov-87 & & $10045-97-3$ & CS-137 & GAMM̄A_GS & USTEST & 0.179 \\
\hline 5658 & PDMS & & sW & & & E095281 & 13-Nov-87 & & 10028-17-8 & TRITIUM & TRITIUM_DIST_LSC & USTEST & 119 \\
\hline 5659 & PDMS & & sW & & & E095281 & 13-Nov-87 & & U-238 & U-238 & UISO_AËA & USTEST & 13.7 \\
\hline 5660 & PDMS & & sW & & & E095281 & 13-Nov-87 & & 15117-96-1 & U-235 & UISO_AEA & USTEST & 0.593 \\
\hline 5661 & PDMS & & sW & & & E095281 & 13-Nov-87 & & $13966-29-5$ & $\mathrm{U}-234$ & UISO_AEA & USTEST & 14.7 \\
\hline 5662 & PDMS & & SW & & & E095619 & 17-Nov-87 & & $10045-97-3$ & CS-137 & GAMMA_GS & PNL-RO & -0.000632 \\
\hline 5663 & PDMS & & SW & & & E095683 & 17-Nov-87 & & $10045-97-3$ & CS-137 & GAMMA_GS & PNL-RO & 0.000485 \\
\hline 5664 & PDMS & & SW & & & E095687 & 17-Nov-87 & & $10045-97-3$ & CS-137 & GAMMA_GS & PNL-RO & 0.00383 \\
\hline 5665 & PDMS & & sW & & & E095691 & 17-Nov-87 & & $10045-97-3$ & CS-137 & GAMMA_GS & PNL-RO & -0.00213 \\
\hline 5666 & PDMS & & sW & & & E095699 & 17-Nov-87 & & $10045-97-3$ & CS-137 & GAMMA_GS & PNL-RO & 0.00845 \\
\hline 5667 & PDMS & & SW & & & E095695 & 17-Nov-87 & & $10045-97-3$ & CS-137 & GAMMA_GS & PNL-RO & 0.00173 \\
\hline 5668 & PDMS & & SW & & & E095684 & 01-Dec-87 & & $10045-97-3$ & CS-137 & GAMMA_GS & PNL-RO & 0.000895 \\
\hline 5669 & PDMS & & SW & & & E095680 & 01-Dec-87 & & $10045-97-3$ & CS-137 & GAMMA_GS & PNL-RO & 0.00247 \\
\hline
\end{tabular}




\begin{tabular}{|c|c|c|c|c|c|c|c|c|c|c|c|c|c|}
\hline & A & $B$ & $\mathrm{C}$ & $\mathrm{D}$ & $\mathrm{E}$ & $\mathrm{F}$ & $\mathrm{G}$ & $\mathrm{H}$ & $\mathrm{I}$ & $\mathrm{J}$ & $\mathrm{K}$ & $\mathrm{L}$ & $\mathrm{M}$ \\
\hline 1 & SAMPLE_NUM_ASSIGNED_TO & SAMPLER & MEDIA & BIOTA_MEDIA & TAG_ID & SAMP_NUM & SAMP_DATE_TIME & SAMP_DATE_TIME_ON & CON_ID & CON_SHORT_NAME & METHOD_NAME & LAB_CODE & VALUE_RPTD \\
\hline 5670 & PDMS & & SW & & & E033477 & 01-Dec-87 & & $10028-17-8$ & TRITIUM & TRITIUM_ELECT_LSC & USTEST & 48.9 \\
\hline 5671 & PDMS & & SW & & & E033477 & 01-Dec-87 & & $10045-97-3$ & CS-137 & GAMMA_GS & USTEST & -0.0964 \\
\hline 5672 & PDMS & & SW & & & E033477 & 01-Dec-87 & & U-238 & U-238 & UISO_AĒA & USTEST & 0.232 \\
\hline 5673 & PDMS & & SW & & & E033477 & 01-Dec-87 & & 15117-96-1 & U-235 & UISO_AEA & USTEST & 0.0281 \\
\hline 5674 & PDMS & & SW & & & E033477 & 01-Dec-87 & & $13966-29-5$ & U-234 & UISO_AEA & USTEST & 0.212 \\
\hline 5675 & PDMS & & SW & & & E033477 & 01-Dec-87 & & $10098-97-2$ & SR-90 & SRISO_SEP_PRECIP_GPC & USTEST & 0.122 \\
\hline 5676 & PDMS & & SW & & & E095692 & 01-Dec-87 & & $10045-97-3$ & CS-137 & GAMMA_GS & PNL-RO & 0.00216 \\
\hline 5677 & PDMS & & SW & & & E095688 & 01-Dec-87 & & $10045-97-3$ & CS-137 & GAMMA_GS & PNL-RO & 0.00577 \\
\hline 5678 & PDMS & & SW & & & E033476 & 01-Dec-87 & & $10028-17-8$ & TRITIUM & TRITIUM ELECT LSC & USTEST & 146 \\
\hline 5679 & PDMS & & SW & & & E033476 & 01-Dec-87 & & $10045-97-3$ & CS-137 & GAMMA_GS & USTEST & -1.23 \\
\hline 5680 & PDMS & & SW & & & E033476 & 01-Dec-87 & & U-238 & U-238 & UISO_AĒA & USTEST & 0.227 \\
\hline 5681 & PDMS & & SW & & & E033476 & 01-Dec-87 & & 15117-96-1 & $\mathrm{U}-235$ & UISO_AEA & USTEST & 0.00347 \\
\hline 5682 & PDMS & & SW & & & E033476 & 01-Dec-87 & & $13966-29-5$ & $\mathrm{U}-234$ & UISO_AEA & USTEST & 0.215 \\
\hline 5683 & PDMS & & SW & & & E033476 & 01-Dec-87 & & $10098-97-2$ & SR-90 & SRISŌSEP_PRECIP_GPC & USTEST & 0.0999 \\
\hline 5684 & PDMS & & SW & & & E095700 & 01-Dec-87 & & $10045-97-3$ & CS-137 & GAMMA_GS & PNL-RO & -0.000398 \\
\hline 5685 & PDMS & & SW & & & E095696 & 01-Dec-87 & & $10045-97-3$ & CS-137 & GAMMA_GS & PNL-RO & 0.00269 \\
\hline 5686 & PDMS & & SW & & & E106220 & 03-Dec-87 & & 15046-84-1 & I-129 & I129_SEP_TEMS & 320 & 0.01299 \\
\hline 5687 & PDMS & & SW & & & E095704 & 07-Dec-87 & & $10045-97-3$ & CS-137 & 901.1_GAMMA_GS & USTEST & -0.512 \\
\hline 5688 & PDMS & & SW & & & E095704 & 07-Dec-87 & & $10028-17-8$ & TRITIUM & 906.0_H3_LSC & USTEST & -85.9 \\
\hline 5689 & PDMS & & SW & & & E095704 & 07-Dec-87 & & $10098-97-2$ & SR-90 & 905.0_SR_GPC & USTEST & 0.255 \\
\hline 5690 & PDMS & & SW & & & E095703 & 07-Dec-87 & & $10045-97-3$ & CS-137 & 901.1_GAMMA_GS & USTEST & 0.0456 \\
\hline 5691 & PDMS & & SW & & & E095703 & 07-Dec-87 & & $10028-17-8$ & TRITIUM & 906.0_H3_LSC & USTEST & 7660 \\
\hline 5692 & PDMS & & SW & & & E095703 & 07-Dec-87 & & $10098-97-2$ & SR-90 & 905.0_SR_GPC & USTEST & 0.0352 \\
\hline 5693 & PDMS & & SW & & & E095702 & 07-Dec-87 & & $10045-97-3$ & CS-137 & 901.1_GAMMA_GS & USTEST & 0.0963 \\
\hline 5694 & PDMS & & SW & & & E095702 & 07-Dec-87 & & 10028-17-8 & TRITIUM & 906.0_H3_LSC & USTEST & -72 \\
\hline 5695 & PDMS & & SW & & & E095702 & 07-Dec-87 & & $10098-97-2$ & SR-90 & 905.0_SR_GPC & USTEST & 0.104 \\
\hline 5696 & PDMS & & sW & & & E095705 & 07-Dec-87 & & $10045-97-3$ & CS-137 & 901.1_GAMMA_GS & USTEST & -1.22 \\
\hline 5697 & PDMS & & SW & & & E095705 & 07-Dec-87 & & $10028-17-8$ & TRITIUM & 906.0_H3_LSC & USTEST & 52.6 \\
\hline 5698 & PDMS & & SW & & & E095705 & 07-Dec-87 & & $10098-97-2$ & SR-90 & 905.0_SR_GPC & USTEST & 0.00523 \\
\hline 5699 & PDMS & & SW & & & E095701 & 09-Dec-87 & & $10045-97-3$ & CS-137 & GAMM̄A_ĒS & USTEST & 0.374 \\
\hline 5700 & PDMS & & SW & & & E095701 & 09-Dec-87 & & 10028-17-8 & TRITIUM & TRITIUM_DIST_LSC & USTEST & 15 \\
\hline 5701 & PDMS & & SW & & & E095701 & 09-Dec-87 & & $\mathrm{U}-238$ & $\mathrm{U}-238$ & UISO_AEA & USTEST & 0.0053 \\
\hline 5702 & PDMS & & SW & & & E095701 & 09-Dec-87 & & 15117-96-1 & $\mathrm{U}-235$ & UISO_AEA & USTEST & -0.0021 \\
\hline 5703 & PDMS & & SW & & & E095701 & 09-Dec-87 & & $13966-29-5$ & $\mathrm{U}-234$ & UISO_AEA & USTEST & 0.0228 \\
\hline 5704 & PDMS & & SW & & & E033478 & 15-Dec-87 & & $10028-17-8$ & TRITIUM & TRITIŪM_ELECT_LSC & USTEST & 71.3 \\
\hline 5705 & PDMS & & SW & & & E033478 & 15-Dec-87 & & $10045-97-3$ & CS-137 & 901.1_GAMMMA_Ḡ & USTEST & -0.0449 \\
\hline 5706 & PDMS & & SW & & & E033478 & 15-Dec-87 & & $10098-97-2$ & SR-90 & 905.0_SR_GPC & USTEST & 0.114 \\
\hline 5707 & PDMS & & SW & & & E096216 & 15-Dec-87 & & $10045-97-3$ & CS-137 & GAMMA_GS & PNL-RO & 0.00308 \\
\hline 5708 & PDMS & & SW & & & E096217 & 15-Dec-87 & & $10045-97-3$ & CS-137 & GAMMA_GS & PNL-RO & -0.000527 \\
\hline 5709 & PDMS & & SW & & & E096218 & 15-Dec-87 & & $10045-97-3$ & CS-137 & GAMMA_GS & PNL-RO & 0.0017 \\
\hline 5710 & PDMS & & SW & & & E096219 & 15-Dec-87 & & $10045-97-3$ & CS-137 & GAMMA_GS & PNL-RO & 0.0022 \\
\hline 5711 & PDMS & & SW & & & E096220 & 15-Dec-87 & & $10045-97-3$ & CS-137 & GAMMA_GS & PNL-RO & -0.000726 \\
\hline 5712 & PDMS & & SW & & & E096221 & 15-Dec-87 & & $10045-97-3$ & CS-137 & GAMMA_GS & PNL-RO & 0.00192 \\
\hline 5713 & PDMS & & SW & & & E033479 & 22-Dec-87 & & $10028-17-8$ & TRITIUM & TRITIUM_ELECT_LSC & USTEST & 156 \\
\hline 5714 & PDMS & & SW & & & E033479 & 22-Dec-87 & & $10045-97-3$ & CS-137 & 901.1_GAMMA_GS & USTEST & -0.778 \\
\hline 5715 & PDMS & & SW & & & E033479 & 22-Dec-87 & & $\mathrm{U}-238$ & $\mathrm{U}-238$ & UISO_IE_PLATE_AEA & USTEST & 0.243 \\
\hline 5716 & PDMS & & SW & & & E033479 & 22-Dec-87 & & 15117-96-1 & $\mathrm{U}-235$ & UISO_IE_PLATE_AEA & USTEST & 0.00444 \\
\hline 5717 & PDMS & & SW & & & E033479 & 22-Dec-87 & & $13966-29-5$ & $\mathrm{U}-234$ & UISO_IE_PLATE_AEA & USTEST & 0.301 \\
\hline 5718 & PDMS & & SW & & & E033479 & 22-Dec-87 & & $10098-97-2$ & SR-90 & 905.0_SR_GPC & USTEST & 0.127 \\
\hline 5719 & PDMS & & sW & & & E033645 & 29-Dec-87 & & 15046-84-1 & I-129 & I129_SEP_TEMS & 320 & 0.0000985 \\
\hline 5720 & PDMS & & SW & & & E033645 & 29-Dec-87 & & PU-239/240 & PU-239/240 & PUISO IE AEA & PNL-RO & 0.0000409 \\
\hline 5721 & PDMS & & SW & & & E033645 & 29-Dec-87 & & $10045-97-3$ & CS-137 & GAMMA_GS & PNL-RO & -0.00295 \\
\hline
\end{tabular}




\begin{tabular}{|c|c|c|c|c|c|c|c|c|c|c|c|c|c|}
\hline & A & $\mathrm{B}$ & $\mathrm{C}$ & $\mathrm{D}$ & $\mathrm{E}$ & $\mathrm{F}$ & $\mathrm{G}$ & $\mathrm{H}$ & $\mathrm{I}$ & $\mathrm{J}$ & $\mathrm{K}$ & $\mathrm{L}$ & $\mathrm{M}$ \\
\hline 1 & SAMPLE_NUM_ASSIGNED_TO & \begin{tabular}{|l|} 
SAMPLER \\
\end{tabular} & MEDIA & BIOTA_MEDIA & TAG_ID & SAMP_NUM & SAMP_DATE_TIME & SAMP_DATE_TIME_ON & CON_ID & CON_SHORT_NAME & METHOD_NAME & LAB_CODE & VALUE_RPTD \\
\hline 5722 & PDMS & & SW & & & E033677 & 29-Dec-87 & & PU-239/240 & PU-239/240 & PUISO_IE_AEA & PNL-RO & 0.0000178 \\
\hline 5723 & PDMS & & sw & & & E033677 & 29-Dec-87 & & $10045-97-3$ & CS-137 & GAMMA_GS & PNL-RO & 0.000417 \\
\hline 5724 & PDMS & & sw & & & E033481 & 29-Dec-87 & & $10028-17-8$ & TRITIUM & TRITIUM_ELECT_LSC & USTEST & 76.6 \\
\hline 5725 & PDMS & & sw & & & E033481 & 29-Dec-87 & & $10045-97-3$ & CS-137 & GAMMA_GS & USTEST & 0.18 \\
\hline \begin{tabular}{|l|l|}
5726 \\
\end{tabular} & PDMS & & sw & & & E033481 & 29-Dec-87 & & U-238 & $\mathrm{U}-238$ & UISO_AEA & USTEST & 0.205 \\
\hline \begin{tabular}{|l|}
5727 \\
\end{tabular} & PDMS & & sW & & & E033481 & 29-Dec-87 & & 15117-96-1 & U-235 & UISO_AEA & USTEST & 0.0117 \\
\hline 5728 & PDMS & & sW & & & E033481 & 29-Dec-87 & & 13966-29-5 & U-234 & UISO_AEA & USTEST & 0.255 \\
\hline 5729 & PDMS & & SW & & & E033481 & 29-Dec-87 & & $10098-97-2$ & SR-90 & SRISO_SEP_PRECIP_GPC & USTEST & 0.138 \\
\hline 5730 & PDMS & & sw & & & E033673 & 29-Dec-87 & & $15046-84-1$ & I-129 & I129_SEP_TEMS & 320 & 0.0000121 \\
\hline \begin{tabular}{|l|}
5731 \\
\end{tabular} & PDMS & & SW & & & E033673 & 29-Dec-87 & & PU-239/240 & PU-239/240 & PUISO_IE_AEA & PNL-RO & 0.000126 \\
\hline 5732 & PDMS & & sW & & & E033670 & 29-Dec-87 & & PU-239/240 & PU-239/240 & PUISO_IE_AEA & PNL-RO & 0.0000282 \\
\hline 5733 & PDMS & & sW & & & E033670 & 29-Dec-87 & & $10045-97-3$ & CS-137 & GAMMA_ḠS & PNL-RO & 0.000734 \\
\hline 5734 & PDMS & & sW & & & E033673 & 29-Dec-87 & & $10045-97-3$ & CS-137 & GAMMA_GS & PNL-RO & 0.00228 \\
\hline 5735 & PDMS & & sW & & & E033480 & 29-Dec-87 & & $10028-17-8$ & TRITIUM & TRITIUM_ELELECT_LSC & USTEST & 122 \\
\hline 5736 & PDMS & & sw & & & E033653 & 29-Dec-87 & & 15046-84-1 & I-129 & I129_SEP_TEMS & 320 & 0.0000875 \\
\hline 5737 & PDMS & & sw & & & E033653 & 29-Dec-87 & & PU-239/240 & PU-239/240 & PUISO_IE_AEA & PNL-RO & 0.000049 \\
\hline 5738 & PDMS & & sw & & & E033649 & 29-Dec-87 & & PU-239/240 & PU-239/240 & PUISO_IE_AEA & PNL-RO & 0.0000282 \\
\hline 5739 & PDMS & & sW & & & E033480 & 29-Dec-87 & & $10045-97-3$ & CS-137 & GAMMA_GS & USTEST & -0.157 \\
\hline 5740 & PDMS & & sW & & & E033649 & 29-Dec-87 & & $10045-97-3$ & CS-137 & GAMMA_GS & PNL-RO & 0.00102 \\
\hline 5741 & PDMS & & sW & & & E033480 & 29-Dec-87 & & U-238 & U-238 & UISO_AĒAA & USTEST & 0.186 \\
\hline 5742 & PDMS & & sw & & & E033480 & 29-Dec-87 & & 15117-96-1 & U-235 & UISO_AEA & USTEST & 0.0295 \\
\hline 5743 & PDMS & & sW & & & E033480 & 29-Dec-87 & & $13966-29-5$ & U-234 & UISO_AEA & USTEST & 0.237 \\
\hline 5744 & PDMS & & SW & & & E033653 & 29-Dec-87 & & $10045-97-3$ & CS-137 & GAMMA_GS & PNL-RO & 0.00229 \\
\hline 5745 & PDMS & & sW & & & E033480 & 29-Dec-87 & & $10098-97-2$ & SR-90 & SRISO_SEEP_PRECIP_GPC & USTEST & 0.136 \\
\hline 5746 & PDMS & & SW & & & E096331 & 12-Jan-88 & & $10045-97-3$ & CS-137 & 901.1_GAMMA_GS & USTEST & -0.449 \\
\hline 5747 & PDMS & & sW & & & E096331 & 12-Jan-88 & & $10028-17-8$ & TRITIUM & 906.0_H3_LSC & USTEST & 6.44 \\
\hline 5748 & PDMS & & sW & & & E096331 & 12-Jan-88 & & $10098-97-2$ & SR-90 & 905.0_SR_GPC & USTEST & -0.0112 \\
\hline 5749 & PDMS & & sW & & & E096462 & 12-Jan-88 & & $10045-97-3$ & CS-137 & GAMMA_ĒS & USTEST & 0.0017 \\
\hline 5750 & PDMS & & sw & & & E096461 & 12-Jan-88 & & $10045-97-3$ & CS-137 & GAMMA_GS & USTEST & 0.00342 \\
\hline 5751 & PDMS & & sW & & & E096460 & 12-Jan-88 & & $10045-97-3$ & CS-137 & GAMMA_GS & USTEST & 0.000236 \\
\hline 5752 & PDMS & & sW & & & E096459 & 12-Jan-88 & & $10045-97-3$ & CS-137 & GAMMA_GS & USTEST & 0.00302 \\
\hline 5753 & PDMS & & sW & & & E096464 & 12-Jan-88 & & $10045-97-3$ & CS-137 & GAMMA_GS & USTEST & 0.00148 \\
\hline 5754 & PDMS & & sW & & & E096463 & 12-Jan-88 & & $10045-97-3$ & CS-137 & GAMMA_GS & USTEST & 0.00249 \\
\hline 5755 & PDMS & & SW & & & E033484 & 13-Jan-88 & & U-238 & $\mathrm{U}-238$ & UISO_AĒAA & USTEST & 18.1 \\
\hline 5756 & PDMS & & sW & & & E033484 & 13-Jan-88 & & 15117-96-1 & U-235 & UISO_AEA & USTEST & 0.72 \\
\hline 5757 & PDMS & & sW & & & E033484 & 13-Jan-88 & & 13966-29-5 & U-234 & UISO_AEA & USTEST & 28.7 \\
\hline 5758 & PDMS & & SW & & & E033484 & 13-Jan-88 & & $10045-97-3$ & CS-137 & GAMMA_GS & USTEST & 0.627 \\
\hline 5759 & PDMS & & sW & & & E033484 & 13-Jan-88 & & 10028-17-8 & TRITIUM & TRITIUM_DIST_LSC & USTEST & -44 \\
\hline 5760 & PDMS & & sW & & & E033797 & 15-Jan-88 & & $10098-97-2$ & SR-90 & 905.0_SR_GPC & USTEST & 0.343 \\
\hline 5761 & PDMS & & SW & & & E033797 & 15-Jan-88 & & $10045-97-3$ & CS-137 & 901.1_GAMMA_GS & USTEST & 0.128 \\
\hline 5762 & PDMS & & sW & & & E033797 & 15-Jan-88 & & 10028-17-8 & TRITIUM & 906.0_H3_LSC & USTEST & 213 \\
\hline 5763 & PDMS & & sW & & & E033486 & 20-Jan-88 & & U-238 & U-238 & UISO_AEA & USTEST & 3.08 \\
\hline 5764 & PDMS & & sW & & & E033486 & 20-Jan-88 & & 15117-96-1 & U-235 & UISO_AEA & USTEST & 0.163 \\
\hline 5765 & PDMS & & sW & & & E033486 & 20-Jan-88 & & $13966-29-5$ & $\mathrm{U}-234$ & UISO_AEA & USTEST & 3.92 \\
\hline 5766 & PDMS & & SW & & & E033486 & 20-Jan-88 & & 10028-17-8 & TRITIUM & TRITIUMM_DIST_LSC & USTEST & -21.5 \\
\hline 5767 & PDMS & & SW & & & E033486 & 20-Jan-88 & & $10045-97-3$ & CS-137 & GAMMA_GS & USTEST & 0.375 \\
\hline 5768 & PDMS & & sW & & & E033485 & 20-Jan-88 & & U-238 & $\mathrm{U}-238$ & UISO_AĒA & USTEST & 0.00563 \\
\hline 5769 & PDMS & & sW & & & E033485 & 20-Jan-88 & & 15117-96-1 & U-235 & UISO_AEA & USTEST & 0.000109 \\
\hline 5770 & PDMS & & sw & & & E033485 & 20-Jan-88 & & $13966-29-5$ & U-234 & UISO_AEA & USTEST & 0.0111 \\
\hline 5771 & PDMS & & sW & & & E033485 & 20-Jan-88 & & 10028-17-8 & TRITIUM & TRITIUM_DIST_LSC & USTEST & -162 \\
\hline 5772 & PDMS & & SW & & & E033485 & 20-Jan-88 & & $10045-97-3$ & CS-137 & GAMMA_GS & USTEST & -0.388 \\
\hline 5773 & PDMS & & SW & & & E096457 & 26-Jan-88 & & $10045-97-3$ & CS-137 & GAMMA_GS & USTEST & \\
\hline
\end{tabular}




\begin{tabular}{|c|c|c|c|c|c|c|c|c|c|c|c|c|c|}
\hline & $\bar{A}$ & $\mathrm{~B}$ & $\mathrm{C}$ & $\bar{D}$ & $\mathrm{E}$ & $\mathrm{F}$ & $\bar{G}$ & $\mathrm{H}$ & 1 & $\mathrm{~J}$ & $\mathrm{~K}$ & $\mathrm{~L}$ & $\bar{M}$ \\
\hline 1 & SAMPLE_NUM_ASSIGNED_TO & SAMPLER & MEDIA & BIOTA_MEDIA & TAG_ID & SAMP_NUM & SAMP_DATE_TIME & SAMP_DATE_TIME_ON & CON_ID & \begin{tabular}{|l|} 
CON_SHORT_NAME \\
\end{tabular} & METHOD_NAME & LAB_CODE & VALUE_RPTD \\
\hline 5774 & PDMS & & SW & & & E096455 & 26-Jan-88 & & $10045-97-3$ & CS-137 & GAMMA_GS & USTEST & \\
\hline 5775 & PDMS & & SW & & & E096531 & 26-Jan-88 & & $10045-97-3$ & CS-137 & GAMMA_GS & USTEST & 0.00427 \\
\hline 5776 & PDMS & & SW & & & E096530 & 26-Jan-88 & & $10045-97-3$ & CS-137 & GAMMA_GS & USTEST & 0.00605 \\
\hline 5777 & PDMS & & SW & & & E096533 & 26-Jan-88 & & $10045-97-3$ & CS-137 & GAMMA_GS & USTEST & 0.000584 \\
\hline 5778 & PDMS & & SW & & & E096532 & 26-Jan-88 & & $10045-97-3$ & CS-137 & GAMMA_GS & USTEST & 0.00255 \\
\hline 5779 & PDMS & & SW & & & E033483 & 02-Feb-88 & & U-238 & $\mathrm{U}-238$ & UISO_AĒA & USTEST & 0.209 \\
\hline 5780 & PDMS & & sW & & & E033483 & 02-Feb-88 & & 15117-96-1 & U-235 & UISO_AEA & USTEST & 0.0129 \\
\hline 5781 & PDMS & & sW & & & E033483 & 02-Feb-88 & & $13966-29-5$ & U-234 & UISO_AEA & USTEST & 0.242 \\
\hline 5782 & PDMS & & sW & & & E033483 & 02-Feb-88 & & $10098-97-2$ & SR-90 & SRISŌSEP_PRECIP_GPC & USTEST & 0.119 \\
\hline 5783 & PDMS & & SW & & & E033483 & 02-Feb-88 & & $10045-97-3$ & CS-137 & GAMMA_GS & USTEST & -0.122 \\
\hline 5784 & PDMS & & SW & & & E033483 & 02-Feb-88 & & $10028-17-8$ & TRITIUM & TRITIUM_ELECT_LSC & USTEST & 67.5 \\
\hline 5785 & PDMS & & SW & & & E033482 & 02-Feb-88 & & U-238 & $\mathrm{U}-238$ & UISO_AEA & USTEST & 0.225 \\
\hline 5786 & PDMS & & sW & & & E033482 & 02-Feb-88 & & 15117-96-1 & U-235 & UISO_AEA & USTEST & 0.0133 \\
\hline 5787 & PDMS & & SW & & & E033482 & $02-F e b-88$ & & 13966-29-5 & U-234 & UISO_AEA & USTEST & 0.275 \\
\hline 5788 & PDMS & & SW & & & E033482 & 02-Feb-88 & & $10098-97-2$ & SR-90 & SRISO_SEP_PRECIP_GPC & USTEST & 0.143 \\
\hline 5789 & PDMS & & SW & & & E033482 & 02-Feb-88 & & $10045-97-3$ & CS-137 & GAMMA_GS & USTEST & 0.057 \\
\hline 5790 & PDMS & & SW & & & E033482 & 02-Feb-88 & & $10028-17-8$ & TRITIUM & TRITIUM_ELECT_LSC & USTEST & 126 \\
\hline 5791 & PDMS & & sW & & & E033654 & 02-Feb-88 & & 10098-97-2 & SR-90 & SRISO_SEP_PRECIP_GPC & USTEST & 1.61 \\
\hline 5792 & PDMS & & sW & & & E033654 & 02-Feb-88 & & $10028-17-8$ & TRITIUM & TRITIUM_DIST_LSC & USTEST & -64.5 \\
\hline 5793 & PDMS & & SW & & & E033654 & $02-F e b-88$ & & $10045-97-3$ & CS-137 & GAMMA_GS & USTEST & 0.512 \\
\hline 5794 & PDMS & & sW & & & E096522 & 02-Feb-88 & & $10028-17-8$ & TRITIUM & TRITIUM_DIST_LSC & USTEST & \\
\hline 5795 & PDMS & & SW & & & E096522 & 02-Feb-88 & & $10045-97-3$ & CS-137 & GAMMA_GS & USTEST & \\
\hline 5796 & PDMS & & SW & & & E096523 & 02-Feb-88 & & U-238 & U-238 & UISO_AEA & USTEST & \\
\hline 5797 & PDMS & & SW & & & E096523 & 02-Feb-88 & & $15117-96-1$ & U-235 & UISO_AEA & USTEST & \\
\hline 5798 & PDMS & & SW & & & E096523 & 02-Feb-88 & & $13966-29-5$ & U-234 & UISO_AEA & USTEST & \\
\hline 5799 & PDMS & & SW & & & E096523 & $02-F e b-88$ & & $10098-97-2$ & SR-90 & SRISO_SEP_PRECIP_GPC & USTEST & \\
\hline 5800 & PDMS & & SW & & & E096523 & $02-F e b-88$ & & $10028-17-8$ & TRITIUM & TRITIUM_DIST_LSC & USTEST & \\
\hline 5801 & PDMS & & SW & & & E096523 & 02-Feb-88 & & $10045-97-3$ & CS-137 & GAMMA_GS & USTEST & \\
\hline 5802 & PDMS & & SW & & & E096614 & 09-Feb-88 & & $10045-97-3$ & CS-137 & GAMMA_GS & USTEST & 0.00094 \\
\hline 5803 & PDMS & & SW & & & E096613 & $09-F e b-88$ & & $10045-97-3$ & CS-137 & GAMMA_GS & USTEST & 0.00262 \\
\hline 5804 & PDMS & & SW & & & E096578 & 09-Feb-88 & & $10045-97-3$ & CS-137 & GAMMA_GS & USTEST & \\
\hline 5805 & PDMS & & SW & & & E096577 & 09-Feb-88 & & $10045-97-3$ & CS-137 & GAMMA_GS & USTEST & \\
\hline 5806 & PDMS & & SW & & & E096615 & 09-Feb-88 & & $10045-97-3$ & CS-137 & GAMMA_GS & USTEST & 0.00301 \\
\hline 5807 & PDMS & & SW & & & E096616 & 09-Feb-88 & & $10045-97-3$ & CS-137 & GAMMA_GS & USTEST & -0.000104 \\
\hline 5808 & PDMS & & SW & & & E033717 & 23-Feb-88 & & $10045-97-3$ & CS-137 & GAMMA_GS & USTEST & -0.0000968 \\
\hline 5809 & PDMS & & SW & & & E033716 & 23-Feb-88 & & $10045-97-3$ & CS-137 & GAMMA_GS & USTEST & 0.00219 \\
\hline 5810 & PDMS & & SW & & & E033719 & 23-Feb-88 & & $10045-97-3$ & CS-137 & GAMMA_GS & USTEST & 0.00112 \\
\hline 5811 & PDMS & & sW & & & E033718 & 23-Feb-88 & & $10045-97-3$ & CS-137 & GAMMA_GS & USTEST & 0.000247 \\
\hline 5812 & PDMS & & SW & & & E033448 & 23-Feb-88 & & $10045-97-3$ & CS-137 & GAMMA_GS & USTEST & 0.00264 \\
\hline 5813 & PDMS & & SW & & & E033720 & 23-Feb-88 & & $10045-97-3$ & CS-137 & GAMMA_GS & USTEST & 0.000194 \\
\hline 5814 & PDMS & & SW & & & E033586 & 29-Feb-88 & & $10045-97-3$ & CS-137 & 901.1_GAMMA_GS & USTEST & 0.171 \\
\hline 5815 & PDMS & & SW & & & E033586 & 29-Feb-88 & & $10028-17-8$ & TRITIUM & 906.0_H3_LSC & USTEST & -103 \\
\hline 5816 & PDMS & & SW & & & E033586 & 29-Feb-88 & & $10098-97-2$ & SR-90 & 905.0_SR_GPC & USTEST & 0.00953 \\
\hline 5817 & PDMS & & SW & & & E033585 & 29-Feb-88 & & $10045-97-3$ & CS-137 & 901.1_GAMMA_GS & USTEST & 0.0803 \\
\hline 5818 & PDMS & & SW & & & E033585 & 29-Feb-88 & & $10028-17-8$ & TRITIUM & 906.0_H3_LSC & USTEST & 5150 \\
\hline 5819 & PDMS & & SW & & & E033585 & 29-Feb-88 & & $10098-97-2$ & SR-90 & 905.0_SR_GPC & USTEST & 0.00397 \\
\hline 5820 & PDMS & & SW & & & E033584 & 29-Feb-88 & & $10045-97-3$ & CS-137 & 901.1_GAMMA_GS & USTEST & 0.365 \\
\hline 5821 & PDMS & & SW & & & E033584 & 29-Feb-88 & & $10028-17-8$ & TRITIUM & 906.0_H3_LSC & USTEST & 4.31 \\
\hline 5822 & PDMS & & SW & & & E033584 & 29-Feb-88 & & 10098-97-2 & SR-90 & 905.0_SR_GPC & USTEST & 0.00853 \\
\hline 5823 & PDMS & & SW & & & E033587 & 29-Feb-88 & & $10028-17-8$ & TRITIUM & 906.0_H3_LSC & USTEST & -59.2 \\
\hline 5824 & PDMS & & SW & & & E033587 & 29-Feb-88 & & $10098-97-2$ & SR-90 & 905.0_SR_GPC & USTEST & -0.00817 \\
\hline 5825 & PDMS & & SW & & & E033587 & $29-F e b-88$ & & $10045-97-3$ & CS-137 & 901.1_GAMMA_GS & USTEST & 0.228 \\
\hline
\end{tabular}




\begin{tabular}{|c|c|c|c|c|c|c|c|c|c|c|c|c|c|}
\hline & $\bar{A}$ & $\mathrm{~B}$ & $\mathrm{C}$ & $\bar{D}$ & $\mathrm{E}$ & $\mathrm{F}$ & $\bar{G}$ & $\mathrm{H}$ & 1 & $\mathrm{~J}$ & $\mathrm{~K}$ & $\bar{L}$ & $\bar{M}$ \\
\hline 1 & SAMPLE_NUM_ASSIGNED_TO & SAMPLER & MEDIA & BIOTA_MEDIA & TAG_ID & SAMP_NUM & SAMP_DATE_TIME & SAMP_DATE_TIME_ON & CON_ID & CON_SHORT_NAME & METHOD_NAME & LAB_CODE & VALUE_RPTD \\
\hline 5826 & PDMS & & SW & & & E033598 & 01-Mar-88 & & $10098-97-2$ & SR-90 & SRISO_SEP_PRECIP_GPC & USTEST & 0.106 \\
\hline 5827 & PDMS & & sW & & & E033598 & 01-Mar-88 & & U-238 & U-238 & UISO_AEA & USTEST & 0.174 \\
\hline 5828 & PDMS & & SW & & & E033598 & 01-Mar-88 & & 15117-96-1 & U-235 & UISO_AEA & USTEST & 0.0091 \\
\hline 5829 & PDMS & & sW & & & E033598 & 01-Mar-88 & & 13966-29-5 & U-234 & UISO AEA & USTEST & 0.256 \\
\hline 5830 & PDMS & & SW & & & E033598 & 01-Mar-88 & & $10045-97-3$ & CS-137 & GAMMA_GS & USTEST & -0.837 \\
\hline 5831 & PDMS & & sw & & & E033598 & 01-Mar-88 & & $10028-17-8$ & TRITIUM & TRITIUM_ELECT_LSC & USTEST & 81.3 \\
\hline 5832 & PDMS & & sW & & & E033597 & 01-Mar-88 & & $10098-97-2$ & SR-90 & SRISO_SËP_PRËCIP_GPC & USTEST & 0.135 \\
\hline 5833 & PDMS & & sW & & & E033597 & 01-Mar-88 & & U-238 & U-238 & UISO_AEEA & USTEST & 0.253 \\
\hline 5834 & PDMS & & sW & & & E033597 & 01-Mar-88 & & 15117-96-1 & U-235 & UISO_AEA & USTEST & 0.0131 \\
\hline 5835 & PDMS & & sW & & & E033597 & 01-Mar-88 & & $13966-29-5$ & U-234 & UISO_AEA & USTEST & 0.274 \\
\hline 5836 & PDMS & & sW & & & E033597 & 01-Mar-88 & & $10045-97-3$ & CS-137 & GAMMA_GS & USTEST & 0.969 \\
\hline 5837 & PDMS & & sw & & & E033597 & 01-Mar-88 & & $10028-17-8$ & TRITIUM & TRITIUM_ELECT_LSC & USTEST & 144 \\
\hline 5838 & PDMS & & sw & & & E107120 & 03-Mar-88 & & $15046-84-1$ & I-129 & I129_SEP_TEMS & 320 & 0.0000084 \\
\hline 5839 & PDMS & & sw & & & E107119 & 03-Mar-88 & & 15046-84-1 & I-129 & I129_SEP_TEMS & 320 & 0.0000329 \\
\hline 5840 & PDMS & & sw & & & E107395 & 03-Mar-88 & & $15046-84-1$ & I-129 & I129_SEP_TEMS & 320 & 0.0000233 \\
\hline 5841 & PDMS & & SW & & & E033582 & 03-Mar-88 & & 15046-84-1 & I-129 & 1129_SEP_TEMS & 320 & 0.0000958 \\
\hline 5842 & PDMS & & sW & & & E033582 & 03-Mar-88 & & $10045-97-3$ & CS-137 & GAMMA_ḠS & USTEST & 0.783 \\
\hline 5843 & PDMS & & sw & & & E033582 & 03-Mar-88 & & U-238 & U-238 & UISO_AĒAA & USTEST & 14.5 \\
\hline 5844 & PDMS & & sw & & & E033582 & 03-Mar-88 & & 15117-96-1 & U-235 & UISO_AEA & USTEST & 0.543 \\
\hline 5845 & PDMS & & sW & & & E033582 & 03-Mar-88 & & $13966-29-5$ & U-234 & UISO_AEA & USTEST & 15.6 \\
\hline 5846 & PDMS & & sW & & & E033582 & 03-Mar-88 & & $10028-17-8$ & TRITIUM & TRITIUUM_DIST_LSC & USTEST & -79.7 \\
\hline 5847 & PDMS & & SW & & & E033581 & 08-Mar-88 & & $10098-97-2$ & SR-90 & 905.0_SR_GPC & USTEST & 0.147 \\
\hline 5848 & PDMS & & sW & & & E033581 & 08-Mar-88 & & $10028-17-8$ & TRITIUM & 906.0_H3_LSC & USTEST & 51.6 \\
\hline 5849 & PDMS & & sw & & & E033581 & 08-Mar-88 & & $10045-97-3$ & CS-137 & 901.1_GAMMA_GS & USTEST & 0.18 \\
\hline 5850 & PDMS & & sW & & & E096976 & 08-Mar-88 & & $10045-97-3$ & CS-137 & GAMMA_GS & USTEST & \\
\hline 5851 & PDMS & & sw & & & E096977 & 08-Mar-88 & & $10045-97-3$ & CS-137 & GAMMA_GS & USTEST & \\
\hline 5852 & PDMS & & sw & & & E096979 & 08-Mar-88 & & $10045-97-3$ & CS-137 & GAMMA_GS & USTEST & \\
\hline 5853 & PDMS & & sw & & & E096978 & 08-Mar-88 & & $10045-97-3$ & CS-137 & GAMMA_GS & USTEST & \\
\hline 5854 & PDMS & & sw & & & E033449 & 08-Mar-88 & & $10045-97-3$ & CS-137 & GAMMA_GS & USTEST & -0.00123 \\
\hline 5855 & PDMS & & sW & & & E033583 & 08-Mar-88 & & $10045-97-3$ & CS-137 & GAMMA_GS & USTEST & 0.000734 \\
\hline 5856 & PDMS & & sw & & & E034156 & 22-Mar-88 & & $10045-97-3$ & CS-137 & GAMMA_GS & USTEST & 0.000332 \\
\hline 5857 & PDMS & & sw & & & E034155 & 22-Mar-88 & & $10045-97-3$ & CS-137 & GAMMA_GS & USTEST & 0.0034 \\
\hline 5858 & PDMS & & sw & & & E034175 & 22-Mar-88 & & $10045-97-3$ & CS-137 & GAMMA_GS & USTEST & 0.000647 \\
\hline 5859 & PDMS & & sW & & & E034174 & 22-Mar-88 & & $10045-97-3$ & CS-137 & GAMMA_GS & USTEST & -0.00112 \\
\hline 5860 & PDMS & & SW & & & E034158 & 22-Mar-88 & & $10045-97-3$ & CS-137 & GAMMA_GS & USTEST & 0.013 \\
\hline 5861 & PDMS & & sW & & & E034157 & 22-Mar-88 & & $10045-97-3$ & CS-137 & GAMMA_GS & USTEST & 0.00169 \\
\hline 5862 & PDMS & & sW & & & E034032 & 29-Mar-88 & & $10045-97-3$ & CS-137 & GAMMA_GS & USTEST & 0.53 \\
\hline 5863 & PDMS & & sW & & & E034032 & 29-Mar-88 & & U-238 & U-238 & UISO_AĒA & USTEST & 0.148 \\
\hline 5864 & PDMS & & sw & & & E034032 & 29-Mar-88 & & 15117-96-1 & U-235 & UISO_AEA & USTEST & 0.00898 \\
\hline 5865 & PDMS & & sw & & & E034032 & 29-Mar-88 & & $13966-29-5$ & $\mathrm{U}-234$ & UISO_AEA & USTEST & 0.187 \\
\hline 5866 & PDMS & & sw & & & E034032 & 29-Mar-88 & & $10098-97-2$ & SR-90 & SRISO_SEP_PRECIP_GPC & USTEST & 0.149 \\
\hline 5867 & PDMS & & sW & & & E034032 & 29-Mar-88 & & $10028-17-8$ & TRITIUM & TRITIUM̄E_ELECT_LSC̄ & USTEST & 89.4 \\
\hline 5868 & PDMS & & sW & & & E034031 & 29-Mar-88 & & $10045-97-3$ & CS-137 & GAMMA_GS & USTEST & 0.472 \\
\hline 5869 & PDMS & & sw & & & E034031 & 29-Mar-88 & & U-238 & U-238 & UISO_AËA & USTEST & 0.242 \\
\hline 5870 & PDMS & & sw & & & E034031 & 29-Mar-88 & & 15117-96-1 & U-235 & UISO_AEA & USTEST & 0.0443 \\
\hline 5871 & PDMS & & sw & & & E034031 & 29-Mar-88 & & $13966-29-5$ & $\mathrm{U}-234$ & UISO_AEA & USTEST & 0.282 \\
\hline 5872 & PDMS & & sw & & & E034031 & 29-Mar-88 & & $10098-97-2$ & SR-90 & SRISO_SEP_PRECIP_GPC & USTEST & 0.168 \\
\hline 5873 & PDMS & & sw & & & E034031 & 29-Mar-88 & & $10028-17-8$ & TRITIUM & TRITIUM_ELECT_LSC & USTEST & 146 \\
\hline 5874 & PDMS & & sW & & & E034007 & 05-Apr-88 & & $10045-97-3$ & CS-137 & 901.1_GAMMA_GS & USTEST & 0.996 \\
\hline 5875 & PDMS & & sW & & & E034007 & 05-Apr-88 & & $10028-17-8$ & TRITIUM & 906.0_H3_LSC & USTEST & 34.4 \\
\hline 5876 & PDMS & & SW & & & E034007 & 05-Apr-88 & & $10098-97-2$ & SR-90 & 905.0_SR_GPC & USTEST & -0.0334 \\
\hline 5877 & PDMS & & SW & & & E034033 & 05-Apr-88 & & $10045-97-3$ & CS-137 & GAMMA GS & USTEST & -0.00021 \\
\hline
\end{tabular}




\begin{tabular}{|c|c|c|c|c|c|c|c|c|c|c|c|c|c|}
\hline & A & $\mathrm{B}$ & $\mathrm{c}$ & $\mathrm{D}$ & $\mathrm{E}$ & $\mathrm{F}$ & G & $\mathrm{H}$ & $\mathrm{I}$ & $\mathrm{J}$ & $\mathrm{K}$ & $\mathrm{L}$ & $\mathrm{M}$ \\
\hline 1 & SAMPLE_NUM_ASSIGNED_TO & SAMPLER & MEDIA & BIOTA_MEDIA & TAG_ID & SAMP_NUM & SAMP_DATE_TIME & SAMP_DATE_TIME_ON & CON_ID & CON_SHORT_NAME & METHOD_NAME & LAB_CODE & \begin{tabular}{|l|} 
VALUE_RPTD \\
\end{tabular} \\
\hline 5878 & PDMS & & SW & & & E034033 & $05-\mathrm{Apr}-88$ & & PU-239/240 & PU-239/240 & PUISO_IE_AEA & USTEST & 0.0000373 \\
\hline 5879 & PDMS & & sW & & & E096795 & 05-Apr-88 & & 15046-84-1 & I-129 & I129_SEP_TEMS & 320 & 0.0001142 \\
\hline 5880 & PDMS & & sW & & & E096795 & 05-Apr-88 & & $10045-97-3$ & CS-137 & GAMMA_GS & USTEST & 0.0000365 \\
\hline 5881 & PDMS & & sW & & & E096795 & 05-Apr-88 & & PU-239/240 & PU-239/240 & PUISO_IE_AEA & USTEST & -0.00000331 \\
\hline \begin{tabular}{|l|}
5882 \\
\end{tabular} & PDMS & & sW & & & E034034 & 05-Apr-88 & & $10045-97-3$ & CS-137 & GAMMA_GS & USTEST & 0.000978 \\
\hline 5883 & PDMS & & sW & & & E034034 & 05-Apr-88 & & PU-239/240 & PU-239/240 & PUISO_IE_AEA & USTEST & 0.0000564 \\
\hline 5884 & PDMS & & SW & & & E096796 & 05-Apr-88 & & 15046-84-1 & I-129 & 1129 SEP_TEMS & 320 & 0.0000084 \\
\hline 5885 & PDMS & & SW & & & E096796 & 05-Apr-88 & & $10045-97-3$ & CS-137 & GAMMA_ḠS & USTEST & 0.000499 \\
\hline 5886 & PDMS & & sW & & & E096796 & 05-Apr-88 & & PU-239/240 & PU-239/240 & PUISO IE AEA & USTEST & 0.0000196 \\
\hline \begin{tabular}{|l|}
5887 \\
\end{tabular} & PDMS & & SW & & & E034035 & 05-Apr-88 & & 10045-97-3 & CS-137 & GAMMA_GSS & USTEST & 0.00125 \\
\hline 5888 & PDMS & & sW & & & E034035 & 05-Apr-88 & & PU-239/240 & PU-239/240 & PUISO_IE_AEA & USTEST & 0.0000575 \\
\hline 5889 & PDMS & & SW & & & E096797 & 05-Apr-88 & & 15046-84-1 & $1-129$ & I129_SEP_TEMS & 320 & 0.0000975 \\
\hline 5890 & PDMS & & SW & & & E096797 & 05-Apr-88 & & $10045-97-3$ & CS-137 & GAMMA_ĒS & USTEST & -0.000741 \\
\hline 5891 & PDMS & & SW & & & E096797 & 05-Apr-88 & & PU-239/240 & PU-239/240 & PUISO IE AEA & USTEST & 0.0000488 \\
\hline 5892 & PDMS & & SW & & & E034006 & 06-Apr-88 & & $10045-97-3$ & CS-137 & GAMMA_GSS & USTEST & 0.0963 \\
\hline 5893 & PDMS & & SW & & & E034006 & 06-Apr-88 & & $10028-17-8$ & TRITIUM & TRITIUM_DIST_LSC & USTEST & 350 \\
\hline \begin{tabular}{|l|}
5894 \\
\end{tabular} & PDMS & & SW & & & E034006 & 06-Apr-88 & & U-238 & $\mathrm{U}-238$ & UISO_AEA & USTEST & 0.0387 \\
\hline \begin{tabular}{|l|}
5895 \\
\end{tabular} & PDMS & & sW & & & E034006 & 06-Apr-88 & & 15117-96-1 & U-235 & UISO_AEA & USTEST & -0.00167 \\
\hline 5896 & PDMS & & SW & & & E034006 & 06-Apr-88 & & 13966-29-5 & $\mathrm{U}-234$ & UISO_AEA & USTEST & 0.0561 \\
\hline 5897 & PDMS & & SW & & & E034036 & 06-Apr-88 & & $10045-97-3$ & CS-137 & GAMMA_GS & USTEST & -0.0456 \\
\hline 5898 & PDMS & & SW & & & E034036 & 06-Apr-88 & & $10028-17-8$ & TRITIUM & TRITIUM_DIST_LSC & USTEST & 350 \\
\hline 5899 & PDMS & & SW & & & E034036 & 06-Apr-88 & & 13966-29-5 & $\mathrm{U}-234$ & UISO_AEA & USTEST & 0.271 \\
\hline 5900 & PDMS & & SW & & & E034036 & 06-Apr-88 & & U-238 & $\mathrm{U}-238$ & UISO_AEA & USTEST & 0.212 \\
\hline 5901 & PDMS & & SW & & & E034036 & 06-Apr-88 & & 15117-96-1 & $\mathrm{U}-235$ & UISO_AEA & USTEST & -0.00308 \\
\hline 5902 & PDMS & & SW & & & E034008 & 08-Apr-88 & & $10028-17-8$ & TRITIUM & 906.0_H3_LSC & USTEST & 567 \\
\hline 5903 & PDMS & & SW & & & E034008 & 08-Apr-88 & & $10045-97-3$ & CS-137 & 901.1_GAMMA_GS & USTEST & -0.228 \\
\hline 5904 & PDMS & & SW & & & E034008 & 08-Apr-88 & & $10098-97-2$ & SR-90 & 905.0_SR_GPC & USTEST & 0.394 \\
\hline 5905 & PDMS & & SW & & & E096794 & 12-Apr-88 & & $10045-97-3$ & CS-137 & 901.1_GAMMA_GS & USTEST & 0.221 \\
\hline 5906 & PDMS & & SW & & & E096794 & 12-Apr-88 & & $10028-17-8$ & TRITIUM & 906.0_H3_LSC & USTEST & 259 \\
\hline \begin{tabular}{|l|l|}
5907 \\
\end{tabular} & PDMS & & SW & & & E096794 & 12-Apr-88 & & U-238 & $\mathrm{U}-238$ & UISO_IE_PLATE_AEA & USTEST & 0.239 \\
\hline 5908 & PDMS & & sW & & & E096794 & 12-Apr-88 & & 15117-96-1 & U-235 & UISO_IE_PLATE_AEA & USTEST & 0.00513 \\
\hline 5909 & PDMS & & SW & & & E096794 & 12-Apr-88 & & $13966-29-5$ & $\mathrm{U}-234$ & UISO_IE_PLATE_AEA & USTEST & 0.332 \\
\hline 5910 & PDMS & & SW & & & E096794 & 12-Apr-88 & & $10098-97-2$ & SR-90 & 905.0_SR_GPC & USTEST & 0.0919 \\
\hline 5911 & PDMS & & SW & & & E096798 & 13-Apr-88 & & $10045-97-3$ & CS-137 & GAMM̄A_ESS & USTEST & -0.352 \\
\hline 5912 & PDMS & & SW & & & E096798 & 13-Apr-88 & & $10028-17-8$ & TRITIUM & TRITIUM_DIST_LSC & USTEST & 225 \\
\hline 5913 & PDMS & & SW & & & E096798 & 13-Apr-88 & & U-238 & U-238 & UISO_AEA & USTEST & 3.48 \\
\hline 5914 & PDMS & & SW & & & E096798 & 13-Apr-88 & & 15117-96-1 & $\mathrm{U}-235$ & UISO_AEA & USTEST & 0.126 \\
\hline 5915 & PDMS & & SW & & & E096798 & 13-Apr-88 & & $13966-29-5$ & $\mathrm{U}-234$ & UISO_AEA & USTEST & 4.16 \\
\hline 5916 & PDMS & & SW & & & E097076 & 13-Apr-88 & & $\mathrm{U}-238$ & $\mathrm{U}-238$ & UISO_AEA & USTEST & \\
\hline \begin{tabular}{|l|l|}
5917 \\
\end{tabular} & PDMS & & SW & & & E097076 & 13-Apr-88 & & 15117-96-1 & U-235 & UISO_AEA & USTEST & \\
\hline 5918 & PDMS & & SW & & & E097076 & 13-Apr-88 & & $13966-29-5$ & $\mathrm{U}-234$ & UISO_AEA & USTEST & \\
\hline 5919 & PDMS & & SW & & & E097076 & 13-Apr-88 & & $10028-17-8$ & TRITIUM & TRITIŪM_DIST_LSC & USTEST & \\
\hline 5920 & PDMS & & SW & & & E097076 & 13-Apr-88 & & $10045-97-3$ & CS-137 & GAMMA_GS & USTEST & \\
\hline 5921 & PDMS & & SW & & & E096924 & 19-Apr-88 & & $10045-97-3$ & CS-137 & GAMMA_GS & USTEST & 0 \\
\hline 5922 & PDMS & & SW & & & E096924 & 19-Apr-88 & & $13966-29-5$ & $\mathrm{U}-234$ & 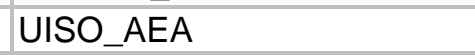 & USTEST & 0.423 \\
\hline 5923 & PDMS & & SW & & & E096924 & 19-Apr-88 & & $10098-97-2$ & SR-90 & SRISŌ_SEP_PRECIP_GPC & USTEST & 0.128 \\
\hline 5924 & PDMS & & SW & & & E096924 & 19-Apr-88 & & $10028-17-8$ & TRITIUM & TRITIUM̄ELEEECT_LSC̄ & USTEST & 181 \\
\hline 5925 & PDMS & & SW & & & E096924 & 19-Apr-88 & & $\mathrm{U}-238$ & $\mathrm{U}-238$ & UISO_AËA & USTEST & 0.37 \\
\hline 5926 & PDMS & & SW & & & E096924 & 19-Apr-88 & & 15117-96-1 & U-235 & UISO_AEA & USTEST & 0.00279 \\
\hline 5927 & PDMS & & SW & & & E096923 & 19-Apr-88 & & $10045-97-3$ & CS-137 & GAMMA_ASS & USTEST & 0.0963 \\
\hline 5928 & PDMS & & SW & & & E096923 & 19-Apr-88 & & 15117-96-1 & U-235 & 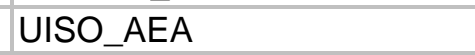 & USTEST & 0.00398 \\
\hline 5929 & PDMS & & SW & & & E096923 & 19-Apr-88 & & $10028-17-8$ & TRITIUM & TRITIÜM_ELECT_LSC & USTEST & 166 \\
\hline
\end{tabular}




\begin{tabular}{|c|c|c|c|c|c|c|c|c|c|c|c|c|c|}
\hline & A & $B$ & $\mathrm{C}$ & $\mathrm{D}$ & $\mathrm{E}$ & $\mathrm{F}$ & G & $\mathrm{H}$ & 1 & $\mathrm{~J}$ & $\mathrm{~K}$ & $\mathrm{~L}$ & $\mathrm{M}$ \\
\hline 1 & SAMPLE_NUM_ASSIGNED_TO & SAMPLER & MEDIA & BIOTA_MEDIA & TAG_ID & SAMP_NUM & SAMP_DATE_TIME & SAMP_DATE_TIME_ON & CON_ID & CON_SHORT_NAME & METHOD_NAME & LAB_CODE & VALUE_RPTD \\
\hline 5930 & PDMS & & SW & & & E096923 & 19-Apr-88 & & U-238 & $U-238$ & UISO_AEA & USTEST & 0.411 \\
\hline 5931 & PDMS & & SW & & & E096923 & 19-Apr-88 & & 13966-29-5 & U-234 & UISO_AEA & USTEST & 0.529 \\
\hline 5932 & PDMS & & SW & & & E096923 & 19-Apr-88 & & $10098-97-2$ & SR-90 & SRISO_SEP_PRECIP_GPC & USTEST & 0.0987 \\
\hline 5933 & PDMS & & SW & & & E096926 & 19-Apr-88 & & $10045-97-3$ & CS-137 & GAMMA_GS & USTEST & 0.000581 \\
\hline 5934 & PDMS & & SW & & & E096925 & 19-Apr-88 & & $10045-97-3$ & CS-137 & GAMMA_GS & USTEST & 0.00568 \\
\hline 5935 & PDMS & & SW & & & E096928 & 19-Apr-88 & & $10045-97-3$ & CS-137 & GAMMA_GS & USTEST & 0.00188 \\
\hline 5936 & PDMS & & SW & & & E096927 & 19-Apr-88 & & $10045-97-3$ & CS-137 & GAMMA_GS & USTEST & 0.00422 \\
\hline 5937 & PDMS & & SW & & & E096929 & 19-Apr-88 & & $10045-97-3$ & CS-137 & GAMMA_GS & USTEST & 0.00187 \\
\hline 5938 & PDMS & & SW & & & E096930 & 19-Apr-88 & & $10045-97-3$ & CS-137 & GAMMA_GS & USTEST & 0.00323 \\
\hline 5939 & PDMS & & SW & & & E097013 & 26-Apr-88 & & $10045-97-3$ & CS-137 & GAMMA_GS & USTEST & 1.43 \\
\hline 5940 & PDMS & & SW & & & E097013 & 26-Apr-88 & & $10098-97-2$ & SR-90 & SRISO_SEP_PRECIP_GPC & USTEST & 0.421 \\
\hline 5941 & PDMS & & SW & & & E097013 & 26-Apr-88 & & $10028-17-8$ & TRITIUM & TRITIUM_DIST_LSC & USTEST & 169 \\
\hline 5942 & PDMS & & SW & & & E097015 & 26-Apr-88 & & $10045-97-3$ & CS-137 & GAMMA_GS & USTEST & -0.152 \\
\hline 5943 & PDMS & & SW & & & E097015 & 26-Apr-88 & & $10028-17-8$ & TRITIUM & TRITIUM_DIST_LSC & USTEST & 5550 \\
\hline $5944 \mathrm{~F}$ & PDMS & & SW & & & E097014 & 26-Apr-88 & & $10045-97-3$ & CS-137 & GAMMA_GS & USTEST & 2.28 \\
\hline 5945 & PDMS & & SW & & & E097014 & 26-Apr-88 & & U-238 & U-238 & UISO_AEAA & USTEST & 99.9 \\
\hline 5946 & PDMS & & SW & & & E097014 & 26-Apr-88 & & $15117-96-1$ & U-235 & UISO_AEA & USTEST & 4.01 \\
\hline 5947 & PDMS & & SW & & & E097014 & 26-Apr-88 & & 13966-29-5 & U-234 & UISO_AEA & USTEST & 106 \\
\hline 5948 & PDMS & & SW & & & E097014 & 26-Apr-88 & & $10098-97-2$ & SR-90 & SRISO_SEP_PRECIP_GPC & USTEST & 2.63 \\
\hline 5949 & PDMS & & SW & & & E097014 & 26-Apr-88 & & $10028-17-8$ & TRITIUM & TRITIUM_DIST_LSC & USTEST & 368 \\
\hline 5950 & PDMS & & SW & & & E097082 & 03-May-88 & & $10045-97-3$ & CS-137 & GAMMA_GS & USTEST & 0.00112 \\
\hline 5951 & PDMS & & SW & & & E097133 & 03-May-88 & & $10045-97-3$ & CS-137 & GAMMA_GS & USTEST & 0.00523 \\
\hline 5952 & PDMS & & SW & & & E097083 & 03-May-88 & & $10045-97-3$ & CS-137 & GAMMA_GS & USTEST & 0.00179 \\
\hline 5953 & PDMS & & SW & & & E097081 & 03-May-88 & & $10045-97-3$ & CS-137 & GAMMA_GS & USTEST & -0.273 \\
\hline 5954 & PDMS & & SW & & & E097081 & 03-May-88 & & U-238 & $\mathrm{U}-238$ & UISO_AEA & USTEST & 0.167 \\
\hline 5955 & PDMS & & SW & & & E097081 & 03-May-88 & & 15117-96-1 & U-235 & UISO_AEA & USTEST & 0.00232 \\
\hline 5956 & PDMS & & SW & & & E097081 & 03-May-88 & & $13966-29-5$ & $\mathrm{U}-234$ & UISO_AEA & USTEST & 0.21 \\
\hline 5957 & PDMS & & SW & & & E097081 & 03-May-88 & & $10098-97-2$ & SR-90 & SRISO_SEP_PRECIP_GPC & USTEST & 0.114 \\
\hline 5958 & PDMS & & SW & & & E097134 & 03-May-88 & & $10045-97-3$ & CS-137 & GAMMA_GS & USTEST & 0.00665 \\
\hline 5959 & PDMS & & SW & & & E097081 & 03-May-88 & & $10028-17-8$ & TRITIUM & TRITIUM_ELECT_LSC & USTEST & 77.6 \\
\hline 5960 & PDMS & & SW & & & E097084 & 03-May-88 & & $10045-97-3$ & CS-137 & GAMMA_GS & USTEST & 0.00151 \\
\hline 5961 & PDMS & & SW & & & E097080 & 03-May-88 & & U-238 & $\mathrm{U}-238$ & UISO_AEA & USTEST & 0.224 \\
\hline 5962 & PDMS & & SW & & & E097080 & 03-May-88 & & 15117-96-1 & U-235 & UISO_AEA & USTEST & 0.0127 \\
\hline 5963 & PDMS & & SW & & & E097080 & 03-May-88 & & $13966-29-5$ & $\mathrm{U}-234$ & UISO_AEA & USTEST & 0.246 \\
\hline 5964 & PDMS & & SW & & & E097136 & 03-May-88 & & $10045-97-3$ & CS-137 & GAMMA_GS & USTEST & 0.00176 \\
\hline 5965 & PDMS & & SW & & & E097080 & 03-May-88 & & $10098-97-2$ & SR-90 & SRISO_SEP_PRECIP_GPC & USTEST & 0.146 \\
\hline 5966 & PDMS & & SW & & & E097080 & 03-May-88 & & $10045-97-3$ & CS-137 & GAMMA_GS & USTEST & -0.198 \\
\hline 5967 & PDMS & & SW & & & E097080 & 03-May-88 & & $10028-17-8$ & TRITIUM & TRITIUM_ELECT_LSC & USTEST & 162 \\
\hline 5968 & PDMS & & SW & & & E099210 & 17-May-88 & & $10045-97-3$ & CS-137 & GAMMA_GS & USTEST & 0.0037 \\
\hline 5969 & PDMS & & SW & & & E103402 & 17-May-88 & & $10045-97-3$ & CS-137 & GAMMA_GS & USTEST & 0.00114 \\
\hline 5970 & PDMS & & SW & & & E099211 & 17-May-88 & & $10045-97-3$ & CS-137 & GAMMA_GS & USTEST & 0.00215 \\
\hline 5971 & PDMS & & SW & & & E103403 & 17-May-88 & & $10045-97-3$ & CS-137 & GAMMA_GS & USTEST & 0.00198 \\
\hline 5972 & PDMS & & SW & & & E099212 & 17-May-88 & & $10045-97-3$ & CS-137 & GAMMA_GS & USTEST & 0.00573 \\
\hline 5973 & PDMS & & SW & & & E103404 & 17-May-88 & & $10045-97-3$ & CS-137 & GAMMA_GS & USTEST & 0.00413 \\
\hline 5974 & PDMS & & SW & & & E103343 & 18-May-88 & & $10045-97-3$ & CS-137 & GAMMA_GS & USTEST & -0.532 \\
\hline 5975 & PDMS & & SW & & & E103343 & 18-May-88 & & 10098-97-2 & SR-90 & SRISO_SEP_PRECIP_GPC & USTEST & 0.197 \\
\hline 5976 & PDMS & & SW & & & E103346 & 23-May-88 & & $10045-97-3$ & CS-137 & 901.1_GAMMA_GS & USTEST & 0.135 \\
\hline 5977 & PDMS & & SW & & & E103346 & 23-May-88 & & $10098-97-2$ & SR-90 & 905.0_SR_GPC & USTEST & -0.0193 \\
\hline 5978 & PDMS & & SW & & & E103346 & 23-May-88 & & $10028-17-8$ & TRITIUM & 906.0_H3_LSC & USTEST & 0 \\
\hline 5979 & PDMS & & SW & & & E103345 & 23-May-88 & & $10098-97-2$ & SR-90 & 905.0_SR_GPC & USTEST & 0.00857 \\
\hline 5980 & PDMS & & SW & & & E103345 & 23-May-88 & & $10045-97-3$ & CS-137 & 901.1_GAMMA_GS & USTEST & -0.0522 \\
\hline 5981 & PDMS & & SW & & & E103345 & 23-Мау-88 & & $10028-17-8$ & TRITIUM & 906.0_H3_LSC & USTEST & 5970 \\
\hline
\end{tabular}




\begin{tabular}{|c|c|c|c|c|c|c|c|c|c|c|c|c|c|}
\hline & A & $\mathrm{B}$ & $\mathrm{C}$ & $\mathrm{D}$ & $\mathrm{E}$ & $\mathrm{F}$ & G & $\mathrm{H}$ & $\mathrm{I}$ & $\mathrm{J}$ & $\mathrm{K}$ & $\mathrm{L}$ & $\mathrm{M}$ \\
\hline 1 & SAMPLE_NUM_ASSIGNED_TO & \begin{tabular}{|l|} 
SAMPLER \\
\end{tabular} & MEDIA & BIOTA_MEDIA & TAG_ID & SAMP_NUM & SAMP_DATE_TIME & SAMP_DATE_TIME_ON & CON_ID & CON_SHORT_NAME & METHOD_NAME & LAB_CODE & VALUE_RPTD \\
\hline 5982 & PDMS & & SW & & & E103344 & 23-Мау-88 & & $10098-97-2$ & SR-90 & 905.0 SR_GPC & USTEST & -0.0171 \\
\hline 5983 & PDMS & & sw & & & E103344 & 23-May-88 & & $10045-97-3$ & CS-137 & 901.1_GAMMA_GS & USTEST & 0.161 \\
\hline 5984 & PDMS & & sw & & & E103344 & 23-May-88 & & $10028-17-8$ & TRITIUM & 906.0_H3_LSC & USTEST & -23.6 \\
\hline 5985 & PDMS & & sw & & & E103347 & 23-May-88 & & $10045-97-3$ & CS-137 & 901.1_GAMMA_GS & USTEST & -0.662 \\
\hline \begin{tabular}{|l|l|}
5986 \\
\end{tabular} & PDMS & & sw & & & E103347 & 23-May-88 & & $10028-17-8$ & TRITIUM & 906.0_H3_LSC & USTEST & -18.2 \\
\hline \begin{tabular}{|l|l|}
5987 \\
\end{tabular} & PDMS & & sw & & & E103347 & 23-May-88 & & $10098-97-2$ & SR-90 & 905.0_SR_GPC & USTEST & -0.0222 \\
\hline 5988 & PDMS & & sW & & & E103415 & 31-May-88 & & $10028-17-8$ & TRITIUM & 906.0 H3_LSC & USTEST & 226 \\
\hline 5989 & PDMS & & SW & & & E103415 & 31-May-88 & & $10045-97-3$ & CS-137 & 901.1_GAMMA_GS & USTEST & -0.285 \\
\hline 5990 & PDMS & & sW & & & E103415 & 31-May-88 & & $10098-97-2$ & SR-90 & 905.0_SR_GPC & USTEST & 0.123 \\
\hline \begin{tabular}{|l|l|}
5991 \\
\end{tabular} & PDMS & & SW & & & E103455 & 31-May-88 & & $10045-97-3$ & CS-137 & GAMMĀA_GS & USTEST & 0.00333 \\
\hline 5992 & PDMS & & sw & & & E103501 & 31-May-88 & & $10045-97-3$ & CS-137 & GAMMA_GS & USTEST & 0.00421 \\
\hline 5993 & PDMS & & sW & & & E103456 & 31-Мау-88 & & $10045-97-3$ & CS-137 & GAMMA_GS & USTEST & 0.00193 \\
\hline 5994 & PDMS & & sW & & & E103417 & 31-Мау-88 & & $10045-97-3$ & CS-137 & GAMMA_GS & USTEST & 0.051 \\
\hline 5995 & PDMS & & sW & & & E103417 & 31-Мау-88 & & $10098-97-2$ & SR-90 & SRISO SEP PRECIP GPC & USTEST & 0.116 \\
\hline 5996 & PDMS & & sw & & & E103417 & 31-May-88 & & U-238 & U-238 & UISO_AEA & USTEST & 0.204 \\
\hline 5997 & PDMS & & sw & & & E103417 & 31-May-88 & & 15117-96-1 & U-235 & UISO_AEA & USTEST & 0.00635 \\
\hline 5998 & PDMS & & sw & & & E103417 & 31-May-88 & & $13966-29-5$ & U-234 & UISO_AEA & USTEST & 0.242 \\
\hline 5999 & PDMS & & sw & & & E103417 & 31-May-88 & & $10028-17-8$ & TRITIUM & TRITIÜM_ELECT_LSC & USTEST & 69.8 \\
\hline 6000 & PDMS & & sW & & & E103502 & 31-Мау-88 & & $10045-97-3$ & CS-137 & GAMMA_GS & USTEST & 0.0046 \\
\hline 6001 & PDMS & & sW & & & E103457 & 31-May-88 & & $10045-97-3$ & CS-137 & GAMMA_GS & USTEST & 0.00121 \\
\hline 6002 & PDMS & & sw & & & E103416 & 31-May-88 & & $10045-97-3$ & CS-137 & GAMMA_GS & USTEST & -0.337 \\
\hline 6003 & PDMS & & sW & & & E103416 & 31-May-88 & & $10098-97-2$ & SR-90 & SRISO_SEP_PRECIP_GPC & USTEST & 0.0894 \\
\hline 6004 & PDMS & & SW & & & E103416 & 31-May-88 & & U-238 & $\mathrm{U}-238$ & UISO_AEEA & USTEST & 0.147 \\
\hline 6005 & PDMS & & sW & & & E103416 & 31-Мау-88 & & 15117-96-1 & U-235 & UISO_AEA & USTEST & 0.00228 \\
\hline 6006 & PDMS & & SW & & & E103416 & 31-Мау-88 & & $13966-29-5$ & U-234 & UISO_AEA & USTEST & 0.229 \\
\hline 6007 & PDMS & & sW & & & E103503 & 31-Мау-88 & & $10045-97-3$ & CS-137 & GAMM̄A_GS & USTEST & 0.00418 \\
\hline 6008 & PDMS & & sW & & & E103416 & 31-May-88 & & $10028-17-8$ & TRITIUM & TRITIUM_ELEECT_LSC & USTEST & 119 \\
\hline 6009 & PDMS & & sW & & & E103592 & 14-Jun-88 & & $10045-97-3$ & CS-137 & GAMMA_GS & USTEST & 0.00499 \\
\hline 6010 & PDMS & & sw & & & E103590 & 14-Jun-88 & & $10045-97-3$ & CS-137 & GAMMA_GS & USTEST & 0.00227 \\
\hline 6011 & PDMS & & sw & & & E103464 & 14-Jun-88 & & $10045-97-3$ & CS-137 & GAMMA_GS & USTEST & \\
\hline 6012 & PDMS & & sW & & & E103463 & 14-Jun-88 & & $10045-97-3$ & CS-137 & GAMMA_GS & USTEST & \\
\hline 6013 & PDMS & & sW & & & E103593 & 14-Jun-88 & & $10045-97-3$ & CS-137 & GAMMA_GS & USTEST & 0.00328 \\
\hline 6014 & PDMS & & sW & & & E103591 & 14-Jun-88 & & $10045-97-3$ & CS-137 & GAMMA_GS & USTEST & 0.0022 \\
\hline 6015 & PDMS & & SW & & & E103869 & 28-Jun-88 & & $10045-97-3$ & CS-137 & 901.1_GAMMMA_GS & USTEST & 0.366 \\
\hline 6016 & PDMS & & sW & & & E103869 & 28-Jun-88 & & $10098-97-2$ & SR-90 & 905.0_SR_GPC & USTEST & -0.00554 \\
\hline 6017 & PDMS & & sW & & & E103869 & 28-Jun-88 & & $10028-17-8$ & TRITIUM & 906.0_H3_LSC & USTEST & -70.9 \\
\hline 6018 & PDMS & & SW & & & E104095 & 28-Jun-88 & & 15046-84-1 & I-129 & I129_SEP_TEMS & 320 & 0.0001098 \\
\hline 6019 & PDMS & & sW & & & E104095 & 28-Jun-88 & & PU-239/240 & PU-239/240 & PUISO_IE_AEA & USTEST & 0.0000282 \\
\hline 6020 & PDMS & & sW & & & E103918 & 28-Jun-88 & & PU-239/240 & PU-239/240 & PUISO_IE_AEA & USTEST & 0.0000452 \\
\hline 6021 & PDMS & & SW & & & E103918 & 28-Jun-88 & & $10045-97-3$ & CS-137 & GAMMA_GES & USTEST & 0.000799 \\
\hline 6022 & PDMS & & sW & & & E104095 & 28-Jun-88 & & $10045-97-3$ & CS-137 & GAMMA_GS & USTEST & 0.00382 \\
\hline 6023 & PDMS & & sW & & & E103867 & 28-Jun-88 & & $10045-97-3$ & CS-137 & GAMMA_GS & USTEST & 0 \\
\hline $6024 \mathrm{~F}$ & PDMS & & sW & & & E103867 & 28-Jun-88 & & U-238 & U-238 & UISO_AĒAA & USTEST & 0.152 \\
\hline 6025 & PDMS & & sW & & & E103867 & 28-Jun-88 & & 15117-96-1 & $\mathrm{U}-235$ & UISO_AEA & USTEST & -0.00289 \\
\hline 6026 & PDMS & & sw & & & E103867 & 28-Jun-88 & & $13966-29-5$ & $\mathrm{U}-234$ & UISO_AEA & USTEST & 0.211 \\
\hline 6027 & PDMS & & SW & & & E103867 & 28-Jun-88 & & 10098-97-2 & SR-90 & SRISŌSEP_PRECIP_GPC & USTEST & 0.109 \\
\hline 6028 & PDMS & & sW & & & E103867 & 28-Jun-88 & & $10028-17-8$ & TRITIUM & TRITIUM̄E_ELECT_LSC̄ & USTEST & 57.6 \\
\hline 6029 & PDMS & & sW & & & E104096 & 28-Jun-88 & & 15046-84-1 & I-129 & I129_SEP_TEMS & 320 & 0.0000454 \\
\hline $6030 \mathrm{~F}$ & PDMS & & sw & & & E104096 & 28-Jun-88 & & PU-239/240 & PU-239/240 & PUISO_IE_AEA & USTEST & 0.0000433 \\
\hline 6031 & PDMS & & SW & & & E103919 & 28-Jun-88 & & PU-239/240 & PU-239/240 & PUISO_IE_AEA & USTEST & 0.0000937 \\
\hline 6032 & PDMS & & SW & & & E103919 & 28-Jun-88 & & $10045-97-3$ & CS-137 & GAMMA_GSS & USTEST & 0.00109 \\
\hline 6033 & PDMS & & SW & & & E104096 & 28-Jun-88 & & $10045-97-3$ & CS-137 & GAMMA_GS & USTEST & 0.00335 \\
\hline
\end{tabular}




\begin{tabular}{|c|c|c|c|c|c|c|c|c|c|c|c|c|c|}
\hline & A & $\mathrm{B}$ & $\mathrm{C}$ & $\mathrm{D}$ & $\mathrm{E}$ & $\mathrm{F}$ & G & $\mathrm{H}$ & $\mathrm{I}$ & $\mathrm{J}$ & $\mathrm{K}$ & $\mathrm{L}$ & $\mathrm{M}$ \\
\hline 1 & SAMPLE_NUM_ASSIGNED_TO & SAMPLER & MEDIA & BIOTA_MEDIA & TAG_ID & SAMP_NUM & SAMP_DATE_TIME & SAMP_DATE_TIME_ON & CON_ID & CON_SHORT_NAME & METHOD_NAME & LAB_CODE & VALUE_RPTD \\
\hline $6034 \mathrm{~F}$ & PDMS & & SW & & & E104097 & 28-Jun-88 & & 15046-84-1 & $1-129$ & I129_SEP_TEMS & 320 & 0.0000931 \\
\hline 6035 & PDMS & & SW & & & E104097 & 28-Jun-88 & & PU-239/240 & PU-239/240 & PUISO_IE_AEA & USTEST & 0.000048 \\
\hline 6036 & PDMS & & SW & & & E103920 & 28-Jun-88 & & PU-239/240 & PU-239/240 & PUISO_IE_AEA & USTEST & 0.000127 \\
\hline $6037 \mathrm{~F}$ & PDMS & & SW & & & E103920 & 28-Jun-88 & & $10045-97-3$ & CS-137 & GAMMA_GS & USTEST & 0.002 \\
\hline 6038 & PDMS & & SW & & & E103866 & 28-Jun-88 & & $10045-97-3$ & CS-137 & GAMMA_GS & USTEST & 0.732 \\
\hline 6039 & PDMS & & SW & & & E103866 & 28-Jun-88 & & U-238 & $\mathrm{U}-238$ & UISO_AEA & USTEST & 0.184 \\
\hline 6040 & PDMS & & SW & & & E103866 & 28-Jun-88 & & 15117-96-1 & U-235 & UISO_AEA & USTEST & 0.00797 \\
\hline 6041 & PDMS & & SW & & & E103866 & 28-Jun-88 & & $13966-29-5$ & U-234 & UISO_AEA & USTEST & 0.209 \\
\hline $6042 \mathrm{~F}$ & PDMS & & SW & & & E103866 & 28-Jun-88 & & $10098-97-2$ & SR-90 & SRISO_SEP_PRECIP_GPC & USTEST & 0.119 \\
\hline 6043 & PDMS & & SW & & & E104097 & 28-Jun-88 & & $10045-97-3$ & CS-137 & GAMMA_GS & USTEST & 0.00699 \\
\hline $6044 \mathrm{~F}$ & PDMS & & SW & & & E103866 & 28-Jun-88 & & $10028-17-8$ & TRITIUM & TRITIUM_ELECT_LSC & USTEST & 124 \\
\hline 6045 & PDMS & & SW & & & E103933 & 29-Jun-88 & & $10045-97-3$ & CS-137 & GAMMA_GS & USTEST & 0.113 \\
\hline 6046 & PDMS & & SW & & & E103933 & 29-Jun-88 & & $10028-17-8$ & TRITIUM & TRITIUM_DIST_LSC & USTEST & 198 \\
\hline 6047 & PDMS & & SW & & & E103933 & 29-Jun-88 & & U-238 & U-238 & UISO_AEA & USTEST & 13.9 \\
\hline 6048 & PDMS & & SW & & & E103933 & 29-Jun-88 & & 15117-96-1 & U-235 & UISO_AEA & USTEST & 0.549 \\
\hline 6049 & PDMS & & SW & & & E103933 & 29-Jun-88 & & $13966-29-5$ & U-234 & UISO_AEA & USTEST & 15 \\
\hline 6050 & PDMS & & SW & & & E103934 & 30-Jun-88 & & U-238 & U-238 & UISO_AEA & USTEST & -0.0293 \\
\hline 6051 & PDMS & & SW & & & E103934 & 30-Jun-88 & & 15117-96-1 & U-235 & UISO_AEA & USTEST & -0.0259 \\
\hline 6052 & PDMS & & SW & & & E103934 & 30-Jun-88 & & 13966-29-5 & U-234 & UISO_AEA & USTEST & -0.0431 \\
\hline 6053 & PDMS & & SW & & & E103934 & 30-Jun-88 & & $10045-97-3$ & CS-137 & GAMMA_GS & USTEST & -0.0871 \\
\hline 6054 & PDMS & & SW & & & E103934 & 30-Jun-88 & & $10028-17-8$ & TRITIUM & TRITIUM_DIST_LSC & USTEST & -76.2 \\
\hline 6055 & PDMS & & SW & & & E103932 & 05-Jul-88 & & $10045-97-3$ & CS-137 & 901.1_GAMMA_GS & USTEST & 0.0305 \\
\hline 6056 & PDMS & & SW & & & E103932 & 05-Jul-88 & & U-238 & U-238 & UISO_IE_PLATE_AEA & USTEST & 0.2 \\
\hline 6057 & PDMS & & SW & & & E103932 & 05-Jul-88 & & 15117-96-1 & U-235 & UISO_IE_PLATE_AEA & USTEST & 0.00191 \\
\hline 6058 & PDMS & & SW & & & E103932 & 05-Jul-88 & & $13966-29-5$ & $\mathrm{U}-234$ & UISO_IE_PLATE_AEA & USTEST & 0.211 \\
\hline 6059 & PDMS & & SW & & & E103932 & 05-Jul-88 & & 10098-97-2 & SR-90 & 905.0_SR_GPC & USTEST & 0.136 \\
\hline 6060 & PDMS & & SW & & & E103932 & 05-Jul-88 & & $10028-17-8$ & TRITIUM & TRITIUM_ELECT_LSC & USTEST & 128 \\
\hline 6061 & PDMS & & SW & & & E103935 & 06-Jul-88 & & $10028-17-8$ & TRITIUM & TRITIUM_DIST_LSC & USTEST & 121 \\
\hline 6062 & PDMS & & SW & & & E103935 & 06-Jul-88 & & U-238 & $\mathrm{U}-238$ & UISO_AEA & USTEST & 3.53 \\
\hline 6063 & PDMS & & SW & & & E103935 & 06-Jul-88 & & $15117-96-1$ & U-235 & UISO_AEA & USTEST & 0.107 \\
\hline 6064 & PDMS & & SW & & & E103935 & 06-Jul-88 & & $13966-29-5$ & $\mathrm{U}-234$ & UISO_AEA & USTEST & 4.19 \\
\hline 6065 & PDMS & & SW & & & E103935 & 06-Jul-88 & & $10045-97-3$ & CS-137 & GAMMA_GS & USTEST & -0.382 \\
\hline 6066 & PDMS & & SW & & & E104092 & 12-Jul-88 & & $10045-97-3$ & CS-137 & GAMMA_GS & USTEST & 0.000799 \\
\hline 6067 & PDMS & & SW & & & E104098 & 12-Jul-88 & & $10045-97-3$ & CS-137 & GAMMA_GS & USTEST & 0.00324 \\
\hline 6068 & PDMS & & SW & & & E104099 & 12-Jul-88 & & $10045-97-3$ & CS-137 & GAMMA_GS & USTEST & 0.00255 \\
\hline 6069 & PDMS & & SW & & & E104093 & 12-Jul-88 & & $10045-97-3$ & CS-137 & GAMMA_GS & USTEST & 0.0032 \\
\hline 6070 & PDMS & & SW & & & E104100 & 12-Jul-88 & & $10045-97-3$ & CS-137 & GAMMA_GS & USTEST & 0.00299 \\
\hline 6071 & PDMS & & SW & & & E104094 & 12-Jul-88 & & $10045-97-3$ & CS-137 & GAMMA_GS & USTEST & 0.00199 \\
\hline 6072 & PDMS & & SW & & & E103936 & 13-Jul-88 & & $10045-97-3$ & CS-137 & GAMMA_GS & USTEST & -0.289 \\
\hline 6073 & PDMS & & SW & & & E103936 & 13-Jul-88 & & $10098-97-2$ & SR-90 & SRISO_SEP_PRECIP_GPC & USTEST & 0.139 \\
\hline $6074 \mathrm{~F}$ & PDMS & & SW & & & E104244 & 19-Jul-88 & & $10045-97-3$ & CS-137 & GAMMA_GS & USTEST & -0.306 \\
\hline 6075 & PDMS & & SW & & & E104244 & 19-Jul-88 & & $10098-97-2$ & SR-90 & SRISO_SEP_PRECIP_GPC & USTEST & 0.689 \\
\hline 6076 & PDMS & & SW & & & E104244 & 19-Jul-88 & & $10028-17-8$ & TRITIUM & TRITIUM_DIST_LSC & USTEST & 127 \\
\hline 6077 F & PDMS & & SW & & & E104247 & 19-Jul-88 & & $10045-97-3$ & CS-137 & GAMMA_GS & USTEST & 1.02 \\
\hline 6078 & PDMS & & SW & & & E104247 & 19-Jul-88 & & $10028-17-8$ & TRITIUM & TRITIUM_DIST_LSC & USTEST & 5200 \\
\hline 6079 & PDMS & & SW & & & E104246 & 19-Jul-88 & & $10045-97-3$ & CS-137 & GAMMA_GS & USTEST & 0.204 \\
\hline 6080 & PDMS & & SW & & & E104246 & 19-Jul-88 & & $10098-97-2$ & SR-90 & SRISO_SEP_PRECIP_GPC & USTEST & 2.36 \\
\hline 6081 & PDMS & & SW & & & E104246 & 19-Jul-88 & & $13966-29-5$ & $\mathrm{U}-234$ & UISO_AEA & USTEST & 153 \\
\hline 6082 & PDMS & & SW & & & E104246 & 19-Jul-88 & & $15117-96-1$ & U-235 & UISO_AEA & USTEST & 5.46 \\
\hline 6083 & PDMS & & SW & & & E104246 & 19-Jul-88 & & U-238 & $\mathrm{U}-238$ & UISO_AEA & USTEST & 146 \\
\hline 6084 & PDMS & & SW & & & E104246 & 19-Jul-88 & & $10028-17-8$ & TRITIUM & TRITIÜM_DIST_LSC & USTEST & 430 \\
\hline 6085 & PDMS & & SW & & & E104291 & 26-Jul-88 & & $10045-97-3$ & CS-137 & GAMMA_GS & USTEST & 0.000232 \\
\hline
\end{tabular}




\begin{tabular}{|c|c|c|c|c|c|c|c|c|c|c|c|c|c|}
\hline & A & $\mathrm{B}$ & $\mathrm{c}$ & $\mathrm{D}$ & $\mathrm{E}$ & $\mathrm{F}$ & G & $\mathrm{H}$ & $\mathrm{I}$ & $\mathrm{J}$ & $\mathrm{K}$ & $\mathrm{L}$ & $\mathrm{M}$ \\
\hline 1 & SAMPLE_NUM_ASSIGNED_TO & SAMPLER & MEDIA & BIOTA_MEDIA & TAG_ID & SAMP_NUM & SAMP_DATE_TIME & SAMP_DATE_TIME_ON & CON_ID & CON_SHORT_NAME & METHOD_NAME & LAB_CODE & \begin{tabular}{|l|} 
VALUE_RPTD \\
\end{tabular} \\
\hline 6086 & PDMS & & SW & & & E104362 & 26-Jul-88 & & $10045-97-3$ & CS-137 & GAMMA GS & USTEST & 0.00319 \\
\hline \begin{tabular}{|c|}
6087 \\
\end{tabular} & PDMS & & sW & & & E104363 & 26-Jul-88 & & $10045-97-3$ & CS-137 & GAMMA_GS & USTEST & 0.00108 \\
\hline 6088 & PDMS & & sW & & & E104292 & 26-Jul-88 & & $10045-97-3$ & CS-137 & GAMMA_GS & USTEST & 0.00318 \\
\hline 6089 & PDMS & & sW & & & E104293 & 26-Jul-88 & & $10045-97-3$ & CS-137 & GAMMA_GS & USTEST & 0.00324 \\
\hline 6090 & PDMS & & sW & & & E104364 & 26-Jul-88 & & $10045-97-3$ & CS-137 & GAMMA_GS & USTEST & 0.00363 \\
\hline \begin{tabular}{|l|}
6091 \\
\end{tabular} & PDMS & & sW & & & E104305 & 02-Aug-88 & & $10045-97-3$ & CS-137 & GAMMA_GS & USTEST & -0.575 \\
\hline 6092 & PDMS & & SW & & & E104305 & 02-Aug-88 & & $13966-29-5$ & U-234 & UISO_AEA & USTEST & 0.127 \\
\hline 6093 & PDMS & & SW & & & E104305 & 02-Aug-88 & & 15117-96-1 & U-235 & UISO_AEA & USTEST & 0.0135 \\
\hline 6094 & PDMS & & sW & & & E104305 & 02-Aug-88 & & $\mathrm{U}-238$ & $\mathrm{U}-238$ & UISO AEA & USTEST & 0.141 \\
\hline 6095 & PDMS & & SW & & & E104305 & 02-Aug-88 & & $10098-97-2$ & SR-90 & SRISO_SEP_PRECIP_GPC & USTEST & 0.117 \\
\hline \begin{tabular}{|c|}
6096 \\
\end{tabular} & PDMS & & sW & & & E104305 & 02-Aug-88 & & $10028-17-8$ & TRITIUM & TRITIUM_ELEECT_LSC & USTEST & 61.3 \\
\hline 6097 & PDMS & & SW & & & E104304 & 02-Aug-88 & & $10045-97-3$ & CS-137 & GAMMA_GS & USTEST & 0.357 \\
\hline 6098 & PDMS & & SW & & & E104304 & 02-Aug-88 & & $13966-29-5$ & $\mathrm{U}-234$ & UISO_AĒA & USTEST & 0.23 \\
\hline 6099 & PDMS & & SW & & & E104304 & 02-Aug-88 & & 15117-96-1 & U-235 & UISO_AEA & USTEST & 0.00955 \\
\hline 6100 & PDMS & & SW & & & E104304 & 02-Aug-88 & & $\mathrm{U}-238$ & $\mathrm{U}-238$ & UISO_AEA & USTEST & 0.208 \\
\hline 6101 & PDMS & & SW & & & E104304 & 02-Aug-88 & & $10098-97-2$ & SR-90 & SRISO_SEP_PRECIP_GPC & USTEST & 0.0982 \\
\hline 6102 & PDMS & & sW & & & E104304 & 02-Aug-88 & & $10028-17-8$ & TRITIUM & TRITIUM_ELEECT_LSC & USTEST & 150 \\
\hline \begin{tabular}{|l|}
6103 \\
\end{tabular} & PDMS & & sW & & & E104680 & 09-Aug-88 & & $10045-97-3$ & CS-137 & GAMMA_GS & USTEST & 0.00481 \\
\hline 6104 & PDMS & & SW & & & E104678 & 09-Aug-88 & & $10045-97-3$ & CS-137 & GAMMA_GS & USTEST & 0.0012 \\
\hline 6105 & PDMS & & SW & & & E104681 & 09-Aug-88 & & $10045-97-3$ & CS-137 & GAMMA_GS & USTEST & 0.00437 \\
\hline 6106 & PDMS & & SW & & & E104679 & 09-Aug-88 & & $10045-97-3$ & CS-137 & GAMMA_GS & USTEST & 0.00246 \\
\hline \begin{tabular}{|l|l|}
6107 \\
\end{tabular} & PDMS & & SW & & & E104251 & 09-Aug-88 & & $10045-97-3$ & CS-137 & GAMMA_GS & USTEST & \\
\hline \begin{tabular}{|l|}
6108 \\
\end{tabular} & PDMS & & SW & & & E104299 & 09-Aug-88 & & $10045-97-3$ & CS-137 & GAMMA_GS & USTEST & \\
\hline 6109 & PDMS & & SW & & & E104570 & 16-Aug-88 & & $10045-97-3$ & CS-137 & 901.1_GAMMA_GS & USTEST & 0.395 \\
\hline 6110 & PDMS & & SW & & & E104570 & 16-Aug-88 & & 10028-17-8 & TRITIUM & 906.0_H3_LSC & USTEST & 128 \\
\hline \begin{tabular}{|l|l|}
6111 \\
\end{tabular} & PDMS & & SW & & & E104570 & 16-Aug-88 & & 10098-97-2 & SR-90 & 905.0_SR_GPC & USTEST & 0.243 \\
\hline 6112 & PDMS & & SW & & & E104569 & 16-Aug-88 & & $10045-97-3$ & CS-137 & 901.1_GAMMA_GS & USTEST & 0.157 \\
\hline 6113 & PDMS & & SW & & & E104569 & 16-Aug-88 & & $10028-17-8$ & TRITIUM & 906.0_H3_LSC & USTEST & 6350 \\
\hline \begin{tabular}{|l|}
6114 \\
\end{tabular} & PDMS & & SW & & & E104569 & 16-Aug-88 & & $10098-97-2$ & SR-90 & 905.0_SR_GPC & USTEST & -0.012 \\
\hline \begin{tabular}{|c|}
6115 \\
\end{tabular} & PDMS & & SW & & & E104568 & 16-Aug-88 & & $10045-97-3$ & CS-137 & 901.1_GAMMA_GS & USTEST & 0.27 \\
\hline 6116 & PDMS & & SW & & & E104568 & 16-Aug-88 & & 10028-17-8 & TRITIUM & 906.0_H3_LSC & USTEST & 42.6 \\
\hline \begin{tabular}{|l|}
6117 \\
\end{tabular} & PDMS & & SW & & & E104568 & 16-Aug-88 & & $10098-97-2$ & SR-90 & 905.0_SR_GPC & USTEST & -0.0172 \\
\hline 6118 & PDMS & & SW & & & E104571 & 16-Aug-88 & & $10045-97-3$ & CS-137 & 901.1_GAMMA_GS & USTEST & 0.696 \\
\hline 6119 & PDMS & & SW & & & E104571 & 16-Aug-88 & & 10028-17-8 & TRITIUM & 906.0_H3_LSC & USTEST & 37.8 \\
\hline 6120 & PDMS & & SW & & & E104571 & 16-Aug-88 & & $10098-97-2$ & SR-90 & 905.0_SR_GPC & USTEST & 0.0139 \\
\hline \begin{tabular}{|l|}
6121 \\
\end{tabular} & PDMS & & SW & & & E104701 & 23-Aug-88 & & $10045-97-3$ & CS-137 & 901.1_GAMMA_GS & USTEST & 0.0482 \\
\hline 6122 & PDMS & & SW & & & E104701 & 23-Aug-88 & & 10098-97-2 & SR-90 & 905.0_SR_GPC & USTEST & 0.113 \\
\hline 6123 & PDMS & & SW & & & E104701 & 23-Aug-88 & & 10028-17-8 & TRITIUM & 906.0_H3_LSC & USTEST & 132 \\
\hline 6124 & PDMS & & SW & & & E104805 & 23-Aug-88 & & $10045-97-3$ & CS-137 & GAMM̄A_GS & USTEST & 0.00351 \\
\hline 6125 & PDMS & & SW & & & E104807 & 23-Aug-88 & & $10045-97-3$ & CS-137 & GAMMA_GS & USTEST & 0.00267 \\
\hline 6126 & PDMS & & SW & & & E104806 & 23-Aug-88 & & $10045-97-3$ & CS-137 & GAMMA_GS & USTEST & 0.00142 \\
\hline \begin{tabular}{|l|l|}
6127 \\
\end{tabular} & PDMS & & SW & & & E104808 & 23-Aug-88 & & $10045-97-3$ & CS-137 & GAMMA_GS & USTEST & 0.000209 \\
\hline \begin{tabular}{|l|}
6128 \\
\end{tabular} & PDMS & & SW & & & E104688 & 23-Aug-88 & & $10045-97-3$ & CS-137 & GAMMA_GS & USTEST & \\
\hline 6129 & PDMS & & SW & & & E104689 & 23-Aug-88 & & $10045-97-3$ & CS-137 & GAMMA_GS & USTEST & \\
\hline 6130 & PDMS & & SW & & & E104909 & 30-Aug-88 & & $10045-97-3$ & CS-137 & GAMMA_GS & USTEST & -0.608 \\
\hline \begin{tabular}{|l|}
6131 \\
\end{tabular} & PDMS & & SW & & & E104909 & 30-Aug-88 & & $13966-29-5$ & $\mathrm{U}-234$ & 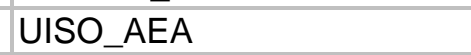 & USTEST & 0.112 \\
\hline 6132 & PDMS & & SW & & & E104909 & 30-Aug-88 & & 15117-96-1 & U-235 & UISO_AEA & USTEST & 0.00442 \\
\hline 6133 & PDMS & & SW & & & E104909 & 30-Aug-88 & & U-238 & U-238 & UISO_AEA & USTEST & 0.11 \\
\hline \begin{tabular}{|l|}
6134 \\
\end{tabular} & PDMS & & SW & & & E104909 & 30-Aug-88 & & $10098-97-2$ & SR-90 & SRISŌ_SEP_PRECIP_GPC & USTEST & 0.0712 \\
\hline 6135 & PDMS & & sW & & & E104909 & 30-Aug-88 & & $10028-17-8$ & TRITIUM & TRITIUM̄ELEEECT_LSC & USTEST & 70.8 \\
\hline 6136 & PDMS & & sW & & & E104908 & 30-Aug-88 & & $10045-97-3$ & CS-137 & GAMMA GS & USTEST & 0.323 \\
\hline 6137 & PDMS & & SW & & & E104908 & 30-Aug-88 & & $13966-29-5$ & $\mathrm{U}-234$ & UISO_AĒA & USTEST & 0.0432 \\
\hline
\end{tabular}




\begin{tabular}{|c|c|c|c|c|c|c|c|c|c|c|c|c|c|}
\hline & A & $\mathrm{B}$ & $\mathrm{c}$ & $\mathrm{D}$ & $\mathrm{E}$ & $\mathrm{F}$ & G & $\mathrm{H}$ & $\mathrm{I}$ & $\mathrm{J}$ & $\mathrm{K}$ & $\mathrm{L}$ & $\mathrm{M}$ \\
\hline 1 & SAMPLE_NUM_ASSIGNED_TO & SAMPLER & MEDIA & BIOTA_MEDIA & TAG_ID & SAMP_NUM & SAMP_DATE_TIME & SAMP_DATE_TIME_ON & CON_ID & CON_SHORT_NAME & METHOD_NAME & LAB_CODE & \begin{tabular}{|l|} 
VALUE_RPTD \\
\end{tabular} \\
\hline 6138 & PDMS & & SW & & & E104908 & 30-Aug-88 & & $15117-96-1$ & U-235 & UISO AEA & USTEST & -0.000778 \\
\hline 6139 & PDMS & & sW & & & E104908 & 30-Aug-88 & & $\mathrm{U}-238$ & U-238 & UISO_AEA & USTEST & 0.0685 \\
\hline 6140 & PDMS & & sW & & & E104908 & 30-Aug-88 & & $10098-97-2$ & SR-90 & SRISO_SEP_PRECIP_GPC & USTEST & 0.0892 \\
\hline \begin{tabular}{|l|}
6141 \\
\end{tabular} & PDMS & & sW & & & E104908 & 30-Aug-88 & & $10028-17-8$ & TRITIUM & TRITIUM_ELECT_LSC & USTEST & 140 \\
\hline \begin{tabular}{|l|}
6142 \\
\end{tabular} & PDMS & & sW & & & E104946 & 06-Sep-88 & & $10045-97-3$ & CS-137 & GAMMA_GS & USTEST & 0.000733 \\
\hline \begin{tabular}{|l|}
6143 \\
\end{tabular} & PDMS & & sW & & & E104948 & 06-Sep-88 & & $10045-97-3$ & CS-137 & GAMMA_GS & USTEST & 0.00204 \\
\hline 6144 & PDMS & & SW & & & E104947 & 06-Sep-88 & & $10045-97-3$ & CS-137 & GAMMA_GS & USTEST & 0.000861 \\
\hline 6145 & PDMS & & SW & & & E104949 & 06-Sep-88 & & $10045-97-3$ & CS-137 & GAMMA_GS & USTEST & -0.00187 \\
\hline \begin{tabular}{|l|}
6146 \\
\end{tabular} & PDMS & & sW & & & E015788 & 06-Sep-88 & & $10045-97-3$ & CS-137 & GAMMA GS & USTEST & \\
\hline 6147 & PDMS & & SW & & & E077822 & 06-Sep-88 & & $10045-97-3$ & CS-137 & GAMMA_GS & USTEST & \\
\hline \begin{tabular}{|l|l|}
6148 & \\
\end{tabular} & PDMS & & sW & & & E104955 & 07-Sep-88 & & $10045-97-3$ & CS-137 & GAMMA_GS & USTEST & 0.034 \\
\hline 6149 & PDMS & & SW & & & E104955 & 07-Sep-88 & & $10098-97-2$ & SR-90 & SRISO_SEEP_PRECIP_GPC & USTEST & 0.143 \\
\hline 6150 & PDMS & & SW & & & E105023 & $20-$ Sep-88 & & $10045-97-3$ & CS-137 & 901.1_GAMMĀGS & USTEST & -0.198 \\
\hline 6151 & PDMS & & SW & & & E105023 & 20-Sep-88 & & $10028-17-8$ & TRITIUM & 906.0 H3 LSC & USTEST & 695 \\
\hline 6152 & PDMS & & SW & & & E105023 & 20-Sep-88 & & $10098-97-2$ & SR-90 & 905.0_SR_GPC & USTEST & -0.0076 \\
\hline 6153 & PDMS & & SW & & & E105112 & 20-Sep-88 & & $10045-97-3$ & CS-137 & GAMMA_GS & USTEST & 0.00274 \\
\hline 6154 & PDMS & & sW & & & E105181 & 20-Sep-88 & & $10045-97-3$ & CS-137 & GAMMA_GS & USTEST & 0.00658 \\
\hline \begin{tabular}{|c|}
6155 \\
\end{tabular} & PDMS & & sW & & & E104953 & 20-Sep-88 & & $10045-97-3$ & CS-137 & GAMMA_GS & USTEST & \\
\hline 6156 & PDMS & & SW & & & E104954 & $20-$ Sep-88 & & $10045-97-3$ & CS-137 & GAMMA_GS & USTEST & \\
\hline \begin{tabular}{|l|l|}
6157 & \\
\end{tabular} & PDMS & & SW & & & E105113 & 20-Sep-88 & & $10045-97-3$ & CS-137 & GAMMA_GS & USTEST & -0.000429 \\
\hline 6158 & PDMS & & SW & & & E105182 & 20-Sep-88 & & $10045-97-3$ & CS-137 & GAMMA_GS & USTEST & 0.00269 \\
\hline 6159 & PDMS & & SW & & & E105022 & 21-Sep-88 & & $10045-97-3$ & CS-137 & GAMMA_GS & USTEST & 0.306 \\
\hline 6160 & PDMS & & SW & & & E105022 & 21-Sep-88 & & $13966-29-5$ & U-234 & UISO_AĒA & USTEST & 1.03 \\
\hline 6161 & PDMS & & SW & & & E105022 & 21-Sep-88 & & $15117-96-1$ & U-235 & UISO_AEA & USTEST & 0.0243 \\
\hline 6162 & PDMS & & SW & & & E105022 & 21-Sep-88 & & $\mathrm{U}-238$ & $\mathrm{U}-238$ & UISO_AEA & USTEST & 0.941 \\
\hline 6163 & PDMS & & SW & & & E105022 & 21-Sep-88 & & 10028-17-8 & TRITIUM & TRITIÜM_DIST_LSC & USTEST & 345 \\
\hline \begin{tabular}{|l|l|}
6164 & \\
\end{tabular} & PDMS & & SW & & & E105021 & 21-Sep-88 & & $10045-97-3$ & CS-137 & GAMMA_GS & USTEST & 0.255 \\
\hline 6165 & PDMS & & SW & & & E105021 & 21-Sep-88 & & $13966-29-5$ & U-234 & 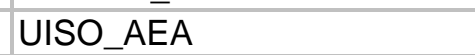 & USTEST & 6.92 \\
\hline \begin{tabular}{|l|}
6166 \\
\end{tabular} & PDMS & & SW & & & E105021 & 21-Sep-88 & & 15117-96-1 & U-235 & UISO_AEA & USTEST & 0.232 \\
\hline \begin{tabular}{|l|l|}
6167 \\
\end{tabular} & PDMS & & SW & & & E105021 & 21-Sep-88 & & U-238 & U-238 & UISO_AEA & USTEST & 5.89 \\
\hline \begin{tabular}{|l|}
6168 \\
\end{tabular} & PDMS & & sW & & & E105021 & 21-Sep-88 & & $10028-17-8$ & TRITIUM & TRITIUM_DIST_LSC & USTEST & 377 \\
\hline 6169 & PDMS & & SW & & & E105024 & 23-Sep-88 & & $10045-97-3$ & CS-137 & 901.1_GAMMMA_GS & USTEST & -0.204 \\
\hline 6170 & PDMS & & SW & & & E105024 & 23-Sep-88 & & 10028-17-8 & TRITIUM & 906.0_H3_LSC & USTEST & 601 \\
\hline \begin{tabular}{|l|}
6171 \\
\end{tabular} & PDMS & & SW & & & E105024 & 23-Sep-88 & & $10098-97-2$ & SR-90 & 905.0_SR_GPC & USTEST & 0.336 \\
\hline 6172 & PDMS & & SW & & & E105197 & 27-Sep-88 & & $10045-97-3$ & CS-137 & 901.1_GAMMA_GS & USTEST & 0.102 \\
\hline \begin{tabular}{|l|}
6173 \\
\end{tabular} & PDMS & & SW & & & E105197 & 27-Sep-88 & & $10028-17-8$ & TRITIUM & TRITIŪM_ELECT_LSC & USTEST & 170 \\
\hline 6174 & PDMS & & SW & & & E105197 & 27-Sep-88 & & $10098-97-2$ & SR-90 & 905.0_SR_GPC & USTEST & 0.117 \\
\hline 6175 & PDMS & & SW & & & E105197 & 27-Sep-88 & & $13966-29-5$ & $\mathrm{U}-234$ & UISO_IE_PLATE_AEA & USTEST & 0.264 \\
\hline 6176 & PDMS & & SW & & & E105197 & 27-Sep-88 & & 15117-96-1 & U-235 & UISO_IE_PLATE_AEA & USTEST & 0.00944 \\
\hline \begin{tabular}{|l|l}
6177 \\
\end{tabular} & PDMS & & SW & & & E105197 & 27-Sep-88 & & $\mathrm{U}-238$ & U-238 & UISO_IE_PLATE_AEA & USTEST & 0.183 \\
\hline 6178 & PDMS & & SW & & & E105196 & 27-Sep-88 & & $10045-97-3$ & CS-137 & GAMM̄A_GS & USTEST & -0.0334 \\
\hline 6179 & PDMS & & SW & & & E105196 & 27-Sep-88 & & $10028-17-8$ & TRITIUM & TRITIUM_ELECT_LSC & USTEST & 66.7 \\
\hline 6180 & PDMS & & SW & & & E105196 & 27-Sep-88 & & $10098-97-2$ & SR-90 & SRISO_SEP_PRECIP_GPC & USTEST & 0.0706 \\
\hline 6181 & PDMS & & SW & & & E105196 & 27-Sep-88 & & $13966-29-5$ & U-234 & UISO_AEA & USTEST & 0.179 \\
\hline 6182 & PDMS & & SW & & & E105196 & 27-Sep-88 & & 15117-96-1 & U-235 & UISO_AEA & USTEST & 0.008 \\
\hline 6183 & PDMS & & SW & & & E105196 & 27-Sep-88 & & $\mathrm{U}-238$ & U-238 & UISO_AEA & USTEST & 0.141 \\
\hline 6184 & PDMS & & SW & & & E105195 & 27-Sep-88 & & $10045-97-3$ & CS-137 & GAMM̄A_GS & USTEST & -0.741 \\
\hline 6185 & PDMS & & SW & & & E105195 & 27-Sep-88 & & $10098-97-2$ & SR-90 & SRISO_SEEP_PRECIP_GPC & USTEST & 0.0938 \\
\hline 6186 & PDMS & & SW & & & E105195 & 27-Sep-88 & & $10028-17-8$ & TRITIUM & TRITIUM̄ELELECT_LSC & USTEST & 138 \\
\hline 6187 & PDMS & & SW & & & E105195 & 27-Sep-88 & & $13966-29-5$ & U-234 & UISO_AEA & USTEST & 0.225 \\
\hline 6188 & PDMS & & SW & & & E105195 & 27-Sep-88 & & 15117-96-1 & U-235 & UISO_AEA & USTEST & -0.00285 \\
\hline 6189 & PDMS & & SW & & & E105195 & 27-Sep-88 & & $\mathrm{U}-238$ & U-238 & UISO_AEA & USTEST & 0.134 \\
\hline
\end{tabular}




\begin{tabular}{|c|c|c|c|c|c|c|c|c|c|c|c|c|c|}
\hline & A & $\mathrm{B}$ & $\mathrm{c}$ & $\mathrm{D}$ & $\mathrm{E}$ & $\mathrm{F}$ & G & $\mathrm{H}$ & $\mathrm{I}$ & $\mathrm{J}$ & $\mathrm{K}$ & $\mathrm{L}$ & $\mathrm{M}$ \\
\hline 1 & SAMPLE_NUM_ASSIGNED_TO & SAMPLER & MEDIA & BIOTA_MEDIA & TAG_ID & SAMP_NUM & SAMP_DATE_TIME & SAMP_DATE_TIME_ON & CON_ID & CON_SHORT_NAME & METHOD_NAME & LAB_CODE & \begin{tabular}{|l|} 
VALUE_RPTD \\
\end{tabular} \\
\hline 6190 & PDMS & & SW & & & E105199 & 28-Sep-88 & & $10028-17-8$ & TRITIUM & TRITIUM_DIST_LSC & USTEST & 93.1 \\
\hline 6191 & PDMS & & sW & & & E105199 & 28-Sep-88 & & $10045-97-3$ & CS-137 & GAMMA_GS & USTEST & -0.151 \\
\hline 6192 & PDMS & & sW & & & E105199 & 28-Sep-88 & & $13966-29-5$ & U-234 & UISO_AEA & USTEST & 3.88 \\
\hline \begin{tabular}{|l|}
6193 \\
\end{tabular} & PDMS & & sW & & & E105199 & 28-Sep-88 & & $15117-96-1$ & U-235 & UISO_AEA & USTEST & 0.103 \\
\hline \begin{tabular}{|l|}
6194 \\
\end{tabular} & PDMS & & sW & & & E105199 & 28-Sep-88 & & $\mathrm{U}-238$ & $\mathrm{U}-238$ & UISO_AEA & USTEST & 3.22 \\
\hline \begin{tabular}{|c|}
6195 \\
\end{tabular} & PDMS & & sW & & & E105246 & $04-$ Oct-88 & & 15046-84-1 & $1-129$ & I129_SEP_TEMS & 320 & 0.0000846 \\
\hline 6196 & PDMS & & SW & & & E105246 & 04-Oct-88 & & PU-239/240 & PU-239/240 & PUISO_IE_AEA & USTEST & 0.0000185 \\
\hline 6197 & PDMS & & SW & & & E105246 & 04-Oct-88 & & $10045-97-3$ & CS-137 & GAMMA_GS & USTEST & 0.00409 \\
\hline 6198 & PDMS & & sW & & & E105244 & 04-Oct-88 & & PU-239/240 & PU-239/240 & PUISO IE AEA & USTEST & 0.0000163 \\
\hline 6199 & PDMS & & SW & & & E105244 & 04-Oct-88 & & $10045-97-3$ & CS-137 & GAMMA_GS & USTEST & 0.00106 \\
\hline 6200 & PDMS & & SW & & & E105141 & 04-Oct-88 & & 15046-84-1 & $1-129$ & I129_SEP_TEMS & 320 & 0.0000058 \\
\hline 6201 & PDMS & & SW & & & E105141 & 04-Oct-88 & & PU-239/240 & PU-239/240 & PUISOO_IE_AEA & USTEST & 0.000101 \\
\hline 6202 & PDMS & & SW & & & E105141 & 04-Oct-88 & & $10045-97-3$ & CS-137 & GAMMA_GS & USTEST & \\
\hline 6203 & PDMS & & SW & & & E105142 & 04-Oct-88 & & PU-239/240 & PU-239/240 & PUISO IE AEA & USTEST & 0.000103 \\
\hline 6204 & PDMS & & SW & & & E105142 & 04-Oct-88 & & $10045-97-3$ & CS-137 & GAMMA_GS & USTEST & \\
\hline 6205 & PDMS & & SW & & & E105247 & 04-Oct-88 & & 15046-84-1 & I-129 & I129_SEP_TEMS & 320 & 0.0001416 \\
\hline \begin{tabular}{|l|}
6206 \\
\end{tabular} & PDMS & & SW & & & E105247 & 04-Oct-88 & & PU-239/240 & PU-239/240 & PUISO_IE_AEA & USTEST & -0.00000514 \\
\hline \begin{tabular}{|l|l|}
6207 \\
\end{tabular} & PDMS & & sW & & & E105245 & 04-Oct-88 & & PU-239/240 & PU-239/240 & PUISO_IE_AEA & USTEST & 0.0000867 \\
\hline \begin{tabular}{|l|}
6208 \\
\end{tabular} & PDMS & & sW & & & E105245 & 04-Oct-88 & & $10045-97-3$ & CS-137 & GAMMA_GS & USTEST & 0.000758 \\
\hline 6209 & PDMS & & SW & & & E105247 & 04-Oct-88 & & $10045-97-3$ & CS-137 & GAMMA_GS & USTEST & 0.00751 \\
\hline 6210 & PDMS & & SW & & & E105262 & 11-Oct-88 & & $10098-97-2$ & SR-90 & SRISO_SEP_PRECIP_GPC & USTEST & 1.53 \\
\hline 6211 & PDMS & & SW & & & E105262 & 11-Oct-88 & & $10028-17-8$ & TRITIUM & TRITIUM_DISTT_LSC & USTEST & 81.1 \\
\hline 6212 & PDMS & & SW & & & E105262 & 11-Oct-88 & & $10045-97-3$ & CS-137 & GAMMA_GS & USTEST & 1.35 \\
\hline 6213 & PDMS & & SW & & & E105185 & 11-Oct-88 & & $10045-97-3$ & CS-137 & GAMMA_GS & USTEST & \\
\hline 6214 & PDMS & & SW & & & E105185 & 11-Oct-88 & & $10028-17-8$ & TRITIUM & TRITIUM_DIST_LSC & USTEST & \\
\hline 6215 & PDMS & & SW & & & E105263 & 11-Oct-88 & & $10098-97-2$ & SR-90 & SRISO_SEP_PERECIP_GPC & USTEST & 2.06 \\
\hline 6216 & PDMS & & SW & & & E105263 & 11-Oct-88 & & $10028-17-8$ & TRITIUM & TRITIUM_DIST_LSC & USTEST & 652 \\
\hline \begin{tabular}{|l|l|}
6217 \\
\end{tabular} & PDMS & & SW & & & E105263 & 11-Oct-88 & & $\mathrm{U}-238$ & $\mathrm{U}-238$ & UISO_AËA & USTEST & 165 \\
\hline 6218 & PDMS & & SW & & & E105263 & 11-Oct-88 & & 15117-96-1 & $\mathrm{U}-235$ & UISO_AEA & USTEST & 6.27 \\
\hline 6219 & PDMS & & SW & & & E105263 & 11-Oct-88 & & $13966-29-5$ & $\mathrm{U}-234$ & UISO_AEA & USTEST & 175 \\
\hline 6220 & PDMS & & sW & & & E105263 & 11-Oct-88 & & $10045-97-3$ & CS-137 & GAMMA_GS & USTEST & -0.157 \\
\hline 6221 & PDMS & & SW & & & E105341 & 18-Oct-88 & & $10028-17-8$ & TRITIUM & TRITIUM_ELEECT_LSC & USTEST & 215 \\
\hline 6222 & PDMS & & SW & & & E105341 & 18-Oct-88 & & $10098-97-2$ & SR-90 & SRISO_SEPP_PRËCIP_GPC & USTEST & 0.138 \\
\hline 6223 & PDMS & & SW & & & E105341 & 18-Oct-88 & & $\mathrm{U}-238$ & $\mathrm{U}-238$ & UISO_AEEA & USTEST & 0.172 \\
\hline 6224 & PDMS & & SW & & & E105341 & 18-Oct-88 & & 15117-96-1 & U-235 & UISO_AEA & USTEST & 0.00906 \\
\hline 6225 & PDMS & & sW & & & E105341 & 18-Oct-88 & & $13966-29-5$ & $\mathrm{U}-234$ & UISO_AEA & USTEST & 0.268 \\
\hline 6226 & PDMS & & SW & & & E105341 & $18-O c t-88$ & & $10045-97-3$ & CS-137 & GAMMA_GS & USTEST & -0.56 \\
\hline \begin{tabular}{|l|l|}
6227 & $r \cdot r$ \\
\end{tabular} & PDMS & & SW & & & E105340 & 18-Oct-88 & & $10045-97-3$ & CS-137 & GAMMA_GS & USTEST & -0.0509 \\
\hline 6228 & PDMS & & SW & & & E105340 & 18-Oct-88 & & $10028-17-8$ & TRITIUM & TRITIUM_ELELECT_LSC & USTEST & 60.2 \\
\hline 6229 & PDMS & & SW & & & E105340 & 18-Oct-88 & & $10098-97-2$ & SR-90 & SRISO_SËP_PRËCIP_GPC & USTEST & 0.124 \\
\hline 6230 & PDMS & & SW & & & E105340 & 18-Oct-88 & & $\mathrm{U}-238$ & $\mathrm{U}-238$ & UISO_AEEA & USTEST & 0.11 \\
\hline 6231 & PDMS & & SW & & & E105340 & 18-Oct-88 & & 15117-96-1 & $\mathrm{U}-235$ & UISO_AEA & USTEST & -0.00518 \\
\hline 6232 & PDMS & & SW & & & E105340 & 18-Oct-88 & & $13966-29-5$ & U-234 & UISO_AEA & USTEST & 0.141 \\
\hline 6233 & PDMS & & SW & & & E105198 & 18-Oct-88 & & $10045-97-3$ & CS-137 & GAMMA_GS & USTEST & \\
\hline 6234 & PDMS & & SW & & & E105259 & 18-Oct-88 & & $10045-97-3$ & CS-137 & GAMMA_GS & USTEST & \\
\hline 6235 & PDMS & & SW & & & E105391 & 18-Oct-88 & & $10045-97-3$ & CS-137 & GAMMA_GS & USTEST & 0.00603 \\
\hline \begin{tabular}{|l|}
6236 \\
\end{tabular} & PDMS & & SW & & & E105389 & 18-Oct-88 & & $10045-97-3$ & CS-137 & GAMMA_GS & USTEST & 0.000254 \\
\hline \begin{tabular}{|l|l|}
6237 \\
\end{tabular} & PDMS & & SW & & & E105392 & 18-Oct-88 & & $10045-97-3$ & CS-137 & GAMMA_GS & USTEST & 0.00895 \\
\hline \begin{tabular}{|l|}
6238 \\
\end{tabular} & PDMS & & SW & & & E105390 & 18-Oct-88 & & $10045-97-3$ & CS-137 & GAMMA_GS & USTEST & 0.00183 \\
\hline 6239 & PDMS & & SW & & & E105778 & 01-Nov-88 & & $10045-97-3$ & CS-137 & GAMMA_GS & USTEST & 0.00245 \\
\hline 6240 & PDMS & & SW & & & E105780 & 01-Nov-88 & & $10045-97-3$ & CS-137 & GAMMA_GS & USTEST & 0.00326 \\
\hline 6241 & PDMS & & SW & & & E105525 & 01-Nov-88 & & $10045-97-3$ & CS-137 & GAMMA_GS & USTEST & \\
\hline
\end{tabular}




\begin{tabular}{|c|c|c|c|c|c|c|c|c|c|c|c|c|c|}
\hline & A & $\mathrm{B}$ & $\mathrm{c}$ & $\mathrm{D}$ & $\mathrm{E}$ & $\mathrm{F}$ & G & $\mathrm{H}$ & $\mathrm{I}$ & $\mathrm{J}$ & $\mathrm{K}$ & $\mathrm{L}$ & $\mathrm{M}$ \\
\hline 1 & SAMPLE_NUM_ASSIGNED_TO & SAMPLER & MEDIA & BIOTA_MEDIA & TAG_ID & SAMP_NUM & SAMP_DATE_TIME & SAMP_DATE_TIME_ON & CON_ID & CON_SHORT_NAME & METHOD_NAME & LAB_CODE & \begin{tabular}{|l|} 
VALUE_RPTD \\
\end{tabular} \\
\hline 6242 & PDMS & & SW & & & E105526 & 01-Nov-88 & & $10045-97-3$ & CS-137 & GAMMA GS & USTEST & \\
\hline 6243 & PDMS & & sW & & & E105716 & 01-Nov-88 & & $10098-97-2$ & SR-90 & SRISO_SEP_PRECIP_GPC & USTEST & 0.0776 \\
\hline \begin{tabular}{|l|}
6244 \\
\end{tabular} & PDMS & & sW & & & E105716 & 01-Nov-88 & & $\mathrm{U}-238$ & U-238 & UISO_AEA & USTEST & 0.187 \\
\hline 6245 & PDMS & & sW & & & E105716 & 01-Nov-88 & & 15117-96-1 & U-235 & UISO_AEA & USTEST & 0.00646 \\
\hline 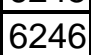 & PDMS & & sW & & & E105716 & 01-Nov-88 & & $13966-29-5$ & $\mathrm{U}-234$ & UISO_AEA & USTEST & 0.175 \\
\hline \begin{tabular}{|c|c|}
6247 \\
\end{tabular} & PDMS & & SW & & & E105716 & 01-Nov-88 & & $10028-17-8$ & TRITIUM & TRITIUM_ELECT_LSC & USTEST & 64.7 \\
\hline 6248 & PDMS & & SW & & & E105716 & 01-Nov-88 & & $10045-97-3$ & CS-137 & GAMMA_GS & USTEST & 0.289 \\
\hline 6249 & PDMS & & SW & & & E105584 & 01-Nov-88 & & $10098-97-2$ & SR-90 & SRISO_SEP_PRECIP_GPC & USTEST & 0.124 \\
\hline 6250 & PDMS & & sW & & & E105584 & 01-Nov-88 & & U-238 & $\mathrm{U}-238$ & UISO_AEA & USTEST & 0.149 \\
\hline 6251 & PDMS & & SW & & & E105584 & 01-Nov-88 & & 15117-96-1 & U-235 & UISO_AEA & USTEST & -0.00518 \\
\hline \begin{tabular}{|l|}
6252 \\
\end{tabular} & PDMS & & sW & & & E105584 & 01-Nov-88 & & $13966-29-5$ & U-234 & UISO_AEA & USTEST & 0.193 \\
\hline 6253 & PDMS & & SW & & & E105584 & 01-Nov-88 & & $10028-17-8$ & TRITIUM & TRITIŪM_ELECT_LSC & USTEST & 132 \\
\hline 6254 & PDMS & & SW & & & E105584 & 01-Nov-88 & & $10045-97-3$ & CS-137 & GAMMA_GS & USTEST & -0.306 \\
\hline 6255 & PDMS & & SW & & & E105781 & 01-Nov-88 & & $10045-97-3$ & CS-137 & GAMMA GS & USTEST & -0.000592 \\
\hline 6256 & PDMS & & SW & & & E105779 & 01-Nov-88 & & $10045-97-3$ & CS-137 & GAMMA_GS & USTEST & 0.00187 \\
\hline \begin{tabular}{|l|l|}
6257 \\
\end{tabular} & PDMS & & SW & & & E105884 & 07-Nov-88 & & $10045-97-3$ & CS-137 & 901.1_GAMMA_GS & USTEST & 0.025 \\
\hline \begin{tabular}{|l|}
6258 \\
\end{tabular} & PDMS & & sW & & & E105884 & 07-Nov-88 & & $10098-97-2$ & SR-90 & 905.0_SR_GPC & USTEST & 0.00754 \\
\hline \begin{tabular}{|l|}
6259 \\
\end{tabular} & PDMS & & sW & & & E105884 & 07-Nov-88 & & $10028-17-8$ & TRITIUM & 906.0_H3_LSC & USTEST & 79.7 \\
\hline 6260 & PDMS & & SW & & & E105883 & 07-Nov-88 & & $10045-97-3$ & CS-137 & 901.1_GAMMA_GS & USTEST & -0.229 \\
\hline 6261 & PDMS & & SW & & & E105883 & 07-Nov-88 & & $10098-97-2$ & SR-90 & 905.0_SR_GPC & USTEST & -0.00265 \\
\hline 6262 & PDMS & & SW & & & E105883 & 07-Nov-88 & & $10028-17-8$ & TRITIUM & 906.0_H3_LSC & USTEST & 11700 \\
\hline 6263 & PDMS & & SW & & & E105882 & 07-Nov-88 & & $10045-97-3$ & CS-137 & 901.1_GAMMA_GS & USTEST & 0.575 \\
\hline \begin{tabular}{|l|l|}
6264 & \\
\end{tabular} & PDMS & & SW & & & E105882 & 07-Nov-88 & & $10098-97-2$ & SR-90 & 905.0_SR_GPC & USTEST & -0.00626 \\
\hline 6265 & PDMS & & SW & & & E105882 & 07-Nov-88 & & 10028-17-8 & TRITIUM & 906.0_H3_LSC & USTEST & 208 \\
\hline 6266 & PDMS & & SW & & & E105885 & 07-Nov-88 & & $10045-97-3$ & CS-137 & 901.1_GAMMA_GS & USTEST & -0.74 \\
\hline \begin{tabular}{|l|l|}
6267 & \\
\end{tabular} & PDMS & & SW & & & E105885 & 07-Nov-88 & & 10028-17-8 & TRITIUM & 906.0_H3_LSC & USTEST & 47.4 \\
\hline \begin{tabular}{|l|}
6268 \\
\end{tabular} & PDMS & & SW & & & E105885 & 07-Nov-88 & & $10098-97-2$ & SR-90 & 905.0_SR_GPC & USTEST & -0.0114 \\
\hline 6269 & PDMS & & SW & & & E105798 & 15-Nov-88 & & $10045-97-3$ & CS-137 & 901.1_GAMMA_GS & USTEST & 0.535 \\
\hline 6270 & PDMS & & SW & & & E105798 & 15-Nov-88 & & $10098-97-2$ & SR-90 & 905.0_SR_GPC & USTEST & 0.107 \\
\hline \begin{tabular}{|l|}
6271 \\
\end{tabular} & PDMS & & SW & & & E105798 & 15-Nov-88 & & $10028-17-8$ & TRITIUM & 906.0_H3_LSC & USTEST & 161 \\
\hline \begin{tabular}{|l|}
6272 \\
\end{tabular} & PDMS & & sW & & & E106002 & 15-Nov-88 & & $10045-97-3$ & CS-137 & GAMMA_GS & USTEST & 0.00336 \\
\hline 6273 & PDMS & & SW & & & E106042 & 15-Nov-88 & & $10045-97-3$ & CS-137 & GAMMA_GS & USTEST & 0.00173 \\
\hline 6274 & PDMS & & SW & & & E106003 & 15-Nov-88 & & $10045-97-3$ & CS-137 & GAMMA_GS & USTEST & 0.00331 \\
\hline 6275 & PDMS & & SW & & & E106043 & 15-Nov-88 & & $10045-97-3$ & CS-137 & GAMMA_GS & USTEST & 0.00221 \\
\hline \begin{tabular}{|l|}
6276 \\
\end{tabular} & PDMS & & SW & & & E106004 & 15-Nov-88 & & $10045-97-3$ & CS-137 & GAMMA_GS & USTEST & 0.00219 \\
\hline \begin{tabular}{|l|l|}
6277 \\
\end{tabular} & PDMS & & SW & & & E106044 & 15-Nov-88 & & $10045-97-3$ & CS-137 & GAMMA_GS & USTEST & 0.00133 \\
\hline 6278 & PDMS & & SW & & & E106157 & 29-Nov-88 & & $10045-97-3$ & CS-137 & GAMMA_GS & USTEST & 0.00237 \\
\hline 6279 & PDMS & & SW & & & E106154 & 29-Nov-88 & & $10045-97-3$ & CS-137 & GAMMA_GS & USTEST & 0.0000639 \\
\hline 6280 & PDMS & & SW & & & E106158 & 29-Nov-88 & & $10045-97-3$ & CS-137 & GAMMA_GS & USTEST & 0.00231 \\
\hline 6281 & PDMS & & SW & & & E106110 & 29-Nov-88 & & $10045-97-3$ & CS-137 & GAMMA_GS & USTEST & -0.306 \\
\hline 6282 & PDMS & & SW & & & E106110 & 29-Nov-88 & & $\mathrm{U}-238$ & $\mathrm{U}-238$ & UISO_AĒA & USTEST & 0.144 \\
\hline 6283 & PDMS & & SW & & & E106110 & 29-Nov-88 & & 15117-96-1 & U-235 & UISO_AEA & USTEST & -0.00117 \\
\hline 6284 & PDMS & & SW & & & E106110 & 29-Nov-88 & & $10028-17-8$ & TRITIUM & TRITIÜM_ELECT_LSC & USTEST & 56.6 \\
\hline 6285 & PDMS & & SW & & & E106110 & 29-Nov-88 & & $13966-29-5$ & $\mathrm{U}-234$ & UISO_AEA & USTEST & 0.212 \\
\hline 6286 & PDMS & & SW & & & E106110 & 29-Nov-88 & & $10098-97-2$ & SR-90 & SRISO_SEP_PRECIP_GPC & USTEST & 0.0992 \\
\hline 6287 & PDMS & & SW & & & E106155 & 29-Nov-88 & & $10045-97-3$ & CS-137 & GAMMĀGS & USTEST & 0.00179 \\
\hline 6288 & PDMS & & SW & & & E106156 & 29-Nov-88 & & $10045-97-3$ & CS-137 & GAMMA_GS & USTEST & 0.000591 \\
\hline 6289 & PDMS & & SW & & & E106109 & 29-Nov-88 & & $10045-97-3$ & CS-137 & GAMMA_GS & USTEST & 0 \\
\hline 6290 & PDMS & & SW & & & E106109 & 29-Nov-88 & & $10028-17-8$ & TRITIUM & TRITIUM_ELECT_LSC & USTEST & 109 \\
\hline 6291 & PDMS & & SW & & & E106109 & 29-Nov-88 & & $\mathrm{U}-238$ & $\mathrm{U}-238$ & UISO_AEA & USTEST & 0.173 \\
\hline 6292 & PDMS & & SW & & & E106109 & 29-Nov-88 & & 15117-96-1 & U-235 & UISO_AEA & USTEST & 0.00711 \\
\hline 6293 & PDMS & & SW & & & E106109 & 29-Nov-88 & & $13966-29-5$ & $\mathrm{U}-234$ & UISO_AEA & USTEST & 0.248 \\
\hline
\end{tabular}




\begin{tabular}{|c|c|c|c|c|c|c|c|c|c|c|c|c|c|}
\hline & $\bar{A}$ & $\mathrm{~B}$ & $\bar{C}$ & $\mathrm{D}$ & $\mathrm{E}$ & $\mathrm{F}$ & $\bar{G}$ & $\mathrm{H}$ & 1 & $\mathrm{~J}$ & $\bar{K}$ & $\bar{L}$ & $\mathrm{M}$ \\
\hline 1 & \begin{tabular}{|l} 
SAMPLE_NUM_ASSIGNED_TO \\
\end{tabular} & \begin{tabular}{|l} 
SAMPLER \\
\end{tabular} & MEDIA & BIOTA_MEDIA & TAG_ID & SAMP_NUM & SAMP_DATE_TIME & SAMP_DATE_TIME_ON & CON_ID & \begin{tabular}{|l} 
CON_SHORT_NAME \\
\end{tabular} & METHOD_NAME & LAB_CODE & VALUE_RPTD \\
\hline 6294 & PDMS & & SW & & & E106109 & 29-Nov-88 & & $10098-97-2$ & SR-90 & SRISO_SEP_PRECIP_GPC & USTEST & 0.0994 \\
\hline 6295 & PDMS & & SW & & & E106159 & 29-Nov-88 & & $10045-97-3$ & CS-137 & GAMMA_GS & USTEST & 0.00331 \\
\hline 6296 & PDMS & & SW & & & E076909 & 13-Dec-88 & & $10098-97-2$ & SR-90 & 905.0_SR_GPC & USTEST & 0.281 \\
\hline 6297 & PDMS & & SW & & & E076909 & 13-Dec-88 & & $10028-17-8$ & TRITIUM & 906.0_H3_LSC & USTEST & 295 \\
\hline 6298 & PDMS & & SW & & & E076909 & 13-Dec-88 & & $10045-97-3$ & CS-137 & 901.1_GAMMA_GS & USTEST & 0.179 \\
\hline 6299 & PDMS & & SW & & & E106225 & 13-Dec-88 & & $10045-97-3$ & CS-137 & 901.1_GAMMA_GS & USTEST & 0.135 \\
\hline 6300 & PDMS & & SW & & & E106225 & 13-Dec-88 & & $10098-97-2$ & SR-90 & 905.0_SR_GPC & USTEST & -0.0143 \\
\hline 6301 & PDMS & & SW & & & E106225 & 13-Dec-88 & & $10028-17-8$ & TRITIUM & 906.0_H3_LSC & USTEST & 115 \\
\hline 6302 & PDMS & & SW & & & E106287 & 13-Dec-88 & & $10045-97-3$ & CS-137 & GAMM̄A_GS & USTEST & 0.00604 \\
\hline 6303 & PDMS & & SW & & & E106284 & 13-Dec-88 & & $10045-97-3$ & CS-137 & GAMMA_GS & USTEST & 0.00287 \\
\hline 6304 & PDMS & & SW & & & E106285 & 13-Dec-88 & & $10045-97-3$ & CS-137 & GAMMA_GS & USTEST & 0.00143 \\
\hline 6305 & PDMS & & SW & & & E106304 & 13-Dec-88 & & $10045-97-3$ & CS-137 & GAMMA_GS & USTEST & 0.00409 \\
\hline 6306 & PDMS & & SW & & & E106286 & 13-Dec-88 & & $10045-97-3$ & CS-137 & GAMMA_GS & USTEST & 0.00361 \\
\hline 6307 & PDMS & & SW & & & E106305 & 13-Dec-88 & & $10045-97-3$ & CS-137 & GAMMA_GS & USTEST & 0.00103 \\
\hline 6308 & PDMS & & SW & & & E106224 & 14-Dec-88 & & $10045-97-3$ & CS-137 & GAMMA_GS & USTEST & -0.877 \\
\hline 6309 & PDMS & & SW & & & E106224 & 14-Dec-88 & & U-238 & $\mathrm{U}-238$ & UISO_AEA & USTEST & 14.4 \\
\hline 6310 & PDMS & & SW & & & E106224 & 14-Dec-88 & & 15117-96-1 & $\mathrm{U}-235$ & UISO_AEA & USTEST & 0.527 \\
\hline 6311 & PDMS & & SW & & & E106224 & 14-Dec-88 & & $13966-29-5$ & $\mathrm{U}-234$ & UISO_AEA & USTEST & 23.2 \\
\hline 6312 & PDMS & & SW & & & E106224 & 14-Dec-88 & & 10028-17-8 & TRITIUM & TRITIÜM_DIST_LSC & USTEST & 176 \\
\hline 6313 & PDMS & & SW & & & E106223 & 14-Dec-88 & & $10045-97-3$ & CS-137 & GAMMA_GS & USTEST & -0.256 \\
\hline 6314 & PDMS & & SW & & & E106223 & 14-Dec-88 & & $\mathrm{U}-238$ & $\mathrm{U}-238$ & UISO_ĀEA & USTEST & 6.76 \\
\hline 6315 & PDMS & & SW & & & E106223 & 14-Dec-88 & & 15117-96-1 & $\mathrm{U}-235$ & UISO_AEA & USTEST & 0.225 \\
\hline 6316 & PDMS & & SW & & & E106223 & 14-Dec-88 & & $13966-29-5$ & $\mathrm{U}-234$ & UISO_AEA & USTEST & 7.37 \\
\hline 6317 & PDMS & & SW & & & E106223 & 14-Dec-88 & & 10028-17-8 & TRITIUM & TRITIUM_DIST_LSC & USTEST & 270 \\
\hline 6318 & PDMS & & SW & & & E106292 & 20-Dec-88 & & 10098-97-2 & SR-90 & 905.0_SR_GPC & USTEST & 0.125 \\
\hline 6319 & PDMS & & SW & & & E106292 & 20-Dec-88 & & $10045-97-3$ & CS-137 & 901.1_GAMMA_GS & USTEST & -0.204 \\
\hline 6320 & PDMS & & SW & & & E106292 & 20-Dec-88 & & U-238 & $\mathrm{U}-238$ & UISO_IE_PLATE_AEA & USTEST & 0.193 \\
\hline 6321 & PDMS & & SW & & & E106292 & 20-Dec-88 & & $15117-96-1$ & U-235 & UISO_IE_PLATE_AEA & USTEST & 0.00696 \\
\hline 6322 & PDMS & & SW & & & E106292 & 20-Dec-88 & & $13966-29-5$ & $\mathrm{U}-234$ & UISO_IE_PLATE_AEA & USTEST & 0.268 \\
\hline 6323 & PDMS & & SW & & & E106292 & 20-Dec-88 & & $10028-17-8$ & TRITIUM & TRITIUM_ELECT_LSC & USTEST & 147 \\
\hline 6324 & PDMS & & SW & & & E106310 & 21-Dec-88 & & $10045-97-3$ & CS-137 & GAMMA_GS & USTEST & 0.57 \\
\hline 6325 & PDMS & & SW & & & E106310 & 21-Dec-88 & & $\mathrm{U}-238$ & $\mathrm{U}-238$ & UISO_AEA & USTEST & 3.44 \\
\hline 6326 & PDMS & & SW & & & E106310 & 21-Dec-88 & & 15117-96-1 & $\mathrm{U}-235$ & UISO_AEA & USTEST & 0.115 \\
\hline 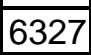 & PDMS & & SW & & & E106310 & 21-Dec-88 & & $13966-29-5$ & $\mathrm{U}-234$ & UISO_AEA & USTEST & 3.78 \\
\hline 6328 & PDMS & & SW & & & E106310 & 21-Dec-88 & & $10028-17-8$ & TRITIUM & TRITIUM_DIST_LSC & USTEST & 167 \\
\hline 6329 & PDMS & & SW & & & E106309 & 21-Dec-88 & & $10045-97-3$ & CS-137 & GAMMA_GS & USTEST & 0.175 \\
\hline 6330 & PDMS & & SW & & & E106309 & 21-Dec-88 & & $\mathrm{U}-238$ & $\mathrm{U}-238$ & UISO_AEA & USTEST & -0.00162 \\
\hline 6331 & PDMS & & SW & & & E106309 & 21-Dec-88 & & 15117-96-1 & U-235 & UISO_AEA & USTEST & -0.00518 \\
\hline 6332 & PDMS & & SW & & & E106309 & 21-Dec-88 & & $13966-29-5$ & U-234 & UISO_AEA & USTEST & -0.000138 \\
\hline 6333 & PDMS & & SW & & & E106309 & 21-Dec-88 & & $10028-17-8$ & TRITIUM & TRITIUM_DIST_LSC & USTEST & 12.9 \\
\hline 6334 & PDMS & & SW & & & E106395 & 27-Dec-88 & & $10045-97-3$ & CS-137 & GAMMA_GS & USTEST & 0.000766 \\
\hline 6335 & PDMS & & SW & & & E106444 & 27-Dec-88 & & 15046-84-1 & I-129 & I129_SEP_TEMS & 320 & 0.0000542 \\
\hline 6336 & PDMS & & SW & & & E106395 & 27-Dec-88 & & PU-239/240 & PU-239/240 & PUISO_IE_AEA & USTEST & 0.0000103 \\
\hline 6337 & PDMS & & SW & & & E106444 & 27-Dec-88 & & PU-239/240 & PU-239/240 & PUISO_IE_AEA & USTEST & 0.0000138 \\
\hline 6338 & PDMS & & SW & & & E106444 & 27-Dec-88 & & $10045-97-3$ & CS-137 & GAMMA_GS & USTEST & 0.00152 \\
\hline 6339 & PDMS & & SW & & & E106445 & 27-Dec-88 & & 15046-84-1 & I-129 & I129_SEP_TEMS & 320 & 0.0000066 \\
\hline 6340 & PDMS & & SW & & & E106445 & 27-Dec-88 & & PU-239/240 & PU-239/240 & PUISO_IE_AEA & USTEST & 0.0000836 \\
\hline 6341 & PDMS & & SW & & & E106445 & 27-Dec-88 & & $10045-97-3$ & CS-137 & GAMMA_GS & USTEST & 0.00402 \\
\hline 6342 & PDMS & & SW & & & E106396 & 27-Dec-88 & & $10045-97-3$ & CS-137 & GAMMA_GS & USTEST & 0.00207 \\
\hline 6343 & PDMS & & SW & & & E106396 & 27-Dec-88 & & PU-239/240 & PU-239/240 & PUISO_IE_AEA & USTEST & 0.00000199 \\
\hline 6344 & PDMS & & SW & & & E106446 & 27-Dec-88 & & 15046-84-1 & I-129 & 1129_SEP_TEMS & 320 & 0.0000686 \\
\hline 6345 & PDMS & & SW & & & E106397 & 27-Dec-88 & & PU-239/240 & PU-239/240 & PUISO_IE_AEA & USTEST & 0.0000165 \\
\hline
\end{tabular}




\begin{tabular}{|c|c|c|c|c|c|c|c|c|c|c|c|c|c|}
\hline & A & $\mathrm{B}$ & $\mathrm{c}$ & $\mathrm{D}$ & $\mathrm{E}$ & $\mathrm{F}$ & G & $\mathrm{H}$ & $\mathrm{I}$ & $\mathrm{J}$ & $\mathrm{K}$ & $\mathrm{L}$ & $\mathrm{M}$ \\
\hline 1 & SAMPLE_NUM_ASSIGNED_TO & SAMPLER & MEDIA & BIOTA_MEDIA & TAG_ID & SAMP_NUM & SAMP_DATE_TIME & SAMP_DATE_TIME_ON & CON_ID & CON_SHORT_NAME & METHOD_NAME & LAB_CODE & \begin{tabular}{|l|} 
VALUE_RPTD \\
\end{tabular} \\
\hline 6346 & PDMS & & SW & & & E106446 & 27-Dec-88 & & PU-239/240 & PU-239/240 & PUISO IE AEA & USTEST & 0.0000161 \\
\hline \begin{tabular}{|l|l|}
6347 & \\
\end{tabular} & PDMS & & sW & & & E106397 & 27-Dec-88 & & $10045-97-3$ & CS-137 & GAMMA_GS & USTEST & 0.00106 \\
\hline 6348 & PDMS & & sW & & & E106446 & 27-Dec-88 & & $10045-97-3$ & CS-137 & GAMMA_GS & USTEST & 0.00276 \\
\hline 6349 & PDMS & & sW & & & E107122 & 30-Dec-88 & & $15046-84-1$ & $1-129$ & I129_SEP_TEMS & 320 & 0.004565 \\
\hline 6350 & PDMS & & sW & & & E106403 & 03-Jan-89 & & $10028-17-8$ & TRITIUM & TRITIUM_ELECT_LSC & USTEST & 71.4 \\
\hline 6351 & PDMS & & SW & & & E106403 & 03-Jan-89 & & $10098-97-2$ & SR-90 & SRISO_SEPP_PRËCIP_GPC & USTEST & 0.0489 \\
\hline 6352 & PDMS & & SW & & & E106403 & 03-Jan-89 & & $10045-97-3$ & CS-137 & GAMMA_GS & USTEST & -0.0523 \\
\hline 6353 & PDMS & & SW & & & E106403 & 03-Jan-89 & & U-238 & U-238 & UISO_AËA & USTEST & 0.203 \\
\hline 6354 & PDMS & & sW & & & E106403 & 03-Jan-89 & & 15117-96-1 & U-235 & UISO AEA & USTEST & 0.00814 \\
\hline 6355 & PDMS & & SW & & & E106403 & 03-Jan-89 & & $13966-29-5$ & U-234 & UISO_AEA & USTEST & 0.268 \\
\hline \begin{tabular}{|l|}
6356 \\
\end{tabular} & PDMS & & sW & & & E106402 & 03-Jan-89 & & $10028-17-8$ & TRITIUM & TRITIUMM_ELECT_LSC & USTEST & 98.5 \\
\hline 6357 & PDMS & & SW & & & E106402 & 03-Jan-89 & & 10098-97-2 & SR-90 & SRISO_SEPP_PRËCIP_GPC & USTEST & 0.0767 \\
\hline 6358 & PDMS & & SW & & & E106402 & 03-Jan-89 & & $10045-97-3$ & CS-137 & GAMMĀGS & USTEST & -0.331 \\
\hline 6359 & PDMS & & SW & & & E106402 & 03-Jan-89 & & U-238 & U-238 & UISO_AĒAA & USTEST & 0.167 \\
\hline 6360 & PDMS & & SW & & & E106402 & 03-Jan-89 & & 15117-96-1 & U-235 & UISO_AEA & USTEST & 0.00368 \\
\hline 6361 & PDMS & & SW & & & E106402 & 03-Jan-89 & & $13966-29-5$ & $\mathrm{U}-234$ & UISO_AEA & USTEST & 0.237 \\
\hline \begin{tabular}{|l|}
6362 \\
\end{tabular} & PDMS & & sW & & & E106404 & 03-Jan-89 & & $10098-97-2$ & SR-90 & SRISO_SEP_PRECIP_GPC & USTEST & 1.95 \\
\hline \begin{tabular}{|c|}
6363 \\
\end{tabular} & PDMS & & sW & & & E106404 & 03-Jan-89 & & $10045-97-3$ & CS-137 & GAMMA_GS & USTEST & -0.763 \\
\hline 6364 & PDMS & & SW & & & E106404 & 03-Jan-89 & & $10028-17-8$ & TRITIUM & TRITIUM_DIST_LSC & USTEST & 3.22 \\
\hline 6365 & PDMS & & SW & & & E106406 & 03-Jan-89 & & $10028-17-8$ & TRITIUM & TRITIUM_DIST_LSC & USTEST & 7120 \\
\hline 6366 & PDMS & & SW & & & E106406 & 03-Jan-89 & & $10045-97-3$ & CS-137 & GAMMA_GS & USTEST & 1.56 \\
\hline \begin{tabular}{|l|l|}
6367 & \\
\end{tabular} & PDMS & & SW & & & E106405 & 03-Jan-89 & & $10098-97-2$ & SR-90 & SRISO_SEPPPRECIP_GPC & USTEST & 0.128 \\
\hline 6368 & PDMS & & SW & & & E106405 & 03-Jan-89 & & $10045-97-3$ & CS-137 & GAMMA_GS & USTEST & 0.393 \\
\hline 6369 & PDMS & & SW & & & E106405 & 03-Jan-89 & & $10028-17-8$ & TRITIUM & TRITIUM_DIST_LSC & USTEST & 608 \\
\hline 6370 & PDMS & & SW & & & E106405 & 03-Jan-89 & & $\mathrm{U}-238$ & $\mathrm{U}-238$ & UISO_AEA & USTEST & 5.23 \\
\hline 6371 & PDMS & & SW & & & E106405 & 03-Jan-89 & & 15117-96-1 & $\mathrm{U}-235$ & UISO_AEA & USTEST & 0.211 \\
\hline 6372 & PDMS & & SW & & & E106405 & 03-Jan-89 & & $13966-29-5$ & U-234 & UISO_AEA & USTEST & 5.61 \\
\hline 6373 & PDMS & & SW & & & E106662 & 10-Jan-89 & & $10045-97-3$ & CS-137 & GAMMA_GS & USTEST & 0.00287 \\
\hline 6374 & PDMS & & SW & & & E106615 & 10-Jan-89 & & $10045-97-3$ & CS-137 & GAMMA_GS & USTEST & 0.00459 \\
\hline 6375 & PDMS & & SW & & & E106663 & 10-Jan-89 & & $10045-97-3$ & CS-137 & GAMMA_GS & USTEST & 0.00195 \\
\hline \begin{tabular}{|l|}
6376 \\
\end{tabular} & PDMS & & SW & & & E106616 & 10-Jan-89 & & $10045-97-3$ & CS-137 & GAMMA_GS & USTEST & 0.00127 \\
\hline \begin{tabular}{|l|}
6377 \\
\end{tabular} & PDMS & & SW & & & E106664 & 10-Jan-89 & & $10045-97-3$ & CS-137 & GAMMA_GS & USTEST & 0.000661 \\
\hline \begin{tabular}{|l|}
6378 \\
\end{tabular} & PDMS & & SW & & & E106617 & 10-Jan-89 & & $10045-97-3$ & CS-137 & GAMMA_GS & USTEST & 0.00123 \\
\hline 6379 & PDMS & & SW & & & E106619 & 24-Jan-89 & & $10045-97-3$ & CS-137 & GAMMA_GS & USTEST & \\
\hline 6380 & PDMS & & SW & & & E106620 & 24-Jan-89 & & $10045-97-3$ & CS-137 & GAMMA_GS & USTEST & \\
\hline \begin{tabular}{|l|}
6381 \\
\end{tabular} & PDMS & & SW & & & E106885 & 24-Jan-89 & & $10045-97-3$ & CS-137 & GAMMA_GS & USTEST & 0.00452 \\
\hline 6382 & PDMS & & SW & & & E106883 & 24-Jan-89 & & $10045-97-3$ & CS-137 & GAMMA_GS & USTEST & 0.000866 \\
\hline 6383 & PDMS & & SW & & & E106886 & 24-Jan-89 & & $10045-97-3$ & CS-137 & GAMMA_GS & USTEST & 0.000708 \\
\hline \begin{tabular}{|l|l|}
6384 \\
\end{tabular} & PDMS & & SW & & & E106884 & 24-Jan-89 & & $10045-97-3$ & CS-137 & GAMMA_GS & USTEST & 0.00102 \\
\hline 6385 & PDMS & & SW & & & E106952 & 27-Jan-89 & & U-238 & U-238 & 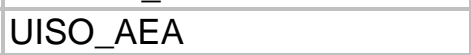 & USTEST & 92.8 \\
\hline 6386 & PDMS & & SW & & & E106952 & 27-Jan-89 & & 15117-96-1 & U-235 & UISO_AEA & USTEST & 3.51 \\
\hline \begin{tabular}{|l|l|}
6387 \\
\end{tabular} & PDMS & & SW & & & E106952 & 27-Jan-89 & & $13966-29-5$ & U-234 & UISO_AEA & USTEST & 96.5 \\
\hline \begin{tabular}{|l|}
6388 \\
\end{tabular} & PDMS & & SW & & & E106952 & 27-Jan-89 & & $10098-97-2$ & SR-90 & SRISO_SEP_PRECIP_GPC & USTEST & 2.06 \\
\hline 6389 & PDMS & & SW & & & E077049 & 30-Jan-89 & & $10045-97-3$ & CS-137 & 901.1_GAMMA_GS & USTEST & 0.0261 \\
\hline 6390 & PDMS & & SW & & & E077049 & 30-Jan-89 & & $10098-97-2$ & SR-90 & 905.0_SR_GPC & USTEST & -0.0198 \\
\hline 6391 & PDMS & & SW & & & E077049 & 30-Jan-89 & & $10028-17-8$ & TRITIUM & 906.0_H3_LSC & USTEST & 234 \\
\hline 6392 & PDMS & & SW & & & E077048 & 30-Jan-89 & & $10045-97-3$ & CS-137 & 901.1_GAMMA_GS & USTEST & -0.352 \\
\hline 6393 & PDMS & & SW & & & E077048 & 30-Jan-89 & & $10098-97-2$ & SR-90 & 905.0_SR_GPC & USTEST & -0.00576 \\
\hline 6394 & PDMS & & SW & & & E077048 & 30-Jan-89 & & $10028-17-8$ & TRITIUM & 906.0_H3_LSC & USTEST & 12200 \\
\hline 6395 & PDMS & & SW & & & E077047 & 30-Jan-89 & & $10098-97-2$ & SR-90 & 905.0_SR_GPC & USTEST & -0.0152 \\
\hline 6396 & PDMS & & SW & & & E077047 & 30-Jan-89 & & $10045-97-3$ & CS-137 & 901.1_GAMMA_GS & USTEST & 0.102 \\
\hline 6397 & PDMS & & SW & & & E077047 & 30-Jan-89 & & $10028-17-8$ & TRITIUM & 906.0_H3_LSC & USTEST & 238 \\
\hline
\end{tabular}




\begin{tabular}{|c|c|c|c|c|c|c|c|c|c|c|c|c|c|}
\hline & $\bar{A}$ & $\mathrm{~B}$ & C & $\mathrm{D}$ & $E$ & $\mathrm{~F}$ & G & $\mathrm{H}$ & $\mathrm{I}$ & $\mathrm{J}$ & $\mathrm{K}$ & $\mathrm{L}$ & $\mathrm{M}$ \\
\hline 1 & SAMPLE_NUM_ASSIGNED_TO & SAMPLER & MEDIA & BIOTA_MEDIA & TAG_ID & SAMP_NUM & SAMP_DATE_TIME & SAMP_DATE_TIME_ON & CON_ID & CON_SHORT_NAME & METHOD_NAME & LAB_CODE & VALUE_RPTD \\
\hline 6398 & PDMS & & SW & & & E077050 & $30-J a n-89$ & & $10045-97-3$ & CS-137 & 901.1_GAMMA_GS & USTEST & $=0.178$ \\
\hline 6399 & PDMS & & sW & & & E077050 & 30-Jan-89 & & $10098-97-2$ & SR-90 & 905.0_SR_GPC & USTEST & -0.017 \\
\hline 6400 & PDMS & & SW & & & E077050 & 30-Jan-89 & & 10028-17-8 & TRITIUM & 906.0_H3_LSC & USTEST & 82.9 \\
\hline 6401 & PDMS & & SW & & & E076903 & 31-Jan-89 & & PU-239/240 & PU-239/240 & PUISO_IE_AEA & USTEST & \\
\hline 6402 & PDMS & & SW & & & E076903 & 31-Jan-89 & & $10045-97-3$ & CS-137 & GAMMA_GSS & USTEST & \\
\hline 6403 & PDMS & & SW & & & E076902 & 31-Jan-89 & & PU-239/240 & PU-239/240 & PUISO_IE_AEA & USTEST & \\
\hline 6404 & PDMS & & SW & & & E076902 & 31-Jan-89 & & $10045-97-3$ & CS-137 & GAMMA_ḠS & USTEST & \\
\hline 6405 & PDMS & & SW & & & E076901 & 31-Jan-89 & & PU-239/240 & PU-239/240 & PUISO_IE_AEA & USTEST & \\
\hline 6406 & PDMS & & SW & & & E076901 & 31-Jan-89 & & $10045-97-3$ & CS-137 & GAMMA_GS & USTEST & \\
\hline 6407 & PDMS & & SW & & & E076906 & 31-Jan-89 & & $10098-97-2$ & SR-90 & SRISO_SEP_PRECIP_GPC & USTEST & 0.0746 \\
\hline 6408 & PDMS & & SW & & & E076906 & 31-Jan-89 & & 14133-76-7 & TC-99 & TC99_SEPE_LSC & USTEST & 0.0263 \\
\hline 6409 & PDMS & & SW & & & E076906 & 31-Jan-89 & & $10028-17-8$ & TRITIUM & TRITIÜM_ËELET_LSC & USTEST & 70 \\
\hline 6410 & PDMS & & SW & & & E076900 & 31-Jan-89 & & PU-239/240 & PU-239/240 & PUISO_IE_AEA & USTEST & \\
\hline 6411 & PDMS & & SW & & & E076900 & 31-Jan-89 & & $10045-97-3$ & CS-137 & GAMMA_ḠS & USTEST & \\
\hline 6412 & PDMS & & SW & & & E076906 & 31-Jan-89 & & $10045-97-3$ & CS-137 & GAMMA_GS & USTEST & -0.392 \\
\hline 6413 & PDMS & & SW & & & E076906 & 31-Jan-89 & & $\mathrm{U}-238$ & U-238 & UISO_AEA & USTEST & 0.24 \\
\hline 6414 & PDMS & & SW & & & E076906 & 31-Jan-89 & & 15117-96-1 & U-235 & UISO_AEA & USTEST & 0.0057 \\
\hline 6415 & PDMS & & SW & & & E076906 & 31-Jan-89 & & $13966-29-5$ & $\mathrm{U}-234$ & UISO_AEA & USTEST & 0.222 \\
\hline 6416 & PDMS & & SW & & & E076905 & 31-Jan-89 & & PU-239/240 & PU-239/240 & PUISO_IE_AEA & USTEST & \\
\hline 6417 & PDMS & & SW & & & E076905 & 31-Jan-89 & & $10045-97-3$ & CS-137 & GAMMA_ESS & USTEST & \\
\hline 6418 & PDMS & & SW & & & E076904 & 31-Jan-89 & & PU-239/240 & PU-239/240 & PUISO_IE_AEA & USTEST & \\
\hline 6419 & PDMS & & SW & & & E076904 & 31-Jan-89 & & $10045-97-3$ & CS-137 & GAMMA_GS & USTEST & \\
\hline 6420 & PDMS & & SW & & & E077045 & 31-Jan-89 & & $10098-97-2$ & SR-90 & SRISO_SEP_PRECIP_GPC & USTEST & 0.116 \\
\hline 6421 & PDMS & & SW & & & E077045 & 31-Jan-89 & & 14133-76-7 & TC-99 & TC99_SEPP_LSC & USTEST & 1.02 \\
\hline 6422 & PDMS & & SW & & & E077045 & 31-Jan-89 & & 10028-17-8 & TRITIUM & TRITIÜM_ËECCT_LSC & USTEST & 125 \\
\hline 6423 & PDMS & & SW & & & E077045 & 31-Jan-89 & & $10045-97-3$ & CS-137 & GAMMA_GS & USTEST & 0.484 \\
\hline 6424 & PDMS & & SW & & & E077045 & 31-Jan-89 & & $\mathrm{U}-238$ & U-238 & UISO_AĒAA & USTEST & 0.265 \\
\hline 6425 & PDMS & & SW & & & E077045 & 31-Jan-89 & & 15117-96-1 & U-235 & UISO_AEA & USTEST & 0.0119 \\
\hline 6426 & PDMS & & SW & & & E077045 & 31-Jan-89 & & $13966-29-5$ & $\mathrm{U}-234$ & UISO_AEA & USTEST & 0.277 \\
\hline 6427 & PDMS & & SW & & & E076908 & 07-Feb-89 & & $10098-97-2$ & SR-90 & 905.0_SR_GPC & USTEST & 0.102 \\
\hline 6428 & PDMS & & SW & & & E076908 & 07-Feb-89 & & $10028-17-8$ & TRITIUM & 906.0_H3_LSC & USTEST & 63 \\
\hline \begin{tabular}{|c|}
6429 \\
\end{tabular} & PDMS & & SW & & & E076908 & 07-Feb-89 & & $10045-97-3$ & CS-137 & 901.1_GAMMA_GS & USTEST & -0.522 \\
\hline 6430 & PDMS & & SW & & & E076978 & 07-Feb-89 & & $10045-97-3$ & CS-137 & GAMMA_GS & USTEST & 0.00147 \\
\hline 6431 & PDMS & & SW & & & E076975 & 07-Feb-89 & & $10045-97-3$ & CS-137 & GAMMA_GS & USTEST & 0.557 \\
\hline 6432 & PDMS & & SW & & & E076979 & 07-Feb-89 & & $10045-97-3$ & CS-137 & GAMMA_GS & USTEST & 0.000805 \\
\hline 6433 & PDMS & & SW & & & E076976 & 07-Feb-89 & & $10045-97-3$ & CS-137 & GAMMA_GS & USTEST & 0.00091 \\
\hline 6434 & PDMS & & SW & & & E076977 & 07-Feb-89 & & $10045-97-3$ & CS-137 & GAMMA_GS & USTEST & -0.000715 \\
\hline 6435 & PDMS & & SW & & & E076980 & 07-Feb-89 & & $10045-97-3$ & CS-137 & GAMMA_GS & USTEST & -0.000579 \\
\hline \begin{tabular}{|c|}
6436 \\
\end{tabular} & PDMS & & SW & & & E107181 & 21-Feb-89 & & $10045-97-3$ & CS-137 & GAMMA_GS & USTEST & 0.00314 \\
\hline \begin{tabular}{|l|l|}
6437 \\
\end{tabular} & PDMS & & SW & & & E107178 & 21-Feb-89 & & $10045-97-3$ & CS-137 & GAMMA_GS & USTEST & 0.00109 \\
\hline 6438 & PDMS & & SW & & & E107182 & 21-Feb-89 & & $10045-97-3$ & CS-137 & GAMMA_GS & USTEST & 0.000468 \\
\hline 6439 & PDMS & & SW & & & E107179 & 21-Feb-89 & & $10045-97-3$ & CS-137 & GAMMA_GS & USTEST & 0.00073 \\
\hline 6440 & PDMS & & SW & & & E107180 & 21-Feb-89 & & $10045-97-3$ & CS-137 & GAMMA_GS & USTEST & 0.00128 \\
\hline 6441 & PDMS & & SW & & & E107183 & 21-Feb-89 & & $10045-97-3$ & CS-137 & GAMMA_GS & USTEST & 0.00135 \\
\hline \begin{tabular}{|c|}
6442 \\
\end{tabular} & PDMS & & SW & & & E107398 & 28-Feb-89 & & 10098-97-2 & SR-90 & SRISO_SEEP_PRECIP_GPC & USTEST & 0.0962 \\
\hline 6443 & PDMS & & SW & & & E107398 & 28-Feb-89 & & $\mathrm{U}-238$ & $\mathrm{U}-238$ & UISO_AEAA & USTEST & 0.154 \\
\hline 6444 & PDMS & & SW & & & E107398 & 28-Feb-89 & & 15117-96-1 & $\mathrm{U}-235$ & UISO_AEA & USTEST & -0.00257 \\
\hline 6445 & PDMS & & SW & & & E107398 & 28-Feb-89 & & $13966-29-5$ & $\mathrm{U}-234$ & UISO_AEA & USTEST & 0.251 \\
\hline 6446 & PDMS & & SW & & & E107398 & 28-Feb-89 & & $10045-97-3$ & CS-137 & GAMMA_GS & USTEST & 0.543 \\
\hline 6447 & PDMS & & SW & & & E107398 & 28-Feb-89 & & 14133-76-7 & TC-99 & TC99_SEP_LSC & USTEST & -2.12 \\
\hline 6448 & PDMS & & SW & & & E107398 & 28-Feb-89 & & 10028-17-8 & TRITIUM & TRITIŪM_ËECET_LSC & USTEST & 64.8 \\
\hline 6449 & PDMS & & SW & & & E107397 & 28-Feb-89 & & $\mathrm{U}-238$ & $\mathrm{U}-238$ & UISO_AËA & USTEST & 0.27 \\
\hline
\end{tabular}




\begin{tabular}{|c|c|c|c|c|c|c|c|c|c|c|c|c|c|}
\hline & A & $\mathrm{B}$ & C & $\mathrm{D}$ & $E$ & $\mathrm{~F}$ & G & $\mathrm{H}$ & $\mathrm{I}$ & $\mathrm{J}$ & $\mathrm{K}$ & $\mathrm{L}$ & $\mathrm{M}$ \\
\hline 1 & SAMPLE_NUM_ASSIGNED_TO & SAMPLER & MEDIA & BIOTA_MEDIA & TAG_ID & SAMP_NUM & SAMP_DATE_TIME & SAMP_DATE_TIME_ON & CON_ID & CON_SHORT_NAME & METHOD_NAME & LAB_CODE & VALUE_RPTD \\
\hline 6450 & PDMS & & SW & & & E107397 & 28 -Feb-89 & & $15117-96-1$ & U-235 & UISO_AEA & USTEST & 0.00418 \\
\hline 6451 & PDMS & & SW & & & E107397 & 28-Feb-89 & & $13966-29-5$ & U-234 & UISO_AEA & USTEST & 0.284 \\
\hline 6452 & PDMS & & SW & & & E107397 & 28-Feb-89 & & $10098-97-2$ & SR-90 & SRISO_SEP_PRECIP_GPC & USTEST & 0.0876 \\
\hline 6453 & PDMS & & SW & & & E107397 & 28-Feb-89 & & $10045-97-3$ & CS-137 & GAMMA_GS & USTEST & 0.28 \\
\hline 6454 & PDMS & & SW & & & E107397 & 28-Feb-89 & & 14133-76-7 & TC-99 & TC99_SEP_LSC & USTEST & -0.916 \\
\hline 6455 & PDMS & & SW & & & E107397 & 28-Feb-89 & & $10028-17-8$ & TRITIUM & TRITIUM_ELECT_LSC & USTEST & 154 \\
\hline 6456 & PDMS & & SW & & & E107502 & 07-Mar-89 & & $10045-97-3$ & CS-137 & 901.1_GAMMA_GS & USTEST & -0.0255 \\
\hline 6457 & PDMS & & SW & & & E107502 & 07-Mar-89 & & $10028-17-8$ & TRITIUM & 906.0_H3_LSC & USTEST & 24.8 \\
\hline 6458 & PDMS & & SW & & & E107502 & 07-Mar-89 & & 10098-97-2 & SR-90 & 905.0_SR_GPC & USTEST & -0.00105 \\
\hline 6459 & PDMS & & SW & & & E107562 & 07-Mar-89 & & $10045-97-3$ & CS-137 & GAMMA_GS & USTEST & 0.00029 \\
\hline 6460 & PDMS & & SW & & & E107565 & 07-Mar-89 & & $10045-97-3$ & CS-137 & GAMMA_GS & USTEST & 0.00233 \\
\hline \begin{tabular}{|l|l|}
6461 \\
\end{tabular} & PDMS & & SW & & & E107563 & 07-Mar-89 & & $10045-97-3$ & CS-137 & GAMMA_GS & USTEST & 0.00151 \\
\hline \begin{tabular}{|c|}
6462 \\
\end{tabular} & PDMS & & SW & & & E107566 & 07-Mar-89 & & $10045-97-3$ & CS-137 & GAMMA_GS & USTEST & 0.00173 \\
\hline 6463 & PDMS & & SW & & & E107564 & 07-Mar-89 & & $10045-97-3$ & CS-137 & GAMMA_GS & USTEST & 0.00207 \\
\hline \begin{tabular}{|l|l|}
6464 \\
\end{tabular} & PDMS & & SW & & & E107567 & 07-Mar-89 & & $10045-97-3$ & CS-137 & GAMMA_GS & USTEST & 0.00304 \\
\hline 6465 & PDMS & & SW & & & E107500 & 08-Mar-89 & & U-238 & U-238 & UISO_AEA & USTEST & 14.9 \\
\hline \begin{tabular}{|l|}
6466 \\
\end{tabular} & PDMS & & SW & & & E107500 & 08-Mar-89 & & 15117-96-1 & U-235 & UISO_AEA & USTEST & 0.38 \\
\hline \begin{tabular}{|l|l|}
6467 \\
\end{tabular} & PDMS & & SW & & & E107500 & 08-Mar-89 & & $13966-29-5$ & $\mathrm{U}-234$ & UISO_AEA & USTEST & 23.6 \\
\hline 6468 & PDMS & & SW & & & E107500 & 08-Mar-89 & & $10028-17-8$ & TRITIUM & TRITIUM_DIST_LSC & USTEST & -89.3 \\
\hline 6469 & PDMS & & SW & & & E107500 & 08-Mar-89 & & $10045-97-3$ & CS-137 & GAMMA_GS & USTEST & 0.0918 \\
\hline \begin{tabular}{|c|}
6470 \\
\end{tabular} & PDMS & & SW & & & E107501 & 09-Mar-89 & & $\mathrm{U}-238$ & $\mathrm{U}-238$ & UISO_AEA & USTEST & 8.46 \\
\hline \begin{tabular}{|l|}
6471 \\
\end{tabular} & PDMS & & SW & & & E107501 & 09-Mar-89 & & 15117-96-1 & U-235 & UISO_AEA & USTEST & 0.293 \\
\hline \begin{tabular}{|l|}
6472 \\
\end{tabular} & PDMS & & SW & & & E107501 & 09-Mar-89 & & $13966-29-5$ & U-234 & UISO_AEA & USTEST & 9.12 \\
\hline \begin{tabular}{|c|}
6473 \\
\end{tabular} & PDMS & & SW & & & E107501 & 09-Mar-89 & & 10028-17-8 & TRITIUM & TRITIÜM_DIST_LSC & USTEST & 172 \\
\hline \begin{tabular}{|c|}
6474 \\
\end{tabular} & PDMS & & SW & & & E107501 & 09-Mar-89 & & $10045-97-3$ & CS-137 & GAMMA_GS & USTEST & 0.171 \\
\hline \begin{tabular}{|l|}
6475 \\
\end{tabular} & PDMS & & SW & & & E107503 & 10-Mar-89 & & 10028-17-8 & TRITIUM & 906.0_H3_LSC & USTEST & 61.3 \\
\hline \begin{tabular}{|l|}
6476 \\
\end{tabular} & PDMS & & SW & & & E107503 & 10-Mar-89 & & $10098-97-2$ & SR-90 & 905.0_SR_GPC & USTEST & 0.305 \\
\hline \begin{tabular}{|l|}
6477 \\
\end{tabular} & PDMS & & SW & & & E107503 & 10-Mar-89 & & $10045-97-3$ & CS-137 & 901.1_GAMMA_GS & USTEST & 0.449 \\
\hline \begin{tabular}{|l|}
6478 \\
\end{tabular} & PDMS & & SW & & & E107576 & 15-Mar-89 & & $10045-97-3$ & CS-137 & GAMMA_GS & USTEST & 0.0478 \\
\hline \begin{tabular}{|c|}
6479 \\
\end{tabular} & PDMS & & SW & & & E107576 & 15-Mar-89 & & 10028-17-8 & TRITIUM & TRITIUM_DIST_LSC & USTEST & 139 \\
\hline \begin{tabular}{|c|}
6480 \\
\end{tabular} & PDMS & & SW & & & E107576 & 15-Mar-89 & & $\mathrm{U}-238$ & U-238 & UISO_AEA & USTEST & 3.27 \\
\hline \begin{tabular}{|c|}
6481 \\
\end{tabular} & PDMS & & SW & & & E107576 & 15-Mar-89 & & 15117-96-1 & U-235 & UISO_AEA & USTEST & 0.126 \\
\hline \begin{tabular}{|l|}
6482 \\
\end{tabular} & PDMS & & SW & & & E107576 & 15-Mar-89 & & $13966-29-5$ & U-234 & UISO_AEA & USTEST & 3.72 \\
\hline \begin{tabular}{|c|}
6483 \\
\end{tabular} & PDMS & & SW & & & E107575 & 15-Mar-89 & & $10045-97-3$ & CS-137 & GAMMA_GS & USTEST & 1.13 \\
\hline \begin{tabular}{|l|}
6484 \\
\end{tabular} & PDMS & & SW & & & E107575 & 15-Mar-89 & & $10028-17-8$ & TRITIUM & TRITIUM_DIST_LSC & USTEST & -43 \\
\hline \begin{tabular}{|c|}
6485 \\
\end{tabular} & PDMS & & SW & & & E107575 & 15-Mar-89 & & $\mathrm{U}-238$ & U-238 & UISO_AEA & USTEST & 0.00883 \\
\hline \begin{tabular}{|l|}
6486 \\
\end{tabular} & PDMS & & SW & & & E107575 & 15-Mar-89 & & 15117-96-1 & U-235 & UISO_AEA & USTEST & -0.00518 \\
\hline \begin{tabular}{|l|l|}
6487 \\
\end{tabular} & PDMS & & SW & & & E107575 & 15-Mar-89 & & $13966-29-5$ & $\mathrm{U}-234$ & UISO_AEA & USTEST & -0.0207 \\
\hline \begin{tabular}{|c|}
6488 \\
\end{tabular} & PDMS & & SW & & & E107828 & 21-Mar-89 & & $10045-97-3$ & CS-137 & GAMM̄A_GS & USTEST & 0.00127 \\
\hline \begin{tabular}{|c|}
6489 \\
\end{tabular} & PDMS & & SW & & & E107825 & 21-Mar-89 & & $10045-97-3$ & CS-137 & GAMMA_GS & USTEST & 0.00365 \\
\hline 6490 & PDMS & & SW & & & E107826 & 21-Mar-89 & & $10045-97-3$ & CS-137 & GAMMA_GS & USTEST & 0.00436 \\
\hline 6491 & PDMS & & SW & & & E107829 & 21-Mar-89 & & $10045-97-3$ & CS-137 & GAMMA_GS & USTEST & 0.0002 \\
\hline 6492 & PDMS & & SW & & & E107827 & 21-Mar-89 & & $10045-97-3$ & CS-137 & GAMMA_GS & USTEST & 0.00413 \\
\hline 6493 & PDMS & & SW & & & E107830 & 21-Mar-89 & & $10045-97-3$ & CS-137 & GAMMA_GS & USTEST & 0.00107 \\
\hline 6494 & PDMS & & SW & & & E107857 & 28-Mar-89 & & $\mathrm{U}-238$ & U-238 & UISO_AĒA & USTEST & 0.23 \\
\hline 6495 & PDMS & & SW & & & E107857 & 28-Mar-89 & & 15117-96-1 & U-235 & UISO_AEA & USTEST & 0.00157 \\
\hline 6496 & PDMS & & SW & & & E107857 & 28-Mar-89 & & $13966-29-5$ & U-234 & UISO_AEA & USTEST & 0.22 \\
\hline 6497 & PDMS & & SW & & & E107857 & 28-Mar-89 & & $10045-97-3$ & CS-137 & GAMMA_GS & USTEST & -0.0933 \\
\hline 6498 & PDMS & & SW & & & E107857 & 28-Mar-89 & & $10098-97-2$ & SR-90 & SRISO_SEP_PRECIP_GPC & USTEST & 0.115 \\
\hline 6499 & PDMS & & SW & & & E107857 & 28-Mar-89 & & 14133-76-7 & TC-99 & TC99_SEP_LSC & USTEST & -1.44 \\
\hline 6500 & PDMS & & SW & & & E107857 & 28-Mar-89 & & 10028-17-8 & TRITIUM & TRITIŪM_ËECET_LSC & USTEST & 65.8 \\
\hline 6501 & PDMS & & SW & & & E107856 & 28-Mar-89 & & $\mathrm{U}-238$ & U-238 & UISO_AEA & USTEST & 0.181 \\
\hline
\end{tabular}




\begin{tabular}{|c|c|c|c|c|c|c|c|c|c|c|c|c|c|}
\hline & A & $\mathrm{B}$ & $\mathrm{c}$ & $\mathrm{D}$ & $\mathrm{E}$ & $\mathrm{F}$ & G & $\mathrm{H}$ & $\mathrm{I}$ & $\mathrm{J}$ & $\mathrm{K}$ & $\mathrm{L}$ & $\mathrm{M}$ \\
\hline 1 & SAMPLE_NUM_ASSIGNED_TO & SAMPLER & MEDIA & BIOTA_MEDIA & TAG_ID & SAMP_NUM & SAMP_DATE_TIME & SAMP_DATE_TIME_ON & CON_ID & CON_SHORT_NAME & METHOD_NAME & LAB_CODE & VALUE_RPTD \\
\hline 6502 & PDMS & & SW & & & E107856 & 28-Mar-89 & & $15117-96-1$ & U-235 & UISO AEA & USTEST & 0.0147 \\
\hline 6503 & PDMS & & sW & & & E107856 & 28-Mar-89 & & $13966-29-5$ & U-234 & UISO_AEA & USTEST & 0.285 \\
\hline 6504 & PDMS & & sW & & & E107856 & 28-Mar-89 & & $10045-97-3$ & CS-137 & GAMMA_GS & USTEST & -0.0334 \\
\hline 6505 & PDMS & & sW & & & E107856 & 28-Mar-89 & & $10098-97-2$ & SR-90 & SRISO_SEP_PRECIP_GPC & USTEST & 0.106 \\
\hline \begin{tabular}{|l|l|}
6506 \\
\end{tabular} & PDMS & & sW & & & E107856 & 28-Mar-89 & & $14133-76-7$ & TC-99 & TC99_SEP_LSC & USTEST & -0.934 \\
\hline 6507 & PDMS & & SW & & & E107856 & 28-Mar-89 & & $10028-17-8$ & TRITIUM & TRITIŪM_ELECTI_LSC & USTEST & 172 \\
\hline 6508 & PDMS & & SW & & & E107858 & 28-Mar-89 & & $10045-97-3$ & CS-137 & GAMMA_GS & USTEST & 4 \\
\hline 6509 & PDMS & & SW & & & E107858 & 28-Mar-89 & & $10028-17-8$ & TRITIUM & TRITIUM_DIST_LSC & USTEST & 77.3 \\
\hline 6510 & PDMS & & sW & & & E107858 & 28-Mar-89 & & $10098-97-2$ & SR-90 & SRISO_SEP_PRECIP_GPC & USTEST & 0.755 \\
\hline 6511 & PDMS & & SW & & & E107859 & 28-Mar-89 & & $\mathrm{U}-238$ & U-238 & UISO_AEA & USTEST & 76.6 \\
\hline 6512 & PDMS & & sW & & & E107859 & 28-Mar-89 & & 15117-96-1 & U-235 & UISO_AEA & USTEST & 1.44 \\
\hline 6513 & PDMS & & SW & & & E107859 & 28-Mar-89 & & $13966-29-5$ & $\mathrm{U}-234$ & UISO_AEA & USTEST & 81.9 \\
\hline 6514 & PDMS & & SW & & & E107859 & 28-Mar-89 & & $10045-97-3$ & CS-137 & GAMMĀA_GS & USTEST & 0 \\
\hline 6515 & PDMS & & SW & & & E107859 & 28-Mar-89 & & $10028-17-8$ & TRITIUM & TRITIUM DIST LSC & USTEST & 548 \\
\hline 6516 & PDMS & & SW & & & E107859 & 28-Mar-89 & & $10098-97-2$ & SR-90 & SRISO_SEP_PRECIP_GPC & USTEST & 1.18 \\
\hline \begin{tabular}{|l|l|}
6517 \\
\end{tabular} & PDMS & & SW & & & E107860 & 29-Mar-89 & & $10045-97-3$ & CS-137 & GAMMA_GS & USTEST & 0.163 \\
\hline 6518 & PDMS & & sW & & & E107860 & 29-Mar-89 & & $10028-17-8$ & TRITIUM & TRITIUM_DIST_LSC & USTEST & 6230 \\
\hline \begin{tabular}{|l|}
6519 \\
\end{tabular} & PDMS & & sW & & & E108030 & 04-Apr-89 & & $10045-97-3$ & CS-137 & GAMMA_GS & USTEST & -0.000521 \\
\hline 6520 & PDMS & & SW & & & E108030 & 04-Apr-89 & & PU-239/240 & PU-239/240 & PUISO_IE_AEA & USTEST & -0.0000128 \\
\hline 6521 & PDMS & & SW & & & E108030 & 04-Apr-89 & & 15046-84-1 & I-129 & I129_SEP_TEMS & 320 & 0.000226 \\
\hline 6522 & PDMS & & SW & & & E108027 & 04-Apr-89 & & $10045-97-3$ & CS-137 & GAMMA_GSS & USTEST & 0.00304 \\
\hline 6523 & PDMS & & SW & & & E108027 & 04-Apr-89 & & PU-239/240 & PU-239/240 & PUISO_IE_AEA & USTEST & 0.00204 \\
\hline \begin{tabular}{|l|}
6524 \\
\end{tabular} & PDMS & & SW & & & E108029 & 04-Apr-89 & & $10045-97-3$ & CS-137 & GAMMA_GS & USTEST & 0.00232 \\
\hline \begin{tabular}{|l|l|}
6525 \\
\end{tabular} & PDMS & & sW & & & E108032 & 04-Apr-89 & & PU-239/240 & PU-239/240 & PUISO_IE_AEA & USTEST & 0.00000459 \\
\hline 6526 & PDMS & & SW & & & E108029 & 04-Apr-89 & & PU-239/240 & PU-239/240 & PUISO_IE_AEA & USTEST & 0.0000163 \\
\hline \begin{tabular}{|l|l|}
6527 \\
\end{tabular} & PDMS & & SW & & & E108032 & 04-Apr-89 & & $10045-97-3$ & CS-137 & GAMMĀESS & USTEST & -0.000172 \\
\hline \begin{tabular}{|l|}
6528 \\
\end{tabular} & PDMS & & SW & & & E108032 & 04-Apr-89 & & 15046-84-1 & I-129 & I129_SEP_TEMS & 320 & 0.0000056 \\
\hline 6529 & PDMS & & SW & & & E108031 & 04-Apr-89 & & $10045-97-3$ & CS-137 & GAMMA_GSS & USTEST & 0.00131 \\
\hline 6530 & PDMS & & SW & & & E108031 & 04-Apr-89 & & PU-239/240 & PU-239/240 & PUISO_IE_AEA & USTEST & 0.00000555 \\
\hline \begin{tabular}{|l|}
6531 \\
\end{tabular} & PDMS & & SW & & & E108031 & 04-Apr-89 & & 15046-84-1 & I-129 & I129_SEP_TEMS & 320 & 0.000109 \\
\hline \begin{tabular}{|l|}
6532 \\
\end{tabular} & PDMS & & sW & & & E108028 & 04-Apr-89 & & $10045-97-3$ & CS-137 & GAMMA_GS & USTEST & 0.00301 \\
\hline 6533 & PDMS & & SW & & & E108028 & 04-Apr-89 & & PU-239/240 & PU-239/240 & PUISO_IE_AEA & USTEST & 0.0000126 \\
\hline 6534 & PDMS & & SW & & & E108041 & 11-Apr-89 & & $\mathrm{U}-238$ & $\mathrm{U}-238$ & UISO_IE_PLATE_AEA & USTEST & 0.211 \\
\hline 6535 & PDMS & & SW & & & E108041 & 11-Apr-89 & & 15117-96-1 & $\mathrm{U}-235$ & UISO_IE_PLATE_AEA & USTEST & -0.00518 \\
\hline 6536 & PDMS & & SW & & & E108041 & 11-Apr-89 & & $13966-29-5$ & $\mathrm{U}-234$ & UISO_IE_PLATE_AEA & USTEST & 0.259 \\
\hline 6537 & PDMS & & SW & & & E108041 & 11-Apr-89 & & $10098-97-2$ & SR-90 & 905.0_SR_GPC & USTEST & 0.121 \\
\hline 6538 & PDMS & & SW & & & E108041 & 11-Apr-89 & & 10028-17-8 & TRITIUM & TRITIÜM_ELECT_LSC & USTEST & 185 \\
\hline 6539 & PDMS & & SW & & & E108041 & 11-Apr-89 & & 14133-76-7 & TC-99 & TC99_SEP_LSC & USTEST & -1.33 \\
\hline 6540 & PDMS & & SW & & & E108041 & 11-Apr-89 & & $10045-97-3$ & CS-137 & 901.1_GAMMA_GS & USTEST & -0.746 \\
\hline 6541 & PDMS & & SW & & & E108044 & 18-Apr-89 & & $10098-97-2$ & SR-90 & SRISŌ_SEP_PRECIP_GPC & USTEST & 0.108 \\
\hline 6542 & PDMS & & SW & & & E108044 & 18-Apr-89 & & $10028-17-8$ & TRITIUM & TRITIŪM_ELEECT_LSC̄ & USTEST & 137 \\
\hline 6543 & PDMS & & SW & & & E108044 & 18-Apr-89 & & U-238 & $\mathrm{U}-238$ & UISO_AËA & USTEST & 0.169 \\
\hline 6544 & PDMS & & SW & & & E108044 & 18-Apr-89 & & 15117-96-1 & U-235 & UISO_AEA & USTEST & 0.0134 \\
\hline 6545 & PDMS & & SW & & & E108044 & 18-Apr-89 & & $13966-29-5$ & $\mathrm{U}-234$ & UISO_AEA & USTEST & 0.282 \\
\hline 6546 & PDMS & & SW & & & E108044 & 18-Apr-89 & & $10045-97-3$ & CS-137 & GAMM̄A_GS & USTEST & 0.661 \\
\hline 6547 & PDMS & & SW & & & E108043 & 18-Apr-89 & & $10098-97-2$ & SR-90 & SRISO_S̄EP_PRECIP_GPC & USTEST & 0.116 \\
\hline 6548 & PDMS & & SW & & & E108043 & 18-Apr-89 & & $10028-17-8$ & TRITIUM & TRITIUM̄ELEEECT_LSC̄ & USTEST & 131 \\
\hline 6549 & PDMS & & SW & & & E108043 & 18-Apr-89 & & $\mathrm{U}-238$ & $\mathrm{U}-238$ & UISO_AËA & USTEST & 0.213 \\
\hline 6550 & PDMS & & SW & & & E108043 & 18-Apr-89 & & 15117-96-1 & U-235 & UISO_AEA & USTEST & 0.0305 \\
\hline 6551 & PDMS & & sW & & & E108043 & 18-Apr-89 & & $13966-29-5$ & U-234 & UISO_AEA & USTEST & 0.245 \\
\hline 6552 & PDMS & & SW & & & E108043 & 18-Apr-89 & & $10045-97-3$ & CS-137 & GAMM̄A_GS & USTEST & -0.526 \\
\hline 6553 & PDMS & & SW & & & E108158 & 18-Apr-89 & & $10045-97-3$ & CS-137 & GAMMA_GS & USTEST & 0.0017 \\
\hline
\end{tabular}




\begin{tabular}{|c|c|c|c|c|c|c|c|c|c|c|c|c|c|}
\hline & A & $\mathrm{B}$ & $\mathrm{c}$ & $\mathrm{D}$ & $\mathrm{E}$ & $\mathrm{F}$ & G & $\mathrm{H}$ & $\mathrm{I}$ & $\mathrm{J}$ & $\mathrm{K}$ & $\mathrm{L}$ & $\mathrm{M}$ \\
\hline 1 & SAMPLE_NUM_ASSIGNED_TO & SAMPLER & MEDIA & BIOTA_MEDIA & TAG_ID & SAMP_NUM & SAMP_DATE_TIME & SAMP_DATE_TIME_ON & CON_ID & CON_SHORT_NAME & METHOD_NAME & LAB_CODE & \begin{tabular}{|l|} 
VALUE_RPTD \\
\end{tabular} \\
\hline 6554 & PDMS & & SW & & & E108155 & 18-Apr-89 & & $10045-97-3$ & CS-137 & GAMMA GS & USTEST & -0.000226 \\
\hline 6555 & PDMS & & sW & & & E108156 & 18-Apr-89 & & $10045-97-3$ & CS-137 & GAMMA_GS & USTEST & 0.000943 \\
\hline 6556 & PDMS & & sW & & & E108159 & 18-Apr-89 & & $10045-97-3$ & CS-137 & GAMMA_GS & USTEST & 0.00132 \\
\hline 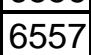 & PDMS & & sW & & & E108157 & 18-Apr-89 & & $10045-97-3$ & CS-137 & GAMMA_GS & USTEST & 0.00271 \\
\hline \begin{tabular}{|l|l|}
6558 \\
\end{tabular} & PDMS & & sW & & & E108160 & 18-Apr-89 & & $10045-97-3$ & CS-137 & GAMMA_GS & USTEST & 0.00012 \\
\hline \begin{tabular}{|c|}
6559 \\
\end{tabular} & PDMS & & sW & & & E108278 & 24-Apr-89 & & $10045-97-3$ & CS-137 & 901.1_GAMMA_GS & USTEST & 0 \\
\hline 6560 & PDMS & & SW & & & E108278 & 24-Apr-89 & & $10098-97-2$ & SR-90 & 905.0_SR_GPC & USTEST & -0.0136 \\
\hline 6561 & PDMS & & SW & & & E108278 & 24-Apr-89 & & $10028-17-8$ & TRITIUM & 906.0_H3_LSC & USTEST & 5.81 \\
\hline 6562 & PDMS & & sW & & & E108277 & 24-Apr-89 & & $10045-97-3$ & CS-137 & 901.1 GAMMA GS & USTEST & -0.153 \\
\hline 6563 & PDMS & & SW & & & E108277 & 24-Apr-89 & & 10098-97-2 & SR-90 & 905.0_SR_GPC & USTEST & -0.0141 \\
\hline \begin{tabular}{|l|}
6564 \\
\end{tabular} & PDMS & & sW & & & E108277 & 24-Apr-89 & & $10028-17-8$ & TRITIUM & 906.0_H3_LSC & USTEST & 8550 \\
\hline 6565 & PDMS & & SW & & & E108276 & 24-Apr-89 & & $10045-97-3$ & CS-137 & 901.1_GAMMA_GS & USTEST & 0.313 \\
\hline 6566 & PDMS & & SW & & & E108276 & 24-Apr-89 & & 10098-97-2 & SR-90 & 905.0_SR_GPC & USTEST & -0.00832 \\
\hline \begin{tabular}{|l|l|}
6567 \\
\end{tabular} & PDMS & & SW & & & E108276 & 24-Apr-89 & & $10028-17-8$ & TRITIUM & 906.0_H3_LSC & USTEST & -79.4 \\
\hline 6568 & PDMS & & SW & & & E108279 & 24-Apr-89 & & $10045-97-3$ & CS-137 & 901.1_GAMMA_GS & USTEST & -0.0974 \\
\hline 6569 & PDMS & & SW & & & E108279 & 24-Apr-89 & & $10098-97-2$ & SR-90 & 905.0_SR_GPC & USTEST & 0.069 \\
\hline 6570 & PDMS & & SW & & & E108279 & 24-Apr-89 & & $10028-17-8$ & TRITIUM & 906.0_H3_LSC & USTEST & 50.3 \\
\hline \begin{tabular}{|l|}
6571 \\
\end{tabular} & PDMS & & sW & & & E108328 & 02-May-89 & & $10028-17-8$ & TRITIUM & 906.0_H3_LSC & USTEST & -26.2 \\
\hline 6572 & PDMS & & SW & & & E108328 & 02-May-89 & & $10098-97-2$ & SR-90 & 905.0_SR_GPC & USTEST & 0.0592 \\
\hline 6573 & PDMS & & SW & & & E108328 & 02-May-89 & & $10045-97-3$ & CS-137 & 901.1_GAMMA_GS & USTEST & -0.157 \\
\hline 6574 & PDMS & & SW & & & E108400 & 02-May-89 & & $10045-97-3$ & CS-137 & GAMMA_GS & USTEST & 0.00203 \\
\hline 6575 & PDMS & & SW & & & E108397 & 02-May-89 & & $10045-97-3$ & CS-137 & GAMMA_GS & USTEST & 0.00301 \\
\hline 6576 & PDMS & & SW & & & E108398 & 02-May-89 & & $10045-97-3$ & CS-137 & GAMMA_GS & USTEST & 0.00118 \\
\hline 6577 & PDMS & & SW & & & E108329 & 02-May-89 & & $\mathrm{U}-238$ & $\mathrm{U}-238$ & UISO_AĒA & USTEST & 0.213 \\
\hline 6578 & PDMS & & SW & & & E108329 & 02-May-89 & & 15117-96-1 & $\mathrm{U}-235$ & UISO_AEA & USTEST & 0.00569 \\
\hline 6579 & PDMS & & SW & & & E108329 & 02-May-89 & & $13966-29-5$ & $\mathrm{U}-234$ & UISO_AEA & USTEST & 0.234 \\
\hline 6580 & PDMS & & SW & & & E108401 & 02-May-89 & & $10045-97-3$ & CS-137 & GAMM̄A_GS & USTEST & 0.00189 \\
\hline 6581 & PDMS & & SW & & & E108329 & 02-May-89 & & $14133-76-7$ & TC-99 & TC99_SEP_LSC & USTEST & 0.617 \\
\hline 6582 & PDMS & & SW & & & E108329 & 02-May-89 & & $10028-17-8$ & TRITIUM & TRITIÜM_ELEECT_LSC & USTEST & 79.4 \\
\hline 6583 & PDMS & & SW & & & E108329 & 02-May-89 & & $10098-97-2$ & SR-90 & SRISO_SEP_PRËCIP_GPC & USTEST & 0.0685 \\
\hline 6584 & PDMS & & sW & & & E108329 & 02-May-89 & & $10045-97-3$ & CS-137 & GAMMA_GS & USTEST & 0.0765 \\
\hline 6585 & PDMS & & SW & & & E108330 & 02-May-89 & & $\mathrm{U}-238$ & $\mathrm{U}-238$ & UISO_AĒA & USTEST & 0.214 \\
\hline 6586 & PDMS & & SW & & & E108330 & 02-May-89 & & 15117-96-1 & $\mathrm{U}-235$ & UISO_AEA & USTEST & 0.00507 \\
\hline 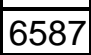 & PDMS & & SW & & & E108330 & 02-May-89 & & $13966-29-5$ & $\mathrm{U}-234$ & UISO_AEA & USTEST & 0.301 \\
\hline 6588 & PDMS & & SW & & & E108330 & 02-May-89 & & 14133-76-7 & TC-99 & TC99_SEP_LSC & USTEST & -1.11 \\
\hline 6589 & PDMS & & SW & & & E108330 & 02-May-89 & & $10028-17-8$ & TRITIUM & TRITIUM_ELECT_LSC & USTEST & 103 \\
\hline 6590 & PDMS & & SW & & & E108330 & 02-May-89 & & $10098-97-2$ & SR-90 & SRISO_SEP_PRECIP_GPC & USTEST & 0.0879 \\
\hline 6591 & PDMS & & SW & & & E108330 & 02-May-89 & & $10045-97-3$ & CS-137 & GAMMA_GS & USTEST & 0.127 \\
\hline 6592 & PDMS & & SW & & & E108402 & 02-May-89 & & $10045-97-3$ & CS-137 & GAMMA_GS & USTEST & -0.00069 \\
\hline 6593 & PDMS & & SW & & & E108399 & 02-May-89 & & $10045-97-3$ & CS-137 & GAMMA_GS & USTEST & 0.000574 \\
\hline 6594 & PDMS & & SW & & & E108606 & 16-May-89 & & $10045-97-3$ & CS-137 & GAMMA_GS & USTEST & 0.00902 \\
\hline 6595 & PDMS & & SW & & & E108609 & 16-May-89 & & $10045-97-3$ & CS-137 & GAMMA_GS & USTEST & 0.00402 \\
\hline 6596 & PDMS & & SW & & & E108610 & 16-May-89 & & $10045-97-3$ & CS-137 & GAMMA_GS & USTEST & 0.000195 \\
\hline 6597 & PDMS & & SW & & & E108607 & 16-May-89 & & $10045-97-3$ & CS-137 & GAMMA_GS & USTEST & 0.00341 \\
\hline 6598 & PDMS & & SW & & & E108611 & 16-May-89 & & $10045-97-3$ & CS-137 & GAMMA_GS & USTEST & 0.00436 \\
\hline 6599 & PDMS & & SW & & & E108608 & 16-May-89 & & $10045-97-3$ & CS-137 & GAMMA_GS & USTEST & 0.00569 \\
\hline 6600 & PDMS & & SW & & & E108786 & 17-May-89 & & $10045-97-3$ & CS-137 & GAMMA_GS & USTEST & 0.734 \\
\hline 6601 & PDMS & & SW & & & E108786 & 17-May-89 & & $10028-17-8$ & TRITIUM & TRITIUM_DIST_LSC & USTEST & -38.7 \\
\hline 6602 & PDMS & & SW & & & E108786 & 17-May-89 & & U-238 & $\mathrm{U}-238$ & UISO_AËA & USTEST & 0.2 \\
\hline 6603 & PDMS & & SW & & & E108786 & 17-May-89 & & $15117-96-1$ & U-235 & UISO_AEA & USTEST & 0.00286 \\
\hline 6604 & PDMS & & SW & & & E108786 & 17-May-89 & & $13966-29-5$ & $\mathrm{U}-234$ & UISO_AEA & USTEST & 0.235 \\
\hline 6605 & PDMS & & SW & & & E108786 & 17-May-89 & & $10098-97-2$ & SR-90 & SRISŌ_SEP_PRECIP_GPC & USTEST & 0.0947 \\
\hline
\end{tabular}




\begin{tabular}{|c|c|c|c|c|c|c|c|c|c|c|c|c|c|}
\hline & A & $\mathrm{B}$ & $\mathrm{c}$ & $\mathrm{D}$ & $\mathrm{E}$ & $\mathrm{F}$ & G & $\mathrm{H}$ & $\mathrm{I}$ & $\mathrm{J}$ & $\mathrm{K}$ & $\mathrm{L}$ & $\mathrm{M}$ \\
\hline 1 & SAMPLE_NUM_ASSIGNED_TO & SAMPLER & MEDIA & BIOTA_MEDIA & TAG_ID & SAMP_NUM & SAMP_DATE_TIME & SAMP_DATE_TIME_ON & CON_ID & CON_SHORT_NAME & METHOD_NAME & LAB_CODE & \begin{tabular}{|l|} 
VALUE_RPTD \\
\end{tabular} \\
\hline 6606 & PDMS & & SW & & & E108905 & 30-Мау-89 & & $10045-97-3$ & CS-137 & 901.1 GAMMA GS & USTEST & 0.228 \\
\hline \begin{tabular}{|l|l|}
6607 & $-2 \cdot r$ \\
\end{tabular} & PDMS & & sW & & & E108905 & 30-May-89 & & $10028-17-8$ & TRITIUM & 906.0_H3_LSC & USTEST & -75.6 \\
\hline 6608 & PDMS & & sW & & & E108905 & 30-May-89 & & $10098-97-2$ & SR-90 & 905.0_SR_GPC & USTEST & -0.00443 \\
\hline 6609 & PDMS & & sW & & & E108954 & 30-May-89 & & $10045-97-3$ & CS-137 & GAMMA_GS & USTEST & 0.00319 \\
\hline \begin{tabular}{|l|}
6610 \\
\end{tabular} & PDMS & & sW & & & E108951 & 30-May-89 & & $10045-97-3$ & CS-137 & GAMMA_GS & USTEST & 0.00144 \\
\hline \begin{tabular}{|l|}
6611 \\
\end{tabular} & PDMS & & sW & & & E108952 & 30-May-89 & & $10045-97-3$ & CS-137 & GAMMA_GS & USTEST & 0.00256 \\
\hline 6612 & PDMS & & SW & & & E108902 & 30-May-89 & & $10045-97-3$ & CS-137 & GAMMA_GS & USTEST & -0.375 \\
\hline 6613 & PDMS & & SW & & & E108902 & 30-May-89 & & $\mathrm{U}-238$ & U-238 & UISO_AEA & USTEST & 0.145 \\
\hline \begin{tabular}{|l|}
6614 \\
\end{tabular} & PDMS & & sW & & & E108902 & 30-May-89 & & 15117-96-1 & U-235 & UISO AEA & USTEST & 0.00133 \\
\hline 6615 & PDMS & & SW & & & E108902 & 30-Мау-89 & & $13966-29-5$ & U-234 & UISO_AEA & USTEST & 0.196 \\
\hline \begin{tabular}{|l|l|}
6616 \\
\end{tabular} & PDMS & & sW & & & E108955 & 30-May-89 & & $10045-97-3$ & CS-137 & GAMMA_GS & USTEST & 0.0025 \\
\hline 6617 & PDMS & & SW & & & E108902 & 30-May-89 & & $10028-17-8$ & TRITIUM & TRITIUM_ELECCT_LSC & USTEST & 55.8 \\
\hline 6618 & PDMS & & SW & & & E108902 & 30-May-89 & & $10098-97-2$ & SR-90 & SRISO_SEPP_PRËCIP_GPC & USTEST & 0.0832 \\
\hline 6619 & PDMS & & SW & & & E108902 & 30-May-89 & & $14133-76-7$ & TC-99 & TC99 SEP LSC & USTEST & -0.448 \\
\hline 6620 & PDMS & & SW & & & E108900 & 30-May-89 & & $10045-97-3$ & CS-137 & GAMMA_GS & USTEST & 0.209 \\
\hline 6621 & PDMS & & SW & & & E108900 & 30-May-89 & & $\mathrm{U}-238$ & $\mathrm{U}-238$ & UISO_AEA & USTEST & 0.162 \\
\hline \begin{tabular}{|l|}
6622 \\
\end{tabular} & PDMS & & sW & & & E108900 & 30-May-89 & & 15117-96-1 & U-235 & UISO_AEA & USTEST & 0.0165 \\
\hline \begin{tabular}{|c|}
6623 \\
\end{tabular} & PDMS & & sW & & & E108900 & 30-May-89 & & $13966-29-5$ & $\mathrm{U}-234$ & UISO_AEA & USTEST & 0.226 \\
\hline 6624 & PDMS & & SW & & & E108956 & 30-May-89 & & $10045-97-3$ & CS-137 & GAMM̄A_GS & USTEST & 0.00209 \\
\hline 6625 & PDMS & & SW & & & E108900 & 30-May-89 & & $10028-17-8$ & TRITIUM & TRITIUM_ELECT_LSC & USTEST & 70.6 \\
\hline 6626 & PDMS & & SW & & & E108900 & 30-May-89 & & $10098-97-2$ & SR-90 & SRISO_SEP_PRËCIP_GPC & USTEST & 0.0774 \\
\hline \begin{tabular}{|l|l|}
6627 & $\cdot r \cdot r$ \\
\end{tabular} & PDMS & & SW & & & E108900 & 30-May-89 & & 14133-76-7 & TC-99 & TC99_SEPE_LSC & USTEST & -0.0991 \\
\hline 6628 & PDMS & & SW & & & E108953 & 30-Мау-89 & & $10045-97-3$ & CS-137 & GAMMA_GS & USTEST & 0.00334 \\
\hline 6629 & PDMS & & SW & & & E112801 & 31-May-89 & & 15046-84-1 & $1-129$ & I129_SEP_TEMS & 320 & 0.0000036 \\
\hline 6630 & PDMS & & SW & & & E112800 & 31-May-89 & & 15046-84-1 & I-129 & I129_SEP_TEMS & 320 & 0.0000452 \\
\hline 6631 & PDMS & & SW & & & E108904 & 01-Jun-89 & & $10045-97-3$ & CS-137 & GAMMA_ḠS & USTEST & 0.0848 \\
\hline 6632 & PDMS & & SW & & & E108904 & 01-Jun-89 & & $10028-17-8$ & TRITIUM & TRITIUM_DIST_LSC & USTEST & -64.1 \\
\hline 6633 & PDMS & & SW & & & E108904 & 01-Jun-89 & & $\mathrm{U}-238$ & $\mathrm{U}-238$ & UISO_AËA & USTEST & -0.00945 \\
\hline 6634 & PDMS & & SW & & & E108904 & 01-Jun-89 & & 15117-96-1 & $\mathrm{U}-235$ & UISO_AEA & USTEST & -0.0259 \\
\hline 6635 & PDMS & & SW & & & E108904 & 01-Jun-89 & & $13966-29-5$ & $\mathrm{U}-234$ & UISO_AEA & USTEST & -0.0145 \\
\hline \begin{tabular}{|l|}
6636 \\
\end{tabular} & PDMS & & sW & & & E108903 & 01-Jun-89 & & $10045-97-3$ & CS-137 & GAMMA_GS & USTEST & -0.843 \\
\hline \begin{tabular}{|c|}
6637 \\
\end{tabular} & PDMS & & SW & & & E108903 & 01-Jun-89 & & $10028-17-8$ & TRITIUM & TRITIUM_DIST_LSC & USTEST & 14.3 \\
\hline 6638 & PDMS & & SW & & & E108903 & 01-Jun-89 & & 15117-96-1 & $\mathrm{U}-235$ & UISO_AËA & USTEST & 0.328 \\
\hline 6639 & PDMS & & SW & & & E108903 & 01-Jun-89 & & $13966-29-5$ & $\mathrm{U}-234$ & UISO_AEA & USTEST & 9.32 \\
\hline 6640 & PDMS & & SW & & & E108903 & 01-Jun-89 & & U-238 & U-238 & UISO_AEA & USTEST & 8.75 \\
\hline 6641 & PDMS & & SW & & & E108965 & 07-Jun-89 & & $\mathrm{U}-238$ & $\mathrm{U}-238$ & UISO_AEA & USTEST & 3.33 \\
\hline 6642 & PDMS & & SW & & & E108965 & 07-Jun-89 & & 15117-96-1 & $\mathrm{U}-235$ & UISO_AEA & USTEST & 0.167 \\
\hline 6643 & PDMS & & SW & & & E108965 & 07-Jun-89 & & $13966-29-5$ & $\mathrm{U}-234$ & UISO_AEA & USTEST & 4.09 \\
\hline 6644 & PDMS & & SW & & & E108965 & 07-Jun-89 & & $10045-97-3$ & CS-137 & GAMM̄A_GS & USTEST & -0.136 \\
\hline 6645 & PDMS & & SW & & & E108965 & 07-Jun-89 & & 10028-17-8 & TRITIUM & TRITIUM_DIST_LSC & USTEST & 194 \\
\hline 6646 & PDMS & & SW & & & E108964 & 07-Jun-89 & & $10028-17-8$ & TRITIUM & TRITIUM_DIST_LSC & USTEST & 131 \\
\hline \begin{tabular}{|l|l|}
6647 \\
\end{tabular} & PDMS & & SW & & & E108964 & 07-Jun-89 & & U-238 & U-238 & UISO_AËA & USTEST & 3.49 \\
\hline 6648 & PDMS & & SW & & & E108964 & 07-Jun-89 & & 15117-96-1 & U-235 & UISO_AEA & USTEST & 0.131 \\
\hline 6649 & PDMS & & SW & & & E108964 & 07-Jun-89 & & $13966-29-5$ & $\mathrm{U}-234$ & UISO_AEA & USTEST & 4.32 \\
\hline \begin{tabular}{|l|}
6650 \\
\end{tabular} & PDMS & & sW & & & E108964 & 07-Jun-89 & & $10045-97-3$ & CS-137 & GAMMA_GS & USTEST & -0.1 \\
\hline 6651 & PDMS & & SW & & & E112802 & 08-Jun-89 & & 15046-84-1 & I-129 & I129_SEP_TEMS & 320 & 0.0000665 \\
\hline 6652 & PDMS & & SW & & & E112803 & 08-Jun-89 & & 15046-84-1 & I-129 & I129_SEP_TEMS & 320 & 0.000161 \\
\hline 6653 & PDMS & & SW & & & E109130 & 09-Jun-89 & & $10028-17-8$ & TRITIUM & 906.0̄_H3_LSC & USTEST & 275 \\
\hline 6654 & PDMS & & SW & & & E109130 & 09-Jun-89 & & $10098-97-2$ & SR-90 & 905.0_SR_GPC & USTEST & 0.428 \\
\hline 6655 & PDMS & & SW & & & E109130 & 09-Jun-89 & & $10045-97-3$ & CS-137 & 901.1_GAMMA_GS & USTEST & 0.261 \\
\hline 6656 & PDMS & & SW & & & E108961 & 13-Jun-89 & & $10045-97-3$ & CS-137 & GAMMA_AS & USTEST & \\
\hline 6657 & PDMS & & SW & & & E108960 & 13-Jun-89 & & $10045-97-3$ & CS-137 & GAMMA_GS & USTEST & \\
\hline
\end{tabular}




\begin{tabular}{|c|c|c|c|c|c|c|c|c|c|c|c|c|c|}
\hline & A & $\mathrm{B}$ & $\mathrm{c}$ & $\mathrm{D}$ & $\mathrm{E}$ & $\mathrm{F}$ & G & $\mathrm{H}$ & $\mathrm{I}$ & $\mathrm{J}$ & $\mathrm{K}$ & $\mathrm{L}$ & $\mathrm{M}$ \\
\hline 1 & SAMPLE_NUM_ASSIGNED_TO & SAMPLER & MEDIA & BIOTA_MEDIA & TAG_ID & SAMP_NUM & SAMP_DATE_TIME & SAMP_DATE_TIME_ON & CON_ID & CON_SHORT_NAME & METHOD_NAME & LAB_CODE & \begin{tabular}{|l|} 
VALUE_RPTD \\
\end{tabular} \\
\hline 6658 & PDMS & & SW & & & E109255 & 13-Jun-89 & & $10045-97-3$ & CS-137 & GAMMA GS & USTEST & 0.0047 \\
\hline 6659 & PDMS & & sW & & & E109254 & 13-Jun-89 & & $10045-97-3$ & CS-137 & GAMMA_GS & USTEST & 0.00139 \\
\hline 6660 & PDMS & & sW & & & E109061 & 13-Jun-89 & & $10045-97-3$ & CS-137 & GAMMA_GS & USTEST & \\
\hline \begin{tabular}{|l|}
6661 \\
\end{tabular} & PDMS & & sW & & & E108962 & 13-Jun-89 & & $10045-97-3$ & CS-137 & GAMMA_GS & USTEST & \\
\hline \begin{tabular}{|l|}
6662 \\
\end{tabular} & PDMS & & sW & & & E109131 & 19-Jun-89 & & $10045-97-3$ & CS-137 & 901.1_GAMMA_GS & USTEST & -0.0674 \\
\hline \begin{tabular}{|l|}
6663 \\
\end{tabular} & PDMS & & sW & & & E109131 & 19-Jun-89 & & $10028-17-8$ & TRITIUM & 906.0_H3_LSC & USTEST & 6710 \\
\hline 6664 & PDMS & & SW & & & E109131 & 19-Jun-89 & & $10098-97-2$ & SR-90 & 905.0_SR_GPC & USTEST & -0.0135 \\
\hline 6665 & PDMS & & SW & & & E109127 & 20-Jun-89 & & $10098-97-2$ & SR-90 & SRISO_SËP_PRECIP_GPC & USTEST & 2.37 \\
\hline 6666 & PDMS & & sW & & & E109127 & 20-Jun-89 & & $10028-17-8$ & TRITIUM & TRITIUM_DIST_LSC & USTEST & 59 \\
\hline 6667 & PDMS & & SW & & & E109127 & 20-Jun-89 & & $10045-97-3$ & CS-137 & GAMMA_GS & USTEST & 1.68 \\
\hline 6668 & PDMS & & sW & & & E109129 & 20-Jun-89 & & $10045-97-3$ & CS-137 & GAMMA_GS & USTEST & -0.924 \\
\hline 6669 & PDMS & & SW & & & E109129 & 20-Jun-89 & & $10028-17-8$ & TRITIUM & TRITIUM_DIST_LSC & USTEST & 6640 \\
\hline 6670 & PDMS & & SW & & & E109128 & 20-Jun-89 & & $\mathrm{U}-238$ & $\mathrm{U}-238$ & UISO_AËA & USTEST & 102 \\
\hline 6671 & PDMS & & SW & & & E109128 & 20-Jun-89 & & 15117-96-1 & U-235 & UISO_AEA & USTEST & 3.99 \\
\hline 6672 & PDMS & & SW & & & E109128 & 20-Jun-89 & & $13966-29-5$ & $\mathrm{U}-234$ & UISO_AEA & USTEST & 112 \\
\hline 6673 & PDMS & & SW & & & E109128 & 20-Jun-89 & & $10098-97-2$ & SR-90 & SRISO_SEP_PRECIP_GPC & USTEST & 13.2 \\
\hline \begin{tabular}{|l|}
6674 \\
\end{tabular} & PDMS & & sW & & & E109128 & 20-Jun-89 & & $10028-17-8$ & TRITIUM & TRITIUM_DIST_LSC & USTEST & 425 \\
\hline \begin{tabular}{|l|}
6675 \\
\end{tabular} & PDMS & & sW & & & E109128 & 20-Jun-89 & & $10045-97-3$ & CS-137 & GAMMA_GS & USTEST & 4.59 \\
\hline 6676 & PDMS & & SW & & & E109365 & 27-Jun-89 & & 15046-84-1 & I-129 & I129_SEP__TEMS & 320 & 0.0000897 \\
\hline 6677 & PDMS & & SW & & & E109365 & 27-Jun-89 & & $10045-97-3$ & CS-137 & GAMMA_GS & USTEST & 0.00121 \\
\hline 6678 & PDMS & & SW & & & E109365 & 27-Jun-89 & & PU-239/240 & PU-239/240 & PUISO_IE_AEA & USTEST & 0.00000778 \\
\hline 6679 & PDMS & & SW & & & E109362 & 27-Jun-89 & & PU-239/240 & PU-239/240 & PUISO_IE_AEA & USTEST & 0.0000017 \\
\hline 6680 & PDMS & & SW & & & E109362 & 27-Jun-89 & & $10045-97-3$ & CS-137 & GAMMA_GS & USTEST & 0.00157 \\
\hline 6681 & PDMS & & SW & & & E109366 & 27-Jun-89 & & 15046-84-1 & I-129 & I129_SEP_TEMS & 320 & 0.000007 \\
\hline 6682 & PDMS & & SW & & & E109267 & 27-Jun-89 & & 14133-76-7 & TC-99 & TC99_SEP_LSC & USTEST & -0.302 \\
\hline 6683 & PDMS & & SW & & & E109366 & 27-Jun-89 & & $10045-97-3$ & CS-137 & GAMM̄A_GS & USTEST & 0.0025 \\
\hline 6684 & PDMS & & SW & & & E109363 & 27-Jun-89 & & $10045-97-3$ & CS-137 & GAMMA_GS & USTEST & 0.00207 \\
\hline 6685 & PDMS & & SW & & & E109267 & 27-Jun-89 & & $10028-17-8$ & TRITIUM & TRITIUM_ELECCT_LSC & USTEST & 58.4 \\
\hline 6686 & PDMS & & SW & & & E109267 & 27-Jun-89 & & $10045-97-3$ & CS-137 & GAMMA_GS & USTEST & 0.0609 \\
\hline \begin{tabular}{|l|l|}
6687 & \\
\end{tabular} & PDMS & & SW & & & E109267 & 27-Jun-89 & & $10098-97-2$ & SR-90 & SRISO_SEP_PRECIP_GPC & USTEST & 0.0529 \\
\hline \begin{tabular}{|l|l|}
6688 & -1 \\
\end{tabular} & PDMS & & sW & & & E109363 & 27-Jun-89 & & PU-239/240 & PU-239/240 & PUISO_IE_AEA & USTEST & 0.0000208 \\
\hline 6689 & PDMS & & SW & & & E109366 & 27-Jun-89 & & PU-239/240 & PU-239/240 & PUISO_IE_AEA & USTEST & 0.0000282 \\
\hline 6690 & PDMS & & SW & & & E109267 & 27-Jun-89 & & $\mathrm{U}-238$ & $\mathrm{U}-238$ & UISO_AEĀ & USTEST & 0.187 \\
\hline 6691 & PDMS & & SW & & & E109267 & 27-Jun-89 & & 15117-96-1 & $\mathrm{U}-235$ & UISO_AEA & USTEST & 0.00799 \\
\hline 6692 & PDMS & & SW & & & E109267 & 27-Jun-89 & & $13966-29-5$ & $\mathrm{U}-234$ & UISO_AEA & USTEST & 0.238 \\
\hline 6693 & PDMS & & sW & & & E109367 & 27-Jun-89 & & 15046-84-1 & I-129 & I129_SEEP_TEMS & 320 & 0.0001546 \\
\hline 6694 & PDMS & & SW & & & E109266 & 27-Jun-89 & & $10045-97-3$ & CS-137 & GAMMA_GS & USTEST & l \\
\hline 6695 & PDMS & & SW & & & E109367 & 27-Jun-89 & & $10045-97-3$ & CS-137 & GAMMA_GS & USTEST & 0.00227 \\
\hline 6696 & PDMS & & SW & & & E109364 & 27-Jun-89 & & $10045-97-3$ & CS-137 & GAMMA_GS & USTEST & 0.00418 \\
\hline 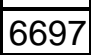 & PDMS & & SW & & & E109266 & 27-Jun-89 & & $10028-17-8$ & TRITIUM & TRITIUM_ELELECT_LSC & USTEST & 158 \\
\hline 6698 & PDMS & & SW & & & E109266 & 27-Jun-89 & & $10098-97-2$ & SR-90 & SRISO_SEPP_PRËCIP_GPC & USTEST & 0.0421 \\
\hline 6699 & PDMS & & SW & & & E109364 & 27-Jun-89 & & PU-239/240 & PU-239/240 & PUISO_IE_AEAA & USTEST & 0.00000278 \\
\hline 6700 & PDMS & & SW & & & E109367 & 27-Jun-89 & & PU-239/240 & PU-239/240 & PUISO_IE_AEA & USTEST & 0.00004 \\
\hline 6701 & PDMS & & SW & & & E109266 & 27-Jun-89 & & $\mathrm{U}-238$ & $\mathrm{U}-238$ & UISO_AEA & USTEST & 0.209 \\
\hline 6702 & PDMS & & SW & & & E109266 & 27-Jun-89 & & 15117-96-1 & U-235 & UISO_AEA & USTEST & -0.000658 \\
\hline 6703 & PDMS & & SW & & & E109266 & 27-Jun-89 & & $13966-29-5$ & $\mathrm{U}-234$ & UISO_AEA & USTEST & 0.249 \\
\hline 6704 & PDMS & & SW & & & E109266 & 27-Jun-89 & & $14133-76-7$ & TC-99 & TC99_SEP_LSC & USTEST & 0.725 \\
\hline 6705 & PDMS & & SW & & & E109268 & 07-Jul-89 & & $10028-17-8$ & TRITIUM & TRITIŪM_ELEECT_LSC & USTEST & 145 \\
\hline 6706 & PDMS & & SW & & & E109268 & 07-Jul-89 & & $14133-76-7$ & TC-99 & TC99_SEP_LSC & USTEST & 3 \\
\hline 6707 & PDMS & & SW & & & E109268 & 07-Jul-89 & & 10098-97-2 & SR-90 & 905.0_SR_GPC & USTEST & 0.0817 \\
\hline 6708 & PDMS & & SW & & & E109268 & 07-Jul-89 & & $\mathrm{U}-238$ & $\mathrm{U}-238$ & UISO_IE_PLATE_AEA & USTEST & 0.198 \\
\hline 6709 & PDMS & & SW & & & E109268 & 07-Jul-89 & & 15117-96-1 & $\mathrm{U}-235$ & UISO_IE_PLATE_AEA & USTEST & 0.00126 \\
\hline
\end{tabular}




\begin{tabular}{|c|c|c|c|c|c|c|c|c|c|c|c|c|c|}
\hline & A & $\mathrm{B}$ & $\mathrm{c}$ & $\mathrm{D}$ & $\mathrm{E}$ & $\mathrm{F}$ & G & $\mathrm{H}$ & $\mathrm{I}$ & $\mathrm{J}$ & $\mathrm{K}$ & $\mathrm{L}$ & $\mathrm{M}$ \\
\hline 1 & SAMPLE_NUM_ASSIGNED_TO & SAMPLER & MEDIA & BIOTA_MEDIA & TAG_ID & SAMP_NUM & SAMP_DATE_TIME & SAMP_DATE_TIME_ON & CON_ID & CON_SHORT_NAME & METHOD_NAME & LAB_CODE & \begin{tabular}{|l|} 
VALUE_RPTD \\
\end{tabular} \\
\hline 6710 & PDMS & & SW & & & E109268 & $07-J u l-89$ & & $13966-29-5$ & U-234 & UISO_IE_PLATE_AEA & USTEST & 0.226 \\
\hline 6711 & PDMS & & sW & & & E109268 & 07-Jul-89 & & $10045-97-3$ & CS-137 & 901.1_GAMMA_GS & USTEST & -0.0387 \\
\hline 6712 & PDMS & & sW & & & E109445 & 11-Jul-89 & & $10045-97-3$ & CS-137 & GAMMA_GS & USTEST & 0.00317 \\
\hline 6713 & PDMS & & sW & & & E109442 & 11-Jul-89 & & $10045-97-3$ & CS-137 & GAMMA_GS & USTEST & 0.00128 \\
\hline 6714 & PDMS & & sW & & & E109443 & 11-Jul-89 & & $10045-97-3$ & CS-137 & GAMMA_GS & USTEST & -0.000585 \\
\hline 6715 & PDMS & & sW & & & E109446 & 11-Jul-89 & & $10045-97-3$ & CS-137 & GAMMA_GS & USTEST & 0.00249 \\
\hline 6716 & PDMS & & SW & & & E109444 & 11-Jul-89 & & $10045-97-3$ & CS-137 & GAMMA_GS & USTEST & 0.00235 \\
\hline 6717 & PDMS & & SW & & & E109447 & 11-Jul-89 & & $10045-97-3$ & CS-137 & GAMMA_GS & USTEST & 0.000429 \\
\hline 6718 & PDMS & & sW & & & E109373 & 12-Jul-89 & & $\mathrm{U}-238$ & U-238 & UISO AEA & USTEST & 0.241 \\
\hline 6719 & PDMS & & SW & & & E109373 & 12-Jul-89 & & 15117-96-1 & U-235 & UISO_AEA & USTEST & 0.0256 \\
\hline 6720 & PDMS & & sW & & & E109373 & 12-Jul-89 & & $13966-29-5$ & $\mathrm{U}-234$ & UISO_AEA & USTEST & 0.315 \\
\hline 6721 & PDMS & & SW & & & E109373 & 12-Jul-89 & & $10098-97-2$ & SR-90 & SRISŌSEP_PRECIP_GPC & USTEST & 0.1 \\
\hline 6722 & PDMS & & SW & & & E109373 & 12-Jul-89 & & $10045-97-3$ & CS-137 & GAMMA_GS & USTEST & 0.0749 \\
\hline 6723 & PDMS & & SW & & & E109373 & 12-Jul-89 & & $10028-17-8$ & TRITIUM & TRITIUM DIST_LSC & USTEST & 27.1 \\
\hline 6724 & PDMS & & SW & & & E109454 & 17-Jul-89 & & $10045-97-3$ & CS-137 & 901.1_GAMMA_GS & USTEST & 0.247 \\
\hline 6725 & PDMS & & SW & & & E109454 & 17-Jul-89 & & $10098-97-2$ & SR-90 & 905.0_SR_GPC & USTEST & 0.00131 \\
\hline \begin{tabular}{|c|}
6726 \\
\end{tabular} & PDMS & & sW & & & E109454 & 17-Jul-89 & & $10028-17-8$ & TRITIUM & 906.0_H3_LSC & USTEST & 19.6 \\
\hline \begin{tabular}{|l|l|}
6727 \\
\end{tabular} & PDMS & & sW & & & E109453 & 17-Jul-89 & & $15046-84-1$ & I-129 & I129_SEP_TEMS & 320 & 0.00547 \\
\hline 6728 & PDMS & & SW & & & E109453 & 17-Jul-89 & & $10045-97-3$ & CS-137 & 901.1_GAMMA_GS & USTEST & -0.546 \\
\hline 6729 & PDMS & & SW & & & E109453 & 17-Jul-89 & & $10098-97-2$ & SR-90 & 905.0_SR_GPC & USTEST & -0.0139 \\
\hline 6730 & PDMS & & SW & & & E109453 & 17-Jul-89 & & $10028-17-8$ & TRITIUM & 906.0_H3_LSC & USTEST & 6740 \\
\hline \begin{tabular}{|c|}
6731 \\
\end{tabular} & PDMS & & SW & & & E109452 & 17-Jul-89 & & $10045-97-3$ & CS-137 & 901.1_GAMMA_GS & USTEST & -0.455 \\
\hline 6732 & PDMS & & SW & & & E109452 & 17-Jul-89 & & $10098-97-2$ & SR-90 & 905.0_SR_GPC & USTEST & -0.0042 \\
\hline 6733 & PDMS & & SW & & & E109452 & 17-Jul-89 & & 10028-17-8 & TRITIUM & 906.0_H3_LSC & USTEST & -56.7 \\
\hline \begin{tabular}{|c|}
6734 \\
\end{tabular} & PDMS & & SW & & & E109455 & 17-Jul-89 & & $10045-97-3$ & CS-137 & 901.1_GAMMA_GS & USTEST & 0.209 \\
\hline \begin{tabular}{|c|}
6735 \\
\end{tabular} & PDMS & & SW & & & E109455 & 17-Jul-89 & & 10098-97-2 & SR-90 & 905.0_SR_GPC & USTEST & -0.00322 \\
\hline \begin{tabular}{|l|}
6736 \\
\end{tabular} & PDMS & & SW & & & E109455 & 17-Jul-89 & & 10028-17-8 & TRITIUM & 906.0_H3_LSC & USTEST & 46.4 \\
\hline \begin{tabular}{|l|}
6737 \\
\end{tabular} & PDMS & & SW & & & E109561 & 25-Jul-89 & & $10045-97-3$ & CS-137 & GAMMA_GS & USTEST & 0.00402 \\
\hline 6738 & PDMS & & SW & & & E109558 & 25-Jul-89 & & $10045-97-3$ & CS-137 & GAMMA_GS & USTEST & 0.002 \\
\hline \begin{tabular}{|l|}
6739 \\
\end{tabular} & PDMS & & SW & & & E109562 & 25-Jul-89 & & $10045-97-3$ & CS-137 & GAMMA_GS & USTEST & 0.00199 \\
\hline \begin{tabular}{|l|}
6740 \\
\end{tabular} & PDMS & & sW & & & E109559 & 25-Jul-89 & & $10045-97-3$ & CS-137 & GAMMA_GS & USTEST & -0.00021 \\
\hline \begin{tabular}{|c|}
6741 \\
\end{tabular} & PDMS & & SW & & & E109560 & 25-Jul-89 & & $10045-97-3$ & CS-137 & GAMMA_GS & USTEST & 0.00285 \\
\hline \begin{tabular}{|c|}
6742 \\
\end{tabular} & PDMS & & SW & & & E109563 & 25-Jul-89 & & $10045-97-3$ & CS-137 & GAMMA_GS & USTEST & 0.00214 \\
\hline \begin{tabular}{|c|}
6743 \\
\end{tabular} & PDMS & & SW & & & E109569 & 01-Aug-89 & & $10045-97-3$ & CS-137 & GAMMA_GS & USTEST & 0.015 \\
\hline 6744 & PDMS & & SW & & & E109569 & 01-Aug-89 & & U-238 & U-238 & UISO_AĒA & USTEST & 0.214 \\
\hline 6745 & PDMS & & sW & & & E109569 & 01-Aug-89 & & 15117-96-1 & U-235 & UISO_AEA & USTEST & 0.0309 \\
\hline \begin{tabular}{|c|}
6746 \\
\end{tabular} & PDMS & & SW & & & E109569 & 01-Aug-89 & & $13966-29-5$ & $\mathrm{U}-234$ & UISO_AEA & USTEST & 0.287 \\
\hline \begin{tabular}{|c|}
6747 \\
\end{tabular} & PDMS & & SW & & & E109569 & 01-Aug-89 & & 10028-17-8 & TRITIUM & TRITIÜM_ELECT_LSC & USTEST & 52.8 \\
\hline 6748 & PDMS & & SW & & & E109569 & 01-Aug-89 & & 14133-76-7 & TC-99 & TC99_SEP_LSC & USTEST & 1.85 \\
\hline 6749 & PDMS & & SW & & & E109569 & 01-Aug-89 & & $10098-97-2$ & SR-90 & SRISŌSEEP_PRECIP_GPC & USTEST & 0.0693 \\
\hline 6750 & PDMS & & SW & & & E109570 & 01-Aug-89 & & $10045-97-3$ & CS-137 & GAMMĀGS & USTEST & 0 \\
\hline 6751 & PDMS & & SW & & & E109570 & 01-Aug-89 & & U-238 & $\mathrm{U}-238$ & UISO_AĒAA & USTEST & 0.201 \\
\hline 6752 & PDMS & & SW & & & E109570 & 01-Aug-89 & & 15117-96-1 & U-235 & UISO_AEA & USTEST & 0.00202 \\
\hline 6753 & PDMS & & SW & & & E109570 & 01-Aug-89 & & $13966-29-5$ & $\mathrm{U}-234$ & UISO_AEA & USTEST & 0.251 \\
\hline 6754 & PDMS & & SW & & & E109570 & 01-Aug-89 & & 10028-17-8 & TRITIUM & TRITIŪM_ELECT_LSC & USTEST & 156 \\
\hline 6755 & PDMS & & SW & & & E109570 & 01-Aug-89 & & 14133-76-7 & TC-99 & TC99_SEP_LSC & USTEST & 4.03 \\
\hline 6756 & PDMS & & SW & & & E109570 & 01-Aug-89 & & $10098-97-2$ & SR-90 & SRISŌSEP_PRECIP_GPC & USTEST & 0.048 \\
\hline 6757 & PDMS & & SW & & & E109571 & 03-Aug-89 & & $10045-97-3$ & CS-137 & 901.1_GAMM̄̄A_GS & USTEST & 0.42 \\
\hline 6758 & PDMS & & SW & & & E109571 & 03-Aug-89 & & $10028-17-8$ & TRITIUM & 906.0_H3_LSC & USTEST & 43.3 \\
\hline 6759 & PDMS & & SW & & & E109571 & 03-Aug-89 & & $10098-97-2$ & SR-90 & 905.0_SR_GPC & USTEST & 0.0747 \\
\hline 6760 & PDMS & & SW & & & E109665 & 08-Aug-89 & & $10045-97-3$ & CS-137 & GAMMA_E_GS & USTEST & 0.00219 \\
\hline 6761 & PDMS & & SW & & & E109667 & 08-Aug-89 & & $10045-97-3$ & CS-137 & GAMMA_GS & USTEST & 0.0037 \\
\hline
\end{tabular}




\begin{tabular}{|c|c|c|c|c|c|c|c|c|c|c|c|c|c|}
\hline & A & $\mathrm{B}$ & $\mathrm{c}$ & $\mathrm{D}$ & $\mathrm{E}$ & $\mathrm{F}$ & G & $\mathrm{H}$ & $\mathrm{I}$ & $\mathrm{J}$ & $\mathrm{K}$ & $\mathrm{L}$ & $\mathrm{M}$ \\
\hline 1 & SAMPLE_NUM_ASSIGNED_TO & SAMPLER & MEDIA & BIOTA_MEDIA & TAG_ID & SAMP_NUM & SAMP_DATE_TIME & SAMP_DATE_TIME_ON & CON_ID & CON_SHORT_NAME & METHOD_NAME & LAB_CODE & \begin{tabular}{|l|} 
VALUE_RPTD \\
\end{tabular} \\
\hline 6762 & PDMS & & SW & & & E109666 & 08-Aug-89 & & $10045-97-3$ & CS-137 & GAMMA GS & USTEST & 0.0017 \\
\hline 6763 & PDMS & & sW & & & E109730 & 08-Aug-89 & & $10045-97-3$ & CS-137 & GAMMA_GS & USTEST & 0.000569 \\
\hline 6764 & PDMS & & sW & & & E109767 & 08-Aug-89 & & $10045-97-3$ & CS-137 & GAMMA_GS & USTEST & \\
\hline 6765 & PDMS & & sW & & & E109766 & 08-Aug-89 & & $10045-97-3$ & CS-137 & GAMMA_GS & USTEST & \\
\hline 6766 & PDMS & & SW & & & E109674 & 14-Aug-89 & & $10045-97-3$ & CS-137 & 901.1_GAMMA_GS & USTEST & -0.225 \\
\hline 6767 & PDMS & & SW & & & E109674 & 14-Aug-89 & & $10028-17-8$ & TRITIUM & 906.0 H3 LSC & USTEST & 8290 \\
\hline 6768 & PDMS & & SW & & & E109674 & 14-Aug-89 & & $10098-97-2$ & SR-90 & 905.0_SR_GPC & USTEST & -0.00682 \\
\hline 6769 & PDMS & & SW & & & E109801 & 22-Aug-89 & & $10028-17-8$ & TRITIUM & 906.0_H3_LSC & USTEST & -31.6 \\
\hline 6770 & PDMS & & sW & & & E109801 & 22-Aug-89 & & $10045-97-3$ & CS-137 & 901.1_GAMMA_GS & USTEST & -0.575 \\
\hline 6771 & PDMS & & SW & & & E109801 & 22-Aug-89 & & 10098-97-2 & SR-90 & 905.0_SR_GPC & USTEST & -0.00378 \\
\hline 6772 & PDMS & & SW & & & E109959 & 22-Aug-89 & & $10045-97-3$ & CS-137 & GAMMA_GS & USTEST & 0.0036 \\
\hline 6773 & PDMS & & SW & & & E109961 & 22-Aug-89 & & $10045-97-3$ & CS-137 & GAMMA_GS & USTEST & 0.00117 \\
\hline 6774 & PDMS & & SW & & & E109672 & 22-Aug-89 & & $10045-97-3$ & CS-137 & GAMMA_GS & USTEST & \\
\hline 6775 & PDMS & & SW & & & E109671 & 22-Aug-89 & & $10045-97-3$ & CS-137 & GAMMA_GS & USTEST & \\
\hline \begin{tabular}{|c|}
6776 \\
\end{tabular} & PDMS & & SW & & & E109962 & 22-Aug-89 & & $10045-97-3$ & CS-137 & GAMMA_GS & USTEST & -0.0000603 \\
\hline \begin{tabular}{|l|l|}
6777 \\
\end{tabular} & PDMS & & SW & & & E109960 & 22-Aug-89 & & $10045-97-3$ & CS-137 & GAMMA_GS & USTEST & 0.00391 \\
\hline \begin{tabular}{|l|}
6778 \\
\end{tabular} & PDMS & & SW & & & E109800 & 23-Aug-89 & & $10028-17-8$ & TRITIUM & TRITIUM_DIST_LSC & USTEST & -29.6 \\
\hline \begin{tabular}{|l|}
6779 \\
\end{tabular} & PDMS & & sW & & & E109800 & 23-Aug-89 & & $10045-97-3$ & CS-137 & GAMMA_GS & USTEST & -2.69 \\
\hline 6780 & PDMS & & SW & & & E109800 & 23-Aug-89 & & $13966-29-5$ & $\mathrm{U}-234$ & UISO_AĒA & USTEST & 29.6 \\
\hline 6781 & PDMS & & SW & & & E109800 & 23-Aug-89 & & 15117-96-1 & U-235 & UISO_AEA & USTEST & 0.721 \\
\hline 6782 & PDMS & & SW & & & E109800 & 23-Aug-89 & & U-238 & U-238 & UISO_AEA & USTEST & 17.3 \\
\hline 6783 & PDMS & & SW & & & E109799 & 23-Aug-89 & & $10028-17-8$ & TRITIUM & TRITIUMM_DIST_LSC & USTEST & 71.7 \\
\hline \begin{tabular}{|l|}
6784 \\
\end{tabular} & PDMS & & SW & & & E109799 & 23-Aug-89 & & $10045-97-3$ & CS-137 & GAMMA_GS & USTEST & 7.69 \\
\hline 6785 & PDMS & & SW & & & E109799 & 23-Aug-89 & & $13966-29-5$ & $\mathrm{U}-234$ & UISO_AĒA & USTEST & \\
\hline 6786 & PDMS & & SW & & & E109799 & 23-Aug-89 & & 15117-96-1 & $\mathrm{U}-235$ & UISO_AEA & USTEST & \\
\hline 6787 & PDMS & & SW & & & E109799 & 23-Aug-89 & & $\mathrm{U}-238$ & $\mathrm{U}-238$ & UISO_AEA & USTEST & \\
\hline 6788 & PDMS & & SW & & & E109802 & 25-Aug-89 & & $10045-97-3$ & CS-137 & 901.1_GAMMA_GS & USTEST & $v$ \\
\hline 6789 & PDMS & & SW & & & E109802 & 25-Aug-89 & & $10028-17-8$ & TRITIUM & 906.0_H3_LSC & USTEST & 224 \\
\hline 6790 & PDMS & & SW & & & E109802 & 25-Aug-89 & & $10098-97-2$ & SR-90 & 905.0_SR_GPC & USTEST & 0.202 \\
\hline 6791 & PDMS & & SW & & & E109798 & 29-Aug-89 & & $10045-97-3$ & CS-137 & GAMMA_G_GS & USTEST & -0.272 \\
\hline 6792 & PDMS & & sW & & & E109798 & 29-Aug-89 & & $14133-76-7$ & TC-99 & TC99_SEP_LSC & USTEST & 0.648 \\
\hline 6793 & PDMS & & SW & & & E109798 & 29-Aug-89 & & $10098-97-2$ & SR-90 & SRISŌSEP_PRECIP_GPC & USTEST & 0.0882 \\
\hline 6794 & PDMS & & SW & & & E109798 & 29-Aug-89 & & $10028-17-8$ & TRITIUM & TRITIUM̄E_ELECT_LSC̄ & USTEST & 62.8 \\
\hline 6795 & PDMS & & SW & & & E109798 & 29-Aug-89 & & $13966-29-5$ & $\mathrm{U}-234$ & UISO_AËA & USTEST & 0.335 \\
\hline 6796 & PDMS & & SW & & & E109798 & 29-Aug-89 & & 15117-96-1 & $\mathrm{U}-235$ & UISO_AEA & USTEST & -0.00124 \\
\hline 6797 & PDMS & & SW & & & E109798 & 29-Aug-89 & & $\mathrm{U}-238$ & $\mathrm{U}-238$ & UISO_AEA & USTEST & 0.196 \\
\hline 6798 & PDMS & & SW & & & E109797 & 29-Aug-89 & & $10045-97-3$ & CS-137 & GAMMA_GS & USTEST & 0.643 \\
\hline 6799 & PDMS & & SW & & & E109797 & 29-Aug-89 & & $13966-29-5$ & $\mathrm{U}-234$ & UISO_AĒA & USTEST & 0.202 \\
\hline 6800 & PDMS & & SW & & & E109797 & 29-Aug-89 & & 15117-96-1 & U-235 & UISO_AEA & USTEST & 0.0193 \\
\hline 6801 & PDMS & & SW & & & E109797 & 29-Aug-89 & & $\mathrm{U}-238$ & $\mathrm{U}-238$ & UISO_AEA & USTEST & 0.251 \\
\hline 6802 & PDMS & & SW & & & E109797 & 29-Aug-89 & & 14133-76-7 & TC-99 & TC99_SEP_LSC & USTEST & 0.648 \\
\hline 6803 & PDMS & & SW & & & E109797 & 29-Aug-89 & & $10098-97-2$ & SR-90 & SRISŌ_SEP_PRECIP_GPC & USTEST & 0.0702 \\
\hline 6804 & PDMS & & SW & & & E109797 & 29-Aug-89 & & $10028-17-8$ & TRITIUM & TRITIUM_ELECT_LSC & USTEST & 165 \\
\hline 6805 & PDMS & & SW & & & E110184 & 30-Aug-89 & & $10028-17-8$ & TRITIUM & TRITIUM_DIST_LSC & USTEST & 80.6 \\
\hline 6806 & PDMS & & SW & & & E110184 & 30-Aug-89 & & $10045-97-3$ & CS-137 & GAMMA_GS & USTEST & 0.0678 \\
\hline 6807 & PDMS & & SW & & & E110184 & 30-Aug-89 & & $13966-29-5$ & $\mathrm{U}-234$ & UISO_AĒA & USTEST & 3.85 \\
\hline 6808 & PDMS & & SW & & & E110184 & 30-Aug-89 & & 15117-96-1 & U-235 & UISO_AEA & USTEST & 0.18 \\
\hline 6809 & PDMS & & SW & & & E110184 & 30-Aug-89 & & U-238 & U-238 & UISO_AEA & USTEST & 3.19 \\
\hline 6810 & PDMS & & SW & & & E110183 & 30-Aug-89 & & $10028-17-8$ & TRITIUM & TRITIŪM_DIST_LSC & USTEST & -122 \\
\hline 6811 & PDMS & & SW & & & E110183 & 30-Aug-89 & & $10045-97-3$ & CS-137 & GAMMA_GS & USTEST & 0.0509 \\
\hline 6812 & PDMS & & SW & & & E110183 & 30-Aug-89 & & $13966-29-5$ & $\mathrm{U}-234$ & UISO_AEAA & USTEST & -0.0176 \\
\hline 6813 & PDMS & & SW & & & E110183 & 30-Aug-89 & & 15117-96-1 & U-235 & UISO_AEA & USTEST & -0.0017 \\
\hline
\end{tabular}




\begin{tabular}{|c|c|c|c|c|c|c|c|c|c|c|c|c|c|}
\hline & A & $\mathrm{B}$ & $\mathrm{c}$ & $\mathrm{D}$ & $\mathrm{E}$ & $\mathrm{F}$ & G & $\mathrm{H}$ & $\mathrm{I}$ & $\mathrm{J}$ & $\mathrm{K}$ & $\mathrm{L}$ & $\mathrm{M}$ \\
\hline 1 & SAMPLE_NUM_ASSIGNED_TO & SAMPLER & MEDIA & BIOTA_MEDIA & TAG_ID & SAMP_NUM & SAMP_DATE_TIME & SAMP_DATE_TIME_ON & CON_ID & CON_SHORT_NAME & METHOD_NAME & LAB_CODE & \begin{tabular}{|l|} 
VALUE_RPTD \\
\end{tabular} \\
\hline 6814 & PDMS & & SW & & & E110183 & 30-Aug-89 & & U-238 & U-238 & UISO AEA & USTEST & -0.00426 \\
\hline 6815 & PDMS & & sW & & & E110229 & 05-Sep-89 & & $10045-97-3$ & CS-137 & GAMMA_GS & USTEST & 0.00325 \\
\hline \begin{tabular}{|c|}
6816 \\
\end{tabular} & PDMS & & sW & & & E110232 & 05-Sep-89 & & $10045-97-3$ & CS-137 & GAMMA_GS & USTEST & 0.00212 \\
\hline \begin{tabular}{|l|l|}
6817 \\
\end{tabular} & PDMS & & sW & & & E110230 & 05-Sep-89 & & $10045-97-3$ & CS-137 & GAMMA_GS & USTEST & 0.00492 \\
\hline \begin{tabular}{|l|}
6818 \\
\end{tabular} & PDMS & & sW & & & E110233 & 05-Sep-89 & & $10045-97-3$ & CS-137 & GAMMA_GS & USTEST & 0.00311 \\
\hline \begin{tabular}{|l|}
6819 \\
\end{tabular} & PDMS & & sW & & & E110231 & 05-Sep-89 & & $10045-97-3$ & CS-137 & GAMMA_GS & USTEST & 0.00356 \\
\hline 6820 & PDMS & & SW & & & E110234 & 05-Sep-89 & & $10045-97-3$ & CS-137 & GAMMA_GS & USTEST & 0.0037 \\
\hline 6821 & PDMS & & SW & & & E110185 & 06-Sep-89 & & $10045-97-3$ & CS-137 & GAMMA_GS & USTEST & 0.246 \\
\hline 6822 & PDMS & & sW & & & E110185 & 06-Sep-89 & & $10028-17-8$ & TRITIUM & TRITIUM DIST LSC & USTEST & -81.5 \\
\hline 6823 & PDMS & & SW & & & E110185 & 06-Sep-89 & & $13966-29-5$ & U-234 & UISO_AEA & USTEST & 0.298 \\
\hline \begin{tabular}{|l|}
6824 \\
\end{tabular} & PDMS & & sW & & & E110185 & 06-Sep-89 & & $15117-96-1$ & U-235 & UISO_AEA & USTEST & 0.00839 \\
\hline 6825 & PDMS & & SW & & & E110185 & 06-Sep-89 & & U-238 & $\mathrm{U}-238$ & UISO_AEA & USTEST & 0.154 \\
\hline 6826 & PDMS & & SW & & & E110185 & 06-Sep-89 & & $10098-97-2$ & SR-90 & SRISŌSSEP_PRECIP_GPC & USTEST & 0.0628 \\
\hline \begin{tabular}{|l|l|}
6827 & $\cdot r \cdot r$ \\
\end{tabular} & PDMS & & SW & & & E110247 & 11-Sep-89 & & $10028-17-8$ & TRITIUM & $906.0 \overline{\mathrm{H}} 3 \mathrm{LSC}$ & USTEST & 8470 \\
\hline 6828 & PDMS & & SW & & & E110247 & 11-Sep-89 & & $10045-97-3$ & CS-137 & 901.1_GAMMA_GS & USTEST & -0.202 \\
\hline 6829 & PDMS & & SW & & & E110247 & 11-Sep-89 & & $10098-97-2$ & SR-90 & 905.0_SR_GPC & USTEST & 0.00064 \\
\hline 6830 & PDMS & & sW & & & E110244 & 12-Sep-89 & & $10045-97-3$ & CS-137 & GAMMA_GS & USTEST & 0.237 \\
\hline \begin{tabular}{|l|}
6831 \\
\end{tabular} & PDMS & & sW & & & E110244 & 12-Sep-89 & & $10028-17-8$ & TRITIUM & TRITIUM_DIST_LSC & USTEST & 147 \\
\hline 6832 & PDMS & & SW & & & E110244 & 12-Sep-89 & & $10098-97-2$ & SR-90 & SRISO_SEP_PERECIP_GPC & USTEST & 0.277 \\
\hline 6833 & PDMS & & SW & & & E110246 & 12-Sep-89 & & $10028-17-8$ & TRITIUM & TRITIUM_DIST_LSC & USTEST & 9200 \\
\hline 6834 & PDMS & & SW & & & E110246 & 12-Sep-89 & & $10045-97-3$ & CS-137 & GAMMA_GS & USTEST & -0.817 \\
\hline 6835 & PDMS & & SW & & & E110245 & 12-Sep-89 & & $10028-17-8$ & TRITIUM & TRITIUM_DIST_LSC & USTEST & 298 \\
\hline 6836 & PDMS & & SW & & & E110245 & 12-Sep-89 & & $10045-97-3$ & CS-137 & GAMMA_GS & USTEST & 1.53 \\
\hline 6837 & PDMS & & SW & & & E110245 & 12-Sep-89 & & 13966-29-5 & $\mathrm{U}-234$ & UISO_AEA & USTEST & 199 \\
\hline 6838 & PDMS & & SW & & & E110245 & 12-Sep-89 & & 15117-96-1 & $\mathrm{U}-235$ & UISO_AEA & USTEST & 21.4 \\
\hline 6839 & PDMS & & SW & & & E110245 & 12-Sep-89 & & U-238 & $\mathrm{U}-238$ & UISO_AEA & USTEST & 197 \\
\hline 6840 & PDMS & & SW & & & E110245 & 12-Sep-89 & & $10098-97-2$ & SR-90 & SRISŌ_SEP_PRECIP_GPC & USTEST & 1.85 \\
\hline 6841 & PDMS & & SW & & & E110417 & 19-Sep-89 & & $10045-97-3$ & CS-137 & GAMMA_GS & USTEST & 0.00261 \\
\hline 6842 & PDMS & & SW & & & E110420 & 19-Sep-89 & & $10045-97-3$ & CS-137 & GAMMA_GS & USTEST & 0.00458 \\
\hline 6843 & PDMS & & SW & & & E110421 & 19-Sep-89 & & $10045-97-3$ & CS-137 & GAMMA_GS & USTEST & 0.00393 \\
\hline \begin{tabular}{|l|}
6844 \\
\end{tabular} & PDMS & & sW & & & E110418 & 19-Sep-89 & & $10045-97-3$ & CS-137 & GAMMA_GS & USTEST & 0.00126 \\
\hline 6845 & PDMS & & SW & & & E110419 & 19-Sep-89 & & $10045-97-3$ & CS-137 & GAMMA_GS & USTEST & 0.00418 \\
\hline 6846 & PDMS & & SW & & & E110422 & 19-Sep-89 & & $10045-97-3$ & CS-137 & GAMMA_GS & USTEST & 0.0049 \\
\hline \begin{tabular}{|l|l|}
6847 \\
\end{tabular} & PDMS & & SW & & & E110341 & 26-Sep-89 & & $10045-97-3$ & CS-137 & 901.1_GAMMMA_GS & USTEST & 0.552 \\
\hline 6848 & PDMS & & SW & & & E110341 & 26-Sep-89 & & $13966-29-5$ & $\mathrm{U}-234$ & UISO_IE_PLATE_AEA & USTEST & 0.317 \\
\hline 6849 & PDMS & & SW & & & E110341 & 26-Sep-89 & & 15117-96-1 & U-235 & UISO_IE_PLATE_AEA & USTEST & 0.0131 \\
\hline 6850 & PDMS & & SW & & & E110341 & 26-Sep-89 & & U-238 & $\mathrm{U}-238$ & UISO_IE_PLATE_AEA & USTEST & 0.304 \\
\hline 6851 & PDMS & & SW & & & E110341 & 26-Sep-89 & & $14133-76-7$ & TC-99 & TC99_SEP_LSC & USTEST & 0.886 \\
\hline 6852 & PDMS & & SW & & & E110341 & 26-Sep-89 & & 10028-17-8 & TRITIUM & TRITIŪM_ELECTT_LSC & USTEST & 195 \\
\hline 6853 & PDMS & & SW & & & E110341 & 26-Sep-89 & & $10098-97-2$ & SR-90 & 905.0_SR_GPC & USTEST & 0.0746 \\
\hline 6854 & PDMS & & SW & & & E110491 & 03-Oct-89 & & PU-239/240 & PU-239/240 & PUISO_IE_AEA & USTEST & 0.0000254 \\
\hline 6855 & PDMS & & SW & & & E110494 & 03-Oct-89 & & 15046-84-1 & I-129 & I129_SEP_TEMS & 320 & 0.0002598 \\
\hline 6856 & PDMS & & SW & & & E110491 & 03-Oct-89 & & $10045-97-3$ & CS-137 & GAMMA_GS & USTEST & 0.00241 \\
\hline 6857 & PDMS & & SW & & & E110494 & 03-Oct-89 & & $10045-97-3$ & CS-137 & GAMMA_GS & USTEST & 0.00407 \\
\hline 6858 & PDMS & & SW & & & E110494 & 03-Oct-89 & & PU-239/240 & PU-239/240 & PUISO_IE_AEA & USTEST & 0.00005 \\
\hline 6859 & PDMS & & SW & & & E110444 & 03-Oct-89 & & $10045-97-3$ & CS-137 & GAMMA_GSS & USTEST & -0.166 \\
\hline 6860 & PDMS & & SW & & & E110444 & 03-Oct-89 & & $14133-76-7$ & TC-99 & TC99_SEPP_LSC & USTEST & -0.945 \\
\hline 6861 & PDMS & & SW & & & E110444 & 03-Oct-89 & & $13966-29-5$ & $\mathrm{U}-234$ & UISO_AEA & USTEST & 0.254 \\
\hline 6862 & PDMS & & SW & & & E110444 & 03-Oct-89 & & 15117-96-1 & U-235 & UISO_AEA & USTEST & 0.000718 \\
\hline 6863 & PDMS & & sW & & & E110444 & 03-Oct-89 & & $\mathrm{U}-238$ & $\mathrm{U}-238$ & UISO_AEA & USTEST & 0.205 \\
\hline \begin{tabular}{|c|}
6864 \\
\end{tabular} & PDMS & & sW & & & E110444 & 03-Oct-89 & & $10098-97-2$ & SR-90 & SRISO_SEP_PRECIP_GPC & USTEST & 0.0725 \\
\hline 6865 & PDMS & & SW & & & E110444 & 03-Oct-89 & & $10028-17-8$ & TRITIUM & TRITIUM_E_ELECT_LSC & USTEST & 73.2 \\
\hline
\end{tabular}




\begin{tabular}{|c|c|c|c|c|c|c|c|c|c|c|c|c|c|}
\hline & A & $\mathrm{B}$ & $\mathrm{c}$ & $\mathrm{D}$ & $\mathrm{E}$ & $\mathrm{F}$ & G & $\mathrm{H}$ & $\mathrm{I}$ & $\mathrm{J}$ & $\mathrm{K}$ & $\mathrm{L}$ & $\mathrm{M}$ \\
\hline 1 & SAMPLE_NUM_ASSIGNED_TO & SAMPLER & MEDIA & BIOTA_MEDIA & TAG_ID & SAMP_NUM & SAMP_DATE_TIME & SAMP_DATE_TIME_ON & CON_ID & CON_SHORT_NAME & METHOD_NAME & LAB_CODE & \begin{tabular}{|l|} 
VALUE_RPTD \\
\end{tabular} \\
\hline 6866 & PDMS & & SW & & & E110490 & 03-Oct-89 & & PU-239/240 & PU-239/240 & PUISO IE AEA & USTEST & 0.0000143 \\
\hline \begin{tabular}{|c|c|}
6867 & \\
\end{tabular} & PDMS & & sW & & & E110493 & 03-Oct-89 & & 15046-84-1 & I-129 & I129_SEP_TEMS & 320 & 0.0000045 \\
\hline 6868 & PDMS & & sW & & & E110490 & 03-Oct-89 & & $10045-97-3$ & CS-137 & GAMMA_GS & USTEST & 0.00137 \\
\hline 6869 & PDMS & & sW & & & E110493 & 03-Oct-89 & & $10045-97-3$ & CS-137 & GAMMA_GS & USTEST & 0.00161 \\
\hline \begin{tabular}{|c|}
6870 \\
\end{tabular} & PDMS & & sW & & & E110493 & 03-Oct-89 & & PU-239/240 & PU-239/240 & PUISO_IE_AEA & USTEST & -0.00000413 \\
\hline \begin{tabular}{|l|}
6871 \\
\end{tabular} & PDMS & & sW & & & E110492 & 03-Oct-89 & & PU-239/240 & PU-239/240 & PUISO_IE_AEA & USTEST & 0.0000347 \\
\hline 6872 & PDMS & & SW & & & E110495 & 03-Oct-89 & & 15046-84-1 & I-129 & 1129 SEP_TEMS & 320 & 0.0001596 \\
\hline 6873 & PDMS & & SW & & & E110492 & 03-Oct-89 & & $10045-97-3$ & CS-137 & GAMMA_GS & USTEST & 0.00146 \\
\hline \begin{tabular}{|c|}
6874 \\
\end{tabular} & PDMS & & sW & & & E110495 & 03-Oct-89 & & $10045-97-3$ & CS-137 & GAMMA GS & USTEST & 0.00493 \\
\hline 6875 & PDMS & & SW & & & E110495 & 03-Oct-89 & & PU-239/240 & PU-239/240 & PUISO_IE_AEA & USTEST & -0.00000419 \\
\hline \begin{tabular}{|l|}
6876 \\
\end{tabular} & PDMS & & sW & & & E110446 & 03-Oct-89 & & $10045-97-3$ & CS-137 & GAMMA_GS & USTEST & -0.259 \\
\hline 6877 & PDMS & & SW & & & E110446 & 03-Oct-89 & & $14133-76-7$ & TC-99 & TC99_SEEP_LSC & USTEST & 1.48 \\
\hline 6878 & PDMS & & SW & & & E110446 & 03-Oct-89 & & 13966-29-5 & $\mathrm{U}-234$ & UISO_AEA & USTEST & 0.236 \\
\hline 6879 & PDMS & & SW & & & E110446 & 03-Oct-89 & & 15117-96-1 & U-235 & UISO_AEA & USTEST & 0.0099 \\
\hline 6880 & PDMS & & SW & & & E110446 & 03-Oct-89 & & U-238 & $\mathrm{U}-238$ & UISO_AEA & USTEST & 0.202 \\
\hline 6881 & PDMS & & SW & & & E110446 & 03-Oct-89 & & $10098-97-2$ & SR-90 & SRISO_SEP_PRECIP_GPC & USTEST & 0.0678 \\
\hline 6882 & PDMS & & sW & & & E110446 & 03-Oct-89 & & $10028-17-8$ & TRITIUM & TRITIUM_ELEECT_LSC & USTEST & 120 \\
\hline \begin{tabular}{|c|}
6883 \\
\end{tabular} & PDMS & & sW & & & E110515 & 09-Oct-89 & & $10028-17-8$ & TRITIUM & 906.0_H3_LSC & USTEST & 35.6 \\
\hline 6884 & PDMS & & SW & & & E110515 & $09-$ Oct-89 & & $10045-97-3$ & CS-137 & 901.1_GAMMA_GS & USTEST & 0.0593 \\
\hline 6885 & PDMS & & SW & & & E110515 & $09-O c t-89$ & & $10098-97-2$ & SR-90 & 905.0_SR_GPC & USTEST & -0.0258 \\
\hline 6886 & PDMS & & SW & & & E110514 & 09-Oct-89 & & $10028-17-8$ & TRITIUM & 906.0_H3_LSC & USTEST & 7660 \\
\hline \begin{tabular}{|l|l|}
6887 \\
\end{tabular} & PDMS & & SW & & & E110514 & 09-Oct-89 & & $10045-97-3$ & CS-137 & 901.1_GAMMA_GS & USTEST & 0.0854 \\
\hline 6888 & PDMS & & SW & & & E110514 & $09-O c t-89$ & & $10098-97-2$ & SR-90 & 905.0_SR_GPC & USTEST & -0.00493 \\
\hline 6889 & PDMS & & SW & & & E110512 & $09-$ Oct-89 & & $10028-17-8$ & TRITIUM & 906.0_H3_LSC & USTEST & 1.87 \\
\hline 6890 & PDMS & & SW & & & E110512 & 09-Oct-89 & & $10045-97-3$ & CS-137 & 901.1_GAMMA_GS & USTEST & -0.351 \\
\hline 6891 & PDMS & & SW & & & E110512 & 09-Oct-89 & & $10098-97-2$ & SR-90 & 905.0_SR_GPC & USTEST & -0.0145 \\
\hline 6892 & PDMS & & SW & & & E110516 & $09-$ Oct-89 & & 10028-17-8 & TRITIUM & 906.0_H3_LSC & USTEST & -12.2 \\
\hline 6893 & PDMS & & SW & & & E110516 & $09-O c t-89$ & & $10098-97-2$ & SR-90 & 905.0_SR_GPC & USTEST & -0.0033 \\
\hline 6894 & PDMS & & SW & & & E110516 & 09-Oct-89 & & $10045-97-3$ & CS-137 & 901.1_GAMMA_GS & USTEST & 0.449 \\
\hline 6895 & PDMS & & SW & & & E110578 & $17-$ Oct-89 & & $10098-97-2$ & SR-90 & 905.0_SR_GPC & USTEST & 0.0958 \\
\hline \begin{tabular}{|l|}
6896 \\
\end{tabular} & PDMS & & sW & & & E110578 & 17-Oct-89 & & $10028-17-8$ & TRITIUM & 906.0_H3_LSC & USTEST & 33.8 \\
\hline \begin{tabular}{|l|}
6897 \\
\end{tabular} & PDMS & & SW & & & E110578 & 17-Oct-89 & & $10045-97-3$ & CS-137 & 901.1_GAMMA_GS & USTEST & 0.019 \\
\hline 6898 & PDMS & & SW & & & E110580 & 17-Oct-89 & & $10045-97-3$ & CS-137 & GAMM̄A_GS & USTEST & -0.102 \\
\hline 6899 & PDMS & & SW & & & E110580 & 17-Oct-89 & & $10098-97-2$ & SR-90 & SRISO_S̄EP_PRECIP_GPC & USTEST & 0.074 \\
\hline 6900 & PDMS & & SW & & & E110580 & 17-Oct-89 & & $10028-17-8$ & TRITIUM & TRITIŪM_ELEECT_LSC̄ & USTEST & 184 \\
\hline 6901 & PDMS & & sW & & & E110580 & $17-$ Oct-89 & & U-238 & $\mathrm{U}-238$ & UISO_AEA & USTEST & 0.18 \\
\hline 6902 & PDMS & & SW & & & E110580 & 17-Oct-89 & & 15117-96-1 & $\mathrm{U}-235$ & UISO_AEA & USTEST & -0.00128 \\
\hline 6903 & PDMS & & SW & & & E110580 & 17-Oct-89 & & $13966-29-5$ & $\mathrm{U}-234$ & UISO_AEA & USTEST & 0.252 \\
\hline 6904 & PDMS & & SW & & & E110579 & 17-Oct-89 & & $10045-97-3$ & CS-137 & GAMM̄A_GS & USTEST & 0.0697 \\
\hline 6905 & PDMS & & SW & & & E110579 & 17-Oct-89 & & $10098-97-2$ & SR-90 & SRISO_S̄EP_PRECIP_GPC & USTEST & 0.0763 \\
\hline 6906 & PDMS & & SW & & & E110579 & 17-Oct-89 & & $10028-17-8$ & TRITIUM & TRITIŪM_ELEECT_LSC̄ & USTEST & 60.9 \\
\hline \begin{tabular}{|c|}
6907 \\
\end{tabular} & PDMS & & SW & & & E110579 & 17-Oct-89 & & U-238 & $\mathrm{U}-238$ & UISO_AËA & USTEST & 0.182 \\
\hline 6908 & PDMS & & SW & & & E110579 & 17-Oct-89 & & 15117-96-1 & U-235 & UISO_AEA & USTEST & 0.00198 \\
\hline 6909 & PDMS & & SW & & & E110579 & 17-Oct-89 & & $13966-29-5$ & $\mathrm{U}-234$ & UISO_AEA & USTEST & 0.221 \\
\hline 6910 & PDMS & & SW & & & E110620 & 17-Oct-89 & & $10045-97-3$ & CS-137 & GAMM̄A_GS & USTEST & 0.00442 \\
\hline \begin{tabular}{|l|}
6911 \\
\end{tabular} & PDMS & & SW & & & E110617 & 17-Oct-89 & & $10045-97-3$ & CS-137 & GAMMA_GS & USTEST & 0.00267 \\
\hline \begin{tabular}{|l|l|}
6912 \\
\end{tabular} & PDMS & & SW & & & E110618 & 17-Oct-89 & & $10045-97-3$ & CS-137 & GAMMA_GS & USTEST & -0.000215 \\
\hline \begin{tabular}{|l|}
6913 \\
\end{tabular} & PDMS & & SW & & & E110621 & 17-Oct-89 & & $10045-97-3$ & CS-137 & GAMMA_GS & USTEST & 0.00111 \\
\hline \begin{tabular}{|l|}
6914 \\
\end{tabular} & PDMS & & SW & & & E110622 & 17-Oct-89 & & $10045-97-3$ & CS-137 & GAMMA_GS & USTEST & -0.00039 \\
\hline 6915 & PDMS & & SW & & & E110619 & 17-Oct-89 & & $10045-97-3$ & CS-137 & GAMMA_GS & USTEST & 0.00311 \\
\hline 6916 & PDMS & & SW & & & E110748 & 31-Oct-89 & & $10045-97-3$ & CS-137 & GAMMA_GS & USTEST & -0.000059 \\
\hline \begin{tabular}{|l|}
6917 \\
\end{tabular} & PDMS & & SW & & & E110745 & 31-Oct-89 & & $10045-97-3$ & CS-137 & GAMMA_GS & USTEST & -0.0000978 \\
\hline
\end{tabular}




\begin{tabular}{|c|c|c|c|c|c|c|c|c|c|c|c|c|c|}
\hline & A & $\mathrm{B}$ & $\mathrm{c}$ & $\mathrm{D}$ & $\mathrm{E}$ & $\mathrm{F}$ & G & $\mathrm{H}$ & $\mathrm{I}$ & $\mathrm{J}$ & $\mathrm{K}$ & $\mathrm{L}$ & $\mathrm{M}$ \\
\hline 1 & SAMPLE_NUM_ASSIGNED_TO & SAMPLER & MEDIA & BIOTA_MEDIA & TAG_ID & SAMP_NUM & SAMP_DATE_TIME & SAMP_DATE_TIME_ON & CON_ID & CON_SHORT_NAME & METHOD_NAME & LAB_CODE & \begin{tabular}{|l|} 
VALUE_RPTD \\
\end{tabular} \\
\hline 6918 & PDMS & & SW & & & E110711 & 31-Oct-89 & & U-238 & U-238 & UISO AEA & USTEST & 0.214 \\
\hline 6919 & PDMS & & sW & & & E110711 & 31-Oct-89 & & 15117-96-1 & U-235 & UISO_AEA & USTEST & 0.00756 \\
\hline 6920 & PDMS & & sW & & & E110711 & 31-Oct-89 & & $13966-29-5$ & U-234 & UISO_AEA & USTEST & 0.258 \\
\hline \begin{tabular}{|l|}
6921 \\
\end{tabular} & PDMS & & sW & & & E110711 & 31-Oct-89 & & $14133-76-7$ & TC-99 & TC99_SEP_LSC & USTEST & 4.06 \\
\hline \begin{tabular}{|l|}
6922 \\
\end{tabular} & PDMS & & sW & & & E110749 & 31-Oct-89 & & $10045-97-3$ & CS-137 & GAMMA_GS & USTEST & 0.00351 \\
\hline \begin{tabular}{|l|}
6923 \\
\end{tabular} & PDMS & & sW & & & E110746 & 31-Oct-89 & & $10045-97-3$ & CS-137 & GAMMA_GS & USTEST & 0.00192 \\
\hline 6924 & PDMS & & SW & & & E110711 & 31-Oct-89 & & $10028-17-8$ & TRITIUM & TRITIUM_ELECT_LSC & USTEST & 55.6 \\
\hline 6925 & PDMS & & SW & & & E110711 & 31-Oct-89 & & $10098-97-2$ & SR-90 & SRISO_SEP_PRËCIP_GPC & USTEST & 0.0691 \\
\hline \begin{tabular}{|c|}
6926 \\
\end{tabular} & PDMS & & sW & & & E110711 & 31-Oct-89 & & $10045-97-3$ & CS-137 & GAMMA_GS & USTEST & 0.776 \\
\hline 6927 & PDMS & & SW & & & E110710 & 31-Oct-89 & & U-238 & U-238 & UISO_AEAA & USTEST & 0.16 \\
\hline 6928 & PDMS & & sW & & & E110710 & 31-Oct-89 & & 15117-96-1 & U-235 & UISO_AEA & USTEST & -0.00312 \\
\hline 6929 & PDMS & & SW & & & E110710 & 31-Oct-89 & & $13966-29-5$ & $\mathrm{U}-234$ & UISO_AEA & USTEST & 0.202 \\
\hline 6930 & PDMS & & SW & & & E110710 & 31-Oct-89 & & $14133-76-7$ & TC-99 & TC99_SEP_LSC & USTEST & 1.98 \\
\hline 6931 & PDMS & & SW & & & E110710 & 31-Oct-89 & & $10028-17-8$ & TRITIUM & TRITIŪM_ELEECT_LSC & USTEST & 103 \\
\hline 6932 & PDMS & & SW & & & E110710 & 31-Oct-89 & & $10098-97-2$ & SR-90 & SRISO_SEP_PRËCIP_GPC & USTEST & 0.0805 \\
\hline 6933 & PDMS & & SW & & & E110710 & 31-Oct-89 & & $10045-97-3$ & CS-137 & GAMMA_GS & USTEST & -0.0075 \\
\hline \begin{tabular}{|c|}
6934 \\
\end{tabular} & PDMS & & sW & & & E110750 & 31-Oct-89 & & $10045-97-3$ & CS-137 & GAMMA_GS & USTEST & 0.000644 \\
\hline \begin{tabular}{|c|}
6935 \\
\end{tabular} & PDMS & & sW & & & E110747 & 31-Oct-89 & & $10045-97-3$ & CS-137 & GAMMA_GS & USTEST & 0.00154 \\
\hline 6936 & PDMS & & SW & & & E110755 & 06-Nov-89 & & $10098-97-2$ & SR-90 & 905.0_SR_GPC & USTEST & -0.0184 \\
\hline \begin{tabular}{|l|l|}
6937 \\
\end{tabular} & PDMS & & SW & & & E110755 & 06-Nov-89 & & $10028-17-8$ & TRITIUM & 906.0_H3_LSC & USTEST & 8030 \\
\hline 6938 & PDMS & & SW & & & E110755 & 06-Nov-89 & & $10045-97-3$ & CS-137 & 901.1_GAMMA_GS & USTEST & 0.575 \\
\hline 6939 & PDMS & & SW & & & E110872 & 14-Nov-89 & & $10098-97-2$ & SR-90 & 905.0_SR_GPC & USTEST & 0.00566 \\
\hline 6940 & PDMS & & SW & & & E110872 & 14-Nov-89 & & $10045-97-3$ & CS-137 & 901.1_GAMMA_GS & USTEST & 0.322 \\
\hline 6941 & PDMS & & SW & & & E110872 & 14-Nov-89 & & $10028-17-8$ & TRITIUM & 906.0_H3_LSC & USTEST & -28.1 \\
\hline 6942 & PDMS & & SW & & & E110956 & 14-Nov-89 & & $10045-97-3$ & CS-137 & GAMMA_ESS & USTEST & 0.00152 \\
\hline 6943 & PDMS & & SW & & & E110953 & 14-Nov-89 & & $10045-97-3$ & CS-137 & GAMMA_GS & USTEST & 0.00128 \\
\hline 6944 & PDMS & & SW & & & E110954 & 14-Nov-89 & & $10045-97-3$ & CS-137 & GAMMA_GS & USTEST & 0.00109 \\
\hline 6945 & PDMS & & SW & & & E110957 & 14-Nov-89 & & $10045-97-3$ & CS-137 & GAMMA_GS & USTEST & 0.00347 \\
\hline 6946 & PDMS & & SW & & & E110955 & 14-Nov-89 & & $10045-97-3$ & CS-137 & GAMMA_GS & USTEST & 0.000922 \\
\hline \begin{tabular}{|l|l|}
6947 \\
\end{tabular} & PDMS & & SW & & & E110958 & 14-Nov-89 & & $10045-97-3$ & CS-137 & GAMMA_GS & USTEST & 0.00143 \\
\hline \begin{tabular}{|l|l|}
6948 \\
\end{tabular} & PDMS & & sW & & & E110871 & 15-Nov-89 & & 15117-96-1 & $\mathrm{U}-235$ & UISO_AEA & USTEST & 0.653 \\
\hline 6949 & PDMS & & SW & & & E110871 & 15-Nov-89 & & $13966-29-5$ & $\mathrm{U}-234$ & UISO_AEA & USTEST & 25.2 \\
\hline 6950 & PDMS & & SW & & & E110871 & 15-Nov-89 & & $\mathrm{U}-238$ & $\mathrm{U}-238$ & UISO_AEA & USTEST & 16.1 \\
\hline 6951 & PDMS & & SW & & & E110871 & 15-Nov-89 & & $10045-97-3$ & CS-137 & GAMM̄A_GS & USTEST & -0.412 \\
\hline 6952 & PDMS & & SW & & & E110871 & 15-Nov-89 & & $10028-17-8$ & TRITIUM & TRITIUM_DIST_LSC & USTEST & 64.6 \\
\hline 6953 & PDMS & & SW & & & E110870 & 15-Nov-89 & & $\mathrm{U}-238$ & U-238 & UISO_AEA & USTEST & 7.61 \\
\hline \begin{tabular}{|l|}
6954 \\
\end{tabular} & PDMS & & sW & & & E110870 & 15-Nov-89 & & 15117-96-1 & U-235 & UISO_AEA & USTEST & 0.374 \\
\hline 6955 & PDMS & & SW & & & E110870 & 15-Nov-89 & & $13966-29-5$ & $\mathrm{U}-234$ & UISO_AEA & USTEST & 8.19 \\
\hline 6956 & PDMS & & SW & & & E110870 & 15-Nov-89 & & $10045-97-3$ & CS-137 & GAMM̄A_GS & USTEST & 0.0536 \\
\hline \begin{tabular}{|l|l|}
6957 \\
\end{tabular} & PDMS & & SW & & & E110870 & 15-Nov-89 & & $10028-17-8$ & TRITIUM & TRITIUM_DIST_LSC & USTEST & 166 \\
\hline 6958 & PDMS & & SW & & & E110873 & 17-Nov-89 & & $10098-97-2$ & SR-90 & 905.0_SR_GPC & USTEST & 0.386 \\
\hline 6959 & PDMS & & SW & & & E110873 & 17-Nov-89 & & $10045-97-3$ & CS-137 & 901.1_GAMMA_GS & USTEST & -0.104 \\
\hline 6960 & PDMS & & SW & & & E110873 & 17-Nov-89 & & $10028-17-8$ & TRITIUM & 906.0_H3_LSC & USTEST & 289 \\
\hline 6961 & PDMS & & SW & & & E110964 & 22-Nov-89 & & $\mathrm{U}-238$ & $\mathrm{U}-238$ & UISO_AEA & USTEST & 2.67 \\
\hline 6962 & PDMS & & SW & & & E110964 & 22-Nov-89 & & 15117-96-1 & U-235 & UISO_AEA & USTEST & 0.106 \\
\hline 6963 & PDMS & & SW & & & E110964 & 22-Nov-89 & & $13966-29-5$ & $\mathrm{U}-234$ & UISO_AEA & USTEST & 3.33 \\
\hline 6964 & PDMS & & SW & & & E110964 & 22-Nov-89 & & $10045-97-3$ & CS-137 & GAMM̄A_GS & USTEST & 0.146 \\
\hline 6965 & PDMS & & SW & & & E110964 & 22-Nov-89 & & $10028-17-8$ & TRITIUM & TRITIUM_DIST_LSC & USTEST & 220 \\
\hline 6966 & PDMS & & SW & & & E110963 & 22-Nov-89 & & $10045-97-3$ & CS-137 & GAMMA_GS & USTEST & -0.297 \\
\hline \begin{tabular}{|l|l|}
6967 \\
\end{tabular} & PDMS & & SW & & & E110963 & 22-Nov-89 & & $\mathrm{U}-238$ & $\mathrm{U}-238$ & UISO_AEAA & USTEST & 0.0138 \\
\hline 6968 & PDMS & & SW & & & E110963 & 22-Nov-89 & & 15117-96-1 & U-235 & UISO_AEA & USTEST & 0.0136 \\
\hline 6969 & PDMS & & SW & & & E110963 & 22-Nov-89 & & 13966-29-5 & $\mathrm{U}-234$ & UISO_AEA & USTEST & -0.0014 \\
\hline
\end{tabular}




\begin{tabular}{|c|c|c|c|c|c|c|c|c|c|c|c|c|c|}
\hline & $\bar{A}$ & $\mathrm{~B}$ & C & $\mathrm{D}$ & $E$ & $\mathrm{~F}$ & G & $\mathrm{H}$ & $\mathrm{I}$ & $\mathrm{J}$ & $\mathrm{K}$ & $\mathrm{L}$ & $\mathrm{M}$ \\
\hline 1 & SAMPLE_NUM_ASSIGNED_TO & SAMPLER & MEDIA & BIOTA_MEDIA & TAG_ID & SAMP_NUM & SAMP_DATE_TIME & SAMP_DATE_TIME_ON & CON_ID & CON_SHORT_NAME & METHOD_NAME & LAB_CODE & VALUE_RPTD \\
\hline 6970 & PDMS & & SW & & & E110963 & 22-Nov-89 & & $10028-17-8$ & TRITIUM & TRITIUM_DIST_LSC & USTEST & $\begin{array}{ll}-39.2 \\
\end{array}$ \\
\hline 6971 & PDMS & & SW & & & E111003 & 28-Nov-89 & & $10045-97-3$ & CS-137 & GAMMA_GS & USTEST & 0.00256 \\
\hline 6972 & PDMS & & SW & & & E111006 & 28-Nov-89 & & $10045-97-3$ & CS-137 & GAMMA_GS & USTEST & 0.00215 \\
\hline 6973 & PDMS & & SW & & & E110961 & 28-Nov-89 & & $10045-97-3$ & CS-137 & GAMMA_GS & USTEST & -0.0525 \\
\hline 6974 & PDMS & & SW & & & E110961 & 28-Nov-89 & & $\mathrm{U}-238$ & U-238 & UISO_AĒA & USTEST & 0.204 \\
\hline 6975 & PDMS & & SW & & & E110961 & 28-Nov-89 & & 15117-96-1 & U-235 & UISO_AEA & USTEST & 0.00945 \\
\hline 6976 & PDMS & & SW & & & E110961 & 28-Nov-89 & & $13966-29-5$ & U-234 & UISO_AEA & USTEST & 0.217 \\
\hline 6977 & PDMS & & SW & & & E110961 & 28-Nov-89 & & $14133-76-7$ & TC-99 & TC99_SEP_LSC & USTEST & -1.09 \\
\hline 6978 & PDMS & & SW & & & E110961 & 28-Nov-89 & & $10028-17-8$ & TRITIUM & TRITIÜM_ELECT_LSC & USTEST & 61 \\
\hline 6979 & PDMS & & SW & & & E110961 & 28-Nov-89 & & $10098-97-2$ & SR-90 & SRISO_SEP_PRECIP_GPC & USTEST & 0.0521 \\
\hline 6980 & PDMS & & SW & & & E111004 & 28-Nov-89 & & $10045-97-3$ & CS-137 & GAMMA_GS & USTEST & 0.00397 \\
\hline 6981 & PDMS & & SW & & & E111007 & 28-Nov-89 & & $10045-97-3$ & CS-137 & GAMMA_GS & USTEST & 0.000778 \\
\hline 6982 & PDMS & & SW & & & E110962 & 28-Nov-89 & & $10045-97-3$ & CS-137 & GAMMA_GS & USTEST & -0.399 \\
\hline 6983 & PDMS & & SW & & & E110962 & 28-Nov-89 & & 15117-96-1 & U-235 & UISO_AĒA & USTEST & -0.00095 \\
\hline 6984 & PDMS & & SW & & & E110962 & 28-Nov-89 & & $13966-29-5$ & U-234 & UISO_AEA & USTEST & 0.0766 \\
\hline 6985 & PDMS & & SW & & & E110962 & 28-Nov-89 & & $14133-76-7$ & TC-99 & TC99_SEP_LSC & USTEST & -1.86 \\
\hline \begin{tabular}{|c|}
6986 \\
\end{tabular} & PDMS & & SW & & & E110962 & 28-Nov-89 & & $10028-17-8$ & TRITIUM & TRITIUMM_ELECT_LSC & USTEST & 108 \\
\hline \begin{tabular}{|c|}
6987 \\
\end{tabular} & PDMS & & SW & & & E110962 & 28-Nov-89 & & $10098-97-2$ & SR-90 & SRISO_SEPPPRËCIP_GPC & USTEST & 0.0339 \\
\hline 6988 & PDMS & & SW & & & E110962 & 28-Nov-89 & & $\mathrm{U}-238$ & U-238 & UISO_AEEA & USTEST & 0.0314 \\
\hline 6989 & PDMS & & SW & & & E111008 & 28-Nov-89 & & $10045-97-3$ & CS-137 & GAMMA_GS & USTEST & 0.00314 \\
\hline 6990 & PDMS & & SW & & & E111005 & 28-Nov-89 & & $10045-97-3$ & CS-137 & GAMMA_GS & USTEST & 0.0014 \\
\hline 6991 & PDMS & & SW & & & E111017 & 04-Dec-89 & & $10028-17-8$ & TRITIUM & 906.0_H3_LSC & USTEST & 7890 \\
\hline 6992 & PDMS & & SW & & & E111017 & 04-Dec-89 & & $10045-97-3$ & CS-137 & 901.1_GAMMA_GS & USTEST & 0.209 \\
\hline 6993 & PDMS & & SW & & & E111017 & 04-Dec-89 & & $10098-97-2$ & SR-90 & 905.0_SR_GPC & USTEST & 0.00628 \\
\hline 6994 & PDMS & & SW & & & E111014 & 05-Dec-89 & & 10028-17-8 & TRITIUM & TRITIUMM_DIST_LSC & USTEST & 165 \\
\hline 6995 & PDMS & & SW & & & E111014 & 05-Dec-89 & & $10098-97-2$ & SR-90 & SRISO_SEP_PERECIP_GPC & USTEST & 0.267 \\
\hline 6996 & PDMS & & SW & & & E111014 & 05-Dec-89 & & $10045-97-3$ & CS-137 & GAMMĀGS & USTEST & 0.139 \\
\hline 6997 & PDMS & & SW & & & E111016 & 05-Dec-89 & & $10045-97-3$ & CS-137 & GAMMA_GS & USTEST & -1.35 \\
\hline 6998 & PDMS & & SW & & & E111016 & 05-Dec-89 & & $10028-17-8$ & TRITIUM & TRITIUM_DIST_LSC & USTEST & 7440 \\
\hline 6999 & PDMS & & SW & & & E111015 & 05-Dec-89 & & $10045-97-3$ & CS-137 & GAMMA_GS & USTEST & 0.0678 \\
\hline 7000 & PDMS & & SW & & & E111015 & 05-Dec-89 & & $\mathrm{U}-238$ & U-238 & UISO_AĒA & USTEST & 110 \\
\hline 7001 & PDMS & & SW & & & E111015 & 05-Dec-89 & & 15117-96-1 & U-235 & UISO_AEA & USTEST & 5.33 \\
\hline 7002 & PDMS & & SW & & & E111015 & 05-Dec-89 & & $13966-29-5$ & U-234 & UISO_AEA & USTEST & 121 \\
\hline 7003 & PDMS & & SW & & & E111015 & 05-Dec-89 & & $10028-17-8$ & TRITIUM & TRITIUM_DIST_LSC & USTEST & 472 \\
\hline 7004 & PDMS & & SW & & & E111015 & 05-Dec-89 & & $10098-97-2$ & SR-90 & SRISO_SEP_PRECIP_GPC & USTEST & 1.45 \\
\hline 7005 & PDMS & & SW & & & E111303 & 12-Dec-89 & & $10045-97-3$ & CS-137 & GAMMA_GS & USTEST & \\
\hline \begin{tabular}{|l|}
7006 \\
\end{tabular} & PDMS & & SW & & & E111302 & 12-Dec-89 & & $10045-97-3$ & CS-137 & GAMMA_GS & USTEST & \\
\hline 7007 & PDMS & & SW & & & E111242 & 12-Dec-89 & & $10045-97-3$ & CS-137 & GAMMA_GS & USTEST & 0.000939 \\
\hline \begin{tabular}{|c|}
7008 \\
\end{tabular} & PDMS & & SW & & & E111152 & 12-Dec-89 & & $10045-97-3$ & CS-137 & GAMMA_GS & USTEST & 0.00512 \\
\hline 7009 & PDMS & & SW & & & E111151 & 12-Dec-89 & & $10045-97-3$ & CS-137 & GAMMA_GS & USTEST & 0.00148 \\
\hline 7010 & PDMS & & SW & & & E111243 & 12-Dec-89 & & $10045-97-3$ & CS-137 & GAMMA_GS & USTEST & 0.00189 \\
\hline 7011 & PDMS & & SW & & & E111159 & 19-Dec-89 & & $10045-97-3$ & CS-137 & 901.1_GAMMA_GS & USTEST & 0.0503 \\
\hline 7012 & PDMS & & SW & & & E111159 & 19-Dec-89 & & $10098-97-2$ & SR-90 & 905.0_SR_GPC & USTEST & 0.0757 \\
\hline 7013 & PDMS & & SW & & & E111159 & 19-Dec-89 & & $10028-17-8$ & TRITIUM & TRITIÜM_ELECT_LSC & USTEST & 119 \\
\hline 7014 & PDMS & & SW & & & E111159 & 19-Dec-89 & & 14133-76-7 & TC-99 & TC99_SEP_LSC & USTEST & -2.74 \\
\hline 7015 & PDMS & & SW & & & E111159 & 19-Dec-89 & & $\mathrm{U}-238$ & U-238 & UISO_IE_PLATE_AEA & USTEST & 0.292 \\
\hline \begin{tabular}{|c|}
7016 \\
\end{tabular} & PDMS & & SW & & & E111159 & 19-Dec-89 & & 15117-96-1 & U-235 & UISO_IE_PLATE_AEA & USTEST & 0.04 \\
\hline 7017 & PDMS & & SW & & & E111159 & 19-Dec-89 & & $13966-29-5$ & U-234 & UISO_IE_PLATE_AEA & USTEST & 0.435 \\
\hline 7018 & PDMS & & SW & & & E111295 & 28-Dec-89 & & PU-239/240 & PU-239/240 & PUISO_IE_AEA & USTEST & 0.0000135 \\
\hline 7019 & PDMS & & SW & & & E111295 & 28-Dec-89 & & $10045-97-3$ & CS-137 & GAMMA_GS & USTEST & 0.0000873 \\
\hline 7020 & PDMS & & SW & & & E111298 & 28-Dec-89 & & $10045-97-3$ & CS-137 & GAMMA_GS & USTEST & 0.00303 \\
\hline 7021 & PDMS & & SW & & & E111298 & 28-Dec-89 & & PU-239/240 & PU-239/240 & PUISO_IE_AEA & USTEST & -0.0000148 \\
\hline
\end{tabular}




\begin{tabular}{|c|c|c|c|c|c|c|c|c|c|c|c|c|c|}
\hline & A & $\mathrm{B}$ & $\mathrm{C}$ & $\mathrm{D}$ & $\mathrm{E}$ & $\mathrm{F}$ & $\bar{G}$ & $\mathrm{H}$ & $\mathrm{I}$ & $\mathrm{J}$ & $\mathrm{K}$ & $\mathrm{L}$ & $\mathrm{M}$ \\
\hline 1 & \begin{tabular}{|l|} 
SAMPE_NUM_ASSIGNED_TO \\
\end{tabular} & SAMPLER & MEDIA & BIOTA_MEDIA & TAG_ID & SAMP_NUM & SAMP_DATE_TIME & SAMP_DATE_TIME_ON & CON_ID & CON_SHORT_NAME & METHOD_NAME & LAB_CODE & VALUE_RPTD \\
\hline 7022 & PDMS & & SW & & & E111298 & 28-Dec-89 & & $15046-84-1$ & $1-129$ & I129_SEP_TEMS & 320 & 0.0000887 \\
\hline 7023 & PDMS & & SW & & & E111299 & 28-Dec-89 & & $10045-97-3$ & CS-137 & GAMMA_Ḡ'S & USTEST & 0.000997 \\
\hline 7024 & PDMS & & SW & & & E111299 & 28-Dec-89 & & PU-239/240 & PU-239/240 & PUISO_IE_AEA & USTEST & -0.0000125 \\
\hline 7025 & PDMS & & SW & & & E111296 & 28-Dec-89 & & PU-239/240 & PU-239/240 & PUISO_IE_AEA & USTEST & 0.0000217 \\
\hline 7026 & PDMS & & SW & & & E111299 & 28-Dec-89 & & 15046-84-1 & I-129 & 1129_SEP_TEMS & 320 & 0.0000042 \\
\hline 7027 & PDMS & & SW & & & E111296 & 28-Dec-89 & & $10045-97-3$ & CS-137 & GAMMA_ḠS & USTEST & 0.00109 \\
\hline 7028 & PDMS & & SW & & & E111297 & 28-Dec-89 & & PU-239/240 & PU-239/240 & PUISO_IE_AEA & USTEST & 0.0000239 \\
\hline 7029 & PDMS & & SW & & & E111297 & 28-Dec-89 & & $10045-97-3$ & CS-137 & GAMMA_GS & USTEST & 0.00176 \\
\hline 7030 & PDMS & & SW & & & E111300 & 28-Dec-89 & & $10045-97-3$ & CS-137 & GAMMA_GS & USTEST & 0.00475 \\
\hline 7031 & PDMS & & SW & & & E111300 & 28-Dec-89 & & PU-239/240 & PU-239/240 & PUISO_IE_AEA & USTEST & 0.0000458 \\
\hline \begin{tabular}{|c|}
7032 \\
\end{tabular} & PDMS & & SW & & & E111300 & 28-Dec-89 & & $15046-84-1$ & $1-129$ & I129_SEP_TEMS & 320 & 0.00005 \\
\hline
\end{tabular}




\begin{tabular}{|c|c|c|c|c|c|c|c|c|c|c|c|c|}
\hline & $\mathrm{N}$ & $\mathrm{O}$ & $\mathrm{P}$ & $\mathrm{Q}$ & $\mathrm{R}$ & $\mathrm{s}$ & $T$ & $\bar{U}$ & $\mathrm{~V}$ & $\mathrm{~W}$ & $\mathrm{X}$ & $\bar{Y}$ \\
\hline 1 & ANAL_UNITS_RPTD & COUNTING_ERROR & TOTAL_ANAL_ERROR & MIN_DETECTABLE_ACTIVITY & PCNT_MOISTURE & LAB_QUALIFIER & REVIEW_QUALIFIER & SAMP_FROM & SAMP_ITEM & SAMP_MTHD & COLL_MTHD & SAMP_SITE_ID \\
\hline 2 & $\mathrm{pCi} / \mathrm{L}$ & 0 & & & & & & SURFACE & & RAW GRAB & UNFILTERED & 444 \\
\hline 3 & ug/L & 0 & & & & & & SANITARY & & CUMULATIVE & UNFILTERED & 385 \\
\hline 4 & UNKNOWN & 0 & & & & U & & RIVER & & RAW GRAB & UNFILTERED & 461 \\
\hline 5 & UNKNOWN & 0 & & & & & & RIVER & & RAW GRAB & UNFILTERED & 456 \\
\hline 6 & $\mathrm{ug} / \mathrm{L}$ & 0 & & & & & & SANITARY & & CUMULATIVE & UNFILTERED & 491 \\
\hline 7 & $\mathrm{pCi} / \mathrm{L}$ & 0 & & & & & & SURFACE & & RAW GRAB & UNFILTERED & 444 \\
\hline 8 & $u g / L$ & 0 & & & & & & RIVER & & RAW GRAB & UNFILTERED & 456 \\
\hline 9 & $\mathrm{ug} / \mathrm{L}$ & 0 & & & & & & RIVER & & RAW GRAB & UNFILTERED & 456 \\
\hline 10 & $\mathrm{ug} / \mathrm{L}$ & 0 & & & & & & SANITARY & & CUMULATIVE & UNFILTERED & 491 \\
\hline 11 & $\mathrm{ug} / \mathrm{L}$ & 0 & & & & & & SANITARY & & CUMULATIVE & UNFILTERED & 385 \\
\hline 12 & UNKNOWN & 0 & & & & & & RIVER & & RAW GRAB & UNFILTERED & 461 \\
\hline 13 & $\mathrm{pCi} / \mathrm{L}$ & 0 & & & & & & RIVER & & CUMULATIVE & UNFILTERED & 463 \\
\hline 14 & UNKNOWN & 0 & & & & & & RIVER & & RAW GRAB & UNFILTERED & 456 \\
\hline 15 & $\mathrm{ug} / \mathrm{L}$ & 0 & & & & & & RIVER & & RAW GRAB & UNFILTERED & 456 \\
\hline 16 & $\mathrm{pCi} / \mathrm{L}$ & 0 & & & & & & SURFACE & & RAW GRAB & UNFILTERED & 121 \\
\hline 17 & $\mathrm{pCi} / \mathrm{L}$ & 0 & & & & & & SURFACE & & RAW GRAB & UNFILTERED & 484 \\
\hline 18 & $\mathrm{pCi} / \mathrm{L}$ & 0 & & & & U & & SURFACE & & RAW GRAB & UNFILTERED & 509 \\
\hline 19 & $\mathrm{pCi} / \mathrm{L}$ & 0 & & & & & & SURFACE & & RAW GRAB & UNFILTERED & 146 \\
\hline 20 & $\mathrm{pCi} / \mathrm{L}$ & 0 & & & & U & & SURFACE & & RAW GRAB & UNFILTERED & 601 \\
\hline 21 & $\mathrm{pCi} / \mathrm{L}$ & 0 & & & & & & SURFACE & & RAW GRAB & UNFILTERED & 413 \\
\hline 22 & $\mathrm{pCi} / \mathrm{L}$ & 0 & & & & & & SURFACE & & RAW GRAB & UNFILTERED & 412 \\
\hline 23 & ug/L & 0 & & & & & & SANITARY & & CUMULATIVE & UNFILTERED & 385 \\
\hline 24 & $\mathrm{pCi} / \mathrm{L}$ & 0 & & & & $U$ & & SURFACE & & CUMULATIVE & UNFILTERED & 60 \\
\hline 25 & $\mathrm{pCi} / \mathrm{L}$ & 0 & & & & & & RIVER & & CUMULATIVE & UNFILTERED & 463 \\
\hline 26 & $\mathrm{pCi} / \mathrm{L}$ & 0 & & & & & & RIVER & & CUMULATIVE & UNFILTERED & 463 \\
\hline 27 & $\mathrm{pCi} / \mathrm{L}$ & 0 & & & & & & RIVER & & CUMULATIVE & UNFILTERED & 463 \\
\hline 28 & $\mathrm{pCi} / \mathrm{L}$ & 0 & & & & & & RIVER & & RAW GRAB & UNFILTERED & 456 \\
\hline 29 & $u g / L$ & 0 & & & & & & RIVER & & RAW GRAB & UNFILTERED & 456 \\
\hline 30 & $\mathrm{pCi} / \mathrm{L}$ & 0 & & & & & & RIVER & & RAW GRAB & UNFILTERED & 456 \\
\hline 31 & ug/L & 0 & & & & & & SANITARY & & CUMULATIVE & UNFILTERED & 491 \\
\hline 32 & UNKNOWN & 0 & & & & & & RIVER & & RAW GRAB & UNFILTERED & 461 \\
\hline 33 & $\mathrm{pCi} / \mathrm{L}$ & 0 & & & & U & & RIVER & & CUMULATIVE & UNFILTERED & 463 \\
\hline 34 & UNKNOWN & 0 & & & & & & RIVER & & RAW GRAB & UNFILTERED & 456 \\
\hline 35 & $u g / L$ & 0 & & & & & & RIVER & & RAW GRAB & UNFILTERED & 456 \\
\hline 36 & $\mathrm{ug} / \mathrm{L}$ & 0 & & & & & & SANITARY & & CUMULATIVE & UNFILTERED & 385 \\
\hline 37 & $\mathrm{ug} / \mathrm{L}$ & 0 & & & & & & SANITARY & & CUMULATIVE & UNFILTERED & 491 \\
\hline 38 & ug/L & 0 & & & & & & SANITARY & & CUMULATIVE & UNFILTERED & 385 \\
\hline 39 & $\mathrm{ug} / \mathrm{L}$ & 0 & & & & & & RIVER & & RAW GRAB & UNFILTERED & 456 \\
\hline 40 & $\mathrm{ug} / \mathrm{L}$ & 0 & & & & & & SANITARY & & CUMULATIVE & UNFILTERED & 491 \\
\hline 41 & $\mathrm{ug} / \mathrm{L}$ & 0 & & & & & & SANITARY & & CUMULATIVE & UNFILTERED & 385 \\
\hline 42 & ug/L & 0 & & & & & & RIVER & & RAW GRAB & UNFILTERED & 456 \\
\hline 43 & $\mathrm{ug} / \mathrm{L}$ & 0 & & & & & & SANITARY & & CUMULATIVE & UNFILTERED & 491 \\
\hline 44 & UNKNOWN & 0 & & & & & & RIVER & & RAW GRAB & UNFILTERED & 461 \\
\hline 45 & UNKNOWN & 0 & & & & & & RIVER & & RAW GRAB & UNFILTERED & 456 \\
\hline 46 & $\mathrm{pCi} / \mathrm{L}$ & 0 & & & & & & RIVER & & CUMULATIVE & UNFILTERED & 463 \\
\hline 47 & $\mathrm{pCi} / \mathrm{L}$ & 0 & & & & & & RIVER & & CUMULATIVE & UNFILTERED & 463 \\
\hline 48 & $\mathrm{pCi} / \mathrm{L}$ & 0 & & & & & & RIVER & & RAW GRAB & UNFILTERED & 456 \\
\hline 49 & ug/L & 0 & & & & & & RIVER & & RAW GRAB & UNFILTERED & 456 \\
\hline 50 & $\mathrm{pCi} / \mathrm{L}$ & 0 & & & & & & RIVER & & RAW GRAB & UNFILTERED & 456 \\
\hline 51 & ug/L & 0 & & & & & & SANITARY & & CUMULATIVE & UNFILTERED & 491 \\
\hline 52 & UNKNOWN & 0 & & & & & & RIVER & & RAW GRAB & UNFILTERED & 461 \\
\hline 53 & UNKNOWN & 0 & & & & & & RIVER & & RAW GRAB & UNFILTERED & 456 \\
\hline
\end{tabular}




\begin{tabular}{|c|c|c|c|c|c|c|c|c|c|c|c|c|}
\hline & $\mathrm{N}$ & $\mathrm{O}$ & $\mathrm{P}$ & $\mathrm{Q}$ & $\mathrm{R}$ & $\mathrm{S}$ & $\mathrm{T}$ & $\mathrm{U}$ & $\mathrm{V}$ & $\mathrm{w}$ & $\mathrm{X}$ & $\mathrm{Y}$ \\
\hline 1 & ANAL_UNITS_RPTD & COUNTING_ERROR & TOTAL_ANAL_ERROR & MIN_DETECTABLE_ACTIVITY & PCNT_MOISTURE & LAB_QUALIFIER & REVIEW_QUALIFIER & SAMP_FROM & SAMP_ITEM & SAMP_MTHD & COLL_MTHD & SAMP_SITE_ID \\
\hline 54 & ug/L & 0 & & & & & & RIVER & & RAW GRAB & UNFILTERED & 456 \\
\hline 55 & ug/L & 0 & & & & $\mathrm{U}$ & & SANITARY & & CUMULATIVE & UNFILTERED & 491 \\
\hline 56 & ug/L & 0 & & & & & & SANITARY & & CUMULATIVE & UNFILTERED & 385 \\
\hline 57 & ug/L & 0 & & & & & & RIVER & & RAW GRAB & UNFILTERED & 456 \\
\hline 58 & ug/L & 0 & & & & & & SANITARY & & CUMULATIVE & UNFILTERED & 491 \\
\hline 59 & ug/L & 0 & & & & & & SANITARY & & CUMULATIVE & UNFILTERED & 385 \\
\hline 60 & UNKNOWN & 0 & & & & & & RIVER & & RAW GRAB & UNFILTERED & 461 \\
\hline 61 & UNKNOWN & 0 & & & & & & RIVER & & RAW GRAB & UNFILTERED & 456 \\
\hline 62 & ug/L & 0 & & & & & & RIVER & & RAW GRAB & UNFILTERED & 456 \\
\hline 63 & $\mathrm{ug} / \mathrm{L}$ & 0 & & & & U & & SANITARY & & CUMULATIVE & UNFILTERED & 491 \\
\hline 64 & $\mathrm{pCi} / \mathrm{L}$ & 0 & & & & & & SURFACE & & RAW GRAB & UNFILTERED & 444 \\
\hline 65 & ug/L & 0 & & & & & & SANITARY & & CUMULATIVE & UNFILTERED & 385 \\
\hline 66 & $\mathrm{pCi} / \mathrm{L}$ & 0 & & & & & & SURFACE & & CUMULATIVE & UNFILTERED & 60 \\
\hline 67 & $\mathrm{pCi} / \mathrm{L}$ & 0 & & & & & & RIVER & & CUMULATIVE & UNFILTERED & 463 \\
\hline 68 & $\mathrm{pCi} / \mathrm{L}$ & 0 & & & & & & RIVER & & CUMULATIVE & UNFILTERED & 463 \\
\hline 69 & $\mathrm{pCi} / \mathrm{L}$ & 0 & & & & & & RIVER & & RAW GRAB & UNFILTERED & 456 \\
\hline 70 & ug/L & 0 & & & & & & RIVER & & RAW GRAB & UNFILTERED & 456 \\
\hline 71 & $\mathrm{pCi} / \mathrm{L}$ & 0 & & & & & & RIVER & & RAW GRAB & UNFILTERED & 456 \\
\hline 72 & ug/L & 0 & & & & & & SANITARY & & CUMULATIVE & UNFILTERED & 491 \\
\hline 73 & ug/L & 0 & & & & & & SANITARY & & CUMULATIVE & UNFILTERED & 385 \\
\hline 74 & UNKNOWN & 0 & & & & & & RIVER & & RAW GRAB & UNFILTERED & 456 \\
\hline 75 & ug/L & 0 & & & & & & SANITARY & & CUMULATIVE & UNFILTERED & 491 \\
\hline 76 & UNKNOWN & 0 & & & & & & RIVER & & RAW GRAB & UNFILTERED & 461 \\
\hline 77 & ug/L & 0 & & & & & & SANITARY & & CUMULATIVE & UNFILTERED & 385 \\
\hline 78 & ug/L & 0 & & & & & & SANITARY & & CUMULATIVE & UNFILTERED & 491 \\
\hline 79 & ug/L & 0 & & & & & & SANITARY & & CUMULATIVE & UNFILTERED & 385 \\
\hline 80 & UNKNOWN & 0 & & & & & & RIVER & & RAW GRAB & UNFILTERED & 461 \\
\hline 81 & UNKNOWN & 0 & & & & & & RIVER & & RAW GRAB & UNFILTERED & 456 \\
\hline 82 & ug/L & 0 & & & & & & SANITARY & & CUMULATIVE & UNFILTERED & 491 \\
\hline 83 & $\mathrm{pCi} / \mathrm{L}$ & 0 & & & & & & SURFACE & & RAW GRAB & UNFILTERED & 444 \\
\hline 84 & $\mathrm{pCi} / \mathrm{L}$ & 0 & & & & & & SURFACE & & RAW GRAB & UNFILTERED & 484 \\
\hline 85 & $\mathrm{pCi} / \mathrm{L}$ & 0 & & & & & & SURFACE & & RAW GRAB & UNFILTERED & 509 \\
\hline 86 & $\mathrm{pCi} / \mathrm{L}$ & 0 & & & & & & SURFACE & & RAW GRAB & UNFILTERED & 146 \\
\hline 87 & $\mathrm{pCi} / \mathrm{L}$ & 0 & & & & & & SURFACE & & RAW GRAB & UNFILTERED & 601 \\
\hline 88 & $\mathrm{pCi} / \mathrm{L}$ & 0 & & & & & & SURFACE & & RAW GRAB & UNFILTERED & 413 \\
\hline 89 & $\mathrm{pCi} / \mathrm{L}$ & 0 & & & & & & SURFACE & & RAW GRAB & UNFILTERED & 412 \\
\hline 90 & ug/L & 0 & & & & & & SANITARY & & CUMULATIVE & UNFILTERED & 385 \\
\hline 91 & ug/L & 0 & & & & & & SANITARY & & CUMULATIVE & UNFILTERED & 491 \\
\hline 92 & ug/L & 0 & & & & & & SANITARY & & CUMULATIVE & UNFILTERED & 385 \\
\hline 93 & $\mathrm{pCi} / \mathrm{L}$ & 0 & & & & U & & RIVER & & CUMULATIVE & UNFILTERED & 463 \\
\hline 94 & $\mathrm{pCi} / \mathrm{L}$ & 0 & & & & & & RIVER & & CUMULATIVE & UNFILTERED & 463 \\
\hline 95 & $\mathrm{pCi} / \mathrm{L}$ & 0 & & & & & & RIVER & & RAW GRAB & UNFILTERED & 456 \\
\hline 96 & $\mathrm{pCi} / \mathrm{L}$ & 0 & & & & & & RIVER & & RAW GRAB & UNFILTERED & 456 \\
\hline 97 & ug/L & 0 & & & & & & SANITARY & & CUMULATIVE & UNFILTERED & 491 \\
\hline 98 & ug/L & 0 & & & & & & SANITARY & & CUMULATIVE & UNFILTERED & 385 \\
\hline 99 & UNKNOWN & 0 & & & & & & RIVER & & RAW GRAB & UNFILTERED & 461 \\
\hline 100 & UNKNOWN & 0 & & & & & & RIVER & & RAW GRAB & UNFILTERED & 456 \\
\hline 101 & ug/L & 0 & & & & & & SANITARY & & CUMULATIVE & UNFILTERED & 491 \\
\hline 102 & ug/L & 0 & & & & & & SANITARY & & CUMULATIVE & UNFILTERED & 385 \\
\hline 103 & ug/L & 0 & & & & & & SANITARY & & CUMULATIVE & UNFILTERED & 491 \\
\hline 104 & ug/L & 0 & & & & & & SANITARY & & CUMULATIVE & UNFILTERED & 385 \\
\hline 105 & UNKNOWN & 0 & & & & & & RIVER & & RAW GRAB & UNFILTERED & 461 \\
\hline
\end{tabular}




\begin{tabular}{|c|c|c|c|c|c|c|c|c|c|c|c|c|c|}
\hline & $\mathrm{N}$ & $\mathrm{O}$ & & $\mathrm{P}$ & $\mathrm{Q}$ & $\mathrm{R}$ & $\mathrm{s}$ & $\mathrm{T}$ & $\bar{U}$ & $\mathrm{~V}$ & $\mathrm{~W}$ & $\mathrm{x}$ & $\bar{Y}$ \\
\hline 1 & ANAL_UNITS_RPTD & COUNTING & ERROR & TOTAL_ANAL_ERROR & MIN_DETECTABLE_ACTIVITT & PCNT_MOISTURE & LAB_QUALIFIER & REVIEW_QUALIFIER & SAMP_FROM & SAMP_ITEM & SAMP_MTHD & COLL_MTHD & SAMP_SITE_ID \\
\hline 106 & UNKNOWN & & 0 & & & & & & RIVER & & RAW GRAB & UNFILTERED & 456 \\
\hline 107 & ug/L & & 0 & & & & & & SANITARY & & CUMULATIVE & UNFILTERED & 491 \\
\hline 108 & $\mathrm{pCi} / \mathrm{L}$ & & 0 & & & & & & SURFACE & & RAW GRAB & UNFILTERED & 444 \\
\hline 109 & $\mathrm{pCi} / \mathrm{L}$ & & 0 & & & & & & SURFACE & & CUMULATIVE & UNFILTERED & 60 \\
\hline 110 & $\mathrm{ug} / \mathrm{L}$ & & 0 & & & & & & SANITARY & & CUMULATIVE & UNFILTERED & 385 \\
\hline 111 & $\mathrm{pCi} / \mathrm{L}$ & & 0 & & & & & & RIVER & & CUMULATIVE & UNFILTERED & 463 \\
\hline 112 & $\mathrm{pCi} / \mathrm{L}$ & & 0 & & & & & & RIVER & & CUMULATIVE & UNFILTERED & 463 \\
\hline 113 & $\mathrm{pCi} / \mathrm{L}$ & & 0 & & & & & & RIVER & & RAW GRAB & UNFILTERED & 456 \\
\hline 114 & $\mathrm{pCi} / \mathrm{L}$ & & 0 & & & & & & RIVER & & RAW GRAB & UNFILTERED & 456 \\
\hline 115 & $\mathrm{ug} / \mathrm{L}$ & & 0 & & & & & & SANITARY & & CUMULATIVE & UNFILTERED & 491 \\
\hline 116 & UNKNOWN & & 0 & & & & & & RIVER & & RAW GRAB & UNFILTERED & 461 \\
\hline 117 & UNKNOWN & & 0 & & & & & & RIVER & & RAW GRAB & UNFILTERED & 456 \\
\hline 118 & ug/L & & 0 & & & & & & SANITARY & & CUMULATIVE & UNFILTERED & 385 \\
\hline 119 & ug/L & & 0 & & & & & & SANITARY & & CUMULATIVE & UNFILTERED & 491 \\
\hline 120 & $\mathrm{ug} / \mathrm{L}$ & & 0 & & & & & & SANITARY & & CUMULATIVE & UNFILTERED & 385 \\
\hline 121 & ug/L & & 0 & & & & & & SANITARY & & CUMULATIVE & UNFILTERED & 491 \\
\hline 122 & ug/L & & 0 & & & & & & SANITARY & & CUMULATIVE & UNFILTERED & 385 \\
\hline 123 & pCi/L & & 0 & & & & & & SURFACE & & CUMULATIVE & UNFILTERED & 60 \\
\hline 124 & UNKNOWN & & 0 & & & & & & RIVER & & RAW GRAB & UNFILTERED & 461 \\
\hline 125 & UNKNOWN & & 0 & & & & & & RIVER & & RAW GRAB & UNFILTERED & 456 \\
\hline 126 & ug/L & & 0 & & & & & & SANITARY & & CUMULATIVE & UNFILTERED & 491 \\
\hline 127 & pCi/L & & 0 & & & & & & SURFACE & & RAW GRAB & UNFILTERED & 444 \\
\hline 128 & $\mathrm{pCi} / \mathrm{L}$ & & 0 & & & & & & SURFACE & & RAW GRAB & UNFILTERED & 67 \\
\hline 129 & ug/L & & 0 & & & & & & SANITARY & & CUMULATIVE & UNFILTERED & 385 \\
\hline 130 & pCi/L & & 0 & & & & & & RIVER & & CUMULATIVE & UNFILTERED & 463 \\
\hline 131 & $\mathrm{pCi} / \mathrm{L}$ & & 0 & & & & & & RIVER & & CUMULATIVE & UNFILTERED & 463 \\
\hline 132 & $\mathrm{pCi} / \mathrm{L}$ & & 0 & & & & & & RIVER & & RAW GRAB & UNFILTERED & 456 \\
\hline 133 & $\mathrm{pCi} / \mathrm{L}$ & & 0 & & & & & & RIVER & & RAW GRAB & UNFILTERED & 456 \\
\hline 134 & $\mathrm{ug} / \mathrm{L}$ & & 0 & & & & & & SANITARY & & CUMULATIVE & UNFILTERED & 491 \\
\hline 135 & ug/L & & 0 & & & & & & SANITARY & & CUMULATIVE & UNFILTERED & 385 \\
\hline 136 & UNKNOWN & & 0 & & & & & & RIVER & & RAW GRAB & UNFILTERED & 461 \\
\hline 137 & UNKNOWN & & 0 & & & & & & RIVER & & RAW GRAB & UNFILTERED & 456 \\
\hline 138 & ug/L & & 0 & & & & & & SANITARY & & CUMULATIVE & UNFILTERED & 491 \\
\hline 139 & $\mathrm{ug} / \mathrm{L}$ & & 0 & & & & & & SANITARY & & CUMULATIVE & UNFILTERED & 385 \\
\hline 140 & ug/L & & 0 & & & & & & SANITARY & & CUMULATIVE & UNFILTERED & 385 \\
\hline 141 & pCi/L & & 0 & & & & & & SURFACE & & CUMULATIVE & UNFILTERED & 60 \\
\hline 142 & $\mathrm{pCi} / \mathrm{L}$ & & 0 & & & & & & SEEP & & RAW GRAB & UNFILTERED & 556 \\
\hline 143 & $\mathrm{pCi} / \mathrm{L}$ & & 0 & & & & & & SURFACE & & RAW GRAB & UNFILTERED & 121 \\
\hline 144 & $\mathrm{pCi} / \mathrm{L}$ & & 0 & & & & & & SURFACE & & RAW GRAB & UNFILTERED & 484 \\
\hline 145 & $\mathrm{pCi} / \mathrm{L}$ & & 0 & & & & & & SURFACE & & RAW GRAB & UNFILTERED & 509 \\
\hline 146 & $\mathrm{pCi} / \mathrm{L}$ & & 0 & & & & & & SURFACE & & RAW GRAB & UNFILTERED & 146 \\
\hline 147 & $\mathrm{pCi} / \mathrm{L}$ & & 0 & & & & & & SURFACE & & RAW GRAB & UNFILTERED & 413 \\
\hline 148 & $\mathrm{pCi} / \mathrm{L}$ & & 0 & & & & & & SURFACE & & RAW GRAB & UNFILTERED & 412 \\
\hline 149 & $\mathrm{ug} / \mathrm{L}$ & & 0 & & & & & & SANITARY & & CUMULATIVE & UNFILTERED & 385 \\
\hline 150 & $\mathrm{ug} / \mathrm{L}$ & & 0 & & & & & & SANITARY & & CUMULATIVE & UNFILTERED & 385 \\
\hline 151 & $\mathrm{pCi} / \mathrm{L}$ & & 0 & & & & & & RIVER & & CUMULATIVE & UNFILTERED & 463 \\
\hline 152 & $\mathrm{pCi} / \mathrm{L}$ & & 0 & & & & & & RIVER & & CUMULATIVE & UNFILTERED & 463 \\
\hline 153 & $\mathrm{pCi} / \mathrm{L}$ & & 0 & & & & & & RIVER & & CUMULATIVE & UNFILTERED & 463 \\
\hline 154 & $\mathrm{pCi} / \mathrm{L}$ & & 0 & & & & & & RIVER & & RAW GRAB & UNFILTERED & 456 \\
\hline 155 & $\mathrm{pCi} / \mathrm{L}$ & & 0 & & & & & & RIVER & & RAW GRAB & UNFILTERED & 456 \\
\hline 156 & $\mathrm{pCi} / \mathrm{L}$ & & 0 & & & & & & RIVER & & RAW GRAB & UNFILTERED & 456 \\
\hline 157 & $\mathrm{ug} / \mathrm{L}$ & & 0 & & & & & & SANITARY & & CUMULATIVE & UNFILTERED & 385 \\
\hline
\end{tabular}




\begin{tabular}{|c|c|c|c|c|c|c|c|c|c|c|c|c|}
\hline & $\mathrm{N}$ & $\mathrm{O}$ & $\mathrm{P}$ & $\mathrm{Q}$ & $\mathrm{R}$ & $\mathrm{S}$ & $T$ & $U$ & $\mathrm{~V}$ & $\overline{\mathrm{W}}$ & $\mathrm{X}$ & $\mathrm{Y}$ \\
\hline 1 & ANAL_UNITS_RPTD & COUNTING_ERROR & TOTAL_ANAL_ERROR & MIN_DETECTABLE_ACTIVITY & PCNT_MOISTURE & LAB_QUALIFIER & REVIEW_QUALIFIER & SAMP_FROM & SAMP_ITEM & SAMP_MTHD & COLL_MTHD & SAMP_SITE_ID \\
\hline 158 & ug/L & 0 & & & & & & SANITARY & & CUMULATIVE & UNFILTERED & 385 \\
\hline 159 & ug/L & 0 & & & & & & SANITARY & & CUMULATIVE & UNFILTERED & 385 \\
\hline 160 & $\mathrm{pCi} / \mathrm{L}$ & 0 & & & & & & SURFACE & & RAW GRAB & UNFILTERED & 444 \\
\hline 161 & $\mathrm{pCi} / \mathrm{L}$ & 0 & & & & & & RIVER & & RAW GRAB & UNFILTERED & 277 \\
\hline 162 & ug/L & 0 & & & & & & SANITARY & & CUMULATIVE & UNFILTERED & 385 \\
\hline 163 & $\mathrm{pCi} / \mathrm{L}$ & 0 & & & & & & RIVER & & CUMULATIVE & UNFILTERED & 463 \\
\hline 164 & $\mathrm{pCi} / \mathrm{L}$ & 0 & & & & & & RIVER & & CUMULATIVE & UNFILTERED & 463 \\
\hline 165 & $\mathrm{pCi} / \mathrm{L}$ & 0 & & & & & & RIVER & & CUMULATIVE & UNFILTERED & 463 \\
\hline 166 & $\mathrm{pCi} / \mathrm{L}$ & 0 & & & & & & RIVER & & RAW GRAB & UNFILTERED & 456 \\
\hline 167 & pCi/L & 0 & & & & & & RIVER & & RAW GRAB & UNFILTERED & 456 \\
\hline 168 & UNKNOWN & 0 & & & & & & RIVER & & RAW GRAB & UNFILTERED & 310 \\
\hline 169 & ug/L & 0 & & & & & & RIVER & & RAW GRAB & UNFILTERED & 463 \\
\hline 170 & ug/L & 0 & & & & & & SANITARY & & CUMULATIVE & UNFILTERED & 385 \\
\hline 171 & ug/L & 0 & & & & & & SANITARY & & CUMULATIVE & UNFILTERED & 385 \\
\hline 172 & ug/L & 0 & & & & & & SANITARY & & CUMULATIVE & UNFILTERED & 385 \\
\hline 173 & pCi/L & 0 & & & & & & SURFACE & & CUMULATIVE & UNFILTERED & 60 \\
\hline 174 & $\mathrm{pCi} / \mathrm{L}$ & 0 & & & & & & SURFACE & & RAW GRAB & UNFILTERED & 444 \\
\hline 175 & ug/L & 0 & & & & & & SANITARY & & CUMULATIVE & UNFILTERED & 385 \\
\hline 176 & pCi/L & 0 & & & & & & RIVER & & CUMULATIVE & UNFILTERED & 463 \\
\hline 177 & pCi/L & 0 & & & & & & RIVER & & CUMULATIVE & UNFILTERED & 463 \\
\hline 178 & pCi/L & 0 & & & & & & RIVER & & CUMULATIVE & UNFILTERED & 463 \\
\hline 179 & pCi/L & 0 & & & & & & RIVER & & RAW GRAB & UNFILTERED & 456 \\
\hline 180 & $\mathrm{pCi} / \mathrm{L}$ & 0 & & & & & & RIVER & & RAW GRAB & UNFILTERED & 456 \\
\hline 181 & pCi/L & 0 & & & & & & RIVER & & RAW GRAB & UNFILTERED & 456 \\
\hline 182 & ug/L & 0 & & & & & & SANITARY & & CUMULATIVE & UNFILTERED & 385 \\
\hline 183 & $\mathrm{pCi} / \mathrm{L}$ & 0 & & & & & & SURFACE & & CUMULATIVE & UNFILTERED & 60 \\
\hline 184 & $\mathrm{pCi} / \mathrm{L}$ & 0 & & & & & & SURFACE & & CUMULATIVE & UNFILTERED & 60 \\
\hline 185 & pCi/L & 0 & & & & & & SURFACE & & RAW GRAB & UNFILTERED & 444 \\
\hline 186 & pCi/L & 0 & & & & & & SURFACE & & RAW GRAB & UNFILTERED & 121 \\
\hline 187 & $\mathrm{pCi} / \mathrm{L}$ & 0 & & & & & & SURFACE & & RAW GRAB & UNFILTERED & 484 \\
\hline 188 & $\mathrm{pCi} / \mathrm{L}$ & 0 & & & & & & SURFACE & & RAW GRAB & UNFILTERED & 509 \\
\hline 189 & pCi/L & 0 & & & & & & SURFACE & & RAW GRAB & UNFILTERED & 146 \\
\hline 190 & $\mathrm{pCi} / \mathrm{L}$ & 0 & & & & & & SURFACE & & RAW GRAB & UNFILTERED & 601 \\
\hline 191 & $\mathrm{pCi} / \mathrm{L}$ & 0 & & & & & & SURFACE & & RAW GRAB & UNFILTERED & 413 \\
\hline 192 & pCi/L & 0 & & & & & & SURFACE & & RAW GRAB & UNFILTERED & 412 \\
\hline 193 & pCi/L & 0 & & & & & & SANITARY & & CUMULATIVE & UNFILTERED & 491 \\
\hline 194 & pCi/L & 0 & & & & & & SANITARY & & CUMULATIVE & UNFILTERED & 491 \\
\hline 195 & pCi/L & 0 & & & & & & RIVER & & CUMULATIVE & UNFILTERED & 463 \\
\hline 196 & pCi/L & 0 & & & & & & RIVER & & CUMULATIVE & UNFILTERED & 463 \\
\hline 197 & $\mathrm{pCi} / \mathrm{L}$ & 0 & & & & & & RIVER & & RAW GRAB & UNFILTERED & 456 \\
\hline 198 & pCi/L & 0 & & & & & & RIVER & & RAW GRAB & UNFILTERED & 456 \\
\hline 199 & pCi/L & 0 & & & & & & RIVER & & RAW GRAB & UNFILTERED & 567 \\
\hline 200 & pCi/L & 0 & & & & & & SANITARY & & CUMULATIVE & UNFILTERED & 491 \\
\hline 201 & pCi/L & 0 & & & & & & SANITARY & & CUMULATIVE & UNFILTERED & 491 \\
\hline 202 & pCi/L & 0 & & & & & & RIVER & & CUMULATIVE & UNFILTERED & 463 \\
\hline 203 & $\mathrm{pCi} / \mathrm{L}$ & 0 & & & & & & RIVER & & RAW GRAB & UNFILTERED & 567 \\
\hline 204 & pCi/L & 0 & & & & $U$ & & SURFACE & & CUMULATIVE & UNFILTERED & 60 \\
\hline 205 & pCi/L & 0 & & & & & & SURFACE & & RAW GRAB & UNFILTERED & 444 \\
\hline 206 & pCi/L & 0 & & & & & & RIVER & & RAW GRAB & UNFILTERED & 567 \\
\hline 207 & pCi/L & 0 & & & & & & SANITARY & & CUMULATIVE & UNFILTERED & 385 \\
\hline 208 & pCi/L & 0 & & & & U & & RIVER & & CUMULATIVE & UNFILTERED & 463 \\
\hline 209 & $\mathrm{pCi} / \mathrm{L}$ & 0 & & & & & & RIVER & & CUMULATIVE & UNFILTERED & 463 \\
\hline
\end{tabular}




\begin{tabular}{|c|c|c|c|c|c|c|c|c|c|c|c|c|}
\hline & $\mathrm{N}$ & $\mathrm{O}$ & $\mathrm{P}$ & $\mathrm{Q}$ & $\mathrm{R}$ & $\mathrm{s}$ & $\mathrm{T}$ & $\bar{U}$ & $\mathrm{v}$ & $\mathrm{W}$ & $\mathrm{x}$ & $\mathrm{Y}$ \\
\hline 1 & ANAL_UNITS_RPTD & COUNTING_ERROR & TOTAL_ANAL_ERROR & MIN_DETECTABLE_ACTIVITY & PCNT_MOISTURE & LAB_QUALIFIER & REVIEW_QUALIFIER & SAMP_FROM & SAMP_ITEM & SAMP_MTHD & COLL_MTHD & SAMP_SITE_ID \\
\hline 210 & $\mathrm{pCi} / \mathrm{L}$ & 0 & & & & & & RIVER & & CUMULATIVE & UNFILTERED & 463 \\
\hline 211 & $\mathrm{pCi} / \mathrm{L}$ & 0 & & & & & & RIVER & & RAW GRAB & UNFILTERED & 456 \\
\hline 212 & $\mathrm{pCi} / \mathrm{L}$ & 0 & & & & U & & RIVER & & RAW GRAB & UNFILTERED & 456 \\
\hline 213 & $\mathrm{pCi} / \mathrm{L}$ & 0 & & & & & & RIVER & & RAW GRAB & UNFILTERED & 456 \\
\hline 214 & $\mathrm{pCi} / \mathrm{L}$ & 0 & & & & & & SANITARY & & CUMULATIVE & UNFILTERED & 491 \\
\hline 215 & $\mathrm{pCi} / \mathrm{L}$ & 0 & & & & & & RIVER & & CUMULATIVE & UNFILTERED & 463 \\
\hline 216 & $\mathrm{pCi} / \mathrm{L}$ & 0 & & & & & & SANITARY & & CUMULATIVE & UNFILTERED & 385 \\
\hline 217 & $\mathrm{pCi} / \mathrm{L}$ & 0 & & & & & & RIVER & & CUMULATIVE & UNFILTERED & 463 \\
\hline 218 & $\mathrm{pCi} / \mathrm{L}$ & 0 & & & & & & RIVER & & CUMULATIVE & UNFILTERED & 463 \\
\hline 219 & $\mathrm{pCi} / \mathrm{L}$ & 0 & & & & & & RIVER & & RAW GRAB & UNFILTERED & 456 \\
\hline 220 & $\mathrm{pCi} / \mathrm{L}$ & 0 & & & & U & & RIVER & & RAW GRAB & UNFILTERED & 456 \\
\hline 221 & $\mathrm{pCi} / \mathrm{L}$ & 0 & & & & & & RIVER & & RAW GRAB & UNFILTERED & 567 \\
\hline 222 & $\mathrm{pCi} / \mathrm{L}$ & 0 & & & & U & & SURFACE & & CUMULATIVE & UNFILTERED & 60 \\
\hline 223 & $\mathrm{pCi} / \mathrm{L}$ & 0 & & & & U & & SURFACE & & RAW GRAB & UNFILTERED & 484 \\
\hline 224 & $\mathrm{pCi} / \mathrm{L}$ & 0 & & & & & & SURFACE & & RAW GRAB & UNFILTERED & 509 \\
\hline 225 & $\mathrm{pCi} / \mathrm{L}$ & 0 & & & & & & SURFACE & & RAW GRAB & UNFILTERED & 146 \\
\hline 226 & $\mathrm{pCi} / \mathrm{L}$ & 0 & & & & & & SURFACE & & RAW GRAB & UNFILTERED & 413 \\
\hline 227 & $\mathrm{pCi} / \mathrm{L}$ & 0 & & & & & & SANITARY & & CUMULATIVE & UNFILTERED & 385 \\
\hline 228 & $\mathrm{pCi} / \mathrm{L}$ & 0 & & & & & & RIVER & & CUMULATIVE & UNFILTERED & 463 \\
\hline 229 & $\mathrm{pCi} / \mathrm{L}$ & 0 & & & & & & RIVER & & CUMULATIVE & UNFILTERED & 463 \\
\hline 230 & $\mathrm{pCi} / \mathrm{L}$ & 0 & & & & & & RIVER & & RAW GRAB & UNFILTERED & 456 \\
\hline 231 & $\mathrm{pCi} / \mathrm{L}$ & 0 & & & & & & RIVER & & RAW GRAB & UNFILTERED & 456 \\
\hline 232 & $\mathrm{pCi} / \mathrm{L}$ & 0 & & & & & & SANITARY & & CUMULATIVE & UNFILTERED & 385 \\
\hline 233 & $\mathrm{pCi} / \mathrm{L}$ & 0 & & & & & & RIVER & & CUMULATIVE & UNFILTERED & 463 \\
\hline 234 & pCi/L & 0 & & & & & & SURFACE & & RAW GRAB & UNFILTERED & 444 \\
\hline 235 & pCi/L & 0 & & & & & & RIVER & & CUMULATIVE & UNFILTERED & 463 \\
\hline 236 & $\mathrm{pCi} / \mathrm{L}$ & 0 & & & & $\mathrm{U}$ & & RIVER & & CUMULATIVE & UNFILTERED & 463 \\
\hline 237 & pCi/L & 0 & & & & & & RIVER & & CUMULATIVE & UNFILTERED & 463 \\
\hline 238 & pCi/L & 0 & & & & & & RIVER & & RAW GRAB & UNFILTERED & 456 \\
\hline 239 & pCi/L & 0 & & & & & & RIVER & & RAW GRAB & UNFILTERED & 456 \\
\hline 240 & $\mathrm{pCi} / \mathrm{L}$ & 0 & & & & & & RIVER & & RAW GRAB & UNFILTERED & 456 \\
\hline 241 & pCi/L & 0 & & & & & & SANITARY & & CUMULATIVE & UNFILTERED & 385 \\
\hline 242 & $\mathrm{pCi} / \mathrm{L}$ & 0 & & & & $U$ & & SURFACE & & CUMULATIVE & UNFILTERED & 60 \\
\hline 243 & $\mathrm{pCi} / \mathrm{L}$ & 0 & & & & & & SURFACE & & RAW GRAB & UNFILTERED & 444 \\
\hline 244 & pCi/L & 0 & & & & & & SANITARY & & CUMULATIVE & UNFILTERED & 491 \\
\hline 245 & pCi/L & 0 & & & & & & SANITARY & & CUMULATIVE & UNFILTERED & 385 \\
\hline 246 & pCi/L & 0 & & & & U & & RIVER & & CUMULATIVE & UNFILTERED & 463 \\
\hline 247 & $\mathrm{pCi} / \mathrm{L}$ & 0 & & & & & & RIVER & & CUMULATIVE & UNFILTERED & 463 \\
\hline 248 & $\mathrm{pCi} / \mathrm{L}$ & 0 & & & & & & RIVER & & RAW GRAB & UNFILTERED & 456 \\
\hline 249 & $\mathrm{pCi} / \mathrm{L}$ & 0 & & & & & & RIVER & & RAW GRAB & UNFILTERED & 456 \\
\hline 250 & $\mathrm{pCi} / \mathrm{L}$ & 0 & & & & & & SANITARY & & CUMULATIVE & UNFILTERED & 385 \\
\hline 251 & pCi/L & 0 & & & & & & RIVER & & RAW GRAB & UNFILTERED & 273 \\
\hline 252 & pCi/L & 0 & & & & & & SURFACE & & RAW GRAB & UNFILTERED & 444 \\
\hline 253 & pCi/L & 0 & & & & $U$ & & SURFACE & & RAW GRAB & UNFILTERED & 67 \\
\hline 254 & pCi/L & 0 & & & & $U$ & & SURFACE & & RAW GRAB & UNFILTERED & 121 \\
\hline 255 & pCi/L & 0 & & & & & & SURFACE & & RAW GRAB & UNFILTERED & 484 \\
\hline 256 & pCi/L & 0 & & & & $U$ & & SURFACE & & RAW GRAB & UNFILTERED & 509 \\
\hline 257 & pCi/L & 0 & & & & & & SURFACE & & RAW GRAB & UNFILTERED & 146 \\
\hline 258 & $\mathrm{pCi} / \mathrm{L}$ & 0 & & & & & & SURFACE & & RAW GRAB & UNFILTERED & 413 \\
\hline 259 & pCi/L & 0 & & & & & & SURFACE & & RAW GRAB & UNFILTERED & 412 \\
\hline 260 & pCi/L & 0 & & & & U & & SANITARY & & CUMULATIVE & UNFILTERED & 491 \\
\hline 261 & $\mathrm{pCi} / \mathrm{L}$ & 0 & & & & & & SANITARY & & CUMULATIVE & UNFILTERED & 385 \\
\hline
\end{tabular}




\begin{tabular}{|c|c|c|c|c|c|c|c|c|c|c|c|c|}
\hline & $\mathrm{N}$ & $\mathrm{O}$ & $\mathrm{P}$ & $\mathrm{Q}$ & $\mathrm{R}$ & $\mathrm{s}$ & $\mathrm{T}$ & $\mathrm{U}$ & $\mathrm{V}$ & $\mathrm{w}$ & $\mathrm{x}$ & $\mathrm{Y}$ \\
\hline 1 & ANAL_UNITS_RPTD & COUNTING_ERROR & TOTAL_ANAL_ERROR & MIN_DETECTABLE_ACTIVITY & PCNT_MOISTURE & LAB_QUALIFIER & REVIEW_QUALIFIER & SAMP_FROM & SAMP_ITEM & SAMP_MTHD & COLL_MTHD & SAMP_SITE_ID \\
\hline 262 & $\mathrm{pCi} / \mathrm{L}$ & 0 & & & & & & RIVER & & CUMULATIVE & UNFILTERED & 463 \\
\hline 263 & $\mathrm{pCi} / \mathrm{L}$ & 0 & & & & & & SANITARY & & CUMULATIVE & UNFILTERED & 491 \\
\hline 264 & $\mathrm{pCi} / \mathrm{L}$ & 0 & & & & & & SANITARY & & CUMULATIVE & UNFILTERED & 385 \\
\hline 265 & $\mathrm{pCi} / \mathrm{L}$ & 0 & & & & $\mathrm{U}$ & & RIVER & & CUMULATIVE & UNFILTERED & 463 \\
\hline 266 & $\mathrm{pCi} / \mathrm{L}$ & 0 & & & & & & RIVER & & CUMULATIVE & UNFILTERED & 463 \\
\hline 267 & $\mathrm{pCi} / \mathrm{L}$ & 0 & & & & & & RIVER & & RAW GRAB & UNFILTERED & 456 \\
\hline 268 & $\mathrm{pCi} / \mathrm{L}$ & 0 & & & & $\mathrm{U}$ & & RIVER & & RAW GRAB & UNFILTERED & 456 \\
\hline 269 & $\mathrm{pCi} / \mathrm{L}$ & 0 & & & & $\mathrm{U}$ & & SURFACE & & CUMULATIVE & UNFILTERED & 60 \\
\hline 270 & $\mathrm{pCi} / \mathrm{L}$ & 0 & & & & & & RIVER & & CUMULATIVE & UNFILTERED & 463 \\
\hline 271 & $\mathrm{pCi} / \mathrm{L}$ & 0 & & & & & & SURFACE & & RAW GRAB & UNFILTERED & 444 \\
\hline 272 & $\mathrm{pCi} / \mathrm{L}$ & 0 & & & & $\mathrm{U}$ & & SANITARY & & CUMULATIVE & UNFILTERED & 491 \\
\hline 273 & $\mathrm{pCi} / \mathrm{L}$ & 0 & & & & U & & SANITARY & & CUMULATIVE & UNFILTERED & 385 \\
\hline 274 & $\mathrm{pCi} / \mathrm{L}$ & 0 & & & & & & RIVER & & CUMULATIVE & UNFILTERED & 463 \\
\hline 275 & $\mathrm{pCi} / \mathrm{L}$ & 0 & & & & & & SANITARY & & CUMULATIVE & UNFILTERED & 385 \\
\hline 276 & $p C i / L$ & 0 & & & & & & RIVER & & RAW GRAB & UNFILTERED & 277 \\
\hline 277 & $\mathrm{pCi} / \mathrm{L}$ & 0 & & & & & & RIVER & & CUMULATIVE & UNFILTERED & 463 \\
\hline 278 & $\mathrm{pCi} / \mathrm{L}$ & 0 & & & & & & RIVER & & CUMULATIVE & UNFILTERED & 463 \\
\hline 279 & $\mathrm{pCi} / \mathrm{L}$ & 0 & & & & & & RIVER & & CUMULATIVE & UNFILTERED & 463 \\
\hline 280 & $\mathrm{pCi} / \mathrm{L}$ & 0 & & & & & & RIVER & & RAW GRAB & UNFILTERED & 456 \\
\hline 281 & $\mathrm{pCi} / \mathrm{L}$ & 0 & & & & & & RIVER & & RAW GRAB & UNFILTERED & 456 \\
\hline 282 & $\mathrm{pCi} / \mathrm{L}$ & 0 & & & & U & & RIVER & & RAW GRAB & UNFILTERED & 456 \\
\hline 283 & $\mathrm{pCi} / \mathrm{L}$ & 0 & & & & & & RIVER & & CUMULATIVE & UNFILTERED & 463 \\
\hline 284 & $\mathrm{pCi} / \mathrm{L}$ & 0 & & & & & & SANITARY & & CUMULATIVE & UNFILTERED & 491 \\
\hline 285 & $\mathrm{pCi} / \mathrm{L}$ & 0 & & & & & & SANITARY & & CUMULATIVE & UNFILTERED & 385 \\
\hline 286 & $\mathrm{pCi} / \mathrm{L}$ & 0 & & & & & & SURFACE & & RAW GRAB & UNFILTERED & 147 \\
\hline 287 & pCi/L & 0 & & & & & & SANITARY & & CUMULATIVE & UNFILTERED & 491 \\
\hline 288 & $\mathrm{pCi} / \mathrm{L}$ & 0 & & & & & & SANITARY & & CUMULATIVE & UNFILTERED & 385 \\
\hline 289 & $\mathrm{pCi} / \mathrm{L}$ & 0 & & & & & & RIVER & & CUMULATIVE & UNFILTERED & 463 \\
\hline 290 & $\mathrm{pCi} / \mathrm{L}$ & 0 & & & & & & RIVER & & CUMULATIVE & UNFILTERED & 463 \\
\hline 291 & $\mathrm{pCi} / \mathrm{L}$ & 0 & & & & & & SURFACE & & RAW GRAB & UNFILTERED & 444 \\
\hline 292 & $\mathrm{pCi} / \mathrm{L}$ & 0 & & & & & & SANITARY & & CUMULATIVE & UNFILTERED & 491 \\
\hline 293 & $\mathrm{pCi} / \mathrm{L}$ & 0 & & & & & & SANITARY & & CUMULATIVE & UNFILTERED & 385 \\
\hline 294 & $\mathrm{pCi} / \mathrm{L}$ & 0 & & & & & & SANITARY & & CUMULATIVE & UNFILTERED & 385 \\
\hline 295 & $\mathrm{pCi} / \mathrm{L}$ & 0 & & & & U & & RIVER & & CUMULATIVE & UNFILTERED & 463 \\
\hline 296 & $\mathrm{pCi} / \mathrm{L}$ & 0 & & & & & & RIVER & & CUMULATIVE & UNFILTERED & 463 \\
\hline 297 & pCi/L & 0 & & & & & & RIVER & & CUMULATIVE & UNFILTERED & 463 \\
\hline 298 & $\mathrm{pCi} / \mathrm{L}$ & 0 & & & & & & RIVER & & RAW GRAB & UNFILTERED & 456 \\
\hline 299 & $\mathrm{pCi} / \mathrm{L}$ & 0 & & & & U & & RIVER & & RAW GRAB & UNFILTERED & 456 \\
\hline 300 & $\mathrm{pCi} / \mathrm{L}$ & 0 & & & & & & SANITARY & & CUMULATIVE & UNFILTERED & 491 \\
\hline 301 & pCi/L & 0 & & & & U & & SANITARY & & CUMULATIVE & UNFILTERED & 385 \\
\hline 302 & $\mathrm{pCi} / \mathrm{L}$ & 0 & & & & & & RIVER & & CUMULATIVE & UNFILTERED & 463 \\
\hline 303 & $\mathrm{pCi} / \mathrm{L}$ & 0 & & & & & & SURFACE & & RAW GRAB & UNFILTERED & 121 \\
\hline 304 & $\mathrm{pCi} / \mathrm{L}$ & 0 & & & & & & SURFACE & & RAW GRAB & UNFILTERED & 484 \\
\hline 305 & $\mathrm{pCi} / \mathrm{L}$ & 0 & & & & & & SURFACE & & RAW GRAB & UNFILTERED & 509 \\
\hline 306 & $\mathrm{pCi} / \mathrm{L}$ & 0 & & & & & & SURFACE & & RAW GRAB & UNFILTERED & 146 \\
\hline 307 & pCi/L & 0 & & & & & & SURFACE & & RAW GRAB & UNFILTERED & 413 \\
\hline 308 & $\mathrm{pCi} / \mathrm{L}$ & 0 & & & & & & SURFACE & & RAW GRAB & UNFILTERED & 412 \\
\hline 309 & $\mathrm{pCi} / \mathrm{L}$ & 0 & & & & & & SURFACE & & RAW GRAB & UNFILTERED & 147 \\
\hline 310 & pCi/L & 0 & & & & & & SURFACE & & RAW GRAB & UNFILTERED & 147 \\
\hline 311 & $\mathrm{pCi} / \mathrm{L}$ & 0 & & & & & & SANITARY & & CUMULATIVE & UNFILTERED & 491 \\
\hline 312 & $\mathrm{pCi} / \mathrm{L}$ & 0 & & & & & & SANITARY & & CUMULATIVE & UNFILTERED & 385 \\
\hline 313 & $\mathrm{pCi} / \mathrm{L}$ & 0 & ) & & & & & SURFACE & & RAW GRAB & UNFILTERED & 444 \\
\hline
\end{tabular}




\begin{tabular}{|c|c|c|c|c|c|c|c|c|c|c|c|c|}
\hline & $\mathrm{N}$ & $\mathrm{O}$ & $\mathrm{P}$ & $\mathrm{Q}$ & $\mathrm{R}$ & $\mathrm{s}$ & $\mathrm{T}$ & $\bar{U}$ & $\mathrm{v}$ & $\mathrm{W}$ & $\mathrm{x}$ & $\mathrm{Y}$ \\
\hline 1 & ANAL_UNITS_RPTD & COUNTING_ERROR & TOTAL_ANAL_ERROR & MIN_DETECTABLE_ACTIVITY & PCNT_MOISTURE & LAB_QUALIFIER & REVIEW_QUALIFIER & SAMP_FROM & SAMP_ITEM & SAMP_MTHD & COLL_MTHD & SAMP_SITE_ID \\
\hline 314 & $\mathrm{pCi} / \mathrm{L}$ & 0 & & & & $U$ & & SURFACE & & RAW GRAB & UNFILTERED & 67 \\
\hline 315 & $\mathrm{pCi} / \mathrm{L}$ & 0 & & & & & & RIVER & & CUMULATIVE & UNFILTERED & 463 \\
\hline 316 & $\mathrm{pCi} / \mathrm{L}$ & 0 & & & & & & SANITARY & & CUMULATIVE & UNFILTERED & 491 \\
\hline 317 & $\mathrm{pCi} / \mathrm{L}$ & 0 & & & & & & SANITARY & & CUMULATIVE & UNFILTERED & 385 \\
\hline 318 & $\mathrm{pCi} / \mathrm{L}$ & 0 & & & & & & SANITARY & & CUMULATIVE & UNFILTERED & 385 \\
\hline 319 & $\mathrm{pCi} / \mathrm{L}$ & 0 & & & & U & & RIVER & & CUMULATIVE & UNFILTERED & 463 \\
\hline 320 & $\mathrm{pCi} / \mathrm{L}$ & 0 & & & & & & RIVER & & CUMULATIVE & UNFILTERED & 463 \\
\hline 321 & $\mathrm{pCi} / \mathrm{L}$ & 0 & & & & & & RIVER & & CUMULATIVE & UNFILTERED & 463 \\
\hline 322 & $\mathrm{pCi} / \mathrm{L}$ & 0 & & & & & & RIVER & & RAW GRAB & UNFILTERED & 456 \\
\hline 323 & $\mathrm{pCi} / \mathrm{L}$ & 0 & & & & & & RIVER & & RAW GRAB & UNFILTERED & 456 \\
\hline 324 & $\mathrm{pCi} / \mathrm{L}$ & 0 & & & & U & & RIVER & & RAW GRAB & UNFILTERED & 456 \\
\hline 325 & $\mathrm{pCi} / \mathrm{L}$ & 0 & & & & & & SANITARY & & CUMULATIVE & UNFILTERED & 491 \\
\hline 326 & pCi/L & 0 & & & & & & SURFACE & & CUMULATIVE & UNFILTERED & 60 \\
\hline 327 & $\mathrm{pCi} / \mathrm{L}$ & 0 & & & & & & SURFACE & & RAW GRAB & UNFILTERED & 147 \\
\hline 328 & pCi/L & 0 & & & & & & SURFACE & & RAW GRAB & UNFILTERED & 444 \\
\hline 329 & $\mathrm{pCi} / \mathrm{L}$ & 0 & & & & & & SANITARY & & CUMULATIVE & UNFILTERED & 385 \\
\hline 330 & pCi/L & 0 & & & & & & SURFACE & & CUMULATIVE & UNFILTERED & 60 \\
\hline 331 & pCi/L & 0 & & & & & & RIVER & & CUMULATIVE & UNFILTERED & 463 \\
\hline 332 & $\mathrm{pCi} / \mathrm{L}$ & 0 & & & & & & RIVER & & CUMULATIVE & UNFILTERED & 463 \\
\hline 333 & $\mathrm{pCi} / \mathrm{L}$ & 0 & & & & & & RIVER & & CUMULATIVE & UNFILTERED & 463 \\
\hline 334 & $\mathrm{pCi} / \mathrm{L}$ & 0 & & & & & & RIVER & & RAW GRAB & UNFILTERED & 456 \\
\hline 335 & $\mathrm{pCi} / \mathrm{L}$ & 0 & & & & & & RIVER & & RAW GRAB & UNFILTERED & 456 \\
\hline 336 & $\mathrm{pCi} / \mathrm{L}$ & 0 & & & & U & & RIVER & & RAW GRAB & UNFILTERED & 456 \\
\hline 337 & $\mathrm{pCi} / \mathrm{L}$ & 0 & & & & & & SURFACE & & RAW GRAB & UNFILTERED & 444 \\
\hline 338 & pCi/L & 0 & & & & & & SURFACE & & RAW GRAB & UNFILTERED & 147 \\
\hline 339 & pCi/L & 0 & & & & & & SANITARY & & CUMULATIVE & UNFILTERED & 491 \\
\hline 340 & $\mathrm{pCi} / \mathrm{L}$ & 0 & & & & & & SANITARY & & CUMULATIVE & UNFILTERED & 385 \\
\hline 341 & $\mathrm{pCi} / \mathrm{L}$ & 0 & & & & U & & RIVER & & CUMULATIVE & UNFILTERED & 463 \\
\hline 342 & $\mathrm{pCi} / \mathrm{L}$ & 0 & & & & & & RIVER & & RAW GRAB & UNFILTERED & 277 \\
\hline 343 & pCi/L & 0 & & & & & & SANITARY & & CUMULATIVE & UNFILTERED & 385 \\
\hline 344 & $\mathrm{pCi} / \mathrm{L}$ & 0 & & & & U & & RIVER & & CUMULATIVE & UNFILTERED & 463 \\
\hline 345 & pCi/L & 0 & & & & & & RIVER & & CUMULATIVE & UNFILTERED & 463 \\
\hline 346 & $\mathrm{pCi} / \mathrm{L}$ & 0 & & & & & & RIVER & & CUMULATIVE & UNFILTERED & 463 \\
\hline 347 & $\mathrm{pCi} / \mathrm{L}$ & 0 & & & & & & RIVER & & RAW GRAB & UNFILTERED & 456 \\
\hline 348 & $\mathrm{pCi} / \mathrm{L}$ & 0 & & & & & & RIVER & & RAW GRAB & UNFILTERED & 456 \\
\hline 349 & $\mathrm{pCi} / \mathrm{L}$ & 0 & & & & U & & RIVER & & RAW GRAB & UNFILTERED & 456 \\
\hline 350 & $\mathrm{pCi} / \mathrm{L}$ & 0 & & & & & & SANITARY & & CUMULATIVE & UNFILTERED & 385 \\
\hline 351 & $\mathrm{pCi} / \mathrm{L}$ & 0 & & & & & & SURFACE & & RAW GRAB & UNFILTERED & 444 \\
\hline 352 & $\mathrm{pCi} / \mathrm{L}$ & 0 & & & & & & SURFACE & & RAW GRAB & UNFILTERED & 121 \\
\hline 353 & $\mathrm{pCi} / \mathrm{L}$ & 0 & & & & & & SURFACE & & RAW GRAB & UNFILTERED & 484 \\
\hline 354 & $\mathrm{pCi} / \mathrm{L}$ & 0 & & & & & & SURFACE & & RAW GRAB & UNFILTERED & 509 \\
\hline 355 & $\mathrm{pCi} / \mathrm{L}$ & 0 & & & & & & SURFACE & & RAW GRAB & UNFILTERED & 146 \\
\hline 356 & $\mathrm{pCi} / \mathrm{L}$ & 0 & & & & & & SURFACE & & RAW GRAB & UNFILTERED & 412 \\
\hline 357 & $\mathrm{pCi} / \mathrm{L}$ & 0 & & & & & & SURFACE & & RAW GRAB & UNFILTERED & 147 \\
\hline 358 & $\mathrm{pCi} / \mathrm{L}$ & 0 & & & & & & SURFACE & & RAW GRAB & UNFILTERED & 147 \\
\hline 359 & pCi/L & 0 & & & & U & & SANITARY & & CUMULATIVE & UNFILTERED & 385 \\
\hline 360 & $\mathrm{pCi} / \mathrm{L}$ & 0 & & & & $U$ & & SURFACE & & CUMULATIVE & UNFILTERED & 60 \\
\hline 361 & $\mathrm{pCi} / \mathrm{L}$ & 0 & & & & & & RIVER & & CUMULATIVE & UNFILTERED & 463 \\
\hline 362 & $\mathrm{pCi} / \mathrm{L}$ & 0 & & & & & & RIVER & & CUMULATIVE & UNFILTERED & 463 \\
\hline 363 & $\mathrm{pCi} / \mathrm{L}$ & 0 & & & & & & RIVER & & CUMULATIVE & UNFILTERED & 463 \\
\hline 364 & $\mathrm{pCi} / \mathrm{L}$ & 0 & & & & U & & RIVER & & CUMULATIVE & UNFILTERED & 463 \\
\hline 365 & $\mathrm{pCi} / \mathrm{L}$ & 0 & & & & & & RIVER & & RAW GRAB & UNFILTERED & 456 \\
\hline
\end{tabular}




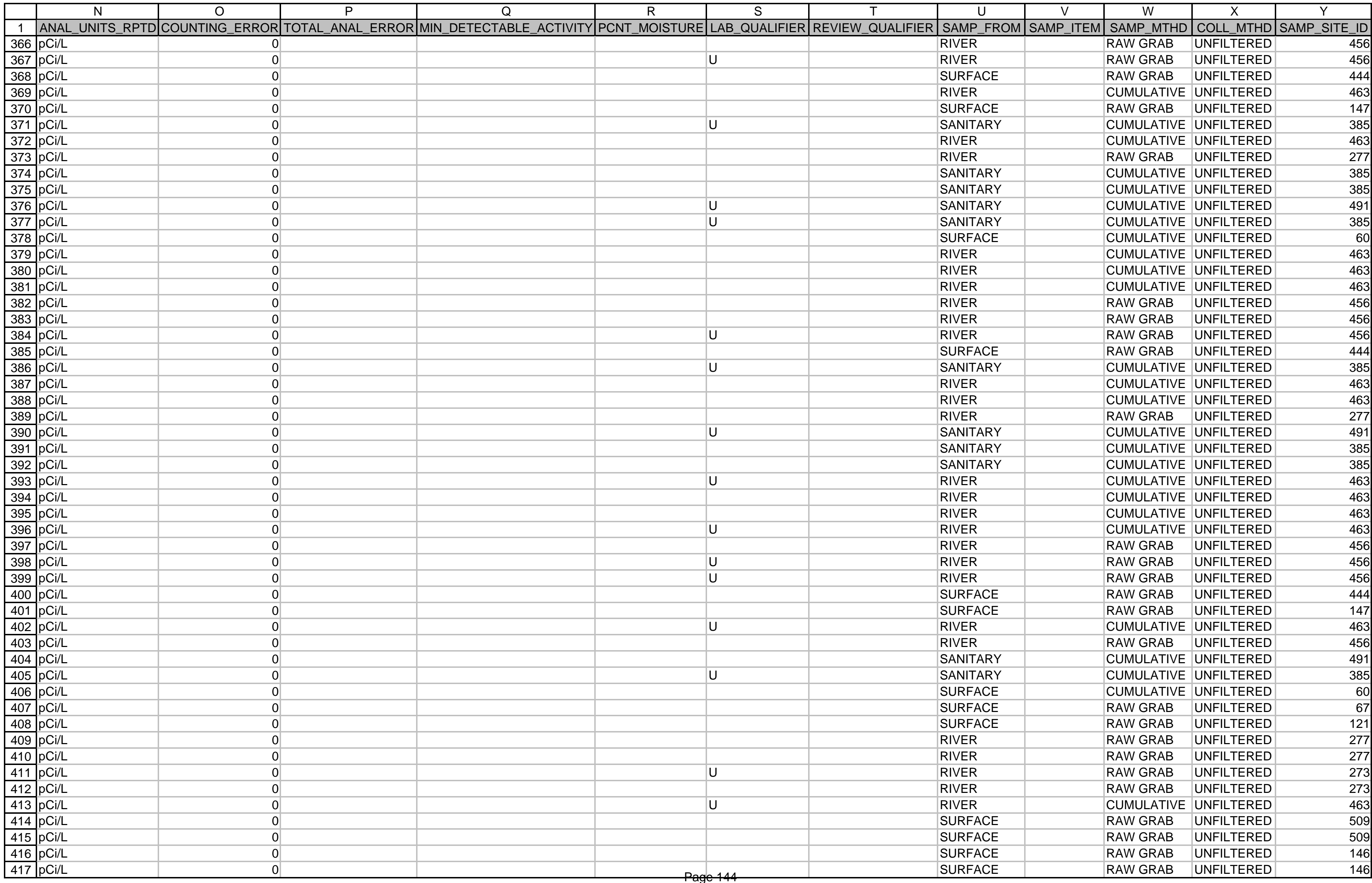




\begin{tabular}{|c|c|c|c|c|c|c|c|c|c|c|c|c|c|}
\hline & $\mathrm{N}$ & $\mathrm{O}$ & & $\mathrm{P}$ & $\mathrm{Q}$ & $\mathrm{R}$ & $\mathrm{s}$ & $\mathrm{T}$ & $\mathrm{U}$ & $\mathrm{V}$ & $\mathrm{W}$ & $\mathrm{x}$ & $\bar{Y}$ \\
\hline 1 & ANAL_UNITS_RPTD & COUNTING & ERROR & TOTAL_ANAL_ERROR & MIN_DETECTABLE_ACTIVITT & PCNT_MOISTURE & LAB_QUALIFIER & REVIEW_QUALIFIER & SAMP_FROM & SAMP_ITEM & SAMP_MTHD & COLL_MTHD & SAMP_SITE_ID \\
\hline 418 & $\mathrm{pCi} / \mathrm{L}$ & & 0 & & & & & & SURFACE & & RAW GRAB & UNFILTERED & 412 \\
\hline 419 & $\mathrm{pCi} / \mathrm{L}$ & & 0 & & & & & & SANITARY & & CUMULATIVE & UNFILTERED & 385 \\
\hline 420 & $\mathrm{pCi} / \mathrm{L}$ & & 0 & & & & & & SURFACE & & RAW GRAB & UNFILTERED & 444 \\
\hline 421 & $\mathrm{pCi} / \mathrm{L}$ & & 0 & & & & & & RIVER & & CUMULATIVE & UNFILTERED & 463 \\
\hline 422 & $\mathrm{pCi} / \mathrm{L}$ & & 0 & & & & & & RIVER & & CUMULATIVE & UNFILTERED & 463 \\
\hline 423 & $\mathrm{pCi} / \mathrm{L}$ & & 0 & & & & $\mathrm{U}$ & & RIVER & & CUMULATIVE & UNFILTERED & 463 \\
\hline 424 & $\mathrm{pCi} / \mathrm{L}$ & & 0 & & & & & & RIVER & & RAW GRAB & UNFILTERED & 456 \\
\hline 425 & $\mathrm{pCi} / \mathrm{L}$ & & 0 & & & & $\mathrm{U}$ & & RIVER & & RAW GRAB & UNFILTERED & 456 \\
\hline 426 & $\mathrm{pCi} / \mathrm{L}$ & & 0 & & & & & & RIVER & & RAW GRAB & UNFILTERED & 456 \\
\hline 427 & $\mathrm{pCi} / \mathrm{L}$ & & 0 & & & & $u$ & & SANITARY & & CUMULATIVE & UNFILTERED & 491 \\
\hline 428 & $\mathrm{pCi} / \mathrm{L}$ & & 0 & & & & $\mathrm{U}$ & & SANITARY & & CUMULATIVE & UNFILTERED & 385 \\
\hline 429 & $\mathrm{pCi} / \mathrm{L}$ & & 0 & & & & & & RIVER & & CUMULATIVE & UNFILTERED & 463 \\
\hline 430 & $\mathrm{pCi} / \mathrm{L}$ & & 0 & & & & U & & SURFACE & & CUMULATIVE & UNFILTERED & 60 \\
\hline 431 & $\mathrm{pCi} / \mathrm{L}$ & & 0 & & & & & & RIVER & & CUMULATIVE & UNFILTERED & 463 \\
\hline 432 & $\mathrm{pCi} / \mathrm{L}$ & & 0 & & & & & & SURFACE & & RAW GRAB & UNFILTERED & 147 \\
\hline 433 & $\mathrm{pCi} / \mathrm{L}$ & & 0 & & & & & & SANITARY & & CUMULATIVE & UNFILTERED & 491 \\
\hline 434 & $\mathrm{pCi} / \mathrm{L}$ & & 0 & & & & $U$ & & SANITARY & & CUMULATIVE & UNFILTERED & 385 \\
\hline 435 & $\mathrm{pCi} / \mathrm{L}$ & & 0 & & & & & & SURFACE & & RAW GRAB & UNFILTERED & 444 \\
\hline 436 & $\mathrm{pCi} / \mathrm{L}$ & & 0 & & & & & & RIVER & & RAW GRAB & UNFILTERED & 277 \\
\hline 437 & $\mathrm{pCi} / \mathrm{L}$ & & 0 & & & & & & RIVER & & CUMULATIVE & UNFILTERED & 463 \\
\hline 438 & $\mathrm{pCi} / \mathrm{L}$ & & 0 & & & & & & RIVER & & CUMULATIVE & UNFILTERED & 463 \\
\hline 439 & $\mathrm{pCi} / \mathrm{L}$ & & 0 & & & & $U$ & & RIVER & & CUMULATIVE & UNFILTERED & 463 \\
\hline 440 & $\mathrm{pCi} / \mathrm{L}$ & & 0 & & & & & & RIVER & & RAW GRAB & UNFILTERED & 456 \\
\hline 441 & $\mathrm{pCi} / \mathrm{L}$ & & 0 & & & & $U$ & & RIVER & & RAW GRAB & UNFILTERED & 456 \\
\hline 442 & $\mathrm{pCi} / \mathrm{L}$ & & 0 & & & & U & & SANITARY & & CUMULATIVE & UNFILTERED & 491 \\
\hline 443 & $\mathrm{pCi} / \mathrm{L}$ & & 0 & & & & & & SANITARY & & CUMULATIVE & UNFILTERED & 385 \\
\hline 444 & $\mathrm{pCi} / \mathrm{L}$ & & 0 & & & & & & RIVER & & CUMULATIVE & UNFILTERED & 463 \\
\hline 445 & $\mathrm{pCi} / \mathrm{L}$ & & 0 & & & & $U$ & & SANITARY & & CUMULATIVE & UNFILTERED & 491 \\
\hline 446 & $\mathrm{pCi} / \mathrm{L}$ & & 0 & & & & $U$ & & SURFACE & & CUMULATIVE & UNFILTERED & 60 \\
\hline 447 & $\mathrm{pCi} / \mathrm{L}$ & & 0 & & & & $U$ & & RIVER & & CUMULATIVE & UNFILTERED & 463 \\
\hline 448 & $\mathrm{pCi} / \mathrm{L}$ & & 0 & & & & & & SURFACE & & RAW GRAB & UNFILTERED & 147 \\
\hline 449 & $\mathrm{pCi} / \mathrm{L}$ & & 0 & & & & & & RIVER & & RAW GRAB & UNFILTERED & 277 \\
\hline 450 & $\mathrm{pCi} / \mathrm{L}$ & & 0 & & & & & & RIVER & & RAW GRAB & UNFILTERED & 277 \\
\hline 451 & $\mathrm{pCi} / \mathrm{L}$ & & 0 & & & & & & RIVER & & CUMULATIVE & UNFILTERED & 553 \\
\hline 452 & $p \mathrm{pi} / \mathrm{L}$ & & 0 & & & & & & SANITARY & & CUMULATIVE & UNFILTERED & 491 \\
\hline 453 & $\mathrm{pCi} / \mathrm{L}$ & & 0 & & & & $U$ & & SANITARY & & CUMULATIVE & UNFILTERED & 385 \\
\hline 454 & $\mathrm{pCi} / \mathrm{L}$ & & 0 & & & & & & RIVER & & CUMULATIVE & UNFILTERED & 463 \\
\hline 455 & $\mathrm{pCi} / \mathrm{L}$ & & 0 & & & & & & SURFACE & & RAW GRAB & UNFILTERED & 444 \\
\hline 456 & $\mathrm{pCi} / \mathrm{L}$ & & 0 & & & & & & RIVER & & CUMULATIVE & UNFILTERED & 553 \\
\hline 457 & $\mathrm{pCi} / \mathrm{L}$ & & 0 & & & & & & SANITARY & & CUMULATIVE & UNFILTERED & 491 \\
\hline 458 & $\mathrm{pCi} / \mathrm{L}$ & & 0 & & & & & & SANITARY & & CUMULATIVE & UNFILTERED & 385 \\
\hline 459 & $\mathrm{pCi} / \mathrm{L}$ & & 0 & & & & & & SANITARY & & CUMULATIVE & UNFILTERED & 385 \\
\hline 460 & $\mathrm{pCi} / \mathrm{L}$ & & 0 & & & & & & RIVER & & CUMULATIVE & UNFILTERED & 463 \\
\hline 461 & $\mathrm{pCi} / \mathrm{L}$ & & 0 & & & & & & RIVER & & CUMULATIVE & UNFILTERED & 463 \\
\hline 462 & $\mathrm{pCi} / \mathrm{L}$ & & 0 & & & & & & RIVER & & CUMULATIVE & UNFILTERED & 463 \\
\hline 463 & $\mathrm{pCi} / \mathrm{L}$ & & 0 & & & & & & RIVER & & RAW GRAB & UNFILTERED & 456 \\
\hline 464 & $\mathrm{pCi} / \mathrm{L}$ & & 0 & & & & & & RIVER & & RAW GRAB & UNFILTERED & 456 \\
\hline 465 & $\mathrm{pCi} / \mathrm{L}$ & & 0 & & & & & & RIVER & & RAW GRAB & UNFILTERED & 456 \\
\hline 466 & $\mathrm{pCi} / \mathrm{L}$ & & 0 & & & & U & & SANITARY & & CUMULATIVE & UNFILTERED & 491 \\
\hline 467 & $\mathrm{pCi} / \mathrm{L}$ & & 0 & & & & & & SANITARY & & CUMULATIVE & UNFILTERED & 385 \\
\hline 468 & $\mathrm{pCi} / \mathrm{L}$ & & 0 & & & & & & SURFACE & & CUMULATIVE & UNFILTERED & 60 \\
\hline 469 & $\mathrm{pCi} / \mathrm{L}$ & & 0 & & & & & & SURFACE & & RAW GRAB & UNFILTERED & 509 \\
\hline
\end{tabular}




\begin{tabular}{|c|c|c|c|c|c|c|c|c|c|c|c|c|}
\hline & $\mathrm{N}$ & $\mathrm{O}$ & $\mathrm{P}$ & $\mathrm{Q}$ & $\mathrm{R}$ & $\mathrm{s}$ & $\mathrm{T}$ & $\bar{U}$ & $\mathrm{v}$ & $\mathrm{w}$ & $\mathrm{x}$ & $\bar{Y}$ \\
\hline 1 & ANAL_UNITS_RPTD & COUNTING_ERROR & TOTAL_ANAL_ERROR & MIN_DETECTABLE_ACTIVIT & PCNT_MOISTURE & LAB_QUALIFIER & REVIEW_QUALIFIER & SAMP_FROM & SAMP_ITEM & SAMP_MTHD & COLL_MTHD & SAMP_SITE_ID \\
\hline 470 & $\mathrm{pCi} / \mathrm{L}$ & 0 & & & & & & SURFACE & & RAW GRAB & UNFILTERED & 509 \\
\hline 471 & $\mathrm{pCi} / \mathrm{L}$ & 0 & & & & & & SURFACE & & RAW GRAB & UNFILTERED & 146 \\
\hline 472 & $\mathrm{pCi} / \mathrm{L}$ & 0 & & & & & & SURFACE & & RAW GRAB & UNFILTERED & 146 \\
\hline 473 & $\mathrm{pCi} / \mathrm{L}$ & 0 & & & & & & SURFACE & & RAW GRAB & UNFILTERED & 412 \\
\hline 474 & $\mathrm{pCi} / \mathrm{L}$ & 0 & & & & & & SURFACE & & RAW GRAB & UNFILTERED & 147 \\
\hline 475 & $\mathrm{pCi} / \mathrm{L}$ & 0 & & & & & & SURFACE & & RAW GRAB & UNFILTERED & 147 \\
\hline 476 & $\mathrm{pCi} / \mathrm{L}$ & 0 & & & & & & RIVER & & CUMULATIVE & UNFILTERED & 553 \\
\hline 477 & $\mathrm{pCi} / \mathrm{L}$ & 0 & & & & & & RIVER & & CUMULATIVE & UNFILTERED & 553 \\
\hline 478 & $\mathrm{pCi} / \mathrm{L}$ & 0 & & & & U & & SANITARY & & CUMULATIVE & UNFILTERED & 491 \\
\hline 479 & $\mathrm{pCi} / \mathrm{L}$ & 0 & & & & U & & SANITARY & & CUMULATIVE & UNFILTERED & 385 \\
\hline 480 & $\mathrm{pCi} / \mathrm{L}$ & 0 & & & & & & RIVER & & CUMULATIVE & UNFILTERED & 463 \\
\hline 481 & $\mathrm{pCi} / \mathrm{L}$ & 0 & & & & & & SURFACE & & RAW GRAB & UNFILTERED & 444 \\
\hline 482 & $\mathrm{pCi} / \mathrm{L}$ & 0 & & & & & & SURFACE & & RAW GRAB & UNFILTERED & 147 \\
\hline 483 & $\mathrm{pCi} / \mathrm{L}$ & 0 & & & & & & RIVER & & RAW GRAB & UNFILTERED & 277 \\
\hline 484 & $p C i / L$ & 0 & & & & & & RIVER & & RAW GRAB & UNFILTERED & 277 \\
\hline 485 & $\mathrm{pCi} / \mathrm{L}$ & 0 & & & & U & & RIVER & & RAW GRAB & UNFILTERED & 277 \\
\hline 486 & $\mathrm{pCi} / \mathrm{L}$ & 0 & & & & & & SANITARY & & CUMULATIVE & UNFILTERED & 491 \\
\hline 487 & $\mathrm{pCi} / \mathrm{L}$ & 0 & & & & & & SANITARY & & CUMULATIVE & UNFILTERED & 385 \\
\hline 488 & $\mathrm{pCi} / \mathrm{L}$ & 0 & & & & & & RIVER & & CUMULATIVE & UNFILTERED & 463 \\
\hline 489 & $\mathrm{pCi} / \mathrm{L}$ & 0 & & & & & & RIVER & & CUMULATIVE & UNFILTERED & 463 \\
\hline 490 & $\mathrm{pCi} / \mathrm{L}$ & 0 & & & & & & RIVER & & CUMULATIVE & UNFILTERED & 463 \\
\hline 491 & $\mathrm{pCi} / \mathrm{L}$ & 0 & & & & & & RIVER & & CUMULATIVE & UNFILTERED & 463 \\
\hline 492 & $\mathrm{pCi} / \mathrm{L}$ & 0 & & & & & & RIVER & & RAW GRAB & UNFILTERED & 456 \\
\hline 493 & $p C i / L$ & 0 & & & & & & RIVER & & RAW GRAB & UNFILTERED & 273 \\
\hline 494 & $\mathrm{pCi} / \mathrm{L}$ & 0 & & & & & & RIVER & & CUMULATIVE & UNFILTERED & 553 \\
\hline 495 & pCi/L & 0 & & & & $\mathrm{U}$ & & SANITARY & & CUMULATIVE & UNFILTERED & 491 \\
\hline 496 & $\mathrm{pCi} / \mathrm{L}$ & 0 & & & & U & & SANITARY & & CUMULATIVE & UNFILTERED & 385 \\
\hline 497 & $\mathrm{pCi} / \mathrm{L}$ & 0 & & & & & & SURFACE & & CUMULATIVE & UNFILTERED & 60 \\
\hline 498 & $\mathrm{pCi} / \mathrm{L}$ & 0 & & & & U & & RIVER & & CUMULATIVE & UNFILTERED & 553 \\
\hline 499 & $\mathrm{pCi} / \mathrm{L}$ & 0 & & & & & & SURFACE & & RAW GRAB & UNFILTERED & 147 \\
\hline 500 & $\mathrm{pCi} / \mathrm{L}$ & 0 & & & & & & SANITARY & & CUMULATIVE & UNFILTERED & 491 \\
\hline 501 & $\mathrm{pCi} / \mathrm{L}$ & 0 & & & & & & SANITARY & & CUMULATIVE & UNFILTERED & 385 \\
\hline 502 & $\mathrm{pCi} / \mathrm{L}$ & 0 & & & & & & RIVER & & CUMULATIVE & UNFILTERED & 463 \\
\hline 503 & $\mathrm{pCi} / \mathrm{L}$ & 0 & & & & U & & SANITARY & & CUMULATIVE & UNFILTERED & 385 \\
\hline 504 & $\mathrm{pCi} / \mathrm{L}$ & 0 & & & & & & SURFACE & & RAW GRAB & UNFILTERED & 444 \\
\hline 505 & pCi/L & 0 & & & & & & RIVER & & RAW GRAB & UNFILTERED & 277 \\
\hline 506 & $\mathrm{pCi} / \mathrm{L}$ & 0 & & & & U & & RIVER & & RAW GRAB & UNFILTERED & 277 \\
\hline 507 & $\mathrm{pCi} / \mathrm{L}$ & 0 & & & & & & RIVER & & RAW GRAB & UNFILTERED & 277 \\
\hline 508 & $\mathrm{pCi} / \mathrm{L}$ & 0 & & & & & & SANITARY & & CUMULATIVE & UNFILTERED & 491 \\
\hline 509 & $\mathrm{pCi} / \mathrm{L}$ & 0 & & & & & & SANITARY & & CUMULATIVE & UNFILTERED & 385 \\
\hline 510 & $\mathrm{pCi} / \mathrm{L}$ & 0 & & & & & & SANITARY & & CUMULATIVE & UNFILTERED & 385 \\
\hline 511 & $\mathrm{pCi} / \mathrm{L}$ & 0 & & & & & & RIVER & & CUMULATIVE & UNFILTERED & 463 \\
\hline 512 & $\mathrm{pCi} / \mathrm{L}$ & 0 & & & & & & RIVER & & CUMULATIVE & UNFILTERED & 553 \\
\hline 513 & $\mathrm{pCi} / \mathrm{L}$ & 0 & & & & & & RIVER & & CUMULATIVE & UNFILTERED & 463 \\
\hline 514 & $\mathrm{pCi} / \mathrm{L}$ & 0 & & & & & & RIVER & & CUMULATIVE & UNFILTERED & 463 \\
\hline 515 & pCi/L & 0 & & & & & & RIVER & & CUMULATIVE & UNFILTERED & 463 \\
\hline 516 & $\mathrm{pCi} / \mathrm{L}$ & 0 & & & & & & RIVER & & CUMULATIVE & UNFILTERED & 463 \\
\hline 517 & $\mathrm{pCi} / \mathrm{L}$ & 0 & & & & & & RIVER & & RAW GRAB & UNFILTERED & 456 \\
\hline 518 & $\mathrm{pCi} / \mathrm{L}$ & 0 & & & & & & SANITARY & & CUMULATIVE & UNFILTERED & 491 \\
\hline 519 & $\mathrm{pCi} / \mathrm{L}$ & 0 & & & & & & SANITARY & & CUMULATIVE & UNFILTERED & 385 \\
\hline 520 & $\mathrm{pCi} / \mathrm{L}$ & 0 & & & & & & SURFACE & & CUMULATIVE & UNFILTERED & 60 \\
\hline 521 & $\mathrm{pCi} / \mathrm{L}$ & 0 & D & & & & & SURFACE & & RAW GRAB & UNFILTERED & 147 \\
\hline
\end{tabular}




\begin{tabular}{|c|c|c|c|c|c|c|c|c|c|c|c|c|}
\hline & $\mathrm{N}$ & $\mathrm{O}$ & $\mathrm{P}$ & $\mathrm{Q}$ & $\mathrm{R}$ & $\mathrm{s}$ & $\mathrm{T}$ & $\bar{U}$ & $\mathrm{v}$ & $\mathrm{W}$ & $\mathrm{x}$ & $\mathrm{Y}$ \\
\hline 1 & ANAL_UNITS_RPTD & COUNTING_ERROR & TOTAL_ANAL_ERROR & MIN_DETECTABLE_ACTIVITY & PCNT_MOISTURE & LAB_QUALIFIER & REVIEW_QUALIFIER & SAMP_FROM & SAMP_ITEM & SAMP_MTHD & COLL_MTHD & SAMP_SITE_ID \\
\hline $522 \mathrm{r}$ & $\mathrm{pCi} / \mathrm{L}$ & 0 & & & & $\mathrm{U}$ & & SANITARY & & CUMULATIVE & UNFILTERED & 491 \\
\hline 523 & $\mathrm{pCi} / \mathrm{L}$ & 0 & & & & $\mathrm{U}$ & & SANITARY & & CUMULATIVE & UNFILTERED & 385 \\
\hline 524 & $\mathrm{pCi} / \mathrm{L}$ & 0 & & & & U & & RIVER & & CUMULATIVE & UNFILTERED & 463 \\
\hline 525 & $\mathrm{pCi} / \mathrm{L}$ & 0 & & & & & & SANITARY & & CUMULATIVE & UNFILTERED & 491 \\
\hline 526 & $\mathrm{pCi} / \mathrm{L}$ & 0 & & & & & & SANITARY & & CUMULATIVE & UNFILTERED & 385 \\
\hline $527 \mathrm{r}$ & $\mathrm{pCi} / \mathrm{L}$ & 0 & & & & & & RIVER & & CUMULATIVE & UNFILTERED & 463 \\
\hline 528 & $\mathrm{pCi} / \mathrm{L}$ & 0 & & & & & & SURFACE & & RAW GRAB & UNFILTERED & 444 \\
\hline 529 & $\mathrm{pCi} / \mathrm{L}$ & 0 & & & & & & RIVER & & RAW GRAB & UNFILTERED & 277 \\
\hline 530 & $\mathrm{pCi} / \mathrm{L}$ & 0 & & & & & & RIVER & & RAW GRAB & UNFILTERED & 277 \\
\hline 531 & $\mathrm{pCi} / \mathrm{L}$ & 0 & & & & & & RIVER & & RAW GRAB & UNFILTERED & 277 \\
\hline 532 & $\mathrm{pCi} / \mathrm{L}$ & 0 & & & & U & & SANITARY & & CUMULATIVE & UNFILTERED & 491 \\
\hline 533 & $\mathrm{pCi} / \mathrm{L}$ & 0 & & & & & & SANITARY & & CUMULATIVE & UNFILTERED & 385 \\
\hline 534 & $\mathrm{pCi} / \mathrm{L}$ & 0 & & & & & & RIVER & & CUMULATIVE & UNFILTERED & 463 \\
\hline 535 & $\mathrm{pCi} / \mathrm{L}$ & 0 & & & & U & & RIVER & & CUMULATIVE & UNFILTERED & 553 \\
\hline 536 & $\mathrm{pCi} / \mathrm{L}$ & 0 & & & & & & RIVER & & CUMULATIVE & UNFILTERED & 553 \\
\hline 537 & $\mathrm{pCi} / \mathrm{L}$ & 0 & & & & & & RIVER & & CUMULATIVE & UNFILTERED & 463 \\
\hline 538 & pCi/L & 0 & & & & & & RIVER & & RAW GRAB & UNFILTERED & 456 \\
\hline 539 & $\mathrm{pCi} / \mathrm{L}$ & 0 & & & & & & SANITARY & & CUMULATIVE & UNFILTERED & 491 \\
\hline 540 & $\mathrm{pCi} / \mathrm{L}$ & 0 & & & & & & SANITARY & & CUMULATIVE & UNFILTERED & 385 \\
\hline 541 & $\mathrm{pCi} / \mathrm{L}$ & 0 & & & & & & SANITARY & & CUMULATIVE & UNFILTERED & 385 \\
\hline 542 & $\mathrm{pCi} / \mathrm{L}$ & 0 & & & & & & RIVER & & CUMULATIVE & UNFILTERED & 463 \\
\hline 543 & $\mathrm{pCi} / \mathrm{L}$ & 0 & & & & & & RIVER & & CUMULATIVE & UNFILTERED & 463 \\
\hline 544 & $\mathrm{pCi} / \mathrm{L}$ & 0 & & & & & & RIVER & & CUMULATIVE & UNFILTERED & 463 \\
\hline 545 & $\mathrm{pCi} / \mathrm{L}$ & 0 & & & & & & SANITARY & & CUMULATIVE & UNFILTERED & 491 \\
\hline 546 & pCi/L & 0 & & & & U & & SANITARY & & CUMULATIVE & UNFILTERED & 385 \\
\hline 547 & pCi/L & 0 & & & & $U$ & & RIVER & & CUMULATIVE & UNFILTERED & 463 \\
\hline 548 & $\mathrm{pCi} / \mathrm{L}$ & 0 & & & & $\mathrm{U}$ & & SURFACE & & RAW GRAB & UNFILTERED & 121 \\
\hline 549 & $\mathrm{pCi} / \mathrm{L}$ & 0 & & & & $U$ & & SURFACE & & RAW GRAB & UNFILTERED & 484 \\
\hline 550 & $\mathrm{pCi} / \mathrm{L}$ & 0 & & & & & & SURFACE & & RAW GRAB & UNFILTERED & 509 \\
\hline 551 & pCi/L & 0 & & & & U & & SURFACE & & RAW GRAB & UNFILTERED & 509 \\
\hline 552 & pCi/L & 0 & & & & & & SURFACE & & RAW GRAB & UNFILTERED & 146 \\
\hline 553 & pCi/L & 0 & & & & & & SURFACE & & RAW GRAB & UNFILTERED & 146 \\
\hline 554 & $\mathrm{pCi} / \mathrm{L}$ & 0 & & & & & & SURFACE & & RAW GRAB & UNFILTERED & 412 \\
\hline 555 & $\mathrm{pCi} / \mathrm{L}$ & 0 & & & & & & SURFACE & & RAW GRAB & UNFILTERED & 147 \\
\hline 556 & $\mathrm{pCi} / \mathrm{L}$ & 0 & & & & & & SURFACE & & RAW GRAB & UNFILTERED & 147 \\
\hline 557 & $\mathrm{pCi} / \mathrm{L}$ & 0 & & & & & & SANITARY & & CUMULATIVE & UNFILTERED & 491 \\
\hline 558 & $\mathrm{pCi} / \mathrm{L}$ & 0 & & & & & & SANITARY & & CUMULATIVE & UNFILTERED & 385 \\
\hline 559 & $\mathrm{pCi} / \mathrm{L}$ & 0 & & & & U & & RIVER & & CUMULATIVE & UNFILTERED & 463 \\
\hline 560 & $\mathrm{pCi} / \mathrm{L}$ & 0 & & & & & & SURFACE & & RAW GRAB & UNFILTERED & 444 \\
\hline 561 & $\mathrm{pCi} / \mathrm{L}$ & 0 & & & & $\mathrm{U}$ & & SANITARY & & CUMULATIVE & UNFILTERED & 491 \\
\hline 562 & $\mathrm{pCi} / \mathrm{L}$ & 0 & & & & U & & SANITARY & & CUMULATIVE & UNFILTERED & 385 \\
\hline 563 & $\mathrm{pCi} / \mathrm{L}$ & 0 & & & & & & RIVER & & CUMULATIVE & UNFILTERED & 463 \\
\hline 564 & pCi/L & 0 & & & & U & & SURFACE & & RAW GRAB & UNFILTERED & 67 \\
\hline 565 & pCi/L & 0 & & & & U & & SANITARY & & CUMULATIVE & UNFILTERED & 491 \\
\hline 566 & $\mathrm{pCi} / \mathrm{L}$ & 0 & & & & & & SANITARY & & CUMULATIVE & UNFILTERED & 385 \\
\hline 567 & $\mathrm{pCi} / \mathrm{L}$ & 0 & & & & U & & SANITARY & & CUMULATIVE & UNFILTERED & 385 \\
\hline 568 & $\mathrm{pCi} / \mathrm{L}$ & 0 & & & & & & RIVER & & CUMULATIVE & UNFILTERED & 463 \\
\hline 569 & $\mathrm{pCi} / \mathrm{L}$ & 0 & & & & U & & RIVER & & CUMULATIVE & UNFILTERED & 463 \\
\hline 570 & $\mathrm{pCi} / \mathrm{L}$ & 0 & & & & & & RIVER & & CUMULATIVE & UNFILTERED & 463 \\
\hline 571 & $\mathrm{pCi} / \mathrm{L}$ & 0 & & & & & & RIVER & & RAW GRAB & UNFILTERED & 456 \\
\hline 572 & $\mathrm{pCi} / \mathrm{L}$ & 0 & & & & & & RIVER & & RAW GRAB & UNFILTERED & 277 \\
\hline 573 & $\mathrm{pCi} / \mathrm{L}$ & 0 & & & & $U_{1}$ & & RIVER & & RAW GRAB & UNFILTERED & 277 \\
\hline
\end{tabular}




\begin{tabular}{|c|c|c|c|c|c|c|c|c|c|c|c|c|c|}
\hline & $\mathrm{N}$ & 0 & & $\mathrm{P}$ & $\mathrm{Q}$ & $\mathrm{R}$ & $\mathrm{s}$ & $\mathrm{T}$ & $\mathrm{U}$ & $\mathrm{V}$ & $\mathrm{W}$ & $\mathrm{x}$ & $\bar{Y}$ \\
\hline 1 & ANAL_UNITS_RPTD & COUNTING & ERROR & TOTAL_ANAL_ERROR & MIN_DETECTABLE_ACTIVITT & PCNT_MOISTURE & LAB_QUALIFIER & REVIEW_QUALIFIER & SAMP_FROM & SAMP_ITEM & SAMP_MTHD & COLL_MTHD & SAMP_SITE_ID \\
\hline 574 & $\mathrm{pCi} / \mathrm{L}$ & & 0 & & & & & & RIVER & & RAW GRAB & UNFILTERED & 277 \\
\hline 575 & $\mathrm{pCi} / \mathrm{L}$ & & 0 & & & & & & RIVER & & RAW GRAB & UNFILTERED & 273 \\
\hline 576 & $\mathrm{pCi} / \mathrm{L}$ & & 0 & & & & & & RIVER & & CUMULATIVE & UNFILTERED & 553 \\
\hline 577 & $\mathrm{pCi} / \mathrm{L}$ & & 0 & & & & $u$ & & SANITARY & & CUMULATIVE & UNFILTERED & 491 \\
\hline 578 & $\mathrm{pCi} / \mathrm{L}$ & & 0 & & & & & & SANITARY & & CUMULATIVE & UNFILTERED & 385 \\
\hline 579 & $\mathrm{pCi} / \mathrm{L}$ & & 0 & & & & & & SURFACE & & CUMULATIVE & UNFILTERED & 60 \\
\hline 580 & $\mathrm{pCi} / \mathrm{L}$ & & 0 & & & & & & RIVER & & CUMULATIVE & UNFILTERED & 463 \\
\hline 581 & $\mathrm{pCi} / \mathrm{L}$ & & 0 & & & & & & RIVER & & CUMULATIVE & UNFILTERED & 463 \\
\hline 582 & $\mathrm{pCi} / \mathrm{L}$ & & 0 & & & & & & SURFACE & & RAW GRAB & UNFILTERED & 147 \\
\hline 583 & $\mathrm{pCi} / \mathrm{L}$ & & 0 & & & & $u$ & & SANITARY & & CUMULATIVE & UNFILTERED & 491 \\
\hline 584 & $\mathrm{pCi} / \mathrm{L}$ & & 0 & & & & & & SANITARY & & CUMULATIVE & UNFILTERED & 385 \\
\hline 585 & $\mathrm{pCi} / \mathrm{L}$ & & 0 & & & & & & RIVER & & CUMULATIVE & UNFILTERED & 463 \\
\hline 586 & $\mathrm{pCi} / \mathrm{L}$ & & 0 & & & & & & SURFACE & & RAW GRAB & UNFILTERED & 444 \\
\hline 587 & $\mathrm{pCi} / \mathrm{L}$ & & 0 & & & & & & RIVER & & RAW GRAB & UNFILTERED & 277 \\
\hline 588 & $\mathrm{pCi} / \mathrm{L}$ & & 0 & & & & U & & RIVER & & RAW GRAB & UNFILTERED & 277 \\
\hline 589 & $\mathrm{pCi} / \mathrm{L}$ & & 0 & & & & & & RIVER & & RAW GRAB & UNFILTERED & 277 \\
\hline 590 & $\mathrm{pCi} / \mathrm{L}$ & & 0 & & & & $U$ & & SANITARY & & CUMULATIVE & UNFILTERED & 491 \\
\hline 591 & $\mathrm{pCi} / \mathrm{L}$ & & 0 & & & & & & SANITARY & & CUMULATIVE & UNFILTERED & 385 \\
\hline 592 & $\mathrm{pCi} / \mathrm{L}$ & & 0 & & & & & & SANITARY & & CUMULATIVE & UNFILTERED & 385 \\
\hline 593 & $\mathrm{pCi} / \mathrm{L}$ & & 0 & & & & $U$ & & RIVER & & CUMULATIVE & UNFILTERED & 553 \\
\hline 594 & $\mathrm{pCi} / \mathrm{L}$ & & 0 & & & & & & RIVER & & CUMULATIVE & UNFILTERED & 553 \\
\hline 595 & $\mathrm{pCi} / \mathrm{L}$ & & 0 & & & & & & SURFACE & & CUMULATIVE & UNFILTERED & 60 \\
\hline 596 & $\mathrm{pCi} / \mathrm{L}$ & & 0 & & & & & & RIVER & & CUMULATIVE & UNFILTERED & 463 \\
\hline 597 & $\mathrm{pCi} / \mathrm{L}$ & & 0 & & & & $U$ & & RIVER & & CUMULATIVE & UNFILTERED & 463 \\
\hline 598 & $\mathrm{pCi} / \mathrm{L}$ & & 0 & & & & & & RIVER & & CUMULATIVE & UNFILTERED & 463 \\
\hline 599 & $\mathrm{pCi} / \mathrm{L}$ & & 0 & & & & & & RIVER & & RAW GRAB & UNFILTERED & 456 \\
\hline 600 & $\mathrm{pCi} / \mathrm{L}$ & & 0 & & & & & & SURFACE & & RAW GRAB & UNFILTERED & 147 \\
\hline 601 & $\mathrm{pCi} / \mathrm{L}$ & & 0 & & & & & & RIVER & & CUMULATIVE & UNFILTERED & 463 \\
\hline 602 & $\mathrm{pCi} / \mathrm{L}$ & & 0 & & & & $U$ & & SANITARY & & CUMULATIVE & UNFILTERED & 491 \\
\hline 603 & $\mathrm{pCi} / \mathrm{L}$ & & 0 & & & & & & SURFACE & & RAW GRAB & UNFILTERED & 444 \\
\hline 604 & $\mathrm{pCi} / \mathrm{L}$ & & 0 & & & & & & RIVER & & RAW GRAB & UNFILTERED & 277 \\
\hline 605 & $\mathrm{pCi} / \mathrm{L}$ & & 0 & & & & & & RIVER & & RAW GRAB & UNFILTERED & 277 \\
\hline 606 & $\mathrm{pCi} / \mathrm{L}$ & & 0 & & & & & & RIVER & & RAW GRAB & UNFILTERED & 277 \\
\hline 607 & $\mathrm{pCi} / \mathrm{L}$ & & 0 & & & & & & SANITARY & & CUMULATIVE & UNFILTERED & 491 \\
\hline 608 & $p \mathrm{pi} / \mathrm{L}$ & & 0 & & & & & & SANITARY & & CUMULATIVE & UNFILTERED & 385 \\
\hline 609 & $\mathrm{pCi} / \mathrm{L}$ & & 0 & & & & & & RIVER & & CUMULATIVE & UNFILTERED & 463 \\
\hline 610 & $\mathrm{pCi} / \mathrm{L}$ & & 0 & & & & & & RIVER & & CUMULATIVE & UNFILTERED & 463 \\
\hline 611 & $\mathrm{pCi} / \mathrm{L}$ & & 0 & & & & & & SANITARY & & CUMULATIVE & UNFILTERED & 491 \\
\hline 612 & $\mathrm{pCi} / \mathrm{L}$ & & 0 & & & & & & SANITARY & & CUMULATIVE & UNFILTERED & 385 \\
\hline 613 & $\mathrm{pCi} / \mathrm{L}$ & & 0 & & & & & & SANITARY & & CUMULATIVE & UNFILTERED & 385 \\
\hline 614 & $\mathrm{pCi} / \mathrm{L}$ & & 0 & & & & & & RIVER & & CUMULATIVE & UNFILTERED & 553 \\
\hline 615 & $\mathrm{pCi} / \mathrm{L}$ & & 0 & & & & $U$ & & RIVER & & CUMULATIVE & UNFILTERED & 553 \\
\hline 616 & $\mathrm{pCi} / \mathrm{L}$ & & 0 & & & & & & RIVER & & CUMULATIVE & UNFILTERED & 463 \\
\hline 617 & $\mathrm{pCi} / \mathrm{L}$ & & 0 & & & & & & RIVER & & CUMULATIVE & UNFILTERED & 463 \\
\hline 618 & $\mathrm{pCi} / \mathrm{L}$ & & 0 & & & & & & RIVER & & CUMULATIVE & UNFILTERED & 463 \\
\hline 619 & $\mathrm{pCi} / \mathrm{L}$ & & 0 & & & & & & RIVER & & RAW GRAB & UNFILTERED & 456 \\
\hline 620 & $\mathrm{pCi} / \mathrm{L}$ & & 0 & & & & $U$ & & SANITARY & & CUMULATIVE & UNFILTERED & 491 \\
\hline 621 & $\mathrm{pCi} / \mathrm{L}$ & & 0 & & & & & & SANITARY & & CUMULATIVE & UNFILTERED & 385 \\
\hline 622 & $\mathrm{pCi} / \mathrm{L}$ & & 0 & & & & & & SURFACE & & CUMULATIVE & UNFILTERED & 60 \\
\hline 623 & $\mathrm{pCi} / \mathrm{L}$ & & 0 & & & & & & RIVER & & CUMULATIVE & UNFILTERED & 463 \\
\hline 624 & $\mathrm{pCi} / \mathrm{L}$ & & 0 & & & & & & SANITARY & & CUMULATIVE & UNFILTERED & 491 \\
\hline 625 & $\mathrm{pCi} / \mathrm{L}$ & & 0 & & & & & & SANITARY & & CUMULATIVE & UNFILTERED & 385 \\
\hline
\end{tabular}




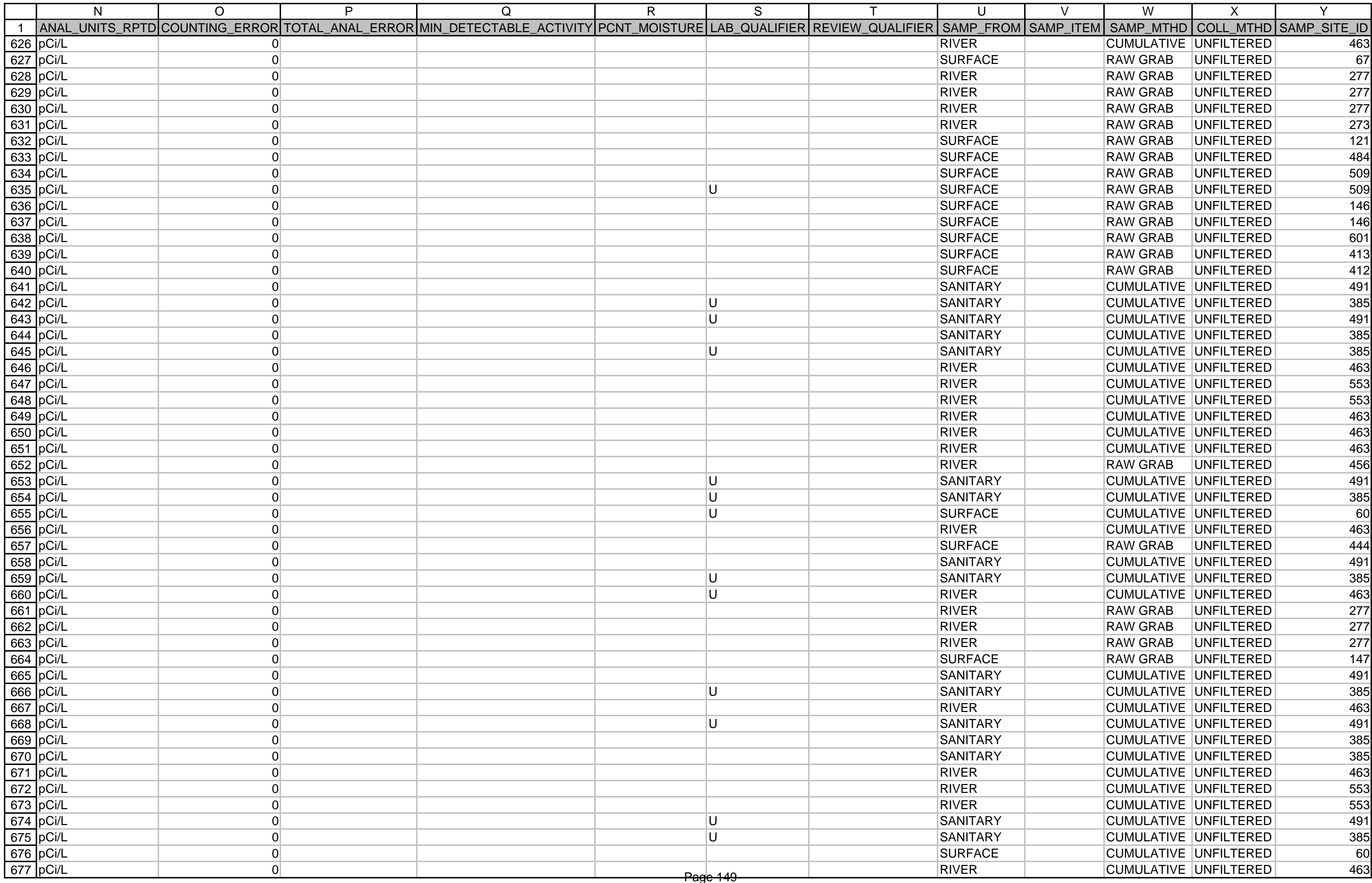




\begin{tabular}{|c|c|c|c|c|c|c|c|c|c|c|c|c|}
\hline & $\mathrm{N}$ & $\mathrm{O}$ & $\mathrm{P}$ & $\mathrm{Q}$ & $\mathrm{R}$ & $\mathrm{s}$ & $\mathrm{T}$ & $\bar{U}$ & $\mathrm{v}$ & $\mathrm{W}$ & $\mathrm{x}$ & $\mathrm{Y}$ \\
\hline 1 & ANAL_UNITS_RPTD & COUNTING_ERROR & TOTAL_ANAL_ERROR & MIN_DETECTABLE_ACTIVITY & PCNT_MOISTURE & LAB_QUALIFIER & REVIEW_QUALIFIER & SAMP_FROM & SAMP_ITEM & SAMP_MTHD & COLL_MTHD & SAMP_SITE_ID \\
\hline 678 & $\mathrm{pCi} / \mathrm{L}$ & 0 & & & & & & RIVER & & CUMULATIVE & UNFILTERED & 463 \\
\hline 679 & $\mathrm{pCi} / \mathrm{L}$ & 0 & & & & $\mathrm{U}$ & & RIVER & & CUMULATIVE & UNFILTERED & 463 \\
\hline 680 & $\mathrm{pCi} / \mathrm{L}$ & 0 & & & & & & RIVER & & CUMULATIVE & UNFILTERED & 463 \\
\hline 681 & $\mathrm{pCi} / \mathrm{L}$ & 0 & & & & & & RIVER & & RAW GRAB & UNFILTERED & 456 \\
\hline 682 & $\mathrm{pCi} / \mathrm{L}$ & 0 & & & & & & SURFACE & & RAW GRAB & UNFILTERED & 444 \\
\hline 683 & $\mathrm{pCi} / \mathrm{L}$ & 0 & & & & & & SANITARY & & CUMULATIVE & UNFILTERED & 491 \\
\hline 684 & pCi/L & 0 & & & & & & SANITARY & & CUMULATIVE & UNFILTERED & 385 \\
\hline 685 & $\mathrm{pCi} / \mathrm{L}$ & 0 & & & & & & RIVER & & CUMULATIVE & UNFILTERED & 463 \\
\hline 686 & $\mathrm{pCi} / \mathrm{L}$ & 0 & & & & & & RIVER & & RAW GRAB & UNFILTERED & 277 \\
\hline 687 & $\mathrm{pCi} / \mathrm{L}$ & 0 & & & & & & RIVER & & RAW GRAB & UNFILTERED & 277 \\
\hline 688 & $\mathrm{pCi} / \mathrm{L}$ & 0 & & & & & & RIVER & & RAW GRAB & UNFILTERED & 277 \\
\hline 689 & $\mathrm{pCi} / \mathrm{L}$ & 0 & & & & & & SURFACE & & RAW GRAB & UNFILTERED & 147 \\
\hline 690 & pCi/L & 0 & & & & $U$ & & SANITARY & & CUMULATIVE & UNFILTERED & 491 \\
\hline 691 & pCi/L & 0 & & & & U & & SANITARY & & CUMULATIVE & UNFILTERED & 385 \\
\hline 692 & pCi/L & 0 & & & & & & RIVER & & CUMULATIVE & UNFILTERED & 463 \\
\hline 693 & pCi/L & 0 & & & & & & SANITARY & & CUMULATIVE & UNFILTERED & 491 \\
\hline 694 & pCi/L & 0 & & & & & & SANITARY & & CUMULATIVE & UNFILTERED & 385 \\
\hline 695 & pCi/L & 0 & & & & & & RIVER & & CUMULATIVE & UNFILTERED & 463 \\
\hline 696 & pCi/L & 0 & & & & & & SANITARY & & CUMULATIVE & UNFILTERED & 491 \\
\hline 697 & pCi/L & 0 & & & & & & SANITARY & & CUMULATIVE & UNFILTERED & 385 \\
\hline 698 & pCi/L & 0 & & & & U & & SANITARY & & CUMULATIVE & UNFILTERED & 385 \\
\hline 699 & pCi/L & 0 & & & & & & RIVER & & CUMULATIVE & UNFILTERED & 553 \\
\hline 700 & pCi/L & 0 & & & & & & RIVER & & CUMULATIVE & UNFILTERED & 553 \\
\hline 701 & pCi/L & 0 & & & & & & RIVER & & CUMULATIVE & UNFILTERED & 463 \\
\hline 702 & pCi/L & 0 & & & & & & RIVER & & CUMULATIVE & UNFILTERED & 463 \\
\hline 703 & pCi/L & 0 & & & & U & & RIVER & & CUMULATIVE & UNFILTERED & 463 \\
\hline 704 & $\mathrm{pCi} / \mathrm{L}$ & 0 & & & & & & RIVER & & RAW GRAB & UNFILTERED & 456 \\
\hline 705 & $\mathrm{pCi} / \mathrm{L}$ & 0 & & & & & & SURFACE & & RAW GRAB & UNFILTERED & 444 \\
\hline 706 & pCi/L & 0 & & & & & & SANITARY & & CUMULATIVE & UNFILTERED & 491 \\
\hline 707 & pCi/L & 0 & & & & U & & SANITARY & & CUMULATIVE & UNFILTERED & 385 \\
\hline 708 & $\mathrm{pCi} / \mathrm{L}$ & 0 & & & & & & RIVER & & CUMULATIVE & UNFILTERED & 463 \\
\hline 709 & pCi/L & 0 & & & & $U$ & & SURFACE & & RAW GRAB & UNFILTERED & 67 \\
\hline 710 & $\mathrm{pCi} / \mathrm{L}$ & 0 & & & & $U$ & & SANITARY & & CUMULATIVE & UNFILTERED & 491 \\
\hline 711 & $\mathrm{pCi} / \mathrm{L}$ & 0 & & & & $\mathrm{U}$ & & SANITARY & & CUMULATIVE & UNFILTERED & 385 \\
\hline 712 & pCi/L & 0 & & & & & & RIVER & & CUMULATIVE & UNFILTERED & 463 \\
\hline 713 & pCi/L & 0 & & & & & & SURFACE & & RAW GRAB & UNFILTERED & 121 \\
\hline 714 & pCi/L & 0 & & & & & & SURFACE & & RAW GRAB & UNFILTERED & 484 \\
\hline 715 & $\mathrm{pCi} / \mathrm{L}$ & 0 & & & & U & & SURFACE & & RAW GRAB & UNFILTERED & 413 \\
\hline 716 & $\mathrm{pCi} / \mathrm{L}$ & 0 & & & & U & & SANITARY & & CUMULATIVE & UNFILTERED & 491 \\
\hline 717 & $\mathrm{pCi} / \mathrm{L}$ & 0 & & & & & & SANITARY & & CUMULATIVE & UNFILTERED & 385 \\
\hline 718 & $\mathrm{pCi} / \mathrm{L}$ & 0 & & & & U & & RIVER & & CUMULATIVE & UNFILTERED & 463 \\
\hline 719 & pCi/L & 0 & & & & & & SURFACE & & RAW GRAB & UNFILTERED & 509 \\
\hline 720 & pCi/L & 0 & & & & & & SURFACE & & RAW GRAB & UNFILTERED & 509 \\
\hline 721 & pCi/L & 0 & & & & & & SURFACE & & RAW GRAB & UNFILTERED & 146 \\
\hline 722 & pCi/L & 0 & & & & & & SURFACE & & RAW GRAB & UNFILTERED & 146 \\
\hline 723 & $\mathrm{pCi} / \mathrm{L}$ & 0 & & & & U & & SURFACE & & RAW GRAB & UNFILTERED & 412 \\
\hline 724 & pCi/L & 0 & & & & $U$ & & SURFACE & & RAW GRAB & UNFILTERED & 147 \\
\hline 725 & pCi/L & 0 & & & & & & SURFACE & & RAW GRAB & UNFILTERED & 147 \\
\hline 726 & $\mathrm{pCi} / \mathrm{L}$ & 0 & & & & & & RIVER & & RAW GRAB & UNFILTERED & 462 \\
\hline 727 & pCi/L & 0 & & & & U & & SANITARY & & CUMULATIVE & UNFILTERED & 491 \\
\hline 728 & pCi/L & 0 & & & & & & SANITARY & & CUMULATIVE & UNFILTERED & 385 \\
\hline 729 & $\mathrm{pCi} / \mathrm{L}$ & 0 & & & & & & SANITARY & & CUMULATIVE & UNFILTERED & 385 \\
\hline
\end{tabular}




\begin{tabular}{|c|c|c|c|c|c|c|c|c|c|c|c|c|}
\hline & $\mathrm{N}$ & $\mathrm{O}$ & $\mathrm{P}$ & $\mathrm{Q}$ & $\mathrm{R}$ & $\mathrm{s}$ & $\mathrm{T}$ & $\mathrm{U}$ & $\mathrm{V}$ & $\mathrm{w}$ & $\mathrm{X}$ & $\mathrm{Y}$ \\
\hline 1 & ANAL_UNITS_RPTD & COUNTING_ERROR & TOTAL_ANAL_ERROR & MIN_DETECTABLE_ACTIVITY & PCNT_MOISTURE & LAB_QUALIFIER & REVIEW_QUALIFIER & SAMP_FROM & SAMP_ITEM & SAMP_MTHD & COLL_MTHD & SAMP_SITE_ID \\
\hline 730 & $\mathrm{pCi} / \mathrm{L}$ & 0 & & & & $U$ & & RIVER & & CUMULATIVE & UNFILTERED & 463 \\
\hline 731 & $\mathrm{pCi} / \mathrm{L}$ & 0 & & & & & & SURFACE & & RAW GRAB & UNFILTERED & 444 \\
\hline 732 & $\mathrm{pCi} / \mathrm{L}$ & 0 & & & & & & RIVER & & CUMULATIVE & UNFILTERED & 553 \\
\hline 733 & $\mathrm{pCi} / \mathrm{L}$ & 0 & & & & & & RIVER & & CUMULATIVE & UNFILTERED & 553 \\
\hline 734 & $\mathrm{pCi} / \mathrm{L}$ & 0 & & & & & & SANITARY & & CUMULATIVE & UNFILTERED & 491 \\
\hline 735 & $\mathrm{pCi} / \mathrm{L}$ & 0 & & & & & & SANITARY & & SAN. GRAB & UNFILTERED & 491 \\
\hline 736 & $\mathrm{pCi} / \mathrm{L}$ & 0 & & & & U & & SANITARY & & CUMULATIVE & UNFILTERED & 385 \\
\hline 737 & $\mathrm{pCi} / \mathrm{L}$ & 0 & & & & & & RIVER & & CUMULATIVE & UNFILTERED & 463 \\
\hline 738 & $\mathrm{pCi} / \mathrm{L}$ & 0 & & & & & & RIVER & & CUMULATIVE & UNFILTERED & 463 \\
\hline 739 & $\mathrm{pCi} / \mathrm{L}$ & 0 & & & & U & & RIVER & & CUMULATIVE & UNFILTERED & 463 \\
\hline 740 & $\mathrm{pCi} / \mathrm{L}$ & 0 & & & & $\mathrm{U}$ & & RIVER & & CUMULATIVE & UNFILTERED & 463 \\
\hline 741 & $\mathrm{pCi} / \mathrm{L}$ & 0 & & & & & & RIVER & & RAW GRAB & UNFILTERED & 456 \\
\hline 742 & $\mathrm{pCi} / \mathrm{L}$ & 0 & & & & & & SANITARY & & CUMULATIVE & UNFILTERED & 491 \\
\hline 743 & $\mathrm{pCi} / \mathrm{L}$ & 0 & & & & & & RIVER & & CUMULATIVE & UNFILTERED & 463 \\
\hline 744 & $\mathrm{pCi} / \mathrm{L}$ & 0 & & & & & & RIVER & & RAW GRAB & UNFILTERED & 462 \\
\hline 745 & $\mathrm{pCi} / \mathrm{L}$ & 0 & & & & & & RIVER & & RAW GRAB & UNFILTERED & 462 \\
\hline 746 & $\mathrm{pCi} / \mathrm{L}$ & 0 & & & & & & SURFACE & & RAW GRAB & UNFILTERED & 147 \\
\hline 747 & $\mathrm{pCi} / \mathrm{L}$ & 0 & & & & & & SANITARY & & CUMULATIVE & UNFILTERED & 491 \\
\hline 748 & $\mathrm{pCi} / \mathrm{L}$ & 0 & & & & & & SANITARY & & SAN. GRAB & UNFILTERED & 491 \\
\hline 749 & $\mathrm{pCi} / \mathrm{L}$ & 0 & & & & & & SANITARY & & CUMULATIVE & UNFILTERED & 385 \\
\hline 750 & $\mathrm{pCi} / \mathrm{L}$ & 0 & & & & $U$ & & RIVER & & CUMULATIVE & UNFILTERED & 463 \\
\hline 751 & $\mathrm{pCi} / \mathrm{L}$ & 0 & & & & & & RIVER & & CUMULATIVE & UNFILTERED & 463 \\
\hline 752 & $\mathrm{pCi} / \mathrm{L}$ & 0 & & & & $U$ & & SANITARY & & CUMULATIVE & UNFILTERED & 491 \\
\hline 753 & $\mathrm{pCi} / \mathrm{L}$ & 0 & & & & & & SANITARY & & CUMULATIVE & UNFILTERED & 385 \\
\hline 754 & $\mathrm{pCi} / \mathrm{L}$ & 0 & & & & $U$ & & SANITARY & & CUMULATIVE & UNFILTERED & 385 \\
\hline 755 & $\mathrm{pCi} / \mathrm{L}$ & 0 & & & & & & SURFACE & & RAW GRAB & UNFILTERED & 444 \\
\hline 756 & $\mathrm{pCi} / \mathrm{L}$ & 0 & & & & & & RIVER & & CUMULATIVE & UNFILTERED & 553 \\
\hline 757 & $\mathrm{pCi} / \mathrm{L}$ & 0 & & & & & & RIVER & & CUMULATIVE & UNFILTERED & 553 \\
\hline 758 & $\mathrm{pCi} / \mathrm{L}$ & 0 & & & & & & SANITARY & & CUMULATIVE & UNFILTERED & 491 \\
\hline 759 & $\mathrm{pCi} / \mathrm{L}$ & 0 & & & & & & SANITARY & & CUMULATIVE & UNFILTERED & 385 \\
\hline 760 & $\mathrm{pCi} / \mathrm{L}$ & 0 & & & & & & RIVER & & CUMULATIVE & UNFILTERED & 463 \\
\hline 761 & $\mathrm{pCi} / \mathrm{L}$ & 0 & & & & & & RIVER & & CUMULATIVE & UNFILTERED & 463 \\
\hline 762 & $\mathrm{pCi} / \mathrm{L}$ & 0 & & & & U & & RIVER & & CUMULATIVE & UNFILTERED & 463 \\
\hline 763 & $\mathrm{pCi} / \mathrm{L}$ & 0 & & & & $U$ & & RIVER & & CUMULATIVE & UNFILTERED & 463 \\
\hline 764 & pCi/L & 0 & & & & & & RIVER & & RAW GRAB & UNFILTERED & 456 \\
\hline 765 & pCi/L & 0 & & & & $U$ & & SANITARY & & CUMULATIVE & UNFILTERED & 491 \\
\hline 766 & pCi/L & 0 & & & & & & SANITARY & & CUMULATIVE & UNFILTERED & 385 \\
\hline 767 & $\mathrm{pCi} / \mathrm{L}$ & 0 & & & & $U$ & & SURFACE & & CUMULATIVE & UNFILTERED & 60 \\
\hline 768 & $\mathrm{pCi} / \mathrm{L}$ & 0 & & & & & & RIVER & & CUMULATIVE & UNFILTERED & 463 \\
\hline 769 & $\mathrm{pCi} / \mathrm{L}$ & 0 & & & & & & SURFACE & & RAW GRAB & UNFILTERED & 147 \\
\hline 770 & $\mathrm{pCi} / \mathrm{L}$ & 0 & & & & & & SANITARY & & CUMULATIVE & UNFILTERED & 491 \\
\hline 771 & $\mathrm{pCi} / \mathrm{L}$ & 0 & & & & $U$ & & SANITARY & & CUMULATIVE & UNFILTERED & 385 \\
\hline 772 & $\mathrm{pCi} / \mathrm{L}$ & 0 & & & & U & & RIVER & & CUMULATIVE & UNFILTERED & 463 \\
\hline 773 & $\mathrm{pCi} / \mathrm{L}$ & 0 & & & & & & RIVER & & RAW GRAB & UNFILTERED & 462 \\
\hline 774 & pCi/L & 0 & & & & & & RIVER & & RAW GRAB & UNFILTERED & 462 \\
\hline 775 & $\mathrm{pCi} / \mathrm{L}$ & 0 & & & & & & RIVER & & RAW GRAB & UNFILTERED & 462 \\
\hline 776 & pCi/L & 0 & & & & & & SANITARY & & CUMULATIVE & UNFILTERED & 491 \\
\hline 777 & pCi/L & 0 & & & & & & SANITARY & & CUMULATIVE & UNFILTERED & 385 \\
\hline 778 & pCi/L & 0 & & & & & & RIVER & & CUMULATIVE & UNFILTERED & 463 \\
\hline 779 & pCi/L & 0 & & & & & & SURFACE & & RAW GRAB & UNFILTERED & 444 \\
\hline 780 & pCi/L & 0 & & & & $U$ & & RIVER & & CUMULATIVE & UNFILTERED & 553 \\
\hline 781 & $\mathrm{pCi} / \mathrm{L}$ & 0 & 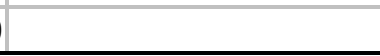 & & & & & RIVER & & CUMULATIVE & UNFILTERED & 463 \\
\hline
\end{tabular}




\begin{tabular}{|c|c|c|c|c|c|c|c|c|c|c|c|c|c|}
\hline & $\mathrm{N}$ & 0 & & $\mathrm{P}$ & $\mathrm{Q}$ & $\mathrm{R}$ & $\mathrm{s}$ & $\mathrm{T}$ & $\mathrm{U}$ & $\mathrm{V}$ & $\mathrm{W}$ & $\mathrm{x}$ & $\bar{Y}$ \\
\hline 1 & ANAL_UNITS_RPTD & COUNTING & ERROR & TOTAL_ANAL_ERROR & MIN_DETECTABLE_ACTIVITT & PCNT_MOISTURE & LAB_QUALIFIER & REVIEW_QUALIFIER & SAMP_FROM & SAMP_ITEM & SAMP_MTHD & COLL_MTHD & SAMP_SITE_ID \\
\hline 782 & $\mathrm{pCi} / \mathrm{L}$ & & 0 & & & & & & RIVER & & CUMULATIVE & UNFILTERED & 463 \\
\hline 783 & $\mathrm{pCi} / \mathrm{L}$ & & 0 & & & & & & RIVER & & CUMULATIVE & UNFILTERED & 463 \\
\hline 784 & $\mathrm{pCi} / \mathrm{L}$ & & 0 & & & & $U$ & & SANITARY & & CUMULATIVE & UNFILTERED & 491 \\
\hline 785 & $\mathrm{pCi} / \mathrm{L}$ & & 0 & & & & & & SANITARY & & CUMULATIVE & UNFILTERED & 385 \\
\hline 786 & $\mathrm{pCi} / \mathrm{L}$ & & 0 & & & & $\mathrm{U}$ & & SANITARY & & CUMULATIVE & UNFILTERED & 385 \\
\hline 787 & $\mathrm{pCi} / \mathrm{L}$ & & 0 & & & & & & RIVER & & CUMULATIVE & UNFILTERED & 463 \\
\hline 788 & $\mathrm{pCi} / \mathrm{L}$ & & 0 & & & & & & RIVER & & RAW GRAB & UNFILTERED & 456 \\
\hline 789 & $\mathrm{pCi} / \mathrm{L}$ & & 0 & & & & & & SANITARY & & CUMULATIVE & UNFILTERED & 491 \\
\hline 790 & $\mathrm{pCi} / \mathrm{L}$ & & 0 & & & & & & SANITARY & & CUMULATIVE & UNFILTERED & 385 \\
\hline 791 & $\mathrm{pCi} / \mathrm{L}$ & & 0 & & & & & & RIVER & & CUMULATIVE & UNFILTERED & 463 \\
\hline 792 & $\mathrm{pCi} / \mathrm{L}$ & & 0 & & & & & & SURFACE & & RAW GRAB & UNFILTERED & 121 \\
\hline 793 & $\mathrm{pCi} / \mathrm{L}$ & & 0 & & & & U & & SURFACE & & RAW GRAB & UNFILTERED & 509 \\
\hline 794 & $\mathrm{pCi} / \mathrm{L}$ & & 0 & & & & & & SURFACE & & RAW GRAB & UNFILTERED & 509 \\
\hline 795 & $\mathrm{pCi} / \mathrm{L}$ & & 0 & & & & & & SURFACE & & RAW GRAB & UNFILTERED & 146 \\
\hline 796 & $\mathrm{pCi} / \mathrm{L}$ & & 0 & & & & & & SURFACE & & RAW GRAB & UNFILTERED & 146 \\
\hline 797 & $\mathrm{pCi} / \mathrm{L}$ & & 0 & & & & & & SURFACE & & RAW GRAB & UNFILTERED & 412 \\
\hline 798 & $\mathrm{pCi} / \mathrm{L}$ & & 0 & & & & & & SURFACE & & RAW GRAB & UNFILTERED & 147 \\
\hline 799 & $\mathrm{pCi} / \mathrm{L}$ & & 0 & & & & & & SURFACE & & RAW GRAB & UNFILTERED & 147 \\
\hline 800 & $\mathrm{pCi} / \mathrm{L}$ & & 0 & & & & & & SANITARY & & CUMULATIVE & UNFILTERED & 491 \\
\hline 801 & $\mathrm{pCi} / \mathrm{L}$ & & 0 & & & & & & SANITARY & & CUMULATIVE & UNFILTERED & 385 \\
\hline 802 & $\mathrm{pCi} / \mathrm{L}$ & & 0 & & & & U & & SURFACE & & CUMULATIVE & UNFILTERED & 60 \\
\hline 803 & $\mathrm{pCi} / \mathrm{L}$ & & 0 & & & & & & RIVER & & CUMULATIVE & UNFILTERED & 463 \\
\hline 804 & $\mathrm{pCi} / \mathrm{L}$ & & 0 & & & & & & RIVER & & RAW GRAB & UNFILTERED & 273 \\
\hline 805 & $\mathrm{pCi} / \mathrm{L}$ & & 0 & & & & & & RIVER & & RAW GRAB & UNFILTERED & 462 \\
\hline 806 & $\mathrm{pCi} / \mathrm{L}$ & & 0 & & & & & & RIVER & & RAW GRAB & UNFILTERED & 462 \\
\hline 807 & $\mathrm{pCi} / \mathrm{L}$ & & 0 & & & & $U$ & & SANITARY & & CUMULATIVE & UNFILTERED & 491 \\
\hline 808 & $\mathrm{pCi} / \mathrm{L}$ & & 0 & & & & & & SANITARY & & CUMULATIVE & UNFILTERED & 385 \\
\hline 809 & $\mathrm{pCi} / \mathrm{L}$ & & 0 & & & & & & RIVER & & CUMULATIVE & UNFILTERED & 463 \\
\hline 810 & $\mathrm{pCi} / \mathrm{L}$ & & 0 & & & & & & SURFACE & & RAW GRAB & UNFILTERED & 444 \\
\hline 811 & $\mathrm{pCi} / \mathrm{L}$ & & 0 & & & & & & SANITARY & & CUMULATIVE & UNFILTERED & 491 \\
\hline 812 & $\mathrm{pCi} / \mathrm{L}$ & & 0 & & & & $U$ & & SANITARY & & CUMULATIVE & UNFILTERED & 385 \\
\hline 813 & $\mathrm{pCi} / \mathrm{L}$ & & 0 & & & & & & SANITARY & & CUMULATIVE & UNFILTERED & 385 \\
\hline 814 & $\mathrm{pCi} / \mathrm{L}$ & & 0 & & & & & & RIVER & & CUMULATIVE & UNFILTERED & 463 \\
\hline 815 & $\mathrm{pCi} / \mathrm{L}$ & & 0 & & & & & & RIVER & & CUMULATIVE & UNFILTERED & 463 \\
\hline 816 & $p \mathrm{pi} / \mathrm{L}$ & & 0 & & & & & & RIVER & & CUMULATIVE & UNFILTERED & 463 \\
\hline 817 & $\mathrm{pCi} / \mathrm{L}$ & & 0 & & & & & & RIVER & & CUMULATIVE & UNFILTERED & 463 \\
\hline 818 & $\mathrm{pCi} / \mathrm{L}$ & & 0 & & & & & & RIVER & & CUMULATIVE & UNFILTERED & 553 \\
\hline 819 & $\mathrm{pCi} / \mathrm{L}$ & & 0 & & & & & & RIVER & & CUMULATIVE & UNFILTERED & 553 \\
\hline 820 & $\mathrm{pCi} / \mathrm{L}$ & & 0 & & & & $U$ & & SANITARY & & CUMULATIVE & UNFILTERED & 491 \\
\hline 821 & $\mathrm{pCi} / \mathrm{L}$ & & 0 & & & & U & & SANITARY & & CUMULATIVE & UNFILTERED & 385 \\
\hline 822 & $\mathrm{pCi} / \mathrm{L}$ & & 0 & & & & & & RIVER & & CUMULATIVE & UNFILTERED & 463 \\
\hline 823 & $\mathrm{pCi} / \mathrm{L}$ & & 0 & & & & & & SURFACE & & RAW GRAB & UNFILTERED & 147 \\
\hline 824 & $p C i / L$ & & 0 & & & & & & SANITARY & & CUMULATIVE & UNFILTERED & 491 \\
\hline 825 & $\mathrm{pCi} / \mathrm{L}$ & & 0 & & & & & & SANITARY & & CUMULATIVE & UNFILTERED & 385 \\
\hline 826 & $\mathrm{pCi} / \mathrm{L}$ & & 0 & & & & $U$ & & RIVER & & CUMULATIVE & UNFILTERED & 463 \\
\hline 827 & $\mathrm{pCi} / \mathrm{L}$ & & 0 & & & & & & RIVER & & RAW GRAB & UNFILTERED & 462 \\
\hline 828 & $\mathrm{pCi} / \mathrm{L}$ & & 0 & & & & & & RIVER & & RAW GRAB & UNFILTERED & 462 \\
\hline 829 & $\mathrm{pCi} / \mathrm{L}$ & & 0 & & & & & & RIVER & & RAW GRAB & UNFILTERED & 462 \\
\hline 830 & $\mathrm{pCi} / \mathrm{L}$ & & 0 & & & & & & SANITARY & & CUMULATIVE & UNFILTERED & 491 \\
\hline 831 & $\mathrm{pCi} / \mathrm{L}$ & & 0 & & & & $U$ & & SANITARY & & CUMULATIVE & UNFILTERED & 385 \\
\hline 832 & $\mathrm{pCi} / \mathrm{L}$ & & 0 & & & & & & RIVER & & CUMULATIVE & UNFILTERED & 463 \\
\hline 833 & $\mathrm{pCi} / \mathrm{L}$ & & 0 & & & & & & SURFACE & & RAW GRAB & UNFILTERED & 444 \\
\hline
\end{tabular}




\begin{tabular}{|c|c|c|c|c|c|c|c|c|c|c|c|c|}
\hline & $\mathrm{N}$ & $\mathrm{O}$ & $\mathrm{P}$ & $\mathrm{Q}$ & $\mathrm{R}$ & $\mathrm{s}$ & $\mathrm{T}$ & $\bar{U}$ & $\mathrm{v}$ & $\mathrm{W}$ & $\mathrm{x}$ & $\mathrm{Y}$ \\
\hline 1 & ANAL_UNITS_RPTD & COUNTING_ERROR & TOTAL_ANAL_ERROR & MIN_DETECTABLE_ACTIVITY & PCNT_MOISTURE & LAB_QUALIFIER & REVIEW_QUALIFIER & SAMP_FROM & SAMP_ITEM & SAMP_MTHD & COLL_MTHD & SAMP_SITE_ID \\
\hline 834 & $\mathrm{pCi} / \mathrm{L}$ & 0 & & & & & & SANITARY & & CUMULATIVE & UNFILTERED & 491 \\
\hline 835 & $\mathrm{pCi} / \mathrm{L}$ & 0 & & & & & & SANITARY & & CUMULATIVE & UNFILTERED & 385 \\
\hline 836 & $\mathrm{pCi} / \mathrm{L}$ & 0 & & & & & & RIVER & & CUMULATIVE & UNFILTERED & 553 \\
\hline 837 & $\mathrm{pCi} / \mathrm{L}$ & 0 & & & & & & RIVER & & CUMULATIVE & UNFILTERED & 553 \\
\hline 838 & $\mathrm{pCi} / \mathrm{L}$ & 0 & & & & & & RIVER & & CUMULATIVE & UNFILTERED & 463 \\
\hline 839 & $\mathrm{pCi} / \mathrm{L}$ & 0 & & & & & & RIVER & & CUMULATIVE & UNFILTERED & 463 \\
\hline 840 & $\mathrm{pCi} / \mathrm{L}$ & 0 & & & & & & RIVER & & CUMULATIVE & UNFILTERED & 463 \\
\hline 841 & $\mathrm{pCi} / \mathrm{L}$ & 0 & & & & $\mathrm{U}$ & & SANITARY & & CUMULATIVE & UNFILTERED & 491 \\
\hline 842 & $\mathrm{pCi} / \mathrm{L}$ & 0 & & & & U & & SANITARY & & CUMULATIVE & UNFILTERED & 385 \\
\hline 843 & $\mathrm{pCi} / \mathrm{L}$ & 0 & & & & & & SURFACE & & RAW GRAB & UNFILTERED & 147 \\
\hline 844 & $\mathrm{pCi} / \mathrm{L}$ & 0 & & & & & & SANITARY & & CUMULATIVE & UNFILTERED & 491 \\
\hline 845 & $\mathrm{pCi} / \mathrm{L}$ & 0 & & & & & & SANITARY & & CUMULATIVE & UNFILTERED & 385 \\
\hline 846 & $\mathrm{pCi} / \mathrm{L}$ & 0 & & & & $U$ & & RIVER & & CUMULATIVE & UNFILTERED & 463 \\
\hline 847 & $\mathrm{pCi} / \mathrm{L}$ & 0 & & & & & & RIVER & & RAW GRAB & UNFILTERED & 462 \\
\hline 848 & $\mathrm{pCi} / \mathrm{L}$ & 0 & & & & & & RIVER & & RAW GRAB & UNFILTERED & 462 \\
\hline 849 & $\mathrm{pCi} / \mathrm{L}$ & 0 & & & & U & & SANITARY & & CUMULATIVE & UNFILTERED & 491 \\
\hline 850 & $\mathrm{pCi} / \mathrm{L}$ & 0 & & & & U & & SANITARY & & CUMULATIVE & UNFILTERED & 385 \\
\hline 851 & $\mathrm{pCi} / \mathrm{L}$ & 0 & & & & U & & RIVER & & CUMULATIVE & UNFILTERED & 463 \\
\hline 852 & $\mathrm{pCi} / \mathrm{L}$ & 0 & & & & & & SURFACE & & RAW GRAB & UNFILTERED & 444 \\
\hline 853 & $\mathrm{pCi} / \mathrm{L}$ & 0 & & & & & & RIVER & & CUMULATIVE & UNFILTERED & 463 \\
\hline 854 & $\mathrm{pCi} / \mathrm{L}$ & 0 & & & & U & & RIVER & & CUMULATIVE & UNFILTERED & 553 \\
\hline 855 & $\mathrm{pCi} / \mathrm{L}$ & 0 & & & & & & RIVER & & CUMULATIVE & UNFILTERED & 553 \\
\hline 856 & $\mathrm{pCi} / \mathrm{L}$ & 0 & & & & & & RIVER & & CUMULATIVE & UNFILTERED & 465 \\
\hline 857 & $\mathrm{pCi} / \mathrm{L}$ & 0 & & & & & & SANITARY & & CUMULATIVE & UNFILTERED & 491 \\
\hline 858 & $\mathrm{pCi} / \mathrm{L}$ & 0 & & & & & & SANITARY & & CUMULATIVE & UNFILTERED & 385 \\
\hline 859 & $\mathrm{pCi} / \mathrm{L}$ & 0 & & & & U & & SANITARY & & CUMULATIVE & UNFILTERED & 385 \\
\hline 860 & $\mathrm{pCi} / \mathrm{L}$ & 0 & & & & & & RIVER & & CUMULATIVE & UNFILTERED & 463 \\
\hline 861 & pCi/L & 0 & & & & & & RIVER & & CUMULATIVE & UNFILTERED & 463 \\
\hline 862 & pCi/L & 0 & & & & U & & RIVER & & CUMULATIVE & UNFILTERED & 463 \\
\hline 863 & pCi/L & 0 & & & & U & & RIVER & & CUMULATIVE & UNFILTERED & 463 \\
\hline 864 & pCi/L & 0 & & & & & & RIVER & & RAW GRAB & UNFILTERED & 456 \\
\hline 865 & pCi/L & 0 & & & & & & SANITARY & & CUMULATIVE & UNFILTERED & 491 \\
\hline 866 & pCi/L & 0 & & & & & & SANITARY & & CUMULATIVE & UNFILTERED & 385 \\
\hline 867 & $\mathrm{pCi} / \mathrm{L}$ & 0 & & & & $\mathrm{U}$ & & SURFACE & & CUMULATIVE & UNFILTERED & 60 \\
\hline 868 & pCi/L & 0 & & & & & & SANITARY & & CUMULATIVE & UNFILTERED & 385 \\
\hline 869 & pCi/L & 0 & & & & & & RIVER & & CUMULATIVE & UNFILTERED & 463 \\
\hline 870 & pCi/L & 0 & & & & & & SURFACE & & RAW GRAB & UNFILTERED & 444 \\
\hline 871 & $\mathrm{pCi} / \mathrm{L}$ & 0 & & & & U & & RIVER & & RAW GRAB & UNFILTERED & 462 \\
\hline 872 & $\mathrm{pCi} / \mathrm{L}$ & 0 & & & & & & RIVER & & RAW GRAB & UNFILTERED & 462 \\
\hline 873 & $\mathrm{pCi} / \mathrm{L}$ & 0 & & & & & & RIVER & & RAW GRAB & UNFILTERED & 462 \\
\hline 874 & $\mathrm{pCi} / \mathrm{L}$ & 0 & & & & U & & SURFACE & & RAW GRAB & UNFILTERED & 121 \\
\hline 875 & pCi/L & 0 & & & & & & SURFACE & & RAW GRAB & UNFILTERED & 509 \\
\hline 876 & pCi/L & 0 & & & & & & SURFACE & & RAW GRAB & UNFILTERED & 509 \\
\hline 877 & pCi/L & 0 & & & & & & SURFACE & & RAW GRAB & UNFILTERED & 146 \\
\hline 878 & pCi/L & 0 & & & & & & SURFACE & & RAW GRAB & UNFILTERED & 146 \\
\hline 879 & $\mathrm{pCi} / \mathrm{L}$ & 0 & & & & U & & SURFACE & & RAW GRAB & UNFILTERED & 413 \\
\hline 880 & $\mathrm{pCi} / \mathrm{L}$ & 0 & & & & & & SURFACE & & RAW GRAB & UNFILTERED & 412 \\
\hline 881 & pCi/L & 0 & & & & U & & SURFACE & & RAW GRAB & UNFILTERED & 147 \\
\hline 882 & pCi/L & 0 & & & & & & SURFACE & & RAW GRAB & UNFILTERED & 147 \\
\hline 883 & pCi/L & 0 & & & & U & & SANITARY & & CUMULATIVE & UNFILTERED & 491 \\
\hline 884 & pCi/L & 0 & & & & U & & SANITARY & & CUMULATIVE & UNFILTERED & 385 \\
\hline 885 & $\mathrm{pCi} / \mathrm{L}$ & 0 & & & & & & RIVER & & CUMULATIVE & UNFILTERED & 463 \\
\hline
\end{tabular}




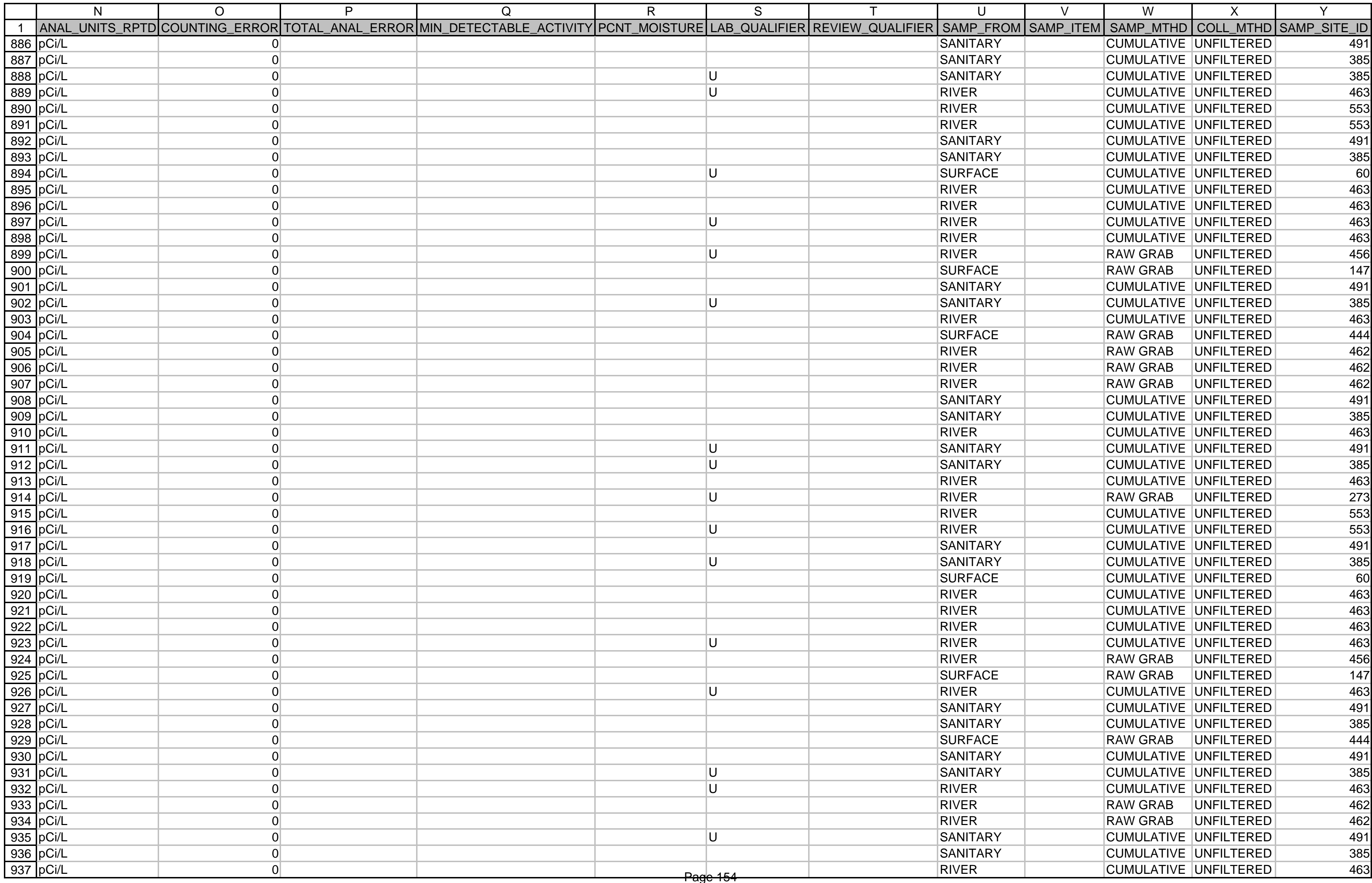




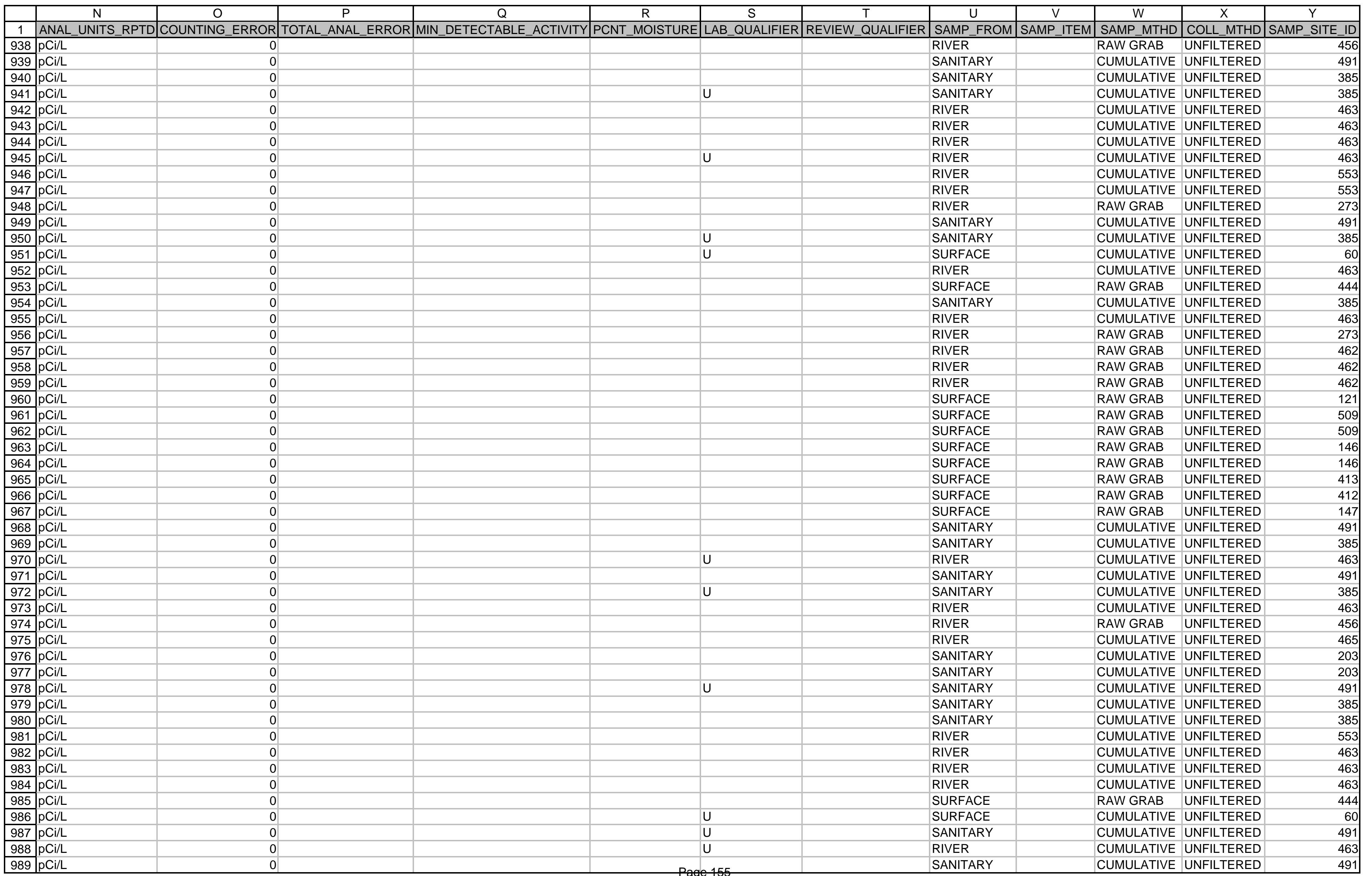




\begin{tabular}{|c|c|c|c|c|c|c|c|c|c|c|c|c|}
\hline & $\mathrm{N}$ & $\mathrm{O}$ & $\mathrm{P}$ & $\mathrm{Q}$ & $\mathrm{R}$ & $\mathrm{s}$ & $\mathrm{T}$ & $\mathrm{U}$ & $\mathrm{V}$ & $\mathrm{W}$ & $\mathrm{X}$ & $\bar{Y}$ \\
\hline 1 & ANAL_UNITS_RPTD & COUNTING_ERROR & TOTAL_ANAL_ERROR & MIN_DETECTABLE_ACTIVITY & PCNT_MOISTURE & LAB_QUALIFIER & REVIEW_QUALIFIER & SAMP_FROM & SAMP_ITEM & SAMP_MTHD & COLL_MTHD & SAMP_SITE_ID \\
\hline 990 & $\mathrm{pCi} / \mathrm{L}$ & 0 & & & & $U$ & & RIVER & & RAW GRAB & UNFILTERED & 273 \\
\hline 991 & $\mathrm{pCi} / \mathrm{L}$ & 0 & & & & & & RIVER & & RAW GRAB & UNFILTERED & 462 \\
\hline 992 & $\mathrm{pCi} / \mathrm{L}$ & 0 & & & & & & RIVER & & RAW GRAB & UNFILTERED & 462 \\
\hline 993 & $\mathrm{pCi} / \mathrm{L}$ & 0 & & & & & & RIVER & & RAW GRAB & UNFILTERED & 462 \\
\hline 994 & $\mathrm{pCi} / \mathrm{L}$ & 0 & & & & U & & SANITARY & & CUMULATIVE & UNFILTERED & 491 \\
\hline 995 & $\mathrm{pCi} / \mathrm{L}$ & 0 & & & & & & RIVER & & CUMULATIVE & UNFILTERED & 553 \\
\hline 996 & $\mathrm{pCi} / \mathrm{L}$ & 0 & & & & & & RIVER & & CUMULATIVE & UNFILTERED & 553 \\
\hline 997 & $\mathrm{pCi} / \mathrm{L}$ & 0 & & & & & & RIVER & & CUMULATIVE & UNFILTERED & 465 \\
\hline 998 & $\mathrm{pCi} / \mathrm{L}$ & 0 & & & & & & SANITARY & & CUMULATIVE & UNFILTERED & 203 \\
\hline 999 & $\mathrm{pCi} / \mathrm{L}$ & 0 & & & & & & SANITARY & & CUMULATIVE & UNFILTERED & 203 \\
\hline 1000 & $\mathrm{pCi} / \mathrm{L}$ & 0 & & & & & & SANITARY & & CUMULATIVE & UNFILTERED & 491 \\
\hline 1001 & $\mathrm{pCi} / \mathrm{L}$ & 0 & & & & & & SANITARY & & CUMULATIVE & UNFILTERED & 385 \\
\hline 1002 & $\mathrm{pCi} / \mathrm{L}$ & 0 & & & & & & SANITARY & & CUMULATIVE & UNFILTERED & 385 \\
\hline 1003 & $\mathrm{pCi} / \mathrm{L}$ & 0 & & & & $U$ & & SURFACE & & CUMULATIVE & UNFILTERED & 60 \\
\hline 1004 & $\mathrm{pCi} / \mathrm{L}$ & 0 & & & & & & RIVER & & CUMULATIVE & UNFILTERED & 463 \\
\hline 1005 & $\mathrm{pCi} / \mathrm{L}$ & 0 & & & & & & RIVER & & CUMULATIVE & UNFILTERED & 463 \\
\hline 1006 & $\mathrm{pCi} / \mathrm{L}$ & 0 & & & & & & RIVER & & CUMULATIVE & UNFILTERED & 463 \\
\hline 1007 & $\mathrm{pCi} / \mathrm{L}$ & 0 & & & & & & RIVER & & CUMULATIVE & UNFILTERED & 463 \\
\hline 1008 & $\mathrm{pCi} / \mathrm{L}$ & 0 & & & & & & RIVER & & RAW GRAB & UNFILTERED & 456 \\
\hline 1009 & $\mathrm{pCi} / \mathrm{L}$ & 0 & & & & & & SURFACE & & RAW GRAB & UNFILTERED & 444 \\
\hline 1010 & $\mathrm{pCi} / \mathrm{L}$ & 0 & & & & $U$ & & SANITARY & & CUMULATIVE & UNFILTERED & 491 \\
\hline 1011 & $\mathrm{pCi} / \mathrm{L}$ & 0 & & & & $U$ & & RIVER & & CUMULATIVE & UNFILTERED & 463 \\
\hline 1012 & $\mathrm{pCi} / \mathrm{L}$ & 0 & & & & & & RIVER & & RAW GRAB & UNFILTERED & 273 \\
\hline 1013 & $\mathrm{pCi} / \mathrm{L}$ & 0 & & & & & & RIVER & & RAW GRAB & UNFILTERED & 462 \\
\hline 1014 & $\mathrm{pCi} / \mathrm{L}$ & 0 & & & & & & RIVER & & RAW GRAB & UNFILTERED & 462 \\
\hline 1015 & $\mathrm{pCi} / \mathrm{L}$ & 0 & & & & $U$ & & SANITARY & & CUMULATIVE & UNFILTERED & 491 \\
\hline 1016 & $\mathrm{pCi} / \mathrm{L}$ & 0 & & & & & & SANITARY & & CUMULATIVE & UNFILTERED & 385 \\
\hline 1017 & $\mathrm{pCi} / \mathrm{L}$ & 0 & & & & & & RIVER & & CUMULATIVE & UNFILTERED & 463 \\
\hline 1018 & $\mathrm{pCi} / \mathrm{L}$ & 0 & & & & & & RIVER & & RAW GRAB & UNFILTERED & 462 \\
\hline 1019 & $\mathrm{pCi} / \mathrm{L}$ & 0 & & & & & & SANITARY & & CUMULATIVE & UNFILTERED & 491 \\
\hline 1020 & $\mathrm{pCi} / \mathrm{L}$ & 0 & & & & & & RIVER & & CUMULATIVE & UNFILTERED & 463 \\
\hline 1021 & $\mathrm{pCi} / \mathrm{L}$ & 0 & & & & & & RIVER & & CUMULATIVE & UNFILTERED & 463 \\
\hline 1022 & $\mathrm{pCi} / \mathrm{L}$ & 0 & & & & & & SANITARY & & CUMULATIVE & UNFILTERED & 491 \\
\hline 1023 & $\mathrm{pCi} / \mathrm{L}$ & 0 & & & & & & SURFACE & & RAW GRAB & UNFILTERED & 444 \\
\hline 1024 & $\mathrm{pCi} / \mathrm{L}$ & 0 & & & & & & RIVER & & CUMULATIVE & UNFILTERED & 553 \\
\hline 1025 & $\mathrm{pCi} / \mathrm{L}$ & 0 & & & & $U$ & & RIVER & & CUMULATIVE & UNFILTERED & 553 \\
\hline 1026 & $\mathrm{pCi} / \mathrm{L}$ & 0 & & & & $U$ & & SANITARY & & CUMULATIVE & UNFILTERED & 203 \\
\hline 1027 & $\mathrm{pCi} / \mathrm{L}$ & 0 & & & & U & & SANITARY & & CUMULATIVE & UNFILTERED & 491 \\
\hline 1028 & $\mathrm{pCi} / \mathrm{L}$ & 0 & & & & & & SANITARY & & CUMULATIVE & UNFILTERED & 385 \\
\hline 1029 & $\mathrm{pCi} / \mathrm{L}$ & 0 & & & & $U$ & & SANITARY & & CUMULATIVE & UNFILTERED & 385 \\
\hline 1030 & $\mathrm{pCi} / \mathrm{L}$ & 0 & & & & $U$ & & SURFACE & & CUMULATIVE & UNFILTERED & 60 \\
\hline 1031 & $\mathrm{pCi} / \mathrm{L}$ & 0 & & & & & & RIVER & & CUMULATIVE & UNFILTERED & 463 \\
\hline 1032 & $\mathrm{pCi} / \mathrm{L}$ & 0 & & & & & & RIVER & & CUMULATIVE & UNFILTERED & 463 \\
\hline 1033 & $\mathrm{pCi} / \mathrm{L}$ & 0 & & & & & & RIVER & & CUMULATIVE & UNFILTERED & 463 \\
\hline 1034 & $\mathrm{pCi} / \mathrm{L}$ & 0 & & & & & & RIVER & & CUMULATIVE & UNFILTERED & 463 \\
\hline 1035 & $\mathrm{pCi} / \mathrm{L}$ & 0 & & & & & & RIVER & & RAW GRAB & UNFILTERED & 456 \\
\hline 1036 & $\mathrm{pCi} / \mathrm{L}$ & 0 & & & & $U$ & & RIVER & & CUMULATIVE & UNFILTERED & 465 \\
\hline 1037 & $\mathrm{pCi} / \mathrm{L}$ & 0 & & & & & & SANITARY & & CUMULATIVE & UNFILTERED & 491 \\
\hline 1038 & $\mathrm{pCi} / \mathrm{L}$ & 0 & & & & & & RIVER & & CUMULATIVE & UNFILTERED & 463 \\
\hline 1039 & $\mathrm{pCi} / \mathrm{L}$ & 0 & & & & $U$ & & RIVER & & RAW GRAB & UNFILTERED & 462 \\
\hline 1040 & $\mathrm{pCi} / \mathrm{L}$ & 0 & & & & & & RIVER & & RAW GRAB & UNFILTERED & 462 \\
\hline 1041 & $\mathrm{pCi} / \mathrm{L}$ & 0 & 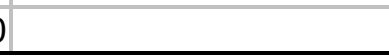 & & & & & RIVER & & RAW GRAB & UNFILTERED & 462 \\
\hline
\end{tabular}




\begin{tabular}{|c|c|c|c|c|c|c|c|c|c|c|c|c|}
\hline & $\mathrm{N}$ & $\mathrm{O}$ & $\mathrm{P}$ & $\mathrm{Q}$ & $\mathrm{R}$ & $\mathrm{s}$ & $T$ & $\mathrm{u}$ & $\mathrm{v}$ & $\mathrm{w}$ & $\mathrm{x}$ & $\bar{Y}$ \\
\hline 1 & ANAL_UNITS_RPTD & COUNTING_ERROR & TOTAL_ANAL_ERROR & MIN_DETECTABLE_ACTIVITY & PCNT_MOISTURE & LAB_QUALIFIER & REVIEW_QUALIFIER & SAMP_FROM & SAMP_ITEM & SAMP_MTHD & COLL_MTHD & SAMP_SITE_ID \\
\hline 1042 & $\mathrm{pCi} / \mathrm{L}$ & 0 & & & & $U$ & & SEEP & & RAW GRAB & UNFILTERED & 556 \\
\hline 1043 & $\mathrm{pCi} / \mathrm{L}$ & 0 & & & & & & SURFACE & & RAW GRAB & UNFILTERED & 121 \\
\hline 1044 & $\mathrm{pCi} / \mathrm{L}$ & 0 & & & & & & SURFACE & & RAW GRAB & UNFILTERED & 509 \\
\hline 1045 & $\mathrm{pCi} / \mathrm{L}$ & 0 & & & & & & SURFACE & & RAW GRAB & UNFILTERED & 509 \\
\hline 1046 & $\mathrm{pCi} / \mathrm{L}$ & 0 & & & & & & SURFACE & & RAW GRAB & UNFILTERED & 412 \\
\hline 1047 & $\mathrm{pCi} / \mathrm{L}$ & 0 & & & & & & SURFACE & & RAW GRAB & UNFILTERED & 147 \\
\hline 1048 & $\mathrm{pCi} / \mathrm{L}$ & 0 & & & & & & SURFACE & & RAW GRAB & UNFILTERED & 147 \\
\hline 1049 & $\mathrm{pCi} / \mathrm{L}$ & 0 & & & & U & & SANITARY & & CUMULATIVE & UNFILTERED & 491 \\
\hline 1050 & $\mathrm{pCi} / \mathrm{L}$ & 0 & & & & $\mathrm{U}$ & & RIVER & & CUMULATIVE & UNFILTERED & 463 \\
\hline 1051 & $\mathrm{pCi} / \mathrm{L}$ & 0 & & & & & & SANITARY & & CUMULATIVE & UNFILTERED & 491 \\
\hline 1052 & $\mathrm{pCi} / \mathrm{L}$ & 0 & & & & & & RIVER & & CUMULATIVE & UNFILTERED & 463 \\
\hline 1053 & $\mathrm{pCi} / \mathrm{L}$ & 0 & & & & & & SURFACE & & RAW GRAB & UNFILTERED & 444 \\
\hline 1054 & $\mathrm{pCi} / \mathrm{L}$ & 0 & & & & & & RIVER & & CUMULATIVE & UNFILTERED & 553 \\
\hline 1055 & $\mathrm{pCi} / \mathrm{L}$ & 0 & & & & & & RIVER & & CUMULATIVE & UNFILTERED & 553 \\
\hline 1056 & $\mathrm{pCi} / \mathrm{L}$ & 0 & & & & & & SANITARY & & CUMULATIVE & UNFILTERED & 203 \\
\hline 1057 & $\mathrm{pCi} / \mathrm{L}$ & 0 & & & & U & & SANITARY & & CUMULATIVE & UNFILTERED & 491 \\
\hline 1058 & $\mathrm{pCi} / \mathrm{L}$ & 0 & & & & & & SANITARY & & CUMULATIVE & UNFILTERED & 385 \\
\hline 1059 & $\mathrm{pCi} / \mathrm{L}$ & 0 & & & & U & & SANITARY & & CUMULATIVE & UNFILTERED & 385 \\
\hline 1060 & $\mathrm{pCi} / \mathrm{L}$ & 0 & & & & U & & SURFACE & & CUMULATIVE & UNFILTERED & 60 \\
\hline 1061 & $\mathrm{pCi} / \mathrm{L}$ & 0 & & & & & & RIVER & & CUMULATIVE & UNFILTERED & 463 \\
\hline 1062 & $\mathrm{pCi} / \mathrm{L}$ & 0 & & & & U & & RIVER & & CUMULATIVE & UNFILTERED & 463 \\
\hline 1063 & $\mathrm{pCi} / \mathrm{L}$ & 0 & & & & & & RIVER & & CUMULATIVE & UNFILTERED & 463 \\
\hline 1064 & $\mathrm{pCi} / \mathrm{L}$ & 0 & & & & & & RIVER & & CUMULATIVE & UNFILTERED & 463 \\
\hline 1065 & $\mathrm{pCi} / \mathrm{L}$ & 0 & & & & & & RIVER & & RAW GRAB & UNFILTERED & 456 \\
\hline 1066 & $\mathrm{pCi} / \mathrm{L}$ & 0 & & & & U & & SANITARY & & CUMULATIVE & UNFILTERED & 491 \\
\hline 1067 & $\mathrm{pCi} / \mathrm{L}$ & 0 & & & & & & RIVER & & CUMULATIVE & UNFILTERED & 463 \\
\hline 1068 & $\mathrm{pCi} / \mathrm{L}$ & 0 & & & & & & SANITARY & & CUMULATIVE & UNFILTERED & 491 \\
\hline 1069 & $\mathrm{pCi} / \mathrm{L}$ & 0 & & & & & & RIVER & & CUMULATIVE & UNFILTERED & 463 \\
\hline 1070 & $\mathrm{pCi} / \mathrm{L}$ & 0 & & & & U & & RIVER & & RAW GRAB & UNFILTERED & 462 \\
\hline 1071 & $\mathrm{pCi} / \mathrm{L}$ & 0 & & & & & & RIVER & & RAW GRAB & UNFILTERED & 462 \\
\hline 1072 & $\mathrm{pCi} / \mathrm{L}$ & 0 & & & & & & RIVER & & RAW GRAB & UNFILTERED & 462 \\
\hline 1073 & $\mathrm{pCi} / \mathrm{L}$ & 0 & & & & & & SEEP & & RAW GRAB & UNFILTERED & 556 \\
\hline 1074 & $\mathrm{pCi} / \mathrm{L}$ & 0 & & & & & & SANITARY & & CUMULATIVE & UNFILTERED & 491 \\
\hline 1075 & $\mathrm{pCi} / \mathrm{L}$ & 0 & & & & & & SURFACE & & RAW GRAB & UNFILTERED & 444 \\
\hline 1076 & $\mathrm{pCi} / \mathrm{L}$ & 0 & & & & & & RIVER & & CUMULATIVE & UNFILTERED & 553 \\
\hline 1077 & $\mathrm{pCi} / \mathrm{L}$ & 0 & & & & & & RIVER & & CUMULATIVE & UNFILTERED & 553 \\
\hline 1078 & $\mathrm{pCi} / \mathrm{L}$ & 0 & & & & & & SANITARY & & CUMULATIVE & UNFILTERED & 203 \\
\hline 1079 & $\mathrm{pCi} / \mathrm{L}$ & 0 & & & & & & SANITARY & & CUMULATIVE & UNFILTERED & 203 \\
\hline 1080 & $\mathrm{pCi} / \mathrm{L}$ & 0 & & & & U & & SANITARY & & CUMULATIVE & UNFILTERED & 491 \\
\hline 1081 & $\mathrm{pCi} / \mathrm{L}$ & 0 & & & & & & SANITARY & & CUMULATIVE & UNFILTERED & 385 \\
\hline 1082 & $\mathrm{pCi} / \mathrm{L}$ & 0 & & & & & & SURFACE & & CUMULATIVE & UNFILTERED & 60 \\
\hline 1083 & $\mathrm{pCi} / \mathrm{L}$ & 0 & & & & & & RIVER & & CUMULATIVE & UNFILTERED & 463 \\
\hline 1084 & $\mathrm{pCi} / \mathrm{L}$ & 0 & & & & & & RIVER & & CUMULATIVE & UNFILTERED & 463 \\
\hline 1085 & $\mathrm{pCi} / \mathrm{L}$ & 0 & & & & & & RIVER & & CUMULATIVE & UNFILTERED & 463 \\
\hline 1086 & $\mathrm{pCi} / \mathrm{L}$ & 0 & & & & & & RIVER & & CUMULATIVE & UNFILTERED & 463 \\
\hline 1087 & $\mathrm{pCi} / \mathrm{L}$ & 0 & & & & & & RIVER & & RAW GRAB & UNFILTERED & 456 \\
\hline 1088 & $\mathrm{pCi} / \mathrm{L}$ & 0 & & & & U & & RIVER & & RAW GRAB & UNFILTERED & 462 \\
\hline 1089 & $\mathrm{pCi} / \mathrm{L}$ & 0 & & & & & & RIVER & & RAW GRAB & UNFILTERED & 462 \\
\hline 1090 & $\mathrm{pCi} / \mathrm{L}$ & 0 & & & & & & RIVER & & RAW GRAB & UNFILTERED & 462 \\
\hline 1091 & $\mathrm{pCi} / \mathrm{L}$ & 0 & & & & & & SEEP & & RAW GRAB & UNFILTERED & 556 \\
\hline 1092 & $\mathrm{pCi} / \mathrm{L}$ & 0 & & & & & & SANITARY & & CUMULATIVE & UNFILTERED & 491 \\
\hline 1093 & $\mathrm{pCi} / \mathrm{L}$ & 0 & & & & & & RIVER & & CUMULATIVE & UNFILTERED & 463 \\
\hline
\end{tabular}




\begin{tabular}{|c|c|c|c|c|c|c|c|c|c|c|c|c|}
\hline & $\mathrm{N}$ & $\mathrm{O}$ & $\mathrm{P}$ & $\mathrm{Q}$ & $\mathrm{R}$ & $\mathrm{s}$ & $\mathrm{T}$ & $\mathrm{U}$ & $\mathrm{V}$ & $\mathrm{W}$ & $\mathrm{X}$ & $\bar{Y}$ \\
\hline 1 & ANAL_UNITS_RPTD & COUNTING_ERROR & TOTAL_ANAL_ERROR & MIN_DETECTABLE_ACTIVITY & PCNT_MOISTURE & LAB_QUALIFIER & REVIEW_QUALIFIER & SAMP_FROM & SAMP_ITEM & SAMP_MTHD & COLL_MTHD & SAMP_SITE_ID \\
\hline 1094 & $\mathrm{pCi} / \mathrm{L}$ & 0 & & & & & & SANITARY & & CUMULATIVE & UNFILTERED & 491 \\
\hline 1095 & $\mathrm{pCi} / \mathrm{L}$ & 0 & & & & & & RIVER & & CUMULATIVE & UNFILTERED & 463 \\
\hline 1096 & $\mathrm{pCi} / \mathrm{L}$ & 0 & & & & & & SURFACE & & RAW GRAB & UNFILTERED & 444 \\
\hline 1097 & $\mathrm{pCi} / \mathrm{L}$ & 0 & & & & $\mathrm{U}$ & & SANITARY & & CUMULATIVE & UNFILTERED & 203 \\
\hline 1098 & $\mathrm{pCi} / \mathrm{L}$ & 0 & & & & & & SANITARY & & CUMULATIVE & UNFILTERED & 203 \\
\hline 1099 & $\mathrm{pCi} / \mathrm{L}$ & 0 & & & & U & & SANITARY & & CUMULATIVE & UNFILTERED & 491 \\
\hline 1100 & $\mathrm{pCi} / \mathrm{L}$ & 0 & & & & & & SANITARY & & CUMULATIVE & UNFILTERED & 385 \\
\hline 1101 & $\mathrm{pCi} / \mathrm{L}$ & 0 & & & & U & & SANITARY & & CUMULATIVE & UNFILTERED & 385 \\
\hline 1102 & $\mathrm{pCi} / \mathrm{L}$ & 0 & & & & U & & SURFACE & & CUMULATIVE & UNFILTERED & 60 \\
\hline 1103 & $\mathrm{pCi} / \mathrm{L}$ & 0 & & & & & & RIVER & & CUMULATIVE & UNFILTERED & 463 \\
\hline 1104 & $\mathrm{pCi} / \mathrm{L}$ & 0 & & & & & & RIVER & & CUMULATIVE & UNFILTERED & 463 \\
\hline 1105 & $\mathrm{pCi} / \mathrm{L}$ & 0 & & & & & & RIVER & & CUMULATIVE & UNFILTERED & 463 \\
\hline 1106 & $\mathrm{pCi} / \mathrm{L}$ & 0 & & & & & & RIVER & & CUMULATIVE & UNFILTERED & 463 \\
\hline 1107 & $\mathrm{pCi} / \mathrm{L}$ & 0 & & & & & & RIVER & & RAW GRAB & UNFILTERED & 456 \\
\hline 1108 & $\mathrm{pCi} / \mathrm{L}$ & 0 & & & & U & & RIVER & & CUMULATIVE & UNFILTERED & 553 \\
\hline 1109 & $\mathrm{pCi} / \mathrm{L}$ & 0 & & & & & & RIVER & & CUMULATIVE & UNFILTERED & 553 \\
\hline 1110 & $\mathrm{pCi} / \mathrm{L}$ & 0 & & & & & & SANITARY & & CUMULATIVE & UNFILTERED & 491 \\
\hline 1111 & $\mathrm{pCi} / \mathrm{L}$ & 0 & & & & & & RIVER & & CUMULATIVE & UNFILTERED & 463 \\
\hline 1112 & $\mathrm{pCi} / \mathrm{L}$ & 0 & & & & U & & SURFACE & & RAW GRAB & UNFILTERED & 121 \\
\hline 1113 & $\mathrm{pCi} / \mathrm{L}$ & 0 & & & & & & SURFACE & & RAW GRAB & UNFILTERED & 509 \\
\hline 1114 & $\mathrm{pCi} / \mathrm{L}$ & 0 & & & & & & SURFACE & & RAW GRAB & UNFILTERED & 509 \\
\hline 1115 & $\mathrm{pCi} / \mathrm{L}$ & 0 & & & & & & SURFACE & & RAW GRAB & UNFILTERED & 146 \\
\hline 1116 & $\mathrm{pCi} / \mathrm{L}$ & 0 & & & & & & SURFACE & & RAW GRAB & UNFILTERED & 146 \\
\hline 1117 & $\mathrm{pCi} / \mathrm{L}$ & 0 & & & & & & SURFACE & & RAW GRAB & UNFILTERED & 412 \\
\hline 1118 & $\mathrm{pCi} / \mathrm{L}$ & 0 & & & & & & SURFACE & & RAW GRAB & UNFILTERED & 147 \\
\hline 1119 & $\mathrm{pCi} / \mathrm{L}$ & 0 & & & & & & SURFACE & & RAW GRAB & UNFILTERED & 147 \\
\hline 1120 & $\mathrm{pCi} / \mathrm{L}$ & 0 & & & & & & SANITARY & & CUMULATIVE & UNFILTERED & 491 \\
\hline 1121 & $\mathrm{pCi} / \mathrm{L}$ & 0 & & & & $U$ & & RIVER & & CUMULATIVE & UNFILTERED & 463 \\
\hline 1122 & $\mathrm{pCi} / \mathrm{L}$ & 0 & & & & & & RIVER & & RAW GRAB & UNFILTERED & 462 \\
\hline 1123 & $\mathrm{pCi} / \mathrm{L}$ & 0 & & & & & & RIVER & & RAW GRAB & UNFILTERED & 462 \\
\hline 1124 & $\mathrm{pCi} / \mathrm{L}$ & 0 & & & & & & RIVER & & RAW GRAB & UNFILTERED & 462 \\
\hline 1125 & $\mathrm{pCi} / \mathrm{L}$ & 0 & & & & $U$ & & SEEP & & RAW GRAB & UNFILTERED & 556 \\
\hline 1126 & $\mathrm{pCi} / \mathrm{L}$ & 0 & & & & & & SANITARY & & CUMULATIVE & UNFILTERED & 491 \\
\hline 1127 & $\mathrm{pCi} / \mathrm{L}$ & 0 & & & & & & RIVER & & CUMULATIVE & UNFILTERED & 463 \\
\hline 1128 & $\mathrm{pCi} / \mathrm{L}$ & 0 & & & & & & SURFACE & & RAW GRAB & UNFILTERED & 444 \\
\hline 1129 & $\mathrm{pCi} / \mathrm{L}$ & 0 & & & & U & & SANITARY & & CUMULATIVE & UNFILTERED & 491 \\
\hline 1130 & $\mathrm{pCi} / \mathrm{L}$ & 0 & & & & & & RIVER & & CUMULATIVE & UNFILTERED & 463 \\
\hline 1131 & $\mathrm{pCi} / \mathrm{L}$ & 0 & & & & & & RIVER & & CUMULATIVE & UNFILTERED & 553 \\
\hline 1132 & $\mathrm{pCi} / \mathrm{L}$ & 0 & & & & & & RIVER & & CUMULATIVE & UNFILTERED & 553 \\
\hline 1133 & $\mathrm{pCi} / \mathrm{L}$ & 0 & & & & & & SANITARY & & CUMULATIVE & UNFILTERED & 203 \\
\hline 1134 & $\mathrm{pCi} / \mathrm{L}$ & 0 & & & & & & SANITARY & & CUMULATIVE & UNFILTERED & 203 \\
\hline 1135 & $\mathrm{pCi} / \mathrm{L}$ & 0 & & & & & & SANITARY & & CUMULATIVE & UNFILTERED & 491 \\
\hline 1136 & $\mathrm{pCi} / \mathrm{L}$ & 0 & & & & & & SANITARY & & CUMULATIVE & UNFILTERED & 385 \\
\hline 1137 & $\mathrm{pCi} / \mathrm{L}$ & 0 & & & & & & SANITARY & & CUMULATIVE & UNFILTERED & 385 \\
\hline 1138 & $\mathrm{pCi} / \mathrm{L}$ & 0 & & & & & & SURFACE & & CUMULATIVE & UNFILTERED & 60 \\
\hline 1139 & $\mathrm{pCi} / \mathrm{L}$ & 0 & & & & & & RIVER & & CUMULATIVE & UNFILTERED & 463 \\
\hline 1140 & $\mathrm{pCi} / \mathrm{L}$ & 0 & & & & U & & RIVER & & CUMULATIVE & UNFILTERED & 463 \\
\hline 1141 & $\mathrm{pCi} / \mathrm{L}$ & 0 & & & & & & RIVER & & CUMULATIVE & UNFILTERED & 463 \\
\hline 1142 & $\mathrm{pCi} / \mathrm{L}$ & 0 & & & & & & RIVER & & CUMULATIVE & UNFILTERED & 463 \\
\hline 1143 & pCi/L & 0 & & & & & & RIVER & & RAW GRAB & UNFILTERED & 456 \\
\hline 1144 & $\mathrm{pCi} / \mathrm{L}$ & 0 & & & & & & SANITARY & & CUMULATIVE & UNFILTERED & 491 \\
\hline 1145 & $\mathrm{pCi} / \mathrm{L}$ & 0 & 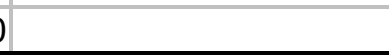 & & & & & RIVER & & CUMULATIVE & UNFILTERED & 463 \\
\hline
\end{tabular}




\begin{tabular}{|c|c|c|c|c|c|c|c|c|c|c|c|c|}
\hline & $\mathrm{N}$ & $\mathrm{O}$ & $\mathrm{P}$ & $\mathrm{Q}$ & $\mathrm{R}$ & $\mathrm{s}$ & $\mathrm{T}$ & $\mathrm{U}$ & $\mathrm{V}$ & $\mathrm{W}$ & $\mathrm{X}$ & $\bar{Y}$ \\
\hline 1 & ANAL_UNITS_RPTD & COUNTING_ERROR & TOTAL_ANAL_ERROR & MIN_DETECTABLE_ACTIVITY & PCNT_MOISTURE & LAB_QUALIFIER & REVIEW_QUALIFIER & SAMP_FROM & SAMP_ITEM & SAMP_MTHD & COLL_MTHD & SAMP_SITE_ID \\
\hline 1146 & $\mathrm{pCi} / \mathrm{L}$ & 0 & & & & & & RIVER & & RAW GRAB & UNFILTERED & 462 \\
\hline 1147 & $\mathrm{pCi} / \mathrm{L}$ & 0 & & & & & & RIVER & & RAW GRAB & UNFILTERED & 462 \\
\hline 1148 & $\mathrm{pCi} / \mathrm{L}$ & 0 & & & & & & RIVER & & RAW GRAB & UNFILTERED & 462 \\
\hline 1149 & $\mathrm{pCi} / \mathrm{L}$ & 0 & & & & & & SEEP & & RAW GRAB & UNFILTERED & 556 \\
\hline 1150 & $\mathrm{pCi} / \mathrm{L}$ & 0 & & & & $\mathrm{U}$ & & SANITARY & & CUMULATIVE & UNFILTERED & 491 \\
\hline 1151 & $\mathrm{pCi} / \mathrm{L}$ & 0 & & & & U & & RIVER & & CUMULATIVE & UNFILTERED & 463 \\
\hline 1152 & $\mathrm{pCi} / \mathrm{L}$ & 0 & & & & & & SURFACE & & RAW GRAB & UNFILTERED & 444 \\
\hline 1153 & $\mathrm{pCi} / \mathrm{L}$ & 0 & & & & & & RIVER & & CUMULATIVE & UNFILTERED & 463 \\
\hline 1154 & $\mathrm{pCi} / \mathrm{L}$ & 0 & & & & & & SANITARY & & CUMULATIVE & UNFILTERED & 491 \\
\hline 1155 & $\mathrm{pCi} / \mathrm{L}$ & 0 & & & & & & RIVER & & CUMULATIVE & UNFILTERED & 553 \\
\hline 1156 & $\mathrm{pCi} / \mathrm{L}$ & 0 & & & & & & RIVER & & CUMULATIVE & UNFILTERED & 553 \\
\hline 1157 & $\mathrm{pCi} / \mathrm{L}$ & 0 & & & & & & RIVER & & CUMULATIVE & UNFILTERED & 463 \\
\hline 1158 & $\mathrm{pCi} / \mathrm{L}$ & 0 & & & & & & RIVER & & CUMULATIVE & UNFILTERED & 463 \\
\hline 1159 & $\mathrm{pCi} / \mathrm{L}$ & 0 & & & & & & RIVER & & CUMULATIVE & UNFILTERED & 463 \\
\hline 1160 & $\mathrm{pCi} / \mathrm{L}$ & 0 & & & & & & RIVER & & CUMULATIVE & UNFILTERED & 463 \\
\hline 1161 & $\mathrm{pCi} / \mathrm{L}$ & 0 & & & & & & RIVER & & RAW GRAB & UNFILTERED & 456 \\
\hline 1162 & $\mathrm{pCi} / \mathrm{L}$ & 0 & & & & & & SANITARY & & CUMULATIVE & UNFILTERED & 203 \\
\hline 1163 & $\mathrm{pCi} / \mathrm{L}$ & 0 & & & & $U$ & & SANITARY & & CUMULATIVE & UNFILTERED & 491 \\
\hline 1164 & $\mathrm{pCi} / \mathrm{L}$ & 0 & & & & & & SANITARY & & CUMULATIVE & UNFILTERED & 385 \\
\hline 1165 & $\mathrm{pCi} / \mathrm{L}$ & 0 & & & & & & SANITARY & & CUMULATIVE & UNFILTERED & 385 \\
\hline 1166 & $\mathrm{pCi} / \mathrm{L}$ & 0 & & & & & & SURFACE & & CUMULATIVE & UNFILTERED & 60 \\
\hline 1167 & $\mathrm{pCi} / \mathrm{L}$ & 0 & & & & & & SANITARY & & CUMULATIVE & UNFILTERED & 491 \\
\hline 1168 & $\mathrm{pCi} / \mathrm{L}$ & 0 & & & & & & RIVER & & CUMULATIVE & UNFILTERED & 463 \\
\hline 1169 & $\mathrm{pCi} / \mathrm{L}$ & 0 & & & & & & RIVER & & RAW GRAB & UNFILTERED & 462 \\
\hline 1170 & $\mathrm{pCi} / \mathrm{L}$ & 0 & & & & $U$ & & RIVER & & RAW GRAB & UNFILTERED & 462 \\
\hline 1171 & $\mathrm{pCi} / \mathrm{L}$ & 0 & & & & & & RIVER & & RAW GRAB & UNFILTERED & 462 \\
\hline 1172 & $\mathrm{pCi} / \mathrm{L}$ & 0 & & & & & & SEEP & & RAW GRAB & UNFILTERED & 556 \\
\hline 1173 & $\mathrm{pCi} / \mathrm{L}$ & 0 & & & & & & SANITARY & & CUMULATIVE & UNFILTERED & 491 \\
\hline 1174 & $\mathrm{pCi} / \mathrm{L}$ & 0 & & & & & & RIVER & & CUMULATIVE & UNFILTERED & 463 \\
\hline 1175 & $\mathrm{pCi} / \mathrm{L}$ & 0 & & & & & & SURFACE & & RAW GRAB & UNFILTERED & 444 \\
\hline 1176 & $\mathrm{pCi} / \mathrm{L}$ & 0 & & & & $U$ & & SANITARY & & CUMULATIVE & UNFILTERED & 491 \\
\hline 1177 & $\mathrm{pCi} / \mathrm{L}$ & 0 & & & & & & RIVER & & CUMULATIVE & UNFILTERED & 463 \\
\hline 1178 & $\mathrm{pCi} / \mathrm{L}$ & 0 & & & & & & SANITARY & & CUMULATIVE & UNFILTERED & 203 \\
\hline 1179 & $\mathrm{pCi} / \mathrm{L}$ & 0 & & & & & & SANITARY & & CUMULATIVE & UNFILTERED & 203 \\
\hline 1180 & $\mathrm{pCi} / \mathrm{L}$ & 0 & & & & & & SANITARY & & CUMULATIVE & UNFILTERED & 491 \\
\hline 1181 & $\mathrm{pCi} / \mathrm{L}$ & 0 & & & & & & SANITARY & & CUMULATIVE & UNFILTERED & 385 \\
\hline 1182 & $\mathrm{pCi} / \mathrm{L}$ & 0 & & & & & & SANITARY & & CUMULATIVE & UNFILTERED & 385 \\
\hline 1183 & $\mathrm{pCi} / \mathrm{L}$ & 0 & & & & & & SURFACE & & CUMULATIVE & UNFILTERED & 60 \\
\hline 1184 & $\mathrm{pCi} / \mathrm{L}$ & 0 & & & & & & RIVER & & CUMULATIVE & UNFILTERED & 463 \\
\hline 1185 & $\mathrm{pCi} / \mathrm{L}$ & 0 & & & & & & RIVER & & CUMULATIVE & UNFILTERED & 463 \\
\hline 1186 & $\mathrm{pCi} / \mathrm{L}$ & 0 & & & & U & & RIVER & & CUMULATIVE & UNFILTERED & 463 \\
\hline 1187 & $\mathrm{pCi} / \mathrm{L}$ & 0 & & & & & & RIVER & & CUMULATIVE & UNFILTERED & 463 \\
\hline 1188 & $\mathrm{pCi} / \mathrm{L}$ & 0 & & & & & & RIVER & & RAW GRAB & UNFILTERED & 456 \\
\hline 1189 & $\mathrm{pCi} / \mathrm{L}$ & 0 & & & & & & RIVER & & RAW GRAB & UNFILTERED & 463 \\
\hline 1190 & $\mathrm{pCi} / \mathrm{L}$ & 0 & & & & & & RIVER & & CUMULATIVE & UNFILTERED & 553 \\
\hline 1191 & $\mathrm{pCi} / \mathrm{L}$ & 0 & & & & & & RIVER & & CUMULATIVE & UNFILTERED & 553 \\
\hline 1192 & $\mathrm{pCi} / \mathrm{L}$ & 0 & & & & $U$ & & RIVER & & CUMULATIVE & UNFILTERED & 553 \\
\hline 1193 & $\mathrm{pCi} / \mathrm{L}$ & 0 & & & & U & & SANITARY & & CUMULATIVE & UNFILTERED & 491 \\
\hline 1194 & $\mathrm{pCi} / \mathrm{L}$ & 0 & & & & & & RIVER & & CUMULATIVE & UNFILTERED & 463 \\
\hline 1195 & pCi/L & 0 & & & & & & SANITARY & & CUMULATIVE & UNFILTERED & 491 \\
\hline 1196 & $\mathrm{pCi} / \mathrm{L}$ & 0 & & & & $U$ & & RIVER & & CUMULATIVE & UNFILTERED & 463 \\
\hline 1197 & $\mathrm{pCi} / \mathrm{L}$ & 0 & 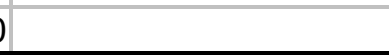 & & & & & SURFACE & & RAW GRAB & UNFILTERED & 444 \\
\hline
\end{tabular}




\begin{tabular}{|c|c|c|c|c|c|c|c|c|c|c|c|c|}
\hline & $\mathrm{N}$ & $\mathrm{O}$ & $P$ & $\mathrm{Q}$ & $\mathrm{R}$ & $\mathrm{s}$ & $\mathrm{T}$ & $\mathrm{U}$ & $\mathrm{V}$ & $\mathrm{W}$ & $\mathrm{x}$ & $\bar{Y}$ \\
\hline 1 & ANAL_UNITS_RPTD & COUNTING_ERROR & TOTAL_ANAL_ERROR & \begin{tabular}{|l|l|} 
MIN_DETECTABLE_ACTIVITY \\
\end{tabular} & PCNT_MOISTURE & LAB_QUALIFIER & REVIEW_QUALIFIER & SAMP_FROM & SAMP_ITEM & SAMP_MTHD & $\mid$ COLL_MTHD & SAMP_SITE_ID \\
\hline 1198 & $\mathrm{pCi} / \mathrm{L}$ & 0 & & & & & & RIVER & & RAW GRAB & UNFILTERED & 462 \\
\hline 1199 & $\mathrm{pCi} / \mathrm{L}$ & 0 & & & & & & RIVER & & RAW GRAB & UNFILTERED & 462 \\
\hline 1200 & $\mathrm{pCi} / \mathrm{L}$ & 0 & & & & & & RIVER & & RAW GRAB & UNFILTERED & 462 \\
\hline 1201 & $\mathrm{pCi} / \mathrm{L}$ & 0 & & & & & & SEEP & & RAW GRAB & UNFILTERED & 556 \\
\hline 1202 & $\mathrm{pCi} / \mathrm{L}$ & 0 & & & & & & SEEP & & RAW GRAB & UNFILTERED & 556 \\
\hline 1203 & $\mathrm{pCi} / \mathrm{L}$ & 0 & & & & & & SANITARY & & CUMULATIVE & UNFILTERED & 491 \\
\hline 1204 & $\mathrm{pCi} / \mathrm{L}$ & 0 & & & & & & RIVER & & CUMULATIVE & UNFILTERED & 463 \\
\hline 1205 & $\mathrm{pCi} / \mathrm{L}$ & 0 & & & & $\mathrm{U}$ & & RIVER & & CUMULATIVE & UNFILTERED & 463 \\
\hline 1206 & $\mathrm{pCi} / \mathrm{L}$ & 0 & & & & & & RIVER & & CUMULATIVE & UNFILTERED & 553 \\
\hline 1207 & $\mathrm{pCi} / \mathrm{L}$ & 0 & & & & & & RIVER & & CUMULATIVE & UNFILTERED & 553 \\
\hline 1208 & $\mathrm{pCi} / \mathrm{L}$ & 0 & & & & & & SANITARY & & CUMULATIVE & UNFILTERED & 203 \\
\hline 1209 & $\mathrm{pCi} / \mathrm{L}$ & 0 & & & & $\mathrm{U}$ & & SANITARY & & CUMULATIVE & UNFILTERED & 491 \\
\hline 1210 & $\mathrm{pCi} / \mathrm{L}$ & 0 & & & & & & SANITARY & & CUMULATIVE & UNFILTERED & 385 \\
\hline \begin{tabular}{|l|}
1211 \\
\end{tabular} & $\mathrm{pCi} / \mathrm{L}$ & 0 & & & & $U$ & & SANITARY & & CUMULATIVE & UNFILTERED & 385 \\
\hline \begin{tabular}{|l|}
1212 \\
\end{tabular} & $\mathrm{pCi} / \mathrm{L}$ & 0 & & & & & & SURFACE & & CUMULATIVE & UNFILTERED & 60 \\
\hline \begin{tabular}{|l|}
1213 \\
\end{tabular} & $\mathrm{pCi} / \mathrm{L}$ & 0 & & & & & & RIVER & & CUMULATIVE & UNFILTERED & 463 \\
\hline 1214 & $\mathrm{pCi} / \mathrm{L}$ & 0 & & & & & & RIVER & & CUMULATIVE & UNFILTERED & 463 \\
\hline \begin{tabular}{|l|}
1215 \\
\end{tabular} & $\mathrm{pCi} / \mathrm{L}$ & 0 & & & & & & RIVER & & CUMULATIVE & UNFILTERED & 463 \\
\hline 1216 & $\mathrm{pCi} / \mathrm{L}$ & 0 & & & & & & RIVER & & RAW GRAB & UNFILTERED & 463 \\
\hline 1217 & $\mathrm{pCi} / \mathrm{L}$ & 0 & & & & & & RIVER & & RAW GRAB & UNFILTERED & 456 \\
\hline 1218 & $\mathrm{pCi} / \mathrm{L}$ & 0 & & & & & & SURFACE & & RAW GRAB & UNFILTERED & 121 \\
\hline \begin{tabular}{|l|}
1219 \\
\end{tabular} & $\mathrm{pCi} / \mathrm{L}$ & 0 & & & & & & SURFACE & & RAW GRAB & UNFILTERED & 509 \\
\hline 1220 & $\mathrm{pCi} / \mathrm{L}$ & 0 & & & & & & SURFACE & & RAW GRAB & UNFILTERED & 509 \\
\hline \begin{tabular}{|l|}
1221 \\
\end{tabular} & $\mathrm{pCi} / \mathrm{L}$ & 0 & & & & & & SURFACE & & RAW GRAB & UNFILTERED & 146 \\
\hline 1222 & $\mathrm{pCi} / \mathrm{L}$ & 0 & & & & & & SURFACE & & RAW GRAB & UNFILTERED & 146 \\
\hline 1223 & $\mathrm{pCi} / \mathrm{L}$ & 0 & & & & & & SURFACE & & RAW GRAB & UNFILTERED & 413 \\
\hline 1224 & $\mathrm{pCi} / \mathrm{L}$ & 0 & & & & & & SURFACE & & RAW GRAB & UNFILTERED & 412 \\
\hline \begin{tabular}{|l|}
1225 \\
\end{tabular} & $\mathrm{pCi} / \mathrm{L}$ & 0 & & & & $U$ & & SURFACE & & RAW GRAB & UNFILTERED & 147 \\
\hline \begin{tabular}{|l|}
1226 \\
\end{tabular} & $\mathrm{pCi} / \mathrm{L}$ & 0 & & & & & & SURFACE & & RAW GRAB & UNFILTERED & 147 \\
\hline \begin{tabular}{|l|}
1227 \\
\end{tabular} & $\mathrm{pCi} / \mathrm{L}$ & 0 & & & & & & RIVER & & CUMULATIVE & UNFILTERED & 463 \\
\hline 1228 & $\mathrm{pCi} / \mathrm{L}$ & 0 & & & & $U$ & & SANITARY & & CUMULATIVE & UNFILTERED & 491 \\
\hline 1229 & $\mathrm{pCi} / \mathrm{L}$ & 0 & & & & & & SURFACE & & RAW GRAB & UNFILTERED & 444 \\
\hline 1230 & $\mathrm{pCi} / \mathrm{L}$ & 0 & & & & & & RIVER & & RAW GRAB & UNFILTERED & 462 \\
\hline \begin{tabular}{|l|}
1231 \\
\end{tabular} & $\mathrm{pCi} / \mathrm{L}$ & 0 & & & & & & RIVER & & RAW GRAB & UNFILTERED & 462 \\
\hline 1232 & $\mathrm{pCi} / \mathrm{L}$ & 0 & & & & & & RIVER & & RAW GRAB & UNFILTERED & 462 \\
\hline 1233 & $\mathrm{pCi} / \mathrm{L}$ & 0 & & & & & & SEEP & & RAW GRAB & UNFILTERED & 556 \\
\hline 1234 & $\mathrm{pCi} / \mathrm{L}$ & 0 & & & & & & SEEP & & RAW GRAB & UNFILTERED & 556 \\
\hline \begin{tabular}{|l|}
1235 \\
\end{tabular} & $\mathrm{pCi} / \mathrm{L}$ & 0 & & & & & & SANITARY & & CUMULATIVE & UNFILTERED & 491 \\
\hline \begin{tabular}{|l|}
1236 \\
\end{tabular} & $\mathrm{pCi} / \mathrm{L}$ & 0 & & & & $U$ & & RIVER & & CUMULATIVE & UNFILTERED & 463 \\
\hline 1237 & $\mathrm{pCi} / \mathrm{L}$ & 0 & & & & $U$ & & SANITARY & & CUMULATIVE & UNFILTERED & 491 \\
\hline 1238 & $\mathrm{pCi} / \mathrm{L}$ & 0 & & & & & & RIVER & & CUMULATIVE & UNFILTERED & 463 \\
\hline 1239 & $\mathrm{pCi} / \mathrm{L}$ & 0 & & & & & & RIVER & & CUMULATIVE & UNFILTERED & 553 \\
\hline 1240 & $\mathrm{pCi} / \mathrm{L}$ & 0 & & & & & & RIVER & & CUMULATIVE & UNFILTERED & 553 \\
\hline \begin{tabular}{|l|}
1241 \\
\end{tabular} & $\mathrm{pCi} / \mathrm{L}$ & 0 & & & & & & SANITARY & & CUMULATIVE & UNFILTERED & 203 \\
\hline 1242 & $\mathrm{pCi} / \mathrm{L}$ & 0 & & & & & & SANITARY & & CUMULATIVE & UNFILTERED & 491 \\
\hline 1243 & $\mathrm{pCi} / \mathrm{L}$ & 0 & & & & & & SANITARY & & CUMULATIVE & UNFILTERED & 385 \\
\hline 1244 & $\mathrm{pCi} / \mathrm{L}$ & 0 & & & & & & SURFACE & & CUMULATIVE & UNFILTERED & 60 \\
\hline 1245 & $\mathrm{pCi} / \mathrm{L}$ & 0 & & & & & & RIVER & & CUMULATIVE & UNFILTERED & 463 \\
\hline \begin{tabular}{|l|}
1246 \\
\end{tabular} & $\mathrm{pCi} / \mathrm{L}$ & 0 & & & & & & RIVER & & CUMULATIVE & UNFILTERED & 463 \\
\hline 1247 . & $\mathrm{pCi} / \mathrm{L}$ & 0 & & & & & & RIVER & & CUMULATIVE & UNFILTERED & 463 \\
\hline 1248 & $\mathrm{pCi} / \mathrm{L}$ & 0 & & & & & & RIVER & & CUMULATIVE & UNFILTERED & 463 \\
\hline 1249 & $\mathrm{pCi} / \mathrm{L}$ & 0 & & & & & & RIVER & & RAW GRAB & UNFILTERED & 463 \\
\hline
\end{tabular}




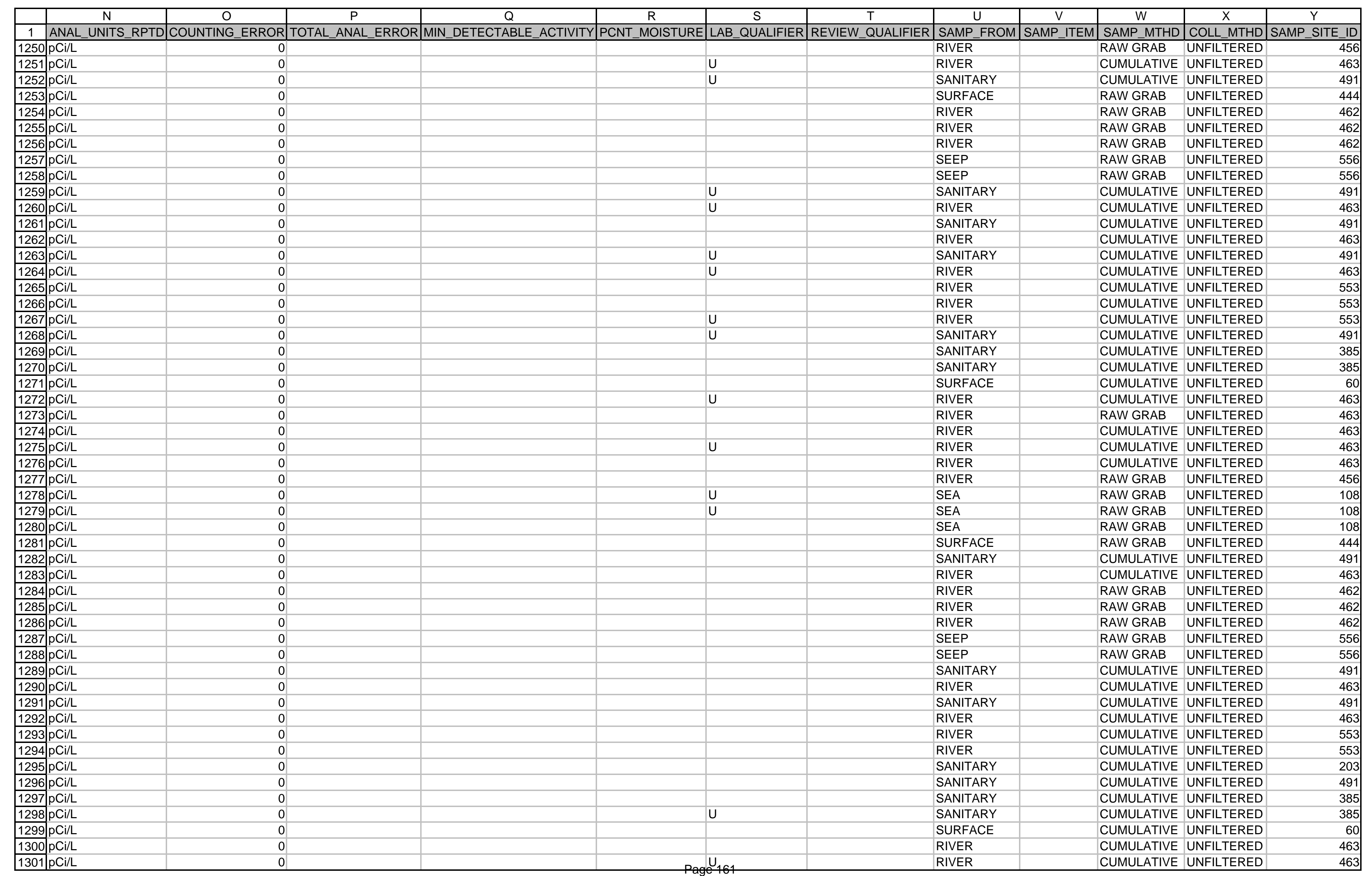




\begin{tabular}{|c|c|c|c|c|c|c|c|c|c|c|c|c|}
\hline & $\mathrm{N}$ & $\mathrm{O}$ & $\mathrm{P}$ & $\mathrm{Q}$ & $\mathrm{R}$ & $\mathrm{s}$ & $\mathrm{T}$ & $\mathrm{u}$ & $\mathrm{v}$ & $\mathrm{w}$ & $\mathrm{x}$ & $\bar{Y}$ \\
\hline 1 & ANAL_UNITS_RPTD & COUNTING_ERROR & TOTAL_ANAL_ERROR & MIN_DETECTABLE_ACTIVITY & PCNT_MOISTURE & LAB_QUALIFIER & REVIEW_QUALIFIER & SAMP_FROM & SAMP_ITEM & SAMP_MTHD & COLL_MTHD & SAMP_SITE_ID \\
\hline 1302 & $\mathrm{pCi} / \mathrm{L}$ & 0 & & & & $U$ & & RIVER & & CUMULATIVE & UNFILTERED & 463 \\
\hline 1303 & $\mathrm{pCi} / \mathrm{L}$ & 0 & & & & & & RIVER & & CUMULATIVE & UNFILTERED & 463 \\
\hline 1304 & $\mathrm{pCi} / \mathrm{L}$ & 0 & & & & & & RIVER & & RAW GRAB & UNFILTERED & 463 \\
\hline 1305 & $\mathrm{pCi} / \mathrm{L}$ & 0 & & & & & & RIVER & & RAW GRAB & UNFILTERED & 456 \\
\hline 1306 & $\mathrm{pCi} / \mathrm{L}$ & 0 & & & & & & SURFACE & & RAW GRAB & UNFILTERED & 444 \\
\hline \begin{tabular}{|l|l|}
1307 \\
\end{tabular} & $\mathrm{pCi} / \mathrm{L}$ & 0 & & & & $\mathrm{U}$ & & SANITARY & & CUMULATIVE & UNFILTERED & 491 \\
\hline 1308 & $\mathrm{pCi} / \mathrm{L}$ & 0 & & & & & & RIVER & & CUMULATIVE & UNFILTERED & 463 \\
\hline 1309 & $\mathrm{pCi} / \mathrm{L}$ & 0 & & & & & & RIVER & & RAW GRAB & UNFILTERED & 462 \\
\hline 1310 & $\mathrm{pCi} / \mathrm{L}$ & 0 & & & & & & RIVER & & RAW GRAB & UNFILTERED & 462 \\
\hline 1311 & $\mathrm{pCi} / \mathrm{L}$ & 0 & & & & & & RIVER & & RAW GRAB & UNFILTERED & 462 \\
\hline 1312 & $\mathrm{pCi} / \mathrm{L}$ & 0 & & & & & & SEEP & & RAW GRAB & UNFILTERED & 556 \\
\hline 1313 & $\mathrm{pCi} / \mathrm{L}$ & 0 & & & & & & SEEP & & RAW GRAB & UNFILTERED & 556 \\
\hline 1314 & $\mathrm{pCi} / \mathrm{L}$ & 0 & & & & & & SURFACE & & RAW GRAB & UNFILTERED & 121 \\
\hline 1315 & $\mathrm{pCi} / \mathrm{L}$ & 0 & & & & & & SURFACE & & RAW GRAB & UNFILTERED & 509 \\
\hline 1316 & $\mathrm{pCi} / \mathrm{L}$ & 0 & & & & & & SURFACE & & RAW GRAB & UNFILTERED & 509 \\
\hline \begin{tabular}{|l|l|}
1317 \\
\end{tabular} & $\mathrm{pCi} / \mathrm{L}$ & 0 & & & & & & SURFACE & & RAW GRAB & UNFILTERED & 146 \\
\hline 1318 & $\mathrm{pCi} / \mathrm{L}$ & 0 & & & & & & SURFACE & & RAW GRAB & UNFILTERED & 146 \\
\hline 1319 & $\mathrm{pCi} / \mathrm{L}$ & 0 & & & & & & SURFACE & & RAW GRAB & UNFILTERED & 412 \\
\hline 1320 & $\mathrm{pCi} / \mathrm{L}$ & 0 & & & & & & SURFACE & & RAW GRAB & UNFILTERED & 147 \\
\hline 1321 & $\mathrm{pCi} / \mathrm{L}$ & 0 & & & & & & SURFACE & & RAW GRAB & UNFILTERED & 147 \\
\hline 1322 & $\mathrm{pCi} / \mathrm{L}$ & 0 & & & & $U$ & & SANITARY & & CUMULATIVE & UNFILTERED & 491 \\
\hline 1323 & $\mathrm{pCi} / \mathrm{L}$ & 0 & & & & $U$ & & RIVER & & CUMULATIVE & UNFILTERED & 463 \\
\hline 1324 & $\mathrm{pCi} / \mathrm{L}$ & 0 & & & & & & SANITARY & & CUMULATIVE & UNFILTERED & 491 \\
\hline 1325 & $\mathrm{pCi} / \mathrm{L}$ & 0 & & & & & & RIVER & & CUMULATIVE & UNFILTERED & 463 \\
\hline 1326 & $\mathrm{pCi} / \mathrm{L}$ & 0 & & & & & & RIVER & & CUMULATIVE & UNFILTERED & 553 \\
\hline \begin{tabular}{|l|l|}
1327 \\
\end{tabular} & $\mathrm{pCi} / \mathrm{L}$ & 0 & & & & & & RIVER & & CUMULATIVE & UNFILTERED & 553 \\
\hline 1328 & $\mathrm{pCi} / \mathrm{L}$ & 0 & & & & & & SANITARY & & CUMULATIVE & UNFILTERED & 203 \\
\hline 1329 & $\mathrm{pCi} / \mathrm{L}$ & 0 & & & & $U$ & & SANITARY & & CUMULATIVE & UNFILTERED & 491 \\
\hline 1330 & $\mathrm{pCi} / \mathrm{L}$ & 0 & & & & & & SANITARY & & CUMULATIVE & UNFILTERED & 385 \\
\hline 1331 & $\mathrm{pCi} / \mathrm{L}$ & 0 & & & & & & SANITARY & & CUMULATIVE & UNFILTERED & 385 \\
\hline 1332 & $\mathrm{pCi} / \mathrm{L}$ & 0 & & & & & & SURFACE & & CUMULATIVE & UNFILTERED & 60 \\
\hline 1333 & $\mathrm{pCi} / \mathrm{L}$ & 0 & & & & & & RIVER & & CUMULATIVE & UNFILTERED & 463 \\
\hline 1334 & $\mathrm{pCi} / \mathrm{L}$ & 0 & & & & & & RIVER & & CUMULATIVE & UNFILTERED & 463 \\
\hline 1335 & $\mathrm{pCi} / \mathrm{L}$ & 0 & & & & & & RIVER & & CUMULATIVE & UNFILTERED & 463 \\
\hline 1336 & $\mathrm{pCi} / \mathrm{L}$ & 0 & & & & & & RIVER & & CUMULATIVE & UNFILTERED & 463 \\
\hline 1337 . & $\mathrm{pCi} / \mathrm{L}$ & 0 & & & & & & RIVER & & RAW GRAB & UNFILTERED & 463 \\
\hline 1338 & $\mathrm{pCi} / \mathrm{L}$ & 0 & & & & & & RIVER & & RAW GRAB & UNFILTERED & 456 \\
\hline 1339 & $\mathrm{pCi} / \mathrm{L}$ & 0 & & & & & & SURFACE & & RAW GRAB & UNFILTERED & 444 \\
\hline 1340 & $\mathrm{pCi} / \mathrm{L}$ & 0 & & & & & & SANITARY & & CUMULATIVE & UNFILTERED & 491 \\
\hline 1341 & $\mathrm{pCi} / \mathrm{L}$ & 0 & & & & $U$ & & RIVER & & CUMULATIVE & UNFILTERED & 463 \\
\hline 1342 & $\mathrm{pCi} / \mathrm{L}$ & 0 & & & & & & RIVER & & RAW GRAB & UNFILTERED & 462 \\
\hline 1343 & $\mathrm{pCi} / \mathrm{L}$ & 0 & & & & & & RIVER & & RAW GRAB & UNFILTERED & 462 \\
\hline 1344 & $\mathrm{pCi} / \mathrm{L}$ & 0 & & & & & & RIVER & & RAW GRAB & UNFILTERED & 462 \\
\hline 1345 & $\mathrm{pCi} / \mathrm{L}$ & 0 & & & & & & SEEP & & RAW GRAB & UNFILTERED & 556 \\
\hline 1346 & $\mathrm{pCi} / \mathrm{L}$ & 0 & & & & $U$ & & SEEP & & RAW GRAB & UNFILTERED & 556 \\
\hline 1347 & $\mathrm{pCi} / \mathrm{L}$ & 0 & & & & & & SANITARY & & CUMULATIVE & UNFILTERED & 491 \\
\hline 1348 & $\mathrm{pCi} / \mathrm{L}$ & 0 & & & & $U$ & & RIVER & & CUMULATIVE & UNFILTERED & 463 \\
\hline 1349 & $\mathrm{pCi} / \mathrm{L}$ & 0 & & & & $U$ & & SANITARY & & CUMULATIVE & UNFILTERED & 491 \\
\hline 1350 & $\mathrm{pCi} / \mathrm{L}$ & 0 & & & & $U$ & & RIVER & & CUMULATIVE & UNFILTERED & 463 \\
\hline 1351 & $\mathrm{pCi} / \mathrm{L}$ & 0 & & & & & & SANITARY & & CUMULATIVE & UNFILTERED & 491 \\
\hline 1352 & $\mathrm{pCi} / \mathrm{L}$ & 0 & & & & & & RIVER & & CUMULATIVE & UNFILTERED & 463 \\
\hline 1353 & $\mathrm{pCi} / \mathrm{L}$ & 0 & & & & & & SURFACE & & RAW GRAB & UNFILTERED & 444 \\
\hline
\end{tabular}




\begin{tabular}{|c|c|c|c|c|c|c|c|c|c|c|c|c|}
\hline & $\mathrm{N}$ & $\mathrm{O}$ & $\mathrm{P}$ & $\mathrm{Q}$ & $\mathrm{R}$ & $\mathrm{s}$ & $\mathrm{T}$ & $U$ & $\mathrm{~V}$ & $\mathrm{w}$ & $\mathrm{x}$ & $\mathrm{Y}$ \\
\hline 1 & ANAL_UNITS_RPTD & COUNTING ERROR & TOTAL ANAL ERROR & \begin{tabular}{|l|l|} 
MIN DETECTABLE ACTIVITY \\
\end{tabular} & PCNT_MOISTURE & LAB QUALIFIER & REVIEW_QUALIFIER & SAMP FROM & SAMP_ITEM & SAMP MTHD & COLL MTHD & SAMP SITE ID \\
\hline 1354 & $\mathrm{pCi} / \mathrm{L}$ & 0 & & & & & & RIVER & & CUMULATIVE & UNFILTERED & 553 \\
\hline 1355 & $\mathrm{pCi} / \mathrm{L}$ & 0 & & & & & & RIVER & & CUMULATIVE & UNFILTERED & 553 \\
\hline 1356 & $\mathrm{pCi} / \mathrm{L}$ & 0 & & & & $u$ & & RIVER & & CUMULATIVE & UNFILTERED & 553 \\
\hline 1357 & $\mathrm{pCi} / \mathrm{L}$ & 0 & & & & U & & SANITARY & & CUMULATIVE & UNFILTERED & 203 \\
\hline 1358 & $\mathrm{pCi} / \mathrm{L}$ & 0 & & & & & & SANITARY & & SAN. GRAB & UNFILTERED & 149 \\
\hline 1359 & $\mathrm{pCi} / \mathrm{L}$ & 0 & & & & $\mathrm{U}$ & & SANITARY & & CUMULATIVE & UNFILTERED & 385 \\
\hline 1360 & $\mathrm{pCi} / \mathrm{L}$ & 0 & & & & $\mathrm{U}$ & & SURFACE & & CUMULATIVE & UNFILTERED & 60 \\
\hline 1361 & $\mathrm{pCi} / \mathrm{L}$ & 0.00000546 & & & & & & RIVER & & CONTINUOUS & RESIN & 307 \\
\hline 1362 & $\mathrm{pCi} / \mathrm{L}$ & 0 & & & & $\mathrm{U}$ & & RIVER & & CUMULATIVE & UNFILTERED & 463 \\
\hline 1363 & $\mathrm{pCi} / \mathrm{L}$ & 0 & & & & & & RIVER & & CUMULATIVE & UNFILTERED & 463 \\
\hline 1364 & $\mathrm{pCi} / \mathrm{L}$ & 0 & & & & & & RIVER & & CUMULATIVE & UNFILTERED & 463 \\
\hline 1365 & $\mathrm{pCi} / \mathrm{L}$ & 0 & & & & & & RIVER & & RAW GRAB & UNFILTERED & 463 \\
\hline 1366 & $\mathrm{pCi} / \mathrm{L}$ & 0 & & & & & & RIVER & & RAW GRAB & UNFILTERED & 456 \\
\hline \begin{tabular}{|l|}
1367 \\
\end{tabular} & $\mathrm{pCi} / \mathrm{L}$ & 0 & & & & $U$ & & SANITARY & & SAN. GRAB & UNFILTERED & 50 \\
\hline 1368 & $\mathrm{pCi} / \mathrm{L}$ & 0 & & & & $U$ & & DRINKING & & SAN. GRAB & UNFILTERED & 186 \\
\hline 1369 & $\mathrm{pCi} / \mathrm{L}$ & 0 & & & & $U$ & & DRINKING & & SAN. GRAB & UNFILTERED & 186 \\
\hline 1370 & $\mathrm{pCi} / \mathrm{L}$ & 0.0000024 & & & & & & RIVER & & CONTINUOUS & RESIN & 252 \\
\hline 1371 & $\mathrm{pCi} / \mathrm{L}$ & 0 & & & & & & SEEP & & RAW GRAB & UNFILTERED & 556 \\
\hline \begin{tabular}{|l|}
1372 \\
\end{tabular} & $\mathrm{pCi} / \mathrm{L}$ & 0 & & & & $U$ & & SEEP & & RAW GRAB & UNFILTERED & 556 \\
\hline \begin{tabular}{|l|}
1373 \\
\end{tabular} & $\mathrm{pCi} / \mathrm{L}$ & 0 & & & & & & SURFACE & & RAW GRAB & UNFILTERED & 121 \\
\hline 1374 & & & & & & $U$ & & SURFACE & & RAW GRAB & UNFILTERED & 146 \\
\hline 1375 & & & & & & $U$ & & SURFACE & & RAW GRAB & UNFILTERED & 146 \\
\hline 1376 & $\mathrm{pCi} / \mathrm{L}$ & 0 & & & & & & SURFACE & & RAW GRAB & UNFILTERED & 412 \\
\hline \begin{tabular}{|l|}
1377 \\
\end{tabular} & $\mathrm{pCi} / \mathrm{L}$ & 0 & & & & & & SURFACE & & RAW GRAB & UNFILTERED & 412 \\
\hline 1378 & $\mathrm{pCi} / \mathrm{L}$ & 0 & & & & & & SURFACE & & RAW GRAB & UNFILTERED & 509 \\
\hline \begin{tabular}{|l|}
1379 \\
\end{tabular} & $\mathrm{pCi} / \mathrm{L}$ & 0 & & & & & & SURFACE & & RAW GRAB & UNFILTERED & 509 \\
\hline 1380 & $\mathrm{pCi} / \mathrm{L}$ & 0 & & & & & & SURFACE & & RAW GRAB & UNFILTERED & 146 \\
\hline 1381 & $\mathrm{pCi} / \mathrm{L}$ & 0 & & & & & & RIVER & & CUMULATIVE & UNFILTERED & 553 \\
\hline 1382 & $\mathrm{pCi} / \mathrm{L}$ & 0 & & & & & & RIVER & & CUMULATIVE & UNFILTERED & 553 \\
\hline 1383 & $\mathrm{pCi} / \mathrm{L}$ & 0 & & & & $U$ & & RIVER & & CUMULATIVE & UNFILTERED & 553 \\
\hline \begin{tabular}{|l|}
1384 \\
\end{tabular} & $\mathrm{pCi} / \mathrm{L}$ & 0 & & & & & & SANITARY & & CUMULATIVE & UNFILTERED & 203 \\
\hline 1385 & $\mathrm{pCi} / \mathrm{L}$ & 0 & & & & & & SANITARY & & SAN. GRAB & UNFILTERED & 50 \\
\hline \begin{tabular}{|l|}
1386 \\
\end{tabular} & $\mathrm{pCi} / \mathrm{L}$ & 0 & & & & & & SANITARY & & SAN. GRAB & UNFILTERED & 50 \\
\hline \begin{tabular}{|l|l}
1387 \\
\end{tabular} & $\mathrm{pCi} / \mathrm{L}$ & 0 & & & & $U$ & & SANITARY & & SAN. GRAB & UNFILTERED & 149 \\
\hline 1388 & $\mathrm{pCi} / \mathrm{L}$ & 0 & & & & & & SANITARY & & CUMULATIVE & UNFILTERED & 385 \\
\hline 1389 & $\mathrm{pCi} / \mathrm{L}$ & 0 & & & & & & SANITARY & & SAN. GRAB & UNFILTERED & 589 \\
\hline 1390 & $\mathrm{pCi} / \mathrm{L}$ & 0 & & & & $\mathrm{U}$ & & SANITARY & & SAN. GRAB & UNFILTERED & 589 \\
\hline 1391 & $\mathrm{pCi} / \mathrm{L}$ & 0 & & & & $U$ & & SURFACE & & CUMULATIVE & UNFILTERED & 60 \\
\hline \begin{tabular}{|l|}
1392 \\
\end{tabular} & $\mathrm{pCi} / \mathrm{L}$ & 0 & & & & & & RIVER & & CUMULATIVE & UNFILTERED & 463 \\
\hline \begin{tabular}{|l|}
1393 \\
\end{tabular} & $\mathrm{pCi} / \mathrm{L}$ & 0 & & & & & & RIVER & & CUMULATIVE & UNFILTERED & 463 \\
\hline \begin{tabular}{|l|}
1394 \\
\end{tabular} & $\mathrm{pCi} / \mathrm{L}$ & 0 & & & & & & RIVER & & CUMULATIVE & UNFILTERED & 463 \\
\hline 1395 & $\mathrm{pCi} / \mathrm{L}$ & 0 & & & & $U$ & & RIVER & & RAW GRAB & UNFILTERED & 463 \\
\hline \begin{tabular}{|l|}
1396 \\
\end{tabular} & $\mathrm{pCi} / \mathrm{L}$ & 0 & & & & & & RIVER & & RAW GRAB & UNFILTERED & 456 \\
\hline 1397 & $\mathrm{pCi} / \mathrm{L}$ & 0 & & & & $\mathrm{U}$ & & DRINKING & & SAN. GRAB & UNFILTERED & 186 \\
\hline \begin{tabular}{|l|}
1398 \\
\end{tabular} & $\mathrm{pCi} / \mathrm{L}$ & 0 & & & & & & DRINKING & & SAN. GRAB & UNFILTERED & 186 \\
\hline 1399 & $\mathrm{pCi} / \mathrm{L}$ & 0 & & & & & & SEEP & & RAW GRAB & UNFILTERED & 556 \\
\hline 1400 & $\mathrm{pCi} / \mathrm{L}$ & 0 & & & & $U$ & & SEEP & & RAW GRAB & UNFILTERED & 556 \\
\hline \begin{tabular}{|l|}
1401 \\
\end{tabular} & $\mathrm{pCi} / \mathrm{L}$ & 0.00000546 & & & & & & RIVER & & CONTINUOUS & RESIN & 307 \\
\hline 1402 & $\mathrm{pCi} / \mathrm{L}$ & 0.0000072 & & & & & & RIVER & & CONTINUOUS & RESIN & 252 \\
\hline 1403 & $\mathrm{pCi} / \mathrm{L}$ & 0 & & & & & & RIVER & & CUMULATIVE & UNFILTERED & 553 \\
\hline 1404 & $\mathrm{pCi} / \mathrm{L}$ & 0 & & & & & & RIVER & & CUMULATIVE & UNFILTERED & 553 \\
\hline 1405 & $\mathrm{pCi} / \mathrm{L}$ & 0 & & & & $u$ & & RIVER & & CUMULATIVE & UNFILTERED & 553 \\
\hline
\end{tabular}




\begin{tabular}{|c|c|c|c|c|c|c|c|c|c|c|c|c|}
\hline & $\mathrm{N}$ & $\mathrm{O}$ & $\mathrm{P}$ & $\mathrm{Q}$ & $\mathrm{R}$ & $\mathrm{s}$ & $T$ & $\mathrm{U}$ & $\mathrm{V}$ & $\mathrm{W}$ & $\mathrm{X}$ & $\bar{Y}$ \\
\hline 1 & ANAL_UNITS_RPTD & COUNTING_ERROR & TOTAL_ANAL_ERROR & MIN_DETECTABLE_ACTIVITY & PCNT_MOISTURE & LAB_QUALIFIER & REVIEW_QUALIFIER & SAMP_FROM & SAMP_ITEM & SAMP MTHD & COLL MTHD & SAMP SITE ID \\
\hline 1406 & $\mathrm{pCi} / \mathrm{L}$ & 0 & & & & & & SANITARY & & CUMULATIVE & UNFILTERED & 203 \\
\hline 1407 & $\mathrm{pCi} / \mathrm{L}$ & 0 & & & & & & SANITARY & & SAN. GRAB & UNFILTERED & 50 \\
\hline 1408 & $\mathrm{pCi} / \mathrm{L}$ & 0 & & & & & & SANITARY & & SAN. GRAB & UNFILTERED & 50 \\
\hline 1409 & $\mathrm{pCi} / \mathrm{L}$ & 0 & & & & & & SANITARY & & SAN. GRAB & UNFILTERED & 149 \\
\hline 1410 & $\mathrm{pCi} / \mathrm{L}$ & 0 & & & & $\mathrm{U}$ & & SANITARY & & CUMULATIVE & UNFILTERED & 385 \\
\hline 1411 & $\mathrm{pCi} / \mathrm{L}$ & 0 & & & & & & SANITARY & & SAN. GRAB & UNFILTERED & 589 \\
\hline 1412 & $\mathrm{pCi} / \mathrm{L}$ & 0 & & & & & & SANITARY & & SAN. GRAB & UNFILTERED & 589 \\
\hline 1413 & $\mathrm{pCi} / \mathrm{L}$ & 0 & & & & U & & SURFACE & & CUMULATIVE & UNFILTERED & 60 \\
\hline 1414 & $\mathrm{pCi} / \mathrm{L}$ & 0 & & & & & & RIVER & & CUMULATIVE & UNFILTERED & 463 \\
\hline 1415 & $\mathrm{pCi} / \mathrm{L}$ & 0 & & & & & & RIVER & & CUMULATIVE & UNFILTERED & 463 \\
\hline 1416 & $\mathrm{pCi} / \mathrm{L}$ & 0 & & & & & & RIVER & & CUMULATIVE & UNFILTERED & 463 \\
\hline 1417 & $\mathrm{pCi} / \mathrm{L}$ & 0 & & & & & & RIVER & & RAW GRAB & UNFILTERED & 463 \\
\hline 1418 & $\mathrm{pCi} / \mathrm{L}$ & 0 & & & & & & RIVER & & RAW GRAB & UNFILTERED & 456 \\
\hline 1419 & $\mathrm{pCi} / \mathrm{L}$ & 0 & & & & & & DRINKING & & SAN. GRAB & UNFILTERED & 186 \\
\hline 1420 & $\mathrm{pCi} / \mathrm{L}$ & 0 & & & & U & & DRINKING & & SAN. GRAB & UNFILTERED & 186 \\
\hline \begin{tabular}{|l|}
1421 \\
\end{tabular} & $\mathrm{pCi} / \mathrm{L}$ & 0 & & & & & & SEEP & & RAW GRAB & UNFILTERED & 556 \\
\hline 1422 & $\mathrm{pCi} / \mathrm{L}$ & 0 & & & & U & & SEEP & & RAW GRAB & UNFILTERED & 556 \\
\hline 1423 & $\mathrm{pCi} / \mathrm{L}$ & 0 & & & & U & & SANITARY & & SAN. GRAB & UNFILTERED & 149 \\
\hline 1424 & $\mathrm{pCi} / \mathrm{L}$ & 0 & & & & & & SURFACE & & CUMULATIVE & UNFILTERED & 60 \\
\hline 1425 & $\mathrm{pCi} / \mathrm{L}$ & 0.0000038 & & & & & & RIVER & & CONTINUOUS & RESIN & 307 \\
\hline 1426 & $\mathrm{pCi} / \mathrm{L}$ & 0 & & & & U & & RIVER & & CUMULATIVE & UNFILTERED & 463 \\
\hline \begin{tabular}{|l|}
1427 \\
\end{tabular} & $\mathrm{pCi} / \mathrm{L}$ & 0 & & & & U & & RIVER & & CUMULATIVE & UNFILTERED & 553 \\
\hline 1428 - & $\mathrm{pCi} / \mathrm{L}$ & 0 & & & & & & RIVER & & CUMULATIVE & UNFILTERED & 553 \\
\hline 1429 & $\mathrm{pCi} / \mathrm{L}$ & 0 & & & & & & RIVER & & CUMULATIVE & UNFILTERED & 553 \\
\hline 1430 & $\mathrm{pCi} / \mathrm{L}$ & 0 & & & & & & RIVER & & RAW GRAB & UNFILTERED & 463 \\
\hline \begin{tabular}{|l|}
1431 \\
\end{tabular} & $\mathrm{pCi} / \mathrm{L}$ & 0 & & & & & & RIVER & & CUMULATIVE & UNFILTERED & 463 \\
\hline 1432 & $\mathrm{pCi} / \mathrm{L}$ & 0 & & & & & & RIVER & & CUMULATIVE & UNFILTERED & 463 \\
\hline 1433 & $\mathrm{pCi} / \mathrm{L}$ & 0 & & & & & & RIVER & & CUMULATIVE & UNFILTERED & 463 \\
\hline 1434 & $\mathrm{pCi} / \mathrm{L}$ & 0 & & & & & & RIVER & & RAW GRAB & UNFILTERED & 456 \\
\hline 1435 & $\mathrm{pCi} / \mathrm{L}$ & 0 & & & & U & & SANITARY & & CUMULATIVE & UNFILTERED & 203 \\
\hline 1436 & $\mathrm{pCi} / \mathrm{L}$ & 0 & & & & & & SANITARY & & SAN. GRAB & UNFILTERED & 50 \\
\hline \begin{tabular}{|l|l|}
1437 \\
\end{tabular} & $\mathrm{pCi} / \mathrm{L}$ & 0 & & & & $U$ & & SANITARY & & SAN. GRAB & UNFILTERED & 50 \\
\hline 1438 & $\mathrm{pCi} / \mathrm{L}$ & 0 & & & & $U$ & & SANITARY & & CUMULATIVE & UNFILTERED & 385 \\
\hline \begin{tabular}{|l|}
1439 \\
\end{tabular} & $\mathrm{pCi} / \mathrm{L}$ & 0 & & & & & & SANITARY & & SAN. GRAB & UNFILTERED & 589 \\
\hline 1440 & $\mathrm{pCi} / \mathrm{L}$ & 0 & & & & U & & SANITARY & & SAN. GRAB & UNFILTERED & 589 \\
\hline \begin{tabular}{|l|l|}
1441 \\
\end{tabular} & $\mathrm{pCi} / \mathrm{L}$ & 0 & & & & U & & SURFACE & & RAW GRAB & UNFILTERED & 121 \\
\hline 1442 & $\mathrm{pCi} / \mathrm{L}$ & 0 & & & & & & SURFACE & & RAW GRAB & UNFILTERED & 509 \\
\hline 1443 & $\mathrm{pCi} / \mathrm{L}$ & 0 & & & & & & SURFACE & & RAW GRAB & UNFILTERED & 509 \\
\hline 1444 & $\mathrm{pCi} / \mathrm{L}$ & 0 & & & & & & SURFACE & & RAW GRAB & UNFILTERED & 146 \\
\hline 1445 & $\mathrm{pCi} / \mathrm{L}$ & 0 & & & & & & SURFACE & & RAW GRAB & UNFILTERED & 146 \\
\hline 1446 & $\mathrm{pCi} / \mathrm{L}$ & 0 & & & & & & SURFACE & & RAW GRAB & UNFILTERED & 413 \\
\hline 1447 & $\mathrm{pCi} / \mathrm{L}$ & 0 & & & & & & SURFACE & & RAW GRAB & UNFILTERED & 413 \\
\hline 1448 & $\mathrm{pCi} / \mathrm{L}$ & 0 & & & & & & SURFACE & & RAW GRAB & UNFILTERED & 412 \\
\hline 1449 & $\mathrm{pCi} / \mathrm{L}$ & 0 & & & & & & SURFACE & & RAW GRAB & UNFILTERED & 412 \\
\hline 1450 & $\mathrm{pCi} / \mathrm{L}$ & 0 & & & & & & SURFACE & & RAW GRAB & UNFILTERED & 147 \\
\hline \begin{tabular}{|l|}
1451 \\
\end{tabular} & $\mathrm{pCi} / \mathrm{L}$ & 0 & & & & & & SURFACE & & RAW GRAB & UNFILTERED & 147 \\
\hline 1452 & $\mathrm{pCi} / \mathrm{L}$ & 0.00000368 & & & & & & RIVER & & CONTINUOUS & RESIN & 252 \\
\hline 1453 & $\mathrm{pCi} / \mathrm{L}$ & 0 & & & & & & SEEP & & RAW GRAB & UNFILTERED & 556 \\
\hline 1454 & $\mathrm{pCi} / \mathrm{L}$ & 0 & & & & & & SEEP & & RAW GRAB & UNFILTERED & 556 \\
\hline 1455 & $\mathrm{pCi} / \mathrm{L}$ & 0 & & & & & & RIVER & & CUMULATIVE & UNFILTERED & 553 \\
\hline 1456 & $\mathrm{pCi} / \mathrm{L}$ & 0 & & & & & & RIVER & & CUMULATIVE & UNFILTERED & 553 \\
\hline 1457 & $\mathrm{pCi} / \mathrm{L}$ & 0 & & & & & & RIVER & & CUMULATIVE & UNFILTERED & 553 \\
\hline
\end{tabular}




\begin{tabular}{|c|c|c|c|c|c|c|c|c|c|c|c|c|}
\hline & $\mathrm{N}$ & $\mathrm{O}$ & $\mathrm{P}$ & $\mathrm{Q}$ & $\mathrm{R}$ & $\mathrm{S}$ & $T$ & $\bar{U}$ & $\mathrm{~V}$ & $\mathrm{~W}$ & $\mathrm{X}$ & $\overline{\mathrm{Y}}$ \\
\hline 1 & ANAL_UNITS_RPTD & COUNTING_ERROR & TOTAL_ANAL_ERROR & MIN_DETECTABLE_ACTIVITY & PCNT_MOISTURE & LAB_QUALIFIER & REVIEW_QUALIFIER & SAMP_FROM & SAMP_ITEM & SAMP_MTHD & COLL_MTHD & SAMP_SITE_ID \\
\hline 1458 & $\mathrm{pCi} / \mathrm{L}$ & 0 & & & & & & SANITARY & & SAN. GRAB & UNFILTERED & 149 \\
\hline 1459 & $\mathrm{pCi} / \mathrm{L}$ & 0 & & & & U & & SANITARY & & CUMULATIVE & UNFILTERED & 485 \\
\hline 1460 & $\mathrm{pCi} / \mathrm{L}$ & 0 & & & & & & SANITARY & & CUMULATIVE & UNFILTERED & 203 \\
\hline 1461 & $\mathrm{pCi} / \mathrm{L}$ & 0 & & & & & & SANITARY & & SAN. GRAB & UNFILTERED & 50 \\
\hline 1462 & $\mathrm{pCi} / \mathrm{L}$ & 0 & & & & & & SANITARY & & SAN. GRAB & UNFILTERED & 50 \\
\hline 1463 & $\mathrm{pCi} / \mathrm{L}$ & 0 & & & & U & & SANITARY & & CUMULATIVE & UNFILTERED & 385 \\
\hline 1464 & $\mathrm{pCi} / \mathrm{L}$ & 0 & & & & & & SANITARY & & SAN. GRAB & UNFILTERED & 589 \\
\hline 1465 & $\mathrm{pCi} / \mathrm{L}$ & 0 & & & & & & SANITARY & & SAN. GRAB & UNFILTERED & 589 \\
\hline 1466 & $\mathrm{pCi} / \mathrm{L}$ & 0 & & & & U & & SURFACE & & CUMULATIVE & UNFILTERED & 60 \\
\hline 1467 & $\mathrm{pCi} / \mathrm{L}$ & 0 & & & & $\mathrm{U}$ & & RIVER & & CUMULATIVE & UNFILTERED & 463 \\
\hline 1468 & $\mathrm{pCi} / \mathrm{L}$ & 0 & & & & & & RIVER & & CUMULATIVE & UNFILTERED & 463 \\
\hline 1469 & $\mathrm{pCi} / \mathrm{L}$ & 0 & & & & & & RIVER & & CUMULATIVE & UNFILTERED & 463 \\
\hline 1470 & $\mathrm{pCi} / \mathrm{L}$ & 0 & & & & & & RIVER & & RAW GRAB & UNFILTERED & 463 \\
\hline 1471 & $\mathrm{pCi} / \mathrm{L}$ & 0 & & & & & & RIVER & & RAW GRAB & UNFILTERED & 456 \\
\hline 1472 & $\mathrm{pCi} / \mathrm{L}$ & 0 & & & & & & DRINKING & & SAN. GRAB & UNFILTERED & 186 \\
\hline 1473 & $\mathrm{pCi} / \mathrm{L}$ & 0 & & & & $U$ & & DRINKING & & SAN. GRAB & UNFILTERED & 186 \\
\hline 1474 & $\mathrm{pCi} / \mathrm{L}$ & 0.00000182 & & & & & & RIVER & & CONTINUOUS & RESIN & 252 \\
\hline 1475 & $\mathrm{pCi} / \mathrm{L}$ & 0 & & & & & & SANITARY & & CUMULATIVE & UNFILTERED & 485 \\
\hline 1476 & $\mathrm{pCi} / \mathrm{L}$ & 0.00000525 & & & & & & RIVER & & CONTINUOUS & RESIN & 307 \\
\hline 1477 & $\mathrm{pCi} / \mathrm{L}$ & 0 & & & & & & SEEP & & RAW GRAB & UNFILTERED & 556 \\
\hline 1478 & $\mathrm{pCi} / \mathrm{L}$ & 0 & & & & & & SEEP & & RAW GRAB & UNFILTERED & 556 \\
\hline 1479 & $\mathrm{pCi} / \mathrm{L}$ & 0 & & & & U & & DRINKING & & SAN. GRAB & UNFILTERED & 186 \\
\hline 1480 & $\mathrm{pCi} / \mathrm{L}$ & 0 & & & & & & DRINKING & & SAN. GRAB & UNFILTERED & 186 \\
\hline 1481 & $\mathrm{pCi} / \mathrm{L}$ & 0 & & & & & & SANITARY & & SAN. GRAB & UNFILTERED & 149 \\
\hline 1482 & $\mathrm{pCi} / \mathrm{L}$ & 0 & & & & & & RIVER & & CUMULATIVE & UNFILTERED & 553 \\
\hline 1483 & $\mathrm{pCi} / \mathrm{L}$ & 0 & & & & & & RIVER & & CUMULATIVE & UNFILTERED & 553 \\
\hline 1484 & $\mathrm{pCi} / \mathrm{L}$ & 0 & & & & & & RIVER & & CUMULATIVE & UNFILTERED & 553 \\
\hline 1485 & $\mathrm{pCi} / \mathrm{L}$ & 0 & & & & & & SANITARY & & CUMULATIVE & UNFILTERED & 203 \\
\hline 1486 & $\mathrm{pCi} / \mathrm{L}$ & 0 & & & & & & SANITARY & & SAN. GRAB & UNFILTERED & 50 \\
\hline 1487 & $\mathrm{pCi} / \mathrm{L}$ & 0 & & & & $U$ & & SANITARY & & SAN. GRAB & UNFILTERED & 50 \\
\hline 1488 & $\mathrm{pCi} / \mathrm{L}$ & 0 & & & & & & SANITARY & & CUMULATIVE & UNFILTERED & 385 \\
\hline 1489 & $\mathrm{pCi} / \mathrm{L}$ & 0 & & & & & & SANITARY & & SAN. GRAB & UNFILTERED & 589 \\
\hline 1490 & $\mathrm{pCi} / \mathrm{L}$ & 31 & & & & U & & SANITARY & & SAN. GRAB & UNFILTERED & 589 \\
\hline 1491 & $\mathrm{pCi} / \mathrm{L}$ & 0 & & & & & & RIVER & & CUMULATIVE & UNFILTERED & 463 \\
\hline 1492 & $\mathrm{pCi} / \mathrm{L}$ & 0 & & & & & & RIVER & & CUMULATIVE & UNFILTERED & 463 \\
\hline 1493 & $\mathrm{pCi} / \mathrm{L}$ & 0 & & & & & & RIVER & & CUMULATIVE & UNFILTERED & 463 \\
\hline 1494 & $\mathrm{pCi} / \mathrm{L}$ & 0 & & & & & & RIVER & & RAW GRAB & UNFILTERED & 463 \\
\hline 1495 & $\mathrm{pCi} / \mathrm{L}$ & 0 & & & & & & RIVER & & RAW GRAB & UNFILTERED & 456 \\
\hline 1496 & $\mathrm{pCi} / \mathrm{L}$ & 0 & & & & U & & SURFACE & & CUMULATIVE & UNFILTERED & 60 \\
\hline 1497 & $\mathrm{pCi} / \mathrm{L}$ & 0 & & & & & & SANITARY & & CUMULATIVE & UNFILTERED & 485 \\
\hline 1498 & $\mathrm{pCi} / \mathrm{L}$ & 0 & & & & U & & SANITARY & & CUMULATIVE & UNFILTERED & 485 \\
\hline 1499 & $\mathrm{pCi} / \mathrm{L}$ & 0.0000308 & & & & & & RIVER & & CONTINUOUS & RESIN & 307 \\
\hline 1500 & $\mathrm{pCi} / \mathrm{L}$ & 0.0000014 & & & & & & RIVER & & CONTINUOUS & RESIN & 252 \\
\hline 1501 & $\mathrm{pCi} / \mathrm{L}$ & 0 & & & & & & SANITARY & & SAN. GRAB & UNFILTERED & 149 \\
\hline 1502 & $\mathrm{pCi} / \mathrm{L}$ & 0 & & & & & & DRINKING & & SAN. GRAB & UNFILTERED & 186 \\
\hline 1503 & $\mathrm{pCi} / \mathrm{L}$ & 0 & & & & & & DRINKING & & SAN. GRAB & UNFILTERED & 186 \\
\hline 1504 & $\mathrm{pCi} / \mathrm{L}$ & 0 & & & & U & & RIVER & & CUMULATIVE & UNFILTERED & 553 \\
\hline 1505 & $\mathrm{pCi} / \mathrm{L}$ & 0 & & & & & & RIVER & & CUMULATIVE & UNFILTERED & 553 \\
\hline 1506 & $\mathrm{pCi} / \mathrm{L}$ & 0 & & & & & & RIVER & & CUMULATIVE & UNFILTERED & 553 \\
\hline 1507 & $\mathrm{pCi} / \mathrm{L}$ & 0 & & & & & & SANITARY & & CUMULATIVE & UNFILTERED & 203 \\
\hline 1508 & $\mathrm{pCi} / \mathrm{L}$ & 0 & & & & & & SANITARY & & CUMULATIVE & UNFILTERED & 485 \\
\hline 1509 & $\mathrm{pCi} / \mathrm{L}$ & 0 & & & & U & & SANITARY & & CUMULATIVE & UNFILTERED & 485 \\
\hline
\end{tabular}




\begin{tabular}{|c|c|c|c|c|c|c|c|c|c|c|c|c|}
\hline & $\mathrm{N}$ & $\mathrm{O}$ & $\mathrm{P}$ & $\mathrm{Q}$ & $\mathrm{R}$ & $\mathrm{s}$ & $\mathrm{T}$ & $\mathrm{U}$ & $\mathrm{V}$ & $\mathrm{W}$ & $x$ & $\mathrm{Y}$ \\
\hline 1 & ANAL_UNITS_RPTD & COUNTING_ERROR & TOTAL_ANAL_ERROR & MIN_DETECTABLE_ACTIVITY & PCNT_MOISTURE & LAB_QUALIFIER & REVIEW_QUALIFIER & SAMP_FROM & SAMP_ITEM & SAMP_MTHD & COLL_MTHD & SAMP_SITE_ID \\
\hline 1510 & $\mathrm{pCi} / \mathrm{L}$ & 0 & & & & & & SANITARY & & SAN. GRAB & UNFILTERED & 50 \\
\hline 1511 & pCi/L & 0 & & & & $\mathrm{U}$ & & SANITARY & & SAN. GRAB & UNFILTERED & 50 \\
\hline 1512 & $\mathrm{pCi} / \mathrm{L}$ & 0 & & & & & & SANITARY & & CUMULATIVE & UNFILTERED & 385 \\
\hline 1513 & $\mathrm{pCi} / \mathrm{L}$ & 0 & & & & & & SANITARY & & SAN. GRAB & UNFILTERED & 589 \\
\hline 1514 & $\mathrm{pCi} / \mathrm{L}$ & 0 & & & & U & & SANITARY & & SAN. GRAB & UNFILTERED & 589 \\
\hline 1515 & $\mathrm{pCi} / \mathrm{L}$ & 0.0000027 & & & & & & RIVER & & CONTINUOUS & RESIN & 307 \\
\hline 1516 & $\mathrm{pCi} / \mathrm{L}$ & 0.00000176 & & & & & & RIVER & & CONTINUOUS & RESIN & 252 \\
\hline 1517 & $\mathrm{pCi} / \mathrm{L}$ & 0 & & & & U & & RIVER & & CUMULATIVE & UNFILTERED & 463 \\
\hline 1518 & $\mathrm{pCi} / \mathrm{L}$ & 0 & & & & & & RIVER & & CUMULATIVE & UNFILTERED & 463 \\
\hline 1519 & $\mathrm{pCi} / \mathrm{L}$ & 0 & & & & U & & RIVER & & CUMULATIVE & UNFILTERED & 463 \\
\hline 1520 & $\mathrm{pCi} / \mathrm{L}$ & 0 & & & & $\mathrm{U}$ & & RIVER & & RAW GRAB & UNFILTERED & 463 \\
\hline 1521 & $\mathrm{pCi} / \mathrm{L}$ & 0 & & & & U & & RIVER & & RAW GRAB & UNFILTERED & 456 \\
\hline 1522 & $\mathrm{pCi} / \mathrm{L}$ & 0 & & & & U & & SURFACE & & CUMULATIVE & UNFILTERED & 60 \\
\hline 1523 & $\mathrm{pCi} / \mathrm{L}$ & 0 & & & & & & SANITARY & & SAN. GRAB & UNFILTERED & 149 \\
\hline 1524 & $\mathrm{pCi} / \mathrm{L}$ & 0 & & & & & & DRINKING & & SAN. GRAB & UNFILTERED & 186 \\
\hline 1525 & $\mathrm{pCi} / \mathrm{L}$ & 0 & & & & & & DRINKING & & SAN. GRAB & UNFILTERED & 186 \\
\hline 1526 & $\mathrm{pCi} / \mathrm{L}$ & 0 & & & & & & SANITARY & & CUMULATIVE & UNFILTERED & 485 \\
\hline 1527 & $\mathrm{pCi} / \mathrm{L}$ & 0 & & & & U & & SANITARY & & CUMULATIVE & UNFILTERED & 485 \\
\hline 1528 & $\mathrm{pCi} / \mathrm{L}$ & 0 & & & & & & SURFACE & & RAW GRAB & UNFILTERED & 121 \\
\hline 1529 & $\mathrm{pCi} / \mathrm{L}$ & 0 & & & & & & SURFACE & & RAW GRAB & UNFILTERED & 509 \\
\hline 1530 & $\mathrm{pCi} / \mathrm{L}$ & 0 & & & & U & & SURFACE & & RAW GRAB & UNFILTERED & 509 \\
\hline 1531 & $\mathrm{pCi} / \mathrm{L}$ & 0 & & & & & & SURFACE & & RAW GRAB & UNFILTERED & 146 \\
\hline 1532 & $\mathrm{pCi} / \mathrm{L}$ & 0 & & & & & & SURFACE & & RAW GRAB & UNFILTERED & 146 \\
\hline 1533 & pCi/L & 0 & & & & & & SURFACE & & RAW GRAB & UNFILTERED & 412 \\
\hline 1534 & $\mathrm{pCi} / \mathrm{L}$ & 0 & & & & & & SURFACE & & RAW GRAB & UNFILTERED & 412 \\
\hline 1535 & $\mathrm{pCi} / \mathrm{L}$ & 0 & & & & U & & SURFACE & & RAW GRAB & UNFILTERED & 147 \\
\hline 1536 & $\mathrm{pCi} / \mathrm{L}$ & 0 & & & & & & SURFACE & & RAW GRAB & UNFILTERED & 147 \\
\hline 1537 & $\mathrm{pCi} / \mathrm{L}$ & 0 & & & & & & RIVER & & CUMULATIVE & UNFILTERED & 553 \\
\hline 1538 & $\mathrm{pCi} / \mathrm{L}$ & 0 & & & & & & RIVER & & CUMULATIVE & UNFILTERED & 553 \\
\hline 1539 & $\mathrm{pCi} / \mathrm{L}$ & 0 & & & & & & SANITARY & & CUMULATIVE & UNFILTERED & 203 \\
\hline 1540 & $\mathrm{pCi} / \mathrm{L}$ & 0 & & & & & & SANITARY & & SAN. GRAB & UNFILTERED & 50 \\
\hline 1541 & $\mathrm{pCi} / \mathrm{L}$ & 0 & & & & & & SANITARY & & SAN. GRAB & UNFILTERED & 50 \\
\hline 1542 & $\mathrm{pCi} / \mathrm{L}$ & 0 & & & & & & SANITARY & & CUMULATIVE & UNFILTERED & 385 \\
\hline 1543 & $\mathrm{pCi} / \mathrm{L}$ & 0 & & & & & & SANITARY & & SAN. GRAB & UNFILTERED & 589 \\
\hline 1544 & $\mathrm{pCi} / \mathrm{L}$ & 0 & & & & & & SANITARY & & SAN. GRAB & UNFILTERED & 589 \\
\hline 1545 & $\mathrm{pCi} / \mathrm{L}$ & 0 & & & & U & & SURFACE & & CUMULATIVE & UNFILTERED & 60 \\
\hline 1546 & $\mathrm{pCi} / \mathrm{L}$ & 0 & & & & & & RIVER & & RAW GRAB & UNFILTERED & 463 \\
\hline 1547 & $\mathrm{pCi} / \mathrm{L}$ & 0 & & & & & & RIVER & & CUMULATIVE & UNFILTERED & 463 \\
\hline 1548 & $\mathrm{pCi} / \mathrm{L}$ & 0 & & & & & & RIVER & & CUMULATIVE & UNFILTERED & 463 \\
\hline 1549 & $\mathrm{pCi} / \mathrm{L}$ & 0 & & & & & & RIVER & & CUMULATIVE & UNFILTERED & 463 \\
\hline 1550 & $\mathrm{pCi} / \mathrm{L}$ & 0 & & & & & & RIVER & & RAW GRAB & UNFILTERED & 456 \\
\hline 1551 & $\mathrm{pCi} / \mathrm{L}$ & 0.0000036 & & & & & & RIVER & & CONTINUOUS & RESIN & 307 \\
\hline 1552 & $\mathrm{pCi} / \mathrm{L}$ & 0.00000199 & & & & & & RIVER & & CONTINUOUS & RESIN & 252 \\
\hline 1553 & $\mathrm{pCi} / \mathrm{L}$ & 0 & & & & & & DRINKING & & SAN. GRAB & UNFILTERED & 186 \\
\hline 1554 & $\mathrm{pCi} / \mathrm{L}$ & 0 & & & & & & DRINKING & & SAN. GRAB & UNFILTERED & 186 \\
\hline 1555 & $\mathrm{pCi} / \mathrm{L}$ & 0 & & & & & & SANITARY & & SAN. GRAB & UNFILTERED & 149 \\
\hline 1556 & $\mathrm{pCi} / \mathrm{L}$ & 0 & & & & & & RIVER & & RAW GRAB & UNFILTERED & 456 \\
\hline 1557 & pCi/L & 0 & & & & & & RIVER & & CUMULATIVE & UNFILTERED & 553 \\
\hline 1558 & $\mathrm{pCi} / \mathrm{L}$ & 0 & & & & & & RIVER & & CUMULATIVE & UNFILTERED & 553 \\
\hline 1559 & pCi/L & 0 & & & & & & RIVER & & CUMULATIVE & UNFILTERED & 553 \\
\hline 1560 & $\mathrm{pCi} / \mathrm{L}$ & 0.0000016 & & & & & & RIVER & & CONTINUOUS & RESIN & 307 \\
\hline 1561 & $\mathrm{pCi} / \mathrm{L}$ & 0.00000103 & & & & & & RIVER & & CONTINUOUS & RESIN & 252 \\
\hline
\end{tabular}




\begin{tabular}{|c|c|c|c|c|c|c|c|c|c|c|c|c|}
\hline & $\mathrm{N}$ & $\mathrm{O}$ & $\mathrm{P}$ & $\mathrm{Q}$ & $\mathrm{R}$ & $\mathrm{s}$ & $T$ & $U$ & $\mathrm{~V}$ & $\mathrm{~W}$ & $\mathrm{x}$ & $\bar{Y}$ \\
\hline \begin{tabular}{|l|l|l}
1 \\
\end{tabular} & ANAL_UNITS_RPTD & COUNTING_ERROR & TOTAL_ANAL_ERROR & MIN_DETECTABLE_ACTIVITY & PCNT_MOISTURE & LAB_QUALIFIER & REVIEW_QUALIFIER & SAMP_FROM & SAMP_ITEM & SAMP_MTHD & COLL_MTHD & SAMP_SITE_ID \\
\hline 1562 & $\mathrm{pCi} / \mathrm{L}$ & 0 & & & & & & RIVER & & RAW GRAB & UNFILTERED & 463 \\
\hline 1563 & $\mathrm{pCi} / \mathrm{L}$ & 0 & & & & $\mathrm{U}$ & & SANITARY & & CUMULATIVE & UNFILTERED & 203 \\
\hline 1564 & $\mathrm{pCi} / \mathrm{L}$ & 0 & & & & & & SANITARY & & CUMULATIVE & UNFILTERED & 485 \\
\hline 1565 & $\mathrm{pCi} / \mathrm{L}$ & 0 & & & & & & SANITARY & & CUMULATIVE & UNFILTERED & 485 \\
\hline 1566 & $\mathrm{pCi} / \mathrm{L}$ & 0 & & & & & & SANITARY & & SAN. GRAB & UNFILTERED & 50 \\
\hline 1567 & $\mathrm{pCi} / \mathrm{L}$ & 0 & & & & $\mathrm{U}$ & & SANITARY & & SAN. GRAB & UNFILTERED & 50 \\
\hline 1568 & $\mathrm{pCi} / \mathrm{L}$ & 0 & & & & & & SANITARY & & CUMULATIVE & UNFILTERED & 385 \\
\hline 1569 & $\mathrm{pCi} / \mathrm{L}$ & 0 & & & & & & SANITARY & & SAN. GRAB & UNFILTERED & 589 \\
\hline 1570 & $\mathrm{pCi} / \mathrm{L}$ & 0 & & & & & & SANITARY & & SAN. GRAB & UNFILTERED & 589 \\
\hline 1571 & $\mathrm{pCi} / \mathrm{L}$ & 0 & & & & & & RIVER & & CUMULATIVE & UNFILTERED & 463 \\
\hline 1572 & $\mathrm{pCi} / \mathrm{L}$ & 0 & & & & & & RIVER & & CUMULATIVE & UNFILTERED & 463 \\
\hline 1573 & $\mathrm{pCi} / \mathrm{L}$ & 0 & & & & & & DRINKING & & SAN. GRAB & UNFILTERED & 186 \\
\hline 1574 & $\mathrm{pCi} / \mathrm{L}$ & 0 & & & & & & SANITARY & & SAN. GRAB & UNFILTERED & 149 \\
\hline 1575 & $\mathrm{pCi} / \mathrm{L}$ & 0 & & & & & & RIVER & & CUMULATIVE & UNFILTERED & 553 \\
\hline 1576 & $\mathrm{pCi} / \mathrm{L}$ & 0 & & & & & & RIVER & & CUMULATIVE & UNFILTERED & 553 \\
\hline 1577 & $\mathrm{pCi} / \mathrm{L}$ & 0 & & & & & & RIVER & & CUMULATIVE & UNFILTERED & 553 \\
\hline 1578 & $\mathrm{pCi} / \mathrm{L}$ & 0 & & & & & & SANITARY & & CUMULATIVE & UNFILTERED & 203 \\
\hline 1579 & $\mathrm{pCi} / \mathrm{L}$ & 0 & & & & & & SANITARY & & CUMULATIVE & UNFILTERED & 485 \\
\hline 1580 & $\mathrm{pCi} / \mathrm{L}$ & 0 & & & & & & SANITARY & & CUMULATIVE & UNFILTERED & 485 \\
\hline \begin{tabular}{|l|}
1581 \\
\end{tabular} & $\mathrm{pCi} / \mathrm{L}$ & 0 & & & & & & SANITARY & & SAN. GRAB & UNFILTERED & 50 \\
\hline 1582 & $\mathrm{pCi} / \mathrm{L}$ & 0 & & & & & & SANITARY & & SAN. GRAB & UNFILTERED & 50 \\
\hline 1583 & $\mathrm{pCi} / \mathrm{L}$ & 0 & & & & & & SANITARY & & CUMULATIVE & UNFILTERED & 385 \\
\hline 1584 & $\mathrm{pCi} / \mathrm{L}$ & 0 & & & & & & SANITARY & & SAN. GRAB & UNFILTERED & 589 \\
\hline 1585 & $\mathrm{pCi} / \mathrm{L}$ & 0 & & & & & & SANITARY & & SAN. GRAB & UNFILTERED & 589 \\
\hline 1586 & $\mathrm{pCi} / \mathrm{L}$ & 0.00000812 & & & & & & RIVER & & CONTINUOUS & RESIN & 307 \\
\hline 1587 & $\mathrm{pCi} / \mathrm{L}$ & 0.00000574 & & & & & & RIVER & & CONTINUOUS & RESIN & 252 \\
\hline 1588 & $\mathrm{pCi} / \mathrm{L}$ & 0 & & & & & & RIVER & & RAW GRAB & UNFILTERED & 463 \\
\hline 1589 & $\mathrm{pCi} / \mathrm{L}$ & 0 & & & & & & RIVER & & CUMULATIVE & UNFILTERED & 463 \\
\hline 1590 & $\mathrm{pCi} / \mathrm{L}$ & 0 & & & & & & RIVER & & CUMULATIVE & UNFILTERED & 463 \\
\hline 1591 & $\mathrm{pCi} / \mathrm{L}$ & 0 & & & & & & RIVER & & CUMULATIVE & UNFILTERED & 463 \\
\hline 1592 & $\mathrm{pCi} / \mathrm{L}$ & 0 & & & & & & RIVER & & RAW GRAB & UNFILTERED & 456 \\
\hline 1593 & $\mathrm{pCi} / \mathrm{L}$ & 0 & & & & & & SANITARY & & SAN. GRAB & UNFILTERED & 149 \\
\hline 1594 & $\mathrm{pCi} / \mathrm{L}$ & 0 & & & & U & & DRINKING & & SAN. GRAB & UNFILTERED & 186 \\
\hline \begin{tabular}{|l|}
1595 \\
\end{tabular} & $\mathrm{pCi} / \mathrm{L}$ & 0 & & & & $U$ & & DRINKING & & SAN. GRAB & UNFILTERED & 186 \\
\hline 1596 & $\mathrm{pCi} / \mathrm{L}$ & 0 & & & & & & SANITARY & & CUMULATIVE & UNFILTERED & 485 \\
\hline 1597 & $\mathrm{pCi} / \mathrm{L}$ & 0 & & & & & & SANITARY & & CUMULATIVE & UNFILTERED & 485 \\
\hline 1598 & $\mathrm{pCi} / \mathrm{L}$ & 0 & & & & & & RIVER & & CUMULATIVE & UNFILTERED & 553 \\
\hline 1599 & $\mathrm{pCi} / \mathrm{L}$ & 0 & & & & & & RIVER & & CUMULATIVE & UNFILTERED & 553 \\
\hline 1600 & $\mathrm{pCi} / \mathrm{L}$ & 0 & & & & $U$ & & RIVER & & CUMULATIVE & UNFILTERED & 553 \\
\hline 1601 & $\mathrm{pCi} / \mathrm{L}$ & 0 & & & & $U$ & & SANITARY & & CUMULATIVE & UNFILTERED & 203 \\
\hline \begin{tabular}{|l|}
1602 \\
\end{tabular} & $\mathrm{pCi} / \mathrm{L}$ & 0 & & & & & & SANITARY & & SAN. GRAB & UNFILTERED & 50 \\
\hline 1603 & $\mathrm{pCi} / \mathrm{L}$ & 0 & & & & $U$ & & SANITARY & & SAN. GRAB & UNFILTERED & 50 \\
\hline 1604 & $\mathrm{pCi} / \mathrm{L}$ & 0 & & & & $U$ & & SANITARY & & CUMULATIVE & UNFILTERED & 385 \\
\hline 1605 & $\mathrm{pCi} / \mathrm{L}$ & 0 & & & & & & SANITARY & & SAN. GRAB & UNFILTERED & 589 \\
\hline 1606 & $\mathrm{pCi} / \mathrm{L}$ & 0 & & & & & & SANITARY & & SAN. GRAB & UNFILTERED & 589 \\
\hline 1607 & $\mathrm{pCi} / \mathrm{L}$ & 0 & & & & & & SURFACE & & RAW GRAB & UNFILTERED & 121 \\
\hline 1608 & $\mathrm{pCi} / \mathrm{L}$ & 0.17 & & & & & & SURFACE & & RAW GRAB & UNFILTERED & 509 \\
\hline 1609 & $\mathrm{pCi} / \mathrm{L}$ & 0 & & & & & & SURFACE & & RAW GRAB & UNFILTERED & 509 \\
\hline 1610 & $\mathrm{pCi} / \mathrm{L}$ & 0 & & & & & & SURFACE & & RAW GRAB & UNFILTERED & 146 \\
\hline 1611 & $\mathrm{pCi} / \mathrm{L}$ & 0.17 & & & & & & SURFACE & & RAW GRAB & UNFILTERED & 146 \\
\hline 1612 & $\mathrm{pCi} / \mathrm{L}$ & 0 & & & & & & SURFACE & & RAW GRAB & UNFILTERED & 412 \\
\hline 1613 & $\mathrm{pCi} / \mathrm{L}$ & 0 & & & & & & SURFACE & & RAW GRAB & UNFILTERED & 412 \\
\hline
\end{tabular}




\begin{tabular}{|c|c|c|c|c|c|c|c|c|c|c|c|c|}
\hline & $\mathrm{N}$ & $\mathrm{O}$ & $\mathrm{P}$ & $\mathrm{Q}$ & $\mathrm{R}$ & $\mathrm{s}$ & $\mathrm{T}$ & $\mathrm{u}$ & $\mathrm{v}$ & $\mathrm{w}$ & $\mathrm{x}$ & $\bar{Y}$ \\
\hline 1 & ANAL_UNITS_RPTD & COUNTING_ERROR & TOTAL_ANAL_ERROR & MIN_DETECTABLE_ACTIVITY & PCNT_MOISTURE & LAB_QUALIFIER & REVIEW_QUALIFIER & SAMP_FROM & SAMP_ITEM & SAMP_MTHD & COLL_MTHD & SAMP_SITE_ID \\
\hline 1614 & $\mathrm{pCi} / \mathrm{L}$ & 0 & & & & $\mathrm{U}$ & & SURFACE & & RAW GRAB & UNFILTERED & 147 \\
\hline 1615 & $\mathrm{pCi} / \mathrm{L}$ & 0 & & & & & & SURFACE & & RAW GRAB & UNFILTERED & 147 \\
\hline 1616 & $\mathrm{pCi} / \mathrm{L}$ & 0.00000354 & & & & & & RIVER & & CONTINUOUS & RESIN & 307 \\
\hline 1617 & $\mathrm{pCi} / \mathrm{L}$ & 0.00000194 & & & & & & RIVER & & CONTINUOUS & RESIN & 252 \\
\hline 1618 & $\mathrm{pCi} / \mathrm{L}$ & 0 & & & & & & RIVER & & CUMULATIVE & UNFILTERED & 463 \\
\hline 1619 & $\mathrm{pCi} / \mathrm{L}$ & 0 & & & & & & RIVER & & CUMULATIVE & UNFILTERED & 463 \\
\hline 1620 & $\mathrm{pCi} / \mathrm{L}$ & 0 & & & & U & & RIVER & & CUMULATIVE & UNFILTERED & 463 \\
\hline 1621 & $\mathrm{pCi} / \mathrm{L}$ & 0 & & & & U & & RIVER & & RAW GRAB & UNFILTERED & 463 \\
\hline 1622 & $\mathrm{pCi} / \mathrm{L}$ & 0 & & & & & & RIVER & & RAW GRAB & UNFILTERED & 456 \\
\hline 1623 & $\mathrm{pCi} / \mathrm{L}$ & 0 & & & & & & DRINKING & & SAN. GRAB & UNFILTERED & 186 \\
\hline 1624 & $\mathrm{pCi} / \mathrm{L}$ & 0 & & & & & & DRINKING & & SAN. GRAB & UNFILTERED & 186 \\
\hline 1625 & $\mathrm{pCi} / \mathrm{L}$ & 0 & & & & & & SANITARY & & SAN. GRAB & UNFILTERED & 149 \\
\hline 1626 & $\mathrm{pCi} / \mathrm{L}$ & 0 & & & & & & RIVER & & RAW GRAB & UNFILTERED & 463 \\
\hline 1627 & $\mathrm{pCi} / \mathrm{L}$ & 0 & & & & U & & RIVER & & CUMULATIVE & UNFILTERED & 463 \\
\hline 1628 & $\mathrm{pCi} / \mathrm{L}$ & 0.068 & & & & & & RIVER & & CUMULATIVE & UNFILTERED & 463 \\
\hline 1629 & $\mathrm{pCi} / \mathrm{L}$ & 0 & & & & & & RIVER & & CUMULATIVE & UNFILTERED & 463 \\
\hline 1630 & $\mathrm{pCi} / \mathrm{L}$ & 0 & & & & & & RIVER & & RAW GRAB & UNFILTERED & 456 \\
\hline 1631 & $\mathrm{pCi} / \mathrm{L}$ & 0 & & & & & & DRINKING & & SAN. GRAB & UNFILTERED & 186 \\
\hline 1632 & $\mathrm{pCi} / \mathrm{L}$ & 0 & & & & & & DRINKING & & SAN. GRAB & UNFILTERED & 186 \\
\hline 1633 & $\mathrm{pCi} / \mathrm{L}$ & 0.068 & & & & & & RIVER & & CUMULATIVE & UNFILTERED & 553 \\
\hline 1634 & $\mathrm{pCi} / \mathrm{L}$ & 0 & & & & & & RIVER & & CUMULATIVE & UNFILTERED & 553 \\
\hline 1635 & $\mathrm{pCi} / \mathrm{L}$ & 0 & & & & & & RIVER & & CUMULATIVE & UNFILTERED & 553 \\
\hline 1636 & $\mathrm{pCi} / \mathrm{L}$ & 0.00000296 & & & & & & RIVER & & CONTINUOUS & RESIN & 307 \\
\hline 1637 & $\mathrm{pCi} / \mathrm{L}$ & 0.0000124 & & & & & & RIVER & & CONTINUOUS & RESIN & 252 \\
\hline 1638 & $\mathrm{pCi} / \mathrm{L}$ & 0 & & & & & & SANITARY & & CUMULATIVE & UNFILTERED & 203 \\
\hline 1639 & $\mathrm{pCi} / \mathrm{L}$ & 0 & & & & & & SANITARY & & SAN. GRAB & UNFILTERED & 50 \\
\hline 1640 & $\mathrm{pCi} / \mathrm{L}$ & 0 & & & & & & SANITARY & & SAN. GRAB & UNFILTERED & 50 \\
\hline 1641 & $\mathrm{pCi} / \mathrm{L}$ & 0 & & & & U & & SANITARY & & SAN. GRAB & UNFILTERED & 149 \\
\hline 1642 & $\mathrm{pCi} / \mathrm{L}$ & 0 & & & & & & SANITARY & & CUMULATIVE & UNFILTERED & 385 \\
\hline 1643 & $\mathrm{pCi} / \mathrm{L}$ & 0 & & & & & & SANITARY & & SAN. GRAB & UNFILTERED & 589 \\
\hline 1644 & $\mathrm{pCi} / \mathrm{L}$ & 0 & & & & & & SANITARY & & SAN. GRAB & UNFILTERED & 589 \\
\hline 1645 & $\mathrm{pCi} / \mathrm{L}$ & 31 & & & & $U$ & & DRINKING & & SAN. GRAB & UNFILTERED & 186 \\
\hline 1646 & $\mathrm{pCi} / \mathrm{L}$ & 150 & & & & U & & DRINKING & & SAN. GRAB & UNFILTERED & 186 \\
\hline 1647 & $\mathrm{pCi} / \mathrm{L}$ & 0.068 & & & & & & RIVER & & CUMULATIVE & UNFILTERED & 553 \\
\hline 1648 & $\mathrm{pCi} / \mathrm{L}$ & 330 & & & & U & & RIVER & & CUMULATIVE & UNFILTERED & 553 \\
\hline 1649 & $\mathrm{pCi} / \mathrm{L}$ & 1.5 & & & & U & & RIVER & & CUMULATIVE & UNFILTERED & 553 \\
\hline 1650 & $\mathrm{pCi} / \mathrm{L}$ & 31 & & & & U & & SANITARY & & CUMULATIVE & UNFILTERED & 203 \\
\hline 1651 & $\mathrm{pCi} / \mathrm{L}$ & 620 & & & & & & SANITARY & & SAN. GRAB & UNFILTERED & 50 \\
\hline 1652 & $\mathrm{pCi} / \mathrm{L}$ & 31 & & & & U & & SANITARY & & SAN. GRAB & UNFILTERED & 50 \\
\hline 1653 & $\mathrm{pCi} / \mathrm{L}$ & 31 & & & & U & & SANITARY & & SAN. GRAB & UNFILTERED & 149 \\
\hline 1654 & $\mathrm{pCi} / \mathrm{L}$ & 31 & & & & U & & SANITARY & & CUMULATIVE & UNFILTERED & 385 \\
\hline 1655 & $\mathrm{pCi} / \mathrm{L}$ & 1700 & & & & & & SANITARY & & SAN. GRAB & UNFILTERED & 589 \\
\hline 1656 & $\mathrm{pCi} / \mathrm{L}$ & 0.00000168 & & & & & & RIVER & & CONTINUOUS & RESIN & 307 \\
\hline 1657 & $\mathrm{pCi} / \mathrm{L}$ & 0.00000214 & & & & & & RIVER & & CONTINUOUS & RESIN & 252 \\
\hline 1658 & $\mathrm{pCi} / \mathrm{L}$ & 350 & & & & U & & RIVER & & CUMULATIVE & UNFILTERED & 463 \\
\hline 1659 & $\mathrm{pCi} / \mathrm{L}$ & 0.068 & & & & & & RIVER & & CUMULATIVE & UNFILTERED & 463 \\
\hline 1660 & $\mathrm{pCi} / \mathrm{L}$ & 1.5 & & & & U & & RIVER & & CUMULATIVE & UNFILTERED & 463 \\
\hline 1661 & $\mathrm{pCi} / \mathrm{L}$ & 290 & & & & U & & RIVER & & RAW GRAB & UNFILTERED & 463 \\
\hline 1662 & $\mathrm{pCi} / \mathrm{L}$ & 280 & & & & U & & RIVER & & RAW GRAB & UNFILTERED & 456 \\
\hline 1663 & $\mathrm{pCi} / \mathrm{L}$ & 31 & & & & U & & SANITARY & & SAN. GRAB & UNFILTERED & 589 \\
\hline 1664 & & & & & & $U$ & & SURFACE & & RAW GRAB & UNFILTERED & 146 \\
\hline 1665 & & & & & & $U_{1}$ & & SURFACE & & RAW GRAB & UNFILTERED & 146 \\
\hline
\end{tabular}




\begin{tabular}{|c|c|c|c|c|c|c|c|c|c|c|c|c|}
\hline & $\mathrm{N}$ & $\mathrm{O}$ & $\mathrm{P}$ & $\mathrm{Q}$ & $\mathrm{R}$ & $\mathrm{s}$ & $\mathrm{T}$ & $\mathrm{U}$ & $\mathrm{V}$ & $\mathrm{W}$ & $\mathrm{x}$ & $\mathrm{Y}$ \\
\hline 1 & ANAL_UNITS_RPTD & COUNTING_ERROR & TOTAL ANAL ERROR & 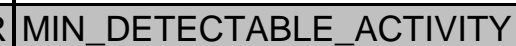 & PCNT_MOISTURE & LAB QUALIFIER & REVIEW_QUALIFIER & SAMP FROM & SAMP_ITEM & SAMP MTHD & COLL_MTHD & SAMP SITE ID \\
\hline 1666 & $\mathrm{pCi} / \mathrm{L}$ & 0 & & & & & & RIVER & & CONTINUOUS & FILTER & 307 \\
\hline 1667 & $\mathrm{pCi} / \mathrm{L}$ & 0 & & & & & & RIVER & & CONTINUOUS & RESIN & 307 \\
\hline 1668 & $\mathrm{pCi} / \mathrm{L}$ & 0 & & & & & & RIVER & & CONTINUOUS & RESIN & 252 \\
\hline 1669 & $\mathrm{pCi} / \mathrm{L}$ & 0 & & & & & & RIVER & & CONTINUOUS & FILTER & 252 \\
\hline 1670 & $\mathrm{pCi} / \mathrm{L}$ & 31 & & & & $U$ & & DRINKING & & SAN. GRAB & UNFILTERED & 186 \\
\hline 1671 & $\mathrm{pCi} / \mathrm{L}$ & 300 & & & & $\mathrm{U}$ & & DRINKING & & SAN. GRAB & UNFILTERED & 186 \\
\hline 1672 & $\mathrm{pCi} / \mathrm{L}$ & 590 & & & & & & SANITARY & & SAN. GRAB & UNFILTERED & 50 \\
\hline 1673 & $\mathrm{pCi} / \mathrm{L}$ & 31 & & & & $\mathrm{U}$ & & SANITARY & & SAN. GRAB & UNFILTERED & 50 \\
\hline 1674 & $\mathrm{pCi} / \mathrm{L}$ & 1800 & & & & & & SANITARY & & SAN. GRAB & UNFILTERED & 589 \\
\hline 1675 & $\mathrm{pCi} / \mathrm{L}$ & 31 & & & & U & & SANITARY & & SAN. GRAB & UNFILTERED & 589 \\
\hline \begin{tabular}{|c|}
1676 \\
\end{tabular} & $\mathrm{pCi} / \mathrm{L}$ & 0 & & & & & & RIVER & & CONTINUOUS & FILTER & 307 \\
\hline 1677 & $\mathrm{pCi} / \mathrm{L}$ & 0 & & & & & & RIVER & & CONTINUOUS & RESIN & 307 \\
\hline \begin{tabular}{|c|}
1678 \\
\end{tabular} & $\mathrm{pCi} / \mathrm{L}$ & 0.000006 & & & & & & RIVER & & CONTINUOUS & RESIN & 307 \\
\hline 1679 & $\mathrm{pCi} / \mathrm{L}$ & 0.00000152 & & & & & & RIVER & & CONTINUOUS & RESIN & 252 \\
\hline 1680 & $\mathrm{pCi} / \mathrm{L}$ & 0 & & & & & & RIVER & & CONTINUOUS & RESIN & 252 \\
\hline \begin{tabular}{|l|}
1681 \\
\end{tabular} & $\mathrm{pCi} / \mathrm{L}$ & 0 & & & & & & RIVER & & CONTINUOUS & FILTER & 252 \\
\hline 1682 & $\mathrm{pCi} / \mathrm{L}$ & 210 & & & & $\mathrm{U}$ & & RIVER & & CUMULATIVE & UNFILTERED & 463 \\
\hline 1683 & $\mathrm{pCi} / \mathrm{L}$ & 31 & & & & $U$ & & RIVER & & CUMULATIVE & UNFILTERED & 463 \\
\hline \begin{tabular}{|l|}
1684 \\
\end{tabular} & $\mathrm{pCi} / \mathrm{L}$ & 170 & & & & & & RIVER & & RAW GRAB & UNFILTERED & 463 \\
\hline 1685 & $\mathrm{pCi} / \mathrm{L}$ & 130 & & & & & & RIVER & & RAW GRAB & UNFILTERED & 456 \\
\hline 1686 & $\mathrm{pCi} / \mathrm{L}$ & 31 & & & & U & & RIVER & & CUMULATIVE & UNFILTERED & 553 \\
\hline 1687 & $\mathrm{pCi} / \mathrm{L}$ & 0 & & & & & & RIVER & & CUMULATIVE & UNFILTERED & 553 \\
\hline 1688 & $\mathrm{pCi} / \mathrm{L}$ & 31 & & & & $U$ & & SANITARY & & CUMULATIVE & UNFILTERED & 203 \\
\hline 1689 & $\mathrm{pCi} / \mathrm{L}$ & 31 & & & & $U$ & & SANITARY & & CUMULATIVE & UNFILTERED & 385 \\
\hline 1690 & $\mathrm{pCi} / \mathrm{L}$ & 15 & & & & $U$ & & SANITARY & & CUMULATIVE & UNFILTERED & 385 \\
\hline \begin{tabular}{|l|}
1691 \\
\end{tabular} & $\mathrm{pCi} / \mathrm{L}$ & 0 & & & & & & RIVER & & CONTINUOUS & RESIN & 307 \\
\hline 1692 & $\mathrm{pCi} / \mathrm{L}$ & 0.00000552 & & & & & & RIVER & & CONTINUOUS & RESIN & 307 \\
\hline 1693 & $\mathrm{pCi} / \mathrm{L}$ & 0 & & & & & & RIVER & & CONTINUOUS & FILTER & 307 \\
\hline \begin{tabular}{|l|}
1694 \\
\end{tabular} & $\mathrm{pCi} / \mathrm{L}$ & 0 & & & & & & RIVER & & CONTINUOUS & RESIN & 252 \\
\hline 1695 & $\mathrm{pCi} / \mathrm{L}$ & 0 & & & & & & RIVER & & CONTINUOUS & FILTER & 252 \\
\hline \begin{tabular}{|l|}
1696 \\
\end{tabular} & $\mathrm{pCi} / \mathrm{L}$ & 31 & & & & $U$ & & DRINKING & & SAN. GRAB & UNFILTERED & 186 \\
\hline \begin{tabular}{|l|}
1697 \\
\end{tabular} & $\mathrm{pCi} / \mathrm{L}$ & 220 & & & & $U$ & & DRINKING & & SAN. GRAB & UNFILTERED & 186 \\
\hline \begin{tabular}{|l|}
1698 \\
\end{tabular} & $\mathrm{pCi} / \mathrm{L}$ & 0.00000224 & & & & & & RIVER & & CONTINUOUS & RESIN & 252 \\
\hline \begin{tabular}{|l|}
1699 \\
\end{tabular} & $\mathrm{pCi} / \mathrm{L}$ & 0 & & & & & & RIVER & & CONTINUOUS & RESIN & 252 \\
\hline 1700 & $\mathrm{pCi} / \mathrm{L}$ & 0 & & & & & & RIVER & & CONTINUOUS & FILTER & 252 \\
\hline \begin{tabular}{|l|}
1701 \\
\end{tabular} & $\mathrm{pCi} / \mathrm{L}$ & 680 & & & & & & SANITARY & & SAN. GRAB & UNFILTERED & 50 \\
\hline 1702 & $\mathrm{pCi} / \mathrm{L}$ & 31 & & & & $\mathrm{U}$ & & SANITARY & & SAN. GRAB & UNFILTERED & 50 \\
\hline 1703 & $\mathrm{pCi} / \mathrm{L}$ & 2500 & & & & & & SANITARY & & SAN. GRAB & UNFILTERED & 589 \\
\hline 1704 & $\mathrm{pCi} / \mathrm{L}$ & 31 & & & & $U$ & & SANITARY & & SAN. GRAB & UNFILTERED & 589 \\
\hline \begin{tabular}{|l|}
1705 \\
\end{tabular} & $\mathrm{pCi} / \mathrm{L}$ & 10 & & & & $U$ & & SURFACE & & RAW GRAB & UNFILTERED & 121 \\
\hline \begin{tabular}{|l|}
1706 \\
\end{tabular} & $\mathrm{pCi} / \mathrm{L}$ & 0.09 & & & & & & SURFACE & & RAW GRAB & UNFILTERED & 509 \\
\hline 1707 | & $\mathrm{pCi} / \mathrm{L}$ & 2.1 & & & & & & SURFACE & & RAW GRAB & UNFILTERED & 509 \\
\hline 1708 | & $\mathrm{pCi} / \mathrm{L}$ & 2.1 & & & & $U$ & & SURFACE & & RAW GRAB & UNFILTERED & 146 \\
\hline 1709 & $\mathrm{pCi} / \mathrm{L}$ & 0.12 & & & & & & SURFACE & & RAW GRAB & UNFILTERED & 146 \\
\hline 1710 & $\mathrm{pCi} / \mathrm{L}$ & 2.1 & & & & & & SURFACE & & RAW GRAB & UNFILTERED & 412 \\
\hline \begin{tabular}{|l|}
1711 \\
\end{tabular} & $\mathrm{pCi} / \mathrm{L}$ & 0.24 & & & & & & SURFACE & & RAW GRAB & UNFILTERED & 412 \\
\hline \begin{tabular}{|l|}
1712 \\
\end{tabular} & $\mathrm{pCi} / \mathrm{L}$ & 6.1 & & & & $U$ & & SURFACE & & RAW GRAB & UNFILTERED & 147 \\
\hline \begin{tabular}{|l|}
1713 \\
\end{tabular} & $\mathrm{pCi} / \mathrm{L}$ & 0.27 & & & & & & SURFACE & & RAW GRAB & UNFILTERED & 147 \\
\hline 1714 & $\mathrm{pCi} / \mathrm{L}$ & 0 & & & & & & RIVER & & CONTINUOUS & RESIN & 307 \\
\hline 1715 & $\mathrm{pCi} / \mathrm{L}$ & 0 & & & & & & RIVER & & CONTINUOUS & FILTER & 307 \\
\hline \begin{tabular}{|l|l|}
1716 \\
\end{tabular} & $\mathrm{pCi} / \mathrm{L}$ & 230 & & & & & & RIVER & & CUMULATIVE & UNFILTERED & 463 \\
\hline \begin{tabular}{|l|}
1717 \\
\end{tabular} & $\mathrm{pCi} / \mathrm{L}$ & 31 & & & & $u$ & & RIVER & & CUMULATIVE & UNFILTERED & 463 \\
\hline
\end{tabular}




\begin{tabular}{|c|c|c|c|c|c|c|c|c|c|c|c|c|}
\hline & $\mathrm{N}$ & $\mathrm{O}$ & $\mathrm{P}$ & $\mathrm{Q}$ & $\mathrm{R}$ & $\mathrm{s}$ & $\mathrm{T}$ & $\mathrm{U}$ & $\mathrm{V}$ & $\mathrm{W}$ & $\mathrm{X}$ & $\bar{Y}$ \\
\hline 1 & ANAL_UNITS_RPTD & COUNTING_ERROR & TOTAL_ANAL_ERROR & MIN_DETECTABLE_ACTIVITY & PCNT_MOISTURE & LAB_QUALIFIER & REVIEW_QUALIFIER & SAMP_FROM & SAMP_ITEM & SAMP_MTHD & COLL_MTHD & SAMP_SITE_ID \\
\hline 1718 & $\mathrm{pCi} / \mathrm{L}$ & 290 & & & & & & RIVER & & RAW GRAB & UNFILTERED & 463 \\
\hline 1719 & $\mathrm{pCi} / \mathrm{L}$ & 370 & & & & & & RIVER & & RAW GRAB & UNFILTERED & 456 \\
\hline 1720 & $\mathrm{pCi} / \mathrm{L}$ & 170 & & & & & & RIVER & & CUMULATIVE & UNFILTERED & 553 \\
\hline 1721 & $\mathrm{pCi} / \mathrm{L}$ & 31 & & & & U & & RIVER & & CUMULATIVE & UNFILTERED & 553 \\
\hline 1722 & $\mathrm{pCi} / \mathrm{L}$ & 31 & & & & U & & SANITARY & & CUMULATIVE & UNFILTERED & 203 \\
\hline 1723 & $\mathrm{pCi} / \mathrm{L}$ & 31 & & & & U & & SANITARY & & CUMULATIVE & UNFILTERED & 385 \\
\hline 1724 & $\mathrm{pCi} / \mathrm{L}$ & 0 & & & & & & RIVER & & CONTINUOUS & RESIN & 307 \\
\hline 1725 & $\mathrm{pCi} / \mathrm{L}$ & 0 & & & & & & RIVER & & CONTINUOUS & FILTER & 307 \\
\hline 1726 & $\mathrm{pCi} / \mathrm{L}$ & 0.068 & & & & U & & DRINKING & & SAN. GRAB & UNFILTERED & 186 \\
\hline 1727 & $\mathrm{pCi} / \mathrm{L}$ & 31 & & & & U & & DRINKING & & SAN. GRAB & UNFILTERED & 186 \\
\hline 1728 & $\mathrm{pCi} / \mathrm{L}$ & 800 & & & & $\mathrm{U}$ & & DRINKING & & SAN. GRAB & UNFILTERED & 186 \\
\hline 1729 & $\mathrm{pCi} / \mathrm{L}$ & 680 & & & & & & SANITARY & & SAN. GRAB & UNFILTERED & 50 \\
\hline 1730 & $\mathrm{pCi} / \mathrm{L}$ & 0.34 & & & & $U$ & & SANITARY & & SAN. GRAB & UNFILTERED & 50 \\
\hline 1731 & $\mathrm{pCi} / \mathrm{L}$ & 31 & & & & $U$ & & SANITARY & & SAN. GRAB & UNFILTERED & 50 \\
\hline 1732 & $\mathrm{pCi} / \mathrm{L}$ & 2300 & & & & & & SANITARY & & SAN. GRAB & UNFILTERED & 589 \\
\hline 1733 & $\mathrm{pCi} / \mathrm{L}$ & 31 & & & & $U$ & & SANITARY & & SAN. GRAB & UNFILTERED & 589 \\
\hline \begin{tabular}{|l|}
1734 \\
\end{tabular} & $\mathrm{pCi} / \mathrm{L}$ & 0 & & & & & & RIVER & & CONTINUOUS & RESIN & 307 \\
\hline 1735 & $\mathrm{pCi} / \mathrm{L}$ & 0 & & & & & & RIVER & & CONTINUOUS & FILTER & 307 \\
\hline 1736 & $\mathrm{pCi} / \mathrm{L}$ & 0 & & & & & & RIVER & & CONTINUOUS & RESIN & 252 \\
\hline \begin{tabular}{|l|}
1737 \\
\end{tabular} & $\mathrm{pCi} / \mathrm{L}$ & 0 & & & & & & RIVER & & CONTINUOUS & FILTER & 252 \\
\hline 1738 & $\mathrm{pCi} / \mathrm{L}$ & 31 & & & & $U$ & & RIVER & & CUMULATIVE & UNFILTERED & 553 \\
\hline 1739 & $\mathrm{pCi} / \mathrm{L}$ & 160 & & & & & & RIVER & & CUMULATIVE & UNFILTERED & 553 \\
\hline 1740 & $\mathrm{pCi} / \mathrm{L}$ & 0.068 & & & & & & RIVER & & CUMULATIVE & UNFILTERED & 553 \\
\hline 1741 & $\mathrm{pCi} / \mathrm{L}$ & 31 & & & & U & & SANITARY & & CUMULATIVE & UNFILTERED & 203 \\
\hline 1742 & $\mathrm{pCi} / \mathrm{L}$ & 31 & & & & $U$ & & SANITARY & & CUMULATIVE & UNFILTERED & 385 \\
\hline 1743 & $\mathrm{pCi} / \mathrm{L}$ & 180 & & & & & & RIVER & & RAW GRAB & UNFILTERED & 463 \\
\hline \begin{tabular}{|l|}
1744 \\
\end{tabular} & $\mathrm{pCi} / \mathrm{L}$ & 130 & & & & & & RIVER & & CUMULATIVE & UNFILTERED & 463 \\
\hline 1745 & $\mathrm{pCi} / \mathrm{L}$ & 0.068 & & & & & & RIVER & & CUMULATIVE & UNFILTERED & 463 \\
\hline 1746 & $\mathrm{pCi} / \mathrm{L}$ & 31 & & & & U & & RIVER & & CUMULATIVE & UNFILTERED & 463 \\
\hline \begin{tabular}{|l|}
1747 \\
\end{tabular} & $\mathrm{pCi} / \mathrm{L}$ & 250 & & & & $U$ & & RIVER & & RAW GRAB & UNFILTERED & 456 \\
\hline 1748 & $\mathrm{pCi} / \mathrm{L}$ & 0 & & & & & & RIVER & & CONTINUOUS & RESIN & 307 \\
\hline 1749 & $\mathrm{pCi} / \mathrm{L}$ & 0.0000044 & & & & & & RIVER & & CONTINUOUS & RESIN & 307 \\
\hline 1750 & $\mathrm{pCi} / \mathrm{L}$ & 0 & & & & & & RIVER & & CONTINUOUS & FILTER & 307 \\
\hline \begin{tabular}{|l|}
1751 \\
\end{tabular} & $\mathrm{pCi} / \mathrm{L}$ & 0.000005 & & & & & & RIVER & & CONTINUOUS & RESIN & 252 \\
\hline \begin{tabular}{|l|}
1752 \\
\end{tabular} & $\mathrm{pCi} / \mathrm{L}$ & 0 & & & & & & RIVER & & CONTINUOUS & RESIN & 252 \\
\hline 1753 & $\mathrm{pCi} / \mathrm{L}$ & 0 & & & & & & RIVER & & CONTINUOUS & FILTER & 252 \\
\hline \begin{tabular}{|l|}
1754 \\
\end{tabular} & $\mathrm{pCi} / \mathrm{L}$ & 31 & & & & $U$ & & DRINKING & & SAN. GRAB & UNFILTERED & 186 \\
\hline 1755 & $\mathrm{pCi} / \mathrm{L}$ & 410 & & & & U & & DRINKING & & SAN. GRAB & UNFILTERED & 186 \\
\hline \begin{tabular}{|l|}
1756 \\
\end{tabular} & $\mathrm{pCi} / \mathrm{L}$ & 1600 & & & & & & SANITARY & & SAN. GRAB & UNFILTERED & 50 \\
\hline \begin{tabular}{|l|}
1757 \\
\end{tabular} & $\mathrm{pCi} / \mathrm{L}$ & 31 & & & & $U$ & & SANITARY & & SAN. GRAB & UNFILTERED & 50 \\
\hline 1758 & $\mathrm{pCi} / \mathrm{L}$ & 3700 & & & & & & SANITARY & & SAN. GRAB & UNFILTERED & 589 \\
\hline 1759 & $\mathrm{pCi} / \mathrm{L}$ & 31 & & & & $U$ & & SANITARY & & SAN. GRAB & UNFILTERED & 589 \\
\hline 1760 & $\mathrm{pCi} / \mathrm{L}$ & 0 & & & & & & RIVER & & CONTINUOUS & RESIN & 307 \\
\hline \begin{tabular}{|l|}
1761 \\
\end{tabular} & $\mathrm{pCi} / \mathrm{L}$ & 0 & & & & & & RIVER & & CONTINUOUS & FILTER & 307 \\
\hline 1762 & $\mathrm{pCi} / \mathrm{L}$ & 0 & & & & & & RIVER & & CONTINUOUS & RESIN & 252 \\
\hline 1763 & $\mathrm{pCi} / \mathrm{L}$ & 0 & & & & & & RIVER & & CONTINUOUS & FILTER & 252 \\
\hline 1764 & $\mathrm{pCi} / \mathrm{L}$ & 31 & & & & $U$ & & RIVER & & CUMULATIVE & UNFILTERED & 553 \\
\hline 1765 & $\mathrm{pCi} / \mathrm{L}$ & 200 & & & & U & & RIVER & & CUMULATIVE & UNFILTERED & 553 \\
\hline 1766 | & $\mathrm{pCi} / \mathrm{L}$ & 31 & & & & $U$ & & SANITARY & & CUMULATIVE & UNFILTERED & 203 \\
\hline 1767 . & $\mathrm{pCi} / \mathrm{L}$ & 31 & & & & $U$ & & SANITARY & & CUMULATIVE & UNFILTERED & 385 \\
\hline 1768 & $\mathrm{pCi} / \mathrm{L}$ & 6.2 & & & & $U$ & & SURFACE & & RAW GRAB & UNFILTERED & 121 \\
\hline 1769 & $\mathrm{pCi} / \mathrm{L}$ & 0.09 & & & & & & SURFACE & & RAW GRAB & UNFILTERED & 509 \\
\hline
\end{tabular}




\begin{tabular}{|c|c|c|c|c|c|c|c|c|c|c|c|c|}
\hline & $\mathrm{N}$ & $\mathrm{O}$ & $\mathrm{P}$ & $\mathrm{Q}$ & $\mathrm{R}$ & $\mathrm{s}$ & $\mathrm{T}$ & $\mathrm{U}$ & $\mathrm{V}$ & $\mathrm{W}$ & $\mathrm{X}$ & $\bar{Y}$ \\
\hline 1 & ANAL_UNITS_RPTD & COUNTING_ERROR & TOTAL_ANAL_ERROR & MIN_DETECTABLE_ACTIVITY & PCNT_MOISTURE & LAB_QUALIFIER & REVIEW_QUALIFIER & SAMP_FROM & SAMP_ITEM & SAMP_MTHD & COLL_MTHD & SAMP_SITE_ID \\
\hline 1770 & $\mathrm{pCi} / \mathrm{L}$ & 2.1 & & & & & & SURFACE & & RAW GRAB & UNFILTERED & 509 \\
\hline 1771 & $\mathrm{pCi} / \mathrm{L}$ & 2.1 & & & & & & SURFACE & & RAW GRAB & UNFILTERED & 146 \\
\hline 1772 & $\mathrm{pCi} / \mathrm{L}$ & 0.09 & & & & & & SURFACE & & RAW GRAB & UNFILTERED & 146 \\
\hline 1773 & $\mathrm{pCi} / \mathrm{L}$ & 2.1 & & & & & & SURFACE & & RAW GRAB & UNFILTERED & 412 \\
\hline 1774 & $\mathrm{pCi} / \mathrm{L}$ & 0.09 & & & & & & SURFACE & & RAW GRAB & UNFILTERED & 412 \\
\hline 1775 & $\mathrm{pCi} / \mathrm{L}$ & 10 & & & & U & & SURFACE & & RAW GRAB & UNFILTERED & 147 \\
\hline 1776 & $\mathrm{pCi} / \mathrm{L}$ & 0.34 & & & & & & SURFACE & & RAW GRAB & UNFILTERED & 147 \\
\hline 1777 & $\mathrm{pCi} / \mathrm{L}$ & 240 & & & & & & RIVER & & RAW GRAB & UNFILTERED & 463 \\
\hline 1778 & $\mathrm{pCi} / \mathrm{L}$ & 160 & & & & & & RIVER & & CUMULATIVE & UNFILTERED & 463 \\
\hline 1779 & $\mathrm{pCi} / \mathrm{L}$ & 31 & & & & U & & RIVER & & CUMULATIVE & UNFILTERED & 463 \\
\hline 1780 & $\mathrm{pCi} / \mathrm{L}$ & 260 & & & & & & RIVER & & RAW GRAB & UNFILTERED & 456 \\
\hline 1781 & $\mathrm{pCi} / \mathrm{L}$ & 0 & & & & & & RIVER & & CONTINUOUS & RESIN & 307 \\
\hline 1782 & $\mathrm{pCi} / \mathrm{L}$ & 0.0000515 & & & & & & RIVER & & CONTINUOUS & RESIN & 307 \\
\hline 1783 & $\mathrm{pCi} / \mathrm{L}$ & 0 & & & & & & RIVER & & CONTINUOUS & FILTER & 307 \\
\hline \begin{tabular}{|c|}
1784 \\
\end{tabular} & $\mathrm{pCi} / \mathrm{L}$ & 0 & & & & & & RIVER & & CONTINUOUS & FILTER & 252 \\
\hline 1785 & $\mathrm{pCi} / \mathrm{L}$ & 0.00000696 & & & & & & RIVER & & CONTINUOUS & RESIN & 252 \\
\hline 1786 & $\mathrm{pCi} / \mathrm{L}$ & 0 & & & & & & RIVER & & CONTINUOUS & RESIN & 252 \\
\hline 1787 & $\mathrm{pCi} / \mathrm{L}$ & 31 & & & & $U$ & & DRINKING & & SAN. GRAB & UNFILTERED & 186 \\
\hline 1788 & $\mathrm{pCi} / \mathrm{L}$ & 160 & & & & $U$ & & DRINKING & & SAN. GRAB & UNFILTERED & 186 \\
\hline 1789 & $\mathrm{pCi} / \mathrm{L}$ & 430 & & & & & & SANITARY & & SAN. GRAB & UNFILTERED & 50 \\
\hline 1790 & $\mathrm{pCi} / \mathrm{L}$ & 31 & & & & $U$ & & SANITARY & & SAN. GRAB & UNFILTERED & 50 \\
\hline \begin{tabular}{|l|}
1791 \\
\end{tabular} & $\mathrm{pCi} / \mathrm{L}$ & 1200 & & & & & & SANITARY & & SAN. GRAB & UNFILTERED & 589 \\
\hline 1792 & $\mathrm{pCi} / \mathrm{L}$ & 31 & & & & $U$ & & SANITARY & & SAN. GRAB & UNFILTERED & 589 \\
\hline 1793 & $\mathrm{pCi} / \mathrm{L}$ & 0 & & & & & & RIVER & & CONTINUOUS & RESIN & 307 \\
\hline 1794 & $\mathrm{pCi} / \mathrm{L}$ & 0 & & & & & & RIVER & & CONTINUOUS & FILTER & 307 \\
\hline 1795 & $\mathrm{pCi} / \mathrm{L}$ & 0 & & & & & & RIVER & & CONTINUOUS & RESIN & 252 \\
\hline 1796 & $\mathrm{pCi} / \mathrm{L}$ & 0 & & & & & & RIVER & & CONTINUOUS & FILTER & 252 \\
\hline \begin{tabular}{|l|}
1797 \\
\end{tabular} & $\mathrm{pCi} / \mathrm{L}$ & 160 & & & & & & RIVER & & CUMULATIVE & UNFILTERED & 463 \\
\hline 1798 & $\mathrm{pCi} / \mathrm{L}$ & 31 & & & & U & & RIVER & & CUMULATIVE & UNFILTERED & 463 \\
\hline 1799 & $\mathrm{pCi} / \mathrm{L}$ & 250 & & & & & & RIVER & & RAW GRAB & UNFILTERED & 463 \\
\hline 1800 & $\mathrm{pCi} / \mathrm{L}$ & 280 & & & & & & RIVER & & RAW GRAB & UNFILTERED & 456 \\
\hline 1801 & $\mathrm{pCi} / \mathrm{L}$ & 410 & & & & $U$ & & RIVER & & CUMULATIVE & UNFILTERED & 553 \\
\hline 1802 & $\mathrm{pCi} / \mathrm{L}$ & 31 & & & & $U$ & & RIVER & & CUMULATIVE & UNFILTERED & 553 \\
\hline 1803 & $\mathrm{pCi} / \mathrm{L}$ & 0 & & & & & & RIVER & & CONTINUOUS & RESIN & 307 \\
\hline 1804 & $\mathrm{pCi} / \mathrm{L}$ & 0.00000344 & & & & & & RIVER & & CONTINUOUS & RESIN & 307 \\
\hline 1805 & $\mathrm{pCi} / \mathrm{L}$ & 0 & & & & & & RIVER & & CONTINUOUS & FILTER & 307 \\
\hline 1806 & $\mathrm{pCi} / \mathrm{L}$ & 0.0000024 & & & & & & RIVER & & CONTINUOUS & RESIN & 252 \\
\hline 1807 & $\mathrm{pCi} / \mathrm{L}$ & 0 & & & & & & RIVER & & CONTINUOUS & RESIN & 252 \\
\hline $\mid 1808$ & $\mathrm{pCi} / \mathrm{L}$ & 0 & & & & & & RIVER & & CONTINUOUS & FILTER & 252 \\
\hline 1809 & $\mathrm{pCi} / \mathrm{L}$ & 31 & & & & $U$ & & SANITARY & & CUMULATIVE & UNFILTERED & 203 \\
\hline 1810 & $\mathrm{pCi} / \mathrm{L}$ & 31 & & & & U & & SANITARY & & CUMULATIVE & UNFILTERED & 385 \\
\hline 1811 & $\mathrm{pCi} / \mathrm{L}$ & 0.14 & & & & $U$ & & DRINKING & & SAN. GRAB & UNFILTERED & 186 \\
\hline 1812 & $\mathrm{pCi} / \mathrm{L}$ & 31 & & & & U & & DRINKING & & SAN. GRAB & UNFILTERED & 186 \\
\hline 1813 & $\mathrm{pCi} / \mathrm{L}$ & 270 & & & & $U$ & & DRINKING & & SAN. GRAB & UNFILTERED & 186 \\
\hline 1814 & $\mathrm{pCi} / \mathrm{L}$ & 750 & & & & & & SANITARY & & SAN. GRAB & UNFILTERED & 50 \\
\hline 1815 & $\mathrm{pCi} / \mathrm{L}$ & 0.14 & & & & U & & SANITARY & & SAN. GRAB & UNFILTERED & 50 \\
\hline 1816 & $\mathrm{pCi} / \mathrm{L}$ & 31 & & & & $U$ & & SANITARY & & SAN. GRAB & UNFILTERED & 50 \\
\hline 1817 & $\mathrm{pCi} / \mathrm{L}$ & 1300 & & & & & & SANITARY & & SAN. GRAB & UNFILTERED & 589 \\
\hline 1818 & $\mathrm{pCi} / \mathrm{L}$ & 31 & & & & $U$ & & SANITARY & & SAN. GRAB & UNFILTERED & 589 \\
\hline 1819 & $\mathrm{pCi} / \mathrm{L}$ & 0.068 & & & & U & & SANITARY & & SAN. GRAB & UNFILTERED & 589 \\
\hline 1820 & $\mathrm{pCi} / \mathrm{L}$ & 0 & & & & & & RIVER & & CONTINUOUS & RESIN & 307 \\
\hline 1821 & $\mathrm{pCi} / \mathrm{L}$ & 0 & & & & & & RIVER & & CONTINUOUS & FILTER & 307 \\
\hline
\end{tabular}




\begin{tabular}{|c|c|c|c|c|c|c|c|c|c|c|c|c|}
\hline & $\mathrm{N}$ & $\mathrm{O}$ & $\mathrm{P}$ & $\mathrm{Q}$ & $\mathrm{R}$ & $\mathrm{s}$ & $\mathrm{T}$ & $\mathrm{u}$ & $\mathrm{v}$ & $\mathrm{w}$ & $\mathrm{x}$ & $\bar{Y}$ \\
\hline 1 & ANAL_UNITS_RPTD & COUNTING_ERROR & TOTAL_ANAL_ERROR & MIN_DETECTABLE_ACTIVITY & PCNT_MOISTURE & LAB_QUALIFIER & REVIEW_QUALIFIER & SAMP_FROM & SAMP_ITEM & SAMP_MTHD & COLL_MTHD & SAMP_SITE_ID \\
\hline 1822 & $\mathrm{pCi} / \mathrm{L}$ & 0 & & & & & & RIVER & & CONTINUOUS & RESIN & 252 \\
\hline 1823 & $\mathrm{pCi} / \mathrm{L}$ & 0 & & & & & & RIVER & & CONTINUOUS & FILTER & 252 \\
\hline 1824 & $\mathrm{pCi} / \mathrm{L}$ & 31 & & & & $\mathrm{U}$ & & RIVER & & CUMULATIVE & UNFILTERED & 553 \\
\hline 1825 & $\mathrm{pCi} / \mathrm{L}$ & 300 & & & & & & RIVER & & CUMULATIVE & UNFILTERED & 553 \\
\hline \begin{tabular}{|l|l|}
1826 \\
\end{tabular} & $\mathrm{pCi} / \mathrm{L}$ & 0.068 & & & & & & RIVER & & CUMULATIVE & UNFILTERED & 553 \\
\hline \begin{tabular}{|l|l|}
1827 & -10
\end{tabular} & $\mathrm{pCi} / \mathrm{L}$ & 31 & & & & $\mathrm{U}$ & & SANITARY & & CUMULATIVE & UNFILTERED & 203 \\
\hline 1828 & $\mathrm{pCi} / \mathrm{L}$ & 31 & & & & $\mathrm{U}$ & & SANITARY & & CUMULATIVE & UNFILTERED & 385 \\
\hline \begin{tabular}{|l|l|}
1829 \\
\end{tabular} & $\mathrm{pCi} / \mathrm{L}$ & 0 & & & & & & RIVER & & CONTINUOUS & RESIN & 307 \\
\hline 1830 & $\mathrm{pCi} / \mathrm{L}$ & 0.0000064 & & & & & & RIVER & & CONTINUOUS & RESIN & 307 \\
\hline 1831 & $\mathrm{pCi} / \mathrm{L}$ & 0 & & & & & & RIVER & & CONTINUOUS & FILTER & 307 \\
\hline 1832 & $\mathrm{pCi} / \mathrm{L}$ & 0.0000022 & & & & & & RIVER & & CONTINUOUS & RESIN & 252 \\
\hline 1833 & $\mathrm{pCi} / \mathrm{L}$ & 0 & & & & & & RIVER & & CONTINUOUS & RESIN & 252 \\
\hline 1834 & $\mathrm{pCi} / \mathrm{L}$ & 0 & & & & & & RIVER & & CONTINUOUS & FILTER & 252 \\
\hline 1835 & $\mathrm{pCi} / \mathrm{L}$ & 330 & & & & & & RIVER & & CUMULATIVE & UNFILTERED & 463 \\
\hline 1836 & $\mathrm{pCi} / \mathrm{L}$ & 0.068 & & & & & & RIVER & & CUMULATIVE & UNFILTERED & 463 \\
\hline \begin{tabular}{|l|}
1837 \\
\end{tabular} & $\mathrm{pCi} / \mathrm{L}$ & 31 & & & & $U$ & & RIVER & & CUMULATIVE & UNFILTERED & 463 \\
\hline 1838 & $\mathrm{pCi} / \mathrm{L}$ & 400 & & & & U & & RIVER & & RAW GRAB & UNFILTERED & 463 \\
\hline 1839 & $\mathrm{pCi} / \mathrm{L}$ & 300 & & & & U & & RIVER & & RAW GRAB & UNFILTERED & 456 \\
\hline 1840 & $\mathrm{pCi} / \mathrm{L}$ & 31 & & & & U & & DRINKING & & SAN. GRAB & UNFILTERED & 186 \\
\hline 1841 & $\mathrm{pCi} / \mathrm{L}$ & 250 & & & & $U$ & & DRINKING & & SAN. GRAB & UNFILTERED & 186 \\
\hline \begin{tabular}{|l|}
1842 \\
\end{tabular} & $\mathrm{pCi} / \mathrm{L}$ & 240 & & & & & & SANITARY & & CUMULATIVE & UNFILTERED & 485 \\
\hline 1843 & $\mathrm{pCi} / \mathrm{L}$ & 31 & & & & $U$ & & SANITARY & & CUMULATIVE & UNFILTERED & 485 \\
\hline 1844 & $\mathrm{pCi} / \mathrm{L}$ & 1100 & & & & & & SANITARY & & SAN. GRAB & UNFILTERED & 50 \\
\hline 1845 & $\mathrm{pCi} / \mathrm{L}$ & 31 & & & & U & & SANITARY & & SAN. GRAB & UNFILTERED & 50 \\
\hline \begin{tabular}{|l|}
1846 \\
\end{tabular} & $\mathrm{pCi} / \mathrm{L}$ & 2000 & & & & & & SANITARY & & SAN. GRAB & UNFILTERED & 589 \\
\hline \begin{tabular}{|l|l|}
1847 \\
\end{tabular} & $\mathrm{pCi} / \mathrm{L}$ & 31 & & & & $U$ & & SANITARY & & SAN. GRAB & UNFILTERED & 589 \\
\hline 1848 & $\mathrm{pCi} / \mathrm{L}$ & 0 & & & & & & RIVER & & CONTINUOUS & RESIN & 307 \\
\hline 1849 & $\mathrm{pCi} / \mathrm{L}$ & 0 & & & & & & RIVER & & CONTINUOUS & FILTER & 307 \\
\hline 1850 & $\mathrm{pCi} / \mathrm{L}$ & 0.000000828 & & & & & & RIVER & & CONTINUOUS & RESIN & 252 \\
\hline \begin{tabular}{|c|}
1851 \\
\end{tabular} & $\mathrm{pCi} / \mathrm{L}$ & 0 & & & & & & RIVER & & CONTINUOUS & RESIN & 252 \\
\hline 1852 & $\mathrm{pCi} / \mathrm{L}$ & 0 & & & & & & RIVER & & CONTINUOUS & FILTER & 252 \\
\hline \begin{tabular}{|l|}
1853 \\
\end{tabular} & $\mathrm{pCi} / \mathrm{L}$ & 380 & & & & $U$ & & RIVER & & RAW GRAB & UNFILTERED & 463 \\
\hline 1854 & $\mathrm{pCi} / \mathrm{L}$ & 7.7 & & & & $U$ & & SURFACE & & RAW GRAB & UNFILTERED & 121 \\
\hline 1855 & $\mathrm{pCi} / \mathrm{L}$ & 0.097 & & & & & & SURFACE & & RAW GRAB & UNFILTERED & 509 \\
\hline 1856 & $\mathrm{pCi} / \mathrm{L}$ & 2.2 & & & & & & SURFACE & & RAW GRAB & UNFILTERED & 509 \\
\hline \begin{tabular}{|l|}
1857 \\
\end{tabular} & $\mathrm{pCi} / \mathrm{L}$ & 2.2 & & & & & & SURFACE & & RAW GRAB & UNFILTERED & 146 \\
\hline 1858 & $\mathrm{pCi} / \mathrm{L}$ & 0.097 & & & & & & SURFACE & & RAW GRAB & UNFILTERED & 146 \\
\hline \begin{tabular}{|l|}
1859 \\
\end{tabular} & $\mathrm{pCi} / \mathrm{L}$ & 2.2 & & & & & & SURFACE & & RAW GRAB & UNFILTERED & 412 \\
\hline $\mid 1860$ & $\mathrm{pCi} / \mathrm{L}$ & 0.097 & & & & & & SURFACE & & RAW GRAB & UNFILTERED & 412 \\
\hline 1861 & $\mathrm{pCi} / \mathrm{L}$ & 10 & & & & $U$ & & SURFACE & & RAW GRAB & UNFILTERED & 147 \\
\hline 1862 & $\mathrm{pCi} / \mathrm{L}$ & 0.34 & & & & & & SURFACE & & RAW GRAB & UNFILTERED & 147 \\
\hline 1863 & $\mathrm{pCi} / \mathrm{L}$ & 160 & & & & & & RIVER & & CUMULATIVE & UNFILTERED & 553 \\
\hline 1864 & $\mathrm{pCi} / \mathrm{L}$ & 31 & & & & U & & RIVER & & CUMULATIVE & UNFILTERED & 553 \\
\hline 1865 & $\mathrm{pCi} / \mathrm{L}$ & 31 & & & & U & & SANITARY & & CUMULATIVE & UNFILTERED & 203 \\
\hline 1866 & $\mathrm{pCi} / \mathrm{L}$ & 220 & & & & $U$ & & SANITARY & & CUMULATIVE & UNFILTERED & 485 \\
\hline 1867 & $\mathrm{pCi} / \mathrm{L}$ & 31 & & & & U & & SANITARY & & CUMULATIVE & UNFILTERED & 485 \\
\hline 1868 & $\mathrm{pCi} / \mathrm{L}$ & 31 & & & & $U$ & & SANITARY & & CUMULATIVE & UNFILTERED & 385 \\
\hline 1869 & $\mathrm{pCi} / \mathrm{L}$ & 0 & & & & & & RIVER & & CONTINUOUS & RESIN & 307 \\
\hline 1870 & $\mathrm{pCi} / \mathrm{L}$ & 0.00000432 & & & & & & RIVER & & CONTINUOUS & RESIN & 307 \\
\hline 1871 & $\mathrm{pCi} / \mathrm{L}$ & 0 & & & & & & RIVER & & CONTINUOUS & FILTER & 307 \\
\hline 1872 & $\mathrm{pCi} / \mathrm{L}$ & 800 & & & & & & RIVER & & CUMULATIVE & UNFILTERED & 463 \\
\hline 1873 & $\mathrm{pCi} / \mathrm{L}$ & 31 & & & & $U_{1}$ & & RIVER & & CUMULATIVE & UNFILTERED & 463 \\
\hline
\end{tabular}




\begin{tabular}{|c|c|c|c|c|c|c|c|c|c|c|c|c|}
\hline & $\mathrm{N}$ & $\mathrm{O}$ & $\mathrm{P}$ & $\mathrm{Q}$ & $\mathrm{R}$ & $\mathrm{s}$ & $\mathrm{T}$ & $\mathrm{u}$ & $\mathrm{v}$ & $\mathrm{w}$ & $\mathrm{x}$ & $\bar{Y}$ \\
\hline 1 & ANAL_UNITS_RPTD & COUNTING_ERROR & TOTAL_ANAL_ERROR & MIN_DETECTABLE_ACTIVITY & PCNT_MOISTURE & LAB_QUALIFIER & REVIEW_QUALIFIER & SAMP_FROM & SAMP_ITEM & SAMP_MTHD & COLL_MTHD & SAMP_SITE_ID \\
\hline 1874 & $\mathrm{pCi} / \mathrm{L}$ & 370 & & & & & & RIVER & & RAW GRAB & UNFILTERED & 456 \\
\hline 1875 & $\mathrm{pCi} / \mathrm{L}$ & 0 & & & & & & RIVER & & CONTINUOUS & FILTER & 307 \\
\hline 1876 & $\mathrm{pCi} / \mathrm{L}$ & 0 & & & & & & RIVER & & CONTINUOUS & RESIN & 307 \\
\hline 1877 & $\mathrm{pCi} / \mathrm{L}$ & 0 & & & & & & RIVER & & CONTINUOUS & RESIN & 252 \\
\hline 1878 & $\mathrm{pCi} / \mathrm{L}$ & 0 & & & & & & RIVER & & CONTINUOUS & FILTER & 252 \\
\hline 1879 & $\mathrm{pCi} / \mathrm{L}$ & 0.09 & & & & $\mathrm{U}$ & & SANITARY & & CUMULATIVE & UNFILTERED & 512 \\
\hline 1880 & $\mathrm{pCi} / \mathrm{L}$ & 0 & & & & & & RIVER & & CONTINUOUS & FILTER & 307 \\
\hline 1881 & $\mathrm{pCi} / \mathrm{L}$ & 0 & & & & & & RIVER & & CONTINUOUS & RESIN & 307 \\
\hline 1882 & $\mathrm{pCi} / \mathrm{L}$ & 0 & & & & & & RIVER & & CONTINUOUS & RESIN & 252 \\
\hline 1883 & $\mathrm{pCi} / \mathrm{L}$ & 0 & & & & & & RIVER & & CONTINUOUS & FILTER & 252 \\
\hline 1884 & $\mathrm{pCi} / \mathrm{L}$ & 0.09 & & & & $\mathrm{U}$ & & DRINKING & & SAN. GRAB & UNFILTERED & 186 \\
\hline 1885 & $\mathrm{pCi} / \mathrm{L}$ & 380 & & & & $\mathrm{U}$ & & DRINKING & & SAN. GRAB & UNFILTERED & 186 \\
\hline 1886 & $\mathrm{pCi} / \mathrm{L}$ & 0.09 & & & & $U$ & & DRINKING & & SAN. GRAB & UNFILTERED & 187 \\
\hline 1887 & $\mathrm{pCi} / \mathrm{L}$ & 360 & & & & & & DRINKING & & SAN. GRAB & UNFILTERED & 187 \\
\hline 1888 & $\mathrm{pCi} / \mathrm{L}$ & 320 & & & & & & RIVER & & CUMULATIVE & UNFILTERED & 553 \\
\hline 1889 & $\mathrm{pCi} / \mathrm{L}$ & 31 & & & & $U$ & & RIVER & & CUMULATIVE & UNFILTERED & 553 \\
\hline 1890 & $\mathrm{pCi} / \mathrm{L}$ & 0.09 & & & & & & SANITARY & & CUMULATIVE & UNFILTERED & 491 \\
\hline 1891 & $\mathrm{pCi} / \mathrm{L}$ & 230 & & & & & & SANITARY & & CUMULATIVE & UNFILTERED & 491 \\
\hline 1892 & $\mathrm{pCi} / \mathrm{L}$ & 31 & & & & $U$ & & SANITARY & & CUMULATIVE & UNFILTERED & 491 \\
\hline 1893 & $\mathrm{pCi} / \mathrm{L}$ & 31 & & & & $U$ & & SANITARY & & CUMULATIVE & UNFILTERED & 512 \\
\hline 1894 & $\mathrm{pCi} / \mathrm{L}$ & 1200 & & & & & & SANITARY & & CUMULATIVE & UNFILTERED & 512 \\
\hline 1895 & $\mathrm{pCi} / \mathrm{L}$ & 31 & & & & $U$ & & SANITARY & & CUMULATIVE & UNFILTERED & 385 \\
\hline 1896 & $\mathrm{pCi} / \mathrm{L}$ & 260 & & & & & & RIVER & & RAW GRAB & UNFILTERED & 463 \\
\hline 1897 & $\mathrm{pCi} / \mathrm{L}$ & 31 & & & & U & & RIVER & & CUMULATIVE & UNFILTERED & 463 \\
\hline 1898 & $\mathrm{pCi} / \mathrm{L}$ & 280 & & & & & & RIVER & & RAW GRAB & UNFILTERED & 456 \\
\hline 1899 & $\mathrm{pCi} / \mathrm{L}$ & 0 & & & & & & RIVER & & CONTINUOUS & RESIN & 307 \\
\hline 1900 & $\mathrm{pCi} / \mathrm{L}$ & 0.00000312 & & & & & & RIVER & & CONTINUOUS & RESIN & 307 \\
\hline 1901 & $\mathrm{pCi} / \mathrm{L}$ & 0 & & & & & & RIVER & & CONTINUOUS & FILTER & 307 \\
\hline 1902 & $\mathrm{pCi} / \mathrm{L}$ & 0.000000602 & & & & & & RIVER & & CONTINUOUS & RESIN & 252 \\
\hline 1903 & $\mathrm{pCi} / \mathrm{L}$ & 0 & & & & & & RIVER & & CONTINUOUS & RESIN & 252 \\
\hline 1904 & $\mathrm{pCi} / \mathrm{L}$ & 0 & & & & & & RIVER & & CONTINUOUS & FILTER & 252 \\
\hline 1905 & $\mathrm{pCi} / \mathrm{L}$ & 0 & & & & & & RIVER & & CONTINUOUS & FILTER & 307 \\
\hline 1906 & $\mathrm{pCi} / \mathrm{L}$ & 0 & & & & & & RIVER & & CONTINUOUS & RESIN & 307 \\
\hline 1907 & $\mathrm{pCi} / \mathrm{L}$ & 0 & & & & & & RIVER & & CONTINUOUS & RESIN & 252 \\
\hline 1908 & $\mathrm{pCi} / \mathrm{L}$ & 0 & & & & & & RIVER & & CONTINUOUS & FILTER & 252 \\
\hline 1909 & $\mathrm{pCi} / \mathrm{L}$ & 31 & & & & U & & RIVER & & CUMULATIVE & UNFILTERED & 553 \\
\hline 1910 & $\mathrm{pCi} / \mathrm{L}$ & 580 & & & & U & & RIVER & & CUMULATIVE & UNFILTERED & 553 \\
\hline 1911 & $\mathrm{pCi} / \mathrm{L}$ & 0.068 & & & & & & RIVER & & CUMULATIVE & UNFILTERED & 553 \\
\hline 1912 & $\mathrm{pCi} / \mathrm{L}$ & 31 & & & & $U$ & & SANITARY & & CUMULATIVE & UNFILTERED & 491 \\
\hline 1913 & $\mathrm{pCi} / \mathrm{L}$ & 31 & & & & $U$ & & SANITARY & & CUMULATIVE & UNFILTERED & 512 \\
\hline 1914 & $\mathrm{pCi} / \mathrm{L}$ & 870 & & & & & & SANITARY & & CUMULATIVE & UNFILTERED & 512 \\
\hline 1915 & $\mathrm{pCi} / \mathrm{L}$ & 31 & & & & $U$ & & SANITARY & & CUMULATIVE & UNFILTERED & 385 \\
\hline 1916 & $\mathrm{pCi} / \mathrm{L}$ & 380 & & & & U & & RIVER & & CUMULATIVE & UNFILTERED & 463 \\
\hline 1917 & $\mathrm{pCi} / \mathrm{L}$ & 0.068 & & & & & & RIVER & & CUMULATIVE & UNFILTERED & 463 \\
\hline 1918 & $\mathrm{pCi} / \mathrm{L}$ & 31 & & & & $U$ & & RIVER & & CUMULATIVE & UNFILTERED & 463 \\
\hline 1919 & $\mathrm{pCi} / \mathrm{L}$ & 430 & & & & $U$ & & RIVER & & RAW GRAB & UNFILTERED & 463 \\
\hline 1920 & $\mathrm{pCi} / \mathrm{L}$ & 440 & & & & $U$ & & RIVER & & RAW GRAB & UNFILTERED & 456 \\
\hline 1921 & $\mathrm{pCi} / \mathrm{L}$ & 0 & & & & & & RIVER & & CONTINUOUS & RESIN & 307 \\
\hline 1922 & $\mathrm{pCi} / \mathrm{L}$ & 0 & & & & & & RIVER & & CONTINUOUS & FILTER & 307 \\
\hline 1923 & $\mathrm{pCi} / \mathrm{L}$ & 0.000000816 & & & & & & RIVER & & CONTINUOUS & RESIN & 252 \\
\hline 1924 & $\mathrm{pCi} / \mathrm{L}$ & 0 & & & & & & RIVER & & CONTINUOUS & RESIN & 252 \\
\hline 1925 & $\mathrm{pCi} / \mathrm{L}$ & 0 & & & & & & RIVER & & CONTINUOUS & FILTER & 252 \\
\hline
\end{tabular}




\begin{tabular}{|c|c|c|c|c|c|c|c|c|c|c|c|c|}
\hline & $\mathrm{N}$ & $\mathrm{O}$ & $\mathrm{P}$ & $\mathrm{Q}$ & $\mathrm{R}$ & $\mathrm{s}$ & $\mathrm{T}$ & $\mathrm{U}$ & $\mathrm{V}$ & $\mathrm{W}$ & $\mathrm{X}$ & $\bar{Y}$ \\
\hline 1 & ANAL_UNITS_RPTD & COUNTING_ERROR & TOTAL_ANAL_ERROR & MIN_DETECTABLE_ACTIVITY & PCNT_MOISTURE & LAB_QUALIFIER & REVIEW_QUALIFIER & SAMP_FROM & SAMP_ITEM & SAMP_MTHD & COLL_MTHD & SAMP_SITE_ID \\
\hline 1926 & $\mathrm{pCi} / \mathrm{L}$ & 0.0000048 & & & & & & RIVER & & CONTINUOUS & RESIN & 307 \\
\hline 1927 & $\mathrm{pCi} / \mathrm{L}$ & 6.1 & & & & U & & RIVER & & CUMULATIVE & UNFILTERED & 463 \\
\hline 1928 & $\mathrm{pCi} / \mathrm{L}$ & 7.7 & & & & $u$ & & SURFACE & & RAW GRAB & UNFILTERED & 121 \\
\hline 1929 & $\mathrm{pCi} / \mathrm{L}$ & 0.097 & & & & & & SURFACE & & RAW GRAB & UNFILTERED & 509 \\
\hline 1930 & $\mathrm{pCi} / \mathrm{L}$ & 2.2 & & & & & & SURFACE & & RAW GRAB & UNFILTERED & 509 \\
\hline 1931 & $\mathrm{pCi} / \mathrm{L}$ & 2.2 & & & & U & & SURFACE & & RAW GRAB & UNFILTERED & 146 \\
\hline 1932 & $\mathrm{pCi} / \mathrm{L}$ & 0.097 & & & & & & SURFACE & & RAW GRAB & UNFILTERED & 146 \\
\hline 1933 & $\mathrm{pCi} / \mathrm{L}$ & 2.3 & & & & & & SURFACE & & RAW GRAB & UNFILTERED & 412 \\
\hline 1934 & $\mathrm{pCi} / \mathrm{L}$ & 0.097 & & & & & & SURFACE & & RAW GRAB & UNFILTERED & 412 \\
\hline 1935 & $\mathrm{pCi} / \mathrm{L}$ & 10 & & & & U & & SURFACE & & RAW GRAB & UNFILTERED & 147 \\
\hline 1936 & $\mathrm{pCi} / \mathrm{L}$ & 0.34 & & & & & & SURFACE & & RAW GRAB & UNFILTERED & 147 \\
\hline 1937 & $\mathrm{pCi} / \mathrm{L}$ & 0 & & & & & & RIVER & & CONTINUOUS & RESIN & 307 \\
\hline 1938 & $\mathrm{pCi} / \mathrm{L}$ & 0 & & & & & & RIVER & & CONTINUOUS & FILTER & 307 \\
\hline 1939 & $\mathrm{pCi} / \mathrm{L}$ & 0 & & & & & & RIVER & & CONTINUOUS & RESIN & 252 \\
\hline 1940 & $\mathrm{pCi} / \mathrm{L}$ & 0 & & & & & & RIVER & & CONTINUOUS & FILTER & 252 \\
\hline 1941 & $\mathrm{pCi} / \mathrm{L}$ & 290 & & & & $U$ & & RIVER & & CUMULATIVE & UNFILTERED & 463 \\
\hline 1942 & $\mathrm{pCi} / \mathrm{L}$ & 31 & & & & $U$ & & RIVER & & CUMULATIVE & UNFILTERED & 463 \\
\hline 1943 & $\mathrm{pCi} / \mathrm{L}$ & 230 & & & & $U$ & & RIVER & & RAW GRAB & UNFILTERED & 463 \\
\hline 1944 & $\mathrm{pCi} / \mathrm{L}$ & 360 & & & & $U$ & & RIVER & & RAW GRAB & UNFILTERED & 456 \\
\hline 1945 & $\mathrm{pCi} / \mathrm{L}$ & 31 & & & & $U$ & & RIVER & & CUMULATIVE & UNFILTERED & 553 \\
\hline 1946 & $\mathrm{pCi} / \mathrm{L}$ & 440 & & & & $U$ & & RIVER & & CUMULATIVE & UNFILTERED & 553 \\
\hline 1947 & $\mathrm{pCi} / \mathrm{L}$ & 31 & & & & $U$ & & SANITARY & & CUMULATIVE & UNFILTERED & 491 \\
\hline 1948 & $\mathrm{pCi} / \mathrm{L}$ & 31 & & & & $U$ & & SANITARY & & CUMULATIVE & UNFILTERED & 512 \\
\hline 1949 & $\mathrm{pCi} / \mathrm{L}$ & 1500 & & & & & & SANITARY & & CUMULATIVE & UNFILTERED & 512 \\
\hline 1950 & $\mathrm{pCi} / \mathrm{L}$ & 31 & & & & $U$ & & SANITARY & & CUMULATIVE & UNFILTERED & 385 \\
\hline 1951 & $\mathrm{pCi} / \mathrm{L}$ & 0 & & & & & & RIVER & & CONTINUOUS & RESIN & 307 \\
\hline 1952 & $\mathrm{pCi} / \mathrm{L}$ & 0.00000215 & & & & & & RIVER & & CONTINUOUS & RESIN & 307 \\
\hline 1953 & $\mathrm{pCi} / \mathrm{L}$ & 0 & & & & & & RIVER & & CONTINUOUS & FILTER & 307 \\
\hline 1954 & $\mathrm{pCi} / \mathrm{L}$ & 0.000000768 & & & & & & RIVER & & CONTINUOUS & RESIN & 252 \\
\hline 1955 & $\mathrm{pCi} / \mathrm{L}$ & 0 & & & & & & RIVER & & CONTINUOUS & RESIN & 252 \\
\hline 1956 & $\mathrm{pCi} / \mathrm{L}$ & 0 & & & & & & RIVER & & CONTINUOUS & FILTER & 252 \\
\hline 1957 & $\mathrm{pCi} / \mathrm{L}$ & 0.13 & & & & & & SURFACE & & RAW GRAB & UNFILTERED & 509 \\
\hline 1958 & $\mathrm{pCi} / \mathrm{L}$ & 0.09 & & & & & & SURFACE & & RAW GRAB & UNFILTERED & 146 \\
\hline 1959 & $\mathrm{pCi} / \mathrm{L}$ & 1.1 & & & & & & SURFACE & & RAW GRAB & UNFILTERED & 147 \\
\hline 1960 & $\mathrm{pCi} / \mathrm{L}$ & 0 & & & & & & RIVER & & CONTINUOUS & RESIN & 307 \\
\hline 1961 & $\mathrm{pCi} / \mathrm{L}$ & 0 & & & & & & RIVER & & CONTINUOUS & FILTER & 307 \\
\hline 1962 & $\mathrm{pCi} / \mathrm{L}$ & 0 & & & & & & RIVER & & CONTINUOUS & RESIN & 252 \\
\hline 1963 & $\mathrm{pCi} / \mathrm{L}$ & 0 & & & & & & RIVER & & CONTINUOUS & FILTER & 252 \\
\hline 1964 & $\mathrm{pCi} / \mathrm{L}$ & 230 & & & & & & RIVER & & CUMULATIVE & UNFILTERED & 553 \\
\hline 1965 & $\mathrm{pCi} / \mathrm{L}$ & 31 & & & & U & & RIVER & & CUMULATIVE & UNFILTERED & 553 \\
\hline 1966 & $\mathrm{pCi} / \mathrm{L}$ & 0.12 & & & & & & SANITARY & & CUMULATIVE & UNFILTERED & 491 \\
\hline 1967 & $\mathrm{pCi} / \mathrm{L}$ & 260 & & & & & & SANITARY & & CUMULATIVE & UNFILTERED & 491 \\
\hline 1968 & $\mathrm{pCi} / \mathrm{L}$ & 31 & & & & U & & SANITARY & & CUMULATIVE & UNFILTERED & 491 \\
\hline 1969 & pCi/L & 0.09 & & & & & & SANITARY & & CUMULATIVE & UNFILTERED & 512 \\
\hline 1970 & $\mathrm{pCi} / \mathrm{L}$ & 31 & & & & U & & SANITARY & & CUMULATIVE & UNFILTERED & 512 \\
\hline 1971 & $\mathrm{pCi} / \mathrm{L}$ & 1300 & & & & & & SANITARY & & CUMULATIVE & UNFILTERED & 512 \\
\hline 1972 & pCi/L & 31 & & & & $U$ & & SANITARY & & CUMULATIVE & UNFILTERED & 385 \\
\hline 1973 & $\mathrm{pCi} / \mathrm{L}$ & 0 & & & & & & RIVER & & CONTINUOUS & RESIN & 307 \\
\hline 1974 & $\mathrm{pCi} / \mathrm{L}$ & 0.00000282 & & & & & & RIVER & & CONTINUOUS & RESIN & 307 \\
\hline 1975 & pCi/L & 0 & & & & & & RIVER & & CONTINUOUS & FILTER & 307 \\
\hline 1976 & $\mathrm{pCi} / \mathrm{L}$ & 0 & & & & & & RIVER & & CONTINUOUS & FILTER & 252 \\
\hline 1977 & $\mathrm{pCi} / \mathrm{L}$ & 0.00000078 & & & & & & RIVER & & CONTINUOUS & RESIN & 252 \\
\hline
\end{tabular}




\begin{tabular}{|c|c|c|c|c|c|c|c|c|c|c|c|c|}
\hline & $\mathrm{N}$ & $\mathrm{O}$ & $\mathrm{P}$ & $\mathrm{Q}$ & $\mathrm{R}$ & $\mathrm{s}$ & $T$ & $\mathrm{u}$ & $\mathrm{v}$ & $\mathrm{w}$ & $\mathrm{x}$ & $\bar{Y}$ \\
\hline 1 & ANAL_UNITS_RPTD & COUNTING_ERROR & TOTAL_ANAL_ERROR & MIN_DETECTABLE_ACTIVITY & PCNT_MOISTURE & LAB_QUALIFIER & REVIEW_QUALIFIER & SAMP_FROM & SAMP_ITEM & SAMP_MTHD & COLL_MTHD & SAMP_SITE_ID \\
\hline 1978 & $\mathrm{pCi} / \mathrm{L}$ & 0 & & & & & & RIVER & & CONTINUOUS & RESIN & 252 \\
\hline 1979 & $\mathrm{pCi} / \mathrm{L}$ & 250 & & & & & & RIVER & & CUMULATIVE & UNFILTERED & 463 \\
\hline 1980 & $\mathrm{pCi} / \mathrm{L}$ & 31 & & & & $\mathrm{U}$ & & RIVER & & CUMULATIVE & UNFILTERED & 463 \\
\hline 1981 & $\mathrm{pCi} / \mathrm{L}$ & 200 & & & & & & RIVER & & RAW GRAB & UNFILTERED & 463 \\
\hline 1982 & $\mathrm{pCi} / \mathrm{L}$ & 280 & & & & & & RIVER & & RAW GRAB & UNFILTERED & 456 \\
\hline 1983 & $\mathrm{pCi} / \mathrm{L}$ & 0 & & & & & & RIVER & & CONTINUOUS & RESIN & 307 \\
\hline 1984 & $\mathrm{pCi} / \mathrm{L}$ & 0 & & & & & & RIVER & & CONTINUOUS & FILTER & 307 \\
\hline 1985 & $\mathrm{pCi} / \mathrm{L}$ & 0 & & & & & & RIVER & & CONTINUOUS & RESIN & 252 \\
\hline 1986 & $\mathrm{pCi} / \mathrm{L}$ & 0 & & & & & & RIVER & & CONTINUOUS & FILTER & 252 \\
\hline 1987 & ug/L & 0 & & & & & & SANITARY & & CUMULATIVE & UNFILTERED & 491 \\
\hline 1988 & ug/L & 0 & & & & & & SANITARY & & CUMULATIVE & UNFILTERED & 385 \\
\hline 1989 & $\mathrm{pCi} / \mathrm{L}$ & 31 & & & & $\mathrm{U}$ & & RIVER & & CUMULATIVE & UNFILTERED & 553 \\
\hline 1990 & $\mathrm{pCi} / \mathrm{L}$ & 270 & & & & & & RIVER & & CUMULATIVE & UNFILTERED & 553 \\
\hline \begin{tabular}{|l|}
1991 \\
\end{tabular} & $\mathrm{pCi} / \mathrm{L}$ & 0.09 & & & & & & RIVER & & CUMULATIVE & UNFILTERED & 553 \\
\hline \begin{tabular}{|l|}
1992 \\
\end{tabular} & $\mathrm{pCi} / \mathrm{L}$ & 0 & & & & & & RIVER & & CONTINUOUS & RESIN & 307 \\
\hline \begin{tabular}{|l|}
1993 \\
\end{tabular} & $\mathrm{pCi} / \mathrm{L}$ & 0.00000312 & & & & & & RIVER & & CONTINUOUS & RESIN & 307 \\
\hline \begin{tabular}{|l|}
1994 \\
\end{tabular} & $\mathrm{pCi} / \mathrm{L}$ & 0 & & & & & & RIVER & & CONTINUOUS & FILTER & 307 \\
\hline \begin{tabular}{|l|}
1995 \\
\end{tabular} & $\mathrm{pCi} / \mathrm{L}$ & 0.00000168 & & & & & & RIVER & & CONTINUOUS & RESIN & 252 \\
\hline 1996 & $\mathrm{pCi} / \mathrm{L}$ & 0 & & & & & & RIVER & & CONTINUOUS & RESIN & 252 \\
\hline \begin{tabular}{|l|}
1997 \\
\end{tabular} & $\mathrm{pCi} / \mathrm{L}$ & 0 & & & & & & RIVER & & CONTINUOUS & FILTER & 252 \\
\hline \begin{tabular}{|l|}
1998 \\
\end{tabular} & $\mathrm{pCi} / \mathrm{L}$ & 31 & & & & $U$ & & SANITARY & & CUMULATIVE & UNFILTERED & 491 \\
\hline 1999 & $\mathrm{pCi} / \mathrm{L}$ & 31 & & & & $U$ & & SANITARY & & CUMULATIVE & UNFILTERED & 512 \\
\hline 2000 & $\mathrm{pCi} / \mathrm{L}$ & 690 & & & & & & SANITARY & & CUMULATIVE & UNFILTERED & 512 \\
\hline 2001 & $\mathrm{pCi} / \mathrm{L}$ & 31 & & & & $U$ & & SANITARY & & CUMULATIVE & UNFILTERED & 385 \\
\hline 2002 & $\mathrm{pCi} / \mathrm{L}$ & 340 & & & & $U$ & & RIVER & & CUMULATIVE & UNFILTERED & 463 \\
\hline 2003 & $\mathrm{pCi} / \mathrm{L}$ & 0.09 & & & & & & RIVER & & CUMULATIVE & UNFILTERED & 463 \\
\hline 2004 & $\mathrm{pCi} / \mathrm{L}$ & 31 & & & & $\mathrm{U}$ & & RIVER & & CUMULATIVE & UNFILTERED & 463 \\
\hline 2005 & $\mathrm{pCi} / \mathrm{L}$ & 340 & & & & & & RIVER & & RAW GRAB & UNFILTERED & 463 \\
\hline 2006 & $\mathrm{pCi} / \mathrm{L}$ & 290 & & & & $U$ & & RIVER & & RAW GRAB & UNFILTERED & 456 \\
\hline \begin{tabular}{|l|}
2007 \\
\end{tabular} & $\mathrm{pCi} / \mathrm{L}$ & 0 & & & & & & RIVER & & CONTINUOUS & RESIN & 307 \\
\hline \begin{tabular}{|l|}
2008 \\
\end{tabular} & $\mathrm{pCi} / \mathrm{L}$ & 0 & & & & & & RIVER & & CONTINUOUS & FILTER & 252 \\
\hline 2009 & $\mathrm{pCi} / \mathrm{L}$ & 0 & & & & & & RIVER & & CONTINUOUS & RESIN & 252 \\
\hline 2010 & $\mathrm{pCi} / \mathrm{L}$ & 0 & & & & & & RIVER & & CONTINUOUS & RESIN & 307 \\
\hline 2011 & $\mathrm{pCi} / \mathrm{L}$ & 0 & & & & & & RIVER & & CONTINUOUS & RESIN & 307 \\
\hline 2012 & $\mathrm{pCi} / \mathrm{L}$ & 0 & & & & & & RIVER & & CONTINUOUS & RESIN & 307 \\
\hline \begin{tabular}{|l|}
2013 \\
\end{tabular} & $\mathrm{pCi} / \mathrm{L}$ & 0.0000038 & & & & & & RIVER & & CONTINUOUS & RESIN & 307 \\
\hline 2014 & $\mathrm{pCi} / \mathrm{L}$ & 0 & & & & & & RIVER & & CONTINUOUS & FILTER & 252 \\
\hline 2015 & $\mathrm{pCi} / \mathrm{L}$ & 0.00000102 & & & & & & RIVER & & CONTINUOUS & RESIN & 252 \\
\hline 2016 & $\mathrm{pCi} / \mathrm{L}$ & 0 & & & & & & RIVER & & CONTINUOUS & RESIN & 252 \\
\hline \begin{tabular}{|l|}
2017 \\
\end{tabular} & $\mathrm{pCi} / \mathrm{L}$ & 170 & & & & & & RIVER & & RAW GRAB & UNFILTERED & 463 \\
\hline 2018 & $\mathrm{pCi} / \mathrm{L}$ & 220 & & & & $U$ & & RIVER & & RAW GRAB & UNFILTERED & 456 \\
\hline 2019 & $\mathrm{pCi} / \mathrm{L}$ & 31 & & & & $U$ & & RIVER & & CUMULATIVE & UNFILTERED & 553 \\
\hline 2020 & $\mathrm{pCi} / \mathrm{L}$ & 230 & & & & & & RIVER & & CUMULATIVE & UNFILTERED & 553 \\
\hline 2021 & $\mathrm{pCi} / \mathrm{L}$ & 31 & & & & $\mathrm{U}$ & & SANITARY & & CUMULATIVE & UNFILTERED & 491 \\
\hline 2022 & $\mathrm{pCi} / \mathrm{L}$ & 31 & & & & $U$ & & SANITARY & & CUMULATIVE & UNFILTERED & 512 \\
\hline 2023 & $\mathrm{pCi} / \mathrm{L}$ & 910 & & & & & & SANITARY & & CUMULATIVE & UNFILTERED & 512 \\
\hline 2024 & $\mathrm{pCi} / \mathrm{L}$ & 31 & & & & $U$ & & SANITARY & & CUMULATIVE & UNFILTERED & 385 \\
\hline 2025 & $\mathrm{pCi} / \mathrm{L}$ & 0 & & & & & & RIVER & & CONTINUOUS & RESIN & 307 \\
\hline 2026 & $\mathrm{pCi} / \mathrm{L}$ & 0 & & & & & & RIVER & & CONTINUOUS & FILTER & 307 \\
\hline 2027 & $\mathrm{pCi} / \mathrm{L}$ & 0 & & & & & & RIVER & & CONTINUOUS & RESIN & 252 \\
\hline 2028 & $\mathrm{pCi} / \mathrm{L}$ & 0 & & & & & & RIVER & & CONTINUOUS & FILTER & 252 \\
\hline 2029 & $\mathrm{pCi} / \mathrm{L}$ & 7.7 & & & & $U_{1}$ & & SURFACE & & RAW GRAB & UNFILTERED & 121 \\
\hline
\end{tabular}




\begin{tabular}{|c|c|c|c|c|c|c|c|c|c|c|c|c|}
\hline & $\mathrm{N}$ & $\mathrm{O}$ & $\mathrm{P}$ & $\mathrm{Q}$ & $\mathrm{R}$ & $\mathrm{s}$ & $T$ & $\mathrm{U}$ & $\mathrm{V}$ & $\mathrm{W}$ & $\mathrm{X}$ & $\bar{Y}$ \\
\hline 1 & ANAL_UNITS_RPTD & COUNTING_ERROR & TOTAL_ANAL_ERROR & MIN_DETECTABLE_ACTIVITY & PCNT_MOISTURE & LAB_QUALIFIER & REVIEW_QUALIFIER & SAMP_FROM & SAMP_ITEM & SAMP MTHD & COLL MTHD & SAMP SITE ID \\
\hline 2030 & $\mathrm{pCi} / \mathrm{L}$ & 0.09 & & & & & & SURFACE & & RAW GRAB & UNFILTERED & 509 \\
\hline 2031 & $\mathrm{pCi} / \mathrm{L}$ & 2.1 & & & & & & SURFACE & & RAW GRAB & UNFILTERED & 509 \\
\hline 2032 & $\mathrm{pCi} / \mathrm{L}$ & 2.1 & & & & & & SURFACE & & RAW GRAB & UNFILTERED & 146 \\
\hline 2033 & $\mathrm{pCi} / \mathrm{L}$ & 0.21 & & & & & & SURFACE & & RAW GRAB & UNFILTERED & 146 \\
\hline 2034 & $\mathrm{pCi} / \mathrm{L}$ & 2.1 & & & & & & SURFACE & & RAW GRAB & UNFILTERED & 412 \\
\hline 2035 & $\mathrm{pCi} / \mathrm{L}$ & 0.28 & & & & & & SURFACE & & RAW GRAB & UNFILTERED & 412 \\
\hline 2036 & $\mathrm{pCi} / \mathrm{L}$ & 10 & & & & & & SURFACE & & RAW GRAB & UNFILTERED & 147 \\
\hline 2037 & $\mathrm{pCi} / \mathrm{L}$ & 0.34 & & & & & & SURFACE & & RAW GRAB & UNFILTERED & 147 \\
\hline 2038 & $\mathrm{pCi} / \mathrm{L}$ & 0 & & & & & & RIVER & & CONTINUOUS & RESIN & 307 \\
\hline 2039 & $\mathrm{pCi} / \mathrm{L}$ & 0.00000232 & & & & & & RIVER & & CONTINUOUS & RESIN & 307 \\
\hline 2040 & $\mathrm{pCi} / \mathrm{L}$ & 0 & & & & & & RIVER & & CONTINUOUS & FILTER & 307 \\
\hline 2041 & $\mathrm{pCi} / \mathrm{L}$ & 0.00000087 & & & & & & RIVER & & CONTINUOUS & RESIN & 252 \\
\hline 2042 & $\mathrm{pCi} / \mathrm{L}$ & 0 & & & & & & RIVER & & CONTINUOUS & RESIN & 252 \\
\hline 2043 & $\mathrm{pCi} / \mathrm{L}$ & 0 & & & & & & RIVER & & CONTINUOUS & FILTER & 252 \\
\hline 2044 & $\mathrm{pCi} / \mathrm{L}$ & 240 & & & & U & & RIVER & & CUMULATIVE & UNFILTERED & 463 \\
\hline 2045 & $\mathrm{pCi} / \mathrm{L}$ & 31 & & & & U & & RIVER & & CUMULATIVE & UNFILTERED & 463 \\
\hline 2046 & $\mathrm{pCi} / \mathrm{L}$ & 160 & & & & & & RIVER & & RAW GRAB & UNFILTERED & 463 \\
\hline 2047 & $\mathrm{pCi} / \mathrm{L}$ & 170 & & & & & & RIVER & & RAW GRAB & UNFILTERED & 456 \\
\hline \begin{tabular}{|l|}
2048 \\
\end{tabular} & $\mathrm{pCi} / \mathrm{L}$ & 31 & & & & $U$ & & RIVER & & CUMULATIVE & UNFILTERED & 553 \\
\hline 2049 & $\mathrm{pCi} / \mathrm{L}$ & 290 & & & & $U$ & & RIVER & & CUMULATIVE & UNFILTERED & 553 \\
\hline 2050 & $\mathrm{pCi} / \mathrm{L}$ & 0.09 & & & & & & RIVER & & CUMULATIVE & UNFILTERED & 553 \\
\hline \begin{tabular}{|l|}
2051 \\
\end{tabular} & $\mathrm{pCi} / \mathrm{L}$ & 0.09 & & & & & & SANITARY & & CUMULATIVE & UNFILTERED & 491 \\
\hline 2052 & $\mathrm{pCi} / \mathrm{L}$ & 250 & & & & U & & SANITARY & & CUMULATIVE & UNFILTERED & 491 \\
\hline 2053 & $\mathrm{pCi} / \mathrm{L}$ & 31 & & & & U & & SANITARY & & CUMULATIVE & UNFILTERED & 491 \\
\hline \begin{tabular}{|l|}
2054 \\
\end{tabular} & $\mathrm{pCi} / \mathrm{L}$ & 0.09 & & & & & & SANITARY & & CUMULATIVE & UNFILTERED & 512 \\
\hline 2055 & $\mathrm{pCi} / \mathrm{L}$ & 31 & & & & $U$ & & SANITARY & & CUMULATIVE & UNFILTERED & 512 \\
\hline 2056 & $\mathrm{pCi} / \mathrm{L}$ & 970 & & & & & & SANITARY & & CUMULATIVE & UNFILTERED & 512 \\
\hline \begin{tabular}{|l|}
2057 \\
\end{tabular} & $\mathrm{pCi} / \mathrm{L}$ & 31 & & & & U & & SANITARY & & CUMULATIVE & UNFILTERED & 385 \\
\hline 2058 & $\mathrm{pCi} / \mathrm{L}$ & 0 & & & & & & RIVER & & CONTINUOUS & RESIN & 307 \\
\hline 2059 & $\mathrm{pCi} / \mathrm{L}$ & 0 & & & & & & RIVER & & CONTINUOUS & FILTER & 307 \\
\hline 2060 & $\mathrm{pCi} / \mathrm{L}$ & 0 & & & & & & RIVER & & CONTINUOUS & FILTER & 252 \\
\hline \begin{tabular}{|l|}
2061 \\
\end{tabular} & $\mathrm{pCi} / \mathrm{L}$ & 0 & & & & & & RIVER & & CONTINUOUS & RESIN & 252 \\
\hline \begin{tabular}{|l|}
2062 \\
\end{tabular} & $\mathrm{pCi} / \mathrm{L}$ & 0 & & & & & & RIVER & & CONTINUOUS & RESIN & 307 \\
\hline 2063 & $\mathrm{pCi} / \mathrm{L}$ & 0 & & & & & & RIVER & & CONTINUOUS & FILTER & 307 \\
\hline 2064 & $\mathrm{pCi} / \mathrm{L}$ & 300 & & & & & & RIVER & & CUMULATIVE & UNFILTERED & 553 \\
\hline 2065 & $\mathrm{pCi} / \mathrm{L}$ & 5.1 & & & & U & & RIVER & & CUMULATIVE & UNFILTERED & 553 \\
\hline 2066 & $\mathrm{pCi} / \mathrm{L}$ & 31 & & & & U & & SANITARY & & CUMULATIVE & UNFILTERED & 491 \\
\hline 2067 & $\mathrm{pCi} / \mathrm{L}$ & 5.1 & & & & U & & SANITARY & & CUMULATIVE & UNFILTERED & 512 \\
\hline 2068 & $\mathrm{pCi} / \mathrm{L}$ & 950 & & & & & & SANITARY & & CUMULATIVE & UNFILTERED & 512 \\
\hline 2069 & $\mathrm{pCi} / \mathrm{L}$ & 31 & & & & U & & SANITARY & & CUMULATIVE & UNFILTERED & 385 \\
\hline 2070 & $\mathrm{pCi} / \mathrm{L}$ & 370 & & & & & & RIVER & & CUMULATIVE & UNFILTERED & 463 \\
\hline 2071 & $\mathrm{pCi} / \mathrm{L}$ & 0.09 & & & & & & RIVER & & CUMULATIVE & UNFILTERED & 463 \\
\hline 2072 & $\mathrm{pCi} / \mathrm{L}$ & 31 & & & & U & & RIVER & & CUMULATIVE & UNFILTERED & 463 \\
\hline 2073 & $\mathrm{pCi} / \mathrm{L}$ & 260 & & & & & & RIVER & & RAW GRAB & UNFILTERED & 463 \\
\hline 2074 & $\mathrm{pCi} / \mathrm{L}$ & 230 & & & & & & RIVER & & RAW GRAB & UNFILTERED & 456 \\
\hline 2075 & $\mathrm{pCi} / \mathrm{L}$ & 0.000088 & & & & U & & RIVER & & CONTINUOUS & RESIN & 307 \\
\hline 2076 & $\mathrm{pCi} / \mathrm{L}$ & 0 & & & & & & RIVER & & CONTINUOUS & RESIN & 307 \\
\hline 2077 & $\mathrm{pCi} / \mathrm{L}$ & 0.00000344 & & & & & & RIVER & & CONTINUOUS & RESIN & 307 \\
\hline 2078 & $\mathrm{pCi} / \mathrm{L}$ & 0.000004 & & & & & & RIVER & & CONTINUOUS & FILTER & 307 \\
\hline 2079 & $\mathrm{pCi} / \mathrm{L}$ & 0 & & & & & & RIVER & & CONTINUOUS & FILTER & 307 \\
\hline 2080 & $\mathrm{pCi} / \mathrm{L}$ & 0.000002 & & & & & & RIVER & & CONTINUOUS & FILTER & 252 \\
\hline 2081 & $\mathrm{pCi} / \mathrm{L}$ & 0 & & & & & & RIVER & & CONTINUOUS & FILTER & 252 \\
\hline
\end{tabular}




\begin{tabular}{|c|c|c|c|c|c|c|c|c|c|c|c|c|}
\hline & $\mathrm{N}$ & $\mathrm{O}$ & $P$ & $\mathrm{Q}$ & $\mathrm{R}$ & $\mathrm{S}$ & $T$ & $\bar{U}$ & $\mathrm{~V}$ & $\mathrm{~W}$ & $\bar{X}$ & $\overline{\mathrm{Y}}$ \\
\hline 1 & ANAL_UNITS_RPTD & COUNTING_ERROR & TOTAL_ANAL_ERROR & MIN_DETECTABLE_ACTIVITY & PCNT_MOISTURE & LAB_QUALIFIER & REVIEW_QUALIFIER & SAMP_FROM & SAMP_ITEM & SAMP_MTHD & COLL_MTHD & SAMP_SITE_ID \\
\hline 2082 & $\mathrm{pCi} / \mathrm{L}$ & 0.000000714 & & & & & & RIVER & & CONTINUOUS & RESIN & 252 \\
\hline 2083 & $\mathrm{pCi} / \mathrm{L}$ & 0.00007 & & & & & & RIVER & & CONTINUOUS & RESIN & 252 \\
\hline 2084 & $\mathrm{pCi} / \mathrm{L}$ & 0 & & & & & & RIVER & & CONTINUOUS & RESIN & 252 \\
\hline 2085 & $\mathrm{pCi} / \mathrm{L}$ & 260 & & & & & & RIVER & & RAW GRAB & UNFILTERED & 463 \\
\hline 2086 & $\mathrm{pCi} / \mathrm{L}$ & 0 & & & & & & RIVER & & CONTINUOUS & RESIN & 307 \\
\hline 2087 & $\mathrm{pCi} / \mathrm{L}$ & 0 & & & & & & RIVER & & CONTINUOUS & FILTER & 307 \\
\hline 2088 & $\mathrm{pCi} / \mathrm{L}$ & 0.00000112 & & & & & & RIVER & & CONTINUOUS & RESIN & 252 \\
\hline 2089 & $\mathrm{pCi} / \mathrm{L}$ & 0 & & & & & & RIVER & & CONTINUOUS & RESIN & 252 \\
\hline 2090 & $\mathrm{pCi} / \mathrm{L}$ & 0 & & & & & & RIVER & & CONTINUOUS & FILTER & 252 \\
\hline 2091 & $\mathrm{pCi} / \mathrm{L}$ & 5.1 & & & & U & & RIVER & & CUMULATIVE & UNFILTERED & 553 \\
\hline 2092 & $\mathrm{pCi} / \mathrm{L}$ & 300 & & & & $\mathrm{U}$ & & RIVER & & CUMULATIVE & UNFILTERED & 553 \\
\hline 2093 & $\mathrm{pCi} / \mathrm{L}$ & 31 & & & & U & & SANITARY & & CUMULATIVE & UNFILTERED & 491 \\
\hline 2094 & $\mathrm{pCi} / \mathrm{L}$ & 5.1 & & & & U & & SANITARY & & CUMULATIVE & UNFILTERED & 512 \\
\hline 2095 & $\mathrm{pCi} / \mathrm{L}$ & 650 & & & & & & SANITARY & & CUMULATIVE & UNFILTERED & 512 \\
\hline 2096 & $\mathrm{pCi} / \mathrm{L}$ & 31 & & & & U & & SANITARY & & CUMULATIVE & UNFILTERED & 385 \\
\hline 2097 & $\mathrm{pCi} / \mathrm{L}$ & 240 & & & & & & RIVER & & CUMULATIVE & UNFILTERED & 463 \\
\hline 2098 & $\mathrm{pCi} / \mathrm{L}$ & 31 & & & & U & & RIVER & & CUMULATIVE & UNFILTERED & 463 \\
\hline 2099 & $\mathrm{pCi} / \mathrm{L}$ & 220 & & & & & & RIVER & & RAW GRAB & UNFILTERED & 456 \\
\hline 2100 & $\mathrm{pCi} / \mathrm{L}$ & 0 & & & & & & RIVER & & CONTINUOUS & FILTER & 307 \\
\hline 2101 & $\mathrm{pCi} / \mathrm{L}$ & 0 & & & & & & RIVER & & CONTINUOUS & RESIN & 307 \\
\hline 2102 & $\mathrm{pCi} / \mathrm{L}$ & 0.00000072 & & & & & & RIVER & & CONTINUOUS & RESIN & 307 \\
\hline 2103 & $\mathrm{pCi} / \mathrm{L}$ & 0 & & & & & & RIVER & & CONTINUOUS & FILTER & 252 \\
\hline 2104 & $\mathrm{pCi} / \mathrm{L}$ & 5.2 & & & & & & SURFACE & & RAW GRAB & UNFILTERED & 121 \\
\hline 2105 & $\mathrm{pCi} / \mathrm{L}$ & 0.09 & & & & & & SURFACE & & RAW GRAB & UNFILTERED & 509 \\
\hline 2106 & $\mathrm{pCi} / \mathrm{L}$ & 2.1 & & & & & & SURFACE & & RAW GRAB & UNFILTERED & 509 \\
\hline 2107 & $\mathrm{pCi} / \mathrm{L}$ & 2.3 & & & & & & SURFACE & & RAW GRAB & UNFILTERED & 146 \\
\hline 2108 & $\mathrm{pCi} / \mathrm{L}$ & 0.09 & & & & & & SURFACE & & RAW GRAB & UNFILTERED & 146 \\
\hline 2109 & $\mathrm{pCi} / \mathrm{L}$ & 2.1 & & & & & & SURFACE & & RAW GRAB & UNFILTERED & 412 \\
\hline 2110 & $\mathrm{pCi} / \mathrm{L}$ & 0.11 & & & & & & SURFACE & & RAW GRAB & UNFILTERED & 412 \\
\hline 2111 & $\mathrm{pCi} / \mathrm{L}$ & 8.2 & & & & U & & SURFACE & & RAW GRAB & UNFILTERED & 147 \\
\hline 2112 & $\mathrm{pCi} / \mathrm{L}$ & 0.27 & & & & & & SURFACE & & RAW GRAB & UNFILTERED & 147 \\
\hline 2113 & $\mathrm{pCi} / \mathrm{L}$ & 0 & & & & & & RIVER & & CONTINUOUS & RESIN & 307 \\
\hline 2114 & $\mathrm{pCi} / \mathrm{L}$ & 0 & & & & & & RIVER & & CONTINUOUS & FILTER & 307 \\
\hline 2115 & $\mathrm{pCi} / \mathrm{L}$ & 0 & & & & & & RIVER & & CONTINUOUS & FILTER & 252 \\
\hline 2116 & $\mathrm{pCi} / \mathrm{L}$ & 0 & & & & & & RIVER & & CONTINUOUS & RESIN & 252 \\
\hline 2117 & $\mathrm{pCi} / \mathrm{L}$ & 0.12 & & & & & & SANITARY & & CUMULATIVE & UNFILTERED & 491 \\
\hline 2118 & $\mathrm{pCi} / \mathrm{L}$ & 240 & & & & & & SANITARY & & CUMULATIVE & UNFILTERED & 491 \\
\hline 2119 & $\mathrm{pCi} / \mathrm{L}$ & 31 & & & & U & & SANITARY & & CUMULATIVE & UNFILTERED & 491 \\
\hline 2120 & $\mathrm{pCi} / \mathrm{L}$ & 0.09 & & & & U & & SANITARY & & CUMULATIVE & UNFILTERED & 512 \\
\hline 2121 & $\mathrm{pCi} / \mathrm{L}$ & 5.1 & & & & U & & SANITARY & & CUMULATIVE & UNFILTERED & 512 \\
\hline 2122 & $\mathrm{pCi} / \mathrm{L}$ & 730 & & & & & & SANITARY & & CUMULATIVE & UNFILTERED & 512 \\
\hline 2123 & $\mathrm{pCi} / \mathrm{L}$ & 31 & & & & U & & SANITARY & & CUMULATIVE & UNFILTERED & 385 \\
\hline 2124 & $\mathrm{pCi} / \mathrm{L}$ & 5.1 & & & & U & & RIVER & & CUMULATIVE & UNFILTERED & 553 \\
\hline 2125 & $\mathrm{pCi} / \mathrm{L}$ & 150 & & & & & & RIVER & & CUMULATIVE & UNFILTERED & 553 \\
\hline 2126 & $\mathrm{pCi} / \mathrm{L}$ & 0.2 & & & & & & RIVER & & CUMULATIVE & UNFILTERED & 553 \\
\hline 2127 & $\mathrm{pCi} / \mathrm{L}$ & 0 & & & & & & RIVER & & CONTINUOUS & RESIN & 307 \\
\hline 2128 & $\mathrm{pCi} / \mathrm{L}$ & 0.000001 & & & & & & RIVER & & CONTINUOUS & RESIN & 307 \\
\hline 2129 & $\mathrm{pCi} / \mathrm{L}$ & 0 & & & & & & RIVER & & CONTINUOUS & FILTER & 307 \\
\hline 2130 & $\mathrm{pCi} / \mathrm{L}$ & 0 & & & & & & RIVER & & CONTINUOUS & FILTER & 252 \\
\hline 2131 & $\mathrm{pCi} / \mathrm{L}$ & 0.00000088 & & & & & & RIVER & & CONTINUOUS & RESIN & 252 \\
\hline 2132 & $\mathrm{pCi} / \mathrm{L}$ & 0 & & & & & & RIVER & & CONTINUOUS & RESIN & 252 \\
\hline 2133 & $\mathrm{pCi} / \mathrm{L}$ & 590 & & & & U & & RIVER & & RAW GRAB & UNFILTERED & 463 \\
\hline
\end{tabular}




\begin{tabular}{|c|c|c|c|c|c|c|c|c|c|c|c|c|}
\hline & $\mathrm{N}$ & $\mathrm{O}$ & $\mathrm{P}$ & $\mathrm{Q}$ & $\mathrm{R}$ & $\mathrm{s}$ & $\mathrm{T}$ & $\mathrm{U}$ & $\mathrm{V}$ & $\mathrm{W}$ & $\mathrm{X}$ & $\bar{Y}$ \\
\hline 1 & ANAL_UNITS_RPTD & COUNTING_ERROR & TOTAL_ANAL_ERROR & MIN_DETECTABLE_ACTIVITY & PCNT_MOISTURE & LAB_QUALIFIER & REVIEW_QUALIFIER & SAMP_FROM & SAMP_ITEM & SAMP_MTHD & COLL_MTHD & SAMP_SITE_ID \\
\hline 2134 & $\mathrm{pCi} / \mathrm{L}$ & 220 & & & & $U$ & & RIVER & & RAW GRAB & UNFILTERED & 456 \\
\hline 2135 & $\mathrm{pCi} / \mathrm{L}$ & 140 & & & & & & RIVER & & CUMULATIVE & UNFILTERED & 463 \\
\hline 2136 & $\mathrm{pCi} / \mathrm{L}$ & 31 & & & & $u$ & & RIVER & & CUMULATIVE & UNFILTERED & 463 \\
\hline 2137 & $\mathrm{pCi} / \mathrm{L}$ & 0 & & & & & & RIVER & & CONTINUOUS & FILTER & 307 \\
\hline 2138 & $\mathrm{pCi} / \mathrm{L}$ & 0 & & & & & & RIVER & & CONTINUOUS & RESIN & 307 \\
\hline 2139 & $\mathrm{pCi} / \mathrm{L}$ & 0 & & & & & & RIVER & & CONTINUOUS & FILTER & 252 \\
\hline 2140 & $\mathrm{pCi} / \mathrm{L}$ & 0 & & & & & & RIVER & & CONTINUOUS & RESIN & 252 \\
\hline 2141 & $\mathrm{pCi} / \mathrm{L}$ & 370 & & & & U & & RIVER & & CUMULATIVE & UNFILTERED & 553 \\
\hline 2142 & $\mathrm{pCi} / \mathrm{L}$ & 5.1 & & & & U & & RIVER & & CUMULATIVE & UNFILTERED & 553 \\
\hline 2143 & $\mathrm{pCi} / \mathrm{L}$ & 240 & & & & & & RIVER & & CUMULATIVE & UNFILTERED & 463 \\
\hline 2144 & $\mathrm{pCi} / \mathrm{L}$ & 0.09 & & & & & & RIVER & & CUMULATIVE & UNFILTERED & 463 \\
\hline 2145 & $\mathrm{pCi} / \mathrm{L}$ & 31 & & & & U & & RIVER & & CUMULATIVE & UNFILTERED & 463 \\
\hline 2146 & $\mathrm{pCi} / \mathrm{L}$ & 31 & & & & $U$ & & SANITARY & & CUMULATIVE & UNFILTERED & 491 \\
\hline 2147 & $\mathrm{pCi} / \mathrm{L}$ & 5.1 & & & & $U$ & & SANITARY & & CUMULATIVE & UNFILTERED & 512 \\
\hline 2148 & $\mathrm{pCi} / \mathrm{L}$ & 900 & & & & & & SANITARY & & CUMULATIVE & UNFILTERED & 512 \\
\hline 2149 & $\mathrm{pCi} / \mathrm{L}$ & 31 & & & & $U$ & & SANITARY & & CUMULATIVE & UNFILTERED & 385 \\
\hline 2150 & $\mathrm{pCi} / \mathrm{L}$ & 0.000118 & & & & & & RIVER & & CONTINUOUS & RESIN & 307 \\
\hline 2151 & $\mathrm{pCi} / \mathrm{L}$ & 0 & & & & & & RIVER & & CONTINUOUS & RESIN & 307 \\
\hline 2152 & $\mathrm{pCi} / \mathrm{L}$ & 0.00000372 & & & & & & RIVER & & CONTINUOUS & RESIN & 307 \\
\hline 2153 & $\mathrm{pCi} / \mathrm{L}$ & 0 & & & & & & RIVER & & CONTINUOUS & FILTER & 307 \\
\hline 2154 & $\mathrm{pCi} / \mathrm{L}$ & 0.000008 & & & & & & RIVER & & CONTINUOUS & FILTER & 307 \\
\hline 2155 & $\mathrm{pCi} / \mathrm{L}$ & 0.000000546 & & & & & & RIVER & & CONTINUOUS & RESIN & 252 \\
\hline 2156 & $\mathrm{pCi} / \mathrm{L}$ & 0.000154 & & & & $U$ & & RIVER & & CONTINUOUS & RESIN & 252 \\
\hline 2157 & $\mathrm{pCi} / \mathrm{L}$ & 0 & & & & & & RIVER & & CONTINUOUS & RESIN & 252 \\
\hline 2158 & $\mathrm{pCi} / \mathrm{L}$ & 0.000004 & & & & & & RIVER & & CONTINUOUS & FILTER & 252 \\
\hline 2159 & $\mathrm{pCi} / \mathrm{L}$ & 0 & & & & & & RIVER & & CONTINUOUS & FILTER & 252 \\
\hline 2160 & $\mathrm{pCi} / \mathrm{L}$ & 250 & & & & & & RIVER & & RAW GRAB & UNFILTERED & 463 \\
\hline 2161 & $\mathrm{pCi} / \mathrm{L}$ & 310 & & & & $U$ & & RIVER & & RAW GRAB & UNFILTERED & 456 \\
\hline 2162 & $\mathrm{pCi} / \mathrm{L}$ & 0.00000104 & & & & & & RIVER & & CONTINUOUS & RESIN & 252 \\
\hline 2163 & $\mathrm{pCi} / \mathrm{L}$ & 0 & & & & & & RIVER & & CONTINUOUS & RESIN & 252 \\
\hline 2164 & $\mathrm{pCi} / \mathrm{L}$ & 0 & & & & & & RIVER & & CONTINUOUS & FILTER & 252 \\
\hline 2165 & $\mathrm{pCi} / \mathrm{L}$ & 0 & & & & & & RIVER & & CONTINUOUS & RESIN & 307 \\
\hline 2166 & $\mathrm{pCi} / \mathrm{L}$ & 0 & & & & & & RIVER & & CONTINUOUS & FILTER & 307 \\
\hline 2167 & $\mathrm{pCi} / \mathrm{L}$ & 0 & & & & & & RIVER & & CONTINUOUS & RESIN & 307 \\
\hline 2168 & $\mathrm{pCi} / \mathrm{L}$ & 0.00000264 & & & & & & RIVER & & CONTINUOUS & RESIN & 307 \\
\hline 2169 & $\mathrm{pCi} / \mathrm{L}$ & 0 & & & & & & RIVER & & CONTINUOUS & FILTER & 307 \\
\hline 2170 & $\mathrm{pCi} / \mathrm{L}$ & 270 & & & & $U$ & & RIVER & & CUMULATIVE & UNFILTERED & 463 \\
\hline 2171 & $\mathrm{pCi} / \mathrm{L}$ & 31 & & & & $U$ & & RIVER & & CUMULATIVE & UNFILTERED & 463 \\
\hline 2172 & $\mathrm{pCi} / \mathrm{L}$ & 290 & & & & U & & RIVER & & RAW GRAB & UNFILTERED & 463 \\
\hline 2173 & $\mathrm{pCi} / \mathrm{L}$ & 540 & & & & U & & RIVER & & RAW GRAB & UNFILTERED & 456 \\
\hline 2174 & $\mathrm{pCi} / \mathrm{L}$ & 240 & & & & & & RIVER & & CUMULATIVE & UNFILTERED & 553 \\
\hline 2175 & $\mathrm{pCi} / \mathrm{L}$ & 5.1 & & & & $U$ & & RIVER & & CUMULATIVE & UNFILTERED & 553 \\
\hline 2176 & $\mathrm{pCi} / \mathrm{L}$ & 31 & & & & U & & SANITARY & & CUMULATIVE & UNFILTERED & 491 \\
\hline 2177 & $\mathrm{pCi} / \mathrm{L}$ & 5.1 & & & & $U$ & & SANITARY & & CUMULATIVE & UNFILTERED & 512 \\
\hline 2178 & $\mathrm{pCi} / \mathrm{L}$ & 440 & & & & & & SANITARY & & CUMULATIVE & UNFILTERED & 512 \\
\hline 2179 & $\mathrm{pCi} / \mathrm{L}$ & 31 & & & & U & & SANITARY & & CUMULATIVE & UNFILTERED & 385 \\
\hline 2180 & $\mathrm{pCi} / \mathrm{L}$ & 0 & & & & & & RIVER & & CONTINUOUS & RESIN & 307 \\
\hline 2181 & $\mathrm{pCi} / \mathrm{L}$ & 7.8 & & & & & & SURFACE & & RAW GRAB & UNFILTERED & 121 \\
\hline 2182 & $\mathrm{pCi} / \mathrm{L}$ & 0.097 & & & & & & SURFACE & & RAW GRAB & UNFILTERED & 509 \\
\hline 2183 & pCi/L & 2.2 & & & & & & SURFACE & & RAW GRAB & UNFILTERED & 509 \\
\hline 2184 & $\mathrm{pCi} / \mathrm{L}$ & 2.3 & & & & & & SURFACE & & RAW GRAB & UNFILTERED & 146 \\
\hline 2185 & $\mathrm{pCi} / \mathrm{L}$ & 0.097 & & & & & & SURFACE & & RAW GRAB & UNFILTERED & 146 \\
\hline
\end{tabular}




\begin{tabular}{|c|c|c|c|c|c|c|c|c|c|c|c|c|}
\hline & $\mathrm{N}$ & $\mathrm{O}$ & $\mathrm{P}$ & $\mathrm{Q}$ & $\mathrm{R}$ & $\mathrm{s}$ & $T$ & $\mathrm{U}$ & $\mathrm{V}$ & $\mathrm{W}$ & $x$ & $\bar{Y}$ \\
\hline 1 & ANAL_UNITS_RPTD & COUNTING_ERROR & TOTAL_ANAL_ERROR & MIN_DETECTABLE_ACTIVITY & PCNT_MOISTURE & LAB_QUALIFIER & REVIEW_QUALIFIER & SAMP_FROM & SAMP_ITEM & SAMP_MTHD & COLL_MTHD & SAMP_SITE_ID \\
\hline 2186 & pCi/L & 2.3 & & & & & & SURFACE & & RAW GRAB & UNFILTERED & 412 \\
\hline 2187 & $\mathrm{pCi} / \mathrm{L}$ & 0.097 & & & & & & SURFACE & & RAW GRAB & UNFILTERED & 412 \\
\hline 2188 & $\mathrm{pCi} / \mathrm{L}$ & 10 & & & & & & SURFACE & & RAW GRAB & UNFILTERED & 147 \\
\hline 2189 & $\mathrm{pCi} / \mathrm{L}$ & 0.34 & & & & & & SURFACE & & RAW GRAB & UNFILTERED & 147 \\
\hline 2190 & $\mathrm{pCi} / \mathrm{L}$ & 0 & & & & & & RIVER & & CONTINUOUS & RESIN & 307 \\
\hline 2191 & $\mathrm{pCi} / \mathrm{L}$ & 0.00000594 & & & & & & RIVER & & CONTINUOUS & RESIN & 307 \\
\hline 2192 & $\mathrm{pCi} / \mathrm{L}$ & 0 & & & & & & RIVER & & CONTINUOUS & FILTER & 307 \\
\hline 2193 & $\mathrm{pCi} / \mathrm{L}$ & 5.1 & & & & U & & RIVER & & CUMULATIVE & UNFILTERED & 553 \\
\hline 2194 & $\mathrm{pCi} / \mathrm{L}$ & 210 & & & & & & RIVER & & CUMULATIVE & UNFILTERED & 553 \\
\hline 2195 & $\mathrm{pCi} / \mathrm{L}$ & 0.097 & & & & & & RIVER & & CUMULATIVE & UNFILTERED & 553 \\
\hline 2196 & $\mathrm{pCi} / \mathrm{L}$ & 0.097 & & & & & & SANITARY & & CUMULATIVE & UNFILTERED & 491 \\
\hline 2197 & $\mathrm{pCi} / \mathrm{L}$ & 230 & & & & & & SANITARY & & CUMULATIVE & UNFILTERED & 491 \\
\hline 2198 & $\mathrm{pCi} / \mathrm{L}$ & 31 & & & & $U$ & & SANITARY & & CUMULATIVE & UNFILTERED & 491 \\
\hline 2199 & $\mathrm{pCi} / \mathrm{L}$ & 0.097 & & & & & & SANITARY & & CUMULATIVE & UNFILTERED & 512 \\
\hline 2200 & $\mathrm{pCi} / \mathrm{L}$ & 5.1 & & & & $U$ & & SANITARY & & CUMULATIVE & UNFILTERED & 512 \\
\hline \begin{tabular}{|l|}
2201 \\
\end{tabular} & $\mathrm{pCi} / \mathrm{L}$ & 410 & & & & & & SANITARY & & CUMULATIVE & UNFILTERED & 512 \\
\hline 2202 & $\mathrm{pCi} / \mathrm{L}$ & 31 & & & & $U$ & & SANITARY & & CUMULATIVE & UNFILTERED & 385 \\
\hline 2203 & $\mathrm{pCi} / \mathrm{L}$ & 180 & & & & & & RIVER & & CUMULATIVE & UNFILTERED & 463 \\
\hline 2204 & $\mathrm{pCi} / \mathrm{L}$ & 31 & & & & U & & RIVER & & CUMULATIVE & UNFILTERED & 463 \\
\hline 2205 & $\mathrm{pCi} / \mathrm{L}$ & 320 & & & & & & RIVER & & RAW GRAB & UNFILTERED & 463 \\
\hline 2206 & pCi/L & 310 & & & & & & RIVER & & RAW GRAB & UNFILTERED & 456 \\
\hline 2207 & pCi/L & 0 & & & & & & RIVER & & CONTINUOUS & RESIN & 307 \\
\hline 2208 & $\mathrm{pCi} / \mathrm{L}$ & 0 & & & & & & RIVER & & CONTINUOUS & FILTER & 307 \\
\hline 2209 & $\mathrm{pCi} / \mathrm{L}$ & 0 & & & & & & RIVER & & CONTINUOUS & RESIN & 252 \\
\hline 2210 & $\mathrm{pCi} / \mathrm{L}$ & 0 & & & & & & RIVER & & CONTINUOUS & FILTER & 252 \\
\hline 2211 & $\mathrm{pCi} / \mathrm{L}$ & 0 & & & & & & RIVER & & CONTINUOUS & RESIN & 307 \\
\hline 2212 & $\mathrm{pCi} / \mathrm{L}$ & 0 & & & & & & RIVER & & CONTINUOUS & FILTER & 307 \\
\hline 2213 & $\mathrm{pCi} / \mathrm{L}$ & 0 & & & & & & RIVER & & CONTINUOUS & RESIN & 252 \\
\hline 2214 & $\mathrm{pCi} / \mathrm{L}$ & 0 & & & & & & RIVER & & CONTINUOUS & FILTER & 252 \\
\hline 2215 & $\mathrm{pCi} / \mathrm{L}$ & 190 & & & & & & RIVER & & CUMULATIVE & UNFILTERED & 553 \\
\hline 2216 & $\mathrm{pCi} / \mathrm{L}$ & 5.1 & & & & $U$ & & RIVER & & CUMULATIVE & UNFILTERED & 553 \\
\hline 2217 & $\mathrm{pCi} / \mathrm{L}$ & 31 & & & & U & & SANITARY & & CUMULATIVE & UNFILTERED & 491 \\
\hline 2218 & $\mathrm{pCi} / \mathrm{L}$ & 5.1 & & & & U & & SANITARY & & CUMULATIVE & UNFILTERED & 512 \\
\hline 2219 & $\mathrm{pCi} / \mathrm{L}$ & 1100 & & & & & & SANITARY & & CUMULATIVE & UNFILTERED & 512 \\
\hline 2220 & $\mathrm{pCi} / \mathrm{L}$ & 31 & & & & $U$ & & SANITARY & & CUMULATIVE & UNFILTERED & 385 \\
\hline 2221 & pCi/L & 240 & & & & & & RIVER & & CUMULATIVE & UNFILTERED & 463 \\
\hline 2222 & $\mathrm{pCi} / \mathrm{L}$ & 0.097 & & & & & & RIVER & & CUMULATIVE & UNFILTERED & 463 \\
\hline 2223 & $\mathrm{pCi} / \mathrm{L}$ & 31 & & & & $U$ & & RIVER & & CUMULATIVE & UNFILTERED & 463 \\
\hline 2224 & $\mathrm{pCi} / \mathrm{L}$ & 230 & & & & & & RIVER & & RAW GRAB & UNFILTERED & 463 \\
\hline 2225 & $\mathrm{pCi} / \mathrm{L}$ & 200 & & & & & & RIVER & & RAW GRAB & UNFILTERED & 456 \\
\hline \begin{tabular}{|l|}
2226 \\
\end{tabular} & $\mathrm{pCi} / \mathrm{L}$ & 0.000006 & & & & & & RIVER & & CONTINUOUS & FILTER & 307 \\
\hline \begin{tabular}{|l|}
2227 \\
\end{tabular} & $\mathrm{pCi} / \mathrm{L}$ & 0 & & & & & & RIVER & & CONTINUOUS & FILTER & 307 \\
\hline 2228 & $\mathrm{pCi} / \mathrm{L}$ & 0.00011 & & & & U & & RIVER & & CONTINUOUS & RESIN & 307 \\
\hline 2229 & $\mathrm{pCi} / \mathrm{L}$ & 0 & & & & & & RIVER & & CONTINUOUS & RESIN & 307 \\
\hline 2230 & $\mathrm{pCi} / \mathrm{L}$ & 0.000006 & & & & & & RIVER & & CONTINUOUS & RESIN & 307 \\
\hline 2231 & $\mathrm{pCi} / \mathrm{L}$ & 0.00000128 & & & & & & RIVER & & CONTINUOUS & RESIN & 252 \\
\hline 2232 & $\mathrm{pCi} / \mathrm{L}$ & 0 & & & & & & RIVER & & CONTINUOUS & RESIN & 252 \\
\hline 2233 & $\mathrm{pCi} / \mathrm{L}$ & 0 & & & & & & RIVER & & CONTINUOUS & RESIN & 252 \\
\hline 2234 & pCi/L & 0.000006 & & & & & & RIVER & & CONTINUOUS & FILTER & 252 \\
\hline 2235 & pCi/L & 0 & & & & & & RIVER & & CONTINUOUS & FILTER & 252 \\
\hline $2236 \mid$ & $\mathrm{pCi} / \mathrm{L}$ & 0 & & & & & & RIVER & & CONTINUOUS & RESIN & 307 \\
\hline 2237 & $\mathrm{pCi} / \mathrm{L}$ & 0 & & & & & & RIVER & & CONTINUOUS & FILTER & 307 \\
\hline
\end{tabular}




\begin{tabular}{|c|c|c|c|c|c|c|c|c|c|c|c|c|}
\hline & $\mathrm{N}$ & $\mathrm{O}$ & $\mathrm{P}$ & $\mathrm{Q}$ & $\mathrm{R}$ & $\mathrm{s}$ & $\mathrm{T}$ & $\mathrm{u}$ & $\mathrm{v}$ & $\mathrm{w}$ & $\mathrm{x}$ & $\bar{Y}$ \\
\hline 1 & ANAL_UNITS_RPTD & COUNTING_ERROR & TOTAL_ANAL_ERROR & MIN_DETECTABLE_ACTIVITY & PCNT_MOISTURE & LAB_QUALIFIER & REVIEW_QUALIFIER & SAMP_FROM & SAMP_ITEM & SAMP_MTHD & COLL_MTHD & SAMP_SITE_ID \\
\hline 2238 & $\mathrm{pCi} / \mathrm{L}$ & 0 & & & & & & RIVER & & CONTINUOUS & RESIN & 252 \\
\hline 2239 & $\mathrm{pCi} / \mathrm{L}$ & 0 & & & & & & RIVER & & CONTINUOUS & FILTER & 252 \\
\hline 2240 & $\mathrm{pCi} / \mathrm{L}$ & 320 & & & & & & RIVER & & CUMULATIVE & UNFILTERED & 463 \\
\hline 2241 & $\mathrm{pCi} / \mathrm{L}$ & 31 & & & & $\mathrm{U}$ & & RIVER & & CUMULATIVE & UNFILTERED & 463 \\
\hline 2242 & $\mathrm{pCi} / \mathrm{L}$ & 330 & & & & & & RIVER & & RAW GRAB & UNFILTERED & 463 \\
\hline 2243 & $\mathrm{pCi} / \mathrm{L}$ & 450 & & & & & & RIVER & & RAW GRAB & UNFILTERED & 456 \\
\hline 2244 & $\mathrm{pCi} / \mathrm{L}$ & 430 & & & & U & & RIVER & & CUMULATIVE & UNFILTERED & 553 \\
\hline 2245 & $\mathrm{pCi} / \mathrm{L}$ & 5.1 & & & & U & & RIVER & & CUMULATIVE & UNFILTERED & 553 \\
\hline 2246 & $\mathrm{pCi} / \mathrm{L}$ & 31 & & & & U & & SANITARY & & CUMULATIVE & UNFILTERED & 491 \\
\hline 2247 & $\mathrm{pCi} / \mathrm{L}$ & 5.1 & & & & U & & SANITARY & & CUMULATIVE & UNFILTERED & 512 \\
\hline 2248 & $\mathrm{pCi} / \mathrm{L}$ & 1600 & & & & & & SANITARY & & CUMULATIVE & UNFILTERED & 512 \\
\hline 2249 & $\mathrm{pCi} / \mathrm{L}$ & 31 & & & & U & & SANITARY & & CUMULATIVE & UNFILTERED & 385 \\
\hline 2250 & $\mathrm{pCi} / \mathrm{L}$ & 0 & & & & & & RIVER & & CONTINUOUS & RESIN & 307 \\
\hline 2251 & $\mathrm{pCi} / \mathrm{L}$ & 0.0000076 & & & & & & RIVER & & CONTINUOUS & RESIN & 307 \\
\hline 2252 & $\mathrm{pCi} / \mathrm{L}$ & 0 & & & & & & RIVER & & CONTINUOUS & FILTER & 307 \\
\hline 2253 & $\mathrm{pCi} / \mathrm{L}$ & 0 & & & & & & RIVER & & CONTINUOUS & RESIN & 252 \\
\hline 2254 & $\mathrm{pCi} / \mathrm{L}$ & 0.00000412 & & & & & & RIVER & & CONTINUOUS & RESIN & 252 \\
\hline 2255 & $\mathrm{pCi} / \mathrm{L}$ & 0 & & & & & & RIVER & & CONTINUOUS & FILTER & 252 \\
\hline 2256 & $\mathrm{pCi} / \mathrm{L}$ & 1400 & & & & & & SANITARY & & CUMULATIVE & UNFILTERED & 512 \\
\hline \begin{tabular}{|l|}
2257 \\
\end{tabular} & $\mathrm{pCi} / \mathrm{L}$ & 5.1 & & & & U & & SURFACE & & RAW GRAB & UNFILTERED & 121 \\
\hline 2258 & $\mathrm{pCi} / \mathrm{L}$ & 0.13 & & & & & & SURFACE & & RAW GRAB & UNFILTERED & 509 \\
\hline 2259 & $\mathrm{pCi} / \mathrm{L}$ & 2.1 & & & & & & SURFACE & & RAW GRAB & UNFILTERED & 509 \\
\hline 2260 & $\mathrm{pCi} / \mathrm{L}$ & 2.1 & & & & U & & SURFACE & & RAW GRAB & UNFILTERED & 146 \\
\hline 2261 & $\mathrm{pCi} / \mathrm{L}$ & 0.09 & & & & & & SURFACE & & RAW GRAB & UNFILTERED & 146 \\
\hline 2262 & $\mathrm{pCi} / \mathrm{L}$ & 2.1 & & & & & & SURFACE & & RAW GRAB & UNFILTERED & 412 \\
\hline 2263 & $\mathrm{pCi} / \mathrm{L}$ & 0.31 & & & & & & SURFACE & & RAW GRAB & UNFILTERED & 412 \\
\hline 2264 & $\mathrm{pCi} / \mathrm{L}$ & 10 & & & & U & & SURFACE & & RAW GRAB & UNFILTERED & 147 \\
\hline 2265 & $\mathrm{pCi} / \mathrm{L}$ & 1.1 & & & & & & SURFACE & & RAW GRAB & UNFILTERED & 147 \\
\hline 2266 & $\mathrm{pCi} / \mathrm{L}$ & 770 & & & & & & SANITARY & & CUMULATIVE & UNFILTERED & 512 \\
\hline 2267 & $\mathrm{pCi} / \mathrm{L}$ & 0 & & & & & & RIVER & & CONTINUOUS & FILTER & 307 \\
\hline 2268 & $\mathrm{pCi} / \mathrm{L}$ & 0 & & & & & & RIVER & & CONTINUOUS & RESIN & 307 \\
\hline 2269 & $\mathrm{pCi} / \mathrm{L}$ & 2400 & & & & & & SANITARY & & CUMULATIVE & UNFILTERED & 512 \\
\hline 2270 & $\mathrm{pCi} / \mathrm{L}$ & 0.09 & & & & & & RIVER & & CUMULATIVE & UNFILTERED & 553 \\
\hline 2271 & $\mathrm{pCi} / \mathrm{L}$ & 210 & & & & & & RIVER & & CUMULATIVE & UNFILTERED & 553 \\
\hline 2272 & $\mathrm{pCi} / \mathrm{L}$ & 5.1 & & & & U & & RIVER & & CUMULATIVE & UNFILTERED & 553 \\
\hline 2273 & $\mathrm{pCi} / \mathrm{L}$ & 0.09 & & & & & & SANITARY & & CUMULATIVE & UNFILTERED & 491 \\
\hline 2274 & $\mathrm{pCi} / \mathrm{L}$ & 170 & & & & & & SANITARY & & CUMULATIVE & UNFILTERED & 491 \\
\hline 2275 & $\mathrm{pCi} / \mathrm{L}$ & 31 & & & & U & & SANITARY & & CUMULATIVE & UNFILTERED & 491 \\
\hline 2276 & $\mathrm{pCi} / \mathrm{L}$ & 0.09 & & & & U & & SANITARY & & CUMULATIVE & UNFILTERED & 512 \\
\hline \begin{tabular}{|l|}
2277 \\
\end{tabular} & $\mathrm{pCi} / \mathrm{L}$ & 5.1 & & & & $U$ & & SANITARY & & CUMULATIVE & UNFILTERED & 512 \\
\hline 2278 & $\mathrm{pCi} / \mathrm{L}$ & 820 & & & & & & SANITARY & & CUMULATIVE & UNFILTERED & 512 \\
\hline 2279 & $\mathrm{pCi} / \mathrm{L}$ & 31 & & & & U & & SANITARY & & CUMULATIVE & UNFILTERED & 385 \\
\hline 2280 & $\mathrm{pCi} / \mathrm{L}$ & 0 & & & & & & RIVER & & CONTINUOUS & RESIN & 307 \\
\hline 2281 & $\mathrm{pCi} / \mathrm{L}$ & 0.00000402 & & & & & & RIVER & & CONTINUOUS & RESIN & 307 \\
\hline 2282 & $\mathrm{pCi} / \mathrm{L}$ & 0 & & & & & & RIVER & & CONTINUOUS & FILTER & 307 \\
\hline 2283 & $\mathrm{pCi} / \mathrm{L}$ & 0 & & & & & & RIVER & & CONTINUOUS & RESIN & 252 \\
\hline 2284 & $\mathrm{pCi} / \mathrm{L}$ & 0.00000184 & & & & & & RIVER & & CONTINUOUS & RESIN & 252 \\
\hline 2285 & $\mathrm{pCi} / \mathrm{L}$ & 0 & & & & & & RIVER & & CONTINUOUS & FILTER & 252 \\
\hline 2286 & $\mathrm{pCi} / \mathrm{L}$ & 280 & & & & U & & RIVER & & CUMULATIVE & UNFILTERED & 463 \\
\hline 2287 & $\mathrm{pCi} / \mathrm{L}$ & 31 & & & & U & & RIVER & & CUMULATIVE & UNFILTERED & 463 \\
\hline 2288 & $\mathrm{pCi} / \mathrm{L}$ & 240 & & & & & & RIVER & & RAW GRAB & UNFILTERED & 463 \\
\hline 2289 & $\mathrm{pCi} / \mathrm{L}$ & 330 & & & & $U_{1}$ & & RIVER & & RAW GRAB & UNFILTERED & 456 \\
\hline
\end{tabular}




\begin{tabular}{|c|c|c|c|c|c|c|c|c|c|c|c|c|}
\hline & $\mathrm{N}$ & $\mathrm{O}$ & $\mathrm{P}$ & $\mathrm{Q}$ & $\mathrm{R}$ & $\mathrm{s}$ & $\mathrm{T}$ & $U$ & $\mathrm{~V}$ & $\mathrm{~W}$ & $\mathrm{X}$ & $\mathrm{Y}$ \\
\hline 1 & ANAL_UNITS_RPTD & COUNTING_ERROR & TOTAL_ANAL_ERROR & MIN_DETECTABLE_ACTIVITY & PCNT_MOISTURE & LAB_QUALIFIER & REVIEW_QUALIFIER & SAMP_FROM & SAMP_ITEM & SAMP_MTHD & COLL_MTHD & SAMP_SITE_ID \\
\hline 2290 & $\mathrm{pCi} / \mathrm{L}$ & 1600 & & & & & & SANITARY & & CUMULATIVE & UNFILTERED & 512 \\
\hline 2291 & $\mathrm{pCi} / \mathrm{L}$ & 2100 & & & & & & SANITARY & & CUMULATIVE & UNFILTERED & 512 \\
\hline 2292 & $\mathrm{pCi} / \mathrm{L}$ & 0 & & & & & & RIVER & & CONTINUOUS & RESIN & 307 \\
\hline 2293 & $\mathrm{pCi} / \mathrm{L}$ & 0 & & & & & & RIVER & & CONTINUOUS & FILTER & 307 \\
\hline 2294 & $\mathrm{pCi} / \mathrm{L}$ & 0 & & & & & & RIVER & & CONTINUOUS & RESIN & 252 \\
\hline 2295 & $\mathrm{pCi} / \mathrm{L}$ & 0 & & & & & & RIVER & & CONTINUOUS & FILTER & 252 \\
\hline 2296 & $\mathrm{pCi} / \mathrm{L}$ & 880 & & & & & & SANITARY & & CUMULATIVE & UNFILTERED & 512 \\
\hline 2297 & $\mathrm{pCi} / \mathrm{L}$ & 230 & & & & & & RIVER & & RAW GRAB & UNFILTERED & 463 \\
\hline 2298 & $\mathrm{pCi} / \mathrm{L}$ & 240 & & & & U & & RIVER & & RAW GRAB & UNFILTERED & 456 \\
\hline 2299 & $\mathrm{pCi} / \mathrm{L}$ & 240 & & & & U & & RIVER & & CUMULATIVE & UNFILTERED & 553 \\
\hline 2300 & $\mathrm{pCi} / \mathrm{L}$ & 2.4 & & & & U & & RIVER & & CUMULATIVE & UNFILTERED & 553 \\
\hline 2301 & $\mathrm{pCi} / \mathrm{L}$ & 14 & & & & $U$ & & SANITARY & & CUMULATIVE & UNFILTERED & 491 \\
\hline 2302 & $\mathrm{pCi} / \mathrm{L}$ & 2.4 & & & & U & & SANITARY & & CUMULATIVE & UNFILTERED & 512 \\
\hline 2303 & $\mathrm{pCi} / \mathrm{L}$ & 620 & & & & & & SANITARY & & CUMULATIVE & UNFILTERED & 512 \\
\hline 2304 & $\mathrm{pCi} / \mathrm{L}$ & 14 & & & & $U$ & & SANITARY & & CUMULATIVE & UNFILTERED & 385 \\
\hline 2305 & $\mathrm{pCi} / \mathrm{L}$ & 0.00005 & & & & & & RIVER & & CONTINUOUS & RESIN & 307 \\
\hline 2306 & $\mathrm{pCi} / \mathrm{L}$ & 0 & & & & & & RIVER & & CONTINUOUS & RESIN & 307 \\
\hline 2307 & $\mathrm{pCi} / \mathrm{L}$ & 0.00000474 & & & & & & RIVER & & CONTINUOUS & RESIN & 307 \\
\hline 2308 & $\mathrm{pCi} / \mathrm{L}$ & 0 & & & & & & RIVER & & CONTINUOUS & FILTER & 307 \\
\hline 2309 & $\mathrm{pCi} / \mathrm{L}$ & 0.000002 & & & & & & RIVER & & CONTINUOUS & FILTER & 307 \\
\hline 2310 & $\mathrm{pCi} / \mathrm{L}$ & 0.000004 & & & & & & RIVER & & CONTINUOUS & FILTER & 252 \\
\hline 2311 & $\mathrm{pCi} / \mathrm{L}$ & 0 & & & & & & RIVER & & CONTINUOUS & FILTER & 252 \\
\hline 2312 & $\mathrm{pCi} / \mathrm{L}$ & 0.00006 & & & & & & RIVER & & CONTINUOUS & RESIN & 252 \\
\hline 2313 & $\mathrm{pCi} / \mathrm{L}$ & 0 & & & & & & RIVER & & CONTINUOUS & RESIN & 252 \\
\hline 2314 & $\mathrm{pCi} / \mathrm{L}$ & 0.00000096 & & & & & & RIVER & & CONTINUOUS & RESIN & 252 \\
\hline 2315 & $\mathrm{pCi} / \mathrm{L}$ & 210 & & & & & & RIVER & & CUMULATIVE & UNFILTERED & 463 \\
\hline 2316 & $\mathrm{pCi} / \mathrm{L}$ & 0.09 & & & & & & RIVER & & CUMULATIVE & UNFILTERED & 463 \\
\hline 2317 & $\mathrm{pCi} / \mathrm{L}$ & 14 & & & & U & & RIVER & & CUMULATIVE & UNFILTERED & 463 \\
\hline 2318 & $\mathrm{pCi} / \mathrm{L}$ & 140 & & & & & & RIVER & & CUMULATIVE & UNFILTERED & 463 \\
\hline 2319 & $\mathrm{pCi} / \mathrm{L}$ & 14 & & & & U & & RIVER & & CUMULATIVE & UNFILTERED & 463 \\
\hline 2320 & $\mathrm{pCi} / \mathrm{L}$ & 0.7 & & & & U & & RIVER & & CONTINUOUS & RESIN & 307 \\
\hline 2321 & $\mathrm{pCi} / \mathrm{L}$ & 0.02 & & & & $U$ & & RIVER & & CONTINUOUS & FILTER & 307 \\
\hline 2322 & $\mathrm{pCi} / \mathrm{L}$ & 0.002 & & & & & & RIVER & & CONTINUOUS & FILTER & 252 \\
\hline 2323 & $\mathrm{pCi} / \mathrm{L}$ & 0.07 & & & & $U$ & & RIVER & & CONTINUOUS & RESIN & 252 \\
\hline 2324 & $\mathrm{pCi} / \mathrm{L}$ & 0.11 & & & & & & RIVER & & CUMULATIVE & UNFILTERED & 553 \\
\hline 2325 & $\mathrm{pCi} / \mathrm{L}$ & 120 & & & & & & RIVER & & CUMULATIVE & UNFILTERED & 553 \\
\hline 2326 & $\mathrm{pCi} / \mathrm{L}$ & 2.4 & & & & U & & RIVER & & CUMULATIVE & UNFILTERED & 553 \\
\hline 2327 & $\mathrm{pCi} / \mathrm{L}$ & 14 & & & & U & & SANITARY & & CUMULATIVE & UNFILTERED & 491 \\
\hline 2328 & $\mathrm{pCi} / \mathrm{L}$ & 2.5 & & & & & & SANITARY & & CUMULATIVE & UNFILTERED & 512 \\
\hline 2329 & $\mathrm{pCi} / \mathrm{L}$ & 810 & & & & & & SANITARY & & CUMULATIVE & UNFILTERED & 512 \\
\hline 2330 & $\mathrm{pCi} / \mathrm{L}$ & 15 & & & & U & & SANITARY & & CUMULATIVE & UNFILTERED & 385 \\
\hline 2331 & $\mathrm{pCi} / \mathrm{L}$ & 0.02 & & & & U & & RIVER & & CONTINUOUS & RESIN & 307 \\
\hline 2332 & $\mathrm{pCi} / \mathrm{L}$ & 0.008 & & & & U & & RIVER & & CONTINUOUS & FILTER & 307 \\
\hline 2333 & $\mathrm{pCi} / \mathrm{L}$ & 0.02 & & & & & & RIVER & & CONTINUOUS & RESIN & 252 \\
\hline 2334 & $\mathrm{pCi} / \mathrm{L}$ & 0.005 & & & & $U$ & & RIVER & & CONTINUOUS & FILTER & 252 \\
\hline 2335 & $\mathrm{pCi} / \mathrm{L}$ & 0.012 & & & & U & & RIVER & & CONTINUOUS & FILTER & 307 \\
\hline 2336 & $\mathrm{pCi} / \mathrm{L}$ & 0.06 & & & & $U$ & & RIVER & & CONTINUOUS & RESIN & 307 \\
\hline 2337 & $\mathrm{pCi} / \mathrm{L}$ & 0.009 & & & & & & RIVER & & CONTINUOUS & RESIN & 252 \\
\hline 2338 & $\mathrm{pCi} / \mathrm{L}$ & 0.007 & & & & U & & RIVER & & CONTINUOUS & FILTER & 252 \\
\hline 2339 & $\mathrm{pCi} / \mathrm{L}$ & 0.09 & & & & & & SURFACE & & RAW GRAB & UNFILTERED & 509 \\
\hline 2340 & $\mathrm{pCi} / \mathrm{L}$ & 1.1 & & & & & & SURFACE & & RAW GRAB & UNFILTERED & 509 \\
\hline 2341 & $\mathrm{pCi} / \mathrm{L}$ & 0.098 & & & & & & SURFACE & & RAW GRAB & UNFILTERED & 146 \\
\hline
\end{tabular}




\begin{tabular}{|c|c|c|c|c|c|c|c|c|c|c|c|c|}
\hline & $\mathrm{N}$ & $\mathrm{O}$ & $\mathrm{P}$ & $\mathrm{Q}$ & $\mathrm{R}$ & $\mathrm{s}$ & $T$ & $\mathrm{U}$ & $\mathrm{V}$ & $\mathrm{W}$ & $\mathrm{X}$ & $\bar{Y}$ \\
\hline 1 & ANAL_UNITS_RPTD & COUNTING_ERROR & TOTAL_ANAL_ERROR & MIN_DETECTABLE_ACTIVITY & PCNT_MOISTURE & LAB_QUALIFIER & REVIEW_QUALIFIER & SAMP_FROM & SAMP_ITEM & SAMP_MTHD & COLL_MTHD & SAMP_SITE_ID \\
\hline 2342 & $\mathrm{pCi} / \mathrm{L}$ & 0.09 & & & & & & SURFACE & & RAW GRAB & UNFILTERED & 146 \\
\hline 2343 & $\mathrm{pCi} / \mathrm{L}$ & 3.7 & & & & & & SURFACE & & RAW GRAB & UNFILTERED & 147 \\
\hline 2344 & $\mathrm{pCi} / \mathrm{L}$ & 0.34 & & & & & & SURFACE & & RAW GRAB & UNFILTERED & 147 \\
\hline 2345 & $\mathrm{pCi} / \mathrm{L}$ & 2.4 & & & & $\mathrm{U}$ & & RIVER & & CUMULATIVE & UNFILTERED & 553 \\
\hline 2346 & $\mathrm{pCi} / \mathrm{L}$ & 220 & & & & & & RIVER & & CUMULATIVE & UNFILTERED & 553 \\
\hline 2347 & $\mathrm{pCi} / \mathrm{L}$ & 0.006 & & & & $U$ & & RIVER & & CONTINUOUS & FILTER & 307 \\
\hline 2348 & $\mathrm{pCi} / \mathrm{L}$ & 0.01 & & & & $\mathrm{U}$ & & RIVER & & CONTINUOUS & RESIN & 307 \\
\hline 2349 & $\mathrm{pCi} / \mathrm{L}$ & 0.004 & & & & & & RIVER & & CONTINUOUS & FILTER & 252 \\
\hline 2350 & $\mathrm{pCi} / \mathrm{L}$ & 0.01 & & & & & & RIVER & & CONTINUOUS & RESIN & 252 \\
\hline 2351 & $\mathrm{pCi} / \mathrm{L}$ & 170 & & & & & & RIVER & & CUMULATIVE & UNFILTERED & 463 \\
\hline 2353 & $\mathrm{pCi} / \mathrm{L}$ & 15 & & & & U & & RIVER & & CUMULATIVE & UNFILTERED & 463 \\
\hline 2354 & $\mathrm{pCi} / \mathrm{L}$ & 0.09 & & & & & & SANITARY & & CUMULATIVE & UNFILTERED & 491 \\
\hline 2355 & $\mathrm{pCi} / \mathrm{L}$ & 170 & & & & & & SANITARY & & CUMULATIVE & UNFILTERED & 491 \\
\hline 2356 & $\mathrm{pCi} / \mathrm{L}$ & 14 & & & & $U$ & & SANITARY & & CUMULATIVE & UNFILTERED & 491 \\
\hline 2357 & $\mathrm{pCi} / \mathrm{L}$ & 0.23 & & & & $U$ & & SANITARY & & CUMULATIVE & UNFILTERED & 512 \\
\hline 2358 & $\mathrm{pCi} / \mathrm{L}$ & 2.4 & & & & $U$ & & SANITARY & & CUMULATIVE & UNFILTERED & 512 \\
\hline 2359 & $\mathrm{pCi} / \mathrm{L}$ & 580 & & & & & & SANITARY & & CUMULATIVE & UNFILTERED & 512 \\
\hline 2360 & $\mathrm{pCi} / \mathrm{L}$ & 14 & & & & $U$ & & SANITARY & & CUMULATIVE & UNFILTERED & 385 \\
\hline \begin{tabular}{|l|}
2361 \\
\end{tabular} & $\mathrm{pCi} / \mathrm{L}$ & 0.12 & & & & $U$ & & RIVER & & CONTINUOUS & RESIN & 307 \\
\hline 2362 & $\mathrm{pCi} / \mathrm{L}$ & 0.02 & & & & $U$ & & RIVER & & CONTINUOUS & FILTER & 307 \\
\hline 2363 & $\mathrm{pCi} / \mathrm{L}$ & 0.34 & & & & $U$ & & RIVER & & CONTINUOUS & FILTER & 252 \\
\hline 2365 & $\mathrm{pCi} / \mathrm{L}$ & 0.0003 & & & & & & RIVER & & CONTINUOUS & RESIN & 307 \\
\hline 2366 & $\mathrm{pCi} / \mathrm{L}$ & 0.01 & & & & $U$ & & RIVER & & CONTINUOUS & RESIN & 307 \\
\hline 2367 & $\mathrm{pCi} / \mathrm{L}$ & & 0.000015 & & & & & RIVER & & CONTINUOUS & RESIN & 307 \\
\hline 2368 & $\mathrm{pCi} / \mathrm{L}$ & 0.0000022 & & & & & & RIVER & & CONTINUOUS & FILTER & 307 \\
\hline 2369 & $\mathrm{pCi} / \mathrm{L}$ & 0.006 & & & & $U$ & & RIVER & & CONTINUOUS & FILTER & 307 \\
\hline 2370 & $\mathrm{pCi} / \mathrm{L}$ & 0.000003 & & & & & & RIVER & & CONTINUOUS & FILTER & 252 \\
\hline 2371 & $\mathrm{pCi} / \mathrm{L}$ & 0.06 & & & & $U$ & & RIVER & & CONTINUOUS & FILTER & 252 \\
\hline 2372 & $\mathrm{pCi} / \mathrm{L}$ & 0.0001 & & & & & & RIVER & & CONTINUOUS & RESIN & 252 \\
\hline 2373 & $\mathrm{pCi} / \mathrm{L}$ & 0.02 & & & & $U$ & & RIVER & & CONTINUOUS & RESIN & 252 \\
\hline 2374 & $\mathrm{pCi} / \mathrm{L}$ & & 0.0000865 & & & & & RIVER & & CONTINUOUS & RESIN & 252 \\
\hline 2375 & $\mathrm{pCi} / \mathrm{L}$ & 2.4 & & & & $U$ & & RIVER & & CUMULATIVE & UNFILTERED & 553 \\
\hline 2376 & $\mathrm{pCi} / \mathrm{L}$ & 110 & & & & U & & RIVER & & CUMULATIVE & UNFILTERED & 553 \\
\hline 2377 & $\mathrm{pCi} / \mathrm{L}$ & 14 & & & & U & & SANITARY & & CUMULATIVE & UNFILTERED & 491 \\
\hline 2378 & $\mathrm{pCi} / \mathrm{L}$ & 2.4 & & & & $U$ & & SANITARY & & CUMULATIVE & UNFILTERED & 512 \\
\hline 2379 & $\mathrm{pCi} / \mathrm{L}$ & 430 & & & & & & SANITARY & & CUMULATIVE & UNFILTERED & 512 \\
\hline 2380 & $\mathrm{pCi} / \mathrm{L}$ & 14 & & & & $U$ & & SANITARY & & CUMULATIVE & UNFILTERED & 385 \\
\hline 2381 & $\mathrm{pCi} / \mathrm{L}$ & 210 & & & & & & RIVER & & CUMULATIVE & UNFILTERED & 463 \\
\hline 2382 & $\mathrm{pCi} / \mathrm{L}$ & 15 & & & & U & & RIVER & & CUMULATIVE & UNFILTERED & 463 \\
\hline 2383 & $\mathrm{pCi} / \mathrm{L}$ & 0.01 & & & & $U$ & & RIVER & & CONTINUOUS & RESIN & 307 \\
\hline 2384 & $\mathrm{pCi} / \mathrm{L}$ & 0.02 & & & & $U$ & & RIVER & & CONTINUOUS & FILTER & 307 \\
\hline 2385 & $\mathrm{pCi} / \mathrm{L}$ & 0.1 & & & & $U$ & & RIVER & & CONTINUOUS & RESIN & 252 \\
\hline 2386 & $\mathrm{pCi} / \mathrm{L}$ & 0.06 & & & & $U$ & & RIVER & & CONTINUOUS & FILTER & 252 \\
\hline 2387 & $\mathrm{pCi} / \mathrm{L}$ & 0.005 & & & & & & RIVER & & CONTINUOUS & FILTER & 307 \\
\hline 2388 & $\mathrm{pCi} / \mathrm{L}$ & 0.01 & & & & $U$ & & RIVER & & CONTINUOUS & RESIN & 307 \\
\hline 2389 & $\mathrm{pCi} / \mathrm{L}$ & 0.007 & & & & & & RIVER & & CONTINUOUS & RESIN & 252 \\
\hline 2390 & $\mathrm{pCi} / \mathrm{L}$ & 0.005 & & & & & & RIVER & & CONTINUOUS & FILTER & 252 \\
\hline 2391 & $\mathrm{pCi} / \mathrm{L}$ & 14 & & & & $U$ & & SANITARY & & CUMULATIVE & UNFILTERED & 491 \\
\hline 2392 & $\mathrm{pCi} / \mathrm{L}$ & 2.4 & & & & $U$ & & SANITARY & & CUMULATIVE & UNFILTERED & 512 \\
\hline 2393 & $\mathrm{pCi} / \mathrm{L}$ & 350 & & & & & & SANITARY & & CUMULATIVE & UNFILTERED & 512 \\
\hline
\end{tabular}




\begin{tabular}{|c|c|c|c|c|c|c|c|c|c|c|c|c|}
\hline & $\mathrm{N}$ & $\mathrm{O}$ & $\mathrm{P}$ & $\mathrm{Q}$ & $\mathrm{R}$ & $\mathrm{s}$ & $\mathrm{T}$ & $\mathrm{U}$ & $\mathrm{V}$ & $\mathrm{W}$ & $\mathrm{X}$ & $\mathrm{Y}$ \\
\hline 1 & ANAL_UNITS_RPTD & COUNTING_ERROR & TOTAL_ANAL_ERROR & MIN_DETECTABLE_ACTIVITY & PCNT_MOISTURE & LAB_QUALIFIER & REVIEW_QUALIFIER & SAMP_FROM & SAMP_ITEM & SAMP_MTHD & COLL_MTHD & SAMP_SITE_ID \\
\hline 2394 & $\mathrm{pCi} / \mathrm{L}$ & 14 & & & & U & & SANITARY & & CUMULATIVE & UNFILTERED & 385 \\
\hline 2395 & $\mathrm{pCi} / \mathrm{L}$ & 200 & & & & & & RIVER & & CUMULATIVE & UNFILTERED & 463 \\
\hline 2396 & $\mathrm{pCi} / \mathrm{L}$ & 0.11 & & & & & & RIVER & & CUMULATIVE & UNFILTERED & 463 \\
\hline 2397 & $\mathrm{pCi} / \mathrm{L}$ & 14 & & & & U & & RIVER & & CUMULATIVE & UNFILTERED & 463 \\
\hline 2398 & $\mathrm{pCi} / \mathrm{L}$ & 2.4 & & & & U & & RIVER & & CUMULATIVE & UNFILTERED & 553 \\
\hline 2399 & $\mathrm{pCi} / \mathrm{L}$ & 260 & & & & U & & RIVER & & CUMULATIVE & UNFILTERED & 553 \\
\hline 2400 & $\mathrm{pCi} / \mathrm{L}$ & 0.11 & & & & & & RIVER & & CUMULATIVE & UNFILTERED & 553 \\
\hline 2401 & $\mathrm{pCi} / \mathrm{L}$ & 0.02 & & & & U & & RIVER & & CONTINUOUS & RESIN & 307 \\
\hline 2402 & $\mathrm{pCi} / \mathrm{L}$ & 0.006 & & & & & & RIVER & & CONTINUOUS & FILTER & 307 \\
\hline 2403 & $\mathrm{pCi} / \mathrm{L}$ & 2.8 & & & & $\mathrm{U}$ & & RIVER & & CONTINUOUS & RESIN & 252 \\
\hline 2404 & $\mathrm{pCi} / \mathrm{L}$ & 0.1 & & & & & & RIVER & & CONTINUOUS & FILTER & 252 \\
\hline 2405 & $\mathrm{pCi} / \mathrm{L}$ & 0.097 & & & & & & SURFACE & & RAW GRAB & UNFILTERED & 509 \\
\hline 2406 & $\mathrm{pCi} / \mathrm{L}$ & 1.1 & & & & & & SURFACE & & RAW GRAB & UNFILTERED & 509 \\
\hline 2407 & $\mathrm{pCi} / \mathrm{L}$ & 1.4 & & & & & & SURFACE & & RAW GRAB & UNFILTERED & 146 \\
\hline 2408 & pCi/L & 0.097 & & & & & & SURFACE & & RAW GRAB & UNFILTERED & 146 \\
\hline 2409 & pCi/L & 2.7 & & & & & & SURFACE & & RAW GRAB & UNFILTERED & 147 \\
\hline 2410 & $\mathrm{pCi} / \mathrm{L}$ & 0.23 & & & & & & SURFACE & & RAW GRAB & UNFILTERED & 147 \\
\hline 2411 & $\mathrm{pCi} / \mathrm{L}$ & 160 & & & & U & & RIVER & & CUMULATIVE & UNFILTERED & 463 \\
\hline 2412 & $\mathrm{pCi} / \mathrm{L}$ & 15 & & & & $U$ & & RIVER & & CUMULATIVE & UNFILTERED & 463 \\
\hline 2413 & $\mathrm{pCi} / \mathrm{L}$ & 0.007 & & & & & & RIVER & & CONTINUOUS & RESIN & 307 \\
\hline 2414 & $\mathrm{pCi} / \mathrm{L}$ & 0.005 & & & & $U$ & & RIVER & & CONTINUOUS & FILTER & 307 \\
\hline 2415 & $\mathrm{pCi} / \mathrm{L}$ & 1.1 & & & & $U$ & & RIVER & & CONTINUOUS & RESIN & 252 \\
\hline 2416 & $\mathrm{pCi} / \mathrm{L}$ & 1 & & & & $U$ & & RIVER & & CONTINUOUS & FILTER & 252 \\
\hline 2417 & pCi/L & 190 & & & & & & RIVER & & CUMULATIVE & UNFILTERED & 553 \\
\hline 2418 & $\mathrm{pCi} / \mathrm{L}$ & 2.4 & & & & $U$ & & RIVER & & CUMULATIVE & UNFILTERED & 553 \\
\hline 2419 & $\mathrm{pCi} / \mathrm{L}$ & 0.14 & & & & U & & RIVER & & CONTINUOUS & RESIN & 307 \\
\hline 2420 & $\mathrm{pCi} / \mathrm{L}$ & 0.004 & & & & & & RIVER & & CONTINUOUS & FILTER & 307 \\
\hline 2421 & $\mathrm{pCi} / \mathrm{L}$ & 0.02 & & & & $U$ & & RIVER & & CONTINUOUS & RESIN & 252 \\
\hline 2422 & $\mathrm{pCi} / \mathrm{L}$ & 0.007 & & & & & & RIVER & & CONTINUOUS & FILTER & 252 \\
\hline 2423 & pCi/L & 0.09 & & & & & & SANITARY & & CUMULATIVE & UNFILTERED & 491 \\
\hline 2424 & $\mathrm{pCi} / \mathrm{L}$ & 240 & & & & & & SANITARY & & CUMULATIVE & UNFILTERED & 491 \\
\hline 2425 & $\mathrm{pCi} / \mathrm{L}$ & 15 & & & & $U$ & & SANITARY & & CUMULATIVE & UNFILTERED & 491 \\
\hline 2426 & $\mathrm{pCi} / \mathrm{L}$ & 0.23 & & & & U & & SANITARY & & CUMULATIVE & UNFILTERED & 512 \\
\hline 2427 & $\mathrm{pCi} / \mathrm{L}$ & 2.4 & & & & $U$ & & SANITARY & & CUMULATIVE & UNFILTERED & 512 \\
\hline 2428 & $\mathrm{pCi} / \mathrm{L}$ & 530 & & & & & & SANITARY & & CUMULATIVE & UNFILTERED & 512 \\
\hline 2429 & $\mathrm{pCi} / \mathrm{L}$ & 0.14 & & & & & & SANITARY & & CUMULATIVE & UNFILTERED & 512 \\
\hline 2430 & $\mathrm{pCi} / \mathrm{L}$ & 14 & & & & $U$ & & SANITARY & & CUMULATIVE & UNFILTERED & 385 \\
\hline 2431 & $\mathrm{pCi} / \mathrm{L}$ & 0.004 & & & & & & RIVER & & CONTINUOUS & FILTER & 307 \\
\hline 2432 & $\mathrm{pCi} / \mathrm{L}$ & 0.004 & & & & & & RIVER & & CONTINUOUS & RESIN & 307 \\
\hline 2433 & $\mathrm{pCi} / \mathrm{L}$ & 0.02 & & & & $U$ & & RIVER & & CONTINUOUS & RESIN & 252 \\
\hline 2434 & $\mathrm{pCi} / \mathrm{L}$ & 0.009 & & & & & & RIVER & & CONTINUOUS & FILTER & 252 \\
\hline 2435 & $\mathrm{pCi} / \mathrm{L}$ & 2.5 & & & & & & RIVER & & CUMULATIVE & UNFILTERED & 553 \\
\hline 2436 & pCi/L & 320 & & & & $U$ & & RIVER & & CUMULATIVE & UNFILTERED & 553 \\
\hline 2437 & $\mathrm{pCi} / \mathrm{L}$ & 15 & & & & $U$ & & SANITARY & & CUMULATIVE & UNFILTERED & 491 \\
\hline 2438 & pCi/L & 2.4 & & & & U & & SANITARY & & CUMULATIVE & UNFILTERED & 512 \\
\hline 2439 & $\mathrm{pCi} / \mathrm{L}$ & 340 & & & & & & SANITARY & & CUMULATIVE & UNFILTERED & 512 \\
\hline 2440 & pCi/L & 15 & & & & $U$ & & SANITARY & & CUMULATIVE & UNFILTERED & 385 \\
\hline 2441 & pCi/L & 0.00004 & & & & $U$ & & RIVER & & CONTINUOUS & RESIN & 307 \\
\hline 2442 & $\mathrm{pCi} / \mathrm{L}$ & 0.01 & & & & & & RIVER & & CONTINUOUS & RESIN & 307 \\
\hline 2443 & pCi/L & & 0.0000095 & & & & & RIVER & & CONTINUOUS & RESIN & 307 \\
\hline 2444 & $\mathrm{pCi} / \mathrm{L}$ & 0.005 & & & & & & RIVER & & CONTINUOUS & FILTER & 307 \\
\hline 2445 & $\mathrm{pCi} / \mathrm{L}$ & 0.0000034 & & & & & & RIVER & & CONTINUOUS & FILTER & 307 \\
\hline
\end{tabular}




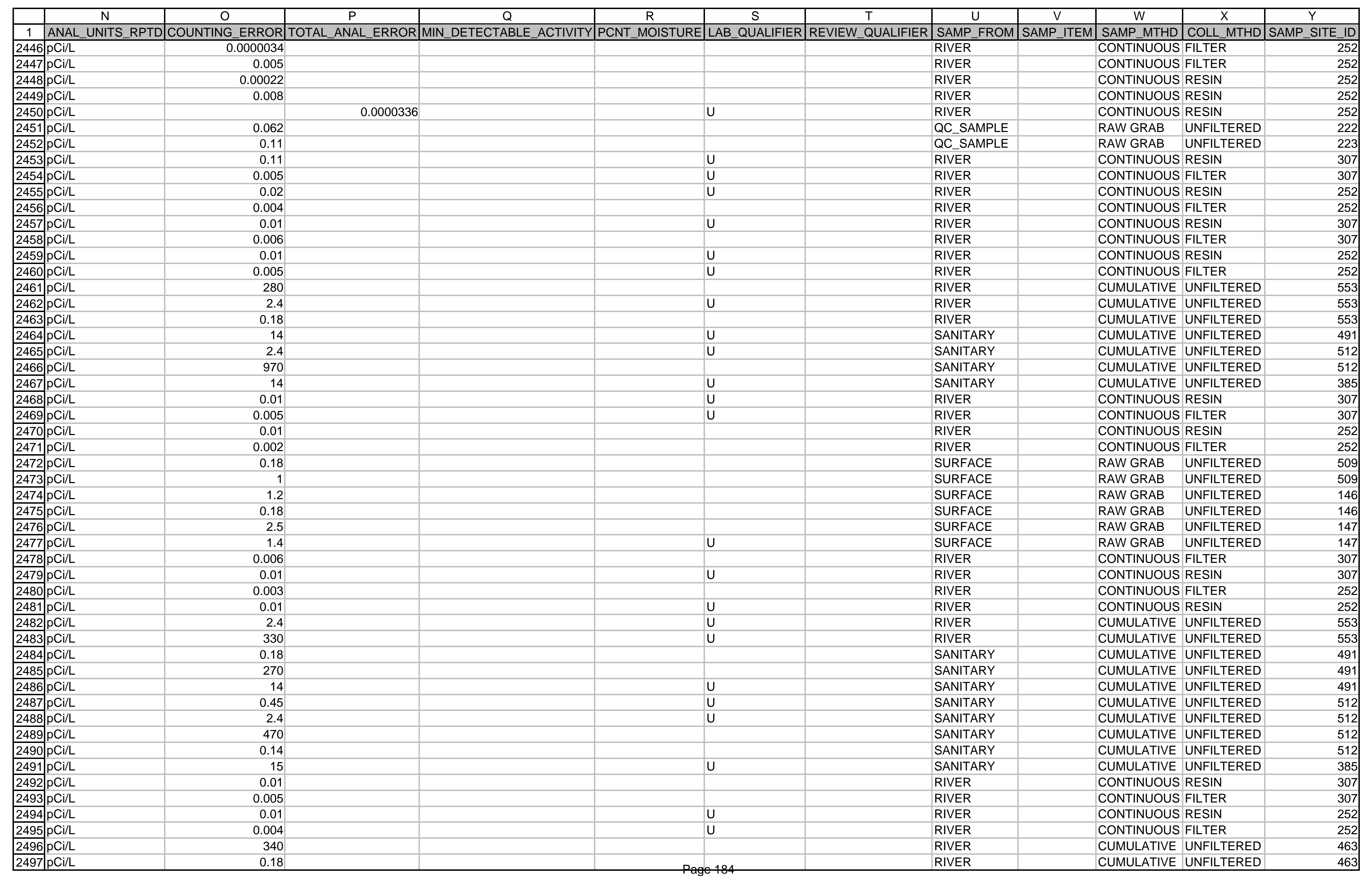




\begin{tabular}{|c|c|c|c|c|c|c|c|c|c|c|c|c|}
\hline & $\mathrm{N}$ & $\mathrm{O}$ & $\mathrm{P}$ & $\mathrm{Q}$ & $\mathrm{R}$ & $\mathrm{S}$ & $T$ & $\bar{U}$ & $\mathrm{~V}$ & $\mathrm{~W}$ & $\mathrm{X}$ & $\overline{\mathrm{Y}}$ \\
\hline 1 & ANAL_UNITS_RPTD & COUNTING_ERROR & $\mid$ TOTAL_ANAL_ERROR | $^{\prime}$ & MIN_DETECTABLE_ACTIVITY & PCNT_MOISTURE & LAB_QUALIFIER & REVIEW_QUALIFIER & SAMP_FROM & SAMP_ITEM & SAMP_MTHD & COLL_MTHD & SAMP_SITE_ID \\
\hline 2498 & $\mathrm{pCi} / \mathrm{L}$ & 15 & & & & $U$ & & RIVER & & CUMULATIVE & UNFILTERED & 463 \\
\hline 2499 & $\mathrm{pCi} / \mathrm{L}$ & 0.006 & & & & & & RIVER & & CONTINUOUS & FILTER & 307 \\
\hline 2500 & $\mathrm{pCi} / \mathrm{L}$ & 0.01 & & & & & & RIVER & & CONTINUOUS & RESIN & 307 \\
\hline 2501 & $\mathrm{pCi} / \mathrm{L}$ & 0.01 & & & & $\mathrm{U}$ & & RIVER & & CONTINUOUS & RESIN & 252 \\
\hline 2502 & $\mathrm{pCi} / \mathrm{L}$ & 0.004 & & & & & & RIVER & & CONTINUOUS & FILTER & 252 \\
\hline 2503 & $\mathrm{pCi} / \mathrm{L}$ & 15 & & & & u & & SANITARY & & CUMULATIVE & UNFILTERED & 385 \\
\hline 2504 & $\mathrm{pCi} / \mathrm{L}$ & 2.4 & & & & $\mathrm{U}$ & & RIVER & & CUMULATIVE & UNFILTERED & 553 \\
\hline 2505 & $\mathrm{pCi} / \mathrm{L}$ & 200 & & & & $\mathrm{U}$ & & RIVER & & CUMULATIVE & UNFILTERED & 553 \\
\hline 2506 & $\mathrm{pCi} / \mathrm{L}$ & 14 & & & & u & & SANITARY & & CUMULATIVE & UNFILTERED & 491 \\
\hline 2507 & $\mathrm{pCi} / \mathrm{L}$ & 2.4 & & & & u & & SANITARY & & CUMULATIVE & UNFILTERED & 512 \\
\hline 2508 & $\mathrm{pCi} / \mathrm{L}$ & 850 & & & & & & SANITARY & & CUMULATIVE & UNFILTERED & 512 \\
\hline 2509 & $\mathrm{pCi} / \mathrm{L}$ & 0.01 & & & & U & & RIVER & & CONTINUOUS & RESIN & 307 \\
\hline 2510 & $\mathrm{pCi} / \mathrm{L}$ & 0.005 & & & & & & RIVER & & CONTINUOUS & FILTER & 307 \\
\hline 2511 & $\mathrm{pCi} / \mathrm{L}$ & 0.01 & & & & U & & RIVER & & CONTINUOUS & FILTER & 252 \\
\hline 2512 & $\mathrm{pCi} / \mathrm{L}$ & 0.01 & & & & U & & RIVER & & CONTINUOUS & RESIN & 252 \\
\hline 2513 & $\mathrm{pCi} / \mathrm{L}$ & 170 & & & & $U$ & & RIVER & & CUMULATIVE & UNFILTERED & 463 \\
\hline 2514 & $\mathrm{pCi} / \mathrm{L}$ & 14 & & & & $U$ & & RIVER & & CUMULATIVE & UNFILTERED & 463 \\
\hline 2515 & $\mathrm{pCi} / \mathrm{L}$ & 0.00003 & & & & U & & RIVER & & CONTINUOUS & RESIN & 307 \\
\hline 2516 & $\mathrm{pCi} / \mathrm{L}$ & & 0.00001 & & & & & RIVER & & CONTINUOUS & RESIN & 307 \\
\hline 2517 & $\mathrm{pCi} / \mathrm{L}$ & 0.000004 & & & & & & RIVER & & CONTINUOUS & FILTER & 307 \\
\hline 2518 & $\mathrm{pCi} / \mathrm{L}$ & 0.00007 & & & & & & RIVER & & CONTINUOUS & RESIN & 252 \\
\hline 2519 & $\mathrm{pCi} / \mathrm{L}$ & & 0.00000084 & & & & & RIVER & & CONTINUOUS & RESIN & 252 \\
\hline 2520 & $\mathrm{pCi} / \mathrm{L}$ & 0.000003 & & & & & & RIVER & & CONTINUOUS & FILTER & 252 \\
\hline 2521 & $\mathrm{pCi} / \mathrm{L}$ & 0.003 & & & & U & & RIVER & & CONTINUOUS & FILTER & 307 \\
\hline 2522 & $\mathrm{pCi} / \mathrm{L}$ & 0.009 & & & & & & RIVER & & CONTINUOUS & RESIN & 307 \\
\hline 2523 & $\mathrm{pCi} / \mathrm{L}$ & 0.01 & & & & & & RIVER & & CONTINUOUS & RESIN & 307 \\
\hline 2524 & $\mathrm{pCi} / \mathrm{L}$ & 0.004 & & & & & & RIVER & & CONTINUOUS & FILTER & 307 \\
\hline 2525 & $\mathrm{pCi} / \mathrm{L}$ & 0.008 & & & & & & RIVER & & CONTINUOUS & FILTER & 252 \\
\hline 2526 & $\mathrm{pCi} / \mathrm{L}$ & 0.01 & & & & & & RIVER & & CONTINUOUS & RESIN & 252 \\
\hline 2527 & $\mathrm{pCi} / \mathrm{L}$ & 110 & & & & & & RIVER & & CUMULATIVE & UNFILTERED & 463 \\
\hline 2528 & $\mathrm{pCi} / \mathrm{L}$ & 14 & & & & U & & RIVER & & CUMULATIVE & UNFILTERED & 463 \\
\hline 2529 & $\mathrm{pCi} / \mathrm{L}$ & 2.4 & & & & U & & RIVER & & CUMULATIVE & UNFILTERED & 553 \\
\hline 2530 & $\mathrm{pCi} / \mathrm{L}$ & 0.19 & & & & & & RIVER & & CUMULATIVE & UNFILTERED & 553 \\
\hline 2531 & $\mathrm{pCi} / \mathrm{L}$ & 150 & & & & & & RIVER & & CUMULATIVE & UNFILTERED & 553 \\
\hline 2532 & $\mathrm{pCi} / \mathrm{L}$ & 14 & & & & U & & SANITARY & & CUMULATIVE & UNFILTERED & 491 \\
\hline 2533 & $\mathrm{pCi} / \mathrm{L}$ & 2.4 & & & & $U$ & & SANITARY & & CUMULATIVE & UNFILTERED & 512 \\
\hline 2534 & $\mathrm{pCi} / \mathrm{L}$ & 570 & & & & & & SANITARY & & CUMULATIVE & UNFILTERED & 512 \\
\hline 2535 & $\mathrm{pCi} / \mathrm{L}$ & 14 & & & & U & & SANITARY & & CUMULATIVE & UNFILTERED & 385 \\
\hline 2536 & $\mathrm{pCi} / \mathrm{L}$ & 0.18 & & & & & & SURFACE & & RAW GRAB & UNFILTERED & 509 \\
\hline 2537 & $\mathrm{pCi} / \mathrm{L}$ & 1.1 & & & & & & SURFACE & & RAW GRAB & UNFILTERED & 509 \\
\hline 2538 & $\mathrm{pCi} / \mathrm{L}$ & 1.2 & & & & & & SURFACE & & RAW GRAB & UNFILTERED & 146 \\
\hline 2539 & $\mathrm{pCi} / \mathrm{L}$ & 0.18 & & & & & & SURFACE & & RAW GRAB & UNFILTERED & 146 \\
\hline 2540 & $\mathrm{pCi} / \mathrm{L}$ & 7.2 & & & & $U$ & & SURFACE & & RAW GRAB & UNFILTERED & 147 \\
\hline 2541 & $\mathrm{pCi} / \mathrm{L}$ & 1.4 & & & & & & SURFACE & & RAW GRAB & UNFILTERED & 147 \\
\hline 2542 & $\mathrm{pCi} / \mathrm{L}$ & 0.004 & & & & & & RIVER & & CONTINUOUS & RESIN & 307 \\
\hline 2543 & $\mathrm{pCi} / \mathrm{L}$ & 0.004 & & & & & & RIVER & & CONTINUOUS & FILTER & 307 \\
\hline 2544 & $\mathrm{pCi} / \mathrm{L}$ & 150 & & & & & & RIVER & & CUMULATIVE & UNFILTERED & 553 \\
\hline 2545 & $\mathrm{pCi} / \mathrm{L}$ & 2.4 & & & & U & & RIVER & & CUMULATIVE & UNFILTERED & 553 \\
\hline 2546 & $\mathrm{pCi} / \mathrm{L}$ & 0.19 & & & & & & SANITARY & & CUMULATIVE & UNFILTERED & 491 \\
\hline 2547 & $\mathrm{pCi} / \mathrm{L}$ & 360 & & & & U & & SANITARY & & CUMULATIVE & UNFILTERED & 491 \\
\hline 2548 & $\mathrm{pCi} / \mathrm{L}$ & 15 & & & & U & & SANITARY & & CUMULATIVE & UNFILTERED & 491 \\
\hline 2549 & $\mathrm{pCi} / \mathrm{L}$ & 0.45 & & & & 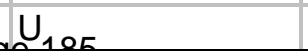 & & SANITARY & & CUMULATIVE & UNFILTERED & 512 \\
\hline
\end{tabular}




\begin{tabular}{|c|c|c|c|c|c|c|c|c|c|c|c|c|}
\hline & $\mathrm{N}$ & $\mathrm{O}$ & $\mathrm{P}$ & $\mathrm{Q}$ & $\mathrm{R}$ & $\mathrm{s}$ & $T$ & $\mathrm{U}$ & $\mathrm{V}$ & $\mathrm{W}$ & $\mathrm{X}$ & $\bar{Y}$ \\
\hline 1 & ANAL_UNITS_RPTD & COUNTING_ERROR & TOTAL_ANAL_ERROR & MIN_DETECTABLE_ACTIVITY & PCNT_MOISTURE & LAB_QUALIFIER & REVIEW_QUALIFIER & SAMP_FROM & SAMP_ITEM & SAMP_MTHD & COLL_MTHD & SAMP_SITE_ID \\
\hline 2550 & pCi/L & 2.4 & & & & $U$ & & SANITARY & & CUMULATIVE & UNFILTERED & 512 \\
\hline 2551 & $\mathrm{pCi} / \mathrm{L}$ & 440 & & & & & & SANITARY & & CUMULATIVE & UNFILTERED & 512 \\
\hline 2552 & $\mathrm{pCi} / \mathrm{L}$ & 0.87 & & & & U & & SANITARY & & CUMULATIVE & UNFILTERED & 512 \\
\hline 2553 & $\mathrm{pCi} / \mathrm{L}$ & 14 & & & & $\mathrm{U}$ & & SANITARY & & CUMULATIVE & UNFILTERED & 385 \\
\hline 2554 & $\mathrm{pCi} / \mathrm{L}$ & 0.006 & & & & & & RIVER & & CONTINUOUS & FILTER & 307 \\
\hline 2555 & $\mathrm{pCi} / \mathrm{L}$ & 0.01 & & & & & & RIVER & & CONTINUOUS & RESIN & 307 \\
\hline 2556 & $\mathrm{pCi} / \mathrm{L}$ & 0.006 & & & & & & RIVER & & CONTINUOUS & FILTER & 252 \\
\hline 2557 & $\mathrm{pCi} / \mathrm{L}$ & 0.01 & & & & & & RIVER & & CONTINUOUS & RESIN & 252 \\
\hline 2558 & $\mathrm{pCi} / \mathrm{L}$ & 190 & & & & & & RIVER & & CUMULATIVE & UNFILTERED & 463 \\
\hline 2559 & $\mathrm{pCi} / \mathrm{L}$ & 0.18 & & & & & & RIVER & & CUMULATIVE & UNFILTERED & 463 \\
\hline 2561 & $\mathrm{pCi} / \mathrm{L}$ & 0.003 & & & & & & RIVER & & CONTINUOUS & FILTER & 307 \\
\hline 2562 & $\mathrm{pCi} / \mathrm{L}$ & 0.01 & & & & $U$ & & RIVER & & CONTINUOUS & RESIN & 307 \\
\hline 2563 & $\mathrm{pCi} / \mathrm{L}$ & 0.009 & & & & $U$ & & RIVER & & CONTINUOUS & RESIN & 252 \\
\hline 2564 & $\mathrm{pCi} / \mathrm{L}$ & 0.003 & & & & & & RIVER & & CONTINUOUS & FILTER & 252 \\
\hline 2565 & $\mathrm{pCi} / \mathrm{L}$ & 0.02 & & & & $U$ & & RIVER & & CONTINUOUS & RESIN & 307 \\
\hline 2566 & $\mathrm{pCi} / \mathrm{L}$ & 0.02 & & & & $U$ & & RIVER & & CONTINUOUS & FILTER & 307 \\
\hline $2567 \mid$ & $\mathrm{pCi} / \mathrm{L}$ & 0.03 & & & & U & & RIVER & & CONTINUOUS & RESIN & 252 \\
\hline 2568 & $\mathrm{pCi} / \mathrm{L}$ & 0.004 & & & & & & RIVER & & CONTINUOUS & FILTER & 252 \\
\hline 2569 & $\mathrm{pCi} / \mathrm{L}$ & 140 & & & & & & RIVER & & CUMULATIVE & UNFILTERED & 463 \\
\hline 2570 & $\mathrm{pCi} / \mathrm{L}$ & 15 & & & & $U$ & & RIVER & & CUMULATIVE & UNFILTERED & 463 \\
\hline \begin{tabular}{|l|}
2571 \\
\end{tabular} & $\mathrm{pCi} / \mathrm{L}$ & 2.4 & & & & $U$ & & RIVER & & CUMULATIVE & UNFILTERED & 553 \\
\hline 2573 & $\mathrm{pCi} / \mathrm{L}$ & 15 & & & & & & SANITARY & & CUMULATIVE & UNFILTERED & 491 \\
\hline 2574 & $\mathrm{pCi} / \mathrm{L}$ & 2.4 & & & & $U$ & & SANITARY & & CUMULATIVE & UNFILTERED & 512 \\
\hline 2575 & $\mathrm{pCi} / \mathrm{L}$ & 750 & & & & & & SANITARY & & CUMULATIVE & UNFILTERED & 512 \\
\hline 2576 & $\mathrm{pCi} / \mathrm{L}$ & 14 & & & & U & & SANITARY & & CUMULATIVE & UNFILTERED & 385 \\
\hline \begin{tabular}{|l|}
2577 \\
\end{tabular} & $\mathrm{pCi} / \mathrm{L}$ & 0.00012 & & & & & & RIVER & & CONTINUOUS & RESIN & 307 \\
\hline 2578 & $\mathrm{pCi} / \mathrm{L}$ & 0.13 & & & & U & & RIVER & & CONTINUOUS & RESIN & 307 \\
\hline 2579 & $\mathrm{pCi} / \mathrm{L}$ & & 0.0000096 & & & & & RIVER & & CONTINUOUS & RESIN & 307 \\
\hline 2580 & $\mathrm{pCi} / \mathrm{L}$ & 0.006 & & & & & & RIVER & & CONTINUOUS & FILTER & 307 \\
\hline \begin{tabular}{|l|}
2581 \\
\end{tabular} & $\mathrm{pCi} / \mathrm{L}$ & 0.0000022 & & & & & & RIVER & & CONTINUOUS & FILTER & 307 \\
\hline \begin{tabular}{|l|}
2582 \\
\end{tabular} & $\mathrm{pCi} / \mathrm{L}$ & 0.006 & & & & & & RIVER & & CONTINUOUS & RESIN & 252 \\
\hline 2583 & $\mathrm{pCi} / \mathrm{L}$ & 0.00015 & & & & $U$ & & RIVER & & CONTINUOUS & RESIN & 252 \\
\hline 2584 & $\mathrm{pCi} / \mathrm{L}$ & & 0.000000948 & & & & & RIVER & & CONTINUOUS & RESIN & 252 \\
\hline 2585 & $\mathrm{pCi} / \mathrm{L}$ & 0.0000032 & & & & & & RIVER & & CONTINUOUS & FILTER & 252 \\
\hline 2586 & $\mathrm{pCi} / \mathrm{L}$ & 0.05 & & & & $U$ & & RIVER & & CONTINUOUS & FILTER & 252 \\
\hline 2587 & $\mathrm{pCi} / \mathrm{L}$ & 15 & & & & U & & SANITARY & & CUMULATIVE & UNFILTERED & 491 \\
\hline 2588 & $\mathrm{pCi} / \mathrm{L}$ & 0.04 & & & & & & RIVER & & CONTINUOUS & RESIN & 307 \\
\hline 2589 & $\mathrm{pCi} / \mathrm{L}$ & 0.009 & & & & & & RIVER & & CONTINUOUS & FILTER & 307 \\
\hline 2590 & $\mathrm{pCi} / \mathrm{L}$ & 0.26 & & & & U & & RIVER & & CUMULATIVE & UNFILTERED & 553 \\
\hline 2591 & $\mathrm{pCi} / \mathrm{L}$ & 71 & & & & & & RIVER & & CUMULATIVE & UNFILTERED & 553 \\
\hline 2592 & $\mathrm{pCi} / \mathrm{L}$ & 15 & & & & $U$ & & RIVER & & CUMULATIVE & UNFILTERED & 553 \\
\hline 2593 & $\mathrm{pCi} / \mathrm{L}$ & 15 & & & & U & & SANITARY & & CUMULATIVE & UNFILTERED & 491 \\
\hline 2594 & $\mathrm{pCi} / \mathrm{L}$ & 2.4 & & & & $U$ & & SANITARY & & CUMULATIVE & UNFILTERED & 512 \\
\hline 2595 & $\mathrm{pCi} / \mathrm{L}$ & 1000 & & & & & & SANITARY & & CUMULATIVE & UNFILTERED & 512 \\
\hline \begin{tabular}{|l|}
2596 \\
\end{tabular} & $\mathrm{pCi} / \mathrm{L}$ & 14 & & & & $U$ & & SANITARY & & CUMULATIVE & UNFILTERED & 385 \\
\hline \begin{tabular}{|l|}
2597 \\
\end{tabular} & $\mathrm{pCi} / \mathrm{L}$ & 290 & & & & & & RIVER & & CUMULATIVE & UNFILTERED & 463 \\
\hline 2598 & $\mathrm{pCi} / \mathrm{L}$ & 14 & & & & U & & RIVER & & CUMULATIVE & UNFILTERED & 463 \\
\hline 2599 & $\mathrm{pCi} / \mathrm{L}$ & 0.004 & & & & & & RIVER & & CONTINUOUS & FILTER & 307 \\
\hline 2600 & $\mathrm{pCi} / \mathrm{L}$ & 0.01 & & & & $U$ & & RIVER & & CONTINUOUS & RESIN & 307 \\
\hline 2601 & $\mathrm{pCi} / \mathrm{L}$ & 0.45 & & & & & & SURFACE & & RAW GRAB & UNFILTERED & 509 \\
\hline
\end{tabular}




\begin{tabular}{|c|c|c|c|c|c|c|c|c|c|c|c|c|}
\hline & $\mathrm{N}$ & $\mathrm{O}$ & $\mathrm{P}$ & $\mathrm{Q}$ & $\mathrm{R}$ & $\mathrm{s}$ & $\mathrm{T}$ & $\mathrm{u}$ & $\mathrm{V}$ & $\mathrm{w}$ & $\mathrm{x}$ & $\bar{Y}$ \\
\hline 1 & ANAL_UNITS_RPTD & COUNTING_ERROR & TOTAL_ANAL_ERROR & MIN_DETECTABLE_ACTIVITY & PCNT_MOISTURE & LAB_QUALIFIER & REVIEW_QUALIFIER & SAMP_FROM & SAMP_ITEM & SAMP_MTHD & COLL_MTHD & SAMP_SITE_ID \\
\hline 2602 & $\mathrm{pCi} / \mathrm{L}$ & 2.5 & & & & & & SURFACE & & RAW GRAB & UNFILTERED & 509 \\
\hline 2603 & $\mathrm{pCi} / \mathrm{L}$ & 16 & & & & & & SURFACE & & RAW GRAB & UNFILTERED & 146 \\
\hline 2604 & $\mathrm{pCi} / \mathrm{L}$ & 0.18 & & & & & & SURFACE & & RAW GRAB & UNFILTERED & 146 \\
\hline 2605 & $\mathrm{pCi} / \mathrm{L}$ & 7.5 & & & & & & SURFACE & & RAW GRAB & UNFILTERED & 147 \\
\hline 2606 & $\mathrm{pCi} / \mathrm{L}$ & 1.4 & & & & $\mathrm{U}$ & & SURFACE & & RAW GRAB & UNFILTERED & 147 \\
\hline 2607 & $\mathrm{pCi} / \mathrm{L}$ & 0.02 & & & & & & RIVER & & CONTINUOUS I & RESIN & 307 \\
\hline 2608 & $\mathrm{pCi} / \mathrm{L}$ & 0.01 & & & & & & RIVER & & CONTINUOUS I & FILTER & 307 \\
\hline 2609 & $\mathrm{pCi} / \mathrm{L}$ & 0.01 & & & & & & RIVER & & CONTINUOUS I & RESIN & 252 \\
\hline 2610 & $\mathrm{pCi} / \mathrm{L}$ & 0.005 & & & & & & RIVER & & CONTINUOUS & FILTER & 252 \\
\hline 2611 & $\mathrm{pCi} / \mathrm{L}$ & 2.4 & & & & $\mathrm{U}$ & & RIVER & & CUMULATIVE & UNFILTERED & 553 \\
\hline 2612 & $\mathrm{pCi} / \mathrm{L}$ & 120 & & & & $\mathrm{U}$ & & RIVER & & CUMULATIVE & UNFILTERED & 553 \\
\hline 2613 & $\mathrm{pCi} / \mathrm{L}$ & 0.18 & & & & & & SANITARY & & CUMULATIVE & UNFILTERED & 491 \\
\hline 2614 & $\mathrm{pCi} / \mathrm{L}$ & 120 & & & & & & SANITARY & & CUMULATIVE & UNFILTERED & 491 \\
\hline 2615 & $\mathrm{pCi} / \mathrm{L}$ & 14 & & & & $U$ & & SANITARY & & CUMULATIVE & UNFILTERED & 491 \\
\hline 2616 & $\mathrm{pCi} / \mathrm{L}$ & 0.48 & & & & U & & SANITARY & & CUMULATIVE & UNFILTERED & 512 \\
\hline \begin{tabular}{|l|l|}
2617 \\
\end{tabular} & $\mathrm{pCi} / \mathrm{L}$ & 2.4 & & & & U & & SANITARY & & CUMULATIVE & UNFILTERED & 512 \\
\hline 2618 & $\mathrm{pCi} / \mathrm{L}$ & 780 & & & & & & SANITARY & & CUMULATIVE & UNFILTERED & 512 \\
\hline \begin{tabular}{|l|}
2619 \\
\end{tabular} & $\mathrm{pCi} / \mathrm{L}$ & 0.8 & & & & & & SANITARY & & CUMULATIVE & UNFILTERED & 512 \\
\hline 2620 & $\mathrm{pCi} / \mathrm{L}$ & 14 & & & & $U$ & & SANITARY & & CUMULATIVE & UNFILTERED & 385 \\
\hline 2621 & $\mathrm{pCi} / \mathrm{L}$ & 110 & & & & & & RIVER & & CUMULATIVE & UNFILTERED & 463 \\
\hline 2622 & $\mathrm{pCi} / \mathrm{L}$ & 0.18 & & & & & & RIVER & & CUMULATIVE & UNFILTERED & 463 \\
\hline 2623 & $\mathrm{pCi} / \mathrm{L}$ & 15 & & & & $U$ & & RIVER & & CUMULATIVE & UNFILTERED & 463 \\
\hline 2624 & $\mathrm{pCi} / \mathrm{L}$ & 0.007 & & & & & & RIVER & & CONTINUOUS & FILTER & 307 \\
\hline 2625 & $\mathrm{pCi} / \mathrm{L}$ & 0.01 & & & & U & & RIVER & & CONTINUOUS & RESIN & 307 \\
\hline 2626 & $\mathrm{pCi} / \mathrm{L}$ & 0.01 & & & & & & RIVER & & CONTINUOUS & RESIN & 252 \\
\hline \begin{tabular}{|l|l|}
2627 \\
\end{tabular} & $\mathrm{pCi} / \mathrm{L}$ & 0.003 & & & & & & RIVER & & CONTINUOUS & FILTER & 252 \\
\hline 2628 & $\mathrm{pCi} / \mathrm{L}$ & 0.01 & & & & $U$ & & RIVER & & CONTINUOUS & RESIN & 307 \\
\hline 2629 & $\mathrm{pCi} / \mathrm{L}$ & 0.005 & & & & & & RIVER & & CONTINUOUS & FILTER & 307 \\
\hline 2630 & $\mathrm{pCi} / \mathrm{L}$ & 0.004 & & & & & & RIVER & & CONTINUOUS & FILTER & 252 \\
\hline 2631 & $\mathrm{pCi} / \mathrm{L}$ & 0.02 & & & & U & & RIVER & & CONTINUOUS & RESIN & 252 \\
\hline 2632 & $\mathrm{pCi} / \mathrm{L}$ & 150 & & & & & & RIVER & & CUMULATIVE & UNFILTERED & 463 \\
\hline 2633 & $\mathrm{pCi} / \mathrm{L}$ & 14 & & & & $U$ & & RIVER & & CUMULATIVE & UNFILTERED & 463 \\
\hline 2634 & $\mathrm{pCi} / \mathrm{L}$ & 110 & & & & & & RIVER & & CUMULATIVE & UNFILTERED & 553 \\
\hline 2635 & $\mathrm{pCi} / \mathrm{L}$ & 1.5 & & & & $U$ & & RIVER & & CUMULATIVE & UNFILTERED & 553 \\
\hline 2636 & $\mathrm{pCi} / \mathrm{L}$ & 1.4 & & & & $U$ & & SANITARY & & CUMULATIVE & UNFILTERED & 491 \\
\hline 2637 & $\mathrm{pCi} / \mathrm{L}$ & 1.4 & & & & U & & SANITARY & & CUMULATIVE & UNFILTERED & 512 \\
\hline 2638 & $\mathrm{pCi} / \mathrm{L}$ & 940 & & & & & & SANITARY & & CUMULATIVE & UNFILTERED & 512 \\
\hline 2639 & $\mathrm{pCi} / \mathrm{L}$ & 1.5 & & & & $U$ & & SANITARY & & CUMULATIVE & UNFILTERED & 385 \\
\hline 2640 & $\mathrm{pCi} / \mathrm{L}$ & 0.0000078 & & & & & & RIVER & & CONTINUOUS & FILTER & 307 \\
\hline 2641 & $\mathrm{pCi} / \mathrm{L}$ & 0.005 & & & & $U$ & & RIVER & & CONTINUOUS & FILTER & 307 \\
\hline 2642 & $\mathrm{pCi} / \mathrm{L}$ & 0.0000247 & & & & & & RIVER & & CONTINUOUS & RESIN & 307 \\
\hline 2643 & $\mathrm{pCi} / \mathrm{L}$ & 0.01 & & & & U & & RIVER & & CONTINUOUS & RESIN & 307 \\
\hline 2644 & $\mathrm{pCi} / \mathrm{L}$ & & 0.000032 & & & & & RIVER & & CONTINUOUS & RESIN & 307 \\
\hline 2645 & $\mathrm{pCi} / \mathrm{L}$ & 0.01 & & & & U & & RIVER & & CONTINUOUS & RESIN & 252 \\
\hline 2646 & $\mathrm{pCi} / \mathrm{L}$ & 0.000013 & & & & & & RIVER & & CONTINUOUS & RESIN & 252 \\
\hline 2647 & $\mathrm{pCi} / \mathrm{L}$ & & 0.0000017 & & & & & RIVER & & CONTINUOUS & RESIN & 252 \\
\hline 2648 & $\mathrm{pCi} / \mathrm{L}$ & 0.00000012 & & & & & & RIVER & & CONTINUOUS & FILTER & 252 \\
\hline 2649 & $\mathrm{pCi} / \mathrm{L}$ & 0.004 & & & & & & RIVER & & CONTINUOUS & FILTER & 252 \\
\hline 2650 & $\mathrm{pCi} / \mathrm{L}$ & 0.03 & & & & $U$ & & RIVER & & CONTINUOUS & RESIN & 307 \\
\hline 2651 & $\mathrm{pCi} / \mathrm{L}$ & 0.02 & & & & U & & RIVER & & CONTINUOUS & FILTER & 307 \\
\hline 2652 & $\mathrm{pCi} / \mathrm{L}$ & 0.02 & & & & $U$ & & RIVER & & CONTINUOUS & RESIN & 252 \\
\hline 2653 & $\mathrm{pCi} / \mathrm{L}$ & 0.005 & & & & $U_{1}$ & & RIVER & & CONTINUOUS & FILTER & 252 \\
\hline
\end{tabular}




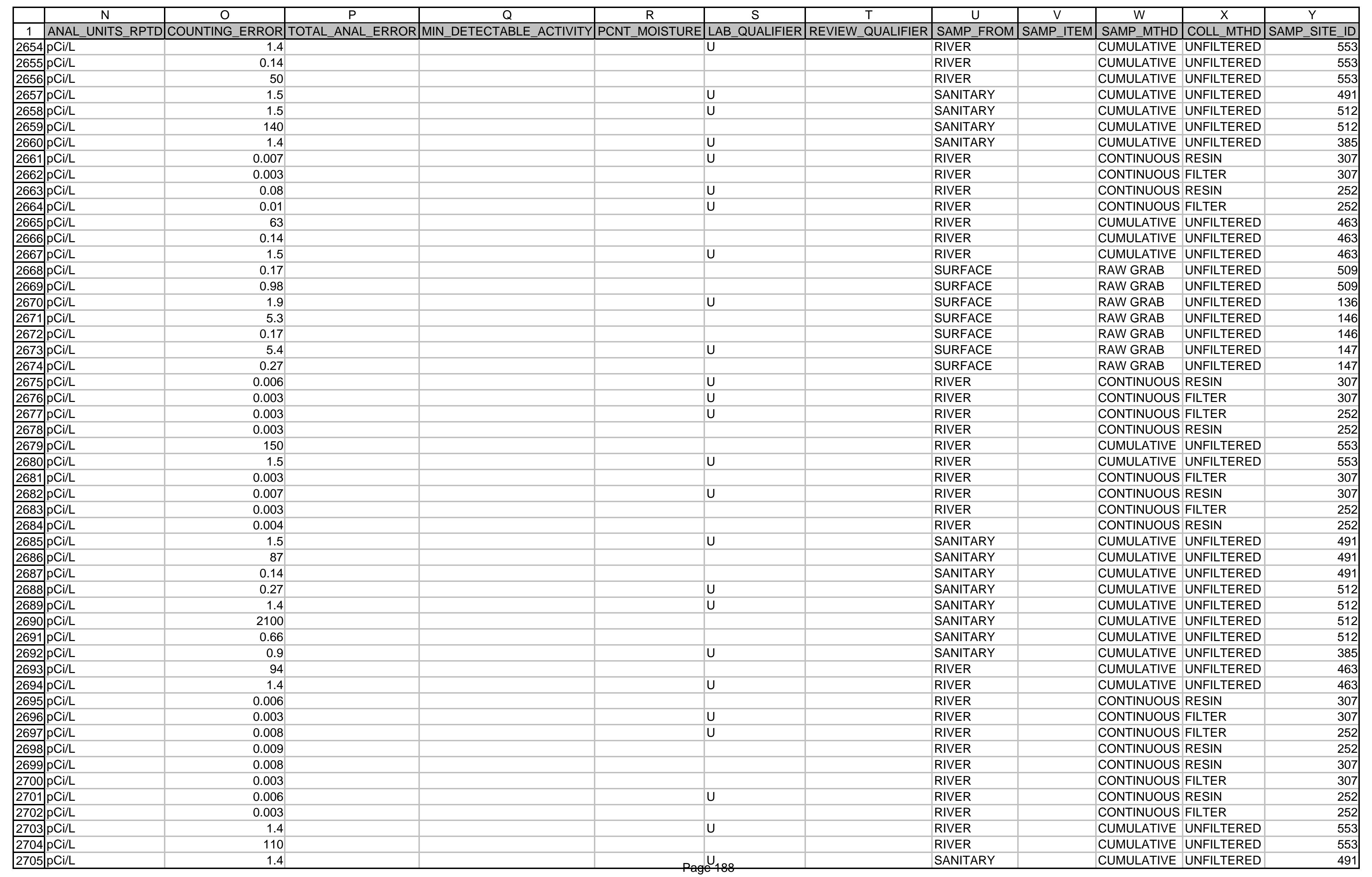




\begin{tabular}{|c|c|c|c|c|c|c|c|c|c|c|c|c|}
\hline & $\mathrm{N}$ & $\mathrm{O}$ & $\mathrm{P}$ & $\mathrm{Q}$ & $\mathrm{R}$ & $\mathrm{s}$ & $\mathrm{T}$ & $\mathrm{u}$ & $\mathrm{v}$ & $\mathrm{w}$ & $\mathrm{x}$ & $\bar{Y}$ \\
\hline 1 & ANAL_UNITS_RPTD & COUNTING_ERROR & TOTAL_ANAL_ERROR & MIN_DETECTABLE_ACTIVITY & PCNT_MOISTURE & LAB_QUALIFIER & REVIEW_QUALIFIER & SAMP_FROM & SAMP_ITEM & SAMP_MTHD & COLL_MTHD & SAMP_SITE_ID \\
\hline 2706 & $\mathrm{pCi} / \mathrm{L}$ & 1.5 & & & & & & SANITARY & & CUMULATIVE & UNFILTERED & 512 \\
\hline 2707 & $\mathrm{pCi} / \mathrm{L}$ & 770 & & & & & & SANITARY & & CUMULATIVE & UNFILTERED & 512 \\
\hline 2708 & $\mathrm{pCi} / \mathrm{L}$ & 0.73 & & & & $\mathrm{U}$ & & SANITARY & & CUMULATIVE & UNFILTERED & 385 \\
\hline 2709 & $\mathrm{pCi} / \mathrm{L}$ & 97 & & & & & & RIVER & & CUMULATIVE & UNFILTERED & 463 \\
\hline 2710 & $\mathrm{pCi} / \mathrm{L}$ & 1.5 & & & & $\mathrm{U}$ & & RIVER & & CUMULATIVE & UNFILTERED & 463 \\
\hline 2711 & $\mathrm{pCi} / \mathrm{L}$ & 0.0000267 & & & & & & RIVER & & CONTINUOUS & RESIN & 307 \\
\hline 2712 & $\mathrm{pCi} / \mathrm{L}$ & 0.006 & & & & & & RIVER & & CONTINUOUS & RESIN & 307 \\
\hline 2713 & $\mathrm{pCi} / \mathrm{L}$ & & 0.0000069 & & & & & RIVER & & CONTINUOUS & RESIN & 307 \\
\hline 2714 & $\mathrm{pCi} / \mathrm{L}$ & 0.002 & & & & & & RIVER & & CONTINUOUS & FILTER & 307 \\
\hline 2715 & $\mathrm{pCi} / \mathrm{L}$ & 0.0000153 & & & & & & RIVER & & CONTINUOUS & FILTER & 307 \\
\hline 2716 & $\mathrm{pCi} / \mathrm{L}$ & 0.0000119 & & & & & & RIVER & & CONTINUOUS & FILTER & 252 \\
\hline 2717 & $\mathrm{pCi} / \mathrm{L}$ & 0.002 & & & & & & RIVER & & CONTINUOUS & FILTER & 252 \\
\hline \begin{tabular}{|l|}
2718 \\
\end{tabular} & $\mathrm{pCi} / \mathrm{L}$ & 0.006 & & & & $U$ & & RIVER & & CONTINUOUS & RESIN & 252 \\
\hline 2719 & $\mathrm{pCi} / \mathrm{L}$ & 0.0000289 & & & & & & RIVER & & CONTINUOUS & RESIN & 252 \\
\hline 2720 & $\mathrm{pCi} / \mathrm{L}$ & & 0.0000021 & & & & & RIVER & & CONTINUOUS & RESIN & 252 \\
\hline \begin{tabular}{|l|}
2721 \\
\end{tabular} & $\mathrm{pCi} / \mathrm{L}$ & 0.006 & & & & $U$ & & RIVER & & CONTINUOUS & RESIN & 307 \\
\hline 2722 & $\mathrm{pCi} / \mathrm{L}$ & 0.002 & & & & & & RIVER & & CONTINUOUS & FILTER & 307 \\
\hline 2723 & $\mathrm{pCi} / \mathrm{L}$ & 0.01 & & & & U & & RIVER & & CONTINUOUS & RESIN & 252 \\
\hline 2724 & $\mathrm{pCi} / \mathrm{L}$ & 0.002 & & & & & & RIVER & & CONTINUOUS & FILTER & 252 \\
\hline 2725 & $\mathrm{pCi} / \mathrm{L}$ & 0.14 & & & & & & RIVER & & CUMULATIVE & UNFILTERED & 553 \\
\hline 2726 & $\mathrm{pCi} / \mathrm{L}$ & 1.4 & & & & $U$ & & RIVER & & CUMULATIVE & UNFILTERED & 553 \\
\hline \begin{tabular}{|l|}
2727 \\
\end{tabular} & $\mathrm{pCi} / \mathrm{L}$ & 240 & & & & $U$ & & RIVER & & CUMULATIVE & UNFILTERED & 553 \\
\hline \begin{tabular}{|l|}
2728 \\
\end{tabular} & $\mathrm{pCi} / \mathrm{L}$ & 1.4 & & & & U & & SANITARY & & CUMULATIVE & UNFILTERED & 491 \\
\hline 2729 & $\mathrm{pCi} / \mathrm{L}$ & 1.4 & & & & $U$ & & SANITARY & & CUMULATIVE & UNFILTERED & 512 \\
\hline 2730 & $\mathrm{pCi} / \mathrm{L}$ & 2100 & & & & & & SANITARY & & CUMULATIVE & UNFILTERED & 512 \\
\hline \begin{tabular}{|l|}
2731 \\
\end{tabular} & $\mathrm{pCi} / \mathrm{L}$ & 0.72 & & & & $U$ & & SANITARY & & CUMULATIVE & UNFILTERED & 385 \\
\hline 2732 & $\mathrm{pCi} / \mathrm{L}$ & 170 & & & & & & RIVER & & CUMULATIVE & UNFILTERED & 463 \\
\hline 2733 & $\mathrm{pCi} / \mathrm{L}$ & 0.14 & & & & & & RIVER & & CUMULATIVE & UNFILTERED & 463 \\
\hline 2734 & $\mathrm{pCi} / \mathrm{L}$ & 1.5 & & & & $U$ & & RIVER & & CUMULATIVE & UNFILTERED & 463 \\
\hline 2735 & $\mathrm{pCi} / \mathrm{L}$ & 0.003 & & & & & & RIVER & & CONTINUOUS & FILTER & 307 \\
\hline \begin{tabular}{|l|}
2736 \\
\end{tabular} & $\mathrm{pCi} / \mathrm{L}$ & 0.06 & & & & U & & RIVER & & CONTINUOUS & RESIN & 252 \\
\hline \begin{tabular}{|l|}
2737 \\
\end{tabular} & $\mathrm{pCi} / \mathrm{L}$ & 0.004 & & & & & & RIVER & & CONTINUOUS & FILTER & 252 \\
\hline 2738 & $\mathrm{pCi} / \mathrm{L}$ & 1.6 & & & & & & SURFACE & & RAW GRAB & UNFILTERED & 509 \\
\hline 2739 & $\mathrm{pCi} / \mathrm{L}$ & 1.1 & & & & & & SURFACE & & RAW GRAB & UNFILTERED & 509 \\
\hline 2740 & $\mathrm{pCi} / \mathrm{L}$ & 1.6 & & & & $U$ & & SURFACE & & RAW GRAB & UNFILTERED & 136 \\
\hline \begin{tabular}{|l|}
2741 \\
\end{tabular} & $\mathrm{pCi} / \mathrm{L}$ & 1.1 & & & & & & SURFACE & & RAW GRAB & UNFILTERED & 146 \\
\hline 2742 & $\mathrm{pCi} / \mathrm{L}$ & 0.14 & & & & & & SURFACE & & RAW GRAB & UNFILTERED & 146 \\
\hline 2743 & $\mathrm{pCi} / \mathrm{L}$ & 3.3 & & & & & & SURFACE & & RAW GRAB & UNFILTERED & 147 \\
\hline 2744 & $\mathrm{pCi} / \mathrm{L}$ & 0.45 & & & & & & SURFACE & & RAW GRAB & UNFILTERED & 147 \\
\hline 2745 & $\mathrm{pCi} / \mathrm{L}$ & 0.003 & & & & & & RIVER & & CONTINUOUS & FILTER & 307 \\
\hline 2746 & $\mathrm{pCi} / \mathrm{L}$ & 0.004 & & & & & & RIVER & & CONTINUOUS & RESIN & 307 \\
\hline 2747 & $\mathrm{pCi} / \mathrm{L}$ & 0.003 & & & & & & RIVER & & CONTINUOUS & FILTER & 252 \\
\hline \begin{tabular}{|l|}
2748 \\
\end{tabular} & $\mathrm{pCi} / \mathrm{L}$ & 0.004 & & & & & & RIVER & & CONTINUOUS & RESIN & 252 \\
\hline \begin{tabular}{|l|}
2749 \\
\end{tabular} & $\mathrm{pCi} / \mathrm{L}$ & 2.9 & & & & U & & RIVER & & CUMULATIVE & UNFILTERED & 553 \\
\hline 2750 & $\mathrm{pCi} / \mathrm{L}$ & 160 & & & & $U$ & & RIVER & & CUMULATIVE & UNFILTERED & 553 \\
\hline 2751 & $\mathrm{pCi} / \mathrm{L}$ & 0.14 & & & & & & SANITARY & & CUMULATIVE & UNFILTERED & 491 \\
\hline \begin{tabular}{|l|}
2752 \\
\end{tabular} & $\mathrm{pCi} / \mathrm{L}$ & 130 & & & & & & SANITARY & & CUMULATIVE & UNFILTERED & 491 \\
\hline 2753 & $\mathrm{pCi} / \mathrm{L}$ & 1.6 & & & & $U$ & & SANITARY & & CUMULATIVE & UNFILTERED & 491 \\
\hline 2754 & $\mathrm{pCi} / \mathrm{L}$ & 0.27 & & & & $U$ & & SANITARY & & CUMULATIVE & UNFILTERED & 512 \\
\hline 2755 & $\mathrm{pCi} / \mathrm{L}$ & 1.4 & & & & U & & SANITARY & & CUMULATIVE & UNFILTERED & 512 \\
\hline \begin{tabular}{|l|}
2756 \\
\end{tabular} & $\mathrm{pCi} / \mathrm{L}$ & 710 & & & & & & SANITARY & & CUMULATIVE & UNFILTERED & 512 \\
\hline 2757 & $\mathrm{pCi} / \mathrm{L}$ & 1.6 & & & & & & SANITARY & & CUMULATIVE & UNFILTERED & 512 \\
\hline
\end{tabular}




\begin{tabular}{|c|c|c|c|c|c|c|c|c|c|c|c|c|}
\hline & $\mathrm{N}$ & $\mathrm{O}$ & $\mathrm{P}$ & $\mathrm{Q}$ & $\mathrm{R}$ & $\mathrm{s}$ & $T$ & $\mathrm{U}$ & $\mathrm{V}$ & $\mathrm{W}$ & $\mathrm{X}$ & $\bar{Y}$ \\
\hline 1 & ANAL_UNITS_RPTD & COUNTING_ERROR & TOTAL_ANAL_ERROR & MIN_DETECTABLE_ACTIVITY & PCNT_MOISTURE & LAB_QUALIFIER & REVIEW_QUALIFIER & SAMP_FROM & SAMP_ITEM & SAMP MTHD & COLL MTHD & SAMP SITE ID \\
\hline 2758 & $\mathrm{pCi} / \mathrm{L}$ & 0.72 & & & & $U$ & & SANITARY & & CUMULATIVE & UNFILTERED & 385 \\
\hline 2759 & $\mathrm{pCi} / \mathrm{L}$ & 140 & & & & & & RIVER & & CUMULATIVE & UNFILTERED & 463 \\
\hline 2760 & $\mathrm{pCi} / \mathrm{L}$ & 1.6 & & & & U & & RIVER & & CUMULATIVE & UNFILTERED & 463 \\
\hline 2761 & $\mathrm{pCi} / \mathrm{L}$ & 0.005 & & & & $\mathrm{U}$ & & RIVER & & CONTINUOUS & RESIN & 307 \\
\hline 2762 & $\mathrm{pCi} / \mathrm{L}$ & 0.003 & & & & & & RIVER & & CONTINUOUS & FILTER & 307 \\
\hline 2763 & $\mathrm{pCi} / \mathrm{L}$ & 0.006 & & & & $U$ & & RIVER & & CONTINUOUS & RESIN & 252 \\
\hline 2764 & $\mathrm{pCi} / \mathrm{L}$ & 0.004 & & & & $\mathrm{U}$ & & RIVER & & CONTINUOUS & FILTER & 252 \\
\hline 2765 & $\mathrm{pCi} / \mathrm{L}$ & 0.0000466 & & & & & & RIVER & & CONTINUOUS & RESIN & 307 \\
\hline 2766 & $\mathrm{pCi} / \mathrm{L}$ & 0.007 & & & & $\mathrm{U}$ & & RIVER & & CONTINUOUS & RESIN & 307 \\
\hline 2767 & $\mathrm{pCi} / \mathrm{L}$ & 0.00000916 & & & & & & RIVER & & CONTINUOUS & FILTER & 307 \\
\hline 2768 & $\mathrm{pCi} / \mathrm{L}$ & 0.002 & & & & & & RIVER & & CONTINUOUS & FILTER & 307 \\
\hline 2769 & $\mathrm{pCi} / \mathrm{L}$ & 0.11 & & & & U & & RIVER & & CONTINUOUS & RESIN & 252 \\
\hline 2770 & $\mathrm{pCi} / \mathrm{L}$ & 0.0000205 & & & & & & RIVER & & CONTINUOUS & RESIN & 252 \\
\hline 2771 & $\mathrm{pCi} / \mathrm{L}$ & 0.0000147 & & & & & & RIVER & & CONTINUOUS & FILTER & 252 \\
\hline 2772 & $\mathrm{pCi} / \mathrm{L}$ & 0.04 & & & & $U$ & & RIVER & & CONTINUOUS & FILTER & 252 \\
\hline 2773 & $\mathrm{pCi} / \mathrm{L}$ & 2 & & & & & & SURFACE & & RAW GRAB & UNFILTERED & 509 \\
\hline 2774 & $\mathrm{pCi} / \mathrm{L}$ & 0.79 & & & & & & SURFACE & & RAW GRAB & UNFILTERED & 509 \\
\hline 2775 & $\mathrm{pCi} / \mathrm{L}$ & 1.4 & & & & U & & RIVER & & CUMULATIVE & UNFILTERED & 553 \\
\hline 2776 & $\mathrm{pCi} / \mathrm{L}$ & 90 & & & & & & RIVER & & CUMULATIVE & UNFILTERED & 553 \\
\hline \begin{tabular}{|l|}
2777 \\
\end{tabular} & $\mathrm{pCi} / \mathrm{L}$ & 1.4 & & & & $U$ & & SANITARY & & CUMULATIVE & UNFILTERED & 491 \\
\hline 2778 & $\mathrm{pCi} / \mathrm{L}$ & 1.6 & & & & U & & SANITARY & & CUMULATIVE & UNFILTERED & 512 \\
\hline 2779 & $\mathrm{pCi} / \mathrm{L}$ & 190 & & & & & & SANITARY & & CUMULATIVE & UNFILTERED & 512 \\
\hline 2780 & $\mathrm{pCi} / \mathrm{L}$ & 0.72 & & & & $U$ & & SANITARY & & CUMULATIVE & UNFILTERED & 385 \\
\hline 2781 & $\mathrm{pCi} / \mathrm{L}$ & 0.006 & & & & $U$ & & RIVER & & CONTINUOUS & RESIN & 307 \\
\hline 2782 & $\mathrm{pCi} / \mathrm{L}$ & & 0.000011 & & & & & RIVER & & CONTINUOUS & RESIN & 307 \\
\hline 2783 & $\mathrm{pCi} / \mathrm{L}$ & 0.002 & & & & & & RIVER & & CONTINUOUS & FILTER & 307 \\
\hline 2784 & $\mathrm{pCi} / \mathrm{L}$ & 0.007 & & & & U & & RIVER & & CONTINUOUS & RESIN & 252 \\
\hline 2785 & $\mathrm{pCi} / \mathrm{L}$ & & 0.0000013 & & & & & RIVER & & CONTINUOUS & RESIN & 252 \\
\hline 2786 & $\mathrm{pCi} / \mathrm{L}$ & 0.003 & & & & & & RIVER & & CONTINUOUS & FILTER & 252 \\
\hline 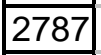 & $\mathrm{pCi} / \mathrm{L}$ & 76 & & & & & & RIVER & & CUMULATIVE & UNFILTERED & 463 \\
\hline 2788 & $\mathrm{pCi} / \mathrm{L}$ & 1.4 & & & & U & & RIVER & & CUMULATIVE & UNFILTERED & 463 \\
\hline 2789 & $\mathrm{pCi} / \mathrm{L}$ & 0.006 & & & & U & & RIVER & & CONTINUOUS & RESIN & 307 \\
\hline 2790 & $\mathrm{pCi} / \mathrm{L}$ & 0.004 & & & & U & & RIVER & & CONTINUOUS & FILTER & 307 \\
\hline 2791 & $\mathrm{pCi} / \mathrm{L}$ & 0.01 & & & & U & & RIVER & & CONTINUOUS & RESIN & 252 \\
\hline \begin{tabular}{|l|}
2792 \\
\end{tabular} & $\mathrm{pCi} / \mathrm{L}$ & 0.001 & & & & & & RIVER & & CONTINUOUS & FILTER & 252 \\
\hline 2793 & $\mathrm{pCi} / \mathrm{L}$ & 0.14 & & & & & & RIVER & & CUMULATIVE & UNFILTERED & 553 \\
\hline 2794 & $\mathrm{pCi} / \mathrm{L}$ & 3.6 & & & & $U$ & & RIVER & & CUMULATIVE & UNFILTERED & 553 \\
\hline 2795 & $\mathrm{pCi} / \mathrm{L}$ & 240 & & & & & & RIVER & & CUMULATIVE & UNFILTERED & 553 \\
\hline 2796 & $\mathrm{pCi} / \mathrm{L}$ & 1.5 & & & & & & SANITARY & & CUMULATIVE & UNFILTERED & 491 \\
\hline 2797 & $\mathrm{pCi} / \mathrm{L}$ & 1.5 & & & & U & & SANITARY & & CUMULATIVE & UNFILTERED & 512 \\
\hline 2798 & $\mathrm{pCi} / \mathrm{L}$ & 590 & & & & & & SANITARY & & CUMULATIVE & UNFILTERED & 512 \\
\hline 2799 & $\mathrm{pCi} / \mathrm{L}$ & 0.72 & & & & $U$ & & SANITARY & & CUMULATIVE & UNFILTERED & 385 \\
\hline 2800 & $\mathrm{pCi} / \mathrm{L}$ & 0.008 & & & & $U$ & & RIVER & & CONTINUOUS & RESIN & 307 \\
\hline 2801 & $\mathrm{pCi} / \mathrm{L}$ & 0.002 & & & & $U$ & & RIVER & & CONTINUOUS & FILTER & 307 \\
\hline 2802 & $\mathrm{pCi} / \mathrm{L}$ & 0.005 & & & & $U$ & & RIVER & & CONTINUOUS & FILTER & 252 \\
\hline 2803 & $\mathrm{pCi} / \mathrm{L}$ & 0.005 & & & & & & RIVER & & CONTINUOUS & RESIN & 252 \\
\hline 2804 & $\mathrm{pCi} / \mathrm{L}$ & 140 & & & & & & RIVER & & CUMULATIVE & UNFILTERED & 463 \\
\hline 2805 & $\mathrm{pCi} / \mathrm{L}$ & 0.14 & & & & & & RIVER & & CUMULATIVE & UNFILTERED & 463 \\
\hline 2806 & $\mathrm{pCi} / \mathrm{L}$ & 1.4 & & & & U & & RIVER & & CUMULATIVE & UNFILTERED & 463 \\
\hline 2807 & $\mathrm{pCi} / \mathrm{L}$ & 0.002 & & & & & & RIVER & & CONTINUOUS & FILTER & 307 \\
\hline 2808 & $\mathrm{pCi} / \mathrm{L}$ & 0.007 & & & & U & & RIVER & & CONTINUOUS & RESIN & 307 \\
\hline 2809 & $\mathrm{pCi} / \mathrm{L}$ & 0.004 & & & & $u$ & & RIVER & & CONTINUOUS & FILTER & 252 \\
\hline
\end{tabular}




\begin{tabular}{|c|c|c|c|c|c|c|c|c|c|c|c|c|}
\hline & $\mathrm{N}$ & $\mathrm{O}$ & $\mathrm{P}$ & $\mathrm{Q}$ & $\mathrm{R}$ & $\mathrm{s}$ & $T$ & $\mathrm{U}$ & $\mathrm{V}$ & $\mathrm{W}$ & $\mathrm{X}$ & $\bar{Y}$ \\
\hline 1 & ANAL_UNITS_RPTD & COUNTING_ERROR & $\mid$ TOTAL_ANAL_ERROR | & MIN_DETECTABLE_ACTIVITY & PCNT_MOISTURE & LAB_QUALIFIER & REVIEW_QUALIFIER & SAMP_FROM & SAMP_ITEM & SAMP_MTHD & COLL_MTHD & SAMP_SITE_ID \\
\hline 2810 & $\mathrm{pCi} / \mathrm{L}$ & 0.006 & & & & $U$ & & RIVER & & CONTINUOUS & RESIN & 252 \\
\hline 2811 & $\mathrm{pCi} / \mathrm{L}$ & 0.14 & & & & & & SURFACE & & RAW GRAB & UNFILTERED & 509 \\
\hline 2812 & $\mathrm{pCi} / \mathrm{L}$ & 1.1 & & & & & & SURFACE & & RAW GRAB & UNFILTERED & 509 \\
\hline 2813 & $\mathrm{pCi} / \mathrm{L}$ & 1.6 & & & & $\mathrm{U}$ & & SURFACE & & RAW GRAB & UNFILTERED & 136 \\
\hline 2814 & $\mathrm{pCi} / \mathrm{L}$ & 1 & & & & & & SURFACE & & RAW GRAB & UNFILTERED & 146 \\
\hline 2815 & $\mathrm{pCi} / \mathrm{L}$ & 0.14 & & & & & & SURFACE & & RAW GRAB & UNFILTERED & 146 \\
\hline 2816 & $\mathrm{pCi} / \mathrm{L}$ & 7.2 & & & & U & & SURFACE & & RAW GRAB & UNFILTERED & 147 \\
\hline 2817 & $\mathrm{pCi} / \mathrm{L}$ & 0.27 & & & & & & SURFACE & & RAW GRAB & UNFILTERED & 147 \\
\hline 2818 & $\mathrm{pCi} / \mathrm{L}$ & 0.14 & & & & & & SANITARY & & CUMULATIVE & UNFILTERED & 491 \\
\hline 2819 & $\mathrm{pCi} / \mathrm{L}$ & 140 & & & & & & SANITARY & & CUMULATIVE & UNFILTERED & 491 \\
\hline 2820 & $\mathrm{pCi} / \mathrm{L}$ & 1.4 & & & & U & & SANITARY & & CUMULATIVE & UNFILTERED & 491 \\
\hline 2821 & $\mathrm{pCi} / \mathrm{L}$ & 0.19 & & & & $U$ & & SANITARY & & CUMULATIVE & UNFILTERED & 512 \\
\hline 2822 & $\mathrm{pCi} / \mathrm{L}$ & 1.6 & & & & $U$ & & SANITARY & & CUMULATIVE & UNFILTERED & 512 \\
\hline 2823 & $\mathrm{pCi} / \mathrm{L}$ & 470 & & & & & & SANITARY & & CUMULATIVE & UNFILTERED & 512 \\
\hline 2824 & $\mathrm{pCi} / \mathrm{L}$ & 1 & & & & & & SANITARY & & CUMULATIVE & UNFILTERED & 512 \\
\hline 2825 & $\mathrm{pCi} / \mathrm{L}$ & 0.81 & & & & $U$ & & SANITARY & & CUMULATIVE & UNFILTERED & 385 \\
\hline 2826 & $\mathrm{pCi} / \mathrm{L}$ & 0.0000172 & & & & & & RIVER & & CONTINUOUS & RESIN & 307 \\
\hline 2827 & $\mathrm{pCi} / \mathrm{L}$ & 0.003 & & & & U & & RIVER & & CONTINUOUS & RESIN & 307 \\
\hline \begin{tabular}{|l|}
2828 \\
\end{tabular} & $\mathrm{pCi} / \mathrm{L}$ & & 0.000012 & & & & & RIVER & & CONTINUOUS & RESIN & 307 \\
\hline 2829 & $\mathrm{pCi} / \mathrm{L}$ & 0.007 & & & & $U$ & & RIVER & & CONTINUOUS & FILTER & 307 \\
\hline 2830 & $\mathrm{pCi} / \mathrm{L}$ & 0.0000052 & & & & & & RIVER & & CONTINUOUS & FILTER & 307 \\
\hline \begin{tabular}{|l|}
2831 \\
\end{tabular} & $\mathrm{pCi} / \mathrm{L}$ & 0.006 & & & & $U$ & & RIVER & & CONTINUOUS & RESIN & 252 \\
\hline 2832 & $\mathrm{pCi} / \mathrm{L}$ & 0.0000444 & & & & & & RIVER & & CONTINUOUS & RESIN & 252 \\
\hline 2833 & $\mathrm{pCi} / \mathrm{L}$ & & 0.0000016 & & & & & RIVER & & CONTINUOUS & RESIN & 252 \\
\hline 2834 & $\mathrm{pCi} / \mathrm{L}$ & 0.0000259 & & & & & & RIVER & & CONTINUOUS & FILTER & 252 \\
\hline 2835 & $\mathrm{pCi} / \mathrm{L}$ & 0.002 & & & & & & RIVER & & CONTINUOUS & FILTER & 252 \\
\hline 2836 & $\mathrm{pCi} / \mathrm{L}$ & 190 & & & & & & RIVER & & CUMULATIVE & UNFILTERED & 463 \\
\hline \begin{tabular}{|l|}
2837 \\
\end{tabular} & $\mathrm{pCi} / \mathrm{L}$ & 1.5 & & & & $U$ & & RIVER & & CUMULATIVE & UNFILTERED & 463 \\
\hline 2838 & $\mathrm{pCi} / \mathrm{L}$ & 1.5 & & & & U & & RIVER & & CUMULATIVE & UNFILTERED & 553 \\
\hline 2839 & $\mathrm{pCi} / \mathrm{L}$ & 200 & & & & & & RIVER & & CUMULATIVE & UNFILTERED & 553 \\
\hline 2840 & $\mathrm{pCi} / \mathrm{L}$ & 0.005 & & & & $U$ & & RIVER & & CONTINUOUS & RESIN & 307 \\
\hline \begin{tabular}{|l|}
2841 \\
\end{tabular} & $\mathrm{pCi} / \mathrm{L}$ & 0.002 & & & & U & & RIVER & & CONTINUOUS & FILTER & 307 \\
\hline \begin{tabular}{|l|}
2842 \\
\end{tabular} & $\mathrm{pCi} / \mathrm{L}$ & 0.005 & & & & U & & RIVER & & CONTINUOUS & RESIN & 252 \\
\hline \begin{tabular}{|l|}
2843 \\
\end{tabular} & $\mathrm{pCi} / \mathrm{L}$ & 0.001 & & & & $U$ & & RIVER & & CONTINUOUS & FILTER & 252 \\
\hline 2844 & $\mathrm{pCi} / \mathrm{L}$ & 0.27 & & & & & & SURFACE & & RAW GRAB & UNFILTERED & 509 \\
\hline 2845 & $\mathrm{pCi} / \mathrm{L}$ & 9.4 & & & & & & SURFACE & & RAW GRAB & UNFILTERED & 509 \\
\hline 2846 & $\mathrm{pCi} / \mathrm{L}$ & 0.002 & & & & & & RIVER & & CONTINUOUS & FILTER & 307 \\
\hline 2847 & $\mathrm{pCi} / \mathrm{L}$ & 0.006 & & & & U & & RIVER & & CONTINUOUS & RESIN & 307 \\
\hline 2848 & $\mathrm{pCi} / \mathrm{L}$ & 0.01 & & & & $U$ & & RIVER & & CONTINUOUS & RESIN & 252 \\
\hline 2849 & $\mathrm{pCi} / \mathrm{L}$ & 0.004 & & & & U & & RIVER & & CONTINUOUS & FILTER & 252 \\
\hline 2850 & $\mathrm{pCi} / \mathrm{L}$ & 1.4 & & & & U & & SANITARY & & CUMULATIVE & UNFILTERED & 491 \\
\hline 2851 & $\mathrm{pCi} / \mathrm{L}$ & 1.9 & & & & U & & SANITARY & & CUMULATIVE & UNFILTERED & 512 \\
\hline 2852 & $\mathrm{pCi} / \mathrm{L}$ & 780 & & & & & & SANITARY & & CUMULATIVE & UNFILTERED & 512 \\
\hline 2853 & $\mathrm{pCi} / \mathrm{L}$ & 1.1 & & & & $U$ & & SANITARY & & CUMULATIVE & UNFILTERED & 385 \\
\hline 2854 & $\mathrm{pCi} / \mathrm{L}$ & 190 & & & & & & RIVER & & CUMULATIVE & UNFILTERED & 553 \\
\hline 2855 & $\mathrm{pCi} / \mathrm{L}$ & 1.5 & & & & $U$ & & RIVER & & CUMULATIVE & UNFILTERED & 553 \\
\hline 2856 & $\mathrm{pCi} / \mathrm{L}$ & 150 & & & & & & RIVER & & CUMULATIVE & UNFILTERED & 463 \\
\hline 2857 & $\mathrm{pCi} / \mathrm{L}$ & 1.5 & & & & U & & RIVER & & CUMULATIVE & UNFILTERED & 463 \\
\hline 2858 & $\mathrm{pCi} / \mathrm{L}$ & 0.02 & & & & U & & RIVER & & CONTINUOUS & RESIN & 307 \\
\hline 2859 & $\mathrm{pCi} / \mathrm{L}$ & & 0.00000953 & & & & & RIVER & & CONTINUOUS & RESIN & 307 \\
\hline 2860 & $\mathrm{pCi} / \mathrm{L}$ & 0.003 & & & & & & RIVER & & CONTINUOUS & FILTER & 307 \\
\hline $2861 \mid$ & $\mathrm{pCi} / \mathrm{L}$ & 0.003 & & & & $u$ & & RIVER & & CONTINUOUS & FILTER & 252 \\
\hline
\end{tabular}




\begin{tabular}{|c|c|c|c|c|c|c|c|c|c|c|c|c|}
\hline & $\mathrm{N}$ & $\mathrm{O}$ & $\mathrm{P}$ & $\mathrm{Q}$ & $\mathrm{R}$ & $\mathrm{s}$ & $T$ & $\mathrm{U}$ & $\mathrm{V}$ & $\mathrm{W}$ & $x$ & $\bar{Y}$ \\
\hline 1 & ANAL_UNITS_RPTD & COUNTING_ERROR & ||$_{\text {TOTAL_ANAL_ERROR | }}$ & MIN_DETECTABLE_ACTIVITY & PCNT_MOISTURE & LAB_QUALIFIER & REVIEW_QUALIFIER & SAMP_FROM & SAMP_ITEM & SAMP_MTHD & COLL_MTHD & SAMP_SITE_ID \\
\hline 2862 & $\mathrm{pCi} / \mathrm{L}$ & 0.007 & & & & $U$ & & RIVER & & CONTINUOUS & RESIN & 252 \\
\hline 2863 & $\mathrm{pCi} / \mathrm{L}$ & & 0.00000131 & & & & & RIVER & & CONTINUOUS & RESIN & 252 \\
\hline 2864 & $\mathrm{pCi} / \mathrm{L}$ & 0.27 & & & & & & SURFACE & & RAW GRAB & UNFILTERED & 509 \\
\hline 2865 & $\mathrm{pCi} / \mathrm{L}$ & 8.8 & & & & & & SURFACE & & RAW GRAB & UNFILTERED & 509 \\
\hline 2866 & $\mathrm{pCi} / \mathrm{L}$ & 0.73 & & & & U & & SANITARY & & CUMULATIVE & UNFILTERED & 385 \\
\hline 2867 & $\mathrm{pCi} / \mathrm{L}$ & 0.05 & & & & U & & RIVER & & CONTINUOUS & RESIN & 307 \\
\hline 2868 & $\mathrm{pCi} / \mathrm{L}$ & 0.002 & & & & & & RIVER & & CONTINUOUS & FILTER & 307 \\
\hline 2869 & $\mathrm{pCi} / \mathrm{L}$ & 0.02 & & & & U & & RIVER & & CONTINUOUS & RESIN & 252 \\
\hline 2870 & $\mathrm{pCi} / \mathrm{L}$ & 0.002 & & & & & & RIVER & & CONTINUOUS & FILTER & 252 \\
\hline 2871 & $\mathrm{pCi} / \mathrm{L}$ & 0.14 & & & & & & SANITARY & & CUMULATIVE & UNFILTERED & 491 \\
\hline 2872 & $\mathrm{pCi} / \mathrm{L}$ & 260 & & & & & & SANITARY & & CUMULATIVE & UNFILTERED & 491 \\
\hline 2873 & $\mathrm{pCi} / \mathrm{L}$ & 0.27 & & & & U & & SANITARY & & CUMULATIVE & UNFILTERED & 512 \\
\hline 2874 & $\mathrm{pCi} / \mathrm{L}$ & 0.28 & & & & $U$ & & SANITARY & & CUMULATIVE & UNFILTERED & 512 \\
\hline 2875 & $\mathrm{pCi} / \mathrm{L}$ & 1400 & & & & & & SANITARY & & CUMULATIVE & UNFILTERED & 512 \\
\hline 2876 & $\mathrm{pCi} / \mathrm{L}$ & 0.14 & & & & & & SANITARY & & CUMULATIVE & UNFILTERED & 385 \\
\hline 2877 & $\mathrm{pCi} / \mathrm{L}$ & 0.007 & & & & $U$ & & RIVER & & CONTINUOUS & RESIN & 307 \\
\hline 2878 & $\mathrm{pCi} / \mathrm{L}$ & & 0.00000264 & & & & & RIVER & & CONTINUOUS & RESIN & 307 \\
\hline 2879 & $\mathrm{pCi} / \mathrm{L}$ & 0.004 & & & & & & RIVER & & CONTINUOUS & FILTER & 307 \\
\hline 2880 & $\mathrm{pCi} / \mathrm{L}$ & 10 & & & & & & RIVER & & CUMULATIVE & UNFILTERED & 252 \\
\hline 2881 & $\mathrm{pCi} / \mathrm{L}$ & 0.14 & & & & & & RIVER & & CUMULATIVE & UNFILTERED & 252 \\
\hline 2882 & pCi/L & 1.5 & & & & $U$ & & RIVER & & CUMULATIVE & UNFILTERED & 252 \\
\hline 2883 & pCi/L & 0.004 & & & & U & & RIVER & & CONTINUOUS & FILTER & 252 \\
\hline 2884 & $\mathrm{pCi} / \mathrm{L}$ & 0.01 & & & & U & & RIVER & & CONTINUOUS & RESIN & 252 \\
\hline 2885 & $\mathrm{pCi} / \mathrm{L}$ & & 0.0000013 & & & & & RIVER & & CONTINUOUS & RESIN & 252 \\
\hline 2886 & $\mathrm{pCi} / \mathrm{L}$ & 12 & & & & & & RIVER & & CUMULATIVE & UNFILTERED & 463 \\
\hline 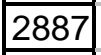 & $\mathrm{pCi} / \mathrm{L}$ & 0.14 & & & & & & RIVER & & CUMULATIVE & UNFILTERED & 463 \\
\hline 2888 & $\mathrm{pCi} / \mathrm{L}$ & 1.5 & & & & $U$ & & RIVER & & CUMULATIVE & UNFILTERED & 463 \\
\hline 2889 & $\mathrm{pCi} / \mathrm{L}$ & 0.17 & & & & & & SURFACE & & RAW GRAB & UNFILTERED & 509 \\
\hline 2890 & $\mathrm{pCi} / \mathrm{L}$ & 3.4 & & & & & & SURFACE & & RAW GRAB & UNFILTERED & 509 \\
\hline 2891 & $\mathrm{pCi} / \mathrm{L}$ & 1.5 & & & & & & SURFACE & & RAW GRAB & UNFILTERED & 136 \\
\hline 2892 & $\mathrm{pCi} / \mathrm{L}$ & 1.1 & & & & & & SURFACE & & RAW GRAB & UNFILTERED & 146 \\
\hline 2893 & $\mathrm{pCi} / \mathrm{L}$ & 0.14 & & & & & & SURFACE & & RAW GRAB & UNFILTERED & 146 \\
\hline 2894 & $\mathrm{pCi} / \mathrm{L}$ & 1.7 & & & & & & SURFACE & & RAW GRAB & UNFILTERED & 147 \\
\hline 2895 & $\mathrm{pCi} / \mathrm{L}$ & 0.97 & & & & & & SURFACE & & RAW GRAB & UNFILTERED & 147 \\
\hline 2896 & $\mathrm{pCi} / \mathrm{L}$ & 0.009 & & & & & & RIVER & & CONTINUOUS & RESIN & 307 \\
\hline 2897 & pCi/L & 0.005 & & & & $U$ & & RIVER & & CONTINUOUS & FILTER & 307 \\
\hline 2898 & $\mathrm{pCi} / \mathrm{L}$ & 0.007 & & & & $U$ & & RIVER & & CONTINUOUS & RESIN & 252 \\
\hline 2899 & $\mathrm{pCi} / \mathrm{L}$ & 0.004 & & & & U & & RIVER & & CONTINUOUS & FILTER & 252 \\
\hline 2900 & $\mathrm{pCi} / \mathrm{L}$ & 0.23 & & & & $U$ & & SANITARY & & CUMULATIVE & UNFILTERED & 512 \\
\hline 2901 & $\mathrm{pCi} / \mathrm{L}$ & 380 & & & & & & SANITARY & & CUMULATIVE & UNFILTERED & 512 \\
\hline \begin{tabular}{|l|}
2902 \\
\end{tabular} & $\mathrm{pCi} / \mathrm{L}$ & 0.72 & & & & U & & SANITARY & & CUMULATIVE & UNFILTERED & 385 \\
\hline 2903 & $\mathrm{pCi} / \mathrm{L}$ & 9.4 & & & & & & RIVER & & CUMULATIVE & UNFILTERED & 252 \\
\hline 2904 & $\mathrm{pCi} / \mathrm{L}$ & 1.4 & & & & $U$ & & RIVER & & CUMULATIVE & UNFILTERED & 252 \\
\hline 2905 & $\mathrm{pCi} / \mathrm{L}$ & 0.004 & & & & U & & RIVER & & CONTINUOUS & RESIN & 307 \\
\hline 2906 & $\mathrm{pCi} / \mathrm{L}$ & & 0.0000128 & & & & & RIVER & & CONTINUOUS & RESIN & 307 \\
\hline 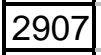 & $\mathrm{pCi} / \mathrm{L}$ & 0.002 & & & & & & RIVER & & CONTINUOUS & FILTER & 307 \\
\hline 2908 & pCi/L & 0.006 & & & & U & & RIVER & & CONTINUOUS & RESIN & 252 \\
\hline 2909 & $\mathrm{pCi} / \mathrm{L}$ & & 0.00000106 & & & & & RIVER & & CONTINUOUS & RESIN & 252 \\
\hline 2910 & pCi/L & 0.003 & & & & & & RIVER & & CONTINUOUS & FILTER & 252 \\
\hline 2911 & pCi/L & 11 & & & & & & RIVER & & CUMULATIVE & UNFILTERED & 463 \\
\hline 2912 & $\mathrm{pCi} / \mathrm{L}$ & 1.5 & & & & $U$ & & RIVER & & CUMULATIVE & UNFILTERED & 463 \\
\hline 2913 & $\mathrm{pCi} / \mathrm{L}$ & 0.42 & & & & & & SURFACE & & RAW GRAB & UNFILTERED & 509 \\
\hline
\end{tabular}




\begin{tabular}{|c|c|c|c|c|c|c|c|c|c|c|c|c|}
\hline & $\mathrm{N}$ & $\mathrm{O}$ & $\mathrm{P}$ & $\mathrm{Q}$ & $\mathrm{R}$ & $\mathrm{s}$ & $T$ & $\mathrm{U}$ & $\mathrm{V}$ & $\mathrm{W}$ & $\mathrm{X}$ & $\bar{Y}$ \\
\hline 1 & ANAL_UNITS_RPTD & COUNTING_ERROR & TOTAL_ANAL_ERROR | & MIN_DETECTABLE_ACTIVITY & PCNT_MOISTURE & LAB_QUALIFIER & REVIEW_QUALIFIER & SAMP_FROM & SAMP_ITEM & SAMP_MTHD & COLL_MTHD & SAMP_SITE_ID \\
\hline 2914 & $\mathrm{pCi} / \mathrm{L}$ & 3.2 & & & & & & SURFACE & & RAW GRAB & UNFILTERED & 509 \\
\hline 2915 & $\mathrm{pCi} / \mathrm{L}$ & 0.19 & & & & U & & DRINKING & & SAN. GRAB & UNFILTERED & 187 \\
\hline 2916 & $\mathrm{pCi} / \mathrm{L}$ & 180 & & & & U & & DRINKING & & SAN. GRAB & UNFILTERED & 187 \\
\hline 2917 & $\mathrm{pCi} / \mathrm{L}$ & 0.92 & & & & & & DRINKING & & RAW GRAB & UNFILTERED & 553 \\
\hline 2918 & $\mathrm{pCi} / \mathrm{L}$ & 0.14 & & & & & & DRINKING & & RAW GRAB & UNFILTERED & 553 \\
\hline 2919 & $\mathrm{pCi} / \mathrm{L}$ & 5 & & & & & & DRINKING & & RAW GRAB & UNFILTERED & 553 \\
\hline 2920 & $\mathrm{pCi} / \mathrm{L}$ & 0.005 & & & & $\mathrm{U}$ & & RIVER & & CONTINUOUS & RESIN & 307 \\
\hline 2921 & $\mathrm{pCi} / \mathrm{L}$ & 0.002 & & & & U & & RIVER & & CONTINUOUS & FILTER & 307 \\
\hline 2922 & $\mathrm{pCi} / \mathrm{L}$ & 0.008 & & & & $\mathrm{U}$ & & RIVER & & CONTINUOUS & RESIN & 252 \\
\hline 2923 & $\mathrm{pCi} / \mathrm{L}$ & 0.004 & & & & & & RIVER & & CONTINUOUS & FILTER & 252 \\
\hline 2924 & $\mathrm{pCi} / \mathrm{L}$ & 0.23 & & & & $\mathrm{U}$ & & SANITARY & & CUMULATIVE & UNFILTERED & 512 \\
\hline 2925 & $\mathrm{pCi} / \mathrm{L}$ & 570 & & & & & & SANITARY & & CUMULATIVE & UNFILTERED & 512 \\
\hline 2926 & $\mathrm{pCi} / \mathrm{L}$ & 0.81 & & & & $U$ & & SANITARY & & CUMULATIVE & UNFILTERED & 385 \\
\hline \begin{tabular}{|l|}
2927 \\
\end{tabular} & $\mathrm{pCi} / \mathrm{L}$ & 0.007 & & & & & & RIVER & & CONTINUOUS & RESIN & 307 \\
\hline 2928 | & $\mathrm{pCi} / \mathrm{L}$ & & 0.00000912 & & & & & RIVER & & CONTINUOUS & RESIN & 307 \\
\hline 2929 & $\mathrm{pCi} / \mathrm{L}$ & 0.002 & & & & $U$ & & RIVER & & CONTINUOUS & FILTER & 307 \\
\hline 2930 & $\mathrm{pCi} / \mathrm{L}$ & 20 & & & & & & RIVER & & CUMULATIVE & UNFILTERED & 252 \\
\hline 2931 & $\mathrm{pCi} / \mathrm{L}$ & 0.002 & & & & & & RIVER & & CONTINUOUS & FILTER & 252 \\
\hline \begin{tabular}{|l|}
2932 \\
\end{tabular} & $\mathrm{pCi} / \mathrm{L}$ & 1.4 & & & & $U$ & & RIVER & & CUMULATIVE & UNFILTERED & 252 \\
\hline 2933 & $\mathrm{pCi} / \mathrm{L}$ & 0.007 & & & & $U$ & & RIVER & & CONTINUOUS & RESIN & 252 \\
\hline 2934 & $\mathrm{pCi} / \mathrm{L}$ & & 0.00000121 & & & & & RIVER & & CONTINUOUS & RESIN & 252 \\
\hline 2935 & $\mathrm{pCi} / \mathrm{L}$ & 10 & & & & & & RIVER & & CUMULATIVE & UNFILTERED & 463 \\
\hline 2936 & $\mathrm{pCi} / \mathrm{L}$ & 1.4 & & & & $U$ & & RIVER & & CUMULATIVE & UNFILTERED & 463 \\
\hline 2937 & $\mathrm{pCi} / \mathrm{L}$ & 0.21 & & & & & & SURFACE & & RAW GRAB & UNFILTERED & 509 \\
\hline 2938 & $\mathrm{pCi} / \mathrm{L}$ & 1.2 & & & & & & SURFACE & & RAW GRAB & UNFILTERED & 509 \\
\hline 2939 & $\mathrm{pCi} / \mathrm{L}$ & 0.00003 & & & & & & RIVER & & CONTINUOUS & RESIN & 307 \\
\hline 2940 & $\mathrm{pCi} / \mathrm{L}$ & 0.01 & & & & U & & RIVER & & CONTINUOUS & RESIN & 307 \\
\hline 2941 & $\mathrm{pCi} / \mathrm{L}$ & & 0.0000049 & & & & & RIVER & & CONTINUOUS & RESIN & 307 \\
\hline 2942 & $\mathrm{pCi} / \mathrm{L}$ & 0.007 & & & & U & & RIVER & & CONTINUOUS & FILTER & 307 \\
\hline 2943 & $\mathrm{pCi} / \mathrm{L}$ & 0.000002 & & & & & & RIVER & & CONTINUOUS & FILTER & 307 \\
\hline 2944 & $\mathrm{pCi} / \mathrm{L}$ & 0.00005 & & & & & & RIVER & & CONTINUOUS & RESIN & 252 \\
\hline 2945 & $\mathrm{pCi} / \mathrm{L}$ & 0.000002 & & & & & & RIVER & & CONTINUOUS & FILTER & 252 \\
\hline \begin{tabular}{|l|}
2946 \\
\end{tabular} & $\mathrm{pCi} / \mathrm{L}$ & 0.045 & & & & U & & SANITARY & & CUMULATIVE & UNFILTERED & 512 \\
\hline \begin{tabular}{|l|}
2947 \\
\end{tabular} & $\mathrm{pCi} / \mathrm{L}$ & 0.34 & & & & $U$ & & SANITARY & & CUMULATIVE & UNFILTERED & 512 \\
\hline 2948 & $\mathrm{pCi} / \mathrm{L}$ & 510 & & & & & & SANITARY & & CUMULATIVE & UNFILTERED & 512 \\
\hline 2949 & $\mathrm{pCi} / \mathrm{L}$ & 0.045 & & & & & & SANITARY & & CUMULATIVE & UNFILTERED & 385 \\
\hline 2950 & $\mathrm{pCi} / \mathrm{L}$ & 0.73 & & & & $U$ & & SANITARY & & CUMULATIVE & UNFILTERED & 385 \\
\hline 2951 & $\mathrm{pCi} / \mathrm{L}$ & 31 & & & & & & RIVER & & CUMULATIVE & UNFILTERED & 252 \\
\hline 2952 & $\mathrm{pCi} / \mathrm{L}$ & 0.045 & & & & & & RIVER & & CUMULATIVE & UNFILTERED & 252 \\
\hline 2953 & $\mathrm{pCi} / \mathrm{L}$ & 1.5 & & & & U & & RIVER & & CUMULATIVE & UNFILTERED & 252 \\
\hline 2954 & $\mathrm{pCi} / \mathrm{L}$ & 0.02 & & & & U & & RIVER & & CONTINUOUS & RESIN & 252 \\
\hline 2955 & $\mathrm{pCi} / \mathrm{L}$ & & 0.0000021 & & & & & RIVER & & CONTINUOUS & RESIN & 252 \\
\hline 2956 & $\mathrm{pCi} / \mathrm{L}$ & 0.009 & & & & $U$ & & RIVER & & CONTINUOUS & FILTER & 252 \\
\hline 2957 & $\mathrm{pCi} / \mathrm{L}$ & 31 & & & & & & RIVER & & CUMULATIVE & UNFILTERED & 463 \\
\hline 2958 & $\mathrm{pCi} / \mathrm{L}$ & 0.045 & & & & & & RIVER & & CUMULATIVE & UNFILTERED & 463 \\
\hline 2959 & $\mathrm{pCi} / \mathrm{L}$ & 1.5 & & & & $U$ & & RIVER & & CUMULATIVE & UNFILTERED & 463 \\
\hline 2960 & $\mathrm{pCi} / \mathrm{L}$ & 0.056 & & & & & & SURFACE & & RAW GRAB & UNFILTERED & 509 \\
\hline 2961 & $\mathrm{pCi} / \mathrm{L}$ & 4.1 & & & & & & SURFACE & & RAW GRAB & UNFILTERED & 509 \\
\hline 2962 & $\mathrm{pCi} / \mathrm{L}$ & 1.4 & & & & U & & SURFACE & & RAW GRAB & UNFILTERED & 136 \\
\hline 2963 & $\mathrm{pCi} / \mathrm{L}$ & 4.7 & & & & & & SURFACE & & RAW GRAB & UNFILTERED & 146 \\
\hline 2964 & $\mathrm{pCi} / \mathrm{L}$ & 0.056 & & & & & & SURFACE & & RAW GRAB & UNFILTERED & 146 \\
\hline 2965 & $\mathrm{pCi} / \mathrm{L}$ & 5.1 & & & & $u$ & & SURFACE & & RAW GRAB & UNFILTERED & 147 \\
\hline
\end{tabular}




\begin{tabular}{|c|c|c|c|c|c|c|c|c|c|c|c|c|}
\hline & $\mathrm{N}$ & $\mathrm{O}$ & $\mathrm{P}$ & $\mathrm{Q}$ & $\mathrm{R}$ & $\mathrm{s}$ & $T$ & $\bar{U}$ & $\mathrm{~V}$ & $\mathrm{~W}$ & $\mathrm{X}$ & $\bar{Y}$ \\
\hline 1 & ANAL_UNITS_RPTD & COUNTING_ERROR & TOTAL_ANAL_ERROR & MIN_DETECTABLE_ACTIVITY & PCNT_MOISTURE & LAB_QUALIFIER & REVIEW_QUALIFIER & SAMP_FROM & SAMP_ITEM & \begin{tabular}{|l|} 
SAMP_MTHD \\
\end{tabular} & COLL_MTHD & SAMP_SITE_ID \\
\hline 2966 & pCi/L & 0.11 & & & & & & SURFACE & & RAW GRAB & UNFILTERED & 147 \\
\hline $2967 \mid$ & $\mathrm{pCi} / \mathrm{L}$ & 0.045 & & & & U & & QC_SAMPLE & & RAW GRAB & UNFILTERED & 223 \\
\hline 2968 & $\mathrm{pCi} / \mathrm{L}$ & 0.009 & & & & U & & RIVER & & CONTINUOUS & RESIN & 307 \\
\hline 2969 & $\mathrm{pCi} / \mathrm{L}$ & 0.005 & & & & & & RIVER & & CONTINUOUS & FILTER & 307 \\
\hline 2970 & $\mathrm{pCi} / \mathrm{L}$ & 0.009 & & & & $U$ & & RIVER & & CONTINUOUS & RESIN & 252 \\
\hline 2971 & $\mathrm{pCi} / \mathrm{L}$ & 0.004 & & & & & & RIVER & & CONTINUOUS & FILTER & 252 \\
\hline 2972 & $\mathrm{pCi} / \mathrm{L}$ & 0.007 & & & & U & & RIVER & & CONTINUOUS & RESIN & 307 \\
\hline 2973 & $\mathrm{pCi} / \mathrm{L}$ & & 0.00000384 & & & & & RIVER & & CONTINUOUS & RESIN & 307 \\
\hline 2974 & $\mathrm{pCi} / \mathrm{L}$ & 0.004 & & & & & & RIVER & & CONTINUOUS & FILTER & 307 \\
\hline 2975 & $\mathrm{pCi} / \mathrm{L}$ & 0.009 & & & & U & & RIVER & & CONTINUOUS & RESIN & 252 \\
\hline 2976 & $\mathrm{pCi} / \mathrm{L}$ & & 0.0000011 & & & & & RIVER & & CONTINUOUS & RESIN & 252 \\
\hline 2977 & $\mathrm{pCi} / \mathrm{L}$ & 0.004 & & & & & & RIVER & & CONTINUOUS & FILTER & 252 \\
\hline 2978 & $\mathrm{pCi} / \mathrm{L}$ & 0.045 & & & & & & SANITARY & & CUMULATIVE & UNFILTERED & 491 \\
\hline 2979 & $\mathrm{pCi} / \mathrm{L}$ & 120 & & & & & & SANITARY & & CUMULATIVE & UNFILTERED & 491 \\
\hline 2980 & $\mathrm{pCi} / \mathrm{L}$ & 0.045 & & & & $U$ & & SANITARY & & CUMULATIVE & UNFILTERED & 512 \\
\hline \begin{tabular}{|l|}
2981 \\
\end{tabular} & $\mathrm{pCi} / \mathrm{L}$ & 390 & & & & & & SANITARY & & CUMULATIVE & UNFILTERED & 512 \\
\hline 2982 & $\mathrm{pCi} / \mathrm{L}$ & 0.72 & & & & $U$ & & SANITARY & & CUMULATIVE & UNFILTERED & 385 \\
\hline 2983 & $\mathrm{pCi} / \mathrm{L}$ & 31 & & & & & & RIVER & & CUMULATIVE & UNFILTERED & 252 \\
\hline 2984 & $\mathrm{pCi} / \mathrm{L}$ & 1.4 & & & & $U$ & & RIVER & & CUMULATIVE & UNFILTERED & 252 \\
\hline 2985 & $\mathrm{pCi} / \mathrm{L}$ & 31 & & & & & & RIVER & & CUMULATIVE & UNFILTERED & 463 \\
\hline 2986 & $\mathrm{pCi} / \mathrm{L}$ & 1.4 & & & & U & & RIVER & & CUMULATIVE & UNFILTERED & 463 \\
\hline \begin{tabular}{|l|}
2987 \\
\end{tabular} & $\mathrm{pCi} / \mathrm{L}$ & 0.045 & & & & & & DRINKING & & SAN. GRAB & UNFILTERED & 187 \\
\hline 2988 & $\mathrm{pCi} / \mathrm{L}$ & 100 & & & & & & DRINKING & & SAN. GRAB & UNFILTERED & 187 \\
\hline 2989 & $\mathrm{pCi} / \mathrm{L}$ & 0.009 & & & & $U$ & & RIVER & & CONTINUOUS & RESIN & 307 \\
\hline 2990 & $\mathrm{pCi} / \mathrm{L}$ & 0.004 & & & & & & RIVER & & CONTINUOUS & FILTER & 307 \\
\hline 2991 & $\mathrm{pCi} / \mathrm{L}$ & 0.01 & & & & $U$ & & RIVER & & CONTINUOUS & RESIN & 252 \\
\hline 2992 & $\mathrm{pCi} / \mathrm{L}$ & 0.006 & & & & & & RIVER & & CONTINUOUS & FILTER & 252 \\
\hline 2993 & $\mathrm{pCi} / \mathrm{L}$ & 0.73 & & & & U & & DRINKING & & RAW GRAB & UNFILTERED & 553 \\
\hline 2994 & $\mathrm{pCi} / \mathrm{L}$ & 0.045 & & & & & & DRINKING & & RAW GRAB & UNFILTERED & 553 \\
\hline 2995 & $\mathrm{pCi} / \mathrm{L}$ & 21 & & & & & & DRINKING & & RAW GRAB & UNFILTERED & 553 \\
\hline 2996 & pCi/L & 0.079 & & & & & & SURFACE & & RAW GRAB & UNFILTERED & 509 \\
\hline \begin{tabular}{|l|}
2997 \\
\end{tabular} & $\mathrm{pCi} / \mathrm{L}$ & 1.1 & & & & & & SURFACE & & RAW GRAB & UNFILTERED & 509 \\
\hline 2998 & $\mathrm{pCi} / \mathrm{L}$ & 0.00003 & & & & & & RIVER & & CONTINUOUS & RESIN & 307 \\
\hline 2999 & $\mathrm{pCi} / \mathrm{L}$ & 0.008 & & & & $U$ & & RIVER & & CONTINUOUS & RESIN & 307 \\
\hline 3000 & $\mathrm{pCi} / \mathrm{L}$ & & 0.00000173 & & & & & RIVER & & CONTINUOUS & RESIN & 307 \\
\hline 3001 & $\mathrm{pCi} / \mathrm{L}$ & 0.003 & & & & $U$ & & RIVER & & CONTINUOUS & FILTER & 307 \\
\hline 3002 & $\mathrm{pCi} / \mathrm{L}$ & 0.000004 & & & & & & RIVER & & CONTINUOUS & FILTER & 307 \\
\hline 3003 & $\mathrm{pCi} / \mathrm{L}$ & 0.000002 & & & & & & RIVER & & CONTINUOUS & FILTER & 252 \\
\hline 3004 & $\mathrm{pCi} / \mathrm{L}$ & 0.008 & & & & & & RIVER & & CONTINUOUS & FILTER & 252 \\
\hline 3005 & $\mathrm{pCi} / \mathrm{L}$ & 0.02 & & & & & & RIVER & & CONTINUOUS & RESIN & 252 \\
\hline 3006 & $\mathrm{pCi} / \mathrm{L}$ & 0.00004 & & & & & & RIVER & & CONTINUOUS & RESIN & 252 \\
\hline 3007 & $\mathrm{pCi} / \mathrm{L}$ & & 0.0000154 & & & $U$ & & RIVER & & CONTINUOUS & RESIN & 252 \\
\hline 3008 & $\mathrm{pCi} / \mathrm{L}$ & 0.045 & & & & $U$ & & SANITARY & & CUMULATIVE & UNFILTERED & 512 \\
\hline 3009 & $\mathrm{pCi} / \mathrm{L}$ & 600 & & & & & & SANITARY & & CUMULATIVE & UNFILTERED & 512 \\
\hline 3010 & $\mathrm{pCi} / \mathrm{L}$ & 0.73 & & & & $U$ & & SANITARY & & CUMULATIVE & UNFILTERED & 385 \\
\hline 3011 & $\mathrm{pCi} / \mathrm{L}$ & 21 & & & & & & RIVER & & CUMULATIVE & UNFILTERED & 252 \\
\hline 3012 & $\mathrm{pCi} / \mathrm{L}$ & 1.5 & & & & $U$ & & RIVER & & CUMULATIVE & UNFILTERED & 252 \\
\hline 3013 & $\mathrm{pCi} / \mathrm{L}$ & 20 & & & & & & RIVER & & CUMULATIVE & UNFILTERED & 463 \\
\hline 3014 & $\mathrm{pCi} / \mathrm{L}$ & 1.4 & & & & U & & RIVER & & CUMULATIVE & UNFILTERED & 463 \\
\hline 3015 & $\mathrm{pCi} / \mathrm{L}$ & 0.005 & & & & & & RIVER & & CONTINUOUS & RESIN & 307 \\
\hline 3016 & $\mathrm{pCi} / \mathrm{L}$ & 0.002 & & & & & & RIVER & & CONTINUOUS & FILTER & 307 \\
\hline 3017 & $\mathrm{pCi} / \mathrm{L}$ & 0.004 & & & & & & RIVER & & CONTINUOUS & FILTER & 252 \\
\hline
\end{tabular}




\begin{tabular}{|c|c|c|c|c|c|c|c|c|c|c|c|c|}
\hline & $\mathrm{N}$ & $\mathrm{O}$ & $\mathrm{P}$ & $\mathrm{Q}$ & $\mathrm{R}$ & $\mathrm{s}$ & $T$ & $\mathrm{U}$ & $\mathrm{V}$ & $\mathrm{W}$ & $x$ & $\bar{Y}$ \\
\hline 1 & ANAL_UNITS_RPTD & COUNTING_ERROR & ||$_{\text {TOTAL_ANAL_ERROR | }}$ & MIN_DETECTABLE_ACTIVITY & PCNT_MOISTURE & LAB_QUALIFIER & REVIEW_QUALIFIER & SAMP_FROM & SAMP_ITEM & SAMP_MTHD & COLL_MTHD & SAMP_SITE_ID \\
\hline 3018 & $\mathrm{pCi} / \mathrm{L}$ & 0.007 & & & & & & RIVER & & CONTINUOUS & RESIN & 252 \\
\hline 3019 & $\mathrm{pCi} / \mathrm{L}$ & 0.063 & & & & & & SURFACE & & RAW GRAB & UNFILTERED & 509 \\
\hline 3020 & $\mathrm{pCi} / \mathrm{L}$ & 3.7 & & & & & & SURFACE & & RAW GRAB & UNFILTERED & 509 \\
\hline 3021 & $\mathrm{pCi} / \mathrm{L}$ & 0.006 & & & & & & RIVER & & CONTINUOUS & RESIN & 307 \\
\hline 3022 & $\mathrm{pCi} / \mathrm{L}$ & 0.003 & & & & & & RIVER & & CONTINUOUS & FILTER & 307 \\
\hline 3023 & $\mathrm{pCi} / \mathrm{L}$ & 0.003 & & & & & & RIVER & & CONTINUOUS & FILTER & 252 \\
\hline 3024 & $\mathrm{pCi} / \mathrm{L}$ & 0.01 & & & & $\mathrm{u}$ & & RIVER & & CONTINUOUS & RESIN & 252 \\
\hline 3025 & $\mathrm{pCi} / \mathrm{L}$ & 0.045 & & & & & & SANITARY & & CUMULATIVE & UNFILTERED & 512 \\
\hline 3026 & $\mathrm{pCi} / \mathrm{L}$ & 0.24 & & & & U & & SANITARY & & CUMULATIVE & UNFILTERED & 512 \\
\hline 3027 & $\mathrm{pCi} / \mathrm{L}$ & 1900 & & & & & & SANITARY & & CUMULATIVE & UNFILTERED & 512 \\
\hline 3028 & $\mathrm{pCi} / \mathrm{L}$ & 0.045 & & & & & & SANITARY & & CUMULATIVE & UNFILTERED & 385 \\
\hline 3029 & $\mathrm{pCi} / \mathrm{L}$ & 0.73 & & & & U & & SANITARY & & CUMULATIVE & UNFILTERED & 385 \\
\hline 3030 & $\mathrm{pCi} / \mathrm{L}$ & 22 & & & & & & RIVER & & CUMULATIVE & UNFILTERED & 252 \\
\hline 3031 & $\mathrm{pCi} / \mathrm{L}$ & 0.045 & & & & & & RIVER & & CUMULATIVE & UNFILTERED & 252 \\
\hline 3032 & $\mathrm{pCi} / \mathrm{L}$ & 1.4 & & & & $U$ & & RIVER & & CUMULATIVE & UNFILTERED & 252 \\
\hline 3033 & $\mathrm{pCi} / \mathrm{L}$ & 22 & & & & & & RIVER & & CUMULATIVE & UNFILTERED & 463 \\
\hline 3034 & $\mathrm{pCi} / \mathrm{L}$ & 0.045 & & & & & & RIVER & & CUMULATIVE & UNFILTERED & 463 \\
\hline 3035 & $\mathrm{pCi} / \mathrm{L}$ & 1.5 & & & & $U$ & & RIVER & & CUMULATIVE & UNFILTERED & 463 \\
\hline 3036 & $\mathrm{pCi} / \mathrm{L}$ & 0.045 & & & & & & SANITARY & & CUMULATIVE & UNFILTERED & 491 \\
\hline 3037 & $\mathrm{pCi} / \mathrm{L}$ & 130 & & & & & & SANITARY & & CUMULATIVE & UNFILTERED & 491 \\
\hline 3038 & pCi/L & 0.002 & & & & & & RIVER & & CONTINUOUS & FILTER & 307 \\
\hline 3039 & pCi/L & 0.006 & & & & U & & RIVER & & CONTINUOUS & RESIN & 307 \\
\hline 3040 & $\mathrm{pCi} / \mathrm{L}$ & & 0.00000168 & & & & & RIVER & & CONTINUOUS & RESIN & 307 \\
\hline 3041 & $\mathrm{pCi} / \mathrm{L}$ & 0.03 & & & & U & & RIVER & & CONTINUOUS & FILTER & 252 \\
\hline 3042 & $\mathrm{pCi} / \mathrm{L}$ & 0.008 & & & & $U$ & & RIVER & & CONTINUOUS & RESIN & 252 \\
\hline 3043 & $\mathrm{pCi} / \mathrm{L}$ & & 0.00000125 & & & & & RIVER & & CONTINUOUS & RESIN & 252 \\
\hline 3044 & $\mathrm{pCi} / \mathrm{L}$ & 0.021 & & & & & & SURFACE & & RAW GRAB & UNFILTERED & 509 \\
\hline 3045 & $\mathrm{pCi} / \mathrm{L}$ & 2.3 & & & & & & SURFACE & & RAW GRAB & UNFILTERED & 509 \\
\hline 3046 & $\mathrm{pCi} / \mathrm{L}$ & 1.4 & & & & U & & SURFACE & & RAW GRAB & UNFILTERED & 136 \\
\hline 3047 & $\mathrm{pCi} / \mathrm{L}$ & 0.92 & & & & & & SURFACE & & RAW GRAB & UNFILTERED & 146 \\
\hline 3048 & $\mathrm{pCi} / \mathrm{L}$ & 0.56 & & & & $U$ & & SURFACE & & RAW GRAB & UNFILTERED & 146 \\
\hline 3049 & $\mathrm{pCi} / \mathrm{L}$ & 0.045 & & & & & & QC_SAMPLE & & RAW GRAB & UNFILTERED & 223 \\
\hline 3050 & $\mathrm{pCi} / \mathrm{L}$ & 0.003 & & & & & & RIVER & & CONTINUOUS & RESIN & 307 \\
\hline 3051 & pCi/L & 0.003 & & & & $U$ & & RIVER & & CONTINUOUS & FILTER & 307 \\
\hline 3052 & pCi/L & 0.006 & & & & & & RIVER & & CONTINUOUS & RESIN & 252 \\
\hline 3053 & pCi/L & 0.003 & & & & $U$ & & RIVER & & CONTINUOUS & FILTER & 252 \\
\hline 3054 & $\mathrm{pCi} / \mathrm{L}$ & 0.05 & & & & $U$ & & DRINKING & & SAN. GRAB & UNFILTERED & 187 \\
\hline 3055 & $\mathrm{pCi} / \mathrm{L}$ & 110 & & & & & & DRINKING & & SAN. GRAB & UNFILTERED & 187 \\
\hline 3056 & $\mathrm{pCi} / \mathrm{L}$ & 0.045 & & & & $U$ & & SANITARY & & CUMULATIVE & UNFILTERED & 512 \\
\hline 3057 & $\mathrm{pCi} / \mathrm{L}$ & 390 & & & & & & SANITARY & & CUMULATIVE & UNFILTERED & 512 \\
\hline 3058 & $\mathrm{pCi} / \mathrm{L}$ & 0.72 & & & & U & & SANITARY & & CUMULATIVE & UNFILTERED & 385 \\
\hline 3059 & pCi/L & 0.008 & & & & $U$ & & RIVER & & CONTINUOUS & RESIN & 307 \\
\hline 3060 & $\mathrm{pCi} / \mathrm{L}$ & & 0.0000057 & & & & & RIVER & & CONTINUOUS & RESIN & 307 \\
\hline 3061 & $\mathrm{pCi} / \mathrm{L}$ & 0.002 & & & & & & RIVER & & CONTINUOUS & FILTER & 307 \\
\hline 3062 & $\mathrm{pCi} / \mathrm{L}$ & 0.003 & & & & $U$ & & RIVER & & CONTINUOUS & FILTER & 252 \\
\hline 3063 & $\mathrm{pCi} / \mathrm{L}$ & 0.009 & & & & U & & RIVER & & CONTINUOUS & RESIN & 252 \\
\hline 3064 & $\mathrm{pCi} / \mathrm{L}$ & & 0.0000013 & & & & & RIVER & & CONTINUOUS & RESIN & 252 \\
\hline 3065 & $\mathrm{pCi} / \mathrm{L}$ & 22 & & & & & & RIVER & & CUMULATIVE & UNFILTERED & 252 \\
\hline 3066 & pCi/L & 1.4 & & & & U & & RIVER & & CUMULATIVE & UNFILTERED & 252 \\
\hline 3067 & pCi/L & 22 & & & & & & RIVER & & CUMULATIVE & UNFILTERED & 463 \\
\hline 3068 & $\mathrm{pCi} / \mathrm{L}$ & 1.4 & & & & $U$ & & RIVER & & CUMULATIVE & UNFILTERED & 463 \\
\hline 3069 & $\mathrm{pCi} / \mathrm{L}$ & 0.13 & & & & & & SURFACE & & RAW GRAB & UNFILTERED & 509 \\
\hline
\end{tabular}




\begin{tabular}{|c|c|c|c|c|c|c|c|c|c|c|c|c|}
\hline & $\mathrm{N}$ & $\mathrm{O}$ & $\mathrm{P}$ & $\mathrm{Q}$ & $\mathrm{R}$ & $\mathrm{S}$ & $T$ & $\bar{U}$ & $\mathrm{~V}$ & $\mathrm{~W}$ & $\mathrm{X}$ & $\overline{\mathrm{Y}}$ \\
\hline 1 & ANAL_UNITS_RPTD & COUNTING_ERROR & $\mid$ TOTAL_ANAL_ERROR | $^{\prime}$ & MIN_DETECTABLE_ACTIVITY & PCNT_MOISTURE & LAB_QUALIFIER & REVIEW_QUALIFIER & SAMP_FROM & SAMP_ITEM & SAMP_MTHD & COLL_MTHD & SAMP_SITE_ID \\
\hline 3070 & $\mathrm{pCi} / \mathrm{L}$ & 1.1 & & & & & & SURFACE & & RAW GRAB & UNFILTERED & 509 \\
\hline 3071 & $\mathrm{pCi} / \mathrm{L}$ & 0.002 & & & & & & RIVER & & CONTINUOUS & FILTER & 307 \\
\hline 3072 & $\mathrm{pCi} / \mathrm{L}$ & 0.01 & & & & & & RIVER & & CONTINUOUS & RESIN & 307 \\
\hline 3073 & $\mathrm{pCi} / \mathrm{L}$ & 0.006 & & & & & & RIVER & & CONTINUOUS & RESIN & 252 \\
\hline 3074 & $\mathrm{pCi} / \mathrm{L}$ & 0.003 & & & & U & & RIVER & & CONTINUOUS & FILTER & 252 \\
\hline 3075 & $\mathrm{pCi} / \mathrm{L}$ & 0.063 & & & & & & DRINKING & & RAW GRAB & UNFILTERED & 553 \\
\hline 3076 & $\mathrm{pCi} / \mathrm{L}$ & 22 & & & & & & DRINKING & & RAW GRAB & UNFILTERED & 553 \\
\hline 3077 & $\mathrm{pCi} / \mathrm{L}$ & 0.054 & & & & U & & SANITARY & & CUMULATIVE & UNFILTERED & 512 \\
\hline 3078 & $\mathrm{pCi} / \mathrm{L}$ & 950 & & & & & & SANITARY & & CUMULATIVE & UNFILTERED & 512 \\
\hline 3079 & $\mathrm{pCi} / \mathrm{L}$ & 0.73 & & & & $\mathrm{U}$ & & SANITARY & & CUMULATIVE & UNFILTERED & 385 \\
\hline 3080 & $\mathrm{pCi} / \mathrm{L}$ & 0.00002 & & & & & & RIVER & & CONTINUOUS & RESIN & 307 \\
\hline 3081 & $\mathrm{pCi} / \mathrm{L}$ & 0.009 & & & & & & RIVER & & CONTINUOUS & RESIN & 307 \\
\hline 3082 & $\mathrm{pCi} / \mathrm{L}$ & & 0.0000182 & & & & & RIVER & & CONTINUOUS & RESIN & 307 \\
\hline 3083 & $\mathrm{pCi} / \mathrm{L}$ & 0.005 & & & & & & RIVER & & CONTINUOUS & FILTER & 307 \\
\hline 3084 & $\mathrm{pCi} / \mathrm{L}$ & 0.000002 & & & & & & RIVER & & CONTINUOUS & FILTER & 307 \\
\hline 3085 & $\mathrm{pCi} / \mathrm{L}$ & 0.000003 & & & & & & RIVER & & CONTINUOUS & FILTER & 252 \\
\hline 3086 & $\mathrm{pCi} / \mathrm{L}$ & 0.004 & & & & & & RIVER & & CONTINUOUS & FILTER & 252 \\
\hline 3087 & $\mathrm{pCi} / \mathrm{L}$ & 0.008 & & & & & & RIVER & & CONTINUOUS & RESIN & 252 \\
\hline 3088 & $\mathrm{pCi} / \mathrm{L}$ & 0.00004 & & & & & & RIVER & & CONTINUOUS & RESIN & 252 \\
\hline 3089 & $\mathrm{pCi} / \mathrm{L}$ & & 0.00000118 & & & & & RIVER & & CONTINUOUS & RESIN & 252 \\
\hline 3090 & $\mathrm{pCi} / \mathrm{L}$ & 22 & & & & & & RIVER & & CUMULATIVE & UNFILTERED & 252 \\
\hline 3091 & $\mathrm{pCi} / \mathrm{L}$ & 1.4 & & & & U & & RIVER & & CUMULATIVE & UNFILTERED & 252 \\
\hline 3092 & $\mathrm{pCi} / \mathrm{L}$ & 24 & & & & & & RIVER & & CUMULATIVE & UNFILTERED & 463 \\
\hline 3093 & $\mathrm{pCi} / \mathrm{L}$ & 1.4 & & & & U & & RIVER & & CUMULATIVE & UNFILTERED & 463 \\
\hline 3094 & $\mathrm{pCi} / \mathrm{L}$ & 0.007 & & & & & & RIVER & & CONTINUOUS & RESIN & 307 \\
\hline 3095 & $\mathrm{pCi} / \mathrm{L}$ & 0.003 & & & & & & RIVER & & CONTINUOUS & FILTER & 307 \\
\hline 3096 & $\mathrm{pCi} / \mathrm{L}$ & 0.003 & & & & & & RIVER & & CONTINUOUS & FILTER & 252 \\
\hline 3097 & $\mathrm{pCi} / \mathrm{L}$ & 0.006 & & & & & & RIVER & & CONTINUOUS & RESIN & 252 \\
\hline 3098 & $\mathrm{pCi} / \mathrm{L}$ & 0.13 & & & & & & SURFACE & & RAW GRAB & UNFILTERED & 509 \\
\hline 3099 & $\mathrm{pCi} / \mathrm{L}$ & 7.4 & & & & & & SURFACE & & RAW GRAB & UNFILTERED & 509 \\
\hline 3100 & $\mathrm{pCi} / \mathrm{L}$ & 0.045 & & & & U & & DRINKING & & SAN. GRAB & UNFILTERED & 487 \\
\hline 3101 & $\mathrm{pCi} / \mathrm{L}$ & 7.3 & & & & U & & DRINKING & & SAN. GRAB & UNFILTERED & 487 \\
\hline 3102 & $\mathrm{pCi} / \mathrm{L}$ & 94 & & & & & & DRINKING & & SAN. GRAB & UNFILTERED & 487 \\
\hline 3103 & $\mathrm{pCi} / \mathrm{L}$ & 0.054 & & & & U & & SANITARY & & CUMULATIVE & UNFILTERED & 512 \\
\hline 3104 & $\mathrm{pCi} / \mathrm{L}$ & 0.19 & & & & U & & SANITARY & & CUMULATIVE & UNFILTERED & 512 \\
\hline 3105 & $\mathrm{pCi} / \mathrm{L}$ & 370 & & & & & & SANITARY & & CUMULATIVE & UNFILTERED & 512 \\
\hline 3106 & $\mathrm{pCi} / \mathrm{L}$ & 0.01 & & & & & & RIVER & & CONTINUOUS & RESIN & 307 \\
\hline 3107 & $\mathrm{pCi} / \mathrm{L}$ & & 0.0000109 & & & & & RIVER & & CONTINUOUS & RESIN & 307 \\
\hline 3108 & $\mathrm{pCi} / \mathrm{L}$ & 0.006 & & & & & & RIVER & & CONTINUOUS & FILTER & 307 \\
\hline 3109 & $\mathrm{pCi} / \mathrm{L}$ & & 0 & 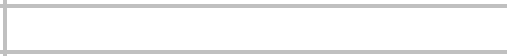 & & & & RIVER & & CONTINUOUS & RESIN & 290 \\
\hline 3110 & $\mathrm{pCi} / \mathrm{L}$ & 0.01 & & & & & & RIVER & & CONTINUOUS & RESIN & 252 \\
\hline 3111 & $\mathrm{pCi} / \mathrm{L}$ & & 0.00000101 & & & & & RIVER & & CONTINUOUS & RESIN & 252 \\
\hline 3112 & $\mathrm{pCi} / \mathrm{L}$ & 0.004 & & & & & & RIVER & & CONTINUOUS & FILTER & 252 \\
\hline 3113 & $\mathrm{pCi} / \mathrm{L}$ & 1.4 & & & & U & & RIVER & & CUMULATIVE & UNFILTERED & 463 \\
\hline 3114 & $\mathrm{pCi} / \mathrm{L}$ & 0.063 & & & & & & SANITARY & & CUMULATIVE & UNFILTERED & 491 \\
\hline 3115 & $\mathrm{pCi} / \mathrm{L}$ & 340 & & & & & & SANITARY & & CUMULATIVE & UNFILTERED & 491 \\
\hline 3116 & $\mathrm{pCi} / \mathrm{L}$ & 0.045 & & & & & & SANITARY & & CUMULATIVE & UNFILTERED & 385 \\
\hline 3117 & $\mathrm{pCi} / \mathrm{L}$ & 0.73 & & & & U & & SANITARY & & CUMULATIVE & UNFILTERED & 385 \\
\hline 3118 & $\mathrm{pCi} / \mathrm{L}$ & 22 & & & & & & RIVER & & CUMULATIVE & UNFILTERED & 290 \\
\hline 3119 & $\mathrm{pCi} / \mathrm{L}$ & 22 & & & & & & RIVER & & CUMULATIVE & UNFILTERED & 252 \\
\hline 3120 & $\mathrm{pCi} / \mathrm{L}$ & 0.063 & & & & & & RIVER & & CUMULATIVE & UNFILTERED & 252 \\
\hline 3121 & $\mathrm{pCi} / \mathrm{L}$ & 1.4 & & & & U & & RIVER & & CUMULATIVE & UNFILTERED & 252 \\
\hline
\end{tabular}




\begin{tabular}{|c|c|c|c|c|c|c|c|c|c|c|c|c|}
\hline & $\mathrm{N}$ & $\mathrm{O}$ & $\mathrm{P}$ & $\mathrm{Q}$ & $\mathrm{R}$ & $\mathrm{s}$ & $\mathrm{T}$ & $\mathrm{u}$ & $\mathrm{v}$ & $\mathrm{w}$ & $\mathrm{x}$ & $\bar{Y}$ \\
\hline 1 & ANAL_UNITS_RPTD & COUNTING_ERROR & TOTAL_ANAL_ERROR & MIN_DETECTABLE_ACTIVITY & PCNT_MOISTURE & LAB_QUALIFIER & REVIEW_QUALIFIER & SAMP_FROM & SAMP_ITEM & SAMP_MTHD & COLL_MTHD & SAMP_SITE_ID \\
\hline 3122 & $\mathrm{pCi} / \mathrm{L}$ & 22 & & & & & & RIVER & & CUMULATIVE & UNFILTERED & 463 \\
\hline 3123 & $\mathrm{pCi} / \mathrm{L}$ & 0.063 & & & & & & RIVER & & CUMULATIVE & UNFILTERED & 463 \\
\hline 3124 & $\mathrm{pCi} / \mathrm{L}$ & 1.4 & & & & $\mathrm{U}$ & & RIVER & & CUMULATIVE & UNFILTERED & 463 \\
\hline 3125 & $\mathrm{pCi} / \mathrm{L}$ & 0.063 & & & & & & DRINKING & & SAN. GRAB & UNFILTERED & 187 \\
\hline 3126 & $\mathrm{pCi} / \mathrm{L}$ & 140 & & & & & & DRINKING & & SAN. GRAB & UNFILTERED & 187 \\
\hline 3127 & $\mathrm{pCi} / \mathrm{L}$ & 0.005 & & & & & & RIVER & & CONTINUOUS & RESIN & 307 \\
\hline 3128 & $\mathrm{pCi} / \mathrm{L}$ & 0.003 & & & & & & RIVER & & CONTINUOUS & FILTER & 307 \\
\hline 3129 & $\mathrm{pCi} / \mathrm{L}$ & 0.006 & & & & & & RIVER & & CONTINUOUS & FILTER & 252 \\
\hline 3130 & $\mathrm{pCi} / \mathrm{L}$ & 0.008 & & & & & & RIVER & & CONTINUOUS & RESIN & 252 \\
\hline 3131 & $\mathrm{pCi} / \mathrm{L}$ & 0.054 & & & & & & SURFACE & & RAW GRAB & UNFILTERED & 509 \\
\hline 3132 & $\mathrm{pCi} / \mathrm{L}$ & 3.9 & & & & & & SURFACE & & RAW GRAB & UNFILTERED & 509 \\
\hline 3133 & $\mathrm{pCi} / \mathrm{L}$ & 20 & & & & $\mathrm{U}$ & & SURFACE & & RAW GRAB & UNFILTERED & 136 \\
\hline 3134 & $\mathrm{pCi} / \mathrm{L}$ & 1.1 & & & & & & SURFACE & & RAW GRAB & UNFILTERED & 146 \\
\hline 3135 & $\mathrm{pCi} / \mathrm{L}$ & 0.054 & & & & & & SURFACE & & RAW GRAB & UNFILTERED & 146 \\
\hline 3136 & $\mathrm{pCi} / \mathrm{L}$ & 2.6 & & & & U & & SURFACE & & RAW GRAB & UNFILTERED & 147 \\
\hline 3137 & $\mathrm{pCi} / \mathrm{L}$ & 0.14 & & & & & & SURFACE & & RAW GRAB & UNFILTERED & 147 \\
\hline 3138 & $\mathrm{pCi} / \mathrm{L}$ & 0.00004 & & & & & & RIVER & & CONTINUOUS & RESIN & 307 \\
\hline 3139 & $\mathrm{pCi} / \mathrm{L}$ & 0.006 & & & & & & RIVER & & CONTINUOUS & RESIN & 307 \\
\hline 3140 & $\mathrm{pCi} / \mathrm{L}$ & & 0.00000938 & & & & & RIVER & & CONTINUOUS & RESIN & 307 \\
\hline 3141 & $\mathrm{pCi} / \mathrm{L}$ & 0.002 & & & & & & RIVER & & CONTINUOUS & FILTER & 307 \\
\hline 3142 & $\mathrm{pCi} / \mathrm{L}$ & 0.000004 & & & & & & RIVER & & CONTINUOUS & FILTER & 307 \\
\hline 3143 & $\mathrm{pCi} / \mathrm{L}$ & & 0 & & & & & RIVER & & CONTINUOUS & RESIN & 290 \\
\hline 3144 & $\mathrm{pCi} / \mathrm{L}$ & 0.008 & & & & & & RIVER & & CONTINUOUS & RESIN & 252 \\
\hline 3145 & $\mathrm{pCi} / \mathrm{L}$ & 0.00003 & & & & & & RIVER & & CONTINUOUS & RESIN & 252 \\
\hline 3146 & $\mathrm{pCi} / \mathrm{L}$ & & 0.000000976 & & & & & RIVER & & CONTINUOUS & RESIN & 252 \\
\hline 3147 & $\mathrm{pCi} / \mathrm{L}$ & 0.000003 & & & & & & RIVER & & CONTINUOUS & FILTER & 252 \\
\hline 3148 & $\mathrm{pCi} / \mathrm{L}$ & 0.004 & & & & & & RIVER & & CONTINUOUS & FILTER & 252 \\
\hline 3149 & $\mathrm{pCi} / \mathrm{L}$ & 0.045 & & & & & & SANITARY & & CUMULATIVE & UNFILTERED & 512 \\
\hline 3150 & $\mathrm{pCi} / \mathrm{L}$ & 360 & & & & & & SANITARY & & CUMULATIVE & UNFILTERED & 512 \\
\hline 3151 & $\mathrm{pCi} / \mathrm{L}$ & 0.054 & & & & & & SANITARY & & CUMULATIVE & UNFILTERED & 385 \\
\hline 3152 & $\mathrm{pCi} / \mathrm{L}$ & 1.4 & & & & U & & SANITARY & & CUMULATIVE & UNFILTERED & 385 \\
\hline 3153 & $\mathrm{pCi} / \mathrm{L}$ & 22 & & & & & & RIVER & & CUMULATIVE & UNFILTERED & 290 \\
\hline 3154 & $\mathrm{pCi} / \mathrm{L}$ & 22 & & & & & & RIVER & & CUMULATIVE & UNFILTERED & 252 \\
\hline 3155 & $\mathrm{pCi} / \mathrm{L}$ & 1.4 & & & & $U$ & & RIVER & & CUMULATIVE & UNFILTERED & 252 \\
\hline 3156 & $\mathrm{pCi} / \mathrm{L}$ & 22 & & & & & & RIVER & & CUMULATIVE & UNFILTERED & 463 \\
\hline 3157 & $\mathrm{pCi} / \mathrm{L}$ & 1.4 & & & & U & & RIVER & & CUMULATIVE & UNFILTERED & 463 \\
\hline 3158 & $\mathrm{pCi} / \mathrm{L}$ & 0.006 & & & & & & RIVER & & CONTINUOUS & RESIN & 307 \\
\hline 3159 & $\mathrm{pCi} / \mathrm{L}$ & 0.003 & & & & & & RIVER & & CONTINUOUS & FILTER & 307 \\
\hline 3160 & $\mathrm{pCi} / \mathrm{L}$ & 0.008 & & & & & & RIVER & & CONTINUOUS & RESIN & 252 \\
\hline 3161 & $\mathrm{pCi} / \mathrm{L}$ & 0.004 & & & & & & RIVER & & CONTINUOUS & FILTER & 252 \\
\hline 3162 & $\mathrm{pCi} / \mathrm{L}$ & 0.72 & & & & $U$ & & DRINKING & & RAW GRAB & UNFILTERED & 553 \\
\hline 3163 & $\mathrm{pCi} / \mathrm{L}$ & 0.054 & & & & & & DRINKING & & RAW GRAB & UNFILTERED & 553 \\
\hline 3164 & $\mathrm{pCi} / \mathrm{L}$ & 82 & & & & & & DRINKING & & RAW GRAB & UNFILTERED & 553 \\
\hline 3165 & $\mathrm{pCi} / \mathrm{L}$ & 18 & & & & & & RIVER & & CUMULATIVE & UNFILTERED & 290 \\
\hline 3166 & $\mathrm{pCi} / \mathrm{L}$ & 17 & & & & & & RIVER & & CUMULATIVE & UNFILTERED & 252 \\
\hline 3167 & $\mathrm{pCi} / \mathrm{L}$ & 0.054 & & & & & & RIVER & & CUMULATIVE & UNFILTERED & 252 \\
\hline 3168 & $\mathrm{pCi} / \mathrm{L}$ & 1.5 & & & & $U$ & & RIVER & & CUMULATIVE & UNFILTERED & 252 \\
\hline 3169 & $\mathrm{pCi} / \mathrm{L}$ & 19 & & & & & & RIVER & & CUMULATIVE & UNFILTERED & 463 \\
\hline 3170 & $\mathrm{pCi} / \mathrm{L}$ & 0.045 & & & & & & RIVER & & CUMULATIVE & UNFILTERED & 463 \\
\hline 3171 & $\mathrm{pCi} / \mathrm{L}$ & 0.65 & & & & U & & RIVER & & CUMULATIVE & UNFILTERED & 463 \\
\hline 3172 & $\mathrm{pCi} / \mathrm{L}$ & 0.045 & & & & & & SANITARY & & CUMULATIVE & UNFILTERED & 385 \\
\hline 3173 & $\mathrm{pCi} / \mathrm{L}$ & 0.73 & & & & $U_{1}$ & & SANITARY & & CUMULATIVE & UNFILTERED & 385 \\
\hline
\end{tabular}




\begin{tabular}{|c|c|c|c|c|c|c|c|c|c|c|c|c|}
\hline & $\mathrm{N}$ & $\mathrm{O}$ & $\mathrm{P}$ & $\mathrm{Q}$ & $\mathrm{R}$ & $\mathrm{s}$ & $\mathrm{T}$ & $\mathrm{U}$ & $\mathrm{V}$ & $\mathrm{W}$ & $\mathrm{X}$ & $\mathrm{Y}$ \\
\hline 1 & ANAL_UNITS_RPTD & COUNTING_ERROR & TOTAL_ANAL_ERROR & MIN_DETECTABLE_ACTIVITY & PCNT_MOISTURE & LAB_QUALIFIER & REVIEW_QUALIFIER & SAMP_FROM & SAMP_ITEM & SAMP_MTHD & COLL_MTHD & SAMP_SITE_ID \\
\hline 3174 & $\mathrm{pCi} / \mathrm{L}$ & 0.008 & & & & & & RIVER & & CONTINUOUS & RESIN & 307 \\
\hline 3175 & $\mathrm{pCi} / \mathrm{L}$ & 0.003 & & & & & & RIVER & & CONTINUOUS & FILTER & 307 \\
\hline 3176 & $\mathrm{pCi} / \mathrm{L}$ & 0.008 & & & & & & RIVER & & CONTINUOUS & RESIN & 252 \\
\hline 3177 & $\mathrm{pCi} / \mathrm{L}$ & 0.004 & & & & & & RIVER & & CONTINUOUS & FILTER & 252 \\
\hline 3178 & $\mathrm{pCi} / \mathrm{L}$ & 18 & & & & & & RIVER & & CUMULATIVE & UNFILTERED & 290 \\
\hline 3179 & $\mathrm{pCi} / \mathrm{L}$ & 0.054 & & & & & & SANITARY & & CUMULATIVE & UNFILTERED & 491 \\
\hline 3180 & $\mathrm{pCi} / \mathrm{L}$ & 86 & & & & & & SANITARY & & CUMULATIVE & UNFILTERED & 491 \\
\hline 3181 & $\mathrm{pCi} / \mathrm{L}$ & 0.0061 & & & & & & RIVER & & CONTINUOUS & RESIN & 307 \\
\hline 3182 & $\mathrm{pCi} / \mathrm{L}$ & & 0.00000492 & & & & & RIVER & & CONTINUOUS & RESIN & 307 \\
\hline 3183 & $\mathrm{pCi} / \mathrm{L}$ & 0.0026 & & & & & & RIVER & & CONTINUOUS & FILTER & 307 \\
\hline 3184 & $\mathrm{pCi} / \mathrm{L}$ & 0.0056 & & & & & & RIVER & & CONTINUOUS & RESIN & 252 \\
\hline 3185 & $\mathrm{pCi} / \mathrm{L}$ & & 0.0000007 & & & & & RIVER & & CONTINUOUS & RESIN & 252 \\
\hline 3186 & $\mathrm{pCi} / \mathrm{L}$ & 0.0024 & & & & & & RIVER & & CONTINUOUS & FILTER & 252 \\
\hline 3187 & $\mathrm{pCi} / \mathrm{L}$ & 20 & & & & & & RIVER & & CUMULATIVE & UNFILTERED & 252 \\
\hline 3188 & $\mathrm{pCi} / \mathrm{L}$ & 16 & & & & & & RIVER & & CUMULATIVE & UNFILTERED & 463 \\
\hline 3189 & $\mathrm{pCi} / \mathrm{L}$ & 0.0072 & & & & & & RIVER & & CONTINUOUS & RESIN & 307 \\
\hline 3190 & $\mathrm{pCi} / \mathrm{L}$ & 0.0034 & & & & & & RIVER & & CONTINUOUS & FILTER & 307 \\
\hline 3191 & & & & & & U & & RIVER & & CONTINUOUS & RESIN & 252 \\
\hline 3192 & & & & & & U & & RIVER & & CONTINUOUS & FILTER & 252 \\
\hline 3193 & & & & & & U & & RIVER & & CONTINUOUS & RESIN & 252 \\
\hline 3194 & $\mathrm{pCi} / \mathrm{L}$ & 0.075 & & & & & & RIVER & & CUMULATIVE & UNFILTERED & 252 \\
\hline 3195 & $\mathrm{pCi} / \mathrm{L}$ & 0.075 & & & & & & RIVER & & CUMULATIVE & UNFILTERED & 463 \\
\hline 3196 & $\mathrm{pCi} / \mathrm{L}$ & 20 & & & & & & QC_SAMPLE & & RAW GRAB & UNFILTERED & 222 \\
\hline 3197 & $\mathrm{pCi} / \mathrm{L}$ & 0.09 & & & & & & QC_SAMPLE & & RAW GRAB & UNFILTERED & 222 \\
\hline 3198 & $\mathrm{pCi} / \mathrm{L}$ & 1.1 & & & & & & QC_SAMPLE & & RAW GRAB & UNFILTERED & 222 \\
\hline 3199 & $\mathrm{pCi} / \mathrm{L}$ & 18 & & & & U & & QC_SAMPLE & & RAW GRAB & UNFILTERED & 223 \\
\hline 3200 & $\mathrm{pCi} / \mathrm{L}$ & 0.045 & & & & & & QC_SAMPLE & & RAW GRAB & UNFILTERED & 223 \\
\hline 3201 & $\mathrm{pCi} / \mathrm{L}$ & 0.52 & & & & U & & QC_SAMPLE & & RAW GRAB & UNFILTERED & 223 \\
\hline 3202 & $\mathrm{pCi} / \mathrm{L}$ & 14 & & & & & & RIVER & & CUMULATIVE & UNFILTERED & 252 \\
\hline 3203 & $\mathrm{pCi} / \mathrm{L}$ & 20 & & & & & & RIVER & & CUMULATIVE & UNFILTERED & 463 \\
\hline 3204 & $\mathrm{pCi} / \mathrm{L}$ & 0.056 & & & & & & SANITARY & & CUMULATIVE & UNFILTERED & 385 \\
\hline 3205 & $\mathrm{pCi} / \mathrm{L}$ & 1.4 & & & & U & & RIVER & & CUMULATIVE & UNFILTERED & 252 \\
\hline 3206 & $\mathrm{pCi} / \mathrm{L}$ & 1.5 & & & & U & & RIVER & & CUMULATIVE & UNFILTERED & 463 \\
\hline 3207 & $\mathrm{pCi} / \mathrm{L}$ & 280 & & & & & & SANITARY & & CUMULATIVE & UNFILTERED & 512 \\
\hline 3208 & $\mathrm{pCi} / \mathrm{L}$ & 0.72 & & & & U & & SANITARY & & CUMULATIVE & UNFILTERED & 385 \\
\hline 3209 & $\mathrm{pCi} / \mathrm{L}$ & 0.008 & & & & & & RIVER & & CONTINUOUS & RESIN & 307 \\
\hline 3210 & $\mathrm{pCi} / \mathrm{L}$ & & 0.0000042 & & & & & RIVER & & CONTINUOUS & RESIN & 307 \\
\hline 3211 & pCi/L & 0.0032 & & & & & & RIVER & & CONTINUOUS & FILTER & 307 \\
\hline 3212 & & & & & & & & RIVER & & CONTINUOUS & RESIN & 252 \\
\hline 3213 & $\mathrm{pCi} / \mathrm{L}$ & 16 & & & & & & RIVER & & CUMULATIVE & UNFILTERED & 463 \\
\hline 3214 & $\mathrm{pCi} / \mathrm{L}$ & 4.3 & & & & & & QC_SAMPLE & & RAW GRAB & UNFILTERED & 225 \\
\hline 3215 & $\mathrm{pCi} / \mathrm{L}$ & 0.14 & & & & & & QC_SAMPLE & & RAW GRAB & UNFILTERED & 225 \\
\hline 3216 & $\mathrm{pCi} / \mathrm{L}$ & 4.1 & & & & & & QC_SAMPLE & & RAW GRAB & UNFILTERED & 541 \\
\hline 3217 & $\mathrm{pCi} / \mathrm{L}$ & 0.045 & & & & U & & QC_SAMPLE & & RAW GRAB & UNFILTERED & 541 \\
\hline 3218 & $\mathrm{pCi} / \mathrm{L}$ & 0.42 & & & & & & SURFACE & & RAW GRAB & UNFILTERED & 509 \\
\hline 3219 & $\mathrm{pCi} / \mathrm{L}$ & 2.9 & & & & & & SURFACE & & RAW GRAB & UNFILTERED & 509 \\
\hline 3220 & $\mathrm{pCi} / \mathrm{L}$ & 1.4 & & & & U & & SURFACE & & RAW GRAB & UNFILTERED & 136 \\
\hline 3221 & $\mathrm{pCi} / \mathrm{L}$ & 3.2 & & & & & & SURFACE & & RAW GRAB & UNFILTERED & 146 \\
\hline 3222 & $\mathrm{pCi} / \mathrm{L}$ & 0.054 & & & & & & SURFACE & & RAW GRAB & UNFILTERED & 146 \\
\hline 3223 & pCi/L & 4.8 & & & & U & & SURFACE & & RAW GRAB & UNFILTERED & 147 \\
\hline 3224 & $\mathrm{pCi} / \mathrm{L}$ & 0.42 & & & & & & SURFACE & & RAW GRAB & UNFILTERED & 147 \\
\hline 3225 & $\mathrm{pCi} / \mathrm{L}$ & 0.0073 & & & & & & RIVER & & CONTINUOUS & RESIN & 307 \\
\hline
\end{tabular}




\begin{tabular}{|c|c|c|c|c|c|c|c|c|c|c|c|c|}
\hline & $\mathrm{N}$ & $\mathrm{O}$ & $\mathrm{P}$ & $\mathrm{Q}$ & $\mathrm{R}$ & $\mathrm{s}$ & $T$ & $\bar{U}$ & $\mathrm{~V}$ & $\mathrm{~W}$ & $\mathrm{X}$ & $\bar{Y}$ \\
\hline 1 & ANAL_UNITS_RPTD & COUNTING_ERROR & TOTAL_ANAL_ERROR|I & MIN_DETECTABLE_ACTIVITY & PCNT_MOISTURE & LAB_QUALIFIER & REVIEW_QUALIFIER & SAMP_FROM & SAMP_ITEM & SAMP MTHD & COLL MTHD & SAMP SITE ID \\
\hline 3226 & $\mathrm{pCi} / \mathrm{L}$ & 0.003 & & & & & & RIVER & & CONTINUOUS & FILTER & 307 \\
\hline 3227. & $\mathrm{pCi} / \mathrm{L}$ & & 0.000000324 & & & & & RIVER & & CONTINUOUS & RESIN & 290 \\
\hline 3228 & $\mathrm{pCi} / \mathrm{L}$ & 0.008 & & & & & & RIVER & & CONTINUOUS & RESIN & 252 \\
\hline 3229 & $\mathrm{pCi} / \mathrm{L}$ & 0.003 & & & & & & RIVER & & CONTINUOUS & FILTER & 252 \\
\hline 3230 & $\mathrm{pCi} / \mathrm{L}$ & 1.4 & & & & $\mathrm{U}$ & & SANITARY & & CUMULATIVE & UNFILTERED & 385 \\
\hline 3231 & $\mathrm{pCi} / \mathrm{L}$ & 16 & & & & & & RIVER & & CUMULATIVE & UNFILTERED & 290 \\
\hline 3232 & $\mathrm{pCi} / \mathrm{L}$ & 18 & & & & & & RIVER & & CUMULATIVE & UNFILTERED & 252 \\
\hline 3233 & $\mathrm{pCi} / \mathrm{L}$ & 1.4 & & & & U & & RIVER & & CUMULATIVE & UNFILTERED & 252 \\
\hline 3234 & $\mathrm{pCi} / \mathrm{L}$ & 22 & & & & & & RIVER & & CUMULATIVE & UNFILTERED & 463 \\
\hline 3235 & $\mathrm{pCi} / \mathrm{L}$ & 1.4 & & & & U & & RIVER & & CUMULATIVE & UNFILTERED & 463 \\
\hline 3236 & $\mathrm{pCi} / \mathrm{L}$ & 0.09 & & & & & & DRINKING & & SAN. GRAB & UNFILTERED & 187 \\
\hline \begin{tabular}{|c|}
3237 \\
\end{tabular} & $\mathrm{pCi} / \mathrm{L}$ & 190 & & & & & & DRINKING & & SAN. GRAB & UNFILTERED & 187 \\
\hline 3238 & $\mathrm{pCi} / \mathrm{L}$ & 0.0083 & & & & & & RIVER & & CONTINUOUS & RESIN & 307 \\
\hline 3239 & $\mathrm{pCi} / \mathrm{L}$ & & 0.0000036 & & & & & RIVER & & CONTINUOUS & RESIN & 307 \\
\hline 3240 & $\mathrm{pCi} / \mathrm{L}$ & 0.0037 & & & & & & RIVER & & CONTINUOUS & FILTER & 307 \\
\hline \begin{tabular}{|l|}
3241 \\
\end{tabular} & $\mathrm{pCi} / \mathrm{L}$ & 20 & & & & & & RIVER & & CUMULATIVE & UNFILTERED & 290 \\
\hline 3242 & $\mathrm{pCi} / \mathrm{L}$ & 0.008 & & & & & & RIVER & & CONTINUOUS & RESIN & 252 \\
\hline 3243 & $\mathrm{pCi} / \mathrm{L}$ & & 0.000092 & & & U & & RIVER & & CONTINUOUS & RESIN & 252 \\
\hline 3244 & $\mathrm{pCi} / \mathrm{L}$ & 0.0036 & & & & & & RIVER & & CONTINUOUS & FILTER & 252 \\
\hline 3245 & $\mathrm{pCi} / \mathrm{L}$ & 20 & & & & & & RIVER & & CUMULATIVE & UNFILTERED & 252 \\
\hline 3246 & $\mathrm{pCi} / \mathrm{L}$ & 20 & & & & & & RIVER & & CUMULATIVE & UNFILTERED & 463 \\
\hline \begin{tabular}{|l|}
3247 \\
\end{tabular} & $\mathrm{pCi} / \mathrm{L}$ & 0.063 & & & & U & & DRINKING & & SAN. GRAB & UNFILTERED & 512 \\
\hline 3248 & $\mathrm{pCi} / \mathrm{L}$ & 290 & & & & & & DRINKING & & SAN. GRAB & UNFILTERED & 512 \\
\hline 3249 & $\mathrm{pCi} / \mathrm{L}$ & 0.4 & & & & U & & DRINKING & & SAN. GRAB & UNFILTERED & 512 \\
\hline 3250 & $\mathrm{pCi} / \mathrm{L}$ & 0.063 & & & & & & SANITARY & & CUMULATIVE & UNFILTERED & 491 \\
\hline \begin{tabular}{|l|}
3251 \\
\end{tabular} & $\mathrm{pCi} / \mathrm{L}$ & 97 & & & & & & SANITARY & & CUMULATIVE & UNFILTERED & 491 \\
\hline 3252 & $\mathrm{pCi} / \mathrm{L}$ & 0.063 & & & & & & RIVER & & CUMULATIVE & UNFILTERED & 252 \\
\hline 3253 & $\mathrm{pCi} / \mathrm{L}$ & 0.063 & & & & U & & RIVER & & CUMULATIVE & UNFILTERED & 463 \\
\hline 3254 & $\mathrm{pCi} / \mathrm{L}$ & 0.44 & & & & U & & SANITARY & & CUMULATIVE & UNFILTERED & 385 \\
\hline 3255 & $\mathrm{pCi} / \mathrm{L}$ & 0.43 & & & & U & & RIVER & & CUMULATIVE & UNFILTERED & 553 \\
\hline 3256 & $\mathrm{pCi} / \mathrm{L}$ & 180 & & & & & & RIVER & & CUMULATIVE & UNFILTERED & 553 \\
\hline \begin{tabular}{|l|}
3257 \\
\end{tabular} & $\mathrm{pCi} / \mathrm{L}$ & 0.063 & & & & & & RIVER & & CUMULATIVE & UNFILTERED & 553 \\
\hline 3258 & $\mathrm{pCi} / \mathrm{L}$ & 0.0044 & & & & & & RIVER & & CONTINUOUS & FILTER & 307 \\
\hline 3259 & $\mathrm{pCi} / \mathrm{L}$ & 0.0064 & & & & & & RIVER & & CONTINUOUS & RESIN & 307 \\
\hline 3260 & $\mathrm{pCi} / \mathrm{L}$ & 20 & & & & & & RIVER & & CUMULATIVE & UNFILTERED & 290 \\
\hline 3261 & $\mathrm{pCi} / \mathrm{L}$ & 0.0059 & & & & & & RIVER & & CONTINUOUS & RESIN & 252 \\
\hline 3262 & $\mathrm{pCi} / \mathrm{L}$ & 20 & & & & & & RIVER & & CUMULATIVE & UNFILTERED & 252 \\
\hline 3263 & $\mathrm{pCi} / \mathrm{L}$ & 0.063 & & & & U & & RIVER & & CUMULATIVE & UNFILTERED & 252 \\
\hline 3264 & $\mathrm{pCi} / \mathrm{L}$ & 0.85 & & & & $U$ & & RIVER & & CUMULATIVE & UNFILTERED & 252 \\
\hline 3265 & $\mathrm{pCi} / \mathrm{L}$ & 0.0028 & & & & & & RIVER & & CONTINUOUS & FILTER & 252 \\
\hline 3266 & $\mathrm{pCi} / \mathrm{L}$ & 20 & & & & & & RIVER & & CUMULATIVE & UNFILTERED & 463 \\
\hline \begin{tabular}{|l|}
3267 \\
\end{tabular} & $\mathrm{pCi} / \mathrm{L}$ & 0.3 & & & & & & RIVER & & CUMULATIVE & UNFILTERED & 463 \\
\hline 3268 & $\mathrm{pCi} / \mathrm{L}$ & 0.88 & & & & U & & RIVER & & CUMULATIVE & UNFILTERED & 463 \\
\hline 3269 & $\mathrm{pCi} / \mathrm{L}$ & 0.44 & & & & U & & DRINKING & & SAN. GRAB & UNFILTERED & 487 \\
\hline 3270 & $\mathrm{pCi} / \mathrm{L}$ & 150 & & & & & & DRINKING & & SAN. GRAB & UNFILTERED & 487 \\
\hline 3271 & $\mathrm{pCi} / \mathrm{L}$ & 20 & & & & & & RIVER & & CUMULATIVE & UNFILTERED & 290 \\
\hline 3272 & $\mathrm{pCi} / \mathrm{L}$ & 0.0049 & & & & & & RIVER & & CONTINUOUS & FILTER & 307 \\
\hline \begin{tabular}{|l|}
3273 \\
\end{tabular} & $\mathrm{pCi} / \mathrm{L}$ & 0.000002 & & & & & & RIVER & & CONTINUOUS & FILTER & 307 \\
\hline 3274 & $\mathrm{pCi} / \mathrm{L}$ & 0.0000055 & & & & $U$ & & RIVER & & CONTINUOUS & RESIN & 307 \\
\hline 3275 & $\mathrm{pCi} / \mathrm{L}$ & 0.0092 & & & & & & RIVER & & CONTINUOUS & RESIN & 307 \\
\hline 3276 & $\mathrm{pCi} / \mathrm{L}$ & & 0.0000009 & & & & & RIVER & & CONTINUOUS & RESIN & 307 \\
\hline 3277 & $\mathrm{pCi} / \mathrm{L}$ & & 0.000000816 & & & & & RIVER & & CONTINUOUS & RESIN & 290 \\
\hline
\end{tabular}




\begin{tabular}{|c|c|c|c|c|c|c|c|c|c|c|c|c|}
\hline & $\mathrm{N}$ & $\mathrm{O}$ & $\mathrm{P}$ & $\mathrm{Q}$ & $\mathrm{R}$ & $\mathrm{s}$ & $T$ & $\mathrm{U}$ & $\mathrm{V}$ & $\mathrm{W}$ & $x$ & $\bar{Y}$ \\
\hline 1 & ANAL_UNITS_RPTD & COUNTING_ERROR & ||$_{\text {TOTAL_ANAL_ERROR | }}$ & MIN_DETECTABLE_ACTIVITY & PCNT_MOISTURE & LAB_QUALIFIER & REVIEW_QUALIFIER & SAMP_FROM & SAMP_ITEM & SAMP_MTHD & COLL_MTHD & SAMP_SITE_ID \\
\hline 3278 & pCi/L & 0.0094 & & & & & & RIVER & & CONTINUOUS & RESIN & 252 \\
\hline 3279 & $\mathrm{pCi} / \mathrm{L}$ & 0.0000053 & & & & $\mathrm{U}$ & & RIVER & & CONTINUOUS & RESIN & 252 \\
\hline 3280 & $\mathrm{pCi} / \mathrm{L}$ & & 0.00000051 & & & & & RIVER & & CONTINUOUS & RESIN & 252 \\
\hline 3281 & $\mathrm{pCi} / \mathrm{L}$ & 0.000002 & & & & & & RIVER & & CONTINUOUS & FILTER & 252 \\
\hline 3282 & $\mathrm{pCi} / \mathrm{L}$ & 0.0042 & & & & & & RIVER & & CONTINUOUS & FILTER & 252 \\
\hline 3283 & $\mathrm{pCi} / \mathrm{L}$ & 20 & & & & & & RIVER & & CUMULATIVE & UNFILTERED & 252 \\
\hline 3284 & $\mathrm{pCi} / \mathrm{L}$ & 20 & & & & & & RIVER & & CUMULATIVE & UNFILTERED & 463 \\
\hline 3285 & $\mathrm{pCi} / \mathrm{L}$ & 0.72 & & & & $\mathrm{U}$ & & SANITARY & & CUMULATIVE & UNFILTERED & 385 \\
\hline 3286 & $\mathrm{pCi} / \mathrm{L}$ & 0.0035 & & & & & & RIVER & & CONTINUOUS & FILTER & 307 \\
\hline 3287 & $\mathrm{pCi} / \mathrm{L}$ & 0.0086 & & & & & & RIVER & & CONTINUOUS & RESIN & 307 \\
\hline 3288 & $\mathrm{pCi} / \mathrm{L}$ & 20 & & & & & & RIVER & & CUMULATIVE & UNFILTERED & 252 \\
\hline 3289 & $\mathrm{pCi} / \mathrm{L}$ & 0.063 & & & & & & RIVER & & CUMULATIVE & UNFILTERED & 252 \\
\hline 3290 & $\mathrm{pCi} / \mathrm{L}$ & 0.72 & & & & $U$ & & RIVER & & CUMULATIVE & UNFILTERED & 252 \\
\hline 3291 & $\mathrm{pCi} / \mathrm{L}$ & 0.0068 & & & & & & RIVER & & CONTINUOUS & RESIN & 252 \\
\hline 3292 & $\mathrm{pCi} / \mathrm{L}$ & 0.0034 & & & & & & RIVER & & CONTINUOUS & FILTER & 252 \\
\hline 3293 & $\mathrm{pCi} / \mathrm{L}$ & 20 & & & & & & RIVER & & CUMULATIVE & UNFILTERED & 463 \\
\hline 3294 & $\mathrm{pCi} / \mathrm{L}$ & 0.063 & & & & & & RIVER & & CUMULATIVE & UNFILTERED & 463 \\
\hline 3295 & $\mathrm{pCi} / \mathrm{L}$ & 0.31 & & & & $U$ & & RIVER & & CUMULATIVE & UNFILTERED & 463 \\
\hline 3296 & $\mathrm{pCi} / \mathrm{L}$ & 20 & & & & & & QC_SAMPLE & & RAW GRAB & UNFILTERED & 222 \\
\hline 3297 & $\mathrm{pCi} / \mathrm{L}$ & 0.13 & & & & & & QC_SAMPLE & & RAW GRAB & UNFILTERED & 222 \\
\hline 3298 & pCi/L & 0.45 & & & & & & QC_SAMPLE & & RAW GRAB & UNFILTERED & 222 \\
\hline 3299 & pCi/L & 32 & & & & U & & QC_SAMPLE & & RAW GRAB & UNFILTERED & 223 \\
\hline 3300 & $\mathrm{pCi} / \mathrm{L}$ & 0.063 & & & & U & & QC_SAMPLE & & RAW GRAB & UNFILTERED & 223 \\
\hline 3301 & $\mathrm{pCi} / \mathrm{L}$ & 0.31 & & & & U & & QC_SAMPLE & & RAW GRAB & UNFILTERED & 223 \\
\hline 3302 & $\mathrm{pCi} / \mathrm{L}$ & 20 & & & & & & RIVER & & CUMULATIVE & UNFILTERED & 290 \\
\hline 3303 & $\mathrm{pCi} / \mathrm{L}$ & 0.0072 & & & & & & RIVER & & CONTINUOUS & RESIN & 307 \\
\hline 3304 & $\mathrm{pCi} / \mathrm{L}$ & & 0.0000026 & & & & & RIVER & & CONTINUOUS & RESIN & 307 \\
\hline 3305 & $\mathrm{pCi} / \mathrm{L}$ & 0.0037 & & & & & & RIVER & & CONTINUOUS & FILTER & 307 \\
\hline 3306 & $\mathrm{pCi} / \mathrm{L}$ & & 0.000000672 & & & & & RIVER & & CONTINUOUS & RESIN & 290 \\
\hline 3307 & $\mathrm{pCi} / \mathrm{L}$ & 0.0078 & & & & & & RIVER & & CONTINUOUS & RESIN & 252 \\
\hline 3308 & $\mathrm{pCi} / \mathrm{L}$ & & 0.00000063 & & & & & RIVER & & CONTINUOUS & RESIN & 252 \\
\hline 3309 & $\mathrm{pCi} / \mathrm{L}$ & 0.0038 & & & & & & RIVER & & CONTINUOUS & FILTER & 252 \\
\hline 3310 & $\mathrm{pCi} / \mathrm{L}$ & 20 & & & & & & RIVER & & CUMULATIVE & UNFILTERED & 252 \\
\hline 3311 & $\mathrm{pCi} / \mathrm{L}$ & 20 & & & & & & RIVER & & CUMULATIVE & UNFILTERED & 463 \\
\hline 3312 & $\mathrm{pCi} / \mathrm{L}$ & 2.5 & & & & & & QC_SAMPLE & & RAW GRAB & UNFILTERED & 451 \\
\hline 3313 & pCi/L & 0.13 & & & & & & QC_SAMPLE & & RAW GRAB & UNFILTERED & 451 \\
\hline 3314 & $\mathrm{pCi} / \mathrm{L}$ & 0.13 & & & & & & QC_SAMPLE & & RAW GRAB & UNFILTERED & 542 \\
\hline 3315 & $\mathrm{pCi} / \mathrm{L}$ & 2.4 & & & & & & QC_SAMPLE & & RAW GRAB & UNFILTERED & 542 \\
\hline 3316 & $\mathrm{pCi} / \mathrm{L}$ & 0.13 & & & & & & QC_SAMPLE & & RAW GRAB & UNFILTERED & 226 \\
\hline 3317 & $\mathrm{pCi} / \mathrm{L}$ & 2.2 & & & & & & QC_SAMPLE & & RAW GRAB & UNFILTERED & 226 \\
\hline 3318 & $\mathrm{pCi} / \mathrm{L}$ & 0.13 & & & & U & & QC_SAMPLE & & RAW GRAB & UNFILTERED & 450 \\
\hline 3319 & $\mathrm{pCi} / \mathrm{L}$ & 1.5 & & & & U & & QC_SAMPLE & & RAW GRAB & UNFILTERED & 450 \\
\hline 3320 & $\mathrm{pCi} / \mathrm{L}$ & 0.13 & & & & & & SURFACE & & RAW GRAB & UNFILTERED & 509 \\
\hline 3321 & $\mathrm{pCi} / \mathrm{L}$ & 2.5 & & & & & & SURFACE & & RAW GRAB & UNFILTERED & 509 \\
\hline 3322 & $\mathrm{pCi} / \mathrm{L}$ & 1.9 & & & & $U$ & & SURFACE & & RAW GRAB & UNFILTERED & 136 \\
\hline 3323 & $\mathrm{pCi} / \mathrm{L}$ & 1.6 & & & & & & SURFACE & & RAW GRAB & UNFILTERED & 146 \\
\hline 3324 & $\mathrm{pCi} / \mathrm{L}$ & 0.063 & & & & & & SURFACE & & RAW GRAB & UNFILTERED & 146 \\
\hline 3325 & $\mathrm{pCi} / \mathrm{L}$ & 1.5 & & & & U & & SURFACE & & RAW GRAB & UNFILTERED & 147 \\
\hline 3326 & pCi/L & 0.13 & & & & & & SURFACE & & RAW GRAB & UNFILTERED & 147 \\
\hline 3327 & pCi/L & 1.1 & & & & & & SANITARY & & CUMULATIVE & UNFILTERED & 385 \\
\hline 3328 & $\mathrm{pCi} / \mathrm{L}$ & 0.0069 & & & & & & RIVER & & CONTINUOUS & RESIN & 307 \\
\hline 3329 & $\mathrm{pCi} / \mathrm{L}$ & 0.0035 & & & & & & RIVER & & CONTINUOUS & FILTER & 307 \\
\hline
\end{tabular}




\begin{tabular}{|c|c|c|c|c|c|c|c|c|c|c|c|c|}
\hline & $\mathrm{N}$ & $\mathrm{O}$ & $\mathrm{P}$ & $\mathrm{Q}$ & $\mathrm{R}$ & $\mathrm{s}$ & $T$ & $\mathrm{U}$ & $\mathrm{V}$ & $\mathrm{W}$ & $\mathrm{X}$ & $\bar{Y}$ \\
\hline 1 & ANAL_UNITS_RPTD & COUNTING_ERROR & TOTAL_ANAL_ERROR & MIN_DETECTABLE_ACTIVITY & PCNT_MOISTURE & LAB_QUALIFIER & REVIEW_QUALIFIER & SAMP_FROM & SAMP_ITEM & SAMP MTHD & COLL MTHD & SAMP SITE ID \\
\hline 3330 & $\mathrm{pCi} / \mathrm{L}$ & 16 & & & & & & RIVER & & CUMULATIVE & UNFILTERED & 290 \\
\hline 3331 & $\mathrm{pCi} / \mathrm{L}$ & 14 & & & & & & RIVER & & CUMULATIVE & UNFILTERED & 252 \\
\hline 3332 & $\mathrm{pCi} / \mathrm{L}$ & 0.063 & & & & & & RIVER & & CUMULATIVE & UNFILTERED & 252 \\
\hline 3333 & $\mathrm{pCi} / \mathrm{L}$ & 0.49 & & & & $\mathrm{U}$ & & RIVER & & CUMULATIVE & UNFILTERED & 252 \\
\hline 3334 & $\mathrm{pCi} / \mathrm{L}$ & 0.0042 & & & & & & RIVER & & CONTINUOUS & FILTER & 252 \\
\hline 3335 & $\mathrm{pCi} / \mathrm{L}$ & 0.009 & & & & & & RIVER & & CONTINUOUS & RESIN & 252 \\
\hline 3336 & $\mathrm{pCi} / \mathrm{L}$ & 20 & & & & & & RIVER & & CUMULATIVE & UNFILTERED & 463 \\
\hline 3337 & $\mathrm{pCi} / \mathrm{L}$ & 0.063 & & & & & & RIVER & & CUMULATIVE & UNFILTERED & 463 \\
\hline 3338 & $\mathrm{pCi} / \mathrm{L}$ & 1 & & & & U & & RIVER & & CUMULATIVE & UNFILTERED & 463 \\
\hline 3339 & $\mathrm{pCi} / \mathrm{L}$ & 22 & & & & & & RIVER & & CUMULATIVE & UNFILTERED & 290 \\
\hline 3340 & $\mathrm{pCi} / \mathrm{L}$ & 18 & & & & & & RIVER & & CUMULATIVE & UNFILTERED & 252 \\
\hline 3341 & $\mathrm{pCi} / \mathrm{L}$ & 20 & & & & & & RIVER & & CUMULATIVE & UNFILTERED & 463 \\
\hline 3342 & $\mathrm{pCi} / \mathrm{L}$ & 0.063 & & & & $U$ & & DRINKING & & SAN. GRAB & UNFILTERED & 187 \\
\hline 3343 & $\mathrm{pCi} / \mathrm{L}$ & 4.2 & & & & & & DRINKING & & SAN. GRAB & UNFILTERED & 187 \\
\hline 3344 & $\mathrm{pCi} / \mathrm{L}$ & 0.0074 & & & & & & RIVER & & CONTINUOUS & RESIN & 307 \\
\hline 3345 & $\mathrm{pCi} / \mathrm{L}$ & & 0.000002 & & & & & RIVER & & CONTINUOUS & RESIN & 307 \\
\hline 3346 & $\mathrm{pCi} / \mathrm{L}$ & 0.0036 & & & & & & RIVER & & CONTINUOUS & FILTER & 307 \\
\hline 3347 & $\mathrm{pCi} / \mathrm{L}$ & & 0.000000672 & & & & & RIVER & & CONTINUOUS & RESIN & 290 \\
\hline 3348 & & & & & & $U$ & & RIVER & & CONTINUOUS & FILTER & 252 \\
\hline 3349 & & & & & & $U$ & & RIVER & & CONTINUOUS & RESIN & 252 \\
\hline 3350 & $\mathrm{pCi} / \mathrm{L}$ & & 0.000000448 & & & & & RIVER & & CONTINUOUS & RESIN & 252 \\
\hline 3351 & $u g / L$ & 0 & & & & & & RIVER & & RAW GRAB & UNFILTERED & 463 \\
\hline 3352 & $u g / L$ & 0 & & & & & & RIVER & & RAW GRAB & UNFILTERED & 456 \\
\hline 3353 & $\mathrm{pCi} / \mathrm{L}$ & 0.0459 & & & & & & DRINKING & & SAN. GRAB & UNFILTERED & 512 \\
\hline 3354 & $\mathrm{pCi} / \mathrm{L}$ & 850 & & & & & & DRINKING & & SAN. GRAB & UNFILTERED & 512 \\
\hline 3355 & $\mathrm{pCi} / \mathrm{L}$ & 0.98 & & & & & & DRINKING & & SAN. GRAB & UNFILTERED & 512 \\
\hline 3356 & $\mathrm{pCi} / \mathrm{L}$ & 0.0000064 & & & & & & RIVER & & CONTINUOUS & RESIN & 307 \\
\hline 3357 & $\mathrm{pCi} / \mathrm{L}$ & 0.000003 & & & & & & RIVER & & CONTINUOUS & FILTER & 307 \\
\hline 3358 & $\mathrm{pCi} / \mathrm{L}$ & 0.0000099 & & & & & & RIVER & & CONTINUOUS & RESIN & 252 \\
\hline 3359 & $\mathrm{pCi} / \mathrm{L}$ & 0.000003 & & & & & & RIVER & & CONTINUOUS & FILTER & 252 \\
\hline 3360 & $\mathrm{pCi} / \mathrm{L}$ & 0.042 & & & & & & SANITARY & & CUMULATIVE & UNFILTERED & 491 \\
\hline 3361 & $\mathrm{pCi} / \mathrm{L}$ & 48 & & & & & & SANITARY & & CUMULATIVE & UNFILTERED & 491 \\
\hline 3362 & $\mathrm{pCi} / \mathrm{L}$ & 1.4 & & & & $U$ & & SANITARY & & CUMULATIVE & UNFILTERED & 385 \\
\hline 3363 & $\mathrm{pCi} / \mathrm{L}$ & 0.037 & & & & & & RIVER & & CUMULATIVE & UNFILTERED & 553 \\
\hline 3364 & $\mathrm{pCi} / \mathrm{L}$ & 48 & & & & & & RIVER & & CUMULATIVE & UNFILTERED & 553 \\
\hline 3365 & $\mathrm{pCi} / \mathrm{L}$ & 0.63 & & & & U & & RIVER & & CUMULATIVE & UNFILTERED & 553 \\
\hline 3366 & $\mathrm{pCi} / \mathrm{L}$ & 0.0072 & & & & & & RIVER & & CONTINUOUS & RESIN & 307 \\
\hline 3367 & $\mathrm{pCi} / \mathrm{L}$ & 0.0035 & & & & & & RIVER & & CONTINUOUS & FILTER & 307 \\
\hline 3368 & $\mathrm{pCi} / \mathrm{L}$ & 16 & & & & & & RIVER & & CUMULATIVE & UNFILTERED & 290 \\
\hline 3369 & $\mathrm{pCi} / \mathrm{L}$ & 14 & & & & & & RIVER & & CUMULATIVE & UNFILTERED & 252 \\
\hline 3370 & $\mathrm{pCi} / \mathrm{L}$ & 0.0631 & & & & & & RIVER & & CUMULATIVE & UNFILTERED & 252 \\
\hline 3371 & $\mathrm{pCi} / \mathrm{L}$ & 1.4 & & & & U & & RIVER & & CUMULATIVE & UNFILTERED & 252 \\
\hline 3372 & & & & & & U & & RIVER & & CONTINUOUS & FILTER & 252 \\
\hline 3373 & & & & & & U & & RIVER & & CONTINUOUS & RESIN & 252 \\
\hline 3374 & $\mathrm{pCi} / \mathrm{L}$ & 16 & & & & & & RIVER & & CUMULATIVE & UNFILTERED & 463 \\
\hline 3375 & $\mathrm{pCi} / \mathrm{L}$ & 0.0631 & & & & & & RIVER & & CUMULATIVE & UNFILTERED & 463 \\
\hline 3376 & $\mathrm{pCi} / \mathrm{L}$ & 1.3 & & & & $U$ & & RIVER & & CUMULATIVE & UNFILTERED & 463 \\
\hline 3377 & $\mathrm{pCi} / \mathrm{L}$ & 11 & & & & U & & DRINKING & & SAN. GRAB & UNFILTERED & 487 \\
\hline 3378 & $\mathrm{pCi} / \mathrm{L}$ & 23 & & & & & & DRINKING & & SAN. GRAB & UNFILTERED & 487 \\
\hline 3379 & $\mathrm{pCi} / \mathrm{L}$ & 0.015 & & & & & & RIVER & & CONTINUOUS & RESIN & 307 \\
\hline 3380 & $\mathrm{pCi} / \mathrm{L}$ & & 0.00000112 & & & & & RIVER & & CONTINUOUS & RESIN & 307 \\
\hline 3381 & $\mathrm{pCi} / \mathrm{L}$ & 0.0073 & & & & & & RIVER & & CONTINUOUS & FILTER & 307 \\
\hline
\end{tabular}




\begin{tabular}{|c|c|c|c|c|c|c|c|c|c|c|c|c|}
\hline & $\mathrm{N}$ & $\mathrm{O}$ & $\mathrm{P}$ & $\mathrm{Q}$ & $\mathrm{R}$ & $\mathrm{s}$ & $T$ & $\mathrm{U}$ & $\mathrm{V}$ & $\mathrm{W}$ & $x$ & $\bar{Y}$ \\
\hline 1 & ANAL_UNITS_RPTD & COUNTING_ERROR & \begin{tabular}{|c|} 
TOTAL_ANAL_ERROR \\
\end{tabular} & MIN_DETECTABLE_ACTIVITY & PCNT_MOISTURE & LAB_QUALIFIER & REVIEW_QUALIFIER & SAMP_FROM & SAMP_ITEM & SAMP_MTHD & COLL_MTHD & SAMP_SITE_ID \\
\hline 3382 & pCi/L & & 0.000000528 & & & & & RIVER & & CONTINUOUS & RESIN & 290 \\
\hline 3383 & $\mathrm{pCi} / \mathrm{L}$ & 0.009 & & & & & & RIVER & & CONTINUOUS & RESIN & 252 \\
\hline 3384 & $\mathrm{pCi} / \mathrm{L}$ & & 0.00000081 & & & & & RIVER & & CONTINUOUS & RESIN & 252 \\
\hline 3385 & $\mathrm{pCi} / \mathrm{L}$ & 0.0045 & & & & & & RIVER & & CONTINUOUS & FILTER & 252 \\
\hline 3386 & $\mathrm{pCi} / \mathrm{L}$ & 14 & & & & & & RIVER & & CUMULATIVE & UNFILTERED & 463 \\
\hline 3387 & $\mathrm{pCi} / \mathrm{L}$ & 0.0631 & & & & U & & RIVER & & CUMULATIVE & UNFILTERED & 463 \\
\hline 3388 & $\mathrm{pCi} / \mathrm{L}$ & 5 & & & & $\mathrm{U}$ & & RIVER & & CUMULATIVE & UNFILTERED & 463 \\
\hline 3389 & $\mathrm{pCi} / \mathrm{L}$ & 188 & & & & $\mathrm{U}$ & & QC_SAMPLE & & RAW GRAB & UNFILTERED & 222 \\
\hline 3390 & $\mathrm{pCi} / \mathrm{L}$ & 18 & & & & & & QC_SAMPLE & & RAW GRAB & UNFILTERED & 222 \\
\hline 3391 & $\mathrm{pCi} / \mathrm{L}$ & 0.0631 & & & & & & QC_SAMPLE & & RAW GRAB & UNFILTERED & 222 \\
\hline 3392 & $\mathrm{pCi} / \mathrm{L}$ & 0.949 & & & & & & QC_SAMPLE & & RAW GRAB & UNFILTERED & 222 \\
\hline 3393 & $\mathrm{pCi} / \mathrm{L}$ & 147 & & & & U & & QC_SAMPLE & & RAW GRAB & UNFILTERED & 223 \\
\hline 3394 & $\mathrm{pCi} / \mathrm{L}$ & 18 & & & & & & QC_SAMPLE & & RAW GRAB & UNFILTERED & 223 \\
\hline 3395 & $\mathrm{pCi} / \mathrm{L}$ & 0.0631 & & & & & & QC_SAMPLE & & RAW GRAB & UNFILTERED & 223 \\
\hline 3396 & $\mathrm{pCi} / \mathrm{L}$ & 0.657 & & & & & & QC_SAMPLE & & RAW GRAB & UNFILTERED & 223 \\
\hline 3397 & $\mathrm{pCi} / \mathrm{L}$ & 0.0037 & & & & & & RIVER & & CONTINUOUS & FILTER & 307 \\
\hline 3398 & $\mathrm{pCi} / \mathrm{L}$ & 0.0067 & & & & & & RIVER & & CONTINUOUS & RESIN & 307 \\
\hline 3399 & $\mathrm{pCi} / \mathrm{L}$ & 0.0047 & & & & & & RIVER & & CONTINUOUS & RESIN & 252 \\
\hline 3400 & $\mathrm{pCi} / \mathrm{L}$ & 14 & & & & & & RIVER & & CUMULATIVE & UNFILTERED & 252 \\
\hline 3401 & $\mathrm{pCi} / \mathrm{L}$ & 0.0631 & & & & & & RIVER & & CUMULATIVE & UNFILTERED & 252 \\
\hline 3402 & pCi/L & 0.0029 & & & & & & RIVER & & CONTINUOUS & FILTER & 252 \\
\hline 3403 & pCi/L & 2.4 & & & & U & & RIVER & & CUMULATIVE & UNFILTERED & 252 \\
\hline 3404 & $\mathrm{pCi} / \mathrm{L}$ & 7.3 & & & & & & SANITARY & & CUMULATIVE & UNFILTERED & 385 \\
\hline 3405 & & & & & & U & & RIVER & & CUMULATIVE & UNFILTERED & 290 \\
\hline 3406 & & & & & & U & & RIVER & & CONTINUOUS & RESIN & 307 \\
\hline 3407 & & & & & & U & & RIVER & & CONTINUOUS & FILTER & 307 \\
\hline 3408 & & & & & & U & & RIVER & & CONTINUOUS & FILTER & 252 \\
\hline 3409 & & & & & & U & & RIVER & & CONTINUOUS & RESIN & 252 \\
\hline 3410 & $\mathrm{pCi} / \mathrm{L}$ & 0.126 & & & & U & & QC_SAMPLE & & RAW GRAB & UNFILTERED & 227 \\
\hline 3411 & $\mathrm{pCi} / \mathrm{L}$ & 0.685 & & & & U & & QC_SAMPLE & & RAW GRAB & UNFILTERED & 227 \\
\hline 3412 & $\mathrm{pCi} / \mathrm{L}$ & 0.126 & & & & U & & QC_SAMPLE & & RAW GRAB & UNFILTERED & 228 \\
\hline 3413 & $\mathrm{pCi} / \mathrm{L}$ & 0.627 & & & & U & & QC_SAMPLE & & RAW GRAB & UNFILTERED & 228 \\
\hline 3414 & $\mathrm{pCi} / \mathrm{L}$ & 0.126 & & & & & & SURFACE & & RAW GRAB & UNFILTERED & 509 \\
\hline 3415 & $\mathrm{pCi} / \mathrm{L}$ & 1.52 & & & & & & SURFACE & & RAW GRAB & UNFILTERED & 509 \\
\hline 3416 & $\mathrm{pCi} / \mathrm{L}$ & 0.281 & & & & & & SURFACE & & RAW GRAB & UNFILTERED & 136 \\
\hline 3417 & pCi/L & 0.793 & & & & & & SURFACE & & RAW GRAB & UNFILTERED & 146 \\
\hline 3418 & $\mathrm{pCi} / \mathrm{L}$ & 0.0631 & & & & & & SURFACE & & RAW GRAB & UNFILTERED & 146 \\
\hline 3419 & & & & & & U & & SURFACE & & RAW GRAB & UNFILTERED & 147 \\
\hline 3420 & & & & & & U & & SURFACE & & RAW GRAB & UNFILTERED & 147 \\
\hline 3421 & $\mathrm{pCi} / \mathrm{L}$ & 0.451 & & & & U & & SANITARY & & CUMULATIVE & UNFILTERED & 385 \\
\hline 3422 & $\mathrm{pCi} / \mathrm{L}$ & 0.012 & & & & & & RIVER & & CONTINUOUS & RESIN & 307 \\
\hline 3423 & $\mathrm{pCi} / \mathrm{L}$ & & 0.00000224 & & & & & RIVER & & CONTINUOUS & RESIN & 307 \\
\hline 3424 & $\mathrm{pCi} / \mathrm{L}$ & 0.0067 & & & & & & RIVER & & CONTINUOUS & FILTER & 307 \\
\hline 3425 & $\mathrm{pCi} / \mathrm{L}$ & & 0.00006 & & & U & & RIVER & & CONTINUOUS & RESIN & 290 \\
\hline 3426 & $\mathrm{pCi} / \mathrm{L}$ & 14 & & & & & & RIVER & & CUMULATIVE & UNFILTERED & 290 \\
\hline 3427 & $\mathrm{pCi} / \mathrm{L}$ & 0.0068 & & & & & & RIVER & & CONTINUOUS & RESIN & 252 \\
\hline 3428 & $\mathrm{pCi} / \mathrm{L}$ & & 0.0000011 & & & & & RIVER & & CONTINUOUS & RESIN & 252 \\
\hline 3429 & $\mathrm{pCi} / \mathrm{L}$ & 0.004 & & & & & & RIVER & & CONTINUOUS & FILTER & 252 \\
\hline 3430 & pCi/L & 14 & & & & & & RIVER & & CUMULATIVE & UNFILTERED & 252 \\
\hline 3431 & pCi/L & 0.0631 & & & & & & RIVER & & CUMULATIVE & UNFILTERED & 252 \\
\hline 3432 & $\mathrm{pCi} / \mathrm{L}$ & 0.476 & & & & U & & RIVER & & CUMULATIVE & UNFILTERED & 252 \\
\hline 3433 & $\mathrm{pCi} / \mathrm{L}$ & 14 & & & & & & RIVER & & CUMULATIVE & UNFILTERED & 463 \\
\hline
\end{tabular}




\begin{tabular}{|c|c|c|c|c|c|c|c|c|c|c|c|c|}
\hline & $\mathrm{N}$ & $\mathrm{O}$ & $\mathrm{P}$ & $\mathrm{Q}$ & $\mathrm{R}$ & $\mathrm{s}$ & $T$ & $U$ & $\mathrm{~V}$ & $\mathrm{~W}$ & $x$ & $\bar{Y}$ \\
\hline 1 & ANAL_UNITS_RPTD & COUNTING_ERROR & TOTAL_ANAL_ERROR & MIN_DETECTABLE_ACTIVITY & PCNT_MOISTURE & LAB_QUALIFIER & REVIEW_QUALIFIER & SAMP_FROM & SAMP_ITEM & \begin{tabular}{|l|} 
SAMP_MTHD \\
\end{tabular} & COLL_MTHD & SAMP_SITE_ID \\
\hline 3434 & $\mathrm{pCi} / \mathrm{L}$ & 0.0631 & & & & $U$ & & RIVER & & CUMULATIVE & UNFILTERED & 463 \\
\hline 3435 & $\mathrm{pCi} / \mathrm{L}$ & 0.519 & & & & U & & RIVER & & CUMULATIVE & UNFILTERED & 463 \\
\hline 3436 & & & & & & $\mathrm{U}$ & & RIVER & & CUMULATIVE & UNFILTERED & 290 \\
\hline 3437 & $\mathrm{pCi} / \mathrm{L}$ & 0.0631 & & & & $\mathrm{U}$ & & DRINKING & & SAN. GRAB & UNFILTERED & 187 \\
\hline 3438 & $\mathrm{pCi} / \mathrm{L}$ & 211 & & & & $U$ & & DRINKING & & SAN. GRAB & UNFILTERED & 187 \\
\hline 3439 & $\mathrm{pCi} / \mathrm{L}$ & 0.0094 & & & & & & RIVER & & CONTINUOUS & RESIN & 307 \\
\hline 3440 & $\mathrm{pCi} / \mathrm{L}$ & 0.0045 & & & & & & RIVER & & CONTINUOUS & FILTER & 307 \\
\hline 3441 & $\mathrm{pCi} / \mathrm{L}$ & 0.0032 & & & & & & RIVER & & CONTINUOUS & FILTER & 252 \\
\hline 3442 & $\mathrm{pCi} / \mathrm{L}$ & 0.0068 & & & & & & RIVER & & CONTINUOUS & RESIN & 252 \\
\hline 3443 & $\mathrm{pCi} / \mathrm{L}$ & 0.043 & & & & & & DRINKING & & SAN. GRAB & UNFILTERED & 512 \\
\hline 3444 & $\mathrm{pCi} / \mathrm{L}$ & 693 & & & & & & DRINKING & & SAN. GRAB & UNFILTERED & 512 \\
\hline 3445 & $\mathrm{pCi} / \mathrm{L}$ & 0.661 & & & & & & DRINKING & & SAN. GRAB & UNFILTERED & 512 \\
\hline \begin{tabular}{|l|}
3446 \\
\end{tabular} & $\mathrm{pCi} / \mathrm{L}$ & 0.0795 & & & & & & SANITARY & & CUMULATIVE & UNFILTERED & 491 \\
\hline \begin{tabular}{|l|}
3447 \\
\end{tabular} & $\mathrm{pCi} / \mathrm{L}$ & 315 & & & & $U$ & & SANITARY & & CUMULATIVE & UNFILTERED & 491 \\
\hline \begin{tabular}{|l|}
3448 \\
\end{tabular} & $\mathrm{pCi} / \mathrm{L}$ & 0.195 & & & & $U$ & & SANITARY & & CUMULATIVE & UNFILTERED & 385 \\
\hline \begin{tabular}{|l|}
3449 \\
\end{tabular} & $\mathrm{pCi} / \mathrm{L}$ & 0.821 & & & & & & SANITARY & & CUMULATIVE & UNFILTERED & 385 \\
\hline 3450 & $\mathrm{pCi} / \mathrm{L}$ & 0.0367 & & & & & & RIVER & & CUMULATIVE & UNFILTERED & 553 \\
\hline \begin{tabular}{|l|}
3451 \\
\end{tabular} & $\mathrm{pCi} / \mathrm{L}$ & 181 & & & & & & RIVER & & CUMULATIVE & UNFILTERED & 553 \\
\hline \begin{tabular}{|l|}
3452 \\
\end{tabular} & $\mathrm{pCi} / \mathrm{L}$ & 0.219 & & & & $U$ & & RIVER & & CUMULATIVE & UNFILTERED & 553 \\
\hline 3453 & $\mathrm{pCi} / \mathrm{L}$ & 0.0000064 & & & & & & RIVER & & CONTINUOUS & RESIN & 307 \\
\hline 3454 & $\mathrm{pCi} / \mathrm{L}$ & 0.0063 & & & & & & RIVER & & CONTINUOUS & RESIN & 307 \\
\hline \begin{tabular}{|l|}
3455 \\
\end{tabular} & $\mathrm{pCi} / \mathrm{L}$ & & 0.000012 & & & & & RIVER & & CONTINUOUS & RESIN & 307 \\
\hline \begin{tabular}{|l|}
3456 \\
\end{tabular} & $\mathrm{pCi} / \mathrm{L}$ & 0.0041 & & & & & & RIVER & & CONTINUOUS & FILTER & 307 \\
\hline \begin{tabular}{|l|}
3457 \\
\end{tabular} & $\mathrm{pCi} / \mathrm{L}$ & 0.000005 & & & & & & RIVER & & CONTINUOUS & FILTER & 307 \\
\hline \begin{tabular}{|l|}
3458 \\
\end{tabular} & $\mathrm{pCi} / \mathrm{L}$ & 0.0054 & & & & & & RIVER & & CONTINUOUS & RESIN & 252 \\
\hline \begin{tabular}{|l|}
3459 \\
\end{tabular} & $\mathrm{pCi} / \mathrm{L}$ & 0.0000065 & & & & & & RIVER & & CONTINUOUS & RESIN & 252 \\
\hline 3460 & $\mathrm{pCi} / \mathrm{L}$ & & 0.000000882 & & & & & RIVER & & CONTINUOUS & RESIN & 252 \\
\hline \begin{tabular}{|l|}
3461 \\
\end{tabular} & $\mathrm{pCi} / \mathrm{L}$ & 14 & & & & & & RIVER & & CUMULATIVE & UNFILTERED & 252 \\
\hline \begin{tabular}{|l|}
3462 \\
\end{tabular} & $\mathrm{pCi} / \mathrm{L}$ & 0.000004 & & & & & & RIVER & & CONTINUOUS & FILTER & 252 \\
\hline \begin{tabular}{|l|}
3463 \\
\end{tabular} & $\mathrm{pCi} / \mathrm{L}$ & 0.0735 & & & & & & RIVER & & CUMULATIVE & UNFILTERED & 252 \\
\hline 3464 & $\mathrm{pCi} / \mathrm{L}$ & 0.0036 & & & & & & RIVER & & CONTINUOUS & FILTER & 252 \\
\hline \begin{tabular}{|l|}
3465 \\
\end{tabular} & $\mathrm{pCi} / \mathrm{L}$ & 0.518 & & & & $U$ & & RIVER & & CUMULATIVE & UNFILTERED & 252 \\
\hline \begin{tabular}{|l|}
3466 \\
\end{tabular} & $\mathrm{pCi} / \mathrm{L}$ & 14 & & & & & & RIVER & & CUMULATIVE & UNFILTERED & 463 \\
\hline \begin{tabular}{|l|}
3467 \\
\end{tabular} & $\mathrm{pCi} / \mathrm{L}$ & 0.0935 & & & & & & RIVER & & CUMULATIVE & UNFILTERED & 463 \\
\hline \begin{tabular}{|l|}
3468 \\
\end{tabular} & $\mathrm{pCi} / \mathrm{L}$ & 0.245 & & & & & & RIVER & & CUMULATIVE & UNFILTERED & 463 \\
\hline \begin{tabular}{|l|}
3469 \\
\end{tabular} & $\mathrm{pCi} / \mathrm{L}$ & 0.249 & & & & $U$ & & DRINKING & & SAN. GRAB & UNFILTERED & 487 \\
\hline 3470 & $\mathrm{pCi} / \mathrm{L}$ & 229 & & & & $\mathrm{U}$ & & DRINKING & & SAN. GRAB & UNFILTERED & 487 \\
\hline \begin{tabular}{|l|}
3471 \\
\end{tabular} & & & & & & $U$ & & RIVER & & CUMULATIVE & UNFILTERED & 290 \\
\hline \begin{tabular}{|l|}
3472 \\
\end{tabular} & $\mathrm{pCi} / \mathrm{L}$ & 0.0057 & & & & & & RIVER & & CONTINUOUS & RESIN & 307 \\
\hline 3473 & $\mathrm{pCi} / \mathrm{L}$ & 0.0026 & & & & & & RIVER & & CONTINUOUS & FILTER & 307 \\
\hline 3474 & & & & & & $U$ & & RIVER & & CONTINUOUS & FILTER & 252 \\
\hline \begin{tabular}{|l|}
3475 \\
\end{tabular} & & & & & & $U$ & & RIVER & & CONTINUOUS & RESIN & 252 \\
\hline \begin{tabular}{|l|}
34766 \\
\end{tabular} & $\mathrm{pCi} / \mathrm{L}$ & 15 & & & & & & RIVER & & CUMULATIVE & UNFILTERED & 463 \\
\hline 3477 & $\mathrm{pCi} / \mathrm{L}$ & 0.0647 & & & & & & RIVER & & CUMULATIVE & UNFILTERED & 463 \\
\hline \begin{tabular}{|l|}
3478 \\
\end{tabular} & $\mathrm{pCi} / \mathrm{L}$ & 0.903 & & & & $U$ & & RIVER & & CUMULATIVE & UNFILTERED & 463 \\
\hline 3479 & $\mathrm{pCi} / \mathrm{L}$ & 0.0662 & & & & & & SANITARY & & CUMULATIVE & UNFILTERED & 385 \\
\hline 3480 & $\mathrm{pCi} / \mathrm{L}$ & 0.168 & & & & $\mathrm{U}$ & & SANITARY & & CUMULATIVE & UNFILTERED & 385 \\
\hline \begin{tabular}{|l|}
3481 \\
\end{tabular} & $\mathrm{pCi} / \mathrm{L}$ & 0.0076 & & & & & & RIVER & & CONTINUOUS & RESIN & 307 \\
\hline \begin{tabular}{|l|}
3482 \\
\end{tabular} & $\mathrm{pCi} / \mathrm{L}$ & & 0.0000136 & & & & & RIVER & & CONTINUOUS & RESIN & 307 \\
\hline \begin{tabular}{|l|}
3483 \\
\end{tabular} & $\mathrm{pCi} / \mathrm{L}$ & 0.0028 & & & & & & RIVER & & CONTINUOUS & FILTER & 307 \\
\hline \begin{tabular}{|l|}
3484 \\
\end{tabular} & $\mathrm{pCi} / \mathrm{L}$ & & 0.00000104 & & & & & RIVER & & CONTINUOUS & RESIN & 290 \\
\hline 3485 & $\mathrm{pCi} / \mathrm{L}$ & 16 & & & & & & RIVER & & CUMULATIVE & UNFILTERED & 290 \\
\hline
\end{tabular}




\begin{tabular}{|c|c|c|c|c|c|c|c|c|c|c|c|c|}
\hline & $\mathrm{N}$ & $\mathrm{O}$ & $\mathrm{P}$ & $\mathrm{Q}$ & $\mathrm{R}$ & $\mathrm{s}$ & $T$ & $\bar{U}$ & $\mathrm{~V}$ & $\mathrm{~W}$ & $\mathrm{X}$ & $\bar{Y}$ \\
\hline 1 & ANAL_UNITS_RPTD & COUNTING_ERROR & \begin{tabular}{|l|} 
TOTAL_ANAL_ERROR \\
\end{tabular} & MIN_DETECTABLE_ACTIVITY & PCNT_MOISTURE & LAB_QUALIFIER & REVIEW_QUALIFIER & SAMP_FROM & SAMP_ITEM & SAMP MTHD & COLL MTHD & SAMP SITE ID \\
\hline 3486 & & & & & & & & RIVER & & CONTINUOUS & RESIN & 252 \\
\hline 3487 & $\mathrm{pCi} / \mathrm{L}$ & 0.013 & & & & & & RIVER & & CONTINUOUS & RESIN & 252 \\
\hline 3488 & $\mathrm{pCi} / \mathrm{L}$ & 15 & & & & & & RIVER & & CUMULATIVE & UNFILTERED & 252 \\
\hline 3489 & $\mathrm{pCi} / \mathrm{L}$ & 0.0498 & & & & & & RIVER & & CUMULATIVE & UNFILTERED & 252 \\
\hline 3490 & $\mathrm{pCi} / \mathrm{L}$ & 0.236 & & & & & & RIVER & & CUMULATIVE & UNFILTERED & 252 \\
\hline 3491 & $\mathrm{pCi} / \mathrm{L}$ & 0.0079 & & & & & & RIVER & & CONTINUOUS & FILTER & 252 \\
\hline 3492 & $\mathrm{pCi} / \mathrm{L}$ & 0.0062 & & & & & & RIVER & & CONTINUOUS & RESIN & 307 \\
\hline 3493 & $\mathrm{pCi} / \mathrm{L}$ & 0.0032 & & & & & & RIVER & & CONTINUOUS & FILTER & 307 \\
\hline 3494 & $\mathrm{pCi} / \mathrm{L}$ & 0.026 & & & & & & RIVER & & CONTINUOUS & RESIN & 252 \\
\hline 3495 & $\mathrm{pCi} / \mathrm{L}$ & 0.012 & & & & & & RIVER & & CONTINUOUS & FILTER & 252 \\
\hline 3496 & $\mathrm{pCi} / \mathrm{L}$ & 0.838 & & & & & & QC_SAMPLE & & RAW GRAB & UNFILTERED & 451 \\
\hline 3497 & $\mathrm{pCi} / \mathrm{L}$ & 0.412 & & & & & & QC_SAMPLE & & RAW GRAB & UNFILTERED & 451 \\
\hline 3498 & $\mathrm{pCi} / \mathrm{L}$ & 0.187 & & & & & & QC_SAMPLE & & RAW GRAB & UNFILTERED & 542 \\
\hline 3499 & $\mathrm{pCi} / \mathrm{L}$ & 1.4 & & & & & & QC_SAMPLE & & RAW GRAB & UNFILTERED & 542 \\
\hline 3500 & $\mathrm{pCi} / \mathrm{L}$ & 0.269 & & & & & & SURFACE & & RAW GRAB & UNFILTERED & 509 \\
\hline 3501 & $\mathrm{pCi} / \mathrm{L}$ & 0.748 & & & & & & SURFACE & & RAW GRAB & UNFILTERED & 509 \\
\hline 3502 & $\mathrm{pCi} / \mathrm{L}$ & 0.493 & & & & $U$ & & SURFACE & & RAW GRAB & UNFILTERED & 136 \\
\hline 3503 & $\mathrm{pCi} / \mathrm{L}$ & 1.09 & & & & & & SURFACE & & RAW GRAB & UNFILTERED & 146 \\
\hline 3504 & $\mathrm{pCi} / \mathrm{L}$ & 0.0753 & & & & & & SURFACE & & RAW GRAB & UNFILTERED & 146 \\
\hline 3505 & $\mathrm{pCi} / \mathrm{L}$ & 0.535 & & & & & & SURFACE & & RAW GRAB & UNFILTERED & 147 \\
\hline 3506 & $\mathrm{pCi} / \mathrm{L}$ & 0.411 & & & & & & SURFACE & & RAW GRAB & UNFILTERED & 147 \\
\hline 3507 & $\mathrm{pCi} / \mathrm{L}$ & 0.0073 & & & & & & RIVER & & CONTINUOUS & RESIN & 307 \\
\hline 3508 & $\mathrm{pCi} / \mathrm{L}$ & & 0.0000096 & & & & & RIVER & & CONTINUOUS & RESIN & 307 \\
\hline 3509 & $\mathrm{pCi} / \mathrm{L}$ & 0.0028 & & & & & & RIVER & & CONTINUOUS & FILTER & 307 \\
\hline 3510 & $\mathrm{pCi} / \mathrm{L}$ & & 0.00000065 & & & & & RIVER & & CONTINUOUS & RESIN & 290 \\
\hline 3511 & $\mathrm{pCi} / \mathrm{L}$ & 12 & & & & & & RIVER & & CUMULATIVE & UNFILTERED & 290 \\
\hline 3512 & $\mathrm{pCi} / \mathrm{L}$ & & 0.00000156 & & & & & RIVER & & CONTINUOUS & RESIN & 252 \\
\hline 3513 & $\mathrm{pCi} / \mathrm{L}$ & 0.0077 & & & & & & RIVER & & CONTINUOUS & RESIN & 252 \\
\hline 3514 & $\mathrm{pCi} / \mathrm{L}$ & 0.0038 & & & & & & RIVER & & CONTINUOUS & FILTER & 252 \\
\hline 3515 & $\mathrm{pCi} / \mathrm{L}$ & 12 & & & & & & RIVER & & CUMULATIVE & UNFILTERED & 252 \\
\hline 3516 & $\mathrm{pCi} / \mathrm{L}$ & 0.135 & & & & & & RIVER & & CUMULATIVE & UNFILTERED & 252 \\
\hline 3517 & $\mathrm{pCi} / \mathrm{L}$ & 0.681 & & & & $U$ & & RIVER & & CUMULATIVE & UNFILTERED & 252 \\
\hline 3518 & $\mathrm{pCi} / \mathrm{L}$ & 14 & & & & & & RIVER & & CUMULATIVE & UNFILTERED & 463 \\
\hline 3519 & $\mathrm{pCi} / \mathrm{L}$ & 0.104 & & & & & & RIVER & & CUMULATIVE & UNFILTERED & 463 \\
\hline 3520 & $\mathrm{pCi} / \mathrm{L}$ & 0.314 & & & & U & & RIVER & & CUMULATIVE & UNFILTERED & 463 \\
\hline 3521 & $\mathrm{pCi} / \mathrm{L}$ & 0.259 & & & & & & SANITARY & & CUMULATIVE & UNFILTERED & 385 \\
\hline 3522 & $\mathrm{pCi} / \mathrm{L}$ & 0.444 & & & & $U$ & & SANITARY & & CUMULATIVE & UNFILTERED & 385 \\
\hline 3523 & $\mathrm{pCi} / \mathrm{L}$ & 0.0223 & & & & U & & DRINKING & & SAN. GRAB & UNFILTERED & 187 \\
\hline 3524 & $\mathrm{pCi} / \mathrm{L}$ & 408 & & & & $U$ & & DRINKING & & SAN. GRAB & UNFILTERED & 187 \\
\hline 3525 & $\mathrm{pCi} / \mathrm{L}$ & 0.0029 & & & & & & RIVER & & CONTINUOUS & FILTER & 307 \\
\hline 3526 & $\mathrm{pCi} / \mathrm{L}$ & 0.0073 & & & & & & RIVER & & CONTINUOUS & RESIN & 307 \\
\hline 3527 & $\mathrm{pCi} / \mathrm{L}$ & 0.0072 & & & & & & RIVER & & CONTINUOUS & FILTER & 252 \\
\hline 3528 & $\mathrm{pCi} / \mathrm{L}$ & 0.02 & & & & & & RIVER & & CONTINUOUS & RESIN & 252 \\
\hline 3529 & $\mathrm{pCi} / \mathrm{L}$ & 0.0623 & & & & & & DRINKING & & SAN. GRAB & UNFILTERED & 512 \\
\hline 3530 & $\mathrm{pCi} / \mathrm{L}$ & 139 & & & & & & DRINKING & & SAN. GRAB & UNFILTERED & 512 \\
\hline 3531 & $\mathrm{pCi} / \mathrm{L}$ & 0.545 & & & & $U$ & & DRINKING & & SAN. GRAB & UNFILTERED & 512 \\
\hline 3532 & $\mathrm{pCi} / \mathrm{L}$ & 0.0562 & & & & & & SANITARY & & CUMULATIVE & UNFILTERED & 491 \\
\hline 3533 & $\mathrm{pCi} / \mathrm{L}$ & 419 & & & & $U$ & & SANITARY & & CUMULATIVE & UNFILTERED & 491 \\
\hline 3534 & $\mathrm{pCi} / \mathrm{L}$ & 0.0469 & & & & & & SANITARY & & CUMULATIVE & UNFILTERED & 385 \\
\hline 3535 & $\mathrm{pCi} / \mathrm{L}$ & 0.594 & & & & $U$ & & SANITARY & & CUMULATIVE & UNFILTERED & 385 \\
\hline 3536 & $\mathrm{pCi} / \mathrm{L}$ & 0.0513 & & & & & & RIVER & & CUMULATIVE & UNFILTERED & 553 \\
\hline 3537 & $\mathrm{pCi} / \mathrm{L}$ & 414 & & & & $\mathrm{U}_{2}$ & & RIVER & & CUMULATIVE & UNFILTERED & 553 \\
\hline
\end{tabular}




\begin{tabular}{|c|c|c|c|c|c|c|c|c|c|c|c|c|}
\hline & $\mathrm{N}$ & $\mathrm{O}$ & $\mathrm{P}$ & $\mathrm{Q}$ & $\mathrm{R}$ & $\mathrm{s}$ & $T$ & $\bar{U}$ & $\mathrm{~V}$ & $\mathrm{~W}$ & $\mathrm{X}$ & $\bar{Y}$ \\
\hline 1 & ANAL_UNITS_RPTD & COUNTING_ERROR & TOTAL_ANAL_ERROR & MIN_DETECTABLE_ACTIVITY & PCNT_MOISTURE & LAB_QUALIFIER & REVIEW_QUALIFIER & SAMP_FROM & SAMP_ITEM & SAMP MTHD & COLL MTHD & SAMP SITE ID \\
\hline 3538 & $\mathrm{pCi} / \mathrm{L}$ & 0.494 & & & & & & RIVER & & CUMULATIVE & UNFILTERED & 553 \\
\hline 3539 & $\mathrm{pCi} / \mathrm{L}$ & 0.0000036 & & & & & & RIVER & & CONTINUOUS & RESIN & 307 \\
\hline 3540 & $\mathrm{pCi} / \mathrm{L}$ & 0.0064 & & & & & & RIVER & & CONTINUOUS & RESIN & 307 \\
\hline 3541 & $\mathrm{pCi} / \mathrm{L}$ & & 0.0000086 & & & & & RIVER & & CONTINUOUS & RESIN & 307 \\
\hline 3542 & $\mathrm{pCi} / \mathrm{L}$ & 0.0035 & & & & & & RIVER & & CONTINUOUS & FILTER & 307 \\
\hline 3543 & $\mathrm{pCi} / \mathrm{L}$ & 0.000003 & & & & & & RIVER & & CONTINUOUS & FILTER & 307 \\
\hline 3544 & $\mathrm{pCi} / \mathrm{L}$ & & 0.00000063 & & & & & RIVER & & CONTINUOUS & RESIN & 290 \\
\hline 3545 & $\mathrm{pCi} / \mathrm{L}$ & 12 & & & & & & RIVER & & CUMULATIVE & UNFILTERED & 290 \\
\hline 3546 & $\mathrm{pCi} / \mathrm{L}$ & & 0.00000133 & & & & & RIVER & & CONTINUOUS & RESIN & 252 \\
\hline 3547 & $\mathrm{pCi} / \mathrm{L}$ & 0.007 & & & & & & RIVER & & CONTINUOUS & RESIN & 252 \\
\hline 3548 & $\mathrm{pCi} / \mathrm{L}$ & 0.0000009 & & & & & & RIVER & & CONTINUOUS & RESIN & 252 \\
\hline 3549 & $\mathrm{pCi} / \mathrm{L}$ & 12 & & & & & & RIVER & & CUMULATIVE & UNFILTERED & 252 \\
\hline 3550 & $\mathrm{pCi} / \mathrm{L}$ & 0.000005 & & & & & & RIVER & & CONTINUOUS & FILTER & 252 \\
\hline \begin{tabular}{|l|}
3551 \\
\end{tabular} & $\mathrm{pCi} / \mathrm{L}$ & 0.0032 & & & & & & RIVER & & CONTINUOUS & FILTER & 252 \\
\hline 3552 & $\mathrm{pCi} / \mathrm{L}$ & 0.0435 & & & & & & RIVER & & CUMULATIVE & UNFILTERED & 252 \\
\hline 3553 & $\mathrm{pCi} / \mathrm{L}$ & 0.597 & & & & $U$ & & RIVER & & CUMULATIVE & UNFILTERED & 252 \\
\hline 3554 & $\mathrm{pCi} / \mathrm{L}$ & 14 & & & & & & RIVER & & CUMULATIVE & UNFILTERED & 463 \\
\hline 3555 & $\mathrm{pCi} / \mathrm{L}$ & 0.0516 & & & & & & RIVER & & CUMULATIVE & UNFILTERED & 463 \\
\hline 3556 & $\mathrm{pCi} / \mathrm{L}$ & 0.561 & & & & $U$ & & RIVER & & CUMULATIVE & UNFILTERED & 463 \\
\hline 3557 & $\mathrm{pCi} / \mathrm{L}$ & 0.55 & & & & $U$ & & DRINKING & & SAN. GRAB & UNFILTERED & 487 \\
\hline 3558 & $\mathrm{pCi} / \mathrm{L}$ & 187 & & & & $U$ & & DRINKING & & SAN. GRAB & UNFILTERED & 487 \\
\hline 3559 & $\mathrm{pCi} / \mathrm{L}$ & 0.0061 & & & & & & RIVER & & CONTINUOUS & RESIN & 307 \\
\hline 3560 & $\mathrm{pCi} / \mathrm{L}$ & 0.0031 & & & & & & RIVER & & CONTINUOUS & FILTER & 307 \\
\hline 3561 & $\mathrm{pCi} / \mathrm{L}$ & 0.0041 & & & & & & RIVER & & CONTINUOUS & FILTER & 252 \\
\hline 3562 & $\mathrm{pCi} / \mathrm{L}$ & 0.0082 & & & & & & RIVER & & CONTINUOUS & RESIN & 252 \\
\hline 3563 & $\mathrm{pCi} / \mathrm{L}$ & 14 & & & & & & RIVER & & CUMULATIVE & UNFILTERED & 463 \\
\hline 3564 & $\mathrm{pCi} / \mathrm{L}$ & 0.05 & & & & & & RIVER & & CUMULATIVE & UNFILTERED & 463 \\
\hline 3565 & $\mathrm{pCi} / \mathrm{L}$ & 0.251 & & & & & & RIVER & & CUMULATIVE & UNFILTERED & 463 \\
\hline 3566 (356 & $\mathrm{pCi} / \mathrm{L}$ & 0.0031 & & & & & & RIVER & & CONTINUOUS & FILTER & 307 \\
\hline 3567 & $\mathrm{pCi} / \mathrm{L}$ & 0.006 & & & & & & RIVER & & CONTINUOUS & RESIN & 307 \\
\hline 3568 & $\mathrm{pCi} / \mathrm{L}$ & & 0.0000088 & & & & & RIVER & & CONTINUOUS & RESIN & 307 \\
\hline 3569 & $\mathrm{pCi} / \mathrm{L}$ & & 0.00000133 & & & & & RIVER & & CONTINUOUS & RESIN & 290 \\
\hline 3570 & $\mathrm{pCi} / \mathrm{L}$ & 12 & & & & & & RIVER & & CUMULATIVE & UNFILTERED & 290 \\
\hline 3571 & $\mathrm{pCi} / \mathrm{L}$ & & 0.000000832 & & & & & RIVER & & CONTINUOUS & RESIN & 252 \\
\hline \begin{tabular}{|l|}
3572 \\
\end{tabular} & $\mathrm{pCi} / \mathrm{L}$ & 0.017 & & & & & & RIVER & & CONTINUOUS & RESIN & 252 \\
\hline 3573 & $\mathrm{pCi} / \mathrm{L}$ & 0.0075 & & & & & & RIVER & & CONTINUOUS & FILTER & 252 \\
\hline 3574 & $\mathrm{pCi} / \mathrm{L}$ & 14 & & & & & & RIVER & & CUMULATIVE & UNFILTERED & 252 \\
\hline 3575 & $\mathrm{pCi} / \mathrm{L}$ & 0.183 & & & & & & RIVER & & CUMULATIVE & UNFILTERED & 252 \\
\hline 3576 & $\mathrm{pCi} / \mathrm{L}$ & 0.568 & & & & $U$ & & RIVER & & CUMULATIVE & UNFILTERED & 252 \\
\hline 3577 & $\mathrm{pCi} / \mathrm{L}$ & 0.048 & & & & & & SANITARY & & CUMULATIVE & UNFILTERED & 385 \\
\hline 3578 & $\mathrm{pCi} / \mathrm{L}$ & 0.178 & & & & U & & SANITARY & & CUMULATIVE & UNFILTERED & 385 \\
\hline 3579 & $\mathrm{pCi} / \mathrm{L}$ & 186 & & & & & & QC_SAMPLE & & RAW GRAB & UNFILTERED & 222 \\
\hline 3580 & $\mathrm{pCi} / \mathrm{L}$ & 0.0781 & & & & & & QC_SAMPLE & & RAW GRAB & UNFILTERED & 222 \\
\hline 3581 & $\mathrm{pCi} / \mathrm{L}$ & 0.465 & & & & & & QC_SAMPLE & & RAW GRAB & UNFILTERED & 222 \\
\hline 3582 & $\mathrm{pCi} / \mathrm{L}$ & 111 & & & & & & QC_SAMPLE & & RAW GRAB & UNFILTERED & 223 \\
\hline 3583 & $\mathrm{pCi} / \mathrm{L}$ & 0.062 & & & & U & & QC_SAMPLE & & RAW GRAB & UNFILTERED & 223 \\
\hline 3584 & $\mathrm{pCi} / \mathrm{L}$ & 0.255 & & & & U & & QC_SAMPLE & & RAW GRAB & UNFILTERED & 223 \\
\hline 3585 & $\mathrm{pCi} / \mathrm{L}$ & 0.0071 & & & & & & RIVER & & CONTINUOUS & RESIN & 307 \\
\hline 3586 & $\mathrm{pCi} / \mathrm{L}$ & 0.0029 & & & & & & RIVER & & CONTINUOUS & FILTER & 307 \\
\hline \begin{tabular}{|l|}
3587 \\
\end{tabular} & $\mathrm{pCi} / \mathrm{L}$ & 13.9 & & & & & & RIVER & & CUMULATIVE & UNFILTERED & 290 \\
\hline 3588 & $\mathrm{pCi} / \mathrm{L}$ & 0.007 & & & & & & RIVER & & CONTINUOUS & RESIN & 252 \\
\hline 3589 & $\mathrm{pCi} / \mathrm{L}$ & 0.0031 & & & & & & RIVER & & CONTINUOUS & FILTER & 252 \\
\hline
\end{tabular}




\begin{tabular}{|c|c|c|c|c|c|c|c|c|c|c|c|c|}
\hline & $\mathrm{N}$ & $\mathrm{O}$ & $\mathrm{P}$ & $\mathrm{Q}$ & $\mathrm{R}$ & $\mathrm{s}$ & $T$ & $\bar{U}$ & $\mathrm{~V}$ & $\mathrm{~W}$ & $\mathrm{X}$ & $\bar{Y}$ \\
\hline 1 & ANAL_UNITS_RPTD & COUNTING_ERROR & TOTAL_ANAL_ERROR & MIN_DETECTABLE_ACTIVITY & PCNT_MOISTURE & LAB_QUALIFIER & REVIEW_QUALIFIER & SAMP_FROM & SAMP_ITEM & SAMP MTHD & COLL MTHD & SAMP SITE ID \\
\hline 3590 & $\mathrm{pCi} / \mathrm{L}$ & 0.0275 & & & & & & SANITARY & & CUMULATIVE & UNFILTERED & 385 \\
\hline 3591 & $\mathrm{pCi} / \mathrm{L}$ & 0.331 & & & & U & & SANITARY & & CUMULATIVE & UNFILTERED & 385 \\
\hline 3592 & $\mathrm{pCi} / \mathrm{L}$ & 0.0081 & & & & & & RIVER & & CONTINUOUS & RESIN & 307 \\
\hline 3593 & $\mathrm{pCi} / \mathrm{L}$ & 0.0031 & & & & & & RIVER & & CONTINUOUS & FILTER & 307 \\
\hline 3594 & $\mathrm{pCi} / \mathrm{L}$ & 0.0066 & & & & & & RIVER & & CONTINUOUS & RESIN & 252 \\
\hline 3595 & $\mathrm{pCi} / \mathrm{L}$ & 13.6 & & & & & & RIVER & & CUMULATIVE & UNFILTERED & 252 \\
\hline 3596 & $\mathrm{pCi} / \mathrm{L}$ & 0.003614 & & & & & & RIVER & & CONTINUOUS & FILTER & 252 \\
\hline 3597 & $\mathrm{pCi} / \mathrm{L}$ & 0.0246 & & & & & & RIVER & & CUMULATIVE & UNFILTERED & 252 \\
\hline 3598 & $\mathrm{pCi} / \mathrm{L}$ & 0.397 & & & & $\mathrm{U}$ & & RIVER & & CUMULATIVE & UNFILTERED & 252 \\
\hline 3599 & $\mathrm{pCi} / \mathrm{L}$ & 13.4 & & & & & & RIVER & & CUMULATIVE & UNFILTERED & 463 \\
\hline 3600 & $\mathrm{pCi} / \mathrm{L}$ & 0.0051 & & & & & & RIVER & & CONTINUOUS & RESIN & 307 \\
\hline 3601 & $\mathrm{pCi} / \mathrm{L}$ & 0.00034 & & & & & & RIVER & & CONTINUOUS & FILTER & 307 \\
\hline 3602 & $\mathrm{pCi} / \mathrm{L}$ & 0.0042 & & & & & & RIVER & & CONTINUOUS & FILTER & 252 \\
\hline 3603 & $\mathrm{pCi} / \mathrm{L}$ & 0.0087 & & & & & & RIVER & & CONTINUOUS & RESIN & 252 \\
\hline 3604 & $\mathrm{pCi} / \mathrm{L}$ & 0.683 & & & & $U$ & & SURFACE & & RAW GRAB & UNFILTERED & 146 \\
\hline 3605 & $\mathrm{pCi} / \mathrm{L}$ & 2 & & & & & & SURFACE & & RAW GRAB & UNFILTERED & 146 \\
\hline 3606 & $\mathrm{pCi} / \mathrm{L}$ & 206 & & & & $U$ & & SURFACE & & RAW GRAB & UNFILTERED & 146 \\
\hline 3607 & $\mathrm{pCi} / \mathrm{L}$ & 0.0288 & & & & & & SANITARY & & CUMULATIVE & UNFILTERED & 491 \\
\hline 3608 & $\mathrm{pCi} / \mathrm{L}$ & 207 & & & & $U$ & & SANITARY & & CUMULATIVE & UNFILTERED & 491 \\
\hline 3609 & $\mathrm{pCi} / \mathrm{L}$ & 0.0271 & & & & & & SANITARY & & CUMULATIVE & UNFILTERED & 385 \\
\hline 3610 & $\mathrm{pCi} / \mathrm{L}$ & 0.585 & & & & U & & SANITARY & & CUMULATIVE & UNFILTERED & 385 \\
\hline 3611 & $\mathrm{pCi} / \mathrm{L}$ & 0.0666 & & & & & & RIVER & & CUMULATIVE & UNFILTERED & 553 \\
\hline 3612 & $\mathrm{pCi} / \mathrm{L}$ & 207 & & & & $U$ & & RIVER & & CUMULATIVE & UNFILTERED & 553 \\
\hline 3613 & $\mathrm{pCi} / \mathrm{L}$ & 0.594 & & & & $U$ & & RIVER & & CUMULATIVE & UNFILTERED & 553 \\
\hline 3614 & $\mathrm{pCi} / \mathrm{L}$ & 0.000154 & & & & $U$ & & RIVER & & CONTINUOUS & RESIN & 307 \\
\hline 3615 & $\mathrm{pCi} / \mathrm{L}$ & 0.006 & & & & & & RIVER & & CONTINUOUS & RESIN & 307 \\
\hline 3616 & $\mathrm{pCi} / \mathrm{L}$ & & 0.00000408 & & & & & RIVER & & CONTINUOUS & RESIN & 307 \\
\hline 3617 & $\mathrm{pCi} / \mathrm{L}$ & 0.0032 & & & & & & RIVER & & CONTINUOUS & FILTER & 307 \\
\hline 3618 & $\mathrm{pCi} / \mathrm{L}$ & 0.000002 & & & & & & RIVER & & CONTINUOUS & FILTER & 307 \\
\hline 3619 & $\mathrm{pCi} / \mathrm{L}$ & 14.7 & & & & & & RIVER & & CUMULATIVE & UNFILTERED & 252 \\
\hline 3620 & $\mathrm{pCi} / \mathrm{L}$ & 0.0318 & & & & & & RIVER & & CUMULATIVE & UNFILTERED & 252 \\
\hline 3621 & $\mathrm{pCi} / \mathrm{L}$ & 0.479 & & & & $U$ & & RIVER & & CUMULATIVE & UNFILTERED & 252 \\
\hline 3622 & $\mathrm{pCi} / \mathrm{L}$ & & 0.0000224 & & & & & RIVER & & CONTINUOUS & RESIN & 252 \\
\hline 3623 & $\mathrm{pCi} / \mathrm{L}$ & 0.0088 & & & & & & RIVER & & CONTINUOUS & RESIN & 252 \\
\hline 3624 & $\mathrm{pCi} / \mathrm{L}$ & 0.00007 & & & & & & RIVER & & CONTINUOUS & RESIN & 252 \\
\hline 3625 & $\mathrm{pCi} / \mathrm{L}$ & 0.000006 & & & & & & RIVER & & CONTINUOUS & FILTER & 252 \\
\hline 3626 & $\mathrm{pCi} / \mathrm{L}$ & 0.00043 & & & & & & RIVER & & CONTINUOUS & FILTER & 252 \\
\hline 3627 & $\mathrm{pCi} / \mathrm{L}$ & 13.6 & & & & & & RIVER & & CUMULATIVE & UNFILTERED & 463 \\
\hline 3628 & $\mathrm{pCi} / \mathrm{L}$ & 0.182 & & & & & & RIVER & & CUMULATIVE & UNFILTERED & 463 \\
\hline 3629 & $\mathrm{pCi} / \mathrm{L}$ & 0.0664 & & & & & & RIVER & & CUMULATIVE & UNFILTERED & 463 \\
\hline 3630 & & & & & & $U$ & & QC_SAMPLE & & RAW GRAB & UNFILTERED & 225 \\
\hline 3631 & & & & & & $U$ & & QC_SAMPLE & & RAW GRAB & UNFILTERED & 225 \\
\hline 3632 & & & & & & $U$ & & RIVER & & CUMULATIVE & UNFILTERED & 553 \\
\hline 3633 & & & & & & $U$ & & RIVER & & CUMULATIVE & UNFILTERED & 553 \\
\hline 3634 & & & & & & $U$ & & RIVER & & CUMULATIVE & UNFILTERED & 553 \\
\hline 3635 & $\mathrm{pCi} / \mathrm{L}$ & 1.35 & & & & & & SURFACE & & RAW GRAB & UNFILTERED & 509 \\
\hline 3636 & $\mathrm{pCi} / \mathrm{L}$ & 0.553 & & & & & & SURFACE & & RAW GRAB & UNFILTERED & 509 \\
\hline 3637 & $\mathrm{pCi} / \mathrm{L}$ & 211 & & & & & & SURFACE & & RAW GRAB & UNFILTERED & 509 \\
\hline 3638 & $\mathrm{pCi} / \mathrm{L}$ & 418 & & & & & & SURFACE & & RAW GRAB & UNFILTERED & 136 \\
\hline 3639 & $\mathrm{pCi} / \mathrm{L}$ & 1.98 & & & & $U$ & & SURFACE & & RAW GRAB & UNFILTERED & 136 \\
\hline 3640 & $\mathrm{pCi} / \mathrm{L}$ & 1.66 & & & & & & SURFACE & & RAW GRAB & UNFILTERED & 146 \\
\hline 3641 & $\mathrm{pCi} / \mathrm{L}$ & 0.646 & & & & & & SURFACE & & RAW GRAB & UNFILTERED & 146 \\
\hline
\end{tabular}




\begin{tabular}{|c|c|c|c|c|c|c|c|c|c|c|c|c|}
\hline & $\mathrm{N}$ & $\mathrm{O}$ & $\mathrm{P}$ & $\mathrm{Q}$ & $\mathrm{R}$ & $\mathrm{s}$ & $T$ & $\mathrm{U}$ & $\mathrm{V}$ & $\overline{\mathrm{W}}$ & $\mathrm{X}$ & $\bar{Y}$ \\
\hline 1 & ANAL_UNITS_RPTD & COUNTING_ERROR & TOTAL_ANAL_ERROR & MIN_DETECTABLE_ACTIVITY & PCNT_MOISTURE & LAB_QUALIFIER & REVIEW_QUALIFIER & SAMP_FROM & SAMP_ITEM & SAMP_MTHD & COLL_MTHD & SAMP_SITE_ID \\
\hline $3642 \mathrm{r}$ & $\mathrm{pCi} / \mathrm{L}$ & 209 & & & & & & SURFACE & & RAW GRAB & UNFILTERED & 146 \\
\hline $3643 \mathrm{r}$ & $\mathrm{pCi} / \mathrm{L}$ & 248 & & & & & & SURFACE & & RAW GRAB & UNFILTERED & 147 \\
\hline $3644 \mathrm{r}$ & $\mathrm{pCi} / \mathrm{L}$ & 3.04 & & & & U & & SURFACE & & RAW GRAB & UNFILTERED & 147 \\
\hline $3645 \mathrm{r}$ & $\mathrm{pCi} / \mathrm{L}$ & 0.0772 & & & & & & SURFACE & & RAW GRAB & UNFILTERED & 147 \\
\hline 3646 & $\mathrm{pCi} / \mathrm{L}$ & 0.567 & & & & U & & DRINKING & & SAN. GRAB & UNFILTERED & 187 \\
\hline $3647 \mathrm{r}$ & $\mathrm{pCi} / \mathrm{L}$ & 214 & & & & $\mathrm{U}$ & & DRINKING & & SAN. GRAB & UNFILTERED & 187 \\
\hline $3648 \mathrm{r}$ & $\mathrm{pCi} / \mathrm{L}$ & 0.0072 & & & & & & RIVER & & CONTINUOUS & RESIN & 307 \\
\hline 3649 & $\mathrm{pCi} / \mathrm{L}$ & 0.0033 & & & & & & RIVER & & CONTINUOUS & FILTER & 307 \\
\hline 3650 & $\mathrm{pCi} / \mathrm{L}$ & 0.0027 & & & & & & RIVER & & CONTINUOUS & FILTER & 252 \\
\hline $3651 \mathrm{r}$ & $\mathrm{pCi} / \mathrm{L}$ & 0.0058 & & & & & & RIVER & & CONTINUOUS & RESIN & 252 \\
\hline $3652 \mathrm{r}$ & $\mathrm{pCi} / \mathrm{L}$ & 0.0547 & & & & & & DRINKING & & SAN. GRAB & UNFILTERED & 512 \\
\hline 3653 & $\mathrm{pCi} / \mathrm{L}$ & 410 & & & & & & DRINKING & & SAN. GRAB & UNFILTERED & 512 \\
\hline 3654 & $\mathrm{pCi} / \mathrm{L}$ & 0.497 & & & & U & & DRINKING & & SAN. GRAB & UNFILTERED & 512 \\
\hline 3655 & $\mathrm{pCi} / \mathrm{L}$ & 0.0374 & & & & & & SANITARY & & CUMULATIVE & UNFILTERED & 385 \\
\hline 3656 & $\mathrm{pCi} / \mathrm{L}$ & 0.935 & & & & & & SANITARY & & CUMULATIVE & UNFILTERED & 385 \\
\hline 3657 & $\mathrm{pCi} / \mathrm{L}$ & 0.0034 & & & & & & RIVER & & CONTINUOUS & FILTER & 307 \\
\hline 3658 & $\mathrm{pCi} / \mathrm{L}$ & 0.0062 & & & & & & RIVER & & CONTINUOUS & RESIN & 307 \\
\hline 3659 & $\mathrm{pCi} / \mathrm{L}$ & 0.013 & & & & & & RIVER & & CONTINUOUS & RESIN & 252 \\
\hline $3660 \mathrm{r}$ & $\mathrm{pCi} / \mathrm{L}$ & 0.0058 & & & & & & RIVER & & CONTINUOUS & FILTER & 252 \\
\hline $3661 \mathrm{r}$ & $\mathrm{pCi} / \mathrm{L}$ & 14.1 & & & & & & RIVER & & CUMULATIVE & UNFILTERED & 252 \\
\hline 3662 & $\mathrm{pCi} / \mathrm{L}$ & 0.0411 & & & & & & RIVER & & CUMULATIVE & UNFILTERED & 252 \\
\hline $3663 \mathrm{r}$ & pCi/L & 0.299 & & & & U & & RIVER & & CUMULATIVE & UNFILTERED & 252 \\
\hline 3664 & $\mathrm{pCi} / \mathrm{L}$ & 14 & & & & & & RIVER & & CUMULATIVE & UNFILTERED & 463 \\
\hline 3665 & pCi/L & 0.0344 & & & & & & RIVER & & CUMULATIVE & UNFILTERED & 463 \\
\hline $3666 \mathrm{r}$ & $\mathrm{pCi} / \mathrm{L}$ & 0.503 & & & & $U$ & & RIVER & & CUMULATIVE & UNFILTERED & 463 \\
\hline 3667 & $\mathrm{pCi} / \mathrm{L}$ & 0.0347 & & & & & & DRINKING & & SAN. GRAB & UNFILTERED & 487 \\
\hline $3668 \mathrm{r}$ & $\mathrm{pCi} / \mathrm{L}$ & 0.226 & & & & $\mathrm{U}$ & & DRINKING & & SAN. GRAB & UNFILTERED & 487 \\
\hline 3669 & $\mathrm{pCi} / \mathrm{L}$ & 213 & & & & U & & DRINKING & & SAN. GRAB & UNFILTERED & 487 \\
\hline 3670 & $\mathrm{pCi} / \mathrm{L}$ & 0.665 & & & & U & & DRINKING & & SAN. GRAB & UNFILTERED & 530 \\
\hline 3671 & & & & & & U & & DRINKING & & SAN. GRAB & UNFILTERED & 530 \\
\hline $3672 \mathrm{r}$ & $\mathrm{pCi} / \mathrm{L}$ & 0.0436 & & & & & & DRINKING & & SAN. GRAB & UNFILTERED & 530 \\
\hline 3673 & $\mathrm{pCi} / \mathrm{L}$ & 0.005 & & & & & & RIVER & & CONTINUOUS & FILTER & 307 \\
\hline 3674 & $\mathrm{pCi} / \mathrm{L}$ & 0.0074 & & & & & & RIVER & & CONTINUOUS & RESIN & 307 \\
\hline 3675 & $\mathrm{pCi} / \mathrm{L}$ & 0.0087 & & & & & & RIVER & & CONTINUOUS & RESIN & 252 \\
\hline 3676 & pCi/L & 0.0042 & & & & & & RIVER & & CONTINUOUS & FILTER & 252 \\
\hline 3677 & $\mathrm{pCi} / \mathrm{L}$ & 206 & & & & U & & DRINKING & & CUMULATIVE & UNFILTERED & 307 \\
\hline 3678 & $\mathrm{pCi} / \mathrm{L}$ & 0.048 & & & & & & DRINKING & & CUMULATIVE & UNFILTERED & 307 \\
\hline 3679 & $\mathrm{pCi} / \mathrm{L}$ & 0.0394 & & & & & & SANITARY & & CUMULATIVE & UNFILTERED & 385 \\
\hline $3680 \mathrm{r}$ & $\mathrm{pCi} / \mathrm{L}$ & 1.1 & & & & $U$ & & SANITARY & & CUMULATIVE & UNFILTERED & 385 \\
\hline $3681 \mathrm{r}$ & $\mathrm{pCi} / \mathrm{L}$ & 0.0066 & & & & & & RIVER & & CONTINUOUS & RESIN & 307 \\
\hline $3682 \mathrm{r}$ & $\mathrm{pCi} / \mathrm{L}$ & 0.0032 & & & & & & RIVER & & CONTINUOUS & FILTER & 307 \\
\hline 3683 & $\mathrm{pCi} / \mathrm{L}$ & 0.013 & & & & & & RIVER & & CONTINUOUS & RESIN & 252 \\
\hline 3684 & $\mathrm{pCi} / \mathrm{L}$ & 15.3 & & & & & & RIVER & & CUMULATIVE & UNFILTERED & 252 \\
\hline 3685 & $\mathrm{pCi} / \mathrm{L}$ & 0.0053 & & & & & & RIVER & & CONTINUOUS & FILTER & 252 \\
\hline $3686 \mathrm{r}$ & $\mathrm{pCi} / \mathrm{L}$ & 0.0353 & & & & & & RIVER & & CUMULATIVE & UNFILTERED & 252 \\
\hline $3687 \mathrm{r}$ & $\mathrm{pCi} / \mathrm{L}$ & 0.839 & & & & U & & RIVER & & CUMULATIVE & UNFILTERED & 252 \\
\hline $3688 \mathrm{r}$ & $\mathrm{pCi} / \mathrm{L}$ & 15.3 & & & & & & RIVER & & CUMULATIVE & UNFILTERED & 463 \\
\hline 3689 & $\mathrm{pCi} / \mathrm{L}$ & 0.0367 & & & & & & RIVER & & CUMULATIVE & UNFILTERED & 463 \\
\hline 3690 & $\mathrm{pCi} / \mathrm{L}$ & 0.879 & & & & U & & RIVER & & CUMULATIVE & UNFILTERED & 463 \\
\hline $3691 \mathrm{r}$ & $\mathrm{pCi} / \mathrm{L}$ & 0.0339 & & & & & & QC_SAMPLE & & RAW GRAB & UNFILTERED & 550 \\
\hline $3692 \mathrm{r}$ & pCi/L & 0.761 & & & & U & & QC_SAMPLE & & RAW GRAB & UNFILTERED & 550 \\
\hline 3693 & $\mathrm{pCi} / \mathrm{L}$ & 8.72 & & & & & & QC_SAMPLE & & RAW GRAB & UNFILTERED & 550 \\
\hline
\end{tabular}




\begin{tabular}{|c|c|c|c|c|c|c|c|c|c|c|c|c|}
\hline & $\mathrm{N}$ & $\mathrm{O}$ & $\mathrm{P}$ & $\mathrm{Q}$ & $\mathrm{R}$ & $\mathrm{s}$ & $T$ & $\mathrm{U}$ & $\mathrm{V}$ & $\mathrm{W}$ & $x$ & $\bar{Y}$ \\
\hline 1 & ANAL_UNITS_RPTD & COUNTING_ERROR & \begin{tabular}{|c|} 
TOTAL_ANAL_ERROR \\
\end{tabular} & MIN_DETECTABLE_ACTIVITY & PCNT_MOISTURE & LAB_QUALIFIER & REVIEW_QUALIFIER & SAMP_FROM & SAMP_ITEM & SAMP_MTHD & COLL_MTHD & SAMP_SITE_ID \\
\hline 3694 & pCi/L & 0.618 & & & & $U$ & & QC_SAMPLE & & RAW GRAB & UNFILTERED & 247 \\
\hline 3695 & $\mathrm{pCi} / \mathrm{L}$ & 0.0443 & & & & & & QC_SAMPLE & & RAW GRAB & UNFILTERED & 247 \\
\hline 3696 & $\mathrm{pCi} / \mathrm{L}$ & 16.8 & & & & & & QC_SAMPLE & & RAW GRAB & UNFILTERED & 247 \\
\hline 3697 & $\mathrm{pCi} / \mathrm{L}$ & 0.0032 & & & & & & RIVER & & CONTINUOUS & FILTER & 307 \\
\hline 3698 & $\mathrm{pCi} / \mathrm{L}$ & 0.0057 & & & & & & RIVER & & CONTINUOUS & RESIN & 307 \\
\hline 3699 & $\mathrm{pCi} / \mathrm{L}$ & 0.0061 & & & & & & RIVER & & CONTINUOUS & RESIN & 252 \\
\hline 3700 & $\mathrm{pCi} / \mathrm{L}$ & 0.0034 & & & & & & RIVER & & CONTINUOUS & FILTER & 252 \\
\hline 3701 & $\mathrm{pCi} / \mathrm{L}$ & 0.045 & & & & & & SANITARY & & CUMULATIVE & UNFILTERED & 385 \\
\hline 3702 & $\mathrm{pCi} / \mathrm{L}$ & 0.639 & & & & U & & SANITARY & & CUMULATIVE & UNFILTERED & 385 \\
\hline 3703 & $\mathrm{pCi} / \mathrm{L}$ & 0.00008 & & & & & & RIVER & & CONTINUOUS & RESIN & 307 \\
\hline 3704 & $\mathrm{pCi} / \mathrm{L}$ & 0.0085 & & & & & & RIVER & & CONTINUOUS & RESIN & 307 \\
\hline 3705 & $\mathrm{pCi} / \mathrm{L}$ & & 0.00000414 & & & & & RIVER & & CONTINUOUS & RESIN & 307 \\
\hline 3706 & $\mathrm{pCi} / \mathrm{L}$ & 0.0032 & & & & & & RIVER & & CONTINUOUS & FILTER & 307 \\
\hline 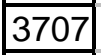 & $\mathrm{pCi} / \mathrm{L}$ & 0.000004 & & & & & & RIVER & & CONTINUOUS & FILTER & 307 \\
\hline 3708 & $\mathrm{pCi} / \mathrm{L}$ & 0.00702 & & & & & & RIVER & & CONTINUOUS & RESIN & 252 \\
\hline 3709 & $\mathrm{pCi} / \mathrm{L}$ & 0.000023 & & & & $U$ & & RIVER & & CONTINUOUS & RESIN & 252 \\
\hline 3710 & $\mathrm{pCi} / \mathrm{L}$ & & 0.00000409 & & & & & RIVER & & CONTINUOUS & RESIN & 252 \\
\hline 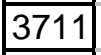 & $\mathrm{pCi} / \mathrm{L}$ & 0.000004 & & & & & & RIVER & & CONTINUOUS & FILTER & 252 \\
\hline \begin{tabular}{|l|}
3712 \\
\end{tabular} & $\mathrm{pCi} / \mathrm{L}$ & 0.003 & & & & & & RIVER & & CONTINUOUS & FILTER & 252 \\
\hline 3713 & $\mathrm{pCi} / \mathrm{L}$ & 15.8 & & & & & & RIVER & & CUMULATIVE & UNFILTERED & 252 \\
\hline 3714 & $\mathrm{pCi} / \mathrm{L}$ & 0.0453 & & & & & & RIVER & & CUMULATIVE & UNFILTERED & 252 \\
\hline 3715 & pCi/L & 0.317 & & & & U & & RIVER & & CUMULATIVE & UNFILTERED & 252 \\
\hline 3716 & $\mathrm{pCi} / \mathrm{L}$ & 20.6 & & & & & & RIVER & & CUMULATIVE & UNFILTERED & 463 \\
\hline 3717 & $\mathrm{pCi} / \mathrm{L}$ & 0.0454 & & & & & & RIVER & & CUMULATIVE & UNFILTERED & 463 \\
\hline 3718 & $\mathrm{pCi} / \mathrm{L}$ & 0.373 & & & & & & RIVER & & CUMULATIVE & UNFILTERED & 463 \\
\hline 3719 & $\mathrm{pCi} / \mathrm{L}$ & 0.0387 & & & & & & RIVER & & CUMULATIVE & UNFILTERED & 553 \\
\hline 3720 & $\mathrm{pCi} / \mathrm{L}$ & 205 & & & & $U$ & & RIVER & & CUMULATIVE & UNFILTERED & 553 \\
\hline \begin{tabular}{|l|}
3721 \\
\end{tabular} & $\mathrm{pCi} / \mathrm{L}$ & 0.682 & & & & $U$ & & RIVER & & CUMULATIVE & UNFILTERED & 553 \\
\hline \begin{tabular}{|l|}
3722 \\
\end{tabular} & $\mathrm{pCi} / \mathrm{L}$ & 0.255 & & & & & & SURFACE & & RAW GRAB & UNFILTERED & 509 \\
\hline 3723 & $\mathrm{pCi} / \mathrm{L}$ & 2.27 & & & & & & SURFACE & & RAW GRAB & UNFILTERED & 509 \\
\hline 3724 & $\mathrm{pCi} / \mathrm{L}$ & 205 & & & & $U$ & & SURFACE & & RAW GRAB & UNFILTERED & 509 \\
\hline 3725 & $\mathrm{pCi} / \mathrm{L}$ & 442 & & & & & & SURFACE & & RAW GRAB & UNFILTERED & 136 \\
\hline 3726 & $\mathrm{pCi} / \mathrm{L}$ & 0.637 & & & & & & SURFACE & & RAW GRAB & UNFILTERED & 136 \\
\hline \begin{tabular}{|l|}
3727 \\
\end{tabular} & $\mathrm{pCi} / \mathrm{L}$ & 2.36 & & & & & & SURFACE & & RAW GRAB & UNFILTERED & 146 \\
\hline 3728 & $\mathrm{pCi} / \mathrm{L}$ & 0.222 & & & & & & SURFACE & & RAW GRAB & UNFILTERED & 146 \\
\hline 3729 & pCi/L & 208 & & & & & & SURFACE & & RAW GRAB & UNFILTERED & 146 \\
\hline 3730 & $\mathrm{pCi} / \mathrm{L}$ & 217 & & & & & & SURFACE & & RAW GRAB & UNFILTERED & 147 \\
\hline \begin{tabular}{|l|}
3731 \\
\end{tabular} & $\mathrm{pCi} / \mathrm{L}$ & 0.747 & & & & & & SURFACE & & RAW GRAB & UNFILTERED & 147 \\
\hline 3732 & $\mathrm{pCi} / \mathrm{L}$ & 0.173 & & & & & & SURFACE & & RAW GRAB & UNFILTERED & 147 \\
\hline 3733 & $\mathrm{pCi} / \mathrm{L}$ & 0.0387 & & & & U & & DRINKING & & SAN. GRAB & UNFILTERED & 144 \\
\hline 3734 & $\mathrm{pCi} / \mathrm{L}$ & 204 & & & & U & & DRINKING & & SAN. GRAB & UNFILTERED & 144 \\
\hline 3735 & pCi/L & 1.16 & & & & U & & DRINKING & & SAN. GRAB & UNFILTERED & 144 \\
\hline 3736 & $\mathrm{pCi} / \mathrm{L}$ & 0.419 & & & & U & & DRINKING & & SAN. GRAB & UNFILTERED & 187 \\
\hline 3737 . & $\mathrm{pCi} / \mathrm{L}$ & 202 & & & & U & & DRINKING & & SAN. GRAB & UNFILTERED & 187 \\
\hline 3738 & $\mathrm{pCi} / \mathrm{L}$ & 0.0067 & & & & & & RIVER & & CONTINUOUS & RESIN & 307 \\
\hline 3739 & $\mathrm{pCi} / \mathrm{L}$ & 0.0029 & & & & & & RIVER & & CONTINUOUS & FILTER & 307 \\
\hline 3740 & $\mathrm{pCi} / \mathrm{L}$ & 0.0056 & & & & & & RIVER & & CONTINUOUS & RESIN & 252 \\
\hline \begin{tabular}{|l|}
3741 \\
\end{tabular} & $\mathrm{pCi} / \mathrm{L}$ & 0.0035 & & & & & & RIVER & & CONTINUOUS & FILTER & 252 \\
\hline 3742 & pCi/L & 0.0286 & & & & U & & DRINKING & & SAN. GRAB & UNFILTERED & 512 \\
\hline 3743 & pCi/L & 422 & & & & & & DRINKING & & SAN. GRAB & UNFILTERED & 512 \\
\hline 3744 & $\mathrm{pCi} / \mathrm{L}$ & 1.61 & & & & & & DRINKING & & SAN. GRAB & UNFILTERED & 512 \\
\hline 3745 & $\mathrm{pCi} / \mathrm{L}$ & 0.0327 & & & & & & SANITARY & & CUMULATIVE & UNFILTERED & 385 \\
\hline
\end{tabular}




\begin{tabular}{|c|c|c|c|c|c|c|c|c|c|c|c|c|}
\hline & $\mathrm{N}$ & $\mathrm{O}$ & $\mathrm{P}$ & $\mathrm{Q}$ & $\mathrm{R}$ & $\mathrm{s}$ & $\mathrm{T}$ & $\mathrm{U}$ & $\mathrm{V}$ & $\mathrm{W}$ & $\mathrm{X}$ & $\bar{Y}$ \\
\hline 1 & ANAL_UNITS_RPTD & COUNTING_ERROR & TOTAL_ANAL_ERROR & MIN_DETECTABLE_ACTIVITY & PCNT_MOISTURE & LAB_QUALIFIER & REVIEW_QUALIFIER & SAMP_FROM & SAMP_ITEM & SAMP_MTHD & COLL_MTHD & SAMP_SITE_ID \\
\hline 3746 & $\mathrm{pCi} / \mathrm{L}$ & 0.83 & & & & $U$ & & SANITARY & & CUMULATIVE & UNFILTERED & 385 \\
\hline 3747 & $\mathrm{pCi} / \mathrm{L}$ & 0.0068 & & & & & & RIVER & & CONTINUOUS & RESIN & 307 \\
\hline 3748 & $\mathrm{pCi} / \mathrm{L}$ & 0.0033 & & & & & & RIVER & & CONTINUOUS & FILTER & 307 \\
\hline 3749 & $\mathrm{pCi} / \mathrm{L}$ & 0.0098 & & & & & & RIVER & & CONTINUOUS & RESIN & 252 \\
\hline 3750 & $\mathrm{pCi} / \mathrm{L}$ & 11.9 & & & & & & RIVER & & CUMULATIVE & UNFILTERED & 252 \\
\hline 3751 & $\mathrm{pCi} / \mathrm{L}$ & 0.0333 & & & & & & RIVER & & CUMULATIVE & UNFILTERED & 252 \\
\hline 3752 & $\mathrm{pCi} / \mathrm{L}$ & 0.872 & & & & U & & RIVER & & CUMULATIVE & UNFILTERED & 252 \\
\hline 3753 & $\mathrm{pCi} / \mathrm{L}$ & 0.0036 & & & & & & RIVER & & CONTINUOUS & FILTER & 252 \\
\hline 3754 & $\mathrm{pCi} / \mathrm{L}$ & 20.9 & & & & & & RIVER & & CUMULATIVE & UNFILTERED & 463 \\
\hline 3755 & $\mathrm{pCi} / \mathrm{L}$ & 0.0395 & & & & & & RIVER & & CUMULATIVE & UNFILTERED & 463 \\
\hline 3756 & $\mathrm{pCi} / \mathrm{L}$ & 1.38 & & & & $\mathrm{U}$ & & RIVER & & CUMULATIVE & UNFILTERED & 463 \\
\hline 3757 & $\mathrm{pCi} / \mathrm{L}$ & 205 & & & & & & DRINKING & & SAN. GRAB & UNFILTERED & 530 \\
\hline 3758 & $\mathrm{pCi} / \mathrm{L}$ & 0.0264 & & & & $U$ & & DRINKING & & SAN. GRAB & UNFILTERED & 487 \\
\hline 3759 & $\mathrm{pCi} / \mathrm{L}$ & 0.581 & & & & $U$ & & DRINKING & & SAN. GRAB & UNFILTERED & 487 \\
\hline 3760 & $\mathrm{pCi} / \mathrm{L}$ & 206 & & & & & & DRINKING & & SAN. GRAB & UNFILTERED & 487 \\
\hline 3761 & $\mathrm{pCi} / \mathrm{L}$ & 0.419 & & & & $U$ & & DRINKING & & SAN. GRAB & UNFILTERED & 530 \\
\hline 3762 & $\mathrm{pCi} / \mathrm{L}$ & 0.0453 & & & & & & DRINKING & & SAN. GRAB & UNFILTERED & 530 \\
\hline 3763 & $\mathrm{pCi} / \mathrm{L}$ & 0.0057 & & & & & & RIVER & & CONTINUOUS & RESIN & 307 \\
\hline 3764 & $\mathrm{pCi} / \mathrm{L}$ & 0.00003 & & & & & & RIVER & & CONTINUOUS & FILTER & 307 \\
\hline 3765 & $\mathrm{pCi} / \mathrm{L}$ & 0.01 & & & & & & RIVER & & CONTINUOUS & RESIN & 252 \\
\hline 3766 & $\mathrm{pCi} / \mathrm{L}$ & 0.0045 & & & & & & RIVER & & CONTINUOUS & FILTER & 252 \\
\hline 3767 & $\mathrm{pCi} / \mathrm{L}$ & 211 & & & & & & DRINKING & & CUMULATIVE & UNFILTERED & 307 \\
\hline 3768 & $\mathrm{pCi} / \mathrm{L}$ & 0.048 & & & & & & DRINKING & & CUMULATIVE & UNFILTERED & 307 \\
\hline 3769 & $\mathrm{pCi} / \mathrm{L}$ & 0.906 & & & & U & & DRINKING & & CUMULATIVE & UNFILTERED & 307 \\
\hline 3770 & $\mathrm{pCi} / \mathrm{L}$ & 0.0037 & & & & & & RIVER & & CONTINUOUS & FILTER & 307 \\
\hline 3771 & $\mathrm{pCi} / \mathrm{L}$ & 0.0073 & & & & & & RIVER & & CONTINUOUS & RESIN & 307 \\
\hline 3772 & $\mathrm{pCi} / \mathrm{L}$ & 12.6 & & & & & & RIVER & & CUMULATIVE & UNFILTERED & 252 \\
\hline 3773 & $\mathrm{pCi} / \mathrm{L}$ & 0.0046 & & & & & & RIVER & & CONTINUOUS & FILTER & 252 \\
\hline 3774 & $\mathrm{pCi} / \mathrm{L}$ & 0.0344 & & & & & & RIVER & & CUMULATIVE & UNFILTERED & 252 \\
\hline 3775 & $\mathrm{pCi} / \mathrm{L}$ & 0.657 & & & & $U$ & & RIVER & & CUMULATIVE & UNFILTERED & 252 \\
\hline 3776 & $\mathrm{pCi} / \mathrm{L}$ & 0.0092 & & & & & & RIVER & & CONTINUOUS & RESIN & 252 \\
\hline 3777 & $\mathrm{pCi} / \mathrm{L}$ & 17.4 & & & & & & RIVER & & CUMULATIVE & UNFILTERED & 463 \\
\hline 3778 & $\mathrm{pCi} / \mathrm{L}$ & 0.0349 & & & & & & RIVER & & CUMULATIVE & UNFILTERED & 463 \\
\hline 3779 & $\mathrm{pCi} / \mathrm{L}$ & 0.464 & & & & $U$ & & RIVER & & CUMULATIVE & UNFILTERED & 463 \\
\hline 3780 & $\mathrm{pCi} / \mathrm{L}$ & 0.0358 & & & & & & SANITARY & & CUMULATIVE & UNFILTERED & 385 \\
\hline 3781 & $\mathrm{pCi} / \mathrm{L}$ & 0.379 & & & & $U$ & & SANITARY & & CUMULATIVE & UNFILTERED & 385 \\
\hline 3782 & $\mathrm{pCi} / \mathrm{L}$ & 0.0079 & & & & & & RIVER & & CONTINUOUS & RESIN & 307 \\
\hline 3783 & $\mathrm{pCi} / \mathrm{L}$ & 0.0029 & & & & & & RIVER & & CONTINUOUS & FILTER & 307 \\
\hline 3784 & $\mathrm{pCi} / \mathrm{L}$ & 0.0061 & & & & & & RIVER & & CONTINUOUS & RESIN & 252 \\
\hline 3785 & $\mathrm{pCi} / \mathrm{L}$ & 0.0032 & & & & & & RIVER & & CONTINUOUS & FILTER & 252 \\
\hline 3786 & $\mathrm{pCi} / \mathrm{L}$ & 0.0529 & & & & & & RIVER & & RAW GRAB & UNFILTERED & 277 \\
\hline 3787 & $\mathrm{pCi} / \mathrm{L}$ & 0.789 & & & & & & RIVER & & RAW GRAB & UNFILTERED & 277 \\
\hline 3788 & $\mathrm{pCi} / \mathrm{L}$ & 210 & & & & & & RIVER & & RAW GRAB & UNFILTERED & 277 \\
\hline 3789 & $\mathrm{pCi} / \mathrm{L}$ & & 0.00000762 & & & & & RIVER & & CONTINUOUS & RESIN & 307 \\
\hline 3790 & $\mathrm{pCi} / \mathrm{L}$ & & 0.00000168 & & & & & RIVER & & CONTINUOUS & RESIN & 252 \\
\hline 3791 & $\mathrm{pCi} / \mathrm{L}$ & 0.049 & & & & & & SANITARY & & CUMULATIVE & UNFILTERED & 385 \\
\hline 3792 & pCi/L & 0.317 & & & & $U$ & & SANITARY & & CUMULATIVE & UNFILTERED & 385 \\
\hline 3793 & $\mathrm{pCi} / \mathrm{L}$ & 0.000006 & & & & & & RIVER & & CONTINUOUS & RESIN & 307 \\
\hline 3794 & $\mathrm{pCi} / \mathrm{L}$ & 0.0074 & & & & & & RIVER & & CONTINUOUS & RESIN & 307 \\
\hline 3795 & pCi/L & 0.000006 & & & & & & RIVER & & CONTINUOUS & FILTER & 307 \\
\hline 3796 & $\mathrm{pCi} / \mathrm{L}$ & 0.0033 & & & & & & RIVER & & CONTINUOUS & FILTER & 307 \\
\hline 3797 & $\mathrm{pCi} / \mathrm{L}$ & 21.5 & & & & & & RIVER & & CUMULATIVE & UNFILTERED & 252 \\
\hline
\end{tabular}




\begin{tabular}{|c|c|c|c|c|c|c|c|c|c|c|c|c|}
\hline & $\mathrm{N}$ & $\mathrm{O}$ & $\mathrm{P}$ & $\mathrm{Q}$ & $\mathrm{R}$ & $\mathrm{s}$ & $\mathrm{T}$ & $\mathrm{u}$ & $\mathrm{v}$ & $\mathrm{w}$ & $\mathrm{x}$ & $\bar{Y}$ \\
\hline 1 & ANAL_UNITS_RPTD & COUNTING_ERROR & TOTAL_ANAL_ERROR & MIN_DETECTABLE_ACTIVITY & PCNT_MOISTURE & LAB_QUALIFIER & REVIEW_QUALIFIER & SAMP_FROM & SAMP_ITEM & SAMP_MTHD & COLL_MTHD & SAMP_SITE_ID \\
\hline 3798 & $\mathrm{pCi} / \mathrm{L}$ & 0.000002 & & & & & & RIVER & & CONTINUOUS & FILTER & 252 \\
\hline 3799 & $\mathrm{pCi} / \mathrm{L}$ & 0.003 & & & & & & RIVER & & CONTINUOUS & FILTER & 252 \\
\hline 3800 & $\mathrm{pCi} / \mathrm{L}$ & 0.0311 & & & & $\mathrm{U}$ & & RIVER & & CUMULATIVE & UNFILTERED & 252 \\
\hline 3801 & $\mathrm{pCi} / \mathrm{L}$ & 0.526 & & & & $\mathrm{U}$ & & RIVER & & CUMULATIVE & UNFILTERED & 252 \\
\hline 3802 & $\mathrm{pCi} / \mathrm{L}$ & 0.0056 & & & & & & RIVER & & CONTINUOUS & RESIN & 252 \\
\hline 3803 & $\mathrm{pCi} / \mathrm{L}$ & 0.000008 & & & & $\mathrm{U}$ & & RIVER & & CONTINUOUS & RESIN & 252 \\
\hline 3804 & $\mathrm{pCi} / \mathrm{L}$ & 20.7 & & & & & & RIVER & & CUMULATIVE & UNFILTERED & 463 \\
\hline 3805 & $\mathrm{pCi} / \mathrm{L}$ & 0.0491 & & & & & & RIVER & & CUMULATIVE & UNFILTERED & 463 \\
\hline 3806 & $\mathrm{pCi} / \mathrm{L}$ & 0.592 & & & & $\mathrm{U}$ & & RIVER & & CUMULATIVE & UNFILTERED & 463 \\
\hline 3807 & $\mathrm{pCi} / \mathrm{L}$ & 0.211 & & & & & & SURFACE & & RAW GRAB & UNFILTERED & 509 \\
\hline 3808 & $\mathrm{pCi} / \mathrm{L}$ & 1 & & & & & & SURFACE & & RAW GRAB & UNFILTERED & 509 \\
\hline 3809 & $\mathrm{pCi} / \mathrm{L}$ & 210 & & & & U & & SURFACE & & RAW GRAB & UNFILTERED & 509 \\
\hline 3810 & $\mathrm{pCi} / \mathrm{L}$ & 421 & & & & & & SURFACE & & RAW GRAB & UNFILTERED & 136 \\
\hline 3811 & $\mathrm{pCi} / \mathrm{L}$ & 1.26 & & & & U & & SURFACE & & RAW GRAB & UNFILTERED & 136 \\
\hline 3812 & $\mathrm{pCi} / \mathrm{L}$ & 4.13 & & & & & & SURFACE & & RAW GRAB & UNFILTERED & 146 \\
\hline 3813 & $\mathrm{pCi} / \mathrm{L}$ & 0.146 & & & & & & SURFACE & & RAW GRAB & UNFILTERED & 146 \\
\hline 3814 & $\mathrm{pCi} / \mathrm{L}$ & 207 & & & & $U$ & & SURFACE & & RAW GRAB & UNFILTERED & 146 \\
\hline 3815 & $\mathrm{pCi} / \mathrm{L}$ & 214 & & & & & & SURFACE & & RAW GRAB & UNFILTERED & 147 \\
\hline 3816 & $\mathrm{pCi} / \mathrm{L}$ & 3.12 & & & & & & SURFACE & & RAW GRAB & UNFILTERED & 147 \\
\hline 3817 & $\mathrm{pCi} / \mathrm{L}$ & 0.234 & & & & & & SURFACE & & RAW GRAB & UNFILTERED & 147 \\
\hline 3818 & $\mathrm{pCi} / \mathrm{L}$ & 0.048 & & & & & & DRINKING & & SAN. GRAB & UNFILTERED & 144 \\
\hline 3819 & $\mathrm{pCi} / \mathrm{L}$ & 209 & & & & U & & DRINKING & & SAN. GRAB & UNFILTERED & 144 \\
\hline 3820 & $\mathrm{pCi} / \mathrm{L}$ & 0.509 & & & & U & & DRINKING & & SAN. GRAB & UNFILTERED & 144 \\
\hline 3821 & $\mathrm{pCi} / \mathrm{L}$ & 0.718 & & & & U & & DRINKING & & SAN. GRAB & UNFILTERED & 187 \\
\hline 3822 & $\mathrm{pCi} / \mathrm{L}$ & 148 & & & & U & & DRINKING & & SAN. GRAB & UNFILTERED & 187 \\
\hline 3823 & $\mathrm{pCi} / \mathrm{L}$ & 0.0098 & & & & & & RIVER & & CONTINUOUS & RESIN & 307 \\
\hline 3824 & $\mathrm{pCi} / \mathrm{L}$ & 0.0038 & & & & & & RIVER & & CONTINUOUS & FILTER & 307 \\
\hline 3825 & $\mathrm{pCi} / \mathrm{L}$ & 0.0044 & & & & & & RIVER & & CONTINUOUS & RESIN & 252 \\
\hline 3826 & $\mathrm{pCi} / \mathrm{L}$ & 0.0028 & & & & & & RIVER & & CONTINUOUS & FILTER & 252 \\
\hline 3827 & $\mathrm{pCi} / \mathrm{L}$ & 0.0432 & & & & & & DRINKING & & SAN. GRAB & UNFILTERED & 512 \\
\hline 3828 & $\mathrm{pCi} / \mathrm{L}$ & 461 & & & & & & DRINKING & & SAN. GRAB & UNFILTERED & 512 \\
\hline 3829 & $\mathrm{pCi} / \mathrm{L}$ & 0.916 & & & & U & & DRINKING & & SAN. GRAB & UNFILTERED & 512 \\
\hline 3830 & $\mathrm{pCi} / \mathrm{L}$ & 0.0432 & & & & & & SANITARY & & CUMULATIVE & UNFILTERED & 385 \\
\hline 3831 & $\mathrm{pCi} / \mathrm{L}$ & 0.577 & & & & U & & SANITARY & & CUMULATIVE & UNFILTERED & 385 \\
\hline 3832 & $\mathrm{pCi} / \mathrm{L}$ & 0.011 & & & & & & RIVER & & CONTINUOUS & RESIN & 307 \\
\hline 3833 & $\mathrm{pCi} / \mathrm{L}$ & 0.006 & & & & & & RIVER & & CONTINUOUS & FILTER & 307 \\
\hline 3834 & $\mathrm{pCi} / \mathrm{L}$ & 0.003 & & & & & & RIVER & & CONTINUOUS & FILTER & 252 \\
\hline 3835 & $\mathrm{pCi} / \mathrm{L}$ & 0.0057 & & & & & & RIVER & & CONTINUOUS & RESIN & 252 \\
\hline 3836 & $\mathrm{pCi} / \mathrm{L}$ & 20.9 & & & & & & RIVER & & CUMULATIVE & UNFILTERED & 252 \\
\hline 3837 & $\mathrm{pCi} / \mathrm{L}$ & 0.059 & & & & & & RIVER & & CUMULATIVE & UNFILTERED & 252 \\
\hline 3838 & $\mathrm{pCi} / \mathrm{L}$ & 0.829 & & & & U & & RIVER & & CUMULATIVE & UNFILTERED & 252 \\
\hline 3839 & $\mathrm{pCi} / \mathrm{L}$ & 14.9 & & & & & & RIVER & & CUMULATIVE & UNFILTERED & 463 \\
\hline 3840 & $\mathrm{pCi} / \mathrm{L}$ & 0.0441 & & & & & & RIVER & & CUMULATIVE & UNFILTERED & 463 \\
\hline 3841 & $\mathrm{pCi} / \mathrm{L}$ & 0.749 & & & & U & & RIVER & & CUMULATIVE & UNFILTERED & 463 \\
\hline 3842 & $\mathrm{pCi} / \mathrm{L}$ & 0.0363 & & & & $U$ & & DRINKING & & SAN. GRAB & UNFILTERED & 487 \\
\hline 3843 & $\mathrm{pCi} / \mathrm{L}$ & 0.634 & & & & & & DRINKING & & SAN. GRAB & UNFILTERED & 487 \\
\hline 3844 & $\mathrm{pCi} / \mathrm{L}$ & 204 & & & & U & & DRINKING & & SAN. GRAB & UNFILTERED & 487 \\
\hline 3845 & $\mathrm{pCi} / \mathrm{L}$ & 0.0068 & & & & & & RIVER & & CONTINUOUS & RESIN & 307 \\
\hline 3846 & $\mathrm{pCi} / \mathrm{L}$ & 0.0041 & & & & & & RIVER & & CONTINUOUS & FILTER & 307 \\
\hline 3847 & & & & & & U & & RIVER & & CONTINUOUS & FILTER & 252 \\
\hline 3848 & & & & & & $U$ & & RIVER & & CONTINUOUS & RESIN & 252 \\
\hline 3849 & $\mathrm{pCi} / \mathrm{L}$ & 0.829 & & & & $\mathrm{U}_{2}$ & & DRINKING & & SAN. GRAB & UNFILTERED & 530 \\
\hline
\end{tabular}




\begin{tabular}{|c|c|c|c|c|c|c|c|c|c|c|c|c|}
\hline & $\mathrm{N}$ & $\mathrm{O}$ & $\mathrm{P}$ & $\mathrm{Q}$ & $\mathrm{R}$ & $\mathrm{s}$ & $T$ & $\mathrm{U}$ & $\mathrm{V}$ & $\mathrm{W}$ & $x$ & $\bar{Y}$ \\
\hline 1 & ANAL_UNITS_RPTD & COUNTING_ERROR & ||$_{\text {TOTAL_ANAL_ERROR | }}$ & MIN_DETECTABLE_ACTIVITY & PCNT_MOISTURE & LAB_QUALIFIER & REVIEW_QUALIFIER & SAMP_FROM & SAMP_ITEM & SAMP_MTHD & COLL_MTHD & SAMP_SITE_ID \\
\hline 3850 & $\mathrm{pCi} / \mathrm{L}$ & 251 & & & & $U$ & & DRINKING & & SAN. GRAB & UNFILTERED & 530 \\
\hline 3851 & $\mathrm{pCi} / \mathrm{L}$ & 0.0776 & & & & & & DRINKING & & SAN. GRAB & UNFILTERED & 530 \\
\hline 3852 & $\mathrm{pCi} / \mathrm{L}$ & 293 & & & & & & DRINKING & & CUMULATIVE & UNFILTERED & 307 \\
\hline 3853 & $\mathrm{pCi} / \mathrm{L}$ & 0.0851 & & & & & & DRINKING & & CUMULATIVE & UNFILTERED & 307 \\
\hline 3854 & $\mathrm{pCi} / \mathrm{L}$ & 1.03 & & & & U & & DRINKING & & CUMULATIVE & UNFILTERED & 307 \\
\hline 3855 & $\mathrm{pCi} / \mathrm{L}$ & 0.0749 & & & & & & QC_SAMPLE & & RAW GRAB & UNFILTERED & 550 \\
\hline 3856 & $\mathrm{pCi} / \mathrm{L}$ & 0.69 & & & & $\mathrm{u}$ & & QC_SAMPLE & & RAW GRAB & UNFILTERED & 550 \\
\hline 3857 & $\mathrm{pCi} / \mathrm{L}$ & 14.6 & & & & & & QC_SAMPLE & & RAW GRAB & UNFILTERED & 550 \\
\hline 3858 & $\mathrm{pCi} / \mathrm{L}$ & 13.7 & & & & & & QC_SAMPLE & & RAW GRAB & UNFILTERED & 247 \\
\hline 3859 & $\mathrm{pCi} / \mathrm{L}$ & 0.669 & & & & & & QC_SAMPLE & & RAW GRAB & UNFILTERED & 247 \\
\hline 3860 & $\mathrm{pCi} / \mathrm{L}$ & 0.0658 & & & & & & QC_SAMPLE & & RAW GRAB & UNFILTERED & 247 \\
\hline 3861 & $\mathrm{pCi} / \mathrm{L}$ & 0.0058 & & & & & & RIVER & & CONTINUOUS & RESIN & 307 \\
\hline 3862 & $\mathrm{pCi} / \mathrm{L}$ & 0.0026 & & & & & & RIVER & & CONTINUOUS & FILTER & 307 \\
\hline 3863 & $\mathrm{pCi} / \mathrm{L}$ & 0.0087 & & & & & & RIVER & & CONTINUOUS & RESIN & 252 \\
\hline 3864 & $\mathrm{pCi} / \mathrm{L}$ & 13.8 & & & & & & RIVER & & CUMULATIVE & UNFILTERED & 252 \\
\hline 3865 & $\mathrm{pCi} / \mathrm{L}$ & 0.0031 & & & & & & RIVER & & CONTINUOUS & FILTER & 252 \\
\hline 3866 & $\mathrm{pCi} / \mathrm{L}$ & 0.0425 & & & & & & RIVER & & CUMULATIVE & UNFILTERED & 252 \\
\hline 3867 & $\mathrm{pCi} / \mathrm{L}$ & 1.26 & & & & $U$ & & RIVER & & CUMULATIVE & UNFILTERED & 252 \\
\hline 3868 & $\mathrm{pCi} / \mathrm{L}$ & 14.4 & & & & & & RIVER & & CUMULATIVE & UNFILTERED & 463 \\
\hline 3869 & $\mathrm{pCi} / \mathrm{L}$ & 0.0681 & & & & & & RIVER & & CUMULATIVE & UNFILTERED & 463 \\
\hline 3870 & $\mathrm{pCi} / \mathrm{L}$ & 0.686 & & & & & & RIVER & & CUMULATIVE & UNFILTERED & 463 \\
\hline 3871 & $\mathrm{pCi} / \mathrm{L}$ & 0.0756 & & & & & & SANITARY & & CUMULATIVE & UNFILTERED & 385 \\
\hline 3872 & $\mathrm{pCi} / \mathrm{L}$ & 1.08 & & & & U & & SANITARY & & CUMULATIVE & UNFILTERED & 385 \\
\hline 3873 & $\mathrm{pCi} / \mathrm{L}$ & 0.0065 & & & & & & RIVER & & CONTINUOUS & RESIN & 307 \\
\hline 3874 & $\mathrm{pCi} / \mathrm{L}$ & 0.0031 & & & & & & RIVER & & CONTINUOUS & FILTER & 307 \\
\hline 3875 & $\mathrm{pCi} / \mathrm{L}$ & 0.0031 & & & & & & RIVER & & CONTINUOUS & FILTER & 252 \\
\hline 3876 & $\mathrm{pCi} / \mathrm{L}$ & 0.0069 & & & & & & RIVER & & CONTINUOUS & RESIN & 252 \\
\hline 3877 & $\mathrm{pCi} / \mathrm{L}$ & 0.000004 & & & & & & RIVER & & CONTINUOUS & FILTER & 307 \\
\hline 3878 & $\mathrm{pCi} / \mathrm{L}$ & 0.0028 & & & & & & RIVER & & CONTINUOUS & FILTER & 307 \\
\hline 3879 & $\mathrm{pCi} / \mathrm{L}$ & 0.000004 & & & & & & RIVER & & CONTINUOUS & RESIN & 307 \\
\hline 3880 & $\mathrm{pCi} / \mathrm{L}$ & 0.0058 & & & & & & RIVER & & CONTINUOUS & RESIN & 307 \\
\hline 3881 & $\mathrm{pCi} / \mathrm{L}$ & & 0.0000122 & & & & & RIVER & & CONTINUOUS & RESIN & 307 \\
\hline 3882 & $\mathrm{pCi} / \mathrm{L}$ & 0.0062 & & & & & & RIVER & & CONTINUOUS & RESIN & 252 \\
\hline 3883 & $\mathrm{pCi} / \mathrm{L}$ & 0.000033 & & & & $U$ & & RIVER & & CONTINUOUS & RESIN & 252 \\
\hline 3884 & $\mathrm{pCi} / \mathrm{L}$ & & 0.00000164 & & & & & RIVER & & CONTINUOUS & RESIN & 252 \\
\hline 3885 & pCi/L & 13.6 & & & & & & RIVER & & CUMULATIVE & UNFILTERED & 252 \\
\hline 3886 & $\mathrm{pCi} / \mathrm{L}$ & 0.0474 & & & & & & RIVER & & CUMULATIVE & UNFILTERED & 252 \\
\hline 3887 & $\mathrm{pCi} / \mathrm{L}$ & 0.391 & & & & $U$ & & RIVER & & CUMULATIVE & UNFILTERED & 252 \\
\hline 3888 & $\mathrm{pCi} / \mathrm{L}$ & 0.000014 & & & & & & RIVER & & CONTINUOUS & FILTER & 252 \\
\hline 3889 & $\mathrm{pCi} / \mathrm{L}$ & 0.0037 & & & & & & RIVER & & CONTINUOUS & FILTER & 252 \\
\hline 3890 & $\mathrm{pCi} / \mathrm{L}$ & 14.5 & & & & & & RIVER & & CUMULATIVE & UNFILTERED & 463 \\
\hline 3891 & $\mathrm{pCi} / \mathrm{L}$ & 0.678 & & & & $U$ & & RIVER & & CUMULATIVE & UNFILTERED & 463 \\
\hline 3892 & $\mathrm{pCi} / \mathrm{L}$ & 0.0471 & & & & & & RIVER & & CUMULATIVE & UNFILTERED & 463 \\
\hline 3893 & $\mathrm{pCi} / \mathrm{L}$ & 0.0436 & & & & & & SANITARY & & CUMULATIVE & UNFILTERED & 385 \\
\hline 3894 & $\mathrm{pCi} / \mathrm{L}$ & 0.5 & & & & $U$ & & SANITARY & & CUMULATIVE & UNFILTERED & 385 \\
\hline 3895 & $\mathrm{pCi} / \mathrm{L}$ & 0.0598 & & & & & & RIVER & & CUMULATIVE & UNFILTERED & 553 \\
\hline 3896 & $\mathrm{pCi} / \mathrm{L}$ & 209 & & & & & & RIVER & & CUMULATIVE & UNFILTERED & 553 \\
\hline 3897 & $\mathrm{pCi} / \mathrm{L}$ & 1.02 & & & & & & RIVER & & CUMULATIVE & UNFILTERED & 553 \\
\hline 3898 & pCi/L & 0.105 & & & & & & SURFACE & & RAW GRAB & UNFILTERED & 509 \\
\hline 3899 & pCi/L & 1.56 & & & & & & SURFACE & & RAW GRAB & UNFILTERED & 509 \\
\hline 3900 & $\mathrm{pCi} / \mathrm{L}$ & 205 & & & & $U$ & & SURFACE & & RAW GRAB & UNFILTERED & 509 \\
\hline 3901 & $\mathrm{pCi} / \mathrm{L}$ & 497 & & & & & & SURFACE & & RAW GRAB & UNFILTERED & 136 \\
\hline
\end{tabular}




\begin{tabular}{|c|c|c|c|c|c|c|c|c|c|c|c|c|}
\hline & $\mathrm{N}$ & $\mathrm{O}$ & $\mathrm{P}$ & $\mathrm{Q}$ & $\mathrm{R}$ & $\mathrm{s}$ & $T$ & $\bar{U}$ & $\mathrm{~V}$ & $\mathrm{~W}$ & $\mathrm{X}$ & $\bar{Y}$ \\
\hline 1 & ANAL_UNITS_RPTD & COUNTING_ERROR & TOTAL_ANAL_ERROR & MIN_DETECTABLE_ACTIVITY & PCNT_MOISTURE & LAB_QUALIFIER & REVIEW_QUALIFIER & SAMP_FROM & SAMP_ITEM & SAMP_MTHD & COLL_MTHD & SAMP_SITE_ID \\
\hline 3902 & $\mathrm{pCi} / \mathrm{L}$ & 1.88 & & & & $U$ & & SURFACE & & RAW GRAB & UNFILTERED & 136 \\
\hline 3903 & $\mathrm{pCi} / \mathrm{L}$ & 3.28 & & & & & & SURFACE & & RAW GRAB & UNFILTERED & 146 \\
\hline 3904 & $\mathrm{pCi} / \mathrm{L}$ & 0.0901 & & & & & & SURFACE & & RAW GRAB & UNFILTERED & 146 \\
\hline 3905 & $\mathrm{pCi} / \mathrm{L}$ & 207 & & & & & & SURFACE & & RAW GRAB & UNFILTERED & 146 \\
\hline 3906 & $\mathrm{pCi} / \mathrm{L}$ & 218 & & & & & & SURFACE & & RAW GRAB & UNFILTERED & 147 \\
\hline 3907 & $\mathrm{pCi} / \mathrm{L}$ & 2.75 & & & & $U$ & & SURFACE & & RAW GRAB & UNFILTERED & 147 \\
\hline 3908 & $\mathrm{pCi} / \mathrm{L}$ & 0.202 & & & & & & SURFACE & & RAW GRAB & UNFILTERED & 147 \\
\hline 3909 & $\mathrm{pCi} / \mathrm{L}$ & 0.037 & & & & & & DRINKING & & SAN. GRAB & UNFILTERED & 144 \\
\hline 3910 & $\mathrm{pCi} / \mathrm{L}$ & 206 & & & & & & DRINKING & & SAN. GRAB & UNFILTERED & 144 \\
\hline 3911 & $\mathrm{pCi} / \mathrm{L}$ & 0.792 & & & & $U$ & & DRINKING & & SAN. GRAB & UNFILTERED & 144 \\
\hline 3913 & $\mathrm{pCi} / \mathrm{L}$ & 217 & & & & U & & DRINKING & & SAN. GRAB & UNFILTERED & 187 \\
\hline 3914 & $\mathrm{pCi} / \mathrm{L}$ & 0.0029 & & & & & & RIVER & & CONTINUOUS & FILTER & 307 \\
\hline 3915 & $\mathrm{pCi} / \mathrm{L}$ & 0.0066 & & & & & & RIVER & & CONTINUOUS & RESIN & 307 \\
\hline 3916 & $\mathrm{pCi} / \mathrm{L}$ & 0.0058 & & & & & & RIVER & & CONTINUOUS & RESIN & 252 \\
\hline 3917 & $\mathrm{pCi} / \mathrm{L}$ & 0.0033 & & & & & & RIVER & & CONTINUOUS & FILTER & 252 \\
\hline 3918 & $\mathrm{pCi} / \mathrm{L}$ & 0.0544 & & & & $U$ & & DRINKING & & SAN. GRAB & UNFILTERED & 512 \\
\hline 3919 & $\mathrm{pCi} / \mathrm{L}$ & 602 & & & & & & DRINKING & & SAN. GRAB & UNFILTERED & 512 \\
\hline 3920 & $\mathrm{pCi} / \mathrm{L}$ & 0.519 & & & & $U$ & & DRINKING & & SAN. GRAB & UNFILTERED & 512 \\
\hline 3921 & $\mathrm{pCi} / \mathrm{L}$ & 0.0076 & & & & & & RIVER & & CONTINUOUS & RESIN & 307 \\
\hline 3922 & $\mathrm{pCi} / \mathrm{L}$ & 0.0041 & & & & & & RIVER & & CONTINUOUS & FILTER & 307 \\
\hline \begin{tabular}{|l|}
3923 \\
\end{tabular} & $\mathrm{pCi} / \mathrm{L}$ & 0.0096 & & & & & & RIVER & & CONTINUOUS & RESIN & 252 \\
\hline 3925 & $\mathrm{pCi} / \mathrm{L}$ & 11.2 & & & & & & RIVER & & CUMULATIVE & UNFILTERED & 252 \\
\hline 3926 & $\mathrm{pCi} / \mathrm{L}$ & 0.0356 & & & & & & RIVER & & CUMULATIVE & UNFILTERED & 252 \\
\hline 3927 & $\mathrm{pCi} / \mathrm{L}$ & 0.421 & & & & $U$ & & RIVER & & CUMULATIVE & UNFILTERED & 252 \\
\hline 3928 . & $\mathrm{pCi} / \mathrm{L}$ & 12.2 & & & & & & RIVER & & CUMULATIVE & UNFILTERED & 463 \\
\hline \begin{tabular}{|l|}
3929 \\
\end{tabular} & $\mathrm{pCi} / \mathrm{L}$ & 0.4 & & & & $U$ & & RIVER & & CUMULATIVE & UNFILTERED & 463 \\
\hline 3930 & $\mathrm{pCi} / \mathrm{L}$ & 0.0426 & & & & & & RIVER & & CUMULATIVE & UNFILTERED & 463 \\
\hline \begin{tabular}{|l|}
3931 \\
\end{tabular} & $\mathrm{pCi} / \mathrm{L}$ & 0.0672 & & & & & & SANITARY & & CUMULATIVE & UNFILTERED & 385 \\
\hline 3932 & $\mathrm{pCi} / \mathrm{L}$ & 0.483 & & & & $U$ & & SANITARY & & CUMULATIVE & UNFILTERED & 385 \\
\hline 3933 & $\mathrm{pCi} / \mathrm{L}$ & 0.0914 & & & & $U$ & & DRINKING & & SAN. GRAB & UNFILTERED & 487 \\
\hline 3934 & $\mathrm{pCi} / \mathrm{L}$ & 0.514 & & & & U & & DRINKING & & SAN. GRAB & UNFILTERED & 487 \\
\hline 3935 & $\mathrm{pCi} / \mathrm{L}$ & 199 & & & & $U$ & & DRINKING & & SAN. GRAB & UNFILTERED & 487 \\
\hline 3936 & $\mathrm{pCi} / \mathrm{L}$ & 0.0081 & & & & & & RIVER & & CONTINUOUS & RESIN & 307 \\
\hline \begin{tabular}{|l|}
3937 \\
\end{tabular} & $\mathrm{pCi} / \mathrm{L}$ & 0.0034 & & & & & & RIVER & & CONTINUOUS & FILTER & 307 \\
\hline 3938 & $\mathrm{pCi} / \mathrm{L}$ & 0.0079 & & & & & & RIVER & & CONTINUOUS & RESIN & 252 \\
\hline 3939 & $\mathrm{pCi} / \mathrm{L}$ & 0.0034 & & & & & & RIVER & & CONTINUOUS & FILTER & 252 \\
\hline 3940 & $\mathrm{pCi} / \mathrm{L}$ & 1.08 & & & & $U$ & & DRINKING & & SAN. GRAB & UNFILTERED & 530 \\
\hline 3941 & $\mathrm{pCi} / \mathrm{L}$ & 199 & & & & U & & DRINKING & & SAN. GRAB & UNFILTERED & 530 \\
\hline \begin{tabular}{|l|}
3942 \\
\end{tabular} & $\mathrm{pCi} / \mathrm{L}$ & 0.113 & & & & & & DRINKING & & SAN. GRAB & UNFILTERED & 530 \\
\hline 3943 & $\mathrm{pCi} / \mathrm{L}$ & 250 & & & & & & DRINKING & & CUMULATIVE & UNFILTERED & 307 \\
\hline 3944 & $\mathrm{pCi} / \mathrm{L}$ & 0.0376 & & & & & & DRINKING & & CUMULATIVE & UNFILTERED & 307 \\
\hline 3945 & $\mathrm{pCi} / \mathrm{L}$ & 0.643 & & & & $U$ & & DRINKING & & CUMULATIVE & UNFILTERED & 307 \\
\hline 3946 & $\mathrm{pCi} / \mathrm{L}$ & 0.0057 & & & & & & RIVER & & CONTINUOUS & RESIN & 307 \\
\hline 3947 & $\mathrm{pCi} / \mathrm{L}$ & 0.0033 & & & & & & RIVER & & CONTINUOUS & FILTER & 307 \\
\hline 3948 & $\mathrm{pCi} / \mathrm{L}$ & 0.0061 & & & & & & RIVER & & CONTINUOUS & RESIN & 252 \\
\hline 3949 & $\mathrm{pCi} / \mathrm{L}$ & 11.9 & & & & & & RIVER & & CUMULATIVE & UNFILTERED & 252 \\
\hline 3950 & $\mathrm{pCi} / \mathrm{L}$ & 0.0031 & & & & & & RIVER & & CONTINUOUS & FILTER & 252 \\
\hline 3951 & $\mathrm{pCi} / \mathrm{L}$ & 0.051 & & & & & & RIVER & & CUMULATIVE & UNFILTERED & 252 \\
\hline 3952 & $\mathrm{pCi} / \mathrm{L}$ & 0.464 & & & & & & RIVER & & CUMULATIVE & UNFILTERED & 252 \\
\hline 3953 & $\mathrm{pCi} / \mathrm{L}$ & 12.7 & & & & & & RIVER & & CUMULATIVE & UNFILTERED & 463 \\
\hline
\end{tabular}




\begin{tabular}{|c|c|c|c|c|c|c|c|c|c|c|c|c|}
\hline & $\mathrm{N}$ & $\mathrm{O}$ & $\mathrm{P}$ & $\mathrm{Q}$ & $\mathrm{R}$ & $\mathrm{s}$ & $T$ & $\mathrm{U}$ & $\mathrm{V}$ & $\overline{\mathrm{W}}$ & $\mathrm{X}$ & $\bar{Y}$ \\
\hline 1 & ANAL_UNITS_RPTD & COUNTING_ERROR & $\mid$ TOTAL_ANAL_ERROR | & MIN_DETECTABLE_ACTIVITY & PCNT_MOISTURE & LAB_QUALIFIER & REVIEW_QUALIFIER & SAMP_FROM & SAMP_ITEM & SAMP_MTHD & COLL_MTHD & SAMP_SITE_ID \\
\hline 3954 & $\mathrm{pCi} / \mathrm{L}$ & 0.566 & & & & $U$ & & RIVER & & CUMULATIVE & UNFILTERED & 463 \\
\hline 3955 & $\mathrm{pCi} / \mathrm{L}$ & 0.0519 & & & & & & RIVER & & CUMULATIVE & UNFILTERED & 463 \\
\hline 3956 & $\mathrm{pCi} / \mathrm{L}$ & 0.0373 & & & & & & SANITARY & & CUMULATIVE & UNFILTERED & 385 \\
\hline 3957 & $\mathrm{pCi} / \mathrm{L}$ & 0.72 & & & & U & & SANITARY & & CUMULATIVE & UNFILTERED & 385 \\
\hline 3958 & $\mathrm{pCi} / \mathrm{L}$ & 0.0051 & & & & & & RIVER & & CONTINUOUS & RESIN & 307 \\
\hline 3959 & $\mathrm{pCi} / \mathrm{L}$ & 0.003 & & & & & & RIVER & & CONTINUOUS & FILTER & 307 \\
\hline 3960 & $\mathrm{pCi} / \mathrm{L}$ & 0.0073 & & & & & & RIVER & & CONTINUOUS & RESIN & 252 \\
\hline 3961 & $\mathrm{pCi} / \mathrm{L}$ & 0.0038 & & & & & & RIVER & & CONTINUOUS & FILTER & 252 \\
\hline 3962 & $\mathrm{pCi} / \mathrm{L}$ & 0.00006 & & & & & & RIVER & & CONTINUOUS & RESIN & 307 \\
\hline 3963 & $\mathrm{pCi} / \mathrm{L}$ & 0.0063 & & & & & & RIVER & & CONTINUOUS & RESIN & 307 \\
\hline 3964 & $\mathrm{pCi} / \mathrm{L}$ & & 0.0000067 & & & & & RIVER & & CONTINUOUS & RESIN & 307 \\
\hline 3965 & $\mathrm{pCi} / \mathrm{L}$ & 0.0027 & & & & & & RIVER & & CONTINUOUS & FILTER & 307 \\
\hline 3966 & $\mathrm{pCi} / \mathrm{L}$ & 0.000002 & & & & & & RIVER & & CONTINUOUS & FILTER & 307 \\
\hline 3967 & $\mathrm{pCi} / \mathrm{L}$ & 0.0073 & & & & & & RIVER & & CONTINUOUS & RESIN & 252 \\
\hline 3968 & $\mathrm{pCi} / \mathrm{L}$ & 0.00018 & & & & & & RIVER & & CONTINUOUS & RESIN & 252 \\
\hline 3969 & $\mathrm{pCi} / \mathrm{L}$ & & 0.0000013 & & & & & RIVER & & CONTINUOUS & RESIN & 252 \\
\hline 3970 & $\mathrm{pCi} / \mathrm{L}$ & 11 & 0 & 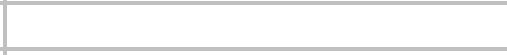 & & & & RIVER & & CUMULATIVE & UNFILTERED & 252 \\
\hline 3971 & $\mathrm{pCi} / \mathrm{L}$ & 0.0594 & & & & & & RIVER & & CUMULATIVE & UNFILTERED & 252 \\
\hline 3972 & $\mathrm{pCi} / \mathrm{L}$ & 0.557 & & & & U & & RIVER & & CUMULATIVE & UNFILTERED & 252 \\
\hline 3973 & $\mathrm{pCi} / \mathrm{L}$ & 0.00001 & & & & U & & RIVER & & CONTINUOUS & FILTER & 252 \\
\hline 3974 & $\mathrm{pCi} / \mathrm{L}$ & 0.0036 & & & & & & RIVER & & CONTINUOUS & FILTER & 252 \\
\hline 3975 & & & & & & U & & RIVER & & CUMULATIVE & UNFILTERED & 463 \\
\hline 3976 & & & & & & U & & RIVER & & CUMULATIVE & UNFILTERED & 463 \\
\hline 3977 & & & & & & U & & RIVER & & CUMULATIVE & UNFILTERED & 463 \\
\hline 3978 & $\mathrm{pCi} / \mathrm{L}$ & 0.125 & & & & & & RIVER & & CUMULATIVE & UNFILTERED & 553 \\
\hline 3979 & $\mathrm{pCi} / \mathrm{L}$ & 208 & & & & & & RIVER & & CUMULATIVE & UNFILTERED & 553 \\
\hline 3980 & $\mathrm{pCi} / \mathrm{L}$ & 0.514 & & & & U & & RIVER & & CUMULATIVE & UNFILTERED & 553 \\
\hline 3981 & $\mathrm{pCi} / \mathrm{L}$ & 0.0036 & & & & & & RIVER & & CONTINUOUS & FILTER & 307 \\
\hline 3982 & $\mathrm{pCi} / \mathrm{L}$ & 0.0076 & & & & & & RIVER & & CONTINUOUS & RESIN & 307 \\
\hline 3983 & & & & & & $U$ & & RIVER & & CONTINUOUS & FILTER & 252 \\
\hline 3984 & & & & & & U & & RIVER & & CONTINUOUS & RESIN & 252 \\
\hline 3985 & $\mathrm{pCi} / \mathrm{L}$ & 0.117 & & & & U & & DRINKING & & SAN. GRAB & UNFILTERED & 144 \\
\hline 3986 & $\mathrm{pCi} / \mathrm{L}$ & 205 & & & & U & & DRINKING & & SAN. GRAB & UNFILTERED & 144 \\
\hline 3987 & $\mathrm{pCi} / \mathrm{L}$ & 1.38 & & & & U & & DRINKING & & SAN. GRAB & UNFILTERED & 144 \\
\hline 3988 & $\mathrm{pCi} / \mathrm{L}$ & 0.978 & & & & U & & DRINKING & & SAN. GRAB & UNFILTERED & 187 \\
\hline 3989 & $\mathrm{pCi} / \mathrm{L}$ & 204 & & & & U & & DRINKING & & SAN. GRAB & UNFILTERED & 187 \\
\hline 3990 & & & & & & U & & RIVER & & CONTINUOUS & FILTER & 307 \\
\hline 3991 & & & & & & U & & RIVER & & CONTINUOUS & RESIN & 307 \\
\hline 3992 & $\mathrm{pCi} / \mathrm{L}$ & 0.0095 & & & & & & RIVER & & CONTINUOUS & RESIN & 252 \\
\hline 3993 & $\mathrm{pCi} / \mathrm{L}$ & 11.1 & 0 & 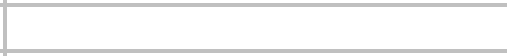 & & & & RIVER & & CUMULATIVE & UNFILTERED & 252 \\
\hline 3994 & $\mathrm{pCi} / \mathrm{L}$ & 0.0044 & & & & & & RIVER & & CONTINUOUS & FILTER & 252 \\
\hline 3995 & $\mathrm{pCi} / \mathrm{L}$ & 0.0621 & & & & & & RIVER & & CUMULATIVE & UNFILTERED & 252 \\
\hline 3996 & $\mathrm{pCi} / \mathrm{L}$ & 1.08 & & & & $U$ & & RIVER & & CUMULATIVE & UNFILTERED & 252 \\
\hline 3997 & $\mathrm{pCi} / \mathrm{L}$ & 12.3 & & & & & & RIVER & & CUMULATIVE & UNFILTERED & 463 \\
\hline 3998 & $\mathrm{pCi} / \mathrm{L}$ & 0.819 & & & & & & RIVER & & CUMULATIVE & UNFILTERED & 463 \\
\hline 3999 & $\mathrm{pCi} / \mathrm{L}$ & 0.0553 & & & & & & RIVER & & CUMULATIVE & UNFILTERED & 463 \\
\hline 4000 & $\mathrm{pCi} / \mathrm{L}$ & 0.162 & & & & & & SURFACE & & RAW GRAB & UNFILTERED & 509 \\
\hline 4001 & $\mathrm{pCi} / \mathrm{L}$ & 3.69 & & & & & & SURFACE & & RAW GRAB & UNFILTERED & 509 \\
\hline 4002 & $\mathrm{pCi} / \mathrm{L}$ & 284 & 396 & & & & & SURFACE & & RAW GRAB & UNFILTERED & 509 \\
\hline 4003 & $\mathrm{pCi} / \mathrm{L}$ & 632 & & & & & & SURFACE & & RAW GRAB & UNFILTERED & 136 \\
\hline 4004 & $\mathrm{pCi} / \mathrm{L}$ & 1.83 & & & & U & & SURFACE & & RAW GRAB & UNFILTERED & 136 \\
\hline 4005 & $\mathrm{pCi} / \mathrm{L}$ & 2.39 & & & & & & SURFACE & & RAW GRAB & UNFILTERED & 146 \\
\hline
\end{tabular}




\begin{tabular}{|c|c|c|c|c|c|c|c|c|c|c|c|c|}
\hline & $\mathrm{N}$ & $\mathrm{O}$ & $\mathrm{P}$ & $\mathrm{Q}$ & $\mathrm{R}$ & $\mathrm{s}$ & $T$ & $\mathrm{U}$ & $\mathrm{V}$ & $\mathrm{W}$ & $\mathrm{X}$ & $\bar{Y}$ \\
\hline 1 & ANAL_UNITS_RPTD & COUNTING_ERROR & TOTAL_ANAL_ERROR & MIN_DETECTABLE_ACTIVITY & PCNT_MOISTURE & LAB_QUALIFIER & REVIEW_QUALIFIER & SAMP_FROM & SAMP_ITEM & \begin{tabular}{|l|} 
SAMP_MTHD \\
\end{tabular} & COLL_MTHD & SAMP_SITE_ID \\
\hline 4006 & $\mathrm{pCi} / \mathrm{L}$ & 0.104 & & & & & & SURFACE & & RAW GRAB & UNFILTERED & 146 \\
\hline 4007 & $\mathrm{pCi} / \mathrm{L}$ & 205 & & & & U & & SURFACE & & RAW GRAB & UNFILTERED & 146 \\
\hline 4008 & $\mathrm{pCi} / \mathrm{L}$ & 224 & & & & & & SURFACE & & RAW GRAB & UNFILTERED & 147 \\
\hline 4009 & $\mathrm{pCi} / \mathrm{L}$ & 1.8 & & & & $\mathrm{U}$ & & SURFACE & & RAW GRAB & UNFILTERED & 147 \\
\hline 4010 & $\mathrm{pCi} / \mathrm{L}$ & 0.257 & 0.373 & & & & & SURFACE & & RAW GRAB & UNFILTERED & 147 \\
\hline 4011 & $\mathrm{pCi} / \mathrm{L}$ & 0.0066 & & & & & & RIVER & & CONTINUOUS & RESIN & 307 \\
\hline 4012 & $\mathrm{pCi} / \mathrm{L}$ & 0.0031 & & & & & & RIVER & & CONTINUOUS & FILTER & 307 \\
\hline 4013 & $\mathrm{pCi} / \mathrm{L}$ & 0.0053 & & & & & & RIVER & & CONTINUOUS & FILTER & 252 \\
\hline 4014 & $\mathrm{pCi} / \mathrm{L}$ & 0.012 & & & & & & RIVER & & CONTINUOUS & RESIN & 252 \\
\hline 4015 & $\mathrm{pCi} / \mathrm{L}$ & 593 & & & & & & DRINKING & & SAN. GRAB & UNFILTERED & 512 \\
\hline 4016 & $\mathrm{pCi} / \mathrm{L}$ & 1.31 & & & & U & & DRINKING & & SAN. GRAB & UNFILTERED & 512 \\
\hline 4017 & $\mathrm{pCi} / \mathrm{L}$ & 0.0554 & 0.0565 & & & & & DRINKING & & SAN. GRAB & UNFILTERED & 512 \\
\hline 4018 & $\mathrm{pCi} / \mathrm{L}$ & 0.0034 & & & & & & RIVER & & CONTINUOUS & FILTER & 307 \\
\hline \begin{tabular}{|l|}
4019 \\
\end{tabular} & $\mathrm{pCi} / \mathrm{L}$ & 0.0074 & & & & & & RIVER & & CONTINUOUS & RESIN & 307 \\
\hline 4020 & $\mathrm{pCi} / \mathrm{L}$ & 12.3 & & & & & & RIVER & & CUMULATIVE & UNFILTERED & 252 \\
\hline \begin{tabular}{|l|}
4021 \\
\end{tabular} & $\mathrm{pCi} / \mathrm{L}$ & 0.0419 & 0.0605 & & & & & RIVER & & CUMULATIVE & UNFILTERED & 252 \\
\hline \begin{tabular}{|l|}
4022 \\
\end{tabular} & $\mathrm{pCi} / \mathrm{L}$ & 1.19 & & & & $U$ & & RIVER & & CUMULATIVE & UNFILTERED & 252 \\
\hline \begin{tabular}{|l|}
4023 \\
\end{tabular} & $\mathrm{pCi} / \mathrm{L}$ & 0.0068 & & & & & & RIVER & & CONTINUOUS & RESIN & 252 \\
\hline \begin{tabular}{|l|}
4024 \\
\end{tabular} & $\mathrm{pCi} / \mathrm{L}$ & 0.0042 & & & & & & RIVER & & CONTINUOUS & FILTER & 252 \\
\hline \begin{tabular}{|l|}
4025 \\
\end{tabular} & $\mathrm{pCi} / \mathrm{L}$ & 12.5 & & & & & & RIVER & & CUMULATIVE & UNFILTERED & 463 \\
\hline 4026 & $\mathrm{pCi} / \mathrm{L}$ & 0.691 & & & & U & & RIVER & & CUMULATIVE & UNFILTERED & 463 \\
\hline \begin{tabular}{|l|}
4027 \\
\end{tabular} & $\mathrm{pCi} / \mathrm{L}$ & 0.0509 & 0.0631 & & & & & RIVER & & CUMULATIVE & UNFILTERED & 463 \\
\hline \begin{tabular}{|l|}
4028 \\
\end{tabular} & $\mathrm{pCi} / \mathrm{L}$ & 0.113 & 0.189 & & & & & DRINKING & & SAN. GRAB & UNFILTERED & 487 \\
\hline \begin{tabular}{|l|}
4029 \\
\end{tabular} & $\mathrm{pCi} / \mathrm{L}$ & 1.46 & & & & $U$ & & DRINKING & & SAN. GRAB & UNFILTERED & 487 \\
\hline 4030 & $\mathrm{pCi} / \mathrm{L}$ & 206 & & & & & & DRINKING & & SAN. GRAB & UNFILTERED & 487 \\
\hline \begin{tabular}{|l|}
4031 \\
\end{tabular} & $\mathrm{pCi} / \mathrm{L}$ & 212 & & & & & & DRINKING & & CUMULATIVE & UNFILTERED & 307 \\
\hline \begin{tabular}{|l|}
4032 \\
\end{tabular} & $\mathrm{pCi} / \mathrm{L}$ & 0.106 & 0.125 & & & & & DRINKING & & CUMULATIVE & UNFILTERED & 307 \\
\hline \begin{tabular}{|l|}
4033 \\
\end{tabular} & $\mathrm{pCi} / \mathrm{L}$ & 1.13 & & & & $U$ & & DRINKING & & CUMULATIVE & UNFILTERED & 307 \\
\hline \begin{tabular}{|l|}
4034 \\
\end{tabular} & $\mathrm{pCi} / \mathrm{L}$ & 1.29 & & & & U & & DRINKING & & SAN. GRAB & UNFILTERED & 530 \\
\hline \begin{tabular}{|l|}
4035 \\
\end{tabular} & $\mathrm{pCi} / \mathrm{L}$ & 203 & & & & $U$ & & DRINKING & & SAN. GRAB & UNFILTERED & 530 \\
\hline \begin{tabular}{|l|}
4036 \\
\end{tabular} & $\mathrm{pCi} / \mathrm{L}$ & 0.0969 & 0.175 & & & & & DRINKING & & SAN. GRAB & UNFILTERED & 530 \\
\hline \begin{tabular}{|l|}
4037 \\
\end{tabular} & $\mathrm{pCi} / \mathrm{L}$ & 0.0028 & & & & & & RIVER & & CONTINUOUS & FILTER & 307 \\
\hline \begin{tabular}{|l|}
4038 \\
\end{tabular} & $\mathrm{pCi} / \mathrm{L}$ & 0.0046 & & & & & & RIVER & & CONTINUOUS & RESIN & 307 \\
\hline \begin{tabular}{|l|}
4039 \\
\end{tabular} & $\mathrm{pCi} / \mathrm{L}$ & 0.011 & & & & & & RIVER & & CONTINUOUS & RESIN & 252 \\
\hline \begin{tabular}{|l|}
4040 \\
\end{tabular} & $\mathrm{pCi} / \mathrm{L}$ & 0.005 & & & & & & RIVER & & CONTINUOUS & FILTER & 252 \\
\hline \begin{tabular}{|l|}
4041 \\
\end{tabular} & $\mathrm{pCi} / \mathrm{L}$ & 0.0755 & 0.0923 & & & & & QC_SAMPLE & & RAW GRAB & UNFILTERED & 550 \\
\hline \begin{tabular}{|l|}
4042 \\
\end{tabular} & $\mathrm{pCi} / \mathrm{L}$ & 0.89 & & & & $U$ & & QC_SAMPLE & & RAW GRAB & UNFILTERED & 550 \\
\hline \begin{tabular}{|l|}
4043 \\
\end{tabular} & $\mathrm{pCi} / \mathrm{L}$ & 11.7 & 0 & & & & & QC_SAMPLE & & RAW GRAB & UNFILTERED & 550 \\
\hline 4044 & $\mathrm{pCi} / \mathrm{L}$ & 10.2 & 0 & & & & & QC_SAMPLE & & RAW GRAB & UNFILTERED & 247 \\
\hline 4045 & $\mathrm{pCi} / \mathrm{L}$ & 0.864 & 0.864 & & & U & & QC_SAMPLE & & RAW GRAB & UNFILTERED & 247 \\
\hline \begin{tabular}{|l|}
4046 \\
\end{tabular} & $\mathrm{pCi} / \mathrm{L}$ & 0.0746 & 0.087 & & & & & QC_SAMPLE & & RAW GRAB & UNFILTERED & 247 \\
\hline \begin{tabular}{|l|l|}
4047 \\
\end{tabular} & $\mathrm{pCi} / \mathrm{L}$ & 0.0033 & & & & & & RIVER & & CONTINUOUS & FILTER & 307 \\
\hline \begin{tabular}{|l|}
4048 \\
\end{tabular} & $\mathrm{pCi} / \mathrm{L}$ & 0.000004 & & & & & & RIVER & & CONTINUOUS & FILTER & 307 \\
\hline \begin{tabular}{|l|}
4049 \\
\end{tabular} & $\mathrm{pCi} / \mathrm{L}$ & 0.02 & & & & & & RIVER & & CONTINUOUS & RESIN & 307 \\
\hline \begin{tabular}{|l|}
4050 \\
\end{tabular} & $\mathrm{pCi} / \mathrm{L}$ & 0.00004 & & & & & & RIVER & & CONTINUOUS & RESIN & 307 \\
\hline \begin{tabular}{|l|}
4051 \\
\end{tabular} & $\mathrm{pCi} / \mathrm{L}$ & 0.0064 & & & & & & RIVER & & CONTINUOUS & RESIN & 307 \\
\hline \begin{tabular}{|l|}
4052 \\
\end{tabular} & $\mathrm{pCi} / \mathrm{L}$ & & 0.0000097 & & & & & RIVER & & CONTINUOUS & RESIN & 307 \\
\hline \begin{tabular}{|l|}
4053 \\
\end{tabular} & & & & & & $U$ & & RIVER & & CONTINUOUS & RESIN & 252 \\
\hline 4054 & $\mathrm{pCi} / \mathrm{L}$ & 0.02 & & & & & & RIVER & & CONTINUOUS & RESIN & 252 \\
\hline \begin{tabular}{|l|}
4055 \\
\end{tabular} & $\mathrm{pCi} / \mathrm{L}$ & 0.00012 & & & & & & RIVER & & CONTINUOUS & RESIN & 252 \\
\hline 4056 & $\mathrm{pCi} / \mathrm{L}$ & & 0.0000015 & & & & & RIVER & & CONTINUOUS & RESIN & 252 \\
\hline 4057 & $\mathrm{pCi} / \mathrm{L}$ & 9.4 & 0 & & & & & RIVER & & CUMULATIVE & UNFILTERED & 252 \\
\hline
\end{tabular}




\begin{tabular}{|c|c|c|c|c|c|c|c|c|c|c|c|c|}
\hline & $\mathrm{N}$ & $\mathrm{O}$ & $\mathrm{P}$ & $\mathrm{Q}$ & $\mathrm{R}$ & $\mathrm{s}$ & $T$ & $\mathrm{U}$ & $\mathrm{V}$ & $\mathrm{W}$ & $\mathrm{X}$ & $\bar{Y}$ \\
\hline 1 & ANAL_UNITS_RPTD & COUNTING_ERROR & TOTAL_ANAL_ERROR & MIN_DETECTABLE_ACTIVITY & PCNT_MOISTURE & LAB_QUALIFIER & REVIEW_QUALIFIER & SAMP_FROM & SAMP_ITEM & \begin{tabular}{|l|} 
SAMP_MTHD \\
\end{tabular} & COLL_MTHD & SAMP_SITE_ID \\
\hline 4058 & pCi/L & 0.058 & 0.0691 & & & & & RIVER & & CUMULATIVE & UNFILTERED & 252 \\
\hline 4059 & $\mathrm{pCi} / \mathrm{L}$ & 0.932 & 0.932 & & & U & & RIVER & & CUMULATIVE & UNFILTERED & 252 \\
\hline 4060 & $\mathrm{pCi} / \mathrm{L}$ & 0.00001 & & & & & & RIVER & & CONTINUOUS & FILTER & 252 \\
\hline 4061 & & & & & & $\mathrm{U}$ & & RIVER & & CONTINUOUS & FILTER & 252 \\
\hline 4062 & $\mathrm{pCi} / \mathrm{L}$ & 10.5 & 0 & & & & & RIVER & & CUMULATIVE & UNFILTERED & 463 \\
\hline 4063 & $\mathrm{pCi} / \mathrm{L}$ & 0.901 & 0.901 & & & $U$ & & RIVER & & CUMULATIVE & UNFILTERED & 463 \\
\hline 4064 & $\mathrm{pCi} / \mathrm{L}$ & 0.0486 & 0.0492 & & & $\mathrm{U}$ & & RIVER & & CUMULATIVE & UNFILTERED & 463 \\
\hline 4065 & $\mathrm{pCi} / \mathrm{L}$ & 0.0797 & 0.101 & & & & & RIVER & & CUMULATIVE & UNFILTERED & 553 \\
\hline 4066 & $\mathrm{pCi} / \mathrm{L}$ & 204 & 237 & & & $\mathrm{U}$ & & RIVER & & CUMULATIVE & UNFILTERED & 553 \\
\hline 4067 & $\mathrm{pCi} / \mathrm{L}$ & 1.03 & 1.03 & & & U & & RIVER & & CUMULATIVE & UNFILTERED & 553 \\
\hline 4068 & $\mathrm{pCi} / \mathrm{L}$ & 0.0073 & & & & & & RIVER & & CONTINUOUS & RESIN & 307 \\
\hline 4069 & $\mathrm{pCi} / \mathrm{L}$ & 0.0036 & & & & & & RIVER & & CONTINUOUS & FILTER & 307 \\
\hline 4070 & $\mathrm{pCi} / \mathrm{L}$ & 0.012 & & & & & & RIVER & & CONTINUOUS & RESIN & 252 \\
\hline 4071 & $\mathrm{pCi} / \mathrm{L}$ & 0.0041 & & & & & & RIVER & & CONTINUOUS & FILTER & 252 \\
\hline 4072 & $\mathrm{pCi} / \mathrm{L}$ & 0.0739 & 0.0743 & & & U & & DRINKING & & SAN. GRAB & UNFILTERED & 144 \\
\hline 4073 & $\mathrm{pCi} / \mathrm{L}$ & 197 & 227 & & & $U$ & & DRINKING & & SAN. GRAB & UNFILTERED & 144 \\
\hline 4074 & $\mathrm{pCi} / \mathrm{L}$ & 0.778 & 0.778 & & & $U$ & & DRINKING & & SAN. GRAB & UNFILTERED & 144 \\
\hline 4075 & $\mathrm{pCi} / \mathrm{L}$ & 0.513 & 0.514 & & & & & DRINKING & & SAN. GRAB & UNFILTERED & 187 \\
\hline 4076 & $\mathrm{pCi} / \mathrm{L}$ & 197 & 228 & & & $U$ & & DRINKING & & SAN. GRAB & UNFILTERED & 187 \\
\hline 4077 & $\mathrm{pCi} / \mathrm{L}$ & 0.0038 & & & & & & RIVER & & CONTINUOUS & FILTER & 307 \\
\hline 4078 & $\mathrm{pCi} / \mathrm{L}$ & 0.006 & & & & & & RIVER & & CONTINUOUS & RESIN & 307 \\
\hline 4079 & $\mathrm{pCi} / \mathrm{L}$ & 0.0084 & & & & & & RIVER & & CONTINUOUS & RESIN & 252 \\
\hline 4080 & $\mathrm{pCi} / \mathrm{L}$ & 10.7 & 10.7 & & & & & RIVER & & CUMULATIVE & UNFILTERED & 252 \\
\hline 4081 & $\mathrm{pCi} / \mathrm{L}$ & 0.0039 & & & & & & RIVER & & CONTINUOUS & FILTER & 252 \\
\hline 4082 & $\mathrm{pCi} / \mathrm{L}$ & 0.0655 & 0.0714 & & & & & RIVER & & CUMULATIVE & UNFILTERED & 252 \\
\hline 4083 & $\mathrm{pCi} / \mathrm{L}$ & 0.723 & 0.723 & & & $U$ & & RIVER & & CUMULATIVE & UNFILTERED & 252 \\
\hline 4084 & $\mathrm{pCi} / \mathrm{L}$ & 10.8 & 10.8 & & & & & RIVER & & CUMULATIVE & UNFILTERED & 463 \\
\hline 4085 & $\mathrm{pCi} / \mathrm{L}$ & 1.07 & 1.07 & & & $U$ & & RIVER & & CUMULATIVE & UNFILTERED & 463 \\
\hline 4086 & $\mathrm{pCi} / \mathrm{L}$ & 0.107 & 0.125 & & & & & RIVER & & CUMULATIVE & UNFILTERED & 463 \\
\hline 4087 & $\mathrm{pCi} / \mathrm{L}$ & 0.697 & 6.05 & & & & & SURFACE & & RAW GRAB & UNFILTERED & 509 \\
\hline 4088 & $\mathrm{pCi} / \mathrm{L}$ & 1.74 & 1.75 & & & & & SURFACE & & RAW GRAB & UNFILTERED & 509 \\
\hline 4089 & $\mathrm{pCi} / \mathrm{L}$ & 351 & 571 & & & & & SURFACE & & RAW GRAB & UNFILTERED & 509 \\
\hline 4090 & $\mathrm{pCi} / \mathrm{L}$ & 523 & 1160 & & & & & SURFACE & & RAW GRAB & UNFILTERED & 136 \\
\hline 4091 & $\mathrm{pCi} / \mathrm{L}$ & 1.81 & 1.81 & & & $U$ & & SURFACE & & RAW GRAB & UNFILTERED & 136 \\
\hline 4092 & $\mathrm{pCi} / \mathrm{L}$ & 3.14 & 3.43 & & & & & SURFACE & & RAW GRAB & UNFILTERED & 146 \\
\hline 4093 & $\mathrm{pCi} / \mathrm{L}$ & 0.215 & 0.888 & & & & & SURFACE & & RAW GRAB & UNFILTERED & 146 \\
\hline 4094 & $\mathrm{pCi} / \mathrm{L}$ & 204 & 237 & & & $U$ & & SURFACE & & RAW GRAB & UNFILTERED & 146 \\
\hline 4095 & $\mathrm{pCi} / \mathrm{L}$ & 220 & 263 & & & & & SURFACE & & RAW GRAB & UNFILTERED & 147 \\
\hline 4096 & $\mathrm{pCi} / \mathrm{L}$ & 1.89 & 1.9 & & & & & SURFACE & & RAW GRAB & UNFILTERED & 147 \\
\hline 4097 & $\mathrm{pCi} / \mathrm{L}$ & 0.0797 & 0.189 & & & & & SURFACE & & RAW GRAB & UNFILTERED & 147 \\
\hline 4098 & $\mathrm{pCi} / \mathrm{L}$ & 0.0429 & 0.0436 & & & U & & DRINKING & & SAN. GRAB & UNFILTERED & 186 \\
\hline 4099 & $\mathrm{pCi} / \mathrm{L}$ & 1.18 & & & & $U$ & & DRINKING & & SAN. GRAB & UNFILTERED & 186 \\
\hline 4100 & $\mathrm{pCi} / \mathrm{L}$ & 200 & 232 & & & $U$ & & DRINKING & & SAN. GRAB & UNFILTERED & 186 \\
\hline 4101 & $\mathrm{pCi} / \mathrm{L}$ & 0.0072 & & & & & & RIVER & & CONTINUOUS & RESIN & 307 \\
\hline 4102 & $\mathrm{pCi} / \mathrm{L}$ & 0.0033 & & & & & & RIVER & & CONTINUOUS & FILTER & 307 \\
\hline 4103 & & & & & & $U$ & & RIVER & & CONTINUOUS & FILTER & 252 \\
\hline 4104 & & & & & & U & & RIVER & & CONTINUOUS & RESIN & 252 \\
\hline 4105 & $\mathrm{pCi} / \mathrm{L}$ & 0.0406 & 0.0444 & & & & & DRINKING & & SAN. GRAB & UNFILTERED & 512 \\
\hline 4106 & $\mathrm{pCi} / \mathrm{L}$ & 511 & 1150 & & & & & DRINKING & & SAN. GRAB & UNFILTERED & 512 \\
\hline 4107 & $\mathrm{pCi} / \mathrm{L}$ & 0.799 & 0.8 & & & U & & DRINKING & & SAN. GRAB & UNFILTERED & 512 \\
\hline 4108 & $\mathrm{pCi} / \mathrm{L}$ & 0.0027 & & & & & & RIVER & & CONTINUOUS & FILTER & 307 \\
\hline 4109 & $\mathrm{pCi} / \mathrm{L}$ & 0.0053 & & & & & & RIVER & & CONTINUOUS & RESIN & 307 \\
\hline
\end{tabular}




\begin{tabular}{|c|c|c|c|c|c|c|c|c|c|c|c|c|}
\hline & $\mathrm{N}$ & $\mathrm{O}$ & $\mathrm{P}$ & $\mathrm{Q}$ & $\mathrm{R}$ & $\mathrm{s}$ & $\mathrm{T}$ & $\mathrm{U}$ & $\mathrm{V}$ & $\mathrm{W}$ & $\mathrm{X}$ & $\bar{Y}$ \\
\hline 1 & ANAL_UNITS_RPTD & COUNTING_ERROR & TOTAL_ANAL_ERROR & MIN_DETECTABLE_ACTIVITY & PCNT_MOISTURE & LAB_QUALIFIER & REVIEW_QUALIFIER & SAMP_FROM & SAMP_ITEM & SAMP_MTHD & COLL_MTHD & SAMP_SITE_ID \\
\hline 4110 & $\mathrm{pCi} / \mathrm{L}$ & 10.7 & 10.7 & & & & & RIVER & & CUMULATIVE & UNFILTERED & 252 \\
\hline 4111 & $\mathrm{pCi} / \mathrm{L}$ & 0.0057 & & & & & & RIVER & & CONTINUOUS & FILTER & 252 \\
\hline 4112 & $\mathrm{pCi} / \mathrm{L}$ & 0.0373 & 0.0414 & & & & & RIVER & & CUMULATIVE & UNFILTERED & 252 \\
\hline 4113 & $\mathrm{pCi} / \mathrm{L}$ & 0.631 & 0.631 & & & U & & RIVER & & CUMULATIVE & UNFILTERED & 252 \\
\hline 4114 & $\mathrm{pCi} / \mathrm{L}$ & 0.012 & & & & & & RIVER & & CONTINUOUS & RESIN & 252 \\
\hline 4115 & $\mathrm{pCi} / \mathrm{L}$ & 11.1 & 11.1 & & & & & RIVER & & CUMULATIVE & UNFILTERED & 463 \\
\hline 4116 & $\mathrm{pCi} / \mathrm{L}$ & 0.0663 & 0.0787 & & & & & RIVER & & CUMULATIVE & UNFILTERED & 463 \\
\hline 4117 & $\mathrm{pCi} / \mathrm{L}$ & 0.801 & 0.801 & & & U & & RIVER & & CUMULATIVE & UNFILTERED & 463 \\
\hline 4118 & $\mathrm{pCi} / \mathrm{L}$ & 0.0564 & 0.0569 & & & U & & DRINKING & & SAN. GRAB & UNFILTERED & 487 \\
\hline 4119 & $\mathrm{pCi} / \mathrm{L}$ & 0.506 & 0.506 & & & U & & DRINKING & & SAN. GRAB & UNFILTERED & 487 \\
\hline 4120 & $\mathrm{pCi} / \mathrm{L}$ & 195 & 225 & & & $U$ & & DRINKING & & SAN. GRAB & UNFILTERED & 487 \\
\hline 4121 & $\mathrm{pCi} / \mathrm{L}$ & 202 & 234 & & & U & & DRINKING & & CUMULATIVE & UNFILTERED & 307 \\
\hline 4122 & $\mathrm{pCi} / \mathrm{L}$ & 0.0475 & 0.0606 & & & & & DRINKING & & CUMULATIVE & UNFILTERED & 307 \\
\hline 4123 & $\mathrm{pCi} / \mathrm{L}$ & 1.23 & 1.23 & & & $U$ & & DRINKING & & CUMULATIVE & UNFILTERED & 307 \\
\hline 4124 & $\mathrm{pCi} / \mathrm{L}$ & 0.692 & 0.692 & & & U & & DRINKING & & SAN. GRAB & UNFILTERED & 530 \\
\hline 4125 & $\mathrm{pCi} / \mathrm{L}$ & 200 & 233 & & & & & DRINKING & & SAN. GRAB & UNFILTERED & 530 \\
\hline 4126 & $\mathrm{pCi} / \mathrm{L}$ & 0.17 & 0.185 & & & & & DRINKING & & SAN. GRAB & UNFILTERED & 530 \\
\hline 4127 & $\mathrm{pCi} / \mathrm{L}$ & 0.0072 & & & & & & RIVER & & CONTINUOUS & RESIN & 307 \\
\hline 4128 & & & & & & & & RIVER & & CONTINUOUS & FILTER & 307 \\
\hline 4129 & $\mathrm{pCi} / \mathrm{L}$ & 0.0034 & & & & & & RIVER & & CONTINUOUS & FILTER & 252 \\
\hline 4130 & $\mathrm{pCi} / \mathrm{L}$ & 0.0074 & & & & & & RIVER & & CONTINUOUS & RESIN & 252 \\
\hline 4131 & & & & & & $U$ & & RIVER & & CONTINUOUS & RESIN & 307 \\
\hline 4132 & $\mathrm{pCi} / \mathrm{L}$ & 0.000066 & & & & $U$ & & RIVER & & CONTINUOUS & RESIN & 307 \\
\hline 4133 & $\mathrm{pCi} / \mathrm{L}$ & 0.0068 & & & & & & RIVER & & CONTINUOUS & RESIN & 307 \\
\hline 4134 & $\mathrm{pCi} / \mathrm{L}$ & & 0.0000049 & & & & & RIVER & & CONTINUOUS & RESIN & 307 \\
\hline 4135 & $\mathrm{pCi} / \mathrm{L}$ & 0.0031 & & & & & & RIVER & & CONTINUOUS & FILTER & 307 \\
\hline 4136 & $\mathrm{pCi} / \mathrm{L}$ & 0.000006 & & & & & & RIVER & & CONTINUOUS & FILTER & 307 \\
\hline 4137 & $\mathrm{pCi} / \mathrm{L}$ & 0.000006 & & & & & & RIVER & & CONTINUOUS & FILTER & 252 \\
\hline 4138 & $\mathrm{pCi} / \mathrm{L}$ & 0.0045 & & & & & & RIVER & & CONTINUOUS & FILTER & 252 \\
\hline 4139 & $\mathrm{pCi} / \mathrm{L}$ & 0.0087 & & & & & & RIVER & & CONTINUOUS & RESIN & 252 \\
\hline 4140 & $\mathrm{pCi} / \mathrm{L}$ & 0.02 & & & & & & RIVER & & CONTINUOUS & RESIN & 252 \\
\hline 4141 & $\mathrm{pCi} / \mathrm{L}$ & 0.0001 & & & & & & RIVER & & CONTINUOUS & RESIN & 252 \\
\hline 4142 & $\mathrm{pCi} / \mathrm{L}$ & & 0.0000019 & & & & & RIVER & & CONTINUOUS & RESIN & 252 \\
\hline 4143 & $\mathrm{pCi} / \mathrm{L}$ & 8.8 & 8.8 & & & & & RIVER & & CUMULATIVE & UNFILTERED & 252 \\
\hline 4144 & $\mathrm{pCi} / \mathrm{L}$ & 0.0467 & 0.0555 & & & & & RIVER & & CUMULATIVE & UNFILTERED & 252 \\
\hline 4145 & $\mathrm{pCi} / \mathrm{L}$ & 0.92 & 0.921 & & & $U$ & & RIVER & & CUMULATIVE & UNFILTERED & 252 \\
\hline 4146 & $\mathrm{pCi} / \mathrm{L}$ & 8.3 & 8.3 & & & & & RIVER & & CUMULATIVE & UNFILTERED & 463 \\
\hline 4147 & $\mathrm{pCi} / \mathrm{L}$ & 0.0561 & 0.0734 & & & & & RIVER & & CUMULATIVE & UNFILTERED & 463 \\
\hline 4148 & $\mathrm{pCi} / \mathrm{L}$ & 0.549 & 0.551 & & & & & RIVER & & CUMULATIVE & UNFILTERED & 463 \\
\hline 4149 & $\mathrm{pCi} / \mathrm{L}$ & 0.0294 & 0.0533 & & & & & RIVER & & CUMULATIVE & UNFILTERED & 553 \\
\hline 4150 & $\mathrm{pCi} / \mathrm{L}$ & 197 & 227 & & & U & & RIVER & & CUMULATIVE & UNFILTERED & 553 \\
\hline 4151 & $\mathrm{pCi} / \mathrm{L}$ & 0.579 & 0.581 & & & & & RIVER & & CUMULATIVE & UNFILTERED & 553 \\
\hline 4152 & $\mathrm{pCi} / \mathrm{L}$ & 0.0059 & & & & & & RIVER & & CONTINUOUS & RESIN & 307 \\
\hline 4153 & $\mathrm{pCi} / \mathrm{L}$ & 0.0032 & & & & & & RIVER & & CONTINUOUS & FILTER & 307 \\
\hline 4154 & $\mathrm{pCi} / \mathrm{L}$ & 0.0077 & & & & & & RIVER & & CONTINUOUS & RESIN & 252 \\
\hline 4155 & $\mathrm{pCi} / \mathrm{L}$ & 0.004 & & & & & & RIVER & & CONTINUOUS & FILTER & 252 \\
\hline 4156 & $\mathrm{pCi} / \mathrm{L}$ & 0.21 & 0.551 & & & & & SURFACE & & RAW GRAB & UNFILTERED & 509 \\
\hline 4157 & $\mathrm{pCi} / \mathrm{L}$ & 1.16 & 1.16 & & & $U$ & & SURFACE & & RAW GRAB & UNFILTERED & 509 \\
\hline 4158 & $\mathrm{pCi} / \mathrm{L}$ & 202 & 236 & & & $U$ & & SURFACE & & RAW GRAB & UNFILTERED & 509 \\
\hline 4159 & $\mathrm{pCi} / \mathrm{L}$ & 0.854 & 0.854 & & & U & & DRINKING & & SAN. GRAB & UNFILTERED & 187 \\
\hline 4160 & $\mathrm{pCi} / \mathrm{L}$ & 198 & 229 & & & $U$ & & DRINKING & & SAN. GRAB & UNFILTERED & 187 \\
\hline 4161 & $\mathrm{pCi} / \mathrm{L}$ & 0.0059 & & & & & & RIVER & & CONTINUOUS & RESIN & 307 \\
\hline
\end{tabular}




\begin{tabular}{|c|c|c|c|c|c|c|c|c|c|c|c|c|}
\hline & $\mathrm{N}$ & $\mathrm{O}$ & $\mathrm{P}$ & $\mathrm{Q}$ & $\mathrm{R}$ & $\mathrm{s}$ & $\mathrm{T}$ & $\mathrm{U}$ & $\mathrm{V}$ & $\mathrm{W}$ & $\mathrm{X}$ & $\bar{Y}$ \\
\hline 1 & ANAL_UNITS_RPTD & COUNTING_ERROR & TOTAL_ANAL_ERROR | & MIN_DETECTABLE_ACTIVITY & PCNT_MOISTURE & LAB_QUALIFIER & REVIEW_QUALIFIER & SAMP_FROM & SAMP_ITEM & SAMP_MTHD & COLL_MTHD & SAMP_SITE_ID \\
\hline 4162 & $\mathrm{pCi} / \mathrm{L}$ & 0.003 & & & & & & RIVER & & CONTINUOUS & FILTER & 307 \\
\hline 4163 & & & & & & & & RIVER & & CONTINUOUS & FILTER & 252 \\
\hline 4164 & & & & & & & & RIVER & & CONTINUOUS & RESIN & 252 \\
\hline 4165 & $\mathrm{pCi} / \mathrm{L}$ & 10 & 10 & & & & & RIVER & & CUMULATIVE & UNFILTERED & 252 \\
\hline 4166 & $\mathrm{pCi} / \mathrm{L}$ & 0.0539 & 0.065 & & & & & RIVER & & CUMULATIVE & UNFILTERED & 252 \\
\hline 4167 & $\mathrm{pCi} / \mathrm{L}$ & 1.02 & 1.02 & & & U & & RIVER & & CUMULATIVE & UNFILTERED & 252 \\
\hline 4168 & $\mathrm{pCi} / \mathrm{L}$ & 10 & 10 & & & & & RIVER & & CUMULATIVE & UNFILTERED & 463 \\
\hline 4169 & $\mathrm{pCi} / \mathrm{L}$ & 0.0829 & 0.0971 & & & & & RIVER & & CUMULATIVE & UNFILTERED & 463 \\
\hline 4170 & $\mathrm{pCi} / \mathrm{L}$ & 0.779 & 0.78 & & & U & & RIVER & & CUMULATIVE & UNFILTERED & 463 \\
\hline 4171 & $\mathrm{pCi} / \mathrm{L}$ & 0.0373 & 0.0376 & & & U & & DRINKING & & SAN. GRAB & UNFILTERED & 186 \\
\hline 4172 & $\mathrm{pCi} / \mathrm{L}$ & 0.866 & 0.866 & & & $\mathrm{U}$ & & DRINKING & & SAN. GRAB & UNFILTERED & 186 \\
\hline 4173 & $\mathrm{pCi} / \mathrm{L}$ & 201 & 235 & & & U & & DRINKING & & SAN. GRAB & UNFILTERED & 186 \\
\hline 4174 & $\mathrm{pCi} / \mathrm{L}$ & 0.0468 & 0.0723 & & & & & DRINKING & & SAN. GRAB & UNFILTERED & 144 \\
\hline 4175 & $\mathrm{pCi} / \mathrm{L}$ & 199 & 232 & & & $U$ & & DRINKING & & SAN. GRAB & UNFILTERED & 144 \\
\hline 4176 & $\mathrm{pCi} / \mathrm{L}$ & 0.972 & 0.973 & & & U & & DRINKING & & SAN. GRAB & UNFILTERED & 144 \\
\hline 4177 & $\mathrm{pCi} / \mathrm{L}$ & 0.195 & 0.689 & & & & & SURFACE & & RAW GRAB & UNFILTERED & 509 \\
\hline 4178 & $\mathrm{pCi} / \mathrm{L}$ & 1.74 & 1.74 & & & $U$ & & SURFACE & & RAW GRAB & UNFILTERED & 509 \\
\hline 4179 & $\mathrm{pCi} / \mathrm{L}$ & 376 & 640 & & & & & SURFACE & & RAW GRAB & UNFILTERED & 509 \\
\hline 4180 & $\mathrm{pCi} / \mathrm{L}$ & 549 & 1300 & & & & & SURFACE & & RAW GRAB & UNFILTERED & 136 \\
\hline 4181 & $\mathrm{pCi} / \mathrm{L}$ & 2.68 & 2.68 & & & $U$ & & SURFACE & & RAW GRAB & UNFILTERED & 136 \\
\hline 4182 & $\mathrm{pCi} / \mathrm{L}$ & 5.68 & 5.91 & & & & & SURFACE & & RAW GRAB & UNFILTERED & 146 \\
\hline 4183 & $\mathrm{pCi} / \mathrm{L}$ & 0.13 & 0.68 & & & & & SURFACE & & RAW GRAB & UNFILTERED & 146 \\
\hline 4184 & $\mathrm{pCi} / \mathrm{L}$ & 201 & 233 & & & & & SURFACE & & RAW GRAB & UNFILTERED & 146 \\
\hline 4185 & $\mathrm{pCi} / \mathrm{L}$ & 212 & 252 & & & & & SURFACE & & RAW GRAB & UNFILTERED & 147 \\
\hline 4186 & $\mathrm{pCi} / \mathrm{L}$ & 3.95 & 3.95 & & & $U$ & & SURFACE & & RAW GRAB & UNFILTERED & 147 \\
\hline 4187 & $\mathrm{pCi} / \mathrm{L}$ & 0.278 & 1.01 & & & & & SURFACE & & RAW GRAB & UNFILTERED & 147 \\
\hline 4188 & $\mathrm{pCi} / \mathrm{L}$ & 0.0073 & & & & & & RIVER & & CONTINUOUS & RESIN & 307 \\
\hline 4189 & $\mathrm{pCi} / \mathrm{L}$ & 0.0038 & & & & & & RIVER & & CONTINUOUS & FILTER & 307 \\
\hline 4190 & $\mathrm{pCi} / \mathrm{L}$ & 0.0078 & & & & & & RIVER & & CONTINUOUS & RESIN & 252 \\
\hline 4191 & $\mathrm{pCi} / \mathrm{L}$ & 0.0036 & & & & & & RIVER & & CONTINUOUS & FILTER & 252 \\
\hline 4192 & $\mathrm{pCi} / \mathrm{L}$ & 0.0458 & 0.0459 & & & $U$ & & DRINKING & & SAN. GRAB & UNFILTERED & 512 \\
\hline 4193 & $\mathrm{pCi} / \mathrm{L}$ & 541 & 1270 & & & & & DRINKING & & SAN. GRAB & UNFILTERED & 512 \\
\hline 4194 & $\mathrm{pCi} / \mathrm{L}$ & 0.816 & 0.816 & & & $U$ & & DRINKING & & SAN. GRAB & UNFILTERED & 512 \\
\hline 4195 & $\mathrm{pCi} / \mathrm{L}$ & 0.0066 & & & & & & RIVER & & CONTINUOUS & RESIN & 307 \\
\hline 4196 & $\mathrm{pCi} / \mathrm{L}$ & 0.0034 & & & & & & RIVER & & CONTINUOUS & FILTER & 307 \\
\hline 4197 & $\mathrm{pCi} / \mathrm{L}$ & 0.0075 & & & & & & RIVER & & CONTINUOUS & RESIN & 252 \\
\hline 4198 & $\mathrm{pCi} / \mathrm{L}$ & 9.4 & 9.4 & & & & & RIVER & & CUMULATIVE & UNFILTERED & 252 \\
\hline 4199 & $\mathrm{pCi} / \mathrm{L}$ & 0.0036 & & & & & & RIVER & & CONTINUOUS & FILTER & 252 \\
\hline 4200 & $\mathrm{pCi} / \mathrm{L}$ & 0.034 & 0.0477 & & & & & RIVER & & CUMULATIVE & UNFILTERED & 252 \\
\hline 4201 & $\mathrm{pCi} / \mathrm{L}$ & 0.446 & 0.446 & & & U & & RIVER & & CUMULATIVE & UNFILTERED & 252 \\
\hline 4202 & $\mathrm{pCi} / \mathrm{L}$ & 9.9 & 9.9 & & & & & RIVER & & CUMULATIVE & UNFILTERED & 463 \\
\hline 4203 & $\mathrm{pCi} / \mathrm{L}$ & 0.0217 & 0.0322 & & & & & RIVER & & CUMULATIVE & UNFILTERED & 463 \\
\hline 4204 & $\mathrm{pCi} / \mathrm{L}$ & 0.6 & 0.6 & & & U & & RIVER & & CUMULATIVE & UNFILTERED & 463 \\
\hline 4205 & $\mathrm{pCi} / \mathrm{L}$ & 0.0453 & 0.046 & & & U & & DRINKING & & SAN. GRAB & UNFILTERED & 487 \\
\hline 4206 & $\mathrm{pCi} / \mathrm{L}$ & 0.535 & 0.535 & & & U & & DRINKING & & SAN. GRAB & UNFILTERED & 487 \\
\hline 4207 & $\mathrm{pCi} / \mathrm{L}$ & 190 & 220 & & & U & & DRINKING & & SAN. GRAB & UNFILTERED & 487 \\
\hline 4208 & $\mathrm{pCi} / \mathrm{L}$ & 193 & 224 & & & $U$ & & DRINKING & & CUMULATIVE & UNFILTERED & 307 \\
\hline 4209 & $\mathrm{pCi} / \mathrm{L}$ & 0.0451 & 0.0605 & & & & & DRINKING & & CUMULATIVE & UNFILTERED & 307 \\
\hline 4210 & $\mathrm{pCi} / \mathrm{L}$ & 0.469 & 0.469 & & & $U$ & & DRINKING & & CUMULATIVE & UNFILTERED & 307 \\
\hline 4211 & $\mathrm{pCi} / \mathrm{L}$ & 0.914 & 0.914 & & & $U$ & & DRINKING & & SAN. GRAB & UNFILTERED & 530 \\
\hline 4212 & & & & & & $U$ & & DRINKING & & SAN. GRAB & UNFILTERED & 530 \\
\hline 4213 & $\mathrm{pCi} / \mathrm{L}$ & 0.0884 & 0.234 & & & & & DRINKING & & SAN. GRAB & UNFILTERED & 530 \\
\hline
\end{tabular}




\begin{tabular}{|c|c|c|c|c|c|c|c|c|c|c|c|c|}
\hline & $\mathrm{N}$ & $\mathrm{O}$ & $\mathrm{P}$ & $\mathrm{Q}$ & $\mathrm{R}$ & $\mathrm{s}$ & $T$ & $\mathrm{U}$ & $\mathrm{V}$ & $\mathrm{W}$ & $\mathrm{X}$ & $\bar{Y}$ \\
\hline 1 & ANAL_UNITS_RPTD & COUNTING_ERROR & TOTAL_ANAL_ERROR & MIN_DETECTABLE_ACTIVITY & PCNT_MOISTURE & LAB_QUALIFIER & REVIEW_QUALIFIER & SAMP_FROM & SAMP_ITEM & SAMP_MTHD & COLL_MTHD & SAMP_SITE_ID \\
\hline 4214 & $\mathrm{pCi} / \mathrm{L}$ & 0.0059 & & & & & & RIVER & & CONTINUOUS & RESIN & 307 \\
\hline 4215 & $\mathrm{pCi} / \mathrm{L}$ & 0.003 & & & & & & RIVER & & CONTINUOUS & FILTER & 307 \\
\hline \begin{tabular}{|l|l|}
4216 \\
\end{tabular} & & & & & & & & RIVER & & CONTINUOUS & FILTER & 252 \\
\hline 4217 & & & & & & & & RIVER & & CONTINUOUS & RESIN & 252 \\
\hline 4218 & $\mathrm{pCi} / \mathrm{L}$ & 0.0816 & 0.0962 & & & & & QC_SAMPLE & & RAW GRAB & UNFILTERED & 550 \\
\hline 4219 & $\mathrm{pCi} / \mathrm{L}$ & 0.63 & 0.631 & & & $U$ & & QC_SAMPLE & & RAW GRAB & UNFILTERED & 550 \\
\hline 4220 & $\mathrm{pCi} / \mathrm{L}$ & 14.1 & 26.7 & & & & & QC_SAMPLE & & RAW GRAB & UNFILTERED & 550 \\
\hline 4221 & $\mathrm{pCi} / \mathrm{L}$ & 13.7 & 24.6 & & & & & QC_SAMPLE & & RAW GRAB & UNFILTERED & 247 \\
\hline 4222 & $\mathrm{pCi} / \mathrm{L}$ & 0.718 & 0.722 & & & & & QC_SAMPLE & & RAW GRAB & UNFILTERED & 247 \\
\hline 4223 & $\mathrm{pCi} / \mathrm{L}$ & 0.122 & 0.132 & & & & & QC_SAMPLE & & RAW GRAB & UNFILTERED & 247 \\
\hline 4224 & $\mathrm{pCi} / \mathrm{L}$ & 0.02 & & & & & & RIVER & & CONTINUOUS & RESIN & 307 \\
\hline 4225 & $\mathrm{pCi} / \mathrm{L}$ & 0.00012 & & & & & & RIVER & & CONTINUOUS & RESIN & 307 \\
\hline \begin{tabular}{|l|}
4226 \\
\end{tabular} & $\mathrm{pCi} / \mathrm{L}$ & 0.0061 & & & & & & RIVER & & CONTINUOUS & RESIN & 307 \\
\hline \begin{tabular}{|l|l|}
4227 \\
\end{tabular} & $\mathrm{pCi} / \mathrm{L}$ & & 0.0000114 & & & & & RIVER & & CONTINUOUS & RESIN & 307 \\
\hline \begin{tabular}{|l|}
4228 \\
\end{tabular} & $\mathrm{pCi} / \mathrm{L}$ & 0.0029 & & & & & & RIVER & & CONTINUOUS & FILTER & 307 \\
\hline \begin{tabular}{|l|}
4229 \\
\end{tabular} & $\mathrm{pCi} / \mathrm{L}$ & 0.000002 & & & & & & RIVER & & CONTINUOUS & FILTER & 307 \\
\hline 4230 & $\mathrm{pCi} / \mathrm{L}$ & 11.6 & 18.8 & & & & & RIVER & & CUMULATIVE & UNFILTERED & 252 \\
\hline \begin{tabular}{|l|}
4231 \\
\end{tabular} & $\mathrm{pCi} / \mathrm{L}$ & 0.000006 & & & & & & RIVER & & CONTINUOUS & FILTER & 252 \\
\hline \begin{tabular}{|l|}
4232 \\
\end{tabular} & $\mathrm{pCi} / \mathrm{L}$ & 0.0029 & & & & & & RIVER & & CONTINUOUS & FILTER & 252 \\
\hline \begin{tabular}{|l|}
4233 \\
\end{tabular} & $\mathrm{pCi} / \mathrm{L}$ & 0.0649 & 0.0734 & & & & & RIVER & & CUMULATIVE & UNFILTERED & 252 \\
\hline 4234 & $\mathrm{pCi} / \mathrm{L}$ & 1.06 & 1.06 & & & $U$ & & RIVER & & CUMULATIVE & UNFILTERED & 252 \\
\hline 4235 & $\mathrm{pCi} / \mathrm{L}$ & 0.0061 & & & & & & RIVER & & CONTINUOUS & RESIN & 252 \\
\hline \begin{tabular}{|l|}
4236 \\
\end{tabular} & $\mathrm{pCi} / \mathrm{L}$ & 0.02 & & & & & & RIVER & & CONTINUOUS & RESIN & 252 \\
\hline \begin{tabular}{|l|l|}
4237 \\
\end{tabular} & $\mathrm{pCi} / \mathrm{L}$ & 0.00008 & & & & & & RIVER & & CONTINUOUS & RESIN & 252 \\
\hline \begin{tabular}{|l|}
4238 \\
\end{tabular} & $\mathrm{pCi} / \mathrm{L}$ & & 0.0000013 & & & & & RIVER & & CONTINUOUS & RESIN & 252 \\
\hline \begin{tabular}{|l|}
4239 \\
\end{tabular} & $\mathrm{pCi} / \mathrm{L}$ & 13.9 & 27 & & & & & RIVER & & CUMULATIVE & UNFILTERED & 463 \\
\hline \begin{tabular}{|l|}
4240 \\
\end{tabular} & $\mathrm{pCi} / \mathrm{L}$ & 0.0486 & 0.0663 & & & & & RIVER & & CUMULATIVE & UNFILTERED & 463 \\
\hline \begin{tabular}{|l|}
4241 \\
\end{tabular} & $\mathrm{pCi} / \mathrm{L}$ & 0.995 & 0.995 & & & $U$ & & RIVER & & CUMULATIVE & UNFILTERED & 463 \\
\hline \begin{tabular}{|l|}
4242 \\
\end{tabular} & $\mathrm{pCi} / \mathrm{L}$ & 0.055 & 0.0645 & & & & & RIVER & & CUMULATIVE & UNFILTERED & 553 \\
\hline \begin{tabular}{|l|}
4243 \\
\end{tabular} & $\mathrm{pCi} / \mathrm{L}$ & 198 & 239 & & & $U$ & & RIVER & & CUMULATIVE & UNFILTERED & 553 \\
\hline \begin{tabular}{|l|l|}
4244 \\
\end{tabular} & $\mathrm{pCi} / \mathrm{L}$ & 0.768 & 0.769 & & & $U$ & & RIVER & & CUMULATIVE & UNFILTERED & 553 \\
\hline \begin{tabular}{|l|}
4245 \\
\end{tabular} & $\mathrm{pCi} / \mathrm{L}$ & 0.0063 & & & & & & RIVER & & CONTINUOUS & RESIN & 307 \\
\hline \begin{tabular}{|l|}
4246 \\
\end{tabular} & $\mathrm{pCi} / \mathrm{L}$ & 0.003 & & & & & & RIVER & & CONTINUOUS & FILTER & 307 \\
\hline \begin{tabular}{|l|l|}
4247 \\
\end{tabular} & $\mathrm{pCi} / \mathrm{L}$ & 0.0096 & & & & & & RIVER & & CONTINUOUS & RESIN & 252 \\
\hline \begin{tabular}{|l|}
4248 \\
\end{tabular} & $\mathrm{pCi} / \mathrm{L}$ & 0.0049 & & & & & & RIVER & & CONTINUOUS & FILTER & 252 \\
\hline \begin{tabular}{|l|}
4249 \\
\end{tabular} & $\mathrm{pCi} / \mathrm{L}$ & 0.0196 & 0.0522 & & & & & DRINKING & & SAN. GRAB & UNFILTERED & 186 \\
\hline 4250 & $\mathrm{pCi} / \mathrm{L}$ & 0.936 & 0.937 & & & $U$ & & DRINKING & & SAN. GRAB & UNFILTERED & 186 \\
\hline \begin{tabular}{|l|}
4251 \\
\end{tabular} & $\mathrm{pCi} / \mathrm{L}$ & 193 & 232 & & & U & & DRINKING & & SAN. GRAB & UNFILTERED & 186 \\
\hline \begin{tabular}{|l|}
4252 \\
\end{tabular} & $\mathrm{pCi} / \mathrm{L}$ & 0.0109 & 0.0125 & & & & & DRINKING & & SAN. GRAB & UNFILTERED & 144 \\
\hline 4253 & $\mathrm{pCi} / \mathrm{L}$ & 192 & 230 & & & U & & DRINKING & & SAN. GRAB & UNFILTERED & 144 \\
\hline \begin{tabular}{|l|}
4254 \\
\end{tabular} & $\mathrm{pCi} / \mathrm{L}$ & 0.88 & 0.881 & & & $U$ & & DRINKING & & SAN. GRAB & UNFILTERED & 144 \\
\hline \begin{tabular}{|l|}
4255 \\
\end{tabular} & $\mathrm{pCi} / \mathrm{L}$ & 0.745 & 0.746 & & & $U$ & & DRINKING & & SAN. GRAB & UNFILTERED & 187 \\
\hline \begin{tabular}{|l|}
4256 \\
\end{tabular} & $\mathrm{pCi} / \mathrm{L}$ & 191 & 228 & & & $U$ & & DRINKING & & SAN. GRAB & UNFILTERED & 187 \\
\hline \begin{tabular}{|l|l|}
4257 \\
\end{tabular} & $\mathrm{pCi} / \mathrm{L}$ & 0.0062 & & & & & & RIVER & & CONTINUOUS & RESIN & 307 \\
\hline \begin{tabular}{|l|}
4258 \\
\end{tabular} & $\mathrm{pCi} / \mathrm{L}$ & 0.0032 & & & & & & RIVER & & CONTINUOUS & FILTER & 307 \\
\hline \begin{tabular}{|l|}
4259 \\
\end{tabular} & $\mathrm{pCi} / \mathrm{L}$ & 12.2 & 20.3 & & & & & RIVER & & CUMULATIVE & UNFILTERED & 252 \\
\hline 4260 & $\mathrm{pCi} / \mathrm{L}$ & 0.0183 & 0.0443 & & & & & RIVER & & CUMULATIVE & UNFILTERED & 252 \\
\hline \begin{tabular}{|l|}
4261 \\
\end{tabular} & $\mathrm{pCi} / \mathrm{L}$ & 0.819 & 0.82 & & & $U$ & & RIVER & & CUMULATIVE & UNFILTERED & 252 \\
\hline \begin{tabular}{|l|}
4262 \\
\end{tabular} & $\mathrm{pCi} / \mathrm{L}$ & 0.0053 & & & & & & RIVER & & CONTINUOUS & RESIN & 252 \\
\hline \begin{tabular}{|l|}
4263 \\
\end{tabular} & $\mathrm{pCi} / \mathrm{L}$ & 0.0026 & & & & & & RIVER & & CONTINUOUS & FILTER & 252 \\
\hline \begin{tabular}{|l|}
4264 \\
\end{tabular} & $\mathrm{pCi} / \mathrm{L}$ & 13.9 & 23.4 & & & & & RIVER & & CUMULATIVE & UNFILTERED & 463 \\
\hline \begin{tabular}{|l|}
4265 \\
\end{tabular} & $\mathrm{pCi} / \mathrm{L}$ & 0.0296 & 0.0698 & & & & & RIVER & & CUMULATIVE & UNFILTERED & 463 \\
\hline
\end{tabular}




\begin{tabular}{|c|c|c|c|c|c|c|c|c|c|c|c|c|}
\hline & $\mathrm{N}$ & $\mathrm{O}$ & $\mathrm{P}$ & $\mathrm{Q}$ & $\mathrm{R}$ & $\mathrm{s}$ & $T$ & $\mathrm{U}$ & $\mathrm{V}$ & $\mathrm{W}$ & $x$ & $\bar{Y}$ \\
\hline 1 & ANAL_UNITS_RPTD & COUNTING_ERROR & TOTAL_ANAL_ERROR & MIN_DETECTABLE_ACTIVITY & PCNT_MOISTURE & LAB_QUALIFIER & REVIEW_QUALIFIER & SAMP_FROM & SAMP_ITEM & \begin{tabular}{|l|} 
SAMP_MTHD \\
\end{tabular} & COLL_MTHD & SAMP_SITE_ID \\
\hline \begin{tabular}{|c|}
4266 \\
\end{tabular} & $\mathrm{pCi} / \mathrm{L}$ & 0.48 & 0.483 & & & & & RIVER & & CUMULATIVE & UNFILTERED & 463 \\
\hline 4267 & $\mathrm{pCi} / \mathrm{L}$ & 171 & 221 & & & U & & RIVER & & CUMULATIVE & UNFILTERED & 553 \\
\hline \begin{tabular}{|l|}
4268 \\
\end{tabular}$-100$ & $\mathrm{pCi} / \mathrm{L}$ & 0.0843 & 0.44 & & & & & SURFACE & & RAW GRAB & UNFILTERED & 509 \\
\hline \begin{tabular}{|l|l|}
4269 \\
\end{tabular} & $\mathrm{pCi} / \mathrm{L}$ & 2.11 & 2.11 & & & $\mathrm{U}$ & & SURFACE & & RAW GRAB & UNFILTERED & 509 \\
\hline 4270 & $\mathrm{pCi} / \mathrm{L}$ & 202 & 248 & & & & & SURFACE & & RAW GRAB & UNFILTERED & 509 \\
\hline \begin{tabular}{|l|}
4271 \\
\end{tabular} & $\mathrm{pCi} / \mathrm{L}$ & 451 & 1130 & & & & & SURFACE & & RAW GRAB & UNFILTERED & 136 \\
\hline \begin{tabular}{|c|}
4272 \\
\end{tabular} & $\mathrm{pCi} / \mathrm{L}$ & 2.84 & 2.84 & & & $\mathrm{U}$ & & SURFACE & & RAW GRAB & UNFILTERED & 136 \\
\hline 4273 & $\mathrm{pCi} / \mathrm{L}$ & 1.58 & 1.61 & & & & & SURFACE & & RAW GRAB & UNFILTERED & 146 \\
\hline \begin{tabular}{|l|}
4274 \\
\end{tabular} & $\mathrm{pCi} / \mathrm{L}$ & 0.0584 & 0.329 & & & & & SURFACE & & RAW GRAB & UNFILTERED & 146 \\
\hline \begin{tabular}{|l|}
4275 \\
\end{tabular} & $\mathrm{pCi} / \mathrm{L}$ & 194 & 233 & & & $\mathrm{U}$ & & SURFACE & & RAW GRAB & UNFILTERED & 146 \\
\hline \begin{tabular}{|l|}
4276 \\
\end{tabular} & $\mathrm{pCi} / \mathrm{L}$ & 207 & 259 & & & & & SURFACE & & RAW GRAB & UNFILTERED & 147 \\
\hline \begin{tabular}{|l|}
4277 \\
\end{tabular} & $\mathrm{pCi} / \mathrm{L}$ & 2.63 & 2.64 & & & & & SURFACE & & RAW GRAB & UNFILTERED & 147 \\
\hline \begin{tabular}{|l|}
4278 \\
\end{tabular} & $\mathrm{pCi} / \mathrm{L}$ & 0.101 & 0.405 & & & & & SURFACE & & RAW GRAB & UNFILTERED & 147 \\
\hline \begin{tabular}{|l|}
4279 \\
\end{tabular} & $\mathrm{pCi} / \mathrm{L}$ & 0.0073 & & & & & & RIVER & & CONTINUOUS & RESIN & 307 \\
\hline \begin{tabular}{|l|}
4280 \\
\end{tabular} & $\mathrm{pCi} / \mathrm{L}$ & 0.0039 & & & & & & RIVER & & CONTINUOUS & FILTER & 307 \\
\hline \begin{tabular}{|l|}
4281 \\
\end{tabular} & $\mathrm{pCi} / \mathrm{L}$ & 0.0031 & & & & & & RIVER & & CONTINUOUS & FILTER & 252 \\
\hline \begin{tabular}{|l|}
4282 \\
\end{tabular} & $\mathrm{pCi} / \mathrm{L}$ & 0.006 & & & & & & RIVER & & CONTINUOUS & RESIN & 252 \\
\hline \begin{tabular}{|l|}
4283 \\
\end{tabular} & $\mathrm{pCi} / \mathrm{L}$ & 0.0331 & 0.0365 & & & & & DRINKING & & SAN. GRAB & UNFILTERED & 512 \\
\hline \begin{tabular}{|l|}
4284 \\
\end{tabular} & $\mathrm{pCi} / \mathrm{L}$ & 484 & 1290 & & & & & DRINKING & & SAN. GRAB & UNFILTERED & 512 \\
\hline \begin{tabular}{|l|}
4285 \\
\end{tabular} & $\mathrm{pCi} / \mathrm{L}$ & 2.06 & 2.06 & & & $U$ & & DRINKING & & SAN. GRAB & UNFILTERED & 512 \\
\hline \begin{tabular}{|l|}
4286 \\
\end{tabular} & $\mathrm{pCi} / \mathrm{L}$ & 0.0056 & & & & & & RIVER & & CONTINUOUS & RESIN & 307 \\
\hline \begin{tabular}{|l|}
4287 \\
\end{tabular} & $\mathrm{pCi} / \mathrm{L}$ & 0.0028 & & & & & & RIVER & & CONTINUOUS & FILTER & 307 \\
\hline \begin{tabular}{|l|}
4288 \\
\end{tabular} & $\mathrm{pCi} / \mathrm{L}$ & 0.0063 & & & & & & RIVER & & CONTINUOUS & RESIN & 252 \\
\hline \begin{tabular}{|l|}
4289 \\
\end{tabular} & $\mathrm{pCi} / \mathrm{L}$ & 12.7 & 20 & & & & & RIVER & & CUMULATIVE & UNFILTERED & 252 \\
\hline \begin{tabular}{|l|}
4290 \\
\end{tabular} & $\mathrm{pCi} / \mathrm{L}$ & 0.0162 & 0.0405 & & & & & RIVER & & CUMULATIVE & UNFILTERED & 252 \\
\hline \begin{tabular}{|l|}
4291 \\
\end{tabular} & $\mathrm{pCi} / \mathrm{L}$ & 0.629 & 0.629 & & & $\mathrm{U}$ & & RIVER & & CUMULATIVE & UNFILTERED & 252 \\
\hline \begin{tabular}{|l|}
4292 \\
\end{tabular} & $\mathrm{pCi} / \mathrm{L}$ & 0.0032 & & & & & & RIVER & & CONTINUOUS & FILTER & 252 \\
\hline \begin{tabular}{|l|}
4293 \\
\end{tabular} & $\mathrm{pCi} / \mathrm{L}$ & 12.6 & 20.7 & & & & & RIVER & & CUMULATIVE & UNFILTERED & 463 \\
\hline \begin{tabular}{|l|}
4294 \\
\end{tabular} & $\mathrm{pCi} / \mathrm{L}$ & 0.0296 & 0.0513 & & & & & RIVER & & CUMULATIVE & UNFILTERED & 463 \\
\hline \begin{tabular}{|l|}
4295 \\
\end{tabular} & $\mathrm{pCi} / \mathrm{L}$ & 0.7 & 0.7 & & & $U$ & & RIVER & & CUMULATIVE & UNFILTERED & 463 \\
\hline \begin{tabular}{|l|}
4296 \\
\end{tabular} & $\mathrm{pCi} / \mathrm{L}$ & 0.0252 & 0.0337 & & & & & DRINKING & & SAN. GRAB & UNFILTERED & 487 \\
\hline \begin{tabular}{|l|}
4297 \\
\end{tabular} & $\mathrm{pCi} / \mathrm{L}$ & 0.968 & 0.969 & & & $U$ & & DRINKING & & SAN. GRAB & UNFILTERED & 487 \\
\hline \begin{tabular}{|l|}
4298 \\
\end{tabular} & $\mathrm{pCi} / \mathrm{L}$ & 197 & 238 & & & U & & DRINKING & & SAN. GRAB & UNFILTERED & 487 \\
\hline \begin{tabular}{|l|}
4299 \\
\end{tabular} & $\mathrm{pCi} / \mathrm{L}$ & 200 & 244 & & & U & & DRINKING & & CUMULATIVE & UNFILTERED & 307 \\
\hline \begin{tabular}{|l|}
4300 \\
\end{tabular} & $\mathrm{pCi} / \mathrm{L}$ & 0.0332 & 0.0608 & & & & & DRINKING & & CUMULATIVE & UNFILTERED & 307 \\
\hline \begin{tabular}{|l|}
4301 \\
\end{tabular} & $\mathrm{pCi} / \mathrm{L}$ & 0.614 & 0.617 & & & & & DRINKING & & CUMULATIVE & UNFILTERED & 307 \\
\hline \begin{tabular}{|l|}
4302 \\
\end{tabular} & $\mathrm{pCi} / \mathrm{L}$ & 0.682 & 0.682 & & & $\mathrm{U}$ & & DRINKING & & SAN. GRAB & UNFILTERED & 530 \\
\hline 4303 & $\mathrm{pCi} / \mathrm{L}$ & 196 & 237 & & & $U$ & & DRINKING & & SAN. GRAB & UNFILTERED & 530 \\
\hline \begin{tabular}{|l|}
4304 \\
\end{tabular} & $\mathrm{pCi} / \mathrm{L}$ & 0.0677 & 0.309 & & & & & DRINKING & & SAN. GRAB & UNFILTERED & 530 \\
\hline \begin{tabular}{|l|}
4305 \\
\end{tabular} & & & & & & & & RIVER & & CONTINUOUS & RESIN & 307 \\
\hline \begin{tabular}{|l|}
4306 \\
\end{tabular} & & & & & & & & RIVER & & CONTINUOUS & FILTER & 307 \\
\hline \begin{tabular}{|l|}
4307 \\
\end{tabular} & $\mathrm{pCi} / \mathrm{L}$ & 0.0045 & & & & & & RIVER & & CONTINUOUS & FILTER & 252 \\
\hline \begin{tabular}{|l|}
4308 \\
\end{tabular} & $\mathrm{pCi} / \mathrm{L}$ & 0.009 & & & & & & RIVER & & CONTINUOUS & RESIN & 252 \\
\hline \begin{tabular}{|l|}
4309 \\
\end{tabular} & $\mathrm{pCi} / \mathrm{L}$ & 0.003 & & & & & & RIVER & & CONTINUOUS & FILTER & 307 \\
\hline \begin{tabular}{|l|}
4310 \\
\end{tabular} & $\mathrm{pCi} / \mathrm{L}$ & 0.000006 & & & & & & RIVER & & CONTINUOUS & FILTER & 307 \\
\hline \begin{tabular}{|l|l|}
4311 \\
\end{tabular} & $\mathrm{pCi} / \mathrm{L}$ & 0.0000408 & & & & $U$ & & RIVER & & CONTINUOUS & RESIN & 307 \\
\hline \begin{tabular}{|l|}
4312 \\
\end{tabular} & $\mathrm{pCi} / \mathrm{L}$ & 0.0057 & & & & & & RIVER & & CONTINUOUS & RESIN & 307 \\
\hline \begin{tabular}{|l|}
4313 \\
\end{tabular} & $\mathrm{pCi} / \mathrm{L}$ & & 0.0000066 & & & & & RIVER & & CONTINUOUS & RESIN & 307 \\
\hline \begin{tabular}{|l|}
4314 \\
\end{tabular} & $\mathrm{pCi} / \mathrm{L}$ & 0.0078 & & & & & & RIVER & & CONTINUOUS & RESIN & 252 \\
\hline \begin{tabular}{|l|}
4315 \\
\end{tabular} & $\mathrm{pCi} / \mathrm{L}$ & 0.000035 & & & & $U$ & & RIVER & & CONTINUOUS & RESIN & 252 \\
\hline \begin{tabular}{|l|}
4316 \\
\end{tabular} & $\mathrm{pCi} / \mathrm{L}$ & & 0.00000096 & & & & & RIVER & & CONTINUOUS & RESIN & 252 \\
\hline \begin{tabular}{|l|l|}
4317 \\
\end{tabular} & $\mathrm{pCi} / \mathrm{L}$ & 13.7 & 23.2 & & & & & RIVER & & CUMULATIVE & UNFILTERED & 252 \\
\hline
\end{tabular}




\begin{tabular}{|c|c|c|c|c|c|c|c|c|c|c|c|c|}
\hline & $\mathrm{N}$ & $\mathrm{O}$ & $\mathrm{P}$ & $\mathrm{Q}$ & $\mathrm{R}$ & $\mathrm{s}$ & $T$ & $\mathrm{U}$ & $\mathrm{V}$ & $\mathrm{W}$ & $\mathrm{X}$ & $\bar{Y}$ \\
\hline 1 & ANAL_UNITS_RPTD & COUNTING_ERROR & TOTAL_ANAL_ERROR & MIN_DETECTABLE_ACTIVITY & PCNT_MOISTURE & LAB_QUALIFIER & REVIEW_QUALIFIER & SAMP_FROM & SAMP_ITEM & SAMP_MTHD & COLL_MTHD & SAMP_SITE_ID \\
\hline 4318 & $\mathrm{pCi} / \mathrm{L}$ & 0.0000044 & & & & & & RIVER & & CONTINUOUS & FILTER & 252 \\
\hline 4319 & $\mathrm{pCi} / \mathrm{L}$ & 0.0037 & & & & & & RIVER & & CONTINUOUS & FILTER & 252 \\
\hline 4320 & $\mathrm{pCi} / \mathrm{L}$ & 0.0233 & 0.0413 & & & & & RIVER & & CUMULATIVE & UNFILTERED & 252 \\
\hline \begin{tabular}{|l|}
4321 \\
\end{tabular} & $\mathrm{pCi} / \mathrm{L}$ & 1 & 1 & & & $\mathrm{U}$ & & RIVER & & CUMULATIVE & UNFILTERED & 252 \\
\hline 4322 & $\mathrm{pCi} / \mathrm{L}$ & 12.8 & 22.4 & & & & & RIVER & & CUMULATIVE & UNFILTERED & 463 \\
\hline 4323 & $\mathrm{pCi} / \mathrm{L}$ & 0.0282 & 0.0488 & & & & & RIVER & & CUMULATIVE & UNFILTERED & 463 \\
\hline 4324 & $\mathrm{pCi} / \mathrm{L}$ & 0.929 & 0.931 & & & $\mathrm{U}$ & & RIVER & & CUMULATIVE & UNFILTERED & 463 \\
\hline 4325 & $\mathrm{pCi} / \mathrm{L}$ & 208 & 265 & & & u & & DRINKING & & CUMULATIVE & UNFILTERED & 307 \\
\hline 4326 & $\mathrm{pCi} / \mathrm{L}$ & 0.055 & 0.156 & & & & & DRINKING & & CUMULATIVE & UNFILTERED & 307 \\
\hline \begin{tabular}{|l|l|}
4327 \\
\end{tabular} & $\mathrm{pCi} / \mathrm{L}$ & 2.68 & 2.69 & & & U & & DRINKING & & CUMULATIVE & UNFILTERED & 307 \\
\hline 4328 & $\mathrm{pCi} / \mathrm{L}$ & 0.0058 & & & & & & RIVER & & CONTINUOUS & RESIN & 307 \\
\hline \begin{tabular}{|l|}
4329 \\
\end{tabular} & $\mathrm{pCi} / \mathrm{L}$ & 0.0027 & & & & & & RIVER & & CONTINUOUS & FILTER & 307 \\
\hline 4330 & $\mathrm{pCi} / \mathrm{L}$ & 0.006 & & & & & & RIVER & & CONTINUOUS & RESIN & 252 \\
\hline \begin{tabular}{|l|l|}
4331 \\
\end{tabular} & $\mathrm{pCi} / \mathrm{L}$ & 0.003 & & & & & & RIVER & & CONTINUOUS & FILTER & 252 \\
\hline \begin{tabular}{|l|}
4332 \\
\end{tabular} & $\mathrm{pCi} / \mathrm{L}$ & 0.0073 & & & & & & RIVER & & CONTINUOUS & RESIN & 307 \\
\hline \begin{tabular}{|l|}
4333 \\
\end{tabular} & $\mathrm{pCi} / \mathrm{L}$ & 0.0038 & & & & & & RIVER & & CONTINUOUS & FILTER & 307 \\
\hline 4334 & $\mathrm{pCi} / \mathrm{L}$ & 0.003 & & & & & & RIVER & & CONTINUOUS & FILTER & 252 \\
\hline \begin{tabular}{|l|}
4335 \\
\end{tabular} & $\mathrm{pCi} / \mathrm{L}$ & 0.0056 & & & & & & RIVER & & CONTINUOUS & RESIN & 252 \\
\hline \begin{tabular}{|l|}
4336 \\
\end{tabular} & $\mathrm{pCi} / \mathrm{L}$ & 0.0073 & & & & & & RIVER & & CONTINUOUS & RESIN & 307 \\
\hline \begin{tabular}{|l|l|}
4337 \\
\end{tabular} & $\mathrm{pCi} / \mathrm{L}$ & 0.004 & & & & & & RIVER & & CONTINUOUS & FILTER & 307 \\
\hline 4338 & $\mathrm{pCi} / \mathrm{L}$ & 13.1 & 21.6 & & & & & RIVER & & CUMULATIVE & UNFILTERED & 252 \\
\hline \begin{tabular}{|l|}
4339 \\
\end{tabular} & $\mathrm{pCi} / \mathrm{L}$ & 0.0038 & & & & & & RIVER & & CONTINUOUS & FILTER & 252 \\
\hline 4340 & $\mathrm{pCi} / \mathrm{L}$ & 0.0221 & 0.0423 & & & & & RIVER & & CUMULATIVE & UNFILTERED & 252 \\
\hline \begin{tabular}{|l|}
4341 \\
\end{tabular} & $\mathrm{pCi} / \mathrm{L}$ & 0.475 & 0.476 & & & & & RIVER & & CUMULATIVE & UNFILTERED & 252 \\
\hline \begin{tabular}{|l|}
4342 \\
\end{tabular} & $\mathrm{pCi} / \mathrm{L}$ & 0.0078 & & & & & & RIVER & & CONTINUOUS & RESIN & 252 \\
\hline \begin{tabular}{|l|}
4343 \\
\end{tabular} & $\mathrm{pCi} / \mathrm{L}$ & 13.6 & 22.2 & & & & & RIVER & & CUMULATIVE & UNFILTERED & 463 \\
\hline \begin{tabular}{|l|l|}
4344 \\
\end{tabular} & $\mathrm{pCi} / \mathrm{L}$ & 0.757 & 0.758 & & & U & & RIVER & & CUMULATIVE & UNFILTERED & 463 \\
\hline \begin{tabular}{|l|}
4345 \\
\end{tabular} & $\mathrm{pCi} / \mathrm{L}$ & 0.0275 & 0.0513 & & & & & RIVER & & CUMULATIVE & UNFILTERED & 463 \\
\hline \begin{tabular}{|l|}
4346 \\
\end{tabular} & $\mathrm{pCi} / \mathrm{L}$ & 0.0077 & & & & & & RIVER & & CONTINUOUS & RESIN & 307 \\
\hline \begin{tabular}{|l|}
4347 \\
\end{tabular} & $\mathrm{pCi} / \mathrm{L}$ & 0.0041 & & & & & & RIVER & & CONTINUOUS & FILTER & 307 \\
\hline \begin{tabular}{|l|}
4348 \\
\end{tabular} & $\mathrm{pCi} / \mathrm{L}$ & 0.0044 & & & & & & RIVER & & CONTINUOUS & FILTER & 252 \\
\hline \begin{tabular}{|l|}
4349 \\
\end{tabular} & $\mathrm{pCi} / \mathrm{L}$ & 0.0084 & & & & & & RIVER & & CONTINUOUS & RESIN & 252 \\
\hline \begin{tabular}{|l|}
4350 \\
\end{tabular} & $\mathrm{pCi} / \mathrm{L}$ & 11.6 & 18.6 & & & & & RIVER & & CUMULATIVE & UNFILTERED & 463 \\
\hline \begin{tabular}{|l|l|}
4351 \\
\end{tabular} & $\mathrm{pCi} / \mathrm{L}$ & 0.0405 & 0.0723 & & & & & DRINKING & & SAN. GRAB & UNFILTERED & 186 \\
\hline \begin{tabular}{|l|}
4352 \\
\end{tabular} & $\mathrm{pCi} / \mathrm{L}$ & 1.38 & 1.38 & & & U & & DRINKING & & SAN. GRAB & UNFILTERED & 186 \\
\hline \begin{tabular}{|l|}
4353 \\
\end{tabular} & $\mathrm{pCi} / \mathrm{L}$ & 174 & 225 & & & $U$ & & DRINKING & & SAN. GRAB & UNFILTERED & 186 \\
\hline 4354 & $\mathrm{pCi} / \mathrm{L}$ & 0.0215 & 0.0251 & & & & & DRINKING & & SAN. GRAB & UNFILTERED & 144 \\
\hline \begin{tabular}{|l|}
4355 \\
\end{tabular} & $\mathrm{pCi} / \mathrm{L}$ & 174 & 226 & & & U & & DRINKING & & SAN. GRAB & UNFILTERED & 144 \\
\hline \begin{tabular}{|l|}
4356 \\
\end{tabular} & $\mathrm{pCi} / \mathrm{L}$ & 0.446 & 0.446 & & & $U$ & & DRINKING & & SAN. GRAB & UNFILTERED & 144 \\
\hline \begin{tabular}{|l|l|}
4357 \\
\end{tabular} & $\mathrm{pCi} / \mathrm{L}$ & 1.04 & 1.04 & & & U & & DRINKING & & SAN. GRAB & UNFILTERED & 187 \\
\hline \begin{tabular}{|l|}
4358 \\
\end{tabular} & $\mathrm{pCi} / \mathrm{L}$ & 173 & 220 & & & U & & DRINKING & & SAN. GRAB & UNFILTERED & 187 \\
\hline \begin{tabular}{|l|}
4359 \\
\end{tabular} & & & & & & & & RIVER & & CONTINUOUS & RESIN & 307 \\
\hline 4360 & & & & & & & & RIVER & & CONTINUOUS & FILTER & 307 \\
\hline \begin{tabular}{|l|}
4361 \\
\end{tabular} & $\mathrm{pCi} / \mathrm{L}$ & 0.0095 & & & & & & RIVER & & CONTINUOUS & RESIN & 252 \\
\hline \begin{tabular}{|l|}
4362 \\
\end{tabular} & $\mathrm{pCi} / \mathrm{L}$ & 11.9 & 18.8 & & & & & RIVER & & CUMULATIVE & UNFILTERED & 252 \\
\hline \begin{tabular}{|l|}
4363 \\
\end{tabular} & $\mathrm{pCi} / \mathrm{L}$ & 0.026 & 0.0537 & & & & & RIVER & & CUMULATIVE & UNFILTERED & 252 \\
\hline \begin{tabular}{|l|l|}
4364 \\
\end{tabular} & $\mathrm{pCi} / \mathrm{L}$ & 0.645 & 0.648 & & & $U$ & & RIVER & & CUMULATIVE & UNFILTERED & 252 \\
\hline \begin{tabular}{|l|}
4365 \\
\end{tabular} & $\mathrm{pCi} / \mathrm{L}$ & 0.0047 & & & & & & RIVER & & CONTINUOUS & FILTER & 252 \\
\hline \begin{tabular}{|l|l|}
4366 \\
\end{tabular} & $\mathrm{pCi} / \mathrm{L}$ & 0.451 & 0.451 & & & & & RIVER & & CUMULATIVE & UNFILTERED & 463 \\
\hline \begin{tabular}{|l|l|}
4367 \\
\end{tabular} & $\mathrm{pCi} / \mathrm{L}$ & 0.0221 & 0.0414 & & & & & RIVER & & CUMULATIVE & UNFILTERED & 463 \\
\hline \begin{tabular}{|l|}
4368 \\
\end{tabular} & $\mathrm{pCi} / \mathrm{L}$ & 0.199 & 0.822 & & & & & SURFACE & & RAW GRAB & UNFILTERED & 509 \\
\hline \begin{tabular}{|l|l|}
4369 \\
\end{tabular} & $\mathrm{pCi} / \mathrm{L}$ & 1.46 & 1.46 & & & & & SURFACE & & RAW GRAB & UNFILTERED & 509 \\
\hline
\end{tabular}




\begin{tabular}{|c|c|c|c|c|c|c|c|c|c|c|c|c|}
\hline & $\mathrm{N}$ & $\mathrm{O}$ & $\mathrm{P}$ & $\mathrm{Q}$ & $\mathrm{R}$ & $\mathrm{s}$ & $T$ & $\mathrm{U}$ & $\mathrm{V}$ & $\mathrm{W}$ & $x$ & $\bar{Y}$ \\
\hline 1 & ANAL_UNITS_RPTD & COUNTING_ERROR & ||$_{\text {TOTAL_ANAL_ERROR | }}$ & MIN_DETECTABLE_ACTIVITY & PCNT_MOISTURE & LAB_QUALIFIER & REVIEW_QUALIFIER & SAMP_FROM & SAMP_ITEM & SAMP_MTHD & COLL_MTHD & SAMP_SITE_ID \\
\hline 4370 & pCi/L & 195 & 269 & & & & & SURFACE & & RAW GRAB & UNFILTERED & 509 \\
\hline 4371 & $\mathrm{pCi} / \mathrm{L}$ & 426 & 1210 & & & & & SURFACE & & RAW GRAB & UNFILTERED & 136 \\
\hline 4372 & $\mathrm{pCi} / \mathrm{L}$ & 1.51 & 1.51 & & & 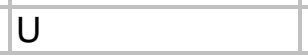 & & SURFACE & & RAW GRAB & UNFILTERED & 136 \\
\hline 4373 & $\mathrm{pCi} / \mathrm{L}$ & 1.56 & 1.61 & & & & & SURFACE & & RAW GRAB & UNFILTERED & 146 \\
\hline 4374 & $\mathrm{pCi} / \mathrm{L}$ & 0.139 & 0.904 & & & & & SURFACE & & RAW GRAB & UNFILTERED & 146 \\
\hline 4375 & $\mathrm{pCi} / \mathrm{L}$ & 177 & 231 & & & U & & SURFACE & & RAW GRAB & UNFILTERED & 146 \\
\hline 4376 & $\mathrm{pCi} / \mathrm{L}$ & 184 & 245 & & & & & SURFACE & & RAW GRAB & UNFILTERED & 147 \\
\hline 4377 & $\mathrm{pCi} / \mathrm{L}$ & 2.44 & 2.44 & & & U & & SURFACE & & RAW GRAB & UNFILTERED & 147 \\
\hline 4378 & $\mathrm{pCi} / \mathrm{L}$ & 0.113 & 0.255 & & & & & SURFACE & & RAW GRAB & UNFILTERED & 147 \\
\hline 4379 & $\mathrm{pCi} / \mathrm{L}$ & 0.0486 & 0.0672 & & & & & DRINKING & & SAN. GRAB & UNFILTERED & 512 \\
\hline 4380 & $\mathrm{pCi} / \mathrm{L}$ & 409 & 1100 & & & & & DRINKING & & SAN. GRAB & UNFILTERED & 512 \\
\hline 4381 & $\mathrm{pCi} / \mathrm{L}$ & 0.433 & 0.434 & & & & & DRINKING & & SAN. GRAB & UNFILTERED & 512 \\
\hline 4382 & $\mathrm{pCi} / \mathrm{L}$ & 0.0454 & 0.052 & & & & & DRINKING & & SAN. GRAB & UNFILTERED & 487 \\
\hline 4383 & $\mathrm{pCi} / \mathrm{L}$ & 0.597 & 0.597 & & & $U$ & & DRINKING & & SAN. GRAB & UNFILTERED & 487 \\
\hline 4384 & $\mathrm{pCi} / \mathrm{L}$ & 167 & 211 & & & $U$ & & DRINKING & & SAN. GRAB & UNFILTERED & 487 \\
\hline 4385 & $\mathrm{pCi} / \mathrm{L}$ & 0.519 & 0.521 & & & & & DRINKING & & SAN. GRAB & UNFILTERED & 530 \\
\hline 4386 & $\mathrm{pCi} / \mathrm{L}$ & 172 & 221 & & & $U$ & & DRINKING & & SAN. GRAB & UNFILTERED & 530 \\
\hline 4387 & $\mathrm{pCi} / \mathrm{L}$ & 0.0836 & 0.225 & & & & & DRINKING & & SAN. GRAB & UNFILTERED & 530 \\
\hline 4388 & $\mathrm{pCi} / \mathrm{L}$ & 0.0057 & & & & & & RIVER & & CONTINUOUS & RESIN & 307 \\
\hline 4389 & $\mathrm{pCi} / \mathrm{L}$ & 0.0028 & & & & & & RIVER & & CONTINUOUS & FILTER & 307 \\
\hline 4390 & $\mathrm{pCi} / \mathrm{L}$ & 0.000296 & & & & U & & RIVER & & CONTINUOUS & RESIN & 252 \\
\hline 4391 & $\mathrm{pCi} / \mathrm{L}$ & 0.0044 & & & & & & RIVER & & CONTINUOUS & RESIN & 252 \\
\hline 4392 & $\mathrm{pCi} / \mathrm{L}$ & 0.0066 & & & & & & RIVER & & CONTINUOUS & RESIN & 252 \\
\hline 4393 & $\mathrm{pCi} / \mathrm{L}$ & 0.0000088 & & & & & & RIVER & & CONTINUOUS & FILTER & 252 \\
\hline 4394 & $\mathrm{pCi} / \mathrm{L}$ & 0.0035 & & & & & & RIVER & & CONTINUOUS & FILTER & 252 \\
\hline 4395 & $\mathrm{pCi} / \mathrm{L}$ & 168 & 214 & & & $U$ & & DRINKING & & CUMULATIVE & UNFILTERED & 307 \\
\hline 4396 & $\mathrm{pCi} / \mathrm{L}$ & 0.0255 & 0.0493 & & & & & DRINKING & & CUMULATIVE & UNFILTERED & 307 \\
\hline 4397 & $\mathrm{pCi} / \mathrm{L}$ & 0.703 & 0.704 & & & $U$ & & DRINKING & & CUMULATIVE & UNFILTERED & 307 \\
\hline 4398 & $\mathrm{pCi} / \mathrm{L}$ & 0.003 & & & & & & RIVER & & CONTINUOUS & FILTER & 307 \\
\hline 4399 & $\mathrm{pCi} / \mathrm{L}$ & 0.0000166 & & & & $U$ & & RIVER & & CONTINUOUS & FILTER & 307 \\
\hline 4400 & $\mathrm{pCi} / \mathrm{L}$ & & $7.2964 \mathrm{E}-06$ & & & & & RIVER & & CONTINUOUS & RESIN & 307 \\
\hline 4401 & $\mathrm{pCi} / \mathrm{L}$ & 0.00038 & & & & & & RIVER & & CONTINUOUS & RESIN & 307 \\
\hline 4402 & $\mathrm{pCi} / \mathrm{L}$ & 0.0038 & & & & & & RIVER & & CONTINUOUS & RESIN & 307 \\
\hline 4403 & $\mathrm{pCi} / \mathrm{L}$ & 0.0062 & & & & & & RIVER & & CONTINUOUS & RESIN & 307 \\
\hline 4404 & & & & & & & & RIVER & & CONTINUOUS & FILTER & 252 \\
\hline 4405 & pCi/L & & $1.4418 \mathrm{E}-06$ & & & & & RIVER & & CONTINUOUS & RESIN & 252 \\
\hline 4406 & & & & & & & & RIVER & & CONTINUOUS & RESIN & 252 \\
\hline 4407 & $\mathrm{pCi} / \mathrm{L}$ & 0.651 & 0.651 & & & $U$ & & DRINKING & & RAW GRAB & UNFILTERED & 553 \\
\hline 4408 & $\mathrm{pCi} / \mathrm{L}$ & 0.0658 & 0.0841 & & & & & DRINKING & & RAW GRAB & UNFILTERED & 553 \\
\hline 4409 & $\mathrm{pCi} / \mathrm{L}$ & 225 & 287 & & & & & DRINKING & & RAW GRAB & UNFILTERED & 553 \\
\hline 4410 & $\mathrm{pCi} / \mathrm{L}$ & 13.3 & 20.3 & & & & & RIVER & & CUMULATIVE & UNFILTERED & 252 \\
\hline 4411 & $\mathrm{pCi} / \mathrm{L}$ & 0.0217 & 0.0433 & & & & & RIVER & & CUMULATIVE & UNFILTERED & 252 \\
\hline 4412 & $\mathrm{pCi} / \mathrm{L}$ & 0.716 & 0.717 & & & U & & RIVER & & CUMULATIVE & UNFILTERED & 252 \\
\hline 4413 & $\mathrm{pCi} / \mathrm{L}$ & 14.3 & 23.3 & & & & & RIVER & & CUMULATIVE & UNFILTERED & 463 \\
\hline 4414 & $\mathrm{pCi} / \mathrm{L}$ & 0.646 & 0.647 & & & U & & RIVER & & CUMULATIVE & UNFILTERED & 463 \\
\hline 4415 & $\mathrm{pCi} / \mathrm{L}$ & 0.025 & 0.0575 & & & & & RIVER & & CUMULATIVE & UNFILTERED & 463 \\
\hline 4416 & $\mathrm{pCi} / \mathrm{L}$ & 0.0184 & 0.0237 & & & & & QC_SAMPLE & & RAW GRAB & UNFILTERED & 550 \\
\hline 4417 & $\mathrm{pCi} / \mathrm{L}$ & 0.368 & 0.369 & & & & & QC_SAMPLE & & RAW GRAB & UNFILTERED & 550 \\
\hline 4418 & pCi/L & 16.1 & 28.9 & & & & & QC_SAMPLE & & RAW GRAB & UNFILTERED & 550 \\
\hline 4419 & pCi/L & 12.9 & 19.6 & & & & & QC_SAMPLE & & RAW GRAB & UNFILTERED & 247 \\
\hline 4420 & $\mathrm{pCi} / \mathrm{L}$ & 1.04 & 1.04 & & & $U$ & & QC_SAMPLE & & RAW GRAB & UNFILTERED & 247 \\
\hline \begin{tabular}{|l|l|}
4421 \\
\end{tabular} & $\mathrm{pCi} / \mathrm{L}$ & 0.0295 & 0.0625 & & & & & QC_SAMPLE & & RAW GRAB & UNFILTERED & 247 \\
\hline
\end{tabular}




\begin{tabular}{|c|c|c|c|c|c|c|c|c|c|c|c|c|}
\hline & $\mathrm{N}$ & $\mathrm{O}$ & $\mathrm{P}$ & $\mathrm{Q}$ & $\mathrm{R}$ & $\mathrm{s}$ & $T$ & $\mathrm{U}$ & $\mathrm{V}$ & $\mathrm{W}$ & $x$ & $\bar{Y}$ \\
\hline 1 & ANAL_UNITS_RPTD & COUNTING_ERROR & ||$_{\text {TOTAL_ANAL_ERROR | }}$ & MIN_DETECTABLE_ACTIVITY & PCNT_MOISTURE & LAB_QUALIFIER & REVIEW_QUALIFIER & SAMP_FROM & SAMP_ITEM & SAMP_MTHD & COLL_MTHD & SAMP_SITE_ID \\
\hline 4422 & $\mathrm{pCi} / \mathrm{L}$ & 0.0051 & & & & & & RIVER & & CONTINUOUS & RESIN & 307 \\
\hline 4423 & $\mathrm{pCi} / \mathrm{L}$ & 0.0028 & & & & & & RIVER & & CONTINUOUS & FILTER & 307 \\
\hline 4424 & $\mathrm{pCi} / \mathrm{L}$ & 0.004 & & & & & & RIVER & & CONTINUOUS & FILTER & 252 \\
\hline 4425 & $\mathrm{pCi} / \mathrm{L}$ & 0.008 & & & & & & RIVER & & CONTINUOUS & RESIN & 252 \\
\hline 4426 & $\mathrm{pCi} / \mathrm{L}$ & 0.0039 & & & & & & RIVER & & CONTINUOUS & RESIN & 307 \\
\hline 4427 & $\mathrm{pCi} / \mathrm{L}$ & 0.0019 & & & & & & RIVER & & CONTINUOUS & FILTER & 307 \\
\hline 4428 & & & & & & & & RIVER & & CONTINUOUS & FILTER & 252 \\
\hline 4429 & & & & & & & & RIVER & & CONTINUOUS & RESIN & 252 \\
\hline 4430 & $\mathrm{pCi} / \mathrm{L}$ & 11.2 & 17.3 & & & & & RIVER & & CUMULATIVE & UNFILTERED & 252 \\
\hline 4431 & $\mathrm{pCi} / \mathrm{L}$ & 0.0456 & 0.0697 & & & & & RIVER & & CUMULATIVE & UNFILTERED & 252 \\
\hline 4432 & $\mathrm{pCi} / \mathrm{L}$ & 1.32 & 1.32 & & & U & & RIVER & & CUMULATIVE & UNFILTERED & 252 \\
\hline 4433 & $\mathrm{pCi} / \mathrm{L}$ & 13.5 & 23.6 & & & & & RIVER & & CUMULATIVE & UNFILTERED & 463 \\
\hline 4434 & $\mathrm{pCi} / \mathrm{L}$ & 0.779 & 0.779 & & & U & & RIVER & & CUMULATIVE & UNFILTERED & 463 \\
\hline 4435 & $\mathrm{pCi} / \mathrm{L}$ & 0.0374 & 0.0637 & & & & & RIVER & & CUMULATIVE & UNFILTERED & 463 \\
\hline 4436 & $\mathrm{pCi} / \mathrm{L}$ & 0.0061 & & & & & & RIVER & & CONTINUOUS & RESIN & 307 \\
\hline \begin{tabular}{|l|l|}
4437 \\
\end{tabular} & $\mathrm{pCi} / \mathrm{L}$ & 0.0034 & & & & & & RIVER & & CONTINUOUS & FILTER & 307 \\
\hline 4438 & & & & & & & & RIVER & & CONTINUOUS & RESIN & 252 \\
\hline 4439 & & & & & & & & RIVER & & CONTINUOUS & FILTER & 252 \\
\hline 4440 & $\mathrm{pCi} / \mathrm{L}$ & 0.0424 & 0.0429 & & & U & & DRINKING & & SAN. GRAB & UNFILTERED & 186 \\
\hline \begin{tabular}{|l|l|}
4441 \\
\end{tabular} & $\mathrm{pCi} / \mathrm{L}$ & 0.779 & 0.779 & & & U & & DRINKING & & SAN. GRAB & UNFILTERED & 186 \\
\hline \begin{tabular}{|l|l|}
4442 \\
\end{tabular} & $\mathrm{pCi} / \mathrm{L}$ & 215 & 269 & & & U & & DRINKING & & SAN. GRAB & UNFILTERED & 186 \\
\hline 4443 & $\mathrm{pCi} / \mathrm{L}$ & 0.0423 & 0.0431 & & & U & & DRINKING & & SAN. GRAB & UNFILTERED & 144 \\
\hline 4444 & $\mathrm{pCi} / \mathrm{L}$ & 210 & 261 & & & U & & DRINKING & & SAN. GRAB & UNFILTERED & 144 \\
\hline 4445 & $\mathrm{pCi} / \mathrm{L}$ & 0.888 & 0.888 & & & U & & DRINKING & & SAN. GRAB & UNFILTERED & 144 \\
\hline 4446 & $\mathrm{pCi} / \mathrm{L}$ & 0.577 & 0.579 & & & & & DRINKING & & SAN. GRAB & UNFILTERED & 187 \\
\hline 4447 & $\mathrm{pCi} / \mathrm{L}$ & 0.038 & 0.0388 & & & U & & DRINKING & & SAN. GRAB & UNFILTERED & 187 \\
\hline 4448 & $\mathrm{pCi} / \mathrm{L}$ & 213 & 266 & & & U & & DRINKING & & SAN. GRAB & UNFILTERED & 187 \\
\hline 4449 & $\mathrm{pCi} / \mathrm{L}$ & 0.004 & & & & & & RIVER & & CONTINUOUS & FILTER & 307 \\
\hline 4450 & $\mathrm{pCi} / \mathrm{L}$ & 0.0075 & & & & & & RIVER & & CONTINUOUS & RESIN & 307 \\
\hline 4451 & $\mathrm{pCi} / \mathrm{L}$ & 0.0044 & & & & & & RIVER & & CONTINUOUS & FILTER & 252 \\
\hline 4452 & $\mathrm{pCi} / \mathrm{L}$ & 0.0087 & & & & & & RIVER & & CONTINUOUS & RESIN & 252 \\
\hline 4453 & $\mathrm{pCi} / \mathrm{L}$ & 0.0933 & 0.158 & & & & & SURFACE & & RAW GRAB & UNFILTERED & 509 \\
\hline 4454 & $\mathrm{pCi} / \mathrm{L}$ & 0.991 & 0.994 & & & & & SURFACE & & RAW GRAB & UNFILTERED & 509 \\
\hline 4455 & $\mathrm{pCi} / \mathrm{L}$ & 220 & 279 & & & & & SURFACE & & RAW GRAB & UNFILTERED & 509 \\
\hline 4456 & $\mathrm{pCi} / \mathrm{L}$ & 517 & 1360 & & & & & SURFACE & & RAW GRAB & UNFILTERED & 136 \\
\hline 4457 & pCi/L & 0.818 & 0.819 & & & U & & SURFACE & & RAW GRAB & UNFILTERED & 136 \\
\hline 4458 & $\mathrm{pCi} / \mathrm{L}$ & 2.62 & 2.81 & & & & & SURFACE & & RAW GRAB & UNFILTERED & 146 \\
\hline 4459 & $\mathrm{pCi} / \mathrm{L}$ & 0.0649 & 0.13 & & & & & SURFACE & & RAW GRAB & UNFILTERED & 146 \\
\hline \begin{tabular}{|l|}
4460 \\
\end{tabular} & $\mathrm{pCi} / \mathrm{L}$ & 221 & 280 & & & & & SURFACE & & RAW GRAB & UNFILTERED & 146 \\
\hline \begin{tabular}{|l|}
4461 \\
\end{tabular} & $\mathrm{pCi} / \mathrm{L}$ & 227 & 293 & & & & & SURFACE & & RAW GRAB & UNFILTERED & 147 \\
\hline \begin{tabular}{|l|}
4462 \\
\end{tabular} & $\mathrm{pCi} / \mathrm{L}$ & 2.59 & 2.59 & & & U & & SURFACE & & RAW GRAB & UNFILTERED & 147 \\
\hline \begin{tabular}{|l|}
4463 \\
\end{tabular} & $\mathrm{pCi} / \mathrm{L}$ & 0.179 & 0.379 & & & & & SURFACE & & RAW GRAB & UNFILTERED & 147 \\
\hline 4464 & $\mathrm{pCi} / \mathrm{L}$ & 0.0259 & 0.0285 & & & & & DRINKING & & SAN. GRAB & UNFILTERED & 512 \\
\hline 4465 & $\mathrm{pCi} / \mathrm{L}$ & 494 & 1240 & & & & & DRINKING & & SAN. GRAB & UNFILTERED & 512 \\
\hline \begin{tabular}{|l|}
4466 \\
\end{tabular} & $\mathrm{pCi} / \mathrm{L}$ & 0.623 & 0.623 & & & U & & DRINKING & & SAN. GRAB & UNFILTERED & 512 \\
\hline \begin{tabular}{|l|l|}
4467 \\
\end{tabular} & $\mathrm{pCi} / \mathrm{L}$ & 0.804 & 0.805 & & & U & & DRINKING & & RAW GRAB & UNFILTERED & 553 \\
\hline \begin{tabular}{|l|}
4468 \\
\end{tabular} & $\mathrm{pCi} / \mathrm{L}$ & 0.0311 & 0.0398 & & & & & DRINKING & & RAW GRAB & UNFILTERED & 553 \\
\hline \begin{tabular}{|l|}
4469 \\
\end{tabular} & $\mathrm{pCi} / \mathrm{L}$ & 212 & 264 & & & U & & DRINKING & & RAW GRAB & UNFILTERED & 553 \\
\hline 4470 & pCi/L & 217 & 273 & & & U & & DRINKING & & SAN. GRAB & UNFILTERED & 530 \\
\hline \begin{tabular}{|l|l|}
4471 \\
\end{tabular} & pCi/L & 0.0249 & 0.0254 & & & U & & DRINKING & & SAN. GRAB & UNFILTERED & 487 \\
\hline \begin{tabular}{|l|}
4472 \\
\end{tabular} & $\mathrm{pCi} / \mathrm{L}$ & 0.712 & 0.712 & & & U & & DRINKING & & SAN. GRAB & UNFILTERED & 487 \\
\hline 4473 & $\mathrm{pCi} / \mathrm{L}$ & 211 & 263 & & & & & DRINKING & & SAN. GRAB & UNFILTERED & 487 \\
\hline
\end{tabular}




\begin{tabular}{|c|c|c|c|c|c|c|c|c|c|c|c|c|}
\hline & $\mathrm{N}$ & $\mathrm{O}$ & $\mathrm{P}$ & $\mathrm{Q}$ & $\mathrm{R}$ & $\mathrm{s}$ & $T$ & $U$ & $\mathrm{~V}$ & $\mathrm{~W}$ & $\mathrm{x}$ & $\bar{Y}$ \\
\hline 1 & ANAL_UNITS_RPTD & COUNTING_ERROR & $\mid$ TOTAL_ANAL_ERROR | $^{\prime}$ & MIN_DETECTABLE_ACTIVITY & PCNT_MOISTURE & LAB_QUALIFIER & REVIEW_QUALIFIER & SAMP_FROM & SAMP_ITEM & SAMP_MTHD & COLL_MTHD & SAMP_SITE_ID \\
\hline 4474 & $\mathrm{pCi} / \mathrm{L}$ & 12 & 19.6 & & & & & RIVER & & CUMULATIVE & UNFILTERED & 252 \\
\hline 4475 & $\mathrm{pCi} / \mathrm{L}$ & 0.0251 & 0.033 & & & & & RIVER & & CUMULATIVE & UNFILTERED & 252 \\
\hline 4476 & $\mathrm{pCi} / \mathrm{L}$ & 0.776 & 0.777 & & & $\mathrm{U}$ & & RIVER & & CUMULATIVE & UNFILTERED & 252 \\
\hline 4447 & $\mathrm{pCi} / \mathrm{L}$ & 12 & 20 & & & & & RIVER & & CUMULATIVE & UNFILTERED & 463 \\
\hline 4478 & $\mathrm{pCi} / \mathrm{L}$ & 0.779 & 0.779 & & & $\mathrm{U}$ & & RIVER & & CUMULATIVE & UNFILTERED & 463 \\
\hline 4479 & $\mathrm{pCi} / \mathrm{L}$ & 0.0499 & 0.0725 & & & & & RIVER & & CUMULATIVE & UNFILTERED & 463 \\
\hline 4480 & $\mathrm{pCi} / \mathrm{L}$ & 0.0753 & 0.171 & & & & & DRINKING & & SAN. GRAB & UNFILTERED & 530 \\
\hline 4481 & $\mathrm{pCi} / \mathrm{L}$ & 0.0071 & & & & & & RIVER & & CONTINUOUS & RESIN & 307 \\
\hline 4482 & $\mathrm{pCi} / \mathrm{L}$ & 0.004 & & & & & & RIVER & & CONTINUOUS & FILTER & 307 \\
\hline 4483 & $\mathrm{pCi} / \mathrm{L}$ & 0.0075 & & & & & & RIVER & & CONTINUOUS & RESIN & 252 \\
\hline 4484 & $\mathrm{pCi} / \mathrm{L}$ & 0.0037 & & & & & & RIVER & & CONTINUOUS & FILTER & 252 \\
\hline 4485 & $\mathrm{pCi} / \mathrm{L}$ & 0.0062 & & & & & & RIVER & & CONTINUOUS & RESIN & 307 \\
\hline 4486 & $\mathrm{pCi} / \mathrm{L}$ & 0.003 & & & & & & RIVER & & CONTINUOUS & FILTER & 307 \\
\hline \begin{tabular}{|l|}
4487 \\
\end{tabular} & $\mathrm{pCi} / \mathrm{L}$ & 0.005 & & & & & & RIVER & & CONTINUOUS & FILTER & 252 \\
\hline \begin{tabular}{|l|}
4488 \\
\end{tabular} & $\mathrm{pCi} / \mathrm{L}$ & 0.0091 & & & & & & RIVER & & CONTINUOUS & RESIN & 252 \\
\hline \begin{tabular}{|l|}
4489 \\
\end{tabular} & $\mathrm{pCi} / \mathrm{L}$ & & $9.7272 \mathrm{E}-06$ & & & & & RIVER & & CONTINUOUS & RESIN & 307 \\
\hline 4490 & $\mathrm{pCi} / \mathrm{L}$ & 0.00018 & & & & $U$ & & RIVER & & CONTINUOUS & RESIN & 307 \\
\hline 4491 & $\mathrm{pCi} / \mathrm{L}$ & 0.003 & & & & & & RIVER & & CONTINUOUS & RESIN & 307 \\
\hline \begin{tabular}{|l|l|}
4492 \\
\end{tabular} & $\mathrm{pCi} / \mathrm{L}$ & 0.0062 & & & & & & RIVER & & CONTINUOUS & RESIN & 307 \\
\hline 4493 & $\mathrm{pCi} / \mathrm{L}$ & 0.0034 & & & & & & RIVER & & CONTINUOUS & FILTER & 307 \\
\hline 4494 & $\mathrm{pCi} / \mathrm{L}$ & 0.0000402 & & & & & & RIVER & & CONTINUOUS & FILTER & 307 \\
\hline 4495 & $\mathrm{pCi} / \mathrm{L}$ & 0.001216 & & & & $\mathrm{U}$ & & RIVER & & CONTINUOUS & RESIN & 252 \\
\hline 4496 & $\mathrm{pCi} / \mathrm{L}$ & 0.0048 & & & & & & RIVER & & CONTINUOUS & RESIN & 252 \\
\hline \begin{tabular}{|l|}
4497 \\
\end{tabular} & $\mathrm{pCi} / \mathrm{L}$ & 0.007 & & & & & & RIVER & & CONTINUOUS & RESIN & 252 \\
\hline 4498 & $\mathrm{pCi} / \mathrm{L}$ & 10.9 & 17.8 & & & & & RIVER & & CUMULATIVE & UNFILTERED & 252 \\
\hline \begin{tabular}{|l|l}
4499 \\
\end{tabular} & $\mathrm{pCi} / \mathrm{L}$ & 0.0000244 & & & & $U$ & & RIVER & & CONTINUOUS & FILTER & 252 \\
\hline 4500 & $\mathrm{pCi} / \mathrm{L}$ & 0.0039 & & & & & & RIVER & & CONTINUOUS & FILTER & 252 \\
\hline 4501 & $\mathrm{pCi} / \mathrm{L}$ & 0.0833 & 0.096 & & & & & RIVER & & CUMULATIVE & UNFILTERED & 252 \\
\hline 4502 & $\mathrm{pCi} / \mathrm{L}$ & 0.735 & 0.736 & & & $U$ & & RIVER & & CUMULATIVE & UNFILTERED & 252 \\
\hline 4503 & $\mathrm{pCi} / \mathrm{L}$ & 12.4 & 21.4 & & & & & RIVER & & CUMULATIVE & UNFILTERED & 463 \\
\hline 4504 & $\mathrm{pCi} / \mathrm{L}$ & 0.506 & 0.507 & & & $U$ & & RIVER & & CUMULATIVE & UNFILTERED & 463 \\
\hline 4505 & $\mathrm{pCi} / \mathrm{L}$ & 0.0622 & 0.0715 & & & & & RIVER & & CUMULATIVE & UNFILTERED & 463 \\
\hline 4506 & $\mathrm{pCi} / \mathrm{L}$ & 217 & 272 & & & & & DRINKING & & CUMULATIVE & UNFILTERED & 307 \\
\hline \begin{tabular}{|l|}
4507 \\
\end{tabular} & $\mathrm{pCi} / \mathrm{L}$ & 0.0352 & 0.0541 & & & & & DRINKING & & CUMULATIVE & UNFILTERED & 307 \\
\hline 4508 & $\mathrm{pCi} / \mathrm{L}$ & 0.533 & 0.533 & & & $\mathrm{U}$ & & DRINKING & & CUMULATIVE & UNFILTERED & 307 \\
\hline \begin{tabular}{|l|}
4509 \\
\end{tabular} & $\mathrm{pCi} / \mathrm{L}$ & 0.0036 & & & & & & RIVER & & CONTINUOUS & FILTER & 307 \\
\hline 4510 & $\mathrm{pCi} / \mathrm{L}$ & 0.0074 & & & & & & RIVER & & CONTINUOUS & RESIN & 307 \\
\hline 4511 & $\mathrm{pCi} / \mathrm{L}$ & 0.0065 & & & & & & RIVER & & CONTINUOUS & RESIN & 252 \\
\hline 4512 & $\mathrm{pCi} / \mathrm{L}$ & 0.0034 & & & & & & RIVER & & CONTINUOUS & FILTER & 252 \\
\hline 4513 & $\mathrm{pCi} / \mathrm{L}$ & & $1.0824 \mathrm{E}-06$ & & & & & RIVER & & CONTINUOUS & RESIN & 252 \\
\hline \begin{tabular}{|l|l|}
4514 \\
\end{tabular} & $\mathrm{pCi} / \mathrm{L}$ & 0.0069 & & & & & & RIVER & & CONTINUOUS & RESIN & 307 \\
\hline \begin{tabular}{|l|}
4515 \\
\end{tabular} & $\mathrm{pCi} / \mathrm{L}$ & 0.0034 & & & & & & RIVER & & CONTINUOUS & FILTER & 307 \\
\hline 4516 & $\mathrm{pCi} / \mathrm{L}$ & 0.0071 & & & & & & RIVER & & CONTINUOUS & RESIN & 252 \\
\hline 4517 & $\mathrm{pCi} / \mathrm{L}$ & 0.0038 & & & & & & RIVER & & CONTINUOUS & FILTER & 252 \\
\hline 4518 & $\mathrm{pCi} / \mathrm{L}$ & 10.9 & 17.5 & & & & & RIVER & & CUMULATIVE & UNFILTERED & 252 \\
\hline 4519 & $\mathrm{pCi} / \mathrm{L}$ & 0.792 & 0.797 & & & & & RIVER & & CUMULATIVE & UNFILTERED & 252 \\
\hline 4520 & $\mathrm{pCi} / \mathrm{L}$ & 0.0503 & 0.0611 & & & & & RIVER & & CUMULATIVE & UNFILTERED & 252 \\
\hline \begin{tabular}{|l|}
4521 \\
\end{tabular} & $\mathrm{pCi} / \mathrm{L}$ & 12 & 21.9 & & & & & RIVER & & CUMULATIVE & UNFILTERED & 463 \\
\hline 4522 & $\mathrm{pCi} / \mathrm{L}$ & 0.0534 & 0.0663 & & & & & RIVER & & CUMULATIVE & UNFILTERED & 463 \\
\hline 4523 & $\mathrm{pCi} / \mathrm{L}$ & 1.39 & 1.4 & & & $U$ & & RIVER & & CUMULATIVE & UNFILTERED & 463 \\
\hline 4524 & $\mathrm{pCi} / \mathrm{L}$ & 0.643 & 0.646 & & & & & DRINKING & & SAN. GRAB & UNFILTERED & 186 \\
\hline 4525 & $\mathrm{pCi} / \mathrm{L}$ & 214 & 268 & & & U & & DRINKING & & SAN. GRAB & UNFILTERED & 186 \\
\hline
\end{tabular}




\begin{tabular}{|c|c|c|c|c|c|c|c|c|c|c|c|c|}
\hline & $\mathrm{N}$ & $\mathrm{O}$ & $\mathrm{P}$ & $\mathrm{Q}$ & $\mathrm{R}$ & $\mathrm{s}$ & $T$ & $\mathrm{U}$ & $\mathrm{V}$ & $\mathrm{W}$ & $\mathrm{X}$ & $\bar{Y}$ \\
\hline 1 & ANAL_UNITS_RPTD & COUNTING_ERROR & TOTAL_ANAL_ERROR & MIN_DETECTABLE_ACTIVITY & PCNT_MOISTURE & LAB_QUALIFIER & REVIEW_QUALIFIER & SAMP_FROM & SAMP_ITEM & SAMP_MTHD & COLL_MTHD & SAMP_SITE_ID \\
\hline 4526 & $\mathrm{pCi} / \mathrm{L}$ & 0.0373 & 0.0504 & & & & & DRINKING & & SAN. GRAB & UNFILTERED & 186 \\
\hline \begin{tabular}{|l|l|}
4527 \\
\end{tabular} & $\mathrm{pCi} / \mathrm{L}$ & 215 & 270 & & & U & & DRINKING & & SAN. GRAB & UNFILTERED & 144 \\
\hline 4528 & $\mathrm{pCi} / \mathrm{L}$ & 0.0702 & 0.0714 & & & U & & DRINKING & & SAN. GRAB & UNFILTERED & 144 \\
\hline 4529 & $\mathrm{pCi} / \mathrm{L}$ & 0.816 & 0.822 & & & & & DRINKING & & SAN. GRAB & UNFILTERED & 144 \\
\hline 4530 & $\mathrm{pCi} / \mathrm{L}$ & 0.0059 & & & & & & RIVER & & CONTINUOUS & RESIN & 307 \\
\hline 4531 & $\mathrm{pCi} / \mathrm{L}$ & 0.0029 & & & & & & RIVER & & CONTINUOUS & FILTER & 307 \\
\hline 4532 & $\mathrm{pCi} / \mathrm{L}$ & 0.007 & & & & & & RIVER & & CONTINUOUS & RESIN & 252 \\
\hline 4533 & $\mathrm{pCi} / \mathrm{L}$ & 0.0035 & & & & & & RIVER & & CONTINUOUS & FILTER & 252 \\
\hline 4534 & $\mathrm{pCi} / \mathrm{L}$ & 1.36 & 1.36 & & & $\mathrm{U}$ & & SURFACE & & RAW GRAB & UNFILTERED & 509 \\
\hline 4535 & $\mathrm{pCi} / \mathrm{L}$ & 0.0744 & 0.115 & & & & & SURFACE & & RAW GRAB & UNFILTERED & 509 \\
\hline 4536 & $\mathrm{pCi} / \mathrm{L}$ & 216 & 272 & & & $U$ & & SURFACE & & RAW GRAB & UNFILTERED & 509 \\
\hline \begin{tabular}{|l|}
4537 \\
\end{tabular} & $\mathrm{pCi} / \mathrm{L}$ & 557 & 1570 & & & & & SURFACE & & RAW GRAB & UNFILTERED & 136 \\
\hline 4538 & $\mathrm{pCi} / \mathrm{L}$ & 1.61 & 1.61 & & & $U$ & & SURFACE & & RAW GRAB & UNFILTERED & 136 \\
\hline \begin{tabular}{|l|}
4539 \\
\end{tabular} & $\mathrm{pCi} / \mathrm{L}$ & 2.04 & 2.1 & & & & & SURFACE & & RAW GRAB & UNFILTERED & 146 \\
\hline \begin{tabular}{|l|}
4540 \\
\end{tabular} & $\mathrm{pCi} / \mathrm{L}$ & 216 & 271 & & & $U$ & & SURFACE & & RAW GRAB & UNFILTERED & 146 \\
\hline \begin{tabular}{|l|}
4541 \\
\end{tabular} & $\mathrm{pCi} / \mathrm{L}$ & 0.126 & 0.488 & & & & & SURFACE & & RAW GRAB & UNFILTERED & 146 \\
\hline 4542 & $\mathrm{pCi} / \mathrm{L}$ & 227 & 293 & & & & & SURFACE & & RAW GRAB & UNFILTERED & 147 \\
\hline \begin{tabular}{|l|}
4543 \\
\end{tabular} & $\mathrm{pCi} / \mathrm{L}$ & 2.27 & 2.28 & & & & & SURFACE & & RAW GRAB & UNFILTERED & 147 \\
\hline 4544 & $\mathrm{pCi} / \mathrm{L}$ & 0.202 & 0.556 & & & & & SURFACE & & RAW GRAB & UNFILTERED & 147 \\
\hline 4545 & $\mathrm{pCi} / \mathrm{L}$ & 505 & 1300 & & & & & DRINKING & & SAN. GRAB & UNFILTERED & 512 \\
\hline \begin{tabular}{|l|}
4546 \\
\end{tabular} & $\mathrm{pCi} / \mathrm{L}$ & 0.0413 & 0.0487 & & & & & DRINKING & & SAN. GRAB & UNFILTERED & 512 \\
\hline \begin{tabular}{|l|}
4547 \\
\end{tabular} & $\mathrm{pCi} / \mathrm{L}$ & 0.738 & 0.738 & & & $U$ & & DRINKING & & SAN. GRAB & UNFILTERED & 512 \\
\hline \begin{tabular}{|l|}
4548 \\
\end{tabular} & $\mathrm{pCi} / \mathrm{L}$ & 1.02 & 1.02 & & & $U$ & & DRINKING & & RAW GRAB & UNFILTERED & 553 \\
\hline \begin{tabular}{|l|}
4549 \\
\end{tabular} & $\mathrm{pCi} / \mathrm{L}$ & 219 & 277 & & & & & DRINKING & & RAW GRAB & UNFILTERED & 553 \\
\hline 4550 & $\mathrm{pCi} / \mathrm{L}$ & 0.0382 & 0.0512 & & & & & DRINKING & & RAW GRAB & UNFILTERED & 553 \\
\hline \begin{tabular}{|l|}
4551 \\
\end{tabular} & $\mathrm{pCi} / \mathrm{L}$ & 0.763 & 0.763 & & & $U$ & & DRINKING & & SAN. GRAB & UNFILTERED & 487 \\
\hline \begin{tabular}{|l|}
4552 \\
\end{tabular} & $\mathrm{pCi} / \mathrm{L}$ & 213 & 265 & & & U & & DRINKING & & SAN. GRAB & UNFILTERED & 487 \\
\hline \begin{tabular}{|l|}
4553 \\
\end{tabular} & $\mathrm{pCi} / \mathrm{L}$ & 0.0309 & 0.0348 & & & & & DRINKING & & SAN. GRAB & UNFILTERED & 487 \\
\hline \begin{tabular}{|l|l|}
4554 \\
\end{tabular} & $\mathrm{pCi} / \mathrm{L}$ & 1.6 & 1.6 & & & U & & DRINKING & & SAN. GRAB & UNFILTERED & 187 \\
\hline \begin{tabular}{|l|}
4555 \\
\end{tabular} & $\mathrm{pCi} / \mathrm{L}$ & 218 & 273 & & & $U$ & & DRINKING & & SAN. GRAB & UNFILTERED & 187 \\
\hline \begin{tabular}{|l|}
45566 \\
\end{tabular} & $\mathrm{pCi} / \mathrm{L}$ & 0.0188 & 0.0196 & & & & & DRINKING & & SAN. GRAB & UNFILTERED & 187 \\
\hline \begin{tabular}{|l|}
4557 \\
\end{tabular} & $\mathrm{pCi} / \mathrm{L}$ & 0.0068 & & & & & & RIVER & & CONTINUOUS & RESIN & 307 \\
\hline \begin{tabular}{|l|}
45588 \\
\end{tabular} & $\mathrm{pCi} / \mathrm{L}$ & 0.0039 & & & & & & RIVER & & CONTINUOUS & FILTER & 307 \\
\hline \begin{tabular}{|l|}
4559 \\
\end{tabular} & $\mathrm{pCi} / \mathrm{L}$ & 0.0055 & & & & & & RIVER & & CONTINUOUS & RESIN & 252 \\
\hline 4560 & $\mathrm{pCi} / \mathrm{L}$ & 0.0026 & & & & & & RIVER & & CONTINUOUS & FILTER & 252 \\
\hline \begin{tabular}{|l|}
4561 \\
\end{tabular} & $\mathrm{pCi} / \mathrm{L}$ & 214 & 268 & & & $U$ & & DRINKING & & SAN. GRAB & UNFILTERED & 530 \\
\hline \begin{tabular}{|l|}
4562 \\
\end{tabular} & $\mathrm{pCi} / \mathrm{L}$ & 0.0722 & 0.16 & & & & & DRINKING & & SAN. GRAB & UNFILTERED & 530 \\
\hline 4563 & $\mathrm{pCi} / \mathrm{L}$ & 0.818 & 0.823 & & & & & DRINKING & & SAN. GRAB & UNFILTERED & 530 \\
\hline 4564 & $\mathrm{pCi} / \mathrm{L}$ & 0.726 & 0.727 & & & $U$ & & RIVER & & CUMULATIVE & UNFILTERED & 252 \\
\hline 4565 & $\mathrm{pCi} / \mathrm{L}$ & 0.0327 & 0.0517 & & & & & RIVER & & CUMULATIVE & UNFILTERED & 252 \\
\hline \begin{tabular}{|l|}
4566 \\
\end{tabular} & $\mathrm{pCi} / \mathrm{L}$ & 10.7 & 17.8 & & & & & RIVER & & CUMULATIVE & UNFILTERED & 252 \\
\hline \begin{tabular}{|l|l|}
4567 \\
\end{tabular} & $\mathrm{pCi} / \mathrm{L}$ & 0.0474 & 0.0557 & & & & & RIVER & & CUMULATIVE & UNFILTERED & 463 \\
\hline \begin{tabular}{|l|}
4568 \\
\end{tabular} & $\mathrm{pCi} / \mathrm{L}$ & 0.844 & 0.845 & & & $U$ & & RIVER & & CUMULATIVE & UNFILTERED & 463 \\
\hline 4569 & $\mathrm{pCi} / \mathrm{L}$ & 12.1 & 21.8 & & & & & RIVER & & CUMULATIVE & UNFILTERED & 463 \\
\hline 4570 & $\mathrm{pCi} / \mathrm{L}$ & 0.000048 & & & & & & RIVER & & CONTINUOUS & RESIN & 307 \\
\hline \begin{tabular}{|l|l|}
4571 & \\
\end{tabular} & $\mathrm{pCi} / \mathrm{L}$ & 0.0000094 & & & & & & RIVER & & CONTINUOUS & FILTER & 307 \\
\hline 4572 & $\mathrm{pCi} / \mathrm{L}$ & 0.0082 & & & & & & RIVER & & CONTINUOUS & RESIN & 307 \\
\hline \begin{tabular}{|l|}
4573 \\
\end{tabular} & $\mathrm{pCi} / \mathrm{L}$ & 0.0074 & & & & & & RIVER & & CONTINUOUS & RESIN & 307 \\
\hline \begin{tabular}{|l|l|}
4574 \\
\end{tabular} & $\mathrm{pCi} / \mathrm{L}$ & 0.0035 & & & & & & RIVER & & CONTINUOUS & FILTER & 307 \\
\hline \begin{tabular}{|l|}
4575 \\
\end{tabular} & $\mathrm{pCi} / \mathrm{L}$ & 0.002 & & & & & & RIVER & & CONTINUOUS & FILTER & 252 \\
\hline \begin{tabular}{|l|}
4576 \\
\end{tabular} & $\mathrm{pCi} / \mathrm{L}$ & 0.0039 & & & & & & RIVER & & CONTINUOUS & RESIN & 252 \\
\hline \begin{tabular}{|l|l|}
4577 \\
\end{tabular} & $\mathrm{pCi} / \mathrm{L}$ & & 0.000018812 & & & & & RIVER & & CONTINUOUS & RESIN & 307 \\
\hline
\end{tabular}




\begin{tabular}{|c|c|c|c|c|c|c|c|c|c|c|c|c|}
\hline & $\mathrm{N}$ & $\mathrm{O}$ & $\mathrm{P}$ & $\mathrm{Q}$ & $\mathrm{R}$ & $\mathrm{s}$ & $\mathrm{T}$ & $\mathrm{U}$ & $\mathrm{V}$ & $\mathrm{W}$ & $\mathrm{x}$ & $\mathrm{Y}$ \\
\hline 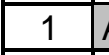 & ANAL_UNITS_RPTD & COUNTING_ERROR & 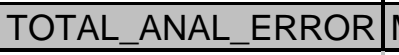 & MIN_DETECTABLE_ACTIVITY & PCNT_MOISTURE & LAB_QUALIFIER & REVIEW_QUALIFIER & SAMP_FROM & SAMP_ITEM & SAMP_MTHD & COLL_MTHD & SAMP_SITE_ID \\
\hline 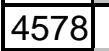 & & & & & & & & RIVER & & CONTINUOUS & RESIN & 307 \\
\hline 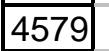 & & & & & & & & RIVER & & CONTINUOUS & FILTER & 307 \\
\hline 4580 & $\mathrm{pCi} / \mathrm{L}$ & & 0.000001188 & & & & & RIVER & & CONTINUOUS & RESIN & 252 \\
\hline $4581 \mathrm{r}$ & $\mathrm{pCi} / \mathrm{L}$ & 0.000176 & & & & & & RIVER & & CONTINUOUS & RESIN & 252 \\
\hline 4582 & $\mathrm{pCi} / \mathrm{L}$ & 0.0000134 & & & & & & RIVER & & CONTINUOUS & FILTER & 252 \\
\hline $4583 \mathrm{r}$ & $\mathrm{pCi} / \mathrm{L}$ & 0.0086 & & & & & & RIVER & & CONTINUOUS & RESIN & 252 \\
\hline 4584 r & $\mathrm{pCi} / \mathrm{L}$ & 0.0076 & & & & & & RIVER & & CONTINUOUS & RESIN & 252 \\
\hline $4585 \mid \mathrm{r}$ & $\mathrm{pCi} / \mathrm{L}$ & 0.004 & & & & & & RIVER & & CONTINUOUS & FILTER & 252 \\
\hline 4586 & $\mathrm{pCi} / \mathrm{L}$ & 0.808 & 0.81 & & & U & & IRRIGATION & & RAW GRAB & UNFILTERED & 440 \\
\hline 4587 & $\mathrm{pCi} / \mathrm{L}$ & 0.0398 & 0.0469 & & & & & IRRIGATION & & RAW GRAB & UNFILTERED & 440 \\
\hline $4588 \mathrm{r}$ & $\mathrm{pCi} / \mathrm{L}$ & 178 & 228 & & & $\mathrm{U}$ & & DRINKING & & CUMULATIVE & UNFILTERED & 307 \\
\hline 4589 & $\mathrm{pCi} / \mathrm{L}$ & 0.801 & 0.801 & & & $\mathrm{U}$ & & DRINKING & & CUMULATIVE & UNFILTERED & 307 \\
\hline $4590 \mathrm{r}$ & $\mathrm{pCi} / \mathrm{L}$ & 0.0478 & 0.0646 & & & & & DRINKING & & CUMULATIVE & UNFILTERED & 307 \\
\hline $4591 \mathrm{r}$ & $\mathrm{pCi} / \mathrm{L}$ & 0.055 & 0.0583 & & & & & RIVER & & CUMULATIVE & UNFILTERED & 252 \\
\hline $4592 \mathrm{r}$ & $\mathrm{pCi} / \mathrm{L}$ & 0.0152 & 0.0153 & & & & & RIVER & & CUMULATIVE & UNFILTERED & 252 \\
\hline 4593 & $\mathrm{pCi} / \mathrm{L}$ & 0.0483 & 0.0505 & & & & & RIVER & & CUMULATIVE & UNFILTERED & 252 \\
\hline 4594 & $\mathrm{pCi} / \mathrm{L}$ & 0.96 & 0.96 & & & $U$ & & RIVER & & CUMULATIVE & UNFILTERED & 252 \\
\hline 4595 & $\mathrm{pCi} / \mathrm{L}$ & 11.3 & 19.2 & & & & & RIVER & & CUMULATIVE & UNFILTERED & 252 \\
\hline $4596 \mathrm{r}$ & $p \mathrm{pi} / \mathrm{L}$ & 0.0316 & 0.0382 & & & & & RIVER & & CUMULATIVE & UNFILTERED & 252 \\
\hline $4597 \mathrm{r}$ & $\mathrm{pCi} / \mathrm{L}$ & 0.8 & 0.801 & & & $U$ & & RIVER & & CUMULATIVE & UNFILTERED & 463 \\
\hline $4598 \mathrm{r}$ & $\mathrm{pCi} / \mathrm{L}$ & 0.0419 & 0.0436 & & & & & RIVER & & CUMULATIVE & UNFILTERED & 463 \\
\hline 4599 & $\mathrm{pCi} / \mathrm{L}$ & 0.0199 & 0.0201 & & & & & RIVER & & CUMULATIVE & UNFILTERED & 463 \\
\hline 4600 & $\mathrm{pCi} / \mathrm{L}$ & 0.0498 & 0.0528 & & & & & RIVER & & CUMULATIVE & UNFILTERED & 463 \\
\hline 4601 & $\mathrm{pCi} / \mathrm{L}$ & 11.1 & 18.4 & & & & & RIVER & & CUMULATIVE & UNFILTERED & 463 \\
\hline $4602 \mathrm{r}$ & $\mathrm{pCi} / \mathrm{L}$ & 0.0374 & 0.049 & & & & & RIVER & & CUMULATIVE & UNFILTERED & 463 \\
\hline 4603 & $\mathrm{pCi} / \mathrm{L}$ & 0.0406 & 0.0479 & & & & & QC_SAMPLE & & RAW GRAB & UNFILTERED & 550 \\
\hline $4604 \mathrm{r}$ & $\mathrm{pCi} / \mathrm{L}$ & 0.746 & 0.746 & & & U & & QC_SAMPLE & & RAW GRAB & UNFILTERED & 550 \\
\hline 4605 & $\mathrm{pCi} / \mathrm{L}$ & 10.1 & 16.7 & & & & & QC_SAMPLE & & RAW GRAB & UNFILTERED & 550 \\
\hline $4606 \mathrm{r}$ & $\mathrm{pCi} / \mathrm{L}$ & 0.0368 & 0.0395 & & & & & QC_SAMPLE & & RAW GRAB & UNFILTERED & 247 \\
\hline 4607 & $\mathrm{pCi} / \mathrm{L}$ & 0.956 & 0.956 & & & $U$ & & QC_SAMPLE & & RAW GRAB & UNFILTERED & 247 \\
\hline 4608 & $\mathrm{pCi} / \mathrm{L}$ & 10.6 & 17.2 & & & & & QC_SAMPLE & & RAW GRAB & UNFILTERED & 247 \\
\hline $4609 \mathrm{r}$ & $p \mathrm{pi} / \mathrm{L}$ & 0.014 & & & & $U$ & & RIVER & & CONTINUOUS & RESIN & 307 \\
\hline $4610 \mathrm{r}$ & $\mathrm{pCi} / \mathrm{L}$ & 0.0067 & & & & U & & RIVER & & CONTINUOUS & FILTER & 307 \\
\hline $4611 \mathrm{r}$ & $\mathrm{pCi} / \mathrm{L}$ & 0.0085 & & & & U & & RIVER & & CONTINUOUS & RESIN & 252 \\
\hline $4612 \mathrm{r}$ & $\mathrm{pCi} / \mathrm{L}$ & 0.0044 & & & & $U$ & & RIVER & & CONTINUOUS & FILTER & 252 \\
\hline $4613 \mathrm{r}$ & $\mathrm{pCi} / \mathrm{L}$ & 0.0037 & & & & U & & RIVER & & CONTINUOUS & FILTER & 307 \\
\hline $4614 \mathrm{r}$ & pCi/L & 0.0075 & & & & $U$ & & RIVER & & CONTINUOUS & RESIN & 307 \\
\hline 4615 & $\mathrm{pCi} / \mathrm{L}$ & 0.0082 & & & & $U$ & & RIVER & & CONTINUOUS & FILTER & 252 \\
\hline 4616 & $\mathrm{pCi} / \mathrm{L}$ & 0.015 & & & & $U$ & & RIVER & & CONTINUOUS & RESIN & 252 \\
\hline $4617 \mathrm{r}$ & $\mathrm{pCi} / \mathrm{L}$ & 10.9 & 18.3 & & & & & RIVER & & CUMULATIVE & UNFILTERED & 252 \\
\hline $4618 \mathrm{r}$ & $\mathrm{pCi} / \mathrm{L}$ & 0.845 & 0.845 & & & $U$ & & RIVER & & CUMULATIVE & UNFILTERED & 252 \\
\hline $4619 \mathrm{r}$ & $\mathrm{pCi} / \mathrm{L}$ & 0.0459 & 0.0584 & & & & & RIVER & & CUMULATIVE & UNFILTERED & 252 \\
\hline 4620 & $\mathrm{pCi} / \mathrm{L}$ & 0.0474 & 0.0506 & & & & & RIVER & & CUMULATIVE & UNFILTERED & 252 \\
\hline $4621 \mathrm{r}$ & $\mathrm{pCi} / \mathrm{L}$ & 0.0127 & 0.0127 & & & & & RIVER & & CUMULATIVE & UNFILTERED & 252 \\
\hline \begin{tabular}{|l|l|}
$4622 \mathrm{r}$ & \\
\end{tabular} & $\mathrm{pCi} / \mathrm{L}$ & 0.047 & 0.0504 & & & & & RIVER & & CUMULATIVE & UNFILTERED & 252 \\
\hline 4623 & $\mathrm{pCi} / \mathrm{L}$ & 10.3 & 17.1 & & & & & RIVER & & CUMULATIVE & UNFILTERED & 463 \\
\hline $4624 \mathrm{r}$ & $p \mathrm{pi} / \mathrm{L}$ & 0.551 & 0.553 & & & & & RIVER & & CUMULATIVE & UNFILTERED & 463 \\
\hline $4625 \mathrm{r}$ & $\mathrm{pCi} / \mathrm{L}$ & 0.0553 & 0.071 & & & & & RIVER & & CUMULATIVE & UNFILTERED & 463 \\
\hline $4626 \mathrm{r}$ & $\mathrm{pCi} / \mathrm{L}$ & 0.052 & 0.057 & & & & & RIVER & & CUMULATIVE & UNFILTERED & 463 \\
\hline $4627 \mathrm{r}$ & $\mathrm{pCi} / \mathrm{L}$ & 0.00747 & 0.00749 & & & $U$ & & RIVER & & CUMULATIVE & UNFILTERED & 463 \\
\hline $4628 \mathrm{r}$ & $\mathrm{pCi} / \mathrm{L}$ & 0.0567 & 0.0629 & & & & & RIVER & & CUMULATIVE & UNFILTERED & 463 \\
\hline $4629 \mid$ & $\mathrm{pCi} / \mathrm{L}$ & 0.0194 & 0.0204 & & & & & DRINKING & & SAN. GRAB & UNFILTERED & 186 \\
\hline
\end{tabular}




\begin{tabular}{|c|c|c|c|c|c|c|c|c|c|c|c|c|}
\hline & $\mathrm{N}$ & $\mathrm{O}$ & $\mathrm{P}$ & $\mathrm{Q}$ & $\mathrm{R}$ & $\mathrm{s}$ & $T$ & $\mathrm{U}$ & $\mathrm{V}$ & $\mathrm{W}$ & $x$ & $\bar{Y}$ \\
\hline 1 & ANAL_UNITS_RPTD & COUNTING_ERROR & ||$_{\text {TOTAL_ANAL_ERROR | }}$ & MIN_DETECTABLE_ACTIVITY & PCNT_MOISTURE & LAB_QUALIFIER & REVIEW_QUALIFIER & SAMP_FROM & SAMP_ITEM & SAMP_MTHD & COLL_MTHD & SAMP_SITE_ID \\
\hline 4630 & pCi/L & 168 & 212 & & & $U$ & & DRINKING & & SAN. GRAB & UNFILTERED & 186 \\
\hline 4631 & $\mathrm{pCi} / \mathrm{L}$ & 0.879 & 0.879 & & & U & & DRINKING & & SAN. GRAB & UNFILTERED & 186 \\
\hline 4632 & $\mathrm{pCi} / \mathrm{L}$ & 0.0397 & 0.0482 & & & & & DRINKING & & SAN. GRAB & UNFILTERED & 144 \\
\hline 4633 & $\mathrm{pCi} / \mathrm{L}$ & 169 & 214 & & & $U$ & & DRINKING & & SAN. GRAB & UNFILTERED & 144 \\
\hline 4634 & $\mathrm{pCi} / \mathrm{L}$ & 1.71 & 1.71 & & & U & & DRINKING & & SAN. GRAB & UNFILTERED & 144 \\
\hline 4635 & $\mathrm{pCi} / \mathrm{L}$ & 0.0194 & 0.0205 & & & & & DRINKING & & SAN. GRAB & UNFILTERED & 187 \\
\hline 4636 & $\mathrm{pCi} / \mathrm{L}$ & 168 & 212 & & & $\mathrm{u}$ & & DRINKING & & SAN. GRAB & UNFILTERED & 187 \\
\hline 4637 & $\mathrm{pCi} / \mathrm{L}$ & 0.899 & 0.899 & & & $\mathrm{U}$ & & DRINKING & & SAN. GRAB & UNFILTERED & 187 \\
\hline 4638 & $\mathrm{pCi} / \mathrm{L}$ & 0.011 & & & & U & & RIVER & & CONTINUOUS & RESIN & 307 \\
\hline 4639 & $\mathrm{pCi} / \mathrm{L}$ & 0.0059 & & & & U & & RIVER & & CONTINUOUS & FILTER & 307 \\
\hline 4640 & & & & & & & & RIVER & & CONTINUOUS & RESIN & 252 \\
\hline 4641 & & & & & & & & RIVER & & CONTINUOUS & FILTER & 252 \\
\hline 4642 & $\mathrm{pCi} / \mathrm{L}$ & 0.119 & 0.171 & & & & & SURFACE & & RAW GRAB & UNFILTERED & 509 \\
\hline 4643 & $\mathrm{pCi} / \mathrm{L}$ & 2.08 & 2.09 & & & & & SURFACE & & RAW GRAB & UNFILTERED & 509 \\
\hline 4644 & $\mathrm{pCi} / \mathrm{L}$ & 181 & 235 & & & & & SURFACE & & RAW GRAB & UNFILTERED & 509 \\
\hline 4645 & $\mathrm{pCi} / \mathrm{L}$ & 2.45 & 2.45 & & & $U$ & & SURFACE & & RAW GRAB & UNFILTERED & 136 \\
\hline \begin{tabular}{|l|}
4646 \\
\end{tabular} & $\mathrm{pCi} / \mathrm{L}$ & 419 & 1160 & & & & & SURFACE & & RAW GRAB & UNFILTERED & 136 \\
\hline 4647 & $\mathrm{pCi} / \mathrm{L}$ & 0.0701 & 0.137 & & & & & SURFACE & & RAW GRAB & UNFILTERED & 146 \\
\hline 4648 & $\mathrm{pCi} / \mathrm{L}$ & 1.72 & 1.75 & & & & & SURFACE & & RAW GRAB & UNFILTERED & 146 \\
\hline 4649 & $\mathrm{pCi} / \mathrm{L}$ & 171 & 216 & & & $U$ & & SURFACE & & RAW GRAB & UNFILTERED & 146 \\
\hline 4650 & $\mathrm{pCi} / \mathrm{L}$ & 0.17 & 0.383 & & & & & SURFACE & & RAW GRAB & UNFILTERED & 147 \\
\hline \begin{tabular}{|l|l|}
4651 \\
\end{tabular} & $\mathrm{pCi} / \mathrm{L}$ & 4.28 & 4.28 & & & $U$ & & SURFACE & & RAW GRAB & UNFILTERED & 147 \\
\hline 4652 & $\mathrm{pCi} / \mathrm{L}$ & 183 & 240 & & & & & SURFACE & & RAW GRAB & UNFILTERED & 147 \\
\hline 4653 & $\mathrm{pCi} / \mathrm{L}$ & 0.0224 & 0.0234 & & & & & DRINKING & & SAN. GRAB & UNFILTERED & 512 \\
\hline 4654 & $\mathrm{pCi} / \mathrm{L}$ & 426 & 1180 & & & & & DRINKING & & SAN. GRAB & UNFILTERED & 512 \\
\hline 4655 & $\mathrm{pCi} / \mathrm{L}$ & 0.894 & 0.895 & & & $U$ & & DRINKING & & SAN. GRAB & UNFILTERED & 512 \\
\hline 4656 & $\mathrm{pCi} / \mathrm{L}$ & 0.0411 & 0.0575 & & & & & DRINKING & & RAW GRAB & UNFILTERED & 553 \\
\hline 4657 & $\mathrm{pCi} / \mathrm{L}$ & 1.67 & 1.67 & & & $U$ & & DRINKING & & RAW GRAB & UNFILTERED & 553 \\
\hline 4658 & $\mathrm{pCi} / \mathrm{L}$ & 172 & 219 & & & U & & DRINKING & & RAW GRAB & UNFILTERED & 553 \\
\hline 4659 & $\mathrm{pCi} / \mathrm{L}$ & 0.0213 & 0.0216 & & & U & & DRINKING & & SAN. GRAB & UNFILTERED & 487 \\
\hline 4660 & $\mathrm{pCi} / \mathrm{L}$ & 168 & 210 & & & U & & DRINKING & & SAN. GRAB & UNFILTERED & 487 \\
\hline 4661 & $\mathrm{pCi} / \mathrm{L}$ & 0.894 & 0.896 & & & & & DRINKING & & SAN. GRAB & UNFILTERED & 487 \\
\hline 4662 & $\mathrm{pCi} / \mathrm{L}$ & 171 & 217 & & & U & & DRINKING & & SAN. GRAB & UNFILTERED & 530 \\
\hline 4663 & $\mathrm{pCi} / \mathrm{L}$ & 0.109 & 0.261 & & & & & DRINKING & & SAN. GRAB & UNFILTERED & 530 \\
\hline 4664 & $\mathrm{pCi} / \mathrm{L}$ & 0.96 & 0.96 & & & $U$ & & DRINKING & & SAN. GRAB & UNFILTERED & 530 \\
\hline 4665 & pCi/L & 0.0046 & & & & $U$ & & RIVER & & CONTINUOUS & FILTER & 307 \\
\hline 4666 & $\mathrm{pCi} / \mathrm{L}$ & 0.0096 & & & & $U$ & & RIVER & & CONTINUOUS & RESIN & 307 \\
\hline 4667 & & & & & & & & RIVER & & CONTINUOUS & FILTER & 252 \\
\hline 4668 & & & & & & & & RIVER & & CONTINUOUS & RESIN & 252 \\
\hline \begin{tabular}{|l|}
4669 \\
\end{tabular} & $\mathrm{pCi} / \mathrm{L}$ & 0.0044 & & & & U & & RIVER & & CONTINUOUS & FILTER & 307 \\
\hline \begin{tabular}{|l|}
4670 \\
\end{tabular} & $\mathrm{pCi} / \mathrm{L}$ & 0.0093 & & & & U & & RIVER & & CONTINUOUS & RESIN & 307 \\
\hline \begin{tabular}{|l|}
4671 \\
\end{tabular} & $\mathrm{pCi} / \mathrm{L}$ & 0.0065 & & & & U & & RIVER & & CONTINUOUS & FILTER & 252 \\
\hline \begin{tabular}{|l|}
4672 \\
\end{tabular} & $\mathrm{pCi} / \mathrm{L}$ & 12 & 20.1 & & & & & RIVER & & CUMULATIVE & UNFILTERED & 252 \\
\hline 4673 & $\mathrm{pCi} / \mathrm{L}$ & 0.046 & 0.0495 & & & & & RIVER & & CUMULATIVE & UNFILTERED & 252 \\
\hline \begin{tabular}{|l|}
4674 \\
\end{tabular} & $\mathrm{pCi} / \mathrm{L}$ & 0.00733 & 0.00734 & & & U & & RIVER & & CUMULATIVE & UNFILTERED & 252 \\
\hline \begin{tabular}{|l|}
4675 \\
\end{tabular} & $\mathrm{pCi} / \mathrm{L}$ & 0.0502 & 0.0549 & & & & & RIVER & & CUMULATIVE & UNFILTERED & 252 \\
\hline \begin{tabular}{|l|}
4676 \\
\end{tabular} & $\mathrm{pCi} / \mathrm{L}$ & 0.0397 & 0.0522 & & & & & RIVER & & CUMULATIVE & UNFILTERED & 252 \\
\hline \begin{tabular}{|l|l|}
4677 \\
\end{tabular} & $\mathrm{pCi} / \mathrm{L}$ & 0.012 & & & & $U$ & & RIVER & & CONTINUOUS & RESIN & 252 \\
\hline 4678 & pCi/L & 0.752 & 0.752 & & & U & & RIVER & & CUMULATIVE & UNFILTERED & 252 \\
\hline 4679 & pCi/L & 0.354 & 0.355 & & & $U$ & & RIVER & & CUMULATIVE & UNFILTERED & 463 \\
\hline 4680 & $\mathrm{pCi} / \mathrm{L}$ & 14.1 & 23 & & & & & RIVER & & CUMULATIVE & UNFILTERED & 463 \\
\hline \begin{tabular}{|l|l|}
4681 \\
\end{tabular} & $\mathrm{pCi} / \mathrm{L}$ & 0.0433 & 0.0478 & & & & & RIVER & & CUMULATIVE & UNFILTERED & 463 \\
\hline
\end{tabular}




\begin{tabular}{|c|c|c|c|c|c|c|c|c|c|c|c|c|}
\hline & $\mathrm{N}$ & $\mathrm{O}$ & $\mathrm{P}$ & $\mathrm{Q}$ & $\mathrm{R}$ & $\mathrm{s}$ & $T$ & $U$ & $\mathrm{~V}$ & $\mathrm{~W}$ & $\mathrm{x}$ & $\bar{Y}$ \\
\hline 1 & ANAL_UNITS_RPTD & COUNTING_ERROR & $\mid$ TOTAL_ANAL_ERROR | & MIN_DETECTABLE_ACTIVITY & PCNT_MOISTURE & LAB_QUALIFIER & REVIEW_QUALIFIER & SAMP_FROM & SAMP_ITEM & SAMP_MTHD & COLL_MTHD & SAMP_SITE_ID \\
\hline \begin{tabular}{|c|}
4682 \\
\end{tabular} & $\mathrm{pCi} / \mathrm{L}$ & 0.029 & 0.0318 & & & & & RIVER & & CUMULATIVE & UNFILTERED & 463 \\
\hline 4683 & $\mathrm{pCi} / \mathrm{L}$ & 0.01 & 0.0101 & & & & & RIVER & & CUMULATIVE & UNFILTERED & 463 \\
\hline 4684 & $\mathrm{pCi} / \mathrm{L}$ & 0.0535 & 0.0618 & & & & & RIVER & & CUMULATIVE & UNFILTERED & 463 \\
\hline 4685 & $5 \mathrm{pCi} / \mathrm{L}$ & 0.0057 & & & & U & & RIVER & & CONTINUOUS & FILTER & 252 \\
\hline \begin{tabular}{|c|}
4686 \\
\end{tabular} & $\mathrm{pCi} / \mathrm{L}$ & 0.01 & & & & $\mathrm{U}$ & & RIVER & & CONTINUOUS & RESIN & 252 \\
\hline \begin{tabular}{|l|l|}
4687 \\
\end{tabular} & & & & & & & & RIVER & & CONTINUOUS & FILTER & 307 \\
\hline 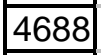 & & & & & & & & RIVER & & CONTINUOUS & RESIN & 307 \\
\hline \begin{tabular}{|c|}
4689 \\
\end{tabular} & $\mathrm{pCi} / \mathrm{L}$ & 0.0483 & 0.0612 & & & & & DRINKING & & CUMULATIVE & UNFILTERED & 307 \\
\hline 4690 & $\mathrm{pCi} / \mathrm{L}$ & 0.936 & 0.936 & & & U & & DRINKING & & CUMULATIVE & UNFILTERED & 307 \\
\hline 4691 & $\mathrm{pCi} / \mathrm{L}$ & 170 & 216 & & & $\mathrm{U}$ & & DRINKING & & CUMULATIVE & UNFILTERED & 307 \\
\hline 4692 & $\mathrm{pCi} / \mathrm{L}$ & & $9.5382 \mathrm{E}-06$ & & & & & RIVER & & CONTINUOUS & RESIN & 307 \\
\hline \begin{tabular}{|l|}
4693 \\
\end{tabular} & $\mathrm{peci} / \mathrm{L}$ & 0.0089 & & & & $U$ & & RIVER & & CONTINUOUS & RESIN & 307 \\
\hline \begin{tabular}{|l|}
4694 \\
\end{tabular} & $\mathrm{pCi} / \mathrm{L}$ & 0.00032 & & & & & & RIVER & & CONTINUOUS & RESIN & 307 \\
\hline \begin{tabular}{|l|}
4695 \\
\end{tabular} & pCi/L & 0.048 & & & & $U$ & & RIVER & & CONTINUOUS & RESIN & 307 \\
\hline \begin{tabular}{|l|}
4696 \\
\end{tabular} & pCi/L & 0.0043 & & & & $U$ & & RIVER & & CONTINUOUS & FILTER & 307 \\
\hline \begin{tabular}{|l|}
4697 \\
\end{tabular} & $\mathrm{pCi} / \mathrm{L}$ & 0.000009 & & & & & & RIVER & & CONTINUOUS & FILTER & 307 \\
\hline \begin{tabular}{|l|}
4698 \\
\end{tabular} & $3 \mathrm{pCi} / \mathrm{L}$ & 0.0063 & & & & $U$ & & RIVER & & CONTINUOUS & FILTER & 252 \\
\hline \begin{tabular}{|l|}
4699 \\
\end{tabular} & $\mathrm{pCi} / \mathrm{L}$ & & $1.2408 \mathrm{E}-06$ & & & & & RIVER & & CONTINUOUS & RESIN & 252 \\
\hline 4700 & $\mathrm{pCi} / \mathrm{L}$ & 12.3 & 20.8 & & & & & RIVER & & CUMULATIVE & UNFILTERED & 252 \\
\hline \begin{tabular}{|l|}
4701 \\
\end{tabular} & $\mathrm{pCi} / \mathrm{L}$ & 0.0462 & 0.0517 & & & & & RIVER & & CUMULATIVE & UNFILTERED & 252 \\
\hline \begin{tabular}{|l|}
4702 \\
\end{tabular} & $\mathrm{pCi} / \mathrm{L}$ & 0.00995 & 0.01 & & & & & RIVER & & CUMULATIVE & UNFILTERED & 252 \\
\hline 4703 & $\mathrm{pCi} / \mathrm{L}$ & 0.0554 & 0.0641 & & & & & RIVER & & CUMULATIVE & UNFILTERED & 252 \\
\hline \begin{tabular}{|l|l|}
4704 \\
\end{tabular} & $4 \mathrm{pCi} / \mathrm{L}$ & 0.0354 & 0.051 & & & & & RIVER & & CUMULATIVE & UNFILTERED & 252 \\
\hline \begin{tabular}{|l|}
4705 \\
\end{tabular} & $5 \mathrm{pCi} / \mathrm{L}$ & 0.011 & & & & $U$ & & RIVER & & CONTINUOUS & RESIN & 252 \\
\hline \begin{tabular}{|l|}
4706 \\
\end{tabular} & $\mathrm{pCi} / \mathrm{L}$ & 0.001 & & & & $\mathrm{U}$ & & RIVER & & CONTINUOUS & RESIN & 252 \\
\hline \begin{tabular}{|l|}
4707 \\
\end{tabular} & $\mathrm{pCi} / \mathrm{L}$ & 0.0000246 & & & & $\mathrm{U}$ & & RIVER & & CONTINUOUS & FILTER & 252 \\
\hline \begin{tabular}{|l|}
4708 \\
\end{tabular} & $\mathrm{pCi} / \mathrm{L}$ & 0.018 & & & & & & RIVER & & CONTINUOUS & RESIN & 252 \\
\hline \begin{tabular}{|l|}
4709 \\
\end{tabular} & $\mathrm{pCi} / \mathrm{L}$ & 0.501 & 0.502 & & & & & RIVER & & CUMULATIVE & UNFILTERED & 252 \\
\hline \begin{tabular}{|l|}
4710 \\
\end{tabular} & $\mathrm{pCi} / \mathrm{L}$ & 0.0334 & 0.0585 & & & & & RIVER & & CUMULATIVE & UNFILTERED & 463 \\
\hline \begin{tabular}{|l|l|}
4711 \\
\end{tabular} & $\mathrm{pCi} / \mathrm{L}$ & 13.3 & 24.7 & & & & & RIVER & & CUMULATIVE & UNFILTERED & 463 \\
\hline \begin{tabular}{|l|}
4712 \\
\end{tabular} & $\mathrm{pCi} / \mathrm{L}$ & 0.0574 & 0.0654 & & & & & RIVER & & CUMULATIVE & UNFILTERED & 463 \\
\hline \begin{tabular}{|l|}
4713 \\
\end{tabular} & $\mathrm{pCCi} / \mathrm{L}$ & 0.0113 & 0.0114 & & & & & RIVER & & CUMULATIVE & UNFILTERED & 463 \\
\hline \begin{tabular}{|l|l|}
4714 \\
\end{tabular} & $\mathrm{pCi} / \mathrm{L}$ & 0.0629 & 0.0736 & & & & & RIVER & & CUMULATIVE & UNFILTERED & 463 \\
\hline \begin{tabular}{|l|}
4715 \\
\end{tabular} & $5 \mathrm{pCi} / \mathrm{L}$ & 1.23 & 1.24 & & & $U$ & & RIVER & & CUMULATIVE & UNFILTERED & 463 \\
\hline \begin{tabular}{|l|}
4716 \\
\end{tabular} & & & & & & & & RIVER & & CONTINUOUS & FILTER & 307 \\
\hline \begin{tabular}{|l|l|}
4717 \\
\end{tabular} & & & & & & & & RIVER & & CONTINUOUS & RESIN & 307 \\
\hline 4718 & $\mathrm{pCi} / \mathrm{L}$ & 0.007 & & & & $U$ & & RIVER & & CONTINUOUS & RESIN & 252 \\
\hline \begin{tabular}{|l|}
4719 \\
\end{tabular} & $\mathrm{pCi} / \mathrm{L}$ & 0.0037 & & & & $\mathrm{U}$ & & RIVER & & CONTINUOUS & FILTER & 252 \\
\hline \begin{tabular}{|l|}
4720 \\
\end{tabular} & $\mathrm{pCi} / \mathrm{L}$ & 0.0077 & & & & $U$ & & RIVER & & CONTINUOUS & RESIN & 307 \\
\hline \begin{tabular}{|l|}
4721 \\
\end{tabular} & $\mathrm{pCi} / \mathrm{L}$ & 0.0037 & & & & $U$ & & RIVER & & CONTINUOUS & FILTER & 307 \\
\hline \begin{tabular}{|l|}
4722 \\
\end{tabular} & $\mathrm{pCi} / \mathrm{L}$ & 0.0099 & & & & $U$ & & RIVER & & CONTINUOUS & RESIN & 252 \\
\hline \begin{tabular}{|l|}
4723 \\
\end{tabular} & $\mathrm{pCi} / \mathrm{L}$ & 0.005 & & & & $U$ & & RIVER & & CONTINUOUS & FILTER & 252 \\
\hline \begin{tabular}{|l|l|}
4724 \\
\end{tabular} & $4 \mathrm{pCi} / \mathrm{L}$ & 0.0484 & 0.0549 & & & & & RIVER & & CUMULATIVE & UNFILTERED & 252 \\
\hline \begin{tabular}{|l|}
4725 \\
\end{tabular} & $\mathrm{pCi} / \mathrm{L}$ & 0.0138 & 0.014 & & & & & RIVER & & CUMULATIVE & UNFILTERED & 252 \\
\hline \begin{tabular}{|l|}
4726 \\
\end{tabular} & $\mathrm{pCi} / \mathrm{L}$ & 0.0522 & 0.06 & & & & & RIVER & & CUMULATIVE & UNFILTERED & 252 \\
\hline \begin{tabular}{|l|}
4727 \\
\end{tabular} & $7 \mathrm{pCi} / \mathrm{L}$ & 0.0328 & 0.0458 & & & & & RIVER & & CUMULATIVE & UNFILTERED & 252 \\
\hline \begin{tabular}{|l|}
4728 \\
\end{tabular} & $\mathrm{pCi} / \mathrm{L}$ & 0.927 & 0.928 & & & $U$ & & RIVER & & CUMULATIVE & UNFILTERED & 252 \\
\hline \begin{tabular}{|l|}
4729 \\
\end{tabular} & $\mathrm{pCi} / \mathrm{L}$ & 10.6 & 17.6 & & & & & RIVER & & CUMULATIVE & UNFILTERED & 252 \\
\hline 4730 & $\mathrm{pCi} / \mathrm{L}$ & 0.513 & 0.514 & & & & & RIVER & & CUMULATIVE & UNFILTERED & 463 \\
\hline \begin{tabular}{|l|}
4731 \\
\end{tabular} & $\mathrm{pCi} / \mathrm{L}$ & 0.054 & 0.0632 & & & & & RIVER & & CUMULATIVE & UNFILTERED & 463 \\
\hline \begin{tabular}{|l|}
4732 \\
\end{tabular} & $\mathrm{pCi} / \mathrm{L}$ & 0.00854 & 0.00855 & & & U & & RIVER & & CUMULATIVE & UNFILTERED & 463 \\
\hline 47733 & $\mathrm{pCi} / \mathrm{L}$ & 0.0576 & 0.0684 & & & & & RIVER & & CUMULATIVE & UNFILTERED & 463 \\
\hline
\end{tabular}




\begin{tabular}{|c|c|c|c|c|c|c|c|c|c|c|c|c|}
\hline & $\mathrm{N}$ & $\mathrm{O}$ & $\mathrm{P}$ & $\mathrm{Q}$ & $\mathrm{R}$ & $\mathrm{s}$ & $T$ & $\mathrm{U}$ & $\mathrm{V}$ & $\mathrm{W}$ & $\mathrm{X}$ & $\bar{Y}$ \\
\hline 1 & ANAL_UNITS_RPTD & COUNTING_ERROR & TOTAL_ANAL_ERROR & MIN_DETECTABLE_ACTIVITY & PCNT_MOISTURE & LAB_QUALIFIER & REVIEW_QUALIFIER & SAMP_FROM & SAMP_ITEM & SAMP_MTHD & COLL_MTHD & SAMP_SITE_ID \\
\hline 4734 & $\mathrm{pCi} / \mathrm{L}$ & 0.0331 & 0.0527 & & & & & RIVER & & CUMULATIVE & UNFILTERED & 463 \\
\hline 4735 & $\mathrm{pCi} / \mathrm{L}$ & 12.7 & 23.8 & & & & & RIVER & & CUMULATIVE & UNFILTERED & 463 \\
\hline 4736 & $\mathrm{pCi} / \mathrm{L}$ & 173 & 232 & & & U & & DRINKING & & SAN. GRAB & UNFILTERED & 186 \\
\hline \begin{tabular}{|l|l|}
4737 \\
\end{tabular} & $\mathrm{pCi} / \mathrm{L}$ & 0.597 & 0.597 & & & $\mathrm{U}$ & & DRINKING & & SAN. GRAB & UNFILTERED & 186 \\
\hline 4738 & $\mathrm{pCi} / \mathrm{L}$ & 0.0342 & 0.0348 & & & $U$ & & DRINKING & & SAN. GRAB & UNFILTERED & 186 \\
\hline 4739 & $\mathrm{pCi} / \mathrm{L}$ & 449 & 1500 & & & & & DRINKING & & SAN. GRAB & UNFILTERED & 512 \\
\hline 4740 & $\mathrm{pCi} / \mathrm{L}$ & 0.413 & 0.413 & & & $\mathrm{U}$ & & DRINKING & & SAN. GRAB & UNFILTERED & 512 \\
\hline \begin{tabular}{|l|l|}
4741 \\
\end{tabular} & $\mathrm{pCi} / \mathrm{L}$ & 0.0421 & 0.0426 & & & U & & DRINKING & & SAN. GRAB & UNFILTERED & 512 \\
\hline \begin{tabular}{|c|}
4742 \\
\end{tabular} & $\mathrm{pCi} / \mathrm{L}$ & 174 & 234 & & & $\mathrm{U}$ & & DRINKING & & SAN. GRAB & UNFILTERED & 144 \\
\hline \begin{tabular}{|c|}
4743 \\
\end{tabular} & $\mathrm{pCi} / \mathrm{L}$ & 0.63 & 0.63 & & & U & & DRINKING & & SAN. GRAB & UNFILTERED & 144 \\
\hline \begin{tabular}{|l|}
4744 \\
\end{tabular} & $\mathrm{pCi} / \mathrm{L}$ & 0.0252 & 0.0254 & & & $U$ & & DRINKING & & SAN. GRAB & UNFILTERED & 144 \\
\hline \begin{tabular}{|l|}
4745 \\
\end{tabular} & $\mathrm{pCi} / \mathrm{L}$ & 174 & 233 & & & $U$ & & DRINKING & & SAN. GRAB & UNFILTERED & 187 \\
\hline \begin{tabular}{|l|}
4746 \\
\end{tabular} & $\mathrm{pCi} / \mathrm{L}$ & 0.83 & 0.83 & & & $U$ & & DRINKING & & SAN. GRAB & UNFILTERED & 187 \\
\hline \begin{tabular}{|l|l|}
4747 \\
\end{tabular} & $\mathrm{pCi} / \mathrm{L}$ & 0.0268 & 0.0274 & & & & & DRINKING & & SAN. GRAB & UNFILTERED & 187 \\
\hline \begin{tabular}{|l|}
4748 \\
\end{tabular} & $\mathrm{pCi} / \mathrm{L}$ & 0.89 & 0.891 & & & $U$ & & DRINKING & & RAW GRAB & UNFILTERED & 553 \\
\hline \begin{tabular}{|l|}
4749 \\
\end{tabular} & $\mathrm{pCi} / \mathrm{L}$ & 199 & 256 & & & $U$ & & DRINKING & & RAW GRAB & UNFILTERED & 553 \\
\hline \begin{tabular}{|l|}
4750 \\
\end{tabular} & $\mathrm{pCi} / \mathrm{L}$ & 0.0365 & 0.0558 & & & & & DRINKING & & RAW GRAB & UNFILTERED & 553 \\
\hline \begin{tabular}{|l|}
4751 \\
\end{tabular} & $\mathrm{pCi} / \mathrm{L}$ & 0.0079 & & & & U & & RIVER & & CONTINUOUS & RESIN & 307 \\
\hline \begin{tabular}{|l|}
4752 \\
\end{tabular} & $\mathrm{pCi} / \mathrm{L}$ & 0.0038 & & & & $U$ & & RIVER & & CONTINUOUS & FILTER & 307 \\
\hline \begin{tabular}{|l|}
4753 \\
\end{tabular} & $\mathrm{pCi} / \mathrm{L}$ & 0.0047 & & & & $U$ & & RIVER & & CONTINUOUS & FILTER & 252 \\
\hline \begin{tabular}{|l|l|}
4754 \\
\end{tabular} & $\mathrm{pCi} / \mathrm{L}$ & 0.0098 & & & & U & & RIVER & & CONTINUOUS & RESIN & 252 \\
\hline \begin{tabular}{|l|}
4755 \\
\end{tabular} & $\mathrm{pCi} / \mathrm{L}$ & 0.0079 & & & & U & & RIVER & & CONTINUOUS & RESIN & 307 \\
\hline \begin{tabular}{|l|l|}
4756 \\
\end{tabular} & $\mathrm{pCi} / \mathrm{L}$ & 0.0038 & & & & U & & RIVER & & CONTINUOUS & FILTER & 307 \\
\hline \begin{tabular}{|l|l|}
4757 \\
\end{tabular} & $\mathrm{pCi} / \mathrm{L}$ & 0.662 & 0.662 & & & $U$ & & RIVER & & CUMULATIVE & UNFILTERED & 252 \\
\hline \begin{tabular}{|l|}
4758 \\
\end{tabular} & $\mathrm{pCi} / \mathrm{L}$ & 0.0522 & 0.0614 & & & & & RIVER & & CUMULATIVE & UNFILTERED & 252 \\
\hline \begin{tabular}{|l|}
4759 \\
\end{tabular} & $\mathrm{pCi} / \mathrm{L}$ & 0.007 & 0.00703 & & & $U$ & & RIVER & & CUMULATIVE & UNFILTERED & 252 \\
\hline 4760 & $\mathrm{pCi} / \mathrm{L}$ & 0.0478 & 0.0549 & & & & & RIVER & & CUMULATIVE & UNFILTERED & 252 \\
\hline \begin{tabular}{|l|}
4761 \\
\end{tabular} & $\mathrm{pCi} / \mathrm{L}$ & 0.0078 & & & & $U$ & & RIVER & & CONTINUOUS & RESIN & 252 \\
\hline \begin{tabular}{|l|}
4762 \\
\end{tabular} & $\mathrm{pCi} / \mathrm{L}$ & 11.1 & 19.1 & & & & & RIVER & & CUMULATIVE & UNFILTERED & 252 \\
\hline \begin{tabular}{|l|}
4763 \\
\end{tabular} & $\mathrm{pCi} / \mathrm{L}$ & 0.0414 & 0.0577 & & & & & RIVER & & CUMULATIVE & UNFILTERED & 252 \\
\hline \begin{tabular}{|l|l|}
4764 \\
\end{tabular} & $\mathrm{pCi} / \mathrm{L}$ & 0.0039 & & & & U & & RIVER & & CONTINUOUS & FILTER & 252 \\
\hline \begin{tabular}{|l|}
4765 \\
\end{tabular} & $\mathrm{pCi} / \mathrm{L}$ & 0.846 & 0.846 & & & U & & RIVER & & CUMULATIVE & UNFILTERED & 463 \\
\hline \begin{tabular}{|l|}
4766 \\
\end{tabular} & $\mathrm{pCi} / \mathrm{L}$ & 0.0519 & 0.0602 & & & & & RIVER & & CUMULATIVE & UNFILTERED & 463 \\
\hline \begin{tabular}{|l|l|}
4767 \\
\end{tabular} & $\mathrm{pCi} / \mathrm{L}$ & 0.00722 & 0.00725 & & & $U$ & & RIVER & & CUMULATIVE & UNFILTERED & 463 \\
\hline \begin{tabular}{|l|}
4768 \\
\end{tabular} & $\mathrm{pCi} / \mathrm{L}$ & 0.0509 & 0.0588 & & & & & RIVER & & CUMULATIVE & UNFILTERED & 463 \\
\hline \begin{tabular}{|l|}
4769 \\
\end{tabular} & $\mathrm{pCi} / \mathrm{L}$ & 13 & 23.8 & & & & & RIVER & & CUMULATIVE & UNFILTERED & 463 \\
\hline \begin{tabular}{|l|}
4770 \\
\end{tabular} & $\mathrm{pCi} / \mathrm{L}$ & 0.0357 & 0.055 & & & & & RIVER & & CUMULATIVE & UNFILTERED & 463 \\
\hline \begin{tabular}{|l|}
4771 \\
\end{tabular} & $\mathrm{pCi} / \mathrm{L}$ & 168 & 221 & & & U & & SURFACE & & RAW GRAB & UNFILTERED & 509 \\
\hline \begin{tabular}{|l|}
4772 \\
\end{tabular} & $\mathrm{pCi} / \mathrm{L}$ & 1.3 & 1.3 & & & U & & SURFACE & & RAW GRAB & UNFILTERED & 509 \\
\hline 4773 & $\mathrm{pCi} / \mathrm{L}$ & 0.0869 & 0.134 & & & & & SURFACE & & RAW GRAB & UNFILTERED & 509 \\
\hline \begin{tabular}{|l|l|}
4774 \\
\end{tabular} & $\mathrm{pCi} / \mathrm{L}$ & 4.31 & 4.31 & & & U & & SURFACE & & RAW GRAB & UNFILTERED & 136 \\
\hline \begin{tabular}{|l|}
4775 \\
\end{tabular} & $\mathrm{pCi} / \mathrm{L}$ & 209 & 322 & & & & & SURFACE & & RAW GRAB & UNFILTERED & 136 \\
\hline \begin{tabular}{|l|l|}
4776 \\
\end{tabular} & $\mathrm{pCi} / \mathrm{L}$ & 167 & 219 & & & $U$ & & SURFACE & & RAW GRAB & UNFILTERED & 146 \\
\hline \begin{tabular}{|l|l|}
4777 \\
\end{tabular} & $\mathrm{pCi} / \mathrm{L}$ & 1.64 & 1.68 & & & & & SURFACE & & RAW GRAB & UNFILTERED & 146 \\
\hline \begin{tabular}{|l|}
4778 \\
\end{tabular} & $\mathrm{pCi} / \mathrm{L}$ & 0.111 & 0.369 & & & & & SURFACE & & RAW GRAB & UNFILTERED & 146 \\
\hline 4779 & $\mathrm{pCi} / \mathrm{L}$ & 178 & 243 & & & & & SURFACE & & RAW GRAB & UNFILTERED & 147 \\
\hline 4780 & $\mathrm{pCi} / \mathrm{L}$ & 2.2 & 2.2 & & & $U$ & & SURFACE & & RAW GRAB & UNFILTERED & 147 \\
\hline \begin{tabular}{|l|}
4781 \\
\end{tabular} & $\mathrm{pCi} / \mathrm{L}$ & 0.205 & 0.478 & & & & & SURFACE & & RAW GRAB & UNFILTERED & 147 \\
\hline \begin{tabular}{|l|}
4782 \\
\end{tabular} & $\mathrm{pCi} / \mathrm{L}$ & 0.8 & 0.8 & & & $U$ & & DRINKING & & SAN. GRAB & UNFILTERED & 487 \\
\hline \begin{tabular}{|l|}
4783 \\
\end{tabular} & $\mathrm{pCi} / \mathrm{L}$ & 165 & 218 & & & $U$ & & DRINKING & & SAN. GRAB & UNFILTERED & 487 \\
\hline \begin{tabular}{|l|l|}
4784 \\
\end{tabular} & $\mathrm{pCi} / \mathrm{L}$ & 0.0321 & 0.0333 & & & & & DRINKING & & SAN. GRAB & UNFILTERED & 487 \\
\hline \begin{tabular}{|l|}
4785 \\
\end{tabular} & $\mathrm{pCi} / \mathrm{L}$ & 0.0074 & & & & $\mathrm{U}$ & & RIVER & & CONTINUOUS & RESIN & 307 \\
\hline
\end{tabular}




\begin{tabular}{|c|c|c|c|c|c|c|c|c|c|c|c|c|}
\hline & $\mathrm{N}$ & $\mathrm{O}$ & $\mathrm{P}$ & $\mathrm{Q}$ & $\mathrm{R}$ & $\mathrm{s}$ & $T$ & $\mathrm{U}$ & $\mathrm{V}$ & $\mathrm{W}$ & $x$ & $\bar{Y}$ \\
\hline 1 & ANAL_UNITS_RPTD & COUNTING_ERROR & ||$_{\text {TOTAL_ANAL_ERROR | }}$ & MIN_DETECTABLE_ACTIVITY & PCNT_MOISTURE & LAB_QUALIFIER & REVIEW_QUALIFIER & SAMP_FROM & SAMP_ITEM & SAMP_MTHD & COLL_MTHD & SAMP_SITE_ID \\
\hline 4786 & $\mathrm{pCi} / \mathrm{L}$ & 0.0038 & & & & $U$ & & RIVER & & CONTINUOUS & FILTER & 307 \\
\hline 4787 & $\mathrm{pCi} / \mathrm{L}$ & 0.0091 & & & & $\mathrm{U}$ & & RIVER & & CONTINUOUS & RESIN & 252 \\
\hline 4788 & $\mathrm{pCi} / \mathrm{L}$ & 0.0046 & & & & U & & RIVER & & CONTINUOUS & FILTER & 252 \\
\hline 4789 & & & & & & & & IRRIGATION & & RAW GRAB & UNFILTERED & 440 \\
\hline 4790 & & & & & & & & IRRIGATION & & RAW GRAB & UNFILTERED & 440 \\
\hline 4791 & $\mathrm{pCi} / \mathrm{L}$ & 166 & 219 & & & U & & SANITARY & & RAW GRAB & UNFILTERED & 525 \\
\hline 4792 & $\mathrm{pCi} / \mathrm{L}$ & 168 & 222 & & & $\mathrm{u}$ & & SANITARY & & RAW GRAB & UNFILTERED & 524 \\
\hline 4793 & $\mathrm{pCi} / \mathrm{L}$ & 163 & 212 & & & U & & SANITARY & & RAW GRAB & UNFILTERED & 95 \\
\hline 4794 & $\mathrm{pCi} / \mathrm{L}$ & 169 & 226 & & & U & & SANITARY & & RAW GRAB & UNFILTERED & 172 \\
\hline 4795 & $\mathrm{pCi} / \mathrm{L}$ & 0.0851 & 0.0854 & & & U & & DRINKING & & CUMULATIVE & UNFILTERED & 307 \\
\hline 4796 & $\mathrm{pCi} / \mathrm{L}$ & 0.0378 & 0.0577 & & & & & DRINKING & & CUMULATIVE & UNFILTERED & 307 \\
\hline \begin{tabular}{|l|l|}
4797 \\
\end{tabular} & $\mathrm{pCi} / \mathrm{L}$ & 0.0621 & 0.0696 & & & & & DRINKING & & CUMULATIVE & UNFILTERED & 307 \\
\hline 4798 & $\mathrm{pCi} / \mathrm{L}$ & 0.012 & 0.012 & & & $U$ & & DRINKING & & CUMULATIVE & UNFILTERED & 307 \\
\hline 4799 & $\mathrm{pCi} / \mathrm{L}$ & 0.0598 & 0.0667 & & & & & DRINKING & & CUMULATIVE & UNFILTERED & 307 \\
\hline 4800 & $\mathrm{pCi} / \mathrm{L}$ & 12.7 & 23.5 & & & & & DRINKING & & CUMULATIVE & UNFILTERED & 307 \\
\hline 4801 & $\mathrm{pCi} / \mathrm{L}$ & 0.0039 & & & & $U$ & & RIVER & & CONTINUOUS & FILTER & 307 \\
\hline 4802 & $\mathrm{pCi} / \mathrm{L}$ & 0.0000072 & & & & & & RIVER & & CONTINUOUS & FILTER & 307 \\
\hline 4803 & $\mathrm{pCi} / \mathrm{L}$ & 0.0077 & & & & $U$ & & RIVER & & CONTINUOUS & RESIN & 307 \\
\hline 4804 & $\mathrm{pCi} / \mathrm{L}$ & & 0.000007296 & & & & & RIVER & & CONTINUOUS & RESIN & 307 \\
\hline 4805 & $\mathrm{pCi} / \mathrm{L}$ & 0.0011 & & & & & & RIVER & & CONTINUOUS & RESIN & 307 \\
\hline 4806 & $\mathrm{pCi} / \mathrm{L}$ & 0.000147 & & & & U & & RIVER & & CONTINUOUS & RESIN & 307 \\
\hline 4807 & pCi/L & & 0.000000891 & & & & & RIVER & & CONTINUOUS & RESIN & 252 \\
\hline 4808 & $\mathrm{pCi} / \mathrm{L}$ & 0.00068 & & & & & & RIVER & & CONTINUOUS & RESIN & 252 \\
\hline 4809 & $\mathrm{pCi} / \mathrm{L}$ & 0.0000416 & & & & U & & RIVER & & CONTINUOUS & RESIN & 252 \\
\hline 4810 & $\mathrm{pCi} / \mathrm{L}$ & 0.000000464 & & & & & & RIVER & & CONTINUOUS & FILTER & 252 \\
\hline 4811 & $\mathrm{pCi} / \mathrm{L}$ & 0.0434 & 0.0569 & & & & & IRRIGATION & & RAW GRAB & UNFILTERED & 440 \\
\hline 4812 & $\mathrm{pCi} / \mathrm{L}$ & 0.988 & 0.988 & & & $U$ & & IRRIGATION & & RAW GRAB & UNFILTERED & 440 \\
\hline 4813 & $\mathrm{pCi} / \mathrm{L}$ & 0.00435 & 0.0049 & & & & & RIVER & & CUMULATIVE & UNFILTERED & 252 \\
\hline 4814 & $\mathrm{pCi} / \mathrm{L}$ & 0.000972 & 0.000979 & & & & & RIVER & & CUMULATIVE & UNFILTERED & 252 \\
\hline 4815 & $\mathrm{pCi} / \mathrm{L}$ & 0.00465 & 0.0053 & & & & & RIVER & & CUMULATIVE & UNFILTERED & 252 \\
\hline 4816 & $\mathrm{pCi} / \mathrm{L}$ & 0.862 & 0.862 & & & $U$ & & RIVER & & CUMULATIVE & UNFILTERED & 252 \\
\hline 4817 & $\mathrm{pCi} / \mathrm{L}$ & 0.0439 & 0.0655 & & & & & RIVER & & CUMULATIVE & UNFILTERED & 252 \\
\hline 4818 & $\mathrm{pCi} / \mathrm{L}$ & 10.3 & 16.8 & & & & & RIVER & & CUMULATIVE & UNFILTERED & 252 \\
\hline 4819 & $\mathrm{pCi} / \mathrm{L}$ & 0.00453 & 0.00513 & & & & & RIVER & & CUMULATIVE & UNFILTERED & 463 \\
\hline 4820 & $\mathrm{pCi} / \mathrm{L}$ & 0.00108 & 0.00108 & & & & & RIVER & & CUMULATIVE & UNFILTERED & 463 \\
\hline 4821 & pCi/L & 0.00449 & 0.00508 & & & & & RIVER & & CUMULATIVE & UNFILTERED & 463 \\
\hline 4822 & $\mathrm{pCi} / \mathrm{L}$ & 0.367 & 0.368 & & & $U$ & & RIVER & & CUMULATIVE & UNFILTERED & 463 \\
\hline 4823 & $\mathrm{pCi} / \mathrm{L}$ & 0.0461 & 0.0653 & & & & & RIVER & & CUMULATIVE & UNFILTERED & 463 \\
\hline \begin{tabular}{|l|l|}
4824 \\
\end{tabular} & $\mathrm{pCi} / \mathrm{L}$ & 10.5 & 17.1 & & & & & RIVER & & CUMULATIVE & UNFILTERED & 463 \\
\hline \begin{tabular}{|l|}
4825 \\
\end{tabular} & $\mathrm{pCi} / \mathrm{L}$ & 0.757 & 0.757 & & & U & & DRINKING & & SAN. GRAB & UNFILTERED & 530 \\
\hline \begin{tabular}{|l|}
4826 \\
\end{tabular} & $\mathrm{pCi} / \mathrm{L}$ & 11 & 19.8 & & & & & DRINKING & & SAN. GRAB & UNFILTERED & 530 \\
\hline \begin{tabular}{|l|l|}
4827 \\
\end{tabular} & pCi/L & 0.077 & 0.211 & & & & & DRINKING & & SAN. GRAB & UNFILTERED & 530 \\
\hline 4828 & & & & & & & & RIVER & & CONTINUOUS & RESIN & 307 \\
\hline \begin{tabular}{|l|}
4829 \\
\end{tabular} & & & & & & & & RIVER & & CONTINUOUS & FILTER & 307 \\
\hline 4830 & $\mathrm{pCi} / \mathrm{L}$ & 0.0068 & & & & $U$ & & RIVER & & CONTINUOUS & FILTER & 252 \\
\hline \begin{tabular}{|l|l|}
4831 \\
\end{tabular} & $\mathrm{pCi} / \mathrm{L}$ & 0.012 & & & & $U$ & & RIVER & & CONTINUOUS & RESIN & 252 \\
\hline \begin{tabular}{|l|}
4832 \\
\end{tabular} & $\mathrm{pCi} / \mathrm{L}$ & 1.2 & 1.2 & & & U & & QC_SAMPLE & & RAW GRAB & UNFILTERED & 247 \\
\hline \begin{tabular}{|l|}
4833 \\
\end{tabular} & $\mathrm{pCi} / \mathrm{L}$ & 0.0502 & 0.0634 & & & & & QC_SAMPLE & & RAW GRAB & UNFILTERED & 247 \\
\hline 4834 & pCi/L & 10.4 & 16.9 & & & & & QC_SAMPLE & & RAW GRAB & UNFILTERED & 247 \\
\hline 4835 & pCi/L & 0.0472 & 0.0526 & & & & & QC_SAMPLE & & RAW GRAB & UNFILTERED & 247 \\
\hline 4836 . & $\mathrm{pCi} / \mathrm{L}$ & 0.00801 & 0.00804 & & & $U$ & & QC_SAMPLE & & RAW GRAB & UNFILTERED & 247 \\
\hline \begin{tabular}{|l|l|}
4837 \\
\end{tabular} & $\mathrm{pCi} / \mathrm{L}$ & 0.0453 & 0.0501 & & & & & QC_SAMPLE & & RAW GRAB & UNFILTERED & 247 \\
\hline
\end{tabular}




\begin{tabular}{|c|c|c|c|c|c|c|c|c|c|c|c|c|}
\hline & $\mathrm{N}$ & $\mathrm{O}$ & $\mathrm{P}$ & $\mathrm{Q}$ & $\mathrm{R}$ & $\mathrm{s}$ & $\mathrm{T}$ & $\mathrm{U}$ & $\mathrm{v}$ & $\mathrm{w}$ & $\mathrm{x}$ & $\mathrm{Y}$ \\
\hline 1 & ANAL_UNITS_RPTD & COUNTING_ERROR & TOTAL_ANAL_ERROR & MIN_DETECTABLE_ACTIVITY & PCNT_MOISTURE & LAB_QUALIFIER & REVIEW_QUALIFIER & SAMP_FROM & SAMP_ITEM & SAMP_MTHD & COLL_MTHD & SAMP_SITE_ID \\
\hline 4838 & $\mathrm{pCi} / \mathrm{L}$ & 0.0045 & & & & $U$ & & RIVER & & CONTINUOUS & FILTER & 307 \\
\hline 4839 & $\mathrm{pCi} / \mathrm{L}$ & 0.0085 & & & & $\mathrm{U}$ & & RIVER & & CONTINUOUS & RESIN & 307 \\
\hline 4840 & $\mathrm{pCi} / \mathrm{L}$ & 0.0086 & & & & $\mathrm{U}$ & & RIVER & & CONTINUOUS & RESIN & 252 \\
\hline 4841 & $\mathrm{pCi} / \mathrm{L}$ & 0.0043 & & & & U & & RIVER & & CONTINUOUS & FILTER & 252 \\
\hline 4842 & $\mathrm{pCi} / \mathrm{L}$ & 172 & 229 & & & $\mathrm{U}$ & & DRINKING & & SAN. GRAB & UNFILTERED & 186 \\
\hline 4843 & $\mathrm{pCi} / \mathrm{L}$ & 0.0293 & 0.0296 & & & U & & DRINKING & & SAN. GRAB & UNFILTERED & 186 \\
\hline 4844 & $\mathrm{pCi} / \mathrm{L}$ & 0.778 & 0.779 & & & U & & DRINKING & & SAN. GRAB & UNFILTERED & 186 \\
\hline 4845 & $\mathrm{pCi} / \mathrm{L}$ & 0.474 & 0.478 & & & & & DRINKING & & SAN. GRAB & UNFILTERED & 512 \\
\hline 4846 & $\mathrm{pCi} / \mathrm{L}$ & 172 & 229 & & & U & & DRINKING & & SAN. GRAB & UNFILTERED & 512 \\
\hline 4847 & $\mathrm{pCi} / \mathrm{L}$ & 0.0354 & 0.0412 & & & & & DRINKING & & SAN. GRAB & UNFILTERED & 512 \\
\hline \begin{tabular}{|l|}
4848 \\
\end{tabular} & $\mathrm{pCi} / \mathrm{L}$ & 0.979 & 0.98 & & & U & & DRINKING & & SAN. GRAB & UNFILTERED & 144 \\
\hline 4849 & $\mathrm{pCi} / \mathrm{L}$ & 0.0356 & 0.0384 & & & & & DRINKING & & SAN. GRAB & UNFILTERED & 144 \\
\hline 4850 & $\mathrm{pCi} / \mathrm{L}$ & 171 & 226 & & & $U$ & & DRINKING & & SAN. GRAB & UNFILTERED & 144 \\
\hline \begin{tabular}{|l|l|}
4851 \\
\end{tabular} & $\mathrm{pCi} / \mathrm{L}$ & 0.026 & 0.0272 & & & & & DRINKING & & SAN. GRAB & UNFILTERED & 187 \\
\hline \begin{tabular}{|l|l|}
4852 \\
\end{tabular} & $\mathrm{pCi} / \mathrm{L}$ & 0.433 & 0.436 & & & & & DRINKING & & SAN. GRAB & UNFILTERED & 187 \\
\hline 4853 & $\mathrm{pCi} / \mathrm{L}$ & 173 & 231 & & & U & & DRINKING & & SAN. GRAB & UNFILTERED & 187 \\
\hline \begin{tabular}{|l|}
4854 \\
\end{tabular} & $\mathrm{pCi} / \mathrm{L}$ & 0.648 & 0.654 & & & & & RIVER & & CUMULATIVE & UNFILTERED & 252 \\
\hline \begin{tabular}{|l|}
4855 \\
\end{tabular} & $\mathrm{pCi} / \mathrm{L}$ & 0.0477 & 0.051 & & & & & RIVER & & CUMULATIVE & UNFILTERED & 252 \\
\hline \begin{tabular}{|l|}
4856 \\
\end{tabular} & $\mathrm{pCi} / \mathrm{L}$ & 0.0143 & 0.0143 & & & U & & RIVER & & CUMULATIVE & UNFILTERED & 252 \\
\hline \begin{tabular}{|l|l|}
4857 \\
\end{tabular} & $\mathrm{pCi} / \mathrm{L}$ & 0.0578 & 0.0632 & & & & & RIVER & & CUMULATIVE & UNFILTERED & 252 \\
\hline 4858 & $\mathrm{pCi} / \mathrm{L}$ & 11 & 17.9 & & & & & RIVER & & CUMULATIVE & UNFILTERED & 252 \\
\hline 4859 & $\mathrm{pCi} / \mathrm{L}$ & 0.0356 & 0.0527 & & & & & RIVER & & CUMULATIVE & UNFILTERED & 252 \\
\hline 4860 & $\mathrm{pCi} / \mathrm{L}$ & 0.793 & 0.805 & & & & & RIVER & & CUMULATIVE & UNFILTERED & 463 \\
\hline \begin{tabular}{|l|l|}
4861 \\
\end{tabular} & $\mathrm{pCi} / \mathrm{L}$ & 0.0725 & 0.0778 & & & & & RIVER & & CUMULATIVE & UNFILTERED & 463 \\
\hline 4862 & $\mathrm{pCi} / \mathrm{L}$ & 0.0137 & 0.0137 & & & U & & RIVER & & CUMULATIVE & UNFILTERED & 463 \\
\hline 4863 & $\mathrm{pCi} / \mathrm{L}$ & 0.0799 & 0.0874 & & & & & RIVER & & CUMULATIVE & UNFILTERED & 463 \\
\hline 4864 & $\mathrm{pCi} / \mathrm{L}$ & 10.8 & 18.1 & & & & & RIVER & & CUMULATIVE & UNFILTERED & 463 \\
\hline 4865 & $\mathrm{pCi} / \mathrm{L}$ & 0.038 & 0.0543 & & & & & RIVER & & CUMULATIVE & UNFILTERED & 463 \\
\hline \begin{tabular}{|l|}
4866 \\
\end{tabular} & $\mathrm{pCi} / \mathrm{L}$ & 0.44 & 0.442 & & & U & & DRINKING & & RAW GRAB & UNFILTERED & 553 \\
\hline 4867 & $\mathrm{pCi} / \mathrm{L}$ & 12.4 & 20.1 & & & & & DRINKING & & RAW GRAB & UNFILTERED & 553 \\
\hline 4868 & $\mathrm{pCi} / \mathrm{L}$ & 0.0592 & 0.0737 & & & & & DRINKING & & RAW GRAB & UNFILTERED & 553 \\
\hline 4869 & $\mathrm{pCi} / \mathrm{L}$ & 0.0066 & & & & U & & RIVER & & CONTINUOUS & FILTER & 307 \\
\hline 4870 & $\mathrm{pCi} / \mathrm{L}$ & 0.014 & & & & U & & RIVER & & CONTINUOUS & RESIN & 307 \\
\hline \begin{tabular}{|l|l|}
4871 \\
\end{tabular} & $\mathrm{pCi} / \mathrm{L}$ & 0.0043 & & & & U & & RIVER & & CONTINUOUS & FILTER & 252 \\
\hline \begin{tabular}{|l|}
4872 \\
\end{tabular} & $\mathrm{pCi} / \mathrm{L}$ & 0.0089 & & & & U & & RIVER & & CONTINUOUS & RESIN & 252 \\
\hline 4873 & & & & & & & & RIVER & & CONTINUOUS & FILTER & 307 \\
\hline 4874 & & & & & & & & RIVER & & CONTINUOUS & RESIN & 307 \\
\hline \begin{tabular}{|l|}
4875 \\
\end{tabular} & $\mathrm{pCi} / \mathrm{L}$ & 0.0039 & & & & & & RIVER & & CONTINUOUS & FILTER & 252 \\
\hline \begin{tabular}{|l|}
4876 \\
\end{tabular} & $\mathrm{pCi} / \mathrm{L}$ & 0.0076 & & & & U & & RIVER & & CONTINUOUS & RESIN & 252 \\
\hline \begin{tabular}{|l|l|}
4877 \\
\end{tabular} & $\mathrm{pCi} / \mathrm{L}$ & 0.0986 & 0.283 & & & & & SURFACE & & RAW GRAB & UNFILTERED & 509 \\
\hline \begin{tabular}{|l|}
4878 \\
\end{tabular} & $\mathrm{pCi} / \mathrm{L}$ & 320 & 527 & & & & & SURFACE & & RAW GRAB & UNFILTERED & 509 \\
\hline 4879 & $\mathrm{pCi} / \mathrm{L}$ & 1.53 & 1.54 & & & U & & SURFACE & & RAW GRAB & UNFILTERED & 509 \\
\hline 4880 & $\mathrm{pCi} / \mathrm{L}$ & 1.85 & 1.86 & & & U & & SURFACE & & RAW GRAB & UNFILTERED & 136 \\
\hline \begin{tabular}{|l|l|}
4881 \\
\end{tabular} & $\mathrm{pCi} / \mathrm{L}$ & 212 & 272 & & & U & & SURFACE & & RAW GRAB & UNFILTERED & 136 \\
\hline 4882 & $\mathrm{pCi} / \mathrm{L}$ & 0.077 & 0.385 & & & & & SURFACE & & RAW GRAB & UNFILTERED & 146 \\
\hline 4883 & $\mathrm{pCi} / \mathrm{L}$ & 208 & 265 & & & U & & SURFACE & & RAW GRAB & UNFILTERED & 146 \\
\hline \begin{tabular}{|l|l|}
4884 \\
\end{tabular} & $\mathrm{pCi} / \mathrm{L}$ & 1.79 & 1.89 & & & & & SURFACE & & RAW GRAB & UNFILTERED & 146 \\
\hline 4885 & $\mathrm{pCi} / \mathrm{L}$ & 0.153 & 0.395 & & & & & SURFACE & & RAW GRAB & UNFILTERED & 147 \\
\hline \begin{tabular}{|l|}
4886 \\
\end{tabular} & $\mathrm{pCi} / \mathrm{L}$ & 1.55 & 1.58 & & & & & SURFACE & & RAW GRAB & UNFILTERED & 147 \\
\hline \begin{tabular}{|l|l|}
4887 \\
\end{tabular} & $\mathrm{pCi} / \mathrm{L}$ & 222 & 291 & & & & & SURFACE & & RAW GRAB & UNFILTERED & 147 \\
\hline \begin{tabular}{|l|}
4888 \\
\end{tabular} & $\mathrm{pCi} / \mathrm{L}$ & 0.821 & 0.821 & & & U & & DRINKING & & SAN. GRAB & UNFILTERED & 530 \\
\hline 4889 & $\mathrm{pCi} / \mathrm{L}$ & 0.0397 & 0.0408 & & & & & DRINKING & & SAN. GRAB & UNFILTERED & 487 \\
\hline
\end{tabular}




\begin{tabular}{|c|c|c|c|c|c|c|c|c|c|c|c|c|}
\hline & $\mathrm{N}$ & $\mathrm{O}$ & $\mathrm{P}$ & $\mathrm{Q}$ & $\mathrm{R}$ & $\mathrm{s}$ & $\mathrm{T}$ & $\mathrm{u}$ & $\mathrm{V}$ & w & $\mathrm{x}$ & $\bar{Y}$ \\
\hline 1 & ANAL_UNITS_RPTD & COUNTING_ERROR & TOTAL_ANAL_ERROR & MIN_DETECTABLE_ACTIVITY & PCNT_MOISTURE & LAB_QUALIFIER & REVIEW_QUALIFIER & SAMP_FROM & SAMP_ITEM & SAMP_MTHD & COLL_MTHD & SAMP_SITE_ID \\
\hline 4890 & $\mathrm{pCi} / \mathrm{L}$ & 203 & 255 & & & $U$ & & DRINKING & & SAN. GRAB & UNFILTERED & 487 \\
\hline 4891 & $\mathrm{pCi} / \mathrm{L}$ & 0.929 & 0.929 & & & $\mathrm{U}$ & & DRINKING & & SAN. GRAB & UNFILTERED & 487 \\
\hline 4892 & & & & & & & & RIVER & & CONTINUOUS & FILTER & 307 \\
\hline 4893 & & & & & & & & RIVER & & CONTINUOUS & RESIN & 307 \\
\hline 4894 & $\mathrm{pCi} / \mathrm{L}$ & 0.0089 & & & & & & RIVER & & CONTINUOUS & RESIN & 252 \\
\hline 4895 & $\mathrm{pCi} / \mathrm{L}$ & 0.0043 & & & & & & RIVER & & CONTINUOUS & FILTER & 252 \\
\hline \begin{tabular}{|c|}
4896 \\
\end{tabular} & $\mathrm{pCi} / \mathrm{L}$ & 0.036 & 0.0481 & & & & & RIVER & & CUMULATIVE & UNFILTERED & 252 \\
\hline 4897 & $\mathrm{pCi} / \mathrm{L}$ & 0.939 & 0.944 & & & U & & RIVER & & CUMULATIVE & UNFILTERED & 252 \\
\hline 4898 & $\mathrm{pCi} / \mathrm{L}$ & 0.0525 & 0.0568 & & & & & RIVER & & CUMULATIVE & UNFILTERED & 252 \\
\hline 4899 & $\mathrm{pCi} / \mathrm{L}$ & 0.011 & 0.0111 & & & $\mathrm{U}$ & & RIVER & & CUMULATIVE & UNFILTERED & 252 \\
\hline 4900 & $\mathrm{pCi} / \mathrm{L}$ & 0.0611 & 0.0678 & & & & & RIVER & & CUMULATIVE & UNFILTERED & 252 \\
\hline 4901 & $\mathrm{pCi} / \mathrm{L}$ & 10.3 & 16.8 & & & & & RIVER & & CUMULATIVE & UNFILTERED & 252 \\
\hline 4902 & $\mathrm{pCi} / \mathrm{L}$ & 0.0349 & 0.0425 & & & & & RIVER & & CUMULATIVE & UNFILTERED & 463 \\
\hline 4903 & $\mathrm{pCi} / \mathrm{L}$ & 10.9 & 18.3 & & & & & RIVER & & CUMULATIVE & UNFILTERED & 463 \\
\hline \begin{tabular}{|l|}
4904 \\
\end{tabular} & $\mathrm{pCi} / \mathrm{L}$ & 0.0435 & 0.0478 & & & & & RIVER & & CUMULATIVE & UNFILTERED & 463 \\
\hline \begin{tabular}{|l|}
4905 \\
\end{tabular} & $\mathrm{pCi} / \mathrm{L}$ & 0.00799 & 0.00802 & & & $U$ & & RIVER & & CUMULATIVE & UNFILTERED & 463 \\
\hline 4906 & $\mathrm{pCi} / \mathrm{L}$ & 0.0482 & 0.0539 & & & & & RIVER & & CUMULATIVE & UNFILTERED & 463 \\
\hline \begin{tabular}{|l|l|}
4907 \\
\end{tabular} & $\mathrm{pCi} / \mathrm{L}$ & 0.597 & 0.597 & & & $U$ & & RIVER & & CUMULATIVE & UNFILTERED & 463 \\
\hline 4908 & $\mathrm{pCi} / \mathrm{L}$ & 0.0856 & 0.0949 & & & & & IRRIGATION & & RAW GRAB & UNFILTERED & 440 \\
\hline \begin{tabular}{|l|}
4909 \\
\end{tabular} & $\mathrm{pCi} / \mathrm{L}$ & 2.16 & 2.17 & & & $U$ & & IRRIGATION & & RAW GRAB & UNFILTERED & 440 \\
\hline 4910 & $\mathrm{pCi} / \mathrm{L}$ & 0.0612 & 0.159 & & & & & DRINKING & & SAN. GRAB & UNFILTERED & 530 \\
\hline \begin{tabular}{|l|l|}
4911 \\
\end{tabular} & $\mathrm{pCi} / \mathrm{L}$ & 11.4 & 20 & & & & & DRINKING & & SAN. GRAB & UNFILTERED & 530 \\
\hline \begin{tabular}{|l|}
4912 \\
\end{tabular} & $\mathrm{pCi} / \mathrm{L}$ & 0.657 & 0.657 & & & U & & DRINKING & & SAN. GRAB & UNFILTERED & 530 \\
\hline \begin{tabular}{|l|}
4913 \\
\end{tabular} & $\mathrm{pCi} / \mathrm{L}$ & 0.0042 & & & & & & RIVER & & CONTINUOUS & FILTER & 307 \\
\hline 4914 & $\mathrm{pCi} / \mathrm{L}$ & 0.0084 & & & & & & RIVER & & CONTINUOUS & RESIN & 307 \\
\hline 4915 & $\mathrm{pCi} / \mathrm{L}$ & 0.0031 & & & & & & RIVER & & CONTINUOUS & FILTER & 252 \\
\hline \begin{tabular}{|l|}
4916 \\
\end{tabular} & $\mathrm{pCi} / \mathrm{L}$ & 0.0064 & & & & $U$ & & RIVER & & CONTINUOUS & RESIN & 252 \\
\hline \begin{tabular}{|l|l|}
4917 \\
\end{tabular} & $\mathrm{pCi} / \mathrm{L}$ & 0.0082 & & & & U & & RIVER & & CONTINUOUS & RESIN & 307 \\
\hline 4918 & $\mathrm{pCi} / \mathrm{L}$ & & 0.000008082 & & & & & RIVER & & CONTINUOUS & RESIN & 307 \\
\hline 4919 & $\mathrm{pCi} / \mathrm{L}$ & 0.00154 & & & & & & RIVER & & CONTINUOUS & RESIN & 307 \\
\hline 4920 & $\mathrm{pCi} / \mathrm{L}$ & 0.0001128 & & & & $U$ & & RIVER & & CONTINUOUS & RESIN & 307 \\
\hline \begin{tabular}{|l|}
4921 \\
\end{tabular} & $\mathrm{pCi} / \mathrm{L}$ & 0.0038 & & & & $U$ & & RIVER & & CONTINUOUS & FILTER & 307 \\
\hline 4922 & $\mathrm{pCi} / \mathrm{L}$ & 0.0000094 & & & & & & RIVER & & CONTINUOUS & FILTER & 307 \\
\hline \begin{tabular}{|l|}
4923 \\
\end{tabular} & $\mathrm{pCi} / \mathrm{L}$ & 0.0039 & & & & $U$ & & RIVER & & CONTINUOUS & FILTER & 252 \\
\hline \begin{tabular}{|l|}
4924 \\
\end{tabular} & $\mathrm{pCi} / \mathrm{L}$ & 0.0000062 & & & & & & RIVER & & CONTINUOUS & FILTER & 252 \\
\hline \begin{tabular}{|l|}
4925 \\
\end{tabular} & $\mathrm{pCi} / \mathrm{L}$ & 0.482 & 0.486 & & & & & RIVER & & CUMULATIVE & UNFILTERED & 252 \\
\hline \begin{tabular}{|l|}
4926 \\
\end{tabular} & $\mathrm{pCi} / \mathrm{L}$ & 0.0359 & 0.044 & & & & & RIVER & & CUMULATIVE & UNFILTERED & 252 \\
\hline \begin{tabular}{|l|l|}
4927 \\
\end{tabular} & $\mathrm{pCi} / \mathrm{L}$ & 0.0386 & 0.0395 & & & & & RIVER & & CUMULATIVE & UNFILTERED & 252 \\
\hline \begin{tabular}{|l|}
4928 \\
\end{tabular} & $\mathrm{pCi} / \mathrm{L}$ & 0.00909 & 0.0091 & & & U & & RIVER & & CUMULATIVE & UNFILTERED & 252 \\
\hline \begin{tabular}{|l|}
4929 \\
\end{tabular} & $\mathrm{pCi} / \mathrm{L}$ & 0.0445 & 0.046 & & & & & RIVER & & CUMULATIVE & UNFILTERED & 252 \\
\hline 4930 & $\mathrm{pCi} / \mathrm{L}$ & 7.07 & 14.5 & & & & & RIVER & & CUMULATIVE & UNFILTERED & 252 \\
\hline 4931 & $\mathrm{pCi} / \mathrm{L}$ & 0.0083 & & & & $U$ & & RIVER & & CONTINUOUS & RESIN & 252 \\
\hline \begin{tabular}{|l|}
4932 \\
\end{tabular} & $\mathrm{pCi} / \mathrm{L}$ & & 0.000000787 & & & & & RIVER & & CONTINUOUS & RESIN & 252 \\
\hline 4933 & $\mathrm{pCi} / \mathrm{L}$ & 0.00066 & & & & & & RIVER & & CONTINUOUS & RESIN & 252 \\
\hline 4934 & $\mathrm{pCi} / \mathrm{L}$ & 0.000116 & & & & $U$ & & RIVER & & CONTINUOUS & RESIN & 252 \\
\hline 4935 & $\mathrm{pCi} / \mathrm{L}$ & 0.823 & 0.823 & & & $U$ & & RIVER & & CUMULATIVE & UNFILTERED & 463 \\
\hline \begin{tabular}{|l|}
4936 \\
\end{tabular} & $\mathrm{pCi} / \mathrm{L}$ & 0.0224 & 0.0348 & & & & & RIVER & & CUMULATIVE & UNFILTERED & 463 \\
\hline \begin{tabular}{|l|l|}
4937 \\
\end{tabular} & $\mathrm{pCi} / \mathrm{L}$ & 0.0562 & 0.0585 & & & & & RIVER & & CUMULATIVE & UNFILTERED & 463 \\
\hline \begin{tabular}{|l|}
4938 \\
\end{tabular} & $\mathrm{pCi} / \mathrm{L}$ & 0.015 & 0.0151 & & & $U$ & & RIVER & & CUMULATIVE & UNFILTERED & 463 \\
\hline 4939 & $\mathrm{pCi} / \mathrm{L}$ & 0.0672 & 0.0711 & & & & & RIVER & & CUMULATIVE & UNFILTERED & 463 \\
\hline 4940 & $\mathrm{pCi} / \mathrm{L}$ & 8.37 & 19.2 & & & & & RIVER & & CUMULATIVE & UNFILTERED & 463 \\
\hline \begin{tabular}{|l|l|}
4941 \\
\end{tabular} & $\mathrm{pCi} / \mathrm{L}$ & 0.916 & 0.917 & & & $\mathrm{U}$ & & DRINKING & & CUMULATIVE & UNFILTERED & 307 \\
\hline
\end{tabular}




\begin{tabular}{|c|c|c|c|c|c|c|c|c|c|c|c|c|}
\hline & $\mathrm{N}$ & $\mathrm{O}$ & $\mathrm{P}$ & $\bar{Q}$ & $\mathrm{R}$ & $\mathrm{s}$ & $\mathrm{T}$ & $\mathrm{u}$ & $\mathrm{v}$ & $\mathrm{w}$ & $\mathrm{x}$ & $\bar{Y}$ \\
\hline 1 & ANAL_UNITS_RPTD & COUNTING_ERROR & \begin{tabular}{|l|} 
TOTAL_ANAL_ERROR \\
\end{tabular} & MIN_DETECTABLE_ACTIVITY & PCNT_MOISTURE & LAB_QUALIFIER & REVIEW_QUALIFIER & SAMP_FROM & SAMP_ITEM & SAMP_MTHD & COLL_MTHD & SAMP_SITE_ID \\
\hline 4942 & $\mathrm{pCi} / \mathrm{L}$ & 0.033 & 0.0393 & & & & & DRINKING & & CUMULATIVE & UNFILTERED & 307 \\
\hline 4943 & $\mathrm{pCi} / \mathrm{L}$ & 0.0768 & 0.0821 & & & & & DRINKING & & CUMULATIVE & UNFILTERED & 307 \\
\hline 4944 & $\mathrm{pCi} / \mathrm{L}$ & 0.0112 & 0.0112 & & & $\mathrm{U}$ & & DRINKING & & CUMULATIVE & UNFILTERED & 307 \\
\hline 4945 & $\mathrm{pCi} / \mathrm{L}$ & 0.0869 & 0.0944 & & & & & DRINKING & & CUMULATIVE & UNFILTERED & 307 \\
\hline 4946 & $\mathrm{pCi} / \mathrm{L}$ & 8.52 & 20.4 & & & & & DRINKING & & CUMULATIVE & UNFILTERED & 307 \\
\hline 4947 & $\mathrm{pCi} / \mathrm{L}$ & 0.0082 & & & & $\mathrm{U}$ & & RIVER & & CONTINUOUS & RESIN & 307 \\
\hline 4948 & $\mathrm{pCi} / \mathrm{L}$ & 0.0038 & & & & & & RIVER & & CONTINUOUS & FILTER & 307 \\
\hline 4949 & $\mathrm{pCi} / \mathrm{L}$ & 0.011 & & & & $\mathrm{U}$ & & RIVER & & CONTINUOUS & RESIN & 252 \\
\hline 4950 & $\mathrm{pCi} / \mathrm{L}$ & 0.0049 & & & & $\mathrm{U}$ & & RIVER & & CONTINUOUS & FILTER & 252 \\
\hline 4951 & $\mathrm{pCi} / \mathrm{L}$ & 0.808 & 0.809 & & & $\mathrm{U}$ & & DRINKING & & SAN. GRAB & UNFILTERED & 186 \\
\hline 4952 & $\mathrm{pCi} / \mathrm{L}$ & 174 & 230 & & & U & & DRINKING & & SAN. GRAB & UNFILTERED & 186 \\
\hline 4953 & $\mathrm{pCi} / \mathrm{L}$ & 0.0269 & 0.0327 & & & & & DRINKING & & SAN. GRAB & UNFILTERED & 186 \\
\hline 4954 & $\mathrm{pCi} / \mathrm{L}$ & 0.944 & 0.944 & & & $U$ & & DRINKING & & SAN. GRAB & UNFILTERED & 512 \\
\hline 4955 & $\mathrm{pCi} / \mathrm{L}$ & 206 & 304 & & & & & DRINKING & & SAN. GRAB & UNFILTERED & 512 \\
\hline 4956 & $\mathrm{pCi} / \mathrm{L}$ & 0.0398 & 0.0436 & & & & & DRINKING & & SAN. GRAB & UNFILTERED & 512 \\
\hline 4957 & $\mathrm{pCi} / \mathrm{L}$ & 0.808 & 0.809 & & & $U$ & & DRINKING & & SAN. GRAB & UNFILTERED & 144 \\
\hline 4958 & $\mathrm{pCi} / \mathrm{L}$ & 175 & 233 & & & U & & DRINKING & & SAN. GRAB & UNFILTERED & 144 \\
\hline 4959 & $\mathrm{pCi} / \mathrm{L}$ & 0.0345 & 0.0354 & & & U & & DRINKING & & SAN. GRAB & UNFILTERED & 144 \\
\hline 4960 & $\mathrm{pCi} / \mathrm{L}$ & 1.28 & 1.28 & & & U & & DRINKING & & SAN. GRAB & UNFILTERED & 187 \\
\hline 4961 & $\mathrm{pCi} / \mathrm{L}$ & 175 & 232 & & & $U$ & & DRINKING & & SAN. GRAB & UNFILTERED & 187 \\
\hline 4962 & $\mathrm{pCi} / \mathrm{L}$ & 0.019 & 0.0196 & & & & & DRINKING & & SAN. GRAB & UNFILTERED & 187 \\
\hline 4963 & $\mathrm{pCi} / \mathrm{L}$ & 0.0429 & 0.0498 & & & & & DRINKING & & RAW GRAB & UNFILTERED & 553 \\
\hline 4964 & $\mathrm{pCi} / \mathrm{L}$ & 0.639 & 0.64 & & & U & & DRINKING & & RAW GRAB & UNFILTERED & 553 \\
\hline 4965 & $\mathrm{pCi} / \mathrm{L}$ & 7.71 & 16.2 & & & & & DRINKING & & RAW GRAB & UNFILTERED & 553 \\
\hline 4966 & $\mathrm{pCi} / \mathrm{L}$ & 0.0087 & & & & $U$ & & RIVER & & CONTINUOUS & RESIN & 307 \\
\hline 4967 & $\mathrm{pCi} / \mathrm{L}$ & 0.0039 & & & & $U$ & & RIVER & & CONTINUOUS & FILTER & 307 \\
\hline 4968 & $\mathrm{pCi} / \mathrm{L}$ & 0.0425 & 0.047 & & & & & RIVER & & CUMULATIVE & UNFILTERED & 252 \\
\hline 4969 & $\mathrm{pCi} / \mathrm{L}$ & 0.00756 & 0.00759 & & & $U$ & & RIVER & & CUMULATIVE & UNFILTERED & 252 \\
\hline 4970 & $\mathrm{pCi} / \mathrm{L}$ & 0.0486 & 0.0551 & & & & & RIVER & & CUMULATIVE & UNFILTERED & 252 \\
\hline 4971 & $\mathrm{pCi} / \mathrm{L}$ & 0.0478 & 0.0578 & & & & & RIVER & & CUMULATIVE & UNFILTERED & 252 \\
\hline 4972 & $\mathrm{pCi} / \mathrm{L}$ & 0.712 & 0.713 & & & U & & RIVER & & CUMULATIVE & UNFILTERED & 252 \\
\hline 4973 & $\mathrm{pCi} / \mathrm{L}$ & 7.06 & 14.4 & & & & & RIVER & & CUMULATIVE & UNFILTERED & 252 \\
\hline 4974 & $\mathrm{pCi} / \mathrm{L}$ & 0.01 & & & & $U$ & & RIVER & & CONTINUOUS & RESIN & 252 \\
\hline 4975 & $\mathrm{pCi} / \mathrm{L}$ & 0.0045 & & & & $U$ & & RIVER & & CONTINUOUS & FILTER & 252 \\
\hline 4976 & $\mathrm{pCi} / \mathrm{L}$ & 0.0433 & 0.0482 & & & & & RIVER & & CUMULATIVE & UNFILTERED & 463 \\
\hline 4977 & $\mathrm{pCi} / \mathrm{L}$ & 0.0103 & 0.0103 & & & U & & RIVER & & CUMULATIVE & UNFILTERED & 463 \\
\hline 4978 & $\mathrm{pCi} / \mathrm{L}$ & 0.0487 & 0.0556 & & & & & RIVER & & CUMULATIVE & UNFILTERED & 463 \\
\hline 4979 & $\mathrm{pCi} / \mathrm{L}$ & 0.0441 & 0.0559 & & & & & RIVER & & CUMULATIVE & UNFILTERED & 463 \\
\hline 4980 & $\mathrm{pCi} / \mathrm{L}$ & 0.45 & 0.453 & & & & & RIVER & & CUMULATIVE & UNFILTERED & 463 \\
\hline 4981 & $\mathrm{pCi} / \mathrm{L}$ & 9.17 & 20.2 & & & & & RIVER & & CUMULATIVE & UNFILTERED & 463 \\
\hline 4982 & $\mathrm{pCi} / \mathrm{L}$ & 0.0047 & & & & $U$ & & RIVER & & CONTINUOUS & FILTER & 307 \\
\hline 4983 & $\mathrm{pCi} / \mathrm{L}$ & 0.0095 & & & & $U$ & & RIVER & & CONTINUOUS & RESIN & 307 \\
\hline 4984 & $\mathrm{pCi} / \mathrm{L}$ & 0.013 & & & & U & & RIVER & & CONTINUOUS & RESIN & 252 \\
\hline 4985 & $\mathrm{pCi} / \mathrm{L}$ & 0.0064 & & & & U & & RIVER & & CONTINUOUS & FILTER & 252 \\
\hline 4986 & $\mathrm{pCi} / \mathrm{L}$ & 2.21 & 2.22 & & & $U$ & & SURFACE & & RAW GRAB & UNFILTERED & 509 \\
\hline 4987 & $\mathrm{pCi} / \mathrm{L}$ & 0.288 & 0.314 & & & $U$ & & SURFACE & & RAW GRAB & UNFILTERED & 509 \\
\hline 4988 & $\mathrm{pCi} / \mathrm{L}$ & 203 & 260 & & & $U$ & & SURFACE & & RAW GRAB & UNFILTERED & 509 \\
\hline 4989 & $\mathrm{pCi} / \mathrm{L}$ & 1.89 & 1.91 & & & & & SURFACE & & RAW GRAB & UNFILTERED & 136 \\
\hline 4990 & $\mathrm{pCi} / \mathrm{L}$ & 217 & 287 & & & & & SURFACE & & RAW GRAB & UNFILTERED & 136 \\
\hline 4991 & $\mathrm{pCi} / \mathrm{L}$ & 2.34 & 2.63 & & & & & SURFACE & & RAW GRAB & UNFILTERED & 146 \\
\hline 4992 & $\mathrm{pCi} / \mathrm{L}$ & 0.161 & 0.878 & & & & & SURFACE & & RAW GRAB & UNFILTERED & 146 \\
\hline 4993 & $\mathrm{pCi} / \mathrm{L}$ & 208 & 268 & & & $U_{2}$ & & SURFACE & & RAW GRAB & UNFILTERED & 146 \\
\hline
\end{tabular}




\begin{tabular}{|c|c|c|c|c|c|c|c|c|c|c|c|c|}
\hline & $\mathrm{N}$ & $\mathrm{O}$ & $\mathrm{P}$ & $\mathrm{Q}$ & $\mathrm{R}$ & $\mathrm{s}$ & $\mathrm{T}$ & $\mathrm{U}$ & $\mathrm{V}$ & $\mathrm{w}$ & $\mathrm{x}$ & $\mathrm{Y}$ \\
\hline 1 & ANAL_UNITS_RPTD & COUNTING_ERROR & TOTAL_ANAL_ERROR & MIN_DETECTABLE_ACTIVITY & PCNT_MOISTURE & LAB_QUALIFIER & REVIEW_QUALIFIER & SAMP_FROM & SAMP_ITEM & SAMP_MTHD & COLL_MTHD & SAMP_SITE_ID \\
\hline 4994 & $\mathrm{pCi} / \mathrm{L}$ & 2.88 & 2.89 & & & $U$ & & SURFACE & & RAW GRAB & UNFILTERED & 147 \\
\hline 4995 & $\mathrm{pCi} / \mathrm{L}$ & 0.189 & 0.511 & & & & & SURFACE & & RAW GRAB & UNFILTERED & 147 \\
\hline 4996 & $\mathrm{pCi} / \mathrm{L}$ & 214 & 281 & & & & & SURFACE & & RAW GRAB & UNFILTERED & 147 \\
\hline 4997 & $\mathrm{pCi} / \mathrm{L}$ & 0.0351 & 0.0398 & & & & & DRINKING & & SAN. GRAB & UNFILTERED & 487 \\
\hline 4998 & $\mathrm{pCi} / \mathrm{L}$ & 0.87 & 0.871 & & & $\mathrm{U}$ & & DRINKING & & SAN. GRAB & UNFILTERED & 487 \\
\hline 4999 & $\mathrm{pCi} / \mathrm{L}$ & 202 & 257 & & & $\mathrm{U}$ & & DRINKING & & SAN. GRAB & UNFILTERED & 487 \\
\hline 5000 & $\mathrm{pCi} / \mathrm{L}$ & 0.0037 & & & & $\mathrm{U}$ & & RIVER & & CONTINUOUS & FILTER & 307 \\
\hline 5001 & $\mathrm{pCi} / \mathrm{L}$ & 0.0079 & & & & $\mathrm{U}$ & & RIVER & & CONTINUOUS & RESIN & 307 \\
\hline 5002 & $\mathrm{pCi} / \mathrm{L}$ & 0.011 & & & & U & & RIVER & & CONTINUOUS & RESIN & 252 \\
\hline 5003 & $\mathrm{pCi} / \mathrm{L}$ & 0.0056 & & & & $U$ & & RIVER & & CONTINUOUS & FILTER & 252 \\
\hline 5004 & $\mathrm{pCi} / \mathrm{L}$ & 0.0406 & 0.0556 & & & & & IRRIGATION & & RAW GRAB & UNFILTERED & 440 \\
\hline 5005 & $\mathrm{pCi} / \mathrm{L}$ & 1.02 & 1.02 & & & U & & IRRIGATION & & RAW GRAB & UNFILTERED & 440 \\
\hline 5006 & $\mathrm{pCi} / \mathrm{L}$ & 0.0961 & 0.183 & & & & & DRINKING & & SAN. GRAB & UNFILTERED & 530 \\
\hline \begin{tabular}{|l|}
5007 \\
\end{tabular} & $\mathrm{pCi} / \mathrm{L}$ & 0.922 & 0.925 & & & U & & DRINKING & & SAN. GRAB & UNFILTERED & 530 \\
\hline 5008 & $\mathrm{pCi} / \mathrm{L}$ & 8.28 & 20.1 & & & & & DRINKING & & SAN. GRAB & UNFILTERED & 530 \\
\hline 5009 & $\mathrm{pCi} / \mathrm{L}$ & 0.0392 & 0.0548 & & & & & RIVER & & CUMULATIVE & UNFILTERED & 252 \\
\hline 5010 & $\mathrm{pCi} / \mathrm{L}$ & 0.037 & 0.0405 & & & & & RIVER & & CUMULATIVE & UNFILTERED & 252 \\
\hline 5011 & $\mathrm{pCi} / \mathrm{L}$ & 0.00554 & 0.00555 & & & U & & RIVER & & CUMULATIVE & UNFILTERED & 252 \\
\hline \begin{tabular}{|l|}
5012 \\
\end{tabular} & $\mathrm{pCi} / \mathrm{L}$ & 0.0417 & 0.0467 & & & & & RIVER & & CUMULATIVE & UNFILTERED & 252 \\
\hline 5013 & $\mathrm{pCi} / \mathrm{L}$ & 0.649 & 0.65 & & & $U$ & & RIVER & & CUMULATIVE & UNFILTERED & 252 \\
\hline \begin{tabular}{|l|l|}
5014 \\
\end{tabular} & $\mathrm{pCi} / \mathrm{L}$ & 8.19 & 17.2 & & & & & RIVER & & CUMULATIVE & UNFILTERED & 252 \\
\hline 5015 & $\mathrm{pCi} / \mathrm{L}$ & 0.0382 & 0.0586 & & & & & RIVER & & CUMULATIVE & UNFILTERED & 463 \\
\hline 5016 & $\mathrm{pCi} / \mathrm{L}$ & 0.0409 & 0.0458 & & & & & RIVER & & CUMULATIVE & UNFILTERED & 463 \\
\hline 5017 & $\mathrm{pCi} / \mathrm{L}$ & 0.00535 & 0.00567 & & & $U$ & & RIVER & & CUMULATIVE & UNFILTERED & 463 \\
\hline 5018 & $\mathrm{pCi} / \mathrm{L}$ & 0.0444 & 0.0507 & & & & & RIVER & & CUMULATIVE & UNFILTERED & 463 \\
\hline \begin{tabular}{|l|}
5019 \\
\end{tabular} & $\mathrm{pCi} / \mathrm{L}$ & 0.764 & 0.767 & & & $\mathrm{U}$ & & RIVER & & CUMULATIVE & UNFILTERED & 463 \\
\hline 5020 & $\mathrm{pCi} / \mathrm{L}$ & 8.29 & 20 & & & & & RIVER & & CUMULATIVE & UNFILTERED & 463 \\
\hline 5021 & $\mathrm{pCi} / \mathrm{L}$ & 0.009 & & & & $\mathrm{U}$ & & RIVER & & CONTINUOUS & RESIN & 307 \\
\hline 5022 & $\mathrm{pCi} / \mathrm{L}$ & 0.0044 & & & & U & & RIVER & & CONTINUOUS & FILTER & 307 \\
\hline 5023 & $\mathrm{pCi} / \mathrm{L}$ & 0.012 & & & & U & & RIVER & & CONTINUOUS & RESIN & 252 \\
\hline 5024 & $\mathrm{pCi} / \mathrm{L}$ & 0.0057 & & & & U & & RIVER & & CONTINUOUS & FILTER & 252 \\
\hline \begin{tabular}{|l|l|}
5025 \\
\end{tabular} & $\mathrm{pCi} / \mathrm{L}$ & 0.0087 & & & & $U$ & & RIVER & & CONTINUOUS & RESIN & 307 \\
\hline \begin{tabular}{|l|l|}
5026 \\
\end{tabular} & $\mathrm{pCi} / \mathrm{L}$ & 0.0041 & & & & $\mathrm{U}$ & & RIVER & & CONTINUOUS & FILTER & 307 \\
\hline \begin{tabular}{|l|}
5027 \\
\end{tabular} & $\mathrm{pCi} / \mathrm{L}$ & 0.0081 & & & & U & & RIVER & & CONTINUOUS & RESIN & 252 \\
\hline 5028 & $\mathrm{pCi} / \mathrm{L}$ & 0.0038 & & & & $U$ & & RIVER & & CONTINUOUS & FILTER & 252 \\
\hline 5029 & $\mathrm{pCi} / \mathrm{L}$ & 0.812 & 0.813 & & & U & & DRINKING & & CUMULATIVE & UNFILTERED & 307 \\
\hline 5030 & $\mathrm{pCi} / \mathrm{L}$ & 0.0351 & 0.0381 & & & & & DRINKING & & CUMULATIVE & UNFILTERED & 307 \\
\hline \begin{tabular}{|l|}
5031 \\
\end{tabular} & $\mathrm{pCi} / \mathrm{L}$ & 0.013 & 0.0132 & & & & & DRINKING & & CUMULATIVE & UNFILTERED & 307 \\
\hline \begin{tabular}{|l|l|}
5032 \\
\end{tabular} & $\mathrm{pCi} / \mathrm{L}$ & 0.042 & 0.0472 & & & & & DRINKING & & CUMULATIVE & UNFILTERED & 307 \\
\hline 5033 & $\mathrm{pCi} / \mathrm{L}$ & 9.31 & 25.6 & & & & & DRINKING & & CUMULATIVE & UNFILTERED & 307 \\
\hline 5034 & $\mathrm{pCi} / \mathrm{L}$ & 0.0275 & 0.0436 & & & & & DRINKING & & CUMULATIVE & UNFILTERED & 307 \\
\hline 5035 & $\mathrm{pCi} / \mathrm{L}$ & 0.909 & 0.912 & & & U & & RIVER & & CUMULATIVE & UNFILTERED & 252 \\
\hline 5036 & $\mathrm{pCi} / \mathrm{L}$ & 0.0548 & 0.0608 & & & & & RIVER & & CUMULATIVE & UNFILTERED & 252 \\
\hline 5037 & $\mathrm{pCi} / \mathrm{L}$ & 0.0114 & 0.0114 & & & $U$ & & RIVER & & CUMULATIVE & UNFILTERED & 252 \\
\hline \begin{tabular}{|l|l|}
5038 \\
\end{tabular} & $\mathrm{pCi} / \mathrm{L}$ & 0.068 & 0.0784 & & & & & RIVER & & CUMULATIVE & UNFILTERED & 252 \\
\hline \begin{tabular}{|l|}
5039 \\
\end{tabular} & $\mathrm{pCi} / \mathrm{L}$ & 0.0279 & 0.0455 & & & & & RIVER & & CUMULATIVE & UNFILTERED & 252 \\
\hline 5040 & $\mathrm{pCi} / \mathrm{L}$ & 7.46 & 15.2 & & & & & RIVER & & CUMULATIVE & UNFILTERED & 252 \\
\hline \begin{tabular}{|l|l|}
5041 \\
\end{tabular} & $\mathrm{pCi} / \mathrm{L}$ & 0.682 & 0.682 & & & $\mathrm{U}$ & & RIVER & & CUMULATIVE & UNFILTERED & 463 \\
\hline \begin{tabular}{|l|}
5042 \\
\end{tabular} & $\mathrm{pCi} / \mathrm{L}$ & 0.0444 & 0.0502 & & & & & RIVER & & CUMULATIVE & UNFILTERED & 463 \\
\hline 5043 & $\mathrm{pCi} / \mathrm{L}$ & 0.00894 & 0.00899 & & & & & RIVER & & CUMULATIVE & UNFILTERED & 463 \\
\hline 5044 & $\mathrm{pCi} / \mathrm{L}$ & 0.0509 & 0.0595 & & & & & RIVER & & CUMULATIVE & UNFILTERED & 463 \\
\hline 5045 & $\mathrm{pCi} / \mathrm{L}$ & 0.03 & 0.0489 & & & & & RIVER & & CUMULATIVE & UNFILTERED & 463 \\
\hline
\end{tabular}




\begin{tabular}{|c|c|c|c|c|c|c|c|c|c|c|c|c|}
\hline & $\mathrm{N}$ & $\mathrm{O}$ & $\mathrm{P}$ & $\mathrm{Q}$ & $\mathrm{R}$ & $\mathrm{S}$ & $T$ & $U$ & $\mathrm{~V}$ & $\mathrm{~W}$ & $\mathrm{X}$ & $\mathrm{Y}$ \\
\hline 1 & ANAL_UNITS_RPTD & COUNTING_ERROR & TOTAL_ANAL_ERROR & MIN_DETECTABLE_ACTIVITY & PCNT_MOISTURE & LAB_QUALIFIER & REVIEW_QUALIFIER & SAMP_FROM & SAMP_ITEM & SAMP_MTHD & COLL_MTHD & SAMP_SITE_ID \\
\hline 5046 & $\mathrm{pCi} / \mathrm{L}$ & 8.23 & 18.7 & & & & & RIVER & & CUMULATIVE & UNFILTERED & 463 \\
\hline $5047 \mathrm{r}$ & $\mathrm{pCi} / \mathrm{L}$ & 0.0075 & & & & $\mathrm{U}$ & & RIVER & & CONTINUOUS & RESIN & 307 \\
\hline $5048 \mathrm{r}$ & pCi/L & 0.0035 & & & & U & & RIVER & & CONTINUOUS & FILTER & 307 \\
\hline 5049 & $\mathrm{pCi} / \mathrm{L}$ & & 0.00001447 & & & & & RIVER & & CONTINUOUS & RESIN & 307 \\
\hline 5050 & $\mathrm{pCi} / \mathrm{L}$ & 0.00152 & & & & & & RIVER & & CONTINUOUS & RESIN & 307 \\
\hline $5051 \mathrm{r}$ & $\mathrm{pCi} / \mathrm{L}$ & 0.000962 & & & & $\mathrm{U}$ & & RIVER & & CONTINUOUS & RESIN & 307 \\
\hline 5052 & $\mathrm{pCi} / \mathrm{L}$ & 0.0000074 & & & & & & RIVER & & CONTINUOUS & FILTER & 307 \\
\hline 5053 & $\mathrm{pCi} / \mathrm{L}$ & 0.0083 & & & & $\mathrm{U}$ & & RIVER & & CONTINUOUS & RESIN & 252 \\
\hline 5054 & $\mathrm{pCi} / \mathrm{L}$ & 0.0039 & & & & $\mathrm{U}$ & & RIVER & & CONTINUOUS & FILTER & 252 \\
\hline 5055 & $\mathrm{pCi} / \mathrm{L}$ & & 0.00000129 & & & & & RIVER & & CONTINUOUS & RESIN & 252 \\
\hline 5056 & $\mathrm{pCi} / \mathrm{L}$ & 0.00066 & & & & & & RIVER & & CONTINUOUS & RESIN & 252 \\
\hline $5057 \mathrm{r}$ & $\mathrm{pCi} / \mathrm{L}$ & 0.000151 & & & & U & & RIVER & & CONTINUOUS & RESIN & 252 \\
\hline 5058 & $\mathrm{pCi} / \mathrm{L}$ & 0.0000088 & & & & & & RIVER & & CONTINUOUS & FILTER & 252 \\
\hline 5059 & $\mathrm{pCi} / \mathrm{L}$ & 0.712 & 0.712 & & & U & & DRINKING & & SAN. GRAB & UNFILTERED & 186 \\
\hline 5060 & $\mathrm{pCi} / \mathrm{L}$ & 221 & 288 & & & U & & DRINKING & & SAN. GRAB & UNFILTERED & 186 \\
\hline $5061 \mathrm{r}$ & $\mathrm{pCi} / \mathrm{L}$ & 0.0252 & 0.0289 & & & & & DRINKING & & SAN. GRAB & UNFILTERED & 186 \\
\hline 5062 & $\mathrm{pCi} / \mathrm{L}$ & 0.962 & 0.963 & & & U & & DRINKING & & SAN. GRAB & UNFILTERED & 512 \\
\hline 5063 & pCi/L & 322 & 560 & & & & & DRINKING & & SAN. GRAB & UNFILTERED & 512 \\
\hline 5064 & $\mathrm{pCi} / \mathrm{L}$ & 0.0277 & 0.0334 & & & & & DRINKING & & SAN. GRAB & UNFILTERED & 512 \\
\hline 5065 & $\mathrm{pCi} / \mathrm{L}$ & 0.742 & 0.742 & & & $U$ & & DRINKING & & SAN. GRAB & UNFILTERED & 144 \\
\hline 5066 & $\mathrm{pCi} / \mathrm{L}$ & 220 & 285 & & & U & & DRINKING & & SAN. GRAB & UNFILTERED & 144 \\
\hline 5067 & pCi/L & 0.0228 & 0.0262 & & & & & DRINKING & & SAN. GRAB & UNFILTERED & 144 \\
\hline 5068 & pCi/L & 0.519 & 0.524 & & & & & DRINKING & & SAN. GRAB & UNFILTERED & 187 \\
\hline 5069 & pCi/L & 218 & 283 & & & U & & DRINKING & & SAN. GRAB & UNFILTERED & 187 \\
\hline 5070 & $\mathrm{pCi} / \mathrm{L}$ & 0.0247 & 0.0253 & & & $U$ & & DRINKING & & SAN. GRAB & UNFILTERED & 187 \\
\hline 5071 & $\mathrm{pCi} / \mathrm{L}$ & 0.776 & 0.777 & & & U & & DRINKING & & RAW GRAB & UNFILTERED & 553 \\
\hline $5072 \mathrm{r}$ & $\mathrm{pCi} / \mathrm{L}$ & 0.0256 & 0.0448 & & & & & DRINKING & & RAW GRAB & UNFILTERED & 553 \\
\hline 5073 & $\mathrm{pCi} / \mathrm{L}$ & 7.56 & 15.8 & & & & & DRINKING & & RAW GRAB & UNFILTERED & 553 \\
\hline 5074 & $\mathrm{pCi} / \mathrm{L}$ & 8.1 & 16.9 & & & & & QC_SAMPLE & & RAW GRAB & UNFILTERED & 550 \\
\hline 5075 & $\mathrm{pCi} / \mathrm{L}$ & 0.948 & 0.948 & & & U & & QC_SAMPLE & & RAW GRAB & UNFILTERED & 550 \\
\hline 5076 & $\mathrm{pCi} / \mathrm{L}$ & 0.048 & 0.056 & & & & & QC_SAMPLE & & RAW GRAB & UNFILTERED & 550 \\
\hline $5077 \mathrm{r}$ & $\mathrm{pCi} / \mathrm{L}$ & 0.0113 & 0.0115 & & & & & QC_SAMPLE & & RAW GRAB & UNFILTERED & 550 \\
\hline 5078 & $\mathrm{pCi} / \mathrm{L}$ & 0.0435 & 0.0495 & & & & & QC_SAMPLE & & RAW GRAB & UNFILTERED & 550 \\
\hline 5079 & $\mathrm{pCi} / \mathrm{L}$ & 0.0257 & 0.043 & & & & & QC_SAMPLE & & RAW GRAB & UNFILTERED & 550 \\
\hline 5080 & pCi/L & 7.92 & 16.9 & & & & & QC_SAMPLE & & RAW GRAB & UNFILTERED & 247 \\
\hline 5081 & pCi/L & 0.926 & 0.929 & & & U & & QC_SAMPLE & & RAW GRAB & UNFILTERED & 247 \\
\hline 5082 & pCi/L & 0.0511 & 0.0601 & & & & & QC_SAMPLE & & RAW GRAB & UNFILTERED & 247 \\
\hline 5083 & $\mathrm{pCi} / \mathrm{L}$ & 0.013 & 0.0131 & & & & & QC_SAMPLE & & RAW GRAB & UNFILTERED & 247 \\
\hline $5084 \mathrm{r}$ & $\mathrm{pCi} / \mathrm{L}$ & 0.0438 & 0.0492 & & & & & QC_SAMPLE & & RAW GRAB & UNFILTERED & 247 \\
\hline 5085 & $\mathrm{pCi} / \mathrm{L}$ & 0.0238 & 0.0422 & & & & & QC_SAMPLE & & RAW GRAB & UNFILTERED & 247 \\
\hline 5086 & $\mathrm{pCi} / \mathrm{L}$ & 0.0093 & & & & U & & RIVER & & CONTINUOUS & RESIN & 307 \\
\hline 5087 & $\mathrm{pCi} / \mathrm{L}$ & 0.0044 & & & & U & & RIVER & & CONTINUOUS & FILTER & 307 \\
\hline 5088 & $\mathrm{pCi} / \mathrm{L}$ & 0.0048 & & & & U & & RIVER & & CONTINUOUS & FILTER & 252 \\
\hline 5089 & $\mathrm{pCi} / \mathrm{L}$ & 0.01 & & & & U & & RIVER & & CONTINUOUS & RESIN & 252 \\
\hline $5090 \mathrm{r}$ & $\mathrm{pCi} / \mathrm{L}$ & 0.0512 & 0.0627 & & & & & RIVER & & CUMULATIVE & UNFILTERED & 252 \\
\hline 5091 & $\mathrm{pCi} / \mathrm{L}$ & 0.08 & 0.0846 & & & & & RIVER & & CUMULATIVE & UNFILTERED & 252 \\
\hline $5092 \mathrm{r}$ & $\mathrm{pCi} / \mathrm{L}$ & 0.0135 & 0.0135 & & & $U$ & & RIVER & & CUMULATIVE & UNFILTERED & 252 \\
\hline 5093 & pCi/L & 0.0753 & 0.0791 & & & & & RIVER & & CUMULATIVE & UNFILTERED & 252 \\
\hline 5094 & $\mathrm{pCi} / \mathrm{L}$ & 0.746 & 0.748 & & & U & & RIVER & & CUMULATIVE & UNFILTERED & 252 \\
\hline $5095 \mathrm{r}$ & pCi/L & 9.04 & 17.5 & & & & & RIVER & & CUMULATIVE & UNFILTERED & 252 \\
\hline 5096 & pCi/L & 0.0471 & 0.06 & & & & & RIVER & & CUMULATIVE & UNFILTERED & 463 \\
\hline 5097 & $\mathrm{pCi} / \mathrm{L}$ & 0.0415 & 0.0475 & & & & & RIVER & & CUMULATIVE & UNFILTERED & 463 \\
\hline
\end{tabular}




\begin{tabular}{|c|c|c|c|c|c|c|c|c|c|c|c|c|}
\hline & $\mathrm{N}$ & $\mathrm{O}$ & $\mathrm{P}$ & $\mathrm{Q}$ & $\mathrm{R}$ & $\mathrm{S}$ & $\mathrm{T}$ & $\mathrm{U}$ & $\mathrm{V}$ & $\mathrm{w}$ & $\mathrm{X}$ & $\mathrm{Y}$ \\
\hline 1 & ANAL_UNITS_RPTD & COUNTING_ERROR & TOTAL_ANAL_ERROR & MIN_DETECTABLE_ACTIVITY & PCNT_MOISTURE & LAB_QUALIFIER & REVIEW_QUALIFIER & SAMP_FROM & SAMP_ITEM & SAMP_MTHD & COLL_MTHD & SAMP_SITE_ID \\
\hline 5098 & $\mathrm{pCi} / \mathrm{L}$ & 0.00597 & 0.00599 & & & $U$ & & RIVER & & CUMULATIVE & UNFILTERED & 463 \\
\hline 5099 & $\mathrm{pCi} / \mathrm{L}$ & 0.0419 & 0.0483 & & & & & RIVER & & CUMULATIVE & UNFILTERED & 463 \\
\hline 5100 & $\mathrm{pCi} / \mathrm{L}$ & 0.597 & 0.597 & & & U & & RIVER & & CUMULATIVE & UNFILTERED & 463 \\
\hline 5101 & $\mathrm{pCi} / \mathrm{L}$ & 8.2 & 18.4 & & & & & RIVER & & CUMULATIVE & UNFILTERED & 463 \\
\hline 5102 & $\mathrm{pCi} / \mathrm{L}$ & 0.0094 & & & & U & & RIVER & & CONTINUOUS & RESIN & 307 \\
\hline 5103 & $\mathrm{pCi} / \mathrm{L}$ & 0.0045 & & & & U & & RIVER & & CONTINUOUS & FILTER & 307 \\
\hline 5104 & $\mathrm{pCi} / \mathrm{L}$ & 0.012 & & & & $\mathrm{u}$ & & RIVER & & CONTINUOUS & RESIN & 252 \\
\hline 5105 & $\mathrm{pCi} / \mathrm{L}$ & 0.0058 & & & & U & & RIVER & & CONTINUOUS & FILTER & 252 \\
\hline 5106 & $\mathrm{pCi} / \mathrm{L}$ & 181 & 242 & & & U & & SURFACE & & RAW GRAB & UNFILTERED & 509 \\
\hline 5107 & $\mathrm{pCi} / \mathrm{L}$ & 0.109 & 0.142 & & & & & SURFACE & & RAW GRAB & UNFILTERED & 509 \\
\hline 5108 & pCi/L & 1.44 & 1.44 & & & U & & SURFACE & & RAW GRAB & UNFILTERED & 509 \\
\hline 5109 & $\mathrm{pCi} / \mathrm{L}$ & 213 & 316 & & & & & SURFACE & & RAW GRAB & UNFILTERED & 136 \\
\hline 5110 & $\mathrm{pCi} / \mathrm{L}$ & 1.87 & 1.87 & & & U & & SURFACE & & RAW GRAB & UNFILTERED & 136 \\
\hline 5111 & $\mathrm{pCi} / \mathrm{L}$ & 181 & 241 & & & U & & SURFACE & & RAW GRAB & UNFILTERED & 146 \\
\hline 5112 & $\mathrm{pCi} / \mathrm{L}$ & 0.169 & 0.54 & & & & & SURFACE & & RAW GRAB & UNFILTERED & 146 \\
\hline 5113 & $\mathrm{pCi} / \mathrm{L}$ & 0.953 & 0.985 & & & & & SURFACE & & RAW GRAB & UNFILTERED & 146 \\
\hline 5114 & $\mathrm{pCi} / \mathrm{L}$ & 1.36 & 9.54 & & & & & SURFACE & & RAW GRAB & UNFILTERED & 147 \\
\hline 5115 & $\mathrm{pCi} / \mathrm{L}$ & 0.264 & 0.444 & & & & & SURFACE & & RAW GRAB & UNFILTERED & 147 \\
\hline 5116 & pCi/L & 1.4 & 10.2 & & & & & SURFACE & & RAW GRAB & UNFILTERED & 147 \\
\hline 5117 & $\mathrm{pCi} / \mathrm{L}$ & 192 & 265 & & & & & SURFACE & & RAW GRAB & UNFILTERED & 147 \\
\hline 5118 & $\mathrm{pCi} / \mathrm{L}$ & 0.285 & 0.498 & & & & & SURFACE & & RAW GRAB & UNFILTERED & 147 \\
\hline 5119 & $\mathrm{pCi} / \mathrm{L}$ & 1.68 & 1.69 & & & U & & SURFACE & & RAW GRAB & UNFILTERED & 147 \\
\hline 5120 & $\mathrm{pCi} / \mathrm{L}$ & 268 & 321 & & & U & & DRINKING & & SAN. GRAB & UNFILTERED & 487 \\
\hline 5121 & pCi/L & 0.948 & 0.948 & & & U & & DRINKING & & SAN. GRAB & UNFILTERED & 487 \\
\hline 5122 & $\mathrm{pCi} / \mathrm{L}$ & 0.0361 & 0.0366 & & & U & & DRINKING & & SAN. GRAB & UNFILTERED & 487 \\
\hline 5123 & $\mathrm{pCi} / \mathrm{L}$ & 0.0093 & & & & U & & RIVER & & CONTINUOUS & RESIN & 307 \\
\hline 5124 & $\mathrm{pCi} / \mathrm{L}$ & 0.0046 & & & & U & & RIVER & & CONTINUOUS & FILTER & 307 \\
\hline 5125 & $\mathrm{pCi} / \mathrm{L}$ & 0.011 & & & & U & & RIVER & & CONTINUOUS & RESIN & 252 \\
\hline 5126 & $\mathrm{pCi} / \mathrm{L}$ & 0.0054 & & & & $U$ & & RIVER & & CONTINUOUS & FILTER & 252 \\
\hline 5127 & & & & & & & & IRRIGATION & & RAW GRAB & UNFILTERED & 440 \\
\hline 5128 & & & & & & & & IRRIGATION & & RAW GRAB & UNFILTERED & 440 \\
\hline 5129 & pCi/L & 1.27 & 1.27 & & & U & & DRINKING & & SAN. GRAB & UNFILTERED & 530 \\
\hline 5130 & $\mathrm{pCi} / \mathrm{L}$ & 0.098 & 0.232 & & & & & DRINKING & & SAN. GRAB & UNFILTERED & 530 \\
\hline 5131 & $\mathrm{pCi} / \mathrm{L}$ & 8.27 & 19.3 & & & & & DRINKING & & SAN. GRAB & UNFILTERED & 530 \\
\hline 5132 & $\mathrm{pCi} / \mathrm{L}$ & 0.0046 & & & & U & & RIVER & & CONTINUOUS & FILTER & 307 \\
\hline 5133 & $\mathrm{pCi} / \mathrm{L}$ & 0.0097 & & & & U & & RIVER & & CONTINUOUS & RESIN & 307 \\
\hline 5134 & $\mathrm{pCi} / \mathrm{L}$ & 0.012 & & & & U & & RIVER & & CONTINUOUS & RESIN & 252 \\
\hline 5135 & pCi/L & 0.006 & & & & U & & RIVER & & CONTINUOUS & FILTER & 252 \\
\hline 5136 & $\mathrm{pCi} / \mathrm{L}$ & 0.584 & 0.585 & & & $U$ & & RIVER & & CUMULATIVE & UNFILTERED & 252 \\
\hline 5137 & $\mathrm{pCi} / \mathrm{L}$ & 0.0666 & 0.0761 & & & & & RIVER & & CUMULATIVE & UNFILTERED & 252 \\
\hline 5138 & $\mathrm{pCi} / \mathrm{L}$ & 0.0382 & 0.0421 & & & & & RIVER & & CUMULATIVE & UNFILTERED & 252 \\
\hline 5139 & $\mathrm{pCi} / \mathrm{L}$ & 0.00675 & 0.00678 & & & $U$ & & RIVER & & CUMULATIVE & UNFILTERED & 252 \\
\hline 5140 & $\mathrm{pCi} / \mathrm{L}$ & 0.0453 & 0.0516 & & & & & RIVER & & CUMULATIVE & UNFILTERED & 252 \\
\hline 5141 & $\mathrm{pCi} / \mathrm{L}$ & 8.29 & 16.8 & & & & & RIVER & & CUMULATIVE & UNFILTERED & 252 \\
\hline 5142 & $\mathrm{pCi} / \mathrm{L}$ & 0.584 & 0.585 & & & U & & RIVER & & CUMULATIVE & UNFILTERED & 463 \\
\hline 5143 & pCi/L & 0.0397 & 0.044 & & & & & RIVER & & CUMULATIVE & UNFILTERED & 463 \\
\hline 5144 & $\mathrm{pCi} / \mathrm{L}$ & 0.0068 & 0.0068 & & & $U$ & & RIVER & & CUMULATIVE & UNFILTERED & 463 \\
\hline 5145 & pCi/L & 0.046 & 0.0526 & & & & & RIVER & & CUMULATIVE & UNFILTERED & 463 \\
\hline 5146 & $\mathrm{pCi} / \mathrm{L}$ & 0.0605 & 0.0773 & & & & & RIVER & & CUMULATIVE & UNFILTERED & 463 \\
\hline 5147 & pCi/L & 8.48 & 17.8 & & & & & RIVER & & CUMULATIVE & UNFILTERED & 463 \\
\hline 5148 & pCi/L & 0.0074 & & & & U & & RIVER & & CONTINUOUS & RESIN & 307 \\
\hline 5149 & $\mathrm{pCi} / \mathrm{L}$ & 0.0036 & & & & $U_{2 x}$ & & RIVER & & CONTINUOUS & FILTER & 307 \\
\hline
\end{tabular}




\begin{tabular}{|c|c|c|c|c|c|c|c|c|c|c|c|c|}
\hline & $\mathrm{N}$ & $\mathrm{O}$ & $\mathrm{P}$ & $\mathrm{Q}$ & $\mathrm{R}$ & $\mathrm{S}$ & $\mathrm{T}$ & $\mathrm{U}$ & $\mathrm{V}$ & $\mathrm{w}$ & $\mathrm{x}$ & $\mathrm{Y}$ \\
\hline 1 & ANAL_UNITS_RPTD & COUNTING_ERROR & TOTAL_ANAL_ERROR & MIN_DETECTABLE_ACTIVITY & PCNT_MOISTURE & LAB_QUALIFIER & REVIEW_QUALIFIER & SAMP_FROM & SAMP_ITEM & SAMP_MTHD & COLL_MTHD & SAMP_SITE_ID \\
\hline 5150 & $\mathrm{pCi} / \mathrm{L}$ & 0.0097 & & & & $U$ & & RIVER & & CONTINUOUS & RESIN & 252 \\
\hline 5151 & $\mathrm{pCi} / \mathrm{L}$ & 0.005 & & & & U & & RIVER & & CONTINUOUS & FILTER & 252 \\
\hline 5152 & $\mathrm{pCi} / \mathrm{L}$ & 0.519 & 0.524 & & & & & DRINKING & & CUMULATIVE & UNFILTERED & 307 \\
\hline 5153 & $\mathrm{pCi} / \mathrm{L}$ & 0.0563 & 0.087 & & & & & DRINKING & & CUMULATIVE & UNFILTERED & 307 \\
\hline 5154 & $\mathrm{pCi} / \mathrm{L}$ & 0.0515 & 0.0609 & & & & & DRINKING & & CUMULATIVE & UNFILTERED & 307 \\
\hline 5155 & $\mathrm{pCi} / \mathrm{L}$ & 0.011 & 0.0111 & & & & & DRINKING & & CUMULATIVE & UNFILTERED & 307 \\
\hline $\mid 5156$ & $\mathrm{pCi} / \mathrm{L}$ & 0.0421 & 0.0472 & & & & & DRINKING & & CUMULATIVE & UNFILTERED & 307 \\
\hline 5157 & $\mathrm{pCi} / \mathrm{L}$ & 8.81 & 19.7 & & & & & DRINKING & & CUMULATIVE & UNFILTERED & 307 \\
\hline 5158 & $\mathrm{pCi} / \mathrm{L}$ & 0.0064 & & & & U & & RIVER & & CONTINUOUS & RESIN & 307 \\
\hline 5159 & $\mathrm{pCi} / \mathrm{L}$ & 0.0031 & & & & U & & RIVER & & CONTINUOUS & FILTER & 307 \\
\hline 5160 & $\mathrm{pCi} / \mathrm{L}$ & & 0.00000724 & & & & & RIVER & & CONTINUOUS & RESIN & 307 \\
\hline 5161 & $\mathrm{pCi} / \mathrm{L}$ & 0.00156 & & & & & & RIVER & & CONTINUOUS & RESIN & 307 \\
\hline 5162 & $\mathrm{pCi} / \mathrm{L}$ & 0.0001348 & & & & U & & RIVER & & CONTINUOUS & RESIN & 307 \\
\hline 5163 & $\mathrm{pCi} / \mathrm{L}$ & 0.000008 & & & & & & RIVER & & CONTINUOUS & FILTER & 307 \\
\hline 5164 & $\mathrm{pCi} / \mathrm{L}$ & 0.0089 & & & & U & & RIVER & & CONTINUOUS & RESIN & 252 \\
\hline 5165 & $\mathrm{pCi} / \mathrm{L}$ & & 0.000000942 & & & & & RIVER & & CONTINUOUS & RESIN & 252 \\
\hline 5166 & $\mathrm{pCi} / \mathrm{L}$ & 0.00072 & & & & & & RIVER & & CONTINUOUS & RESIN & 252 \\
\hline 5167 & $\mathrm{pCi} / \mathrm{L}$ & 0.0001438 & & & & U & & RIVER & & CONTINUOUS & RESIN & 252 \\
\hline 5168 & $\mathrm{pCi} / \mathrm{L}$ & 1.76 & 1.77 & & & U & & RIVER & & CUMULATIVE & UNFILTERED & 252 \\
\hline 5169 & $\mathrm{pCi} / \mathrm{L}$ & 0.0043 & & & & $U$ & & RIVER & & CONTINUOUS & FILTER & 252 \\
\hline 5170 & $\mathrm{pCi} / \mathrm{L}$ & 0.0584 & 0.0757 & & & & & RIVER & & CUMULATIVE & UNFILTERED & 252 \\
\hline 5171 & $\mathrm{pCi} / \mathrm{L}$ & 0.049 & 0.0567 & & & & & RIVER & & CUMULATIVE & UNFILTERED & 252 \\
\hline 5172 & $\mathrm{pCi} / \mathrm{L}$ & 0.0097 & 0.00973 & & & U & & RIVER & & CUMULATIVE & UNFILTERED & 252 \\
\hline 5173 & $\mathrm{pCi} / \mathrm{L}$ & 0.0429 & 0.0477 & & & & & RIVER & & CUMULATIVE & UNFILTERED & 252 \\
\hline 5174 & $\mathrm{pCi} / \mathrm{L}$ & 0.000009 & & & & & & RIVER & & CONTINUOUS & FILTER & 252 \\
\hline 5175 & $\mathrm{pCi} / \mathrm{L}$ & 7.52 & 15.6 & & & & & RIVER & & CUMULATIVE & UNFILTERED & 252 \\
\hline 5176 & $\mathrm{pCi} / \mathrm{L}$ & 0.658 & 0.658 & & & U & & RIVER & & CUMULATIVE & UNFILTERED & 463 \\
\hline 5177 & $\mathrm{pCi} / \mathrm{L}$ & 0.0796 & 0.1 & & & & & RIVER & & CUMULATIVE & UNFILTERED & 463 \\
\hline 5178 & $\mathrm{pCi} / \mathrm{L}$ & 0.0474 & 0.0551 & & & & & RIVER & & CUMULATIVE & UNFILTERED & 463 \\
\hline 5179 & $\mathrm{pCi} / \mathrm{L}$ & 0.012 & 0.0121 & & & & & RIVER & & CUMULATIVE & UNFILTERED & 463 \\
\hline 5180 & $\mathrm{pCi} / \mathrm{L}$ & 0.0437 & 0.0497 & & & & & RIVER & & CUMULATIVE & UNFILTERED & 463 \\
\hline 5181 & $\mathrm{pCi} / \mathrm{L}$ & 8.09 & 17.7 & & & & & RIVER & & CUMULATIVE & UNFILTERED & 463 \\
\hline 5182 & $\mathrm{pCi} / \mathrm{L}$ & 180 & 240 & & & U & & DRINKING & & SAN. GRAB & UNFILTERED & 186 \\
\hline 5183 & $\mathrm{pCi} / \mathrm{L}$ & 1.22 & 1.22 & & & U & & DRINKING & & SAN. GRAB & UNFILTERED & 186 \\
\hline 5184 & $\mathrm{pCi} / \mathrm{L}$ & 0.0256 & 0.0261 & & & U & & DRINKING & & SAN. GRAB & UNFILTERED & 186 \\
\hline 5185 & $\mathrm{pCi} / \mathrm{L}$ & 206 & 301 & & & & & DRINKING & & SAN. GRAB & UNFILTERED & 512 \\
\hline 5186 & $\mathrm{pCi} / \mathrm{L}$ & 0.877 & 0.882 & & & & & DRINKING & & SAN. GRAB & UNFILTERED & 512 \\
\hline 5187 & $\mathrm{pCi} / \mathrm{L}$ & 0.025 & 0.0251 & & & U & & DRINKING & & SAN. GRAB & UNFILTERED & 512 \\
\hline 5188 & $\mathrm{pCi} / \mathrm{L}$ & 0.838 & 0.84 & & & $U$ & & DRINKING & & SAN. GRAB & UNFILTERED & 144 \\
\hline 5189 & $\mathrm{pCi} / \mathrm{L}$ & 181 & 242 & & & $U$ & & DRINKING & & SAN. GRAB & UNFILTERED & 144 \\
\hline 5190 & $\mathrm{pCi} / \mathrm{L}$ & 0.0302 & 0.033 & & & & & DRINKING & & SAN. GRAB & UNFILTERED & 144 \\
\hline 5191 & $\mathrm{pCi} / \mathrm{L}$ & 179 & 239 & & & $U$ & & DRINKING & & SAN. GRAB & UNFILTERED & 187 \\
\hline 5192 & $\mathrm{pCi} / \mathrm{L}$ & 1.24 & 1.24 & & & U & & DRINKING & & SAN. GRAB & UNFILTERED & 187 \\
\hline 5193 & $\mathrm{pCi} / \mathrm{L}$ & 0.0238 & 0.0239 & & & U & & DRINKING & & SAN. GRAB & UNFILTERED & 187 \\
\hline 5194 & $\mathrm{pCi} / \mathrm{L}$ & 1.2 & 1.2 & & & U & & DRINKING & & RAW GRAB & UNFILTERED & 553 \\
\hline 5195 & $\mathrm{pCi} / \mathrm{L}$ & 10.8 & 17.8 & & & & & DRINKING & & RAW GRAB & UNFILTERED & 553 \\
\hline 5196 & $\mathrm{pCi} / \mathrm{L}$ & 0.0405 & 0.0527 & & & & & DRINKING & & RAW GRAB & UNFILTERED & 553 \\
\hline 5197 & $\mathrm{pCi} / \mathrm{L}$ & 0.0096 & & & & U & & RIVER & & CONTINUOUS & RESIN & 307 \\
\hline 5198 & $\mathrm{pCi} / \mathrm{L}$ & 0.0054 & & & & 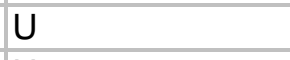 & & RIVER & & CONTINUOUS & FILTER & 307 \\
\hline 5199 & $\mathrm{pCi} / \mathrm{L}$ & 0.011 & & & & U & & RIVER & & CONTINUOUS & RESIN & 252 \\
\hline 5200 & $\mathrm{pCi} / \mathrm{L}$ & 0.0045 & & & & U & & RIVER & & CONTINUOUS & FILTER & 252 \\
\hline 5201 & $\mathrm{pCi} / \mathrm{L}$ & 0.0056 & & & & $\mathrm{U}_{23}$ & & RIVER & & CONTINUOUS & FILTER & 463 \\
\hline
\end{tabular}




\begin{tabular}{|c|c|c|c|c|c|c|c|c|c|c|c|c|}
\hline & $\mathrm{N}$ & $\mathrm{O}$ & $\mathrm{P}$ & $\mathrm{Q}$ & $\mathrm{R}$ & $\mathrm{s}$ & $\mathrm{T}$ & $\mathrm{u}$ & $\mathrm{V}$ & w & $\mathrm{x}$ & $\bar{Y}$ \\
\hline 1 & ANAL_UNITS_RPTD & COUNTING_ERROR & $\mid$ TOTAL_ANAL_ERROR & MIN_DETECTABLE_ACTIVITY & PCNT_MOISTURE & LAB_QUALIFIER & REVIEW_QUALIFIER & SAMP_FROM & SAMP_ITEM & SAMP_MTHD & COLL_MTHD & SAMP_SITE_ID \\
\hline 5202 & $\mathrm{pCi} / \mathrm{L}$ & 0.011 & & & & $U$ & & RIVER & & CONTINUOUS & RESIN & 463 \\
\hline 5203 & $\mathrm{pCi} / \mathrm{L}$ & 0.0083 & & & & $\mathrm{U}$ & & RIVER & & CONTINUOUS & RESIN & 307 \\
\hline 5204 & $\mathrm{pCi} / \mathrm{L}$ & 0.0038 & & & & $\mathrm{U}$ & & RIVER & & CONTINUOUS & FILTER & 307 \\
\hline 5205 & $\mathrm{pCi} / \mathrm{L}$ & 0.012 & & & & $\mathrm{U}$ & & RIVER & & CONTINUOUS & RESIN & 252 \\
\hline 5206 & $\mathrm{pCi} / \mathrm{L}$ & 0.0056 & & & & $\mathrm{U}$ & & RIVER & & CONTINUOUS & FILTER & 252 \\
\hline 5207 & $\mathrm{pCi} / \mathrm{L}$ & 0.014 & & & & $\mathrm{U}$ & & RIVER & & CONTINUOUS & RESIN & 463 \\
\hline 5208 & $\mathrm{pCi} / \mathrm{L}$ & 0.0067 & & & & $\mathrm{U}$ & & RIVER & & CONTINUOUS & FILTER & 463 \\
\hline 5209 & $\mathrm{pCi} / \mathrm{L}$ & 0.987 & 0.987 & & & U & & RIVER & & CUMULATIVE & UNFILTERED & 252 \\
\hline 5210 & $\mathrm{pCi} / \mathrm{L}$ & 0.0451 & 0.052 & & & & & RIVER & & CUMULATIVE & UNFILTERED & 252 \\
\hline 5211 & $\mathrm{pCi} / \mathrm{L}$ & 0.0106 & 0.0107 & & & & & RIVER & & CUMULATIVE & UNFILTERED & 252 \\
\hline 5212 & $\mathrm{pCi} / \mathrm{L}$ & 0.0394 & 0.044 & & & & & RIVER & & CUMULATIVE & UNFILTERED & 252 \\
\hline 5213 & $\mathrm{pCi} / \mathrm{L}$ & 9.19 & 15.1 & & & & & RIVER & & CUMULATIVE & UNFILTERED & 252 \\
\hline 5214 & $\mathrm{pCi} / \mathrm{L}$ & 0.0389 & 0.0504 & & & & & RIVER & & CUMULATIVE & UNFILTERED & 252 \\
\hline 5215 & $\mathrm{pCi} / \mathrm{L}$ & 1.12 & 1.12 & & & $U$ & & RIVER & & CUMULATIVE & UNFILTERED & 463 \\
\hline \begin{tabular}{|l|}
5216 \\
\end{tabular} & $\mathrm{pCi} / \mathrm{L}$ & 0.0474 & 0.0554 & & & & & RIVER & & CUMULATIVE & UNFILTERED & 463 \\
\hline \begin{tabular}{|l|}
5217 \\
\end{tabular} & $\mathrm{pCi} / \mathrm{L}$ & 0.0105 & 0.0106 & & & & & RIVER & & CUMULATIVE & UNFILTERED & 463 \\
\hline \begin{tabular}{|l|}
5218 \\
\end{tabular} & $\mathrm{pCi} / \mathrm{L}$ & 0.0457 & 0.053 & & & & & RIVER & & CUMULATIVE & UNFILTERED & 463 \\
\hline 5219 & $\mathrm{pCi} / \mathrm{L}$ & 11.3 & 19.3 & & & & & RIVER & & CUMULATIVE & UNFILTERED & 463 \\
\hline 5220 & $\mathrm{pCi} / \mathrm{L}$ & 0.0435 & 0.0559 & & & & & RIVER & & CUMULATIVE & UNFILTERED & 463 \\
\hline 5221 & $\mathrm{pCi} / \mathrm{L}$ & 0.649 & 0.649 & & & $U$ & & DRINKING & & SAN. GRAB & UNFILTERED & 487 \\
\hline 5222 & $\mathrm{pCi} / \mathrm{L}$ & 172 & 226 & & & $U$ & & DRINKING & & SAN. GRAB & UNFILTERED & 487 \\
\hline 5223 & $\mathrm{pCi} / \mathrm{L}$ & 0.034 & 0.0355 & & & & & DRINKING & & SAN. GRAB & UNFILTERED & 487 \\
\hline 5224 & $\mathrm{pCi} / \mathrm{L}$ & 0.0064 & & & & U & & RIVER & & CONTINUOUS & RESIN & 307 \\
\hline \begin{tabular}{|l|}
5225 \\
\end{tabular} & $\mathrm{pCi} / \mathrm{L}$ & 0.0032 & & & & $U$ & & RIVER & & CONTINUOUS & FILTER & 307 \\
\hline 5226 & $\mathrm{pCi} / \mathrm{L}$ & 0.0062 & & & & $U$ & & RIVER & & CONTINUOUS & RESIN & 252 \\
\hline 5227 & $\mathrm{pCi} / \mathrm{L}$ & 0.0033 & & & & $U$ & & RIVER & & CONTINUOUS & FILTER & 252 \\
\hline 5228 & $\mathrm{pCi} / \mathrm{L}$ & 0.0082 & & & & $U$ & & RIVER & & CONTINUOUS & RESIN & 463 \\
\hline 5229 & $\mathrm{pCi} / \mathrm{L}$ & 0.004 & & & & U & & RIVER & & CONTINUOUS & FILTER & 463 \\
\hline 5230 & $\mathrm{pCi} / \mathrm{L}$ & 0.535 & 0.535 & & & U & & DRINKING & & SAN. GRAB & UNFILTERED & 530 \\
\hline 5231 & $\mathrm{pCi} / \mathrm{L}$ & 0.0678 & 0.183 & & & & & DRINKING & & SAN. GRAB & UNFILTERED & 530 \\
\hline 5232 & $\mathrm{pCi} / \mathrm{L}$ & 11.5 & 20.1 & & & & & DRINKING & & SAN. GRAB & UNFILTERED & 530 \\
\hline 5233 & $\mathrm{pCi} / \mathrm{L}$ & 0.0032 & & & & $U$ & & RIVER & & CONTINUOUS & FILTER & 307 \\
\hline 5234 & $\mathrm{pCi} / \mathrm{L}$ & 0.0067 & & & & $U$ & & RIVER & & CONTINUOUS & RESIN & 307 \\
\hline 5235 & $\mathrm{pCi} / \mathrm{L}$ & 0.0072 & & & & $U$ & & RIVER & & CONTINUOUS & RESIN & 252 \\
\hline 5236 & $\mathrm{pCi} / \mathrm{L}$ & 0.0035 & & & & $U$ & & RIVER & & CONTINUOUS & FILTER & 252 \\
\hline \begin{tabular}{|l|}
5237 \\
\end{tabular} & $\mathrm{pCi} / \mathrm{L}$ & 0.004 & & & & U & & RIVER & & CONTINUOUS & FILTER & 463 \\
\hline 5238 & $\mathrm{pCi} / \mathrm{L}$ & 0.0082 & & & & U & & RIVER & & CONTINUOUS & RESIN & 463 \\
\hline 5239 & $\mathrm{pCi} / \mathrm{L}$ & 9.39 & 14.8 & & & & & RIVER & & CUMULATIVE & UNFILTERED & 252 \\
\hline 5240 & $\mathrm{pCi} / \mathrm{L}$ & 0.0341 & 0.0496 & & & & & RIVER & & CUMULATIVE & UNFILTERED & 252 \\
\hline 5241 & $\mathrm{pCi} / \mathrm{L}$ & 0.0424 & 0.047 & & & & & RIVER & & CUMULATIVE & UNFILTERED & 252 \\
\hline 5242 & $\mathrm{pCi} / \mathrm{L}$ & 0.00596 & 0.00597 & & & $U$ & & RIVER & & CUMULATIVE & UNFILTERED & 252 \\
\hline 5243 & $\mathrm{pCi} / \mathrm{L}$ & 0.0497 & 0.057 & & & & & RIVER & & CUMULATIVE & UNFILTERED & 252 \\
\hline 5244 & $\mathrm{pCi} / \mathrm{L}$ & 0.941 & 0.941 & & & U & & RIVER & & CUMULATIVE & UNFILTERED & 252 \\
\hline 5245 & $\mathrm{pCi} / \mathrm{L}$ & 10.2 & 17.9 & & & & & RIVER & & CUMULATIVE & UNFILTERED & 463 \\
\hline 5246 & $\mathrm{pCi} / \mathrm{L}$ & 0.0343 & 0.0536 & & & & & RIVER & & CUMULATIVE & UNFILTERED & 463 \\
\hline 5247 & $\mathrm{pCi} / \mathrm{L}$ & 0.0541 & 0.0638 & & & & & RIVER & & CUMULATIVE & UNFILTERED & 463 \\
\hline 5248 & $\mathrm{pCi} / \mathrm{L}$ & 0.0173 & 0.0177 & & & & & RIVER & & CUMULATIVE & UNFILTERED & 463 \\
\hline 5249 & $\mathrm{pCi} / \mathrm{L}$ & 0.0609 & 0.0746 & & & & & RIVER & & CUMULATIVE & UNFILTERED & 463 \\
\hline 5250 & $\mathrm{pCi} / \mathrm{L}$ & 1.13 & 1.14 & & & $U$ & & RIVER & & CUMULATIVE & UNFILTERED & 463 \\
\hline 5251 & $\mathrm{pCi} / \mathrm{L}$ & 177 & 235 & & & $U$ & & SURFACE & & RAW GRAB & UNFILTERED & 509 \\
\hline 5252 & $\mathrm{pCi} / \mathrm{L}$ & 0.0854 & 0.12 & & & & & SURFACE & & RAW GRAB & UNFILTERED & 509 \\
\hline 5253 & $\mathrm{pCi} / \mathrm{L}$ & 2.1 & 2.1 & & & $\mathrm{U}_{2}$ & & SURFACE & & RAW GRAB & UNFILTERED & 509 \\
\hline
\end{tabular}




\begin{tabular}{|c|c|c|c|c|c|c|c|c|c|c|c|c|}
\hline & $\mathrm{N}$ & $\mathrm{O}$ & $\mathrm{P}$ & $\mathrm{Q}$ & $\mathrm{R}$ & $\mathrm{s}$ & $T$ & $\bar{U}$ & $\mathrm{~V}$ & $\mathrm{~W}$ & $\mathrm{X}$ & $\bar{Y}$ \\
\hline 1 & ANAL_UNITS_RPTD & COUNTING_ERROR & TOTAL_ANAL_ERROR & MIN_DETECTABLE_ACTIVITY & PCNT_MOISTURE & LAB_QUALIFIER & REVIEW_QUALIFIER & SAMP_FROM & SAMP_ITEM & SAMP_MTHD & COLL_MTHD & SAMP_SITE_ID \\
\hline 5254 & $\mathrm{pCi} / \mathrm{L}$ & 1.99 & 2 & & & $U$ & & SURFACE & & RAW GRAB & UNFILTERED & 136 \\
\hline 5255 & $\mathrm{pCi} / \mathrm{L}$ & 215 & 331 & & & & & SURFACE & & RAW GRAB & UNFILTERED & 136 \\
\hline 5256 & $\mathrm{pCi} / \mathrm{L}$ & 0.0859 & 0.201 & & & & & SURFACE & & RAW GRAB & UNFILTERED & 146 \\
\hline 5257 & $\mathrm{pCi} / \mathrm{L}$ & 179 & 238 & & & $\mathrm{U}$ & & SURFACE & & RAW GRAB & UNFILTERED & 146 \\
\hline 5258 & $\mathrm{pCi} / \mathrm{L}$ & 2.33 & 2.67 & & & & & SURFACE & & RAW GRAB & UNFILTERED & 146 \\
\hline 5259 & $\mathrm{pCi} / \mathrm{L}$ & 0.821 & 7.3 & & & & & SURFACE & & RAW GRAB & UNFILTERED & 147 \\
\hline 5260 & $\mathrm{pCi} / \mathrm{L}$ & 0.167 & 0.343 & & & & & SURFACE & & RAW GRAB & UNFILTERED & 147 \\
\hline 5261 & $\mathrm{pCi} / \mathrm{L}$ & 0.846 & 7.74 & & & & & SURFACE & & RAW GRAB & UNFILTERED & 147 \\
\hline 5262 & $\mathrm{pCi} / \mathrm{L}$ & 0.957 & 0.965 & & & & & SURFACE & & RAW GRAB & UNFILTERED & 147 \\
\hline 5263 & $\mathrm{pCi} / \mathrm{L}$ & 0.177 & 0.499 & & & & & SURFACE & & RAW GRAB & UNFILTERED & 147 \\
\hline 5264 & $\mathrm{pCi} / \mathrm{L}$ & 184 & 251 & & & & & SURFACE & & RAW GRAB & UNFILTERED & 147 \\
\hline 5265 & & & & & & & & RIVER & & CONTINUOUS & FILTER & 307 \\
\hline 5266 & & & & & & & & RIVER & & CONTINUOUS & RESIN & 307 \\
\hline 5267 & $\mathrm{pCi} / \mathrm{L}$ & 0.013 & & & & U & & RIVER & & CONTINUOUS & RESIN & 252 \\
\hline 5268 & $\mathrm{pCi} / \mathrm{L}$ & 0.0063 & & & & $U$ & & RIVER & & CONTINUOUS & FILTER & 252 \\
\hline 5269 & $\mathrm{pCi} / \mathrm{L}$ & 0.0038 & & & & U & & RIVER & & CONTINUOUS & FILTER & 463 \\
\hline 5270 & $\mathrm{pCi} / \mathrm{L}$ & 0.008 & & & & U & & RIVER & & CONTINUOUS & RESIN & 463 \\
\hline 5271 & $\mathrm{pCi} / \mathrm{L}$ & 0.0024 & & & & U & & RIVER & & CONTINUOUS & FILTER & 307 \\
\hline 5272 & $\mathrm{pCi} / \mathrm{L}$ & 0.0047 & & & & U & & RIVER & & CONTINUOUS & RESIN & 307 \\
\hline 5273 & $\mathrm{pCi} / \mathrm{L}$ & 0.0094 & & & & $U$ & & RIVER & & CONTINUOUS & RESIN & 252 \\
\hline 5274 & $\mathrm{pCi} / \mathrm{L}$ & 0.0045 & & & & U & & RIVER & & CONTINUOUS & FILTER & 252 \\
\hline 5275 & $\mathrm{pCi} / \mathrm{L}$ & 0.0038 & & & & U & & RIVER & & CONTINUOUS & FILTER & 463 \\
\hline 5276 & $\mathrm{pCi} / \mathrm{L}$ & 0.0079 & & & & U & & RIVER & & CONTINUOUS & RESIN & 463 \\
\hline 5277 & $\mathrm{pCi} / \mathrm{L}$ & 0.0198 & 0.0208 & & & & & DRINKING & & SAN. GRAB & UNFILTERED & 186 \\
\hline 5278 & $\mathrm{pCi} / \mathrm{L}$ & 182 & 243 & & & U & & DRINKING & & SAN. GRAB & UNFILTERED & 186 \\
\hline 5279 & $\mathrm{pCi} / \mathrm{L}$ & 0.965 & 0.965 & & & U & & DRINKING & & SAN. GRAB & UNFILTERED & 186 \\
\hline 5280 & $\mathrm{pCi} / \mathrm{L}$ & 0.0341 & 0.0176 & & & U & & DRINKING & & SAN. GRAB & UNFILTERED & 512 \\
\hline 5281 & $\mathrm{pCi} / \mathrm{L}$ & 246 & 413 & & & & & DRINKING & & SAN. GRAB & UNFILTERED & 512 \\
\hline 5282 & $\mathrm{pCi} / \mathrm{L}$ & 1.11 & 1.11 & & & U & & DRINKING & & SAN. GRAB & UNFILTERED & 512 \\
\hline 5283 & $\mathrm{pCi} / \mathrm{L}$ & 0.0381 & 0.0386 & & & U & & DRINKING & & SAN. GRAB & UNFILTERED & 144 \\
\hline 5284 & $\mathrm{pCi} / \mathrm{L}$ & 184 & 246 & & & U & & DRINKING & & SAN. GRAB & UNFILTERED & 144 \\
\hline 5285 & $\mathrm{pCi} / \mathrm{L}$ & 1.01 & 1.02 & & & U & & DRINKING & & SAN. GRAB & UNFILTERED & 144 \\
\hline 5286 & $\mathrm{pCi} / \mathrm{L}$ & 0.0225 & 0.0237 & & & U & & DRINKING & & SAN. GRAB & UNFILTERED & 187 \\
\hline 5287 & $\mathrm{pCi} / \mathrm{L}$ & 182 & 242 & & & U & & DRINKING & & SAN. GRAB & UNFILTERED & 187 \\
\hline 5288 & $\mathrm{pCi} / \mathrm{L}$ & 0.926 & 0.929 & & & U & & DRINKING & & SAN. GRAB & UNFILTERED & 187 \\
\hline 5289 & $\mathrm{pCi} / \mathrm{L}$ & 0.0413 & 0.0603 & & & & & RIVER & & CUMULATIVE & UNFILTERED & 252 \\
\hline 5290 & $\mathrm{pCi} / \mathrm{L}$ & 0.036 & 0.0391 & & & & & RIVER & & CUMULATIVE & UNFILTERED & 252 \\
\hline 5291 & $\mathrm{pCi} / \mathrm{L}$ & 0.011 & 0.0111 & & & & & RIVER & & CUMULATIVE & UNFILTERED & 252 \\
\hline 5292 & $\mathrm{pCi} / \mathrm{L}$ & 0.0455 & 0.0521 & & & & & RIVER & & CUMULATIVE & UNFILTERED & 252 \\
\hline 5293 & $\mathrm{pCi} / \mathrm{L}$ & 8.83 & 13.9 & & & & & RIVER & & CUMULATIVE & UNFILTERED & 252 \\
\hline 5294 & $\mathrm{pCi} / \mathrm{L}$ & 1.08 & 1.08 & & & U & & RIVER & & CUMULATIVE & UNFILTERED & 252 \\
\hline 5295 & $\mathrm{pCi} / \mathrm{L}$ & 0.0431 & 0.0629 & & & & & RIVER & & CUMULATIVE & UNFILTERED & 463 \\
\hline 5296 & $\mathrm{pCi} / \mathrm{L}$ & 0.043 & 0.0484 & & & & & RIVER & & CUMULATIVE & UNFILTERED & 463 \\
\hline 5297 & $\mathrm{pCi} / \mathrm{L}$ & 0.00875 & 0.0088 & & & & & RIVER & & CUMULATIVE & UNFILTERED & 463 \\
\hline 5298 & $\mathrm{pCi} / \mathrm{L}$ & 0.049 & 0.057 & & & & & RIVER & & CUMULATIVE & UNFILTERED & 463 \\
\hline 5299 & $\mathrm{pCi} / \mathrm{L}$ & 9.97 & 17.7 & & & & & RIVER & & CUMULATIVE & UNFILTERED & 463 \\
\hline 5300 & $\mathrm{pCi} / \mathrm{L}$ & 0.723 & 0.724 & & & $U$ & & RIVER & & CUMULATIVE & UNFILTERED & 463 \\
\hline 5301 & $\mathrm{pCi} / \mathrm{L}$ & 0.0349 & 0.0514 & & & & & DRINKING & & RAW GRAB & UNFILTERED & 553 \\
\hline 5302 & $\mathrm{pCi} / \mathrm{L}$ & 0.84 & 0.841 & & & U & & DRINKING & & RAW GRAB & UNFILTERED & 553 \\
\hline 5303 & $\mathrm{pCi} / \mathrm{L}$ & 9.91 & 15.7 & & & & & DRINKING & & RAW GRAB & UNFILTERED & 553 \\
\hline 5304 & $\mathrm{pCi} / \mathrm{L}$ & 0.00000584 & & & & & & RIVER & & CONTINUOUS & FILTER & 307 \\
\hline 5305 & $\mathrm{pCi} / \mathrm{L}$ & 0.0000496 & & & & & & RIVER & & CONTINUOUS & RESIN & 307 \\
\hline
\end{tabular}




\begin{tabular}{|c|c|c|c|c|c|c|c|c|c|c|c|c|}
\hline & $\mathrm{N}$ & $\mathrm{O}$ & $\mathrm{P}$ & $\mathrm{Q}$ & $\mathrm{R}$ & $\mathrm{s}$ & $\mathrm{T}$ & $\mathrm{U}$ & $\mathrm{V}$ & $\mathrm{w}$ & $\mathrm{X}$ & $\mathrm{Y}$ \\
\hline 1 & ANAL_UNITS_RPTD & COUNTING_ERROR & \begin{tabular}{|l|} 
TOTAL_ANAL_ERROR \\
\end{tabular} & MIN_DETECTABLE_ACTIVITY & PCNT_MOISTURE & LAB_QUALIFIER & REVIEW_QUALIFIER & SAMP_FROM & SAMP_ITEM & SAMP_MTHD & COLL_MTHD & SAMP_SITE_ID \\
\hline $\mid 5306$ & $\mathrm{pCi} / \mathrm{L}$ & & 0.00000894 & & & & & RIVER & & CONTINUOUS & RESIN & 307 \\
\hline 5307 & $\mathrm{pCi} / \mathrm{L}$ & 0.0085 & & & & U & & RIVER & & CONTINUOUS & RESIN & 307 \\
\hline 5308 & $\mathrm{pCi} / \mathrm{L}$ & 0.0044 & & & & U & & RIVER & & CONTINUOUS & FILTER & 307 \\
\hline 5309 & $\mathrm{pCi} / \mathrm{L}$ & 0.000066 & & & & & & RIVER & & CONTINUOUS & RESIN & 252 \\
\hline 5310 & $\mathrm{pCi} / \mathrm{L}$ & & 0.000000376 & & & & & RIVER & & CONTINUOUS & RESIN & 252 \\
\hline 5311 & $\mathrm{pCi} / \mathrm{L}$ & 0.0084 & & & & U & & RIVER & & CONTINUOUS & RESIN & 252 \\
\hline 5312 & $\mathrm{pCi} / \mathrm{L}$ & 0.00000212 & & & & & & RIVER & & CONTINUOUS & FILTER & 252 \\
\hline 5313 & $\mathrm{pCi} / \mathrm{L}$ & 0.0041 & & & & U & & RIVER & & CONTINUOUS & FILTER & 252 \\
\hline 5314 & $\mathrm{pCi} / \mathrm{L}$ & 0.0000078 & & & & & & RIVER & & CONTINUOUS & FILTER & 463 \\
\hline 5315 & $\mathrm{pCi} / \mathrm{L}$ & 0.0068 & & & & $\mathrm{U}$ & & RIVER & & CONTINUOUS & FILTER & 463 \\
\hline 5316 & $\mathrm{pCi} / \mathrm{L}$ & 0.0000574 & & & & & & RIVER & & CONTINUOUS & RESIN & 463 \\
\hline 5317 & $\mathrm{pCi} / \mathrm{L}$ & & 0.00000904 & & & & & RIVER & & CONTINUOUS & RESIN & 463 \\
\hline 5318 & $\mathrm{pCi} / \mathrm{L}$ & 0.01 & & & & U & & RIVER & & CONTINUOUS & RESIN & 463 \\
\hline 5319 & $\mathrm{pCi} / \mathrm{L}$ & 0.0448 & 0.0509 & & & & & DRINKING & & CUMULATIVE & UNFILTERED & 307 \\
\hline 5320 & $\mathrm{pCi} / \mathrm{L}$ & 0.0128 & 0.013 & & & & & DRINKING & & CUMULATIVE & UNFILTERED & 307 \\
\hline 5321 & $\mathrm{pCi} / \mathrm{L}$ & 0.0506 & 0.0595 & & & & & DRINKING & & CUMULATIVE & UNFILTERED & 307 \\
\hline 5322 & $\mathrm{pCi} / \mathrm{L}$ & 0.977 & 0.979 & & & U & & DRINKING & & CUMULATIVE & UNFILTERED & 307 \\
\hline 5323 & $\mathrm{pCi} / \mathrm{L}$ & 11.8 & 21.5 & & & & & DRINKING & & CUMULATIVE & UNFILTERED & 307 \\
\hline 5324 & $\mathrm{pCi} / \mathrm{L}$ & 0.0384 & 0.0546 & & & & & DRINKING & & CUMULATIVE & UNFILTERED & 307 \\
\hline 5325 & $\mathrm{pCi} / \mathrm{L}$ & 0.809 & 0.809 & & & U & & QC_SAMPLE & & RAW GRAB & UNFILTERED & 550 \\
\hline 5326 & $\mathrm{pCi} / \mathrm{L}$ & 6.25 & 18.6 & & & & & QC_SAMPLE & & RAW GRAB & UNFILTERED & 550 \\
\hline 5327 & $\mathrm{pCi} / \mathrm{L}$ & 0.0363 & 0.0436 & & & & & QC_SAMPLE & & RAW GRAB & UNFILTERED & 550 \\
\hline 5328 & $\mathrm{pCi} / \mathrm{L}$ & 0.0608 & 0.0755 & & & & & QC_SAMPLE & & RAW GRAB & UNFILTERED & 550 \\
\hline 5329 & $\mathrm{pCi} / \mathrm{L}$ & 0.017 & 0.0173 & & & & & QC_SAMPLE & & RAW GRAB & UNFILTERED & 550 \\
\hline 5330 & $\mathrm{pCi} / \mathrm{L}$ & 0.0682 & 0.0887 & & & & & QC_SAMPLE & & RAW GRAB & UNFILTERED & 550 \\
\hline 5331 & $\mathrm{pCi} / \mathrm{L}$ & 5.73 & 17 & & & & & QC_SAMPLE & & RAW GRAB & UNFILTERED & 247 \\
\hline 5332 & $\mathrm{pCi} / \mathrm{L}$ & 0.0404 & 0.0483 & & & & & QC_SAMPLE & & RAW GRAB & UNFILTERED & 247 \\
\hline 5333 & $\mathrm{pCi} / \mathrm{L}$ & 0.0587 & 0.072 & & & & & QC_SAMPLE & & RAW GRAB & UNFILTERED & 247 \\
\hline 5334 & $\mathrm{pCi} / \mathrm{L}$ & 0.0116 & 0.0118 & & & & & QC_SAMPLE & & RAW GRAB & UNFILTERED & 247 \\
\hline 5335 & $\mathrm{pCi} / \mathrm{L}$ & 0.0694 & 0.0909 & & & & & QC_SAMPLE & & RAW GRAB & UNFILTERED & 247 \\
\hline 5336 & $\mathrm{pCi} / \mathrm{L}$ & 1.08 & 1.08 & & & U & & QC_SAMPLE & & RAW GRAB & UNFILTERED & 247 \\
\hline 5337 & $\mathrm{pCi} / \mathrm{L}$ & 0.0068 & & & & U & & RIVER & & CONTINUOUS & RESIN & 307 \\
\hline 5338 & $\mathrm{pCi} / \mathrm{L}$ & 0.0034 & & & & U & & RIVER & & CONTINUOUS & FILTER & 307 \\
\hline 5339 & $\mathrm{pCi} / \mathrm{L}$ & 0.0063 & & & & U & & RIVER & & CONTINUOUS & RESIN & 252 \\
\hline 5340 & $\mathrm{pCi} / \mathrm{L}$ & 0.0031 & & & & U & & RIVER & & CONTINUOUS & FILTER & 252 \\
\hline 5341 & $\mathrm{pCi} / \mathrm{L}$ & 0.0055 & & & & U & & RIVER & & CONTINUOUS & FILTER & 463 \\
\hline 5342 & $\mathrm{pCi} / \mathrm{L}$ & 0.011 & & & & U & & RIVER & & CONTINUOUS & RESIN & 463 \\
\hline 5343 & $\mathrm{pCi} / \mathrm{L}$ & 0.775 & 0.776 & & & U & & RIVER & & CUMULATIVE & UNFILTERED & 252 \\
\hline 5344 & $\mathrm{pCi} / \mathrm{L}$ & 0.0354 & 0.0472 & & & & & RIVER & & CUMULATIVE & UNFILTERED & 252 \\
\hline 5345 & $\mathrm{pCi} / \mathrm{L}$ & 0.0419 & 0.047 & & & & & RIVER & & CUMULATIVE & UNFILTERED & 252 \\
\hline 5346 & $\mathrm{pCi} / \mathrm{L}$ & 0.00683 & 0.00685 & & & U & & RIVER & & CUMULATIVE & UNFILTERED & 252 \\
\hline 5347 & $\mathrm{pCi} / \mathrm{L}$ & 0.0457 & 0.0521 & & & & & RIVER & & CUMULATIVE & UNFILTERED & 252 \\
\hline 5348 & $\mathrm{pCi} / \mathrm{L}$ & 5.8 & 14.7 & & & & & RIVER & & CUMULATIVE & UNFILTERED & 252 \\
\hline 5349 & $\mathrm{pCi} / \mathrm{L}$ & 0.712 & 0.715 & & & U & & RIVER & & CUMULATIVE & UNFILTERED & 463 \\
\hline 5350 & $\mathrm{pCi} / \mathrm{L}$ & 0.0426 & 0.0586 & & & & & RIVER & & CUMULATIVE & UNFILTERED & 463 \\
\hline 5351 & $\mathrm{pCi} / \mathrm{L}$ & 0.0384 & 0.0428 & & & & & RIVER & & CUMULATIVE & UNFILTERED & 463 \\
\hline 5352 & $\mathrm{pCi} / \mathrm{L}$ & 0.0101 & 0.0102 & & & & & RIVER & & CUMULATIVE & UNFILTERED & 463 \\
\hline 5353 & $\mathrm{pCi} / \mathrm{L}$ & 0.0466 & 0.0547 & & & & & RIVER & & CUMULATIVE & UNFILTERED & 463 \\
\hline 5354 & $\mathrm{pCi} / \mathrm{L}$ & 5.59 & 15.5 & & & & & RIVER & & CUMULATIVE & UNFILTERED & 463 \\
\hline 5355 & $\mathrm{pCi} / \mathrm{L}$ & 0.866 & 0.866 & & & U & & DRINKING & & SAN. GRAB & UNFILTERED & 487 \\
\hline 5356 & $\mathrm{pCi} / \mathrm{L}$ & 218 & 287 & & & U & & DRINKING & & SAN. GRAB & UNFILTERED & 487 \\
\hline 5357| & $\mathrm{pCi} / \mathrm{L}$ & 0.0432 & 0.0462 & & & $U_{2}$ & & DRINKING & & SAN. GRAB & UNFILTERED & 487 \\
\hline
\end{tabular}




\begin{tabular}{|c|c|c|c|c|c|c|c|c|c|c|c|c|}
\hline & $\mathrm{N}$ & $\mathrm{O}$ & $\mathrm{P}$ & $\mathrm{Q}$ & $\mathrm{R}$ & $\mathrm{s}$ & $T$ & $\mathrm{U}$ & $\mathrm{V}$ & $\mathrm{W}$ & $\mathrm{X}$ & $\bar{Y}$ \\
\hline 1 & ANAL_UNITS_RPTD & COUNTING_ERROR & TOTAL_ANAL_ERROR & MIN_DETECTABLE_ACTIVITY & PCNT_MOISTURE & LAB_QUALIFIER & REVIEW_QUALIFIER & SAMP_FROM & SAMP_ITEM & SAMP_MTHD & COLL_MTHD & SAMP_SITE_ID \\
\hline 5358 & $\mathrm{pCi} / \mathrm{L}$ & 0.0067 & & & & $U$ & & RIVER & & CONTINUOUS & RESIN & 307 \\
\hline 5359 & $\mathrm{pCi} / \mathrm{L}$ & 0.0033 & & & & U & & RIVER & & CONTINUOUS & FILTER & 307 \\
\hline 5360 & $\mathrm{pCi} / \mathrm{L}$ & 0.0074 & & & & U & & RIVER & & CONTINUOUS & RESIN & 252 \\
\hline 5361 & $\mathrm{pCi} / \mathrm{L}$ & 0.0036 & & & & $\mathrm{U}$ & & RIVER & & CONTINUOUS & FILTER & 252 \\
\hline 5362 & $\mathrm{pCi} / \mathrm{L}$ & 0.0038 & & & & $U$ & & RIVER & & CONTINUOUS & FILTER & 463 \\
\hline 5363 & $\mathrm{pCi} / \mathrm{L}$ & 0.0076 & & & & $\mathrm{U}$ & & RIVER & & CONTINUOUS & RESIN & 463 \\
\hline 5364 & $\mathrm{pCi} / \mathrm{L}$ & 1.02 & 1.02 & & & $\mathrm{U}$ & & DRINKING & & SAN. GRAB & UNFILTERED & 530 \\
\hline 5365 & $\mathrm{pCi} / \mathrm{L}$ & 0.0611 & 0.152 & & & & & DRINKING & & SAN. GRAB & UNFILTERED & 530 \\
\hline 5366 & $\mathrm{pCi} / \mathrm{L}$ & 6.13 & 15.4 & & & & & DRINKING & & SAN. GRAB & UNFILTERED & 530 \\
\hline 5367 & $\mathrm{pCi} / \mathrm{L}$ & 0.0071 & & & & $U$ & & RIVER & & CONTINUOUS & RESIN & 307 \\
\hline 5369 & & & & & & & & RIVER & & CONTINUOUS & RESIN & 252 \\
\hline 5370 & $\mathrm{pCi} / \mathrm{L}$ & 0.0072 & & & & $U$ & & RIVER & & CONTINUOUS & FILTER & 463 \\
\hline \begin{tabular}{|l|}
5371 \\
\end{tabular} & $\mathrm{pCi} / \mathrm{L}$ & 0.013 & & & & $U$ & & RIVER & & CONTINUOUS & RESIN & 463 \\
\hline \begin{tabular}{|l|}
5372 \\
\end{tabular} & $\mathrm{pCi} / \mathrm{L}$ & 0.956 & 0.959 & & & $U$ & & IRRIGATION & & RAW GRAB & UNFILTERED & 440 \\
\hline \begin{tabular}{|l|}
5373 \\
\end{tabular} & $\mathrm{pCi} / \mathrm{L}$ & 0.0475 & 0.0642 & & & & & IRRIGATION & & RAW GRAB & UNFILTERED & 440 \\
\hline 5374 & $\mathrm{pCi} / \mathrm{L}$ & 1.44 & 1.45 & & & $U$ & & SURFACE & & RAW GRAB & UNFILTERED & 509 \\
\hline 5375 & $\mathrm{pCi} / \mathrm{L}$ & 0.0743 & 0.0873 & & & & & SURFACE & & RAW GRAB & UNFILTERED & 509 \\
\hline 5376 & $\mathrm{pCi} / \mathrm{L}$ & 217 & 281 & & & $U$ & & SURFACE & & RAW GRAB & UNFILTERED & 509 \\
\hline 5377 & $\mathrm{pCi} / \mathrm{L}$ & 1.62 & 1.62 & & & $U$ & & SURFACE & & RAW GRAB & UNFILTERED & 136 \\
\hline 5378 & $\mathrm{pCi} / \mathrm{L}$ & 363 & 719 & & & & & SURFACE & & RAW GRAB & UNFILTERED & 136 \\
\hline 5379 & $\mathrm{pCi} / \mathrm{L}$ & 0.877 & 0.882 & & & & & SURFACE & & RAW GRAB & UNFILTERED & 146 \\
\hline \begin{tabular}{|l|}
5381 \\
\end{tabular} & $\mathrm{pCi} / \mathrm{L}$ & 218 & 284 & & & $U$ & & SURFACE & & RAW GRAB & UNFILTERED & 146 \\
\hline 5382 & $\mathrm{pCi} / \mathrm{L}$ & 2.32 & 2.32 & & & $U$ & & SURFACE & & RAW GRAB & UNFILTERED & 147 \\
\hline 5383 & $\mathrm{pCi} / \mathrm{L}$ & 0.242 & 0.526 & & & & & SURFACE & & RAW GRAB & UNFILTERED & 147 \\
\hline 5384 & $\mathrm{pCi} / \mathrm{L}$ & 227 & 301 & & & & & SURFACE & & RAW GRAB & UNFILTERED & 147 \\
\hline 5385 & $\mathrm{pCi} / \mathrm{L}$ & 2.71 & 9.23 & & & & & SURFACE & & RAW GRAB & UNFILTERED & 147 \\
\hline 5386 & $\mathrm{pCi} / \mathrm{L}$ & 0.546 & 0.652 & & & & & SURFACE & & RAW GRAB & UNFILTERED & 147 \\
\hline \begin{tabular}{|l|}
5387 \\
\end{tabular} & $\mathrm{pCi} / \mathrm{L}$ & 2.82 & 9.99 & & & & & SURFACE & & RAW GRAB & UNFILTERED & 147 \\
\hline 5388 & $\mathrm{pCi} / \mathrm{L}$ & 0.0067 & & & & $U$ & & RIVER & & CONTINUOUS & RESIN & 307 \\
\hline 5389 & $\mathrm{pCi} / \mathrm{L}$ & 0.0034 & & & & $U$ & & RIVER & & CONTINUOUS & FILTER & 307 \\
\hline 5390 & $\mathrm{pCi} / \mathrm{L}$ & 0.0027 & & & & U & & RIVER & & CONTINUOUS & FILTER & 252 \\
\hline 5391 & $\mathrm{pCi} / \mathrm{L}$ & 0.0385 & 0.042 & & & & & RIVER & & CUMULATIVE & UNFILTERED & 252 \\
\hline 5392 & $\mathrm{pCi} / \mathrm{L}$ & 0.0134 & 0.0134 & & & $U$ & & RIVER & & CUMULATIVE & UNFILTERED & 252 \\
\hline 5393 & $\mathrm{pCi} / \mathrm{L}$ & 0.0435 & 0.0483 & & & & & RIVER & & CUMULATIVE & UNFILTERED & 252 \\
\hline 5394 & $\mathrm{pCi} / \mathrm{L}$ & 1.24 & 1.24 & & & $U$ & & RIVER & & CUMULATIVE & UNFILTERED & 252 \\
\hline 5395 & $\mathrm{pCi} / \mathrm{L}$ & 0.005 & & & & U & & RIVER & & CONTINUOUS & RESIN & 252 \\
\hline 5396 & $\mathrm{pCi} / \mathrm{L}$ & 0.0434 & 0.0627 & & & & & RIVER & & CUMULATIVE & UNFILTERED & 252 \\
\hline \begin{tabular}{|l|l|}
5397 \\
\end{tabular} & $\mathrm{pCi} / \mathrm{L}$ & 6.04 & 13.4 & & & & & RIVER & & CUMULATIVE & UNFILTERED & 252 \\
\hline 5398 & $\mathrm{pCi} / \mathrm{L}$ & 0.0077 & & & & $U$ & & RIVER & & CONTINUOUS & RESIN & 463 \\
\hline 5399 & $\mathrm{pCi} / \mathrm{L}$ & 0.038 & 0.0416 & & & & & RIVER & & CUMULATIVE & UNFILTERED & 463 \\
\hline 5400 & $\mathrm{pCi} / \mathrm{L}$ & 0.00917 & 0.00922 & & & & & RIVER & & CUMULATIVE & UNFILTERED & 463 \\
\hline \begin{tabular}{|l|}
5401 \\
\end{tabular} & $\mathrm{pCi} / \mathrm{L}$ & 0.0348 & 0.0373 & & & & & RIVER & & CUMULATIVE & UNFILTERED & 463 \\
\hline \begin{tabular}{|l|}
5402 \\
\end{tabular} & $\mathrm{pCi} / \mathrm{L}$ & 0.689 & 0.691 & & & $U$ & & RIVER & & CUMULATIVE & UNFILTERED & 463 \\
\hline 5403 & $\mathrm{pCi} / \mathrm{L}$ & 0.0038 & & & & U & & RIVER & & CONTINUOUS & FILTER & 463 \\
\hline \begin{tabular}{|l|}
5407 \\
\end{tabular} & $\mathrm{pCi} / \mathrm{L}$ & 0.0072 & & & & $U$ & & RIVER & & CONTINUOUS & RESIN & 307 \\
\hline \begin{tabular}{|l|}
5408 \\
\end{tabular} & $\mathrm{pCi} / \mathrm{L}$ & 0.012 & & & & $U$ & & RIVER & & CONTINUOUS & RESIN & 252 \\
\hline 5409 & $\mathrm{pCi} / \mathrm{L}$ & 0.0061 & & & & $\mathrm{U}$ & & RIVER & & CONTINUOUS & FILTER & 252 \\
\hline
\end{tabular}




\begin{tabular}{|c|c|c|c|c|c|c|c|c|c|c|c|c|}
\hline & $\mathrm{N}$ & $\mathrm{O}$ & $\mathrm{P}$ & $\mathrm{Q}$ & $\mathrm{R}$ & $\mathrm{S}$ & $\mathrm{T}$ & $\mathrm{U}$ & $\mathrm{V}$ & $\mathrm{W}$ & $\mathrm{X}$ & $\mathrm{Y}$ \\
\hline 1 & ANAL_UNITS_RPTD & COUNTING_ERROR & TOTAL_ANAL_ERROR & MIN_DETECTABLE_ACTIVITY & PCNT_MOISTURE & LAB_QUALIFIER & REVIEW_QUALIFIER & SAMP_FROM & SAMP_ITEM & SAMP_MTHD & COLL_MTHD & SAMP_SITE_ID \\
\hline 5410 & $\mathrm{pCi} / \mathrm{L}$ & 0.01 & & & & $U$ & & RIVER & & CONTINUOUS & RESIN & 463 \\
\hline 5411 & $\mathrm{pCi} / \mathrm{L}$ & 0.0049 & & & & U & & RIVER & & CONTINUOUS & FILTER & 463 \\
\hline 5412 & $\mathrm{pCi} / \mathrm{L}$ & 0.734 & 0.736 & & & U & & DRINKING & & SAN. GRAB & UNFILTERED & 186 \\
\hline 5413 & $\mathrm{pCi} / \mathrm{L}$ & 0.0382 & 0.042 & & & & & DRINKING & & SAN. GRAB & UNFILTERED & 186 \\
\hline 5414 & $\mathrm{pCi} / \mathrm{L}$ & 124 & 210 & & & u & & DRINKING & & SAN. GRAB & UNFILTERED & 186 \\
\hline 5415 & $\mathrm{pCi} / \mathrm{L}$ & 1.06 & 1.06 & & & U & & DRINKING & & SAN. GRAB & UNFILTERED & 512 \\
\hline $\mid 5416$ & $\mathrm{pCi} / \mathrm{L}$ & 0.0416 & 0.0427 & & & $\mathrm{u}$ & & DRINKING & & SAN. GRAB & UNFILTERED & 512 \\
\hline 5417 & $\mathrm{pCi} / \mathrm{L}$ & 192 & 324 & & & & & DRINKING & & SAN. GRAB & UNFILTERED & 512 \\
\hline 5418 & $\mathrm{pCi} / \mathrm{L}$ & 0.657 & 0.657 & & & U & & DRINKING & & SAN. GRAB & UNFILTERED & 144 \\
\hline 5419 & $\mathrm{pCi} / \mathrm{L}$ & 0.0309 & 0.0323 & & & U & & DRINKING & & SAN. GRAB & UNFILTERED & 144 \\
\hline 5420 & $\mathrm{pCi} / \mathrm{L}$ & 126 & 212 & & & U & & DRINKING & & SAN. GRAB & UNFILTERED & 144 \\
\hline 5421 & $\mathrm{pCi} / \mathrm{L}$ & 0.798 & 0.804 & & & & & DRINKING & & SAN. GRAB & UNFILTERED & 187 \\
\hline 5422 & $\mathrm{pCi} / \mathrm{L}$ & 0.0341 & 0.0347 & & & U & & DRINKING & & SAN. GRAB & UNFILTERED & 187 \\
\hline 5423 & $\mathrm{pCi} / \mathrm{L}$ & 127 & 213 & & & U & & DRINKING & & SAN. GRAB & UNFILTERED & 187 \\
\hline 5424 & $\mathrm{pCi} / \mathrm{L}$ & 0.758 & 0.77 & & & & & DRINKING & & RAW GRAB & UNFILTERED & 553 \\
\hline 5425 & $\mathrm{pCi} / \mathrm{L}$ & 0.043 & 0.0544 & & & & & DRINKING & & RAW GRAB & UNFILTERED & 553 \\
\hline 5426 & $\mathrm{pCi} / \mathrm{L}$ & 6.27 & 13.9 & & & & & DRINKING & & RAW GRAB & UNFILTERED & 553 \\
\hline 5427 & $\mathrm{pCi} / \mathrm{L}$ & 0.0000054 & & & & & & RIVER & & CONTINUOUS & FILTER & 307 \\
\hline 5428 & $\mathrm{pCi} / \mathrm{L}$ & 0.0035 & & & & U & & RIVER & & CONTINUOUS & FILTER & 307 \\
\hline 5429 & $\mathrm{pCi} / \mathrm{L}$ & 0.0000306 & & & & & & RIVER & & CONTINUOUS & RESIN & 307 \\
\hline 5430 & $\mathrm{pCi} / \mathrm{L}$ & & 0.00000679 & & & & & RIVER & & CONTINUOUS & RESIN & 307 \\
\hline 5431 & $\mathrm{pCi} / \mathrm{L}$ & 0.0066 & & & & U & & RIVER & & CONTINUOUS & RESIN & 307 \\
\hline 5432 & $\mathrm{pCi} / \mathrm{L}$ & 0.000046 & & & & & & RIVER & & CONTINUOUS & RESIN & 252 \\
\hline 5433 & $\mathrm{pCi} / \mathrm{L}$ & 0.0000078 & & & & & & RIVER & & CONTINUOUS & FILTER & 252 \\
\hline 5434 & $\mathrm{pCi} / \mathrm{L}$ & & 0.000000529 & & & & & RIVER & & CONTINUOUS & RESIN & 252 \\
\hline 5435 & $\mathrm{pCi} / \mathrm{L}$ & 0.0079 & & & & U & & RIVER & & CONTINUOUS & RESIN & 252 \\
\hline 5436 & $\mathrm{pCi} / \mathrm{L}$ & 0.0041 & & & & U & & RIVER & & CONTINUOUS & FILTER & 252 \\
\hline 5437 & $\mathrm{pCi} / \mathrm{L}$ & 0.0452 & 0.0511 & & & & & RIVER & & CUMULATIVE & UNFILTERED & 252 \\
\hline 5438 & $\mathrm{pCi} / \mathrm{L}$ & 0.828 & 0.831 & & & U & & RIVER & & CUMULATIVE & UNFILTERED & 252 \\
\hline 5439 & $\mathrm{pCi} / \mathrm{L}$ & 0.045 & 0.0575 & & & & & RIVER & & CUMULATIVE & UNFILTERED & 252 \\
\hline 5440 & $\mathrm{pCi} / \mathrm{L}$ & 0.0388 & 0.0425 & & & & & RIVER & & CUMULATIVE & UNFILTERED & 252 \\
\hline 5441 & $\mathrm{pCi} / \mathrm{L}$ & 0.011 & 0.0112 & & & & & RIVER & & CUMULATIVE & UNFILTERED & 252 \\
\hline 5442 & $\mathrm{pCi} / \mathrm{L}$ & 5.17 & 12 & & & & & RIVER & & CUMULATIVE & UNFILTERED & 252 \\
\hline 5443 & $\mathrm{pCi} / \mathrm{L}$ & 0.0000536 & & & & & & RIVER & & CONTINUOUS & RESIN & 463 \\
\hline 5444 & $\mathrm{pCi} / \mathrm{L}$ & 0.0000088 & & & & & & RIVER & & CONTINUOUS & FILTER & 463 \\
\hline 5445 & $\mathrm{pCi} / \mathrm{L}$ & & 0.00000594 & & & & & RIVER & & CONTINUOUS & RESIN & 463 \\
\hline 5446 & $\mathrm{pCi} / \mathrm{L}$ & 0.0057 & & & & U & & RIVER & & CONTINUOUS & FILTER & 463 \\
\hline 5447 & $\mathrm{pCi} / \mathrm{L}$ & 0.011 & & & & U & & RIVER & & CONTINUOUS & RESIN & 463 \\
\hline 5448 & $\mathrm{pCi} / \mathrm{L}$ & 0.0391 & 0.0438 & & & & & RIVER & & CUMULATIVE & UNFILTERED & 463 \\
\hline 5449 & $\mathrm{pCi} / \mathrm{L}$ & 0.00842 & 0.00854 & & & U & & RIVER & & CUMULATIVE & UNFILTERED & 463 \\
\hline 5450 & $\mathrm{pCi} / \mathrm{L}$ & 0.0476 & 0.0555 & & & & & RIVER & & CUMULATIVE & UNFILTERED & 463 \\
\hline 5451 & $\mathrm{pCi} / \mathrm{L}$ & 0.496 & 0.496 & & & $U$ & & RIVER & & CUMULATIVE & UNFILTERED & 463 \\
\hline 5452 & $\mathrm{pCi} / \mathrm{L}$ & 0.0473 & 0.0574 & & & & & RIVER & & CUMULATIVE & UNFILTERED & 463 \\
\hline 5453 & $\mathrm{pCi} / \mathrm{L}$ & 6.08 & 15 & & & & & RIVER & & CUMULATIVE & UNFILTERED & 463 \\
\hline 5454 & $\mathrm{pCi} / \mathrm{L}$ & 0.319 & 0.32 & & & U & & DRINKING & & CUMULATIVE & UNFILTERED & 307 \\
\hline 5455 & $\mathrm{pCi} / \mathrm{L}$ & 0.0436 & 0.0518 & & & & & DRINKING & & CUMULATIVE & UNFILTERED & 307 \\
\hline 5456 & $\mathrm{pCi} / \mathrm{L}$ & 0.0489 & 0.0546 & & & & & DRINKING & & CUMULATIVE & UNFILTERED & 307 \\
\hline 5457 & $\mathrm{pCi} / \mathrm{L}$ & 0.00937 & 0.00951 & & & U & & DRINKING & & CUMULATIVE & UNFILTERED & 307 \\
\hline 5458 & $\mathrm{pCi} / \mathrm{L}$ & 0.0494 & 0.0557 & & & & & DRINKING & & CUMULATIVE & UNFILTERED & 307 \\
\hline 5459 & $\mathrm{pCi} / \mathrm{L}$ & 6.03 & 15.1 & & & & & DRINKING & & CUMULATIVE & UNFILTERED & 307 \\
\hline 5460 & $\mathrm{pCi} / \mathrm{L}$ & 0.004 & & & & U & & RIVER & & CONTINUOUS & FILTER & 307 \\
\hline 5461 & $\mathrm{pCi} / \mathrm{L}$ & 0.0077 & & & & 1 & & RIVER & & CONTINUOUS & RESIN & 307 \\
\hline
\end{tabular}




\begin{tabular}{|c|c|c|c|c|c|c|c|c|c|c|c|c|}
\hline & $\mathrm{N}$ & $\mathrm{O}$ & $\mathrm{P}$ & $\mathrm{Q}$ & $\mathrm{R}$ & $\mathrm{s}$ & $T$ & $\bar{U}$ & $\mathrm{~V}$ & $\mathrm{w}$ & $\mathrm{X}$ & $\mathrm{Y}$ \\
\hline 1 & ANAL_UNITS_RPTD & COUNTING_ERROR & TOTAL_ANAL_ERROR & MIN_DETECTABLE_ACTIVITY & PCNT_MOISTURE & LAB_QUALIFIER & REVIEW_QUALIFIER & SAMP_FROM & SAMP_ITEM & SAMP_MTHD & COLL_MTHD & SAMP_SITE_ID \\
\hline 5462 & $\mathrm{pCi} / \mathrm{L}$ & 0.0083 & & & & $U$ & & RIVER & & CONTINUOUS & RESIN & 252 \\
\hline 5463 & $\mathrm{pCi} / \mathrm{L}$ & 0.0042 & & & & $\mathrm{U}$ & & RIVER & & CONTINUOUS & FILTER & 252 \\
\hline 5464 & $\mathrm{pCi} / \mathrm{L}$ & 0.0064 & & & & U & & RIVER & & CONTINUOUS & FILTER & 463 \\
\hline 5465 & $\mathrm{pCi} / \mathrm{L}$ & 0.013 & & & & U & & RIVER & & CONTINUOUS & RESIN & 463 \\
\hline 5466 & $\mathrm{pCi} / \mathrm{L}$ & 0.922 & 0.923 & & & U & & IRRIGATION & & RAW GRAB & UNFILTERED & 440 \\
\hline 5467 & $\mathrm{pCi} / \mathrm{L}$ & 0.054 & 0.0657 & & & & & IRRIGATION & & RAW GRAB & UNFILTERED & 440 \\
\hline 5468 & $\mathrm{pCi} / \mathrm{L}$ & 0.8 & 0.8 & & & U & & DRINKING & & SAN. GRAB & UNFILTERED & 487 \\
\hline 5469 & $\mathrm{pCi} / \mathrm{L}$ & 158 & 260 & & & U & & DRINKING & & SAN. GRAB & UNFILTERED & 487 \\
\hline 5470 & $\mathrm{pCi} / \mathrm{L}$ & 0.0281 & 0.0287 & & & U & & DRINKING & & SAN. GRAB & UNFILTERED & 487 \\
\hline 5471 & $\mathrm{pCi} / \mathrm{L}$ & 0.0071 & & & & $\mathrm{U}$ & & RIVER & & CONTINUOUS & RESIN & 307 \\
\hline 5472 & $\mathrm{pCi} / \mathrm{L}$ & 0.0037 & & & & U & & RIVER & & CONTINUOUS & FILTER & 307 \\
\hline 5473 & $\mathrm{pCi} / \mathrm{L}$ & 0.0415 & 0.0461 & & & & & RIVER & & CUMULATIVE & UNFILTERED & 252 \\
\hline 5474 & $\mathrm{pCi} / \mathrm{L}$ & 0.00842 & 0.00857 & & & U & & RIVER & & CUMULATIVE & UNFILTERED & 252 \\
\hline 5475 & $\mathrm{pCi} / \mathrm{L}$ & 0.0452 & 0.051 & & & & & RIVER & & CUMULATIVE & UNFILTERED & 252 \\
\hline 5476 & $\mathrm{pCi} / \mathrm{L}$ & 0.712 & 0.713 & & & U & & RIVER & & CUMULATIVE & UNFILTERED & 252 \\
\hline 5477 & $\mathrm{pCi} / \mathrm{L}$ & 0.0042 & & & & U & & RIVER & & CONTINUOUS & FILTER & 252 \\
\hline 5478 & $\mathrm{pCi} / \mathrm{L}$ & 0.0308 & 0.0425 & & & & & RIVER & & CUMULATIVE & UNFILTERED & 252 \\
\hline 5479 & $\mathrm{pCi} / \mathrm{L}$ & 5.26 & 12 & & & & & RIVER & & CUMULATIVE & UNFILTERED & 252 \\
\hline 5480 & $\mathrm{pCi} / \mathrm{L}$ & 0.0084 & & & & U & & RIVER & & CONTINUOUS & RESIN & 252 \\
\hline 5481 & $\mathrm{pCi} / \mathrm{L}$ & 0.01 & & & & U & & RIVER & & CONTINUOUS & RESIN & 463 \\
\hline 5482 & $\mathrm{pCi} / \mathrm{L}$ & 0.0496 & 0.0564 & & & & & RIVER & & CUMULATIVE & UNFILTERED & 463 \\
\hline 5483 & $\mathrm{pCi} / \mathrm{L}$ & 0.00775 & 0.00789 & & & U & & RIVER & & CUMULATIVE & UNFILTERED & 463 \\
\hline 5484 & $\mathrm{pCi} / \mathrm{L}$ & 0.0528 & 0.0609 & & & & & RIVER & & CUMULATIVE & UNFILTERED & 463 \\
\hline 5485 & $\mathrm{pCi} / \mathrm{L}$ & 0.411 & 0.413 & & & & & RIVER & & CUMULATIVE & UNFILTERED & 463 \\
\hline 5486 & $\mathrm{pCi} / \mathrm{L}$ & 0.0049 & & & & U & & RIVER & & CONTINUOUS & FILTER & 463 \\
\hline 5487 & $\mathrm{pCi} / \mathrm{L}$ & 0.0337 & 0.0449 & & & & & RIVER & & CUMULATIVE & UNFILTERED & 463 \\
\hline 5488 & $\mathrm{pCi} / \mathrm{L}$ & 6.22 & 15.2 & & & & & RIVER & & CUMULATIVE & UNFILTERED & 463 \\
\hline 5489 & $\mathrm{pCi} / \mathrm{L}$ & 1.05 & 1.05 & & & U & & DRINKING & & SAN. GRAB & UNFILTERED & 530 \\
\hline 5490 & $\mathrm{pCi} / \mathrm{L}$ & 0.0596 & 0.128 & & & & & DRINKING & & SAN. GRAB & UNFILTERED & 530 \\
\hline 5491 & $\mathrm{pCi} / \mathrm{L}$ & 5.77 & 13.7 & & & & & DRINKING & & SAN. GRAB & UNFILTERED & 530 \\
\hline 5492 & $\mathrm{pCi} / \mathrm{L}$ & 0.006 & & & & U & & RIVER & & CONTINUOUS & RESIN & 307 \\
\hline 5493 & $\mathrm{pCi} / \mathrm{L}$ & 0.0031 & & & & U & & RIVER & & CONTINUOUS & FILTER & 307 \\
\hline 5494 & $\mathrm{pCi} / \mathrm{L}$ & 0.012 & & & & $U$ & & RIVER & & CONTINUOUS & RESIN & 252 \\
\hline 5495 & $\mathrm{pCi} / \mathrm{L}$ & 0.0061 & & & & U & & RIVER & & CONTINUOUS & FILTER & 252 \\
\hline 5496 & $\mathrm{pCi} / \mathrm{L}$ & 0.0041 & & & & U & & RIVER & & CONTINUOUS & FILTER & 463 \\
\hline 5497 & $\mathrm{pCi} / \mathrm{L}$ & 0.0078 & & & & U & & RIVER & & CONTINUOUS & RESIN & 463 \\
\hline 5498 & $\mathrm{pCi} / \mathrm{L}$ & 1.86 & 1.87 & & & U & & SURFACE & & RAW GRAB & UNFILTERED & 509 \\
\hline 5499 & $\mathrm{pCi} / \mathrm{L}$ & 131 & 216 & & & U & & SURFACE & & RAW GRAB & UNFILTERED & 509 \\
\hline 5500 & $\mathrm{pCi} / \mathrm{L}$ & 0.0954 & 0.151 & & & & & SURFACE & & RAW GRAB & UNFILTERED & 509 \\
\hline 5501 & & & & & & & & SURFACE & & RAW GRAB & UNFILTERED & 136 \\
\hline 5502 & & & & & & & & SURFACE & & RAW GRAB & UNFILTERED & 136 \\
\hline 5503 & $\mathrm{pCi} / \mathrm{L}$ & 4.94 & 7.04 & & & & & SURFACE & & RAW GRAB & UNFILTERED & 146 \\
\hline 5504 & $\mathrm{pCi} / \mathrm{L}$ & 134 & 219 & & & & & SURFACE & & RAW GRAB & UNFILTERED & 146 \\
\hline 5505 & $\mathrm{pCi} / \mathrm{L}$ & 0.109 & 0.384 & & & & & SURFACE & & RAW GRAB & UNFILTERED & 146 \\
\hline 5506 & $\mathrm{pCi} / \mathrm{L}$ & 4.24 & 16.9 & & & & & SURFACE & & RAW GRAB & UNFILTERED & 147 \\
\hline 5507 & $\mathrm{pCi} / \mathrm{L}$ & 0.79 & 0.973 & & & & & SURFACE & & RAW GRAB & UNFILTERED & 147 \\
\hline 5508 & $\mathrm{pCi} / \mathrm{L}$ & 4.36 & 17.8 & & & & & SURFACE & & RAW GRAB & UNFILTERED & 147 \\
\hline 5509 & $\mathrm{pCi} / \mathrm{L}$ & 142 & 230 & & & & & SURFACE & & RAW GRAB & UNFILTERED & 147 \\
\hline 5510 & $\mathrm{pCi} / \mathrm{L}$ & 3.05 & 3.05 & & & U & & SURFACE & & RAW GRAB & UNFILTERED & 147 \\
\hline 5511 & $\mathrm{pCi} / \mathrm{L}$ & 0.264 & 0.767 & & & & & SURFACE & & RAW GRAB & UNFILTERED & 147 \\
\hline 5512 & $\mathrm{pCi} / \mathrm{L}$ & 0.01 & & & & U & & RIVER & & CONTINUOUS & RESIN & 307 \\
\hline 5513 & $\mathrm{pCi} / \mathrm{L}$ & 0.0049 & & & & $U_{2}$ & & RIVER & & CONTINUOUS & FILTER & 307 \\
\hline
\end{tabular}




\begin{tabular}{|c|c|c|c|c|c|c|c|c|c|c|c|c|}
\hline & $\mathrm{N}$ & $\mathrm{O}$ & $\mathrm{P}$ & $\bar{Q}$ & $\bar{R}$ & $\mathrm{~s}$ & $\mathrm{~T}$ & $\bar{U}$ & $\bar{v}$ & $\mathrm{~W}$ & $\bar{x}$ & $\bar{Y}$ \\
\hline 1 & ANAL_UNITS_RPTD & COUNTING_ERROR & \begin{tabular}{|l|} 
TOTAL_ANAL_ERROR \\
\end{tabular} & MIN_DETECTABLE_ACTIVITY & PCNT_MOISTURE & LAB_QUALIFIER & REVIEW_QUALIFIER & SAMP_FROM & SAMP_ITEM & SAMP_MTHD & COLL_MTHD & SAMP_SITE_ID \\
\hline 5514 & & & & & & & & RIVER & & CONTINUOUS & RESIN & 252 \\
\hline 5515 & $\mathrm{pCi} / \mathrm{L}$ & 0.0042 & & & & $\mathrm{U}$ & & RIVER & & CONTINUOUS & FILTER & 463 \\
\hline 5516 & $\mathrm{pCi} / \mathrm{L}$ & 0.0083 & & & & $\mathrm{U}$ & & RIVER & & CONTINUOUS & RESIN & 463 \\
\hline 5517 & $\mathrm{pCi} / \mathrm{L}$ & 8.4 & 19.7 & & & & & RIVER & & CUMULATIVE & UNFILTERED & 252 \\
\hline 5518 & $\mathrm{pCi} / \mathrm{L}$ & 0.764 & 0.765 & & & $\mathrm{U}$ & & RIVER & & CUMULATIVE & UNFILTERED & 252 \\
\hline 5519 & $\mathrm{pCi} / \mathrm{L}$ & 0.0357 & 0.0391 & & & & & RIVER & & CUMULATIVE & UNFILTERED & 252 \\
\hline 5520 & $\mathrm{pCi} / \mathrm{L}$ & 0.0102 & 0.0104 & & & & & RIVER & & CUMULATIVE & UNFILTERED & 252 \\
\hline \begin{tabular}{|l|l|}
5521 \\
\end{tabular} & $\mathrm{pCi} / \mathrm{L}$ & 0.0377 & 0.0419 & & & & & RIVER & & CUMULATIVE & UNFILTERED & 252 \\
\hline 5522 & $\mathrm{pCi} / \mathrm{L}$ & 0.0337 & 0.0501 & & & & & RIVER & & CUMULATIVE & UNFILTERED & 252 \\
\hline 5523 & $\mathrm{pCi} / \mathrm{L}$ & 9.18 & 21.9 & & & & & RIVER & & CUMULATIVE & UNFILTERED & 463 \\
\hline 5524 & $\mathrm{pCi} / \mathrm{L}$ & 1.04 & 1.04 & & & $\mathrm{U}$ & & RIVER & & CUMULATIVE & UNFILTERED & 463 \\
\hline \begin{tabular}{|l|}
5525 \\
\end{tabular} & $\mathrm{pCi} / \mathrm{L}$ & 0.0383 & 0.0427 & & & & & RIVER & & CUMULATIVE & UNFILTERED & 463 \\
\hline \begin{tabular}{|l|}
5526 \\
\end{tabular} & $\mathrm{pCi} / \mathrm{L}$ & 0.0114 & 0.0116 & & & & & RIVER & & CUMULATIVE & UNFILTERED & 463 \\
\hline \begin{tabular}{|l|}
5527 \\
\end{tabular} & $\mathrm{pCi} / \mathrm{L}$ & 0.0415 & 0.0471 & & & & & RIVER & & CUMULATIVE & UNFILTERED & 463 \\
\hline 5528 & $\mathrm{pCi} / \mathrm{L}$ & 0.0301 & 0.0454 & & & & & RIVER & & CUMULATIVE & UNFILTERED & 463 \\
\hline \begin{tabular}{|l|}
5529 \\
\end{tabular} & & & & & & & & RIVER & & CONTINUOUS & RESIN & 307 \\
\hline 5530 & & & & & & & & RIVER & & CONTINUOUS & FILTER & 307 \\
\hline 5531 & $\mathrm{pCi} / \mathrm{L}$ & 0.0017 & & & & U & & RIVER & & CONTINUOUS & FILTER & 252 \\
\hline \begin{tabular}{|l|}
5532 \\
\end{tabular} & $\mathrm{pCi} / \mathrm{L}$ & 0.0033 & & & & U & & RIVER & & CONTINUOUS & RESIN & 252 \\
\hline \begin{tabular}{|l|}
5533 \\
\end{tabular} & $\mathrm{pCi} / \mathrm{L}$ & 0.0049 & & & & U & & RIVER & & CONTINUOUS & RESIN & 463 \\
\hline \begin{tabular}{|l|}
5534 \\
\end{tabular} & $\mathrm{pCi} / \mathrm{L}$ & 0.0025 & & & & U & & RIVER & & CONTINUOUS & FILTER & 463 \\
\hline 5535 & $\mathrm{pCi} / \mathrm{L}$ & 0.648 & 0.649 & & & $U$ & & IRRIGATION & & RAW GRAB & UNFILTERED & 440 \\
\hline 5536 & $\mathrm{pCi} / \mathrm{L}$ & 0.0461 & 0.0652 & & & & & IRRIGATION & & RAW GRAB & UNFILTERED & 440 \\
\hline \begin{tabular}{|l|}
5537 \\
\end{tabular} & $\mathrm{pCi} / \mathrm{L}$ & 131 & 215 & & & $U$ & & DRINKING & & SAN. GRAB & UNFILTERED & 186 \\
\hline 5538 & $\mathrm{pCi} / \mathrm{L}$ & 1.1 & 1.11 & & & & & DRINKING & & SAN. GRAB & UNFILTERED & 186 \\
\hline 5539 & $\mathrm{pCi} / \mathrm{L}$ & 0.0274 & 0.0294 & & & U & & DRINKING & & SAN. GRAB & UNFILTERED & 186 \\
\hline 5540 & $\mathrm{pCi} / \mathrm{L}$ & 216 & 380 & & & & & DRINKING & & SAN. GRAB & UNFILTERED & 512 \\
\hline \begin{tabular}{|l|}
5541 \\
\end{tabular} & $\mathrm{pCi} / \mathrm{L}$ & 0.876 & 0.877 & & & U & & DRINKING & & SAN. GRAB & UNFILTERED & 512 \\
\hline 5542 & $\mathrm{pCi} / \mathrm{L}$ & 0.0213 & 0.0221 & & & $U$ & & DRINKING & & SAN. GRAB & UNFILTERED & 512 \\
\hline 5543 & $\mathrm{pCi} / \mathrm{L}$ & 128 & 212 & & & $U$ & & DRINKING & & SAN. GRAB & UNFILTERED & 144 \\
\hline 5544 & $\mathrm{pCi} / \mathrm{L}$ & 0.362 & 0.363 & & & U & & DRINKING & & SAN. GRAB & UNFILTERED & 144 \\
\hline 5545 & $\mathrm{pCi} / \mathrm{L}$ & 0.0221 & 0.0262 & & & U & & DRINKING & & SAN. GRAB & UNFILTERED & 144 \\
\hline 5546 & $\mathrm{pCi} / \mathrm{L}$ & 128 & 212 & & & U & & DRINKING & & SAN. GRAB & UNFILTERED & 187 \\
\hline \begin{tabular}{|l|}
5547 \\
\end{tabular} & $\mathrm{pCi} / \mathrm{L}$ & 1.04 & 1.04 & & & U & & DRINKING & & SAN. GRAB & UNFILTERED & 187 \\
\hline \begin{tabular}{|l|}
5548 \\
\end{tabular} & $\mathrm{pCi} / \mathrm{L}$ & 0.0185 & 0.0194 & & & U & & DRINKING & & SAN. GRAB & UNFILTERED & 187 \\
\hline \begin{tabular}{|l|l|}
5549 \\
\end{tabular} & $\mathrm{pCi} / \mathrm{L}$ & 9 & 20.9 & & & & & DRINKING & & RAW GRAB & UNFILTERED & 553 \\
\hline 5550 & $\mathrm{pCi} / \mathrm{L}$ & 0.745 & 0.748 & & & $U$ & & DRINKING & & RAW GRAB & UNFILTERED & 553 \\
\hline 5551 & $\mathrm{pCi} / \mathrm{L}$ & 0.045 & 0.0619 & & & & & DRINKING & & RAW GRAB & UNFILTERED & 553 \\
\hline \begin{tabular}{|l|}
5552 \\
\end{tabular} & $\mathrm{pCi} / \mathrm{L}$ & 0.012 & & & & U & & RIVER & & CONTINUOUS & RESIN & 307 \\
\hline 5553 & $\mathrm{pCi} / \mathrm{L}$ & 0.0056 & & & & U & & RIVER & & CONTINUOUS & FILTER & 307 \\
\hline 5554 & $\mathrm{pCi} / \mathrm{L}$ & 0.0036 & & & & $U$ & & RIVER & & CONTINUOUS & RESIN & 252 \\
\hline 5555 & $\mathrm{pCi} / \mathrm{L}$ & 0.0018 & & & & & & RIVER & & CONTINUOUS & FILTER & 252 \\
\hline 5556 & $\mathrm{pCi} / \mathrm{L}$ & 0.0042 & & & & $U$ & & RIVER & & CONTINUOUS & FILTER & 463 \\
\hline \begin{tabular}{|l|}
5557 \\
\end{tabular} & $\mathrm{pCi} / \mathrm{L}$ & 0.0095 & & & & $U$ & & RIVER & & CONTINUOUS & RESIN & 463 \\
\hline 5558 & $\mathrm{pCi} / \mathrm{L}$ & 0.0464 & 0.053 & & & & & DRINKING & & CUMULATIVE & UNFILTERED & 307 \\
\hline 5559 & $\mathrm{pCi} / \mathrm{L}$ & 0.00679 & 0.00694 & & & $U$ & & DRINKING & & CUMULATIVE & UNFILTERED & 307 \\
\hline 5560 & $\mathrm{pCi} / \mathrm{L}$ & 0.0517 & 0.0612 & & & & & DRINKING & & CUMULATIVE & UNFILTERED & 307 \\
\hline \begin{tabular}{|l|}
5561 \\
\end{tabular} & $\mathrm{pCi} / \mathrm{L}$ & 10.3 & 25.7 & & & & & DRINKING & & CUMULATIVE & UNFILTERED & 307 \\
\hline \begin{tabular}{|l|l|}
5562 \\
\end{tabular} & $\mathrm{pCi} / \mathrm{L}$ & 0.79 & 0.791 & & & U & & DRINKING & & CUMULATIVE & UNFILTERED & 307 \\
\hline 5563 & $\mathrm{pCi} / \mathrm{L}$ & 0.0478 & 0.0613 & & & & & DRINKING & & CUMULATIVE & UNFILTERED & 307 \\
\hline 5564 & $\mathrm{pCi} / \mathrm{L}$ & 0.0414 & 0.0458 & & & & & RIVER & & CUMULATIVE & UNFILTERED & 252 \\
\hline 5565 & $\mathrm{pCi} / \mathrm{L}$ & 0.016 & 0.0163 & & & & & RIVER & & CUMULATIVE & UNFILTERED & 252 \\
\hline
\end{tabular}




\begin{tabular}{|c|c|c|c|c|c|c|c|c|c|c|c|c|}
\hline & $\mathrm{N}$ & $\mathrm{O}$ & $\mathrm{P}$ & $\mathrm{Q}$ & $\mathrm{R}$ & $\mathrm{s}$ & $\mathrm{T}$ & $\mathrm{U}$ & $\mathrm{V}$ & $\mathrm{w}$ & $\mathrm{X}$ & $\mathrm{Y}$ \\
\hline 1 & ANAL_UNITS_RPTD & COUNTING_ERROR & TOTAL_ANAL_ERROR & MIN_DETECTABLE_ACTIVITY & PCNT_MOISTURE & LAB_QUALIFIER & REVIEW_QUALIFIER & SAMP_FROM & SAMP_ITEM & SAMP_MTHD & COLL_MTHD & SAMP_SITE_ID \\
\hline 5566 & $\mathrm{pCi} / \mathrm{L}$ & 0.0497 & 0.057 & & & & & RIVER & & CUMULATIVE & UNFILTERED & 252 \\
\hline 5567 & $\mathrm{pCi} / \mathrm{L}$ & 8.09 & 18.7 & & & & & RIVER & & CUMULATIVE & UNFILTERED & 252 \\
\hline 5568 & $\mathrm{pCi} / \mathrm{L}$ & 0.929 & 0.932 & & & U & & RIVER & & CUMULATIVE & UNFILTERED & 252 \\
\hline 5569 & $\mathrm{pCi} / \mathrm{L}$ & 0.0528 & 0.0706 & & & & & RIVER & & CUMULATIVE & UNFILTERED & 252 \\
\hline 5570 & $\mathrm{pCi} / \mathrm{L}$ & 0.0382 & 0.0423 & & & & & RIVER & & CUMULATIVE & UNFILTERED & 463 \\
\hline 5571 & $\mathrm{pCi} / \mathrm{L}$ & 0.0104 & 0.0105 & & & U & & RIVER & & CUMULATIVE & UNFILTERED & 463 \\
\hline 5572 & $\mathrm{pCi} / \mathrm{L}$ & 0.0447 & 0.0511 & & & & & RIVER & & CUMULATIVE & UNFILTERED & 463 \\
\hline 5573 & $\mathrm{pCi} / \mathrm{L}$ & 9.74 & 24.2 & & & & & RIVER & & CUMULATIVE & UNFILTERED & 463 \\
\hline 5574 & $\mathrm{pCi} / \mathrm{L}$ & 0.486 & 0.49 & & & & & RIVER & & CUMULATIVE & UNFILTERED & 463 \\
\hline 5575 & $\mathrm{pCi} / \mathrm{L}$ & 0.0469 & 0.0609 & & & & & RIVER & & CUMULATIVE & UNFILTERED & 463 \\
\hline 5576 & $\mathrm{pCi} / \mathrm{L}$ & & 0.00000963 & & & & & RIVER & & CONTINUOUS & RESIN & 307 \\
\hline 5577 & $\mathrm{pCi} / \mathrm{L}$ & 0.0000084 & & & & & & RIVER & & CONTINUOUS & FILTER & 307 \\
\hline 5578 & $\mathrm{pCi} / \mathrm{L}$ & 0.0000368 & & & & & & RIVER & & CONTINUOUS & RESIN & 307 \\
\hline 5579 & $\mathrm{pCi} / \mathrm{L}$ & 0.0057 & & & & U & & RIVER & & CONTINUOUS & RESIN & 307 \\
\hline 5580 & $\mathrm{pCi} / \mathrm{L}$ & 0.0029 & & & & U & & RIVER & & CONTINUOUS & FILTER & 307 \\
\hline 5581 & $\mathrm{pCi} / \mathrm{L}$ & 0.0000426 & & & & & & RIVER & & CONTINUOUS & RESIN & 252 \\
\hline 5582 & $\mathrm{pCi} / \mathrm{L}$ & & 0.00000122 & & & & & RIVER & & CONTINUOUS & RESIN & 252 \\
\hline 5583 & $\mathrm{pCi} / \mathrm{L}$ & 0.0066 & & & & U & & RIVER & & CONTINUOUS & RESIN & 252 \\
\hline 5584 & $\mathrm{pCi} / \mathrm{L}$ & 0.0000056 & & & & & & RIVER & & CONTINUOUS & FILTER & 252 \\
\hline 5585 & $\mathrm{pCi} / \mathrm{L}$ & 0.0032 & & & & $U$ & & RIVER & & CONTINUOUS & FILTER & 252 \\
\hline 5586 & $\mathrm{pCi} / \mathrm{L}$ & & 0.00000926 & & & & & RIVER & & CONTINUOUS & RESIN & 463 \\
\hline 5587 & $\mathrm{pCi} / \mathrm{L}$ & 0.0000372 & & & & & & RIVER & & CONTINUOUS & RESIN & 463 \\
\hline 5588 & $\mathrm{pCi} / \mathrm{L}$ & 0.0000128 & & & & & & RIVER & & CONTINUOUS & FILTER & 463 \\
\hline 5589 & $\mathrm{pCi} / \mathrm{L}$ & 0.0024 & & & & U & & RIVER & & CONTINUOUS & FILTER & 463 \\
\hline 5590 & $\mathrm{pCi} / \mathrm{L}$ & 0.0042 & & & & U & & RIVER & & CONTINUOUS & RESIN & 463 \\
\hline 5591 & $\mathrm{pCi} / \mathrm{L}$ & 0.0269 & 0.0281 & & & U & & DRINKING & & SAN. GRAB & UNFILTERED & 487 \\
\hline 5592 & $\mathrm{pCi} / \mathrm{L}$ & 132 & 221 & & & U & & DRINKING & & SAN. GRAB & UNFILTERED & 487 \\
\hline 5593 & $\mathrm{pCi} / \mathrm{L}$ & 1.17 & 1.17 & & & U & & DRINKING & & SAN. GRAB & UNFILTERED & 487 \\
\hline 5594 & $\mathrm{pCi} / \mathrm{L}$ & 1.03 & 1.03 & & & U & & QC_SAMPLE & & RAW GRAB & UNFILTERED & 550 \\
\hline 5595 & $\mathrm{pCi} / \mathrm{L}$ & 0.0459 & 0.0518 & & & & & QC_SAMPLE & & RAW GRAB & UNFILTERED & 550 \\
\hline 5596 & $\mathrm{pCi} / \mathrm{L}$ & 0.0119 & 0.012 & & & U & & QC_SAMPLE & & RAW GRAB & UNFILTERED & 550 \\
\hline 5597 & $\mathrm{pCi} / \mathrm{L}$ & 0.0443 & 0.0497 & & & & & QC_SAMPLE & & RAW GRAB & UNFILTERED & 550 \\
\hline 5598 & $\mathrm{pCi} / \mathrm{L}$ & 0.0347 & 0.0505 & & & & & QC_SAMPLE & & RAW GRAB & UNFILTERED & 550 \\
\hline 5599 & $\mathrm{pCi} / \mathrm{L}$ & 5.34 & 18.2 & & & & & QC_SAMPLE & & RAW GRAB & UNFILTERED & 550 \\
\hline 5600 & $\mathrm{pCi} / \mathrm{L}$ & 0.861 & 0.861 & & & U & & QC_SAMPLE & & RAW GRAB & UNFILTERED & 247 \\
\hline 5601 & $\mathrm{pCi} / \mathrm{L}$ & 0.0104 & 0.0105 & & & & & QC_SAMPLE & & RAW GRAB & UNFILTERED & 247 \\
\hline 5602 & $\mathrm{pCi} / \mathrm{L}$ & 0.0467 & 0.0529 & & & & & QC_SAMPLE & & RAW GRAB & UNFILTERED & 247 \\
\hline 5603 & $\mathrm{pCi} / \mathrm{L}$ & 0.0408 & 0.045 & & & & & QC_SAMPLE & & RAW GRAB & UNFILTERED & 247 \\
\hline 5604 & $\mathrm{pCi} / \mathrm{L}$ & 0.0388 & 0.0535 & & & & & QC_SAMPLE & & RAW GRAB & UNFILTERED & 247 \\
\hline 5605 & $\mathrm{pCi} / \mathrm{L}$ & 5.31 & 18.1 & & & & & QC_SAMPLE & & RAW GRAB & UNFILTERED & 247 \\
\hline 5606 & $\mathrm{pCi} / \mathrm{L}$ & 0.0023 & & & & U & & RIVER & & CONTINUOUS & FILTER & 307 \\
\hline 5607 & $\mathrm{pCi} / \mathrm{L}$ & 0.0068 & & & & U & & RIVER & & CONTINUOUS & RESIN & 307 \\
\hline 5608 & $\mathrm{pCi} / \mathrm{L}$ & 0.01 & & & & U & & RIVER & & CONTINUOUS & RESIN & 252 \\
\hline 5609 & $\mathrm{pCi} / \mathrm{L}$ & 0.0036 & & & & U & & RIVER & & CONTINUOUS & FILTER & 252 \\
\hline 5610 & $\mathrm{pCi} / \mathrm{L}$ & 0.0024 & & & & U & & RIVER & & CONTINUOUS & FILTER & 463 \\
\hline 5611 & $\mathrm{pCi} / \mathrm{L}$ & 0.0066 & & & & U & & RIVER & & CONTINUOUS & RESIN & 463 \\
\hline 5612 & $\mathrm{pCi} / \mathrm{L}$ & 8.61 & 28.8 & & & & & DRINKING & & SAN. GRAB & UNFILTERED & 530 \\
\hline 5613 & $\mathrm{pCi} / \mathrm{L}$ & 0.0559 & 0.121 & & & & & DRINKING & & SAN. GRAB & UNFILTERED & 530 \\
\hline 5614 & $\mathrm{pCi} / \mathrm{L}$ & 0.565 & 0.565 & & & U & & DRINKING & & SAN. GRAB & UNFILTERED & 530 \\
\hline 5615 & $\mathrm{pCi} / \mathrm{L}$ & 0.0042 & & & & U & & RIVER & & CONTINUOUS & RESIN & 307 \\
\hline 5616 & $\mathrm{pCi} / \mathrm{L}$ & 0.0033 & & & & U & & RIVER & & CONTINUOUS & FILTER & 307 \\
\hline 5617 & $\mathrm{pCi} / \mathrm{L}$ & 5.69 & 17.2 & & & & & RIVER & & CUMULATIVE & UNFILTERED & 252 \\
\hline
\end{tabular}




\begin{tabular}{|c|c|c|c|c|c|c|c|c|c|c|c|c|}
\hline & $\mathrm{N}$ & $\mathrm{O}$ & $\mathrm{P}$ & $\mathrm{Q}$ & $\mathrm{R}$ & $\mathrm{s}$ & $\mathrm{T}$ & $\mathrm{u}$ & $\mathrm{V}$ & w & $\mathrm{x}$ & $\bar{Y}$ \\
\hline 1 & ANAL_UNITS_RPTD & COUNTING_ERROR & $\mid$ TOTAL_ANAL_ERROR & MIN_DETECTABLE_ACTIVITY & PCNT_MOISTURE & LAB_QUALIFIER & REVIEW_QUALIFIER & SAMP_FROM & SAMP_ITEM & SAMP_MTHD & COLL_MTHD & SAMP_SITE_ID \\
\hline 5618 & $\mathrm{pCi} / \mathrm{L}$ & 0.058 & 0.0685 & & & & & RIVER & & CUMULATIVE & UNFILTERED & 252 \\
\hline 5619 & $\mathrm{pCi} / \mathrm{L}$ & 0.00775 & 0.00789 & & & $\mathrm{U}$ & & RIVER & & CUMULATIVE & UNFILTERED & 252 \\
\hline 5620 & $\mathrm{pCi} / \mathrm{L}$ & 0.0434 & 0.0482 & & & & & RIVER & & CUMULATIVE & UNFILTERED & 252 \\
\hline 5621 & $\mathrm{pCi} / \mathrm{L}$ & 0.0451 & 0.0582 & & & & & RIVER & & CUMULATIVE & UNFILTERED & 252 \\
\hline \begin{tabular}{|l|}
5622 \\
\end{tabular} & $\mathrm{pCi} / \mathrm{L}$ & 0.483 & 0.488 & & & & & RIVER & & CUMULATIVE & UNFILTERED & 252 \\
\hline 5623 & $\mathrm{pCi} / \mathrm{L}$ & 0.0037 & & & & $\mathrm{U}$ & & RIVER & & CONTINUOUS & FILTER & 252 \\
\hline 5624 & $\mathrm{pCi} / \mathrm{L}$ & 0.0045 & & & & & & RIVER & & CONTINUOUS & RESIN & 252 \\
\hline 5625 & $\mathrm{pCi} / \mathrm{L}$ & 0.0086 & & & & $\mathrm{U}$ & & RIVER & & CONTINUOUS & FILTER & 463 \\
\hline 5626 & $\mathrm{pCi} / \mathrm{L}$ & 0.0092 & & & & $\mathrm{U}$ & & RIVER & & CONTINUOUS & RESIN & 463 \\
\hline 5627 & $\mathrm{pCi} / \mathrm{L}$ & 6.59 & 21.3 & & & & & RIVER & & CUMULATIVE & UNFILTERED & 463 \\
\hline 5628 & $\mathrm{pCi} / \mathrm{L}$ & 0.904 & 0.908 & & & U & & RIVER & & CUMULATIVE & UNFILTERED & 463 \\
\hline 5629 & $\mathrm{pCi} / \mathrm{L}$ & 0.0402 & 0.0446 & & & & & RIVER & & CUMULATIVE & UNFILTERED & 463 \\
\hline 5630 & $\mathrm{pCi} / \mathrm{L}$ & 0.0106 & 0.0108 & & & $U$ & & RIVER & & CUMULATIVE & UNFILTERED & 463 \\
\hline 5631 & $\mathrm{pCi} / \mathrm{L}$ & 0.0473 & 0.0544 & & & & & RIVER & & CUMULATIVE & UNFILTERED & 463 \\
\hline \begin{tabular}{|l|}
5632 \\
\end{tabular} & $\mathrm{pCi} / \mathrm{L}$ & 0.0411 & 0.0546 & & & & & RIVER & & CUMULATIVE & UNFILTERED & 463 \\
\hline 5633 & $\mathrm{pCi} / \mathrm{L}$ & 1.92 & 1.92 & & & $U$ & & SURFACE & & RAW GRAB & UNFILTERED & 509 \\
\hline 5634 & $\mathrm{pCi} / \mathrm{L}$ & 0.0809 & 0.114 & & & & & SURFACE & & RAW GRAB & UNFILTERED & 509 \\
\hline 5635 & $\mathrm{pCi} / \mathrm{L}$ & 130 & 220 & & & $U$ & & SURFACE & & RAW GRAB & UNFILTERED & 509 \\
\hline 5636 & $\mathrm{pCi} / \mathrm{L}$ & 1.82 & 1.82 & & & $U$ & & SURFACE & & RAW GRAB & UNFILTERED & 136 \\
\hline \begin{tabular}{|l|}
5637 \\
\end{tabular} & $\mathrm{pCi} / \mathrm{L}$ & 225 & 408 & & & & & SURFACE & & RAW GRAB & UNFILTERED & 136 \\
\hline 5638 & & & & & & & & SURFACE & & RAW GRAB & UNFILTERED & 146 \\
\hline 5639 & & & & & & & & SURFACE & & RAW GRAB & UNFILTERED & 146 \\
\hline \begin{tabular}{|l|l|}
5640 \\
\end{tabular} & & & & & & & & SURFACE & & RAW GRAB & UNFILTERED & 146 \\
\hline \begin{tabular}{|l|l|}
5641 \\
\end{tabular} & $\mathrm{pCi} / \mathrm{L}$ & 1.99 & 1.99 & & & U & & SURFACE & & RAW GRAB & UNFILTERED & 147 \\
\hline 5642 & $\mathrm{pCi} / \mathrm{L}$ & 3.4 & 12.8 & & & & & SURFACE & & RAW GRAB & UNFILTERED & 147 \\
\hline 5643 & $\mathrm{pCi} / \mathrm{L}$ & 0.817 & 1.08 & & & & & SURFACE & & RAW GRAB & UNFILTERED & 147 \\
\hline 5644 & $\mathrm{pCi} / \mathrm{L}$ & 3.46 & 13.2 & & & & & SURFACE & & RAW GRAB & UNFILTERED & 147 \\
\hline 5645 & $\mathrm{pCi} / \mathrm{L}$ & 0.129 & 0.602 & & & & & SURFACE & & RAW GRAB & UNFILTERED & 147 \\
\hline 5646 & $\mathrm{pCi} / \mathrm{L}$ & 143 & 234 & & & & & SURFACE & & RAW GRAB & UNFILTERED & 147 \\
\hline 5647 & $\mathrm{pCi} / \mathrm{L}$ & 0.703 & 0.712 & & & & & SANITARY & & RAW GRAB & UNFILTERED & 525 \\
\hline 5648 & $\mathrm{pCi} / \mathrm{L}$ & 129 & 220 & & & $U$ & & SANITARY & & RAW GRAB & UNFILTERED & 525 \\
\hline 5649 & $\mathrm{pCi} / \mathrm{L}$ & 0.46 & 1.94 & & & & & SANITARY & & RAW GRAB & UNFILTERED & 525 \\
\hline 5650 & $\mathrm{pCi} / \mathrm{L}$ & 0.112 & 0.16 & & & & & SANITARY & & RAW GRAB & UNFILTERED & 525 \\
\hline 5651 & $\mathrm{pCi} / \mathrm{L}$ & 0.564 & 2.9 & & & & & SANITARY & & RAW GRAB & UNFILTERED & 525 \\
\hline 5652 & $\mathrm{pCi} / \mathrm{L}$ & 1.06 & 1.07 & & & & & SANITARY & & RAW GRAB & UNFILTERED & 524 \\
\hline 5653 & $\mathrm{pCi} / \mathrm{L}$ & 133 & 224 & & & U & & SANITARY & & RAW GRAB & UNFILTERED & 524 \\
\hline 5654 & $\mathrm{pCi} / \mathrm{L}$ & 0.158 & 0.348 & & & & & SANITARY & & RAW GRAB & UNFILTERED & 524 \\
\hline \begin{tabular}{|l|}
5655 \\
\end{tabular} & $\mathrm{pCi} / \mathrm{L}$ & 0.0311 & 0.0334 & & & & & SANITARY & & RAW GRAB & UNFILTERED & 524 \\
\hline 5656 & $\mathrm{pCi} / \mathrm{L}$ & 0.172 & 0.403 & & & & & SANITARY & & RAW GRAB & UNFILTERED & 524 \\
\hline \begin{tabular}{|l|}
5657 \\
\end{tabular} & $\mathrm{pCi} / \mathrm{L}$ & 1.72 & 1.72 & & & $U$ & & SANITARY & & RAW GRAB & UNFILTERED & 172 \\
\hline \begin{tabular}{|l|}
5658 \\
\end{tabular} & $\mathrm{pCi} / \mathrm{L}$ & 136 & 227 & & & $U$ & & SANITARY & & RAW GRAB & UNFILTERED & 172 \\
\hline 5659 & $\mathrm{pCi} / \mathrm{L}$ & 0.33 & 1.35 & & & & & SANITARY & & RAW GRAB & UNFILTERED & 172 \\
\hline 5660 & $\mathrm{pCi} / \mathrm{L}$ & 0.069 & 0.0892 & & & & & SANITARY & & RAW GRAB & UNFILTERED & 172 \\
\hline 5661 & $\mathrm{pCi} / \mathrm{L}$ & 0.342 & 1.44 & & & & & SANITARY & & RAW GRAB & UNFILTERED & 172 \\
\hline 5662 & $\mathrm{pCi} / \mathrm{L}$ & 0.0039 & & & & $U$ & & RIVER & & CONTINUOUS & RESIN & 307 \\
\hline 5663 & $\mathrm{pCi} / \mathrm{L}$ & 0.002 & & & & $U$ & & RIVER & & CONTINUOUS & FILTER & 307 \\
\hline 5664 & $\mathrm{pCi} / \mathrm{L}$ & 0.0046 & & & & $U$ & & RIVER & & CONTINUOUS & RESIN & 252 \\
\hline 5665 & $\mathrm{pCi} / \mathrm{L}$ & 0.0022 & & & & $U$ & & RIVER & & CONTINUOUS & FILTER & 252 \\
\hline 5666 & $\mathrm{pCi} / \mathrm{L}$ & 0.0064 & & & & & & RIVER & & CONTINUOUS & RESIN & 463 \\
\hline \begin{tabular}{|l|}
5667 \\
\end{tabular} & $\mathrm{pCi} / \mathrm{L}$ & 0.0033 & & & & U & & RIVER & & CONTINUOUS & FILTER & 463 \\
\hline 5668 & $\mathrm{pCi} / \mathrm{L}$ & 0.002 & & & & U & & RIVER & & CONTINUOUS & FILTER & 307 \\
\hline 5669 & $\mathrm{pCi} / \mathrm{L}$ & 0.0038 & & & & $U_{2}$ & & RIVER & & CONTINUOUS & RESIN & 307 \\
\hline
\end{tabular}




\begin{tabular}{|c|c|c|c|c|c|c|c|c|c|c|c|c|}
\hline & $\mathrm{N}$ & $\mathrm{O}$ & $\mathrm{P}$ & $\mathrm{Q}$ & $\mathrm{R}$ & $\mathrm{s}$ & $\mathrm{T}$ & $\mathrm{u}$ & $\mathrm{V}$ & w & $\mathrm{x}$ & $\bar{Y}$ \\
\hline 1 & ANAL_UNITS_RPTD & COUNTING_ERROR & TOTAL_ANAL_ERROR & MIN_DETECTABLE_ACTIVITY & PCNT_MOISTURE & LAB_QUALIFIER & REVIEW_QUALIFIER & SAMP_FROM & SAMP_ITEM & SAMP_MTHD & COLL_MTHD & SAMP_SITE_ID \\
\hline 5670 & $\mathrm{pCi} / \mathrm{L}$ & 5.5 & 16.8 & & & & & RIVER & & CUMULATIVE & UNFILTERED & 252 \\
\hline 5671 & $\mathrm{pCi} / \mathrm{L}$ & 0.795 & 0.795 & & & u & & RIVER & & CUMULATIVE & UNFILTERED & 252 \\
\hline 5672 & $\mathrm{pCi} / \mathrm{L}$ & 0.0578 & 0.0629 & & & & & RIVER & & CUMULATIVE & UNFILTERED & 252 \\
\hline 5673 & $\mathrm{pCi} / \mathrm{L}$ & 0.0223 & 0.0225 & & & & & RIVER & & CUMULATIVE & UNFILTERED & 252 \\
\hline 5674 & $\mathrm{pCi} / \mathrm{L}$ & 0.0582 & 0.0627 & & & & & RIVER & & CUMULATIVE & UNFILTERED & 252 \\
\hline 5675 & $\mathrm{pCi} / \mathrm{L}$ & 0.04 & 0.0537 & & & & & RIVER & & CUMULATIVE & UNFILTERED & 252 \\
\hline 5676 & $\mathrm{pCi} / \mathrm{L}$ & 0.0072 & & & & U & & RIVER & & CONTINUOUS & FILTER & 252 \\
\hline 5677 & $\mathrm{pCi} / \mathrm{L}$ & 0.01 & & & & U & & RIVER & & CONTINUOUS & RESIN & 252 \\
\hline 5678 & $\mathrm{pCi} / \mathrm{L}$ & 7.05 & 23.7 & & & & & RIVER & & CUMULATIVE & UNFILTERED & 463 \\
\hline 5679 & $\mathrm{pCi} / \mathrm{L}$ & 1.18 & 1.18 & & & U & & RIVER & & CUMULATIVE & UNFILTERED & 463 \\
\hline 5680 & $\mathrm{pCi} / \mathrm{L}$ & 0.0514 & 0.0567 & & & & & RIVER & & CUMULATIVE & UNFILTERED & 463 \\
\hline 5681 & $\mathrm{pCi} / \mathrm{L}$ & 0.0112 & 0.0112 & & & U & & RIVER & & CUMULATIVE & UNFILTERED & 463 \\
\hline \begin{tabular}{|l|}
5682 \\
\end{tabular} & $\mathrm{pCi} / \mathrm{L}$ & 0.0505 & 0.0556 & & & & & RIVER & & CUMULATIVE & UNFILTERED & 463 \\
\hline 5683 & $\mathrm{pCi} / \mathrm{L}$ & 0.0383 & 0.0487 & & & & & RIVER & & CUMULATIVE & UNFILTERED & 463 \\
\hline 5684 & $\mathrm{pCi} / \mathrm{L}$ & 0.0047 & & & & U & & RIVER & & CONTINUOUS & RESIN & 463 \\
\hline 5685 & $\mathrm{pCi} / \mathrm{L}$ & 0.0026 & & & & & & RIVER & & CONTINUOUS & FILTER & 463 \\
\hline \begin{tabular}{|l|}
5686 \\
\end{tabular} & $\mathrm{pCi} / \mathrm{L}$ & & 0.000442 & & & & & DRINKING & & SAN. GRAB & UNFILTERED & 512 \\
\hline \begin{tabular}{|l|}
5687 \\
\end{tabular} & $\mathrm{pCi} / \mathrm{L}$ & 1.22 & 1.22 & & & U & & DRINKING & & SAN. GRAB & UNFILTERED & 186 \\
\hline 5688 & $\mathrm{pCi} / \mathrm{L}$ & 124 & 209 & & & U & & DRINKING & & SAN. GRAB & UNFILTERED & 186 \\
\hline 5689 & $\mathrm{pCi} / \mathrm{L}$ & 0.0635 & 0.0952 & & & & & DRINKING & & SAN. GRAB & UNFILTERED & 186 \\
\hline 5690 & $\mathrm{pCi} / \mathrm{L}$ & 0.619 & 0.619 & & & U & & DRINKING & & SAN. GRAB & UNFILTERED & 512 \\
\hline \begin{tabular}{|l|}
5691 \\
\end{tabular} & $\mathrm{pCi} / \mathrm{L}$ & 266 & 537 & & & & & DRINKING & & SAN. GRAB & UNFILTERED & 512 \\
\hline 5692 & $\mathrm{pCi} / \mathrm{L}$ & 0.0493 & 0.0516 & & & U & & DRINKING & & SAN. GRAB & UNFILTERED & 512 \\
\hline \begin{tabular}{|l|}
5693 \\
\end{tabular} & $\mathrm{pCi} / \mathrm{L}$ & 0.924 & 0.924 & & & $U$ & & DRINKING & & SAN. GRAB & UNFILTERED & 144 \\
\hline \begin{tabular}{|l|}
5694 \\
\end{tabular} & $\mathrm{pCi} / \mathrm{L}$ & 125 & 209 & & & U & & DRINKING & & SAN. GRAB & UNFILTERED & 144 \\
\hline 5695 & $\mathrm{pCi} / \mathrm{L}$ & 0.0492 & 0.0588 & & & & & DRINKING & & SAN. GRAB & UNFILTERED & 144 \\
\hline 5696 & $\mathrm{pCi} / \mathrm{L}$ & 1.32 & 1.33 & & & U & & DRINKING & & SAN. GRAB & UNFILTERED & 187 \\
\hline \begin{tabular}{|l|}
5697 \\
\end{tabular} & $\mathrm{pCi} / \mathrm{L}$ & 129 & 213 & & & U & & DRINKING & & SAN. GRAB & UNFILTERED & 187 \\
\hline 5698 & $\mathrm{pCi} / \mathrm{L}$ & 0.0431 & 0.0439 & & & U & & DRINKING & & SAN. GRAB & UNFILTERED & 187 \\
\hline \begin{tabular}{|l|}
5699 \\
\end{tabular} & $\mathrm{pCi} / \mathrm{L}$ & 1.63 & 1.63 & & & U & & SANITARY & & RAW GRAB & UNFILTERED & 95 \\
\hline 5700 & $\mathrm{pCi} / \mathrm{L}$ & 127 & 212 & & & U & & SANITARY & & RAW GRAB & UNFILTERED & 95 \\
\hline \begin{tabular}{|l|}
5701 \\
\end{tabular} & $\mathrm{pCi} / \mathrm{L}$ & 0.0107 & 0.0119 & & & U & & SANITARY & & RAW GRAB & UNFILTERED & 95 \\
\hline 5702 & $\mathrm{pCi} / \mathrm{L}$ & 0 & 0.00584 & & & U & & SANITARY & & RAW GRAB & UNFILTERED & 95 \\
\hline 5703 & $\mathrm{pCi} / \mathrm{L}$ & 0.0165 & 0.0178 & & & & & SANITARY & & RAW GRAB & UNFILTERED & 95 \\
\hline 5704 & $\mathrm{pCi} / \mathrm{L}$ & 6.08 & 18.4 & & & & & DRINKING & & RAW GRAB & UNFILTERED & 553 \\
\hline 5705 & $\mathrm{pCi} / \mathrm{L}$ & 0.925 & 0.925 & & & U & & DRINKING & & RAW GRAB & UNFILTERED & 553 \\
\hline \begin{tabular}{|l|}
5706 \\
\end{tabular} & $\mathrm{pCi} / \mathrm{L}$ & 0.038 & 0.0506 & & & & & DRINKING & & RAW GRAB & UNFILTERED & 553 \\
\hline \begin{tabular}{|l|}
5707 \\
\end{tabular} & $\mathrm{pCi} / \mathrm{L}$ & 0.0039 & & & & U & & RIVER & & CONTINUOUS & RESIN & 307 \\
\hline 5708 & $\mathrm{pCi} / \mathrm{L}$ & 0.002 & & & & U & & RIVER & & CONTINUOUS & FILTER & 307 \\
\hline 5709 & $\mathrm{pCi} / \mathrm{L}$ & 0.0035 & & & & U & & RIVER & & CONTINUOUS & RESIN & 252 \\
\hline 5710 & $\mathrm{pCi} / \mathrm{L}$ & 0.0026 & & & & $U$ & & RIVER & & CONTINUOUS & FILTER & 252 \\
\hline 5711 & $\mathrm{pCi} / \mathrm{L}$ & 0.0037 & & & & U & & RIVER & & CONTINUOUS & FILTER & 463 \\
\hline 5712 & $\mathrm{pCi} / \mathrm{L}$ & 0.0075 & & & & U & & RIVER & & CONTINUOUS & RESIN & 463 \\
\hline 5713 & $\mathrm{pCi} / \mathrm{L}$ & 7.22 & 24.5 & & & & & DRINKING & & CUMULATIVE & UNFILTERED & 307 \\
\hline 5714 & $\mathrm{pCi} / \mathrm{L}$ & 0.906 & 0.909 & & & U & & DRINKING & & CUMULATIVE & UNFILTERED & 307 \\
\hline 5715 & $\mathrm{pCi} / \mathrm{L}$ & 0.047 & 0.0531 & & & & & DRINKING & & CUMULATIVE & UNFILTERED & 307 \\
\hline 5716 & $\mathrm{pCi} / \mathrm{L}$ & 0.00756 & 0.0077 & & & U & & DRINKING & & CUMULATIVE & UNFILTERED & 307 \\
\hline 5717 & $\mathrm{pCi} / \mathrm{L}$ & 0.0524 & 0.0606 & & & & & DRINKING & & CUMULATIVE & UNFILTERED & 307 \\
\hline 5718 & $\mathrm{pCi} / \mathrm{L}$ & 0.0408 & 0.0549 & & & & & DRINKING & & CUMULATIVE & UNFILTERED & 307 \\
\hline 5719 & $\mathrm{pCi} / \mathrm{L}$ & & 0.0000065 & & & & & RIVER & & CONTINUOUS & RESIN & 307 \\
\hline 5720 & $\mathrm{pCi} / \mathrm{L}$ & 0.0000248 & & & & & & RIVER & & CONTINUOUS & RESIN & 307 \\
\hline 5721 & $\mathrm{pCi} / \mathrm{L}$ & 0.0039 & & & & $\mathrm{U}$ & & RIVER & & CONTINUOUS & RESIN & 307 \\
\hline
\end{tabular}




\begin{tabular}{|c|c|c|c|c|c|c|c|c|c|c|c|c|}
\hline & $\mathrm{N}$ & $\mathrm{O}$ & $P$ & $\mathrm{Q}$ & $\mathrm{R}$ & $\mathrm{s}$ & $T$ & $\mathrm{U}$ & $\mathrm{V}$ & $\mathrm{W}$ & $\mathrm{X}$ & $\mathrm{Y}$ \\
\hline 1 & ANAL_UNITS_RPTD & COUNTING_ERROR & TOTAL_ANAL_ERROR/I & MIN_DETECTABLE_ACTIVITY & PCNT_MOISTURE & LAB_QUALIFIER & REVIEW_QUALIFIER & SAMP_FROM & SAMP_ITEM & SAMP MTHD & COLL MTHD & SAMP SITE ID \\
\hline 5722 & pCi/L & 0.0000062 & & & & & & RIVER & & CONTINUOUS & FILTER & 307 \\
\hline 5723 & $\mathrm{pCi} / \mathrm{L}$ & 0.0021 & & & & $\mathrm{U}$ & & RIVER & & CONTINUOUS & FILTER & 307 \\
\hline 5724 & $\mathrm{pCi} / \mathrm{L}$ & 6.04 & 18.7 & & & & & RIVER & & CUMULATIVE & UNFILTERED & 252 \\
\hline 5725 & $\mathrm{pCi} / \mathrm{L}$ & 0.805 & 0.805 & & & $U$ & & RIVER & & CUMULATIVE & UNFILTERED & 252 \\
\hline 5726 & $\mathrm{pCi} / \mathrm{L}$ & 0.0411 & 0.0461 & & & & & RIVER & & CUMULATIVE & UNFILTERED & 252 \\
\hline 5727 & $\mathrm{pCi} / \mathrm{L}$ & 0.0104 & 0.0106 & & & & & RIVER & & CUMULATIVE & UNFILTERED & 252 \\
\hline 5728 & $\mathrm{pCi} / \mathrm{L}$ & 0.0466 & 0.0533 & & & & & RIVER & & CUMULATIVE & UNFILTERED & 252 \\
\hline 5729 & $\mathrm{pCi} / \mathrm{L}$ & 0.048 & 0.0623 & & & & & RIVER & & CUMULATIVE & UNFILTERED & 252 \\
\hline 5730 & $\mathrm{pCi} / \mathrm{L}$ & & 0.000000871 & & & & & RIVER & & CONTINUOUS & RESIN & 252 \\
\hline 5731 & $\mathrm{pCi} / \mathrm{L}$ & 0.000042 & & & & & & RIVER & & CONTINUOUS & RESIN & 252 \\
\hline 5732 & $\mathrm{pCi} / \mathrm{L}$ & 0.0000066 & & & & & & RIVER & & CONTINUOUS & FILTER & 252 \\
\hline 5733 & $\mathrm{pCi} / \mathrm{L}$ & 0.0034 & & & & U & & RIVER & & CONTINUOUS & FILTER & 252 \\
\hline \begin{tabular}{|l|}
5734 \\
\end{tabular} & $\mathrm{pCi} / \mathrm{L}$ & 0.0071 & & & & $U$ & & RIVER & & CONTINUOUS & RESIN & 252 \\
\hline \begin{tabular}{|l|}
5735 \\
\end{tabular} & $\mathrm{pCi} / \mathrm{L}$ & 6.97 & 22 & & & & & RIVER & & CUMULATIVE & UNFILTERED & 463 \\
\hline 5736 & $\mathrm{pCi} / \mathrm{L}$ & & 0.00000543 & & & & & RIVER & & CONTINUOUS & RESIN & 463 \\
\hline \begin{tabular}{|l|}
5737 \\
\end{tabular} & $\mathrm{pCi} / \mathrm{L}$ & 0.0000352 & & & & & & RIVER & & CONTINUOUS & RESIN & 463 \\
\hline 5738 & $\mathrm{pCi} / \mathrm{L}$ & 0.0000062 & & & & & & RIVER & & CONTINUOUS & FILTER & 463 \\
\hline \begin{tabular}{|l|}
5739 \\
\end{tabular} & $\mathrm{pCi} / \mathrm{L}$ & 1.17 & 1.17 & & & $U$ & & RIVER & & CUMULATIVE & UNFILTERED & 463 \\
\hline 5740 & $\mathrm{pCi} / \mathrm{L}$ & 0.0031 & & & & $U$ & & RIVER & & CONTINUOUS & FILTER & 463 \\
\hline \begin{tabular}{|l|}
5741 \\
\end{tabular} & $\mathrm{pCi} / \mathrm{L}$ & 0.0426 & 0.0468 & & & & & RIVER & & CUMULATIVE & UNFILTERED & 463 \\
\hline \begin{tabular}{|l|}
5742 \\
\end{tabular} & $\mathrm{pCi} / \mathrm{L}$ & 0.0169 & 0.0172 & & & & & RIVER & & CUMULATIVE & UNFILTERED & 463 \\
\hline \begin{tabular}{|l|}
5743 \\
\end{tabular} & $\mathrm{pCi} / \mathrm{L}$ & 0.048 & 0.054 & & & & & RIVER & & CUMULATIVE & UNFILTERED & 463 \\
\hline 5744 & $\mathrm{pCi} / \mathrm{L}$ & 0.006 & & & & $U$ & & RIVER & & CONTINUOUS & RESIN & 463 \\
\hline 5745 & $\mathrm{pCi} / \mathrm{L}$ & 0.0431 & 0.0581 & & & & & RIVER & & CUMULATIVE & UNFILTERED & 463 \\
\hline \begin{tabular}{|l|}
5746 \\
\end{tabular} & $\mathrm{pCi} / \mathrm{L}$ & 1 & 1 & & & $U$ & & DRINKING & & SAN. GRAB & UNFILTERED & 487 \\
\hline \begin{tabular}{|l|}
5747 \\
\end{tabular} & $\mathrm{pCi} / \mathrm{L}$ & 127 & 280 & & & $U$ & & DRINKING & & SAN. GRAB & UNFILTERED & 487 \\
\hline \begin{tabular}{|l|}
5748 \\
\end{tabular} & $\mathrm{pCi} / \mathrm{L}$ & 0.019 & 0.0197 & & & $U$ & & DRINKING & & SAN. GRAB & UNFILTERED & 487 \\
\hline \begin{tabular}{|l|}
5749 \\
\end{tabular} & $\mathrm{pCi} / \mathrm{L}$ & 0.00143 & 0.00144 & & & & & RIVER & & CONTINUOUS & FILTER & 307 \\
\hline 5750 & $\mathrm{pCi} / \mathrm{L}$ & 0.00329 & 0.0033 & & & & & RIVER & & CONTINUOUS & RESIN & 307 \\
\hline \begin{tabular}{|l|}
5751 \\
\end{tabular} & $\mathrm{pCi} / \mathrm{L}$ & 0.00137 & 0.00137 & & & $U$ & & RIVER & & CONTINUOUS & FILTER & 252 \\
\hline \begin{tabular}{|l|}
5752 \\
\end{tabular} & $\mathrm{pCi} / \mathrm{L}$ & 0.00232 & 0.00234 & & & & & RIVER & & CONTINUOUS & RESIN & 252 \\
\hline 5753 & $\mathrm{pCi} / \mathrm{L}$ & 0.00301 & 0.00302 & & & $U$ & & RIVER & & CONTINUOUS & RESIN & 463 \\
\hline \begin{tabular}{|l|}
5754 \\
\end{tabular} & $\mathrm{pCi} / \mathrm{L}$ & 0.00161 & 0.00163 & & & & & RIVER & & CONTINUOUS & FILTER & 463 \\
\hline \begin{tabular}{|l|}
5755 \\
\end{tabular} & $\mathrm{pCi} / \mathrm{L}$ & 0.386 & 1.77 & & & & & SANITARY & & RAW GRAB & UNFILTERED & 525 \\
\hline \begin{tabular}{|l|}
5756 \\
\end{tabular} & $\mathrm{pCi} / \mathrm{L}$ & 0.0772 & 0.103 & & & & & SANITARY & & RAW GRAB & UNFILTERED & 525 \\
\hline \begin{tabular}{|l|}
5757 \\
\end{tabular} & $\mathrm{pCi} / \mathrm{L}$ & 0.486 & 2.78 & & & & & SANITARY & & RAW GRAB & UNFILTERED & 525 \\
\hline \begin{tabular}{|l|}
5758 \\
\end{tabular} & $\mathrm{pCi} / \mathrm{L}$ & 0.926 & 0.929 & & & $U$ & & SANITARY & & RAW GRAB & UNFILTERED & 525 \\
\hline 5759 & $\mathrm{pCi} / \mathrm{L}$ & 125 & 277 & & & U & & SANITARY & & RAW GRAB & UNFILTERED & 525 \\
\hline 5760 & $\mathrm{pCi} / \mathrm{L}$ & 0.0513 & 0.105 & & & & & DRINKING & & SAN. GRAB & UNFILTERED & 530 \\
\hline \begin{tabular}{|l|}
5761 \\
\end{tabular} & $\mathrm{pCi} / \mathrm{L}$ & 0.567 & 0.567 & & & U & & DRINKING & & SAN. GRAB & UNFILTERED & 530 \\
\hline \begin{tabular}{|l|}
5762 \\
\end{tabular} & $\mathrm{pCi} / \mathrm{L}$ & 131 & 289 & & & U & & DRINKING & & SAN. GRAB & UNFILTERED & 530 \\
\hline 5763 & $\mathrm{pCi} / \mathrm{L}$ & 0.161 & 0.336 & & & & & SANITARY & & RAW GRAB & UNFILTERED & 524 \\
\hline 5764 & $\mathrm{pCi} / \mathrm{L}$ & 0.0372 & 0.0404 & & & & & SANITARY & & RAW GRAB & UNFILTERED & 524 \\
\hline 5765 & $\mathrm{pCi} / \mathrm{L}$ & 0.181 & 0.417 & & & & & SANITARY & & RAW GRAB & UNFILTERED & 524 \\
\hline 5766 & $\mathrm{pCi} / \mathrm{L}$ & 163 & 351 & & & $U$ & & SANITARY & & RAW GRAB & UNFILTERED & 524 \\
\hline \begin{tabular}{|l|}
5767 \\
\end{tabular} & $\mathrm{pCi} / \mathrm{L}$ & 1.03 & 1.03 & & & U & & SANITARY & & RAW GRAB & UNFILTERED & 524 \\
\hline 5768 & pCi/L & 0.0117 & 0.0128 & & & U & & SANITARY & & RAW GRAB & UNFILTERED & 95 \\
\hline 5769 & $\mathrm{pCi} / \mathrm{L}$ & 0.00443 & 0.00463 & & & U & & SANITARY & & RAW GRAB & UNFILTERED & 95 \\
\hline 5770 & pCi/L & 0.016 & 0.0171 & & & U & & SANITARY & & RAW GRAB & UNFILTERED & 95 \\
\hline \begin{tabular}{|l|}
5771 \\
\end{tabular} & $\mathrm{pCi} / \mathrm{L}$ & 158 & 344 & & & $U$ & & SANITARY & & RAW GRAB & UNFILTERED & 95 \\
\hline \begin{tabular}{|l|}
5772 \\
\end{tabular} & $\mathrm{pCi} / \mathrm{L}$ & 1.05 & 1.05 & & & U & & SANITARY & & RAW GRAB & UNFILTERED & 95 \\
\hline 5773 & & & & & & & & RIVER & & CONTINUOUS & FILTER & 307 \\
\hline
\end{tabular}




\begin{tabular}{|c|c|c|c|c|c|c|c|c|c|c|c|c|}
\hline & $\mathrm{N}$ & $\mathrm{O}$ & $\mathrm{P}$ & $\mathrm{Q}$ & $\mathrm{R}$ & $\mathrm{s}$ & $T$ & $\bar{U}$ & $\mathrm{~V}$ & $\mathrm{~W}$ & $\mathrm{X}$ & $\bar{Y}$ \\
\hline 1 & ANAL_UNITS_RPTD & COUNTING_ERROR & TOTAL_ANAL_ERROR & MIN_DETECTABLE_ACTIVITY & PCNT_MOISTURE & LAB_QUALIFIER & REVIEW_QUALIFIER & SAMP_FROM & SAMP_ITEM & SAMP_MTHD & COLL_MTHD & SAMP_SITE_ID \\
\hline 5774 & & & & & & & & RIVER & & CONTINUOUS & RESIN & 307 \\
\hline 5775 & $\mathrm{pCi} / \mathrm{L}$ & 0.00235 & 0.00239 & & & & & RIVER & & CONTINUOUS & FILTER & 252 \\
\hline 5776 & $\mathrm{pCi} / \mathrm{L}$ & 0.00361 & 0.00366 & & & & & RIVER & & CONTINUOUS & RESIN & 252 \\
\hline 5777 & $\mathrm{pCi} / \mathrm{L}$ & 0.00328 & 0.00328 & & & U & & RIVER & & CONTINUOUS & RESIN & 463 \\
\hline 5778 & $\mathrm{pCi} / \mathrm{L}$ & 0.00143 & 0.00146 & & & & & RIVER & & CONTINUOUS & FILTER & 463 \\
\hline 5779 & $\mathrm{pCi} / \mathrm{L}$ & 0.0433 & 0.0483 & & & & & RIVER & & CUMULATIVE & UNFILTERED & 252 \\
\hline 5780 & $\mathrm{pCi} / \mathrm{L}$ & 0.0113 & 0.0115 & & & & & RIVER & & CUMULATIVE & UNFILTERED & 252 \\
\hline 5781 & $\mathrm{pCi} / \mathrm{L}$ & 0.0467 & 0.0529 & & & & & RIVER & & CUMULATIVE & UNFILTERED & 252 \\
\hline 5782 & $\mathrm{pCi} / \mathrm{L}$ & 0.0348 & 0.0493 & & & & & RIVER & & CUMULATIVE & UNFILTERED & 252 \\
\hline 5783 & $\mathrm{pCi} / \mathrm{L}$ & 0.667 & 0.667 & & & U & & RIVER & & CUMULATIVE & UNFILTERED & 252 \\
\hline 5784 & $\mathrm{pCi} / \mathrm{L}$ & 5.73 & 17.4 & & & & & RIVER & & CUMULATIVE & UNFILTERED & 252 \\
\hline 5785 & $\mathrm{pCi} / \mathrm{L}$ & 0.0454 & 0.051 & & & & & RIVER & & CUMULATIVE & UNFILTERED & 463 \\
\hline 5786 & $\mathrm{pCi} / \mathrm{L}$ & 0.0116 & 0.0118 & & & & & RIVER & & CUMULATIVE & UNFILTERED & 463 \\
\hline 5787 & $\mathrm{pCi} / \mathrm{L}$ & 0.0503 & 0.0576 & & & & & RIVER & & CUMULATIVE & UNFILTERED & 463 \\
\hline 5788 & $\mathrm{pCi} / \mathrm{L}$ & 0.0385 & 0.0567 & & & & & RIVER & & CUMULATIVE & UNFILTERED & 463 \\
\hline 5789 & $\mathrm{pCi} / \mathrm{L}$ & 1.19 & 1.2 & & & U & & RIVER & & CUMULATIVE & UNFILTERED & 463 \\
\hline 5790 & $\mathrm{pCi} / \mathrm{L}$ & 6.72 & 21.6 & & & & & RIVER & & CUMULATIVE & UNFILTERED & 463 \\
\hline 5791 & $\mathrm{pCi} / \mathrm{L}$ & 0.115 & 0.434 & & & & & SURFACE & & RAW GRAB & UNFILTERED & 509 \\
\hline 5792 & $\mathrm{pCi} / \mathrm{L}$ & 162 & 348 & & & U & & SURFACE & & RAW GRAB & UNFILTERED & 509 \\
\hline 5793 & $\mathrm{pCi} / \mathrm{L}$ & 1.15 & 1.15 & & & U & & SURFACE & & RAW GRAB & UNFILTERED & 509 \\
\hline 5794 & & & & & & & & SURFACE & & RAW GRAB & UNFILTERED & 136 \\
\hline 5795 & & & & & & & & SURFACE & & RAW GRAB & UNFILTERED & 136 \\
\hline 5796 & & & & & & & & SURFACE & & RAW GRAB & UNFILTERED & 147 \\
\hline 5797 & & & & & & & & SURFACE & & RAW GRAB & UNFILTERED & 147 \\
\hline 5798 & & & & & & & & SURFACE & & RAW GRAB & UNFILTERED & 147 \\
\hline 5799 & & & & & & & & SURFACE & & RAW GRAB & UNFILTERED & 147 \\
\hline 5800 & & & & & & & & SURFACE & & RAW GRAB & UNFILTERED & 147 \\
\hline 5801 & & & & & & & & SURFACE & & RAW GRAB & UNFILTERED & 147 \\
\hline 5802 & $\mathrm{pCi} / \mathrm{L}$ & 0.000845 & 0.00085 & & & & & RIVER & & CONTINUOUS & FILTER & 307 \\
\hline 5803 & $\mathrm{pCi} / \mathrm{L}$ & 0.00169 & 0.00171 & & & & & RIVER & & CONTINUOUS & RESIN & 307 \\
\hline 5804 & & & & & & & & RIVER & & CONTINUOUS & FILTER & 252 \\
\hline 5805 & & & & & & & & RIVER & & CONTINUOUS & RESIN & 252 \\
\hline 5806 & $\mathrm{pCi} / \mathrm{L}$ & 0.00189 & 0.00191 & & & & & RIVER & & CONTINUOUS & FILTER & 463 \\
\hline 5807 & $\mathrm{pCi} / \mathrm{L}$ & 0.00232 & 0.00232 & & & U & & RIVER & & CONTINUOUS & RESIN & 463 \\
\hline 5808 & $\mathrm{pCi} / \mathrm{L}$ & 0.000876 & 0.000876 & & & U & & RIVER & & CONTINUOUS & FILTER & 307 \\
\hline 5809 & $\mathrm{pCi} / \mathrm{L}$ & 0.00181 & 0.00182 & & & & & RIVER & & CONTINUOUS & RESIN & 307 \\
\hline 5810 & $\mathrm{pCi} / \mathrm{L}$ & 0.00155 & 0.00156 & & & U & & RIVER & & CONTINUOUS & FILTER & 252 \\
\hline 5811 & $\mathrm{pCi} / \mathrm{L}$ & 0.00324 & 0.00324 & & & U & & RIVER & & CONTINUOUS & RESIN & 252 \\
\hline 5812 & $\mathrm{pCi} / \mathrm{L}$ & 0.00154 & 0.00156 & & & & & RIVER & & CONTINUOUS & FILTER & 463 \\
\hline 5813 & $\mathrm{pCi} / \mathrm{L}$ & 0.0036 & 0.0036 & & & U & & RIVER & & CONTINUOUS & RESIN & 463 \\
\hline 5814 & $\mathrm{pCi} / \mathrm{L}$ & 0.925 & 0.925 & & & U & & DRINKING & & SAN. GRAB & UNFILTERED & 186 \\
\hline 5815 & $\mathrm{pCi} / \mathrm{L}$ & 161 & 347 & & & $U$ & & DRINKING & & SAN. GRAB & UNFILTERED & 186 \\
\hline 5816 & $\mathrm{pCi} / \mathrm{L}$ & 0.0328 & 0.0342 & & & U & & DRINKING & & SAN. GRAB & UNFILTERED & 186 \\
\hline 5817 & $\mathrm{pCi} / \mathrm{L}$ & 0.844 & 0.844 & & & U & & DRINKING & & SAN. GRAB & UNFILTERED & 512 \\
\hline 5818 & $\mathrm{pCi} / \mathrm{L}$ & 277 & 664 & & & & & DRINKING & & SAN. GRAB & UNFILTERED & 512 \\
\hline 5819 & $\mathrm{pCi} / \mathrm{L}$ & 0.0298 & 0.0311 & & & U & & DRINKING & & SAN. GRAB & UNFILTERED & 512 \\
\hline 5820 & $\mathrm{pCi} / \mathrm{L}$ & 0.93 & 0.931 & & & $U$ & & DRINKING & & SAN. GRAB & UNFILTERED & 144 \\
\hline 5821 & $\mathrm{pCi} / \mathrm{L}$ & 165 & 352 & & & U & & DRINKING & & SAN. GRAB & UNFILTERED & 144 \\
\hline 5822 & $\mathrm{pCi} / \mathrm{L}$ & 0.0303 & 0.0315 & & & 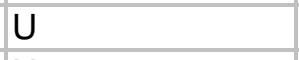 & & DRINKING & & SAN. GRAB & UNFILTERED & 144 \\
\hline 5823 & $\mathrm{pCi} / \mathrm{L}$ & 162 & 349 & & & U & & DRINKING & & SAN. GRAB & UNFILTERED & 187 \\
\hline 5824 & $\mathrm{pCi} / \mathrm{L}$ & 0.0281 & 0.0286 & & & $U$ & & DRINKING & & SAN. GRAB & UNFILTERED & 187 \\
\hline 5825 & $\mathrm{pCi} / \mathrm{L}$ & 0.671 & 0.671 & & & $U_{2}$ & & DRINKING & & SAN. GRAB & UNFILTERED & 187 \\
\hline
\end{tabular}




\begin{tabular}{|c|c|c|c|c|c|c|c|c|c|c|c|c|}
\hline & $\mathrm{N}$ & $\mathrm{O}$ & $\mathrm{P}$ & $\mathrm{Q}$ & $\mathrm{R}$ & $\mathrm{s}$ & $\mathrm{T}$ & $\mathrm{U}$ & $\mathrm{V}$ & $\mathrm{w}$ & $\mathrm{x}$ & $\mathrm{Y}$ \\
\hline 1 & ANAL_UNITS_RPTD & COUNTING_ERROR & TOTAL_ANAL_ERROR & MIN_DETECTABLE_ACTIVITY & PCNT_MOISTURE & LAB_QUALIFIER & REVIEW_QUALIFIER & SAMP_FROM & SAMP_ITEM & SAMP_MTHD & COLL_MTHD & SAMP_SITE_ID \\
\hline 5826 & $\mathrm{pCi} / \mathrm{L}$ & 0.033 & 0.0466 & & & & & RIVER & & CUMULATIVE & UNFILTERED & 252 \\
\hline 5827 & $\mathrm{pCi} / \mathrm{L}$ & 0.0411 & 0.0449 & & & & & RIVER & & CUMULATIVE & UNFILTERED & 252 \\
\hline 5828 & $\mathrm{pCi} / \mathrm{L}$ & 0.01 & 0.0102 & & & U & & RIVER & & CUMULATIVE & UNFILTERED & 252 \\
\hline 5829 & $\mathrm{pCi} / \mathrm{L}$ & 0.0491 & 0.0557 & & & & & RIVER & & CUMULATIVE & UNFILTERED & 252 \\
\hline 5830 & $\mathrm{pCi} / \mathrm{L}$ & 1.26 & 1.26 & & & $\mathrm{U}$ & & RIVER & & CUMULATIVE & UNFILTERED & 252 \\
\hline 5831 & $\mathrm{pCi} / \mathrm{L}$ & 5.92 & 18.3 & & & & & RIVER & & CUMULATIVE & UNFILTERED & 252 \\
\hline 5832 & $\mathrm{pCi} / \mathrm{L}$ & 0.0365 & 0.054 & & & & & RIVER & & CUMULATIVE & UNFILTERED & 463 \\
\hline 5833 & $\mathrm{pCi} / \mathrm{L}$ & 0.0487 & 0.055 & & & & & RIVER & & CUMULATIVE & UNFILTERED & 463 \\
\hline 5834 & $\mathrm{pCi} / \mathrm{L}$ & 0.0115 & 0.0117 & & & & & RIVER & & CUMULATIVE & UNFILTERED & 463 \\
\hline 5835 & $\mathrm{pCi} / \mathrm{L}$ & 0.0504 & 0.0576 & & & & & RIVER & & CUMULATIVE & UNFILTERED & 463 \\
\hline 5836 & $\mathrm{pCi} / \mathrm{L}$ & 0.721 & 0.727 & & & & & RIVER & & CUMULATIVE & UNFILTERED & 463 \\
\hline 5837 & $\mathrm{pCi} / \mathrm{L}$ & 6.98 & 23 & & & & & RIVER & & CUMULATIVE & UNFILTERED & 463 \\
\hline 5838 & $\mathrm{pCi} / \mathrm{L}$ & & 0.00000185 & & & & & SANITARY & & RAW GRAB & UNFILTERED & 525 \\
\hline 5839 & $\mathrm{pCi} / \mathrm{L}$ & & 0.00000467 & & & & & SANITARY & & RAW GRAB & UNFILTERED & 524 \\
\hline 5840 & $\mathrm{pCi} / \mathrm{L}$ & & 0.0000179 & & & & & SANITARY & & RAW GRAB & UNFILTERED & 95 \\
\hline 5841 & $\mathrm{pCi} / \mathrm{L}$ & & 0.0000144 & & & & & SANITARY & & RAW GRAB & UNFILTERED & 172 \\
\hline 5842 & $\mathrm{pCi} / \mathrm{L}$ & 1.01 & 1.01 & & & U & & SANITARY & & RAW GRAB & UNFILTERED & 172 \\
\hline 5843 & $\mathrm{pCi} / \mathrm{L}$ & 0.343 & 1.42 & & & & & SANITARY & & RAW GRAB & UNFILTERED & 172 \\
\hline 5844 & $\mathrm{pCi} / \mathrm{L}$ & 0.0666 & 0.0845 & & & & & SANITARY & & RAW GRAB & UNFILTERED & 172 \\
\hline 5845 & $\mathrm{pCi} / \mathrm{L}$ & 0.357 & 1.53 & & & & & SANITARY & & RAW GRAB & UNFILTERED & 172 \\
\hline 5846 & $\mathrm{pCi} / \mathrm{L}$ & 161 & 347 & & & U & & SANITARY & & RAW GRAB & UNFILTERED & 172 \\
\hline 5847 & $\mathrm{pCi} / \mathrm{L}$ & 0.0426 & 0.0606 & & & & & DRINKING & & RAW GRAB & UNFILTERED & 553 \\
\hline 5848 & $\mathrm{pCi} / \mathrm{L}$ & 165 & 354 & & & U & & DRINKING & & RAW GRAB & UNFILTERED & 553 \\
\hline 5849 & $\mathrm{pCi} / \mathrm{L}$ & 0.804 & 0.804 & & & U & & DRINKING & & RAW GRAB & UNFILTERED & 553 \\
\hline 5850 & & & & & & & & RIVER & & CONTINUOUS & RESIN & 307 \\
\hline 5851 & & & & & & & & RIVER & & CONTINUOUS & FILTER & 307 \\
\hline 5852 & & & & & & & & RIVER & & CONTINUOUS & FILTER & 252 \\
\hline 5853 & & & & & & & & RIVER & & CONTINUOUS & RESIN & 252 \\
\hline 5854 & $\mathrm{pCi} / \mathrm{L}$ & 0.0034 & 0.00341 & & & U & & RIVER & & CONTINUOUS & RESIN & 463 \\
\hline 5855 & $\mathrm{pCi} / \mathrm{L}$ & 0.0018 & 0.0018 & & & U & & RIVER & & CONTINUOUS & FILTER & 463 \\
\hline 5856 & $\mathrm{pCi} / \mathrm{L}$ & 0.00115 & 0.00115 & & & U & & RIVER & & CONTINUOUS & FILTER & 307 \\
\hline 5857 & $\mathrm{pCi} / \mathrm{L}$ & 0.00191 & 0.00194 & & & & & RIVER & & CONTINUOUS & RESIN & 307 \\
\hline 5858 & $\mathrm{pCi} / \mathrm{L}$ & 0.000896 & 0.000899 & & & $U$ & & RIVER & & CONTINUOUS & FILTER & 252 \\
\hline 5859 & $\mathrm{pCi} / \mathrm{L}$ & 0.00211 & 0.00212 & & & U & & RIVER & & CONTINUOUS & RESIN & 252 \\
\hline 5860 & $\mathrm{pCi} / \mathrm{L}$ & 0.00593 & 0.00607 & & & & & RIVER & & CONTINUOUS & RESIN & 463 \\
\hline 5861 & $\mathrm{pCi} / \mathrm{L}$ & 0.00366 & 0.00366 & & & U & & RIVER & & CONTINUOUS & FILTER & 463 \\
\hline 5862 & $\mathrm{pCi} / \mathrm{L}$ & 0.783 & 0.785 & & & U & & RIVER & & CUMULATIVE & UNFILTERED & 252 \\
\hline 5863 & $\mathrm{pCi} / \mathrm{L}$ & 0.0389 & 0.0423 & & & & & RIVER & & CUMULATIVE & UNFILTERED & 252 \\
\hline 5864 & $\mathrm{pCi} / \mathrm{L}$ & 0.0116 & 0.0119 & & & U & & RIVER & & CUMULATIVE & UNFILTERED & 252 \\
\hline 5865 & $\mathrm{pCi} / \mathrm{L}$ & 0.0445 & 0.0498 & & & & & RIVER & & CUMULATIVE & UNFILTERED & 252 \\
\hline 5866 & $\mathrm{pCi} / \mathrm{L}$ & 0.0308 & 0.0533 & & & & & RIVER & & CUMULATIVE & UNFILTERED & 252 \\
\hline 5867 & $\mathrm{pCi} / \mathrm{L}$ & 6.17 & 18.9 & & & & & RIVER & & CUMULATIVE & UNFILTERED & 252 \\
\hline 5868 & $\mathrm{pCi} / \mathrm{L}$ & 0.543 & 0.545 & & & U & & RIVER & & CUMULATIVE & UNFILTERED & 463 \\
\hline 5869 & $\mathrm{pCi} / \mathrm{L}$ & 0.0466 & 0.0529 & & & & & RIVER & & CUMULATIVE & UNFILTERED & 463 \\
\hline 5870 & $\mathrm{pCi} / \mathrm{L}$ & 0.0202 & 0.0209 & & & & & RIVER & & CUMULATIVE & UNFILTERED & 463 \\
\hline 5871 & $\mathrm{pCi} / \mathrm{L}$ & 0.0515 & 0.0598 & & & & & RIVER & & CUMULATIVE & UNFILTERED & 463 \\
\hline 5872 & $\mathrm{pCi} / \mathrm{L}$ & 0.0323 & 0.0583 & & & & & RIVER & & CUMULATIVE & UNFILTERED & 463 \\
\hline 5873 & $\mathrm{pCi} / \mathrm{L}$ & 6.96 & 23 & & & & & RIVER & & CUMULATIVE & UNFILTERED & 463 \\
\hline 5874 & $\mathrm{pCi} / \mathrm{L}$ & 0.81 & 0.816 & & & & & DRINKING & & SAN. GRAB & UNFILTERED & 487 \\
\hline 5875 & $\mathrm{pCi} / \mathrm{L}$ & 152 & 320 & & & U & & DRINKING & & SAN. GRAB & UNFILTERED & 487 \\
\hline 5876 & $\mathrm{pCi} / \mathrm{L}$ & 0.0331 & 0.0341 & & & U & & DRINKING & & SAN. GRAB & UNFILTERED & 487 \\
\hline 5877 & $\mathrm{pCi} / \mathrm{L}$ & 0.000725 & 0.000725 & & & $U_{2}$ & & RIVER & & CONTINUOUS & FILTER & 307 \\
\hline
\end{tabular}




\begin{tabular}{|c|c|c|c|c|c|c|c|c|c|c|c|c|}
\hline & $\mathrm{N}$ & $\mathrm{O}$ & $\mathrm{P}$ & $\mathrm{Q}$ & $\mathrm{R}$ & $\mathrm{s}$ & $\mathrm{T}$ & $\mathrm{U}$ & $\mathrm{V}$ & $\mathrm{w}$ & $\mathrm{x}$ & $\mathrm{Y}$ \\
\hline 1 & ANAL_UNITS_RPTD & COUNTING_ERROR & TOTAL_ANAL_ERROR & MIN_DETECTABLE_ACTIVITY & PCNT_MOISTURE & LAB_QUALIFIER & REVIEW_QUALIFIER & SAMP_FROM & SAMP_ITEM & SAMP_MTHD & COLL_MTHD & SAMP_SITE_ID \\
\hline 5878 & $\mathrm{pCi} / \mathrm{L}$ & 0.000027 & 0.0000273 & & & & & RIVER & & CONTINUOUS & FILTER & 307 \\
\hline 5879 & $\mathrm{pCi} / \mathrm{L}$ & & 0.0000137 & & & & & RIVER & & CONTINUOUS & RESIN & 307 \\
\hline 5880 & $\mathrm{pCi} / \mathrm{L}$ & 0.00143 & 0.00143 & & & $U$ & & RIVER & & CONTINUOUS & RESIN & 307 \\
\hline 5881 & $\mathrm{pCi} / \mathrm{L}$ & 0 & 0.0000622 & & & $\mathrm{U}$ & & RIVER & & CONTINUOUS & RESIN & 307 \\
\hline 5882 & $\mathrm{pCi} / \mathrm{L}$ & 0.000967 & 0.000972 & & & & & RIVER & & CONTINUOUS & FILTER & 252 \\
\hline 5883 & $\mathrm{pCi} / \mathrm{L}$ & 0.0000466 & 0.0000471 & & & & & RIVER & & CONTINUOUS & FILTER & 252 \\
\hline 5884 & $\mathrm{pCi} / \mathrm{L}$ & & 0.00000136 & & & & & RIVER & & CONTINUOUS & RESIN & 252 \\
\hline 5885 & $\mathrm{pCi} / \mathrm{L}$ & 0.00198 & 0.00198 & & & $\mathrm{U}$ & & RIVER & & CONTINUOUS & RESIN & 252 \\
\hline 5886 & $\mathrm{pCi} / \mathrm{L}$ & 0.0000474 & 0.0000477 & & & U & & RIVER & & CONTINUOUS & RESIN & 252 \\
\hline 5887 & $\mathrm{pCi} / \mathrm{L}$ & 0.000892 & 0.0009 & & & & & RIVER & & CONTINUOUS & FILTER & 463 \\
\hline 5888 & pCi/L & 0.0000324 & 0.000033 & & & & & RIVER & & CONTINUOUS & FILTER & 463 \\
\hline 5889 & $\mathrm{pCi} / \mathrm{L}$ & & 0.00000995 & & & & & RIVER & & CONTINUOUS & RESIN & 463 \\
\hline 5890 & $\mathrm{pCi} / \mathrm{L}$ & 0.00165 & 0.00165 & & & $\mathrm{U}$ & & RIVER & & CONTINUOUS & RESIN & 463 \\
\hline 5891 & $\mathrm{pCi} / \mathrm{L}$ & 0.000107 & 0.000107 & & & U & & RIVER & & CONTINUOUS & RESIN & 463 \\
\hline 5892 & $\mathrm{pCi} / \mathrm{L}$ & 0.924 & 0.924 & & & U & & SANITARY & & RAW GRAB & UNFILTERED & 525 \\
\hline 5893 & $\mathrm{pCi} / \mathrm{L}$ & 161 & 336 & & & & & SANITARY & & RAW GRAB & UNFILTERED & 525 \\
\hline 5894 & $\mathrm{pCi} / \mathrm{L}$ & 0.0153 & 0.0162 & & & & & SANITARY & & RAW GRAB & UNFILTERED & 525 \\
\hline 5895 & $\mathrm{pCi} / \mathrm{L}$ & 0.00241 & 0.00275 & & & U & & SANITARY & & RAW GRAB & UNFILTERED & 525 \\
\hline 5896 & $\mathrm{pCi} / \mathrm{L}$ & 0.0182 & 0.02 & & & & & SANITARY & & RAW GRAB & UNFILTERED & 525 \\
\hline 5897 & $\mathrm{pCi} / \mathrm{L}$ & 0.591 & 0.591 & & & $U$ & & SANITARY & & RAW GRAB & UNFILTERED & 172 \\
\hline 5898 & $\mathrm{pCi} / \mathrm{L}$ & 160 & 335 & & & & & SANITARY & & RAW GRAB & UNFILTERED & 172 \\
\hline 5899 & $\mathrm{pCi} / \mathrm{L}$ & 0.0501 & 0.0581 & & & & & SANITARY & & RAW GRAB & UNFILTERED & 172 \\
\hline 5900 & $\mathrm{pCi} / \mathrm{L}$ & 0.0439 & 0.0492 & & & & & SANITARY & & RAW GRAB & UNFILTERED & 172 \\
\hline \begin{tabular}{|l|}
5901 \\
\end{tabular} & pCi/L & 0.0042 & 0.00482 & & & $U$ & & SANITARY & & RAW GRAB & UNFILTERED & 172 \\
\hline \begin{tabular}{|l|}
5902 \\
\end{tabular} & pCi/L & 167 & 347 & & & & & DRINKING & & SAN. GRAB & UNFILTERED & 530 \\
\hline \begin{tabular}{|l|}
5903 \\
\end{tabular} & $\mathrm{pCi} / \mathrm{L}$ & 0.911 & 0.911 & & & $\mathrm{U}$ & & DRINKING & & SAN. GRAB & UNFILTERED & 530 \\
\hline \begin{tabular}{|l|}
5904 \\
\end{tabular} & $\mathrm{pCi} / \mathrm{L}$ & 0.0528 & 0.117 & & & & & DRINKING & & SAN. GRAB & UNFILTERED & 530 \\
\hline \begin{tabular}{|l|}
5905 \\
\end{tabular} & $\mathrm{pCi} / \mathrm{L}$ & 1.08 & 1.08 & & & $\mathrm{U}$ & & DRINKING & & CUMULATIVE & UNFILTERED & 307 \\
\hline \begin{tabular}{|l|l|}
5906 \\
\end{tabular} & $\mathrm{pCi} / \mathrm{L}$ & 162 & 342 & & & U & & DRINKING & & CUMULATIVE & UNFILTERED & 307 \\
\hline \begin{tabular}{|l|}
5907 \\
\end{tabular} & $\mathrm{pCi} / \mathrm{L}$ & 0.0456 & 0.0518 & & & & & DRINKING & & CUMULATIVE & UNFILTERED & 307 \\
\hline 5908 & $\mathrm{pCi} / \mathrm{L}$ & 0.00922 & 0.00956 & & & U & & DRINKING & & CUMULATIVE & UNFILTERED & 307 \\
\hline \begin{tabular}{|l|}
5909 \\
\end{tabular} & $\mathrm{pCi} / \mathrm{L}$ & 0.0541 & 0.0644 & & & & & DRINKING & & CUMULATIVE & UNFILTERED & 307 \\
\hline 5910 & $\mathrm{pCi} / \mathrm{L}$ & 0.0313 & 0.0428 & & & & & DRINKING & & CUMULATIVE & UNFILTERED & 307 \\
\hline 5911 & $\mathrm{pCi} / \mathrm{L}$ & 0.666 & 0.667 & & & U & & SANITARY & & RAW GRAB & UNFILTERED & 524 \\
\hline 5912 & $\mathrm{pCi} / \mathrm{L}$ & 159 & 337 & & & $U$ & & SANITARY & & RAW GRAB & UNFILTERED & 524 \\
\hline 5913 & $\mathrm{pCi} / \mathrm{L}$ & 0.173 & 0.377 & & & & & SANITARY & & RAW GRAB & UNFILTERED & 524 \\
\hline 5914 & $\mathrm{pCi} / \mathrm{L}$ & 0.0335 & 0.0358 & & & & & SANITARY & & RAW GRAB & UNFILTERED & 524 \\
\hline 5915 & $\mathrm{pCi} / \mathrm{L}$ & 0.19 & 0.444 & & & & & SANITARY & & RAW GRAB & UNFILTERED & 524 \\
\hline 5916 & & & & & & & & SANITARY & & RAW GRAB & UNFILTERED & 95 \\
\hline \begin{tabular}{|l|l|}
5917 \\
\end{tabular} & & & & & & & & SANITARY & & RAW GRAB & UNFILTERED & 95 \\
\hline 5918 & & & & & & & & SANITARY & & RAW GRAB & UNFILTERED & 95 \\
\hline 5919 & & & & & & & & SANITARY & & RAW GRAB & UNFILTERED & 95 \\
\hline 5920 & & & & & & & & SANITARY & & RAW GRAB & UNFILTERED & 95 \\
\hline 5921 & $\mathrm{pCi} / \mathrm{L}$ & 0.881 & 0.881 & & & U & & QC_SAMPLE & & RAW GRAB & UNFILTERED & 550 \\
\hline \begin{tabular}{|l|l|}
5922 \\
\end{tabular} & $\mathrm{pCi} / \mathrm{L}$ & 0.0693 & 0.0824 & & & & & QC_SAMPLE & & RAW GRAB & UNFILTERED & 550 \\
\hline 5923 & $\mathrm{pCi} / \mathrm{L}$ & 0.0406 & 0.0559 & & & & & QC_SAMPLE & & RAW GRAB & UNFILTERED & 550 \\
\hline \begin{tabular}{|l|}
5924 \\
\end{tabular} & $\mathrm{pCi} / \mathrm{L}$ & 7.36 & 25.6 & & & & & QC_SAMPLE & & RAW GRAB & UNFILTERED & 550 \\
\hline \begin{tabular}{|l|l|}
5925 \\
\end{tabular} & $\mathrm{pCi} / \mathrm{L}$ & 0.0638 & 0.0742 & & & & & QC_SAMPLE & & RAW GRAB & UNFILTERED & 550 \\
\hline \begin{tabular}{|l|l|}
5926 \\
\end{tabular} & $\mathrm{pCi} / \mathrm{L}$ & 0.0092 & 0.00953 & & & U & & QC_SAMPLE & & RAW GRAB & UNFILTERED & 550 \\
\hline 5927. & pCi/L & 0.924 & 0.924 & & & $U$ & & QC_SAMPLE & & RAW GRAB & UNFILTERED & 247 \\
\hline \begin{tabular}{|l|l|}
5928 \\
\end{tabular} & $\mathrm{pCi} / \mathrm{L}$ & 0.00916 & 0.00949 & & & U & & QC_SAMPLE & & RAW GRAB & UNFILTERED & 247 \\
\hline 5929 & $\mathrm{pCi} / \mathrm{L}$ & 7.23 & 24.4 & & & & & QC_SAMPLE & & RAW GRAB & UNFILTERED & 247 \\
\hline
\end{tabular}




\begin{tabular}{|c|c|c|c|c|c|c|c|c|c|c|c|c|}
\hline & $\mathrm{N}$ & $\mathrm{O}$ & $\mathrm{P}$ & $\mathrm{Q}$ & $\mathrm{R}$ & $\mathrm{s}$ & $\mathrm{T}$ & $\mathrm{U}$ & $\mathrm{V}$ & $\mathrm{W}$ & $\mathrm{X}$ & $\mathrm{Y}$ \\
\hline 1 & ANAL_UNITS_RPTD & COUNTING_ERROR & TOTAL_ANAL_ERROR & MIN_DETECTABLE_ACTIVITY & PCNT_MOISTURE & LAB_QUALIFIER & REVIEW_QUALIFIER & SAMP_FROM & SAMP_ITEM & SAMP_MTHD & COLL_MTHD & SAMP_SITE_ID \\
\hline 5930 & $\mathrm{pCi} / \mathrm{L}$ & 0.0626 & 0.075 & & & & & QC_SAMPLE & & RAW GRAB & UNFILTERED & 247 \\
\hline 5931 & $\mathrm{pCi} / \mathrm{L}$ & 0.0711 & 0.0892 & & & & & QC_SAMPLE & & RAW GRAB & UNFILTERED & 247 \\
\hline 5932 & $\mathrm{pCi} / \mathrm{L}$ & 0.0376 & 0.0495 & & & & & QC_SAMPLE & & RAW GRAB & UNFILTERED & 247 \\
\hline 5933 & $\mathrm{pCi} / \mathrm{L}$ & 0.0013 & 0.0013 & & & $\mathrm{U}$ & & RIVER & & CONTINUOUS & FILTER & 307 \\
\hline 5934 & $\mathrm{pCi} / \mathrm{L}$ & 0.00347 & 0.00352 & & & & & RIVER & & CONTINUOUS & RESIN & 307 \\
\hline 5935 & $\mathrm{pCi} / \mathrm{L}$ & 0.00252 & 0.00253 & & & U & & RIVER & & CONTINUOUS & FILTER & 252 \\
\hline 5936 & $\mathrm{pCi} / \mathrm{L}$ & 0.00378 & 0.0038 & & & & & RIVER & & CONTINUOUS & RESIN & 252 \\
\hline 5937 & $\mathrm{pCi} / \mathrm{L}$ & 0.00151 & 0.00152 & & & & & RIVER & & CONTINUOUS & FILTER & 463 \\
\hline 5938 & $\mathrm{pCi} / \mathrm{L}$ & 0.00272 & 0.00274 & & & & & RIVER & & CONTINUOUS & RESIN & 463 \\
\hline 5939 & $\mathrm{pCi} / \mathrm{L}$ & 1.78 & 1.78 & & & U & & SURFACE & & RAW GRAB & UNFILTERED & 509 \\
\hline 5940 & $\mathrm{pCi} / \mathrm{L}$ & 0.0786 & 0.121 & & & & & SURFACE & & RAW GRAB & UNFILTERED & 509 \\
\hline 5941 & $\mathrm{pCi} / \mathrm{L}$ & 162 & 344 & & & U & & SURFACE & & RAW GRAB & UNFILTERED & 509 \\
\hline 5942 & $\mathrm{pCi} / \mathrm{L}$ & 0.614 & 0.614 & & & U & & SURFACE & & RAW GRAB & UNFILTERED & 136 \\
\hline 5943 & $\mathrm{pCi} / \mathrm{L}$ & 276 & 677 & & & & & SURFACE & & RAW GRAB & UNFILTERED & 136 \\
\hline 5944 & $\mathrm{pCi} / \mathrm{L}$ & 2.12 & 2.13 & & & & & SURFACE & & RAW GRAB & UNFILTERED & 147 \\
\hline 5945 & $\mathrm{pCi} / \mathrm{L}$ & 2.91 & 10 & & & & & SURFACE & & RAW GRAB & UNFILTERED & 147 \\
\hline 5946 & $\mathrm{pCi} / \mathrm{L}$ & 0.587 & 0.704 & & & & & SURFACE & & RAW GRAB & UNFILTERED & 147 \\
\hline 5947 & $\mathrm{pCi} / \mathrm{L}$ & 3 & 10.6 & & & & & SURFACE & & RAW GRAB & UNFILTERED & 147 \\
\hline 5948 & $\mathrm{pCi} / \mathrm{L}$ & 0.18 & 0.559 & & & & & SURFACE & & RAW GRAB & UNFILTERED & 147 \\
\hline 5949 & $\mathrm{pCi} / \mathrm{L}$ & 167 & 354 & & & & & SURFACE & & RAW GRAB & UNFILTERED & 147 \\
\hline 5950 & $\mathrm{pCi} / \mathrm{L}$ & 0.00123 & 0.00123 & & & U & & RIVER & & CONTINUOUS & FILTER & 307 \\
\hline 5951 & $\mathrm{pCi} / \mathrm{L}$ & 0.00313 & 0.00318 & & & & & RIVER & & CONTINUOUS & RESIN & 307 \\
\hline 5952 & $\mathrm{pCi} / \mathrm{L}$ & 0.0019 & 0.00191 & & & U & & RIVER & & CONTINUOUS & FILTER & 252 \\
\hline 5953 & $\mathrm{pCi} / \mathrm{L}$ & 0.922 & 0.922 & & & U & & RIVER & & CUMULATIVE & UNFILTERED & 252 \\
\hline 5954 & $\mathrm{pCi} / \mathrm{L}$ & 0.0424 & 0.0463 & & & & & RIVER & & CUMULATIVE & UNFILTERED & 252 \\
\hline 5955 & $\mathrm{pCi} / \mathrm{L}$ & 0.0132 & 0.0135 & & & U & & RIVER & & CUMULATIVE & UNFILTERED & 252 \\
\hline 5956 & $\mathrm{pCi} / \mathrm{L}$ & 0.0482 & 0.054 & & & & & RIVER & & CUMULATIVE & UNFILTERED & 252 \\
\hline 5957 & $\mathrm{pCi} / \mathrm{L}$ & 0.0349 & 0.0495 & & & & & RIVER & & CUMULATIVE & UNFILTERED & 252 \\
\hline 5958 & $\mathrm{pCi} / \mathrm{L}$ & 0.00401 & 0.00406 & & & & & RIVER & & CONTINUOUS & RESIN & 252 \\
\hline 5959 & $\mathrm{pCi} / \mathrm{L}$ & 5.95 & 18.6 & & & & & RIVER & & CUMULATIVE & UNFILTERED & 252 \\
\hline 5960 & $\mathrm{pCi} / \mathrm{L}$ & 0.00176 & 0.00176 & & & U & & RIVER & & CONTINUOUS & FILTER & 463 \\
\hline 5961 & $\mathrm{pCi} / \mathrm{L}$ & 0.0461 & 0.0517 & & & & & RIVER & & CUMULATIVE & UNFILTERED & 463 \\
\hline 5962 & $\mathrm{pCi} / \mathrm{L}$ & 0.0127 & 0.013 & & & $\mathrm{U}$ & & RIVER & & CUMULATIVE & UNFILTERED & 463 \\
\hline 5963 & $\mathrm{pCi} / \mathrm{L}$ & 0.0495 & 0.0565 & & & & & RIVER & & CUMULATIVE & UNFILTERED & 463 \\
\hline 5964 & $\mathrm{pCi} / \mathrm{L}$ & 0.00242 & 0.00242 & & & U & & RIVER & & CONTINUOUS & RESIN & 463 \\
\hline 5965 & $\mathrm{pCi} / \mathrm{L}$ & 0.0356 & 0.0554 & & & & & RIVER & & CUMULATIVE & UNFILTERED & 463 \\
\hline 5966 & $\mathrm{pCi} / \mathrm{L}$ & 0.735 & 0.735 & & & U & & RIVER & & CUMULATIVE & UNFILTERED & 463 \\
\hline 5967 & $\mathrm{pCi} / \mathrm{L}$ & 7.08 & 24.7 & & & & & RIVER & & CUMULATIVE & UNFILTERED & 463 \\
\hline 5968 & $\mathrm{pCi} / \mathrm{L}$ & 0.00275 & 0.00277 & & & & & RIVER & & CONTINUOUS & FILTER & 307 \\
\hline 5969 & $\mathrm{pCi} / \mathrm{L}$ & 0.00498 & 0.00498 & & & $U$ & & RIVER & & CONTINUOUS & RESIN & 307 \\
\hline 5970 & $\mathrm{pCi} / \mathrm{L}$ & 0.00282 & 0.00283 & & & $U$ & & RIVER & & CONTINUOUS & FILTER & 252 \\
\hline 5971 & $\mathrm{pCi} / \mathrm{L}$ & 0.00574 & 0.00574 & & & $U$ & & RIVER & & CONTINUOUS & RESIN & 252 \\
\hline 5972 & $\mathrm{pCi} / \mathrm{L}$ & 0.00169 & 0.00178 & & & & & RIVER & & CONTINUOUS & FILTER & 463 \\
\hline 5973 & $\mathrm{pCi} / \mathrm{L}$ & 0.00287 & 0.0029 & & & & & RIVER & & CONTINUOUS & RESIN & 463 \\
\hline 5974 & $\mathrm{pCi} / \mathrm{L}$ & 0.813 & 0.814 & & & $U$ & & IRRIGATION & & RAW GRAB & UNFILTERED & 440 \\
\hline 5975 & $\mathrm{pCi} / \mathrm{L}$ & 0.0448 & 0.0626 & & & & & IRRIGATION & & RAW GRAB & UNFILTERED & 440 \\
\hline 5976 & $\mathrm{pCi} / \mathrm{L}$ & 0.582 & 0.583 & & & $U$ & & DRINKING & & SAN. GRAB & UNFILTERED & 186 \\
\hline 5977 & $\mathrm{pCi} / \mathrm{L}$ & 0.00848 & 0.00922 & & & U & & DRINKING & & SAN. GRAB & UNFILTERED & 186 \\
\hline 5978 & $\mathrm{pCi} / \mathrm{L}$ & 125 & 275 & & & $\mathrm{U}$ & & DRINKING & & SAN. GRAB & UNFILTERED & 186 \\
\hline 5979 & $\mathrm{pCi} / \mathrm{L}$ & 0.0123 & 0.0148 & & & $U$ & & DRINKING & & SAN. GRAB & UNFILTERED & 512 \\
\hline 5980 & $\mathrm{pCi} / \mathrm{L}$ & 0.946 & 0.946 & & & $U$ & & DRINKING & & SAN. GRAB & UNFILTERED & 512 \\
\hline 5981 & $\mathrm{pCi} / \mathrm{L}$ & 238 & 651 & & & & & DRINKING & & SAN. GRAB & UNFILTERED & 512 \\
\hline
\end{tabular}




\begin{tabular}{|c|c|c|c|c|c|c|c|c|c|c|c|c|}
\hline & $\mathrm{N}$ & $\mathrm{O}$ & $\mathrm{P}$ & $\mathrm{Q}$ & $\mathrm{R}$ & $\mathrm{s}$ & $\mathrm{T}$ & $\mathrm{u}$ & $\mathrm{V}$ & w & $\mathrm{x}$ & $\bar{Y}$ \\
\hline 1 & ANAL_UNITS_RPTD & COUNTING_ERROR & $\mid$ TOTAL_ANAL_ERROR & MIN_DETECTABLE_ACTIVITY & PCNT_MOISTURE & LAB_QUALIFIER & REVIEW_QUALIFIER & SAMP_FROM & SAMP_ITEM & SAMP_MTHD & COLL_MTHD & SAMP_SITE_ID \\
\hline 5982 & $\mathrm{pCi} / \mathrm{L}$ & 0.00905 & 0.00985 & & & $U$ & & DRINKING & & SAN. GRAB & UNFILTERED & 144 \\
\hline 5983 & $\mathrm{pCi} / \mathrm{L}$ & 0.778 & 0.779 & & & $\mathrm{U}$ & & DRINKING & & SAN. GRAB & UNFILTERED & 144 \\
\hline 5984 & $\mathrm{pCi} / \mathrm{L}$ & 124 & 274 & & & $\mathrm{U}$ & & DRINKING & & SAN. GRAB & UNFILTERED & 144 \\
\hline 5985 & $\mathrm{pCi} / \mathrm{L}$ & 1.16 & 1.16 & & & $\mathrm{U}$ & & DRINKING & & SAN. GRAB & UNFILTERED & 187 \\
\hline \begin{tabular}{|l|l|}
5986 \\
\end{tabular} & $\mathrm{pCi} / \mathrm{L}$ & 124 & 274 & & & $\mathrm{U}$ & & DRINKING & & SAN. GRAB & UNFILTERED & 187 \\
\hline 5987 & $\mathrm{pCi} / \mathrm{L}$ & 0.00757 & 0.00838 & & & $\mathrm{U}$ & & DRINKING & & SAN. GRAB & UNFILTERED & 187 \\
\hline 5988 & $\mathrm{pCi} / \mathrm{L}$ & 133 & 294 & & & $\mathrm{U}$ & & DRINKING & & RAW GRAB & UNFILTERED & 553 \\
\hline 5989 & $\mathrm{pCi} / \mathrm{L}$ & 0.805 & 0.805 & & & $\mathrm{U}$ & & DRINKING & & RAW GRAB & UNFILTERED & 553 \\
\hline 5990 & $\mathrm{pCi} / \mathrm{L}$ & 0.0359 & 0.0515 & & & & & DRINKING & & RAW GRAB & UNFILTERED & 553 \\
\hline 5991 & $\mathrm{pCi} / \mathrm{L}$ & 0.00148 & 0.00151 & & & & & RIVER & & CONTINUOUS & FILTER & 307 \\
\hline \begin{tabular}{|l|}
5992 \\
\end{tabular} & $\mathrm{pCi} / \mathrm{L}$ & 0.00288 & 0.00291 & & & & & RIVER & & CONTINUOUS & RESIN & 307 \\
\hline 5993 & $\mathrm{pCi} / \mathrm{L}$ & 0.00136 & 0.00138 & & & & & RIVER & & CONTINUOUS & FILTER & 252 \\
\hline 5994 & $\mathrm{pCi} / \mathrm{L}$ & 0.945 & 0.945 & & & $U$ & & RIVER & & CUMULATIVE & UNFILTERED & 252 \\
\hline 5995 & $\mathrm{pCi} / \mathrm{L}$ & 0.0376 & 0.0515 & & & & & RIVER & & CUMULATIVE & UNFILTERED & 252 \\
\hline 5996 & $\mathrm{pCi} / \mathrm{L}$ & 0.0447 & 0.0497 & & & & & RIVER & & CUMULATIVE & UNFILTERED & 252 \\
\hline \begin{tabular}{|l|}
5997 \\
\end{tabular} & $\mathrm{pCi} / \mathrm{L}$ & 0.0103 & 0.0106 & & & $U$ & & RIVER & & CUMULATIVE & UNFILTERED & 252 \\
\hline \begin{tabular}{|l|}
5998 \\
\end{tabular} & $\mathrm{pCi} / \mathrm{L}$ & 0.0495 & 0.0564 & & & & & RIVER & & CUMULATIVE & UNFILTERED & 252 \\
\hline \begin{tabular}{|l|}
5999 \\
\end{tabular} & $\mathrm{pCi} / \mathrm{L}$ & 5.73 & 18 & & & & & RIVER & & CUMULATIVE & UNFILTERED & 252 \\
\hline 6000 & $\mathrm{pCi} / \mathrm{L}$ & 0.00263 & 0.00267 & & & & & RIVER & & CONTINUOUS & RESIN & 252 \\
\hline 6001 & $\mathrm{pCi} / \mathrm{L}$ & 0.00156 & 0.00156 & & & $U$ & & RIVER & & CONTINUOUS & FILTER & 463 \\
\hline 6002 & $\mathrm{pCi} / \mathrm{L}$ & 1.05 & 1.05 & & & $U$ & & RIVER & & CUMULATIVE & UNFILTERED & 463 \\
\hline 6003 & $\mathrm{pCi} / \mathrm{L}$ & 0.032 & 0.0429 & & & & & RIVER & & CUMULATIVE & UNFILTERED & 463 \\
\hline 6004 & $\mathrm{pCi} / \mathrm{L}$ & 0.0346 & 0.0382 & & & & & RIVER & & CUMULATIVE & UNFILTERED & 463 \\
\hline 6005 & $\mathrm{pCi} / \mathrm{L}$ & 0.00746 & 0.00785 & & & U & & RIVER & & CUMULATIVE & UNFILTERED & 463 \\
\hline 6006 & $\mathrm{pCi} / \mathrm{L}$ & 0.0437 & 0.0505 & & & & & RIVER & & CUMULATIVE & UNFILTERED & 463 \\
\hline 6007 & $\mathrm{pCi} / \mathrm{L}$ & 0.00256 & 0.00259 & & & & & RIVER & & CONTINUOUS & RESIN & 463 \\
\hline 6008 & $\mathrm{pCi} / \mathrm{L}$ & 6.51 & 21.4 & & & & & RIVER & & CUMULATIVE & UNFILTERED & 463 \\
\hline 6009 & $\mathrm{pCi} / \mathrm{L}$ & 0.00292 & 0.00296 & & & & & RIVER & & CONTINUOUS & RESIN & 307 \\
\hline 6010 & $\mathrm{pCi} / \mathrm{L}$ & 0.0015 & 0.00151 & & & & & RIVER & & CONTINUOUS & FILTER & 307 \\
\hline \begin{tabular}{|l|}
6011 \\
\end{tabular} & & & & & & & & RIVER & & CONTINUOUS & FILTER & 252 \\
\hline 6012 & & & & & & & & RIVER & & CONTINUOUS & RESIN & 252 \\
\hline 6013 & $\mathrm{pCi} / \mathrm{L}$ & 0.00276 & 0.00278 & & & & & RIVER & & CONTINUOUS & RESIN & 463 \\
\hline 6014 & $\mathrm{pCi} / \mathrm{L}$ & 0.00146 & 0.00148 & & & & & RIVER & & CONTINUOUS & FILTER & 463 \\
\hline 6015 & $\mathrm{pCi} / \mathrm{L}$ & 1.03 & 1.03 & & & $U$ & & DRINKING & & SAN. GRAB & UNFILTERED & 487 \\
\hline 6016 & $\mathrm{pCi} / \mathrm{L}$ & 0.0246 & 0.0251 & & & $U$ & & DRINKING & & SAN. GRAB & UNFILTERED & 487 \\
\hline \begin{tabular}{|l|}
6017 \\
\end{tabular} & $\mathrm{pCi} / \mathrm{L}$ & 154 & 330 & & & U & & DRINKING & & SAN. GRAB & UNFILTERED & 487 \\
\hline 6018 & $\mathrm{pCi} / \mathrm{L}$ & & 0.0000134 & & & & & RIVER & & CONTINUOUS & RESIN & 307 \\
\hline 6019 & $\mathrm{pCi} / \mathrm{L}$ & 0.0000489 & 0.0000491 & & & $U$ & & RIVER & & CONTINUOUS & RESIN & 307 \\
\hline 6020 & $\mathrm{pCi} / \mathrm{L}$ & 0.0000404 & 0.0000407 & & & & & RIVER & & CONTINUOUS & FILTER & 307 \\
\hline 6021 & $\mathrm{pCi} / \mathrm{L}$ & 0.00125 & 0.00125 & & & $U$ & & RIVER & & CONTINUOUS & FILTER & 307 \\
\hline 6022 & $\mathrm{pCi} / \mathrm{L}$ & 0.00308 & 0.0031 & & & & & RIVER & & CONTINUOUS & RESIN & 307 \\
\hline 6023 & $\mathrm{pCi} / \mathrm{L}$ & 1.22 & 1.22 & & & U & & RIVER & & CUMULATIVE & UNFILTERED & 252 \\
\hline 6024 & $\mathrm{pCi} / \mathrm{L}$ & 0.0395 & 0.0429 & & & & & RIVER & & CUMULATIVE & UNFILTERED & 252 \\
\hline 6025 & $\mathrm{pCi} / \mathrm{L}$ & 0.00795 & 0.00829 & & & U & & RIVER & & CUMULATIVE & UNFILTERED & 252 \\
\hline 6026 & $\mathrm{pCi} / \mathrm{L}$ & 0.0463 & 0.0523 & & & & & RIVER & & CUMULATIVE & UNFILTERED & 252 \\
\hline \begin{tabular}{|l|}
6027 \\
\end{tabular} & $\mathrm{pCi} / \mathrm{L}$ & 0.0326 & 0.0467 & & & & & RIVER & & CUMULATIVE & UNFILTERED & 252 \\
\hline 6028 & $\mathrm{pCi} / \mathrm{L}$ & 5.43 & 17 & & & & & RIVER & & CUMULATIVE & UNFILTERED & 252 \\
\hline 6029 & $\mathrm{pCi} / \mathrm{L}$ & & 0.00000545 & & & & & RIVER & & CONTINUOUS & RESIN & 252 \\
\hline 6030 & $\mathrm{pCi} / \mathrm{L}$ & 0.0000694 & 0.0000698 & & & $U$ & & RIVER & & CONTINUOUS & RESIN & 252 \\
\hline 6031 & $\mathrm{pCi} / \mathrm{L}$ & 0.0000579 & 0.0000587 & & & & & RIVER & & CONTINUOUS & FILTER & 252 \\
\hline 6032 & $\mathrm{pCi} / \mathrm{L}$ & 0.00186 & 0.00186 & & & U & & RIVER & & CONTINUOUS & FILTER & 252 \\
\hline 6033 & $\mathrm{pCi} / \mathrm{L}$ & 0.00434 & 0.00435 & & & $\mathrm{U}_{2}$ & & RIVER & & CONTINUOUS & RESIN & 252 \\
\hline
\end{tabular}




\begin{tabular}{|c|c|c|c|c|c|c|c|c|c|c|c|c|}
\hline & $\mathrm{N}$ & $\mathrm{O}$ & $\mathrm{P}$ & $\mathrm{Q}$ & $\mathrm{R}$ & $\mathrm{s}$ & $\mathrm{T}$ & $\mathrm{u}$ & $\mathrm{V}$ & w & $\mathrm{x}$ & $\bar{Y}$ \\
\hline 1 & ANAL_UNITS_RPTD & COUNTING_ERROR & $\mid$ TOTAL_ANAL_ERROR & MIN_DETECTABLE_ACTIVITY & PCNT_MOISTURE & LAB_QUALIFIER & $\begin{array}{l}\text { REVIEW_QUALIFIER } \\
\end{array}$ & SAMP_FROM & SAMP_ITEM & SAMP_MTHD & COLL_MTHD & SAMP_SITE_ID \\
\hline 6034 & $\mathrm{pCi} / \mathrm{L}$ & & 0.0000102 & & & & & RIVER & & CONTINUOUS & RESIN & 463 \\
\hline 6035 & $\mathrm{pCi} / \mathrm{L}$ & 0.0000646 & 0.0000649 & & & u & & RIVER & & CONTINUOUS & RESIN & 463 \\
\hline 6036 & $\mathrm{pCi} / \mathrm{L}$ & 0.0000558 & 0.0000574 & & & & & RIVER & & CONTINUOUS & FILTER & 463 \\
\hline 6037 & $\mathrm{pCi} / \mathrm{L}$ & 0.00192 & 0.00193 & & & & & RIVER & & CONTINUOUS & FILTER & 463 \\
\hline 6038 & $\mathrm{pCi} / \mathrm{L}$ & 0.912 & 0.915 & & & u & & RIVER & & CUMULATIVE & UNFILTERED & 463 \\
\hline 6039 & $\mathrm{pCi} / \mathrm{L}$ & 0.0416 & 0.046 & & & & & RIVER & & CUMULATIVE & UNFILTERED & 463 \\
\hline 6040 & $\mathrm{pCi} / \mathrm{L}$ & 0.0107 & 0.0111 & & & u & & RIVER & & CUMULATIVE & UNFILTERED & 463 \\
\hline 6041 & $\mathrm{pCi} / \mathrm{L}$ & 0.0451 & 0.0511 & & & & & RIVER & & CUMULATIVE & UNFILTERED & 463 \\
\hline 6042 & $\mathrm{pCi} / \mathrm{L}$ & 0.0299 & 0.0465 & & & & & RIVER & & CUMULATIVE & UNFILTERED & 463 \\
\hline 6043 & $\mathrm{pCi} / \mathrm{L}$ & 0.0038 & 0.00386 & & & & & RIVER & & CONTINUOUS & RESIN & 463 \\
\hline 6044 & $\mathrm{pCi} / \mathrm{L}$ & 6.43 & 21.7 & & & & & RIVER & & CUMULATIVE & UNFILTERED & 463 \\
\hline 6045 & $\mathrm{pCi} / \mathrm{L}$ & 0.698 & 0.698 & & & U & & SANITARY & & RAW GRAB & UNFILTERED & 172 \\
\hline 6046 & $\mathrm{pCi} / \mathrm{L}$ & 161 & 343 & & & U & & SANITARY & & RAW GRAB & UNFILTERED & 172 \\
\hline 6047 & $\mathrm{pCi} / \mathrm{L}$ & 0.369 & 1.39 & & & & & SANITARY & & RAW GRAB & UNFILTERED & 172 \\
\hline 6048 & $\mathrm{pCi} / \mathrm{L}$ & 0.0741 & 0.0915 & & & & & SANITARY & & RAW GRAB & UNFILTERED & 172 \\
\hline \begin{tabular}{|l|}
6049 \\
\end{tabular} & $\mathrm{pCi} / \mathrm{L}$ & 0.385 & 1.51 & & & & & SANITARY & & RAW GRAB & UNFILTERED & 172 \\
\hline 6050 & $\mathrm{pCi} / \mathrm{L}$ & 0.0411 & 0.0507 & & & U & & SANITARY & & RAW GRAB & UNFILTERED & 525 \\
\hline \begin{tabular}{|l|}
6051 \\
\end{tabular} & $\mathrm{pCi} / \mathrm{L}$ & 0 & 0.0375 & & & U & & SANITARY & & RAW GRAB & UNFILTERED & 525 \\
\hline 6052 & $\mathrm{pCi} / \mathrm{L}$ & 0.0671 & 0.0821 & & & u & & SANITARY & & RAW GRAB & UNFILTERED & 525 \\
\hline 6053 & $\mathrm{pCi} / \mathrm{L}$ & 0.766 & 0.766 & & & u & & SANITARY & & RAW GRAB & UNFILTERED & 525 \\
\hline 6054 & $\mathrm{pCi} / \mathrm{L}$ & 153 & 329 & & & U & & SANITARY & & RAW GRAB & UNFILTERED & 525 \\
\hline 6055 & $\mathrm{pCi} / \mathrm{L}$ & 0.507 & 0.507 & & & U & & DRINKING & & CUMULATIVE & UNFILTERED & 307 \\
\hline \begin{tabular}{|l|}
6056 \\
\end{tabular} & $\mathrm{pCi} / \mathrm{L}$ & 0.0453 & 0.0501 & & & & & DRINKING & & CUMULATIVE & UNFILTERED & 307 \\
\hline \begin{tabular}{|l|}
6057 \\
\end{tabular} & $\mathrm{pCi} / \mathrm{L}$ & 0.00819 & 0.00854 & & & u & & DRINKING & & CUMULATIVE & UNFILTERED & 307 \\
\hline 6058 & $\mathrm{pCi} / \mathrm{L}$ & 0.047 & 0.0529 & & & & & DRINKING & & CUMULATIVE & UNFILTERED & 307 \\
\hline 6059 & $\mathrm{pCi} / \mathrm{L}$ & 0.0436 & 0.0594 & & & & & DRINKING & & CUMULATIVE & UNFILTERED & 307 \\
\hline 6060 & $\mathrm{pCi} / \mathrm{L}$ & 6.47 & 22 & & & & & DRINKING & & CUMULATIVE & UNFILTERED & 307 \\
\hline 6061 & $\mathrm{pCi} / \mathrm{L}$ & 156 & 332 & & & U & & SANITARY & & RAW GRAB & UNFILTERED & 524 \\
\hline 6062 & $\mathrm{pCi} / \mathrm{L}$ & 0.177 & 0.382 & & & & & SANITARY & & RAW GRAB & UNFILTERED & 524 \\
\hline 6063 & $\mathrm{pCi} / \mathrm{L}$ & 0.0314 & 0.0333 & & & & & SANITARY & & RAW GRAB & UNFILTERED & 524 \\
\hline 6064 & $\mathrm{pCi} / \mathrm{L}$ & 0.193 & 0.447 & & & & & SANITARY & & RAW GRAB & UNFILTERED & 524 \\
\hline 6065 & $\mathrm{pCi} / \mathrm{L}$ & 1.32 & 1.32 & & & U & & SANITARY & & RAW GRAB & UNFILTERED & 524 \\
\hline 6066 & $\mathrm{pCi} / \mathrm{L}$ & 0.00145 & 0.00145 & & & U & & RIVER & & CONTINUOUS & FILTER & 307 \\
\hline \begin{tabular}{|l|}
6067 \\
\end{tabular} & $\mathrm{pCi} / \mathrm{L}$ & 0.00268 & 0.0027 & & & & & RIVER & & CONTINUOUS & RESIN & 307 \\
\hline 6068 & $\mathrm{pCi} / \mathrm{L}$ & 0.00452 & 0.00452 & & & U & & RIVER & & CONTINUOUS & RESIN & 252 \\
\hline \begin{tabular}{|l|}
6069 \\
\end{tabular} & $\mathrm{pCi} / \mathrm{L}$ & 0.00216 & 0.00219 & & & & & RIVER & & CONTINUOUS & FILTER & 252 \\
\hline 6070 & $\mathrm{pCi} / \mathrm{L}$ & 0.00326 & 0.00328 & & & u & & RIVER & & CONTINUOUS & RESIN & 463 \\
\hline 6071 & $\mathrm{pCi} / \mathrm{L}$ & 0.00167 & 0.00168 & & & & & RIVER & & CONTINUOUS & FILTER & 463 \\
\hline 6072 & $\mathrm{pCi} / \mathrm{L}$ & 0.84 & 0.84 & & & U & & IRRIGATION & & RAW GRAB & UNFILTERED & 440 \\
\hline 6073 & $\mathrm{pCi} / \mathrm{L}$ & 0.0367 & 0.049 & & & & & IRRIGATION & & RAW GRAB & UNFILTERED & 440 \\
\hline 6074 & $\mathrm{pCi} / \mathrm{L}$ & 2.06 & 2.06 & & & U & & SURFACE & & RAW GRAB & UNFILTERED & 509 \\
\hline 6075 & $\mathrm{pCi} / \mathrm{L}$ & 0.105 & 0.179 & & & & & SURFACE & & RAW GRAB & UNFILTERED & 509 \\
\hline 6076 & $\mathrm{pCi} / \mathrm{L}$ & 130 & 239 & & & U & & SURFACE & & RAW GRAB & UNFILTERED & 509 \\
\hline 6077 & $\mathrm{pCi} / \mathrm{L}$ & 1.95 & 1.96 & & & U & & SURFACE & & RAW GRAB & UNFILTERED & 136 \\
\hline 6078 & $\mathrm{pCi} / \mathrm{L}$ & 259 & 567 & & & & & SURFACE & & RAW GRAB & UNFILTERED & 136 \\
\hline 6079 & $\mathrm{pCi} / \mathrm{L}$ & 1.78 & 1.78 & & & U & & SURFACE & & RAW GRAB & UNFILTERED & 147 \\
\hline 6080 & $\mathrm{pCi} / \mathrm{L}$ & 0.156 & 0.501 & & & & & SURFACE & & RAW GRAB & UNFILTERED & 147 \\
\hline \begin{tabular}{|l|}
6081 \\
\end{tabular} & $\mathrm{pCi} / \mathrm{L}$ & 3.66 & 15.1 & & & & & SURFACE & & RAW GRAB & UNFILTERED & 147 \\
\hline 6082 & $\mathrm{pCi} / \mathrm{L}$ & 0.696 & 0.873 & & & & & SURFACE & & RAW GRAB & UNFILTERED & 147 \\
\hline \begin{tabular}{|l|}
6083 \\
\end{tabular} & $\mathrm{pCi} / \mathrm{L}$ & 3.57 & 14.4 & & & & & SURFACE & & RAW GRAB & UNFILTERED & 147 \\
\hline 6084 & $\mathrm{pCi} / \mathrm{L}$ & 142 & 256 & & & & & SURFACE & & RAW GRAB & UNFILTERED & 147 \\
\hline 6085 & $\mathrm{pCi} / \mathrm{L}$ & 0.00103 & 0.00103 & & & $\mathrm{U}$ & & RIVER & & CONTINUOUS & FILTER & 307 \\
\hline
\end{tabular}




\begin{tabular}{|c|c|c|c|c|c|c|c|c|c|c|c|c|}
\hline & $\mathrm{N}$ & $\mathrm{O}$ & $\mathrm{P}$ & $\mathrm{Q}$ & $\mathrm{R}$ & $\mathrm{s}$ & $T$ & $\mathrm{U}$ & $\mathrm{V}$ & $\mathrm{W}$ & $x$ & $\bar{Y}$ \\
\hline 1 & ANAL_UNITS_RPTD & COUNTING_ERROR & \begin{tabular}{|c|} 
TOTAL_ANAL_ERROR \\
\end{tabular} & MIN_DETECTABLE_ACTIVITY & PCNT_MOISTURE & LAB_QUALIFIER & REVIEW_QUALIFIER & SAMP_FROM & SAMP_ITEM & SAMP_MTHD & COLL_MTHD & SAMP_SITE_ID \\
\hline 6086 & pCi/L & 0.00177 & 0.0018 & & & & & RIVER & & CONTINUOUS & RESIN & 307 \\
\hline 6087 & $\mathrm{pCi} / \mathrm{L}$ & 0.00376 & 0.00376 & & & $\mathrm{U}$ & & RIVER & & CONTINUOUS & RESIN & 252 \\
\hline 6088 & $\mathrm{pCi} / \mathrm{L}$ & 0.00202 & 0.00204 & & & & & RIVER & & CONTINUOUS & FILTER & 252 \\
\hline 6089 & $\mathrm{pCi} / \mathrm{L}$ & 0.00191 & 0.00194 & & & & & RIVER & & CONTINUOUS & FILTER & 463 \\
\hline 6090 & $\mathrm{pCi} / \mathrm{L}$ & 0.00435 & 0.00437 & & & U & & RIVER & & CONTINUOUS & RESIN & 463 \\
\hline 6091 & $\mathrm{pCi} / \mathrm{L}$ & 0.97 & 0.972 & & & U & & RIVER & & CUMULATIVE & UNFILTERED & 252 \\
\hline 6092 & $\mathrm{pCi} / \mathrm{L}$ & 0.0374 & 0.041 & & & & & RIVER & & CUMULATIVE & UNFILTERED & 252 \\
\hline 6093 & $\mathrm{pCi} / \mathrm{L}$ & 0.0132 & 0.0135 & & & $\mathrm{U}$ & & RIVER & & CUMULATIVE & UNFILTERED & 252 \\
\hline 6094 & $\mathrm{pCi} / \mathrm{L}$ & 0.0385 & 0.0416 & & & & & RIVER & & CUMULATIVE & UNFILTERED & 252 \\
\hline 6095 & $\mathrm{pCi} / \mathrm{L}$ & 0.0334 & 0.0489 & & & & & RIVER & & CUMULATIVE & UNFILTERED & 252 \\
\hline 6096 & $\mathrm{pCi} / \mathrm{L}$ & 5.88 & 25 & & & & & RIVER & & CUMULATIVE & UNFILTERED & 252 \\
\hline 6097 & $\mathrm{pCi} / \mathrm{L}$ & 1.13 & 1.13 & & & U & & RIVER & & CUMULATIVE & UNFILTERED & 463 \\
\hline 6098 & $\mathrm{pCi} / \mathrm{L}$ & 0.0465 & 0.0531 & & & & & RIVER & & CUMULATIVE & UNFILTERED & 463 \\
\hline \begin{tabular}{|l|}
6099 \\
\end{tabular} & $\mathrm{pCi} / \mathrm{L}$ & 0.0111 & 0.0115 & & & $U$ & & RIVER & & CUMULATIVE & UNFILTERED & 463 \\
\hline 6100 & $\mathrm{pCi} / \mathrm{L}$ & 0.0431 & 0.0483 & & & & & RIVER & & CUMULATIVE & UNFILTERED & 463 \\
\hline \begin{tabular}{|l|}
6101 \\
\end{tabular} & $\mathrm{pCi} / \mathrm{L}$ & 0.034 & 0.0459 & & & & & RIVER & & CUMULATIVE & UNFILTERED & 463 \\
\hline 6102 & $\mathrm{pCi} / \mathrm{L}$ & 7.14 & 30.2 & & & & & RIVER & & CUMULATIVE & UNFILTERED & 463 \\
\hline 6103 & $\mathrm{pCi} / \mathrm{L}$ & 0.00277 & 0.00281 & & & & & RIVER & & CONTINUOUS & RESIN & 307 \\
\hline 6104 & $\mathrm{pCi} / \mathrm{L}$ & 0.00141 & 0.00142 & & & U & & RIVER & & CONTINUOUS & FILTER & 307 \\
\hline \begin{tabular}{|l|}
6105 \\
\end{tabular} & $\mathrm{pCi} / \mathrm{L}$ & 0.003 & 0.00303 & & & & & RIVER & & CONTINUOUS & RESIN & 252 \\
\hline 6106 & pCi/L & 0.0018 & 0.00182 & & & & & RIVER & & CONTINUOUS & FILTER & 252 \\
\hline \begin{tabular}{|l|}
6107 \\
\end{tabular} & & & & & & & & RIVER & & CONTINUOUS & FILTER & 463 \\
\hline 6108 & & & & & & & & RIVER & & CONTINUOUS & RESIN & 463 \\
\hline 6109 & $\mathrm{pCi} / \mathrm{L}$ & 0.623 & 0.625 & & & U & & DRINKING & & SAN. GRAB & UNFILTERED & 186 \\
\hline 6110 & $\mathrm{pCi} / \mathrm{L}$ & 130 & 238 & & & $U$ & & DRINKING & & SAN. GRAB & UNFILTERED & 186 \\
\hline \begin{tabular}{|l|l|}
6111 \\
\end{tabular} & $\mathrm{pCi} / \mathrm{L}$ & 0.0406 & 0.067 & & & & & DRINKING & & SAN. GRAB & UNFILTERED & 186 \\
\hline \begin{tabular}{|l|}
6112 \\
\end{tabular} & $\mathrm{pCi} / \mathrm{L}$ & 0.991 & 0.991 & & & $U$ & & DRINKING & & SAN. GRAB & UNFILTERED & 512 \\
\hline \begin{tabular}{|l|}
6113 \\
\end{tabular} & $\mathrm{pCi} / \mathrm{L}$ & 280 & 647 & & & & & DRINKING & & SAN. GRAB & UNFILTERED & 512 \\
\hline \begin{tabular}{|l|}
6114 \\
\end{tabular} & $\mathrm{pCi} / \mathrm{L}$ & 0.0362 & 0.0364 & & & U & & DRINKING & & SAN. GRAB & UNFILTERED & 512 \\
\hline 6115 & $\mathrm{pCi} / \mathrm{L}$ & 0.696 & 0.697 & & & U & & DRINKING & & SAN. GRAB & UNFILTERED & 144 \\
\hline 6116 & $\mathrm{pCi} / \mathrm{L}$ & 126 & 234 & & & U & & DRINKING & & SAN. GRAB & UNFILTERED & 144 \\
\hline \begin{tabular}{|l|l|}
6117 \\
\end{tabular} & $\mathrm{pCi} / \mathrm{L}$ & 0.0287 & 0.029 & & & U & & DRINKING & & SAN. GRAB & UNFILTERED & 144 \\
\hline 6118 & $\mathrm{pCi} / \mathrm{L}$ & 1.02 & 1.02 & & & U & & DRINKING & & SAN. GRAB & UNFILTERED & 187 \\
\hline 6119 & $\mathrm{pCi} / \mathrm{L}$ & 126 & 233 & & & U & & DRINKING & & SAN. GRAB & UNFILTERED & 187 \\
\hline 6120 & $\mathrm{pCi} / \mathrm{L}$ & 0.04 & 0.0413 & & & U & & DRINKING & & SAN. GRAB & UNFILTERED & 187 \\
\hline \begin{tabular}{|l|l|}
6121 \\
\end{tabular} & pCi/L & 0.653 & 0.653 & & & U & & DRINKING & & RAW GRAB & UNFILTERED & 553 \\
\hline 6122 & $\mathrm{pCi} / \mathrm{L}$ & 0.0449 & 0.0566 & & & & & DRINKING & & RAW GRAB & UNFILTERED & 553 \\
\hline 6123 & $\mathrm{pCi} / \mathrm{L}$ & 131 & 239 & & & $U$ & & DRINKING & & RAW GRAB & UNFILTERED & 553 \\
\hline 6124 & $\mathrm{pCi} / \mathrm{L}$ & 0.00185 & 0.00188 & & & & & RIVER & & CONTINUOUS & FILTER & 307 \\
\hline 6125 & $\mathrm{pCi} / \mathrm{L}$ & 0.00271 & 0.00273 & & & U & & RIVER & & CONTINUOUS & RESIN & 307 \\
\hline \begin{tabular}{|l|}
6126 \\
\end{tabular} & $\mathrm{pCi} / \mathrm{L}$ & 0.00305 & 0.00306 & & & U & & RIVER & & CONTINUOUS & FILTER & 252 \\
\hline 6127 & pCi/L & 0.00633 & 0.00633 & & & $U$ & & RIVER & & CONTINUOUS & RESIN & 252 \\
\hline 6128 & & & & & & & & RIVER & & CONTINUOUS & FILTER & 463 \\
\hline 6129 & & & & & & & & RIVER & & CONTINUOUS & RESIN & 463 \\
\hline 6130 & $\mathrm{pCi} / \mathrm{L}$ & 1.55 & 1.55 & & & U & & RIVER & & CUMULATIVE & UNFILTERED & 252 \\
\hline \begin{tabular}{|l|}
6131 \\
\end{tabular} & $\mathrm{pCi} / \mathrm{L}$ & 0.033 & 0.0366 & & & & & RIVER & & CUMULATIVE & UNFILTERED & 252 \\
\hline \begin{tabular}{|l|}
6132 \\
\end{tabular} & $\mathrm{pCi} / \mathrm{L}$ & 0.00859 & 0.00894 & & & $U$ & & RIVER & & CUMULATIVE & UNFILTERED & 252 \\
\hline \begin{tabular}{|l|}
6133 \\
\end{tabular} & $\mathrm{pCi} / \mathrm{L}$ & 0.0312 & 0.0338 & & & & & RIVER & & CUMULATIVE & UNFILTERED & 252 \\
\hline 6134 & pCi/L & 0.0358 & 0.0449 & & & & & RIVER & & CUMULATIVE & UNFILTERED & 252 \\
\hline 6135 & pCi/L & 6.02 & 25.4 & & & & & RIVER & & CUMULATIVE & UNFILTERED & 252 \\
\hline 6136 & $\mathrm{pCi} / \mathrm{L}$ & 1.09 & 1.1 & & & $U$ & & RIVER & & CUMULATIVE & UNFILTERED & 463 \\
\hline 6137 & $\mathrm{pCi} / \mathrm{L}$ & 0.0249 & 0.0273 & & & & & RIVER & & CUMULATIVE & UNFILTERED & 463 \\
\hline
\end{tabular}




\begin{tabular}{|c|c|c|c|c|c|c|c|c|c|c|c|c|}
\hline & $\mathrm{N}$ & $\mathrm{O}$ & $\mathrm{P}$ & $\mathrm{Q}$ & $\mathrm{R}$ & $\mathrm{S}$ & $\mathrm{T}$ & $\mathrm{U}$ & $\mathrm{V}$ & $\mathrm{w}$ & $\mathrm{X}$ & $\mathrm{Y}$ \\
\hline 1 & ANAL_UNITS_RPTD & COUNTING_ERROR & TOTAL_ANAL_ERROR & MIN_DETECTABLE_ACTIVITY & PCNT_MOISTURE & LAB_QUALIFIER & REVIEW_QUALIFIER & SAMP_FROM & SAMP_ITEM & SAMP_MTHD & COLL_MTHD & SAMP_SITE_ID \\
\hline 6138 & $\mathrm{pCi} / \mathrm{L}$ & 0.00623 & 0.00666 & & & $U$ & & RIVER & & CUMULATIVE & UNFILTERED & 463 \\
\hline 6139 & $\mathrm{pCi} / \mathrm{L}$ & 0.0282 & 0.0298 & & & & & RIVER & & CUMULATIVE & UNFILTERED & 463 \\
\hline 6140 & $\mathrm{pCi} / \mathrm{L}$ & 0.0338 & 0.0441 & & & & & RIVER & & CUMULATIVE & UNFILTERED & 463 \\
\hline 6141 & $\mathrm{pCi} / \mathrm{L}$ & 6.95 & 29.5 & & & & & RIVER & & CUMULATIVE & UNFILTERED & 463 \\
\hline 6142 & $\mathrm{pCi} / \mathrm{L}$ & 0.000613 & 0.000618 & & & & & RIVER & & CONTINUOUS & FILTER & 307 \\
\hline 6143 & $\mathrm{pCi} / \mathrm{L}$ & 0.00128 & 0.0013 & & & & & RIVER & & CONTINUOUS & RESIN & 307 \\
\hline 6144 & $\mathrm{pCi} / \mathrm{L}$ & 0.00183 & 0.00183 & & & $\mathrm{u}$ & & RIVER & & CONTINUOUS & FILTER & 252 \\
\hline 6145 & $\mathrm{pCi} / \mathrm{L}$ & 0.00436 & 0.00436 & & & U & & RIVER & & CONTINUOUS & RESIN & 252 \\
\hline 6146 & & & & & & & & RIVER & & CONTINUOUS & FILTER & 463 \\
\hline 6147 & & & & & & & & RIVER & & CONTINUOUS & RESIN & 463 \\
\hline 6148 & $\mathrm{pCi} / \mathrm{L}$ & 1.11 & 1.11 & & & $\mathrm{U}$ & & IRRIGATION & & RAW GRAB & UNFILTERED & 440 \\
\hline 6149 & $\mathrm{pCi} / \mathrm{L}$ & 0.0405 & 0.0523 & & & & & IRRIGATION & & RAW GRAB & UNFILTERED & 440 \\
\hline 6150 & $\mathrm{pCi} / \mathrm{L}$ & 0.734 & 0.734 & & & U & & DRINKING & & SAN. GRAB & UNFILTERED & 487 \\
\hline 6151 & $\mathrm{pCi} / \mathrm{L}$ & 163 & 408 & & & & & DRINKING & & SAN. GRAB & UNFILTERED & 487 \\
\hline 6152 & $\mathrm{pCi} / \mathrm{L}$ & 0.0208 & 0.0217 & & & U & & DRINKING & & SAN. GRAB & UNFILTERED & 487 \\
\hline 6153 & $\mathrm{pCi} / \mathrm{L}$ & 0.00171 & 0.00173 & & & & & RIVER & & CONTINUOUS & FILTER & 307 \\
\hline 6154 & $\mathrm{pCi} / \mathrm{L}$ & 0.00282 & 0.00289 & & & & & RIVER & & CONTINUOUS & RESIN & 307 \\
\hline 6155 & & & & & & & & RIVER & & CONTINUOUS & RESIN & 252 \\
\hline 6156 & & & & & & & & RIVER & & CONTINUOUS & FILTER & 252 \\
\hline 6157 & $\mathrm{pCi} / \mathrm{L}$ & 0.00142 & 0.00142 & & & $U$ & & RIVER & & CONTINUOUS & FILTER & 463 \\
\hline 6158 & $\mathrm{pCi} / \mathrm{L}$ & 0.00312 & 0.00313 & & & U & & RIVER & & CONTINUOUS & RESIN & 463 \\
\hline 6159 & $\mathrm{pCi} / \mathrm{L}$ & 1.02 & 1.02 & & & U & & SANITARY & & RAW GRAB & UNFILTERED & 525 \\
\hline 6160 & $\mathrm{pCi} / \mathrm{L}$ & 0.102 & 0.145 & & & & & SANITARY & & RAW GRAB & UNFILTERED & 525 \\
\hline 6161 & pCi/L & 0.0184 & 0.0187 & & & & & SANITARY & & RAW GRAB & UNFILTERED & 525 \\
\hline 6162 & pCi/L & 0.0968 & 0.134 & & & & & SANITARY & & RAW GRAB & UNFILTERED & 525 \\
\hline 6163 & $\mathrm{pCi} / \mathrm{L}$ & 156 & 390 & & & U & & SANITARY & & RAW GRAB & UNFILTERED & 525 \\
\hline 6164 & $\mathrm{pCi} / \mathrm{L}$ & 1.21 & 1.21 & & & U & & SANITARY & & RAW GRAB & UNFILTERED & 172 \\
\hline 6165 & $\mathrm{pCi} / \mathrm{L}$ & 0.251 & 0.716 & & & & & SANITARY & & RAW GRAB & UNFILTERED & 172 \\
\hline 6166 & $\mathrm{pCi} / \mathrm{L}$ & 0.0463 & 0.0517 & & & & & SANITARY & & RAW GRAB & UNFILTERED & 172 \\
\hline 6167 & $\mathrm{pCi} / \mathrm{L}$ & 0.231 & 0.615 & & & & & SANITARY & & RAW GRAB & UNFILTERED & 172 \\
\hline 6168 & pCi/L & 156 & 391 & & & U & & SANITARY & & RAW GRAB & UNFILTERED & 172 \\
\hline 6169 & pCi/L & 0.911 & 0.911 & & & U & & DRINKING & & SAN. GRAB & UNFILTERED & 530 \\
\hline 6170 & $\mathrm{pCi} / \mathrm{L}$ & 161 & 403 & & & & & DRINKING & & SAN. GRAB & UNFILTERED & 530 \\
\hline 6171 & $\mathrm{pCi} / \mathrm{L}$ & 0.0522 & 0.104 & & & & & DRINKING & & SAN. GRAB & UNFILTERED & 530 \\
\hline 6172 & $\mathrm{pCi} / \mathrm{L}$ & 0.89 & 0.89 & & & U & & DRINKING & & CUMULATIVE & UNFILTERED & 307 \\
\hline 6173 & $\mathrm{pCi} / \mathrm{L}$ & 5.56 & 19.1 & & & & & DRINKING & & CUMULATIVE & UNFILTERED & 307 \\
\hline 6174 & pCi/L & 0.0364 & 0.0509 & & & & & DRINKING & & CUMULATIVE & UNFILTERED & 307 \\
\hline 6175 & pCi/L & 0.0579 & 0.0651 & & & & & DRINKING & & CUMULATIVE & UNFILTERED & 307 \\
\hline 6176 & pCi/L & 0.0131 & 0.0134 & & & U & & DRINKING & & CUMULATIVE & UNFILTERED & 307 \\
\hline 6177 & $\mathrm{pCi} / \mathrm{L}$ & 0.0486 & 0.0526 & & & & & DRINKING & & CUMULATIVE & UNFILTERED & 307 \\
\hline 6178 & $\mathrm{pCi} / \mathrm{L}$ & 0.76 & 0.76 & & & U & & RIVER & & CUMULATIVE & UNFILTERED & 252 \\
\hline 6179 & $\mathrm{pCi} / \mathrm{L}$ & 4.02 & 11 & & & & & RIVER & & CUMULATIVE & UNFILTERED & 252 \\
\hline 6180 & $\mathrm{pCi} / \mathrm{L}$ & 0.0329 & 0.041 & & & & & RIVER & & CUMULATIVE & UNFILTERED & 252 \\
\hline 6181 & $\mathrm{pCi} / \mathrm{L}$ & 0.0426 & 0.0476 & & & & & RIVER & & CUMULATIVE & UNFILTERED & 252 \\
\hline 6182 & $\mathrm{pCi} / \mathrm{L}$ & 0.0124 & 0.0127 & & & U & & RIVER & & CUMULATIVE & UNFILTERED & 252 \\
\hline 6183 & $\mathrm{pCi} / \mathrm{L}$ & 0.0368 & 0.04 & & & & & RIVER & & CUMULATIVE & UNFILTERED & 252 \\
\hline 6184 & pCi/L & 1.3 & 1.3 & & & U & & RIVER & & CUMULATIVE & UNFILTERED & 463 \\
\hline 6185 & $\mathrm{pCi} / \mathrm{L}$ & 0.0337 & 0.045 & & & & & RIVER & & CUMULATIVE & UNFILTERED & 463 \\
\hline 6186 & $\mathrm{pCi} / \mathrm{L}$ & 5.18 & 16.5 & & & & & RIVER & & CUMULATIVE & UNFILTERED & 463 \\
\hline 6187 & pCi/L & 0.0481 & 0.0544 & & & & & RIVER & & CUMULATIVE & UNFILTERED & 463 \\
\hline 6188 & pCi/L & 0.00809 & 0.00843 & & & U & & RIVER & & CUMULATIVE & UNFILTERED & 463 \\
\hline 6189 & $\mathrm{pCi} / \mathrm{L}$ & 0.0371 & 0.0401 & & & & & RIVER & & CUMULATIVE & UNFILTERED & 463 \\
\hline
\end{tabular}




\begin{tabular}{|c|c|c|c|c|c|c|c|c|c|c|c|c|}
\hline & $\mathrm{N}$ & $\mathrm{O}$ & $\mathrm{P}$ & $\mathrm{Q}$ & $\mathrm{R}$ & $\mathrm{s}$ & $T$ & $\mathrm{U}$ & $\mathrm{V}$ & $\mathrm{W}$ & $\mathrm{X}$ & $\bar{Y}$ \\
\hline 1 & ANAL_UNITS_RPTD & COUNTING_ERROR & $\mid$ TOTAL_ANAL_ERROR | & MIN_DETECTABLE_ACTIVITY & PCNT_MOISTURE & LAB_QUALIFIER & REVIEW_QUALIFIER & SAMP_FROM & SAMP_ITEM & SAMP_MTHD & COLL_MTHD & SAMP_SITE_ID \\
\hline 6190 & $\mathrm{pCi} / \mathrm{L}$ & 150 & 376 & & & $U$ & & SANITARY & & RAW GRAB & UNFILTERED & 524 \\
\hline 6191 & $\mathrm{pCi} / \mathrm{L}$ & 0.826 & 0.826 & & & U & & SANITARY & & RAW GRAB & UNFILTERED & 524 \\
\hline 6192 & $\mathrm{pCi} / \mathrm{L}$ & 0.175 & 0.41 & & & & & SANITARY & & RAW GRAB & UNFILTERED & 524 \\
\hline 6193 & $\mathrm{pCi} / \mathrm{L}$ & 0.0297 & 0.0314 & & & & & SANITARY & & RAW GRAB & UNFILTERED & 524 \\
\hline 6194 & $\mathrm{pCi} / \mathrm{L}$ & 0.159 & 0.346 & & & & & SANITARY & & RAW GRAB & UNFILTERED & 524 \\
\hline 6195 & $\mathrm{pCi} / \mathrm{L}$ & & 0.00000931 & & & & & RIVER & & CONTINUOUS & RESIN & 307 \\
\hline \begin{tabular}{|c|}
6196 \\
\end{tabular} & $\mathrm{pCi} / \mathrm{L}$ & 0.0000442 & 0.0000443 & & & $\mathrm{U}$ & & RIVER & & CONTINUOUS & RESIN & 307 \\
\hline \begin{tabular}{|c|c|}
6197 \\
\end{tabular} & $\mathrm{pCi} / \mathrm{L}$ & 0.00308 & 0.0031 & & & & & RIVER & & CONTINUOUS & RESIN & 307 \\
\hline 6198 & $\mathrm{pCi} / \mathrm{L}$ & 0.0000199 & 0.00002 & & & $\mathrm{U}$ & & RIVER & & CONTINUOUS & FILTER & 307 \\
\hline 6199 & $\mathrm{pCi} / \mathrm{L}$ & 0.00161 & 0.00161 & & & U & & RIVER & & CONTINUOUS & FILTER & 307 \\
\hline 6200 & $\mathrm{pCi} / \mathrm{L}$ & & 0.00000109 & & & & & RIVER & & CONTINUOUS & RESIN & 252 \\
\hline \begin{tabular}{|l|}
6201 \\
\end{tabular} & $\mathrm{pCi} / \mathrm{L}$ & 0.00016 & 0.000161 & & & $U$ & & RIVER & & CONTINUOUS & RESIN & 252 \\
\hline 6202 & & & & & & & & RIVER & & CONTINUOUS & RESIN & 252 \\
\hline 6203 & $\mathrm{pCi} / \mathrm{L}$ & 0.0000792 & 0.0000799 & & & & & RIVER & & CONTINUOUS & FILTER & 252 \\
\hline 6204 & & & & & & & & RIVER & & CONTINUOUS & FILTER & 252 \\
\hline 6205 & $\mathrm{pCi} / \mathrm{L}$ & & 0.0000161 & & & & & RIVER & & CONTINUOUS & RESIN & 463 \\
\hline 6206 & $\mathrm{pCi} / \mathrm{L}$ & 0.0000572 & 0.0000574 & & & $U$ & & RIVER & & CONTINUOUS & RESIN & 463 \\
\hline 6207 & $\mathrm{pCi} / \mathrm{L}$ & 0.0000656 & 0.0000663 & & & & & RIVER & & CONTINUOUS & FILTER & 463 \\
\hline \begin{tabular}{|c|}
6208 \\
\end{tabular} & $\mathrm{pCi} / \mathrm{L}$ & 0.00151 & 0.00152 & & & $U$ & & RIVER & & CONTINUOUS & FILTER & 463 \\
\hline 6209 & $\mathrm{pCi} / \mathrm{L}$ & 0.00368 & 0.00376 & & & & & RIVER & & CONTINUOUS & RESIN & 463 \\
\hline 6210 & $\mathrm{pCi} / \mathrm{L}$ & 0.0997 & 0.312 & & & & & SURFACE & & RAW GRAB & UNFILTERED & 509 \\
\hline \begin{tabular}{|l|}
6211 \\
\end{tabular} & $\mathrm{pCi} / \mathrm{L}$ & 131 & 294 & & & $U$ & & SURFACE & & RAW GRAB & UNFILTERED & 509 \\
\hline 6212 & $\mathrm{pCi} / \mathrm{L}$ & 1.03 & 1.04 & & & & & SURFACE & & RAW GRAB & UNFILTERED & 509 \\
\hline 6213 & & & & & & & & SURFACE & & RAW GRAB & UNFILTERED & 136 \\
\hline 6214 & & & & & & & & SURFACE & & RAW GRAB & UNFILTERED & 136 \\
\hline \begin{tabular}{|c|}
6215 \\
\end{tabular} & $\mathrm{pCi} / \mathrm{L}$ & 0.142 & 0.418 & & & & & SURFACE & & RAW GRAB & UNFILTERED & 147 \\
\hline 6216 & $\mathrm{pCi} / \mathrm{L}$ & 146 & 324 & & & & & SURFACE & & RAW GRAB & UNFILTERED & 147 \\
\hline \begin{tabular}{|l|l|}
6217 \\
\end{tabular} & $\mathrm{pCi} / \mathrm{L}$ & 4.23 & 16.8 & & & & & SURFACE & & RAW GRAB & UNFILTERED & 147 \\
\hline 6218 & $\mathrm{pCi} / \mathrm{L}$ & 0.829 & 1.04 & & & & & SURFACE & & RAW GRAB & UNFILTERED & 147 \\
\hline \begin{tabular}{|l|}
6219 \\
\end{tabular} & $\mathrm{pCi} / \mathrm{L}$ & 4.36 & 17.8 & & & & & SURFACE & & RAW GRAB & UNFILTERED & 147 \\
\hline 6220 & $\mathrm{pCi} / \mathrm{L}$ & 1.56 & 1.56 & & & $U$ & & SURFACE & & RAW GRAB & UNFILTERED & 147 \\
\hline \begin{tabular}{|l|}
6221 \\
\end{tabular} & $\mathrm{pCi} / \mathrm{L}$ & 6.08 & 22.7 & & & & & QC_SAMPLE & & RAW GRAB & UNFILTERED & 550 \\
\hline \begin{tabular}{|c|}
6222 \\
\end{tabular} & $\mathrm{pCi} / \mathrm{L}$ & 0.0395 & 0.0567 & & & & & QC_SAMPLE & & RAW GRAB & UNFILTERED & 550 \\
\hline 6223 & $\mathrm{pCi} / \mathrm{L}$ & 0.0459 & 0.0497 & & & & & QC_SAMPLE & & RAW GRAB & UNFILTERED & 550 \\
\hline 6224 & $\mathrm{pCi} / \mathrm{L}$ & 0.0151 & 0.0153 & & & U & & QC_SAMPLE & & RAW GRAB & UNFILTERED & 550 \\
\hline \begin{tabular}{|l|}
6225 \\
\end{tabular} & $\mathrm{pCi} / \mathrm{L}$ & 0.0575 & 0.0649 & & & & & QC_SAMPLE & & RAW GRAB & UNFILTERED & 550 \\
\hline \begin{tabular}{|l|}
6226 \\
\end{tabular} & $\mathrm{pCi} / \mathrm{L}$ & 1.07 & 1.07 & & & $U$ & & QC_SAMPLE & & RAW GRAB & UNFILTERED & 550 \\
\hline \begin{tabular}{|l|}
6227 \\
\end{tabular} & $\mathrm{pCi} / \mathrm{L}$ & 0.922 & 0.922 & & & U & & QC_SAMPLE & & RAW GRAB & UNFILTERED & 247 \\
\hline 6228 & $\mathrm{pCi} / \mathrm{L}$ & 3.87 & 10.5 & & & & & QC_SAMPLE & & RAW GRAB & UNFILTERED & 247 \\
\hline 6229 & $\mathrm{pCi} / \mathrm{L}$ & 0.0364 & 0.0519 & & & & & QC_SAMPLE & & RAW GRAB & UNFILTERED & 247 \\
\hline 6230 & $\mathrm{pCi} / \mathrm{L}$ & 0.0364 & 0.0388 & & & & & QC_SAMPLE & & RAW GRAB & UNFILTERED & 247 \\
\hline \begin{tabular}{|l|}
6231 \\
\end{tabular} & $\mathrm{pCi} / \mathrm{L}$ & 0.00759 & 0.00839 & & & $U$ & & QC_SAMPLE & & RAW GRAB & UNFILTERED & 247 \\
\hline 6232 & $\mathrm{pCi} / \mathrm{L}$ & 0.0419 & 0.0458 & & & & & QC_SAMPLE & & RAW GRAB & UNFILTERED & 247 \\
\hline 6233 & & & & & & & & RIVER & & CONTINUOUS & RESIN & 307 \\
\hline 6234 & & & & & & & & RIVER & & CONTINUOUS & FILTER & 307 \\
\hline 6235 & $\mathrm{pCi} / \mathrm{L}$ & 0.0031 & 0.00315 & & & & & RIVER & & CONTINUOUS & RESIN & 252 \\
\hline \begin{tabular}{|l|}
6236 \\
\end{tabular} & $\mathrm{pCi} / \mathrm{L}$ & 0.00128 & 0.00129 & & & $U$ & & RIVER & & CONTINUOUS & FILTER & 252 \\
\hline \begin{tabular}{|l|l|}
6237 \\
\end{tabular} & $\mathrm{pCi} / \mathrm{L}$ & 0.00293 & 0.00306 & & & & & RIVER & & CONTINUOUS & RESIN & 463 \\
\hline 6238 & $\mathrm{pCi} / \mathrm{L}$ & 0.00146 & 0.00147 & & & & & RIVER & & CONTINUOUS & FILTER & 463 \\
\hline 6239 & $\mathrm{pCi} / \mathrm{L}$ & 0.00151 & 0.00153 & & & & & RIVER & & CONTINUOUS & FILTER & 307 \\
\hline 6240 & $\mathrm{pCi} / \mathrm{L}$ & 0.00255 & 0.00257 & & & & & RIVER & & CONTINUOUS & RESIN & 307 \\
\hline 6241 & & & & & & & & RIVER & & CONTINUOUS & RESIN & 252 \\
\hline
\end{tabular}




\begin{tabular}{|c|c|c|c|c|c|c|c|c|c|c|c|c|}
\hline & $\mathrm{N}$ & $\mathrm{O}$ & $\mathrm{P}$ & $\mathrm{Q}$ & $\mathrm{R}$ & $\mathrm{s}$ & $\mathrm{T}$ & $\mathrm{U}$ & $\mathrm{V}$ & $\mathrm{W}$ & $\mathrm{x}$ & $\mathrm{Y}$ \\
\hline 1 & ANAL_UNITS_RPTD & COUNTING_ERROR & TOTAL_ANAL_ERROR & MIN_DETECTABLE_ACTIVITY & PCNT_MOISTURE & LAB_QUALIFIER & REVIEW_QUALIFIER & SAMP_FROM & SAMP_ITEM & SAMP_MTHD & COLL_MTHD & SAMP_SITE_ID \\
\hline 6242 & & & & & & & & RIVER & & CONTINUOUS & FILTER & 252 \\
\hline 6243 & $\mathrm{pCi} / \mathrm{L}$ & 0.0302 & 0.0397 & & & & & RIVER & & CUMULATIVE & UNFILTERED & 252 \\
\hline 6244 & $\mathrm{pCi} / \mathrm{L}$ & 0.0432 & 0.0476 & & & & & RIVER & & CUMULATIVE & UNFILTERED & 252 \\
\hline 6245 & $\mathrm{pCi} / \mathrm{L}$ & 0.0123 & 0.0126 & & & $\mathrm{U}$ & & RIVER & & CUMULATIVE & UNFILTERED & 252 \\
\hline 6246 & $\mathrm{pCi} / \mathrm{L}$ & 0.0429 & 0.0478 & & & & & RIVER & & CUMULATIVE & UNFILTERED & 252 \\
\hline 6247 & $\mathrm{pCi} / \mathrm{L}$ & 4.08 & 10.9 & & & & & RIVER & & CUMULATIVE & UNFILTERED & 252 \\
\hline 6248 & $\mathrm{pCi} / \mathrm{L}$ & 0.938 & 0.939 & & & $\mathrm{U}$ & & RIVER & & CUMULATIVE & UNFILTERED & 252 \\
\hline 6249 & $\mathrm{pCi} / \mathrm{L}$ & 0.0343 & 0.0507 & & & & & RIVER & & CUMULATIVE & UNFILTERED & 463 \\
\hline 6250 & $\mathrm{pCi} / \mathrm{L}$ & 0.0411 & 0.0443 & & & & & RIVER & & CUMULATIVE & UNFILTERED & 463 \\
\hline 6251 & $\mathrm{pCi} / \mathrm{L}$ & 0 & 0.00773 & & & $\mathrm{U}$ & & RIVER & & CUMULATIVE & UNFILTERED & 463 \\
\hline 6252 & $\mathrm{pCi} / \mathrm{L}$ & 0.0465 & 0.0518 & & & & & RIVER & & CUMULATIVE & UNFILTERED & 463 \\
\hline 6253 & $\mathrm{pCi} / \mathrm{L}$ & 5.09 & 16 & & & & & RIVER & & CUMULATIVE & UNFILTERED & 463 \\
\hline 6254 & $\mathrm{pCi} / \mathrm{L}$ & 0.889 & 0.889 & & & U & & RIVER & & CUMULATIVE & UNFILTERED & 463 \\
\hline 6255 & $\mathrm{pCi} / \mathrm{L}$ & 0.00176 & 0.00176 & & & $U$ & & RIVER & & CONTINUOUS & RESIN & 463 \\
\hline \begin{tabular}{|l|}
6256 \\
\end{tabular} & $\mathrm{pCi} / \mathrm{L}$ & 0.000932 & 0.000951 & & & & & RIVER & & CONTINUOUS & FILTER & 463 \\
\hline 6257 & $\mathrm{pCi} / \mathrm{L}$ & 0.58 & 0.58 & & & $U$ & & DRINKING & & SAN. GRAB & UNFILTERED & 186 \\
\hline 6258 & $\mathrm{pCi} / \mathrm{L}$ & 0.0203 & 0.0215 & & & $U$ & & DRINKING & & SAN. GRAB & UNFILTERED & 186 \\
\hline 6259 & $\mathrm{pCi} / \mathrm{L}$ & 131 & 293 & & & $U$ & & DRINKING & & SAN. GRAB & UNFILTERED & 186 \\
\hline 6260 & $p \mathrm{pi} / \mathrm{L}$ & 0.694 & 0.695 & & & $U$ & & DRINKING & & SAN. GRAB & UNFILTERED & 512 \\
\hline 6261 & $\mathrm{pCi} / \mathrm{L}$ & 0.0228 & 0.0234 & & & $U$ & & DRINKING & & SAN. GRAB & UNFILTERED & 512 \\
\hline 6262 & $\mathrm{pCi} / \mathrm{L}$ & 271 & 1030 & & & & & DRINKING & & SAN. GRAB & UNFILTERED & 512 \\
\hline 6263 & $\mathrm{pCi} / \mathrm{L}$ & 0.669 & 0.672 & & & U & & DRINKING & & SAN. GRAB & UNFILTERED & 144 \\
\hline 6264 & $\mathrm{pCi} / \mathrm{L}$ & 0.0209 & 0.0216 & & & $U$ & & DRINKING & & SAN. GRAB & UNFILTERED & 144 \\
\hline 6265 & $\mathrm{pCi} / \mathrm{L}$ & 134 & 299 & & & $U$ & & DRINKING & & SAN. GRAB & UNFILTERED & 144 \\
\hline 6266 & $\mathrm{pCi} / \mathrm{L}$ & 0.829 & 0.832 & & & $U$ & & DRINKING & & SAN. GRAB & UNFILTERED & 187 \\
\hline 6267 & $\mathrm{pCi} / \mathrm{L}$ & 130 & 291 & & & $U$ & & DRINKING & & SAN. GRAB & UNFILTERED & 187 \\
\hline 6268 & $\mathrm{pCi} / \mathrm{L}$ & 0.0222 & 0.0226 & & & U & & DRINKING & & SAN. GRAB & UNFILTERED & 187 \\
\hline 6269 & $\mathrm{pCi} / \mathrm{L}$ & 0.514 & 0.517 & & & & & DRINKING & & RAW GRAB & UNFILTERED & 553 \\
\hline 6270 & $\mathrm{pCi} / \mathrm{L}$ & 0.0347 & 0.0477 & & & & & DRINKING & & RAW GRAB & UNFILTERED & 553 \\
\hline \begin{tabular}{|l|}
6271 \\
\end{tabular} & $\mathrm{pCi} / \mathrm{L}$ & 131 & 286 & & & $U$ & & DRINKING & & RAW GRAB & UNFILTERED & 553 \\
\hline 6272 & $\mathrm{pCi} / \mathrm{L}$ & 0.0027 & 0.00272 & & & & & RIVER & & CONTINUOUS & RESIN & 307 \\
\hline 6273 & $p \mathrm{pi} / \mathrm{L}$ & 0.00135 & 0.00136 & & & & & RIVER & & CONTINUOUS & FILTER & 307 \\
\hline 6274 & $\mathrm{pCi} / \mathrm{L}$ & 0.00341 & 0.00342 & & & U & & RIVER & & CONTINUOUS & RESIN & 252 \\
\hline 6275 & $\mathrm{pCi} / \mathrm{L}$ & 0.00154 & 0.00156 & & & & & RIVER & & CONTINUOUS & FILTER & 252 \\
\hline 6276 & $\mathrm{pCi} / \mathrm{L}$ & 0.00329 & 0.0033 & & & $U$ & & RIVER & & CONTINUOUS & RESIN & 463 \\
\hline \begin{tabular}{|l|l|}
6277 \\
\end{tabular} & $\mathrm{pCi} / \mathrm{L}$ & 0.00169 & 0.00169 & & & U & & RIVER & & CONTINUOUS & FILTER & 463 \\
\hline 6278 & pCi/L & 0.00258 & 0.00259 & & & $U$ & & RIVER & & CONTINUOUS & RESIN & 307 \\
\hline 6279 & $\mathrm{pCi} / \mathrm{L}$ & 0.00134 & 0.00134 & & & $U$ & & RIVER & & CONTINUOUS & FILTER & 307 \\
\hline 6280 & $\mathrm{pCi} / \mathrm{L}$ & 0.00362 & 0.00363 & & & $U$ & & RIVER & & CONTINUOUS & RESIN & 252 \\
\hline 6281 & $\mathrm{pCi} / \mathrm{L}$ & 0.612 & 0.612 & & & $U$ & & RIVER & & CUMULATIVE & UNFILTERED & 252 \\
\hline 6282 & $\mathrm{pCi} / \mathrm{L}$ & 0.0359 & 0.0393 & & & & & RIVER & & CUMULATIVE & UNFILTERED & 252 \\
\hline 6283 & $\mathrm{pCi} / \mathrm{L}$ & 0.00568 & 0.00615 & & & U & & RIVER & & CUMULATIVE & UNFILTERED & 252 \\
\hline 6284 & $\mathrm{pCi} / \mathrm{L}$ & 3.97 & 10.7 & & & & & RIVER & & CUMULATIVE & UNFILTERED & 252 \\
\hline 6285 & $\mathrm{pCi} / \mathrm{L}$ & 0.0438 & 0.0499 & & & & & RIVER & & CUMULATIVE & UNFILTERED & 252 \\
\hline 6286 & $\mathrm{pCi} / \mathrm{L}$ & 0.0411 & 0.0517 & & & & & RIVER & & CUMULATIVE & UNFILTERED & 252 \\
\hline 6287 & $\mathrm{pCi} / \mathrm{L}$ & 0.00171 & 0.00172 & & & & & RIVER & & CONTINUOUS & FILTER & 252 \\
\hline 6288 & $\mathrm{pCi} / \mathrm{L}$ & 0.0018 & 0.00181 & & & U & & RIVER & & CONTINUOUS & FILTER & 463 \\
\hline 6289 & pCi/L & 0.573 & 0.573 & & & U & & RIVER & & CUMULATIVE & UNFILTERED & 463 \\
\hline 6290 & $\mathrm{pCi} / \mathrm{L}$ & 4.8 & 14.7 & & & & & RIVER & & CUMULATIVE & UNFILTERED & 463 \\
\hline 6291 & $\mathrm{pCi} / \mathrm{L}$ & 0.0391 & 0.0433 & & & & & RIVER & & CUMULATIVE & UNFILTERED & 463 \\
\hline 6292 & $\mathrm{pCi} / \mathrm{L}$ & 0.01 & 0.0104 & & & U & & RIVER & & CUMULATIVE & UNFILTERED & 463 \\
\hline 6293 & $\mathrm{pCi} / \mathrm{L}$ & 0.0471 & 0.0544 & & & & & RIVER & & CUMULATIVE & UNFILTERED & 463 \\
\hline
\end{tabular}




\begin{tabular}{|c|c|c|c|c|c|c|c|c|c|c|c|c|}
\hline & $\mathrm{N}$ & $\mathrm{O}$ & $\mathrm{P}$ & $\mathrm{Q}$ & $\mathrm{R}$ & $\mathrm{s}$ & $\mathrm{T}$ & $\mathrm{U}$ & $\mathrm{V}$ & $\mathrm{W}$ & $\mathrm{X}$ & $\mathrm{Y}$ \\
\hline 1 & ANAL_UNITS_RPTD & COUNTING_ERROR & TOTAL_ANAL_ERROR & MIN_DETECTABLE_ACTIVITY & PCNT_MOISTURE & LAB_QUALIFIER & REVIEW_QUALIFIER & SAMP_FROM & SAMP_ITEM & SAMP_MTHD & COLL_MTHD & SAMP_SITE_ID \\
\hline 6294 & $\mathrm{pCi} / \mathrm{L}$ & 0.0431 & 0.0531 & & & & & RIVER & & CUMULATIVE & UNFILTERED & 463 \\
\hline 6295 & $\mathrm{pCi} / \mathrm{L}$ & 0.00289 & 0.00291 & & & & & RIVER & & CONTINUOUS & RESIN & 463 \\
\hline 6296 & $\mathrm{pCi} / \mathrm{L}$ & 0.0605 & 0.0996 & & & & & DRINKING & & SAN. GRAB & UNFILTERED & 530 \\
\hline 6297 & $\mathrm{pCi} / \mathrm{L}$ & 133 & 281 & & & & & DRINKING & & SAN. GRAB & UNFILTERED & 530 \\
\hline 6298 & $\mathrm{pCi} / \mathrm{L}$ & 0.843 & 0.843 & & & $\mathrm{U}$ & & DRINKING & & SAN. GRAB & UNFILTERED & 530 \\
\hline 6299 & $\mathrm{pCi} / \mathrm{L}$ & 0.561 & 0.562 & & & U & & DRINKING & & SAN. GRAB & UNFILTERED & 487 \\
\hline 6300 & $\mathrm{pCi} / \mathrm{L}$ & 0.0183 & 0.0189 & & & $\mathrm{U}$ & & DRINKING & & SAN. GRAB & UNFILTERED & 487 \\
\hline 6301 & $\mathrm{pCi} / \mathrm{L}$ & 126 & 274 & & & U & & DRINKING & & SAN. GRAB & UNFILTERED & 487 \\
\hline 6302 & $\mathrm{pCi} / \mathrm{L}$ & 0.00293 & 0.00299 & & & & & RIVER & & CONTINUOUS & RESIN & 307 \\
\hline 6303 & $\mathrm{pCi} / \mathrm{L}$ & 0.0014 & 0.00143 & & & & & RIVER & & CONTINUOUS & FILTER & 307 \\
\hline 6304 & $\mathrm{pCi} / \mathrm{L}$ & 0.00138 & 0.00139 & & & & & RIVER & & CONTINUOUS & FILTER & 252 \\
\hline 6305 & $\mathrm{pCi} / \mathrm{L}$ & 0.00348 & 0.00351 & & & & & RIVER & & CONTINUOUS & RESIN & 252 \\
\hline 6306 & $\mathrm{pCi} / \mathrm{L}$ & 0.00204 & 0.00207 & & & & & RIVER & & CONTINUOUS & FILTER & 463 \\
\hline 6307 & $\mathrm{pCi} / \mathrm{L}$ & 0.0054 & 0.0054 & & & U & & RIVER & & CONTINUOUS & RESIN & 463 \\
\hline 6308 & $\mathrm{pCi} / \mathrm{L}$ & 0.904 & 0.909 & & & U & & SANITARY & & RAW GRAB & UNFILTERED & 525 \\
\hline 6309 & $\mathrm{pCi} / \mathrm{L}$ & 0.825 & 1.63 & & & & & SANITARY & & RAW GRAB & UNFILTERED & 525 \\
\hline 6310 & $\mathrm{pCi} / \mathrm{L}$ & 0.161 & 0.17 & & & & & SANITARY & & RAW GRAB & UNFILTERED & 525 \\
\hline 6311 & $\mathrm{pCi} / \mathrm{L}$ & 1.05 & 2.49 & & & & & SANITARY & & RAW GRAB & UNFILTERED & 525 \\
\hline 6312 & $\mathrm{pCi} / \mathrm{L}$ & 129 & 277 & & & U & & SANITARY & & RAW GRAB & UNFILTERED & 525 \\
\hline 6313 & $\mathrm{pCi} / \mathrm{L}$ & 0.914 & 0.914 & & & U & & SANITARY & & RAW GRAB & UNFILTERED & 172 \\
\hline 6314 & $\mathrm{pCi} / \mathrm{L}$ & 0.256 & 0.706 & & & & & SANITARY & & RAW GRAB & UNFILTERED & 172 \\
\hline 6315 & $\mathrm{pCi} / \mathrm{L}$ & 0.0472 & 0.0523 & & & & & SANITARY & & RAW GRAB & UNFILTERED & 172 \\
\hline 6316 & $\mathrm{pCi} / \mathrm{L}$ & 0.268 & 0.766 & & & & & SANITARY & & RAW GRAB & UNFILTERED & 172 \\
\hline 6317 & $\mathrm{pCi} / \mathrm{L}$ & 131 & 282 & & & U & & SANITARY & & RAW GRAB & UNFILTERED & 172 \\
\hline 6318 & $\mathrm{pCi} / \mathrm{L}$ & 0.0387 & 0.0539 & & & & & DRINKING & & CUMULATIVE & UNFILTERED & 307 \\
\hline 6319 & $\mathrm{pCi} / \mathrm{L}$ & 0.52 & 0.52 & & & U & & DRINKING & & CUMULATIVE & UNFILTERED & 307 \\
\hline 6320 & $\mathrm{pCi} / \mathrm{L}$ & 0.0448 & 0.0494 & & & & & DRINKING & & CUMULATIVE & UNFILTERED & 307 \\
\hline 6321 & $\mathrm{pCi} / \mathrm{L}$ & 0.0109 & 0.0112 & & & U & & DRINKING & & CUMULATIVE & UNFILTERED & 307 \\
\hline 6322 & $\mathrm{pCi} / \mathrm{L}$ & 0.0532 & 0.0609 & & & & & DRINKING & & CUMULATIVE & UNFILTERED & 307 \\
\hline 6323 & $\mathrm{pCi} / \mathrm{L}$ & 5.34 & 17.6 & & & & & DRINKING & & CUMULATIVE & UNFILTERED & 307 \\
\hline 6324 & $\mathrm{pCi} / \mathrm{L}$ & 0.679 & 0.682 & & & U & & SANITARY & & RAW GRAB & UNFILTERED & 524 \\
\hline 6325 & $\mathrm{pCi} / \mathrm{L}$ & 0.201 & 0.398 & & & & & SANITARY & & RAW GRAB & UNFILTERED & 524 \\
\hline 6326 & $\mathrm{pCi} / \mathrm{L}$ & 0.0376 & 0.0395 & & & & & SANITARY & & RAW GRAB & UNFILTERED & 524 \\
\hline 6327 & $\mathrm{pCi} / \mathrm{L}$ & 0.211 & 0.433 & & & & & SANITARY & & RAW GRAB & UNFILTERED & 524 \\
\hline 6328 & $\mathrm{pCi} / \mathrm{L}$ & 129 & 279 & & & U & & SANITARY & & RAW GRAB & UNFILTERED & 524 \\
\hline 6329 & $\mathrm{pCi} / \mathrm{L}$ & 0.604 & 0.604 & & & U & & SANITARY & & RAW GRAB & UNFILTERED & 519 \\
\hline 6330 & $\mathrm{pCi} / \mathrm{L}$ & 0.0114 & 0.0128 & & & U & & SANITARY & & RAW GRAB & UNFILTERED & 519 \\
\hline 6331 & $\mathrm{pCi} / \mathrm{L}$ & 0 & 0.00884 & & & U & & SANITARY & & RAW GRAB & UNFILTERED & 519 \\
\hline 6332 & $\mathrm{pCi} / \mathrm{L}$ & 0.018 & 0.0204 & & & U & & SANITARY & & RAW GRAB & UNFILTERED & 519 \\
\hline 6333 & $\mathrm{pCi} / \mathrm{L}$ & 124 & 272 & & & U & & SANITARY & & RAW GRAB & UNFILTERED & 519 \\
\hline 6334 & $\mathrm{pCi} / \mathrm{L}$ & 0.000693 & 0.000698 & & & & & RIVER & & CONTINUOUS & FILTER & 307 \\
\hline 6335 & $\mathrm{pCi} / \mathrm{L}$ & & 0.00000607 & & & & & RIVER & & CONTINUOUS & RESIN & 307 \\
\hline 6336 & $\mathrm{pCi} / \mathrm{L}$ & 0.0000088 & 0.00000892 & & & & & RIVER & & CONTINUOUS & FILTER & 307 \\
\hline 6337 & $\mathrm{pCi} / \mathrm{L}$ & 0.0000348 & 0.000035 & & & U & & RIVER & & CONTINUOUS & RESIN & 307 \\
\hline 6338 & $\mathrm{pCi} / \mathrm{L}$ & 0.00156 & 0.00156 & & & U & & RIVER & & CONTINUOUS & RESIN & 307 \\
\hline 6339 & $\mathrm{pCi} / \mathrm{L}$ & & 0.000000818 & & & & & RIVER & & CONTINUOUS & RESIN & 252 \\
\hline 6340 & $\mathrm{pCi} / \mathrm{L}$ & 0.000132 & 0.000132 & & & U & & RIVER & & CONTINUOUS & RESIN & 252 \\
\hline 6341 & $\mathrm{pCi} / \mathrm{L}$ & 0.00385 & 0.00387 & & & & & RIVER & & CONTINUOUS & RESIN & 252 \\
\hline 6342 & $\mathrm{pCi} / \mathrm{L}$ & 0.00131 & 0.00133 & & & & & RIVER & & CONTINUOUS & FILTER & 252 \\
\hline 6343 & $\mathrm{pCi} / \mathrm{L}$ & 0.00000746 & 0.00000753 & & & U & & RIVER & & CONTINUOUS & FILTER & 252 \\
\hline 6344 & $\mathrm{pCi} / \mathrm{L}$ & & 0.00000727 & & & & & RIVER & & CONTINUOUS & RESIN & 463 \\
\hline 6345 & $\mathrm{pCi} / \mathrm{L}$ & 0.0000113 & 0.0000115 & & & & & RIVER & & CONTINUOUS & FILTER & 463 \\
\hline
\end{tabular}




\begin{tabular}{|c|c|c|c|c|c|c|c|c|c|c|c|c|}
\hline & $\mathrm{N}$ & $\mathrm{O}$ & $\mathrm{P}$ & $\mathrm{Q}$ & $\mathrm{R}$ & $\mathrm{s}$ & $\mathrm{T}$ & $\mathrm{u}$ & $\mathrm{V}$ & w & $\mathrm{x}$ & $\bar{Y}$ \\
\hline 1 & ANAL_UNITS_RPTD & COUNTING_ERROR & TOTAL_ANAL_ERROR & MIN_DETECTABLE_ACTIVITY & PCNT_MOISTURE & LAB_QUALIFIER & $\begin{array}{l}\text { REVIEW_QUALIFIER } \\
\end{array}$ & SAMP_FROM & SAMP_ITEM & SAMP_MTHD & COLL_MTHD & SAMP_SITE_ID \\
\hline 6346 & $\mathrm{pCi} / \mathrm{L}$ & 0.0000409 & 0.0000411 & & & $U$ & & RIVER & & CONTINUOUS & RESIN & 463 \\
\hline 6347 & $\mathrm{pCi} / \mathrm{L}$ & 0.00165 & 0.00165 & & & $\mathrm{U}$ & & RIVER & & CONTINUOUS & FILTER & 463 \\
\hline 6348 & $\mathrm{pCi} / \mathrm{L}$ & 0.00271 & 0.00272 & & & & & RIVER & & CONTINUOUS & RESIN & 463 \\
\hline 6349 & $\mathrm{pCi} / \mathrm{L}$ & & 0.000931 & & & & & DRINKING & & SAN. GRAB & UNFILTERED & 512 \\
\hline 6350 & $\mathrm{pCi} / \mathrm{L}$ & 4.57 & 12.3 & & & & & RIVER & & CUMULATIVE & UNFILTERED & 252 \\
\hline 6351 & $\mathrm{pCi} / \mathrm{L}$ & 0.0284 & 0.0339 & & & & & RIVER & & CUMULATIVE & UNFILTERED & 252 \\
\hline 6352 & $\mathrm{pCi} / \mathrm{L}$ & 0.563 & 0.563 & & & $\mathrm{U}$ & & RIVER & & CUMULATIVE & UNFILTERED & 252 \\
\hline 6353 & $\mathrm{pCi} / \mathrm{L}$ & 0.048 & 0.0527 & & & & & RIVER & & CUMULATIVE & UNFILTERED & 252 \\
\hline 6354 & $\mathrm{pCi} / \mathrm{L}$ & 0.0119 & 0.0122 & & & $\mathrm{U}$ & & RIVER & & CUMULATIVE & UNFILTERED & 252 \\
\hline 6355 & $\mathrm{pCi} / \mathrm{L}$ & 0.0556 & 0.0631 & & & & & RIVER & & CUMULATIVE & UNFILTERED & 252 \\
\hline \begin{tabular}{|l|}
6356 \\
\end{tabular} & $\mathrm{pCi} / \mathrm{L}$ & 4.66 & 13.7 & & & & & RIVER & & CUMULATIVE & UNFILTERED & 463 \\
\hline \begin{tabular}{|l|l|}
6357 \\
\end{tabular} & $\mathrm{pCi} / \mathrm{L}$ & 0.029 & 0.0386 & & & & & RIVER & & CUMULATIVE & UNFILTERED & 463 \\
\hline 6358 & $\mathrm{pCi} / \mathrm{L}$ & 0.581 & 0.582 & & & $U$ & & RIVER & & CUMULATIVE & UNFILTERED & 463 \\
\hline \begin{tabular}{|l|}
6359 \\
\end{tabular} & $\mathrm{pCi} / \mathrm{L}$ & 0.0461 & 0.0498 & & & & & RIVER & & CUMULATIVE & UNFILTERED & 463 \\
\hline 6360 & $\mathrm{pCi} / \mathrm{L}$ & 0.0102 & 0.0105 & & & U & & RIVER & & CUMULATIVE & UNFILTERED & 463 \\
\hline \begin{tabular}{|l|}
6361 \\
\end{tabular} & $\mathrm{pCi} / \mathrm{L}$ & 0.0554 & 0.0618 & & & & & RIVER & & CUMULATIVE & UNFILTERED & 463 \\
\hline 6362 & $\mathrm{pCi} / \mathrm{L}$ & 0.163 & 0.427 & & & & & SURFACE & & RAW GRAB & UNFILTERED & 509 \\
\hline \begin{tabular}{|l|}
6363 \\
\end{tabular} & $\mathrm{pCi} / \mathrm{L}$ & 1.54 & 1.55 & & & U & & SURFACE & & RAW GRAB & UNFILTERED & 509 \\
\hline 6364 & $\mathrm{pCi} / \mathrm{L}$ & 152 & 299 & & & $U$ & & SURFACE & & RAW GRAB & UNFILTERED & 509 \\
\hline 6365 & $\mathrm{pCi} / \mathrm{L}$ & 313 & 756 & & & & & SURFACE & & RAW GRAB & UNFILTERED & 136 \\
\hline 6366 & $\mathrm{pCi} / \mathrm{L}$ & 1.81 & 1.82 & & & $U$ & & SURFACE & & RAW GRAB & UNFILTERED & 136 \\
\hline \begin{tabular}{|l|}
6367 \\
\end{tabular} & $\mathrm{pCi} / \mathrm{L}$ & 0.0545 & 0.0649 & & & & & SURFACE & & RAW GRAB & UNFILTERED & 147 \\
\hline \begin{tabular}{|l|}
6368 \\
\end{tabular} & $\mathrm{pCi} / \mathrm{L}$ & 2.78 & 2.78 & & & U & & SURFACE & & RAW GRAB & UNFILTERED & 147 \\
\hline \begin{tabular}{|l|}
6369 \\
\end{tabular} & $\mathrm{pCi} / \mathrm{L}$ & 172 & 331 & & & & & SURFACE & & RAW GRAB & UNFILTERED & 147 \\
\hline 6370 & $\mathrm{pCi} / \mathrm{L}$ & 0.696 & 0.867 & & & & & SURFACE & & RAW GRAB & UNFILTERED & 147 \\
\hline 6371 & $\mathrm{pCi} / \mathrm{L}$ & 0.152 & 0.155 & & & & & SURFACE & & RAW GRAB & UNFILTERED & 147 \\
\hline \begin{tabular}{|l|}
6372 \\
\end{tabular} & $\mathrm{pCi} / \mathrm{L}$ & 0.717 & 0.914 & & & & & SURFACE & & RAW GRAB & UNFILTERED & 147 \\
\hline 6373 & $\mathrm{pCi} / \mathrm{L}$ & 0.00272 & 0.00274 & & & & & RIVER & & CONTINUOUS & RESIN & 307 \\
\hline \begin{tabular}{|l|}
6374 \\
\end{tabular} & $\mathrm{pCi} / \mathrm{L}$ & 0.00152 & 0.00159 & & & & & RIVER & & CONTINUOUS & FILTER & 307 \\
\hline 6375 & $\mathrm{pCi} / \mathrm{L}$ & 0.00302 & 0.00302 & & & U & & RIVER & & CONTINUOUS & RESIN & 252 \\
\hline \begin{tabular}{|l|}
6376 \\
\end{tabular} & $\mathrm{pCi} / \mathrm{L}$ & 0.0016 & 0.00161 & & & $U$ & & RIVER & & CONTINUOUS & FILTER & 252 \\
\hline \begin{tabular}{|l|l|}
6377 \\
\end{tabular} & $\mathrm{pCi} / \mathrm{L}$ & 0.00211 & 0.00211 & & & $U$ & & RIVER & & CONTINUOUS & RESIN & 463 \\
\hline \begin{tabular}{|l|}
6378 \\
\end{tabular} & $\mathrm{pCi} / \mathrm{L}$ & 0.00096 & 0.000968 & & & & & RIVER & & CONTINUOUS & FILTER & 463 \\
\hline 6379 & & & & & & & & RIVER & & CONTINUOUS & RESIN & 307 \\
\hline 6380 & & & & & & & & RIVER & & CONTINUOUS & FILTER & 307 \\
\hline \begin{tabular}{|l|}
6381 \\
\end{tabular} & $\mathrm{pCi} / \mathrm{L}$ & 0.00285 & 0.00289 & & & & & RIVER & & CONTINUOUS & RESIN & 252 \\
\hline \begin{tabular}{|l|}
6382 \\
\end{tabular} & $\mathrm{pCi} / \mathrm{L}$ & 0.00127 & 0.00127 & & & U & & RIVER & & CONTINUOUS & FILTER & 252 \\
\hline 6383 & $\mathrm{pCi} / \mathrm{L}$ & 0.00236 & 0.00236 & & & U & & RIVER & & CONTINUOUS & RESIN & 463 \\
\hline 6384 & $\mathrm{pCi} / \mathrm{L}$ & 0.00134 & 0.00135 & & & $U$ & & RIVER & & CONTINUOUS & FILTER & 463 \\
\hline 6385 & $\mathrm{pCi} / \mathrm{L}$ & 2.93 & 9.45 & & & & & SURFACE & & RAW GRAB & UNFILTERED & 147 \\
\hline \begin{tabular}{|l|}
6386 \\
\end{tabular} & $\mathrm{pCi} / \mathrm{L}$ & 0.573 & 0.669 & & & & & SURFACE & & RAW GRAB & UNFILTERED & 147 \\
\hline \begin{tabular}{|l|l|}
6387 \\
\end{tabular} & $\mathrm{pCi} / \mathrm{L}$ & 2.99 & 9.82 & & & & & SURFACE & & RAW GRAB & UNFILTERED & 147 \\
\hline \begin{tabular}{|l|}
6388 \\
\end{tabular} & $\mathrm{pCi} / \mathrm{L}$ & 0.426 & 0.625 & & & & & SURFACE & & RAW GRAB & UNFILTERED & 147 \\
\hline 6389 & $\mathrm{pCi} / \mathrm{L}$ & 0.605 & 0.605 & & & U & & DRINKING & & SAN. GRAB & UNFILTERED & 186 \\
\hline 6390 & $\mathrm{pCi} / \mathrm{L}$ & 0.019 & 0.0195 & & & $U$ & & DRINKING & & SAN. GRAB & UNFILTERED & 186 \\
\hline 6391 & $\mathrm{pCi} / \mathrm{L}$ & 130 & 276 & & & $U$ & & DRINKING & & SAN. GRAB & UNFILTERED & 186 \\
\hline 6392 & $\mathrm{pCi} / \mathrm{L}$ & 0.707 & 0.708 & & & $U$ & & DRINKING & & SAN. GRAB & UNFILTERED & 512 \\
\hline \begin{tabular}{|l|}
6393 \\
\end{tabular} & $\mathrm{pCi} / \mathrm{L}$ & 0.019 & 0.0197 & & & $U$ & & DRINKING & & SAN. GRAB & UNFILTERED & 512 \\
\hline 6394 & $\mathrm{pCi} / \mathrm{L}$ & 320 & 1080 & & & & & DRINKING & & SAN. GRAB & UNFILTERED & 512 \\
\hline 6395 & $\mathrm{pCi} / \mathrm{L}$ & 0.0185 & 0.019 & & & U & & DRINKING & & SAN. GRAB & UNFILTERED & 144 \\
\hline 6396 & $\mathrm{pCi} / \mathrm{L}$ & 0.498 & 0.498 & & & $U$ & & DRINKING & & SAN. GRAB & UNFILTERED & 144 \\
\hline \begin{tabular}{|l|}
6397 \\
\end{tabular} & $\mathrm{pCi} / \mathrm{L}$ & 130 & 276 & & & $U_{2}$ & & DRINKING & & SAN. GRAB & UNFILTERED & 144 \\
\hline
\end{tabular}




\begin{tabular}{|c|c|c|c|c|c|c|c|c|c|c|c|c|}
\hline & $\mathrm{N}$ & $\mathrm{O}$ & $\mathrm{P}$ & $\mathrm{Q}$ & $\mathrm{R}$ & $\mathrm{S}$ & $\mathrm{T}$ & $\mathrm{U}$ & $\mathrm{V}$ & $\mathrm{w}$ & $\mathrm{X}$ & $\mathrm{Y}$ \\
\hline 1 & ANAL_UNITS_RPTD & COUNTING_ERROR & \begin{tabular}{|l|} 
TOTAL_ANAL_ERROR \\
\end{tabular} & MIN_DETECTABLE_ACTIVITY & PCNT_MOISTURE & LAB_QUALIFIER & REVIEW_QUALIFIER & SAMP_FROM & SAMP_ITEM & SAMP_MTHD & COLL_MTHD & SAMP_SITE_ID \\
\hline 6398 & $\mathrm{pCi} / \mathrm{L}$ & 0.494 & 0.494 & & & $U$ & & DRINKING & & SAN. GRAB & UNFILTERED & 187 \\
\hline 6399 & $\mathrm{pCi} / \mathrm{L}$ & 0.0217 & 0.022 & & & $\mathrm{U}$ & & DRINKING & & SAN. GRAB & UNFILTERED & 187 \\
\hline 6400 & $\mathrm{pCi} / \mathrm{L}$ & 125 & 267 & & & U & & DRINKING & & SAN. GRAB & UNFILTERED & 187 \\
\hline 6401 & & & & & & $\mathrm{U}$ & & RIVER & & CONTINUOUS & FILTER & 307 \\
\hline 6402 & & & & & & $\mathrm{U}$ & & RIVER & & CONTINUOUS & FILTER & 307 \\
\hline 6403 & & & & & & U & & RIVER & & CONTINUOUS & RESIN & 307 \\
\hline 6404 & & & & & & $\mathrm{U}$ & & RIVER & & CONTINUOUS & RESIN & 307 \\
\hline 6405 & & & & & & U & & RIVER & & CONTINUOUS & FILTER & 252 \\
\hline 6406 & & & & & & U & & RIVER & & CONTINUOUS & FILTER & 252 \\
\hline 6407 & $\mathrm{pCi} / \mathrm{L}$ & 0.0303 & 0.0393 & & & & & RIVER & & CUMULATIVE & UNFILTERED & 252 \\
\hline 6408 & $\mathrm{pCi} / \mathrm{L}$ & 1.12 & 6.23 & & & $\mathrm{U}$ & & RIVER & & CUMULATIVE & UNFILTERED & 252 \\
\hline 6409 & $\mathrm{pCi} / \mathrm{L}$ & 4.14 & 11.4 & & & & & RIVER & & CUMULATIVE & UNFILTERED & 252 \\
\hline 6410 & & & & & & $U$ & & RIVER & & CONTINUOUS & RESIN & 252 \\
\hline 6411 & & & & & & U & & RIVER & & CONTINUOUS & RESIN & 252 \\
\hline 6412 & $\mathrm{pCi} / \mathrm{L}$ & 0.64 & 0.642 & & & $U$ & & RIVER & & CUMULATIVE & UNFILTERED & 252 \\
\hline 6413 & $\mathrm{pCi} / \mathrm{L}$ & 0.053 & 0.0588 & & & & & RIVER & & CUMULATIVE & UNFILTERED & 252 \\
\hline 6414 & $\mathrm{pCi} / \mathrm{L}$ & 0.0109 & 0.0112 & & & U & & RIVER & & CUMULATIVE & UNFILTERED & 252 \\
\hline 6415 & $\mathrm{pCi} / \mathrm{L}$ & 0.0516 & 0.0576 & & & & & RIVER & & CUMULATIVE & UNFILTERED & 252 \\
\hline 6416 & & & & & & U & & RIVER & & CONTINUOUS & RESIN & 463 \\
\hline 6417 & & & & & & $U$ & & RIVER & & CONTINUOUS & RESIN & 463 \\
\hline 6418 & & & & & & U & & RIVER & & CONTINUOUS & FILTER & 463 \\
\hline 6419 & & & & & & U & & RIVER & & CONTINUOUS & FILTER & 463 \\
\hline 6420 & $\mathrm{pCi} / \mathrm{L}$ & 0.0398 & 0.053 & & & & & RIVER & & CUMULATIVE & UNFILTERED & 463 \\
\hline 6421 & $\mathrm{pCi} / \mathrm{L}$ & 1.18 & 6.58 & & & U & & RIVER & & CUMULATIVE & UNFILTERED & 463 \\
\hline 6422 & $\mathrm{pCi} / \mathrm{L}$ & 5.03 & 15.8 & & & & & RIVER & & CUMULATIVE & UNFILTERED & 463 \\
\hline 6423 & $\mathrm{pCi} / \mathrm{L}$ & 0.616 & 0.618 & & & U & & RIVER & & CUMULATIVE & UNFILTERED & 463 \\
\hline 6424 & $\mathrm{pCi} / \mathrm{L}$ & 0.069 & 0.0753 & & & & & RIVER & & CUMULATIVE & UNFILTERED & 463 \\
\hline 6425 & $\mathrm{pCi} / \mathrm{L}$ & 0.0242 & 0.0244 & & & U & & RIVER & & CUMULATIVE & UNFILTERED & 463 \\
\hline 6426 & $\mathrm{pCi} / \mathrm{L}$ & 0.0746 & 0.0816 & & & & & RIVER & & CUMULATIVE & UNFILTERED & 463 \\
\hline 6427 & $\mathrm{pCi} / \mathrm{L}$ & 0.0357 & 0.0483 & & & & & DRINKING & & RAW GRAB & UNFILTERED & 553 \\
\hline 6428 & $\mathrm{pCi} / \mathrm{L}$ & 97.3 & 181 & & & U & & DRINKING & & RAW GRAB & UNFILTERED & 553 \\
\hline 6429 & $\mathrm{pCi} / \mathrm{L}$ & 0.644 & 0.646 & & & U & & DRINKING & & RAW GRAB & UNFILTERED & 553 \\
\hline 6430 & $\mathrm{pCi} / \mathrm{L}$ & 0.00356 & 0.00356 & & & $\mathrm{U}$ & & RIVER & & CONTINUOUS & RESIN & 307 \\
\hline 6431 & $\mathrm{pCi} / \mathrm{L}$ & 0.0113 & 0.0568 & & & & & RIVER & & CONTINUOUS & FILTER & 307 \\
\hline 6432 & $\mathrm{pCi} / \mathrm{L}$ & 0.0048 & 0.0048 & & & U & & RIVER & & CONTINUOUS & RESIN & 252 \\
\hline 6433 & $\mathrm{pCi} / \mathrm{L}$ & 0.00219 & 0.00219 & & & U & & RIVER & & CONTINUOUS & FILTER & 252 \\
\hline 6434 & $\mathrm{pCi} / \mathrm{L}$ & 0.00182 & 0.00182 & & & U & & RIVER & & CONTINUOUS & FILTER & 463 \\
\hline 6435 & $\mathrm{pCi} / \mathrm{L}$ & 0.00347 & 0.00347 & & & U & & RIVER & & CONTINUOUS & RESIN & 463 \\
\hline 6436 & $\mathrm{pCi} / \mathrm{L}$ & 0.00217 & 0.00219 & & & & & RIVER & & CONTINUOUS & RESIN & 307 \\
\hline 6437 & $\mathrm{pCi} / \mathrm{L}$ & 0.00121 & 0.00122 & & & U & & RIVER & & CONTINUOUS & FILTER & 307 \\
\hline 6438 & $\mathrm{pCi} / \mathrm{L}$ & 0.00254 & 0.00254 & & & U & & RIVER & & CONTINUOUS & RESIN & 252 \\
\hline 6439 & $\mathrm{pCi} / \mathrm{L}$ & 0.00129 & 0.0013 & & & $U$ & & RIVER & & CONTINUOUS & FILTER & 252 \\
\hline 6440 & $\mathrm{pCi} / \mathrm{L}$ & 0.00117 & 0.00118 & & & & & RIVER & & CONTINUOUS & FILTER & 463 \\
\hline 6441 & $\mathrm{pCi} / \mathrm{L}$ & 0.0025 & 0.0025 & & & U & & RIVER & & CONTINUOUS & RESIN & 463 \\
\hline 6442 & $\mathrm{pCi} / \mathrm{L}$ & 0.0326 & 0.0449 & & & & & RIVER & & CUMULATIVE & UNFILTERED & 252 \\
\hline 6443 & $\mathrm{pCi} / \mathrm{L}$ & 0.0418 & 0.0452 & & & & & RIVER & & CUMULATIVE & UNFILTERED & 252 \\
\hline 6444 & $\mathrm{pCi} / \mathrm{L}$ & 0.00522 & 0.00573 & & & U & & RIVER & & CUMULATIVE & UNFILTERED & 252 \\
\hline 6445 & $\mathrm{pCi} / \mathrm{L}$ & 0.054 & 0.0609 & & & & & RIVER & & CUMULATIVE & UNFILTERED & 252 \\
\hline 6446 & $\mathrm{pCi} / \mathrm{L}$ & 0.541 & 0.544 & & & U & & RIVER & & CUMULATIVE & UNFILTERED & 252 \\
\hline 6447 & $\mathrm{pCi} / \mathrm{L}$ & 1.08 & 6.09 & & & U & & RIVER & & CUMULATIVE & UNFILTERED & 252 \\
\hline 6448 & $\mathrm{pCi} / \mathrm{L}$ & 4.19 & 11.3 & & & & & RIVER & & CUMULATIVE & UNFILTERED & 252 \\
\hline 6449 & $\mathrm{pCi} / \mathrm{L}$ & 0.0515 & 0.0586 & & & & & RIVER & & CUMULATIVE & UNFILTERED & 463 \\
\hline
\end{tabular}




\begin{tabular}{|c|c|c|c|c|c|c|c|c|c|c|c|c|}
\hline & $\mathrm{N}$ & $\mathrm{O}$ & $\mathrm{P}$ & $\mathrm{Q}$ & $\mathrm{R}$ & $\mathrm{s}$ & $\mathrm{T}$ & $\mathrm{U}$ & $\mathrm{V}$ & $\mathrm{w}$ & $\mathrm{X}$ & $\mathrm{Y}$ \\
\hline 1 & ANAL_UNITS_RPTD & COUNTING_ERROR & TOTAL_ANAL_ERROR & MIN_DETECTABLE_ACTIVITY & PCNT_MOISTURE & LAB_QUALIFIER & REVIEW_QUALIFIER & SAMP_FROM & SAMP_ITEM & SAMP_MTHD & COLL_MTHD & SAMP_SITE_ID \\
\hline 6450 & $\mathrm{pCi} / \mathrm{L}$ & 0.00936 & 0.00969 & & & $U$ & & RIVER & & CUMULATIVE & UNFILTERED & 463 \\
\hline 6451 & $\mathrm{pCi} / \mathrm{L}$ & 0.054 & 0.0622 & & & & & RIVER & & CUMULATIVE & UNFILTERED & 463 \\
\hline 6452 & $\mathrm{pCi} / \mathrm{L}$ & 0.0296 & 0.0407 & & & & & RIVER & & CUMULATIVE & UNFILTERED & 463 \\
\hline 6453 & $\mathrm{pCi} / \mathrm{L}$ & 0.632 & 0.632 & & & $\mathrm{U}$ & & RIVER & & CUMULATIVE & UNFILTERED & 463 \\
\hline 6454 & $\mathrm{pCi} / \mathrm{L}$ & 1.15 & 6.35 & & & $\mathrm{U}$ & & RIVER & & CUMULATIVE & UNFILTERED & 463 \\
\hline 6455 & $\mathrm{pCi} / \mathrm{L}$ & 5.5 & 18.3 & & & & & RIVER & & CUMULATIVE & UNFILTERED & 463 \\
\hline 6456 & $\mathrm{pCi} / \mathrm{L}$ & 0.632 & 0.632 & & & $\mathrm{U}$ & & DRINKING & & SAN. GRAB & UNFILTERED & 487 \\
\hline 6457 & $\mathrm{pCi} / \mathrm{L}$ & 124 & 271 & & & U & & DRINKING & & SAN. GRAB & UNFILTERED & 487 \\
\hline 6458 & $\mathrm{pCi} / \mathrm{L}$ & 0.0243 & 0.025 & & & U & & DRINKING & & SAN. GRAB & UNFILTERED & 487 \\
\hline 6459 & $\mathrm{pCi} / \mathrm{L}$ & 0.00126 & 0.00126 & & & U & & RIVER & & CONTINUOUS & FILTER & 307 \\
\hline 6460 & pCi/L & 0.00313 & 0.00314 & & & U & & RIVER & & CONTINUOUS & RESIN & 307 \\
\hline 6461 & $\mathrm{pCi} / \mathrm{L}$ & 0.00126 & 0.00127 & & & & & RIVER & & CONTINUOUS & FILTER & 252 \\
\hline 6462 & $\mathrm{pCi} / \mathrm{L}$ & 0.0026 & 0.00261 & & & U & & RIVER & & CONTINUOUS & RESIN & 252 \\
\hline 6463 & $\mathrm{pCi} / \mathrm{L}$ & 0.00123 & 0.00125 & & & & & RIVER & & CONTINUOUS & FILTER & 463 \\
\hline 6464 & $\mathrm{pCi} / \mathrm{L}$ & 0.00249 & 0.00251 & & & & & RIVER & & CONTINUOUS & RESIN & 463 \\
\hline 6465 & $\mathrm{pCi} / \mathrm{L}$ & 0.858 & 1.69 & & & & & SANITARY & & RAW GRAB & UNFILTERED & 525 \\
\hline 6466 & $\mathrm{pCi} / \mathrm{L}$ & 0.141 & 0.147 & & & & & SANITARY & & RAW GRAB & UNFILTERED & 525 \\
\hline 6467 & $\mathrm{pCi} / \mathrm{L}$ & 1.08 & 2.55 & & & & & SANITARY & & RAW GRAB & UNFILTERED & 525 \\
\hline 6468 & pCi/L & 120 & 265 & & & U & & SANITARY & & RAW GRAB & UNFILTERED & 525 \\
\hline 6469 & $\mathrm{pCi} / \mathrm{L}$ & 0.651 & 0.651 & & & U & & SANITARY & & RAW GRAB & UNFILTERED & 525 \\
\hline 6470 & $\mathrm{pCi} / \mathrm{L}$ & 0.279 & 0.865 & & & & & SANITARY & & RAW GRAB & UNFILTERED & 172 \\
\hline 6471 & pCi/L & 0.0524 & 0.0598 & & & & & SANITARY & & RAW GRAB & UNFILTERED & 172 \\
\hline 6472 & $\mathrm{pCi} / \mathrm{L}$ & 0.29 & 0.93 & & & & & SANITARY & & RAW GRAB & UNFILTERED & 172 \\
\hline 6473 & pCi/L & 127 & 278 & & & U & & SANITARY & & RAW GRAB & UNFILTERED & 172 \\
\hline 6474 & $\mathrm{pCi} / \mathrm{L}$ & 0.654 & 0.654 & & & U & & SANITARY & & RAW GRAB & UNFILTERED & 172 \\
\hline 6475 & $\mathrm{pCi} / \mathrm{L}$ & 125 & 273 & & & U & & DRINKING & & SAN. GRAB & UNFILTERED & 530 \\
\hline 6476 & $\mathrm{pCi} / \mathrm{L}$ & 0.0489 & 0.0942 & & & & & DRINKING & & SAN. GRAB & UNFILTERED & 530 \\
\hline 6477 & $\mathrm{pCi} / \mathrm{L}$ & 0.53 & 0.532 & & & U & & DRINKING & & SAN. GRAB & UNFILTERED & 530 \\
\hline 6478 & $\mathrm{pCi} / \mathrm{L}$ & 0.642 & 0.642 & & & U & & SANITARY & & RAW GRAB & UNFILTERED & 524 \\
\hline 6479 & $\mathrm{pCi} / \mathrm{L}$ & 127 & 277 & & & U & & SANITARY & & RAW GRAB & UNFILTERED & 524 \\
\hline 6480 & $\mathrm{pCi} / \mathrm{L}$ & 0.178 & 0.366 & & & & & SANITARY & & RAW GRAB & UNFILTERED & 524 \\
\hline 6481 & pCi/L & 0.0356 & 0.0378 & & & & & SANITARY & & RAW GRAB & UNFILTERED & 524 \\
\hline 6482 & $\mathrm{pCi} / \mathrm{L}$ & 0.19 & 0.411 & & & & & SANITARY & & RAW GRAB & UNFILTERED & 524 \\
\hline 6483 & $\mathrm{pCi} / \mathrm{L}$ & 0.892 & 0.899 & & & & & SANITARY & & RAW GRAB & UNFILTERED & 519 \\
\hline 6484 & $\mathrm{pCi} / \mathrm{L}$ & 121 & 267 & & & U & & SANITARY & & RAW GRAB & UNFILTERED & 519 \\
\hline 6485 & $\mathrm{pCi} / \mathrm{L}$ & 0.0138 & 0.0151 & & & U & & SANITARY & & RAW GRAB & UNFILTERED & 519 \\
\hline 6486 & pCi/L & 0 & 0.00695 & & & U & & SANITARY & & RAW GRAB & UNFILTERED & 519 \\
\hline 6487 & pCi/L & 0.00436 & 0.0104 & & & U & & SANITARY & & RAW GRAB & UNFILTERED & 519 \\
\hline 6488 & pCi/L & 0.00286 & 0.00286 & & & U & & RIVER & & CONTINUOUS & RESIN & 307 \\
\hline 6489 & $\mathrm{pCi} / \mathrm{L}$ & 0.00183 & 0.00187 & & & & & RIVER & & CONTINUOUS & FILTER & 307 \\
\hline 6490 & $\mathrm{pCi} / \mathrm{L}$ & 0.00164 & 0.0017 & & & & & RIVER & & CONTINUOUS & FILTER & 252 \\
\hline 6491 & $\mathrm{pCi} / \mathrm{L}$ & 0.00293 & 0.00293 & & & U & & RIVER & & CONTINUOUS & RESIN & 252 \\
\hline 6492 & $\mathrm{pCi} / \mathrm{L}$ & 0.00155 & 0.0016 & & & & & RIVER & & CONTINUOUS & FILTER & 463 \\
\hline 6493 & pCi/L & 0.00379 & 0.00379 & & & U & & RIVER & & CONTINUOUS & RESIN & 463 \\
\hline 6494 & $\mathrm{pCi} / \mathrm{L}$ & 0.081 & 0.0859 & & & & & RIVER & & CUMULATIVE & UNFILTERED & 252 \\
\hline 6495 & $\mathrm{pCi} / \mathrm{L}$ & 0.0302 & 0.0303 & & & U & & RIVER & & CUMULATIVE & UNFILTERED & 252 \\
\hline 6496 & pCi/L & 0.0833 & 0.0883 & & & & & RIVER & & CUMULATIVE & UNFILTERED & 252 \\
\hline 6497 & pCi/L & 0.497 & 0.497 & & & U & & RIVER & & CUMULATIVE & UNFILTERED & 252 \\
\hline 6498 & $\mathrm{pCi} / \mathrm{L}$ & 0.0383 & 0.0516 & & & & & RIVER & & CUMULATIVE & UNFILTERED & 252 \\
\hline 6499 & pCi/L & 1.07 & 5.94 & & & U & & RIVER & & CUMULATIVE & UNFILTERED & 252 \\
\hline 6500 & $\mathrm{pCi} / \mathrm{L}$ & 4.11 & 11.2 & & & & & RIVER & & CUMULATIVE & UNFILTERED & 252 \\
\hline 6501 & $\mathrm{pCi} / \mathrm{L}$ & 0.0621 & 0.0657 & & & & & RIVER & & CUMULATIVE & UNFILTERED & 463 \\
\hline
\end{tabular}




\begin{tabular}{|c|c|c|c|c|c|c|c|c|c|c|c|c|}
\hline & $\mathrm{N}$ & $\mathrm{O}$ & $\mathrm{P}$ & $\mathrm{Q}$ & $\mathrm{R}$ & $\mathrm{s}$ & $T$ & $U$ & $\mathrm{~V}$ & $\mathrm{w}$ & $\mathrm{X}$ & $\mathrm{Y}$ \\
\hline 1 & ANAL_UNITS_RPTD & COUNTING_ERROR & \begin{tabular}{|c|} 
TOTAL_ANAL_ERROR \\
\end{tabular} & MIN_DETECTABLE_ACTIVITY & PCNT_MOISTURE & LAB_QUALIFIER & REVIEW_QUALIFIER & SAMP_FROM & SAMP_ITEM & SAMP_MTHD & COLL_MTHD & SAMP_SITE_ID \\
\hline 6502 & $\mathrm{pCi} / \mathrm{L}$ & 0.0199 & 0.0201 & & & $U$ & & RIVER & & CUMULATIVE & UNFILTERED & 463 \\
\hline 6503 & $\mathrm{pCi} / \mathrm{L}$ & 0.0782 & 0.0855 & & & & & RIVER & & CUMULATIVE & UNFILTERED & 463 \\
\hline 6504 & $\mathrm{pCi} / \mathrm{L}$ & 0.537 & 0.537 & & & U & & RIVER & & CUMULATIVE & UNFILTERED & 463 \\
\hline 6505 & $\mathrm{pCi} / \mathrm{L}$ & 0.0338 & 0.0466 & & & & & RIVER & & CUMULATIVE & UNFILTERED & 463 \\
\hline 6506 & $\mathrm{pCi} / \mathrm{L}$ & 1.11 & 6.14 & & & U & & RIVER & & CUMULATIVE & UNFILTERED & 463 \\
\hline 6507 & $\mathrm{pCi} / \mathrm{L}$ & 5.75 & 19.7 & & & & & RIVER & & CUMULATIVE & UNFILTERED & 463 \\
\hline 6508 & $\mathrm{pCi} / \mathrm{L}$ & 1.81 & 1.86 & & & & & SURFACE & & RAW GRAB & UNFILTERED & 509 \\
\hline 6509 & $\mathrm{pCi} / \mathrm{L}$ & 123 & 269 & & & U & & SURFACE & & RAW GRAB & UNFILTERED & 509 \\
\hline 6510 & $\mathrm{pCi} / \mathrm{L}$ & 0.0766 & 0.178 & & & & & SURFACE & & RAW GRAB & UNFILTERED & 509 \\
\hline 6511 & $\mathrm{pCi} / \mathrm{L}$ & 4.38 & 9.74 & & & & & SURFACE & & RAW GRAB & UNFILTERED & 147 \\
\hline 6512 & $\mathrm{pCi} / \mathrm{L}$ & 0.635 & 0.658 & & & & & SURFACE & & RAW GRAB & UNFILTERED & 147 \\
\hline 6513 & $\mathrm{pCi} / \mathrm{L}$ & 4.53 & 10.4 & & & & & SURFACE & & RAW GRAB & UNFILTERED & 147 \\
\hline 6514 & $\mathrm{pCi} / \mathrm{L}$ & 2.17 & 2.17 & & & U & & SURFACE & & RAW GRAB & UNFILTERED & 147 \\
\hline 6515 & $\mathrm{pCi} / \mathrm{L}$ & 135 & 293 & & & & & SURFACE & & RAW GRAB & UNFILTERED & 147 \\
\hline 6516 & $\mathrm{pCi} / \mathrm{L}$ & 0.108 & 0.264 & & & & & SURFACE & & RAW GRAB & UNFILTERED & 147 \\
\hline 6517 & $\mathrm{pCi} / \mathrm{L}$ & 1.72 & 1.72 & & & U & & SURFACE & & RAW GRAB & UNFILTERED & 136 \\
\hline 6518 & $\mathrm{pCi} / \mathrm{L}$ & 236 & 660 & & & & & SURFACE & & RAW GRAB & UNFILTERED & 136 \\
\hline 6519 & $\mathrm{pCi} / \mathrm{L}$ & 0.00274 & 0.00274 & & & U & & RIVER & & CONTINUOUS & RESIN & 307 \\
\hline 6520 & $\mathrm{pCi} / \mathrm{L}$ & 0.0000372 & 0.0000374 & & & U & & RIVER & & CONTINUOUS & RESIN & 307 \\
\hline 6521 & $\mathrm{pCi} / \mathrm{L}$ & & 0.0000235 & & & & & RIVER & & CONTINUOUS & RESIN & 307 \\
\hline 6522 & $\mathrm{pCi} / \mathrm{L}$ & 0.00162 & 0.00165 & & & & & RIVER & & CONTINUOUS & FILTER & 307 \\
\hline 6523 & $\mathrm{pCi} / \mathrm{L}$ & 0.000111 & 0.000245 & & & & & RIVER & & CONTINUOUS & FILTER & 307 \\
\hline 6524 & $\mathrm{pCi} / \mathrm{L}$ & 0.00196 & 0.00198 & & & & & RIVER & & CONTINUOUS & FILTER & 252 \\
\hline 6525 & $\mathrm{pCi} / \mathrm{L}$ & 0.0000182 & 0.0000187 & & & U & & RIVER & & CONTINUOUS & RESIN & 252 \\
\hline 6526 & $\mathrm{pCi} / \mathrm{L}$ & 0.00000957 & 0.00000977 & & & & & RIVER & & CONTINUOUS & FILTER & 252 \\
\hline 6527 & $\mathrm{pCi} / \mathrm{L}$ & 0.00242 & 0.00242 & & & U & & RIVER & & CONTINUOUS & RESIN & 252 \\
\hline 6528 & $\mathrm{pCi} / \mathrm{L}$ & & 0.000000101 & & & & & RIVER & & CONTINUOUS & RESIN & 252 \\
\hline 6529 & $\mathrm{pCi} / \mathrm{L}$ & 0.00226 & 0.00227 & & & U & & RIVER & & CONTINUOUS & RESIN & 463 \\
\hline 6530 & $\mathrm{pCi} / \mathrm{L}$ & 0.0000194 & 0.0000198 & & & U & & RIVER & & CONTINUOUS & RESIN & 463 \\
\hline 6531 & $\mathrm{pCi} / \mathrm{L}$ & & 0.0000061 & & & & & RIVER & & CONTINUOUS & RESIN & 463 \\
\hline 6532 & $\mathrm{pCi} / \mathrm{L}$ & 0.0014 & 0.00143 & & & & & RIVER & & CONTINUOUS & FILTER & 463 \\
\hline 6533 & $\mathrm{pCi} / \mathrm{L}$ & 0.00000786 & 0.00000801 & & & & & RIVER & & CONTINUOUS & FILTER & 463 \\
\hline 6534 & $\mathrm{pCi} / \mathrm{L}$ & 0.0453 & 0.0505 & & & & & DRINKING & & CUMULATIVE & UNFILTERED & 307 \\
\hline 6535 & $\mathrm{pCi} / \mathrm{L}$ & 0.00622 & 0.007 & & & U & & DRINKING & & CUMULATIVE & UNFILTERED & 307 \\
\hline 6536 & $\mathrm{pCi} / \mathrm{L}$ & 0.0501 & 0.0577 & & & & & DRINKING & & CUMULATIVE & UNFILTERED & 307 \\
\hline 6537 & $\mathrm{pCi} / \mathrm{L}$ & 0.0394 & 0.0542 & & & & & DRINKING & & CUMULATIVE & UNFILTERED & 307 \\
\hline 6538 & $\mathrm{pCi} / \mathrm{L}$ & 5.91 & 20.8 & & & & & DRINKING & & CUMULATIVE & UNFILTERED & 307 \\
\hline 6539 & $\mathrm{pCi} / \mathrm{L}$ & 1.13 & 6.25 & & & $U$ & & DRINKING & & CUMULATIVE & UNFILTERED & 307 \\
\hline 6540 & $\mathrm{pCi} / \mathrm{L}$ & 0.576 & 0.581 & & & U & & DRINKING & & CUMULATIVE & UNFILTERED & 307 \\
\hline 6541 & $\mathrm{pCi} / \mathrm{L}$ & 0.0339 & 0.0484 & & & & & QC_SAMPLE & & RAW GRAB & UNFILTERED & 550 \\
\hline 6542 & $\mathrm{pCi} / \mathrm{L}$ & 5.23 & 16.8 & & & & & QC_SAMPLE & & RAW GRAB & UNFILTERED & 550 \\
\hline 6543 & $\mathrm{pCi} / \mathrm{L}$ & 0.0521 & 0.0557 & & & & & QC_SAMPLE & & RAW GRAB & UNFILTERED & 550 \\
\hline 6544 & $\mathrm{pCi} / \mathrm{L}$ & 0.0167 & 0.0169 & & & U & & QC_SAMPLE & & RAW GRAB & UNFILTERED & 550 \\
\hline 6545 & $\mathrm{pCi} / \mathrm{L}$ & 0.0683 & 0.0757 & & & & & QC_SAMPLE & & RAW GRAB & UNFILTERED & 550 \\
\hline 6546 & $\mathrm{pCi} / \mathrm{L}$ & 0.602 & 0.605 & & & & & QC_SAMPLE & & RAW GRAB & UNFILTERED & 550 \\
\hline 6547 & $\mathrm{pCi} / \mathrm{L}$ & 0.0385 & 0.0522 & & & & & QC_SAMPLE & & RAW GRAB & UNFILTERED & 247 \\
\hline 6548 & $\mathrm{pCi} / \mathrm{L}$ & 5.12 & 16.4 & & & & & QC_SAMPLE & & RAW GRAB & UNFILTERED & 247 \\
\hline 6549 & $\mathrm{pCi} / \mathrm{L}$ & 0.0753 & 0.0797 & & & & & QC_SAMPLE & & RAW GRAB & UNFILTERED & 247 \\
\hline 6550 & $\mathrm{pCi} / \mathrm{L}$ & 0.0337 & 0.034 & & & U & & QC_SAMPLE & & RAW GRAB & UNFILTERED & 247 \\
\hline 6551 & $\mathrm{pCi} / \mathrm{L}$ & 0.085 & 0.0907 & & & & & QC_SAMPLE & & RAW GRAB & UNFILTERED & 247 \\
\hline 6552 & $\mathrm{pCi} / \mathrm{L}$ & 0.668 & 0.67 & & & U & & QC_SAMPLE & & RAW GRAB & UNFILTERED & 247 \\
\hline 6553 & $\mathrm{pCi} / \mathrm{L}$ & 0.00238 & 0.00239 & & & $U_{26}$ & & RIVER & & CONTINUOUS & RESIN & 307 \\
\hline
\end{tabular}




\begin{tabular}{|c|c|c|c|c|c|c|c|c|c|c|c|c|c|}
\hline & $\mathrm{N}$ & $\mathrm{O}$ & $\mathrm{P}$ & & $\mathrm{Q}$ & $\mathrm{R}$ & $\mathrm{s}$ & $\mathrm{T}$ & $\mathrm{U}$ & $\mathrm{V}$ & $\mathrm{W}$ & $\mathrm{X}$ & $\mathrm{Y}$ \\
\hline 1 & ANAL_UNITS_RPTD & COUNTING_ERROR & TOTAL_ANAL & ERROR & MIN_DETECTABLE_ACTIVITY & PCNT_MOISTURE & LAB_QUALIFIER & REVIEW_QUALIFIER & SAMP_FROM & SAMP_ITEM & SAMP_MTHD & COLL_MTHD & SAMP_SITE_ID \\
\hline 6554 & $\mathrm{pCi} / \mathrm{L}$ & 0.00134 & & 0.00134 & & & $U$ & & RIVER & & CONTINUOUS & FILTER & 307 \\
\hline 6555 & $\mathrm{pCi} / \mathrm{L}$ & 0.00153 & & 0.00153 & & & U & & RIVER & & CONTINUOUS & FILTER & 252 \\
\hline 6556 & $\mathrm{pCi} / \mathrm{L}$ & 0.00283 & & 0.00283 & & & $U$ & & RIVER & & CONTINUOUS & RESIN & 252 \\
\hline 6557 & $\mathrm{pCi} / \mathrm{L}$ & 0.0016 & & 0.00162 & & & & & RIVER & & CONTINUOUS & FILTER & 463 \\
\hline 6558 & $\mathrm{pCi} / \mathrm{L}$ & 0.00341 & & 0.00341 & & & $\mathrm{U}$ & & RIVER & & CONTINUOUS I & RESIN & 463 \\
\hline 6559 & $\mathrm{pCi} / \mathrm{L}$ & 0.483 & & 0.483 & & & U & & DRINKING & & SAN. GRAB & UNFILTERED & 186 \\
\hline 6560 & $\mathrm{pCi} / \mathrm{L}$ & 0.0207 & & 0.0211 & & & $\mathrm{u}$ & & DRINKING & & SAN. GRAB & UNFILTERED & 186 \\
\hline 6561 & $\mathrm{pCi} / \mathrm{L}$ & 97.2 & & 180 & & & U & & DRINKING & & SAN. GRAB & UNFILTERED & 186 \\
\hline 6562 & $\mathrm{pCi} / \mathrm{L}$ & 0.585 & & 0.585 & & & U & & DRINKING & & SAN. GRAB & UNFILTERED & 512 \\
\hline 6563 & $\mathrm{pCi} / \mathrm{L}$ & 0.018 & & 0.0185 & & & $U$ & & DRINKING & & SAN. GRAB & UNFILTERED & 512 \\
\hline 6564 & $\mathrm{pCi} / \mathrm{L}$ & 270 & & 759 & & & & & DRINKING & & SAN. GRAB & UNFILTERED & 512 \\
\hline 6565 & $\mathrm{pCi} / \mathrm{L}$ & 0.677 & & 0.678 & & & $\mathrm{U}$ & & DRINKING & & SAN. GRAB & UNFILTERED & 144 \\
\hline 6566 & $\mathrm{pCi} / \mathrm{L}$ & 0.019 & & 0.0195 & & & U & & DRINKING & & SAN. GRAB & UNFILTERED & 144 \\
\hline 6567 & $\mathrm{pCi} / \mathrm{L}$ & 93.3 & & 175 & & & U & & DRINKING & & SAN. GRAB & UNFILTERED & 144 \\
\hline 6568 & $\mathrm{pCi} / \mathrm{L}$ & 0.555 & & 0.555 & & & U & & DRINKING & & SAN. GRAB & UNFILTERED & 187 \\
\hline 6569 & $\mathrm{pCi} / \mathrm{L}$ & 0.0293 & & 0.0344 & & & & & DRINKING & & SAN. GRAB & UNFILTERED & 187 \\
\hline 6570 & $\mathrm{pCi} / \mathrm{L}$ & 98.2 & & 182 & & & U & & DRINKING & & SAN. GRAB & UNFILTERED & 187 \\
\hline 6571 & $\mathrm{pCi} / \mathrm{L}$ & 97.1 & & 184 & & & u & & DRINKING & & RAW GRAB & UNFILTERED & 553 \\
\hline 6572 & $\mathrm{pCi} / \mathrm{L}$ & 0.0272 & & 0.0344 & & & & & DRINKING & & RAW GRAB & UNFILTERED & 553 \\
\hline 6573 & $\mathrm{pCi} / \mathrm{L}$ & 0.652 & & 0.653 & & & $\mathrm{U}$ & & DRINKING & & RAW GRAB & UNFILTERED & 553 \\
\hline 6574 & $\mathrm{pCi} / \mathrm{L}$ & 0.00288 & & 0.00289 & & & U & & RIVER & & CONTINUOUS & RESIN & 307 \\
\hline 6575 & pCi/L & 0.00119 & & 0.00122 & & & & & RIVER & & CONTINUOUS & FILTER & 307 \\
\hline 6576 & $\mathrm{pCi} / \mathrm{L}$ & 0.0016 & & 0.00161 & & & U & & RIVER & & CONTINUOUS & FILTER & 252 \\
\hline 6577 & pCi/L & 0.0443 & & 0.0497 & & & & & RIVER & & CUMULATIVE & UNFILTERED & 252 \\
\hline 6578 & $\mathrm{pCi} / \mathrm{L}$ & 0.00972 & & 0.01 & & & U & & RIVER & & CUMULATIVE & UNFILTERED & 252 \\
\hline 6579 & $\mathrm{pCi} / \mathrm{L}$ & 0.0476 & & 0.0543 & & & & & RIVER & & CUMULATIVE & UNFILTERED & 252 \\
\hline 6580 & $\mathrm{pCi} / \mathrm{L}$ & 0.00313 & & 0.00314 & & & U & & RIVER & & CONTINUOUS & RESIN & 252 \\
\hline 6581 & $\mathrm{pCi} / \mathrm{L}$ & 1.13 & & 6.09 & & & U & & RIVER & & CUMULATIVE & UNFILTERED & 252 \\
\hline 6582 & $\mathrm{pCi} / \mathrm{L}$ & 4.26 & & 12 & & & & & RIVER & & CUMULATIVE & UNFILTERED & 252 \\
\hline 6583 & $\mathrm{pCi} / \mathrm{L}$ & 0.0281 & & 0.0371 & & & & & RIVER & & CUMULATIVE & UNFILTERED & 252 \\
\hline 6584 & $\mathrm{pCi} / \mathrm{L}$ & 0.695 & & 0.695 & & & u & & RIVER & & CUMULATIVE & UNFILTERED & 252 \\
\hline 6585 & $\mathrm{pCi} / \mathrm{L}$ & 0.0436 & & 0.049 & & & & & RIVER & & CUMULATIVE & UNFILTERED & 463 \\
\hline 6586 & $\mathrm{pCi} / \mathrm{L}$ & 0.0108 & & 0.0111 & & & $\mathrm{U}$ & & RIVER & & CUMULATIVE & UNFILTERED & 463 \\
\hline 6587 & $\mathrm{pCi} / \mathrm{L}$ & 0.0518 & & 0.061 & & & & & RIVER & & CUMULATIVE & UNFILTERED & 463 \\
\hline 6588 & $\mathrm{pCi} / \mathrm{L}$ & 1.11 & & 6.11 & & & U & & RIVER & & CUMULATIVE & UNFILTERED & 463 \\
\hline 6589 & $\mathrm{pCi} / \mathrm{L}$ & 4.66 & & 13.9 & & & & & RIVER & & CUMULATIVE & UNFILTERED & 463 \\
\hline 6590 & $\mathrm{pCi} / \mathrm{L}$ & 0.0314 & & 0.042 & & & & & RIVER & & CUMULATIVE & UNFILTERED & 463 \\
\hline 6591 & $\mathrm{pCi} / \mathrm{L}$ & 0.657 & & 0.657 & & & U & & RIVER & & CUMULATIVE & UNFILTERED & 463 \\
\hline 6592 & $\mathrm{pCi} / \mathrm{L}$ & 0.00242 & & 0.00242 & & & U & & RIVER & & CONTINUOUS & RESIN & 463 \\
\hline 6593 & $\mathrm{pCi} / \mathrm{L}$ & 0.0017 & & 0.0017 & & & U & & RIVER & & CONTINUOUS & FILTER & 463 \\
\hline 6594 & $\mathrm{pCi} / \mathrm{L}$ & 0.00207 & & 0.00226 & & & & & RIVER & & CONTINUOUS & FILTER & 307 \\
\hline 6595 & $\mathrm{pCi} / \mathrm{L}$ & 0.00324 & & 0.00327 & & & & & RIVER & & CONTINUOUS & RESIN & 307 \\
\hline 6596 & $\mathrm{pCi} / \mathrm{L}$ & 0.00247 & & 0.00247 & & & U & & RIVER & & CONTINUOUS & RESIN & 252 \\
\hline 6597 & pCi/L & 0.00126 & & 0.00131 & & & & & RIVER & & CONTINUOUS & FILTER & 252 \\
\hline 6598 & $\mathrm{pCi} / \mathrm{L}$ & 0.00339 & & 0.00341 & & & & & RIVER & & CONTINUOUS & RESIN & 463 \\
\hline 6599 & $\mathrm{pCi} / \mathrm{L}$ & 0.00178 & & 0.00186 & & & & & RIVER & & CONTINUOUS & FILTER & 463 \\
\hline 6600 & $\mathrm{pCi} / \mathrm{L}$ & 0.608 & & 0.612 & & & & & IRRIGATION & & RAW GRAB & UNFILTERED & 440 \\
\hline 6601 & $\mathrm{pCi} / \mathrm{L}$ & 97.2 & & 184 & & & U & & IRRIGATION & & RAW GRAB & UNFILTERED & 440 \\
\hline 6602 & $\mathrm{pCi} / \mathrm{L}$ & 0.0418 & & 0.0468 & & & & & IRRIGATION & & RAW GRAB & UNFILTERED & 440 \\
\hline 6603 & pCi/L & 0.00804 & & 0.00841 & & & U & & IRRIGATION & & RAW GRAB & UNFILTERED & 440 \\
\hline 6604 & $\mathrm{pCi} / \mathrm{L}$ & 0.0459 & & 0.0528 & & & & & IRRIGATION & & RAW GRAB & UNFILTERED & 440 \\
\hline 6605 & $\mathrm{pCi} / \mathrm{L}$ & 0.0259 & & 0.0346 & & & & & IRRIGATION & & RAW GRAB & UNFILTERED & 440 \\
\hline
\end{tabular}




\begin{tabular}{|c|c|c|c|c|c|c|c|c|c|c|c|c|}
\hline & $\mathrm{N}$ & $\mathrm{O}$ & $\mathrm{P}$ & $\mathrm{Q}$ & $\mathrm{R}$ & $\mathrm{S}$ & $\mathrm{T}$ & $\mathrm{U}$ & $\mathrm{V}$ & $\mathrm{w}$ & $\mathrm{X}$ & $\mathrm{Y}$ \\
\hline 1 & ANAL_UNITS_RPTD & COUNTING_ERROR & \begin{tabular}{|l|} 
TOTAL_ANAL_ERROR \\
\end{tabular} & MIN_DETECTABLE_ACTIVITY & PCNT_MOISTURE & LAB_QUALIFIER & REVIEW_QUALIFIER & SAMP_FROM & SAMP_ITEM & SAMP_MTHD & COLL_MTHD & SAMP_SITE_ID \\
\hline 6606 & $\mathrm{pCi} / \mathrm{L}$ & 0.821 & 0.821 & & & $U$ & & DRINKING & & SAN. GRAB & UNFILTERED & 487 \\
\hline 6607 & $\mathrm{pCi} / \mathrm{L}$ & 98.7 & 190 & & & U & & DRINKING & & SAN. GRAB & UNFILTERED & 487 \\
\hline 6608 & $\mathrm{pCi} / \mathrm{L}$ & 0.0175 & 0.0182 & & & $U$ & & DRINKING & & SAN. GRAB & UNFILTERED & 487 \\
\hline 6609 & $\mathrm{pCi} / \mathrm{L}$ & 0.00242 & 0.00244 & & & & & RIVER & & CONTINUOUS & RESIN & 307 \\
\hline 6610 & $\mathrm{pCi} / \mathrm{L}$ & 0.00132 & 0.00132 & & & & & RIVER & & CONTINUOUS & FILTER & 307 \\
\hline 6611 & $\mathrm{pCi} / \mathrm{L}$ & 0.00152 & 0.00154 & & & & & RIVER & & CONTINUOUS & FILTER & 252 \\
\hline 6612 & $\mathrm{pCi} / \mathrm{L}$ & 0.638 & 0.639 & & & $\mathrm{U}$ & & RIVER & & CUMULATIVE & UNFILTERED & 252 \\
\hline 6613 & $\mathrm{pCi} / \mathrm{L}$ & 0.0371 & 0.0405 & & & & & RIVER & & CUMULATIVE & UNFILTERED & 252 \\
\hline 6614 & $\mathrm{pCi} / \mathrm{L}$ & 0.0097 & 0.01 & & & u & & RIVER & & CUMULATIVE & UNFILTERED & 252 \\
\hline 6615 & $\mathrm{pCi} / \mathrm{L}$ & 0.044 & 0.0496 & & & & & RIVER & & CUMULATIVE & UNFILTERED & 252 \\
\hline 6616 & $\mathrm{pCi} / \mathrm{L}$ & 0.00252 & 0.00253 & & & U & & RIVER & & CONTINUOUS & RESIN & 252 \\
\hline 6617 & $\mathrm{pCi} / \mathrm{L}$ & 3.85 & 10.1 & & & & & RIVER & & CUMULATIVE & UNFILTERED & 252 \\
\hline 6618 & $\mathrm{pCi} / \mathrm{L}$ & 0.0321 & 0.0423 & & & & & RIVER & & CUMULATIVE & UNFILTERED & 252 \\
\hline 6619 & $\mathrm{pCi} / \mathrm{L}$ & 1.09 & 6.04 & & & U & & RIVER & & CUMULATIVE & UNFILTERED & 252 \\
\hline 6620 & $\mathrm{pCi} / \mathrm{L}$ & 0.875 & 0.875 & & & $U$ & & RIVER & & CUMULATIVE & UNFILTERED & 463 \\
\hline 6621 & $\mathrm{pCi} / \mathrm{L}$ & 0.039 & 0.0428 & & & & & RIVER & & CUMULATIVE & UNFILTERED & 463 \\
\hline 6622 & $\mathrm{pCi} / \mathrm{L}$ & 0.015 & 0.0153 & & & & & RIVER & & CUMULATIVE & UNFILTERED & 463 \\
\hline 6623 & $\mathrm{pCi} / \mathrm{L}$ & 0.0473 & 0.0537 & & & & & RIVER & & CUMULATIVE & UNFILTERED & 463 \\
\hline 6624 & pCi/L & 0.0032 & 0.00321 & & & U & & RIVER & & CONTINUOUS & RESIN & 463 \\
\hline 6625 & $\mathrm{pCi} / \mathrm{L}$ & 4.12 & 11.2 & & & & & RIVER & & CUMULATIVE & UNFILTERED & 463 \\
\hline 6626 & $\mathrm{pCi} / \mathrm{L}$ & 0.0295 & 0.0394 & & & & & RIVER & & CUMULATIVE & UNFILTERED & 463 \\
\hline 6627 & pCi/L & 1.13 & 6.19 & & & U & & RIVER & & CUMULATIVE & UNFILTERED & 463 \\
\hline 6628 & $\mathrm{pCi} / \mathrm{L}$ & 0.00215 & 0.00217 & & & & & RIVER & & CONTINUOUS & FILTER & 463 \\
\hline 6629 & pCi/L & & 0.00000158 & & & & & SANITARY & & RAW GRAB & UNFILTERED & 525 \\
\hline 6630 & $\mathrm{pCi} / \mathrm{L}$ & & 0.00000588 & & & & & SANITARY & & RAW GRAB & UNFILTERED & 172 \\
\hline 6631 & $\mathrm{pCi} / \mathrm{L}$ & 0.63 & 0.63 & & & U & & SANITARY & & RAW GRAB & UNFILTERED & 525 \\
\hline 6632 & $\mathrm{pCi} / \mathrm{L}$ & 101 & 191 & & & U & & SANITARY & & RAW GRAB & UNFILTERED & 525 \\
\hline 6633 & $\mathrm{pCi} / \mathrm{L}$ & 0.0587 & 0.0659 & & & U & & SANITARY & & RAW GRAB & UNFILTERED & 525 \\
\hline 6634 & $\mathrm{pCi} / \mathrm{L}$ & 0 & 0.0353 & & & U & & SANITARY & & RAW GRAB & UNFILTERED & 525 \\
\hline 6635 & $\mathrm{pCi} / \mathrm{L}$ & 0.0665 & 0.0819 & & & U & & SANITARY & & RAW GRAB & UNFILTERED & 525 \\
\hline 6636 & $\mathrm{pCi} / \mathrm{L}$ & 0.728 & 0.733 & & & U & & SANITARY & & RAW GRAB & UNFILTERED & 172 \\
\hline 6637 & pCi/L & 102 & 194 & & & U & & SANITARY & & RAW GRAB & UNFILTERED & 172 \\
\hline 6638 & $\mathrm{pCi} / \mathrm{L}$ & 0.054 & 0.0628 & & & & & SANITARY & & RAW GRAB & UNFILTERED & 172 \\
\hline 6639 & $\mathrm{pCi} / \mathrm{L}$ & 0.286 & 0.943 & & & & & SANITARY & & RAW GRAB & UNFILTERED & 172 \\
\hline 6640 & $\mathrm{pCi} / \mathrm{L}$ & 0.277 & 0.887 & & & & & SANITARY & & RAW GRAB & UNFILTERED & 172 \\
\hline 6641 & $\mathrm{pCi} / \mathrm{L}$ & 0.161 & 0.356 & & & & & SANITARY & & RAW GRAB & UNFILTERED & 524 \\
\hline 6642 & pCi/L & 0.0374 & 0.0409 & & & & & SANITARY & & RAW GRAB & UNFILTERED & 524 \\
\hline 6643 & $\mathrm{pCi} / \mathrm{L}$ & 0.179 & 0.429 & & & & & SANITARY & & RAW GRAB & UNFILTERED & 524 \\
\hline 6644 & pCi/L & 0.525 & 0.525 & & & U & & SANITARY & & RAW GRAB & UNFILTERED & 524 \\
\hline 6645 & $\mathrm{pCi} / \mathrm{L}$ & 102 & 185 & & & & & SANITARY & & RAW GRAB & UNFILTERED & 524 \\
\hline 6646 & $\mathrm{pCi} / \mathrm{L}$ & 99 & 181 & & & U & & SANITARY & & RAW GRAB & UNFILTERED & 519 \\
\hline 6647 & $\mathrm{pCi} / \mathrm{L}$ & 0.169 & 0.375 & & & & & SANITARY & & RAW GRAB & UNFILTERED & 519 \\
\hline 6648 & $\mathrm{pCi} / \mathrm{L}$ & 0.0337 & 0.0362 & & & & & SANITARY & & RAW GRAB & UNFILTERED & 519 \\
\hline 6649 & pCi/L & 0.188 & 0.455 & & & & & SANITARY & & RAW GRAB & UNFILTERED & 519 \\
\hline 6650 & $\mathrm{pCi} / \mathrm{L}$ & 0.649 & 0.649 & & & U & & SANITARY & & RAW GRAB & UNFILTERED & 519 \\
\hline 6651 & $\mathrm{pCi} / \mathrm{L}$ & & 0.00000585 & & & & & SANITARY & & RAW GRAB & UNFILTERED & 524 \\
\hline 6652 & $\mathrm{pCi} / \mathrm{L}$ & & 0.0000132 & & & & & SANITARY & & RAW GRAB & UNFILTERED & 519 \\
\hline 6653 & $\mathrm{pCi} / \mathrm{L}$ & 105 & 190 & & & & & DRINKING & & SAN. GRAB & UNFILTERED & 530 \\
\hline 6654 & $\mathrm{pCi} / \mathrm{L}$ & 0.0592 & 0.126 & & & & & DRINKING & & SAN. GRAB & UNFILTERED & 530 \\
\hline 6655 & pCi/L & 0.669 & 0.67 & & & U & & DRINKING & & SAN. GRAB & UNFILTERED & 530 \\
\hline 6656 & & & & & & & & RIVER & & CONTINUOUS & FILTER & 307 \\
\hline 6657 & & & & & & & & RIVER & & CONTINUOUS & RESIN & 307 \\
\hline
\end{tabular}




\begin{tabular}{|c|c|c|c|c|c|c|c|c|c|c|c|c|}
\hline & $\mathrm{N}$ & $\mathrm{O}$ & $\mathrm{P}$ & $\mathrm{Q}$ & $\mathrm{R}$ & $\mathrm{s}$ & $\mathrm{T}$ & $\mathrm{u}$ & $\mathrm{V}$ & w & $\mathrm{x}$ & $\bar{Y}$ \\
\hline 1 & ANAL_UNITS_RPTD & COUNTING_ERROR & $\mid$ TOTAL_ANAL_ERROR & MIN_DETECTABLE_ACTIVITY & PCNT_MOISTURE & LAB_QUALIFIER & REVIEW_QUALIFIER & SAMP_FROM & SAMP_ITEM & SAMP_MTHD & COLL_MTHD & SAMP_SITE_ID \\
\hline 6658 & $\mathrm{pCi} / \mathrm{L}$ & 0.00241 & 0.00245 & & & & & RIVER & & CONTINUOUS & RESIN & 252 \\
\hline 6659 & $\mathrm{pCi} / \mathrm{L}$ & 0.00153 & 0.00153 & & & $\mathrm{U}$ & & RIVER & & CONTINUOUS & FILTER & 252 \\
\hline 6660 & & & & & & & & RIVER & & CONTINUOUS & RESIN & 463 \\
\hline 6661 & & & & & & & & RIVER & & CONTINUOUS & FILTER & 463 \\
\hline 6662 & $\mathrm{pCi} / \mathrm{L}$ & 0.505 & 0.505 & & & $\mathrm{U}$ & & DRINKING & & SAN. GRAB & UNFILTERED & 512 \\
\hline 6663 & $\mathrm{pCi} / \mathrm{L}$ & 246 & 636 & & & & & DRINKING & & SAN. GRAB & UNFILTERED & 512 \\
\hline 6664 & $\mathrm{pCi} / \mathrm{L}$ & 0.0198 & 0.0202 & & & $\mathrm{U}$ & & DRINKING & & SAN. GRAB & UNFILTERED & 512 \\
\hline 6665 & $\mathrm{pCi} / \mathrm{L}$ & 0.194 & 0.485 & & & & & SURFACE & & RAW GRAB & UNFILTERED & 509 \\
\hline \begin{tabular}{|c|}
6666 \\
\end{tabular} & $\mathrm{pCi} / \mathrm{L}$ & 101 & 192 & & & $\mathrm{U}$ & & SURFACE & & RAW GRAB & UNFILTERED & 509 \\
\hline \begin{tabular}{|l|l|}
6667 & -10 \\
\end{tabular} & $\mathrm{pCi} / \mathrm{L}$ & 1.43 & 1.44 & & & & & SURFACE & & RAW GRAB & UNFILTERED & 509 \\
\hline 6668 & $\mathrm{pCi} / \mathrm{L}$ & 2.61 & 2.61 & & & $\mathrm{U}$ & & SURFACE & & RAW GRAB & UNFILTERED & 136 \\
\hline 6669 & $\mathrm{pCi} / \mathrm{L}$ & 245 & 631 & & & & & SURFACE & & RAW GRAB & UNFILTERED & 136 \\
\hline 6670 & $\mathrm{pCi} / \mathrm{L}$ & 3.45 & 10.7 & & & & & SURFACE & & RAW GRAB & UNFILTERED & 147 \\
\hline 6671 & $\mathrm{pCi} / \mathrm{L}$ & 0.69 & 0.799 & & & & & SURFACE & & RAW GRAB & UNFILTERED & 147 \\
\hline \begin{tabular}{|l|}
6672 \\
\end{tabular} & $\mathrm{pCi} / \mathrm{L}$ & 3.62 & 11.8 & & & & & SURFACE & & RAW GRAB & UNFILTERED & 147 \\
\hline \begin{tabular}{|l|}
6673 \\
\end{tabular} & $\mathrm{pCi} / \mathrm{L}$ & 0.547 & 2.59 & & & & & SURFACE & & RAW GRAB & UNFILTERED & 147 \\
\hline 6674 & $\mathrm{pCi} / \mathrm{L}$ & 115 & 213 & & & & & SURFACE & & RAW GRAB & UNFILTERED & 147 \\
\hline \begin{tabular}{|l|}
6675 \\
\end{tabular} & $\mathrm{pCi} / \mathrm{L}$ & 2.99 & 2.99 & & & & & SURFACE & & RAW GRAB & UNFILTERED & 147 \\
\hline 6676 & $\mathrm{pCi} / \mathrm{L}$ & & 0.00000538 & & & & & RIVER & & CONTINUOUS & RESIN & 307 \\
\hline \begin{tabular}{|l|l|}
6677 \\
\end{tabular} & $\mathrm{pCi} / \mathrm{L}$ & 0.00276 & 0.00276 & & & $U$ & & RIVER & & CONTINUOUS & RESIN & 307 \\
\hline \begin{tabular}{|l|}
6678 \\
\end{tabular} & $\mathrm{pCi} / \mathrm{L}$ & 0.0000243 & 0.0000246 & & & $U$ & & RIVER & & CONTINUOUS & RESIN & 307 \\
\hline 6679 & $\mathrm{pCi} / \mathrm{L}$ & 0.0000114 & 0.0000115 & & & $U$ & & RIVER & & CONTINUOUS & FILTER & 307 \\
\hline 6680 & $\mathrm{pCi} / \mathrm{L}$ & 0.00129 & 0.0013 & & & & & RIVER & & CONTINUOUS & FILTER & 307 \\
\hline \begin{tabular}{|l|}
6681 \\
\end{tabular} & $\mathrm{pCi} / \mathrm{L}$ & & 0.00000245 & & & & & RIVER & & CONTINUOUS & RESIN & 252 \\
\hline \begin{tabular}{|l|}
6682 \\
\end{tabular} & $\mathrm{pCi} / \mathrm{L}$ & 1.08 & 5.82 & & & $U$ & & RIVER & & CUMULATIVE & UNFILTERED & 252 \\
\hline 6683 & $\mathrm{pCi} / \mathrm{L}$ & 0.00246 & 0.00248 & & & & & RIVER & & CONTINUOUS & RESIN & 252 \\
\hline \begin{tabular}{|l|}
6684 \\
\end{tabular} & $\mathrm{pCi} / \mathrm{L}$ & 0.00129 & 0.0013 & & & & & RIVER & & CONTINUOUS & FILTER & 252 \\
\hline 6685 & $\mathrm{pCi} / \mathrm{L}$ & 3.95 & 10.2 & & & & & RIVER & & CUMULATIVE & UNFILTERED & 252 \\
\hline 6686 & $\mathrm{pCi} / \mathrm{L}$ & 0.73 & 0.73 & & & $U$ & & RIVER & & CUMULATIVE & UNFILTERED & 252 \\
\hline \begin{tabular}{|l|}
6687 \\
\end{tabular} & $\mathrm{pCi} / \mathrm{L}$ & 0.0257 & 0.0322 & & & & & RIVER & & CUMULATIVE & UNFILTERED & 252 \\
\hline \begin{tabular}{|l|}
6688 \\
\end{tabular} & $\mathrm{pCi} / \mathrm{L}$ & 0.0000272 & 0.0000274 & & & $U$ & & RIVER & & CONTINUOUS & FILTER & 252 \\
\hline 6689 & $\mathrm{pCi} / \mathrm{L}$ & 0.0000378 & 0.0000382 & & & $U$ & & RIVER & & CONTINUOUS & RESIN & 252 \\
\hline 6690 & $\mathrm{pCi} / \mathrm{L}$ & 0.0432 & 0.0477 & & & & & RIVER & & CUMULATIVE & UNFILTERED & 252 \\
\hline 6691 & $\mathrm{pCi} / \mathrm{L}$ & 0.0124 & 0.0127 & & & $U$ & & RIVER & & CUMULATIVE & UNFILTERED & 252 \\
\hline 6692 & $\mathrm{pCi} / \mathrm{L}$ & 0.0487 & 0.0555 & & & & & RIVER & & CUMULATIVE & UNFILTERED & 252 \\
\hline \begin{tabular}{|l|}
6693 \\
\end{tabular} & $\mathrm{pCi} / \mathrm{L}$ & & 0.00000866 & & & & & RIVER & & CONTINUOUS & RESIN & 463 \\
\hline \begin{tabular}{|l|}
6694 \\
\end{tabular} & $\mathrm{pCi} / \mathrm{L}$ & 0.499 & 0.499 & & & U & & RIVER & & CUMULATIVE & UNFILTERED & 463 \\
\hline 6695 & $\mathrm{pCi} / \mathrm{L}$ & 0.00237 & 0.00238 & & & U & & RIVER & & CONTINUOUS & RESIN & 463 \\
\hline 66 & $\mathrm{pCi} / \mathrm{L}$ & 0.00178 & 0.00183 & & & & & RIVER & & CONTINUOUS & FILTER & 463 \\
\hline \begin{tabular}{|l|}
6697 \\
\end{tabular} & $\mathrm{pCi} / \mathrm{L}$ & 5.44 & 18.1 & & & & & RIVER & & CUMULATIVE & UNFILTERED & 463 \\
\hline 6698 & $\mathrm{pCi} / \mathrm{L}$ & 0.0243 & 0.0294 & & & & & RIVER & & CUMULATIVE & UNFILTERED & 463 \\
\hline \begin{tabular}{|l|}
66999 \\
\end{tabular} & $\mathrm{pCi} / \mathrm{L}$ & 0.00000886 & 0.00000892 & & & U & & RIVER & & CONTINUOUS & FILTER & 463 \\
\hline 6700 & $\mathrm{pCi} / \mathrm{L}$ & 0.000154 & 0.000154 & & & U & & RIVER & & CONTINUOUS & RESIN & 463 \\
\hline 6701 & $\mathrm{pCi} / \mathrm{L}$ & 0.0448 & 0.0499 & & & & & RIVER & & CUMULATIVE & UNFILTERED & 463 \\
\hline 6702 & $\mathrm{pCi} / \mathrm{L}$ & 0.0128 & 0.013 & & & $U$ & & RIVER & & CUMULATIVE & UNFILTERED & 463 \\
\hline 6703 & $\mathrm{pCi} / \mathrm{L}$ & 0.0504 & 0.0574 & & & & & RIVER & & CUMULATIVE & UNFILTERED & 463 \\
\hline 6704 & $\mathrm{pCi} / \mathrm{L}$ & 1.1 & 5.91 & & & $U$ & & RIVER & & CUMULATIVE & UNFILTERED & 463 \\
\hline 6705 & $\mathrm{pCi} / \mathrm{L}$ & 5.44 & 17.7 & & & & & DRINKING & & CUMULATIVE & UNFILTERED & 307 \\
\hline 6706 & $\mathrm{pCi} / \mathrm{L}$ & 0.376 & 6.34 & & & $U$ & & DRINKING & & CUMULATIVE & UNFILTERED & 307 \\
\hline \begin{tabular}{|l|}
6707 \\
\end{tabular} & $\mathrm{pCi} / \mathrm{L}$ & 0.0284 & 0.0396 & & & & & DRINKING & & CUMULATIVE & UNFILTERED & 307 \\
\hline 6708 & $\mathrm{pCi} / \mathrm{L}$ & 0.043 & 0.0478 & & & & & DRINKING & & CUMULATIVE & UNFILTERED & 307 \\
\hline 6709 & $\mathrm{pCi} / \mathrm{L}$ & 0.00744 & 0.00782 & & & $U_{2}$ & & DRINKING & & CUMULATIVE & UNFILTERED & 307 \\
\hline
\end{tabular}




\begin{tabular}{|c|c|c|c|c|c|c|c|c|c|c|c|c|}
\hline & $\mathrm{N}$ & $\mathrm{O}$ & $\mathrm{P}$ & $\mathrm{Q}$ & $\mathrm{R}$ & $\mathrm{s}$ & $T$ & $\mathrm{U}$ & $\mathrm{V}$ & $\mathrm{W}$ & $x$ & $\bar{Y}$ \\
\hline 1 & ANAL_UNITS_RPTD & COUNTING_ERROR & ||$_{\text {TOTAL_ANAL_ERROR | }}$ & MIN_DETECTABLE_ACTIVITY & PCNT_MOISTURE & LAB_QUALIFIER & REVIEW_QUALIFIER & SAMP_FROM & SAMP_ITEM & SAMP_MTHD & COLL_MTHD & SAMP_SITE_ID \\
\hline 6710 & $\mathrm{pCi} / \mathrm{L}$ & 0.0467 & 0.0532 & & & & & DRINKING & & CUMULATIVE & UNFILTERED & 307 \\
\hline 6711 & $\mathrm{pCi} / \mathrm{L}$ & 0.415 & 0.415 & & & U & & DRINKING & & CUMULATIVE & UNFILTERED & 307 \\
\hline 6712 & $\mathrm{pCi} / \mathrm{L}$ & 0.00262 & 0.00264 & & & & & RIVER & & CONTINUOUS & RESIN & 307 \\
\hline 6713 & $\mathrm{pCi} / \mathrm{L}$ & 0.0014 & 0.00141 & & & U & & RIVER & & CONTINUOUS & FILTER & 307 \\
\hline 6714 & $\mathrm{pCi} / \mathrm{L}$ & 0.00187 & 0.00187 & & & U & & RIVER & & CONTINUOUS & FILTER & 252 \\
\hline 6715 & $\mathrm{pCi} / \mathrm{L}$ & 0.00253 & 0.00254 & & & U & & RIVER & & CONTINUOUS & RESIN & 252 \\
\hline 6716 & $\mathrm{pCi} / \mathrm{L}$ & 0.0016 & 0.00161 & & & & & RIVER & & CONTINUOUS & FILTER & 463 \\
\hline 6717 & $\mathrm{pCi} / \mathrm{L}$ & 0.00417 & 0.00417 & & & U & & RIVER & & CONTINUOUS & RESIN & 463 \\
\hline 6718 & $\mathrm{pCi} / \mathrm{L}$ & 0.0562 & 0.0619 & & & & & IRRIGATION & & RAW GRAB & UNFILTERED & 440 \\
\hline 6719 & $\mathrm{pCi} / \mathrm{L}$ & 0.0231 & 0.0234 & & & & & IRRIGATION & & RAW GRAB & UNFILTERED & 440 \\
\hline 6720 & $\mathrm{pCi} / \mathrm{L}$ & 0.062 & 0.0711 & & & & & IRRIGATION & & RAW GRAB & UNFILTERED & 440 \\
\hline \begin{tabular}{|l|}
6721 \\
\end{tabular} & $\mathrm{pCi} / \mathrm{L}$ & 0.0338 & 0.0417 & & & & & IRRIGATION & & RAW GRAB & UNFILTERED & 440 \\
\hline \begin{tabular}{|l|}
6722 \\
\end{tabular} & $\mathrm{pCi} / \mathrm{L}$ & 0.557 & 0.557 & & & $U$ & & IRRIGATION & & RAW GRAB & UNFILTERED & 440 \\
\hline \begin{tabular}{|l|}
6723 \\
\end{tabular} & $\mathrm{pCi} / \mathrm{L}$ & 94.2 & 174 & & & $U$ & & IRRIGATION & & RAW GRAB & UNFILTERED & 440 \\
\hline 6724 & $\mathrm{pCi} / \mathrm{L}$ & 0.558 & 0.558 & & & $U$ & & DRINKING & & SAN. GRAB & UNFILTERED & 186 \\
\hline 6725 & $\mathrm{pCi} / \mathrm{L}$ & 0.0198 & 0.0207 & & & $U$ & & DRINKING & & SAN. GRAB & UNFILTERED & 186 \\
\hline 6726 & $\mathrm{pCi} / \mathrm{L}$ & 148 & 293 & & & $U$ & & DRINKING & & SAN. GRAB & UNFILTERED & 186 \\
\hline \begin{tabular}{|c|}
6727 \\
\end{tabular} & $\mathrm{pCi} / \mathrm{L}$ & & 0.00081 & & & & & DRINKING & & SAN. GRAB & UNFILTERED & 512 \\
\hline \begin{tabular}{|l|}
6728 \\
\end{tabular} & $\mathrm{pCi} / \mathrm{L}$ & 0.817 & 0.818 & & & U & & DRINKING & & SAN. GRAB & UNFILTERED & 512 \\
\hline \begin{tabular}{|l|}
6729 \\
\end{tabular} & $\mathrm{pCi} / \mathrm{L}$ & 0.0264 & 0.0267 & & & $U$ & & DRINKING & & SAN. GRAB & UNFILTERED & 512 \\
\hline 6730 & $\mathrm{pCi} / \mathrm{L}$ & 301 & 723 & & & & & DRINKING & & SAN. GRAB & UNFILTERED & 512 \\
\hline 6731 & $\mathrm{pCi} / \mathrm{L}$ & 0.72 & 0.721 & & & U & & DRINKING & & SAN. GRAB & UNFILTERED & 144 \\
\hline 6732 & $\mathrm{pCi} / \mathrm{L}$ & 0.0298 & 0.0302 & & & U & & DRINKING & & SAN. GRAB & UNFILTERED & 144 \\
\hline 6733 & $\mathrm{pCi} / \mathrm{L}$ & 145 & 289 & & & U & & DRINKING & & SAN. GRAB & UNFILTERED & 144 \\
\hline 6734 & $\mathrm{pCi} / \mathrm{L}$ & 0.661 & 0.661 & & & $U$ & & DRINKING & & SAN. GRAB & UNFILTERED & 187 \\
\hline \begin{tabular}{|l|}
6735 \\
\end{tabular} & $\mathrm{pCi} / \mathrm{L}$ & 0.0216 & 0.0222 & & & $U$ & & DRINKING & & SAN. GRAB & UNFILTERED & 187 \\
\hline \begin{tabular}{|l|}
6736 \\
\end{tabular} & $\mathrm{pCi} / \mathrm{L}$ & 149 & 294 & & & U & & DRINKING & & SAN. GRAB & UNFILTERED & 187 \\
\hline \begin{tabular}{|l|l|}
6737 \\
\end{tabular} & $\mathrm{pCi} / \mathrm{L}$ & 0.00246 & 0.00249 & & & & & RIVER & & CONTINUOUS & RESIN & 307 \\
\hline 6738 & $\mathrm{pCi} / \mathrm{L}$ & 0.00141 & 0.00143 & & & & & RIVER & & CONTINUOUS & FILTER & 307 \\
\hline 6739 & $\mathrm{pCi} / \mathrm{L}$ & 0.00251 & 0.00252 & & & $U$ & & RIVER & & CONTINUOUS & RESIN & 252 \\
\hline 6740 & $\mathrm{pCi} / \mathrm{L}$ & 0.00142 & 0.00142 & & & U & & RIVER & & CONTINUOUS & FILTER & 252 \\
\hline \begin{tabular}{|l|l|}
6741 \\
\end{tabular} & $\mathrm{pCi} / \mathrm{L}$ & 0.00138 & 0.00141 & & & & & RIVER & & CONTINUOUS & FILTER & 463 \\
\hline \begin{tabular}{|l|}
6742 \\
\end{tabular} & $\mathrm{pCi} / \mathrm{L}$ & 0.00257 & 0.00258 & & & U & & RIVER & & CONTINUOUS & RESIN & 463 \\
\hline \begin{tabular}{|l|}
6743 \\
\end{tabular} & $\mathrm{pCi} / \mathrm{L}$ & 0.49 & 0.49 & & & U & & RIVER & & CUMULATIVE & UNFILTERED & 252 \\
\hline 6744 & $\mathrm{pCi} / \mathrm{L}$ & 0.0581 & 0.0629 & & & & & RIVER & & CUMULATIVE & UNFILTERED & 252 \\
\hline 6745 & $\mathrm{pCi} / \mathrm{L}$ & 0.027 & 0.0273 & & & & & RIVER & & CUMULATIVE & UNFILTERED & 252 \\
\hline 6746 & $\mathrm{pCi} / \mathrm{L}$ & 0.0684 & 0.0759 & & & & & RIVER & & CUMULATIVE & UNFILTERED & 252 \\
\hline \begin{tabular}{|l|l|}
6747 \\
\end{tabular} & $\mathrm{pCi} / \mathrm{L}$ & 3.48 & 10.7 & & & & & RIVER & & CUMULATIVE & UNFILTERED & 252 \\
\hline 6748 & $\mathrm{pCi} / \mathrm{L}$ & 1.1 & 6 & & & $U$ & & RIVER & & CUMULATIVE & UNFILTERED & 252 \\
\hline 6749 & $\mathrm{pCi} / \mathrm{L}$ & 0.0288 & 0.0369 & & & & & RIVER & & CUMULATIVE & UNFILTERED & 252 \\
\hline 6750 & $\mathrm{pCi} / \mathrm{L}$ & 0.678 & 0.678 & & & U & & RIVER & & CUMULATIVE & UNFILTERED & 463 \\
\hline \begin{tabular}{|l|l|}
6751 \\
\end{tabular} & $\mathrm{pCi} / \mathrm{L}$ & 0.0453 & 0.0501 & & & & & RIVER & & CUMULATIVE & UNFILTERED & 463 \\
\hline 6752 & $\mathrm{pCi} / \mathrm{L}$ & 0.0107 & 0.011 & & & U & & RIVER & & CUMULATIVE & UNFILTERED & 463 \\
\hline 6753 & $\mathrm{pCi} / \mathrm{L}$ & 0.0512 & 0.0584 & & & & & RIVER & & CUMULATIVE & UNFILTERED & 463 \\
\hline 6754 & $\mathrm{pCi} / \mathrm{L}$ & 4.82 & 18.8 & & & & & RIVER & & CUMULATIVE & UNFILTERED & 463 \\
\hline 6755 & $\mathrm{pCi} / \mathrm{L}$ & 1.21 & 6.49 & & & U & & RIVER & & CUMULATIVE & UNFILTERED & 463 \\
\hline \begin{tabular}{|l|}
6756 \\
\end{tabular} & $\mathrm{pCi} / \mathrm{L}$ & 0.0258 & 0.0319 & & & & & RIVER & & CUMULATIVE & UNFILTERED & 463 \\
\hline \begin{tabular}{|l|l|}
6757 \\
\end{tabular} & $\mathrm{pCi} / \mathrm{L}$ & 0.7 & 0.701 & & & $U$ & & DRINKING & & RAW GRAB & UNFILTERED & 553 \\
\hline 6758 & $\mathrm{pCi} / \mathrm{L}$ & 122 & 270 & & & U & & DRINKING & & RAW GRAB & UNFILTERED & 553 \\
\hline 6759 & $\mathrm{pCi} / \mathrm{L}$ & 0.03 & 0.0387 & & & & & DRINKING & & RAW GRAB & UNFILTERED & 553 \\
\hline 6760 & $\mathrm{pCi} / \mathrm{L}$ & 0.0013 & 0.00131 & & & & & RIVER & & CONTINUOUS & FILTER & 307 \\
\hline 6761 & $\mathrm{pCi} / \mathrm{L}$ & 0.0026 & 0.00262 & & & & & RIVER & & CONTINUOUS & RESIN & 307 \\
\hline
\end{tabular}




\begin{tabular}{|c|c|c|c|c|c|c|c|c|c|c|c|c|}
\hline & $\mathrm{N}$ & $\mathrm{O}$ & $\mathrm{P}$ & $\mathrm{Q}$ & $\mathrm{R}$ & $\mathrm{S}$ & $\mathrm{T}$ & $\mathrm{U}$ & $\mathrm{V}$ & $\mathrm{W}$ & $\mathrm{X}$ & $\mathrm{Y}$ \\
\hline 1 & ANAL_UNITS_RPTD & COUNTING_ERROR & TOTAL_ANAL_ERROR & MIN_DETECTABLE_ACTIVITY & PCNT_MOISTURE & LAB_QUALIFIER & REVIEW_QUALIFIER & SAMP_FROM & SAMP_ITEM & SAMP_MTHD & COLL_MTHD & SAMP_SITE_ID \\
\hline 6762 & $\mathrm{pCi} / \mathrm{L}$ & 0.00123 & 0.00124 & & & & & RIVER & & CONTINUOUS & FILTER & 252 \\
\hline 6763 & $\mathrm{pCi} / \mathrm{L}$ & 0.00268 & 0.00268 & & & U & & RIVER & & CONTINUOUS & RESIN & 252 \\
\hline 6764 & & & & & & & & RIVER & & CONTINUOUS & RESIN & 463 \\
\hline 6765 & & & & & & & & RIVER & & CONTINUOUS & FILTER & 463 \\
\hline 6766 & $\mathrm{pCi} / \mathrm{L}$ & 0.547 & 0.547 & & & u & & DRINKING & & SAN. GRAB & UNFILTERED & 512 \\
\hline 6767 & $\mathrm{pCi} / \mathrm{L}$ & 272 & 754 & & & & & DRINKING & & SAN. GRAB & UNFILTERED & 512 \\
\hline 6768 & $\mathrm{pCi} / \mathrm{L}$ & 0.0215 & 0.022 & & & $\mathrm{U}$ & & DRINKING & & SAN. GRAB & UNFILTERED & 512 \\
\hline 6769 & $\mathrm{pCi} / \mathrm{L}$ & 86.8 & 178 & & & U & & DRINKING & & SAN. GRAB & UNFILTERED & 487 \\
\hline 6770 & $\mathrm{pCi} / \mathrm{L}$ & 0.775 & 0.777 & & & U & & DRINKING & & SAN. GRAB & UNFILTERED & 487 \\
\hline 6771 & $\mathrm{pCi} / \mathrm{L}$ & 0.0207 & 0.0214 & & & U & & DRINKING & & SAN. GRAB & UNFILTERED & 487 \\
\hline 6772 & $\mathrm{pCi} / \mathrm{L}$ & 0.00162 & 0.00165 & & & & & RIVER & & CONTINUOUS & FILTER & 307 \\
\hline 6773 & $\mathrm{pCi} / \mathrm{L}$ & 0.00127 & 0.00128 & & & U & & RIVER & & CONTINUOUS & RESIN & 307 \\
\hline 6774 & & & & & & & & RIVER & & CONTINUOUS & FILTER & 252 \\
\hline 6775 & & & & & & & & RIVER & & CONTINUOUS & RESIN & 252 \\
\hline 6776 & $\mathrm{pCi} / \mathrm{L}$ & 0.000997 & 0.000997 & & & U & & RIVER & & CONTINUOUS & RESIN & 463 \\
\hline 6777 & $\mathrm{pCi} / \mathrm{L}$ & 0.00132 & 0.00137 & & & & & RIVER & & CONTINUOUS & FILTER & 463 \\
\hline 6778 & $\mathrm{pCi} / \mathrm{L}$ & 86.2 & 178 & & & U & & SANITARY & & RAW GRAB & UNFILTERED & 525 \\
\hline 6779 & $\mathrm{pCi} / \mathrm{L}$ & 6.39 & 6.39 & & & U & & SANITARY & & RAW GRAB & UNFILTERED & 525 \\
\hline 6780 & $\mathrm{pCi} / \mathrm{L}$ & 1.72 & 3.67 & & & & & SANITARY & & RAW GRAB & UNFILTERED & 525 \\
\hline 6781 & $\mathrm{pCi} / \mathrm{L}$ & 0.299 & 0.31 & & & & & SANITARY & & RAW GRAB & UNFILTERED & 525 \\
\hline 6782 & $\mathrm{pCi} / \mathrm{L}$ & 1.32 & 2.31 & & & & & SANITARY & & RAW GRAB & UNFILTERED & 525 \\
\hline 6783 & $\mathrm{pCi} / \mathrm{L}$ & 89.1 & 183 & & & U & & SANITARY & & RAW GRAB & UNFILTERED & 172 \\
\hline 6784 & $\mathrm{pCi} / \mathrm{L}$ & 5.64 & 5.69 & & & & & SANITARY & & RAW GRAB & UNFILTERED & 172 \\
\hline 6785 & & & & & & & & SANITARY & & RAW GRAB & UNFILTERED & 172 \\
\hline 6786 & & & & & & & & SANITARY & & RAW GRAB & UNFILTERED & 172 \\
\hline 6787 & & & & & & & & SANITARY & & RAW GRAB & UNFILTERED & 172 \\
\hline 6788 & $\mathrm{pCi} / \mathrm{L}$ & 0.558 & 0.558 & & & U & & DRINKING & & SAN. GRAB & UNFILTERED & 530 \\
\hline 6789 & $\mathrm{pCi} / \mathrm{L}$ & 104 & 188 & & & & & DRINKING & & SAN. GRAB & UNFILTERED & 530 \\
\hline 6790 & $\mathrm{pCi} / \mathrm{L}$ & 0.0404 & 0.0704 & & & & & DRINKING & & SAN. GRAB & UNFILTERED & 530 \\
\hline 6791 & $\mathrm{pCi} / \mathrm{L}$ & 0.57 & 0.571 & & & U & & RIVER & & CUMULATIVE & UNFILTERED & 252 \\
\hline 6792 & $\mathrm{pCi} / \mathrm{L}$ & 1.08 & 5.86 & & & U & & RIVER & & CUMULATIVE & UNFILTERED & 252 \\
\hline 6793 & pCi/L & 0.037 & 0.0472 & & & & & RIVER & & CUMULATIVE & UNFILTERED & 252 \\
\hline 6794 & $\mathrm{pCi} / \mathrm{L}$ & 4.21 & 11.3 & & & & & RIVER & & CUMULATIVE & UNFILTERED & 252 \\
\hline 6795 & $\mathrm{pCi} / \mathrm{L}$ & 0.0767 & 0.0857 & & & & & RIVER & & CUMULATIVE & UNFILTERED & 252 \\
\hline 6796 & $\mathrm{pCi} / \mathrm{L}$ & 0.00787 & 0.00822 & & & U & & RIVER & & CUMULATIVE & UNFILTERED & 252 \\
\hline 6797 & $\mathrm{pCi} / \mathrm{L}$ & 0.0573 & 0.0615 & & & & & RIVER & & CUMULATIVE & UNFILTERED & 252 \\
\hline 6798 & pCi/L & 0.56 & 0.563 & & & & & RIVER & & CUMULATIVE & UNFILTERED & 463 \\
\hline 6799 & pCi/L & 0.0474 & 0.053 & & & & & RIVER & & CUMULATIVE & UNFILTERED & 463 \\
\hline 6800 & pCi/L & 0.0155 & 0.0158 & & & & & RIVER & & CUMULATIVE & UNFILTERED & 463 \\
\hline 6801 & $\mathrm{pCi} / \mathrm{L}$ & 0.0509 & 0.0572 & & & & & RIVER & & CUMULATIVE & UNFILTERED & 463 \\
\hline 6802 & $\mathrm{pCi} / \mathrm{L}$ & 1.08 & 5.86 & & & U & & RIVER & & CUMULATIVE & UNFILTERED & 463 \\
\hline 6803 & $\mathrm{pCi} / \mathrm{L}$ & 0.0279 & 0.0366 & & & & & RIVER & & CUMULATIVE & UNFILTERED & 463 \\
\hline 6804 & $\mathrm{pCi} / \mathrm{L}$ & 5.79 & 19.4 & & & & & RIVER & & CUMULATIVE & UNFILTERED & 463 \\
\hline 6805 & pCi/L & 99.1 & 178 & & & U & & SANITARY & & RAW GRAB & UNFILTERED & 524 \\
\hline 6806 & $\mathrm{pCi} / \mathrm{L}$ & 1.24 & 1.24 & & & U & & SANITARY & & RAW GRAB & UNFILTERED & 524 \\
\hline 6807 & $\mathrm{pCi} / \mathrm{L}$ & 0.175 & 0.408 & & & & & SANITARY & & RAW GRAB & UNFILTERED & 524 \\
\hline 6808 & $\mathrm{pCi} / \mathrm{L}$ & 0.0382 & 0.0421 & & & & & SANITARY & & RAW GRAB & UNFILTERED & 524 \\
\hline 6809 & pCi/L & 0.159 & 0.343 & & & & & SANITARY & & RAW GRAB & UNFILTERED & 524 \\
\hline 6810 & $\mathrm{pCi} / \mathrm{L}$ & 90.1 & 167 & & & U & & SANITARY & & RAW GRAB & UNFILTERED & 519 \\
\hline 6811 & pCi/L & 1.07 & 1.07 & & & U & & SANITARY & & RAW GRAB & UNFILTERED & 519 \\
\hline 6812 & pCi/L & 0.00604 & 0.0112 & & & U & & SANITARY & & RAW GRAB & UNFILTERED & 519 \\
\hline 6813 & $\mathrm{pCi} / \mathrm{L}$ & 0.00697 & 0.00736 & & & 10 & & SANITARY & & RAW GRAB & UNFILTERED & 519 \\
\hline
\end{tabular}




\begin{tabular}{|c|c|c|c|c|c|c|c|c|c|c|c|c|}
\hline & $\mathrm{N}$ & $\mathrm{O}$ & $\mathrm{P}$ & $\mathrm{Q}$ & $\mathrm{R}$ & $\mathrm{S}$ & $\mathrm{T}$ & $\mathrm{U}$ & $\mathrm{V}$ & $\mathrm{w}$ & $\mathrm{X}$ & $\mathrm{Y}$ \\
\hline 1 & ANAL_UNITS_RPTD & COUNTING_ERROR & TOTAL_ANAL_ERROR & MIN_DETECTABLE_ACTIVITY & PCNT_MOISTURE & LAB_QUALIFIER & REVIEW_QUALIFIER & SAMP_FROM & SAMP_ITEM & SAMP_MTHD & COLL_MTHD & SAMP_SITE_ID \\
\hline 6814 & $\mathrm{pCi} / \mathrm{L}$ & 0.00779 & 0.00981 & & & $U$ & & SANITARY & & RAW GRAB & UNFILTERED & 519 \\
\hline 6815 & $\mathrm{pCi} / \mathrm{L}$ & 0.00146 & 0.0015 & & & & & RIVER & & CONTINUOUS & FILTER & 307 \\
\hline 6816 & $\mathrm{pCi} / \mathrm{L}$ & 0.00232 & 0.00233 & & & U & & RIVER & & CONTINUOUS & RESIN & 307 \\
\hline 6817 & $\mathrm{pCi} / \mathrm{L}$ & 0.00161 & 0.00168 & & & & & RIVER & & CONTINUOUS & FILTER & 252 \\
\hline 6818 & $\mathrm{pCi} / \mathrm{L}$ & 0.00219 & 0.00221 & & & & & RIVER & & CONTINUOUS & RESIN & 252 \\
\hline 6819 & $\mathrm{pCi} / \mathrm{L}$ & 0.00187 & 0.0019 & & & & & RIVER & & CONTINUOUS & FILTER & 463 \\
\hline 6820 & $\mathrm{pCi} / \mathrm{L}$ & 0.0024 & 0.00243 & & & & & RIVER & & CONTINUOUS & RESIN & 463 \\
\hline 6821 & $\mathrm{pCi} / \mathrm{L}$ & 0.853 & 0.854 & & & U & & IRRIGATION & & RAW GRAB & UNFILTERED & 440 \\
\hline 6822 & $\mathrm{pCi} / \mathrm{L}$ & 99.4 & 188 & & & U & & IRRIGATION & & RAW GRAB & UNFILTERED & 440 \\
\hline 6823 & $\mathrm{pCi} / \mathrm{L}$ & 0.0539 & 0.0628 & & & & & IRRIGATION & & RAW GRAB & UNFILTERED & 440 \\
\hline 6824 & $\mathrm{pCi} / \mathrm{L}$ & 0.0128 & 0.0131 & & & U & & IRRIGATION & & RAW GRAB & UNFILTERED & 440 \\
\hline 6825 & $\mathrm{pCi} / \mathrm{L}$ & 0.0394 & 0.043 & & & & & IRRIGATION & & RAW GRAB & UNFILTERED & 440 \\
\hline 6826 & $\mathrm{pCi} / \mathrm{L}$ & 0.0388 & 0.0435 & & & & & IRRIGATION & & RAW GRAB & UNFILTERED & 440 \\
\hline 6827 & $\mathrm{pCi} / \mathrm{L}$ & 272 & 750 & & & & & DRINKING & & SAN. GRAB & UNFILTERED & 512 \\
\hline $\mid 6828$ & $\mathrm{pCi} / \mathrm{L}$ & 0.639 & 0.639 & & & U & & DRINKING & & SAN. GRAB & UNFILTERED & 512 \\
\hline 6829 & $\mathrm{pCi} / \mathrm{L}$ & 0.0249 & 0.0258 & & & U & & DRINKING & & SAN. GRAB & UNFILTERED & 512 \\
\hline 6830 & $\mathrm{pCi} / \mathrm{L}$ & 2.84 & 2.84 & & & U & & SURFACE & & RAW GRAB & UNFILTERED & 509 \\
\hline 6831 & $\mathrm{pCi} / \mathrm{L}$ & 101 & 181 & & & U & & SURFACE & & RAW GRAB & UNFILTERED & 509 \\
\hline 6832 & $\mathrm{pCi} / \mathrm{L}$ & 0.077 & 0.111 & & & & & SURFACE & & RAW GRAB & UNFILTERED & 509 \\
\hline 6833 & $\mathrm{pCi} / \mathrm{L}$ & 287 & 804 & & & & & SURFACE & & RAW GRAB & UNFILTERED & 136 \\
\hline 6834 & $\mathrm{pCi} / \mathrm{L}$ & 2.36 & 2.36 & & & U & & SURFACE & & RAW GRAB & UNFILTERED & 136 \\
\hline 6835 & $\mathrm{pCi} / \mathrm{L}$ & 108 & 190 & & & & & SURFACE & & RAW GRAB & UNFILTERED & 147 \\
\hline 6836 & $\mathrm{pCi} / \mathrm{L}$ & 2.38 & 2.39 & & & U & & SURFACE & & RAW GRAB & UNFILTERED & 147 \\
\hline 6837 & $\mathrm{pCi} / \mathrm{L}$ & 8.65 & 21.2 & & & & & SURFACE & & RAW GRAB & UNFILTERED & 147 \\
\hline 6838 & $\mathrm{pCi} / \mathrm{L}$ & 2.84 & 3.52 & & & & & SURFACE & & RAW GRAB & UNFILTERED & 147 \\
\hline 6839 & $\mathrm{pCi} / \mathrm{L}$ & 8.59 & 20.9 & & & & & SURFACE & & RAW GRAB & UNFILTERED & 147 \\
\hline 6840 & $\mathrm{pCi} / \mathrm{L}$ & 0.144 & 0.468 & & & & & SURFACE & & RAW GRAB & UNFILTERED & 147 \\
\hline 6841 & $\mathrm{pCi} / \mathrm{L}$ & 0.0019 & 0.00191 & & & & & RIVER & & CONTINUOUS & FILTER & 307 \\
\hline 6842 & $\mathrm{pCi} / \mathrm{L}$ & 0.00415 & 0.00417 & & & & & RIVER & & CONTINUOUS & RESIN & 307 \\
\hline 6843 & $\mathrm{pCi} / \mathrm{L}$ & 0.00251 & 0.00254 & & & & & RIVER & & CONTINUOUS & RESIN & 252 \\
\hline 6844 & $\mathrm{pCi} / \mathrm{L}$ & 0.00135 & 0.00136 & & & U & & RIVER & & CONTINUOUS & FILTER & 252 \\
\hline 6845 & $\mathrm{pCi} / \mathrm{L}$ & 0.00196 & 0.002 & & & & & RIVER & & CONTINUOUS & FILTER & 463 \\
\hline 6846 & $\mathrm{pCi} / \mathrm{L}$ & 0.00325 & 0.00329 & & & & & RIVER & & CONTINUOUS & RESIN & 463 \\
\hline 6847 & $\mathrm{pCi} / \mathrm{L}$ & 0.563 & 0.566 & & & U & & DRINKING & & CUMULATIVE & UNFILTERED & 307 \\
\hline 6848 & $\mathrm{pCi} / \mathrm{L}$ & 0.0557 & 0.0652 & & & & & DRINKING & & CUMULATIVE & UNFILTERED & 307 \\
\hline 6849 & $\mathrm{pCi} / \mathrm{L}$ & 0.0158 & 0.0161 & & & U & & DRINKING & & CUMULATIVE & UNFILTERED & 307 \\
\hline 6850 & $\mathrm{pCi} / \mathrm{L}$ & 0.0542 & 0.0625 & & & & & DRINKING & & CUMULATIVE & UNFILTERED & 307 \\
\hline 6851 & $\mathrm{pCi} / \mathrm{L}$ & 1.08 & 5.87 & & & U & & DRINKING & & CUMULATIVE & UNFILTERED & 307 \\
\hline 6852 & $\mathrm{pCi} / \mathrm{L}$ & 6.07 & 21.3 & & & & & DRINKING & & CUMULATIVE & UNFILTERED & 307 \\
\hline 6853 & $\mathrm{pCi} / \mathrm{L}$ & 0.0361 & 0.0439 & & & & & DRINKING & & CUMULATIVE & UNFILTERED & 307 \\
\hline 6854 & $\mathrm{pCi} / \mathrm{L}$ & 0.0000136 & 0.0000139 & & & & & RIVER & & CONTINUOUS & FILTER & 307 \\
\hline 6855 & $\mathrm{pCi} / \mathrm{L}$ & & 0.0000177 & & & & & RIVER & & CONTINUOUS & RESIN & 307 \\
\hline 6856 & $\mathrm{pCi} / \mathrm{L}$ & 0.00195 & 0.00197 & & & & & RIVER & & CONTINUOUS & FILTER & 307 \\
\hline 6857 & $\mathrm{pCi} / \mathrm{L}$ & 0.0027 & 0.00273 & & & & & RIVER & & CONTINUOUS & RESIN & 307 \\
\hline 6858 & $\mathrm{pCi} / \mathrm{L}$ & 0.0000486 & 0.000049 & & & & & RIVER & & CONTINUOUS & RESIN & 307 \\
\hline 6859 & $\mathrm{pCi} / \mathrm{L}$ & 0.804 & 0.805 & & & U & & RIVER & & CUMULATIVE & UNFILTERED & 252 \\
\hline 6860 & $\mathrm{pCi} / \mathrm{L}$ & 1.06 & 5.79 & & & U & & RIVER & & CUMULATIVE & UNFILTERED & 252 \\
\hline 6861 & $\mathrm{pCi} / \mathrm{L}$ & 0.0467 & 0.0543 & & & & & RIVER & & CUMULATIVE & UNFILTERED & 252 \\
\hline 6862 & $\mathrm{pCi} / \mathrm{L}$ & 0.00681 & 0.00722 & & & U & & RIVER & & CUMULATIVE & UNFILTERED & 252 \\
\hline 6863 & $\mathrm{pCi} / \mathrm{L}$ & 0.0414 & 0.0467 & & & & & RIVER & & CUMULATIVE & UNFILTERED & 252 \\
\hline 6864 & $\mathrm{pCi} / \mathrm{L}$ & 0.0348 & 0.0427 & & & & & RIVER & & CUMULATIVE & UNFILTERED & 252 \\
\hline 6865 & $\mathrm{pCi} / \mathrm{L}$ & 4.23 & 11.3 & & & & & RIVER & & CUMULATIVE & UNFILTERED & 252 \\
\hline
\end{tabular}




\begin{tabular}{|c|c|c|c|c|c|c|c|c|c|c|c|c|}
\hline & $\mathrm{N}$ & $\mathrm{O}$ & $\mathrm{P}$ & $\mathrm{Q}$ & $\mathrm{R}$ & $\mathrm{s}$ & $\mathrm{T}$ & $\mathrm{U}$ & $\mathrm{V}$ & $\mathrm{w}$ & $\mathrm{X}$ & $\mathrm{Y}$ \\
\hline 1 & ANAL_UNITS_RPTD & COUNTING_ERROR & TOTAL_ANAL_ERROR & MIN_DETECTABLE_ACTIVITY & PCNT_MOISTURE & LAB_QUALIFIER & REVIEW_QUALIFIER & SAMP_FROM & SAMP_ITEM & SAMP_MTHD & COLL_MTHD & SAMP_SITE_ID \\
\hline 6866 & $\mathrm{pCi} / \mathrm{L}$ & 0.0000127 & 0.0000129 & & & & & RIVER & & CONTINUOUS & FILTER & 252 \\
\hline 6867 & $\mathrm{pCi} / \mathrm{L}$ & & 0.000000918 & & & & & RIVER & & CONTINUOUS & RESIN & 252 \\
\hline 6868 & $\mathrm{pCi} / \mathrm{L}$ & 0.00125 & 0.00126 & & & & & RIVER & & CONTINUOUS & FILTER & 252 \\
\hline 6869 & $\mathrm{pCi} / \mathrm{L}$ & 0.00217 & 0.00217 & & & U & & RIVER & & CONTINUOUS & RESIN & 252 \\
\hline 6870 & $\mathrm{pCi} / \mathrm{L}$ & 0 & 0.0000243 & & & $\mathrm{U}$ & & RIVER & & CONTINUOUS & RESIN & 252 \\
\hline 6871 & $\mathrm{pCi} / \mathrm{L}$ & 0.000018 & 0.0000185 & & & & & RIVER & & CONTINUOUS & FILTER & 463 \\
\hline 6872 & $\mathrm{pCi} / \mathrm{L}$ & & 0.0000067 & & & & & RIVER & & CONTINUOUS & RESIN & 463 \\
\hline 6873 & $\mathrm{pCi} / \mathrm{L}$ & 0.00144 & 0.00145 & & & & & RIVER & & CONTINUOUS & FILTER & 463 \\
\hline 6874 & $\mathrm{pCi} / \mathrm{L}$ & 0.00366 & 0.0037 & & & & & RIVER & & CONTINUOUS & RESIN & 463 \\
\hline 6875 & $\mathrm{pCi} / \mathrm{L}$ & 0.000024 & 0.0000257 & & & U & & RIVER & & CONTINUOUS & RESIN & 463 \\
\hline 6876 & $\mathrm{pCi} / \mathrm{L}$ & 0.595 & 0.595 & & & U & & RIVER & & CUMULATIVE & UNFILTERED & 463 \\
\hline 6877 & $\mathrm{pCi} / \mathrm{L}$ & 1.12 & 6.1 & & & U & & RIVER & & CUMULATIVE & UNFILTERED & 463 \\
\hline 6878 & $\mathrm{pCi} / \mathrm{L}$ & 0.0472 & 0.054 & & & & & RIVER & & CUMULATIVE & UNFILTERED & 463 \\
\hline 6879 & $\mathrm{pCi} / \mathrm{L}$ & 0.0114 & 0.0117 & & & U & & RIVER & & CUMULATIVE & UNFILTERED & 463 \\
\hline 6880 & $\mathrm{pCi} / \mathrm{L}$ & 0.0435 & 0.0485 & & & & & RIVER & & CUMULATIVE & UNFILTERED & 463 \\
\hline 6881 & $\mathrm{pCi} / \mathrm{L}$ & 0.0335 & 0.0409 & & & & & RIVER & & CUMULATIVE & UNFILTERED & 463 \\
\hline 6882 & $\mathrm{pCi} / \mathrm{L}$ & 5.06 & 15.2 & & & & & RIVER & & CUMULATIVE & UNFILTERED & 463 \\
\hline 6883 & $\mathrm{pCi} / \mathrm{L}$ & 102 & 185 & & & U & & DRINKING & & SAN. GRAB & UNFILTERED & 186 \\
\hline 6884 & $\mathrm{pCi} / \mathrm{L}$ & 0.527 & 0.527 & & & U & & DRINKING & & SAN. GRAB & UNFILTERED & 186 \\
\hline 6885 & $\mathrm{pCi} / \mathrm{L}$ & 0.0217 & 0.0224 & & & U & & DRINKING & & SAN. GRAB & UNFILTERED & 186 \\
\hline 6886 & $\mathrm{pCi} / \mathrm{L}$ & 270 & 700 & & & & & DRINKING & & SAN. GRAB & UNFILTERED & 512 \\
\hline 6887 & $\mathrm{pCi} / \mathrm{L}$ & 0.669 & 0.669 & & & U & & DRINKING & & SAN. GRAB & UNFILTERED & 512 \\
\hline 6888 & $\mathrm{pCi} / \mathrm{L}$ & 0.0268 & 0.0272 & & & $U$ & & DRINKING & & SAN. GRAB & UNFILTERED & 512 \\
\hline 6889 & $\mathrm{pCi} / \mathrm{L}$ & 101 & 184 & & & U & & DRINKING & & SAN. GRAB & UNFILTERED & 144 \\
\hline 6890 & $\mathrm{pCi} / \mathrm{L}$ & 0.609 & 0.61 & & & U & & DRINKING & & SAN. GRAB & UNFILTERED & 144 \\
\hline 6891 & $\mathrm{pCi} / \mathrm{L}$ & 0.0229 & 0.0233 & & & U & & DRINKING & & SAN. GRAB & UNFILTERED & 144 \\
\hline 6892 & $\mathrm{pCi} / \mathrm{L}$ & 99.1 & 183 & & & U & & DRINKING & & SAN. GRAB & UNFILTERED & 187 \\
\hline 6893 & $\mathrm{pCi} / \mathrm{L}$ & 0.027 & 0.0277 & & & U & & DRINKING & & SAN. GRAB & UNFILTERED & 187 \\
\hline 6894 & $\mathrm{pCi} / \mathrm{L}$ & 0.53 & 0.532 & & & U & & DRINKING & & SAN. GRAB & UNFILTERED & 187 \\
\hline 6895 & $\mathrm{pCi} / \mathrm{L}$ & 0.0399 & 0.0499 & & & & & DRINKING & & RAW GRAB & UNFILTERED & 553 \\
\hline 6896 & $\mathrm{pCi} / \mathrm{L}$ & 129 & 236 & & & U & & DRINKING & & RAW GRAB & UNFILTERED & 553 \\
\hline 6897 & $\mathrm{pCi} / \mathrm{L}$ & 0.62 & 0.62 & & & U & & DRINKING & & RAW GRAB & UNFILTERED & 553 \\
\hline 6898 & $\mathrm{pCi} / \mathrm{L}$ & 0.539 & 0.539 & & & U & & QC_SAMPLE & & RAW GRAB & UNFILTERED & 550 \\
\hline 6899 & $\mathrm{pCi} / \mathrm{L}$ & 0.0348 & 0.043 & & & & & QC_SAMPLE & & RAW GRAB & UNFILTERED & 550 \\
\hline 6900 & $\mathrm{pCi} / \mathrm{L}$ & 10.7 & 22.8 & & & & & QC_SAMPLE & & RAW GRAB & UNFILTERED & 550 \\
\hline 6901 & $\mathrm{pCi} / \mathrm{L}$ & 0.0404 & 0.0447 & & & & & QC_SAMPLE & & RAW GRAB & UNFILTERED & 550 \\
\hline 6902 & $\mathrm{pCi} / \mathrm{L}$ & 0.00781 & 0.00816 & & & U & & QC_SAMPLE & & RAW GRAB & UNFILTERED & 550 \\
\hline 6903 & $\mathrm{pCi} / \mathrm{L}$ & 0.0463 & 0.0539 & & & & & QC_SAMPLE & & RAW GRAB & UNFILTERED & 550 \\
\hline 6904 & $\mathrm{pCi} / \mathrm{L}$ & 0.758 & 0.758 & & & $U$ & & QC_SAMPLE & & RAW GRAB & UNFILTERED & 247 \\
\hline 6905 & $\mathrm{pCi} / \mathrm{L}$ & 0.0378 & 0.0453 & & & & & QC_SAMPLE & & RAW GRAB & UNFILTERED & 247 \\
\hline 6906 & $\mathrm{pCi} / \mathrm{L}$ & 6.97 & 12.3 & & & & & QC_SAMPLE & & RAW GRAB & UNFILTERED & 247 \\
\hline 6907 & $\mathrm{pCi} / \mathrm{L}$ & 0.0377 & 0.0423 & & & & & QC_SAMPLE & & RAW GRAB & UNFILTERED & 247 \\
\hline 6908 & $\mathrm{pCi} / \mathrm{L}$ & 0.00716 & 0.00756 & & & U & & QC_SAMPLE & & RAW GRAB & UNFILTERED & 247 \\
\hline 6909 & $\mathrm{pCi} / \mathrm{L}$ & 0.0421 & 0.0487 & & & & & QC_SAMPLE & & RAW GRAB & UNFILTERED & 247 \\
\hline 6910 & $\mathrm{pCi} / \mathrm{L}$ & 0.00311 & 0.00314 & & & & & RIVER & & CONTINUOUS & RESIN & 307 \\
\hline 6911 & $\mathrm{pCi} / \mathrm{L}$ & 0.00107 & 0.0011 & & & & & RIVER & & CONTINUOUS & FILTER & 307 \\
\hline 6912 & $\mathrm{pCi} / \mathrm{L}$ & 0.00117 & 0.00117 & & & U & & RIVER & & CONTINUOUS & FILTER & 252 \\
\hline 6913 & $\mathrm{pCi} / \mathrm{L}$ & 0.00217 & 0.00217 & & & U & & RIVER & & CONTINUOUS & RESIN & 252 \\
\hline 6914 & $\mathrm{pCi} / \mathrm{L}$ & 0.00246 & 0.00246 & & & U & & RIVER & & CONTINUOUS & RESIN & 463 \\
\hline 6915 & $\mathrm{pCi} / \mathrm{L}$ & 0.00162 & 0.00165 & & & & & RIVER & & CONTINUOUS & FILTER & 463 \\
\hline 6916 & $\mathrm{pCi} / \mathrm{L}$ & 0.00282 & 0.00282 & & & U & & RIVER & & CONTINUOUS & RESIN & 307 \\
\hline 6917 & $\mathrm{pCi} / \mathrm{L}$ & 0.0013 & 0.0013 & & & 10 & & RIVER & & CONTINUOUS & FILTER & 307 \\
\hline
\end{tabular}




\begin{tabular}{|c|c|c|c|c|c|c|c|c|c|c|c|c|}
\hline & $\mathrm{N}$ & $\mathrm{O}$ & $\mathrm{P}$ & $\mathrm{Q}$ & $\mathrm{R}$ & $\mathrm{S}$ & $\mathrm{T}$ & $\mathrm{U}$ & $\mathrm{V}$ & $\mathrm{w}$ & $\mathrm{X}$ & $\mathrm{Y}$ \\
\hline 1 & ANAL_UNITS_RPTD & COUNTING_ERROR & TOTAL_ANAL_ERROR & MIN_DETECTABLE_ACTIVITY & PCNT_MOISTURE & LAB_QUALIFIER & REVIEW_QUALIFIER & SAMP_FROM & SAMP_ITEM & SAMP_MTHD & COLL_MTHD & SAMP_SITE_ID \\
\hline 6918 & $\mathrm{pCi} / \mathrm{L}$ & 0.0481 & 0.0532 & & & & & RIVER & & CUMULATIVE & UNFILTERED & 252 \\
\hline 6919 & $\mathrm{pCi} / \mathrm{L}$ & 0.0114 & 0.0117 & & & U & & RIVER & & CUMULATIVE & UNFILTERED & 252 \\
\hline 6920 & $\mathrm{pCi} / \mathrm{L}$ & 0.0535 & 0.0607 & & & & & RIVER & & CUMULATIVE & UNFILTERED & 252 \\
\hline 6921 & $\mathrm{pCi} / \mathrm{L}$ & 1.16 & 6.18 & & & $\mathrm{U}$ & & RIVER & & CUMULATIVE & UNFILTERED & 252 \\
\hline 6922 & $\mathrm{pCi} / \mathrm{L}$ & 0.00244 & 0.00247 & & & & & RIVER & & CONTINUOUS & RESIN & 252 \\
\hline 6923 & $\mathrm{pCi} / \mathrm{L}$ & 0.00145 & 0.00146 & & & & & RIVER & & CONTINUOUS & FILTER & 252 \\
\hline 6924 & $\mathrm{pCi} / \mathrm{L}$ & 3.94 & 10.7 & & & & & RIVER & & CUMULATIVE & UNFILTERED & 252 \\
\hline 6925 & $\mathrm{pCi} / \mathrm{L}$ & 0.0316 & 0.0423 & & & & & RIVER & & CUMULATIVE & UNFILTERED & 252 \\
\hline 6926 & $\mathrm{pCi} / \mathrm{L}$ & 0.777 & 0.781 & & & U & & RIVER & & CUMULATIVE & UNFILTERED & 252 \\
\hline 6927 & $\mathrm{pCi} / \mathrm{L}$ & 0.0382 & 0.042 & & & & & RIVER & & CUMULATIVE & UNFILTERED & 463 \\
\hline 6928 & $\mathrm{pCi} / \mathrm{L}$ & 0.00412 & 0.00475 & & & U & & RIVER & & CUMULATIVE & UNFILTERED & 463 \\
\hline 6929 & $\mathrm{pCi} / \mathrm{L}$ & 0.043 & 0.0489 & & & & & RIVER & & CUMULATIVE & UNFILTERED & 463 \\
\hline 6930 & $\mathrm{pCi} / \mathrm{L}$ & 1.16 & 6.08 & & & U & & RIVER & & CUMULATIVE & UNFILTERED & 463 \\
\hline 6931 & $\mathrm{pCi} / \mathrm{L}$ & 4.67 & 14.1 & & & & & RIVER & & CUMULATIVE & UNFILTERED & 463 \\
\hline 6932 & $\mathrm{pCi} / \mathrm{L}$ & 0.0301 & 0.0431 & & & & & RIVER & & CUMULATIVE & UNFILTERED & 463 \\
\hline 6933 & $\mathrm{pCi} / \mathrm{L}$ & 0.57 & 0.57 & & & U & & RIVER & & CUMULATIVE & UNFILTERED & 463 \\
\hline 6934 & $\mathrm{pCi} / \mathrm{L}$ & 0.00296 & 0.00296 & & & U & & RIVER & & CONTINUOUS & RESIN & 463 \\
\hline 6935 & $\mathrm{pCi} / \mathrm{L}$ & 0.002 & 0.00201 & & & U & & RIVER & & CONTINUOUS & FILTER & 463 \\
\hline 6936 & $\mathrm{pCi} / \mathrm{L}$ & 0.0226 & 0.0232 & & & U & & DRINKING & & SAN. GRAB & UNFILTERED & 512 \\
\hline 6937 & $\mathrm{pCi} / \mathrm{L}$ & 322 & 742 & & & & & DRINKING & & SAN. GRAB & UNFILTERED & 512 \\
\hline 6938 & $\mathrm{pCi} / \mathrm{L}$ & 0.775 & 0.777 & & & U & & DRINKING & & SAN. GRAB & UNFILTERED & 512 \\
\hline 6939 & $\mathrm{pCi} / \mathrm{L}$ & 0.022 & 0.0229 & & & U & & DRINKING & & SAN. GRAB & UNFILTERED & 487 \\
\hline 6940 & $\mathrm{pCi} / \mathrm{L}$ & 0.767 & 0.768 & & & U & & DRINKING & & SAN. GRAB & UNFILTERED & 487 \\
\hline 6941 & $\mathrm{pCi} / \mathrm{L}$ & 96.7 & 177 & & & U & & DRINKING & & SAN. GRAB & UNFILTERED & 487 \\
\hline 6942 & $\mathrm{pCi} / \mathrm{L}$ & 0.0032 & 0.0032 & & & U & & RIVER & & CONTINUOUS & RESIN & 307 \\
\hline 6943 & $\mathrm{pCi} / \mathrm{L}$ & 0.00111 & 0.00111 & & & & & RIVER & & CONTINUOUS & FILTER & 307 \\
\hline 6944 & $\mathrm{pCi} / \mathrm{L}$ & 0.00142 & 0.00142 & & & U & & RIVER & & CONTINUOUS & FILTER & 252 \\
\hline 6945 & $\mathrm{pCi} / \mathrm{L}$ & 0.00354 & 0.00355 & & & U & & RIVER & & CONTINUOUS & RESIN & 252 \\
\hline 6946 & $\mathrm{pCi} / \mathrm{L}$ & 0.0015 & 0.0015 & & & U & & RIVER & & CONTINUOUS & FILTER & 463 \\
\hline 6947 & $\mathrm{pCi} / \mathrm{L}$ & 0.0038 & 0.0038 & & & U & & RIVER & & CONTINUOUS & RESIN & 463 \\
\hline 6948 & $\mathrm{pCi} / \mathrm{L}$ & 0.162 & 0.175 & & & & & SANITARY & & RAW GRAB & UNFILTERED & 525 \\
\hline 6949 & $\mathrm{pCi} / \mathrm{L}$ & 0.977 & 2.59 & & & & & SANITARY & & RAW GRAB & UNFILTERED & 525 \\
\hline 6950 & $\mathrm{pCi} / \mathrm{L}$ & 0.782 & 1.72 & & & & & SANITARY & & RAW GRAB & UNFILTERED & 525 \\
\hline 6951 & $\mathrm{pCi} / \mathrm{L}$ & 0.656 & 0.657 & & & U & & SANITARY & & RAW GRAB & UNFILTERED & 525 \\
\hline 6952 & $\mathrm{pCi} / \mathrm{L}$ & 101 & 182 & & & U & & SANITARY & & RAW GRAB & UNFILTERED & 525 \\
\hline 6953 & $\mathrm{pCi} / \mathrm{L}$ & 0.274 & 0.793 & & & & & SANITARY & & RAW GRAB & UNFILTERED & 172 \\
\hline 6954 & $\mathrm{pCi} / \mathrm{L}$ & 0.0615 & 0.0717 & & & & & SANITARY & & RAW GRAB & UNFILTERED & 172 \\
\hline 6955 & $\mathrm{pCi} / \mathrm{L}$ & 0.284 & 0.85 & & & & & SANITARY & & RAW GRAB & UNFILTERED & 172 \\
\hline 6956 & $\mathrm{pCi} / \mathrm{L}$ & 0.719 & 0.719 & & & U & & SANITARY & & RAW GRAB & UNFILTERED & 172 \\
\hline 6957 & $\mathrm{pCi} / \mathrm{L}$ & 105 & 187 & & & U & & SANITARY & & RAW GRAB & UNFILTERED & 172 \\
\hline 6958 & $\mathrm{pCi} / \mathrm{L}$ & 0.0562 & 0.114 & & & & & DRINKING & & SAN. GRAB & UNFILTERED & 530 \\
\hline 6959 & $\mathrm{pCi} / \mathrm{L}$ & 0.556 & 0.556 & & & U & & DRINKING & & SAN. GRAB & UNFILTERED & 530 \\
\hline 6960 & $\mathrm{pCi} / \mathrm{L}$ & 111 & 194 & & & & & DRINKING & & SAN. GRAB & UNFILTERED & 530 \\
\hline 6961 & $\mathrm{pCi} / \mathrm{L}$ & 0.238 & 0.378 & & & & & SANITARY & & RAW GRAB & UNFILTERED & 524 \\
\hline 6962 & $\mathrm{pCi} / \mathrm{L}$ & 0.0506 & 0.0521 & & & & & SANITARY & & RAW GRAB & UNFILTERED & 524 \\
\hline 6963 & $\mathrm{pCi} / \mathrm{L}$ & 0.266 & 0.453 & & & & & SANITARY & & RAW GRAB & UNFILTERED & 524 \\
\hline 6964 & $\mathrm{pCi} / \mathrm{L}$ & 0.705 & 0.705 & & & $U$ & & SANITARY & & RAW GRAB & UNFILTERED & 524 \\
\hline 6965 & $\mathrm{pCi} / \mathrm{L}$ & 127 & 272 & & & U & & SANITARY & & RAW GRAB & UNFILTERED & 524 \\
\hline 6966 & $\mathrm{pCi} / \mathrm{L}$ & 0.87 & 0.87 & & & 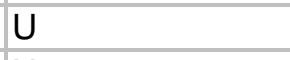 & & SANITARY & & RAW GRAB & UNFILTERED & 519 \\
\hline 6967 & $\mathrm{pCi} / \mathrm{L}$ & 0.0201 & 0.0211 & & & U & & SANITARY & & RAW GRAB & UNFILTERED & 519 \\
\hline 6968 & $\mathrm{pCi} / \mathrm{L}$ & 0.0161 & 0.0164 & & & U & & SANITARY & & RAW GRAB & UNFILTERED & 519 \\
\hline 6969 & $\mathrm{pCi} / \mathrm{L}$ & 0.017 & 0.0195 & & & $U_{2}$ & & SANITARY & & RAW GRAB & UNFILTERED & 519 \\
\hline
\end{tabular}




\begin{tabular}{|c|c|c|c|c|c|c|c|c|c|c|c|c|}
\hline & $\mathrm{N}$ & $\mathrm{O}$ & $\mathrm{P}$ & $\mathrm{Q}$ & $\mathrm{R}$ & $\mathrm{s}$ & $\mathrm{T}$ & $\mathrm{u}$ & $\mathrm{V}$ & w & $\mathrm{x}$ & $\bar{Y}$ \\
\hline 1 & ANAL_UNITS_RPTD & COUNTING_ERROR & $\mid$ TOTAL_ANAL_ERROR & MIN_DETECTABLE_ACTIVITY & PCNT_MOISTURE & LAB_QUALIFIER & REVIEW_QUALIFIER & SAMP_FROM & SAMP_ITEM & SAMP_MTHD & COLL_MTHD & SAMP_SITE_ID \\
\hline 6970 & $\mathrm{pCi} / \mathrm{L}$ & 120 & 259 & & & $U$ & & SANITARY & & RAW GRAB & UNFILTERED & 519 \\
\hline 6971 & $\mathrm{pCi} / \mathrm{L}$ & 0.00132 & 0.00135 & & & & & RIVER & & CONTINUOUS & FILTER & 307 \\
\hline 6972 & $\mathrm{pCi} / \mathrm{L}$ & 0.00263 & 0.00264 & & & $\mathrm{U}$ & & RIVER & & CONTINUOUS & RESIN & 307 \\
\hline \begin{tabular}{|l|l|}
6973 \\
\end{tabular} & $\mathrm{pCi} / \mathrm{L}$ & 0.704 & 0.704 & & & $\mathrm{U}$ & & RIVER & & CUMULATIVE & UNFILTERED & 252 \\
\hline \begin{tabular}{|l|}
6974 \\
\end{tabular} & $\mathrm{pCi} / \mathrm{L}$ & 0.0426 & 0.0477 & & & & & RIVER & & CUMULATIVE & UNFILTERED & 252 \\
\hline 6975 & $\mathrm{pCi} / \mathrm{L}$ & 0.0111 & 0.0114 & & & $\mathrm{U}$ & & RIVER & & CUMULATIVE & UNFILTERED & 252 \\
\hline 6976 & $\mathrm{pCi} / \mathrm{L}$ & 0.0456 & 0.0518 & & & & & RIVER & & CUMULATIVE & UNFILTERED & 252 \\
\hline \begin{tabular}{|l|}
6977 \\
\end{tabular} & $\mathrm{pCi} / \mathrm{L}$ & 1.06 & 5.78 & & & U & & RIVER & & CUMULATIVE & UNFILTERED & 252 \\
\hline 6978 & $\mathrm{pCi} / \mathrm{L}$ & 3.96 & 10.4 & & & & & RIVER & & CUMULATIVE & UNFILTERED & 252 \\
\hline 6979 & $\mathrm{pCi} / \mathrm{L}$ & 0.037 & 0.0414 & & & & & RIVER & & CUMULATIVE & UNFILTERED & 252 \\
\hline \begin{tabular}{|l|}
6980 \\
\end{tabular} & $\mathrm{pCi} / \mathrm{L}$ & 0.00185 & 0.00189 & & & & & RIVER & & CONTINUOUS & FILTER & 252 \\
\hline \begin{tabular}{|l|}
6981 \\
\end{tabular} & $\mathrm{pCi} / \mathrm{L}$ & 0.00288 & 0.00288 & & & $U$ & & RIVER & & CONTINUOUS & RESIN & 252 \\
\hline \begin{tabular}{|c|}
6982 \\
\end{tabular} & $\mathrm{pCi} / \mathrm{L}$ & 0.693 & 0.694 & & & $U$ & & RIVER & & CUMULATIVE & UNFILTERED & 463 \\
\hline 6983 & $\mathrm{pCi} / \mathrm{L}$ & 0.00846 & 0.00879 & & & $U$ & & RIVER & & CUMULATIVE & UNFILTERED & 463 \\
\hline 6984 & $\mathrm{pCi} / \mathrm{L}$ & 0.0314 & 0.034 & & & & & RIVER & & CUMULATIVE & UNFILTERED & 463 \\
\hline 6985 & $\mathrm{pCi} / \mathrm{L}$ & 1.05 & 5.72 & & & $U$ & & RIVER & & CUMULATIVE & UNFILTERED & 463 \\
\hline \begin{tabular}{|l|}
6986 \\
\end{tabular} & $\mathrm{pCi} / \mathrm{L}$ & 4.77 & 14.1 & & & & & RIVER & & CUMULATIVE & UNFILTERED & 463 \\
\hline \begin{tabular}{|l|}
6987 \\
\end{tabular} & $\mathrm{pCi} / \mathrm{L}$ & 0.0374 & 0.0398 & & & $U$ & & RIVER & & CUMULATIVE & UNFILTERED & 463 \\
\hline \begin{tabular}{|c|}
6988 \\
\end{tabular} & $\mathrm{pCi} / \mathrm{L}$ & 0.0296 & 0.0305 & & & & & RIVER & & CUMULATIVE & UNFILTERED & 463 \\
\hline 6989 & $\mathrm{pCi} / \mathrm{L}$ & 0.00252 & 0.00254 & & & & & RIVER & & CONTINUOUS & RESIN & 463 \\
\hline 6990 & $\mathrm{pCi} / \mathrm{L}$ & 0.000835 & 0.000847 & & & & & RIVER & & CONTINUOUS & FILTER & 463 \\
\hline 6991 & $\mathrm{pCi} / \mathrm{L}$ & 266 & 721 & & & & & DRINKING & & SAN. GRAB & UNFILTERED & 512 \\
\hline \begin{tabular}{|l|}
6992 \\
\end{tabular} & $\mathrm{pCi} / \mathrm{L}$ & 0.547 & 0.548 & & & U & & DRINKING & & SAN. GRAB & UNFILTERED & 512 \\
\hline \begin{tabular}{|l|}
6993 \\
\end{tabular} & $\mathrm{pCi} / \mathrm{L}$ & 0.0221 & 0.0232 & & & U & & DRINKING & & SAN. GRAB & UNFILTERED & 512 \\
\hline \begin{tabular}{|c|}
6994 \\
\end{tabular} & $\mathrm{pCi} / \mathrm{L}$ & 107 & 200 & & & $U$ & & SURFACE & & RAW GRAB & UNFILTERED & 509 \\
\hline \begin{tabular}{|c|}
69955 \\
\end{tabular} & $\mathrm{pCi} / \mathrm{L}$ & 0.0719 & 0.0911 & & & & & SURFACE & & RAW GRAB & UNFILTERED & 509 \\
\hline \begin{tabular}{|l|}
6996 \\
\end{tabular} & $\mathrm{pCi} / \mathrm{L}$ & 1.52 & 1.52 & & & $U$ & & SURFACE & & RAW GRAB & UNFILTERED & 509 \\
\hline \begin{tabular}{|l|}
6997 \\
\end{tabular} & $\mathrm{pCi} / \mathrm{L}$ & 2.53 & 2.53 & & & $U$ & & SURFACE & & RAW GRAB & UNFILTERED & 136 \\
\hline 6998 & $\mathrm{pCi} / \mathrm{L}$ & 260 & 689 & & & & & SURFACE & & RAW GRAB & UNFILTERED & 136 \\
\hline \begin{tabular}{|l|}
6999 \\
\end{tabular} & $\mathrm{pCi} / \mathrm{L}$ & 2.21 & 2.21 & & & U & & SURFACE & & RAW GRAB & UNFILTERED & 147 \\
\hline 7000 & $\mathrm{pCi} / \mathrm{L}$ & 7.29 & 13.2 & & & & & SURFACE & & RAW GRAB & UNFILTERED & 147 \\
\hline \begin{tabular}{|l|}
7001 \\
\end{tabular} & $\mathrm{pCi} / \mathrm{L}$ & 1.67 & 1.76 & & & & & SURFACE & & RAW GRAB & UNFILTERED & 147 \\
\hline \begin{tabular}{|l|}
7002 \\
\end{tabular} & $\mathrm{pCi} / \mathrm{L}$ & 7.67 & 14.4 & & & & & SURFACE & & RAW GRAB & UNFILTERED & 147 \\
\hline 7003 & $\mathrm{pCi} / \mathrm{L}$ & 120 & 218 & & & & & SURFACE & & RAW GRAB & UNFILTERED & 147 \\
\hline 7004 & $\mathrm{pCi} / \mathrm{L}$ & 0.142 & 0.315 & & & & & SURFACE & & RAW GRAB & UNFILTERED & 147 \\
\hline 7005 & & & & & & & & RIVER & & CONTINUOUS & RESIN & 307 \\
\hline \begin{tabular}{|l|}
7006 \\
\end{tabular} & & & & & & & & RIVER & & CONTINUOUS & FILTER & 307 \\
\hline \begin{tabular}{|l|}
7007 \\
\end{tabular} & $\mathrm{pCi} / \mathrm{L}$ & 0.00191 & 0.00191 & & & $U$ & & RIVER & & CONTINUOUS & FILTER & 252 \\
\hline \begin{tabular}{|c|}
7008 \\
\end{tabular} & $\mathrm{pCi} / \mathrm{L}$ & 0.00284 & 0.00289 & & & & & RIVER & & CONTINUOUS & RESIN & 252 \\
\hline 7009 & $\mathrm{pCi} / \mathrm{L}$ & 0.00185 & 0.00186 & & & $U$ & & RIVER & & CONTINUOUS & FILTER & 463 \\
\hline 7010 & $\mathrm{pCi} / \mathrm{L}$ & 0.00255 & 0.00256 & & & $U$ & & RIVER & & CONTINUOUS & RESIN & 463 \\
\hline 7011 & $\mathrm{pCi} / \mathrm{L}$ & 0.482 & 0.482 & & & $U$ & & DRINKING & & CUMULATIVE & UNFILTERED & 307 \\
\hline 7012 & $\mathrm{pCi} / \mathrm{L}$ & 0.0325 & 0.04 & & & & & DRINKING & & CUMULATIVE & UNFILTERED & 307 \\
\hline 7013 & $\mathrm{pCi} / \mathrm{L}$ & 4.92 & 14.9 & & & & & DRINKING & & CUMULATIVE & UNFILTERED & 307 \\
\hline 7014 & $\mathrm{pCi} / \mathrm{L}$ & 1.18 & 6.63 & & & $U$ & & DRINKING & & CUMULATIVE & UNFILTERED & 307 \\
\hline 7015 & $\mathrm{pCi} / \mathrm{L}$ & 0.0558 & 0.063 & & & & & DRINKING & & CUMULATIVE & UNFILTERED & 307 \\
\hline 7016 & $\mathrm{pCi} / \mathrm{L}$ & 0.0206 & 0.0211 & & & & & DRINKING & & CUMULATIVE & UNFILTERED & 307 \\
\hline 7017 & $\mathrm{pCi} / \mathrm{L}$ & 0.0681 & 0.0808 & & & & & DRINKING & & CUMULATIVE & UNFILTERED & 307 \\
\hline 7018 & $\mathrm{pCi} / \mathrm{L}$ & 0.00000859 & 0.00000876 & & & & & RIVER & & CONTINUOUS & FILTER & 307 \\
\hline 7019 & $\mathrm{pCi} / \mathrm{L}$ & 0.00111 & 0.00111 & & & U & & RIVER & & CONTINUOUS & FILTER & 307 \\
\hline 7020 & $\mathrm{pCi} / \mathrm{L}$ & 0.00173 & 0.00175 & & & & & RIVER & & CONTINUOUS & RESIN & 307 \\
\hline 7021 & $\mathrm{pCi} / \mathrm{L}$ & 0.0000368 & 0.000037 & & & $\mathrm{U}$ & & RIVER & & CONTINUOUS & RESIN & 307 \\
\hline
\end{tabular}




\begin{tabular}{|c|c|c|c|c|c|c|c|c|c|c|c|c|}
\hline & $\mathrm{N}$ & $\mathrm{O}$ & $P$ & $\mathrm{Q}$ & $\mathrm{R}$ & $\mathrm{s}$ & $T$ & $\mathrm{U}$ & $\overline{\mathrm{V}}$ & $\mathrm{W}$ & $\mathrm{X}$ & $\bar{Y}$ \\
\hline 1 & ANAL_UNITS_RPTD & COUNTING_ERROR & TOTAL_ANAL_ERROR & MIN_DETECTABLE_ACTIVITY & PCNT_MOISTURE & LAB_QUALIFIER & REVIEW_QUALIFIER & SAMP_FROM & SAMP_ITEM & SAMP_MTHD & COLL_MTHD & SAMP_SITE_ID \\
\hline 7022 & $\mathrm{pCi} / \mathrm{L}$ & & 0.00000337 & & & & & RIVER & & CONTINUOUS & RESIN & 307 \\
\hline 7023 & $\mathrm{pCi} / \mathrm{L}$ & 0.00162 & 0.00163 & & & $\mathrm{U}$ & & RIVER & & CONTINUOUS & RESIN & 252 \\
\hline 7024 & $\mathrm{pCi} / \mathrm{L}$ & 0.0000177 & 0.000018 & & & $\mathrm{U}$ & & RIVER & & CONTINUOUS & RESIN & 252 \\
\hline 7025 & $\mathrm{pCi} / \mathrm{L}$ & 0.00000971 & 0.00001 & & & & & RIVER & & CONTINUOUS & FILTER & 252 \\
\hline 7026 & $\mathrm{pCi} / \mathrm{L}$ & & 0.00000047 & & & & & RIVER & & CONTINUOUS & RESIN & 252 \\
\hline 7027 & $\mathrm{pCi} / \mathrm{L}$ & 0.000815 & 0.000823 & & & & & RIVER & & CONTINUOUS & FILTER & 252 \\
\hline 7028 & $\mathrm{pCi} / \mathrm{L}$ & 0.0000127 & 0.000013 & & & & & RIVER & & CONTINUOUS & FILTER & 463 \\
\hline 7029 & $\mathrm{pCi} / \mathrm{L}$ & 0.00134 & 0.00135 & & & & & RIVER & & CONTINUOUS & FILTER & 463 \\
\hline 7030 & $\mathrm{pCi} / \mathrm{L}$ & 0.00347 & 0.0035 & & & & & RIVER & & CONTINUOUS & RESIN & 463 \\
\hline 7031 & $\mathrm{pCi} / \mathrm{L}$ & 0.0000504 & 0.0000508 & & & $\mathrm{U}$ & & RIVER & & CONTINUOUS & RESIN & 463 \\
\hline 7032 & $\mathrm{pCi} / \mathrm{L}$ & & 0.0000037 & & & & & RIVER & & CONTINUOUS & RESIN & 463 \\
\hline
\end{tabular}




\begin{tabular}{|c|c|c|c|}
\hline & $\mathrm{Z}$ & $\overline{\mathrm{AA}}$ & $\mathrm{AB}$ \\
\hline 1 & SAMP_SITE_NAME & WELL_NAME & SAMPLE_LOCATION \\
\hline 2 & $100 \mathrm{~F}$ TRENCH & & \\
\hline 3 & RICHLAND SAN PLANT & & \\
\hline 4 & 100 F AREA-RIVER & & \\
\hline 5 & VERNITA & & \\
\hline 6 & 300 SAN WATER PLANT & & \\
\hline 7 & 100 F TRENCH & & \\
\hline 8 & VERNITA & & \\
\hline 9 & VERNITA & & \\
\hline 10 & 300 SAN WATER PLANT & & \\
\hline 11 & RICHLAND SAN PLANT & & \\
\hline 12 & 100 F AREA-RIVER & & \\
\hline 13 & RICH.PMPHS HRM 46.4 & & \\
\hline 14 & VERNITA & & \\
\hline 15 & VERNITA & & \\
\hline 16 & 216 B 63 DITCH 200 E & & \\
\hline 17 & 222-S POND $200 \mathrm{~W}$ & & \\
\hline 18 & B POND NORTH SIDE & & \\
\hline 19 & GABLE POND N END & & \\
\hline 20 & REDOX POND & & \\
\hline 21 & T POND & & \\
\hline 22 & U POND & & \\
\hline 23 & RICHLAND SAN PLANT & & \\
\hline 24 & 300 POND & & \\
\hline 25 & RICH.PMPHS HRM 46.4 & & \\
\hline 26 & RICH.PMPHS HRM 46.4 & & \\
\hline 27 & RICH.PMPHS HRM 46.4 & & \\
\hline 28 & VERNITA & & \\
\hline 29 & VERNITA & & \\
\hline 30 & VERNITA & & \\
\hline 31 & 300 SAN WATER PLANT & & \\
\hline 32 & 100 F AREA-RIVER & & \\
\hline 33 & RICH.PMPHS HRM 46.4 & & \\
\hline 34 & VERNITA & & \\
\hline 35 & VERNITA & & \\
\hline 36 & RICHLAND SAN PLANT & & \\
\hline 37 & 300 SAN WATER PLANT & & \\
\hline 38 & RICHLAND SAN PLANT & & \\
\hline 39 & VERNITA & & \\
\hline 40 & 300 SAN WATER PLANT & & \\
\hline 41 & RICHLAND SAN PLANT & & \\
\hline 42 & VERNITA & & \\
\hline 43 & 300 SAN WATER PLANT & & \\
\hline 44 & $100 \mathrm{~F}$ AREA-RIVER & & \\
\hline 45 & VERNITA & & \\
\hline 46 & RICH.PMPHS HRM 46.4 & & \\
\hline 47 & RICH.PMPHS HRM 46.4 & & \\
\hline 48 & VERNITA & & \\
\hline 49 & VERNITA & & \\
\hline 50 & VERNITA & & \\
\hline 51 & 300 SAN WATER PLANT & & \\
\hline 52 & 100 F AREA-RIVER & & \\
\hline 53 & VERNITA & & \\
\hline
\end{tabular}




\begin{tabular}{|c|l|l|l|}
\hline & \multicolumn{1}{|c|}{ Z } & AA & AB \\
\hline 1 & \multicolumn{1}{|c|}{ SAMP_SITE_NAME } & WELL_NAME & SAMPLE_LOCATION \\
\hline 54 & VERNITA & & \\
\hline 55 & 300 SAN WATER PLANT & & \\
\hline 56 & RICHLAND SAN PLANT & & \\
\hline 57 & VERNITA & & \\
\hline 58 & 300 SAN WATER PLANT & & \\
\hline 59 & RICHLAND SAN PLANT & & \\
\hline 60 & 100 F AREA-RIVER & & \\
\hline 61 & VERNITA & & \\
\hline 62 & VERNITA & & \\
\hline 63 & 300 SAN WATER PLANT & & \\
\hline 64 & 100 F TRENCH & & \\
\hline 65 & RICHLAND SAN PLANT & & \\
\hline 66 & 300 POND & & \\
\hline 67 & RICH.PMPHS HRM 46.4 & & \\
\hline 68 & RICH.PMPHS HRM 46.4 & & \\
\hline 69 & VERNITA & & \\
\hline 70 & VERNITA & & \\
\hline 71 & VERNITA & & \\
\hline 72 & 300 SAN WATER PLANT & & \\
\hline 73 & RICHLAND SAN PLANT & & \\
\hline 74 & VERNITA & & \\
\hline 75 & 300 SAN WATER PLANT & & \\
\hline 76 & 100 F AREA-RIVER & & \\
\hline 77 & RICHLAND SAN PLANT & & \\
\hline 78 & 300 SAN WATER PLANT & & \\
\hline 79 & RICHLAND SAN PLANT & & \\
\hline 80 & 100 F AREA-RIVER & & \\
\hline 81 & VERNITA & & \\
\hline 82 & 300 SAN WATER PLANT & & \\
\hline 83 & 100 F TRENCH & & \\
\hline 84 & 222 S POND 200 W & & \\
\hline 85 & B POND NORTH SIDE & & \\
\hline 86 & GABLE POND N END & & \\
\hline 87 & REDOX POND & & \\
\hline 88 & T POND & & \\
\hline 89 & U POND & & \\
\hline 90 & RICHLAND SAN PLANT & & \\
\hline 91 & 300 SAN WATER PLANT & & \\
\hline 92 & RICHLAND SAN PLANT & & \\
\hline 93 & RICH.PMPHS HRM 46.4 & & \\
\hline 94 & RICH.PMPHS HRM 46.4 & & \\
\hline 95 & VERNITA & & \\
\hline 96 & VERNITA & & \\
\hline 97 & 300 SAN WATER PLANT & & \\
\hline 98 & RICHLAND SAN PLANT & & \\
\hline 99 & 100 F AREA-RIVER & & \\
\hline 100 & VERNITA & & \\
\hline 101 & 300 SAN WATER PLANT & & \\
\hline 102 & RICHLAND SAN PLANT & & \\
\hline 103 & 300 SAN WATER PLANT & & \\
\hline 104 & RICHLAND SAN PLANT & & \\
\hline 105 & 100 F AREA-RIVER & & \\
\hline & & & \\
\hline
\end{tabular}




\begin{tabular}{|c|c|c|c|}
\hline & $\mathrm{Z}$ & $\overline{\mathrm{AA}}$ & $\mathrm{AB}$ \\
\hline 1 & SAMP_SITE_NAME & WELL_NAME & SAMPLE_LOCATION \\
\hline 106 & VERNITA & & \\
\hline 107 & 300 SAN WATER PLANT & & \\
\hline 108 & $100 \mathrm{~F}$ TRENCH & & \\
\hline 109 & 300 POND & & \\
\hline 110 & RICHLAND SAN PLANT & & \\
\hline 111 & RICH.PMPHS HRM 46.4 & & \\
\hline 112 & RICH.PMPHS HRM 46.4 & & \\
\hline 113 & VERNITA & & \\
\hline 114 & VERNITA & & \\
\hline 115 & 300 SAN WATER PLANT & & \\
\hline 116 & 100 F AREA-RIVER & & \\
\hline 117 & VERNITA & & \\
\hline 118 & RICHLAND SAN PLANT & & \\
\hline 119 & 300 SAN WATER PLANT & & \\
\hline 120 & RICHLAND SAN PLANT & & \\
\hline 121 & 300 SAN WATER PLANT & & \\
\hline 122 & RICHLAND SAN PLANT & & \\
\hline 123 & 300 POND & & \\
\hline 124 & 100 F AREA-RIVER & & \\
\hline 125 & VERNITA & & \\
\hline 126 & 300 SAN WATER PLANT & & \\
\hline 127 & $100 \mathrm{~F}$ TRENCH & & \\
\hline 128 & 331 POND 300 AREA & & \\
\hline 129 & RICHLAND SAN PLANT & & \\
\hline 130 & RICH.PMPHS HRM 46.4 & & \\
\hline 131 & RICH.PMPHS HRM 46.4 & & \\
\hline 132 & VERNITA & & \\
\hline 133 & VERNITA & & \\
\hline 134 & 300 SAN WATER PLANT & & \\
\hline 135 & RICHLAND SAN PLANT & & \\
\hline 136 & 100 F AREA-RIVER & & \\
\hline 137 & VERNITA & & \\
\hline 138 & 300 SAN WATER PLANT & & \\
\hline 139 & RICHLAND SAN PLANT & & \\
\hline 140 & RICHLAND SAN PLANT & & \\
\hline 141 & 300 POND & & \\
\hline 142 & 100 N TRENCH SPRING & & \\
\hline 143 & 216 B 63 DITCH 200 E & & \\
\hline 144 & 222-S POND $200 \mathrm{~W}$ & & \\
\hline 145 & B POND NORTH SIDE & & \\
\hline 146 & GABLE POND N END & & \\
\hline 147 & TPOND & & \\
\hline 148 & U POND & & \\
\hline 149 & RICHLAND SAN PLANT & & \\
\hline 150 & RICHLAND SAN PLANT & & \\
\hline 151 & RICH.PMPHS HRM 46.4 & & \\
\hline 152 & RICH.PMPHS HRM 46.4 & & \\
\hline 153 & RICH.PMPHS HRM 46.4 & & \\
\hline 154 & VERNITA & & \\
\hline 155 & VERNITA & & \\
\hline 156 & VERNITA & & \\
\hline 157 & RICHLAND & & \\
\hline
\end{tabular}




\begin{tabular}{|c|c|c|c|}
\hline & $\mathrm{Z}$ & $\overline{\mathrm{AA}}$ & $\mathrm{AB}$ \\
\hline 1 & SAMP_SITE_NAME & WELL_NAME & SAMPLE_LOCATION \\
\hline 158 & RICHLAND SAN PLANT & & \\
\hline 159 & RICHLAND SAN PLANT & & \\
\hline 160 & $100 \mathrm{~F}$ TRENCH & & \\
\hline 161 & 100 D RESERVOIR & & \\
\hline 162 & RICHLAND SAN PLANT & & \\
\hline 163 & RICH.PMPHS HRM 46.4 & & \\
\hline 164 & RICH.PMPHS HRM 46.4 & & \\
\hline 165 & RICH.PMPHS HRM 46.4 & & \\
\hline 166 & VERNITA & & \\
\hline 167 & VERNITA & & \\
\hline 168 & PORT OF BENTON SPR & & \\
\hline 169 & RICH.PMPHS HRM 46.4 & & \\
\hline 170 & RICHLAND SAN PLANT & & \\
\hline 171 & RICHLAND SAN PLANT & & \\
\hline 172 & RICHLAND SAN PLANT & & \\
\hline 173 & 300 POND & & \\
\hline 174 & $100 \mathrm{~F}$ TRENCH & & \\
\hline 175 & RICHLAND SAN PLANT & & \\
\hline 176 & RICH.PMPHS HRM 46.4 & & \\
\hline 177 & RICH.PMPHS HRM 46.4 & & \\
\hline 178 & RICH.PMPHS HRM 46.4 & & \\
\hline 179 & VERNITA & & \\
\hline 180 & VERNITA & & \\
\hline 181 & VERNITA & & \\
\hline 182 & RICHLAND SAN PLANT & & \\
\hline 183 & 300 POND & & \\
\hline 184 & 300 POND & & \\
\hline 185 & $100 \mathrm{~F}$ TRENCH & & \\
\hline 186 & 216 B 63 DITCH 200 E & & \\
\hline 187 & 222-S POND $200 \mathrm{~W}$ & & \\
\hline 188 & B POND NORTH SIDE & & \\
\hline 189 & GABLE POND N END & & \\
\hline 190 & REDOX POND & & \\
\hline 191 & TPOND & & \\
\hline 192 & U POND & & \\
\hline 193 & 300 SAN WATER PLANT & & \\
\hline 194 & 300 SAN WATER PLANT & & \\
\hline 195 & RICH.PMPHS HRM 46.4 & & \\
\hline 196 & RICH.PMPHS HRM 46.4 & & \\
\hline 197 & VERNITA & & \\
\hline 198 & VERNITA & & \\
\hline 199 & ABOVE 300 AREA SPR & & \\
\hline 200 & 300 SAN WATER PLANT & & \\
\hline 201 & 300 SAN WATER PLANT & & \\
\hline 202 & RICH.PMPHS HRM 46.4 & & \\
\hline 203 & ABOVE 300 AREA SPR & & \\
\hline 204 & 300 POND & & \\
\hline 205 & $100 \mathrm{~F}$ TRENCH & & \\
\hline 206 & ABOVE 300 AREA SPR & & \\
\hline 207 & RICHLAND SAN PLANT & & \\
\hline 208 & RICH.PMPHS HRM 46.4 & & \\
\hline 209 & RICH.PMPHS HRM 46.4 & & \\
\hline
\end{tabular}




\begin{tabular}{|c|c|c|c|}
\hline & $\bar{Z}$ & $\overline{\mathrm{AA}}$ & $\mathrm{AB}$ \\
\hline 1 & SAMP_SITE_NAME & WELL_NAME & SAMPLE_LOCATION \\
\hline 210 & RICH.PMPHS HRM 46.4 & & \\
\hline 211 & VERNITA & & \\
\hline 212 & VERNITA & & \\
\hline 213 & VERNITA & & \\
\hline 214 & 300 SAN WATER PLANT & & \\
\hline 215 & RICH.PMPHS HRM 46.4 & & \\
\hline 216 & RICHLAND SAN PLANT & & \\
\hline 217 & RICH.PMPHS HRM 46.4 & & \\
\hline 218 & RICH.PMPHS HRM 46.4 & & \\
\hline 219 & VERNITA & & \\
\hline 220 & VERNITA & & \\
\hline 221 & ABOVE 300 AREA SPR & & \\
\hline 222 & 300 POND & & \\
\hline 223 & 222-S POND $200 \mathrm{~W}$ & & \\
\hline 224 & B POND NORTH SIDE & & \\
\hline 225 & GABLE POND N END & & \\
\hline 226 & T POND & & \\
\hline 227 & RICHLAND SAN PLANT & & \\
\hline 228 & RICH.PMPHS HRM 46.4 & & \\
\hline 229 & RICH.PMPHS HRM 46.4 & & \\
\hline 230 & VERNITA & & \\
\hline 231 & VERNITA & & \\
\hline 232 & RICHLAND SAN PLANT & & \\
\hline 233 & RICH.PMPHS HRM 46.4 & & \\
\hline 234 & 100 F TRENCH & & \\
\hline 235 & RICH.PMPHS HRM 46.4 & & \\
\hline 236 & RICH.PMPHS HRM 46.4 & & \\
\hline 237 & RICH.PMPHS HRM 46.4 & & \\
\hline 238 & VERNITA & & \\
\hline 239 & VERNITA & & \\
\hline 240 & VERNITA & & \\
\hline 241 & RICHLAND SAN PLANT & & \\
\hline 242 & 300 POND & & \\
\hline 243 & $100 \mathrm{~F}$ TRENCH & & \\
\hline 244 & 300 SAN WATER PLANT & & \\
\hline 245 & RICHLAND SAN PLANT & & \\
\hline 246 & RICH.PMPHS HRM 46.4 & & \\
\hline 247 & RICH.PMPHS HRM 46.4 & & \\
\hline 248 & VERNITA & & \\
\hline 249 & VERNITA & & \\
\hline 250 & RICHLAND SAN PLANT & & \\
\hline 251 & BELOW $181 \mathrm{~N}$ & & \\
\hline 252 & $100 \mathrm{~F}$ TRENCH & & \\
\hline 253 & 331 POND 300 AREA & & \\
\hline 254 & 216 B 63 DITCH 200 E & & \\
\hline 255 & 222-S POND $200 \mathrm{~W}$ & & \\
\hline 256 & B POND NORTH SIDE & & \\
\hline 257 & GABLE POND N END & & \\
\hline 258 & T POND & & \\
\hline 259 & U POND & & \\
\hline 260 & 300 SAN WATER PLANT & & \\
\hline 261 & RICHLAND SAN PLANT & & \\
\hline
\end{tabular}




\begin{tabular}{|c|c|c|c|}
\hline & $\mathrm{Z}$ & $\overline{\mathrm{AA}}$ & $\mathrm{AB}$ \\
\hline 1 & SAMP_SITE_NAME & WELL_NAME & SAMPLE_LOCATION \\
\hline 262 & RICH.PMPHS HRM 46.4 & & \\
\hline 263 & 300 SAN WATER PLANT & & \\
\hline 264 & RICHLAND SAN PLANT & & \\
\hline 265 & RICH.PMPHS HRM 46.4 & & \\
\hline 266 & RICH.PMPHS HRM 46.4 & & \\
\hline 267 & VERNITA & & \\
\hline 268 & VERNITA & & \\
\hline 269 & 300 POND & & \\
\hline 270 & RICH.PMPHS HRM 46.4 & & \\
\hline 271 & 100 F TRENCH & & \\
\hline 272 & 300 SAN WATER PLANT & & \\
\hline 273 & RICHLAND SAN PLANT & & \\
\hline 274 & RICH.PMPHS HRM 46.4 & & \\
\hline 275 & RICHLAND SAN PLANT & & \\
\hline 276 & 100 D RESERVOIR & & \\
\hline 277 & RICH.PMPHS HRM 46.4 & & \\
\hline 278 & RICH.PMPHS HRM 46.4 & & \\
\hline 279 & RICH.PMPHS HRM 46.4 & & \\
\hline 280 & VERNITA & & \\
\hline 281 & VERNITA & & \\
\hline 282 & VERNITA & & \\
\hline 283 & RICH.PMPHS HRM 46.4 & & \\
\hline 284 & 300 SAN WATER PLANT & & \\
\hline 285 & RICHLAND SAN PLANT & & \\
\hline 286 & WEST LAKE & & \\
\hline 287 & 300 SAN WATER PLANT & & \\
\hline 288 & RICHLAND SAN PLANT & & \\
\hline 289 & RICH.PMPHS HRM 46.4 & & \\
\hline 290 & RICH.PMPHS HRM 46.4 & & \\
\hline 291 & $100 \mathrm{~F}$ TRENCH & & \\
\hline 292 & 300 SAN WATER PLANT & & \\
\hline 293 & RICHLAND SAN PLANT & & \\
\hline 294 & RICHLAND SAN PLANT & & \\
\hline 295 & RICH.PMPHS HRM 46.4 & & \\
\hline 296 & RICH.PMPHS HRM 46.4 & & \\
\hline 297 & RICH.PMPHS HRM 46.4 & & \\
\hline 298 & VERNITA & & \\
\hline 299 & VERNITA & & \\
\hline 300 & 300 SAN WATER PLANT & & \\
\hline 301 & RICHLAND SAN PLANT & & \\
\hline 302 & RICH.PMPHS HRM 46.4 & & \\
\hline 303 & 216 B 63 DITCH 200 E & & \\
\hline 304 & 222-S POND $200 \mathrm{~W}$ & & \\
\hline 305 & B POND NORTH SIDE & & \\
\hline 306 & GABLE POND N END & & \\
\hline 307 & T POND & & \\
\hline 308 & U POND & & \\
\hline 309 & WEST LAKE & & \\
\hline 310 & WEST LAKE & & \\
\hline 311 & 300 SAN WATER PLANT & & \\
\hline 312 & RICHLAND SAN PLANT & & \\
\hline 313 & $100 \mathrm{~F}$ TRENCH & & \\
\hline
\end{tabular}




\begin{tabular}{|c|c|c|c|}
\hline & $\mathrm{Z}$ & $\overline{\mathrm{AA}}$ & $\mathrm{AB}$ \\
\hline 1 & SAMP_SITE_NAME & WELL_NAME & SAMPLE_LOCATION \\
\hline 314 & 331 POND 300 AREA & & \\
\hline 315 & RICH.PMPHS HRM 46.4 & & \\
\hline 316 & 300 SAN WATER PLANT & & \\
\hline 317 & RICHLAND SAN PLANT & & \\
\hline 318 & RICHLAND SAN PLANT & & \\
\hline 319 & RICH.PMPHS HRM 46.4 & & \\
\hline 320 & RICH.PMPHS HRM 46.4 & & \\
\hline 321 & RICH.PMPHS HRM 46.4 & & \\
\hline 322 & VERNITA & & \\
\hline 323 & VERNITA & & \\
\hline 324 & VERNITA & & \\
\hline 325 & 300 SAN WATER PLANT & & \\
\hline 326 & 300 POND & & \\
\hline 327 & WEST LAKE & & \\
\hline 328 & $100 \mathrm{~F}$ TRENCH & & \\
\hline 329 & RICHLAND SAN PLANT & & \\
\hline 330 & 300 POND & & \\
\hline 331 & RICH.PMPHS HRM 46.4 & & \\
\hline 332 & RICH.PMPHS HRM 46.4 & & \\
\hline 333 & RICH.PMPHS HRM 46.4 & & \\
\hline 334 & VERNITA & & \\
\hline 335 & VERNITA & & \\
\hline 336 & VERNITA & & \\
\hline 337 & $100 \mathrm{~F}$ TRENCH & & \\
\hline 338 & WEST LAKE & & \\
\hline 339 & 300 SAN WATER PLANT & & \\
\hline 340 & RICHLAND SAN PLANT & & \\
\hline 341 & RICH.PMPHS HRM 46.4 & & \\
\hline 342 & 100 D RESERVOIR & & \\
\hline 343 & RICHLAND SAN PLANT & & \\
\hline 344 & RICH.PMPHS HRM 46.4 & & \\
\hline 345 & RICH.PMPHS HRM 46.4 & & \\
\hline 346 & RICH.PMPHS HRM 46.4 & & \\
\hline 347 & VERNITA & & \\
\hline 348 & VERNITA & & \\
\hline 349 & VERNITA & & \\
\hline 350 & RICHLAND SAN PLANT & & \\
\hline 351 & $100 \mathrm{~F}$ TRENCH & & \\
\hline 352 & 216 B 63 DITCH 200 E & & \\
\hline 353 & 222-S POND $200 \mathrm{~W}$ & & \\
\hline 354 & B POND NORTH SIDE & & \\
\hline 355 & GABLE POND N END & & \\
\hline 356 & U POND & & \\
\hline 357 & WEST LAKE & & \\
\hline 358 & WEST LAKE & & \\
\hline 359 & RICHLAND SAN PLANT & & \\
\hline 360 & 300 POND & & \\
\hline 361 & RICH.PMPHS HRM 46.4 & & \\
\hline 362 & RICH.PMPHS HRM 46.4 & & \\
\hline 363 & RICH.PMPHS HRM 46.4 & & \\
\hline 364 & RICH.PMPHS HRM 46.4 & & \\
\hline 365 & VERNITA & & \\
\hline
\end{tabular}




\begin{tabular}{|c|c|c|c|}
\hline & $Z$ & $\mathrm{AA}$ & $\mathrm{AB}$ \\
\hline 1 & SAMP_SITE_NAME & WELL_NAME & SAMPLE_LOCATION \\
\hline 366 & VERNITA & & \\
\hline 367 & VERNITA & & \\
\hline 368 & $100 \mathrm{~F}$ TRENCH & & \\
\hline 369 & RICH.PMPHS HRM 46.4 & & \\
\hline 370 & WEST LAKE & & \\
\hline 371 & RICHLAND SAN PLANT & & \\
\hline 372 & RICH.PMPHS HRM 46.4 & & \\
\hline 373 & 100 D RESERVOIR & & \\
\hline 374 & RICHLAND SAN PLANT & & \\
\hline 375 & RICHLAND SAN PLANT & & \\
\hline 376 & 300 SAN WATER PLANT & & \\
\hline 377 & RICHLAND SAN PLANT & & \\
\hline 378 & 300 POND & & \\
\hline 379 & RICH.PMPHS HRM 46.4 & & \\
\hline 380 & RICH.PMPHS HRM 46.4 & & \\
\hline 381 & RICH.PMPHS HRM 46.4 & & \\
\hline 382 & VERNITA & & \\
\hline 383 & VERNITA & & \\
\hline 384 & VERNITA & & \\
\hline 385 & $100 \mathrm{~F}$ TRENCH & & \\
\hline 386 & RICHLAND SAN PLANT & & \\
\hline 387 & RICH.PMPHS HRM 46.4 & & \\
\hline 388 & RICH.PMPHS HRM 46.4 & & \\
\hline 389 & 100 D RESERVOIR & & \\
\hline 390 & 300 SAN WATER PLANT & & \\
\hline 391 & RICHLAND SAN PLANT & & \\
\hline 392 & RICHLAND SAN PLANT & & \\
\hline 393 & RICH.PMPHS HRM 46.4 & & \\
\hline 394 & RICH.PMPHS HRM 46.4 & & \\
\hline 395 & RICH.PMPHS HRM 46.4 & & \\
\hline 396 & RICH.PMPHS HRM 46.4 & & \\
\hline 397 & VERNITA & & \\
\hline 398 & VERNITA & & \\
\hline 399 & VERNITA & & \\
\hline 400 & $100 \mathrm{~F}$ TRENCH & & \\
\hline 401 & WEST LAKE & & \\
\hline 402 & RICH.PMPHS HRM 46.4 & & \\
\hline 403 & VERNITA & & \\
\hline 404 & 300 SAN WATER PLANT & & \\
\hline 405 & RICHLAND SAN PLANT & & \\
\hline 406 & 300 POND & & \\
\hline 407 & 331 POND 300 AREA & & \\
\hline 408 & 216 B 63 DITCH 200 E & & \\
\hline 409 & 100 D RESERVOIR & & \\
\hline 410 & 100 D RESERVOIR & & \\
\hline 411 & BELOW $181 \mathrm{~N}$ & & \\
\hline 412 & BELOW $181 \mathrm{~N}$ & & \\
\hline 413 & RICH.PMPHS HRM 46.4 & & \\
\hline 414 & B POND NORTH SIDE & & \\
\hline 415 & B POND NORTH SIDE & & \\
\hline 416 & GABLE POND N END & & \\
\hline 417 & GABLE POND N END & & \\
\hline
\end{tabular}




\begin{tabular}{|c|c|c|c|}
\hline & $\mathrm{z}$ & $\mathrm{AA}$ & $\mathrm{AB}$ \\
\hline 1 & SAMP_SITE_NAME & WELL_NAME & SAMPLE_LOCATION \\
\hline 418 & U POND & & \\
\hline 419 & RICHLAND SAN PLANT & & \\
\hline 420 & 100 F TRENCH & & \\
\hline 421 & RICH.PMPHS HRM 46.4 & & \\
\hline 422 & RICH.PMPHS HRM 46.4 & & \\
\hline 423 & RICH.PMPHS HRM 46.4 & & \\
\hline 424 & VERNITA & & \\
\hline 425 & VERNITA & & \\
\hline 426 & VERNITA & & \\
\hline 427 & 300 SAN WATER PLANT & & \\
\hline 428 & RICHLAND SAN PLANT & & \\
\hline 429 & RICH.PMPHS HRM 46.4 & & \\
\hline 430 & 300 POND & & \\
\hline 431 & RICH.PMPHS HRM 46.4 & & \\
\hline 432 & WEST LAKE & & \\
\hline 433 & 300 SAN WATER PLANT & & \\
\hline 434 & RICHLAND SAN PLANT & & \\
\hline 435 & 100 F TRENCH & & \\
\hline 436 & 100 D RESERVOIR & & \\
\hline 437 & RICH.PMPHS HRM 46.4 & & \\
\hline 438 & RICH.PMPHS HRM 46.4 & & \\
\hline 439 & RICH.PMPHS HRM 46.4 & & \\
\hline 440 & VERNITA & & \\
\hline 441 & VERNITA & & \\
\hline 442 & 300 SAN WATER PLANT & & \\
\hline 443 & RICHLAND SAN PLANT & & \\
\hline 444 & RICH.PMPHS HRM 46.4 & & \\
\hline 445 & 300 SAN WATER PLANT & & \\
\hline 446 & 300 POND & & \\
\hline 447 & RICH.PMPHS HRM 46.4 & & \\
\hline 448 & WEST LAKE & & \\
\hline 449 & 100 D RESERVOIR & & \\
\hline 450 & 100 D RESERVOIR & & \\
\hline 451 & 100 B AREA-RIVER & & \\
\hline 452 & 300 SAN WATER PLANT & & \\
\hline 453 & RICHLAND SAN PLANT & & \\
\hline 454 & RICH.PMPHS HRM 46.4 & & \\
\hline 455 & 100 F TRENCH & & \\
\hline 456 & 100 B AREA-RIVER & & \\
\hline 457 & 300 SAN WATER PLANT & & \\
\hline 458 & RICHLAND SAN PLANT & & \\
\hline 459 & RICHLAND SAN PLANT & & \\
\hline 460 & RICH.PMPHS HRM 46.4 & & \\
\hline 461 & RICH.PMPHS HRM 46.4 & & \\
\hline 462 & RICH.PMPHS HRM 46.4 & & \\
\hline 463 & VERNITA & & \\
\hline 464 & VERNITA & & \\
\hline 465 & VERNITA & & \\
\hline 466 & 300 SAN WATER PLANT & & \\
\hline 467 & RICHLAND SAN PLANT & & \\
\hline 468 & 300 POND & & \\
\hline 469 & B POND NORT & & \\
\hline
\end{tabular}




\begin{tabular}{|c|c|c|c|}
\hline & $\bar{Z}$ & $\overline{\mathrm{AA}}$ & $\mathrm{AB}$ \\
\hline 1 & SAMP_SITE_NAME & WELL_NAME & SAMPLE_LOCATION \\
\hline 470 & B POND NORTH SIDE & & \\
\hline 471 & GABLE POND N END & & \\
\hline 472 & GABLE POND N END & & \\
\hline 473 & U POND & & \\
\hline 474 & WEST LAKE & & \\
\hline 475 & WEST LAKE & & \\
\hline 476 & 100 B AREA-RIVER & & \\
\hline 477 & 100 B AREA-RIVER & & \\
\hline 478 & 300 SAN WATER PLANT & & \\
\hline$\overline{479}$ & RICHLAND SAN PLANT & & \\
\hline 480 & RICH.PMPHS HRM 46.4 & & \\
\hline 481 & 100 F TRENCH & & \\
\hline 482 & WEST LAKE & & \\
\hline 483 & 100 D RESERVOIR & & \\
\hline 484 & 100 D RESERVOIR & & \\
\hline 485 & 100 D RESERVOIR & & \\
\hline 486 & 300 SAN WATER PLANT & & \\
\hline 487 & RICHLAND SAN PLANT & & \\
\hline 488 & RICH.PMPHS HRM 46.4 & & \\
\hline 489 & RICH.PMPHS HRM 46.4 & & \\
\hline 490 & RICH.PMPHS HRM 46.4 & & \\
\hline 491 & RICH.PMPHS HRM 46.4 & & \\
\hline 492 & VERNITA & & \\
\hline 493 & BELOW $181 \mathrm{~N}$ & & \\
\hline 494 & 100 B AREA-RIVER & & \\
\hline 495 & 300 SAN WATER PLANT & & \\
\hline 496 & RICHLAND SAN PLANT & & \\
\hline 497 & 300 POND & & \\
\hline 498 & 100 B AREA-RIVER & & \\
\hline 499 & WEST LAKE & & \\
\hline 500 & 300 SAN WATER PLANT & & \\
\hline 501 & RICHLAND SAN PLANT & & \\
\hline 502 & RICH.PMPHS HRM 46.4 & & \\
\hline 503 & RICHLAND SAN PLANT & & \\
\hline 504 & $100 \mathrm{~F}$ TRENCH & & \\
\hline 505 & 100 D RESERVOIR & & \\
\hline 506 & 100 D RESERVOIR & & \\
\hline 507 & 100 D RESERVOIR & & \\
\hline 508 & 300 SAN WATER PLANT & & \\
\hline 509 & RICHLAND SAN PLANT & & \\
\hline 510 & RICHLAND SAN PLANT & & \\
\hline 511 & RICH.PMPHS HRM 46.4 & & \\
\hline 512 & 100 B AREA-RIVER & & \\
\hline 513 & RICH.PMPHS HRM 46.4 & & \\
\hline 514 & RICH.PMPHS HRM 46.4 & & \\
\hline 515 & RICH.PMPHS HRM 46.4 & & \\
\hline 516 & RICH.PMPHS HRM 46.4 & & \\
\hline 517 & VERNITA & & \\
\hline 518 & 300 SAN WATER PLANT & & \\
\hline 519 & RICHLAND SAN PLANT & & \\
\hline 520 & 300 POND & & \\
\hline$\overline{521}$ & WEST LAKE & & \\
\hline
\end{tabular}




\begin{tabular}{|c|l|l|l|}
\hline & \multicolumn{1}{|c|}{$Z$} & A & AB \\
\hline 1 & SAMP_SITE_NAME & WELL_NAME & SAMPLE_LOCATION \\
\hline 522 & 300 SAN WATER PLANT & & \\
\hline 523 & RICHLAND SAN PLANT & & \\
\hline 524 & RICH.PMPHS HRM 46.4 & & \\
\hline 525 & 300 SAN WATER PLANT & & \\
\hline 526 & RICHLAND SAN PLANT & & \\
\hline 527 & RICH.PMPHS HRM 46.4 & & \\
\hline 528 & 100 F TRENCH & & \\
\hline 529 & 100 D RESERVOIR & & \\
\hline 530 & 100 D RESERVOIR & & \\
\hline 531 & 100 D RESERVOIR & & \\
\hline 532 & 300 SAN WATER PLANT & & \\
\hline 533 & RICHLAND SAN PLANT & & \\
\hline 534 & RICH.PMPHS HRM 46.4 & & \\
\hline 535 & 100 B AREA-RIVER & & \\
\hline 536 & 100 B AREA-RIVER & & \\
\hline 537 & RICH.PMPHS HRM 46.4 & & \\
\hline 538 & VERNITA & & \\
\hline 539 & 300 SAN WATER PLANT & & \\
\hline 540 & RICHLAND SAN PLANT & & \\
\hline 541 & RICHLAND SAN PLANT & & \\
\hline 542 & RICH.PMPHS HRM 46.4 & & \\
\hline 543 & RICH.PMPHS HRM 46.4 & & \\
\hline 544 & RICH.PMPHS HRM 46.4 & & \\
\hline 545 & 300 SAN WATER PLANT & & \\
\hline 546 & RICHLAND SAN PLANT & & \\
\hline 547 & RICH.PMPHS HRM 46.4 & & \\
\hline 548 & 216 B 63 DITCH 200 E & & \\
\hline 549 & 222 SS POND 200 W & & \\
\hline 550 & B POND NORTH SIDE & & \\
\hline 551 & B POND NORTH SIDE & & \\
\hline 552 & GABLE POND N END & & \\
\hline 553 & GABLE POND N END & & \\
\hline 554 & U POND & & \\
\hline 555 & WESTT LAKE & & \\
\hline 556 & WESST LAKE & & \\
\hline 557 & 300 SAN WATER PLANT & & \\
\hline 558 & RICHLAND SAN PLANT & & \\
\hline 559 & RICH.PMPHS HRM 46.4 & & \\
\hline 560 & 100 F TRENCH & & \\
\hline 561 & 300 SAN WATER PLANT & & \\
\hline 562 & RICHLAND SAN PLANT & & \\
\hline 563 & RICH.PMPHS HRM 46.4 & & \\
\hline 564 & 331 POND 300 AREA & & \\
\hline 565 & 300 SAN WATER PLANT & & \\
\hline 566 & RICHLAND SAN PLANT & & \\
\hline 567 & RICHLAND SAN PLANT & & \\
\hline 568 & RICH.PMPHS HRM 46.4 & & \\
\hline 569 & RICH.PMPHS HRM 46.4 & & \\
\hline 570 & RICH.PMPHS HRM 46.4 & & \\
\hline 571 & VERNITA & & \\
\hline 572 & 100 D RESERVOIR & & \\
\hline 573 & 100 D RESERVOIR & & \\
\hline & & & \\
\hline
\end{tabular}




\begin{tabular}{|c|l|l|l|}
\hline & \multicolumn{1}{|c|}{ Z } & \multicolumn{1}{|c|}{ AA } & \multicolumn{1}{|c|}{ AB } \\
\hline 1 & \multicolumn{1}{|c|}{ SAMP_SITE_NAME } & WELL_NAME & SAMPLE_LOCATION \\
\hline 574 & 100 D RESERVOIR & & \\
\hline 575 & BELOW 181 N & & \\
\hline 576 & 100 B AREA-RIVER & & \\
\hline 577 & 300 SAN WATER PLANT & & \\
\hline 578 & RICHLAND SAN PLANT & & \\
\hline 579 & 300 POND & & \\
\hline 580 & RICH.PMPHS HRM 46.4 & & \\
\hline 581 & RICH.PMPHS HRM 46.4 & & \\
\hline 582 & WEST LAKE & & \\
\hline 583 & 300 SAN WATER PLANT & & \\
\hline 584 & RICHLAND SAN PLANT & & \\
\hline 585 & RICH.PMPHS HRM 46.4 & & \\
\hline 586 & 100 F TRENCH & & \\
\hline 587 & 100 D RESERVOIR & & \\
\hline 588 & 100 D RESERVOIR & & \\
\hline 589 & 100 D RESERVOIR & & \\
\hline 590 & 300 SAN WATER PLANT & & \\
\hline 591 & RICHLAND SAN PLANT & & \\
\hline 592 & RICHLAND SAN PLANT & & \\
\hline 593 & 100 B AREA-RIVER & & \\
\hline 594 & 100 B AREA-RIVER & & \\
\hline 595 & 300 POND & & \\
\hline 596 & RICH.PMPHS HRM 46.4 & & \\
\hline 597 & RICH.PMPHS HRM 46.4 & & \\
\hline 598 & RICH.PMPHS HRM 46.4 & & \\
\hline 599 & VERNITA & & \\
\hline 600 & WEST LAKE & & \\
\hline 601 & RICH.PMPHS HRM 46.4 & & \\
\hline 602 & 300 SAN WATER PLANT & & \\
\hline 603 & 100 F TRENCH & & \\
\hline 604 & 100 D RESERVOIR & & \\
\hline 605 & 100 D RESERVOIR & & \\
\hline 606 & 100 D RESERVOIR & & \\
\hline 607 & 300 SAN WATER PLANT & & \\
\hline 608 & RICHLAND SAN PLANT & & \\
\hline 609 & RICH.PMPHS HRM 46.4 & & \\
\hline 610 & RICH.PMPHS HRM 46.4 & & \\
\hline 611 & 300 SAN WATER PLANT & & \\
\hline 612 & RICHLAND SAN PLANT & & \\
\hline 613 & RICHLAND SAN PLANT & & \\
\hline 614 & 100 B AREA-RIVER & & \\
\hline 615 & 100 B AREA-RIVER & & \\
\hline 616 & RICH.PMPHS HRM 46.4 & & \\
\hline 617 & RICH.PMPHS HRM 46.4 & & \\
\hline 618 & RICH.PMPHS HRM 46.4 & & \\
\hline 619 & VERNITA & & \\
\hline 620 & 300 SAN WATER PLANT & & \\
\hline 621 & RICHLAND SAN PLANT & & \\
\hline 622 & 300 POND & & \\
\hline 623 & RICH.PMPHS HRM 46.4 & & \\
\hline 624 & 300 SAN WATER PLANT & & \\
\hline 625 & RICHLAND SAN PLANT & & \\
\hline & & & \\
\hline
\end{tabular}




\begin{tabular}{|c|c|c|c|}
\hline & $\mathrm{Z}$ & $\overline{\mathrm{AA}}$ & $\mathrm{AB}$ \\
\hline 1 & SAMP_SITE_NAME & WELL_NAME & SAMPLE_LOCATION \\
\hline 626 & RICH.PMPHS HRM 46.4 & & \\
\hline 627 & 331 POND 300 AREA & & \\
\hline 628 & 100 D RESERVOIR & & \\
\hline 629 & 100 D RESERVOIR & & \\
\hline 630 & 100 D RESERVOIR & & \\
\hline 631 & BELOW $181 \mathrm{~N}$ & & \\
\hline 632 & 216 B 63 DITCH 200 E & & \\
\hline 633 & 222-S POND $200 \mathrm{~W}$ & & \\
\hline 634 & B POND NORTH SIDE & & \\
\hline 635 & B POND NORTH SIDE & & \\
\hline 636 & GABLE POND N END & & \\
\hline 637 & GABLE POND N END & & \\
\hline 638 & REDOX POND & & \\
\hline 639 & TPOND & & \\
\hline 640 & U POND & & \\
\hline 641 & 300 SAN WATER PLANT & & \\
\hline 642 & RICHLAND SAN PLANT & & \\
\hline 643 & 300 SAN WATER PLANT & & \\
\hline 644 & RICHLAND SAN PLANT & & \\
\hline 645 & RICHLAND SAN PLANT & & \\
\hline 646 & RICH.PMPHS HRM 46.4 & & \\
\hline 647 & 100 B AREA-RIVER & & \\
\hline 648 & 100 B AREA-RIVER & & \\
\hline 649 & RICH.PMPHS HRM 46.4 & & \\
\hline 650 & RICH.PMPHS HRM 46.4 & & \\
\hline 651 & RICH.PMPHS HRM 46.4 & & \\
\hline 652 & VERNITA & & \\
\hline 653 & 300 SAN WATER PLANT & & \\
\hline 654 & RICHLAND SAN PLANT & & \\
\hline 655 & 300 POND & & \\
\hline 656 & RICH.PMPHS HRM 46.4 & & \\
\hline 657 & 100 F TRENCH & & \\
\hline 658 & 300 SAN WATER PLANT & & \\
\hline 659 & RICHLAND SAN PLANT & & \\
\hline 660 & RICH.PMPHS HRM 46.4 & & \\
\hline 661 & 100 D RESERVOIR & & \\
\hline 662 & 100 D RESERVOIR & & \\
\hline 663 & 100 D RESERVOIR & & \\
\hline 664 & WEST LAKE & & \\
\hline 665 & 300 SAN WATER PLANT & & \\
\hline 666 & RICHLAND SAN PLANT & & \\
\hline 667 & RICH.PMPHS HRM 46.4 & & \\
\hline 668 & 300 SAN WATER PLANT & & \\
\hline 669 & RICHLAND SAN PLANT & & \\
\hline 670 & RICHLAND SAN PLANT & & \\
\hline 671 & RICH.PMPHS HRM 46.4 & & \\
\hline 672 & 100 B AREA-RIVER & & \\
\hline 673 & 100 B AREA-RIVER & & \\
\hline 674 & 300 SAN WATER PLANT & & \\
\hline 675 & RICHLAND SAN PLANT & & \\
\hline 676 & 300 POND & & \\
\hline 677 & RICH.PMPHS HRM 46.4 & & \\
\hline
\end{tabular}




\begin{tabular}{|c|c|c|c|}
\hline & $\mathrm{Z}$ & $\overline{\mathrm{AA}}$ & $\mathrm{AB}$ \\
\hline 1 & SAMP_SITE_NAME & WELL_NAME & SAMPLE_LOCATION \\
\hline 678 & RICH.PMPHS HRM 46.4 & & \\
\hline 679 & RICH.PMPHS HRM 46.4 & & \\
\hline 680 & RICH.PMPHS HRM 46.4 & & \\
\hline 681 & VERNITA & & \\
\hline 682 & $100 \mathrm{~F}$ TRENCH & & \\
\hline 683 & 300 SAN WATER PLANT & & \\
\hline 684 & RICHLAND SAN PLANT & & \\
\hline 685 & RICH.PMPHS HRM 46.4 & & \\
\hline 686 & 100 D RESERVOIR & & \\
\hline 687 & 100 D RESERVOIR & & \\
\hline 688 & 100 D RESERVOIR & & \\
\hline 689 & WEST LAKE & & \\
\hline 690 & 300 SAN WATER PLANT & & \\
\hline 691 & RICHLAND SAN PLANT & & \\
\hline 692 & RICH.PMPHS HRM 46.4 & & \\
\hline 693 & 300 SAN WATER PLANT & & \\
\hline 694 & RICHLAND SAN PLANT & & \\
\hline 695 & RICH.PMPHS HRM 46.4 & & \\
\hline 696 & 300 SAN WATER PLANT & & \\
\hline 697 & RICHLAND SAN PLANT & & \\
\hline 698 & RICHLAND SAN PLANT & & \\
\hline 699 & 100 B AREA-RIVER & & \\
\hline 700 & 100 B AREA-RIVER & & \\
\hline 701 & RICH.PMPHS HRM 46.4 & & \\
\hline 702 & RICH.PMPHS HRM 46.4 & & \\
\hline 703 & RICH.PMPHS HRM 46.4 & & \\
\hline 704 & VERNITA & & \\
\hline 705 & $100 \mathrm{~F}$ TRENCH & & \\
\hline 706 & 300 SAN WATER PLANT & & \\
\hline 707 & RICHLAND SAN PLANT & & \\
\hline 708 & RICH.PMPHS HRM 46.4 & & \\
\hline 709 & 331 POND 300 AREA & & \\
\hline 710 & 300 SAN WATER PLANT & & \\
\hline 711 & RICHLAND SAN PLANT & & \\
\hline 712 & RICH.PMPHS HRM 46.4 & & \\
\hline 713 & 216 B 63 DITCH 200 E & & \\
\hline 714 & 222-S POND $200 \mathrm{~W}$ & & \\
\hline 715 & T POND & & \\
\hline 716 & 300 SAN WATER PLANT & & \\
\hline 717 & RICHLAND SAN PLANT & & \\
\hline 718 & RICH.PMPHS HRM 46.4 & & \\
\hline 719 & B POND NORTH SIDE & & \\
\hline 720 & B POND NORTH SIDE & & \\
\hline 721 & GABLE POND N END & & \\
\hline 722 & GABLE POND N END & & \\
\hline 723 & U POND & & \\
\hline 724 & WEST LAKE & & \\
\hline 725 & WEST LAKE & & \\
\hline 726 & HANF POWERLINE XING & & \\
\hline 727 & 300 SAN WATER PLANT & & \\
\hline 728 & RICHLAND SAN PLANT & & \\
\hline 729 & RICHLAND SAN PLANT & & \\
\hline
\end{tabular}




\begin{tabular}{|c|c|c|c|}
\hline & $\mathrm{z}$ & $\mathrm{AA}$ & $\mathrm{AB}$ \\
\hline 1 & SAMP_SITE_NAME & WELL_NAME & SAMPLE_LOCATION \\
\hline 730 & RICH.PMPHS HRM 46.4 & & \\
\hline 731 & $100 \mathrm{~F}$ TRENCH & & \\
\hline 732 & 100 B AREA-RIVER & & \\
\hline 733 & 100 B AREA-RIVER & & \\
\hline 734 & 300 SAN WATER PLANT & & \\
\hline 735 & 300 SAN WATER PLANT & & \\
\hline 736 & RICHLAND SAN PLANT & & \\
\hline 737 & RICH.PMPHS HRM 46.4 & & \\
\hline 738 & RICH.PMPHS HRM 46.4 & & \\
\hline 739 & RICH.PMPHS HRM 46.4 & & \\
\hline 740 & RICH.PMPHS HRM 46.4 & & \\
\hline 741 & VERNITA & & \\
\hline 742 & 300 SAN WATER PLANT & & \\
\hline 743 & RICH.PMPHS HRM 46.4 & & \\
\hline 744 & HANF POWERLINE XING & & \\
\hline 745 & HANF POWERLINE XING & & \\
\hline 746 & WEST LAKE & & \\
\hline 747 & 300 SAN WATER PLANT & & \\
\hline 748 & 300 SAN WATER PLANT & & \\
\hline 749 & RICHLAND SAN PLANT & & \\
\hline 750 & RICH.PMPHS HRM 46.4 & & \\
\hline 751 & RICH.PMPHS HRM 46.4 & & \\
\hline 752 & 300 SAN WATER PLANT & & \\
\hline 753 & RICHLAND SAN PLANT & & \\
\hline 754 & RICHLAND SAN PLANT & & \\
\hline 755 & 100 F TRENCH & & \\
\hline 756 & 100 B AREA-RIVER & & \\
\hline 757 & 100 B AREA-RIVER & & \\
\hline 758 & 300 SAN WATER PLANT & & \\
\hline 759 & RICHLAND SAN PLANT & & \\
\hline 760 & RICH.PMPHS HRM 46.4 & & \\
\hline 761 & RICH.PMPHS HRM 46.4 & & \\
\hline 762 & RICH.PMPHS HRM 46.4 & & \\
\hline 763 & RICH.PMPHS HRM 46.4 & & \\
\hline 764 & VERNITA & & \\
\hline 765 & 300 SAN WATER PLANT & & \\
\hline 766 & RICHLAND SAN PLANT & & \\
\hline 767 & 300 POND & & \\
\hline 768 & RICH.PMPHS HRM 46.4 & & \\
\hline 769 & WEST LAKE & & \\
\hline 770 & 300 SAN WATER PLANT & & \\
\hline 771 & RICHLAND SAN PLANT & & \\
\hline 772 & RICH.PMPHS HRM 46.4 & & \\
\hline 773 & HANF POWERLINE XING & & \\
\hline 774 & HANF POWERLINE XING & & \\
\hline 775 & HANF POWERLINE XING & & \\
\hline 776 & 300 SAN WATER PLANT & & \\
\hline 777 & RICHLAND SAN PLANT & & \\
\hline 778 & RICH.PMPHS HRM 46.4 & & \\
\hline 779 & $100 \mathrm{~F}$ TRENCH & & \\
\hline 780 & 100 B AREA-RIVER & & \\
\hline 781 & RICH.PMPHS HRM 46.4 & & \\
\hline
\end{tabular}




\begin{tabular}{|c|c|c|c|}
\hline & $\mathrm{Z}$ & $\overline{\mathrm{AA}}$ & $\mathrm{AB}$ \\
\hline 1 & SAMP_SITE_NAME & WELL_NAME & SAMPLE_LOCATION \\
\hline 782 & RICH.PMPHS HRM 46.4 & & \\
\hline 783 & RICH.PMPHS HRM 46.4 & & \\
\hline 784 & 300 SAN WATER PLANT & & \\
\hline 785 & RICHLAND SAN PLANT & & \\
\hline 786 & RICHLAND SAN PLANT & & \\
\hline 787 & RICH.PMPHS HRM 46.4 & & \\
\hline 788 & VERNITA & & \\
\hline 789 & 300 SAN WATER PLANT & & \\
\hline 790 & RICHLAND SAN PLANT & & \\
\hline 791 & RICH.PMPHS HRM 46.4 & & \\
\hline 792 & 216 B 63 DITCH 200 E & & \\
\hline 793 & B POND NORTH SIDE & & \\
\hline 794 & B POND NORTH SIDE & & \\
\hline 795 & GABLE POND N END & & \\
\hline 796 & GABLE POND N END & & \\
\hline 797 & U POND & & \\
\hline 798 & WEST LAKE & & \\
\hline 799 & WEST LAKE & & \\
\hline 800 & 300 SAN WATER PLANT & & \\
\hline 801 & RICHLAND SAN PLANT & & \\
\hline 802 & 300 POND & & \\
\hline 803 & RICH.PMPHS HRM 46.4 & & \\
\hline 804 & BELOW $181 \mathrm{~N}$ & & \\
\hline 805 & HANF POWERLINE XING & & \\
\hline 806 & HANF POWERLINE XING & & \\
\hline 807 & 300 SAN WATER PLANT & & \\
\hline 808 & RICHLAND SAN PLANT & & \\
\hline 809 & RICH.PMPHS HRM 46.4 & & \\
\hline 810 & 100 F TRENCH & & \\
\hline 811 & 300 SAN WATER PLANT & & \\
\hline 812 & RICHLAND SAN PLANT & & \\
\hline 813 & RICHLAND SAN PLANT & & \\
\hline 814 & RICH.PMPHS HRM 46.4 & & \\
\hline 815 & RICH.PMPHS HRM 46.4 & & \\
\hline 816 & RICH.PMPHS HRM 46.4 & & \\
\hline 817 & RICH.PMPHS HRM 46.4 & & \\
\hline 818 & 100 B AREA-RIVER & & \\
\hline 819 & 100 B AREA-RIVER & & \\
\hline 820 & 300 SAN WATER PLANT & & \\
\hline 821 & RICHLAND SAN PLANT & & \\
\hline 822 & RICH.PMPHS HRM 46.4 & & \\
\hline 823 & WEST LAKE & & \\
\hline 824 & 300 SAN WATER PLANT & & \\
\hline 825 & RICHLAND SAN PLANT & & \\
\hline 826 & RICH.PMPHS HRM 46.4 & & \\
\hline 827 & HANF POWERLINE XING & & \\
\hline 828 & HANF POWERLINE XING & & \\
\hline 829 & HANF POWERLINE XING & & \\
\hline 830 & 300 SAN WATER PLANT & & \\
\hline 831 & RICHLAND SAN PLANT & & \\
\hline 832 & RICH.PMPHS HRM 46.4 & & \\
\hline 833 & 100 F TRENCH & & \\
\hline
\end{tabular}




\begin{tabular}{|c|l|l|l|}
\hline & \multicolumn{1}{|c|}{$Z$} & \multicolumn{1}{|c|}{ Z } & AB \\
\hline 1 & SAMP_SITE_NAME & WELL_NAME & SAMPLE_LOCATION \\
\hline 834 & 300 SAN WATER PLANT & & \\
\hline 835 & RICHLAND SAN PLANT & & \\
\hline 836 & 100 B AREA-RIVER & & \\
\hline 837 & 100 B AREA-RIVER & & \\
\hline 838 & RICH.PMPHS HRM 46.4 & & \\
\hline 839 & RICH.PMPHS HRM 46.4 & & \\
\hline 840 & RICH.PMPHS HRM 46.4 & & \\
\hline 841 & 300 SAN WATER PLANT & & \\
\hline 842 & RICHLAND SAN PLANT & & \\
\hline 843 & WEST LAKE & & \\
\hline 844 & 300 SAN WATER PLANT & & \\
\hline 845 & RICHLAND SAN PLANT & & \\
\hline 846 & RICH.PMPHS HRM 46.4 & & \\
\hline 847 & HANF POWERLINE XING & & \\
\hline 848 & HANF POWERLINE XING & & \\
\hline 849 & 300 SAN WATER PLANT & & \\
\hline 850 & RICHLAND SAN PLANT & & \\
\hline 851 & RICH.PMPHS HRM 46.4 & & \\
\hline 852 & 100 F TRENCH & & \\
\hline 853 & RICH.PMPHS HRM 46.4 & & \\
\hline 854 & 100 B AREA-RIVER & & \\
\hline 855 & 100 B AREA-RIVER & & \\
\hline 856 & BONNEVILLE DAM-RIVER & & \\
\hline 857 & 300 SAN WATER PLANT & & \\
\hline 858 & RICHLAND SAN PLANT & & \\
\hline 859 & RICHLAND SAN PLANT & & \\
\hline 860 & RICH.PMPHS HRM 46.4 & & \\
\hline 861 & RICH.PMPHS HRM 46.4 & & \\
\hline 862 & RICH.PMPHS HRM 46.4 & & \\
\hline 863 & RICH.PMPHS HRM 46.4 & & \\
\hline 864 & VERNITA & & \\
\hline 865 & 300 SAN WATER PLANT & & \\
\hline 866 & RICHLAND SAN PLANT & & \\
\hline 867 & 300 POND & & \\
\hline 868 & RICHLAND SAN PLANT & & \\
\hline 869 & RICH.PMPHS HRM 46.4 & & \\
\hline 870 & 100 F TRENCH & & \\
\hline 871 & HANF POWERLINE XING & & \\
\hline 872 & HANF POWERLINE XING & & \\
\hline 873 & HANF POWERLINE XING & & \\
\hline 874 & 216 B 63 DITCH 200 E & & \\
\hline 875 & B POND NORTH SIDE & & \\
\hline 876 & B POND NORTH SIDE & & \\
\hline 877 & GABLE POND N END & & \\
\hline 878 & GABLE POND N END & & \\
\hline 879 & T POND & & \\
\hline 880 & U POND & & \\
\hline 881 & WEST LAKE & & \\
\hline 882 & WEST LAKE & & \\
\hline 883 & 300 SAN WATER PLANT & & \\
\hline 884 & RICHLAND SAN PLANT & & \\
\hline 885 & RICH.PMPHS HRM 46.4 & & \\
\hline & & & \\
\hline
\end{tabular}




\begin{tabular}{|c|c|c|c|}
\hline & $Z$ & $\mathrm{AA}$ & $\mathrm{AB}$ \\
\hline 1 & SAMP_SITE_NAME & WELL_NAME & SAMPLE_LOCATION \\
\hline 886 & 300 SAN WATER PLANT & & \\
\hline 887 & RICHLAND SAN PLANT & & \\
\hline 888 & RICHLAND SAN PLANT & & \\
\hline 889 & RICH.PMPHS HRM 46.4 & & \\
\hline 890 & 100 B AREA-RIVER & & \\
\hline 891 & 100 B AREA-RIVER & & \\
\hline 892 & 300 SAN WATER PLANT & & \\
\hline 893 & RICHLAND SAN PLANT & & \\
\hline 894 & 300 POND & & \\
\hline 895 & RICH.PMPHS HRM 46.4 & & \\
\hline 896 & RICH.PMPHS HRM 46.4 & & \\
\hline 897 & RICH.PMPHS HRM 46.4 & & \\
\hline 898 & RICH.PMPHS HRM 46.4 & & \\
\hline 899 & VERNITA & & \\
\hline 900 & WEST LAKE & & \\
\hline 901 & 300 SAN WATER PLANT & & \\
\hline 902 & RICHLAND SAN PLANT & & \\
\hline 903 & RICH.PMPHS HRM 46.4 & & \\
\hline 904 & 100 F TRENCH & & \\
\hline 905 & HANF POWERLINE XING & & \\
\hline 906 & HANF POWERLINE XING & & \\
\hline 907 & HANF POWERLINE XING & & \\
\hline 908 & 300 SAN WATER PLANT & & \\
\hline 909 & RICHLAND SAN PLANT & & \\
\hline 910 & RICH.PMPHS HRM 46.4 & & \\
\hline 911 & 300 SAN WATER PLANT & & \\
\hline 912 & RICHLAND SAN PLANT & & \\
\hline 913 & RICH.PMPHS HRM 46.4 & & \\
\hline 914 & BELOW $181 \mathrm{~N}$ & & \\
\hline 915 & 100 B AREA-RIVER & & \\
\hline 916 & 100 B AREA-RIVER & & \\
\hline 917 & 300 SAN WATER PLANT & & \\
\hline 918 & RICHLAND SAN PLANT & & \\
\hline 919 & 300 POND & & \\
\hline 920 & RICH.PMPHS HRM 46.4 & & \\
\hline 921 & RICH.PMPHS HRM 46.4 & & \\
\hline 922 & RICH.PMPHS HRM 46.4 & & \\
\hline 923 & RICH.PMPHS HRM 46.4 & & \\
\hline 924 & VERNITA & & \\
\hline 925 & WEST LAKE & & \\
\hline 926 & RICH.PMPHS HRM 46.4 & & \\
\hline 927 & 300 SAN WATER PLANT & & \\
\hline 928 & RICHLAND SAN PLANT & & \\
\hline 929 & $100 \mathrm{~F}$ TRENCH & & \\
\hline 930 & 300 SAN WATER PLANT & & \\
\hline 931 & RICHLAND SAN PLANT & & \\
\hline 932 & RICH.PMPHS HRM 46.4 & & \\
\hline 933 & HANF POWERLINE XING & & \\
\hline 934 & HANF POWERLINE XING & & \\
\hline 935 & 300 SAN WATER PLANT & & \\
\hline 936 & RICHLAND SAN PLANT & & \\
\hline 937 & RICH.PMPHS HRM 46.4 & & \\
\hline
\end{tabular}




\begin{tabular}{|c|c|c|c|}
\hline & $Z$ & $\mathrm{AA}$ & $\mathrm{AB}$ \\
\hline 1 & SAMP_SITE_NAME & WELL_NAME & SAMPLE_LOCATION \\
\hline 938 & VERNITA & & \\
\hline 939 & 300 SAN WATER PLANT & & \\
\hline 940 & RICHLAND SAN PLANT & & \\
\hline 941 & RICHLAND SAN PLANT & & \\
\hline 942 & RICH.PMPHS HRM 46.4 & & \\
\hline 943 & RICH.PMPHS HRM 46.4 & & \\
\hline 944 & RICH.PMPHS HRM 46.4 & & \\
\hline 945 & RICH.PMPHS HRM 46.4 & & \\
\hline 946 & 100 B AREA-RIVER & & \\
\hline 947 & 100 B AREA-RIVER & & \\
\hline 948 & BELOW $181 \mathrm{~N}$ & & \\
\hline 949 & 300 SAN WATER PLANT & & \\
\hline 950 & RICHLAND SAN PLANT & & \\
\hline 951 & 300 POND & & \\
\hline 952 & RICH.PMPHS HRM 46.4 & & \\
\hline 953 & $100 \mathrm{~F}$ TRENCH & & \\
\hline 954 & RICHLAND SAN PLANT & & \\
\hline 955 & RICH.PMPHS HRM 46.4 & & \\
\hline 956 & BELOW $181 \mathrm{~N}$ & & \\
\hline 957 & HANF POWERLINE XING & & \\
\hline 958 & HANF POWERLINE XING & & \\
\hline 959 & HANF POWERLINE XING & & \\
\hline 960 & 216 B 63 DITCH 200 E & & \\
\hline 961 & B POND NORTH SIDE & & \\
\hline 962 & B POND NORTH SIDE & & \\
\hline 963 & GABLE POND N END & & \\
\hline 964 & GABLE POND N END & & \\
\hline 965 & T POND & & \\
\hline 966 & U POND & & \\
\hline 967 & WEST LAKE & & \\
\hline 968 & 300 SAN WATER PLANT & & \\
\hline 969 & RICHLAND SAN PLANT & & \\
\hline 970 & RICH.PMPHS HRM 46.4 & & \\
\hline 971 & 300 SAN WATER PLANT & & \\
\hline 972 & RICHLAND SAN PLANT & & \\
\hline 973 & RICH.PMPHS HRM 46.4 & & \\
\hline 974 & VERNITA & & \\
\hline 975 & BONNEVILLE DAM-RIVER & & \\
\hline 976 & 181 N BLDG 100 N AREA & & \\
\hline 977 & 181 N BLDG 100N AREA & & \\
\hline 978 & 300 SAN WATER PLANT & & \\
\hline 979 & RICHLAND SAN PLANT & & \\
\hline 980 & RICHLAND SAN PLANT & & \\
\hline 981 & 100 B AREA-RIVER & & \\
\hline 982 & RICH.PMPHS HRM 46.4 & & \\
\hline 983 & RICH.PMPHS HRM 46.4 & & \\
\hline 984 & RICH.PMPHS HRM 46.4 & & \\
\hline 985 & $100 \mathrm{~F}$ TRENCH & & \\
\hline 986 & 300 POND & & \\
\hline 987 & 300 SAN WATER PLANT & & \\
\hline 988 & RICH.PMPHS HRM 46.4 & & \\
\hline 989 & 300 SAN WATER PLANT & & \\
\hline
\end{tabular}




\begin{tabular}{|c|c|c|c|}
\hline & $\bar{Z}$ & $\overline{\mathrm{AA}}$ & $\overline{A B}$ \\
\hline 1 & SAMP_SITE_NAME & WELL_NAME & SAMPLE_LOCATION \\
\hline 990 & BELOW $181 \mathrm{~N}$ & & \\
\hline 991 & HANF POWERLINE XING & & \\
\hline 992 & HANF POWERLINE XING & & \\
\hline \begin{tabular}{|l|}
993 \\
\end{tabular} & HANF POWERLINE XING & & \\
\hline 994 & 300 SAN WATER PLANT & & \\
\hline 995 & 100 B AREA-RIVER & & \\
\hline 996 & 100 B AREA-RIVER & & \\
\hline 997 & BONNEVILLE DAM-RIVER & & \\
\hline 998 & 181 N BLDG 100N AREA & & \\
\hline 999 & 181 N BLDG 100N AREA & & \\
\hline 1000 & 300 SAN WATER PLANT & & \\
\hline 1001 & RICHLAND SAN PLANT & & \\
\hline 1002 & RICHLAND SAN PLANT & & \\
\hline 1003 & 300 POND & & \\
\hline 1004 & RICH.PMPHS HRM 46.4 & & \\
\hline 1005 & RICH.PMPHS HRM 46.4 & & \\
\hline 1006 & RICH.PMPHS HRM 46.4 & & \\
\hline 1007 & RICH.PMPHS HRM 46.4 & & \\
\hline 1008 & VERNITA & & \\
\hline 1009 & 100 F TRENCH & & \\
\hline 1010 & 300 SAN WATER PLANT & & \\
\hline 1011 & RICH.PMPHS HRM 46.4 & & \\
\hline 1012 & BELOW $181 \mathrm{~N}$ & & \\
\hline 1013 & HANF POWERLINE XING & & \\
\hline 1014 & HANF POWERLINE XING & & \\
\hline 1015 & 300 SAN WATER PLANT & & \\
\hline 1016 & RICHLAND SAN PLANT & & \\
\hline 1017 & RICH.PMPHS HRM 46.4 & & \\
\hline 1018 & HANF POWERLINE XING & & \\
\hline 1019 & 300 SAN WATER PLANT & & \\
\hline 1020 & RICH.PMPHS HRM 46.4 & & \\
\hline 1021 & RICH.PMPHS HRM 46.4 & & \\
\hline 1022 & 300 SAN WATER PLANT & & \\
\hline 1023 & 100 F TRENCH & & \\
\hline 1024 & 100 B AREA-RIVER & & \\
\hline 1025 & 100 B AREA-RIVER & & \\
\hline 1026 & 181 N BLDG 100N AREA & & \\
\hline 1027 & 300 SAN WATER PLANT & & \\
\hline 1028 & RICHLAND SAN PLANT & & \\
\hline 1029 & RICHLAND SAN PLANT & & \\
\hline 1030 & 300 POND & & \\
\hline 1031 & RICH.PMPHS HRM 46.4 & & \\
\hline 1032 & RICH.PMPHS HRM 46.4 & & \\
\hline 1033 & RICH.PMPHS HRM 46.4 & & \\
\hline 1034 & RICH.PMPHS HRM 46.4 & & \\
\hline 1035 & VERNITA & & \\
\hline 1036 & BONNEVILLE DAM-RIVER & & \\
\hline 1037 & 300 SAN WATER PLANT & & \\
\hline 1038 & RICH.PMPHS HRM 46.4 & & \\
\hline 1039 & HANF POWERLINE XING & & \\
\hline 1040 & HANF POWERLINE XING & & \\
\hline 1041 & HANF POWERLINE XING & & \\
\hline
\end{tabular}




\begin{tabular}{|c|c|c|c|}
\hline & $\mathrm{Z}$ & $\overline{\mathrm{AA}}$ & $\mathrm{AB}$ \\
\hline 1 & SAMP_SITE_NAME & WELL_NAME & SAMPLE_LOCATION \\
\hline 1042 & 100 N TRENCH SPRING & & \\
\hline 1043 & 216 B 63 DITCH 200 E & & \\
\hline 1044 & B POND NORTH SIDE & & \\
\hline 1045 & B POND NORTH SIDE & & \\
\hline 1046 & U POND & & \\
\hline 1047 & WEST LAKE & & \\
\hline 1048 & WEST LAKE & & \\
\hline 1049 & 300 SAN WATER PLANT & & \\
\hline 1050 & RICH.PMPHS HRM 46.4 & & \\
\hline 1051 & 300 SAN WATER PLANT & & \\
\hline 1052 & RICH.PMPHS HRM 46.4 & & \\
\hline 1053 & 100 F TRENCH & & \\
\hline 1054 & 100 B AREA-RIVER & & \\
\hline 1055 & 100 B AREA-RIVER & & \\
\hline 1056 & 181 N BLDG $100 N$ AREA & & \\
\hline 1057 & 300 SAN WATER PLANT & & \\
\hline 1058 & RICHLAND SAN PLANT & & \\
\hline 1059 & RICHLAND SAN PLANT & & \\
\hline 1060 & 300 POND & & \\
\hline 1061 & RICH.PMPHS HRM 46.4 & & \\
\hline 1062 & RICH.PMPHS HRM 46.4 & & \\
\hline 1063 & RICH.PMPHS HRM 46.4 & & \\
\hline 1064 & RICH.PMPHS HRM 46.4 & & \\
\hline 1065 & VERNITA & & \\
\hline 1066 & 300 SAN WATER PLANT & & \\
\hline 1067 & RICH.PMPHS HRM 46.4 & & \\
\hline 1068 & 300 SAN WATER PLANT & & \\
\hline 1069 & RICH.PMPHS HRM 46.4 & & \\
\hline 1070 & HANF POWERLINE XING & & \\
\hline 1071 & HANF POWERLINE XING & & \\
\hline 1072 & HANF POWERLINE XING & & \\
\hline 1073 & 100 N TRENCH SPRING & & \\
\hline 1074 & 300 SAN WATER PLANT & & \\
\hline 1075 & 100 F TRENCH & & \\
\hline 1076 & 100 B AREA-RIVER & & \\
\hline 1077 & 100 B AREA-RIVER & & \\
\hline 1078 & 181 N BLDG 100N AREA & & \\
\hline 1079 & 181 N BLDG 100N AREA & & \\
\hline 1080 & 300 SAN WATER PLANT & & \\
\hline 1081 & RICHLAND SAN PLANT & & \\
\hline 1082 & 300 POND & & \\
\hline 1083 & RICH.PMPHS HRM 46.4 & & \\
\hline 1084 & RICH.PMPHS HRM 46.4 & & \\
\hline 1085 & RICH.PMPHS HRM 46.4 & & \\
\hline 1086 & RICH.PMPHS HRM 46.4 & & \\
\hline 1087 & VERNITA & & \\
\hline 1088 & HANF POWERLINE XING & & \\
\hline 1089 & HANF POWERLINE XING & & \\
\hline 1090 & HANF POWERLINE XING & & \\
\hline 1091 & 100 N TRENCH SPRING & & \\
\hline 1092 & 300 SAN WATER PLANT & & \\
\hline 1093 & RICH.PMPHS HRM 46.4 & & \\
\hline
\end{tabular}




\begin{tabular}{|c|c|c|c|}
\hline & $\bar{Z}$ & $\overline{\mathrm{AA}}$ & $\overline{A B}$ \\
\hline 1 & SAMP_SITE_NAME & WELL_NAME & SAMPLE_LOCATION \\
\hline 1094 & 300 SAN WATER PLANT & & \\
\hline 1095 & RICH.PMPHS HRM 46.4 & & \\
\hline 1096 & $100 \mathrm{~F}$ TRENCH & & \\
\hline 1097 & 181 N BLDG 100N AREA & & \\
\hline 1098 & 181 N BLDG 100N AREA & & \\
\hline 1099 & 300 SAN WATER PLANT & & \\
\hline 1100 & RICHLAND SAN PLANT & & \\
\hline 1101 & RICHLAND SAN PLANT & & \\
\hline 1102 & 300 POND & & \\
\hline 1103 & RICH.PMPHS HRM 46.4 & & \\
\hline 1104 & RICH.PMPHS HRM 46.4 & & \\
\hline 1105 & RICH.PMPHS HRM 46.4 & & \\
\hline 1106 & RICH.PMPHS HRM 46.4 & & \\
\hline 1107 & VERNITA & & \\
\hline 1108 & 100 B AREA-RIVER & & \\
\hline 1109 & 100 B AREA-RIVER & & \\
\hline 1110 & 300 SAN WATER PLANT & & \\
\hline 1111 & RICH.PMPHS HRM 46.4 & & \\
\hline 1112 & 216 B 63 DITCH 200 E & & \\
\hline 1113 & B POND NORTH SIDE & & \\
\hline 1114 & B POND NORTH SIDE & & \\
\hline 1115 & GABLE POND N END & & \\
\hline 1116 & GABLE POND N END & & \\
\hline 1117 & U POND & & \\
\hline 1118 & WEST LAKE & & \\
\hline 1119 & WEST LAKE & & \\
\hline 1120 & 300 SAN WATER PLANT & & \\
\hline 1121 & RICH.PMPHS HRM 46.4 & & \\
\hline 1122 & HANF POWERLINE XING & & \\
\hline 1123 & HANF POWERLINE XING & & \\
\hline 1124 & HANF POWERLINE XING & & \\
\hline 1125 & 100 N TRENCH SPRING & & \\
\hline 1126 & 300 SAN WATER PLANT & & \\
\hline 1127 & RICH.PMPHS HRM 46.4 & & \\
\hline 1128 & $100 \mathrm{~F}$ TRENCH & & \\
\hline 1129 & 300 SAN WATER PLANT & & \\
\hline 1130 & RICH.PMPHS HRM 46.4 & & \\
\hline 1131 & 100 B AREA-RIVER & & \\
\hline 1132 & 100 B AREA-RIVER & & \\
\hline 1133 & 181 N BLDG 100 N AREA & & \\
\hline 1134 & 181 N BLDG 100 N AREA & & \\
\hline 1135 & 300 SAN WATER PLANT & & \\
\hline 1136 & RICHLAND SAN PLANT & & \\
\hline 1137 & RICHLAND SAN PLANT & & \\
\hline 1138 & 300 POND & & \\
\hline 1139 & RICH.PMPHS HRM 46.4 & & \\
\hline 1140 & RICH.PMPHS HRM 46.4 & & \\
\hline 1141 & RICH.PMPHS HRM 46.4 & & \\
\hline 1142 & RICH.PMPHS HRM 46.4 & & \\
\hline 1143 & VERNITA & & \\
\hline 1144 & 300 SAN WATER PLANT & & \\
\hline 1145 & RICH.PMPHS HRM 46.4 & & \\
\hline
\end{tabular}




\begin{tabular}{|c|c|c|c|}
\hline & $\bar{Z}$ & $\overline{\mathrm{AA}}$ & $\overline{A B}$ \\
\hline 1 & SAMP_SITE_NAME & WELL_NAME & SAMPLE_LOCATION \\
\hline 1146 & HANF POWERLINE XING & & \\
\hline 1147 & HANF POWERLINE XING & & \\
\hline 1148 & HANF POWERLINE XING & & \\
\hline 1149 & 100 N TRENCH SPRING & & \\
\hline 1150 & 300 SAN WATER PLANT & & \\
\hline 1151 & RICH.PMPHS HRM 46.4 & & \\
\hline 1152 & 100 F TRENCH & & \\
\hline 1153 & RICH.PMPHS HRM 46.4 & & \\
\hline 1154 & 300 SAN WATER PLANT & & \\
\hline 1155 & 100 B AREA-RIVER & & \\
\hline 1156 & 100 B AREA-RIVER & & \\
\hline 1157 & RICH.PMPHS HRM 46.4 & & \\
\hline 1158 & RICH.PMPHS HRM 46.4 & & \\
\hline 1159 & RICH.PMPHS HRM 46.4 & & \\
\hline 1160 & RICH.PMPHS HRM 46.4 & & \\
\hline 1161 & VERNITA & & \\
\hline 1162 & 181 N BLDG $100 N$ AREA & & \\
\hline 1163 & 300 SAN WATER PLANT & & \\
\hline 1164 & RICHLAND SAN PLANT & & \\
\hline 1165 & RICHLAND SAN PLANT & & \\
\hline 1166 & 300 POND & & \\
\hline 1167 & 300 SAN WATER PLANT & & \\
\hline 1168 & RICH.PMPHS HRM 46.4 & & \\
\hline 1169 & HANF POWERLINE XING & & \\
\hline 1170 & HANF POWERLINE XING & & \\
\hline 1171 & HANF POWERLINE XING & & \\
\hline 1172 & 100 N TRENCH SPRING & & \\
\hline 1173 & 300 SAN WATER PLANT & & \\
\hline 1174 & RICH.PMPHS HRM 46.4 & & \\
\hline 1175 & $100 \mathrm{~F}$ TRENCH & & \\
\hline 1176 & 300 SAN WATER PLANT & & \\
\hline 1177 & RICH.PMPHS HRM 46.4 & & \\
\hline 1178 & 181 N BLDG 100N AREA & & \\
\hline 1179 & 181 N BLDG 100 N AREA & & \\
\hline 1180 & 300 SAN WATER PLANT & & \\
\hline 1181 & RICHLAND SAN PLANT & & \\
\hline 1182 & RICHLAND SAN PLANT & & \\
\hline 1183 & 300 POND & & \\
\hline 1184 & RICH.PMPHS HRM 46.4 & & \\
\hline 1185 & RICH.PMPHS HRM 46.4 & & \\
\hline 1186 & RICH.PMPHS HRM 46.4 & & \\
\hline 1187 & RICH.PMPHS HRM 46.4 & & \\
\hline 1188 & VERNITA & & \\
\hline 1189 & RICH.PMPHS HRM 46.4 & & \\
\hline 1190 & 100 B AREA-RIVER & & \\
\hline 1191 & 100 B AREA-RIVER & & \\
\hline 1192 & 100 B AREA-RIVER & & \\
\hline 1193 & 300 SAN WATER PLANT & & \\
\hline 1194 & RICH.PMPHS HRM 46.4 & & \\
\hline 1195 & 300 SAN WATER PLANT & & \\
\hline 1196 & RICH.PMPHS HRM 46.4 & & \\
\hline 1197 & $100 \mathrm{~F}$ TRENCH & & \\
\hline
\end{tabular}




\begin{tabular}{|c|c|c|c|}
\hline & $\mathrm{Z}$ & $\overline{\mathrm{AA}}$ & $\mathrm{AB}$ \\
\hline 1 & SAMP_SITE_NAME & WELL_NAME & SAMPLE_LOCATION \\
\hline 1198 & HANF POWERLINE XING & & \\
\hline 1199 & HANF POWERLINE XING & & \\
\hline 1200 & HANF POWERLINE XING & & \\
\hline 1201 & 100 N TRENCH SPRING & & \\
\hline 1202 & 100 N TRENCH SPRING & & \\
\hline 1203 & 300 SAN WATER PLANT & & \\
\hline 1204 & RICH.PMPHS HRM 46.4 & & \\
\hline 1205 & RICH.PMPHS HRM 46.4 & & \\
\hline 1206 & 100 B AREA-RIVER & & \\
\hline 1207 & 100 B AREA-RIVER & & \\
\hline 1208 & 181 N BLDG 100N AREA & & \\
\hline 1209 & 300 SAN WATER PLANT & & \\
\hline 1210 & RICHLAND SAN PLANT & & \\
\hline 1211 & RICHLAND SAN PLANT & & \\
\hline 1212 & 300 POND & & \\
\hline 1213 & RICH.PMPHS HRM 46.4 & & \\
\hline 1214 & RICH.PMPHS HRM 46.4 & & \\
\hline 1215 & RICH.PMPHS HRM 46.4 & & \\
\hline 1216 & RICH.PMPHS HRM 46.4 & & \\
\hline 1217 & VERNITA & & \\
\hline 1218 & 216 B 63 DITCH 200 E & & \\
\hline 1219 & B POND NORTH SIDE & & \\
\hline 1220 & B POND NORTH SIDE & & \\
\hline 1221 & GABLE POND N END & & \\
\hline 1222 & GABLE POND N END & & \\
\hline 1223 & T POND & & \\
\hline 1224 & U POND & & \\
\hline 1225 & WEST LAKE & & \\
\hline 1226 & WEST LAKE & & \\
\hline 1227. & RICH.PMPHS HRM 46.4 & & \\
\hline 1228 & 300 SAN WATER PLANT & & \\
\hline 1229 & 100 F TRENCH & & \\
\hline 1230 & HANF POWERLINE XING & & \\
\hline 1231 & HANF POWERLINE XING & & \\
\hline 1232 & HANF POWERLINE XING & & \\
\hline 1233 & 100 N TRENCH SPRING & & \\
\hline 1234 & 100 N TRENCH SPRING & & \\
\hline 1235 & 300 SAN WATER PLANT & & \\
\hline 1236 & RICH.PMPHS HRM 46.4 & & \\
\hline 1237 & 300 SAN WATER PLANT & & \\
\hline 1238 & RICH.PMPHS HRM 46.4 & & \\
\hline 1239 & 100 B AREA-RIVER & & \\
\hline 1240 & 100 B AREA-RIVER & & \\
\hline 1241 & 181 N BLDG 100N AREA & & \\
\hline 1242 & 300 SAN WATER PLANT & & \\
\hline 1243 & RICHLAND SAN PLANT & & \\
\hline 1244 & 300 POND & & \\
\hline 1245 & RICH.PMPHS HRM 46.4 & & \\
\hline 1246 & RICH.PMPHS HRM 46.4 & & \\
\hline 1247 & RICH.PMPHS HRM 46.4 & & \\
\hline 1248 & RICH.PMPHS HRM 46.4 & & \\
\hline 1249 & RICH.PMPHS HRM 46.4 & & \\
\hline
\end{tabular}




\begin{tabular}{|c|c|c|c|}
\hline & $\bar{Z}$ & $\overline{\mathrm{AA}}$ & $\overline{A B}$ \\
\hline 1 & SAMP_SITE_NAME & WELL_NAME & SAMPLE_LOCATION \\
\hline 1250 & VERNITA & & \\
\hline 1251 & RICH.PMPHS HRM 46.4 & & \\
\hline 1252 & 300 SAN WATER PLANT & & \\
\hline 1253 & $100 \mathrm{~F}$ TRENCH & & \\
\hline 1254 & HANF POWERLINE XING & & \\
\hline 1255 & HANF POWERLINE XING & & \\
\hline 1256 & HANF POWERLINE XING & & \\
\hline 1257 & 100 N TRENCH SPRING & & \\
\hline 1258 & 100 N TRENCH SPRING & & \\
\hline 1259 & 300 SAN WATER PLANT & & \\
\hline 1260 & RICH.PMPHS HRM 46.4 & & \\
\hline 1261 & 300 SAN WATER PLANT & & \\
\hline 1262 & RICH.PMPHS HRM 46.4 & & \\
\hline 1263 & 300 SAN WATER PLANT & & \\
\hline 1264 & RICH.PMPHS HRM 46.4 & & \\
\hline 1265 & 100 B AREA-RIVER & & \\
\hline 1266 & 100 B AREA-RIVER & & \\
\hline 1267 & 100 B AREA-RIVER & & \\
\hline 1268 & 300 SAN WATER PLANT & & \\
\hline 1269 & RICHLAND SAN PLANT & & \\
\hline 1270 & RICHLAND SAN PLANT & & \\
\hline 1271 & 300 POND & & \\
\hline 1272 & RICH.PMPHS HRM 46.4 & & \\
\hline 1273 & RICH.PMPHS HRM 46.4 & & \\
\hline 1274 & RICH.PMPHS HRM 46.4 & & \\
\hline 1275 & RICH.PMPHS HRM 46.4 & & \\
\hline 1276 & RICH.PMPHS HRM 46.4 & & \\
\hline 1277 & VERNITA & & \\
\hline 1278 & SEQUIM BAY & & \\
\hline 1279 & SEQUIM BAY & & \\
\hline 1280 & SEQUIM BAY & & \\
\hline 1281 & $100 \mathrm{~F}$ TRENCH & & \\
\hline 1282 & 300 SAN WATER PLANT & & \\
\hline 1283 & RICH.PMPHS HRM 46.4 & & \\
\hline 1284 & HANF POWERLINE XING & & \\
\hline 1285 & HANF POWERLINE XING & & \\
\hline 1286 & HANF POWERLINE XING & & \\
\hline 1287 & 100 N TRENCH SPRING & & \\
\hline 1288 & 100 N TRENCH SPRING & & \\
\hline 1289 & 300 SAN WATER PLANT & & \\
\hline 1290 & RICH.PMPHS HRM 46.4 & & \\
\hline 1291 & 300 SAN WATER PLANT & & \\
\hline 1292 & RICH.PMPHS HRM 46.4 & & \\
\hline 1293 & 100 B AREA-RIVER & & \\
\hline 1294 & 100 B AREA-RIVER & & \\
\hline 1295 & 181 N BLDG 100N AREA & & \\
\hline 1296 & 300 SAN WATER PLANT & & \\
\hline 1297 & RICHLAND SAN PLANT & & \\
\hline 1298 & RICHLAND SAN PLANT & & \\
\hline 1299 & 300 POND & & \\
\hline 1300 & RICH.PMPHS HRM 46.4 & & \\
\hline 1301 & RICH.PMPHS HRM 46.4 & & \\
\hline
\end{tabular}




\begin{tabular}{|c|c|c|c|}
\hline & $\mathrm{Z}$ & $\overline{\mathrm{AA}}$ & $\mathrm{AB}$ \\
\hline 1 & SAMP_SITE_NAME & WELL_NAME & SAMPLE_LOCATION \\
\hline 1302 & RICH.PMPHS HRM 46.4 & & \\
\hline 1303 & RICH.PMPHS HRM 46.4 & & \\
\hline 1304 & RICH.PMPHS HRM 46.4 & & \\
\hline 1305 & VERNITA & & \\
\hline 1306 & $100 \mathrm{~F}$ TRENCH & & \\
\hline 1307 & 300 SAN WATER PLANT & & \\
\hline 1308 & RICH.PMPHS HRM 46.4 & & \\
\hline 1309 & HANF POWERLINE XING & & \\
\hline 1310 & HANF POWERLINE XING & & \\
\hline 1311 & HANF POWERLINE XING & & \\
\hline 1312 & 100 N TRENCH SPRING & & \\
\hline 1313 & 100 N TRENCH SPRING & & \\
\hline 1314 & 216 B 63 DITCH 200 E & & \\
\hline 1315 & B POND NORTH SIDE & & \\
\hline 1316 & B POND NORTH SIDE & & \\
\hline 1317 & GABLE POND N END & & \\
\hline 1318 & GABLE POND N END & & \\
\hline 1319 & U POND & & \\
\hline 1320 & WEST LAKE & & \\
\hline 1321 & WEST LAKE & & \\
\hline 1322 & 300 SAN WATER PLANT & & \\
\hline 1323 & RICH.PMPHS HRM 46.4 & & \\
\hline 1324 & 300 SAN WATER PLANT & & \\
\hline 1325 & RICH.PMPHS HRM 46.4 & & \\
\hline 1326 & 100 B AREA-RIVER & & \\
\hline 1327 & 100 B AREA-RIVER & & \\
\hline 1328 & 181 N BLDG 100N AREA & & \\
\hline 1329 & 300 SAN WATER PLANT & & \\
\hline 1330 & RICHLAND SAN PLANT & & \\
\hline 1331 & RICHLAND SAN PLANT & & \\
\hline 1332 & 300 POND & & \\
\hline 1333 & RICH.PMPHS HRM 46.4 & & \\
\hline 1334 & RICH.PMPHS HRM 46.4 & & \\
\hline 1335 & RICH.PMPHS HRM 46.4 & & \\
\hline 1336 & RICH.PMPHS HRM 46.4 & & \\
\hline 1337 & RICH.PMPHS HRM 46.4 & & \\
\hline 1338 & VERNITA & & \\
\hline 1339 & $100 \mathrm{~F}$ TRENCH & & \\
\hline 1340 & 300 SAN WATER PLANT & & \\
\hline 1341 & RICH.PMPHS HRM 46.4 & & \\
\hline 1342 & HANF POWERLINE XING & & \\
\hline 1343 & HANF POWERLINE XING & & \\
\hline 1344 & HANF POWERLINE XING & & \\
\hline 1345 & 100 N TRENCH SPRING & & \\
\hline 1346 & 100 N TRENCH SPRING & & \\
\hline 1347 & 300 SAN WATER PLANT & & \\
\hline 1348 & RICH.PMPHS HRM 46.4 & & \\
\hline 1349 & 300 SAN WATER PLANT & & \\
\hline 1350 & RICH.PMPHS HRM 46.4 & & \\
\hline 1351 & 300 SAN WATER PLANT & & \\
\hline 1352 & RICH.PMPHS HRM 46.4 & & \\
\hline 1353 & $100 \mathrm{~F}$ TRENCH & & \\
\hline
\end{tabular}




\begin{tabular}{|c|c|c|c|}
\hline & $\mathrm{Z}$ & $\overline{\mathrm{AA}}$ & $\mathrm{AB}$ \\
\hline 1 & SAMP_SITE_NAME & WELL_NAME & SAMPLE_LOCATION \\
\hline 1354 & 100 B AREA-RIVER & & \\
\hline 1355 & 100 B AREA-RIVER & & \\
\hline 1356 & 100 B AREA-RIVER & & \\
\hline 1357 & 181 N BLDG 100N AREA & & \\
\hline 1358 & HANFORD TOWNSITE & & \\
\hline 1359 & RICHLAND SAN PLANT & & \\
\hline 1360 & 300 POND & & \\
\hline 1361 & 300 AREA & & \\
\hline 1362 & RICH.PMPHS HRM 46.4 & & \\
\hline 1363 & RICH.PMPHS HRM 46.4 & & \\
\hline 1364 & RICH.PMPHS HRM 46.4 & & \\
\hline 1365 & RICH.PMPHS HRM 46.4 & & \\
\hline 1366 & VERNITA & & \\
\hline 1367 & FFTF (699 S 00-07) & & \\
\hline 1368 & ALE HDQTRS & & \\
\hline 1369 & ALE HDQTRS & & \\
\hline 1370 & PRIEST RAPIDS-RIVER & & \\
\hline 1371 & 100 N TRENCH SPRING & & \\
\hline 1372 & 100 N TRENCH SPRING & & \\
\hline 1373 & 216 B 63 DITCH 200 E & & \\
\hline 1374 & GABLE POND N END & & \\
\hline 1375 & GABLE POND N END & & \\
\hline 1376 & U POND & & \\
\hline 1377 & U POND & & \\
\hline 1378 & B POND NORTH SIDE & & \\
\hline 1379 & B POND NORTH SIDE & & \\
\hline 1380 & GABLE POND N END & & \\
\hline 1381 & 100 B AREA-RIVER & & \\
\hline 1382 & 100 B AREA-RIVER & & \\
\hline 1383 & 100 B AREA-RIVER & & \\
\hline 1384 & 181 N BLDG 100N AREA & & \\
\hline 1385 & FFTF (699 S 00-07) & & \\
\hline 1386 & FFTF (699 S 00-07) & & \\
\hline 1387 & HANFORD TOWNSITE & & \\
\hline 1388 & RICHLAND SAN PLANT & & \\
\hline 1389 & WPPSS NO. 2 AREA & & \\
\hline 1390 & WPPSS NO. 2 AREA & & \\
\hline 1391 & 300 POND & & \\
\hline 1392 & RICH.PMPHS HRM 46.4 & & \\
\hline 1393 & RICH.PMPHS HRM 46.4 & & \\
\hline 1394 & RICH.PMPHS HRM 46.4 & & \\
\hline 1395 & RICH.PMPHS HRM 46.4 & & \\
\hline 1396 & VERNITA & & \\
\hline 1397 & ALE HDQTRS & & \\
\hline 1398 & ALE HDQTRS & & \\
\hline 1399 & 100 N TRENCH SPRING & & \\
\hline 1400 & 100 N TRENCH SPRING & & \\
\hline 1401 & 300 AREA & & \\
\hline 1402 & PRIEST RAPIDS-RIVER & & \\
\hline 1403 & 100 B AREA-RIVER & & \\
\hline 1404 & 100 B AREA-RIVER & & \\
\hline 1405 & 100 B AREA-RIVER & & \\
\hline
\end{tabular}




\begin{tabular}{|c|c|c|c|}
\hline & $\bar{Z}$ & $\overline{\mathrm{AA}}$ & $\overline{A B}$ \\
\hline 1 & SAMP_SITE_NAME & WELL_NAME & SAMPLE_LOCATION \\
\hline 1406 & 181 N BLDG 100N AREA & & \\
\hline 1407 & FFTF (699 S 00-07) & & \\
\hline 1408 & FFTF (699 S 00-07) & & \\
\hline 1409 & HANFORD TOWNSITE & & \\
\hline 1410 & RICHLAND SAN PLANT & & \\
\hline 1411 & WPPSS NO. 2 AREA & & \\
\hline 1412 & WPPSS NO. 2 AREA & & \\
\hline 1413 & 300 POND & & \\
\hline 1414 & RICH.PMPHS HRM 46.4 & & \\
\hline 1415 & RICH.PMPHS HRM 46.4 & & \\
\hline 1416 & RICH.PMPHS HRM 46.4 & & \\
\hline 1417 & RICH.PMPHS HRM 46.4 & & \\
\hline 1418 & VERNITA & & \\
\hline 1419 & ALE HDQTRS & & \\
\hline 1420 & ALE HDQTRS & & \\
\hline 1421 & 100 N TRENCH SPRING & & \\
\hline 1422 & 100 N TRENCH SPRING & & \\
\hline 1423 & HANFORD TOWNSITE & & \\
\hline 1424 & 300 POND & & \\
\hline 1425 & 300 AREA & & \\
\hline 1426 & RICH.PMPHS HRM 46.4 & & \\
\hline 1427 & 100 B AREA-RIVER & & \\
\hline 1428 & 100 B AREA-RIVER & & \\
\hline 1429 & 100 B AREA-RIVER & & \\
\hline 1430 & RICH.PMPHS HRM 46.4 & & \\
\hline 1431 & RICH.PMPHS HRM 46.4 & & \\
\hline 1432 & RICH.PMPHS HRM 46.4 & & \\
\hline 1433 & RICH.PMPHS HRM 46.4 & & \\
\hline 1434 & VERNITA & & \\
\hline 1435 & 181 N BLDG 100N AREA & & \\
\hline 1436 & FFTF (699 S 00-07) & & \\
\hline 1437 & FFTF (699 S 00-07) & & \\
\hline 1438 & RICHLAND SAN PLANT & & \\
\hline 1439 & WPPSS NO. 2 AREA & & \\
\hline 1440 & WPPSS NO. 2 AREA & & \\
\hline 1441 & 216 B 63 DITCH 200 E & & \\
\hline 1442 & B POND NORTH SIDE & & \\
\hline 1443 & B POND NORTH SIDE & & \\
\hline 1444 & GABLE POND N END & & \\
\hline 1445 & GABLE POND N END & & \\
\hline 1446 & TPOND & & \\
\hline 1447 & TPOND & & \\
\hline 1448 & U POND & & \\
\hline 1449 & U POND & & \\
\hline 1450 & WEST LAKE & & \\
\hline 1451 & WEST LAKE & & \\
\hline 1452 & PRIEST RAPIDS-RIVER & & \\
\hline 1453 & 100 N TRENCH SPRING & & \\
\hline 1454 & 100 N TRENCH SPRING & & \\
\hline 1455 & 100 B AREA-RIVER & & \\
\hline 1456 & 100 B AREA-RIVER & & \\
\hline 1457 & 100 B AREA-RIVER & & \\
\hline
\end{tabular}




\begin{tabular}{|c|c|c|c|}
\hline & $\bar{Z}$ & $\overline{\mathrm{AA}}$ & $\overline{A B}$ \\
\hline 1 & SAMP_SITE_NAME & WELL_NAME & SAMPLE_LOCATION \\
\hline 1458 & HANFORD TOWNSITE & & \\
\hline 1459 & 309 WELL (399 04-05) & & \\
\hline 1460 & 181 N BLDG $100 \mathrm{~N}$ AREA & & \\
\hline 1461 & FFTF (699 S 00-07) & & \\
\hline 1462 & FFTF (699 S 00-07) & & \\
\hline 1463 & RICHLAND SAN PLANT & & \\
\hline 1464 & WPPSS NO. 2 AREA & & \\
\hline 1465 & WPPSS NO. 2 AREA & & \\
\hline 1466 & 300 POND & & \\
\hline 1467 & RICH.PMPHS HRM 46.4 & & \\
\hline 1468 & RICH.PMPHS HRM 46.4 & & \\
\hline 1469 & RICH.PMPHS HRM 46.4 & & \\
\hline 1470 & RICH.PMPHS HRM 46.4 & & \\
\hline 1471 & VERNITA & & \\
\hline 1472 & ALE HDQTRS & & \\
\hline 1473 & ALE HDQTRS & & \\
\hline 1474 & PRIEST RAPIDS-RIVER & & \\
\hline 1475 & 309 WELL (399 04-05) & & \\
\hline 1476 & 300 AREA & & \\
\hline 1477 & 100 N TRENCH SPRING & & \\
\hline 1478 & 100 N TRENCH SPRING & & \\
\hline 1479 & ALE HDQTRS & & \\
\hline 1480 & ALE HDQTRS & & \\
\hline 1481 & HANFORD TOWNSITE & & \\
\hline 1482 & 100 B AREA-RIVER & & \\
\hline 1483 & 100 B AREA-RIVER & & \\
\hline 1484 & 100 B AREA-RIVER & & \\
\hline 1485 & 181 N BLDG $100 N$ AREA & & \\
\hline 1486 & FFTF (699 S 00-07) & & \\
\hline 1487 & FFTF (699 S 00-07) & & \\
\hline 1488 & RICHLAND SAN PLANT & & \\
\hline 1489 & WPPSS NO. 2 AREA & & \\
\hline 1490 & WPPSS NO. 2 AREA & & \\
\hline 1491 & RICH.PMPHS HRM 46.4 & & \\
\hline 1492 & RICH.PMPHS HRM 46.4 & & \\
\hline 1493 & RICH.PMPHS HRM 46.4 & & \\
\hline 1494 & RICH.PMPHS HRM 46.4 & & \\
\hline 1495 & VERNITA & & \\
\hline 1496 & 300 POND & & \\
\hline 1497 & 309 WELL (399 04-05) & & \\
\hline 1498 & 309 WELL (399 04-05) & & \\
\hline 1499 & 300 AREA & & \\
\hline 1500 & PRIEST RAPIDS-RIVER & & \\
\hline 1501 & HANFORD TOWNSITE & & \\
\hline 1502 & ALE HDQTRS & & \\
\hline 1503 & ALE HDQTRS & & \\
\hline 1504 & 100 B AREA-RIVER & & \\
\hline 1505 & 100 B AREA-RIVER & & \\
\hline 1506 & 100 B AREA-RIVER & & \\
\hline 1507 & 181 N BLDG 100N AREA & & \\
\hline 1508 & 309 WELL (399 04-05) & & \\
\hline 1509 & 309 WELL (399 04-05) & & \\
\hline
\end{tabular}




\begin{tabular}{|c|c|c|c|}
\hline & $\bar{Z}$ & $\overline{\mathrm{AA}}$ & $\overline{A B}$ \\
\hline 1 & SAMP_SITE_NAME & WELL_NAME & SAMPLE_LOCATION \\
\hline 1510 & FFTF (699 S 00-07) & & \\
\hline 1511 & FFTF (699 S 00-07) & & \\
\hline 1512 & RICHLAND SAN PLANT & & \\
\hline 1513 & WPPSS NO. 2 AREA & & \\
\hline 1514 & WPPSS NO. 2 AREA & & \\
\hline 1515 & 300 AREA & & \\
\hline 1516 & PRIEST RAPIDS-RIVER & & \\
\hline 1517 & RICH.PMPHS HRM 46.4 & & \\
\hline 1518 & RICH.PMPHS HRM 46.4 & & \\
\hline 1519 & RICH.PMPHS HRM 46.4 & & \\
\hline 1520 & RICH.PMPHS HRM 46.4 & & \\
\hline 1521 & VERNITA & & \\
\hline 1522 & 300 POND & & \\
\hline 1523 & HANFORD TOWNSITE & & \\
\hline 1524 & ALE HDQTRS & & \\
\hline 1525 & ALE HDQTRS & & \\
\hline 1526 & 309 WELL (399 04-05) & & \\
\hline 1527 & 309 WELL (399 04-05) & & \\
\hline 1528 & 216 B 63 DITCH 200 E & & \\
\hline 1529 & B POND NORTH SIDE & & \\
\hline 1530 & B POND NORTH SIDE & & \\
\hline 1531 & GABLE POND N END & & \\
\hline 1532 & GABLE POND N END & & \\
\hline 1533 & U POND & & \\
\hline 1534 & U POND & & \\
\hline 1535 & WEST LAKE & & \\
\hline 1536 & WEST LAKE & & \\
\hline 1537 & 100 B AREA-RIVER & & \\
\hline 1538 & 100 B AREA-RIVER & & \\
\hline 1539 & 181 N BLDG $100 N$ AREA & & \\
\hline 1540 & FFTF (699 S 00-07) & & \\
\hline 1541 & FFTF (699 S 00-07) & & \\
\hline 1542 & RICHLAND SAN PLANT & & \\
\hline 1543 & WPPSS NO. 2 AREA & & \\
\hline 1544 & WPPSS NO. 2 AREA & & \\
\hline 1545 & 300 POND & & \\
\hline 1546 & RICH.PMPHS HRM 46.4 & & \\
\hline 1547 & RICH.PMPHS HRM 46.4 & & \\
\hline 1548 & RICH.PMPHS HRM 46.4 & & \\
\hline 1549 & RICH.PMPHS HRM 46.4 & & \\
\hline 1550 & VERNITA & & \\
\hline 1551 & 300 AREA & & \\
\hline 1552 & PRIEST RAPIDS-RIVER & & \\
\hline 1553 & ALE HDQTRS & & \\
\hline 1554 & ALE HDQTRS & & \\
\hline 1555 & HANFORD TOWNSITE & & \\
\hline 1556 & VERNITA & & \\
\hline 1557 & 100 B AREA-RIVER & & \\
\hline 1558 & 100 B AREA-RIVER & & \\
\hline 1559 & 100 B AREA-RIVER & & \\
\hline 1560 & 300 AREA & & \\
\hline 1561 & PRIEST RAF & & \\
\hline
\end{tabular}




\begin{tabular}{|c|c|c|c|}
\hline & $\mathrm{Z}$ & $\overline{\mathrm{AA}}$ & $\mathrm{AB}$ \\
\hline 1 & SAMP_SITE_NAME & WELL_NAME & SAMPLE_LOCATION \\
\hline 1562 & RICH.PMPHS HRM 46.4 & & \\
\hline 1563 & 181 N BLDG $100 N$ AREA & & \\
\hline 1564 & 309 WELL (399 04-05) & & \\
\hline 1565 & 309 WELL (399 04-05) & & \\
\hline 1566 & FFTF (699 S 00-07) & & \\
\hline 1567 & FFTF (699 S 00-07) & & \\
\hline 1568 & RICHLAND SAN PLANT & & \\
\hline 1569 & WPPSS NO. 2 AREA & & \\
\hline 1570 & WPPSS NO. 2 AREA & & \\
\hline 1571 & RICH.PMPHS HRM 46.4 & & \\
\hline 1572 & RICH.PMPHS HRM 46.4 & & \\
\hline 1573 & ALE HDQTRS & & \\
\hline 1574 & HANFORD TOWNSITE & & \\
\hline 1575 & 100 B AREA-RIVER & & \\
\hline 1576 & 100 B AREA-RIVER & & \\
\hline 1577 & 100 B AREA-RIVER & & \\
\hline 1578 & 181 N BLDG $100 N$ AREA & & \\
\hline 1579 & 309 WELL (399 04-05) & & \\
\hline 1580 & 309 WELL (399 04-05) & & \\
\hline 1581 & FFTF (699 S 00-07) & & \\
\hline 1582 & FFTF (699 S 00-07) & & \\
\hline 1583 & RICHLAND SAN PLANT & & \\
\hline 1584 & WPPSS NO. 2 AREA & & \\
\hline 1585 & WPPSS NO. 2 AREA & & \\
\hline 1586 & 300 AREA & & \\
\hline 1587 & PRIEST RAPIDS-RIVER & & \\
\hline 1588 & RICH.PMPHS HRM 46.4 & & \\
\hline 1589 & RICH.PMPHS HRM 46.4 & & \\
\hline 1590 & RICH.PMPHS HRM 46.4 & & \\
\hline 1591 & RICH.PMPHS HRM 46.4 & & \\
\hline 1592 & VERNITA & & \\
\hline 1593 & HANFORD TOWNSITE & & \\
\hline 1594 & ALE HDQTRS & & \\
\hline 1595 & ALE HDQTRS & & \\
\hline 1596 & 309 WELL (399 04-05) & & \\
\hline 1597 & 309 WELL (399 04-05) & & \\
\hline 1598 & 100 B AREA-RIVER & & \\
\hline 1599 & 100 B AREA-RIVER & & \\
\hline 1600 & 100 B AREA-RIVER & & \\
\hline 1601 & 181 N BLDG 100 N AREA & & \\
\hline 1602 & FFTF (699 S 00-07) & & \\
\hline 1603 & FFTF (699 S 00-07) & & \\
\hline 1604 & RICHLAND SAN PLANT & & \\
\hline 1605 & WPPSS NO. 2 AREA & & \\
\hline 1606 & WPPSS NO. 2 AREA & & \\
\hline 1607 & 216 B 63 DITCH 200 E & & \\
\hline 1608 & B POND NORTH SIDE & & \\
\hline 1609 & B POND NORTH SIDE & & \\
\hline 1610 & GABLE POND N END & & \\
\hline 1611 & GABLE POND N END & & \\
\hline 1612 & U POND & & \\
\hline 1613 & U POND & & \\
\hline
\end{tabular}




\begin{tabular}{|c|c|c|c|}
\hline & $\mathrm{Z}$ & $\overline{\mathrm{AA}}$ & $\mathrm{AB}$ \\
\hline 1 & SAMP_SITE_NAME & WELL_NAME & SAMPLE_LOCATION \\
\hline 1614 & WEST LAKE & & \\
\hline 1615 & WEST LAKE & & \\
\hline 1616 & 300 AREA & & \\
\hline \begin{tabular}{|c|}
1617 \\
\end{tabular} & PRIEST RAPIDS-RIVER & & \\
\hline 1618 & RICH.PMPHS HRM 46.4 & & \\
\hline 1619 & RICH.PMPHS HRM 46.4 & & \\
\hline 1620 & RICH.PMPHS HRM 46.4 & & \\
\hline 1621 & RICH.PMPHS HRM 46.4 & & \\
\hline 1622 & VERNITA & & \\
\hline 1623 & ALE HDQTRS & & \\
\hline 1624 & ALE HDQTRS & & \\
\hline 1625 & HANFORD TOWNSITE & & \\
\hline 1626 & RICH.PMPHS HRM 46.4 & & \\
\hline 1627 & RICH.PMPHS HRM 46.4 & & \\
\hline 1628 & RICH.PMPHS HRM 46.4 & & \\
\hline 1629 & RICH.PMPHS HRM 46.4 & & \\
\hline 1630 & VERNITA & & \\
\hline 1631 & ALE HDQTRS & & \\
\hline 1632 & ALE HDQTRS & & \\
\hline 1633 & 100 B AREA-RIVER & & \\
\hline 1634 & 100 B AREA-RIVER & & \\
\hline 1635 & 100 B AREA-RIVER & & \\
\hline 1636 & 300 AREA & & \\
\hline 1637 . & PRIEST RAPIDS-RIVER & & \\
\hline 1638 & 181 N BLDG 100 N AREA & & \\
\hline 1639 & FFTF (699 S 00-07) & & \\
\hline 1640 & FFTF (699 S 00-07) & & \\
\hline 1641 & HANFORD TOWNSITE & & \\
\hline 1642 & RICHLAND SAN PLANT & & \\
\hline 1643 & WPPSS NO. 2 AREA & & \\
\hline 1644 & WPPSS NO. 2 AREA & & \\
\hline 1645 & ALE HDQTRS & & \\
\hline 1646 & ALE HDQTRS & & \\
\hline 1647 & 100 B AREA-RIVER & & \\
\hline 1648 & 100 B AREA-RIVER & & \\
\hline 1649 & 100 B AREA-RIVER & & \\
\hline 1650 & 181 N BLDG 100 N AREA & & \\
\hline 1651 & FFTF (699 S 00-07) & & \\
\hline 1652 & FFTF (699 S 00-07) & & \\
\hline 1653 & HANFORD TOWNSITE & & \\
\hline 1654 & RICHLAND SAN PLANT & & \\
\hline 1655 & WPPSS NO. 2 AREA & & \\
\hline 1656 & 300 AREA & & \\
\hline 1657 & PRIEST RAPIDS-RIVER & & \\
\hline 1658 & RICH.PMPHS HRM 46.4 & & \\
\hline 1659 & RICH.PMPHS HRM 46.4 & & \\
\hline 1660 & RICH.PMPHS HRM 46.4 & & \\
\hline 1661 & RICH.PMPHS HRM 46.4 & & \\
\hline 1662 & VERNITA & & \\
\hline 1663 & WPPSS NO. 2 AREA & & \\
\hline 1664 & GABLE POND N END & & \\
\hline 1665 & GABLE POND N END & & \\
\hline
\end{tabular}




\begin{tabular}{|c|c|c|c|}
\hline & $\bar{Z}$ & $\overline{\mathrm{AA}}$ & $\overline{A B}$ \\
\hline 1 & SAMP_SITE_NAME & WELL_NAME & SAMPLE_LOCATION \\
\hline 1666 & 300 AREA & & \\
\hline 1667 & 300 AREA & & \\
\hline 1668 & PRIEST RAPIDS-RIVER & & \\
\hline 1669 & PRIEST RAPIDS-RIVER & & \\
\hline 1670 & ALE HDQTRS & & \\
\hline 1671 & ALE HDQTRS & & \\
\hline 1672 & FFTF (699 S 00-07) & & \\
\hline 1673 & FFTF (699 S 00-07) & & \\
\hline 1674 & WPPSS NO. 2 AREA & & \\
\hline 1675 & WPPSS NO. 2 AREA & & \\
\hline 1676 & 300 AREA & & \\
\hline 1677 & 300 AREA & & \\
\hline 1678 & 300 AREA & & \\
\hline 1679 & PRIEST RAPIDS-RIVER & & \\
\hline 1680 & PRIEST RAPIDS-RIVER & & \\
\hline 1681 & PRIEST RAPIDS-RIVER & & \\
\hline 1682 & RICH.PMPHS HRM 46.4 & & \\
\hline 1683 & RICH.PMPHS HRM 46.4 & & \\
\hline 1684 & RICH.PMPHS HRM 46.4 & & \\
\hline 1685 & VERNITA & & \\
\hline 1686 & 100 B AREA-RIVER & & \\
\hline 1687. & 100 B AREA-RIVER & & \\
\hline 1688 & 181 N BLDG 100N AREA & & \\
\hline 1689 & RICHLAND SAN PLANT & & \\
\hline 1690 & RICHLAND SAN PLANT & & \\
\hline 1691 & 300 AREA & & \\
\hline 1692 & 300 AREA & & \\
\hline 1693 & 300 AREA & & \\
\hline 1694 & PRIEST RAPIDS-RIVER & & \\
\hline 1695 & PRIEST RAPIDS-RIVER & & \\
\hline 1696 & ALE HDQTRS & & \\
\hline 1697 & ALE HDQTRS & & \\
\hline 1698 & PRIEST RAPIDS-RIVER & & \\
\hline 1699 & PRIEST RAPIDS-RIVER & & \\
\hline 1700 & PRIEST RAPIDS-RIVER & & \\
\hline 1701 & FFTF (699 S 00-07) & & \\
\hline 1702 & FFTF (699 S 00-07) & & \\
\hline 1703 & WPPSS NO. 2 AREA & & \\
\hline 1704 & WPPSS NO. 2 AREA & & \\
\hline 1705 & 216 B 63 DITCH 200 E & & \\
\hline 1706 & B POND NORTH SIDE & & \\
\hline 1707 & B POND NORTH SIDE & & \\
\hline 1708 & GABLE POND N END & & \\
\hline 1709 & GABLE POND N END & & \\
\hline 1710 & U POND & & \\
\hline 1711 & U POND & & \\
\hline 1712 & WEST LAKE & & \\
\hline 1713 & WEST LAKE & & \\
\hline 1714 & 300 AREA & & \\
\hline 1715 & 300 AREA & & \\
\hline 1716 & RICH.PMPHS HRM 46.4 & & \\
\hline 1717 & RICH.PMPHS HRM 46.4 & & \\
\hline
\end{tabular}




\begin{tabular}{|c|c|c|c|}
\hline & $\mathrm{Z}$ & $\overline{\mathrm{AA}}$ & $\mathrm{AB}$ \\
\hline 1 & SAMP_SITE_NAME & WELL_NAME & SAMPLE_LOCATION \\
\hline 1718 & RICH.PMPHS HRM 46.4 & & \\
\hline 1719 & VERNITA & & \\
\hline 1720 & 100 B AREA-RIVER & & \\
\hline 1721. & 100 B AREA-RIVER & & \\
\hline 1722 & 181 N BLDG $100 \mathrm{~N}$ AREA & & \\
\hline 1723 & RICHLAND SAN PLANT & & \\
\hline 1724 & 300 AREA & & \\
\hline 1725 & 300 AREA & & \\
\hline 1726 & ALE HDQTRS & & \\
\hline 1727 & ALE HDQTRS & & \\
\hline 1728 & ALE HDQTRS & & \\
\hline 1729 & FFTF (699 S 00-07) & & \\
\hline 1730 & FFTF (699 S 00-07) & & \\
\hline 1731 & FFTF (699 S 00-07) & & \\
\hline 1732 & WPPSS NO. 2 AREA & & \\
\hline 1733 & WPPSS NO. 2 AREA & & \\
\hline 1734 & 300 AREA & & \\
\hline 1735 & 300 AREA & & \\
\hline 1736 & PRIEST RAPIDS-RIVER & & \\
\hline 1737 & PRIEST RAPIDS-RIVER & & \\
\hline 1738 & 100 B AREA-RIVER & & \\
\hline 1739 & 100 B AREA-RIVER & & \\
\hline 1740. & 100 B AREA-RIVER & & \\
\hline 1741. & 181 N BLDG 100N AREA & & \\
\hline 1742 & RICHLAND SAN PLANT & & \\
\hline 1743 & RICH.PMPHS HRM 46.4 & & \\
\hline 1744 & RICH.PMPHS HRM 46.4 & & \\
\hline 1745 & RICH.PMPHS HRM 46.4 & & \\
\hline 1746 & RICH.PMPHS HRM 46.4 & & \\
\hline 1747 & VERNITA & & \\
\hline 1748 & 300 AREA & & \\
\hline 1749 & 300 AREA & & \\
\hline 1750 & 300 AREA & & \\
\hline 1751 & PRIEST RAPIDS-RIVER & & \\
\hline 1752 & PRIEST RAPIDS-RIVER & & \\
\hline 1753 & PRIEST RAPIDS-RIVER & & \\
\hline 1754 & ALE HDQTRS & & \\
\hline 1755 & ALE HDQTRS & & \\
\hline 1756 & FFTF (699 S 00-07) & & \\
\hline 1757 & FFTF (699 S 00-07) & & \\
\hline 1758 & WPPSS NO. 2 AREA & & \\
\hline 1759 & WPPSS NO. 2 AREA & & \\
\hline 1760 & 300 AREA & & \\
\hline 1761 & 300 AREA & & \\
\hline 1762 & PRIEST RAPIDS-RIVER & & \\
\hline 1763 & PRIEST RAPIDS-RIVER & & \\
\hline 1764 & 100 B AREA-RIVER & & \\
\hline 1765 & 100 B AREA-RIVER & & \\
\hline 1766 & 181 N BLDG 100N AREA & & \\
\hline 1767 & RICHLAND SAN PLANT & & \\
\hline 1768 & 216 B 63 DITCH 200 E & & \\
\hline 1769 & B POND NORTH SIDE & & \\
\hline
\end{tabular}




\begin{tabular}{|c|c|c|c|}
\hline & $\bar{Z}$ & $\overline{\mathrm{AA}}$ & $\overline{A B}$ \\
\hline 1 & SAMP_SITE_NAME & WELL_NAME & SAMPLE_LOCATION \\
\hline 1770 & B POND NORTH SIDE & & \\
\hline 1771 & GABLE POND N END & & \\
\hline 1772 & GABLE POND N END & & \\
\hline 1773 & U POND & & \\
\hline 1774 & U POND & & \\
\hline 1775 & WEST LAKE & & \\
\hline 1776 & WEST LAKE & & \\
\hline 1777 . & RICH.PMPHS HRM 46.4 & & \\
\hline 1778 & RICH.PMPHS HRM 46.4 & & \\
\hline 1779 & RICH.PMPHS HRM 46.4 & & \\
\hline 1780 & VERNITA & & \\
\hline 1781 & 300 AREA & & \\
\hline 1782 & 300 AREA & & \\
\hline 1783 & 300 AREA & & \\
\hline 1784 & PRIEST RAPIDS-RIVER & & \\
\hline 1785 & PRIEST RAPIDS-RIVER & & \\
\hline 1786 & PRIEST RAPIDS-RIVER & & \\
\hline 1787 & ALE HDQTRS & & \\
\hline 1788 & ALE HDQTRS & & \\
\hline 1789 & FFTF (699 S 00-07) & & \\
\hline 1790 & FFTF (699 S 00-07) & & \\
\hline 1791 & WPPSS NO. 2 AREA & & \\
\hline 1792 & WPPSS NO. 2 AREA & & \\
\hline 1793 & 300 AREA & & \\
\hline 1794 & 300 AREA & & \\
\hline 1795 & PRIEST RAPIDS-RIVER & & \\
\hline 1796 & PRIEST RAPIDS-RIVER & & \\
\hline 1797 & RICH.PMPHS HRM 46.4 & & \\
\hline 1798 & RICH.PMPHS HRM 46.4 & & \\
\hline 1799 & RICH.PMPHS HRM 46.4 & & \\
\hline 1800 & VERNITA & & \\
\hline 1801 & 100 B AREA-RIVER & & \\
\hline 1802 & 100 B AREA-RIVER & & \\
\hline 1803 & 300 AREA & & \\
\hline 1804 & 300 AREA & & \\
\hline 1805 & 300 AREA & & \\
\hline 1806 & PRIEST RAPIDS-RIVER & & \\
\hline 1807 & PRIEST RAPIDS-RIVER & & \\
\hline 1808 & PRIEST RAPIDS-RIVER & & \\
\hline 1809 & 181 N BLDG 100N AREA & & \\
\hline 1810 & RICHLAND SAN PLANT & & \\
\hline 1811 & ALE HDQTRS & & \\
\hline 1812 & ALE HDQTRS & & \\
\hline 1813 & ALE HDQTRS & & \\
\hline 1814 & FFTF (699 S 00-07) & & \\
\hline 1815 & FFTF (699 S 00-07) & & \\
\hline 1816 & FFTF (699 S 00-07) & & \\
\hline 1817 & WPPSS NO. 2 AREA & & \\
\hline 1818 & WPPSS NO. 2 AREA & & \\
\hline 1819 & WPPSS NO. 2 AREA & & \\
\hline 1820 & 300 AREA & & \\
\hline 1821 & 300 AREA & & \\
\hline
\end{tabular}




\begin{tabular}{|c|c|c|c|}
\hline & $\mathrm{Z}$ & $\overline{\mathrm{AA}}$ & $\mathrm{AB}$ \\
\hline 1 & SAMP_SITE_NAME & WELL_NAME & SAMPLE_LOCATION \\
\hline 1822 & PRIEST RAPIDS-RIVER & & \\
\hline 1823 & PRIEST RAPIDS-RIVER & & \\
\hline 1824 & 100 B AREA-RIVER & & \\
\hline 1825 & 100 B AREA-RIVER & & \\
\hline 1826 & 100 B AREA-RIVER & & \\
\hline 1827 & 181 N BLDG 100 N AREA & & \\
\hline 1828 & RICHLAND SAN PLANT & & \\
\hline 1829 & 300 AREA & & \\
\hline 1830 & 300 AREA & & \\
\hline 1831 & 300 AREA & & \\
\hline 1832 & PRIEST RAPIDS-RIVER & & \\
\hline 1833 & PRIEST RAPIDS-RIVER & & \\
\hline 1834 & PRIEST RAPIDS-RIVER & & \\
\hline 1835 & RICH.PMPHS HRM 46.4 & & \\
\hline 1836 & RICH.PMPHS HRM 46.4 & & \\
\hline 1837 & RICH.PMPHS HRM 46.4 & & \\
\hline 1838 & RICH.PMPHS HRM 46.4 & & \\
\hline 1839 & VERNITA & & \\
\hline 1840 & ALE HDQTRS & & \\
\hline 1841 & ALE HDQTRS & & \\
\hline 1842 & 309 WELL (399 04-05) & & \\
\hline 1843 & 309 WELL (399 04-05) & & \\
\hline 1844 & FFTF (699 S 00-07) & & \\
\hline 1845 & FFTF (699 S 00-07) & & \\
\hline 1846 & WPPSS NO. 2 AREA & & \\
\hline 1847 & WPPSS NO. 2 AREA & & \\
\hline 1848 & 300 AREA & & \\
\hline 1849 & 300 AREA & & \\
\hline 1850 & PRIEST RAPIDS-RIVER & & \\
\hline 1851 & PRIEST RAPIDS-RIVER & & \\
\hline 1852 & PRIEST RAPIDS-RIVER & & \\
\hline 1853 & RICH.PMPHS HRM 46.4 & & \\
\hline 1854 & 216 B 63 DITCH 200 E & & \\
\hline 1855 & B POND NORTH SIDE & & \\
\hline 1856 & B POND NORTH SIDE & & \\
\hline 1857 & GABLE POND N END & & \\
\hline 1858 & GABLE POND N END & & \\
\hline 1859 & U POND & & \\
\hline 1860 & U POND & & \\
\hline 1861 & WEST LAKE & & \\
\hline 1862 & WEST LAKE & & \\
\hline 1863 & 100 B AREA-RIVER & & \\
\hline 1864 & 100 B AREA-RIVER & & \\
\hline 1865 & 181 N BLDG 100N AREA & & \\
\hline 1866 & 309 WELL (399 04-05) & & \\
\hline 1867. & 309 WELL (399 04-05) & & \\
\hline 1868 & RICHLAND SAN PLANT & & \\
\hline 1869 & 300 AREA & & \\
\hline 1870 & 300 AREA & & \\
\hline 1871 & 300 AREA & & \\
\hline 1872 & RICH.PMPHS HRM 46.4 & & \\
\hline 1873 & RICH.PMPHS HRM 46.4 & & \\
\hline
\end{tabular}




\begin{tabular}{|c|c|c|c|}
\hline & $\bar{Z}$ & $\overline{\mathrm{AA}}$ & $\overline{A B}$ \\
\hline 1 & SAMP_SITE_NAME & WELL_NAME & SAMPLE_LOCATION \\
\hline 1874 & VERNITA & & \\
\hline 1875 & 300 AREA & & \\
\hline 1876 & 300 AREA & & \\
\hline 1877 & PRIEST RAPIDS-RIVER & & \\
\hline 1878 & PRIEST RAPIDS-RIVER & & \\
\hline 1879 & FFTF & & \\
\hline 1880 & 300 AREA & & \\
\hline 1881 & 300 AREA & & \\
\hline 1882 & PRIEST RAPIDS-RIVER & & \\
\hline 1883 & PRIEST RAPIDS-RIVER & & \\
\hline 1884 & ALE HDQTRS & & \\
\hline 1885 & ALE HDQTRS & & \\
\hline 1886 & OBSEVATORY-RTLSNK MT & & \\
\hline 1887 & OBSEVATORY-RTLSNK MT & & \\
\hline 1888 & 100 B AREA-RIVER & & \\
\hline 1889 & 100 B AREA-RIVER & & \\
\hline 1890 & 300 SAN WATER PLANT & & \\
\hline 1891 & 300 SAN WATER PLANT & & \\
\hline 1892 & 300 SAN WATER PLANT & & \\
\hline 1893 & FFTF & & \\
\hline 1894 & FFTF & & \\
\hline 1895 & RICHLAND SAN PLANT & & \\
\hline 1896 & RICH.PMPHS HRM 46.4 & & \\
\hline 1897 & RICH.PMPHS HRM 46.4 & & \\
\hline 1898 & VERNITA & & \\
\hline 1899 & 300 AREA & & \\
\hline 1900 & 300 AREA & & \\
\hline 1901 & 300 AREA & & \\
\hline 1902 & PRIEST RAPIDS-RIVER & & \\
\hline 1903 & PRIEST RAPIDS-RIVER & & \\
\hline 1904 & PRIEST RAPIDS-RIVER & & \\
\hline 1905 & 300 AREA & & \\
\hline 1906 & 300 AREA & & \\
\hline 1907 & PRIEST RAPIDS-RIVER & & \\
\hline 1908 & PRIEST RAPIDS-RIVER & & \\
\hline 1909 & 100 B AREA-RIVER & & \\
\hline 1910 & 100 B AREA-RIVER & & \\
\hline 1911 & 100 B AREA-RIVER & & \\
\hline 1912 & 300 SAN WATER PLANT & & \\
\hline 1913 & FFTF & & \\
\hline 1914 & FFTF & & \\
\hline 1915 & RICHLAND SAN PLANT & & \\
\hline 1916 & RICH.PMPHS HRM 46.4 & & \\
\hline 1917 & RICH.PMPHS HRM 46.4 & & \\
\hline 1918 & RICH.PMPHS HRM 46.4 & & \\
\hline 1919 & RICH.PMPHS HRM 46.4 & & \\
\hline 1920 & VERNITA & & \\
\hline 1921 & 300 AREA & & \\
\hline 1922 & 300 AREA & & \\
\hline 1923 & PRIEST RAPIDS-RIVER & & \\
\hline 1924 & PRIEST RAPIDS-RIVER & & \\
\hline 1925 & PRIEST RAPIDS-RIVER & & \\
\hline
\end{tabular}




\begin{tabular}{|c|c|c|c|}
\hline & $\bar{Z}$ & $\overline{\mathrm{AA}}$ & $\overline{A B}$ \\
\hline 1 & SAMP_SITE_NAME & WELL_NAME & SAMPLE_LOCATION \\
\hline 1926 & 300 AREA & & \\
\hline 1927 & RICH.PMPHS HRM 46.4 & & \\
\hline 1928 & 216 B 63 DITCH 200 E & & \\
\hline 1929 & B POND NORTH SIDE & & \\
\hline 1930 & B POND NORTH SIDE & & \\
\hline 1931 & GABLE POND N END & & \\
\hline 1932 & GABLE POND N END & & \\
\hline 1933 & U POND & & \\
\hline 1934 & U POND & & \\
\hline 1935 & WEST LAKE & & \\
\hline 1936 & WEST LAKE & & \\
\hline 1937 & 300 AREA & & \\
\hline 1938 & 300 AREA & & \\
\hline 1939 & PRIEST RAPIDS-RIVER & & \\
\hline 1940 & PRIEST RAPIDS-RIVER & & \\
\hline 1941 & RICH.PMPHS HRM 46.4 & & \\
\hline 1942 & RICH.PMPHS HRM 46.4 & & \\
\hline 1943 & RICH.PMPHS HRM 46.4 & & \\
\hline 1944 & VERNITA & & \\
\hline 1945 & 100 B AREA-RIVER & & \\
\hline 1946 & 100 B AREA-RIVER & & \\
\hline 1947 & 300 SAN WATER PLANT & & \\
\hline 1948 & FFTF & & \\
\hline 1949 & FFTF & & \\
\hline 1950 & RICHLAND SAN PLANT & & \\
\hline 1951 & 300 AREA & & \\
\hline 1952 & 300 AREA & & \\
\hline 1953 & 300 AREA & & \\
\hline 1954 & PRIEST RAPIDS-RIVER & & \\
\hline 1955 & PRIEST RAPIDS-RIVER & & \\
\hline 1956 & PRIEST RAPIDS-RIVER & & \\
\hline 1957 & B POND NORTH SIDE & & \\
\hline 1958 & GABLE POND N END & & \\
\hline 1959 & WEST LAKE & & \\
\hline 1960 & 300 AREA & & \\
\hline 1961 & 300 AREA & & \\
\hline 1962 & PRIEST RAPIDS-RIVER & & \\
\hline 1963 & PRIEST RAPIDS-RIVER & & \\
\hline 1964. & 100 B AREA-RIVER & & \\
\hline 1965 & 100 B AREA-RIVER & & \\
\hline 1966 & 300 SAN WATER PLANT & & \\
\hline 1967 & 300 SAN WATER PLANT & & \\
\hline 1968 & 300 SAN WATER PLANT & & \\
\hline 1969 & FFTF & & \\
\hline 1970 & FFTF & & \\
\hline 1971 & FFTF & & \\
\hline 1972 & RICHLAND SAN PLANT & & \\
\hline 1973 & 300 AREA & & \\
\hline 1974 : & 300 AREA & & \\
\hline 1975 & 300 AREA & & \\
\hline 1976 & PRIEST RAPIDS-RIVER & & \\
\hline$|1977|$ & PRIEST RAPIDS-RIVER & & \\
\hline
\end{tabular}




\begin{tabular}{|c|l|l|l|}
\hline & \multicolumn{1}{|c|}{$Z$} & A & AB \\
\hline 1 & SAMP_SITE_NAME & WELL_NAME & SAMPLE_LOCATION \\
\hline 1978 & PRIEST RAPIDS-RIVER & & \\
\hline 1979 & RICH.PMPHS HRM 46.4 & & \\
\hline 1980 & RICH.PMPHS HRM 46.4 & & \\
\hline 1981 & RICH.PMPHS HRM 46.4 & & \\
\hline 1982 & VERNITA & & \\
\hline 1983 & 300 AREA & & \\
\hline 1984 & 300 AREA & & \\
\hline 1985 & PRIEST RAPIDS-RIVER & & \\
\hline 1986 & PRIEST RAPIDS-RIVER & & \\
\hline 1987 & 300 SAN WATER PLANT & & \\
\hline 1988 & RICHLAND SAN PLANT & & \\
\hline 1989 & 100 B AREA-RIVER & & \\
\hline 1990 & 100 B AREA-RIVER & & \\
\hline 1991 & 100 B AREA-RIVER & & \\
\hline 1992 & 300 AREA & & \\
\hline 1993 & 300 AREA & & \\
\hline 1994 & 300 AREA & & \\
\hline 1995 & PRIEST RAPIDS-RIVER & & \\
\hline 1996 & PRIEST RAPIDS-RIVER & & \\
\hline 1997 & PRIEST RAPIDS-RIVER & & \\
\hline 1998 & 300 SAN WATER PLANT & & \\
\hline 1999 & FFTF & & \\
\hline 2000 & FFTF & & \\
\hline 2001 & RICHLAND SAN PLANT & & \\
\hline 2002 & RICH.PMPHS HRM 46.4 & & \\
\hline 2003 & RICH.PMPHS HRM 46.4 & & \\
\hline 2004 & RICH.PMPHS HRM 46.4 & & \\
\hline 2005 & RICH.PMPHS HRM 46.4 & & \\
\hline 2006 & VERNITA & & \\
\hline 2007 & 300 AREA & & \\
\hline 2008 & PRIEST RAPIDS-RIVER & & \\
\hline 2009 & PRIEST RAPIDS-RIVER & & \\
\hline 2010 & 300 AREA & & \\
\hline 2011 & 300 AREA & & \\
\hline 2012 & 300 AREA & & \\
\hline 2013 & 300 AREA & & \\
\hline 2014 & PRIEST RAPIDS-RIVER & & \\
\hline 2015 & PRIEST RAPIDS-RIVER & & \\
\hline 2016 & PRIEST RAPIDS-RIVER & & \\
\hline 2017 & RICH.PMPHS HRM 46.4 & & \\
\hline 2018 & VERNITA & & \\
\hline 2019 & 100 B AREA-RIVER & & \\
\hline 2020 & 100 B AREA-RIVER & & \\
\hline 2021 & 300 SAN WATER PLANT & & \\
\hline 2022 & FFTF & & \\
\hline 2023 & FFTF & & \\
\hline 2024 & RICHLAND SAN PLANT & & \\
\hline 2025 & 300 AREA & & \\
\hline 2026 & 300 AREA & & \\
\hline 2027 & PRIEST RAPIDS-RIVER & & \\
\hline 2028 & PRIEST RAPIDS-RIVER & & \\
\hline 20216 B 63 DITCH 200E & & & \\
\hline
\end{tabular}




\begin{tabular}{|c|c|c|c|}
\hline & $\bar{Z}$ & $\overline{\mathrm{AA}}$ & $\overline{A B}$ \\
\hline 1 & SAMP_SITE_NAME & WELL_NAME & SAMPLE_LOCATION \\
\hline 2030 & B POND NORTH SIDE & & \\
\hline 2031 & B POND NORTH SIDE & & \\
\hline 2032 & GABLE POND N END & & \\
\hline 2033 & GABLE POND N END & & \\
\hline 2034 & U POND & & \\
\hline 2035 & U POND & & \\
\hline 2036 & WEST LAKE & & \\
\hline 2037 & WEST LAKE & & \\
\hline 2038 & 300 AREA & & \\
\hline 2039 & 300 AREA & & \\
\hline 2040 & 300 AREA & & \\
\hline 2041 & PRIEST RAPIDS-RIVER & & \\
\hline 2042 & PRIEST RAPIDS-RIVER & & \\
\hline 2043 & PRIEST RAPIDS-RIVER & & \\
\hline 2044 & RICH.PMPHS HRM 46.4 & & \\
\hline 2045 & RICH.PMPHS HRM 46.4 & & \\
\hline 2046 & RICH.PMPHS HRM 46.4 & & \\
\hline 2047 & VERNITA & & \\
\hline 2048 & 100 B AREA-RIVER & & \\
\hline 2049 & 100 B AREA-RIVER & & \\
\hline 2050 & 100 B AREA-RIVER & & \\
\hline 2051 & 300 SAN WATER PLANT & & \\
\hline 2052 & 300 SAN WATER PLANT & & \\
\hline 2053 & 300 SAN WATER PLANT & & \\
\hline 2054 & FFTF & & \\
\hline 2055 & FFTF & & \\
\hline 2056 & FFTF & & \\
\hline 2057 & RICHLAND SAN PLANT & & \\
\hline 2058 & 300 AREA & & \\
\hline 2059 & 300 AREA & & \\
\hline 2060 & PRIEST RAPIDS-RIVER & & \\
\hline 2061 & PRIEST RAPIDS-RIVER & & \\
\hline 2062 & 300 AREA & & \\
\hline 2063 & 300 AREA & & \\
\hline 2064 & 100 B AREA-RIVER & & \\
\hline 2065 & 100 B AREA-RIVER & & \\
\hline 2066 & 300 SAN WATER PLANT & & \\
\hline 2067 & FFTF & & \\
\hline 2068 & FFTF & & \\
\hline 2069 & RICHLAND SAN PLANT & & \\
\hline 2070 & RICH.PMPHS HRM 46.4 & & \\
\hline 2071 & RICH.PMPHS HRM 46.4 & & \\
\hline 2072 & RICH.PMPHS HRM 46.4 & & \\
\hline 2073 & RICH.PMPHS HRM 46.4 & & \\
\hline 2074 & VERNITA & & \\
\hline 2075 & 300 AREA & & \\
\hline 2076 & 300 AREA & & \\
\hline 2077 & 300 AREA & & \\
\hline 2078 & 300 AREA & & \\
\hline 2079 & 300 AREA & & \\
\hline 2080 & PRIEST RAPIDS-RIVER & & \\
\hline 2081 & PRIEST RAPIDS-RIVER & & \\
\hline
\end{tabular}




\begin{tabular}{|c|c|c|c|}
\hline & $\mathrm{Z}$ & $\overline{\mathrm{AA}}$ & $\mathrm{AB}$ \\
\hline 1 & SAMP_SITE_NAME & WELL_NAME & SAMPLE_LOCATION \\
\hline 2082 & PRIEST RAPIDS-RIVER & & \\
\hline 2083 & PRIEST RAPIDS-RIVER & & \\
\hline 2084 & PRIEST RAPIDS-RIVER & & \\
\hline 2085 & RICH.PMPHS HRM 46.4 & & \\
\hline 2086 & 300 AREA & & \\
\hline 2087 & 300 AREA & & \\
\hline 2088 & PRIEST RAPIDS-RIVER & & \\
\hline 2089 & PRIEST RAPIDS-RIVER & & \\
\hline 2090 & PRIEST RAPIDS-RIVER & & \\
\hline 2091 & 100 B AREA-RIVER & & \\
\hline 2092 & 100 B AREA-RIVER & & \\
\hline 2093 & 300 SAN WATER PLANT & & \\
\hline 2094 & FFTF & & \\
\hline 2095 & FFTF & & \\
\hline 2096 & RICHLAND SAN PLANT & & \\
\hline 2097 & RICH.PMPHS HRM 46.4 & & \\
\hline 2098 & RICH.PMPHS HRM 46.4 & & \\
\hline 2099 & VERNITA & & \\
\hline 2100 & 300 AREA & & \\
\hline 2101 & 300 AREA & & \\
\hline 2102 & 300 AREA & & \\
\hline 2103 & PRIEST RAPIDS-RIVER & & \\
\hline 2104 & 216 B 63 DITCH 200 E & & \\
\hline 2105 & B POND NORTH SIDE & & \\
\hline 2106 & B POND NORTH SIDE & & \\
\hline \begin{tabular}{|l|l|}
2107 & -1 \\
\end{tabular} & GABLE POND N END & & \\
\hline 2108 & GABLE POND N END & & \\
\hline 2109 & U POND & & \\
\hline 2110 & U POND & & \\
\hline 2111 & WEST LAKE & & \\
\hline 2112 & WEST LAKE & & \\
\hline 2113 & 300 AREA & & \\
\hline 2114 & 300 AREA & & \\
\hline 2115 & PRIEST RAPIDS-RIVER & & \\
\hline 2116 & PRIEST RAPIDS-RIVER & & \\
\hline 2117 & 300 SAN WATER PLANT & & \\
\hline 2118 & 300 SAN WATER PLANT & & \\
\hline 2119 & 300 SAN WATER PLANT & & \\
\hline 2120 & FFTF & & \\
\hline 2121 & FFTF & & \\
\hline 2122 & FFTF & & \\
\hline 2123 & RICHLAND SAN PLANT & & \\
\hline 2124 & 100 B AREA-RIVER & & \\
\hline 2125 & 100 B AREA-RIVER & & \\
\hline 2126 & 100 B AREA-RIVER & & \\
\hline 2127 & 300 AREA & & \\
\hline 2128 & 300 AREA & & \\
\hline 2129 & 300 AREA & & \\
\hline 2130 & PRIEST RAPIDS-RIVER & & \\
\hline 2131 & PRIEST RAPIDS-RIVER & & \\
\hline 2132 & PRIEST RAPIDS-RIVER & & \\
\hline 2133 & RICH.PMPHS HRM 46.4 & & \\
\hline
\end{tabular}




\begin{tabular}{|c|c|c|c|}
\hline & $\mathrm{Z}$ & $\overline{\mathrm{AA}}$ & $\mathrm{AB}$ \\
\hline 1 & SAMP_SITE_NAME & WELL_NAME & SAMPLE_LOCATION \\
\hline 2134 & VERNITA & & \\
\hline 2135 & RICH.PMPHS HRM 46.4 & & \\
\hline 2136 & RICH.PMPHS HRM 46.4 & & \\
\hline 2137 & 300 AREA & & \\
\hline 2138 & 300 AREA & & \\
\hline 2139 & PRIEST RAPIDS-RIVER & & \\
\hline 2140 & PRIEST RAPIDS-RIVER & & \\
\hline 2141 & 100 B AREA-RIVER & & \\
\hline 2142 & 100 B AREA-RIVER & & \\
\hline 2143 & RICH.PMPHS HRM 46.4 & & \\
\hline 2144 & RICH.PMPHS HRM 46.4 & & \\
\hline 2145 & RICH.PMPHS HRM 46.4 & & \\
\hline 2146 & 300 SAN WATER PLANT & & \\
\hline 2147 & FFTF & & \\
\hline 2148 & FFTF & & \\
\hline 2149 & RICHLAND SAN PLANT & & \\
\hline 2150 & 300 AREA & & \\
\hline 2151 & 300 AREA & & \\
\hline 2152 & 300 AREA & & \\
\hline 2153 & 300 AREA & & \\
\hline 2154 & 300 AREA & & \\
\hline 2155 & PRIEST RAPIDS-RIVER & & \\
\hline 2156 & PRIEST RAPIDS-RIVER & & \\
\hline 2157 & PRIEST RAPIDS-RIVER & & \\
\hline 2158 & PRIEST RAPIDS-RIVER & & \\
\hline 2159 & PRIEST RAPIDS-RIVER & & \\
\hline 2160 & RICH.PMPHS HRM 46.4 & & \\
\hline 2161 & VERNITA & & \\
\hline 2162 & PRIEST RAPIDS-RIVER & & \\
\hline 2163 & PRIEST RAPIDS-RIVER & & \\
\hline 2164 & PRIEST RAPIDS-RIVER & & \\
\hline 2165 & 300 AREA & & \\
\hline 2166 & 300 AREA & & \\
\hline \begin{tabular}{|l|l|}
2167 & -1 \\
\end{tabular} & 300 AREA & & \\
\hline 2168 & 300 AREA & & \\
\hline 2169 & 300 AREA & & \\
\hline 2170 & RICH.PMPHS HRM 46.4 & & \\
\hline 2171 & RICH.PMPHS HRM 46.4 & & \\
\hline 2172 & RICH.PMPHS HRM 46.4 & & \\
\hline 2173 & VERNITA & & \\
\hline 2174 & 100 B AREA-RIVER & & \\
\hline 2175 & 100 B AREA-RIVER & & \\
\hline 2176 & 300 SAN WATER PLANT & & \\
\hline 2177 & FFTF & & \\
\hline 2178 & FFTF & & \\
\hline 2179 & RICHLAND SAN PLANT & & \\
\hline 2180 & 300 AREA & & \\
\hline 2181 & 216 B 63 DITCH 200 E & & \\
\hline 2182 & B POND NORTH SIDE & & \\
\hline 2183 & B POND NORTH SIDE & & \\
\hline 2184 & GABLE POND N END & & \\
\hline 2185 & GABLE POND N END & & \\
\hline
\end{tabular}




\begin{tabular}{|c|c|c|c|}
\hline & $\bar{Z}$ & $\overline{\mathrm{AA}}$ & $\overline{A B}$ \\
\hline 1 & SAMP_SITE_NAME & WELL_NAME & SAMPLE_LOCATION \\
\hline 2186 & U POND & & \\
\hline 2187 & U POND & & \\
\hline 2188 & WEST LAKE & & \\
\hline 2189 & WEST LAKE & & \\
\hline 2190 & 300 AREA & & \\
\hline 2191 & 300 AREA & & \\
\hline 2192 & 300 AREA & & \\
\hline 2193 & 100 B AREA-RIVER & & \\
\hline 2194 & 100 B AREA-RIVER & & \\
\hline 2195 & 100 B AREA-RIVER & & \\
\hline 2196 & 300 SAN WATER PLANT & & \\
\hline 2197 & 300 SAN WATER PLANT & & \\
\hline 2198 & 300 SAN WATER PLANT & & \\
\hline 2199 & FFTF & & \\
\hline 2200 & FFTF & & \\
\hline 2201 & FFTF & & \\
\hline 2202 & RICHLAND SAN PLANT & & \\
\hline 2203 & RICH.PMPHS HRM 46.4 & & \\
\hline 2204 & RICH.PMPHS HRM 46.4 & & \\
\hline 2205 & RICH.PMPHS HRM 46.4 & & \\
\hline 2206 & VERNITA & & \\
\hline 2207 & 300 AREA & & \\
\hline 2208 & 300 AREA & & \\
\hline 2209 & PRIEST RAPIDS-RIVER & & \\
\hline 2210 & PRIEST RAPIDS-RIVER & & \\
\hline 2211 & 300 AREA & & \\
\hline 2212 & 300 AREA & & \\
\hline 2213 & PRIEST RAPIDS-RIVER & & \\
\hline 2214 & PRIEST RAPIDS-RIVER & & \\
\hline 2215 & 100 B AREA-RIVER & & \\
\hline 2216 & 100 B AREA-RIVER & & \\
\hline 2217 & 300 SAN WATER PLANT & & \\
\hline 2218 & FFTF & & \\
\hline 2219 & FFTF & & \\
\hline 2220 & RICHLAND SAN PLANT & & \\
\hline 2221 & RICH.PMPHS HRM 46.4 & & \\
\hline 2222 & RICH.PMPHS HRM 46.4 & & \\
\hline 2223 & RICH.PMPHS HRM 46.4 & & \\
\hline 2224 & RICH.PMPHS HRM 46.4 & & \\
\hline 2225 & VERNITA & & \\
\hline 2226 & 300 AREA & & \\
\hline 2227 & 300 AREA & & \\
\hline 2228 & 300 AREA & & \\
\hline 2229 & 300 AREA & & \\
\hline 2230 & 300 AREA & & \\
\hline 2231 & PRIEST RAPIDS-RIVER & & \\
\hline 2232 & PRIEST RAPIDS-RIVER & & \\
\hline 2233 & PRIEST RAPIDS-RIVER & & \\
\hline 2234 & PRIEST RAPIDS-RIVER & & \\
\hline 2235 & PRIEST RAPIDS-RIVER & & \\
\hline 2236 & 300 AREA & & \\
\hline 2237 & 300 AREA & & \\
\hline
\end{tabular}




\begin{tabular}{|c|c|c|c|}
\hline & $\bar{Z}$ & $\overline{\mathrm{AA}}$ & $\overline{A B}$ \\
\hline 1 & SAMP_SITE_NAME & WELL_NAME & SAMPLE_LOCATION \\
\hline 2238 & PRIEST RAPIDS-RIVER & & \\
\hline 2239 & PRIEST RAPIDS-RIVER & & \\
\hline 2240 & RICH.PMPHS HRM 46.4 & & \\
\hline 2241 & RICH.PMPHS HRM 46.4 & & \\
\hline 2242 & RICH.PMPHS HRM 46.4 & & \\
\hline 2243 & VERNITA & & \\
\hline 2244 & 100 B AREA-RIVER & & \\
\hline 2245 & 100 B AREA-RIVER & & \\
\hline 2246 & 300 SAN WATER PLANT & & \\
\hline 2247 & FFTF & & \\
\hline 2248 & FFTF & & \\
\hline 2249 & RICHLAND SAN PLANT & & \\
\hline 2250 & 300 AREA & & \\
\hline 2251 & 300 AREA & & \\
\hline 2252 & 300 AREA & & \\
\hline 2253 & PRIEST RAPIDS-RIVER & & \\
\hline 2254 & PRIEST RAPIDS-RIVER & & \\
\hline 2255 & PRIEST RAPIDS-RIVER & & \\
\hline 2256 & FFTF & & \\
\hline 2257 & 216 B 63 DITCH 200 E & & \\
\hline 2258 & B POND NORTH SIDE & & \\
\hline 2259 & B POND NORTH SIDE & & \\
\hline 2260 & GABLE POND N END & & \\
\hline 2261 & GABLE POND N END & & \\
\hline 2262 & U POND & & \\
\hline 2263 & U POND & & \\
\hline 2264 & WEST LAKE & & \\
\hline 2265 & WEST LAKE & & \\
\hline 2266 & FFTF & & \\
\hline 2267 & 300 AREA & & \\
\hline 2268 & 300 AREA & & \\
\hline 2269 & FFTF & & \\
\hline 2270 & 100 B AREA-RIVER & & \\
\hline 2271 & 100 B AREA-RIVER & & \\
\hline 2272 & 100 B AREA-RIVER & & \\
\hline 2273 & 300 SAN WATER PLANT & & \\
\hline 2274 & 300 SAN WATER PLANT & & \\
\hline 2275 & 300 SAN WATER PLANT & & \\
\hline 2276 & FFTF & & \\
\hline 2277 & FFTF & & \\
\hline 2278 & FFTF & & \\
\hline 2279 & RICHLAND SAN PLANT & & \\
\hline 2280 & 300 AREA & & \\
\hline 2281 & 300 AREA & & \\
\hline 2282 & 300 AREA & & \\
\hline 2283 & PRIEST RAPIDS-RIVER & & \\
\hline 2284 & PRIEST RAPIDS-RIVER & & \\
\hline 2285 & PRIEST RAPIDS-RIVER & & \\
\hline 2286 & RICH.PMPHS HRM 46.4 & & \\
\hline 2287 & RICH.PMPHS HRM 46.4 & & \\
\hline 2288 & RICH.PMPHS HRM 46.4 & & \\
\hline 2289 & VERNITA & & \\
\hline
\end{tabular}




\begin{tabular}{|c|c|c|c|}
\hline & $\bar{Z}$ & $\overline{\mathrm{AA}}$ & $\overline{A B}$ \\
\hline 1 & SAMP_SITE_NAME & WELL_NAME & SAMPLE_LOCATION \\
\hline 2290 & FFTF & & \\
\hline 2291 & FFTF & & \\
\hline 2292 & 300 AREA & & \\
\hline 2293 & 300 AREA & & \\
\hline 2294 & PRIEST RAPIDS-RIVER & & \\
\hline 2295 & PRIEST RAPIDS-RIVER & & \\
\hline 2296 & FFTF & & \\
\hline 2297 & RICH.PMPHS HRM 46.4 & & \\
\hline 2298 & VERNITA & & \\
\hline 2299 & 100 B AREA-RIVER & & \\
\hline 2300 & 100 B AREA-RIVER & & \\
\hline 2301 & 300 SAN WATER PLANT & & \\
\hline 2302 & FFTF & & \\
\hline 2303 & FFTF & & \\
\hline 2304 & RICHLAND SAN PLANT & & \\
\hline 2305 & 300 AREA & & \\
\hline 2306 & 300 AREA & & \\
\hline 2307 & 300 AREA & & \\
\hline 2308 & 300 AREA & & \\
\hline 2309 & 300 AREA & & \\
\hline 2310 & PRIEST RAPIDS-RIVER & & \\
\hline 2311 & PRIEST RAPIDS-RIVER & & \\
\hline 2312 & PRIEST RAPIDS-RIVER & & \\
\hline 2313 & PRIEST RAPIDS-RIVER & & \\
\hline 2314 & PRIEST RAPIDS-RIVER & & \\
\hline 2315 & RICH.PMPHS HRM 46.4 & & \\
\hline 2316 & RICH.PMPHS HRM 46.4 & & \\
\hline 2317 & RICH.PMPHS HRM 46.4 & & \\
\hline 2318 & RICH.PMPHS HRM 46.4 & & \\
\hline 2319 & RICH.PMPHS HRM 46.4 & & \\
\hline 2320 & 300 AREA & & \\
\hline 2321 & 300 AREA & & \\
\hline 2322 & PRIEST RAPIDS-RIVER & & \\
\hline 2323 & PRIEST RAPIDS-RIVER & & \\
\hline 2324 & 100 B AREA-RIVER & & \\
\hline 2325 & 100 B AREA-RIVER & & \\
\hline 2326 & 100 B AREA-RIVER & & \\
\hline 2327 & 300 SAN WATER PLANT & & \\
\hline \begin{tabular}{|c|}
2328 \\
23
\end{tabular} & FFTF & & \\
\hline 2329 & FFTF & & \\
\hline 2330 & RICHLAND SAN PLANT & & \\
\hline 2331 & 300 AREA & & \\
\hline 2332 & 300 AREA & & \\
\hline 2333 & PRIEST RAPIDS-RIVER & & \\
\hline 2334 & PRIEST RAPIDS-RIVER & & \\
\hline 2335 & 300 AREA & & \\
\hline 2336 & 300 AREA & & \\
\hline 2337 & PRIEST RAPIDS-RIVER & & \\
\hline 2338 & PRIEST RAPIDS-RIVER & & \\
\hline 2339 & B POND NORTH SIDE & & \\
\hline 2340 & B POND NORTH SIDE & & \\
\hline 2341 & GABLE POND N END & & \\
\hline
\end{tabular}




\begin{tabular}{|c|c|c|c|}
\hline & $\bar{Z}$ & $\overline{\mathrm{AA}}$ & $\overline{A B}$ \\
\hline 1 & SAMP_SITE_NAME & WELL_NAME & SAMPLE_LOCATION \\
\hline 2342 & GABLE POND N END & & \\
\hline 2343 & WEST LAKE & & \\
\hline 2344 & WEST LAKE & & \\
\hline 2345 & 100 B AREA-RIVER & & \\
\hline 2346 & 100 B AREA-RIVER & & \\
\hline 2347 & 300 AREA & & \\
\hline 2348 & 300 AREA & & \\
\hline 2349 & PRIEST RAPIDS-RIVER & & \\
\hline 2350 & PRIEST RAPIDS-RIVER & & \\
\hline 2351 & RICH.PMPHS HRM 46.4 & & \\
\hline 2352 & RICH.PMPHS HRM 46.4 & & \\
\hline 2353 & RICH.PMPHS HRM 46.4 & & \\
\hline 2354 & 300 SAN WATER PLANT & & \\
\hline 2355 & 300 SAN WATER PLANT & & \\
\hline 2356 & 300 SAN WATER PLANT & & \\
\hline 2357 & FFTF & & \\
\hline 2358 & FFTF & & \\
\hline 2359 & FFTF & & \\
\hline 2360 & RICHLAND SAN PLANT & & \\
\hline 2361 & 300 AREA & & \\
\hline 2362 & 300 AREA & & \\
\hline 2363 & PRIEST RAPIDS-RIVER & & \\
\hline 2364 & PRIEST RAPIDS-RIVER & & \\
\hline 2365 & 300 AREA & & \\
\hline 2366 & 300 AREA & & \\
\hline 2367 & 300 AREA & & \\
\hline 2368 & 300 AREA & & \\
\hline 2369 & 300 AREA & & \\
\hline 2370 & PRIEST RAPIDS-RIVER & & \\
\hline 2371 & PRIEST RAPIDS-RIVER & & \\
\hline 2372 & PRIEST RAPIDS-RIVER & & \\
\hline 2373 & PRIEST RAPIDS-RIVER & & \\
\hline 2374 & PRIEST RAPIDS-RIVER & & \\
\hline 2375 & 100 B AREA-RIVER & & \\
\hline 2376 & 100 B AREA-RIVER & & \\
\hline 2377 & 300 SAN WATER PLANT & & \\
\hline 2378 & FFTF & & \\
\hline 2379 & FFTF & & \\
\hline 2380 & RICHLAND SAN PLANT & & \\
\hline 2381 & RICH.PMPHS HRM 46.4 & & \\
\hline 2382 & RICH.PMPHS HRM 46.4 & & \\
\hline 2383 & 300 AREA & & \\
\hline 2384 & 300 AREA & & \\
\hline 2385 & PRIEST RAPIDS-RIVER & & \\
\hline 2386 & PRIEST RAPIDS-RIVER & & \\
\hline 2387 & 300 AREA & & \\
\hline 2388 & 300 AREA & & \\
\hline 2389 & PRIEST RAPIDS-RIVER & & \\
\hline 2390 & PRIEST RAPIDS-RIVER & & \\
\hline 2391 & 300 SAN WATER PLANT & & \\
\hline 2392 & FFTF & & \\
\hline 2393 & FFTF & & \\
\hline
\end{tabular}




\begin{tabular}{|c|c|c|c|}
\hline & $\bar{Z}$ & $\overline{\mathrm{AA}}$ & $\overline{A B}$ \\
\hline 1 & SAMP_SITE_NAME & WELL_NAME & SAMPLE_LOCATION \\
\hline 2394 & RICHLAND SAN PLANT & & \\
\hline 2395 & RICH.PMPHS HRM 46.4 & & \\
\hline 2396 & RICH.PMPHS HRM 46.4 & & \\
\hline 2397 & RICH.PMPHS HRM 46.4 & & \\
\hline 2398 & 100 B AREA-RIVER & & \\
\hline 2399 & 100 B AREA-RIVER & & \\
\hline 2400 & 100 B AREA-RIVER & & \\
\hline 2401 & 300 AREA & & \\
\hline 2402 & 300 AREA & & \\
\hline 2403 & PRIEST RAPIDS-RIVER & & \\
\hline 2404 & PRIEST RAPIDS-RIVER & & \\
\hline 2405 & B POND NORTH SIDE & & \\
\hline 2406 & B POND NORTH SIDE & & \\
\hline 2407 & GABLE POND N END & & \\
\hline 2408 & GABLE POND N END & & \\
\hline 2409 & WEST LAKE & & \\
\hline 2410 & WEST LAKE & & \\
\hline 2411 & RICH.PMPHS HRM 46.4 & & \\
\hline 2412 & RICH.PMPHS HRM 46.4 & & \\
\hline 2413 & 300 AREA & & \\
\hline 2414 & 300 AREA & & \\
\hline 2415 & PRIEST RAPIDS-RIVER & & \\
\hline 2416 & PRIEST RAPIDS-RIVER & & \\
\hline 2417. & 100 B AREA-RIVER & & \\
\hline 2418 & 100 B AREA-RIVER & & \\
\hline 2419 & 300 AREA & & \\
\hline 2420 & 300 AREA & & \\
\hline 2421 & PRIEST RAPIDS-RIVER & & \\
\hline 2422 & PRIEST RAPIDS-RIVER & & \\
\hline 2423 & 300 SAN WATER PLANT & & \\
\hline 2424 & 300 SAN WATER PLANT & & \\
\hline 2425 & 300 SAN WATER PLANT & & \\
\hline 2426 & FFTF & & \\
\hline 2427 & FFTF & & \\
\hline 2428 & FFTF & & \\
\hline 2429 & FFTF & & \\
\hline 2430 & RICHLAND SAN PLANT & & \\
\hline 2431 & 300 AREA & & \\
\hline 2432 & 300 AREA & & \\
\hline 2433 & PRIEST RAPIDS-RIVER & & \\
\hline 2434 & PRIEST RAPIDS-RIVER & & \\
\hline 2435 & 100 B AREA-RIVER & & \\
\hline 2436 & 100 B AREA-RIVER & & \\
\hline 2437 & 300 SAN WATER PLANT & & \\
\hline 2438 & FFTF & & \\
\hline 2439 & FFTF & & \\
\hline 2440 & RICHLAND SAN PLANT & & \\
\hline 2441 & 300 AREA & & \\
\hline 2442 & 300 AREA & & \\
\hline 2443 & 300 AREA & & \\
\hline 2444 & 300 AREA & & \\
\hline 2445 & 300 AREA & & \\
\hline
\end{tabular}




\begin{tabular}{|c|c|c|c|}
\hline & $\mathrm{Z}$ & $\mathrm{AA}$ & $A B$ \\
\hline 1 & SAMP_SITE_NAME & WELL_NAME & SAMPLE_LOCATION \\
\hline 2446 & PRIEST RAPIDS-RIVER & & \\
\hline 2447 & PRIEST RAPIDS-RIVER & & \\
\hline 2448 & PRIEST RAPIDS-RIVER & & \\
\hline 2449 & PRIEST RAPIDS-RIVER & & \\
\hline 2450 & PRIEST RAPIDS-RIVER & & \\
\hline 2451 & 331 FARMS-300 AREA & & \\
\hline 2452 & MERRILL FARM-W RICH. & & \\
\hline 2453 & 300 AREA & & \\
\hline 2454 & 300 AREA & & \\
\hline 2455 & PRIEST RAPIDS-RIVER & & \\
\hline 2456 & PRIEST RAPIDS-RIVER & & \\
\hline 2457 & 300 AREA & & \\
\hline 2458 & 300 AREA & & \\
\hline 2459 & PRIEST RAPIDS-RIVER & & \\
\hline 2460 & PRIEST RAPIDS-RIVER & & \\
\hline 2461 & 100 B AREA-RIVER & & \\
\hline 2462 & 100 B AREA-RIVER & & \\
\hline 2463 & 100 B AREA-RIVER & & \\
\hline 2464 & 300 SAN WATER PLANT & & \\
\hline 2465 & FFTF & & \\
\hline 2466 & FFTF & & \\
\hline 2467 & RICHLAND SAN PLANT & & \\
\hline 2468 & 300 AREA & & \\
\hline 2469 & 300 AREA & & \\
\hline 2470 & PRIEST RAPIDS-RIVER & & \\
\hline 2471 & PRIEST RAPIDS-RIVER & & \\
\hline 2472 & B POND NORTH SIDE & & \\
\hline 2473 & B POND NORTH SIDE & & \\
\hline 2474 & GABLE POND N END & & \\
\hline 2475 & GABLE POND N END & & \\
\hline 2476 & WEST LAKE & & \\
\hline 2477 & WEST LAKE & & \\
\hline 2478 & 300 AREA & & \\
\hline 2479 & 300 AREA & & \\
\hline 2480 & PRIEST RAPIDS-RIVER & & \\
\hline 2481 & PRIEST RAPIDS-RIVER & & \\
\hline 2482 & 100 B AREA-RIVER & & \\
\hline 2483 & 100 B AREA-RIVER & & \\
\hline 2484 & 300 SAN WATER PLANT & & \\
\hline 2485 & 300 SAN WATER PLANT & & \\
\hline 2486 & 300 SAN WATER PLANT & & \\
\hline 2487 & FFTF & & \\
\hline 2488 & FFTF & & \\
\hline 2489 & FFTF & & \\
\hline 2490 & FFTF & & \\
\hline 2491 & RICHLAND SAN PLANT & & \\
\hline 2492 & 300 AREA & & \\
\hline 2493 & 300 AREA & & \\
\hline 2494 & PRIEST RAPIDS-RIVER & & \\
\hline 2495 & PRIEST RAPIDS-RIVER & & \\
\hline 2496 & RICH.PMPHS HRM 46.4 & & \\
\hline 2497 & RICH.PMPHS HRM 46.4 & & \\
\hline
\end{tabular}




\begin{tabular}{|c|c|c|c|}
\hline & $\bar{Z}$ & $\overline{\mathrm{AA}}$ & $\overline{A B}$ \\
\hline 1 & SAMP_SITE_NAME & WELL_NAME & SAMPLE_LOCATION \\
\hline 2498 & RICH.PMPHS HRM 46.4 & & \\
\hline 2499 & 300 AREA & & \\
\hline 2500 & 300 AREA & & \\
\hline 2501 & PRIEST RAPIDS-RIVER & & \\
\hline 2502 & PRIEST RAPIDS-RIVER & & \\
\hline 2503 & RICHLAND SAN PLANT & & \\
\hline 2504 & 100 B AREA-RIVER & & \\
\hline 2505 & 100 B AREA-RIVER & & \\
\hline 2506 & 300 SAN WATER PLANT & & \\
\hline 2507 & FFTF & & \\
\hline 2508 & FFTF & & \\
\hline 2509 & 300 AREA & & \\
\hline 2510 & 300 AREA & & \\
\hline 2511 & PRIEST RAPIDS-RIVER & & \\
\hline 2512 & PRIEST RAPIDS-RIVER & & \\
\hline 2513 & RICH.PMPHS HRM 46.4 & & \\
\hline 2514 & RICH.PMPHS HRM 46.4 & & \\
\hline 2515 & 300 AREA & & \\
\hline 2516 & 300 AREA & & \\
\hline 2517 & 300 AREA & & \\
\hline 2518 & PRIEST RAPIDS-RIVER & & \\
\hline 2519 & PRIEST RAPIDS-RIVER & & \\
\hline 2520 & PRIEST RAPIDS-RIVER & & \\
\hline 2521 & 300 AREA & & \\
\hline 2522 & 300 AREA & & \\
\hline 2523 & 300 AREA & & \\
\hline 2524 & 300 AREA & & \\
\hline 2525 & PRIEST RAPIDS-RIVER & & \\
\hline 2526 & PRIEST RAPIDS-RIVER & & \\
\hline 2527 & RICH.PMPHS HRM 46.4 & & \\
\hline 2528 & RICH.PMPHS HRM 46.4 & & \\
\hline 2529 & 100 B AREA-RIVER & & \\
\hline 2530 & 100 B AREA-RIVER & & \\
\hline 2531 & 100 B AREA-RIVER & & \\
\hline 2532 & 300 SAN WATER PLANT & & \\
\hline 2533 & FFTF & & \\
\hline 2534 & FFTF & & \\
\hline 2535 & RICHLAND SAN PLANT & & \\
\hline 2536 & B POND NORTH SIDE & & \\
\hline 2537 & B POND NORTH SIDE & & \\
\hline 2538 & GABLE POND N END & & \\
\hline 2539 & GABLE POND N END & & \\
\hline 2540 & WEST LAKE & & \\
\hline 2541 & WEST LAKE & & \\
\hline 2542 & 300 AREA & & \\
\hline 2543 & 300 AREA & & \\
\hline 2544 & 100 B AREA-RIVER & & \\
\hline 2545 & 100 B AREA-RIVER & & \\
\hline 2546 & 300 SAN WATER PLANT & & \\
\hline 2547 & 300 SAN WATER PLANT & & \\
\hline 2548 & 300 SAN WATER PLANT & & \\
\hline 2549 & FFTF & & \\
\hline
\end{tabular}




\begin{tabular}{|c|c|c|c|}
\hline & $\bar{Z}$ & $\overline{\mathrm{AA}}$ & $\overline{A B}$ \\
\hline 1 & SAMP_SITE_NAME & WELL_NAME & SAMPLE_LOCATION \\
\hline 2550 & FFTF & & \\
\hline 2551 & FFTF & & \\
\hline 2552 & FFTF & & \\
\hline 2553 & RICHLAND SAN PLANT & & \\
\hline 2554 & 300 AREA & & \\
\hline 2555 & 300 AREA & & \\
\hline 2556 & PRIEST RAPIDS-RIVER & & \\
\hline 2557 & PRIEST RAPIDS-RIVER & & \\
\hline 2558 & RICH.PMPHS HRM 46.4 & & \\
\hline 2559 & RICH.PMPHS HRM 46.4 & & \\
\hline 2560 & RICH.PMPHS HRM 46.4 & & \\
\hline 2561 & 300 AREA & & \\
\hline 2562 & 300 AREA & & \\
\hline 2563 & PRIEST RAPIDS-RIVER & & \\
\hline 2564 & PRIEST RAPIDS-RIVER & & \\
\hline 2565 & 300 AREA & & \\
\hline 2566 & 300 AREA & & \\
\hline 2567 & PRIEST RAPIDS-RIVER & & \\
\hline 2568 & PRIEST RAPIDS-RIVER & & \\
\hline 2569 & RICH.PMPHS HRM 46.4 & & \\
\hline 2570 & RICH.PMPHS HRM 46.4 & & \\
\hline 2571 & 100 B AREA-RIVER & & \\
\hline 2572 & 100 B AREA-RIVER & & \\
\hline 2573 & 300 SAN WATER PLANT & & \\
\hline 2574 & FFTF & & \\
\hline 2575 & FFTF & & \\
\hline 2576 & RICHLAND SAN PLANT & & \\
\hline 2577 & 300 AREA & & \\
\hline 2578 & 300 AREA & & \\
\hline 2579 & 300 AREA & & \\
\hline 2580 & 300 AREA & & \\
\hline 2581 & 300 AREA & & \\
\hline 2582 & PRIEST RAPIDS-RIVER & & \\
\hline 2583 & PRIEST RAPIDS-RIVER & & \\
\hline 2584 & PRIEST RAPIDS-RIVER & & \\
\hline 2585 & PRIEST RAPIDS-RIVER & & \\
\hline 2586 & PRIEST RAPIDS-RIVER & & \\
\hline 2587 & 300 SAN WATER PLANT & & \\
\hline \begin{tabular}{|l|}
2588 \\
58
\end{tabular} & 300 AREA & & \\
\hline 2589 & 300 AREA & & \\
\hline 2590 & 100 B AREA-RIVER & & \\
\hline 2591 & 100 B AREA-RIVER & & \\
\hline 2592 & 100 B AREA-RIVER & & \\
\hline 2593 & 300 SAN WATER PLANT & & \\
\hline 2594 & FFTF & & \\
\hline 2595 & FFTF & & \\
\hline 2596 & RICHLAND SAN PLANT & & \\
\hline 2597 & RICH.PMPHS HRM 46.4 & & \\
\hline 2598 & RICH.PMPHS HRM 46.4 & & \\
\hline 2599 & 300 AREA & & \\
\hline 2600 & 300 AREA & & \\
\hline 2601 & B POND NC & & \\
\hline
\end{tabular}




\begin{tabular}{|c|l|l|l|}
\hline & \multicolumn{1}{|c|}{$Z$} & A & AB \\
\hline 1 & SAMP_SITE_NAME & WELL_NAME & SAMPLE_LOCATION \\
\hline 2602 & B POND NORTH SIDE & & \\
\hline 2603 & PABLE POND N END & & \\
\hline 2604 & GABLE POND N END & & \\
\hline 2605 & WEST LAKE & & \\
\hline 2606 & WEST LAKE & & \\
\hline 2607 & 300 AREA & & \\
\hline 2608 & 300 AREA & & \\
\hline 2609 & PRIEST RAPIDS-RIVER & & \\
\hline 2610 & PRIEST RAPIDS-RIVER & & \\
\hline 2611 & 100 B AREA-RIVER & & \\
\hline 2612 & 100 B AREA-RIVER & & \\
\hline 2613 & 300 SAN WATER PLANT & & \\
\hline 2614 & 300 SAN WATER PLANT & & \\
\hline 2615 & 300 SAN WATER PLANT & & \\
\hline 2616 & FFTF & & \\
\hline 2617 & FFTF & & \\
\hline 2618 & FFTF & & \\
\hline 2619 & FFTF & & \\
\hline 2620 & RICHLAND SAN PLANT & & \\
\hline 2621 & RICH.PMPHS HRM 46.4 & & \\
\hline 2622 & RICH.PMPHS HRM 46.4 & & \\
\hline 2623 & RICH.PMPHS HRM 46.4 & & \\
\hline 2624 & 300 AREA & & \\
\hline 2625 & 300 AREA & & \\
\hline 2626 & PRIEST RAPIDS-RIVER & & \\
\hline 2627 & PRIEST RAPIDS-RIVER & & \\
\hline 2628 & 300 AREA & & \\
\hline 2629 & 300 AREA & & \\
\hline 2630 & PRIEST RAPIDS-RIVER & & \\
\hline 2631 & PRIEST RAPIDS-RIVER & & \\
\hline 2632 & RICH.PMPHS HRM 46.4 & & \\
\hline 2633 & RICH.PMPHS HRM 46.4 & & \\
\hline 2634 & 100 B AREA-RIVER & & \\
\hline 2635 & 100 B AREA-RIVER & & \\
\hline 2636 & 300 SAN WATER PLANT & & \\
\hline 2637 & FFTF & & \\
\hline 2638 & FFTF & & \\
\hline 2639 & RICHLAND SAN PLANT & & \\
\hline 2640 & 300 AREA & & \\
\hline 2641 & 300 AREA & & \\
\hline 2642 & 300 AREA & & \\
\hline 2643 & 300 AREA & & \\
\hline 2644 & 300 AREA & & \\
\hline 2645 & PRIEST RAPIDS-RIVER & & \\
\hline 2646 & PRIEST RAPIDS-RIVER & & \\
\hline 2647 & PRIEST RAPIDS-RIVER & & \\
\hline 2648 & PRIEST RAPIDS-RIVER & & \\
\hline 2649 & PRIEST RAPIDS-RIVER & & \\
\hline 2650 & 300 AREA & & \\
\hline 2651 & 300 AREA & & \\
\hline 2652 & PRIEST RAPIDS-RIVER & & \\
\hline 2653 & PRIEST RAPIDS-RIVER & & \\
\hline
\end{tabular}




\begin{tabular}{|c|c|c|c|}
\hline & $\mathrm{Z}$ & $\overline{\mathrm{AA}}$ & $\mathrm{AB}$ \\
\hline 1 & SAMP_SITE_NAME & WELL_NAME & SAMPLE_LOCATION \\
\hline 2654 & 100 B AREA-RIVER & & \\
\hline 2655 & 100 B AREA-RIVER & & \\
\hline 2656 & 100 B AREA-RIVER & & \\
\hline 2657 & 300 SAN WATER PLANT & & \\
\hline 2658 & FFTF & & \\
\hline 2659 & FFTF & & \\
\hline 2660 & RICHLAND SAN PLANT & & \\
\hline 2661 & 300 AREA & & \\
\hline 2662 & 300 AREA & & \\
\hline 2663 & PRIEST RAPIDS-RIVER & & \\
\hline 2664 & PRIEST RAPIDS-RIVER & & \\
\hline 2665 & RICH.PMPHS HRM 46.4 & & \\
\hline 2666 & RICH.PMPHS HRM 46.4 & & \\
\hline 2667 & RICH.PMPHS HRM 46.4 & & \\
\hline 2668 & B POND NORTH SIDE & & \\
\hline 2669 & B POND NORTH SIDE & & \\
\hline 2670 & FFTF POND & & \\
\hline 2671 & GABLE POND N END & & \\
\hline 2672 & GABLE POND N END & & \\
\hline 2673 & WEST LAKE & & \\
\hline 2674 & WEST LAKE & & \\
\hline 2675 & 300 AREA & & \\
\hline 2676 & 300 AREA & & \\
\hline 2677 & PRIEST RAPIDS-RIVER & & \\
\hline 2678 & PRIEST RAPIDS-RIVER & & \\
\hline 2679 & 100 B AREA-RIVER & & \\
\hline 2680 & 100 B AREA-RIVER & & \\
\hline 2681 & 300 AREA & & \\
\hline 2682 & 300 AREA & & \\
\hline 2683 & PRIEST RAPIDS-RIVER & & \\
\hline 2684 & PRIEST RAPIDS-RIVER & & \\
\hline 2685 & 300 SAN WATER PLANT & & \\
\hline 2686 & 300 SAN WATER PLANT & & \\
\hline 2687 & 300 SAN WATER PLANT & & \\
\hline 2688 & FFTF & & \\
\hline 2689 & FFTF & & \\
\hline 2690 & FFTF & & \\
\hline 2691 & FFTF & & \\
\hline 2692 & RICHLAND SAN PLANT & & \\
\hline 2693 & RICH.PMPHS HRM 46.4 & & \\
\hline 2694 & RICH.PMPHS HRM 46.4 & & \\
\hline 2695 & 300 AREA & & \\
\hline 2696 & 300 AREA & & \\
\hline 2697 & PRIEST RAPIDS-RIVER & & \\
\hline 2698 & PRIEST RAPIDS-RIVER & & \\
\hline 2699 & 300 AREA & & \\
\hline 2700 & 300 AREA & & \\
\hline 2701 & PRIEST RAPIDS-RIVER & & \\
\hline 2702 & PRIEST RAPIDS-RIVER & & \\
\hline 2703 & 100 B AREA-RIVER & & \\
\hline 2704 & 100 B AREA-RIVER & & \\
\hline 2705 & 300 SAN WATER PLANT & & \\
\hline
\end{tabular}




\begin{tabular}{|c|c|c|c|}
\hline & $\bar{Z}$ & $\overline{\mathrm{AA}}$ & $\overline{A B}$ \\
\hline 1 & SAMP_SITE_NAME & WELL_NAME & SAMPLE_LOCATION \\
\hline 2706 & FFTF & & \\
\hline 2707 & FFTF & & \\
\hline 2708 & RICHLAND SAN PLANT & & \\
\hline 2709 & RICH.PMPHS HRM 46.4 & & \\
\hline 2710 & RICH.PMPHS HRM 46.4 & & \\
\hline 2711 & 300 AREA & & \\
\hline 2712 & 300 AREA & & \\
\hline 2713 & 300 AREA & & \\
\hline 2714 & 300 AREA & & \\
\hline 2715 & 300 AREA & & \\
\hline 2716 & PRIEST RAPIDS-RIVER & & \\
\hline 2717 & PRIEST RAPIDS-RIVER & & \\
\hline 2718 & PRIEST RAPIDS-RIVER & & \\
\hline 2719 & PRIEST RAPIDS-RIVER & & \\
\hline 2720 & PRIEST RAPIDS-RIVER & & \\
\hline 2721 & 300 AREA & & \\
\hline 2722 & 300 AREA & & \\
\hline 2723 & PRIEST RAPIDS-RIVER & & \\
\hline 2724 & PRIEST RAPIDS-RIVER & & \\
\hline 2725 & 100 B AREA-RIVER & & \\
\hline 2726 & 100 B AREA-RIVER & & \\
\hline 2727 & 100 B AREA-RIVER & & \\
\hline 2728 & 300 SAN WATER PLANT & & \\
\hline 2729 & FFTF & & \\
\hline 2730 & FFTF & & \\
\hline 2731 & RICHLAND SAN PLANT & & \\
\hline 2732 & RICH.PMPHS HRM 46.4 & & \\
\hline 2733 & RICH.PMPHS HRM 46.4 & & \\
\hline 2734 & RICH.PMPHS HRM 46.4 & & \\
\hline 2735 & 300 AREA & & \\
\hline 2736 & PRIEST RAPIDS-RIVER & & \\
\hline 2737 & PRIEST RAPIDS-RIVER & & \\
\hline 2738 & B POND NORTH SIDE & & \\
\hline 2739 & B POND NORTH SIDE & & \\
\hline 2740 & FFTF POND & & \\
\hline 2741 & GABLE POND N END & & \\
\hline 2742 & GABLE POND N END & & \\
\hline 2743 & WEST LAKE & & \\
\hline 2744 & WEST LAKE & & \\
\hline 2745 & 300 AREA & & \\
\hline 2746 & 300 AREA & & \\
\hline 2747 & PRIEST RAPIDS-RIVER & & \\
\hline 2748 & PRIEST RAPIDS-RIVER & & \\
\hline 2749 & 100 B AREA-RIVER & & \\
\hline 2750 & 100 B AREA-RIVER & & \\
\hline 2751 & 300 SAN WATER PLANT & & \\
\hline 2752 & 300 SAN WATER PLANT & & \\
\hline 2753 & 300 SAN WATER PLANT & & \\
\hline 2754 & FFTF & & \\
\hline 2755 & FFTF & & \\
\hline 2756 & FFTF & & \\
\hline 2757 & FFTF & & \\
\hline
\end{tabular}




\begin{tabular}{|c|c|c|c|}
\hline & $\bar{Z}$ & $\overline{\mathrm{AA}}$ & $\overline{A B}$ \\
\hline 1 & SAMP_SITE_NAME & WELL_NAME & SAMPLE_LOCATION \\
\hline 2758 & RICHLAND SAN PLANT & & \\
\hline 2759 & RICH.PMPHS HRM 46.4 & & \\
\hline 2760 & RICH.PMPHS HRM 46.4 & & \\
\hline 2761 & 300 AREA & & \\
\hline 2762 & 300 AREA & & \\
\hline 2763 & PRIEST RAPIDS-RIVER & & \\
\hline 2764 & PRIEST RAPIDS-RIVER & & \\
\hline 2765 & 300 AREA & & \\
\hline 2766 & 300 AREA & & \\
\hline 2767 & 300 AREA & & \\
\hline 2768 & 300 AREA & & \\
\hline 2769 & PRIEST RAPIDS-RIVER & & \\
\hline 2770 & PRIEST RAPIDS-RIVER & & \\
\hline 2771 & PRIEST RAPIDS-RIVER & & \\
\hline 2772 & PRIEST RAPIDS-RIVER & & \\
\hline 2773 & B POND NORTH SIDE & & \\
\hline 2774 & B POND NORTH SIDE & & \\
\hline 2775 & 100 B AREA-RIVER & & \\
\hline 2776 & 100 B AREA-RIVER & & \\
\hline 2777 & 300 SAN WATER PLANT & & \\
\hline 2778 & FFTF & & \\
\hline 2779 & FFTF & & \\
\hline 2780 & RICHLAND SAN PLANT & & \\
\hline 2781 & 300 AREA & & \\
\hline 2782 & 300 AREA & & \\
\hline 2783 & 300 AREA & & \\
\hline 2784 & PRIEST RAPIDS-RIVER & & \\
\hline 2785 & PRIEST RAPIDS-RIVER & & \\
\hline 2786 & PRIEST RAPIDS-RIVER & & \\
\hline 2787 & RICH.PMPHS HRM 46.4 & & \\
\hline 2788 & RICH.PMPHS HRM 46.4 & & \\
\hline 2789 & 300 AREA & & \\
\hline 2790 & 300 AREA & & \\
\hline 2791 & PRIEST RAPIDS-RIVER & & \\
\hline 2792 & PRIEST RAPIDS-RIVER & & \\
\hline 2793 & 100 B AREA-RIVER & & \\
\hline 2794 & 100 B AREA-RIVER & & \\
\hline 2795 & 100 B AREA-RIVER & & \\
\hline 2796 & 300 SAN WATER PLANT & & \\
\hline 2797 & FFTF & & \\
\hline 2798 & FFTF & & \\
\hline 2799 & RICHLAND SAN PLANT & & \\
\hline 2800 & 300 AREA & & \\
\hline 2801 & 300 AREA & & \\
\hline 2802 & PRIEST RAPIDS-RIVER & & \\
\hline 2803 & PRIEST RAPIDS-RIVER & & \\
\hline 2804 & RICH.PMPHS HRM 46.4 & & \\
\hline 2805 & RICH.PMPHS HRM 46.4 & & \\
\hline 2806 & RICH.PMPHS HRM 46.4 & & \\
\hline 2807 & 300 AREA & & \\
\hline 2808 & 300 AREA & & \\
\hline 2809 & PRIEST RAI & & \\
\hline
\end{tabular}




\begin{tabular}{|c|c|c|c|}
\hline & $\bar{Z}$ & $\overline{\mathrm{AA}}$ & $\overline{A B}$ \\
\hline 1 & SAMP_SITE_NAME & WELL_NAME & SAMPLE_LOCATION \\
\hline 2810 & PRIEST RAPIDS-RIVER & & \\
\hline 2811 & B POND NORTH SIDE & & \\
\hline 2812 & B POND NORTH SIDE & & \\
\hline 2813 & FFTF POND & & \\
\hline 2814 & GABLE POND N END & & \\
\hline 2815 & GABLE POND N END & & \\
\hline 2816 & WEST LAKE & & \\
\hline 2817 & WEST LAKE & & \\
\hline 2818 & 300 SAN WATER PLANT & & \\
\hline 2819 & 300 SAN WATER PLANT & & \\
\hline 2820 & 300 SAN WATER PLANT & & \\
\hline 2821 & FFTF & & \\
\hline 2822 & FFTF & & \\
\hline 2823 & FFTF & & \\
\hline 2824 & FFTF & & \\
\hline 2825 & RICHLAND SAN PLANT & & \\
\hline 2826 & 300 AREA & & \\
\hline 2827 & 300 AREA & & \\
\hline 2828 & 300 AREA & & \\
\hline 2829 & 300 AREA & & \\
\hline 2830 & 300 AREA & & \\
\hline 2831 & PRIEST RAPIDS-RIVER & & \\
\hline 2832 & PRIEST RAPIDS-RIVER & & \\
\hline 2833 & PRIEST RAPIDS-RIVER & & \\
\hline 2834 & PRIEST RAPIDS-RIVER & & \\
\hline 2835 & PRIEST RAPIDS-RIVER & & \\
\hline 2836 & RICH.PMPHS HRM 46.4 & & \\
\hline 2837 & RICH.PMPHS HRM 46.4 & & \\
\hline 2838 & 100 B AREA-RIVER & & \\
\hline 2839 & 100 B AREA-RIVER & & \\
\hline 2840 & 300 AREA & & \\
\hline 2841 & 300 AREA & & \\
\hline 2842 & PRIEST RAPIDS-RIVER & & \\
\hline 2843 & PRIEST RAPIDS-RIVER & & \\
\hline 2844 & B POND NORTH SIDE & & \\
\hline 2845 & B POND NORTH SIDE & & \\
\hline 2846 & 300 AREA & & \\
\hline 2847 & 300 AREA & & \\
\hline 2848 & PRIEST RAPIDS-RIVER & & \\
\hline 2849 & PRIEST RAPIDS-RIVER & & \\
\hline 2850 & 300 SAN WATER PLANT & & \\
\hline 2851 & FFTF & & \\
\hline 2852 & FFTF & & \\
\hline 2853 & RICHLAND SAN PLANT & & \\
\hline 2854 & 100 B AREA-RIVER & & \\
\hline 2855 & 100 B AREA-RIVER & & \\
\hline 2856 & RICH.PMPHS HRM 46.4 & & \\
\hline 2857 & RICH.PMPHS HRM 46.4 & & \\
\hline 2858 & 300 AREA & & \\
\hline 2859 & 300 AREA & & \\
\hline 2860 & 300 AREA & & \\
\hline 2861 & PRIEST RAP & & \\
\hline
\end{tabular}




\begin{tabular}{|c|c|c|c|}
\hline & $\mathrm{Z}$ & $\overline{\mathrm{AA}}$ & $\mathrm{AB}$ \\
\hline 1 & SAMP_SITE_NAME & WELL_NAME & SAMPLE_LOCATION \\
\hline 2862 & PRIEST RAPIDS-RIVER & & \\
\hline 2863 & PRIEST RAPIDS-RIVER & & \\
\hline 2864 & B POND NORTH SIDE & & \\
\hline 2865 & B POND NORTH SIDE & & \\
\hline 2866 & RICHLAND SAN PLANT & & \\
\hline 2867 & 300 AREA & & \\
\hline 2868 & 300 AREA & & \\
\hline 2869 & PRIEST RAPIDS-RIVER & & \\
\hline 2870 & PRIEST RAPIDS-RIVER & & \\
\hline 2871 & 300 SAN WATER PLANT & & \\
\hline 2872 & 300 SAN WATER PLANT & & \\
\hline 2873 & FFTF & & \\
\hline 2874 & FFTF & & \\
\hline 2875 & FFTF & & \\
\hline 2876 & RICHLAND SAN PLANT & & \\
\hline 2877 & 300 AREA & & \\
\hline 2878 & 300 AREA & & \\
\hline 2879 & 300 AREA & & \\
\hline 2880 & PRIEST RAPIDS-RIVER & & \\
\hline 2881 & PRIEST RAPIDS-RIVER & & \\
\hline 2882 & PRIEST RAPIDS-RIVER & & \\
\hline 2883 & PRIEST RAPIDS-RIVER & & \\
\hline 2884 & PRIEST RAPIDS-RIVER & & \\
\hline 2885 & PRIEST RAPIDS-RIVER & & \\
\hline 2886 & RICH.PMPHS HRM 46.4 & & \\
\hline \begin{tabular}{|l|l|}
2887 & -1 \\
\end{tabular} & RICH.PMPHS HRM 46.4 & & \\
\hline 2888 & RICH.PMPHS HRM 46.4 & & \\
\hline 2889 & B POND NORTH SIDE & & \\
\hline 2890 & B POND NORTH SIDE & & \\
\hline 2891 & FFTF POND & & \\
\hline 2892 & GABLE POND N END & & \\
\hline 2893 & GABLE POND N END & & \\
\hline 2894 & WEST LAKE & & \\
\hline 2895 & WEST LAKE & & \\
\hline 2896 & 300 AREA & & \\
\hline 2897 & 300 AREA & & \\
\hline 2898 & PRIEST RAPIDS-RIVER & & \\
\hline 2899 & PRIEST RAPIDS-RIVER & & \\
\hline 2900 & FFTF & & \\
\hline 2901 & FFTF & & \\
\hline 2902 & RICHLAND SAN PLANT & & \\
\hline 2903 & PRIEST RAPIDS-RIVER & & \\
\hline 2904 & PRIEST RAPIDS-RIVER & & \\
\hline 2905 & 300 AREA & & \\
\hline 2906 & 300 AREA & & \\
\hline 2907 & 300 AREA & & \\
\hline 2908 & PRIEST RAPIDS-RIVER & & \\
\hline 2909 & PRIEST RAPIDS-RIVER & & \\
\hline 2910 & PRIEST RAPIDS-RIVER & & \\
\hline 2911 & RICH.PMPHS HRM 46.4 & & \\
\hline 2912 & RICH.PMPHS HRM 46.4 & & \\
\hline 2913 & B POND NORTH SIDE & & \\
\hline
\end{tabular}




\begin{tabular}{|c|c|c|c|}
\hline & $\mathrm{Z}$ & $\overline{\mathrm{AA}}$ & $\mathrm{AB}$ \\
\hline 1 & SAMP_SITE_NAME & WELL_NAME & SAMPLE_LOCATION \\
\hline 2914 & B POND NORTH SIDE & & \\
\hline 2915 & OBSEVATORY-RTLSNK MT & & \\
\hline 2916 & OBSEVATORY-RTLSNK MT & & \\
\hline 2917 & 100 B AREA-RIVER & & \\
\hline 2918 & 100 B AREA-RIVER & & \\
\hline 2919 & 100 B AREA-RIVER & & \\
\hline 2920 & 300 AREA & & \\
\hline 2921 & 300 AREA & & \\
\hline 2922 & PRIEST RAPIDS-RIVER & & \\
\hline 2923 & PRIEST RAPIDS-RIVER & & \\
\hline 2924 & FFTF & & \\
\hline 2925 & FFTF & & \\
\hline 2926 & RICHLAND SAN PLANT & & \\
\hline 2927 & 300 AREA & & \\
\hline 2928 & 300 AREA & & \\
\hline 2929 & 300 AREA & & \\
\hline 2930 & PRIEST RAPIDS-RIVER & & \\
\hline 2931 & PRIEST RAPIDS-RIVER & & \\
\hline 2932 & PRIEST RAPIDS-RIVER & & \\
\hline 2933 & PRIEST RAPIDS-RIVER & & \\
\hline 2934 & PRIEST RAPIDS-RIVER & & \\
\hline 2935 & RICH.PMPHS HRM 46.4 & & \\
\hline 2936 & RICH.PMPHS HRM 46.4 & & \\
\hline 2937 & B POND NORTH SIDE & & \\
\hline 2938 & B POND NORTH SIDE & & \\
\hline 2939 & 300 AREA & & \\
\hline 2940 & 300 AREA & & \\
\hline 2941 & 300 AREA & & \\
\hline 2942 & 300 AREA & & \\
\hline 2943 & 300 AREA & & \\
\hline 2944 & PRIEST RAPIDS-RIVER & & \\
\hline 2945 & PRIEST RAPIDS-RIVER & & \\
\hline 2946 & FFTF & & \\
\hline 2947 & FFTF & & \\
\hline 2948 & FFTF & & \\
\hline 2949 & RICHLAND SAN PLANT & & \\
\hline 2950 & RICHLAND SAN PLANT & & \\
\hline 2951 & PRIEST RAPIDS-RIVER & & \\
\hline 2952 & PRIEST RAPIDS-RIVER & & \\
\hline 2953 & PRIEST RAPIDS-RIVER & & \\
\hline 2954 & PRIEST RAPIDS-RIVER & & \\
\hline 2955 & PRIEST RAPIDS-RIVER & & \\
\hline 2956 & PRIEST RAPIDS-RIVER & & \\
\hline 2957 & RICH.PMPHS HRM 46.4 & & \\
\hline 2958 & RICH.PMPHS HRM 46.4 & & \\
\hline 2959 & RICH.PMPHS HRM 46.4 & & \\
\hline 2960 & B POND NORTH SIDE & & \\
\hline 2961 & B POND NORTH SIDE & & \\
\hline 2962 & FFTF POND & & \\
\hline 2963 & GABLE POND N END & & \\
\hline 2964 & GABLE POND N END & & \\
\hline 2965 & WEST LAKE & & \\
\hline
\end{tabular}




\begin{tabular}{|c|c|c|c|}
\hline \multicolumn{1}{|c|}{$Z$} & A & AB \\
\hline 1 & SAMP_SITE_NAME & WELL_NAME & SAMPLE_LOCATION \\
\hline 2966 & WEST LAKE & & \\
\hline 2967 & MERRILL FARM-W RICH. & & \\
\hline 2968 & 300 AREA & & \\
\hline 2969 & 300 AREA & & \\
\hline 2970 & PRIEST RAPIDS-RIVER & & \\
\hline 2971 & PRIEST RAPIDS-RIVER & & \\
\hline 2972 & 300 AREA & & \\
\hline 2973 & 300 AREA & & \\
\hline 2974 & 300 AREA & & \\
\hline 2975 & PRIEST RAPIDS-RIVER & & \\
\hline 2976 & PRIEST RAPIDS-RIVER & & \\
\hline 2977 & PRIEST RAPIDS-RIVER & & \\
\hline 2978 & 300 SAN WATER PLANT & & \\
\hline 2979 & 300 SAN WATER PLANT & & \\
\hline 2980 & FFTF & & \\
\hline 2981 & FFTF & & \\
\hline 2982 & RICHLAND SAN PLANT & & \\
\hline 2983 & PRIEST RAPIDS-RIVER & & \\
\hline 2984 & PRIEST RAPIDS-RIVER & & \\
\hline 2985 & RICH.PMPHS HRM 46.4 & & \\
\hline 2986 & RICH.PMPHS HRM 46.4 & & \\
\hline 2987 & OBSEVATORY-RTLSNK MT & & \\
\hline 2988 & OBSEVATORY-RTLSNK MT & & \\
\hline 2989 & 300 AREA & & \\
\hline 2990 & 300 AREA & & \\
\hline 2991 & PRIEST RAPIDS-RIVER & & \\
\hline 2992 & PRIEST RAPIDS-RIVER & & \\
\hline 2993 & 100 B AREA-RIVER & & \\
\hline 2994 & 100 B AREA-RIVER & & \\
\hline 2995 & 100 B AREA-RIVER & & \\
\hline 2996 & B POND NORTH SIDE & & \\
\hline 2997 & B POND NORTH SIDE & & \\
\hline 2998 & 300 AREA & & \\
\hline 2999 & 300 AREA & & \\
\hline 3000 & 300 AREA & & \\
\hline 3001 & 300 AREA & & \\
\hline 3002 & 300 AREA & & \\
\hline 3003 & PRIEST RAPIDS-RIVER & & \\
\hline 3004 & PRIEST RAPIDS-RIVER & & \\
\hline 3005 & PRIEST RAPIDS-RIVER & & \\
\hline 3006 & PRIEST RAPIDS-RIVER & & \\
\hline 3007 & PRIEST RAPIDS-RIVER & & \\
\hline 3008 & FFTF & \\
\hline 3009 & FFTF & & \\
\hline 3010 & RICHLAND SAN PLANT & & \\
\hline 3011 & PRIEST RAPIDS-RIVER & & \\
\hline 3012 & PRIEST RAPIDS-RIVER & & \\
\hline 3013 & RICH.PMPHS HRM 46.4 & & \\
\hline 3014 & RICH.PMPHS HRM 46.4 & & \\
\hline 3015 & 300 AREA & & \\
\hline 3016 & 300 AREA & & \\
\hline 3017 & PRIEST RAPIDS-RIVER & & \\
\hline
\end{tabular}




\begin{tabular}{|c|c|c|c|}
\hline & \multicolumn{1}{|c|}{$Z$} & A & AB \\
\hline 1 & SAMP_SITE_NAME & WELL_NAME & SAMPLE_LOCATION \\
\hline 3018 & PRIEST RAPIDS-RIVER & & \\
\hline 3019 & B POND NORTH SIDE & & \\
\hline 3020 & B POND NORTH SIDE & & \\
\hline 3021 & 300 AREA & & \\
\hline 3022 & 300 AREA & & \\
\hline 3023 & PRIEST RAPIDS-RIVER & & \\
\hline 3024 & PRIEST RAPIDS-RIVER & & \\
\hline 3025 & FFTF & & \\
\hline 3026 & FFTF & & \\
\hline 3027 & FFTF & & \\
\hline 3028 & RICHLAND SAN PLANT & & \\
\hline 3029 & RICHLAND SAN PLANT & & \\
\hline 3030 & PRIEST RAPIDS-RIVER & & \\
\hline 3031 & PRIEST RAPIDS-RIVER & & \\
\hline 3032 & PRIEST RAPIDS-RIVER & & \\
\hline 3033 & RICH.PMPHS HRM 46.4 & & \\
\hline 3034 & RICH.PMPHS HRM 46.4 & & \\
\hline 3035 & RICH.PMPHS HRM 46.4 & & \\
\hline 3036 & 300 SAN WATER PLANT & & \\
\hline 3037 & 300 SAN WATER PLANT & & \\
\hline 3038 & 300 AREA & & \\
\hline 3039 & 300 AREA & & \\
\hline 3040 & 300 AREA & & \\
\hline 3041 & PRIEST RAPIDS-RIVER & & \\
\hline 3042 & PRIEST RAPIDS-RIVER & & \\
\hline 3043 & PRIEST RAPIDS-RIVER & & \\
\hline 3044 & B POND NORTH SIDE & & \\
\hline 3045 & B POND NORTH SIDE & & \\
\hline 3046 & FFTF POND & & \\
\hline 3047 & GABLE POND N END & & \\
\hline 3048 & GABLE POND N END & & \\
\hline 3049 & MERRILL FARM-W RICH. & & \\
\hline 3050 & 300 AREA & & \\
\hline 3051 & 300 AREA & & \\
\hline 3052 & PRIEST RAPIDS-RIVER & & \\
\hline 3053 & PRIEST RAPIDS-RIVER & & \\
\hline 3054 & OBSEVATORY-RTLSNK MT & & \\
\hline 3055 & OBSEVATORY-RTLSNK MT & & \\
\hline 3056 & FFTF & & \\
\hline 3057 & FFTF & & \\
\hline 3058 & RICHLAND SAN PLANT & & \\
\hline 3059 & 300 AREA & \\
\hline 3060 & 300 AREA & & \\
\hline 3061 & 300 AREA & \\
\hline 3062 & PRIEST RAPIDS-RIVER & & \\
\hline 3063 & PRIEST RAPIDS-RIVER & & \\
\hline 3064 & PRIEST RAPIDS-RIVER & & \\
\hline 3065 & PRIEST RAPIDS-RIVER & & \\
\hline 3066 & PRIEST RAPIDS-RIVER & & \\
\hline 3067 & RICH.PMPHS HRM 46.4 & & \\
\hline 3068 & RICH.PMPHS HRM 46.4 & & \\
\hline 306 POND NORTH SIDE & & & \\
\hline
\end{tabular}




\begin{tabular}{|c|c|c|c|}
\hline & \multicolumn{1}{|c|}{$Z$} & A & AB \\
\hline 1 & SAMP_SITE_NAME & WELL_NAME & SAMPLE_LOCATION \\
\hline 3070 & B POND NORTH SIDE & & \\
\hline 3071 & 300 AREA & & \\
\hline 3072 & 300 AREA & & \\
\hline 3073 & PRIEST RAPIDS-RIVER & & \\
\hline 3074 & PRIEST RAPIDS-RIVER & & \\
\hline 3075 & 100 B AREA-RIVER & & \\
\hline 3076 & 100 B AREA-RIVER & & \\
\hline 3077 & FFTF & & \\
\hline 3078 & FFTF & & \\
\hline 3079 & RICHLAND SAN PLANT & & \\
\hline 3080 & 300 AREA & & \\
\hline 3081 & 300 AREA & & \\
\hline 3082 & 300 AREA & & \\
\hline 3083 & 300 AREA & & \\
\hline 3084 & 300 AREA & & \\
\hline 3085 & PRIEST RAPIDS-RIVER & & \\
\hline 3086 & PRIEST RAPIDS-RIVER & & \\
\hline 3087 & PRIEST RAPIDS-RIVER & & \\
\hline 3088 & PRIEST RAPIDS-RIVER & & \\
\hline 3089 & PRIEST RAPIDS-RIVER & & \\
\hline 3090 & PRIEST RAPIDS-RIVER & & \\
\hline 3091 & PRIEST RAPIDS-RIVER & & \\
\hline 3092 & RICH.PMPHS HRM 46.4 & & \\
\hline 3093 & RICH.PMPHS HRM 46.4 & & \\
\hline 3094 & 300 AREA & & \\
\hline 3095 & 300 AREA & & \\
\hline 3096 & PRIEST RAPIDS-RIVER & & \\
\hline 3097 & PRIEST RAPIDS-RIVER & & \\
\hline 3098 & B POND NORTH SIDE & & \\
\hline 3099 & B POND NORTH SIDE & & \\
\hline 3100 & YAKIMA BARRICADE & & \\
\hline 3101 & YAKIMA BARRICADE & & \\
\hline 3102 & YAKIMA BARRICADE & & \\
\hline 3103 & FFTF & & \\
\hline 3104 & FFTF & & \\
\hline 3105 & FFTF & & \\
\hline 3106 & 300 AREA & & \\
\hline 3107 & 300 AREA & & \\
\hline 3108 & 300 AREA & & \\
\hline 3109 & HANFORD TOWNSITE-RIV & & \\
\hline 3110 & PRIEST RAPIDS-RIVER & & \\
\hline 3111 & PRIEST RAPIDS-RIVER & & \\
\hline 3112 & PRIEST RAPIDS-RIVER & & \\
\hline 3113 & RICH.PMPHS HRM 46.4 & & \\
\hline 3114 & 300 SAN WATER PLANT & & \\
\hline 3115 & 300 SAN WATER PLANT & & \\
\hline 3116 & RICHLAND SAN PLANT & & \\
\hline 3117 & RICHLAND SAN PLANT & & \\
\hline 3118 & HANFORD TOWNSITE-RIV & & \\
\hline 3119 & PRIEST RAPIDS-RIVER & & \\
\hline 3120 & PRIEST RAPIDS-RIVER & & \\
\hline 3121 & PRIEST RAPIDS-RIVER & & \\
\hline
\end{tabular}




\begin{tabular}{|c|c|c|c|}
\hline & $\mathrm{Z}$ & $\overline{\mathrm{AA}}$ & $\mathrm{AB}$ \\
\hline 1 & SAMP_SITE_NAME & WELL_NAME & SAMPLE_LOCATION \\
\hline 3122 & RICH.PMPHS HRM 46.4 & & \\
\hline 3123 & RICH.PMPHS HRM 46.4 & & \\
\hline 3124 & RICH.PMPHS HRM 46.4 & & \\
\hline 3125 & OBSEVATORY-RTLSNK MT & & \\
\hline 3126 & OBSEVATORY-RTLSNK MT & & \\
\hline 3127 & 300 AREA & & \\
\hline 3128 & 300 AREA & & \\
\hline 3129 & PRIEST RAPIDS-RIVER & & \\
\hline 3130 & PRIEST RAPIDS-RIVER & & \\
\hline 3131 & B POND NORTH SIDE & & \\
\hline 3132 & B POND NORTH SIDE & & \\
\hline 3133 & FFTF POND & & \\
\hline 3134 & GABLE POND N END & & \\
\hline 3135 & GABLE POND N END & & \\
\hline 3136 & WEST LAKE & & \\
\hline 3137 & WEST LAKE & & \\
\hline 3138 & 300 AREA & & \\
\hline 3139 & 300 AREA & & \\
\hline 3140 & 300 AREA & & \\
\hline 3141 & 300 AREA & & \\
\hline 3142 & 300 AREA & & \\
\hline 3143 & HANFORD TOWNSITE-RIV & & \\
\hline 3144 & PRIEST RAPIDS-RIVER & & \\
\hline 3145 & PRIEST RAPIDS-RIVER & & \\
\hline 3146 & PRIEST RAPIDS-RIVER & & \\
\hline \begin{tabular}{|l|l|}
3147 & -1 \\
\end{tabular} & PRIEST RAPIDS-RIVER & & \\
\hline 3148 & PRIEST RAPIDS-RIVER & & \\
\hline 3149 & FFTF & & \\
\hline 3150 & FFTF & & \\
\hline 3151 & RICHLAND SAN PLANT & & \\
\hline 3152 & RICHLAND SAN PLANT & & \\
\hline 3153 & HANFORD TOWNSITE-RIV & & \\
\hline 3154 & PRIEST RAPIDS-RIVER & & \\
\hline 3155 & PRIEST RAPIDS-RIVER & & \\
\hline 3156 & RICH.PMPHS HRM 46.4 & & \\
\hline 3157 & RICH.PMPHS HRM 46.4 & & \\
\hline 3158 & 300 AREA & & \\
\hline 3159 & 300 AREA & & \\
\hline 3160 & PRIEST RAPIDS-RIVER & & \\
\hline 3161 & PRIEST RAPIDS-RIVER & & \\
\hline 3162 & 100 B AREA-RIVER & & \\
\hline 3163 & 100 B AREA-RIVER & & \\
\hline 3164 & 100 B AREA-RIVER & & \\
\hline 3165 & HANFORD TOWNSITE-RIV & & \\
\hline 3166 & PRIEST RAPIDS-RIVER & & \\
\hline 3167 & PRIEST RAPIDS-RIVER & & \\
\hline 3168 & PRIEST RAPIDS-RIVER & & \\
\hline 3169 & RICH.PMPHS HRM 46.4 & & \\
\hline 3170 & RICH.PMPHS HRM 46.4 & & \\
\hline 3171 & RICH.PMPHS HRM 46.4 & & \\
\hline 3172 & RICHLAND SAN PLANT & & \\
\hline 3173 & RICHLAND SAN PLANT & & \\
\hline
\end{tabular}




\begin{tabular}{|c|c|c|c|}
\hline & $\bar{Z}$ & $\overline{\mathrm{AA}}$ & $\overline{A B}$ \\
\hline 1 & SAMP_SITE_NAME & WELL_NAME & SAMPLE_LOCATION \\
\hline 3174 & 300 AREA & & \\
\hline 3175 & 300 AREA & & \\
\hline 3176 & PRIEST RAPIDS-RIVER & & \\
\hline 3177 & PRIEST RAPIDS-RIVER & & \\
\hline 3178 & HANFORD TOWNSITE-RIV & & \\
\hline 3179 & 300 SAN WATER PLANT & & \\
\hline 3180 & 300 SAN WATER PLANT & & \\
\hline 3181 & 300 AREA & & \\
\hline 3182 & 300 AREA & & \\
\hline 3183 & 300 AREA & & \\
\hline 3184 & PRIEST RAPIDS-RIVER & & \\
\hline 3185 & PRIEST RAPIDS-RIVER & & \\
\hline 3186 & PRIEST RAPIDS-RIVER & & \\
\hline 3187 & PRIEST RAPIDS-RIVER & & \\
\hline 3188 & RICH.PMPHS HRM 46.4 & & \\
\hline 3189 & 300 AREA & & \\
\hline 3190 & 300 AREA & & \\
\hline 3191 & PRIEST RAPIDS-RIVER & & \\
\hline 3192 & PRIEST RAPIDS-RIVER & & \\
\hline 3193 & PRIEST RAPIDS-RIVER & & \\
\hline 3194 & PRIEST RAPIDS-RIVER & & \\
\hline 3195 & RICH.PMPHS HRM 46.4 & & \\
\hline 3196 & 331 FARMS-300 AREA & & \\
\hline 3197 & 331 FARMS-300 AREA & & \\
\hline 3198 & 331 FARMS-300 AREA & & \\
\hline 3199 & MERRILL FARM-W RICH. & & \\
\hline 3200 & MERRILL FARM-W RICH. & & \\
\hline 3201 & MERRILL FARM-W RICH. & & \\
\hline 3202 & PRIEST RAPIDS-RIVER & & \\
\hline 3203 & RICH.PMPHS HRM 46.4 & & \\
\hline 3204 & RICHLAND SAN PLANT & & \\
\hline 3205 & PRIEST RAPIDS-RIVER & & \\
\hline 3206 & RICH.PMPHS HRM 46.4 & & \\
\hline 3207 & FFTF & & \\
\hline 3208 & RICHLAND SAN PLANT & & \\
\hline 3209 & 300 AREA & & \\
\hline 3210 & 300 AREA & & \\
\hline 3211 & 300 AREA & & \\
\hline 3212 & PRIEST RAPIDS-RIVER & & \\
\hline 3213 & RICH.PMPHS HRM 46.4 & & \\
\hline 3214 & GABLE POND A & & \\
\hline 3215 & GABLE POND A & & \\
\hline 3216 & GABLE POND B & & \\
\hline 3217 & GABLE POND B & & \\
\hline 3218 & B POND NORTH SIDE & & \\
\hline 3219 & B POND NORTH SIDE & & \\
\hline 3220 & FFTF POND & & \\
\hline 3221 & GABLE POND N END & & \\
\hline 3222 & GABLE POND N END & & \\
\hline 3223 & WEST LAKE & & \\
\hline 3224 & WEST LAKE & & \\
\hline 3225 & 300 AREA & & \\
\hline
\end{tabular}




\begin{tabular}{|c|c|c|c|}
\hline & $\bar{Z}$ & $\overline{\mathrm{AA}}$ & $\overline{A B}$ \\
\hline 1 & SAMP_SITE_NAME & WELL_NAME & SAMPLE_LOCATION \\
\hline 3226 & 300 AREA & & \\
\hline 3227 & HANFORD TOWNSITE-RIV & & \\
\hline 3228 & PRIEST RAPIDS-RIVER & & \\
\hline 3229 & PRIEST RAPIDS-RIVER & & \\
\hline 3230 & RICHLAND SAN PLANT & & \\
\hline 3231 & HANFORD TOWNSITE-RIV & & \\
\hline 3232 & PRIEST RAPIDS-RIVER & & \\
\hline 3233 & PRIEST RAPIDS-RIVER & & \\
\hline 3234 & RICH.PMPHS HRM 46.4 & & \\
\hline 3235 & RICH.PMPHS HRM 46.4 & & \\
\hline 3236 & OBSEVATORY-RTLSNK MT & & \\
\hline 3237 & OBSEVATORY-RTLSNK MT & & \\
\hline 3238 & 300 AREA & & \\
\hline 3239 & 300 AREA & & \\
\hline 3240 & 300 AREA & & \\
\hline 3241 & HANFORD TOWNSITE-RIV & & \\
\hline 3242 & PRIEST RAPIDS-RIVER & & \\
\hline 3243 & PRIEST RAPIDS-RIVER & & \\
\hline 3244 & PRIEST RAPIDS-RIVER & & \\
\hline 3245 & PRIEST RAPIDS-RIVER & & \\
\hline 3246 & RICH.PMPHS HRM 46.4 & & \\
\hline 3247 & FFTF & & \\
\hline 3248 & FFTF & & \\
\hline 3249 & FFTF & & \\
\hline 3250 & 300 SAN WATER PLANT & & \\
\hline 3251 & 300 SAN WATER PLANT & & \\
\hline 3252 & PRIEST RAPIDS-RIVER & & \\
\hline 3253 & RICH.PMPHS HRM 46.4 & & \\
\hline 3254 & RICHLAND SAN PLANT & & \\
\hline 3255 & 100 B AREA-RIVER & & \\
\hline 3256 & 100 B AREA-RIVER & & \\
\hline 3257 & 100 B AREA-RIVER & & \\
\hline 3258 & 300 AREA & & \\
\hline 3259 & 300 AREA & & \\
\hline 3260 & HANFORD TOWNSITE-RIV & & \\
\hline 3261 & PRIEST RAPIDS-RIVER & & \\
\hline \begin{tabular}{|l|l|}
3262 \\
\end{tabular} & PRIEST RAPIDS-RIVER & & \\
\hline \begin{tabular}{|c|}
3263 \\
25
\end{tabular} & PRIEST RAPIDS-RIVER & & \\
\hline 3264 & PRIEST RAPIDS-RIVER & & \\
\hline 3265 & PRIEST RAPIDS-RIVER & & \\
\hline 3266 & RICH.PMPHS HRM 46.4 & & \\
\hline \begin{tabular}{|l|l|}
3267 & \\
\end{tabular} & RICH.PMPHS HRM 46.4 & & \\
\hline 3268 & RICH.PMPHS HRM 46.4 & & \\
\hline 3269 & YAKIMA BARRICADE & & \\
\hline 3270 & YAKIMA BARRICADE & & \\
\hline 3271 & HANFORD TOWNSITE-RIV & & \\
\hline 3272 & 300 AREA & & \\
\hline 3273 & 300 AREA & & \\
\hline 3274 & 300 AREA & & \\
\hline 3275 & 300 AREA & & \\
\hline 3276 & 300 AREA & & \\
\hline 3277 & HANFORD TOWNSITE-RIV & & \\
\hline
\end{tabular}




\begin{tabular}{|c|c|c|c|}
\hline & \multicolumn{1}{|c|}{$Z$} & A & AB \\
\hline 1 & SAMP_SITE_NAME & WELL_NAME & SAMPLE_LOCATION \\
\hline 3278 & PRIEST RAPIDS-RIVER & & \\
\hline 3279 & PRIEST RAPIDS-RIVER & & \\
\hline 3280 & PRIEST RAPIDS-RIVER & & \\
\hline 3281 & PRIEST RAPIDS-RIVER & & \\
\hline 3282 & PRIEST RAPIDS-RIVER & & \\
\hline 3283 & PRIEST RAPIDS-RIVER & & \\
\hline 3284 & RICH.PMPHS HRM 46.4 & & \\
\hline 3285 & RICHLAND SAN PLANT & & \\
\hline 3286 & 300 AREA & & \\
\hline 3287 & 300 AREA & & \\
\hline 3288 & PRIEST RAPIDS-RIVER & & \\
\hline 3289 & PRIEST RAPIDS-RIVER & & \\
\hline 3290 & PRIEST RAPIDS-RIVER & & \\
\hline 3291 & PRIEST RAPIDS-RIVER & & \\
\hline 3292 & PRIEST RAPIDS-RIVER & & \\
\hline 3293 & RICH.PMPHS HRM 46.4 & & \\
\hline 3294 & RICH.PMPHS HRM 46.4 & & \\
\hline 3295 & RICH.PMPHS HRM 46.4 & & \\
\hline 3296 & 331 FARMS-300 AREA & & \\
\hline 3297 & 331 FARMS-300 AREA & & \\
\hline 3298 & 331 FARMS-300 AREA & & \\
\hline 3299 & MERRILL FARM-W RICH. & & \\
\hline 3300 & MERRILL FARM-W RICH. & & \\
\hline 3301 & MERRILL FARM-W RICH. & & \\
\hline 3302 & HANFORD TOWNSITE-RIV & & \\
\hline 3303 & 300 AREA & & \\
\hline 3304 & 300 AREA & & \\
\hline 3305 & 300 AREA & & \\
\hline 3306 & HANFORD TOWNSITE-RIV & & \\
\hline 3307 & PRIEST RAPIDS-RIVER & & \\
\hline 3308 & PRIEST RAPIDS-RIVER & & \\
\hline 3309 & PRIEST RAPIDS-RIVER & & \\
\hline 3310 & PRIEST RAPIDS-RIVER & & \\
\hline 3311 & RICH.PMPHS HRM 46.4 & & \\
\hline 3312 & B POND A & & \\
\hline 3313 & B POND A & & \\
\hline 3314 & B POND B & & \\
\hline 3315 & B POND B & & \\
\hline 3316 & WEST LAKE A & & \\
\hline 3317 & WEST LAKE A & & \\
\hline 3318 & WEST LAKE B & & \\
\hline 3319 & WEST LAKE B & & \\
\hline 3320 & B POND NORTH SIDE & & \\
\hline 3321 & B POND NORTH SIDE & & \\
\hline 3322 & FFTF POND & \\
\hline 3323 & GABLE POND N END & & \\
\hline 3324 & GABLE POND N END & & \\
\hline 3325 & WEST LAKE & & \\
\hline 3326 & WEST LAKE & & \\
\hline 3327 & RICHLAND SAN PLANT & & \\
\hline 3328 & 300 AREA & & \\
\hline 3329 & 300 AREA & & \\
& & & \\
\hline
\end{tabular}




\begin{tabular}{|c|c|c|c|}
\hline & $\bar{Z}$ & $\overline{\mathrm{AA}}$ & $\overline{A B}$ \\
\hline 1 & SAMP_SITE_NAME & WELL_NAME & SAMPLE_LOCATION \\
\hline 3330 & HANFORD TOWNSITE-RIV & & \\
\hline 3331 & PRIEST RAPIDS-RIVER & & \\
\hline 3332 & PRIEST RAPIDS-RIVER & & \\
\hline 3333 & PRIEST RAPIDS-RIVER & & \\
\hline 3334 & PRIEST RAPIDS-RIVER & & \\
\hline 3335 & PRIEST RAPIDS-RIVER & & \\
\hline 3336 & RICH.PMPHS HRM 46.4 & & \\
\hline 3337 & RICH.PMPHS HRM 46.4 & & \\
\hline 3338 & RICH.PMPHS HRM 46.4 & & \\
\hline 3339 & HANFORD TOWNSITE-RIV & & \\
\hline 3340 & PRIEST RAPIDS-RIVER & & \\
\hline 3341 & RICH.PMPHS HRM 46.4 & & \\
\hline 3342 & OBSEVATORY-RTLSNK MT & & \\
\hline 3343 & OBSEVATORY-RTLSNK MT & & \\
\hline 3344 & 300 AREA & & \\
\hline 3345 & 300 AREA & & \\
\hline 3346 & 300 AREA & & \\
\hline 3347 & HANFORD TOWNSITE-RIV & & \\
\hline 3348 & PRIEST RAPIDS-RIVER & & \\
\hline 3349 & PRIEST RAPIDS-RIVER & & \\
\hline 3350 & PRIEST RAPIDS-RIVER & & \\
\hline 3351 & RICH.PMPHS HRM 46.4 & & \\
\hline 3352 & VERNITA & & \\
\hline 3353 & FFTF & & \\
\hline 3354 & FFTF & & \\
\hline 3355 & FFTF & & \\
\hline 3356 & 300 AREA & & \\
\hline 3357 & 300 AREA & & \\
\hline 3358 & PRIEST RAPIDS-RIVER & & \\
\hline 3359 & PRIEST RAPIDS-RIVER & & \\
\hline 3360 & 300 SAN WATER PLANT & & \\
\hline 3361 & 300 SAN WATER PLANT & & \\
\hline 3362 & RICHLAND SAN PLANT & & \\
\hline 3363 & 100 B AREA-RIVER & & \\
\hline 3364 & 100 B AREA-RIVER & & \\
\hline 3365 & 100 B AREA-RIVER & & \\
\hline 3366 & 300 AREA & & \\
\hline 3367 & 300 AREA & & \\
\hline 3368 & HANFORD TOWNSITE-RIV & & \\
\hline 3369 & PRIEST RAPIDS-RIVER & & \\
\hline 3370 & PRIEST RAPIDS-RIVER & & \\
\hline 3371 & PRIEST RAPIDS-RIVER & & \\
\hline 3372 & PRIEST RAPIDS-RIVER & & \\
\hline 3373 & PRIEST RAPIDS-RIVER & & \\
\hline 3374 & RICH.PMPHS HRM 46.4 & & \\
\hline 3375 & RICH.PMPHS HRM 46.4 & & \\
\hline 3376 & RICH.PMPHS HRM 46.4 & & \\
\hline 3377 & YAKIMA BARRICADE & & \\
\hline 3378 & YAKIMA BARRICADE & & \\
\hline 3379 & 300 AREA & & \\
\hline 3380 & 300 AREA & & \\
\hline 3381 & 300 AREA & & \\
\hline
\end{tabular}




\begin{tabular}{|c|c|c|c|}
\hline & $\mathrm{Z}$ & $\overline{\mathrm{AA}}$ & $\mathrm{AB}$ \\
\hline 1 & SAMP_SITE_NAME & WELL_NAME & SAMPLE_LOCATION \\
\hline 3382 & HANFORD TOWNSITE-RIV & & \\
\hline 3383 & PRIEST RAPIDS-RIVER & & \\
\hline 3384 & PRIEST RAPIDS-RIVER & & \\
\hline 3385 & PRIEST RAPIDS-RIVER & & \\
\hline 3386 & RICH.PMPHS HRM 46.4 & & \\
\hline \begin{tabular}{|l|l|}
3387 & $r$ \\
\end{tabular} & RICH.PMPHS HRM 46.4 & & \\
\hline 3388 & RICH.PMPHS HRM 46.4 & & \\
\hline 3389 & 331 FARMS-300 AREA & & \\
\hline 3390 & 331 FARMS-300 AREA & & \\
\hline 3391 & 331 FARMS-300 AREA & & \\
\hline 3392 & 331 FARMS-300 AREA & & \\
\hline 3393 & MERRILL FARM-W RICH. & & \\
\hline 3394 & MERRILL FARM-W RICH. & & \\
\hline 3395 & MERRILL FARM-W RICH. & & \\
\hline 3396 & MERRILL FARM-W RICH. & & \\
\hline 3397 & 300 AREA & & \\
\hline 3398 & 300 AREA & & \\
\hline 3399 & PRIEST RAPIDS-RIVER & & \\
\hline 3400 & PRIEST RAPIDS-RIVER & & \\
\hline 3401 & PRIEST RAPIDS-RIVER & & \\
\hline 3402 & PRIEST RAPIDS-RIVER & & \\
\hline 3403 & PRIEST RAPIDS-RIVER & & \\
\hline 3404 & RICHLAND SAN PLANT & & \\
\hline 3405 & HANFORD TOWNSITE-RIV & & \\
\hline 3406 & 300 AREA & & \\
\hline 3407 & 300 AREA & & \\
\hline 3408 & PRIEST RAPIDS-RIVER & & \\
\hline 3409 & PRIEST RAPIDS-RIVER & & \\
\hline 3410 & FFTF POND A & & \\
\hline 3411 & FFTF POND A & & \\
\hline 3412 & FFTF POND B & & \\
\hline 3413 & FFTF POND B & & \\
\hline 3414 & B POND NORTH SIDE & & \\
\hline 3415 & B POND NORTH SIDE & & \\
\hline 3416 & FFTF POND & & \\
\hline 3417 & GABLE POND N END & & \\
\hline 3418 & GABLE POND N END & & \\
\hline 3419 & WEST LAKE & & \\
\hline 3420 & WEST LAKE & & \\
\hline 3421 & RICHLAND SAN PLANT & & \\
\hline 3422 & 300 AREA & & \\
\hline 3423 & 300 AREA & & \\
\hline 3424 & 300 AREA & & \\
\hline 3425 & HANFORD TOWNSITE-RIV & & \\
\hline 3426 & HANFORD TOWNSITE-RIV & & \\
\hline 3427 & PRIEST RAPIDS-RIVER & & \\
\hline 3428 & PRIEST RAPIDS-RIVER & & \\
\hline 3429 & PRIEST RAPIDS-RIVER & & \\
\hline 3430 & PRIEST RAPIDS-RIVER & & \\
\hline 3431 & PRIEST RAPIDS-RIVER & & \\
\hline 3432 & PRIEST RAPIDS-RIVER & & \\
\hline 3433 & RICH.PMPHS HRM 46.4 & & \\
\hline
\end{tabular}




\begin{tabular}{|c|c|c|c|}
\hline & $\bar{Z}$ & $\overline{\mathrm{AA}}$ & $\overline{A B}$ \\
\hline 1 & SAMP_SITE_NAME & WELL_NAME & SAMPLE_LOCATION \\
\hline 3434 & RICH.PMPHS HRM 46.4 & & \\
\hline 3435 & RICH.PMPHS HRM 46.4 & & \\
\hline 3436 & HANFORD TOWNSITE-RIV & & \\
\hline 3437 & OBSEVATORY-RTLSNK MT & & \\
\hline 3438 & OBSEVATORY-RTLSNK MT & & \\
\hline 3439 & 300 AREA & & \\
\hline 3440 & 300 AREA & & \\
\hline 3441 & PRIEST RAPIDS-RIVER & & \\
\hline \begin{tabular}{|c|}
3442 \\
\end{tabular} & PRIEST RAPIDS-RIVER & & \\
\hline 3443 & FFTF & & \\
\hline 3444 & FFTF & & \\
\hline 3445 & FFTF & & \\
\hline 3446 & 300 SAN WATER PLANT & & \\
\hline \begin{tabular}{|l|l|}
3447 & \\
\end{tabular} & 300 SAN WATER PLANT & & \\
\hline \begin{tabular}{|c|}
3448 \\
\end{tabular} & RICHLAND SAN PLANT & & \\
\hline 3449 & RICHLAND SAN PLANT & & \\
\hline 3450 & 100 B AREA-RIVER & & \\
\hline 3451 & 100 B AREA-RIVER & & \\
\hline 3452 & 100 B AREA-RIVER & & \\
\hline 3453 & 300 AREA & & \\
\hline 3454 & 300 AREA & & \\
\hline 3455 & 300 AREA & & \\
\hline \begin{tabular}{|c|}
3456 \\
\end{tabular} & 300 AREA & & \\
\hline \begin{tabular}{|l|l|}
3457 & \\
\end{tabular} & 300 AREA & & \\
\hline \begin{tabular}{|l|l|}
3458 \\
\end{tabular} & PRIEST RAPIDS-RIVER & & \\
\hline 3459 & PRIEST RAPIDS-RIVER & & \\
\hline 3460 & PRIEST RAPIDS-RIVER & & \\
\hline 3461 & PRIEST RAPIDS-RIVER & & \\
\hline \begin{tabular}{|c|}
3462 \\
\end{tabular} & PRIEST RAPIDS-RIVER & & \\
\hline \begin{tabular}{|c|}
3463 \\
\end{tabular} & PRIEST RAPIDS-RIVER & & \\
\hline 3464 & PRIEST RAPIDS-RIVER & & \\
\hline 3465 & PRIEST RAPIDS-RIVER & & \\
\hline 3466 & RICH.PMPHS HRM 46.4 & & \\
\hline \begin{tabular}{|l|l|}
3467 & \\
\end{tabular} & RICH.PMPHS HRM 46.4 & & \\
\hline \begin{tabular}{|c|}
3468 \\
\end{tabular} & RICH.PMPHS HRM 46.4 & & \\
\hline 3469 & YAKIMA BARRICADE & & \\
\hline 3470 & YAKIMA BARRICADE & & \\
\hline \begin{tabular}{|l|l|}
3471 & 1 \\
\end{tabular} & HANFORD TOWNSITE-RIV & & \\
\hline \begin{tabular}{|c|}
3472 \\
\end{tabular} & 300 AREA & & \\
\hline 3473 & 300 AREA & & \\
\hline 3474 & PRIEST RAPIDS-RIVER & & \\
\hline \begin{tabular}{|c|}
3475 \\
\end{tabular} & PRIEST RAPIDS-RIVER & & \\
\hline 3476 & RICH.PMPHS HRM 46.4 & & \\
\hline 3477 & RICH.PMPHS HRM 46.4 & & \\
\hline 3478 & RICH.PMPHS HRM 46.4 & & \\
\hline 3479 & RICHLAND SAN PLANT & & \\
\hline 3480 & RICHLAND SAN PLANT & & \\
\hline 3481 & 300 AREA & & \\
\hline \begin{tabular}{|c|}
3482 \\
\end{tabular} & 300 AREA & & \\
\hline \begin{tabular}{|c|}
3483 \\
\end{tabular} & 300 AREA & & \\
\hline 3484 & HANFORD TOWNSITE-RIV & & \\
\hline 3485 & HANFORD TOWNSITE-RIV & & \\
\hline
\end{tabular}




\begin{tabular}{|c|c|c|c|}
\hline & $\bar{Z}$ & $\overline{\mathrm{AA}}$ & $\overline{A B}$ \\
\hline 1 & SAMP_SITE_NAME & WELL_NAME & SAMPLE_LOCATION \\
\hline 3486 & PRIEST RAPIDS-RIVER & & \\
\hline 3487 & PRIEST RAPIDS-RIVER & & \\
\hline 3488 & PRIEST RAPIDS-RIVER & & \\
\hline 3489 & PRIEST RAPIDS-RIVER & & \\
\hline 3490 & PRIEST RAPIDS-RIVER & & \\
\hline 3491 & PRIEST RAPIDS-RIVER & & \\
\hline \begin{tabular}{|c|}
3492 \\
\end{tabular} & 300 AREA & & \\
\hline 3493 & 300 AREA & & \\
\hline 3494 & PRIEST RAPIDS-RIVER & & \\
\hline 3495 & PRIEST RAPIDS-RIVER & & \\
\hline 3496 & B POND A & & \\
\hline 3497 & B POND A & & \\
\hline 3498 & B POND B & & \\
\hline 3499 & B POND B & & \\
\hline 3500 & B POND NORTH SIDE & & \\
\hline 3501 & B POND NORTH SIDE & & \\
\hline \begin{tabular}{|l|l|}
3502 \\
\end{tabular} & FFTF POND & & \\
\hline 3503 & GABLE POND N END & & \\
\hline 3504 & GABLE POND N END & & \\
\hline 3505 & WEST LAKE & & \\
\hline \begin{tabular}{|c|}
3506 \\
\end{tabular} & WEST LAKE & & \\
\hline \begin{tabular}{|l|l|}
3507 \\
\end{tabular} & 300 AREA & & \\
\hline \begin{tabular}{|c|}
3508 \\
\end{tabular} & 300 AREA & & \\
\hline \begin{tabular}{|l|l|}
3509 \\
\end{tabular} & 300 AREA & & \\
\hline 3510 & HANFORD TOWNSITE-RIV & & \\
\hline 3511 & HANFORD TOWNSITE-RIV & & \\
\hline 3512 & PRIEST RAPIDS-RIVER & & \\
\hline 3513 & PRIEST RAPIDS-RIVER & & \\
\hline 3514 & PRIEST RAPIDS-RIVER & & \\
\hline 3515 & PRIEST RAPIDS-RIVER & & \\
\hline 3516 & PRIEST RAPIDS-RIVER & & \\
\hline 3517 & PRIEST RAPIDS-RIVER & & \\
\hline 3518 & RICH.PMPHS HRM 46.4 & & \\
\hline 3519 & RICH.PMPHS HRM 46.4 & & \\
\hline 3520 & RICH.PMPHS HRM 46.4 & & \\
\hline 3521 & RICHLAND SAN PLANT & & \\
\hline 3522 & RICHLAND SAN PLANT & & \\
\hline 3523 & OBSEVATORY-RTLSNK MT & & \\
\hline \begin{tabular}{|c|c|}
3524 \\
52
\end{tabular} & OBSEVATORY-RTLSNK MT & & \\
\hline 3525 & 300 AREA & & \\
\hline 3526 & 300 AREA & & \\
\hline \begin{tabular}{|l|l|}
3527 & \\
\end{tabular} & PRIEST RAPIDS-RIVER & & \\
\hline 3528 & PRIEST RAPIDS-RIVER & & \\
\hline 3529 & FFTF & & \\
\hline 3530 & FFTF & & \\
\hline 3531 & FFTF & & \\
\hline 3532 & 300 SAN WATER PLANT & & \\
\hline 3533 & 300 SAN WATER PLANT & & \\
\hline 3534 & RICHLAND SAN PLANT & & \\
\hline 3535 & RICHLAND SAN PLANT & & \\
\hline 3536 & 100 B AREA-RIVER & & \\
\hline \begin{tabular}{|l|}
3537 \\
\end{tabular} & 100 B AREA-RIVER & & \\
\hline
\end{tabular}




\begin{tabular}{|c|c|c|c|}
\hline & $\mathrm{Z}$ & $\overline{\mathrm{AA}}$ & $\mathrm{AB}$ \\
\hline 1 & SAMP_SITE_NAME & WELL_NAME & SAMPLE_LOCATION \\
\hline 3538 & 100 B AREA-RIVER & & \\
\hline 3539 & 300 AREA & & \\
\hline 3540 & 300 AREA & & \\
\hline 3541 & 300 AREA & & \\
\hline 3542 & 300 AREA & & \\
\hline 3543 & 300 AREA & & \\
\hline 3544 & HANFORD TOWNSITE-RIV & & \\
\hline 3545 & HANFORD TOWNSITE-RIV & & \\
\hline 3546 & PRIEST RAPIDS-RIVER & & \\
\hline 3547 & PRIEST RAPIDS-RIVER & & \\
\hline 3548 & PRIEST RAPIDS-RIVER & & \\
\hline 3549 & PRIEST RAPIDS-RIVER & & \\
\hline 3550 & PRIEST RAPIDS-RIVER & & \\
\hline 3551 & PRIEST RAPIDS-RIVER & & \\
\hline 3552 & PRIEST RAPIDS-RIVER & & \\
\hline 3553 & PRIEST RAPIDS-RIVER & & \\
\hline 3554 & RICH.PMPHS HRM 46.4 & & \\
\hline 3555 & RICH.PMPHS HRM 46.4 & & \\
\hline 3556 & RICH.PMPHS HRM 46.4 & & \\
\hline 3557 & YAKIMA BARRICADE & & \\
\hline 3558 & YAKIMA BARRICADE & & \\
\hline 3559 & 300 AREA & & \\
\hline 3560 & 300 AREA & & \\
\hline 3561 & PRIEST RAPIDS-RIVER & & \\
\hline 3562 & PRIEST RAPIDS-RIVER & & \\
\hline 3563 & RICH.PMPHS HRM 46.4 & & \\
\hline 3564 & RICH.PMPHS HRM 46.4 & & \\
\hline 3565 & RICH.PMPHS HRM 46.4 & & \\
\hline 3566 & 300 AREA & & \\
\hline 3567 & 300 AREA & & \\
\hline 3568 & 300 AREA & & \\
\hline 3569 & HANFORD TOWNSITE-RIV & & \\
\hline 3570 & HANFORD TOWNSITE-RIV & & \\
\hline 3571 & PRIEST RAPIDS-RIVER & & \\
\hline 3572 & PRIEST RAPIDS-RIVER & & \\
\hline 3573 & PRIEST RAPIDS-RIVER & & \\
\hline 3574 & PRIEST RAPIDS-RIVER & & \\
\hline 3575 & PRIEST RAPIDS-RIVER & & \\
\hline 3576 & PRIEST RAPIDS-RIVER & & \\
\hline 3577 & RICHLAND SAN PLANT & & \\
\hline 3578 & RICHLAND SAN PLANT & & \\
\hline 3579 & 331 FARMS-300 AREA & & \\
\hline 3580 & 331 FARMS-300 AREA & & \\
\hline 3581 & 331 FARMS-300 AREA & & \\
\hline 3582 & MERRILL FARM-W RICH. & & \\
\hline 3583 & MERRILL FARM-W RICH. & & \\
\hline 3584 & MERRILL FARM-W RICH. & & \\
\hline 3585 & 300 AREA & & \\
\hline 3586 & 300 AREA & & \\
\hline 3587 & HANFORD TOWNSITE-RIV & & \\
\hline 3588 & PRIEST RAPIDS-RIVER & & \\
\hline 3589 & PRIEST RAPIDS-RIVER & & \\
\hline
\end{tabular}




\begin{tabular}{|c|c|c|c|}
\hline & $\bar{Z}$ & $\overline{\mathrm{AA}}$ & $\overline{A B}$ \\
\hline 1 & SAMP_SITE_NAME & WELL_NAME & SAMPLE_LOCATION \\
\hline 3590 & RICHLAND SAN PLANT & & \\
\hline 3591 & RICHLAND SAN PLANT & & \\
\hline 3592 & 300 AREA & & \\
\hline \begin{tabular}{|c|}
3593 \\
\end{tabular} & 300 AREA & & \\
\hline 3594 & PRIEST RAPIDS-RIVER & & \\
\hline 3595 & PRIEST RAPIDS-RIVER & & \\
\hline 3596 & PRIEST RAPIDS-RIVER & & \\
\hline 3597 & PRIEST RAPIDS-RIVER & & \\
\hline 3598 & PRIEST RAPIDS-RIVER & & \\
\hline 3599 & RICH.PMPHS HRM 46.4 & & \\
\hline 3600 & 300 AREA & & \\
\hline 3601 & 300 AREA & & \\
\hline 3602 & PRIEST RAPIDS-RIVER & & \\
\hline 3603 & PRIEST RAPIDS-RIVER & & \\
\hline 3604 & GABLE POND N END & & \\
\hline 3605 & GABLE POND N END & & \\
\hline 3606 & GABLE POND N END & & \\
\hline 3607 & 300 SAN WATER PLANT & & \\
\hline 3608 & 300 SAN WATER PLANT & & \\
\hline 3609 & RICHLAND SAN PLANT & & \\
\hline 3610 & RICHLAND SAN PLANT & & \\
\hline 3611 & 100 B AREA-RIVER & & \\
\hline 3612 & 100 B AREA-RIVER & & \\
\hline 3613 & 100 B AREA-RIVER & & \\
\hline 3614 & 300 AREA & & \\
\hline 3615 & 300 AREA & & \\
\hline 3616 & 300 AREA & & \\
\hline 3617 & 300 AREA & & \\
\hline 3618 & 300 AREA & & \\
\hline 3619 & PRIEST RAPIDS-RIVER & & \\
\hline 3620 & PRIEST RAPIDS-RIVER & & \\
\hline 3621 & PRIEST RAPIDS-RIVER & & \\
\hline 3622 & PRIEST RAPIDS-RIVER & & \\
\hline 3623 & PRIEST RAPIDS-RIVER & & \\
\hline 3624 & PRIEST RAPIDS-RIVER & & \\
\hline 3625 & PRIEST RAPIDS-RIVER & & \\
\hline 3626 & PRIEST RAPIDS-RIVER & & \\
\hline 3627 & RICH.PMPHS HRM 46.4 & & \\
\hline 3628 & RICH.PMPHS HRM 46.4 & & \\
\hline 3629 & RICH.PMPHS HRM 46.4 & & \\
\hline 3630 & GABLE POND A & & \\
\hline 3631 & GABLE POND A & & \\
\hline 3632 & 100 B AREA-RIVER & & \\
\hline 3633 & 100 B AREA-RIVER & & \\
\hline 3634 & 100 B AREA-RIVER & & \\
\hline 3635 & B POND NORTH SIDE & & \\
\hline 3636 & B POND NORTH SIDE & & \\
\hline 3637 & B POND NORTH SIDE & & \\
\hline 3638 & FFTF POND & & \\
\hline 3639 & FFTF POND & & \\
\hline 3640 & GABLE POND N END & & \\
\hline 3641 & GABLE POND N END & & \\
\hline
\end{tabular}




\begin{tabular}{|c|c|c|c|}
\hline & $\bar{Z}$ & $\overline{\mathrm{AA}}$ & $\overline{A B}$ \\
\hline 1 & SAMP_SITE_NAME & WELL_NAME & SAMPLE_LOCATION \\
\hline 3642 & GABLE POND N END & & \\
\hline 3643 & WEST LAKE & & \\
\hline 3644 & WEST LAKE & & \\
\hline 3645 & WEST LAKE & & \\
\hline 3646 & OBSEVATORY-RTLSNK MT & & \\
\hline 3647 & OBSEVATORY-RTLSNK MT & & \\
\hline 3648 & 300 AREA & & \\
\hline 3649 & 300 AREA & & \\
\hline 3650 & PRIEST RAPIDS-RIVER & & \\
\hline 3651 & PRIEST RAPIDS-RIVER & & \\
\hline 3652 & FFTF & & \\
\hline 3653 & FFTF & & \\
\hline 3654 & FFTF & & \\
\hline 3655 & RICHLAND SAN PLANT & & \\
\hline 3656 & RICHLAND SAN PLANT & & \\
\hline \begin{tabular}{|c|}
3657 \\
\end{tabular} & 300 AREA & & \\
\hline 3658 & 300 AREA & & \\
\hline 3659 & PRIEST RAPIDS-RIVER & & \\
\hline 3660 & PRIEST RAPIDS-RIVER & & \\
\hline 3661 & PRIEST RAPIDS-RIVER & & \\
\hline 3662 & PRIEST RAPIDS-RIVER & & \\
\hline 3663 & PRIEST RAPIDS-RIVER & & \\
\hline 3664 & RICH.PMPHS HRM 46.4 & & \\
\hline 3665 & RICH.PMPHS HRM 46.4 & & \\
\hline 3666 & RICH.PMPHS HRM 46.4 & & \\
\hline 3667 & YAKIMA BARRICADE & & \\
\hline 3668 & YAKIMA BARRICADE & & \\
\hline 3669 & YAKIMA BARRICADE & & \\
\hline 3670 & 100 D AREA & & \\
\hline 3671 & 100 D AREA & & \\
\hline 3672 & 100 D AREA & & \\
\hline 3673 & 300 AREA & & \\
\hline 3674 & 300 AREA & & \\
\hline 3675 & PRIEST RAPIDS-RIVER & & \\
\hline 3676 & PRIEST RAPIDS-RIVER & & \\
\hline 3677 & 300 AREA & & \\
\hline 3678 & 300 AREA & & \\
\hline 3679 & RICHLAND SAN PLANT & & \\
\hline 3680 & RICHLAND SAN PLANT & & \\
\hline 3681 & 300 AREA & & \\
\hline 3682 & 300 AREA & & \\
\hline 3683 & PRIEST RAPIDS-RIVER & & \\
\hline 3684 & PRIEST RAPIDS-RIVER & & \\
\hline 3685 & PRIEST RAPIDS-RIVER & & \\
\hline 3686 & PRIEST RAPIDS-RIVER & & \\
\hline 3687 & PRIEST RAPIDS-RIVER & & \\
\hline 3688 & RICH.PMPHS HRM 46.4 & & \\
\hline 3689 & RICH.PMPHS HRM 46.4 & & \\
\hline 3690 & RICH.PMPHS HRM 46.4 & & \\
\hline 3691 & LOWER REACH RIVER & & \\
\hline 3692 & LOWER REACH RIVER & & \\
\hline 3693 & LOWER REACH RIVER & & \\
\hline
\end{tabular}




\begin{tabular}{|c|c|c|c|}
\hline & $\mathrm{Z}$ & $\overline{\mathrm{AA}}$ & $\mathrm{AB}$ \\
\hline 1 & SAMP_SITE_NAME & WELL_NAME & SAMPLE_LOCATION \\
\hline 3694 & UPPER REACH RIVER & & \\
\hline 3695 & UPPER REACH RIVER & & \\
\hline 3696 & UPPER REACH RIVER & & \\
\hline 3697 & 300 AREA & & \\
\hline 3698 & 300 AREA & & \\
\hline 3699 & PRIEST RAPIDS-RIVER & & \\
\hline 3700 & PRIEST RAPIDS-RIVER & & \\
\hline 3701 & RICHLAND SAN PLANT & & \\
\hline 3702 & RICHLAND SAN PLANT & & \\
\hline 3703 & 300 AREA & & \\
\hline 3704 & 300 AREA & & \\
\hline 3705 & 300 AREA & & \\
\hline 3706 & 300 AREA & & \\
\hline 3707 & 300 AREA & & \\
\hline 3708 & PRIEST RAPIDS-RIVER & & \\
\hline 3709 & PRIEST RAPIDS-RIVER & & \\
\hline 3710 & PRIEST RAPIDS-RIVER & & \\
\hline 3711 & PRIEST RAPIDS-RIVER & & \\
\hline 3712 & PRIEST RAPIDS-RIVER & & \\
\hline 3713 & PRIEST RAPIDS-RIVER & & \\
\hline 3714 & PRIEST RAPIDS-RIVER & & \\
\hline 3715 & PRIEST RAPIDS-RIVER & & \\
\hline 3716 & RICH.PMPHS HRM 46.4 & & \\
\hline 3717 & RICH.PMPHS HRM 46.4 & & \\
\hline 3718 & RICH.PMPHS HRM 46.4 & & \\
\hline 3719 & 100 B AREA-RIVER & & \\
\hline 3720 & 100 B AREA-RIVER & & \\
\hline 3721 & 100 B AREA-RIVER & & \\
\hline 3722 & B POND NORTH SIDE & & \\
\hline 3723 & B POND NORTH SIDE & & \\
\hline 3724 & B POND NORTH SIDE & & \\
\hline 3725 & FFTF POND & & \\
\hline 3726 & FFTF POND & & \\
\hline 3727 & GABLE POND N END & & \\
\hline 3728 & GABLE POND N END & & \\
\hline 3729 & GABLE POND N END & & \\
\hline 3730 & WEST LAKE & & \\
\hline 3731 & WEST LAKE & & \\
\hline 3732 & WEST LAKE & & \\
\hline 3733 & FIRING RANGE & & \\
\hline 3734 & FIRING RANGE & & \\
\hline 3735 & FIRING RANGE & & \\
\hline 3736 & OBSEVATORY-RTLSNK MT & & \\
\hline 3737 & OBSEVATORY-RTLSNK MT & & \\
\hline 3738 & 300 AREA & & \\
\hline 3739 & 300 AREA & & \\
\hline 3740 & PRIEST RAPIDS-RIVER & & \\
\hline 3741 & PRIEST RAPIDS-RIVER & & \\
\hline 3742 & FFTF & & \\
\hline 3743 & FFTF & & \\
\hline 3744 & FFTF & & \\
\hline 3745 & $\mathrm{RICHL}$ & & \\
\hline
\end{tabular}




\begin{tabular}{|c|c|c|c|}
\hline & \multicolumn{1}{|c|}{$Z$} & A & AB \\
\hline 1 & SAMP_SITE_NAME & WELL_NAME & SAMPLE_LOCATION \\
\hline 3746 & RICHLAND SAN PLANT & & \\
\hline 3747 & 300 AREA & & \\
\hline 3748 & 300 AREA & & \\
\hline 3749 & PRIEST RAPIDS-RIVER & & \\
\hline 3750 & PRIEST RAPIDS-RIVER & & \\
\hline 3751 & PRIEST RAPIDS-RIVER & & \\
\hline 3752 & PRIEST RAPIDS-RIVER & & \\
\hline 3753 & PRIEST RAPIDS-RIVER & & \\
\hline 3754 & RICH.PMPHS HRM 46.4 & & \\
\hline 3755 & RICH.PMPHS HRM 46.4 & & \\
\hline 3756 & RICH.PMPHS HRM 46.4 & & \\
\hline 3757 & 100 D AREA & & \\
\hline 3758 & YAKIMA BARRICADE & & \\
\hline 3759 & YAKIMA BARRICADE & & \\
\hline 3760 & YAKIMA BARRICADE & & \\
\hline 3761 & 100 D AREA & & \\
\hline 3762 & 100 D AREA & & \\
\hline 3763 & 300 AREA & & \\
\hline 3764 & 300 AREA & & \\
\hline 3765 & PRIEST RAPIDS-RIVER & & \\
\hline 3766 & PRIEST RAPIDS-RIVER & & \\
\hline 3767 & 300 AREA & & \\
\hline 3768 & 300 AREA & & \\
\hline 3769 & 300 AREA & & \\
\hline 3770 & 300 AREA & & \\
\hline 3771 & 300 AREA & & \\
\hline 3772 & PRIEST RAPIDS-RIVER & & \\
\hline 3773 & PRIEST RAPIDS-RIVER & & \\
\hline 3774 & PRIEST RAPIDS-RIVER & & \\
\hline 3775 & PRIEST RAPIDS-RIVER & & \\
\hline 3776 & PRIEST RAPIDS-RIVER & & \\
\hline 3777 & RICH.PMPHS HRM 46.4 & & \\
\hline 3778 & RICH.PMPHS HRM 46.4 & & \\
\hline 3779 & RICH.PMPHS HRM 46.4 & & \\
\hline 3780 & RICHLAND SAN PLANT & & \\
\hline 3781 & RICHLAND SAN PLANT & & \\
\hline 3782 & 300 AREA & & \\
\hline 3783 & 300 AREA & & \\
\hline 3784 & PRIEST RAPIDS-RIVER & & \\
\hline 3785 & PRIEST RAPIDS-RIVER & & \\
\hline 3786 & 100 D RESERVOIR & & \\
\hline 3787 & 100 D RESERVOIR & & \\
\hline 3788 & 100 D RESERVOIR & & \\
\hline 3789 & 300 AREA & & \\
\hline 3790 & PRIEST RAPIDS-RIVER & & \\
\hline 3791 & RICHLAND SAN PLANT & & \\
\hline 3792 & RICHLAND SAN PLANT & & \\
\hline 3793 & 300 AREA & & \\
\hline 3794 & 300 AREA & & \\
\hline 3795 & 300 AREA & & \\
\hline 3796 & 300 AREA & & \\
\hline 3797 & PRIEST RAPIDS-RIVER & & \\
\hline
\end{tabular}




\begin{tabular}{|c|c|c|c|}
\hline & $\mathrm{Z}$ & $\overline{\mathrm{AA}}$ & $\mathrm{AB}$ \\
\hline 1 & SAMP_SITE_NAME & WELL_NAME & SAMPLE_LOCATION \\
\hline 3798 & PRIEST RAPIDS-RIVER & & \\
\hline 3799 & PRIEST RAPIDS-RIVER & & \\
\hline 3800 & PRIEST RAPIDS-RIVER & & \\
\hline 3801 & PRIEST RAPIDS-RIVER & & \\
\hline 3802 & PRIEST RAPIDS-RIVER & & \\
\hline 3803 & PRIEST RAPIDS-RIVER & & \\
\hline 3804 & RICH.PMPHS HRM 46.4 & & \\
\hline 3805 & RICH.PMPHS HRM 46.4 & & \\
\hline 3806 & RICH.PMPHS HRM 46.4 & & \\
\hline 3807 & B POND NORTH SIDE & & \\
\hline 3808 & B POND NORTH SIDE & & \\
\hline 3809 & B POND NORTH SIDE & & \\
\hline 3810 & FFTF POND & & \\
\hline 3811 & FFTF POND & & \\
\hline 3812 & GABLE POND N END & & \\
\hline 3813 & GABLE POND N END & & \\
\hline 3814 & GABLE POND N END & & \\
\hline 3815 & WEST LAKE & & \\
\hline 3816 & WEST LAKE & & \\
\hline 3817 & WEST LAKE & & \\
\hline 3818 & FIRING RANGE & & \\
\hline 3819 & FIRING RANGE & & \\
\hline 3820 & FIRING RANGE & & \\
\hline 3821 & OBSEVATORY-RTLSNK MT & & \\
\hline 3822 & OBSEVATORY-RTLSNK MT & & \\
\hline 3823 & 300 AREA & & \\
\hline 3824 & 300 AREA & & \\
\hline 3825 & PRIEST RAPIDS-RIVER & & \\
\hline 3826 & PRIEST RAPIDS-RIVER & & \\
\hline 3827 & FFTF & & \\
\hline 3828 & FFTF & & \\
\hline 3829 & FFTF & & \\
\hline 3830 & RICHLAND SAN PLANT & & \\
\hline 3831 & RICHLAND SAN PLANT & & \\
\hline 3832 & 300 AREA & & \\
\hline 3833 & 300 AREA & & \\
\hline 3834 & PRIEST RAPIDS-RIVER & & \\
\hline 3835 & PRIEST RAPIDS-RIVER & & \\
\hline 3836 & PRIEST RAPIDS-RIVER & & \\
\hline 3837 & PRIEST RAPIDS-RIVER & & \\
\hline 3838 & PRIEST RAPIDS-RIVER & & \\
\hline 3839 & RICH.PMPHS HRM 46.4 & & \\
\hline 3840 & RICH.PMPHS HRM 46.4 & & \\
\hline 3841 & RICH.PMPHS HRM 46.4 & & \\
\hline 3842 & YAKIMA BARRICADE & & \\
\hline 3843 & YAKIMA BARRICADE & & \\
\hline 3844 & YAKIMA BARRICADE & & \\
\hline 3845 & 300 AREA & & \\
\hline 3846 & 300 AREA & & \\
\hline 3847 & PRIEST RAPIDS-RIVER & & \\
\hline 3848 & PRIEST RAPIDS-RIVER & & \\
\hline 3849 & 100 D AREA & & \\
\hline
\end{tabular}




\begin{tabular}{|c|c|c|c|}
\hline & \multicolumn{1}{|c|}{$Z$} & A & AB \\
\hline 1 & SAMP_SITE_NAME & WELL_NAME & SAMPLE_LOCATION \\
\hline 3850 & 100 D AREA & & \\
\hline 3851 & 100 D AREA & & \\
\hline 3852 & 300 AREA & & \\
\hline 3853 & 300 AREA & & \\
\hline 3854 & 300 AREA & & \\
\hline 3855 & LOWER REACH RIVER & & \\
\hline 3856 & LOWER REACH RIVER & & \\
\hline 3857 & LOWER REACH RIVER & & \\
\hline 3858 & UPPER REACH RIVER & & \\
\hline 3859 & UPPER REACH RIVER & & \\
\hline 3860 & UPPER REACH RIVER & & \\
\hline 3861 & 300 AREA & & \\
\hline 3862 & 300 AREA & & \\
\hline 3863 & PRIEST RAPIDS-RIVER & & \\
\hline 3864 & PRIEST RAPIDS-RIVER & & \\
\hline 3865 & PRIEST RAPIDS-RIVER & & \\
\hline 3866 & PRIEST RAPIDS-RIVER & & \\
\hline 3867 & PRIEST RAPIDS-RIVER & & \\
\hline 3868 & RICH.PMPHS HRM 46.4 & & \\
\hline 3869 & RICH.PMPHS HRM 46.4 & & \\
\hline 3870 & RICH.PMPHS HRM 46.4 & & \\
\hline 3871 & RICHLAND SAN PLANT & & \\
\hline 3872 & RICHLAND SAN PLANT & & \\
\hline 3873 & 300 AREA & & \\
\hline 3874 & 300 AREA & & \\
\hline 3875 & PRIEST RAPIDS-RIVER & & \\
\hline 3876 & PRIEST RAPIDS-RIVER & & \\
\hline 3877 & 300 AREA & & \\
\hline 3878 & 300 AREA & & \\
\hline 3879 & 300 AREA & & \\
\hline 3880 & 300 AREA & & \\
\hline 3881 & 300 AREA & & \\
\hline 3882 & PRIEST RAPIDS-RIVER & & \\
\hline 3883 & PRIEST RAPIDS-RIVER & & \\
\hline 3884 & PRIEST RAPIDS-RIVER & & \\
\hline 3885 & PRIEST RAPIDS-RIVER & & \\
\hline 3886 & PRIEST RAPIDS-RIVER & & \\
\hline 3887 & PRIEST RAPIDS-RIVER & & \\
\hline 3888 & PRIEST RAPIDS-RIVER & & \\
\hline 3889 & PRIEST RAPIDS-RIVER & & \\
\hline 3890 & RICH.PMPHS HRM 46.4 & & \\
\hline 3891 & RICH.PMPHS HRM 46.4 & & \\
\hline 3892 & RICH.PMPHS HRM 46.4 & & \\
\hline 3893 & RICHLAND SAN PLANT & & \\
\hline 3894 & RICHLAND SAN PLANT & & \\
\hline 3895 & 100 B AREA-RIVER & & \\
\hline 3896 & 100 B AREA-RIVER & & \\
\hline 3897 & 100 B AREA-RIVER & & \\
\hline 3898 & B POND NORTH SIDE & & \\
\hline 3899 & P POND NORTH SIDE & & \\
\hline 3900 & B POND NORTH SIDE & & \\
\hline 3901 & FFTF POND & & \\
\hline
\end{tabular}




\begin{tabular}{|c|c|c|c|}
\hline & $\bar{Z}$ & $\mathrm{AA}$ & $A B$ \\
\hline 1 & SAMP_SITE_NAME & WELL_NAME & SAMPLE_LOCATION \\
\hline 3902 & FFTF POND & & \\
\hline 3903 & GABLE POND N END & & \\
\hline 3904 & GABLE POND N END & & \\
\hline 3905 & GABLE POND N END & & \\
\hline 3906 & WEST LAKE & & \\
\hline 3907 & WEST LAKE & & \\
\hline 3908 & WEST LAKE & & \\
\hline 3909 & FIRING RANGE & & \\
\hline 3910 & FIRING RANGE & & \\
\hline 3911 & FIRING RANGE & & \\
\hline 3912 & OBSEVATORY-RTLSNK MT & & \\
\hline 3913 & OBSEVATORY-RTLSNK MT & & \\
\hline 3914 & 300 AREA & & \\
\hline 3915 & 300 AREA & & \\
\hline 3916 & PRIEST RAPIDS-RIVER & & \\
\hline 3917 & PRIEST RAPIDS-RIVER & & \\
\hline 3918 & FFTF & & \\
\hline 3919 & FFTF & & \\
\hline 3920 & FFTF & & \\
\hline 3921 & 300 AREA & & \\
\hline 3922. & 300 AREA & & \\
\hline 3923 & PRIEST RAPIDS-RIVER & & \\
\hline 3924 & PRIEST RAPIDS-RIVER & & \\
\hline 3925 & PRIEST RAPIDS-RIVER & & \\
\hline 3926 & PRIEST RAPIDS-RIVER & & \\
\hline 3927 & PRIEST RAPIDS-RIVER & & \\
\hline 3928 & RICH.PMPHS HRM 46.4 & & \\
\hline 3929 & RICH.PMPHS HRM 46.4 & & \\
\hline 3930 & RICH.PMPHS HRM 46.4 & & \\
\hline 3931 & RICHLAND SAN PLANT & & \\
\hline 3932 & RICHLAND SAN PLANT & & \\
\hline 3933 & YAKIMA BARRICADE & & \\
\hline 3934 & YAKIMA BARRICADE & & \\
\hline 3935 & YAKIMA BARRICADE & & \\
\hline 3936 & 300 AREA & & \\
\hline 3937 & 300 AREA & & \\
\hline 3938 & PRIEST RAPIDS-RIVER & & \\
\hline 3939 & PRIEST RAPIDS-RIVER & & \\
\hline 3940 & 100 D AREA & & \\
\hline 3941. & 100 D AREA & & \\
\hline 3942 & 100 D AREA & & \\
\hline 3943 & 300 AREA & & \\
\hline 3944 & 300 AREA & & \\
\hline 3945 & 300 AREA & & \\
\hline 3946 & 300 AREA & & \\
\hline 3947 & 300 AREA & & \\
\hline 3948 & PRIEST RAPIDS-RIVER & & \\
\hline 3949 & PRIEST RAPIDS-RIVER & & \\
\hline 3950 & PRIEST RAPIDS-RIVER & & \\
\hline 3951 & PRIEST RAPIDS-RIVER & & \\
\hline 3952 & PRIEST RAPIDS-RIVER & & \\
\hline 3953 & RICH.PMPHS HRM 46.4 & & \\
\hline
\end{tabular}




\begin{tabular}{|c|c|c|c|}
\hline & $\mathrm{Z}$ & $\overline{\mathrm{AA}}$ & $\mathrm{AB}$ \\
\hline 1 & SAMP_SITE_NAME & WELL_NAME & SAMPLE_LOCATION \\
\hline 3954 & RICH.PMPHS HRM 46.4 & & \\
\hline 3955 & RICH.PMPHS HRM 46.4 & & \\
\hline 3956 & RICHLAND SAN PLANT & & \\
\hline 3957 & RICHLAND SAN PLANT & & \\
\hline 3958 & 300 AREA & & \\
\hline 3959 & 300 AREA & & \\
\hline 3960 & PRIEST RAPIDS-RIVER & & \\
\hline 3961 & PRIEST RAPIDS-RIVER & & \\
\hline 3962 & 300 AREA & & \\
\hline 3963 & 300 AREA & & \\
\hline 3964 & 300 AREA & & \\
\hline 3965 & 300 AREA & & \\
\hline 3966 & 300 AREA & & \\
\hline 3967 & PRIEST RAPIDS-RIVER & & \\
\hline 3968 & PRIEST RAPIDS-RIVER & & \\
\hline 3969 & PRIEST RAPIDS-RIVER & & \\
\hline 3970 & PRIEST RAPIDS-RIVER & & \\
\hline 3971 & PRIEST RAPIDS-RIVER & & \\
\hline 3972 & PRIEST RAPIDS-RIVER & & \\
\hline 3973 & PRIEST RAPIDS-RIVER & & \\
\hline 3974 & PRIEST RAPIDS-RIVER & & \\
\hline 3975 & RICH.PMPHS HRM 46.4 & & \\
\hline 3976 & RICH.PMPHS HRM 46.4 & & \\
\hline 3977 & RICH.PMPHS HRM 46.4 & & \\
\hline 3978 & 100 B AREA-RIVER & & \\
\hline 3979 & 100 B AREA-RIVER & & \\
\hline 3980 & 100 B AREA-RIVER & & \\
\hline 3981 & 300 AREA & & \\
\hline 3982 & 300 AREA & & \\
\hline 3983 & PRIEST RAPIDS-RIVER & & \\
\hline 3984 & PRIEST RAPIDS-RIVER & & \\
\hline 3985 & FIRING RANGE & & \\
\hline 3986 & FIRING RANGE & & \\
\hline 3987 & FIRING RANGE & & \\
\hline 3988 & OBSEVATORY-RTLSNK MT & & \\
\hline 3989 & OBSEVATORY-RTLSNK MT & & \\
\hline 3990 & 300 AREA & & \\
\hline 3991 & 300 AREA & & \\
\hline 3992 & PRIEST RAPIDS-RIVER & & \\
\hline 3993 & PRIEST RAPIDS-RIVER & & \\
\hline 3994 & PRIEST RAPIDS-RIVER & & \\
\hline 3995 & PRIEST RAPIDS-RIVER & & \\
\hline 3996 & PRIEST RAPIDS-RIVER & & \\
\hline 3997 & RICH.PMPHS HRM 46.4 & & \\
\hline 3998 & RICH.PMPHS HRM 46.4 & & \\
\hline 3999 & RICH.PMPHS HRM 46.4 & & \\
\hline 4000 & B POND NORTH SIDE & & \\
\hline 4001 & B POND NORTH SIDE & & \\
\hline 4002 & B POND NORTH SIDE & & \\
\hline 4003 & FFTF POND & & \\
\hline 4004 & FFTF POND & & \\
\hline 4005 & GABLE POND & & \\
\hline
\end{tabular}




\begin{tabular}{|c|l|l|l|}
\hline \multicolumn{1}{|c|}{$Z$} & A & AB \\
\hline 1 & SAMP_SITE_NAME & WELL_NAME & SAMPLE_LOCATION \\
\hline 4006 & GABLE POND N END & & \\
\hline 4007 & GABLE POND N END & & \\
\hline 4008 & WEST LAKE & & \\
\hline 4009 & WEST LAKE & & \\
\hline 4010 & WEST LAKE & & \\
\hline 4011 & 300 AREA & & \\
\hline 4012 & 300 AREA & & \\
\hline 4013 & PRIEST RAPIDS-RIVER & & \\
\hline 4014 & PRIEST RAPIDS-RIVER & & \\
\hline 4015 & FFTF & & \\
\hline 4016 & FFTF & & \\
\hline 4017 & FFTF & & \\
\hline 4018 & 300 AREA & & \\
\hline 4019 & 300 AREA & & \\
\hline 4020 & PRIEST RAPIDS-RIVER & & \\
\hline 4021 & PRIEST RAPIDS-RIVER & & \\
\hline 4022 & PRIEST RAPIDS-RIVER & & \\
\hline 4023 & PRIEST RAPIDS-RIVER & & \\
\hline 4024 & PRIEST RAPIDS-RIVER & & \\
\hline 4025 & RICH.PMPHS HRM 46.4 & & \\
\hline 4026 & RICH.PMPHS HRM 46.4 & & \\
\hline 4027 & RICH.PMPHS HRM 46.4 & & \\
\hline 4028 & YAKIMA BARRICADE & & \\
\hline 4029 & YAKIMA BARRICADE & & \\
\hline 4030 & YAKIMA BARRICADE & & \\
\hline 4031 & 300 AREA & & \\
\hline 4032 & 300 AREA & & \\
\hline 4033 & 300 AREA & & \\
\hline 4034 & 100 D AREA & & \\
\hline 4035 & 100 D AREA & & \\
\hline 4036 & 100 D AREA & & \\
\hline 4037 & 300 AREA & & \\
\hline 4038 & 300 AREA & & \\
\hline 4039 & PRIEST RAPIDS-RIVER & & \\
\hline 4040 & PRIEST RAPIDS-RIVER & & \\
\hline 4041 & LOWER REACH RIVER & & \\
\hline 4042 & LOWER REACH RIVER & & \\
\hline 4043 & LOWER REACH RIVER & & \\
\hline 4044 & UPPER REACH RIVER & & \\
\hline 4045 & UPPER REACH RIVER & & \\
\hline 4046 & UPPER REACH RIVER & & \\
\hline 4047 & 300 AREA & & \\
\hline 4048 & 300 AREA & & \\
\hline 4049 & 300 AREA & & \\
\hline 4050 & 300 AREA & & \\
\hline 4051 & 300 AREA & & \\
\hline 4052 & 300 AREA & & \\
\hline 4053 & PRIEST RAPIDS-RIVER & & \\
\hline 4054 & PRIEST RAPIDS-RIVER & & \\
\hline 4055 & PRIEST RAPIDS-RIVER & & \\
\hline 4056 & PRIEST RAPIDS-RIVER & & \\
\hline 4057 & PRIEST RAPIDS-RIVER & & \\
\hline
\end{tabular}




\begin{tabular}{|c|c|c|c|}
\hline & $\mathrm{Z}$ & $\overline{\mathrm{AA}}$ & $\mathrm{AB}$ \\
\hline 1 & SAMP_SITE_NAME & WELL_NAME & SAMPLE_LOCATION \\
\hline 4058 & PRIEST RAPIDS-RIVER & & \\
\hline 4059 & PRIEST RAPIDS-RIVER & & \\
\hline 4060 & PRIEST RAPIDS-RIVER & & \\
\hline 4061 & PRIEST RAPIDS-RIVER & & \\
\hline 4062 & RICH.PMPHS HRM 46.4 & & \\
\hline 4063 & RICH.PMPHS HRM 46.4 & & \\
\hline 4064 & RICH.PMPHS HRM 46.4 & & \\
\hline 4065 & 100 B AREA-RIVER & & \\
\hline 4066 & 100 B AREA-RIVER & & \\
\hline 4067 & 100 B AREA-RIVER & & \\
\hline 4068 & 300 AREA & & \\
\hline 4069 & 300 AREA & & \\
\hline 4070 & PRIEST RAPIDS-RIVER & & \\
\hline 4071 & PRIEST RAPIDS-RIVER & & \\
\hline 4072 & FIRING RANGE & & \\
\hline 4073 & FIRING RANGE & & \\
\hline 4074 & FIRING RANGE & & \\
\hline 4075 & OBSEVATORY-RTLSNK MT & & \\
\hline 4076 & OBSEVATORY-RTLSNK MT & & \\
\hline 4077 & 300 AREA & & \\
\hline 4078 & 300 AREA & & \\
\hline 4079 & PRIEST RAPIDS-RIVER & & \\
\hline 4080 & PRIEST RAPIDS-RIVER & & \\
\hline 4081 & PRIEST RAPIDS-RIVER & & \\
\hline 4082 & PRIEST RAPIDS-RIVER & & \\
\hline 4083 & PRIEST RAPIDS-RIVER & & \\
\hline 4084 & RICH.PMPHS HRM 46.4 & & \\
\hline 4085 & RICH.PMPHS HRM 46.4 & & \\
\hline 4086 & RICH.PMPHS HRM 46.4 & & \\
\hline 4087 & B POND NORTH SIDE & & \\
\hline 4088 & B POND NORTH SIDE & & \\
\hline 4089 & B POND NORTH SIDE & & \\
\hline 4090 & FFTF POND & & \\
\hline 4091 & FFTF POND & & \\
\hline 4092 & GABLE POND N END & & \\
\hline 4093 & GABLE POND N END & & \\
\hline 4094 & GABLE POND N END & & \\
\hline 4095 & WEST LAKE & & \\
\hline 4096 & WEST LAKE & & \\
\hline 4097 & WEST LAKE & & \\
\hline 4098 & ALE HDQTRS & & \\
\hline 4099 & ALE HDQTRS & & \\
\hline 4100 & ALE HDQTRS & & \\
\hline 4101 & 300 AREA & & \\
\hline 4102 & 300 AREA & & \\
\hline 4103 & PRIEST RAPIDS-RIVER & & \\
\hline 4104 & PRIEST RAPIDS-RIVER & & \\
\hline 4105 & FFTF & & \\
\hline 4106 & FFTF & & \\
\hline 4107 & FFTF & & \\
\hline 4108 & 300 AREA & & \\
\hline 4109 & 300 AREA & & \\
\hline
\end{tabular}




\begin{tabular}{|c|c|c|c|}
\hline & $\mathrm{Z}$ & $\overline{\mathrm{AA}}$ & $\mathrm{AB}$ \\
\hline 1 & SAMP_SITE_NAME & WELL_NAME & SAMPLE_LOCATION \\
\hline 4110 & PRIEST RAPIDS-RIVER & & \\
\hline 4111 & PRIEST RAPIDS-RIVER & & \\
\hline 4112 & PRIEST RAPIDS-RIVER & & \\
\hline 4113 & PRIEST RAPIDS-RIVER & & \\
\hline 4114 & PRIEST RAPIDS-RIVER & & \\
\hline 4115 & RICH.PMPHS HRM 46.4 & & \\
\hline 4116 & RICH.PMPHS HRM 46.4 & & \\
\hline 4117 & RICH.PMPHS HRM 46.4 & & \\
\hline 4118 & YAKIMA BARRICADE & & \\
\hline 4119 & YAKIMA BARRICADE & & \\
\hline 4120 & YAKIMA BARRICADE & & \\
\hline 4121 & 300 AREA & & \\
\hline 4122 & 300 AREA & & \\
\hline 4123 & 300 AREA & & \\
\hline 4124 & 100 D AREA & & \\
\hline 4125 & 100 D AREA & & \\
\hline 4126 & 100 D AREA & & \\
\hline 4127 & 300 AREA & & \\
\hline 4128 & 300 AREA & & \\
\hline 4129 & PRIEST RAPIDS-RIVER & & \\
\hline 4130 & PRIEST RAPIDS-RIVER & & \\
\hline 4131 & 300 AREA & & \\
\hline 4132 & 300 AREA & & \\
\hline 4133 & 300 AREA & & \\
\hline 4134 & 300 AREA & & \\
\hline 4135 & 300 AREA & & \\
\hline 4136 & 300 AREA & & \\
\hline \begin{tabular}{|l|l|}
4137 & -1 \\
\end{tabular} & PRIEST RAPIDS-RIVER & & \\
\hline 4138 & PRIEST RAPIDS-RIVER & & \\
\hline 4139 & PRIEST RAPIDS-RIVER & & \\
\hline 4140 & PRIEST RAPIDS-RIVER & & \\
\hline 4141 & PRIEST RAPIDS-RIVER & & \\
\hline 4142 & PRIEST RAPIDS-RIVER & & \\
\hline 4143 & PRIEST RAPIDS-RIVER & & \\
\hline 4144 & PRIEST RAPIDS-RIVER & & \\
\hline 4145 & PRIEST RAPIDS-RIVER & & \\
\hline 4146 & RICH.PMPHS HRM 46.4 & & \\
\hline 4147 & RICH.PMPHS HRM 46.4 & & \\
\hline 4148 & RICH.PMPHS HRM 46.4 & & \\
\hline 4149 & 100 B AREA-RIVER & & \\
\hline 4150 & 100 B AREA-RIVER & & \\
\hline 4151 & 100 B AREA-RIVER & & \\
\hline 4152 & 300 AREA & & \\
\hline 4153 & 300 AREA & & \\
\hline 4154 & PRIEST RAPIDS-RIVER & & \\
\hline 4155 & PRIEST RAPIDS-RIVER & & \\
\hline 4156 & B POND NORTH SIDE & & \\
\hline 4157 & B POND NORTH SIDE & & \\
\hline 4158 & B POND NORTH SIDE & & \\
\hline 4159 & OBSEVATORY-RTLSNK MT & & \\
\hline 4160 & OBSEVATORY-RTLSNK MT & & \\
\hline 4161 & 300 AREA & & \\
\hline
\end{tabular}




\begin{tabular}{|c|c|c|c|}
\hline & $\bar{Z}$ & $\overline{\mathrm{AA}}$ & $\overline{A B}$ \\
\hline 1 & SAMP_SITE_NAME & WELL_NAME & SAMPLE_LOCATION \\
\hline 4162 & 300 AREA & & \\
\hline 4163 & PRIEST RAPIDS-RIVER & & \\
\hline 4164 & PRIEST RAPIDS-RIVER & & \\
\hline 4165 & PRIEST RAPIDS-RIVER & & \\
\hline 4166 & PRIEST RAPIDS-RIVER & & \\
\hline 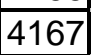 & PRIEST RAPIDS-RIVER & & \\
\hline 4168 & RICH.PMPHS HRM 46.4 & & \\
\hline 4169 & RICH.PMPHS HRM 46.4 & & \\
\hline 4170 & RICH.PMPHS HRM 46.4 & & \\
\hline 4171 & ALE HDQTRS & & \\
\hline 4172 & ALE HDQTRS & & \\
\hline 4173 & ALE HDQTRS & & \\
\hline 4174 & FIRING RANGE & & \\
\hline 4175 & FIRING RANGE & & \\
\hline 4176 & FIRING RANGE & & \\
\hline 4177 & B POND NORTH SIDE & & \\
\hline 4178 & B POND NORTH SIDE & & \\
\hline 4179 & B POND NORTH SIDE & & \\
\hline 4180 & FFTF POND & & \\
\hline 4181 & FFTF POND & & \\
\hline 4182 & GABLE POND N END & & \\
\hline 4183 & GABLE POND N END & & \\
\hline 4184 & GABLE POND N END & & \\
\hline 4185 & WEST LAKE & & \\
\hline 4186 & WEST LAKE & & \\
\hline \begin{tabular}{|l|l|}
4187 & - -1 \\
\end{tabular} & WEST LAKE & & \\
\hline 4188 & 300 AREA & & \\
\hline 4189 & 300 AREA & & \\
\hline 4190 & PRIEST RAPIDS-RIVER & & \\
\hline 4191 & PRIEST RAPIDS-RIVER & & \\
\hline 4192 & FFTF & & \\
\hline 4193 & FFTF & & \\
\hline 4194 & FFTF & & \\
\hline 4195 & 300 AREA & & \\
\hline 4196 & 300 AREA & & \\
\hline 4197 & PRIEST RAPIDS-RIVER & & \\
\hline 4198 & PRIEST RAPIDS-RIVER & & \\
\hline 4199 & PRIEST RAPIDS-RIVER & & \\
\hline 4200 & PRIEST RAPIDS-RIVER & & \\
\hline 4201 & PRIEST RAPIDS-RIVER & & \\
\hline 4202 & RICH.PMPHS HRM 46.4 & & \\
\hline 4203 & RICH.PMPHS HRM 46.4 & & \\
\hline 4204 & RICH.PMPHS HRM 46.4 & & \\
\hline 4205 & YAKIMA BARRICADE & & \\
\hline 4206 & YAKIMA BARRICADE & & \\
\hline 4207 & YAKIMA BARRICADE & & \\
\hline 4208 & 300 AREA & & \\
\hline 4209 & 300 AREA & & \\
\hline 4210 & 300 AREA & & \\
\hline 4211 & 100 D AREA & & \\
\hline 4212 & 100 D AREA & & \\
\hline 4213 & 100 D AREA & & \\
\hline
\end{tabular}




\begin{tabular}{|c|c|c|c|}
\hline & $\mathrm{Z}$ & $\overline{\mathrm{AA}}$ & $\mathrm{AB}$ \\
\hline 1 & SAMP_SITE_NAME & WELL_NAME & SAMPLE_LOCATION \\
\hline 4214 & 300 AREA & & \\
\hline 4215 & 300 AREA & & \\
\hline 4216 & PRIEST RAPIDS-RIVER & & \\
\hline \begin{tabular}{|l|l|}
4217 \\
\end{tabular} & PRIEST RAPIDS-RIVER & & \\
\hline 4218 & LOWER REACH RIVER & & \\
\hline 4219 & LOWER REACH RIVER & & \\
\hline 4220 & LOWER REACH RIVER & & \\
\hline 4221 & UPPER REACH RIVER & & \\
\hline 4222 & UPPER REACH RIVER & & \\
\hline 4223 & UPPER REACH RIVER & & \\
\hline 4224 & 300 AREA & & \\
\hline 4225 & 300 AREA & & \\
\hline 4226 & 300 AREA & & \\
\hline 4227 & 300 AREA & & \\
\hline 4228 & 300 AREA & & \\
\hline 4229 & 300 AREA & & \\
\hline 4230 & PRIEST RAPIDS-RIVER & & \\
\hline 4231 & PRIEST RAPIDS-RIVER & & \\
\hline 4232 & PRIEST RAPIDS-RIVER & & \\
\hline 4233 & PRIEST RAPIDS-RIVER & & \\
\hline 4234 & PRIEST RAPIDS-RIVER & & \\
\hline 4235 & PRIEST RAPIDS-RIVER & & \\
\hline 4236 & PRIEST RAPIDS-RIVER & & \\
\hline 4237 & PRIEST RAPIDS-RIVER & & \\
\hline 4238 & PRIEST RAPIDS-RIVER & & \\
\hline 4239 & RICH.PMPHS HRM 46.4 & & \\
\hline 4240 & RICH.PMPHS HRM 46.4 & & \\
\hline 4241 & RICH.PMPHS HRM 46.4 & & \\
\hline 4242 & 100 B AREA-RIVER & & \\
\hline 4243 & 100 B AREA-RIVER & & \\
\hline 4244 & 100 B AREA-RIVER & & \\
\hline 4245 & 300 AREA & & \\
\hline 4246 & 300 AREA & & \\
\hline \begin{tabular}{|l|l|}
4247 \\
\end{tabular} & PRIEST RAPIDS-RIVER & & \\
\hline 4248 & PRIEST RAPIDS-RIVER & & \\
\hline 4249 & ALE HDQTRS & & \\
\hline 4250 & ALE HDQTRS & & \\
\hline 4251 & ALE HDQTRS & & \\
\hline 4252 & FIRING RANGE & & \\
\hline 4253 & FIRING RANGE & & \\
\hline 4254 & FIRING RANGE & & \\
\hline 4255 & OBSEVATORY-RTLSNK MT & & \\
\hline 4256 & OBSEVATORY-RTLSNK MT & & \\
\hline 4257 & 300 AREA & & \\
\hline 4258 & 300 AREA & & \\
\hline 4259 & PRIEST RAPIDS-RIVER & & \\
\hline 4260 & PRIEST RAPIDS-RIVER & & \\
\hline 4261 & PRIEST RAPIDS-RIVER & & \\
\hline 4262 & PRIEST RAPIDS-RIVER & & \\
\hline 4263 & PRIEST RAPIDS-RIVER & & \\
\hline 4264 & RICH.PMPHS HRM 46.4 & & \\
\hline 4265 & RICH.PMPHS HRM 46.4 & & \\
\hline
\end{tabular}




\begin{tabular}{|c|l|l|l|}
\hline & \multicolumn{1}{|c|}{$Z$} & A & AB \\
\hline 1 & SAMP_SITE_NAME & WELL_NAME & SAMPLE_LOCATION \\
\hline 4266 & RICH.PMPHS HRM 46.4 & & \\
\hline 4267 & 100 B AREA-RIVER & & \\
\hline 4268 & B POND NORTH SIDE & & \\
\hline 4269 & B POND NORTH SIDE & & \\
\hline 4270 & B POND NORTH SIDE & & \\
\hline 4271 & FFTF POND & & \\
\hline 4272 & FFTF POND & & \\
\hline 4273 & GABLE POND N END & & \\
\hline 4274 & GABLE POND N END & & \\
\hline 4275 & GABLE POND N END & & \\
\hline 4276 & WEST LAKE & & \\
\hline 4277 & WEST LAKE & & \\
\hline 4278 & WEST LAKE & & \\
\hline 4279 & 300 AREA & & \\
\hline 4280 & 300 AREA & & \\
\hline 4281 & PRIEST RAPIDS-RIVER & & \\
\hline 4282 & PRIEST RAPIDS-RIVER & & \\
\hline 4283 & FFTF & & \\
\hline 4284 & FFTF & & \\
\hline 4285 & FFTF & & \\
\hline 4286 & 300 AREA & & \\
\hline 4287 & 300 AREA & & \\
\hline 4288 & PRIEST RAPIDS-RIVER & & \\
\hline 4289 & PRIEST RAPIDS-RIVER & & \\
\hline 4290 & PRIEST RAPIDS-RIVER & & \\
\hline 4291 & PRIEST RAPIDS-RIVER & & \\
\hline 4292 & PRIEST RAPIDS-RIVER & & \\
\hline 4293 & RICH.PMPHS HRM 46.4 & & \\
\hline 4294 & RICH.PMPHS HRM 46.4 & & \\
\hline 4295 & RICH.PMPHS HRM 46.4 & & \\
\hline 4296 & YAKIMA BARRICADE & & \\
\hline 4297 & YAKIMA BARRICADE & & \\
\hline 4298 & YAKIMA BARRICADE & & \\
\hline 4299 & 300 AREA & & \\
\hline 4300 & 300 AREA & & \\
\hline 4301 & 300 AREA & & \\
\hline 4302 & 100 D AREA & & \\
\hline 4303 & 100 D AREA & & \\
\hline 4304 & 100 D AREA & & \\
\hline 4305 & 300 AREA & & \\
\hline 4306 & 300 AREA & & \\
\hline 4307 & PRIEST RAPIDS-RIVER & & \\
\hline 4308 & PRIEST RAPIDS-RIVER & & \\
\hline 4309 & 300 AREA & & \\
\hline 4310 & 300 AREA & & \\
\hline 4311 & 300 AREA & & \\
\hline 4312 & 300 AREA & & \\
\hline 4313 & 300 AREA & & \\
\hline 4314 & PRIEST RAPIDS-RIVER & & \\
\hline 4315 & PRIEST RAPIDS-RIVER & & \\
\hline 4316 & PRIEST RAPIDS-RIVER & & \\
\hline 4317 & PRIEST RAPIDS-RIVER & & \\
\hline
\end{tabular}




\begin{tabular}{|c|c|c|c|}
\hline & \multicolumn{1}{|c|}{$Z$} & A & AB \\
\hline 1 & SAMP_SITE_NAME & WELL_NAME & SAMPLE_LOCATION \\
\hline 4318 & PRIEST RAPIDS-RIVER & & \\
\hline 4319 & PRIEST RAPIDS-RIVER & & \\
\hline 4320 & PRIEST RAPIDS-RIVER & & \\
\hline 4321 & PRIEST RAPIDS-RIVER & & \\
\hline 4322 & RICH.PMPHS HRM 46.4 & & \\
\hline 4323 & RICH.PMPHS HRM 46.4 & & \\
\hline 4324 & RICH.PMPHS HRM 46.4 & & \\
\hline 4325 & 300 AREA & & \\
\hline 4326 & 300 AREA & & \\
\hline 4327 & 300 AREA & & \\
\hline 4328 & 300 AREA & & \\
\hline 4329 & 300 AREA & & \\
\hline 4330 & PRIEST RAPIDS-RIVER & & \\
\hline 4331 & PRIEST RAPIDS-RIVER & & \\
\hline 4332 & 300 AREA & & \\
\hline 4333 & 300 AREA & & \\
\hline 4334 & PRIEST RAPIDS-RIVER & & \\
\hline 4335 & PRIEST RAPIDS-RIVER & & \\
\hline 4336 & 300 AREA & & \\
\hline 4337 & 300 AREA & & \\
\hline 4338 & PRIEST RAPIDS-RIVER & & \\
\hline 4339 & PRIEST RAPIDS-RIVER & & \\
\hline 4340 & PRIEST RAPIDS-RIVER & & \\
\hline 4341 & PRIEST RAPIDS-RIVER & & \\
\hline 4342 & PRIEST RAPIDS-RIVER & & \\
\hline 4343 & RICH.PMPHS HRM 46.4 & & \\
\hline 4344 & RICH.PMPHS HRM 46.4 & & \\
\hline 4345 & RICH.PMPHS HRM 46.4 & & \\
\hline 4346 & 300 AREA & & \\
\hline 4347 & 300 AREA & & \\
\hline 4348 & PRIEST RAPIDS-RIVER & & \\
\hline 4349 & PRIEST RAPIDS-RIVER & & \\
\hline 4350 & RICH.PMPHS HRM 46.4 & & \\
\hline 4351 & ALE HDQTRS & & \\
\hline 4352 & ALE HDQTRS & & \\
\hline 4353 & ALE HDQTRS & & \\
\hline 4354 & FIRING RANGE & & \\
\hline 4355 & FIRING RANGE & & \\
\hline 4356 & FIRING RANGE & & \\
\hline 4357 & OBSEVATORY-RTLSNK MT & & \\
\hline 4358 & OBSEVATORY-RTLSNK MT & & \\
\hline 4359 & 300 AREA & & \\
\hline 4360 & 300 AREA & & \\
\hline 4361 & PRIEST RAPIDS-RIVER & & \\
\hline 4362 & PRIEST RAPIDS-RIVER & & \\
\hline 4363 & PRIEST RAPIDS-RIVER & & \\
\hline 4364 & PRIEST RAPIDS-RIVER & & \\
\hline 4365 & PRIEST RAPIDS-RIVER & & \\
\hline 4366 & RICH.PMPHS HRM 46.4 & & \\
\hline 4367 & RICH.PMPHS HRM 46.4 & & \\
\hline 4368 & P POND NORTH SIDE & & \\
\hline 4369 & P POND NORTH SIDE & & \\
\hline
\end{tabular}




\begin{tabular}{|c|l|l|l|}
\hline & \multicolumn{1}{|c|}{$Z$} & A & AB \\
\hline 1 & SAMP_SITE_NAME & WELL_NAME & SAMPLE_LOCATION \\
\hline 4370 & B POND NORTH SIDE & & \\
\hline 4371 & FFTF POND & & \\
\hline 4372 & FFTF POND & & \\
\hline 4373 & GABLE POND N END & & \\
\hline 4374 & GABLE POND N END & & \\
\hline 4375 & GABLE POND N END & & \\
\hline 4376 & WEST LAKE & & \\
\hline 4377 & WEST LAKE & & \\
\hline 4378 & WEST LAKE & & \\
\hline 4379 & FFTF & & \\
\hline 4380 & FFTF & & \\
\hline 4381 & FFTF & & \\
\hline 4382 & YAKIMA BARRICADE & & \\
\hline 4383 & YAKIMA BARRICADE & & \\
\hline 4384 & YAKIMA BARRICADE & & \\
\hline 4385 & 100 D AREA & & \\
\hline 4386 & 100 D AREA & & \\
\hline 4387 & 100 D AREA & & \\
\hline 4388 & 300 AREA & & \\
\hline 4389 & 300 AREA & & \\
\hline 4390 & PRIEST RAPIDS-RIVER & & \\
\hline 4391 & PRIEST RAPIDS-RIVER & & \\
\hline 4392 & PRIEST RAPIDS-RIVER & & \\
\hline 4393 & PRIEST RAPIDS-RIVER & & \\
\hline 4394 & PRIEST RAPIDS-RIVER & & \\
\hline 4395 & 300 AREA & & \\
\hline 4396 & 300 AREA & & \\
\hline 4397 & 300 AREA & & \\
\hline 4398 & 300 AREA & & \\
\hline 4399 & 300 AREA & & \\
\hline 4400 & 300 AREA & & \\
\hline 4401 & 300 AREA & & \\
\hline 4402 & 300 AREA & & \\
\hline 4403 & 300 AREA & & \\
\hline 4404 & PRIEST RAPIDS-RIVER & & \\
\hline 4405 & PRIEST RAPIDS-RIVER & & \\
\hline 4406 & PRIEST RAPIDS-RIVER & & \\
\hline 4407 & 100 B AREA-RIVER & & \\
\hline 4408 & 100 B AREA-RIVER & & \\
\hline 4409 & 100 B AREA-RIVER & & \\
\hline 4410 & PRIEST RAPIDS-RIVER & & \\
\hline 4411 & PRIEST RAPIDS-RIVER & & \\
\hline 4412 & PRIEST RAPIDS-RIVER & & \\
\hline 4413 & RICH.PMPHS HRM 46.4 & & \\
\hline 4414 & RICH.PMPHS HRM 46.4 & & \\
\hline 4415 & RICH.PMPHS HRM 46.4 & & \\
\hline 4416 & LOWER REACH RIVER & & \\
\hline 4417 & LOWER REACH RIVER & & \\
\hline 4418 & LOWER REACH RIVER & & \\
\hline 4419 & UPPER REACH RIVER & & \\
\hline 4420 & UPPER REACH RIVER & & \\
\hline 4421 & UPPER REACH RIVER & & \\
\hline
\end{tabular}




\begin{tabular}{|c|c|c|c|}
\hline & $\bar{Z}$ & $\overline{\mathrm{AA}}$ & $\overline{A B}$ \\
\hline 1 & SAMP_SITE_NAME & WELL_NAME & SAMPLE_LOCATION \\
\hline 4422 & 300 AREA & & \\
\hline 4423 & 300 AREA & & \\
\hline 4424 & PRIEST RAPIDS-RIVER & & \\
\hline 4425 & PRIEST RAPIDS-RIVER & & \\
\hline 4426 & 300 AREA & & \\
\hline 4427 & 300 AREA & & \\
\hline 4428 & PRIEST RAPIDS-RIVER & & \\
\hline 4429 & PRIEST RAPIDS-RIVER & & \\
\hline 4430 & PRIEST RAPIDS-RIVER & & \\
\hline 4431 & PRIEST RAPIDS-RIVER & & \\
\hline 4432 & PRIEST RAPIDS-RIVER & & \\
\hline 4433 & RICH.PMPHS HRM 46.4 & & \\
\hline 4434 & RICH.PMPHS HRM 46.4 & & \\
\hline 4435 & RICH.PMPHS HRM 46.4 & & \\
\hline 4436 & 300 AREA & & \\
\hline 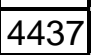 & 300 AREA & & \\
\hline 4438 & PRIEST RAPIDS-RIVER & & \\
\hline 4439 & PRIEST RAPIDS-RIVER & & \\
\hline 4440 & ALE HDQTRS & & \\
\hline 4441 & ALE HDQTRS & & \\
\hline 4442 & ALE HDQTRS & & \\
\hline 4443 & FIRING RANGE & & \\
\hline 4444 & FIRING RANGE & & \\
\hline 4445 & FIRING RANGE & & \\
\hline 4446 & OBSEVATORY-RTLSNK MT & & \\
\hline 4447 & OBSEVATORY-RTLSNK MT & & \\
\hline 4448 & OBSEVATORY-RTLSNK MT & & \\
\hline 4449 & 300 AREA & & \\
\hline 4450 & 300 AREA & & \\
\hline 4451 & PRIEST RAPIDS-RIVER & & \\
\hline 4452 & PRIEST RAPIDS-RIVER & & \\
\hline 4453 & B POND NORTH SIDE & & \\
\hline 4454 & B POND NORTH SIDE & & \\
\hline 4455 & B POND NORTH SIDE & & \\
\hline 4456 & FFTF POND & & \\
\hline 4457 & FFTF POND & & \\
\hline 4458 & GABLE POND N END & & \\
\hline 4459 & GABLE POND N END & & \\
\hline 4460 & GABLE POND N END & & \\
\hline 4461 & WEST LAKE & & \\
\hline 4462 & WEST LAKE & & \\
\hline 4463 & WEST LAKE & & \\
\hline 4464 & FFTF & & \\
\hline 4465 & FFTF & & \\
\hline 4466 & FFTF & & \\
\hline \begin{tabular}{|l|l|}
4467 & -1 \\
\end{tabular} & 100 B AREA-RIVER & & \\
\hline 4468 & 100 B AREA-RIVER & & \\
\hline 4469 & 100 B AREA-RIVER & & \\
\hline 4470 & 100 D AREA & & \\
\hline 4471 & YAKIMA BARRICADE & & \\
\hline 4472 & YAKIMA BARRICADE & & \\
\hline 4473 & YAKIMA BARRICADE & & \\
\hline
\end{tabular}




\begin{tabular}{|c|c|c|c|}
\hline & $\mathrm{Z}$ & $\overline{\mathrm{AA}}$ & $\mathrm{AB}$ \\
\hline 1 & SAMP_SITE_NAME & WELL_NAME & SAMPLE_LOCATION \\
\hline 4474 & PRIEST RAPIDS-RIVER & & \\
\hline 4475 & PRIEST RAPIDS-RIVER & & \\
\hline 4476 & PRIEST RAPIDS-RIVER & & \\
\hline 4477 & RICH.PMPHS HRM 46.4 & & \\
\hline 4478 & RICH.PMPHS HRM 46.4 & & \\
\hline 4479 & RICH.PMPHS HRM 46.4 & & \\
\hline 4480 & 100 D AREA & & \\
\hline 4481 & 300 AREA & & \\
\hline 4482 & 300 AREA & & \\
\hline 4483 & PRIEST RAPIDS-RIVER & & \\
\hline 4484 & PRIEST RAPIDS-RIVER & & \\
\hline 4485 & 300 AREA & & \\
\hline 4486 & 300 AREA & & \\
\hline \begin{tabular}{|l|l|}
4487 & -1 \\
\end{tabular} & PRIEST RAPIDS-RIVER & & \\
\hline 4488 & PRIEST RAPIDS-RIVER & & \\
\hline 4489 & 300 AREA & & \\
\hline 4490 & 300 AREA & & \\
\hline 4491 & 300 AREA & & \\
\hline 4492 & 300 AREA & & \\
\hline 4493 & 300 AREA & & \\
\hline 4494 & 300 AREA & & \\
\hline 4495 & PRIEST RAPIDS-RIVER & & \\
\hline 4496 & PRIEST RAPIDS-RIVER & & \\
\hline 4497 & PRIEST RAPIDS-RIVER & & \\
\hline 4498 & PRIEST RAPIDS-RIVER & & \\
\hline 4499 & PRIEST RAPIDS-RIVER & & \\
\hline 4500 & PRIEST RAPIDS-RIVER & & \\
\hline 4501 & PRIEST RAPIDS-RIVER & & \\
\hline 4502 & PRIEST RAPIDS-RIVER & & \\
\hline 4503 & RICH.PMPHS HRM 46.4 & & \\
\hline 4504 & RICH.PMPHS HRM 46.4 & & \\
\hline 4505 & RICH.PMPHS HRM 46.4 & & \\
\hline 4506 & 300 AREA & & \\
\hline \begin{tabular}{|l|l|}
4507 \\
\end{tabular} & 300 AREA & & \\
\hline 4508 & 300 AREA & & \\
\hline 4509 & 300 AREA & & \\
\hline 4510 & 300 AREA & & \\
\hline 4511 & PRIEST RAPIDS-RIVER & & \\
\hline 4512 & PRIEST RAPIDS-RIVER & & \\
\hline 4513 & PRIEST RAPIDS-RIVER & & \\
\hline 4514 & 300 AREA & & \\
\hline 4515 & 300 AREA & & \\
\hline 4516 & PRIEST RAPIDS-RIVER & & \\
\hline 4517 & PRIEST RAPIDS-RIVER & & \\
\hline 4518 & PRIEST RAPIDS-RIVER & & \\
\hline 4519 & PRIEST RAPIDS-RIVER & & \\
\hline 4520 & PRIEST RAPIDS-RIVER & & \\
\hline 4521 & RICH.PMPHS HRM 46.4 & & \\
\hline 4522 & RICH.PMPHS HRM 46.4 & & \\
\hline 4523 & RICH.PMPHS HRM 46.4 & & \\
\hline 4524 & ALE HDQTRS & & \\
\hline 4525 & ALE HDQTRS & & \\
\hline
\end{tabular}




\begin{tabular}{|c|l|l|l|}
\hline \multicolumn{1}{|c|}{$Z$} & AA & AB \\
\hline 1 & SAMP_SITE_NAME & WELL_NAME & SAMPLE_LOCATION \\
\hline 4526 & ALE HDQTRS & & \\
\hline 4527 & FIRING RANGE & & \\
\hline 4528 & FIRING RANGE & & \\
\hline 4529 & FIRING RANGE & & \\
\hline 4530 & 300 AREA & & \\
\hline 4531 & 300 AREA & & \\
\hline 4532 & PRIEST RAPIDS-RIVER & & \\
\hline 4533 & PRIEST RAPIDS-RIVER & & \\
\hline 4534 & B POND NORTH SIDE & & \\
\hline 4535 & B POND NORTH SIDE & & \\
\hline 4536 & B POND NORTH SIDE & & \\
\hline 4537 & FFTF POND & & \\
\hline 4538 & FFTF POND & & \\
\hline 4539 & GABLE POND N END & & \\
\hline 4540 & GABLE POND N END & & \\
\hline 4541 & GABLE POND N END & & \\
\hline 4542 & WEST LAKE & & \\
\hline 4543 & WEST LAKE & & \\
\hline 4544 & WEST LAKE & & \\
\hline 4545 & FFTF & & \\
\hline 4546 & FFTF & & \\
\hline 4547 & FFTF & & \\
\hline 4548 & 100 B AREA-RIVER & & \\
\hline 4549 & 100 B AREA-RIVER & & \\
\hline 4550 & 100 B AREA-RIVER & & \\
\hline 4551 & YAKIMA BARRICADE & & \\
\hline 4552 & YAKIMA BARRICADE & & \\
\hline 4553 & YAKIMA BARRICADE & & \\
\hline 4554 & OBSEVATORY-RTLSNK MT & & \\
\hline 4555 & OBSEVATORY-RTLSNK MT & & \\
\hline 4556 & OBSEVATORY-RTLSNK MT & & \\
\hline 4557 & 300 AREA & & \\
\hline 4558 & 300 AREA & & \\
\hline 4559 & PRIEST RAPIDS-RIVER & & \\
\hline 4560 & PRIEST RAPIDS-RIVER & & \\
\hline 4561 & 100 D AREA & & \\
\hline 4562 & 100 D AREA & & \\
\hline 4563 & 100 D AREA & & \\
\hline 4564 & PRIEST RAPIDS-RIVER & & \\
\hline 4565 & PRIEST RAPIDS-RIVER & & \\
\hline 4566 & PRIEST RAPIDS-RIVER & & \\
\hline 4567 & RICH.PMPHS HRM 46.4 & & \\
\hline 4568 & RICH.PMPHS HRM 46.4 & & \\
\hline 4569 & RICH.PMPHS HRM 46.4 & & \\
\hline 4570 & 300 AREA & & \\
\hline 4571 & 300 AREA & & \\
\hline 4572 & 300 AREA & & \\
\hline 4573 & 300 AREA & & \\
\hline 4574 & 300 AREA & & \\
\hline 4575 & PRIEST RAPIDS-RIVER & & \\
\hline 4576 & PRIEST RAPIDS-RIVER & & \\
\hline 4577 & 300 AREA & & \\
\hline
\end{tabular}




\begin{tabular}{|c|c|c|c|}
\hline & $\mathrm{Z}$ & $\overline{\mathrm{AA}}$ & $\mathrm{AB}$ \\
\hline 1 & SAMP_SITE_NAME & WELL_NAME & SAMPLE_LOCATION \\
\hline 4578 & 300 AREA & & \\
\hline 4579 & 300 AREA & & \\
\hline 4580 & PRIEST RAPIDS-RIVER & & \\
\hline 4581 & PRIEST RAPIDS-RIVER & & \\
\hline 4582 & PRIEST RAPIDS-RIVER & & \\
\hline 4583 & PRIEST RAPIDS-RIVER & & \\
\hline 4584 & PRIEST RAPIDS-RIVER & & \\
\hline 4585 & PRIEST RAPIDS-RIVER & & \\
\hline 4586 & RIVERVIEW CANAL & & \\
\hline \begin{tabular}{|l|l|}
4587 & -1 \\
\end{tabular} & RIVERVIEW CANAL & & \\
\hline 4588 & 300 AREA & & \\
\hline 4589 & 300 AREA & & \\
\hline 4590 & 300 AREA & & \\
\hline 4591 & PRIEST RAPIDS-RIVER & & \\
\hline 4592 & PRIEST RAPIDS-RIVER & & \\
\hline 4593 & PRIEST RAPIDS-RIVER & & \\
\hline 4594 & PRIEST RAPIDS-RIVER & & \\
\hline 4595 & PRIEST RAPIDS-RIVER & & \\
\hline 4596 & PRIEST RAPIDS-RIVER & & \\
\hline 4597 & RICH.PMPHS HRM 46.4 & & \\
\hline 4598 & RICH.PMPHS HRM 46.4 & & \\
\hline 4599 & RICH.PMPHS HRM 46.4 & & \\
\hline 4600 & RICH.PMPHS HRM 46.4 & & \\
\hline 4601 & RICH.PMPHS HRM 46.4 & & \\
\hline 4602 & RICH.PMPHS HRM 46.4 & & \\
\hline 4603 & LOWER REACH RIVER & & \\
\hline 4604 & LOWER REACH RIVER & & \\
\hline 4605 & LOWER REACH RIVER & & \\
\hline 4606 & UPPER REACH RIVER & & \\
\hline 4607 & UPPER REACH RIVER & & \\
\hline 4608 & UPPER REACH RIVER & & \\
\hline 4609 & 300 AREA & & \\
\hline 4610 & 300 AREA & & \\
\hline 4611 & PRIEST RAPIDS-RIVER & & \\
\hline 4612 & PRIEST RAPIDS-RIVER & & \\
\hline 4613 & 300 AREA & & \\
\hline 4614 & 300 AREA & & \\
\hline 4615 & PRIEST RAPIDS-RIVER & & \\
\hline 4616 & PRIEST RAPIDS-RIVER & & \\
\hline \begin{tabular}{|l|}
4617 \\
\end{tabular} & PRIEST RAPIDS-RIVER & & \\
\hline 4618 & PRIEST RAPIDS-RIVER & & \\
\hline 4619 & PRIEST RAPIDS-RIVER & & \\
\hline 4620 & PRIEST RAPIDS-RIVER & & \\
\hline 4621 & PRIEST RAPIDS-RIVER & & \\
\hline 4622 & PRIEST RAPIDS-RIVER & & \\
\hline 4623 & RICH.PMPHS HRM 46.4 & & \\
\hline 4624 & RICH.PMPHS HRM 46.4 & & \\
\hline 4625 & RICH.PMPHS HRM 46.4 & & \\
\hline 4626 & RICH.PMPHS HRM 46.4 & & \\
\hline \begin{tabular}{|l|l|}
4627 \\
\end{tabular} & RICH.PMPHS HRM 46.4 & & \\
\hline 4628 & RICH.PMPHS HRM 46.4 & & \\
\hline 4629 & ALE HDQTRS & & \\
\hline
\end{tabular}




\begin{tabular}{|c|l|l|l|}
\hline & \multicolumn{1}{|c|}{$Z$} & A & AB \\
\hline 1 & SAMP_SITE_NAME & WELL_NAME & SAMPLE_LOCATION \\
\hline 4630 & ALE HDQTRS & & \\
\hline 4631 & ALE HDQTRS & & \\
\hline 4632 & FIRING RANGE & & \\
\hline 4633 & FIRING RANGE & & \\
\hline 4634 & FIRING RANGE & & \\
\hline 4635 & OBSEVATORY-RTLSNK MT & & \\
\hline 4636 & OBSEVATORY-RTLSNK MT & & \\
\hline 4637 & OBSEVATORY-RTLSNK MT & & \\
\hline 4638 & 300 AREA & & \\
\hline 4639 & 300 AREA & & \\
\hline 4640 & PRIEST RAPIDS-RIVER & & \\
\hline 4641 & PRIEST RAPIDS-RIVER & & \\
\hline 4642 & B POND NORTH SIDE & & \\
\hline 4643 & B POND NORTH SIDE & & \\
\hline 4644 & B POND NORTH SIDE & & \\
\hline 4645 & FFTF POND & & \\
\hline 4646 & FFTF POND & & \\
\hline 4647 & GABLE POND N END & & \\
\hline 4648 & GABLE POND N END & & \\
\hline 4649 & GABLE POND N END & & \\
\hline 4650 & WEST LAKE & & \\
\hline 4651 & WEST LAKE & & \\
\hline 4652 & WEST LAKE & & \\
\hline 4653 & FFTF & & \\
\hline 4654 & FFTF & & \\
\hline 4655 & FFTF & & \\
\hline 4656 & 100 B AREA-RIVER & & \\
\hline 4657 & 100 B AREA-RIVER & & \\
\hline 4658 & 100 B AREA-RIVER & & \\
\hline 4659 & YAKIMA BARRICADE & & \\
\hline 4660 & YAKIMA BARRICADE & & \\
\hline 4661 & YAKIMA BARRICADE & & \\
\hline 4662 & 100 D AREA & & \\
\hline 4663 & 100 D AREA & & \\
\hline 4664 & 100 D AREA & & \\
\hline 4665 & 300 AREA & & \\
\hline 4666 & 300 AREA & & \\
\hline 4667 & PRIEST RAPIDS-RIVER & & \\
\hline 4668 & PRIEST RAPIDS-RIVER & & \\
\hline 4669 & 300 AREA & & \\
\hline 4670 & 300 AREA & & \\
\hline 4671 & PRIEST RAPIDS-RIVER & & \\
\hline 4672 & PRIEST RAPIDS-RIVER & & \\
\hline 4673 & PRIEST RAPIDS-RIVER & & \\
\hline 4674 & PRIEST RAPIDS-RIVER & & \\
\hline 4675 & PRIEST RAPIDS-RIVER & & \\
\hline 4676 & PRIEST RAPIDS-RIVER & & \\
\hline 4677 & PRIEST RAPIDS-RIVER & & \\
\hline 4678 & PRIEST RAPIDS-RIVER & & \\
\hline 4679 & RICH.PMPHS HRM 46.4 & & \\
\hline 4680 & RICH.PMPHS HRM 46.4 & & \\
\hline 4681 & RICH.PMPHS HRM 46.4 & & \\
\hline
\end{tabular}




\begin{tabular}{|c|c|c|c|}
\hline & $\mathrm{Z}$ & $\mathrm{AA}$ & $\mathrm{AB}$ \\
\hline 1 & SAMP_SITE_NAME & WELL_NAME & SAMPLE_LOCATION \\
\hline 4682 & RICH.PMPHS HRM 46.4 & & \\
\hline 4683 & RICH.PMPHS HRM 46.4 & & \\
\hline 4684 & RICH.PMPHS HRM 46.4 & & \\
\hline 4685 & PRIEST RAPIDS-RIVER & & \\
\hline 4686 & PRIEST RAPIDS-RIVER & & \\
\hline \begin{tabular}{|l|l|}
4687 & -1 \\
\end{tabular} & 300 AREA & & \\
\hline 4688 & 300 AREA & & \\
\hline 4689 & 300 AREA & & \\
\hline 4690 & 300 AREA & & \\
\hline 4691 & 300 AREA & & \\
\hline 4692 & 300 AREA & & \\
\hline 4693 & 300 AREA & & \\
\hline 4694 & 300 AREA & & \\
\hline 4695 & 300 AREA & & \\
\hline 4696 & 300 AREA & & \\
\hline \begin{tabular}{|l|l|}
4697 \\
\end{tabular} & 300 AREA & & \\
\hline \begin{tabular}{|c|}
4698 \\
\end{tabular} & PRIEST RAPIDS-RIVER & & \\
\hline 4699 & PRIEST RAPIDS-RIVER & & \\
\hline 4700 & PRIEST RAPIDS-RIVER & & \\
\hline 4701 & PRIEST RAPIDS-RIVER & & \\
\hline 4702 & PRIEST RAPIDS-RIVER & & \\
\hline 4703 & PRIEST RAPIDS-RIVER & & \\
\hline 4704 & PRIEST RAPIDS-RIVER & & \\
\hline 4705 & PRIEST RAPIDS-RIVER & & \\
\hline 4706 & PRIEST RAPIDS-RIVER & & \\
\hline \begin{tabular}{|l|l|}
4707 \\
\end{tabular} & PRIEST RAPIDS-RIVER & & \\
\hline 4708 & PRIEST RAPIDS-RIVER & & \\
\hline 4709 & PRIEST RAPIDS-RIVER & & \\
\hline 4710 & RICH.PMPHS HRM 46.4 & & \\
\hline 4711 & RICH.PMPHS HRM 46.4 & & \\
\hline 4712 & RICH.PMPHS HRM 46.4 & & \\
\hline 4713 & RICH.PMPHS HRM 46.4 & & \\
\hline 4714 & RICH.PMPHS HRM 46.4 & & \\
\hline 4715 & RICH.PMPHS HRM 46.4 & & \\
\hline 4716 & 300 AREA & & \\
\hline \begin{tabular}{|l|l|}
4717 \\
\end{tabular} & 300 AREA & & \\
\hline 4718 & PRIEST RAPIDS-RIVER & & \\
\hline 4719 & PRIEST RAPIDS-RIVER & & \\
\hline 4720 & 300 AREA & & \\
\hline 4721 & 300 AREA & & \\
\hline 4722 & PRIEST RAPIDS-RIVER & & \\
\hline 4723 & PRIEST RAPIDS-RIVER & & \\
\hline 4724 & PRIEST RAPIDS-RIVER & & \\
\hline 4725 & PRIEST RAPIDS-RIVER & & \\
\hline 4726 & PRIEST RAPIDS-RIVER & & \\
\hline \begin{tabular}{|l|l|}
4727 \\
\end{tabular} & PRIEST RAPIDS-RIVER & & \\
\hline \begin{tabular}{|l|l|}
4728 \\
\end{tabular} & PRIEST RAPIDS-RIVER & & \\
\hline 4729 & PRIEST RAPIDS-RIVER & & \\
\hline 4730 & RICH.PMPHS HRM 46.4 & & \\
\hline 4731 & RICH.PMPHS HRM 46.4 & & \\
\hline 4732 & RICH.PMPHS HRM 46.4 & & \\
\hline 4733 & RICH.PMPHS HRM 46.4 & & \\
\hline
\end{tabular}




\begin{tabular}{|c|c|c|c|}
\hline & $\mathrm{Z}$ & $\overline{\mathrm{AA}}$ & $\mathrm{AB}$ \\
\hline 1 & SAMP_SITE_NAME & WELL_NAME & SAMPLE_LOCATION \\
\hline 4734 & RICH.PMPHS HRM 46.4 & & \\
\hline 4735 & RICH.PMPHS HRM 46.4 & & \\
\hline 4736 & ALE HDQTRS & & \\
\hline 4737 & ALE HDQTRS & & \\
\hline 4738 & ALE HDQTRS & & \\
\hline 4739 & FFTF & & \\
\hline 4740 & FFTF & & \\
\hline 4741 & FFTF & & \\
\hline 4742 & FIRING RANGE & & \\
\hline 4743 & FIRING RANGE & & \\
\hline 4744 & FIRING RANGE & & \\
\hline 4745 & OBSEVATORY-RTLSNK MT & & \\
\hline 4746 & OBSEVATORY-RTLSNK MT & & \\
\hline 4747 & OBSEVATORY-RTLSNK MT & & \\
\hline 4748 & 100 B AREA-RIVER & & \\
\hline 4749 & 100 B AREA-RIVER & & \\
\hline 4750 & 100 B AREA-RIVER & & \\
\hline 4751 & 300 AREA & & \\
\hline 4752 & 300 AREA & & \\
\hline 4753 & PRIEST RAPIDS-RIVER & & \\
\hline 4754 & PRIEST RAPIDS-RIVER & & \\
\hline 4755 & 300 AREA & & \\
\hline 4756 & 300 AREA & & \\
\hline 4757 & PRIEST RAPIDS-RIVER & & \\
\hline 4758 & PRIEST RAPIDS-RIVER & & \\
\hline 4759 & PRIEST RAPIDS-RIVER & & \\
\hline 4760 & PRIEST RAPIDS-RIVER & & \\
\hline 4761 & PRIEST RAPIDS-RIVER & & \\
\hline 4762 & PRIEST RAPIDS-RIVER & & \\
\hline 4763 & PRIEST RAPIDS-RIVER & & \\
\hline 4764 & PRIEST RAPIDS-RIVER & & \\
\hline 4765 & RICH.PMPHS HRM 46.4 & & \\
\hline 4766 & RICH.PMPHS HRM 46.4 & & \\
\hline 4767 & RICH.PMPHS HRM 46.4 & & \\
\hline 4768 & RICH.PMPHS HRM 46.4 & & \\
\hline 4769 & RICH.PMPHS HRM 46.4 & & \\
\hline 4770 & RICH.PMPHS HRM 46.4 & & \\
\hline 4771 & B POND NORTH SIDE & & \\
\hline 4772 & B POND NORTH SIDE & & \\
\hline 4773 & B POND NORTH SIDE & & \\
\hline 4774 & FFTF POND & & \\
\hline 4775 & FFTF POND & & \\
\hline 4776 & GABLE POND N END & & \\
\hline 4777 & GABLE POND N END & & \\
\hline 4778 & GABLE POND N END & & \\
\hline 4779 & WEST LAKE & & \\
\hline 4780 & WEST LAKE & & \\
\hline 4781 & WEST LAKE & & \\
\hline 4782 & YAKIMA BARRICADE & & \\
\hline 4783 & YAKIMA BARRICADE & & \\
\hline 4784 & YAKIMA BARRICADE & & \\
\hline 4785 & 300 AREA & & \\
\hline
\end{tabular}




\begin{tabular}{|c|c|c|c|}
\hline & $\mathrm{Z}$ & $\overline{\mathrm{AA}}$ & $\mathrm{AB}$ \\
\hline 1 & SAMP_SITE_NAME & WELL_NAME & SAMPLE_LOCATION \\
\hline 4786 & 300 AREA & & \\
\hline \begin{tabular}{|l|l|}
4787 & -1 \\
\end{tabular} & PRIEST RAPIDS-RIVER & & \\
\hline 4788 & PRIEST RAPIDS-RIVER & & \\
\hline 4789 & RIVERVIEW CANAL & & \\
\hline 4790 & RIVERVIEW CANAL & & \\
\hline 4791 & ALEXANDER FARM & & \\
\hline 4792 & MATHEWS CORNER & & \\
\hline 4793 & WEBBER RANCH & & \\
\hline 4794 & WHITE BLUFFS ASSOC & & \\
\hline 4795 & 300 AREA & & \\
\hline 4796 & 300 AREA & & \\
\hline \begin{tabular}{|l|l|}
4797 \\
\end{tabular} & 300 AREA & & \\
\hline \begin{tabular}{|l|l|}
4798 \\
\end{tabular} & 300 AREA & & \\
\hline 4799 & 300 AREA & & \\
\hline 4800 & 300 AREA & & \\
\hline 4801 & 300 AREA & & \\
\hline 4802 & 300 AREA & & \\
\hline 4803 & 300 AREA & & \\
\hline 4804 & 300 AREA & & \\
\hline 4805 & 300 AREA & & \\
\hline 4806 & 300 AREA & & \\
\hline \begin{tabular}{|l|l|}
4807 \\
\end{tabular} & PRIEST RAPIDS-RIVER & & \\
\hline 4808 & PRIEST RAPIDS-RIVER & & \\
\hline 4809 & PRIEST RAPIDS-RIVER & & \\
\hline 4810 & PRIEST RAPIDS-RIVER & & \\
\hline 4811 & RIVERVIEW CANAL & & \\
\hline 4812 & RIVERVIEW CANAL & & \\
\hline 4813 & PRIEST RAPIDS-RIVER & & \\
\hline 4814 & PRIEST RAPIDS-RIVER & & \\
\hline 4815 & PRIEST RAPIDS-RIVER & & \\
\hline 4816 & PRIEST RAPIDS-RIVER & & \\
\hline \begin{tabular}{|l|l|}
4817 \\
\end{tabular} & PRIEST RAPIDS-RIVER & & \\
\hline 4818 & PRIEST RAPIDS-RIVER & & \\
\hline 4819 & RICH.PMPHS HRM 46.4 & & \\
\hline 4820 & RICH.PMPHS HRM 46.4 & & \\
\hline 4821 & RICH.PMPHS HRM 46.4 & & \\
\hline 4822 & RICH.PMPHS HRM 46.4 & & \\
\hline 4823 & RICH.PMPHS HRM 46.4 & & \\
\hline 4824 & RICH.PMPHS HRM 46.4 & & \\
\hline 4825 & 100 D AREA & & \\
\hline 4826 & 100 D AREA & & \\
\hline \begin{tabular}{|l|l|}
4827 & -1 \\
\end{tabular} & 100 D AREA & & \\
\hline 4828 & 300 AREA & & \\
\hline 4829 & 300 AREA & & \\
\hline 4830 & PRIEST RAPIDS-RIVER & & \\
\hline 4831 & PRIEST RAPIDS-RIVER & & \\
\hline 4832 & UPPER REACH RIVER & & \\
\hline 4833 & UPPER REACH RIVER & & \\
\hline 4834 & UPPER REACH RIVER & & \\
\hline 4835 & UPPER REACH RIVER & & \\
\hline 4836 & UPPER REACH RIVER & & \\
\hline \begin{tabular}{|l|l|}
4837 & -20 \\
\end{tabular} & UPPER REACH RIVER & & \\
\hline
\end{tabular}




\begin{tabular}{|c|c|c|c|}
\hline & $\bar{Z}$ & $\overline{\mathrm{AA}}$ & $\overline{A B}$ \\
\hline 1 & SAMP_SITE_NAME & WELL_NAME & SAMPLE_LOCATION \\
\hline 4838 & 300 AREA & & \\
\hline 4839 & 300 AREA & & \\
\hline 4840 & PRIEST RAPIDS-RIVER & & \\
\hline 4841 & PRIEST RAPIDS-RIVER & & \\
\hline 4842 & ALE HDQTRS & & \\
\hline 4843 & ALE HDQTRS & & \\
\hline 4844 & ALE HDQTRS & & \\
\hline 4845 & FFTF & & \\
\hline 4846 & FFTF & & \\
\hline 4847 & FFTF & & \\
\hline 4848 & FIRING RANGE & & \\
\hline 4849 & FIRING RANGE & & \\
\hline 4850 & FIRING RANGE & & \\
\hline 4851 & OBSEVATORY-RTLSNK MT & & \\
\hline 4852 & OBSEVATORY-RTLSNK MT & & \\
\hline 4853 & OBSEVATORY-RTLSNK MT & & \\
\hline 4854 & PRIEST RAPIDS-RIVER & & \\
\hline 4855 & PRIEST RAPIDS-RIVER & & \\
\hline 4856 & PRIEST RAPIDS-RIVER & & \\
\hline 4857 & PRIEST RAPIDS-RIVER & & \\
\hline 4858 & PRIEST RAPIDS-RIVER & & \\
\hline 4859 & PRIEST RAPIDS-RIVER & & \\
\hline 4860 & RICH.PMPHS HRM 46.4 & & \\
\hline 4861 & RICH.PMPHS HRM 46.4 & & \\
\hline 4862 & RICH.PMPHS HRM 46.4 & & \\
\hline 4863 & RICH.PMPHS HRM 46.4 & & \\
\hline 4864 & RICH.PMPHS HRM 46.4 & & \\
\hline 4865 & RICH.PMPHS HRM 46.4 & & \\
\hline 4866 & 100 B AREA-RIVER & & \\
\hline 4867 & 100 B AREA-RIVER & & \\
\hline 4868 & 100 B AREA-RIVER & & \\
\hline 4869 & 300 AREA & & \\
\hline 4870 & 300 AREA & & \\
\hline 4871 & PRIEST RAPIDS-RIVER & & \\
\hline 4872 & PRIEST RAPIDS-RIVER & & \\
\hline 4873 & 300 AREA & & \\
\hline 4874 & 300 AREA & & \\
\hline 4875 & PRIEST RAPIDS-RIVER & & \\
\hline 4876 & PRIEST RAPIDS-RIVER & & \\
\hline \begin{tabular}{|l|l|}
4877 \\
\end{tabular} & B POND NORTH SIDE & & \\
\hline 4878 & B POND NORTH SIDE & & \\
\hline 4879 & B POND NORTH SIDE & & \\
\hline 4880 & FFTF POND & & \\
\hline 4881 & FFTF POND & & \\
\hline 4882 & GABLE POND N END & & \\
\hline 4883 & GABLE POND N END & & \\
\hline 4884 & GABLE POND N END & & \\
\hline 4885 & WEST LAKE & & \\
\hline 4886 & WEST LAKE & & \\
\hline 4887 & WEST LAKE & & \\
\hline 4888 & 100 D AREA & & \\
\hline 4889 & YAKIMA BARF & & \\
\hline
\end{tabular}




\begin{tabular}{|c|c|c|c|}
\hline & $\mathrm{Z}$ & $\overline{\mathrm{AA}}$ & $\mathrm{AB}$ \\
\hline 1 & SAMP_SITE_NAME & WELL_NAME & SAMPLE_LOCATION \\
\hline 4890 & YAKIMA BARRICADE & & \\
\hline 4891 & YAKIMA BARRICADE & & \\
\hline 4892 & 300 AREA & & \\
\hline 4893 & 300 AREA & & \\
\hline 4894 & PRIEST RAPIDS-RIVER & & \\
\hline 4895 & PRIEST RAPIDS-RIVER & & \\
\hline 4896 & PRIEST RAPIDS-RIVER & & \\
\hline 4897 & PRIEST RAPIDS-RIVER & & \\
\hline 4898 & PRIEST RAPIDS-RIVER & & \\
\hline 4899 & PRIEST RAPIDS-RIVER & & \\
\hline 4900 & PRIEST RAPIDS-RIVER & & \\
\hline 4901 & PRIEST RAPIDS-RIVER & & \\
\hline 4902 & RICH.PMPHS HRM 46.4 & & \\
\hline |4903 & RICH.PMPHS HRM 46.4 & & \\
\hline 4904 & RICH.PMPHS HRM 46.4 & & \\
\hline 4905 & RICH.PMPHS HRM 46.4 & & \\
\hline 4906 & RICH.PMPHS HRM 46.4 & & \\
\hline 4907 & RICH.PMPHS HRM 46.4 & & \\
\hline 4908 & RIVERVIEW CANAL & & \\
\hline 4909 & RIVERVIEW CANAL & & \\
\hline 4910 & 100 D AREA & & \\
\hline 4911. & 100 D AREA & & \\
\hline 4912. & 100 D AREA & & \\
\hline 4913 & 300 AREA & & \\
\hline 4914 & 300 AREA & & \\
\hline 4915 & PRIEST RAPIDS-RIVER & & \\
\hline 4916 & PRIEST RAPIDS-RIVER & & \\
\hline 4917 & 300 AREA & & \\
\hline 4918 & 300 AREA & & \\
\hline 4919 & 300 AREA & & \\
\hline 4920 & 300 AREA & & \\
\hline 4921 & 300 AREA & & \\
\hline 4922 & 300 AREA & & \\
\hline 4923 & PRIEST RAPIDS-RIVER & & \\
\hline 4924 & PRIEST RAPIDS-RIVER & & \\
\hline 4925 & PRIEST RAPIDS-RIVER & & \\
\hline 4926 & PRIEST RAPIDS-RIVER & & \\
\hline 4927 & PRIEST RAPIDS-RIVER & & \\
\hline 4928 & PRIEST RAPIDS-RIVER & & \\
\hline 4929 & PRIEST RAPIDS-RIVER & & \\
\hline 4930 & PRIEST RAPIDS-RIVER & & \\
\hline 4931 & PRIEST RAPIDS-RIVER & & \\
\hline 4932 & PRIEST RAPIDS-RIVER & & \\
\hline 4933 & PRIEST RAPIDS-RIVER & & \\
\hline 4934 & PRIEST RAPIDS-RIVER & & \\
\hline 4935 & RICH.PMPHS HRM 46.4 & & \\
\hline 4936 & RICH.PMPHS HRM 46.4 & & \\
\hline 4937 & RICH.PMPHS HRM 46.4 & & \\
\hline 4938 & RICH.PMPHS HRM 46.4 & & \\
\hline 4939 & RICH.PMPHS HRM 46.4 & & \\
\hline 4940 & RICH.PMPHS HRM 46.4 & & \\
\hline 4941 & 300 AREA & & \\
\hline
\end{tabular}




\begin{tabular}{|c|c|c|c|}
\hline & $\mathrm{Z}$ & $\overline{\mathrm{AA}}$ & $\mathrm{AB}$ \\
\hline 1 & SAMP_SITE_NAME & WELL_NAME & SAMPLE_LOCATION \\
\hline 4942 & 300 AREA & & \\
\hline 4943 & 300 AREA & & \\
\hline 4944 & 300 AREA & & \\
\hline 4945 & 300 AREA & & \\
\hline 4946 & 300 AREA & & \\
\hline 4947 & 300 AREA & & \\
\hline 4948 & 300 AREA & & \\
\hline 4949 & PRIEST RAPIDS-RIVER & & \\
\hline 4950 & PRIEST RAPIDS-RIVER & & \\
\hline 4951 & ALE HDQTRS & & \\
\hline 4952 & ALE HDQTRS & & \\
\hline 4953 & ALE HDQTRS & & \\
\hline 4954 & FFTF & & \\
\hline 4955 & FFTF & & \\
\hline 4956 & FFTF & & \\
\hline 4957 & FIRING RANGE & & \\
\hline 4958 & FIRING RANGE & & \\
\hline 4959 & FIRING RANGE & & \\
\hline 4960 & OBSEVATORY-RTLSNK MT & & \\
\hline 4961 & OBSEVATORY-RTLSNK MT & & \\
\hline 4962 & OBSEVATORY-RTLSNK MT & & \\
\hline 4963 & 100 B AREA-RIVER & & \\
\hline 4964 & 100 B AREA-RIVER & & \\
\hline 4965 & 100 B AREA-RIVER & & \\
\hline 4966 & 300 AREA & & \\
\hline \begin{tabular}{|l|l|}
4967 & -10 \\
\end{tabular} & 300 AREA & & \\
\hline 4968 & PRIEST RAPIDS-RIVER & & \\
\hline 4969 & PRIEST RAPIDS-RIVER & & \\
\hline 4970 & PRIEST RAPIDS-RIVER & & \\
\hline 4971 & PRIEST RAPIDS-RIVER & & \\
\hline 4972 & PRIEST RAPIDS-RIVER & & \\
\hline 4973 & PRIEST RAPIDS-RIVER & & \\
\hline 4974 & PRIEST RAPIDS-RIVER & & \\
\hline 4975 & PRIEST RAPIDS-RIVER & & \\
\hline 4976 & RICH.PMPHS HRM 46.4 & & \\
\hline 4977 & RICH.PMPHS HRM 46.4 & & \\
\hline 4978 & RICH.PMPHS HRM 46.4 & & \\
\hline 4979 & RICH.PMPHS HRM 46.4 & & \\
\hline 4980 & RICH.PMPHS HRM 46.4 & & \\
\hline 4981 & RICH.PMPHS HRM 46.4 & & \\
\hline 4982 & 300 AREA & & \\
\hline 4983 & 300 AREA & & \\
\hline 4984 & PRIEST RAPIDS-RIVER & & \\
\hline 4985 & PRIEST RAPIDS-RIVER & & \\
\hline 4986 & B POND NORTH SIDE & & \\
\hline \begin{tabular}{|l|l|}
4987 \\
\end{tabular} & B POND NORTH SIDE & & \\
\hline 4988 & B POND NORTH SIDE & & \\
\hline 4989 & FFTF POND & & \\
\hline 4990 & FFTF POND & & \\
\hline 4991 & GABLE POND N END & & \\
\hline 4992 & GABLE POND N END & & \\
\hline 4993 & GABLE POND N END & & \\
\hline
\end{tabular}




\begin{tabular}{|c|c|c|c|}
\hline & $\bar{Z}$ & $\overline{\mathrm{AA}}$ & $\overline{A B}$ \\
\hline 1 & SAMP_SITE_NAME & WELL_NAME & SAMPLE_LOCATION \\
\hline 4994 & WEST LAKE & & \\
\hline 4995 & WEST LAKE & & \\
\hline 4996 & WEST LAKE & & \\
\hline 4997 & YAKIMA BARRICADE & & \\
\hline 4998 & YAKIMA BARRICADE & & \\
\hline 4999 & YAKIMA BARRICADE & & \\
\hline 5000 & 300 AREA & & \\
\hline 5001 & 300 AREA & & \\
\hline 5002 & PRIEST RAPIDS-RIVER & & \\
\hline 5003 & PRIEST RAPIDS-RIVER & & \\
\hline 5004 & RIVERVIEW CANAL & & \\
\hline 5005 & RIVERVIEW CANAL & & \\
\hline 5006 & 100 D AREA & & \\
\hline 5007 & 100 D AREA & & \\
\hline 5008 & 100 D AREA & & \\
\hline 5009 & PRIEST RAPIDS-RIVER & & \\
\hline 5010 & PRIEST RAPIDS-RIVER & & \\
\hline 5011 & PRIEST RAPIDS-RIVER & & \\
\hline 5012 & PRIEST RAPIDS-RIVER & & \\
\hline 5013 & PRIEST RAPIDS-RIVER & & \\
\hline 5014 & PRIEST RAPIDS-RIVER & & \\
\hline 5015 & RICH.PMPHS HRM 46.4 & & \\
\hline 5016 & RICH.PMPHS HRM 46.4 & & \\
\hline 5017 & RICH.PMPHS HRM 46.4 & & \\
\hline 5018 & RICH.PMPHS HRM 46.4 & & \\
\hline 5019 & RICH.PMPHS HRM 46.4 & & \\
\hline 5020 & RICH.PMPHS HRM 46.4 & & \\
\hline 5021 & 300 AREA & & \\
\hline 5022 & 300 AREA & & \\
\hline 5023 & PRIEST RAPIDS-RIVER & & \\
\hline 5024 & PRIEST RAPIDS-RIVER & & \\
\hline 5025 & 300 AREA & & \\
\hline 5026 & 300 AREA & & \\
\hline 5027 & PRIEST RAPIDS-RIVER & & \\
\hline 5028 & PRIEST RAPIDS-RIVER & & \\
\hline 5029 & 300 AREA & & \\
\hline 5030 & 300 AREA & & \\
\hline 5031 & 300 AREA & & \\
\hline 5032 & 300 AREA & & \\
\hline 5033 & 300 AREA & & \\
\hline 5034 & 300 AREA & & \\
\hline 5035 & PRIEST RAPIDS-RIVER & & \\
\hline 5036 & PRIEST RAPIDS-RIVER & & \\
\hline 5037 & PRIEST RAPIDS-RIVER & & \\
\hline 5038 & PRIEST RAPIDS-RIVER & & \\
\hline 5039 & PRIEST RAPIDS-RIVER & & \\
\hline 5040 & PRIEST RAPIDS-RIVER & & \\
\hline 5041 & RICH.PMPHS HRM 46.4 & & \\
\hline 5042 & RICH.PMPHS HRM 46.4 & & \\
\hline 5043 & RICH.PMPHS HRM 46.4 & & \\
\hline 5044 & RICH.PMPHS HRM 46.4 & & \\
\hline 5045 & RICH.PMPHS HRM 46.4 & & \\
\hline
\end{tabular}




\begin{tabular}{|c|c|c|c|}
\hline & $\mathrm{z}$ & $\mathrm{AA}$ & $\mathrm{AB}$ \\
\hline 1 & SAMP_SITE_NAME & WELL_NAME & SAMPLE_LOCATION \\
\hline 5046 & RICH.PMPHS HRM 46.4 & & \\
\hline 5047 & 300 AREA & & \\
\hline 5048 & 300 AREA & & \\
\hline 5049 & 300 AREA & & \\
\hline 5050 & 300 AREA & & \\
\hline 5051 & 300 AREA & & \\
\hline 5052 & 300 AREA & & \\
\hline 5053 & PRIEST RAPIDS-RIVER & & \\
\hline 5054 & PRIEST RAPIDS-RIVER & & \\
\hline 5055 & PRIEST RAPIDS-RIVER & & \\
\hline 5056 & PRIEST RAPIDS-RIVER & & \\
\hline 5057 & PRIEST RAPIDS-RIVER & & \\
\hline 5058 & PRIEST RAPIDS-RIVER & & \\
\hline 5059 & ALE HDQTRS & & \\
\hline 5060 & ALE HDQTRS & & \\
\hline 5061 & ALE HDQTRS & & \\
\hline 5062 & FFTF & & \\
\hline 5063 & FFTF & & \\
\hline 5064 & FFTF & & \\
\hline 5065 & FIRING RANGE & & \\
\hline 5066 & FIRING RANGE & & \\
\hline 5067 & FIRING RANGE & & \\
\hline 5068 & OBSEVATORY-RTLSNK MT & & \\
\hline \begin{tabular}{|l|l|l|}
5069 \\
\end{tabular} & OBSEVATORY-RTLSNK MT & & \\
\hline 5070 & OBSEVATORY-RTLSNK MT & & \\
\hline 5071 & 100 B AREA-RIVER & & \\
\hline 5072 & 100 B AREA-RIVER & & \\
\hline 5073 & 100 B AREA-RIVER & & \\
\hline 5074 & LOWER REACH RIVER & & \\
\hline 5075 & LOWER REACH RIVER & & \\
\hline 5076 & LOWER REACH RIVER & & \\
\hline 5077 & LOWER REACH RIVER & & \\
\hline 5078 & LOWER REACH RIVER & & \\
\hline 5079 & LOWER REACH RIVER & & \\
\hline 5080 & UPPER REACH RIVER & & \\
\hline 5081 & UPPER REACH RIVER & & \\
\hline 5082 & UPPER REACH RIVER & & \\
\hline 5083 & UPPER REACH RIVER & & \\
\hline \begin{tabular}{|l|l|}
5084 \\
\end{tabular} & UPPER REACH RIVER & & \\
\hline 5085 & UPPER REACH RIVER & & \\
\hline 5086 & 300 AREA & & \\
\hline 5087 & 300 AREA & & \\
\hline 5088 & PRIEST RAPIDS-RIVER & & \\
\hline 5089 & PRIEST RAPIDS-RIVER & & \\
\hline 5090 & PRIEST RAPIDS-RIVER & & \\
\hline 5091 & PRIEST RAPIDS-RIVER & & \\
\hline 5092 & PRIEST RAPIDS-RIVER & & \\
\hline 5093 & PRIEST RAPIDS-RIVER & & \\
\hline 5094 & PRIEST RAPIDS-RIVER & & \\
\hline 5095 & PRIEST RAPIDS-RIVER & & \\
\hline 5096 & RICH.PMPHS HRM 46.4 & & \\
\hline \begin{tabular}{|l|l|}
5097 \\
\end{tabular} & RICH.PMPHS HRM 46.4 & & \\
\hline
\end{tabular}




\begin{tabular}{|c|c|c|c|}
\hline & $\mathrm{Z}$ & $\overline{\mathrm{AA}}$ & $\mathrm{AB}$ \\
\hline 1 & SAMP_SITE_NAME & WELL_NAME & SAMPLE_LOCATION \\
\hline 5098 & RICH.PMPHS HRM 46.4 & & \\
\hline 5099 & RICH.PMPHS HRM 46.4 & & \\
\hline 5100 & RICH.PMPHS HRM 46.4 & & \\
\hline 5101 & RICH.PMPHS HRM 46.4 & & \\
\hline 5102 & 300 AREA & & \\
\hline 5103 & 300 AREA & & \\
\hline 5104 & PRIEST RAPIDS-RIVER & & \\
\hline 5105 & PRIEST RAPIDS-RIVER & & \\
\hline 5106 & B POND NORTH SIDE & & \\
\hline 5107 & B POND NORTH SIDE & & \\
\hline 5108 & B POND NORTH SIDE & & \\
\hline 5109 & FFTF POND & & \\
\hline 5110 & FFTF POND & & \\
\hline 5111 & GABLE POND N END & & \\
\hline 5112 & GABLE POND N END & & \\
\hline 5113 & GABLE POND N END & & \\
\hline 5114 & WEST LAKE & & \\
\hline 5115 & WEST LAKE & & \\
\hline 5116 & WEST LAKE & & \\
\hline 5117 & WEST LAKE & & \\
\hline 5118 & WEST LAKE & & \\
\hline 5119 & WEST LAKE & & \\
\hline 5120 & YAKIMA BARRICADE & & \\
\hline 5121 & YAKIMA BARRICADE & & \\
\hline 5122 & YAKIMA BARRICADE & & \\
\hline 5123 & 300 AREA & & \\
\hline 5124 & 300 AREA & & \\
\hline 5125 & PRIEST RAPIDS-RIVER & & \\
\hline 5126 & PRIEST RAPIDS-RIVER & & \\
\hline 5127 & RIVERVIEW CANAL & & \\
\hline 5128 & RIVERVIEW CANAL & & \\
\hline 5129 & 100 D AREA & & \\
\hline 5130 & 100 D AREA & & \\
\hline 5131 & 100 D AREA & & \\
\hline 5132 & 300 AREA & & \\
\hline 5133 & 300 AREA & & \\
\hline 5134 & PRIEST RAPIDS-RIVER & & \\
\hline 5135 & PRIEST RAPIDS-RIVER & & \\
\hline 5136 & PRIEST RAPIDS-RIVER & & \\
\hline 5137 & PRIEST RAPIDS-RIVER & & \\
\hline 5138 & PRIEST RAPIDS-RIVER & & \\
\hline 5139 & PRIEST RAPIDS-RIVER & & \\
\hline 5140 & PRIEST RAPIDS-RIVER & & \\
\hline 5141 & PRIEST RAPIDS-RIVER & & \\
\hline 5142 & RICH.PMPHS HRM 46.4 & & \\
\hline 5143 & RICH.PMPHS HRM 46.4 & & \\
\hline 5144 & RICH.PMPHS HRM 46.4 & & \\
\hline 5145 & RICH.PMPHS HRM 46.4 & & \\
\hline 5146 & RICH.PMPHS HRM 46.4 & & \\
\hline 5147 & RICH.PMPHS HRM 46.4 & & \\
\hline 5148 & 300 AREA & & \\
\hline 5149 & 300 AREA & & \\
\hline
\end{tabular}




\begin{tabular}{|c|c|c|c|}
\hline & \multicolumn{1}{|c|}{$Z$} & A & AB \\
\hline 1 & SAMP_SITE_NAME & WELL_NAME & SAMPLE_LOCATION \\
\hline 5150 & PRIEST RAPIDS-RIVER & & \\
\hline 5151 & PRIEST RAPIDS-RIVER & & \\
\hline 5152 & 300 AREA & & \\
\hline 5153 & 300 AREA & & \\
\hline 5154 & 300 AREA & & \\
\hline 5155 & 300 AREA & & \\
\hline 5156 & 300 AREA & & \\
\hline 5157 & 300 AREA & & \\
\hline 5158 & 300 AREA & & \\
\hline 5159 & 300 AREA & & \\
\hline 5160 & 300 AREA & & \\
\hline 5161 & 300 AREA & & \\
\hline 5162 & 300 AREA & & \\
\hline 5163 & 300 AREA & & \\
\hline 5164 & PRIEST RAPIDS-RIVER & & \\
\hline 5165 & PRIEST RAPIDS-RIVER & & \\
\hline 5166 & PRIEST RAPIDS-RIVER & & \\
\hline 5167 & PRIEST RAPIDS-RIVER & & \\
\hline 5168 & PRIEST RAPIDS-RIVER & & \\
\hline 5169 & PRIEST RAPIDS-RIVER & & \\
\hline 5170 & PRIEST RAPIDS-RIVER & & \\
\hline 5171 & PRIEST RAPIDS-RIVER & & \\
\hline 5172 & PRIEST RAPIDS-RIVER & & \\
\hline 5173 & PRIEST RAPIDS-RIVER & & \\
\hline 5174 & PRIEST RAPIDS-RIVER & & \\
\hline 5175 & PRIEST RAPIDS-RIVER & & \\
\hline 5176 & RICH.PMPHS HRM 46.4 & & \\
\hline 5177 & RICH.PMPHS HRM 46.4 & & \\
\hline 5178 & RICH.PMPHS HRM 46.4 & & \\
\hline 5179 & RICH.PMPHS HRM 46.4 & & \\
\hline 5180 & RICH.PMPHS HRM 46.4 & & \\
\hline 5181 & RICH.PMPHS HRM 46.4 & & \\
\hline 5182 & ALE HDQTRS & & \\
\hline 5183 & ALE HDQTRS & & \\
\hline 5184 & ALE HDQTRS & & \\
\hline 5185 & FFTF & & \\
\hline 5186 & FFTF & & \\
\hline 5187 & FFTF & & \\
\hline 5188 & FIRING RANGE & & \\
\hline 5189 & FIRING RANGE & & \\
\hline 5190 & FIRING RANGE & & \\
\hline 5191 & OBSEVATOORY-RTLSNK MT & & \\
\hline 5192 & OBSEVATOORY-RTLSNK MT & & \\
\hline 5193 & OBSEVATORY-RTLSNK MT & & \\
\hline 5194 & 100 B AREA-RIVER & & \\
\hline 5195 & 100 B AREA-RIVER & & \\
\hline 5196 & 100 B AREA-RIVER & & \\
\hline 5197 & 300 AREA & & \\
\hline 5198 & 300 AREA & & \\
\hline 5199 & PRIEST RAPIDS-RIVER & & \\
\hline 5200 & PRIEST RAPIDS-RIVER & & \\
\hline 5201 & RICH.PMPHS HRM 46.4 & & \\
\hline
\end{tabular}




\begin{tabular}{|c|c|c|c|}
\hline & $\mathrm{Z}$ & $\overline{\mathrm{AA}}$ & $\mathrm{AB}$ \\
\hline 1 & SAMP_SITE_NAME & WELL_NAME & SAMPLE_LOCATION \\
\hline 5202 & RICH.PMPHS HRM 46.4 & & \\
\hline 5203 & 300 AREA & & \\
\hline 5204 & 300 AREA & & \\
\hline 5205 & PRIEST RAPIDS-RIVER & & \\
\hline 5206 & PRIEST RAPIDS-RIVER & & \\
\hline 5207 & RICH.PMPHS HRM 46.4 & & \\
\hline 5208 & RICH.PMPHS HRM 46.4 & & \\
\hline 5209 & PRIEST RAPIDS-RIVER & & \\
\hline 5210 & PRIEST RAPIDS-RIVER & & \\
\hline 5211 & PRIEST RAPIDS-RIVER & & \\
\hline 5212 & PRIEST RAPIDS-RIVER & & \\
\hline 5213 & PRIEST RAPIDS-RIVER & & \\
\hline 5214 & PRIEST RAPIDS-RIVER & & \\
\hline 5215 & RICH.PMPHS HRM 46.4 & & \\
\hline 5216 & RICH.PMPHS HRM 46.4 & & \\
\hline \begin{tabular}{|l|l|}
5217 \\
\end{tabular} & RICH.PMPHS HRM 46.4 & & \\
\hline 5218 & RICH.PMPHS HRM 46.4 & & \\
\hline 5219 & RICH.PMPHS HRM 46.4 & & \\
\hline 5220 & RICH.PMPHS HRM 46.4 & & \\
\hline 5221 & YAKIMA BARRICADE & & \\
\hline 5222 & YAKIMA BARRICADE & & \\
\hline 5223 & YAKIMA BARRICADE & & \\
\hline 5224 & 300 AREA & & \\
\hline 5225 & 300 AREA & & \\
\hline 5226 & PRIEST RAPIDS-RIVER & & \\
\hline 5227 & PRIEST RAPIDS-RIVER & & \\
\hline 5228 & RICH.PMPHS HRM 46.4 & & \\
\hline 5229 & RICH.PMPHS HRM 46.4 & & \\
\hline 5230 & 100 D AREA & & \\
\hline 5231 & 100 D AREA & & \\
\hline 5232 & 100 D AREA & & \\
\hline 5233 & 300 AREA & & \\
\hline 5234 & 300 AREA & & \\
\hline 5235 & PRIEST RAPIDS-RIVER & & \\
\hline 5236 & PRIEST RAPIDS-RIVER & & \\
\hline 5237 & RICH.PMPHS HRM 46.4 & & \\
\hline 5238 & RICH.PMPHS HRM 46.4 & & \\
\hline 5239 & PRIEST RAPIDS-RIVER & & \\
\hline 5240 & PRIEST RAPIDS-RIVER & & \\
\hline 5241 & PRIEST RAPIDS-RIVER & & \\
\hline 5242 & PRIEST RAPIDS-RIVER & & \\
\hline 5243 & PRIEST RAPIDS-RIVER & & \\
\hline 5244 & PRIEST RAPIDS-RIVER & & \\
\hline 5245 & RICH.PMPHS HRM 46.4 & & \\
\hline 5246 & RICH.PMPHS HRM 46.4 & & \\
\hline 5247 & RICH.PMPHS HRM 46.4 & & \\
\hline 5248 & RICH.PMPHS HRM 46.4 & & \\
\hline 5249 & RICH.PMPHS HRM 46.4 & & \\
\hline 5250 & RICH.PMPHS HRM 46.4 & & \\
\hline 5251 & B POND NORTH SIDE & & \\
\hline 5252 & B POND NORTH SIDE & & \\
\hline 5253 & B POND NORTH SIDE & & \\
\hline
\end{tabular}




\begin{tabular}{|c|c|c|c|}
\hline & $\bar{Z}$ & $\overline{\mathrm{AA}}$ & $\overline{A B}$ \\
\hline 1 & SAMP_SITE_NAME & WELL_NAME & SAMPLE_LOCATION \\
\hline 5254 & FFTF POND & & \\
\hline 5255 & FFTF POND & & \\
\hline 5256 & GABLE POND N END & & \\
\hline 5257 & GABLE POND N END & & \\
\hline 5258 & GABLE POND N END & & \\
\hline 5259 & WEST LAKE & & \\
\hline 5260 & WEST LAKE & & \\
\hline 5261 & WEST LAKE & & \\
\hline 5262 & WEST LAKE & & \\
\hline 5263 & WEST LAKE & & \\
\hline 5264 & WEST LAKE & & \\
\hline 5265 & 300 AREA & & \\
\hline 5266 & 300 AREA & & \\
\hline 5267 & PRIEST RAPIDS-RIVER & & \\
\hline 5268 & PRIEST RAPIDS-RIVER & & \\
\hline 5269 & RICH.PMPHS HRM 46.4 & & \\
\hline 5270 & RICH.PMPHS HRM 46.4 & & \\
\hline 5271 & 300 AREA & & \\
\hline 5272 & 300 AREA & & \\
\hline 5273 & PRIEST RAPIDS-RIVER & & \\
\hline 5274 & PRIEST RAPIDS-RIVER & & \\
\hline 5275 & RICH.PMPHS HRM 46.4 & & \\
\hline 5276 & RICH.PMPHS HRM 46.4 & & \\
\hline 5277 & ALE HDQTRS & & \\
\hline 5278 & ALE HDQTRS & & \\
\hline 5279 & ALE HDQTRS & & \\
\hline 5280 & FFTF & & \\
\hline 5281 & FFTF & & \\
\hline 5282 & FFTF & & \\
\hline 5283 & FIRING RANGE & & \\
\hline 5284 & FIRING RANGE & & \\
\hline 5285 & FIRING RANGE & & \\
\hline 5286 & OBSEVATORY-RTLSNK MT & & \\
\hline 5287 & OBSEVATORY-RTLSNK MT & & \\
\hline 5288 & OBSEVATORY-RTLSNK MT & & \\
\hline 5289 & PRIEST RAPIDS-RIVER & & \\
\hline 5290 & PRIEST RAPIDS-RIVER & & \\
\hline 5291 & PRIEST RAPIDS-RIVER & & \\
\hline 5292 & PRIEST RAPIDS-RIVER & & \\
\hline 5293 & PRIEST RAPIDS-RIVER & & \\
\hline 5294 & PRIEST RAPIDS-RIVER & & \\
\hline 5295 & RICH.PMPHS HRM 46.4 & & \\
\hline 5296 & RICH.PMPHS HRM 46.4 & & \\
\hline 5297 & RICH.PMPHS HRM 46.4 & & \\
\hline 5298 & RICH.PMPHS HRM 46.4 & & \\
\hline 5299 & RICH.PMPHS HRM 46.4 & & \\
\hline 5300 & RICH.PMPHS HRM 46.4 & & \\
\hline 5301 & 100 B AREA-RIVER & & \\
\hline 5302 & 100 B AREA-RIVER & & \\
\hline 5303 & 100 B AREA-RIVER & & \\
\hline 5304 & 300 AREA & & \\
\hline 5305 & 300 AREA & & \\
\hline
\end{tabular}




\begin{tabular}{|c|c|c|c|}
\hline & \multicolumn{1}{|c|}{$Z$} & A & AB \\
\hline 1 & SAMP_SITE_NAME & WELL_NAME & SAMPLE_LOCATION \\
\hline 5306 & 300 AREA & & \\
\hline 5307 & 300 AREA & & \\
\hline 5308 & 300 AREA & & \\
\hline 5309 & PRIEST RAPIDS-RIVER & & \\
\hline 5310 & PRIEST RAPIDS-RIVER & & \\
\hline 5311 & PRIEST RAPIDS-RIVER & & \\
\hline 5312 & PRIEST RAPIDS-RIVER & & \\
\hline 5313 & PRIEST RAPIDS-RIVER & & \\
\hline 5314 & RICH.PMPHS HRM 46.4 & & \\
\hline 5315 & RICH.PMPHS HRM 46.4 & & \\
\hline 5316 & RICH.PMPHS HRM 46.4 & & \\
\hline 5317 & RICH.PMPHS HRM 46.4 & & \\
\hline 5318 & RICH.PMPHS HRM 46.4 & & \\
\hline 5319 & 300 AREA & & \\
\hline 5320 & 300 AREA & & \\
\hline 5321 & 300 AREA & & \\
\hline 5322 & 300 AREA & & \\
\hline 5323 & 300 AREA & & \\
\hline 5324 & 300 AREA & & \\
\hline 5325 & LOWER REACH RIVER & & \\
\hline 5326 & LOWER REACH RIVER & & \\
\hline 5327 & LOWER REACH RIVER & & \\
\hline 5328 & LOWER REACH RIVER & & \\
\hline 5329 & LOWER REACH RIVER & & \\
\hline 5330 & LOWER REACH RIVER & & \\
\hline 5331 & UPPER REACH RIVER & & \\
\hline 5332 & UPPER REACH RIVER & & \\
\hline 5333 & UPPER REACH RIVER & & \\
\hline 5334 & UPPER REACH RIVER & & \\
\hline 5335 & UPPER REACH RIVER & & \\
\hline 5336 & UPPER REACH RIVER & & \\
\hline 5337 & 300 AREA & & \\
\hline 5338 & 300 AREA & & \\
\hline 5339 & PRIEST RAPIDS-RIVER & & \\
\hline 5340 & PRIEST RAPIDS-RIVER & & \\
\hline 5341 & RICH.PMPHS HRM 46.4 & & \\
\hline 5342 & RICH.PMPHS HRM 46.4 & & \\
\hline 5343 & PRIEST RAPIDS-RIVER & & \\
\hline 5344 & PRIEST RAPIDS-RIVER & & \\
\hline 5345 & PRIEST RAPIDS-RIVER & & \\
\hline 5346 & PRIEST RAPIDS-RIVER & & \\
\hline 5347 & PRIEST RAPIDS-RIVER & & \\
\hline 5348 & PRIEST RAPIDS-RIVER & & \\
\hline 5349 & RICH.PMPHS HRM 46.4 & & \\
\hline 5350 & RICH.PMPHS HRM 46.4 & & \\
\hline 5351 & RICH.PMPHS HRM 46.4 & & \\
\hline 5352 & RICH.PMPHS HRM 46.4 & & \\
\hline 5353 & RICH.PMPHS HRM 46.4 & & \\
\hline 5354 & RICH.PMPHS HRM 46.4 & & \\
\hline 5355 & YAKIMA BARRICADE & & \\
\hline 5356 & YAKIMA BARRICADE & & \\
\hline 5357 & YAKIMA BARRICADE & & \\
\hline
\end{tabular}




\begin{tabular}{|c|c|c|c|}
\hline & $\bar{Z}$ & $\overline{\mathrm{AA}}$ & $\overline{A B}$ \\
\hline 1 & SAMP_SITE_NAME & WELL_NAME & SAMPLE_LOCATION \\
\hline 5358 & 300 AREA & & \\
\hline \begin{tabular}{|l|l|}
5359 \\
\end{tabular} & 300 AREA & & \\
\hline 5360 & PRIEST RAPIDS-RIVER & & \\
\hline 5361 & PRIEST RAPIDS-RIVER & & \\
\hline 5362 & RICH.PMPHS HRM 46.4 & & \\
\hline 5363 & RICH.PMPHS HRM 46.4 & & \\
\hline \begin{tabular}{|l|l|}
5364 & \\
\end{tabular} & 100 D AREA & & \\
\hline 5365 & 100 D AREA & & \\
\hline \begin{tabular}{|l|l|}
5366 \\
\end{tabular} & 100 D AREA & & \\
\hline \begin{tabular}{|l|l|}
5367 \\
\end{tabular} & 300 AREA & & \\
\hline 5368 & 300 AREA & & \\
\hline 5369 & PRIEST RAPIDS-RIVER & & \\
\hline 5370 & RICH.PMPHS HRM 46.4 & & \\
\hline \begin{tabular}{|l|l|}
5371 \\
\end{tabular} & RICH.PMPHS HRM 46.4 & & \\
\hline \begin{tabular}{|l|l|}
5372 \\
\end{tabular} & RIVERVIEW CANAL & & \\
\hline \begin{tabular}{|l|}
5373 \\
\end{tabular} & RIVERVIEW CANAL & & \\
\hline \begin{tabular}{|l|l|}
5374 \\
\end{tabular} & B POND NORTH SIDE & & \\
\hline 5375 & B POND NORTH SIDE & & \\
\hline 5376 & B POND NORTH SIDE & & \\
\hline 5377 & FFTF POND & & \\
\hline \begin{tabular}{|l|l|}
5378 \\
\end{tabular} & FFTF POND & & \\
\hline 5379 & GABLE POND N END & & \\
\hline 5380 & GABLE POND N END & & \\
\hline 5381 & GABLE POND N END & & \\
\hline \begin{tabular}{|l|l|}
5382 \\
\end{tabular} & WEST LAKE & & \\
\hline 5383 & WEST LAKE & & \\
\hline 5384 & WEST LAKE & & \\
\hline 5385 & WEST LAKE & & \\
\hline 5386 & WEST LAKE & & \\
\hline \begin{tabular}{|l|l|}
5387 \\
\end{tabular} & WEST LAKE & & \\
\hline 5388 & 300 AREA & & \\
\hline 5389 & 300 AREA & & \\
\hline 5390 & PRIEST RAPIDS-RIVER & & \\
\hline 5391 & PRIEST RAPIDS-RIVER & & \\
\hline 5392 & PRIEST RAPIDS-RIVER & & \\
\hline \begin{tabular}{|l|l|}
5393 \\
\end{tabular} & PRIEST RAPIDS-RIVER & & \\
\hline \begin{tabular}{|l|l|}
5394 \\
\end{tabular} & PRIEST RAPIDS-RIVER & & \\
\hline 5395 & PRIEST RAPIDS-RIVER & & \\
\hline 5396 & PRIEST RAPIDS-RIVER & & \\
\hline 5397 & PRIEST RAPIDS-RIVER & & \\
\hline 5398 & RICH.PMPHS HRM 46.4 & & \\
\hline 5399 & RICH.PMPHS HRM 46.4 & & \\
\hline 5400 & RICH.PMPHS HRM 46.4 & & \\
\hline \begin{tabular}{|l|l|}
5401 \\
\end{tabular} & RICH.PMPHS HRM 46.4 & & \\
\hline 5402 & RICH.PMPHS HRM 46.4 & & \\
\hline 5403 & RICH.PMPHS HRM 46.4 & & \\
\hline 5404 & RICH.PMPHS HRM 46.4 & & \\
\hline 5405 & RICH.PMPHS HRM 46.4 & & \\
\hline \begin{tabular}{|l|l|}
5406 \\
\end{tabular} & 300 AREA & & \\
\hline \begin{tabular}{|l|l|}
5407 \\
\end{tabular} & 300 AREA & & \\
\hline 5408 & PRIEST RAPIDS-RIVER & & \\
\hline 5409 & PRIEST RAPIDS-RIVER & & \\
\hline
\end{tabular}




\begin{tabular}{|c|c|c|c|}
\hline & $\bar{Z}$ & $\overline{\mathrm{AA}}$ & $\mathrm{AB}$ \\
\hline 1 & SAMP_SITE_NAME & WELL_NAME & SAMPLE_LOCATION \\
\hline 5410 & RICH.PMPHS HRM 46.4 & & \\
\hline 5411 & RICH.PMPHS HRM 46.4 & & \\
\hline 5412 & ALE HDQTRS & & \\
\hline 5413 & ALE HDQTRS & & \\
\hline 5414 & ALE HDQTRS & & \\
\hline 5415 & FFTF & & \\
\hline 5416 & FFTF & & \\
\hline 5417 & FFTF & & \\
\hline 5418 & FIRING RANGE & & \\
\hline 5419 & FIRING RANGE & & \\
\hline 5420 & FIRING RANGE & & \\
\hline 5421 & OBSEVATORY-RTLSNK MT & & \\
\hline 5422 & OBSEVATORY-RTLSNK MT & & \\
\hline 5423 & OBSEVATORY-RTLSNK MT & & \\
\hline 5424 & 100 B AREA-RIVER & & \\
\hline 5425 & 100 B AREA-RIVER & & \\
\hline 5426 & 100 B AREA-RIVER & & \\
\hline 5427 & 300 AREA & & \\
\hline 5428 & 300 AREA & & \\
\hline 5429 & 300 AREA & & \\
\hline 5430 & 300 AREA & & \\
\hline 5431 & 300 AREA & & \\
\hline 5432 & PRIEST RAPIDS-RIVER & & \\
\hline 5433 & PRIEST RAPIDS-RIVER & & \\
\hline 5434 & PRIEST RAPIDS-RIVER & & \\
\hline 5435 & PRIEST RAPIDS-RIVER & & \\
\hline 5436 & PRIEST RAPIDS-RIVER & & \\
\hline 5437 & PRIEST RAPIDS-RIVER & & \\
\hline 5438 & PRIEST RAPIDS-RIVER & & \\
\hline 5439 & PRIEST RAPIDS-RIVER & & \\
\hline 5440 & PRIEST RAPIDS-RIVER & & \\
\hline 5441 & PRIEST RAPIDS-RIVER & & \\
\hline 5442 & PRIEST RAPIDS-RIVER & & \\
\hline 5443 & RICH.PMPHS HRM 46.4 & & \\
\hline 5444 & RICH.PMPHS HRM 46.4 & & \\
\hline 5445 & RICH.PMPHS HRM 46.4 & & \\
\hline 5446 & RICH.PMPHS HRM 46.4 & & \\
\hline 5447 & RICH.PMPHS HRM 46.4 & & \\
\hline 5448 & RICH.PMPHS HRM 46.4 & & \\
\hline 5449 & RICH.PMPHS HRM 46.4 & & \\
\hline 5450 & RICH.PMPHS HRM 46.4 & & \\
\hline 5451 & RICH.PMPHS HRM 46.4 & & \\
\hline 5452 & RICH.PMPHS HRM 46.4 & & \\
\hline 5453 & RICH.PMPHS HRM 46.4 & & \\
\hline 5454 & 300 AREA & & \\
\hline 5455 & 300 AREA & & \\
\hline 5456 & 300 AREA & & \\
\hline 5457 & 300 AREA & & \\
\hline 5458 & 300 AREA & & \\
\hline 5459 & 300 AREA & & \\
\hline 5460 & 300 AREA & & \\
\hline 5461 & 300 AREA & & \\
\hline
\end{tabular}




\begin{tabular}{|c|c|c|c|}
\hline & $\bar{Z}$ & $\overline{\mathrm{AA}}$ & $\overline{A B}$ \\
\hline 1 & SAMP_SITE_NAME & WELL_NAME & SAMPLE_LOCATION \\
\hline 5462 & PRIEST RAPIDS-RIVER & & \\
\hline 5463 & PRIEST RAPIDS-RIVER & & \\
\hline 5464 & RICH.PMPHS HRM 46.4 & & \\
\hline 5465 & RICH.PMPHS HRM 46.4 & & \\
\hline 5466 & RIVERVIEW CANAL & & \\
\hline 5467 & RIVERVIEW CANAL & & \\
\hline 5468 & YAKIMA BARRICADE & & \\
\hline 5469 & YAKIMA BARRICADE & & \\
\hline 5470 & YAKIMA BARRICADE & & \\
\hline 5471 & 300 AREA & & \\
\hline 5472 & 300 AREA & & \\
\hline 5473 & PRIEST RAPIDS-RIVER & & \\
\hline 5474 & PRIEST RAPIDS-RIVER & & \\
\hline 5475 & PRIEST RAPIDS-RIVER & & \\
\hline 5476 & PRIEST RAPIDS-RIVER & & \\
\hline 5477 & PRIEST RAPIDS-RIVER & & \\
\hline 5478 & PRIEST RAPIDS-RIVER & & \\
\hline 5479 & PRIEST RAPIDS-RIVER & & \\
\hline 5480 & PRIEST RAPIDS-RIVER & & \\
\hline 5481 & RICH.PMPHS HRM 46.4 & & \\
\hline 5482 & RICH.PMPHS HRM 46.4 & & \\
\hline 5483 & RICH.PMPHS HRM 46.4 & & \\
\hline 5484 & RICH.PMPHS HRM 46.4 & & \\
\hline 5485 & RICH.PMPHS HRM 46.4 & & \\
\hline \begin{tabular}{|l|l|}
5486 \\
\end{tabular} & RICH.PMPHS HRM 46.4 & & \\
\hline \begin{tabular}{|l|l|}
5487 \\
\end{tabular} & RICH.PMPHS HRM 46.4 & & \\
\hline 5488 & RICH.PMPHS HRM 46.4 & & \\
\hline 5489 & 100 D AREA & & \\
\hline 5490 & 100 D AREA & & \\
\hline 5491 & 100 D AREA & & \\
\hline 5492 & 300 AREA & & \\
\hline 5493 & 300 AREA & & \\
\hline 5494 & PRIEST RAPIDS-RIVER & & \\
\hline 5495 & PRIEST RAPIDS-RIVER & & \\
\hline 5496 & RICH.PMPHS HRM 46.4 & & \\
\hline 5497 & RICH.PMPHS HRM 46.4 & & \\
\hline 5498 & B POND NORTH SIDE & & \\
\hline 5499 & B POND NORTH SIDE & & \\
\hline 5500 & B POND NORTH SIDE & & \\
\hline 5501 & FFTF POND & & \\
\hline 5502 & FFTF POND & & \\
\hline 5503 & GABLE POND N END & & \\
\hline 5504 & GABLE POND N END & & \\
\hline 5505 & GABLE POND N END & & \\
\hline 5506 & WEST LAKE & & \\
\hline 5507 & WEST LAKE & & \\
\hline 5508 & WEST LAKE & & \\
\hline 5509 & WEST LAKE & & \\
\hline 5510 & WEST LAKE & & \\
\hline 5511 & WEST LAKE & & \\
\hline 5512 & 300 AREA & & \\
\hline 5513 & 300 AREA & & \\
\hline
\end{tabular}




\begin{tabular}{|c|c|c|c|}
\hline & $\bar{Z}$ & $\overline{\mathrm{AA}}$ & $\overline{A B}$ \\
\hline 1 & SAMP_SITE_NAME & WELL_NAME & SAMPLE_LOCATION \\
\hline 5514 & PRIEST RAPIDS-RIVER & & \\
\hline 5515 & RICH.PMPHS HRM 46.4 & & \\
\hline 5516 & RICH.PMPHS HRM 46.4 & & \\
\hline 5517 & PRIEST RAPIDS-RIVER & & \\
\hline 5518 & PRIEST RAPIDS-RIVER & & \\
\hline 5519 & PRIEST RAPIDS-RIVER & & \\
\hline 5520 & PRIEST RAPIDS-RIVER & & \\
\hline 5521 & PRIEST RAPIDS-RIVER & & \\
\hline 5522 & PRIEST RAPIDS-RIVER & & \\
\hline 55 & RICH.PMPHS HRM 46.4 & & \\
\hline 5524 & RICH.PMPHS HRM 46.4 & & \\
\hline 5525 & RICH.PMPHS HRM 46.4 & & \\
\hline 5526 & RICH.PMPHS HRM 46.4 & & \\
\hline 5527 & RICH.PMPHS HRM 46.4 & & \\
\hline 5528 & RICH.PMPHS HRM 46.4 & & \\
\hline 5529 & 300 AREA & & \\
\hline 5530 & 300 AREA & & \\
\hline 5531 & PRIEST RAPIDS-RIVER & & \\
\hline 5532 & PRIEST RAPIDS-RIVER & & \\
\hline 5533 & RICH.PMPHS HRM 46.4 & & \\
\hline 5534 & RICH.PMPHS HRM 46.4 & & \\
\hline 5535 & RIVERVIEW CANAL & & \\
\hline 5536 & RIVERVIEW CANAL & & \\
\hline \begin{tabular}{|l|l|}
5537 \\
\end{tabular} & ALE HDQTRS & & \\
\hline 5538 & ALE HDQTRS & & \\
\hline 5539 & ALE HDQTRS & & \\
\hline 5540 & FFTF & & \\
\hline 5541 & FFTF & & \\
\hline 5542 & FFTF & & \\
\hline 5543 & FIRING RANGE & & \\
\hline 5544 & FIRING RANGE & & \\
\hline 5545 & FIRING RANGE & & \\
\hline 5546 & OBSEVATORY-RTLSNK MT & & \\
\hline 5547 & OBSEVATORY-RTLSNK MT & & \\
\hline 5548 & OBSEVATORY-RTLSNK MT & & \\
\hline 5549 & 100 B AREA-RIVER & & \\
\hline 5550 & 100 B AREA-RIVER & & \\
\hline 5551 & 100 B AREA-RIVER & & \\
\hline 5552 & 300 AREA & & \\
\hline 5553 & 300 AREA & & \\
\hline 5554 & PRIEST RAPIDS-RIVER & & \\
\hline 5555 & PRIEST RAPIDS-RIVER & & \\
\hline 5556 & RICH.PMPHS HRM 46.4 & & \\
\hline \begin{tabular}{|l|l|}
5557 \\
\end{tabular} & RICH.PMPHS HRM 46.4 & & \\
\hline 5558 & 300 AREA & & \\
\hline 5559 & 300 AREA & & \\
\hline 5560 & 300 AREA & & \\
\hline 5561 & 300 AREA & & \\
\hline \begin{tabular}{|l|l|}
5562 \\
\end{tabular} & 300 AREA & & \\
\hline 5563 & 300 AREA & & \\
\hline 5564 & PRIEST RAPIDS-RIVER & & \\
\hline \begin{tabular}{|c|}
5565 \\
\end{tabular} & PRIEST RAPIDS-RIVER & & \\
\hline
\end{tabular}




\begin{tabular}{|c|c|c|c|}
\hline & $\mathrm{Z}$ & $\mathrm{AA}$ & $A B$ \\
\hline 1 & SAMP_SITE_NAME & WELL_NAME & SAMPLE_LOCATION \\
\hline 5566 & PRIEST RAPIDS-RIVER & & \\
\hline 5567 & PRIEST RAPIDS-RIVER & & \\
\hline 5568 & PRIEST RAPIDS-RIVER & & \\
\hline 5569 & PRIEST RAPIDS-RIVER & & \\
\hline 5570 & RICH.PMPHS HRM 46.4 & & \\
\hline 5571 & RICH.PMPHS HRM 46.4 & & \\
\hline 5572 & RICH.PMPHS HRM 46.4 & & \\
\hline 5573 & RICH.PMPHS HRM 46.4 & & \\
\hline 5574 & RICH.PMPHS HRM 46.4 & & \\
\hline 5575 & RICH.PMPHS HRM 46.4 & & \\
\hline 5576 & 300 AREA & & \\
\hline 5577 & 300 AREA & & \\
\hline 5578 & 300 AREA & & \\
\hline 5579 & 300 AREA & & \\
\hline 5580 & 300 AREA & & \\
\hline 5581 & PRIEST RAPIDS-RIVER & & \\
\hline 5582 & PRIEST RAPIDS-RIVER & & \\
\hline 5583 & PRIEST RAPIDS-RIVER & & \\
\hline 5584 & PRIEST RAPIDS-RIVER & & \\
\hline 5585 & PRIEST RAPIDS-RIVER & & \\
\hline 5586 & RICH.PMPHS HRM 46.4 & & \\
\hline 5587 & RICH.PMPHS HRM 46.4 & & \\
\hline 5588 & RICH.PMPHS HRM 46.4 & & \\
\hline 5589 & RICH.PMPHS HRM 46.4 & & \\
\hline 5590 & RICH.PMPHS HRM 46.4 & & \\
\hline 5591 & YAKIMA BARRICADE & & \\
\hline 5592 & YAKIMA BARRICADE & & \\
\hline 5593 & YAKIMA BARRICADE & & \\
\hline 5594 & LOWER REACH RIVER & & \\
\hline 5595 & LOWER REACH RIVER & & \\
\hline 5596 & LOWER REACH RIVER & & \\
\hline 5597 & LOWER REACH RIVER & & \\
\hline 5598 & LOWER REACH RIVER & & \\
\hline 5599 & LOWER REACH RIVER & & \\
\hline 5600 & UPPER REACH RIVER & & \\
\hline 5601 & UPPER REACH RIVER & & \\
\hline 5602 & UPPER REACH RIVER & & \\
\hline 5603 & UPPER REACH RIVER & & \\
\hline 5604 & UPPER REACH RIVER & & \\
\hline 5605 & UPPER REACH RIVER & & \\
\hline 5606 & 300 AREA & & \\
\hline 5607 & 300 AREA & & \\
\hline 5608 & PRIEST RAPIDS-RIVER & & \\
\hline 5609 & PRIEST RAPIDS-RIVER & & \\
\hline 5610 & RICH.PMPHS HRM 46.4 & & \\
\hline 5611 & RICH.PMPHS HRM 46.4 & & \\
\hline 5612 & 100 D AREA & & \\
\hline 5613 & 100 D AREA & & \\
\hline 5614 & 100 D AREA & & \\
\hline 5615 & 300 AREA & & \\
\hline 5616 & 300 AREA & & \\
\hline 5617 & PRIEST RAPIDS-RIVER & & \\
\hline
\end{tabular}




\begin{tabular}{|c|c|c|c|}
\hline & $\bar{Z}$ & $\overline{\mathrm{AA}}$ & $\overline{A B}$ \\
\hline 1 & SAMP_SITE_NAME & WELL_NAME & SAMPLE_LOCATION \\
\hline 5618 & PRIEST RAPIDS-RIVER & & \\
\hline 5619 & PRIEST RAPIDS-RIVER & & \\
\hline 5620 & PRIEST RAPIDS-RIVER & & \\
\hline 5621 & PRIEST RAPIDS-RIVER & & \\
\hline 5622 & PRIEST RAPIDS-RIVER & & \\
\hline 5623 & PRIEST RAPIDS-RIVER & & \\
\hline 5624 & PRIEST RAPIDS-RIVER & & \\
\hline 5625 & RICH.PMPHS HRM 46.4 & & \\
\hline 5626 & RICH.PMPHS HRM 46.4 & & \\
\hline 5627 & RICH.PMPHS HRM 46.4 & & \\
\hline 5628 & RICH.PMPHS HRM 46.4 & & \\
\hline 5629 & RICH.PMPHS HRM 46.4 & & \\
\hline 5630 & RICH.PMPHS HRM 46.4 & & \\
\hline 5631 & RICH.PMPHS HRM 46.4 & & \\
\hline 5632 & RICH.PMPHS HRM 46.4 & & \\
\hline 5633 & B POND NORTH SIDE & & \\
\hline 5634 & B POND NORTH SIDE & & \\
\hline 5635 & B POND NORTH SIDE & & \\
\hline 5636 & FFTF POND & & \\
\hline 5637 & FFTF POND & & \\
\hline 5638 & GABLE POND N END & & \\
\hline 5639 & GABLE POND N END & & \\
\hline 5640 & GABLE POND N END & & \\
\hline 5641 & WEST LAKE & & \\
\hline 5642 & WEST LAKE & & \\
\hline 5643 & WEST LAKE & & \\
\hline 5644 & WEST LAKE & & \\
\hline 5645 & WEST LAKE & & \\
\hline 5646 & WEST LAKE & & \\
\hline 5647 & ALEXANDER FARM & & \\
\hline 5648 & ALEXANDER FARM & & \\
\hline 5649 & ALEXANDER FARM & & \\
\hline 5650 & ALEXANDER FARM & & \\
\hline 5651 & ALEXANDER FARM & & \\
\hline 5652 & MATHEWS CORNER & & \\
\hline 5653 & MATHEWS CORNER & & \\
\hline 5654 & MATHEWS CORNER & & \\
\hline 5655 & MATHEWS CORNER & & \\
\hline 5656 & MATHEWS CORNER & & \\
\hline 5657 & WHITE BLUFFS ASSOC & & \\
\hline 5658 & WHITE BLUFFS ASSOC & & \\
\hline 5659 & WHITE BLUFFS ASSOC & & \\
\hline 5660 & WHITE BLUFFS ASSOC & & \\
\hline 5661 & WHITE BLUFFS ASSOC & & \\
\hline 5662 & 300 AREA & & \\
\hline 5663 & 300 AREA & & \\
\hline 5664 & PRIEST RAPIDS-RIVER & & \\
\hline 5665 & PRIEST RAPIDS-RIVER & & \\
\hline 5666 & RICH.PMPHS HRM 46.4 & & \\
\hline 5667 & RICH.PMPHS HRM 46.4 & & \\
\hline 5668 & 300 AREA & & \\
\hline 5669 & 300 AREA & & \\
\hline
\end{tabular}




\begin{tabular}{|c|c|c|c|}
\hline & $\bar{Z}$ & $\overline{\mathrm{AA}}$ & $\overline{A B}$ \\
\hline 1 & SAMP_SITE_NAME & WELL_NAME & SAMPLE_LOCATION \\
\hline 5670 & PRIEST RAPIDS-RIVER & & \\
\hline 5671 & PRIEST RAPIDS-RIVER & & \\
\hline 5672 & PRIEST RAPIDS-RIVER & & \\
\hline 5673 & PRIEST RAPIDS-RIVER & & \\
\hline 5674 & PRIEST RAPIDS-RIVER & & \\
\hline 5675 & PRIEST RAPIDS-RIVER & & \\
\hline \begin{tabular}{|l|l|}
5676 \\
\end{tabular} & PRIEST RAPIDS-RIVER & & \\
\hline \begin{tabular}{|l|l|}
5677 \\
\end{tabular} & PRIEST RAPIDS-RIVER & & \\
\hline \begin{tabular}{|l|l|}
5678 \\
\end{tabular} & RICH.PMPHS HRM 46.4 & & \\
\hline \begin{tabular}{|l|l|}
5679 \\
\end{tabular} & RICH.PMPHS HRM 46.4 & & \\
\hline 5680 & RICH.PMPHS HRM 46.4 & & \\
\hline 5681 & RICH.PMPHS HRM 46.4 & & \\
\hline \begin{tabular}{|c|c|}
5682 \\
\end{tabular} & RICH.PMPHS HRM 46.4 & & \\
\hline 5683 & RICH.PMPHS HRM 46.4 & & \\
\hline 5684 & RICH.PMPHS HRM 46.4 & & \\
\hline 5685 & RICH.PMPHS HRM 46.4 & & \\
\hline \begin{tabular}{|c|}
5686 \\
\end{tabular} & FFTF & & \\
\hline 5687 & ALE HDQTRS & & \\
\hline 5688 & ALE HDQTRS & & \\
\hline 5689 & ALE HDQTRS & & \\
\hline 5690 & FFTF & & \\
\hline 5691 & FFTF & & \\
\hline \begin{tabular}{|l|l|}
5692 \\
\end{tabular} & FFTF & & \\
\hline \begin{tabular}{|c|c|}
5693 \\
\end{tabular} & FIRING RANGE & & \\
\hline 5694 & FIRING RANGE & & \\
\hline 5695 & FIRING RANGE & & \\
\hline 5696 & OBSEVATORY-RTLSNK MT & & \\
\hline 5697 & OBSEVATORY-RTLSNK MT & & \\
\hline 5698 & OBSEVATORY-RTLSNK MT & & \\
\hline \begin{tabular}{|l|l|}
5699 \\
\end{tabular} & WEBBER RANCH & & \\
\hline 5700 & WEBBER RANCH & & \\
\hline 5701 & WEBBER RANCH & & \\
\hline 5702 & WEBBER RANCH & & \\
\hline 5703 & WEBBER RANCH & & \\
\hline 5704 & 100 B AREA-RIVER & & \\
\hline 5705 & 100 B AREA-RIVER & & \\
\hline 5706 & 100 B AREA-RIVER & & \\
\hline \begin{tabular}{|l|l|}
5707 \\
\end{tabular} & 300 AREA & & \\
\hline 5708 & 300 AREA & & \\
\hline 5709 & PRIEST RAPIDS-RIVER & & \\
\hline 5710 & PRIEST RAPIDS-RIVER & & \\
\hline 5711 & RICH.PMPHS HRM 46.4 & & \\
\hline \begin{tabular}{|l|l|}
5712 \\
\end{tabular} & RICH.PMPHS HRM 46.4 & & \\
\hline 5713 & 300 AREA & & \\
\hline 5714 & 300 AREA & & \\
\hline 5715 & 300 AREA & & \\
\hline 5716 & 300 AREA & & \\
\hline 5717 & 300 AREA & & \\
\hline 5718 & 300 AREA & & \\
\hline 5719 & 300 AREA & & \\
\hline 5720 & 300 AREA & & \\
\hline \begin{tabular}{|l|l|}
5721 \\
\end{tabular} & 300 AREA & & \\
\hline
\end{tabular}




\begin{tabular}{|c|c|c|c|}
\hline & $\mathrm{Z}$ & $\mathrm{AA}$ & $A B$ \\
\hline 1 & SAMP_SITE_NAME & WELL_NAME & SAMPLE_LOCATION \\
\hline 5722 & 300 AREA & & \\
\hline 5723 & 300 AREA & & \\
\hline 5724 & PRIEST RAPIDS-RIVER & & \\
\hline 5725 & PRIEST RAPIDS-RIVER & & \\
\hline 5726 & PRIEST RAPIDS-RIVER & & \\
\hline 5727 & PRIEST RAPIDS-RIVER & & \\
\hline 5728 & PRIEST RAPIDS-RIVER & & \\
\hline 5729 & PRIEST RAPIDS-RIVER & & \\
\hline 5730 & PRIEST RAPIDS-RIVER & & \\
\hline 5731 & PRIEST RAPIDS-RIVER & & \\
\hline 5732 & PRIEST RAPIDS-RIVER & & \\
\hline 5733 & PRIEST RAPIDS-RIVER & & \\
\hline 5734 & PRIEST RAPIDS-RIVER & & \\
\hline 5735 & RICH.PMPHS HRM 46.4 & & \\
\hline 5736 & RICH.PMPHS HRM 46.4 & & \\
\hline 5737 & RICH.PMPHS HRM 46.4 & & \\
\hline 5738 & RICH.PMPHS HRM 46.4 & & \\
\hline 5739 & RICH.PMPHS HRM 46.4 & & \\
\hline 5740 & RICH.PMPHS HRM 46.4 & & \\
\hline 5741 & RICH.PMPHS HRM 46.4 & & \\
\hline 5742 & RICH.PMPHS HRM 46.4 & & \\
\hline 5743 & RICH.PMPHS HRM 46.4 & & \\
\hline 5744 & RICH.PMPHS HRM 46.4 & & \\
\hline 5745 & RICH.PMPHS HRM 46.4 & & \\
\hline 5746 & YAKIMA BARRICADE & & \\
\hline 5747 & YAKIMA BARRICADE & & \\
\hline 5748 & YAKIMA BARRICADE & & \\
\hline 5749 & 300 AREA & & \\
\hline 5750 & 300 AREA & & \\
\hline 5751 & PRIEST RAPIDS-RIVER & & \\
\hline 5752 & PRIEST RAPIDS-RIVER & & \\
\hline 5753 & RICH.PMPHS HRM 46.4 & & \\
\hline 5754 & RICH.PMPHS HRM 46.4 & & \\
\hline 5755 & ALEXANDER FARM & & \\
\hline 5756 & ALEXANDER FARM & & \\
\hline 5757 & ALEXANDER FARM & & \\
\hline 5758 & ALEXANDER FARM & & \\
\hline 5759 & ALEXANDER FARM & & \\
\hline 5760 & 100 D AREA & & \\
\hline 5761 & 100 D AREA & & \\
\hline 5762 & 100 D AREA & & \\
\hline 5763 & MATHEWS CORNER & & \\
\hline 5764 & MATHEWS CORNER & & \\
\hline 5765 & MATHEWS CORNER & & \\
\hline 5766 & MATHEWS CORNER & & \\
\hline 5767 & MATHEWS CORNER & & \\
\hline 5768 & WEBBER RANCH & & \\
\hline 5769 & WEBBER RANCH & & \\
\hline 5770 & WEBBER RANCH & & \\
\hline 5771 & WEBBER RANCH & & \\
\hline 5772 & WEBBER RANCH & & \\
\hline 5773 & 300 AREA & & \\
\hline
\end{tabular}




\begin{tabular}{|c|c|c|c|}
\hline & $\bar{Z}$ & $\mathrm{AA}$ & $\mathrm{AB}$ \\
\hline 1 & SAMP_SITE_NAME & WELL_NAME & SAMPLE_LOCATION \\
\hline 5774 & 300 AREA & & \\
\hline 5775 & PRIEST RAPIDS-RIVER & & \\
\hline 5776 & PRIEST RAPIDS-RIVER & & \\
\hline 5777 & RICH.PMPHS HRM 46.4 & & \\
\hline 5778 & RICH.PMPHS HRM 46.4 & & \\
\hline 5779 & PRIEST RAPIDS-RIVER & & \\
\hline 5780 & PRIEST RAPIDS-RIVER & & \\
\hline 5781 & PRIEST RAPIDS-RIVER & & \\
\hline 5782 & PRIEST RAPIDS-RIVER & & \\
\hline 5783 & PRIEST RAPIDS-RIVER & & \\
\hline 5784 & PRIEST RAPIDS-RIVER & & \\
\hline 5785 & RICH.PMPHS HRM 46.4 & & \\
\hline 5786 & RICH.PMPHS HRM 46.4 & & \\
\hline 5787 & RICH.PMPHS HRM 46.4 & & \\
\hline 5788 & RICH.PMPHS HRM 46.4 & & \\
\hline 5789 & RICH.PMPHS HRM 46.4 & & \\
\hline 5790 & RICH.PMPHS HRM 46.4 & & \\
\hline 5791 & B POND NORTH SIDE & & \\
\hline 5792 & B POND NORTH SIDE & & \\
\hline 5793 & B POND NORTH SIDE & & \\
\hline 5794 & FFTF POND & & \\
\hline 5795 & FFTF POND & & \\
\hline 5796 & WEST LAKE & & \\
\hline 5797 & WEST LAKE & & \\
\hline 5798 & WEST LAKE & & \\
\hline 5799 & WEST LAKE & & \\
\hline 5800 & WEST LAKE & & \\
\hline 5801 & WEST LAKE & & \\
\hline 5802 & 300 AREA & & \\
\hline 5803 & 300 AREA & & \\
\hline 5804 & PRIEST RAPIDS-RIVER & & \\
\hline 5805 & PRIEST RAPIDS-RIVER & & \\
\hline 5806 & RICH.PMPHS HRM 46.4 & & \\
\hline 5807 & RICH.PMPHS HRM 46.4 & & \\
\hline 5808 & 300 AREA & & \\
\hline 5809 & 300 AREA & & \\
\hline 5810 & PRIEST RAPIDS-RIVER & & \\
\hline 5811 & PRIEST RAPIDS-RIVER & & \\
\hline 5812 & RICH.PMPHS HRM 46.4 & & \\
\hline 5813 & RICH.PMPHS HRM 46.4 & & \\
\hline 5814 & ALE HDQTRS & & \\
\hline 5815 & ALE HDQTRS & & \\
\hline 5816 & ALE HDQTRS & & \\
\hline 5817 & FFTF & & \\
\hline 5818 & FFTF & & \\
\hline 5819 & FFTF & & \\
\hline 5820 & FIRING RANGE & & \\
\hline 5821 & FIRING RANGE & & \\
\hline 5822 & FIRING RANGE & & \\
\hline 5823 & OBSEVATORY-RTLSNK MT & & \\
\hline 5824 & OBSEVATORY-RTLSNK MT & & \\
\hline 5825 & OBSEVATORY-RTLSNK MT & & \\
\hline
\end{tabular}




\begin{tabular}{|c|c|c|c|}
\hline & $\mathrm{Z}$ & $\overline{\mathrm{AA}}$ & $\mathrm{AB}$ \\
\hline 1 & SAMP_SITE_NAME & WELL_NAME & SAMPLE_LOCATION \\
\hline 5826 & PRIEST RAPIDS-RIVER & & \\
\hline 5827 & PRIEST RAPIDS-RIVER & & \\
\hline $\mid 5828$ & PRIEST RAPIDS-RIVER & & \\
\hline 5829 & PRIEST RAPIDS-RIVER & & \\
\hline 5830 & PRIEST RAPIDS-RIVER & & \\
\hline 5831 & PRIEST RAPIDS-RIVER & & \\
\hline 5832 & RICH.PMPHS HRM 46.4 & & \\
\hline 5833 & RICH.PMPHS HRM 46.4 & & \\
\hline 5834 & RICH.PMPHS HRM 46.4 & & \\
\hline 5835 & RICH.PMPHS HRM 46.4 & & \\
\hline 5836 & RICH.PMPHS HRM 46.4 & & \\
\hline 5837 & RICH.PMPHS HRM 46.4 & & \\
\hline 5838 & ALEXANDER FARM & & \\
\hline 5839 & MATHEWS CORNER & & \\
\hline 5840 & WEBBER RANCH & & \\
\hline 5841 & WHITE BLUFFS ASSOC & & \\
\hline 5842 & WHITE BLUFFS ASSOC & & \\
\hline 5843 & WHITE BLUFFS ASSOC & & \\
\hline 5844 & WHITE BLUFFS ASSOC & & \\
\hline 5845 & WHITE BLUFFS ASSOC & & \\
\hline 5846 & WHITE BLUFFS ASSOC & & \\
\hline 5847 & 100 B AREA-RIVER & & \\
\hline 5848 & 100 B AREA-RIVER & & \\
\hline 5849 & 100 B AREA-RIVER & & \\
\hline 5850 & 300 AREA & & \\
\hline 5851 & 300 AREA & & \\
\hline 5852 & PRIEST RAPIDS-RIVER & & \\
\hline 5853 & PRIEST RAPIDS-RIVER & & \\
\hline 5854 & RICH.PMPHS HRM 46.4 & & \\
\hline 5855 & RICH.PMPHS HRM 46.4 & & \\
\hline 5856 & 300 AREA & & \\
\hline 5857 & 300 AREA & & \\
\hline 5858 & PRIEST RAPIDS-RIVER & & \\
\hline 5859 & PRIEST RAPIDS-RIVER & & \\
\hline 5860 & RICH.PMPHS HRM 46.4 & & \\
\hline 5861 & RICH.PMPHS HRM 46.4 & & \\
\hline 5862 & PRIEST RAPIDS-RIVER & & \\
\hline 5863 & PRIEST RAPIDS-RIVER & & \\
\hline 5864 & PRIEST RAPIDS-RIVER & & \\
\hline 5865 & PRIEST RAPIDS-RIVER & & \\
\hline 5866 & PRIEST RAPIDS-RIVER & & \\
\hline 5867 & PRIEST RAPIDS-RIVER & & \\
\hline 5868 & RICH.PMPHS HRM 46.4 & & \\
\hline 5869 & RICH.PMPHS HRM 46.4 & & \\
\hline 5870 & RICH.PMPHS HRM 46.4 & & \\
\hline 5871 & RICH.PMPHS HRM 46.4 & & \\
\hline 5872 & RICH.PMPHS HRM 46.4 & & \\
\hline 5873 & RICH.PMPHS HRM 46.4 & & \\
\hline 5874 & YAKIMA BARRICADE & & \\
\hline 5875 & YAKIMA BARRICADE & & \\
\hline 5876 & YAKIMA BARRICADE & & \\
\hline 5877 & 300 AREA & & \\
\hline
\end{tabular}




\begin{tabular}{|c|c|c|c|}
\hline & $\bar{Z}$ & $\overline{\mathrm{AA}}$ & $\overline{A B}$ \\
\hline 1 & SAMP_SITE_NAME & WELL_NAME & SAMPLE_LOCATION \\
\hline 5878 & 300 AREA & & \\
\hline 5879 & 300 AREA & & \\
\hline 5880 & 300 AREA & & \\
\hline 5881 & 300 AREA & & \\
\hline 5882 & PRIEST RAPIDS-RIVER & & \\
\hline 5883 & PRIEST RAPIDS-RIVER & & \\
\hline \begin{tabular}{|l|l|}
5884 \\
\end{tabular} & PRIEST RAPIDS-RIVER & & \\
\hline 5885 & PRIEST RAPIDS-RIVER & & \\
\hline \begin{tabular}{|l|l|}
5886 \\
\end{tabular} & PRIEST RAPIDS-RIVER & & \\
\hline \begin{tabular}{|l|l|}
5887 \\
\end{tabular} & RICH.PMPHS HRM 46.4 & & \\
\hline \begin{tabular}{|l|l|}
5888 \\
\end{tabular} & RICH.PMPHS HRM 46.4 & & \\
\hline 5889 & RICH.PMPHS HRM 46.4 & & \\
\hline 5890 & RICH.PMPHS HRM 46.4 & & \\
\hline 5891 & RICH.PMPHS HRM 46.4 & & \\
\hline \begin{tabular}{|l|l|}
5892 \\
\end{tabular} & ALEXANDER FARM & & \\
\hline \begin{tabular}{|l|l|}
5893 \\
\end{tabular} & ALEXANDER FARM & & \\
\hline \begin{tabular}{|l|l|}
5894 \\
\end{tabular} & ALEXANDER FARM & & \\
\hline 5895 & ALEXANDER FARM & & \\
\hline 5896 & ALEXANDER FARM & & \\
\hline 5897 & WHITE BLUFFS ASSOC & & \\
\hline 5898 & WHITE BLUFFS ASSOC & & \\
\hline 5899 & WHITE BLUFFS ASSOC & & \\
\hline 5900 & WHITE BLUFFS ASSOC & & \\
\hline 5901 & WHITE BLUFFS ASSOC & & \\
\hline 5902 & 100 D AREA & & \\
\hline 5903 & 100 D AREA & & \\
\hline 5904 & 100 D AREA & & \\
\hline 5905 & 300 AREA & & \\
\hline \begin{tabular}{|l|l|}
5906 \\
\end{tabular} & 300 AREA & & \\
\hline \begin{tabular}{|l|l|}
5907 \\
\end{tabular} & 300 AREA & & \\
\hline 5908 & 300 AREA & & \\
\hline 5909 & 300 AREA & & \\
\hline 5910 & 300 AREA & & \\
\hline 5911 & MATHEWS CORNER & & \\
\hline \begin{tabular}{|l|l|}
5912 \\
\end{tabular} & MATHEWS CORNER & & \\
\hline \begin{tabular}{|l|l|}
5913 \\
\end{tabular} & MATHEWS CORNER & & \\
\hline \begin{tabular}{|l|l|}
5914 \\
\end{tabular} & MATHEWS CORNER & & \\
\hline 5915 & MATHEWS CORNER & & \\
\hline 5916 & WEBBER RANCH & & \\
\hline 5917 & WEBBER RANCH & & \\
\hline 5918 & WEBBER RANCH & & \\
\hline \begin{tabular}{|l|l|}
5919 \\
\end{tabular} & WEBBER RANCH & & \\
\hline 5920 & WEBBER RANCH & & \\
\hline \begin{tabular}{|l|l|}
5921 \\
\end{tabular} & LOWER REACH RIVER & & \\
\hline 5922 & LOWER REACH RIVER & & \\
\hline 5923 & LOWER REACH RIVER & & \\
\hline 5924 & LOWER REACH RIVER & & \\
\hline 5925 & LOWER REACH RIVER & & \\
\hline 5926 & LOWER REACH RIVER & & \\
\hline \begin{tabular}{|l|l|}
5927 \\
\end{tabular} & UPPER REACH RIVER & & \\
\hline 5928 & UPPER REACH RIVER & & \\
\hline \begin{tabular}{|l|l|}
5929 \\
\end{tabular} & UPPER REACH RIVER & & \\
\hline
\end{tabular}




\begin{tabular}{|c|c|c|c|}
\hline & $\mathrm{Z}$ & $\overline{\mathrm{AA}}$ & $\mathrm{AB}$ \\
\hline 1 & SAMP_SITE_NAME & WELL_NAME & SAMPLE_LOCATION \\
\hline 5930 & UPPER REACH RIVER & & \\
\hline 5931 & UPPER REACH RIVER & & \\
\hline 5932 & UPPER REACH RIVER & & \\
\hline 5933 & 300 AREA & & \\
\hline 5934 & 300 AREA & & \\
\hline 5935 & PRIEST RAPIDS-RIVER & & \\
\hline 5936 & PRIEST RAPIDS-RIVER & & \\
\hline 5937 & RICH.PMPHS HRM 46.4 & & \\
\hline 5938 & RICH.PMPHS HRM 46.4 & & \\
\hline 5939 & B POND NORTH SIDE & & \\
\hline 5940 & B POND NORTH SIDE & & \\
\hline 5941 & B POND NORTH SIDE & & \\
\hline 5942 & FFTF POND & & \\
\hline 5943 & FFTF POND & & \\
\hline 5944 & WEST LAKE & & \\
\hline 5945 & WEST LAKE & & \\
\hline 5946 & WEST LAKE & & \\
\hline 5947 & WEST LAKE & & \\
\hline 5948 & WEST LAKE & & \\
\hline 5949 & WEST LAKE & & \\
\hline 5950 & 300 AREA & & \\
\hline 5951 & 300 AREA & & \\
\hline 5952 & PRIEST RAPIDS-RIVER & & \\
\hline 5953 & PRIEST RAPIDS-RIVER & & \\
\hline 5954 & PRIEST RAPIDS-RIVER & & \\
\hline 5955 & PRIEST RAPIDS-RIVER & & \\
\hline 5956 & PRIEST RAPIDS-RIVER & & \\
\hline 5957 & PRIEST RAPIDS-RIVER & & \\
\hline 5958 & PRIEST RAPIDS-RIVER & & \\
\hline 5959 & PRIEST RAPIDS-RIVER & & \\
\hline 5960 & RICH.PMPHS HRM 46.4 & & \\
\hline 5961 & RICH.PMPHS HRM 46.4 & & \\
\hline 5962 & RICH.PMPHS HRM 46.4 & & \\
\hline 5963 & RICH.PMPHS HRM 46.4 & & \\
\hline 5964 & RICH.PMPHS HRM 46.4 & & \\
\hline 5965 & RICH.PMPHS HRM 46.4 & & \\
\hline 5966 & RICH.PMPHS HRM 46.4 & & \\
\hline 5967 & RICH.PMPHS HRM 46.4 & & \\
\hline 5968 & 300 AREA & & \\
\hline 5969 & 300 AREA & & \\
\hline 5970 & PRIEST RAPIDS-RIVER & & \\
\hline 5971 & PRIEST RAPIDS-RIVER & & \\
\hline 5972 & RICH.PMPHS HRM 46.4 & & \\
\hline 5973 & RICH.PMPHS HRM 46.4 & & \\
\hline 5974 & RIVERVIEW CANAL & & \\
\hline 5975 & RIVERVIEW CANAL & & \\
\hline 5976 & ALE HDQTRS & & \\
\hline 5977 & ALE HDQTRS & & \\
\hline 5978 & ALE HDQTRS & & \\
\hline 5979 & FFTF & & \\
\hline 5980 & FFTF & & \\
\hline 5981 & FFTF & & \\
\hline
\end{tabular}




\begin{tabular}{|c|c|c|c|}
\hline & \multicolumn{1}{|c|}{$Z$} & A & AB \\
\hline 1 & SAMP_SITE_NAME & WELL_NAME & SAMPLE_LOCATION \\
\hline 5982 & FIRING RANGE & & \\
\hline 5983 & FIRING RANGE & & \\
\hline 5984 & FIRING RANGE & & \\
\hline 5985 & OBSEVATORY-RTLSNK MT & & \\
\hline 5986 & OBSEVATORY-RTLSNK MT & & \\
\hline 5987 & OBSEVATORY-RTLSNK MT & & \\
\hline 5988 & 100 B AREA-RIVER & & \\
\hline 5989 & 100 B AREA-RIVER & & \\
\hline 5990 & 100 B AREA-RIVER & & \\
\hline 5991 & 300 AREA & & \\
\hline 5992 & 300 AREA & & \\
\hline 5993 & PRIEST RAPIDS-RIVER & & \\
\hline 5994 & PRIEST RAPIDS-RIVER & & \\
\hline 5995 & PRIEST RAPIDS-RIVER & & \\
\hline 5996 & PRIEST RAPIDS-RIVER & & \\
\hline 5997 & PRIEST RAPIDS-RIVER & & \\
\hline 5998 & PRIEST RAPIDS-RIVER & & \\
\hline 5999 & PRIEST RAPIDS-RIVER & & \\
\hline 6000 & PRIEST RAPIDS-RIVER & & \\
\hline 6001 & RICH.PMPHS HRM 46.4 & & \\
\hline 6002 & RICH.PMPHS HRM 46.4 & & \\
\hline 6003 & RICH.PMPHS HRM 46.4 & & \\
\hline 6004 & RICH.PMPHS HRM 46.4 & & \\
\hline 6005 & RICH.PMPHS HRM 46.4 & & \\
\hline 6006 & RICH.PMPHS HRM 46.4 & & \\
\hline 6007 & RICH.PMPHS HRM 46.4 & & \\
\hline 6008 & RICH.PMPHS HRM 46.4 & & \\
\hline 6009 & 300 AREA & & \\
\hline 6010 & 300 AREA & & \\
\hline 6011 & PRIEST RAPIDS-RIVER & & \\
\hline 6012 & PRIEST RAPIDS-RIVER & & \\
\hline 6013 & RICH.PMPHS HRM 46.4 & & \\
\hline 6014 & RICH.PMPHS HRM 46.4 & & \\
\hline 6015 & YAKIMA BARRICADE & & \\
\hline 6016 & YAKIMA BARRICADE & & \\
\hline 6017 & YAKIMA BARRICADE & & \\
\hline 6018 & 300 AREA & & \\
\hline 6019 & 300 AREA & & \\
\hline 6020 & 300 AREA & & \\
\hline 6021 & 300 AREA & & \\
\hline 6022 & 300 AREA & & \\
\hline 6023 & PRIEST RAPIDS-RIVER & & \\
\hline 6024 & PRIEST RAPIDS-RIVER & & \\
\hline 6025 & PRIEST RAPIDS-RIVER & & \\
\hline 6026 & PRIEST RAPIDS-RIVER & & \\
\hline 6027 & PRIEST RAPIDS-RIVER & & \\
\hline 6028 & PRIEST RAPIDS-RIVER & & \\
\hline 6029 & PRIEST RAPIDS-RIVER & & \\
\hline 6030 & PRIEST RAPIDS-RIVER & & \\
\hline 6031 & PRIEST RAPIDS-RIVER & & \\
\hline 6032 & PRIEST RAPIDS-RIVER & & \\
\hline 6033 & PRIEST RAPIDS-RIVER & & \\
\hline
\end{tabular}




\begin{tabular}{|c|c|c|c|}
\hline & $\mathrm{Z}$ & $\overline{\mathrm{AA}}$ & $\mathrm{AB}$ \\
\hline 1 & SAMP_SITE_NAME & WELL_NAME & SAMPLE_LOCATION \\
\hline 6034 & RICH.PMPHS HRM 46.4 & & \\
\hline 6035 & RICH.PMPHS HRM 46.4 & & \\
\hline 6036 & RICH.PMPHS HRM 46.4 & & \\
\hline 6037 & RICH.PMPHS HRM 46.4 & & \\
\hline 6038 & RICH.PMPHS HRM 46.4 & & \\
\hline 6039 & RICH.PMPHS HRM 46.4 & & \\
\hline 6040 & RICH.PMPHS HRM 46.4 & & \\
\hline 6041 & RICH.PMPHS HRM 46.4 & & \\
\hline 6042 & RICH.PMPHS HRM 46.4 & & \\
\hline 6043 & RICH.PMPHS HRM 46.4 & & \\
\hline 6044 & RICH.PMPHS HRM 46.4 & & \\
\hline 6045 & WHITE BLUFFS ASSOC & & \\
\hline 6046 & WHITE BLUFFS ASSOC & & \\
\hline 6047 & WHITE BLUFFS ASSOC & & \\
\hline 6048 & WHITE BLUFFS ASSOC & & \\
\hline 6049 & WHITE BLUFFS ASSOC & & \\
\hline 6050 & ALEXANDER FARM & & \\
\hline 6051 & ALEXANDER FARM & & \\
\hline 6052 & ALEXANDER FARM & & \\
\hline 6053 & ALEXANDER FARM & & \\
\hline 6054 & ALEXANDER FARM & & \\
\hline 6055 & 300 AREA & & \\
\hline 6056 & 300 AREA & & \\
\hline 6057 & 300 AREA & & \\
\hline 6058 & 300 AREA & & \\
\hline 6059 & 300 AREA & & \\
\hline 6060 & 300 AREA & & \\
\hline 6061 & MATHEWS CORNER & & \\
\hline 6062 & MATHEWS CORNER & & \\
\hline 6063 & MATHEWS CORNER & & \\
\hline 6064 & MATHEWS CORNER & & \\
\hline 6065 & MATHEWS CORNER & & \\
\hline 6066 & 300 AREA & & \\
\hline 6067 & 300 AREA & & \\
\hline 6068 & PRIEST RAPIDS-RIVER & & \\
\hline 6069 & PRIEST RAPIDS-RIVER & & \\
\hline 6070 & RICH.PMPHS HRM 46.4 & & \\
\hline 6071 & RICH.PMPHS HRM 46.4 & & \\
\hline 6072 & RIVERVIEW CANAL & & \\
\hline 6073 & RIVERVIEW CANAL & & \\
\hline 6074 & B POND NORTH SIDE & & \\
\hline 6075 & B POND NORTH SIDE & & \\
\hline 6076 & B POND NORTH SIDE & & \\
\hline 6077 & FFTF POND & & \\
\hline 6078 & FFTF POND & & \\
\hline 6079 & WEST LAKE & & \\
\hline 6080 & WEST LAKE & & \\
\hline 6081 & WEST LAKE & & \\
\hline 6082 & WEST LAKE & & \\
\hline 6083 & WEST LAKE & & \\
\hline 6084 & WEST LAKE & & \\
\hline 6085 & 300 AREA & & \\
\hline
\end{tabular}




\begin{tabular}{|c|c|c|c|}
\hline & $\bar{Z}$ & $\overline{\mathrm{AA}}$ & $\overline{A B}$ \\
\hline 1 & SAMP_SITE_NAME & WELL_NAME & SAMPLE_LOCATION \\
\hline 6086 & 300 AREA & & \\
\hline 6087 & PRIEST RAPIDS-RIVER & & \\
\hline 6088 & PRIEST RAPIDS-RIVER & & \\
\hline 6089 & RICH.PMPHS HRM 46.4 & & \\
\hline 6090 & RICH.PMPHS HRM 46.4 & & \\
\hline 6091 & PRIEST RAPIDS-RIVER & & \\
\hline 6092 & PRIEST RAPIDS-RIVER & & \\
\hline 6093 & PRIEST RAPIDS-RIVER & & \\
\hline 6094 & PRIEST RAPIDS-RIVER & & \\
\hline 6095 & PRIEST RAPIDS-RIVER & & \\
\hline 6096 & PRIEST RAPIDS-RIVER & & \\
\hline 6097 & RICH.PMPHS HRM 46.4 & & \\
\hline 6098 & RICH.PMPHS HRM 46.4 & & \\
\hline 6099 & RICH.PMPHS HRM 46.4 & & \\
\hline 6100 & RICH.PMPHS HRM 46.4 & & \\
\hline 6101 & RICH.PMPHS HRM 46.4 & & \\
\hline 6102 & RICH.PMPHS HRM 46.4 & & \\
\hline 6103 & 300 AREA & & \\
\hline 6104 & 300 AREA & & \\
\hline 6105 & PRIEST RAPIDS-RIVER & & \\
\hline 6106 & PRIEST RAPIDS-RIVER & & \\
\hline 6107 & RICH.PMPHS HRM 46.4 & & \\
\hline 6108 & RICH.PMPHS HRM 46.4 & & \\
\hline 6109 & ALE HDQTRS & & \\
\hline 6110 & ALE HDQTRS & & \\
\hline 6111 & ALE HDQTRS & & \\
\hline 6112 & FFTF & & \\
\hline 6113 & FFTF & & \\
\hline 6114 & FFTF & & \\
\hline 6115 & FIRING RANGE & & \\
\hline 6116 & FIRING RANGE & & \\
\hline 6117 & FIRING RANGE & & \\
\hline 6118 & OBSEVATORY-RTLSNK MT & & \\
\hline 6119 & OBSEVATORY-RTLSNK MT & & \\
\hline 6120 & OBSEVATORY-RTLSNK MT & & \\
\hline 6121 & 100 B AREA-RIVER & & \\
\hline 6122 & 100 B AREA-RIVER & & \\
\hline 6123 & 100 B AREA-RIVER & & \\
\hline 6124 & 300 AREA & & \\
\hline 6125 & 300 AREA & & \\
\hline 6126 & PRIEST RAPIDS-RIVER & & \\
\hline 6127 & PRIEST RAPIDS-RIVER & & \\
\hline 6128 & RICH.PMPHS HRM 46.4 & & \\
\hline 6129 & RICH.PMPHS HRM 46.4 & & \\
\hline 6130 & PRIEST RAPIDS-RIVER & & \\
\hline 6131 & PRIEST RAPIDS-RIVER & & \\
\hline 6132 & PRIEST RAPIDS-RIVER & & \\
\hline 6133 & PRIEST RAPIDS-RIVER & & \\
\hline 6134 & PRIEST RAPIDS-RIVER & & \\
\hline 6135 & PRIEST RAPIDS-RIVER & & \\
\hline 6136 & RICH.PMPHS HRM 46.4 & & \\
\hline \begin{tabular}{|c|}
6137 \\
\end{tabular} & RICH.PMPHS HRM 46.4 & & \\
\hline
\end{tabular}




\begin{tabular}{|c|c|c|c|}
\hline & $\mathrm{Z}$ & $\overline{\mathrm{AA}}$ & $\mathrm{AB}$ \\
\hline 1 & SAMP_SITE_NAME & WELL_NAME & SAMPLE_LOCATION \\
\hline 6138 & RICH.PMPHS HRM 46.4 & & \\
\hline 6139 & RICH.PMPHS HRM 46.4 & & \\
\hline 6140 & RICH.PMPHS HRM 46.4 & & \\
\hline 6141 & RICH.PMPHS HRM 46.4 & & \\
\hline 6142 & 300 AREA & & \\
\hline 6143 & 300 AREA & & \\
\hline 6144 & PRIEST RAPIDS-RIVER & & \\
\hline 6145 & PRIEST RAPIDS-RIVER & & \\
\hline 6146 & RICH.PMPHS HRM 46.4 & & \\
\hline \begin{tabular}{|c|}
6147 \\
\end{tabular} & RICH.PMPHS HRM 46.4 & & \\
\hline 6148 & RIVERVIEW CANAL & & \\
\hline 6149 & RIVERVIEW CANAL & & \\
\hline 6150 & YAKIMA BARRICADE & & \\
\hline 6151 & YAKIMA BARRICADE & & \\
\hline 6152 & YAKIMA BARRICADE & & \\
\hline 6153 & 300 AREA & & \\
\hline 6154 & 300 AREA & & \\
\hline 6155 & PRIEST RAPIDS-RIVER & & \\
\hline 6156 & PRIEST RAPIDS-RIVER & & \\
\hline 6157 & RICH.PMPHS HRM 46.4 & & \\
\hline 6158 & RICH.PMPHS HRM 46.4 & & \\
\hline 6159 & ALEXANDER FARM & & \\
\hline 6160 & ALEXANDER FARM & & \\
\hline 6161 & ALEXANDER FARM & & \\
\hline 6162 & ALEXANDER FARM & & \\
\hline 6163 & ALEXANDER FARM & & \\
\hline 6164 & WHITE BLUFFS ASSOC & & \\
\hline 6165 & WHITE BLUFFS ASSOC & & \\
\hline 6166 & WHITE BLUFFS ASSOC & & \\
\hline 6167 & WHITE BLUFFS ASSOC & & \\
\hline 6168 & WHITE BLUFFS ASSOC & & \\
\hline 6169 & 100 D AREA & & \\
\hline 6170 & 100 D AREA & & \\
\hline 6171 & 100 D AREA & & \\
\hline 6172 & 300 AREA & & \\
\hline 6173 & 300 AREA & & \\
\hline 6174 & 300 AREA & & \\
\hline 6175 & 300 AREA & & \\
\hline 6176 & 300 AREA & & \\
\hline \begin{tabular}{|l|l|}
6177 \\
\end{tabular} & 300 AREA & & \\
\hline 6178 & PRIEST RAPIDS-RIVER & & \\
\hline 6179 & PRIEST RAPIDS-RIVER & & \\
\hline 6180 & PRIEST RAPIDS-RIVER & & \\
\hline 6181 & PRIEST RAPIDS-RIVER & & \\
\hline 6182 & PRIEST RAPIDS-RIVER & & \\
\hline 6183 & PRIEST RAPIDS-RIVER & & \\
\hline 6184 & RICH.PMPHS HRM 46.4 & & \\
\hline 6185 & RICH.PMPHS HRM 46.4 & & \\
\hline 6186 & RICH.PMPHS HRM 46.4 & & \\
\hline 6187 & RICH.PMPHS HRM 46.4 & & \\
\hline 6188 & RICH.PMPHS HRM 46.4 & & \\
\hline 6189 & RICH.PMPHS HRM 46.4 & & \\
\hline
\end{tabular}




\begin{tabular}{|c|c|c|c|}
\hline & $\bar{Z}$ & $\overline{\mathrm{AA}}$ & $\mathrm{AB}$ \\
\hline 1 & SAMP_SITE_NAME & WELL_NAME & SAMPLE_LOCATION \\
\hline 6190 & MATHEWS CORNER & & \\
\hline 6191 & MATHEWS CORNER & & \\
\hline 6192 & MATHEWS CORNER & & \\
\hline 6193 & MATHEWS CORNER & & \\
\hline 6194 & MATHEWS CORNER & & \\
\hline 6195 & 300 AREA & & \\
\hline 6196 & 300 AREA & & \\
\hline 6197 & 300 AREA & & \\
\hline 6198 & 300 AREA & & \\
\hline 6199 & 300 AREA & & \\
\hline 6200 & PRIEST RAPIDS-RIVER & & \\
\hline 6201 & PRIEST RAPIDS-RIVER & & \\
\hline 6202 & PRIEST RAPIDS-RIVER & & \\
\hline 6203 & PRIEST RAPIDS-RIVER & & \\
\hline 6204 & PRIEST RAPIDS-RIVER & & \\
\hline 6205 & RICH.PMPHS HRM 46.4 & & \\
\hline 6206 & RICH.PMPHS HRM 46.4 & & \\
\hline 6207 & RICH.PMPHS HRM 46.4 & & \\
\hline 6208 & RICH.PMPHS HRM 46.4 & & \\
\hline 6209 & RICH.PMPHS HRM 46.4 & & \\
\hline 6210 & B POND NORTH SIDE & & \\
\hline 6211 & B POND NORTH SIDE & & \\
\hline 6212 & B POND NORTH SIDE & & \\
\hline 6213 & FFTF POND & & \\
\hline 6214 & FFTF POND & & \\
\hline 6215 & WEST LAKE & & \\
\hline 6216 & WEST LAKE & & \\
\hline 6217 & WEST LAKE & & \\
\hline 6218 & WEST LAKE & & \\
\hline 6219 & WEST LAKE & & \\
\hline 6220 & WEST LAKE & & \\
\hline 6221 & LOWER REACH RIVER & & \\
\hline 6222 & LOWER REACH RIVER & & \\
\hline 6223 & LOWER REACH RIVER & & \\
\hline 6224 & LOWER REACH RIVER & & \\
\hline 6225 & LOWER REACH RIVER & & \\
\hline 6226 & LOWER REACH RIVER & & \\
\hline 6227 & UPPER REACH RIVER & & \\
\hline 6228 & UPPER REACH RIVER & & \\
\hline 6229 & UPPER REACH RIVER & & \\
\hline 6230 & UPPER REACH RIVER & & \\
\hline 6231 & UPPER REACH RIVER & & \\
\hline 6232 & UPPER REACH RIVER & & \\
\hline 6233 & 300 AREA & & \\
\hline 6234 & 300 AREA & & \\
\hline 6235 & PRIEST RAPIDS-RIVER & & \\
\hline 6236 & PRIEST RAPIDS-RIVER & & \\
\hline 6237 & RICH.PMPHS HRM 46.4 & & \\
\hline 6238 & RICH.PMPHS HRM 46.4 & & \\
\hline 6239 & 300 AREA & & \\
\hline 6240 & 300 AREA & & \\
\hline 6241 & PRIEST RA & & \\
\hline
\end{tabular}




\begin{tabular}{|c|c|c|c|}
\hline & $\bar{Z}$ & $\mathrm{AA}$ & $\mathrm{AB}$ \\
\hline 1 & SAMP_SITE_NAME & WELL_NAME & SAMPLE_LOCATION \\
\hline 6242 & PRIEST RAPIDS-RIVER & & \\
\hline 6243 & PRIEST RAPIDS-RIVER & & \\
\hline 6244 & PRIEST RAPIDS-RIVER & & \\
\hline 6245 & PRIEST RAPIDS-RIVER & & \\
\hline 6246 & PRIEST RAPIDS-RIVER & & \\
\hline 6247 & PRIEST RAPIDS-RIVER & & \\
\hline 6248 & PRIEST RAPIDS-RIVER & & \\
\hline 6249 & RICH.PMPHS HRM 46.4 & & \\
\hline 6250 & RICH.PMPHS HRM 46.4 & & \\
\hline 6251 & RICH.PMPHS HRM 46.4 & & \\
\hline 6252 & RICH.PMPHS HRM 46.4 & & \\
\hline 6253 & RICH.PMPHS HRM 46.4 & & \\
\hline 6254 & RICH.PMPHS HRM 46.4 & & \\
\hline 6255 & RICH.PMPHS HRM 46.4 & & \\
\hline 6256 & RICH.PMPHS HRM 46.4 & & \\
\hline 6257 & ALE HDQTRS & & \\
\hline 6258 & ALE HDQTRS & & \\
\hline 6259 & ALE HDQTRS & & \\
\hline 6260 & FFTF & & \\
\hline 6261 & FFTF & & \\
\hline 6262 & FFTF & & \\
\hline 6263 & FIRING RANGE & & \\
\hline 6264 & FIRING RANGE & & \\
\hline 6265 & FIRING RANGE & & \\
\hline 6266 & OBSEVATORY-RTLSNK MT & & \\
\hline 6267 & OBSEVATORY-RTLSNK MT & & \\
\hline 6268 & OBSEVATORY-RTLSNK MT & & \\
\hline 6269 & 100 B AREA-RIVER & & \\
\hline 6270 & 100 B AREA-RIVER & & \\
\hline 6271. & 100 B AREA-RIVER & & \\
\hline 6272 & 300 AREA & & \\
\hline 6273 & 300 AREA & & \\
\hline 6274 & PRIEST RAPIDS-RIVER & & \\
\hline 6275 & PRIEST RAPIDS-RIVER & & \\
\hline 6276 & RICH.PMPHS HRM 46.4 & & \\
\hline 6277 & RICH.PMPHS HRM 46.4 & & \\
\hline 6278 & 300 AREA & & \\
\hline 6279 & 300 AREA & & \\
\hline 6280 & PRIEST RAPIDS-RIVER & & \\
\hline 6281 & PRIEST RAPIDS-RIVER & & \\
\hline 6282 & PRIEST RAPIDS-RIVER & & \\
\hline 6283 & PRIEST RAPIDS-RIVER & & \\
\hline 6284 & PRIEST RAPIDS-RIVER & & \\
\hline 6285 & PRIEST RAPIDS-RIVER & & \\
\hline 6286 & PRIEST RAPIDS-RIVER & & \\
\hline 6287 & PRIEST RAPIDS-RIVER & & \\
\hline 6288 & RICH.PMPHS HRM 46.4 & & \\
\hline 6289 & RICH.PMPHS HRM 46.4 & & \\
\hline 6290 & RICH.PMPHS HRM 46.4 & & \\
\hline 6291 & RICH.PMPHS HRM 46.4 & & \\
\hline 6292 & RICH.PMPHS HRM 46.4 & & \\
\hline 6293 & RICH.PMPHS HRM 46.4 & & \\
\hline
\end{tabular}




\begin{tabular}{|c|c|c|c|}
\hline & $\bar{Z}$ & $\overline{\mathrm{AA}}$ & $\overline{A B}$ \\
\hline 1 & SAMP_SITE_NAME & WELL_NAME & SAMPLE_LOCATION \\
\hline 6294 & RICH.PMPHS HRM 46.4 & & \\
\hline 6295 & RICH.PMPHS HRM 46.4 & & \\
\hline 6296 & 100 D AREA & & \\
\hline 6297 & 100 D AREA & & \\
\hline 6298 & 100 D AREA & & \\
\hline 6299 & YAKIMA BARRICADE & & \\
\hline 6300 & YAKIMA BARRICADE & & \\
\hline 6301 & YAKIMA BARRICADE & & \\
\hline 6302 & 300 AREA & & \\
\hline 6303 & 300 AREA & & \\
\hline 6304 & PRIEST RAPIDS-RIVER & & \\
\hline 6305 & PRIEST RAPIDS-RIVER & & \\
\hline 6306 & RICH.PMPHS HRM 46.4 & & \\
\hline 6307 & RICH.PMPHS HRM 46.4 & & \\
\hline 6308 & ALEXANDER FARM & & \\
\hline 6309 & ALEXANDER FARM & & \\
\hline 6310 & ALEXANDER FARM & & \\
\hline 6311 & ALEXANDER FARM & & \\
\hline 6312 & ALEXANDER FARM & & \\
\hline 6313 & WHITE BLUFFS ASSOC & & \\
\hline 6314 & WHITE BLUFFS ASSOC & & \\
\hline 6315 & WHITE BLUFFS ASSOC & & \\
\hline 6316 & WHITE BLUFFS ASSOC & & \\
\hline 6317 & WHITE BLUFFS ASSOC & & \\
\hline \begin{tabular}{|l|}
6318 \\
318
\end{tabular} & 300 AREA & & \\
\hline 6319 & 300 AREA & & \\
\hline 6320 & 300 AREA & & \\
\hline 6321 & 300 AREA & & \\
\hline 6322 & 300 AREA & & \\
\hline 6323 & 300 AREA & & \\
\hline 6324 & MATHEWS CORNER & & \\
\hline 6325 & MATHEWS CORNER & & \\
\hline 6326 & MATHEWS CORNER & & \\
\hline 6327 & MATHEWS CORNER & & \\
\hline 6328 & MATHEWS CORNER & & \\
\hline 6329 & RINGOLD HATCHERY & & \\
\hline 6330 & RINGOLD HATCHERY & & \\
\hline 6331 & RINGOLD HATCHERY & & \\
\hline \begin{tabular}{|c|}
6332 \\
\end{tabular} & RINGOLD HATCHERY & & \\
\hline 6333 & RINGOLD HATCHERY & & \\
\hline 6334 & 300 AREA & & \\
\hline 6335 & 300 AREA & & \\
\hline 6336 & 300 AREA & & \\
\hline 6337 & 300 AREA & & \\
\hline 6338 & 300 AREA & & \\
\hline 6339 & PRIEST RAPIDS-RIVER & & \\
\hline 6340 & PRIEST RAPIDS-RIVER & & \\
\hline 6341 & PRIEST RAPIDS-RIVER & & \\
\hline 6342 & PRIEST RAPIDS-RIVER & & \\
\hline 6343 & PRIEST RAPIDS-RIVER & & \\
\hline 6344 & RICH.PMPHS HRM 46.4 & & \\
\hline 6345 & RICH.PMPHS HRM 46.4 & & \\
\hline
\end{tabular}




\begin{tabular}{|c|c|c|c|}
\hline & $\bar{Z}$ & $\overline{\mathrm{AA}}$ & $\overline{A B}$ \\
\hline 1 & SAMP_SITE_NAME & WELL_NAME & SAMPLE_LOCATION \\
\hline 6346 & RICH.PMPHS HRM 46.4 & & \\
\hline \begin{tabular}{|c|}
6347 \\
\end{tabular} & RICH.PMPHS HRM 46.4 & & \\
\hline 6348 & RICH.PMPHS HRM 46.4 & & \\
\hline 6349 & FFTF & & \\
\hline 6350 & PRIEST RAPIDS-RIVER & & \\
\hline \begin{tabular}{|c|}
6351 \\
\end{tabular} & PRIEST RAPIDS-RIVER & & \\
\hline \begin{tabular}{|c|}
6352 \\
\end{tabular} & PRIEST RAPIDS-RIVER & & \\
\hline \begin{tabular}{|c|}
6353 \\
\end{tabular} & PRIEST RAPIDS-RIVER & & \\
\hline 6354 & PRIEST RAPIDS-RIVER & & \\
\hline \begin{tabular}{|c|}
6355 \\
\end{tabular} & PRIEST RAPIDS-RIVER & & \\
\hline \begin{tabular}{|c|}
6356 \\
\end{tabular} & RICH.PMPHS HRM 46.4 & & \\
\hline \begin{tabular}{|l|l|}
6357 & $-2 \cdot 1$ \\
\end{tabular} & RICH.PMPHS HRM 46.4 & & \\
\hline \begin{tabular}{|c|}
6358 \\
\end{tabular} & RICH.PMPHS HRM 46.4 & & \\
\hline \begin{tabular}{|c|}
6359 \\
\end{tabular} & RICH.PMPHS HRM 46.4 & & \\
\hline 6360 & RICH.PMPHS HRM 46.4 & & \\
\hline 6361 & RICH.PMPHS HRM 46.4 & & \\
\hline \begin{tabular}{|c|}
6362 \\
\end{tabular} & B POND NORTH SIDE & & \\
\hline 6363 & B POND NORTH SIDE & & \\
\hline 6364 & B POND NORTH SIDE & & \\
\hline 6365 & FFTF POND & & \\
\hline \begin{tabular}{|l|l|}
6366 \\
\end{tabular} & FFTF POND & & \\
\hline \begin{tabular}{|l|l|}
6367 \\
\end{tabular} & WEST LAKE & & \\
\hline \begin{tabular}{|c|}
6368 \\
\end{tabular} & WEST LAKE & & \\
\hline \begin{tabular}{|c|}
6369 \\
630
\end{tabular} & WEST LAKE & & \\
\hline 6370 & WEST LAKE & & \\
\hline \begin{tabular}{|l|}
6371 \\
\end{tabular} & WEST LAKE & & \\
\hline \begin{tabular}{|c|}
6372 \\
\end{tabular} & WEST LAKE & & \\
\hline \begin{tabular}{|c|}
6373 \\
\end{tabular} & 300 AREA & & \\
\hline \begin{tabular}{|l|l|}
6374 \\
\end{tabular} & 300 AREA & & \\
\hline 6375 & PRIEST RAPIDS-RIVER & & \\
\hline 6376 & PRIEST RAPIDS-RIVER & & \\
\hline \begin{tabular}{|l|l|}
6377 & -12 \\
\end{tabular} & RICH.PMPHS HRM 46.4 & & \\
\hline \begin{tabular}{|l|l|}
6378 \\
\end{tabular} & RICH.PMPHS HRM 46.4 & & \\
\hline \begin{tabular}{|l|l|}
6379 \\
\end{tabular} & 300 AREA & & \\
\hline 6380 & 300 AREA & & \\
\hline \begin{tabular}{|c|}
6381 \\
\end{tabular} & PRIEST RAPIDS-RIVER & & \\
\hline \begin{tabular}{|c|}
6382 \\
\end{tabular} & PRIEST RAPIDS-RIVER & & \\
\hline 6383 & RICH.PMPHS HRM 46.4 & & \\
\hline \begin{tabular}{|c|}
6384 \\
630
\end{tabular} & RICH.PMPHS HRM 46.4 & & \\
\hline 6385 & WEST LAKE & & \\
\hline \begin{tabular}{|l|l|}
6386 \\
\end{tabular} & WEST LAKE & & \\
\hline \begin{tabular}{|l|}
6387 \\
$32-10$
\end{tabular} & WEST LAKE & & \\
\hline \begin{tabular}{|c|}
6388 \\
\end{tabular} & WEST LAKE & & \\
\hline 6389 & ALE HDQTRS & & \\
\hline 6390 & ALE HDQTRS & & \\
\hline 6391 & ALE HDQTRS & & \\
\hline 6392 & FFTF & & \\
\hline 6393 & FFTF & & \\
\hline 6394 & FFTF & & \\
\hline 6395 & FIRING RANGE & & \\
\hline 6396 & FIRING RANGE & & \\
\hline \begin{tabular}{|l|}
6397 \\
630
\end{tabular} & FIRING RANGE & & \\
\hline
\end{tabular}




\begin{tabular}{|c|c|c|c|}
\hline & $\mathrm{Z}$ & $\overline{\mathrm{AA}}$ & $\mathrm{AB}$ \\
\hline 1 & SAMP_SITE_NAME & WELL_NAME & SAMPLE_LOCATION \\
\hline 6398 & OBSEVATORY-RTLSNK MT & & \\
\hline 6399 & OBSEVATORY-RTLSNK MT & & \\
\hline 6400 & OBSEVATORY-RTLSNK MT & & \\
\hline 6401 & 300 AREA & & \\
\hline 6402 & 300 AREA & & \\
\hline 6403 & 300 AREA & & \\
\hline 6404 & 300 AREA & & \\
\hline 6405 & PRIEST RAPIDS-RIVER & & \\
\hline 6406 & PRIEST RAPIDS-RIVER & & \\
\hline 6407 & PRIEST RAPIDS-RIVER & & \\
\hline 6408 & PRIEST RAPIDS-RIVER & & \\
\hline 6409 & PRIEST RAPIDS-RIVER & & \\
\hline 6410 & PRIEST RAPIDS-RIVER & & \\
\hline 6411 & PRIEST RAPIDS-RIVER & & \\
\hline 6412 & PRIEST RAPIDS-RIVER & & \\
\hline 6413 & PRIEST RAPIDS-RIVER & & \\
\hline 6414 & PRIEST RAPIDS-RIVER & & \\
\hline 6415 & PRIEST RAPIDS-RIVER & & \\
\hline 6416 & RICH.PMPHS HRM 46.4 & & \\
\hline 6417 & RICH.PMPHS HRM 46.4 & & \\
\hline 6418 & RICH.PMPHS HRM 46.4 & & \\
\hline 6419 & RICH.PMPHS HRM 46.4 & & \\
\hline 6420 & RICH.PMPHS HRM 46.4 & & \\
\hline 6421 & RICH.PMPHS HRM 46.4 & & \\
\hline 6422 & RICH.PMPHS HRM 46.4 & & \\
\hline 6423 & RICH.PMPHS HRM 46.4 & & \\
\hline 6424 & RICH.PMPHS HRM 46.4 & & \\
\hline 6425 & RICH.PMPHS HRM 46.4 & & \\
\hline 6426 & RICH.PMPHS HRM 46.4 & & \\
\hline 6427 & 100 B AREA-RIVER & & \\
\hline 6428 & 100 B AREA-RIVER & & \\
\hline 6429 & 100 B AREA-RIVER & & \\
\hline 6430 & 300 AREA & & \\
\hline 6431 & 300 AREA & & \\
\hline 6432 & PRIEST RAPIDS-RIVER & & \\
\hline 6433 & PRIEST RAPIDS-RIVER & & \\
\hline 6434 & RICH.PMPHS HRM 46.4 & & \\
\hline 6435 & RICH.PMPHS HRM 46.4 & & \\
\hline 6436 & 300 AREA & & \\
\hline 6437 & 300 AREA & & \\
\hline 6438 & PRIEST RAPIDS-RIVER & & \\
\hline 6439 & PRIEST RAPIDS-RIVER & & \\
\hline 6440 & RICH.PMPHS HRM 46.4 & & \\
\hline 6441 & RICH.PMPHS HRM 46.4 & & \\
\hline 6442 & PRIEST RAPIDS-RIVER & & \\
\hline 6443 & PRIEST RAPIDS-RIVER & & \\
\hline 6444 & PRIEST RAPIDS-RIVER & & \\
\hline 6445 & PRIEST RAPIDS-RIVER & & \\
\hline 6446 & PRIEST RAPIDS-RIVER & & \\
\hline 6447 & PRIEST RAPIDS-RIVER & & \\
\hline 6448 & PRIEST RAPIDS-RIVER & & \\
\hline 6449 & RICH.PMPHS HRM 46.4 & & \\
\hline
\end{tabular}




\begin{tabular}{|c|c|c|c|}
\hline & $\bar{Z}$ & $\overline{\mathrm{AA}}$ & $\overline{A B}$ \\
\hline 1 & SAMP_SITE_NAME & WELL_NAME & SAMPLE_LOCATION \\
\hline 6450 & RICH.PMPHS HRM 46.4 & & \\
\hline 6451 & RICH.PMPHS HRM 46.4 & & \\
\hline 6452 & RICH.PMPHS HRM 46.4 & & \\
\hline 6453 & RICH.PMPHS HRM 46.4 & & \\
\hline 6454 & RICH.PMPHS HRM 46.4 & & \\
\hline 6455 & RICH.PMPHS HRM 46.4 & & \\
\hline 6456 & YAKIMA BARRICADE & & \\
\hline 6457 & YAKIMA BARRICADE & & \\
\hline 6458 & YAKIMA BARRICADE & & \\
\hline 6459 & 300 AREA & & \\
\hline 6460 & 300 AREA & & \\
\hline 6461 & PRIEST RAPIDS-RIVER & & \\
\hline 6462 & PRIEST RAPIDS-RIVER & & \\
\hline 6463 & RICH.PMPHS HRM 46.4 & & \\
\hline 6464 & RICH.PMPHS HRM 46.4 & & \\
\hline 6465 & ALEXANDER FARM & & \\
\hline 6466 & ALEXANDER FARM & & \\
\hline 6467 & ALEXANDER FARM & & \\
\hline 6468 & ALEXANDER FARM & & \\
\hline 6469 & ALEXANDER FARM & & \\
\hline 6470 & WHITE BLUFFS ASSOC & & \\
\hline 6471 & WHITE BLUFFS ASSOC & & \\
\hline 6472 & WHITE BLUFFS ASSOC & & \\
\hline 6473 & WHITE BLUFFS ASSOC & & \\
\hline 6474 & WHITE BLUFFS ASSOC & & \\
\hline 6475 & 100 D AREA & & \\
\hline 6476 & 100 D AREA & & \\
\hline 6477 & 100 D AREA & & \\
\hline 6478 & MATHEWS CORNER & & \\
\hline 6479 & MATHEWS CORNER & & \\
\hline 6480 & MATHEWS CORNER & & \\
\hline 6481 & MATHEWS CORNER & & \\
\hline 6482 & MATHEWS CORNER & & \\
\hline 6483 & RINGOLD HATCHERY & & \\
\hline 6484 & RINGOLD HATCHERY & & \\
\hline 6485 & RINGOLD HATCHERY & & \\
\hline 6486 & RINGOLD HATCHERY & & \\
\hline 6487 & RINGOLD HATCHERY & & \\
\hline 6488 & 300 AREA & & \\
\hline 6489 & 300 AREA & & \\
\hline 6490 & PRIEST RAPIDS-RIVER & & \\
\hline 6491 & PRIEST RAPIDS-RIVER & & \\
\hline 6492 & RICH.PMPHS HRM 46.4 & & \\
\hline 6493 & RICH.PMPHS HRM 46.4 & & \\
\hline 6494 & PRIEST RAPIDS-RIVER & & \\
\hline 6495 & PRIEST RAPIDS-RIVER & & \\
\hline 6496 & PRIEST RAPIDS-RIVER & & \\
\hline 6497 & PRIEST RAPIDS-RIVER & & \\
\hline 6498 & PRIEST RAPIDS-RIVER & & \\
\hline 6499 & PRIEST RAPIDS-RIVER & & \\
\hline 6500 & PRIEST RAPIDS-RIVER & & \\
\hline 6501 & RICH.PMPHS HRM 46.4 & & \\
\hline
\end{tabular}




\begin{tabular}{|c|l|l|l|}
\hline \multicolumn{1}{|c|}{$Z$} & A & AB & AB \\
\hline 1 & SAMP_SITE_NAME & WELL_NAME & SAMPLE_LOCATION \\
\hline 6502 & RICH.PMPHS HRM 46.4 & & \\
\hline 6503 & RICH.PMPHS HRM 46.4 & & \\
\hline 6504 & RICH.PMPHS HRM 46.4 & & \\
\hline 6505 & RICH.PMPHS HRM 46.4 & & \\
\hline 6506 & RICH.PMPHS HRM 46.4 & & \\
\hline 6507 & RICH.PMPHS HRM 46.4 & & \\
\hline 6508 & B POND NORTH SIDE & & \\
\hline 6509 & B POND NORTH SIDE & & \\
\hline 6510 & B POND NORTH SIDE & & \\
\hline 6511 & WEST LAKE & & \\
\hline 6512 & WEST LAKE & & \\
\hline 6513 & WEST LAKE & & \\
\hline 6514 & WEST LAKE & & \\
\hline 6515 & WEST LAKE & & \\
\hline 6516 & WEST LAKE & & \\
\hline 6517 & FFTF POND & & \\
\hline 6518 & FFTF POND & & \\
\hline 6519 & 300 AREA & & \\
\hline 6520 & 300 AREA & & \\
\hline 6521 & 300 AREA & & \\
\hline 6522 & 300 AREA & & \\
\hline 6523 & 300 AREA & & \\
\hline 6524 & PRIEST RAPIDS-RIVER & & \\
\hline 6525 & PRIEST RAPIDS-RIVER & & \\
\hline 6526 & PRIEST RAPIDS-RIVER & & \\
\hline 6527 & PRIEST RAPIDS-RIVER & & \\
\hline 6528 & PRIEST RAPIDS-RIVER & & \\
\hline 6529 & RICH.PMPHS HRM 46.4 & & \\
\hline 6530 & RICH.PMPHS HRM 46.4 & & \\
\hline 6531 & RICH.PMPHS HRM 46.4 & & \\
\hline 6532 & RICH.PMPHS HRM 46.4 & & \\
\hline 6533 & RICH.PMPHS HRM 46.4 & & \\
\hline 6534 & 300 AREA & & \\
\hline 6535 & 300 AREA & & \\
\hline 6536 & 300 AREA & & \\
\hline 6537 & 300 AREA & & \\
\hline 6538 & 300 AREA & & \\
\hline 6539 & 300 AREA & & \\
\hline 6540 & 300 AREA & & \\
\hline 6541 & LOWER REACH RIVER & & \\
\hline 6542 & LOWER REACH RIVER & & \\
\hline 6543 & LOWER REACH RIVER & & \\
\hline 6544 & LOWER REACH RIVER & & \\
\hline 6545 & LOWER REACH RIVER & & \\
\hline 6546 & LOWER REACH RIVER & & \\
\hline 6547 & UPPER REACH RIVER & & \\
\hline 6548 & UPPER REACH RIVER & & \\
\hline 6549 & UPPER REACH RIVER & & \\
\hline 6550 & UPPER REACH RIVER & & \\
\hline 6551 & UPPER REACH RIVER & & \\
\hline 6552 & UPPER REACH RIVER & & \\
\hline 6553 & 300 AREA & & \\
\hline
\end{tabular}




\begin{tabular}{|c|c|c|c|}
\hline & $\bar{Z}$ & $\overline{\mathrm{AA}}$ & $\overline{A B}$ \\
\hline 1 & SAMP_SITE_NAME & WELL_NAME & SAMPLE_LOCATION \\
\hline 6554 & 300 AREA & & \\
\hline 6555 & PRIEST RAPIDS-RIVER & & \\
\hline 6556 & PRIEST RAPIDS-RIVER & & \\
\hline 6557 & RICH.PMPHS HRM 46.4 & & \\
\hline 6558 & RICH.PMPHS HRM 46.4 & & \\
\hline 6559 & ALE HDQTRS & & \\
\hline 6560 & ALE HDQTRS & & \\
\hline $6561 \mid$ & ALE HDQTRS & & \\
\hline \begin{tabular}{|c|}
6562 \\
\end{tabular} & FFTF & & \\
\hline |6563 & FFTF & & \\
\hline \begin{tabular}{|c|}
6564 \\
\end{tabular} & FFTF & & \\
\hline 6565 & FIRING RANGE & & \\
\hline \begin{tabular}{|c|}
6566 \\
\end{tabular} & FIRING RANGE & & \\
\hline \begin{tabular}{|l|l|}
6567 \\
\end{tabular} & FIRING RANGE & & \\
\hline \begin{tabular}{|c|}
6568 \\
\end{tabular} & OBSEVATORY-RTLSNK MT & & \\
\hline 6569 & OBSEVATORY-RTLSNK MT & & \\
\hline 6570 & OBSEVATORY-RTLSNK MT & & \\
\hline 6571 & 100 B AREA-RIVER & & \\
\hline 6572 & 100 B AREA-RIVER & & \\
\hline 6573 & 100 B AREA-RIVER & & \\
\hline 6574 & 300 AREA & & \\
\hline 6575 & 300 AREA & & \\
\hline \begin{tabular}{|c|}
6576 \\
\end{tabular} & PRIEST RAPIDS-RIVER & & \\
\hline \begin{tabular}{|c|}
6577 \\
\end{tabular} & PRIEST RAPIDS-RIVER & & \\
\hline 6578 & PRIEST RAPIDS-RIVER & & \\
\hline 6579 & PRIEST RAPIDS-RIVER & & \\
\hline 6580 & PRIEST RAPIDS-RIVER & & \\
\hline 6581 & PRIEST RAPIDS-RIVER & & \\
\hline \begin{tabular}{|c|}
6582 \\
\end{tabular} & PRIEST RAPIDS-RIVER & & \\
\hline 6583 & PRIEST RAPIDS-RIVER & & \\
\hline 6584 & PRIEST RAPIDS-RIVER & & \\
\hline 6585 & RICH.PMPHS HRM 46.4 & & \\
\hline 6586 & RICH.PMPHS HRM 46.4 & & \\
\hline \begin{tabular}{|c|}
6587 \\
\end{tabular} & RICH.PMPHS HRM 46.4 & & \\
\hline 6588 & RICH.PMPHS HRM 46.4 & & \\
\hline 6589 & RICH.PMPHS HRM 46.4 & & \\
\hline 6590 & RICH.PMPHS HRM 46.4 & & \\
\hline 6591 & RICH.PMPHS HRM 46.4 & & \\
\hline 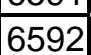 & RICH.PMPHS HRM 46.4 & & \\
\hline 6593 & RICH.PMPHS HRM 46.4 & & \\
\hline 6594 & 300 AREA & & \\
\hline \begin{tabular}{|c|}
6595 \\
\end{tabular} & 300 AREA & & \\
\hline 6596 & PRIEST RAPIDS-RIVER & & \\
\hline 6597 & PRIEST RAPIDS-RIVER & & \\
\hline 6598 & RICH.PMPHS HRM 46.4 & & \\
\hline 6599 & RICH.PMPHS HRM 46.4 & & \\
\hline 6600 & RIVERVIEW CANAL & & \\
\hline 6601 & RIVERVIEW CANAL & & \\
\hline \begin{tabular}{|c|}
6602 \\
\end{tabular} & RIVERVIEW CANAL & & \\
\hline \begin{tabular}{|c|c|}
6603 \\
\end{tabular} & RIVERVIEW CANAL & & \\
\hline 6604 & RIVERVIEW CANAL & & \\
\hline \begin{tabular}{|c|}
6605 \\
\end{tabular} & RIVERVIEW CANAL & & \\
\hline
\end{tabular}




\begin{tabular}{|c|c|c|c|}
\hline & $\mathrm{Z}$ & $\overline{\mathrm{AA}}$ & $\mathrm{AB}$ \\
\hline 1 & SAMP_SITE_NAME & WELL_NAME & SAMPLE_LOCATION \\
\hline 6606 & YAKIMA BARRICADE & & \\
\hline 6607 & YAKIMA BARRICADE & & \\
\hline 6608 & YAKIMA BARRICADE & & \\
\hline 6609 & 300 AREA & & \\
\hline 6610 & 300 AREA & & \\
\hline 6611 & PRIEST RAPIDS-RIVER & & \\
\hline 6612 & PRIEST RAPIDS-RIVER & & \\
\hline 6613 & PRIEST RAPIDS-RIVER & & \\
\hline 6614 & PRIEST RAPIDS-RIVER & & \\
\hline 6615 & PRIEST RAPIDS-RIVER & & \\
\hline 6616 & PRIEST RAPIDS-RIVER & & \\
\hline 6617 & PRIEST RAPIDS-RIVER & & \\
\hline 6618 & PRIEST RAPIDS-RIVER & & \\
\hline 6619 & PRIEST RAPIDS-RIVER & & \\
\hline 6620 & RICH.PMPHS HRM 46.4 & & \\
\hline 6621 & RICH.PMPHS HRM 46.4 & & \\
\hline 6622 & RICH.PMPHS HRM 46.4 & & \\
\hline 6623 & RICH.PMPHS HRM 46.4 & & \\
\hline 6624 & RICH.PMPHS HRM 46.4 & & \\
\hline 6625 & RICH.PMPHS HRM 46.4 & & \\
\hline 6626 & RICH.PMPHS HRM 46.4 & & \\
\hline 6627 & RICH.PMPHS HRM 46.4 & & \\
\hline 6628 & RICH.PMPHS HRM 46.4 & & \\
\hline 6629 & ALEXANDER FARM & & \\
\hline 6630 & WHITE BLUFFS ASSOC & & \\
\hline 6631 & ALEXANDER FARM & & \\
\hline 6632 & ALEXANDER FARM & & \\
\hline 6633 & ALEXANDER FARM & & \\
\hline 6634 & ALEXANDER FARM & & \\
\hline 6635 & ALEXANDER FARM & & \\
\hline 6636 & WHITE BLUFFS ASSOC & & \\
\hline 6637 & WHITE BLUFFS ASSOC & & \\
\hline 6638 & WHITE BLUFFS ASSOC & & \\
\hline 6639 & WHITE BLUFFS ASSOC & & \\
\hline 6640 & WHITE BLUFFS ASSOC & & \\
\hline 6641 & MATHEWS CORNER & & \\
\hline 6642 & MATHEWS CORNER & & \\
\hline 6643 & MATHEWS CORNER & & \\
\hline 6644 & MATHEWS CORNER & & \\
\hline 6645 & MATHEWS CORNER & & \\
\hline 6646 & RINGOLD HATCHERY & & \\
\hline 6647 & RINGOLD HATCHERY & & \\
\hline 6648 & RINGOLD HATCHERY & & \\
\hline 6649 & RINGOLD HATCHERY & & \\
\hline 6650 & RINGOLD HATCHERY & & \\
\hline 6651 & MATHEWS CORNER & & \\
\hline 6652 & RINGOLD HATCHERY & & \\
\hline 6653 & 100 D AREA & & \\
\hline 6654 & 100 D AREA & & \\
\hline 6655 & 100 D AREA & & \\
\hline 6656 & 300 AREA & & \\
\hline 6657 & 300 AREA & & \\
\hline
\end{tabular}




\begin{tabular}{|c|c|c|c|}
\hline & $\bar{Z}$ & $\overline{\mathrm{AA}}$ & $\overline{A B}$ \\
\hline 1 & SAMP_SITE_NAME & WELL_NAME & SAMPLE_LOCATION \\
\hline 6658 & PRIEST RAPIDS-RIVER & & \\
\hline 6659 & PRIEST RAPIDS-RIVER & & \\
\hline 6660 & RICH.PMPHS HRM 46.4 & & \\
\hline 6661 & RICH.PMPHS HRM 46.4 & & \\
\hline 6662 & FFTF & & \\
\hline 6663 & FFTF & & \\
\hline $6664 \mid$ & FFTF & & \\
\hline 6665 & B POND NORTH SIDE & & \\
\hline 6666 & B POND NORTH SIDE & & \\
\hline 6667 & B POND NORTH SIDE & & \\
\hline 6668 & FFTF POND & & \\
\hline 6669 & FFTF POND & & \\
\hline 6670 & WEST LAKE & & \\
\hline 6671 & WEST LAKE & & \\
\hline 6672 & WEST LAKE & & \\
\hline 6673 & WEST LAKE & & \\
\hline 6674 & WEST LAKE & & \\
\hline 6675 & WEST LAKE & & \\
\hline 6676 & 300 AREA & & \\
\hline 6677 & 300 AREA & & \\
\hline 6678 & 300 AREA & & \\
\hline 6679 & 300 AREA & & \\
\hline 6680 & 300 AREA & & \\
\hline 6681 & PRIEST RAPIDS-RIVER & & \\
\hline 6682 & PRIEST RAPIDS-RIVER & & \\
\hline 6683 & PRIEST RAPIDS-RIVER & & \\
\hline 6684 & PRIEST RAPIDS-RIVER & & \\
\hline 6685 & PRIEST RAPIDS-RIVER & & \\
\hline 6686 & PRIEST RAPIDS-RIVER & & \\
\hline$|6687|$ & PRIEST RAPIDS-RIVER & & \\
\hline 6688 & PRIEST RAPIDS-RIVER & & \\
\hline 6689 & PRIEST RAPIDS-RIVER & & \\
\hline 6690 & PRIEST RAPIDS-RIVER & & \\
\hline 6691 & PRIEST RAPIDS-RIVER & & \\
\hline $\mid 6692$ & PRIEST RAPIDS-RIVER & & \\
\hline 6693 & RICH.PMPHS HRM 46.4 & & \\
\hline 6694 & RICH.PMPHS HRM 46.4 & & \\
\hline 6695 & RICH.PMPHS HRM 46.4 & & \\
\hline 6696 & RICH.PMPHS HRM 46.4 & & \\
\hline 6697 & RICH.PMPHS HRM 46.4 & & \\
\hline 6698 & RICH.PMPHS HRM 46.4 & & \\
\hline 6699 & RICH.PMPHS HRM 46.4 & & \\
\hline 6700 & RICH.PMPHS HRM 46.4 & & \\
\hline 6701 & RICH.PMPHS HRM 46.4 & & \\
\hline 6702 & RICH.PMPHS HRM 46.4 & & \\
\hline 6703 & RICH.PMPHS HRM 46.4 & & \\
\hline 6704 & RICH.PMPHS HRM 46.4 & & \\
\hline 6705 & 300 AREA & & \\
\hline 6706 & 300 AREA & & \\
\hline 6707 & 300 AREA & & \\
\hline 6708 & 300 AREA & & \\
\hline 6709 & 300 AREA & & \\
\hline
\end{tabular}




\begin{tabular}{|c|c|c|c|}
\hline & $\bar{Z}$ & $\overline{\mathrm{AA}}$ & $\overline{A B}$ \\
\hline 1 & SAMP_SITE_NAME & WELL_NAME & SAMPLE_LOCATION \\
\hline 6710 & 300 AREA & & \\
\hline 6711 & 300 AREA & & \\
\hline 6712 & 300 AREA & & \\
\hline 6713 & 300 AREA & & \\
\hline 6714 & PRIEST RAPIDS-RIVER & & \\
\hline 6715 & PRIEST RAPIDS-RIVER & & \\
\hline 6716 & RICH.PMPHS HRM 46.4 & & \\
\hline 6717 & RICH.PMPHS HRM 46.4 & & \\
\hline 6718 & RIVERVIEW CANAL & & \\
\hline 6719 & RIVERVIEW CANAL & & \\
\hline 6720 & RIVERVIEW CANAL & & \\
\hline 6721 & RIVERVIEW CANAL & & \\
\hline 6722 & RIVERVIEW CANAL & & \\
\hline 6723 & RIVERVIEW CANAL & & \\
\hline 6724 & ALE HDQTRS & & \\
\hline 6725 & ALE HDQTRS & & \\
\hline 6726 & ALE HDQTRS & & \\
\hline 6727 & FFTF & & \\
\hline 6728 & FFTF & & \\
\hline 6729 & FFTF & & \\
\hline 6730 & FFTF & & \\
\hline 6731 & FIRING RANGE & & \\
\hline 6732 & FIRING RANGE & & \\
\hline 6733 & FIRING RANGE & & \\
\hline 6734 & OBSEVATORY-RTLSNK MT & & \\
\hline 6735 & OBSEVATORY-RTLSNK MT & & \\
\hline 6736 & OBSEVATORY-RTLSNK MT & & \\
\hline 6737 & 300 AREA & & \\
\hline 6738 & 300 AREA & & \\
\hline 6739 & PRIEST RAPIDS-RIVER & & \\
\hline 6740 & PRIEST RAPIDS-RIVER & & \\
\hline 6741 & RICH.PMPHS HRM 46.4 & & \\
\hline 6742 & RICH.PMPHS HRM 46.4 & & \\
\hline 6743 & PRIEST RAPIDS-RIVER & & \\
\hline 6744 & PRIEST RAPIDS-RIVER & & \\
\hline 6745 & PRIEST RAPIDS-RIVER & & \\
\hline 6746 & PRIEST RAPIDS-RIVER & & \\
\hline 6747 & PRIEST RAPIDS-RIVER & & \\
\hline 6748 & PRIEST RAPIDS-RIVER & & \\
\hline 6749 & PRIEST RAPIDS-RIVER & & \\
\hline 6750 & RICH.PMPHS HRM 46.4 & & \\
\hline 6751 & RICH.PMPHS HRM 46.4 & & \\
\hline 6752 & RICH.PMPHS HRM 46.4 & & \\
\hline 6753 & RICH.PMPHS HRM 46.4 & & \\
\hline 6754 & RICH.PMPHS HRM 46.4 & & \\
\hline 6755 & RICH.PMPHS HRM 46.4 & & \\
\hline 6756 & RICH.PMPHS HRM 46.4 & & \\
\hline 6757 & 100 B AREA-RIVER & & \\
\hline 6758 & 100 B AREA-RIVER & & \\
\hline 6759 & 100 B AREA-RIVER & & \\
\hline 6760 & 300 AREA & & \\
\hline 6761 & 300 AREA & & \\
\hline
\end{tabular}




\begin{tabular}{|c|c|c|c|}
\hline & $\bar{Z}$ & $\overline{\mathrm{AA}}$ & $\overline{A B}$ \\
\hline 1 & SAMP_SITE_NAME & \begin{tabular}{|l|} 
WELL_NAME \\
\end{tabular} & SAMPLE_LOCATION \\
\hline 6762 & PRIEST RAPIDS-RIVER & & \\
\hline 6763 & PRIEST RAPIDS-RIVER & & \\
\hline 6764 & RICH.PMPHS HRM 46.4 & & \\
\hline 6765 & RICH.PMPHS HRM 46.4 & & \\
\hline 6766 & FFTF & & \\
\hline \begin{tabular}{|l|l|}
6767 \\
\end{tabular} & FFTF & & \\
\hline \begin{tabular}{|c|}
6768 \\
\end{tabular} & FFTF & & \\
\hline \begin{tabular}{|c|}
6769 \\
\end{tabular} & YAKIMA BARRICADE & & \\
\hline 6770 & YAKIMA BARRICADE & & \\
\hline 6771 & YAKIMA BARRICADE & & \\
\hline \begin{tabular}{|l|l|}
6772 \\
\end{tabular} & 300 AREA & & \\
\hline 6773 & 300 AREA & & \\
\hline 6774 & PRIEST RAPIDS-RIVER & & \\
\hline \begin{tabular}{|c|}
6775 \\
\end{tabular} & PRIEST RAPIDS-RIVER & & \\
\hline 6776 & RICH.PMPHS HRM 46.4 & & \\
\hline 6777 & RICH.PMPHS HRM 46.4 & & \\
\hline 6778 & ALEXANDER FARM & & \\
\hline 6779 & ALEXANDER FARM & & \\
\hline 6780 & ALEXANDER FARM & & \\
\hline 6781 & ALEXANDER FARM & & \\
\hline \begin{tabular}{|c|}
6782 \\
\end{tabular} & ALEXANDER FARM & & \\
\hline 6783 & WHITE BLUFFS ASSOC & & \\
\hline \begin{tabular}{|c|}
6784 \\
\end{tabular} & WHITE BLUFFS ASSOC & & \\
\hline \begin{tabular}{|c|}
6785 \\
\end{tabular} & WHITE BLUFFS ASSOC & & \\
\hline 6786 & WHITE BLUFFS ASSOC & & \\
\hline \begin{tabular}{|l|}
6787 \\
\end{tabular} & WHITE BLUFFS ASSOC & & \\
\hline 6788 & 100 D AREA & & \\
\hline 6789 & 100 D AREA & & \\
\hline 6790 & 100 D AREA & & \\
\hline 6791 & PRIEST RAPIDS-RIVER & & \\
\hline 6792 & PRIEST RAPIDS-RIVER & & \\
\hline 6793 & PRIEST RAPIDS-RIVER & & \\
\hline 6794 & PRIEST RAPIDS-RIVER & & \\
\hline 6795 & PRIEST RAPIDS-RIVER & & \\
\hline 6796 & PRIEST RAPIDS-RIVER & & \\
\hline \begin{tabular}{|l|l|}
6797 \\
\end{tabular} & PRIEST RAPIDS-RIVER & & \\
\hline \begin{tabular}{|c|}
6798 \\
\end{tabular} & RICH.PMPHS HRM 46.4 & & \\
\hline \begin{tabular}{|l|l|}
6799 \\
\end{tabular} & RICH.PMPHS HRM 46.4 & & \\
\hline 6800 & RICH.PMPHS HRM 46.4 & & \\
\hline 6801 & RICH.PMPHS HRM 46.4 & & \\
\hline \begin{tabular}{|c|}
6802 \\
\end{tabular} & RICH.PMPHS HRM 46.4 & & \\
\hline \begin{tabular}{|c|}
6803 \\
\end{tabular} & RICH.PMPHS HRM 46.4 & & \\
\hline \begin{tabular}{|c|}
6804 \\
\end{tabular} & RICH.PMPHS HRM 46.4 & & \\
\hline \begin{tabular}{|c|}
6805 \\
\end{tabular} & MATHEWS CORNER & & \\
\hline 6806 & MATHEWS CORNER & & \\
\hline 6807 & MATHEWS CORNER & & \\
\hline 6808 & MATHEWS CORNER & & \\
\hline 6809 & MATHEWS CORNER & & \\
\hline \begin{tabular}{|c|}
6810 \\
\end{tabular} & RINGOLD HATCHERY & & \\
\hline \begin{tabular}{|c|}
6811 \\
\end{tabular} & RINGOLD HATCHERY & & \\
\hline 6812 & RINGOLD HATCHERY & & \\
\hline \begin{tabular}{|c|}
6813 \\
3
\end{tabular} & RINGOLD HATCHERY & & \\
\hline
\end{tabular}




\begin{tabular}{|c|c|c|c|}
\hline & $\bar{Z}$ & $\overline{\mathrm{AA}}$ & $\overline{A B}$ \\
\hline 1 & SAMP_SITE_NAME & WELL_NAME & SAMPLE_LOCATION \\
\hline 6814 & RINGOLD HATCHERY & & \\
\hline 6815 & 300 AREA & & \\
\hline 6816 & 300 AREA & & \\
\hline 6817 & PRIEST RAPIDS-RIVER & & \\
\hline $\mid 6818$ & PRIEST RAPIDS-RIVER & & \\
\hline 6819 & RICH.PMPHS HRM 46.4 & & \\
\hline 6820 & RICH.PMPHS HRM 46.4 & & \\
\hline 6821 & RIVERVIEW CANAL & & \\
\hline 6822 & RIVERVIEW CANAL & & \\
\hline $\mid 6823$ & RIVERVIEW CANAL & & \\
\hline 6824 & RIVERVIEW CANAL & & \\
\hline 6825 & RIVERVIEW CANAL & & \\
\hline 6826 & RIVERVIEW CANAL & & \\
\hline 6827 & FFTF & & \\
\hline 6828 & FFTF & & \\
\hline 6829 & FFTF & & \\
\hline 6830 & B POND NORTH SIDE & & \\
\hline 6831 & B POND NORTH SIDE & & \\
\hline 6832 & B POND NORTH SIDE & & \\
\hline 6833 & FFTF POND & & \\
\hline 6834 & FFTF POND & & \\
\hline 6835 & WEST LAKE & & \\
\hline 6836 & WEST LAKE & & \\
\hline 6837 & WEST LAKE & & \\
\hline 6838 & WEST LAKE & & \\
\hline 6839 & WEST LAKE & & \\
\hline 6840 & WEST LAKE & & \\
\hline 6841 & 300 AREA & & \\
\hline 6842 & 300 AREA & & \\
\hline 6843 & PRIEST RAPIDS-RIVER & & \\
\hline 6844 & PRIEST RAPIDS-RIVER & & \\
\hline 6845 & RICH.PMPHS HRM 46.4 & & \\
\hline 6846 & RICH.PMPHS HRM 46.4 & & \\
\hline 6847 & 300 AREA & & \\
\hline 6848 & 300 AREA & & \\
\hline 6849 & 300 AREA & & \\
\hline 6850 & 300 AREA & & \\
\hline 6851 & 300 AREA & & \\
\hline 6852 & 300 AREA & & \\
\hline 6853 & 300 AREA & & \\
\hline 6854 & 300 AREA & & \\
\hline 6855 & 300 AREA & & \\
\hline 6856 & 300 AREA & & \\
\hline 6857 & 300 AREA & & \\
\hline 6858 & 300 AREA & & \\
\hline 6859 & PRIEST RAPIDS-RIVER & & \\
\hline 6860 & PRIEST RAPIDS-RIVER & & \\
\hline 6861 & PRIEST RAPIDS-RIVER & & \\
\hline 6862 & PRIEST RAPIDS-RIVER & & \\
\hline 6863 & PRIEST RAPIDS-RIVER & & \\
\hline 6864 & PRIEST RAPIDS-RIVER & & \\
\hline 6865 & PRIEST RAPIDS-RIVER & & \\
\hline
\end{tabular}




\begin{tabular}{|c|c|c|c|}
\hline & $\mathrm{Z}$ & $\overline{\mathrm{AA}}$ & $\mathrm{AB}$ \\
\hline 1 & SAMP_SITE_NAME & WELL_NAME & SAMPLE_LOCATION \\
\hline 6866 & PRIEST RAPIDS-RIVER & & \\
\hline \begin{tabular}{|c|}
6867 \\
\end{tabular} & PRIEST RAPIDS-RIVER & & \\
\hline 6868 & PRIEST RAPIDS-RIVER & & \\
\hline 6869 & PRIEST RAPIDS-RIVER & & \\
\hline 6870 & PRIEST RAPIDS-RIVER & & \\
\hline 6871 & RICH.PMPHS HRM 46.4 & & \\
\hline 6872 & RICH.PMPHS HRM 46.4 & & \\
\hline 6873 & RICH.PMPHS HRM 46.4 & & \\
\hline 6874 & RICH.PMPHS HRM 46.4 & & \\
\hline 6875 & RICH.PMPHS HRM 46.4 & & \\
\hline 6876 & RICH.PMPHS HRM 46.4 & & \\
\hline 6877 & RICH.PMPHS HRM 46.4 & & \\
\hline 6878 & RICH.PMPHS HRM 46.4 & & \\
\hline 6879 & RICH.PMPHS HRM 46.4 & & \\
\hline 6880 & RICH.PMPHS HRM 46.4 & & \\
\hline 6881 & RICH.PMPHS HRM 46.4 & & \\
\hline 6882 & RICH.PMPHS HRM 46.4 & & \\
\hline 6883 & ALE HDQTRS & & \\
\hline 6884 & ALE HDQTRS & & \\
\hline 6885 & ALE HDQTRS & & \\
\hline 6886 & FFTF & & \\
\hline 6887 & FFTF & & \\
\hline 6888 & FFTF & & \\
\hline 6889 & FIRING RANGE & & \\
\hline 6890 & FIRING RANGE & & \\
\hline 6891 & FIRING RANGE & & \\
\hline 6892 & OBSEVATORY-RTLSNK MT & & \\
\hline 6893 & OBSEVATORY-RTLSNK MT & & \\
\hline 6894 & OBSEVATORY-RTLSNK MT & & \\
\hline 6895 & 100 B AREA-RIVER & & \\
\hline 6896 & 100 B AREA-RIVER & & \\
\hline 6897 & 100 B AREA-RIVER & & \\
\hline 6898 & LOWER REACH RIVER & & \\
\hline 6899 & LOWER REACH RIVER & & \\
\hline 6900 & LOWER REACH RIVER & & \\
\hline 6901 & LOWER REACH RIVER & & \\
\hline 6902 & LOWER REACH RIVER & & \\
\hline 6903 & LOWER REACH RIVER & & \\
\hline 6904 & UPPER REACH RIVER & & \\
\hline 6905 & UPPER REACH RIVER & & \\
\hline 6906 & UPPER REACH RIVER & & \\
\hline 6907 & UPPER REACH RIVER & & \\
\hline 6908 & UPPER REACH RIVER & & \\
\hline 6909 & UPPER REACH RIVER & & \\
\hline 6910 & 300 AREA & & \\
\hline 6911 & 300 AREA & & \\
\hline 6912 & PRIEST RAPIDS-RIVER & & \\
\hline 6913 & PRIEST RAPIDS-RIVER & & \\
\hline 6914 & RICH.PMPHS HRM 46.4 & & \\
\hline 6915 & RICH.PMPHS HRM 46.4 & & \\
\hline 6916 & 300 AREA & & \\
\hline \begin{tabular}{|l|l|}
6917 \\
\end{tabular} & 300 AREA & & \\
\hline
\end{tabular}




\begin{tabular}{|c|c|c|c|}
\hline & $\bar{Z}$ & $\overline{\mathrm{AA}}$ & $\overline{A B}$ \\
\hline 1 & SAMP_SITE_NAME & WELL_NAME & SAMPLE_LOCATION \\
\hline 6918 & PRIEST RAPIDS-RIVER & & \\
\hline 6919 & PRIEST RAPIDS-RIVER & & \\
\hline 6920 & PRIEST RAPIDS-RIVER & & \\
\hline 6921 & PRIEST RAPIDS-RIVER & & \\
\hline 6922 & PRIEST RAPIDS-RIVER & & \\
\hline 6923 & PRIEST RAPIDS-RIVER & & \\
\hline \begin{tabular}{|l|}
6924 \\
\end{tabular} & PRIEST RAPIDS-RIVER & & \\
\hline \begin{tabular}{|c|}
6925 \\
\end{tabular} & PRIEST RAPIDS-RIVER & & \\
\hline \begin{tabular}{|l|l|}
6926 \\
\end{tabular} & PRIEST RAPIDS-RIVER & & \\
\hline \begin{tabular}{|c|}
6927 \\
2020
\end{tabular} & RICH.PMPHS HRM 46.4 & & \\
\hline \begin{tabular}{|l|l|}
6928 \\
\end{tabular} & RICH.PMPHS HRM 46.4 & & \\
\hline 6929 & RICH.PMPHS HRM 46.4 & & \\
\hline 6930 & RICH.PMPHS HRM 46.4 & & \\
\hline 6931 & RICH.PMPHS HRM 46.4 & & \\
\hline \begin{tabular}{|c|}
6932 \\
\end{tabular} & RICH.PMPHS HRM 46.4 & & \\
\hline 6933 & RICH.PMPHS HRM 46.4 & & \\
\hline \begin{tabular}{|c|}
6934 \\
\end{tabular} & RICH.PMPHS HRM 46.4 & & \\
\hline 6935 & RICH.PMPHS HRM 46.4 & & \\
\hline 6936 & FFTF & & \\
\hline \begin{tabular}{|l|l|}
6937 & -10 \\
\end{tabular} & FFTF & & \\
\hline \begin{tabular}{|c|}
6938 \\
\end{tabular} & FFTF & & \\
\hline 6939 & YAKIMA BARRICADE & & \\
\hline \begin{tabular}{|c|}
6940 \\
\end{tabular} & YAKIMA BARRICADE & & \\
\hline \begin{tabular}{|c|}
6941 \\
\end{tabular} & YAKIMA BARRICADE & & \\
\hline 6942 & 300 AREA & & \\
\hline 6943 & 300 AREA & & \\
\hline 6944 & PRIEST RAPIDS-RIVER & & \\
\hline \begin{tabular}{|c|c|}
6945 \\
\end{tabular} & PRIEST RAPIDS-RIVER & & \\
\hline \begin{tabular}{|l|l|}
6946 \\
\end{tabular} & RICH.PMPHS HRM 46.4 & & \\
\hline \begin{tabular}{|l|l|}
6947 \\
\end{tabular} & RICH.PMPHS HRM 46.4 & & \\
\hline 6948 & ALEXANDER FARM & & \\
\hline 6949 & ALEXANDER FARM & & \\
\hline 6950 & ALEXANDER FARM & & \\
\hline 6951 & ALEXANDER FARM & & \\
\hline 6952 & ALEXANDER FARM & & \\
\hline \begin{tabular}{|c|}
6953 \\
\end{tabular} & WHITE BLUFFS ASSOC & & \\
\hline \begin{tabular}{|c|}
6954 \\
\end{tabular} & WHITE BLUFFS ASSOC & & \\
\hline 6955 & WHITE BLUFFS ASSOC & & \\
\hline \begin{tabular}{|c|}
6956 \\
\end{tabular} & WHITE BLUFFS ASSOC & & \\
\hline \begin{tabular}{|c|}
6957 \\
\end{tabular} & WHITE BLUFFS ASSOC & & \\
\hline 6958 & 100 D AREA & & \\
\hline \begin{tabular}{|c|}
6959 \\
\end{tabular} & 100 D AREA & & \\
\hline \begin{tabular}{|c|}
6960 \\
\end{tabular} & 100 D AREA & & \\
\hline \begin{tabular}{|l|l|}
6961 \\
\end{tabular} & MATHEWS CORNER & & \\
\hline 6962 & MATHEWS CORNER & & \\
\hline 6963 & MATHEWS CORNER & & \\
\hline 6964 & MATHEWS CORNER & & \\
\hline 6965 & MATHEWS CORNER & & \\
\hline \begin{tabular}{|l|l|}
6966 \\
\end{tabular} & RINGOLD HATCHERY & & \\
\hline \begin{tabular}{|l|l|}
6967 \\
\end{tabular} & RINGOLD HATCHERY & & \\
\hline 6968 & RINGOLD HATCHERY & & \\
\hline \begin{tabular}{|c|}
6969 \\
\end{tabular} & RINGOLD HATCHERY & & \\
\hline
\end{tabular}




\begin{tabular}{|c|c|c|c|}
\hline & $\bar{Z}$ & $\overline{\mathrm{AA}}$ & $\overline{A B}$ \\
\hline 1 & SAMP_SITE_NAME & WELL_NAME & SAMPLE_LOCATION \\
\hline 6970 & RINGOLD HATCHERY & & \\
\hline 6971 & 300 AREA & & \\
\hline 6972 & 300 AREA & & \\
\hline 6973 & PRIEST RAPIDS-RIVER & & \\
\hline 6974 & PRIEST RAPIDS-RIVER & & \\
\hline 6975 & PRIEST RAPIDS-RIVER & & \\
\hline 6976 & PRIEST RAPIDS-RIVER & & \\
\hline \begin{tabular}{|c|}
6977 \\
\end{tabular} & PRIEST RAPIDS-RIVER & & \\
\hline 6978 & PRIEST RAPIDS-RIVER & & \\
\hline 6979 & PRIEST RAPIDS-RIVER & & \\
\hline 6980 & PRIEST RAPIDS-RIVER & & \\
\hline 6981 & PRIEST RAPIDS-RIVER & & \\
\hline 6982 & RICH.PMPHS HRM 46.4 & & \\
\hline 6983 & RICH.PMPHS HRM 46.4 & & \\
\hline 6984 & RICH.PMPHS HRM 46.4 & & \\
\hline 6985 & RICH.PMPHS HRM 46.4 & & \\
\hline \begin{tabular}{|c|}
6986 \\
\end{tabular} & RICH.PMPHS HRM 46.4 & & \\
\hline 6987 & RICH.PMPHS HRM 46.4 & & \\
\hline 6988 & RICH.PMPHS HRM 46.4 & & \\
\hline 6989 & RICH.PMPHS HRM 46.4 & & \\
\hline 6990 & RICH.PMPHS HRM 46.4 & & \\
\hline 6991 & FFTF & & \\
\hline 6992 & FFTF & & \\
\hline 6993 & FFTF & & \\
\hline 6994 & B POND NORTH SIDE & & \\
\hline 6995 & B POND NORTH SIDE & & \\
\hline 6996 & B POND NORTH SIDE & & \\
\hline \begin{tabular}{|c|}
6997 \\
\end{tabular} & FFTF POND & & \\
\hline \begin{tabular}{|c|}
6998 \\
\end{tabular} & FFTF POND & & \\
\hline 6999 & WEST LAKE & & \\
\hline 7000 & WEST LAKE & & \\
\hline 7001 & WEST LAKE & & \\
\hline 7002 & WEST LAKE & & \\
\hline 7003 & WEST LAKE & & \\
\hline 7004 & WEST LAKE & & \\
\hline 7005 & 300 AREA & & \\
\hline 7006 & 300 AREA & & \\
\hline 7007 & PRIEST RAPIDS-RIVER & & \\
\hline 7008 & PRIEST RAPIDS-RIVER & & \\
\hline 7009 & RICH.PMPHS HRM 46.4 & & \\
\hline 7010 & RICH.PMPHS HRM 46.4 & & \\
\hline 7011 & 300 AREA & & \\
\hline 7012 & 300 AREA & & \\
\hline 7013 & 300 AREA & & \\
\hline 7014 & 300 AREA & & \\
\hline 7015 & 300 AREA & & \\
\hline 7016 & 300 AREA & & \\
\hline 7017 & 300 AREA & & \\
\hline 7018 & 300 AREA & & \\
\hline 7019 & 300 AREA & & \\
\hline 7020 & 300 AREA & & \\
\hline 7021 & 300 AREA & & \\
\hline
\end{tabular}




\begin{tabular}{|c|c|c|c|}
\hline & $\bar{Z}$ & $\mathrm{AA}$ & $\overline{A B}$ \\
\hline 1 & SAMP_SITE_NAME & WELL_NAME & SAMPLE_LOCATION \\
\hline 7022 & 300 AREA & & \\
\hline 7023 & PRIEST RAPIDS-RIVER & & \\
\hline 7024 & PRIEST RAPIDS-RIVER & & \\
\hline 7025 & PRIEST RAPIDS-RIVER & & \\
\hline 7026 & PRIEST RAPIDS-RIVER & & \\
\hline 7027 & PRIEST RAPIDS-RIVER & & \\
\hline 7028 & RICH.PMPHS HRM 46.4 & & \\
\hline 7029 & RICH.PMPHS HRM 46.4 & & \\
\hline 7030 & RICH.PMPHS HRM 46.4 & & \\
\hline 7031 & RICH.PMPHS HRM 46.4 & & \\
\hline 7032 & RICH.PMPHS HRM 46.4 & & \\
\hline
\end{tabular}




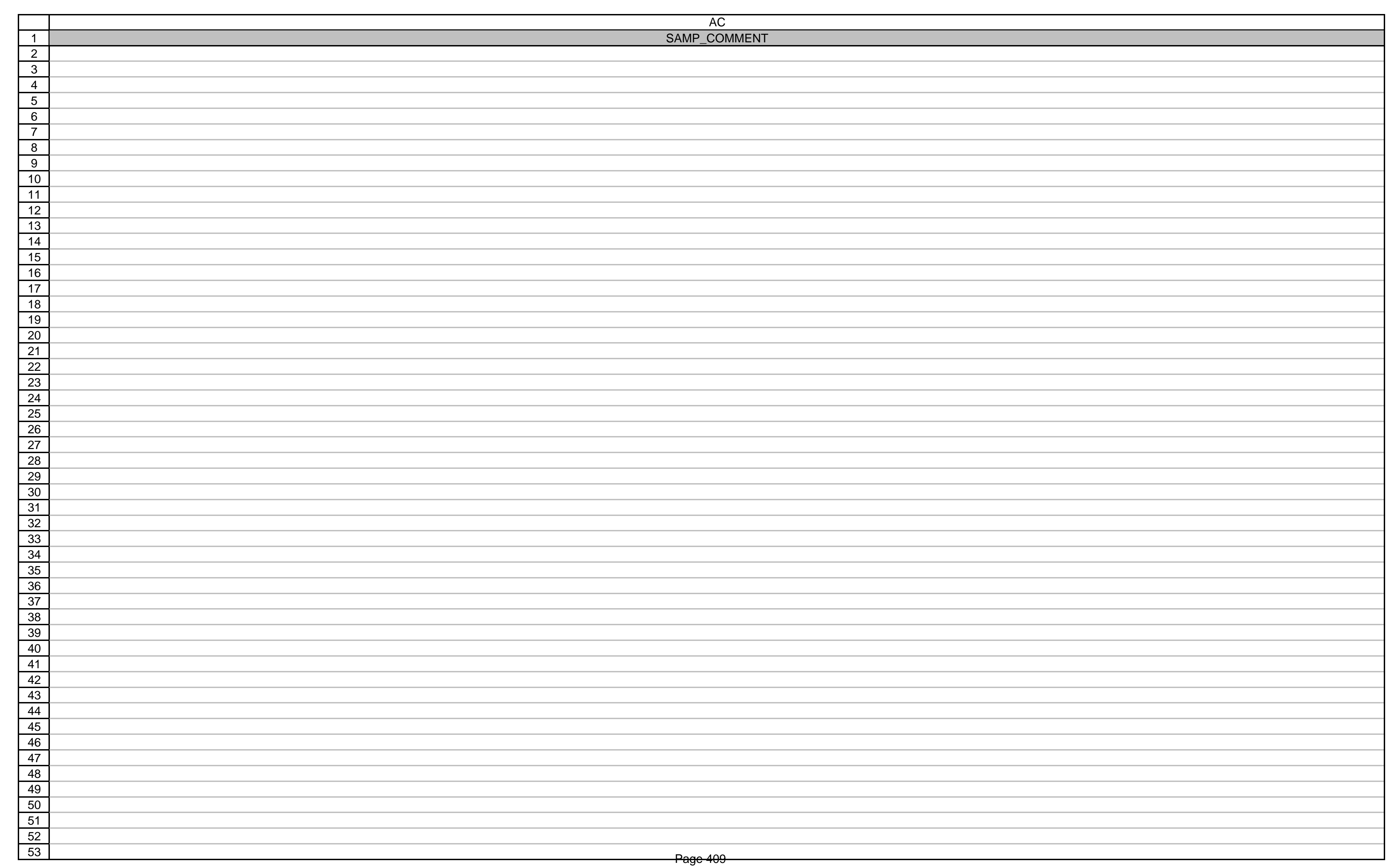




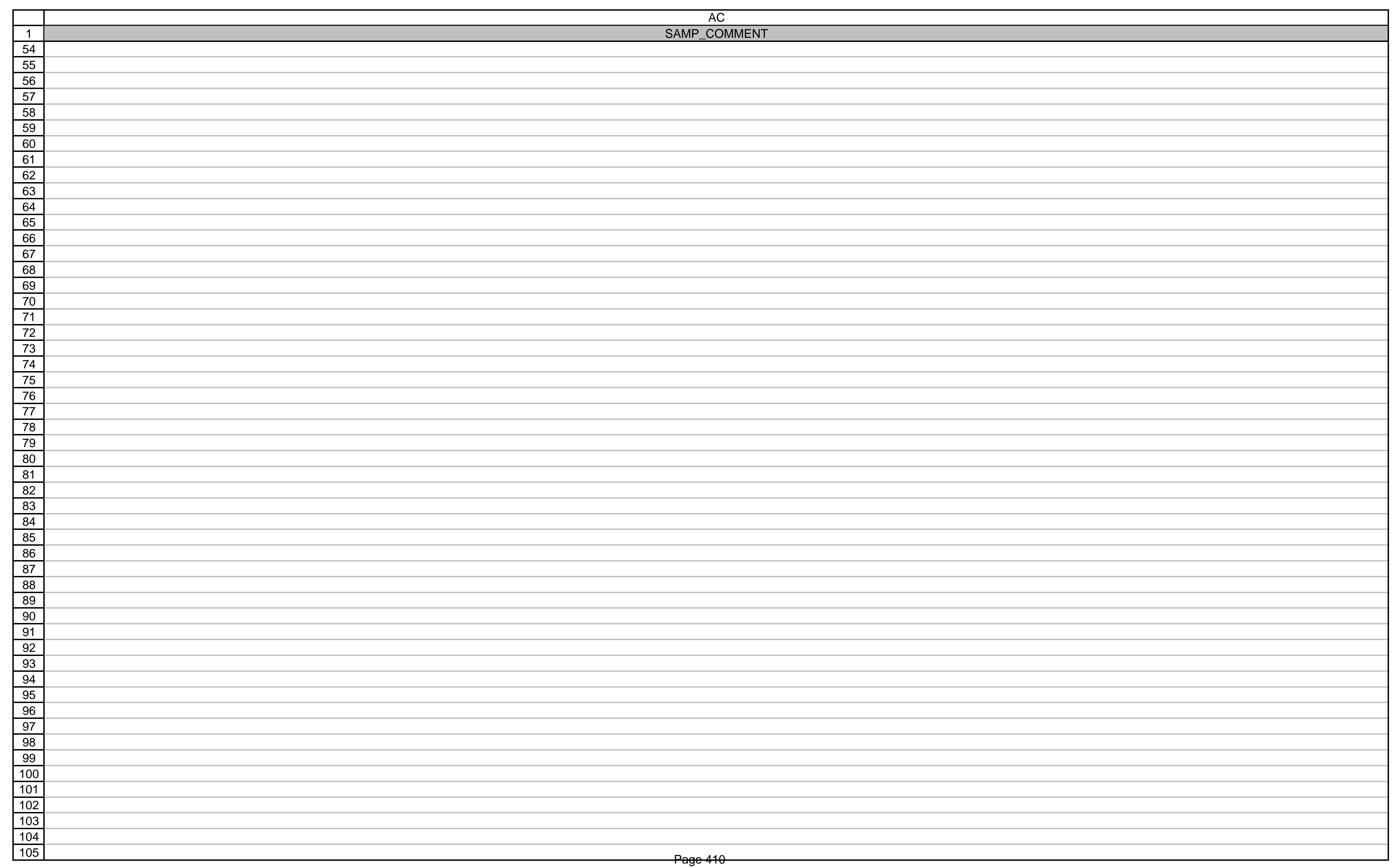




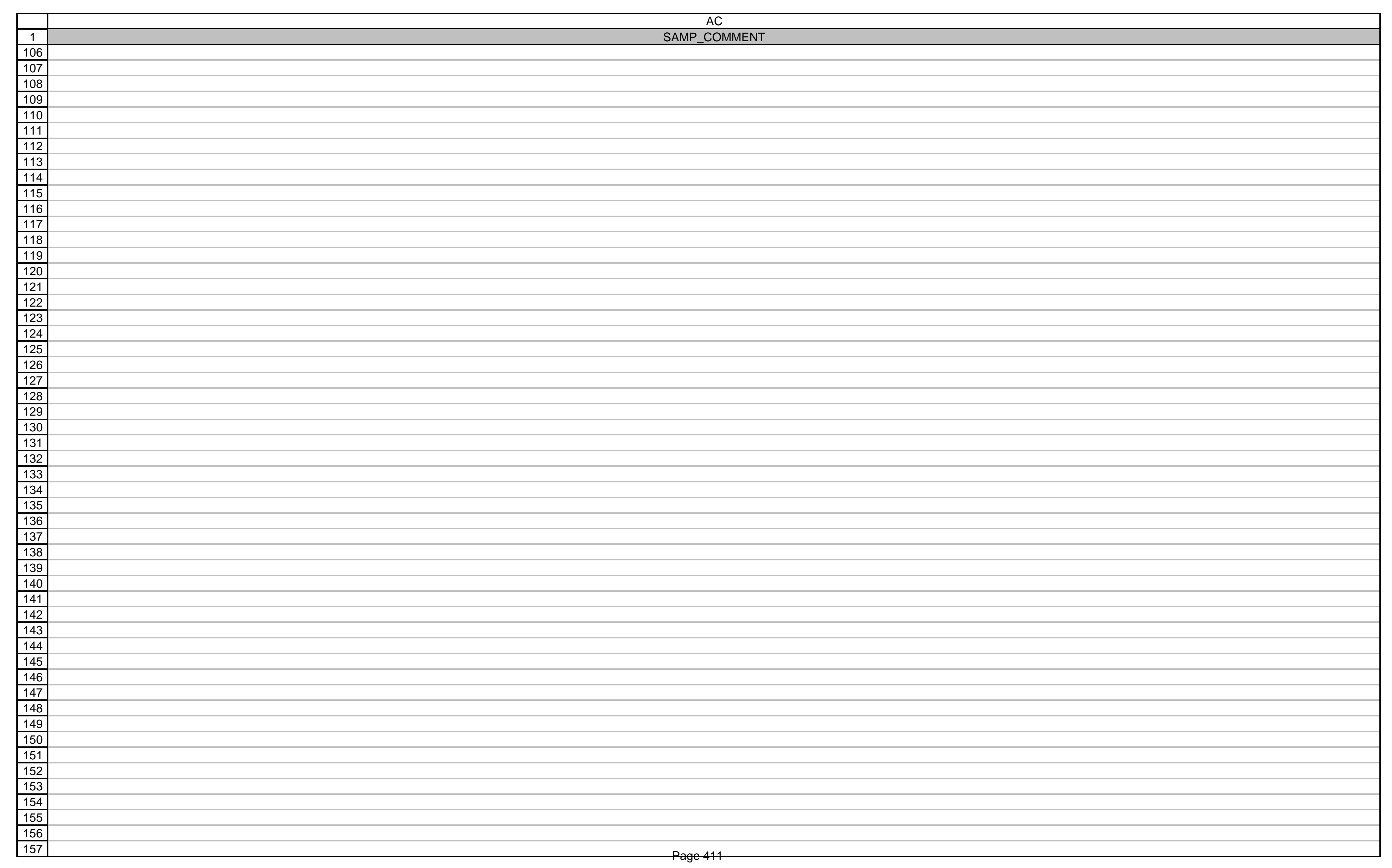




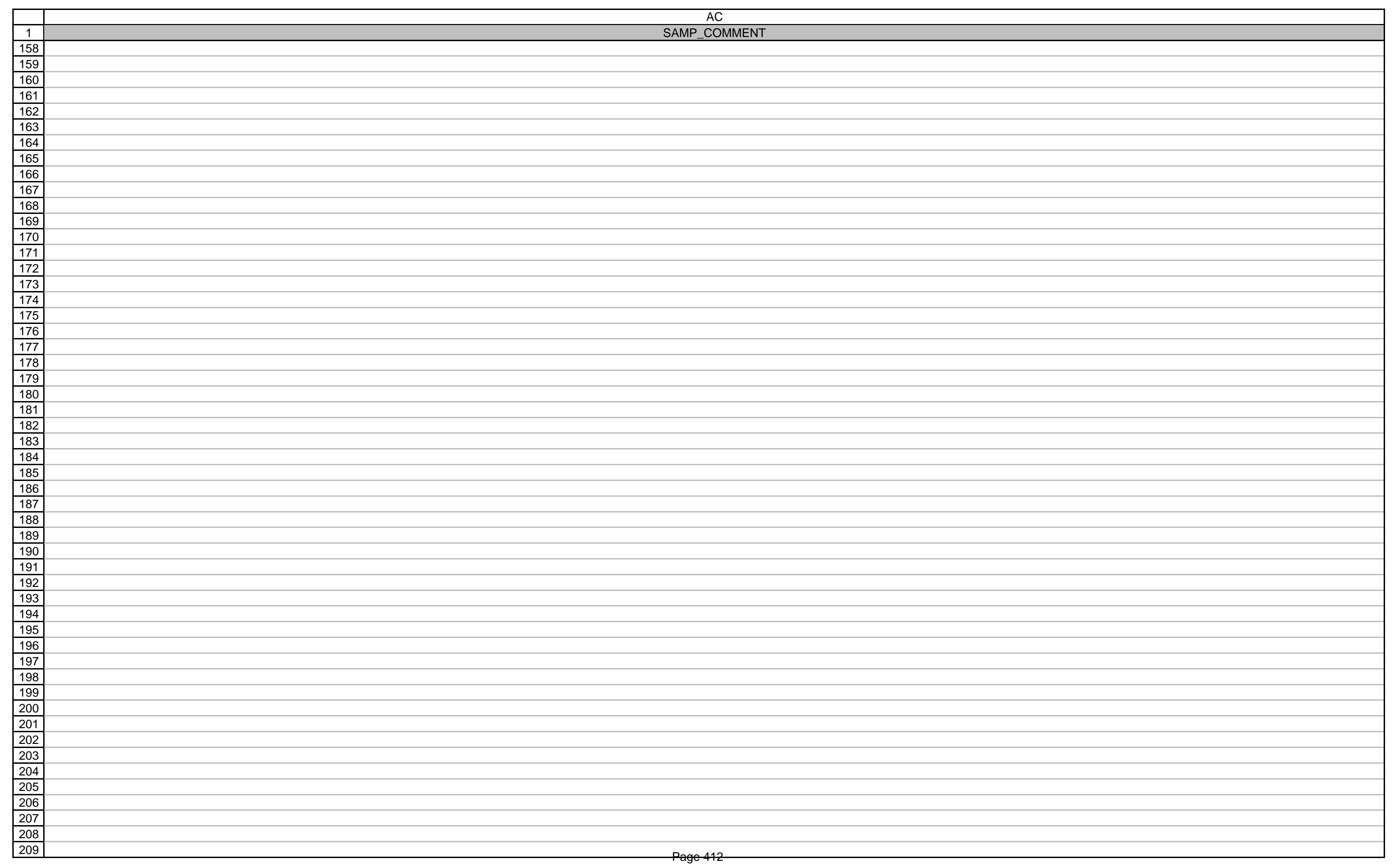




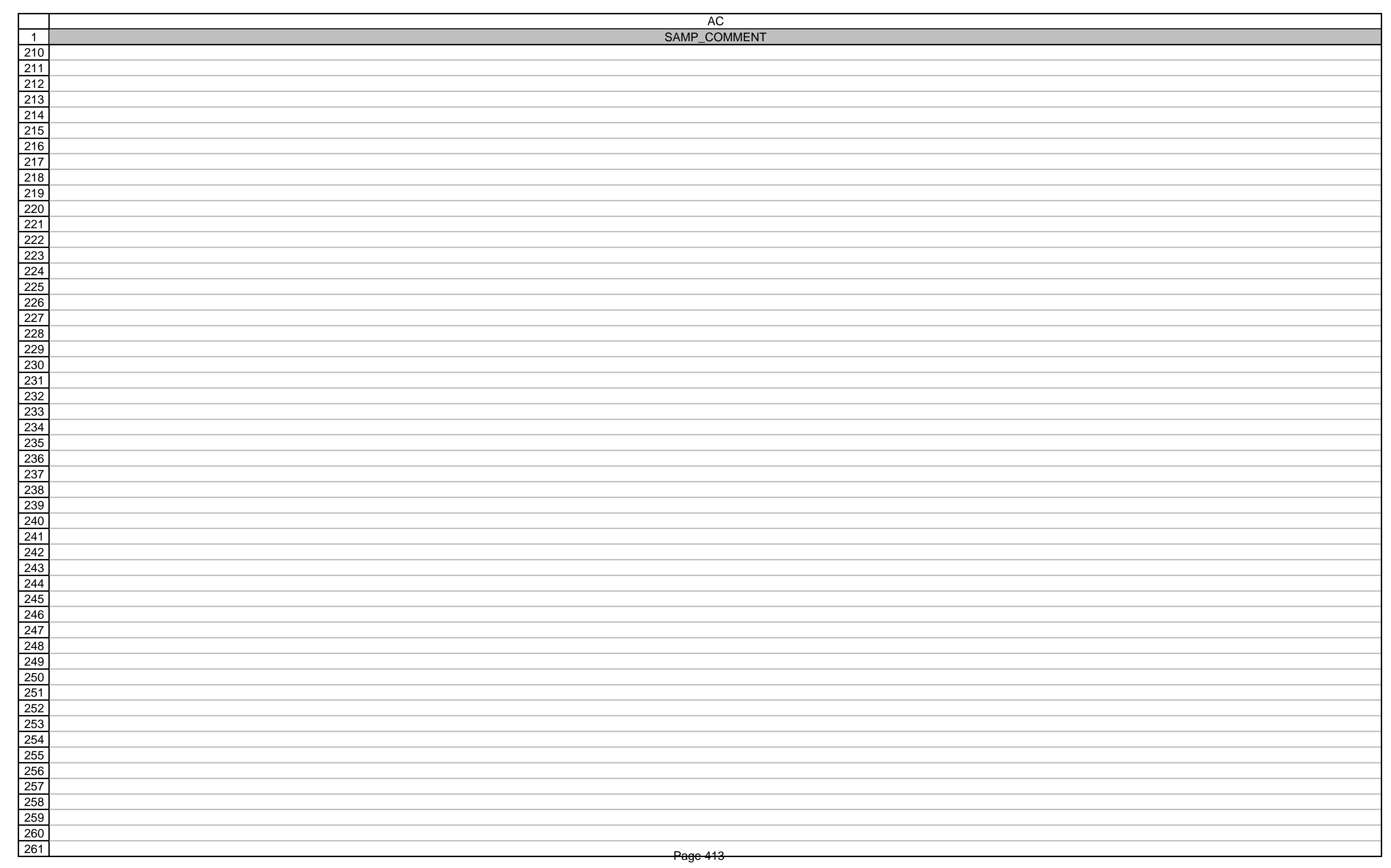




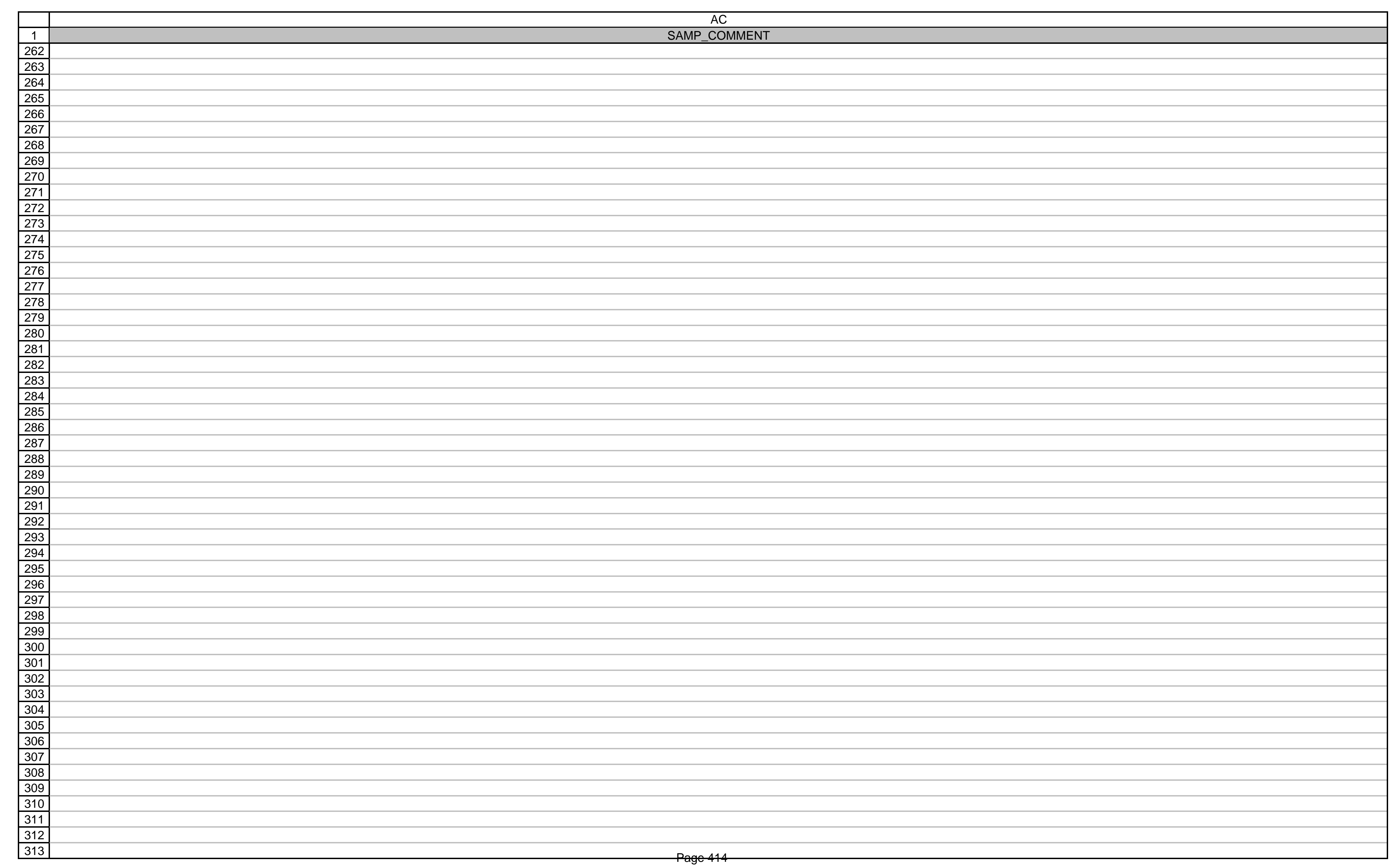




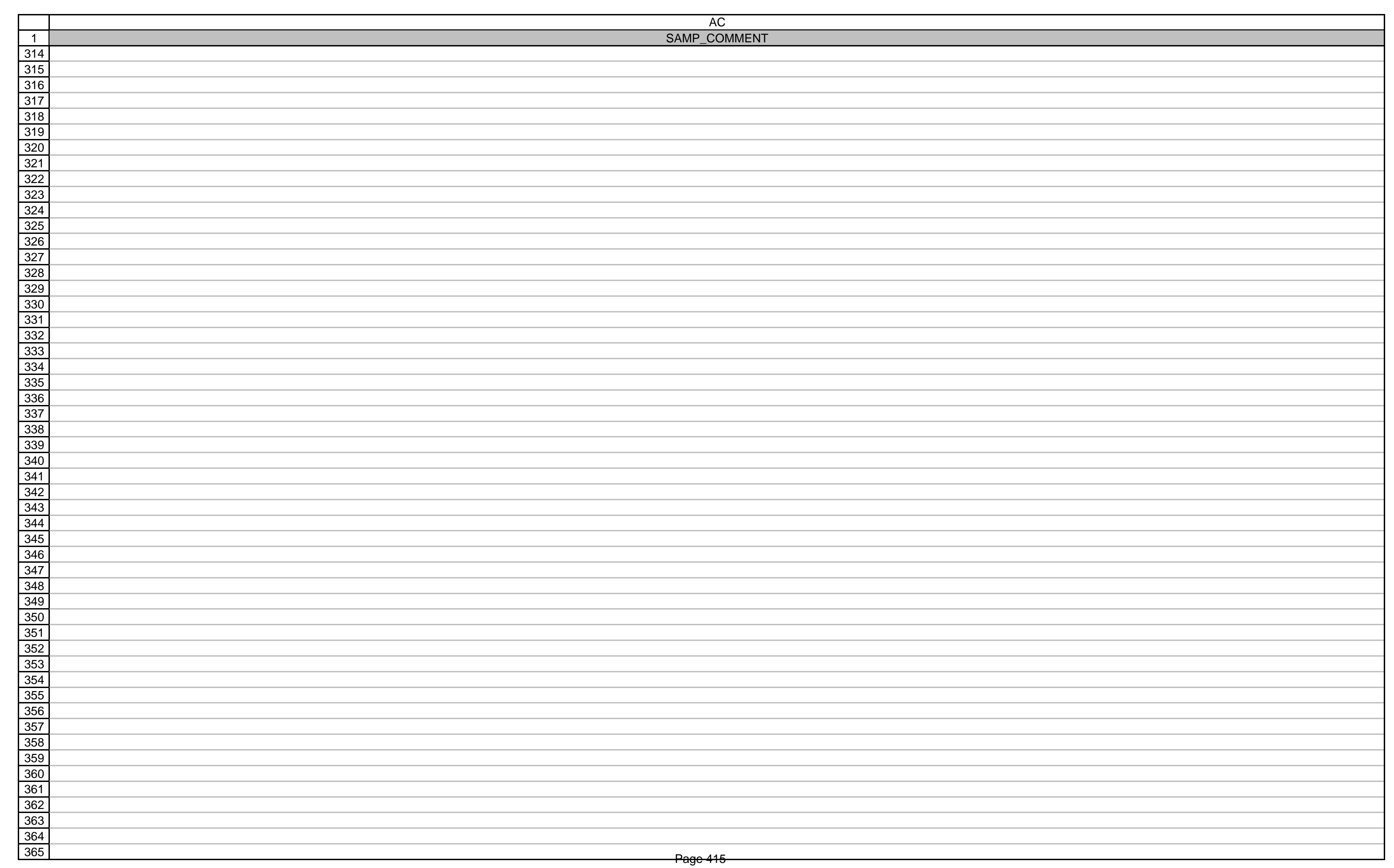




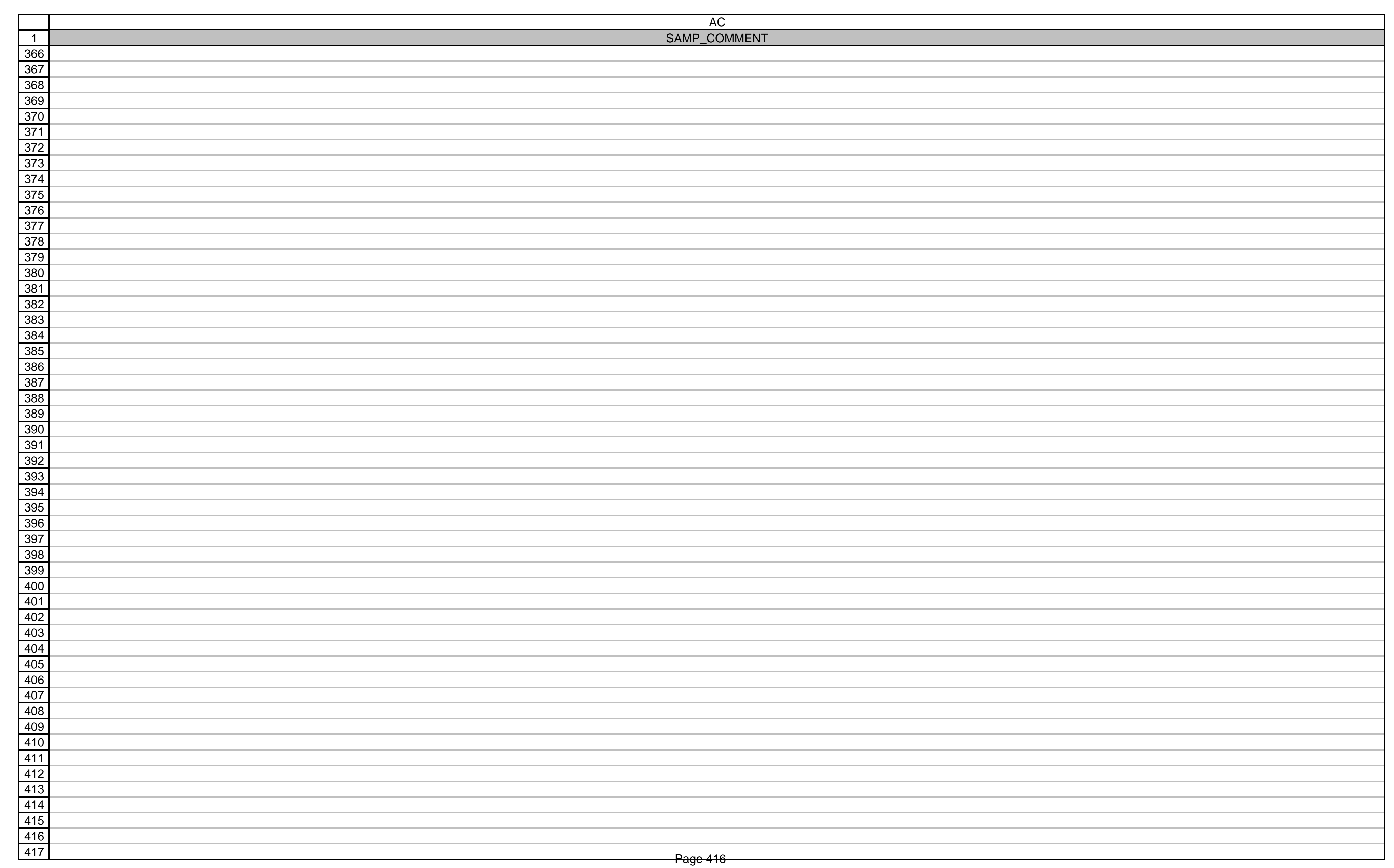




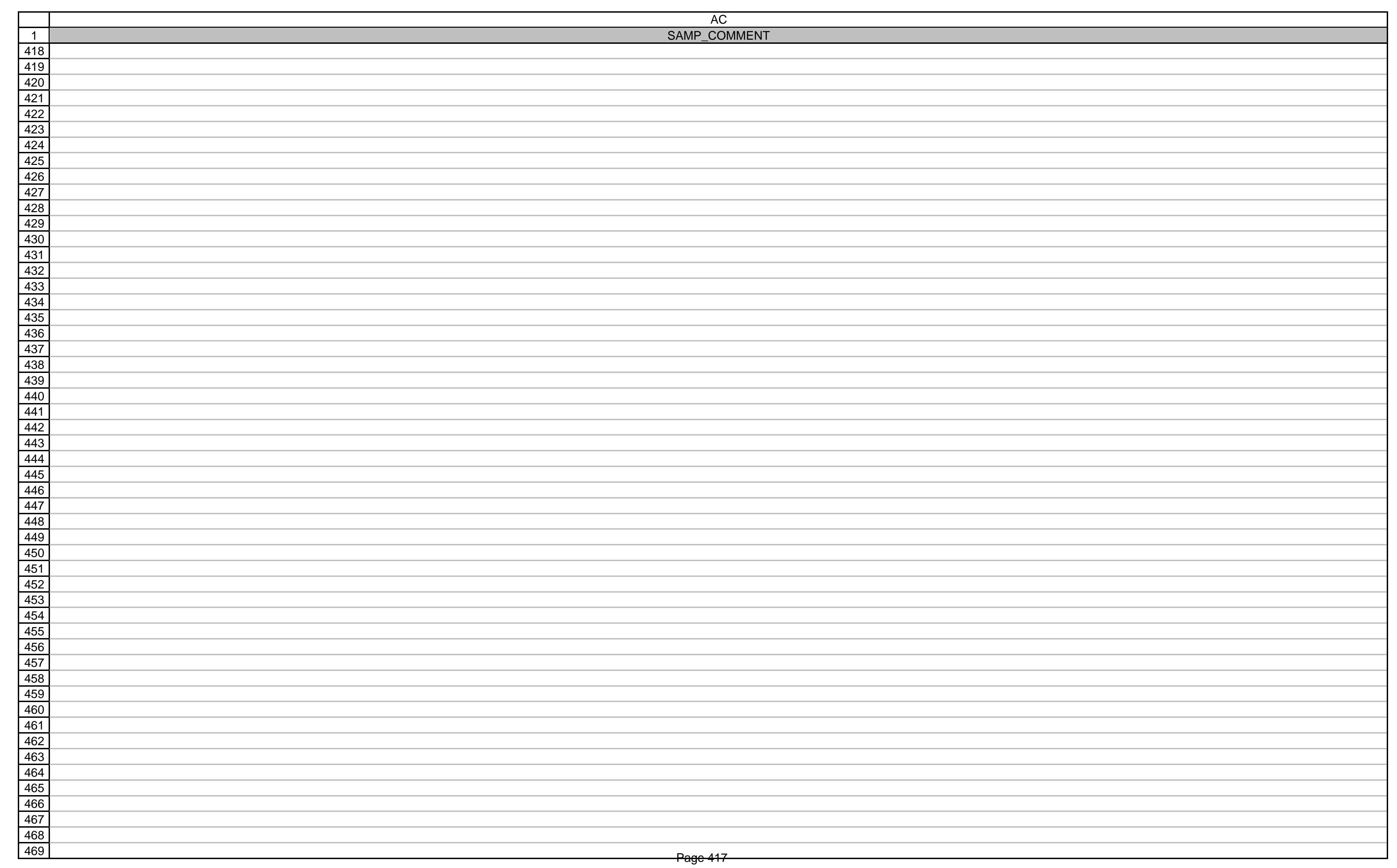




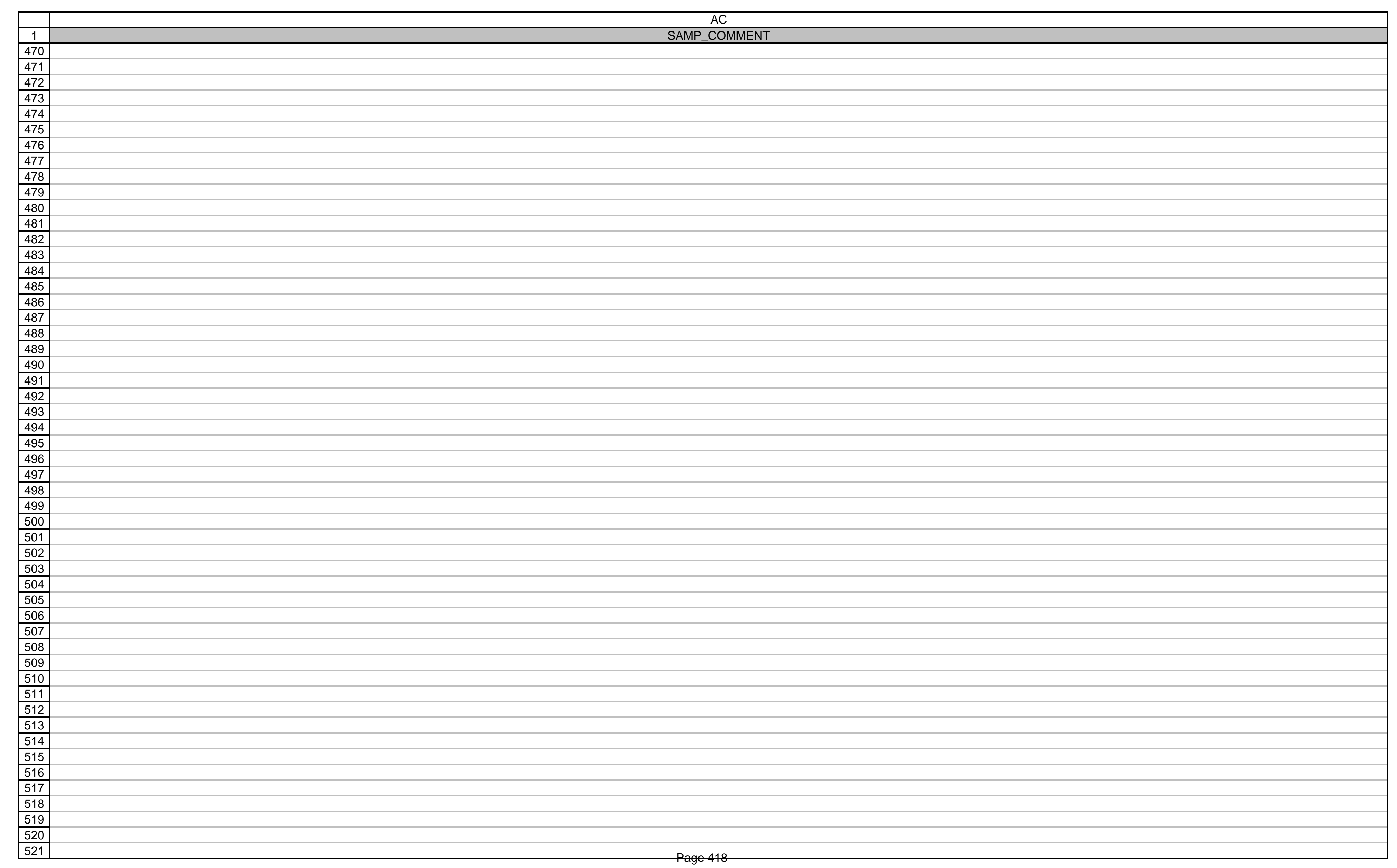




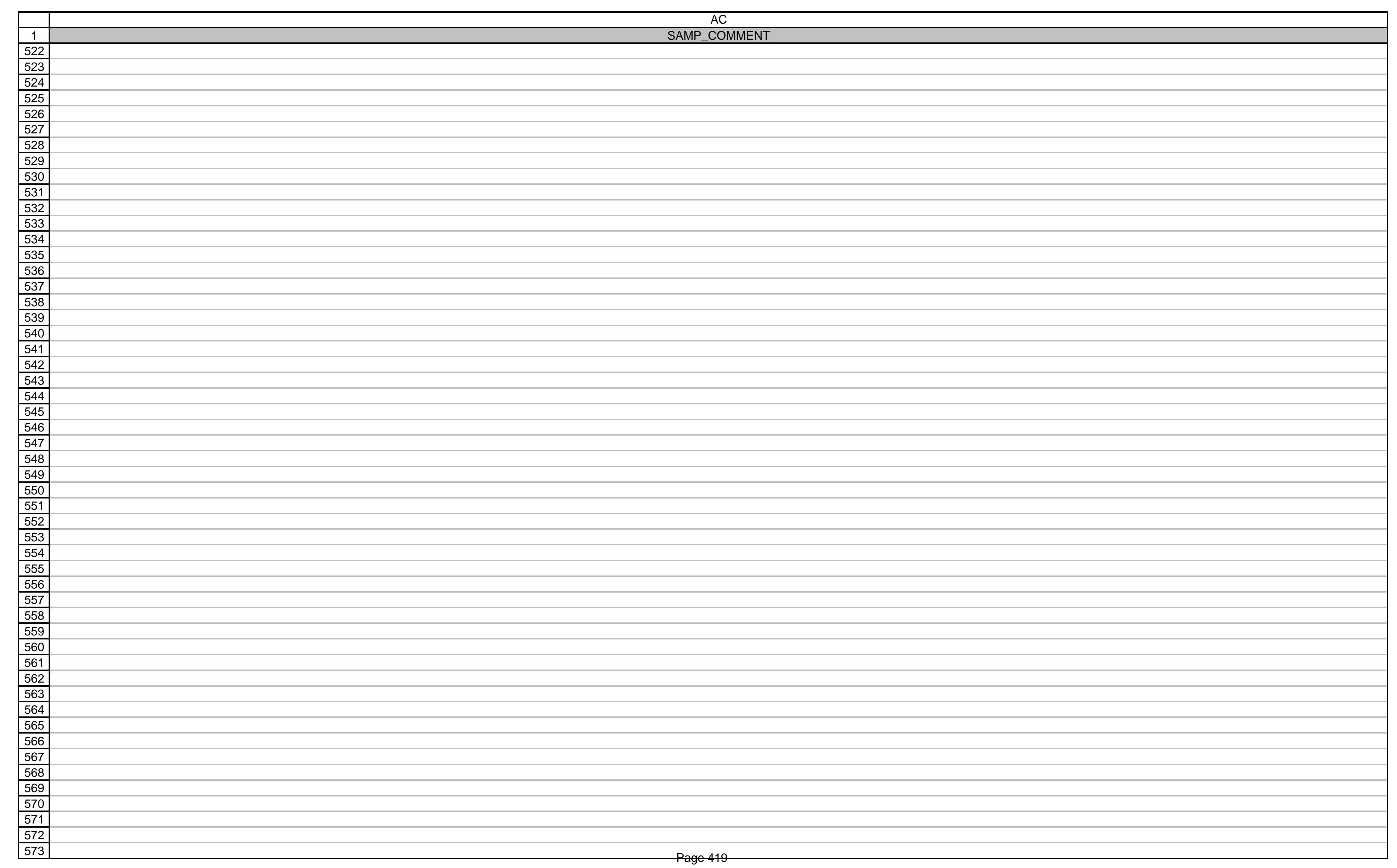




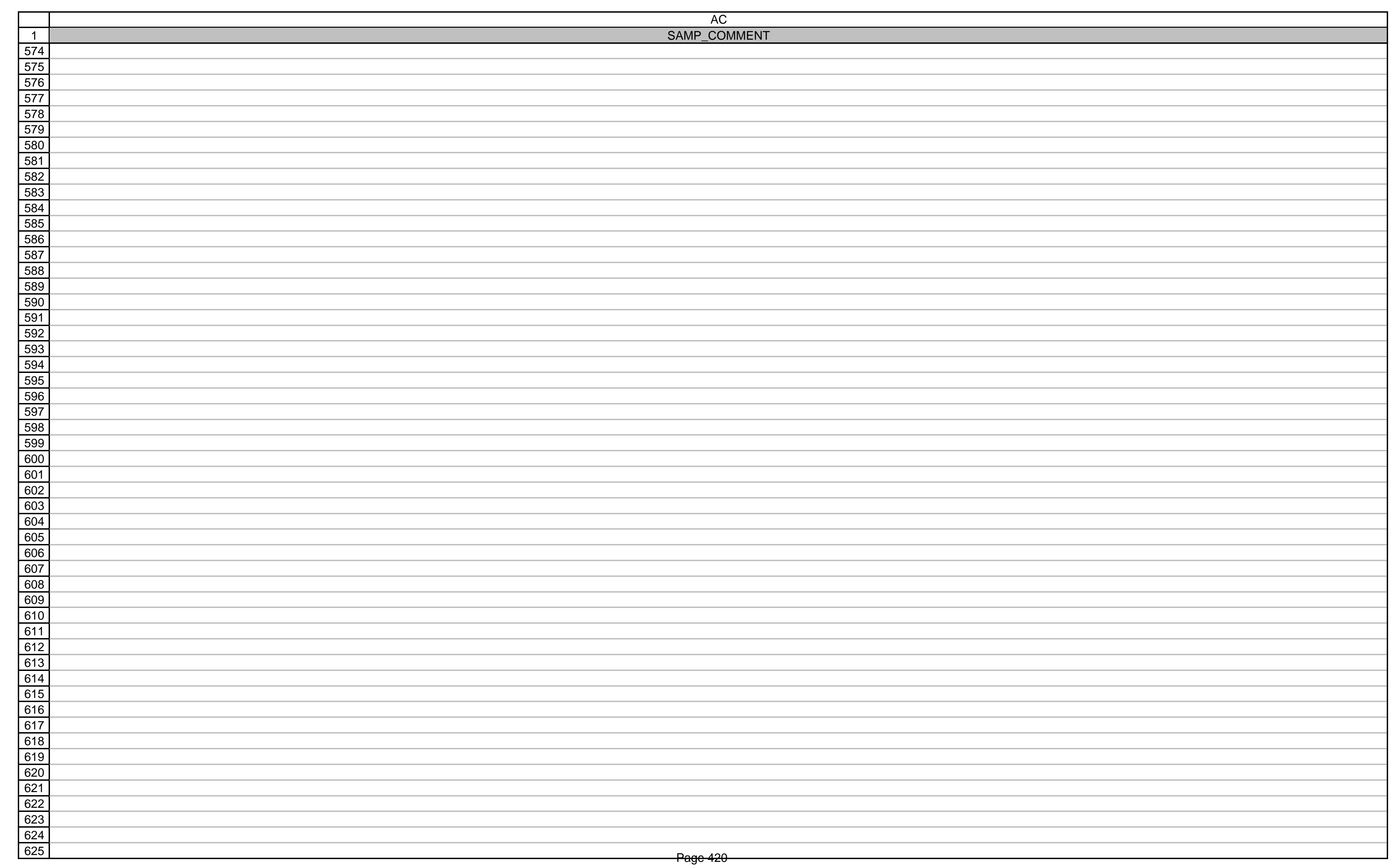




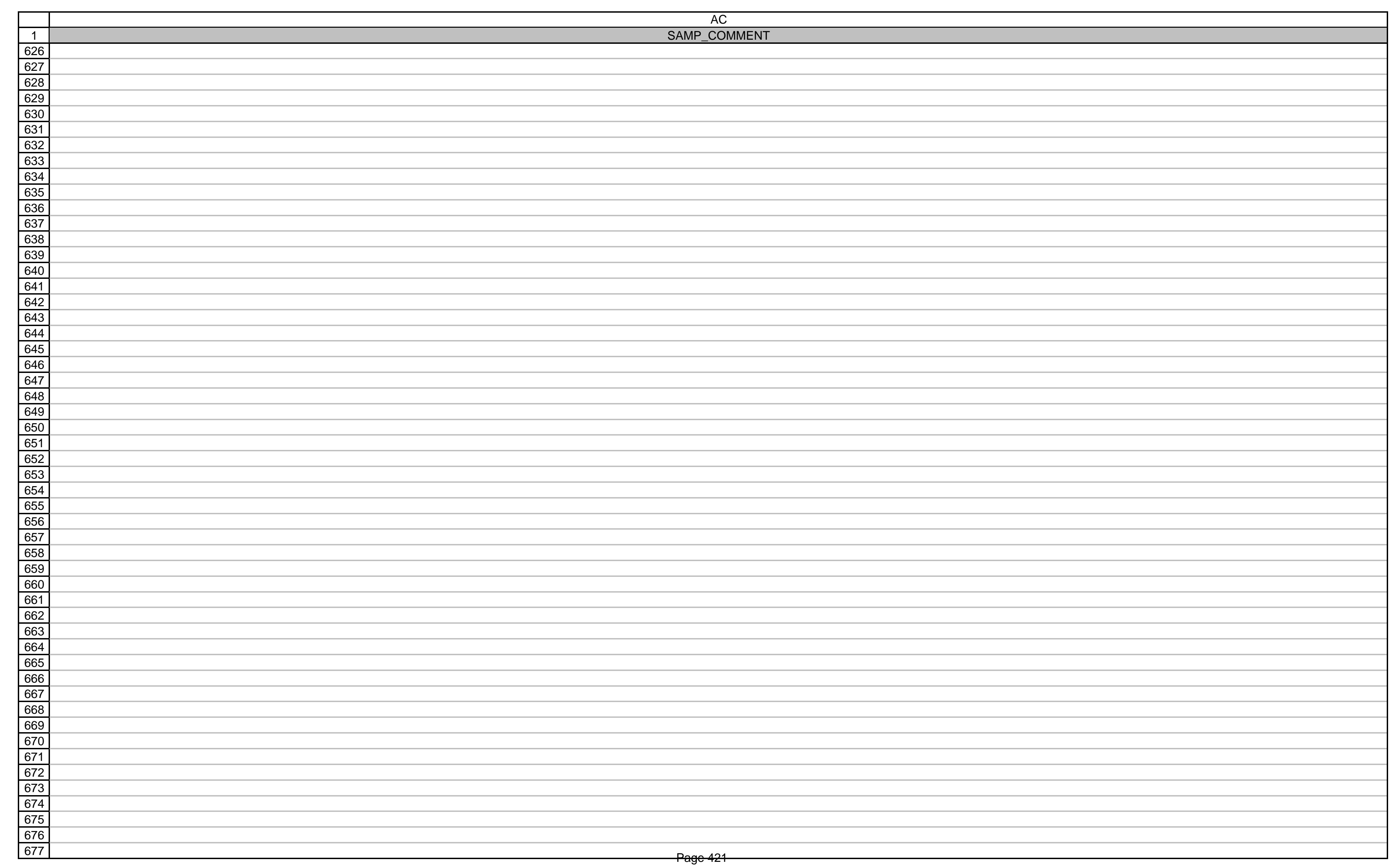




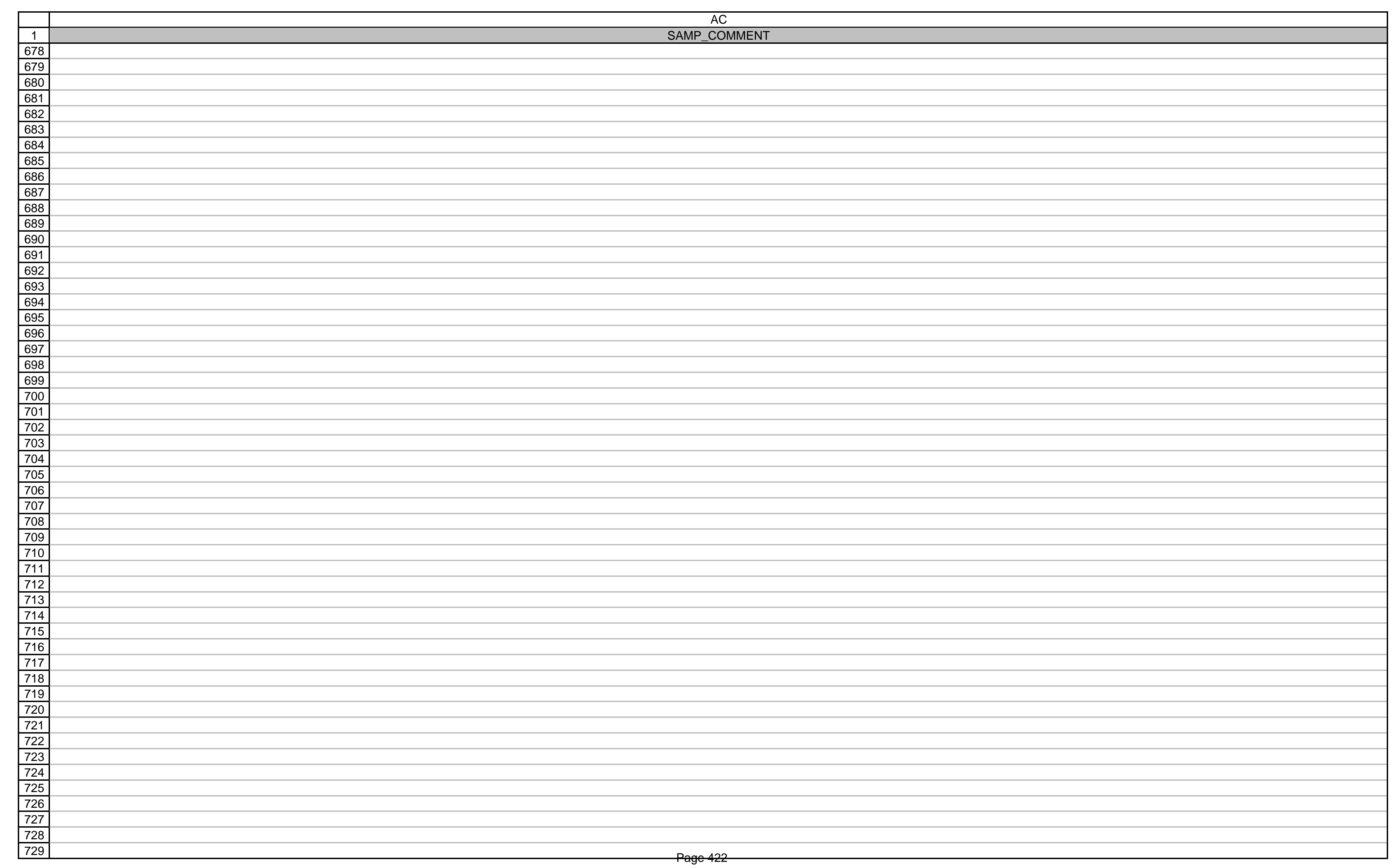




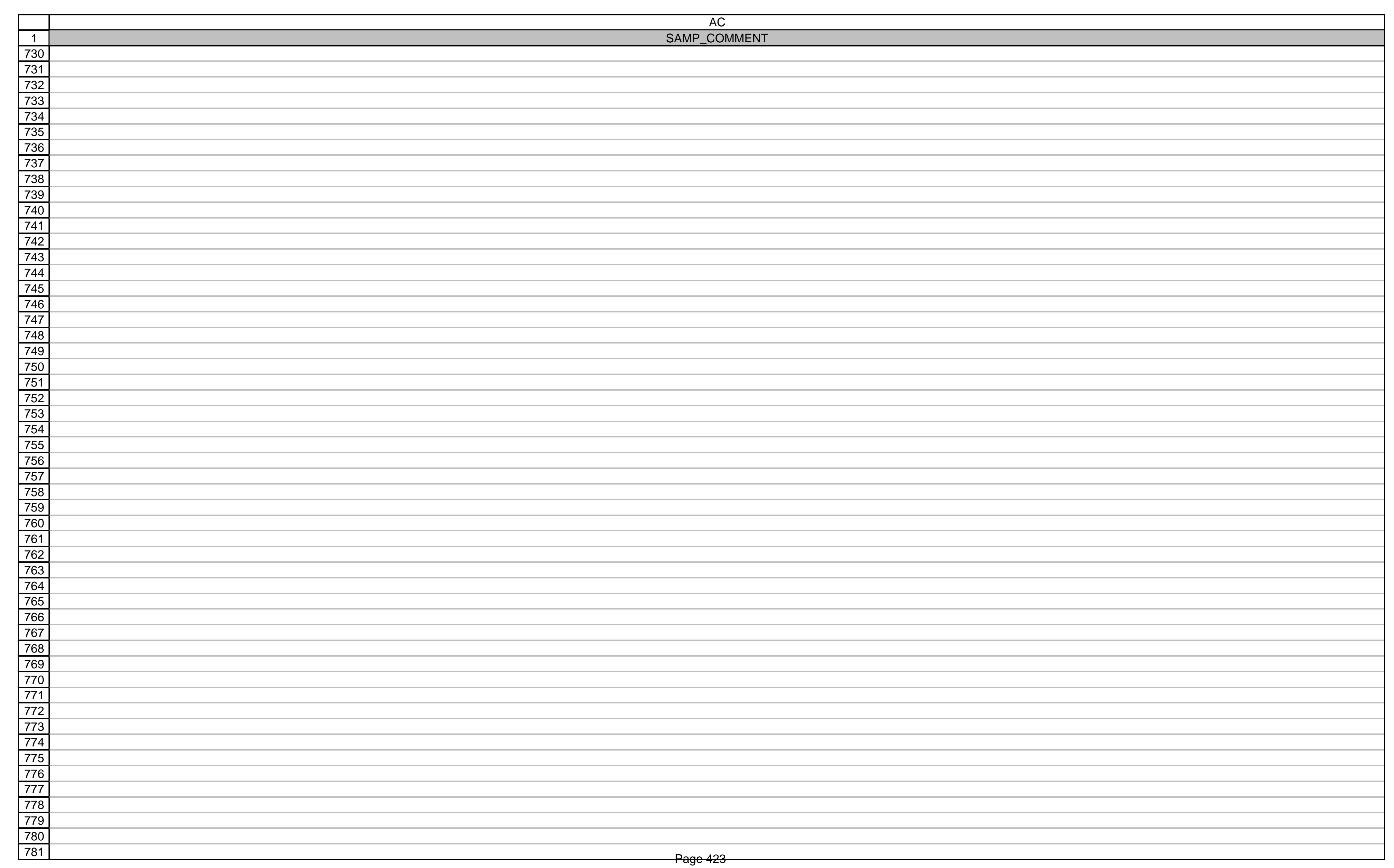




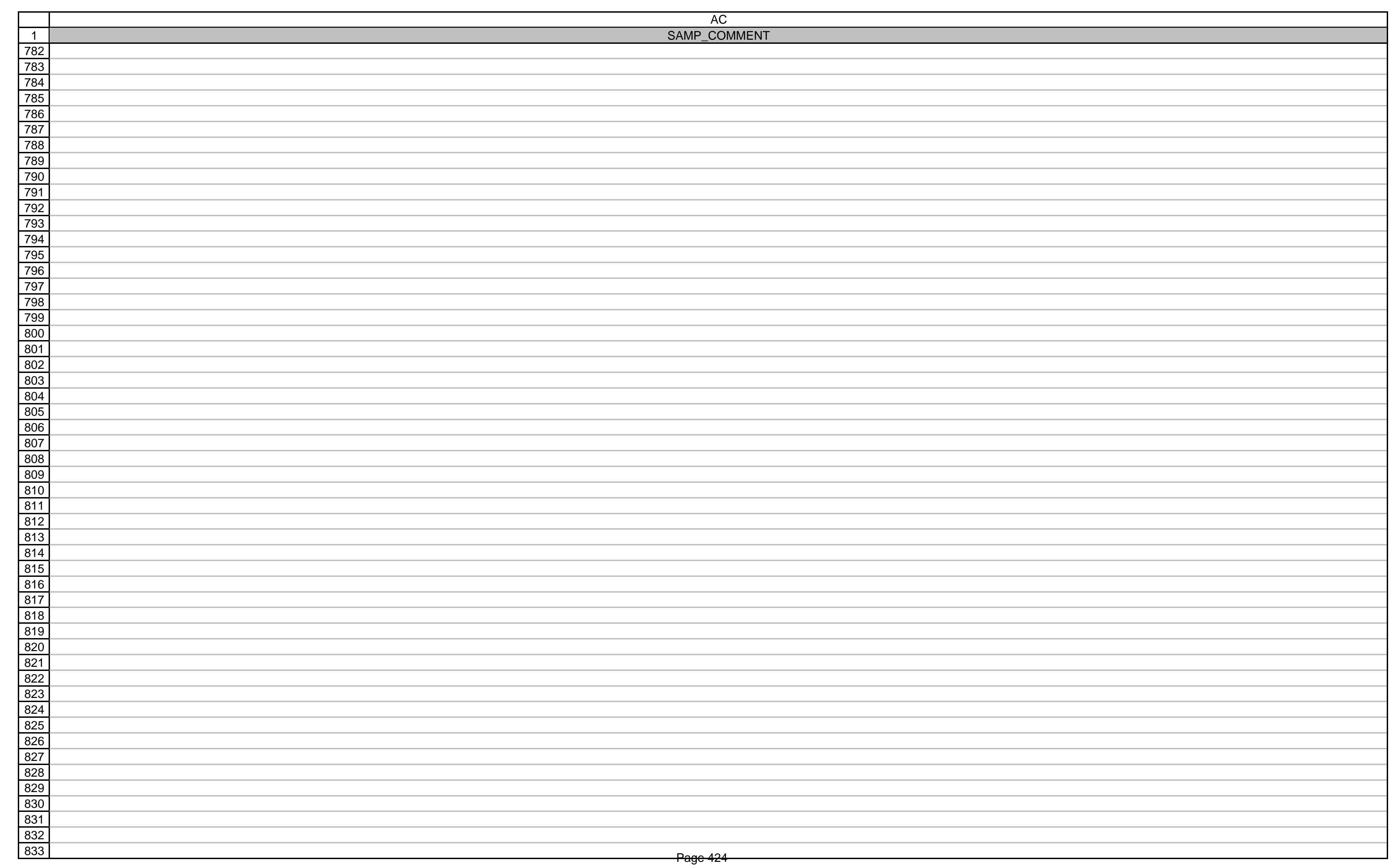




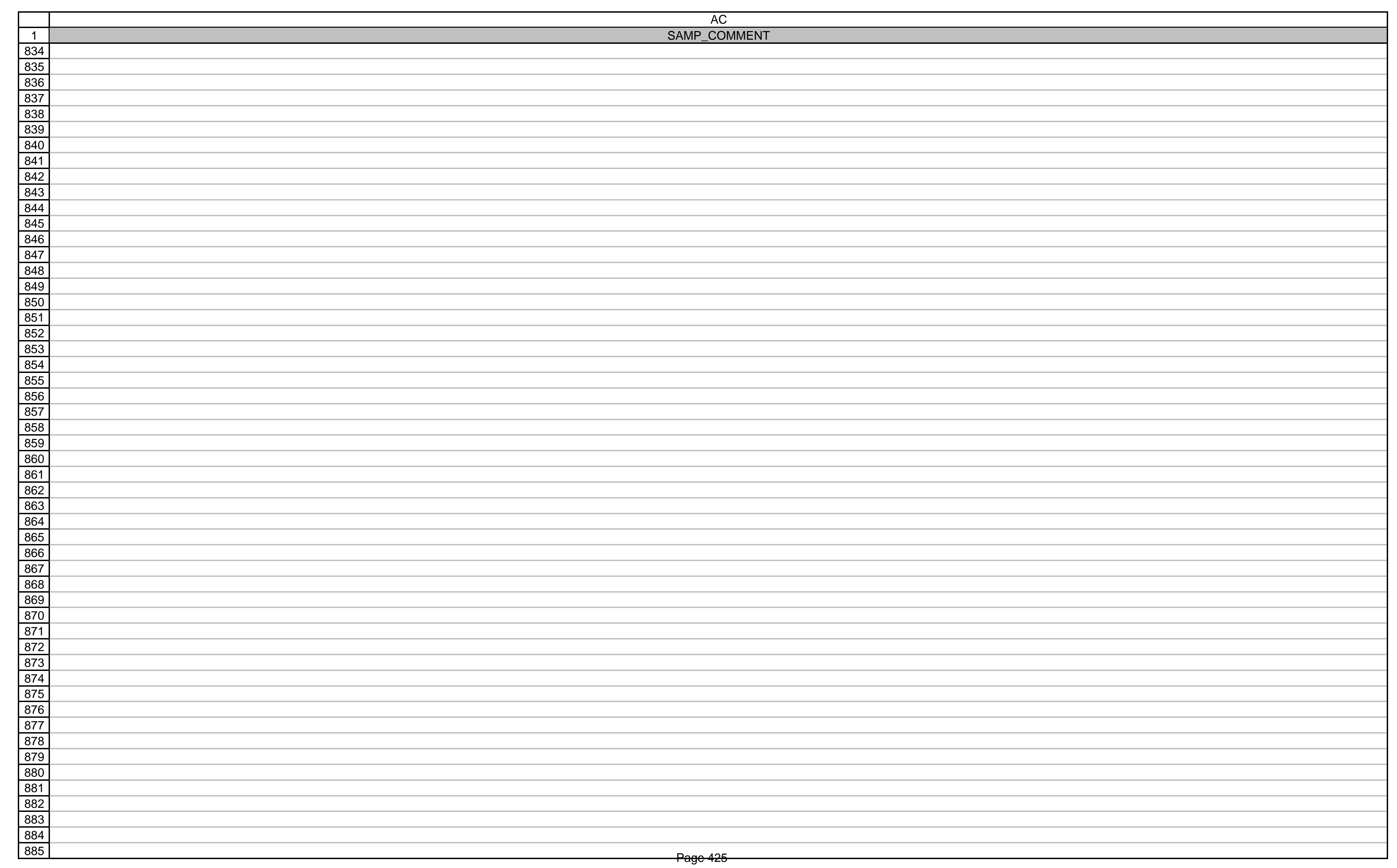




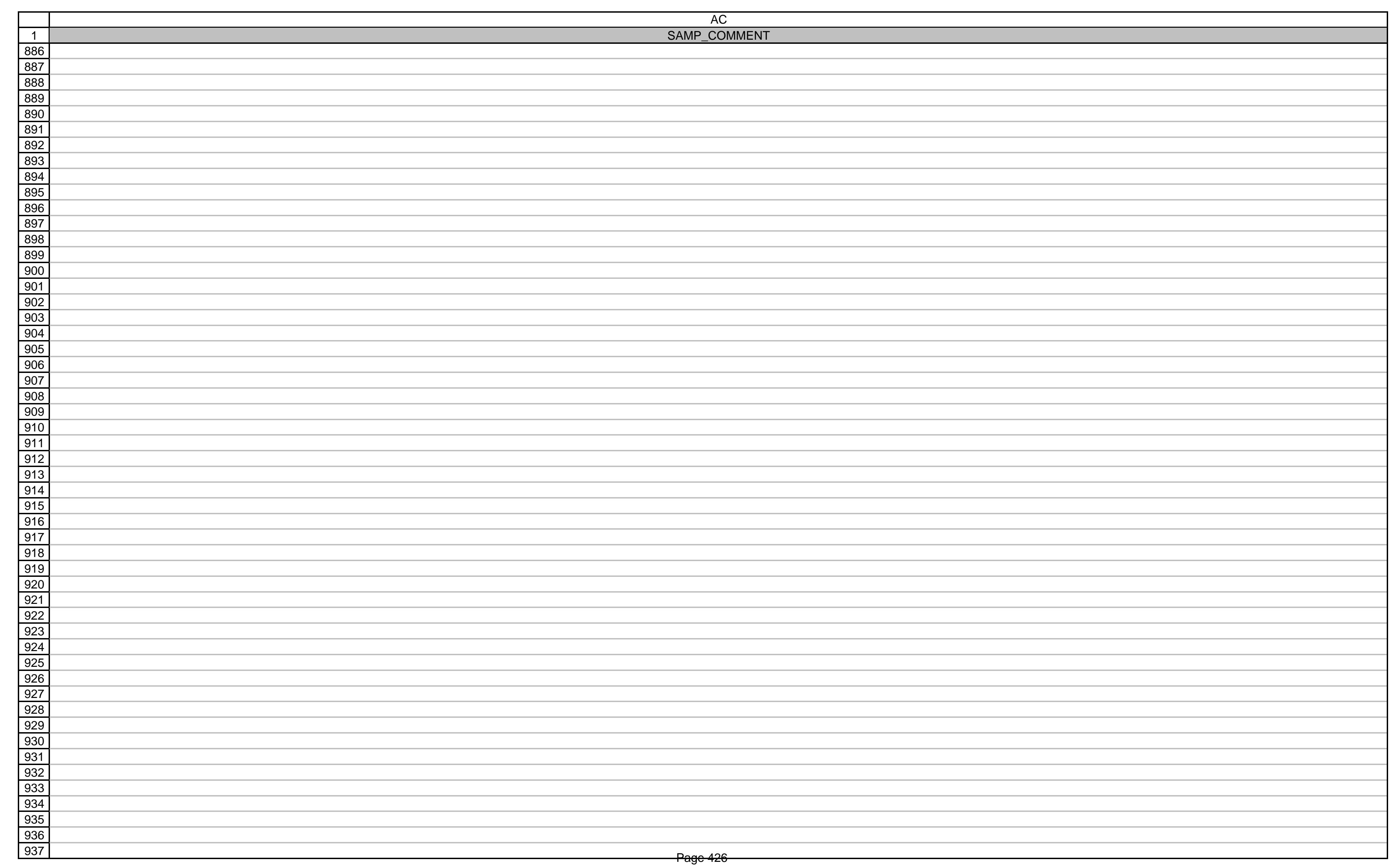




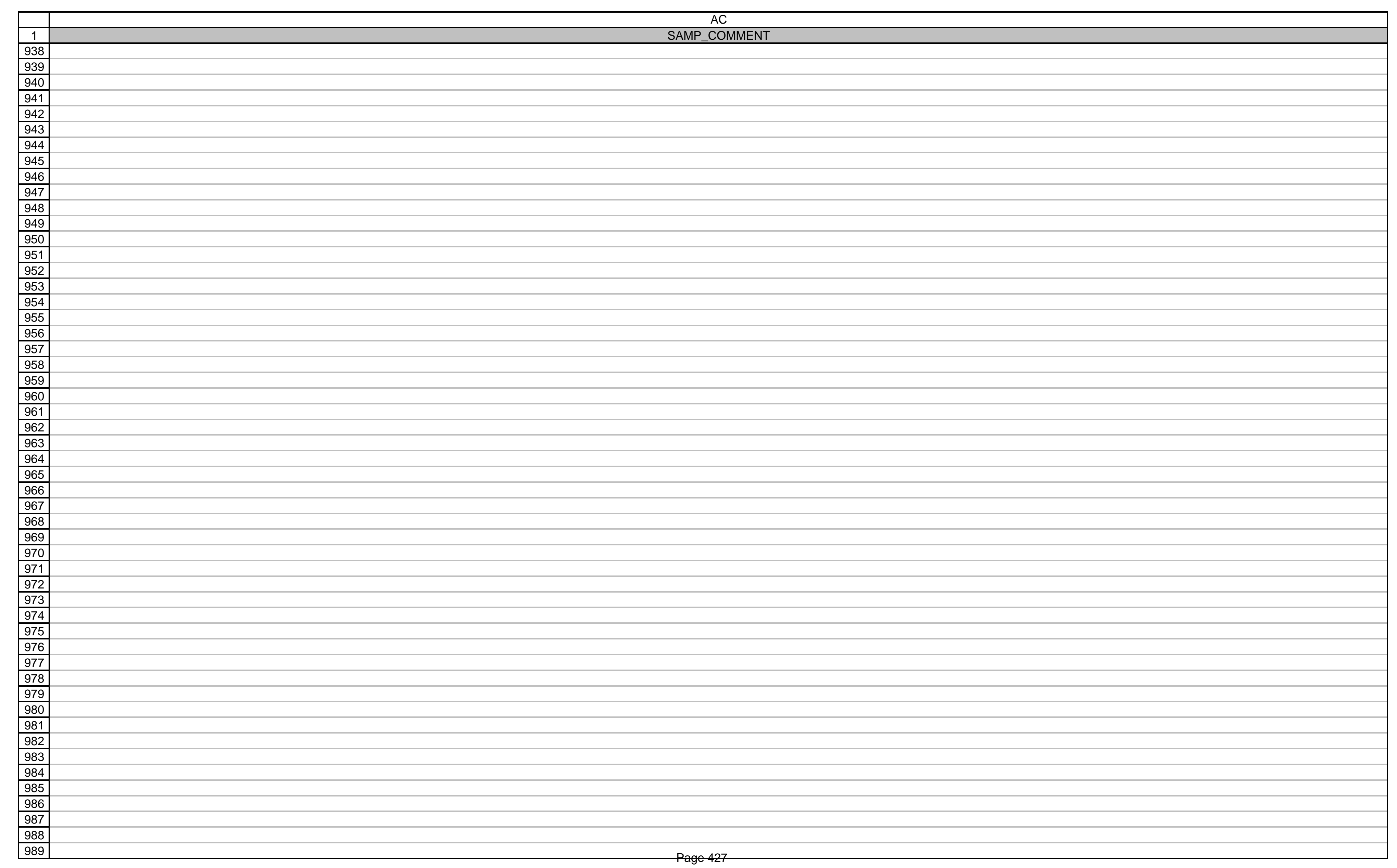




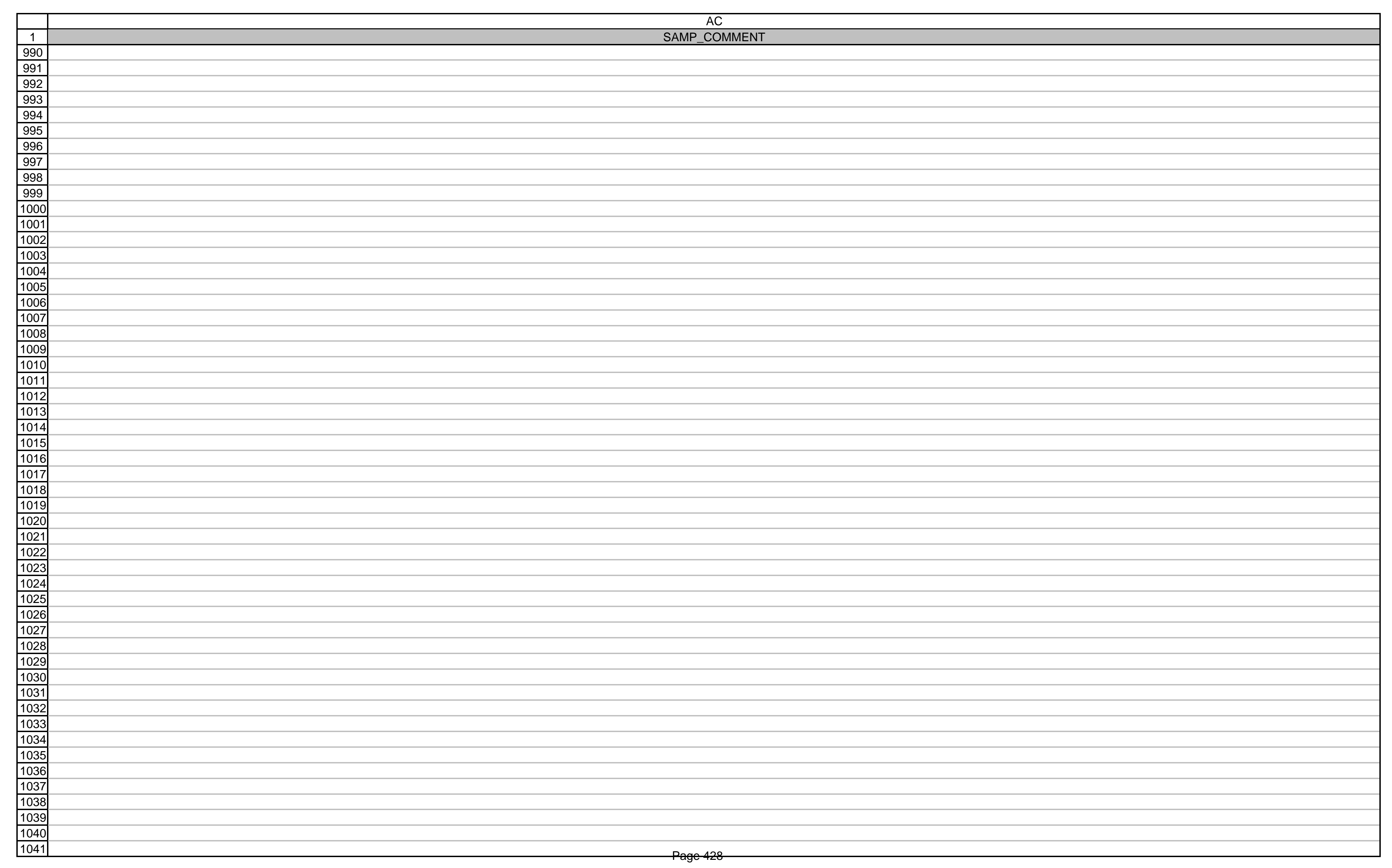




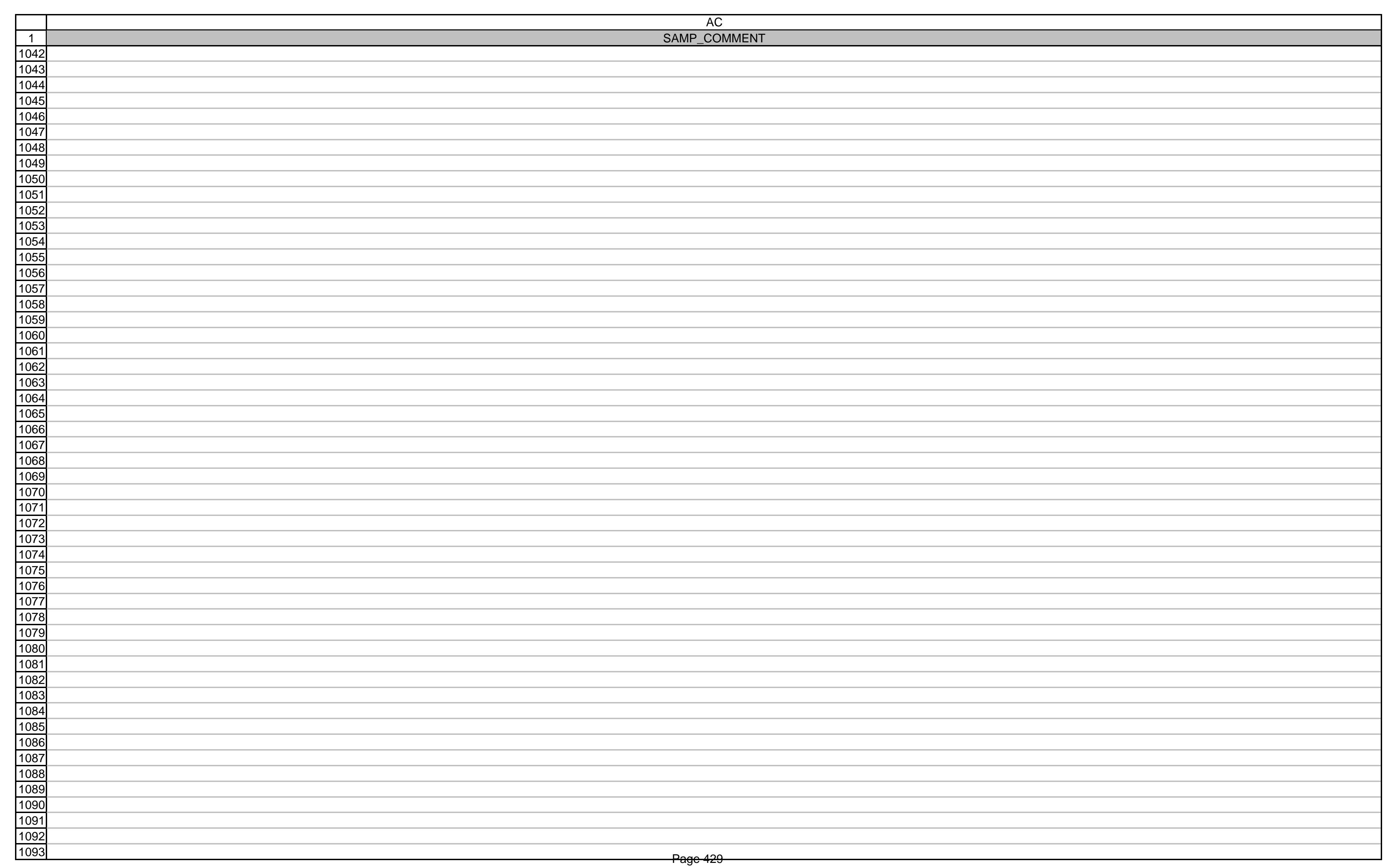




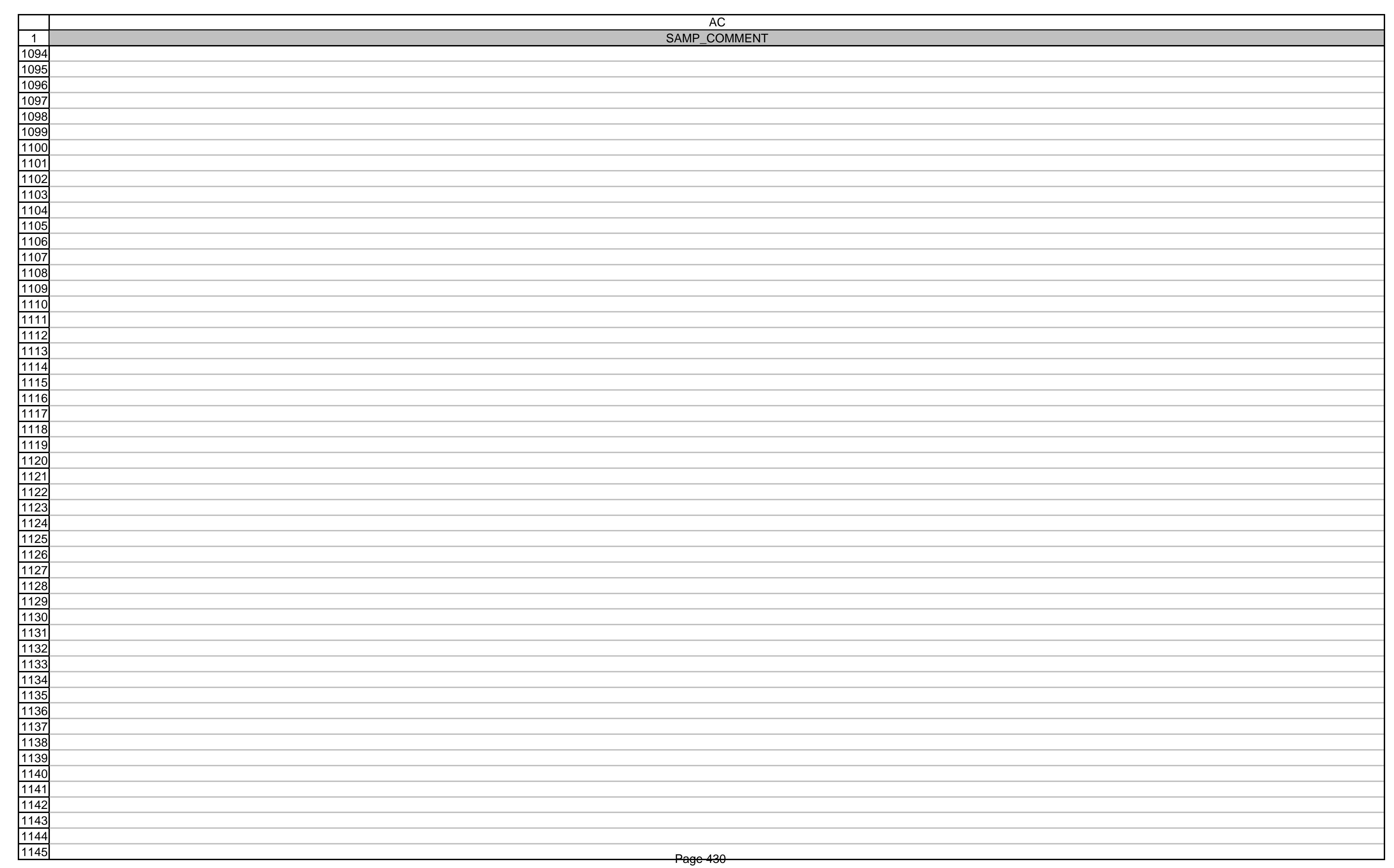




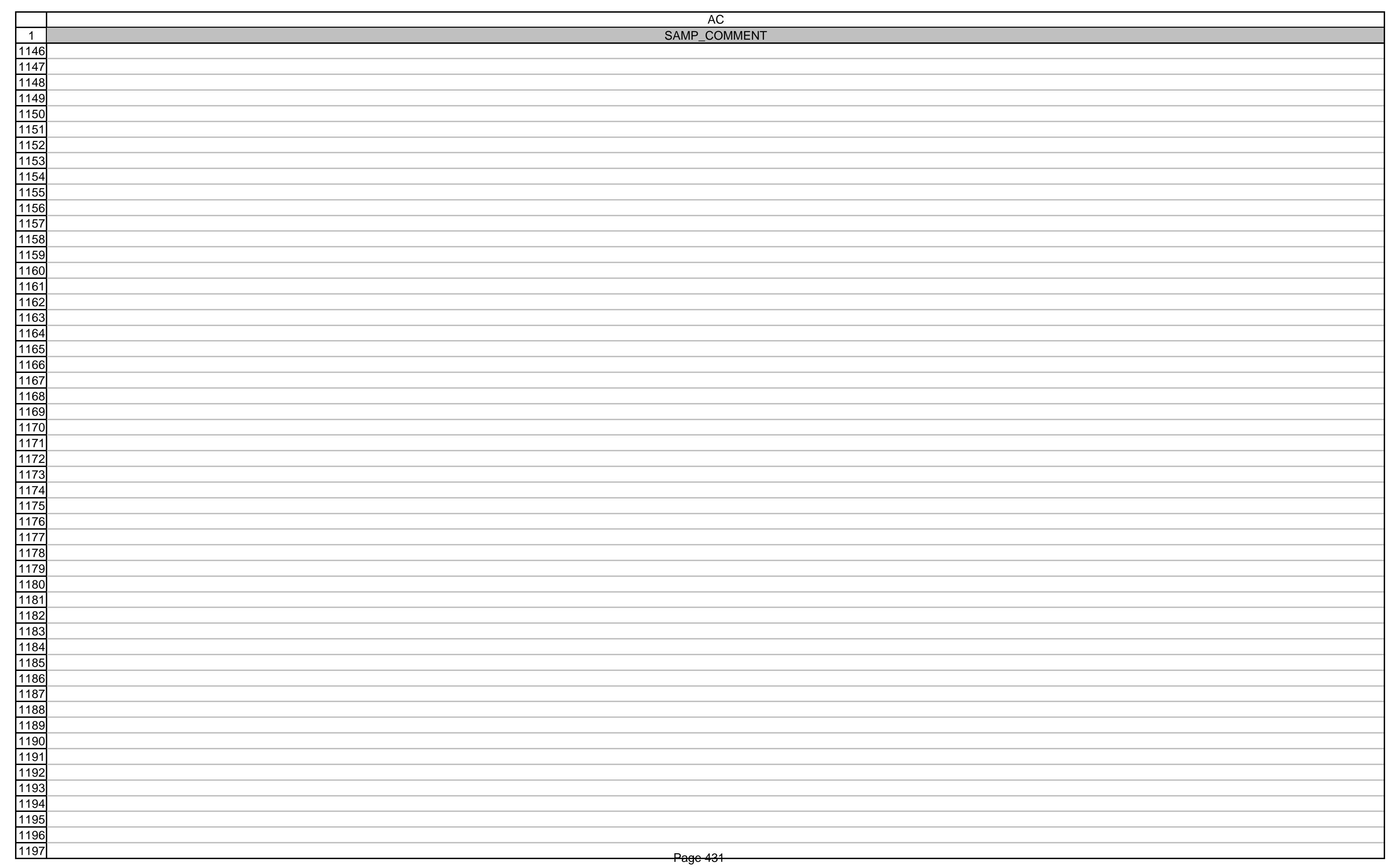




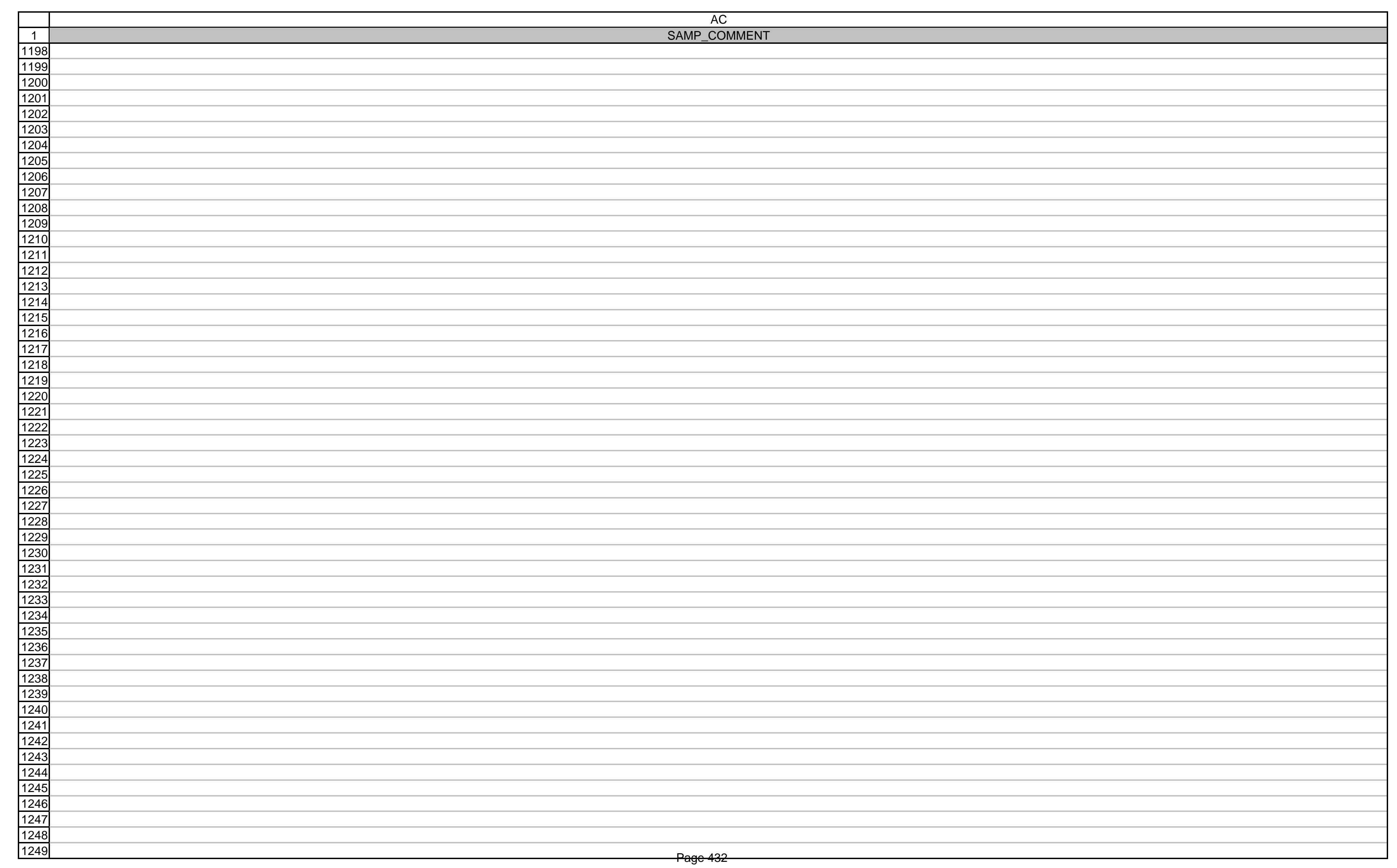




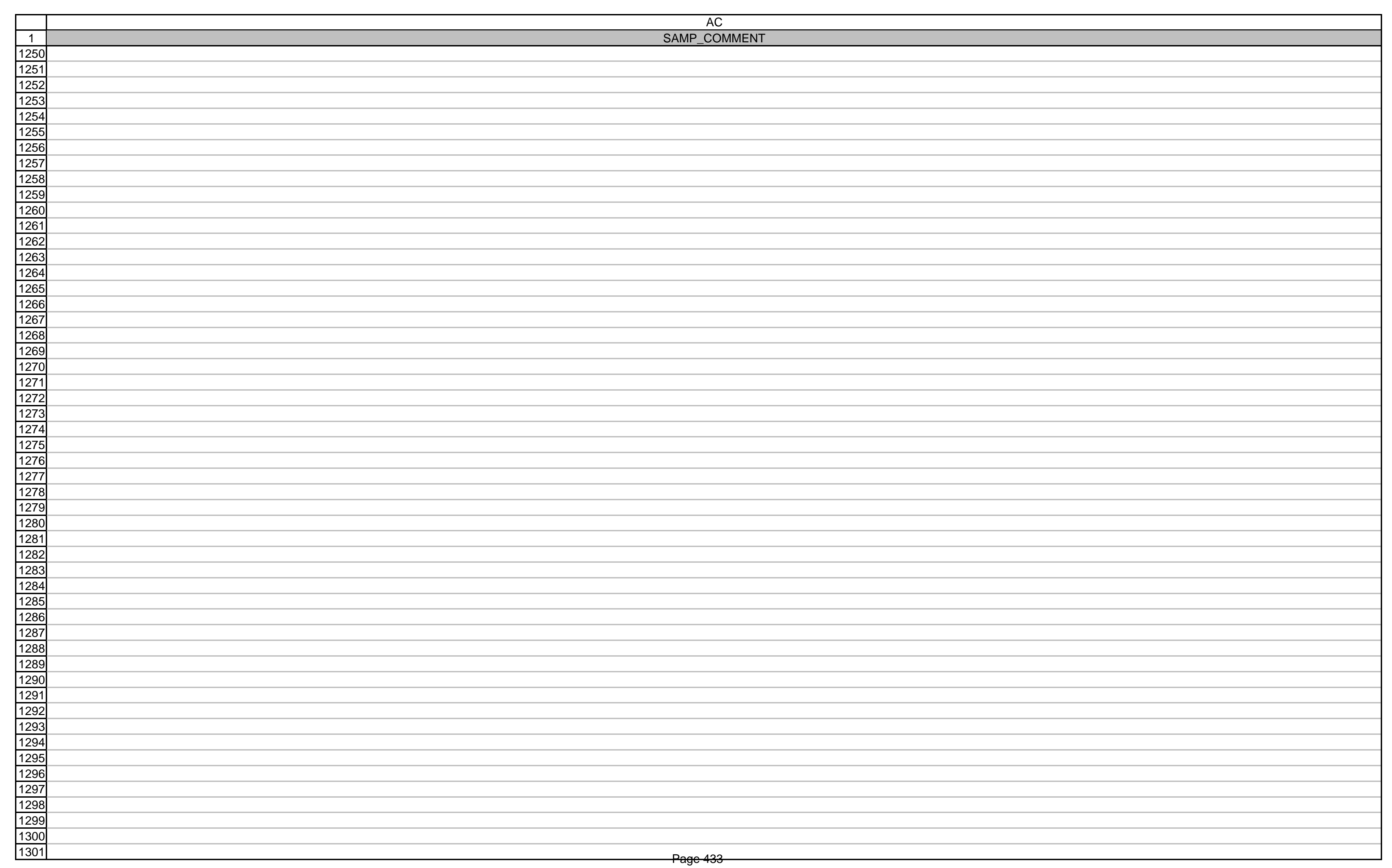




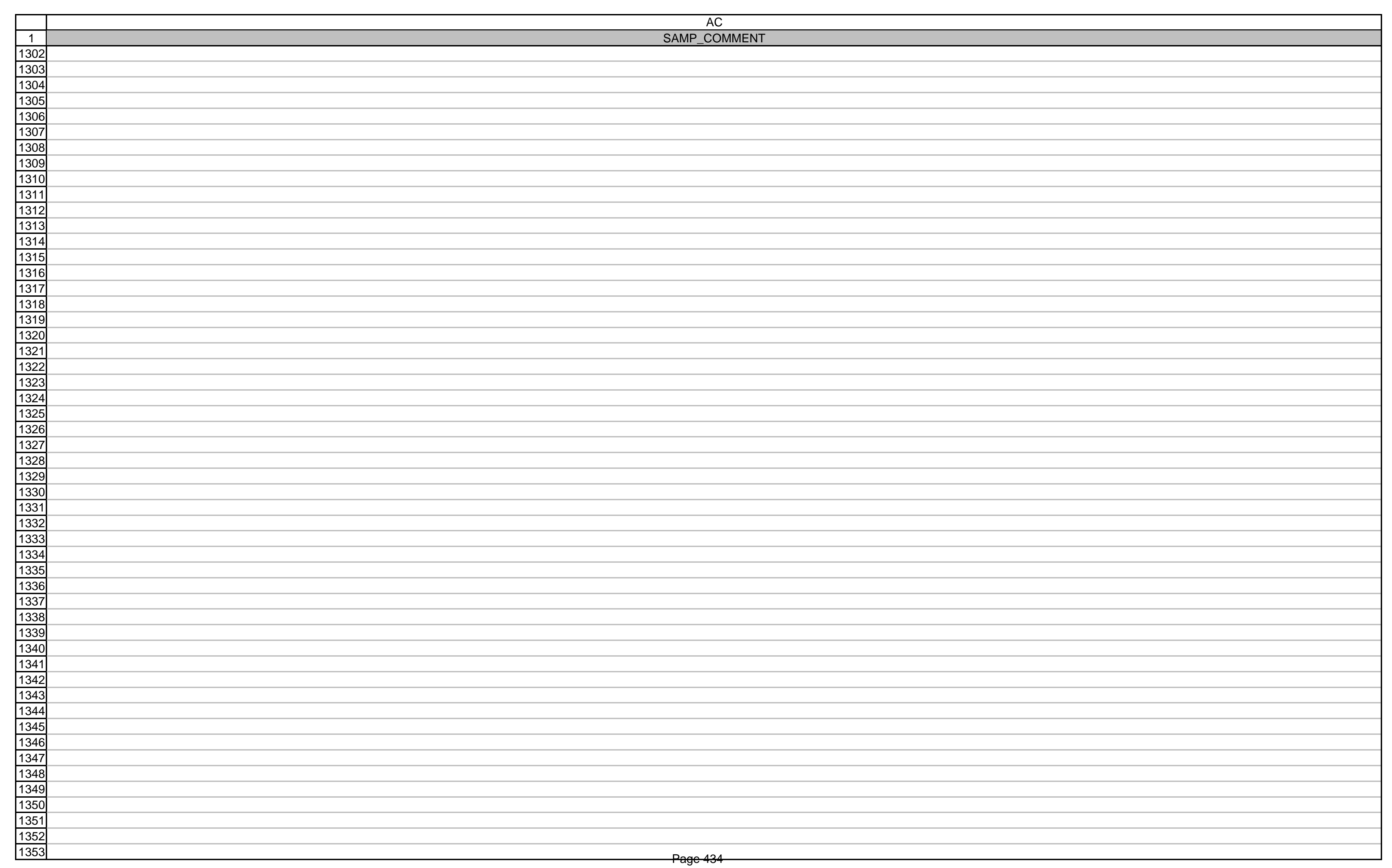




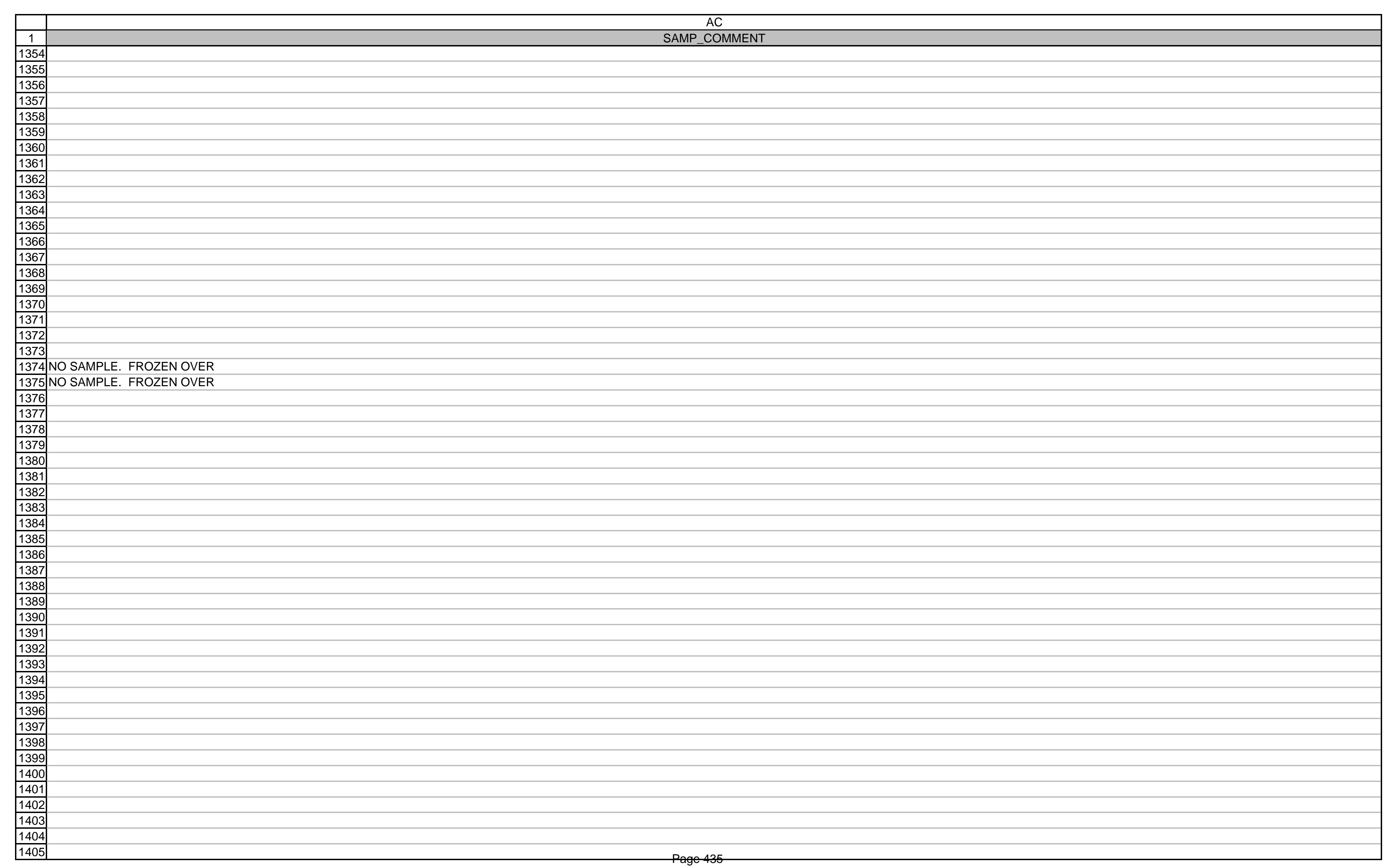




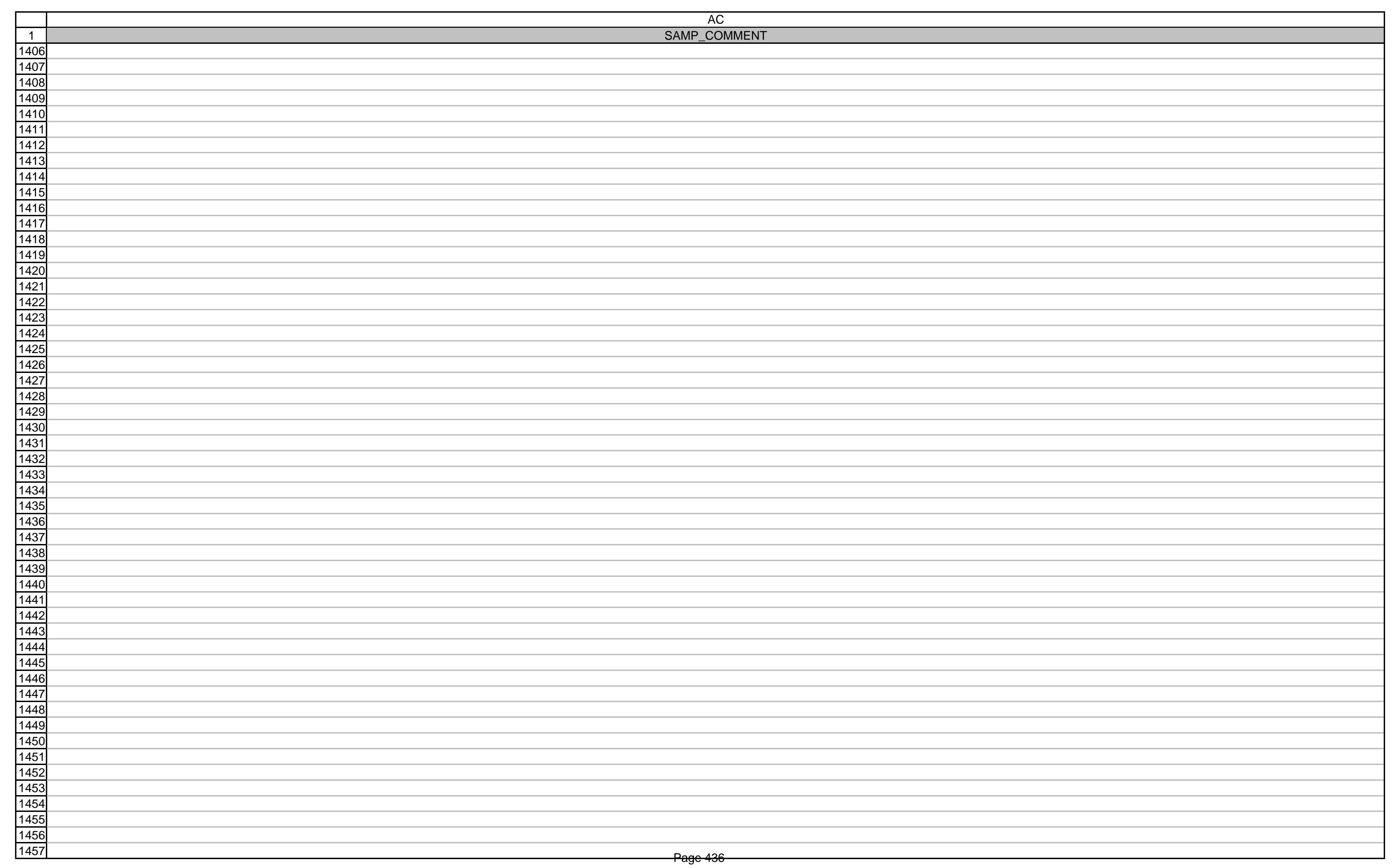




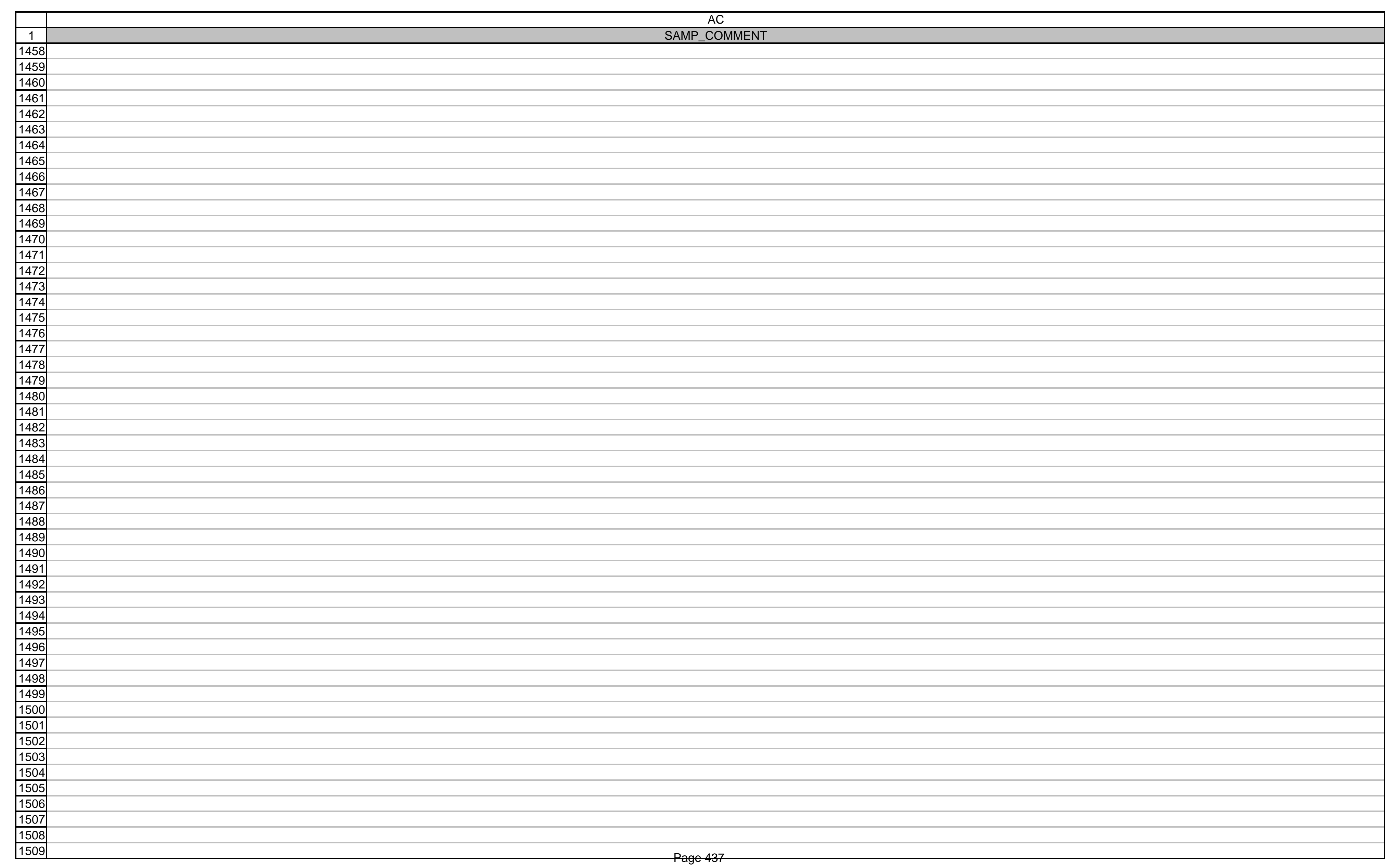




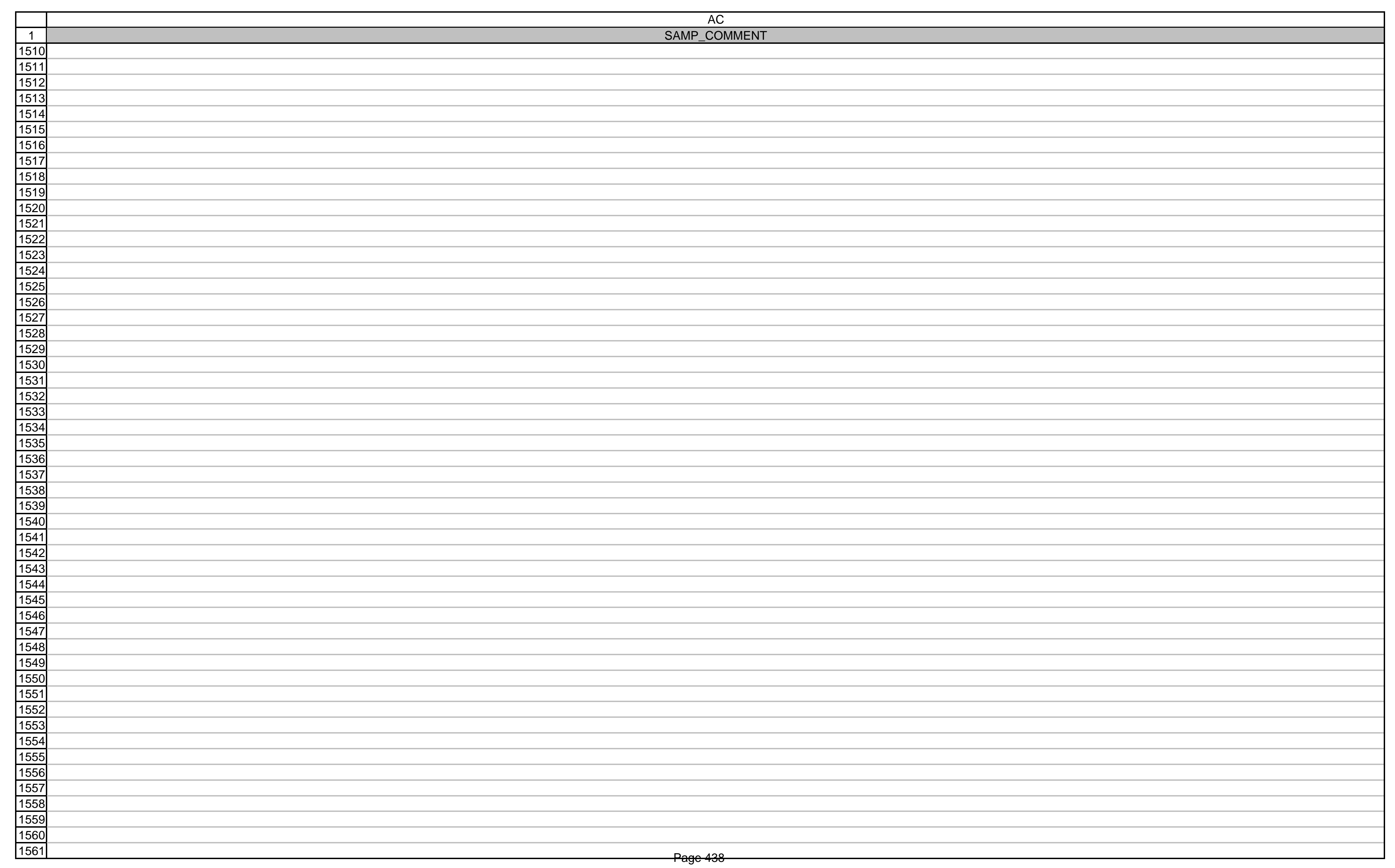




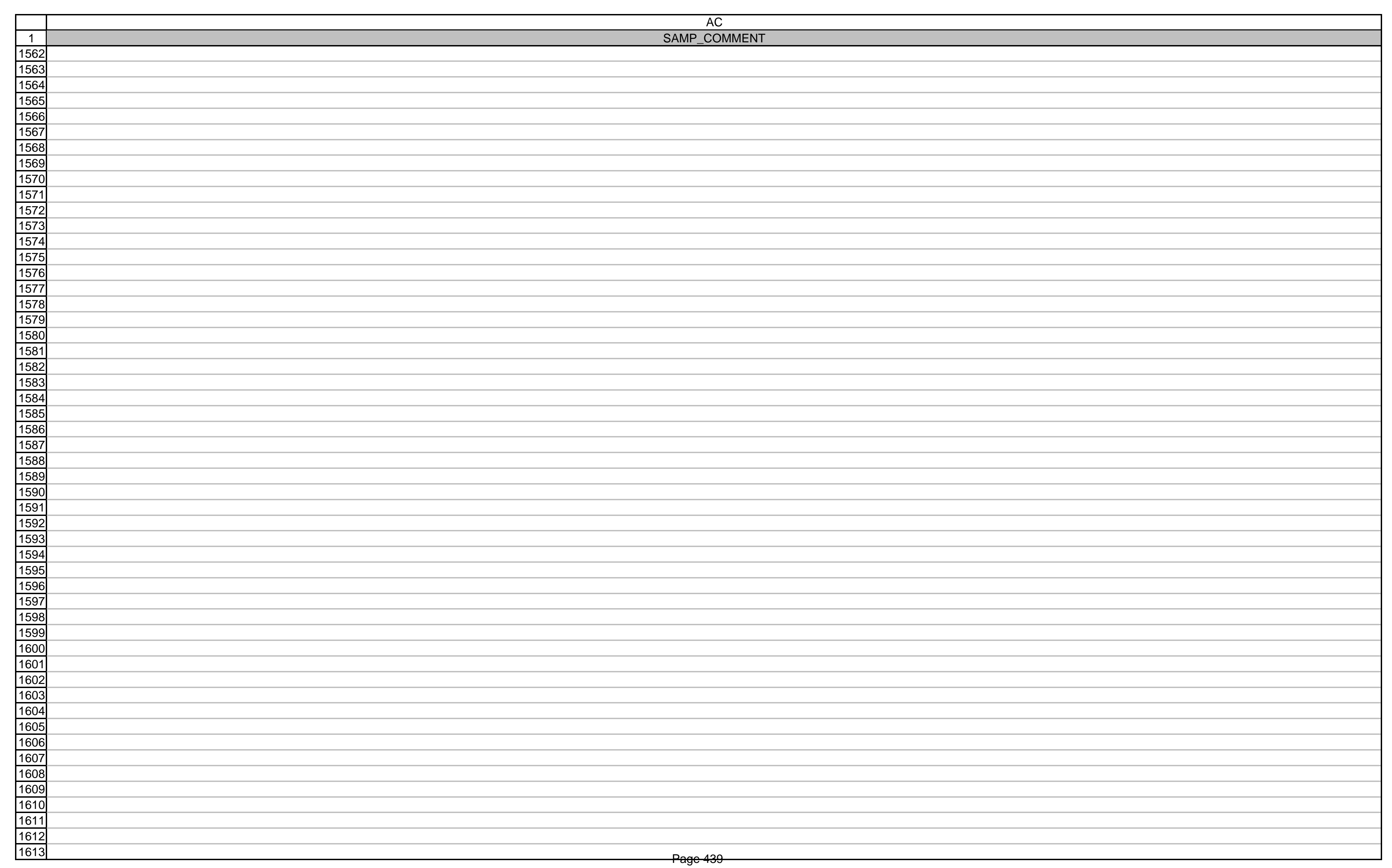




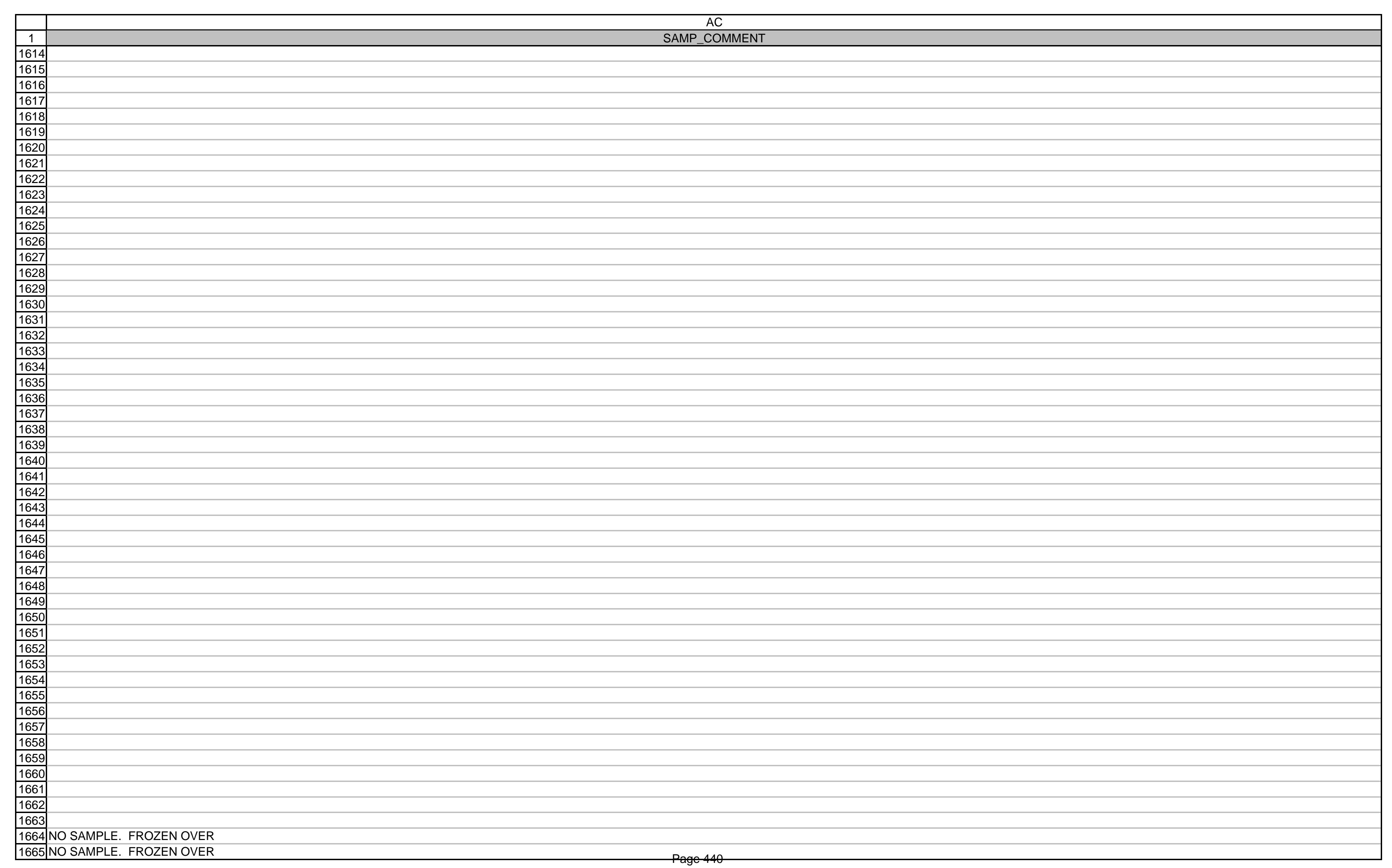




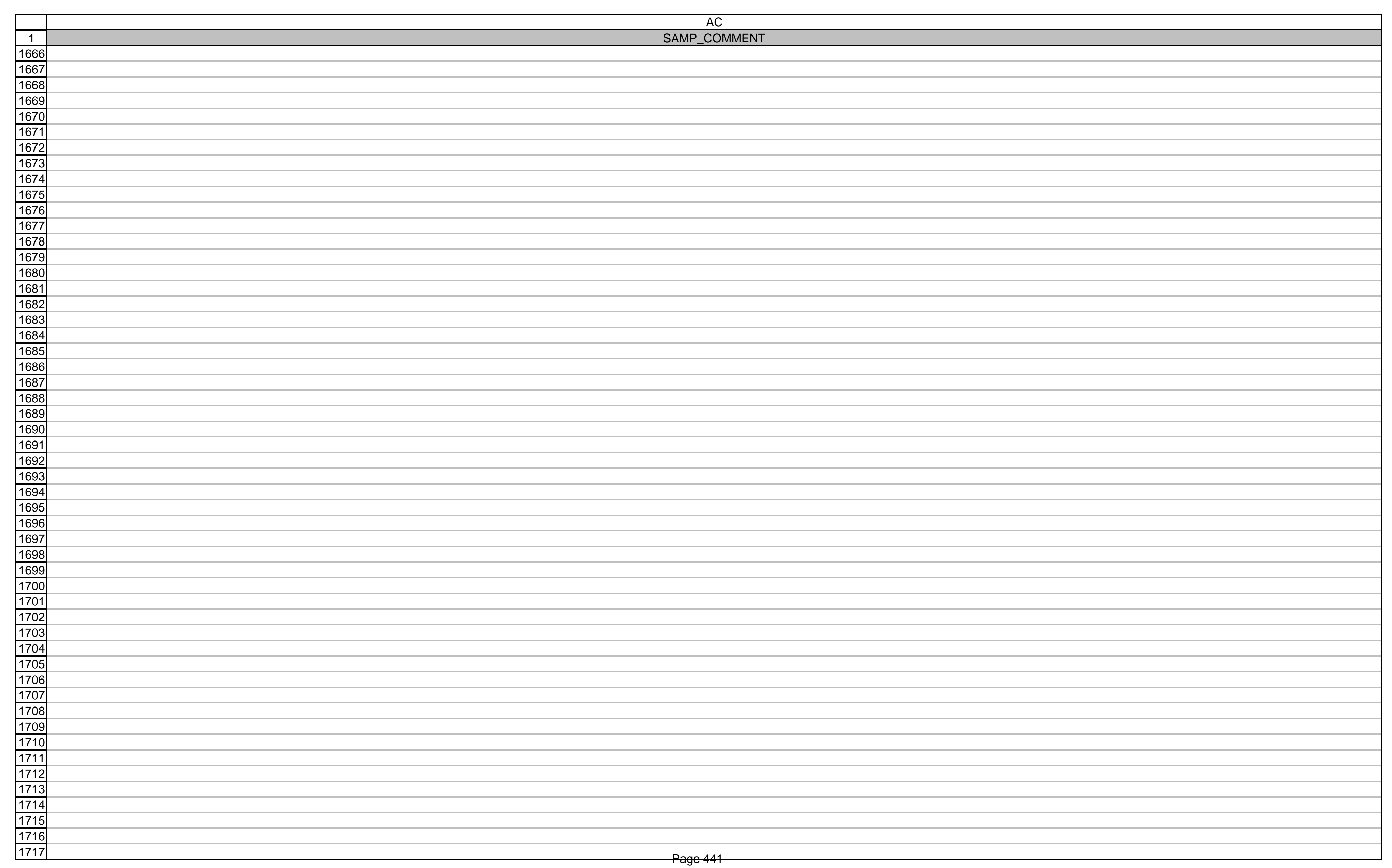




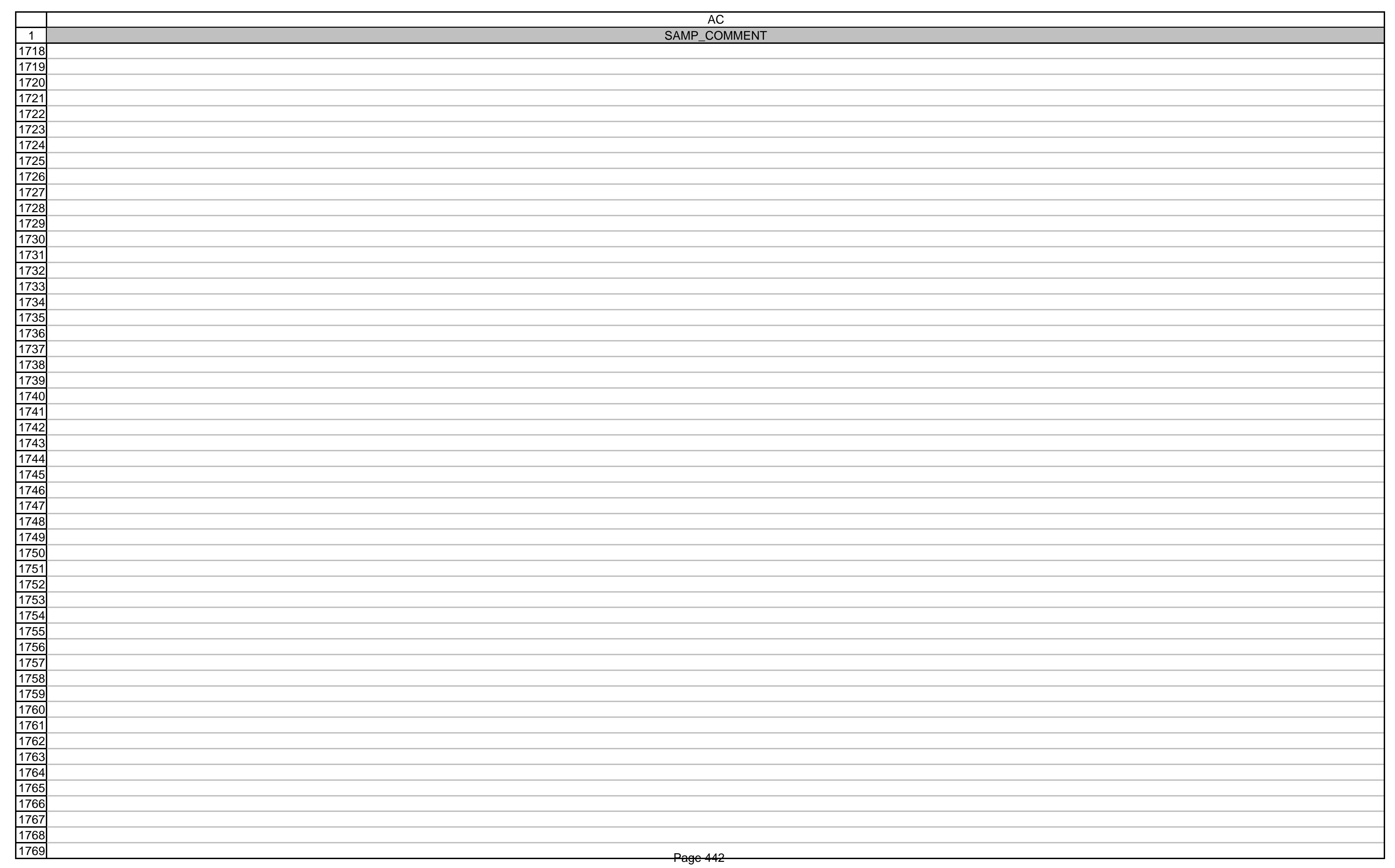




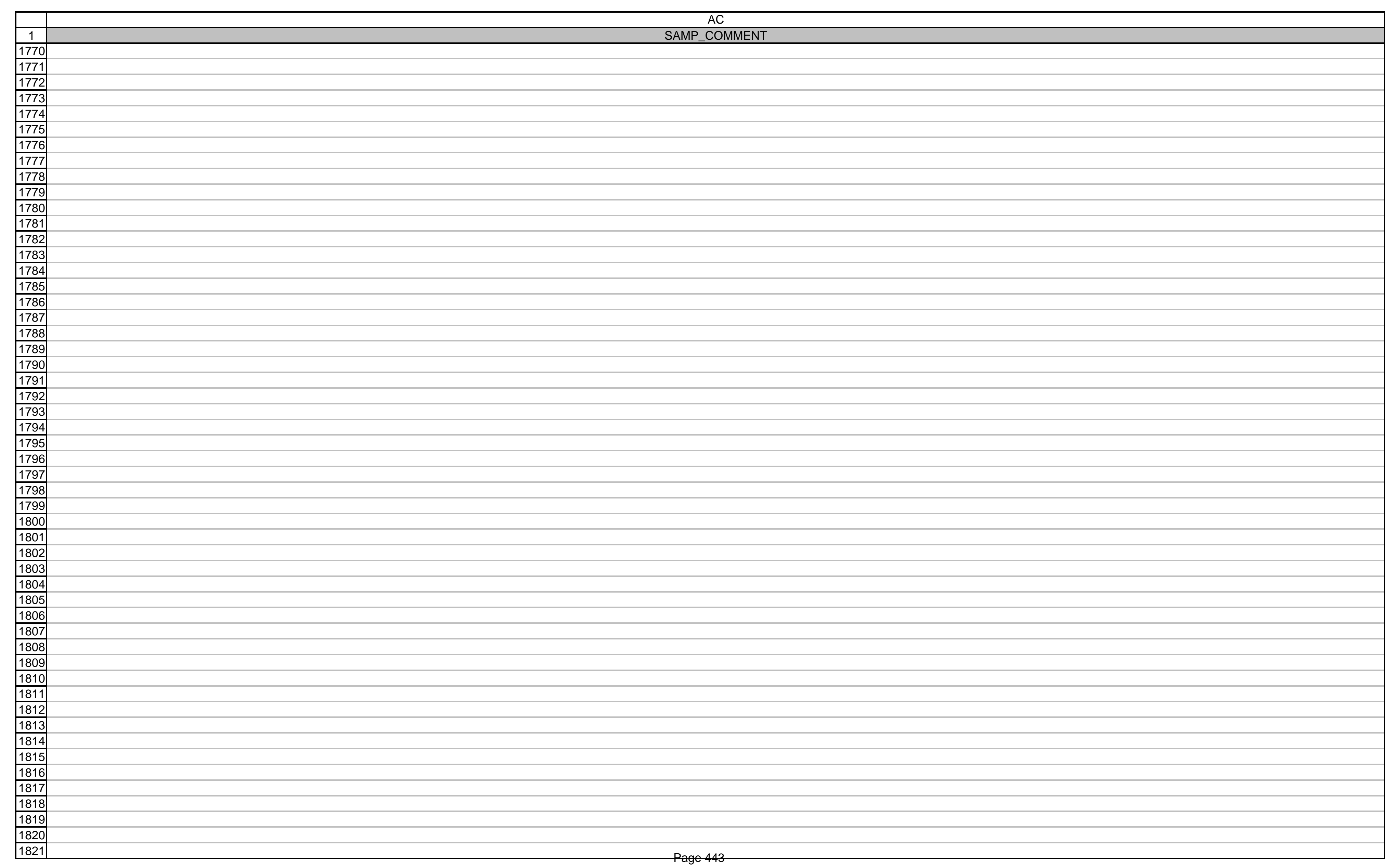




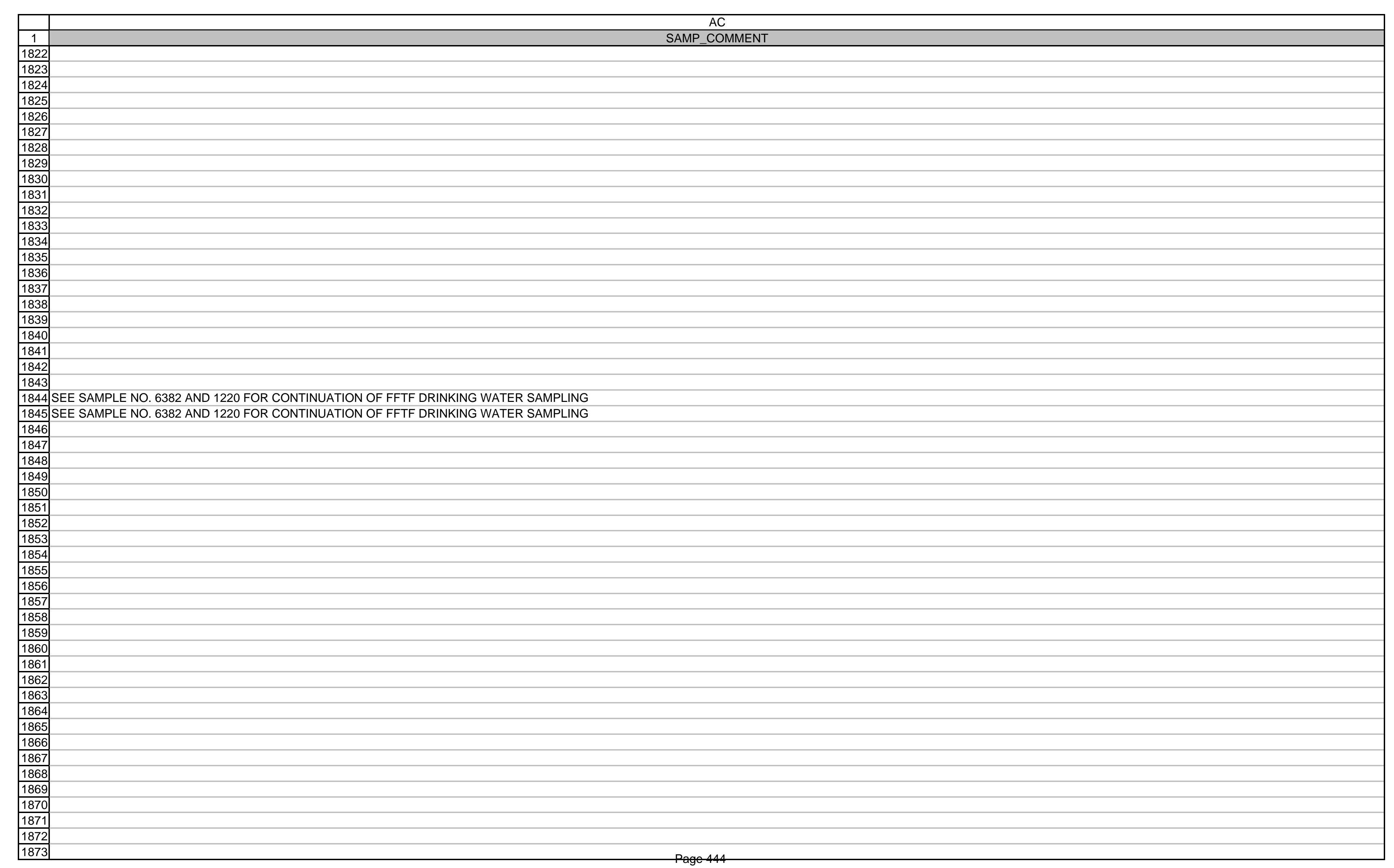




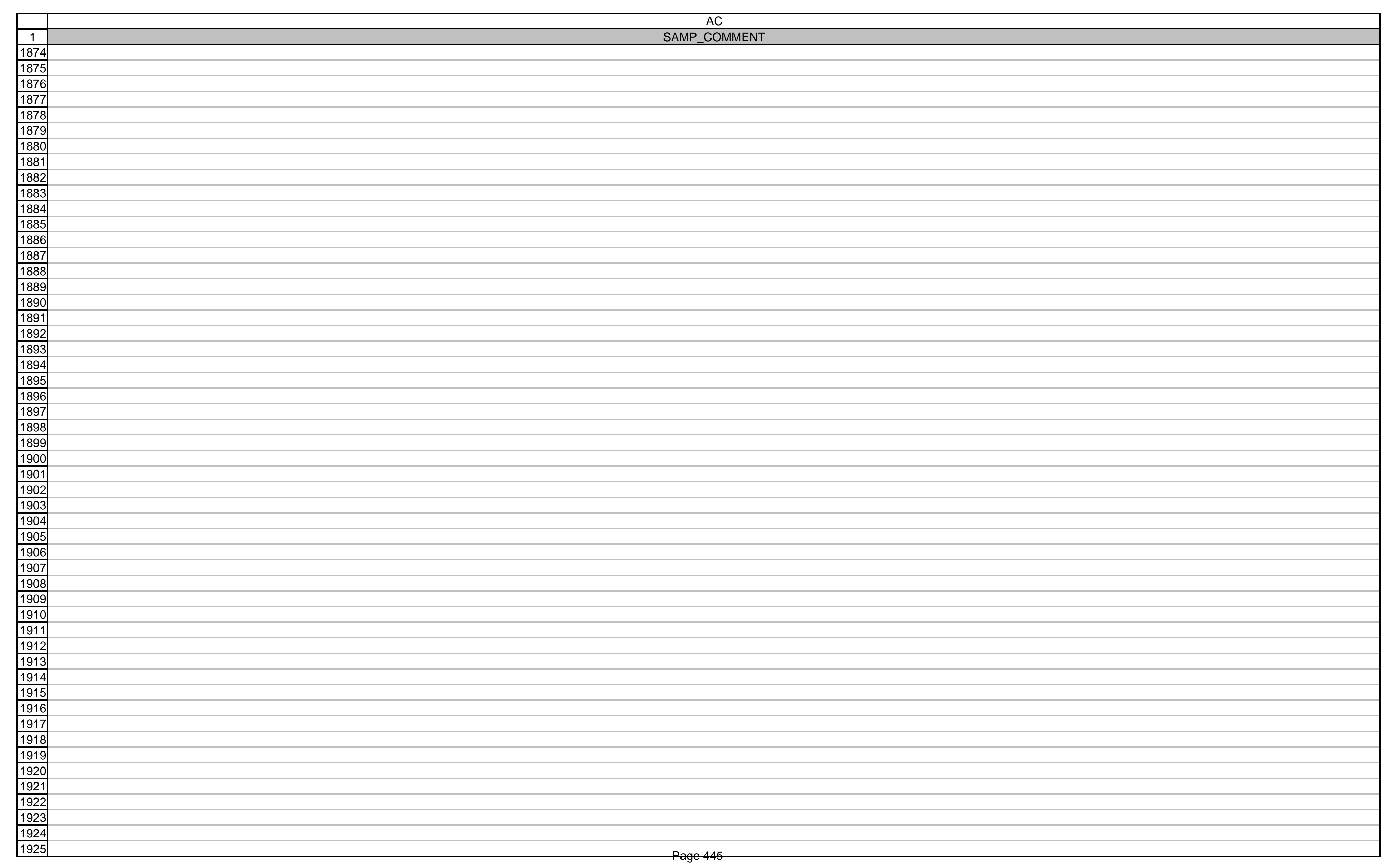




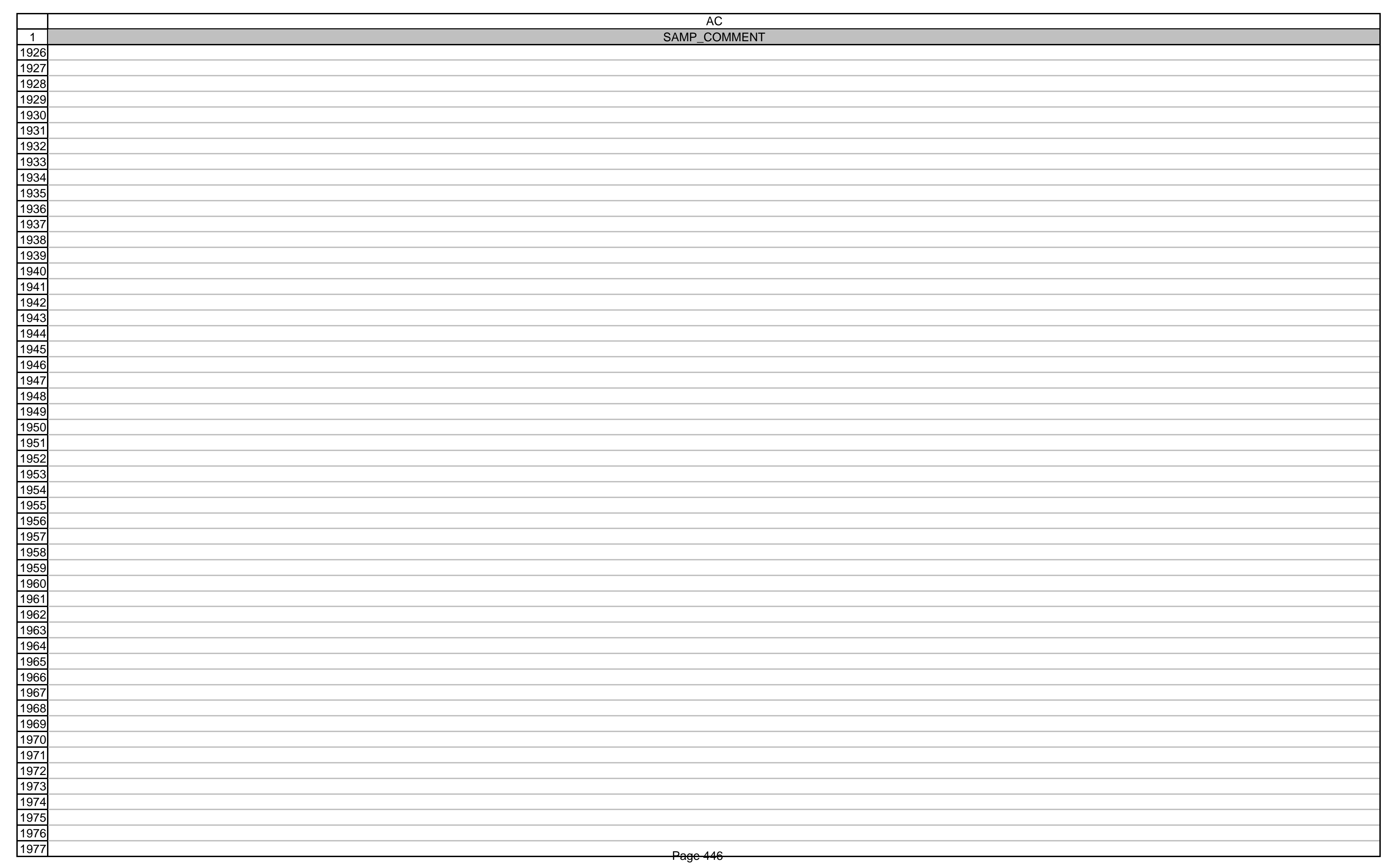




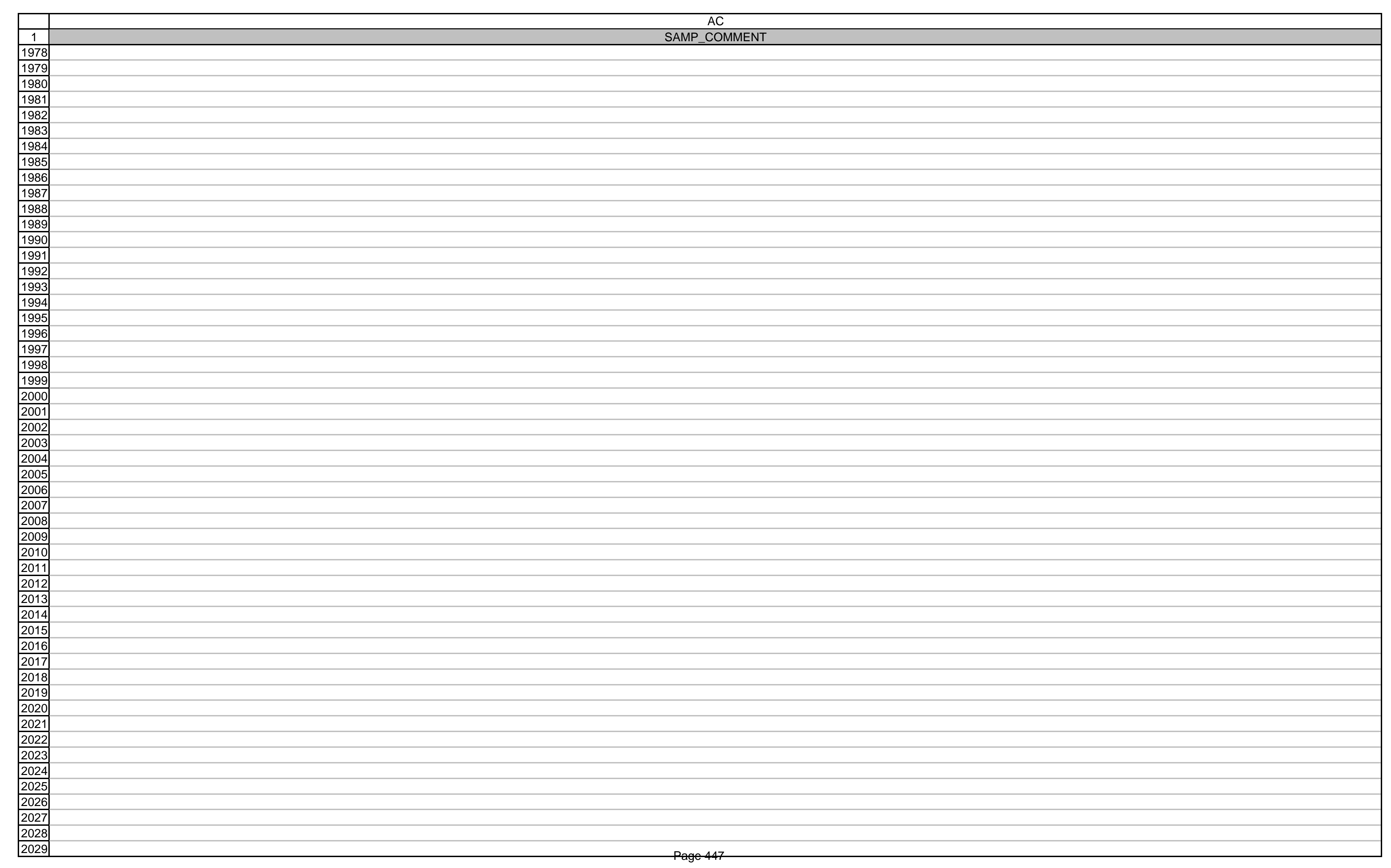




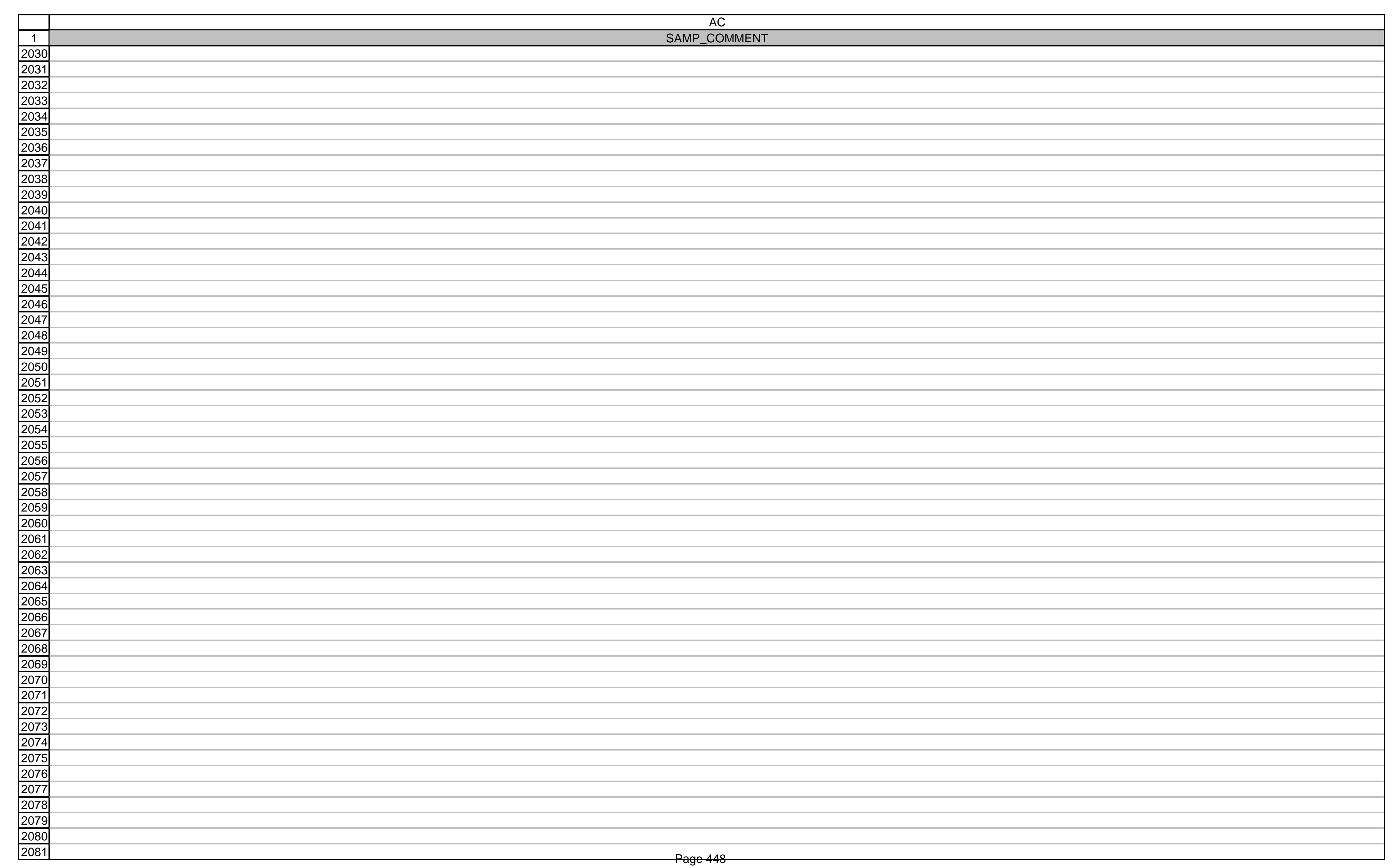




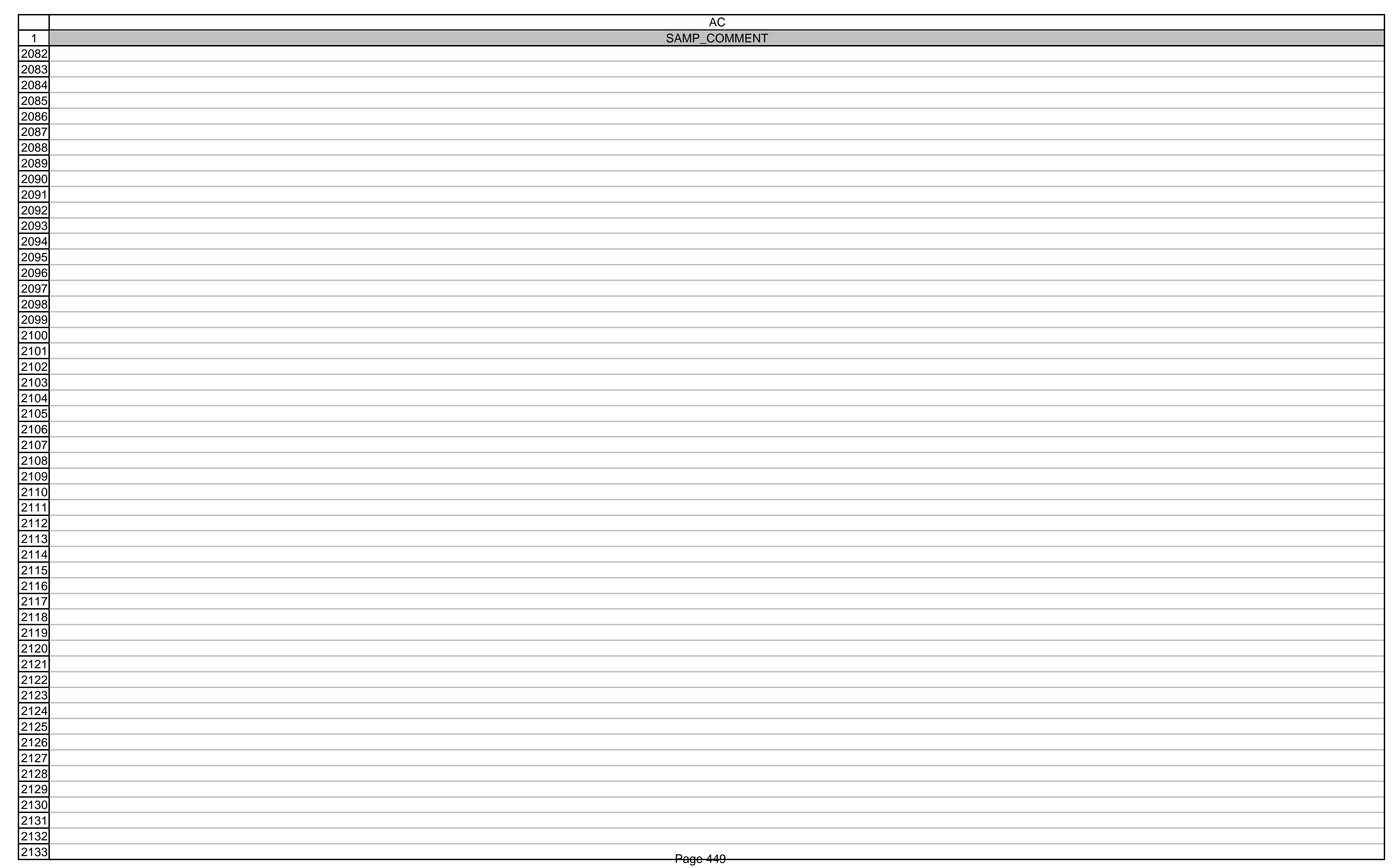




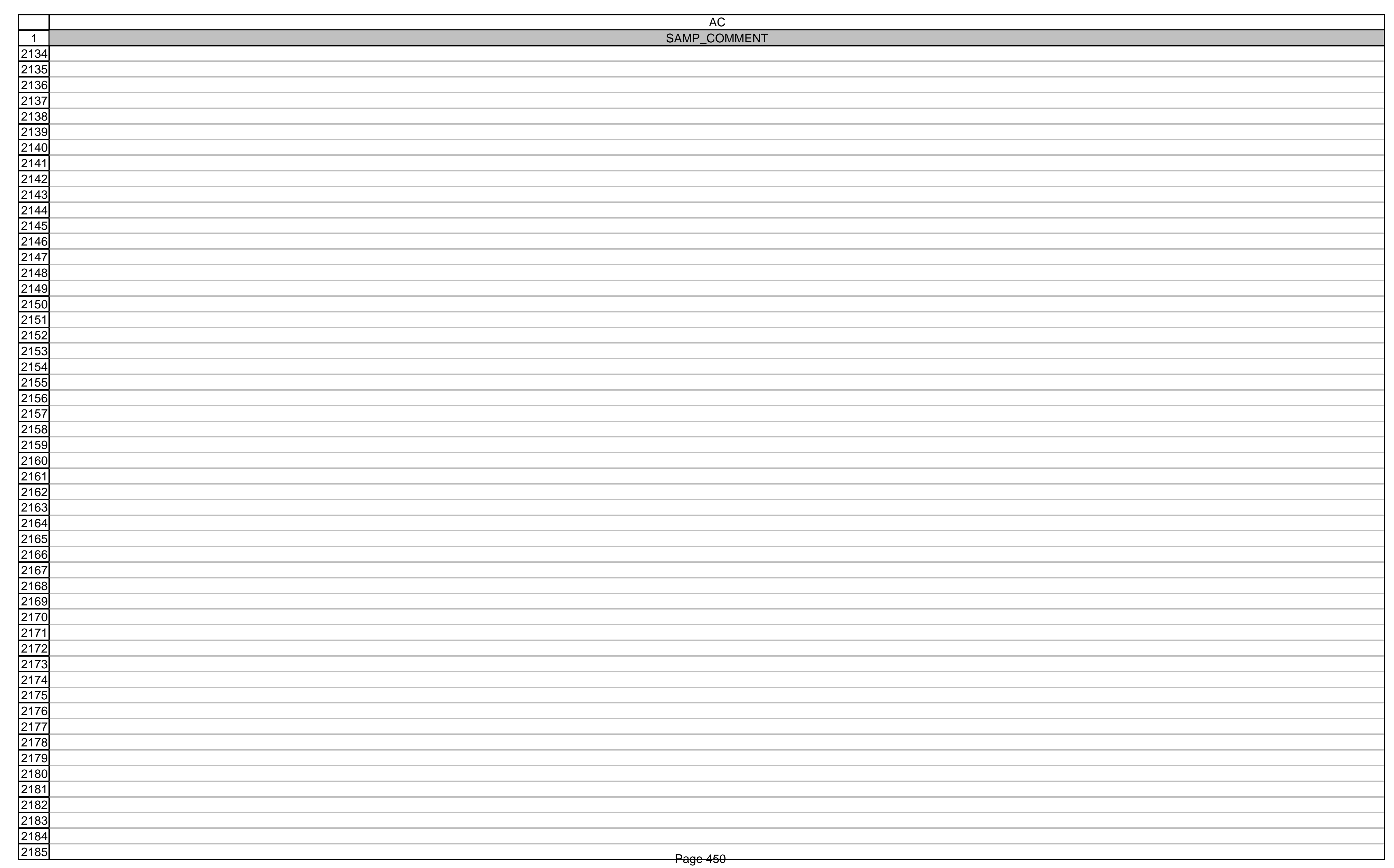




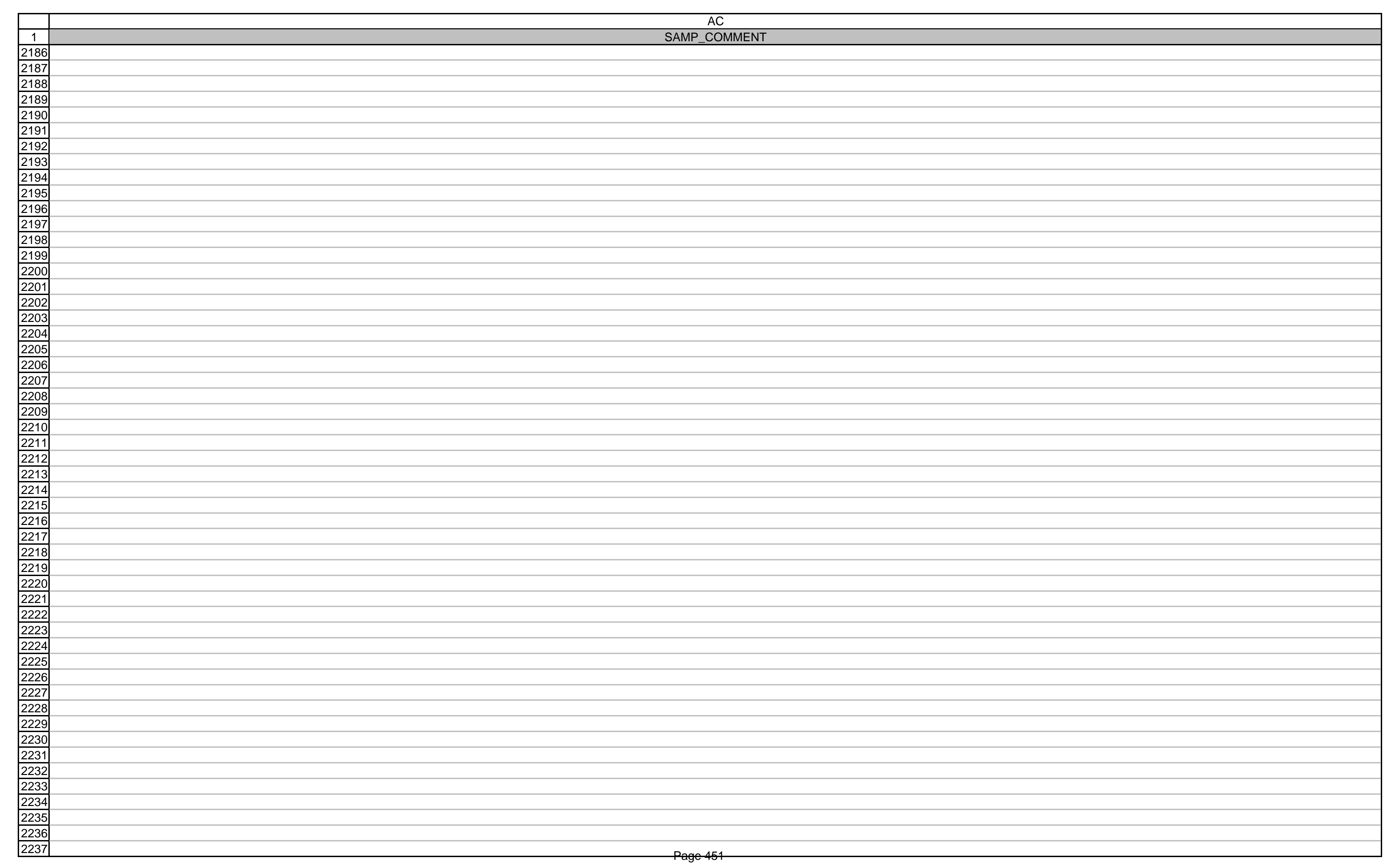




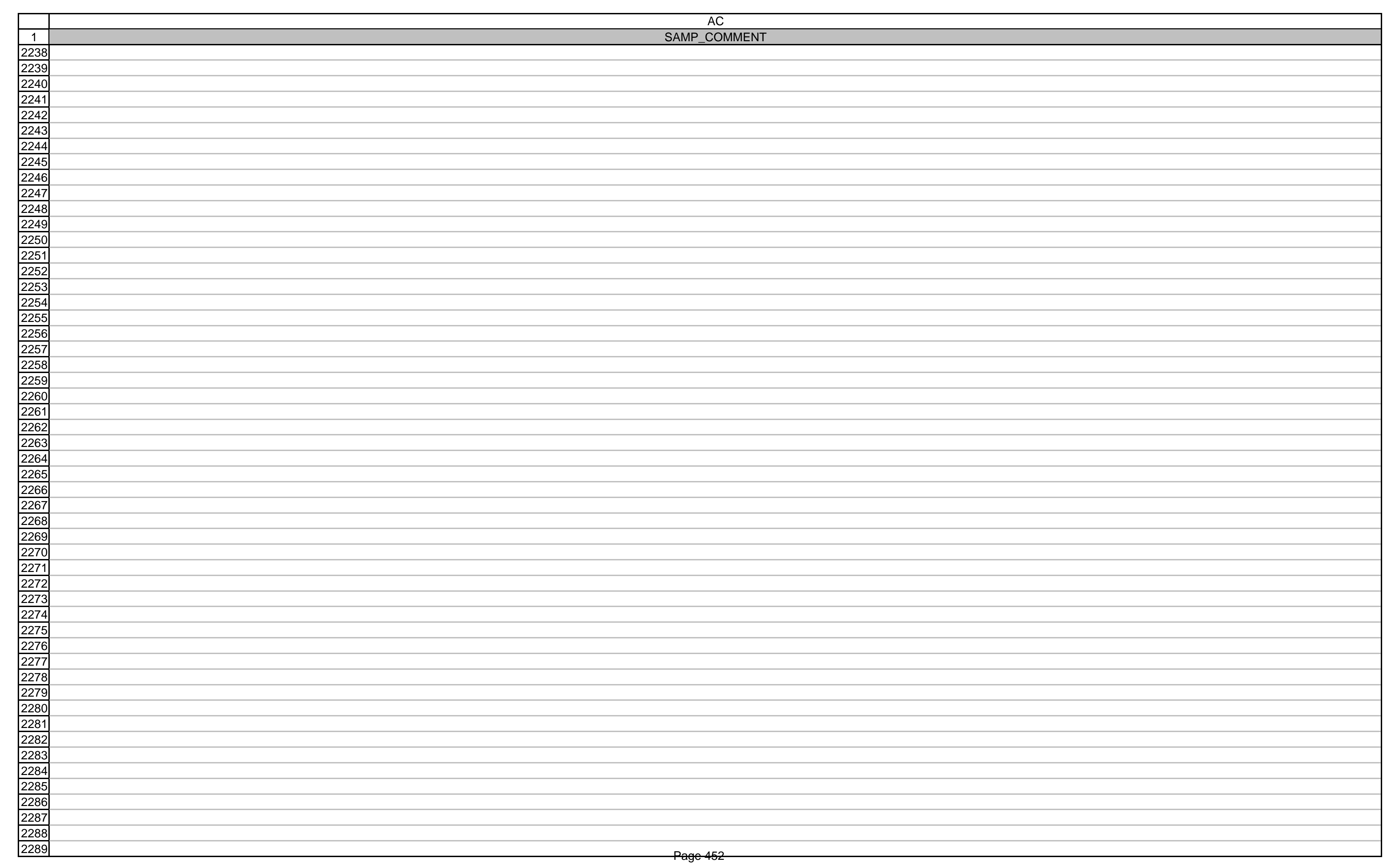




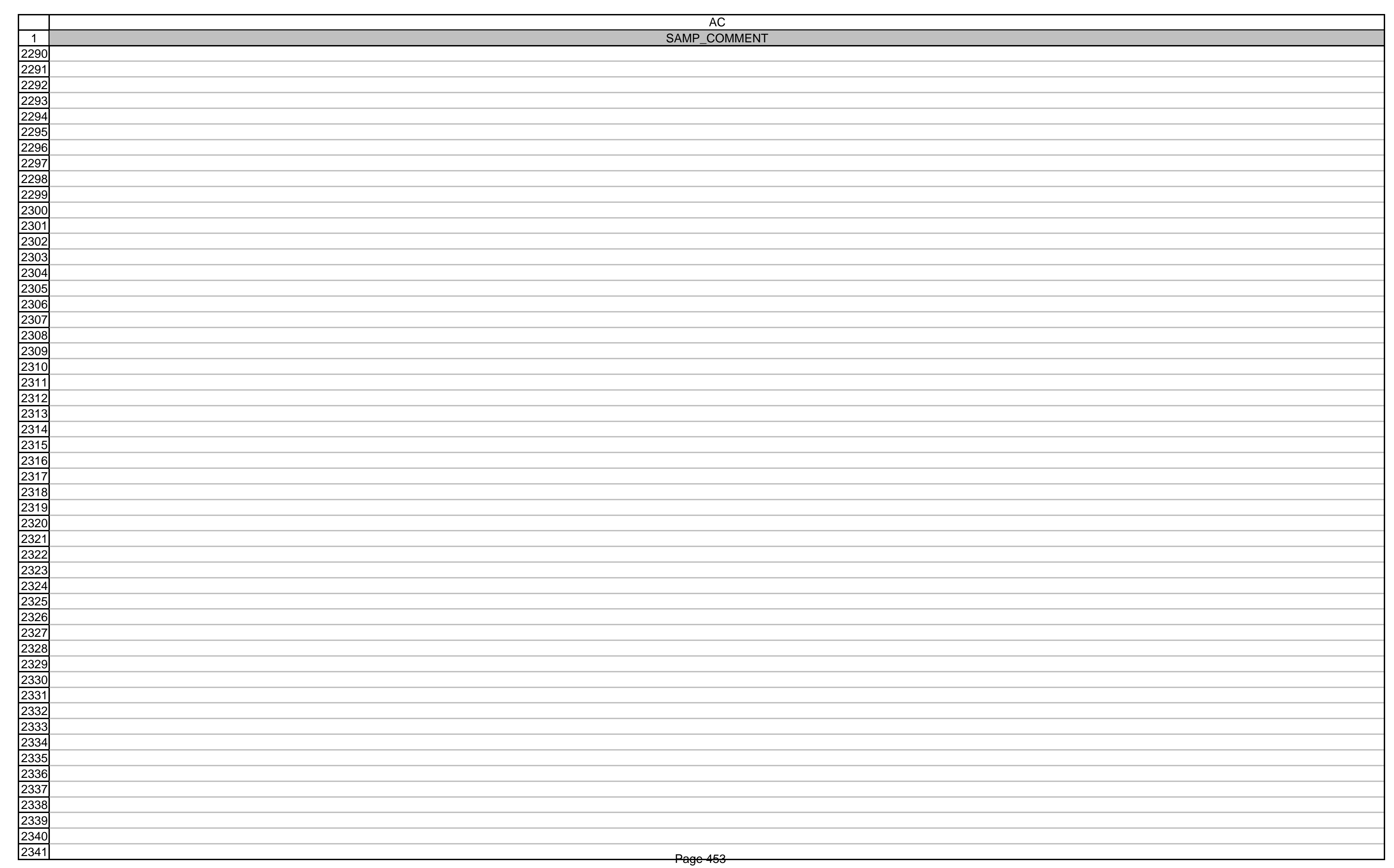




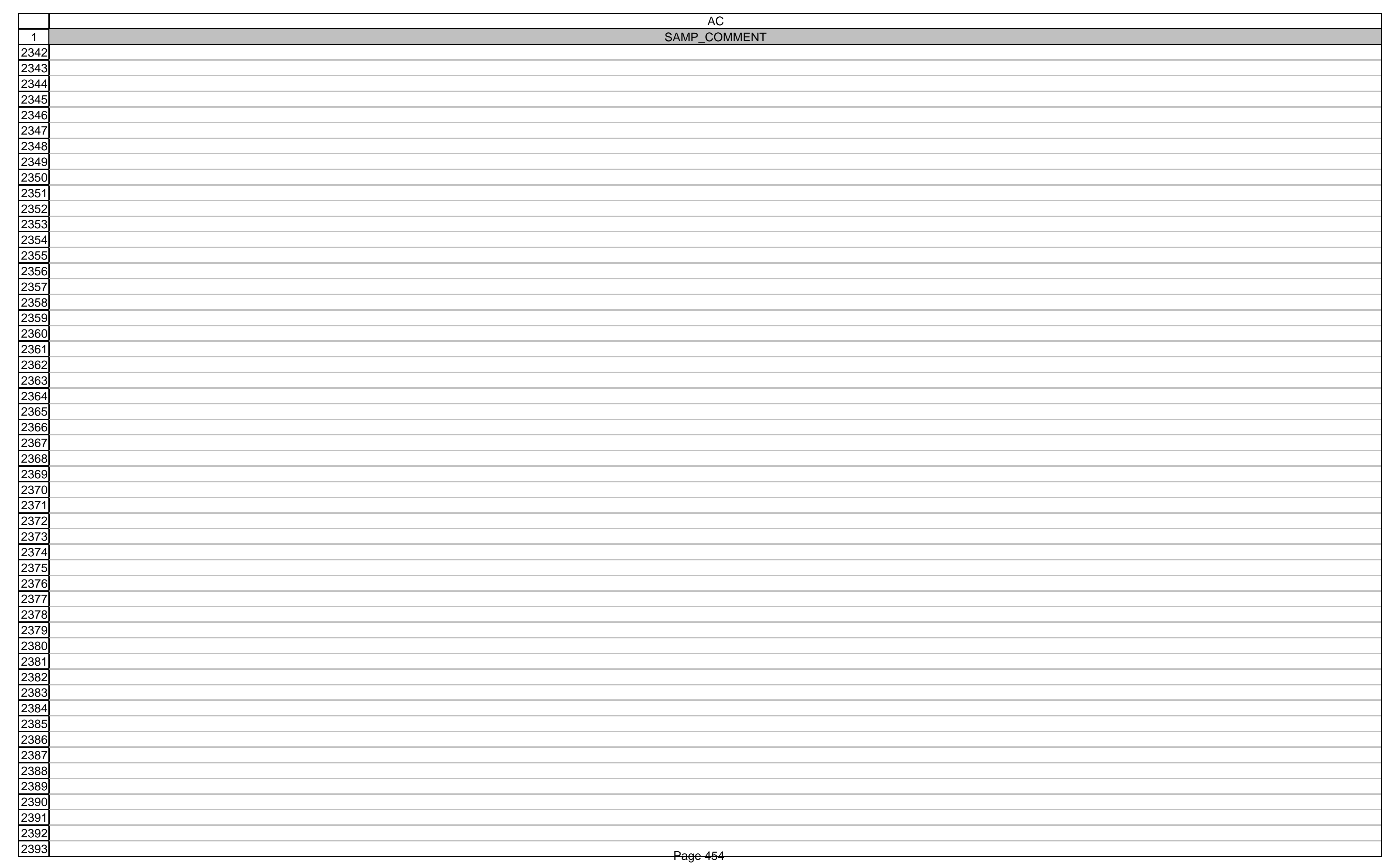




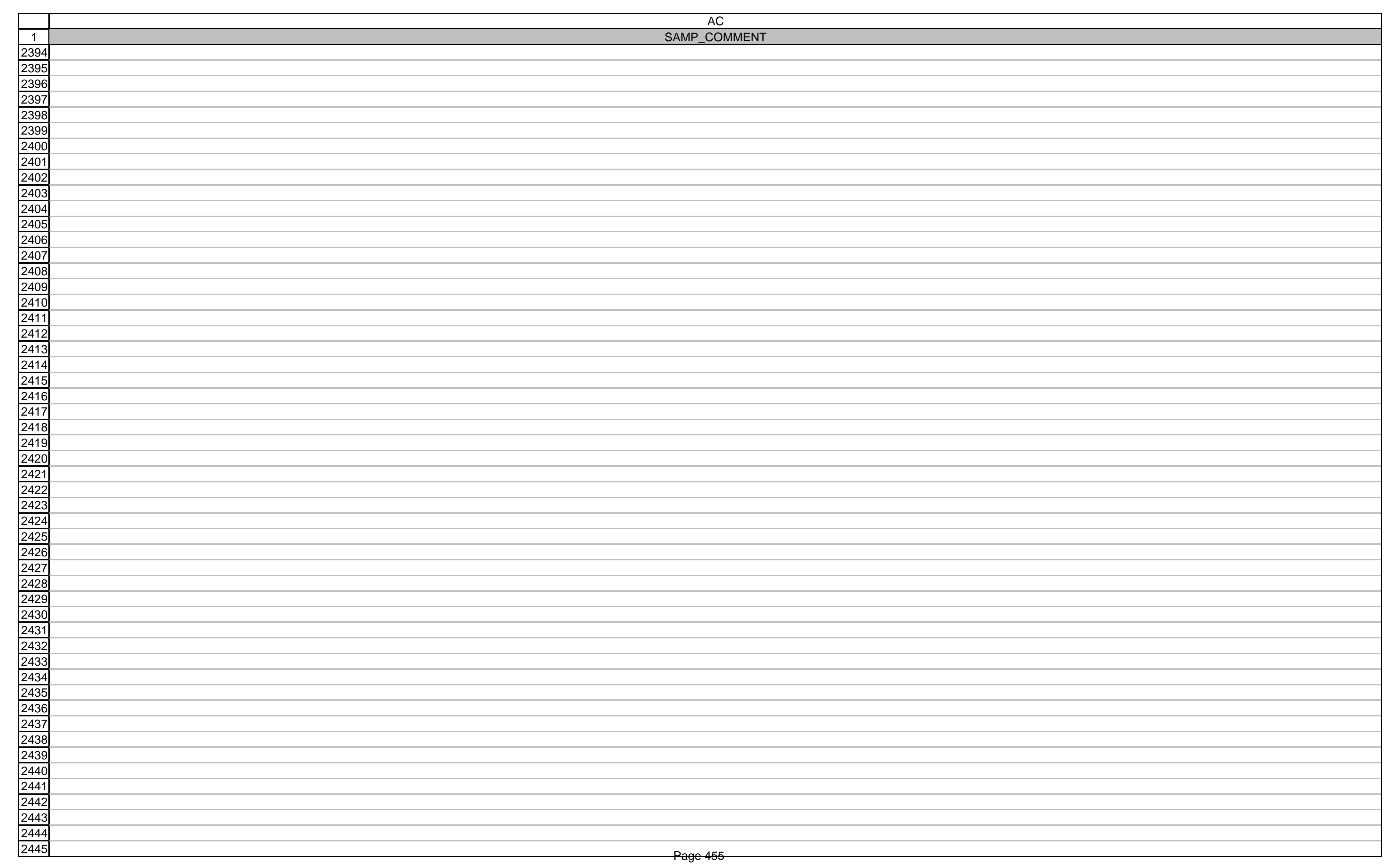




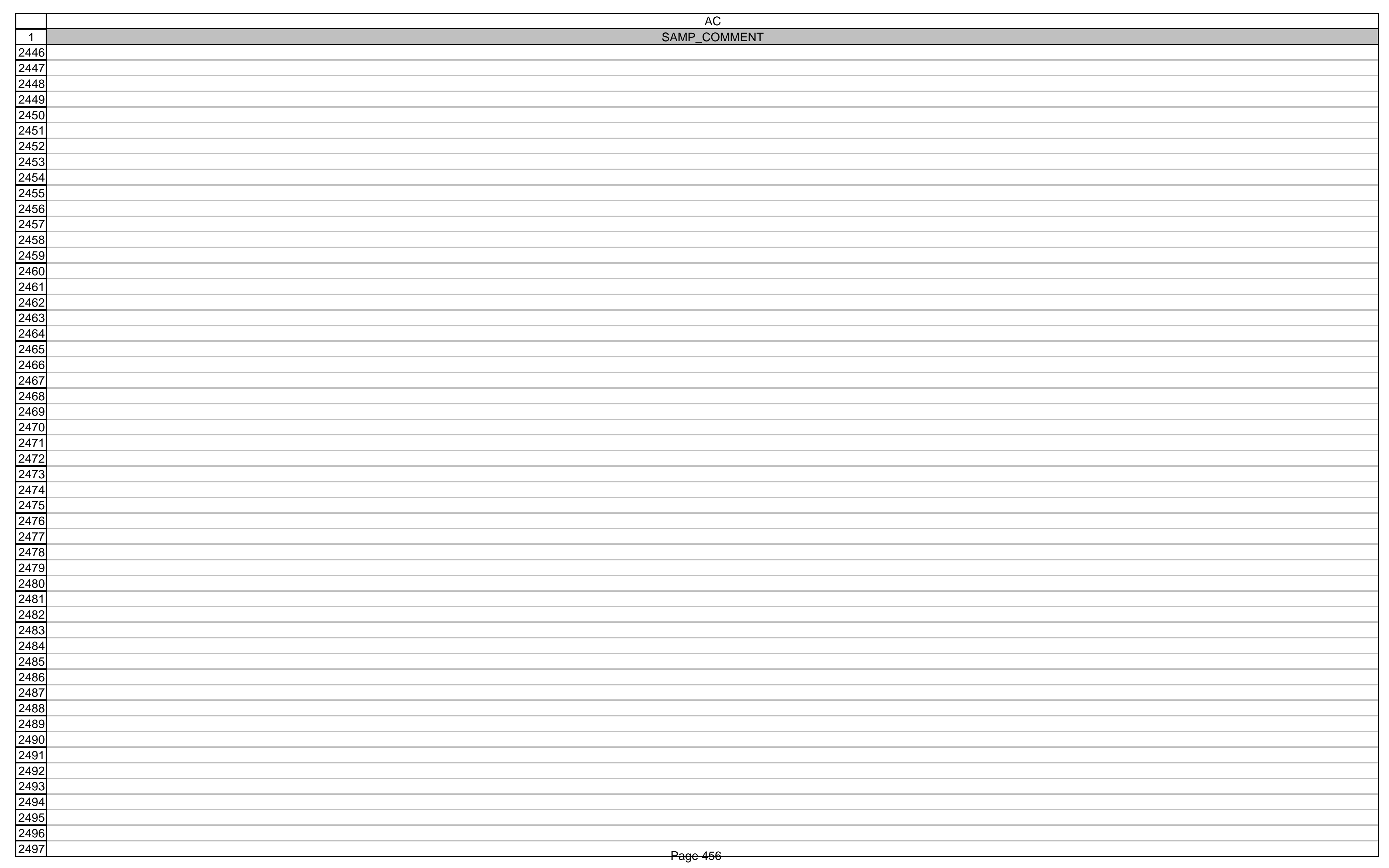




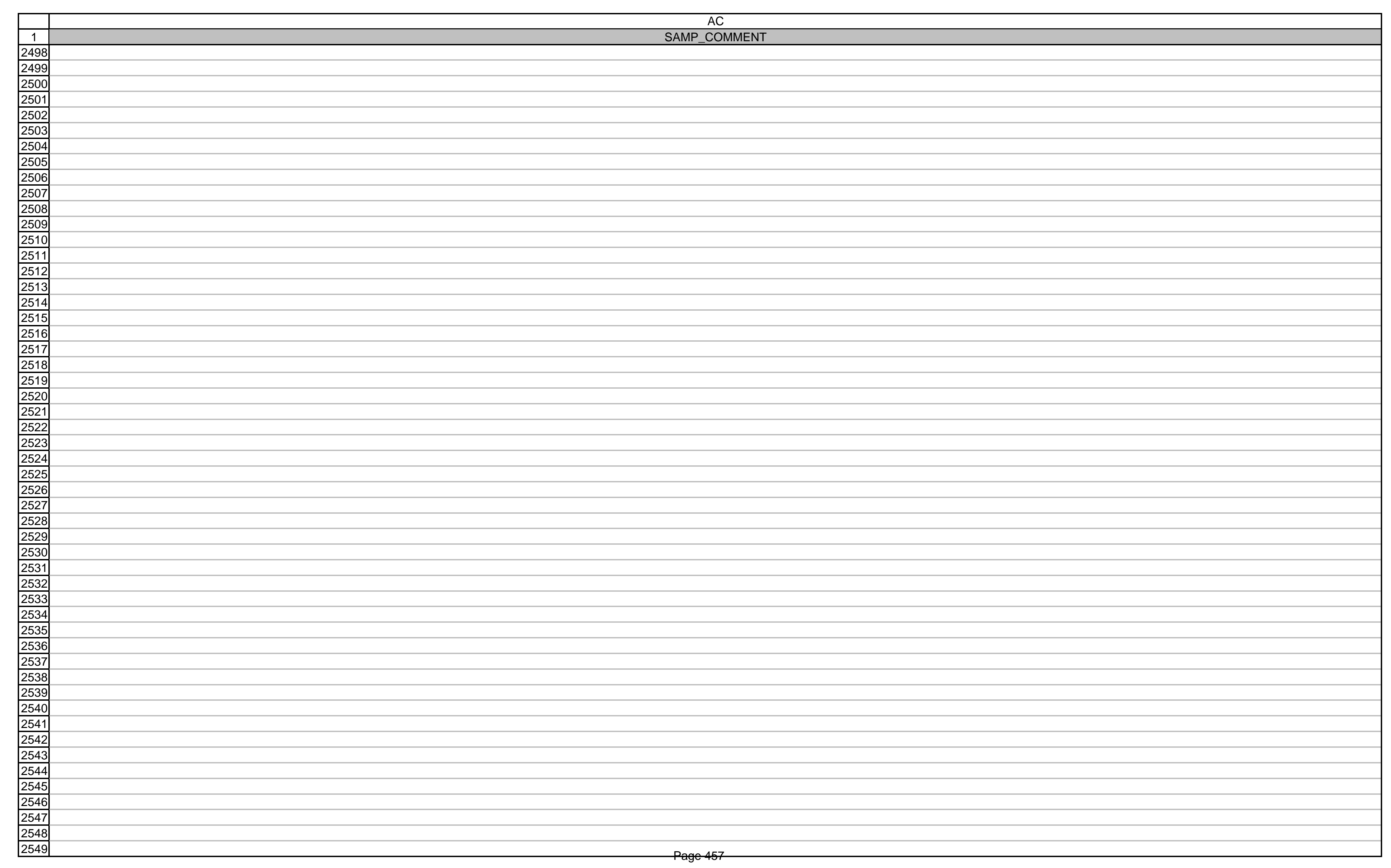




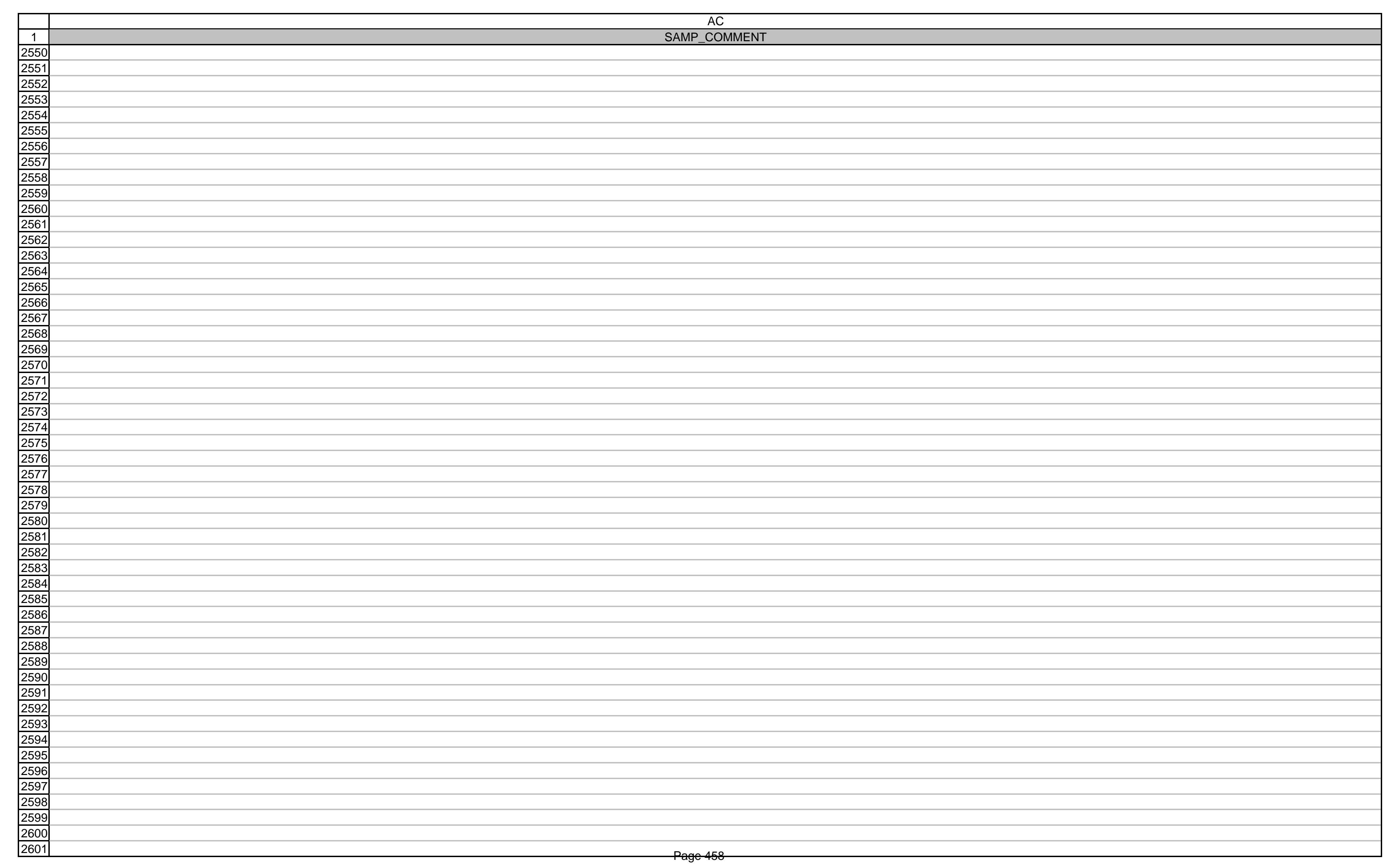




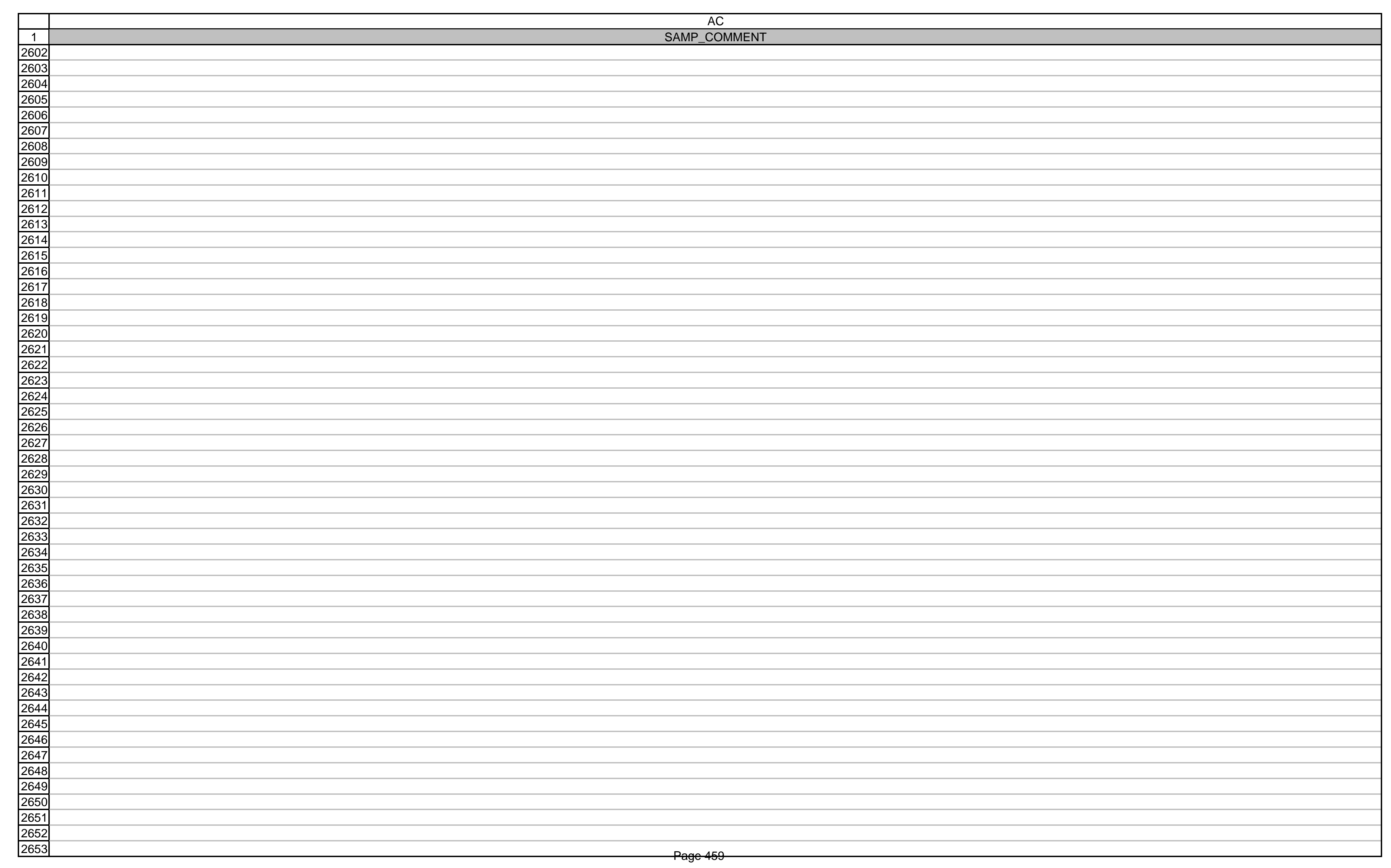




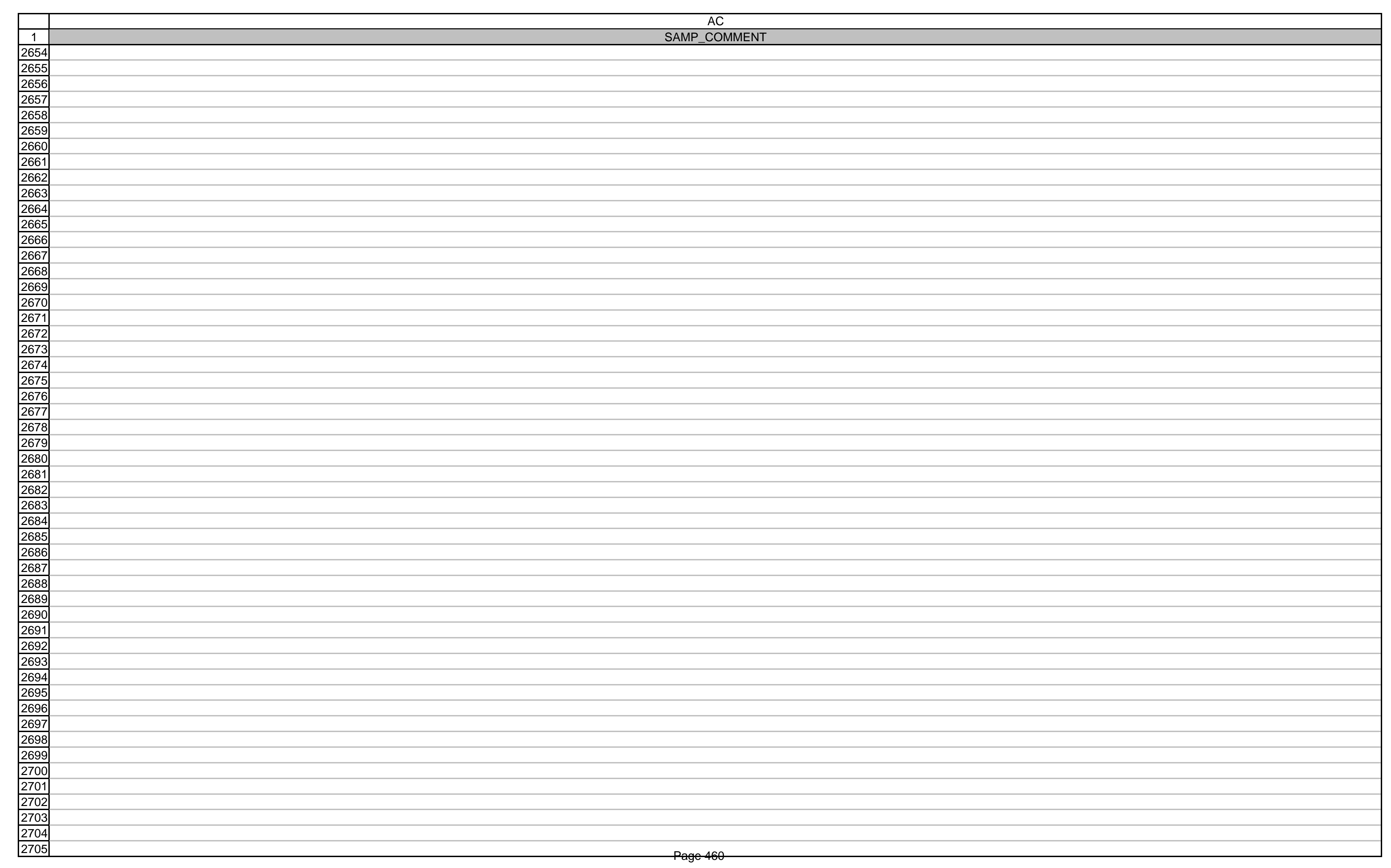




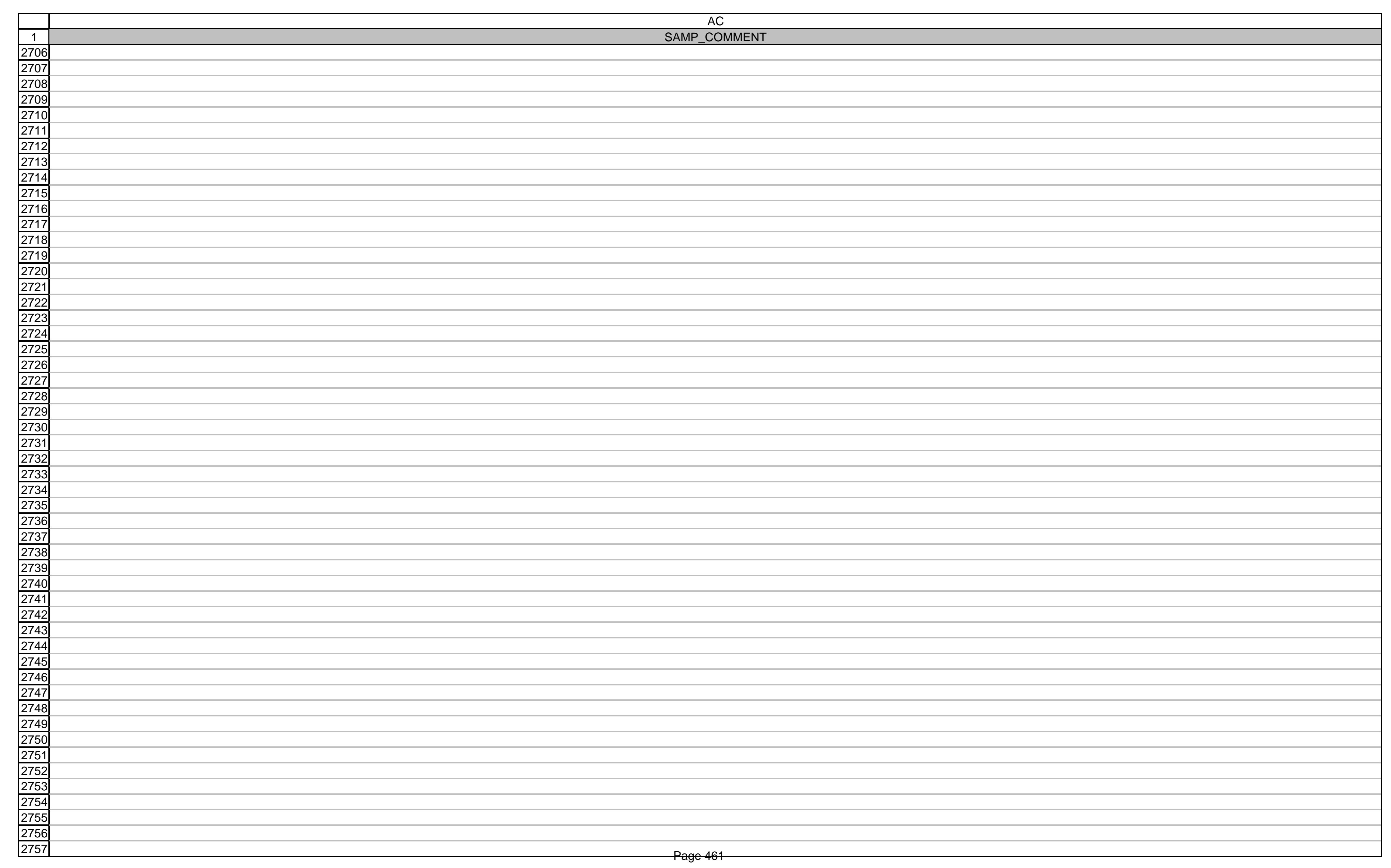




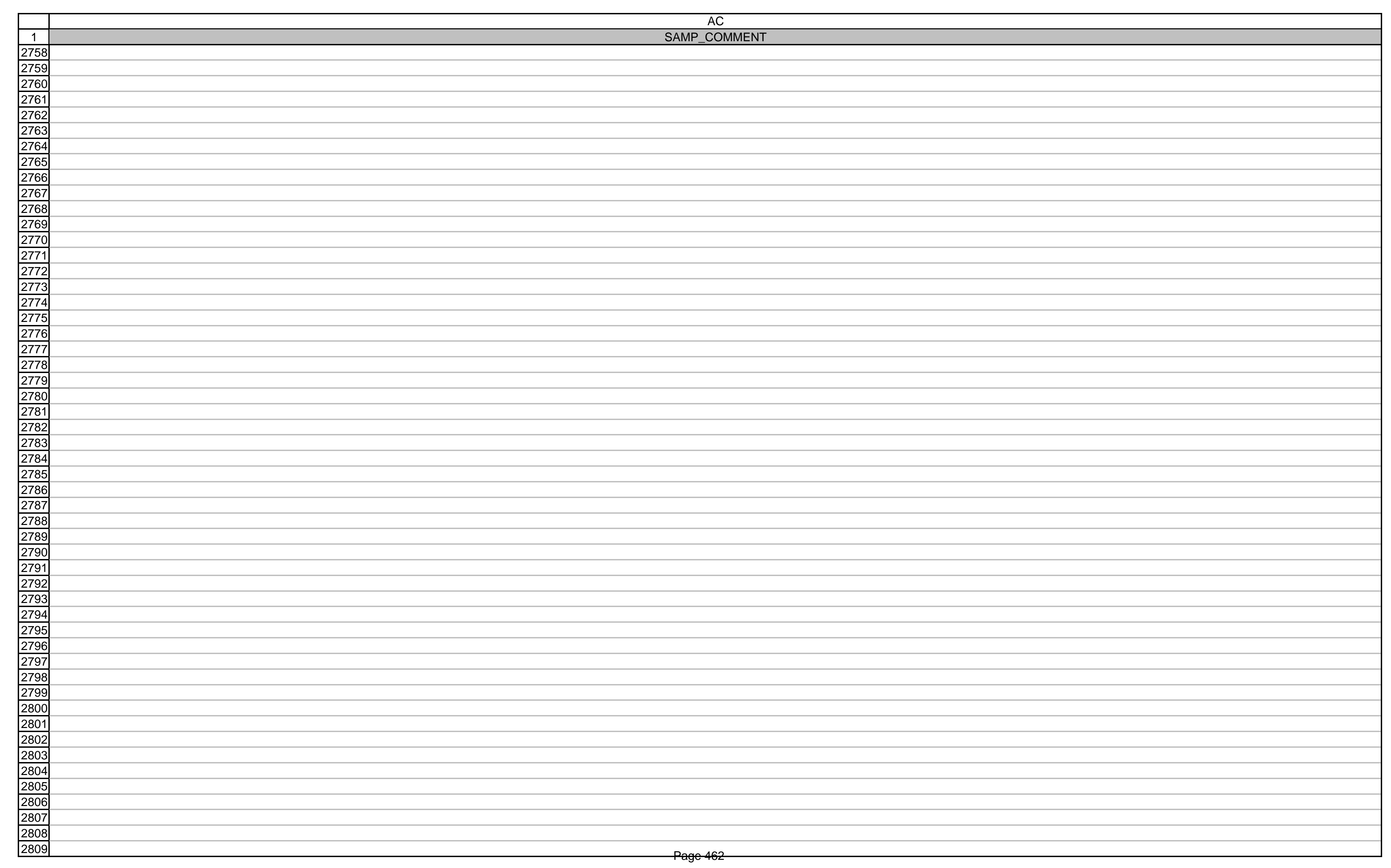




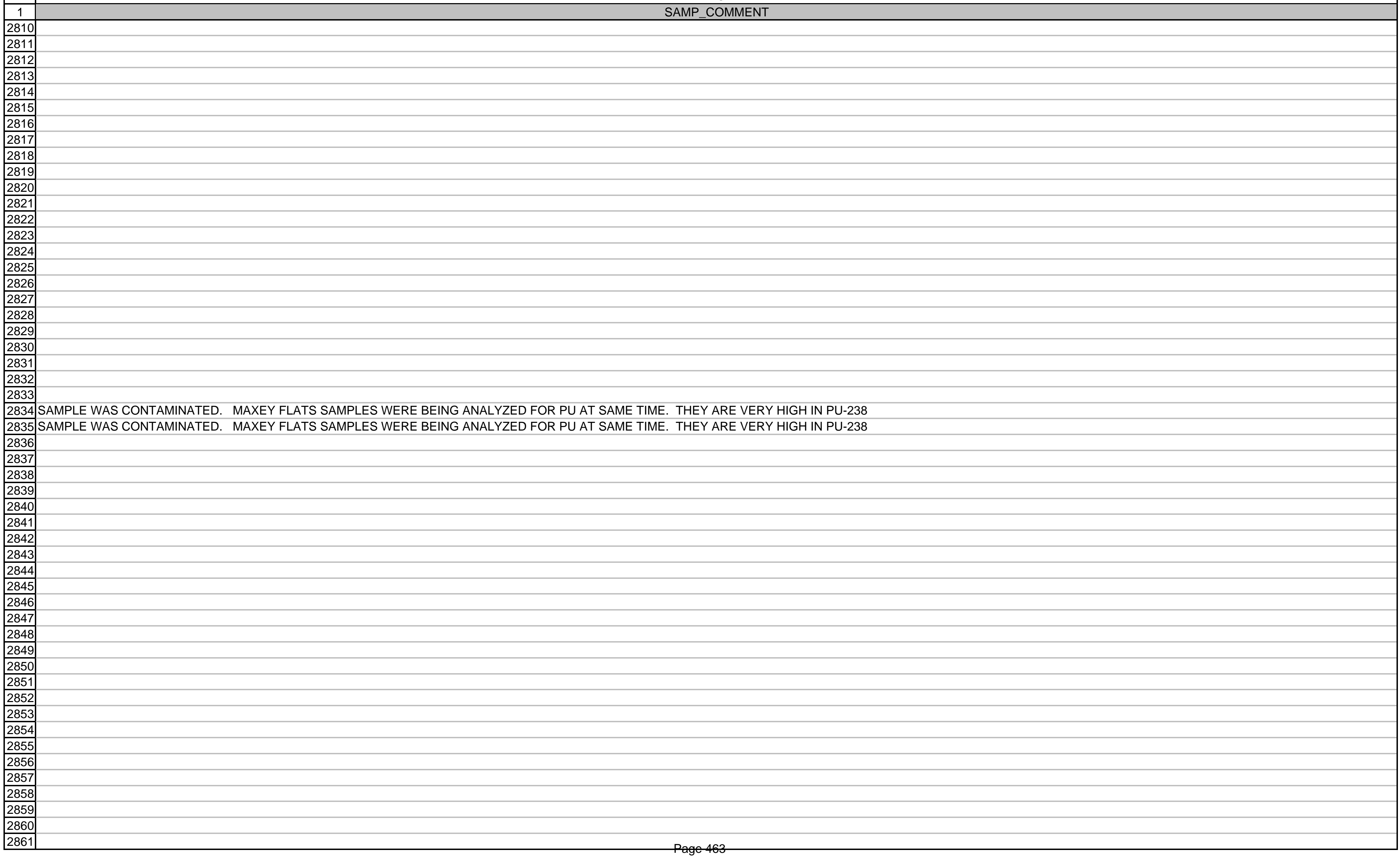




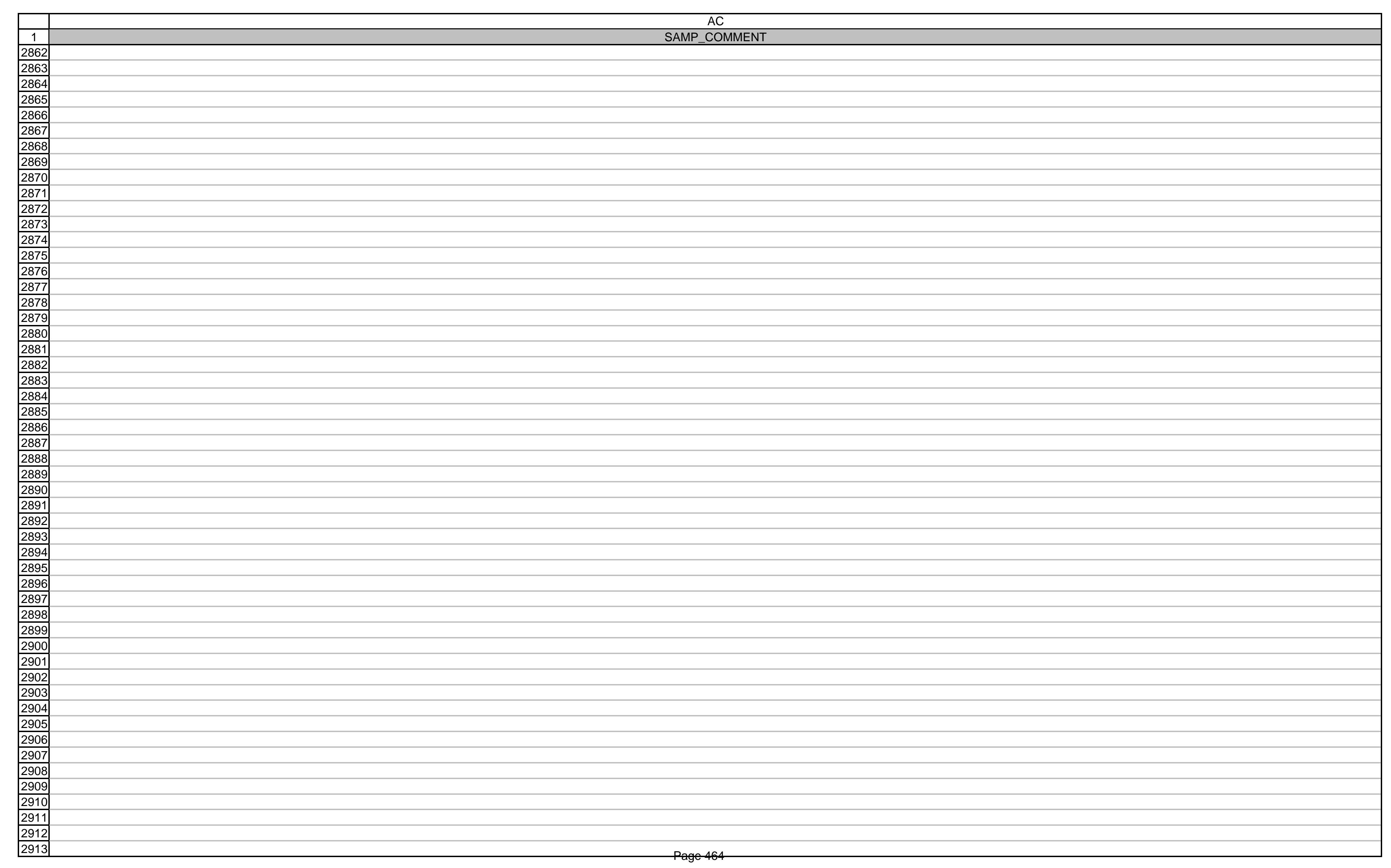




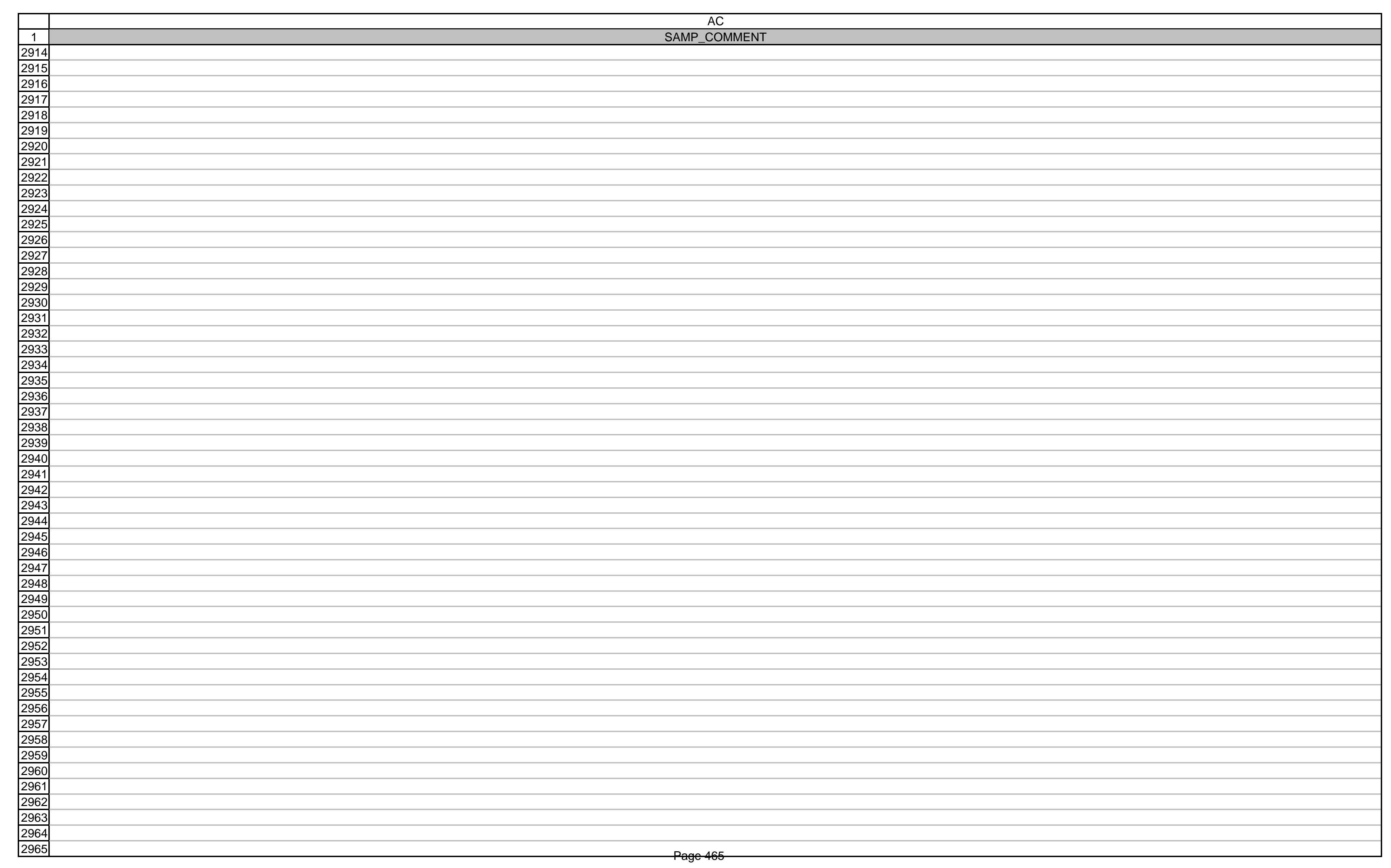




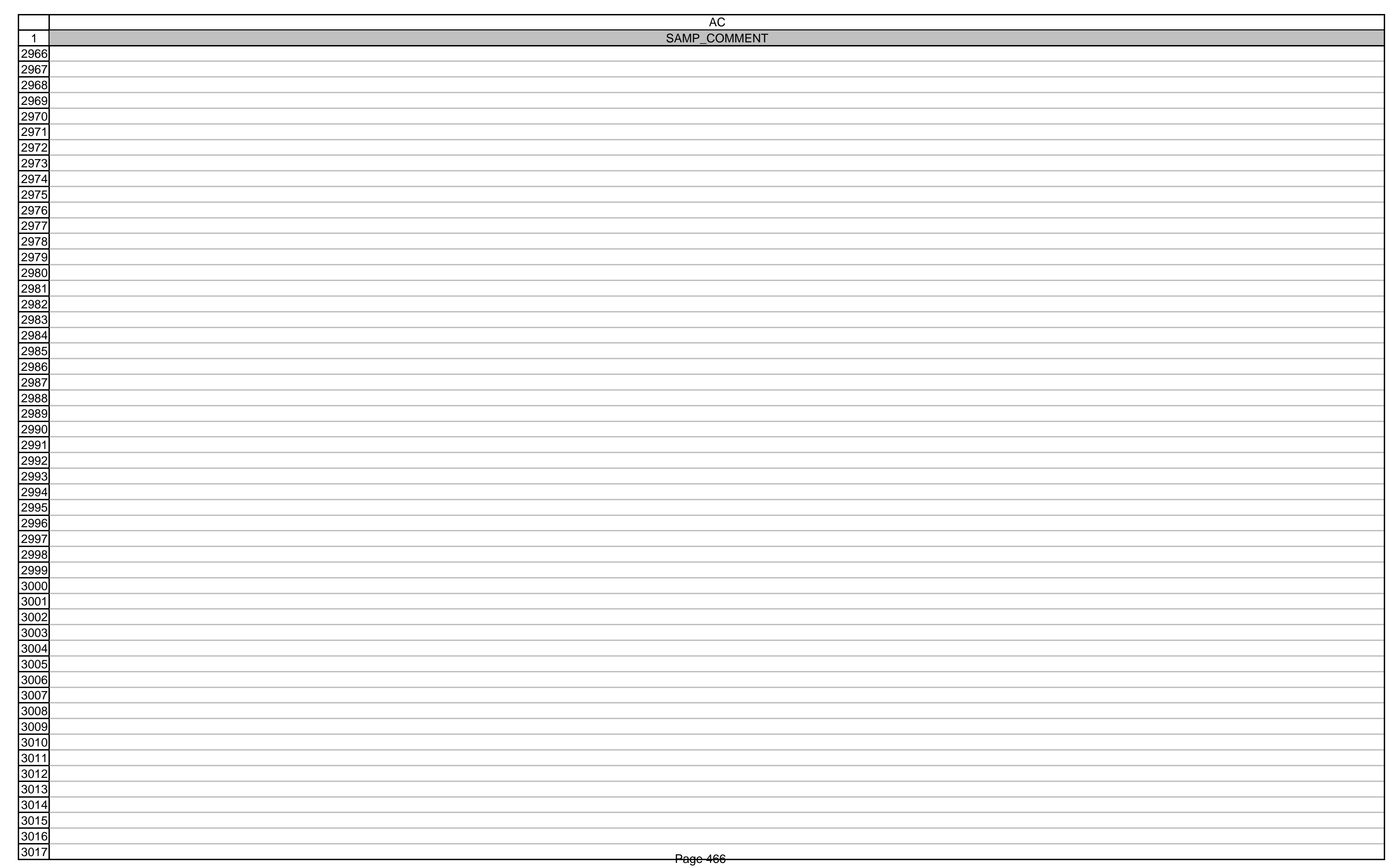




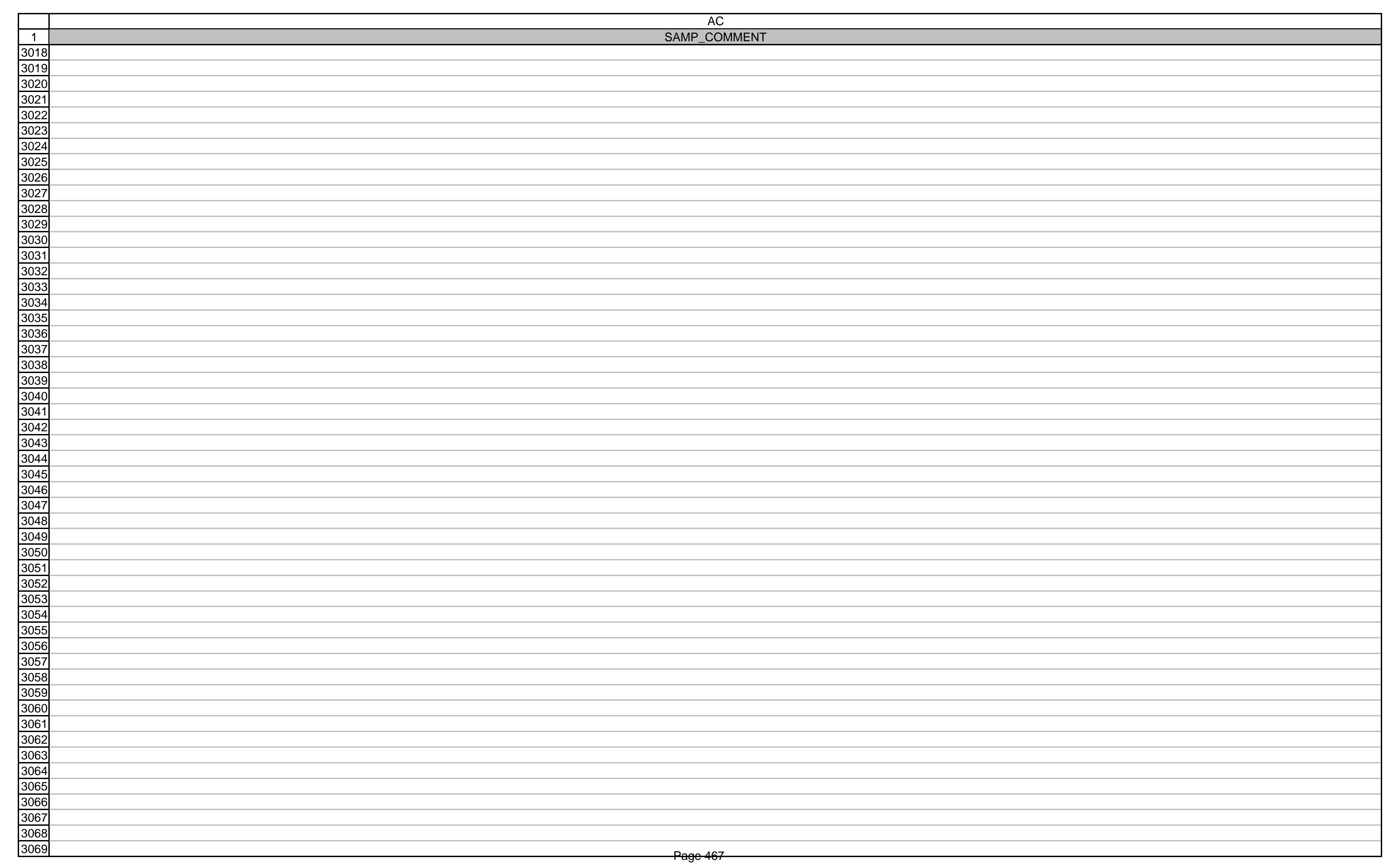




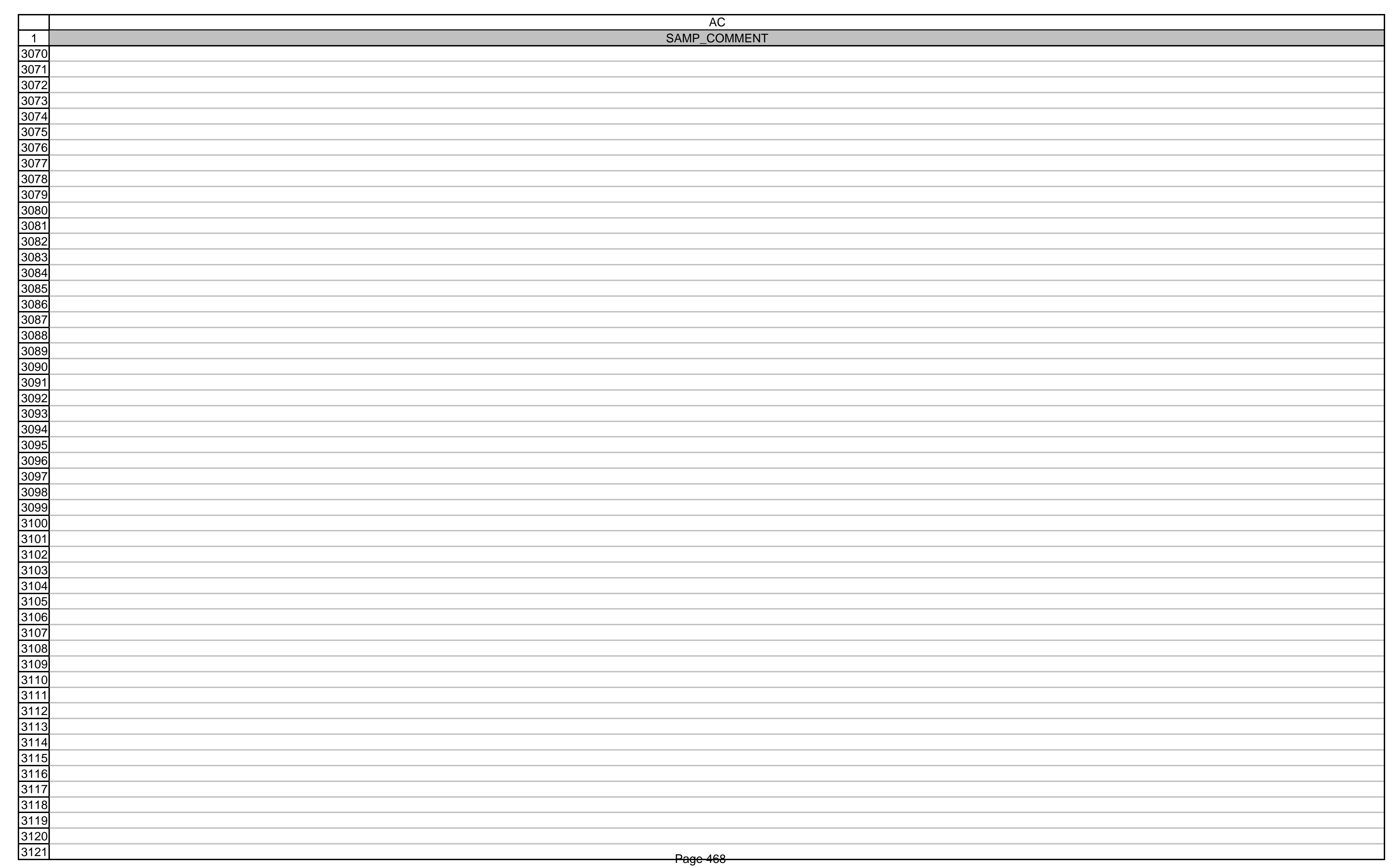




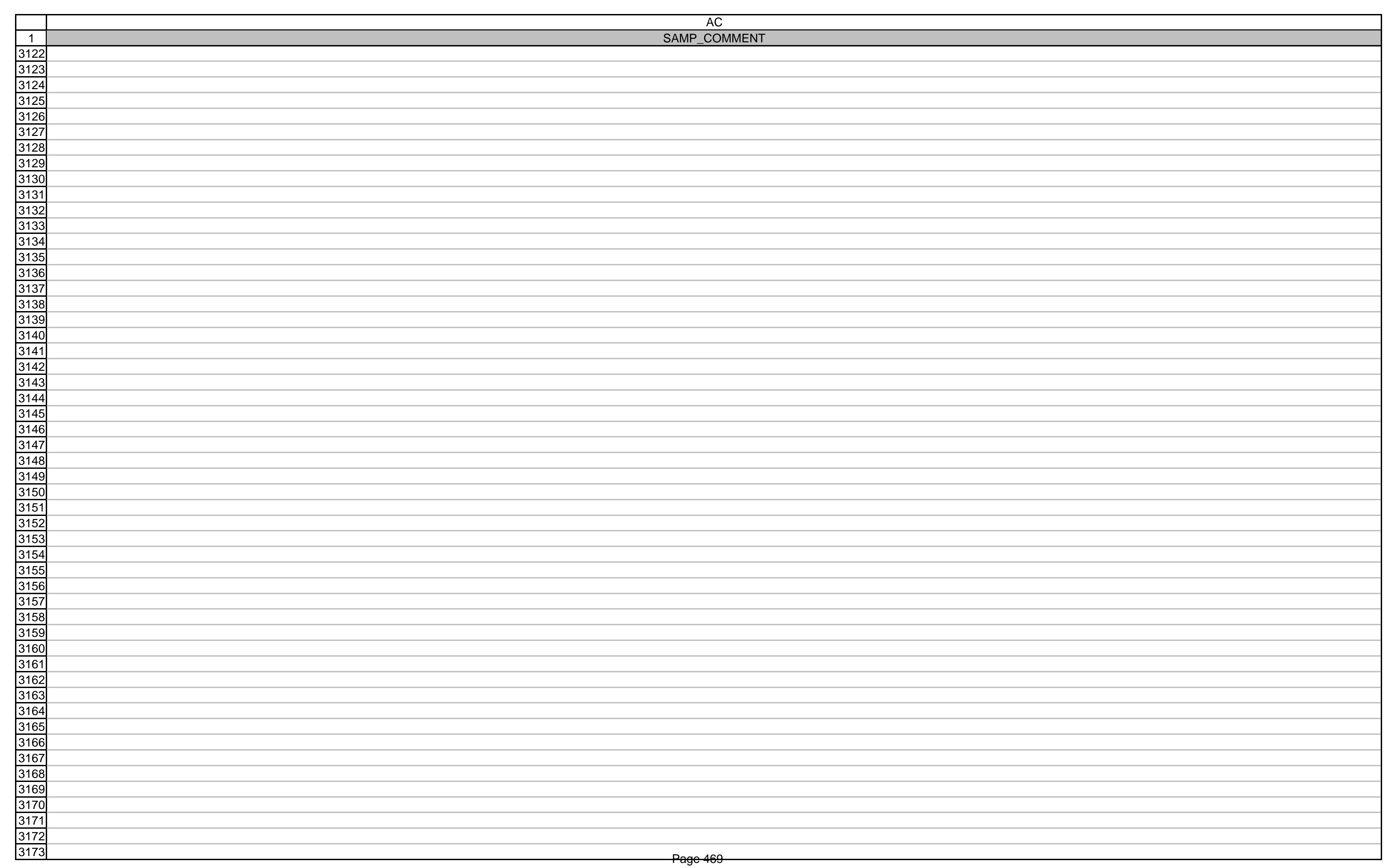


3191 SAMPLE SIZE WAS 15\% OF THAT EXPECTED AND AS A RESULT,ANALYTICAL DETECTION LEVELS AND ERROR WERE HIGH SOTHAT DATA WAS NOT COMPARABLE TO OTHER SAMPLE COLLECTED 3192 SAMPLE SIZE WAS 15\% OF THAT EXPECTED AND AS A RESULT,ANALYTICAL DETECTION LEVELS AND ERROR WERE HIGH SOTHAT DATA WAS NOT COMPARABLE TO OTHER SAMPLE COLLECTED 3193 SAMPLE SIZE WAS 15\% OF THAT EXPECTED AND AS A RESULT,ANALYTICAL DETECTION LEVELS AND ERROR WERE HIGH SOTHAT DATA WAS NOT COMPARABLE TO OTHER SAMPLE COLLECTED

\begin{tabular}{l}
3193 \\
3194 \\
\hline 3195
\end{tabular} 1 LITER ONLY. LINES WERE DISCONNECTED.

3195 NO SAMPLE 12/22-12/29 WATER LINES FROZE 


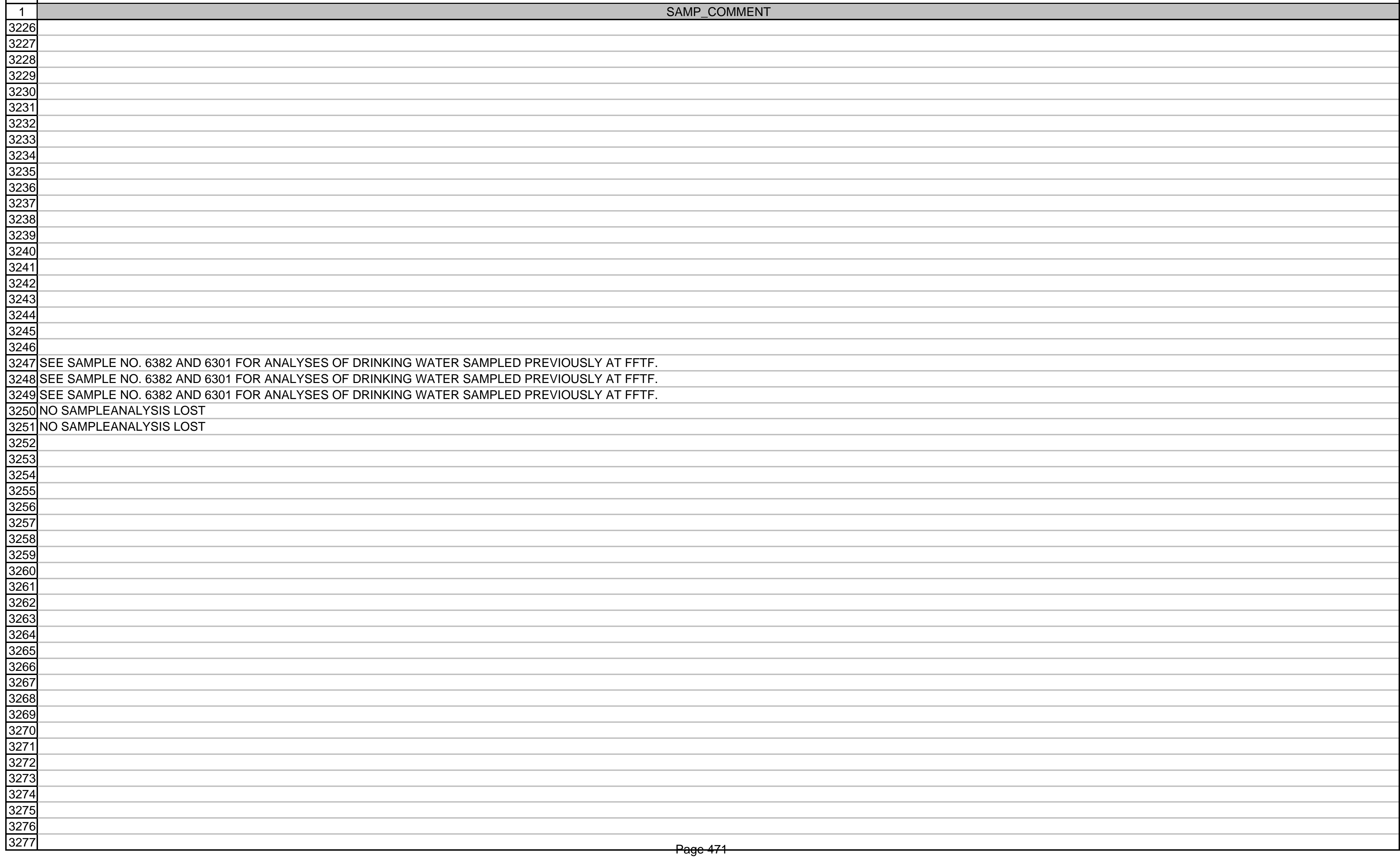




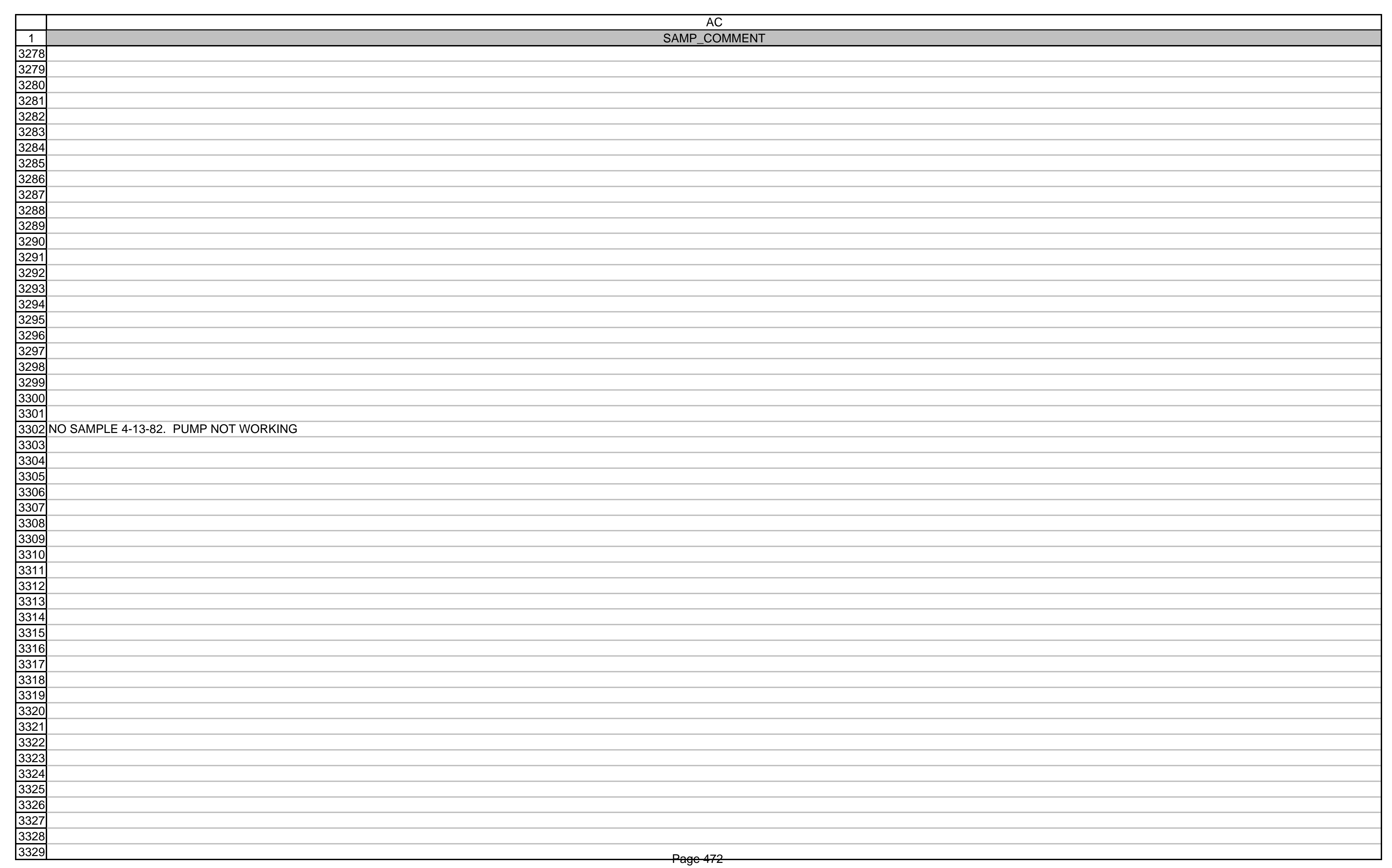




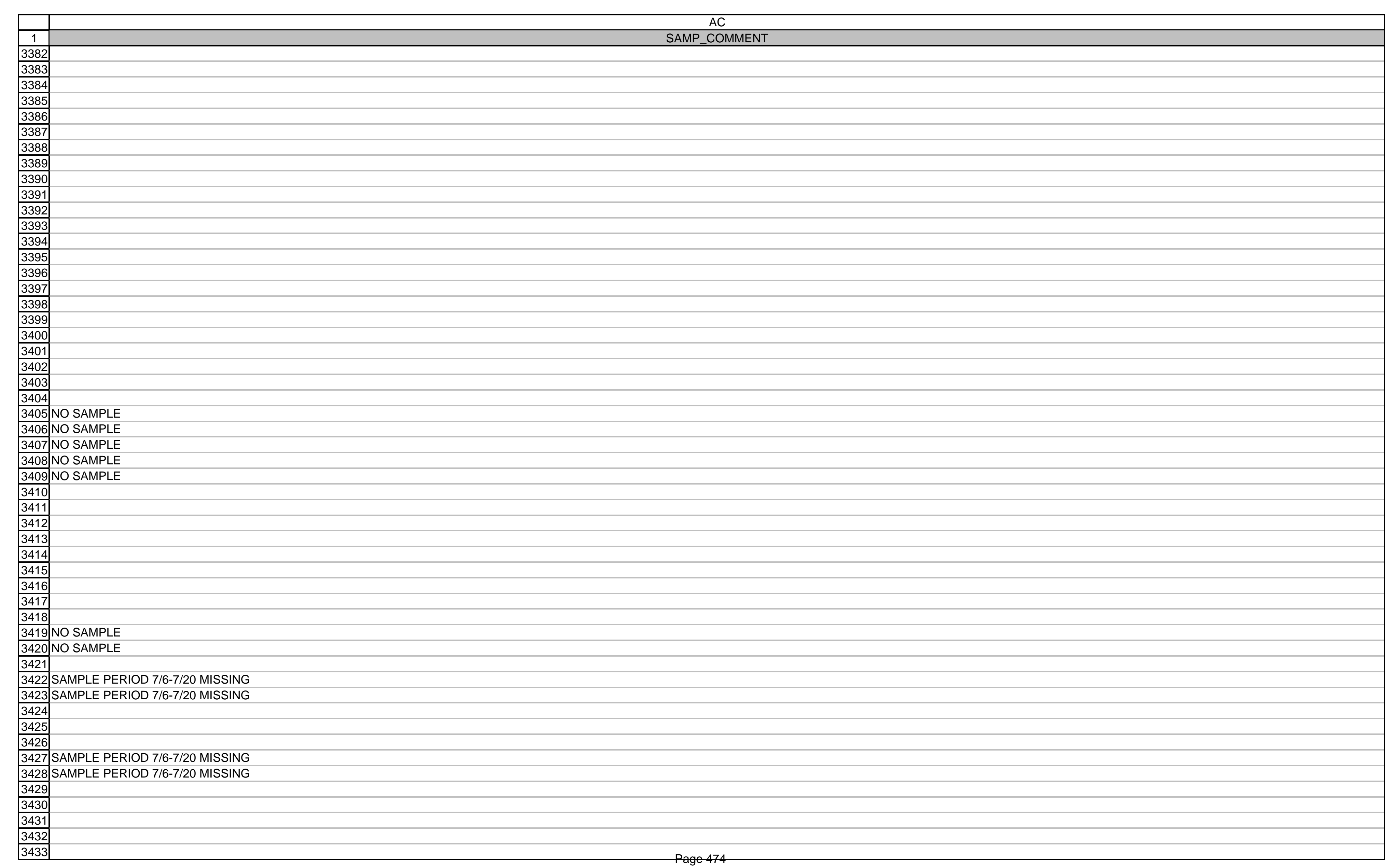


3474 NO SAMPLE. PUMP DOWN

3475 NO SAMPLE. PUMP DOWN

3476

3478

3480 
3486 INVALID SAMPLE. SAMPLE VOLUME LOW

3487 INVALID SAMPLE. SAMPLE VOLUME LOW

3488

3490

3491

\begin{tabular}{|l|}
\hline 3492 \\
\hline 3493 \\
\hline 3494 \\
\hline
\end{tabular}

3494 SAMPLE FAILED DURING SAMPLING PERIOD. SAMPLE SIZE WAS 270 L COMPARED TO EXPECTED $1000 \mathrm{~L}$.

3495 SAAMPLE FAILED DURING SAMPLING PERIOD. SAMPLE SIZE WAS $270 \mathrm{~L}$ VS EXPECTED $1000 \mathrm{~L}$

3496

3497

3498

3500

\begin{tabular}{|l|}
\hline 3501 \\
\hline 3502 \\
\hline 3503
\end{tabular}

3502

3504

\begin{tabular}{|l|}
3504 \\
\hline 3505 \\
\hline 3506 \\
\hline 3507
\end{tabular}

3506

3512 SAMPLE VOLUME LOW FOR HALF OF MONTH

3513 SAMPLE VOLUME LOW FOR HALF OF MONTH

3514

351

3516

3518 


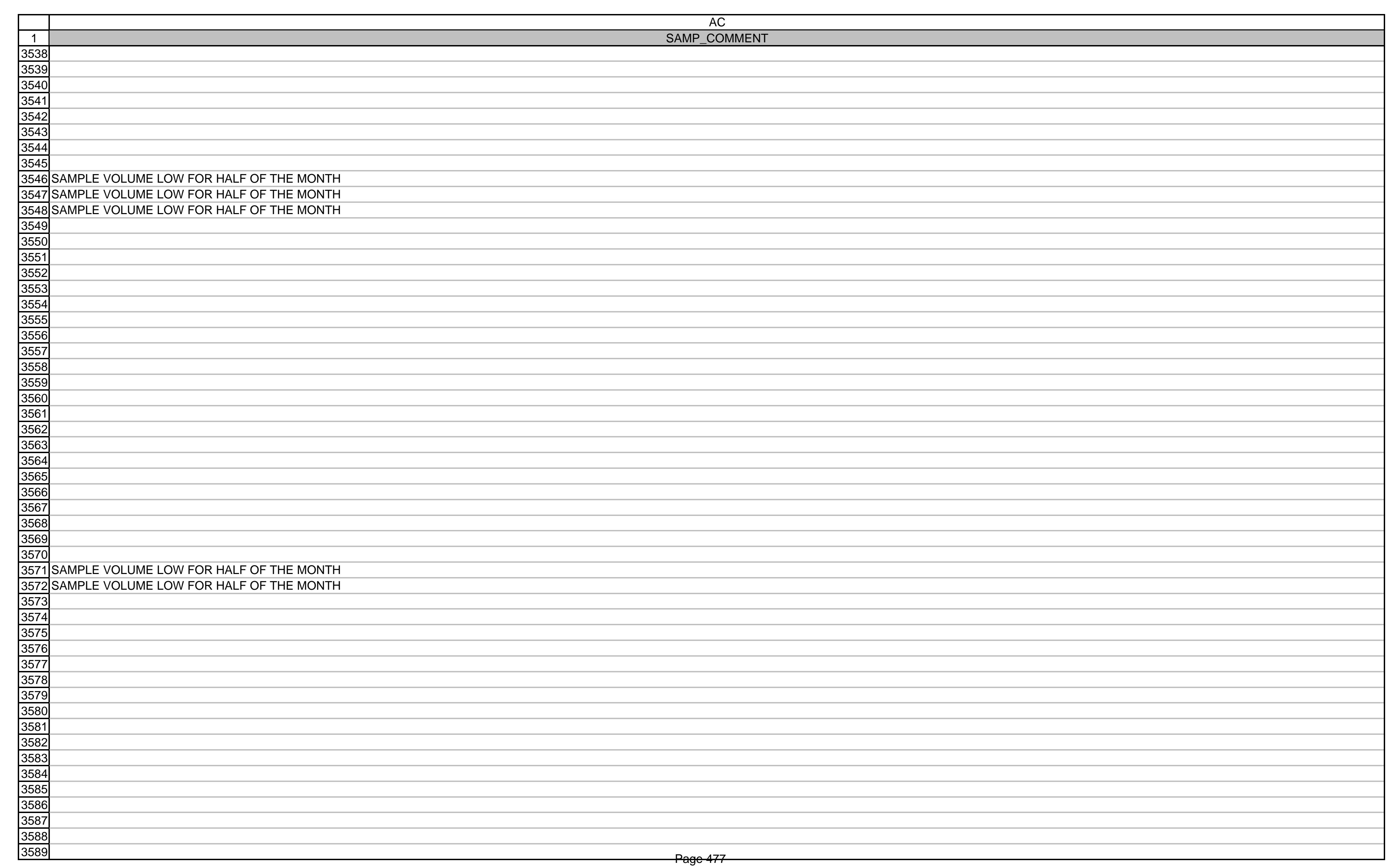




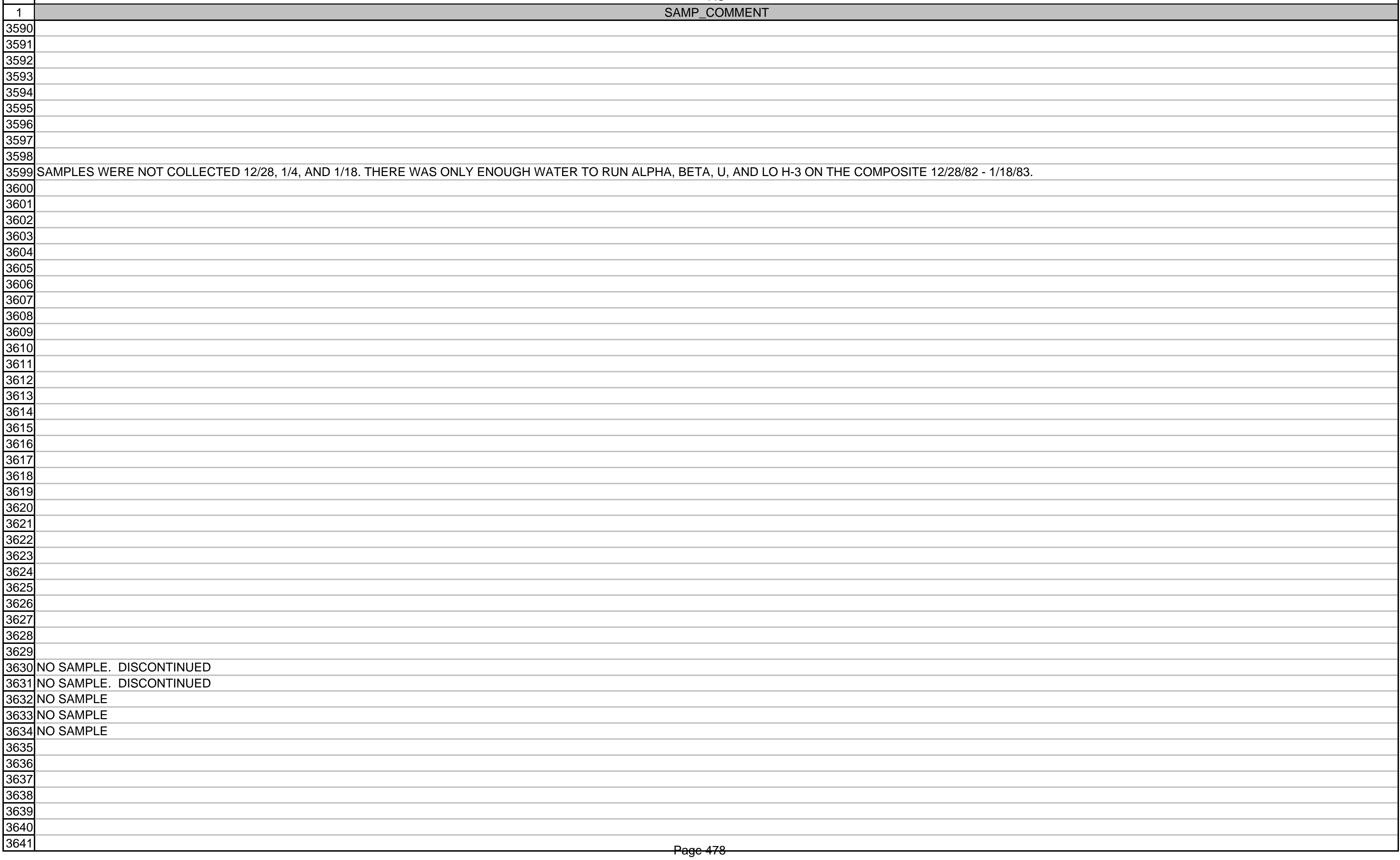




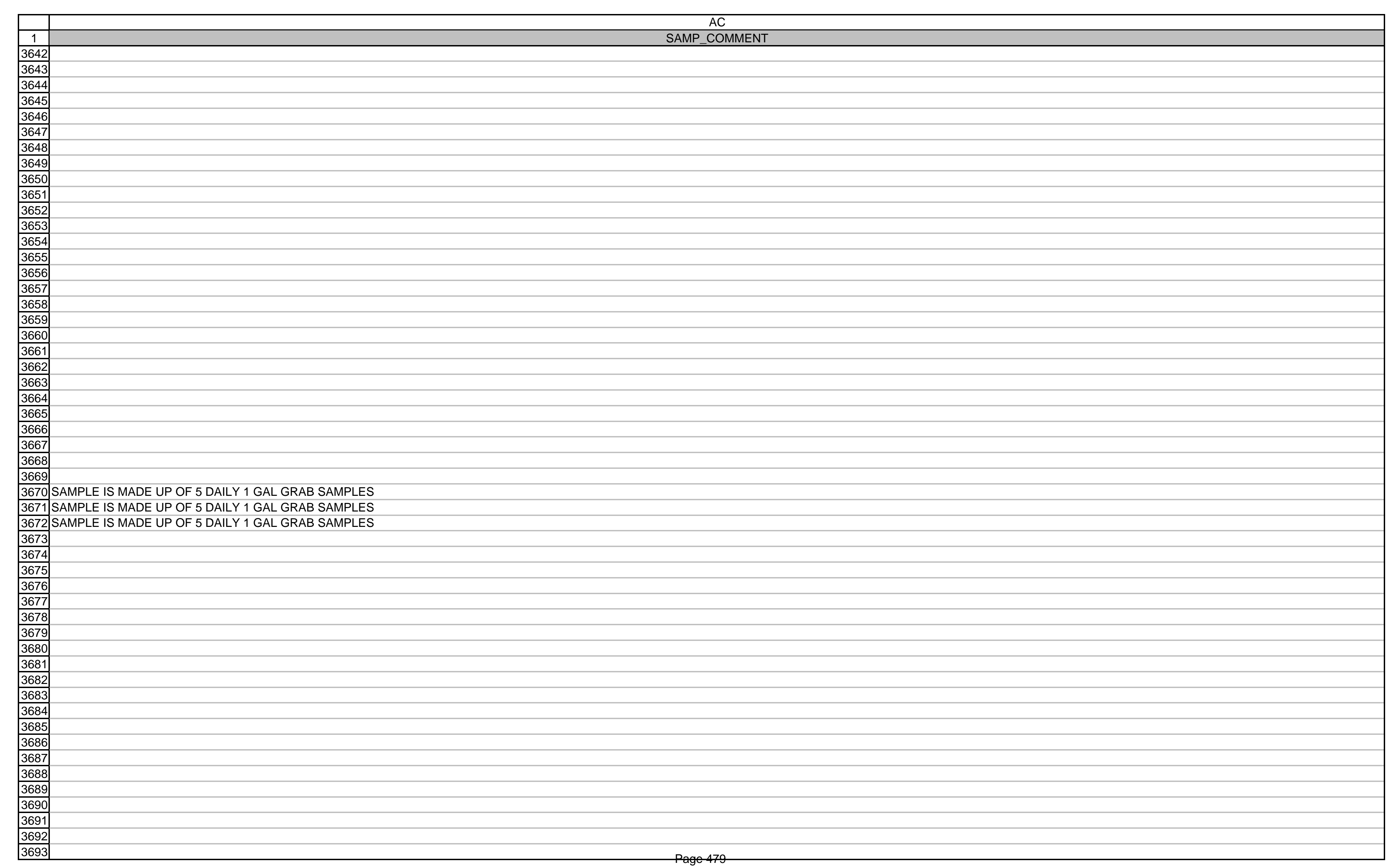




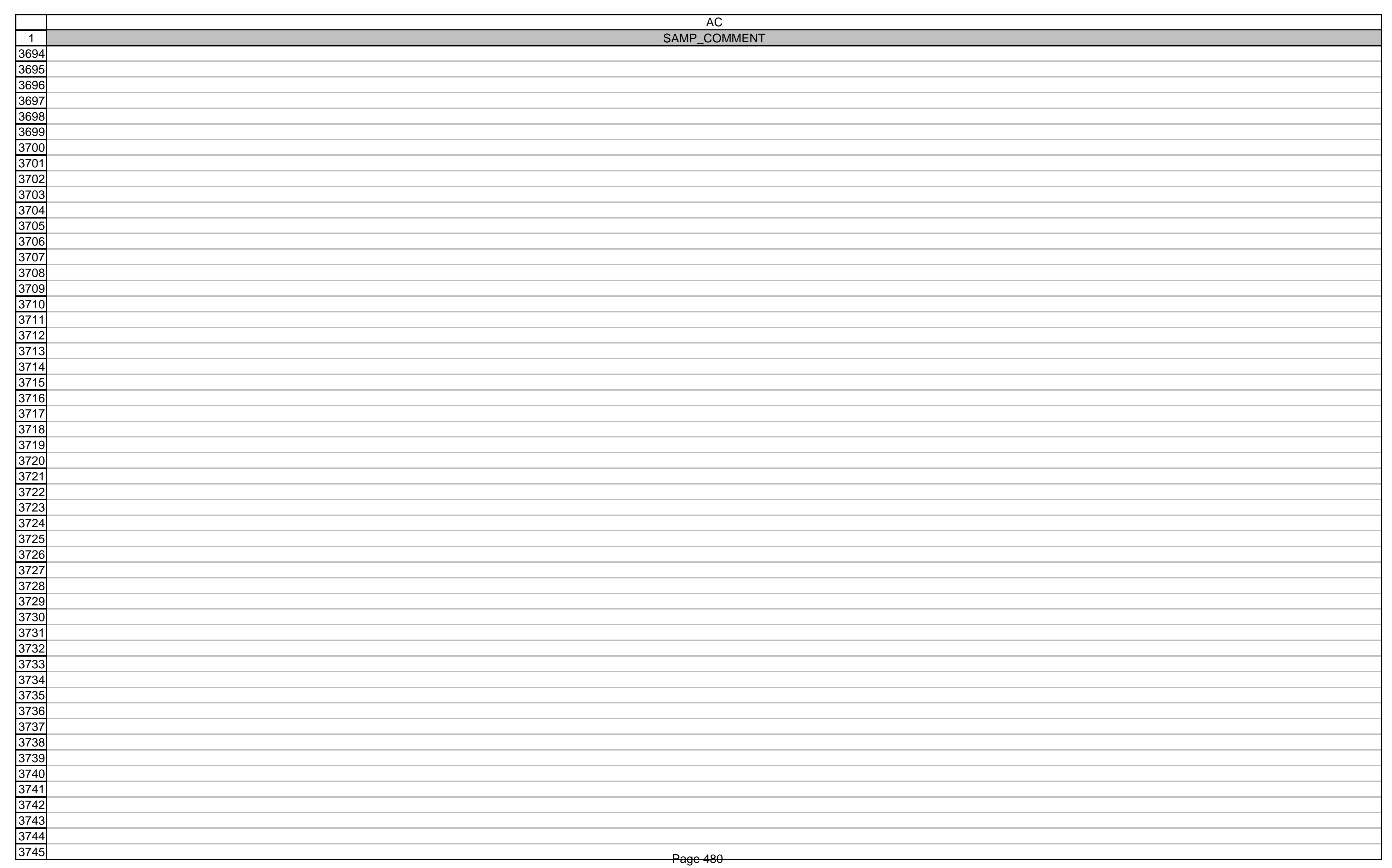




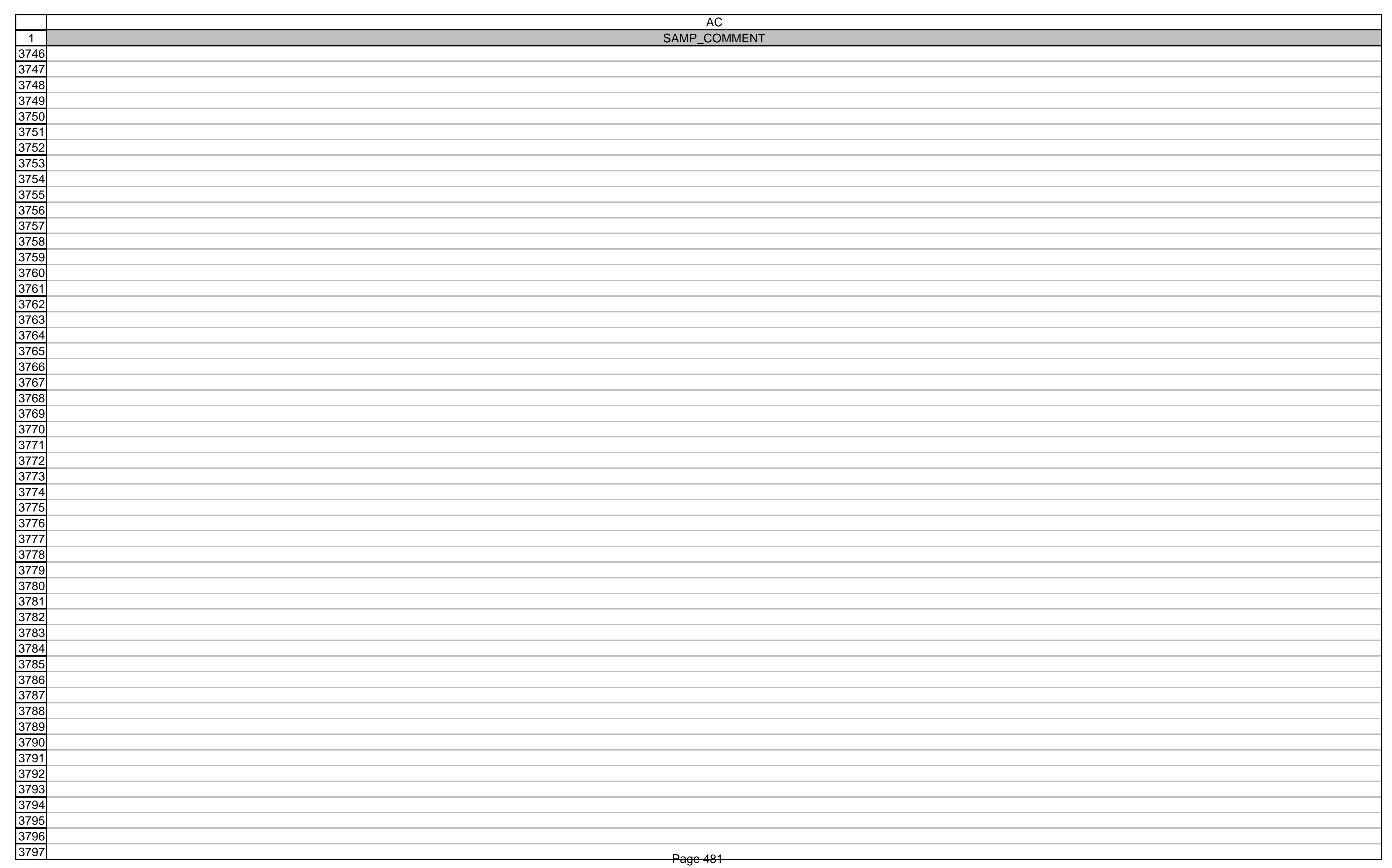




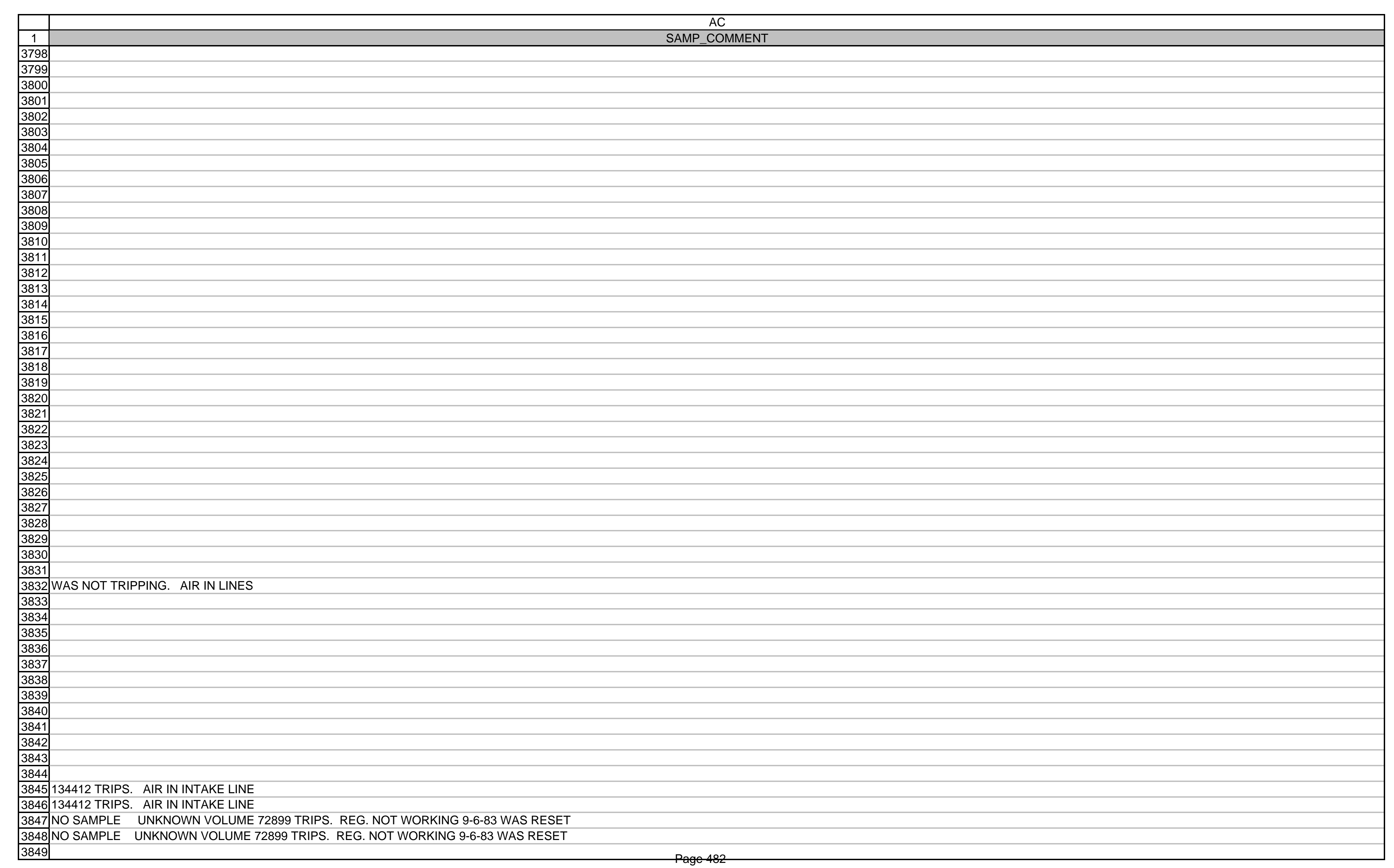




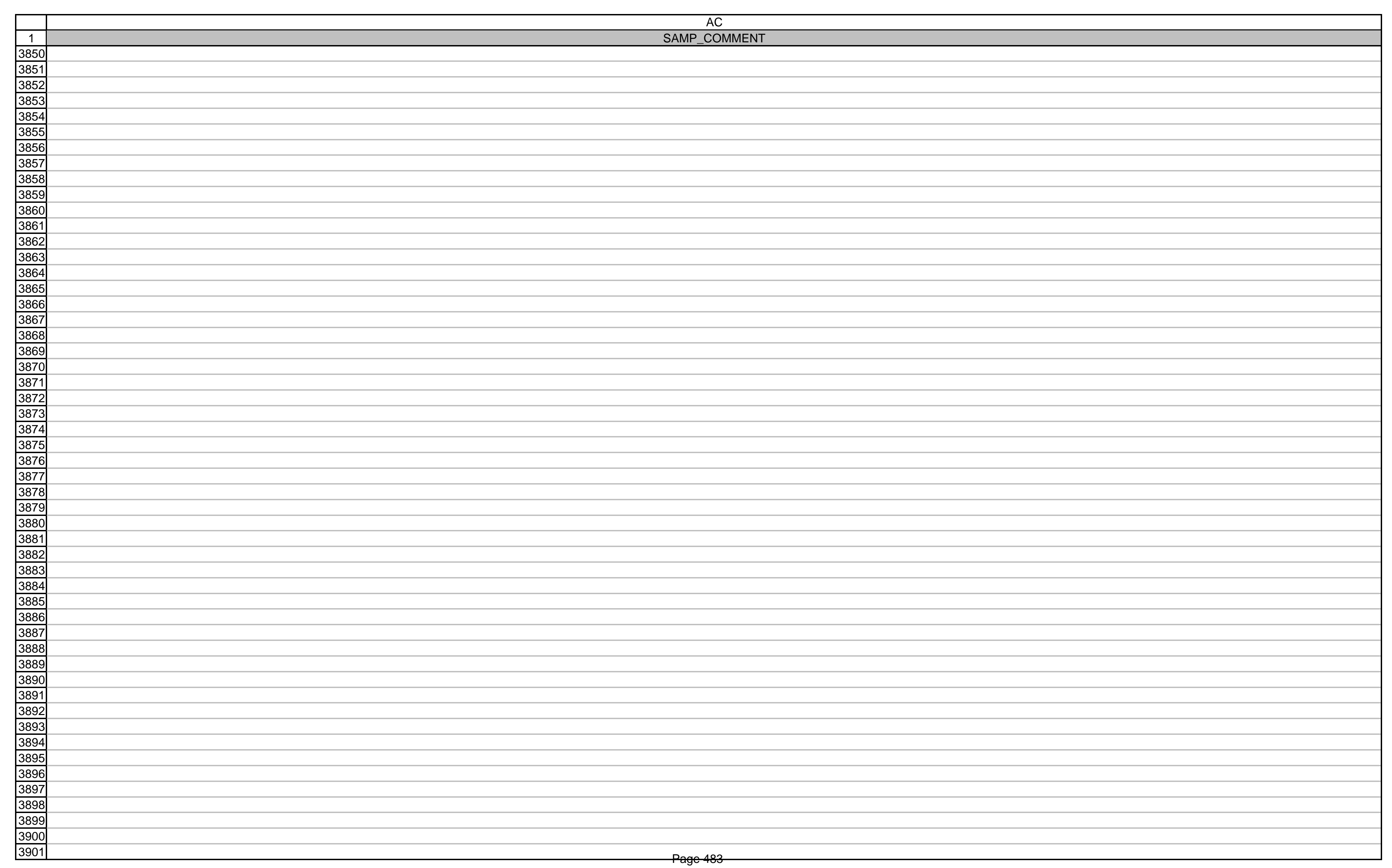




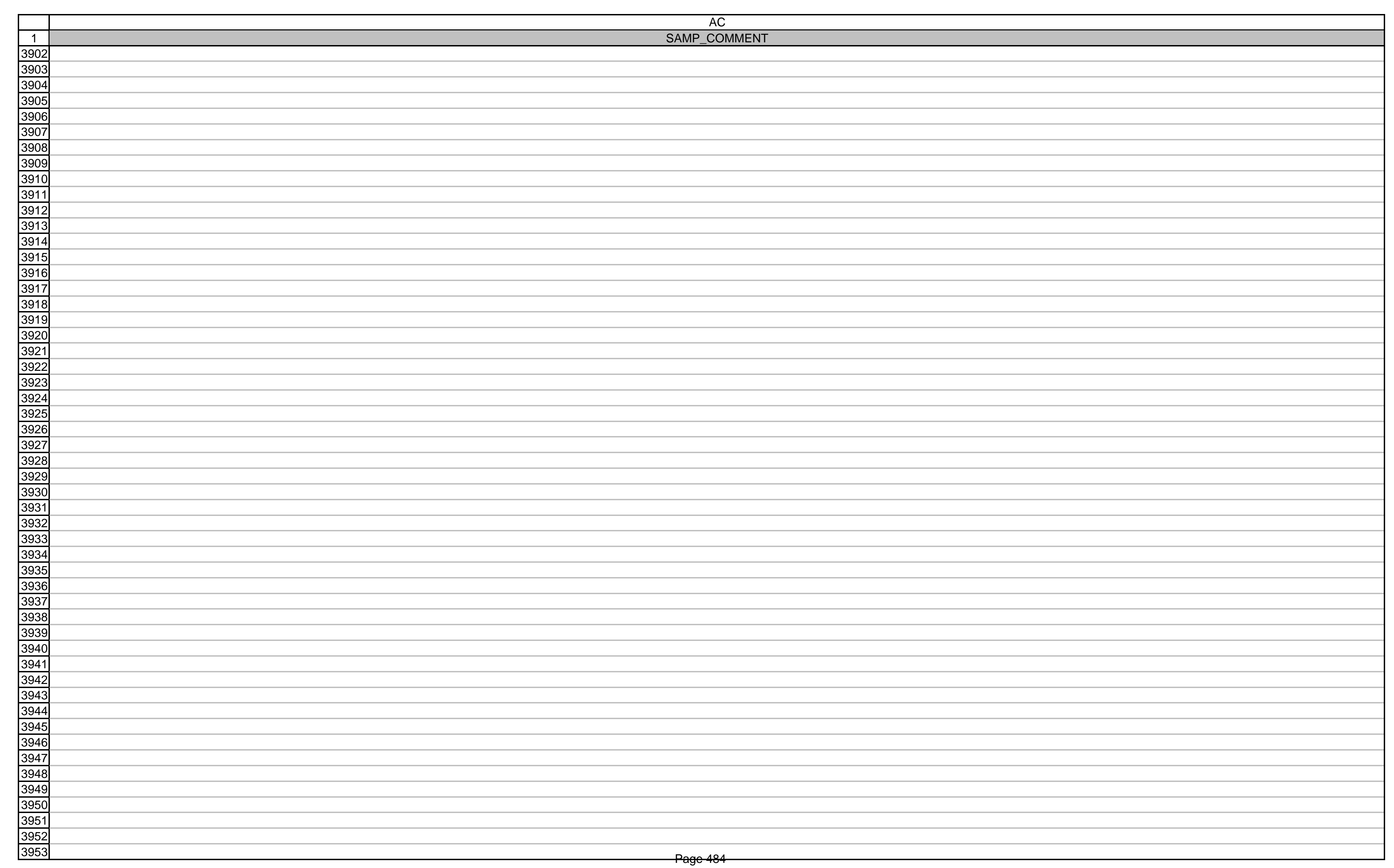




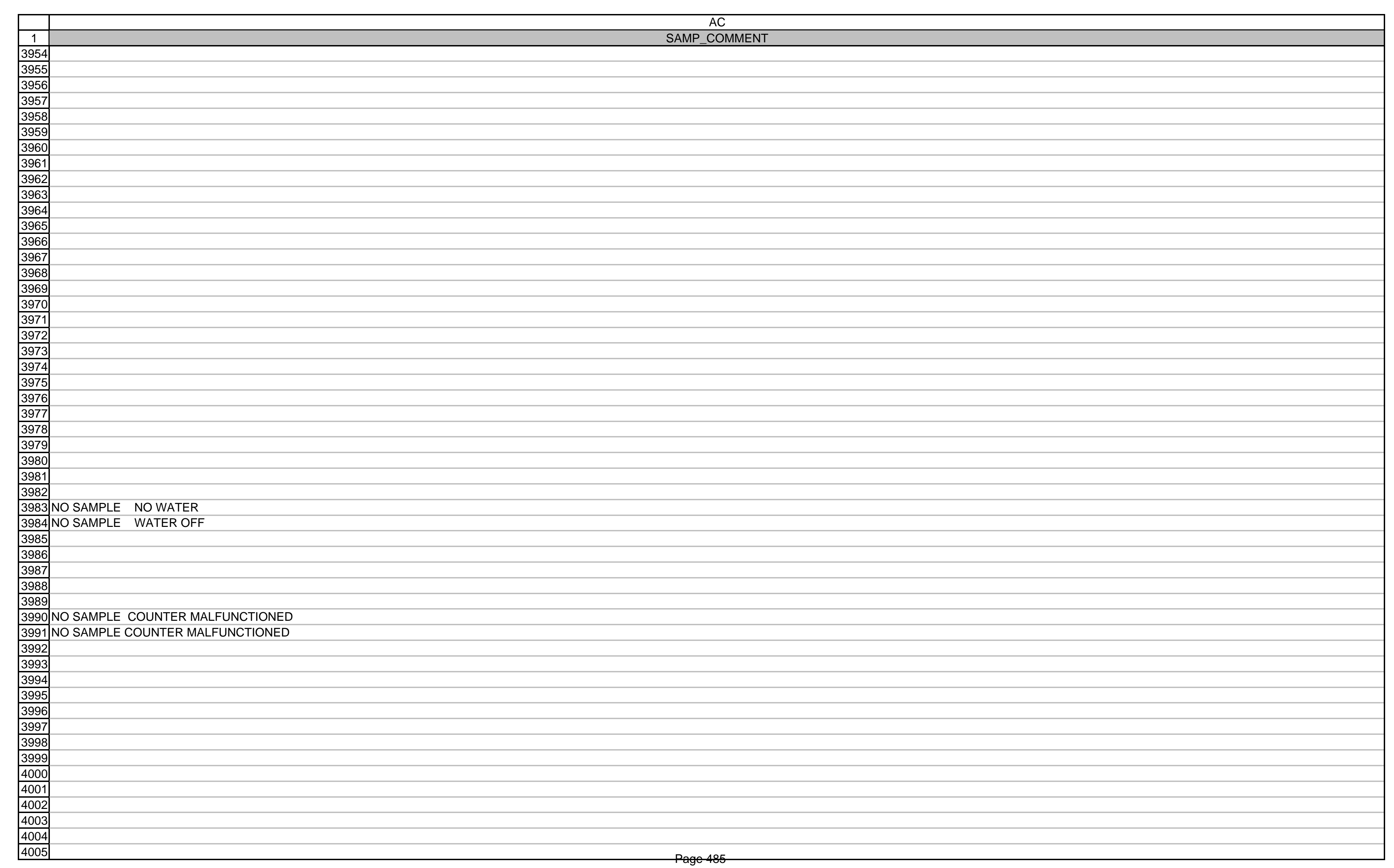




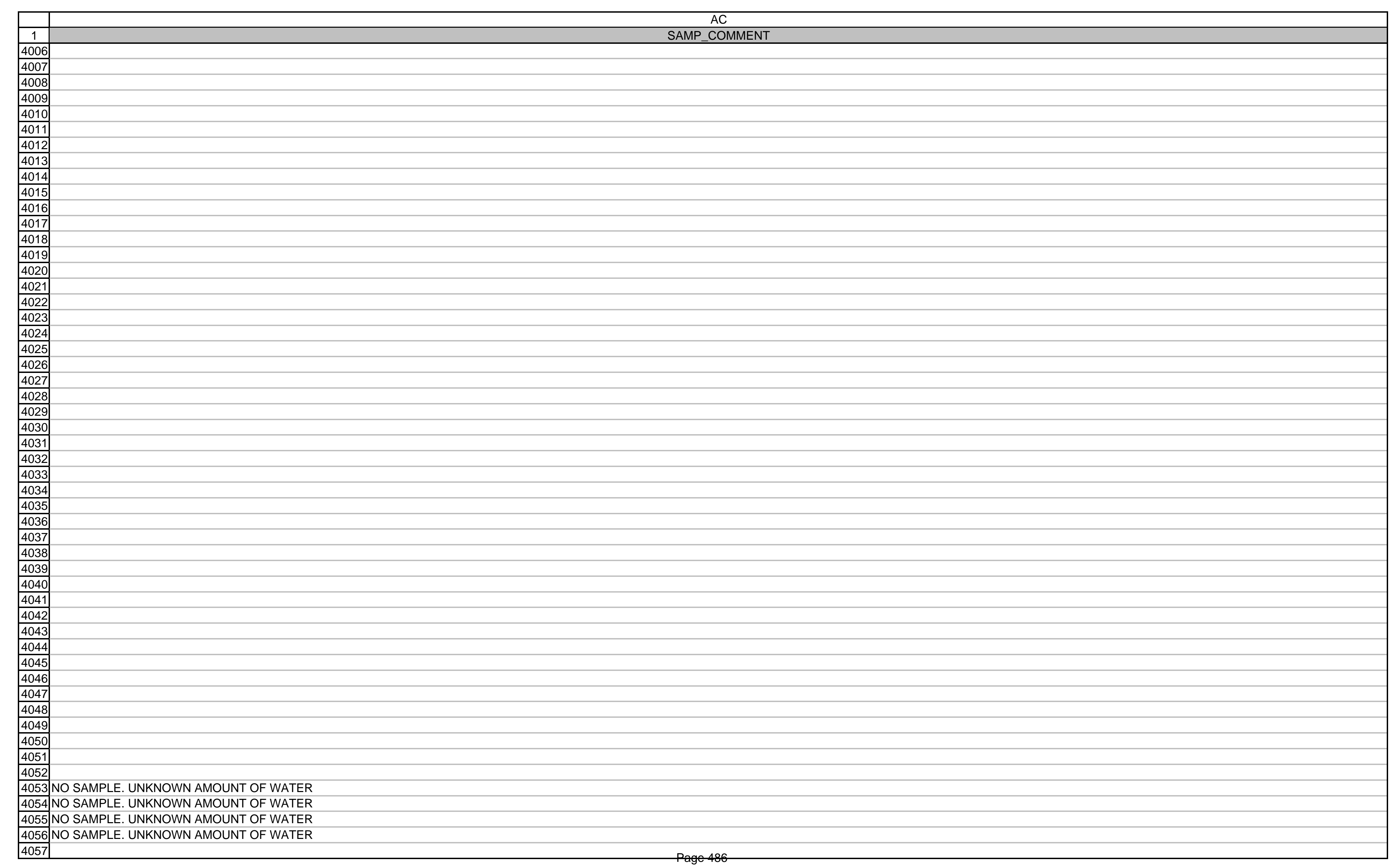




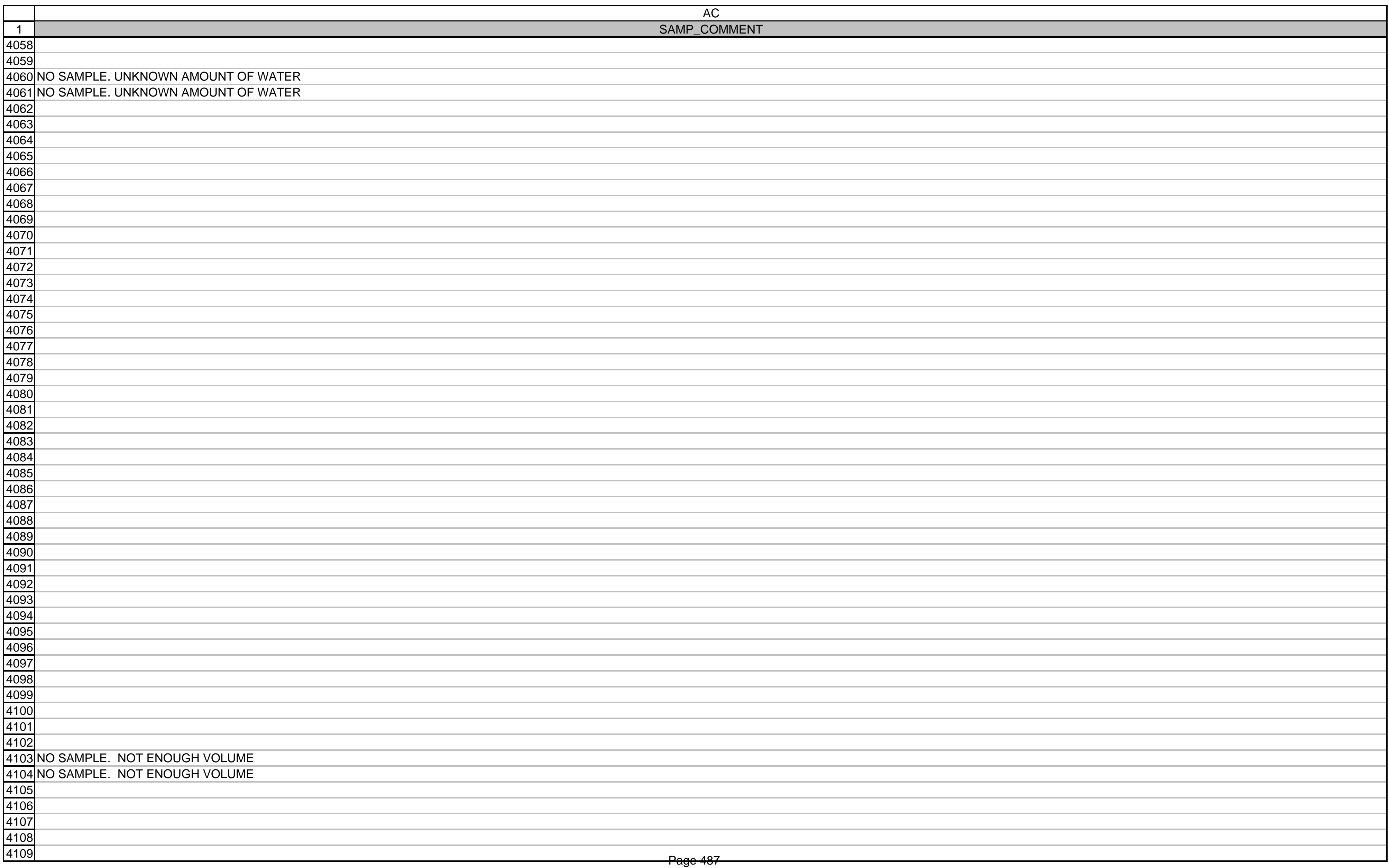




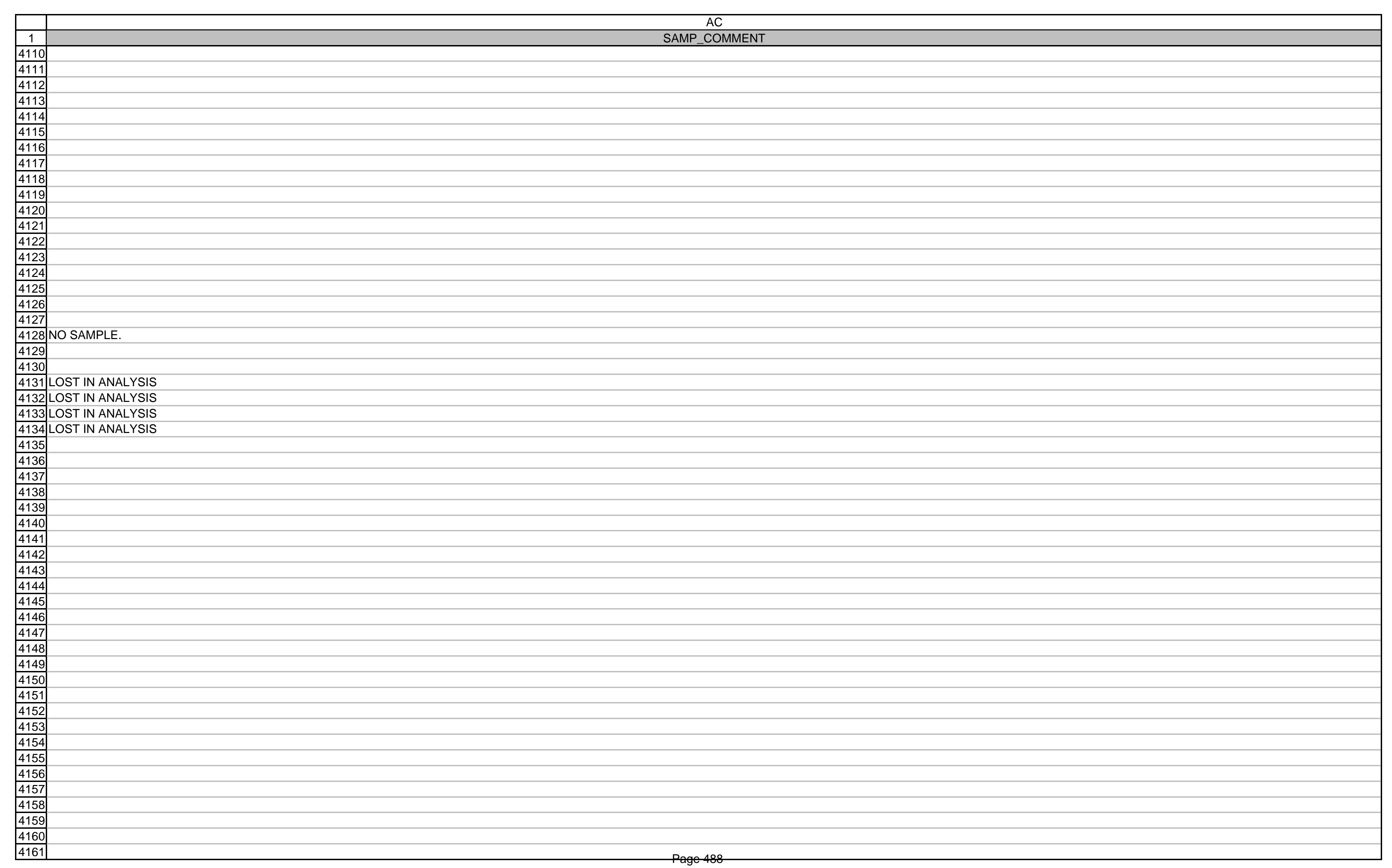




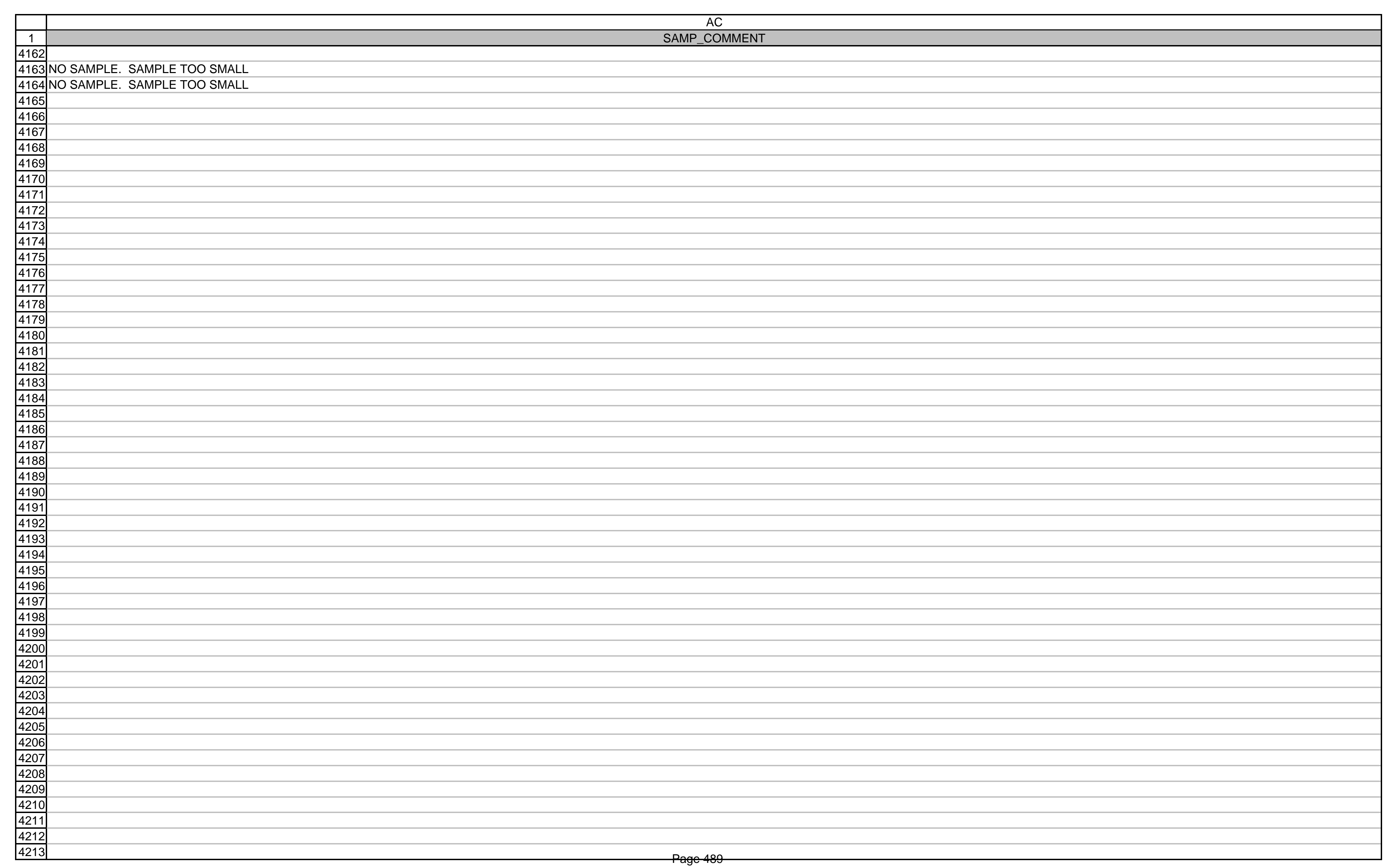




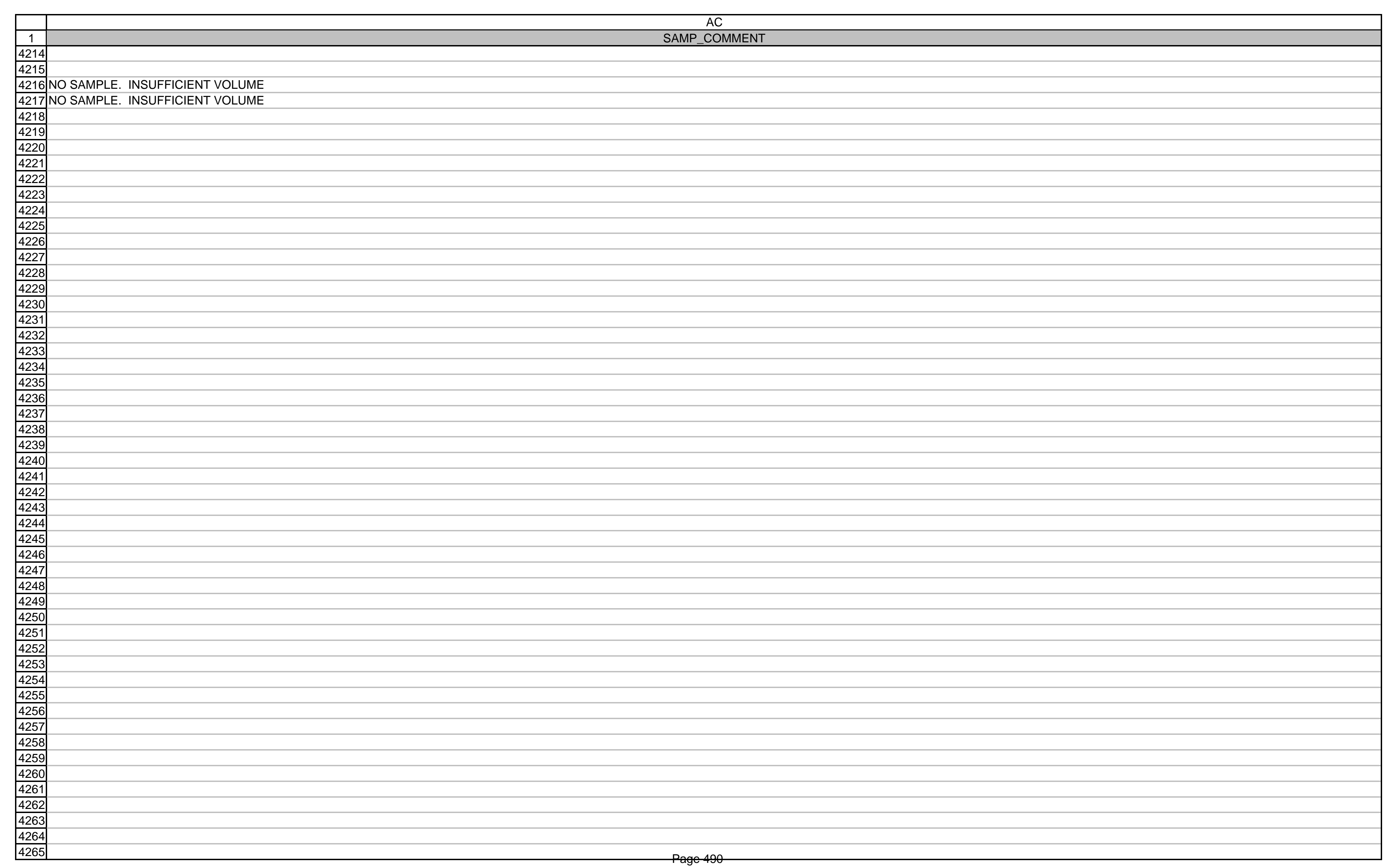




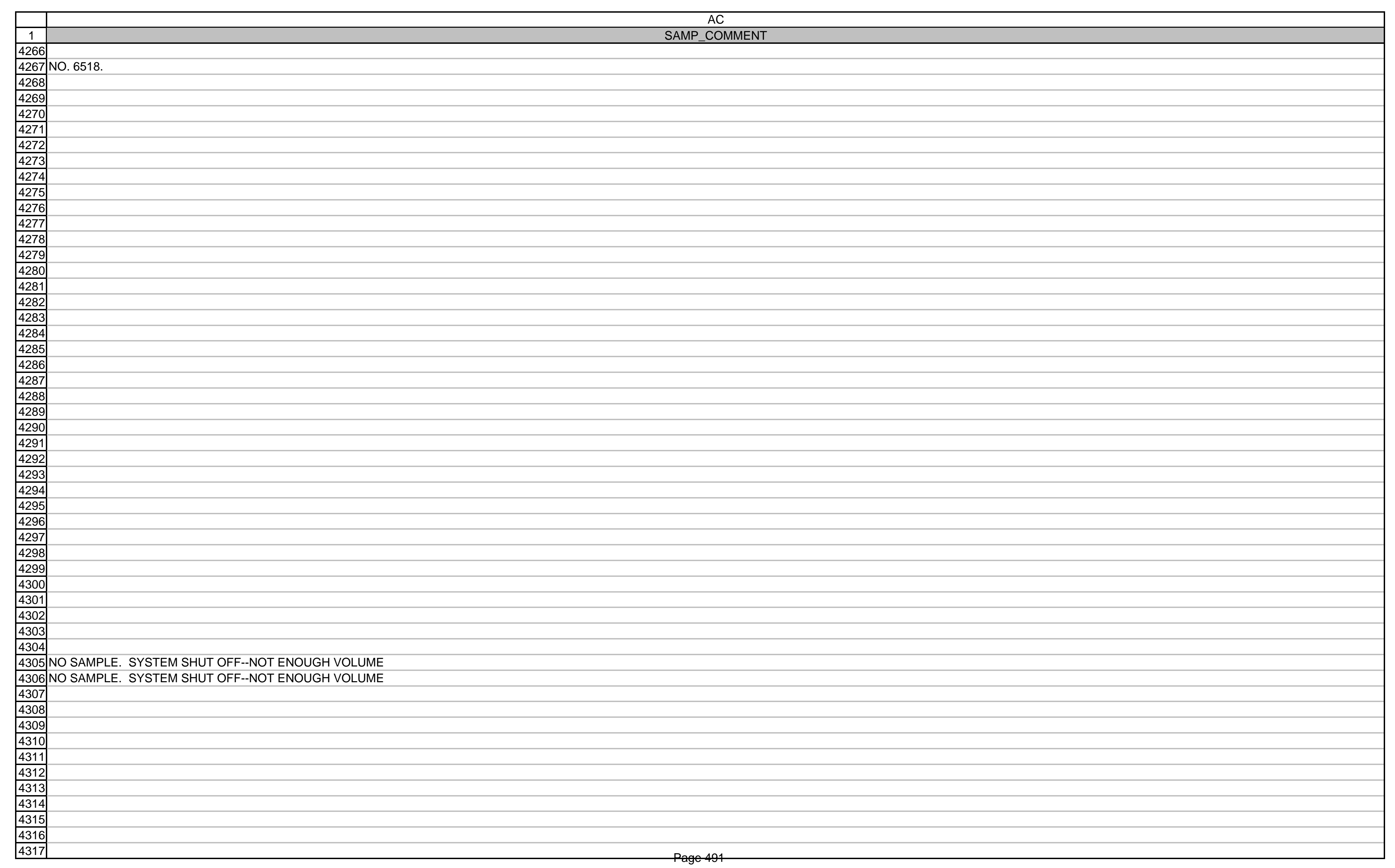




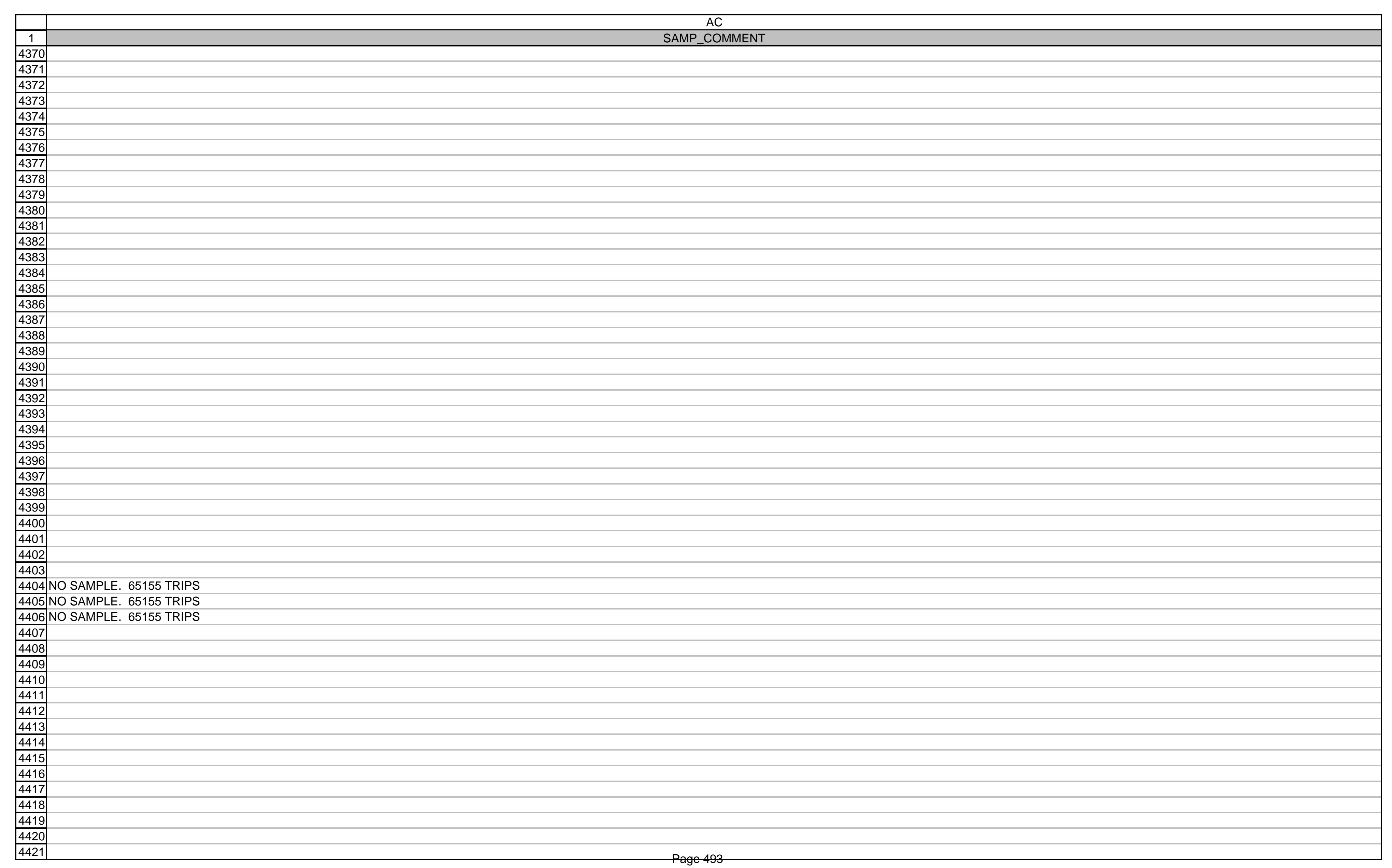




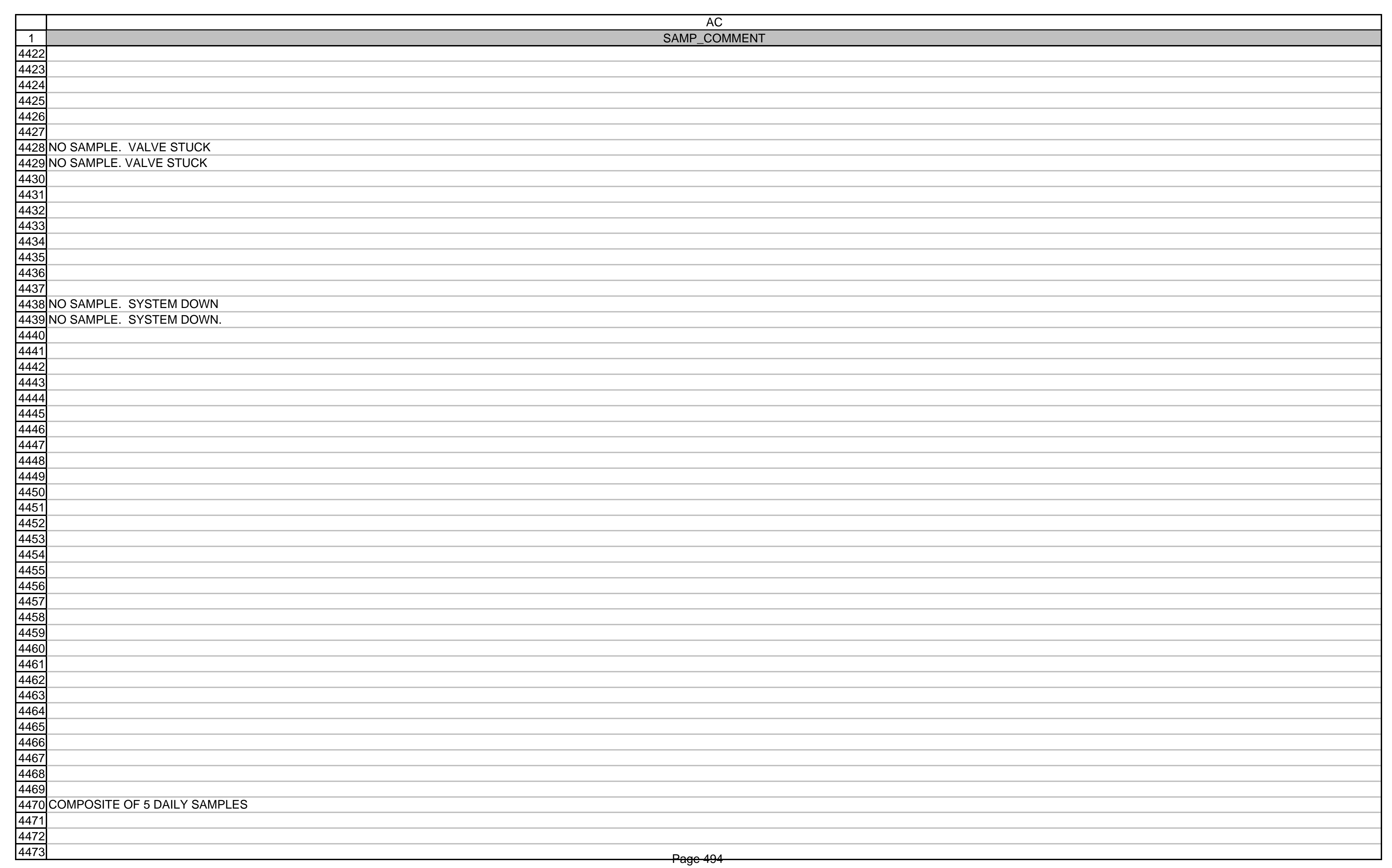




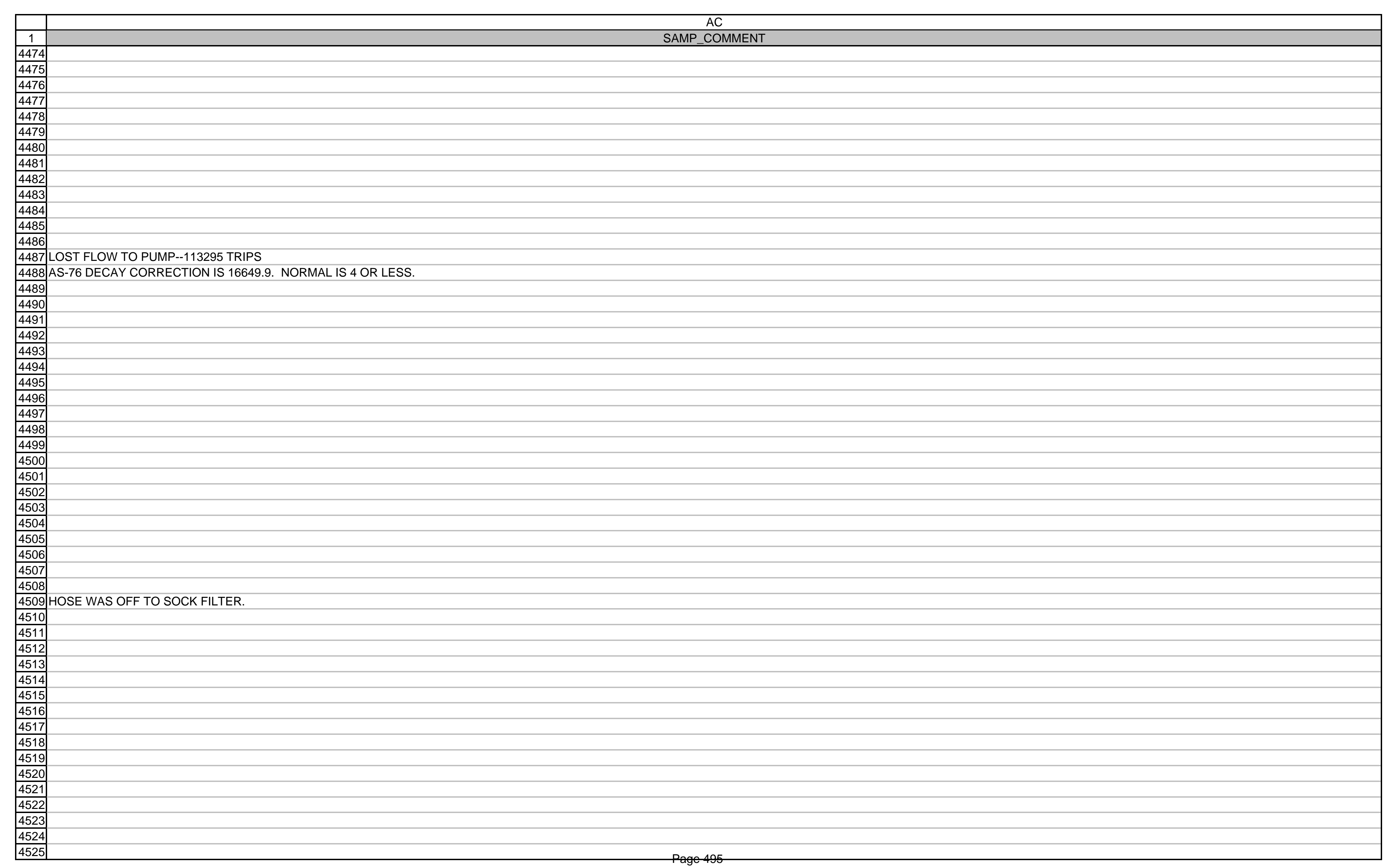




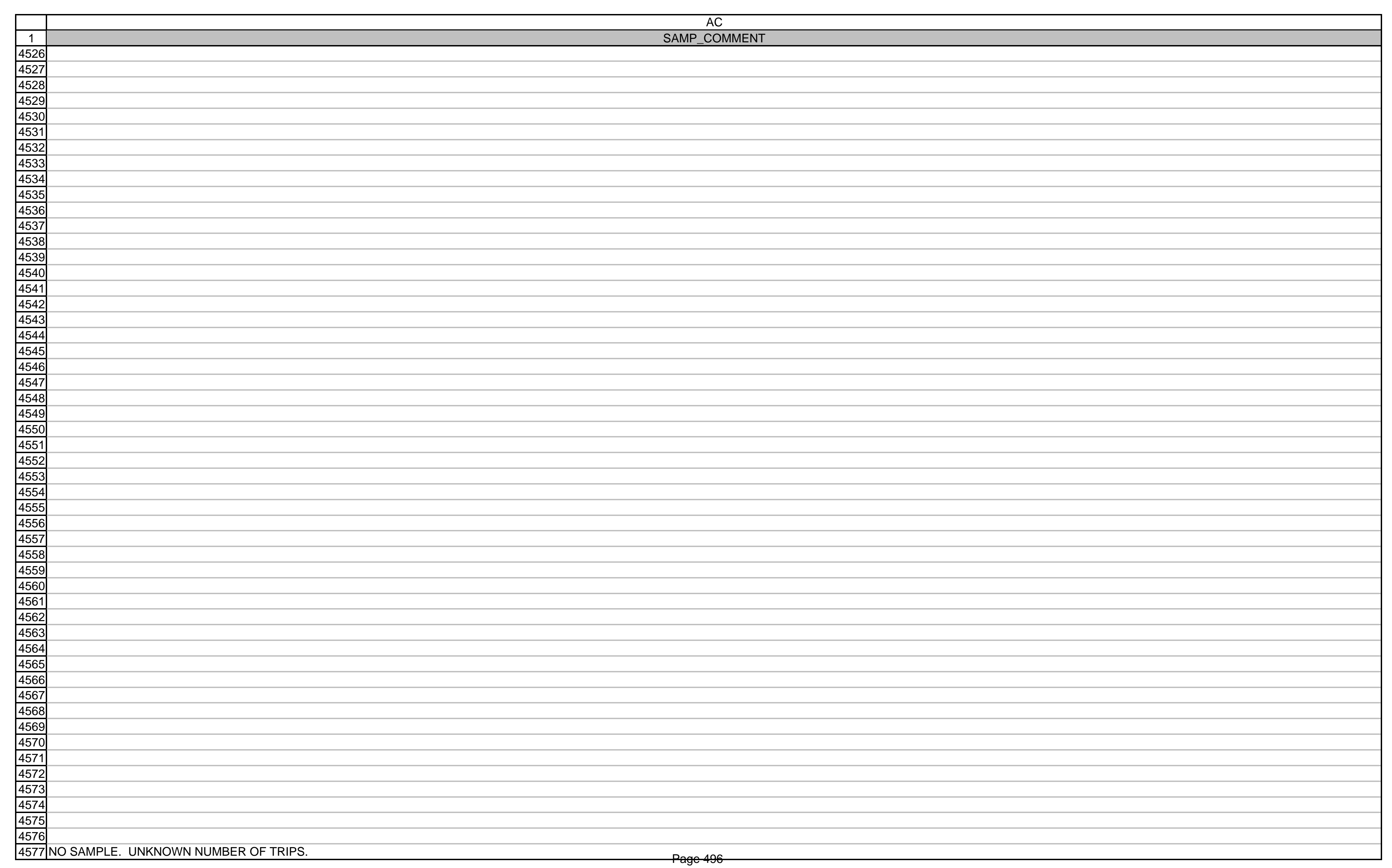


\begin{tabular}{|c|cc}
\hline & AC \\
\hline 1 & SAMP_COMMENT \\
\hline
\end{tabular}

4578 NO SAMPLE. UNKNOWN NUMBER OF TRIPS.

4579 NO SAMPLE.

4580

4581

4582

4583 4584

4584 4585

458

4587

4588

4589

4590

4591

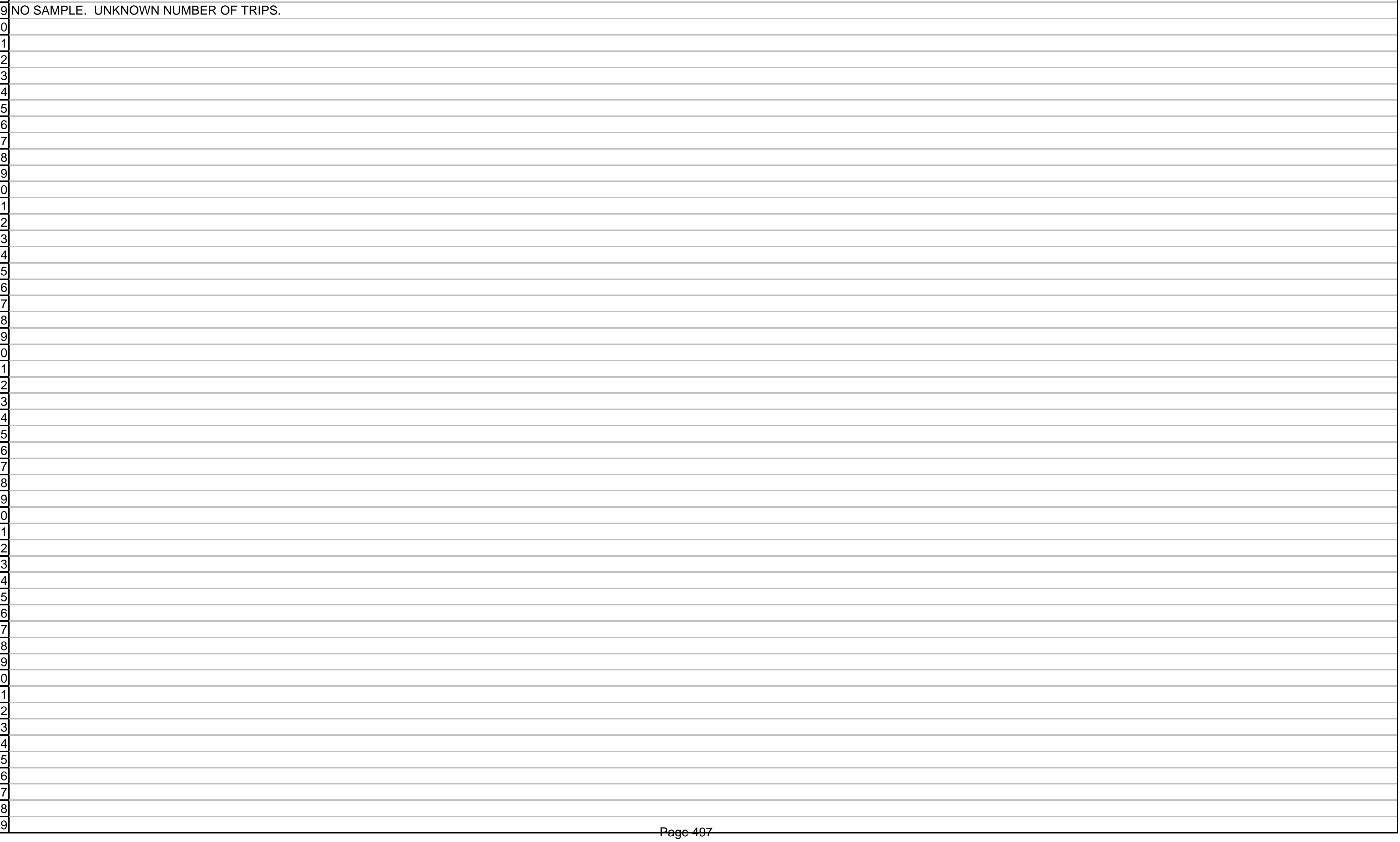




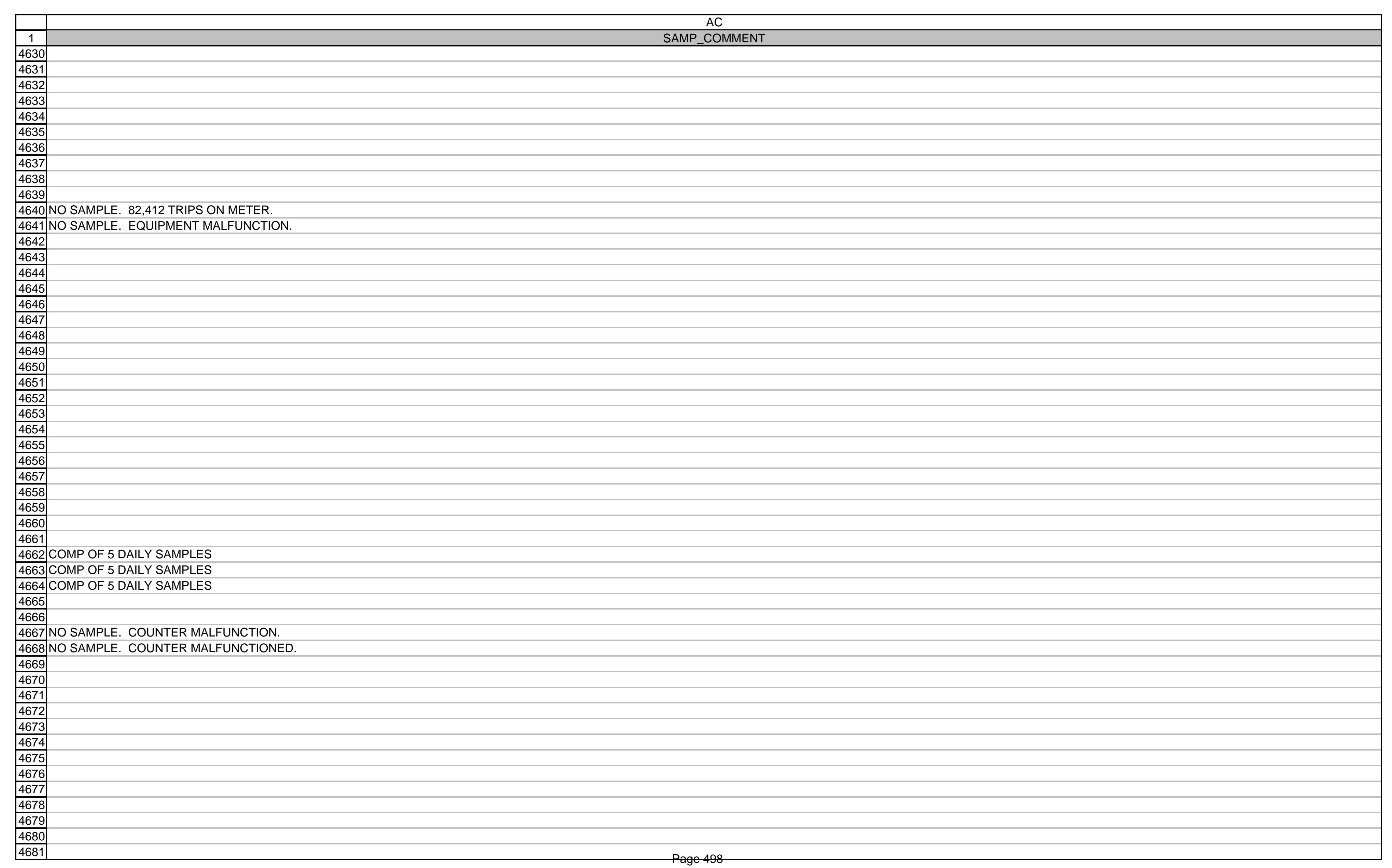




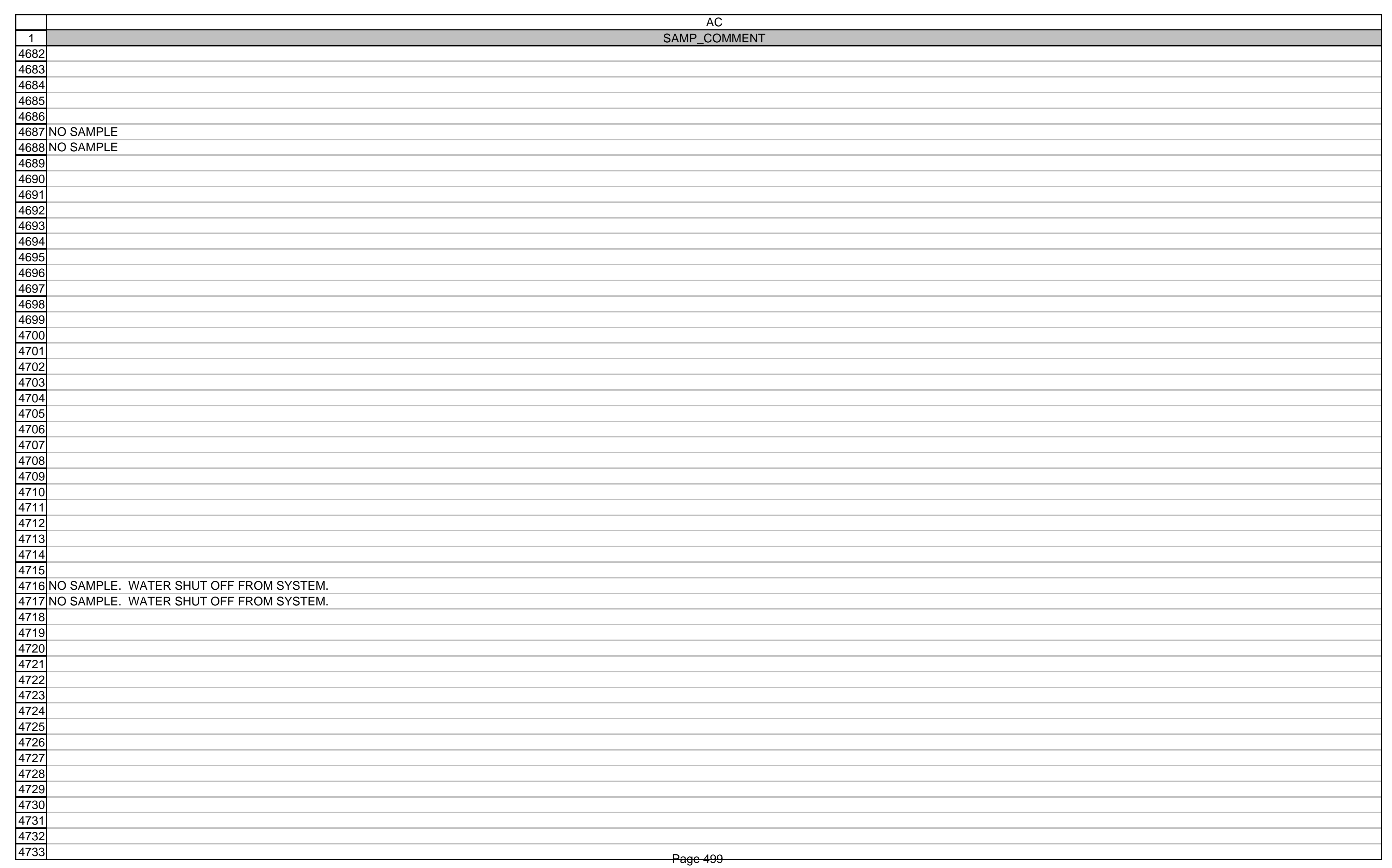




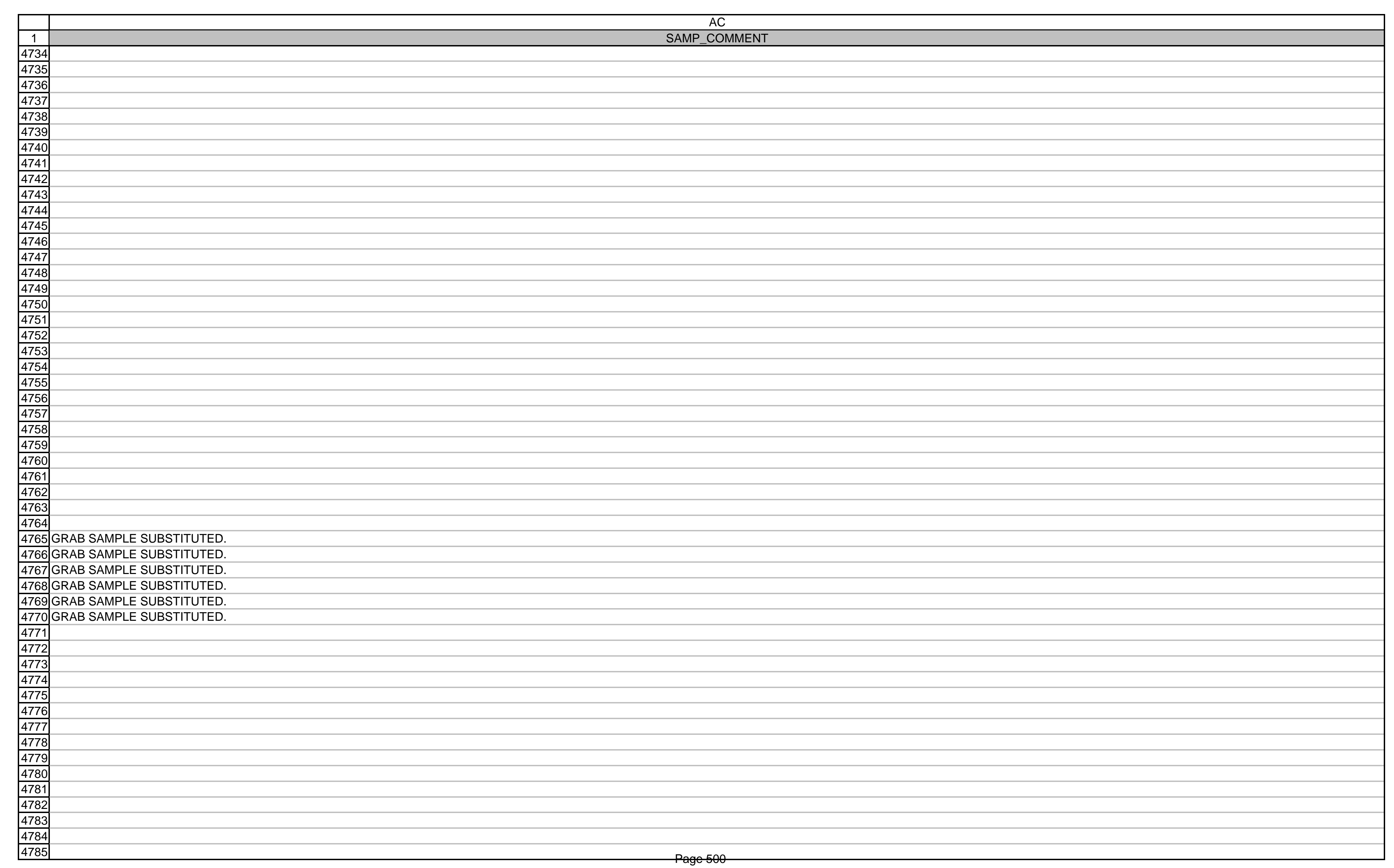




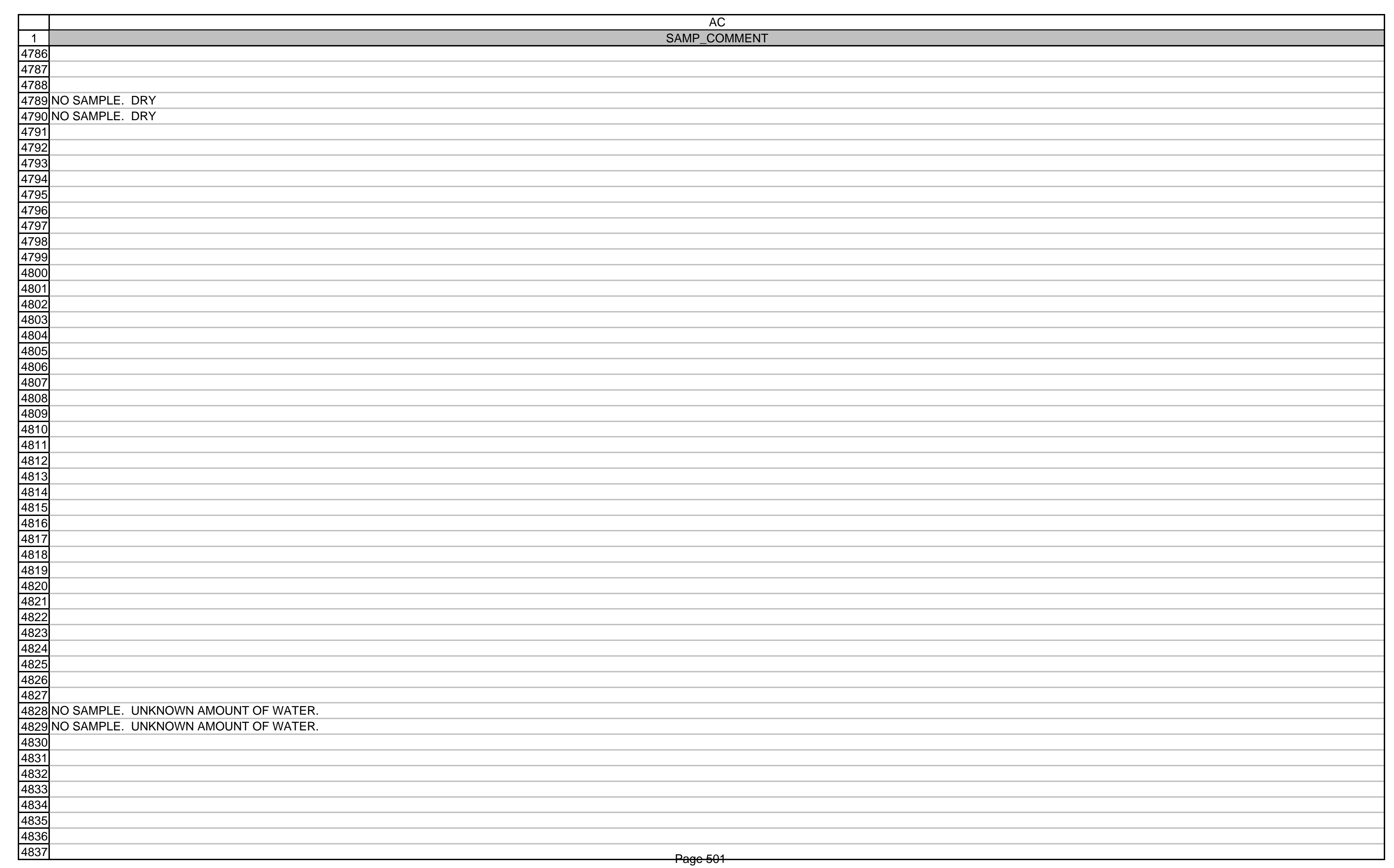




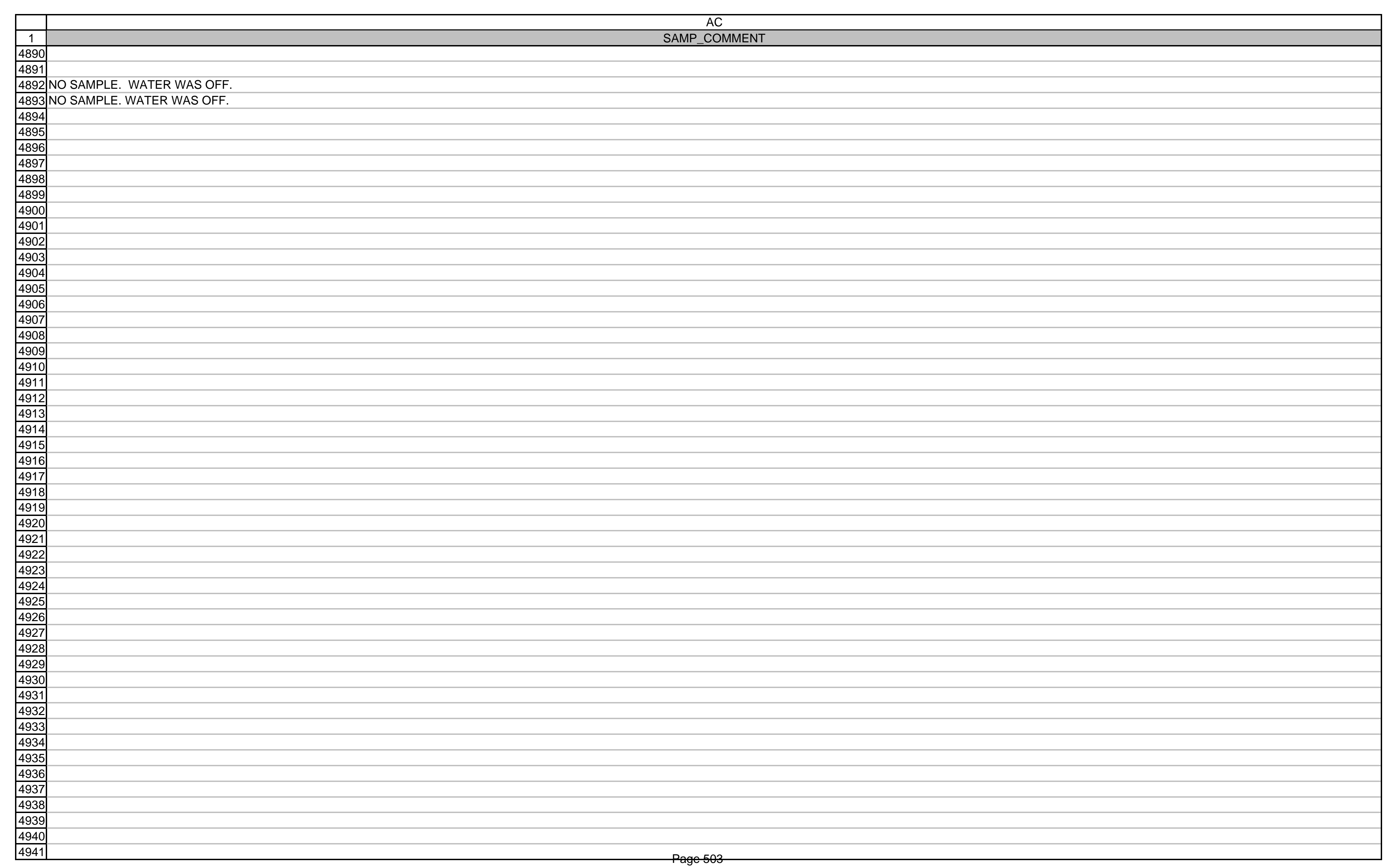




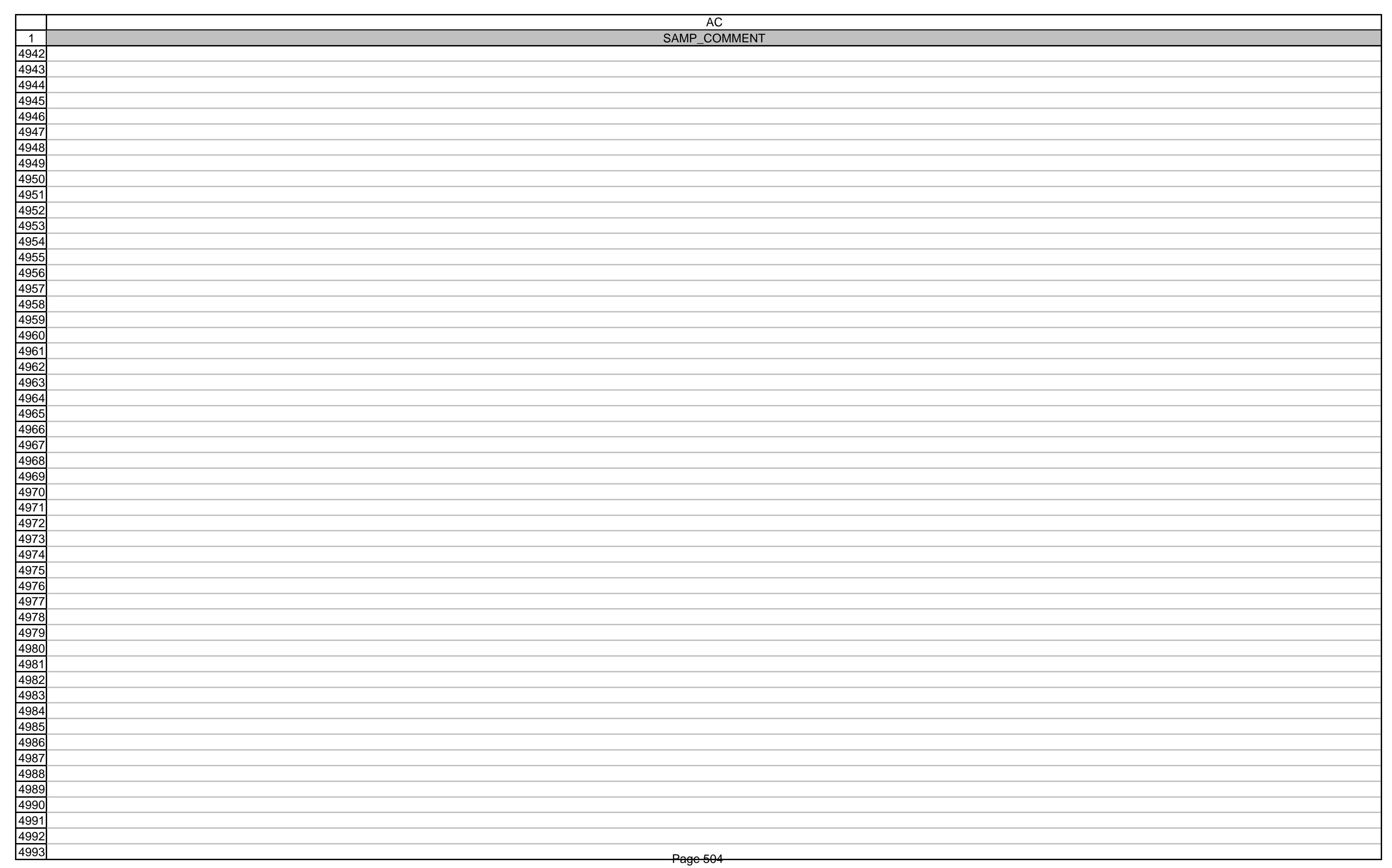




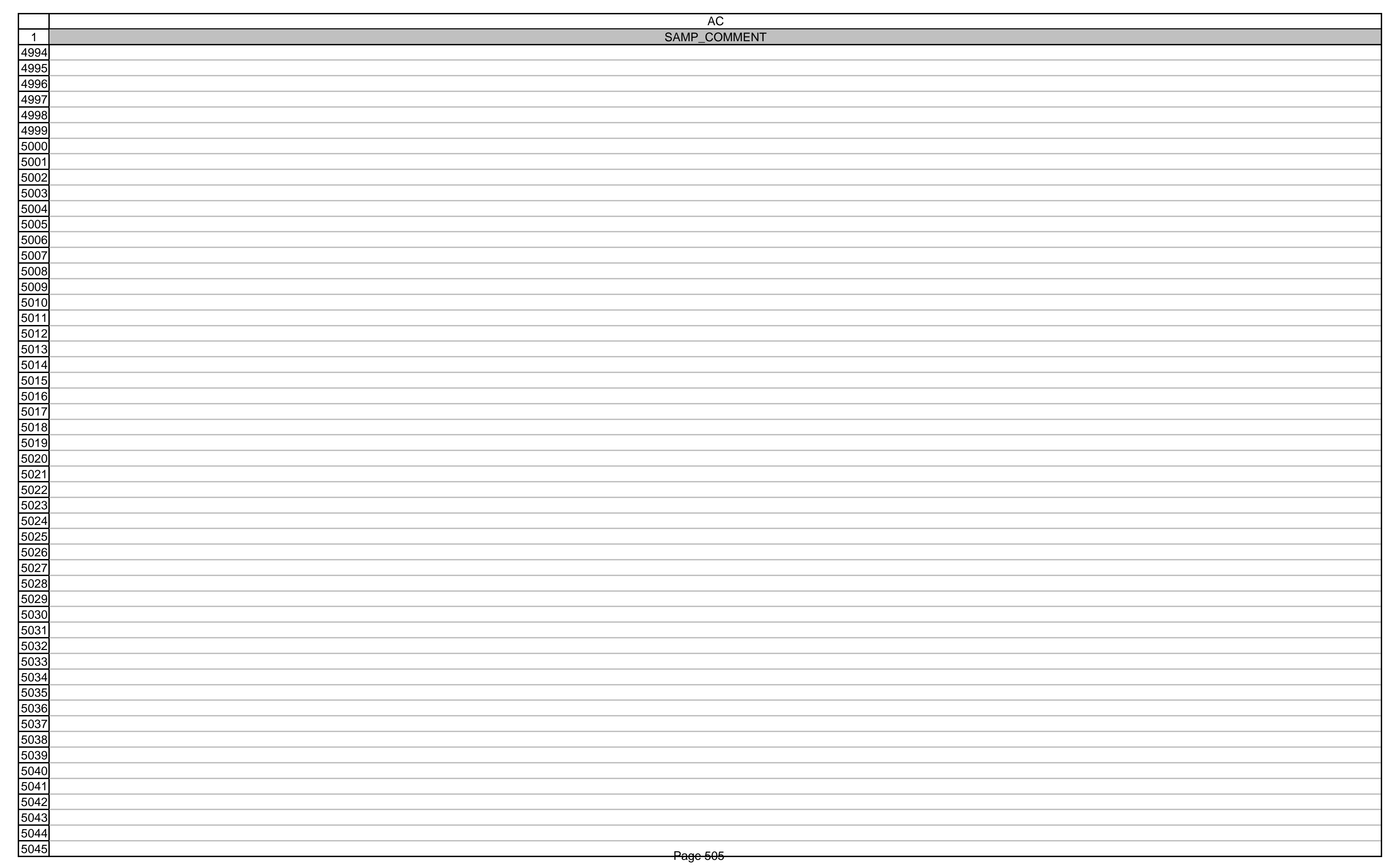




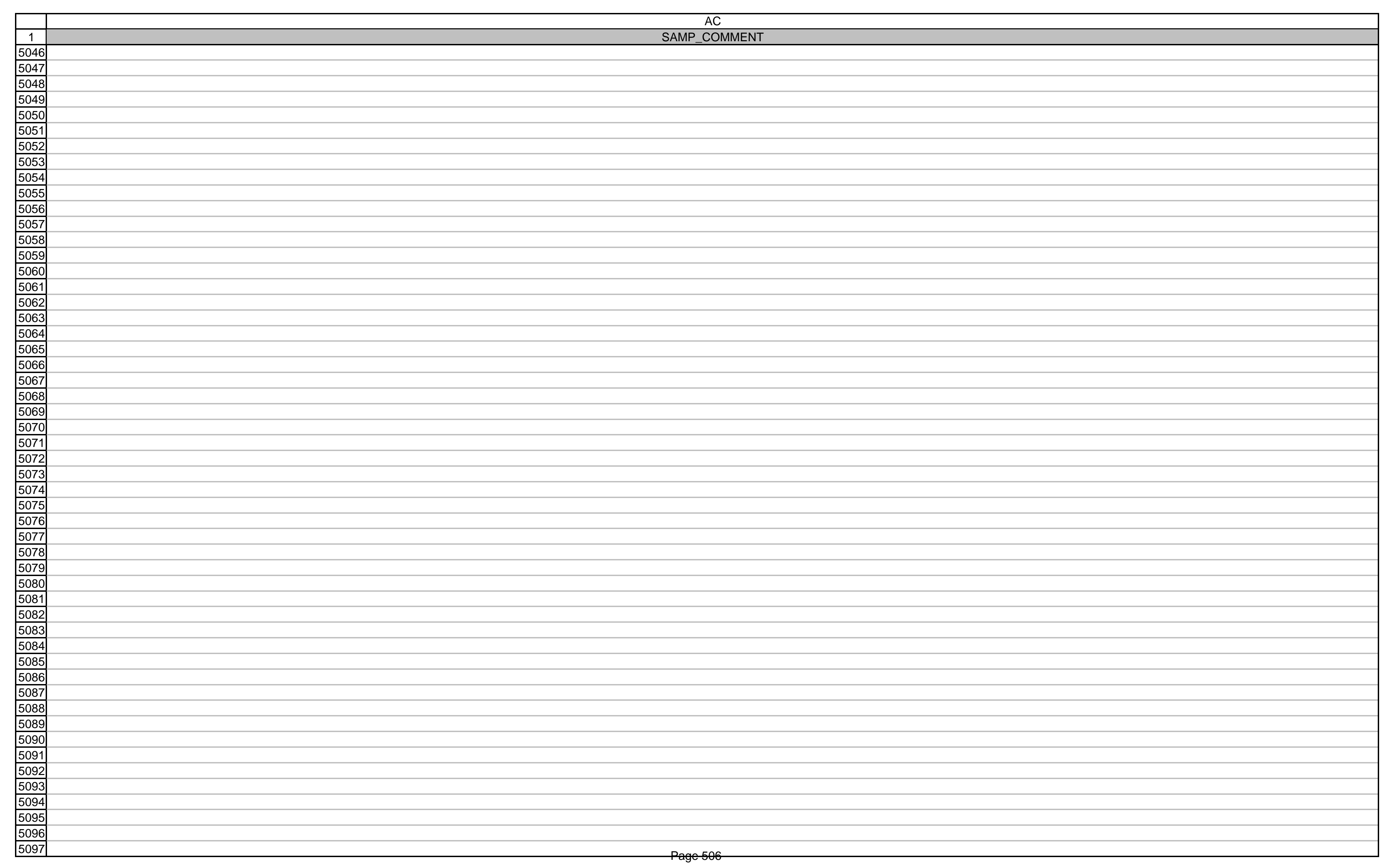




$$
F
$$




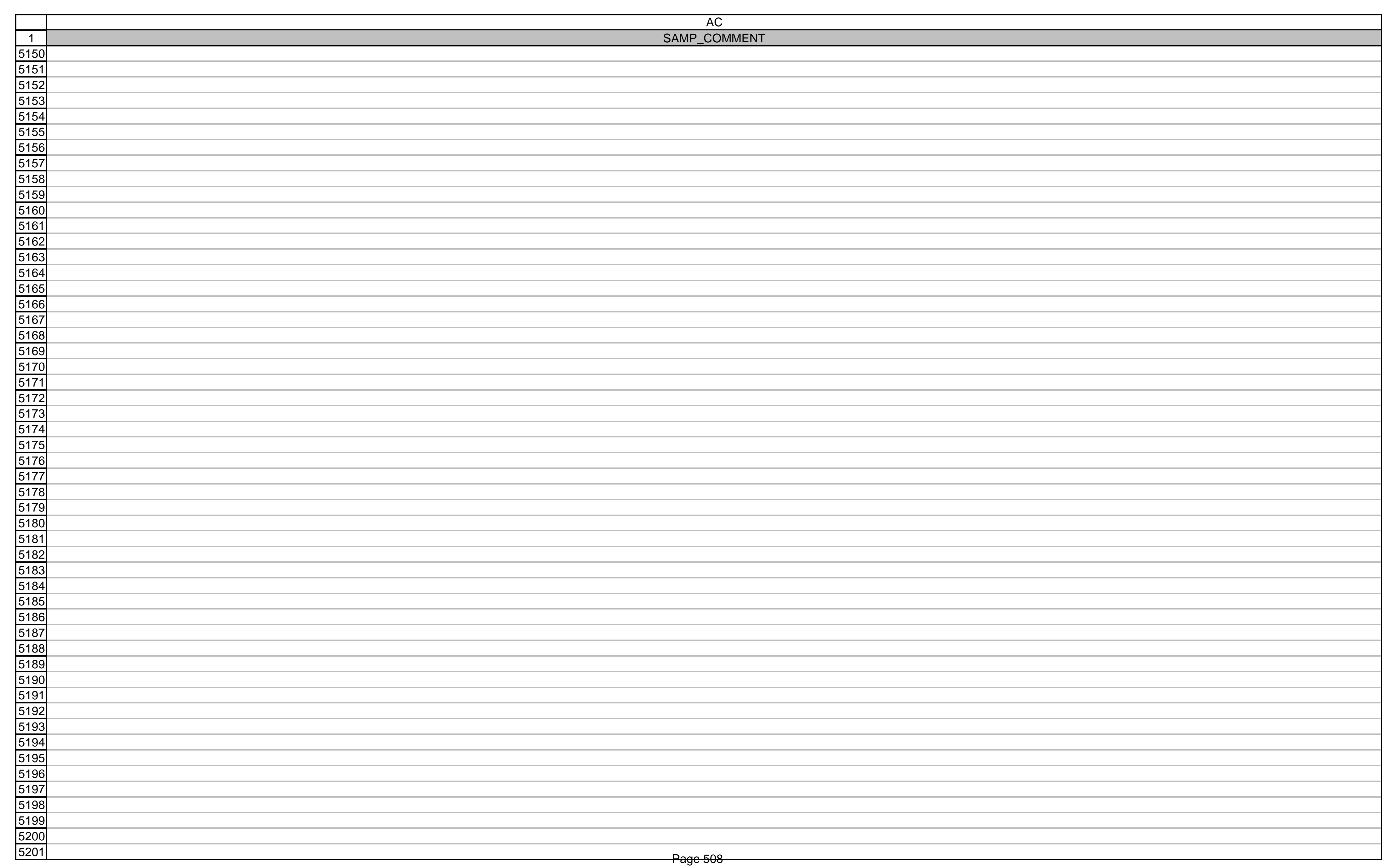




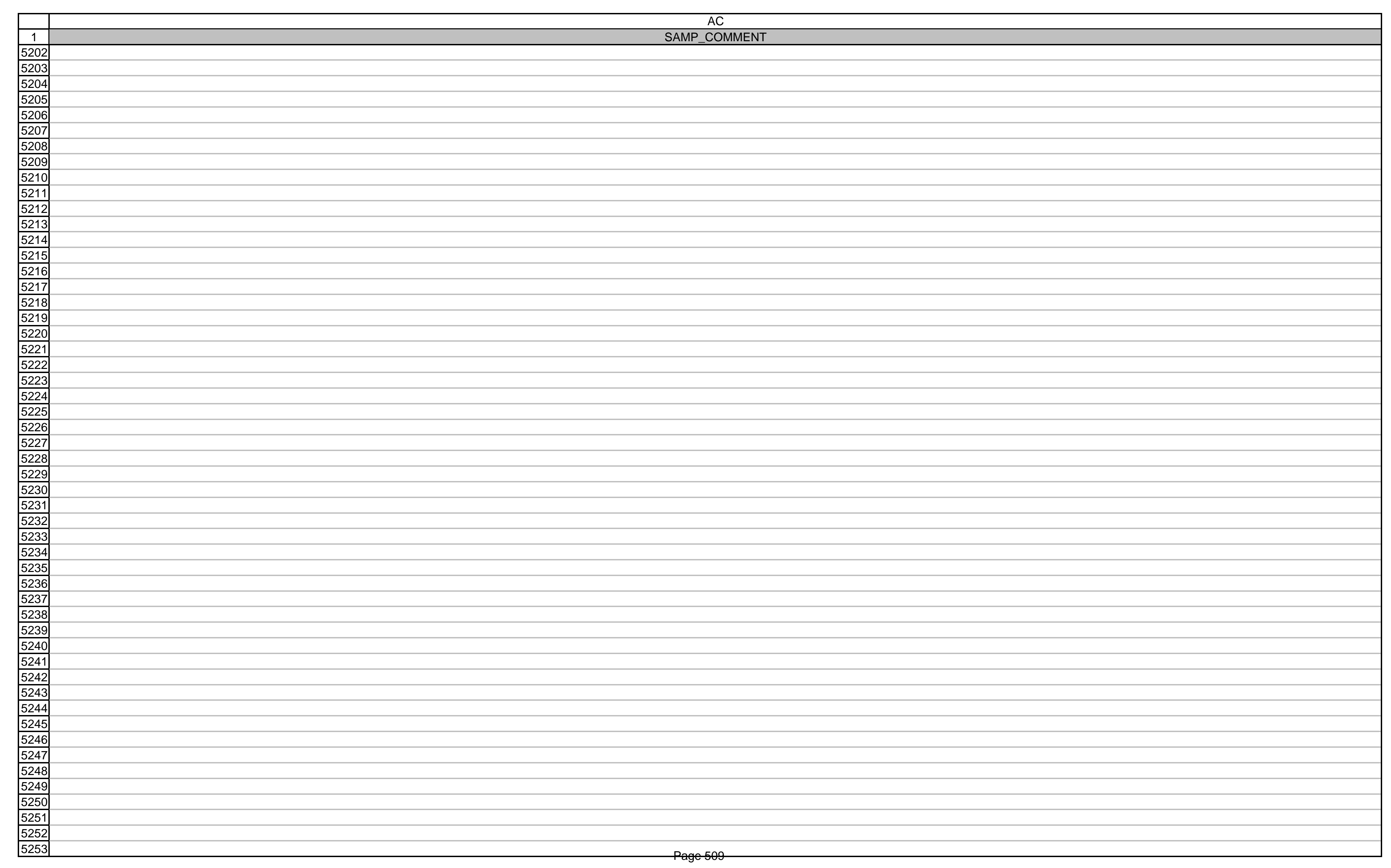




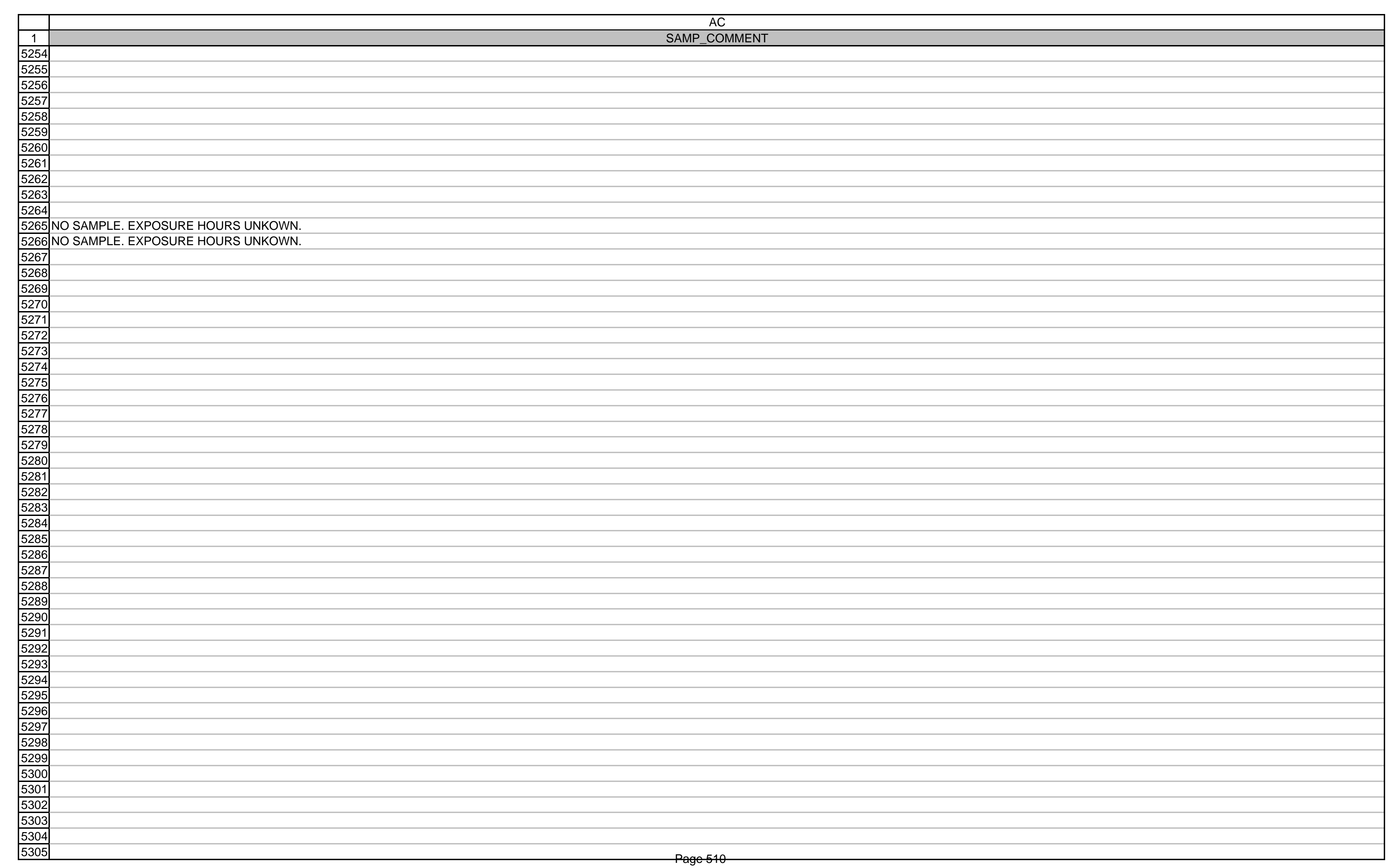




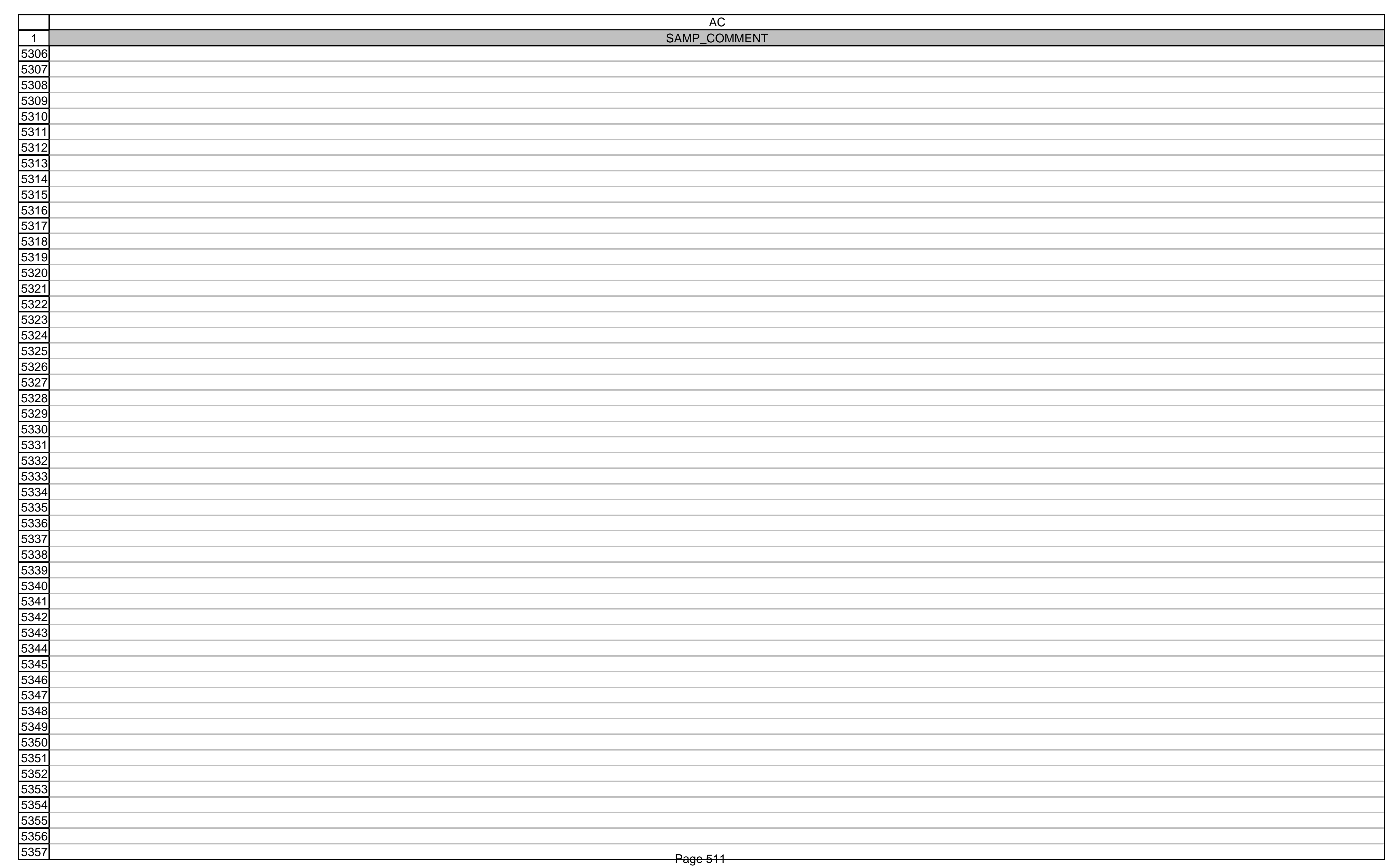




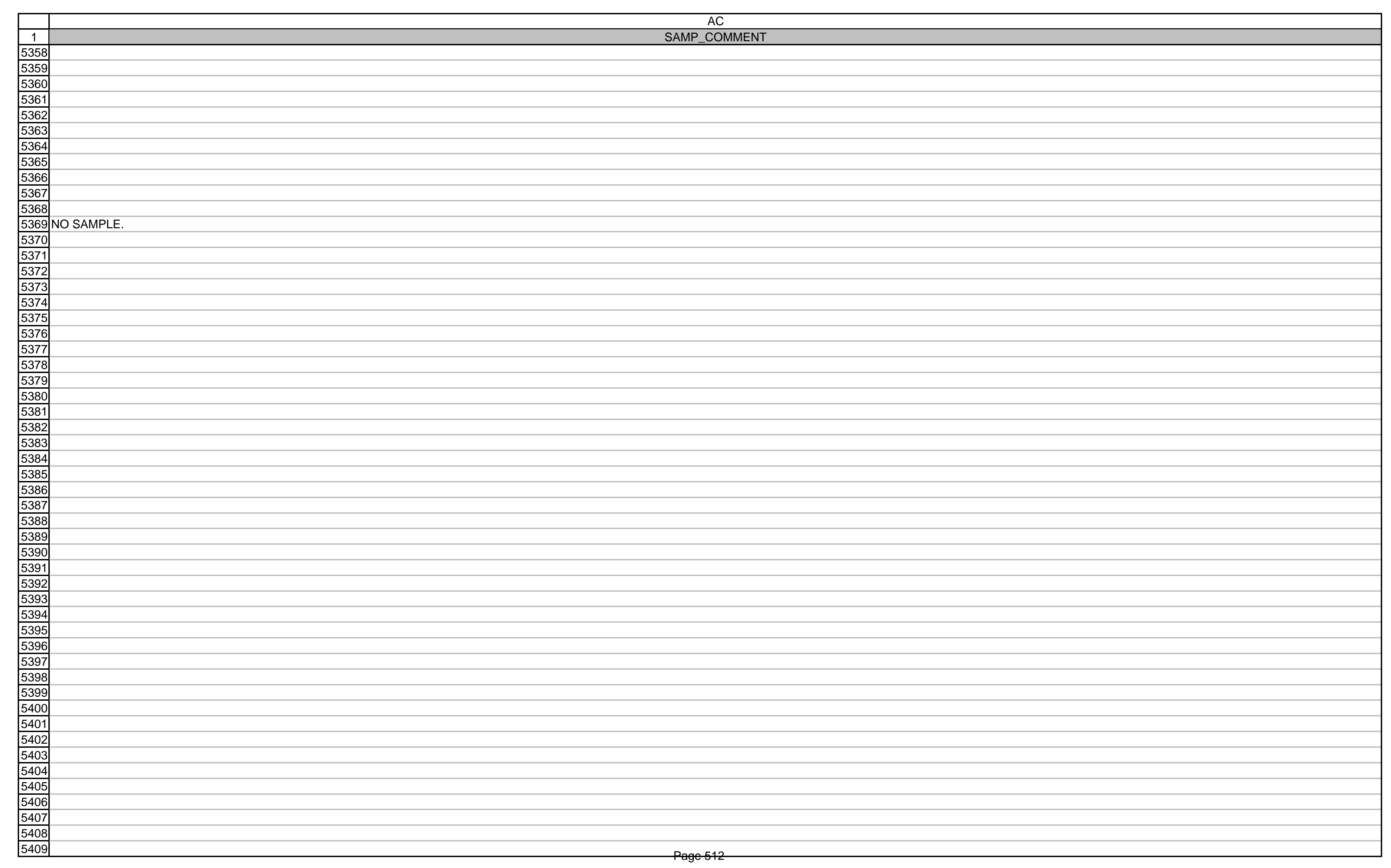




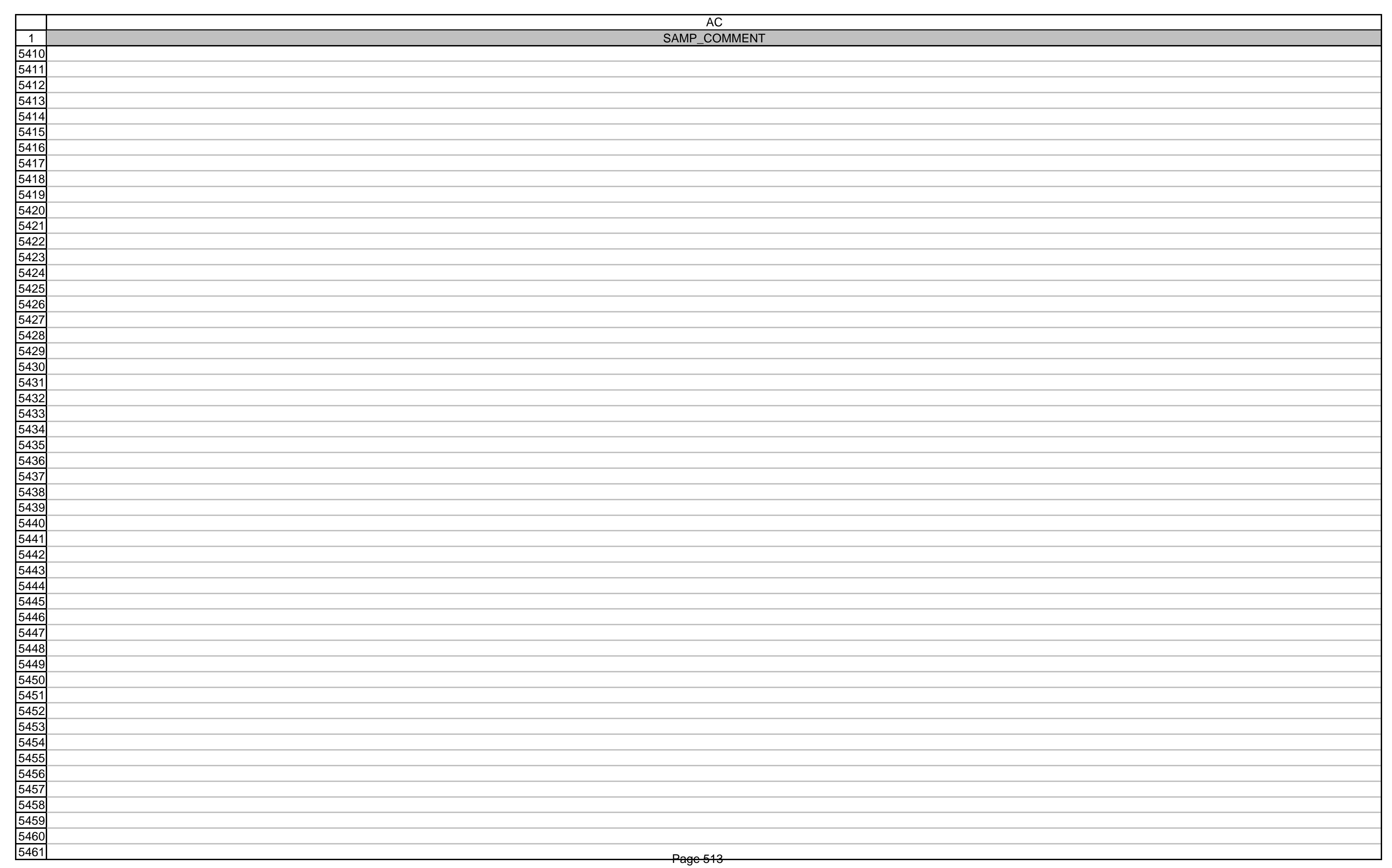




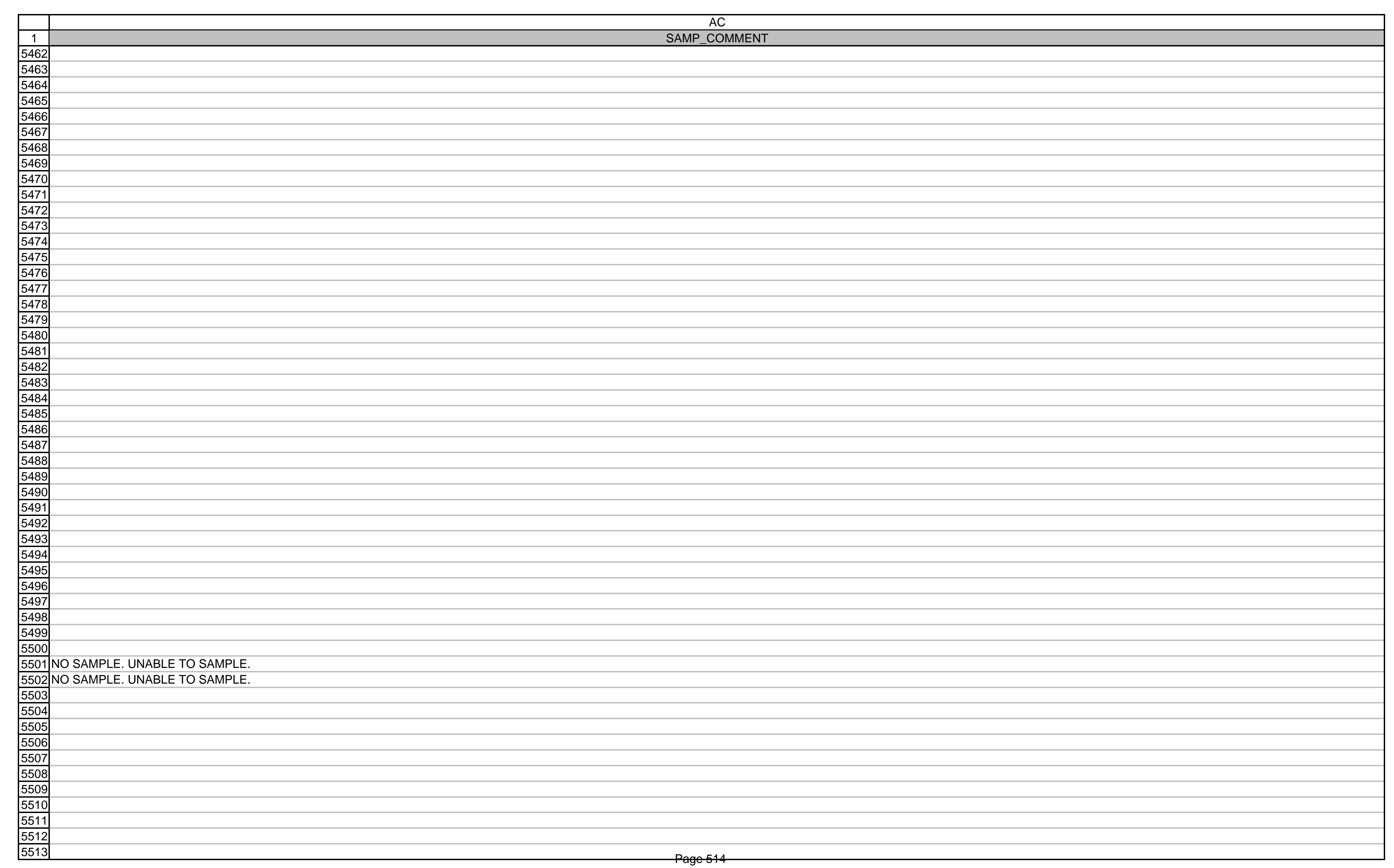




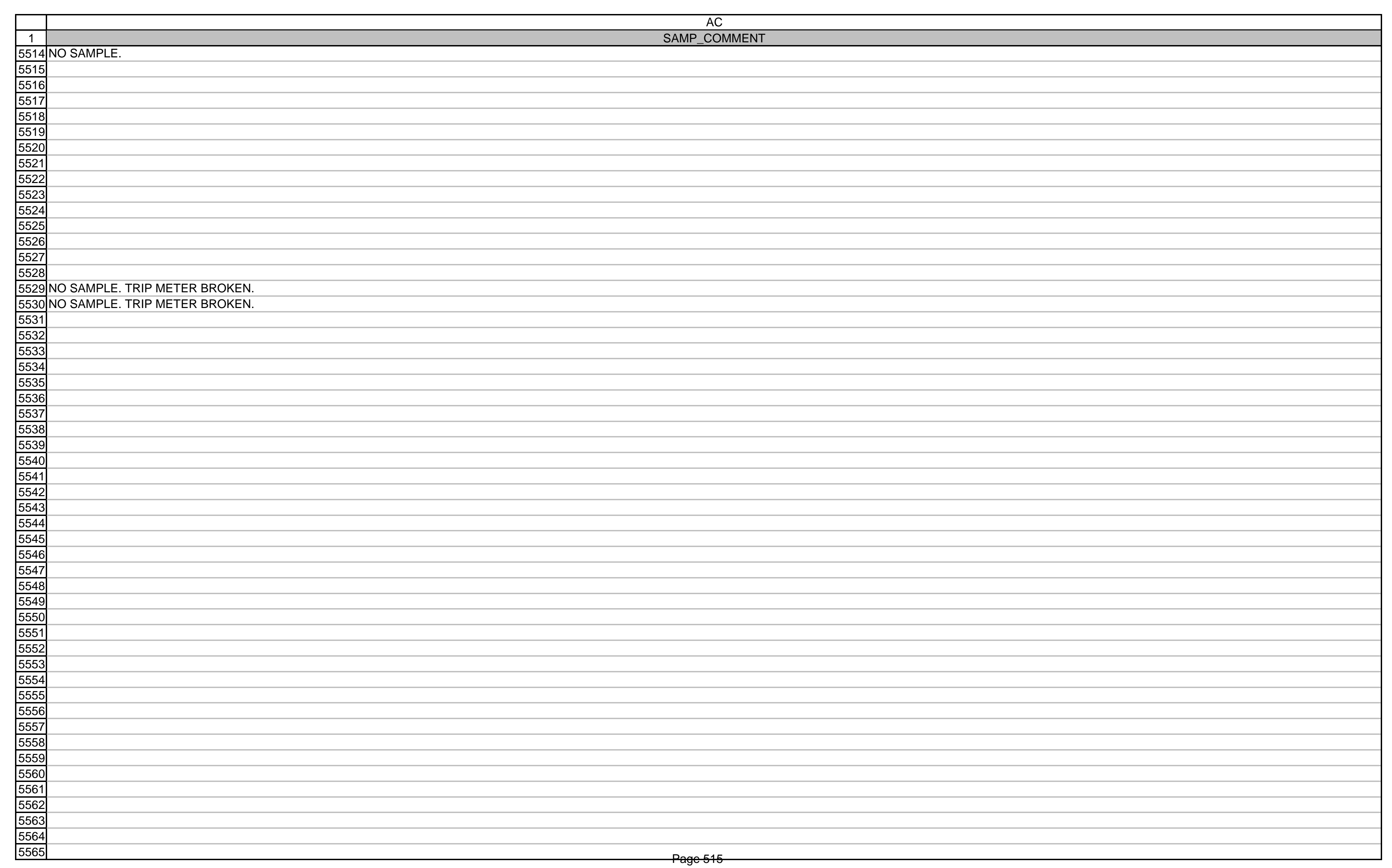




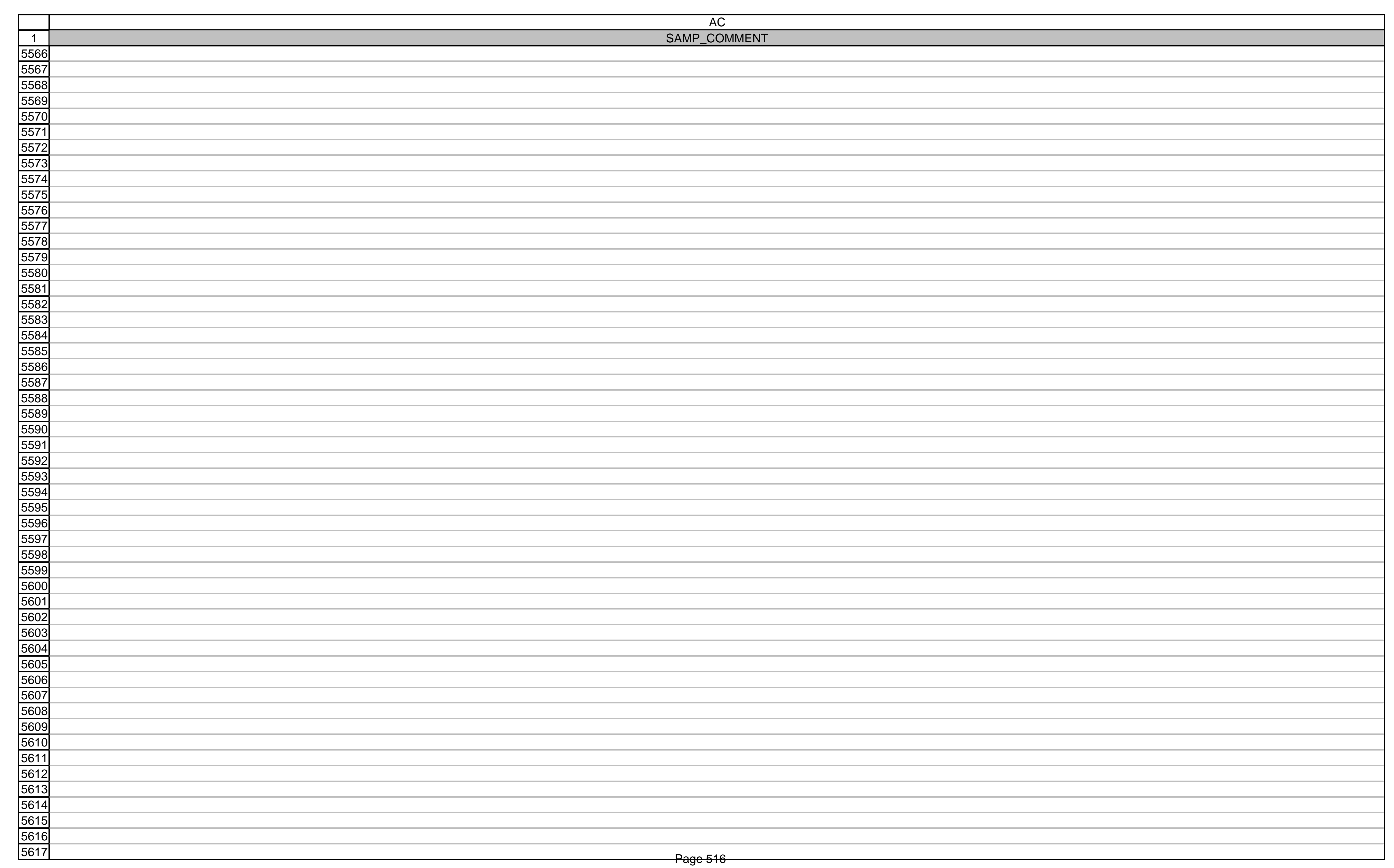




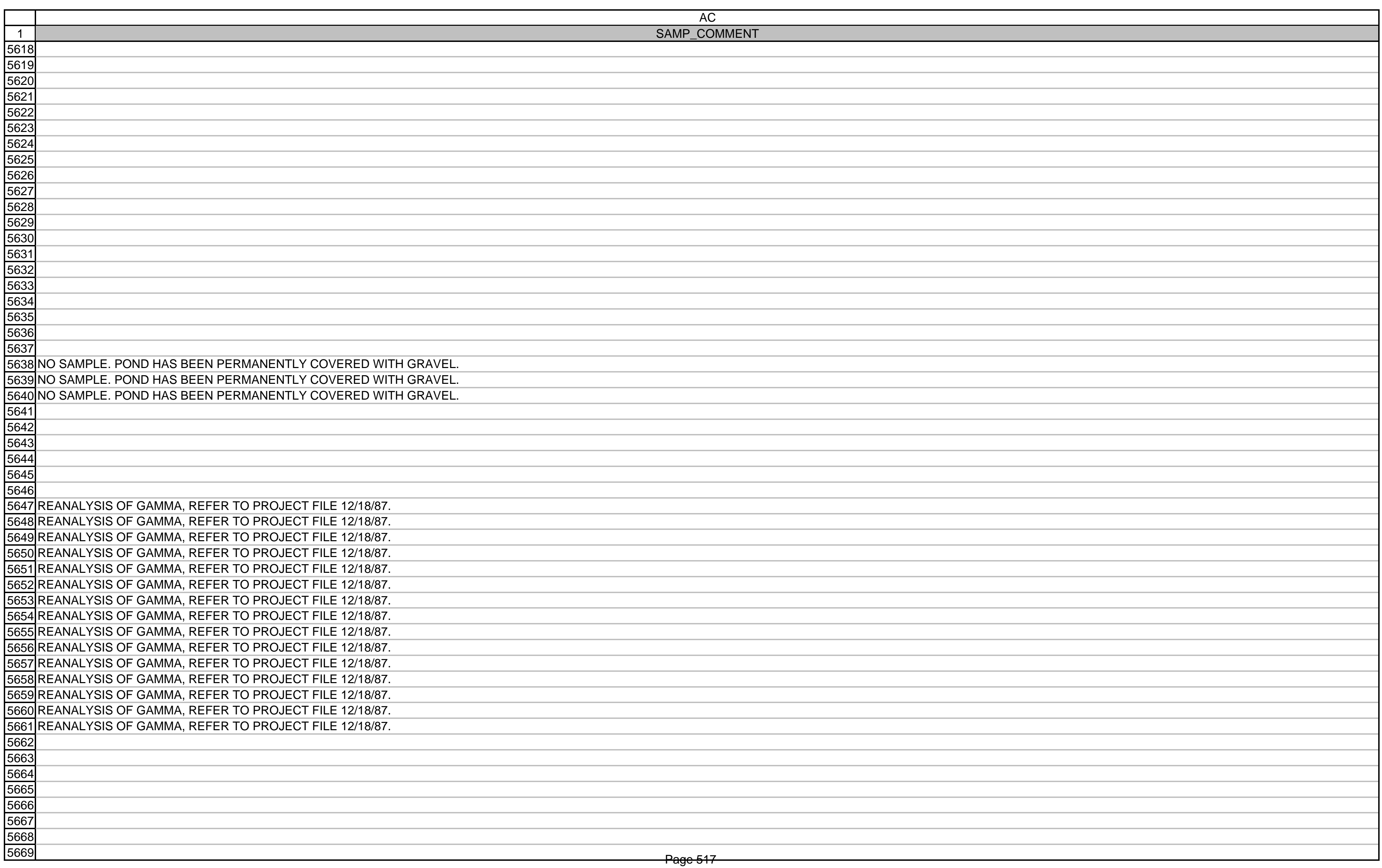




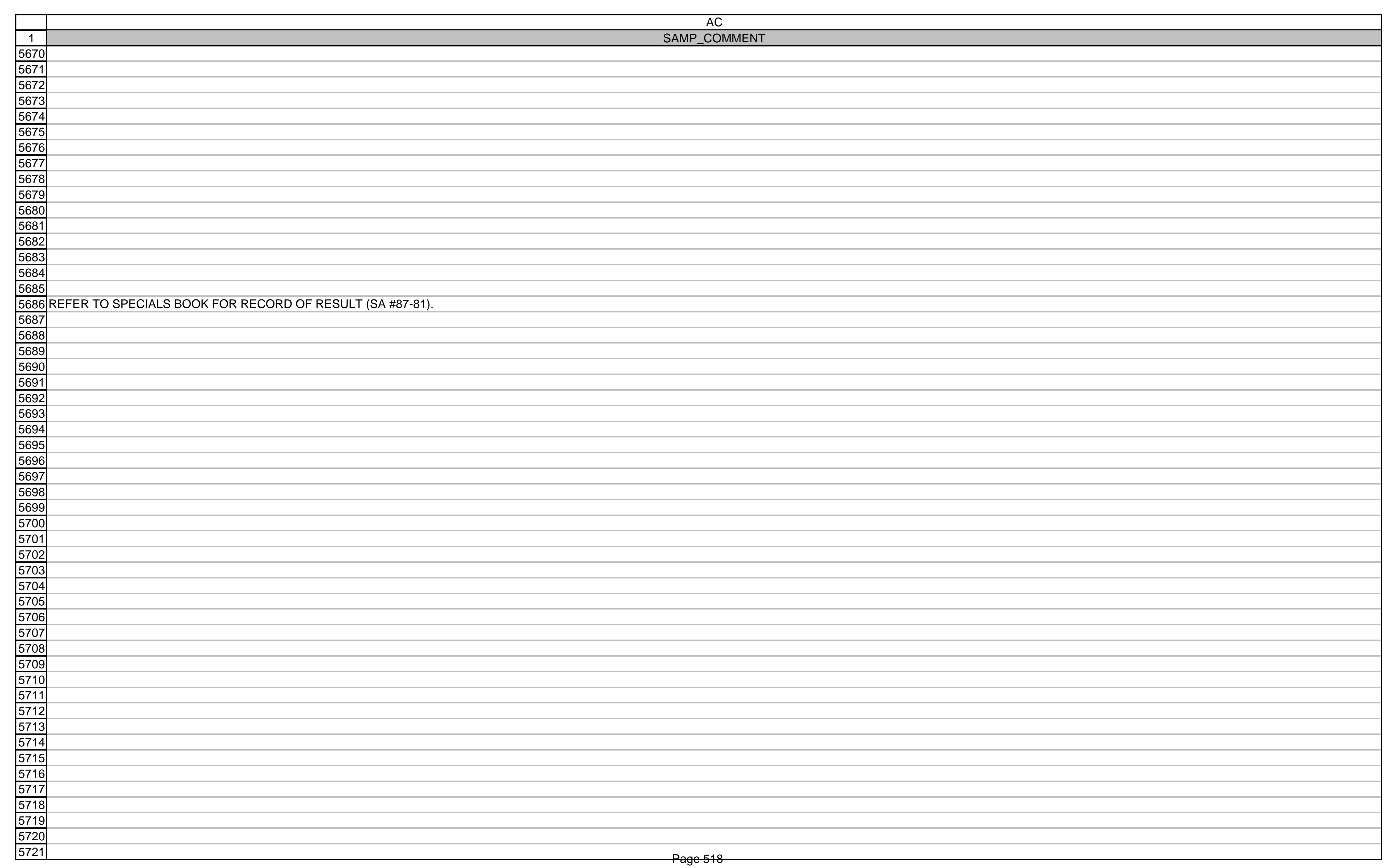




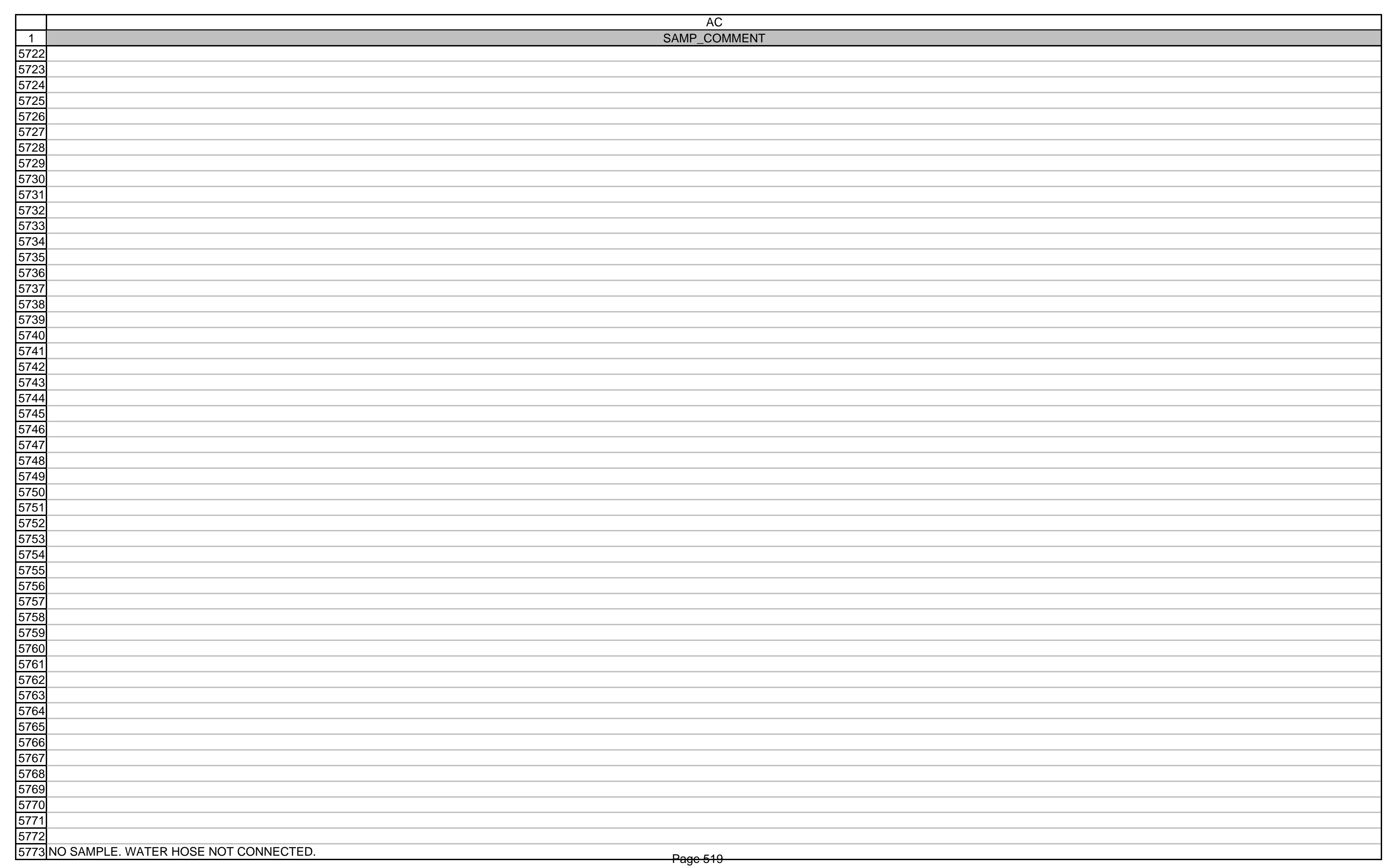





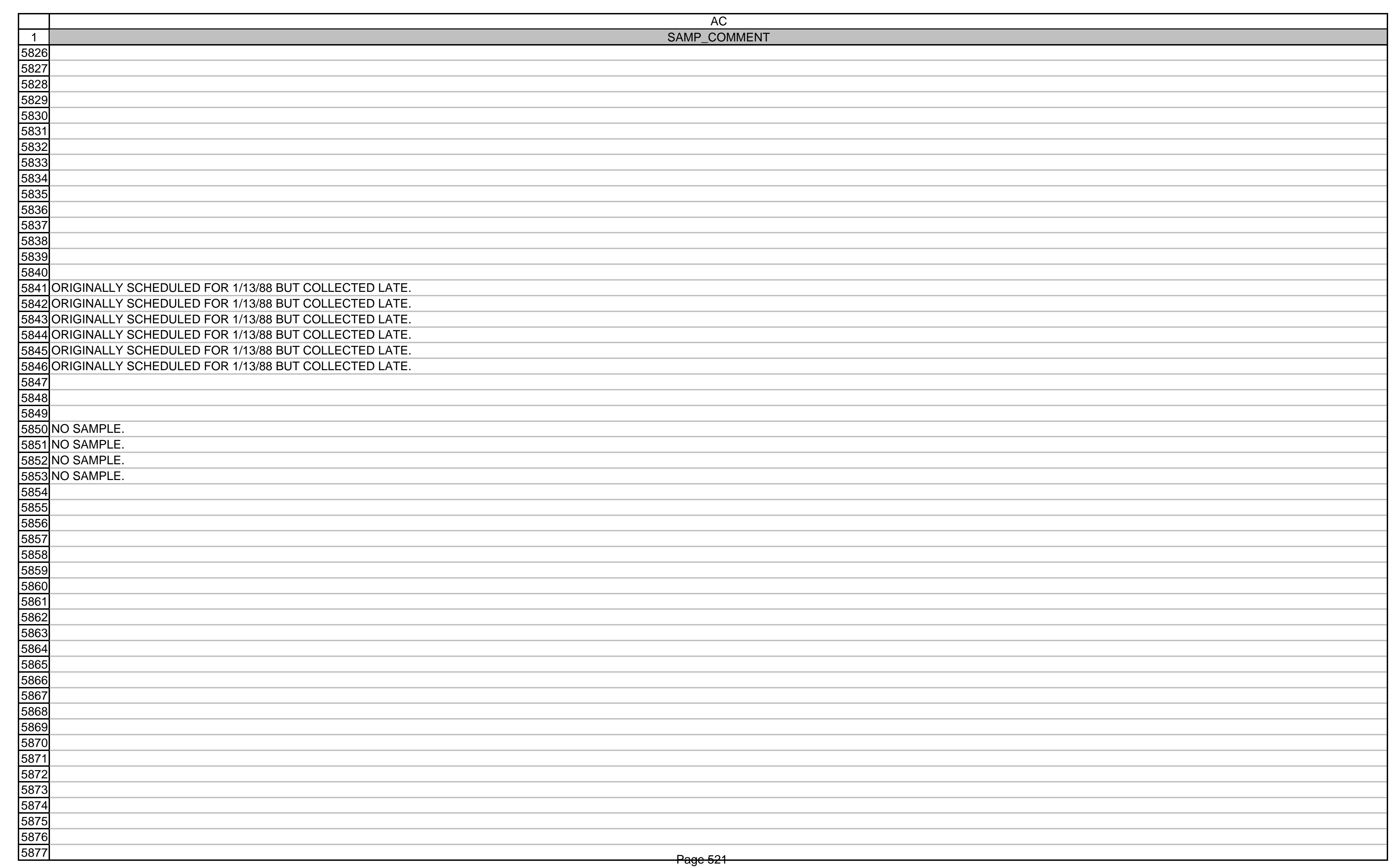




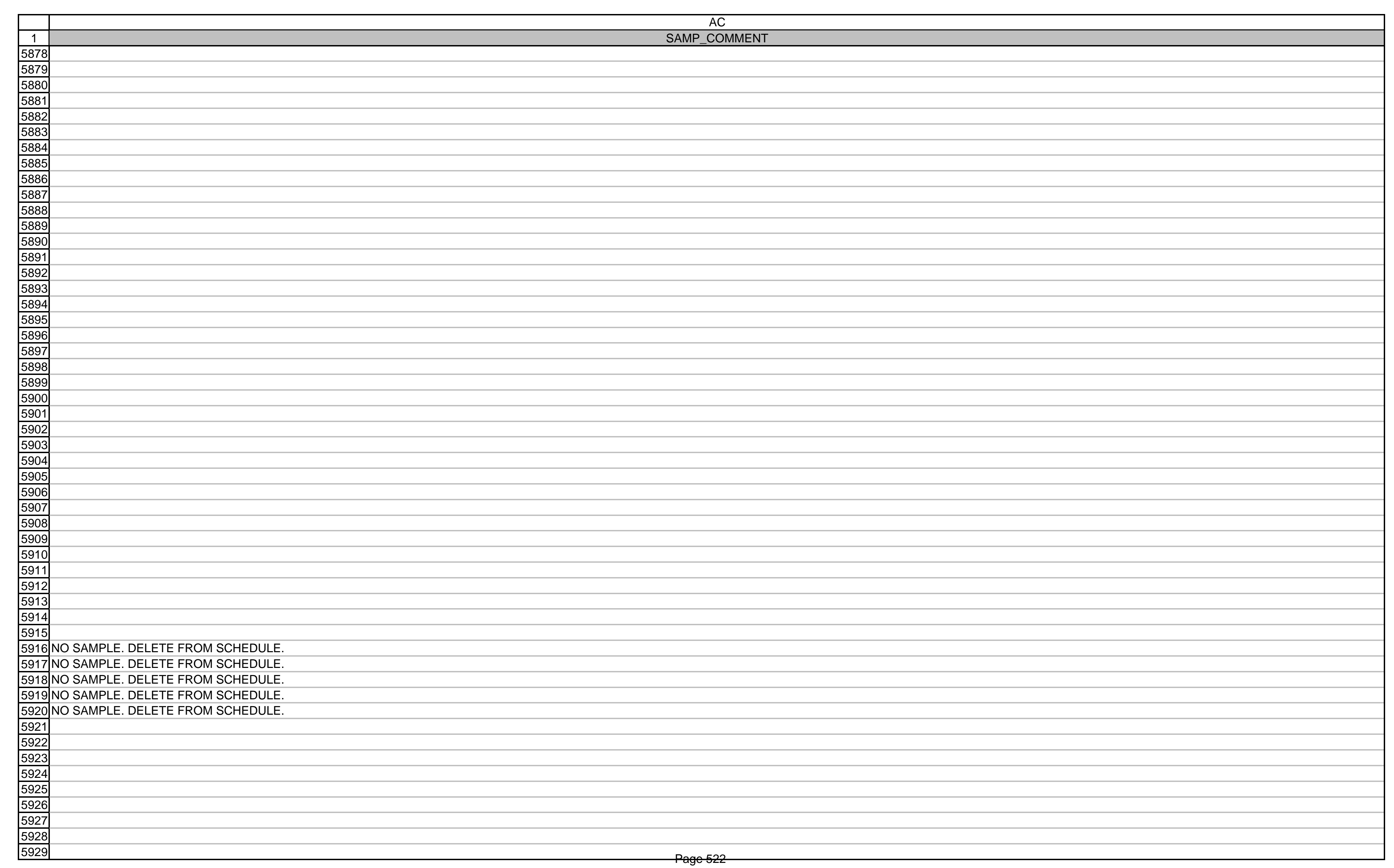




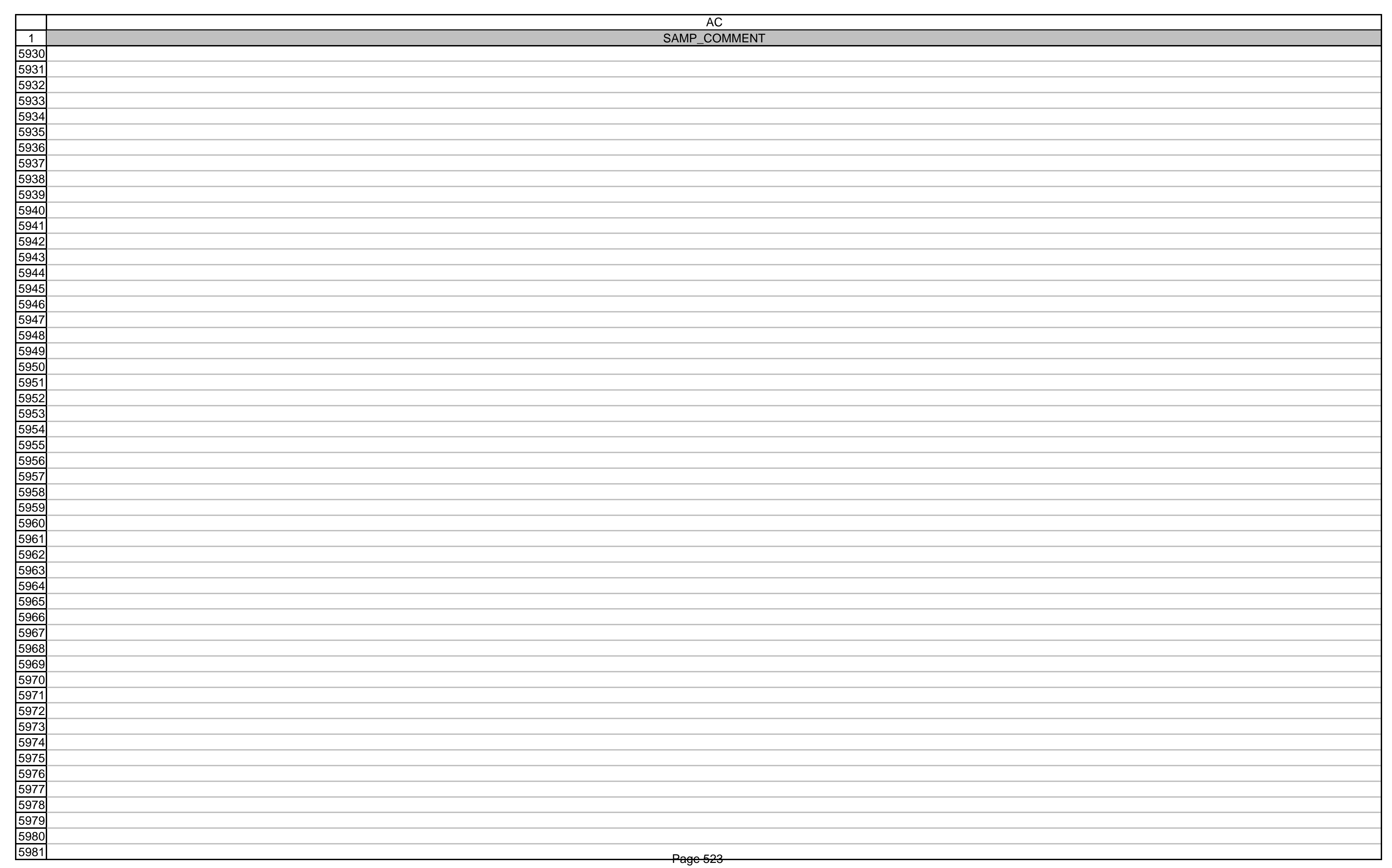




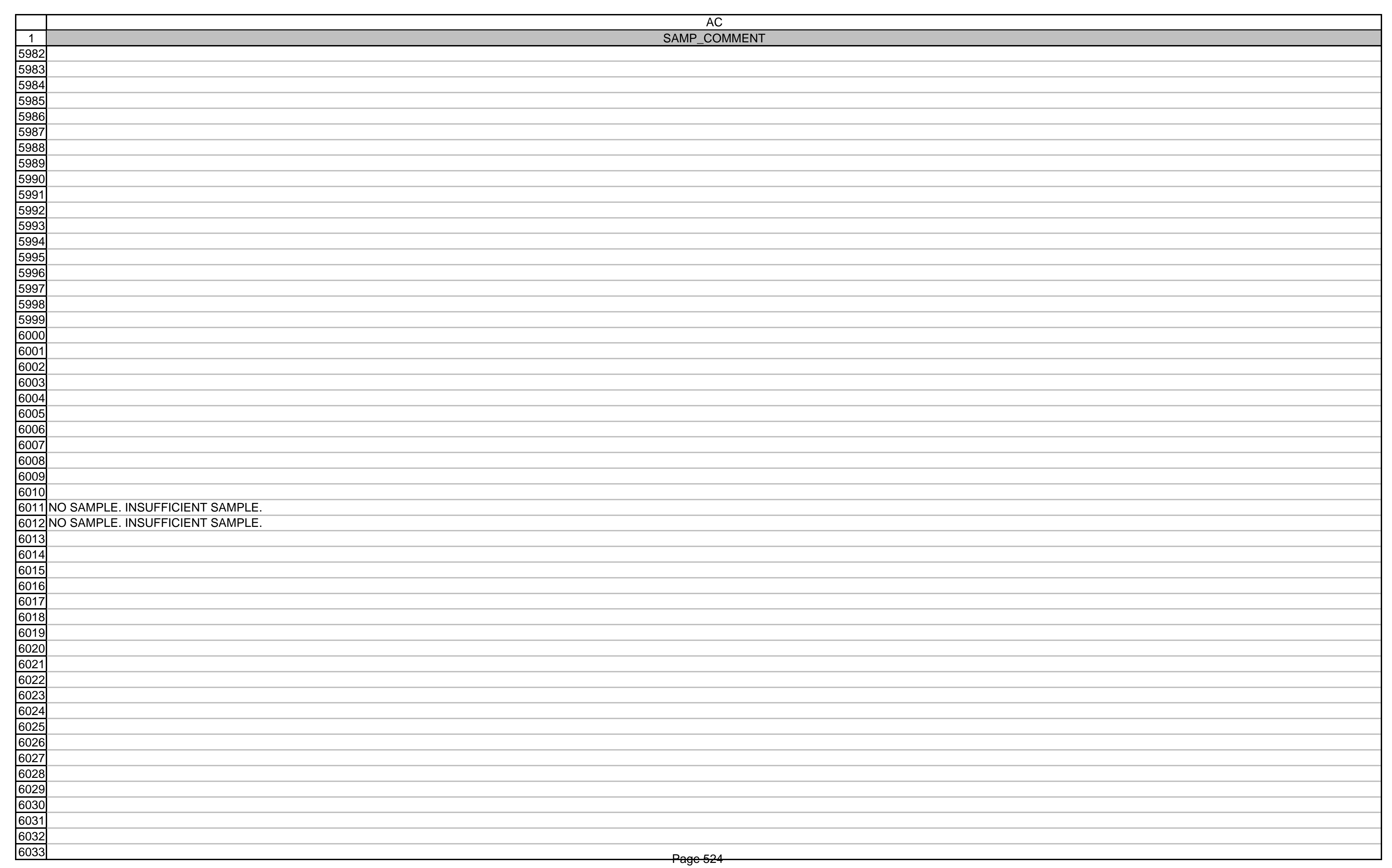




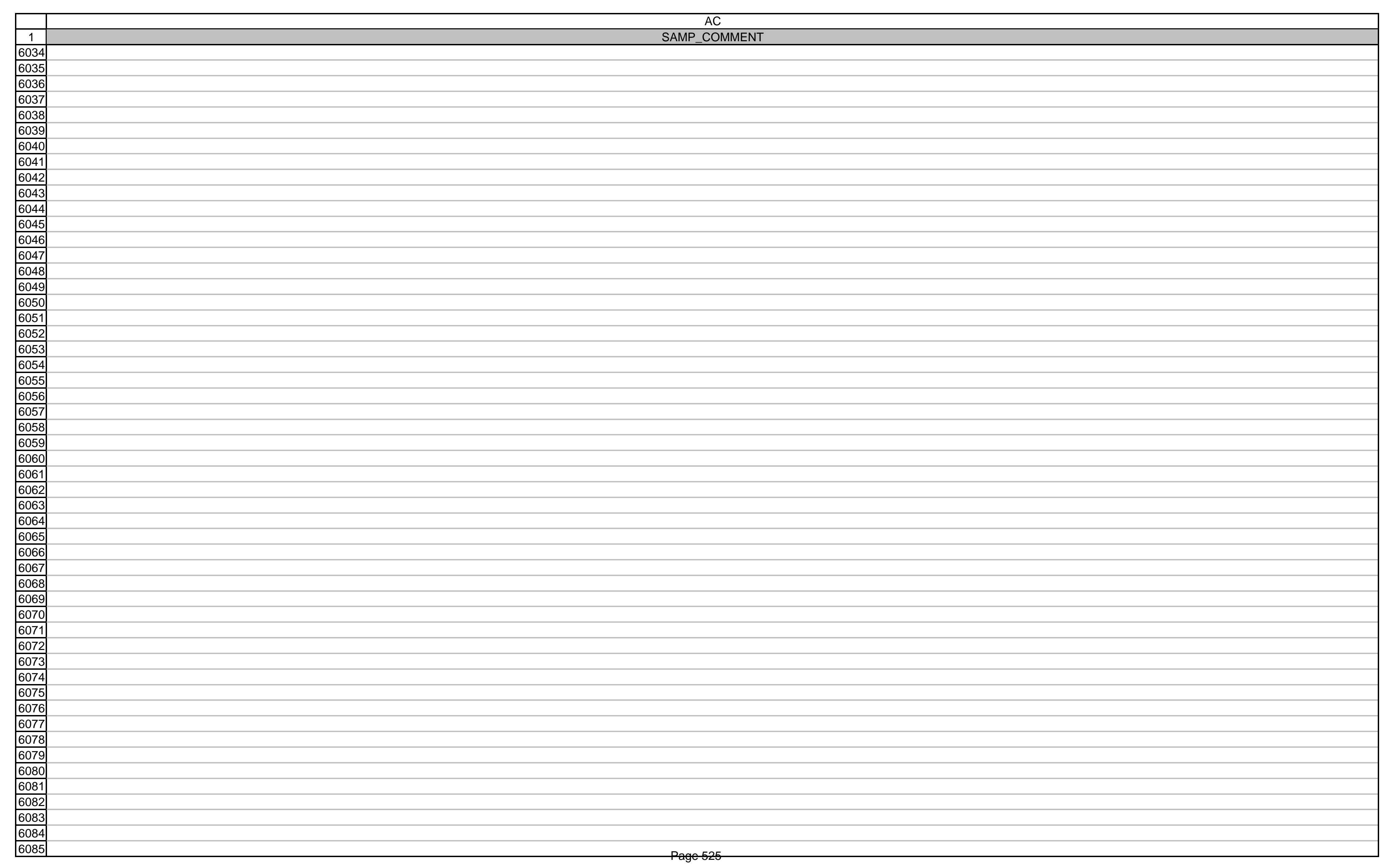




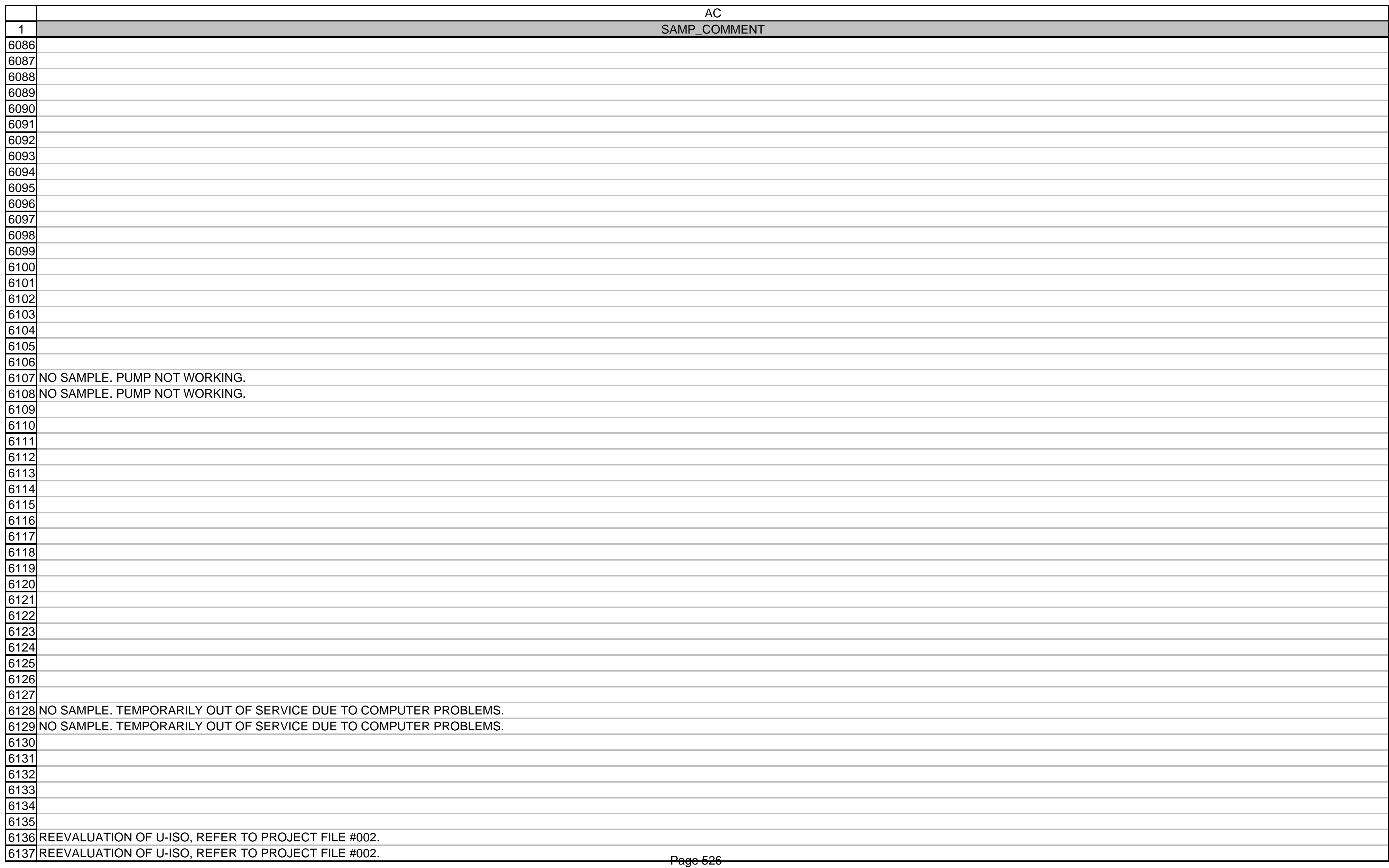


6138 REEVALUATION OF U-ISO, REFER TO PROJECT FILE \#002.

6139 REEVALUATION OF U-ISO, REFER TO PROJECT FILE \#002

6140 REEVALUATION OF U-ISO, REFER TO PROJECT FILE \#002.

6141 REEVALUATION OF U-ISO REFER TO PROJECT FILE $\# 002$.

6142

6143

6144

6145

6146 NO SAMPLE. TEMPORARILY OUT OF SERVICE DUE TO COMPUTER PROBLEMS.

\begin{tabular}{|l|l|}
6147 & NO SAMPLE. TEMPORARILY OUT OF SERVICE DUE TO COMPUTER PROBLEMS \\
\hline 6148 &
\end{tabular}

6148

6149

6150

6151

6152

6152

6154

6155 NO SAMPLE.

6156 NO SAMPLE.

\begin{tabular}{|l|}
6157 \\
\hline 6158 \\
\hline
\end{tabular}

6158

6159

6160

6161

6162

6163

616

6165

\begin{tabular}{|l|}
\hline 6166 \\
\hline 6167 \\
\hline
\end{tabular}

\begin{tabular}{|l|}
\hline 6167 \\
\hline 6168 \\
\hline
\end{tabular}

\begin{tabular}{|l|}
\hline 6167 \\
\hline 6168 \\
\hline 6169
\end{tabular}

\begin{tabular}{|l|}
\hline 6168 \\
\hline 6169 \\
\hline 6170
\end{tabular}

\begin{tabular}{|l|}
\hline 6170 \\
\hline 6171 \\
\hline
\end{tabular}

\begin{tabular}{|l|}
\hline 6171 \\
\hline 6172 \\
\hline
\end{tabular}

\begin{tabular}{|l|}
\hline 6171 \\
\hline 6172 \\
\hline 6173
\end{tabular}

$\begin{array}{r}6172 \\ 6173 \\ \hline\end{array}$

6173

6174

$\frac{6175}{6176}$

\begin{tabular}{|l|}
6176 \\
\hline 6177 \\
\hline 6178
\end{tabular}

\begin{tabular}{|l|l|}
6177 \\
\hline 6178 \\
\hline
\end{tabular}

\begin{tabular}{|l|}
6178 \\
\hline 6179 \\
\hline
\end{tabular}

\begin{tabular}{|l|}
\hline 6179 \\
\hline 6180 \\
\hline 6181 \\
\hline
\end{tabular}

6180

\begin{tabular}{|l|}
6181 \\
\hline 6182 \\
\hline 6
\end{tabular}

6182

6183

\begin{tabular}{|l|}
6184 \\
\hline 6185 \\
\hline 6186
\end{tabular}

6185 


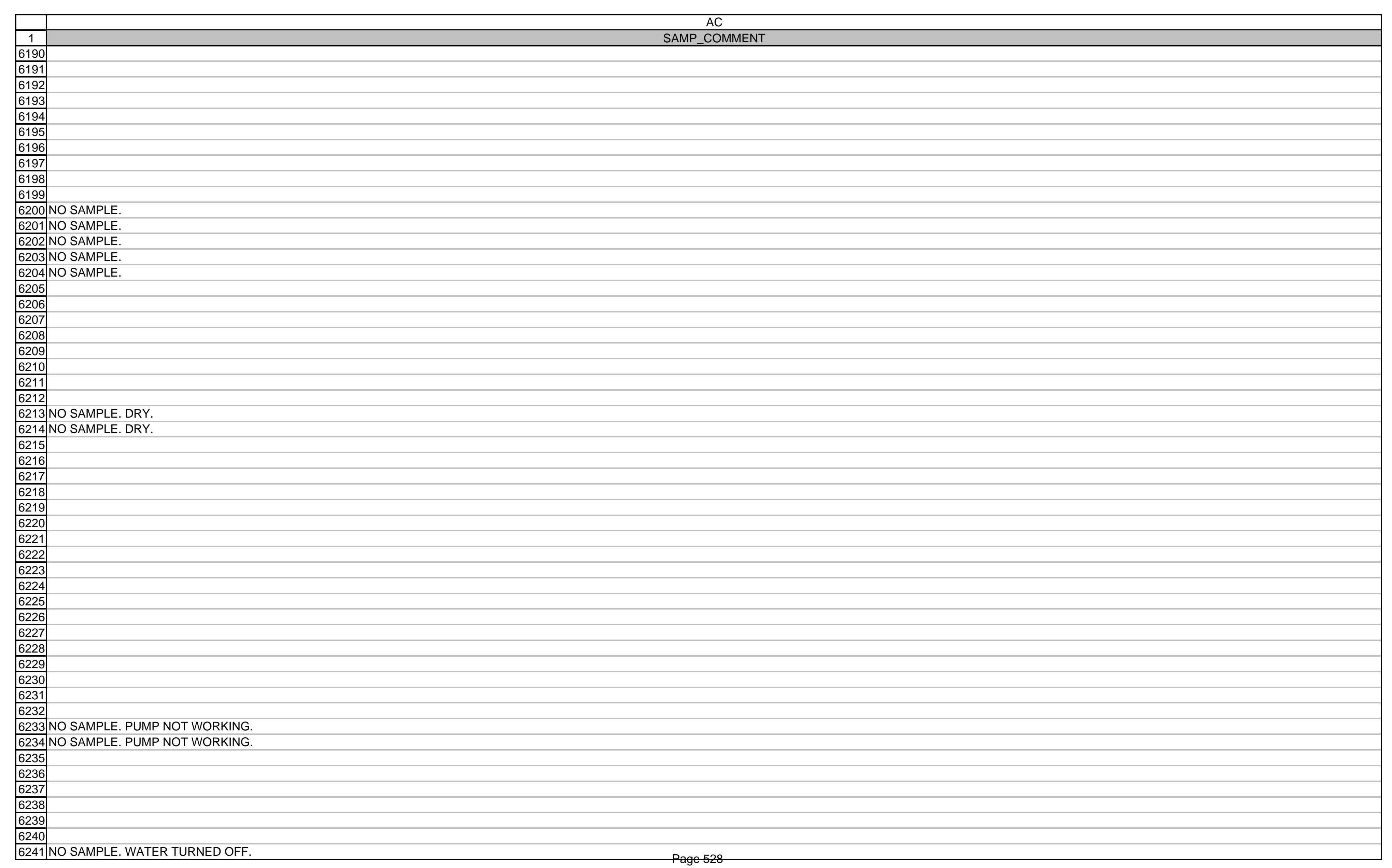




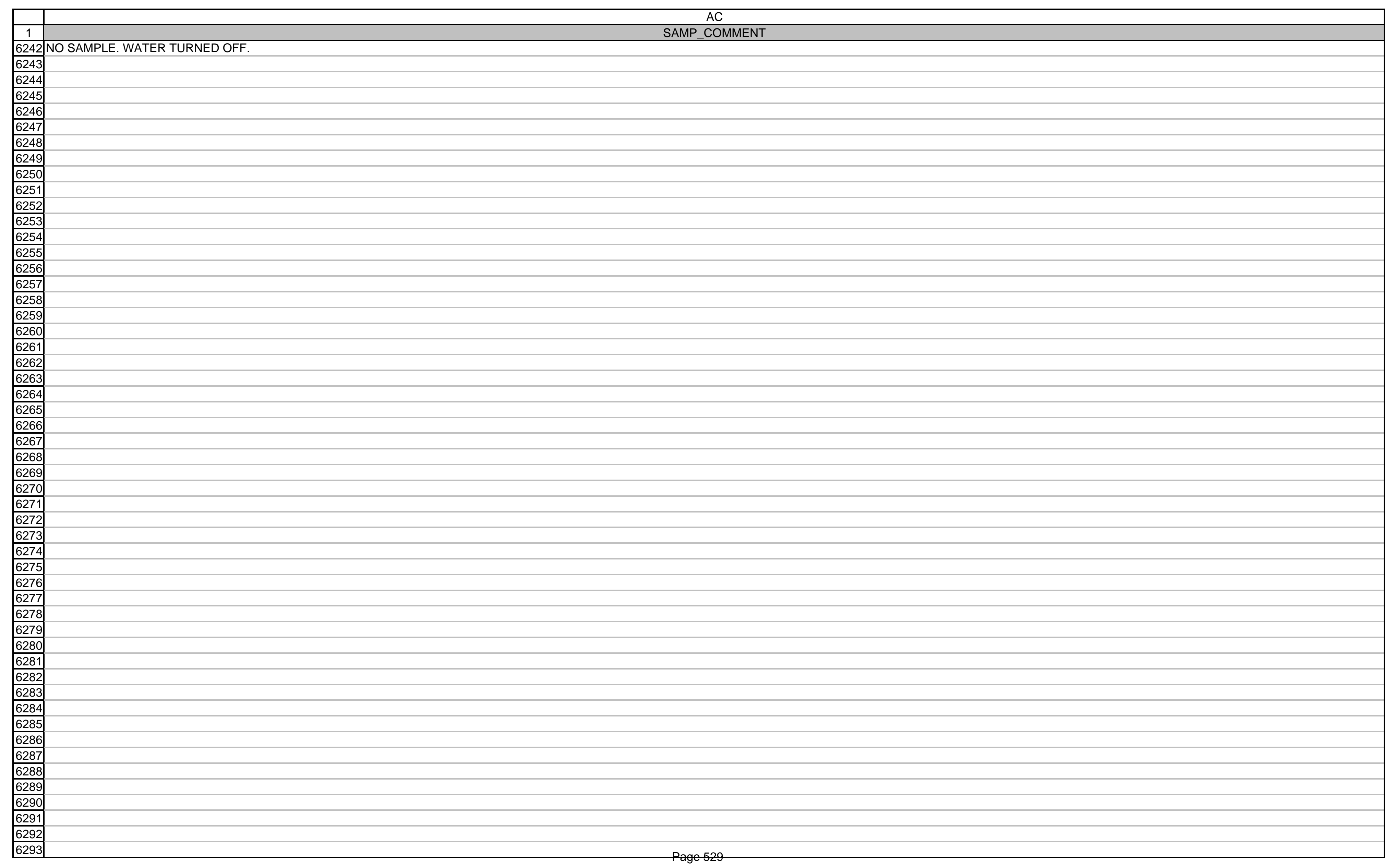




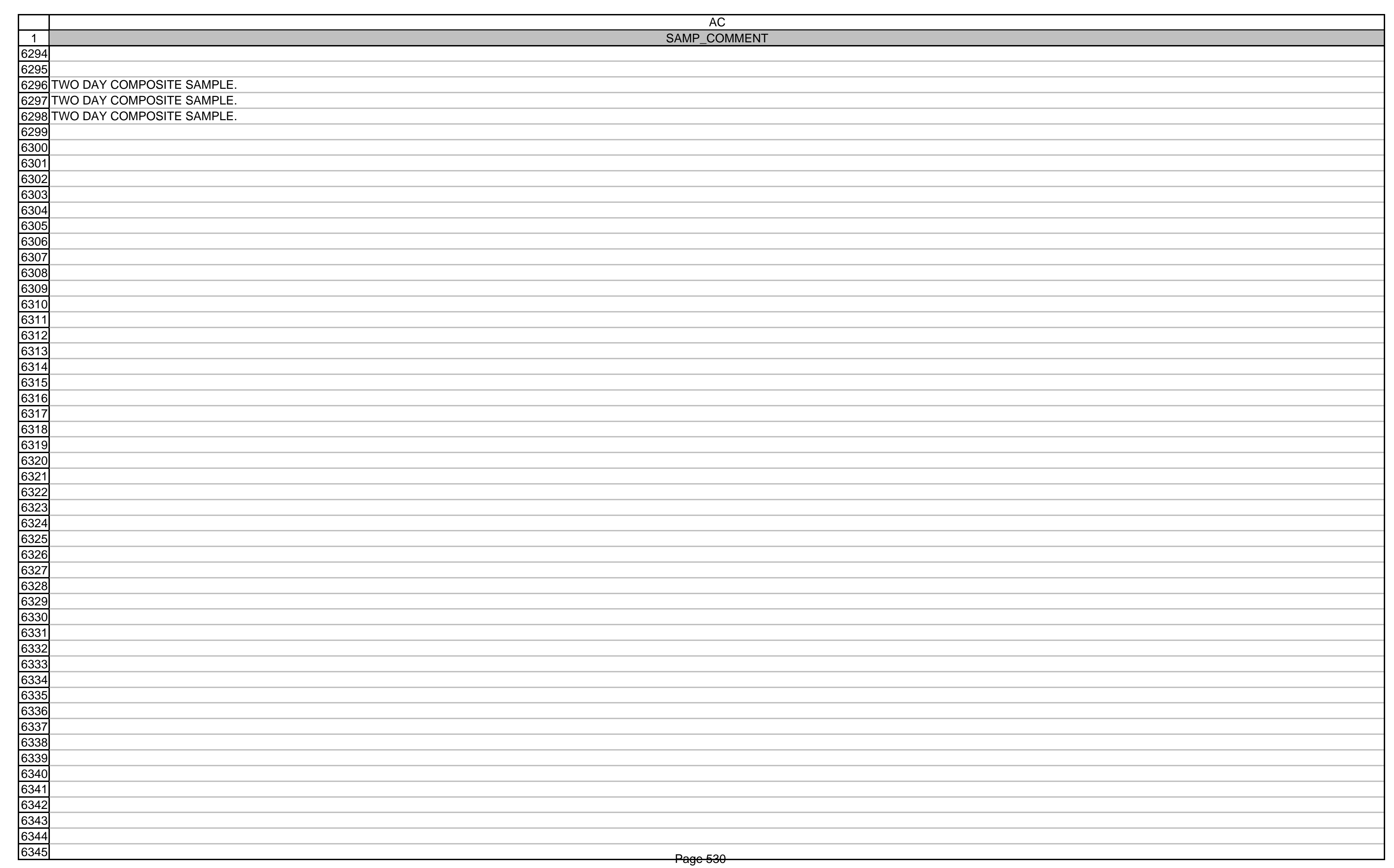


6367 REANALYSIS OF U-ISO, REFER TO PROJECT FILE \#001. RE-EVALUATION OF SR90, REFER TO PROJECT FILE \#003. 6368 REANALYSIS OF U-ISO, REFER TO PROJECT FILE \#001. RE-EVALUATION OF SR90, REFER TO PROJECT FILE \#003. 6369 REANALYSIS OF U-ISO, REFER TO PROJECT FILE \#001. RE-EVALUATION OF SR90, REFER TO PROJECT FILE \#003. 6370 REANALYSIS OF U-ISO, REFER TO PROJECT FILE \#001. RE-EVALUATION OF SR90, REFER TO PROJECT FILE \#003.

6371 REANALYSIS OF U-ISO, REFER TO PROJECT FILE \#001. RE-EVALUATION OF SR90, REFER TO PROJECT FILE \#003. 6372 REANALYSIS OF U-ISO, REFER TO PROJECT FILE \#001. RE-EVALUATION OF SR90, REFER TO PROJECT FILE \#003. 
6404 SAMPLER NOT IN SERVICE-NO RESIN COLUMNS.

6405 SAMPLER NOT IN SERVICE-NO RESIN COLUMN.

6406 SAMPLER NOT IN SERVICE-NO RESIN COLUMN.

\begin{tabular}{|l|}
\hline 6407 \\
\hline 6408 \\
\hline 6409 \\
\hline
\end{tabular}

6409

6410 SAMPLER NOT IN SERVICE-NO RESIN COLUMNS.

6411 SAMPLER NOT IN SERVICE-NO RESIN COLUMNS.

\begin{tabular}{|l|}
\hline 6412 \\
\hline 6413 \\
\hline 6414 \\
\hline
\end{tabular}

\begin{tabular}{|l|}
\hline 6413 \\
\hline 6414 \\
\hline 6415 \\
\hline
\end{tabular}

6414

6416 SAMPLER NOT IN SERVICE-NO RESIN COLUMNS.

6417 SAMPLER NOT IN SERVICE-NO RESIN COLUMNS.

6417 SAMPLER NOT IN SERVICE-NO RESIN COLUMNS.

\begin{tabular}{l|l}
6418 & SAMPLER NOT IN SERICE-NO RESIN COLUMNS. \\
\hline 6419 & SAMPLER NOT IN SERVICE-NO RESIN COLUMNS.
\end{tabular}

6420

6421

6422

6423

6424

\begin{tabular}{|l|}
\hline 6425 \\
\hline 6426 \\
\hline 6427 \\
\hline
\end{tabular}

6427

6428

6429

\begin{tabular}{|l|}
6429 \\
\hline 6431 \\
\hline
\end{tabular}

\begin{tabular}{|l|}
\hline 6431 \\
\hline 6432 \\
\hline 6433 \\
\hline
\end{tabular}

\begin{tabular}{|l|}
6432 \\
\hline 6433 \\
\hline 6434 \\
\hline
\end{tabular}

6433

6434

6435

6436 
6478 LOCATION PREVIOUSLY KNOWN AS W15 UPDATED TO MATHEWS CORNER.(SAME LOCATION MORE PRECISE NAME PER R. DIRKES) 6479 LOCATION PREVIOUSLY KNOWN AS W15 UPDATED TO MATHEWS CORNER.(SAME LOCATION MORE PRECISE NAME PER R. DIRKES) 6480 LOCATION PREVIOUSLY KNOWN AS W15 UPDATED TO MATHEWS CORNER.(SAME LOCATION MORE PRECISE NAME PER R. DIRKES) 6481 LOCATION PREVIOUSLY KNOWN AS W15 UPDATED TO MATHEWS CORNER.(SAME LOCATION MORE PRECISE NAME PER R. DIRKES) 6482 LOCATION PREVIOUSLY KNOWN AS W15 UPDATED TO MATHEWS CORNER.(SAME LOCATION MORE PRECISE NAME PER R. DIRKES) 


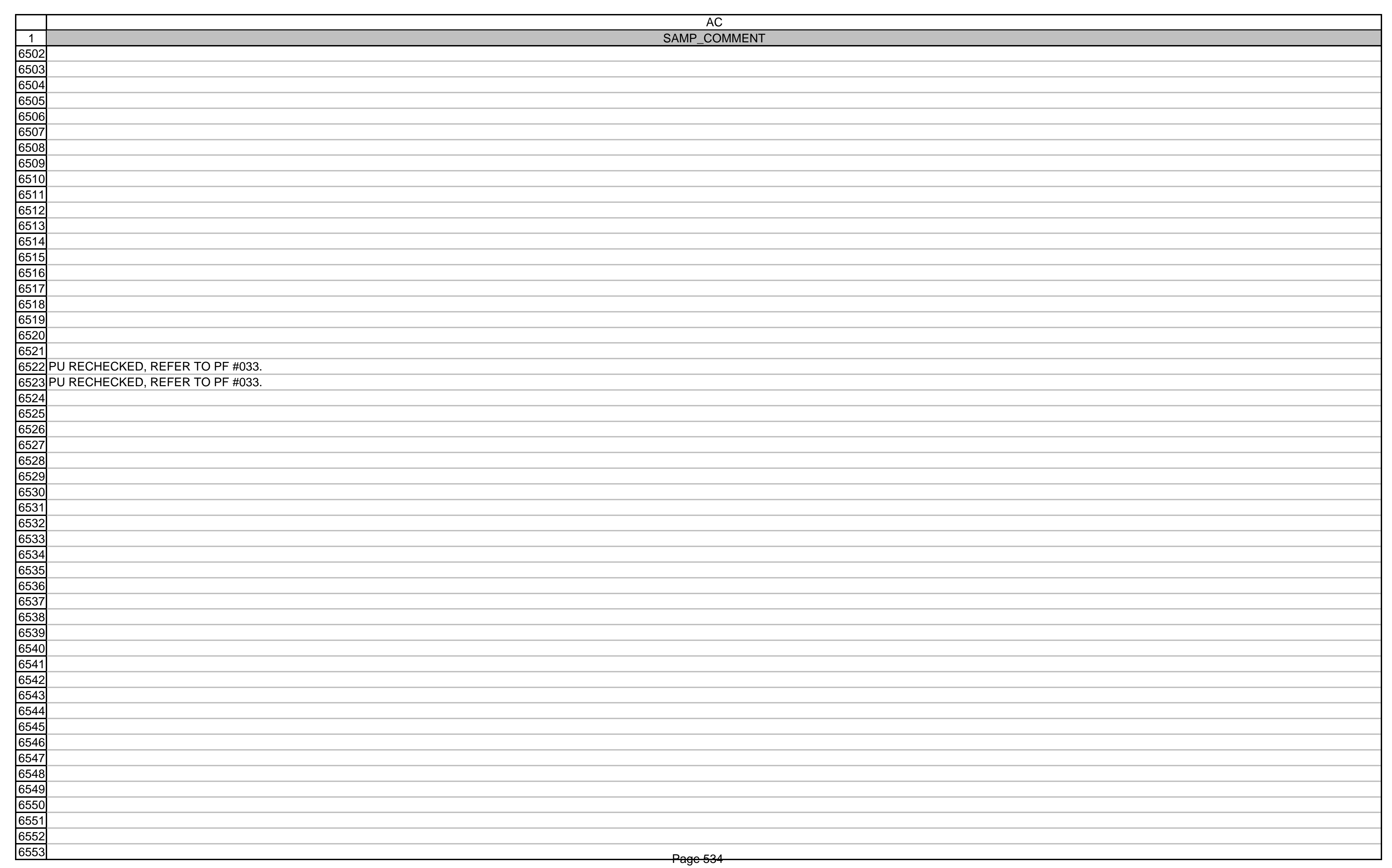




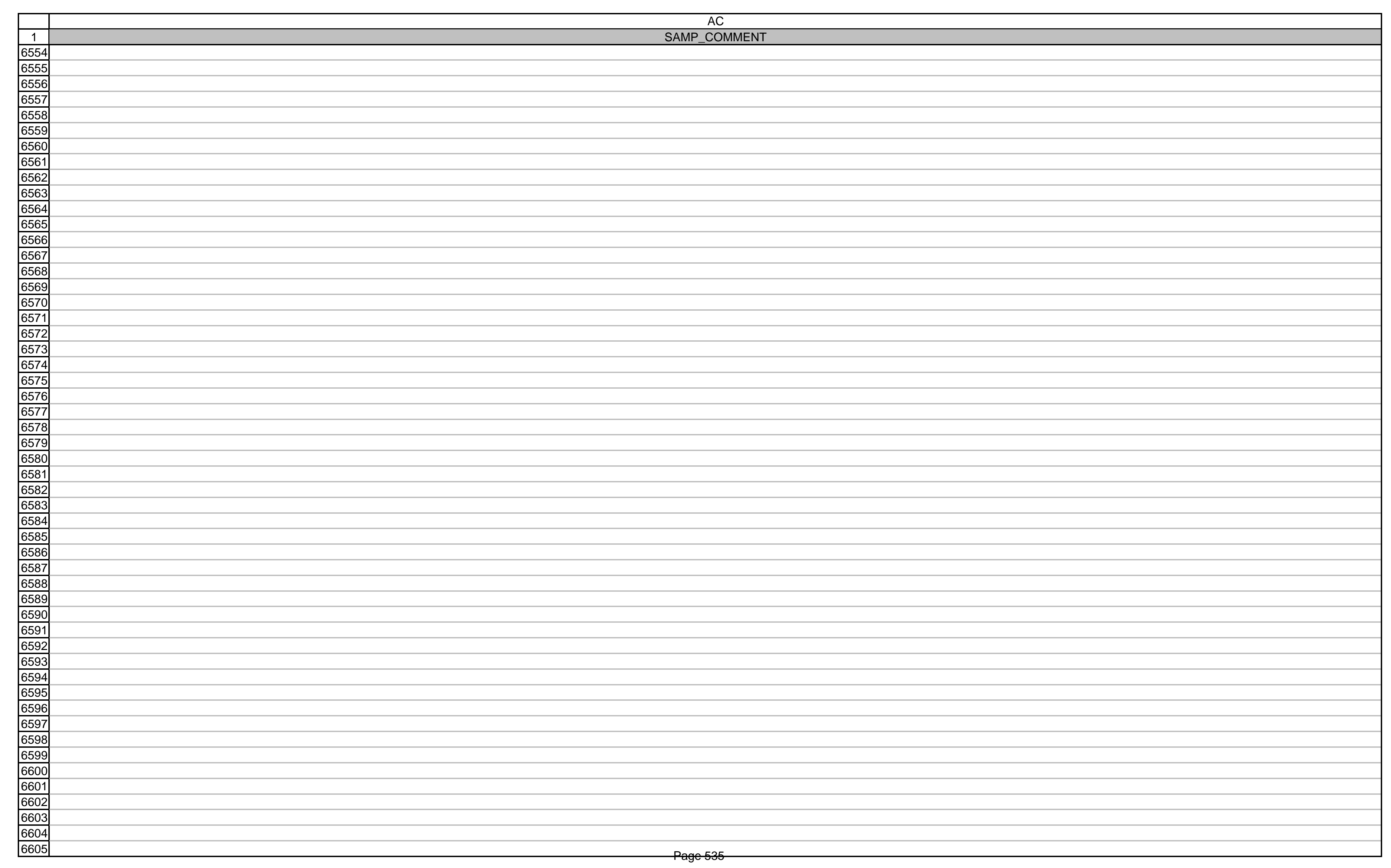




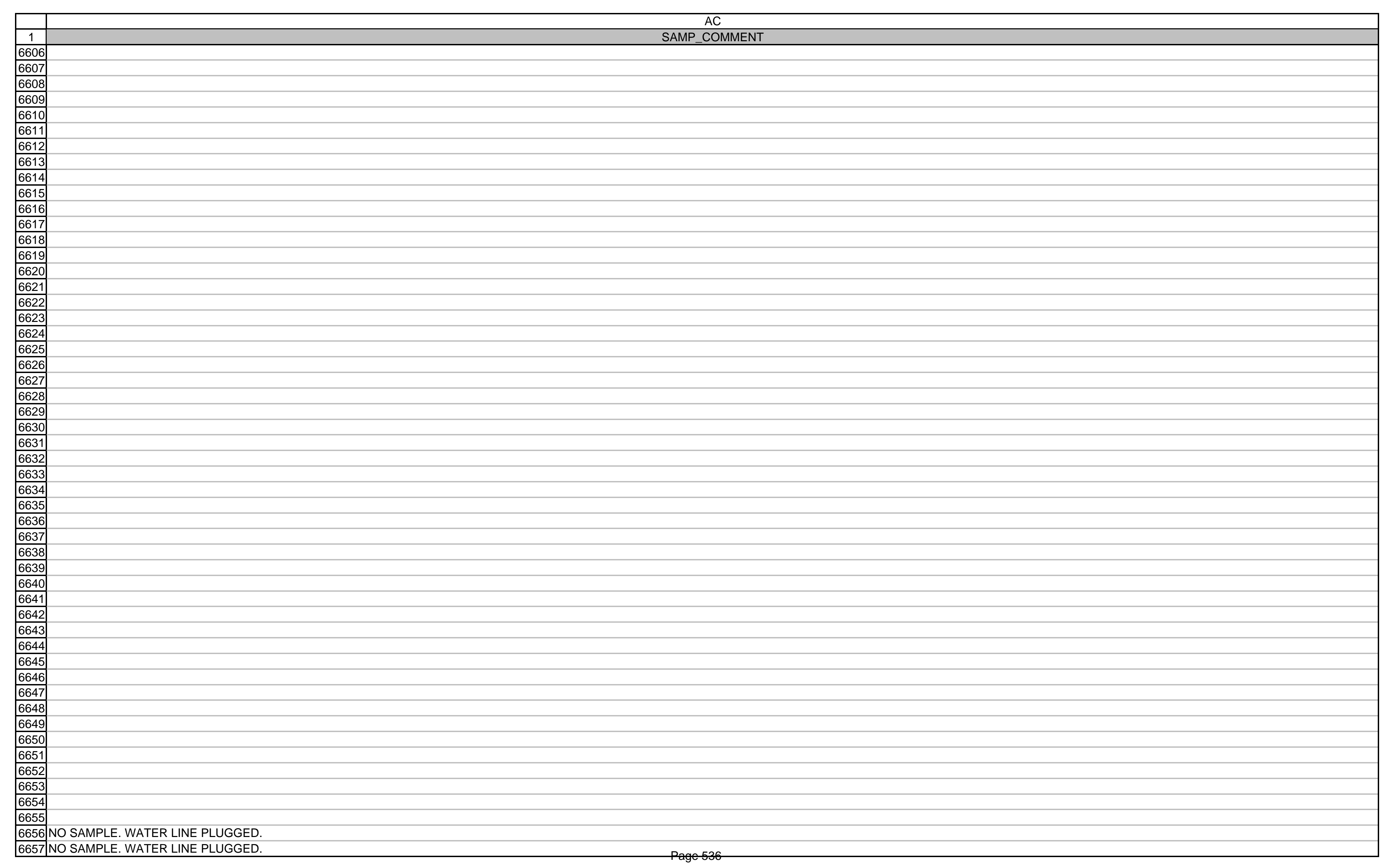




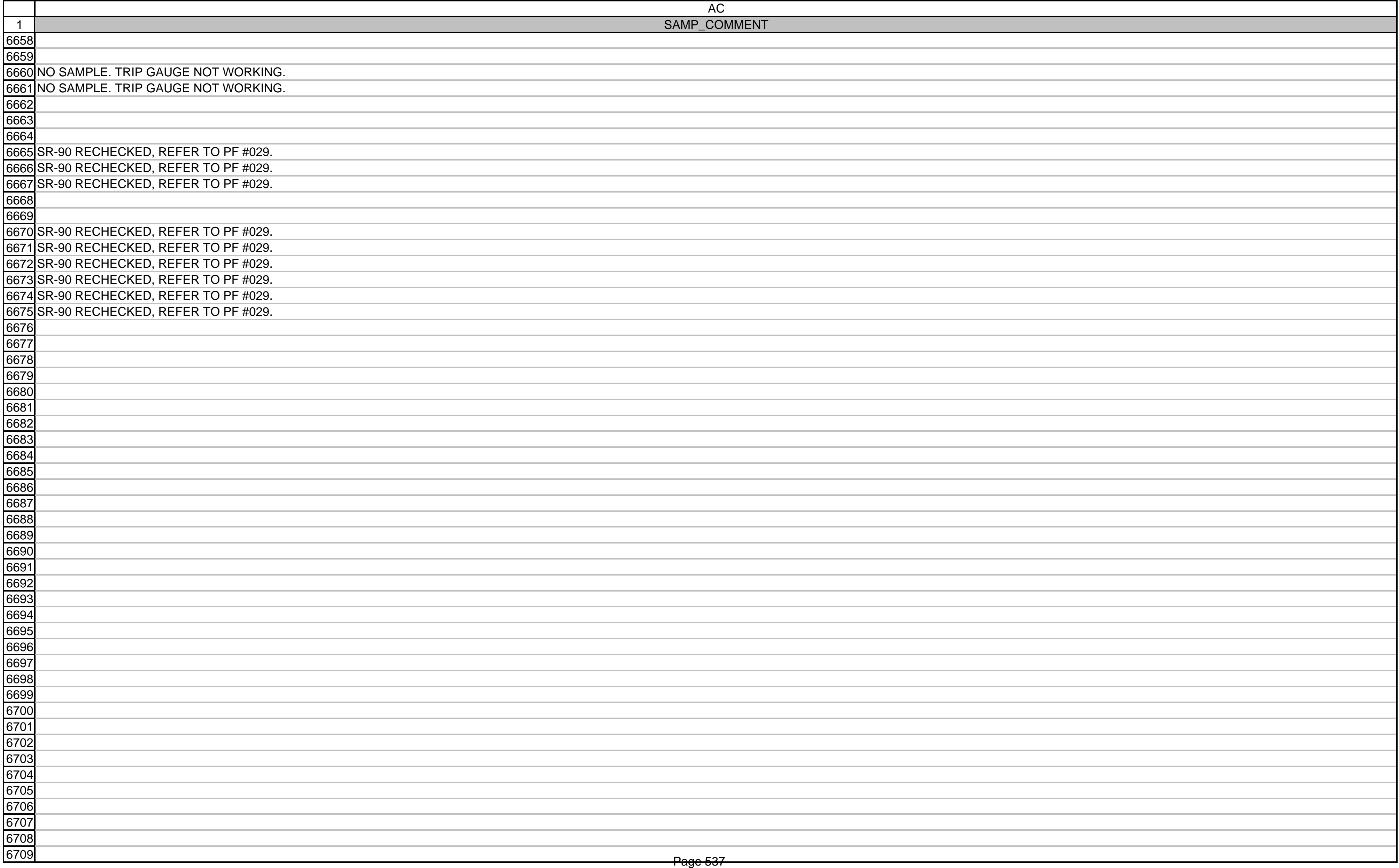




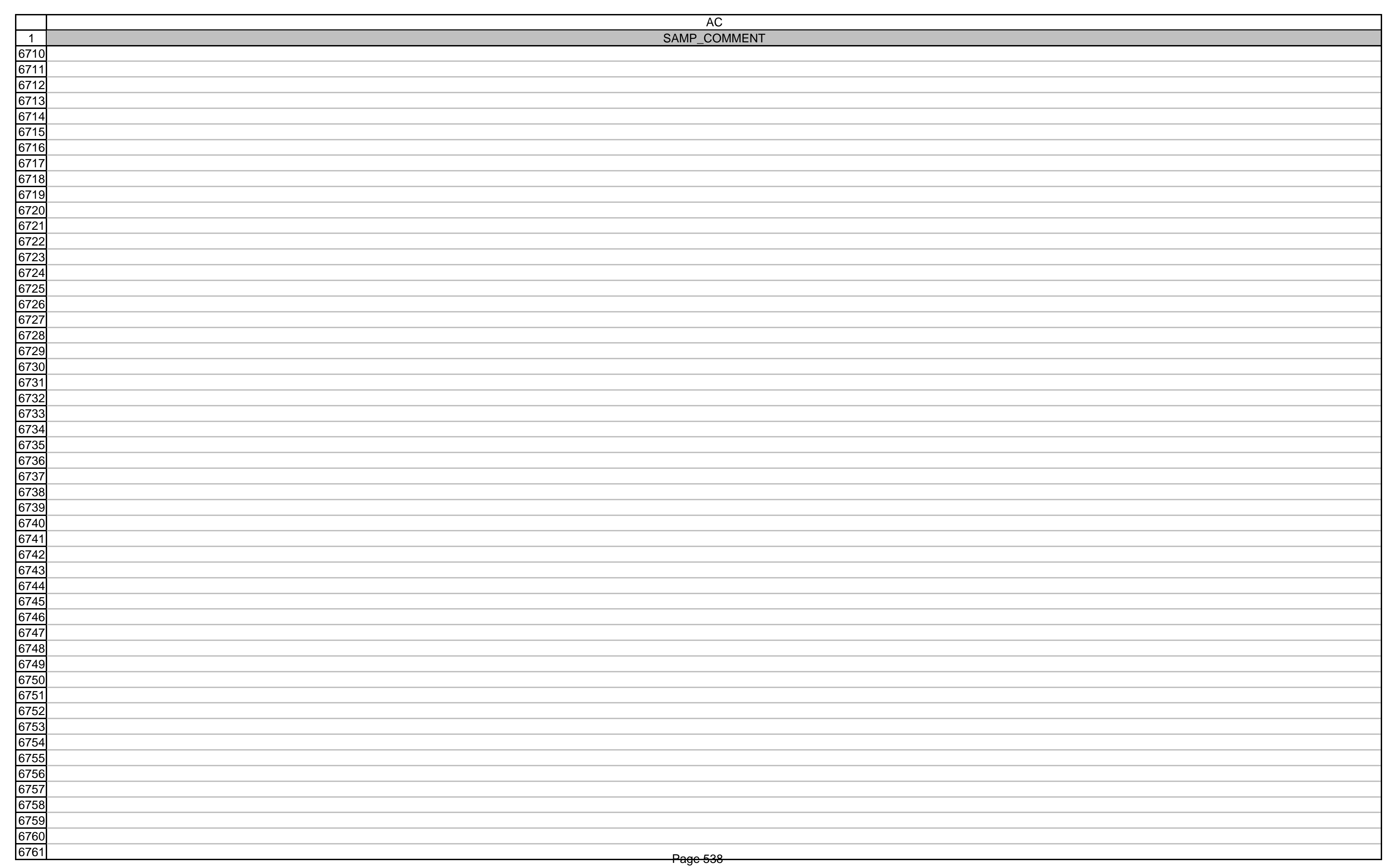


6763 NO SAMPLE. SYSTEM PLUGGED.

\begin{tabular}{|l|l|}
6764 & NO SAMPLE. SYSTEM PLUGGED. \\
\hline 6765 & NO SAMPLE. SYSTEM PLUGGED. \\
\hline
\end{tabular}

\begin{tabular}{|l|}
\hline 6765 \\
\hline 6766 \\
\hline 6767 \\
\hline 6768 \\
\hline
\end{tabular}

\begin{tabular}{|l|}
\hline 6767 \\
\hline 6768 \\
\hline 6770 \\
\hline
\end{tabular}

\begin{tabular}{|l|}
\hline 6769 \\
\hline 6770 \\
\hline 6771 \\
\hline 6772
\end{tabular}

6771

6772

6773

6774 NO SAMPLE. WATER NOT FLOWING.

6775 NO SAMPLE. WATER NOT FLOWING.

\begin{tabular}{|l|}
\hline 6776 \\
\hline 6777 \\
\hline 6778 \\
\hline
\end{tabular}

\begin{tabular}{|l|}
\hline 6777 \\
\hline 6778 \\
\hline 6779 \\
\hline
\end{tabular}

\begin{tabular}{|l|}
\hline 6778 \\
\hline 6779 \\
\hline 6780
\end{tabular}

6779

\begin{tabular}{|l|}
6780 \\
\hline 6781 \\
\hline 6782 \\
\hline
\end{tabular}

6781

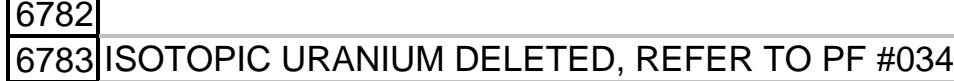

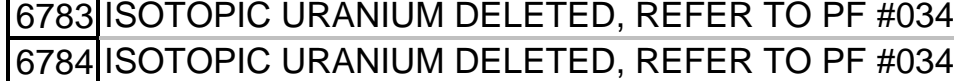

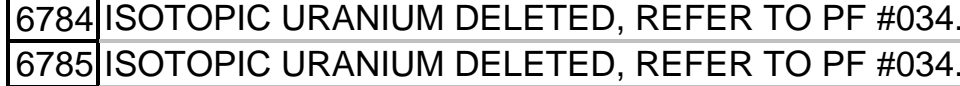

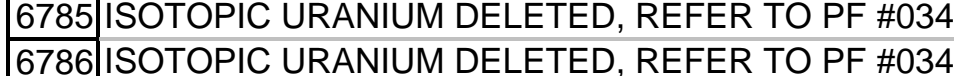

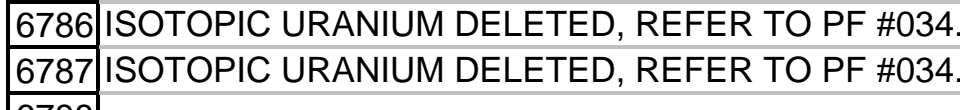

6788

6789

\begin{tabular}{|l|}
6790 \\
\hline 6791 \\
\hline
\end{tabular}

6791

\begin{tabular}{l}
6792 \\
\hline 6793 \\
\hline 6721
\end{tabular}

6793

\begin{tabular}{|l|}
6794 \\
\hline 6795 \\
\hline
\end{tabular}

\begin{tabular}{|l|}
6794 \\
\hline 6795 \\
\hline 6796 \\
\hline
\end{tabular}

$\frac{6795}{6796}$

6796

\begin{tabular}{l}
6797 \\
\hline 6798 \\
\hline 6799 \\
\hline
\end{tabular}

6798

\begin{tabular}{|l|}
\hline 6799 \\
\hline 6800 \\
\hline 6801 \\
\hline 6802 \\
\hline
\end{tabular}

6801

$\begin{array}{r}6802 \\ \hline 6803 \\ \hline 6804 \\ \hline\end{array}$ 
6835 ALPHA MODIFIED 12/21/89, REFER TO PF \#023. ISO. U RECHECKED(2/16/90), REFER TO PF \#031. 6836 ALPHA MODIFIED 12/21/89, REFER TO PF \#023. ISO. U RECHECKED(2/16/90), REFER TO PF \#031. 6837 ALPHA MODIFIED 12/21/89, REFER TO PF \#023. ISO. U RECHECKED(2/16/90), REFER TO PF \#031. 6838 ALPHA MODIFIED 12/21/89, REFER TO PF \#023. ISO. U RECHECKED(2/16/90), REFER TO PF \#031. 6839 ALPHA MODIFIED 12/21/89, REFER TO PF \#023. ISO. U RECHECKED(2/16/90), REFER TO PF \#031. 6840 ALPHA MODIFIED 12/21/89, REFER TO PF \#023. ISO. U RECHECKED(2/16/90), REFER TO PF \#031. 


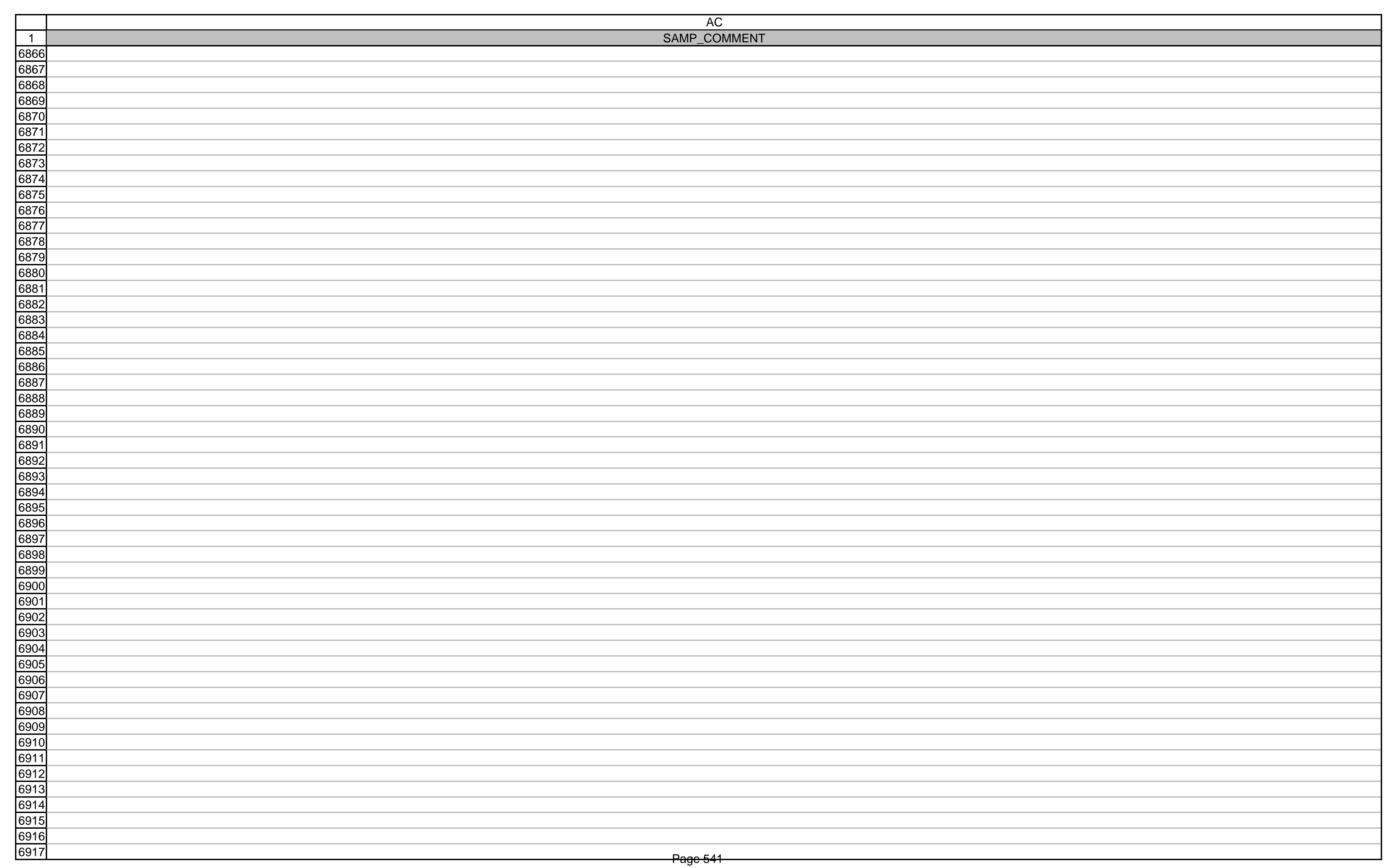




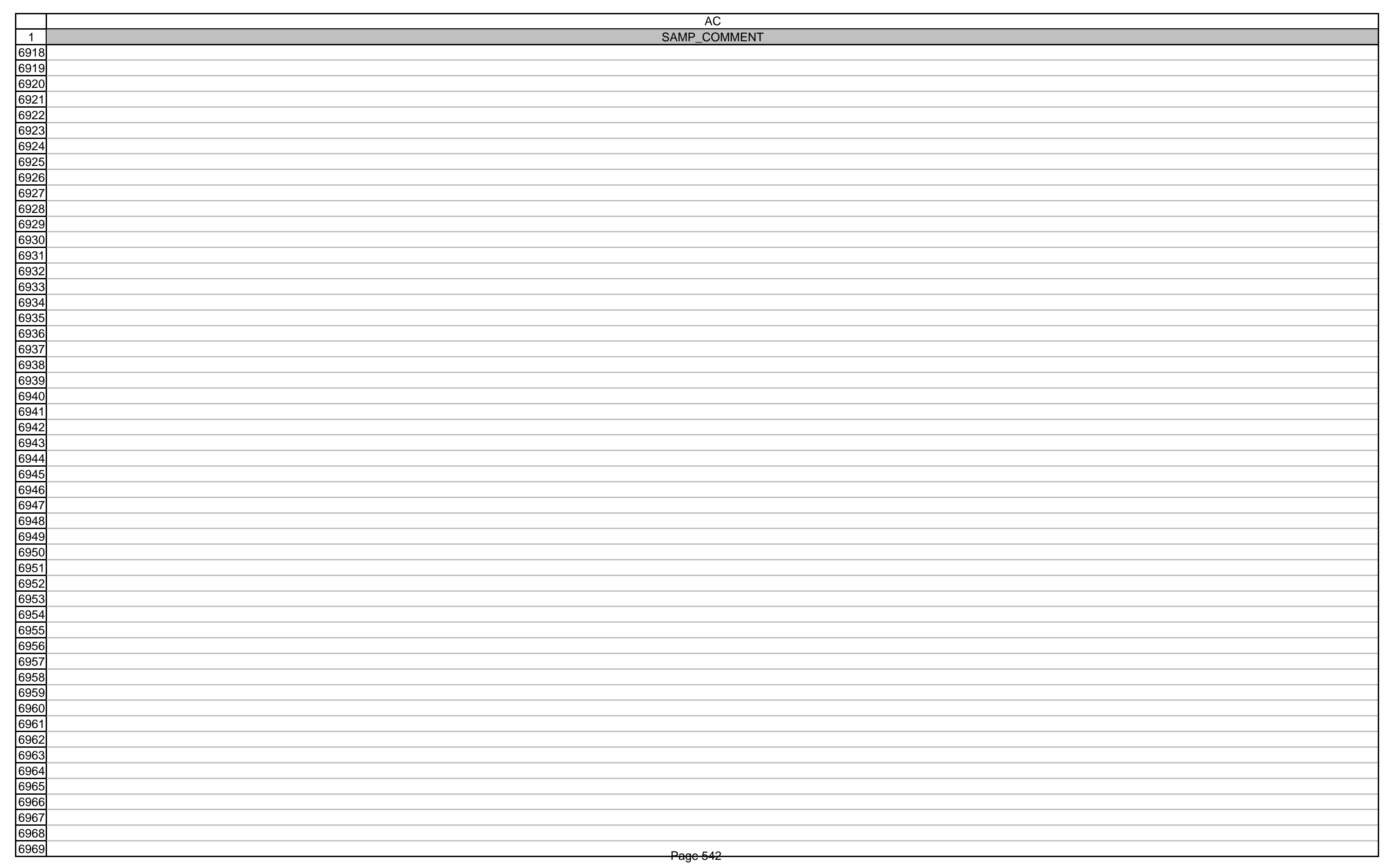


6973 ALPHA MODIFIED (2/8/90), REFER TO PF \#034.

6973 ALPHA MODIFIED (2/8/90), REFER TO PF \#034.

6974 ALPHA MODIFIED (2/8/90), REFER TO PF \#034.

6975 ALPHA MODIFIED (2/8/90), REFER TO PF \#034.

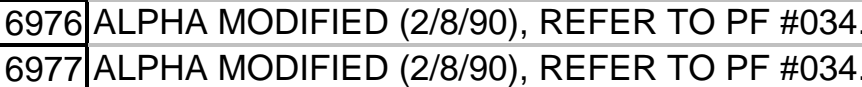

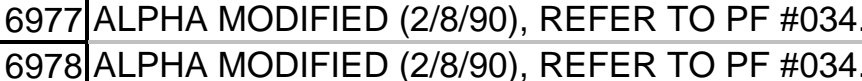

6978 ALPHA MODIFIED (2/8/90), REFER TO PF \#034.

6980

6981

6982

6983

6984

6984

$\frac{6985}{6986}$

6987

6987

6988

6989

6990

\begin{tabular}{|l|}
6991 \\
\hline 6992 \\
\hline 6993 \\
\hline
\end{tabular}

\begin{tabular}{|l|}
6992 \\
\hline 6993 \\
\hline 6994 \\
\hline
\end{tabular}

6994

6995

\begin{tabular}{l}
6996 \\
\hline 6997 \\
\hline 6998 \\
\hline
\end{tabular}

6997

\begin{tabular}{|l|}
\hline 6998 \\
\hline 6999 \\
\hline 7000 \\
\hline
\end{tabular}

7000

\begin{tabular}{|l|}
7000 \\
\hline 7001 \\
\hline 7002 \\
\hline
\end{tabular}

\begin{tabular}{|l|}
7001 \\
\hline 7002 \\
\hline 7003 \\
\hline
\end{tabular}

\begin{tabular}{l}
7002 \\
\hline 7003 \\
\hline 7004 \\
\hline
\end{tabular}

\begin{tabular}{|l|}
\hline 7003 \\
\hline 7004 \\
\hline 7005 \\
\hline
\end{tabular}

7005 NO SAMPLE. NO WATER FLOW.

7006 NO SAMPLE. NO WATER FLOW.

\begin{tabular}{|l|}
\hline 7006 \\
\hline 7007 \\
\hline 7008 \\
\hline 7009 \\
\hline
\end{tabular}

7009 


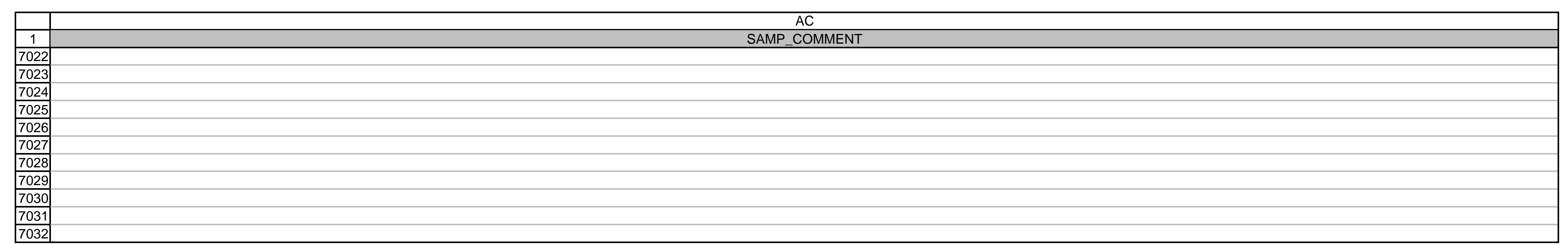




\begin{tabular}{|c|c|c|c|c|c|c|}
\hline & $\mathrm{AD}$ & $\mathrm{AE}$ & $\mathrm{AF}$ & $\mathrm{AG}$ & $\mathrm{AH}$ & $\mathrm{Al}$ \\
\hline 1 & RESULT_COMMENT & COLL_SAMP_SIZE & COLL_SAMP_SIZE_UNITS & DIST_CLASS & OWNER_ID & COLLECTION_PURPOSE \\
\hline 2 & & & & & SESPMNT & $R$ \\
\hline 3 & & & & & SESPMNT & $\mathrm{R}$ \\
\hline 4 & & & & & SESPMNT & $\mathrm{R}$ \\
\hline 5 & & & & OFFSITE & SESPMNT & $\mathrm{R}$ \\
\hline 6 & & & & & SESPMNT & $\mathrm{R}$ \\
\hline 7 & & & & & SESPMNT & $\mathrm{R}$ \\
\hline 8 & & & & OFFSITE & SESPMNT & $\mathrm{R}$ \\
\hline 9 & & & & OFFSITE & SESPMNT & $\mathrm{R}$ \\
\hline 10 & & & & & SESPMNT & $\mathrm{R}$ \\
\hline 11 & & & & & SESPMNT & $\mathrm{R}$ \\
\hline 12 & & & & & SESPMNT & $\mathrm{R}$ \\
\hline 13 & & & & OFFSITE & SESPMNT & $\mathrm{R}$ \\
\hline 14 & & & & OFFSITE & SESPMNT & $\mathrm{R}$ \\
\hline 15 & & & & OFFSITE & SESPMNT & $\mathrm{R}$ \\
\hline 16 & & & & & SESPMNT & $\mathrm{R}$ \\
\hline 17 & & & & & SESPMNT & $\mathrm{R}$ \\
\hline 18 & & & & ONSITE & SESPMNT & $\mathrm{R}$ \\
\hline 19 & & & & & SESPMNT & $\mathrm{R}$ \\
\hline 20 & & & & & SESPMNT & $\mathrm{R}$ \\
\hline 21 & & & & & SESPMNT & $\mathrm{R}$ \\
\hline 22 & & & & & SESPMNT & $\mathrm{R}$ \\
\hline 23 & & & & & SESPMNT & $\mathrm{R}$ \\
\hline 24 & & & & & SESPMNT & $\mathrm{R}$ \\
\hline 25 & & & & OFFSITE & SESPMNT & $\mathrm{R}$ \\
\hline 26 & & & & OFFSITE & SESPMNT & $\mathrm{R}$ \\
\hline 27 & & & & OFFSITE & SESPMNT & $\mathrm{R}$ \\
\hline 28 & & & & OFFSITE & SESPMNT & $\mathrm{R}$ \\
\hline 29 & & & & OFFSITE & SESPMNT & $\mathrm{R}$ \\
\hline 30 & & & & OFFSITE & SESPMNT & $\mathrm{R}$ \\
\hline 31 & & & & & SESPMNT & $\mathrm{R}$ \\
\hline 32 & & & & & SESPMNT & $\mathrm{R}$ \\
\hline 33 & & & & OFFSITE & SESPMNT & $\mathrm{R}$ \\
\hline 34 & & & & OFFSITE & SESPMNT & $\mathrm{R}$ \\
\hline 35 & & & & OFFSITE & SESPMNT & $\mathrm{R}$ \\
\hline 36 & & & & & SESPMNT & $\mathrm{R}$ \\
\hline 37 & & & & & SESPMNT & $\mathrm{R}$ \\
\hline 38 & & & & & SESPMNT & $\mathrm{R}$ \\
\hline 39 & & & & OFFSITE & SESPMNT & $\mathrm{R}$ \\
\hline 40 & & & & & SESPMNT & $\mathrm{R}$ \\
\hline 41 & & & & & SESPMNT & $\mathrm{R}$ \\
\hline 42 & & & & OFFSITE & SESPMNT & $\mathrm{R}$ \\
\hline 43 & & & & & SESPMNT & $\mathrm{R}$ \\
\hline 44 & & & & & SESPMNT & $\mathrm{R}$ \\
\hline 45 & & & & OFFSITE & SESPMNT & $\mathrm{R}$ \\
\hline 46 & & & & OFFSITE & SESPMNT & $\mathrm{R}$ \\
\hline 47 & & & & OFFSITE & SESPMNT & $\mathrm{R}$ \\
\hline 48 & & & & OFFSITE & SESPMNT & $\mathrm{R}$ \\
\hline 49 & & & & OFFSITE & SESPMNT & $\mathrm{R}$ \\
\hline 50 & & & & OFFSITE & SESPMNT & $\mathrm{R}$ \\
\hline 51 & & & & & SESPMNT & $\mathrm{R}$ \\
\hline 52 & & & & & SESPMNT & $\mathrm{R}$ \\
\hline 53 & & & & OFFSITE & SESBMNT & $R$ \\
\hline
\end{tabular}




\begin{tabular}{|c|c|c|c|c|c|c|}
\hline & $A D$ & $\mathrm{AE}$ & $\mathrm{AF}$ & $\mathrm{AG}$ & $\mathrm{AH}$ & $\mathrm{Al}$ \\
\hline 1 & RESULT_COMMENT & COLL_SAMP_SIZE & COLL_SAMP_SIZE_UNITS & DIST_CLASS & OWNER_ID & COLLECTION_PURPOSE \\
\hline 54 & & & & OFFSITE & SESPMNT & $\mathrm{R}$ \\
\hline 55 & & & & & SESPMNT & $\mathrm{R}$ \\
\hline 56 & & & & & SESPMNT & $\mathrm{R}$ \\
\hline 57 & & & & OFFSITE & SESPMNT & $\mathrm{R}$ \\
\hline 58 & & & & & SESPMNT & $\mathrm{R}$ \\
\hline 59 & & & & & SESPMNT & $\mathrm{R}$ \\
\hline 60 & & & & & SESPMNT & $\mathrm{R}$ \\
\hline 61 & & & & OFFSITE & SESPMNT & $\mathrm{R}$ \\
\hline 62 & & & & OFFSITE & SESPMNT & $\mathrm{R}$ \\
\hline 63 & & & & & SESPMNT & $\mathrm{R}$ \\
\hline 64 & & & & & SESPMNT & $\mathrm{R}$ \\
\hline 65 & & & & & SESPMNT & $\mathrm{R}$ \\
\hline 66 & & & & & SESPMNT & $\mathrm{R}$ \\
\hline 67 & & & & OFFSITE & SESPMNT & $\mathrm{R}$ \\
\hline 68 & & & & OFFSITE & SESPMNT & $\mathrm{R}$ \\
\hline 69 & & & & OFFSITE & SESPMNT & $\mathrm{R}$ \\
\hline 70 & & & & OFFSITE & SESPMNT & $\mathrm{R}$ \\
\hline 71 & & & & OFFSITE & SESPMNT & $\mathrm{R}$ \\
\hline 72 & & & & & SESPMNT & $\mathrm{R}$ \\
\hline 73 & & & & & SESPMNT & $\mathrm{R}$ \\
\hline 74 & & & & OFFSITE & SESPMNT & $\mathrm{R}$ \\
\hline 75 & & & & & SESPMNT & $\mathrm{R}$ \\
\hline 76 & & & & & SESPMNT & $\mathrm{R}$ \\
\hline 77 & & & & & SESPMNT & $\mathrm{R}$ \\
\hline 78 & & & & & SESPMNT & $\mathrm{R}$ \\
\hline 79 & & & & & SESPMNT & $\mathrm{R}$ \\
\hline 80 & & & & & SESPMNT & $\mathrm{R}$ \\
\hline 81 & & & & OFFSITE & SESPMNT & $\mathrm{R}$ \\
\hline 82 & & & & & SESPMNT & $\mathrm{R}$ \\
\hline 83 & & & & & SESPMNT & $R$ \\
\hline 84 & & & & & SESPMNT & $\mathrm{R}$ \\
\hline 85 & & & & ONSITE & SESPMNT & $\mathrm{R}$ \\
\hline 86 & & & & & SESPMNT & $\mathrm{R}$ \\
\hline 87 & & & & & SESPMNT & $\mathrm{R}$ \\
\hline 88 & & & & & SESPMNT & $\mathrm{R}$ \\
\hline 89 & & & & & SESPMNT & $\mathrm{R}$ \\
\hline 90 & & & & & SESPMNT & $\mathrm{R}$ \\
\hline 91 & & & & & SESPMNT & $\mathrm{R}$ \\
\hline 92 & & & & & SESPMNT & $\mathrm{R}$ \\
\hline 93 & & & & OFFSITE & SESPMNT & $R$ \\
\hline 94 & & & & OFFSITE & SESPMNT & $\mathrm{R}$ \\
\hline 95 & & & & OFFSITE & SESPMNT & $\mathrm{R}$ \\
\hline 96 & & & & OFFSITE & SESPMNT & $\mathrm{R}$ \\
\hline 97 & & & & & SESPMNT & $\mathrm{R}$ \\
\hline 98 & & & & & SESPMNT & $\mathrm{R}$ \\
\hline 99 & & & & & SESPMNT & $\mathrm{R}$ \\
\hline 100 & & & & OFFSITE & SESPMNT & $\mathrm{R}$ \\
\hline 101 & & & & & SESPMNT & $\mathrm{R}$ \\
\hline 102 & & & & & SESPMNT & $\mathrm{R}$ \\
\hline 103 & & & & & SESPMNT & $\mathrm{R}$ \\
\hline 104 & & & & & SESPMNT & $\mathrm{R}$ \\
\hline 105 & & & & & SESBMNT & $R$ \\
\hline
\end{tabular}




\begin{tabular}{|c|c|c|c|c|c|c|}
\hline & $A D$ & $\mathrm{AE}$ & $\mathrm{AF}$ & $A G$ & $\mathrm{AH}$ & $\mathrm{Al}$ \\
\hline 1 & RESULT_COMMENT & COLL_SAMP_SIZE & COLL_SAMP_SIZE_UNITS & DIST_CLASS & OWNER_ID & COLLECTION_PURPOSE \\
\hline 106 & & & & OFFSITE & SESPMNT & $\mathrm{R}$ \\
\hline 107 & & & & & SESPMNT & $\mathrm{R}$ \\
\hline 108 & & & & & SESPMNT & $\mathrm{R}$ \\
\hline 109 & & & & & SESPMNT & $\mathrm{R}$ \\
\hline 110 & & & & & SESPMNT & $\mathrm{R}$ \\
\hline 111 & & & & OFFSITE & SESPMNT & $\mathrm{R}$ \\
\hline 112 & & & & OFFSITE & SESPMNT & $\mathrm{R}$ \\
\hline 113 & & & & OFFSITE & SESPMNT & $\mathrm{R}$ \\
\hline 114 & & & & OFFSITE & SESPMNT & $\mathrm{R}$ \\
\hline 115 & & & & & SESPMNT & $\mathrm{R}$ \\
\hline 116 & & & & & SESPMNT & $\mathrm{R}$ \\
\hline 117 & & & & OFFSITE & SESPMNT & $\mathrm{R}$ \\
\hline 118 & & & & & SESPMNT & $\mathrm{R}$ \\
\hline 119 & & & & & SESPMNT & $\mathrm{R}$ \\
\hline 120 & & & & & SESPMNT & $\mathrm{R}$ \\
\hline 121 & & & & & SESPMNT & $\mathrm{R}$ \\
\hline 122 & & & & & SESPMNT & $\mathrm{R}$ \\
\hline 123 & & & & & SESPMNT & $\mathrm{R}$ \\
\hline 124 & & & & & SESPMNT & $\mathrm{R}$ \\
\hline 125 & & & & OFFSITE & SESPMNT & $\mathrm{R}$ \\
\hline 126 & & & & & SESPMNT & $\mathrm{R}$ \\
\hline 127 & & & & & SESPMNT & $\mathrm{R}$ \\
\hline 128 & & & & & SESPMNT & $\mathrm{R}$ \\
\hline 129 & & & & & SESPMNT & $\mathrm{R}$ \\
\hline 130 & & & & OFFSITE & SESPMNT & $\mathrm{R}$ \\
\hline 131 & & & & OFFSITE & SESPMNT & $\mathrm{R}$ \\
\hline 132 & & & & OFFSITE & SESPMNT & $\mathrm{R}$ \\
\hline 133 & & & & OFFSITE & SESPMNT & $\mathrm{R}$ \\
\hline 134 & & & & & SESPMNT & $\mathrm{R}$ \\
\hline 135 & & & & & SESPMNT & $\mathrm{R}$ \\
\hline 136 & & & & & SESPMNT & $\mathrm{R}$ \\
\hline 137 & & & & OFFSITE & SESPMNT & $\mathrm{R}$ \\
\hline 138 & & & & & SESPMNT & $\mathrm{R}$ \\
\hline 139 & & & & & SESPMNT & $\mathrm{R}$ \\
\hline 140 & & & & & SESPMNT & $\mathrm{R}$ \\
\hline 141 & & & & & SESPMNT & $\mathrm{R}$ \\
\hline 142 & & & & RIVER_SHORELINE & SESPMNT & $\mathrm{R}$ \\
\hline 143 & & & & & SESPMNT & $\mathrm{R}$ \\
\hline 144 & & & & & SESPMNT & $\mathrm{R}$ \\
\hline 145 & & & & ONSITE & SESPMNT & $\mathrm{R}$ \\
\hline 146 & & & & & SESPMNT & $\mathrm{R}$ \\
\hline 147 & & & & & SESPMNT & $\mathrm{R}$ \\
\hline 148 & & & & & SESPMNT & $\mathrm{R}$ \\
\hline 149 & & & & & SESPMNT & $\mathrm{R}$ \\
\hline 150 & & & & & SESPMNT & $\mathrm{R}$ \\
\hline 151 & & & & OFFSITE & SESPMNT & $\mathrm{R}$ \\
\hline 152 & & & & OFFSITE & SESPMNT & $\mathrm{R}$ \\
\hline 153 & & & & OFFSITE & SESPMNT & $\mathrm{R}$ \\
\hline 154 & & & & OFFSITE & SESPMNT & $\mathrm{R}$ \\
\hline 155 & & & & OFFSITE & SESPMNT & $\mathrm{R}$ \\
\hline 156 & & & & OFFSITE & SESPMNT & $\mathrm{R}$ \\
\hline 157 & & & & & SESBMNT & ${ }_{7} R$ \\
\hline
\end{tabular}




\begin{tabular}{|c|c|c|c|c|c|c|}
\hline & $A D$ & $\mathrm{AE}$ & $\mathrm{AF}$ & $A G$ & $\mathrm{AH}$ & $\mathrm{Al}$ \\
\hline 1 & RESULT_COMMENT & COLL_SAMP_SIZE & COLL_SAMP_SIZE_UNITS & DIST_CLASS & OWNER_ID & COLLECTION_PURPOSE \\
\hline 158 & & & & & SESPMNT & $\mathrm{R}$ \\
\hline 159 & & & & & SESPMNT & $\mathrm{R}$ \\
\hline 160 & & & & & SESPMNT & $\mathrm{R}$ \\
\hline 161 & & & & & SESPMNT & $\mathrm{R}$ \\
\hline 162 & & & & & SESPMNT & $\mathrm{R}$ \\
\hline 163 & & & & OFFSITE & SESPMNT & $\mathrm{R}$ \\
\hline 164 & & & & OFFSITE & SESPMNT & $\mathrm{R}$ \\
\hline 165 & & & & OFFSITE & SESPMNT & $\mathrm{R}$ \\
\hline 166 & & & & OFFSITE & SESPMNT & $\mathrm{R}$ \\
\hline 167 & & & & OFFSITE & SESPMNT & $\mathrm{R}$ \\
\hline 168 & & & & & SESPMNT & $\mathrm{R}$ \\
\hline 169 & & & & OFFSITE & SESPMNT & $\mathrm{R}$ \\
\hline 170 & & & & & SESPMNT & $\mathrm{R}$ \\
\hline 171 & & & & & SESPMNT & $\mathrm{R}$ \\
\hline 172 & & & & & SESPMNT & $\mathrm{R}$ \\
\hline 173 & & & & & SESPMNT & $\mathrm{R}$ \\
\hline 174 & & & & & SESPMNT & $\mathrm{R}$ \\
\hline 175 & & & & & SESPMNT & $\mathrm{R}$ \\
\hline 176 & & & & OFFSITE & SESPMNT & $\mathrm{R}$ \\
\hline 177 & & & & OFFSITE & SESPMNT & $\mathrm{R}$ \\
\hline 178 & & & & OFFSITE & SESPMNT & $\mathrm{R}$ \\
\hline 179 & & & & OFFSITE & SESPMNT & $\mathrm{R}$ \\
\hline 180 & & & & OFFSITE & SESPMNT & $\mathrm{R}$ \\
\hline 181 & & & & OFFSITE & SESPMNT & $\mathrm{R}$ \\
\hline 182 & & & & & SESPMNT & $\mathrm{R}$ \\
\hline 183 & & & & & SESPMNT & $\mathrm{R}$ \\
\hline 184 & & & & & SESPMNT & $\mathrm{R}$ \\
\hline 185 & & & & & SESPMNT & $\mathrm{R}$ \\
\hline 186 & & & & & SESPMNT & $\mathrm{R}$ \\
\hline 187 & & & & & SESPMNT & $\mathrm{R}$ \\
\hline 188 & & & & ONSITE & SESPMNT & $\mathrm{R}$ \\
\hline 189 & & & & & SESPMNT & $\mathrm{R}$ \\
\hline 190 & & & & & SESPMNT & $\mathrm{R}$ \\
\hline 191 & & & & & SESPMNT & $\mathrm{R}$ \\
\hline 192 & & & & & SESPMNT & $\mathrm{R}$ \\
\hline 193 & & & & & SESPMNT & $\mathrm{R}$ \\
\hline 194 & & & & & SESPMNT & $\mathrm{R}$ \\
\hline 195 & & & & OFFSITE & SESPMNT & $\mathrm{R}$ \\
\hline 196 & & & & OFFSITE & SESPMNT & $\mathrm{R}$ \\
\hline 197 & & & & OFFSITE & SESPMNT & $\mathrm{R}$ \\
\hline 198 & & & & OFFSITE & SESPMNT & $\mathrm{R}$ \\
\hline 199 & & & & & SESPMNT & $\mathrm{R}$ \\
\hline 200 & & & & & SESPMNT & $\mathrm{R}$ \\
\hline 201 & & & & & SESPMNT & $\mathrm{R}$ \\
\hline 202 & & & & OFFSITE & SESPMNT & $\mathrm{R}$ \\
\hline 203 & & & & & SESPMNT & $\mathrm{R}$ \\
\hline 204 & & & & & SESPMNT & $\mathrm{R}$ \\
\hline 205 & & & & & SESPMNT & $\mathrm{R}$ \\
\hline 206 & & & & & SESPMNT & $\mathrm{R}$ \\
\hline 207 & & & & & SESPMNT & $R$ \\
\hline 208 & & & & OFFSITE & SESPMNT & $\mathrm{R}$ \\
\hline 209 & & & & OFFSITE & SESBMNT & ${ }_{8}^{R}$ \\
\hline
\end{tabular}




\begin{tabular}{|c|c|c|c|c|c|c|}
\hline & $A D$ & $\mathrm{AE}$ & $\mathrm{AF}$ & $A G$ & $\mathrm{AH}$ & $\mathrm{Al}$ \\
\hline 1 & RESULT_COMMENT & COLL_SAMP_SIZE & COLL_SAMP_SIZE_UNITS & DIST_CLASS & OWNER_ID & COLLECTION_PURPOSE \\
\hline 210 & & & & OFFSITE & SESPMNT & $\mathrm{R}$ \\
\hline 211 & & & & OFFSITE & SESPMNT & $\mathrm{R}$ \\
\hline 212 & & & & OFFSITE & SESPMNT & $\mathrm{R}$ \\
\hline 213 & & & & OFFSITE & SESPMNT & $\mathrm{R}$ \\
\hline 214 & & & & & SESPMNT & $\mathrm{R}$ \\
\hline 215 & & & & OFFSITE & SESPMNT & $\mathrm{R}$ \\
\hline 216 & & & & & SESPMNT & $\mathrm{R}$ \\
\hline 217 & & & & OFFSITE & SESPMNT & $\mathrm{R}$ \\
\hline 218 & & & & OFFSITE & SESPMNT & $\mathrm{R}$ \\
\hline 219 & & & & OFFSITE & SESPMNT & $\mathrm{R}$ \\
\hline 220 & & & & OFFSITE & SESPMNT & $\mathrm{R}$ \\
\hline 221 & & & & & SESPMNT & $\mathrm{R}$ \\
\hline 222 & & & & & SESPMNT & $\mathrm{R}$ \\
\hline 223 & & & & & SESPMNT & $\mathrm{R}$ \\
\hline 224 & & & & ONSITE & SESPMNT & $\mathrm{R}$ \\
\hline 225 & & & & & SESPMNT & $\mathrm{R}$ \\
\hline 226 & & & & & SESPMNT & $\mathrm{R}$ \\
\hline 227 & & & & & SESPMNT & $\mathrm{R}$ \\
\hline 228 & & & & OFFSITE & SESPMNT & $\mathrm{R}$ \\
\hline 229 & & & & OFFSITE & SESPMNT & $\mathrm{R}$ \\
\hline 230 & & & & OFFSITE & SESPMNT & $\mathrm{R}$ \\
\hline 231 & & & & OFFSITE & SESPMNT & $\mathrm{R}$ \\
\hline 232 & & & & & SESPMNT & $\mathrm{R}$ \\
\hline 233 & & & & OFFSITE & SESPMNT & $\mathrm{R}$ \\
\hline 234 & & & & & SESPMNT & $\mathrm{R}$ \\
\hline 235 & & & & OFFSITE & SESPMNT & $\mathrm{R}$ \\
\hline 236 & & & & OFFSITE & SESPMNT & $\mathrm{R}$ \\
\hline 237 & & & & OFFSITE & SESPMNT & $\mathrm{R}$ \\
\hline 238 & & & & OFFSITE & SESPMNT & $\mathrm{R}$ \\
\hline 239 & & & & OFFSITE & SESPMNT & $\mathrm{R}$ \\
\hline 240 & & & & OFFSITE & SESPMNT & $\mathrm{R}$ \\
\hline 241 & & & & & SESPMNT & $\mathrm{R}$ \\
\hline 242 & & & & & SESPMNT & $\mathrm{R}$ \\
\hline 243 & & & & & SESPMNT & $\mathrm{R}$ \\
\hline 244 & & & & & SESPMNT & $\mathrm{R}$ \\
\hline 245 & & & & & SESPMNT & $\mathrm{R}$ \\
\hline 246 & & & & OFFSITE & SESPMNT & $\mathrm{R}$ \\
\hline 247 & & & & OFFSITE & SESPMNT & $\mathrm{R}$ \\
\hline 248 & & & & OFFSITE & SESPMNT & $\mathrm{R}$ \\
\hline 249 & & & & OFFSITE & SESPMNT & $\mathrm{R}$ \\
\hline 250 & & & & & SESPMNT & $\mathrm{R}$ \\
\hline 251 & & & & & SESPMNT & $\mathrm{R}$ \\
\hline 252 & & & & & SESPMNT & $\mathrm{R}$ \\
\hline 253 & & & & & SESPMNT & $\mathrm{R}$ \\
\hline 254 & & & & & SESPMNT & $\mathrm{R}$ \\
\hline 255 & & & & & SESPMNT & $\mathrm{R}$ \\
\hline 256 & & & & ONSITE & SESPMNT & $\mathrm{R}$ \\
\hline 257 & & & & & SESPMNT & $\mathrm{R}$ \\
\hline 258 & & & & & SESPMNT & $\mathrm{R}$ \\
\hline 259 & & & & & SESPMNT & $R$ \\
\hline 260 & & & & & SESPMNT & $\mathrm{R}$ \\
\hline 261 & & & & & SESBMNT & ${ }_{0}^{R}$ \\
\hline
\end{tabular}




\begin{tabular}{|c|c|c|c|c|c|c|}
\hline & $A D$ & $\mathrm{AE}$ & $\mathrm{AF}$ & $A G$ & $\mathrm{AH}$ & $\mathrm{Al}$ \\
\hline 1 & RESULT_COMMENT & COLL_SAMP_SIZE & COLL_SAMP_SIZE_UNITS & DIST_CLASS & OWNER_ID & COLLECTION_PURPOSE \\
\hline 262 & & & & OFFSITE & SESPMNT & $\mathrm{R}$ \\
\hline 263 & & & & & SESPMNT & $\mathrm{R}$ \\
\hline 264 & & & & & SESPMNT & $\mathrm{R}$ \\
\hline 265 & & & & OFFSITE & SESPMNT & $\mathrm{R}$ \\
\hline 266 & & & & OFFSITE & SESPMNT & $\mathrm{R}$ \\
\hline 267 & & & & OFFSITE & SESPMNT & $\mathrm{R}$ \\
\hline 268 & & & & OFFSITE & SESPMNT & $\mathrm{R}$ \\
\hline 269 & & & & & SESPMNT & $\mathrm{R}$ \\
\hline 270 & & & & OFFSITE & SESPMNT & $\mathrm{R}$ \\
\hline 271 & & & & & SESPMNT & $\mathrm{R}$ \\
\hline 272 & & & & & SESPMNT & $\mathrm{R}$ \\
\hline 273 & & & & & SESPMNT & $\mathrm{R}$ \\
\hline 274 & & & & OFFSITE & SESPMNT & $\mathrm{R}$ \\
\hline 275 & & & & & SESPMNT & $\mathrm{R}$ \\
\hline 276 & & & & & SESPMNT & $\mathrm{R}$ \\
\hline 277 & & & & OFFSITE & SESPMNT & $\mathrm{R}$ \\
\hline 278 & & & & OFFSITE & SESPMNT & $\mathrm{R}$ \\
\hline 279 & & & & OFFSITE & SESPMNT & $\mathrm{R}$ \\
\hline 280 & & & & OFFSITE & SESPMNT & $\mathrm{R}$ \\
\hline 281 & & & & OFFSITE & SESPMNT & $\mathrm{R}$ \\
\hline 282 & & & & OFFSITE & SESPMNT & $\mathrm{R}$ \\
\hline 283 & & & & OFFSITE & SESPMNT & $\mathrm{R}$ \\
\hline 284 & & & & & SESPMNT & $\mathrm{R}$ \\
\hline 285 & & & & & SESPMNT & $\mathrm{R}$ \\
\hline 286 & & & & ONSITE & SESPMNT & $\mathrm{R}$ \\
\hline 287 & & & & & SESPMNT & $\mathrm{R}$ \\
\hline 288 & & & & & SESPMNT & $\mathrm{R}$ \\
\hline 289 & & & & OFFSITE & SESPMNT & $\mathrm{R}$ \\
\hline 290 & & & & OFFSITE & SESPMNT & $\mathrm{R}$ \\
\hline 291 & & & & & SESPMNT & $\mathrm{R}$ \\
\hline 292 & & & & & SESPMNT & $\mathrm{R}$ \\
\hline 293 & & & & & SESPMNT & $\mathrm{R}$ \\
\hline 294 & & & & & SESPMNT & $\mathrm{R}$ \\
\hline 295 & & & & OFFSITE & SESPMNT & $\mathrm{R}$ \\
\hline 296 & & & & OFFSITE & SESPMNT & $\mathrm{R}$ \\
\hline 297 & & & & OFFSITE & SESPMNT & $\mathrm{R}$ \\
\hline 298 & & & & OFFSITE & SESPMNT & $\mathrm{R}$ \\
\hline 299 & & & & OFFSITE & SESPMNT & $\mathrm{R}$ \\
\hline 300 & & & & & SESPMNT & $\mathrm{R}$ \\
\hline 301 & & & & & SESPMNT & $\mathrm{R}$ \\
\hline 302 & & & & OFFSITE & SESPMNT & $\mathrm{R}$ \\
\hline 303 & & & & & SESPMNT & $\mathrm{R}$ \\
\hline 304 & & & & & SESPMNT & $\mathrm{R}$ \\
\hline 305 & & & & ONSITE & SESPMNT & $\mathrm{R}$ \\
\hline 306 & & & & & SESPMNT & $\mathrm{R}$ \\
\hline 307 & & & & & SESPMNT & $\mathrm{R}$ \\
\hline 308 & & & & & SESPMNT & $\mathrm{R}$ \\
\hline 309 & & & & ONSITE & SESPMNT & $\mathrm{R}$ \\
\hline 310 & & & & ONSITE & SESPMNT & $\mathrm{R}$ \\
\hline 311 & & & & & SESPMNT & $R$ \\
\hline 312 & & & & & SESPMNT & $\mathrm{R}$ \\
\hline 313 & & & & & SESBMNT & $R$ \\
\hline
\end{tabular}




\begin{tabular}{|c|c|c|c|c|c|c|}
\hline & $\mathrm{AD}$ & $\mathrm{AE}$ & $\mathrm{AF}$ & AG & $\mathrm{AH}$ & $\mathrm{Al}$ \\
\hline 1 & RESULT_COMMENT & COLL_SAMP_SIZE & COLL_SAMP_SIZE_UNITS & DIST_CLASS & OWNER_ID & COLLECTION_PURPOSE \\
\hline 314 & & & & & SESPMNT & $\mathrm{R}$ \\
\hline 315 & & & & OFFSITE & SESPMNT & $\mathrm{R}$ \\
\hline 316 & & & & & SESPMNT & $\mathrm{R}$ \\
\hline 317 & & & & & SESPMNT & $\mathrm{R}$ \\
\hline 318 & & & & & SESPMNT & $\mathrm{R}$ \\
\hline 319 & & & & OFFSITE & SESPMNT & $\mathrm{R}$ \\
\hline 320 & & & & OFFSITE & SESPMNT & $\mathrm{R}$ \\
\hline 321 & & & & OFFSITE & SESPMNT & $\mathrm{R}$ \\
\hline 322 & & & & OFFSITE & SESPMNT & $\mathrm{R}$ \\
\hline 323 & & & & OFFSITE & SESPMNT & $\mathrm{R}$ \\
\hline 324 & & & & OFFSITE & SESPMNT & $\mathrm{R}$ \\
\hline 325 & & & & & SESPMNT & $\mathrm{R}$ \\
\hline 326 & & & & & SESPMNT & $\mathrm{R}$ \\
\hline 327 & & & & ONSITE & SESPMNT & $\mathrm{R}$ \\
\hline 328 & & & & & SESPMNT & $\mathrm{R}$ \\
\hline 329 & & & & & SESPMNT & $\mathrm{R}$ \\
\hline 330 & & & & & SESPMNT & $\mathrm{R}$ \\
\hline 331 & & & & OFFSITE & SESPMNT & $\mathrm{R}$ \\
\hline 332 & & & & OFFSITE & SESPMNT & $\mathrm{R}$ \\
\hline 333 & & & & OFFSITE & SESPMNT & $\mathrm{R}$ \\
\hline 334 & & & & OFFSITE & SESPMNT & $\mathrm{R}$ \\
\hline 335 & & & & OFFSITE & SESPMNT & $\mathrm{R}$ \\
\hline 336 & & & & OFFSITE & SESPMNT & $\mathrm{R}$ \\
\hline 337 & & & & & SESPMNT & $\mathrm{R}$ \\
\hline 338 & & & & ONSITE & SESPMNT & $\mathrm{R}$ \\
\hline 339 & & & & & SESPMNT & $\mathrm{R}$ \\
\hline 340 & & & & & SESPMNT & $\mathrm{R}$ \\
\hline 341 & & & & OFFSITE & SESPMNT & $\mathrm{R}$ \\
\hline 342 & & & & & SESPMNT & $\mathrm{R}$ \\
\hline 343 & & & & & SESPMNT & $\mathrm{R}$ \\
\hline 344 & & & & OFFSITE & SESPMNT & $\mathrm{R}$ \\
\hline 345 & & & & OFFSITE & SESPMNT & $\mathrm{R}$ \\
\hline 346 & & & & OFFSITE & SESPMNT & $\mathrm{R}$ \\
\hline 347 & & & & OFFSITE & SESPMNT & $\mathrm{R}$ \\
\hline 348 & & & & OFFSITE & SESPMNT & $\mathrm{R}$ \\
\hline 349 & & & & OFFSITE & SESPMNT & $\mathrm{R}$ \\
\hline 350 & & & & & SESPMNT & $\mathrm{R}$ \\
\hline 351 & & & & & SESPMNT & $\mathrm{R}$ \\
\hline 352 & & & & & SESPMNT & $\mathrm{R}$ \\
\hline 353 & & & & & SESPMNT & $\mathrm{R}$ \\
\hline 354 & & & & ONSITE & SESPMNT & $\mathrm{R}$ \\
\hline 355 & & & & & SESPMNT & $\mathrm{R}$ \\
\hline 356 & & & & & SESPMNT & $\mathrm{R}$ \\
\hline 357 & & & & ONSITE & SESPMNT & $\mathrm{R}$ \\
\hline 358 & & & & ONSITE & SESPMNT & $\mathrm{R}$ \\
\hline 359 & & & & & SESPMNT & $\mathrm{R}$ \\
\hline 360 & & & & & SESPMNT & $\mathrm{R}$ \\
\hline 361 & & & & OFFSITE & SESPMNT & $\mathrm{R}$ \\
\hline 362 & & & & OFFSITE & SESPMNT & $\mathrm{R}$ \\
\hline 363 & & & & OFFSITE & SESPMNT & $\mathrm{R}$ \\
\hline 364 & & & & OFFSITE & SESPMNT & $\mathrm{R}$ \\
\hline 365 & & & & OFFSITE & SESBMNT & $R$ \\
\hline
\end{tabular}




\begin{tabular}{|c|c|c|c|c|c|c|}
\hline & $A D$ & $\mathrm{AE}$ & $\mathrm{AF}$ & $A G$ & $\mathrm{AH}$ & $\mathrm{Al}$ \\
\hline 1 & RESULT_COMMENT & COLL_SAMP_SIZE & COLL_SAMP_SIZE_UNITS & DIST_CLASS & OWNER_ID & COLLECTION_PURPOSE \\
\hline 366 & & & & OFFSITE & SESPMNT & $\mathrm{R}$ \\
\hline 367 & & & & OFFSITE & SESPMNT & $\mathrm{R}$ \\
\hline 368 & & & & & SESPMNT & $\mathrm{R}$ \\
\hline 369 & & & & OFFSITE & SESPMNT & $\mathrm{R}$ \\
\hline 370 & & & & ONSITE & SESPMNT & $\mathrm{R}$ \\
\hline 371 & & & & & SESPMNT & $\mathrm{R}$ \\
\hline 372 & & & & OFFSITE & SESPMNT & $\mathrm{R}$ \\
\hline 373 & & & & & SESPMNT & $\mathrm{R}$ \\
\hline 374 & & & & & SESPMNT & $\mathrm{R}$ \\
\hline 375 & & & & & SESPMNT & $\mathrm{R}$ \\
\hline 376 & & & & & SESPMNT & $\mathrm{R}$ \\
\hline 377 & & & & & SESPMNT & $\mathrm{R}$ \\
\hline 378 & & & & & SESPMNT & $\mathrm{R}$ \\
\hline 379 & & & & OFFSITE & SESPMNT & $\mathrm{R}$ \\
\hline 380 & & & & OFFSITE & SESPMNT & $\mathrm{R}$ \\
\hline 381 & & & & OFFSITE & SESPMNT & $\mathrm{R}$ \\
\hline 382 & & & & OFFSITE & SESPMNT & $\mathrm{R}$ \\
\hline 383 & & & & OFFSITE & SESPMNT & $\mathrm{R}$ \\
\hline 384 & & & & OFFSITE & SESPMNT & $\mathrm{R}$ \\
\hline 385 & & & & & SESPMNT & $\mathrm{R}$ \\
\hline 386 & & & & & SESPMNT & $\mathrm{R}$ \\
\hline 387 & & & & OFFSITE & SESPMNT & $\mathrm{R}$ \\
\hline 388 & & & & OFFSITE & SESPMNT & $\mathrm{R}$ \\
\hline 389 & & & & & SESPMNT & $\mathrm{R}$ \\
\hline 390 & & & & & SESPMNT & $\mathrm{R}$ \\
\hline 391 & & & & & SESPMNT & $\mathrm{R}$ \\
\hline 392 & & & & & SESPMNT & $\mathrm{R}$ \\
\hline 393 & & & & OFFSITE & SESPMNT & $\mathrm{R}$ \\
\hline 394 & & & & OFFSITE & SESPMNT & $\mathrm{R}$ \\
\hline 395 & & & & OFFSITE & SESPMNT & $\mathrm{R}$ \\
\hline 396 & & & & OFFSITE & SESPMNT & $\mathrm{R}$ \\
\hline 397 & & & & OFFSITE & SESPMNT & $\mathrm{R}$ \\
\hline 398 & & & & OFFSITE & SESPMNT & $\mathrm{R}$ \\
\hline 399 & & & & OFFSITE & SESPMNT & $\mathrm{R}$ \\
\hline 400 & & & & & SESPMNT & $\mathrm{R}$ \\
\hline 401 & & & & ONSITE & SESPMNT & $\mathrm{R}$ \\
\hline 402 & & & & OFFSITE & SESPMNT & $\mathrm{R}$ \\
\hline 403 & & & & OFFSITE & SESPMNT & $\mathrm{R}$ \\
\hline 404 & & & & & SESPMNT & $\mathrm{R}$ \\
\hline 405 & & & & & SESPMNT & $\mathrm{R}$ \\
\hline 406 & & & & & SESPMNT & $\mathrm{R}$ \\
\hline 407 & & & & & SESPMNT & $\mathrm{R}$ \\
\hline 408 & & & & & SESPMNT & $\mathrm{R}$ \\
\hline 409 & & & & & SESPMNT & $\mathrm{R}$ \\
\hline 410 & & & & & SESPMNT & $\mathrm{R}$ \\
\hline 411 & & & & & SESPMNT & $\mathrm{R}$ \\
\hline 412 & & & & & SESPMNT & $\mathrm{R}$ \\
\hline 413 & & & & OFFSITE & SESPMNT & $\mathrm{R}$ \\
\hline 414 & & & & ONSITE & SESPMNT & $\mathrm{R}$ \\
\hline 415 & & & & ONSITE & SESPMNT & $R$ \\
\hline 416 & & & & & SESPMNT & $\mathrm{R}$ \\
\hline 417 & & & & & SESBMNT & ${ }_{2}^{R}$ \\
\hline
\end{tabular}




\begin{tabular}{|c|c|c|c|c|c|c|}
\hline & $\mathrm{AD}$ & $\mathrm{AE}$ & $\mathrm{AF}$ & AG & $\mathrm{AH}$ & $\overline{\mathrm{Al}}$ \\
\hline 1 & RESULT_COMMENT & COLL_SAMP_SIZE & COLL_SAMP_SIZE_UNITS & DIST_CLASS & OWNER_ID & COLLECTION_PURPOSE \\
\hline 4 & & & & & SESPMNT & $\mathrm{R}$ \\
\hline 419 & & & & & SESPMNT & $\mathrm{R}$ \\
\hline 420 & & & & & SESPMNT & $\mathrm{R}$ \\
\hline 421 & & & & OFFSITE & SESPMNT & $\mathrm{R}$ \\
\hline 422 & & & & OFFSITE & SESPMNT & $\mathrm{R}$ \\
\hline 423 & & & & OFFSITE & SESPMNT & $\mathrm{R}$ \\
\hline 424 & & & & OFFSITE & SESPMNT & $\mathrm{R}$ \\
\hline 425 & & & & OFFSITE & SESPMNT & $\mathrm{R}$ \\
\hline 426 & & & & OFFSITE & SESPMNT & $\mathrm{R}$ \\
\hline 427 & & & & & SESPMNT & $\mathrm{R}$ \\
\hline 428 & & & & & SESPMNT & $\mathrm{R}$ \\
\hline 429 & & & & OFFSITE & SESPMNT & $\mathrm{R}$ \\
\hline 430 & & & & & SESPMNT & $\mathrm{R}$ \\
\hline 431 & & & & OFFSITE & SESPMNT & $\mathrm{R}$ \\
\hline 432 & & & & ONSITE & SESPMNT & $\mathrm{R}$ \\
\hline 433 & & & & & SESPMNT & $\mathrm{R}$ \\
\hline 434 & & & & & SESPMNT & $\mathrm{R}$ \\
\hline 435 & & & & & SESPMNT & $\mathrm{R}$ \\
\hline 436 & & & & & SESPMNT & $\mathrm{R}$ \\
\hline 437 & & & & OFFSITE & SESPMNT & $\mathrm{R}$ \\
\hline 438 & & & & OFFSITE & SESPMNT & $R$ \\
\hline 439 & & & & OFFSITE & SESPMNT & $\mathrm{R}$ \\
\hline 440 & & & & OFFSITE & SESPMNT & $\mathrm{R}$ \\
\hline 441 & & & & OFFSITE & SESPMNT & $\mathrm{R}$ \\
\hline 442 & & & & & SESPMNT & $\mathrm{R}$ \\
\hline 443 & & & & & SESPMNT & $\mathrm{R}$ \\
\hline 444 & & & & OFFSITE & SESPMNT & $R$ \\
\hline 445 & & & & & SESPMNT & $\mathrm{R}$ \\
\hline 446 & & & & & SESPMNT & $\mathrm{R}$ \\
\hline \begin{tabular}{|l|}
447 \\
\end{tabular} & & & & OFFSITE & SESPMNT & $\mathrm{R}$ \\
\hline 448 & & & & ONSITE & SESPMNT & $\mathrm{R}$ \\
\hline 449 & & & & & SESPMNT & $\mathrm{R}$ \\
\hline 450 & & & & & SESPMNT & $\mathrm{R}$ \\
\hline 451 & & & & ONSITE & SESPMNT & $R$ \\
\hline 452 & & & & & SESPMNT & $\mathrm{R}$ \\
\hline 453 & & & & & SESPMNT & $R$ \\
\hline 454 & & & & OFFSITE & SESPMNT & $R$ \\
\hline 455 & & & & & SESPMNT & $R$ \\
\hline \begin{tabular}{|l|}
456 \\
\end{tabular} & & & & ONSITE & SESPMNT & $R$ \\
\hline 457 & & & & & SESPMNT & $R$ \\
\hline 458 & & & & & SESPMNT & $R$ \\
\hline 459 & & & & & SESPMNT & $R$ \\
\hline 460 & & & & OFFSITE & SESPMNT & $R$ \\
\hline 461 & & & & OFFSITE & SESPMNT & $\mathrm{R}$ \\
\hline 462 & & & & OFFSITE & SESPMNT & $R$ \\
\hline 463 & & & & OFFSITE & SESPMNT & $\mathrm{R}$ \\
\hline 464 & & & & OFFSITE & SESPMNT & $R$ \\
\hline 465 & & & & OFFSITE & SESPMNT & $R$ \\
\hline 466 & & & & & SESPMNT & $R$ \\
\hline 467 & & & & & SESPMNT & $R$ \\
\hline 468 & & & & & SESPMNT & $\mathrm{R}$ \\
\hline 469 & & & & ONSITE & SESBMNT & ${ }_{2} R$ \\
\hline
\end{tabular}




\begin{tabular}{|c|c|c|c|c|c|c|}
\hline & $\mathrm{AD}$ & $\mathrm{AE}$ & $\mathrm{AF}$ & AG & $\mathrm{AH}$ & $\mathrm{Al}$ \\
\hline 1 & RESULT_COMMENT & COLL_SAMP_SIZE & COLL_SAMP_SIZE_UNITS & DIST_CLASS & OWNER_ID & COLLECTION_PURPOSE \\
\hline 470 & & & & ONSITE & SESPMNT & $\mathrm{R}$ \\
\hline 471 & & & & & SESPMNT & $\mathrm{R}$ \\
\hline 472 & & & & & SESPMNT & $\mathrm{R}$ \\
\hline 473 & & & & & SESPMNT & $\mathrm{R}$ \\
\hline 474 & & & & ONSITE & SESPMNT & $\mathrm{R}$ \\
\hline 475 & & & & ONSITE & SESPMNT & $\mathrm{R}$ \\
\hline 476 & & & & ONSITE & SESPMNT & $\mathrm{R}$ \\
\hline 477 & & & & ONSITE & SESPMNT & $\mathrm{R}$ \\
\hline 478 & & & & & SESPMNT & $\mathrm{R}$ \\
\hline 479 & & & & & SESPMNT & $\mathrm{R}$ \\
\hline 480 & & & & OFFSITE & SESPMNT & $\mathrm{R}$ \\
\hline 481 & & & & & SESPMNT & $R$ \\
\hline 482 & & & & ONSITE & SESPMNT & $\mathrm{R}$ \\
\hline 483 & & & & & SESPMNT & $\mathrm{R}$ \\
\hline 484 & & & & & SESPMNT & $R$ \\
\hline 485 & & & & & SESPMNT & $\mathrm{R}$ \\
\hline 486 & & & & & SESPMNT & $\mathrm{R}$ \\
\hline 487 & & & & & SESPMNT & $\mathrm{R}$ \\
\hline 488 & & & & OFFSITE & SESPMNT & $\mathrm{R}$ \\
\hline 489 & & & & OFFSITE & SESPMNT & $\mathrm{R}$ \\
\hline 490 & & & & OFFSITE & SESPMNT & $R$ \\
\hline 491 & & & & OFFSITE & SESPMNT & $\mathrm{R}$ \\
\hline 492 & & & & OFFSITE & SESPMNT & $\mathrm{R}$ \\
\hline 493 & & & & & SESPMNT & $\mathrm{R}$ \\
\hline 494 & & & & ONSITE & SESPMNT & $\mathrm{R}$ \\
\hline 495 & & & & & SESPMNT & $\mathrm{R}$ \\
\hline 496 & & & & & SESPMNT & $\mathrm{R}$ \\
\hline 40 & & & & & SESPMNT & $\mathrm{R}$ \\
\hline 498 & & & & ONSITE & SESPMNT & $\mathrm{R}$ \\
\hline 499 & & & & ONSITE & SESPMNT & $\mathrm{R}$ \\
\hline 500 & & & & & SESPMNT & $\mathrm{R}$ \\
\hline 501 & & & & & SESPMNT & $\mathrm{R}$ \\
\hline 502 & & & & OFFSITE & SESPMNT & $\mathrm{R}$ \\
\hline 503 & & & & & SESPMNT & $R$ \\
\hline 504 & & & & & SESPMNT & $\mathrm{R}$ \\
\hline 505 & & & & & SESPMNT & $R$ \\
\hline 506 & & & & & SESPMNT & $R$ \\
\hline 507 & & & & & SESPMNT & $R$ \\
\hline 508 & & & & & SESPMNT & $\mathrm{R}$ \\
\hline 509 & & & & & SESPMNT & $\mathrm{R}$ \\
\hline 510 & & & & & SESPMNT & $R$ \\
\hline 511 & & & & OFFSITE & SESPMNT & $R$ \\
\hline 512 & & & & ONSITE & SESPMNT & $R$ \\
\hline 513 & & & & OFFSITE & SESPMNT & $\mathrm{R}$ \\
\hline 514 & & & & OFFSITE & SESPMNT & $\mathrm{R}$ \\
\hline 515 & & & & OFFSITE & SESPMNT & $\mathrm{R}$ \\
\hline 516 & & & & OFFSITE & SESPMNT & $\mathrm{R}$ \\
\hline 517 & & & & OFFSITE & SESPMNT & $\mathrm{R}$ \\
\hline 518 & & & & & SESPMNT & $\mathrm{R}$ \\
\hline 519 & & & & & SESPMNT & $R$ \\
\hline 520 & & & & & SESPMNT & $\mathrm{R}$ \\
\hline 521 & & & & ONSITE & SESBMNT & $R$ \\
\hline
\end{tabular}




\begin{tabular}{|c|c|c|c|c|c|c|}
\hline & $\mathrm{AD}$ & $\mathrm{AE}$ & $\mathrm{AF}$ & $A G$ & $\mathrm{AH}$ & $\mathrm{Al}$ \\
\hline 1 & RESULT_COMMENT & COLL_SAMP_SIZE & COLL_SAMP_SIZE_UNITS & DIST_CLASS & OWNER_ID & COLLECTION_PURPOSE \\
\hline 522 & & & & & SESPMNT & $\mathrm{R}$ \\
\hline 523 & & & & & SESPMNT & $\mathrm{R}$ \\
\hline 524 & & & & OFFSITE & SESPMNT & $\mathrm{R}$ \\
\hline 525 & & & & & SESPMNT & $\mathrm{R}$ \\
\hline 526 & & & & & SESPMNT & $\mathrm{R}$ \\
\hline 527 & & & & OFFSITE & SESPMNT & $\mathrm{R}$ \\
\hline 528 & & & & & SESPMNT & $\mathrm{R}$ \\
\hline 529 & & & & & SESPMNT & $\mathrm{R}$ \\
\hline 530 & & & & & SESPMNT & $\mathrm{R}$ \\
\hline 531 & & & & & SESPMNT & $\mathrm{R}$ \\
\hline 532 & & & & & SESPMNT & $\mathrm{R}$ \\
\hline 533 & & & & & SESPMNT & $\mathrm{R}$ \\
\hline 534 & & & & OFFSITE & SESPMNT & $\mathrm{R}$ \\
\hline 535 & & & & ONSITE & SESPMNT & $\mathrm{R}$ \\
\hline 536 & & & & ONSITE & SESPMNT & $\mathrm{R}$ \\
\hline 537 & & & & OFFSITE & SESPMNT & $\mathrm{R}$ \\
\hline 538 & & & & OFFSITE & SESPMNT & $\mathrm{R}$ \\
\hline 539 & & & & & SESPMNT & $\mathrm{R}$ \\
\hline 540 & & & & & SESPMNT & $\mathrm{R}$ \\
\hline 541 & & & & & SESPMNT & $\mathrm{R}$ \\
\hline 542 & & & & OFFSITE & SESPMNT & $\mathrm{R}$ \\
\hline 543 & & & & OFFSITE & SESPMNT & $\mathrm{R}$ \\
\hline 544 & & & & OFFSITE & SESPMNT & $\mathrm{R}$ \\
\hline 545 & & & & & SESPMNT & $\mathrm{R}$ \\
\hline 546 & & & & & SESPMNT & $\mathrm{R}$ \\
\hline 547 & & & & OFFSITE & SESPMNT & $\mathrm{R}$ \\
\hline 548 & & & & & SESPMNT & $\mathrm{R}$ \\
\hline 549 & & & & & SESPMNT & $\mathrm{R}$ \\
\hline 550 & & & & ONSITE & SESPMNT & $\mathrm{R}$ \\
\hline 551 & & & & ONSITE & SESPMNT & $\mathrm{R}$ \\
\hline 552 & & & & & SESPMNT & $\mathrm{R}$ \\
\hline 553 & & & & & SESPMNT & $\mathrm{R}$ \\
\hline 554 & & & & & SESPMNT & $\mathrm{R}$ \\
\hline 555 & & & & ONSITE & SESPMNT & $\mathrm{R}$ \\
\hline 556 & & & & ONSITE & SESPMNT & $R$ \\
\hline 557 & & & & & SESPMNT & $\mathrm{R}$ \\
\hline 558 & & & & & SESPMNT & $R$ \\
\hline 559 & & & & OFFSITE & SESPMNT & $\mathrm{R}$ \\
\hline 560 & & & & & SESPMNT & $\mathrm{R}$ \\
\hline 561 & & & & & SESPMNT & $\mathrm{R}$ \\
\hline 562 & & & & & SESPMNT & $\mathrm{R}$ \\
\hline 563 & & & & OFFSITE & SESPMNT & $R$ \\
\hline 564 & & & & & SESPMNT & $\mathrm{R}$ \\
\hline 565 & & & & & SESPMNT & $R$ \\
\hline 566 & & & & & SESPMNT & $R$ \\
\hline 567 & & & & & SESPMNT & $R$ \\
\hline 568 & & & & OFFSITE & SESPMNT & $\mathrm{R}$ \\
\hline 569 & & & & OFFSITE & SESPMNT & $R$ \\
\hline 570 & & & & OFFSITE & SESPMNT & $\mathrm{R}$ \\
\hline 571 & & & & OFFSITE & SESPMNT & $\mathrm{R}$ \\
\hline 572 & & & & & SESPMNT & $\mathrm{R}$ \\
\hline 573 & & & & & SESBMNT & $R$ \\
\hline
\end{tabular}




\begin{tabular}{|c|c|c|c|c|c|c|}
\hline & $\mathrm{AD}$ & $\mathrm{AE}$ & $\mathrm{AF}$ & AG & $\mathrm{AH}$ & $\mathrm{Al}$ \\
\hline 1 & RESULT_COMMENT & COLL_SAMP_SIZE & COLL_SAMP_SIZE_UNITS & DIST_CLASS & OWNER_ID & COLLECTION_PURPOSE \\
\hline 574 & & & & & SESPMNT & $\mathrm{R}$ \\
\hline 575 & & & & & SESPMNT & $\mathrm{R}$ \\
\hline 576 & & & & ONSITE & SESPMNT & $\mathrm{R}$ \\
\hline 577 & & & & & SESPMNT & $\mathrm{R}$ \\
\hline 578 & & & & & SESPMNT & $\mathrm{R}$ \\
\hline 579 & & & & & SESPMNT & $\mathrm{R}$ \\
\hline 580 & & & & OFFSITE & SESPMNT & $\mathrm{R}$ \\
\hline 581 & & & & OFFSITE & SESPMNT & $\mathrm{R}$ \\
\hline 582 & & & & ONSITE & SESPMNT & $\mathrm{R}$ \\
\hline 583 & & & & & SESPMNT & $\mathrm{R}$ \\
\hline 584 & & & & & SESPMNT & $\mathrm{R}$ \\
\hline 585 & & & & OFFSITE & SESPMNT & $\mathrm{R}$ \\
\hline 586 & & & & & SESPMNT & $\mathrm{R}$ \\
\hline 587 & & & & & SESPMNT & $\mathrm{R}$ \\
\hline 588 & & & & & SESPMNT & $\mathrm{R}$ \\
\hline 589 & & & & & SESPMNT & $\mathrm{R}$ \\
\hline 590 & & & & & SESPMNT & $\mathrm{R}$ \\
\hline 591 & & & & & SESPMNT & $\mathrm{R}$ \\
\hline 592 & & & & & SESPMNT & $\mathrm{R}$ \\
\hline 593 & & & & ONSITE & SESPMNT & $\mathrm{R}$ \\
\hline 594 & & & & ONSITE & SESPMNT & $\mathrm{R}$ \\
\hline 595 & & & & & SESPMNT & $\mathrm{R}$ \\
\hline 596 & & & & OFFSITE & SESPMNT & $\mathrm{R}$ \\
\hline 597 & & & & OFFSITE & SESPMNT & $\mathrm{R}$ \\
\hline 598 & & & & OFFSITE & SESPMNT & $\mathrm{R}$ \\
\hline 599 & & & & OFFSITE & SESPMNT & $\mathrm{R}$ \\
\hline 600 & & & & ONSITE & SESPMNT & $\mathrm{R}$ \\
\hline 601 & & & & OFFSITE & SESPMNT & $\mathrm{R}$ \\
\hline 602 & & & & & SESPMNT & $\mathrm{R}$ \\
\hline 603 & & & & & SESPMNT & $\mathrm{R}$ \\
\hline 604 & & & & & SESPMNT & $\mathrm{R}$ \\
\hline 605 & & & & & SESPMNT & $\mathrm{R}$ \\
\hline 606 & & & & & SESPMNT & $\mathrm{R}$ \\
\hline 607 & & & & & SESPMNT & $\mathrm{R}$ \\
\hline 608 & & & & & SESPMNT & $\mathrm{R}$ \\
\hline 609 & & & & OFFSITE & SESPMNT & $\mathrm{R}$ \\
\hline 610 & & & & OFFSITE & SESPMNT & $\mathrm{R}$ \\
\hline 611 & & & & & SESPMNT & $\mathrm{R}$ \\
\hline \begin{tabular}{|l|}
612 \\
\end{tabular} & & & & & SESPMNT & $\mathrm{R}$ \\
\hline 613 & & & & & SESPMNT & $\mathrm{R}$ \\
\hline 614 & & & & ONSITE & SESPMNT & $\mathrm{R}$ \\
\hline 615 & & & & ONSITE & SESPMNT & $\mathrm{R}$ \\
\hline 616 & & & & OFFSITE & SESPMNT & $\mathrm{R}$ \\
\hline 617 & & & & OFFSITE & SESPMNT & $\mathrm{R}$ \\
\hline 618 & & & & OFFSITE & SESPMNT & $\mathrm{R}$ \\
\hline 619 & & & & OFFSITE & SESPMNT & $\mathrm{R}$ \\
\hline 620 & & & & & SESPMNT & $\mathrm{R}$ \\
\hline 621 & & & & & SESPMNT & $\mathrm{R}$ \\
\hline 622 & & & & & SESPMNT & $\mathrm{R}$ \\
\hline 623 & & & & OFFSITE & SESPMNT & $\mathrm{R}$ \\
\hline 624 & & & & & SESPMNT & $\mathrm{R}$ \\
\hline 625 & & & & & SESBMNT & $R$ \\
\hline
\end{tabular}




\begin{tabular}{|c|c|c|c|c|c|c|}
\hline & $\mathrm{AD}$ & $\mathrm{AE}$ & $\mathrm{AF}$ & AG & $\mathrm{AH}$ & $\mathrm{Al}$ \\
\hline 1 & RESULT_COMMENT & COLL_SAMP_SIZE & COLL_SAMP_SIZE_UNITS & DIST_CLASS & OWNER_ID & COLLECTION_PURPOSE \\
\hline 626 & & & & OFFSITE & SESPMNT & $\mathrm{R}$ \\
\hline 627 & & & & & SESPMNT & $\mathrm{R}$ \\
\hline 628 & & & & & SESPMNT & $\mathrm{R}$ \\
\hline 629 & & & & & SESPMNT & $\mathrm{R}$ \\
\hline 630 & & & & & SESPMNT & $\mathrm{R}$ \\
\hline 631 & & & & & SESPMNT & $\mathrm{R}$ \\
\hline 632 & & & & & SESPMNT & $\mathrm{R}$ \\
\hline 633 & & & & & SESPMNT & $\mathrm{R}$ \\
\hline 634 & & & & ONSITE & SESPMNT & $\mathrm{R}$ \\
\hline 635 & & & & ONSITE & SESPMNT & $\mathrm{R}$ \\
\hline 636 & & & & & SESPMNT & $\mathrm{R}$ \\
\hline 637 & & & & & SESPMNT & $R$ \\
\hline 638 & & & & & SESPMNT & $\mathrm{R}$ \\
\hline 639 & & & & & SESPMNT & $\mathrm{R}$ \\
\hline 640 & & & & & SESPMNT & $R$ \\
\hline 641 & & & & & SESPMNT & $\mathrm{R}$ \\
\hline 642 & & & & & SESPMNT & $\mathrm{R}$ \\
\hline 643 & & & & & SESPMNT & $\mathrm{R}$ \\
\hline 644 & & & & & SESPMNT & $\mathrm{R}$ \\
\hline 645 & & & & & SESPMNT & $\mathrm{R}$ \\
\hline 646 & & & & OFFSITE & SESPMNT & $R$ \\
\hline 647 & & & & ONSITE & SESPMNT & $\mathrm{R}$ \\
\hline 648 & & & & ONSITE & SESPMNT & $\mathrm{R}$ \\
\hline 649 & & & & OFFSITE & SESPMNT & $\mathrm{R}$ \\
\hline 650 & & & & OFFSITE & SESPMNT & $\mathrm{R}$ \\
\hline 651 & & & & OFFSITE & SESPMNT & $\mathrm{R}$ \\
\hline 652 & & & & OFFSITE & SESPMNT & $\mathrm{R}$ \\
\hline 653 & & & & & SESPMNT & $\mathrm{R}$ \\
\hline 654 & & & & & SESPMNT & $\mathrm{R}$ \\
\hline 655 & & & & & SESPMNT & $\mathrm{R}$ \\
\hline 656 & & & & OFFSITE & SESPMNT & $\mathrm{R}$ \\
\hline 657 & & & & & SESPMNT & $\mathrm{R}$ \\
\hline 658 & & & & & SESPMNT & $\mathrm{R}$ \\
\hline 659 & & & & & SESPMNT & $\mathrm{R}$ \\
\hline 660 & & & & OFFSITE & SESPMNT & $\mathrm{R}$ \\
\hline 661 & & & & & SESPMNT & $R$ \\
\hline 662 & & & & & SESPMNT & $R$ \\
\hline 663 & & & & & SESPMNT & $R$ \\
\hline 664 & & & & ONSITE & SESPMNT & $R$ \\
\hline 665 & & & & & SESPMNT & $\mathrm{R}$ \\
\hline 666 & & & & & SESPMNT & $R$ \\
\hline \begin{tabular}{|l|}
667 \\
\end{tabular} & & & & OFFSITE & SESPMNT & $R$ \\
\hline 668 & & & & & SESPMNT & $R$ \\
\hline 669 & & & & & SESPMNT & $\mathrm{R}$ \\
\hline 670 & & & & & SESPMNT & $\mathrm{R}$ \\
\hline 671 & & & & OFFSITE & SESPMNT & $\mathrm{R}$ \\
\hline 672 & & & & ONSITE & SESPMNT & $R$ \\
\hline 673 & & & & ONSITE & SESPMNT & $\mathrm{R}$ \\
\hline 674 & & & & & SESPMNT & $\mathrm{R}$ \\
\hline 675 & & & & & SESPMNT & $R$ \\
\hline 676 & & & & & SESPMNT & $\mathrm{R}$ \\
\hline 677 & & & & OFFSITE & SESBMNT & $R$ \\
\hline
\end{tabular}




\begin{tabular}{|c|c|c|c|c|c|c|}
\hline & $A D$ & $\mathrm{AE}$ & $\mathrm{AF}$ & $A G$ & $\mathrm{AH}$ & $\mathrm{Al}$ \\
\hline 1 & RESULT_COMMENT & COLL_SAMP_SIZE & COLL_SAMP_SIZE_UNITS & DIST_CLASS & OWNER_ID & COLLECTION_PURPOSE \\
\hline 678 & & & & OFFSITE & SESPMNT & $R$ \\
\hline 679 & & & & OFFSITE & SESPMNT & $\mathrm{R}$ \\
\hline 680 & & & & OFFSITE & SESPMNT & $\mathrm{R}$ \\
\hline 681 & & & & OFFSITE & SESPMNT & $\mathrm{R}$ \\
\hline 682 & & & & & SESPMNT & $\mathrm{R}$ \\
\hline 683 & & & & & SESPMNT & $\mathrm{R}$ \\
\hline 684 & & & & & SESPMNT & $\mathrm{R}$ \\
\hline 685 & & & & OFFSITE & SESPMNT & $\mathrm{R}$ \\
\hline 686 & & & & & SESPMNT & $\mathrm{R}$ \\
\hline 687 & & & & & SESPMNT & $\mathrm{R}$ \\
\hline 688 & & & & & SESPMNT & $\mathrm{R}$ \\
\hline 689 & & & & ONSITE & SESPMNT & $\mathrm{R}$ \\
\hline 690 & & & & & SESPMNT & $\mathrm{R}$ \\
\hline 691 & & & & & SESPMNT & $\mathrm{R}$ \\
\hline \begin{tabular}{|c|}
692 \\
\end{tabular} & & & & OFFSITE & SESPMNT & $\mathrm{R}$ \\
\hline 693 & & & & & SESPMNT & $\mathrm{R}$ \\
\hline 694 & & & & & SESPMNT & $\mathrm{R}$ \\
\hline 695 & & & & OFFSITE & SESPMNT & $\mathrm{R}$ \\
\hline 696 & & & & & SESPMNT & $\mathrm{R}$ \\
\hline \begin{tabular}{|c|}
697 \\
\end{tabular} & & & & & SESPMNT & $\mathrm{R}$ \\
\hline 698 & & & & & SESPMNT & $\mathrm{R}$ \\
\hline 699 & & & & ONSITE & SESPMNT & $\mathrm{R}$ \\
\hline 700 & & & & ONSITE & SESPMNT & $\mathrm{R}$ \\
\hline 701 & & & & OFFSITE & SESPMNT & $\mathrm{R}$ \\
\hline 702 & & & & OFFSITE & SESPMNT & $\mathrm{R}$ \\
\hline 703 & & & & OFFSITE & SESPMNT & $\mathrm{R}$ \\
\hline 704 & & & & OFFSITE & SESPMNT & $\mathrm{R}$ \\
\hline 705 & & & & & SESPMNT & $\mathrm{R}$ \\
\hline 706 & & & & & SESPMNT & $\mathrm{R}$ \\
\hline 707 & & & & & SESPMNT & $\mathrm{R}$ \\
\hline 708 & & & & OFFSITE & SESPMNT & $\mathrm{R}$ \\
\hline 709 & & & & & SESPMNT & $\mathrm{R}$ \\
\hline 710 & & & & & SESPMNT & $\mathrm{R}$ \\
\hline \begin{tabular}{l|l|}
711 \\
\end{tabular} & & & & & SESPMNT & $\mathrm{R}$ \\
\hline 712 & & & & OFFSITE & SESPMNT & $\mathrm{R}$ \\
\hline 713 & & & & & SESPMNT & $\mathrm{R}$ \\
\hline 714 & & & & & SESPMNT & $\mathrm{R}$ \\
\hline 715 & & & & & SESPMNT & $\mathrm{R}$ \\
\hline \begin{tabular}{|l|}
716 \\
\end{tabular} & & & & & SESPMNT & $\mathrm{R}$ \\
\hline 717 & & & & & SESPMNT & $\mathrm{R}$ \\
\hline 718 & & & & OFFSITE & SESPMNT & $\mathrm{R}$ \\
\hline 719 & & & & ONSITE & SESPMNT & $\mathrm{R}$ \\
\hline 720 & & & & ONSITE & SESPMNT & $\mathrm{R}$ \\
\hline 721 & & & & & SESPMNT & $\mathrm{R}$ \\
\hline 722 & & & & & SESPMNT & $\mathrm{R}$ \\
\hline 723 & & & & & SESPMNT & $\mathrm{R}$ \\
\hline 724 & & & & ONSITE & SESPMNT & $\mathrm{R}$ \\
\hline 725 & & & & ONSITE & SESPMNT & $\mathrm{R}$ \\
\hline 726 & & & & RIVER_SHORELINE & SESPMNT & $\mathrm{R}$ \\
\hline 727 & & & & & SESPMNT & $\mathrm{R}$ \\
\hline 728 & & & & & SESPMNT & $\mathrm{R}$ \\
\hline 729 & & & & & SESBMNT & ${ }_{0}^{R}$ \\
\hline
\end{tabular}




\begin{tabular}{|c|c|c|c|c|c|c|}
\hline & $A D$ & $\mathrm{AE}$ & $\mathrm{AF}$ & $A G$ & $\mathrm{AH}$ & $\mathrm{Al}$ \\
\hline 1 & RESULT_COMMENT & COLL_SAMP_SIZE & COLL_SAMP_SIZE_UNITS & DIST_CLASS & OWNER_ID & COLLECTION_PURPOSE \\
\hline 730 & & & & OFFSITE & SESPMNT & $R$ \\
\hline 731 & & & & & SESPMNT & $\mathrm{R}$ \\
\hline 732 & & & & ONSITE & SESPMNT & $\mathrm{R}$ \\
\hline 733 & & & & ONSITE & SESPMNT & $\mathrm{R}$ \\
\hline 734 & & & & & SESPMNT & $\mathrm{R}$ \\
\hline 735 & & & & & SESPMNT & $\mathrm{R}$ \\
\hline 736 & & & & & SESPMNT & $\mathrm{R}$ \\
\hline 737 & & & & OFFSITE & SESPMNT & $\mathrm{R}$ \\
\hline 738 & & & & OFFSITE & SESPMNT & $\mathrm{R}$ \\
\hline 739 & & & & OFFSITE & SESPMNT & $\mathrm{R}$ \\
\hline 740 & & & & OFFSITE & SESPMNT & $\mathrm{R}$ \\
\hline 741 & & & & OFFSITE & SESPMNT & $\mathrm{R}$ \\
\hline 742 & & & & & SESPMNT & $\mathrm{R}$ \\
\hline 743 & & & & OFFSITE & SESPMNT & $\mathrm{R}$ \\
\hline 744 & & & & RIVER_SHORELINE & SESPMNT & $\mathrm{R}$ \\
\hline 745 & & & & RIVER_SHORELINE & SESPMNT & $\mathrm{R}$ \\
\hline 746 & & & & ONSITE & SESPMNT & $\mathrm{R}$ \\
\hline 747 & & & & & SESPMNT & $\mathrm{R}$ \\
\hline 748 & & & & & SESPMNT & $\mathrm{R}$ \\
\hline 749 & & & & & SESPMNT & $\mathrm{R}$ \\
\hline 750 & & & & OFFSITE & SESPMNT & $\mathrm{R}$ \\
\hline 751 & & & & OFFSITE & SESPMNT & $\mathrm{R}$ \\
\hline 752 & & & & & SESPMNT & $\mathrm{R}$ \\
\hline 753 & & & & & SESPMNT & $\mathrm{R}$ \\
\hline 754 & & & & & SESPMNT & $\mathrm{R}$ \\
\hline 755 & & & & & SESPMNT & $\mathrm{R}$ \\
\hline 756 & & & & ONSITE & SESPMNT & $\mathrm{R}$ \\
\hline 757 & & & & ONSITE & SESPMNT & $\mathrm{R}$ \\
\hline 758 & & & & & SESPMNT & $\mathrm{R}$ \\
\hline 759 & & & & & SESPMNT & $\mathrm{R}$ \\
\hline 760 & & & & OFFSITE & SESPMNT & $\mathrm{R}$ \\
\hline 761 & & & & OFFSITE & SESPMNT & $\mathrm{R}$ \\
\hline 762 & & & & OFFSITE & SESPMNT & $\mathrm{R}$ \\
\hline 763 & & & & OFFSITE & SESPMNT & $\mathrm{R}$ \\
\hline 764 & & & & OFFSITE & SESPMNT & $\mathrm{R}$ \\
\hline 765 & & & & & SESPMNT & $\mathrm{R}$ \\
\hline 766 & & & & & SESPMNT & $\mathrm{R}$ \\
\hline 767 & & & & & SESPMNT & $\mathrm{R}$ \\
\hline 768 & & & & OFFSITE & SESPMNT & $\mathrm{R}$ \\
\hline 769 & & & & ONSITE & SESPMNT & $\mathrm{R}$ \\
\hline 770 & & & & & SESPMNT & $\mathrm{R}$ \\
\hline 771 & & & & & SESPMNT & $\mathrm{R}$ \\
\hline 772 & & & & OFFSITE & SESPMNT & $\mathrm{R}$ \\
\hline 773 & & & & RIVER_SHORELINE & SESPMNT & $\mathrm{R}$ \\
\hline 774 & & & & RIVER SHORELINE & SESPMNT & $\mathrm{R}$ \\
\hline 775 & & & & RIVER_SHORELINE & SESPMNT & $\mathrm{R}$ \\
\hline 776 & & & & & SESPMNT & $\mathrm{R}$ \\
\hline 777 & & & & & SESPMNT & $\mathrm{R}$ \\
\hline 778 & & & & OFFSITE & SESPMNT & $\mathrm{R}$ \\
\hline 779 & & & & & SESPMNT & $\mathrm{R}$ \\
\hline 780 & & & & ONSITE & SESPMNT & $\mathrm{R}$ \\
\hline 781 & & & & OFFSITE & SESBMNT & ${ }_{0}^{R}$ \\
\hline
\end{tabular}




\begin{tabular}{|c|c|c|c|c|c|c|}
\hline & $A D$ & $\mathrm{AE}$ & $\mathrm{AF}$ & $A G$ & $\mathrm{AH}$ & $\mathrm{Al}$ \\
\hline 1 & RESULT_COMMENT & COLL_SAMP_SIZE & COLL_SAMP_SIZE_UNITS & DIST_CLASS & OWNER_ID & COLLECTION_PURPOSE \\
\hline 782 & & & & OFFSITE & SESPMNT & $\mathrm{R}$ \\
\hline 783 & & & & OFFSITE & SESPMNT & $\mathrm{R}$ \\
\hline 784 & & & & & SESPMNT & $\mathrm{R}$ \\
\hline 785 & & & & & SESPMNT & $\mathrm{R}$ \\
\hline 786 & & & & & SESPMNT & $\mathrm{R}$ \\
\hline 787 & & & & OFFSITE & SESPMNT & $\mathrm{R}$ \\
\hline 788 & & & & OFFSITE & SESPMNT & $\mathrm{R}$ \\
\hline 789 & & & & & SESPMNT & $\mathrm{R}$ \\
\hline 790 & & & & & SESPMNT & $\mathrm{R}$ \\
\hline 791 & & & & OFFSITE & SESPMNT & $\mathrm{R}$ \\
\hline 792 & & & & & SESPMNT & $\mathrm{R}$ \\
\hline 793 & & & & ONSITE & SESPMNT & $\mathrm{R}$ \\
\hline 794 & & & & ONSITE & SESPMNT & $\mathrm{R}$ \\
\hline 795 & & & & & SESPMNT & $\mathrm{R}$ \\
\hline 796 & & & & & SESPMNT & $\mathrm{R}$ \\
\hline 797 & & & & & SESPMNT & $\mathrm{R}$ \\
\hline 798 & & & & ONSITE & SESPMNT & $\mathrm{R}$ \\
\hline 799 & & & & ONSITE & SESPMNT & $\mathrm{R}$ \\
\hline 800 & & & & & SESPMNT & $\mathrm{R}$ \\
\hline 801 & & & & & SESPMNT & $\mathrm{R}$ \\
\hline 802 & & & & & SESPMNT & $\mathrm{R}$ \\
\hline 803 & & & & OFFSITE & SESPMNT & $\mathrm{R}$ \\
\hline 804 & & & & & SESPMNT & $\mathrm{R}$ \\
\hline 805 & & & & RIVER_SHORELINE & SESPMNT & $\mathrm{R}$ \\
\hline 806 & & & & RIVER_SHORELINE & SESPMNT & $\mathrm{R}$ \\
\hline 807 & & & & & SESPMNT & $\mathrm{R}$ \\
\hline 808 & & & & & SESPMNT & $\mathrm{R}$ \\
\hline 809 & & & & OFFSITE & SESPMNT & $\mathrm{R}$ \\
\hline 810 & & & & & SESPMNT & $\mathrm{R}$ \\
\hline 811 & & & & & SESPMNT & $\mathrm{R}$ \\
\hline 812 & & & & & SESPMNT & $\mathrm{R}$ \\
\hline 813 & & & & & SESPMNT & $\mathrm{R}$ \\
\hline 814 & & & & OFFSITE & SESPMNT & $\mathrm{R}$ \\
\hline 815 & & & & OFFSITE & SESPMNT & $\mathrm{R}$ \\
\hline 816 & & & & OFFSITE & SESPMNT & $\mathrm{R}$ \\
\hline 817 & & & & OFFSITE & SESPMNT & $\mathrm{R}$ \\
\hline 818 & & & & ONSITE & SESPMNT & $\mathrm{R}$ \\
\hline 819 & & & & ONSITE & SESPMNT & $\mathrm{R}$ \\
\hline 820 & & & & & SESPMNT & $\mathrm{R}$ \\
\hline 821 & & & & & SESPMNT & $\mathrm{R}$ \\
\hline 822 & & & & OFFSITE & SESPMNT & $\mathrm{R}$ \\
\hline 823 & & & & ONSITE & SESPMNT & $\mathrm{R}$ \\
\hline 824 & & & & & SESPMNT & $\mathrm{R}$ \\
\hline 825 & & & & & SESPMNT & $\mathrm{R}$ \\
\hline 826 & & & & OFFSITE & SESPMNT & $\mathrm{R}$ \\
\hline 827 & & & & RIVER_SHORELINE & SESPMNT & $\mathrm{R}$ \\
\hline 828 & & & & RIVER_SHORELINE & SESPMNT & $\mathrm{R}$ \\
\hline 829 & & & & RIVER_SHORELINE & SESPMNT & $\mathrm{R}$ \\
\hline 830 & & & & & SESPMNT & $\mathrm{R}$ \\
\hline 831 & & & & & SESPMNT & $\mathrm{R}$ \\
\hline 832 & & & & OFFSITE & SESPMNT & $\mathrm{R}$ \\
\hline 833 & & & & & SESBMNT & $R$ \\
\hline
\end{tabular}




\begin{tabular}{|c|c|c|c|c|c|c|}
\hline & $\mathrm{AD}$ & $\mathrm{AE}$ & $\mathrm{AF}$ & AG & $\mathrm{AH}$ & $\overline{\mathrm{Al}}$ \\
\hline 1 & RESULT_COMMENT & COLL_SAMP_SIZE & COLL_SAMP_SIZE_UNITS & DIST_CLASS & OWNER_ID & COLLECTION_PURPOSE \\
\hline 834 & & & & & SESPMNT & $\mathrm{R}$ \\
\hline 835 & & & & & SESPMNT & $\mathrm{R}$ \\
\hline 836 & & & & ONSITE & SESPMNT & $\mathrm{R}$ \\
\hline 837 & & & & ONSITE & SESPMNT & $\mathrm{R}$ \\
\hline 838 & & & & OFFSITE & SESPMNT & $\mathrm{R}$ \\
\hline 839 & & & & OFFSITE & SESPMNT & $\mathrm{R}$ \\
\hline 840 & & & & OFFSITE & SESPMNT & $\mathrm{R}$ \\
\hline 841 & & & & & SESPMNT & $\mathrm{R}$ \\
\hline 842 & & & & & SESPMNT & $\mathrm{R}$ \\
\hline 843 & & & & ONSITE & SESPMNT & $\mathrm{R}$ \\
\hline 844 & & & & & SESPMNT & $\mathrm{R}$ \\
\hline 845 & & & & & SESPMNT & $\mathrm{R}$ \\
\hline 846 & & & & OFFSITE & SESPMNT & $\mathrm{R}$ \\
\hline 847 & & & & RIVER_SHORELINE & SESPMNT & $\mathrm{R}$ \\
\hline 848 & & & & RIVER_SHORELINE & SESPMNT & $\mathrm{R}$ \\
\hline 849 & & & & & SESPMNT & $\mathrm{R}$ \\
\hline 850 & & & & & SESPMNT & $\mathrm{R}$ \\
\hline 851 & & & & OFFSITE & SESPMNT & $\mathrm{R}$ \\
\hline 852 & & & & & SESPMNT & $\mathrm{R}$ \\
\hline 853 & & & & OFFSITE & SESPMNT & $\mathrm{R}$ \\
\hline 854 & & & & ONSITE & SESPMNT & $R$ \\
\hline 855 & & & & ONSITE & SESPMNT & $\mathrm{R}$ \\
\hline 856 & & & & & SESPMNT & $\mathrm{R}$ \\
\hline 857 & & & & & SESPMNT & $\mathrm{R}$ \\
\hline 858 & & & & & SESPMNT & $\mathrm{R}$ \\
\hline 859 & & & & & SESPMNT & $\mathrm{R}$ \\
\hline 860 & & & & OFFSITE & SESPMNT & $R$ \\
\hline 861 & & & & OFFSITE & SESPMNT & $\mathrm{R}$ \\
\hline 862 & & & & OFFSITE & SESPMNT & $\mathrm{R}$ \\
\hline 863 & & & & OFFSITE & SESPMNT & $\mathrm{R}$ \\
\hline 864 & & & & OFFSITE & SESPMNT & $\mathrm{R}$ \\
\hline 865 & & & & & SESPMNT & $\mathrm{R}$ \\
\hline 866 & & & & & SESPMNT & $\mathrm{R}$ \\
\hline 867 & & & & & SESPMNT & $\mathrm{R}$ \\
\hline 868 & & & & & SESPMNT & $\mathrm{R}$ \\
\hline 869 & & & & OFFSITE & SESPMNT & $\mathrm{R}$ \\
\hline 870 & & & & & SESPMNT & $\mathrm{R}$ \\
\hline 871 & & & & RIVER_SHORELINE & SESPMNT & $\mathrm{R}$ \\
\hline 872 & & & & RIVER_SHORELINE & SESPMNT & $\mathrm{R}$ \\
\hline 873 & & & & RIVER_SHORELINE & SESPMNT & $R$ \\
\hline 874 & & & & & SESPMNT & $\mathrm{R}$ \\
\hline 875 & & & & ONSITE & SESPMNT & $\mathrm{R}$ \\
\hline 876 & & & & ONSITE & SESPMNT & $\mathrm{R}$ \\
\hline 877 & & & & & SESPMNT & $\mathrm{R}$ \\
\hline 878 & & & & & SESPMNT & $\mathrm{R}$ \\
\hline 879 & & & & & SESPMNT & $\mathrm{R}$ \\
\hline 880 & & & & & SESPMNT & $\mathrm{R}$ \\
\hline 881 & & & & ONSITE & SESPMNT & $\mathrm{R}$ \\
\hline 882 & & & & ONSITE & SESPMNT & $\mathrm{R}$ \\
\hline 883 & & & & & SESPMNT & $\mathrm{R}$ \\
\hline 884 & & & & & SESPMNT & $\mathrm{R}$ \\
\hline 885 & & & & OFFSITE & SESBMNT & $R$ \\
\hline
\end{tabular}




\begin{tabular}{|c|c|c|c|c|c|c|}
\hline & $\mathrm{AD}$ & $\mathrm{AE}$ & $\mathrm{AF}$ & AG & $\mathrm{AH}$ & $\mathrm{Al}$ \\
\hline 1 & RESULT_COMMENT & COLL_SAMP_SIZE & COLL_SAMP_SIZE_UNITS & DIST_CLASS & OWNER_ID & COLLECTION_PURPOSE \\
\hline 886 & & & & & SESPMNT & $\mathrm{R}$ \\
\hline 887 & & & & & SESPMNT & $\mathrm{R}$ \\
\hline 888 & & & & & SESPMNT & $\mathrm{R}$ \\
\hline 889 & & & & OFFSITE & SESPMNT & $\mathrm{R}$ \\
\hline 890 & & & & ONSITE & SESPMNT & $\mathrm{R}$ \\
\hline 891 & & & & ONSITE & SESPMNT & $\mathrm{R}$ \\
\hline 892 & & & & & SESPMNT & $\mathrm{R}$ \\
\hline 893 & & & & & SESPMNT & $\mathrm{R}$ \\
\hline 894 & & & & & SESPMNT & $\mathrm{R}$ \\
\hline 895 & & & & OFFSITE & SESPMNT & $\mathrm{R}$ \\
\hline 896 & & & & OFFSITE & SESPMNT & $\mathrm{R}$ \\
\hline 897 & & & & OFFSITE & SESPMNT & $R$ \\
\hline 898 & & & & OFFSITE & SESPMNT & $\mathrm{R}$ \\
\hline 899 & & & & OFFSITE & SESPMNT & $\mathrm{R}$ \\
\hline 900 & & & & ONSITE & SESPMNT & $R$ \\
\hline 901 & & & & & SESPMNT & $\mathrm{R}$ \\
\hline 902 & & & & & SESPMNT & $\mathrm{R}$ \\
\hline 903 & & & & OFFSITE & SESPMNT & $\mathrm{R}$ \\
\hline 904 & & & & & SESPMNT & $\mathrm{R}$ \\
\hline 905 & & & & RIVER_SHORELINE & SESPMNT & $\mathrm{R}$ \\
\hline 906 & & & & RIVER_SHORELINE & SESPMNT & $R$ \\
\hline 907 & & & & RIVER_SHORELINE & SESPMNT & $\mathrm{R}$ \\
\hline 908 & & & & & SESPMNT & $\mathrm{R}$ \\
\hline 909 & & & & & SESPMNT & $\mathrm{R}$ \\
\hline 910 & & & & OFFSITE & SESPMNT & $\mathrm{R}$ \\
\hline 911 & & & & & SESPMNT & $\mathrm{R}$ \\
\hline 912 & & & & & SESPMNT & $\mathrm{R}$ \\
\hline 913 & & & & OFFSITE & SESPMNT & $\mathrm{R}$ \\
\hline 914 & & & & & SESPMNT & $\mathrm{R}$ \\
\hline 915 & & & & ONSITE & SESPMNT & $\mathrm{R}$ \\
\hline 916 & & & & ONSITE & SESPMNT & $\mathrm{R}$ \\
\hline 917 & & & & & SESPMNT & $\mathrm{R}$ \\
\hline 918 & & & & & SESPMNT & $\mathrm{R}$ \\
\hline 919 & & & & & SESPMNT & $\mathrm{R}$ \\
\hline 920 & & & & OFFSITE & SESPMNT & $\mathrm{R}$ \\
\hline 921 & & & & OFFSITE & SESPMNT & $R$ \\
\hline 922 & & & & OFFSITE & SESPMNT & $\mathrm{R}$ \\
\hline 923 & & & & OFFSITE & SESPMNT & $\mathrm{R}$ \\
\hline 924 & & & & OFFSITE & SESPMNT & $R$ \\
\hline 925 & & & & ONSITE & SESPMNT & $\mathrm{R}$ \\
\hline 926 & & & & OFFSITE & SESPMNT & $\mathrm{R}$ \\
\hline \begin{tabular}{|l|}
927 \\
\end{tabular} & & & & & SESPMNT & $\mathrm{R}$ \\
\hline 928 & & & & & SESPMNT & $R$ \\
\hline 929 & & & & & SESPMNT & $\mathrm{R}$ \\
\hline 930 & & & & & SESPMNT & $\mathrm{R}$ \\
\hline 931 & & & & & SESPMNT & $\mathrm{R}$ \\
\hline 932 & & & & OFFSITE & SESPMNT & $\mathrm{R}$ \\
\hline 933 & & & & RIVER_SHORELINE & SESPMNT & $\mathrm{R}$ \\
\hline 934 & & & & RIVER_SHORELINE & SESPMNT & $\mathrm{R}$ \\
\hline 935 & & & & & SESPMNT & $\mathrm{R}$ \\
\hline 936 & & & & & SESPMNT & $\mathrm{R}$ \\
\hline 937 & & & & OFFSITE & SESBMNT & $R$ \\
\hline
\end{tabular}




\begin{tabular}{|c|c|c|c|c|c|c|}
\hline & $A D$ & $\mathrm{AE}$ & $\mathrm{AF}$ & $A G$ & $\mathrm{AH}$ & $\mathrm{Al}$ \\
\hline 1 & RESULT_COMMENT & COLL_SAMP_SIZE & COLL_SAMP_SIZE_UNITS & DIST_CLASS & OWNER_ID & COLLECTION_PURPOSE \\
\hline 938 & & & & OFFSITE & SESPMNT & $R$ \\
\hline 939 & & & & & SESPMNT & $\mathrm{R}$ \\
\hline 940 & & & & & SESPMNT & $\mathrm{R}$ \\
\hline 941 & & & & & SESPMNT & $\mathrm{R}$ \\
\hline 942 & & & & OFFSITE & SESPMNT & $\mathrm{R}$ \\
\hline 943 & & & & OFFSITE & SESPMNT & $\mathrm{R}$ \\
\hline 944 & & & & OFFSITE & SESPMNT & $\mathrm{R}$ \\
\hline 945 & & & & OFFSITE & SESPMNT & $\mathrm{R}$ \\
\hline 946 & & & & ONSITE & SESPMNT & $\mathrm{R}$ \\
\hline 947 & & & & ONSITE & SESPMNT & $\mathrm{R}$ \\
\hline 948 & & & & & SESPMNT & $\mathrm{R}$ \\
\hline 949 & & & & & SESPMNT & $\mathrm{R}$ \\
\hline 950 & & & & & SESPMNT & $\mathrm{R}$ \\
\hline 951 & & & & & SESPMNT & $\mathrm{R}$ \\
\hline 952 & & & & OFFSITE & SESPMNT & $\mathrm{R}$ \\
\hline 953 & & & & & SESPMNT & $\mathrm{R}$ \\
\hline 954 & & & & & SESPMNT & $\mathrm{R}$ \\
\hline 955 & & & & OFFSITE & SESPMNT & $\mathrm{R}$ \\
\hline 956 & & & & & SESPMNT & $\mathrm{R}$ \\
\hline 957 & & & & RIVER_SHORELINE & SESPMNT & $\mathrm{R}$ \\
\hline 958 & & & & RIVER_SHORELINE & SESPMNT & $\mathrm{R}$ \\
\hline 959 & & & & RIVER_SHORELINE & SESPMNT & $\mathrm{R}$ \\
\hline 960 & & & & & SESPMNT & $\mathrm{R}$ \\
\hline 961 & & & & ONSITE & SESPMNT & $\mathrm{R}$ \\
\hline 962 & & & & ONSITE & SESPMNT & $\mathrm{R}$ \\
\hline 963 & & & & & SESPMNT & $\mathrm{R}$ \\
\hline 964 & & & & & SESPMNT & $\mathrm{R}$ \\
\hline 965 & & & & & SESPMNT & $\mathrm{R}$ \\
\hline 966 & & & & & SESPMNT & $\mathrm{R}$ \\
\hline \begin{tabular}{|l|}
967 \\
\end{tabular} & & & & ONSITE & SESPMNT & $\mathrm{R}$ \\
\hline 968 & & & & & SESPMNT & $\mathrm{R}$ \\
\hline 969 & & & & & SESPMNT & $\mathrm{R}$ \\
\hline 970 & & & & OFFSITE & SESPMNT & $\mathrm{R}$ \\
\hline 971 & & & & & SESPMNT & $\mathrm{R}$ \\
\hline 972 & & & & & SESPMNT & $\mathrm{R}$ \\
\hline 973 & & & & OFFSITE & SESPMNT & $\mathrm{R}$ \\
\hline 974 & & & & OFFSITE & SESPMNT & $\mathrm{R}$ \\
\hline 975 & & & & & SESPMNT & $\mathrm{R}$ \\
\hline 976 & & & & & SESPMNT & $\mathrm{R}$ \\
\hline 977 & & & & & SESPMNT & $\mathrm{R}$ \\
\hline 978 & & & & & SESPMNT & $\mathrm{R}$ \\
\hline 979 & & & & & SESPMNT & $\mathrm{R}$ \\
\hline 980 & & & & & SESPMNT & $\mathrm{R}$ \\
\hline 981 & & & & ONSITE & SESPMNT & $\mathrm{R}$ \\
\hline 982 & & & & OFFSITE & SESPMNT & $\mathrm{R}$ \\
\hline 983 & & & & OFFSITE & SESPMNT & $\mathrm{R}$ \\
\hline 984 & & & & OFFSITE & SESPMNT & $\mathrm{R}$ \\
\hline 985 & & & & & SESPMNT & $\mathrm{R}$ \\
\hline 986 & & & & & SESPMNT & $\mathrm{R}$ \\
\hline 987 & & & & & SESPMNT & $R$ \\
\hline 988 & & & & OFFSITE & SESPMNT & $\mathrm{R}$ \\
\hline 989 & & & & & SESBMNT & ${ }_{2}^{R}$ \\
\hline
\end{tabular}




\begin{tabular}{|c|c|c|c|c|c|c|}
\hline & $A D$ & $\mathrm{AE}$ & $\mathrm{AF}$ & $\mathrm{AG}$ & $\mathrm{AH}$ & $\mathrm{Al}$ \\
\hline 1 & RESULT_COMMENT & COLL_SAMP_SIZE & COLL_SAMP_SIZE_UNITS & DIST_CLASS & OWNER_ID & COLLECTION_PURPOSE \\
\hline 990 & & & & & SESPMNT & $\mathrm{R}$ \\
\hline 991 & & & & RIVER_SHORELINE & SESPMNT & $\mathrm{R}$ \\
\hline 992 & & & & RIVER_SHORELINE & SESPMNT & $\mathrm{R}$ \\
\hline 993 & & & & RIVER_SHORELINE & SESPMNT & $\mathrm{R}$ \\
\hline \begin{tabular}{|l|l|}
994 \\
\end{tabular} & & & & & SESPMNT & $\mathrm{R}$ \\
\hline 995 & & & & ONSITE & SESPMNT & $\mathrm{R}$ \\
\hline 996 & & & & ONSITE & SESPMNT & $\mathrm{R}$ \\
\hline 997 & & & & & SESPMNT & $\mathrm{R}$ \\
\hline 998 & & & & & SESPMNT & $\mathrm{R}$ \\
\hline 999 & & & & & SESPMNT & $\mathrm{R}$ \\
\hline 1000 & & & & & SESPMNT & $\mathrm{R}$ \\
\hline 1001 & & & & & SESPMNT & $\mathrm{R}$ \\
\hline 1002 & & & & & SESPMNT & $\mathrm{R}$ \\
\hline 1003 & & & & & SESPMNT & $\mathrm{R}$ \\
\hline 1004 & & & & OFFSITE & SESPMNT & $\mathrm{R}$ \\
\hline 1005 & & & & OFFSITE & SESPMNT & $\mathrm{R}$ \\
\hline 1006 & & & & OFFSITE & SESPMNT & $\mathrm{R}$ \\
\hline 1007 & & & & OFFSITE & SESPMNT & $\mathrm{R}$ \\
\hline 1008 & & & & OFFSITE & SESPMNT & $\mathrm{R}$ \\
\hline 1009 & & & & & SESPMNT & $\mathrm{R}$ \\
\hline 1010 & & & & & SESPMNT & $\mathrm{R}$ \\
\hline 1011 & & & & OFFSITE & SESPMNT & $\mathrm{R}$ \\
\hline 1012 & & & & & SESPMNT & $\mathrm{R}$ \\
\hline 1013 & & & & RIVER_SHORELINE & SESPMNT & $\mathrm{R}$ \\
\hline 1014 & & & & RIVER_SHORELINE & SESPMNT & $\mathrm{R}$ \\
\hline 1015 & & & & & SESPMNT & $\mathrm{R}$ \\
\hline 1016 & & & & & SESPMNT & $\mathrm{R}$ \\
\hline 1017 & & & & OFFSITE & SESPMNT & $\mathrm{R}$ \\
\hline 1018 & & & & RIVER_SHORELINE & SESPMNT & $\mathrm{R}$ \\
\hline 1019 & & & & & SESPMNT & $\mathrm{R}$ \\
\hline 1020 & & & & OFFSITE & SESPMNT & $\mathrm{R}$ \\
\hline 1021 & & & & OFFSITE & SESPMNT & $\mathrm{R}$ \\
\hline \begin{tabular}{|c|}
1022 \\
\end{tabular} & & & & & SESPMNT & $\mathrm{R}$ \\
\hline 1023 & & & & & SESPMNT & $\mathrm{R}$ \\
\hline 1024 & & & & ONSITE & SESPMNT & $\mathrm{R}$ \\
\hline 1025 & & & & ONSITE & SESPMNT & $\mathrm{R}$ \\
\hline 1026 & & & & & SESPMNT & $\mathrm{R}$ \\
\hline 1027 & & & & & SESPMNT & $\mathrm{R}$ \\
\hline 1028 & & & & & SESPMNT & $\mathrm{R}$ \\
\hline 1029 & & & & & SESPMNT & $\mathrm{R}$ \\
\hline 1030 & & & & & SESPMNT & $\mathrm{R}$ \\
\hline 1031 & & & & OFFSITE & SESPMNT & $\mathrm{R}$ \\
\hline 1032 & & & & OFFSITE & SESPMNT & $\mathrm{R}$ \\
\hline 1033 & & & & OFFSITE & SESPMNT & $\mathrm{R}$ \\
\hline 1034 & & & & OFFSITE & SESPMNT & $\mathrm{R}$ \\
\hline 1035 & & & & OFFSITE & SESPMNT & $\mathrm{R}$ \\
\hline 1036 & & & & & SESPMNT & $\mathrm{R}$ \\
\hline 1037 & & & & & SESPMNT & $\mathrm{R}$ \\
\hline 1038 & & & & OFFSITE & SESPMNT & $\mathrm{R}$ \\
\hline 1039 & & & & RIVER_SHORELINE & SESPMNT & $\mathrm{R}$ \\
\hline 1040 & & & & RIVER_SHORELINE & SESPMNT & $\mathrm{R}$ \\
\hline 1041 & & & & RIVER_SHORELINE & SESBMNT & ${ }_{4} R$ \\
\hline
\end{tabular}




\begin{tabular}{|c|c|c|c|c|c|c|}
\hline & $A D$ & $\mathrm{AE}$ & $\mathrm{AF}$ & $A G$ & $\mathrm{AH}$ & $\mathrm{Al}$ \\
\hline 1 & RESULT_COMMENT & COLL_SAMP_SIZE & COLL_SAMP_SIZE_UNITS & DIST_CLASS & OWNER_ID & COLLECTION_PURPOSE \\
\hline 1042 & & & & RIVER_SHORELINE & SESPMNT & $\mathrm{R}$ \\
\hline 1043 & & & & & SESPMNT & $\mathrm{R}$ \\
\hline 1044 & & & & ONSITE & SESPMNT & $\mathrm{R}$ \\
\hline 1045 & & & & ONSITE & SESPMNT & $\mathrm{R}$ \\
\hline 1046 & & & & & SESPMNT & $\mathrm{R}$ \\
\hline 1047 & & & & ONSITE & SESPMNT & $\mathrm{R}$ \\
\hline 1048 & & & & ONSITE & SESPMNT & $\mathrm{R}$ \\
\hline 1049 & & & & & SESPMNT & $\mathrm{R}$ \\
\hline 1050 & & & & OFFSITE & SESPMNT & $\mathrm{R}$ \\
\hline 1051 & & & & & SESPMNT & $\mathrm{R}$ \\
\hline $\mid 1052$ & & & & OFFSITE & SESPMNT & $\mathrm{R}$ \\
\hline 1053 & & & & & SESPMNT & $\mathrm{R}$ \\
\hline 1054 & & & & ONSITE & SESPMNT & $\mathrm{R}$ \\
\hline 1055 & & & & ONSITE & SESPMNT & $\mathrm{R}$ \\
\hline 1056 & & & & & SESPMNT & $\mathrm{R}$ \\
\hline 1057 & & & & & SESPMNT & $\mathrm{R}$ \\
\hline 1058 & & & & & SESPMNT & $\mathrm{R}$ \\
\hline 1059 & & & & & SESPMNT & $\mathrm{R}$ \\
\hline 1060 & & & & & SESPMNT & $\mathrm{R}$ \\
\hline 1061 & & & & OFFSITE & SESPMNT & $\mathrm{R}$ \\
\hline 1062 & & & & OFFSITE & SESPMNT & $\mathrm{R}$ \\
\hline 1063 & & & & OFFSITE & SESPMNT & $\mathrm{R}$ \\
\hline 1064 & & & & OFFSITE & SESPMNT & $\mathrm{R}$ \\
\hline 1065 & & & & OFFSITE & SESPMNT & $\mathrm{R}$ \\
\hline 1066 & & & & & SESPMNT & $\mathrm{R}$ \\
\hline 1067 & & & & OFFSITE & SESPMNT & $\mathrm{R}$ \\
\hline 1068 & & & & & SESPMNT & $\mathrm{R}$ \\
\hline 1069 & & & & OFFSITE & SESPMNT & $\mathrm{R}$ \\
\hline 1070 & & & & RIVER_SHORELINE & SESPMNT & $\mathrm{R}$ \\
\hline 1071 & & & & RIVER_SHORELINE & SESPMNT & $\mathrm{R}$ \\
\hline 1072 & & & & RIVER_SHORELINE & SESPMNT & $\mathrm{R}$ \\
\hline 1073 & & & & RIVER_SHORELINE & SESPMNT & $\mathrm{R}$ \\
\hline 1074 & & & & & SESPMNT & $\mathrm{R}$ \\
\hline 1075 & & & & & SESPMNT & $\mathrm{R}$ \\
\hline 1076 & & & & ONSITE & SESPMNT & $\mathrm{R}$ \\
\hline 1077 & & & & ONSITE & SESPMNT & $\mathrm{R}$ \\
\hline 1078 & & & & & SESPMNT & $\mathrm{R}$ \\
\hline 1079 & & & & & SESPMNT & $\mathrm{R}$ \\
\hline 1080 & & & & & SESPMNT & $\mathrm{R}$ \\
\hline 1081 & & & & & SESPMNT & $\mathrm{R}$ \\
\hline 1082 & & & & & SESPMNT & $\mathrm{R}$ \\
\hline 1083 & & & & OFFSITE & SESPMNT & $\mathrm{R}$ \\
\hline 1084 & & & & OFFSITE & SESPMNT & $\mathrm{R}$ \\
\hline 1085 & & & & OFFSITE & SESPMNT & $\mathrm{R}$ \\
\hline 1086 & & & & OFFSITE & SESPMNT & $\mathrm{R}$ \\
\hline 1087 & & & & OFFSITE & SESPMNT & $\mathrm{R}$ \\
\hline 1088 & & & & RIVER_SHORELINE & SESPMNT & $\mathrm{R}$ \\
\hline 1089 & & & & RIVER_SHORELINE & SESPMNT & $\mathrm{R}$ \\
\hline 1090 & & & & RIVER_SHORELINE & SESPMNT & $\mathrm{R}$ \\
\hline 1091 & & & & RIVER_SHORELINE & SESPMNT & $\mathrm{R}$ \\
\hline 1092 & & & & & SESPMNT & $\mathrm{R}$ \\
\hline 1093 & & & & OFFSITE & SESBMNT & $R$ \\
\hline
\end{tabular}




\begin{tabular}{|c|c|c|c|c|c|c|}
\hline & $A D$ & $\mathrm{AE}$ & $\mathrm{AF}$ & $A G$ & $\mathrm{AH}$ & $\mathrm{Al}$ \\
\hline 1 & RESULT_COMMENT & COLL_SAMP_SIZE & COLL_SAMP_SIZE_UNITS & DIST_CLASS & OWNER_ID & COLLECTION_PURPOSE \\
\hline 1094 & & & & & SESPMNT & $\mathrm{R}$ \\
\hline 1095 & & & & OFFSITE & SESPMNT & $\mathrm{R}$ \\
\hline 1096 & & & & & SESPMNT & $\mathrm{R}$ \\
\hline 1097 & & & & & SESPMNT & $\mathrm{R}$ \\
\hline 1098 & & & & & SESPMNT & $\mathrm{R}$ \\
\hline 1099 & & & & & SESPMNT & $\mathrm{R}$ \\
\hline 1100 & & & & & SESPMNT & $\mathrm{R}$ \\
\hline 1101 & & & & & SESPMNT & $\mathrm{R}$ \\
\hline 1102 & & & & & SESPMNT & $\mathrm{R}$ \\
\hline 1103 & & & & OFFSITE & SESPMNT & $\mathrm{R}$ \\
\hline 1104 & & & & OFFSITE & SESPMNT & $\mathrm{R}$ \\
\hline 1105 & & & & OFFSITE & SESPMNT & $\mathrm{R}$ \\
\hline 1106 & & & & OFFSITE & SESPMNT & $\mathrm{R}$ \\
\hline 1107 & & & & OFFSITE & SESPMNT & $\mathrm{R}$ \\
\hline 1108 & & & & ONSITE & SESPMNT & $\mathrm{R}$ \\
\hline 1109 & & & & ONSITE & SESPMNT & $\mathrm{R}$ \\
\hline 1110 & & & & & SESPMNT & $\mathrm{R}$ \\
\hline 1111 & & & & OFFSITE & SESPMNT & $\mathrm{R}$ \\
\hline 1112 & & & & & SESPMNT & $\mathrm{R}$ \\
\hline 1113 & & & & ONSITE & SESPMNT & $\mathrm{R}$ \\
\hline 1114 & & & & ONSITE & SESPMNT & $\mathrm{R}$ \\
\hline 1115 & & & & & SESPMNT & $\mathrm{R}$ \\
\hline 1116 & & & & & SESPMNT & $\mathrm{R}$ \\
\hline 11117 & & & & & SESPMNT & $\mathrm{R}$ \\
\hline 1118 & & & & ONSITE & SESPMNT & $\mathrm{R}$ \\
\hline 1119 & & & & ONSITE & SESPMNT & $\mathrm{R}$ \\
\hline 1120 & & & & & SESPMNT & $\mathrm{R}$ \\
\hline 1121 & & & & OFFSITE & SESPMNT & $\mathrm{R}$ \\
\hline 1122 & & & & RIVER_SHORELINE & SESPMNT & $\mathrm{R}$ \\
\hline 1123 & & & & RIVER_SHORELINE & SESPMNT & $\mathrm{R}$ \\
\hline 1124 & & & & RIVER_SHORELINE & SESPMNT & $\mathrm{R}$ \\
\hline 1125 & & & & RIVER_SHORELINE & SESPMNT & $\mathrm{R}$ \\
\hline 1126 & & & & & SESPMNT & $\mathrm{R}$ \\
\hline 1127 & & & & OFFSITE & SESPMNT & $\mathrm{R}$ \\
\hline 1128 & & & & & SESPMNT & $\mathrm{R}$ \\
\hline 1129 & & & & & SESPMNT & $\mathrm{R}$ \\
\hline 1130 & & & & OFFSITE & SESPMNT & $\mathrm{R}$ \\
\hline 1131 & & & & ONSITE & SESPMNT & $\mathrm{R}$ \\
\hline 1132 & & & & ONSITE & SESPMNT & $\mathrm{R}$ \\
\hline 1133 & & & & & SESPMNT & $\mathrm{R}$ \\
\hline 1134 & & & & & SESPMNT & $\mathrm{R}$ \\
\hline 1135 & & & & & SESPMNT & $\mathrm{R}$ \\
\hline 1136 & & & & & SESPMNT & $\mathrm{R}$ \\
\hline \begin{tabular}{|l|l|}
1137 \\
\end{tabular} & & & & & SESPMNT & $\mathrm{R}$ \\
\hline 1138 & & & & & SESPMNT & $\mathrm{R}$ \\
\hline 1139 & & & & OFFSITE & SESPMNT & $\mathrm{R}$ \\
\hline 1140 & & & & OFFSITE & SESPMNT & $\mathrm{R}$ \\
\hline 1141 & & & & OFFSITE & SESPMNT & $\mathrm{R}$ \\
\hline 1142 & & & & OFFSITE & SESPMNT & $\mathrm{R}$ \\
\hline 1143 & & & & OFFSITE & SESPMNT & $\mathrm{R}$ \\
\hline 1144 & & & & & SESPMNT & $\mathrm{R}$ \\
\hline \begin{tabular}{|c|}
1145 \\
\end{tabular} & & & & OFFSITE & SESBMNT & $\mathrm{R}$ \\
\hline
\end{tabular}




\begin{tabular}{|c|c|c|c|c|c|c|}
\hline & $\mathrm{AD}$ & $\mathrm{AE}$ & $\mathrm{AF}$ & AG & $\mathrm{AH}$ & $\mathrm{Al}$ \\
\hline 1 & RESULT_COMMENT & COLL_SAMP_SIZE & COLL_SAMP_SIZE_UNITS & DIST_CLASS & OWNER_ID & COLLECTION_PURPOSE \\
\hline 1146 & & & & RIVER_SHORELINE & SESPMNT & $\mathrm{R}$ \\
\hline 1147 & & & & RIVER_SHORELINE & SESPMNT & $\mathrm{R}$ \\
\hline 1148 & & & & RIVER_SHORELINE & SESPMNT & $\mathrm{R}$ \\
\hline 1149 & & & & RIVER_SHORELINE & SESPMNT & $\mathrm{R}$ \\
\hline 1150 & & & & & SESPMNT & $\mathrm{R}$ \\
\hline 1151 & & & & OFFSITE & SESPMNT & $\mathrm{R}$ \\
\hline 1152 & & & & & SESPMNT & $\mathrm{R}$ \\
\hline 1153 & & & & OFFSITE & SESPMNT & $\mathrm{R}$ \\
\hline 1154 & & & & & SESPMNT & $\mathrm{R}$ \\
\hline 1155 & & & & ONSITE & SESPMNT & $\mathrm{R}$ \\
\hline 1156 & & & & ONSITE & SESPMNT & $\mathrm{R}$ \\
\hline 1157 & & & & OFFSITE & SESPMNT & $\mathrm{R}$ \\
\hline 1158 & & & & OFFSITE & SESPMNT & $\mathrm{R}$ \\
\hline 1159 & & & & OFFSITE & SESPMNT & $\mathrm{R}$ \\
\hline 1160 & & & & OFFSITE & SESPMNT & $\mathrm{R}$ \\
\hline 1161 & & & & OFFSITE & SESPMNT & $\mathrm{R}$ \\
\hline 1162 & & & & & SESPMNT & $\mathrm{R}$ \\
\hline 1163 & & & & & SESPMNT & $\mathrm{R}$ \\
\hline 1164 & & & & & SESPMNT & $\mathrm{R}$ \\
\hline 1165 & & & & & SESPMNT & $\mathrm{R}$ \\
\hline 1166 & & & & & SESPMNT & $R$ \\
\hline 1167 & & & & & SESPMNT & $\mathrm{R}$ \\
\hline 1168 & & & & OFFSITE & SESPMNT & $\mathrm{R}$ \\
\hline 1169 & & & & RIVER_SHORELINE & SESPMNT & $\mathrm{R}$ \\
\hline 1170 & & & & RIVER_SHORELINE & SESPMNT & $\mathrm{R}$ \\
\hline 1171 & & & & RIVER_SHORELINE & SESPMNT & $\mathrm{R}$ \\
\hline 1172 & & & & RIVER_SHORELINE & SESPMNT & $\mathrm{R}$ \\
\hline 1173 & & & & & SESPMNT & $\mathrm{R}$ \\
\hline 1174 & & & & OFFSITE & SESPMNT & $\mathrm{R}$ \\
\hline 1175 & & & & & SESPMNT & $\mathrm{R}$ \\
\hline 1176 & & & & & SESPMNT & $\mathrm{R}$ \\
\hline 1177 & & & & OFFSITE & SESPMNT & $\mathrm{R}$ \\
\hline 1178 & & & & & SESPMNT & $R$ \\
\hline 1179 & & & & & SESPMNT & $\mathrm{R}$ \\
\hline 1180 & & & & & SESPMNT & $\mathrm{R}$ \\
\hline 1181 & & & & & SESPMNT & $\mathrm{R}$ \\
\hline 1182 & & & & & SESPMNT & $\mathrm{R}$ \\
\hline \begin{tabular}{|l|l|}
1183 \\
\end{tabular} & & & & & SESPMNT & $R$ \\
\hline 1184 & & & & OFFSITE & SESPMNT & $R$ \\
\hline 1185 & & & & OFFSITE & SESPMNT & $R$ \\
\hline 1186 & & & & OFFSITE & SESPMNT & $R$ \\
\hline 1187 & & & & OFFSITE & SESPMNT & $\mathrm{R}$ \\
\hline 1188 & & & & OFFSITE & SESPMNT & $R$ \\
\hline 1189 & & & & OFFSITE & SESPMNT & $\mathrm{R}$ \\
\hline 1190 & & & & ONSITE & SESPMNT & $R$ \\
\hline 1191 & & & & ONSITE & SESPMNT & $R$ \\
\hline 1192 & & & & ONSITE & SESPMNT & $R$ \\
\hline 1193 & & & & & SESPMNT & $\mathrm{R}$ \\
\hline 1194 & & & & OFFSITE & SESPMNT & $\mathrm{R}$ \\
\hline 1195 & & & & & SESPMNT & $\mathrm{R}$ \\
\hline 1196 & & & & OFFSITE & SESPMNT & $R$ \\
\hline \begin{tabular}{|l|}
1197 \\
\end{tabular} & & & & & SESBMNT & $R$ \\
\hline
\end{tabular}




\begin{tabular}{|c|c|c|c|c|c|c|}
\hline & $A D$ & $\mathrm{AE}$ & $\mathrm{AF}$ & $A G$ & $\mathrm{AH}$ & $\mathrm{Al}$ \\
\hline 1 & RESULT_COMMENT & COLL_SAMP_SIZE & COLL_SAMP_SIZE_UNITS & DIST_CLASS & OWNER_ID & COLLECTION_PURPOSE \\
\hline 1198 & & & & RIVER_SHORELINE & SESPMNT & $\mathrm{R}$ \\
\hline 1199 & & & & RIVER_SHORELINE & SESPMNT & $\mathrm{R}$ \\
\hline 1200 & & & & RIVER_SHORELINE & SESPMNT & $\mathrm{R}$ \\
\hline 1201 & & & & RIVER_SHORELINE & SESPMNT & $\mathrm{R}$ \\
\hline 1202 & & & & RIVER_SHORELINE & SESPMNT & $\mathrm{R}$ \\
\hline 1203 & & & & & SESPMNT & $\mathrm{R}$ \\
\hline 1204 & & & & OFFSITE & SESPMNT & $\mathrm{R}$ \\
\hline 1205 & & & & OFFSITE & SESPMNT & $\mathrm{R}$ \\
\hline \begin{tabular}{|l|l|}
1206 \\
\end{tabular} & & & & ONSITE & SESPMNT & $\mathrm{R}$ \\
\hline \begin{tabular}{|c|}
1207 \\
\end{tabular} & & & & ONSITE & SESPMNT & $\mathrm{R}$ \\
\hline 1208 & & & & & SESPMNT & $\mathrm{R}$ \\
\hline 1209 & & & & & SESPMNT & $\mathrm{R}$ \\
\hline 1210 & & & & & SESPMNT & $\mathrm{R}$ \\
\hline 1211 & & & & & SESPMNT & $\mathrm{R}$ \\
\hline 1212 & & & & & SESPMNT & $\mathrm{R}$ \\
\hline 1213 & & & & OFFSITE & SESPMNT & $\mathrm{R}$ \\
\hline 1214 & & & & OFFSITE & SESPMNT & $\mathrm{R}$ \\
\hline 1215 & & & & OFFSITE & SESPMNT & $\mathrm{R}$ \\
\hline 1216 & & & & OFFSITE & SESPMNT & $\mathrm{R}$ \\
\hline 1217 & & & & OFFSITE & SESPMNT & $\mathrm{R}$ \\
\hline 1218 & & & & & SESPMNT & $\mathrm{R}$ \\
\hline 1219 & & & & ONSITE & SESPMNT & $\mathrm{R}$ \\
\hline 1220 & & & & ONSITE & SESPMNT & $\mathrm{R}$ \\
\hline 1221 & & & & & SESPMNT & $\mathrm{R}$ \\
\hline 1222 & & & & & SESPMNT & $\mathrm{R}$ \\
\hline 1223 & & & & & SESPMNT & $\mathrm{R}$ \\
\hline 1224 & & & & & SESPMNT & $\mathrm{R}$ \\
\hline 1225 & & & & ONSITE & SESPMNT & $\mathrm{R}$ \\
\hline \begin{tabular}{|c|}
1226 \\
\end{tabular} & & & & ONSITE & SESPMNT & $\mathrm{R}$ \\
\hline \begin{tabular}{|l|l|}
1227 \\
\end{tabular} & & & & OFFSITE & SESPMNT & $\mathrm{R}$ \\
\hline 1228 & & & & & SESPMNT & $\mathrm{R}$ \\
\hline 1229 & & & & & SESPMNT & $\mathrm{R}$ \\
\hline 1230 & & & & RIVER_SHORELINE & SESPMNT & $\mathrm{R}$ \\
\hline 1231 & & & & RIVER_SHORELINE & SESPMNT & $\mathrm{R}$ \\
\hline 1232 & & & & RIVER_SHORELINE & SESPMNT & $\mathrm{R}$ \\
\hline 1233 & & & & RIVER_SHORELINE & SESPMNT & $\mathrm{R}$ \\
\hline 1234 & & & & RIVER_SHORELINE & SESPMNT & $\mathrm{R}$ \\
\hline 1235 & & & & & SESPMNT & $\mathrm{R}$ \\
\hline 1236 & & & & OFFSITE & SESPMNT & $\mathrm{R}$ \\
\hline 1237 & & & & & SESPMNT & $\mathrm{R}$ \\
\hline 1238 & & & & OFFSITE & SESPMNT & $\mathrm{R}$ \\
\hline 1239 & & & & ONSITE & SESPMNT & $\mathrm{R}$ \\
\hline 1240 & & & & ONSITE & SESPMNT & $\mathrm{R}$ \\
\hline 1241 & & & & & SESPMNT & $\mathrm{R}$ \\
\hline 1242 & & & & & SESPMNT & $\mathrm{R}$ \\
\hline 1243 & & & & & SESPMNT & $\mathrm{R}$ \\
\hline 1244 & & & & & SESPMNT & $\mathrm{R}$ \\
\hline 1245 & & & & OFFSITE & SESPMNT & $\mathrm{R}$ \\
\hline \begin{tabular}{|l|l|}
1246 \\
\end{tabular} & & & & OFFSITE & SESPMNT & $\mathrm{R}$ \\
\hline \begin{tabular}{|l|l|}
1247 \\
\end{tabular} & & & & OFFSITE & SESPMNT & $\mathrm{R}$ \\
\hline 1248 & & & & OFFSITE & SESPMNT & $\mathrm{R}$ \\
\hline 1249 & & & & OFFSITE & SESBMNT & $R$ \\
\hline
\end{tabular}




\begin{tabular}{|c|c|c|c|c|c|c|}
\hline & $\mathrm{AD}$ & $\mathrm{AE}$ & $\mathrm{AF}$ & $\mathrm{AG}$ & $\mathrm{AH}$ & $\mathrm{Al}$ \\
\hline 1 & RESULT_COMMENT & COLL_SAMP_SIZE & COLL_SAMP_SIZE_UNITS & DIST_CLASS & OWNER_ID & COLLECTION_PURPOSE \\
\hline 1250 & & & & OFFSITE & SESPMNT & $\mathrm{R}$ \\
\hline 1251 & & & & OFFSITE & SESPMNT & $\mathrm{R}$ \\
\hline 1252 & & & & & SESPMNT & $\mathrm{R}$ \\
\hline 1253 & & & & & SESPMNT & $\mathrm{R}$ \\
\hline 1254 & & & & RIVER_SHORELINE & SESPMNT & $\mathrm{R}$ \\
\hline 1255 & & & & RIVER_SHORELINE & SESPMNT & $\mathrm{R}$ \\
\hline 1256 & & & & RIVER_SHORELINE & SESPMNT & $\mathrm{R}$ \\
\hline 1257 & & & & RIVER_SHORELINE & SESPMNT & $\mathrm{R}$ \\
\hline 1258 & & & & RIVER_SHORELINE & SESPMNT & $\mathrm{R}$ \\
\hline 1259 & & & & & SESPMNT & $\mathrm{R}$ \\
\hline 1260 & & & & OFFSITE & SESPMNT & $\mathrm{R}$ \\
\hline 1261 & & & & & SESPMNT & $\mathrm{R}$ \\
\hline 1262 & & & & OFFSITE & SESPMNT & $\mathrm{R}$ \\
\hline 1263 & & & & & SESPMNT & $\mathrm{R}$ \\
\hline 1264 & & & & OFFSITE & SESPMNT & $\mathrm{R}$ \\
\hline 1265 & & & & ONSITE & SESPMNT & $\mathrm{R}$ \\
\hline 1266 & & & & ONSITE & SESPMNT & $\mathrm{R}$ \\
\hline 1267 & & & & ONSITE & SESPMNT & $\mathrm{R}$ \\
\hline 1268 & & & & & SESPMNT & $\mathrm{R}$ \\
\hline 1269 & & & & & SESPMNT & $\mathrm{R}$ \\
\hline 1270 & & & & & SESPMNT & $\mathrm{R}$ \\
\hline 1271 & & & & & SESPMNT & $\mathrm{R}$ \\
\hline 1272 & & & & OFFSITE & SESPMNT & $\mathrm{R}$ \\
\hline 1273 & & & & OFFSITE & SESPMNT & $\mathrm{R}$ \\
\hline 1274 & & & & OFFSITE & SESPMNT & $\mathrm{R}$ \\
\hline 1275 & & & & OFFSITE & SESPMNT & $\mathrm{R}$ \\
\hline 1276 & & & & OFFSITE & SESPMNT & $\mathrm{R}$ \\
\hline \begin{tabular}{|l|}
1277 \\
\end{tabular} & & & & OFFSITE & SESPMNT & $\mathrm{R}$ \\
\hline 1278 & & & & & SESPMNT & $\mathrm{R}$ \\
\hline 1279 & & & & & SESPMNT & $\mathrm{R}$ \\
\hline 1280 & & & & & SESPMNT & $\mathrm{R}$ \\
\hline 1281 & & & & & SESPMNT & $\mathrm{R}$ \\
\hline 1282 & & & & & SESPMNT & $\mathrm{R}$ \\
\hline \begin{tabular}{|c|}
1283 \\
\end{tabular} & & & & OFFSITE & SESPMNT & $\mathrm{R}$ \\
\hline $\mid 1284$ & & & & RIVER_SHORELINE & SESPMNT & $\mathrm{R}$ \\
\hline 1285 & & & & RIVER_SHORELINE & SESPMNT & $\mathrm{R}$ \\
\hline \begin{tabular}{|l|l|}
1286 \\
\end{tabular} & & & & RIVER_SHORELINE & SESPMNT & $\mathrm{R}$ \\
\hline \begin{tabular}{|l|}
1287 \\
\end{tabular} & & & & RIVER_SHORELINE & SESPMNT & $\mathrm{R}$ \\
\hline \begin{tabular}{|l|l|}
1288 \\
\end{tabular} & & & & RIVER_SHORELINE & SESPMNT & $\mathrm{R}$ \\
\hline \begin{tabular}{|l|l|}
1289 \\
\end{tabular} & & & & & SESPMNT & $\mathrm{R}$ \\
\hline \begin{tabular}{|l|}
1290 \\
\end{tabular} & & & & OFFSITE & SESPMNT & $\mathrm{R}$ \\
\hline \begin{tabular}{|l|}
1291 \\
\end{tabular} & & & & & SESPMNT & $\mathrm{R}$ \\
\hline \begin{tabular}{|l|}
1292 \\
\end{tabular} & & & & OFFSITE & SESPMNT & $\mathrm{R}$ \\
\hline \begin{tabular}{|l|l|}
1293 \\
\end{tabular} & & & & ONSITE & SESPMNT & $\mathrm{R}$ \\
\hline \begin{tabular}{|l|}
1294 \\
\end{tabular} & & & & ONSITE & SESPMNT & $\mathrm{R}$ \\
\hline \begin{tabular}{|l|l|}
1295 \\
\end{tabular} & & & & & SESPMNT & $\mathrm{R}$ \\
\hline \begin{tabular}{|l|l|}
1296 \\
\end{tabular} & & & & & SESPMNT & $\mathrm{R}$ \\
\hline \begin{tabular}{|l|}
1297 \\
\end{tabular} & & & & & SESPMNT & $\mathrm{R}$ \\
\hline \begin{tabular}{|l|}
1298 \\
\end{tabular} & & & & & SESPMNT & $\mathrm{R}$ \\
\hline \begin{tabular}{|l|l|}
1299 \\
\end{tabular} & & & & & SESPMNT & $\mathrm{R}$ \\
\hline 1300 & & & & OFFSITE & SESPMNT & $\mathrm{R}$ \\
\hline \begin{tabular}{|l|}
1301 \\
\end{tabular} & & & & OFFSITE & SESBMNT & $R$ \\
\hline
\end{tabular}




\begin{tabular}{|c|c|c|c|c|c|c|}
\hline & $A D$ & $\mathrm{AE}$ & $\mathrm{AF}$ & $A G$ & $\mathrm{AH}$ & $\mathrm{Al}$ \\
\hline 1 & RESULT_COMMENT & COLL_SAMP_SIZE & COLL_SAMP_SIZE_UNITS & DIST_CLASS & OWNER_ID & COLLECTION_PURPOSE \\
\hline 1302 & & & & OFFSITE & SESPMNT & $\mathrm{R}$ \\
\hline 1303 & & & & OFFSITE & SESPMNT & $\mathrm{R}$ \\
\hline 1304 & & & & OFFSITE & SESPMNT & $\mathrm{R}$ \\
\hline 1305 & & & & OFFSITE & SESPMNT & $\mathrm{R}$ \\
\hline 1306 & & & & & SESPMNT & $\mathrm{R}$ \\
\hline 1307 & & & & & SESPMNT & $\mathrm{R}$ \\
\hline 1308 & & & & OFFSITE & SESPMNT & $\mathrm{R}$ \\
\hline 1309 & & & & RIVER_SHORELINE & SESPMNT & $\mathrm{R}$ \\
\hline 1310 & & & & RIVER_SHORELINE & SESPMNT & $\mathrm{R}$ \\
\hline 1311 & & & & RIVER_SHORELINE & SESPMNT & $\mathrm{R}$ \\
\hline 1312 & & & & RIVER_SHORELINE & SESPMNT & $\mathrm{R}$ \\
\hline 1313 & & & & RIVER_SHORELINE & SESPMNT & $\mathrm{R}$ \\
\hline 1314 & & & & & SESPMNT & $\mathrm{R}$ \\
\hline 1315 & & & & ONSITE & SESPMNT & $\mathrm{R}$ \\
\hline 1316 & & & & ONSITE & SESPMNT & $\mathrm{R}$ \\
\hline 1317 & & & & & SESPMNT & $\mathrm{R}$ \\
\hline 1318 & & & & & SESPMNT & $\mathrm{R}$ \\
\hline 1319 & & & & & SESPMNT & $\mathrm{R}$ \\
\hline 1320 & & & & ONSITE & SESPMNT & $\mathrm{R}$ \\
\hline \begin{tabular}{|c|}
1321 \\
\end{tabular} & & & & ONSITE & SESPMNT & $\mathrm{R}$ \\
\hline 1322 & & & & & SESPMNT & $\mathrm{R}$ \\
\hline 1323 & & & & OFFSITE & SESPMNT & $\mathrm{R}$ \\
\hline 1324 & & & & & SESPMNT & $\mathrm{R}$ \\
\hline 1325 & & & & OFFSITE & SESPMNT & $\mathrm{R}$ \\
\hline 1326 & & & & ONSITE & SESPMNT & $\mathrm{R}$ \\
\hline 1327 & & & & ONSITE & SESPMNT & $\mathrm{R}$ \\
\hline 1328 & & & & & SESPMNT & $\mathrm{R}$ \\
\hline 1329 & & & & & SESPMNT & $\mathrm{R}$ \\
\hline 1330 & & & & & SESPMNT & $\mathrm{R}$ \\
\hline 1331 & & & & & SESPMNT & $\mathrm{R}$ \\
\hline 1332 & & & & & SESPMNT & $\mathrm{R}$ \\
\hline 1333 & & & & OFFSITE & SESPMNT & $\mathrm{R}$ \\
\hline 1334 & & & & OFFSITE & SESPMNT & $\mathrm{R}$ \\
\hline 1335 & & & & OFFSITE & SESPMNT & $\mathrm{R}$ \\
\hline \begin{tabular}{|c|}
1336 \\
\end{tabular} & & & & OFFSITE & SESPMNT & $\mathrm{R}$ \\
\hline \begin{tabular}{|c|}
1337 \\
\end{tabular} & & & & OFFSITE & SESPMNT & $\mathrm{R}$ \\
\hline 1338 & & & & OFFSITE & SESPMNT & $\mathrm{R}$ \\
\hline 1339 & & & & & SESPMNT & $\mathrm{R}$ \\
\hline 1340 & & & & & SESPMNT & $\mathrm{R}$ \\
\hline 1341 & & & & OFFSITE & SESPMNT & $\mathrm{R}$ \\
\hline 1342 & & & & RIVER_SHORELINE & SESPMNT & $\mathrm{R}$ \\
\hline 1343 & & & & RIVER_SHORELINE & SESPMNT & $\mathrm{R}$ \\
\hline 1344 & & & & RIVER_SHORELINE & SESPMNT & $\mathrm{R}$ \\
\hline 1345 & & & & RIVER_SHORELINE & SESPMNT & $\mathrm{R}$ \\
\hline 1346 & & & & RIVER_SHORELINE & SESPMNT & $\mathrm{R}$ \\
\hline 1347 & & & & & SESPMNT & $\mathrm{R}$ \\
\hline 1348 & & & & OFFSITE & SESPMNT & $\mathrm{R}$ \\
\hline 1349 & & & & & SESPMNT & $\mathrm{R}$ \\
\hline 1350 & & & & OFFSITE & SESPMNT & $\mathrm{R}$ \\
\hline 1351 & & & & & SESPMNT & $\mathrm{R}$ \\
\hline 1352 & & & & OFFSITE & SESPMNT & $\mathrm{R}$ \\
\hline 1353 & & & & & SESBMNT & $R$ \\
\hline
\end{tabular}




\begin{tabular}{|c|c|c|c|c|c|c|}
\hline & $A D$ & $\mathrm{AE}$ & $\mathrm{AF}$ & $A G$ & $\mathrm{AH}$ & $\mathrm{Al}$ \\
\hline 1 & RESULT_COMMENT & COLL_SAMP_SIZE & COLL_SAMP_SIZE_UNITS & DIST_CLASS & OWNER_ID & COLLECTION_PURPOSE \\
\hline 1354 & & & & ONSITE & SESPMNT & $\mathrm{R}$ \\
\hline 1355 & & & & ONSITE & SESPMNT & $\mathrm{R}$ \\
\hline 1356 & & & & ONSITE & SESPMNT & $\mathrm{R}$ \\
\hline 1357 & & & & & SESPMNT & $\mathrm{R}$ \\
\hline 1358 & & & & ONSITE & SESPMNT & $\mathrm{R}$ \\
\hline 1359 & & & & & SESPMNT & $\mathrm{R}$ \\
\hline 1360 & & & & & SESPMNT & $\mathrm{R}$ \\
\hline 1361 & & & & ONSITE & SESPMNT & $\mathrm{R}$ \\
\hline 1362 & & & & OFFSITE & SESPMNT & $\mathrm{R}$ \\
\hline 1363 & & & & OFFSITE & SESPMNT & $\mathrm{R}$ \\
\hline 1364 & & & & OFFSITE & SESPMNT & $\mathrm{R}$ \\
\hline 1365 & & & & OFFSITE & SESPMNT & $\mathrm{R}$ \\
\hline 1366 & & & & OFFSITE & SESPMNT & $\mathrm{R}$ \\
\hline 1367 & & & & & SESPMNT & $\mathrm{R}$ \\
\hline 1368 & & & & ONSITE & SESPMNT & $\mathrm{R}$ \\
\hline 1369 & & & & ONSITE & SESPMNT & $\mathrm{R}$ \\
\hline 1370 & & & & OFFSITE & SESPMNT & $\mathrm{R}$ \\
\hline 1371 & & & & RIVER_SHORELINE & SESPMNT & $\mathrm{R}$ \\
\hline 1372 & & & & RIVER_SHORELINE & SESPMNT & $\mathrm{R}$ \\
\hline 1373 & & & & & SESPMNT & $\mathrm{R}$ \\
\hline 1374 & See sample record for comment. & & & & SESPMNT & $\mathrm{R}$ \\
\hline 1375 & See sample record for comment. & & & & SESPMNT & $\mathrm{R}$ \\
\hline 1376 & & & & & SESPMNT & $\mathrm{R}$ \\
\hline 1377 & & & & & SESPMNT & $\mathrm{R}$ \\
\hline 1378 & & & & ONSITE & SESPMNT & $\mathrm{R}$ \\
\hline 1379 & & & & ONSITE & SESPMNT & $\mathrm{R}$ \\
\hline 1380 & & & & & SESPMNT & $\mathrm{R}$ \\
\hline 1381 & & & & ONSITE & SESPMNT & $\mathrm{R}$ \\
\hline 1382 & & & & ONSITE & SESPMNT & $\mathrm{R}$ \\
\hline 1383 & & & & ONSITE & SESPMNT & $\mathrm{R}$ \\
\hline 1384 & & & & & SESPMNT & $\mathrm{R}$ \\
\hline 1385 & & & & & SESPMNT & $\mathrm{R}$ \\
\hline 1386 & & & & & SESPMNT & $\mathrm{R}$ \\
\hline \begin{tabular}{|c|}
1387 \\
\end{tabular} & & & & ONSITE & SESPMNT & $\mathrm{R}$ \\
\hline 1388 & & & & & SESPMNT & $\mathrm{R}$ \\
\hline 1389 & & & & & SESPMNT & $\mathrm{R}$ \\
\hline 1390 & & & & & SESPMNT & $\mathrm{R}$ \\
\hline 1391 & & & & & SESPMNT & $\mathrm{R}$ \\
\hline 1392 & & & & OFFSITE & SESPMNT & $\mathrm{R}$ \\
\hline 1393 & & & & OFFSITE & SESPMNT & $\mathrm{R}$ \\
\hline 1394 & & & & OFFSITE & SESPMNT & $\mathrm{R}$ \\
\hline 1395 & & & & OFFSITE & SESPMNT & $\mathrm{R}$ \\
\hline 1396 & & & & OFFSITE & SESPMNT & $\mathrm{R}$ \\
\hline \begin{tabular}{|c|}
1397 \\
\end{tabular} & & & & ONSITE & SESPMNT & $\mathrm{R}$ \\
\hline 1398 & & & & ONSITE & SESPMNT & $\mathrm{R}$ \\
\hline 1399 & & & & RIVER_SHORELINE & SESPMNT & $\mathrm{R}$ \\
\hline 1400 & & & & RIVER_SHORELINE & SESPMNT & $\mathrm{R}$ \\
\hline 1401 & & & & ONSITE & SESPMNT & $\mathrm{R}$ \\
\hline 1402 & & & & OFFSITE & SESPMNT & $\mathrm{R}$ \\
\hline 1403 & & & & ONSITE & SESPMNT & $\mathrm{R}$ \\
\hline 1404 & & & & ONSITE & SESPMNT & $\mathrm{R}$ \\
\hline 1405 & & & & ONSITE & SESBMNT & $R$ \\
\hline
\end{tabular}




\begin{tabular}{|c|c|c|c|c|c|c|}
\hline & $A D$ & $\mathrm{AE}$ & $\mathrm{AF}$ & $A G$ & $\mathrm{AH}$ & $\mathrm{Al}$ \\
\hline 1 & RESULT_COMMENT & COLL_SAMP_SIZE & COLL_SAMP_SIZE_UNITS & DIST_CLASS & OWNER_ID & COLLECTION_PURPOSE \\
\hline 1406 & & & & & SESPMNT & $\mathrm{R}$ \\
\hline 1407 & & & & & SESPMNT & $\mathrm{R}$ \\
\hline 1408 & & & & & SESPMNT & $\mathrm{R}$ \\
\hline 1409 & & & & ONSITE & SESPMNT & $\mathrm{R}$ \\
\hline 1410 & & & & & SESPMNT & $\mathrm{R}$ \\
\hline 1411 & & & & & SESPMNT & $\mathrm{R}$ \\
\hline 1412 & & & & & SESPMNT & $\mathrm{R}$ \\
\hline 1413 & & & & & SESPMNT & $\mathrm{R}$ \\
\hline 1414 & & & & OFFSITE & SESPMNT & $\mathrm{R}$ \\
\hline 1415 & & & & OFFSITE & SESPMNT & $\mathrm{R}$ \\
\hline 1416 & & & & OFFSITE & SESPMNT & $\mathrm{R}$ \\
\hline 1417 & & & & OFFSITE & SESPMNT & $\mathrm{R}$ \\
\hline 1418 & & & & OFFSITE & SESPMNT & $\mathrm{R}$ \\
\hline 1419 & & & & ONSITE & SESPMNT & $\mathrm{R}$ \\
\hline 1420 & & & & ONSITE & SESPMNT & $\mathrm{R}$ \\
\hline 1421 & & & & RIVER_SHORELINE & SESPMNT & $\mathrm{R}$ \\
\hline 1422 & & & & RIVER_SHORELINE & SESPMNT & $\mathrm{R}$ \\
\hline 1423 & & & & ONSITE & SESPMNT & $\mathrm{R}$ \\
\hline 1424 & & & & & SESPMNT & $\mathrm{R}$ \\
\hline 1425 & & & & ONSITE & SESPMNT & $\mathrm{R}$ \\
\hline 1426 & & & & OFFSITE & SESPMNT & $\mathrm{R}$ \\
\hline 1427 & & & & ONSITE & SESPMNT & $\mathrm{R}$ \\
\hline 1428 & & & & ONSITE & SESPMNT & $\mathrm{R}$ \\
\hline 1429 & & & & ONSITE & SESPMNT & $\mathrm{R}$ \\
\hline 1430 & & & & OFFSITE & SESPMNT & $\mathrm{R}$ \\
\hline 1431 & & & & OFFSITE & SESPMNT & $\mathrm{R}$ \\
\hline 1432 & & & & OFFSITE & SESPMNT & $\mathrm{R}$ \\
\hline 1433 & & & & OFFSITE & SESPMNT & $\mathrm{R}$ \\
\hline 1434 & & & & OFFSITE & SESPMNT & $\mathrm{R}$ \\
\hline 1435 & & & & & SESPMNT & $\mathrm{R}$ \\
\hline 1436 & & & & & SESPMNT & $\mathrm{R}$ \\
\hline 1437 & & & & & SESPMNT & $\mathrm{R}$ \\
\hline 1438 & & & & & SESPMNT & $\mathrm{R}$ \\
\hline 1439 & & & & & SESPMNT & $\mathrm{R}$ \\
\hline 1440 & & & & & SESPMNT & $\mathrm{R}$ \\
\hline \begin{tabular}{|c|}
1441 \\
\end{tabular} & & & & & SESPMNT & $\mathrm{R}$ \\
\hline 1442 & & & & ONSITE & SESPMNT & $\mathrm{R}$ \\
\hline 1443 & & & & ONSITE & SESPMNT & $\mathrm{R}$ \\
\hline 1444 & & & & & SESPMNT & $\mathrm{R}$ \\
\hline 1445 & & & & & SESPMNT & $\mathrm{R}$ \\
\hline 1446 & & & & & SESPMNT & $\mathrm{R}$ \\
\hline \begin{tabular}{|l|l|}
1447 \\
\end{tabular} & & & & & SESPMNT & $\mathrm{R}$ \\
\hline 1448 & & & & & SESPMNT & $\mathrm{R}$ \\
\hline 1449 & & & & & SESPMNT & $\mathrm{R}$ \\
\hline 1450 & & & & ONSITE & SESPMNT & $\mathrm{R}$ \\
\hline 1451 & & & & ONSITE & SESPMNT & $\mathrm{R}$ \\
\hline 1452 & & & & OFFSITE & SESPMNT & $\mathrm{R}$ \\
\hline 1453 & & & & RIVER_SHORELINE & SESPMNT & $\mathrm{R}$ \\
\hline 1454 & & & & RIVER_SHORELINE & SESPMNT & $\mathrm{R}$ \\
\hline 1455 & & & & ONSITE & SESPMNT & $\mathrm{R}$ \\
\hline 1456 & & & & ONSITE & SESPMNT & $\mathrm{R}$ \\
\hline \begin{tabular}{|l|l|}
1457 \\
\end{tabular} & & & & ONSITE & SESBMNT & $R$ \\
\hline
\end{tabular}




\begin{tabular}{|c|c|c|c|c|c|c|}
\hline & $\mathrm{AD}$ & $\mathrm{AE}$ & $\mathrm{AF}$ & AG & $\mathrm{AH}$ & $\mathrm{Al}$ \\
\hline 1 & RESULT_COMMENT & COLL_SAMP_SIZE & COLL_SAMP_SIZE_UNITS & DIST_CLASS & OWNER_ID & COLLECTION_PURPOSE \\
\hline 1458 & & & & ONSITE & SESPMNT & $\mathrm{R}$ \\
\hline 1459 & & & & & SESPMNT & $\mathrm{R}$ \\
\hline 1460 & & & & & SESPMNT & $\mathrm{R}$ \\
\hline 1461 & & & & & SESPMNT & $\mathrm{R}$ \\
\hline 1462 & & & & & SESPMNT & $\mathrm{R}$ \\
\hline 1463 & & & & & SESPMNT & $\mathrm{R}$ \\
\hline 1464 & & & & & SESPMNT & $\mathrm{R}$ \\
\hline 1465 & & & & & SESPMNT & $\mathrm{R}$ \\
\hline 1466 & & & & & SESPMNT & $\mathrm{R}$ \\
\hline \begin{tabular}{|l|l|}
1467 & -1 \\
\end{tabular} & & & & OFFSITE & SESPMNT & $\mathrm{R}$ \\
\hline 1468 & & & & OFFSITE & SESPMNT & $\mathrm{R}$ \\
\hline 1469 & & & & OFFSITE & SESPMNT & $\mathrm{R}$ \\
\hline 1470 & & & & OFFSITE & SESPMNT & $\mathrm{R}$ \\
\hline 1471 & & & & OFFSITE & SESPMNT & $\mathrm{R}$ \\
\hline 1472 & & & & ONSITE & SESPMNT & $\mathrm{R}$ \\
\hline \begin{tabular}{|c|}
1473 \\
\end{tabular} & & & & ONSITE & SESPMNT & $\mathrm{R}$ \\
\hline 1474 & & & & OFFSITE & SESPMNT & $\mathrm{R}$ \\
\hline 1475 & & & & & SESPMNT & $\mathrm{R}$ \\
\hline 1476 & & & & ONSITE & SESPMNT & $\mathrm{R}$ \\
\hline \begin{tabular}{|l|}
1477 \\
\end{tabular} & & & & RIVER_SHORELINE & SESPMNT & $\mathrm{R}$ \\
\hline 1478 & & & & RIVER_SHORELINE & SESPMNT & $\mathrm{R}$ \\
\hline \begin{tabular}{|l|l|}
1479 \\
\end{tabular} & & & & ONSITE & SESPMNT & $\mathrm{R}$ \\
\hline 1480 & & & & ONSITE & SESPMNT & $\mathrm{R}$ \\
\hline 1481 & & & & ONSITE & SESPMNT & $\mathrm{R}$ \\
\hline 1482 & & & & ONSITE & SESPMNT & $\mathrm{R}$ \\
\hline \begin{tabular}{|c|}
1483 \\
\end{tabular} & & & & ONSITE & SESPMNT & $\mathrm{R}$ \\
\hline 1484 & & & & ONSITE & SESPMNT & $\mathrm{R}$ \\
\hline 1485 & & & & & SESPMNT & $\mathrm{R}$ \\
\hline 1486 & & & & & SESPMNT & $\mathrm{R}$ \\
\hline 1487 & & & & & SESPMNT & $\mathrm{R}$ \\
\hline 1488 & & & & & SESPMNT & $\mathrm{R}$ \\
\hline $\mid 1489$ & & & & & SESPMNT & $\mathrm{R}$ \\
\hline 1490 & & & & & SESPMNT & $\mathrm{R}$ \\
\hline \begin{tabular}{|l|}
1491 \\
\end{tabular} & & & & OFFSITE & SESPMNT & $\mathrm{R}$ \\
\hline \begin{tabular}{|l|}
1492 \\
\end{tabular} & & & & OFFSITE & SESPMNT & $\mathrm{R}$ \\
\hline \begin{tabular}{|l|}
1493 \\
\end{tabular} & & & & OFFSITE & SESPMNT & $\mathrm{R}$ \\
\hline 1494 & & & & OFFSITE & SESPMNT & $\mathrm{R}$ \\
\hline 1495 & & & & OFFSITE & SESPMNT & $\mathrm{R}$ \\
\hline \begin{tabular}{|l|}
1496 \\
\end{tabular} & & & & & SESPMNT & $\mathrm{R}$ \\
\hline \begin{tabular}{|l|}
1497 \\
\end{tabular} & & & & & SESPMNT & $\mathrm{R}$ \\
\hline \begin{tabular}{|l|}
1498 \\
\end{tabular} & & & & & SESPMNT & $\mathrm{R}$ \\
\hline \begin{tabular}{|l|}
1499 \\
\end{tabular} & & & & ONSITE & SESPMNT & $\mathrm{R}$ \\
\hline 1500 & & & & OFFSITE & SESPMNT & $\mathrm{R}$ \\
\hline 1501 & & & & ONSITE & SESPMNT & $\mathrm{R}$ \\
\hline \begin{tabular}{|l|}
1502 \\
\end{tabular} & & & & ONSITE & SESPMNT & $\mathrm{R}$ \\
\hline 1503 & & & & ONSITE & SESPMNT & $\mathrm{R}$ \\
\hline \begin{tabular}{|l|}
1504 \\
\end{tabular} & & & & ONSITE & SESPMNT & $\mathrm{R}$ \\
\hline 1505 & & & & ONSITE & SESPMNT & $\mathrm{R}$ \\
\hline 1506 & & & & ONSITE & SESPMNT & $\mathrm{R}$ \\
\hline \begin{tabular}{|l|}
1507 \\
\end{tabular} & & & & & SESPMNT & $\mathrm{R}$ \\
\hline 1508 & & & & & SESPMNT & $\mathrm{R}$ \\
\hline \begin{tabular}{|l|}
1509 \\
\end{tabular} & & & & & SESBMNT & $R$ \\
\hline
\end{tabular}




\begin{tabular}{|c|c|c|c|c|c|c|}
\hline & $A D$ & $\mathrm{AE}$ & $\mathrm{AF}$ & $A G$ & $\mathrm{AH}$ & $\mathrm{Al}$ \\
\hline 1 & RESULT_COMMENT & COLL_SAMP_SIZE & COLL_SAMP_SIZE_UNITS & DIST_CLASS & OWNER_ID & COLLECTION_PURPOSE \\
\hline 1510 & & & & & SESPMNT & $\mathrm{R}$ \\
\hline 1511 & & & & & SESPMNT & $\mathrm{R}$ \\
\hline 1512 & & & & & SESPMNT & $\mathrm{R}$ \\
\hline 1513 & & & & & SESPMNT & $\mathrm{R}$ \\
\hline 1514 & & & & & SESPMNT & $\mathrm{R}$ \\
\hline 1515 & & & & ONSITE & SESPMNT & $\mathrm{R}$ \\
\hline 1516 & & & & OFFSITE & SESPMNT & $\mathrm{R}$ \\
\hline 1517 & & & & OFFSITE & SESPMNT & $\mathrm{R}$ \\
\hline 1518 & & & & OFFSITE & SESPMNT & $\mathrm{R}$ \\
\hline 1519 & & & & OFFSITE & SESPMNT & $\mathrm{R}$ \\
\hline 1520 & & & & OFFSITE & SESPMNT & $\mathrm{R}$ \\
\hline 1521 & & & & OFFSITE & SESPMNT & $\mathrm{R}$ \\
\hline 1522 & & & & & SESPMNT & $\mathrm{R}$ \\
\hline 1523 & & & & ONSITE & SESPMNT & $\mathrm{R}$ \\
\hline 1524 & & & & ONSITE & SESPMNT & $\mathrm{R}$ \\
\hline 1525 & & & & ONSITE & SESPMNT & $\mathrm{R}$ \\
\hline 1526 & & & & & SESPMNT & $\mathrm{R}$ \\
\hline \begin{tabular}{|c|}
1527 \\
\end{tabular} & & & & & SESPMNT & $\mathrm{R}$ \\
\hline 1528 & & & & & SESPMNT & $\mathrm{R}$ \\
\hline 1529 & & & & ONSITE & SESPMNT & $\mathrm{R}$ \\
\hline 1530 & & & & ONSITE & SESPMNT & $\mathrm{R}$ \\
\hline 1531 & & & & & SESPMNT & $\mathrm{R}$ \\
\hline 1532 & & & & & SESPMNT & $\mathrm{R}$ \\
\hline 1533 & & & & & SESPMNT & $\mathrm{R}$ \\
\hline 1534 & & & & & SESPMNT & $\mathrm{R}$ \\
\hline 1535 & & & & ONSITE & SESPMNT & $\mathrm{R}$ \\
\hline 1536 & & & & ONSITE & SESPMNT & $\mathrm{R}$ \\
\hline \begin{tabular}{|l|}
1537 \\
\end{tabular} & & & & ONSITE & SESPMNT & $\mathrm{R}$ \\
\hline 1538 & & & & ONSITE & SESPMNT & $\mathrm{R}$ \\
\hline 1539 & & & & & SESPMNT & $\mathrm{R}$ \\
\hline 1540 & & & & & SESPMNT & $\mathrm{R}$ \\
\hline \begin{tabular}{|l|l|}
1541 \\
\end{tabular} & & & & & SESPMNT & $\mathrm{R}$ \\
\hline 1542 & & & & & SESPMNT & $\mathrm{R}$ \\
\hline \begin{tabular}{|l|}
1543 \\
\end{tabular} & & & & & SESPMNT & $\mathrm{R}$ \\
\hline 1544 & & & & & SESPMNT & $\mathrm{R}$ \\
\hline \begin{tabular}{|l|}
1545 \\
\end{tabular} & & & & & SESPMNT & $\mathrm{R}$ \\
\hline \begin{tabular}{|l|}
1546 \\
\end{tabular} & & & & OFFSITE & SESPMNT & $\mathrm{R}$ \\
\hline \begin{tabular}{|l|}
1547 \\
\end{tabular} & & & & OFFSITE & SESPMNT & $R$ \\
\hline 1548 & & & & OFFSITE & SESPMNT & $\mathrm{R}$ \\
\hline 1549 & & & & OFFSITE & SESPMNT & $\mathrm{R}$ \\
\hline 1550 & & & & OFFSITE & SESPMNT & $\mathrm{R}$ \\
\hline \begin{tabular}{|l|l|}
1551 \\
\end{tabular} & & & & ONSITE & SESPMNT & $\mathrm{R}$ \\
\hline 1552 & & & & OFFSITE & SESPMNT & $\mathrm{R}$ \\
\hline 1553 & & & & ONSITE & SESPMNT & $\mathrm{R}$ \\
\hline 1554 & & & & ONSITE & SESPMNT & $R$ \\
\hline 1555 & & & & ONSITE & SESPMNT & $\mathrm{R}$ \\
\hline 1556 & & & & OFFSITE & SESPMNT & $\mathrm{R}$ \\
\hline \begin{tabular}{|l|}
1557 \\
\end{tabular} & & & & ONSITE & SESPMNT & $\mathrm{R}$ \\
\hline 1558 & & & & ONSITE & SESPMNT & $\mathrm{R}$ \\
\hline 1559 & & & & ONSITE & SESPMNT & $\mathrm{R}$ \\
\hline 1560 & & & & ONSITE & SESPMNT & $\mathrm{R}$ \\
\hline \begin{tabular}{|l|}
1561 \\
\end{tabular} & & & & OFFSITE & SESBMNT & $R$ \\
\hline
\end{tabular}




\begin{tabular}{|c|c|c|c|c|c|c|}
\hline & $\mathrm{AD}$ & $\mathrm{AE}$ & $\mathrm{AF}$ & AG & $\mathrm{AH}$ & $\mathrm{Al}$ \\
\hline 1 & RESULT_COMMENT & COLL_SAMP_SIZE & COLL_SAMP_SIZE_UNITS & DIST_CLASS & OWNER_ID & COLLECTION_PURPOSE \\
\hline 1562 & & & & OFFSITE & SESPMNT & $\mathrm{R}$ \\
\hline \begin{tabular}{|c|}
1563 \\
\end{tabular} & & & & & SESPMNT & $\mathrm{R}$ \\
\hline 1564 & & & & & SESPMNT & $\mathrm{R}$ \\
\hline 1565 & & & & & SESPMNT & $\mathrm{R}$ \\
\hline 1566 & & & & & SESPMNT & $\mathrm{R}$ \\
\hline \begin{tabular}{|l|}
1567 \\
\end{tabular} & & & & & SESPMNT & $\mathrm{R}$ \\
\hline 1568 & & & & & SESPMNT & $\mathrm{R}$ \\
\hline 1569 & & & & & SESPMNT & $\mathrm{R}$ \\
\hline 1570 & & & & & SESPMNT & $\mathrm{R}$ \\
\hline 1571 & & & & OFFSITE & SESPMNT & $\mathrm{R}$ \\
\hline 1572 & & & & OFFSITE & SESPMNT & $\mathrm{R}$ \\
\hline 1573 & & & & ONSITE & SESPMNT & $\mathrm{R}$ \\
\hline 1574 & & & & ONSITE & SESPMNT & $\mathrm{R}$ \\
\hline 1575 & & & & ONSITE & SESPMNT & $\mathrm{R}$ \\
\hline 1576 & & & & ONSITE & SESPMNT & $\mathrm{R}$ \\
\hline \begin{tabular}{|c|}
1577 \\
\end{tabular} & & & & ONSITE & SESPMNT & $\mathrm{R}$ \\
\hline 1578 & & & & & SESPMNT & $\mathrm{R}$ \\
\hline 1579 & & & & & SESPMNT & $\mathrm{R}$ \\
\hline 1580 & & & & & SESPMNT & $\mathrm{R}$ \\
\hline 1581 & & & & & SESPMNT & $\mathrm{R}$ \\
\hline \begin{tabular}{|c|}
1582 \\
\end{tabular} & & & & & SESPMNT & $\mathrm{R}$ \\
\hline 1583 & & & & & SESPMNT & $\mathrm{R}$ \\
\hline 1584 & & & & & SESPMNT & $\mathrm{R}$ \\
\hline 1585 & & & & & SESPMNT & $\mathrm{R}$ \\
\hline 1586 & & & & ONSITE & SESPMNT & $\mathrm{R}$ \\
\hline \begin{tabular}{|c|}
1587 \\
\end{tabular} & & & & OFFSITE & SESPMNT & $\mathrm{R}$ \\
\hline 1588 & & & & OFFSITE & SESPMNT & $\mathrm{R}$ \\
\hline 1589 & & & & OFFSITE & SESPMNT & $\mathrm{R}$ \\
\hline 1590 & & & & OFFSITE & SESPMNT & $\mathrm{R}$ \\
\hline 1591 & & & & OFFSITE & SESPMNT & $\mathrm{R}$ \\
\hline 1592 & & & & OFFSITE & SESPMNT & $\mathrm{R}$ \\
\hline 1593 & & & & ONSITE & SESPMNT & $\mathrm{R}$ \\
\hline 1594 & & & & ONSITE & SESPMNT & $\mathrm{R}$ \\
\hline 1595 & & & & ONSITE & SESPMNT & $\mathrm{R}$ \\
\hline \begin{tabular}{|l|}
1596 \\
\end{tabular} & & & & & SESPMNT & $\mathrm{R}$ \\
\hline 1597 & & & & & SESPMNT & $\mathrm{R}$ \\
\hline 1598 & & & & ONSITE & SESPMNT & $\mathrm{R}$ \\
\hline 1599 & & & & ONSITE & SESPMNT & $\mathrm{R}$ \\
\hline \begin{tabular}{|l|}
1600 \\
\end{tabular} & & & & ONSITE & SESPMNT & $\mathrm{R}$ \\
\hline \begin{tabular}{|l|}
1601 \\
\end{tabular} & & & & & SESPMNT & $\mathrm{R}$ \\
\hline \begin{tabular}{|l|}
1602 \\
\end{tabular} & & & & & SESPMNT & $\mathrm{R}$ \\
\hline \begin{tabular}{|l|}
1603 \\
\end{tabular} & & & & & SESPMNT & $\mathrm{R}$ \\
\hline 1604 & & & & & SESPMNT & $\mathrm{R}$ \\
\hline 1605 & & & & & SESPMNT & $\mathrm{R}$ \\
\hline \begin{tabular}{|l|}
1606 \\
\end{tabular} & & & & & SESPMNT & $\mathrm{R}$ \\
\hline \begin{tabular}{|l|}
1607 \\
\end{tabular} & & & & & SESPMNT & $\mathrm{R}$ \\
\hline \begin{tabular}{|c|}
1608 \\
\end{tabular} & & & & ONSITE & SESPMNT & $\mathrm{R}$ \\
\hline \begin{tabular}{|l|}
1609 \\
\end{tabular} & & & & ONSITE & SESPMNT & $\mathrm{R}$ \\
\hline 1610 & & & & & SESPMNT & $\mathrm{R}$ \\
\hline 1611 & & & & & SESPMNT & $\mathrm{R}$ \\
\hline \begin{tabular}{|l|}
1612 \\
\end{tabular} & & & & & SESPMNT & $\mathrm{R}$ \\
\hline \begin{tabular}{|l|}
1613 \\
\end{tabular} & & & & & SESBMNT & $R$ \\
\hline
\end{tabular}




\begin{tabular}{|c|c|c|c|c|c|c|}
\hline & $A D$ & $\mathrm{AE}$ & $\mathrm{AF}$ & $A G$ & $\mathrm{AH}$ & $\mathrm{Al}$ \\
\hline 1 & RESULT_COMMENT & COLL_SAMP_SIZE & COLL_SAMP_SIZE_UNITS & DIST_CLASS & OWNER_ID & COLLECTION_PURPOSE \\
\hline 1614 & & & & ONSITE & SESPMNT & $\mathrm{R}$ \\
\hline $16 \mid$ & & & & ONSITE & SESPMNT & $\mathrm{R}$ \\
\hline \begin{tabular}{|l|l|}
1616 \\
\end{tabular} & & & & ONSITE & SESPMNT & $\mathrm{R}$ \\
\hline \begin{tabular}{|l|l|}
1617 \\
\end{tabular} & & & & OFFSITE & SESPMNT & $\mathrm{R}$ \\
\hline 1618 & & & & OFFSITE & SESPMNT & $\mathrm{R}$ \\
\hline 1619 & & & & OFFSITE & SESPMNT & $\mathrm{R}$ \\
\hline 1620 & & & & OFFSITE & SESPMNT & $\mathrm{R}$ \\
\hline \begin{tabular}{|c|}
1621 \\
\end{tabular} & & & & OFFSITE & SESPMNT & $\mathrm{R}$ \\
\hline 1622 & & & & OFFSITE & SESPMNT & $\mathrm{R}$ \\
\hline 1623 & & & & ONSITE & SESPMNT & $\mathrm{R}$ \\
\hline 1624 & & & & ONSITE & SESPMNT & $\mathrm{R}$ \\
\hline 1625 & & & & ONSITE & SESPMNT & $\mathrm{R}$ \\
\hline 1626 & & & & OFFSITE & SESPMNT & $\mathrm{R}$ \\
\hline \begin{tabular}{|l|l|}
1627 & \\
\end{tabular} & & & & OFFSITE & SESPMNT & $\mathrm{R}$ \\
\hline \begin{tabular}{|c|}
1628 \\
\end{tabular} & & & & OFFSITE & SESPMNT & $\mathrm{R}$ \\
\hline \begin{tabular}{|c|}
1629 \\
\end{tabular} & & & & OFFSITE & SESPMNT & $\mathrm{R}$ \\
\hline 1630 & & & & OFFSITE & SESPMNT & $\mathrm{R}$ \\
\hline 1631 & & & & ONSITE & SESPMNT & $\mathrm{R}$ \\
\hline 1632 & & & & ONSITE & SESPMNT & $\mathrm{R}$ \\
\hline 1633 & & & & ONSITE & SESPMNT & $\mathrm{R}$ \\
\hline 1634 & & & & ONSITE & SESPMNT & $\mathrm{R}$ \\
\hline 1635 & & & & ONSITE & SESPMNT & $\mathrm{R}$ \\
\hline 1636 & & & & ONSITE & SESPMNT & $\mathrm{R}$ \\
\hline 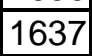 & & & & OFFSITE & SESPMNT & $\mathrm{R}$ \\
\hline 1638 & & & & & SESPMNT & $\mathrm{R}$ \\
\hline 1639 & & & & & SESPMNT & $\mathrm{R}$ \\
\hline 1640 & & & & & SESPMNT & $\mathrm{R}$ \\
\hline 1641 & & & & ONSITE & SESPMNT & $\mathrm{R}$ \\
\hline 1642 & & & & & SESPMNT & $\mathrm{R}$ \\
\hline 1643 & & & & & SESPMNT & $\mathrm{R}$ \\
\hline 1644 & & & & & SESPMNT & $\mathrm{R}$ \\
\hline 1645 & & & & ONSITE & SESPMNT & $\mathrm{R}$ \\
\hline 1646 & & & & ONSITE & SESPMNT & $\mathrm{R}$ \\
\hline \begin{tabular}{|l|l|}
1647 \\
\end{tabular} & & & & ONSITE & SESPMNT & $\mathrm{R}$ \\
\hline 1648 & & & & ONSITE & SESPMNT & $\mathrm{R}$ \\
\hline 1649 & & & & ONSITE & SESPMNT & $\mathrm{R}$ \\
\hline 1650 & & & & & SESPMNT & $\mathrm{R}$ \\
\hline 1651 & & & & & SESPMNT & $\mathrm{R}$ \\
\hline 1652 & & & & & SESPMNT & $\mathrm{R}$ \\
\hline 1653 & & & & ONSITE & SESPMNT & $\mathrm{R}$ \\
\hline 1654 & & & & & SESPMNT & $\mathrm{R}$ \\
\hline 1655 & & & & & SESPMNT & $\mathrm{R}$ \\
\hline 1656 & & & & ONSITE & SESPMNT & $\mathrm{R}$ \\
\hline 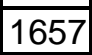 & & & & OFFSITE & SESPMNT & $\mathrm{R}$ \\
\hline 1658 & & & & OFFSITE & SESPMNT & $\mathrm{R}$ \\
\hline 1659 & & & & OFFSITE & SESPMNT & $\mathrm{R}$ \\
\hline 1660 & & & & OFFSITE & SESPMNT & $\mathrm{R}$ \\
\hline 1661 & & & & OFFSITE & SESPMNT & $\mathrm{R}$ \\
\hline 1662 & & & & OFFSITE & SESPMNT & $\mathrm{R}$ \\
\hline 1663 & & & & & SESPMNT & $\mathrm{R}$ \\
\hline 1664 & See sample record for comment. & & & & SESPMNT & $\mathrm{R}$ \\
\hline 1665 & See sample record for comment. & & & & SESBMNT & $R$ \\
\hline
\end{tabular}




\begin{tabular}{|c|c|c|c|c|c|c|}
\hline & $A D$ & $\mathrm{AE}$ & $\mathrm{AF}$ & $A G$ & $\mathrm{AH}$ & $\mathrm{Al}$ \\
\hline 1 & RESULT_COMMENT & COLL_SAMP_SIZE & COLL_SAMP_SIZE_UNITS & DIST_CLASS & OWNER_ID & COLLECTION_PURPOSE \\
\hline 1666 & & & & ONSITE & SESPMNT & $\mathrm{R}$ \\
\hline \begin{tabular}{|c|}
1667 \\
\end{tabular} & & & & ONSITE & SESPMNT & $\mathrm{R}$ \\
\hline 1668 & & & & OFFSITE & SESPMNT & $\mathrm{R}$ \\
\hline 1669 & & & & OFFSITE & SESPMNT & $\mathrm{R}$ \\
\hline 1670 & & & & ONSITE & SESPMNT & $\mathrm{R}$ \\
\hline 1671 & & & & ONSITE & SESPMNT & $\mathrm{R}$ \\
\hline 1672 & & & & & SESPMNT & $\mathrm{R}$ \\
\hline 1673 & & & & & SESPMNT & $\mathrm{R}$ \\
\hline 1674 & & & & & SESPMNT & $\mathrm{R}$ \\
\hline 1675 & & & & & SESPMNT & $\mathrm{R}$ \\
\hline \begin{tabular}{|c|}
1676 \\
\end{tabular} & & & & ONSITE & SESPMNT & $\mathrm{R}$ \\
\hline 1677 & & & & ONSITE & SESPMNT & $\mathrm{R}$ \\
\hline 1678 & & & & ONSITE & SESPMNT & $\mathrm{R}$ \\
\hline 1679 & & & & OFFSITE & SESPMNT & $\mathrm{R}$ \\
\hline 1680 & & & & OFFSITE & SESPMNT & $\mathrm{R}$ \\
\hline 1681 & & & & OFFSITE & SESPMNT & $\mathrm{R}$ \\
\hline 1682 & & & & OFFSITE & SESPMNT & $\mathrm{R}$ \\
\hline 1683 & & & & OFFSITE & SESPMNT & $\mathrm{R}$ \\
\hline 1684 & & & & OFFSITE & SESPMNT & $\mathrm{R}$ \\
\hline 1685 & & & & OFFSITE & SESPMNT & $\mathrm{R}$ \\
\hline 1686 & & & & ONSITE & SESPMNT & $\mathrm{R}$ \\
\hline 1687 & & & & ONSITE & SESPMNT & $\mathrm{R}$ \\
\hline 1688 & & & & & SESPMNT & $\mathrm{R}$ \\
\hline 1689 & & & & & SESPMNT & $\mathrm{R}$ \\
\hline 1690 & & & & & SESPMNT & $\mathrm{R}$ \\
\hline 1691 & & & & ONSITE & SESPMNT & $\mathrm{R}$ \\
\hline \begin{tabular}{|l|}
1692 \\
\end{tabular} & & & & ONSITE & SESPMNT & $\mathrm{R}$ \\
\hline 1693 & & & & ONSITE & SESPMNT & $\mathrm{R}$ \\
\hline 1694 & & & & OFFSITE & SESPMNT & $\mathrm{R}$ \\
\hline \begin{tabular}{|c|}
1695 \\
\end{tabular} & & & & OFFSITE & SESPMNT & $\mathrm{R}$ \\
\hline \begin{tabular}{|l|}
1696 \\
\end{tabular} & & & & ONSITE & SESPMNT & $\mathrm{R}$ \\
\hline \begin{tabular}{|l|}
1697 \\
\end{tabular} & & & & ONSITE & SESPMNT & $\mathrm{R}$ \\
\hline 1698 & & & & OFFSITE & SESPMNT & $\mathrm{R}$ \\
\hline 1699 & & & & OFFSITE & SESPMNT & $\mathrm{R}$ \\
\hline 1700 & & & & OFFSITE & SESPMNT & $\mathrm{R}$ \\
\hline \begin{tabular}{|l|}
1701 \\
\end{tabular} & & & & & SESPMNT & $\mathrm{R}$ \\
\hline \begin{tabular}{|l|}
1702 \\
\end{tabular} & & & & & SESPMNT & $\mathrm{R}$ \\
\hline 1703 & & & & & SESPMNT & $R$ \\
\hline 1704 & & & & & SESPMNT & $\mathrm{R}$ \\
\hline 1705 & & & & & SESPMNT & $\mathrm{R}$ \\
\hline 1706 & & & & ONSITE & SESPMNT & $\mathrm{R}$ \\
\hline \begin{tabular}{|l|l|}
1707 \\
\end{tabular} & & & & ONSITE & SESPMNT & $\mathrm{R}$ \\
\hline 1708 & & & & & SESPMNT & $\mathrm{R}$ \\
\hline \begin{tabular}{|l|}
1709 \\
\end{tabular} & & & & & SESPMNT & $\mathrm{R}$ \\
\hline 1710 & & & & & SESPMNT & $R$ \\
\hline \begin{tabular}{|l|l|}
1711 \\
\end{tabular} & & & & & SESPMNT & $\mathrm{R}$ \\
\hline 1712 & & & & ONSITE & SESPMNT & $\mathrm{R}$ \\
\hline 1713 & & & & ONSITE & SESPMNT & $\mathrm{R}$ \\
\hline \begin{tabular}{|l|l|}
1714 \\
\end{tabular} & & & & ONSITE & SESPMNT & $\mathrm{R}$ \\
\hline \begin{tabular}{|l|l|}
1715 \\
\end{tabular} & & & & ONSITE & SESPMNT & $\mathrm{R}$ \\
\hline \begin{tabular}{|l|}
1716 \\
\end{tabular} & & & & OFFSITE & SESPMNT & $\mathrm{R}$ \\
\hline \begin{tabular}{|l|l|}
1717 \\
\end{tabular} & & & & OFFSITE & SESBMNT & $R$ \\
\hline
\end{tabular}




\begin{tabular}{|c|c|c|c|c|c|c|}
\hline & $A D$ & $\mathrm{AE}$ & $\mathrm{AF}$ & $A G$ & $\mathrm{AH}$ & $\mathrm{Al}$ \\
\hline 1 & RESULT_COMMENT & COLL_SAMP_SIZE & COLL_SAMP_SIZE_UNITS & DIST_CLASS & OWNER_ID & COLLECTION_PURPOSE \\
\hline 1718 & & & & OFFSITE & SESPMNT & $\mathrm{R}$ \\
\hline 1719 & & & & OFFSITE & SESPMNT & $\mathrm{R}$ \\
\hline 1720 & & & & ONSITE & SESPMNT & $\mathrm{R}$ \\
\hline 1721 & & & & ONSITE & SESPMNT & $\mathrm{R}$ \\
\hline 1722 & & & & & SESPMNT & $\mathrm{R}$ \\
\hline 1723 & & & & & SESPMNT & $\mathrm{R}$ \\
\hline 1724 & & & & ONSITE & SESPMNT & $\mathrm{R}$ \\
\hline 1725 & & & & ONSITE & SESPMNT & $\mathrm{R}$ \\
\hline 1726 & & & & ONSITE & SESPMNT & $\mathrm{R}$ \\
\hline \begin{tabular}{|l|l|l|l|l}
1727 \\
\end{tabular} & & & & ONSITE & SESPMNT & $\mathrm{R}$ \\
\hline 1728 & & & & ONSITE & SESPMNT & $\mathrm{R}$ \\
\hline 1729 & & & & & SESPMNT & $\mathrm{R}$ \\
\hline 1730 & & & & & SESPMNT & $\mathrm{R}$ \\
\hline 1731 & & & & & SESPMNT & $\mathrm{R}$ \\
\hline 1732 & & & & & SESPMNT & $\mathrm{R}$ \\
\hline 1733 & & & & & SESPMNT & $\mathrm{R}$ \\
\hline 1734 & & & & ONSITE & SESPMNT & $\mathrm{R}$ \\
\hline 1735 & & & & ONSITE & SESPMNT & $\mathrm{R}$ \\
\hline 1736 & & & & OFFSITE & SESPMNT & $\mathrm{R}$ \\
\hline 1737 & & & & OFFSITE & SESPMNT & $\mathrm{R}$ \\
\hline 1738 & & & & ONSITE & SESPMNT & $\mathrm{R}$ \\
\hline 1739 & & & & ONSITE & SESPMNT & $\mathrm{R}$ \\
\hline 1740 & & & & ONSITE & SESPMNT & $\mathrm{R}$ \\
\hline 1741 & & & & & SESPMNT & $\mathrm{R}$ \\
\hline 1742 & & & & & SESPMNT & $\mathrm{R}$ \\
\hline 1743 & & & & OFFSITE & SESPMNT & $\mathrm{R}$ \\
\hline 1744 & & & & OFFSITE & SESPMNT & $\mathrm{R}$ \\
\hline 1745 & & & & OFFSITE & SESPMNT & $\mathrm{R}$ \\
\hline 1746 & & & & OFFSITE & SESPMNT & $\mathrm{R}$ \\
\hline \begin{tabular}{|l|l|}
1747 \\
\end{tabular} & & & & OFFSITE & SESPMNT & $\mathrm{R}$ \\
\hline \begin{tabular}{|l|}
1748 \\
\end{tabular} & & & & ONSITE & SESPMNT & $\mathrm{R}$ \\
\hline \begin{tabular}{|l|l|}
1749 \\
\end{tabular} & & & & ONSITE & SESPMNT & $\mathrm{R}$ \\
\hline 1750 & & & & ONSITE & SESPMNT & $\mathrm{R}$ \\
\hline \begin{tabular}{|l|l|}
1751 \\
\end{tabular} & & & & OFFSITE & SESPMNT & $\mathrm{R}$ \\
\hline 1752 & & & & OFFSITE & SESPMNT & $\mathrm{R}$ \\
\hline \begin{tabular}{|l|}
1753 \\
\end{tabular} & & & & OFFSITE & SESPMNT & $\mathrm{R}$ \\
\hline \begin{tabular}{|l|l|}
1754 \\
\end{tabular} & & & & ONSITE & SESPMNT & $\mathrm{R}$ \\
\hline \begin{tabular}{|l|l|}
1755 \\
\end{tabular} & & & & ONSITE & SESPMNT & $R$ \\
\hline \begin{tabular}{|l|}
1756 \\
\end{tabular} & & & & & SESPMNT & $\mathrm{R}$ \\
\hline \begin{tabular}{|l|}
1757 \\
\end{tabular} & & & & & SESPMNT & $\mathrm{R}$ \\
\hline 1758 & & & & & SESPMNT & $\mathrm{R}$ \\
\hline 1759 & & & & & SESPMNT & $\mathrm{R}$ \\
\hline 1760 & & & & ONSITE & SESPMNT & $\mathrm{R}$ \\
\hline \begin{tabular}{|l|l|}
1761 \\
\end{tabular} & & & & ONSITE & SESPMNT & $\mathrm{R}$ \\
\hline \begin{tabular}{|l|}
1762 \\
\end{tabular} & & & & OFFSITE & SESPMNT & $R$ \\
\hline \begin{tabular}{|l|}
1763 \\
\end{tabular} & & & & OFFSITE & SESPMNT & $\mathrm{R}$ \\
\hline 1764 & & & & ONSITE & SESPMNT & $\mathrm{R}$ \\
\hline 1765 & & & & ONSITE & SESPMNT & $\mathrm{R}$ \\
\hline \begin{tabular}{|l|}
1766 \\
\end{tabular} & & & & & SESPMNT & $\mathrm{R}$ \\
\hline \begin{tabular}{|l|l|}
1767 \\
\end{tabular} & & & & & SESPMNT & $\mathrm{R}$ \\
\hline \begin{tabular}{|l|}
1768 \\
\end{tabular} & & & & & SESPMNT & $\mathrm{R}$ \\
\hline \begin{tabular}{|l|}
1769 \\
\end{tabular} & & & & ONSITE & SESBMNT & $R$ \\
\hline
\end{tabular}




\begin{tabular}{|c|c|c|c|c|c|c|}
\hline & $\mathrm{AD}$ & $\mathrm{AE}$ & $\mathrm{AF}$ & AG & $\mathrm{AH}$ & $\mathrm{Al}$ \\
\hline 1 & RESULT_COMMENT & COLL_SAMP_SIZE & COLL_SAMP_SIZE_UNITS & DIST_CLASS & OWNER_ID & COLLECTION_PURPOSE \\
\hline 1770 & & & & ONSITE & SESPMNT & $\mathrm{R}$ \\
\hline 1771 & & & & & SESPMNT & $\mathrm{R}$ \\
\hline 1772 & & & & & SESPMNT & $\mathrm{R}$ \\
\hline 1773 & & & & & SESPMNT & $\mathrm{R}$ \\
\hline 1774 & & & & & SESPMNT & $\mathrm{R}$ \\
\hline 1775 & & & & ONSITE & SESPMNT & $\mathrm{R}$ \\
\hline 1776 & & & & ONSITE & SESPMNT & $\mathrm{R}$ \\
\hline 1777 & & & & OFFSITE & SESPMNT & $\mathrm{R}$ \\
\hline 1778 & & & & OFFSITE & SESPMNT & $\mathrm{R}$ \\
\hline $\mid 1779$ & & & & OFFSITE & SESPMNT & $\mathrm{R}$ \\
\hline 1780 & & & & OFFSITE & SESPMNT & $\mathrm{R}$ \\
\hline 1781 & & & & ONSITE & SESPMNT & $\mathrm{R}$ \\
\hline 1782 & & & & ONSITE & SESPMNT & $\mathrm{R}$ \\
\hline 1783 & & & & ONSITE & SESPMNT & $\mathrm{R}$ \\
\hline 1784 & & & & OFFSITE & SESPMNT & $\mathrm{R}$ \\
\hline 1785 & & & & OFFSITE & SESPMNT & $\mathrm{R}$ \\
\hline 1786 & & & & OFFSITE & SESPMNT & $\mathrm{R}$ \\
\hline \begin{tabular}{|l|l}
1787 \\
\end{tabular} & & & & ONSITE & SESPMNT & $\mathrm{R}$ \\
\hline 1788 & & & & ONSITE & SESPMNT & $\mathrm{R}$ \\
\hline 1789 & & & & & SESPMNT & $\mathrm{R}$ \\
\hline 1790 & & & & & SESPMNT & $\mathrm{R}$ \\
\hline 1791 & & & & & SESPMNT & $\mathrm{R}$ \\
\hline 1792 & & & & & SESPMNT & $\mathrm{R}$ \\
\hline 1793 & & & & ONSITE & SESPMNT & $\mathrm{R}$ \\
\hline 1794 & & & & ONSITE & SESPMNT & $\mathrm{R}$ \\
\hline 1795 & & & & OFFSITE & SESPMNT & $\mathrm{R}$ \\
\hline 1796 & & & & OFFSITE & SESPMNT & $\mathrm{R}$ \\
\hline \begin{tabular}{|c|}
1797 \\
\end{tabular} & & & & OFFSITE & SESPMNT & $\mathrm{R}$ \\
\hline 1798 & & & & OFFSITE & SESPMNT & $\mathrm{R}$ \\
\hline 1799 & & & & OFFSITE & SESPMNT & $\mathrm{R}$ \\
\hline 1800 & & & & OFFSITE & SESPMNT & $\mathrm{R}$ \\
\hline 1801 & & & & ONSITE & SESPMNT & $\mathrm{R}$ \\
\hline \begin{tabular}{|l|}
1802 \\
\end{tabular} & & & & ONSITE & SESPMNT & $\mathrm{R}$ \\
\hline \begin{tabular}{|l|}
1803 \\
\end{tabular} & & & & ONSITE & SESPMNT & $\mathrm{R}$ \\
\hline 1804 & & & & ONSITE & SESPMNT & $\mathrm{R}$ \\
\hline 1805 & & & & ONSITE & SESPMNT & $\mathrm{R}$ \\
\hline 1806 & & & & OFFSITE & SESPMNT & $\mathrm{R}$ \\
\hline \begin{tabular}{|l|l|}
1807 \\
\end{tabular} & & & & OFFSITE & SESPMNT & $\mathrm{R}$ \\
\hline \begin{tabular}{|l|}
1808 \\
\end{tabular} & & & & OFFSITE & SESPMNT & $\mathrm{R}$ \\
\hline \begin{tabular}{|l|}
1809 \\
\end{tabular} & & & & & SESPMNT & $\mathrm{R}$ \\
\hline 1810 & & & & & SESPMNT & $\mathrm{R}$ \\
\hline \begin{tabular}{|l|l|}
1811 \\
\end{tabular} & & & & ONSITE & SESPMNT & $\mathrm{R}$ \\
\hline \begin{tabular}{|l|}
1812 \\
\end{tabular} & & & & ONSITE & SESPMNT & $\mathrm{R}$ \\
\hline \begin{tabular}{|l|}
1813 \\
\end{tabular} & & & & ONSITE & SESPMNT & $\mathrm{R}$ \\
\hline \begin{tabular}{|l|l|}
1814 \\
\end{tabular} & & & & & SESPMNT & $\mathrm{R}$ \\
\hline 1815 & & & & & SESPMNT & $\mathrm{R}$ \\
\hline \begin{tabular}{|l|}
1816 \\
\end{tabular} & & & & & SESPMNT & $\mathrm{R}$ \\
\hline \begin{tabular}{|l|}
1817 \\
\end{tabular} & & & & & SESPMNT & $\mathrm{R}$ \\
\hline 1818 & & & & & SESPMNT & $\mathrm{R}$ \\
\hline \begin{tabular}{|l|}
1819 \\
\end{tabular} & & & & & SESPMNT & $\mathrm{R}$ \\
\hline 1820 & & & & ONSITE & SESPMNT & $\mathrm{R}$ \\
\hline 1821 & & & & ONSITE & SESBMNT & $R$ \\
\hline
\end{tabular}




\begin{tabular}{|c|c|c|c|c|c|c|}
\hline & $A D$ & $\mathrm{AE}$ & $\mathrm{AF}$ & $A G$ & $\mathrm{AH}$ & $\mathrm{Al}$ \\
\hline 1 & RESULT_COMMENT & COLL_SAMP_SIZE & COLL_SAMP_SIZE_UNITS & DIST_CLASS & OWNER_ID & COLLECTION_PURPOSE \\
\hline 1822 & & & & OFFSITE & SESPMNT & $\mathrm{R}$ \\
\hline 1823 & & & & OFFSITE & SESPMNT & $\mathrm{R}$ \\
\hline 1824 & & & & ONSITE & SESPMNT & $\mathrm{R}$ \\
\hline 1825 & & & & ONSITE & SESPMNT & $\mathrm{R}$ \\
\hline 1826 & & & & ONSITE & SESPMNT & $\mathrm{R}$ \\
\hline $\mid 1827$ & & & & & SESPMNT & $\mathrm{R}$ \\
\hline 1828 & & & & & SESPMNT & $\mathrm{R}$ \\
\hline 1829 & & & & ONSITE & SESPMNT & $\mathrm{R}$ \\
\hline 1830 & & & & ONSITE & SESPMNT & $\mathrm{R}$ \\
\hline 1831 & & & & ONSITE & SESPMNT & $\mathrm{R}$ \\
\hline 1832 & & & & OFFSITE & SESPMNT & $\mathrm{R}$ \\
\hline 1833 & & & & OFFSITE & SESPMNT & $\mathrm{R}$ \\
\hline 1834 & & & & OFFSITE & SESPMNT & $\mathrm{R}$ \\
\hline 1835 & & & & OFFSITE & SESPMNT & $\mathrm{R}$ \\
\hline 1836 & & & & OFFSITE & SESPMNT & $\mathrm{R}$ \\
\hline \begin{tabular}{|c|c|}
1837 \\
\end{tabular} & & & & OFFSITE & SESPMNT & $\mathrm{R}$ \\
\hline 1838 & & & & OFFSITE & SESPMNT & $\mathrm{R}$ \\
\hline 1839 & & & & OFFSITE & SESPMNT & $\mathrm{R}$ \\
\hline 1840 & & & & ONSITE & SESPMNT & $\mathrm{R}$ \\
\hline 1841 & & & & ONSITE & SESPMNT & $\mathrm{R}$ \\
\hline 1842 & & & & & SESPMNT & $\mathrm{R}$ \\
\hline 1843 & & & & & SESPMNT & $\mathrm{R}$ \\
\hline 1844 & See sample record for comment. & & & & SESPMNT & $\mathrm{R}$ \\
\hline 1845 & See sample record for comment. & & & & SESPMNT & $\mathrm{R}$ \\
\hline 1846 & & & & & SESPMNT & $\mathrm{R}$ \\
\hline \begin{tabular}{|l|l|}
1847 \\
\end{tabular} & & & & & SESPMNT & $\mathrm{R}$ \\
\hline 1848 & & & & ONSITE & SESPMNT & $\mathrm{R}$ \\
\hline \begin{tabular}{|l|}
1849 \\
\end{tabular} & & & & ONSITE & SESPMNT & $\mathrm{R}$ \\
\hline 1850 & & & & OFFSITE & SESPMNT & $\mathrm{R}$ \\
\hline 1851 & & & & OFFSITE & SESPMNT & $\mathrm{R}$ \\
\hline \begin{tabular}{|l|}
1852 \\
\end{tabular} & & & & OFFSITE & SESPMNT & $\mathrm{R}$ \\
\hline \begin{tabular}{|l|}
1853 \\
\end{tabular} & & & & OFFSITE & SESPMNT & $\mathrm{R}$ \\
\hline 1854 & & & & & SESPMNT & $\mathrm{R}$ \\
\hline \begin{tabular}{|l|}
1855 \\
\end{tabular} & & & & ONSITE & SESPMNT & $\mathrm{R}$ \\
\hline \begin{tabular}{|l|}
1856 \\
\end{tabular} & & & & ONSITE & SESPMNT & $\mathrm{R}$ \\
\hline \begin{tabular}{|l|}
1857 \\
\end{tabular} & & & & & SESPMNT & $\mathrm{R}$ \\
\hline 1858 & & & & & SESPMNT & $\mathrm{R}$ \\
\hline \begin{tabular}{|l|l|}
1859 \\
\end{tabular} & & & & & SESPMNT & $\mathrm{R}$ \\
\hline 1860 & & & & & SESPMNT & $\mathrm{R}$ \\
\hline \begin{tabular}{|l|}
1861 \\
\end{tabular} & & & & ONSITE & SESPMNT & $\mathrm{R}$ \\
\hline \begin{tabular}{|l|}
1862 \\
\end{tabular} & & & & ONSITE & SESPMNT & $\mathrm{R}$ \\
\hline 1863 & & & & ONSITE & SESPMNT & $\mathrm{R}$ \\
\hline 1864 & & & & ONSITE & SESPMNT & $\mathrm{R}$ \\
\hline 1865 & & & & & SESPMNT & $\mathrm{R}$ \\
\hline \begin{tabular}{|l|l|}
1866 \\
\end{tabular} & & & & & SESPMNT & $\mathrm{R}$ \\
\hline \begin{tabular}{|l|l|}
1867 \\
\end{tabular} & & & & & SESPMNT & $\mathrm{R}$ \\
\hline 1868 & & & & & SESPMNT & $\mathrm{R}$ \\
\hline 1869 & & & & ONSITE & SESPMNT & $\mathrm{R}$ \\
\hline 1870 & & & & ONSITE & SESPMNT & $R$ \\
\hline \begin{tabular}{|l|}
1871 \\
\end{tabular} & & & & ONSITE & SESPMNT & $\mathrm{R}$ \\
\hline \begin{tabular}{|l|}
1872 \\
\end{tabular} & & & & OFFSITE & SESPMNT & $\mathrm{R}$ \\
\hline \begin{tabular}{|l|}
1873 \\
\end{tabular} & & & & OFFSITE & SESBMNT $_{\text {O }}$ & $R$ \\
\hline
\end{tabular}




\begin{tabular}{|c|c|c|c|c|c|c|}
\hline & $\mathrm{AD}$ & $\mathrm{AE}$ & $\mathrm{AF}$ & $A G$ & $\mathrm{AH}$ & $\mathrm{Al}$ \\
\hline 1 & RESULT_COMMENT & COLL_SAMP_SIZE & COLL_SAMP_SIZE_UNITS & DIST_CLASS & OWNER_ID & COLLECTION_PURPOSE \\
\hline 1874 & & & & OFFSITE & SESPMNT & $R$ \\
\hline 1875 & & & & ONSITE & SESPMNT & $\mathrm{R}$ \\
\hline 1876 & & & & ONSITE & SESPMNT & $\mathrm{R}$ \\
\hline 1877 & & & & OFFSITE & SESPMNT & $\mathrm{R}$ \\
\hline 1878 & & & & OFFSITE & SESPMNT & $\mathrm{R}$ \\
\hline 1879 & & & & ONSITE & SESPMNT & $\mathrm{R}$ \\
\hline 1880 & & & & ONSITE & SESPMNT & $\mathrm{R}$ \\
\hline 1881 & & & & ONSITE & SESPMNT & $\mathrm{R}$ \\
\hline 1882 & & & & OFFSITE & SESPMNT & $\mathrm{R}$ \\
\hline 1883 & & & & OFFSITE & SESPMNT & $\mathrm{R}$ \\
\hline 1884 & & & & ONSITE & SESPMNT & $\mathrm{R}$ \\
\hline 1885 & & & & ONSITE & SESPMNT & $\mathrm{R}$ \\
\hline 1886 & & & & ONSITE & SESPMNT & $\mathrm{R}$ \\
\hline 1887 & & & & ONSITE & SESPMNT & $\mathrm{R}$ \\
\hline 1888 & & & & ONSITE & SESPMNT & $\mathrm{R}$ \\
\hline 1889 & & & & ONSITE & SESPMNT & $\mathrm{R}$ \\
\hline 1890 & & & & & SESPMNT & $\mathrm{R}$ \\
\hline 1891 & & & & & SESPMNT & $\mathrm{R}$ \\
\hline 1892 & & & & & SESPMNT & $\mathrm{R}$ \\
\hline 1893 & & & & ONSITE & SESPMNT & $\mathrm{R}$ \\
\hline 1894 & & & & ONSITE & SESPMNT & $\mathrm{R}$ \\
\hline 1895 & & & & & SESPMNT & $\mathrm{R}$ \\
\hline 1896 & & & & OFFSITE & SESPMNT & $\mathrm{R}$ \\
\hline 1897 & & & & OFFSITE & SESPMNT & $\mathrm{R}$ \\
\hline 1898 & & & & OFFSITE & SESPMNT & $\mathrm{R}$ \\
\hline 1899 & & & & ONSITE & SESPMNT & $\mathrm{R}$ \\
\hline 1900 & & & & ONSITE & SESPMNT & $\mathrm{R}$ \\
\hline 1901 & & & & ONSITE & SESPMNT & $\mathrm{R}$ \\
\hline 1902 & & & & OFFSITE & SESPMNT & $\mathrm{R}$ \\
\hline 1903 & & & & OFFSITE & SESPMNT & $\mathrm{R}$ \\
\hline 1904 & & & & OFFSITE & SESPMNT & $\mathrm{R}$ \\
\hline 1905 & & & & ONSITE & SESPMNT & $R$ \\
\hline 1906 & & & & ONSITE & SESPMNT & $R$ \\
\hline 1907 & & & & OFFSITE & SESPMNT & $R$ \\
\hline 1908 & & & & OFFSITE & SESPMNT & $\mathrm{R}$ \\
\hline 1909 & & & & ONSITE & SESPMNT & $R$ \\
\hline 1910 & & & & ONSITE & SESPMNT & $R$ \\
\hline 1911 & & & & ONSITE & SESPMNT & $R$ \\
\hline 1912 & & & & & SESPMNT & $R$ \\
\hline 1913 & & & & ONSITE & SESPMNT & $R$ \\
\hline 1914 & & & & ONSITE & SESPMNT & $\mathrm{R}$ \\
\hline 1915 & & & & & SESPMNT & $\mathrm{R}$ \\
\hline 1916 & & & & OFFSITE & SESPMNT & $R$ \\
\hline 1917 & & & & OFFSITE & SESPMNT & $R$ \\
\hline 1918 & & & & OFFSITE & SESPMNT & $R$ \\
\hline 1919 & & & & OFFSITE & SESPMNT & $R$ \\
\hline 1920 & & & & OFFSITE & SESPMNT & $\mathrm{R}$ \\
\hline 1921 & & & & ONSITE & SESPMNT & $R$ \\
\hline 1922 & & & & ONSITE & SESPMNT & $\mathrm{R}$ \\
\hline 1923 & & & & OFFSITE & SESPMNT & $R$ \\
\hline 1924 & & & & OFFSITE & SESPMNT & $R$ \\
\hline 1925 & & & & OFFSITE & SESBMNT & $R$ \\
\hline
\end{tabular}




\begin{tabular}{|c|c|c|c|c|c|c|}
\hline & $\mathrm{AD}$ & $\mathrm{AE}$ & $\mathrm{AF}$ & $A G$ & $\mathrm{AH}$ & $\mathrm{Al}$ \\
\hline 1 & RESULT_COMMENT & COLL_SAMP_SIZE & COLL_SAMP_SIZE_UNITS & DIST_CLASS & OWNER_ID & COLLECTION_PURPOSE \\
\hline 1926 & & & & ONSITE & SESPMNT & $R$ \\
\hline 1927 & & & & OFFSITE & SESPMNT & $\mathrm{R}$ \\
\hline 1928 & & & & & SESPMNT & $\mathrm{R}$ \\
\hline 1929 & & & & ONSITE & SESPMNT & $\mathrm{R}$ \\
\hline 1930 & & & & ONSITE & SESPMNT & $\mathrm{R}$ \\
\hline 1931 & & & & & SESPMNT & $\mathrm{R}$ \\
\hline 1932 & & & & & SESPMNT & $\mathrm{R}$ \\
\hline 1933 & & & & & SESPMNT & $\mathrm{R}$ \\
\hline 1934 & & & & & SESPMNT & $\mathrm{R}$ \\
\hline 1935 & & & & ONSITE & SESPMNT & $\mathrm{R}$ \\
\hline 1936 & & & & ONSITE & SESPMNT & $\mathrm{R}$ \\
\hline 1937 & & & & ONSITE & SESPMNT & $\mathrm{R}$ \\
\hline 1938 & & & & ONSITE & SESPMNT & $\mathrm{R}$ \\
\hline 1939 & & & & OFFSITE & SESPMNT & $\mathrm{R}$ \\
\hline 1940 & & & & OFFSITE & SESPMNT & $\mathrm{R}$ \\
\hline 1941 & & & & OFFSITE & SESPMNT & $\mathrm{R}$ \\
\hline 1942 & & & & OFFSITE & SESPMNT & $\mathrm{R}$ \\
\hline 1943 & & & & OFFSITE & SESPMNT & $\mathrm{R}$ \\
\hline 1944 & & & & OFFSITE & SESPMNT & $\mathrm{R}$ \\
\hline 1945 & & & & ONSITE & SESPMNT & $\mathrm{R}$ \\
\hline 1946 & & & & ONSITE & SESPMNT & $\mathrm{R}$ \\
\hline 1947 & & & & & SESPMNT & $\mathrm{R}$ \\
\hline 1948 & & & & ONSITE & SESPMNT & $\mathrm{R}$ \\
\hline 1949 & & & & ONSITE & SESPMNT & $\mathrm{R}$ \\
\hline 1950 & & & & & SESPMNT & $\mathrm{R}$ \\
\hline 1951 & & & & ONSITE & SESPMNT & $\mathrm{R}$ \\
\hline 1952 & & & & ONSITE & SESPMNT & $\mathrm{R}$ \\
\hline 1953 & & & & ONSITE & SESPMNT & $\mathrm{R}$ \\
\hline 1954 & & & & OFFSITE & SESPMNT & $\mathrm{R}$ \\
\hline 1955 & & & & OFFSITE & SESPMNT & $\mathrm{R}$ \\
\hline 1956 & & & & OFFSITE & SESPMNT & $\mathrm{R}$ \\
\hline 1957 & & & & ONSITE & SESPMNT & $R$ \\
\hline 1958 & & & & & SESPMNT & $R$ \\
\hline 1959 & & & & ONSITE & SESPMNT & $\mathrm{R}$ \\
\hline 1960 & & & & ONSITE & SESPMNT & $\mathrm{R}$ \\
\hline 1961 & & & & ONSITE & SESPMNT & $R$ \\
\hline 1962 & & & & OFFSITE & SESPMNT & $\mathrm{R}$ \\
\hline 1963 & & & & OFFSITE & SESPMNT & $\mathrm{R}$ \\
\hline 1964 & & & & ONSITE & SESPMNT & $R$ \\
\hline 1965 & & & & ONSITE & SESPMNT & $\mathrm{R}$ \\
\hline 1966 & & & & & SESPMNT & $\mathrm{R}$ \\
\hline 1967 & & & & & SESPMNT & $\mathrm{R}$ \\
\hline \begin{tabular}{|l|l|}
1968 \\
\end{tabular} & & & & & SESPMNT & $R$ \\
\hline 1969 & & & & ONSITE & SESPMNT & $\mathrm{R}$ \\
\hline 1970 & & & & ONSITE & SESPMNT & $R$ \\
\hline 1971 & & & & ONSITE & SESPMNT & $R$ \\
\hline 1972 & & & & & SESPMNT & $\mathrm{R}$ \\
\hline 1973 & & & & ONSITE & SESPMNT & $\mathrm{R}$ \\
\hline 1974 & & & & ONSITE & SESPMNT & $\mathrm{R}$ \\
\hline 1975 & & & & ONSITE & SESPMNT & $R$ \\
\hline 1976 & & & & OFFSITE & SESPMNT & $R$ \\
\hline 1977 & & & & OFFSITE & SESBMNT & $R$ \\
\hline
\end{tabular}




\begin{tabular}{|c|c|c|c|c|c|c|}
\hline & $A D$ & $\mathrm{AE}$ & $\mathrm{AF}$ & $A G$ & $\mathrm{AH}$ & $\mathrm{Al}$ \\
\hline 1 & RESULT_COMMENT & COLL_SAMP_SIZE & COLL_SAMP_SIZE_UNITS & DIST_CLASS & OWNER_ID & COLLECTION_PURPOSE \\
\hline 1978 & & & & OFFSITE & SESPMNT & $\mathrm{R}$ \\
\hline 1979 & & & & OFFSITE & SESPMNT & $\mathrm{R}$ \\
\hline 1980 & & & & OFFSITE & SESPMNT & $\mathrm{R}$ \\
\hline 1981 & & & & OFFSITE & SESPMNT & $\mathrm{R}$ \\
\hline 1982 & & & & OFFSITE & SESPMNT & $\mathrm{R}$ \\
\hline 1983 & & & & ONSITE & SESPMNT & $\mathrm{R}$ \\
\hline 1984 & & & & ONSITE & SESPMNT & $\mathrm{R}$ \\
\hline 1985 & & & & OFFSITE & SESPMNT & $\mathrm{R}$ \\
\hline 1986 & & & & OFFSITE & SESPMNT & $\mathrm{R}$ \\
\hline 1987 & & & & & SESPMNT & $\mathrm{R}$ \\
\hline 1988 & & & & & SESPMNT & $\mathrm{R}$ \\
\hline 1989 & & & & ONSITE & SESPMNT & $\mathrm{R}$ \\
\hline 1990 & & & & ONSITE & SESPMNT & $\mathrm{R}$ \\
\hline 1991 & & & & ONSITE & SESPMNT & $\mathrm{R}$ \\
\hline 1992 & & & & ONSITE & SESPMNT & $\mathrm{R}$ \\
\hline 1993 & & & & ONSITE & SESPMNT & $\mathrm{R}$ \\
\hline 1994 & & & & ONSITE & SESPMNT & $\mathrm{R}$ \\
\hline 1995 & & & & OFFSITE & SESPMNT & $\mathrm{R}$ \\
\hline 1996 & & & & OFFSITE & SESPMNT & $\mathrm{R}$ \\
\hline 1997 & & & & OFFSITE & SESPMNT & $\mathrm{R}$ \\
\hline 1998 & & & & & SESPMNT & $\mathrm{R}$ \\
\hline 1999 & & & & ONSITE & SESPMNT & $\mathrm{R}$ \\
\hline 2000 & & & & ONSITE & SESPMNT & $\mathrm{R}$ \\
\hline 2001 & & & & & SESPMNT & $\mathrm{R}$ \\
\hline 2002 & & & & OFFSITE & SESPMNT & $\mathrm{R}$ \\
\hline 2003 & & & & OFFSITE & SESPMNT & $\mathrm{R}$ \\
\hline 2004 & & & & OFFSITE & SESPMNT & $\mathrm{R}$ \\
\hline 2005 & & & & OFFSITE & SESPMNT & $\mathrm{R}$ \\
\hline 2006 & & & & OFFSITE & SESPMNT & $\mathrm{R}$ \\
\hline \begin{tabular}{|l|}
2007 \\
\end{tabular} & & & & ONSITE & SESPMNT & $\mathrm{R}$ \\
\hline 2008 & & & & OFFSITE & SESPMNT & $\mathrm{R}$ \\
\hline 2009 & & & & OFFSITE & SESPMNT & $\mathrm{R}$ \\
\hline 2010 & & & & ONSITE & SESPMNT & $\mathrm{R}$ \\
\hline 2011 & & & & ONSITE & SESPMNT & $\mathrm{R}$ \\
\hline 2012 & & & & ONSITE & SESPMNT & $\mathrm{R}$ \\
\hline 2013 & & & & ONSITE & SESPMNT & $\mathrm{R}$ \\
\hline 2014 & & & & OFFSITE & SESPMNT & $\mathrm{R}$ \\
\hline 2015 & & & & OFFSITE & SESPMNT & $R$ \\
\hline 2016 & & & & OFFSITE & SESPMNT & $\mathrm{R}$ \\
\hline \begin{tabular}{|l|}
2017 \\
\end{tabular} & & & & OFFSITE & SESPMNT & $\mathrm{R}$ \\
\hline 2018 & & & & OFFSITE & SESPMNT & $\mathrm{R}$ \\
\hline 2019 & & & & ONSITE & SESPMNT & $\mathrm{R}$ \\
\hline 2020 & & & & ONSITE & SESPMNT & $\mathrm{R}$ \\
\hline \begin{tabular}{|l|}
2021 \\
\end{tabular} & & & & & SESPMNT & $\mathrm{R}$ \\
\hline 2022 & & & & ONSITE & SESPMNT & $R$ \\
\hline 2023 & & & & ONSITE & SESPMNT & $\mathrm{R}$ \\
\hline 2024 & & & & & SESPMNT & $\mathrm{R}$ \\
\hline 2025 & & & & ONSITE & SESPMNT & $\mathrm{R}$ \\
\hline \begin{tabular}{|l|}
2026 \\
\end{tabular} & & & & ONSITE & SESPMNT & $\mathrm{R}$ \\
\hline \begin{tabular}{|l|}
2027 \\
\end{tabular} & & & & OFFSITE & SESPMNT & $\mathrm{R}$ \\
\hline 2028 & & & & OFFSITE & SESPMNT & $R$ \\
\hline \begin{tabular}{|l|}
2029 \\
\end{tabular} & & & & & SESBMNT & ${ }_{2} R$ \\
\hline
\end{tabular}




\begin{tabular}{|c|c|c|c|c|c|c|}
\hline & $\mathrm{AD}$ & $\mathrm{AE}$ & $\mathrm{AF}$ & $A G$ & $\mathrm{AH}$ & $\mathrm{Al}$ \\
\hline 1 & RESULT_COMMENT & COLL_SAMP_SIZE & COLL_SAMP_SIZE_UNITS & DIST_CLASS & OWNER_ID & COLLECTION_PURPOSE \\
\hline 2030 & & & & ONSITE & SESPMNT & $R$ \\
\hline 2031 & & & & ONSITE & SESPMNT & $\mathrm{R}$ \\
\hline 2032 & & & & & SESPMNT & $\mathrm{R}$ \\
\hline 2033 & & & & & SESPMNT & $\mathrm{R}$ \\
\hline 2034 & & & & & SESPMNT & $\mathrm{R}$ \\
\hline 2035 & & & & & SESPMNT & $\mathrm{R}$ \\
\hline 2036 & & & & ONSITE & SESPMNT & $\mathrm{R}$ \\
\hline 2037 & & & & ONSITE & SESPMNT & $\mathrm{R}$ \\
\hline 2038 & & & & ONSITE & SESPMNT & $\mathrm{R}$ \\
\hline 2039 & & & & ONSITE & SESPMNT & $\mathrm{R}$ \\
\hline 2040 & & & & ONSITE & SESPMNT & $\mathrm{R}$ \\
\hline 2041 & & & & OFFSITE & SESPMNT & $\mathrm{R}$ \\
\hline 2042 & & & & OFFSITE & SESPMNT & $\mathrm{R}$ \\
\hline 2043 & & & & OFFSITE & SESPMNT & $\mathrm{R}$ \\
\hline 2044 & & & & OFFSITE & SESPMNT & $\mathrm{R}$ \\
\hline 2045 & & & & OFFSITE & SESPMNT & $\mathrm{R}$ \\
\hline 2046 & & & & OFFSITE & SESPMNT & $\mathrm{R}$ \\
\hline 2047 & & & & OFFSITE & SESPMNT & $\mathrm{R}$ \\
\hline 2048 & & & & ONSITE & SESPMNT & $\mathrm{R}$ \\
\hline 2049 & & & & ONSITE & SESPMNT & $\mathrm{R}$ \\
\hline 2050 & & & & ONSITE & SESPMNT & $\mathrm{R}$ \\
\hline 2051 & & & & & SESPMNT & $\mathrm{R}$ \\
\hline 2052 & & & & & SESPMNT & $\mathrm{R}$ \\
\hline 2053 & & & & & SESPMNT & $\mathrm{R}$ \\
\hline 2054 & & & & ONSITE & SESPMNT & $\mathrm{R}$ \\
\hline 2055 & & & & ONSITE & SESPMNT & $\mathrm{R}$ \\
\hline 2056 & & & & ONSITE & SESPMNT & $\mathrm{R}$ \\
\hline 2057 & & & & & SESPMNT & $\mathrm{R}$ \\
\hline 2058 & & & & ONSITE & SESPMNT & $\mathrm{R}$ \\
\hline 2059 & & & & ONSITE & SESPMNT & $\mathrm{R}$ \\
\hline 2060 & & & & OFFSITE & SESPMNT & $\mathrm{R}$ \\
\hline 2061 & & & & OFFSITE & SESPMNT & $R$ \\
\hline 2062 & & & & ONSITE & SESPMNT & $R$ \\
\hline 2063 & & & & ONSITE & SESPMNT & $\mathrm{R}$ \\
\hline 2064 & & & & ONSITE & SESPMNT & $\mathrm{R}$ \\
\hline 2065 & & & & ONSITE & SESPMNT & $R$ \\
\hline 2066 & & & & & SESPMNT & $R$ \\
\hline 2067 & & & & ONSITE & SESPMNT & $\mathrm{R}$ \\
\hline 2068 & & & & ONSITE & SESPMNT & $\mathrm{R}$ \\
\hline 2069 & & & & & SESPMNT & $\mathrm{R}$ \\
\hline 2070 & & & & OFFSITE & SESPMNT & $\mathrm{R}$ \\
\hline 2071 & & & & OFFSITE & SESPMNT & $\mathrm{R}$ \\
\hline 2072 & & & & OFFSITE & SESPMNT & $R$ \\
\hline 2073 & & & & OFFSITE & SESPMNT & $\mathrm{R}$ \\
\hline 2074 & & & & OFFSITE & SESPMNT & $R$ \\
\hline 2075 & & & & ONSITE & SESPMNT & $R$ \\
\hline 2076 & & & & ONSITE & SESPMNT & $\mathrm{R}$ \\
\hline 2077 & & & & ONSITE & SESPMNT & $\mathrm{R}$ \\
\hline 2078 & & & & ONSITE & SESPMNT & $\mathrm{R}$ \\
\hline 2079 & & & & ONSITE & SESPMNT & $R$ \\
\hline 2080 & & & & OFFSITE & SESPMNT & $R$ \\
\hline 2081 & & & & OFFSITE & SESBMNT & $R$ \\
\hline
\end{tabular}




\begin{tabular}{|c|c|c|c|c|c|c|}
\hline & $\mathrm{AD}$ & $\mathrm{AE}$ & $\mathrm{AF}$ & $\mathrm{AG}$ & $\mathrm{AH}$ & $\mathrm{Al}$ \\
\hline 1 & RESULT_COMMENT & COLL_SAMP_SIZE & COLL_SAMP_SIZE_UNITS & DIST_CLASS & OWNER_ID & COLLECTION_PURPOSE \\
\hline 2082 & & & & OFFSITE & SESPMNT & $R$ \\
\hline 2083 & & & & OFFSITE & SESPMNT & $\mathrm{R}$ \\
\hline 2084 & & & & OFFSITE & SESPMNT & $\mathrm{R}$ \\
\hline 2085 & & & & OFFSITE & SESPMNT & $\mathrm{R}$ \\
\hline 2086 & & & & ONSITE & SESPMNT & $\mathrm{R}$ \\
\hline \begin{tabular}{|c|}
2087 \\
\end{tabular} & & & & ONSITE & SESPMNT & $\mathrm{R}$ \\
\hline \begin{tabular}{|c|}
2088 \\
\end{tabular} & & & & OFFSITE & SESPMNT & $\mathrm{R}$ \\
\hline 2089 & & & & OFFSITE & SESPMNT & $\mathrm{R}$ \\
\hline 2090 & & & & OFFSITE & SESPMNT & $\mathrm{R}$ \\
\hline 2091 & & & & ONSITE & SESPMNT & $\mathrm{R}$ \\
\hline 2092 & & & & ONSITE & SESPMNT & $\mathrm{R}$ \\
\hline 2093 & & & & & SESPMNT & $\mathrm{R}$ \\
\hline 2094 & & & & ONSITE & SESPMNT & $\mathrm{R}$ \\
\hline 2095 & & & & ONSITE & SESPMNT & $\mathrm{R}$ \\
\hline 2096 & & & & & SESPMNT & $\mathrm{R}$ \\
\hline \begin{tabular}{|l|l|}
2097 \\
\end{tabular} & & & & OFFSITE & SESPMNT & $\mathrm{R}$ \\
\hline 2098 & & & & OFFSITE & SESPMNT & $\mathrm{R}$ \\
\hline 2099 & & & & OFFSITE & SESPMNT & $\mathrm{R}$ \\
\hline 2100 & & & & ONSITE & SESPMNT & $\mathrm{R}$ \\
\hline 2101 & & & & ONSITE & SESPMNT & $\mathrm{R}$ \\
\hline 2102 & & & & ONSITE & SESPMNT & $\mathrm{R}$ \\
\hline 22103 & & & & OFFSITE & SESPMNT & $\mathrm{R}$ \\
\hline 2104 & & & & & SESPMNT & $\mathrm{R}$ \\
\hline 2105 & & & & ONSITE & SESPMNT & $\mathrm{R}$ \\
\hline 2106 & & & & ONSITE & SESPMNT & $\mathrm{R}$ \\
\hline \begin{tabular}{|l|}
2107 \\
\end{tabular} & & & & & SESPMNT & $\mathrm{R}$ \\
\hline 2108 & & & & & SESPMNT & $\mathrm{R}$ \\
\hline 2109 & & & & & SESPMNT & $\mathrm{R}$ \\
\hline 2110 & & & & & SESPMNT & $\mathrm{R}$ \\
\hline 2111 & & & & ONSITE & SESPMNT & $\mathrm{R}$ \\
\hline 2112 & & & & ONSITE & SESPMNT & $\mathrm{R}$ \\
\hline \begin{tabular}{|l|}
2113 \\
\end{tabular} & & & & ONSITE & SESPMNT & $\mathrm{R}$ \\
\hline \begin{tabular}{|l|}
2114 \\
\end{tabular} & & & & ONSITE & SESPMNT & $\mathrm{R}$ \\
\hline 2115 & & & & OFFSITE & SESPMNT & $\mathrm{R}$ \\
\hline \begin{tabular}{|l|}
2116 \\
\end{tabular} & & & & OFFSITE & SESPMNT & $\mathrm{R}$ \\
\hline \begin{tabular}{|l|}
2117 \\
\end{tabular} & & & & & SESPMNT & $\mathrm{R}$ \\
\hline 2118 & & & & & SESPMNT & $\mathrm{R}$ \\
\hline 2119 & & & & & SESPMNT & $\mathrm{R}$ \\
\hline 2120 & & & & ONSITE & SESPMNT & $\mathrm{R}$ \\
\hline 2121 & & & & ONSITE & SESPMNT & $\mathrm{R}$ \\
\hline \begin{tabular}{|l|}
2122 \\
\end{tabular} & & & & ONSITE & SESPMNT & $\mathrm{R}$ \\
\hline \begin{tabular}{|l|}
2123 \\
\end{tabular} & & & & & SESPMNT & $\mathrm{R}$ \\
\hline \begin{tabular}{|l|}
2124 \\
\end{tabular} & & & & ONSITE & SESPMNT & $\mathrm{R}$ \\
\hline \begin{tabular}{|l|}
2125 \\
\end{tabular} & & & & ONSITE & SESPMNT & $\mathrm{R}$ \\
\hline 2126 & & & & ONSITE & SESPMNT & $\mathrm{R}$ \\
\hline \begin{tabular}{|l|}
2127 \\
\end{tabular} & & & & ONSITE & SESPMNT & $\mathrm{R}$ \\
\hline \begin{tabular}{|l|l|}
2128 \\
\end{tabular} & & & & ONSITE & SESPMNT & $\mathrm{R}$ \\
\hline 2129 & & & & ONSITE & SESPMNT & $R$ \\
\hline \begin{tabular}{|l|}
2130 \\
\end{tabular} & & & & OFFSITE & SESPMNT & $\mathrm{R}$ \\
\hline 2131 & & & & OFFSITE & SESPMNT & $\mathrm{R}$ \\
\hline 2132 & & & & OFFSITE & SESPMNT & $\mathrm{R}$ \\
\hline 2133 & & & & OFFSITE & SESBMNT & $R$ \\
\hline
\end{tabular}




\begin{tabular}{|c|c|c|c|c|c|c|}
\hline & $A D$ & $\mathrm{AE}$ & $\mathrm{AF}$ & $A G$ & $\mathrm{AH}$ & $\mathrm{Al}$ \\
\hline 1 & RESULT_COMMENT & COLL_SAMP_SIZE & COLL_SAMP_SIZE_UNITS & DIST_CLASS & OWNER_ID & COLLECTION_PURPOSE \\
\hline 2134 & & & & OFFSITE & SESPMNT & $\mathrm{R}$ \\
\hline 2135 & & & & OFFSITE & SESPMNT & $\mathrm{R}$ \\
\hline 2136 & & & & OFFSITE & SESPMNT & $\mathrm{R}$ \\
\hline 2137 & & & & ONSITE & SESPMNT & $\mathrm{R}$ \\
\hline 2138 & & & & ONSITE & SESPMNT & $\mathrm{R}$ \\
\hline 2139 & & & & OFFSITE & SESPMNT & $\mathrm{R}$ \\
\hline 2140 & & & & OFFSITE & SESPMNT & $\mathrm{R}$ \\
\hline 2141 & & & & ONSITE & SESPMNT & $\mathrm{R}$ \\
\hline 2142 & & & & ONSITE & SESPMNT & $\mathrm{R}$ \\
\hline 2143 & & & & OFFSITE & SESPMNT & $\mathrm{R}$ \\
\hline 2144 & & & & OFFSITE & SESPMNT & $\mathrm{R}$ \\
\hline 2145 & & & & OFFSITE & SESPMNT & $\mathrm{R}$ \\
\hline 2146 & & & & & SESPMNT & $\mathrm{R}$ \\
\hline 2147 & & & & ONSITE & SESPMNT & $\mathrm{R}$ \\
\hline 2148 & & & & ONSITE & SESPMNT & $\mathrm{R}$ \\
\hline 2149 & & & & & SESPMNT & $\mathrm{R}$ \\
\hline 2150 & & & & ONSITE & SESPMNT & $\mathrm{R}$ \\
\hline 2151 & & & & ONSITE & SESPMNT & $\mathrm{R}$ \\
\hline 2152 & & & & ONSITE & SESPMNT & $\mathrm{R}$ \\
\hline 2153 & & & & ONSITE & SESPMNT & $\mathrm{R}$ \\
\hline 2154 & & & & ONSITE & SESPMNT & $\mathrm{R}$ \\
\hline 2155 & & & & OFFSITE & SESPMNT & $\mathrm{R}$ \\
\hline 2156 & & & & OFFSITE & SESPMNT & $\mathrm{R}$ \\
\hline 2157 & & & & OFFSITE & SESPMNT & $\mathrm{R}$ \\
\hline 2158 & & & & OFFSITE & SESPMNT & $\mathrm{R}$ \\
\hline 2159 & & & & OFFSITE & SESPMNT & $\mathrm{R}$ \\
\hline 2160 & & & & OFFSITE & SESPMNT & $\mathrm{R}$ \\
\hline 2161 & & & & OFFSITE & SESPMNT & $\mathrm{R}$ \\
\hline \begin{tabular}{|l|}
2162 \\
\end{tabular} & & & & OFFSITE & SESPMNT & $\mathrm{R}$ \\
\hline \begin{tabular}{|l|}
2163 \\
\end{tabular} & & & & OFFSITE & SESPMNT & $\mathrm{R}$ \\
\hline 2164 & & & & OFFSITE & SESPMNT & $\mathrm{R}$ \\
\hline 2165 & & & & ONSITE & SESPMNT & $\mathrm{R}$ \\
\hline 2166 & & & & ONSITE & SESPMNT & $\mathrm{R}$ \\
\hline \begin{tabular}{|l|}
2167 \\
\end{tabular} & & & & ONSITE & SESPMNT & $\mathrm{R}$ \\
\hline 2168 & & & & ONSITE & SESPMNT & $\mathrm{R}$ \\
\hline \begin{tabular}{|l|}
2169 \\
\end{tabular} & & & & ONSITE & SESPMNT & $\mathrm{R}$ \\
\hline 2170 & & & & OFFSITE & SESPMNT & $\mathrm{R}$ \\
\hline \begin{tabular}{|l|}
2171 \\
\end{tabular} & & & & OFFSITE & SESPMNT & $R$ \\
\hline 2172 & & & & OFFSITE & SESPMNT & $\mathrm{R}$ \\
\hline 2173 & & & & OFFSITE & SESPMNT & $\mathrm{R}$ \\
\hline 2174 & & & & ONSITE & SESPMNT & $\mathrm{R}$ \\
\hline 2175 & & & & ONSITE & SESPMNT & $\mathrm{R}$ \\
\hline \begin{tabular}{|l|}
2176 \\
\end{tabular} & & & & & SESPMNT & $\mathrm{R}$ \\
\hline \begin{tabular}{|l|}
2177 \\
\end{tabular} & & & & ONSITE & SESPMNT & $\mathrm{R}$ \\
\hline \begin{tabular}{|l|}
2178 \\
\end{tabular} & & & & ONSITE & SESPMNT & $R$ \\
\hline 2179 & & & & & SESPMNT & $\mathrm{R}$ \\
\hline 2180 & & & & ONSITE & SESPMNT & $\mathrm{R}$ \\
\hline 2181 & & & & & SESPMNT & $\mathrm{R}$ \\
\hline \begin{tabular}{|l|}
2182 \\
\end{tabular} & & & & ONSITE & SESPMNT & $\mathrm{R}$ \\
\hline 2183 & & & & ONSITE & SESPMNT & $\mathrm{R}$ \\
\hline 2184 & & & & & SESPMNT & $R$ \\
\hline \begin{tabular}{|l|}
2185 \\
\end{tabular} & & & & & SESBMNT & $R$ \\
\hline
\end{tabular}




\begin{tabular}{|c|c|c|c|c|c|c|}
\hline & $\mathrm{AD}$ & $\mathrm{AE}$ & $\mathrm{AF}$ & AG & $\mathrm{AH}$ & $\overline{\mathrm{Al}}$ \\
\hline 1 & RESULT_COMMENT & COLL_SAMP_SIZE & COLL_SAMP_SIZE_UNITS & DIST_CLASS & OWNER_ID & COLLECTION_PURPOSE \\
\hline 2186 & & & & & SESPMNT & $\mathrm{R}$ \\
\hline 2187 & & & & & SESPMNT & $\mathrm{R}$ \\
\hline 2188 & & & & ONSITE & SESPMNT & $\mathrm{R}$ \\
\hline 2189 & & & & ONSITE & SESPMNT & $\mathrm{R}$ \\
\hline 2190 & & & & ONSITE & SESPMNT & $\mathrm{R}$ \\
\hline 2191 & & & & ONSITE & SESPMNT & $\mathrm{R}$ \\
\hline 2192 & & & & ONSITE & SESPMNT & $\mathrm{R}$ \\
\hline 2193 & & & & ONSITE & SESPMNT & $\mathrm{R}$ \\
\hline 2194 & & & & ONSITE & SESPMNT & $\mathrm{R}$ \\
\hline 2195 & & & & ONSITE & SESPMNT & $\mathrm{R}$ \\
\hline 2196 & & & & & SESPMNT & $\mathrm{R}$ \\
\hline 2197 & & & & & SESPMNT & $\mathrm{R}$ \\
\hline 2198 & & & & & SESPMNT & $\mathrm{R}$ \\
\hline 2199 & & & & ONSITE & SESPMNT & $\mathrm{R}$ \\
\hline 2200 & & & & ONSITE & SESPMNT & $\mathrm{R}$ \\
\hline 2201 & & & & ONSITE & SESPMNT & $\mathrm{R}$ \\
\hline 2202 & & & & & SESPMNT & $\mathrm{R}$ \\
\hline 2203 & & & & OFFSITE & SESPMNT & $\mathrm{R}$ \\
\hline 2204 & & & & OFFSITE & SESPMNT & $\mathrm{R}$ \\
\hline 2205 & & & & OFFSITE & SESPMNT & $\mathrm{R}$ \\
\hline 2206 & & & & OFFSITE & SESPMNT & $R$ \\
\hline 2207 & & & & ONSITE & SESPMNT & $\mathrm{R}$ \\
\hline 2208 & & & & ONSITE & SESPMNT & $\mathrm{R}$ \\
\hline 2209 & & & & OFFSITE & SESPMNT & $\mathrm{R}$ \\
\hline 2210 & & & & OFFSITE & SESPMNT & $\mathrm{R}$ \\
\hline 2211 & & & & ONSITE & SESPMNT & $\mathrm{R}$ \\
\hline 2212 & & & & ONSITE & SESPMNT & $R$ \\
\hline 2213 & & & & OFFSITE & SESPMNT & $\mathrm{R}$ \\
\hline 2214 & & & & OFFSITE & SESPMNT & $\mathrm{R}$ \\
\hline 2215 & & & & ONSITE & SESPMNT & $\mathrm{R}$ \\
\hline 2216 & & & & ONSITE & SESPMNT & $R$ \\
\hline 2217 & & & & & SESPMNT & $R$ \\
\hline 2218 & & & & ONSITE & SESPMNT & $R$ \\
\hline 2219 & & & & ONSITE & SESPMNT & $R$ \\
\hline 2220 & & & & & SESPMNT & $R$ \\
\hline 2221 & & & & OFFSITE & SESPMNT & $\mathrm{R}$ \\
\hline 2222 & & & & OFFSITE & SESPMNT & $R$ \\
\hline 2223 & & & & OFFSITE & SESPMNT & $R$ \\
\hline 2224 & & & & OFFSITE & SESPMNT & $R$ \\
\hline 2225 & & & & OFFSITE & SESPMNT & $R$ \\
\hline 2226 & & & & ONSITE & SESPMNT & $R$ \\
\hline 2227 & & & & ONSITE & SESPMNT & $R$ \\
\hline 2228 & & & & ONSITE & SESPMNT & $R$ \\
\hline 2229 & & & & ONSITE & SESPMNT & $R$ \\
\hline 2230 & & & & ONSITE & SESPMNT & $R$ \\
\hline 2231 & & & & OFFSITE & SESPMNT & $R$ \\
\hline 2232 & & & & OFFSITE & SESPMNT & $\mathrm{R}$ \\
\hline 2233 & & & & OFFSITE & SESPMNT & $\mathrm{R}$ \\
\hline 2234 & & & & OFFSITE & SESPMNT & $R$ \\
\hline 2235 & & & & OFFSITE & SESPMNT & $R$ \\
\hline 2236 & & & & ONSITE & SESPMNT & $\mathrm{R}$ \\
\hline 2237 & & & & ONSITE & SESBMNT & $R$ \\
\hline
\end{tabular}




\begin{tabular}{|c|c|c|c|c|c|c|}
\hline & $\mathrm{AD}$ & $\mathrm{AE}$ & $\mathrm{AF}$ & $A G$ & $\mathrm{AH}$ & $\mathrm{Al}$ \\
\hline 1 & RESULT_COMMENT & COLL_SAMP_SIZE & COLL_SAMP_SIZE_UNITS & DIST_CLASS & OWNER_ID & COLLECTION_PURPOSE \\
\hline 2238 & & & & OFFSITE & SESPMNT & $\mathrm{R}$ \\
\hline 2239 & & & & OFFSITE & SESPMNT & $\mathrm{R}$ \\
\hline 2240 & & & & OFFSITE & SESPMNT & $\mathrm{R}$ \\
\hline 2241 & & & & OFFSITE & SESPMNT & $\mathrm{R}$ \\
\hline 2242 & & & & OFFSITE & SESPMNT & $\mathrm{R}$ \\
\hline 2243 & & & & OFFSITE & SESPMNT & $\mathrm{R}$ \\
\hline 2244 & & & & ONSITE & SESPMNT & $\mathrm{R}$ \\
\hline 2245 & & & & ONSITE & SESPMNT & $\mathrm{R}$ \\
\hline 2246 & & & & & SESPMNT & $\mathrm{R}$ \\
\hline 2247 & & & & ONSITE & SESPMNT & $\mathrm{R}$ \\
\hline 2248 & & & & ONSITE & SESPMNT & $\mathrm{R}$ \\
\hline 2249 & & & & & SESPMNT & $\mathrm{R}$ \\
\hline 2250 & & & & ONSITE & SESPMNT & $\mathrm{R}$ \\
\hline 2251 & & & & ONSITE & SESPMNT & $\mathrm{R}$ \\
\hline 2252 & & & & ONSITE & SESPMNT & $\mathrm{R}$ \\
\hline 2253 & & & & OFFSITE & SESPMNT & $\mathrm{R}$ \\
\hline 2254 & & & & OFFSITE & SESPMNT & $\mathrm{R}$ \\
\hline 2255 & & & & OFFSITE & SESPMNT & $\mathrm{R}$ \\
\hline 2256 & & & & ONSITE & SESPMNT & $\mathrm{R}$ \\
\hline 2257 & & & & & SESPMNT & $\mathrm{R}$ \\
\hline 2258 & & & & ONSITE & SESPMNT & $\mathrm{R}$ \\
\hline 2259 & & & & ONSITE & SESPMNT & $\mathrm{R}$ \\
\hline 2260 & & & & & SESPMNT & $\mathrm{R}$ \\
\hline 2261 & & & & & SESPMNT & $\mathrm{R}$ \\
\hline 2262 & & & & & SESPMNT & $\mathrm{R}$ \\
\hline 2263 & & & & & SESPMNT & $\mathrm{R}$ \\
\hline 2264 & & & & ONSITE & SESPMNT & $\mathrm{R}$ \\
\hline 2265 & & & & ONSITE & SESPMNT & $\mathrm{R}$ \\
\hline 2266 & & & & ONSITE & SESPMNT & $\mathrm{R}$ \\
\hline 2267 & & & & ONSITE & SESPMNT & $\mathrm{R}$ \\
\hline 2268 & & & & ONSITE & SESPMNT & $\mathrm{R}$ \\
\hline 2269 & & & & ONSITE & SESPMNT & $R$ \\
\hline 2270 & & & & ONSITE & SESPMNT & $R$ \\
\hline 2271 & & & & ONSITE & SESPMNT & $\mathrm{R}$ \\
\hline 2272 & & & & ONSITE & SESPMNT & $\mathrm{R}$ \\
\hline 2273 & & & & & SESPMNT & $R$ \\
\hline 2274 & & & & & SESPMNT & $R$ \\
\hline 2275 & & & & & SESPMNT & $\mathrm{R}$ \\
\hline 2276 & & & & ONSITE & SESPMNT & $\mathrm{R}$ \\
\hline 2277 & & & & ONSITE & SESPMNT & $\mathrm{R}$ \\
\hline 2278 & & & & ONSITE & SESPMNT & $\mathrm{R}$ \\
\hline 2279 & & & & & SESPMNT & $\mathrm{R}$ \\
\hline 2280 & & & & ONSITE & SESPMNT & $R$ \\
\hline 2281 & & & & ONSITE & SESPMNT & $\mathrm{R}$ \\
\hline 2282 & & & & ONSITE & SESPMNT & $R$ \\
\hline 2283 & & & & OFFSITE & SESPMNT & $R$ \\
\hline 2284 & & & & OFFSITE & SESPMNT & $\mathrm{R}$ \\
\hline 2285 & & & & OFFSITE & SESPMNT & $\mathrm{R}$ \\
\hline 2286 & & & & OFFSITE & SESPMNT & $\mathrm{R}$ \\
\hline 2287 & & & & OFFSITE & SESPMNT & $R$ \\
\hline 2288 & & & & OFFSITE & SESPMNT & $R$ \\
\hline 2289 & & & & OFFSITE & SESBMNT & $R$ \\
\hline
\end{tabular}




\begin{tabular}{|c|c|c|c|c|c|c|}
\hline & $A D$ & $\mathrm{AE}$ & $\mathrm{AF}$ & $A G$ & $\mathrm{AH}$ & $\mathrm{Al}$ \\
\hline 1 & RESULT_COMMENT & COLL_SAMP_SIZE & COLL_SAMP_SIZE_UNITS & DIST_CLASS & OWNER_ID & COLLECTION_PURPOSE \\
\hline 2290 & & & & ONSITE & SESPMNT & $\mathrm{R}$ \\
\hline 2291 & & & & ONSITE & SESPMNT & $\mathrm{R}$ \\
\hline 2292 & & & & ONSITE & SESPMNT & $\mathrm{R}$ \\
\hline 2293 & & & & ONSITE & SESPMNT & $\mathrm{R}$ \\
\hline 2294 & & & & OFFSITE & SESPMNT & $\mathrm{R}$ \\
\hline 2295 & & & & OFFSITE & SESPMNT & $\mathrm{R}$ \\
\hline 2296 & & & & ONSITE & SESPMNT & $\mathrm{R}$ \\
\hline 2297 & & & & OFFSITE & SESPMNT & $\mathrm{R}$ \\
\hline 2298 & & & & OFFSITE & SESPMNT & $\mathrm{R}$ \\
\hline 2299 & & & & ONSITE & SESPMNT & $\mathrm{R}$ \\
\hline 2300 & & & & ONSITE & SESPMNT & $\mathrm{R}$ \\
\hline 2301 & & & & & SESPMNT & $\mathrm{R}$ \\
\hline 2302 & & & & ONSITE & SESPMNT & $\mathrm{R}$ \\
\hline 2303 & & & & ONSITE & SESPMNT & $\mathrm{R}$ \\
\hline 2304 & & & & & SESPMNT & $\mathrm{R}$ \\
\hline 2305 & & & & ONSITE & SESPMNT & $\mathrm{R}$ \\
\hline 2306 & & & & ONSITE & SESPMNT & $\mathrm{R}$ \\
\hline 2307 & & & & ONSITE & SESPMNT & $\mathrm{R}$ \\
\hline 2308 & & & & ONSITE & SESPMNT & $\mathrm{R}$ \\
\hline 2309 & & & & ONSITE & SESPMNT & $\mathrm{R}$ \\
\hline 2310 & & & & OFFSITE & SESPMNT & $\mathrm{R}$ \\
\hline 2311 & & & & OFFSITE & SESPMNT & $\mathrm{R}$ \\
\hline 2312 & & & & OFFSITE & SESPMNT & $\mathrm{R}$ \\
\hline 2313 & & & & OFFSITE & SESPMNT & $\mathrm{R}$ \\
\hline 2314 & & & & OFFSITE & SESPMNT & $\mathrm{R}$ \\
\hline 2315 & & & & OFFSITE & SESPMNT & $\mathrm{R}$ \\
\hline 2316 & & & & OFFSITE & SESPMNT & $\mathrm{R}$ \\
\hline \begin{tabular}{|l|}
2317 \\
\end{tabular} & & & & OFFSITE & SESPMNT & $\mathrm{R}$ \\
\hline 2318 & & & & OFFSITE & SESPMNT & $\mathrm{R}$ \\
\hline 2319 & & & & OFFSITE & SESPMNT & $R$ \\
\hline 2320 & & & & ONSITE & SESPMNT & $\mathrm{R}$ \\
\hline 2321 & & & & ONSITE & SESPMNT & $\mathrm{R}$ \\
\hline \begin{tabular}{|l|}
2322 \\
\end{tabular} & & & & OFFSITE & SESPMNT & $\mathrm{R}$ \\
\hline 2323 & & & & OFFSITE & SESPMNT & $\mathrm{R}$ \\
\hline 2324 & & & & ONSITE & SESPMNT & $\mathrm{R}$ \\
\hline \begin{tabular}{|l|}
2325 \\
\end{tabular} & & & & ONSITE & SESPMNT & $\mathrm{R}$ \\
\hline \begin{tabular}{|l|}
2326 \\
\end{tabular} & & & & ONSITE & SESPMNT & $\mathrm{R}$ \\
\hline \begin{tabular}{|l|}
2327 \\
\end{tabular} & & & & & SESPMNT & $\mathrm{R}$ \\
\hline 2328 & & & & ONSITE & SESPMNT & $\mathrm{R}$ \\
\hline 2329 & & & & ONSITE & SESPMNT & $\mathrm{R}$ \\
\hline 2330 & & & & & SESPMNT & $\mathrm{R}$ \\
\hline \begin{tabular}{|l|}
2331 \\
\end{tabular} & & & & ONSITE & SESPMNT & $\mathrm{R}$ \\
\hline 2332 & & & & ONSITE & SESPMNT & $\mathrm{R}$ \\
\hline 2333 & & & & OFFSITE & SESPMNT & $\mathrm{R}$ \\
\hline 2334 & & & & OFFSITE & SESPMNT & $\mathrm{R}$ \\
\hline 2335 & & & & ONSITE & SESPMNT & $\mathrm{R}$ \\
\hline 2336 & & & & ONSITE & SESPMNT & $\mathrm{R}$ \\
\hline \begin{tabular}{|l|}
2337 \\
\end{tabular} & & & & OFFSITE & SESPMNT & $\mathrm{R}$ \\
\hline 2338 & & & & OFFSITE & SESPMNT & $\mathrm{R}$ \\
\hline 2339 & & & & ONSITE & SESPMNT & $R$ \\
\hline 2340 & & & & ONSITE & $\begin{array}{l}\text { SESPMNT } \\
\end{array}$ & $\mathrm{R}$ \\
\hline 2341 & & & & & SESBMNT & $R$ \\
\hline
\end{tabular}




\begin{tabular}{|c|c|c|c|c|c|c|}
\hline & $\mathrm{AD}$ & $\mathrm{AE}$ & $\mathrm{AF}$ & $\mathrm{AG}$ & $\mathrm{AH}$ & $\mathrm{Al}$ \\
\hline 1 & RESULT_COMMENT & COLL_SAMP_SIZE & COLL_SAMP_SIZE_UNITS & DIST_CLASS & OWNER_ID & COLLECTION_PURPOSE \\
\hline 2342 & & & & & SESPMNT & $\mathrm{R}$ \\
\hline 2343 & & & & ONSITE & SESPMNT & $\mathrm{R}$ \\
\hline 2344 & & & & ONSITE & SESPMNT & $\mathrm{R}$ \\
\hline 2345 & & & & ONSITE & SESPMNT & $\mathrm{R}$ \\
\hline 2346 & & & & ONSITE & SESPMNT & $\mathrm{R}$ \\
\hline 2347 & & & & ONSITE & SESPMNT & $\mathrm{R}$ \\
\hline 2348 & & & & ONSITE & SESPMNT & $\mathrm{R}$ \\
\hline 2349 & & & & OFFSITE & SESPMNT & $\mathrm{R}$ \\
\hline 2350 & & & & OFFSITE & SESPMNT & $\mathrm{R}$ \\
\hline 2351 & & & & OFFSITE & SESPMNT & $\mathrm{R}$ \\
\hline 2352 & & & & OFFSITE & SESPMNT & $\mathrm{R}$ \\
\hline 2353 & & & & OFFSITE & SESPMNT & $\mathrm{R}$ \\
\hline 2354 & & & & & SESPMNT & $\mathrm{R}$ \\
\hline 2355 & & & & & SESPMNT & $\mathrm{R}$ \\
\hline 2356 & & & & & SESPMNT & $\mathrm{R}$ \\
\hline 2357 & & & & ONSITE & SESPMNT & $\mathrm{R}$ \\
\hline 2358 & & & & ONSITE & SESPMNT & $\mathrm{R}$ \\
\hline 2359 & & & & ONSITE & SESPMNT & $\mathrm{R}$ \\
\hline 2360 & & & & & SESPMNT & $\mathrm{R}$ \\
\hline 2361 & & & & ONSITE & SESPMNT & $\mathrm{R}$ \\
\hline 2362 & & & & ONSITE & SESPMNT & $\mathrm{R}$ \\
\hline 2363 & & & & OFFSITE & SESPMNT & $\mathrm{R}$ \\
\hline 2364 & & & & OFFSITE & SESPMNT & $\mathrm{R}$ \\
\hline 2365 & & & & ONSITE & SESPMNT & $\mathrm{R}$ \\
\hline 2366 & & & & ONSITE & SESPMNT & $\mathrm{R}$ \\
\hline \begin{tabular}{|l|}
2367 \\
\end{tabular} & & & & ONSITE & SESPMNT & $\mathrm{R}$ \\
\hline 2368 & & & & ONSITE & SESPMNT & $\mathrm{R}$ \\
\hline 2369 & & & & ONSITE & SESPMNT & $\mathrm{R}$ \\
\hline 2370 & & & & OFFSITE & SESPMNT & $\mathrm{R}$ \\
\hline 2371 & & & & OFFSITE & SESPMNT & $\mathrm{R}$ \\
\hline 2372 & & & & OFFSITE & SESPMNT & $\mathrm{R}$ \\
\hline 2373 & & & & OFFSITE & SESPMNT & $\mathrm{R}$ \\
\hline 2374 & & & & OFFSITE & SESPMNT & $\mathrm{R}$ \\
\hline 2375 & & & & ONSITE & SESPMNT & $\mathrm{R}$ \\
\hline 2376 & & & & ONSITE & SESPMNT & $\mathrm{R}$ \\
\hline \begin{tabular}{|l|}
2377 \\
\end{tabular} & & & & & SESPMNT & $\mathrm{R}$ \\
\hline \begin{tabular}{|l|}
2378 \\
\end{tabular} & & & & ONSITE & SESPMNT & $\mathrm{R}$ \\
\hline \begin{tabular}{|l|}
2379 \\
\end{tabular} & & & & ONSITE & SESPMNT & $\mathrm{R}$ \\
\hline \begin{tabular}{|l|}
2380 \\
\end{tabular} & & & & & SESPMNT & $\mathrm{R}$ \\
\hline \begin{tabular}{|l|}
2381 \\
\end{tabular} & & & & OFFSITE & SESPMNT & $\mathrm{R}$ \\
\hline \begin{tabular}{|l|}
2382 \\
\end{tabular} & & & & OFFSITE & SESPMNT & $\mathrm{R}$ \\
\hline \begin{tabular}{|l|}
2383 \\
\end{tabular} & & & & ONSITE & SESPMNT & $\mathrm{R}$ \\
\hline \begin{tabular}{|l|}
2384 \\
\end{tabular} & & & & ONSITE & SESPMNT & $\mathrm{R}$ \\
\hline \begin{tabular}{|l|}
2385 \\
\end{tabular} & & & & OFFSITE & SESPMNT & $\mathrm{R}$ \\
\hline \begin{tabular}{|l|}
2386 \\
\end{tabular} & & & & OFFSITE & SESPMNT & $\mathrm{R}$ \\
\hline \begin{tabular}{|l|}
2387 \\
\end{tabular} & & & & ONSITE & SESPMNT & $\mathrm{R}$ \\
\hline \begin{tabular}{|l|}
2388 \\
\end{tabular} & & & & ONSITE & SESPMNT & $\mathrm{R}$ \\
\hline \begin{tabular}{|l|}
2389 \\
\end{tabular} & & & & OFFSITE & SESPMNT & $\mathrm{R}$ \\
\hline \begin{tabular}{|l|}
2390 \\
\end{tabular} & & & & OFFSITE & SESPMNT & $\mathrm{R}$ \\
\hline \begin{tabular}{|l|}
2391 \\
\end{tabular} & & & & & SESPMNT & $\mathrm{R}$ \\
\hline \begin{tabular}{|l|}
2392 \\
\end{tabular} & & & & ONSITE & SESPMNT & $\mathrm{R}$ \\
\hline \begin{tabular}{|l|}
2393 \\
\end{tabular} & & & & ONSITE & SESBMNT & $R$ \\
\hline
\end{tabular}




\begin{tabular}{|c|c|c|c|c|c|c|}
\hline & $A D$ & $\mathrm{AE}$ & $\mathrm{AF}$ & $A G$ & $\mathrm{AH}$ & $\mathrm{Al}$ \\
\hline 1 & RESULT_COMMENT & COLL_SAMP_SIZE & COLL_SAMP_SIZE_UNITS & DIST_CLASS & OWNER_ID & COLLECTION_PURPOSE \\
\hline 2394 & & & & & SESPMNT & $\mathrm{R}$ \\
\hline 2395 & & & & OFFSITE & SESPMNT & $\mathrm{R}$ \\
\hline 2396 & & & & OFFSITE & SESPMNT & $\mathrm{R}$ \\
\hline 2397 & & & & OFFSITE & SESPMNT & $\mathrm{R}$ \\
\hline 2398 & & & & ONSITE & SESPMNT & $\mathrm{R}$ \\
\hline 2399 & & & & ONSITE & SESPMNT & $\mathrm{R}$ \\
\hline 2400 & & & & ONSITE & SESPMNT & $\mathrm{R}$ \\
\hline 2401 & & & & ONSITE & SESPMNT & $\mathrm{R}$ \\
\hline 2402 & & & & ONSITE & SESPMNT & $\mathrm{R}$ \\
\hline 2403 & & & & OFFSITE & SESPMNT & $\mathrm{R}$ \\
\hline 2404 & & & & OFFSITE & SESPMNT & $\mathrm{R}$ \\
\hline 2405 & & & & ONSITE & SESPMNT & $\mathrm{R}$ \\
\hline 2406 & & & & ONSITE & SESPMNT & $\mathrm{R}$ \\
\hline 2407 & & & & & SESPMNT & $\mathrm{R}$ \\
\hline 2408 & & & & & SESPMNT & $\mathrm{R}$ \\
\hline 2409 & & & & ONSITE & SESPMNT & $\mathrm{R}$ \\
\hline 2410 & & & & ONSITE & SESPMNT & $\mathrm{R}$ \\
\hline 2411 & & & & OFFSITE & SESPMNT & $\mathrm{R}$ \\
\hline 2412 & & & & OFFSITE & SESPMNT & $\mathrm{R}$ \\
\hline 2413 & & & & ONSITE & SESPMNT & $\mathrm{R}$ \\
\hline 2414 & & & & ONSITE & SESPMNT & $\mathrm{R}$ \\
\hline 2415 & & & & OFFSITE & SESPMNT & $\mathrm{R}$ \\
\hline 2416 & & & & OFFSITE & SESPMNT & $\mathrm{R}$ \\
\hline 2417 & & & & ONSITE & SESPMNT & $\mathrm{R}$ \\
\hline 2418 & & & & ONSITE & SESPMNT & $\mathrm{R}$ \\
\hline 2419 & & & & ONSITE & SESPMNT & $\mathrm{R}$ \\
\hline 2420 & & & & ONSITE & SESPMNT & $\mathrm{R}$ \\
\hline 2421 & & & & OFFSITE & SESPMNT & $\mathrm{R}$ \\
\hline \begin{tabular}{|l|}
2422 \\
\end{tabular} & & & & OFFSITE & SESPMNT & $\mathrm{R}$ \\
\hline 2423 & & & & & SESPMNT & $R$ \\
\hline 2424 & & & & & SESPMNT & $\mathrm{R}$ \\
\hline 2425 & & & & & SESPMNT & $\mathrm{R}$ \\
\hline 2426 & & & & ONSITE & SESPMNT & $\mathrm{R}$ \\
\hline \begin{tabular}{|l|}
2427 \\
\end{tabular} & & & & ONSITE & SESPMNT & $\mathrm{R}$ \\
\hline 2428 & & & & ONSITE & SESPMNT & $\mathrm{R}$ \\
\hline 2429 & & & & ONSITE & SESPMNT & $\mathrm{R}$ \\
\hline 2430 & & & & & SESPMNT & $\mathrm{R}$ \\
\hline 2431 & & & & ONSITE & SESPMNT & $\mathrm{R}$ \\
\hline 2432 & & & & ONSITE & SESPMNT & $\mathrm{R}$ \\
\hline 2433 & & & & OFFSITE & SESPMNT & $\mathrm{R}$ \\
\hline 2434 & & & & OFFSITE & SESPMNT & $\mathrm{R}$ \\
\hline 2435 & & & & ONSITE & SESPMNT & $\mathrm{R}$ \\
\hline 2436 & & & & ONSITE & SESPMNT & $\mathrm{R}$ \\
\hline \begin{tabular}{|l|l|}
2437 \\
\end{tabular} & & & & & SESPMNT & $\mathrm{R}$ \\
\hline 2438 & & & & ONSITE & SESPMNT & $\mathrm{R}$ \\
\hline 2439 & & & & ONSITE & SESPMNT & $\mathrm{R}$ \\
\hline 2440 & & & & & SESPMNT & $\mathrm{R}$ \\
\hline \begin{tabular}{|l|}
2441 \\
\end{tabular} & & & & ONSITE & SESPMNT & $\mathrm{R}$ \\
\hline \begin{tabular}{|l|}
2442 \\
\end{tabular} & & & & ONSITE & SESPMNT & $\mathrm{R}$ \\
\hline 2443 & & & & ONSITE & SESPMNT & $R$ \\
\hline \begin{tabular}{|l|}
2444 \\
\end{tabular} & & & & ONSITE & $\begin{array}{l}\text { SESPMNT } \\
\end{array}$ & $\mathrm{R}$ \\
\hline 2445 & & & & ONSITE & SESBMNT & $R$ \\
\hline
\end{tabular}




\begin{tabular}{|c|c|c|c|c|c|c|}
\hline & $A D$ & $\mathrm{AE}$ & $\mathrm{AF}$ & $A G$ & $\mathrm{AH}$ & $\mathrm{Al}$ \\
\hline 1 & RESULT_COMMENT & COLL_SAMP_SIZE & COLL_SAMP_SIZE_UNITS & DIST_CLASS & OWNER_ID & COLLECTION_PURPOSE \\
\hline 2446 & & & & OFFSITE & SESPMNT & $\mathrm{R}$ \\
\hline 2447 & & & & OFFSITE & SESPMNT & $\mathrm{R}$ \\
\hline \begin{tabular}{|c|}
2448 \\
\end{tabular} & & & & OFFSITE & SESPMNT & $\mathrm{R}$ \\
\hline 2449 & & & & OFFSITE & SESPMNT & $\mathrm{R}$ \\
\hline 2450 & & & & OFFSITE & SESPMNT & $\mathrm{R}$ \\
\hline 2451 & & & & & SESPSPEC & $\mathrm{s}$ \\
\hline 2452 & & & & & SESPSPEC & $\mathrm{s}$ \\
\hline 2453 & & & & ONSITE & SESPMNT & $\mathrm{R}$ \\
\hline 2454 & & & & ONSITE & SESPMNT & $\mathrm{R}$ \\
\hline 2455 & & & & OFFSITE & SESPMNT & $\mathrm{R}$ \\
\hline 2456 & & & & OFFSITE & SESPMNT & $\mathrm{R}$ \\
\hline 2457 & & & & ONSITE & SESPMNT & $\mathrm{R}$ \\
\hline 2458 & & & & ONSITE & SESPMNT & $\mathrm{R}$ \\
\hline 2459 & & & & OFFSITE & SESPMNT & $\mathrm{R}$ \\
\hline 2460 & & & & OFFSITE & SESPMNT & $\mathrm{R}$ \\
\hline 2461 & & & & ONSITE & SESPMNT & $\mathrm{R}$ \\
\hline 2462 & & & & ONSITE & SESPMNT & $\mathrm{R}$ \\
\hline 2463 & & & & ONSITE & SESPMNT & $\mathrm{R}$ \\
\hline 2464 & & & & & SESPMNT & $\mathrm{R}$ \\
\hline 2465 & & & & ONSITE & SESPMNT & $\mathrm{R}$ \\
\hline 2466 & & & & ONSITE & SESPMNT & $\mathrm{R}$ \\
\hline 2467 & & & & & SESPMNT & $\mathrm{R}$ \\
\hline 2468 & & & & ONSITE & SESPMNT & $\mathrm{R}$ \\
\hline 2469 & & & & ONSITE & SESPMNT & $\mathrm{R}$ \\
\hline 2470 & & & & OFFSITE & SESPMNT & $\mathrm{R}$ \\
\hline 2471 & & & & OFFSITE & SESPMNT & $\mathrm{R}$ \\
\hline 2472 & & & & ONSITE & SESPMNT & $\mathrm{R}$ \\
\hline 2473 & & & & ONSITE & SESPMNT & $\mathrm{R}$ \\
\hline 2474 & & & & & SESPMNT & $\mathrm{R}$ \\
\hline 2475 & & & & & SESPMNT & $R$ \\
\hline 2476 & & & & ONSITE & SESPMNT & $\mathrm{R}$ \\
\hline 2477 & & & & ONSITE & SESPMNT & $\mathrm{R}$ \\
\hline 2478 & & & & ONSITE & SESPMNT & $\mathrm{R}$ \\
\hline 2479 & & & & ONSITE & SESPMNT & $\mathrm{R}$ \\
\hline 2480 & & & & OFFSITE & SESPMNT & $\mathrm{R}$ \\
\hline 2481 & & & & OFFSITE & SESPMNT & $\mathrm{R}$ \\
\hline 2482 & & & & ONSITE & SESPMNT & $\mathrm{R}$ \\
\hline 2483 & & & & ONSITE & SESPMNT & $\mathrm{R}$ \\
\hline 2484 & & & & & SESPMNT & $\mathrm{R}$ \\
\hline 2485 & & & & & SESPMNT & $\mathrm{R}$ \\
\hline 2486 & & & & & SESPMNT & $\mathrm{R}$ \\
\hline \begin{tabular}{|l|l|}
2487 \\
\end{tabular} & & & & ONSITE & SESPMNT & $\mathrm{R}$ \\
\hline 2488 & & & & ONSITE & SESPMNT & $\mathrm{R}$ \\
\hline 2489 & & & & ONSITE & SESPMNT & $\mathrm{R}$ \\
\hline 2490 & & & & ONSITE & SESPMNT & $\mathrm{R}$ \\
\hline 2491 & & & & & SESPMNT & $\mathrm{R}$ \\
\hline 2492 & & & & ONSITE & SESPMNT & $\mathrm{R}$ \\
\hline 2493 & & & & ONSITE & SESPMNT & $\mathrm{R}$ \\
\hline 2494 & & & & OFFSITE & SESPMNT & $\mathrm{R}$ \\
\hline 2495 & & & & OFFSITE & SESPMNT & $R$ \\
\hline 2496 & & & & OFFSITE & $\begin{array}{l}\text { SESPMNT } \\
\end{array}$ & $\mathrm{R}$ \\
\hline 2497 & & & & OFFSITE & SESBMNT & ${ }_{2}^{R}$ \\
\hline
\end{tabular}




\begin{tabular}{|c|c|c|c|c|c|c|}
\hline & $\mathrm{AD}$ & $\mathrm{AE}$ & $\mathrm{AF}$ & AG & $\mathrm{AH}$ & $\mathrm{Al}$ \\
\hline 1 & RESULT_COMMENT & COLL_SAMP_SIZE & COLL_SAMP_SIZE_UNITS & DIST_CLASS & OWNER_ID & COLLECTION_PURPOSE \\
\hline 2498 & & & & OFFSITE & SESPMNT & $\mathrm{R}$ \\
\hline 2499 & & & & ONSITE & SESPMNT & $\mathrm{R}$ \\
\hline 2500 & & & & ONSITE & SESPMNT & $\mathrm{R}$ \\
\hline 2501 & & & & OFFSITE & SESPMNT & $\mathrm{R}$ \\
\hline 2502 & & & & OFFSITE & SESPMNT & $\mathrm{R}$ \\
\hline 2503 & & & & & SESPMNT & $\mathrm{R}$ \\
\hline 2504 & & & & ONSITE & SESPMNT & $\mathrm{R}$ \\
\hline 2505 & & & & ONSITE & SESPMNT & $\mathrm{R}$ \\
\hline 2506 & & & & & SESPMNT & $\mathrm{R}$ \\
\hline 2507 & & & & ONSITE & SESPMNT & $\mathrm{R}$ \\
\hline 2508 & & & & ONSITE & SESPMNT & $\mathrm{R}$ \\
\hline \begin{tabular}{|l|}
2509 \\
\end{tabular} & & & & ONSITE & SESPMNT & $R$ \\
\hline \begin{tabular}{|l|}
2510 \\
\end{tabular} & & & & ONSITE & SESPMNT & $\mathrm{R}$ \\
\hline \begin{tabular}{|l|}
2511 \\
\end{tabular} & & & & OFFSITE & SESPMNT & $\mathrm{R}$ \\
\hline \begin{tabular}{|l|}
2512 \\
\end{tabular} & & & & OFFSITE & SESPMNT & $R$ \\
\hline \begin{tabular}{|l|}
2513 \\
\end{tabular} & & & & OFFSITE & SESPMNT & $\mathrm{R}$ \\
\hline \begin{tabular}{|l|}
2514 \\
\end{tabular} & & & & OFFSITE & SESPMNT & $\mathrm{R}$ \\
\hline 2515 & & & & ONSITE & SESPMNT & $\mathrm{R}$ \\
\hline \begin{tabular}{|l|}
2516 \\
\end{tabular} & & & & ONSITE & SESPMNT & $\mathrm{R}$ \\
\hline \begin{tabular}{|l|}
2517 \\
\end{tabular} & & & & ONSITE & SESPMNT & $\mathrm{R}$ \\
\hline \begin{tabular}{|l|}
2518 \\
\end{tabular} & & & & OFFSITE & SESPMNT & $R$ \\
\hline \begin{tabular}{|l|}
2519 \\
\end{tabular} & & & & OFFSITE & SESPMNT & $\mathrm{R}$ \\
\hline \begin{tabular}{|l|}
2520 \\
\end{tabular} & & & & OFFSITE & SESPMNT & $\mathrm{R}$ \\
\hline 2521 & & & & ONSITE & SESPMNT & $\mathrm{R}$ \\
\hline 2522 & & & & ONSITE & SESPMNT & $\mathrm{R}$ \\
\hline \begin{tabular}{|l|}
2523 \\
\end{tabular} & & & & ONSITE & SESPMNT & $\mathrm{R}$ \\
\hline \begin{tabular}{|l|}
2524 \\
\end{tabular} & & & & ONSITE & SESPMNT & $\mathrm{R}$ \\
\hline \begin{tabular}{|l|}
2525 \\
\end{tabular} & & & & OFFSITE & SESPMNT & $\mathrm{R}$ \\
\hline \begin{tabular}{|l|}
2526 \\
\end{tabular} & & & & OFFSITE & SESPMNT & $\mathrm{R}$ \\
\hline \begin{tabular}{|l|}
2527 \\
\end{tabular} & & & & OFFSITE & SESPMNT & $R$ \\
\hline \begin{tabular}{|l|}
2528 \\
\end{tabular} & & & & OFFSITE & SESPMNT & $R$ \\
\hline \begin{tabular}{|l|}
2529 \\
\end{tabular} & & & & ONSITE & SESPMNT & $R$ \\
\hline \begin{tabular}{|l|}
2530 \\
\end{tabular} & & & & ONSITE & SESPMNT & $R$ \\
\hline \begin{tabular}{|l|}
2531 \\
\end{tabular} & & & & ONSITE & SESPMNT & $R$ \\
\hline \begin{tabular}{|l|}
2532 \\
\end{tabular} & & & & & SESPMNT & $R$ \\
\hline \begin{tabular}{|l|}
2533 \\
\end{tabular} & & & & ONSITE & SESPMNT & $R$ \\
\hline \begin{tabular}{|l|}
2534 \\
\end{tabular} & & & & ONSITE & SESPMNT & $R$ \\
\hline \begin{tabular}{|l|}
2535 \\
\end{tabular} & & & & & SESPMNT & $R$ \\
\hline \begin{tabular}{|l|}
2536 \\
\end{tabular} & & & & ONSITE & SESPMNT & $R$ \\
\hline \begin{tabular}{|l|}
2537 \\
\end{tabular} & & & & ONSITE & SESPMNT & $R$ \\
\hline \begin{tabular}{|l|}
2538 \\
\end{tabular} & & & & & SESPMNT & $R$ \\
\hline \begin{tabular}{|l|}
2539 \\
\end{tabular} & & & & & SESPMNT & $R$ \\
\hline \begin{tabular}{|l|}
2540 \\
\end{tabular} & & & & ONSITE & SESPMNT & $R$ \\
\hline \begin{tabular}{|l|}
2541 \\
\end{tabular} & & & & ONSITE & SESPMNT & $R$ \\
\hline \begin{tabular}{|l|}
2542 \\
\end{tabular} & & & & ONSITE & SESPMNT & $R$ \\
\hline \begin{tabular}{|l|}
2543 \\
\end{tabular} & & & & ONSITE & SESPMNT & $R$ \\
\hline \begin{tabular}{|l|}
2544 \\
\end{tabular} & & & & ONSITE & SESPMNT & $R$ \\
\hline 2545 & & & & ONSITE & SESPMNT & $R$ \\
\hline \begin{tabular}{|l|}
2546 \\
\end{tabular} & & & & & SESPMNT & $\mathrm{R}$ \\
\hline \begin{tabular}{|l|}
2547 \\
\end{tabular} & & & & & SESPMNT & $R$ \\
\hline \begin{tabular}{|l|}
2548 \\
\end{tabular} & & & & & SESPMNT & $R$ \\
\hline \begin{tabular}{|l|}
2549 \\
\end{tabular} & & & & ONSITE & SESBMNT & $R$ \\
\hline
\end{tabular}




\begin{tabular}{|c|c|c|c|c|c|c|}
\hline & $\mathrm{AD}$ & $\mathrm{AE}$ & $\mathrm{AF}$ & $\mathrm{AG}$ & $\mathrm{AH}$ & $\mathrm{Al}$ \\
\hline 1 & RESULT_COMMENT & COLL_SAMP_SIZE & COLL_SAMP_SIZE_UNITS & DIST_CLASS & OWNER_ID & COLLECTION_PURPOSE \\
\hline 2550 & & & & ONSITE & SESPMNT & $\mathrm{R}$ \\
\hline 2551 & & & & ONSITE & SESPMNT & $\mathrm{R}$ \\
\hline 2552 & & & & ONSITE & SESPMNT & $\mathrm{R}$ \\
\hline 2553 & & & & & SESPMNT & $\mathrm{R}$ \\
\hline 2554 & & & & ONSITE & SESPMNT & $\mathrm{R}$ \\
\hline 2555 & & & & ONSITE & SESPMNT & $\mathrm{R}$ \\
\hline 2556 & & & & OFFSITE & SESPMNT & $\mathrm{R}$ \\
\hline 2557 & & & & OFFSITE & SESPMNT & $\mathrm{R}$ \\
\hline 2558 & & & & OFFSITE & SESPMNT & $\mathrm{R}$ \\
\hline 2559 & & & & OFFSITE & SESPMNT & $\mathrm{R}$ \\
\hline 2560 & & & & OFFSITE & SESPMNT & $\mathrm{R}$ \\
\hline 2561 & & & & ONSITE & SESPMNT & $\mathrm{R}$ \\
\hline 2562 & & & & ONSITE & SESPMNT & $\mathrm{R}$ \\
\hline 2563 & & & & OFFSITE & SESPMNT & $\mathrm{R}$ \\
\hline 2564 & & & & OFFSITE & SESPMNT & $\mathrm{R}$ \\
\hline 2565 & & & & ONSITE & SESPMNT & $\mathrm{R}$ \\
\hline 2566 & & & & ONSITE & SESPMNT & $\mathrm{R}$ \\
\hline 2567 & & & & OFFSITE & SESPMNT & $\mathrm{R}$ \\
\hline 2568 & & & & OFFSITE & SESPMNT & $\mathrm{R}$ \\
\hline 2569 & & & & OFFSITE & SESPMNT & $\mathrm{R}$ \\
\hline 2570 & & & & OFFSITE & SESPMNT & $\mathrm{R}$ \\
\hline 2571 & & & & ONSITE & SESPMNT & $\mathrm{R}$ \\
\hline 2572 & & & & ONSITE & SESPMNT & $\mathrm{R}$ \\
\hline 2573 & & & & & SESPMNT & $\mathrm{R}$ \\
\hline 2574 & & & & ONSITE & SESPMNT & $\mathrm{R}$ \\
\hline 2575 & & & & ONSITE & SESPMNT & $\mathrm{R}$ \\
\hline 2576 & & & & & SESPMNT & $\mathrm{R}$ \\
\hline \begin{tabular}{|l|}
2577 \\
\end{tabular} & & & & ONSITE & SESPMNT & $\mathrm{R}$ \\
\hline \begin{tabular}{|l|}
2578 \\
\end{tabular} & & & & ONSITE & SESPMNT & $\mathrm{R}$ \\
\hline \begin{tabular}{|l|}
2579 \\
\end{tabular} & & & & ONSITE & SESPMNT & $\mathrm{R}$ \\
\hline \begin{tabular}{|l|}
2580 \\
\end{tabular} & & & & ONSITE & SESPMNT & $\mathrm{R}$ \\
\hline \begin{tabular}{|l|}
2581 \\
\end{tabular} & & & & ONSITE & SESPMNT & $\mathrm{R}$ \\
\hline \begin{tabular}{|l|}
2582 \\
\end{tabular} & & & & OFFSITE & SESPMNT & $\mathrm{R}$ \\
\hline \begin{tabular}{|l|}
2583 \\
\end{tabular} & & & & OFFSITE & SESPMNT & $\mathrm{R}$ \\
\hline \begin{tabular}{|l|}
2584 \\
\end{tabular} & & & & OFFSITE & SESPMNT & $\mathrm{R}$ \\
\hline \begin{tabular}{|l|}
2585 \\
\end{tabular} & & & & OFFSITE & SESPMNT & $\mathrm{R}$ \\
\hline \begin{tabular}{|l|}
2586 \\
\end{tabular} & & & & OFFSITE & SESPMNT & $\mathrm{R}$ \\
\hline \begin{tabular}{|l|}
2587 \\
\end{tabular} & & & & & SESPMNT & $\mathrm{R}$ \\
\hline \begin{tabular}{|l|}
2588 \\
\end{tabular} & & & & ONSITE & SESPMNT & $\mathrm{R}$ \\
\hline \begin{tabular}{|l|}
2589 \\
\end{tabular} & & & & ONSITE & SESPMNT & $\mathrm{R}$ \\
\hline \begin{tabular}{|l|}
2590 \\
\end{tabular} & & & & ONSITE & SESPMNT & $\mathrm{R}$ \\
\hline \begin{tabular}{|l|}
2591 \\
\end{tabular} & & & & ONSITE & SESPMNT & $\mathrm{R}$ \\
\hline \begin{tabular}{|l|}
2592 \\
\end{tabular} & & & & ONSITE & SESPMNT & $\mathrm{R}$ \\
\hline \begin{tabular}{|l|}
2593 \\
\end{tabular} & & & & & SESPMNT & $\mathrm{R}$ \\
\hline \begin{tabular}{|l|}
2594 \\
\end{tabular} & & & & ONSITE & SESPMNT & $\mathrm{R}$ \\
\hline \begin{tabular}{|l|l}
2595 \\
\end{tabular} & & & & ONSITE & SESPMNT & $\mathrm{R}$ \\
\hline \begin{tabular}{|l|}
2596 \\
\end{tabular} & & & & & SESPMNT & $\mathrm{R}$ \\
\hline \begin{tabular}{|l|}
2597 \\
\end{tabular} & & & & OFFSITE & SESPMNT & $\mathrm{R}$ \\
\hline \begin{tabular}{|l|}
2598 \\
\end{tabular} & & & & OFFSITE & SESPMNT & $\mathrm{R}$ \\
\hline \begin{tabular}{|l|}
2599 \\
\end{tabular} & & & & ONSITE & SESPMNT & $\mathrm{R}$ \\
\hline \begin{tabular}{|l|}
2600 \\
\end{tabular} & & & & ONSITE & SESPMNT & $\mathrm{R}$ \\
\hline \begin{tabular}{|l|}
2601 \\
\end{tabular} & & & & ONSITE & SESBMNT & $R$ \\
\hline
\end{tabular}




\begin{tabular}{|c|c|c|c|c|c|c|}
\hline & $A D$ & $\mathrm{AE}$ & $\mathrm{AF}$ & $A G$ & $\mathrm{AH}$ & $\mathrm{Al}$ \\
\hline 1 & RESULT_COMMENT & COLL_SAMP_SIZE & COLL_SAMP_SIZE_UNITS & DIST_CLASS & OWNER_ID & COLLECTION_PURPOSE \\
\hline 2602 & & & & ONSITE & SESPMNT & $\mathrm{R}$ \\
\hline 2603 & & & & & SESPMNT & $\mathrm{R}$ \\
\hline 2604 & & & & & SESPMNT & $\mathrm{R}$ \\
\hline 2605 & & & & ONSITE & SESPMNT & $\mathrm{R}$ \\
\hline 2606 & & & & ONSITE & SESPMNT & $\mathrm{R}$ \\
\hline 2607 & & & & ONSITE & SESPMNT & $\mathrm{R}$ \\
\hline 2608 & & & & ONSITE & SESPMNT & $\mathrm{R}$ \\
\hline 2609 & & & & OFFSITE & SESPMNT & $\mathrm{R}$ \\
\hline 2610 & & & & OFFSITE & SESPMNT & $\mathrm{R}$ \\
\hline 2611 & & & & ONSITE & SESPMNT & $\mathrm{R}$ \\
\hline 2612 & & & & ONSITE & SESPMNT & $\mathrm{R}$ \\
\hline 2613 & & & & & SESPMNT & $\mathrm{R}$ \\
\hline 2614 & & & & & SESPMNT & $\mathrm{R}$ \\
\hline 2615 & & & & & SESPMNT & $\mathrm{R}$ \\
\hline 2616 & & & & ONSITE & SESPMNT & $\mathrm{R}$ \\
\hline 2617 & & & & ONSITE & SESPMNT & $\mathrm{R}$ \\
\hline 2618 & & & & ONSITE & SESPMNT & $\mathrm{R}$ \\
\hline 2619 & & & & ONSITE & SESPMNT & $\mathrm{R}$ \\
\hline 2620 & & & & & SESPMNT & $\mathrm{R}$ \\
\hline 2621 & & & & OFFSITE & SESPMNT & $\mathrm{R}$ \\
\hline 2622 & & & & OFFSITE & SESPMNT & $\mathrm{R}$ \\
\hline 2623 & & & & OFFSITE & SESPMNT & $\mathrm{R}$ \\
\hline 2624 & & & & ONSITE & SESPMNT & $\mathrm{R}$ \\
\hline 2625 & & & & ONSITE & SESPMNT & $\mathrm{R}$ \\
\hline 2626 & & & & OFFSITE & SESPMNT & $\mathrm{R}$ \\
\hline 2627 & & & & OFFSITE & SESPMNT & $\mathrm{R}$ \\
\hline \begin{tabular}{|l|}
2628 \\
\end{tabular} & & & & ONSITE & SESPMNT & $\mathrm{R}$ \\
\hline 2629 & & & & ONSITE & SESPMNT & $\mathrm{R}$ \\
\hline 2630 & & & & OFFSITE & SESPMNT & $\mathrm{R}$ \\
\hline 2631 & & & & OFFSITE & SESPMNT & $R$ \\
\hline 2632 & & & & OFFSITE & SESPMNT & $\mathrm{R}$ \\
\hline 2633 & & & & OFFSITE & SESPMNT & $\mathrm{R}$ \\
\hline 2634 & & & & ONSITE & SESPMNT & $\mathrm{R}$ \\
\hline 2635 & & & & ONSITE & SESPMNT & $\mathrm{R}$ \\
\hline \begin{tabular}{|l|}
2636 \\
\end{tabular} & & & & & SESPMNT & $\mathrm{R}$ \\
\hline \begin{tabular}{|l|}
2637 \\
\end{tabular} & & & & ONSITE & SESPMNT & $\mathrm{R}$ \\
\hline 2638 & & & & ONSITE & SESPMNT & $\mathrm{R}$ \\
\hline 2639 & & & & & SESPMNT & $\mathrm{R}$ \\
\hline 2640 & & & & ONSITE & SESPMNT & $\mathrm{R}$ \\
\hline 2641 & & & & ONSITE & SESPMNT & $\mathrm{R}$ \\
\hline \begin{tabular}{|l|}
2642 \\
\end{tabular} & & & & ONSITE & SESPMNT & $\mathrm{R}$ \\
\hline 2643 & & & & ONSITE & SESPMNT & $\mathrm{R}$ \\
\hline 2644 & & & & ONSITE & SESPMNT & $\mathrm{R}$ \\
\hline \begin{tabular}{|l|}
2645 \\
\end{tabular} & & & & OFFSITE & SESPMNT & $\mathrm{R}$ \\
\hline 2646 & & & & OFFSITE & SESPMNT & $\mathrm{R}$ \\
\hline \begin{tabular}{|l|}
2647 \\
\end{tabular} & & & & OFFSITE & SESPMNT & $\mathrm{R}$ \\
\hline 2648 & & & & OFFSITE & SESPMNT & $\mathrm{R}$ \\
\hline \begin{tabular}{|l|}
2649 \\
\end{tabular} & & & & OFFSITE & SESPMNT & $\mathrm{R}$ \\
\hline 2650 & & & & ONSITE & SESPMNT & $\mathrm{R}$ \\
\hline 2651 & & & & ONSITE & SESPMNT & $R$ \\
\hline \begin{tabular}{|l|}
2652 \\
\end{tabular} & & & & OFFSITE & $\begin{array}{l}\text { SESPMNT } \\
\end{array}$ & $\mathrm{R}$ \\
\hline 2653 & & & & OFFSITE & SESBMNT & $R$ \\
\hline
\end{tabular}




\begin{tabular}{|c|c|c|c|c|c|c|}
\hline & $A D$ & $\mathrm{AE}$ & $\mathrm{AF}$ & $A G$ & $\mathrm{AH}$ & $\mathrm{Al}$ \\
\hline 1 & RESULT_COMMENT & COLL_SAMP_SIZE & COLL_SAMP_SIZE_UNITS & DIST_CLASS & OWNER_ID & COLLECTION_PURPOSE \\
\hline 2654 & & & & ONSITE & SESPMNT & $\mathrm{R}$ \\
\hline 2655 & & & & ONSITE & SESPMNT & $\mathrm{R}$ \\
\hline 2656 & & & & ONSITE & SESPMNT & $\mathrm{R}$ \\
\hline 2657 & & & & & SESPMNT & $\mathrm{R}$ \\
\hline 2658 & & & & ONSITE & SESPMNT & $\mathrm{R}$ \\
\hline 2659 & & & & ONSITE & SESPMNT & $\mathrm{R}$ \\
\hline 2660 & & & & & SESPMNT & $\mathrm{R}$ \\
\hline 2661 & & & & ONSITE & SESPMNT & $\mathrm{R}$ \\
\hline 2662 & & & & ONSITE & SESPMNT & $\mathrm{R}$ \\
\hline 2663 & & & & OFFSITE & SESPMNT & $\mathrm{R}$ \\
\hline 2664 & & & & OFFSITE & SESPMNT & $\mathrm{R}$ \\
\hline 2665 & & & & OFFSITE & SESPMNT & $\mathrm{R}$ \\
\hline 2666 & & & & OFFSITE & SESPMNT & $\mathrm{R}$ \\
\hline 2667 & & & & OFFSITE & SESPMNT & $\mathrm{R}$ \\
\hline 2668 & & & & ONSITE & SESPMNT & $\mathrm{R}$ \\
\hline 2669 & & & & ONSITE & SESPMNT & $\mathrm{R}$ \\
\hline 2670 & & & & ONSITE & SESPMNT & $\mathrm{R}$ \\
\hline 2671 & & & & & SESPMNT & $\mathrm{R}$ \\
\hline 2672 & & & & & SESPMNT & $\mathrm{R}$ \\
\hline 2673 & & & & ONSITE & SESPMNT & $\mathrm{R}$ \\
\hline 2674 & & & & ONSITE & SESPMNT & $\mathrm{R}$ \\
\hline 2675 & & & & ONSITE & SESPMNT & $\mathrm{R}$ \\
\hline 2676 & & & & ONSITE & SESPMNT & $\mathrm{R}$ \\
\hline 2677 & & & & OFFSITE & SESPMNT & $\mathrm{R}$ \\
\hline 2678 & & & & OFFSITE & SESPMNT & $\mathrm{R}$ \\
\hline 2679 & & & & ONSITE & SESPMNT & $\mathrm{R}$ \\
\hline 2680 & & & & ONSITE & SESPMNT & $\mathrm{R}$ \\
\hline 2681 & & & & ONSITE & SESPMNT & $\mathrm{R}$ \\
\hline \begin{tabular}{|l|}
2682 \\
\end{tabular} & & & & ONSITE & SESPMNT & $\mathrm{R}$ \\
\hline 2683 & & & & OFFSITE & SESPMNT & $R$ \\
\hline \begin{tabular}{|l|}
2684 \\
\end{tabular} & & & & OFFSITE & SESPMNT & $\mathrm{R}$ \\
\hline 2685 & & & & & SESPMNT & $\mathrm{R}$ \\
\hline 2686 & & & & & SESPMNT & $\mathrm{R}$ \\
\hline \begin{tabular}{|l|}
2687 \\
\end{tabular} & & & & & SESPMNT & $\mathrm{R}$ \\
\hline 2688 & & & & ONSITE & SESPMNT & $\mathrm{R}$ \\
\hline \begin{tabular}{|l|}
2689 \\
\end{tabular} & & & & ONSITE & SESPMNT & $\mathrm{R}$ \\
\hline 2690 & & & & ONSITE & SESPMNT & $\mathrm{R}$ \\
\hline 2691 & & & & ONSITE & SESPMNT & $\mathrm{R}$ \\
\hline 2692 & & & & & SESPMNT & $\mathrm{R}$ \\
\hline 2693 & & & & OFFSITE & SESPMNT & $\mathrm{R}$ \\
\hline \begin{tabular}{|l|}
2694 \\
\end{tabular} & & & & OFFSITE & SESPMNT & $\mathrm{R}$ \\
\hline 2695 & & & & ONSITE & SESPMNT & $\mathrm{R}$ \\
\hline 2696 & & & & ONSITE & SESPMNT & $\mathrm{R}$ \\
\hline \begin{tabular}{|l|}
2697 \\
\end{tabular} & & & & OFFSITE & SESPMNT & $\mathrm{R}$ \\
\hline 2698 & & & & OFFSITE & SESPMNT & $\mathrm{R}$ \\
\hline 2699 & & & & ONSITE & SESPMNT & $\mathrm{R}$ \\
\hline 2700 & & & & ONSITE & SESPMNT & $\mathrm{R}$ \\
\hline 2701 & & & & OFFSITE & SESPMNT & $\mathrm{R}$ \\
\hline \begin{tabular}{|l|}
2702 \\
\end{tabular} & & & & OFFSITE & SESPMNT & $\mathrm{R}$ \\
\hline 2703 & & & & ONSITE & SESPMNT & $R$ \\
\hline 2704 & & & & ONSITE & $\begin{array}{l}\text { SESPMNT } \\
\end{array}$ & $\mathrm{R}$ \\
\hline 2705 & & & & & SESBMNT & $R$ \\
\hline
\end{tabular}




\begin{tabular}{|c|c|c|c|c|c|c|}
\hline & $A D$ & $\mathrm{AE}$ & $\mathrm{AF}$ & $A G$ & $\mathrm{AH}$ & $\mathrm{Al}$ \\
\hline 1 & RESULT_COMMENT & COLL_SAMP_SIZE & COLL_SAMP_SIZE_UNITS & DIST_CLASS & OWNER_ID & COLLECTION_PURPOSE \\
\hline 2706 & & & & ONSITE & SESPMNT & $\mathrm{R}$ \\
\hline 2707 & & & & ONSITE & SESPMNT & $\mathrm{R}$ \\
\hline 2708 & & & & & SESPMNT & $\mathrm{R}$ \\
\hline 2709 & & & & OFFSITE & SESPMNT & $\mathrm{R}$ \\
\hline 2710 & & & & OFFSITE & SESPMNT & $\mathrm{R}$ \\
\hline 2711 & & & & ONSITE & SESPMNT & $\mathrm{R}$ \\
\hline 2712 & & & & ONSITE & SESPMNT & $\mathrm{R}$ \\
\hline 2713 & & & & ONSITE & SESPMNT & $\mathrm{R}$ \\
\hline 2714 & & & & ONSITE & SESPMNT & $\mathrm{R}$ \\
\hline 2715 & & & & ONSITE & SESPMNT & $\mathrm{R}$ \\
\hline 2716 & & & & OFFSITE & SESPMNT & $\mathrm{R}$ \\
\hline 2717 & & & & OFFSITE & SESPMNT & $\mathrm{R}$ \\
\hline 2718 & & & & OFFSITE & SESPMNT & $\mathrm{R}$ \\
\hline 2719 & & & & OFFSITE & SESPMNT & $\mathrm{R}$ \\
\hline 2720 & & & & OFFSITE & SESPMNT & $\mathrm{R}$ \\
\hline 2721 & & & & ONSITE & SESPMNT & $\mathrm{R}$ \\
\hline \begin{tabular}{|l|}
2722 \\
\end{tabular} & & & & ONSITE & SESPMNT & $\mathrm{R}$ \\
\hline 2723 & & & & OFFSITE & SESPMNT & $\mathrm{R}$ \\
\hline 2724 & & & & OFFSITE & SESPMNT & $\mathrm{R}$ \\
\hline 2725 & & & & ONSITE & SESPMNT & $\mathrm{R}$ \\
\hline 2726 & & & & ONSITE & SESPMNT & $\mathrm{R}$ \\
\hline 2727 & & & & ONSITE & SESPMNT & $\mathrm{R}$ \\
\hline 2728 & & & & & SESPMNT & $\mathrm{R}$ \\
\hline 2729 & & & & ONSITE & SESPMNT & $\mathrm{R}$ \\
\hline 2730 & & & & ONSITE & SESPMNT & $\mathrm{R}$ \\
\hline \begin{tabular}{|l|}
2731 \\
\end{tabular} & & & & & SESPMNT & $\mathrm{R}$ \\
\hline \begin{tabular}{|l|}
2732 \\
\end{tabular} & & & & OFFSITE & SESPMNT & $\mathrm{R}$ \\
\hline 2733 & & & & OFFSITE & SESPMNT & $\mathrm{R}$ \\
\hline 2734 & & & & OFFSITE & SESPMNT & $\mathrm{R}$ \\
\hline 2735 & & & & ONSITE & SESPMNT & $\mathrm{R}$ \\
\hline 2736 & & & & OFFSITE & SESPMNT & $\mathrm{R}$ \\
\hline \begin{tabular}{|l|}
2737 \\
\end{tabular} & & & & OFFSITE & SESPMNT & $\mathrm{R}$ \\
\hline 2738 | & & & & ONSITE & SESPMNT & $\mathrm{R}$ \\
\hline \begin{tabular}{|l|}
2739 \\
\end{tabular} & & & & ONSITE & SESPMNT & $\mathrm{R}$ \\
\hline 2740 & & & & ONSITE & SESPMNT & $\mathrm{R}$ \\
\hline \begin{tabular}{|l|}
2741 \\
\end{tabular} & & & & & SESPMNT & $R$ \\
\hline \begin{tabular}{|l|}
2742 \\
\end{tabular} & & & & & SESPMNT & $\mathrm{R}$ \\
\hline 2743 & & & & ONSITE & SESPMNT & $\mathrm{R}$ \\
\hline \begin{tabular}{|l|}
2744 \\
\end{tabular} & & & & ONSITE & SESPMNT & $\mathrm{R}$ \\
\hline 2745 & & & & ONSITE & SESPMNT & $\mathrm{R}$ \\
\hline \begin{tabular}{|l|}
2746 \\
\end{tabular} & & & & ONSITE & SESPMNT & $\mathrm{R}$ \\
\hline \begin{tabular}{|l|}
2747 \\
\end{tabular} & & & & OFFSITE & SESPMNT & $\mathrm{R}$ \\
\hline 2748 & & & & OFFSITE & SESPMNT & $\mathrm{R}$ \\
\hline 2749 & & & & ONSITE & SESPMNT & $\mathrm{R}$ \\
\hline 2750 & & & & ONSITE & SESPMNT & $\mathrm{R}$ \\
\hline 2751 & & & & & SESPMNT & $\mathrm{R}$ \\
\hline \begin{tabular}{|l|}
2752 \\
\end{tabular} & & & & & SESPMNT & $\mathrm{R}$ \\
\hline 2753 & & & & & SESPMNT & $\mathrm{R}$ \\
\hline 2754 & & & & ONSITE & SESPMNT & $R$ \\
\hline 2755 & & & & ONSITE & SESPMNT & $\mathrm{R}$ \\
\hline $2756 \mid$ & & & & ONSITE & SESPMNT & $\mathrm{R}$ \\
\hline \begin{tabular}{|l|}
2757 \\
\end{tabular} & & & & ONSITE & SESBMNT & $R$ \\
\hline
\end{tabular}




\begin{tabular}{|c|c|c|c|c|c|c|}
\hline & $A D$ & $\mathrm{AE}$ & $\mathrm{AF}$ & $A G$ & $\mathrm{AH}$ & $\mathrm{Al}$ \\
\hline 1 & RESULT_COMMENT & COLL_SAMP_SIZE & COLL_SAMP_SIZE_UNITS & DIST_CLASS & OWNER_ID & COLLECTION_PURPOSE \\
\hline 2758 & & & & & SESPMNT & $\mathrm{R}$ \\
\hline 2759 & & & & OFFSITE & SESPMNT & $\mathrm{R}$ \\
\hline 2760 & & & & OFFSITE & SESPMNT & $\mathrm{R}$ \\
\hline 2761 & & & & ONSITE & SESPMNT & $\mathrm{R}$ \\
\hline 2762 & & & & ONSITE & SESPMNT & $\mathrm{R}$ \\
\hline 2763 & & & & OFFSITE & SESPMNT & $\mathrm{R}$ \\
\hline 2764 & & & & OFFSITE & SESPMNT & $\mathrm{R}$ \\
\hline 2765 & & & & ONSITE & SESPMNT & $\mathrm{R}$ \\
\hline \begin{tabular}{|l|}
2766 \\
\end{tabular} & & & & ONSITE & SESPMNT & $\mathrm{R}$ \\
\hline \begin{tabular}{|l|l|}
2767 \\
\end{tabular} & & & & ONSITE & SESPMNT & $\mathrm{R}$ \\
\hline 2768 & & & & ONSITE & SESPMNT & $\mathrm{R}$ \\
\hline 2769 & & & & OFFSITE & SESPMNT & $\mathrm{R}$ \\
\hline 2770 & & & & OFFSITE & SESPMNT & $\mathrm{R}$ \\
\hline 2771 & & & & OFFSITE & SESPMNT & $\mathrm{R}$ \\
\hline 2772 & & & & OFFSITE & SESPMNT & $\mathrm{R}$ \\
\hline 2773 & & & & ONSITE & SESPMNT & $\mathrm{R}$ \\
\hline 2774 & & & & ONSITE & SESPMNT & $\mathrm{R}$ \\
\hline 2775 & & & & ONSITE & SESPMNT & $\mathrm{R}$ \\
\hline 2776 & & & & ONSITE & SESPMNT & $\mathrm{R}$ \\
\hline 2777 & & & & & SESPMNT & $\mathrm{R}$ \\
\hline 2778 & & & & ONSITE & SESPMNT & $\mathrm{R}$ \\
\hline 2779 & & & & ONSITE & SESPMNT & $\mathrm{R}$ \\
\hline 2780 & & & & & SESPMNT & $\mathrm{R}$ \\
\hline 2781 & & & & ONSITE & SESPMNT & $\mathrm{R}$ \\
\hline 2782 & & & & ONSITE & SESPMNT & $\mathrm{R}$ \\
\hline 2783 & & & & ONSITE & SESPMNT & $\mathrm{R}$ \\
\hline \begin{tabular}{|l|l|}
2784 \\
\end{tabular} & & & & OFFSITE & SESPMNT & $\mathrm{R}$ \\
\hline 2785 & & & & OFFSITE & SESPMNT & $\mathrm{R}$ \\
\hline \begin{tabular}{|l|}
2786 \\
\end{tabular} & & & & OFFSITE & SESPMNT & $\mathrm{R}$ \\
\hline \begin{tabular}{|l|}
2787 \\
\end{tabular} & & & & OFFSITE & SESPMNT & $R$ \\
\hline 2788 & & & & OFFSITE & SESPMNT & $\mathrm{R}$ \\
\hline 2789 & & & & ONSITE & SESPMNT & $\mathrm{R}$ \\
\hline 2790 & & & & ONSITE & SESPMNT & $\mathrm{R}$ \\
\hline 2791 & & & & OFFSITE & SESPMNT & $\mathrm{R}$ \\
\hline \begin{tabular}{|l|}
2792 \\
\end{tabular} & & & & OFFSITE & SESPMNT & $\mathrm{R}$ \\
\hline 2793 & & & & ONSITE & SESPMNT & $\mathrm{R}$ \\
\hline 2794 & & & & ONSITE & SESPMNT & $\mathrm{R}$ \\
\hline 2795 & & & & ONSITE & SESPMNT & $\mathrm{R}$ \\
\hline 2796 & & & & & SESPMNT & $\mathrm{R}$ \\
\hline 2797 & & & & ONSITE & SESPMNT & $\mathrm{R}$ \\
\hline 2798 & & & & ONSITE & SESPMNT & $\mathrm{R}$ \\
\hline 2799 & & & & & SESPMNT & $\mathrm{R}$ \\
\hline 2800 & & & & ONSITE & SESPMNT & $\mathrm{R}$ \\
\hline 2801 & & & & ONSITE & SESPMNT & $\mathrm{R}$ \\
\hline 2802 & & & & OFFSITE & SESPMNT & $\mathrm{R}$ \\
\hline 2803 & & & & OFFSITE & SESPMNT & $\mathrm{R}$ \\
\hline 2804 & & & & OFFSITE & SESPMNT & $\mathrm{R}$ \\
\hline 2805 & & & & OFFSITE & SESPMNT & $\mathrm{R}$ \\
\hline \begin{tabular}{|l|}
2806 \\
\end{tabular} & & & & OFFSITE & SESPMNT & $\mathrm{R}$ \\
\hline \begin{tabular}{|l|}
2807 \\
\end{tabular} & & & & ONSITE & SESPMNT & $R$ \\
\hline 2808 & & & & ONSITE & $\begin{array}{l}\text { SESPMNT } \\
\end{array}$ & $\mathrm{R}$ \\
\hline 2809 & & & & OFFSITE & SESBMNT & $R$ \\
\hline
\end{tabular}




\begin{tabular}{|c|c|c|c|c|c|c|}
\hline & $A D$ & $\mathrm{AE}$ & $\mathrm{AF}$ & $A G$ & $\mathrm{AH}$ & $\mathrm{Al}$ \\
\hline 1 & RESULT_COMMENT & COLL_SAMP_SIZE & COLL_SAMP_SIZE_UNITS & DIST_CLASS & OWNER_ID & COLLECTION_PURPOSE \\
\hline 2810 & & & & OFFSITE & SESPMNT & $\mathrm{R}$ \\
\hline 2811 & & & & ONSITE & SESPMNT & $\mathrm{R}$ \\
\hline \begin{tabular}{|l|}
2812 \\
\end{tabular} & & & & ONSITE & SESPMNT & $\mathrm{R}$ \\
\hline $\mid 2813$ & & & & ONSITE & SESPMNT & $\mathrm{R}$ \\
\hline 2814 & & & & & SESPMNT & $\mathrm{R}$ \\
\hline \begin{tabular}{|l|}
2815 \\
\end{tabular} & & & & & SESPMNT & $\mathrm{R}$ \\
\hline 2816 & & & & ONSITE & SESPMNT & $\mathrm{R}$ \\
\hline \begin{tabular}{|l|l|}
2817 & -1 \\
\end{tabular} & & & & ONSITE & SESPMNT & $\mathrm{R}$ \\
\hline 2818 & & & & & SESPMNT & $\mathrm{R}$ \\
\hline 2819 & & & & & SESPMNT & $\mathrm{R}$ \\
\hline 2820 & & & & & SESPMNT & $\mathrm{R}$ \\
\hline 2821 & & & & ONSITE & SESPMNT & $\mathrm{R}$ \\
\hline 2822 & & & & ONSITE & SESPMNT & $\mathrm{R}$ \\
\hline 2823 & & & & ONSITE & SESPMNT & $\mathrm{R}$ \\
\hline \begin{tabular}{|l|}
2824 \\
\end{tabular} & & & & ONSITE & SESPMNT & $\mathrm{R}$ \\
\hline 2825 & & & & & SESPMNT & $\mathrm{R}$ \\
\hline 2826 & & & & ONSITE & SESPMNT & $\mathrm{R}$ \\
\hline \begin{tabular}{|l|l|}
2827 & \\
\end{tabular} & & & & ONSITE & SESPMNT & $\mathrm{R}$ \\
\hline \begin{tabular}{|l|}
2828 \\
\end{tabular} & & & & ONSITE & SESPMNT & $\mathrm{R}$ \\
\hline 2829 & & & & ONSITE & SESPMNT & $\mathrm{R}$ \\
\hline 2830 & & & & ONSITE & SESPMNT & $\mathrm{R}$ \\
\hline 2831 & & & & OFFSITE & SESPMNT & $\mathrm{R}$ \\
\hline 2832 & & & & OFFSITE & SESPMNT & $\mathrm{R}$ \\
\hline 2833 & & & & OFFSITE & SESPMNT & $\mathrm{R}$ \\
\hline 2834 & See sample record for comment. & & & OFFSITE & SESPMNT & $\mathrm{R}$ \\
\hline 2835 & See sample record for comment. & & & OFFSITE & SESPMNT & $\mathrm{R}$ \\
\hline 2836 & & & & OFFSITE & SESPMNT & $\mathrm{R}$ \\
\hline \begin{tabular}{|l|l|}
2837 & \\
\end{tabular} & & & & OFFSITE & SESPMNT & $\mathrm{R}$ \\
\hline \begin{tabular}{|l|}
2838 \\
\end{tabular} & & & & ONSITE & SESPMNT & $\mathrm{R}$ \\
\hline 2839 & & & & ONSITE & SESPMNT & $\mathrm{R}$ \\
\hline 2840 & & & & ONSITE & SESPMNT & $\mathrm{R}$ \\
\hline 2841 & & & & ONSITE & SESPMNT & $\mathrm{R}$ \\
\hline 2842 & & & & OFFSITE & SESPMNT & $\mathrm{R}$ \\
\hline 2843 & & & & OFFSITE & SESPMNT & $\mathrm{R}$ \\
\hline 2844 & & & & ONSITE & SESPMNT & $\mathrm{R}$ \\
\hline 2845 & & & & ONSITE & SESPMNT & $\mathrm{R}$ \\
\hline 2846 & & & & ONSITE & SESPMNT & $\mathrm{R}$ \\
\hline \begin{tabular}{|l|l|}
2847 & \\
\end{tabular} & & & & ONSITE & SESPMNT & $\mathrm{R}$ \\
\hline 2848 & & & & OFFSITE & SESPMNT & $\mathrm{R}$ \\
\hline 2849 & & & & OFFSITE & SESPMNT & $\mathrm{R}$ \\
\hline 2850 & & & & & SESPMNT & $\mathrm{R}$ \\
\hline 2851 & & & & ONSITE & SESPMNT & $\mathrm{R}$ \\
\hline \begin{tabular}{|l|}
2852 \\
\end{tabular} & & & & ONSITE & SESPMNT & $\mathrm{R}$ \\
\hline 2853 & & & & & SESPMNT & $\mathrm{R}$ \\
\hline 2854 & & & & ONSITE & SESPMNT & $\mathrm{R}$ \\
\hline 2855 & & & & ONSITE & SESPMNT & $\mathrm{R}$ \\
\hline 2856 & & & & OFFSITE & SESPMNT & $\mathrm{R}$ \\
\hline 2857. & & & & OFFSITE & SESPMNT & $\mathrm{R}$ \\
\hline 2858 & & & & ONSITE & SESPMNT & $\mathrm{R}$ \\
\hline 2859 & & & & ONSITE & SESPMNT & $\mathrm{R}$ \\
\hline 2860 & & & & ONSITE & SESPMNT & $\mathrm{R}$ \\
\hline 2861 & & & & OFFSITE & SESBMNT & ${ }_{0}^{R}$ \\
\hline
\end{tabular}




\begin{tabular}{|c|c|c|c|c|c|c|}
\hline & $A D$ & $\mathrm{AE}$ & $\mathrm{AF}$ & $A G$ & $\mathrm{AH}$ & $\mathrm{Al}$ \\
\hline 1 & RESULT_COMMENT & COLL_SAMP_SIZE & COLL_SAMP_SIZE_UNITS & DIST_CLASS & OWNER_ID & COLLECTION_PURPOSE \\
\hline 2862 & & & & OFFSITE & SESPMNT & $\mathrm{R}$ \\
\hline 2863 & & & & OFFSITE & SESPMNT & $\mathrm{R}$ \\
\hline 2864 & & & & ONSITE & SESPMNT & $\mathrm{R}$ \\
\hline 2865 & & & & ONSITE & SESPMNT & $\mathrm{R}$ \\
\hline 2866 & & & & & SESPMNT & $\mathrm{R}$ \\
\hline 2867 & & & & ONSITE & SESPMNT & $\mathrm{R}$ \\
\hline 2868 & & & & ONSITE & SESPMNT & $\mathrm{R}$ \\
\hline 2869 & & & & OFFSITE & SESPMNT & $\mathrm{R}$ \\
\hline 2870 & & & & OFFSITE & SESPMNT & $\mathrm{R}$ \\
\hline 2871 & & & & & SESPMNT & $\mathrm{R}$ \\
\hline 2872 & & & & & SESPMNT & $\mathrm{R}$ \\
\hline 2873 & & & & ONSITE & SESPMNT & $\mathrm{R}$ \\
\hline 2874 & & & & ONSITE & SESPMNT & $\mathrm{R}$ \\
\hline 2875 & & & & ONSITE & SESPMNT & $\mathrm{R}$ \\
\hline 2876 & & & & & SESPMNT & $\mathrm{R}$ \\
\hline 2877 & & & & ONSITE & SESPMNT & $\mathrm{R}$ \\
\hline 2878 & & & & ONSITE & SESPMNT & $\mathrm{R}$ \\
\hline 2879 & & & & ONSITE & SESPMNT & $\mathrm{R}$ \\
\hline 2880 & & & & OFFSITE & SESPMNT & $\mathrm{R}$ \\
\hline 2881 & & & & OFFSITE & SESPMNT & $\mathrm{R}$ \\
\hline 2882 & & & & OFFSITE & SESPMNT & $\mathrm{R}$ \\
\hline 2883 & & & & OFFSITE & SESPMNT & $\mathrm{R}$ \\
\hline 2884 & & & & OFFSITE & SESPMNT & $\mathrm{R}$ \\
\hline 2885 & & & & OFFSITE & SESPMNT & $\mathrm{R}$ \\
\hline 2886 & & & & OFFSITE & SESPMNT & $\mathrm{R}$ \\
\hline \begin{tabular}{|l|}
2887 \\
\end{tabular} & & & & OFFSITE & SESPMNT & $\mathrm{R}$ \\
\hline \begin{tabular}{|l|}
2888 \\
\end{tabular} & & & & OFFSITE & SESPMNT & $\mathrm{R}$ \\
\hline 2889 & & & & ONSITE & SESPMNT & $\mathrm{R}$ \\
\hline 2890 & & & & ONSITE & SESPMNT & $\mathrm{R}$ \\
\hline 2891 & & & & ONSITE & SESPMNT & $R$ \\
\hline 2892 & & & & & SESPMNT & $\mathrm{R}$ \\
\hline \begin{tabular}{|l|}
2893 \\
\end{tabular} & & & & & SESPMNT & $\mathrm{R}$ \\
\hline 2894 & & & & ONSITE & SESPMNT & $\mathrm{R}$ \\
\hline 2895 & & & & ONSITE & SESPMNT & $\mathrm{R}$ \\
\hline \begin{tabular}{|l|}
2896 \\
\end{tabular} & & & & ONSITE & SESPMNT & $\mathrm{R}$ \\
\hline \begin{tabular}{|l|}
2897 \\
\end{tabular} & & & & ONSITE & SESPMNT & $\mathrm{R}$ \\
\hline 2898 & & & & OFFSITE & SESPMNT & $\mathrm{R}$ \\
\hline 2899 & & & & OFFSITE & SESPMNT & $\mathrm{R}$ \\
\hline 2900 & & & & ONSITE & SESPMNT & $\mathrm{R}$ \\
\hline 2901 & & & & ONSITE & SESPMNT & $\mathrm{R}$ \\
\hline 2902 & & & & & SESPMNT & $\mathrm{R}$ \\
\hline 2903 & & & & OFFSITE & SESPMNT & $\mathrm{R}$ \\
\hline 2904 & & & & OFFSITE & SESPMNT & $\mathrm{R}$ \\
\hline 2905 & & & & ONSITE & SESPMNT & $\mathrm{R}$ \\
\hline 2906 & & & & ONSITE & SESPMNT & $\mathrm{R}$ \\
\hline 2907 & & & & ONSITE & SESPMNT & $\mathrm{R}$ \\
\hline 2908 年 & & & & OFFSITE & SESPMNT & $\mathrm{R}$ \\
\hline 2909 & & & & OFFSITE & SESPMNT & $\mathrm{R}$ \\
\hline 2910 & & & & OFFSITE & SESPMNT & $\mathrm{R}$ \\
\hline 2911 & & & & OFFSITE & SESPMNT & $R$ \\
\hline \begin{tabular}{|l|}
2912 \\
\end{tabular} & & & & OFFSITE & SESPMNT & $\mathrm{R}$ \\
\hline 2913 & & & & ONSITE & SESBMNT & $R$ \\
\hline
\end{tabular}




\begin{tabular}{|c|c|c|c|c|c|c|}
\hline & $\mathrm{AD}$ & $\mathrm{AE}$ & $\mathrm{AF}$ & AG & $\mathrm{AH}$ & $\mathrm{Al}$ \\
\hline 1 & RESULT_COMMENT & COLL_SAMP_SIZE & COLL_SAMP_SIZE_UNITS & DIST_CLASS & OWNER_ID & COLLECTION_PURPOSE \\
\hline 2914 & & & & ONSITE & SESPMNT & $\mathrm{R}$ \\
\hline 2915 & & & & ONSITE & SESPMNT & $\mathrm{R}$ \\
\hline 2916 & & & & ONSITE & SESPMNT & $\mathrm{R}$ \\
\hline 2917 & & & & ONSITE & SESPMNT & $\mathrm{R}$ \\
\hline 2918 & & & & ONSITE & SESPMNT & $\mathrm{R}$ \\
\hline 2919 & & & & ONSITE & SESPMNT & $\mathrm{R}$ \\
\hline 2920 & & & & ONSITE & SESPMNT & $\mathrm{R}$ \\
\hline 2921 & & & & ONSITE & SESPMNT & $\mathrm{R}$ \\
\hline 2922 & & & & OFFSITE & SESPMNT & $\mathrm{R}$ \\
\hline 2923 & & & & OFFSITE & SESPMNT & $\mathrm{R}$ \\
\hline 2924 & & & & ONSITE & SESPMNT & $\mathrm{R}$ \\
\hline \begin{tabular}{|l|}
2925 \\
\end{tabular} & & & & ONSITE & SESPMNT & $R$ \\
\hline 2926 & & & & & SESPMNT & $\mathrm{R}$ \\
\hline \begin{tabular}{|l|}
2927 \\
\end{tabular} & & & & ONSITE & SESPMNT & $\mathrm{R}$ \\
\hline \begin{tabular}{|l|}
2928 \\
\end{tabular} & & & & ONSITE & SESPMNT & $R$ \\
\hline 2929 & & & & ONSITE & SESPMNT & $\mathrm{R}$ \\
\hline 2930 & & & & OFFSITE & SESPMNT & $\mathrm{R}$ \\
\hline 2931 & & & & OFFSITE & SESPMNT & $\mathrm{R}$ \\
\hline 2932 & & & & OFFSITE & SESPMNT & $\mathrm{R}$ \\
\hline 2933 & & & & OFFSITE & SESPMNT & $\mathrm{R}$ \\
\hline 2934 & & & & OFFSITE & SESPMNT & $R$ \\
\hline 2935 & & & & OFFSITE & SESPMNT & $\mathrm{R}$ \\
\hline 2936 & & & & OFFSITE & SESPMNT & $\mathrm{R}$ \\
\hline \begin{tabular}{|l|l|}
2937 \\
\end{tabular} & & & & ONSITE & SESPMNT & $\mathrm{R}$ \\
\hline 2938 & & & & ONSITE & SESPMNT & $\mathrm{R}$ \\
\hline 2939 & & & & ONSITE & SESPMNT & $\mathrm{R}$ \\
\hline 2940 & & & & ONSITE & SESPMNT & $\mathrm{R}$ \\
\hline 2941 & & & & ONSITE & SESPMNT & $\mathrm{R}$ \\
\hline \begin{tabular}{|l|l|}
2942 \\
\end{tabular} & & & & ONSITE & SESPMNT & $\mathrm{R}$ \\
\hline \begin{tabular}{|l|l|}
2943 \\
\end{tabular} & & & & ONSITE & SESPMNT & $R$ \\
\hline 2944 & & & & OFFSITE & SESPMNT & $R$ \\
\hline \begin{tabular}{|l|}
2945 \\
\end{tabular} & & & & OFFSITE & SESPMNT & $R$ \\
\hline \begin{tabular}{|l|}
2946 \\
\end{tabular} & & & & ONSITE & SESPMNT & $R$ \\
\hline \begin{tabular}{|l|}
2947 \\
\end{tabular} & & & & ONSITE & SESPMNT & $R$ \\
\hline \begin{tabular}{|l|}
2948 \\
\end{tabular} & & & & ONSITE & SESPMNT & $R$ \\
\hline 2949 & & & & & SESPMNT & $\mathrm{R}$ \\
\hline 2950 & & & & & SESPMNT & $R$ \\
\hline 2951 & & & & OFFSITE & SESPMNT & $R$ \\
\hline \begin{tabular}{|l|}
2952 \\
\end{tabular} & & & & OFFSITE & SESPMNT & $R$ \\
\hline 2953 & & & & OFFSITE & SESPMNT & $R$ \\
\hline 2954 & & & & OFFSITE & SESPMNT & $R$ \\
\hline 2955 & & & & OFFSITE & SESPMNT & $R$ \\
\hline 2956 & & & & OFFSITE & SESPMNT & $R$ \\
\hline $2957 \mid$ & & & & OFFSITE & SESPMNT & $\mathrm{R}$ \\
\hline 2958 & & & & OFFSITE & SESPMNT & $R$ \\
\hline 2959 & & & & OFFSITE & SESPMNT & $\mathrm{R}$ \\
\hline 2960 & & & & ONSITE & SESPMNT & $R$ \\
\hline 2961 & & & & ONSITE & SESPMNT & $R$ \\
\hline 2962 & & & & ONSITE & SESPMNT & $\mathrm{R}$ \\
\hline \begin{tabular}{|l|l|}
2963 \\
\end{tabular} & & & & & SESPMNT & $R$ \\
\hline 2964 & & & & & SESPMNT & $\mathrm{R}$ \\
\hline 2965 & & & & ONSITE & SESBMNT & $R$ \\
\hline
\end{tabular}




\begin{tabular}{|c|c|c|c|c|c|c|}
\hline & $\mathrm{AD}$ & $\mathrm{AE}$ & $\mathrm{AF}$ & $A G$ & $\mathrm{AH}$ & $\mathrm{Al}$ \\
\hline 1 & RESULT_COMMENT & COLL_SAMP_SIZE & COLL_SAMP_SIZE_UNITS & DIST_CLASS & OWNER_ID & COLLECTION_PURPOSE \\
\hline 2966 & & & & ONSITE & SESPMNT & $R$ \\
\hline 2967 & & & & & SESPSPEC & $\mathrm{s}$ \\
\hline 2968 & & & & ONSITE & SESPMNT & $\mathrm{R}$ \\
\hline 2969 & & & & ONSITE & SESPMNT & $\mathrm{R}$ \\
\hline 2970 & & & & OFFSITE & SESPMNT & $\mathrm{R}$ \\
\hline 2971 & & & & OFFSITE & SESPMNT & $\mathrm{R}$ \\
\hline 2972 & & & & ONSITE & SESPMNT & $\mathrm{R}$ \\
\hline 2973 & & & & ONSITE & SESPMNT & $\mathrm{R}$ \\
\hline 2974 & & & & ONSITE & SESPMNT & $\mathrm{R}$ \\
\hline 2975 & & & & OFFSITE & SESPMNT & $\mathrm{R}$ \\
\hline 2976 & & & & OFFSITE & SESPMNT & $\mathrm{R}$ \\
\hline 2977 & & & & OFFSITE & SESPMNT & $\mathrm{R}$ \\
\hline 2978 & & & & & SESPMNT & $\mathrm{R}$ \\
\hline 2979 & & & & & SESPMNT & $\mathrm{R}$ \\
\hline 2980 & & & & ONSITE & SESPMNT & $\mathrm{R}$ \\
\hline 2981 & & & & ONSITE & SESPMNT & $\mathrm{R}$ \\
\hline 2982 & & & & & SESPMNT & $\mathrm{R}$ \\
\hline 2983 & & & & OFFSITE & SESPMNT & $\mathrm{R}$ \\
\hline 2984 & & & & OFFSITE & SESPMNT & $\mathrm{R}$ \\
\hline 2985 & & & & OFFSITE & SESPMNT & $\mathrm{R}$ \\
\hline 2986 & & & & OFFSITE & SESPMNT & $\mathrm{R}$ \\
\hline 2987 & & & & ONSITE & SESPMNT & $\mathrm{R}$ \\
\hline 2988 & & & & ONSITE & SESPMNT & $\mathrm{R}$ \\
\hline 2989 & & & & ONSITE & SESPMNT & $\mathrm{R}$ \\
\hline 2990 & & & & ONSITE & SESPMNT & $\mathrm{R}$ \\
\hline 2991 & & & & OFFSITE & SESPMNT & $\mathrm{R}$ \\
\hline 2992 & & & & OFFSITE & SESPMNT & $\mathrm{R}$ \\
\hline 2993 & & & & ONSITE & SESPMNT & $\mathrm{R}$ \\
\hline 2994 & & & & ONSITE & SESPMNT & $\mathrm{R}$ \\
\hline 2995 & & & & ONSITE & SESPMNT & $\mathrm{R}$ \\
\hline 2996 & & & & ONSITE & SESPMNT & $\mathrm{R}$ \\
\hline 2997 & & & & ONSITE & SESPMNT & $R$ \\
\hline 2998 & & & & ONSITE & SESPMNT & $R$ \\
\hline 2999 & & & & ONSITE & SESPMNT & $\mathrm{R}$ \\
\hline 3000 & & & & ONSITE & SESPMNT & $\mathrm{R}$ \\
\hline 3001 & & & & ONSITE & SESPMNT & $R$ \\
\hline 3002 & & & & ONSITE & SESPMNT & $R$ \\
\hline 3003 & & & & OFFSITE & SESPMNT & $\mathrm{R}$ \\
\hline 3004 & & & & OFFSITE & SESPMNT & $\mathrm{R}$ \\
\hline 3005 & & & & OFFSITE & SESPMNT & $\mathrm{R}$ \\
\hline 3006 & & & & OFFSITE & SESPMNT & $\mathrm{R}$ \\
\hline 3007 & & & & OFFSITE & SESPMNT & $\mathrm{R}$ \\
\hline 3008 & & & & ONSITE & SESPMNT & $R$ \\
\hline 3009 & & & & ONSITE & SESPMNT & $\mathrm{R}$ \\
\hline 3010 & & & & & SESPMNT & $R$ \\
\hline 3011 & & & & OFFSITE & SESPMNT & $R$ \\
\hline 3012 & & & & OFFSITE & SESPMNT & $\mathrm{R}$ \\
\hline \begin{tabular}{|l|l|}
3013 \\
\end{tabular} & & & & OFFSITE & SESPMNT & $\mathrm{R}$ \\
\hline 3014 & & & & OFFSITE & SESPMNT & $\mathrm{R}$ \\
\hline 3015 & & & & ONSITE & SESPMNT & $R$ \\
\hline 3016 & & & & ONSITE & SESPMNT & $R$ \\
\hline 3017 & & & & OFFSITE & SESBMNT & $R$ \\
\hline
\end{tabular}




\begin{tabular}{|c|c|c|c|c|c|c|}
\hline & $A D$ & $\mathrm{AE}$ & $\mathrm{AF}$ & $\mathrm{AG}$ & $\mathrm{AH}$ & $\mathrm{Al}$ \\
\hline 1 & RESULT_COMMENT & COLL_SAMP_SIZE & COLL_SAMP_SIZE_UNITS & DIST_CLASS & OWNER_ID & COLLECTION_PURPOSE \\
\hline 3018 & & & & OFFSITE & SESPMNT & $\mathrm{R}$ \\
\hline 3019 & & & & ONSITE & SESPMNT & $\mathrm{R}$ \\
\hline 3020 & & & & ONSITE & SESPMNT & $\mathrm{R}$ \\
\hline 3021 & & & & ONSITE & SESPMNT & $\mathrm{R}$ \\
\hline \begin{tabular}{|c|}
3022 \\
\end{tabular} & & & & ONSITE & SESPMNT & $\mathrm{R}$ \\
\hline 3023 & & & & OFFSITE & SESPMNT & $\mathrm{R}$ \\
\hline 3024 & & & & OFFSITE & SESPMNT & $\mathrm{R}$ \\
\hline 3025 & & & & ONSITE & SESPMNT & $\mathrm{R}$ \\
\hline 3026 & & & & ONSITE & SESPMNT & $\mathrm{R}$ \\
\hline 3027 & & & & ONSITE & SESPMNT & $\mathrm{R}$ \\
\hline 3028 & & & & & SESPMNT & $\mathrm{R}$ \\
\hline 3029 & & & & & SESPMNT & $\mathrm{R}$ \\
\hline 3030 & & & & OFFSITE & SESPMNT & $\mathrm{R}$ \\
\hline 3031 & & & & OFFSITE & SESPMNT & $\mathrm{R}$ \\
\hline 3032 & & & & OFFSITE & SESPMNT & $\mathrm{R}$ \\
\hline 3033 & & & & OFFSITE & SESPMNT & $\mathrm{R}$ \\
\hline 3034 & & & & OFFSITE & SESPMNT & $\mathrm{R}$ \\
\hline 3035 & & & & OFFSITE & SESPMNT & $\mathrm{R}$ \\
\hline 3036 & & & & & SESPMNT & $\mathrm{R}$ \\
\hline 3037 & & & & & SESPMNT & $\mathrm{R}$ \\
\hline \begin{tabular}{|c|}
3038 \\
\end{tabular} & & & & ONSITE & SESPMNT & $\mathrm{R}$ \\
\hline 3039 & & & & ONSITE & SESPMNT & $\mathrm{R}$ \\
\hline 3040 & & & & ONSITE & SESPMNT & $\mathrm{R}$ \\
\hline 3041 & & & & OFFSITE & SESPMNT & $\mathrm{R}$ \\
\hline 3042 & & & & OFFSITE & SESPMNT & $\mathrm{R}$ \\
\hline \begin{tabular}{|c|}
3043 \\
\end{tabular} & & & & OFFSITE & SESPMNT & $\mathrm{R}$ \\
\hline 3044 & & & & ONSITE & SESPMNT & $\mathrm{R}$ \\
\hline 3045 & & & & ONSITE & SESPMNT & $\mathrm{R}$ \\
\hline 3046 & & & & ONSITE & SESPMNT & $\mathrm{R}$ \\
\hline \begin{tabular}{|l|}
3047 \\
\end{tabular} & & & & & SESPMNT & $\mathrm{R}$ \\
\hline 3048 & & & & & SESPMNT & $\mathrm{R}$ \\
\hline \begin{tabular}{|l|l|}
3049 \\
\end{tabular} & & & & & SESPSPEC & S \\
\hline 3050 & & & & ONSITE & SESPMNT & $\mathrm{R}$ \\
\hline 3051 & & & & ONSITE & SESPMNT & $\mathrm{R}$ \\
\hline 3052 & & & & OFFSITE & SESPMNT & $\mathrm{R}$ \\
\hline \begin{tabular}{|l|l|}
3053 \\
\end{tabular} & & & & OFFSITE & SESPMNT & $\mathrm{R}$ \\
\hline 3054 & & & & ONSITE & SESPMNT & $\mathrm{R}$ \\
\hline 3055 & & & & ONSITE & SESPMNT & $\mathrm{R}$ \\
\hline 3056 & & & & ONSITE & SESPMNT & $\mathrm{R}$ \\
\hline 3057 & & & & ONSITE & SESPMNT & $\mathrm{R}$ \\
\hline \begin{tabular}{|l|}
3058 \\
\end{tabular} & & & & & SESPMNT & $\mathrm{R}$ \\
\hline \begin{tabular}{|l|l|}
3059 \\
\end{tabular} & & & & ONSITE & SESPMNT & $\mathrm{R}$ \\
\hline 3060 & & & & ONSITE & SESPMNT & $\mathrm{R}$ \\
\hline 3061 & & & & ONSITE & SESPMNT & $\mathrm{R}$ \\
\hline \begin{tabular}{|l|}
3062 \\
\end{tabular} & & & & OFFSITE & SESPMNT & $\mathrm{R}$ \\
\hline \begin{tabular}{|l|l|}
3063 \\
\end{tabular} & & & & OFFSITE & SESPMNT & $R$ \\
\hline 3064 & & & & OFFSITE & SESPMNT & $\mathrm{R}$ \\
\hline 3065 & & & & OFFSITE & SESPMNT & $\mathrm{R}$ \\
\hline 3066 & & & & OFFSITE & SESPMNT & $\mathrm{R}$ \\
\hline 3067 & & & & OFFSITE & SESPMNT & $\mathrm{R}$ \\
\hline 3068 & & & & OFFSITE & SESPMNT & $\mathrm{R}$ \\
\hline 3069 & & & & ONSITE & SESBMNT & $R$ \\
\hline
\end{tabular}




\begin{tabular}{|c|c|c|c|c|c|c|}
\hline & $A D$ & $\mathrm{AE}$ & $\mathrm{AF}$ & $A G$ & $\mathrm{AH}$ & $\mathrm{Al}$ \\
\hline 1 & RESULT_COMMENT & COLL_SAMP_SIZE & COLL_SAMP_SIZE_UNITS & DIST_CLASS & OWNER_ID & COLLECTION_PURPOSE \\
\hline 3070 & & & & ONSITE & SESPMNT & $\mathrm{R}$ \\
\hline 3071 & & & & ONSITE & SESPMNT & $\mathrm{R}$ \\
\hline 3072 & & & & ONSITE & SESPMNT & $\mathrm{R}$ \\
\hline 3073 & & & & OFFSITE & SESPMNT & $\mathrm{R}$ \\
\hline 3074 & & & & OFFSITE & SESPMNT & $\mathrm{R}$ \\
\hline 3075 & & & & ONSITE & SESPMNT & $\mathrm{R}$ \\
\hline 3076 & & & & ONSITE & SESPMNT & $\mathrm{R}$ \\
\hline 3077 & & & & ONSITE & SESPMNT & $\mathrm{R}$ \\
\hline 3078 & & & & ONSITE & SESPMNT & $\mathrm{R}$ \\
\hline 3079 & & & & & SESPMNT & $\mathrm{R}$ \\
\hline 3080 & & & & ONSITE & SESPMNT & $\mathrm{R}$ \\
\hline 3081 & & & & ONSITE & SESPMNT & $\mathrm{R}$ \\
\hline 3082 & & & & ONSITE & SESPMNT & $\mathrm{R}$ \\
\hline 3083 & & & & ONSITE & SESPMNT & $\mathrm{R}$ \\
\hline 3084 & & & & ONSITE & SESPMNT & $\mathrm{R}$ \\
\hline 3085 & & & & OFFSITE & SESPMNT & $\mathrm{R}$ \\
\hline 3086 & & & & OFFSITE & SESPMNT & $\mathrm{R}$ \\
\hline 3087 & & & & OFFSITE & SESPMNT & $\mathrm{R}$ \\
\hline 3088 & & & & OFFSITE & SESPMNT & $\mathrm{R}$ \\
\hline 3089 & & & & OFFSITE & SESPMNT & $\mathrm{R}$ \\
\hline 3090 & & & & OFFSITE & SESPMNT & $\mathrm{R}$ \\
\hline 3091 & & & & OFFSITE & SESPMNT & $\mathrm{R}$ \\
\hline 3092 & & & & OFFSITE & SESPMNT & $\mathrm{R}$ \\
\hline 3093 & & & & OFFSITE & SESPMNT & $\mathrm{R}$ \\
\hline 3094 & & & & ONSITE & SESPMNT & $\mathrm{R}$ \\
\hline 3095 & & & & ONSITE & SESPMNT & $\mathrm{R}$ \\
\hline 3096 & & & & OFFSITE & SESPMNT & $\mathrm{R}$ \\
\hline 3097 & & & & OFFSITE & SESPMNT & $\mathrm{R}$ \\
\hline 3098 & & & & ONSITE & SESPMNT & $\mathrm{R}$ \\
\hline 3099 & & & & ONSITE & SESPMNT & $\mathrm{R}$ \\
\hline 3100 & & & & PERIMETER & SESPMNT & $\mathrm{R}$ \\
\hline 3101 & & & & PERIMETER & SESPMNT & $\mathrm{R}$ \\
\hline 3102 & & & & PERIMETER & SESPMNT & $\mathrm{R}$ \\
\hline 3103 & & & & ONSITE & SESPMNT & $\mathrm{R}$ \\
\hline 3104 & & & & ONSITE & SESPMNT & $\mathrm{R}$ \\
\hline 3105 & & & & ONSITE & SESPMNT & $\mathrm{R}$ \\
\hline 3106 & & & & ONSITE & SESPMNT & $\mathrm{R}$ \\
\hline 3107 & & & & ONSITE & SESPMNT & $R$ \\
\hline 3108 & & & & ONSITE & SESPMNT & $\mathrm{R}$ \\
\hline 3109 & & & & & SESPMNT & $\mathrm{R}$ \\
\hline 3110 & & & & OFFSITE & SESPMNT & $\mathrm{R}$ \\
\hline 3111 & & & & OFFSITE & SESPMNT & $\mathrm{R}$ \\
\hline 3112 & & & & OFFSITE & SESPMNT & $\mathrm{R}$ \\
\hline 3113 & & & & OFFSITE & SESPMNT & $\mathrm{R}$ \\
\hline 3114 & & & & & SESPMNT & $R$ \\
\hline 3115 & & & & & SESPMNT & $\mathrm{R}$ \\
\hline 3116 & & & & & SESPMNT & $\mathrm{R}$ \\
\hline 3117 & & & & & SESPMNT & $\mathrm{R}$ \\
\hline 3118 & & & & & SESPMNT & $\mathrm{R}$ \\
\hline 3119 & & & & OFFSITE & SESPMNT & $\mathrm{R}$ \\
\hline 3120 & & & & OFFSITE & SESPMNT & $\mathrm{R}$ \\
\hline 3121 & & & & OFFSITE & SESBMNT & ${ }_{1} R$ \\
\hline
\end{tabular}




\begin{tabular}{|c|c|c|c|c|c|c|}
\hline & $A D$ & $\mathrm{AE}$ & $\mathrm{AF}$ & $A G$ & $\mathrm{AH}$ & $\mathrm{Al}$ \\
\hline 1 & RESULT_COMMENT & COLL_SAMP_SIZE & COLL_SAMP_SIZE_UNITS & DIST_CLASS & OWNER_ID & COLLECTION_PURPOSE \\
\hline 3122 & & & & OFFSITE & SESPMNT & $\mathrm{R}$ \\
\hline 3123 & & & & OFFSITE & SESPMNT & $\mathrm{R}$ \\
\hline 3124 & & & & OFFSITE & SESPMNT & $\mathrm{R}$ \\
\hline 3125 & & & & ONSITE & SESPMNT & $\mathrm{R}$ \\
\hline 3126 & & & & ONSITE & SESPMNT & $\mathrm{R}$ \\
\hline 3127 & & & & ONSITE & SESPMNT & $\mathrm{R}$ \\
\hline 3128 & & & & ONSITE & SESPMNT & $\mathrm{R}$ \\
\hline 3129 & & & & OFFSITE & SESPMNT & $\mathrm{R}$ \\
\hline 3130 & & & & OFFSITE & SESPMNT & $\mathrm{R}$ \\
\hline 3131 & & & & ONSITE & SESPMNT & $\mathrm{R}$ \\
\hline 3132 & & & & ONSITE & SESPMNT & $\mathrm{R}$ \\
\hline 3133 & & & & ONSITE & SESPMNT & $\mathrm{R}$ \\
\hline 3134 & & & & & SESPMNT & $\mathrm{R}$ \\
\hline 3135 & & & & & SESPMNT & $\mathrm{R}$ \\
\hline 3136 & & & & ONSITE & SESPMNT & $\mathrm{R}$ \\
\hline \begin{tabular}{|c|}
3137 \\
\end{tabular} & & & & ONSITE & SESPMNT & $\mathrm{R}$ \\
\hline 3138 & & & & ONSITE & SESPMNT & $\mathrm{R}$ \\
\hline 3139 & & & & ONSITE & SESPMNT & $\mathrm{R}$ \\
\hline 3140 & & & & ONSITE & SESPMNT & $\mathrm{R}$ \\
\hline 3141 & & & & ONSITE & SESPMNT & $\mathrm{R}$ \\
\hline 3142 & & & & ONSITE & SESPMNT & $\mathrm{R}$ \\
\hline 3143 & & & & & SESPMNT & $\mathrm{R}$ \\
\hline 3144 & & & & OFFSITE & SESPMNT & $\mathrm{R}$ \\
\hline 3145 & & & & OFFSITE & SESPMNT & $\mathrm{R}$ \\
\hline 3146 & & & & OFFSITE & SESPMNT & $\mathrm{R}$ \\
\hline \begin{tabular}{|l|}
3147 \\
\end{tabular} & & & & OFFSITE & SESPMNT & $\mathrm{R}$ \\
\hline \begin{tabular}{|l|}
3148 \\
\end{tabular} & & & & OFFSITE & SESPMNT & $\mathrm{R}$ \\
\hline \begin{tabular}{|l|}
3149 \\
\end{tabular} & & & & ONSITE & SESPMNT & $\mathrm{R}$ \\
\hline 3150 & & & & ONSITE & SESPMNT & $\mathrm{R}$ \\
\hline 3151 & & & & & SESPMNT & $\mathrm{R}$ \\
\hline 3152 & & & & & SESPMNT & $\mathrm{R}$ \\
\hline 3153 & & & & & SESPMNT & $\mathrm{R}$ \\
\hline 3154 & & & & OFFSITE & SESPMNT & $\mathrm{R}$ \\
\hline 3155 & & & & OFFSITE & SESPMNT & $\mathrm{R}$ \\
\hline \begin{tabular}{|l|}
3156 \\
\end{tabular} & & & & OFFSITE & SESPMNT & $\mathrm{R}$ \\
\hline 3157 & & & & OFFSITE & SESPMNT & $\mathrm{R}$ \\
\hline 3158 & & & & ONSITE & SESPMNT & $\mathrm{R}$ \\
\hline 3159 & & & & ONSITE & SESPMNT & $R$ \\
\hline 3160 & & & & OFFSITE & SESPMNT & $\mathrm{R}$ \\
\hline \begin{tabular}{|l|}
3161 \\
\end{tabular} & & & & OFFSITE & SESPMNT & $\mathrm{R}$ \\
\hline \begin{tabular}{|l|}
3162 \\
\end{tabular} & & & & ONSITE & SESPMNT & $\mathrm{R}$ \\
\hline \begin{tabular}{|l|}
3163 \\
\end{tabular} & & & & ONSITE & SESPMNT & $\mathrm{R}$ \\
\hline 3164 & & & & ONSITE & SESPMNT & $\mathrm{R}$ \\
\hline 3165 & & & & & SESPMNT & $\mathrm{R}$ \\
\hline 3166 & & & & OFFSITE & SESPMNT & $R$ \\
\hline \begin{tabular}{|l|}
3167 \\
\end{tabular} & & & & OFFSITE & SESPMNT & $\mathrm{R}$ \\
\hline 3168 & & & & OFFSITE & SESPMNT & $\mathrm{R}$ \\
\hline \begin{tabular}{|l|}
3169 \\
\end{tabular} & & & & OFFSITE & SESPMNT & $\mathrm{R}$ \\
\hline 3170 & & & & OFFSITE & SESPMNT & $\mathrm{R}$ \\
\hline 3171 & & & & OFFSITE & SESPMNT & $\mathrm{R}$ \\
\hline 3172 & & & & & SESPMNT & $R$ \\
\hline 3173 & & & & & SESBMNT & $R$ \\
\hline
\end{tabular}




\begin{tabular}{|c|c|c|c|c|c|c|}
\hline & $A D$ & $\mathrm{AE}$ & $\mathrm{AF}$ & $A G$ & $\mathrm{AH}$ & $\mathrm{Al}$ \\
\hline 1 & RESULT_COMMENT & COLL_SAMP_SIZE & COLL_SAMP_SIZE_UNITS & DIST_CLASS & OWNER_ID & COLLECTION_PURPOSE \\
\hline 3174 & & & & ONSITE & SESPMNT & $\mathrm{R}$ \\
\hline \begin{tabular}{|l|}
3175 \\
\end{tabular} & & & & ONSITE & SESPMNT & $\mathrm{R}$ \\
\hline \begin{tabular}{|l|}
3176 \\
\end{tabular} & & & & OFFSITE & SESPMNT & $\mathrm{R}$ \\
\hline \begin{tabular}{|l|l|}
3177 \\
\end{tabular} & & & & OFFSITE & SESPMNT & $\mathrm{R}$ \\
\hline 3178 & & & & & SESPMNT & $\mathrm{R}$ \\
\hline 3179 & & & & & SESPMNT & $\mathrm{R}$ \\
\hline 3180 & & & & & SESPMNT & $\mathrm{R}$ \\
\hline 3181 & & & & ONSITE & SESPMNT & $\mathrm{R}$ \\
\hline 3182 & & & & ONSITE & SESPMNT & $\mathrm{R}$ \\
\hline 3183 & & & & ONSITE & SESPMNT & $\mathrm{R}$ \\
\hline 3184 & & & & OFFSITE & SESPMNT & $\mathrm{R}$ \\
\hline 3185 & & & & OFFSITE & SESPMNT & $\mathrm{R}$ \\
\hline 3186 & & & & OFFSITE & SESPMNT & $\mathrm{R}$ \\
\hline \begin{tabular}{|l|l|}
3187 & \\
\end{tabular} & & & & OFFSITE & SESPMNT & $\mathrm{R}$ \\
\hline \begin{tabular}{|l|l|}
3188 & \\
\end{tabular} & & & & OFFSITE & SESPMNT & $\mathrm{R}$ \\
\hline \begin{tabular}{|l|}
3189 \\
\end{tabular} & & & & ONSITE & SESPMNT & $\mathrm{R}$ \\
\hline 3190 & & & & ONSITE & SESPMNT & $\mathrm{R}$ \\
\hline 3191 & Result reported for CS-137.41+/-.051 & & & OFFSITE & SESPMNT & $\mathrm{R}$ \\
\hline 3192 & Result reported for CS-137. $17+/-.024$ & & & OFFSITE & SESPMNT & $\mathrm{R}$ \\
\hline 3193 & See sample record for comment. & & & OFFSITE & SESPMNT & $\mathrm{R}$ \\
\hline 3194 & See sample record for comment. & & & OFFSITE & SESPMNT & $\mathrm{R}$ \\
\hline 3195 & See sample record for comment. & & & OFFSITE & SESPMNT & $\mathrm{R}$ \\
\hline 3196 & & & & & SESPSPEC & $\mathrm{s}$ \\
\hline \begin{tabular}{|l|l|}
3197 & \\
\end{tabular} & & & & & SESPSPEC & $\mathrm{s}$ \\
\hline 3198 & & & & & SESPSPEC & $\mathrm{s}$ \\
\hline 3199 & & & & & SESPSPEC & $\mathrm{s}$ \\
\hline 3200 & & & & & SESPSPEC & $\mathrm{s}$ \\
\hline 3201 & & & & & SESPSPEC & $\mathrm{s}$ \\
\hline \begin{tabular}{|l|}
3202 \\
\end{tabular} & & & & OFFSITE & SESPMNT & $\mathrm{R}$ \\
\hline 3203 & & & & OFFSITE & SESPMNT & $\mathrm{R}$ \\
\hline 3204 & & & & & SESPMNT & $\mathrm{R}$ \\
\hline 3205 & & & & OFFSITE & SESPMNT & $\mathrm{R}$ \\
\hline 3206 & & & & OFFSITE & SESPMNT & $\mathrm{R}$ \\
\hline 3207 & See sample record for comment. & & & ONSITE & SESPMNT & $\mathrm{R}$ \\
\hline 3208 & & & & & SESPMNT & $\mathrm{R}$ \\
\hline 3209 & & & & ONSITE & SESPMNT & $\mathrm{R}$ \\
\hline 3210 & & & & ONSITE & SESPMNT & $\mathrm{R}$ \\
\hline 3211 & & & & ONSITE & SESPMNT & $\mathrm{R}$ \\
\hline 3212 & & & & OFFSITE & SESPMNT & $\mathrm{R}$ \\
\hline 3213 & & & & OFFSITE & SESPMNT & $\mathrm{R}$ \\
\hline \begin{tabular}{|l|}
3214 \\
\end{tabular} & & & & & SESPSPEC & $\mathrm{s}$ \\
\hline 3215 & & & & & SESPSPEC & $\mathrm{s}$ \\
\hline \begin{tabular}{|l|}
3216 \\
\end{tabular} & & & & & SESPSPEC & $\mathrm{s}$ \\
\hline \begin{tabular}{|l|l|}
3217 & \\
\end{tabular} & & & & & SESPSPEC & $\mathrm{s}$ \\
\hline 3218 & & & & ONSITE & SESPMNT & $\mathrm{R}$ \\
\hline 3219 & & & & ONSITE & SESPMNT & $\mathrm{R}$ \\
\hline 3220 & & & & ONSITE & SESPMNT & $\mathrm{R}$ \\
\hline 3221 & & & & & SESPMNT & $\mathrm{R}$ \\
\hline 3222 & & & & & SESPMNT & $\mathrm{R}$ \\
\hline 3223 & & & & ONSITE & SESPMNT & $R$ \\
\hline \begin{tabular}{|l|l|}
3224 \\
\end{tabular} & & & & ONSITE & SESPMNT & $\mathrm{R}$ \\
\hline 3225 & & & & ONSITE & SESBMNT & $\mathrm{R}$ \\
\hline
\end{tabular}




\begin{tabular}{|c|c|c|c|c|c|c|}
\hline & $\mathrm{AD}$ & $\mathrm{AE}$ & $\mathrm{AF}$ & $\mathrm{AG}$ & $\mathrm{AH}$ & $\mathrm{Al}$ \\
\hline 1 & RESULT_COMMENT & COLL_SAMP_SIZE & COLL_SAMP_SIZE_UNITS & DIST_CLASS & OWNER_ID & COLLECTION_PURPOSE \\
\hline 3226 & & & & ONSITE & SESPMNT & $\mathrm{R}$ \\
\hline 3227 & & & & & SESPMNT & $\mathrm{R}$ \\
\hline 3228 & & & & OFFSITE & SESPMNT & $\mathrm{R}$ \\
\hline 3229 & & & & OFFSITE & SESPMNT & $\mathrm{R}$ \\
\hline 3230 & & & & & SESPMNT & $\mathrm{R}$ \\
\hline 3231 & & & & & SESPMNT & $\mathrm{R}$ \\
\hline 3232 & & & & OFFSITE & SESPMNT & $\mathrm{R}$ \\
\hline 3233 & & & & OFFSITE & SESPMNT & $\mathrm{R}$ \\
\hline 3234 & & & & OFFSITE & SESPMNT & $\mathrm{R}$ \\
\hline 3235 & & & & OFFSITE & SESPMNT & $\mathrm{R}$ \\
\hline 3236 & & & & ONSITE & SESPMNT & $\mathrm{R}$ \\
\hline 3237 & & & & ONSITE & SESPMNT & $\mathrm{R}$ \\
\hline 3238 & & & & ONSITE & SESPMNT & $\mathrm{R}$ \\
\hline 3239 & & & & ONSITE & SESPMNT & $\mathrm{R}$ \\
\hline 3240 & & & & ONSITE & SESPMNT & $\mathrm{R}$ \\
\hline 3241 & & & & & SESPMNT & $\mathrm{R}$ \\
\hline 3242 & & & & OFFSITE & SESPMNT & $\mathrm{R}$ \\
\hline 3243 & & & & OFFSITE & SESPMNT & $\mathrm{R}$ \\
\hline 3244 & & & & OFFSITE & SESPMNT & $\mathrm{R}$ \\
\hline 3245 & & & & OFFSITE & SESPMNT & $\mathrm{R}$ \\
\hline 3246 & & & & OFFSITE & SESPMNT & $\mathrm{R}$ \\
\hline 3247 : & See sample record for comment. & & & ONSITE & SESPMNT & $\mathrm{R}$ \\
\hline 3248 & See sample record for comment. & & & ONSITE & SESPMNT & $\mathrm{R}$ \\
\hline 3249 & See sample record for comment. & & & ONSITE & SESPMNT & $\mathrm{R}$ \\
\hline 3250 & See sample record for comment. & & & & SESPMNT & $\mathrm{R}$ \\
\hline 3251 & See sample record for comment. & & & & SESPMNT & $\mathrm{R}$ \\
\hline 3252 & & & & OFFSITE & SESPMNT & $\mathrm{R}$ \\
\hline 3253 & & & & OFFSITE & SESPMNT & $\mathrm{R}$ \\
\hline 3254 & & & & & SESPMNT & $\mathrm{R}$ \\
\hline 3255 & & & & ONSITE & SESPMNT & $\mathrm{R}$ \\
\hline 3256 & & & & ONSITE & SESPMNT & $\mathrm{R}$ \\
\hline 3257 & & & & ONSITE & SESPMNT & $\mathrm{R}$ \\
\hline 3258 & & & & ONSITE & SESPMNT & $\mathrm{R}$ \\
\hline 3259 & & & & ONSITE & SESPMNT & $\mathrm{R}$ \\
\hline 3260 & & & & & SESPMNT & $\mathrm{R}$ \\
\hline 3261 & & & & OFFSITE & SESPMNT & $\mathrm{R}$ \\
\hline 3262 & & & & OFFSITE & SESPMNT & $\mathrm{R}$ \\
\hline 3263 & & & & OFFSITE & SESPMNT & $\mathrm{R}$ \\
\hline \begin{tabular}{|l|l|}
3264 \\
\end{tabular} & & & & OFFSITE & SESPMNT & $\mathrm{R}$ \\
\hline \begin{tabular}{|l|l|}
3265 \\
\end{tabular} & & & & OFFSITE & SESPMNT & $\mathrm{R}$ \\
\hline \begin{tabular}{|l|l|}
3266 \\
\end{tabular} & & & & OFFSITE & SESPMNT & $\mathrm{R}$ \\
\hline \begin{tabular}{|l|}
3267 \\
\end{tabular} & & & & OFFSITE & SESPMNT & $\mathrm{R}$ \\
\hline \begin{tabular}{|l|l|}
3268 \\
\end{tabular} & & & & OFFSITE & SESPMNT & $\mathrm{R}$ \\
\hline \begin{tabular}{|l|l|}
3269 \\
\end{tabular} & & & & PERIMETER & SESPMNT & $\mathrm{R}$ \\
\hline \begin{tabular}{|l|l|}
3270 \\
\end{tabular} & & & & PERIMETER & SESPMNT & $\mathrm{R}$ \\
\hline \begin{tabular}{|l|}
3271 \\
\end{tabular} & & & & & SESPMNT & $\mathrm{R}$ \\
\hline \begin{tabular}{|l|}
3272 \\
\end{tabular} & & & & ONSITE & SESPMNT & $\mathrm{R}$ \\
\hline \begin{tabular}{|l|}
3273 \\
\end{tabular} & & & & ONSITE & SESPMNT & $\mathrm{R}$ \\
\hline \begin{tabular}{|l|}
3274 \\
\end{tabular} & & & & ONSITE & SESPMNT & $\mathrm{R}$ \\
\hline 3275 & & & & ONSITE & SESPMNT & $\mathrm{R}$ \\
\hline \begin{tabular}{|l|l|}
3276 \\
\end{tabular} & & & & ONSITE & SESPMNT & $\mathrm{R}$ \\
\hline \begin{tabular}{|l|l|}
3277 \\
\end{tabular} & & & & & SESBMNT & $\mathrm{R}$ \\
\hline
\end{tabular}




\begin{tabular}{|c|c|c|c|c|c|c|}
\hline & $\mathrm{AD}$ & $\mathrm{AE}$ & $\mathrm{AF}$ & AG & $\mathrm{AH}$ & $\mathrm{Al}$ \\
\hline 1 & RESULT_COMMENT & COLL_SAMP_SIZE & COLL_SAMP_SIZE_UNITS & DIST_CLASS & OWNER_ID & COLLECTION_PURPOSE \\
\hline 3278 & & & & OFFSITE & SESPMNT & $R$ \\
\hline 3279 & & & & OFFSITE & SESPMNT & $\mathrm{R}$ \\
\hline 3280 & & & & OFFSITE & SESPMNT & $\mathrm{R}$ \\
\hline 3281 & & & & OFFSITE & SESPMNT & $\mathrm{R}$ \\
\hline 3282 & & & & OFFSITE & SESPMNT & $\mathrm{R}$ \\
\hline 3283 & & & & OFFSITE & SESPMNT & $\mathrm{R}$ \\
\hline 3284 & & & & OFFSITE & SESPMNT & $\mathrm{R}$ \\
\hline 3285 & & & & & SESPMNT & $\mathrm{R}$ \\
\hline 3286 & & & & ONSITE & SESPMNT & $\mathrm{R}$ \\
\hline 3287 & & & & ONSITE & SESPMNT & $\mathrm{R}$ \\
\hline 3288 & & & & OFFSITE & SESPMNT & $\mathrm{R}$ \\
\hline 3289 & & & & OFFSITE & SESPMNT & $\mathrm{R}$ \\
\hline 3290 & & & & OFFSITE & SESPMNT & $\mathrm{R}$ \\
\hline 3291 & & & & OFFSITE & SESPMNT & $\mathrm{R}$ \\
\hline 3292 & & & & OFFSITE & SESPMNT & $\mathrm{R}$ \\
\hline 3293 & & & & OFFSITE & SESPMNT & $\mathrm{R}$ \\
\hline 3294 & & & & OFFSITE & SESPMNT & $\mathrm{R}$ \\
\hline 3295 & & & & OFFSITE & SESPMNT & $\mathrm{R}$ \\
\hline 3296 & & & & & SESPSPEC & S \\
\hline 3297 & & & & & SESPSPEC & $\mathrm{S}$ \\
\hline 3298 & & & & & SESPSPEC & $\mathrm{S}$ \\
\hline 3299 & & & & & SESPSPEC & $\mathrm{S}$ \\
\hline 3300 & & & & & SESPSPEC & $\mathrm{s}$ \\
\hline 3301 & & & & & SESPSPEC & $\mathrm{s}$ \\
\hline 3302 & See sample record for comment. & & & & SESPMNT & $\mathrm{R}$ \\
\hline 3303 & & & & ONSITE & SESPMNT & $\mathrm{R}$ \\
\hline 3304 & & & & ONSITE & SESPMNT & $\mathrm{R}$ \\
\hline 3305 & & & & ONSITE & SESPMNT & $\mathrm{R}$ \\
\hline 3306 & & & & & SESPMNT & $\mathrm{R}$ \\
\hline 3307 & & & & OFFSITE & SESPMNT & $\mathrm{R}$ \\
\hline 3308 & & & & OFFSITE & SESPMNT & $\mathrm{R}$ \\
\hline 3309 & & & & OFFSITE & SESPMNT & $\mathrm{R}$ \\
\hline 3310 & & & & OFFSITE & SESPMNT & $\mathrm{R}$ \\
\hline 3311 & & & & OFFSITE & SESPMNT & $\mathrm{R}$ \\
\hline 3312 & & & & & SESPSPEC & $\mathrm{S}$ \\
\hline 3313 & & & & & SESPSPEC & S \\
\hline 3314 & & & & & SESPSPEC & S \\
\hline 3315 & & & & & SESPSPEC & S \\
\hline 3316 & & & & & SESPSPEC & S \\
\hline 3317 & & & & & SESPSPEC & $S$ \\
\hline \begin{tabular}{|l|}
3318 \\
\end{tabular} & & & & & SESPSPEC & $\mathrm{s}$ \\
\hline 3319 & & & & & SESPSPEC & $\mathrm{S}$ \\
\hline 3320 & & & & ONSITE & SESPMNT & $\mathrm{R}$ \\
\hline 3321 & & & & ONSITE & SESPMNT & $\mathrm{R}$ \\
\hline 3322 & & & & ONSITE & SESPMNT & $\mathrm{R}$ \\
\hline 3323 & & & & & SESPMNT & $\mathrm{R}$ \\
\hline 3324 & & & & & SESPMNT & $\mathrm{R}$ \\
\hline 3325 & & & & ONSITE & SESPMNT & $\mathrm{R}$ \\
\hline 3326 & & & & ONSITE & SESPMNT & $\mathrm{R}$ \\
\hline \begin{tabular}{|l|}
3327 \\
\end{tabular} & & & & & SESPMNT & $\mathrm{R}$ \\
\hline 3328 & & & & ONSITE & SESPMNT & $\mathrm{R}$ \\
\hline 3329 & & & & ONSITE & SESBMNT & ${ }_{0} R$ \\
\hline
\end{tabular}




\begin{tabular}{|c|c|c|c|c|c|c|}
\hline & $\mathrm{AD}$ & $\mathrm{AE}$ & $\mathrm{AF}$ & $\mathrm{AG}$ & $\mathrm{AH}$ & $\mathrm{Al}$ \\
\hline 1 & RESULT_COMMENT & COLL_SAMP_SIZE & COLL_SAMP_SIZE_UNITS & DIST_CLASS & OWNER_ID & COLLECTION_PURPOSE \\
\hline 3330 & & & & & SESPMNT & $\mathrm{R}$ \\
\hline 3331 & & & & OFFSITE & SESPMNT & $\mathrm{R}$ \\
\hline 3332 & & & & OFFSITE & SESPMNT & $\mathrm{R}$ \\
\hline 3333 & & & & OFFSITE & SESPMNT & $\mathrm{R}$ \\
\hline 3334 & & & & OFFSITE & SESPMNT & $\mathrm{R}$ \\
\hline 3335 & & & & OFFSITE & SESPMNT & $\mathrm{R}$ \\
\hline 3336 & & & & OFFSITE & SESPMNT & $\mathrm{R}$ \\
\hline 3337 & & & & OFFSITE & SESPMNT & $\mathrm{R}$ \\
\hline 3338 & & & & OFFSITE & SESPMNT & $\mathrm{R}$ \\
\hline 3339 & See sample record for comment. & & & & SESPMNT & $\mathrm{R}$ \\
\hline 3340 & & & & OFFSITE & SESPMNT & $\mathrm{R}$ \\
\hline 3341 & & & & OFFSITE & SESPMNT & $\mathrm{R}$ \\
\hline 3342 & & & & ONSITE & SESPMNT & $\mathrm{R}$ \\
\hline 3343 & & & & ONSITE & SESPMNT & $\mathrm{R}$ \\
\hline 3344 & & & & ONSITE & SESPMNT & $\mathrm{R}$ \\
\hline 3345 & & & & ONSITE & SESPMNT & $\mathrm{R}$ \\
\hline 3346 & & & & ONSITE & SESPMNT & $\mathrm{R}$ \\
\hline 3347 & & & & & SESPMNT & $\mathrm{R}$ \\
\hline 3348 & See sample record for comment. & & & OFFSITE & SESPMNT & $\mathrm{R}$ \\
\hline 3349 & See sample record for comment. & & & OFFSITE & SESPMNT & $\mathrm{R}$ \\
\hline 3350 & See sample record for comment. & & & OFFSITE & SESPMNT & $\mathrm{R}$ \\
\hline 3351 & & & & OFFSITE & SESPMNT & $\mathrm{R}$ \\
\hline 3352 & & & & OFFSITE & SESPMNT & $\mathrm{R}$ \\
\hline 3353 & & & & ONSITE & SESPMNT & $\mathrm{R}$ \\
\hline 3354 & & & & ONSITE & SESPMNT & $\mathrm{R}$ \\
\hline 3355 & & & & ONSITE & SESPMNT & $\mathrm{R}$ \\
\hline 3356 & & & & ONSITE & SESPMNT & $\mathrm{R}$ \\
\hline \begin{tabular}{|c|}
3357 \\
\end{tabular} & & & & ONSITE & SESPMNT & $\mathrm{R}$ \\
\hline 3358 & & & & OFFSITE & SESPMNT & $\mathrm{R}$ \\
\hline 3359 & & & & OFFSITE & SESPMNT & $\mathrm{R}$ \\
\hline 3360 & & & & & SESPMNT & $\mathrm{R}$ \\
\hline 3361 & & & & & SESPMNT & $\mathrm{R}$ \\
\hline 3362 & & & & & SESPMNT & $\mathrm{R}$ \\
\hline 3363 & & & & ONSITE & SESPMNT & $\mathrm{R}$ \\
\hline 3364 & & & & ONSITE & SESPMNT & $\mathrm{R}$ \\
\hline 3365 & & & & ONSITE & SESPMNT & $\mathrm{R}$ \\
\hline 3366 & & & & ONSITE & SESPMNT & $\mathrm{R}$ \\
\hline 3367 & & & & ONSITE & SESPMNT & $\mathrm{R}$ \\
\hline 3368 & & & & & SESPMNT & $\mathrm{R}$ \\
\hline 3369 & & & & OFFSITE & SESPMNT & $\mathrm{R}$ \\
\hline 3370 & & & & OFFSITE & SESPMNT & $\mathrm{R}$ \\
\hline 3371 & & & & OFFSITE & SESPMNT & $\mathrm{R}$ \\
\hline 3372 & See sample record for comment. & & & OFFSITE & SESPMNT & $\mathrm{R}$ \\
\hline 3373 & See sample record for comment. & & & OFFSITE & SESPMNT & $\mathrm{R}$ \\
\hline 3374 & & & & OFFSITE & SESPMNT & $\mathrm{R}$ \\
\hline 3375 & & & & OFFSITE & SESPMNT & $\mathrm{R}$ \\
\hline 3376 & & & & OFFSITE & SESPMNT & $\mathrm{R}$ \\
\hline 3377 & & & & PERIMETER & SESPMNT & $\mathrm{R}$ \\
\hline 3378 & & & & PERIMETER & SESPMNT & $\mathrm{R}$ \\
\hline 3379 & See sample record for comment. & & & ONSITE & SESPMNT & $\mathrm{R}$ \\
\hline 3380 & See sample record for comment. & & & ONSITE & SESPMNT & $\mathrm{R}$ \\
\hline 3381 & & & & ONSITE & SESBMNT & $R$ \\
\hline
\end{tabular}




\begin{tabular}{|c|c|c|c|c|c|c|}
\hline & $\mathrm{AD}$ & $\mathrm{AE}$ & $\mathrm{AF}$ & $\mathrm{AG}$ & $\mathrm{AH}$ & $\mathrm{Al}$ \\
\hline 1 & RESULT_COMMENT & COLL_SAMP_SIZE & COLL_SAMP_SIZE_UNITS & DIST_CLASS & OWNER_ID & COLLECTION_PURPOSE \\
\hline 3382 & & & & & SESPMNT & $R$ \\
\hline 3383 & & & & OFFSITE & SESPMNT & $\mathrm{R}$ \\
\hline 3384 & & & & OFFSITE & SESPMNT & $\mathrm{R}$ \\
\hline 3385 & & & & OFFSITE & SESPMNT & $\mathrm{R}$ \\
\hline 3386 & & & & OFFSITE & SESPMNT & $\mathrm{R}$ \\
\hline 3387 & & & & OFFSITE & SESPMNT & $\mathrm{R}$ \\
\hline 3388 & & & & OFFSITE & SESPMNT & $\mathrm{R}$ \\
\hline 3389 & & & & & SESPSPEC & $\mathrm{S}$ \\
\hline 3390 & & & & & SESPSPEC & S \\
\hline 3391 & & & & & SESPSPEC & S \\
\hline 3392 & & & & & SESPSPEC & S \\
\hline 3393 & & & & & SESPSPEC & S \\
\hline 3394 & & & & & SESPSPEC & $\mathrm{S}$ \\
\hline 3395 & & & & & SESPSPEC & $\mathrm{S}$ \\
\hline 3396 & & & & & SESPSPEC & $\mathrm{S}$ \\
\hline 3397 & & & & ONSITE & SESPMNT & $\mathrm{R}$ \\
\hline 3398 & & & & ONSITE & SESPMNT & $\mathrm{R}$ \\
\hline 3399 & & & & OFFSITE & SESPMNT & $\mathrm{R}$ \\
\hline 3400 & & & & OFFSITE & SESPMNT & $\mathrm{R}$ \\
\hline 3401 & & & & OFFSITE & SESPMNT & $\mathrm{R}$ \\
\hline 3402 & & & & OFFSITE & SESPMNT & $\mathrm{R}$ \\
\hline 3403 & & & & OFFSITE & SESPMNT & $\mathrm{R}$ \\
\hline 3404 & & & & & SESPMNT & $\mathrm{R}$ \\
\hline 3405 & See sample record for comment. & & & & SESPMNT & $\mathrm{R}$ \\
\hline 3406 & See sample record for comment. & & & ONSITE & SESPMNT & $\mathrm{R}$ \\
\hline 3407 & See sample record for comment. & & & ONSITE & SESPMNT & $\mathrm{R}$ \\
\hline 3408 & See sample record for comment. & & & OFFSITE & SESPMNT & $\mathrm{R}$ \\
\hline 3409 & See sample record for comment. & & & OFFSITE & SESPMNT & $\mathrm{R}$ \\
\hline 3410 & & & & & SESPSPEC & $\mathrm{S}$ \\
\hline 3411 & & & & & SESPSPEC & $\mathrm{s}$ \\
\hline 3412 & & & & & SESPSPEC & $\mathrm{S}$ \\
\hline 3413 & & & & & SESPSPEC & S \\
\hline 3414 & & & & ONSITE & SESPMNT & $\mathrm{R}$ \\
\hline 3415 & & & & ONSITE & SESPMNT & $\mathrm{R}$ \\
\hline 3416 & & & & ONSITE & SESPMNT & $\mathrm{R}$ \\
\hline 3417 & & & & & SESPMNT & $\mathrm{R}$ \\
\hline 3418 & & & & & SESPMNT & $\mathrm{R}$ \\
\hline 3419 & See sample record for comment. & & & ONSITE & SESPMNT & $\mathrm{R}$ \\
\hline $3420 \leq$ & See sample record for comment. & & & ONSITE & SESPMNT & $\mathrm{R}$ \\
\hline \begin{tabular}{|l|}
3421 \\
\end{tabular} & & & & & SESPMNT & $\mathrm{R}$ \\
\hline \begin{tabular}{|l|l|l}
3422 & $\mathrm{~s}$ \\
\end{tabular} & See sample record for comment. & & & ONSITE & SESPMNT & $\mathrm{R}$ \\
\hline $3423 \leq$ & See sample record for comment. & & & ONSITE & SESPMNT & $\mathrm{R}$ \\
\hline \begin{tabular}{|l|}
3424 \\
\end{tabular} & & & & ONSITE & SESPMNT & $\mathrm{R}$ \\
\hline 3425 & & & & & SESPMNT & $\mathrm{R}$ \\
\hline \begin{tabular}{|l|l|}
3426 \\
\end{tabular} & & & & & SESPMNT & $\mathrm{R}$ \\
\hline 3427 s & See sample record for comment. & & & OFFSITE & SESPMNT & $\mathrm{R}$ \\
\hline 3428 s & See sample record for comment. & & & OFFSITE & SESPMNT & $\mathrm{R}$ \\
\hline \begin{tabular}{|l|l|}
3429 \\
\end{tabular} & & & & OFFSITE & SESPMNT & $\mathrm{R}$ \\
\hline \begin{tabular}{|l|l|}
3430 \\
\end{tabular} & & & & OFFSITE & SESPMNT & $\mathrm{R}$ \\
\hline \begin{tabular}{|l|l|}
3431 \\
\end{tabular} & & & & OFFSITE & SESPMNT & $\mathrm{R}$ \\
\hline 3432 & & & & OFFSITE & SESPMNT & $\mathrm{R}$ \\
\hline \begin{tabular}{|l|l|l|l|l|}
3433 \\
\end{tabular} & & & & OFFSITE & SESBMNT & $R$ \\
\hline
\end{tabular}




\begin{tabular}{|c|c|c|c|c|c|c|}
\hline & $\mathrm{AD}$ & $\mathrm{AE}$ & $\mathrm{AF}$ & $\mathrm{AG}$ & $\mathrm{AH}$ & $\mathrm{Al}$ \\
\hline 1 & RESULT_COMMENT & COLL_SAMP_SIZE & COLL_SAMP_SIZE_UNITS & DIST_CLASS & OWNER_ID & COLLECTION_PURPOSE \\
\hline 3434 & & & & OFFSITE & SESPMNT & $\mathrm{R}$ \\
\hline 3435 & & & & OFFSITE & SESPMNT & $\mathrm{R}$ \\
\hline 3436 & See sample record for comment. & & & & SESPMNT & $\mathrm{R}$ \\
\hline 3437 & & & & ONSITE & SESPMNT & $\mathrm{R}$ \\
\hline 3438 & & & & ONSITE & SESPMNT & $\mathrm{R}$ \\
\hline 3439 & & & & ONSITE & SESPMNT & $\mathrm{R}$ \\
\hline 3440 & & & & ONSITE & SESPMNT & $\mathrm{R}$ \\
\hline 3441 & & & & OFFSITE & SESPMNT & $\mathrm{R}$ \\
\hline 3442 & & & & OFFSITE & SESPMNT & $\mathrm{R}$ \\
\hline 3443 & & & & ONSITE & SESPMNT & $\mathrm{R}$ \\
\hline 3444 & & & & ONSITE & SESPMNT & $\mathrm{R}$ \\
\hline 3445 & & & & ONSITE & SESPMNT & $\mathrm{R}$ \\
\hline 3446 & & & & & SESPMNT & $\mathrm{R}$ \\
\hline 3447 & & & & & SESPMNT & $\mathrm{R}$ \\
\hline 3448 & & & & & SESPMNT & $\mathrm{R}$ \\
\hline 3449 & & & & & SESPMNT & $\mathrm{R}$ \\
\hline 3450 & & & & ONSITE & SESPMNT & $\mathrm{R}$ \\
\hline 3451 & & & & ONSITE & SESPMNT & $\mathrm{R}$ \\
\hline 3452 & & & & ONSITE & SESPMNT & $\mathrm{R}$ \\
\hline 3453 & & & & ONSITE & SESPMNT & $\mathrm{R}$ \\
\hline 3454 & & & & ONSITE & SESPMNT & $\mathrm{R}$ \\
\hline 3455 & & & & ONSITE & SESPMNT & $\mathrm{R}$ \\
\hline 3456 & & & & ONSITE & SESPMNT & $\mathrm{R}$ \\
\hline 3457 & & & & ONSITE & SESPMNT & $\mathrm{R}$ \\
\hline 3458 & & & & OFFSITE & SESPMNT & $\mathrm{R}$ \\
\hline 3459 & & & & OFFSITE & SESPMNT & $\mathrm{R}$ \\
\hline 3460 & & & & OFFSITE & SESPMNT & $\mathrm{R}$ \\
\hline 3461 & & & & OFFSITE & SESPMNT & $\mathrm{R}$ \\
\hline 3462 & & & & OFFSITE & SESPMNT & $\mathrm{R}$ \\
\hline 3463 & & & & OFFSITE & SESPMNT & $\mathrm{R}$ \\
\hline 3464 & & & & OFFSITE & SESPMNT & $\mathrm{R}$ \\
\hline 3465 & & & & OFFSITE & SESPMNT & $\mathrm{R}$ \\
\hline 3466 & & & & OFFSITE & SESPMNT & $\mathrm{R}$ \\
\hline \begin{tabular}{|c|}
3467 \\
\end{tabular} & & & & OFFSITE & SESPMNT & $\mathrm{R}$ \\
\hline 3468 & & & & OFFSITE & SESPMNT & $\mathrm{R}$ \\
\hline 3469 & & & & PERIMETER & SESPMNT & $\mathrm{R}$ \\
\hline 3470 & & & & PERIMETER & SESPMNT & $\mathrm{R}$ \\
\hline 3471 & See sample record for comment. & & & & SESPMNT & $\mathrm{R}$ \\
\hline \begin{tabular}{|l|}
3472 \\
\end{tabular} & & & & ONSITE & SESPMNT & $\mathrm{R}$ \\
\hline \begin{tabular}{|l|l|}
3473 \\
\end{tabular} & & & & ONSITE & SESPMNT & $\mathrm{R}$ \\
\hline \begin{tabular}{|l|l|l|}
3474 & \\
\end{tabular} & See sample record for comment. & & & OFFSITE & SESPMNT & $\mathrm{R}$ \\
\hline $3475 \leq$ & See sample record for comment. & & & OFFSITE & SESPMNT & $\mathrm{R}$ \\
\hline \begin{tabular}{|l|}
3476 \\
\end{tabular} & & & & OFFSITE & SESPMNT & $\mathrm{R}$ \\
\hline \begin{tabular}{|l|l|}
3477 \\
\end{tabular} & & & & OFFSITE & SESPMNT & $\mathrm{R}$ \\
\hline \begin{tabular}{|l|l|}
3478 \\
\end{tabular} & & & & OFFSITE & SESPMNT & $\mathrm{R}$ \\
\hline \begin{tabular}{|l|}
3479 \\
\end{tabular} & & & & & SESPMNT & $\mathrm{R}$ \\
\hline \begin{tabular}{|l|l|}
3480 \\
\end{tabular} & & & & & SESPMNT & $\mathrm{R}$ \\
\hline \begin{tabular}{|l|l|}
3481 \\
\end{tabular} & & & & ONSITE & SESPMNT & $\mathrm{R}$ \\
\hline \begin{tabular}{|l|l|}
3482 \\
\end{tabular} & & & & ONSITE & SESPMNT & $\mathrm{R}$ \\
\hline \begin{tabular}{|l|l|}
3483 \\
\end{tabular} & & & & ONSITE & SESPMNT & $\mathrm{R}$ \\
\hline 3484 & & & & & SESPMNT & $\mathrm{R}$ \\
\hline \begin{tabular}{|l|l|l|l|l|l|l}
3485 \\
\end{tabular} & & & & & SESBMNT & $\mathrm{R}$ \\
\hline
\end{tabular}




\begin{tabular}{|c|c|c|c|c|c|c|}
\hline & $A D$ & $\mathrm{AE}$ & $\mathrm{AF}$ & $A G$ & $\mathrm{AH}$ & $\mathrm{Al}$ \\
\hline 1 & RESULT_COMMENT & COLL_SAMP_SIZE & COLL_SAMP_SIZE_UNITS & DIST_CLASS & OWNER_ID & COLLECTION_PURPOSE \\
\hline 3486 & See sample record for comment. & & & OFFSITE & SESPMNT & $R$ \\
\hline \begin{tabular}{|l|l|}
3487 & \\
\end{tabular} & See sample record for comment. & & & OFFSITE & SESPMNT & $\mathrm{R}$ \\
\hline \begin{tabular}{|c|}
3488 \\
\end{tabular} & & & & OFFSITE & SESPMNT & $\mathrm{R}$ \\
\hline 3489 & & & & OFFSITE & SESPMNT & $\mathrm{R}$ \\
\hline 3490 & & & & OFFSITE & SESPMNT & $\mathrm{R}$ \\
\hline 3491 & & & & OFFSITE & SESPMNT & $\mathrm{R}$ \\
\hline 3492 & & & & ONSITE & SESPMNT & $\mathrm{R}$ \\
\hline \begin{tabular}{|l|}
3493 \\
\end{tabular} & & & & ONSITE & SESPMNT & $\mathrm{R}$ \\
\hline 3494 & See sample record for comment. & & & OFFSITE & SESPMNT & $\mathrm{R}$ \\
\hline 3495 & See sample record for comment. & & & OFFSITE & SESPMNT & $\mathrm{R}$ \\
\hline 3496 & & & & & SESPSPEC & $\mathrm{s}$ \\
\hline \begin{tabular}{|l|l|}
3497 \\
\end{tabular} & & & & & SESPSPEC & $\mathrm{s}$ \\
\hline 3498 & & & & & SESPSPEC & $\mathrm{s}$ \\
\hline 3499 & & & & & SESPSPEC & $\mathrm{s}$ \\
\hline 3500 & & & & ONSITE & SESPMNT & $\mathrm{R}$ \\
\hline \begin{tabular}{|l|}
3501 \\
\end{tabular} & & & & ONSITE & SESPMNT & $\mathrm{R}$ \\
\hline 3502 & & & & ONSITE & SESPMNT & $\mathrm{R}$ \\
\hline 3503 & & & & & SESPMNT & $\mathrm{R}$ \\
\hline 3504 & & & & & SESPMNT & $\mathrm{R}$ \\
\hline \begin{tabular}{|c|}
3505 \\
\end{tabular} & & & & ONSITE & SESPMNT & $\mathrm{R}$ \\
\hline \begin{tabular}{|l|}
3506 \\
\end{tabular} & & & & ONSITE & SESPMNT & $\mathrm{R}$ \\
\hline \begin{tabular}{|l|l|}
3507 & \\
\end{tabular} & & & & ONSITE & SESPMNT & $\mathrm{R}$ \\
\hline 3508 & & & & ONSITE & SESPMNT & $\mathrm{R}$ \\
\hline 3509 & & & & ONSITE & SESPMNT & $\mathrm{R}$ \\
\hline 3510 & & & & & SESPMNT & $\mathrm{R}$ \\
\hline 3511 & & & & & SESPMNT & $\mathrm{R}$ \\
\hline \begin{tabular}{|l|}
3512 \\
\end{tabular} & See sample record for comment. & & & OFFSITE & SESPMNT & $\mathrm{R}$ \\
\hline 3513 & See sample record for comment. & & & OFFSITE & SESPMNT & $\mathrm{R}$ \\
\hline \begin{tabular}{|l|}
3514 \\
\end{tabular} & & & & OFFSITE & SESPMNT & $\mathrm{R}$ \\
\hline \begin{tabular}{|l|}
3515 \\
\end{tabular} & & & & OFFSITE & SESPMNT & $\mathrm{R}$ \\
\hline 3516 & & & & OFFSITE & SESPMNT & $\mathrm{R}$ \\
\hline \begin{tabular}{|l|l|}
3517 & \\
\end{tabular} & & & & OFFSITE & SESPMNT & $\mathrm{R}$ \\
\hline 3518 & & & & OFFSITE & SESPMNT & $\mathrm{R}$ \\
\hline 3519 & & & & OFFSITE & SESPMNT & $\mathrm{R}$ \\
\hline 3520 & & & & OFFSITE & SESPMNT & $\mathrm{R}$ \\
\hline 3521 & & & & & SESPMNT & $\mathrm{R}$ \\
\hline 3522 & & & & & SESPMNT & $\mathrm{R}$ \\
\hline 3523 & & & & ONSITE & SESPMNT & $\mathrm{R}$ \\
\hline 3524 & & & & ONSITE & SESPMNT & $\mathrm{R}$ \\
\hline 3525 & & & & ONSITE & SESPMNT & $\mathrm{R}$ \\
\hline \begin{tabular}{|l|}
3526 \\
\end{tabular} & & & & ONSITE & SESPMNT & $\mathrm{R}$ \\
\hline \begin{tabular}{|l|l}
3527 & \\
\end{tabular} & & & & OFFSITE & SESPMNT & $\mathrm{R}$ \\
\hline \begin{tabular}{|l|}
3528 \\
\end{tabular} & & & & OFFSITE & SESPMNT & $\mathrm{R}$ \\
\hline 3529 & & & & ONSITE & SESPMNT & $\mathrm{R}$ \\
\hline 3530 & & & & ONSITE & SESPMNT & $\mathrm{R}$ \\
\hline 3531 & & & & ONSITE & SESPMNT & $\mathrm{R}$ \\
\hline 3532 & & & & & SESPMNT & $\mathrm{R}$ \\
\hline 3533 & & & & & SESPMNT & $\mathrm{R}$ \\
\hline 3534 & & & & & SESPMNT & $\mathrm{R}$ \\
\hline 3535 & & & & & SESPMNT & $\mathrm{R}$ \\
\hline 3536 & & & & ONSITE & SESPMNT & $\mathrm{R}$ \\
\hline \begin{tabular}{|l|l|}
3537 & $-r$ \\
\end{tabular} & & & & ONSITE & SESBMNT & ${ }_{2} R$ \\
\hline
\end{tabular}




\begin{tabular}{|c|c|c|c|c|c|c|}
\hline & $\mathrm{AD}$ & $\mathrm{AE}$ & $\mathrm{AF}$ & $A G$ & $\mathrm{AH}$ & $\mathrm{Al}$ \\
\hline 1 & RESULT_COMMENT & COLL_SAMP_SIZE & COLL_SAMP_SIZE_UNITS & DIST_CLASS & OWNER_ID & COLLECTION_PURPOSE \\
\hline 3538 & & & & ONSITE & SESPMNT & $\mathrm{R}$ \\
\hline 3539 & & & & ONSITE & SESPMNT & $\mathrm{R}$ \\
\hline 3540 & & & & ONSITE & SESPMNT & $\mathrm{R}$ \\
\hline 3541 & & & & ONSITE & SESPMNT & $\mathrm{R}$ \\
\hline 3542 & & & & ONSITE & SESPMNT & $\mathrm{R}$ \\
\hline 3543 & & & & ONSITE & SESPMNT & $\mathrm{R}$ \\
\hline 3544 & & & & & SESPMNT & $\mathrm{R}$ \\
\hline 3545 & & & & & SESPMNT & $\mathrm{R}$ \\
\hline 3546 & See sample record for comment. & & & OFFSITE & SESPMNT & $\mathrm{R}$ \\
\hline 3547 & See sample record for comment. & & & OFFSITE & SESPMNT & $\mathrm{R}$ \\
\hline 3548 & See sample record for comment. & & & OFFSITE & SESPMNT & $\mathrm{R}$ \\
\hline 3549 & & & & OFFSITE & SESPMNT & $\mathrm{R}$ \\
\hline 3550 & & & & OFFSITE & SESPMNT & $\mathrm{R}$ \\
\hline 3551 & & & & OFFSITE & SESPMNT & $\mathrm{R}$ \\
\hline 3552 & & & & OFFSITE & SESPMNT & $\mathrm{R}$ \\
\hline 3553 & & & & OFFSITE & SESPMNT & $\mathrm{R}$ \\
\hline 3554 & & & & OFFSITE & SESPMNT & $\mathrm{R}$ \\
\hline 3555 & & & & OFFSITE & SESPMNT & $\mathrm{R}$ \\
\hline 3556 & & & & OFFSITE & SESPMNT & $\mathrm{R}$ \\
\hline 3557 & & & & PERIMETER & SESPMNT & $\mathrm{R}$ \\
\hline 3558 & & & & PERIMETER & SESPMNT & $\mathrm{R}$ \\
\hline 3559 & & & & ONSITE & SESPMNT & $\mathrm{R}$ \\
\hline 3560 & & & & ONSITE & SESPMNT & $\mathrm{R}$ \\
\hline 3561 & & & & OFFSITE & SESPMNT & $\mathrm{R}$ \\
\hline 3562 & & & & OFFSITE & SESPMNT & $\mathrm{R}$ \\
\hline 3563 & & & & OFFSITE & SESPMNT & $\mathrm{R}$ \\
\hline 3564 & & & & OFFSITE & SESPMNT & $\mathrm{R}$ \\
\hline 3565 & & & & OFFSITE & SESPMNT & $\mathrm{R}$ \\
\hline 3566 & & & & ONSITE & SESPMNT & $\mathrm{R}$ \\
\hline 3567 & & & & ONSITE & SESPMNT & $\mathrm{R}$ \\
\hline 3568 & & & & ONSITE & SESPMNT & $\mathrm{R}$ \\
\hline 3569 & & & & & SESPMNT & $\mathrm{R}$ \\
\hline 3570 & & & & & SESPMNT & $\mathrm{R}$ \\
\hline 3571 & See sample record for comment. & & & OFFSITE & SESPMNT & $\mathrm{R}$ \\
\hline 3572 & See sample record for comment. & & & OFFSITE & SESPMNT & $\mathrm{R}$ \\
\hline 3573 & & & & OFFSITE & SESPMNT & $\mathrm{R}$ \\
\hline 3574 & & & & OFFSITE & SESPMNT & $\mathrm{R}$ \\
\hline 3575 & & & & OFFSITE & SESPMNT & $\mathrm{R}$ \\
\hline 3576 & & & & OFFSITE & SESPMNT & $\mathrm{R}$ \\
\hline 3577 & & & & & SESPMNT & $\mathrm{R}$ \\
\hline 3578 & & & & & SESPMNT & $\mathrm{R}$ \\
\hline 3579 & & & & & SESPSPEC & $\mathrm{s}$ \\
\hline 3580 & & & & & SESPSPEC & $\mathrm{s}$ \\
\hline 3581 & & & & & SESPSPEC & $\mathrm{s}$ \\
\hline 3582 & & & & & SESPSPEC & $\mathrm{s}$ \\
\hline 3583 & & & & & SESPSPEC & $\mathrm{s}$ \\
\hline 3584 & & & & & SESPSPEC & $\mathrm{S}$ \\
\hline 3585 & & & & ONSITE & SESPMNT & $\mathrm{R}$ \\
\hline 3586 & & & & ONSITE & SESPMNT & $\mathrm{R}$ \\
\hline 3587 & & & & & SESPMNT & $\mathrm{R}$ \\
\hline 3588 & & & & OFFSITE & SESPMNT & $\mathrm{R}$ \\
\hline 3589 & & & & OFFSITE & SESBMNT & $R$ \\
\hline
\end{tabular}




\begin{tabular}{|c|c|c|c|c|c|c|}
\hline & $\mathrm{AD}$ & $\mathrm{AE}$ & $\mathrm{AF}$ & $\mathrm{AG}$ & $\mathrm{AH}$ & $\mathrm{Al}$ \\
\hline 1 & RESULT_COMMENT & COLL_SAMP_SIZE & COLL_SAMP_SIZE_UNITS & DIST_CLASS & OWNER_ID & COLLECTION_PURPOSE \\
\hline 3590 & & & & & SESPMNT & $\mathrm{R}$ \\
\hline 3591 & & & & & SESPMNT & $\mathrm{R}$ \\
\hline 3592 & & & & ONSITE & SESPMNT & $\mathrm{R}$ \\
\hline 3593 & & & & ONSITE & SESPMNT & $\mathrm{R}$ \\
\hline 3594 & & & & OFFSITE & SESPMNT & $\mathrm{R}$ \\
\hline 3595 & & & & OFFSITE & SESPMNT & $\mathrm{R}$ \\
\hline 3596 & & & & OFFSITE & SESPMNT & $\mathrm{R}$ \\
\hline 3597 & & & & OFFSITE & SESPMNT & $\mathrm{R}$ \\
\hline 3598 & & & & OFFSITE & SESPMNT & $\mathrm{R}$ \\
\hline 3599 & See sample record for comment. & & & OFFSITE & SESPMNT & $\mathrm{R}$ \\
\hline 3600 & & & & ONSITE & SESPMNT & $\mathrm{R}$ \\
\hline 3601 & & & & ONSITE & SESPMNT & $\mathrm{R}$ \\
\hline 3602 & & & & OFFSITE & SESPMNT & $\mathrm{R}$ \\
\hline 3603 & & & & OFFSITE & SESPMNT & $\mathrm{R}$ \\
\hline 3604 & & & & & SESPMNT & $\mathrm{R}$ \\
\hline 3605 & & & & & SESPMNT & $\mathrm{R}$ \\
\hline 3606 & & & & & SESPMNT & $\mathrm{R}$ \\
\hline 3607 & & & & & SESPMNT & $\mathrm{R}$ \\
\hline 3608 & & & & & SESPMNT & $\mathrm{R}$ \\
\hline 3609 & & & & & SESPMNT & $\mathrm{R}$ \\
\hline 3610 & & & & & SESPMNT & $\mathrm{R}$ \\
\hline 3611 & & & & ONSITE & SESPMNT & $\mathrm{R}$ \\
\hline 3612 & & & & ONSITE & SESPMNT & $\mathrm{R}$ \\
\hline 3613 & & & & ONSITE & SESPMNT & $\mathrm{R}$ \\
\hline 3614 & & & & ONSITE & SESPMNT & $\mathrm{R}$ \\
\hline 3615 & & & & ONSITE & SESPMNT & $\mathrm{R}$ \\
\hline 3616 & & & & ONSITE & SESPMNT & $\mathrm{R}$ \\
\hline 3617 & & & & ONSITE & SESPMNT & $\mathrm{R}$ \\
\hline 3618 & & & & ONSITE & SESPMNT & $\mathrm{R}$ \\
\hline 3619 & & & & OFFSITE & SESPMNT & $\mathrm{R}$ \\
\hline 3620 & & & & OFFSITE & SESPMNT & $\mathrm{R}$ \\
\hline 3621 & & & & OFFSITE & SESPMNT & $\mathrm{R}$ \\
\hline 3622 & & & & OFFSITE & SESPMNT & $\mathrm{R}$ \\
\hline 3623 & & & & OFFSITE & SESPMNT & $\mathrm{R}$ \\
\hline 3624 & & & & OFFSITE & SESPMNT & $\mathrm{R}$ \\
\hline 3625 & & & & OFFSITE & SESPMNT & $\mathrm{R}$ \\
\hline 3626 & & & & OFFSITE & SESPMNT & $\mathrm{R}$ \\
\hline \begin{tabular}{|c|}
3627 \\
\end{tabular} & & & & OFFSITE & SESPMNT & $\mathrm{R}$ \\
\hline \begin{tabular}{|l|l|}
3628 \\
\end{tabular} & & & & OFFSITE & SESPMNT & $\mathrm{R}$ \\
\hline \begin{tabular}{|l|l|}
3629 \\
\end{tabular} & & & & OFFSITE & SESPMNT & $\mathrm{R}$ \\
\hline \begin{tabular}{|l|l|l}
3630 & \\
\end{tabular} & See sample record for comment. & & & & SESPSPEC & $\mathrm{s}$ \\
\hline $3631 \leq$ & See sample record for comment. & & & & SESPSPEC & $\mathrm{s}$ \\
\hline $3632 \leq$ & See sample record for comment. & & & ONSITE & SESPMNT & $\mathrm{R}$ \\
\hline $3633 \leqslant$ & See sample record for comment. & & & ONSITE & SESPMNT & $\mathrm{R}$ \\
\hline 3634 s & See sample record for comment. & & & ONSITE & SESPMNT & $\mathrm{R}$ \\
\hline \begin{tabular}{|l|l|l}
3635 \\
\end{tabular} & & & & ONSITE & SESPMNT & $\mathrm{R}$ \\
\hline \begin{tabular}{|l|l|}
3636 \\
\end{tabular} & & & & ONSITE & SESPMNT & $\mathrm{R}$ \\
\hline \begin{tabular}{|l|}
3637 \\
\end{tabular} & & & & ONSITE & SESPMNT & $\mathrm{R}$ \\
\hline \begin{tabular}{|l|}
3638 \\
\end{tabular} & & & & ONSITE & SESPMNT & $\mathrm{R}$ \\
\hline \begin{tabular}{|l|l|}
3639 \\
\end{tabular} & & & & ONSITE & SESPMNT & $\mathrm{R}$ \\
\hline 3640 & & & & & SESPMNT & $\mathrm{R}$ \\
\hline \begin{tabular}{|l|l|}
3641 & -1 \\
\end{tabular} & & & & & SESBMNT & $R$ \\
\hline
\end{tabular}




\begin{tabular}{|c|c|c|c|c|c|c|}
\hline & $A D$ & $\mathrm{AE}$ & $\mathrm{AF}$ & $A G$ & $\mathrm{AH}$ & $\mathrm{Al}$ \\
\hline 1 & RESULT_COMMENT & COLL_SAMP_SIZE & COLL_SAMP_SIZE_UNITS & DIST_CLASS & OWNER_ID & COLLECTION_PURPOSE \\
\hline 3642 & & & & & SESPMNT & $\mathrm{R}$ \\
\hline 3643 & & & & ONSITE & SESPMNT & $\mathrm{R}$ \\
\hline 3644 & & & & ONSITE & SESPMNT & $\mathrm{R}$ \\
\hline 3645 & & & & ONSITE & SESPMNT & $\mathrm{R}$ \\
\hline 3646 & & & & ONSITE & SESPMNT & $\mathrm{R}$ \\
\hline 3647 & & & & ONSITE & SESPMNT & $\mathrm{R}$ \\
\hline 3648 & & & & ONSITE & SESPMNT & $\mathrm{R}$ \\
\hline 3649 & & & & ONSITE & SESPMNT & $\mathrm{R}$ \\
\hline 3650 & & & & OFFSITE & SESPMNT & $\mathrm{R}$ \\
\hline 3651 & & & & OFFSITE & SESPMNT & $\mathrm{R}$ \\
\hline 3652 & & & & ONSITE & SESPMNT & $\mathrm{R}$ \\
\hline 3653 & & & & ONSITE & SESPMNT & $\mathrm{R}$ \\
\hline 3654 & & & & ONSITE & SESPMNT & $\mathrm{R}$ \\
\hline 3655 & & & & & SESPMNT & $\mathrm{R}$ \\
\hline 3656 & & & & & SESPMNT & $\mathrm{R}$ \\
\hline 3657 & & & & ONSITE & SESPMNT & $\mathrm{R}$ \\
\hline 3658 & & & & ONSITE & SESPMNT & $\mathrm{R}$ \\
\hline 3659 & & & & OFFSITE & SESPMNT & $\mathrm{R}$ \\
\hline 3660 & & & & OFFSITE & SESPMNT & $\mathrm{R}$ \\
\hline 3661 & & & & OFFSITE & SESPMNT & $\mathrm{R}$ \\
\hline 3662 & & & & OFFSITE & SESPMNT & $\mathrm{R}$ \\
\hline 3663 & & & & OFFSITE & SESPMNT & $\mathrm{R}$ \\
\hline 3664 & & & & OFFSITE & SESPMNT & $\mathrm{R}$ \\
\hline 3665 & & & & OFFSITE & SESPMNT & $\mathrm{R}$ \\
\hline 3666 & & & & OFFSITE & SESPMNT & $\mathrm{R}$ \\
\hline 3667 & & & & PERIMETER & SESPMNT & $\mathrm{R}$ \\
\hline 3668 & & & & PERIMETER & SESPMNT & $\mathrm{R}$ \\
\hline 3669 & & & & PERIMETER & SESPMNT & $\mathrm{R}$ \\
\hline 3670 & See sample record for comment. & & & ONSITE & SESPMNT & $\mathrm{R}$ \\
\hline 3671 & See sample record for comment. & & & ONSITE & SESPMNT & $\mathrm{R}$ \\
\hline 3672 & See sample record for comment. & & & ONSITE & SESPMNT & $\mathrm{R}$ \\
\hline 3673 & & & & ONSITE & SESPMNT & $\mathrm{R}$ \\
\hline 3674 & & & & ONSITE & SESPMNT & $\mathrm{R}$ \\
\hline 3675 & & & & OFFSITE & SESPMNT & $\mathrm{R}$ \\
\hline 3676 & & & & OFFSITE & SESPMNT & $\mathrm{R}$ \\
\hline 3677 & & & & ONSITE & SESPMNT & $\mathrm{R}$ \\
\hline 3678 & & & & ONSITE & SESPMNT & $\mathrm{R}$ \\
\hline 3679 & & & & & SESPMNT & $\mathrm{R}$ \\
\hline 3680 & & & & & SESPMNT & $\mathrm{R}$ \\
\hline 3681 & & & & ONSITE & SESPMNT & $\mathrm{R}$ \\
\hline 3682 & & & & ONSITE & SESPMNT & $\mathrm{R}$ \\
\hline 3683 & & & & OFFSITE & SESPMNT & $\mathrm{R}$ \\
\hline 3684 & & & & OFFSITE & SESPMNT & $\mathrm{R}$ \\
\hline 3685 & & & & OFFSITE & SESPMNT & $\mathrm{R}$ \\
\hline 3686 & & & & OFFSITE & SESPMNT & $\mathrm{R}$ \\
\hline 3687 & & & & OFFSITE & SESPMNT & $\mathrm{R}$ \\
\hline 3688 & & & & OFFSITE & SESPMNT & $\mathrm{R}$ \\
\hline 3689 & & & & OFFSITE & SESPMNT & $\mathrm{R}$ \\
\hline 3690 & & & & OFFSITE & SESPMNT & $\mathrm{R}$ \\
\hline 3691 & & & & OFFSITE & SESPSPEC & $\mathrm{s}$ \\
\hline 3692 & & & & OFFSITE & SESPSPEC & $\mathrm{s}$ \\
\hline 3693 & & & & OFFSITE & SESBSPEC & 5 \\
\hline
\end{tabular}




\begin{tabular}{|c|c|c|c|c|c|c|}
\hline & $A D$ & $\mathrm{AE}$ & $\mathrm{AF}$ & $A G$ & $\mathrm{AH}$ & $\mathrm{Al}$ \\
\hline 1 & RESULT_COMMENT & COLL_SAMP_SIZE & COLL_SAMP_SIZE_UNITS & DIST_CLASS & OWNER_ID & COLLECTION_PURPOSE \\
\hline 3694 & & & & OFFSITE & SESPSPEC & $S$ \\
\hline 3695 & & & & OFFSITE & SESPSPEC & $\mathrm{s}$ \\
\hline 3696 & & & & OFFSITE & SESPSPEC & $\mathrm{s}$ \\
\hline 3697 & & & & ONSITE & SESPMNT & $\mathrm{R}$ \\
\hline 3698 & & & & ONSITE & SESPMNT & $\mathrm{R}$ \\
\hline 3699 & & & & OFFSITE & SESPMNT & $\mathrm{R}$ \\
\hline 3700 & & & & OFFSITE & SESPMNT & $\mathrm{R}$ \\
\hline 3701 & & & & & SESPMNT & $\mathrm{R}$ \\
\hline 3702 & & & & & SESPMNT & $\mathrm{R}$ \\
\hline 3703 & & & & ONSITE & SESPMNT & $\mathrm{R}$ \\
\hline 3704 & & & & ONSITE & SESPMNT & $\mathrm{R}$ \\
\hline 3705 & & & & ONSITE & SESPMNT & $\mathrm{R}$ \\
\hline 3706 & & & & ONSITE & SESPMNT & $\mathrm{R}$ \\
\hline 3707 & & & & ONSITE & SESPMNT & $\mathrm{R}$ \\
\hline 3708 & & & & OFFSITE & SESPMNT & $\mathrm{R}$ \\
\hline 3709 & & & & OFFSITE & SESPMNT & $\mathrm{R}$ \\
\hline 3710 & & & & OFFSITE & SESPMNT & $\mathrm{R}$ \\
\hline 3711 & & & & OFFSITE & SESPMNT & $\mathrm{R}$ \\
\hline 3712 & & & & OFFSITE & SESPMNT & $\mathrm{R}$ \\
\hline 3713 & & & & OFFSITE & SESPMNT & $\mathrm{R}$ \\
\hline 3714 & & & & OFFSITE & SESPMNT & $\mathrm{R}$ \\
\hline 3715 & & & & OFFSITE & SESPMNT & $\mathrm{R}$ \\
\hline 3716 & & & & OFFSITE & SESPMNT & $\mathrm{R}$ \\
\hline 3717 & & & & OFFSITE & SESPMNT & $\mathrm{R}$ \\
\hline 3718 & & & & OFFSITE & SESPMNT & $\mathrm{R}$ \\
\hline 3719 & & & & ONSITE & SESPMNT & $\mathrm{R}$ \\
\hline 3720 & & & & ONSITE & SESPMNT & $\mathrm{R}$ \\
\hline 3721 & & & & ONSITE & SESPMNT & $\mathrm{R}$ \\
\hline 3722 & & & & ONSITE & SESPMNT & $\mathrm{R}$ \\
\hline 3723 & & & & ONSITE & SESPMNT & $R$ \\
\hline 3724 & & & & ONSITE & SESPMNT & $\mathrm{R}$ \\
\hline 3725 & & & & ONSITE & SESPMNT & $\mathrm{R}$ \\
\hline 3726 & & & & ONSITE & SESPMNT & $\mathrm{R}$ \\
\hline \begin{tabular}{|l|}
3727 \\
\end{tabular} & & & & & SESPMNT & $\mathrm{R}$ \\
\hline 3728 & & & & & SESPMNT & $\mathrm{R}$ \\
\hline 3729 & & & & & SESPMNT & $\mathrm{R}$ \\
\hline 3730 & & & & ONSITE & SESPMNT & $\mathrm{R}$ \\
\hline 3731 & & & & ONSITE & SESPMNT & $\mathrm{R}$ \\
\hline 3732 & & & & ONSITE & SESPMNT & $\mathrm{R}$ \\
\hline 3733 & & & & ONSITE & SESPMNT & $\mathrm{R}$ \\
\hline 3734 & & & & ONSITE & SESPMNT & $\mathrm{R}$ \\
\hline 3735 & & & & ONSITE & SESPMNT & $\mathrm{R}$ \\
\hline 3736 & & & & ONSITE & SESPMNT & $R$ \\
\hline \begin{tabular}{|l|l}
3737 \\
\end{tabular} & & & & ONSITE & SESPMNT & $\mathrm{R}$ \\
\hline 3738 & & & & ONSITE & SESPMNT & $\mathrm{R}$ \\
\hline 3739 & & & & ONSITE & SESPMNT & $\mathrm{R}$ \\
\hline 3740 & & & & OFFSITE & SESPMNT & $\mathrm{R}$ \\
\hline 3741 & & & & OFFSITE & SESPMNT & $\mathrm{R}$ \\
\hline 3742 & & & & ONSITE & SESPMNT & $\mathrm{R}$ \\
\hline 3743 & & & & ONSITE & SESPMNT & $R$ \\
\hline 3744 & & & & ONSITE & SESPMNT & $\mathrm{R}$ \\
\hline 3745 & & & & & SESBMNT & $R$ \\
\hline
\end{tabular}




\begin{tabular}{|c|c|c|c|c|c|c|}
\hline & $A D$ & $\mathrm{AE}$ & $\mathrm{AF}$ & $A G$ & $\mathrm{AH}$ & $\mathrm{Al}$ \\
\hline 1 & RESULT_COMMENT & COLL_SAMP_SIZE & COLL_SAMP_SIZE_UNITS & DIST_CLASS & OWNER_ID & COLLECTION_PURPOSE \\
\hline \begin{tabular}{|c|}
3746 \\
\end{tabular} & & & & & SESPMNT & $\mathrm{R}$ \\
\hline 3747 & & & & ONSITE & SESPMNT & $\mathrm{R}$ \\
\hline 3748 & & & & ONSITE & SESPMNT & $\mathrm{R}$ \\
\hline 3749 & & & & OFFSITE & SESPMNT & $\mathrm{R}$ \\
\hline 3750 & & & & OFFSITE & SESPMNT & $\mathrm{R}$ \\
\hline 3751 & & & & OFFSITE & SESPMNT & $\mathrm{R}$ \\
\hline 3752 & & & & OFFSITE & SESPMNT & $\mathrm{R}$ \\
\hline 3753 & & & & OFFSITE & SESPMNT & $\mathrm{R}$ \\
\hline 3754 & & & & OFFSITE & SESPMNT & $\mathrm{R}$ \\
\hline 3755 & & & & OFFSITE & SESPMNT & $\mathrm{R}$ \\
\hline 3756 & & & & OFFSITE & SESPMNT & $\mathrm{R}$ \\
\hline 3757 & & & & ONSITE & SESPMNT & $\mathrm{R}$ \\
\hline 3758 & & & & PERIMETER & SESPMNT & $\mathrm{R}$ \\
\hline 3759 & & & & PERIMETER & SESPMNT & $\mathrm{R}$ \\
\hline 3760 & & & & PERIMETER & SESPMNT & $\mathrm{R}$ \\
\hline 3761 & & & & ONSITE & SESPMNT & $\mathrm{R}$ \\
\hline 3762 & & & & ONSITE & SESPMNT & $\mathrm{R}$ \\
\hline 3763 & & & & ONSITE & SESPMNT & $\mathrm{R}$ \\
\hline 3764 & & & & ONSITE & SESPMNT & $\mathrm{R}$ \\
\hline 3765 & & & & OFFSITE & SESPMNT & $\mathrm{R}$ \\
\hline 3766 & & & & OFFSITE & SESPMNT & $\mathrm{R}$ \\
\hline 3767 & & & & ONSITE & SESPMNT & $\mathrm{R}$ \\
\hline 3768 & & & & ONSITE & SESPMNT & $\mathrm{R}$ \\
\hline 3769 & & & & ONSITE & SESPMNT & $\mathrm{R}$ \\
\hline 3770 & & & & ONSITE & SESPMNT & $\mathrm{R}$ \\
\hline 3771 & & & & ONSITE & SESPMNT & $\mathrm{R}$ \\
\hline \begin{tabular}{|l|}
3772 \\
\end{tabular} & & & & OFFSITE & SESPMNT & $\mathrm{R}$ \\
\hline \begin{tabular}{|l|}
3773 \\
\end{tabular} & & & & OFFSITE & SESPMNT & $\mathrm{R}$ \\
\hline 3774 & & & & OFFSITE & SESPMNT & $\mathrm{R}$ \\
\hline 3775 & & & & OFFSITE & SESPMNT & $R$ \\
\hline 3776 & & & & OFFSITE & SESPMNT & $\mathrm{R}$ \\
\hline \begin{tabular}{|l|}
3777 \\
\end{tabular} & & & & OFFSITE & SESPMNT & $\mathrm{R}$ \\
\hline 3778 & & & & OFFSITE & SESPMNT & $\mathrm{R}$ \\
\hline 3779 & & & & OFFSITE & SESPMNT & $\mathrm{R}$ \\
\hline 3780 & & & & & SESPMNT & $\mathrm{R}$ \\
\hline 3781 & & & & & SESPMNT & $\mathrm{R}$ \\
\hline 3782 & & & & ONSITE & SESPMNT & $\mathrm{R}$ \\
\hline 3783 & & & & ONSITE & SESPMNT & $\mathrm{R}$ \\
\hline 3784 & & & & OFFSITE & SESPMNT & $\mathrm{R}$ \\
\hline 3785 & & & & OFFSITE & SESPMNT & $\mathrm{R}$ \\
\hline 3786 & & & & & SESPMNT & $\mathrm{R}$ \\
\hline \begin{tabular}{|l|}
3787 \\
\end{tabular} & & & & & SESPMNT & $\mathrm{R}$ \\
\hline \begin{tabular}{|c|}
3788 \\
\end{tabular} & & & & & SESPMNT & $\mathrm{R}$ \\
\hline 3789 & & & & ONSITE & SESPMNT & $\mathrm{R}$ \\
\hline 3790 & & & & OFFSITE & SESPMNT & $\mathrm{R}$ \\
\hline 3791 & & & & & SESPMNT & $\mathrm{R}$ \\
\hline 3792 & & & & & SESPMNT & $\mathrm{R}$ \\
\hline 3793 & & & & ONSITE & SESPMNT & $\mathrm{R}$ \\
\hline 3794 & & & & ONSITE & SESPMNT & $\mathrm{R}$ \\
\hline 3795 & & & & ONSITE & SESPMNT & $R$ \\
\hline 3796 & & & & ONSITE & $\begin{array}{l}\text { SESPMNT } \\
\end{array}$ & $\mathrm{R}$ \\
\hline 3797 & & & & OFFSITE & SESBMNT & $\mathrm{R}$ \\
\hline
\end{tabular}




\begin{tabular}{|c|c|c|c|c|c|c|}
\hline & $A D$ & $\mathrm{AE}$ & $\mathrm{AF}$ & $A G$ & $\mathrm{AH}$ & $\mathrm{Al}$ \\
\hline 1 & RESULT_COMMENT & COLL_SAMP_SIZE & COLL_SAMP_SIZE_UNITS & DIST_CLASS & OWNER_ID & COLLECTION_PURPOSE \\
\hline 3798 & & & & OFFSITE & SESPMNT & $\mathrm{R}$ \\
\hline \begin{tabular}{|l|}
3799 \\
\end{tabular} & & & & OFFSITE & SESPMNT & $\mathrm{R}$ \\
\hline 3800 & & & & OFFSITE & SESPMNT & $\mathrm{R}$ \\
\hline 3801 & & & & OFFSITE & SESPMNT & $\mathrm{R}$ \\
\hline 3802 & & & & OFFSITE & SESPMNT & $\mathrm{R}$ \\
\hline 3803 & & & & OFFSITE & SESPMNT & $\mathrm{R}$ \\
\hline \begin{tabular}{|l|}
3804 \\
\end{tabular} & & & & OFFSITE & SESPMNT & $\mathrm{R}$ \\
\hline 3805 & & & & OFFSITE & SESPMNT & $\mathrm{R}$ \\
\hline 3806 & & & & OFFSITE & SESPMNT & $\mathrm{R}$ \\
\hline \begin{tabular}{|l|l|}
3807 & - \\
\end{tabular} & & & & ONSITE & SESPMNT & $\mathrm{R}$ \\
\hline 3808 & & & & ONSITE & SESPMNT & $\mathrm{R}$ \\
\hline 3809 & & & & ONSITE & SESPMNT & $\mathrm{R}$ \\
\hline 3810 & & & & ONSITE & SESPMNT & $\mathrm{R}$ \\
\hline \begin{tabular}{|l|}
3811 \\
\end{tabular} & & & & ONSITE & SESPMNT & $\mathrm{R}$ \\
\hline \begin{tabular}{|l|}
3812 \\
\end{tabular} & & & & & SESPMNT & $\mathrm{R}$ \\
\hline 3813 & & & & & SESPMNT & $\mathrm{R}$ \\
\hline \begin{tabular}{|l|}
3814 \\
\end{tabular} & & & & & SESPMNT & $\mathrm{R}$ \\
\hline 3815 & & & & ONSITE & SESPMNT & $\mathrm{R}$ \\
\hline 3816 & & & & ONSITE & SESPMNT & $\mathrm{R}$ \\
\hline \begin{tabular}{|l|l|}
3817 \\
\end{tabular} & & & & ONSITE & SESPMNT & $\mathrm{R}$ \\
\hline \begin{tabular}{|l|}
3818 \\
\end{tabular} & & & & ONSITE & SESPMNT & $\mathrm{R}$ \\
\hline 3819 & & & & ONSITE & SESPMNT & $\mathrm{R}$ \\
\hline 3820 & & & & ONSITE & SESPMNT & $\mathrm{R}$ \\
\hline 3821 & & & & ONSITE & SESPMNT & $\mathrm{R}$ \\
\hline 3822 & & & & ONSITE & SESPMNT & $\mathrm{R}$ \\
\hline 3823 & & & & ONSITE & SESPMNT & $\mathrm{R}$ \\
\hline \begin{tabular}{|l|}
3824 \\
\end{tabular} & & & & ONSITE & SESPMNT & $\mathrm{R}$ \\
\hline \begin{tabular}{|l|}
3825 \\
\end{tabular} & & & & OFFSITE & SESPMNT & $\mathrm{R}$ \\
\hline 3826 & & & & OFFSITE & SESPMNT & $\mathrm{R}$ \\
\hline \begin{tabular}{|l|l|}
3827 \\
\end{tabular} & & & & ONSITE & SESPMNT & $\mathrm{R}$ \\
\hline 3828 & & & & ONSITE & SESPMNT & $\mathrm{R}$ \\
\hline 3829 & & & & ONSITE & SESPMNT & $\mathrm{R}$ \\
\hline 3830 & & & & & SESPMNT & $\mathrm{R}$ \\
\hline 3831 & & & & & SESPMNT & $\mathrm{R}$ \\
\hline \begin{tabular}{|l|}
3832 \\
\end{tabular} & See sample record for comment. & & & ONSITE & SESPMNT & $\mathrm{R}$ \\
\hline 3833 & & & & ONSITE & SESPMNT & $\mathrm{R}$ \\
\hline 3834 & & & & OFFSITE & SESPMNT & $\mathrm{R}$ \\
\hline 3835 & & & & OFFSITE & SESPMNT & $\mathrm{R}$ \\
\hline 3836 & & & & OFFSITE & SESPMNT & $\mathrm{R}$ \\
\hline \begin{tabular}{|l|l|}
3837 & \\
\end{tabular} & & & & OFFSITE & SESPMNT & $\mathrm{R}$ \\
\hline 3838 & & & & OFFSITE & SESPMNT & $\mathrm{R}$ \\
\hline 3839 & & & & OFFSITE & SESPMNT & $\mathrm{R}$ \\
\hline 3840 & & & & OFFSITE & SESPMNT & $\mathrm{R}$ \\
\hline 3841 & & & & OFFSITE & SESPMNT & $\mathrm{R}$ \\
\hline 3842 & & & & PERIMETER & SESPMNT & $\mathrm{R}$ \\
\hline 3843 & & & & PERIMETER & SESPMNT & $\mathrm{R}$ \\
\hline 3844 & & & & PERIMETER & SESPMNT & $\mathrm{R}$ \\
\hline 3845 & See sample record for comment. & & & ONSITE & SESPMNT & $\mathrm{R}$ \\
\hline 3846 & See sample record for comment. & & & ONSITE & SESPMNT & $\mathrm{R}$ \\
\hline \begin{tabular}{|l|l|}
3847 & \\
\end{tabular} & See sample record for comment. & & & OFFSITE & SESPMNT & $\mathrm{R}$ \\
\hline 3848 & See sample record for comment. & & & OFFSITE & SESPMNT & $\mathrm{R}$ \\
\hline 3849 & & & & ONSITE & SESBMNT & ${ }_{0} R$ \\
\hline
\end{tabular}




\begin{tabular}{|c|c|c|c|c|c|c|}
\hline & $A D$ & $\mathrm{AE}$ & $\mathrm{AF}$ & $A G$ & $\mathrm{AH}$ & $\mathrm{Al}$ \\
\hline 1 & RESULT_COMMENT & COLL_SAMP_SIZE & COLL_SAMP_SIZE_UNITS & DIST_CLASS & OWNER_ID & COLLECTION_PURPOSE \\
\hline 3850 & & & & ONSITE & SESPMNT & $\mathrm{R}$ \\
\hline 3851 & & & & ONSITE & SESPMNT & $\mathrm{R}$ \\
\hline \begin{tabular}{|c|}
3852 \\
\end{tabular} & & & & ONSITE & SESPMNT & $\mathrm{R}$ \\
\hline $\mid 3853$ & & & & ONSITE & SESPMNT & $\mathrm{R}$ \\
\hline 3854 & & & & ONSITE & SESPMNT & $\mathrm{R}$ \\
\hline \begin{tabular}{|c|c|}
3855 \\
\end{tabular} & & & & OFFSITE & SESPSPEC & $\mathrm{s}$ \\
\hline 3856 & & & & OFFSITE & SESPSPEC & $\mathrm{s}$ \\
\hline \begin{tabular}{|c|}
3857 \\
\end{tabular} & & & & OFFSITE & SESPSPEC & $\mathrm{s}$ \\
\hline 3858 & & & & OFFSITE & SESPSPEC & $\mathrm{s}$ \\
\hline 3859 & & & & OFFSITE & SESPSPEC & $\mathrm{s}$ \\
\hline 3860 & & & & OFFSITE & SESPSPEC & $\mathrm{s}$ \\
\hline 3861 & & & & ONSITE & SESPMNT & $\mathrm{R}$ \\
\hline 3862 & & & & ONSITE & SESPMNT & $\mathrm{R}$ \\
\hline \begin{tabular}{|c|}
3863 \\
\end{tabular} & & & & OFFSITE & SESPMNT & $\mathrm{R}$ \\
\hline 3864 & & & & OFFSITE & SESPMNT & $\mathrm{R}$ \\
\hline 3865 & & & & OFFSITE & SESPMNT & $\mathrm{R}$ \\
\hline 3866 & & & & OFFSITE & SESPMNT & $\mathrm{R}$ \\
\hline 3867 & & & & OFFSITE & SESPMNT & $\mathrm{R}$ \\
\hline 3868 & & & & OFFSITE & SESPMNT & $\mathrm{R}$ \\
\hline 3869 & & & & OFFSITE & SESPMNT & $\mathrm{R}$ \\
\hline 3870 & & & & OFFSITE & SESPMNT & $\mathrm{R}$ \\
\hline 3871 & & & & & SESPMNT & $\mathrm{R}$ \\
\hline 3872 & & & & & SESPMNT & $\mathrm{R}$ \\
\hline 3873 & & & & ONSITE & SESPMNT & $\mathrm{R}$ \\
\hline 3874 & & & & ONSITE & SESPMNT & $\mathrm{R}$ \\
\hline 3875 & & & & OFFSITE & SESPMNT & $\mathrm{R}$ \\
\hline \begin{tabular}{|l|}
3876 \\
\end{tabular} & & & & OFFSITE & SESPMNT & $\mathrm{R}$ \\
\hline \begin{tabular}{|l|}
3877 \\
\end{tabular} & & & & ONSITE & SESPMNT & $\mathrm{R}$ \\
\hline 3878 & & & & ONSITE & SESPMNT & $\mathrm{R}$ \\
\hline \begin{tabular}{|l|}
3879 \\
\end{tabular} & & & & ONSITE & SESPMNT & $\mathrm{R}$ \\
\hline 3880 & & & & ONSITE & SESPMNT & $\mathrm{R}$ \\
\hline \begin{tabular}{|l|}
3881 \\
\end{tabular} & & & & ONSITE & SESPMNT & $\mathrm{R}$ \\
\hline \begin{tabular}{|l|}
3882 \\
\end{tabular} & & & & OFFSITE & SESPMNT & $\mathrm{R}$ \\
\hline 3883 & & & & OFFSITE & SESPMNT & $\mathrm{R}$ \\
\hline \begin{tabular}{|l|}
3884 \\
\end{tabular} & & & & OFFSITE & SESPMNT & $\mathrm{R}$ \\
\hline 3885 & & & & OFFSITE & SESPMNT & $\mathrm{R}$ \\
\hline 3886 & & & & OFFSITE & SESPMNT & $\mathrm{R}$ \\
\hline 3887 & & & & OFFSITE & SESPMNT & $\mathrm{R}$ \\
\hline 3888 & & & & OFFSITE & SESPMNT & $\mathrm{R}$ \\
\hline 3889 & & & & OFFSITE & SESPMNT & $\mathrm{R}$ \\
\hline 3890 & & & & OFFSITE & SESPMNT & $\mathrm{R}$ \\
\hline 3891 & & & & OFFSITE & SESPMNT & $\mathrm{R}$ \\
\hline \begin{tabular}{|l|}
3892 \\
\end{tabular} & & & & OFFSITE & SESPMNT & $\mathrm{R}$ \\
\hline 3893 & & & & & SESPMNT & $\mathrm{R}$ \\
\hline \begin{tabular}{|l|}
3894 \\
\end{tabular} & & & & & SESPMNT & $\mathrm{R}$ \\
\hline 3895 & & & & ONSITE & SESPMNT & $\mathrm{R}$ \\
\hline 3896 & & & & ONSITE & SESPMNT & $\mathrm{R}$ \\
\hline 3897 & & & & ONSITE & SESPMNT & $\mathrm{R}$ \\
\hline 3898 & & & & ONSITE & SESPMNT & $\mathrm{R}$ \\
\hline 3899 & & & & ONSITE & SESPMNT & $R$ \\
\hline 3900 & & & & ONSITE & SESPMNT & $\mathrm{R}$ \\
\hline 3901 & & & & ONSITE & SESBMNT & ${ }_{0}^{R}$ \\
\hline
\end{tabular}




\begin{tabular}{|c|c|c|c|c|c|c|}
\hline & $A D$ & $\mathrm{AE}$ & $\mathrm{AF}$ & $A G$ & $\mathrm{AH}$ & $\mathrm{Al}$ \\
\hline 1 & RESULT_COMMENT & COLL_SAMP_SIZE & COLL_SAMP_SIZE_UNITS & DIST_CLASS & OWNER_ID & COLLECTION_PURPOSE \\
\hline 3902 & & & & ONSITE & SESPMNT & $\mathrm{R}$ \\
\hline 3903 & & & & & SESPMNT & $\mathrm{R}$ \\
\hline 3904 & & & & & SESPMNT & $\mathrm{R}$ \\
\hline 3905 & & & & & SESPMNT & $\mathrm{R}$ \\
\hline 3906 & & & & ONSITE & SESPMNT & $\mathrm{R}$ \\
\hline 3907 & & & & ONSITE & SESPMNT & $\mathrm{R}$ \\
\hline 3908 & & & & ONSITE & SESPMNT & $\mathrm{R}$ \\
\hline 3909 & & & & ONSITE & SESPMNT & $\mathrm{R}$ \\
\hline 3910 & & & & ONSITE & SESPMNT & $\mathrm{R}$ \\
\hline 3911 & & & & ONSITE & SESPMNT & $\mathrm{R}$ \\
\hline 3912 & & & & ONSITE & SESPMNT & $\mathrm{R}$ \\
\hline 3913 & & & & ONSITE & SESPMNT & $\mathrm{R}$ \\
\hline 3914 & & & & ONSITE & SESPMNT & $\mathrm{R}$ \\
\hline 3915 & & & & ONSITE & SESPMNT & $\mathrm{R}$ \\
\hline 3916 & & & & OFFSITE & SESPMNT & $\mathrm{R}$ \\
\hline 3917 & & & & OFFSITE & SESPMNT & $\mathrm{R}$ \\
\hline 3918 & & & & ONSITE & SESPMNT & $\mathrm{R}$ \\
\hline 3919 & & & & ONSITE & SESPMNT & $\mathrm{R}$ \\
\hline 3920 & & & & ONSITE & SESPMNT & $\mathrm{R}$ \\
\hline 3921 & & & & ONSITE & SESPMNT & $\mathrm{R}$ \\
\hline 3922 & & & & ONSITE & SESPMNT & $\mathrm{R}$ \\
\hline 3923 & & & & OFFSITE & SESPMNT & $\mathrm{R}$ \\
\hline 3924 & & & & OFFSITE & SESPMNT & $\mathrm{R}$ \\
\hline 3925 & & & & OFFSITE & SESPMNT & $\mathrm{R}$ \\
\hline 3926 & & & & OFFSITE & SESPMNT & $\mathrm{R}$ \\
\hline 3927 & & & & OFFSITE & SESPMNT & $\mathrm{R}$ \\
\hline 3928 & & & & OFFSITE & SESPMNT & $\mathrm{R}$ \\
\hline 3929 & & & & OFFSITE & SESPMNT & $\mathrm{R}$ \\
\hline 3930 & & & & OFFSITE & SESPMNT & $\mathrm{R}$ \\
\hline 3931 & & & & & SESPMNT & $\mathrm{R}$ \\
\hline 3932 & & & & & SESPMNT & $\mathrm{R}$ \\
\hline 3933 & & & & PERIMETER & SESPMNT & $\mathrm{R}$ \\
\hline 3934 & & & & PERIMETER & SESPMNT & $\mathrm{R}$ \\
\hline 3935 & & & & PERIMETER & SESPMNT & $\mathrm{R}$ \\
\hline 3936 & & & & ONSITE & SESPMNT & $\mathrm{R}$ \\
\hline \begin{tabular}{|l|}
3937 \\
\end{tabular} & & & & ONSITE & SESPMNT & $\mathrm{R}$ \\
\hline 3938 & & & & OFFSITE & SESPMNT & $\mathrm{R}$ \\
\hline 3939 & & & & OFFSITE & SESPMNT & $R$ \\
\hline 3940 & & & & ONSITE & SESPMNT & $\mathrm{R}$ \\
\hline 3941 & & & & ONSITE & SESPMNT & $\mathrm{R}$ \\
\hline 3942 & & & & ONSITE & SESPMNT & $\mathrm{R}$ \\
\hline 3943 & & & & ONSITE & SESPMNT & $\mathrm{R}$ \\
\hline 3944 & & & & ONSITE & SESPMNT & $\mathrm{R}$ \\
\hline 3945 & & & & ONSITE & SESPMNT & $\mathrm{R}$ \\
\hline 3946 & & & & ONSITE & SESPMNT & $R$ \\
\hline 3947 & & & & ONSITE & SESPMNT & $\mathrm{R}$ \\
\hline 3948 & & & & OFFSITE & SESPMNT & $\mathrm{R}$ \\
\hline 3949 & & & & OFFSITE & SESPMNT & $\mathrm{R}$ \\
\hline 3950 & & & & OFFSITE & SESPMNT & $\mathrm{R}$ \\
\hline 3951 & & & & OFFSITE & SESPMNT & $\mathrm{R}$ \\
\hline 3952 & & & & OFFSITE & SESPMNT & $\mathrm{R}$ \\
\hline 3953 & & & & OFFSITE & SESBMNT & $R$ \\
\hline
\end{tabular}




\begin{tabular}{|c|c|c|c|c|c|c|}
\hline & $A D$ & $\mathrm{AE}$ & $\mathrm{AF}$ & $A G$ & $\mathrm{AH}$ & $\mathrm{Al}$ \\
\hline 1 & RESULT_COMMENT & COLL_SAMP_SIZE & COLL_SAMP_SIZE_UNITS & DIST_CLASS & OWNER_ID & COLLECTION_PURPOSE \\
\hline 3954 & & & & OFFSITE & SESPMNT & $\mathrm{R}$ \\
\hline \begin{tabular}{|l|}
3955 \\
\end{tabular} & & & & OFFSITE & SESPMNT & $\mathrm{R}$ \\
\hline \begin{tabular}{|l|}
3956 \\
\end{tabular} & & & & & SESPMNT & $\mathrm{R}$ \\
\hline \begin{tabular}{|l|l|}
3957 \\
\end{tabular} & & & & & SESPMNT & $\mathrm{R}$ \\
\hline 3958 & & & & ONSITE & SESPMNT & $\mathrm{R}$ \\
\hline 3959 & & & & ONSITE & SESPMNT & $\mathrm{R}$ \\
\hline 3960 & & & & OFFSITE & SESPMNT & $\mathrm{R}$ \\
\hline 3961 & & & & OFFSITE & SESPMNT & $\mathrm{R}$ \\
\hline 3962 & & & & ONSITE & SESPMNT & $\mathrm{R}$ \\
\hline 3963 & & & & ONSITE & SESPMNT & $\mathrm{R}$ \\
\hline 3964 & & & & ONSITE & SESPMNT & $\mathrm{R}$ \\
\hline 3965 & & & & ONSITE & SESPMNT & $\mathrm{R}$ \\
\hline 3966 & & & & ONSITE & SESPMNT & $\mathrm{R}$ \\
\hline \begin{tabular}{|l|l|}
3967 & \\
\end{tabular} & & & & OFFSITE & SESPMNT & $\mathrm{R}$ \\
\hline \begin{tabular}{|l|l|}
3968 \\
\end{tabular} & & & & OFFSITE & SESPMNT & $\mathrm{R}$ \\
\hline \begin{tabular}{|l|}
3969 \\
\end{tabular} & & & & OFFSITE & SESPMNT & $\mathrm{R}$ \\
\hline 3970 & & & & OFFSITE & SESPMNT & $\mathrm{R}$ \\
\hline 3971 & & & & OFFSITE & SESPMNT & $\mathrm{R}$ \\
\hline 3972 & & & & OFFSITE & SESPMNT & $\mathrm{R}$ \\
\hline \begin{tabular}{|l|}
3973 \\
\end{tabular} & & & & OFFSITE & SESPMNT & $\mathrm{R}$ \\
\hline \begin{tabular}{|l|}
3974 \\
\end{tabular} & & & & OFFSITE & SESPMNT & $\mathrm{R}$ \\
\hline 3975 & & & & OFFSITE & SESPMNT & $\mathrm{R}$ \\
\hline 3976 & & & & OFFSITE & SESPMNT & $\mathrm{R}$ \\
\hline \begin{tabular}{|l|l|}
3977 & -1 \\
\end{tabular} & & & & OFFSITE & SESPMNT & $\mathrm{R}$ \\
\hline 3978 & & & & ONSITE & SESPMNT & $\mathrm{R}$ \\
\hline 3979 & & & & ONSITE & SESPMNT & $\mathrm{R}$ \\
\hline 3980 & & & & ONSITE & SESPMNT & $\mathrm{R}$ \\
\hline 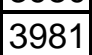 & & & & ONSITE & SESPMNT & $\mathrm{R}$ \\
\hline \begin{tabular}{|l|}
3982 \\
\end{tabular} & & & & ONSITE & SESPMNT & $\mathrm{R}$ \\
\hline \begin{tabular}{|l|}
3983 \\
\end{tabular} & See sample record for comment. & & & OFFSITE & SESPMNT & $\mathrm{R}$ \\
\hline 3984 & See sample record for comment. & & & OFFSITE & SESPMNT & $\mathrm{R}$ \\
\hline 3985 & & & & ONSITE & SESPMNT & $\mathrm{R}$ \\
\hline 3986 & & & & ONSITE & SESPMNT & $\mathrm{R}$ \\
\hline \begin{tabular}{|l|l|}
3987 & \\
\end{tabular} & & & & ONSITE & SESPMNT & $\mathrm{R}$ \\
\hline \begin{tabular}{|l|l|}
3988 \\
\end{tabular} & & & & ONSITE & SESPMNT & $\mathrm{R}$ \\
\hline 3989 & & & & ONSITE & SESPMNT & $\mathrm{R}$ \\
\hline 3990 & See sample record for comment. & & & ONSITE & SESPMNT & $\mathrm{R}$ \\
\hline 3991 & See sample record for comment. & & & ONSITE & SESPMNT & $\mathrm{R}$ \\
\hline 3992 & & & & OFFSITE & SESPMNT & $\mathrm{R}$ \\
\hline 3993 & & & & OFFSITE & SESPMNT & $\mathrm{R}$ \\
\hline 3994 & & & & OFFSITE & SESPMNT & $\mathrm{R}$ \\
\hline 3995 & & & & OFFSITE & SESPMNT & $\mathrm{R}$ \\
\hline \begin{tabular}{|l|}
3996 \\
\end{tabular} & & & & OFFSITE & SESPMNT & $\mathrm{R}$ \\
\hline 3997. & & & & OFFSITE & SESPMNT & $\mathrm{R}$ \\
\hline 3998 & & & & OFFSITE & SESPMNT & $\mathrm{R}$ \\
\hline 3999 & & & & OFFSITE & SESPMNT & $\mathrm{R}$ \\
\hline 4000 & & & & ONSITE & SESPMNT & $\mathrm{R}$ \\
\hline 4001 & & & & ONSITE & SESPMNT & $\mathrm{R}$ \\
\hline 4002 & & & & ONSITE & SESPMNT & $\mathrm{R}$ \\
\hline 4003 & & & & ONSITE & SESPMNT & $\mathrm{R}$ \\
\hline 4004 & & & & ONSITE & SESPMNT & $\mathrm{R}$ \\
\hline 4005 & & & & & SESBMNT & $R$ \\
\hline
\end{tabular}




\begin{tabular}{|c|c|c|c|c|c|c|}
\hline & $A D$ & $\mathrm{AE}$ & $\mathrm{AF}$ & $A G$ & $\mathrm{AH}$ & $\mathrm{Al}$ \\
\hline 1 & RESULT_COMMENT & COLL_SAMP_SIZE & COLL_SAMP_SIZE_UNITS & DIST_CLASS & OWNER_ID & COLLECTION_PURPOSE \\
\hline 4006 & & & & & SESPMNT & $\mathrm{R}$ \\
\hline 4007 & & & & & SESPMNT & $\mathrm{R}$ \\
\hline 4008 & & & & ONSITE & SESPMNT & $\mathrm{R}$ \\
\hline 4009 & & & & ONSITE & SESPMNT & $\mathrm{R}$ \\
\hline 4010 & & & & ONSITE & SESPMNT & $\mathrm{R}$ \\
\hline 4011 & & & & ONSITE & SESPMNT & $\mathrm{R}$ \\
\hline 4012 & & & & ONSITE & SESPMNT & $\mathrm{R}$ \\
\hline 4013 & & & & OFFSITE & SESPMNT & $\mathrm{R}$ \\
\hline 4014 & & & & OFFSITE & SESPMNT & $\mathrm{R}$ \\
\hline \begin{tabular}{|l|}
4015 \\
\end{tabular} & & & & ONSITE & SESPMNT & $\mathrm{R}$ \\
\hline \begin{tabular}{|l|}
4016 \\
\end{tabular} & & & & ONSITE & SESPMNT & $\mathrm{R}$ \\
\hline 4017 & & & & ONSITE & SESPMNT & $\mathrm{R}$ \\
\hline 4018 & & & & ONSITE & SESPMNT & $\mathrm{R}$ \\
\hline 4019 & & & & ONSITE & SESPMNT & $\mathrm{R}$ \\
\hline 4020 & & & & OFFSITE & SESPMNT & $\mathrm{R}$ \\
\hline 4021 & & & & OFFSITE & SESPMNT & $\mathrm{R}$ \\
\hline 4022 & & & & OFFSITE & SESPMNT & $\mathrm{R}$ \\
\hline 4023 & & & & OFFSITE & SESPMNT & $\mathrm{R}$ \\
\hline 4024 & & & & OFFSITE & SESPMNT & $\mathrm{R}$ \\
\hline 4025 & & & & OFFSITE & SESPMNT & $\mathrm{R}$ \\
\hline \begin{tabular}{|l|}
4026 \\
\end{tabular} & & & & OFFSITE & SESPMNT & $\mathrm{R}$ \\
\hline \begin{tabular}{|l|l|}
4027 \\
\end{tabular} & & & & OFFSITE & SESPMNT & $\mathrm{R}$ \\
\hline 4028 & & & & PERIMETER & SESPMNT & $\mathrm{R}$ \\
\hline 4029 & & & & PERIMETER & SESPMNT & $\mathrm{R}$ \\
\hline 4030 & & & & PERIMETER & SESPMNT & $\mathrm{R}$ \\
\hline 4031 & & & & ONSITE & SESPMNT & $\mathrm{R}$ \\
\hline 4032 & & & & ONSITE & SESPMNT & $\mathrm{R}$ \\
\hline 4033 & & & & ONSITE & SESPMNT & $\mathrm{R}$ \\
\hline 4034 & & & & ONSITE & SESPMNT & $\mathrm{R}$ \\
\hline 4035 & & & & ONSITE & SESPMNT & $\mathrm{R}$ \\
\hline \begin{tabular}{|c|}
4036 \\
\end{tabular} & & & & ONSITE & SESPMNT & $\mathrm{R}$ \\
\hline 4037 & & & & ONSITE & SESPMNT & $\mathrm{R}$ \\
\hline 4038 & & & & ONSITE & SESPMNT & $\mathrm{R}$ \\
\hline \begin{tabular}{|c|}
4039 \\
\end{tabular} & & & & OFFSITE & SESPMNT & $\mathrm{R}$ \\
\hline 4040 & & & & OFFSITE & SESPMNT & $\mathrm{R}$ \\
\hline 4041 & & & & OFFSITE & SESPSPEC & $\mathrm{s}$ \\
\hline 4042 & & & & OFFSITE & SESPSPEC & $\mathrm{s}$ \\
\hline 4043 & & & & OFFSITE & SESPSPEC & $\mathrm{s}$ \\
\hline 4044 & & & & OFFSITE & SESPSPEC & $\mathrm{s}$ \\
\hline 4045 & & & & OFFSITE & SESPSPEC & $\mathrm{s}$ \\
\hline \begin{tabular}{|l|}
4046 \\
\end{tabular} & & & & OFFSITE & SESPSPEC & $\mathrm{s}$ \\
\hline 4047 & & & & ONSITE & SESPMNT & $\mathrm{R}$ \\
\hline 4048 & & & & ONSITE & SESPMNT & $\mathrm{R}$ \\
\hline 4049 & & & & ONSITE & SESPMNT & $\mathrm{R}$ \\
\hline 4050 & & & & ONSITE & SESPMNT & $\mathrm{R}$ \\
\hline \begin{tabular}{|c|}
4051 \\
\end{tabular} & & & & ONSITE & SESPMNT & $\mathrm{R}$ \\
\hline 4052 & & & & ONSITE & SESPMNT & $\mathrm{R}$ \\
\hline 4053 & See sample record for comment. & & & OFFSITE & SESPMNT & $\mathrm{R}$ \\
\hline 4054 & See sample record for comment. & & & OFFSITE & SESPMNT & $\mathrm{R}$ \\
\hline 4055 & See sample record for comment. & & & OFFSITE & SESPMNT & $\mathrm{R}$ \\
\hline \begin{tabular}{|c|}
4056 \\
\end{tabular} & See sample record for comment. & & & OFFSITE & SESPMNT & $\mathrm{R}$ \\
\hline \begin{tabular}{|l|}
4057 \\
\end{tabular} & & & & OFFSITE & SESBMNT & ${ }_{2} R$ \\
\hline
\end{tabular}




\begin{tabular}{|c|c|c|c|c|c|c|}
\hline & $\mathrm{AD}$ & $\mathrm{AE}$ & $\mathrm{AF}$ & $A G$ & $\mathrm{AH}$ & $\mathrm{Al}$ \\
\hline 1 & RESULT_COMMENT & COLL_SAMP_SIZE & COLL_SAMP_SIZE_UNITS & DIST_CLASS & OWNER_ID & COLLECTION_PURPOSE \\
\hline 4058 & & & & OFFSITE & SESPMNT & $R$ \\
\hline 4059 & & & & OFFSITE & SESPMNT & $\mathrm{R}$ \\
\hline 4060 & See sample record for comment. & & & OFFSITE & SESPMNT & $\mathrm{R}$ \\
\hline 4061 & See sample record for comment. & & & OFFSITE & SESPMNT & $\mathrm{R}$ \\
\hline 4062 & & & & OFFSITE & SESPMNT & $\mathrm{R}$ \\
\hline 4063 & & & & OFFSITE & SESPMNT & $\mathrm{R}$ \\
\hline 4064 & & & & OFFSITE & SESPMNT & $\mathrm{R}$ \\
\hline 4065 & & & & ONSITE & SESPMNT & $R$ \\
\hline 4066 & & & & ONSITE & SESPMNT & $\mathrm{R}$ \\
\hline 4067 & & & & ONSITE & SESPMNT & $\mathrm{R}$ \\
\hline 4068 & & & & ONSITE & SESPMNT & $\mathrm{R}$ \\
\hline 4069 & & & & ONSITE & SESPMNT & $\mathrm{R}$ \\
\hline 4070 & & & & OFFSITE & SESPMNT & $\mathrm{R}$ \\
\hline 4071 & & & & OFFSITE & SESPMNT & $\mathrm{R}$ \\
\hline 4072 & & & & ONSITE & SESPMNT & $\mathrm{R}$ \\
\hline 4073 & & & & ONSITE & SESPMNT & $\mathrm{R}$ \\
\hline 4074 & & & & ONSITE & SESPMNT & $\mathrm{R}$ \\
\hline 4075 & & & & ONSITE & SESPMNT & $\mathrm{R}$ \\
\hline 4076 & & & & ONSITE & SESPMNT & $\mathrm{R}$ \\
\hline 4077 & & & & ONSITE & SESPMNT & $\mathrm{R}$ \\
\hline 4078 & & & & ONSITE & SESPMNT & $\mathrm{R}$ \\
\hline 4079 & & & & OFFSITE & SESPMNT & $\mathrm{R}$ \\
\hline 4080 & & & & OFFSITE & SESPMNT & $\mathrm{R}$ \\
\hline 4081 & & & & OFFSITE & SESPMNT & $\mathrm{R}$ \\
\hline 4082 & & & & OFFSITE & SESPMNT & $\mathrm{R}$ \\
\hline 4083 & & & & OFFSITE & SESPMNT & $\mathrm{R}$ \\
\hline 4084 & & & & OFFSITE & SESPMNT & $\mathrm{R}$ \\
\hline 4085 & & & & OFFSITE & SESPMNT & $\mathrm{R}$ \\
\hline 4086 & & & & OFFSITE & SESPMNT & $\mathrm{R}$ \\
\hline 4087 & & & & ONSITE & SESPMNT & $\mathrm{R}$ \\
\hline 4088 & & & & ONSITE & SESPMNT & $\mathrm{R}$ \\
\hline 4089 & & & & ONSITE & SESPMNT & $\mathrm{R}$ \\
\hline 4090 & & & & ONSITE & SESPMNT & $\mathrm{R}$ \\
\hline 4091 & & & & ONSITE & SESPMNT & $\mathrm{R}$ \\
\hline 4092 & & & & & SESPMNT & $\mathrm{R}$ \\
\hline 4093 & & & & & SESPMNT & $\mathrm{R}$ \\
\hline 4094 & & & & & SESPMNT & $\mathrm{R}$ \\
\hline 4095 & & & & ONSITE & SESPMNT & $\mathrm{R}$ \\
\hline 4096 & & & & ONSITE & SESPMNT & $\mathrm{R}$ \\
\hline 4097 & & & & ONSITE & SESPMNT & $\mathrm{R}$ \\
\hline 4098 & & & & ONSITE & SESPMNT & $\mathrm{R}$ \\
\hline 4099 & & & & ONSITE & SESPMNT & $\mathrm{R}$ \\
\hline 4100 & & & & ONSITE & SESPMNT & $\mathrm{R}$ \\
\hline 4101 & & & & ONSITE & SESPMNT & $\mathrm{R}$ \\
\hline 4102 & & & & ONSITE & SESPMNT & $\mathrm{R}$ \\
\hline 4103 & See sample record for comment. & & & OFFSITE & SESPMNT & $\mathrm{R}$ \\
\hline 4104 & See sample record for comment. & & & OFFSITE & SESPMNT & $\mathrm{R}$ \\
\hline 4105 & & & & ONSITE & SESPMNT & $\mathrm{R}$ \\
\hline 4106 & & & & ONSITE & SESPMNT & $\mathrm{R}$ \\
\hline 4107 & & & & ONSITE & SESPMNT & $\mathrm{R}$ \\
\hline 4108 & & & & ONSITE & SESPMNT & $\mathrm{R}$ \\
\hline 4109 & & & & ONSITE & SESBMNT & $R$ \\
\hline
\end{tabular}




\begin{tabular}{|c|c|c|c|c|c|c|}
\hline & $A D$ & $\mathrm{AE}$ & $\mathrm{AF}$ & $A G$ & $\mathrm{AH}$ & $\mathrm{Al}$ \\
\hline 1 & RESULT_COMMENT & COLL_SAMP_SIZE & COLL_SAMP_SIZE_UNITS & DIST_CLASS & OWNER_ID & COLLECTION_PURPOSE \\
\hline 4110 & & & & OFFSITE & SESPMNT & $\mathrm{R}$ \\
\hline \begin{tabular}{|l|}
4111 \\
\end{tabular} & & & & OFFSITE & SESPMNT & $\mathrm{R}$ \\
\hline \begin{tabular}{|l|}
4112 \\
\end{tabular} & & & & OFFSITE & SESPMNT & $\mathrm{R}$ \\
\hline 4113 & & & & OFFSITE & SESPMNT & $\mathrm{R}$ \\
\hline \begin{tabular}{|l|l|}
4114 \\
\end{tabular} & & & & OFFSITE & SESPMNT & $\mathrm{R}$ \\
\hline \begin{tabular}{|l|l|l|}
4115 \\
\end{tabular} & & & & OFFSITE & SESPMNT & $\mathrm{R}$ \\
\hline \begin{tabular}{|l|}
4116 \\
\end{tabular} & & & & OFFSITE & SESPMNT & $\mathrm{R}$ \\
\hline \begin{tabular}{|l|l|}
4117 \\
\end{tabular} & & & & OFFSITE & SESPMNT & $\mathrm{R}$ \\
\hline \begin{tabular}{|l|}
4118 \\
\end{tabular} & & & & PERIMETER & SESPMNT & $\mathrm{R}$ \\
\hline \begin{tabular}{|l|}
4119 \\
\end{tabular} & & & & PERIMETER & SESPMNT & $\mathrm{R}$ \\
\hline 4120 & & & & PERIMETER & SESPMNT & $\mathrm{R}$ \\
\hline \begin{tabular}{|l|}
4121 \\
\end{tabular} & & & & ONSITE & SESPMNT & $\mathrm{R}$ \\
\hline 4122 & & & & ONSITE & SESPMNT & $\mathrm{R}$ \\
\hline \begin{tabular}{|l|l|}
4123 \\
\end{tabular} & & & & ONSITE & SESPMNT & $\mathrm{R}$ \\
\hline \begin{tabular}{|l|l|}
4124 \\
\end{tabular} & & & & ONSITE & SESPMNT & $\mathrm{R}$ \\
\hline \begin{tabular}{|l|l|}
4125 \\
\end{tabular} & & & & ONSITE & SESPMNT & $\mathrm{R}$ \\
\hline \begin{tabular}{|l|l|}
4126 \\
\end{tabular} & & & & ONSITE & SESPMNT & $\mathrm{R}$ \\
\hline \begin{tabular}{|l|}
4127 \\
\end{tabular} & & & & ONSITE & SESPMNT & $\mathrm{R}$ \\
\hline \begin{tabular}{|l|l|}
4128 \\
\end{tabular} & See sample record for comment. & & & ONSITE & SESPMNT & $\mathrm{R}$ \\
\hline \begin{tabular}{|l|l|}
4129 \\
\end{tabular} & & & & OFFSITE & SESPMNT & $\mathrm{R}$ \\
\hline 4130 & & & & OFFSITE & SESPMNT & $\mathrm{R}$ \\
\hline \begin{tabular}{|l|l|}
4131 \\
\end{tabular} & See sample record for comment. & & & ONSITE & SESPMNT & $\mathrm{R}$ \\
\hline 4132 & See sample record for comment. & & & ONSITE & SESPMNT & $\mathrm{R}$ \\
\hline 4133 & See sample record for comment. & & & ONSITE & SESPMNT & $\mathrm{R}$ \\
\hline \begin{tabular}{|l|}
4134 \\
\end{tabular} & See sample record for comment. & & & ONSITE & SESPMNT & $\mathrm{R}$ \\
\hline \begin{tabular}{|l|l|l|l}
4135 \\
\end{tabular} & & & & ONSITE & SESPMNT & $\mathrm{R}$ \\
\hline \begin{tabular}{|l|l|}
4136 \\
\end{tabular} & & & & ONSITE & SESPMNT & $\mathrm{R}$ \\
\hline \begin{tabular}{|l|l|}
4137 \\
\end{tabular} & & & & OFFSITE & SESPMNT & $\mathrm{R}$ \\
\hline \begin{tabular}{|l|l|}
4138 \\
\end{tabular} & & & & OFFSITE & SESPMNT & $\mathrm{R}$ \\
\hline \begin{tabular}{|l|l|}
4139 \\
\end{tabular} & & & & OFFSITE & SESPMNT & $\mathrm{R}$ \\
\hline \begin{tabular}{|l|}
4140 \\
\end{tabular} & & & & OFFSITE & SESPMNT & $\mathrm{R}$ \\
\hline \begin{tabular}{|l|l|}
4141 \\
\end{tabular} & & & & OFFSITE & SESPMNT & $\mathrm{R}$ \\
\hline \begin{tabular}{|l|l|l|}
4142 \\
\end{tabular} & & & & OFFSITE & SESPMNT & $\mathrm{R}$ \\
\hline \begin{tabular}{|l|l|l|l}
4143 \\
\end{tabular} & & & & OFFSITE & SESPMNT & $\mathrm{R}$ \\
\hline \begin{tabular}{|l|l|}
4144 \\
\end{tabular} & & & & OFFSITE & SESPMNT & $\mathrm{R}$ \\
\hline \begin{tabular}{|l|l|l|l|}
4145 \\
\end{tabular} & & & & OFFSITE & SESPMNT & $\mathrm{R}$ \\
\hline \begin{tabular}{|l|l|}
4146 \\
\end{tabular} & & & & OFFSITE & SESPMNT & $\mathrm{R}$ \\
\hline \begin{tabular}{|l|l|}
4147 \\
\end{tabular} & & & & OFFSITE & SESPMNT & $\mathrm{R}$ \\
\hline \begin{tabular}{|l|l|l|l}
4148 \\
\end{tabular} & & & & OFFSITE & SESPMNT & $\mathrm{R}$ \\
\hline \begin{tabular}{|l|l|l|l|}
4149 \\
\end{tabular} & & & & ONSITE & SESPMNT & $\mathrm{R}$ \\
\hline 4150 & & & & ONSITE & SESPMNT & $\mathrm{R}$ \\
\hline \begin{tabular}{|l|l|}
4151 \\
\end{tabular} & & & & ONSITE & SESPMNT & $\mathrm{R}$ \\
\hline \begin{tabular}{|l|l|}
4152 \\
\end{tabular} & & & & ONSITE & SESPMNT & $\mathrm{R}$ \\
\hline \begin{tabular}{|l|}
4153 \\
\end{tabular} & & & & ONSITE & SESPMNT & $\mathrm{R}$ \\
\hline \begin{tabular}{|l|l|}
4154 \\
\end{tabular} & & & & OFFSITE & SESPMNT & $\mathrm{R}$ \\
\hline \begin{tabular}{|l|l|l|l|}
4155 \\
\end{tabular} & & & & OFFSITE & SESPMNT & $\mathrm{R}$ \\
\hline \begin{tabular}{|l|l|l|l|l|}
4156 \\
\end{tabular} & & & & ONSITE & SESPMNT & $\mathrm{R}$ \\
\hline \begin{tabular}{|l|l|}
4157 \\
\end{tabular} & & & & ONSITE & SESPMNT & $\mathrm{R}$ \\
\hline \begin{tabular}{|l|}
4158 \\
\end{tabular} & & & & ONSITE & SESPMNT & $\mathrm{R}$ \\
\hline \begin{tabular}{|l|l|}
4159 \\
\end{tabular} & & & & ONSITE & SESPMNT & $\mathrm{R}$ \\
\hline \begin{tabular}{|l|}
4160 \\
\end{tabular} & & & & ONSITE & SESPMNT & $\mathrm{R}$ \\
\hline \begin{tabular}{|l|}
4161 \\
\end{tabular} & & & & ONSITE & SESBMNT & $R$ \\
\hline
\end{tabular}




\begin{tabular}{|c|c|c|c|c|c|c|}
\hline & $\mathrm{AD}$ & $\mathrm{AE}$ & $\mathrm{AF}$ & $\mathrm{AG}$ & $\mathrm{AH}$ & $\mathrm{Al}$ \\
\hline 1 & RESULT_COMMENT & COLL_SAMP_SIZE & COLL_SAMP_SIZE_UNITS & DIST_CLASS & OWNER_ID & COLLECTION_PURPOSE \\
\hline 4162 & & & & ONSITE & SESPMNT & $\mathrm{R}$ \\
\hline 4163 & See sample record for comment. & & & OFFSITE & SESPMNT & $\mathrm{R}$ \\
\hline 4164 & See sample record for comment. & & & OFFSITE & SESPMNT & $\mathrm{R}$ \\
\hline 4165 & & & & OFFSITE & SESPMNT & $\mathrm{R}$ \\
\hline 4166 & & & & OFFSITE & SESPMNT & $\mathrm{R}$ \\
\hline 4167 & & & & OFFSITE & SESPMNT & $\mathrm{R}$ \\
\hline 4168 & & & & OFFSITE & SESPMNT & $\mathrm{R}$ \\
\hline 4169 & & & & OFFSITE & SESPMNT & $\mathrm{R}$ \\
\hline 4170 & & & & OFFSITE & SESPMNT & $\mathrm{R}$ \\
\hline 4171 & & & & ONSITE & SESPMNT & $\mathrm{R}$ \\
\hline 4172 & & & & ONSITE & SESPMNT & $\mathrm{R}$ \\
\hline 4173 & & & & ONSITE & SESPMNT & $\mathrm{R}$ \\
\hline 4174 & & & & ONSITE & SESPMNT & $\mathrm{R}$ \\
\hline 4175 & & & & ONSITE & SESPMNT & $\mathrm{R}$ \\
\hline 4176 & & & & ONSITE & SESPMNT & $\mathrm{R}$ \\
\hline 4177 & & & & ONSITE & SESPMNT & $\mathrm{R}$ \\
\hline 4178 & & & & ONSITE & SESPMNT & $\mathrm{R}$ \\
\hline 4179 & & & & ONSITE & SESPMNT & $\mathrm{R}$ \\
\hline 4180 & & & & ONSITE & SESPMNT & $\mathrm{R}$ \\
\hline 4181 & & & & ONSITE & SESPMNT & $\mathrm{R}$ \\
\hline 4182 & & & & & SESPMNT & $\mathrm{R}$ \\
\hline 4183 & & & & & SESPMNT & $\mathrm{R}$ \\
\hline 4184 & & & & & SESPMNT & $\mathrm{R}$ \\
\hline 4185 & & & & ONSITE & SESPMNT & $\mathrm{R}$ \\
\hline \begin{tabular}{|l|}
4186 \\
\end{tabular} & & & & ONSITE & SESPMNT & $R$ \\
\hline \begin{tabular}{|l|}
4187 \\
\end{tabular} & & & & ONSITE & SESPMNT & $R$ \\
\hline \begin{tabular}{|l|l|}
4188 \\
\end{tabular} & & & & ONSITE & SESPMNT & $R$ \\
\hline \begin{tabular}{|l|l|}
4189 \\
\end{tabular} & & & & ONSITE & SESPMNT & $\mathrm{R}$ \\
\hline \begin{tabular}{|l|}
4190 \\
\end{tabular} & & & & OFFSITE & SESPMNT & $\mathrm{R}$ \\
\hline \begin{tabular}{|l|}
4191 \\
\end{tabular} & & & & OFFSITE & SESPMNT & $R$ \\
\hline \begin{tabular}{|l|}
4192 \\
\end{tabular} & & & & ONSITE & SESPMNT & $\mathrm{R}$ \\
\hline \begin{tabular}{|l|l|}
4193 \\
\end{tabular} & & & & ONSITE & SESPMNT & $\mathrm{R}$ \\
\hline \begin{tabular}{|l|l|}
4194 \\
\end{tabular} & & & & ONSITE & SESPMNT & $R$ \\
\hline \begin{tabular}{|l|l|}
4195 \\
\end{tabular} & & & & ONSITE & SESPMNT & $\mathrm{R}$ \\
\hline \begin{tabular}{|l|}
4196 \\
\end{tabular} & & & & ONSITE & SESPMNT & $\mathrm{R}$ \\
\hline \begin{tabular}{|l|l|}
4197 \\
\end{tabular} & & & & OFFSITE & SESPMNT & $\mathrm{R}$ \\
\hline \begin{tabular}{|l|}
4198 \\
\end{tabular} & & & & OFFSITE & SESPMNT & $R$ \\
\hline \begin{tabular}{|l|}
4199 \\
\end{tabular} & & & & OFFSITE & SESPMNT & $R$ \\
\hline \begin{tabular}{|l|}
4200 \\
\end{tabular} & & & & OFFSITE & SESPMNT & $R$ \\
\hline \begin{tabular}{|l|}
4201 \\
\end{tabular} & & & & OFFSITE & SESPMNT & $R$ \\
\hline \begin{tabular}{|l|}
4202 \\
\end{tabular} & & & & OFFSITE & SESPMNT & $R$ \\
\hline \begin{tabular}{|l|l|}
4203 \\
\end{tabular} & & & & OFFSITE & SESPMNT & $\mathrm{R}$ \\
\hline 4204 & & & & OFFSITE & SESPMNT & $\mathrm{R}$ \\
\hline \begin{tabular}{|l|l|}
4205 \\
\end{tabular} & & & & PERIMETER & SESPMNT & $R$ \\
\hline \begin{tabular}{|l|}
4206 \\
\end{tabular} & & & & PERIMETER & SESPMNT & $R$ \\
\hline \begin{tabular}{|l|}
4207 \\
\end{tabular} & & & & PERIMETER & SESPMNT & $R$ \\
\hline \begin{tabular}{|l|}
4208 \\
\end{tabular} & & & & ONSITE & SESPMNT & $\mathrm{R}$ \\
\hline \begin{tabular}{|l|}
4209 \\
\end{tabular} & & & & ONSITE & SESPMNT & $\mathrm{R}$ \\
\hline 4210 & & & & ONSITE & SESPMNT & $\mathrm{R}$ \\
\hline \begin{tabular}{|l|}
4211 \\
\end{tabular} & & & & ONSITE & SESPMNT & $\mathrm{R}$ \\
\hline \begin{tabular}{|l|}
4212 \\
\end{tabular} & & & & ONSITE & SESPMNT & $R$ \\
\hline \begin{tabular}{|l|}
4213 \\
\end{tabular} & & & & ONSITE & SESBMNT & $R$ \\
\hline
\end{tabular}




\begin{tabular}{|c|c|c|c|c|c|c|}
\hline & $A D$ & $\mathrm{AE}$ & $\mathrm{AF}$ & $A G$ & $\mathrm{AH}$ & $\mathrm{Al}$ \\
\hline 1 & RESULT_COMMENT & COLL_SAMP_SIZE & COLL_SAMP_SIZE_UNITS & DIST_CLASS & OWNER_ID & COLLECTION_PURPOSE \\
\hline \begin{tabular}{|l|}
4214 \\
\end{tabular} & & & & ONSITE & SESPMNT & $\mathrm{R}$ \\
\hline \begin{tabular}{|l|}
4215 \\
\end{tabular} & & & & ONSITE & SESPMNT & $\mathrm{R}$ \\
\hline \begin{tabular}{|l|l|l|l|l|l|}
4216 \\
\end{tabular} & See sample record for comment. & & & OFFSITE & SESPMNT & $\mathrm{R}$ \\
\hline \begin{tabular}{|l|l|}
4217 \\
\end{tabular} & See sample record for comment. & & & OFFSITE & SESPMNT & $\mathrm{R}$ \\
\hline \begin{tabular}{|l|}
4218 \\
\end{tabular} & & & & OFFSITE & SESPSPEC & $\mathrm{s}$ \\
\hline \begin{tabular}{|l|}
4219 \\
\end{tabular} & & & & OFFSITE & SESPSPEC & $\mathrm{s}$ \\
\hline \begin{tabular}{|l|}
4220 \\
\end{tabular} & & & & OFFSITE & SESPSPEC & $\mathrm{s}$ \\
\hline \begin{tabular}{|l|}
4221 \\
\end{tabular} & & & & OFFSITE & SESPSPEC & $\mathrm{s}$ \\
\hline 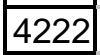 & & & & OFFSITE & SESPSPEC & $\mathrm{s}$ \\
\hline 4223 & & & & OFFSITE & SESPSPEC & $\mathrm{S}$ \\
\hline \begin{tabular}{|c|}
4224 \\
\end{tabular} & & & & ONSITE & SESPMNT & $\mathrm{R}$ \\
\hline \begin{tabular}{|l|l|l|l}
4225 \\
\end{tabular} & & & & ONSITE & SESPMNT & $\mathrm{R}$ \\
\hline \begin{tabular}{|l|l|}
4226 \\
\end{tabular} & & & & ONSITE & SESPMNT & $\mathrm{R}$ \\
\hline \begin{tabular}{|l|l|}
4227 \\
\end{tabular} & & & & ONSITE & SESPMNT & $\mathrm{R}$ \\
\hline \begin{tabular}{|l|}
4228 \\
\end{tabular}$-100$ & & & & ONSITE & SESPMNT & $\mathrm{R}$ \\
\hline \begin{tabular}{|l|l|}
4229 \\
\end{tabular} & & & & ONSITE & SESPMNT & $\mathrm{R}$ \\
\hline 4230 & & & & OFFSITE & SESPMNT & $\mathrm{R}$ \\
\hline \begin{tabular}{|l|}
4231 \\
\end{tabular} & & & & OFFSITE & SESPMNT & $\mathrm{R}$ \\
\hline 4232 & & & & OFFSITE & SESPMNT & $\mathrm{R}$ \\
\hline \begin{tabular}{|l|l|}
4233 \\
\end{tabular} & & & & OFFSITE & SESPMNT & $\mathrm{R}$ \\
\hline \begin{tabular}{|l|l|}
4234 \\
\end{tabular} & & & & OFFSITE & SESPMNT & $\mathrm{R}$ \\
\hline 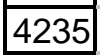 & & & & OFFSITE & SESPMNT & $\mathrm{R}$ \\
\hline \begin{tabular}{|l|}
4236 \\
\end{tabular} & & & & OFFSITE & SESPMNT & $\mathrm{R}$ \\
\hline \begin{tabular}{|l|l|}
4237 \\
\end{tabular} & & & & OFFSITE & SESPMNT & $\mathrm{R}$ \\
\hline \begin{tabular}{|l|}
4238 \\
\end{tabular} & & & & OFFSITE & SESPMNT & $\mathrm{R}$ \\
\hline \begin{tabular}{|l|}
4239 \\
\end{tabular} & & & & OFFSITE & SESPMNT & $\mathrm{R}$ \\
\hline \begin{tabular}{|l|l|}
4240 \\
\end{tabular} & & & & OFFSITE & SESPMNT & $\mathrm{R}$ \\
\hline \begin{tabular}{|l|}
4241 \\
\end{tabular} & & & & OFFSITE & SESPMNT & $\mathrm{R}$ \\
\hline \begin{tabular}{|l|}
4242 \\
\end{tabular} & & & & ONSITE & SESPMNT & $\mathrm{R}$ \\
\hline \begin{tabular}{|l|}
4243 \\
\end{tabular} & & & & ONSITE & SESPMNT & $\mathrm{R}$ \\
\hline \begin{tabular}{|l|}
4244 \\
\end{tabular} & & & & ONSITE & SESPMNT & $\mathrm{R}$ \\
\hline \begin{tabular}{|l|}
4245 \\
\end{tabular} & & & & ONSITE & SESPMNT & $\mathrm{R}$ \\
\hline \begin{tabular}{|l|}
4246 \\
\end{tabular} & & & & ONSITE & SESPMNT & $\mathrm{R}$ \\
\hline \begin{tabular}{|l|}
4247 \\
\end{tabular} & & & & OFFSITE & SESPMNT & $\mathrm{R}$ \\
\hline \begin{tabular}{|l|l|}
4248 \\
\end{tabular} & & & & OFFSITE & SESPMNT & $\mathrm{R}$ \\
\hline \begin{tabular}{|l|}
4249 \\
\end{tabular} & & & & ONSITE & SESPMNT & $\mathrm{R}$ \\
\hline \begin{tabular}{|l|}
4250 \\
\end{tabular} & & & & ONSITE & SESPMNT & $\mathrm{R}$ \\
\hline \begin{tabular}{|l|}
4251 \\
\end{tabular} & & & & ONSITE & SESPMNT & $R$ \\
\hline \begin{tabular}{|l|}
4252 \\
\end{tabular} & & & & ONSITE & SESPMNT & $\mathrm{R}$ \\
\hline \begin{tabular}{|l|}
4253 \\
\end{tabular} & & & & ONSITE & SESPMNT & $\mathrm{R}$ \\
\hline \begin{tabular}{|l|l|}
4254 \\
\end{tabular} & & & & ONSITE & SESPMNT & $\mathrm{R}$ \\
\hline \begin{tabular}{|l|}
4255 \\
\end{tabular} & & & & ONSITE & SESPMNT & $\mathrm{R}$ \\
\hline \begin{tabular}{|l|}
4256 \\
\end{tabular} & & & & ONSITE & SESPMNT & $\mathrm{R}$ \\
\hline \begin{tabular}{|l|}
4257 \\
\end{tabular} & & & & ONSITE & SESPMNT & $\mathrm{R}$ \\
\hline \begin{tabular}{|l|}
4258 \\
\end{tabular} & & & & ONSITE & SESPMNT & $R$ \\
\hline \begin{tabular}{|l|}
4259 \\
\end{tabular} & & & & OFFSITE & SESPMNT & $\mathrm{R}$ \\
\hline \begin{tabular}{|l|}
4260 \\
\end{tabular} & & & & OFFSITE & SESPMNT & $\mathrm{R}$ \\
\hline \begin{tabular}{|l|l|}
4261 \\
\end{tabular} & & & & OFFSITE & SESPMNT & $\mathrm{R}$ \\
\hline \begin{tabular}{|l|}
4262 \\
\end{tabular} & & & & OFFSITE & SESPMNT & $\mathrm{R}$ \\
\hline \begin{tabular}{|l|}
4263 \\
\end{tabular} & & & & OFFSITE & SESPMNT & $\mathrm{R}$ \\
\hline \begin{tabular}{|l|}
4264 \\
\end{tabular} & & & & OFFSITE & SESPMNT & $\mathrm{R}$ \\
\hline \begin{tabular}{|l|}
4265 \\
\end{tabular} & & & & OFFSITE & SESBMNT & $R$ \\
\hline
\end{tabular}




\begin{tabular}{|c|c|c|c|c|c|c|}
\hline & $\mathrm{AD}$ & $\mathrm{AE}$ & AF & AG & $\mathrm{AH}$ & $\mathrm{Al}$ \\
\hline 1 & RESULT_COMMENT & COLL_SAMP_SIZE & COLL_SAMP_SIZE_UNITS & DIST_CLASS & OWNER_ID & COLLECTION_PURPOSE \\
\hline 4266 & & & & OFFSITE & SESPMNT & $\mathrm{R}$ \\
\hline 4267 & See sample record for comment. & & & ONSITE & SESPMNT & $\mathrm{R}$ \\
\hline 4268 & & & & ONSITE & SESPMNT & $\mathrm{R}$ \\
\hline 4269 & & & & ONSITE & SESPMNT & $\mathrm{R}$ \\
\hline 4270 & & & & ONSITE & SESPMNT & $\mathrm{R}$ \\
\hline 4271 & & & & ONSITE & SESPMNT & $\mathrm{R}$ \\
\hline 4272 & & & & ONSITE & SESPMNT & $\mathrm{R}$ \\
\hline 4273 & & & & & SESPMNT & $\mathrm{R}$ \\
\hline 4274 & & & & & SESPMNT & $\mathrm{R}$ \\
\hline 4275 & & & & & SESPMNT & $\mathrm{R}$ \\
\hline 4276 & & & & ONSITE & SESPMNT & $\mathrm{R}$ \\
\hline 4277 & & & & ONSITE & SESPMNT & $\mathrm{R}$ \\
\hline 4278 & & & & ONSITE & SESPMNT & $\mathrm{R}$ \\
\hline 4279 & & & & ONSITE & SESPMNT & $\mathrm{R}$ \\
\hline 4280 & & & & ONSITE & SESPMNT & $\mathrm{R}$ \\
\hline 4281 & & & & OFFSITE & SESPMNT & $\mathrm{R}$ \\
\hline \begin{tabular}{|c|c|}
4282 & -10 \\
\end{tabular} & & & & OFFSITE & SESPMNT & $\mathrm{R}$ \\
\hline 4283 & & & & ONSITE & SESPMNT & $\mathrm{R}$ \\
\hline 4284 & & & & ONSITE & SESPMNT & $\mathrm{R}$ \\
\hline 4285 & & & & ONSITE & SESPMNT & $\mathrm{R}$ \\
\hline 4286 & & & & ONSITE & SESPMNT & $\mathrm{R}$ \\
\hline 4287 & & & & ONSITE & SESPMNT & $\mathrm{R}$ \\
\hline 4288 & & & & OFFSITE & SESPMNT & $\mathrm{R}$ \\
\hline 4289 & & & & OFFSITE & SESPMNT & $\mathrm{R}$ \\
\hline 4290 & & & & OFFSITE & SESPMNT & $\mathrm{R}$ \\
\hline \begin{tabular}{|c|}
4291 \\
\end{tabular} & & & & OFFSITE & SESPMNT & $\mathrm{R}$ \\
\hline \begin{tabular}{|c|}
4292 \\
\end{tabular} & & & & OFFSITE & SESPMNT & $\mathrm{R}$ \\
\hline \begin{tabular}{|c|}
4293 \\
\end{tabular} & & & & OFFSITE & SESPMNT & $\mathrm{R}$ \\
\hline 4294 & & & & OFFSITE & SESPMNT & $\mathrm{R}$ \\
\hline \begin{tabular}{|c|c|}
4295 \\
\end{tabular} & & & & OFFSITE & SESPMNT & $\mathrm{R}$ \\
\hline 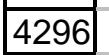 & & & & PERIMETER & SESPMNT & $\mathrm{R}$ \\
\hline 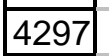 & & & & PERIMETER & SESPMNT & $\mathrm{R}$ \\
\hline 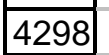 & & & & PERIMETER & SESPMNT & $\mathrm{R}$ \\
\hline \begin{tabular}{|c|}
4299 \\
\end{tabular} & & & & ONSITE & SESPMNT & $\mathrm{R}$ \\
\hline 4300 & & & & ONSITE & SESPMNT & $\mathrm{R}$ \\
\hline 4301 & & & & ONSITE & SESPMNT & $\mathrm{R}$ \\
\hline 4302 & & & & ONSITE & SESPMNT & $\mathrm{R}$ \\
\hline 4303 & & & & ONSITE & SESPMNT & $\mathrm{R}$ \\
\hline \begin{tabular}{|l|l|}
4304 \\
\end{tabular} & & & & ONSITE & SESPMNT & $\mathrm{R}$ \\
\hline $4305 \leq$ & See sample record for comment. & & & ONSITE & SESPMNT & $\mathrm{R}$ \\
\hline \begin{tabular}{|l|l|}
4306 & 5 \\
\end{tabular} & See sample record for comment. & & & ONSITE & SESPMNT & $\mathrm{R}$ \\
\hline \begin{tabular}{|l|l|}
4307 \\
\end{tabular} & & & & OFFSITE & SESPMNT & $\mathrm{R}$ \\
\hline \begin{tabular}{|l|l|}
4308 \\
\end{tabular} & & & & OFFSITE & SESPMNT & $\mathrm{R}$ \\
\hline \begin{tabular}{|l|l|}
4309 \\
\end{tabular} & & & & ONSITE & SESPMNT & $\mathrm{R}$ \\
\hline \begin{tabular}{|l|}
4310 \\
\end{tabular} & & & & ONSITE & SESPMNT & $\mathrm{R}$ \\
\hline \begin{tabular}{|l|l|}
4311 \\
\end{tabular} & & & & ONSITE & SESPMNT & $\mathrm{R}$ \\
\hline \begin{tabular}{|l|}
4312 \\
\end{tabular} & & & & ONSITE & SESPMNT & $\mathrm{R}$ \\
\hline \begin{tabular}{|l|l|}
4313 \\
\end{tabular} & & & & ONSITE & SESPMNT & $\mathrm{R}$ \\
\hline \begin{tabular}{|l|}
4314 \\
\end{tabular} & & & & OFFSITE & SESPMNT & $\mathrm{R}$ \\
\hline \begin{tabular}{|l|l|l|l|}
4315 \\
\end{tabular} & & & & OFFSITE & SESPMNT & $\mathrm{R}$ \\
\hline \begin{tabular}{|l|l|l|}
4316 \\
\end{tabular} & & & & OFFSITE & SESPMNT & $\mathrm{R}$ \\
\hline \begin{tabular}{|l|}
4317 \\
\end{tabular} & & & & OFFSITE & SESBMNT & $\mathrm{R}$ \\
\hline
\end{tabular}




\begin{tabular}{|c|c|c|c|c|c|c|}
\hline & $A D$ & $\mathrm{AE}$ & $\mathrm{AF}$ & $A G$ & $\mathrm{AH}$ & $\mathrm{Al}$ \\
\hline 1 & RESULT_COMMENT & COLL_SAMP_SIZE & COLL_SAMP_SIZE_UNITS & DIST_CLASS & OWNER_ID & COLLECTION_PURPOSE \\
\hline \begin{tabular}{|l|}
4318 \\
\end{tabular} & & & & OFFSITE & SESPMNT & $\mathrm{R}$ \\
\hline 4319 & & & & OFFSITE & SESPMNT & $\mathrm{R}$ \\
\hline 4320 & & & & OFFSITE & SESPMNT & $\mathrm{R}$ \\
\hline \begin{tabular}{|l|}
4321 \\
\end{tabular} & & & & OFFSITE & SESPMNT & $\mathrm{R}$ \\
\hline 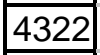 & & & & OFFSITE & SESPMNT & $\mathrm{R}$ \\
\hline \begin{tabular}{|l|}
4323 \\
\end{tabular} & & & & OFFSITE & SESPMNT & $\mathrm{R}$ \\
\hline \begin{tabular}{|l|}
4324 \\
\end{tabular} & & & & OFFSITE & SESPMNT & $\mathrm{R}$ \\
\hline \begin{tabular}{|l|}
4325 \\
\end{tabular}$-\operatorname{ra}$ & & & & ONSITE & SESPMNT & $\mathrm{R}$ \\
\hline \begin{tabular}{|l|l}
4326 \\
\end{tabular} & & & & ONSITE & SESPMNT & $\mathrm{R}$ \\
\hline \begin{tabular}{|l|l|}
4327 \\
\end{tabular} & & & & ONSITE & SESPMNT & $\mathrm{R}$ \\
\hline \begin{tabular}{|c|}
4328 \\
\end{tabular} & & & & ONSITE & SESPMNT & $\mathrm{R}$ \\
\hline \begin{tabular}{|l|}
4329 \\
\end{tabular} & & & & ONSITE & SESPMNT & $\mathrm{R}$ \\
\hline 4330 & & & & OFFSITE & SESPMNT & $\mathrm{R}$ \\
\hline \begin{tabular}{|l|}
4331 \\
\end{tabular} & & & & OFFSITE & SESPMNT & $\mathrm{R}$ \\
\hline \begin{tabular}{|l|}
4332 \\
\end{tabular} & & & & ONSITE & SESPMNT & $\mathrm{R}$ \\
\hline 4333 & & & & ONSITE & SESPMNT & $\mathrm{R}$ \\
\hline \begin{tabular}{|l|}
4334 \\
\end{tabular} & & & & OFFSITE & SESPMNT & $\mathrm{R}$ \\
\hline \begin{tabular}{|l|}
4335 \\
\end{tabular} & & & & OFFSITE & SESPMNT & $\mathrm{R}$ \\
\hline \begin{tabular}{|l|}
4336 \\
\end{tabular} & & & & ONSITE & SESPMNT & $\mathrm{R}$ \\
\hline \begin{tabular}{|l|l|}
4337 \\
\end{tabular} & & & & ONSITE & SESPMNT & $\mathrm{R}$ \\
\hline \begin{tabular}{|l|}
4338 \\
\end{tabular} & See sample record for comment. & & & OFFSITE & SESPMNT & $\mathrm{R}$ \\
\hline \begin{tabular}{|l|}
4339 \\
\end{tabular} & & & & OFFSITE & SESPMNT & $\mathrm{R}$ \\
\hline 4340 & See sample record for comment. & & & OFFSITE & SESPMNT & $\mathrm{R}$ \\
\hline \begin{tabular}{|l|l}
4341 \\
\end{tabular} & See sample record for comment. & & & OFFSITE & SESPMNT & $\mathrm{R}$ \\
\hline 4342 & & & & OFFSITE & SESPMNT & $\mathrm{R}$ \\
\hline \begin{tabular}{|l|l|}
4343 \\
\end{tabular} & See sample record for comment. & & & OFFSITE & SESPMNT & $\mathrm{R}$ \\
\hline \begin{tabular}{|l|}
4344 \\
\end{tabular} & See sample record for comment. & & & OFFSITE & SESPMNT & $\mathrm{R}$ \\
\hline \begin{tabular}{|l|l|}
4345 \\
\end{tabular} & See sample record for comment. & & & OFFSITE & SESPMNT & $\mathrm{R}$ \\
\hline \begin{tabular}{|l|l|}
4346 \\
\end{tabular} & & & & ONSITE & SESPMNT & $\mathrm{R}$ \\
\hline \begin{tabular}{|l|l|}
4347 \\
\end{tabular} & & & & ONSITE & SESPMNT & $\mathrm{R}$ \\
\hline \begin{tabular}{|l|}
4348 \\
\end{tabular} & & & & OFFSITE & SESPMNT & $\mathrm{R}$ \\
\hline \begin{tabular}{|l|}
4349 \\
\end{tabular} & & & & OFFSITE & SESPMNT & $\mathrm{R}$ \\
\hline 4350 & & & & OFFSITE & SESPMNT & $\mathrm{R}$ \\
\hline \begin{tabular}{|l|}
4351 \\
\end{tabular} & & & & ONSITE & SESPMNT & $\mathrm{R}$ \\
\hline \begin{tabular}{|l|}
4352 \\
\end{tabular} & & & & ONSITE & SESPMNT & $\mathrm{R}$ \\
\hline 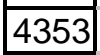 & & & & ONSITE & SESPMNT & $\mathrm{R}$ \\
\hline \begin{tabular}{|l|}
4354 \\
\end{tabular} & & & & ONSITE & SESPMNT & $\mathrm{R}$ \\
\hline \begin{tabular}{|l|l|l}
4355 \\
\end{tabular} & & & & ONSITE & SESPMNT & $\mathrm{R}$ \\
\hline \begin{tabular}{|l|l|}
4356 \\
\end{tabular} & & & & ONSITE & SESPMNT & $\mathrm{R}$ \\
\hline \begin{tabular}{|l|l|}
4357 \\
\end{tabular} & & & & ONSITE & SESPMNT & $\mathrm{R}$ \\
\hline \begin{tabular}{|l|l|}
4358 \\
\end{tabular} & & & & ONSITE & SESPMNT & $\mathrm{R}$ \\
\hline \begin{tabular}{|l|}
4359 \\
\end{tabular} & See sample record for comment. & & & ONSITE & SESPMNT & $\mathrm{R}$ \\
\hline 4360 & See sample record for comment. & & & ONSITE & SESPMNT & $\mathrm{R}$ \\
\hline \begin{tabular}{|l|}
4361 \\
\end{tabular} & & & & OFFSITE & SESPMNT & $\mathrm{R}$ \\
\hline \begin{tabular}{|l|}
4362 \\
\end{tabular} & & & & OFFSITE & SESPMNT & $\mathrm{R}$ \\
\hline \begin{tabular}{|l|}
4363 \\
\end{tabular} & & & & OFFSITE & SESPMNT & $\mathrm{R}$ \\
\hline \begin{tabular}{|l|}
4364 \\
\end{tabular} & & & & OFFSITE & SESPMNT & $\mathrm{R}$ \\
\hline \begin{tabular}{|l|}
4365 \\
\end{tabular} & & & & OFFSITE & SESPMNT & $\mathrm{R}$ \\
\hline \begin{tabular}{|l|}
4366 \\
\end{tabular} & & & & OFFSITE & SESPMNT & $\mathrm{R}$ \\
\hline \begin{tabular}{|l|}
4367 \\
\end{tabular} & & & & OFFSITE & SESPMNT & $R$ \\
\hline \begin{tabular}{|l|}
4368 \\
\end{tabular} & & & & ONSITE & SESPMNT & $\mathrm{R}$ \\
\hline \begin{tabular}{|l|}
4369 \\
\end{tabular} & & & & ONSITE & SESBMNT & $R$ \\
\hline
\end{tabular}




\begin{tabular}{|c|c|c|c|c|c|c|}
\hline & $A D$ & $\mathrm{AE}$ & $\mathrm{AF}$ & $A G$ & $\mathrm{AH}$ & $\mathrm{Al}$ \\
\hline 1 & RESULT_COMMENT & COLL_SAMP_SIZE & COLL_SAMP_SIZE_UNITS & DIST_CLASS & OWNER_ID & COLLECTION_PURPOSE \\
\hline \begin{tabular}{|c|}
4370 \\
\end{tabular} & & & & ONSITE & SESPMNT & $\mathrm{R}$ \\
\hline \begin{tabular}{|l|}
4371 \\
\end{tabular} & & & & ONSITE & SESPMNT & $\mathrm{R}$ \\
\hline \begin{tabular}{|l|}
4372 \\
\end{tabular} & & & & ONSITE & SESPMNT & $\mathrm{R}$ \\
\hline 4373 & & & & & SESPMNT & $\mathrm{R}$ \\
\hline \begin{tabular}{|l|}
4374 \\
\end{tabular} & & & & & SESPMNT & $\mathrm{R}$ \\
\hline \begin{tabular}{|l|}
4375 \\
\end{tabular} & & & & & SESPMNT & $\mathrm{R}$ \\
\hline \begin{tabular}{|l|}
4376 \\
\end{tabular} & & & & ONSITE & SESPMNT & $\mathrm{R}$ \\
\hline \begin{tabular}{|l|l|}
4377 \\
\end{tabular} & & & & ONSITE & SESPMNT & $\mathrm{R}$ \\
\hline \begin{tabular}{|l|}
4378 \\
\end{tabular} & & & & ONSITE & SESPMNT & $\mathrm{R}$ \\
\hline \begin{tabular}{|l|}
4379 \\
\end{tabular} & & & & ONSITE & SESPMNT & $\mathrm{R}$ \\
\hline \begin{tabular}{|l|l}
4380 \\
\end{tabular} & & & & ONSITE & SESPMNT & $\mathrm{R}$ \\
\hline \begin{tabular}{|l|l}
4381 \\
\end{tabular} & & & & ONSITE & SESPMNT & $\mathrm{R}$ \\
\hline 4382 & & & & PERIMETER & SESPMNT & $\mathrm{R}$ \\
\hline \begin{tabular}{|l|l|}
4383 \\
\end{tabular} & & & & PERIMETER & SESPMNT & $\mathrm{R}$ \\
\hline \begin{tabular}{|l|l|}
4384 \\
\end{tabular} & & & & PERIMETER & SESPMNT & $\mathrm{R}$ \\
\hline \begin{tabular}{|l|l|}
4385 \\
\end{tabular} & & & & ONSITE & SESPMNT & $\mathrm{R}$ \\
\hline \begin{tabular}{|l|}
4386 \\
\end{tabular} & & & & ONSITE & SESPMNT & $\mathrm{R}$ \\
\hline \begin{tabular}{|l|l|}
4387 \\
\end{tabular} & & & & ONSITE & SESPMNT & $\mathrm{R}$ \\
\hline \begin{tabular}{|l|}
4388 \\
\end{tabular} & & & & ONSITE & SESPMNT & $\mathrm{R}$ \\
\hline \begin{tabular}{|l|l|}
4389 \\
\end{tabular} & & & & ONSITE & SESPMNT & $\mathrm{R}$ \\
\hline 4390 & & & & OFFSITE & SESPMNT & $\mathrm{R}$ \\
\hline \begin{tabular}{|l|}
4391 \\
\end{tabular} & & & & OFFSITE & SESPMNT & $\mathrm{R}$ \\
\hline \begin{tabular}{|l|}
4392 \\
\end{tabular} & & & & OFFSITE & SESPMNT & $\mathrm{R}$ \\
\hline 4393 & & & & OFFSITE & SESPMNT & $\mathrm{R}$ \\
\hline \begin{tabular}{|l|}
4394 \\
\end{tabular} & & & & OFFSITE & SESPMNT & $\mathrm{R}$ \\
\hline \begin{tabular}{|l|l|l}
4395 \\
\end{tabular} & & & & ONSITE & SESPMNT & $\mathrm{R}$ \\
\hline \begin{tabular}{|l|l|}
4396 \\
\end{tabular} & & & & ONSITE & SESPMNT & $\mathrm{R}$ \\
\hline \begin{tabular}{|l|l|}
4397 \\
\end{tabular} & & & & ONSITE & SESPMNT & $\mathrm{R}$ \\
\hline \begin{tabular}{|l|l|}
4398 \\
\end{tabular} & & & & ONSITE & SESPMNT & $\mathrm{R}$ \\
\hline \begin{tabular}{|l|}
4399 \\
\end{tabular} & & & & ONSITE & SESPMNT & $\mathrm{R}$ \\
\hline 4400 & & & & ONSITE & SESPMNT & $\mathrm{R}$ \\
\hline \begin{tabular}{|l|l|}
4401 \\
\end{tabular} & & & & ONSITE & SESPMNT & $\mathrm{R}$ \\
\hline 4402 & & & & ONSITE & SESPMNT & $\mathrm{R}$ \\
\hline \begin{tabular}{|l|l|}
4403 \\
\end{tabular} & & & & ONSITE & SESPMNT & $\mathrm{R}$ \\
\hline \begin{tabular}{|l|l|}
4404 \\
\end{tabular} & See sample record for comment. & & & OFFSITE & SESPMNT & $\mathrm{R}$ \\
\hline \begin{tabular}{|l|l|}
4405 \\
\end{tabular} & See sample record for comment. & & & OFFSITE & SESPMNT & $\mathrm{R}$ \\
\hline \begin{tabular}{|l|l|}
4406 \\
\end{tabular} & See sample record for comment. & & & OFFSITE & SESPMNT & $\mathrm{R}$ \\
\hline \begin{tabular}{|l|l|}
4407 \\
\end{tabular} & & & & ONSITE & SESPMNT & $\mathrm{R}$ \\
\hline \begin{tabular}{|c|}
4408 \\
\end{tabular} & & & & ONSITE & SESPMNT & $\mathrm{R}$ \\
\hline \begin{tabular}{|l|l|l|l|}
4409 \\
\end{tabular} & & & & ONSITE & SESPMNT & $\mathrm{R}$ \\
\hline 4410 & & & & OFFSITE & SESPMNT & $\mathrm{R}$ \\
\hline \begin{tabular}{|l|l|}
4411 \\
\end{tabular} & & & & OFFSITE & SESPMNT & $\mathrm{R}$ \\
\hline 4412 & & & & OFFSITE & SESPMNT & $\mathrm{R}$ \\
\hline 4413 & & & & OFFSITE & SESPMNT & $\mathrm{R}$ \\
\hline 4414 & & & & OFFSITE & SESPMNT & $\mathrm{R}$ \\
\hline \begin{tabular}{|l|l}
4415 \\
\end{tabular} & & & & OFFSITE & SESPMNT & $\mathrm{R}$ \\
\hline \begin{tabular}{|l|l|l}
4416 \\
\end{tabular} & & & & OFFSITE & SESPSPEC & $\mathrm{s}$ \\
\hline \begin{tabular}{|l|l|}
4417 \\
\end{tabular} & & & & OFFSITE & SESPSPEC & $\mathrm{s}$ \\
\hline \begin{tabular}{|l|}
4418 \\
\end{tabular} & & & & OFFSITE & SESPSPEC & $\mathrm{s}$ \\
\hline \begin{tabular}{|l|}
4419 \\
\end{tabular} & & & & OFFSITE & SESPSPEC & $\mathrm{s}$ \\
\hline 4420 & & & & OFFSITE & SESPSPEC & $\mathrm{S}$ \\
\hline \begin{tabular}{|l|}
4421 \\
\end{tabular} & & & & OFFSITE & SESBSPEC & $0^{5}$ \\
\hline
\end{tabular}




\begin{tabular}{|c|c|c|c|c|c|c|}
\hline & $A D$ & $\mathrm{AE}$ & $\mathrm{AF}$ & $A G$ & $\mathrm{AH}$ & $\mathrm{Al}$ \\
\hline 1 & RESULT_COMMENT & COLL_SAMP_SIZE & COLL_SAMP_SIZE_UNITS & DIST_CLASS & OWNER_ID & COLLECTION_PURPOSE \\
\hline \begin{tabular}{|l|}
4422 \\
\end{tabular} & & & & ONSITE & SESPMNT & $\mathrm{R}$ \\
\hline 4423 & & & & ONSITE & SESPMNT & $\mathrm{R}$ \\
\hline \begin{tabular}{|l|l|}
4424 \\
\end{tabular} & & & & OFFSITE & SESPMNT & $\mathrm{R}$ \\
\hline \begin{tabular}{|l|l|}
4425 \\
\end{tabular} & & & & OFFSITE & SESPMNT & $\mathrm{R}$ \\
\hline \begin{tabular}{|l|l|}
4426 \\
\end{tabular} & & & & ONSITE & SESPMNT & $\mathrm{R}$ \\
\hline \begin{tabular}{|l|l|}
4427 & -10 \\
\end{tabular} & & & & ONSITE & SESPMNT & $\mathrm{R}$ \\
\hline 4428 & See sample record for comment. & & & OFFSITE & SESPMNT & $\mathrm{R}$ \\
\hline \begin{tabular}{|l|l|}
4429 \\
\end{tabular} & See sample record for comment. & & & OFFSITE & SESPMNT & $\mathrm{R}$ \\
\hline 4430 & & & & OFFSITE & SESPMNT & $\mathrm{R}$ \\
\hline 4431 & & & & OFFSITE & SESPMNT & $\mathrm{R}$ \\
\hline 4432 & & & & OFFSITE & SESPMNT & $\mathrm{R}$ \\
\hline 4433 & & & & OFFSITE & SESPMNT & $\mathrm{R}$ \\
\hline 4434 & & & & OFFSITE & SESPMNT & $\mathrm{R}$ \\
\hline \begin{tabular}{|l|l|}
4435 \\
\end{tabular} & & & & OFFSITE & SESPMNT & $\mathrm{R}$ \\
\hline \begin{tabular}{|l|l|}
4436 \\
\end{tabular} & & & & ONSITE & SESPMNT & $\mathrm{R}$ \\
\hline \begin{tabular}{|l|l|}
4437 \\
\end{tabular} & & & & ONSITE & SESPMNT & $\mathrm{R}$ \\
\hline \begin{tabular}{|l|}
4438 \\
\end{tabular} & See sample record for comment. & & & OFFSITE & SESPMNT & $\mathrm{R}$ \\
\hline 4439 & See sample record for comment. & & & OFFSITE & SESPMNT & $\mathrm{R}$ \\
\hline 4440 & & & & ONSITE & SESPMNT & $\mathrm{R}$ \\
\hline \begin{tabular}{|l|l|}
4441 \\
\end{tabular} & & & & ONSITE & SESPMNT & $\mathrm{R}$ \\
\hline \begin{tabular}{|l|l|}
4442 \\
\end{tabular} & & & & ONSITE & SESPMNT & $\mathrm{R}$ \\
\hline 4443 & & & & ONSITE & SESPMNT & $\mathrm{R}$ \\
\hline 4444 & & & & ONSITE & SESPMNT & $\mathrm{R}$ \\
\hline 4445 & & & & ONSITE & SESPMNT & $\mathrm{R}$ \\
\hline \begin{tabular}{|l|l|}
4446 \\
\end{tabular} & & & & ONSITE & SESPMNT & $\mathrm{R}$ \\
\hline \begin{tabular}{|l|l|}
4447 & \\
\end{tabular} & & & & ONSITE & SESPMNT & $\mathrm{R}$ \\
\hline 4448 & & & & ONSITE & SESPMNT & $\mathrm{R}$ \\
\hline 4449 & & & & ONSITE & SESPMNT & $\mathrm{R}$ \\
\hline 4450 & & & & ONSITE & SESPMNT & $\mathrm{R}$ \\
\hline \begin{tabular}{|l|l|}
4451 \\
\end{tabular} & & & & OFFSITE & SESPMNT & $\mathrm{R}$ \\
\hline 4452 & & & & OFFSITE & SESPMNT & $\mathrm{R}$ \\
\hline 4453 & & & & ONSITE & SESPMNT & $\mathrm{R}$ \\
\hline 4454 & & & & ONSITE & SESPMNT & $\mathrm{R}$ \\
\hline 4455 & & & & ONSITE & SESPMNT & $\mathrm{R}$ \\
\hline \begin{tabular}{|l|l|}
4456 \\
\end{tabular} & & & & ONSITE & SESPMNT & $\mathrm{R}$ \\
\hline 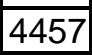 & & & & ONSITE & SESPMNT & $\mathrm{R}$ \\
\hline 4458 & & & & & SESPMNT & $\mathrm{R}$ \\
\hline 4459 & & & & & SESPMNT & $\mathrm{R}$ \\
\hline 4460 & & & & & SESPMNT & $\mathrm{R}$ \\
\hline 4461 & & & & ONSITE & SESPMNT & $\mathrm{R}$ \\
\hline \begin{tabular}{|l|l|}
4462 \\
\end{tabular} & & & & ONSITE & SESPMNT & $\mathrm{R}$ \\
\hline 4463 & & & & ONSITE & SESPMNT & $\mathrm{R}$ \\
\hline \begin{tabular}{|l|l|}
4464 \\
\end{tabular} & & & & ONSITE & SESPMNT & $\mathrm{R}$ \\
\hline 4465 & & & & ONSITE & SESPMNT & $\mathrm{R}$ \\
\hline 4466 & & & & ONSITE & SESPMNT & $\mathrm{R}$ \\
\hline \begin{tabular}{|l|l|}
4467 & $-2 \cdot 1$ \\
\end{tabular} & & & & ONSITE & SESPMNT & $\mathrm{R}$ \\
\hline 4468 & & & & ONSITE & SESPMNT & $\mathrm{R}$ \\
\hline \begin{tabular}{|l|l|}
4469 \\
\end{tabular} & & & & ONSITE & SESPMNT & $\mathrm{R}$ \\
\hline 4470 & See sample record for comment. & & & ONSITE & SESPMNT & $\mathrm{R}$ \\
\hline 4471 & & & & PERIMETER & SESPMNT & $\mathrm{R}$ \\
\hline 4472 & & & & PERIMETER & SESPMNT & $\mathrm{R}$ \\
\hline \begin{tabular}{|l|}
4473 \\
\end{tabular} & & & & PERIMETER & SESBMNT & $R$ \\
\hline
\end{tabular}




\begin{tabular}{|c|c|c|c|c|c|c|}
\hline & $A D$ & $\mathrm{AE}$ & $\mathrm{AF}$ & $A G$ & $\mathrm{AH}$ & $\mathrm{Al}$ \\
\hline 1 & RESULT_COMMENT & COLL_SAMP_SIZE & COLL_SAMP_SIZE_UNITS & DIST_CLASS & OWNER_ID & COLLECTION_PURPOSE \\
\hline \begin{tabular}{|l|}
4474 \\
\end{tabular} & & & & OFFSITE & SESPMNT & $\mathrm{R}$ \\
\hline \begin{tabular}{|l|}
4475 \\
\end{tabular} & & & & OFFSITE & SESPMNT & $\mathrm{R}$ \\
\hline \begin{tabular}{|l|l|}
4476 \\
\end{tabular} & & & & OFFSITE & SESPMNT & $\mathrm{R}$ \\
\hline \begin{tabular}{|l|l|}
4477 \\
\end{tabular} & & & & OFFSITE & SESPMNT & $\mathrm{R}$ \\
\hline 4478 & & & & OFFSITE & SESPMNT & $\mathrm{R}$ \\
\hline 4479 & & & & OFFSITE & SESPMNT & $\mathrm{R}$ \\
\hline 4480 & & & & ONSITE & SESPMNT & $\mathrm{R}$ \\
\hline \begin{tabular}{|l|l|}
4481 \\
\end{tabular} & & & & ONSITE & SESPMNT & $\mathrm{R}$ \\
\hline 4482 & & & & ONSITE & SESPMNT & $\mathrm{R}$ \\
\hline 4483 & & & & OFFSITE & SESPMNT & $\mathrm{R}$ \\
\hline 4484 & & & & OFFSITE & SESPMNT & $\mathrm{R}$ \\
\hline 4485 & & & & ONSITE & SESPMNT & $\mathrm{R}$ \\
\hline 4486 & & & & ONSITE & SESPMNT & $\mathrm{R}$ \\
\hline \begin{tabular}{|l|l|}
4487 \\
\end{tabular} & See sample record for comment. & & & OFFSITE & SESPMNT & $\mathrm{R}$ \\
\hline 4488 & See sample record for comment. & & & OFFSITE & SESPMNT & $\mathrm{R}$ \\
\hline \begin{tabular}{|l|l|}
4489 \\
\end{tabular} & & & & ONSITE & SESPMNT & $\mathrm{R}$ \\
\hline 4490 & & & & ONSITE & SESPMNT & $\mathrm{R}$ \\
\hline 4491 & & & & ONSITE & SESPMNT & $\mathrm{R}$ \\
\hline \begin{tabular}{|l|l|}
4492 \\
\end{tabular} & & & & ONSITE & SESPMNT & $\mathrm{R}$ \\
\hline 4493 & & & & ONSITE & SESPMNT & $\mathrm{R}$ \\
\hline 4494 & & & & ONSITE & SESPMNT & $\mathrm{R}$ \\
\hline 4495 & & & & OFFSITE & SESPMNT & $\mathrm{R}$ \\
\hline 4496 & & & & OFFSITE & SESPMNT & $\mathrm{R}$ \\
\hline \begin{tabular}{|l|l|}
4497 \\
\end{tabular} & & & & OFFSITE & SESPMNT & $\mathrm{R}$ \\
\hline 4498 & & & & OFFSITE & SESPMNT & $\mathrm{R}$ \\
\hline 4499 & & & & OFFSITE & SESPMNT & $\mathrm{R}$ \\
\hline 4500 & & & & OFFSITE & SESPMNT & $\mathrm{R}$ \\
\hline 4501 & & & & OFFSITE & SESPMNT & $\mathrm{R}$ \\
\hline \begin{tabular}{|l|l|}
4502 \\
\end{tabular} & & & & OFFSITE & SESPMNT & $\mathrm{R}$ \\
\hline 4503 & & & & OFFSITE & SESPMNT & $\mathrm{R}$ \\
\hline 4504 & & & & OFFSITE & SESPMNT & $\mathrm{R}$ \\
\hline 4505 & & & & OFFSITE & SESPMNT & $\mathrm{R}$ \\
\hline 4506 & & & & ONSITE & SESPMNT & $\mathrm{R}$ \\
\hline \begin{tabular}{|l|l|}
4507 & -2 \\
\end{tabular} & & & & ONSITE & SESPMNT & $\mathrm{R}$ \\
\hline 4508 & & & & ONSITE & SESPMNT & $\mathrm{R}$ \\
\hline 4509 & See sample record for comment. & & & ONSITE & SESPMNT & $\mathrm{R}$ \\
\hline 4510 & & & & ONSITE & SESPMNT & $\mathrm{R}$ \\
\hline 4511 & & & & OFFSITE & SESPMNT & $\mathrm{R}$ \\
\hline 4512 & & & & OFFSITE & SESPMNT & $\mathrm{R}$ \\
\hline 4513 & & & & OFFSITE & SESPMNT & $\mathrm{R}$ \\
\hline \begin{tabular}{|l|}
4514 \\
\end{tabular} & & & & ONSITE & SESPMNT & $\mathrm{R}$ \\
\hline \begin{tabular}{|l|}
4515 \\
\end{tabular} & & & & ONSITE & SESPMNT & $\mathrm{R}$ \\
\hline \begin{tabular}{|l|l|}
4516 \\
\end{tabular} & & & & OFFSITE & SESPMNT & $\mathrm{R}$ \\
\hline \begin{tabular}{|l|l|}
4517 & $-2 \cdot r$ \\
\end{tabular} & & & & OFFSITE & SESPMNT & $\mathrm{R}$ \\
\hline 4518 & & & & OFFSITE & SESPMNT & $\mathrm{R}$ \\
\hline 4519 & & & & OFFSITE & SESPMNT & $\mathrm{R}$ \\
\hline 4520 & & & & OFFSITE & SESPMNT & $\mathrm{R}$ \\
\hline 4521 & & & & OFFSITE & SESPMNT & $\mathrm{R}$ \\
\hline 4522 & & & & OFFSITE & SESPMNT & $\mathrm{R}$ \\
\hline 4523 & & & & OFFSITE & SESPMNT & $\mathrm{R}$ \\
\hline \begin{tabular}{|l|l|}
4524 \\
\end{tabular} & & & & ONSITE & SESPMNT & $\mathrm{R}$ \\
\hline \begin{tabular}{|l|}
4525 \\
\end{tabular} & & & & ONSITE & SESBMNT & $R$ \\
\hline
\end{tabular}




\begin{tabular}{|c|c|c|c|c|c|c|}
\hline & $A D$ & $\mathrm{AE}$ & $\mathrm{AF}$ & $A G$ & $\mathrm{AH}$ & $\mathrm{Al}$ \\
\hline 1 & RESULT_COMMENT & COLL_SAMP_SIZE & COLL_SAMP_SIZE_UNITS & DIST_CLASS & OWNER_ID & COLLECTION_PURPOSE \\
\hline 4526 & & & & ONSITE & SESPMNT & $\mathrm{R}$ \\
\hline \begin{tabular}{|l|l|}
4527 \\
\end{tabular} & & & & ONSITE & SESPMNT & $\mathrm{R}$ \\
\hline 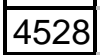 & & & & ONSITE & SESPMNT & $\mathrm{R}$ \\
\hline 4529 & & & & ONSITE & SESPMNT & $\mathrm{R}$ \\
\hline 4530 & & & & ONSITE & SESPMNT & $\mathrm{R}$ \\
\hline \begin{tabular}{|l|}
4531 \\
\end{tabular} & & & & ONSITE & SESPMNT & $\mathrm{R}$ \\
\hline \begin{tabular}{|l|}
4532 \\
\end{tabular} & & & & OFFSITE & SESPMNT & $\mathrm{R}$ \\
\hline 4533 & & & & OFFSITE & SESPMNT & $\mathrm{R}$ \\
\hline \begin{tabular}{|l|}
4534 \\
\end{tabular} & & & & ONSITE & SESPMNT & $\mathrm{R}$ \\
\hline \begin{tabular}{|l|}
4535 \\
\end{tabular} & & & & ONSITE & SESPMNT & $\mathrm{R}$ \\
\hline 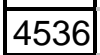 & & & & ONSITE & SESPMNT & $\mathrm{R}$ \\
\hline \begin{tabular}{|l|l|}
4537 \\
\end{tabular} & & & & ONSITE & SESPMNT & $\mathrm{R}$ \\
\hline \begin{tabular}{|c|}
4538 \\
\end{tabular} & & & & ONSITE & SESPMNT & $\mathrm{R}$ \\
\hline \begin{tabular}{|l|l|}
4539 \\
\end{tabular} & & & & & SESPMNT & $\mathrm{R}$ \\
\hline 4540 & & & & & SESPMNT & $\mathrm{R}$ \\
\hline \begin{tabular}{|l|}
4541 \\
\end{tabular} & & & & & SESPMNT & $\mathrm{R}$ \\
\hline \begin{tabular}{|c|}
4542 \\
\end{tabular} & & & & ONSITE & SESPMNT & $\mathrm{R}$ \\
\hline 4543 & & & & ONSITE & SESPMNT & $\mathrm{R}$ \\
\hline \begin{tabular}{|l|l|}
4544 \\
\end{tabular} & & & & ONSITE & SESPMNT & $\mathrm{R}$ \\
\hline 4545 & & & & ONSITE & SESPMNT & $\mathrm{R}$ \\
\hline \begin{tabular}{|l|l|}
4546 \\
\end{tabular} & & & & ONSITE & SESPMNT & $\mathrm{R}$ \\
\hline \begin{tabular}{|l|l|}
4547 \\
\end{tabular} & & & & ONSITE & SESPMNT & $\mathrm{R}$ \\
\hline \begin{tabular}{|l|}
4548 \\
\end{tabular} & & & & ONSITE & SESPMNT & $\mathrm{R}$ \\
\hline 4549 & & & & ONSITE & SESPMNT & $\mathrm{R}$ \\
\hline 4550 & & & & ONSITE & SESPMNT & $\mathrm{R}$ \\
\hline \begin{tabular}{|l|}
4551 \\
\end{tabular} & & & & PERIMETER & SESPMNT & $\mathrm{R}$ \\
\hline \begin{tabular}{|l|}
4552 \\
\end{tabular} & & & & PERIMETER & SESPMNT & $\mathrm{R}$ \\
\hline \begin{tabular}{|l|l|}
4553 \\
\end{tabular} & & & & PERIMETER & SESPMNT & $\mathrm{R}$ \\
\hline \begin{tabular}{|l|}
4554 \\
\end{tabular} & & & & ONSITE & SESPMNT & $\mathrm{R}$ \\
\hline \begin{tabular}{|l|}
4555 \\
\end{tabular} & & & & ONSITE & SESPMNT & $\mathrm{R}$ \\
\hline \begin{tabular}{|l|}
4556 \\
\end{tabular} & & & & ONSITE & SESPMNT & $\mathrm{R}$ \\
\hline \begin{tabular}{|l|}
4557 \\
\end{tabular} & & & & ONSITE & SESPMNT & $\mathrm{R}$ \\
\hline \begin{tabular}{|l|}
4558 \\
\end{tabular} & & & & ONSITE & SESPMNT & $\mathrm{R}$ \\
\hline \begin{tabular}{|l|}
4559 \\
\end{tabular} & & & & OFFSITE & SESPMNT & $\mathrm{R}$ \\
\hline \begin{tabular}{|l|}
4560 \\
\end{tabular} & & & & OFFSITE & SESPMNT & $\mathrm{R}$ \\
\hline \begin{tabular}{|l|}
4561 \\
\end{tabular} & & & & ONSITE & SESPMNT & $\mathrm{R}$ \\
\hline \begin{tabular}{|l|}
4562 \\
\end{tabular} & & & & ONSITE & SESPMNT & $\mathrm{R}$ \\
\hline \begin{tabular}{|l|}
4563 \\
\end{tabular} & & & & ONSITE & SESPMNT & $R$ \\
\hline \begin{tabular}{|l|}
4564 \\
\end{tabular} & & & & OFFSITE & SESPMNT & $\mathrm{R}$ \\
\hline \begin{tabular}{|l|}
4565 \\
\end{tabular} & & & & OFFSITE & SESPMNT & $\mathrm{R}$ \\
\hline \begin{tabular}{|l|}
4566 \\
\end{tabular} & & & & OFFSITE & SESPMNT & $\mathrm{R}$ \\
\hline \begin{tabular}{|l|}
4567 \\
\end{tabular} & & & & OFFSITE & SESPMNT & $\mathrm{R}$ \\
\hline \begin{tabular}{|l|}
4568 \\
\end{tabular} & & & & OFFSITE & SESPMNT & $\mathrm{R}$ \\
\hline \begin{tabular}{|l|}
4569 \\
\end{tabular} & & & & OFFSITE & SESPMNT & $\mathrm{R}$ \\
\hline \begin{tabular}{|l|}
4570 \\
\end{tabular} & & & & ONSITE & SESPMNT & $R$ \\
\hline \begin{tabular}{|l|}
4571 \\
\end{tabular} & & & & ONSITE & SESPMNT & $\mathrm{R}$ \\
\hline \begin{tabular}{|l|}
4572 \\
\end{tabular} & & & & ONSITE & SESPMNT & $\mathrm{R}$ \\
\hline \begin{tabular}{|l|}
4573 \\
\end{tabular} & & & & ONSITE & SESPMNT & $\mathrm{R}$ \\
\hline \begin{tabular}{|l|}
4574 \\
\end{tabular} & & & & ONSITE & SESPMNT & $\mathrm{R}$ \\
\hline \begin{tabular}{|l|}
4575 \\
\end{tabular} & & & & OFFSITE & SESPMNT & $\mathrm{R}$ \\
\hline \begin{tabular}{|l|}
4576 \\
\end{tabular} & & & & OFFSITE & SESPMNT & $\mathrm{R}$ \\
\hline \begin{tabular}{|l|}
4577 \\
\end{tabular} & See sample recc & & & ONSITE & SESBMNT & ${ }_{2} R$ \\
\hline
\end{tabular}




\begin{tabular}{|c|c|c|c|c|c|c|}
\hline & $A D$ & $\mathrm{AE}$ & $\mathrm{AF}$ & $A G$ & $\mathrm{AH}$ & $\mathrm{Al}$ \\
\hline 1 & RESULT_COMMENT & COLL_SAMP_SIZE & COLL_SAMP_SIZE_UNITS & DIST_CLASS & OWNER_ID & COLLECTION_PURPOSE \\
\hline \begin{tabular}{|c|}
4578 \\
\end{tabular} & See sample record for comment. & & & ONSITE & SESPMNT & $\mathrm{R}$ \\
\hline 4579 & See sample record for comment. & & & ONSITE & SESPMNT & $\mathrm{R}$ \\
\hline 4580 & & & & OFFSITE & SESPMNT & $\mathrm{R}$ \\
\hline 4581 & & & & OFFSITE & SESPMNT & $\mathrm{R}$ \\
\hline 4582 & & & & OFFSITE & SESPMNT & $\mathrm{R}$ \\
\hline \begin{tabular}{|l|l|}
4583 \\
\end{tabular} & & & & OFFSITE & SESPMNT & $\mathrm{R}$ \\
\hline \begin{tabular}{|l|}
4584 \\
\end{tabular} & & & & OFFSITE & SESPMNT & $\mathrm{R}$ \\
\hline \begin{tabular}{|l|l|}
4585 \\
\end{tabular} & & & & OFFSITE & SESPMNT & $\mathrm{R}$ \\
\hline \begin{tabular}{|l|}
4586 \\
\end{tabular} & & & & OFFSITE & SESPMNT & $\mathrm{R}$ \\
\hline \begin{tabular}{|l|l|}
4587 \\
\end{tabular} & & & & OFFSITE & SESPMNT & $\mathrm{R}$ \\
\hline \begin{tabular}{|l|}
4588 \\
\end{tabular} & & & & ONSITE & SESPMNT & $\mathrm{R}$ \\
\hline \begin{tabular}{|l|}
4589 \\
\end{tabular} & & & & ONSITE & SESPMNT & $\mathrm{R}$ \\
\hline 4590 & & & & ONSITE & SESPMNT & $\mathrm{R}$ \\
\hline \begin{tabular}{|l|l|}
4591 \\
\end{tabular} & & & & OFFSITE & SESPMNT & $\mathrm{R}$ \\
\hline 4592 & & & & OFFSITE & SESPMNT & $\mathrm{R}$ \\
\hline \begin{tabular}{|l|l|}
4593 \\
\end{tabular} & & & & OFFSITE & SESPMNT & $\mathrm{R}$ \\
\hline 4594 & & & & OFFSITE & SESPMNT & $\mathrm{R}$ \\
\hline \begin{tabular}{|l|}
4595 \\
\end{tabular} & & & & OFFSITE & SESPMNT & $\mathrm{R}$ \\
\hline \begin{tabular}{|l|}
4596 \\
\end{tabular} & & & & OFFSITE & SESPMNT & $\mathrm{R}$ \\
\hline \begin{tabular}{|l|l|}
4597 \\
\end{tabular} & & & & OFFSITE & SESPMNT & $\mathrm{R}$ \\
\hline \begin{tabular}{|l|l|}
4598 \\
\end{tabular} & & & & OFFSITE & SESPMNT & $\mathrm{R}$ \\
\hline \begin{tabular}{|l|}
4599 \\
\end{tabular} & & & & OFFSITE & SESPMNT & $\mathrm{R}$ \\
\hline 4600 & & & & OFFSITE & SESPMNT & $\mathrm{R}$ \\
\hline 4601 & & & & OFFSITE & SESPMNT & $\mathrm{R}$ \\
\hline \begin{tabular}{|l|}
4602 \\
\end{tabular} & & & & OFFSITE & SESPMNT & $\mathrm{R}$ \\
\hline \begin{tabular}{|l|}
4603 \\
\end{tabular} & & & & OFFSITE & SESPSPEC & $\mathrm{S}$ \\
\hline \begin{tabular}{|l|l|}
4604 \\
\end{tabular} & & & & OFFSITE & SESPSPEC & $\mathrm{s}$ \\
\hline \begin{tabular}{|l|}
4605 \\
\end{tabular} & & & & OFFSITE & SESPSPEC & $\mathrm{s}$ \\
\hline \begin{tabular}{|l|}
4606 \\
\end{tabular} & & & & OFFSITE & SESPSPEC & $\mathrm{s}$ \\
\hline \begin{tabular}{|l|}
4607 \\
\end{tabular} & & & & OFFSITE & SESPSPEC & $\mathrm{S}$ \\
\hline \begin{tabular}{|l|}
4608 \\
\end{tabular} & & & & OFFSITE & SESPSPEC & $\mathrm{S}$ \\
\hline \begin{tabular}{|l|}
4609 \\
\end{tabular} & & & & ONSITE & SESPMNT & $\mathrm{R}$ \\
\hline \begin{tabular}{|l|}
4610 \\
\end{tabular} & & & & ONSITE & SESPMNT & $\mathrm{R}$ \\
\hline \begin{tabular}{|l|}
4611 \\
\end{tabular} & & & & OFFSITE & SESPMNT & $\mathrm{R}$ \\
\hline \begin{tabular}{|l|}
4612 \\
\end{tabular} & & & & OFFSITE & SESPMNT & $\mathrm{R}$ \\
\hline \begin{tabular}{|l|}
4613 \\
\end{tabular} & & & & ONSITE & SESPMNT & $\mathrm{R}$ \\
\hline \begin{tabular}{|l|}
4614 \\
\end{tabular} & & & & ONSITE & SESPMNT & $\mathrm{R}$ \\
\hline \begin{tabular}{|l|}
4615 \\
\end{tabular} & & & & OFFSITE & SESPMNT & $R$ \\
\hline \begin{tabular}{|l|}
4616 \\
\end{tabular} & & & & OFFSITE & SESPMNT & $\mathrm{R}$ \\
\hline \begin{tabular}{|l|}
4617 \\
\end{tabular} & & & & OFFSITE & SESPMNT & $\mathrm{R}$ \\
\hline \begin{tabular}{|l|}
4618 \\
\end{tabular} & & & & OFFSITE & SESPMNT & $\mathrm{R}$ \\
\hline \begin{tabular}{|l|}
4619 \\
\end{tabular} & & & & OFFSITE & SESPMNT & $\mathrm{R}$ \\
\hline \begin{tabular}{|l|}
4620 \\
\end{tabular} & & & & OFFSITE & SESPMNT & $\mathrm{R}$ \\
\hline \begin{tabular}{|l|}
4621 \\
\end{tabular} & & & & OFFSITE & SESPMNT & $\mathrm{R}$ \\
\hline \begin{tabular}{|l|}
4622 \\
\end{tabular} & & & & OFFSITE & SESPMNT & $R$ \\
\hline \begin{tabular}{|l|}
4623 \\
\end{tabular} & & & & OFFSITE & SESPMNT & $\mathrm{R}$ \\
\hline \begin{tabular}{|l|}
4624 \\
\end{tabular} & & & & OFFSITE & SESPMNT & $\mathrm{R}$ \\
\hline \begin{tabular}{|l|}
4625 \\
\end{tabular} & & & & OFFSITE & SESPMNT & $\mathrm{R}$ \\
\hline \begin{tabular}{|l|}
4626 \\
\end{tabular} & & & & OFFSITE & SESPMNT & $\mathrm{R}$ \\
\hline \begin{tabular}{|l|}
4627 \\
\end{tabular} & & & & OFFSITE & SESPMNT & $\mathrm{R}$ \\
\hline \begin{tabular}{|l|}
4628 \\
\end{tabular} & & & & OFFSITE & SESPMNT & $\mathrm{R}$ \\
\hline \begin{tabular}{|l|}
4629 \\
\end{tabular} & & & & ONSITE & SESBMNT & ${ }_{2} R$ \\
\hline
\end{tabular}




\begin{tabular}{|c|c|c|c|c|c|c|}
\hline & $\mathrm{AD}$ & $\mathrm{AE}$ & $\mathrm{AF}$ & $\mathrm{AG}$ & $\mathrm{AH}$ & $\mathrm{Al}$ \\
\hline 1 & RESULT_COMMENT & COLL_SAMP_SIZE & COLL_SAMP_SIZE_UNITS & DIST_CLASS & OWNER_ID & COLLECTION_PURPOSE \\
\hline 4630 & & & & ONSITE & SESPMNT & $\mathrm{R}$ \\
\hline 4631 & & & & ONSITE & SESPMNT & $\mathrm{R}$ \\
\hline 4632 & & & & ONSITE & SESPMNT & $\mathrm{R}$ \\
\hline 4633 & & & & ONSITE & SESPMNT & $\mathrm{R}$ \\
\hline 4634 & & & & ONSITE & SESPMNT & $\mathrm{R}$ \\
\hline 4635 & & & & ONSITE & SESPMNT & $\mathrm{R}$ \\
\hline 4636 & & & & ONSITE & SESPMNT & $\mathrm{R}$ \\
\hline 4637 & & & & ONSITE & SESPMNT & $\mathrm{R}$ \\
\hline 4638 & & & & ONSITE & SESPMNT & $\mathrm{R}$ \\
\hline 4639 & & & & ONSITE & SESPMNT & $\mathrm{R}$ \\
\hline 4640 & See sample record for comment. & & & OFFSITE & SESPMNT & $\mathrm{R}$ \\
\hline 4641 & See sample record for comment. & & & OFFSITE & SESPMNT & $\mathrm{R}$ \\
\hline 4642 & & & & ONSITE & SESPMNT & $\mathrm{R}$ \\
\hline 4643 & & & & ONSITE & SESPMNT & $\mathrm{R}$ \\
\hline 4644 & & & & ONSITE & SESPMNT & $\mathrm{R}$ \\
\hline 4645 & & & & ONSITE & SESPMNT & $\mathrm{R}$ \\
\hline 4646 & & & & ONSITE & SESPMNT & $\mathrm{R}$ \\
\hline 4647 & & & & & SESPMNT & $\mathrm{R}$ \\
\hline 4648 & & & & & SESPMNT & $\mathrm{R}$ \\
\hline 4649 & & & & & SESPMNT & $\mathrm{R}$ \\
\hline 4650 & & & & ONSITE & SESPMNT & $\mathrm{R}$ \\
\hline 4651 & & & & ONSITE & SESPMNT & $\mathrm{R}$ \\
\hline 4652 & & & & ONSITE & SESPMNT & $\mathrm{R}$ \\
\hline 4653 & & & & ONSITE & SESPMNT & $\mathrm{R}$ \\
\hline 4654 & & & & ONSITE & SESPMNT & $\mathrm{R}$ \\
\hline \begin{tabular}{|c|}
4655 \\
\end{tabular} & & & & ONSITE & SESPMNT & $\mathrm{R}$ \\
\hline 4656 & & & & ONSITE & SESPMNT & $\mathrm{R}$ \\
\hline \begin{tabular}{|c|}
4657 \\
\end{tabular} & & & & ONSITE & SESPMNT & $\mathrm{R}$ \\
\hline 4658 & & & & ONSITE & SESPMNT & $\mathrm{R}$ \\
\hline 4659 & & & & PERIMETER & SESPMNT & $\mathrm{R}$ \\
\hline 4660 & & & & PERIMETER & SESPMNT & $\mathrm{R}$ \\
\hline \begin{tabular}{|c|}
4661 \\
\end{tabular} & & & & PERIMETER & SESPMNT & $\mathrm{R}$ \\
\hline 4662 & See sample record for comment. & & & ONSITE & SESPMNT & $\mathrm{R}$ \\
\hline 4663 & See sample record for comment. & & & ONSITE & SESPMNT & $\mathrm{R}$ \\
\hline 4664 & See sample record for comment. & & & ONSITE & SESPMNT & $\mathrm{R}$ \\
\hline 4665 & & & & ONSITE & SESPMNT & $\mathrm{R}$ \\
\hline \begin{tabular}{|c|}
4666 \\
\end{tabular} & & & & ONSITE & SESPMNT & $\mathrm{R}$ \\
\hline 4667 & See sample record for comment. & & & OFFSITE & SESPMNT & $\mathrm{R}$ \\
\hline 4668 & See sample record for comment. & & & OFFSITE & SESPMNT & $\mathrm{R}$ \\
\hline 4669 & & & & ONSITE & SESPMNT & $\mathrm{R}$ \\
\hline 4670 & & & & ONSITE & SESPMNT & $\mathrm{R}$ \\
\hline \begin{tabular}{|l|}
4671 \\
\end{tabular} & & & & OFFSITE & SESPMNT & $\mathrm{R}$ \\
\hline \begin{tabular}{|l|}
4672 \\
\end{tabular} & & & & OFFSITE & SESPMNT & $\mathrm{R}$ \\
\hline 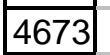 & & & & OFFSITE & SESPMNT & $\mathrm{R}$ \\
\hline 4674 & & & & OFFSITE & SESPMNT & $\mathrm{R}$ \\
\hline 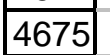 & & & & OFFSITE & SESPMNT & $\mathrm{R}$ \\
\hline 4676 & & & & OFFSITE & SESPMNT & $\mathrm{R}$ \\
\hline 4677 & & & & OFFSITE & SESPMNT & $\mathrm{R}$ \\
\hline \begin{tabular}{|l|l|}
$4678-200$ \\
\end{tabular} & & & & OFFSITE & SESPMNT & $\mathrm{R}$ \\
\hline \begin{tabular}{|l|l|}
4679 \\
\end{tabular} & & & & OFFSITE & SESPMNT & $\mathrm{R}$ \\
\hline \begin{tabular}{|l|}
4680 \\
\end{tabular} & & & & OFFSITE & SESPMNT & $R$ \\
\hline \begin{tabular}{|l|}
4681 \\
\end{tabular} & & & & OFFSITE & SESBMNT & $R$ \\
\hline
\end{tabular}




\begin{tabular}{|c|c|c|c|c|c|c|}
\hline & $A D$ & $\mathrm{AE}$ & $\mathrm{AF}$ & $A G$ & $\mathrm{AH}$ & $\mathrm{Al}$ \\
\hline 1 & RESULT_COMMENT & COLL_SAMP_SIZE & COLL_SAMP_SIZE_UNITS & DIST_CLASS & OWNER_ID & COLLECTION_PURPOSE \\
\hline 4682 & & & & OFFSITE & SESPMNT & $\mathrm{R}$ \\
\hline 4683 & & & & OFFSITE & SESPMNT & $\mathrm{R}$ \\
\hline $\mid 4684$ & & & & OFFSITE & SESPMNT & $\mathrm{R}$ \\
\hline 4685 & & & & OFFSITE & SESPMNT & $\mathrm{R}$ \\
\hline \begin{tabular}{|c|}
4686 \\
\end{tabular} & & & & OFFSITE & SESPMNT & $\mathrm{R}$ \\
\hline 4687 & See sample record for comment. & & & ONSITE & SESPMNT & $\mathrm{R}$ \\
\hline 4688 & See sample record for comment. & & & ONSITE & SESPMNT & $\mathrm{R}$ \\
\hline 4689 & & & & ONSITE & SESPMNT & $\mathrm{R}$ \\
\hline 4690 & & & & ONSITE & SESPMNT & $\mathrm{R}$ \\
\hline 4691 & & & & ONSITE & SESPMNT & $\mathrm{R}$ \\
\hline 4692 & & & & ONSITE & SESPMNT & $\mathrm{R}$ \\
\hline 4693 & & & & ONSITE & SESPMNT & $\mathrm{R}$ \\
\hline 4694 & & & & ONSITE & SESPMNT & $\mathrm{R}$ \\
\hline 4695 & & & & ONSITE & SESPMNT & $\mathrm{R}$ \\
\hline \begin{tabular}{|c|}
4696 \\
\end{tabular} & & & & ONSITE & SESPMNT & $\mathrm{R}$ \\
\hline \begin{tabular}{|c|}
4697 \\
\end{tabular} & & & & ONSITE & SESPMNT & $\mathrm{R}$ \\
\hline 4698 & & & & OFFSITE & SESPMNT & $\mathrm{R}$ \\
\hline 4699 & & & & OFFSITE & SESPMNT & $\mathrm{R}$ \\
\hline 4700 & & & & OFFSITE & SESPMNT & $\mathrm{R}$ \\
\hline 4701 & & & & OFFSITE & SESPMNT & $\mathrm{R}$ \\
\hline \begin{tabular}{|c|}
4702 \\
\end{tabular} & & & & OFFSITE & SESPMNT & $\mathrm{R}$ \\
\hline 4703 & & & & OFFSITE & SESPMNT & $\mathrm{R}$ \\
\hline 4704 & & & & OFFSITE & SESPMNT & $\mathrm{R}$ \\
\hline 4705 & & & & OFFSITE & SESPMNT & $\mathrm{R}$ \\
\hline \begin{tabular}{|l|l|}
4706 \\
\end{tabular} & & & & OFFSITE & SESPMNT & $\mathrm{R}$ \\
\hline 4707 & & & & OFFSITE & SESPMNT & $\mathrm{R}$ \\
\hline 4708 & & & & OFFSITE & SESPMNT & $\mathrm{R}$ \\
\hline 4709 & & & & OFFSITE & SESPMNT & $\mathrm{R}$ \\
\hline 4710 & & & & OFFSITE & SESPMNT & $\mathrm{R}$ \\
\hline \begin{tabular}{|l|l|l|}
4711 \\
\end{tabular} & & & & OFFSITE & SESPMNT & $\mathrm{R}$ \\
\hline 4712 & & & & OFFSITE & SESPMNT & $\mathrm{R}$ \\
\hline 4713 & & & & OFFSITE & SESPMNT & $\mathrm{R}$ \\
\hline 4714 & & & & OFFSITE & SESPMNT & $\mathrm{R}$ \\
\hline 4715 & & & & OFFSITE & SESPMNT & $\mathrm{R}$ \\
\hline 4716 & See sample record for comment. & & & ONSITE & SESPMNT & $\mathrm{R}$ \\
\hline 4717 & See sample record for comment. & & & ONSITE & SESPMNT & $\mathrm{R}$ \\
\hline 4718 & See sample record for comment. & & & OFFSITE & SESPMNT & $\mathrm{R}$ \\
\hline 4719 & & & & OFFSITE & SESPMNT & $\mathrm{R}$ \\
\hline 4720 & & & & ONSITE & SESPMNT & $\mathrm{R}$ \\
\hline 4721 & & & & ONSITE & SESPMNT & $\mathrm{R}$ \\
\hline 4722 & & & & OFFSITE & SESPMNT & $\mathrm{R}$ \\
\hline 4723 & & & & OFFSITE & SESPMNT & $\mathrm{R}$ \\
\hline 4724 & & & & OFFSITE & SESPMNT & $\mathrm{R}$ \\
\hline 4725 & & & & OFFSITE & SESPMNT & $\mathrm{R}$ \\
\hline \begin{tabular}{|l|l|l|l}
4726 \\
\end{tabular} & & & & OFFSITE & SESPMNT & $\mathrm{R}$ \\
\hline \begin{tabular}{|l|l|}
4727 \\
\end{tabular} & & & & OFFSITE & SESPMNT & $\mathrm{R}$ \\
\hline 4728 & & & & OFFSITE & SESPMNT & $\mathrm{R}$ \\
\hline 4729 & & & & OFFSITE & SESPMNT & $\mathrm{R}$ \\
\hline 4730 & & & & OFFSITE & SESPMNT & $\mathrm{R}$ \\
\hline \begin{tabular}{|c|}
4731 \\
\end{tabular} & & & & OFFSITE & SESPMNT & $\mathrm{R}$ \\
\hline \begin{tabular}{|l|}
4732 \\
\end{tabular} & & & & OFFSITE & SESPMNT & $\mathrm{R}$ \\
\hline \begin{tabular}{|l|}
4733 \\
\end{tabular} & & & & OFFSITE & SESBMNT & $R$ \\
\hline
\end{tabular}




\begin{tabular}{|c|c|c|c|c|c|c|}
\hline & $A D$ & $\mathrm{AE}$ & $\mathrm{AF}$ & $A G$ & $\mathrm{AH}$ & $\mathrm{Al}$ \\
\hline 1 & RESULT_COMMENT & COLL_SAMP_SIZE & COLL_SAMP_SIZE_UNITS & DIST_CLASS & OWNER_ID & COLLECTION_PURPOSE \\
\hline 4734 & & & & OFFSITE & SESPMNT & $\mathrm{R}$ \\
\hline 4735 & & & & OFFSITE & SESPMNT & $\mathrm{R}$ \\
\hline \begin{tabular}{|l|l|}
4736 \\
\end{tabular} & & & & ONSITE & SESPMNT & $\mathrm{R}$ \\
\hline \begin{tabular}{|l|l|}
4737 \\
\end{tabular} & & & & ONSITE & SESPMNT & $\mathrm{R}$ \\
\hline 4738 & & & & ONSITE & SESPMNT & $\mathrm{R}$ \\
\hline 4739 & & & & ONSITE & SESPMNT & $\mathrm{R}$ \\
\hline 4740 & & & & ONSITE & SESPMNT & $\mathrm{R}$ \\
\hline \begin{tabular}{|l|l|l|}
4741 \\
\end{tabular} & & & & ONSITE & SESPMNT & $\mathrm{R}$ \\
\hline \begin{tabular}{|c|}
4742 \\
\end{tabular} & & & & ONSITE & SESPMNT & $\mathrm{R}$ \\
\hline 4743 & & & & ONSITE & SESPMNT & $\mathrm{R}$ \\
\hline 4744 & & & & ONSITE & SESPMNT & $\mathrm{R}$ \\
\hline 4745 & & & & ONSITE & SESPMNT & $\mathrm{R}$ \\
\hline 4746 & & & & ONSITE & SESPMNT & $\mathrm{R}$ \\
\hline 4747 & & & & ONSITE & SESPMNT & $\mathrm{R}$ \\
\hline 4748 & & & & ONSITE & SESPMNT & $\mathrm{R}$ \\
\hline 4749 & & & & ONSITE & SESPMNT & $\mathrm{R}$ \\
\hline 4750 & & & & ONSITE & SESPMNT & $\mathrm{R}$ \\
\hline \begin{tabular}{|c|c|}
4751 \\
\end{tabular} & & & & ONSITE & SESPMNT & $\mathrm{R}$ \\
\hline 4752 & & & & ONSITE & SESPMNT & $\mathrm{R}$ \\
\hline 4753 & & & & OFFSITE & SESPMNT & $\mathrm{R}$ \\
\hline 4754 & & & & OFFSITE & SESPMNT & $\mathrm{R}$ \\
\hline 4755 & & & & ONSITE & SESPMNT & $\mathrm{R}$ \\
\hline \begin{tabular}{|l|l|}
4756 \\
\end{tabular} & & & & ONSITE & SESPMNT & $\mathrm{R}$ \\
\hline \begin{tabular}{|l|l|}
4757 \\
\end{tabular} & & & & OFFSITE & SESPMNT & $\mathrm{R}$ \\
\hline 4758 & & & & OFFSITE & SESPMNT & $\mathrm{R}$ \\
\hline 4759 & & & & OFFSITE & SESPMNT & $\mathrm{R}$ \\
\hline 4760 & & & & OFFSITE & SESPMNT & $\mathrm{R}$ \\
\hline 4761 & & & & OFFSITE & SESPMNT & $\mathrm{R}$ \\
\hline 4762 & & & & OFFSITE & SESPMNT & $\mathrm{R}$ \\
\hline 4763 & & & & OFFSITE & SESPMNT & $\mathrm{R}$ \\
\hline 4764 & & & & OFFSITE & SESPMNT & $\mathrm{R}$ \\
\hline 4765 & See sample record for comment. & & & OFFSITE & SESPMNT & $\mathrm{R}$ \\
\hline 4766 & See sample record for comment. & & & OFFSITE & SESPMNT & $\mathrm{R}$ \\
\hline 4767 & See sample record for comment. & & & OFFSITE & SESPMNT & $\mathrm{R}$ \\
\hline 4768 & See sample record for comment. & & & OFFSITE & SESPMNT & $\mathrm{R}$ \\
\hline 4769 & See sample record for comment. & & & OFFSITE & SESPMNT & $\mathrm{R}$ \\
\hline 4770 & See sample record for comment. & & & OFFSITE & SESPMNT & $\mathrm{R}$ \\
\hline \begin{tabular}{|c|c|}
4771 \\
\end{tabular} & & & & ONSITE & SESPMNT & $\mathrm{R}$ \\
\hline 4772 & & & & ONSITE & SESPMNT & $\mathrm{R}$ \\
\hline 4773 & & & & ONSITE & SESPMNT & $\mathrm{R}$ \\
\hline 4774 & & & & ONSITE & SESPMNT & $\mathrm{R}$ \\
\hline 4775 & & & & ONSITE & SESPMNT & $\mathrm{R}$ \\
\hline \begin{tabular}{|l|l|}
4776 \\
\end{tabular} & & & & & SESPMNT & $\mathrm{R}$ \\
\hline 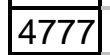 & & & & & SESPMNT & $\mathrm{R}$ \\
\hline 4778 & & & & & SESPMNT & $\mathrm{R}$ \\
\hline 4779 & & & & ONSITE & SESPMNT & $\mathrm{R}$ \\
\hline 4780 & & & & ONSITE & SESPMNT & $\mathrm{R}$ \\
\hline 4781 & & & & ONSITE & SESPMNT & $\mathrm{R}$ \\
\hline \begin{tabular}{|l|l|}
4782 \\
\end{tabular} & & & & PERIMETER & SESPMNT & $\mathrm{R}$ \\
\hline 4783 & & & & PERIMETER & SESPMNT & $\mathrm{R}$ \\
\hline \begin{tabular}{|l|l|}
4784 \\
\end{tabular} & & & & PERIMETER & SESPMNT & $\mathrm{R}$ \\
\hline \begin{tabular}{|l|}
4785 \\
\end{tabular} & & & & ONSITE & SESBMNT & $R$ \\
\hline
\end{tabular}




\begin{tabular}{|c|c|c|c|c|c|c|}
\hline & $A D$ & $\mathrm{AE}$ & $\mathrm{AF}$ & $A G$ & $\mathrm{AH}$ & $\mathrm{Al}$ \\
\hline 1 & RESULT_COMMENT & COLL_SAMP_SIZE & COLL_SAMP_SIZE_UNITS & DIST_CLASS & OWNER_ID & COLLECTION_PURPOSE \\
\hline \begin{tabular}{|l|}
4786 \\
\end{tabular} & & & & ONSITE & SESPMNT & $\mathrm{R}$ \\
\hline \begin{tabular}{|l|l|}
4787 \\
\end{tabular} & & & & OFFSITE & SESPMNT & $\mathrm{R}$ \\
\hline \begin{tabular}{|l|}
4788 \\
\end{tabular} & & & & OFFSITE & SESPMNT & $\mathrm{R}$ \\
\hline \begin{tabular}{|l|}
4789 \\
\end{tabular} & See sample record for comment. & & & OFFSITE & SESPMNT & $\mathrm{R}$ \\
\hline 4790 & See sample record for comment. & & & OFFSITE & SESPMNT & $\mathrm{R}$ \\
\hline \begin{tabular}{|l|}
4791 \\
\end{tabular} & & & & OFFSITE & SESPMNT & $\mathrm{R}$ \\
\hline \begin{tabular}{|l|}
4792 \\
\end{tabular} & & & & OFFSITE & SESPMNT & $\mathrm{R}$ \\
\hline \begin{tabular}{|l|}
4793 \\
\end{tabular} & & & & & SESPMNT & $\mathrm{R}$ \\
\hline \begin{tabular}{|l|}
4794 \\
\end{tabular} & & & & & SESPMNT & $\mathrm{R}$ \\
\hline \begin{tabular}{|l|l|}
4795 \\
\end{tabular} & & & & ONSITE & SESPMNT & $\mathrm{R}$ \\
\hline \begin{tabular}{|l|l|l|l|l|}
4796 \\
\end{tabular} & & & & ONSITE & SESPMNT & $\mathrm{R}$ \\
\hline \begin{tabular}{|l|l|}
4797 \\
\end{tabular} & & & & ONSITE & SESPMNT & $\mathrm{R}$ \\
\hline \begin{tabular}{|l|l|}
4798 \\
\end{tabular} & & & & ONSITE & SESPMNT & $\mathrm{R}$ \\
\hline \begin{tabular}{|l|l|}
4799 \\
\end{tabular} & & & & ONSITE & SESPMNT & $\mathrm{R}$ \\
\hline 4800 & & & & ONSITE & SESPMNT & $\mathrm{R}$ \\
\hline \begin{tabular}{|l|}
4801 \\
\end{tabular} & & & & ONSITE & SESPMNT & $\mathrm{R}$ \\
\hline \begin{tabular}{|l|l|}
4802 \\
\end{tabular} & & & & ONSITE & SESPMNT & $\mathrm{R}$ \\
\hline \begin{tabular}{|c|}
4803 \\
\end{tabular} & & & & ONSITE & SESPMNT & $\mathrm{R}$ \\
\hline 4804 & & & & ONSITE & SESPMNT & $\mathrm{R}$ \\
\hline 4805 & & & & ONSITE & SESPMNT & $\mathrm{R}$ \\
\hline \begin{tabular}{|l|l|}
4806 \\
\end{tabular} & & & & ONSITE & SESPMNT & $\mathrm{R}$ \\
\hline \begin{tabular}{|l|l|}
4807 \\
\end{tabular} & & & & OFFSITE & SESPMNT & $\mathrm{R}$ \\
\hline 4808 & & & & OFFSITE & SESPMNT & $\mathrm{R}$ \\
\hline 4809 & & & & OFFSITE & SESPMNT & $\mathrm{R}$ \\
\hline \begin{tabular}{|l|}
4810 \\
\end{tabular} & & & & OFFSITE & SESPMNT & $\mathrm{R}$ \\
\hline \begin{tabular}{|l|l|l|}
4811 \\
\end{tabular} & & & & OFFSITE & SESPMNT & $\mathrm{R}$ \\
\hline 4812 & & & & OFFSITE & SESPMNT & $\mathrm{R}$ \\
\hline \begin{tabular}{|l|l|}
4813 \\
\end{tabular} & & & & OFFSITE & SESPMNT & $\mathrm{R}$ \\
\hline \begin{tabular}{|l|l|}
4814 \\
\end{tabular} & & & & OFFSITE & SESPMNT & $\mathrm{R}$ \\
\hline \begin{tabular}{|l|l|}
4815 \\
\end{tabular} & & & & OFFSITE & SESPMNT & $\mathrm{R}$ \\
\hline \begin{tabular}{|l|}
4816 \\
\end{tabular} & & & & OFFSITE & SESPMNT & $\mathrm{R}$ \\
\hline \begin{tabular}{|l|l|}
4817 \\
\end{tabular} & & & & OFFSITE & SESPMNT & $\mathrm{R}$ \\
\hline \begin{tabular}{|l|l|}
4818 \\
\end{tabular} & & & & OFFSITE & SESPMNT & $\mathrm{R}$ \\
\hline \begin{tabular}{|l|}
4819 \\
\end{tabular} & & & & OFFSITE & SESPMNT & $\mathrm{R}$ \\
\hline 4820 & & & & OFFSITE & SESPMNT & $\mathrm{R}$ \\
\hline \begin{tabular}{|l|}
4821 \\
\end{tabular} & & & & OFFSITE & SESPMNT & $\mathrm{R}$ \\
\hline 4822 & & & & OFFSITE & SESPMNT & $\mathrm{R}$ \\
\hline 4823 & & & & OFFSITE & SESPMNT & $\mathrm{R}$ \\
\hline \begin{tabular}{|l|l|}
4824 \\
\end{tabular} & & & & OFFSITE & SESPMNT & $\mathrm{R}$ \\
\hline \begin{tabular}{|l|l|}
4825 \\
\end{tabular} & & & & ONSITE & SESPMNT & $\mathrm{R}$ \\
\hline \begin{tabular}{|l|l|}
4826 \\
\end{tabular} & & & & ONSITE & SESPMNT & $\mathrm{R}$ \\
\hline \begin{tabular}{|l|l|}
4827 \\
\end{tabular} & & & & ONSITE & SESPMNT & $\mathrm{R}$ \\
\hline \begin{tabular}{|l|l|}
4828 \\
\end{tabular} & See sample record for comment. & & & ONSITE & SESPMNT & $\mathrm{R}$ \\
\hline 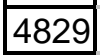 & See sample record for comment. & & & ONSITE & SESPMNT & $\mathrm{R}$ \\
\hline \begin{tabular}{|c|}
4830 \\
\end{tabular} & & & & OFFSITE & SESPMNT & $\mathrm{R}$ \\
\hline \begin{tabular}{|l|}
4831 \\
\end{tabular} & & & & OFFSITE & SESPMNT & $\mathrm{R}$ \\
\hline 4832 & & & & OFFSITE & SESPSPEC & $\mathrm{s}$ \\
\hline \begin{tabular}{|l|l|}
4833 \\
\end{tabular} & & & & OFFSITE & SESPSPEC & $\mathrm{s}$ \\
\hline \begin{tabular}{|l|}
4834 \\
\end{tabular} & & & & OFFSITE & SESPSPEC & $\mathrm{s}$ \\
\hline 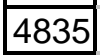 & & & & OFFSITE & SESPSPEC & $\mathrm{s}$ \\
\hline \begin{tabular}{|l|l}
4836 \\
\end{tabular} & & & & OFFSITE & SESPSPEC & $\mathrm{S}$ \\
\hline \begin{tabular}{|l|}
4837 \\
\end{tabular} & & & & OFFSITE & SESBSPEC & 5 \\
\hline
\end{tabular}




\begin{tabular}{|c|c|c|c|c|c|c|}
\hline & $\mathrm{AD}$ & $\mathrm{AE}$ & AF & AG & $\mathrm{AH}$ & $\mathrm{Al}$ \\
\hline 1 & RESULT_COMMENT & COLL_SAMP_SIZE & COLL_SAMP_SIZE_UNITS & DIST_CLASS & OWNER_ID & COLLECTION_PURPOSE \\
\hline 4838 & & & & ONSITE & SESPMNT & $\mathrm{R}$ \\
\hline $\mid 4839$ & & & & ONSITE & SESPMNT & $\mathrm{R}$ \\
\hline 4840 & & & & OFFSITE & SESPMNT & $\mathrm{R}$ \\
\hline 4841 & & & & OFFSITE & SESPMNT & $\mathrm{R}$ \\
\hline 4842 & & & & ONSITE & SESPMNT & $\mathrm{R}$ \\
\hline 4843 & & & & ONSITE & SESPMNT & $\mathrm{R}$ \\
\hline 4844 & & & & ONSITE & SESPMNT & $\mathrm{R}$ \\
\hline 4845 & See sample record for comment. & & & ONSITE & SESPMNT & $\mathrm{R}$ \\
\hline 4846 & See sample record for comment. & & & ONSITE & SESPMNT & $\mathrm{R}$ \\
\hline 4847 & See sample record for comment. & & & ONSITE & SESPMNT & $\mathrm{R}$ \\
\hline 4848 & & & & ONSITE & SESPMNT & $\mathrm{R}$ \\
\hline 4849 & & & & ONSITE & SESPMNT & $\mathrm{R}$ \\
\hline 4850 & & & & ONSITE & SESPMNT & $\mathrm{R}$ \\
\hline \begin{tabular}{|c|}
4851 \\
\end{tabular} & & & & ONSITE & SESPMNT & $\mathrm{R}$ \\
\hline \begin{tabular}{|c|}
4852 \\
\end{tabular} & & & & ONSITE & SESPMNT & $\mathrm{R}$ \\
\hline \begin{tabular}{|c|}
4853 \\
\end{tabular} & & & & ONSITE & SESPMNT & $\mathrm{R}$ \\
\hline 4854 & & & & OFFSITE & SESPMNT & $\mathrm{R}$ \\
\hline 4855 & & & & OFFSITE & SESPMNT & $\mathrm{R}$ \\
\hline 4856 & & & & OFFSITE & SESPMNT & $\mathrm{R}$ \\
\hline \begin{tabular}{|c|}
4857 \\
\end{tabular} & & & & OFFSITE & SESPMNT & $\mathrm{R}$ \\
\hline \begin{tabular}{|c|c|}
4858 \\
\end{tabular} & & & & OFFSITE & SESPMNT & $\mathrm{R}$ \\
\hline 4859 & & & & OFFSITE & SESPMNT & $\mathrm{R}$ \\
\hline 4860 & & & & OFFSITE & SESPMNT & $\mathrm{R}$ \\
\hline 4861 & & & & OFFSITE & SESPMNT & $\mathrm{R}$ \\
\hline 4862 & & & & OFFSITE & SESPMNT & $\mathrm{R}$ \\
\hline \begin{tabular}{|c|}
4863 \\
\end{tabular} & & & & OFFSITE & SESPMNT & $\mathrm{R}$ \\
\hline 4864 & & & & OFFSITE & SESPMNT & $\mathrm{R}$ \\
\hline 4865 & & & & OFFSITE & SESPMNT & $\mathrm{R}$ \\
\hline \begin{tabular}{|c|}
4866 \\
\end{tabular} & & & & ONSITE & SESPMNT & $\mathrm{R}$ \\
\hline \begin{tabular}{|c|}
4867 \\
\end{tabular} & & & & ONSITE & SESPMNT & $\mathrm{R}$ \\
\hline 4868 & & & & ONSITE & SESPMNT & $\mathrm{R}$ \\
\hline \begin{tabular}{|c|}
4869 \\
\end{tabular} & & & & ONSITE & SESPMNT & $\mathrm{R}$ \\
\hline 4870 & & & & ONSITE & SESPMNT & $\mathrm{R}$ \\
\hline \begin{tabular}{|c|}
4871 \\
\end{tabular} & & & & OFFSITE & SESPMNT & $\mathrm{R}$ \\
\hline \begin{tabular}{|c|}
4872 \\
\end{tabular} & & & & OFFSITE & SESPMNT & $\mathrm{R}$ \\
\hline 4873 & See sample record for comment. & & & ONSITE & SESPMNT & $\mathrm{R}$ \\
\hline 4874 & See sample record for comment. & & & ONSITE & SESPMNT & $\mathrm{R}$ \\
\hline \begin{tabular}{|c|}
4875 \\
\end{tabular} & & & & OFFSITE & SESPMNT & $\mathrm{R}$ \\
\hline \begin{tabular}{|l|l|}
4876 \\
\end{tabular} & & & & OFFSITE & SESPMNT & $\mathrm{R}$ \\
\hline \begin{tabular}{|l|l|}
4877 & -10 \\
\end{tabular} & & & & ONSITE & SESPMNT & $\mathrm{R}$ \\
\hline \begin{tabular}{|l|l|}
4878 \\
\end{tabular} & & & & ONSITE & SESPMNT & $\mathrm{R}$ \\
\hline \begin{tabular}{|l|}
4879 \\
\end{tabular} & & & & ONSITE & SESPMNT & $\mathrm{R}$ \\
\hline \begin{tabular}{|l|}
4880 \\
\end{tabular} & & & & ONSITE & SESPMNT & $\mathrm{R}$ \\
\hline \begin{tabular}{|l|l|}
4881 \\
\end{tabular} & & & & ONSITE & SESPMNT & $\mathrm{R}$ \\
\hline \begin{tabular}{|l|l|}
4882 \\
\end{tabular} & & & & & SESPMNT & $\mathrm{R}$ \\
\hline \begin{tabular}{|l|l|}
4883 \\
\end{tabular} & & & & & SESPMNT & $\mathrm{R}$ \\
\hline \begin{tabular}{|l|l|}
4884 \\
\end{tabular} & & & & & SESPMNT & $\mathrm{R}$ \\
\hline \begin{tabular}{|l|l|}
4885 \\
\end{tabular} & & & & ONSITE & SESPMNT & $\mathrm{R}$ \\
\hline \begin{tabular}{|l|l|}
4886 \\
\end{tabular} & & & & ONSITE & SESPMNT & $\mathrm{R}$ \\
\hline \begin{tabular}{|l|l|}
4887 \\
\end{tabular} & & & & ONSITE & SESPMNT & $\mathrm{R}$ \\
\hline \begin{tabular}{|l|l|l|l|l}
4888 \\
\end{tabular} & & & & ONSITE & SESPMNT & $\mathrm{R}$ \\
\hline \begin{tabular}{|l|}
4889 \\
\end{tabular} & & & & PERIMETER & SESBMNT & $\mathrm{R}$ \\
\hline
\end{tabular}




\begin{tabular}{|c|c|c|c|c|c|c|}
\hline & $\mathrm{AD}$ & $\mathrm{AE}$ & $\mathrm{AF}$ & AG & $\mathrm{AH}$ & $\mathrm{Al}$ \\
\hline 1 & RESULT_COMMENT & COLL_SAMP_SIZE & COLL_SAMP_SIZE_UNITS & DIST_CLASS & OWNER_ID & COLLECTION_PURPOSE \\
\hline 4890 & & & & PERIMETER & SESPMNT & $\mathrm{R}$ \\
\hline 4891 & & & & PERIMETER & SESPMNT & $\mathrm{R}$ \\
\hline 4892 & See sample record for comment. & & & ONSITE & SESPMNT & $\mathrm{R}$ \\
\hline 4893 & See sample record for comment. & & & ONSITE & SESPMNT & $\mathrm{R}$ \\
\hline 4894 & & & & OFFSITE & SESPMNT & $\mathrm{R}$ \\
\hline 4895 & & & & OFFSITE & SESPMNT & $\mathrm{R}$ \\
\hline 4896 & & & & OFFSITE & SESPMNT & $\mathrm{R}$ \\
\hline \begin{tabular}{|l|l|}
4897 \\
\end{tabular} & & & & OFFSITE & SESPMNT & $\mathrm{R}$ \\
\hline 4898 & & & & OFFSITE & SESPMNT & $\mathrm{R}$ \\
\hline 4899 & & & & OFFSITE & SESPMNT & $\mathrm{R}$ \\
\hline 4900 & & & & OFFSITE & SESPMNT & $\mathrm{R}$ \\
\hline 4901 & & & & OFFSITE & SESPMNT & $R$ \\
\hline 4902 & & & & OFFSITE & SESPMNT & $\mathrm{R}$ \\
\hline 4903 & & & & OFFSITE & SESPMNT & $\mathrm{R}$ \\
\hline 4904 & & & & OFFSITE & SESPMNT & $\mathrm{R}$ \\
\hline 4905 & & & & OFFSITE & SESPMNT & $\mathrm{R}$ \\
\hline 4906 & & & & OFFSITE & SESPMNT & $\mathrm{R}$ \\
\hline 4907 & & & & OFFSITE & SESPMNT & $\mathrm{R}$ \\
\hline 4908 & & & & OFFSITE & SESPMNT & $\mathrm{R}$ \\
\hline 4909 & & & & OFFSITE & SESPMNT & $\mathrm{R}$ \\
\hline 4910 & & & & ONSITE & SESPMNT & $R$ \\
\hline 4911 & & & & ONSITE & SESPMNT & $\mathrm{R}$ \\
\hline 4912 & & & & ONSITE & SESPMNT & $\mathrm{R}$ \\
\hline 4913 & & & & ONSITE & SESPMNT & $\mathrm{R}$ \\
\hline \begin{tabular}{|l|l|}
4914 \\
\end{tabular} & & & & ONSITE & SESPMNT & $R$ \\
\hline \begin{tabular}{|l|}
4915 \\
\end{tabular} & & & & OFFSITE & SESPMNT & $R$ \\
\hline 4916 & & & & OFFSITE & SESPMNT & $R$ \\
\hline \begin{tabular}{|l|}
4917 \\
\end{tabular} & & & & ONSITE & SESPMNT & $R$ \\
\hline \begin{tabular}{|l|}
4918 \\
\end{tabular} & & & & ONSITE & SESPMNT & $R$ \\
\hline 4919 & & & & ONSITE & SESPMNT & $R$ \\
\hline 4920 & & & & ONSITE & SESPMNT & $R$ \\
\hline \begin{tabular}{|l|l|}
4921 \\
\end{tabular} & & & & ONSITE & SESPMNT & $R$ \\
\hline \begin{tabular}{|l|}
4922 \\
\end{tabular} & & & & ONSITE & SESPMNT & $\mathrm{R}$ \\
\hline \begin{tabular}{|l|}
4923 \\
\end{tabular} & & & & OFFSITE & SESPMNT & $R$ \\
\hline \begin{tabular}{|l|l|}
4924 \\
\end{tabular} & & & & OFFSITE & SESPMNT & $R$ \\
\hline \begin{tabular}{|l|}
4925 \\
\end{tabular} & & & & OFFSITE & SESPMNT & $\mathrm{R}$ \\
\hline \begin{tabular}{|l|}
4926 \\
\end{tabular} & & & & OFFSITE & SESPMNT & $R$ \\
\hline \begin{tabular}{|l|l|}
4927 \\
\end{tabular} & & & & OFFSITE & SESPMNT & $R$ \\
\hline \begin{tabular}{|l|}
4928 \\
\end{tabular} & & & & OFFSITE & SESPMNT & $R$ \\
\hline \begin{tabular}{|l|}
4929 \\
\end{tabular} & & & & OFFSITE & SESPMNT & $R$ \\
\hline 4930 & & & & OFFSITE & SESPMNT & $R$ \\
\hline 4931 & & & & OFFSITE & SESPMNT & $R$ \\
\hline \begin{tabular}{|l|}
4932 \\
\end{tabular} & & & & OFFSITE & SESPMNT & $R$ \\
\hline \begin{tabular}{|l|}
4933 \\
\end{tabular} & & & & OFFSITE & SESPMNT & $\mathrm{R}$ \\
\hline \begin{tabular}{|l|l|}
4934 \\
\end{tabular} & & & & OFFSITE & SESPMNT & $\mathrm{R}$ \\
\hline \begin{tabular}{|l|}
4935 \\
\end{tabular} & & & & OFFSITE & SESPMNT & $\mathrm{R}$ \\
\hline 4936 & & & & OFFSITE & SESPMNT & $R$ \\
\hline \begin{tabular}{|l|}
4937 \\
\end{tabular} & & & & OFFSITE & SESPMNT & $\mathrm{R}$ \\
\hline \begin{tabular}{|l|}
4938 \\
\end{tabular} & & & & OFFSITE & SESPMNT & $\mathrm{R}$ \\
\hline \begin{tabular}{|l|}
4939 \\
\end{tabular} & & & & OFFSITE & SESPMNT & $R$ \\
\hline 4940 & & & & OFFSITE & SESPMNT & $\mathrm{R}$ \\
\hline \begin{tabular}{|l|}
4941 \\
\end{tabular} & & & & ONSITE & SESBMNT & $R$ \\
\hline
\end{tabular}




\begin{tabular}{|c|c|c|c|c|c|c|}
\hline & $A D$ & $\mathrm{AE}$ & $\mathrm{AF}$ & $A G$ & $\mathrm{AH}$ & $\mathrm{Al}$ \\
\hline 1 & RESULT_COMMENT & COLL_SAMP_SIZE & COLL_SAMP_SIZE_UNITS & DIST_CLASS & OWNER_ID & COLLECTION_PURPOSE \\
\hline 4942 & & & & ONSITE & SESPMNT & $\mathrm{R}$ \\
\hline 4943 & & & & ONSITE & SESPMNT & $\mathrm{R}$ \\
\hline 4944 & & & & ONSITE & SESPMNT & $\mathrm{R}$ \\
\hline 4945 & & & & ONSITE & SESPMNT & $\mathrm{R}$ \\
\hline \begin{tabular}{|l|l|}
4946 \\
\end{tabular} & & & & ONSITE & SESPMNT & $\mathrm{R}$ \\
\hline 4947 & & & & ONSITE & SESPMNT & $\mathrm{R}$ \\
\hline 4948 & & & & ONSITE & SESPMNT & $\mathrm{R}$ \\
\hline 4949 & & & & OFFSITE & SESPMNT & $\mathrm{R}$ \\
\hline 4950 & & & & OFFSITE & SESPMNT & $\mathrm{R}$ \\
\hline \begin{tabular}{|c|c|}
4951 \\
\end{tabular} & & & & ONSITE & SESPMNT & $\mathrm{R}$ \\
\hline \begin{tabular}{|c|}
4952 \\
\end{tabular} & & & & ONSITE & SESPMNT & $\mathrm{R}$ \\
\hline 4953 & & & & ONSITE & SESPMNT & $\mathrm{R}$ \\
\hline 4954 & & & & ONSITE & SESPMNT & $\mathrm{R}$ \\
\hline 4955 & & & & ONSITE & SESPMNT & $\mathrm{R}$ \\
\hline \begin{tabular}{|c|}
4956 \\
\end{tabular} & & & & ONSITE & SESPMNT & $\mathrm{R}$ \\
\hline \begin{tabular}{|c|}
4957 \\
\end{tabular} & & & & ONSITE & SESPMNT & $\mathrm{R}$ \\
\hline 4958 & & & & ONSITE & SESPMNT & $\mathrm{R}$ \\
\hline 4959 & & & & ONSITE & SESPMNT & $\mathrm{R}$ \\
\hline 4960 & & & & ONSITE & SESPMNT & $\mathrm{R}$ \\
\hline 4961 & & & & ONSITE & SESPMNT & $\mathrm{R}$ \\
\hline \begin{tabular}{|c|c|}
4962 \\
\end{tabular} & & & & ONSITE & SESPMNT & $\mathrm{R}$ \\
\hline 4963 & & & & ONSITE & SESPMNT & $\mathrm{R}$ \\
\hline 4964 & & & & ONSITE & SESPMNT & $\mathrm{R}$ \\
\hline 4965 & & & & ONSITE & SESPMNT & $\mathrm{R}$ \\
\hline \begin{tabular}{|l|}
4966 \\
\end{tabular} & & & & ONSITE & SESPMNT & $\mathrm{R}$ \\
\hline \begin{tabular}{|l|}
4967 \\
\end{tabular} & & & & ONSITE & SESPMNT & $\mathrm{R}$ \\
\hline \begin{tabular}{|l|}
4968 \\
\end{tabular} & & & & OFFSITE & SESPMNT & $\mathrm{R}$ \\
\hline 4969 & & & & OFFSITE & SESPMNT & $\mathrm{R}$ \\
\hline 4970 & & & & OFFSITE & SESPMNT & $\mathrm{R}$ \\
\hline \begin{tabular}{|l|l|}
4971 \\
\end{tabular} & & & & OFFSITE & SESPMNT & $\mathrm{R}$ \\
\hline \begin{tabular}{|l|}
4972 \\
\end{tabular} & & & & OFFSITE & SESPMNT & $\mathrm{R}$ \\
\hline \begin{tabular}{|l|}
4973 \\
\end{tabular} & & & & OFFSITE & SESPMNT & $\mathrm{R}$ \\
\hline \begin{tabular}{|l|l|}
4974 \\
\end{tabular} & & & & OFFSITE & SESPMNT & $\mathrm{R}$ \\
\hline \begin{tabular}{|l|}
4975 \\
\end{tabular} & & & & OFFSITE & SESPMNT & $\mathrm{R}$ \\
\hline \begin{tabular}{|l|}
4976 \\
\end{tabular} & & & & OFFSITE & SESPMNT & $\mathrm{R}$ \\
\hline \begin{tabular}{|l|l|}
4977 \\
\end{tabular} & & & & OFFSITE & SESPMNT & $\mathrm{R}$ \\
\hline \begin{tabular}{|l|}
4978 \\
\end{tabular} & & & & OFFSITE & SESPMNT & $\mathrm{R}$ \\
\hline \begin{tabular}{|l|}
4979 \\
\end{tabular} & & & & OFFSITE & SESPMNT & $R$ \\
\hline 4980 & & & & OFFSITE & SESPMNT & $\mathrm{R}$ \\
\hline \begin{tabular}{|l|l|}
4981 \\
\end{tabular} & & & & OFFSITE & SESPMNT & $\mathrm{R}$ \\
\hline \begin{tabular}{|l|}
4982 \\
\end{tabular} & & & & ONSITE & SESPMNT & $\mathrm{R}$ \\
\hline \begin{tabular}{|l|}
4983 \\
\end{tabular} & & & & ONSITE & SESPMNT & $\mathrm{R}$ \\
\hline \begin{tabular}{|l|l|}
4984 \\
\end{tabular} & & & & OFFSITE & SESPMNT & $\mathrm{R}$ \\
\hline \begin{tabular}{|l|}
4985 \\
\end{tabular} & & & & OFFSITE & SESPMNT & $\mathrm{R}$ \\
\hline \begin{tabular}{|l|}
4986 \\
\end{tabular} & & & & ONSITE & SESPMNT & $R$ \\
\hline \begin{tabular}{|l|l|}
4987 \\
\end{tabular} & & & & ONSITE & SESPMNT & $\mathrm{R}$ \\
\hline \begin{tabular}{|l|}
4988 \\
\end{tabular} & & & & ONSITE & SESPMNT & $\mathrm{R}$ \\
\hline 4989 & & & & ONSITE & SESPMNT & $\mathrm{R}$ \\
\hline 4990 & & & & ONSITE & SESPMNT & $\mathrm{R}$ \\
\hline \begin{tabular}{|l|l|}
4991 \\
\end{tabular} & & & & & SESPMNT & $\mathrm{R}$ \\
\hline \begin{tabular}{|l|l}
4992 \\
\end{tabular} & & & & & SESPMNT & $R$ \\
\hline \begin{tabular}{|l|}
4993 \\
\end{tabular} & & & & & SESBMNT & $R$ \\
\hline
\end{tabular}




\begin{tabular}{|c|c|c|c|c|c|c|}
\hline & $\mathrm{AD}$ & $\mathrm{AE}$ & $\mathrm{AF}$ & AG & $\mathrm{AH}$ & $\mathrm{Al}$ \\
\hline 1 & RESULT_COMMENT & COLL_SAMP_SIZE & COLL_SAMP_SIZE_UNITS & DIST_CLASS & OWNER_ID & COLLECTION_PURPOSE \\
\hline \begin{tabular}{|c|}
4994 \\
\end{tabular} & & & & ONSITE & SESPMNT & $\mathrm{R}$ \\
\hline \begin{tabular}{|l|l|}
4995 \\
\end{tabular} & & & & ONSITE & SESPMNT & $\mathrm{R}$ \\
\hline \begin{tabular}{|l|l|}
4996 \\
\end{tabular} & & & & ONSITE & SESPMNT & $\mathrm{R}$ \\
\hline \begin{tabular}{|l|l|}
4997 \\
\end{tabular} & & & & PERIMETER & SESPMNT & $\mathrm{R}$ \\
\hline 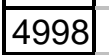 & & & & PERIMETER & SESPMNT & $\mathrm{R}$ \\
\hline \begin{tabular}{|l|l|}
4999 \\
\end{tabular} & & & & PERIMETER & SESPMNT & $\mathrm{R}$ \\
\hline 5000 & & & & ONSITE & SESPMNT & $\mathrm{R}$ \\
\hline 5001 & & & & ONSITE & SESPMNT & $\mathrm{R}$ \\
\hline 5002 & & & & OFFSITE & SESPMNT & $\mathrm{R}$ \\
\hline \begin{tabular}{|l|l|}
5003 \\
\end{tabular} & & & & OFFSITE & SESPMNT & $\mathrm{R}$ \\
\hline 5004 & & & & OFFSITE & SESPMNT & $\mathrm{R}$ \\
\hline 5005 & & & & OFFSITE & SESPMNT & $R$ \\
\hline 5006 & & & & ONSITE & SESPMNT & $\mathrm{R}$ \\
\hline \begin{tabular}{|l|}
5007 \\
\end{tabular} & & & & ONSITE & SESPMNT & $\mathrm{R}$ \\
\hline 5008 & & & & ONSITE & SESPMNT & $R$ \\
\hline \begin{tabular}{|c|}
5009 \\
\end{tabular} & & & & OFFSITE & SESPMNT & $\mathrm{R}$ \\
\hline \begin{tabular}{|l|}
5010 \\
\end{tabular} & & & & OFFSITE & SESPMNT & $\mathrm{R}$ \\
\hline 5011 & & & & OFFSITE & SESPMNT & $\mathrm{R}$ \\
\hline 5012 & & & & OFFSITE & SESPMNT & $\mathrm{R}$ \\
\hline 5013 & & & & OFFSITE & SESPMNT & $\mathrm{R}$ \\
\hline 5014 & & & & OFFSITE & SESPMNT & $R$ \\
\hline 5015 & & & & OFFSITE & SESPMNT & $\mathrm{R}$ \\
\hline 5016 & & & & OFFSITE & SESPMNT & $\mathrm{R}$ \\
\hline \begin{tabular}{|l|l|}
5017 \\
\end{tabular} & & & & OFFSITE & SESPMNT & $\mathrm{R}$ \\
\hline \begin{tabular}{|l|}
5018 \\
\end{tabular} & & & & OFFSITE & SESPMNT & $\mathrm{R}$ \\
\hline 5019 & & & & OFFSITE & SESPMNT & $\mathrm{R}$ \\
\hline 5020 & & & & OFFSITE & SESPMNT & $\mathrm{R}$ \\
\hline 5021 & & & & ONSITE & SESPMNT & $\mathrm{R}$ \\
\hline \begin{tabular}{|l|}
5022 \\
\end{tabular} & & & & ONSITE & SESPMNT & $\mathrm{R}$ \\
\hline \begin{tabular}{|l|}
5023 \\
\end{tabular} & & & & OFFSITE & SESPMNT & $R$ \\
\hline 5024 & & & & OFFSITE & SESPMNT & $R$ \\
\hline \begin{tabular}{|l|}
5025 \\
\end{tabular} & & & & ONSITE & SESPMNT & $R$ \\
\hline 5026 & & & & ONSITE & SESPMNT & $R$ \\
\hline \begin{tabular}{|l|}
5027 \\
\end{tabular} & & & & OFFSITE & SESPMNT & $R$ \\
\hline \begin{tabular}{|l|}
5028 \\
\end{tabular} & & & & OFFSITE & SESPMNT & $R$ \\
\hline \begin{tabular}{|l|}
5029 \\
\end{tabular} & & & & ONSITE & SESPMNT & $R$ \\
\hline 5030 & & & & ONSITE & SESPMNT & $R$ \\
\hline \begin{tabular}{|l|}
5031 \\
\end{tabular} & & & & ONSITE & SESPMNT & $R$ \\
\hline 5032 & & & & ONSITE & SESPMNT & $R$ \\
\hline 5033 & & & & ONSITE & SESPMNT & $R$ \\
\hline 5034 & & & & ONSITE & SESPMNT & $R$ \\
\hline \begin{tabular}{|l|}
5035 \\
\end{tabular} & & & & OFFSITE & SESPMNT & $R$ \\
\hline \begin{tabular}{|l|}
5036 \\
\end{tabular} & & & & OFFSITE & SESPMNT & $R$ \\
\hline \begin{tabular}{|l|}
5037 \\
\end{tabular} & & & & OFFSITE & SESPMNT & $\mathrm{R}$ \\
\hline \begin{tabular}{|l|}
5038 \\
\end{tabular} & & & & OFFSITE & SESPMNT & $R$ \\
\hline \begin{tabular}{|l|}
5039 \\
\end{tabular} & & & & OFFSITE & SESPMNT & $R$ \\
\hline 5040 & & & & OFFSITE & SESPMNT & $R$ \\
\hline 5041 & & & & OFFSITE & SESPMNT & $R$ \\
\hline 5042 & & & & OFFSITE & SESPMNT & $\mathrm{R}$ \\
\hline \begin{tabular}{|l|}
5043 \\
\end{tabular} & & & & OFFSITE & SESPMNT & $R$ \\
\hline 5044 & & & & OFFSITE & SESPMNT & $R$ \\
\hline \begin{tabular}{|l|l|}
5045 \\
\end{tabular} & & & & OFFSITE & SESBMNT & $R$ \\
\hline
\end{tabular}




\begin{tabular}{|c|c|c|c|c|c|c|}
\hline & $A D$ & $\mathrm{AE}$ & $\mathrm{AF}$ & $A G$ & $\mathrm{AH}$ & $\mathrm{Al}$ \\
\hline 1 & RESULT_COMMENT & COLL_SAMP_SIZE & COLL_SAMP_SIZE_UNITS & DIST_CLASS & OWNER_ID & COLLECTION_PURPOSE \\
\hline 5046 & & & & OFFSITE & SESPMNT & $\mathrm{R}$ \\
\hline 5047 & & & & ONSITE & SESPMNT & $\mathrm{R}$ \\
\hline 5048 & & & & ONSITE & SESPMNT & $\mathrm{R}$ \\
\hline 5049 & & & & ONSITE & SESPMNT & $\mathrm{R}$ \\
\hline 5050 & & & & ONSITE & SESPMNT & $\mathrm{R}$ \\
\hline 5051 & & & & ONSITE & SESPMNT & $\mathrm{R}$ \\
\hline 5052 & & & & ONSITE & SESPMNT & $\mathrm{R}$ \\
\hline 5053 & & & & OFFSITE & SESPMNT & $\mathrm{R}$ \\
\hline 5054 & & & & OFFSITE & SESPMNT & $\mathrm{R}$ \\
\hline 5055 & & & & OFFSITE & SESPMNT & $\mathrm{R}$ \\
\hline 5056 & & & & OFFSITE & SESPMNT & $\mathrm{R}$ \\
\hline 5057 & & & & OFFSITE & SESPMNT & $\mathrm{R}$ \\
\hline 5058 & & & & OFFSITE & SESPMNT & $\mathrm{R}$ \\
\hline 5059 & & & & ONSITE & SESPMNT & $\mathrm{R}$ \\
\hline 5060 & & & & ONSITE & SESPMNT & $\mathrm{R}$ \\
\hline 5061 & & & & ONSITE & SESPMNT & $\mathrm{R}$ \\
\hline 5062 & & & & ONSITE & SESPMNT & $\mathrm{R}$ \\
\hline 5063 & & & & ONSITE & SESPMNT & $\mathrm{R}$ \\
\hline 5064 & & & & ONSITE & SESPMNT & $\mathrm{R}$ \\
\hline 5065 & & & & ONSITE & SESPMNT & $\mathrm{R}$ \\
\hline 5066 & & & & ONSITE & SESPMNT & $\mathrm{R}$ \\
\hline 5067 & & & & ONSITE & SESPMNT & $\mathrm{R}$ \\
\hline 5068 & & & & ONSITE & SESPMNT & $\mathrm{R}$ \\
\hline 5069 & & & & ONSITE & SESPMNT & $\mathrm{R}$ \\
\hline 5070 & & & & ONSITE & SESPMNT & $\mathrm{R}$ \\
\hline 5071 & & & & ONSITE & SESPMNT & $\mathrm{R}$ \\
\hline 5072 & & & & ONSITE & SESPMNT & $\mathrm{R}$ \\
\hline 5073 & & & & ONSITE & SESPMNT & $\mathrm{R}$ \\
\hline 5074 & & & & OFFSITE & SESPSPEC & $\mathrm{s}$ \\
\hline 5075 & & & & OFFSITE & SESPSPEC & $\mathrm{S}$ \\
\hline 5076 & & & & OFFSITE & SESPSPEC & $\mathrm{S}$ \\
\hline 5077 & & & & OFFSITE & SESPSPEC & $\mathrm{s}$ \\
\hline 5078 & & & & OFFSITE & SESPSPEC & $\mathrm{S}$ \\
\hline \begin{tabular}{|l|}
5079 \\
\end{tabular} & & & & OFFSITE & SESPSPEC & $\mathrm{S}$ \\
\hline 5080 & & & & OFFSITE & SESPSPEC & $\mathrm{S}$ \\
\hline \begin{tabular}{|l|}
5081 \\
\end{tabular} & & & & OFFSITE & SESPSPEC & $\mathrm{S}$ \\
\hline 5082 & & & & OFFSITE & SESPSPEC & $\mathrm{s}$ \\
\hline 5083 & & & & OFFSITE & SESPSPEC & $\mathrm{S}$ \\
\hline 5084 & & & & OFFSITE & SESPSPEC & $\mathrm{s}$ \\
\hline 5085 & & & & OFFSITE & SESPSPEC & $\mathrm{s}$ \\
\hline 5086 & & & & ONSITE & SESPMNT & $\mathrm{R}$ \\
\hline \begin{tabular}{|l|}
5087 \\
\end{tabular} & & & & ONSITE & SESPMNT & $\mathrm{R}$ \\
\hline 5088 & & & & OFFSITE & SESPMNT & $\mathrm{R}$ \\
\hline 5089 & & & & OFFSITE & SESPMNT & $\mathrm{R}$ \\
\hline 5090 & & & & OFFSITE & SESPMNT & $\mathrm{R}$ \\
\hline 5091 & & & & OFFSITE & SESPMNT & $\mathrm{R}$ \\
\hline \begin{tabular}{|l|}
5092 \\
\end{tabular} & & & & OFFSITE & SESPMNT & $\mathrm{R}$ \\
\hline 5093 & & & & OFFSITE & SESPMNT & $\mathrm{R}$ \\
\hline \begin{tabular}{|l|}
5094 \\
\end{tabular} & & & & OFFSITE & SESPMNT & $\mathrm{R}$ \\
\hline 5095 & & & & OFFSITE & SESPMNT & $R$ \\
\hline 5096 & & & & OFFSITE & SESPMNT & $\mathrm{R}$ \\
\hline \begin{tabular}{|l|}
5097 \\
\end{tabular} & & & & OFFSITE & SESBMNT & ${ }_{2} R$ \\
\hline
\end{tabular}




\begin{tabular}{|c|c|c|c|c|c|c|}
\hline & $A D$ & $\mathrm{AE}$ & $\mathrm{AF}$ & $A G$ & $\mathrm{AH}$ & $\mathrm{Al}$ \\
\hline 1 & RESULT_COMMENT & COLL_SAMP_SIZE & COLL_SAMP_SIZE_UNITS & DIST_CLASS & OWNER_ID & COLLECTION_PURPOSE \\
\hline 5098 & & & & OFFSITE & SESPMNT & $\mathrm{R}$ \\
\hline 5099 & & & & OFFSITE & SESPMNT & $\mathrm{R}$ \\
\hline 5100 & & & & OFFSITE & SESPMNT & $\mathrm{R}$ \\
\hline 5101 & & & & OFFSITE & SESPMNT & $\mathrm{R}$ \\
\hline 5102 & & & & ONSITE & SESPMNT & $\mathrm{R}$ \\
\hline 5103 & & & & ONSITE & SESPMNT & $\mathrm{R}$ \\
\hline 5104 & & & & OFFSITE & SESPMNT & $\mathrm{R}$ \\
\hline 5105 & & & & OFFSITE & SESPMNT & $\mathrm{R}$ \\
\hline 5106 & & & & ONSITE & SESPMNT & $\mathrm{R}$ \\
\hline 5107 & & & & ONSITE & SESPMNT & $\mathrm{R}$ \\
\hline 5108 & & & & ONSITE & SESPMNT & $\mathrm{R}$ \\
\hline 5109 & & & & ONSITE & SESPMNT & $\mathrm{R}$ \\
\hline 5110 & & & & ONSITE & SESPMNT & $\mathrm{R}$ \\
\hline 5111 & & & & & SESPMNT & $\mathrm{R}$ \\
\hline 5112 & & & & & SESPMNT & $\mathrm{R}$ \\
\hline 5113 & & & & & SESPMNT & $\mathrm{R}$ \\
\hline 5114 & & & & ONSITE & SESPMNT & $\mathrm{R}$ \\
\hline 5115 & & & & ONSITE & SESPMNT & $\mathrm{R}$ \\
\hline 5116 & & & & ONSITE & SESPMNT & $\mathrm{R}$ \\
\hline 5117 & & & & ONSITE & SESPMNT & $\mathrm{R}$ \\
\hline 5118 & & & & ONSITE & SESPMNT & $\mathrm{R}$ \\
\hline 5119 & & & & ONSITE & SESPMNT & $\mathrm{R}$ \\
\hline 5120 & & & & PERIMETER & SESPMNT & $\mathrm{R}$ \\
\hline \begin{tabular}{|l|}
5121 \\
\end{tabular} & & & & PERIMETER & SESPMNT & $\mathrm{R}$ \\
\hline 5122 & & & & PERIMETER & SESPMNT & $\mathrm{R}$ \\
\hline 5123 & & & & ONSITE & SESPMNT & $\mathrm{R}$ \\
\hline 5124 & & & & ONSITE & SESPMNT & $\mathrm{R}$ \\
\hline 5125 & & & & OFFSITE & SESPMNT & $\mathrm{R}$ \\
\hline 5126 & & & & OFFSITE & SESPMNT & $\mathrm{R}$ \\
\hline 5127 & See sample record for comment. & & & OFFSITE & SESPMNT & $\mathrm{R}$ \\
\hline 5128 & See sample record for comment. & & & OFFSITE & SESPMNT & $\mathrm{R}$ \\
\hline 5129 & & & & ONSITE & SESPMNT & $\mathrm{R}$ \\
\hline 5130 & & & & ONSITE & SESPMNT & $\mathrm{R}$ \\
\hline 5131 & & & & ONSITE & SESPMNT & $\mathrm{R}$ \\
\hline 5132 & & & & ONSITE & SESPMNT & $\mathrm{R}$ \\
\hline 5133 & & & & ONSITE & SESPMNT & $\mathrm{R}$ \\
\hline 5134 & & & & OFFSITE & SESPMNT & $\mathrm{R}$ \\
\hline 5135 & & & & OFFSITE & SESPMNT & $\mathrm{R}$ \\
\hline 5136 & & & & OFFSITE & SESPMNT & $\mathrm{R}$ \\
\hline 5137 & & & & OFFSITE & SESPMNT & $\mathrm{R}$ \\
\hline 5138 & & & & OFFSITE & SESPMNT & $\mathrm{R}$ \\
\hline 5139 & & & & OFFSITE & SESPMNT & $\mathrm{R}$ \\
\hline 5140 & & & & OFFSITE & SESPMNT & $\mathrm{R}$ \\
\hline 5141 & & & & OFFSITE & SESPMNT & $\mathrm{R}$ \\
\hline 5142 & & & & OFFSITE & SESPMNT & $\mathrm{R}$ \\
\hline 5143 & & & & OFFSITE & SESPMNT & $\mathrm{R}$ \\
\hline 5144 & & & & OFFSITE & SESPMNT & $\mathrm{R}$ \\
\hline 5145 & & & & OFFSITE & SESPMNT & $\mathrm{R}$ \\
\hline 5146 & & & & OFFSITE & SESPMNT & $\mathrm{R}$ \\
\hline 5147 & & & & OFFSITE & SESPMNT & $\mathrm{R}$ \\
\hline 5148 & & & & ONSITE & SESPMNT & $\mathrm{R}$ \\
\hline 5149 & & & & ONSITE & SESBMNT & ${ }_{2} R$ \\
\hline
\end{tabular}




\begin{tabular}{|c|c|c|c|c|c|c|}
\hline & $A D$ & $\mathrm{AE}$ & $\mathrm{AF}$ & $A G$ & $\mathrm{AH}$ & $\mathrm{Al}$ \\
\hline 1 & RESULT_COMMENT & COLL_SAMP_SIZE & COLL_SAMP_SIZE_UNITS & DIST_CLASS & OWNER_ID & COLLECTION_PURPOSE \\
\hline 5150 & & & & OFFSITE & SESPMNT & $\mathrm{R}$ \\
\hline 5151 & & & & OFFSITE & SESPMNT & $\mathrm{R}$ \\
\hline 5152 & & & & ONSITE & SESPMNT & $\mathrm{R}$ \\
\hline 5153 & & & & ONSITE & SESPMNT & $\mathrm{R}$ \\
\hline 5154 & & & & ONSITE & SESPMNT & $\mathrm{R}$ \\
\hline 5155 & & & & ONSITE & SESPMNT & $\mathrm{R}$ \\
\hline 5156 & & & & ONSITE & SESPMNT & $\mathrm{R}$ \\
\hline 5157 & & & & ONSITE & SESPMNT & $\mathrm{R}$ \\
\hline 5158 & & & & ONSITE & SESPMNT & $\mathrm{R}$ \\
\hline 5159 & & & & ONSITE & SESPMNT & $\mathrm{R}$ \\
\hline 5160 & & & & ONSITE & SESPMNT & $\mathrm{R}$ \\
\hline 5161 & & & & ONSITE & SESPMNT & $\mathrm{R}$ \\
\hline 5162 & & & & ONSITE & SESPMNT & $\mathrm{R}$ \\
\hline 5163 & & & & ONSITE & SESPMNT & $\mathrm{R}$ \\
\hline 5164 & & & & OFFSITE & SESPMNT & $\mathrm{R}$ \\
\hline 5165 & & & & OFFSITE & SESPMNT & $\mathrm{R}$ \\
\hline 5166 & & & & OFFSITE & SESPMNT & $\mathrm{R}$ \\
\hline \begin{tabular}{|c|}
5167 \\
\end{tabular} & & & & OFFSITE & SESPMNT & $\mathrm{R}$ \\
\hline 5168 & & & & OFFSITE & SESPMNT & $\mathrm{R}$ \\
\hline 5169 & & & & OFFSITE & SESPMNT & $\mathrm{R}$ \\
\hline 5170 & & & & OFFSITE & SESPMNT & $\mathrm{R}$ \\
\hline 5171 & & & & OFFSITE & SESPMNT & $\mathrm{R}$ \\
\hline 5172 & & & & OFFSITE & SESPMNT & $\mathrm{R}$ \\
\hline 5173 & & & & OFFSITE & SESPMNT & $\mathrm{R}$ \\
\hline 5174 & & & & OFFSITE & SESPMNT & $\mathrm{R}$ \\
\hline 5175 & & & & OFFSITE & SESPMNT & $\mathrm{R}$ \\
\hline 5176 & & & & OFFSITE & SESPMNT & $\mathrm{R}$ \\
\hline 5177 & & & & OFFSITE & SESPMNT & $\mathrm{R}$ \\
\hline 5178 & & & & OFFSITE & SESPMNT & $\mathrm{R}$ \\
\hline 5179 & & & & OFFSITE & SESPMNT & $\mathrm{R}$ \\
\hline 5180 & & & & OFFSITE & SESPMNT & $\mathrm{R}$ \\
\hline 5181 & & & & OFFSITE & SESPMNT & $\mathrm{R}$ \\
\hline 5182 & & & & ONSITE & SESPMNT & $\mathrm{R}$ \\
\hline 5183 & & & & ONSITE & SESPMNT & $\mathrm{R}$ \\
\hline 5184 & & & & ONSITE & SESPMNT & $\mathrm{R}$ \\
\hline 5185 & & & & ONSITE & SESPMNT & $\mathrm{R}$ \\
\hline 5186 & & & & ONSITE & SESPMNT & $\mathrm{R}$ \\
\hline \begin{tabular}{|l|l|}
5187 \\
\end{tabular} & & & & ONSITE & SESPMNT & $R$ \\
\hline 5188 & & & & ONSITE & SESPMNT & $\mathrm{R}$ \\
\hline 5189 & & & & ONSITE & SESPMNT & $\mathrm{R}$ \\
\hline 5190 & & & & ONSITE & SESPMNT & $\mathrm{R}$ \\
\hline 5191 & & & & ONSITE & SESPMNT & $\mathrm{R}$ \\
\hline 5192 & & & & ONSITE & SESPMNT & $\mathrm{R}$ \\
\hline 5193 & & & & ONSITE & SESPMNT & $\mathrm{R}$ \\
\hline 5194 & & & & ONSITE & SESPMNT & $R$ \\
\hline 5195 & & & & ONSITE & SESPMNT & $\mathrm{R}$ \\
\hline 5196 & & & & ONSITE & SESPMNT & $\mathrm{R}$ \\
\hline 5197 & & & & ONSITE & SESPMNT & $\mathrm{R}$ \\
\hline \begin{tabular}{|l|}
5198 \\
\end{tabular} & & & & ONSITE & SESPMNT & $\mathrm{R}$ \\
\hline 5199 & & & & OFFSITE & SESPMNT & $\mathrm{R}$ \\
\hline 5200 & & & & OFFSITE & SESPMNT & $\mathrm{R}$ \\
\hline 5201 & & & & OFFSITE & SESBMNT & ${ }_{1} R$ \\
\hline
\end{tabular}




\begin{tabular}{|c|c|c|c|c|c|c|}
\hline & $\mathrm{AD}$ & $\mathrm{AE}$ & $\mathrm{AF}$ & $\mathrm{AG}$ & $\mathrm{AH}$ & $\mathrm{Al}$ \\
\hline 1 & RESULT_COMMENT & COLL_SAMP_SIZE & COLL_SAMP_SIZE_UNITS & DIST_CLASS & OWNER_ID & COLLECTION_PURPOSE \\
\hline 5202 & & & & OFFSITE & SESPMNT & $\mathrm{R}$ \\
\hline 5203 & & & & ONSITE & SESPMNT & $\mathrm{R}$ \\
\hline 5204 & & & & ONSITE & SESPMNT & $\mathrm{R}$ \\
\hline 5205 & & & & OFFSITE & SESPMNT & $\mathrm{R}$ \\
\hline 5206 & & & & OFFSITE & SESPMNT & $\mathrm{R}$ \\
\hline 5207 & & & & OFFSITE & SESPMNT & $\mathrm{R}$ \\
\hline 5208 & & & & OFFSITE & SESPMNT & $\mathrm{R}$ \\
\hline 5209 & & & & OFFSITE & SESPMNT & $\mathrm{R}$ \\
\hline 5210 & & & & OFFSITE & SESPMNT & $\mathrm{R}$ \\
\hline 5211 & & & & OFFSITE & SESPMNT & $\mathrm{R}$ \\
\hline 5212 & & & & OFFSITE & SESPMNT & $\mathrm{R}$ \\
\hline 5213 & & & & OFFSITE & SESPMNT & $\mathrm{R}$ \\
\hline 5214 & & & & OFFSITE & SESPMNT & $\mathrm{R}$ \\
\hline 5215 & & & & OFFSITE & SESPMNT & $\mathrm{R}$ \\
\hline 5216 & & & & OFFSITE & SESPMNT & $\mathrm{R}$ \\
\hline 5217 & & & & OFFSITE & SESPMNT & $\mathrm{R}$ \\
\hline 5218 & & & & OFFSITE & SESPMNT & $\mathrm{R}$ \\
\hline 5219 & & & & OFFSITE & SESPMNT & $\mathrm{R}$ \\
\hline 5220 & & & & OFFSITE & SESPMNT & $\mathrm{R}$ \\
\hline 5221 & & & & PERIMETER & SESPMNT & $\mathrm{R}$ \\
\hline 5222 & & & & PERIMETER & SESPMNT & $\mathrm{R}$ \\
\hline 5223 & & & & PERIMETER & SESPMNT & $\mathrm{R}$ \\
\hline 5224 & & & & ONSITE & SESPMNT & $\mathrm{R}$ \\
\hline 5225 & & & & ONSITE & SESPMNT & $\mathrm{R}$ \\
\hline 5226 & & & & OFFSITE & SESPMNT & $\mathrm{R}$ \\
\hline \begin{tabular}{|l|}
5227 \\
\end{tabular} & & & & OFFSITE & SESPMNT & $\mathrm{R}$ \\
\hline 5228 & & & & OFFSITE & SESPMNT & $\mathrm{R}$ \\
\hline 5229 & & & & OFFSITE & SESPMNT & $\mathrm{R}$ \\
\hline 5230 & & & & ONSITE & SESPMNT & $\mathrm{R}$ \\
\hline 5231 & & & & ONSITE & SESPMNT & $\mathrm{R}$ \\
\hline \begin{tabular}{|l|}
5232 \\
\end{tabular} & & & & ONSITE & SESPMNT & $\mathrm{R}$ \\
\hline 5233 & & & & ONSITE & SESPMNT & $\mathrm{R}$ \\
\hline 5234 & & & & ONSITE & SESPMNT & $\mathrm{R}$ \\
\hline 5235 & & & & OFFSITE & SESPMNT & $\mathrm{R}$ \\
\hline 5236 & & & & OFFSITE & SESPMNT & $\mathrm{R}$ \\
\hline \begin{tabular}{|l|l|}
5237 \\
\end{tabular} & & & & OFFSITE & SESPMNT & $\mathrm{R}$ \\
\hline 5238 & & & & OFFSITE & SESPMNT & $\mathrm{R}$ \\
\hline 5239 & & & & OFFSITE & SESPMNT & $\mathrm{R}$ \\
\hline \begin{tabular}{|l|}
5240 \\
\end{tabular} & & & & OFFSITE & SESPMNT & $\mathrm{R}$ \\
\hline \begin{tabular}{|l|}
5241 \\
\end{tabular} & & & & OFFSITE & SESPMNT & $\mathrm{R}$ \\
\hline \begin{tabular}{|l|}
5242 \\
\end{tabular} & & & & OFFSITE & SESPMNT & $\mathrm{R}$ \\
\hline \begin{tabular}{|l|}
5243 \\
\end{tabular} & & & & OFFSITE & SESPMNT & $\mathrm{R}$ \\
\hline \begin{tabular}{|l|}
5244 \\
\end{tabular} & & & & OFFSITE & SESPMNT & $\mathrm{R}$ \\
\hline \begin{tabular}{|l|}
5245 \\
\end{tabular} & & & & OFFSITE & SESPMNT & $\mathrm{R}$ \\
\hline \begin{tabular}{|l|}
5246 \\
\end{tabular} & & & & OFFSITE & SESPMNT & $\mathrm{R}$ \\
\hline \begin{tabular}{|l|l|}
5247 \\
\end{tabular} & & & & OFFSITE & SESPMNT & $\mathrm{R}$ \\
\hline \begin{tabular}{|l|}
5248 \\
\end{tabular} & & & & OFFSITE & SESPMNT & $\mathrm{R}$ \\
\hline \begin{tabular}{|l|}
5249 \\
\end{tabular} & & & & OFFSITE & SESPMNT & $\mathrm{R}$ \\
\hline 5250 & & & & OFFSITE & SESPMNT & $\mathrm{R}$ \\
\hline \begin{tabular}{|l|}
5251 \\
\end{tabular} & & & & ONSITE & SESPMNT & $\mathrm{R}$ \\
\hline \begin{tabular}{|l|}
5252 \\
\end{tabular} & & & & ONSITE & SESPMNT & $\mathrm{R}$ \\
\hline \begin{tabular}{|l|}
5253 \\
\end{tabular} & & & & ONSITE & SESBMNT & $R$ \\
\hline
\end{tabular}




\begin{tabular}{|c|c|c|c|c|c|c|}
\hline & $A D$ & $\mathrm{AE}$ & $\mathrm{AF}$ & $A G$ & $\mathrm{AH}$ & $\mathrm{Al}$ \\
\hline 1 & RESULT_COMMENT & COLL_SAMP_SIZE & COLL_SAMP_SIZE_UNITS & DIST_CLASS & OWNER_ID & COLLECTION_PURPOSE \\
\hline 5254 & & & & ONSITE & SESPMNT & $\mathrm{R}$ \\
\hline 5255 & & & & ONSITE & SESPMNT & $\mathrm{R}$ \\
\hline 5256 & & & & & SESPMNT & $\mathrm{R}$ \\
\hline \begin{tabular}{|l|l|}
5257 \\
\end{tabular} & & & & & SESPMNT & $\mathrm{R}$ \\
\hline 5258 & & & & & SESPMNT & $\mathrm{R}$ \\
\hline 5259 & & & & ONSITE & SESPMNT & $\mathrm{R}$ \\
\hline 5260 & & & & ONSITE & SESPMNT & $\mathrm{R}$ \\
\hline 5261 & & & & ONSITE & SESPMNT & $\mathrm{R}$ \\
\hline 5262 & & & & ONSITE & SESPMNT & $\mathrm{R}$ \\
\hline 5263 & & & & ONSITE & SESPMNT & $\mathrm{R}$ \\
\hline 5264 & & & & ONSITE & SESPMNT & $\mathrm{R}$ \\
\hline 5265 & See sample record for comment. & & & ONSITE & SESPMNT & $\mathrm{R}$ \\
\hline 5266 & See sample record for comment. & & & ONSITE & SESPMNT & $\mathrm{R}$ \\
\hline \begin{tabular}{|l|l|}
5267 \\
\end{tabular} & & & & OFFSITE & SESPMNT & $\mathrm{R}$ \\
\hline 5268 & & & & OFFSITE & SESPMNT & $\mathrm{R}$ \\
\hline 5269 & & & & OFFSITE & SESPMNT & $\mathrm{R}$ \\
\hline 5270 & & & & OFFSITE & SESPMNT & $\mathrm{R}$ \\
\hline 5271 & & & & ONSITE & SESPMNT & $\mathrm{R}$ \\
\hline 5272 & & & & ONSITE & SESPMNT & $\mathrm{R}$ \\
\hline 5273 & & & & OFFSITE & SESPMNT & $\mathrm{R}$ \\
\hline 5274 & & & & OFFSITE & SESPMNT & $\mathrm{R}$ \\
\hline 5275 & & & & OFFSITE & SESPMNT & $\mathrm{R}$ \\
\hline 5276 & & & & OFFSITE & SESPMNT & $\mathrm{R}$ \\
\hline 5277 & & & & ONSITE & SESPMNT & $\mathrm{R}$ \\
\hline 5278 & & & & ONSITE & SESPMNT & $\mathrm{R}$ \\
\hline \begin{tabular}{|l|}
5279 \\
\end{tabular} & & & & ONSITE & SESPMNT & $\mathrm{R}$ \\
\hline 5280 & & & & ONSITE & SESPMNT & $\mathrm{R}$ \\
\hline \begin{tabular}{|l|}
5281 \\
\end{tabular} & & & & ONSITE & SESPMNT & $\mathrm{R}$ \\
\hline 5282 & & & & ONSITE & SESPMNT & $\mathrm{R}$ \\
\hline 5283 & & & & ONSITE & SESPMNT & $R$ \\
\hline 5284 & & & & ONSITE & SESPMNT & $\mathrm{R}$ \\
\hline \begin{tabular}{|l|}
5285 \\
\end{tabular} & & & & ONSITE & SESPMNT & $\mathrm{R}$ \\
\hline 5286 & & & & ONSITE & SESPMNT & $\mathrm{R}$ \\
\hline \begin{tabular}{|l|}
5287 \\
\end{tabular} & & & & ONSITE & SESPMNT & $\mathrm{R}$ \\
\hline 5288 & & & & ONSITE & SESPMNT & $\mathrm{R}$ \\
\hline 5289 & & & & OFFSITE & SESPMNT & $\mathrm{R}$ \\
\hline 5290 & & & & OFFSITE & SESPMNT & $\mathrm{R}$ \\
\hline 5291 & & & & OFFSITE & SESPMNT & $\mathrm{R}$ \\
\hline 5292 & & & & OFFSITE & SESPMNT & $\mathrm{R}$ \\
\hline 5293 & & & & OFFSITE & SESPMNT & $\mathrm{R}$ \\
\hline 5294 & & & & OFFSITE & SESPMNT & $\mathrm{R}$ \\
\hline 5295 & & & & OFFSITE & SESPMNT & $\mathrm{R}$ \\
\hline 5296 & & & & OFFSITE & SESPMNT & $\mathrm{R}$ \\
\hline \begin{tabular}{|l|}
5297 \\
\end{tabular} & & & & OFFSITE & SESPMNT & $\mathrm{R}$ \\
\hline \begin{tabular}{|l|}
5298 \\
\end{tabular} & & & & OFFSITE & SESPMNT & $\mathrm{R}$ \\
\hline \begin{tabular}{|l|}
5299 \\
\end{tabular} & & & & OFFSITE & SESPMNT & $\mathrm{R}$ \\
\hline 5300 & & & & OFFSITE & SESPMNT & $\mathrm{R}$ \\
\hline 5301 & & & & ONSITE & SESPMNT & $\mathrm{R}$ \\
\hline 5302 & & & & ONSITE & SESPMNT & $\mathrm{R}$ \\
\hline 5303 & & & & ONSITE & SESPMNT & $R$ \\
\hline 5304 & & & & ONSITE & SESPMNT & $\mathrm{R}$ \\
\hline 5305 & & & & ONSITE & SESBMNT & $R$ \\
\hline
\end{tabular}




\begin{tabular}{|c|c|c|c|c|c|c|}
\hline & $A D$ & $\mathrm{AE}$ & $\mathrm{AF}$ & $A G$ & $\mathrm{AH}$ & $\mathrm{Al}$ \\
\hline 1 & RESULT_COMMENT & COLL_SAMP_SIZE & COLL_SAMP_SIZE_UNITS & DIST_CLASS & OWNER_ID & COLLECTION_PURPOSE \\
\hline 5306 & & & & ONSITE & SESPMNT & $\mathrm{R}$ \\
\hline 5307 & & & & ONSITE & SESPMNT & $\mathrm{R}$ \\
\hline 5308 & & & & ONSITE & SESPMNT & $\mathrm{R}$ \\
\hline 5309 & & & & OFFSITE & SESPMNT & $\mathrm{R}$ \\
\hline 5310 & & & & OFFSITE & SESPMNT & $\mathrm{R}$ \\
\hline 5311 & & & & OFFSITE & SESPMNT & $\mathrm{R}$ \\
\hline 5312 & & & & OFFSITE & SESPMNT & $\mathrm{R}$ \\
\hline 5313 & & & & OFFSITE & SESPMNT & $\mathrm{R}$ \\
\hline 5314 & & & & OFFSITE & SESPMNT & $\mathrm{R}$ \\
\hline 5315 & & & & OFFSITE & SESPMNT & $\mathrm{R}$ \\
\hline 5316 & & & & OFFSITE & SESPMNT & $\mathrm{R}$ \\
\hline 5317 & & & & OFFSITE & SESPMNT & $\mathrm{R}$ \\
\hline 5318 & & & & OFFSITE & SESPMNT & $\mathrm{R}$ \\
\hline 5319 & & & & ONSITE & SESPMNT & $\mathrm{R}$ \\
\hline 5320 & & & & ONSITE & SESPMNT & $\mathrm{R}$ \\
\hline 5321 & & & & ONSITE & SESPMNT & $\mathrm{R}$ \\
\hline 5322 & & & & ONSITE & SESPMNT & $\mathrm{R}$ \\
\hline 5323 & & & & ONSITE & SESPMNT & $\mathrm{R}$ \\
\hline 5324 & & & & ONSITE & SESPMNT & $\mathrm{R}$ \\
\hline 5325 & & & & OFFSITE & SESPSPEC & $\mathrm{s}$ \\
\hline 5326 & & & & OFFSITE & SESPSPEC & $\mathrm{s}$ \\
\hline 5327 & & & & OFFSITE & SESPSPEC & $\mathrm{S}$ \\
\hline 5328 & & & & OFFSITE & SESPSPEC & $\mathrm{s}$ \\
\hline 5329 & & & & OFFSITE & SESPSPEC & $\mathrm{s}$ \\
\hline 5330 & & & & OFFSITE & SESPSPEC & $\mathrm{S}$ \\
\hline 5331 & & & & OFFSITE & SESPSPEC & $\mathrm{s}$ \\
\hline 5332 & & & & OFFSITE & SESPSPEC & $\mathrm{s}$ \\
\hline 5333 & & & & OFFSITE & SESPSPEC & $\mathrm{s}$ \\
\hline 5334 & & & & OFFSITE & SESPSPEC & $\mathrm{s}$ \\
\hline 5335 & & & & OFFSITE & SESPSPEC & $\mathrm{S}$ \\
\hline 5336 & & & & OFFSITE & SESPSPEC & $\mathrm{S}$ \\
\hline 5337 & & & & ONSITE & SESPMNT & $\mathrm{R}$ \\
\hline 5338 & & & & ONSITE & SESPMNT & $\mathrm{R}$ \\
\hline 5339 & & & & OFFSITE & SESPMNT & $\mathrm{R}$ \\
\hline 5340 & & & & OFFSITE & SESPMNT & $\mathrm{R}$ \\
\hline \begin{tabular}{|l|l|}
5341 \\
\end{tabular} & & & & OFFSITE & SESPMNT & $\mathrm{R}$ \\
\hline 5342 & & & & OFFSITE & SESPMNT & $\mathrm{R}$ \\
\hline 5343 & & & & OFFSITE & SESPMNT & $\mathrm{R}$ \\
\hline 5344 & & & & OFFSITE & SESPMNT & $\mathrm{R}$ \\
\hline 5345 & & & & OFFSITE & SESPMNT & $\mathrm{R}$ \\
\hline 5346 & & & & OFFSITE & SESPMNT & $\mathrm{R}$ \\
\hline \begin{tabular}{|l|}
5347 \\
\end{tabular} & & & & OFFSITE & SESPMNT & $\mathrm{R}$ \\
\hline 5348 & & & & OFFSITE & SESPMNT & $\mathrm{R}$ \\
\hline 5349 & & & & OFFSITE & SESPMNT & $\mathrm{R}$ \\
\hline 5350 & & & & OFFSITE & SESPMNT & $\mathrm{R}$ \\
\hline 5351 & & & & OFFSITE & SESPMNT & $\mathrm{R}$ \\
\hline \begin{tabular}{|l|}
5352 \\
\end{tabular} & & & & OFFSITE & SESPMNT & $\mathrm{R}$ \\
\hline 5353 & & & & OFFSITE & SESPMNT & $\mathrm{R}$ \\
\hline 5354 & & & & OFFSITE & SESPMNT & $\mathrm{R}$ \\
\hline 5355 & & & & PERIMETER & SESPMNT & $R$ \\
\hline 5356 & & & & PERIMETER & $\begin{array}{l}\text { SESPMNT } \\
\end{array}$ & $\mathrm{R}$ \\
\hline \begin{tabular}{|l|}
5357 \\
\end{tabular} & & & & PERIMETER & SESBMNT & $\mathrm{R}$ \\
\hline
\end{tabular}




\begin{tabular}{|c|c|c|c|c|c|c|}
\hline & $A D$ & $\mathrm{AE}$ & $\mathrm{AF}$ & $A G$ & $\mathrm{AH}$ & $\mathrm{Al}$ \\
\hline 1 & RESULT_COMMENT & COLL_SAMP_SIZE & COLL_SAMP_SIZE_UNITS & DIST_CLASS & OWNER_ID & COLLECTION_PURPOSE \\
\hline 5358 & & & & ONSITE & SESPMNT & $\mathrm{R}$ \\
\hline 5359 & & & & ONSITE & SESPMNT & $\mathrm{R}$ \\
\hline 5360 & & & & OFFSITE & SESPMNT & $\mathrm{R}$ \\
\hline 5361 & & & & OFFSITE & SESPMNT & $\mathrm{R}$ \\
\hline 5362 & & & & OFFSITE & SESPMNT & $\mathrm{R}$ \\
\hline 5363 & & & & OFFSITE & SESPMNT & $\mathrm{R}$ \\
\hline 5364 & & & & ONSITE & SESPMNT & $\mathrm{R}$ \\
\hline 5365 & & & & ONSITE & SESPMNT & $\mathrm{R}$ \\
\hline 5366 & & & & ONSITE & SESPMNT & $\mathrm{R}$ \\
\hline \begin{tabular}{|c|c|}
5367 \\
\end{tabular} & & & & ONSITE & SESPMNT & $\mathrm{R}$ \\
\hline 5368 & & & & ONSITE & SESPMNT & $\mathrm{R}$ \\
\hline 5369 & See sample record for comment. & & & OFFSITE & SESPMNT & $\mathrm{R}$ \\
\hline 5370 & & & & OFFSITE & SESPMNT & $\mathrm{R}$ \\
\hline 5371 & & & & OFFSITE & SESPMNT & $\mathrm{R}$ \\
\hline 5372 & & & & OFFSITE & SESPMNT & $\mathrm{R}$ \\
\hline 5373 & & & & OFFSITE & SESPMNT & $\mathrm{R}$ \\
\hline 5374 & & & & ONSITE & SESPMNT & $\mathrm{R}$ \\
\hline 5375 & & & & ONSITE & SESPMNT & $\mathrm{R}$ \\
\hline 5376 & & & & ONSITE & SESPMNT & $\mathrm{R}$ \\
\hline 5377 & & & & ONSITE & SESPMNT & $\mathrm{R}$ \\
\hline 5378 & & & & ONSITE & SESPMNT & $\mathrm{R}$ \\
\hline 5379 & & & & & SESPMNT & $\mathrm{R}$ \\
\hline 5380 & & & & & SESPMNT & $\mathrm{R}$ \\
\hline 5381 & & & & & SESPMNT & $\mathrm{R}$ \\
\hline 5382 & & & & ONSITE & SESPMNT & $\mathrm{R}$ \\
\hline 5383 & & & & ONSITE & SESPMNT & $\mathrm{R}$ \\
\hline 5384 & & & & ONSITE & SESPMNT & $\mathrm{R}$ \\
\hline 5385 & & & & ONSITE & SESPMNT & $\mathrm{R}$ \\
\hline 5386 & & & & ONSITE & SESPMNT & $\mathrm{R}$ \\
\hline \begin{tabular}{|l|l|}
5387 \\
\end{tabular} & & & & ONSITE & SESPMNT & $R$ \\
\hline 5388 & & & & ONSITE & SESPMNT & $\mathrm{R}$ \\
\hline 5389 & & & & ONSITE & SESPMNT & $\mathrm{R}$ \\
\hline 5390 & & & & OFFSITE & SESPMNT & $\mathrm{R}$ \\
\hline 5391 & & & & OFFSITE & SESPMNT & $\mathrm{R}$ \\
\hline 5392 & & & & OFFSITE & SESPMNT & $\mathrm{R}$ \\
\hline 5393 & & & & OFFSITE & SESPMNT & $\mathrm{R}$ \\
\hline 5394 & & & & OFFSITE & SESPMNT & $\mathrm{R}$ \\
\hline 5395 & & & & OFFSITE & SESPMNT & $\mathrm{R}$ \\
\hline 5396 & & & & OFFSITE & SESPMNT & $\mathrm{R}$ \\
\hline 5397 & & & & OFFSITE & SESPMNT & $\mathrm{R}$ \\
\hline 5398 & & & & OFFSITE & SESPMNT & $\mathrm{R}$ \\
\hline 5399 & & & & OFFSITE & SESPMNT & $\mathrm{R}$ \\
\hline 5400 & & & & OFFSITE & SESPMNT & $\mathrm{R}$ \\
\hline 5401 & & & & OFFSITE & SESPMNT & $\mathrm{R}$ \\
\hline 5402 & & & & OFFSITE & SESPMNT & $\mathrm{R}$ \\
\hline 5403 & & & & OFFSITE & SESPMNT & $\mathrm{R}$ \\
\hline 5404 & & & & OFFSITE & SESPMNT & $\mathrm{R}$ \\
\hline 5405 & & & & OFFSITE & SESPMNT & $\mathrm{R}$ \\
\hline 5406 & & & & ONSITE & SESPMNT & $\mathrm{R}$ \\
\hline \begin{tabular}{|l|l|}
5407 \\
\end{tabular} & & & & ONSITE & SESPMNT & $R$ \\
\hline 5408 & & & & OFFSITE & SESPMNT & $\mathrm{R}$ \\
\hline 5409 & & & & OFFSITE & SESBMNT & ${ }_{0} R$ \\
\hline
\end{tabular}




\begin{tabular}{|c|c|c|c|c|c|c|}
\hline & $\mathrm{AD}$ & $\mathrm{AE}$ & $\mathrm{AF}$ & AG & $\mathrm{AH}$ & $\overline{\mathrm{Al}}$ \\
\hline 1 & RESULT_COMMENT & COLL_SAMP_SIZE & COLL_SAMP_SIZE_UNITS & DIST_CLASS & OWNER_ID & COLLECTION_PURPOSE \\
\hline 5410 & & & & OFFSITE & SESPMNT & $\mathrm{R}$ \\
\hline 5411 & & & & OFFSITE & SESPMNT & $\mathrm{R}$ \\
\hline 5412 & & & & ONSITE & SESPMNT & $\mathrm{R}$ \\
\hline 5413 & & & & ONSITE & SESPMNT & $\mathrm{R}$ \\
\hline 5414 & & & & ONSITE & SESPMNT & $\mathrm{R}$ \\
\hline 5415 & & & & ONSITE & SESPMNT & $\mathrm{R}$ \\
\hline 5416 & & & & ONSITE & SESPMNT & $\mathrm{R}$ \\
\hline 5417 & & & & ONSITE & SESPMNT & $\mathrm{R}$ \\
\hline 5418 & & & & ONSITE & SESPMNT & $\mathrm{R}$ \\
\hline 5419 & & & & ONSITE & SESPMNT & $\mathrm{R}$ \\
\hline 5420 & & & & ONSITE & SESPMNT & $\mathrm{R}$ \\
\hline 5421 & & & & ONSITE & SESPMNT & $\mathrm{R}$ \\
\hline 5422 & & & & ONSITE & SESPMNT & $\mathrm{R}$ \\
\hline 5423 & & & & ONSITE & SESPMNT & $\mathrm{R}$ \\
\hline 5424 & & & & ONSITE & SESPMNT & $\mathrm{R}$ \\
\hline 5425 & & & & ONSITE & SESPMNT & $\mathrm{R}$ \\
\hline 5426 & & & & ONSITE & SESPMNT & $\mathrm{R}$ \\
\hline 5427 & & & & ONSITE & SESPMNT & $\mathrm{R}$ \\
\hline 5428 & & & & ONSITE & SESPMNT & $\mathrm{R}$ \\
\hline 5429 & & & & ONSITE & SESPMNT & $\mathrm{R}$ \\
\hline 5430 & & & & ONSITE & SESPMNT & $R$ \\
\hline 5431 & & & & ONSITE & SESPMNT & $\mathrm{R}$ \\
\hline 5432 & & & & OFFSITE & SESPMNT & $\mathrm{R}$ \\
\hline 5433 & & & & OFFSITE & SESPMNT & $\mathrm{R}$ \\
\hline 5434 & & & & OFFSITE & SESPMNT & $\mathrm{R}$ \\
\hline 5435 & & & & OFFSITE & SESPMNT & $\mathrm{R}$ \\
\hline 5436 & & & & OFFSITE & SESPMNT & $R$ \\
\hline 5437 & & & & OFFSITE & SESPMNT & $\mathrm{R}$ \\
\hline 5438 & & & & OFFSITE & SESPMNT & $\mathrm{R}$ \\
\hline 5439 & & & & OFFSITE & SESPMNT & $R$ \\
\hline 5440 & & & & OFFSITE & SESPMNT & $R$ \\
\hline 5441 & & & & OFFSITE & SESPMNT & $R$ \\
\hline 5442 & & & & OFFSITE & SESPMNT & $R$ \\
\hline 5443 & & & & OFFSITE & SESPMNT & $R$ \\
\hline 5444 & & & & OFFSITE & SESPMNT & $R$ \\
\hline 5445 & & & & OFFSITE & SESPMNT & $\mathrm{R}$ \\
\hline 5446 & & & & OFFSITE & SESPMNT & $R$ \\
\hline 5447 & & & & OFFSITE & SESPMNT & $R$ \\
\hline 5448 & & & & OFFSITE & SESPMNT & $R$ \\
\hline 5449 & & & & OFFSITE & SESPMNT & $R$ \\
\hline 5450 & & & & OFFSITE & SESPMNT & $R$ \\
\hline 5451 & & & & OFFSITE & SESPMNT & $R$ \\
\hline 5452 & & & & OFFSITE & SESPMNT & $R$ \\
\hline 5453 & & & & OFFSITE & SESPMNT & $R$ \\
\hline 5454 & & & & ONSITE & SESPMNT & $R$ \\
\hline 5455 & & & & ONSITE & SESPMNT & $R$ \\
\hline 5456 & & & & ONSITE & SESPMNT & $R$ \\
\hline 5457 & & & & ONSITE & SESPMNT & $R$ \\
\hline 5458 & & & & ONSITE & SESPMNT & $R$ \\
\hline 5459 & & & & ONSITE & SESPMNT & $R$ \\
\hline 5460 & & & & ONSITE & SESPMNT & $\mathrm{R}$ \\
\hline 5461 & & & & ONSITE & SESBMNT & $R$ \\
\hline
\end{tabular}




\begin{tabular}{|c|c|c|c|c|c|c|}
\hline & $A D$ & $\mathrm{AE}$ & $\mathrm{AF}$ & $A G$ & $\mathrm{AH}$ & $\mathrm{Al}$ \\
\hline 1 & RESULT_COMMENT & COLL_SAMP_SIZE & COLL_SAMP_SIZE_UNITS & DIST_CLASS & OWNER_ID & COLLECTION_PURPOSE \\
\hline 5462 & & & & OFFSITE & SESPMNT & $\mathrm{R}$ \\
\hline 5463 & & & & OFFSITE & SESPMNT & $\mathrm{R}$ \\
\hline 5464 & & & & OFFSITE & SESPMNT & $\mathrm{R}$ \\
\hline 5465 & & & & OFFSITE & SESPMNT & $\mathrm{R}$ \\
\hline 5466 & & & & OFFSITE & SESPMNT & $\mathrm{R}$ \\
\hline 5467 & & & & OFFSITE & SESPMNT & $\mathrm{R}$ \\
\hline 5468 & & & & PERIMETER & SESPMNT & $\mathrm{R}$ \\
\hline 5469 & & & & PERIMETER & SESPMNT & $\mathrm{R}$ \\
\hline 5470 & & & & PERIMETER & SESPMNT & $\mathrm{R}$ \\
\hline 5471 & & & & ONSITE & SESPMNT & $\mathrm{R}$ \\
\hline 5472 & & & & ONSITE & SESPMNT & $\mathrm{R}$ \\
\hline 5473 & & & & OFFSITE & SESPMNT & $\mathrm{R}$ \\
\hline 5474 & & & & OFFSITE & SESPMNT & $\mathrm{R}$ \\
\hline 5475 & & & & OFFSITE & SESPMNT & $\mathrm{R}$ \\
\hline 5476 & & & & OFFSITE & SESPMNT & $\mathrm{R}$ \\
\hline 5477 & & & & OFFSITE & SESPMNT & $\mathrm{R}$ \\
\hline 5478 & & & & OFFSITE & SESPMNT & $\mathrm{R}$ \\
\hline 5479 & & & & OFFSITE & SESPMNT & $\mathrm{R}$ \\
\hline 5480 & & & & OFFSITE & SESPMNT & $\mathrm{R}$ \\
\hline 5481 & & & & OFFSITE & SESPMNT & $\mathrm{R}$ \\
\hline 5482 & & & & OFFSITE & SESPMNT & $\mathrm{R}$ \\
\hline 5483 & & & & OFFSITE & SESPMNT & $\mathrm{R}$ \\
\hline 5484 & & & & OFFSITE & SESPMNT & $\mathrm{R}$ \\
\hline 5485 & & & & OFFSITE & SESPMNT & $\mathrm{R}$ \\
\hline 5486 & & & & OFFSITE & SESPMNT & $\mathrm{R}$ \\
\hline 5487 & & & & OFFSITE & SESPMNT & $\mathrm{R}$ \\
\hline 5488 & & & & OFFSITE & SESPMNT & $\mathrm{R}$ \\
\hline 5489 & & & & ONSITE & SESPMNT & $\mathrm{R}$ \\
\hline 5490 & & & & ONSITE & SESPMNT & $\mathrm{R}$ \\
\hline 5491 & & & & ONSITE & SESPMNT & $\mathrm{R}$ \\
\hline 5492 & & & & ONSITE & SESPMNT & $\mathrm{R}$ \\
\hline 5493 & & & & ONSITE & SESPMNT & $\mathrm{R}$ \\
\hline 5494 & & & & OFFSITE & SESPMNT & $\mathrm{R}$ \\
\hline 5495 & & & & OFFSITE & SESPMNT & $\mathrm{R}$ \\
\hline 5496 & & & & OFFSITE & SESPMNT & $\mathrm{R}$ \\
\hline 5497 & & & & OFFSITE & SESPMNT & $\mathrm{R}$ \\
\hline 5498 & & & & ONSITE & SESPMNT & $\mathrm{R}$ \\
\hline 5499 & & & & ONSITE & SESPMNT & $\mathrm{R}$ \\
\hline 5500 & & & & ONSITE & SESPMNT & $\mathrm{R}$ \\
\hline 5501 & See sample record for comment. & & & ONSITE & SESPMNT & $\mathrm{R}$ \\
\hline 5502 & See sample record for comment. & & & ONSITE & SESPMNT & $\mathrm{R}$ \\
\hline 5503 & & & & & SESPMNT & $\mathrm{R}$ \\
\hline 5504 & & & & & SESPMNT & $\mathrm{R}$ \\
\hline 5505 & & & & & SESPMNT & $\mathrm{R}$ \\
\hline 5506 & & & & ONSITE & SESPMNT & $\mathrm{R}$ \\
\hline 5507 & & & & ONSITE & SESPMNT & $\mathrm{R}$ \\
\hline 5508 & & & & ONSITE & SESPMNT & $\mathrm{R}$ \\
\hline 5509 & & & & ONSITE & SESPMNT & $\mathrm{R}$ \\
\hline 5510 & & & & ONSITE & SESPMNT & $\mathrm{R}$ \\
\hline 5511 & & & & ONSITE & SESPMNT & $\mathrm{R}$ \\
\hline 5512 & & & & ONSITE & SESPMNT & $\mathrm{R}$ \\
\hline 5513 & & & & ONSITE & SESBMNT & $R$ \\
\hline
\end{tabular}




\begin{tabular}{|c|c|c|c|c|c|c|}
\hline & $\mathrm{AD}$ & $\mathrm{AE}$ & $\mathrm{AF}$ & AG & $\mathrm{AH}$ & $\overline{\mathrm{Al}}$ \\
\hline 1 & RESULT_COMMENT & COLL_SAMP_SIZE & COLL_SAMP_SIZE_UNITS & DIST_CLASS & OWNER_ID & COLLECTION_PURPOSE \\
\hline 5514 & See sample record for comment. & & & OFFSITE & SESPMNT & $\mathrm{R}$ \\
\hline 5515 & & & & OFFSITE & SESPMNT & $\mathrm{R}$ \\
\hline 5516 & & & & OFFSITE & SESPMNT & $\mathrm{R}$ \\
\hline 5517 & & & & OFFSITE & SESPMNT & $\mathrm{R}$ \\
\hline 5518 & & & & OFFSITE & SESPMNT & $\mathrm{R}$ \\
\hline 5519 & & & & OFFSITE & SESPMNT & $\mathrm{R}$ \\
\hline 5520 & & & & OFFSITE & SESPMNT & $\mathrm{R}$ \\
\hline 5521 & & & & OFFSITE & SESPMNT & $\mathrm{R}$ \\
\hline 5522 & & & & OFFSITE & SESPMNT & $\mathrm{R}$ \\
\hline 5523 & & & & OFFSITE & SESPMNT & $\mathrm{R}$ \\
\hline 5524 & & & & OFFSITE & SESPMNT & $\mathrm{R}$ \\
\hline 5525 & & & & OFFSITE & SESPMNT & $\mathrm{R}$ \\
\hline 5526 & & & & OFFSITE & SESPMNT & $\mathrm{R}$ \\
\hline 5527 & & & & OFFSITE & SESPMNT & $\mathrm{R}$ \\
\hline 5528 & & & & OFFSITE & SESPMNT & $\mathrm{R}$ \\
\hline 5529 & See sample record for comment. & & & ONSITE & SESPMNT & $\mathrm{R}$ \\
\hline 5530 & See sample record for comment. & & & ONSITE & SESPMNT & $\mathrm{R}$ \\
\hline 5531 & & & & OFFSITE & SESPMNT & $\mathrm{R}$ \\
\hline 5532 & & & & OFFSITE & SESPMNT & $\mathrm{R}$ \\
\hline 5533 & & & & OFFSITE & SESPMNT & $\mathrm{R}$ \\
\hline 5534 & & & & OFFSITE & SESPMNT & $\mathrm{R}$ \\
\hline 5535 & & & & OFFSITE & SESPMNT & $\mathrm{R}$ \\
\hline 5536 & & & & OFFSITE & SESPMNT & $\mathrm{R}$ \\
\hline \begin{tabular}{|l|}
5537 \\
\end{tabular} & & & & ONSITE & SESPMNT & $R$ \\
\hline 5538 & & & & ONSITE & SESPMNT & $R$ \\
\hline 5539 & & & & ONSITE & SESPMNT & $R$ \\
\hline 5540 & & & & ONSITE & SESPMNT & $R$ \\
\hline \begin{tabular}{|l|}
5541 \\
\end{tabular} & & & & ONSITE & SESPMNT & $\mathrm{R}$ \\
\hline \begin{tabular}{|l|}
5542 \\
\end{tabular} & & & & ONSITE & SESPMNT & $\mathrm{R}$ \\
\hline \begin{tabular}{|l|}
5543 \\
\end{tabular} & & & & ONSITE & SESPMNT & $R$ \\
\hline \begin{tabular}{|l|}
5544 \\
\end{tabular} & & & & ONSITE & SESPMNT & $R$ \\
\hline 5545 & & & & ONSITE & SESPMNT & $R$ \\
\hline 554 & & & & ONSITE & SESPMNT & $R$ \\
\hline \begin{tabular}{|l|}
5547 \\
\end{tabular} & & & & ONSITE & SESPMNT & $R$ \\
\hline 5548 & & & & ONSITE & SESPMNT & $\mathrm{R}$ \\
\hline 5549 & & & & ONSITE & SESPMNT & $R$ \\
\hline 5550 & & & & ONSITE & SESPMNT & $\mathrm{R}$ \\
\hline \begin{tabular}{|l|}
5551 \\
\end{tabular} & & & & ONSITE & SESPMNT & $R$ \\
\hline \begin{tabular}{|l|}
5552 \\
\end{tabular} & & & & ONSITE & SESPMNT & $R$ \\
\hline \begin{tabular}{|l|}
5553 \\
\end{tabular} & & & & ONSITE & SESPMNT & $R$ \\
\hline \begin{tabular}{|l|}
5554 \\
\end{tabular} & & & & OFFSITE & SESPMNT & $R$ \\
\hline \begin{tabular}{|l|}
5555 \\
\end{tabular} & & & & OFFSITE & SESPMNT & $R$ \\
\hline \begin{tabular}{|l|}
5556 \\
\end{tabular} & & & & OFFSITE & SESPMNT & $R$ \\
\hline 5557 & & & & OFFSITE & SESPMNT & $\mathrm{R}$ \\
\hline 5558 & & & & ONSITE & SESPMNT & $R$ \\
\hline 5559 & & & & ONSITE & SESPMNT & $R$ \\
\hline 5560 & & & & ONSITE & SESPMNT & $\mathrm{R}$ \\
\hline 5561 & & & & ONSITE & SESPMNT & $\mathrm{R}$ \\
\hline 5562 & & & & ONSITE & SESPMNT & $R$ \\
\hline 5563 & & & & ONSITE & SESPMNT & $\mathrm{R}$ \\
\hline \begin{tabular}{|l|l|}
5564 \\
\end{tabular} & & & & OFFSITE & SESPMNT & $R$ \\
\hline 5565 & & & & OFFSITE & SESBMNT & $R$ \\
\hline
\end{tabular}




\begin{tabular}{|c|c|c|c|c|c|c|}
\hline & $\mathrm{AD}$ & $\mathrm{AE}$ & AF & AG & $\mathrm{AH}$ & $\mathrm{Al}$ \\
\hline 1 & RESULT_COMMENT & COLL_SAMP_SIZE & COLL_SAMP_SIZE_UNITS & DIST_CLASS & OWNER_ID & COLLECTION_PURPOSE \\
\hline 5566 & & & & OFFSITE & SESPMNT & $R$ \\
\hline 5567 & & & & OFFSITE & SESPMNT & $\mathrm{R}$ \\
\hline \begin{tabular}{|l|l|l|}
5568 \\
\end{tabular} & & & & OFFSITE & SESPMNT & $\mathrm{R}$ \\
\hline 5569 & & & & OFFSITE & SESPMNT & $\mathrm{R}$ \\
\hline 5570 & & & & OFFSITE & SESPMNT & $\mathrm{R}$ \\
\hline 5571 & & & & OFFSITE & SESPMNT & $\mathrm{R}$ \\
\hline 5572 & & & & OFFSITE & SESPMNT & $\mathrm{R}$ \\
\hline 5573 & & & & OFFSITE & SESPMNT & $\mathrm{R}$ \\
\hline 5574 & & & & OFFSITE & SESPMNT & $\mathrm{R}$ \\
\hline 5575 & & & & OFFSITE & SESPMNT & $\mathrm{R}$ \\
\hline 5576 & & & & ONSITE & SESPMNT & $\mathrm{R}$ \\
\hline 5577 & & & & ONSITE & SESPMNT & $\mathrm{R}$ \\
\hline 5578 & & & & ONSITE & SESPMNT & $\mathrm{R}$ \\
\hline 5579 & & & & ONSITE & SESPMNT & $\mathrm{R}$ \\
\hline 5580 & & & & ONSITE & SESPMNT & $\mathrm{R}$ \\
\hline 5581 & & & & OFFSITE & SESPMNT & $\mathrm{R}$ \\
\hline 5582 & & & & OFFSITE & SESPMNT & $\mathrm{R}$ \\
\hline \begin{tabular}{|c|}
5583 \\
\end{tabular} & & & & OFFSITE & SESPMNT & $\mathrm{R}$ \\
\hline 5584 & & & & OFFSITE & SESPMNT & $\mathrm{R}$ \\
\hline 5585 & & & & OFFSITE & SESPMNT & $\mathrm{R}$ \\
\hline 5586 & & & & OFFSITE & SESPMNT & $\mathrm{R}$ \\
\hline 5587 & & & & OFFSITE & SESPMNT & $\mathrm{R}$ \\
\hline 5588 & & & & OFFSITE & SESPMNT & $\mathrm{R}$ \\
\hline 5589 & & & & OFFSITE & SESPMNT & $\mathrm{R}$ \\
\hline 5590 & & & & OFFSITE & SESPMNT & $\mathrm{R}$ \\
\hline 5591 & & & & PERIMETER & SESPMNT & $\mathrm{R}$ \\
\hline \begin{tabular}{|l|l|}
5592 \\
\end{tabular} & & & & PERIMETER & SESPMNT & $\mathrm{R}$ \\
\hline \begin{tabular}{|l|l|}
5593 \\
\end{tabular} & & & & PERIMETER & SESPMNT & $\mathrm{R}$ \\
\hline \begin{tabular}{|l|}
5594 \\
\end{tabular} & & & & OFFSITE & SESPSPEC & S \\
\hline \begin{tabular}{|l|l|}
5595 \\
\end{tabular} & & & & OFFSITE & SESPSPEC & S \\
\hline \begin{tabular}{|l|l|}
5596 \\
\end{tabular} & & & & OFFSITE & SESPSPEC & S \\
\hline \begin{tabular}{|l|l|}
5597 \\
\end{tabular} & & & & OFFSITE & SESPSPEC & S \\
\hline \begin{tabular}{|l|l|}
5598 \\
\end{tabular} & & & & OFFSITE & SESPSPEC & S \\
\hline \begin{tabular}{|l|l|}
5599 \\
\end{tabular} & & & & OFFSITE & SESPSPEC & S \\
\hline \begin{tabular}{|l|}
5600 \\
5
\end{tabular} & & & & OFFSITE & SESPSPEC & $S$ \\
\hline 5601 & & & & OFFSITE & SESPSPEC & S \\
\hline 5602 & & & & OFFSITE & SESPSPEC & S \\
\hline \begin{tabular}{|l|l|}
5603 \\
\end{tabular} & & & & OFFSITE & SESPSPEC & S \\
\hline 5604 & & & & OFFSITE & SESPSPEC & S \\
\hline 5605 & & & & OFFSITE & SESPSPEC & S \\
\hline 5606 & & & & ONSITE & SESPMNT & $R$ \\
\hline \begin{tabular}{|l|}
5607 \\
\end{tabular} & & & & ONSITE & SESPMNT & $\mathrm{R}$ \\
\hline \begin{tabular}{|l|l|}
5608 \\
\end{tabular} & & & & OFFSITE & SESPMNT & $\mathrm{R}$ \\
\hline \begin{tabular}{|l|}
5609 \\
\end{tabular} & & & & OFFSITE & SESPMNT & $\mathrm{R}$ \\
\hline 5610 & & & & OFFSITE & SESPMNT & $\mathrm{R}$ \\
\hline 5611 & & & & OFFSITE & SESPMNT & $\mathrm{R}$ \\
\hline 5612 & & & & ONSITE & SESPMNT & $\mathrm{R}$ \\
\hline \begin{tabular}{|l|l|}
5613 \\
\end{tabular} & & & & ONSITE & SESPMNT & $\mathrm{R}$ \\
\hline 5614 & & & & ONSITE & SESPMNT & $\mathrm{R}$ \\
\hline \begin{tabular}{|l|l|}
5615 \\
\end{tabular} & & & & ONSITE & SESPMNT & $\mathrm{R}$ \\
\hline \begin{tabular}{|l|}
5616 \\
\end{tabular} & & & & ONSITE & SESPMNT & $\mathrm{R}$ \\
\hline \begin{tabular}{|l|}
5617 \\
\end{tabular} & & & & OFFSITE & SESBMNT & $R$ \\
\hline
\end{tabular}




\begin{tabular}{|c|c|c|c|c|c|c|}
\hline & $A D$ & $\mathrm{AE}$ & $\mathrm{AF}$ & $A G$ & $\mathrm{AH}$ & $\mathrm{Al}$ \\
\hline 1 & RESULT_COMMENT & COLL_SAMP_SIZE & COLL_SAMP_SIZE_UNITS & DIST_CLASS & OWNER_ID & COLLECTION_PURPOSE \\
\hline 5618 & & & & OFFSITE & SESPMNT & $\mathrm{R}$ \\
\hline 5619 & & & & OFFSITE & SESPMNT & $\mathrm{R}$ \\
\hline 5620 & & & & OFFSITE & SESPMNT & $\mathrm{R}$ \\
\hline 5621 & & & & OFFSITE & SESPMNT & $\mathrm{R}$ \\
\hline \begin{tabular}{|l|l|}
5622 \\
\end{tabular} & & & & OFFSITE & SESPMNT & $\mathrm{R}$ \\
\hline \begin{tabular}{|l|}
5623 \\
\end{tabular} & & & & OFFSITE & SESPMNT & $\mathrm{R}$ \\
\hline 5624 & & & & OFFSITE & SESPMNT & $\mathrm{R}$ \\
\hline \begin{tabular}{|l|l|}
5625 \\
\end{tabular} & & & & OFFSITE & SESPMNT & $\mathrm{R}$ \\
\hline 5626 & & & & OFFSITE & SESPMNT & $\mathrm{R}$ \\
\hline \begin{tabular}{|l|}
5627 \\
\end{tabular} & & & & OFFSITE & SESPMNT & $\mathrm{R}$ \\
\hline \begin{tabular}{|c|}
5628 \\
\end{tabular} & & & & OFFSITE & SESPMNT & $\mathrm{R}$ \\
\hline \begin{tabular}{|l|}
5629 \\
\end{tabular} & & & & OFFSITE & SESPMNT & $\mathrm{R}$ \\
\hline 5630 & & & & OFFSITE & SESPMNT & $\mathrm{R}$ \\
\hline 5631 & & & & OFFSITE & SESPMNT & $\mathrm{R}$ \\
\hline \begin{tabular}{|l|}
5632 \\
\end{tabular} & & & & OFFSITE & SESPMNT & $\mathrm{R}$ \\
\hline \begin{tabular}{|c|}
5633 \\
\end{tabular} & & & & ONSITE & SESPMNT & $\mathrm{R}$ \\
\hline 5634 & & & & ONSITE & SESPMNT & $\mathrm{R}$ \\
\hline \begin{tabular}{|c|}
5635 \\
\end{tabular} & & & & ONSITE & SESPMNT & $\mathrm{R}$ \\
\hline \begin{tabular}{|c|}
5636 \\
\end{tabular} & & & & ONSITE & SESPMNT & $\mathrm{R}$ \\
\hline \begin{tabular}{|c|}
5637 \\
\end{tabular} & & & & ONSITE & SESPMNT & $\mathrm{R}$ \\
\hline 5638 s & See sample record for comment. & & & & SESPMNT & $\mathrm{R}$ \\
\hline$|5639| \leq$ & See sample record for comment. & & & & SESPMNT & $\mathrm{R}$ \\
\hline $5640 \leq$ & See sample record for comment. & & & & SESPMNT & $\mathrm{R}$ \\
\hline 5641 & & & & ONSITE & SESPMNT & $\mathrm{R}$ \\
\hline \begin{tabular}{|l|l|}
5642 \\
\end{tabular} & & & & ONSITE & SESPMNT & $\mathrm{R}$ \\
\hline \begin{tabular}{|l|l|}
5643 \\
\end{tabular} & & & & ONSITE & SESPMNT & $\mathrm{R}$ \\
\hline 5644 & & & & ONSITE & SESPMNT & $\mathrm{R}$ \\
\hline \begin{tabular}{|l|l|}
5645 \\
\end{tabular} & & & & ONSITE & SESPMNT & $\mathrm{R}$ \\
\hline \begin{tabular}{|c|}
5646 \\
\end{tabular} & & & & ONSITE & SESPMNT & $\mathrm{R}$ \\
\hline 5647 s & See sample record for comment. & & & OFFSITE & SESPMNT & $\mathrm{R}$ \\
\hline 5648 & See sample record for comment. & & & OFFSITE & SESPMNT & $\mathrm{R}$ \\
\hline$|5649| s$ & See sample record for comment. & & & OFFSITE & SESPMNT & $\mathrm{R}$ \\
\hline$|5650| \leq$ & See sample record for comment. & & & OFFSITE & SESPMNT & $\mathrm{R}$ \\
\hline$|5651| \leq$ & See sample record for comment. & & & OFFSITE & SESPMNT & $\mathrm{R}$ \\
\hline \begin{tabular}{|c|c|c|}
5652 & \\
\end{tabular} & See sample record for comment. & & & OFFSITE & SESPMNT & $\mathrm{R}$ \\
\hline 5653 & See sample record for comment. & & & OFFSITE & SESPMNT & $\mathrm{R}$ \\
\hline 5654 s & See sample record for comment. & & & OFFSITE & SESPMNT & $\mathrm{R}$ \\
\hline 5655 & See sample record for comment. & & & OFFSITE & SESPMNT & $\mathrm{R}$ \\
\hline 5656 & See sample record for comment. & & & OFFSITE & SESPMNT & $\mathrm{R}$ \\
\hline 5657 s & See sample record for comment. & & & & SESPMNT & $\mathrm{R}$ \\
\hline 5658 & See sample record for comment. & & & & SESPMNT & $\mathrm{R}$ \\
\hline 5659 & See sample record for comment. & & & & SESPMNT & $\mathrm{R}$ \\
\hline $5660 \leq$ & See sample record for comment. & & & & SESPMNT & $\mathrm{R}$ \\
\hline 5661 s & See sample record for comment. & & & & SESPMNT & $\mathrm{R}$ \\
\hline \begin{tabular}{|c|c|}
5662 \\
\end{tabular} & & & & ONSITE & SESPMNT & $\mathrm{R}$ \\
\hline \begin{tabular}{|c|c|}
5663 \\
\end{tabular} & & & & ONSITE & SESPMNT & $\mathrm{R}$ \\
\hline \begin{tabular}{|c|}
5664 \\
\end{tabular} & & & & OFFSITE & SESPMNT & $\mathrm{R}$ \\
\hline \begin{tabular}{|c|}
5665 \\
\end{tabular} & & & & OFFSITE & SESPMNT & $\mathrm{R}$ \\
\hline 5666 & & & & OFFSITE & SESPMNT & $\mathrm{R}$ \\
\hline \begin{tabular}{|l|}
5667 \\
\end{tabular} & & & & OFFSITE & SESPMNT & $\mathrm{R}$ \\
\hline \begin{tabular}{|l|}
5668 \\
\end{tabular} & & & & ONSITE & SESPMNT & $R$ \\
\hline 5669 & & & & ONSITE & SESBMNT & $R$ \\
\hline
\end{tabular}




\begin{tabular}{|c|c|c|c|c|c|c|}
\hline & $A D$ & $\mathrm{AE}$ & $\mathrm{AF}$ & $A G$ & $\mathrm{AH}$ & $\mathrm{Al}$ \\
\hline 1 & RESULT_COMMENT & COLL_SAMP_SIZE & COLL_SAMP_SIZE_UNITS & DIST_CLASS & OWNER_ID & COLLECTION_PURPOSE \\
\hline 5670 & & & & OFFSITE & SESPMNT & $\mathrm{R}$ \\
\hline 5671 & & & & OFFSITE & SESPMNT & $\mathrm{R}$ \\
\hline 5672 & & & & OFFSITE & SESPMNT & $\mathrm{R}$ \\
\hline 5673 & & & & OFFSITE & SESPMNT & $\mathrm{R}$ \\
\hline 5674 & & & & OFFSITE & SESPMNT & $\mathrm{R}$ \\
\hline 5675 & & & & OFFSITE & SESPMNT & $\mathrm{R}$ \\
\hline 5676 & & & & OFFSITE & SESPMNT & $\mathrm{R}$ \\
\hline 5677 & & & & OFFSITE & SESPMNT & $\mathrm{R}$ \\
\hline 5678 & & & & OFFSITE & SESPMNT & $\mathrm{R}$ \\
\hline 5679 & & & & OFFSITE & SESPMNT & $\mathrm{R}$ \\
\hline 5680 & & & & OFFSITE & SESPMNT & $\mathrm{R}$ \\
\hline 5681 & & & & OFFSITE & SESPMNT & $\mathrm{R}$ \\
\hline 5682 & & & & OFFSITE & SESPMNT & $\mathrm{R}$ \\
\hline 5683 & & & & OFFSITE & SESPMNT & $\mathrm{R}$ \\
\hline 5684 & & & & OFFSITE & SESPMNT & $\mathrm{R}$ \\
\hline 5685 & & & & OFFSITE & SESPMNT & $\mathrm{R}$ \\
\hline 5686 & See sample record for comment. & & & ONSITE & SESPMNT & $\mathrm{R}$ \\
\hline 5687 & & & & ONSITE & SESPMNT & $\mathrm{R}$ \\
\hline 5688 & & & & ONSITE & SESPMNT & $\mathrm{R}$ \\
\hline 5689 & & & & ONSITE & SESPMNT & $\mathrm{R}$ \\
\hline 5690 & & & & ONSITE & SESPMNT & $\mathrm{R}$ \\
\hline 5691 & & & & ONSITE & SESPMNT & $\mathrm{R}$ \\
\hline 5692 & & & & ONSITE & SESPMNT & $\mathrm{R}$ \\
\hline 5693 & & & & ONSITE & SESPMNT & $\mathrm{R}$ \\
\hline 5694 & & & & ONSITE & SESPMNT & $\mathrm{R}$ \\
\hline 5695 & & & & ONSITE & SESPMNT & $\mathrm{R}$ \\
\hline 5696 & & & & ONSITE & SESPMNT & $\mathrm{R}$ \\
\hline 5697 & & & & ONSITE & SESPMNT & $\mathrm{R}$ \\
\hline 5698 & & & & ONSITE & SESPMNT & $\mathrm{R}$ \\
\hline 5699 & & & & & SESPMNT & $R$ \\
\hline 5700 & & & & & SESPMNT & $\mathrm{R}$ \\
\hline 5701 & & & & & SESPMNT & $\mathrm{R}$ \\
\hline \begin{tabular}{|l|}
5702 \\
\end{tabular} & & & & & SESPMNT & $\mathrm{R}$ \\
\hline 5703 & & & & & SESPMNT & $\mathrm{R}$ \\
\hline 5704 & & & & ONSITE & SESPMNT & $\mathrm{R}$ \\
\hline 5705 & & & & ONSITE & SESPMNT & $\mathrm{R}$ \\
\hline 5706 & & & & ONSITE & SESPMNT & $\mathrm{R}$ \\
\hline 5707 & & & & ONSITE & SESPMNT & $\mathrm{R}$ \\
\hline 5708 & & & & ONSITE & SESPMNT & $\mathrm{R}$ \\
\hline 5709 & & & & OFFSITE & SESPMNT & $\mathrm{R}$ \\
\hline 5710 & & & & OFFSITE & SESPMNT & $\mathrm{R}$ \\
\hline \begin{tabular}{|l|l|}
5711 \\
\end{tabular} & & & & OFFSITE & SESPMNT & $\mathrm{R}$ \\
\hline 5712 & & & & OFFSITE & SESPMNT & $\mathrm{R}$ \\
\hline 5713 & & & & ONSITE & SESPMNT & $\mathrm{R}$ \\
\hline 5714 & & & & ONSITE & SESPMNT & $\mathrm{R}$ \\
\hline 5715 & & & & ONSITE & SESPMNT & $\mathrm{R}$ \\
\hline 5716 & & & & ONSITE & SESPMNT & $\mathrm{R}$ \\
\hline \begin{tabular}{|l|l|}
5717 \\
\end{tabular} & & & & ONSITE & SESPMNT & $\mathrm{R}$ \\
\hline 5718 & & & & ONSITE & SESPMNT & $\mathrm{R}$ \\
\hline 5719 & & & & ONSITE & SESPMNT & $R$ \\
\hline 5720 & & & & ONSITE & $\begin{array}{l}\text { SESPMNT } \\
\end{array}$ & $\mathrm{R}$ \\
\hline 5721 & & & & ONSITE & SESBMNT & $R$ \\
\hline
\end{tabular}




\begin{tabular}{|c|c|c|c|c|c|c|}
\hline & $A D$ & $\mathrm{AE}$ & $\mathrm{AF}$ & $A G$ & $\mathrm{AH}$ & $\mathrm{Al}$ \\
\hline 1 & RESULT_COMMENT & COLL_SAMP_SIZE & COLL_SAMP_SIZE_UNITS & DIST_CLASS & OWNER_ID & COLLECTION_PURPOSE \\
\hline 5722 & & & & ONSITE & SESPMNT & $\mathrm{R}$ \\
\hline 5723 & & & & ONSITE & SESPMNT & $\mathrm{R}$ \\
\hline 5724 & & & & OFFSITE & SESPMNT & $\mathrm{R}$ \\
\hline 5725 & & & & OFFSITE & SESPMNT & $\mathrm{R}$ \\
\hline 5726 & & & & OFFSITE & SESPMNT & $\mathrm{R}$ \\
\hline 5727 & & & & OFFSITE & SESPMNT & $\mathrm{R}$ \\
\hline 5728 & & & & OFFSITE & SESPMNT & $\mathrm{R}$ \\
\hline 5729 & & & & OFFSITE & SESPMNT & $\mathrm{R}$ \\
\hline 5730 & & & & OFFSITE & SESPMNT & $\mathrm{R}$ \\
\hline 5731 & & & & OFFSITE & SESPMNT & $\mathrm{R}$ \\
\hline 5732 & & & & OFFSITE & SESPMNT & $\mathrm{R}$ \\
\hline 5733 & & & & OFFSITE & SESPMNT & $\mathrm{R}$ \\
\hline 5734 & & & & OFFSITE & SESPMNT & $\mathrm{R}$ \\
\hline 5735 & & & & OFFSITE & SESPMNT & $\mathrm{R}$ \\
\hline 5736 & & & & OFFSITE & SESPMNT & $\mathrm{R}$ \\
\hline 5737 & & & & OFFSITE & SESPMNT & $\mathrm{R}$ \\
\hline 5738 & & & & OFFSITE & SESPMNT & $\mathrm{R}$ \\
\hline 5739 & & & & OFFSITE & SESPMNT & $\mathrm{R}$ \\
\hline 5740 & & & & OFFSITE & SESPMNT & $\mathrm{R}$ \\
\hline 5741 & & & & OFFSITE & SESPMNT & $\mathrm{R}$ \\
\hline 5742 & & & & OFFSITE & SESPMNT & $\mathrm{R}$ \\
\hline 5743 & & & & OFFSITE & SESPMNT & $\mathrm{R}$ \\
\hline 5744 & & & & OFFSITE & SESPMNT & $\mathrm{R}$ \\
\hline 5745 & & & & OFFSITE & SESPMNT & $\mathrm{R}$ \\
\hline 5746 & & & & PERIMETER & SESPMNT & $\mathrm{R}$ \\
\hline 5747 & & & & PERIMETER & SESPMNT & $\mathrm{R}$ \\
\hline 5748 & & & & PERIMETER & SESPMNT & $\mathrm{R}$ \\
\hline 5749 & & & & ONSITE & SESPMNT & $\mathrm{R}$ \\
\hline 5750 & & & & ONSITE & SESPMNT & $\mathrm{R}$ \\
\hline 5751 & & & & OFFSITE & SESPMNT & $\mathrm{R}$ \\
\hline 5752 & & & & OFFSITE & SESPMNT & $\mathrm{R}$ \\
\hline 5753 & & & & OFFSITE & SESPMNT & $\mathrm{R}$ \\
\hline 5754 & & & & OFFSITE & SESPMNT & $\mathrm{R}$ \\
\hline 5755 & & & & OFFSITE & SESPMNT & $\mathrm{R}$ \\
\hline 5756 & & & & OFFSITE & SESPMNT & $\mathrm{R}$ \\
\hline 5757 & & & & OFFSITE & SESPMNT & $\mathrm{R}$ \\
\hline 5758 & & & & OFFSITE & SESPMNT & $\mathrm{R}$ \\
\hline 5759 & & & & OFFSITE & SESPMNT & $\mathrm{R}$ \\
\hline 5760 & & & & ONSITE & SESPMNT & $\mathrm{R}$ \\
\hline 5761 & & & & ONSITE & SESPMNT & $\mathrm{R}$ \\
\hline 5762 & & & & ONSITE & SESPMNT & $\mathrm{R}$ \\
\hline 5763 & & & & OFFSITE & SESPMNT & $\mathrm{R}$ \\
\hline 5764 & & & & OFFSITE & SESPMNT & $\mathrm{R}$ \\
\hline 5765 & & & & OFFSITE & SESPMNT & $\mathrm{R}$ \\
\hline 5766 & & & & OFFSITE & SESPMNT & $\mathrm{R}$ \\
\hline 5767 & & & & OFFSITE & SESPMNT & $\mathrm{R}$ \\
\hline 5768 & & & & & SESPMNT & $\mathrm{R}$ \\
\hline 5769 & & & & & SESPMNT & $\mathrm{R}$ \\
\hline 5770 & & & & & SESPMNT & $\mathrm{R}$ \\
\hline 5771 & & & & & SESPMNT & $\mathrm{R}$ \\
\hline 5772 & & & & & SESPMNT & $\mathrm{R}$ \\
\hline 5773 & See sample record for comment. & & & ONSITE & SESBMNT & $R$ \\
\hline
\end{tabular}




\begin{tabular}{|c|c|c|c|c|c|c|}
\hline & $A D$ & $\mathrm{AE}$ & $\mathrm{AF}$ & $A G$ & $\mathrm{AH}$ & $\mathrm{Al}$ \\
\hline 1 & RESULT_COMMENT & COLL_SAMP_SIZE & COLL_SAMP_SIZE_UNITS & DIST_CLASS & OWNER_ID & COLLECTION_PURPOSE \\
\hline 5774 & See sample record for comment. & & & ONSITE & SESPMNT & $\mathrm{R}$ \\
\hline 5775 & & & & OFFSITE & SESPMNT & $\mathrm{R}$ \\
\hline 5776 & & & & OFFSITE & SESPMNT & $\mathrm{R}$ \\
\hline 5777 & & & & OFFSITE & SESPMNT & $\mathrm{R}$ \\
\hline 5778 & & & & OFFSITE & SESPMNT & $\mathrm{R}$ \\
\hline 5779 & & & & OFFSITE & SESPMNT & $\mathrm{R}$ \\
\hline 5780 & & & & OFFSITE & SESPMNT & $\mathrm{R}$ \\
\hline 5781 & & & & OFFSITE & SESPMNT & $\mathrm{R}$ \\
\hline 5782 & & & & OFFSITE & SESPMNT & $\mathrm{R}$ \\
\hline 5783 & & & & OFFSITE & SESPMNT & $\mathrm{R}$ \\
\hline 5784 & & & & OFFSITE & SESPMNT & $\mathrm{R}$ \\
\hline 5785 & & & & OFFSITE & SESPMNT & $\mathrm{R}$ \\
\hline 5786 & & & & OFFSITE & SESPMNT & $\mathrm{R}$ \\
\hline 5787 & & & & OFFSITE & SESPMNT & $\mathrm{R}$ \\
\hline 5788 & & & & OFFSITE & SESPMNT & $\mathrm{R}$ \\
\hline 5789 & & & & OFFSITE & SESPMNT & $\mathrm{R}$ \\
\hline 5790 & & & & OFFSITE & SESPMNT & $\mathrm{R}$ \\
\hline 5791 & & & & ONSITE & SESPMNT & $\mathrm{R}$ \\
\hline 5792 & & & & ONSITE & SESPMNT & $\mathrm{R}$ \\
\hline 5793 & & & & ONSITE & SESPMNT & $\mathrm{R}$ \\
\hline 5794 & See sample record for comment. & & & ONSITE & SESPMNT & $\mathrm{R}$ \\
\hline 5795 & See sample record for comment. & & & ONSITE & SESPMNT & $\mathrm{R}$ \\
\hline 5796 & See sample record for comment. & & & ONSITE & SESPMNT & $\mathrm{R}$ \\
\hline 5797 & See sample record for comment. & & & ONSITE & SESPMNT & $\mathrm{R}$ \\
\hline 5798 & See sample record for comment. & & & ONSITE & SESPMNT & $\mathrm{R}$ \\
\hline 5799 & See sample record for comment. & & & ONSITE & SESPMNT & $\mathrm{R}$ \\
\hline 5800 & See sample record for comment. & & & ONSITE & SESPMNT & $\mathrm{R}$ \\
\hline 5801 & See sample record for comment. & & & ONSITE & SESPMNT & $\mathrm{R}$ \\
\hline 5802 & & & & ONSITE & SESPMNT & $\mathrm{R}$ \\
\hline 5803 & & & & ONSITE & SESPMNT & $\mathrm{R}$ \\
\hline 5804 & See sample record for comment. & & & OFFSITE & SESPMNT & $\mathrm{R}$ \\
\hline 5805 & See sample record for comment. & & & OFFSITE & SESPMNT & $\mathrm{R}$ \\
\hline 5806 & & & & OFFSITE & SESPMNT & $\mathrm{R}$ \\
\hline 5807 & & & & OFFSITE & SESPMNT & $\mathrm{R}$ \\
\hline 5808 & & & & ONSITE & SESPMNT & $\mathrm{R}$ \\
\hline 5809 & & & & ONSITE & SESPMNT & $\mathrm{R}$ \\
\hline 5810 & & & & OFFSITE & SESPMNT & $\mathrm{R}$ \\
\hline 5811 & & & & OFFSITE & SESPMNT & $\mathrm{R}$ \\
\hline 5812 & & & & OFFSITE & SESPMNT & $\mathrm{R}$ \\
\hline 5813 & & & & OFFSITE & SESPMNT & $\mathrm{R}$ \\
\hline 5814 & & & & ONSITE & SESPMNT & $\mathrm{R}$ \\
\hline 5815 & & & & ONSITE & SESPMNT & $\mathrm{R}$ \\
\hline 5816 & & & & ONSITE & SESPMNT & $\mathrm{R}$ \\
\hline 5817 & & & & ONSITE & SESPMNT & $\mathrm{R}$ \\
\hline 5818 & & & & ONSITE & SESPMNT & $\mathrm{R}$ \\
\hline 5819 & & & & ONSITE & SESPMNT & $\mathrm{R}$ \\
\hline 5820 & & & & ONSITE & SESPMNT & $\mathrm{R}$ \\
\hline 5821 & & & & ONSITE & SESPMNT & $\mathrm{R}$ \\
\hline \begin{tabular}{|l|}
5822 \\
\end{tabular} & & & & ONSITE & SESPMNT & $\mathrm{R}$ \\
\hline 5823 & & & & ONSITE & SESPMNT & $R$ \\
\hline \begin{tabular}{|l|}
5824 \\
\end{tabular} & & & & ONSITE & $\begin{array}{l}\text { SESPMNT } \\
\end{array}$ & $\mathrm{R}$ \\
\hline 5825 & & & & ONSITE & SESBMNT & $\mathrm{R}$ \\
\hline
\end{tabular}




\begin{tabular}{|c|c|c|c|c|c|c|}
\hline & $\mathrm{AD}$ & $\mathrm{AE}$ & $\mathrm{AF}$ & $A G$ & $\mathrm{AH}$ & $\mathrm{Al}$ \\
\hline 1 & RESULT_COMMENT & COLL_SAMP_SIZE & COLL_SAMP_SIZE_UNITS & DIST_CLASS & OWNER_ID & COLLECTION_PURPOSE \\
\hline 5826 & & & & OFFSITE & SESPMNT & $\mathrm{R}$ \\
\hline 5827 & & & & OFFSITE & SESPMNT & $\mathrm{R}$ \\
\hline 5828 & & & & OFFSITE & SESPMNT & $\mathrm{R}$ \\
\hline 5829 & & & & OFFSITE & SESPMNT & $\mathrm{R}$ \\
\hline 5830 & & & & OFFSITE & SESPMNT & $\mathrm{R}$ \\
\hline 5831 & & & & OFFSITE & SESPMNT & $\mathrm{R}$ \\
\hline 5832 & & & & OFFSITE & SESPMNT & $\mathrm{R}$ \\
\hline 5833 & & & & OFFSITE & SESPMNT & $\mathrm{R}$ \\
\hline 5834 & & & & OFFSITE & SESPMNT & $\mathrm{R}$ \\
\hline 5835 & & & & OFFSITE & SESPMNT & $\mathrm{R}$ \\
\hline 5836 & & & & OFFSITE & SESPMNT & $\mathrm{R}$ \\
\hline 5837 & & & & OFFSITE & SESPMNT & $\mathrm{R}$ \\
\hline 5838 & & & & OFFSITE & SESPMNT & $\mathrm{R}$ \\
\hline 5839 & & & & OFFSITE & SESPMNT & $\mathrm{R}$ \\
\hline 5840 & & & & & SESPMNT & $\mathrm{R}$ \\
\hline 5841 & See sample record for comment. & & & & SESPMNT & $\mathrm{R}$ \\
\hline 5842 & See sample record for comment. & & & & SESPMNT & $\mathrm{R}$ \\
\hline 5843 & See sample record for comment. & & & & SESPMNT & $\mathrm{R}$ \\
\hline 5844 & See sample record for comment. & & & & SESPMNT & $\mathrm{R}$ \\
\hline 5845 & See sample record for comment. & & & & SESPMNT & $\mathrm{R}$ \\
\hline 5846 & See sample record for comment. & & & & SESPMNT & $\mathrm{R}$ \\
\hline 5847 & & & & ONSITE & SESPMNT & $\mathrm{R}$ \\
\hline 5848 & & & & ONSITE & SESPMNT & $\mathrm{R}$ \\
\hline 5849 & & & & ONSITE & SESPMNT & $\mathrm{R}$ \\
\hline 5850 & See sample record for comment. & & & ONSITE & SESPMNT & $\mathrm{R}$ \\
\hline 5851 & See sample record for comment. & & & ONSITE & SESPMNT & $\mathrm{R}$ \\
\hline 5852 & See sample record for comment. & & & OFFSITE & SESPMNT & $\mathrm{R}$ \\
\hline 5853 & See sample record for comment. & & & OFFSITE & SESPMNT & $\mathrm{R}$ \\
\hline 5854 & & & & OFFSITE & SESPMNT & $\mathrm{R}$ \\
\hline 5855 & & & & OFFSITE & SESPMNT & $\mathrm{R}$ \\
\hline 5856 & & & & ONSITE & SESPMNT & $\mathrm{R}$ \\
\hline 5857 & & & & ONSITE & SESPMNT & $\mathrm{R}$ \\
\hline 5858 & & & & OFFSITE & SESPMNT & $\mathrm{R}$ \\
\hline 5859 & & & & OFFSITE & SESPMNT & $\mathrm{R}$ \\
\hline 5860 & & & & OFFSITE & SESPMNT & $\mathrm{R}$ \\
\hline 5861 & & & & OFFSITE & SESPMNT & $\mathrm{R}$ \\
\hline 5862 & & & & OFFSITE & SESPMNT & $\mathrm{R}$ \\
\hline 5863 & & & & OFFSITE & SESPMNT & $\mathrm{R}$ \\
\hline 5864 & & & & OFFSITE & SESPMNT & $\mathrm{R}$ \\
\hline 5865 & & & & OFFSITE & SESPMNT & $\mathrm{R}$ \\
\hline 5866 & & & & OFFSITE & SESPMNT & $\mathrm{R}$ \\
\hline 5867 & & & & OFFSITE & SESPMNT & $\mathrm{R}$ \\
\hline 5868 & & & & OFFSITE & SESPMNT & $\mathrm{R}$ \\
\hline 5869 & & & & OFFSITE & SESPMNT & $\mathrm{R}$ \\
\hline 5870 & & & & OFFSITE & SESPMNT & $\mathrm{R}$ \\
\hline 5871 & & & & OFFSITE & SESPMNT & $\mathrm{R}$ \\
\hline 5872 & & & & OFFSITE & SESPMNT & $\mathrm{R}$ \\
\hline 5873 & & & & OFFSITE & SESPMNT & $\mathrm{R}$ \\
\hline 5874 & & & & PERIMETER & SESPMNT & $\mathrm{R}$ \\
\hline 5875 & & & & PERIMETER & SESPMNT & $\mathrm{R}$ \\
\hline 5876 & & & & PERIMETER & SESPMNT & $R$ \\
\hline 5877 & & & & ONSITE & SESBMNT & $R$ \\
\hline
\end{tabular}




\begin{tabular}{|c|c|c|c|c|c|c|}
\hline & $A D$ & $\mathrm{AE}$ & $\mathrm{AF}$ & $A G$ & $\mathrm{AH}$ & $\mathrm{Al}$ \\
\hline 1 & RESULT_COMMENT & COLL_SAMP_SIZE & COLL_SAMP_SIZE_UNITS & DIST_CLASS & OWNER_ID & COLLECTION_PURPOSE \\
\hline 5878 & & & & ONSITE & SESPMNT & $\mathrm{R}$ \\
\hline 5879 & & & & ONSITE & SESPMNT & $\mathrm{R}$ \\
\hline 5880 & & & & ONSITE & SESPMNT & $\mathrm{R}$ \\
\hline 5881 & & & & ONSITE & SESPMNT & $\mathrm{R}$ \\
\hline 5882 & & & & OFFSITE & SESPMNT & $\mathrm{R}$ \\
\hline 5883 & & & & OFFSITE & SESPMNT & $\mathrm{R}$ \\
\hline 5884 & & & & OFFSITE & SESPMNT & $\mathrm{R}$ \\
\hline 5885 & & & & OFFSITE & SESPMNT & $\mathrm{R}$ \\
\hline 5886 & & & & OFFSITE & SESPMNT & $\mathrm{R}$ \\
\hline \begin{tabular}{|c|c|}
5887 \\
\end{tabular} & & & & OFFSITE & SESPMNT & $\mathrm{R}$ \\
\hline 5888 & & & & OFFSITE & SESPMNT & $\mathrm{R}$ \\
\hline 5889 & & & & OFFSITE & SESPMNT & $\mathrm{R}$ \\
\hline 5890 & & & & OFFSITE & SESPMNT & $\mathrm{R}$ \\
\hline 5891 & & & & OFFSITE & SESPMNT & $\mathrm{R}$ \\
\hline 5892 & & & & OFFSITE & SESPMNT & $\mathrm{R}$ \\
\hline 5893 & & & & OFFSITE & SESPMNT & $\mathrm{R}$ \\
\hline 5894 & & & & OFFSITE & SESPMNT & $\mathrm{R}$ \\
\hline 5895 & & & & OFFSITE & SESPMNT & $\mathrm{R}$ \\
\hline 5896 & & & & OFFSITE & SESPMNT & $\mathrm{R}$ \\
\hline 5897 & & & & & SESPMNT & $\mathrm{R}$ \\
\hline 5898 & & & & & SESPMNT & $\mathrm{R}$ \\
\hline 5899 & & & & & SESPMNT & $\mathrm{R}$ \\
\hline 5900 & & & & & SESPMNT & $\mathrm{R}$ \\
\hline 5901 & & & & & SESPMNT & $\mathrm{R}$ \\
\hline 5902 & & & & ONSITE & SESPMNT & $\mathrm{R}$ \\
\hline 5903 & & & & ONSITE & SESPMNT & $\mathrm{R}$ \\
\hline 5904 & & & & ONSITE & SESPMNT & $\mathrm{R}$ \\
\hline 5905 & & & & ONSITE & SESPMNT & $\mathrm{R}$ \\
\hline 5906 & & & & ONSITE & SESPMNT & $\mathrm{R}$ \\
\hline 5907 & & & & ONSITE & SESPMNT & $\mathrm{R}$ \\
\hline 5908 & & & & ONSITE & SESPMNT & $\mathrm{R}$ \\
\hline 5909 & & & & ONSITE & SESPMNT & $\mathrm{R}$ \\
\hline 5910 & & & & ONSITE & SESPMNT & $\mathrm{R}$ \\
\hline 5911 & & & & OFFSITE & SESPMNT & $\mathrm{R}$ \\
\hline 5912 & & & & OFFSITE & SESPMNT & $\mathrm{R}$ \\
\hline 5913 & & & & OFFSITE & SESPMNT & $\mathrm{R}$ \\
\hline 5914 & & & & OFFSITE & SESPMNT & $\mathrm{R}$ \\
\hline 5915 & & & & OFFSITE & SESPMNT & $\mathrm{R}$ \\
\hline 5916 & See sample record for comment. & & & & SESPMNT & $\mathrm{R}$ \\
\hline 5917 & See sample record for comment. & & & & SESPMNT & $\mathrm{R}$ \\
\hline 5918 & See sample record for comment. & & & & SESPMNT & $\mathrm{R}$ \\
\hline 5919 & See sample record for comment. & & & & SESPMNT & $\mathrm{R}$ \\
\hline 5920 & See sample record for comment. & & & & SESPMNT & $\mathrm{R}$ \\
\hline 5921 & & & & OFFSITE & SESPSPEC & $\mathrm{s}$ \\
\hline 5922 & & & & OFFSITE & SESPSPEC & $\mathrm{s}$ \\
\hline 5923 & & & & OFFSITE & SESPSPEC & $\mathrm{s}$ \\
\hline 5924 & & & & OFFSITE & SESPSPEC & $\mathrm{s}$ \\
\hline 5925 & & & & OFFSITE & SESPSPEC & $\mathrm{s}$ \\
\hline 5926 & & & & OFFSITE & SESPSPEC & $\mathrm{s}$ \\
\hline 5927 & & & & OFFSITE & SESPSPEC & $\mathrm{s}$ \\
\hline 5928 & & & & OFFSITE & SESPSPEC & $\mathrm{s}$ \\
\hline 5929 & & & & OFFSITE & SESBSPEC & $0^{5}$ \\
\hline
\end{tabular}




\begin{tabular}{|c|c|c|c|c|c|c|}
\hline & $\mathrm{AD}$ & $\mathrm{AE}$ & $\mathrm{AF}$ & AG & $\mathrm{AH}$ & $\overline{\mathrm{Al}}$ \\
\hline 1 & RESULT_COMMENT & COLL_SAMP_SIZE & COLL_SAMP_SIZE_UNITS & DIST_CLASS & OWNER_ID & COLLECTION_PURPOSE \\
\hline 5930 & & & & OFFSITE & SESPSPEC & S \\
\hline 5931 & & & & OFFSITE & SESPSPEC & $\mathrm{S}$ \\
\hline 5932 & & & & OFFSITE & SESPSPEC & $\mathrm{S}$ \\
\hline 5933 & & & & ONSITE & SESPMNT & $\mathrm{R}$ \\
\hline 5934 & & & & ONSITE & SESPMNT & $\mathrm{R}$ \\
\hline \begin{tabular}{|c|}
5935 \\
503
\end{tabular} & & & & OFFSITE & SESPMNT & $\mathrm{R}$ \\
\hline 5936 & & & & OFFSITE & SESPMNT & $\mathrm{R}$ \\
\hline 5937 & & & & OFFSITE & SESPMNT & $\mathrm{R}$ \\
\hline 5938 & & & & OFFSITE & SESPMNT & $\mathrm{R}$ \\
\hline 5939 & & & & ONSITE & SESPMNT & $\mathrm{R}$ \\
\hline 5940 & & & & ONSITE & SESPMNT & $\mathrm{R}$ \\
\hline \begin{tabular}{|l|}
5941 \\
\end{tabular} & & & & ONSITE & SESPMNT & $\mathrm{R}$ \\
\hline \begin{tabular}{|l|l|}
5942 \\
\end{tabular} & & & & ONSITE & SESPMNT & $\mathrm{R}$ \\
\hline \begin{tabular}{|l|l|}
5943 \\
\end{tabular} & & & & ONSITE & SESPMNT & $\mathrm{R}$ \\
\hline \begin{tabular}{|l|l|}
5944 \\
\end{tabular} & & & & ONSITE & SESPMNT & $\mathrm{R}$ \\
\hline \begin{tabular}{|l|}
5945 \\
\end{tabular} & & & & ONSITE & SESPMNT & $\mathrm{R}$ \\
\hline \begin{tabular}{|l|}
5946 \\
\end{tabular} & & & & ONSITE & SESPMNT & $\mathrm{R}$ \\
\hline \begin{tabular}{|l|l|}
5947 \\
\end{tabular} & & & & ONSITE & SESPMNT & $\mathrm{R}$ \\
\hline \begin{tabular}{|c|}
5948 \\
\end{tabular} & & & & ONSITE & SESPMNT & $\mathrm{R}$ \\
\hline \begin{tabular}{|l|l|}
5949 \\
\end{tabular} & & & & ONSITE & SESPMNT & $\mathrm{R}$ \\
\hline 5950 & & & & ONSITE & SESPMNT & $R$ \\
\hline \begin{tabular}{|l|}
5951 \\
\end{tabular} & & & & ONSITE & SESPMNT & $\mathrm{R}$ \\
\hline 5952 & & & & OFFSITE & SESPMNT & $\mathrm{R}$ \\
\hline 5953 & & & & OFFSITE & SESPMNT & $\mathrm{R}$ \\
\hline 5954 & & & & OFFSITE & SESPMNT & $\mathrm{R}$ \\
\hline \begin{tabular}{|c|}
5955 \\
\end{tabular} & & & & OFFSITE & SESPMNT & $\mathrm{R}$ \\
\hline \begin{tabular}{|c|}
5956 \\
\end{tabular} & & & & OFFSITE & SESPMNT & $R$ \\
\hline \begin{tabular}{|c|}
5957 \\
\end{tabular} & & & & OFFSITE & SESPMNT & $\mathrm{R}$ \\
\hline \begin{tabular}{|c|}
5958 \\
\end{tabular} & & & & OFFSITE & SESPMNT & $\mathrm{R}$ \\
\hline \begin{tabular}{|c|}
5959 \\
\end{tabular} & & & & OFFSITE & SESPMNT & $R$ \\
\hline 5960 & & & & OFFSITE & SESPMNT & $R$ \\
\hline \begin{tabular}{|l|}
5961 \\
\end{tabular} & & & & OFFSITE & SESPMNT & $R$ \\
\hline \begin{tabular}{|l|}
5962 \\
\end{tabular} & & & & OFFSITE & SESPMNT & $R$ \\
\hline \begin{tabular}{|l|l|}
5963 \\
\end{tabular} & & & & OFFSITE & SESPMNT & $R$ \\
\hline 5964 & & & & OFFSITE & SESPMNT & $\mathrm{R}$ \\
\hline \begin{tabular}{|l|l|}
5965 \\
\end{tabular} & & & & OFFSITE & SESPMNT & $\mathrm{R}$ \\
\hline \begin{tabular}{|l|}
5966 \\
\end{tabular} & & & & OFFSITE & SESPMNT & $R$ \\
\hline \begin{tabular}{|l|}
5967 \\
\end{tabular} & & & & OFFSITE & SESPMNT & $R$ \\
\hline \begin{tabular}{|l|l|}
5968 \\
\end{tabular} & & & & ONSITE & SESPMNT & $R$ \\
\hline 5969 & & & & ONSITE & SESPMNT & $R$ \\
\hline 5970 & & & & OFFSITE & SESPMNT & $R$ \\
\hline 5971 & & & & OFFSITE & SESPMNT & $R$ \\
\hline 5972 & & & & OFFSITE & SESPMNT & $R$ \\
\hline \begin{tabular}{|l|l|}
5973 \\
\end{tabular} & & & & OFFSITE & SESPMNT & $R$ \\
\hline 5974 & & & & OFFSITE & SESPMNT & $R$ \\
\hline 5975 & & & & OFFSITE & SESPMNT & $R$ \\
\hline 5976 & & & & ONSITE & SESPMNT & $\mathrm{R}$ \\
\hline \begin{tabular}{|l|}
5977 \\
\end{tabular} & & & & ONSITE & SESPMNT & $\mathrm{R}$ \\
\hline \begin{tabular}{|l|l|}
5978 \\
\end{tabular} & & & & ONSITE & SESPMNT & $R$ \\
\hline \begin{tabular}{|l|l|}
5979 \\
\end{tabular} & & & & ONSITE & SESPMNT & $R$ \\
\hline 5980 & & & & ONSITE & SESPMNT & $\mathrm{R}$ \\
\hline 5981 & & & & ONSITE & SESBMNT & $R$ \\
\hline
\end{tabular}




\begin{tabular}{|c|c|c|c|c|c|c|}
\hline & $A D$ & $\mathrm{AE}$ & $\mathrm{AF}$ & $A G$ & $\mathrm{AH}$ & $\mathrm{Al}$ \\
\hline 1 & RESULT_COMMENT & COLL_SAMP_SIZE & COLL_SAMP_SIZE_UNITS & DIST_CLASS & OWNER_ID & COLLECTION_PURPOSE \\
\hline 5982 & & & & ONSITE & SESPMNT & $\mathrm{R}$ \\
\hline 5983 & & & & ONSITE & SESPMNT & $\mathrm{R}$ \\
\hline 5984 & & & & ONSITE & SESPMNT & $\mathrm{R}$ \\
\hline 5985 & & & & ONSITE & SESPMNT & $\mathrm{R}$ \\
\hline 5986 & & & & ONSITE & SESPMNT & $\mathrm{R}$ \\
\hline 5987 & & & & ONSITE & SESPMNT & $\mathrm{R}$ \\
\hline 5988 & & & & ONSITE & SESPMNT & $\mathrm{R}$ \\
\hline 5989 & & & & ONSITE & SESPMNT & $\mathrm{R}$ \\
\hline 5990 & & & & ONSITE & SESPMNT & $\mathrm{R}$ \\
\hline 5991 & & & & ONSITE & SESPMNT & $\mathrm{R}$ \\
\hline 5992 & & & & ONSITE & SESPMNT & $\mathrm{R}$ \\
\hline 5993 & & & & OFFSITE & SESPMNT & $\mathrm{R}$ \\
\hline 5994 & & & & OFFSITE & SESPMNT & $\mathrm{R}$ \\
\hline 5995 & & & & OFFSITE & SESPMNT & $\mathrm{R}$ \\
\hline 5996 & & & & OFFSITE & SESPMNT & $\mathrm{R}$ \\
\hline 5997 & & & & OFFSITE & SESPMNT & $\mathrm{R}$ \\
\hline 5998 & & & & OFFSITE & SESPMNT & $\mathrm{R}$ \\
\hline 5999 & & & & OFFSITE & SESPMNT & $\mathrm{R}$ \\
\hline 6000 & & & & OFFSITE & SESPMNT & $\mathrm{R}$ \\
\hline 6001 & & & & OFFSITE & SESPMNT & $\mathrm{R}$ \\
\hline 6002 & & & & OFFSITE & SESPMNT & $\mathrm{R}$ \\
\hline 6003 & & & & OFFSITE & SESPMNT & $\mathrm{R}$ \\
\hline 6004 & & & & OFFSITE & SESPMNT & $\mathrm{R}$ \\
\hline 6005 & & & & OFFSITE & SESPMNT & $\mathrm{R}$ \\
\hline 6006 & & & & OFFSITE & SESPMNT & $\mathrm{R}$ \\
\hline 6007 & & & & OFFSITE & SESPMNT & $\mathrm{R}$ \\
\hline 6008 & & & & OFFSITE & SESPMNT & $\mathrm{R}$ \\
\hline 6009 & & & & ONSITE & SESPMNT & $\mathrm{R}$ \\
\hline 6010 & & & & ONSITE & SESPMNT & $\mathrm{R}$ \\
\hline 6011 & See sample record for comment. & & & OFFSITE & SESPMNT & $\mathrm{R}$ \\
\hline 6012 & See sample record for comment. & & & OFFSITE & SESPMNT & $\mathrm{R}$ \\
\hline 6013 & & & & OFFSITE & SESPMNT & $\mathrm{R}$ \\
\hline 6014 & & & & OFFSITE & SESPMNT & $\mathrm{R}$ \\
\hline 6015 & & & & PERIMETER & SESPMNT & $\mathrm{R}$ \\
\hline 6016 & & & & PERIMETER & SESPMNT & $\mathrm{R}$ \\
\hline 6017 & & & & PERIMETER & SESPMNT & $\mathrm{R}$ \\
\hline 6018 & & & & ONSITE & SESPMNT & $\mathrm{R}$ \\
\hline 6019 & & & & ONSITE & SESPMNT & $\mathrm{R}$ \\
\hline 6020 & & & & ONSITE & SESPMNT & $\mathrm{R}$ \\
\hline 6021 & & & & ONSITE & SESPMNT & $\mathrm{R}$ \\
\hline \begin{tabular}{|c|c|}
6022 \\
\end{tabular} & & & & ONSITE & SESPMNT & $\mathrm{R}$ \\
\hline 6023 & & & & OFFSITE & SESPMNT & $\mathrm{R}$ \\
\hline 6024 & & & & OFFSITE & SESPMNT & $\mathrm{R}$ \\
\hline 6025 & & & & OFFSITE & SESPMNT & $\mathrm{R}$ \\
\hline 6026 & & & & OFFSITE & SESPMNT & $\mathrm{R}$ \\
\hline 6027 & & & & OFFSITE & SESPMNT & $\mathrm{R}$ \\
\hline 6028 & & & & OFFSITE & SESPMNT & $\mathrm{R}$ \\
\hline 6029 & & & & OFFSITE & SESPMNT & $\mathrm{R}$ \\
\hline 6030 & & & & OFFSITE & SESPMNT & $\mathrm{R}$ \\
\hline 6031 & & & & OFFSITE & SESPMNT & $\mathrm{R}$ \\
\hline 6032 & & & & OFFSITE & SESPMNT & $\mathrm{R}$ \\
\hline 6033 & & & & OFFSITE & SESBMNT & ${ }_{2} R$ \\
\hline
\end{tabular}




\begin{tabular}{|c|c|c|c|c|c|c|}
\hline & $\mathrm{AD}$ & $\mathrm{AE}$ & $\mathrm{AF}$ & AG & $\mathrm{AH}$ & $\mathrm{Al}$ \\
\hline 1 & RESULT_COMMENT & COLL_SAMP_SIZE & COLL_SAMP_SIZE_UNITS & DIST_CLASS & OWNER_ID & COLLECTION_PURPOSE \\
\hline 6034 & & & & OFFSITE & SESPMNT & $\mathrm{R}$ \\
\hline 6035 & & & & OFFSITE & SESPMNT & $\mathrm{R}$ \\
\hline 6036 & & & & OFFSITE & SESPMNT & $\mathrm{R}$ \\
\hline 6037 & & & & OFFSITE & SESPMNT & $\mathrm{R}$ \\
\hline 6038 & & & & OFFSITE & SESPMNT & $\mathrm{R}$ \\
\hline 6039 & & & & OFFSITE & SESPMNT & $\mathrm{R}$ \\
\hline 6040 & & & & OFFSITE & SESPMNT & $\mathrm{R}$ \\
\hline 6041 & & & & OFFSITE & SESPMNT & $\mathrm{R}$ \\
\hline 6042 & & & & OFFSITE & SESPMNT & $\mathrm{R}$ \\
\hline 6043 & & & & OFFSITE & SESPMNT & $\mathrm{R}$ \\
\hline 6044 & & & & OFFSITE & SESPMNT & $\mathrm{R}$ \\
\hline 6045 & & & & & SESPMNT & $\mathrm{R}$ \\
\hline 6046 & & & & & SESPMNT & $\mathrm{R}$ \\
\hline 6047 & & & & & SESPMNT & $\mathrm{R}$ \\
\hline 6048 & & & & & SESPMNT & $\mathrm{R}$ \\
\hline 6049 & & & & & SESPMNT & $\mathrm{R}$ \\
\hline 6050 & & & & OFFSITE & SESPMNT & $\mathrm{R}$ \\
\hline 6051 & & & & OFFSITE & SESPMNT & $\mathrm{R}$ \\
\hline 6052 & & & & OFFSITE & SESPMNT & $\mathrm{R}$ \\
\hline 6053 & & & & OFFSITE & SESPMNT & $\mathrm{R}$ \\
\hline 6054 & & & & OFFSITE & SESPMNT & $\mathrm{R}$ \\
\hline 6055 & & & & ONSITE & SESPMNT & $\mathrm{R}$ \\
\hline 6056 & & & & ONSITE & SESPMNT & $\mathrm{R}$ \\
\hline 6057 & & & & ONSITE & SESPMNT & $\mathrm{R}$ \\
\hline 6058 & & & & ONSITE & SESPMNT & $\mathrm{R}$ \\
\hline \begin{tabular}{|c|}
6059 \\
\end{tabular} & & & & ONSITE & SESPMNT & $\mathrm{R}$ \\
\hline 6060 & & & & ONSITE & SESPMNT & $\mathrm{R}$ \\
\hline 6061 & & & & OFFSITE & SESPMNT & $\mathrm{R}$ \\
\hline 6062 & & & & OFFSITE & SESPMNT & $\mathrm{R}$ \\
\hline 6063 & & & & OFFSITE & SESPMNT & $\mathrm{R}$ \\
\hline 6064 & & & & OFFSITE & SESPMNT & $\mathrm{R}$ \\
\hline 6065 & & & & OFFSITE & SESPMNT & $\mathrm{R}$ \\
\hline 6066 & & & & ONSITE & SESPMNT & $\mathrm{R}$ \\
\hline \begin{tabular}{|l|l|}
6067 \\
\end{tabular} & & & & ONSITE & SESPMNT & $\mathrm{R}$ \\
\hline 6068 & & & & OFFSITE & SESPMNT & $\mathrm{R}$ \\
\hline 6069 & & & & OFFSITE & SESPMNT & $\mathrm{R}$ \\
\hline 6070 & & & & OFFSITE & SESPMNT & $\mathrm{R}$ \\
\hline \begin{tabular}{|l|}
6071 \\
\end{tabular} & & & & OFFSITE & SESPMNT & $\mathrm{R}$ \\
\hline \begin{tabular}{|l|}
6072 \\
\end{tabular} & & & & OFFSITE & SESPMNT & $\mathrm{R}$ \\
\hline \begin{tabular}{|l|}
6073 \\
\end{tabular} & & & & OFFSITE & SESPMNT & $\mathrm{R}$ \\
\hline \begin{tabular}{|l|}
6074 \\
\end{tabular} & & & & ONSITE & SESPMNT & $\mathrm{R}$ \\
\hline \begin{tabular}{|l|}
6075 \\
\end{tabular} & & & & ONSITE & SESPMNT & $\mathrm{R}$ \\
\hline \begin{tabular}{|l|}
6076 \\
\end{tabular} & & & & ONSITE & SESPMNT & $\mathrm{R}$ \\
\hline \begin{tabular}{|l|}
6077 \\
\end{tabular} & & & & ONSITE & SESPMNT & $\mathrm{R}$ \\
\hline \begin{tabular}{|l|}
6078 \\
\end{tabular} & & & & ONSITE & SESPMNT & $\mathrm{R}$ \\
\hline \begin{tabular}{|l|}
6079 \\
\end{tabular} & & & & ONSITE & SESPMNT & $\mathrm{R}$ \\
\hline \begin{tabular}{|l|}
6080 \\
\end{tabular} & & & & ONSITE & SESPMNT & $\mathrm{R}$ \\
\hline \begin{tabular}{|l|}
6081 \\
\end{tabular} & & & & ONSITE & SESPMNT & $\mathrm{R}$ \\
\hline \begin{tabular}{|l|}
6082 \\
\end{tabular} & & & & ONSITE & SESPMNT & $\mathrm{R}$ \\
\hline \begin{tabular}{|l|}
6083 \\
\end{tabular} & & & & ONSITE & SESPMNT & $\mathrm{R}$ \\
\hline \begin{tabular}{|l|}
6084 \\
\end{tabular} & & & & ONSITE & SESPMNT & $\mathrm{R}$ \\
\hline \begin{tabular}{|l|l|}
6085 \\
\end{tabular} & & & & ONSITE & SESBMNT & $R$ \\
\hline
\end{tabular}




\begin{tabular}{|c|c|c|c|c|c|c|}
\hline & $\mathrm{AD}$ & $\mathrm{AE}$ & $\mathrm{AF}$ & AG & $\mathrm{AH}$ & $\mathrm{Al}$ \\
\hline 1 & RESULT_COMMENT & COLL_SAMP_SIZE & COLL_SAMP_SIZE_UNITS & DIST_CLASS & OWNER_ID & COLLECTION_PURPOSE \\
\hline 6086 & & & & ONSITE & SESPMNT & $\mathrm{R}$ \\
\hline \begin{tabular}{|c|c|}
6087 \\
\end{tabular} & & & & OFFSITE & SESPMNT & $\mathrm{R}$ \\
\hline 6088 & & & & OFFSITE & SESPMNT & $\mathrm{R}$ \\
\hline 6089 & & & & OFFSITE & SESPMNT & $\mathrm{R}$ \\
\hline 6090 & & & & OFFSITE & SESPMNT & $\mathrm{R}$ \\
\hline 6091 & & & & OFFSITE & SESPMNT & $\mathrm{R}$ \\
\hline 6092 & & & & OFFSITE & SESPMNT & $\mathrm{R}$ \\
\hline 6093 & & & & OFFSITE & SESPMNT & $\mathrm{R}$ \\
\hline 6094 & & & & OFFSITE & SESPMNT & $\mathrm{R}$ \\
\hline 6095 & & & & OFFSITE & SESPMNT & $\mathrm{R}$ \\
\hline \begin{tabular}{|c|}
6096 \\
\end{tabular} & & & & OFFSITE & SESPMNT & $\mathrm{R}$ \\
\hline \begin{tabular}{|c|}
6097 \\
\end{tabular} & & & & OFFSITE & SESPMNT & $\mathrm{R}$ \\
\hline \begin{tabular}{|c|}
6098 \\
\end{tabular} & & & & OFFSITE & SESPMNT & $\mathrm{R}$ \\
\hline 6099 & & & & OFFSITE & SESPMNT & $\mathrm{R}$ \\
\hline 6100 & & & & OFFSITE & SESPMNT & $\mathrm{R}$ \\
\hline 6101 & & & & OFFSITE & SESPMNT & $\mathrm{R}$ \\
\hline 6102 & & & & OFFSITE & SESPMNT & $\mathrm{R}$ \\
\hline \begin{tabular}{|c|}
6103 \\
\end{tabular} & & & & ONSITE & SESPMNT & $\mathrm{R}$ \\
\hline \begin{tabular}{|c|}
6104 \\
\end{tabular} & & & & ONSITE & SESPMNT & $\mathrm{R}$ \\
\hline 6105 & & & & OFFSITE & SESPMNT & $\mathrm{R}$ \\
\hline 6106 & & & & OFFSITE & SESPMNT & $\mathrm{R}$ \\
\hline 6107 s & See sample record for comment. & & & OFFSITE & SESPMNT & $\mathrm{R}$ \\
\hline 6108 & See sample record for comment. & & & OFFSITE & SESPMNT & $\mathrm{R}$ \\
\hline 6109 & & & & ONSITE & SESPMNT & $\mathrm{R}$ \\
\hline 6110 & & & & ONSITE & SESPMNT & $\mathrm{R}$ \\
\hline 6111 & & & & ONSITE & SESPMNT & $\mathrm{R}$ \\
\hline \begin{tabular}{|c|}
6112 \\
\end{tabular} & & & & ONSITE & SESPMNT & $\mathrm{R}$ \\
\hline \begin{tabular}{|c|c|}
6113 \\
\end{tabular} & & & & ONSITE & SESPMNT & $\mathrm{R}$ \\
\hline 6114 & & & & ONSITE & SESPMNT & $\mathrm{R}$ \\
\hline 6115 & & & & ONSITE & SESPMNT & $\mathrm{R}$ \\
\hline \begin{tabular}{|c|}
6116 \\
\end{tabular} & & & & ONSITE & SESPMNT & $\mathrm{R}$ \\
\hline 6117 & & & & ONSITE & SESPMNT & $\mathrm{R}$ \\
\hline 6118 & & & & ONSITE & SESPMNT & $\mathrm{R}$ \\
\hline 6119 & & & & ONSITE & SESPMNT & $\mathrm{R}$ \\
\hline 6120 & & & & ONSITE & SESPMNT & $\mathrm{R}$ \\
\hline 6121 & & & & ONSITE & SESPMNT & $\mathrm{R}$ \\
\hline \begin{tabular}{|c|}
6122 \\
\end{tabular} & & & & ONSITE & SESPMNT & $\mathrm{R}$ \\
\hline \begin{tabular}{|c|}
6123 \\
\end{tabular} & & & & ONSITE & SESPMNT & $\mathrm{R}$ \\
\hline 6124 & & & & ONSITE & SESPMNT & $\mathrm{R}$ \\
\hline \begin{tabular}{|c|}
6125 \\
\end{tabular} & & & & ONSITE & SESPMNT & $\mathrm{R}$ \\
\hline \begin{tabular}{|c|}
6126 \\
\end{tabular} & & & & OFFSITE & SESPMNT & $\mathrm{R}$ \\
\hline \begin{tabular}{|c|}
6127 \\
\end{tabular} & & & & OFFSITE & SESPMNT & $\mathrm{R}$ \\
\hline $\mid 6128$ & See sample record for comment. & & & OFFSITE & SESPMNT & $\mathrm{R}$ \\
\hline 6129 & See sample record for comment. & & & OFFSITE & SESPMNT & $\mathrm{R}$ \\
\hline 6130 & & & & OFFSITE & SESPMNT & $\mathrm{R}$ \\
\hline 6131 & & & & OFFSITE & SESPMNT & $\mathrm{R}$ \\
\hline 6132 & & & & OFFSITE & SESPMNT & $\mathrm{R}$ \\
\hline 6133 & & & & OFFSITE & SESPMNT & $\mathrm{R}$ \\
\hline 6134 & & & & OFFSITE & SESPMNT & $\mathrm{R}$ \\
\hline 6135 & & & & OFFSITE & SESPMNT & $\mathrm{R}$ \\
\hline 6136 & See sample record for comment. & & & OFFSITE & SESPMNT & $\mathrm{R}$ \\
\hline 6137 s & See sample record for comment. & & & OFFSITE & SESBMNT & $R$ \\
\hline
\end{tabular}




\begin{tabular}{|c|c|c|c|c|c|c|}
\hline & $A D$ & $\mathrm{AE}$ & $\mathrm{AF}$ & AG & $\mathrm{AH}$ & $\mathrm{Al}$ \\
\hline 1 & RESULT_COMMENT & COLL_SAMP_SIZE & COLL_SAMP_SIZE_UNITS & DIST_CLASS & OWNER_ID & COLLECTION_PURPOSE \\
\hline 6138 & See sample record for comment. & & & OFFSITE & SESPMNT & $\mathrm{R}$ \\
\hline 6139 & See sample record for comment. & & & OFFSITE & SESPMNT & $\mathrm{R}$ \\
\hline 6140 & See sample record for comment. & & & OFFSITE & SESPMNT & $\mathrm{R}$ \\
\hline 6141 & See sample record for comment. & & & OFFSITE & SESPMNT & $\mathrm{R}$ \\
\hline 6142 & & & & ONSITE & SESPMNT & $\mathrm{R}$ \\
\hline 6143 & & & & ONSITE & SESPMNT & $\mathrm{R}$ \\
\hline 6144 & & & & OFFSITE & SESPMNT & $\mathrm{R}$ \\
\hline \begin{tabular}{|l|l|}
6145 \\
\end{tabular} & & & & OFFSITE & SESPMNT & $\mathrm{R}$ \\
\hline \begin{tabular}{|l|l|}
6146 \\
\end{tabular} & See sample record for comment. & & & OFFSITE & SESPMNT & $\mathrm{R}$ \\
\hline \begin{tabular}{|l|l|}
6147 \\
\end{tabular} & See sample record for comment. & & & OFFSITE & SESPMNT & $\mathrm{R}$ \\
\hline \begin{tabular}{|c|}
6148 \\
\end{tabular} & & & & OFFSITE & SESPMNT & $\mathrm{R}$ \\
\hline \begin{tabular}{|c|}
6149 \\
\end{tabular} & & & & OFFSITE & SESPMNT & $\mathrm{R}$ \\
\hline 6150 & & & & PERIMETER & SESPMNT & $\mathrm{R}$ \\
\hline \begin{tabular}{|c|}
6151 \\
615
\end{tabular} & & & & PERIMETER & SESPMNT & $\mathrm{R}$ \\
\hline \begin{tabular}{|c|}
6152 \\
\end{tabular} & & & & PERIMETER & SESPMNT & $\mathrm{R}$ \\
\hline \begin{tabular}{|c|}
6153 \\
\end{tabular} & & & & ONSITE & SESPMNT & $\mathrm{R}$ \\
\hline \begin{tabular}{|c|}
6154 \\
\end{tabular} & & & & ONSITE & SESPMNT & $\mathrm{R}$ \\
\hline \begin{tabular}{|c|}
6155 \\
\end{tabular} & See sample record for comment. & & & OFFSITE & SESPMNT & $\mathrm{R}$ \\
\hline \begin{tabular}{|c|}
6156 \\
\end{tabular} & See sample record for comment. & & & OFFSITE & SESPMNT & $\mathrm{R}$ \\
\hline \begin{tabular}{|l|l|}
6157 & \\
\end{tabular} & & & & OFFSITE & SESPMNT & $\mathrm{R}$ \\
\hline \begin{tabular}{|c|}
6158 \\
\end{tabular} & & & & OFFSITE & SESPMNT & $\mathrm{R}$ \\
\hline \begin{tabular}{|c|}
6159 \\
\end{tabular} & & & & OFFSITE & SESPMNT & $\mathrm{R}$ \\
\hline \begin{tabular}{|l|}
6160 \\
\end{tabular} & & & & OFFSITE & SESPMNT & $\mathrm{R}$ \\
\hline \begin{tabular}{|l|}
6161 \\
\end{tabular} & & & & OFFSITE & SESPMNT & $R$ \\
\hline \begin{tabular}{|l|}
6162 \\
\end{tabular} & & & & OFFSITE & SESPMNT & $\mathrm{R}$ \\
\hline \begin{tabular}{|l|}
6163 \\
\end{tabular} & & & & OFFSITE & SESPMNT & $\mathrm{R}$ \\
\hline \begin{tabular}{|l|}
6164 \\
616
\end{tabular} & & & & & SESPMNT & $\mathrm{R}$ \\
\hline \begin{tabular}{|l|}
6165 \\
\end{tabular} & & & & & SESPMNT & $\mathrm{R}$ \\
\hline \begin{tabular}{|l|}
6166 \\
\end{tabular} & & & & & SESPMNT & $\mathrm{R}$ \\
\hline \begin{tabular}{|l|l|}
6167 \\
\end{tabular} & & & & & SESPMNT & $\mathrm{R}$ \\
\hline \begin{tabular}{|l|}
6168 \\
\end{tabular} & & & & & SESPMNT & $\mathrm{R}$ \\
\hline \begin{tabular}{|l|}
6169 \\
\end{tabular} & & & & ONSITE & SESPMNT & $\mathrm{R}$ \\
\hline \begin{tabular}{|l|}
6170 \\
\end{tabular} & & & & ONSITE & SESPMNT & $\mathrm{R}$ \\
\hline \begin{tabular}{|l|}
6171 \\
\end{tabular} & & & & ONSITE & SESPMNT & $\mathrm{R}$ \\
\hline \begin{tabular}{|l|}
6172 \\
\end{tabular} & & & & ONSITE & SESPMNT & $\mathrm{R}$ \\
\hline \begin{tabular}{|l|}
6173 \\
\end{tabular} & & & & ONSITE & SESPMNT & $\mathrm{R}$ \\
\hline \begin{tabular}{|l|l|}
6174 \\
\end{tabular} & & & & ONSITE & SESPMNT & $\mathrm{R}$ \\
\hline \begin{tabular}{|l|}
6175 \\
\end{tabular} & & & & ONSITE & SESPMNT & $\mathrm{R}$ \\
\hline \begin{tabular}{|l|}
6176 \\
\end{tabular} & & & & ONSITE & SESPMNT & $\mathrm{R}$ \\
\hline \begin{tabular}{|l|}
6177 \\
1
\end{tabular} & & & & ONSITE & SESPMNT & $\mathrm{R}$ \\
\hline \begin{tabular}{|l|}
6178 \\
\end{tabular} & & & & OFFSITE & SESPMNT & $\mathrm{R}$ \\
\hline \begin{tabular}{|l|}
6179 \\
\end{tabular} & & & & OFFSITE & SESPMNT & $\mathrm{R}$ \\
\hline \begin{tabular}{|l|}
6180 \\
\end{tabular} & & & & OFFSITE & SESPMNT & $\mathrm{R}$ \\
\hline \begin{tabular}{|l|}
6181 \\
\end{tabular} & & & & OFFSITE & SESPMNT & $\mathrm{R}$ \\
\hline \begin{tabular}{|l|}
6182 \\
\end{tabular} & & & & OFFSITE & SESPMNT & $\mathrm{R}$ \\
\hline 6183 & & & & OFFSITE & SESPMNT & $\mathrm{R}$ \\
\hline \begin{tabular}{|l|}
6184 \\
\end{tabular} & & & & OFFSITE & SESPMNT & $\mathrm{R}$ \\
\hline \begin{tabular}{|l|}
6185 \\
\end{tabular} & & & & OFFSITE & SESPMNT & $\mathrm{R}$ \\
\hline \begin{tabular}{|l|}
6186 \\
\end{tabular} & & & & OFFSITE & SESPMNT & $\mathrm{R}$ \\
\hline \begin{tabular}{|l|l|}
6187 \\
\end{tabular} & & & & OFFSITE & SESPMNT & $\mathrm{R}$ \\
\hline \begin{tabular}{|l|}
6188 \\
\end{tabular} & & & & OFFSITE & SESPMNT & $\mathrm{R}$ \\
\hline \begin{tabular}{|l|}
6189 \\
\end{tabular} & & & & OFFSITE & SESBMNT & $R$ \\
\hline
\end{tabular}




\begin{tabular}{|c|c|c|c|c|c|c|}
\hline & $A D$ & $\mathrm{AE}$ & $\mathrm{AF}$ & $A G$ & $\mathrm{AH}$ & $\mathrm{Al}$ \\
\hline 1 & RESULT_COMMENT & COLL_SAMP_SIZE & COLL_SAMP_SIZE_UNITS & DIST_CLASS & OWNER_ID & COLLECTION_PURPOSE \\
\hline 6190 & & & & OFFSITE & SESPMNT & $\mathrm{R}$ \\
\hline \begin{tabular}{|l|}
6191 \\
\end{tabular} & & & & OFFSITE & SESPMNT & $\mathrm{R}$ \\
\hline \begin{tabular}{|l|l|}
6192 \\
\end{tabular} & & & & OFFSITE & SESPMNT & $\mathrm{R}$ \\
\hline \begin{tabular}{|c|}
6193 \\
\end{tabular} & & & & OFFSITE & SESPMNT & $\mathrm{R}$ \\
\hline 6194 & & & & OFFSITE & SESPMNT & $\mathrm{R}$ \\
\hline 6195 & & & & ONSITE & SESPMNT & $\mathrm{R}$ \\
\hline \begin{tabular}{|l|l|}
6196 \\
\end{tabular} & & & & ONSITE & SESPMNT & $\mathrm{R}$ \\
\hline \begin{tabular}{|l|l|}
6197 \\
\end{tabular} & & & & ONSITE & SESPMNT & $\mathrm{R}$ \\
\hline 6198 & & & & ONSITE & SESPMNT & $\mathrm{R}$ \\
\hline 6199 & & & & ONSITE & SESPMNT & $\mathrm{R}$ \\
\hline 6200 & See sample record for comment. & & & OFFSITE & SESPMNT & $\mathrm{R}$ \\
\hline 6201 & See sample record for comment. & & & OFFSITE & SESPMNT & $\mathrm{R}$ \\
\hline 6202 & See sample record for comment. & & & OFFSITE & SESPMNT & $\mathrm{R}$ \\
\hline \begin{tabular}{|l|}
6203 \\
\end{tabular} & See sample record for comment. & & & OFFSITE & SESPMNT & $\mathrm{R}$ \\
\hline \begin{tabular}{|l|}
6204 \\
\end{tabular} & See sample record for comment. & & & OFFSITE & SESPMNT & $\mathrm{R}$ \\
\hline \begin{tabular}{|l|l|}
6205 \\
\end{tabular} & & & & OFFSITE & SESPMNT & $\mathrm{R}$ \\
\hline \begin{tabular}{|c|}
6206 \\
\end{tabular} & & & & OFFSITE & SESPMNT & $\mathrm{R}$ \\
\hline \begin{tabular}{|l|l|}
6207 & -1 \\
\end{tabular} & & & & OFFSITE & SESPMNT & $\mathrm{R}$ \\
\hline 6208 & & & & OFFSITE & SESPMNT & $\mathrm{R}$ \\
\hline \begin{tabular}{|l|}
6209 \\
\end{tabular} & & & & OFFSITE & SESPMNT & $\mathrm{R}$ \\
\hline 6210 & & & & ONSITE & SESPMNT & $\mathrm{R}$ \\
\hline 6211 & & & & ONSITE & SESPMNT & $\mathrm{R}$ \\
\hline 6212 & & & & ONSITE & SESPMNT & $\mathrm{R}$ \\
\hline 6213 & See sample record for comment. & & & ONSITE & SESPMNT & $\mathrm{R}$ \\
\hline \begin{tabular}{|c|}
6214 \\
\end{tabular} & See sample record for comment. & & & ONSITE & SESPMNT & $\mathrm{R}$ \\
\hline \begin{tabular}{|c|}
6215 \\
\end{tabular} & & & & ONSITE & SESPMNT & $\mathrm{R}$ \\
\hline \begin{tabular}{|l|l|}
6216 \\
\end{tabular} & & & & ONSITE & SESPMNT & $\mathrm{R}$ \\
\hline \begin{tabular}{|l|l|}
6217 \\
\end{tabular} & & & & ONSITE & SESPMNT & $\mathrm{R}$ \\
\hline \begin{tabular}{|l|}
6218 \\
\end{tabular} & & & & ONSITE & SESPMNT & $\mathrm{R}$ \\
\hline \begin{tabular}{|l|}
6219 \\
\end{tabular} & & & & ONSITE & SESPMNT & $\mathrm{R}$ \\
\hline 6220 & & & & ONSITE & SESPMNT & $\mathrm{R}$ \\
\hline 6221 & & & & OFFSITE & SESPSPEC & $\mathrm{s}$ \\
\hline 6222 & & & & OFFSITE & SESPSPEC & $\mathrm{s}$ \\
\hline 6223 & & & & OFFSITE & SESPSPEC & $\mathrm{s}$ \\
\hline \begin{tabular}{|l|}
6224 \\
\end{tabular} & & & & OFFSITE & SESPSPEC & $\mathrm{s}$ \\
\hline 6225 & & & & OFFSITE & SESPSPEC & $\mathrm{s}$ \\
\hline 6226 & & & & OFFSITE & SESPSPEC & $\mathrm{s}$ \\
\hline \begin{tabular}{|l|l|}
6227 & $-r \cdot r$ \\
\end{tabular} & & & & OFFSITE & SESPSPEC & $\mathrm{s}$ \\
\hline 6228 & & & & OFFSITE & SESPSPEC & $\mathrm{s}$ \\
\hline 6229 & & & & OFFSITE & SESPSPEC & $\mathrm{s}$ \\
\hline 6230 & & & & OFFSITE & SESPSPEC & $\mathrm{s}$ \\
\hline 6231 & & & & OFFSITE & SESPSPEC & $\mathrm{s}$ \\
\hline \begin{tabular}{|l|}
6232 \\
\end{tabular} & & & & OFFSITE & SESPSPEC & $\mathrm{s}$ \\
\hline 6233 & See sample record for comment. & & & ONSITE & SESPMNT & $\mathrm{R}$ \\
\hline 6234 & See sample record for comment. & & & ONSITE & SESPMNT & $\mathrm{R}$ \\
\hline 6235 & & & & OFFSITE & SESPMNT & $\mathrm{R}$ \\
\hline 6236 & & & & OFFSITE & SESPMNT & $\mathrm{R}$ \\
\hline \begin{tabular}{|l|l|}
6237 \\
\end{tabular} & & & & OFFSITE & SESPMNT & $\mathrm{R}$ \\
\hline 6238 & & & & OFFSITE & SESPMNT & $\mathrm{R}$ \\
\hline 6239 & & & & ONSITE & SESPMNT & $\mathrm{R}$ \\
\hline 6240 & & & & ONSITE & SESPMNT & $\mathrm{R}$ \\
\hline 6241 & See sample record for comment. & & & OFFSITE & SESBMNT & $R$ \\
\hline
\end{tabular}




\begin{tabular}{|c|c|c|c|c|c|c|}
\hline & $A D$ & $\mathrm{AE}$ & $\mathrm{AF}$ & $A G$ & $\mathrm{AH}$ & $\mathrm{Al}$ \\
\hline 1 & RESULT_COMMENT & COLL_SAMP_SIZE & COLL_SAMP_SIZE_UNITS & DIST_CLASS & OWNER_ID & COLLECTION_PURPOSE \\
\hline \begin{tabular}{|c|}
6242 \\
\end{tabular} & See sample record for comment. & & & OFFSITE & SESPMNT & $\mathrm{R}$ \\
\hline 6243 & & & & OFFSITE & SESPMNT & $\mathrm{R}$ \\
\hline 6244 & & & & OFFSITE & SESPMNT & $\mathrm{R}$ \\
\hline 6245 & & & & OFFSITE & SESPMNT & $\mathrm{R}$ \\
\hline \begin{tabular}{|c|}
6246 \\
\end{tabular} & & & & OFFSITE & SESPMNT & $\mathrm{R}$ \\
\hline \begin{tabular}{|c|c|}
6247 \\
\end{tabular} & & & & OFFSITE & SESPMNT & $\mathrm{R}$ \\
\hline \begin{tabular}{|c|}
6248 \\
\end{tabular} & & & & OFFSITE & SESPMNT & $\mathrm{R}$ \\
\hline \begin{tabular}{|c|}
6249 \\
\end{tabular} & & & & OFFSITE & SESPMNT & $\mathrm{R}$ \\
\hline 6250 & & & & OFFSITE & SESPMNT & $\mathrm{R}$ \\
\hline 6251 & & & & OFFSITE & SESPMNT & $\mathrm{R}$ \\
\hline 6252 & & & & OFFSITE & SESPMNT & $\mathrm{R}$ \\
\hline 6253 & & & & OFFSITE & SESPMNT & $\mathrm{R}$ \\
\hline 6254 & & & & OFFSITE & SESPMNT & $\mathrm{R}$ \\
\hline 6255 & & & & OFFSITE & SESPMNT & $\mathrm{R}$ \\
\hline \begin{tabular}{|l|l|}
6256 \\
\end{tabular} & & & & OFFSITE & SESPMNT & $\mathrm{R}$ \\
\hline \begin{tabular}{|l|}
6257 \\
\end{tabular} & & & & ONSITE & SESPMNT & $\mathrm{R}$ \\
\hline \begin{tabular}{|c|}
6258 \\
\end{tabular}$-1-20$ & & & & ONSITE & SESPMNT & $\mathrm{R}$ \\
\hline 6259 & & & & ONSITE & SESPMNT & $\mathrm{R}$ \\
\hline 6260 & & & & ONSITE & SESPMNT & $\mathrm{R}$ \\
\hline 6261 & & & & ONSITE & SESPMNT & $\mathrm{R}$ \\
\hline \begin{tabular}{|c|}
6262 \\
\end{tabular} & & & & ONSITE & SESPMNT & $\mathrm{R}$ \\
\hline \begin{tabular}{|c|}
6263 \\
\end{tabular} & & & & ONSITE & SESPMNT & $\mathrm{R}$ \\
\hline 6264 & & & & ONSITE & SESPMNT & $\mathrm{R}$ \\
\hline 6265 & & & & ONSITE & SESPMNT & $\mathrm{R}$ \\
\hline \begin{tabular}{|l|}
6266 \\
\end{tabular} & & & & ONSITE & SESPMNT & $\mathrm{R}$ \\
\hline \begin{tabular}{|l|}
6267 \\
\end{tabular} & & & & ONSITE & SESPMNT & $\mathrm{R}$ \\
\hline \begin{tabular}{|l|}
6268 \\
\end{tabular} & & & & ONSITE & SESPMNT & $\mathrm{R}$ \\
\hline \begin{tabular}{|l|l|}
6269 \\
\end{tabular} & & & & ONSITE & SESPMNT & $\mathrm{R}$ \\
\hline \begin{tabular}{|l|}
6270 \\
\end{tabular} & & & & ONSITE & SESPMNT & $\mathrm{R}$ \\
\hline \begin{tabular}{|l|}
6271 \\
\end{tabular} & & & & ONSITE & SESPMNT & $\mathrm{R}$ \\
\hline \begin{tabular}{|l|}
6272 \\
\end{tabular} & & & & ONSITE & SESPMNT & $\mathrm{R}$ \\
\hline \begin{tabular}{|l|}
6273 \\
\end{tabular} & & & & ONSITE & SESPMNT & $\mathrm{R}$ \\
\hline \begin{tabular}{|l|}
6274 \\
\end{tabular} & & & & OFFSITE & SESPMNT & $\mathrm{R}$ \\
\hline \begin{tabular}{|l|}
6275 \\
\end{tabular} & & & & OFFSITE & SESPMNT & $\mathrm{R}$ \\
\hline \begin{tabular}{|l|}
6276 \\
\end{tabular} & & & & OFFSITE & SESPMNT & $\mathrm{R}$ \\
\hline \begin{tabular}{|l|}
6277 \\
\end{tabular} & & & & OFFSITE & SESPMNT & $\mathrm{R}$ \\
\hline \begin{tabular}{|l|}
6278 \\
\end{tabular} & & & & ONSITE & SESPMNT & $\mathrm{R}$ \\
\hline \begin{tabular}{|l|}
6279 \\
\end{tabular} & & & & ONSITE & SESPMNT & $R$ \\
\hline 6280 & & & & OFFSITE & SESPMNT & $\mathrm{R}$ \\
\hline \begin{tabular}{|l|}
6281 \\
\end{tabular} & & & & OFFSITE & SESPMNT & $\mathrm{R}$ \\
\hline \begin{tabular}{|l|}
6282 \\
\end{tabular} & & & & OFFSITE & SESPMNT & $\mathrm{R}$ \\
\hline \begin{tabular}{|l|l|}
6283 \\
\end{tabular} & & & & OFFSITE & SESPMNT & $\mathrm{R}$ \\
\hline \begin{tabular}{|l|}
6284 \\
\end{tabular} & & & & OFFSITE & SESPMNT & $\mathrm{R}$ \\
\hline \begin{tabular}{|l|}
6285 \\
\end{tabular} & & & & OFFSITE & SESPMNT & $\mathrm{R}$ \\
\hline \begin{tabular}{|l|}
6286 \\
\end{tabular} & & & & OFFSITE & SESPMNT & $R$ \\
\hline \begin{tabular}{|l|}
6287 \\
\end{tabular} & & & & OFFSITE & SESPMNT & $\mathrm{R}$ \\
\hline \begin{tabular}{|l|}
6288 \\
\end{tabular} & & & & OFFSITE & SESPMNT & $\mathrm{R}$ \\
\hline \begin{tabular}{|l|l|}
6289 \\
\end{tabular} & & & & OFFSITE & SESPMNT & $\mathrm{R}$ \\
\hline \begin{tabular}{|l|}
6290 \\
\end{tabular} & & & & OFFSITE & SESPMNT & $\mathrm{R}$ \\
\hline \begin{tabular}{|l|}
6291 \\
\end{tabular} & & & & OFFSITE & SESPMNT & $\mathrm{R}$ \\
\hline \begin{tabular}{|l|}
6292 \\
\end{tabular} & & & & OFFSITE & SESPMNT & $R$ \\
\hline \begin{tabular}{|l|}
6293 \\
\end{tabular} & & & & OFFSITE & SESBMNT & $R$ \\
\hline
\end{tabular}




\begin{tabular}{|c|c|c|c|c|c|c|}
\hline & $A D$ & $\mathrm{AE}$ & $\mathrm{AF}$ & $A G$ & $\mathrm{AH}$ & $\mathrm{Al}$ \\
\hline 1 & RESULT_COMMENT & COLL_SAMP_SIZE & COLL_SAMP_SIZE_UNITS & DIST_CLASS & OWNER_ID & COLLECTION_PURPOSE \\
\hline 6294 & & & & OFFSITE & SESPMNT & $\mathrm{R}$ \\
\hline \begin{tabular}{|l|}
6295 \\
\end{tabular} & & & & OFFSITE & SESPMNT & $\mathrm{R}$ \\
\hline \begin{tabular}{|l|l|}
6296 \\
\end{tabular} & See sample record for comment. & & & ONSITE & SESPMNT & $\mathrm{R}$ \\
\hline \begin{tabular}{|l|l|}
6297 \\
\end{tabular} & See sample record for comment. & & & ONSITE & SESPMNT & $\mathrm{R}$ \\
\hline 6298 & See sample record for comment. & & & ONSITE & SESPMNT & $\mathrm{R}$ \\
\hline 6299 & & & & PERIMETER & SESPMNT & $\mathrm{R}$ \\
\hline 6300 & & & & PERIMETER & SESPMNT & $\mathrm{R}$ \\
\hline 6301 & & & & PERIMETER & SESPMNT & $\mathrm{R}$ \\
\hline 6302 & & & & ONSITE & SESPMNT & $\mathrm{R}$ \\
\hline 6303 & & & & ONSITE & SESPMNT & $\mathrm{R}$ \\
\hline 6304 & & & & OFFSITE & SESPMNT & $\mathrm{R}$ \\
\hline 6305 & & & & OFFSITE & SESPMNT & $\mathrm{R}$ \\
\hline 6306 & & & & OFFSITE & SESPMNT & $\mathrm{R}$ \\
\hline \begin{tabular}{|l|l|}
6307 & \\
\end{tabular} & & & & OFFSITE & SESPMNT & $\mathrm{R}$ \\
\hline \begin{tabular}{|l|l|}
6308 \\
\end{tabular} & & & & OFFSITE & SESPMNT & $\mathrm{R}$ \\
\hline \begin{tabular}{|l|l|}
6309 \\
\end{tabular} & & & & OFFSITE & SESPMNT & $\mathrm{R}$ \\
\hline \begin{tabular}{|l|}
6310 \\
\end{tabular} & & & & OFFSITE & SESPMNT & $\mathrm{R}$ \\
\hline 6311 & & & & OFFSITE & SESPMNT & $\mathrm{R}$ \\
\hline 6312 & & & & OFFSITE & SESPMNT & $\mathrm{R}$ \\
\hline \begin{tabular}{|c|}
6313 \\
\end{tabular} & & & & & SESPMNT & $\mathrm{R}$ \\
\hline \begin{tabular}{|l|}
6314 \\
\end{tabular} & & & & & SESPMNT & $\mathrm{R}$ \\
\hline \begin{tabular}{|l|}
6315 \\
\end{tabular} & & & & & SESPMNT & $\mathrm{R}$ \\
\hline 6316 & & & & & SESPMNT & $\mathrm{R}$ \\
\hline \begin{tabular}{|l|l|}
6317 \\
\end{tabular} & & & & & SESPMNT & $\mathrm{R}$ \\
\hline 6318 & & & & ONSITE & SESPMNT & $\mathrm{R}$ \\
\hline \begin{tabular}{|l|}
6319 \\
\end{tabular} & & & & ONSITE & SESPMNT & $\mathrm{R}$ \\
\hline \begin{tabular}{|l|}
6320 \\
\end{tabular} & & & & ONSITE & SESPMNT & $\mathrm{R}$ \\
\hline \begin{tabular}{|l|}
6321 \\
\end{tabular} & & & & ONSITE & SESPMNT & $\mathrm{R}$ \\
\hline \begin{tabular}{|l|}
6322 \\
\end{tabular} & & & & ONSITE & SESPMNT & $\mathrm{R}$ \\
\hline \begin{tabular}{|l|}
6323 \\
\end{tabular} & & & & ONSITE & SESPMNT & $\mathrm{R}$ \\
\hline \begin{tabular}{|l|l|}
6324 \\
\end{tabular} & & & & OFFSITE & SESPMNT & $\mathrm{R}$ \\
\hline \begin{tabular}{|l|}
6325 \\
\end{tabular} & & & & OFFSITE & SESPMNT & $\mathrm{R}$ \\
\hline \begin{tabular}{|l|}
6326 \\
\end{tabular} & & & & OFFSITE & SESPMNT & $\mathrm{R}$ \\
\hline \begin{tabular}{|l|}
6327 \\
\end{tabular} & & & & OFFSITE & SESPMNT & $\mathrm{R}$ \\
\hline \begin{tabular}{|l|}
6328 \\
\end{tabular} & & & & OFFSITE & SESPMNT & $\mathrm{R}$ \\
\hline \begin{tabular}{|l|}
6329 \\
\end{tabular} & & & & PERIMETER & SESPMNT & $\mathrm{R}$ \\
\hline 6330 & & & & PERIMETER & SESPMNT & $\mathrm{R}$ \\
\hline \begin{tabular}{|l|}
6331 \\
\end{tabular} & & & & PERIMETER & SESPMNT & $\mathrm{R}$ \\
\hline \begin{tabular}{|l|}
6332 \\
\end{tabular} & & & & PERIMETER & SESPMNT & $\mathrm{R}$ \\
\hline 6333 & & & & PERIMETER & SESPMNT & $\mathrm{R}$ \\
\hline \begin{tabular}{|l|}
6334 \\
\end{tabular} & & & & ONSITE & SESPMNT & $\mathrm{R}$ \\
\hline \begin{tabular}{|l|}
6335 \\
\end{tabular} & & & & ONSITE & SESPMNT & $\mathrm{R}$ \\
\hline \begin{tabular}{|l|}
6336 \\
\end{tabular} & & & & ONSITE & SESPMNT & $\mathrm{R}$ \\
\hline \begin{tabular}{|l|l|}
6337 \\
\end{tabular} & & & & ONSITE & SESPMNT & $\mathrm{R}$ \\
\hline \begin{tabular}{|l|}
6338 \\
\end{tabular} & & & & ONSITE & SESPMNT & $\mathrm{R}$ \\
\hline \begin{tabular}{|l|}
6339 \\
\end{tabular} & & & & OFFSITE & SESPMNT & $\mathrm{R}$ \\
\hline 6340 & & & & OFFSITE & SESPMNT & $\mathrm{R}$ \\
\hline \begin{tabular}{|l|}
6341 \\
\end{tabular} & & & & OFFSITE & SESPMNT & $\mathrm{R}$ \\
\hline \begin{tabular}{|l|}
6342 \\
\end{tabular} & & & & OFFSITE & SESPMNT & $\mathrm{R}$ \\
\hline 6343 & & & & OFFSITE & SESPMNT & $R$ \\
\hline \begin{tabular}{|l|l|}
6344 \\
\end{tabular} & & & & OFFSITE & SESPMNT & $\mathrm{R}$ \\
\hline 6345 & & & & OFFSITE & SESBMNT & $\mathrm{R}$ \\
\hline
\end{tabular}




\begin{tabular}{|c|c|c|c|c|c|c|}
\hline & $A D$ & $\mathrm{AE}$ & $\mathrm{AF}$ & $A G$ & $\mathrm{AH}$ & $\mathrm{Al}$ \\
\hline 1 & RESULT_COMMENT & COLL_SAMP_SIZE & COLL_SAMP_SIZE_UNITS & DIST_CLASS & OWNER_ID & COLLECTION_PURPOSE \\
\hline \begin{tabular}{|c|}
6346 \\
\end{tabular} & & & & OFFSITE & SESPMNT & $\mathrm{R}$ \\
\hline \begin{tabular}{|l|l|}
6347 \\
\end{tabular} & & & & OFFSITE & SESPMNT & $\mathrm{R}$ \\
\hline \begin{tabular}{|l|}
6348 \\
\end{tabular} & & & & OFFSITE & SESPMNT & $\mathrm{R}$ \\
\hline 6349 & & & & ONSITE & SESPMNT & $\mathrm{R}$ \\
\hline 6350 & & & & OFFSITE & SESPMNT & $\mathrm{R}$ \\
\hline 6351 & & & & OFFSITE & SESPMNT & $\mathrm{R}$ \\
\hline \begin{tabular}{|l|}
6352 \\
\end{tabular} & & & & OFFSITE & SESPMNT & $\mathrm{R}$ \\
\hline 6353 & & & & OFFSITE & SESPMNT & $\mathrm{R}$ \\
\hline 6354 & & & & OFFSITE & SESPMNT & $\mathrm{R}$ \\
\hline 6355 & & & & OFFSITE & SESPMNT & $\mathrm{R}$ \\
\hline 6356 & & & & OFFSITE & SESPMNT & $\mathrm{R}$ \\
\hline \begin{tabular}{|c|}
6357 \\
\end{tabular} & & & & OFFSITE & SESPMNT & $\mathrm{R}$ \\
\hline 6358 & & & & OFFSITE & SESPMNT & $\mathrm{R}$ \\
\hline \begin{tabular}{|c|}
6359 \\
\end{tabular} & & & & OFFSITE & SESPMNT & $\mathrm{R}$ \\
\hline \begin{tabular}{|l|}
6360 \\
\end{tabular} & & & & OFFSITE & SESPMNT & $\mathrm{R}$ \\
\hline \begin{tabular}{|l|}
6361 \\
\end{tabular} & & & & OFFSITE & SESPMNT & $\mathrm{R}$ \\
\hline 6362 & & & & ONSITE & SESPMNT & $\mathrm{R}$ \\
\hline 6363 & & & & ONSITE & SESPMNT & $\mathrm{R}$ \\
\hline 6364 & & & & ONSITE & SESPMNT & $\mathrm{R}$ \\
\hline \begin{tabular}{|l|l|}
6365 \\
\end{tabular} & & & & ONSITE & SESPMNT & $\mathrm{R}$ \\
\hline \begin{tabular}{|l|}
6366 \\
\end{tabular} & & & & ONSITE & SESPMNT & $\mathrm{R}$ \\
\hline \begin{tabular}{|l|l|}
6367 & -1 \\
\end{tabular} & See sample record for comment. & & & ONSITE & SESPMNT & $\mathrm{R}$ \\
\hline 6368 & See sample record for comment. & & & ONSITE & SESPMNT & $\mathrm{R}$ \\
\hline 6369 & See sample record for comment. & & & ONSITE & SESPMNT & $\mathrm{R}$ \\
\hline 6370 & See sample record for comment. & & & ONSITE & SESPMNT & $\mathrm{R}$ \\
\hline \begin{tabular}{|c|}
6371 \\
\end{tabular} & See sample record for comment. & & & ONSITE & SESPMNT & $\mathrm{R}$ \\
\hline \begin{tabular}{|l|}
6372 \\
\end{tabular} & See sample record for comment. & & & ONSITE & SESPMNT & $\mathrm{R}$ \\
\hline \begin{tabular}{|l|}
6373 \\
\end{tabular} & & & & ONSITE & SESPMNT & $\mathrm{R}$ \\
\hline \begin{tabular}{|l|}
6374 \\
\end{tabular} & & & & ONSITE & SESPMNT & $\mathrm{R}$ \\
\hline 6375 & & & & OFFSITE & SESPMNT & $\mathrm{R}$ \\
\hline \begin{tabular}{|c|}
6376 \\
\end{tabular} & & & & OFFSITE & SESPMNT & $\mathrm{R}$ \\
\hline 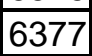 & & & & OFFSITE & SESPMNT & $\mathrm{R}$ \\
\hline 6378 & & & & OFFSITE & SESPMNT & $\mathrm{R}$ \\
\hline 6379 & See sample record for comment. & & & ONSITE & SESPMNT & $\mathrm{R}$ \\
\hline 6380 & See sample record for comment. & & & ONSITE & SESPMNT & $\mathrm{R}$ \\
\hline 6381 & & & & OFFSITE & SESPMNT & $\mathrm{R}$ \\
\hline 6382 & & & & OFFSITE & SESPMNT & $\mathrm{R}$ \\
\hline 6383 & & & & OFFSITE & SESPMNT & $\mathrm{R}$ \\
\hline 6384 & & & & OFFSITE & SESPMNT & $\mathrm{R}$ \\
\hline 6385 & & & & ONSITE & SESPMNT & $\mathrm{R}$ \\
\hline \begin{tabular}{|c|}
6386 \\
\end{tabular} & & & & ONSITE & SESPMNT & $\mathrm{R}$ \\
\hline \begin{tabular}{|l|l|}
6387 & -10 \\
\end{tabular} & & & & ONSITE & SESPMNT & $\mathrm{R}$ \\
\hline 6388 & & & & ONSITE & SESPMNT & $\mathrm{R}$ \\
\hline 6389 & & & & ONSITE & SESPMNT & $\mathrm{R}$ \\
\hline 6390 & & & & ONSITE & SESPMNT & $\mathrm{R}$ \\
\hline 6391 & & & & ONSITE & SESPMNT & $\mathrm{R}$ \\
\hline 6392 & & & & ONSITE & SESPMNT & $\mathrm{R}$ \\
\hline 6393 & & & & ONSITE & SESPMNT & $\mathrm{R}$ \\
\hline 6394 & & & & ONSITE & SESPMNT & $\mathrm{R}$ \\
\hline 6395 & & & & ONSITE & SESPMNT & $R$ \\
\hline 6396 & & & & ONSITE & $\begin{array}{l}\text { SESPMNT } \\
\end{array}$ & $\mathrm{R}$ \\
\hline \begin{tabular}{|l|l|}
6397 \\
\end{tabular} & & & & ONSITE & SESBMNT & $R$ \\
\hline
\end{tabular}




\begin{tabular}{|c|c|c|c|c|c|c|}
\hline & $\mathrm{AD}$ & $\mathrm{AE}$ & $\mathrm{AF}$ & AG & $\mathrm{AH}$ & $\mathrm{Al}$ \\
\hline 1 & RESULT_COMMENT & COLL_SAMP_SIZE & COLL_SAMP_SIZE_UNITS & DIST_CLASS & OWNER_ID & COLLECTION_PURPOSE \\
\hline 6398 & & & & ONSITE & SESPMNT & $\mathrm{R}$ \\
\hline \begin{tabular}{|c|c|}
6399 \\
\end{tabular} & & & & ONSITE & SESPMNT & $\mathrm{R}$ \\
\hline 6400 & & & & ONSITE & SESPMNT & $\mathrm{R}$ \\
\hline $6401 \leq$ & See sample record for comment. & & & ONSITE & SESPMNT & $\mathrm{R}$ \\
\hline 6402 s & See sample record for comment. & & & ONSITE & SESPMNT & $\mathrm{R}$ \\
\hline $6403 \mid$ & See sample record for comment. & & & ONSITE & SESPMNT & $\mathrm{R}$ \\
\hline 6404 s & See sample record for comment. & & & ONSITE & SESPMNT & $\mathrm{R}$ \\
\hline 6405 & See sample record for comment. & & & OFFSITE & SESPMNT & $\mathrm{R}$ \\
\hline 6406: & See sample record for comment. & & & OFFSITE & SESPMNT & $\mathrm{R}$ \\
\hline \begin{tabular}{|c|c|}
6407 & -3 \\
\end{tabular} & & & & OFFSITE & SESPMNT & $\mathrm{R}$ \\
\hline \begin{tabular}{|c|}
6408 \\
\end{tabular} & & & & OFFSITE & SESPMNT & $\mathrm{R}$ \\
\hline 6409 & & & & OFFSITE & SESPMNT & $\mathrm{R}$ \\
\hline $6410 \leq$ & See sample record for comment. & & & OFFSITE & SESPMNT & $\mathrm{R}$ \\
\hline 6411 s & See sample record for comment. & & & OFFSITE & SESPMNT & $\mathrm{R}$ \\
\hline \begin{tabular}{|c|c|}
6412 \\
\end{tabular} & & & & OFFSITE & SESPMNT & $\mathrm{R}$ \\
\hline \begin{tabular}{|c|c|c|}
6413 \\
\end{tabular} & & & & OFFSITE & SESPMNT & $\mathrm{R}$ \\
\hline 6414 & & & & OFFSITE & SESPMNT & $\mathrm{R}$ \\
\hline \begin{tabular}{|c|c|}
6415 \\
\end{tabular} & & & & OFFSITE & SESPMNT & $\mathrm{R}$ \\
\hline 6416 s & See sample record for comment. & & & OFFSITE & SESPMNT & $\mathrm{R}$ \\
\hline 6417 s & See sample record for comment. & & & OFFSITE & SESPMNT & $\mathrm{R}$ \\
\hline 6418 s & See sample record for comment. & & & OFFSITE & SESPMNT & $\mathrm{R}$ \\
\hline 6419 s & See sample record for comment. & & & OFFSITE & SESPMNT & $\mathrm{R}$ \\
\hline 6420 & & & & OFFSITE & SESPMNT & $\mathrm{R}$ \\
\hline 6421 & & & & OFFSITE & SESPMNT & $\mathrm{R}$ \\
\hline \begin{tabular}{|c|c|c|}
6422 \\
\end{tabular} & & & & OFFSITE & SESPMNT & $\mathrm{R}$ \\
\hline \begin{tabular}{|c|}
6423 \\
\end{tabular} & & & & OFFSITE & SESPMNT & $\mathrm{R}$ \\
\hline 6424 & & & & OFFSITE & SESPMNT & $\mathrm{R}$ \\
\hline \begin{tabular}{|c|}
6425 \\
\end{tabular} & & & & OFFSITE & SESPMNT & $\mathrm{R}$ \\
\hline \begin{tabular}{|c|}
6426 \\
\end{tabular} & & & & OFFSITE & SESPMNT & $\mathrm{R}$ \\
\hline \begin{tabular}{|c|c|}
6427 \\
\end{tabular} & & & & ONSITE & SESPMNT & $\mathrm{R}$ \\
\hline \begin{tabular}{|c|}
6428 \\
\end{tabular} & & & & ONSITE & SESPMNT & $\mathrm{R}$ \\
\hline 6429 & & & & ONSITE & SESPMNT & $\mathrm{R}$ \\
\hline 6430 & & & & ONSITE & SESPMNT & $\mathrm{R}$ \\
\hline 6431 & & & & ONSITE & SESPMNT & $\mathrm{R}$ \\
\hline \begin{tabular}{|c|}
6432 \\
\end{tabular} & & & & OFFSITE & SESPMNT & $\mathrm{R}$ \\
\hline \begin{tabular}{|c|c|c|}
6433 \\
\end{tabular} & & & & OFFSITE & SESPMNT & $\mathrm{R}$ \\
\hline 6434 & & & & OFFSITE & SESPMNT & $\mathrm{R}$ \\
\hline \begin{tabular}{|c|c|}
6435 \\
\end{tabular} & & & & OFFSITE & SESPMNT & $\mathrm{R}$ \\
\hline \begin{tabular}{|c|}
6436 \\
\end{tabular} & & & & ONSITE & SESPMNT & $\mathrm{R}$ \\
\hline \begin{tabular}{|c|}
6437 \\
\end{tabular} & & & & ONSITE & SESPMNT & $\mathrm{R}$ \\
\hline \begin{tabular}{|c|}
6438 \\
\end{tabular} & & & & OFFSITE & SESPMNT & $\mathrm{R}$ \\
\hline 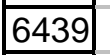 & & & & OFFSITE & SESPMNT & $\mathrm{R}$ \\
\hline \begin{tabular}{|c|}
6440 \\
\end{tabular} & & & & OFFSITE & SESPMNT & $\mathrm{R}$ \\
\hline \begin{tabular}{|c|}
6441 \\
\end{tabular} & & & & OFFSITE & SESPMNT & $\mathrm{R}$ \\
\hline 6442 & & & & OFFSITE & SESPMNT & $\mathrm{R}$ \\
\hline \begin{tabular}{|c|}
6443 \\
\end{tabular} & & & & OFFSITE & SESPMNT & $\mathrm{R}$ \\
\hline 6444 & & & & OFFSITE & SESPMNT & $\mathrm{R}$ \\
\hline 6445 & & & & OFFSITE & SESPMNT & $\mathrm{R}$ \\
\hline \begin{tabular}{|l|}
6446 \\
\end{tabular} & & & & OFFSITE & SESPMNT & $\mathrm{R}$ \\
\hline \begin{tabular}{|l|l|}
6447 \\
\end{tabular} & & & & OFFSITE & SESPMNT & $\mathrm{R}$ \\
\hline \begin{tabular}{|l|l|}
6448 \\
\end{tabular} & & & & OFFSITE & SESPMNT & $R$ \\
\hline \begin{tabular}{|l|l|}
6449 \\
\end{tabular} & & & & OFFSITE & SESBMNT & $R$ \\
\hline
\end{tabular}




\begin{tabular}{|c|c|c|c|c|c|c|}
\hline & $A D$ & $\mathrm{AE}$ & $\mathrm{AF}$ & $A G$ & $\mathrm{AH}$ & $\mathrm{Al}$ \\
\hline 1 & RESULT_COMMENT & COLL_SAMP_SIZE & COLL_SAMP_SIZE_UNITS & DIST_CLASS & OWNER_ID & COLLECTION_PURPOSE \\
\hline 6450 & & & & OFFSITE & SESPMNT & $\mathrm{R}$ \\
\hline \begin{tabular}{|l|}
6451 \\
\end{tabular} & & & & OFFSITE & SESPMNT & $\mathrm{R}$ \\
\hline \begin{tabular}{|l|}
6452 \\
\end{tabular} & & & & OFFSITE & SESPMNT & $\mathrm{R}$ \\
\hline 6453 & & & & OFFSITE & SESPMNT & $\mathrm{R}$ \\
\hline 6454 & & & & OFFSITE & SESPMNT & $\mathrm{R}$ \\
\hline 6455 & & & & OFFSITE & SESPMNT & $\mathrm{R}$ \\
\hline \begin{tabular}{|l|l|}
6456 \\
\end{tabular} & & & & PERIMETER & SESPMNT & $\mathrm{R}$ \\
\hline 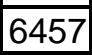 & & & & PERIMETER & SESPMNT & $\mathrm{R}$ \\
\hline 6458 & & & & PERIMETER & SESPMNT & $\mathrm{R}$ \\
\hline 6459 & & & & ONSITE & SESPMNT & $\mathrm{R}$ \\
\hline 6460 & & & & ONSITE & SESPMNT & $\mathrm{R}$ \\
\hline 6461 & & & & OFFSITE & SESPMNT & $\mathrm{R}$ \\
\hline 6462 & & & & OFFSITE & SESPMNT & $\mathrm{R}$ \\
\hline \begin{tabular}{|c|}
6463 \\
\end{tabular} & & & & OFFSITE & SESPMNT & $\mathrm{R}$ \\
\hline \begin{tabular}{|l|}
6464 \\
\end{tabular} & & & & OFFSITE & SESPMNT & $\mathrm{R}$ \\
\hline \begin{tabular}{|l|l|}
6465 \\
\end{tabular} & & & & OFFSITE & SESPMNT & $\mathrm{R}$ \\
\hline 6466 & & & & OFFSITE & SESPMNT & $\mathrm{R}$ \\
\hline \begin{tabular}{|l|l|}
6467 & $-2 \cdot 1$ \\
\end{tabular} & & & & OFFSITE & SESPMNT & $\mathrm{R}$ \\
\hline 6468 & & & & OFFSITE & SESPMNT & $\mathrm{R}$ \\
\hline \begin{tabular}{|l|}
6469 \\
\end{tabular} & & & & OFFSITE & SESPMNT & $\mathrm{R}$ \\
\hline \begin{tabular}{|c|}
6470 \\
\end{tabular} & & & & & SESPMNT & $\mathrm{R}$ \\
\hline 6471 & & & & & SESPMNT & $\mathrm{R}$ \\
\hline 6472 & & & & & SESPMNT & $\mathrm{R}$ \\
\hline 6473 & & & & & SESPMNT & $\mathrm{R}$ \\
\hline 6474 & & & & & SESPMNT & $\mathrm{R}$ \\
\hline 6475 & & & & ONSITE & SESPMNT & $\mathrm{R}$ \\
\hline \begin{tabular}{|l|}
6476 \\
\end{tabular} & & & & ONSITE & SESPMNT & $\mathrm{R}$ \\
\hline \begin{tabular}{|l|l|}
6477 \\
\end{tabular} & & & & ONSITE & SESPMNT & $\mathrm{R}$ \\
\hline \begin{tabular}{|l|}
6478 \\
\end{tabular} & See sample record for comment. & & & OFFSITE & SESPMNT & $\mathrm{R}$ \\
\hline 6479 & See sample record for comment. & & & OFFSITE & SESPMNT & $\mathrm{R}$ \\
\hline 6480 & See sample record for comment. & & & OFFSITE & SESPMNT & $\mathrm{R}$ \\
\hline 6481 & See sample record for comment. & & & OFFSITE & SESPMNT & $\mathrm{R}$ \\
\hline 6482 & See sample record for comment. & & & OFFSITE & SESPMNT & $\mathrm{R}$ \\
\hline 6483 & & & & PERIMETER & SESPMNT & $\mathrm{R}$ \\
\hline \begin{tabular}{|l|}
6484 \\
\end{tabular} & & & & PERIMETER & SESPMNT & $\mathrm{R}$ \\
\hline 6485 & & & & PERIMETER & SESPMNT & $\mathrm{R}$ \\
\hline 6486 & & & & PERIMETER & SESPMNT & $\mathrm{R}$ \\
\hline \begin{tabular}{|l|l|}
6487 & $-2 \cdot r$ \\
\end{tabular} & & & & PERIMETER & SESPMNT & $\mathrm{R}$ \\
\hline 6488 & & & & ONSITE & SESPMNT & $\mathrm{R}$ \\
\hline 6489 & & & & ONSITE & SESPMNT & $\mathrm{R}$ \\
\hline 6490 & & & & OFFSITE & SESPMNT & $\mathrm{R}$ \\
\hline \begin{tabular}{|l|}
6491 \\
\end{tabular} & & & & OFFSITE & SESPMNT & $\mathrm{R}$ \\
\hline 6492 & & & & OFFSITE & SESPMNT & $\mathrm{R}$ \\
\hline 6493 & & & & OFFSITE & SESPMNT & $\mathrm{R}$ \\
\hline 6494 & & & & OFFSITE & SESPMNT & $\mathrm{R}$ \\
\hline 6495 & & & & OFFSITE & SESPMNT & $\mathrm{R}$ \\
\hline 6496 & & & & OFFSITE & SESPMNT & $\mathrm{R}$ \\
\hline \begin{tabular}{|l|l|}
6497 \\
\end{tabular} & & & & OFFSITE & SESPMNT & $\mathrm{R}$ \\
\hline 6498 & & & & OFFSITE & SESPMNT & $\mathrm{R}$ \\
\hline 6499 & & & & OFFSITE & SESPMNT & $\mathrm{R}$ \\
\hline 6500 & & & & OFFSITE & SESPMNT & $\mathrm{R}$ \\
\hline 6501 & & & & OFFSITE & SESBMNT & $R$ \\
\hline
\end{tabular}




\begin{tabular}{|c|c|c|c|c|c|c|}
\hline & $A D$ & $\mathrm{AE}$ & $\mathrm{AF}$ & $A G$ & $\mathrm{AH}$ & $\mathrm{Al}$ \\
\hline 1 & RESULT_COMMENT & COLL_SAMP_SIZE & COLL_SAMP_SIZE_UNITS & DIST_CLASS & OWNER_ID & COLLECTION_PURPOSE \\
\hline 6502 & & & & OFFSITE & SESPMNT & $\mathrm{R}$ \\
\hline 6503 & & & & OFFSITE & SESPMNT & $\mathrm{R}$ \\
\hline 6504 & & & & OFFSITE & SESPMNT & $\mathrm{R}$ \\
\hline 6505 & & & & OFFSITE & SESPMNT & $\mathrm{R}$ \\
\hline \begin{tabular}{|c|}
6506 \\
\end{tabular} & & & & OFFSITE & SESPMNT & $\mathrm{R}$ \\
\hline 6507 & & & & OFFSITE & SESPMNT & $\mathrm{R}$ \\
\hline 6508 & & & & ONSITE & SESPMNT & $\mathrm{R}$ \\
\hline 6509 & & & & ONSITE & SESPMNT & $\mathrm{R}$ \\
\hline 6510 & & & & ONSITE & SESPMNT & $\mathrm{R}$ \\
\hline 6511 & & & & ONSITE & SESPMNT & $\mathrm{R}$ \\
\hline $\mid 6512$ & & & & ONSITE & SESPMNT & $\mathrm{R}$ \\
\hline 6513 & & & & ONSITE & SESPMNT & $\mathrm{R}$ \\
\hline 6514 & & & & ONSITE & SESPMNT & $\mathrm{R}$ \\
\hline 6515 & & & & ONSITE & SESPMNT & $\mathrm{R}$ \\
\hline \begin{tabular}{|c|}
6516 \\
\end{tabular} & & & & ONSITE & SESPMNT & $\mathrm{R}$ \\
\hline 6517 & & & & ONSITE & SESPMNT & $\mathrm{R}$ \\
\hline 6518 & & & & ONSITE & SESPMNT & $\mathrm{R}$ \\
\hline 6519 & & & & ONSITE & SESPMNT & $\mathrm{R}$ \\
\hline 6520 & & & & ONSITE & SESPMNT & $\mathrm{R}$ \\
\hline 6521 & & & & ONSITE & SESPMNT & $\mathrm{R}$ \\
\hline 6522 & See sample record for comment. & & & ONSITE & SESPMNT & $\mathrm{R}$ \\
\hline 6523 & See sample record for comment. & & & ONSITE & SESPMNT & $\mathrm{R}$ \\
\hline 6524 & & & & OFFSITE & SESPMNT & $\mathrm{R}$ \\
\hline 6525 & & & & OFFSITE & SESPMNT & $\mathrm{R}$ \\
\hline \begin{tabular}{|l|}
6526 \\
\end{tabular} & & & & OFFSITE & SESPMNT & $\mathrm{R}$ \\
\hline \begin{tabular}{|l|}
6527 \\
\end{tabular} & & & & OFFSITE & SESPMNT & $\mathrm{R}$ \\
\hline \begin{tabular}{|l|}
6528 \\
\end{tabular} & & & & OFFSITE & SESPMNT & $\mathrm{R}$ \\
\hline 6529 & & & & OFFSITE & SESPMNT & $\mathrm{R}$ \\
\hline 6530 & & & & OFFSITE & SESPMNT & $\mathrm{R}$ \\
\hline \begin{tabular}{|l|}
6531 \\
\end{tabular} & & & & OFFSITE & SESPMNT & $\mathrm{R}$ \\
\hline \begin{tabular}{|l|}
6532 \\
\end{tabular} & & & & OFFSITE & SESPMNT & $\mathrm{R}$ \\
\hline \begin{tabular}{|l|}
6533 \\
\end{tabular} & & & & OFFSITE & SESPMNT & $\mathrm{R}$ \\
\hline \begin{tabular}{|l|}
6534 \\
\end{tabular} & & & & ONSITE & SESPMNT & $\mathrm{R}$ \\
\hline 6535 & & & & ONSITE & SESPMNT & $\mathrm{R}$ \\
\hline 6536 & & & & ONSITE & SESPMNT & $\mathrm{R}$ \\
\hline \begin{tabular}{|l|}
6537 \\
\end{tabular} & & & & ONSITE & SESPMNT & $\mathrm{R}$ \\
\hline \begin{tabular}{|l|}
6538 \\
\end{tabular} & & & & ONSITE & SESPMNT & $\mathrm{R}$ \\
\hline \begin{tabular}{|l|}
6539 \\
\end{tabular} & & & & ONSITE & SESPMNT & $R$ \\
\hline 6540 & & & & ONSITE & SESPMNT & $\mathrm{R}$ \\
\hline 6541 & & & & OFFSITE & SESPSPEC & $\mathrm{s}$ \\
\hline 6542 & & & & OFFSITE & SESPSPEC & $\mathrm{s}$ \\
\hline 6543 & & & & OFFSITE & SESPSPEC & $\mathrm{s}$ \\
\hline \begin{tabular}{|l|}
6544 \\
\end{tabular} & & & & OFFSITE & SESPSPEC & $\mathrm{s}$ \\
\hline \begin{tabular}{|l|}
6545 \\
\end{tabular} & & & & OFFSITE & SESPSPEC & $\mathrm{S}$ \\
\hline \begin{tabular}{|l|}
6546 \\
\end{tabular} & & & & OFFSITE & SESPSPEC & $\mathrm{s}$ \\
\hline \begin{tabular}{|l|l|}
6547 \\
\end{tabular} & & & & OFFSITE & SESPSPEC & $\mathrm{S}$ \\
\hline 6548 & & & & OFFSITE & SESPSPEC & $\mathrm{S}$ \\
\hline 6549 & & & & OFFSITE & SESPSPEC & $\mathrm{s}$ \\
\hline \begin{tabular}{|l|}
6550 \\
\end{tabular} & & & & OFFSITE & SESPSPEC & $\mathrm{S}$ \\
\hline \begin{tabular}{|l|l|}
6551 \\
\end{tabular} & & & & OFFSITE & SESPSPEC & $\mathrm{S}$ \\
\hline \begin{tabular}{|l|}
6552 \\
\end{tabular} & & & & OFFSITE & SESPSPEC & $\mathrm{S}$ \\
\hline \begin{tabular}{|l|}
6553 \\
\end{tabular} & & & & ONSITE & SESBMNT & $R$ \\
\hline
\end{tabular}




\begin{tabular}{|c|c|c|c|c|c|c|}
\hline & $\mathrm{AD}$ & $\mathrm{AE}$ & $\mathrm{AF}$ & $\mathrm{AG}$ & $\mathrm{AH}$ & $\mathrm{Al}$ \\
\hline 1 & RESULT_COMMENT & COLL_SAMP_SIZE & COLL_SAMP_SIZE_UNITS & DIST_CLASS & OWNER_ID & COLLECTION_PURPOSE \\
\hline 6554 & & & & ONSITE & SESPMNT & $\mathrm{R}$ \\
\hline 6555 & & & & OFFSITE & SESPMNT & $\mathrm{R}$ \\
\hline 6556 & & & & OFFSITE & SESPMNT & $\mathrm{R}$ \\
\hline 6557 & & & & OFFSITE & SESPMNT & $\mathrm{R}$ \\
\hline 6558 & & & & OFFSITE & SESPMNT & $\mathrm{R}$ \\
\hline 6559 & & & & ONSITE & SESPMNT & $\mathrm{R}$ \\
\hline 6560 & & & & ONSITE & SESPMNT & $\mathrm{R}$ \\
\hline 6561 & & & & ONSITE & SESPMNT & $\mathrm{R}$ \\
\hline 6562 & & & & ONSITE & SESPMNT & $\mathrm{R}$ \\
\hline \begin{tabular}{|c|}
6563 \\
\end{tabular} & & & & ONSITE & SESPMNT & $\mathrm{R}$ \\
\hline 6564 & & & & ONSITE & SESPMNT & $\mathrm{R}$ \\
\hline 6565 & & & & ONSITE & SESPMNT & $\mathrm{R}$ \\
\hline 65 & & & & ONSITE & SESPMNT & $\mathrm{R}$ \\
\hline \begin{tabular}{|c|}
6567 \\
\end{tabular} & & & & ONSITE & SESPMNT & $\mathrm{R}$ \\
\hline \begin{tabular}{|c|}
6568 \\
\end{tabular} & & & & ONSITE & SESPMNT & $\mathrm{R}$ \\
\hline \begin{tabular}{|c|}
6569 \\
\end{tabular} & & & & ONSITE & SESPMNT & $\mathrm{R}$ \\
\hline 6570 & & & & ONSITE & SESPMNT & $\mathrm{R}$ \\
\hline 6571 & & & & ONSITE & SESPMNT & $\mathrm{R}$ \\
\hline 6572 & & & & ONSITE & SESPMNT & $\mathrm{R}$ \\
\hline 6573 & & & & ONSITE & SESPMNT & $\mathrm{R}$ \\
\hline 6574 & & & & ONSITE & SESPMNT & $\mathrm{R}$ \\
\hline 6575 & & & & ONSITE & SESPMNT & $\mathrm{R}$ \\
\hline 6576 & & & & OFFSITE & SESPMNT & $\mathrm{R}$ \\
\hline 6577 & & & & OFFSITE & SESPMNT & $R$ \\
\hline \begin{tabular}{|l|l|}
6578 \\
\end{tabular} & & & & OFFSITE & SESPMNT & $\mathrm{R}$ \\
\hline \begin{tabular}{|l|l|}
6579 \\
\end{tabular} & & & & OFFSITE & SESPMNT & $\mathrm{R}$ \\
\hline \begin{tabular}{|l|l|}
6580 \\
\end{tabular} & & & & OFFSITE & SESPMNT & $\mathrm{R}$ \\
\hline \begin{tabular}{|l|}
6581 \\
\end{tabular} & & & & OFFSITE & SESPMNT & $\mathrm{R}$ \\
\hline \begin{tabular}{|l|}
6582 \\
\end{tabular} & & & & OFFSITE & SESPMNT & $R$ \\
\hline \begin{tabular}{|l|l|}
6583 \\
\end{tabular} & & & & OFFSITE & SESPMNT & $\mathrm{R}$ \\
\hline 6584 & & & & OFFSITE & SESPMNT & $\mathrm{R}$ \\
\hline \begin{tabular}{|l|l|}
6585 \\
\end{tabular} & & & & OFFSITE & SESPMNT & $\mathrm{R}$ \\
\hline \begin{tabular}{|l|l|}
6586 \\
\end{tabular} & & & & OFFSITE & SESPMNT & $\mathrm{R}$ \\
\hline \begin{tabular}{|l|l|}
6587 \\
\end{tabular} & & & & OFFSITE & SESPMNT & $\mathrm{R}$ \\
\hline \begin{tabular}{|l|l|}
6588 \\
\end{tabular} & & & & OFFSITE & SESPMNT & $\mathrm{R}$ \\
\hline \begin{tabular}{|l|l|}
6589 \\
\end{tabular} & & & & OFFSITE & SESPMNT & $\mathrm{R}$ \\
\hline 6590 & & & & OFFSITE & SESPMNT & $\mathrm{R}$ \\
\hline 6591 & & & & OFFSITE & SESPMNT & $\mathrm{R}$ \\
\hline \begin{tabular}{|l|l|}
6592 \\
\end{tabular} & & & & OFFSITE & SESPMNT & $\mathrm{R}$ \\
\hline \begin{tabular}{|l|l|}
6593 \\
\end{tabular} & & & & OFFSITE & SESPMNT & $\mathrm{R}$ \\
\hline \begin{tabular}{|l|}
6594 \\
\end{tabular} & & & & ONSITE & SESPMNT & $R$ \\
\hline \begin{tabular}{|l|l|}
6595 \\
\end{tabular} & & & & ONSITE & SESPMNT & $\mathrm{R}$ \\
\hline \begin{tabular}{|l|l|}
6596 \\
\end{tabular} & & & & OFFSITE & SESPMNT & $\mathrm{R}$ \\
\hline \begin{tabular}{|l|}
6597 \\
\end{tabular} & & & & OFFSITE & SESPMNT & $\mathrm{R}$ \\
\hline \begin{tabular}{|l|l|}
6598 \\
\end{tabular} & & & & OFFSITE & SESPMNT & $\mathrm{R}$ \\
\hline \begin{tabular}{|l|l|}
6599 \\
\end{tabular} & & & & OFFSITE & SESPMNT & $\mathrm{R}$ \\
\hline 6600 & & & & OFFSITE & SESPMNT & $\mathrm{R}$ \\
\hline \begin{tabular}{|l|}
6601 \\
\end{tabular} & & & & OFFSITE & SESPMNT & $\mathrm{R}$ \\
\hline 6602 & & & & OFFSITE & SESPMNT & $\mathrm{R}$ \\
\hline \begin{tabular}{|l|l|}
6603 \\
\end{tabular} & & & & OFFSITE & SESPMNT & $\mathrm{R}$ \\
\hline \begin{tabular}{|l|}
6604 \\
\end{tabular} & & & & OFFSITE & SESPMNT & $\mathrm{R}$ \\
\hline \begin{tabular}{|c|}
6605 \\
\end{tabular} & & & & OFFSITE & SESBMNT & $R$ \\
\hline
\end{tabular}




\begin{tabular}{|c|c|c|c|c|c|c|}
\hline & $A D$ & $\mathrm{AE}$ & $\mathrm{AF}$ & $A G$ & $\mathrm{AH}$ & $\mathrm{Al}$ \\
\hline 1 & RESULT_COMMENT & COLL_SAMP_SIZE & COLL_SAMP_SIZE_UNITS & DIST_CLASS & OWNER_ID & COLLECTION_PURPOSE \\
\hline \begin{tabular}{|c|}
6606 \\
\end{tabular} & & & & PERIMETER & SESPMNT & $\mathrm{R}$ \\
\hline \begin{tabular}{|l|l|}
6607 \\
\end{tabular} & & & & PERIMETER & SESPMNT & $\mathrm{R}$ \\
\hline \begin{tabular}{|l|}
6608 \\
\end{tabular} & & & & PERIMETER & SESPMNT & $\mathrm{R}$ \\
\hline 6609 & & & & ONSITE & SESPMNT & $\mathrm{R}$ \\
\hline 6610 & & & & ONSITE & SESPMNT & $\mathrm{R}$ \\
\hline \begin{tabular}{|l|}
6611 \\
\end{tabular} & & & & OFFSITE & SESPMNT & $\mathrm{R}$ \\
\hline 6612 & & & & OFFSITE & SESPMNT & $\mathrm{R}$ \\
\hline 6613 & & & & OFFSITE & SESPMNT & $\mathrm{R}$ \\
\hline 6614 & & & & OFFSITE & SESPMNT & $\mathrm{R}$ \\
\hline 6615 & & & & OFFSITE & SESPMNT & $\mathrm{R}$ \\
\hline \begin{tabular}{|l|l|}
6616 \\
\end{tabular} & & & & OFFSITE & SESPMNT & $\mathrm{R}$ \\
\hline \begin{tabular}{|l|}
6617 \\
\end{tabular} & & & & OFFSITE & SESPMNT & $\mathrm{R}$ \\
\hline 6618 & & & & OFFSITE & SESPMNT & $\mathrm{R}$ \\
\hline \begin{tabular}{|l|}
6619 \\
\end{tabular} & & & & OFFSITE & SESPMNT & $\mathrm{R}$ \\
\hline \begin{tabular}{|l|}
6620 \\
\end{tabular} & & & & OFFSITE & SESPMNT & $\mathrm{R}$ \\
\hline \begin{tabular}{|l|}
6621 \\
\end{tabular} & & & & OFFSITE & SESPMNT & $\mathrm{R}$ \\
\hline 6622 & & & & OFFSITE & SESPMNT & $\mathrm{R}$ \\
\hline 6623 & & & & OFFSITE & SESPMNT & $\mathrm{R}$ \\
\hline \begin{tabular}{|l|}
6624 \\
\end{tabular} & & & & OFFSITE & SESPMNT & $\mathrm{R}$ \\
\hline \begin{tabular}{|l|}
6625 \\
\end{tabular} & & & & OFFSITE & SESPMNT & $\mathrm{R}$ \\
\hline \begin{tabular}{|l|}
6626 \\
\end{tabular} & & & & OFFSITE & SESPMNT & $\mathrm{R}$ \\
\hline \begin{tabular}{|l|}
6627 \\
\end{tabular} & & & & OFFSITE & SESPMNT & $\mathrm{R}$ \\
\hline 6628 & & & & OFFSITE & SESPMNT & $\mathrm{R}$ \\
\hline 6629 & & & & OFFSITE & SESPMNT & $\mathrm{R}$ \\
\hline 6630 & & & & & SESPMNT & $\mathrm{R}$ \\
\hline \begin{tabular}{|l|}
6631 \\
\end{tabular} & & & & OFFSITE & SESPMNT & $\mathrm{R}$ \\
\hline \begin{tabular}{|l|}
6632 \\
\end{tabular} & & & & OFFSITE & SESPMNT & $\mathrm{R}$ \\
\hline \begin{tabular}{|l|}
6633 \\
\end{tabular} & & & & OFFSITE & SESPMNT & $\mathrm{R}$ \\
\hline \begin{tabular}{|l|}
6634 \\
\end{tabular} & & & & OFFSITE & SESPMNT & $\mathrm{R}$ \\
\hline 6635 & & & & OFFSITE & SESPMNT & $\mathrm{R}$ \\
\hline 6636 & & & & & SESPMNT & $\mathrm{R}$ \\
\hline \begin{tabular}{|l|}
6637 \\
\end{tabular} & & & & & SESPMNT & $\mathrm{R}$ \\
\hline 6638 & & & & & SESPMNT & $\mathrm{R}$ \\
\hline 6639 & & & & & SESPMNT & $\mathrm{R}$ \\
\hline 6640 & & & & & SESPMNT & $\mathrm{R}$ \\
\hline 6641 & & & & OFFSITE & SESPMNT & $\mathrm{R}$ \\
\hline 6642 & & & & OFFSITE & SESPMNT & $\mathrm{R}$ \\
\hline 6643 & & & & OFFSITE & SESPMNT & $\mathrm{R}$ \\
\hline 6644 & & & & OFFSITE & SESPMNT & $\mathrm{R}$ \\
\hline 6645 & & & & OFFSITE & SESPMNT & $\mathrm{R}$ \\
\hline \begin{tabular}{|c|}
6646 \\
\end{tabular} & & & & PERIMETER & SESPMNT & $\mathrm{R}$ \\
\hline \begin{tabular}{|l|}
6647 \\
\end{tabular} & & & & PERIMETER & SESPMNT & $\mathrm{R}$ \\
\hline 6648 & & & & PERIMETER & SESPMNT & $\mathrm{R}$ \\
\hline 6649 & & & & PERIMETER & SESPMNT & $\mathrm{R}$ \\
\hline 6650 & & & & PERIMETER & SESPMNT & $\mathrm{R}$ \\
\hline 6651 & & & & OFFSITE & SESPMNT & $\mathrm{R}$ \\
\hline 6652 & & & & PERIMETER & SESPMNT & $\mathrm{R}$ \\
\hline 6653 & & & & ONSITE & SESPMNT & $\mathrm{R}$ \\
\hline 6654 & & & & ONSITE & SESPMNT & $\mathrm{R}$ \\
\hline 6655 & & & & ONSITE & SESPMNT & $\mathrm{R}$ \\
\hline 6656 & See sample record for comment. & & & ONSITE & SESPMNT & $\mathrm{R}$ \\
\hline 6657 & See sample record for comment. & & & ONSITE & SESBMNT & ${ }_{2}^{R}$ \\
\hline
\end{tabular}




\begin{tabular}{|c|c|c|c|c|c|c|}
\hline & $A D$ & $\mathrm{AE}$ & $\mathrm{AF}$ & $A G$ & $\mathrm{AH}$ & $\mathrm{Al}$ \\
\hline 1 & RESULT_COMMENT & COLL_SAMP_SIZE & COLL_SAMP_SIZE_UNITS & DIST_CLASS & OWNER_ID & COLLECTION_PURPOSE \\
\hline 6658 & & & & OFFSITE & SESPMNT & $R$ \\
\hline \begin{tabular}{|l|}
6659 \\
\end{tabular} & & & & OFFSITE & SESPMNT & $\mathrm{R}$ \\
\hline \begin{tabular}{|c|}
6660 \\
\end{tabular} & See sample record for comment. & & & OFFSITE & SESPMNT & $\mathrm{R}$ \\
\hline 6661 & See sample record for comment. & & & OFFSITE & SESPMNT & $\mathrm{R}$ \\
\hline 6662 & & & & ONSITE & SESPMNT & $\mathrm{R}$ \\
\hline 6663 & & & & ONSITE & SESPMNT & $\mathrm{R}$ \\
\hline 6664 & & & & ONSITE & SESPMNT & $\mathrm{R}$ \\
\hline \begin{tabular}{|l|}
6665 \\
\end{tabular} & See sample record for comment. & & & ONSITE & SESPMNT & $\mathrm{R}$ \\
\hline 6666 & See sample record for comment. & & & ONSITE & SESPMNT & $\mathrm{R}$ \\
\hline \begin{tabular}{|l|l|}
6667 & -1 \\
\end{tabular} & See sample record for comment. & & & ONSITE & SESPMNT & $\mathrm{R}$ \\
\hline 6668 & & & & ONSITE & SESPMNT & $\mathrm{R}$ \\
\hline 6669 & & & & ONSITE & SESPMNT & $\mathrm{R}$ \\
\hline 6670 & See sample record for comment. & & & ONSITE & SESPMNT & $\mathrm{R}$ \\
\hline \begin{tabular}{|l|}
6671 \\
\end{tabular} & See sample record for comment. & & & ONSITE & SESPMNT & $\mathrm{R}$ \\
\hline \begin{tabular}{|l|l|}
6672 \\
\end{tabular} & See sample record for comment. & & & ONSITE & SESPMNT & $\mathrm{R}$ \\
\hline \begin{tabular}{|l|}
6673 \\
\end{tabular} & See sample record for comment. & & & ONSITE & SESPMNT & $\mathrm{R}$ \\
\hline 6674 & See sample record for comment. & & & ONSITE & SESPMNT & $\mathrm{R}$ \\
\hline 6675 & See sample record for comment. & & & ONSITE & SESPMNT & $\mathrm{R}$ \\
\hline 6676 & & & & ONSITE & SESPMNT & $\mathrm{R}$ \\
\hline \begin{tabular}{|l|l|}
6677 & \\
\end{tabular} & & & & ONSITE & SESPMNT & $\mathrm{R}$ \\
\hline \begin{tabular}{|c|}
6678 \\
\end{tabular} & & & & ONSITE & SESPMNT & $\mathrm{R}$ \\
\hline 6679 & & & & ONSITE & SESPMNT & $\mathrm{R}$ \\
\hline 6680 & & & & ONSITE & SESPMNT & $\mathrm{R}$ \\
\hline \begin{tabular}{|l|}
6681 \\
\end{tabular} & & & & OFFSITE & SESPMNT & $\mathrm{R}$ \\
\hline \begin{tabular}{|l|}
6682 \\
\end{tabular} & & & & OFFSITE & SESPMNT & $\mathrm{R}$ \\
\hline \begin{tabular}{|l|}
6683 \\
\end{tabular} & & & & OFFSITE & SESPMNT & $\mathrm{R}$ \\
\hline \begin{tabular}{|l|}
6684 \\
\end{tabular} & & & & OFFSITE & SESPMNT & $\mathrm{R}$ \\
\hline \begin{tabular}{|l|}
6685 \\
\end{tabular} & & & & OFFSITE & SESPMNT & $\mathrm{R}$ \\
\hline \begin{tabular}{|l|}
6686 \\
\end{tabular} & & & & OFFSITE & SESPMNT & $\mathrm{R}$ \\
\hline \begin{tabular}{|l|l|}
6687 \\
\end{tabular} & & & & OFFSITE & SESPMNT & $R$ \\
\hline \begin{tabular}{|l|}
6688 \\
\end{tabular} & & & & OFFSITE & SESPMNT & $\mathrm{R}$ \\
\hline 6689 & & & & OFFSITE & SESPMNT & $\mathrm{R}$ \\
\hline 6690 & & & & OFFSITE & SESPMNT & $\mathrm{R}$ \\
\hline \begin{tabular}{|l|}
6691 \\
\end{tabular} & & & & OFFSITE & SESPMNT & $\mathrm{R}$ \\
\hline 6692 & & & & OFFSITE & SESPMNT & $\mathrm{R}$ \\
\hline \begin{tabular}{|l|}
6693 \\
\end{tabular} & & & & OFFSITE & SESPMNT & $\mathrm{R}$ \\
\hline 6694 & & & & OFFSITE & SESPMNT & $\mathrm{R}$ \\
\hline \begin{tabular}{|l|}
6695 \\
\end{tabular} & & & & OFFSITE & SESPMNT & $\mathrm{R}$ \\
\hline \begin{tabular}{|l|}
6696 \\
\end{tabular} & & & & OFFSITE & SESPMNT & $\mathrm{R}$ \\
\hline \begin{tabular}{|l|}
6697 \\
\end{tabular} & & & & OFFSITE & SESPMNT & $\mathrm{R}$ \\
\hline \begin{tabular}{|l|}
6698 \\
\end{tabular} & & & & OFFSITE & SESPMNT & $\mathrm{R}$ \\
\hline \begin{tabular}{|l|}
6699 \\
\end{tabular} & & & & OFFSITE & SESPMNT & $\mathrm{R}$ \\
\hline 6700 & & & & OFFSITE & SESPMNT & $\mathrm{R}$ \\
\hline \begin{tabular}{|l|}
6701 \\
\end{tabular} & & & & OFFSITE & SESPMNT & $\mathrm{R}$ \\
\hline \begin{tabular}{|l|}
6702 \\
\end{tabular} & & & & OFFSITE & SESPMNT & $\mathrm{R}$ \\
\hline 6703 & & & & OFFSITE & SESPMNT & $\mathrm{R}$ \\
\hline 6704 & & & & OFFSITE & SESPMNT & $\mathrm{R}$ \\
\hline 6705 & & & & ONSITE & SESPMNT & $\mathrm{R}$ \\
\hline \begin{tabular}{|l|}
6706 \\
\end{tabular} & & & & ONSITE & SESPMNT & $\mathrm{R}$ \\
\hline \begin{tabular}{|l|l|}
6707 \\
\end{tabular} & & & & ONSITE & SESPMNT & $R$ \\
\hline 6708 & & & & ONSITE & $\begin{array}{l}\text { SESPMNT } \\
\end{array}$ & $\mathrm{R}$ \\
\hline 6709 & & & & ONSITE & SESBMNT & $\mathrm{R}$ \\
\hline
\end{tabular}




\begin{tabular}{|c|c|c|c|c|c|c|}
\hline & $A D$ & $\mathrm{AE}$ & $\mathrm{AF}$ & $A G$ & $\mathrm{AH}$ & $\mathrm{Al}$ \\
\hline 1 & RESULT_COMMENT & COLL_SAMP_SIZE & COLL_SAMP_SIZE_UNITS & DIST_CLASS & OWNER_ID & COLLECTION_PURPOSE \\
\hline 6710 & & & & ONSITE & SESPMNT & $\mathrm{R}$ \\
\hline 6711 & & & & ONSITE & SESPMNT & $\mathrm{R}$ \\
\hline 6712 & & & & ONSITE & SESPMNT & $\mathrm{R}$ \\
\hline 6713 & & & & ONSITE & SESPMNT & $\mathrm{R}$ \\
\hline 6714 & & & & OFFSITE & SESPMNT & $\mathrm{R}$ \\
\hline 6715 & & & & OFFSITE & SESPMNT & $\mathrm{R}$ \\
\hline \begin{tabular}{|c|}
6716 \\
\end{tabular} & & & & OFFSITE & SESPMNT & $\mathrm{R}$ \\
\hline \begin{tabular}{|c|c|}
6717 \\
\end{tabular} & & & & OFFSITE & SESPMNT & $\mathrm{R}$ \\
\hline 6718 & & & & OFFSITE & SESPMNT & $\mathrm{R}$ \\
\hline 6719 & & & & OFFSITE & SESPMNT & $\mathrm{R}$ \\
\hline 6720 & & & & OFFSITE & SESPMNT & $\mathrm{R}$ \\
\hline 6721 & & & & OFFSITE & SESPMNT & $\mathrm{R}$ \\
\hline 6722 & & & & OFFSITE & SESPMNT & $\mathrm{R}$ \\
\hline 6723 & & & & OFFSITE & SESPMNT & $\mathrm{R}$ \\
\hline 6724 & & & & ONSITE & SESPMNT & $\mathrm{R}$ \\
\hline 6725 & & & & ONSITE & SESPMNT & $\mathrm{R}$ \\
\hline \begin{tabular}{|c|}
6726 \\
\end{tabular} & & & & ONSITE & SESPMNT & $\mathrm{R}$ \\
\hline \begin{tabular}{|c|c|}
6727 \\
\end{tabular} & & & & ONSITE & SESPMNT & $\mathrm{R}$ \\
\hline 6728 & & & & ONSITE & SESPMNT & $\mathrm{R}$ \\
\hline 6729 & & & & ONSITE & SESPMNT & $\mathrm{R}$ \\
\hline 6730 & & & & ONSITE & SESPMNT & $\mathrm{R}$ \\
\hline \begin{tabular}{|c|}
6731 \\
\end{tabular} & & & & ONSITE & SESPMNT & $\mathrm{R}$ \\
\hline 6732 & & & & ONSITE & SESPMNT & $\mathrm{R}$ \\
\hline 6733 & & & & ONSITE & SESPMNT & $\mathrm{R}$ \\
\hline 6734 & & & & ONSITE & SESPMNT & $\mathrm{R}$ \\
\hline 6735 & & & & ONSITE & SESPMNT & $\mathrm{R}$ \\
\hline 6736 & & & & ONSITE & SESPMNT & $\mathrm{R}$ \\
\hline \begin{tabular}{|l|}
6737 \\
\end{tabular} & & & & ONSITE & SESPMNT & $\mathrm{R}$ \\
\hline 6738 & & & & ONSITE & SESPMNT & $\mathrm{R}$ \\
\hline \begin{tabular}{|l|}
6739 \\
\end{tabular} & & & & OFFSITE & SESPMNT & $\mathrm{R}$ \\
\hline 6740 & & & & OFFSITE & SESPMNT & $\mathrm{R}$ \\
\hline \begin{tabular}{|l|l|}
6741 \\
\end{tabular} & & & & OFFSITE & SESPMNT & $\mathrm{R}$ \\
\hline 6742 & & & & OFFSITE & SESPMNT & $\mathrm{R}$ \\
\hline 6743 & & & & OFFSITE & SESPMNT & $\mathrm{R}$ \\
\hline 6744 & & & & OFFSITE & SESPMNT & $\mathrm{R}$ \\
\hline \begin{tabular}{|l|}
6745 \\
\end{tabular} & & & & OFFSITE & SESPMNT & $\mathrm{R}$ \\
\hline \begin{tabular}{|l|}
6746 \\
\end{tabular} & & & & OFFSITE & SESPMNT & $\mathrm{R}$ \\
\hline \begin{tabular}{|l|l|}
6747 \\
\end{tabular} & & & & OFFSITE & SESPMNT & $R$ \\
\hline 6748 & & & & OFFSITE & SESPMNT & $\mathrm{R}$ \\
\hline 6749 & & & & OFFSITE & SESPMNT & $\mathrm{R}$ \\
\hline 6750 & & & & OFFSITE & SESPMNT & $\mathrm{R}$ \\
\hline 6751 & & & & OFFSITE & SESPMNT & $\mathrm{R}$ \\
\hline \begin{tabular}{|l|}
6752 \\
\end{tabular} & & & & OFFSITE & SESPMNT & $\mathrm{R}$ \\
\hline \begin{tabular}{|l|}
6753 \\
\end{tabular} & & & & OFFSITE & SESPMNT & $\mathrm{R}$ \\
\hline \begin{tabular}{|l|l|}
6754 \\
\end{tabular} & & & & OFFSITE & SESPMNT & $R$ \\
\hline \begin{tabular}{|l|}
6755 \\
\end{tabular} & & & & OFFSITE & SESPMNT & $\mathrm{R}$ \\
\hline 6756 & & & & OFFSITE & SESPMNT & $\mathrm{R}$ \\
\hline \begin{tabular}{|l|}
6757 \\
\end{tabular} & & & & ONSITE & SESPMNT & $\mathrm{R}$ \\
\hline \begin{tabular}{|l|}
6758 \\
\end{tabular} & & & & ONSITE & SESPMNT & $\mathrm{R}$ \\
\hline \begin{tabular}{|l|}
6759 \\
\end{tabular} & & & & ONSITE & SESPMNT & $\mathrm{R}$ \\
\hline 6760 & & & & ONSITE & SESPMNT & $\mathrm{R}$ \\
\hline \begin{tabular}{|l|l|}
6761 \\
\end{tabular} & & & & ONSITE & SESBMNT & $R$ \\
\hline
\end{tabular}




\begin{tabular}{|c|c|c|c|c|c|c|}
\hline & $A D$ & $\mathrm{AE}$ & $\mathrm{AF}$ & $A G$ & $\mathrm{AH}$ & $\mathrm{Al}$ \\
\hline 1 & RESULT_COMMENT & COLL_SAMP_SIZE & COLL_SAMP_SIZE_UNITS & DIST_CLASS & OWNER_ID & COLLECTION_PURPOSE \\
\hline \begin{tabular}{|c|}
6762 \\
\end{tabular} & & & & OFFSITE & SESPMNT & $\mathrm{R}$ \\
\hline 6763 & & & & OFFSITE & SESPMNT & $\mathrm{R}$ \\
\hline 6764 & See sample record for comment. & & & OFFSITE & SESPMNT & $\mathrm{R}$ \\
\hline 6765 & See sample record for comment. & & & OFFSITE & SESPMNT & $\mathrm{R}$ \\
\hline \begin{tabular}{|c|}
6766 \\
\end{tabular} & & & & ONSITE & SESPMNT & $\mathrm{R}$ \\
\hline 6767 & & & & ONSITE & SESPMNT & $\mathrm{R}$ \\
\hline 6768 & & & & ONSITE & SESPMNT & $\mathrm{R}$ \\
\hline 6769 & & & & PERIMETER & SESPMNT & $\mathrm{R}$ \\
\hline 6770 & & & & PERIMETER & SESPMNT & $\mathrm{R}$ \\
\hline 6771 & & & & PERIMETER & SESPMNT & $\mathrm{R}$ \\
\hline 6772 & & & & ONSITE & SESPMNT & $\mathrm{R}$ \\
\hline 6773 & & & & ONSITE & SESPMNT & $\mathrm{R}$ \\
\hline 6774 & See sample record for comment. & & & OFFSITE & SESPMNT & $\mathrm{R}$ \\
\hline 6775 & See sample record for comment. & & & OFFSITE & SESPMNT & $\mathrm{R}$ \\
\hline \begin{tabular}{|c|}
6776 \\
\end{tabular} & & & & OFFSITE & SESPMNT & $\mathrm{R}$ \\
\hline \begin{tabular}{|c|c|}
6777 \\
\end{tabular} & & & & OFFSITE & SESPMNT & $\mathrm{R}$ \\
\hline 6778 & & & & OFFSITE & SESPMNT & $\mathrm{R}$ \\
\hline 6779 & & & & OFFSITE & SESPMNT & $\mathrm{R}$ \\
\hline 6780 & & & & OFFSITE & SESPMNT & $\mathrm{R}$ \\
\hline 6781 & & & & OFFSITE & SESPMNT & $\mathrm{R}$ \\
\hline \begin{tabular}{|c|}
6782 \\
\end{tabular} & & & & OFFSITE & SESPMNT & $\mathrm{R}$ \\
\hline 6783 & See sample record for comment. & & & & SESPMNT & $\mathrm{R}$ \\
\hline 6784 & See sample record for comment. & & & & SESPMNT & $\mathrm{R}$ \\
\hline 6785 & See sample record for comment. & & & & SESPMNT & $\mathrm{R}$ \\
\hline 6786 & See sample record for comment. & & & & SESPMNT & $\mathrm{R}$ \\
\hline 6787 & See sample record for comment. & & & & SESPMNT & $\mathrm{R}$ \\
\hline 6788 & & & & ONSITE & SESPMNT & $\mathrm{R}$ \\
\hline 6789 & & & & ONSITE & SESPMNT & $\mathrm{R}$ \\
\hline 6790 & & & & ONSITE & SESPMNT & $\mathrm{R}$ \\
\hline 6791 & & & & OFFSITE & SESPMNT & $\mathrm{R}$ \\
\hline 6792 & & & & OFFSITE & SESPMNT & $\mathrm{R}$ \\
\hline 6793 & & & & OFFSITE & SESPMNT & $\mathrm{R}$ \\
\hline 6794 & & & & OFFSITE & SESPMNT & $\mathrm{R}$ \\
\hline 6795 & & & & OFFSITE & SESPMNT & $\mathrm{R}$ \\
\hline 6796 & & & & OFFSITE & SESPMNT & $\mathrm{R}$ \\
\hline \begin{tabular}{|c|c|}
6797 \\
\end{tabular} & & & & OFFSITE & SESPMNT & $\mathrm{R}$ \\
\hline 6798 & & & & OFFSITE & SESPMNT & $\mathrm{R}$ \\
\hline 6799 & & & & OFFSITE & SESPMNT & $\mathrm{R}$ \\
\hline 6800 & & & & OFFSITE & SESPMNT & $\mathrm{R}$ \\
\hline 6801 & & & & OFFSITE & SESPMNT & $\mathrm{R}$ \\
\hline 6802 & & & & OFFSITE & SESPMNT & $\mathrm{R}$ \\
\hline 6803 & & & & OFFSITE & SESPMNT & $\mathrm{R}$ \\
\hline 6804 & & & & OFFSITE & SESPMNT & $\mathrm{R}$ \\
\hline 6805 & & & & OFFSITE & SESPMNT & $\mathrm{R}$ \\
\hline \begin{tabular}{|c|}
6806 \\
\end{tabular} & & & & OFFSITE & SESPMNT & $\mathrm{R}$ \\
\hline \begin{tabular}{|c|c|}
6807 \\
\end{tabular} & & & & OFFSITE & SESPMNT & $\mathrm{R}$ \\
\hline 6808 & & & & OFFSITE & SESPMNT & $\mathrm{R}$ \\
\hline 6809 & & & & OFFSITE & SESPMNT & $\mathrm{R}$ \\
\hline 6810 & & & & PERIMETER & SESPMNT & $\mathrm{R}$ \\
\hline 6811 & & & & PERIMETER & SESPMNT & $\mathrm{R}$ \\
\hline \begin{tabular}{|l|}
6812 \\
\end{tabular} & & & & PERIMETER & SESPMNT & $\mathrm{R}$ \\
\hline \begin{tabular}{|l|}
6813 \\
\end{tabular} & & & & PERIMETER & SESBMN- & $R$ \\
\hline
\end{tabular}




\begin{tabular}{|c|c|c|c|c|c|c|}
\hline & $A D$ & $\mathrm{AE}$ & $\mathrm{AF}$ & $A G$ & $\mathrm{AH}$ & $\mathrm{Al}$ \\
\hline 1 & RESULT_COMMENT & COLL_SAMP_SIZE & COLL_SAMP_SIZE_UNITS & DIST_CLASS & OWNER_ID & COLLECTION_PURPOSE \\
\hline 6814 & & & & PERIMETER & SESPMNT & $\mathrm{R}$ \\
\hline \begin{tabular}{|l|}
6815 \\
\end{tabular} & & & & ONSITE & SESPMNT & $\mathrm{R}$ \\
\hline \begin{tabular}{|c|}
6816 \\
\end{tabular} & & & & ONSITE & SESPMNT & $\mathrm{R}$ \\
\hline 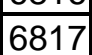 & & & & OFFSITE & SESPMNT & $\mathrm{R}$ \\
\hline 6818 & & & & OFFSITE & SESPMNT & $\mathrm{R}$ \\
\hline 6819 & & & & OFFSITE & SESPMNT & $\mathrm{R}$ \\
\hline 6820 & & & & OFFSITE & SESPMNT & $\mathrm{R}$ \\
\hline \begin{tabular}{|l|}
6821 \\
\end{tabular} & & & & OFFSITE & SESPMNT & $\mathrm{R}$ \\
\hline 6822 & & & & OFFSITE & SESPMNT & $\mathrm{R}$ \\
\hline 6823 & & & & OFFSITE & SESPMNT & $\mathrm{R}$ \\
\hline 6824 & & & & OFFSITE & SESPMNT & $\mathrm{R}$ \\
\hline 6825 & & & & OFFSITE & SESPMNT & $\mathrm{R}$ \\
\hline 6826 & & & & OFFSITE & SESPMNT & $\mathrm{R}$ \\
\hline \begin{tabular}{|l|l|}
6827 & $-2 \cdot r$ \\
\end{tabular} & & & & ONSITE & SESPMNT & $\mathrm{R}$ \\
\hline \begin{tabular}{|l|l|}
6828 \\
\end{tabular} & & & & ONSITE & SESPMNT & $\mathrm{R}$ \\
\hline \begin{tabular}{|l|}
6829 \\
\end{tabular} & & & & ONSITE & SESPMNT & $\mathrm{R}$ \\
\hline \begin{tabular}{|c|}
6830 \\
\end{tabular} & & & & ONSITE & SESPMNT & $\mathrm{R}$ \\
\hline 6831 & & & & ONSITE & SESPMNT & $\mathrm{R}$ \\
\hline 6832 & & & & ONSITE & SESPMNT & $\mathrm{R}$ \\
\hline \begin{tabular}{|c|}
6833 \\
\end{tabular} & & & & ONSITE & SESPMNT & $\mathrm{R}$ \\
\hline \begin{tabular}{|l|}
6834 \\
\end{tabular} & & & & ONSITE & SESPMNT & $\mathrm{R}$ \\
\hline \begin{tabular}{|l|}
6835 \\
\end{tabular} & See sample record for comment. & & & ONSITE & SESPMNT & $\mathrm{R}$ \\
\hline 6836 & See sample record for comment. & & & ONSITE & SESPMNT & $\mathrm{R}$ \\
\hline \begin{tabular}{|l|l|}
6837 & $-2 \cdot r$ \\
\end{tabular} & See sample record for comment. & & & ONSITE & SESPMNT & $\mathrm{R}$ \\
\hline 6838 & See sample record for comment. & & & ONSITE & SESPMNT & $\mathrm{R}$ \\
\hline 6839 & See sample record for comment. & & & ONSITE & SESPMNT & $\mathrm{R}$ \\
\hline 6840 & See sample record for comment. & & & ONSITE & SESPMNT & $\mathrm{R}$ \\
\hline \begin{tabular}{|l|}
6841 \\
\end{tabular} & & & & ONSITE & SESPMNT & $\mathrm{R}$ \\
\hline \begin{tabular}{|l|}
6842 \\
\end{tabular} & & & & ONSITE & SESPMNT & $\mathrm{R}$ \\
\hline \begin{tabular}{|l|}
6843 \\
\end{tabular} & & & & OFFSITE & SESPMNT & $\mathrm{R}$ \\
\hline 6844 & & & & OFFSITE & SESPMNT & $\mathrm{R}$ \\
\hline \begin{tabular}{|c|}
6845 \\
\end{tabular} & & & & OFFSITE & SESPMNT & $\mathrm{R}$ \\
\hline 6846 & & & & OFFSITE & SESPMNT & $\mathrm{R}$ \\
\hline \begin{tabular}{|l|l|}
6847 \\
\end{tabular} & & & & ONSITE & SESPMNT & $\mathrm{R}$ \\
\hline \begin{tabular}{|c|}
6848 \\
\end{tabular} & & & & ONSITE & SESPMNT & $\mathrm{R}$ \\
\hline 6849 & & & & ONSITE & SESPMNT & $\mathrm{R}$ \\
\hline 6850 & & & & ONSITE & SESPMNT & $\mathrm{R}$ \\
\hline 6851 & & & & ONSITE & SESPMNT & $\mathrm{R}$ \\
\hline 6852 & & & & ONSITE & SESPMNT & $\mathrm{R}$ \\
\hline 6853 & & & & ONSITE & SESPMNT & $\mathrm{R}$ \\
\hline \begin{tabular}{|c|}
6854 \\
\end{tabular} & & & & ONSITE & SESPMNT & $\mathrm{R}$ \\
\hline 6855 & & & & ONSITE & SESPMNT & $\mathrm{R}$ \\
\hline \begin{tabular}{|c|}
6856 \\
\end{tabular} & & & & ONSITE & SESPMNT & $\mathrm{R}$ \\
\hline \begin{tabular}{|l|}
6857 \\
\end{tabular} & & & & ONSITE & SESPMNT & $\mathrm{R}$ \\
\hline 6858 & & & & ONSITE & SESPMNT & $\mathrm{R}$ \\
\hline 6859 & & & & OFFSITE & SESPMNT & $\mathrm{R}$ \\
\hline 6860 & & & & OFFSITE & SESPMNT & $\mathrm{R}$ \\
\hline 6861 & & & & OFFSITE & SESPMNT & $\mathrm{R}$ \\
\hline 6862 & & & & OFFSITE & SESPMNT & $\mathrm{R}$ \\
\hline 6863 & & & & OFFSITE & SESPMNT & $R$ \\
\hline \begin{tabular}{|l|l|}
6864 \\
\end{tabular} & & & & OFFSITE & SESPMNT & $\mathrm{R}$ \\
\hline 6865 & & & & OFFSITE & SESBMNT & $\mathrm{R}$ \\
\hline
\end{tabular}




\begin{tabular}{|c|c|c|c|c|c|c|}
\hline & $\mathrm{AD}$ & $\mathrm{AE}$ & $\mathrm{AF}$ & AG & $\mathrm{AH}$ & $\mathrm{Al}$ \\
\hline 1 & RESULT_COMMENT & COLL_SAMP_SIZE & COLL_SAMP_SIZE_UNITS & DIST_CLASS & OWNER_ID & COLLECTION_PURPOSE \\
\hline \begin{tabular}{|c|}
6866 \\
\end{tabular} & & & & OFFSITE & SESPMNT & $\mathrm{R}$ \\
\hline \begin{tabular}{|c|c|}
6867 & -1 \\
\end{tabular} & & & & OFFSITE & SESPMNT & $\mathrm{R}$ \\
\hline 6868 & & & & OFFSITE & SESPMNT & $\mathrm{R}$ \\
\hline 6869 & & & & OFFSITE & SESPMNT & $\mathrm{R}$ \\
\hline 6870 & & & & OFFSITE & SESPMNT & $\mathrm{R}$ \\
\hline \begin{tabular}{|l|}
6871 \\
\end{tabular} & & & & OFFSITE & SESPMNT & $\mathrm{R}$ \\
\hline 6872 & & & & OFFSITE & SESPMNT & $\mathrm{R}$ \\
\hline 6873 & & & & OFFSITE & SESPMNT & $\mathrm{R}$ \\
\hline 6874 & & & & OFFSITE & SESPMNT & $\mathrm{R}$ \\
\hline \begin{tabular}{|c|c|}
6875 \\
\end{tabular} & & & & OFFSITE & SESPMNT & $\mathrm{R}$ \\
\hline \begin{tabular}{|c|}
6876 \\
\end{tabular} & & & & OFFSITE & SESPMNT & $\mathrm{R}$ \\
\hline \begin{tabular}{|l|l|}
6877 & -1 \\
\end{tabular} & & & & OFFSITE & SESPMNT & $R$ \\
\hline 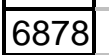 & & & & OFFSITE & SESPMNT & $\mathrm{R}$ \\
\hline \begin{tabular}{|c|}
6879 \\
\end{tabular} & & & & OFFSITE & SESPMNT & $\mathrm{R}$ \\
\hline \begin{tabular}{|c|}
6880 \\
\end{tabular} & & & & OFFSITE & SESPMNT & $R$ \\
\hline \begin{tabular}{|c|}
6881 \\
\end{tabular} & & & & OFFSITE & SESPMNT & $\mathrm{R}$ \\
\hline \begin{tabular}{|c|}
6882 \\
\end{tabular} & & & & OFFSITE & SESPMNT & $\mathrm{R}$ \\
\hline \begin{tabular}{|c|}
6883 \\
\end{tabular} & & & & ONSITE & SESPMNT & $\mathrm{R}$ \\
\hline \begin{tabular}{|c|}
6884 \\
\end{tabular} & & & & ONSITE & SESPMNT & $\mathrm{R}$ \\
\hline \begin{tabular}{|c|c|}
6885 \\
\end{tabular} & & & & ONSITE & SESPMNT & $\mathrm{R}$ \\
\hline \begin{tabular}{|c|}
6886 \\
\end{tabular} & & & & ONSITE & SESPMNT & $R$ \\
\hline \begin{tabular}{|c|}
6887 \\
\end{tabular} & & & & ONSITE & SESPMNT & $\mathrm{R}$ \\
\hline \begin{tabular}{|c|}
6888 \\
\end{tabular} & & & & ONSITE & SESPMNT & $\mathrm{R}$ \\
\hline \begin{tabular}{|c|}
6889 \\
\end{tabular} & & & & ONSITE & SESPMNT & $\mathrm{R}$ \\
\hline \begin{tabular}{|c|}
6890 \\
\end{tabular} & & & & ONSITE & SESPMNT & $\mathrm{R}$ \\
\hline \begin{tabular}{|l|}
6891 \\
\end{tabular} & & & & ONSITE & SESPMNT & $\mathrm{R}$ \\
\hline \begin{tabular}{|c|}
6892 \\
\end{tabular} & & & & ONSITE & SESPMNT & $\mathrm{R}$ \\
\hline \begin{tabular}{|c|c|}
6893 \\
\end{tabular} & & & & ONSITE & SESPMNT & $\mathrm{R}$ \\
\hline \begin{tabular}{|c|}
6894 \\
\end{tabular} & & & & ONSITE & SESPMNT & $\mathrm{R}$ \\
\hline \begin{tabular}{|c|}
6895 \\
\end{tabular} & & & & ONSITE & SESPMNT & $R$ \\
\hline \begin{tabular}{|l|l|}
6896 \\
\end{tabular} & & & & ONSITE & SESPMNT & $R$ \\
\hline \begin{tabular}{|l|}
6897 \\
\end{tabular} & & & & ONSITE & SESPMNT & $R$ \\
\hline \begin{tabular}{|l|}
6898 \\
\end{tabular} & & & & OFFSITE & SESPSPEC & S \\
\hline \begin{tabular}{|l|}
6899 \\
\end{tabular} & & & & OFFSITE & SESPSPEC & $\mathrm{S}$ \\
\hline \begin{tabular}{|l|}
6900 \\
\end{tabular} & & & & OFFSITE & SESPSPEC & S \\
\hline 6901 & & & & OFFSITE & SESPSPEC & $S$ \\
\hline \begin{tabular}{|l|l|}
6902 \\
\end{tabular} & & & & OFFSITE & SESPSPEC & S \\
\hline \begin{tabular}{|l|l|}
6903 \\
\end{tabular} & & & & OFFSITE & SESPSPEC & S \\
\hline 6904 & & & & OFFSITE & SESPSPEC & S \\
\hline \begin{tabular}{|l|l|}
6905 \\
\end{tabular} & & & & OFFSITE & SESPSPEC & S \\
\hline \begin{tabular}{|l|}
6906 \\
\end{tabular} & & & & OFFSITE & SESPSPEC & S \\
\hline \begin{tabular}{|l|}
6907 \\
\end{tabular} & & & & OFFSITE & SESPSPEC & S \\
\hline \begin{tabular}{|l|}
6908 \\
\end{tabular} & & & & OFFSITE & SESPSPEC & $\mathrm{S}$ \\
\hline \begin{tabular}{|l|l|}
6909 \\
\end{tabular} & & & & OFFSITE & SESPSPEC & S \\
\hline \begin{tabular}{|l|}
6910 \\
\end{tabular} & & & & ONSITE & SESPMNT & $R$ \\
\hline \begin{tabular}{|l|}
6911 \\
\end{tabular} & & & & ONSITE & SESPMNT & $R$ \\
\hline \begin{tabular}{|l|}
6912 \\
\end{tabular} & & & & OFFSITE & SESPMNT & $R$ \\
\hline \begin{tabular}{|l|l|}
6913 \\
\end{tabular} & & & & OFFSITE & SESPMNT & $R$ \\
\hline 6914 & & & & OFFSITE & SESPMNT & $\mathrm{R}$ \\
\hline \begin{tabular}{|l|l|}
6915 \\
\end{tabular} & & & & OFFSITE & SESPMNT & $R$ \\
\hline \begin{tabular}{|l|l|}
6916 \\
\end{tabular} & & & & ONSITE & SESPMNT & $R$ \\
\hline \begin{tabular}{|l|l|}
6917 \\
\end{tabular} & & & & ONSITE & SESBMNT & $R$ \\
\hline
\end{tabular}




\begin{tabular}{|c|c|c|c|c|c|c|}
\hline & $A D$ & $\mathrm{AE}$ & $\mathrm{AF}$ & $A G$ & $\mathrm{AH}$ & $\mathrm{Al}$ \\
\hline 1 & RESULT_COMMENT & COLL_SAMP_SIZE & COLL_SAMP_SIZE_UNITS & DIST_CLASS & OWNER_ID & COLLECTION_PURPOSE \\
\hline 6918 & & & & OFFSITE & SESPMNT & $\mathrm{R}$ \\
\hline 6919 & & & & OFFSITE & SESPMNT & $\mathrm{R}$ \\
\hline 6920 & & & & OFFSITE & SESPMNT & $\mathrm{R}$ \\
\hline 6921 & & & & OFFSITE & SESPMNT & $\mathrm{R}$ \\
\hline 6922 & & & & OFFSITE & SESPMNT & $\mathrm{R}$ \\
\hline 6923 & & & & OFFSITE & SESPMNT & $\mathrm{R}$ \\
\hline 6924 & & & & OFFSITE & SESPMNT & $\mathrm{R}$ \\
\hline 6925 & & & & OFFSITE & SESPMNT & $\mathrm{R}$ \\
\hline \begin{tabular}{|c|}
6926 \\
\end{tabular} & & & & OFFSITE & SESPMNT & $\mathrm{R}$ \\
\hline \begin{tabular}{|c|c|c|}
6927 \\
\end{tabular} & & & & OFFSITE & SESPMNT & $\mathrm{R}$ \\
\hline 6928 & & & & OFFSITE & SESPMNT & $\mathrm{R}$ \\
\hline 6929 & & & & OFFSITE & SESPMNT & $\mathrm{R}$ \\
\hline 6930 & & & & OFFSITE & SESPMNT & $\mathrm{R}$ \\
\hline 6931 & & & & OFFSITE & SESPMNT & $\mathrm{R}$ \\
\hline 6932 & & & & OFFSITE & SESPMNT & $\mathrm{R}$ \\
\hline 6933 & & & & OFFSITE & SESPMNT & $\mathrm{R}$ \\
\hline 6934 & & & & OFFSITE & SESPMNT & $\mathrm{R}$ \\
\hline 6935 & & & & OFFSITE & SESPMNT & $\mathrm{R}$ \\
\hline 6936 & & & & ONSITE & SESPMNT & $\mathrm{R}$ \\
\hline 6937 & & & & ONSITE & SESPMNT & $\mathrm{R}$ \\
\hline 6938 & & & & ONSITE & SESPMNT & $\mathrm{R}$ \\
\hline 6939 & & & & PERIMETER & SESPMNT & $\mathrm{R}$ \\
\hline 6940 & & & & PERIMETER & SESPMNT & $\mathrm{R}$ \\
\hline 6941 & & & & PERIMETER & SESPMNT & $\mathrm{R}$ \\
\hline \begin{tabular}{|l|l|}
6942 \\
\end{tabular} & & & & ONSITE & SESPMNT & $\mathrm{R}$ \\
\hline \begin{tabular}{|l|}
6943 \\
\end{tabular} & & & & ONSITE & SESPMNT & $\mathrm{R}$ \\
\hline 6944 & & & & OFFSITE & SESPMNT & $\mathrm{R}$ \\
\hline 6945 & & & & OFFSITE & SESPMNT & $\mathrm{R}$ \\
\hline \begin{tabular}{|l|}
6946 \\
\end{tabular} & & & & OFFSITE & SESPMNT & $\mathrm{R}$ \\
\hline \begin{tabular}{|l|l|}
6947 \\
\end{tabular} & & & & OFFSITE & SESPMNT & $\mathrm{R}$ \\
\hline \begin{tabular}{|l|}
6948 \\
\end{tabular} & & & & OFFSITE & SESPMNT & $\mathrm{R}$ \\
\hline \begin{tabular}{|l|}
6949 \\
\end{tabular} & & & & OFFSITE & SESPMNT & $\mathrm{R}$ \\
\hline 6950 & & & & OFFSITE & SESPMNT & $\mathrm{R}$ \\
\hline 6951 & & & & OFFSITE & SESPMNT & $\mathrm{R}$ \\
\hline 6952 & & & & OFFSITE & SESPMNT & $\mathrm{R}$ \\
\hline \begin{tabular}{|l|}
6953 \\
\end{tabular} & & & & & SESPMNT & $\mathrm{R}$ \\
\hline \begin{tabular}{|l|l|}
6954 \\
\end{tabular} & & & & & SESPMNT & $\mathrm{R}$ \\
\hline \begin{tabular}{|l|l|}
6955 \\
\end{tabular} & & & & & SESPMNT & $R$ \\
\hline \begin{tabular}{|l|}
6956 \\
\end{tabular} & & & & & SESPMNT & $\mathrm{R}$ \\
\hline \begin{tabular}{|l|}
6957 \\
\end{tabular} & & & & & SESPMNT & $\mathrm{R}$ \\
\hline 6958 & & & & ONSITE & SESPMNT & $\mathrm{R}$ \\
\hline 6959 & & & & ONSITE & SESPMNT & $\mathrm{R}$ \\
\hline 6960 & & & & ONSITE & SESPMNT & $\mathrm{R}$ \\
\hline \begin{tabular}{|l|l|}
6961 \\
\end{tabular} & & & & OFFSITE & SESPMNT & $\mathrm{R}$ \\
\hline \begin{tabular}{|l|}
6962 \\
\end{tabular} & & & & OFFSITE & SESPMNT & $R$ \\
\hline \begin{tabular}{|l|}
6963 \\
\end{tabular} & & & & OFFSITE & SESPMNT & $\mathrm{R}$ \\
\hline 6964 & & & & OFFSITE & SESPMNT & $\mathrm{R}$ \\
\hline 6965 & & & & OFFSITE & SESPMNT & $\mathrm{R}$ \\
\hline \begin{tabular}{|l|}
6966 \\
\end{tabular} & & & & PERIMETER & SESPMNT & $\mathrm{R}$ \\
\hline \begin{tabular}{|l|l|}
6967 \\
\end{tabular} & & & & PERIMETER & SESPMNT & $\mathrm{R}$ \\
\hline \begin{tabular}{|l|}
6968 \\
\end{tabular} & & & & PERIMETER & SESPMNT & $\mathrm{R}$ \\
\hline \begin{tabular}{|l|}
6969 \\
\end{tabular} & & & & PERIMETER & SESBMNT & $R$ \\
\hline
\end{tabular}




\begin{tabular}{|c|c|c|c|c|c|c|}
\hline & $A D$ & $\mathrm{AE}$ & $\mathrm{AF}$ & $A G$ & $\mathrm{AH}$ & $\mathrm{Al}$ \\
\hline 1 & RESULT_COMMENT & COLL_SAMP_SIZE & COLL_SAMP_SIZE_UNITS & DIST_CLASS & OWNER_ID & COLLECTION_PURPOSE \\
\hline 6970 & & & & PERIMETER & SESPMNT & $\mathrm{R}$ \\
\hline 6971 & & & & ONSITE & SESPMNT & $\mathrm{R}$ \\
\hline 6972 & & & & ONSITE & SESPMNT & $\mathrm{R}$ \\
\hline 6973 & See sample record for comment. & & & OFFSITE & SESPMNT & $\mathrm{R}$ \\
\hline 6974 & See sample record for comment. & & & OFFSITE & SESPMNT & $\mathrm{R}$ \\
\hline 6975 & See sample record for comment. & & & OFFSITE & SESPMNT & $\mathrm{R}$ \\
\hline 6976 & See sample record for comment. & & & OFFSITE & SESPMNT & $\mathrm{R}$ \\
\hline 6977 & See sample record for comment. & & & OFFSITE & SESPMNT & $\mathrm{R}$ \\
\hline 6978 & See sample record for comment. & & & OFFSITE & SESPMNT & $\mathrm{R}$ \\
\hline 6979 & See sample record for comment. & & & OFFSITE & SESPMNT & $\mathrm{R}$ \\
\hline 6980 & & & & OFFSITE & SESPMNT & $\mathrm{R}$ \\
\hline 6981 & & & & OFFSITE & SESPMNT & $\mathrm{R}$ \\
\hline 6982 & & & & OFFSITE & SESPMNT & $\mathrm{R}$ \\
\hline 6983 & & & & OFFSITE & SESPMNT & $\mathrm{R}$ \\
\hline 6984 & & & & OFFSITE & SESPMNT & $\mathrm{R}$ \\
\hline 6985 & & & & OFFSITE & SESPMNT & $\mathrm{R}$ \\
\hline \begin{tabular}{|c|}
6986 \\
\end{tabular} & & & & OFFSITE & SESPMNT & $\mathrm{R}$ \\
\hline \begin{tabular}{|c|c|}
6987 \\
\end{tabular} & & & & OFFSITE & SESPMNT & $\mathrm{R}$ \\
\hline 6988 & & & & OFFSITE & SESPMNT & $\mathrm{R}$ \\
\hline 6989 & & & & OFFSITE & SESPMNT & $\mathrm{R}$ \\
\hline 6990 & & & & OFFSITE & SESPMNT & $\mathrm{R}$ \\
\hline 6991 & & & & ONSITE & SESPMNT & $\mathrm{R}$ \\
\hline 6992 & & & & ONSITE & SESPMNT & $\mathrm{R}$ \\
\hline 6993 & & & & ONSITE & SESPMNT & $\mathrm{R}$ \\
\hline 6994 & & & & ONSITE & SESPMNT & $\mathrm{R}$ \\
\hline 6995 & & & & ONSITE & SESPMNT & $\mathrm{R}$ \\
\hline 6996 & & & & ONSITE & SESPMNT & $\mathrm{R}$ \\
\hline 6997 & & & & ONSITE & SESPMNT & $\mathrm{R}$ \\
\hline 6998 & & & & ONSITE & SESPMNT & $\mathrm{R}$ \\
\hline 6999 & & & & ONSITE & SESPMNT & $\mathrm{R}$ \\
\hline 7000 & & & & ONSITE & SESPMNT & $\mathrm{R}$ \\
\hline 7001 & & & & ONSITE & SESPMNT & $\mathrm{R}$ \\
\hline 7002 & & & & ONSITE & SESPMNT & $\mathrm{R}$ \\
\hline 7003 & & & & ONSITE & SESPMNT & $\mathrm{R}$ \\
\hline 7004 & & & & ONSITE & SESPMNT & $\mathrm{R}$ \\
\hline 7005 & See sample record for comment. & & & ONSITE & SESPMNT & $\mathrm{R}$ \\
\hline 7006 & See sample record for comment. & & & ONSITE & SESPMNT & $\mathrm{R}$ \\
\hline 7007 & & & & OFFSITE & SESPMNT & $\mathrm{R}$ \\
\hline 7008 & & & & OFFSITE & SESPMNT & $\mathrm{R}$ \\
\hline 7009 & & & & OFFSITE & SESPMNT & $\mathrm{R}$ \\
\hline 7010 & & & & OFFSITE & SESPMNT & $\mathrm{R}$ \\
\hline 7011 & & & & ONSITE & SESPMNT & $\mathrm{R}$ \\
\hline 7012 & & & & ONSITE & SESPMNT & $\mathrm{R}$ \\
\hline 7013 & & & & ONSITE & SESPMNT & $\mathrm{R}$ \\
\hline 7014 & & & & ONSITE & SESPMNT & $\mathrm{R}$ \\
\hline 7015 & & & & ONSITE & SESPMNT & $\mathrm{R}$ \\
\hline 7016 & & & & ONSITE & SESPMNT & $\mathrm{R}$ \\
\hline 7017 & & & & ONSITE & SESPMNT & $\mathrm{R}$ \\
\hline 7018 & & & & ONSITE & SESPMNT & $\mathrm{R}$ \\
\hline 7019 & & & & ONSITE & SESPMNT & $\mathrm{R}$ \\
\hline 7020 & & & & ONSITE & SESPMNT & $\mathrm{R}$ \\
\hline 7021 & & & & ONSITE & SESBMNT & $R$ \\
\hline
\end{tabular}




\begin{tabular}{|c|c|c|c|c|c|c|}
\hline & AD & AE & AF & AG & AH & Al \\
\hline 1 & RESULT_COMMENT & COLL_SAMP_SIZE & COLL_SAMP_SIZE_UNITS & DIST_CLASS & OWNER_ID & COLLECTION_PURPOSE \\
\hline 7022 & & & ONSITE & SESPMNT & R \\
\hline 7023 & & & OFFSITE & SESPMNT & R \\
\hline 7024 & & & OFFSITE & SESPPMNT & R \\
\hline 7025 & & & OFFSITE & SESPPMNT & R \\
\hline 7026 & & & OFFSITE & SESPPMNT & R \\
\hline 7027 & & & OFFSITE & SESPMNT & R \\
\hline 7028 & & & OFFSITE & SESPMNT & R \\
\hline 7029 & & & OFFSITE & SESPMNT & R \\
\hline 7030 & & & OFFSITE & SESPMNT & R \\
\hline 7031 & & & OFFSITE & SESPMNT & R \\
\hline 7032 & & & OFFSITE & SESPMNT & R \\
\hline
\end{tabular}


88 - 90 Sediment 


\begin{tabular}{|c|c|c|c|c|c|c|c|c|c|c|}
\hline & $\mathrm{A}$ & $\mathrm{B}$ & $\mathrm{C}$ & $\mathrm{D}$ & $E$ & $\mathrm{~F}$ & $\mathrm{G}$ & $\mathrm{H}$ & $\mathrm{I}$ & $\mathrm{J}$ \\
\hline 1 & SAMPLE_NUM_ASSIGNED_TO & SAMPLER & MEDIA & BIOTA_MEDIA & TAG_ID & SAMP_NUM & SAMP_DATE_TIME & SAMP_DATE_TIME_ON & CON_ID & CON_SHORT_NAME \\
\hline 2 & PDMS & & SO & & & E105046 & 07-Sep-88 & & $10045-97-3$ & CS-137 \\
\hline 3 & PDMS & & SO & & & E105045 & 07-Sep-88 & & $10045-97-3$ & CS-137 \\
\hline 4 & PDMS & & SO & & & E105044 & 07-Sep-88 & & $10045-97-3$ & CS-137 \\
\hline 5 & PDMS & & SO & & & E105043 & 07-Sep-88 & & $10045-97-3$ & CS-137 \\
\hline 6 & PDMS & & SO & & & E105050 & 15-Sep-88 & & $10045-97-3$ & CS-137 \\
\hline 7 & PDMS & & SO & & & E105049 & 15-Sep-88 & & $10045-97-3$ & CS-137 \\
\hline 8 & PDMS & & SO & & & E105048 & 15-Sep-88 & & $10045-97-3$ & CS-137 \\
\hline 9 & PDMS & & SO & & & E105047 & 15-Sep-88 & & $10045-97-3$ & CS-137 \\
\hline 10 & PDMS & & SO & & & E111107 & 08-Dec-89 & & $10045-97-3$ & CS-137 \\
\hline 11 & PDMS & & SO & & & E111106 & 08-Dec-89 & & $10045-97-3$ & CS-137 \\
\hline 12 & PDMS & & SO & & & E111104 & 08-Dec-89 & & $10045-97-3$ & CS-137 \\
\hline 13 & PDMS & & SO & & & E111103 & 08-Dec-89 & & $10045-97-3$ & CS-137 \\
\hline 14 & PDMS & & SO & & & E111164 & 14-Dec-89 & & $10045-97-3$ & CS-137 \\
\hline 15 & PDMS & & SO & & & E111163 & 14-Dec-89 & & $10045-97-3$ & CS-137 \\
\hline 16 & PDMS & & SO & & & E111162 & 14-Dec-89 & & $10045-97-3$ & CS-137 \\
\hline 17 & PDMS & & SO & & & E111161 & 14-Dec-89 & & $10045-97-3$ & CS-137 \\
\hline 18 & PDMS & & SO & & & E111253 & 21-Dec-89 & & $10045-97-3$ & CS-137 \\
\hline 19 & PDMS & & SO & & & E111252 & 21-Dec-89 & & $10045-97-3$ & CS-137 \\
\hline 20 & PDMS & & SO & & & E111251 & 21-Dec-89 & & $10045-97-3$ & CS-137 \\
\hline 21 & PDMS & & SO & & & E111156 & 21-Dec-89 & & $10045-97-3$ & CS-137 \\
\hline 22 & PDMS & & SO & & & E105046 & 07-Sep-88 & & PU-239/240 & PU-239/240 \\
\hline 23 & PDMS & & SO & & & E105045 & 07-Sep-88 & & PU-239/240 & PU-239/240 \\
\hline 24 & PDMS & & SO & & & E105044 & 07-Sep-88 & & PU-239/240 & PU-239/240 \\
\hline 25 & PDMS & & SO & & & E105043 & 07-Sep-88 & & PU-239/240 & PU-239/240 \\
\hline 26 & PDMS & & SO & & & E105050 & 15-Sep-88 & & PU-239/240 & PU-239/240 \\
\hline 27 & PDMS & & SO & & & E105049 & 15-Sep-88 & & PU-239/240 & PU-239/240 \\
\hline 28 & PDMS & & SO & & & E105048 & 15-Sep-88 & & PU-239/240 & PU-239/240 \\
\hline 29 & PDMS & & SO & & & E105047 & 15-Sep-88 & & PU-239/240 & PU-239/240 \\
\hline 30 & PDMS & & SO & & & E111107 & 08-Dec-89 & & PU-239/240 & PU-239/240 \\
\hline 31 & PDMS & & SO & & & E111106 & 08-Dec-89 & & PU-239/240 & PU-239/240 \\
\hline 32 & PDMS & & SO & & & E111104 & 08-Dec-89 & & PU-239/240 & PU-239/240 \\
\hline 33 & PDMS & & SO & & & E111103 & 08-Dec-89 & & PU-239/240 & PU-239/240 \\
\hline 34 & PDMS & & SO & & & E111164 & 14-Dec-89 & & PU-239/240 & PU-239/240 \\
\hline 35 & PDMS & & SO & & & E111163 & 14-Dec-89 & & PU-239/240 & PU-239/240 \\
\hline 36 & PDMS & & SO & & & E111162 & 14-Dec-89 & & PU-239/240 & PU-239/240 \\
\hline 37 & PDMS & & SO & & & E111161 & 14-Dec-89 & & PU-239/240 & PU-239/240 \\
\hline 38 & PDMS & & SO & & & E111253 & 21-Dec-89 & & PU-239/240 & PU-239/240 \\
\hline 39 & PDMS & & SO & & & E111252 & 21-Dec-89 & & PU-239/240 & PU-239/240 \\
\hline
\end{tabular}




\begin{tabular}{|c|c|c|c|c|c|c|c|c|c|c|}
\hline & $A$ & $B$ & $\mathrm{C}$ & $\mathrm{D}$ & $E$ & $\mathrm{~F}$ & $G$ & $\mathrm{H}$ & $\mathrm{I}$ & $\mathrm{J}$ \\
\hline 1 & SAMPLE NUM ASSIGNED TO & SAMPLER & MEDIA & BIOTA MEDIA & TAG ID & SAMP NUM & SAMP DATE TIME & SAMP DATE TIME ON & CON ID & CON SHORT NAME \\
\hline 40 & PDMS & & SO & & & E111251 & 21-Dec-89 & & PU-239/240 & PU-239/240 \\
\hline 41 & PDMS & & SO & & & E111156 & 21-Dec-89 & & PU-239/240 & PU-239/240 \\
\hline 42 & PDMS & & SO & & & E105046 & 07-Sep-88 & & $10098-97-2$ & SR-90 \\
\hline 43 & PDMS & & SO & & & E105045 & 07-Sep-88 & & $10098-97-2$ & SR-90 \\
\hline 44 & PDMS & & SO & & & E105044 & 07-Sep-88 & & 10098-97-2 & SR-90 \\
\hline 45 & PDMS & & SO & & & E105043 & 07-Sep-88 & & $10098-97-2$ & SR-90 \\
\hline 46 & PDMS & & SO & & & E105050 & 15-Sep-88 & & $10098-97-2$ & SR-90 \\
\hline 47 & PDMS & & SO & & & E105049 & 15-Sep-88 & & 10098-97-2 & SR-90 \\
\hline 48 & PDMS & & SO & & & E105048 & 15-Sep-88 & & $10098-97-2$ & SR-90 \\
\hline 49 & PDMS & & SO & & & E105047 & $15-$ Sep-88 & & $10098-97-2$ & SR-90 \\
\hline 50 & PDMS & & SO & & & E111107 & 08-Dec-89 & & $10098-97-2$ & SR-90 \\
\hline 51 & PDMS & & SO & & & E111106 & 08-Dec-89 & & $10098-97-2$ & SR-90 \\
\hline 52 & PDMS & & SO & & & E111104 & 08-Dec-89 & & $10098-97-2$ & SR-90 \\
\hline 53 & PDMS & & SO & & & E111103 & 08-Dec-89 & & $10098-97-2$ & SR-90 \\
\hline 54 & PDMS & & SO & & & E111164 & 14-Dec-89 & & $10098-97-2$ & SR-90 \\
\hline 55 & PDMS & & SO & & & E111163 & 14-Dec-89 & & $10098-97-2$ & SR-90 \\
\hline 56 & PDMS & & SO & & & E111162 & 14-Dec-89 & & $10098-97-2$ & SR-90 \\
\hline 57 & PDMS & & SO & & & E111161 & 14-Dec-89 & & $10098-97-2$ & SR-90 \\
\hline 58 & PDMS & & SO & & & E111253 & 21-Dec-89 & & $10098-97-2$ & SR-90 \\
\hline 59 & PDMS & & SO & & & E111252 & 21-Dec-89 & & $10098-97-2$ & SR-90 \\
\hline 60 & PDMS & & SO & & & E111251 & 21-Dec-89 & & $10098-97-2$ & SR-90 \\
\hline 61 & PDMS & & SO & & & E111156 & 21-Dec-89 & & $10098-97-2$ & SR-90 \\
\hline 62 & PDMS & & SO & & & E111253 & 21-Dec-89 & & $13966-29-5$ & U-234 \\
\hline 63 & PDMS & & SO & & & E111252 & 21-Dec-89 & & $13966-29-5$ & U-234 \\
\hline 64 & PDMS & & SO & & & E111251 & 21-Dec-89 & & 13966-29-5 & U-234 \\
\hline 65 & PDMS & & SO & & & E105046 & 07-Sep-88 & & $15117-96-1$ & U-235 \\
\hline 66 & PDMS & & SO & & & E105045 & 07-Sep-88 & & 15117-96-1 & U-235 \\
\hline 67 & PDMS & & SO & & & E105044 & 07-Sep-88 & & $15117-96-1$ & U-235 \\
\hline 68 & PDMS & & SO & & & E105043 & 07-Sep-88 & & $15117-96-1$ & U-235 \\
\hline 69 & PDMS & & SO & & & E105050 & 15-Sep-88 & & 15117-96-1 & U-235 \\
\hline 70 & PDMS & & SO & & & E105049 & 15-Sep-88 & & $15117-96-1$ & U-235 \\
\hline 71 & PDMS & & SO & & & E105048 & 15-Sep-88 & & $15117-96-1$ & U-235 \\
\hline 72 & PDMS & & SO & & & E105047 & 15-Sep-88 & & $15117-96-1$ & U-235 \\
\hline 73 & PDMS & & SO & & & E111107 & 08-Dec-89 & & $15117-96-1$ & U-235 \\
\hline 74 & PDMS & & SO & & & E111106 & 08-Dec-89 & & $15117-96-1$ & U-235 \\
\hline 75 & PDMS & & SO & & & E111104 & 08-Dec-89 & & $15117-96-1$ & $\mathrm{U}-235$ \\
\hline 76 & PDMS & & SO & & & E111103 & 08-Dec-89 & & $15117-96-1$ & U-235 \\
\hline 77 & PDMS & & SO & & & E111164 & 14-Dec-89 & & $15117-96-1$ & U-235 \\
\hline
\end{tabular}




\begin{tabular}{|c|c|c|c|c|c|c|c|c|c|c|}
\hline & A & $B$ & $\mathrm{C}$ & $\mathrm{D}$ & $\mathrm{E}$ & $\mathrm{F}$ & G & $\mathrm{H}$ & $\mathrm{I}$ & $\mathrm{J}$ \\
\hline 1 & SAMPLE_NUM_ASSIGNED_TO & SAMPLER & MEDIA & BIOTA_MEDIA & TAG_ID & SAMP_NUM & SAMP_DATE_TIME & SAMP_DATE_TIME_ON & CON_ID & CON_SHORT_NAME \\
\hline 78 & PDMS & & So & & & E111163 & 14-Dec-89 & & $15117-96-1$ & U-235 \\
\hline 79 & PDMS & & SO & & & E111162 & 14-Dec-89 & & 15117-96-1 & U-235 \\
\hline 80 & PDMS & & SO & & & E111161 & 14-Dec-89 & & $15117-96-1$ & U-235 \\
\hline 81 & PDMS & & SO & & & E111253 & 21-Dec-89 & & $15117-96-1$ & $\mathrm{U}-235$ \\
\hline 82 & PDMS & & SO & & & E111253 & 21-Dec-89 & & $15117-96-1$ & $\mathrm{U}-235$ \\
\hline 83 & PDMS & & SO & & & E111252 & 21-Dec-89 & & 15117-96-1 & U-235 \\
\hline 84 & PDMS & & SO & & & E111252 & 21-Dec-89 & & $15117-96-1$ & $\mathrm{U}-235$ \\
\hline 85 & PDMS & & SO & & & E111251 & 21-Dec-89 & & 15117-96-1 & U-235 \\
\hline \begin{tabular}{|l}
86 \\
\end{tabular} & PDMS & & SO & & & E111251 & 21-Dec-89 & & $15117-96-1$ & U-235 \\
\hline \begin{tabular}{|l}
87 \\
\end{tabular} & PDMS & & SO & & & E111156 & 21-Dec-89 & & $15117-96-1$ & U-235 \\
\hline \begin{tabular}{|l}
88 \\
\end{tabular} & PDMS & & SO & & & E105046 & 07-Sep-88 & & U-238 & $\mathrm{U}-238$ \\
\hline \begin{tabular}{|l|}
89 \\
\end{tabular} & PDMS & & SO & & & E105045 & 07-Sep-88 & & U-238 & U-238 \\
\hline \begin{tabular}{|l}
90 \\
\end{tabular} & PDMS & & SO & & & E105044 & 07-Sep-88 & & U-238 & U-238 \\
\hline \begin{tabular}{|l|}
91 \\
\end{tabular} & PDMS & & SO & & & E105043 & 07-Sep-88 & & U-238 & U-238 \\
\hline \begin{tabular}{|l|}
92 \\
\end{tabular} & PDMS & & SO & & & E105050 & 15-Sep-88 & & U-238 & U-238 \\
\hline 93 & PDMS & & SO & & & E105049 & 15-Sep-88 & & U-238 & U-238 \\
\hline \begin{tabular}{|l}
94 \\
\end{tabular} & PDMS & & SO & & & E105048 & 15-Sep-88 & & U-238 & U-238 \\
\hline \begin{tabular}{|l}
95 \\
95 \\
\end{tabular} & PDMS & & SO & & & E105047 & 15-Sep-88 & & U-238 & U-238 \\
\hline 96 & PDMS & & SO & & & E111107 & 08-Dec-89 & & U-238 & U-238 \\
\hline \begin{tabular}{|l}
97 \\
\end{tabular} & PDMS & & SO & & & E111106 & 08-Dec-89 & & U-238 & U-238 \\
\hline \begin{tabular}{|l}
98 \\
\end{tabular} & PDMS & & SO & & & E111104 & 08-Dec-89 & & U-238 & U-238 \\
\hline \begin{tabular}{|l}
99 \\
\end{tabular} & PDMS & & SO & & & E111103 & 08-Dec-89 & & U-238 & U-238 \\
\hline 100 & PDMS & & SO & & & E111164 & 14-Dec-89 & & U-238 & U-238 \\
\hline 101 & PDMS & & SO & & & E111163 & 14-Dec-89 & & U-238 & U-238 \\
\hline $\begin{array}{l}102 \\
10\end{array}$ & PDMS & & SO & & & E111162 & 14-Dec-89 & & U-238 & U-238 \\
\hline 103 & PDMS & & SO & & & E111161 & 14-Dec-89 & & U-238 & U-238 \\
\hline 104 & PDMS & & SO & & & E111253 & 21-Dec-89 & & U-238 & U-238 \\
\hline 105 & PDMS & & SO & & & E111253 & 21-Dec-89 & & U-238 & U-238 \\
\hline $\begin{array}{l}106 \\
\end{array}$ & PDMS & & SO & & & E111252 & 21-Dec-89 & & U-238 & U-238 \\
\hline 107 & PDMS & & SO & & & E111252 & 21-Dec-89 & & U-238 & U-238 \\
\hline 108 & PDMS & & SO & & & E111251 & 21-Dec-89 & & U-238 & U-238 \\
\hline \begin{tabular}{|l}
109 \\
\end{tabular} & PDMS & & SO & & & E111251 & 21-Dec-89 & & U-238 & $\mathrm{U}-238$ \\
\hline 110 & PDMS & & SO & & & E111156 & 21-Dec-89 & & U-238 & $\mathrm{U}-238$ \\
\hline
\end{tabular}




\begin{tabular}{|c|c|c|c|c|c|c|c|c|c|}
\hline & $\mathrm{K}$ & $\mathrm{L}$ & $\mathrm{M}$ & $\mathrm{N}$ & $\mathrm{O}$ & $P$ & $\mathrm{Q}$ & $\mathrm{R}$ & $\mathrm{S}$ \\
\hline 1 & METHOD_NAME & LAB_CODE & VALUE_RPTD & ANAL_UNITS_RPTD & COUNTING_ERROR & TOTAL_ANAL_ERROR & MIN_DETECTABLE_ACTIVITY & PCNT_MOISTURE & LAB_QUALIFIER \\
\hline 2 & GAMMA_GS & USTEST & 0.792 & $\mathrm{pCi} / \mathrm{g}$ & 0.048 & 0.0927 & & & \\
\hline 3 & GAMMA_GS & USTEST & 0.628 & $\mathrm{pCi} / \mathrm{g}$ & 0.0357 & 0.0722 & & & \\
\hline 4 & GAMMA_GS & USTEST & 0.641 & $\mathrm{pCi} / \mathrm{g}$ & 0.0479 & 0.0801 & & & \\
\hline 5 & GAMMA_GS & USTEST & 0.683 & $\mathrm{pCi} / \mathrm{g}$ & 0.0392 & 0.0788 & & & \\
\hline 6 & GAMMA_GS & USTEST & 0.267 & $\mathrm{pCi} / \mathrm{g}$ & 0.0294 & 0.0397 & & & \\
\hline 7 & GAMMA_GS & USTEST & 0.239 & $\mathrm{pCi} / \mathrm{g}$ & 0.0229 & 0.0331 & & & \\
\hline 8 & GAMMA_GS & USTEST & 0.255 & $\mathrm{pCi} / \mathrm{g}$ & 0.0299 & 0.0393 & & & \\
\hline 9 & GAMMA_GS & USTEST & 0.284 & $\mathrm{pCi} / \mathrm{g}$ & 0.0314 & 0.0423 & & & \\
\hline 11 & GAMMA_GS & USTEST & 0.791 & $\mathrm{pCi} / \mathrm{g}$ & 0.0414 & 0.0893 & & & \\
\hline 12 & GAMMA_GS & USTEST & 0.554 & $\mathrm{pCi} / \mathrm{g}$ & 0.0365 & 0.0664 & & & \\
\hline 13 & GAMMA_GS & USTEST & 0.621 & $\mathrm{pCi} / \mathrm{g}$ & 0.0448 & 0.0766 & & & \\
\hline 14 & GAMMA_GS & USTEST & 0.298 & $\mathrm{pCi} / \mathrm{g}$ & 0.0318 & 0.0436 & & & \\
\hline 15 & GAMMA_GS & USTEST & 0.189 & $\mathrm{pCi} / \mathrm{g}$ & 0.0248 & 0.0312 & & & \\
\hline 16 & GAMMA_GS & USTEST & 0.287 & $\mathrm{pCi} / \mathrm{g}$ & 0.031 & 0.0422 & & & \\
\hline 17 & GAMMA_GS & USTEST & 0.285 & $\mathrm{pCi} / \mathrm{g}$ & 0.0319 & 0.0428 & & & \\
\hline 18 & GAMMA_GS & USTEST & 0.21 & $\mathrm{pCi} / \mathrm{g}$ & 0.03 & 0.0366 & & & \\
\hline 19 & GAMMA_GS & USTEST & 0.231 & $\mathrm{pCi} / \mathrm{g}$ & 0.0279 & 0.0362 & & & \\
\hline 21 & GAMMA_GS & USTEST & & & & & & & \\
\hline 22 & PUISO_PLATE_AEA & USTEST & 0.0108 & $\mathrm{pCi} / \mathrm{g}$ & 0.00116 & 0.00161 & & & \\
\hline 23 & PUISO_PLATE_AEA & USTEST & 0.0105 & $\mathrm{pCi} / \mathrm{g}$ & 0.00135 & 0.00178 & & & \\
\hline 24 & PUISO_PLATE_AEA & USTEST & 0.00922 & $\mathrm{pCi} / \mathrm{g}$ & 0.00109 & 0.00145 & & & \\
\hline 25 & PUISO_PLATE_AEA & USTEST & 0.00984 & $\mathrm{pCi} / \mathrm{g}$ & 0.00105 & 0.00145 & & & \\
\hline 26 & PUISO_PLATE_AEA & USTEST & 0.00277 & $\mathrm{pCi} / \mathrm{g}$ & 0.000658 & 0.000723 & & & \\
\hline 27 & PUISO_PLATE_AEA & USTEST & 0.00221 & $\mathrm{pCi} / \mathrm{g}$ & 0.000473 & 0.000523 & & & \\
\hline 28 & PUISO_PLATE_AEA & USTEST & 0.00268 & $\mathrm{pCi} / \mathrm{g}$ & 0.000501 & 0.000567 & & & \\
\hline 29 & PUISO_PLATE_AEA & USTEST & 0.00149 & $\mathrm{pCi} / \mathrm{g}$ & 0.000333 & 0.000364 & & & \\
\hline 30 & PUISO_PLATE_AEA & USTEST & 0.0112 & $\mathrm{pCi} / \mathrm{g}$ & 0.00118 & 0.00165 & & & \\
\hline 31 & PUISO_PLATE_AEA & USTEST & 0.0133 & $\mathrm{pCi} / \mathrm{g}$ & 0.00162 & 0.00222 & & & \\
\hline 32 & PUISO_PLATE_AEA & USTEST & 0.00771 & $\mathrm{pCi} / \mathrm{g}$ & 0.00113 & 0.00141 & & & \\
\hline 33 & PUISO_PLATE_AEA & USTEST & 0.0216 & $\mathrm{pCi} / \mathrm{g}$ & 0.00193 & 0.00307 & & & \\
\hline 34 & PUISO_PLATE_AEA & USTEST & 0.00228 & $\mathrm{pCi} / \mathrm{g}$ & 0.000801 & 0.00085 & & & \\
\hline 35 & PUISO_PLATE_AEA & USTEST & 0.00142 & $\mathrm{pCi} / \mathrm{g}$ & 0.000476 & 0.000502 & & & \\
\hline 36 & PUISO_PLATE_AEA & USTEST & 0.00272 & $\mathrm{pCi} / \mathrm{g}$ & 0.000621 & 0.000686 & & & \\
\hline 37 & PUISO_PLATE_AEA & USTEST & 0.00242 & $\mathrm{pCi} / \mathrm{g}$ & 0.000549 & 0.000605 & & & \\
\hline 38 & PUISO_PLATE_AEA & USTEST & 0.00346 & $\mathrm{pCi} / \mathrm{g}$ & 0.000571 & 0.000667 & & & \\
\hline
\end{tabular}


O

P

Q

$S$

\begin{tabular}{|c|c|c|c|c|c|c|c|c|c|}
\hline & $\mathrm{K}$ & $\mathrm{L}$ & M & $\mathrm{N}$ & $\mathrm{O}$ & $\mathrm{P}$ & Q & $\mathrm{R}$ & S \\
\hline 1 & METHOD_NAME & LAB_CODE & VALUE_RPTD & ANAL_UNITS_RPTD & COUNTING_ERROR & TOTAL_ANAL_ERROR & MIN_DETECTABLE_ACTIVITY & PCNT_MOISTURE & LAB_QUALIFIER \\
\hline 40 & PUISO_PLATE_AEA & USTEST & 0.000825 & $\mathrm{pCi} / \mathrm{g}$ & 0.000279 & 0.000292 & & & \\
\hline 41 & PUISO_PLATE_AEA & USTEST & & & & & & & \\
\hline 42 & SRTOT_SEP_PRECIP_GPC & USTEST & 0.0364 & $\mathrm{pCi} / \mathrm{g}$ & 0.00455 & 0.0116 & & & \\
\hline 43 & ]SRTOT_SEP_PRECIP_GPC & USTEST & 0.0473 & $\mathrm{pCi} / \mathrm{g}$ & 0.00601 & 0.0111 & & & \\
\hline 44 & SRTOT_SEP_PRECIP_GPC & USTEST & 0.0411 & $\mathrm{pCi} / \mathrm{g}$ & 0.00631 & 0.0104 & & & \\
\hline 45 & SRTOT_SEP_PRECIP_GPC & USTEST & 0.0577 & $\mathrm{pCi} / \mathrm{g}$ & 0.00634 & 0.0128 & & & \\
\hline 46 & SRTOT_SEP_PRECIP_GPC & USTEST & 0.00652 & $\mathrm{pCi} / \mathrm{g}$ & 0.00309 & 0.00358 & & & \\
\hline 47 & SRTOT_SEP_PRECIP_GPC & USTEST & 0.00484 & $\mathrm{pCi} / \mathrm{g}$ & 0.00372 & 0.00406 & & & \\
\hline 48 & SRTOT_SEP_PRECIP_GPC & USTEST & 0.0715 & $\mathrm{pCi} / \mathrm{g}$ & 0.00622 & 0.015 & & & \\
\hline 49 & SRTOT_SEP_PRECIP_GPC & USTEST & 0.0198 & $\mathrm{pCi} / \mathrm{g}$ & 0.00434 & 0.006 & & & \\
\hline 50 & SRTOT_SEP_PRECIP_GPC & USTEST & 0.0241 & $\mathrm{pCi} / \mathrm{g}$ & 0.00414 & 0.00762 & & & \\
\hline 51 & SRTOT_SEP_PRECIP_GPC & USTEST & 0.0271 & $\mathrm{pCi} / \mathrm{g}$ & 0.00707 & 0.00887 & & & \\
\hline 52 & SRTOT_SEP_PRECIP_GPC & USTEST & 0.0316 & $\mathrm{pCi} / \mathrm{g}$ & 0.00485 & 0.0077 & & & \\
\hline 53 & SRTOT_SEP_PRECIP_GPC & USTEST & 0.0639 & $\mathrm{pCi} / \mathrm{g}$ & 0.00756 & 0.014 & & & \\
\hline 54 & SRTOT_SEP_PRECIP_GPC & USTEST & 0.0138 & $\mathrm{pCi} / \mathrm{g}$ & 0.00325 & 0.00429 & & & \\
\hline 55 & SRTOT_SEP_PRECIP_GPC & USTEST & 0.014 & $\mathrm{pCi} / \mathrm{g}$ & 0.00478 & 0.00588 & & & \\
\hline 56 & ISRTOT_SEP_PRECIP_GPC & USTEST & 0.0162 & $\mathrm{pCi} / \mathrm{g}$ & 0.00497 & 0.0065 & & & \\
\hline 57 & SRTOT_SEP_PRECIP_GPC & USTEST & 0.011 & $\mathrm{pCi} / \mathrm{g}$ & 0.00445 & 0.0054 & & & \\
\hline 58 & SRTOT_SEP_PRECIP_GPC & USTEST & 0.0205 & $\mathrm{pCi} / \mathrm{g}$ & 0.00579 & 0.00704 & & & \\
\hline 59 & SRTOT_SEP_PRECIP_GPC & USTEST & 0.0052 & $\mathrm{pCi} / \mathrm{g}$ & 0.00289 & 0.00321 & & & \\
\hline 60 & SRTOT_SEP_PRECIP_GPC & USTEST & 0.00632 & $\mathrm{pCi} / \mathrm{g}$ & 0.00432 & 0.00459 & & & \\
\hline 61 & SRTOT_SEP_PRECIP_GPC & USTEST & & & & & & & \\
\hline 62 & UISO_AEA & USTEST & 0.955 & $\mathrm{pCi} / \mathrm{g}$ & 0.102 & 0.139 & & & \\
\hline 63 & UISO_AEA & USTEST & 0.684 & $\mathrm{pCi} / \mathrm{g}$ & 0.0877 & 0.111 & & & \\
\hline 64 & UISO_AEA & USTEST & 0.633 & $\mathrm{pCi} / \mathrm{g}$ & 0.0917 & 0.113 & & & \\
\hline 65 & GAMMA_GS & USTEST & -0.087 & $\mathrm{pCi} / \mathrm{g}$ & 0.163 & 0.163 & & & $\mathrm{U}$ \\
\hline 66 & GAMMA_GS & USTEST & 0.217 & $\mathrm{pCi} / \mathrm{g}$ & 0.144 & 0.145 & & & \\
\hline 67 & GAMMA_GS & USTEST & 0.0693 & $\mathrm{pCi} / \mathrm{g}$ & 0.0928 & 0.0931 & & & $\mathrm{U}$ \\
\hline 68 & GAMMA_GS & USTEST & -0.0182 & $\mathrm{pCi} / \mathrm{g}$ & 0.137 & 0.137 & & & $U$ \\
\hline 69 & GAMMA_GS & USTEST & 0.00669 & $\mathrm{pCi} / \mathrm{g}$ & 0.117 & 0.117 & & & $\mathrm{U}$ \\
\hline 70 & GAMMA_GS & USTEST & 0.0554 & $\mathrm{pCi} / \mathrm{g}$ & 0.101 & 0.101 & & & $U$ \\
\hline 71 & GAMMA_GS & USTEST & 0.0971 & $\mathrm{pCi} / \mathrm{g}$ & 0.147 & 0.147 & & & $\mathrm{U}$ \\
\hline 72 & GAMMA_GS & USTEST & 0.0927 & $\mathrm{pCi} / \mathrm{g}$ & 0.103 & 0.103 & & & $\mathrm{U}$ \\
\hline 73 & GAMMA_GS & USTEST & 0.0169 & $\mathrm{pCi} / \mathrm{g}$ & 0.165 & 0.165 & & & U \\
\hline 74 & GAMMA_GS & USTEST & 0.2 & $\mathrm{pCi} / \mathrm{g}$ & 0.148 & 0.149 & & & \\
\hline 75 & GAMMA_GS & USTEST & 0.0874 & $\mathrm{pCi} / \mathrm{g}$ & 0.1 & 0.101 & & & $U$ \\
\hline 76 & GAMMA_GS & USTEST & -0.043 & $\mathrm{pCi} / \mathrm{g}$ & 0.102 & 0.102 & & & $U$ \\
\hline 77 & GAMMA_GS & USTEST & 0.0324 & $\mathrm{pCi} / \mathrm{g}$ & 0.135 & 0.135 & & & $\mathrm{U}$ \\
\hline
\end{tabular}




\begin{tabular}{|c|c|c|c|c|c|c|c|c|c|}
\hline & $\mathrm{K}$ & $\mathrm{L}$ & $\mathrm{M}$ & $\mathrm{N}$ & $\mathrm{O}$ & $\mathrm{P}$ & $\mathrm{Q}$ & $\mathrm{R}$ & $\mathrm{S}$ \\
\hline 1 & METHOD_NAME & LAB_CODE & VALUE_RPTD & ANAL_UNITS_RPTD & |COUNTING_ERROR & TOTAL_ANAL_ERROR & MIN_DETECTABLE_ACTIVITY & PCNT_MOISTURE & LAB_QUALIFIER \\
\hline 78 & GAMMA_GS & USTEST & 0.0999 & $\mathrm{pCi} / \mathrm{g}$ & 0.0872 & 0.0878 & & & \\
\hline 79 & GAMMA_GS & USTEST & 0.0277 & $\mathrm{pCi} / \mathrm{g}$ & 0.134 & 0.134 & & & U \\
\hline 80 & GAMMA_GS & USTEST & -0.00858 & $\mathrm{pCi} / \mathrm{g}$ & 0.113 & 0.113 & & & $\mathrm{U}$ \\
\hline 81 & GAMMA_GS & USTEST & 0.0626 & $\mathrm{pCi} / \mathrm{g}$ & 0.103 & 0.103 & & & $\mathrm{U}$ \\
\hline 82 & UISO_AĒA & USTEST & 0.0194 & $\mathrm{pCi} / \mathrm{g}$ & 0.0158 & 0.0165 & & & \\
\hline 83 & GAMMA_GS & USTEST & 0.0856 & $\mathrm{pCi} / \mathrm{g}$ & 0.14 & 0.14 & & & $\mathrm{U}$ \\
\hline 84 & UISO_AEA & USTEST & 0.0406 & $\mathrm{pCi} / \mathrm{g}$ & 0.0242 & 0.025 & & & \\
\hline 85 & GAMMA_GS & USTEST & 0.0904 & $\mathrm{pCi} / \mathrm{g}$ & 0.089 & 0.0894 & & & \\
\hline 87 & GAMMA_GS & USTEST & & & & & & & \\
\hline 88 & GAMMA_GS & USTEST & 0.74 & $\mathrm{pCi} / \mathrm{g}$ & 0.416 & 0.422 & & & \\
\hline 89 & GAMMA_GS & USTEST & 0.886 & $\mathrm{pCi} / \mathrm{g}$ & 0.487 & 0.5 & & & \\
\hline 90 & GAMMA_GS & USTEST & 0.628 & $\mathrm{pCi} / \mathrm{g}$ & 0.31 & 0.335 & & & \\
\hline 91 & GAMMA_GS & USTEST & 0.857 & $\mathrm{pCi} / \mathrm{g}$ & 0.47 & 0.482 & & & \\
\hline 92 & GAMMA_GS & USTEST & 0.789 & $\mathrm{pCi} / \mathrm{g}$ & 0.382 & 0.39 & & & \\
\hline 93 & GAMMA_GS & USTEST & 0.734 & $\mathrm{pCi} / \mathrm{g}$ & 0.322 & 0.33 & & & \\
\hline 94 & GAMMA_GS & USTEST & 0.739 & $\mathrm{pCi} / \mathrm{g}$ & 0.481 & 0.487 & & & \\
\hline 95 & GAMMA_GS & USTEST & 0.67 & $\mathrm{pCi} / \mathrm{g}$ & 0.358 & 0.369 & & & \\
\hline 97 & GAMMA_GS & USTEST & 0.785 & $\mathrm{pCi} / \mathrm{g}$ & 0.408 & 0.408 & & & \\
\hline 98 & GAMMA_GS & USTEST & 0.612 & $\mathrm{pCi} / \mathrm{g}$ & 0.313 & 0.313 & & & \\
\hline 99 & GAMMA_GS & USTEST & 0.749 & $\mathrm{pCi} / \mathrm{g}$ & 0.292 & 0.292 & & & \\
\hline 100 & GAMMA_GS & USTEST & 0.907 & $\mathrm{pCi} / \mathrm{g}$ & 0.505 & 0.513 & & & \\
\hline 101 & GAMMA_GS & USTEST & 0.599 & $\mathrm{pCi} / \mathrm{g}$ & 0.315 & 0.324 & & & \\
\hline 102 & GAMMA_GS & USTEST & 0.721 & $\mathrm{pCi} / \mathrm{g}$ & 0.493 & 0.498 & & & \\
\hline 103 & GAMMA_GS & USTEST & 0.815 & $\mathrm{pCi} / \mathrm{g}$ & 0.332 & 0.332 & & & \\
\hline 104 & GAMMA_GS & USTEST & 0.696 & $\mathrm{pCi} / \mathrm{g}$ & 0.28 & 0.28 & & & \\
\hline 105 & UISO_AEA & USTEST & 0.992 & $\mathrm{pCi} / \mathrm{g}$ & 0.103 & 0.142 & & & \\
\hline 106 & GAMMA_GS & USTEST & 0.583 & $\mathrm{pCi} / \mathrm{g}$ & 0.526 & 0.529 & & & \\
\hline 107 & UISO_AEA & USTEST & 0.573 & $\mathrm{pCi} / \mathrm{g}$ & 0.0807 & 0.0988 & & & \\
\hline 108 & GAMMA_GS & USTEST & 0.639 & $\mathrm{pCi} / \mathrm{g}$ & 0.215 & 0.215 & & & \\
\hline 109 & UISO_AEA & USTEST & 0.697 & $\mathrm{pCi} / \mathrm{g}$ & 0.0953 & 0.118 & & & \\
\hline 110 & GAMMA_GS & USTEST & & & & & & & \\
\hline
\end{tabular}




\begin{tabular}{|c|c|c|c|c|c|c|c|c|c|}
\hline & $\mathrm{T}$ & $\bar{U}$ & $\mathrm{~V}$ & $\mathrm{~W}$ & $\mathrm{X}$ & $\bar{Y}$ & $Z$ & $\overline{A A}$ & $A B$ \\
\hline 1 & REVIEW_QUALIFIER & SAMP_FROM & SAMP_ITEM & SAMP_MTHD & COLL_MTHD & SAMP_SITE_ID & SAMP_SITE_NAME & WELL_NAME & SAMPLE_LOCATION \\
\hline 2 & & SUB_SURFACE & SEDIMENT & & & 104 & MCNARY-WASH. SHORE & & \\
\hline 3 & & SUB_SURFACE & SEDIMENT & & & 427 & MCNARY-2/3 OR. SHORE & & \\
\hline 4 & & SUB_SURFACE & SEDIMENT & & & 103 & 3 MCNARY-1/3 OR. SHORE & & \\
\hline 5 & & SUB_SURFACE & SEDIMENT & & & 102 & MCNARY-OREGON SHORE & & \\
\hline 6 & & SUB_SURFACE & SEDIMENT & & & 256 & PRD-YAKIMA CNTY SHOR & & \\
\hline 7 & & SUB_SURFACE & SEDIMENT & & & 255 & PRD-2/3 GRANT SHORE & & \\
\hline 8 & & SUB SURFACE & SEDIMENT & & & 552 & PRD-1/3 GRANT SHORE & & \\
\hline 9 & & SUB_SURFACE & SEDIMENT & & & 254 & PRD-GRANT COUNTY & & \\
\hline 11 & & SUB SURFACE & SEDIMENT & & & 427 & MCNARY-2/3 OR. SHORE & & \\
\hline 12 & & SUB_SURFACE & SEDIMENT & & & 103 & 3 MCNARY-1/3 OR. SHORE & & \\
\hline 13 & & SUB_SURFACE & SEDIMENT & & & 102 & MCNARY-OREGON SHORE & & \\
\hline 14 & & SUB_SURFACE & SEDIMENT & & & 256 & PRD-YAKIMA CNTY SHOR & & \\
\hline 15 & & SUB_SURFACE & SEDIMENT & & & 255 & PRD-2/3 GRANT SHORE & & \\
\hline 16 & & SUB_SURFACE & SEDIMENT & & & 552 & PRD-1/3 GRANT SHORE & & \\
\hline 17 & & SUB_SURFACE & SEDIMENT & & & 254 & PRD-GRANT COUNTY & & \\
\hline 18 & & SUB_SURFACE & SEDIMENT & & & 563 & 3 HANFORD SLOUGH & & \\
\hline 19 & & SUB_SURFACE & SEDIMENT & & & 195 & 100 F SLOUGH & & \\
\hline 21 & & SUB_SURFACE & SEDIMENT & & & 311 & RICHLAND-RIVER & & \\
\hline 22 & & SUB_SURFACE & SEDIMENT & & & 104 & MCNARY-WASH. SHORE & & \\
\hline 23 & & SUB_SURFACE & SEDIMENT & & & 427 & MCNARY-2/3 OR. SHORE & & \\
\hline 24 & & SUB_SURFACE & SEDIMENT & & & 103 & 3 MCNARY-1/3 OR. SHORE & & \\
\hline 25 & & SUB SURFACE & SEDIMENT & & & 102 & MCNARY-OREGON SHORE & & \\
\hline 26 & & SUB_SURFACE & SEDIMENT & & & 256 & PRD-YAKIMA CNTY SHOR & & \\
\hline 27 & & SUB_SURFACE & SEDIMENT & & & 255 & PRD-2/3 GRANT SHORE & & \\
\hline 28 & & SUB_SURFACE & SEDIMENT & & & 552 & PRD-1/3 GRANT SHORE & & \\
\hline 29 & & SUB_SURFACE & SEDIMENT & & & 254 & PRD-GRANT COUNTY & & \\
\hline 30 & & SUB_SURFACE & SEDIMENT & & & 104 & MCNARY-WASH. SHORE & & \\
\hline 31 & & SUB_SURFACE & SEDIMENT & & & 427 & MCNARY-2/3 OR. SHORE & & \\
\hline 32 & & SUB_SURFACE & SEDIMENT & & & 103 & 3 MCNARY-1/3 OR. SHORE & & \\
\hline 33 & & SUB_SURFACE & SEDIMENT & & & 102 & MCNARY-OREGON SHORE & & \\
\hline 34 & & SUB SURFACE & SEDIMENT & & & 256 & PRD-YAKIMA CNTY SHOR & & \\
\hline 35 & & SUB_SURFACE & SEDIMENT & & & 255 & PRD-2/3 GRANT SHORE & & \\
\hline 36 & & SUB_SURFACE & SEDIMENT & & & 552 & PRD-1/3 GRANT SHORE & & \\
\hline 37 & & SUB_SURFACE & SEDIMENT & & & 254 & PRD-GRANT COUNTY & & \\
\hline 38 & & SUB_SURFACE & SEDIMENT & & & 563 & 3 HANFORD SLOUGH & & \\
\hline
\end{tabular}




\begin{tabular}{|c|c|c|c|c|c|c|c|c|c|}
\hline & $T$ & $\mathrm{U}$ & $\mathrm{V}$ & $\mathrm{W}$ & $\mathrm{X}$ & $\mathrm{Y}$ & $Z$ & $\mathrm{AA}$ & $A B$ \\
\hline 1 & REVIEW_QUALIFIER & SAMP_FROM & SAMP_ITEM & SAMP_MTHD & COLL_MTHD & SAMP_SITE_IL & SAMP_SITE_NAME & WELL_NAME & SAMPLE_LOCATION \\
\hline 40 & & SUB_SURFACE & SEDIMENT & & & & 1 WHITE BLUFFS SLOUGH & & \\
\hline 41 & & SUB_SURFACE & SEDIMENT & & & 31 & 1 RICHLAND-RIVER & & \\
\hline 42 & & SUB_SURFACE & SEDIMENT & & & 10 & 4 MCNARY-WASH. SHORE & & \\
\hline 43 & & SUB_SURFACE & SEDIMENT & & & 42 & 7 MCNARY-2/3 OR. SHORE & & \\
\hline 44 & & SUB_SURFACE & SEDIMENT & & & 10 & 3 MCNARY-1/3 OR. SHORE & & \\
\hline 45 & & SUB_SURFACE & SEDIMENT & & & 10 & 2 MCNARY-OREGON SHORE & & \\
\hline 46 & & SUB_SURFACE & SEDIMENT & & & 25 & 6 PRD-YAKIMA CNTY SHOR & & \\
\hline 47 & & SUB_SURFACE & SEDIMENT & & & 25 & 5 PRD-2/3 GRANT SHORE & & \\
\hline 49 & & SUB_SURFACE & SEDIMENT & & & 25 & 4 PRD-GRANT COUNTY & & \\
\hline 50 & & SUB_SURFACE & SEDIMENT & & & 10 & 4 MCNARY-WASH. SHORE & & \\
\hline 51 & & SUB_SURFACE & SEDIMENT & & & 42 & 7 MCNARY-2/3 OR. SHORE & & \\
\hline 52 & & SUB_SURFACE & SEDIMENT & & & 10 & 3 MCNARY-1/3 OR. SHORE & & \\
\hline 53 & & SUB_SURFACE & SEDIMENT & & & 10 & 2 MCNARY-OREGON SHORE & & \\
\hline 54 & & SUB_SURFACE & SEDIMENT & & & 25 & 6 PRD-YAKIMA CNTY SHOR & & \\
\hline 55 & & SUB_SURFACE & SEDIMENT & & & 25 & 5 PRD-2/3 GRANT SHORE & & \\
\hline 56 & & SUB_SURFACE & SEDIMENT & & & 55 & 2 PRD-1/3 GRANT SHORE & & \\
\hline 57 & & SUB_SURFACE & SEDIMENT & & & 25 & 4 PRD-GRANT COUNTY & & \\
\hline 59 & & SUB_SURFACE & SEDIMENT & & & 19 & 5100 F SLOUGH & & \\
\hline 60 & & SUB_SURFACE & SEDIMENT & & & 56 & 1 WHITE BLUFFS SLOUGH & & \\
\hline 61 & & SUB_SURFACE & SEDIMENT & & & 31 & 1 RICHLAND-RIVER & & \\
\hline 62 & & SUB_SURFACE & SEDIMENT & & & 56 & 3 HANFORD SLOUGH & & \\
\hline 63 & & SUB_SURFACE & SEDIMENT & & & 19 & 5100 F SLOUGH & & \\
\hline 64 & & SUB_SURFACE & SEDIMENT & & & 56 & 1 WHITE BLUFFS SLOUGH & & \\
\hline 65 & & SUB_SURFACE & SEDIMENT & & & 10 & 4 MCNARY-WASH. SHORE & & \\
\hline 66 & & SUB_SURFACE & SEDIMENT & & & 42 & 7 MCNARY-2/3 OR. SHORE & & \\
\hline 67 & & SUB_SURFACE & SEDIMENT & & & 10 & 3 MCNARY-1/3 OR. SHORE & & \\
\hline 68 & & SUB_SURFACE & SEDIMENT & & & 10 & 2 MCNARY-OREGON SHORE & & \\
\hline 69 & & SUB_SURFACE & SEDIMENT & & & 25 & 6 PRD-YAKIMA CNTY SHOR & & \\
\hline 70 & & SUB_SURFACE & SEDIMENT & & & 25 & 5 PRD-2/3 GRANT SHORE & & \\
\hline 71 & & SUB_SURFACE & SEDIMENT & & & 55 & 2 PRD-1/3 GRANT SHORE & & \\
\hline 72 & & SUB_SURFACE & SEDIMENT & & & 25 & 4 PRD-GRANT COUNTY & & \\
\hline 73 & & SUB_SURFACE & SEDIMENT & & & 10 & 4 MCNARY-WASH. SHORE & & \\
\hline 74 & & SUB_SURFACE & SEDIMENT & & & 42 & 7 MCNARY-2/3 OR. SHORE & & \\
\hline 75 & & SUB_SURFACE & SEDIMENT & & & 10 & 3 MCNARY-1/3 OR. SHORE & & \\
\hline 76 & & SUB_SURFACE & SEDIMENT & & & 10 & 2 MCNARY-OREGON SHORE & & \\
\hline
\end{tabular}




\begin{tabular}{|c|c|c|c|c|c|c|c|c|c|}
\hline & $\mathrm{T}$ & $\mathrm{U}$ & $\mathrm{V}$ & $\mathrm{W}$ & $\mathrm{X}$ & $\mathrm{Y}$ & $Z$ & AA & $A B$ \\
\hline 1 & REVIEW_QUALIFIER & SAMP_FROM & SAMP_ITEM & SAMP_MTHD & COLL_MTHD & SAMP_SITE_ID & SAMP_SITE_NAME & WELL_NAME & SAMPLE_LOCATION \\
\hline 78 & & SUB_SURFACE & SEDIMENT & & & 255 & 5 PRD-2/3 GRANT SHORE & & \\
\hline 79 & & SUB_SURFACE & SEDIMENT & & & 552 & 2 PRD-1/3 GRANT SHORE & & \\
\hline 80 & & SUB_SURFACE & SEDIMENT & & & 254 & 4 PRD-GRANT COUNTY & & \\
\hline 81 & & SUB_SURFACE & SEDIMENT & & & 563 & 3 HANFORD SLOUGH & & \\
\hline 82 & & SUB_SURFACE & SEDIMENT & & & 563 & 3 HANFORD SLOUGH & & \\
\hline 83 & & SUB_SURFACE & SEDIMENT & & & 195 & 5100 F SLOUGH & & \\
\hline \begin{tabular}{|l|}
84 \\
\end{tabular} & & SUB_SURFACE & SEDIMENT & & & 195 & 5100 F SLOUGH & & \\
\hline 85 & & SUB_SURFACE & SEDIMENT & & & 561 & 1 WHITE BLUFFS SLOUGH & & \\
\hline 87 & & SUB_SURFACE & SEDIMENT & & & 311 & 1 RICHLAND-RIVER & & \\
\hline 88 & & SUB_SURFACE & SEDIMENT & & & 104 & 4 MCNARY-WASH. SHORE & & \\
\hline 89 & & SUB_SURFACE & SEDIMENT & & & 427 & 7 MCNARY-2/3 OR. SHORE & & \\
\hline 90 & & SUB_SURFACE & SEDIMENT & & & 103 & 3 MCNARY-1/3 OR. SHORE & & \\
\hline 91 & & SUB_SURFACE & SEDIMENT & & & 102 & 2 MCNARY-OREGON SHORE & & \\
\hline 92 & & SUB_SURFACE & SEDIMENT & & & 256 & 6 PRD-YAKIMA CNTY SHOR & & \\
\hline 93 & & SUB_SURFACE & SEDIMENT & & & 255 & 5 PRD-2/3 GRANT SHORE & & \\
\hline 94 & & SUB_SURFACE & SEDIMENT & & & 552 & 2 PRD-1/3 GRANT SHORE & & \\
\hline 95 & & SUB_SURFACE & SEDIMENT & & & 254 & 4 PRD-GRANT COUNTY & & \\
\hline 97 & & SUB_SURFACE & SEDIMENT & & & 427 & 7 MCNARY-2/3 OR. SHORE & & \\
\hline 98 & & SUB_SURFACE & SEDIMENT & & & 103 & 3 MCNARY-1/3 OR. SHORE & & \\
\hline 99 & & SUB_SURFACE & SEDIMENT & & & 102 & 2 MCNARY-OREGON SHORE & & \\
\hline 100 & & SUB_SURFACE & SEDIMENT & & & 256 & 6 PRD-YAKIMA CNTY SHOR & & \\
\hline 101 & & SUB_SURFACE & SEDIMENT & & & 255 & 5 PRD-2/3 GRANT SHORE & & \\
\hline 102 & & SUB_SURFACE & SEDIMENT & & & 552 & 2 PRD-1/3 GRANT SHORE & & \\
\hline 103 & & SUB_SURFACE & SEDIMENT & & & 254 & 4 PRD-GRANT COUNTY & & \\
\hline 104 & & SUB_SURFACE & SEDIMENT & & & 563 & 3 HANFORD SLOUGH & & \\
\hline 105 & & SUB_SURFACE & SEDIMENT & & & 563 & 3 HANFORD SLOUGH & & \\
\hline 106 & & SUB_SURFACE & SEDIMENT & & & 195 & 5100 F SLOUGH & & \\
\hline 107 & & SUB_SURFACE & SEDIMENT & & & 195 & 5100 F SLOUGH & & \\
\hline 108 & & SUB_SURFACE & SEDIMENT & & & 561 & 1 WHITE BLUFFS SLOUGH & & \\
\hline 109 & & SUB_SURFACE & SEDIMENT & & & 561 & 1 WHITE BLUFFS SLOUGH & & \\
\hline 110 & & SUB_SURFACE & SEDIMENT & & & 311 & 1 RICHLAND-RIVER & & \\
\hline
\end{tabular}

Page 9 
2 WASHINGTON SHORE

3 2/3 FROM OREGON SHORE

4 1/3 FROM OREGON SHORE

5 OREGON SHORE

6 YAKIMA COUNTY SHORE

7 2/3 FROM GRANT COUNTY SHORE

1/3 FROM GRANT COUNTY SHORE. REFER TO UST RECHECK REPORT 12/27/88 CONCERNING SR-90.

9 GRANT COUNTY SHORE. REFER TO UST RECHECK REPORT 12/27/88 CONCERNING SR-90.

10 WASHINGTON SHORE

11 2/3 FROM OREGON SHORE

12 1/3 FROM OREGON SHORE

13 OREGON SHORE

14 YAKIMA COUNTY SHORE

15 2/3 FROM GRANT COUNTY SHORE

16 1/3 FROM GRANT COUNTY SHORE

\begin{tabular}{|r|l}
\hline 17 & GRANT COUNTY SHORE \\
\hline 18 &
\end{tabular}

18

19

20

21 NO SAMPLE.

22 WASHINGTON SHORE

23 2/3 FROM OREGON SHORE

\begin{tabular}{l|l}
24 & $1 / 3$ FROM OREGON SHORE \\
\hline 25 & OREGON SHORE
\end{tabular}

25 OREGON SHORE

26 YAKIMA COUNTY SHORE

27 2/3 FROM GRANT COUNTY SHORE

28 1/3 FROM GRANT COUNTY SHORE. REFER TO UST RECHECK REPORT 12/27/88 CONCERNING SR-90.

29 GRANT COUNTY SHORE. REFER TO UST RECHECK REPORT 12/27/88 CONCERNING SR-90.

30 WASHINGTON SHORE

31 2/3 FROM OREGON SHORE

32 1/3 FROM OREGON SHORE

33 OREGON SHORE

34 YAKIMA COUNTY SHORE

35 2/3 FROM GRANT COUNTY SHORE

36 1/3 FROM GRANT COUNTY SHORE

37 GRANT COUNTY SHORE
RESULT COMMENT

See sample record for comment.

See sample record for comment. See sample record for comment.

See sample record for comment.

See sample record for comment. See sample record for comment. See sample record for comment. See sample record for comment. See sample record for comment. See sample record for comment. See sample record for comment. See sample record for comment. See sample record for comment. See sample record for comment. See sample record for comment. See sample record for comment.

See sample record for comment. See sample record for comment. See sample record for comment. See sample record for comment. See sample record for comment. See sample record for comment. See sample record for comment. See sample record for comment. See sample record for comment. See sample record for comment. See sample record for comment. See sample record for comment. See sample record for comment. See sample record for comment. See sample record for comment. See sample record for comment. See sample record for comment.

\begin{tabular}{|l|l|}
\hline & \\
\hline & \\
\hline & \\
\hline & \\
\hline & \\
\hline & \\
\hline & \\
\hline & \\
\hline
\end{tabular}


\begin{tabular}{r|l}
1 & \\
\hline 40 & \\
\hline 41 & NO SAMPLE. \\
\hline 42 & WASHINGTON
\end{tabular}

42 WASHINGTON SHORE

43 2/3 FROM OREGON SHORE

\begin{tabular}{|l|l}
44 & $1 / 3$ \\
\hline 45 & FROM OREGON SHORE
\end{tabular}

45 OREGON SHORE

46 YAKIMA COUNTY SHORE

47 2/3 FROM GRANT COUNTY SHORE

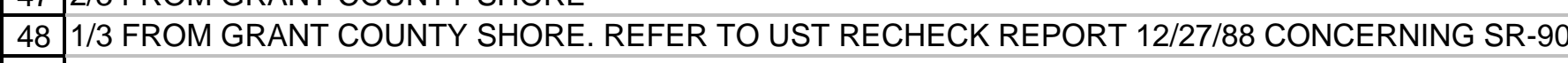

49 GRANT COUNTY SHORE. REFER TO UST RECHECK REPORT 12/27/88 CONCERNING SR-90.

50 WASHINGTON SHORE

\begin{tabular}{|l|l}
51 & $2 / 3$ FROM OREGON SHORE \\
\hline 52 & $1 / 3$ FOM OREGON SHORE
\end{tabular}

\begin{tabular}{|l|l}
\hline 52 & $1 / 3$ FROM OREGON SHORE \\
\hline 53 & OREGON SHORE
\end{tabular}

53 OREGON SHORE

54 YAKIMA COUNTY SHORE

\begin{tabular}{l|l}
55 & 2/3 FROM GRANT COUNTY SHORE \\
\hline 56 & $1 / 3$ FROM GRANT COUNTY SHORE
\end{tabular}

$\begin{array}{ll}56 & 1 / 3 \text { FROM GRANT COUNTY SHORE }\end{array}$

57 GRANT COUNTY SHORE

65 WASHINGTON SHORE

66 2/3 FROM OREGON SHORE

67 1/3 FROM OREGON SHORE

68 OREGON SHORE

69 YAKIMA COUNTY SHORE

\begin{tabular}{l|l}
70 & $2 / 3$ FROM GRANT COUNTY SHORE \\
\hline 71 & S/3 FROM GRANT COUNTY SHORE.
\end{tabular}

71 1/3 FROM GRANT COUNTY SHORE. REFER TO UST RECHECK REPORT 12/27/88 CONCERNING SR-90. 72 GRANT COUNTY SHORE. REFER TO UST RECHECK REPORT 12/27/88 CONCERNING SR-90.

73 WASHINGTON SHORE

74 2/3 FROM OREGON SHORE

75 1/3 FROM OREGON SHORE

76 OREGON SHORE

77 YAKIMA COUNTY SHORE
See sample record for comment. See sample record for comment. See sample record for comment. See sample record for comment. See sample record for comment. See sample record for comment. See sample record for comment. See sample record for comment. See sample record for comment. See sample record for comment. See sample record for comment. See sample record for comment. See sample record for comment. See sample record for comment. See sample record for comment. See sample record for comment. See sample record for comment.

See sample record for comment.

See sample record for comment. See sample record for comment. See sample record for comment. See sample record for comment. See sample record for comment. See sample record for comment. See sample record for comment. See sample record for comment. See sample record for comment. See sample record for comment. See sample record for comment. See sample record for comment. See sample record for comment.

\begin{tabular}{|l|l|}
\hline & \\
\hline & \\
\hline & \\
\hline & \\
\hline & \\
\hline & \\
\hline & \\
\hline & \\
\hline & \\
\hline
\end{tabular}


AC

AD

AE

AF

\begin{tabular}{|c|c|c|c|c|}
\hline & & & & \\
\hline 1 & SAMP_COMMENT & RESULT_COMMENT & COLL_SAMP_SIZE & COLL_SAMP_SIZE_UNITS \\
\hline 78 & 2/3 FROM GRANT COUNTY SHORE & See sample record for comment. & & \\
\hline 79 & 1/3 FROM GRANT COUNTY SHORE & See sample record for comment. & & \\
\hline 80 & GRANT COUNTY SHORE & See sample record for comment. & & \\
\hline 81 & & & & \\
\hline 82 & & & & \\
\hline 83 & & & & \\
\hline 84 & & & & \\
\hline 85 & & & & \\
\hline 86 & & & & \\
\hline 87 & NO SAMPLE. & See sample record for comment. & & \\
\hline 88 & WASHINGTON SHORE & See sample record for comment. & & \\
\hline 89 & 2/3 FROM OREGON SHORE & See sample record for comment. & & \\
\hline 90 & 1/3 FROM OREGON SHORE & See sample record for comment. & & \\
\hline 91 & OREGON SHORE & See sample record for comment. & & \\
\hline 92 & YAKIMA COUNTY SHORE & See sample record for comment. & & \\
\hline 93 & 2/3 FROM GRANT COUNTY SHORE & See sample record for comment. & & \\
\hline 94 & 1/3 FROM GRANT COUNTY SHORE. REFER TO UST RECHECK REPORT 12/27/88 CONCERNING SR-90. & See sample record for comment. & & \\
\hline 95 & GRANT COUNTY SHORE. REFER TO UST RECHECK REPORT 12/27/88 CONCERNING SR-90. & See sample record for comment. & & \\
\hline 96 & WASHINGTON SHORE & See sample record for comment. & & \\
\hline 97 & 2/3 FROM OREGON SHORE & See sample record for comment. & & \\
\hline 98 & 1/3 FROM OREGON SHORE & See sample record for comment. & & \\
\hline 99 & OREGON SHORE & See sample record for comment. & & \\
\hline 100 & YAKIMA COUNTY SHORE & See sample record for comment. & & \\
\hline 101 & 2/3 FROM GRANT COUNTY SHORE & See sample record for comment. & & \\
\hline 102 & 1/3 FROM GRANT COUNTY SHORE & See sample record for comment. & & \\
\hline 103 & GRANT COUNTY SHORE & See sample record for comment. & & \\
\hline 104 & & & & \\
\hline 105 & & & & \\
\hline 106 & & & & \\
\hline 107 & & & & \\
\hline 108 & & & & \\
\hline 109 & & & & \\
\hline 110 & NO SAMPLE. & See sample record for comment. & & \\
\hline
\end{tabular}




\begin{tabular}{|c|c|c|c|}
\hline & AG & $\mathrm{AH}$ & $\mathrm{Al}$ \\
\hline 1 & DIST_CLASS & OWNER_ID & COLLECTION_PURPOSE \\
\hline 2 & OFFSITE & SESPMNT & $\mathrm{R}$ \\
\hline 3 & OFFSITE & SESPMNT & $\mathrm{R}$ \\
\hline 4 & OFFSITE & SESPMNT & $\mathrm{R}$ \\
\hline 5 & OFFSITE & SESPMNT & $\mathrm{R}$ \\
\hline 6 & OFFSITE & SESPMNT & $\mathrm{R}$ \\
\hline 7 & OFFSITE & SESPMNT & $\mathrm{R}$ \\
\hline 8 & OFFSITE & SESPMNT & $\mathrm{R}$ \\
\hline 9 & OFFSITE & SESPMNT & $\mathrm{R}$ \\
\hline 10 & OFFSITE & SESPMNT & $\mathrm{R}$ \\
\hline 11 & OFFSITE & SESPMNT & $\mathrm{R}$ \\
\hline 12 & OFFSITE & SESPMNT & $\mathrm{R}$ \\
\hline 13 & OFFSITE & SESPMNT & $\mathrm{R}$ \\
\hline 14 & OFFSITE & SESPMNT & $\mathrm{R}$ \\
\hline 15 & OFFSITE & SESPMNT & $\mathrm{R}$ \\
\hline 16 & OFFSITE & SESPMNT & $\mathrm{R}$ \\
\hline 17 & OFFSITE & SESPMNT & $\mathrm{R}$ \\
\hline 18 & RIVER_SHORELINE & SESPMNT & $\mathrm{R}$ \\
\hline 19 & ONSITE & SESPMNT & $\mathrm{R}$ \\
\hline 20 & RIVER_SHORELINE & SESPMNT & $\mathrm{R}$ \\
\hline 21 & RIVER_SHORELINE & SESPMNT & $\mathrm{R}$ \\
\hline 22 & OFFSITE & SESPMNT & $\mathrm{R}$ \\
\hline 23 & OFFSITE & SESPMNT & $\mathrm{R}$ \\
\hline 24 & OFFSITE & SESPMNT & $\mathrm{R}$ \\
\hline 25 & OFFSITE & SESPMNT & $\mathrm{R}$ \\
\hline 26 & OFFSITE & SESPMNT & $\mathrm{R}$ \\
\hline 27 & OFFSITE & SESPMNT & $\mathrm{R}$ \\
\hline 28 & OFFSITE & SESPMNT & $\mathrm{R}$ \\
\hline 29 & OFFSITE & SESPMNT & $\mathrm{R}$ \\
\hline 30 & OFFSITE & SESPMNT & $\mathrm{R}$ \\
\hline 31 & OFFSITE & SESPMNT & $\mathrm{R}$ \\
\hline 32 & OFFSITE & SESPMNT & $\mathrm{R}$ \\
\hline 33 & OFFSITE & SESPMNT & $\mathrm{R}$ \\
\hline 34 & OFFSITE & SESPMNT & $\mathrm{R}$ \\
\hline 35 & OFFSITE & SESPMNT & $\mathrm{R}$ \\
\hline 36 & OFFSITE & SESPMNT & $\mathrm{R}$ \\
\hline 37 & OFFSITE & SESPMNT & $\mathrm{R}$ \\
\hline 38 & RIVER_SHORELINE & SESPMNT & $\mathrm{R}$ \\
\hline 39 & ONSITE & SESPMNT & $\mathrm{R}$ \\
\hline
\end{tabular}




\begin{tabular}{|c|c|c|c|}
\hline & AG & $\mathrm{AH}$ & $\mathrm{Al}$ \\
\hline 1 & DIST_CLASS & OWNER_ID & COLLECTION_PURPOSE \\
\hline 40 & RIVER_SHORELINE & SESPMNT & $\mathrm{R}$ \\
\hline 41 & RIVER_SHORELINE & SESPMNT & $\mathrm{R}$ \\
\hline 42 & OFFSITE & SESPMNT & $\mathrm{R}$ \\
\hline 43 & OFFSITE & SESPMNT & $\mathrm{R}$ \\
\hline 44 & OFFSITE & SESPMNT & $\mathrm{R}$ \\
\hline 45 & OFFSITE & SESPMNT & $\mathrm{R}$ \\
\hline 46 & OFFSITE & SESPMNT & $\mathrm{R}$ \\
\hline 47 & OFFSITE & SESPMNT & $\mathrm{R}$ \\
\hline 48 & OFFSITE & SESPMNT & $\mathrm{R}$ \\
\hline 49 & OFFSITE & SESPMNT & $\mathrm{R}$ \\
\hline 50 & OFFSITE & SESPMNT & $\mathrm{R}$ \\
\hline 51 & OFFSITE & SESPMNT & $\mathrm{R}$ \\
\hline 52 & OFFSITE & SESPMNT & $\mathrm{R}$ \\
\hline 53 & OFFSITE & SESPMNT & $\mathrm{R}$ \\
\hline 54 & OFFSITE & SESPMNT & $\mathrm{R}$ \\
\hline 55 & OFFSITE & SESPMNT & $\mathrm{R}$ \\
\hline 56 & OFFSITE & SESPMNT & $\mathrm{R}$ \\
\hline 57 & OFFSITE & SESPMNT & $\mathrm{R}$ \\
\hline 58 & RIVER_SHORELINE & SESPMNT & $\mathrm{R}$ \\
\hline 59 & ONSITE & SESPMNT & $\mathrm{R}$ \\
\hline 60 & RIVER_SHORELINE & SESPMNT & $\mathrm{R}$ \\
\hline 61 & RIVER_SHORELINE & SESPMNT & $\mathrm{R}$ \\
\hline 62 & RIVER_SHORELINE & SESPMNT & $\mathrm{R}$ \\
\hline 63 & ONSITE & SESPMNT & $\mathrm{R}$ \\
\hline 64 & RIVER_SHORELINE & SESPMNT & $\mathrm{R}$ \\
\hline 65 & OFFSITE & SESPMNT & $\mathrm{R}$ \\
\hline 66 & OFFSITE & SESPMNT & $\mathrm{R}$ \\
\hline 67 & OFFSITE & SESPMNT & $\mathrm{R}$ \\
\hline 68 & OFFSITE & SESPMNT & $\mathrm{R}$ \\
\hline 69 & OFFSITE & SESPMNT & $\mathrm{R}$ \\
\hline 70 & OFFSITE & SESPMNT & $\mathrm{R}$ \\
\hline 71 & OFFSITE & SESPMNT & $\mathrm{R}$ \\
\hline 72 & OFFSITE & SESPMNT & $\mathrm{R}$ \\
\hline 73 & OFFSITE & SESPMNT & $\mathrm{R}$ \\
\hline 74 & OFFSITE & SESPMNT & $\mathrm{R}$ \\
\hline 75 & OFFSITE & SESPMNT & $\mathrm{R}$ \\
\hline 76 & OFFSITE & SESPMNT & $\mathrm{R}$ \\
\hline 77 & OFFSITE & SESPMNT & $\mathrm{R}$ \\
\hline
\end{tabular}




\begin{tabular}{|c|c|c|c|}
\hline & $A G$ & $\overline{\mathrm{AH}}$ & $\mathrm{Al}$ \\
\hline 1 & DIST_CLASS & OWNER_ID & COLLECTION_PURPOSE \\
\hline 78 & OFFSITE & SESPMNT & $\mathrm{R}$ \\
\hline 79 & OFFSITE & SESPMNT & $\mathrm{R}$ \\
\hline 80 & OFFSITE & SESPMNT & $\mathrm{R}$ \\
\hline 81 & RIVER_SHORELINE & SESPMNT & $\mathrm{R}$ \\
\hline 82 & RIVER_SHORELINE & SESPMNT & $\mathrm{R}$ \\
\hline 83 & ONSITE & SESPMNT & $\mathrm{R}$ \\
\hline 84 & ONSITE & SESPMNT & $\mathrm{R}$ \\
\hline 85 & RIVER_SHORELINE & SESPMNT & $\mathrm{R}$ \\
\hline 86 & RIVER_SHORELINE & SESPMNT & $\mathrm{R}$ \\
\hline 87 & RIVER_SHORELINE & SESPMNT & $\mathrm{R}$ \\
\hline 88 & OFFSITE & SESPMNT & $\mathrm{R}$ \\
\hline 89 & OFFSITE & SESPMNT & $\mathrm{R}$ \\
\hline 90 & OFFSITE & SESPMNT & $\mathrm{R}$ \\
\hline 91 & OFFSITE & SESPMNT & $\mathrm{R}$ \\
\hline 92 & OFFSITE & SESPMNT & $\mathrm{R}$ \\
\hline 93 & OFFSITE & SESPMNT & $\mathrm{R}$ \\
\hline 94 & OFFSITE & SESPMNT & $\mathrm{R}$ \\
\hline 95 & OFFSITE & SESPMNT & $\mathrm{R}$ \\
\hline 96 & OFFSITE & SESPMNT & $\mathrm{R}$ \\
\hline 97 & OFFSITE & SESPMNT & $\mathrm{R}$ \\
\hline 98 & OFFSITE & SESPMNT & $\mathrm{R}$ \\
\hline 99 & OFFSITE & SESPMNT & $\mathrm{R}$ \\
\hline 100 & OFFSITE & SESPMNT & $\mathrm{R}$ \\
\hline 101 & OFFSITE & SESPMNT & $\mathrm{R}$ \\
\hline 102 & OFFSITE & SESPMNT & $\mathrm{R}$ \\
\hline 103 & OFFSITE & SESPMNT & $\mathrm{R}$ \\
\hline 104 & RIVER_SHORELINE & SESPMNT & $\mathrm{R}$ \\
\hline 105 & RIVER_SHORELINE & SESPMNT & $\mathrm{R}$ \\
\hline 106 & ONSITE & SESPMNT & $\mathrm{R}$ \\
\hline 107 & ONSITE & SESPMNT & $\mathrm{R}$ \\
\hline 108 & RIVER_SHORELINE & SESPMNT & $\mathrm{R}$ \\
\hline 109 & RIVER_SHORELINE & SESPMNT & $\mathrm{R}$ \\
\hline 110 & RIVER_SHORELINE & SESPMNT & $\mathrm{R}$ \\
\hline
\end{tabular}


90 - 00 ALL SEEPS 


\begin{tabular}{|c|c|c|c|c|c|c|c|c|c|c|}
\hline & A & $\mathrm{B}$ & $\mathrm{C}$ & $\mathrm{D}$ & $\mathrm{E}$ & $\mathrm{F}$ & $\mathrm{G}$ & $\mathrm{H}$ & $\mathrm{I}$ & $\mathrm{J}$ \\
\hline 1 & SAMPLE_NUM_ASSIGNED_TO & SAMPLER & MEDIA & BIOTA_MEDIA & TAG_ID & SAMP_NUM & SAMP_DATE_TIME & SAMP_DATE_TIME_ON & CON_ID & CON_SHORT_NAME \\
\hline 2 & & R MAHOOD & SW & & & B06KR8 & 17-Sep-91 & & $7440-47-3$ & CR \\
\hline 3 & & R MAHOOD & SW & & & B06KR9 & 17-Sep-91 & & $7440-47-3$ & CR \\
\hline 4 & & R MAHOOD & SW & & & B06KR8 & 17-Sep-91 & & $10045-97-3$ & CS-137 \\
\hline 5 & & R MAHOOD & SW & & & B06KR8 & 17-Sep-91 & & $10098-97-2$ & SR-90 \\
\hline 6 & & R MAHOOD & SW & & & B06KR8 & 17-Sep-91 & & $10028-17-8$ & TRITIUM \\
\hline 7 & & R MAHOOD & SW & & & B06KR5 & 18-Sep-91 & & $7440-47-3$ & $\mathrm{CR}$ \\
\hline 8 & & R MAHOOD & SW & & & B06KR6 & 18-Sep-91 & & $7440-47-3$ & $\mathrm{CR}$ \\
\hline 9 & & R MAHOOD & SW & & & B06KR5 & 18-Sep-91 & & $10045-97-3$ & CS-137 \\
\hline 10 & & R MAHOOD & SW & & & B06KR5 & 18-Sep-91 & & $10098-97-2$ & SR-90 \\
\hline 11 & & R MAHOOD & SW & & & B06KR5 & 18-Sep-91 & & $10028-17-8$ & TRITIUM \\
\hline 12 & & R MAHOOD & SW & & & B06KR3 & 18-Sep-91 & & $7440-47-3$ & $\mathrm{CR}$ \\
\hline 13 & & R MAHOOD & SW & & & B06KR4 & 18-Sep-91 & & $7440-47-3$ & $\mathrm{CR}$ \\
\hline 14 & & R MAHOOD & SW & & & B06KR3 & 18-Sep-91 & & $10045-97-3$ & CS-137 \\
\hline 15 & & R MAHOOD & SW & & & B06KR3 & 18-Sep-91 & & $10098-97-2$ & SR-90 \\
\hline 16 & & R MAHOOD & SW & & & B06KR3 & 18-Sep-91 & & $10028-17-8$ & TRITIUM \\
\hline 17 & & R MAHOOD & SW & & & B06KS1 & 19-Sep-91 & & $7440-47-3$ & CR \\
\hline 18 & & R MAHOOD & SW & & & B06KS2 & 19-Sep-91 & & $7440-47-3$ & CR \\
\hline 19 & & R MAHOOD & SW & & & B06KS1 & 19-Sep-91 & & $10045-97-3$ & CS-137 \\
\hline 20 & & R MAHOOD & SW & & & B06KS1 & 19-Sep-91 & & $10098-97-2$ & SR-90 \\
\hline 21 & & R MAHOOD & SW & & & B06KS1 & 19-Sep-91 & & $10028-17-8$ & TRITIUM \\
\hline 22 & & R MAHOOD & SW & & & B06KX4 & 19-Sep-91 & & $7440-47-3$ & CR \\
\hline 23 & & R MAHOOD & SW & & & B06KX3 & 19-Sep-91 & & $7440-47-3$ & CR \\
\hline 24 & & R MAHOOD & SW & & & B06KX3 & 19-Sep-91 & & $10045-97-3$ & CS-137 \\
\hline 25 & & R MAHOOD & SW & & & B06KX3 & 19-Sep-91 & & $10098-97-2$ & SR-90 \\
\hline 26 & & R MAHOOD & SW & & & B06KX3 & 19-Sep-91 & & $14133-76-7$ & TC-99 \\
\hline 27 & & R MAHOOD & SW & & & B06KX3 & 19-Sep-91 & & $10028-17-8$ & TRITIUM \\
\hline 28 & & R MAHOOD & SW & & & B06KY1 & 20-Sep-91 & & $7440-47-3$ & CR \\
\hline 29 & & R MAHOOD & SW & & & B06KY2 & 20-Sep-91 & & $7440-47-3$ & CR \\
\hline 30 & & R MAHOOD & SW & & & B06L43 & 20-Sep-91 & & $7440-47-3$ & CR \\
\hline 31 & & R MAHOOD & SW & & & B06L44 & 20-Sep-91 & & $7440-47-3$ & CR \\
\hline 32 & & R MAHOOD & SW & & & B06KY1 & 20-Sep-91 & & $10045-97-3$ & CS-137 \\
\hline 33 & & R MAHOOD & SW & & & B06L43 & 20-Sep-91 & & $10045-97-3$ & CS-137 \\
\hline 34 & & R MAHOOD & SW & & & B06KY1 & 20-Sep-91 & & $10098-97-2$ & SR-90 \\
\hline 35 & & R MAHOOD & SW & & & B06L43 & 20-Sep-91 & & $10098-97-2$ & SR-90 \\
\hline 36 & & R MAHOOD & SW & & & B06KY1 & 20-Sep-91 & & $14133-76-7$ & TC-99 \\
\hline 37 & & R MAHOOD & SW & & & B06L43 & 20-Sep-91 & & $14133-76-7$ & TC-99 \\
\hline 38 & & R MAHOOD & SW & & & B06KY1 & 20-Sep-91 & & $10028-17-8$ & TRITIUM \\
\hline 39 & & R MAHOOD & SW & & & B06L43 & 20-Sep-91 & & $10028-17-8$ & TRITIUM \\
\hline
\end{tabular}




\begin{tabular}{|c|c|c|c|c|c|c|c|c|c|c|}
\hline & A & $\mathrm{B}$ & $\mathrm{C}$ & $\mathrm{D}$ & $\mathrm{E}$ & $\mathrm{F}$ & $\mathrm{G}$ & $\mathrm{H}$ & $\mathrm{I}$ & $\mathrm{J}$ \\
\hline 1 & SAMPLE_NUM_ASSIGNED_TO & SAMPLER & MEDIA & BIOTA_MEDIA & TAG_ID & SAMP_NUM & SAMP_DATE_TIME & SAMP_DATE_TIME_ON & CON_ID & CON_SHORT_NAME \\
\hline 40 & & R MAHOOD & SW & & & B06L48 & 20-Sep-91 & & $7440-47-3$ & CR \\
\hline 41 & & R MAHOOD & SW & & & B06L49 & 20-Sep-91 & & $7440-47-3$ & CR \\
\hline 42 & & R MAHOOD & SW & & & B06L48 & 20-Sep-91 & & $10045-97-3$ & CS-137 \\
\hline 43 & & R MAHOOD & SW & & & B06L48 & 20-Sep-91 & & $10098-97-2$ & SR-90 \\
\hline 44 & & R MAHOOD & SW & & & B06L48 & 20-Sep-91 & & 14133-76-7 & TC-99 \\
\hline 45 & & R MAHOOD & SW & & & B06L48 & 20-Sep-91 & & $10028-17-8$ & TRITIUM \\
\hline 46 & & R MAHOOD & SW & & & B06L46 & 20-Sep-91 & & $7440-47-3$ & $\mathrm{CR}$ \\
\hline 47 & & R MAHOOD & SW & & & B06L47 & 20-Sep-91 & & $7440-47-3$ & $\mathrm{CR}$ \\
\hline 48 & & R MAHOOD & SW & & & B06L46 & 20-Sep-91 & & $10045-97-3$ & CS-137 \\
\hline 49 & & R MAHOOD & SW & & & B06L46 & 20-Sep-91 & & $10098-97-2$ & SR-90 \\
\hline 50 & & R MAHOOD & SW & & & B06L46 & 20-Sep-91 & & $14133-76-7$ & TC-99 \\
\hline 51 & & R MAHOOD & SW & & & B06L46 & 20-Sep-91 & & $10028-17-8$ & TRITIUM \\
\hline 52 & & R MAHOOD & SW & & & B06KS4 & 25-Sep-91 & & $7440-47-3$ & $\mathrm{CR}$ \\
\hline 53 & & R MAHOOD & SW & & & B06KS5 & 25-Sep-91 & & $7440-47-3$ & $\mathrm{CR}$ \\
\hline 54 & & R MAHOOD & SW & & & B06KS4 & 25-Sep-91 & & $10045-97-3$ & CS-137 \\
\hline 55 & & R MAHOOD & SW & & & B06KS4 & 25-Sep-91 & & $10098-97-2$ & SR-90 \\
\hline 56 & & R MAHOOD & SW & & & B06KS4 & 25-Sep-91 & & $10028-17-8$ & TRITIUM \\
\hline 57 & & R MAHOOD & SW & & & B06KS6 & 25-Sep-91 & & $7440-47-3$ & CR \\
\hline 58 & & R MAHOOD & SW & & & B06KS7 & 25-Sep-91 & & $7440-47-3$ & $\mathrm{CR}$ \\
\hline 59 & & R MAHOOD & SW & & & B06KS6 & 25-Sep-91 & & $10045-97-3$ & CS-137 \\
\hline 60 & & R MAHOOD & SW & & & B06KS6 & 25-Sep-91 & & $10098-97-2$ & SR-90 \\
\hline 61 & & R MAHOOD & SW & & & B06KS6 & 25-Sep-91 & & $10028-17-8$ & TRITIUM \\
\hline 62 & & R MAHOOD & SW & & & B06L51 & 25-Sep-91 & & $7440-47-3$ & CR \\
\hline 63 & & R MAHOOD & SW & & & B06L52 & 25-Sep-91 & & $7440-47-3$ & $\mathrm{CR}$ \\
\hline 64 & & R MAHOOD & SW & & & B06L51 & 25-Sep-91 & & $10045-97-3$ & CS-137 \\
\hline 65 & & R MAHOOD & SW & & & B06L51 & 25-Sep-91 & & $10098-97-2$ & SR-90 \\
\hline 66 & & R MAHOOD & SW & & & B06L51 & 25-Sep-91 & & 14133-76-7 & TC-99 \\
\hline 67 & & R MAHOOD & SW & & & B06L51 & 25-Sep-91 & & $10028-17-8$ & TRITIUM \\
\hline 68 & & R MAHOOD & SW & & & B06L53 & 25-Sep-91 & & $7440-47-3$ & $\mathrm{CR}$ \\
\hline 69 & & R MAHOOD & SW & & & B06L54 & 25-Sep-91 & & $7440-47-3$ & CR \\
\hline 70 & & R MAHOOD & SW & & & B06L53 & 25-Sep-91 & & $10045-97-3$ & CS-137 \\
\hline 71 & & R MAHOOD & SW & & & B06L53 & 25-Sep-91 & & $10098-97-2$ & SR-90 \\
\hline 72 & & R MAHOOD & SW & & & B06L53 & 25-Sep-91 & & $14133-76-7$ & TC-99 \\
\hline 73 & & R MAHOOD & SW & & & B06L53 & 25-Sep-91 & & $10028-17-8$ & TRITIUM \\
\hline 74 & & R MAHOOD & SW & & & B06KX6 & 26-Sep-91 & & $7440-47-3$ & $\mathrm{CR}$ \\
\hline 75 & & R MAHOOD & SW & & & B06KX7 & 26-Sep-91 & & $7440-47-3$ & $\mathrm{CR}$ \\
\hline 76 & & R MAHOOD & SW & & & B06KX6 & 26-Sep-91 & & $10045-97-3$ & CS-137 \\
\hline 77 & & R MAHOOD & SW & & & B06KX6 & 26-Sep-91 & & $10098-97-2$ & SR-90 \\
\hline
\end{tabular}




\begin{tabular}{|c|c|c|c|c|c|c|c|c|c|c|}
\hline & $\mathrm{A}$ & $B$ & $C$ & $\mathrm{D}$ & $E$ & $\mathrm{~F}$ & $\mathrm{G}$ & $\mathrm{H}$ & $\mathrm{I}$ & $\mathrm{J}$ \\
\hline 1 & SAMPLE_NUM_ASSIGNED_TO & SAMPLER & MEDIA & BIOTA_MEDIA & TAG_ID & SAMP_NUM & SAMP_DATE_TIME & SAMP_DATE_TIME_ON & CON_ID & CON_SHORT_NAME \\
\hline 78 & & R MAHOOD & SW & & & B06KX6 & 26-Sep-91 & & 14133-76-7 & TC-99 \\
\hline 79 & & R MAHOOD & SW & & & B06KX6 & 26-Sep-91 & & $10028-17-8$ & TRITIUM \\
\hline 80 & & R MAHOOD & SW & & & B06KX8 & 26-Sep-91 & & $7440-47-3$ & CR \\
\hline 81 & & R MAHOOD & SW & & & B06KX9 & 26-Sep-91 & & $7440-47-3$ & CR \\
\hline 82 & & R MAHOOD & SW & & & B06KX8 & 26-Sep-91 & & $10045-97-3$ & CS-137 \\
\hline 83 & & R MAHOOD & SW & & & B06KX8 & 26-Sep-91 & & $10098-97-2$ & SR-90 \\
\hline 84 & & R MAHOOD & SW & & & B06KX8 & 26-Sep-91 & & $14133-76-7$ & TC-99 \\
\hline 85 & & R MAHOOD & SW & & & B06KX8 & 26-Sep-91 & & $10028-17-8$ & TRITIUM \\
\hline 86 & & R MAHOOD & SW & & & B06L56 & 26-Sep-91 & & $7440-47-3$ & CR \\
\hline 87 & & R MAHOOD & SW & & & B06L57 & 26-Sep-91 & & $7440-47-3$ & CR \\
\hline 88 & & R MAHOOD & SW & & & B06L56 & 26-Sep-91 & & $10045-97-3$ & CS-137 \\
\hline 89 & & R MAHOOD & SW & & & B06L56 & 26-Sep-91 & & $10098-97-2$ & SR-90 \\
\hline 90 & & R MAHOOD & SW & & & B06L56 & 26-Sep-91 & & $14133-76-7$ & TC-99 \\
\hline 91 & & R MAHOOD & SW & & & B06L56 & 26-Sep-91 & & $10028-17-8$ & TRITIUM \\
\hline 92 & & R MAHOOD & SW & & & B06L58 & 26-Sep-91 & & $7440-47-3$ & CR \\
\hline 93 & & R MAHOOD & SW & & & B06L59 & 26-Sep-91 & & $7440-47-3$ & CR \\
\hline 94 & & R MAHOOD & SW & & & B06L58 & 26-Sep-91 & & $10045-97-3$ & CS-137 \\
\hline 95 & & R MAHOOD & SW & & & B06L58 & 26-Sep-91 & & $10098-97-2$ & SR-90 \\
\hline 96 & & R MAHOOD & SW & & & B06L58 & 26-Sep-91 & & $14133-76-7$ & TC-99 \\
\hline 97 & & R MAHOOD & SW & & & B06L58 & 26-Sep-91 & & $10028-17-8$ & TRITIUM \\
\hline 98 & & R MAHOOD & SW & & & B06L71 & 27-Sep-91 & & $7440-47-3$ & CR \\
\hline 99 & & R MAHOOD & SW & & & B06L72 & 27-Sep-91 & & $7440-47-3$ & CR \\
\hline 100 & & R MAHOOD & SW & & & B06L71 & 27-Sep-91 & & $10045-97-3$ & CS-137 \\
\hline 101 & & R MAHOOD & SW & & & B06L71 & 27-Sep-91 & & $10098-97-2$ & SR-90 \\
\hline 102 & & R MAHOOD & SW & & & B06L71 & 27-Sep-91 & & $10028-17-8$ & TRITIUM \\
\hline 103 & & R MAHOOD & SW & & & B06L73 & 27-Sep-91 & & $7440-47-3$ & CR \\
\hline 104 & & R MAHOOD & SW & & & B06L74 & 27-Sep-91 & & $7440-47-3$ & CR \\
\hline 105 & & R MAHOOD & SW & & & B06L73 & 27-Sep-91 & & $10045-97-3$ & CS-137 \\
\hline 106 & & R MAHOOD & SW & & & B06L73 & 27-Sep-91 & & $10098-97-2$ & SR-90 \\
\hline 107 & & R MAHOOD & SW & & & B06L73 & 27-Sep-91 & & $10028-17-8$ & TRITIUM \\
\hline 108 & & R MAHOOD & SW & & & B06L66 & 27-Sep-91 & & $7440-47-3$ & CR \\
\hline 109 & & R MAHOOD & SW & & & B06L67 & 27-Sep-91 & & $7440-47-3$ & CR \\
\hline 110 & & R MAHOOD & SW & & & B06L66 & 27-Sep-91 & & $10045-97-3$ & CS-137 \\
\hline 111 & & R MAHOOD & SW & & & B06L66 & 27-Sep-91 & & $10098-97-2$ & SR-90 \\
\hline 112 & & R MAHOOD & SW & & & B06L66 & 27-Sep-91 & & $10028-17-8$ & TRITIUM \\
\hline 113 & & R MAHOOD & SW & & & B06L68 & 27-Sep-91 & & $7440-47-3$ & $\mathrm{CR}$ \\
\hline 114 & & R MAHOOD & SW & & & B06L69 & 27-Sep-91 & & $7440-47-3$ & CR \\
\hline 115 & & R MAHOOD & SW & & & B06L68 & 27-Sep-91 & & $10045-97-3$ & CS-137 \\
\hline
\end{tabular}




\begin{tabular}{|c|c|c|c|c|c|c|c|c|c|c|}
\hline & A & $B$ & $C$ & $\mathrm{D}$ & $E$ & $\mathrm{~F}$ & $G$ & $\mathrm{H}$ & $\mathrm{I}$ & $\mathrm{J}$ \\
\hline 1 & SAMPLE_NUM_ASSIGNED_TO & SAMPLER & MEDIA & BIOTA_MEDIA & TAG_ID & SAMP_NUM & SAMP_DATE_TIME & SAMP_DATE_TIME_ON & CON_ID & CON_SHORT_NAME \\
\hline 116 & & R MAHOOD & SW & & & B06L68 & 27-Sep-91 & & $10098-97-2$ & SR-90 \\
\hline 117 & & R MAHOOD & SW & & & B06L68 & 27-Sep-91 & & $10028-17-8$ & TRITIUM \\
\hline 118 & & R MAHOOD & SW & & & B06L96 & 28-Sep-91 & & $7440-47-3$ & CR \\
\hline 119 & & R MAHOOD & SW & & & B06L97 & 28-Sep-91 & & $7440-47-3$ & CR \\
\hline 120 & & R MAHOOD & SW & & & B06L96 & 28-Sep-91 & & $10045-97-3$ & CS-137 \\
\hline 121 & & R MAHOOD & SW & & & B06L96 & 28-Sep-91 & & $10098-97-2$ & SR-90 \\
\hline 122 & & R MAHOOD & SW & & & B06L96 & 28-Sep-91 & & 10028-17-8 & TRITIUM \\
\hline 123 & & R MAHOOD & SW & & & B06L98 & 28-Sep-91 & & $7440-47-3$ & CR \\
\hline 124 & & R MAHOOD & SW & & & B06L99 & 28-Sep-91 & & $7440-47-3$ & CR \\
\hline 125 & & R MAHOOD & SW & & & B06L98 & 28-Sep-91 & & $10045-97-3$ & CS-137 \\
\hline 126 & & R MAHOOD & SW & & & B06L98 & 28-Sep-91 & & $10098-97-2$ & SR-90 \\
\hline 127 & & R MAHOOD & SW & & & B06L98 & 28-Sep-91 & & $10028-17-8$ & TRITIUM \\
\hline 128 & & R MAHOOD & SW & & & B06L91 & 28-Sep-91 & & $7440-47-3$ & CR \\
\hline 129 & & R MAHOOD & SW & & & B06L92 & 28-Sep-91 & & $7440-47-3$ & CR \\
\hline 130 & & R MAHOOD & SW & & & B06L91 & 28-Sep-91 & & $10045-97-3$ & CS-137 \\
\hline 131 & & R MAHOOD & SW & & & B06L91 & 28-Sep-91 & & $10098-97-2$ & SR-90 \\
\hline 132 & & R MAHOOD & SW & & & B06L91 & 28-Sep-91 & & 10028-17-8 & TRITIUM \\
\hline 133 & & R MAHOOD & SW & & & B06L93 & 28-Sep-91 & & $7440-47-3$ & CR \\
\hline 134 & & R MAHOOD & SW & & & B06L94 & 28-Sep-91 & & $7440-47-3$ & CR \\
\hline 135 & & R MAHOOD & SW & & & B06L93 & 28-Sep-91 & & $10045-97-3$ & CS-137 \\
\hline 136 & & R MAHOOD & SW & & & B06L93 & 28-Sep-91 & & $10098-97-2$ & SR-90 \\
\hline 137 & & R MAHOOD & SW & & & B06L93 & 28-Sep-91 & & 10028-17-8 & TRITIUM \\
\hline 138 & & R MAHOOD & SW & & & B06L81 & 29-Sep-91 & & $7440-47-3$ & CR \\
\hline 139 & & R MAHOOD & SW & & & B06L82 & 29-Sep-91 & & $7440-47-3$ & CR \\
\hline 140 & & R MAHOOD & SW & & & B06L83 & 29-Sep-91 & & $7440-47-3$ & CR \\
\hline 141 & & R MAHOOD & SW & & & B06L81 & 29-Sep-91 & & $10045-97-3$ & CS-137 \\
\hline 142 & & R MAHOOD & SW & & & B06L83 & 29-Sep-91 & & $10045-97-3$ & CS-137 \\
\hline 143 & & R MAHOOD & SW & & & B06L81 & 29-Sep-91 & & $10098-97-2$ & SR-90 \\
\hline 144 & & R MAHOOD & SW & & & B06L83 & 29-Sep-91 & & $10098-97-2$ & SR-90 \\
\hline 145 & & R MAHOOD & SW & & & B06L81 & 29-Sep-91 & & $10028-17-8$ & TRITIUM \\
\hline 146 & & R MAHOOD & SW & & & B06L83 & 29-Sep-91 & & $10028-17-8$ & TRITIUM \\
\hline 147 & & R MAHOOD & SW & & & B06L84 & 29-Sep-91 & & $7440-47-3$ & $\mathrm{CR}$ \\
\hline 148 & & R MAHOOD & SW & & & B06L85 & 29-Sep-91 & & $7440-47-3$ & $\mathrm{CR}$ \\
\hline 149 & & R MAHOOD & SW & & & B06L86 & 29-Sep-91 & & $7440-47-3$ & CR \\
\hline 150 & & R MAHOOD & SW & & & B06L85 & 29-Sep-91 & & $10045-97-3$ & CS-137 \\
\hline 151 & & R MAHOOD & SW & & & B06L85 & 29-Sep-91 & & $10098-97-2$ & SR-90 \\
\hline 152 & & R MAHOOD & SW & & & B06L85 & 29-Sep-91 & & $10028-17-8$ & TRITIUM \\
\hline 153 & & R MAHOOD & SW & & & B06L87 & 29-Sep-91 & & $7440-47-3$ & $\mathrm{CR}$ \\
\hline
\end{tabular}




\begin{tabular}{|c|c|c|c|c|c|c|c|c|c|c|}
\hline & A & $\mathrm{B}$ & $\mathrm{C}$ & $\mathrm{D}$ & $\mathrm{E}$ & $\mathrm{F}$ & $\mathrm{G}$ & $\mathrm{H}$ & $\mathrm{I}$ & $\mathrm{J}$ \\
\hline 1 & SAMPLE_NUM_ASSIGNED_TO & SAMPLER & MEDIA & BIOTA_MEDIA & TAG_ID & SAMP_NUM & SAMP_DATE_TIME & SAMP_DATE_TIME_ON & CON_ID & CON_SHORT_NAME \\
\hline 154 & & R MAHOOD & SW & & & B06L88 & 29-Sep-91 & & $7440-47-3$ & CR \\
\hline 155 & & R MAHOOD & SW & & & B06L87 & 29-Sep-91 & & $10045-97-3$ & CS-137 \\
\hline 156 & & R MAHOOD & SW & & & B06L87 & 29-Sep-91 & & $10098-97-2$ & SR-90 \\
\hline 157 & & R MAHOOD & SW & & & B06L87 & 29-Sep-91 & & $10028-17-8$ & TRITIUM \\
\hline 158 & & R MAHOOD & SW & & & B06L76 & 30-Sep-91 & & $7440-47-3$ & CR \\
\hline 159 & & R MAHOOD & SW & & & B06L77 & 30-Sep-91 & & $7440-47-3$ & CR \\
\hline 160 & & R MAHOOD & SW & & & B06L76 & 30-Sep-91 & & $10045-97-3$ & CS-137 \\
\hline 161 & & R MAHOOD & SW & & & B06L76 & 30-Sep-91 & & 10098-97-2 & SR-90 \\
\hline 162 & & R MAHOOD & SW & & & B06L76 & 30-Sep-91 & & $10028-17-8$ & TRITIUM \\
\hline 163 & & R MAHOOD & SW & & & B06L79 & 30-Sep-91 & & $7440-47-3$ & CR \\
\hline 164 & & R MAHOOD & SW & & & B06L78 & 30-Sep-91 & & $7440-47-3$ & CR \\
\hline 165 & & R MAHOOD & SW & & & B06L78 & 30-Sep-91 & & $10045-97-3$ & CS-137 \\
\hline 166 & & R MAHOOD & SW & & & B06L78 & 30-Sep-91 & & $10098-97-2$ & SR-90 \\
\hline 167 & & R MAHOOD & SW & & & B06L78 & 30-Sep-91 & & $10028-17-8$ & TRITIUM \\
\hline 168 & & R MAHOOD & SW & & & B06LK1 & 02-Oct-91 & & $7440-47-3$ & CR \\
\hline 169 & & R MAHOOD & SW & & & B06LK2 & 02-Oct-91 & & $7440-47-3$ & CR \\
\hline 170 & & R MAHOOD & SW & & & B06LK1 & 02-Oct-91 & & $10045-97-3$ & CS-137 \\
\hline 171 & & R MAHOOD & SW & & & B06LK1 & 02-Oct-91 & & $10098-97-2$ & SR-90 \\
\hline 172 & & R MAHOOD & SW & & & B06LK1 & 02-Oct-91 & & $10028-17-8$ & TRITIUM \\
\hline 173 & & R MAHOOD & SW & & & B06LK3 & 02-Oct-91 & & $7440-47-3$ & CR \\
\hline 174 & & R MAHOOD & SW & & & B06LK4 & 02-Oct-91 & & $7440-47-3$ & CR \\
\hline 175 & & R MAHOOD & SW & & & B06LK3 & 02-Oct-91 & & $10045-97-3$ & CS-137 \\
\hline 176 & & R MAHOOD & SW & & & B06LK3 & 02-Oct-91 & & $10098-97-2$ & SR-90 \\
\hline 177 & & R MAHOOD & SW & & & B06LK3 & $02-$ Oct-91 & & $10028-17-8$ & TRITIUM \\
\hline 178 & & R MAHOOD & SW & & & B06LB1 & 02-Oct-91 & & $7440-47-3$ & CR \\
\hline 179 & & R MAHOOD & SW & & & B06LB2 & 02-Oct-91 & & $7440-47-3$ & CR \\
\hline 180 & & R MAHOOD & SW & & & B06LB1 & 02-Oct-91 & & $10045-97-3$ & CS-137 \\
\hline 181 & & R MAHOOD & SW & & & B06LB1 & 02-Oct-91 & & $10098-97-2$ & SR-90 \\
\hline 182 & & R MAHOOD & SW & & & B06LB1 & 02-Oct-91 & & $10028-17-8$ & TRITIUM \\
\hline 183 & & R MAHOOD & SW & & & B06LJ3 & 02-Oct-91 & & $7440-47-3$ & CR \\
\hline 184 & & R MAHOOD & SW & & & B06LJ4 & 02-Oct-91 & & $7440-47-3$ & CR \\
\hline 185 & & R MAHOOD & SW & & & B06LJ3 & 02-Oct-91 & & $10045-97-3$ & CS-137 \\
\hline 186 & & R MAHOOD & SW & & & B06LJ3 & 02-Oct-91 & & $10098-97-2$ & SR-90 \\
\hline 187 & & R MAHOOD & SW & & & B06LJ3 & 02-Oct-91 & & $10028-17-8$ & TRITIUM \\
\hline 188 & & R MAHOOD & SW & & & B06LJ5 & 02-Oct-91 & & $7440-47-3$ & CR \\
\hline 189 & & R MAHOOD & SW & & & B06LJ6 & 02-Oct-91 & & $7440-47-3$ & CR \\
\hline 190 & & R MAHOOD & SW & & & B06LJ7 & $02-$ Oct-91 & & $7440-47-3$ & CR \\
\hline 191 & & R MAHOOD & SW & & & B06LJ8 & $02-$ Oct-91 & & $7440-47-3$ & CR \\
\hline
\end{tabular}




\begin{tabular}{|c|c|c|c|c|c|c|c|c|c|c|}
\hline & A & $\mathrm{B}$ & $\mathrm{C}$ & $\mathrm{D}$ & $\mathrm{E}$ & $\mathrm{F}$ & $\mathrm{G}$ & $\mathrm{H}$ & $\mathrm{I}$ & $\mathrm{J}$ \\
\hline 1 & SAMPLE_NUM_ASSIGNED_TO & SAMPLER & MEDIA & BIOTA_MEDIA & TAG_ID & SAMP_NUM & SAMP_DATE_TIME & SAMP_DATE_TIME_ON & CON_ID & CON_SHORT_NAME \\
\hline 192 & & R MAHOOD & SW & & & B06LJ5 & 02-Oct-91 & & $10045-97-3$ & CS-137 \\
\hline 193 & & R MAHOOD & SW & & & B06LJ7 & 02-Oct-91 & & $10045-97-3$ & CS-137 \\
\hline 194 & & R MAHOOD & SW & & & B06LJ5 & 02-Oct-91 & & $10098-97-2$ & SR-90 \\
\hline 195 & & R MAHOOD & SW & & & B06LJ7 & 02-Oct-91 & & $10098-97-2$ & SR-90 \\
\hline 196 & & R MAHOOD & SW & & & B06LJ5 & 02-Oct-91 & & $10028-17-8$ & TRITIUM \\
\hline 197 & & R MAHOOD & SW & & & B06LJ7 & 02-Oct-91 & & $10028-17-8$ & TRITIUM \\
\hline 198 & & R MAHOOD & SW & & & B06KV1 & $15-$ Oct-91 & & $7440-47-3$ & $\mathrm{CR}$ \\
\hline 199 & & R MAHOOD & SW & & & B06KV1 & 15-Oct-91 & & $10045-97-3$ & CS-137 \\
\hline 200 & & R MAHOOD & SW & & & B06KV1 & 15-Oct-91 & & $10098-97-2$ & SR-90 \\
\hline 201 & & R MAHOOD & SW & & & B06KV1 & $15-$ Oct-91 & & $14133-76-7$ & TC-99 \\
\hline 202 & & R MAHOOD & SW & & & B06KV1 & $15-$ Oct-91 & & $10028-17-8$ & TRITIUM \\
\hline 203 & & R MAHOOD & SW & & & B06KV0 & 15-Oct-91 & & $7440-47-3$ & CR \\
\hline 204 & & R MAHOOD & SW & & & B06KT9 & 15-Oct-91 & & $7440-47-3$ & CR \\
\hline 205 & & R MAHOOD & SW & & & B06KT9 & 15-Oct-91 & & $10045-97-3$ & CS-137 \\
\hline 206 & & R MAHOOD & SW & & & B06KT9 & 15-Oct-91 & & $10098-97-2$ & SR-90 \\
\hline 207 & & R MAHOOD & SW & & & B06KT9 & 15-Oct-91 & & $14133-76-7$ & TC-99 \\
\hline 208 & & R MAHOOD & SW & & & B06KT9 & 15-Oct-91 & & $10028-17-8$ & TRITIUM \\
\hline 209 & & R MAHOOD & SW & & & B06KV5 & 15-Oct-91 & & $7440-47-3$ & CR \\
\hline 210 & & R MAHOOD & SW & & & B06KV6 & 15-Oct-91 & & $7440-47-3$ & CR \\
\hline 211 & & R MAHOOD & SW & & & B06KV5 & 15-Oct-91 & & $10045-97-3$ & CS-137 \\
\hline 212 & & R MAHOOD & SW & & & B06KV5 & 15-Oct-91 & & $10098-97-2$ & SR-90 \\
\hline 213 & & R MAHOOD & SW & & & B06KV5 & $15-$ Oct-91 & & $14133-76-7$ & TC-99 \\
\hline 214 & & R MAHOOD & SW & & & B06KV5 & 15-Oct-91 & & $10028-17-8$ & TRITIUM \\
\hline 215 & & R MAHOOD & SW & & & B06KV3 & $15-O c t-91$ & & $7440-47-3$ & $\mathrm{CR}$ \\
\hline 216 & & R MAHOOD & SW & & & B06KV4 & 15-Oct-91 & & $7440-47-3$ & CR \\
\hline 217 & & R MAHOOD & SW & & & B06KV3 & 15 -Oct-91 & & $10045-97-3$ & CS-137 \\
\hline 218 & & R MAHOOD & SW & & & B06KV3 & 15-Oct-91 & & $10098-97-2$ & SR-90 \\
\hline 219 & & R MAHOOD & SW & & & B06KV3 & 15-Oct-91 & & $14133-76-7$ & TC-99 \\
\hline 220 & & R MAHOOD & SW & & & B06KV3 & $15-$ Oct-91 & & $10028-17-8$ & TRITIUM \\
\hline 221 & & R MAHOOD & SW & & & B06KT1 & 16-Oct-91 & & $7440-47-3$ & CR \\
\hline 222 & & R MAHOOD & SW & & & B06KT2 & 16-Oct-91 & & $7440-47-3$ & CR \\
\hline 223 & & R MAHOOD & SW & & & B06KT1 & 16-Oct-91 & & $10045-97-3$ & CS-137 \\
\hline 224 & & R MAHOOD & SW & & & B06KT1 & 16-Oct-91 & & $10098-97-2$ & SR-90 \\
\hline 225 & & R MAHOOD & SW & & & B06KT1 & 16 -Oct-91 & & $14133-76-7$ & TC-99 \\
\hline 226 & & R MAHOOD & SW & & & B06KT1 & 16-Oct-91 & & $10028-17-8$ & TRITIUM \\
\hline 227 & & R MAHOOD & SW & & & B06KS9 & 16-Oct-91 & & $7440-47-3$ & CR \\
\hline 228 & & R MAHOOD & SW & & & B06KT0 & 16-Oct-91 & & $7440-47-3$ & CR \\
\hline 229 & & R MAHOOD & SW & & & B06KS9 & $16-$ Oct-91 & & $10045-97-3$ & CS-137 \\
\hline
\end{tabular}




\begin{tabular}{|c|c|c|c|c|c|c|c|c|c|c|}
\hline & A & $\mathrm{B}$ & $\mathrm{C}$ & $\mathrm{D}$ & $\mathrm{E}$ & $\mathrm{F}$ & $\mathrm{G}$ & $\mathrm{H}$ & $\mathrm{I}$ & $\mathrm{J}$ \\
\hline 1 & SAMPLE_NUM_ASSIGNED_TO & SAMPLER & MEDIA & BIOTA_MEDIA & TAG_ID & SAMP_NUM & SAMP_DATE_TIME & SAMP_DATE_TIME_ON & CON_ID & CON_SHORT_NAME \\
\hline 230 & & R MAHOOD & SW & & & B06KS9 & 16-Oct-91 & & $10098-97-2$ & SR-90 \\
\hline 231 & & R MAHOOD & SW & & & B06KS9 & 16-Oct-91 & & $14133-76-7$ & TC-99 \\
\hline 232 & & R MAHOOD & SW & & & B06KS9 & 16-Oct-91 & & $10028-17-8$ & TRITIUM \\
\hline 233 & & R MAHOOD & SW & & & B06KX0 & 17-Oct-91 & & $7440-47-3$ & CR \\
\hline 234 & & R MAHOOD & SW & & & B06KX1 & 17-Oct-91 & & $7440-47-3$ & CR \\
\hline 235 & & R MAHOOD & SW & & & B06KX0 & 17-Oct-91 & & $10045-97-3$ & CS-137 \\
\hline 236 & & R MAHOOD & SW & & & B06KX0 & $17-$ Oct-91 & & $10098-97-2$ & SR-90 \\
\hline 237 & & R MAHOOD & SW & & & B06KX0 & 17-Oct-91 & & $14133-76-7$ & TC-99 \\
\hline 238 & & R MAHOOD & SW & & & B06KX0 & 17-Oct-91 & & $10028-17-8$ & TRITIUM \\
\hline 239 & & R MAHOOD & SW & & & B06KW8 & $17-$ Oct-91 & & $7440-47-3$ & CR \\
\hline 240 & & R MAHOOD & SW & & & B06KW9 & $17-$ Oct-91 & & $7440-47-3$ & CR \\
\hline 241 & & R MAHOOD & SW & & & B06KW8 & 17-Oct-91 & & $10045-97-3$ & CS-137 \\
\hline 242 & & R MAHOOD & SW & & & B06KW8 & 17-Oct-91 & & $10098-97-2$ & SR-90 \\
\hline 243 & & R MAHOOD & SW & & & B06KW8 & 17-Oct-91 & & $14133-76-7$ & TC-99 \\
\hline 244 & & R MAHOOD & SW & & & B06KW8 & 17-Oct-91 & & $10028-17-8$ & TRITIUM \\
\hline 245 & & R MAHOOD & SW & & & B06KW5 & 18-Oct-91 & & $7440-47-3$ & CR \\
\hline 246 & & R MAHOOD & SW & & & B06KW6 & 18-Oct-91 & & $7440-47-3$ & CR \\
\hline 247 & & R MAHOOD & SW & & & B06KW5 & 18-Oct-91 & & $10045-97-3$ & CS-137 \\
\hline 248 & & R MAHOOD & SW & & & B06KW5 & 18-Oct-91 & & $10098-97-2$ & SR-90 \\
\hline 249 & & R MAHOOD & SW & & & B06KW5 & 18-Oct-91 & & $14133-76-7$ & TC-99 \\
\hline 250 & & R MAHOOD & SW & & & B06KW5 & 18-Oct-91 & & $10028-17-8$ & TRITIUM \\
\hline 251 & & R MAHOOD & SW & & & B06KW3 & 18-Oct-91 & & $7440-47-3$ & CR \\
\hline 252 & & R MAHOOD & SW & & & B06KW4 & 18-Oct-91 & & $7440-47-3$ & CR \\
\hline 253 & & R MAHOOD & SW & & & B06KW3 & 18-Oct-91 & & $10045-97-3$ & CS-137 \\
\hline 254 & & R MAHOOD & SW & & & B06KW3 & 18-Oct-91 & & $10098-97-2$ & SR-90 \\
\hline 255 & & R MAHOOD & SW & & & B06KW3 & 18-Oct-91 & & $14133-76-7$ & TC-99 \\
\hline 256 & & R MAHOOD & SW & & & B06KW3 & 18-Oct-91 & & $10028-17-8$ & TRITIUM \\
\hline 257 & & R MAHOOD & SW & & & B06KWO & 18-Oct-91 & & $7440-47-3$ & CR \\
\hline 258 & & R MAHOOD & SW & & & B06KW1 & 18-Oct-91 & & $7440-47-3$ & CR \\
\hline 259 & & R MAHOOD & SW & & & B06KWO & 18-Oct-91 & & $10045-97-3$ & CS-137 \\
\hline 260 & & R MAHOOD & SW & & & B06KWO & 18-Oct-91 & & $10098-97-2$ & SR-90 \\
\hline 261 & & R MAHOOD & SW & & & B06KWO & 18-Oct-91 & & $14133-76-7$ & TC-99 \\
\hline 262 & & R MAHOOD & SW & & & B06KWO & 18-Oct-91 & & $10028-17-8$ & TRITIUM \\
\hline 263 & & R MAHOOD & SW & & & B06KV8 & 18-Oct-91 & & $7440-47-3$ & CR \\
\hline 264 & & R MAHOOD & SW & & & B06KV9 & 18-Oct-91 & & $7440-47-3$ & CR \\
\hline 265 & & R MAHOOD & SW & & & B06KV8 & 18-Oct-91 & & $10045-97-3$ & CS-137 \\
\hline 266 & & R MAHOOD & SW & & & B06KV8 & 18-Oct-91 & & $10098-97-2$ & SR-90 \\
\hline 267 & & R MAHOOD & SW & & & B06KV8 & 18 -Oct-91 & & $14133-76-7$ & TC-99 \\
\hline
\end{tabular}




\begin{tabular}{|c|c|c|c|c|c|c|c|c|c|c|}
\hline & A & $B$ & $C$ & $\mathrm{D}$ & $E$ & $\mathrm{~F}$ & $G$ & $\mathrm{H}$ & $\mathrm{I}$ & $\mathrm{J}$ \\
\hline 1 & SAMPLE_NUM_ASSIGNED_TO & SAMPLER & MEDIA & BIOTA_MEDIA & TAG_ID & SAMP_NUM & SAMP_DATE_TIME & SAMP_DATE_TIME_ON & CON_ID & CON_SHORT_NAME \\
\hline 268 & & R MAHOOD & SW & & & B06KV8 & 18-Oct-91 & & $10028-17-8$ & TRITIUM \\
\hline 269 & & R MAHOOD & SW & & & B06KT6 & 18-Oct-91 & & $7440-47-3$ & CR \\
\hline 270 & & R MAHOOD & SW & & & B06KT7 & 18-Oct-91 & & $7440-47-3$ & CR \\
\hline 271 & & R MAHOOD & SW & & & B06KT6 & 18-Oct-91 & & $10045-97-3$ & CS-137 \\
\hline 272 & & R MAHOOD & SW & & & B06KT6 & 18-Oct-91 & & $10098-97-2$ & SR-90 \\
\hline 273 & & R MAHOOD & SW & & & B06KT6 & 18-Oct-91 & & $14133-76-7$ & TC-99 \\
\hline 274 & & R MAHOOD & SW & & & B06KT6 & 18-Oct-91 & & $10028-17-8$ & TRITIUM \\
\hline 275 & & R MAHOOD & SW & & & B06KT4 & 18-Oct-91 & & $7440-47-3$ & CR \\
\hline 276 & & R MAHOOD & SW & & & B06KT5 & 18-Oct-91 & & $7440-47-3$ & CR \\
\hline 277 & & R MAHOOD & SW & & & B06KT4 & 18-Oct-91 & & $10045-97-3$ & CS-137 \\
\hline 278 & & R MAHOOD & SW & & & B06KT4 & 18-Oct-91 & & $10098-97-2$ & SR-90 \\
\hline 279 & & R MAHOOD & SW & & & B06KT4 & 18-Oct-91 & & $14133-76-7$ & TC-99 \\
\hline 280 & & R MAHOOD & SW & & & B06KT4 & 18-Oct-91 & & 10028-17-8 & TRITIUM \\
\hline 281 & & R MAHOOD & SW & & & B06L62 & 21-Oct-91 & & $7440-47-3$ & CR \\
\hline 282 & & R MAHOOD & SW & & & B06L63 & 21-Oct-91 & & $7440-47-3$ & CR \\
\hline 283 & & R MAHOOD & SW & & & B06L64 & 21-Oct-91 & & $7440-47-3$ & CR \\
\hline 284 & & R MAHOOD & SW & & & B06L61 & 21-Oct-91 & & $7440-47-3$ & CR \\
\hline 285 & & R MAHOOD & SW & & & B06L63 & 21-Oct-91 & & $10045-97-3$ & CS-137 \\
\hline 286 & & R MAHOOD & SW & & & B06L61 & 21-Oct-91 & & $10045-97-3$ & CS-137 \\
\hline 287 & & R MAHOOD & SW & & & B06L63 & 21-Oct-91 & & $10098-97-2$ & SR-90 \\
\hline 288 & & R MAHOOD & SW & & & B06L61 & 21-Oct-91 & & $10098-97-2$ & SR-90 \\
\hline 289 & & R MAHOOD & SW & & & B06L63 & 21-Oct-91 & & $14133-76-7$ & TC-99 \\
\hline 290 & & R MAHOOD & SW & & & B06L61 & 21-Oct-91 & & $14133-76-7$ & TC-99 \\
\hline 291 & & R MAHOOD & SW & & & B06L63 & 21-Oct-91 & & $10028-17-8$ & TRITIUM \\
\hline 292 & & R MAHOOD & SW & & & B06L61 & 21-Oct-91 & & $10028-17-8$ & TRITIUM \\
\hline 293 & PDMS & & SW & & & E118362 & 09-Dec-91 & & $10045-97-3$ & CS-137 \\
\hline 294 & PDMS & & SW & & & E118362 & 09-Dec-91 & & $14133-76-7$ & TC-99 \\
\hline 295 & PDMS & & SW & & & E118362 & 09-Dec-91 & & $10028-17-8$ & TRITIUM \\
\hline 296 & PDMS & & SW & & & E118379 & 17-Dec-91 & & $10045-97-3$ & CS-137 \\
\hline 297 & PDMS & & SW & & & E118379 & 17-Dec-91 & & $10098-97-2$ & SR-90 \\
\hline 298 & PDMS & & SW & & & E118379 & 17-Dec-91 & & $14133-76-7$ & TC-99 \\
\hline 299 & PDMS & & SW & & & E118379 & 17-Dec-91 & & $10028-17-8$ & TRITIUM \\
\hline 300 & PDMS & & SW & & & E118812 & 31-Dec-91 & & $10045-97-3$ & CS-137 \\
\hline 301 & PDMS & & SW & & & E118812 & 31-Dec-91 & & $14133-76-7$ & TC-99 \\
\hline 302 & PDMS & & SW & & & E118812 & 31-Dec-91 & & $10028-17-8$ & TRITIUM \\
\hline 303 & PDMS & & SW & & & E118812 & 31-Dec-91 & & $13966-29-5$ & U-234 \\
\hline 304 & PDMS & & SW & & & E118812 & 31-Dec-91 & & 15117-96-1 & U-235 \\
\hline 305 & PDMS & & SW & & & E118812 & 31-Dec-91 & & U-238 & U-238 \\
\hline
\end{tabular}




\begin{tabular}{|c|c|c|c|c|c|c|c|c|c|c|}
\hline & A & $\mathrm{B}$ & $\mathrm{C}$ & $\mathrm{D}$ & $\mathrm{E}$ & $\mathrm{F}$ & $\mathrm{G}$ & $\mathrm{H}$ & $\mathrm{I}$ & $\mathrm{J}$ \\
\hline 1 & SAMPLE_NUM_ASSIGNED_TO & SAMPLER & MEDIA & BIOTA_MEDIA & TAG_ID & SAMP_NUM & SAMP_DATE_TIME & SAMP_DATE_TIME_ON & CON_ID & CON_SHORT_NAME \\
\hline 306 & PDMS & & SW & & & E121556 & 14-Sep-92 & & $10045-97-3$ & CS-137 \\
\hline 307 & PDMS & & SW & & & E121556 & 14-Sep-92 & & 10028-17-8 & TRITIUM \\
\hline 308 & PDMS & & SW & & & E121556 & 14-Sep-92 & & $13966-29-5$ & U-234 \\
\hline 309 & PDMS & & SW & & & E121556 & 14-Sep-92 & & 15117-96-1 & U-235 \\
\hline 310 & PDMS & & SW & & & E121556 & 14-Sep-92 & & $\mathrm{U}-238$ & $\mathrm{U}-238$ \\
\hline 311 & & & SW & & & B01324 & 21-Sep-92 & & $56-23-5$ & CARBTET \\
\hline 312 & & & SW & & & B01371 & 21-Sep-92 & & $56-23-5$ & CARBTET \\
\hline 313 & & & SW & & & B076Q3 & 21-Sep-92 & & $56-23-5$ & CARBTET \\
\hline 314 & & & SW & & & B01325 & 21-Sep-92 & & $56-23-5$ & CARBTET \\
\hline 315 & & & SW & & & B01324 & 21-Sep-92 & & $7440-47-3$ & CR \\
\hline 316 & & & SW & & & B01371 & 21-Sep-92 & & $7440-47-3$ & CR \\
\hline 317 & & & SW & & & B076H5 & 21-Sep-92 & & $7440-47-3$ & CR \\
\hline 318 & & & SW & & & B076Q3 & 21-Sep-92 & & $7440-47-3$ & CR \\
\hline 319 & & & SW & & & B076Q4 & 21-Sep-92 & & $7440-47-3$ & CR \\
\hline 320 & & & SW & & & B076H1 & 21-Sep-92 & & $7440-47-3$ & CR \\
\hline 321 & & & SW & & & B01325 & 21-Sep-92 & & $7440-47-3$ & CR \\
\hline 322 & & & SW & & & $\mathrm{B} 076 \mathrm{H} 3$ & 21-Sep-92 & & $7440-47-3$ & CR \\
\hline 323 & & & SW & & & B01324 & 21-Sep-92 & & $10045-97-3$ & CS-137 \\
\hline 324 & & & SW & & & B01371 & 21-Sep-92 & & $10045-97-3$ & CS-137 \\
\hline 325 & & & SW & & & B076Q3 & 21-Sep-92 & & $10045-97-3$ & CS-137 \\
\hline 326 & & & SW & & & B01325 & 21-Sep-92 & & $10045-97-3$ & CS-137 \\
\hline 327 & & & SW & & & B076Q3 & 21-Sep-92 & & PU-239/240 & PU-239/240 \\
\hline 328 & & & SW & & & B01324 & 21-Sep-92 & & $10098-97-2$ & SR-90 \\
\hline 329 & & & SW & & & B01371 & 21-Sep-92 & & $10098-97-2$ & SR-90 \\
\hline 330 & & & SW & & & B076Q3 & 21-Sep-92 & & 10098-97-2 & SR-90 \\
\hline 331 & & & SW & & & B01325 & 21-Sep-92 & & $10098-97-2$ & SR-90 \\
\hline 332 & & & SW & & & B01324 & 21-Sep-92 & & 14133-76-7 & TC-99 \\
\hline 333 & & & SW & & & B01371 & 21-Sep-92 & & $14133-76-7$ & TC-99 \\
\hline 334 & & & SW & & & B076Q3 & 21-Sep-92 & & $14133-76-7$ & TC-99 \\
\hline 335 & & & SW & & & B01325 & 21-Sep-92 & & 14133-76-7 & TC-99 \\
\hline 336 & & & SW & & & B01324 & 21-Sep-92 & & 10028-17-8 & TRITIUM \\
\hline 337 & & & SW & & & B01371 & 21-Sep-92 & & $10028-17-8$ & TRITIUM \\
\hline 338 & & & SW & & & B076Q3 & 21-Sep-92 & & $10028-17-8$ & TRITIUM \\
\hline 339 & & & SW & & & B01325 & 21-Sep-92 & & $10028-17-8$ & TRITIUM \\
\hline 340 & & & SW & & & B01324 & 21-Sep-92 & & $15117-96-1$ & U-235 \\
\hline 341 & & & SW & & & B01371 & 21-Sep-92 & & $15117-96-1$ & $\mathrm{U}-235$ \\
\hline 342 & & & SW & & & B076Q3 & 21-Sep-92 & & $15117-96-1$ & U-235 \\
\hline 343 & & & SW & & & B01325 & 21-Sep-92 & & $15117-96-1$ & U-235 \\
\hline
\end{tabular}




\begin{tabular}{|c|c|c|c|c|c|c|c|c|c|c|}
\hline & $\mathrm{A}$ & $B$ & $\mathrm{C}$ & $\mathrm{D}$ & $E$ & $\mathrm{~F}$ & $\mathrm{G}$ & $\mathrm{H}$ & $\mathrm{I}$ & $\mathrm{J}$ \\
\hline 1 & SAMPLE_NUM_ASSIGNED_TO & SAMPLER & MEDIA & BIOTA_MEDIA & TAG_ID & SAMP_NUM & SAMP_DATE_TIME & SAMP_DATE_TIME_ON & CON_ID & CON_SHORT_NAME \\
\hline 344 & & & SW & & & B01324 & 21-Sep-92 & & U-238 & U-238 \\
\hline 345 & & & SW & & & B01371 & 21-Sep-92 & & U-238 & U-238 \\
\hline 346 & & & SW & & & B076Q3 & 21-Sep-92 & & U-238 & U-238 \\
\hline 347 & & & SW & & & B01325 & 21-Sep-92 & & U-238 & U-238 \\
\hline 348 & & & SW & & & B01372 & 21-Sep-92 & & $56-23-5$ & CARBTET \\
\hline 349 & & & SW & & & B01373 & 21-Sep-92 & & $56-23-5$ & CARBTET \\
\hline 350 & & & SW & & & B01374 & 21-Sep-92 & & $56-23-5$ & CARBTET \\
\hline 351 & & & SW & & & B076P7 & 21-Sep-92 & & $56-23-5$ & CARBTET \\
\hline 352 & & & SW & & & B01372 & 21-Sep-92 & & $7440-47-3$ & CR \\
\hline 353 & & & SW & & & B01373 & 21-Sep-92 & & $7440-47-3$ & CR \\
\hline 354 & & & SW & & & B01374 & 21-Sep-92 & & $7440-47-3$ & CR \\
\hline 355 & & & SW & & & $\mathrm{B} 076 \mathrm{H} 7$ & 21-Sep-92 & & $7440-47-3$ & CR \\
\hline 356 & & & SW & & & B076H9 & 21-Sep-92 & & $7440-47-3$ & CR \\
\hline 357 & & & SW & & & B076J1 & 21-Sep-92 & & $7440-47-3$ & CR \\
\hline 358 & & & SW & & & B076P7 & 21-Sep-92 & & $7440-47-3$ & CR \\
\hline 359 & & & SW & & & B076P8 & 21-Sep-92 & & $7440-47-3$ & CR \\
\hline 360 & & & SW & & & B01372 & 21-Sep-92 & & $10045-97-3$ & CS-137 \\
\hline 361 & & & SW & & & B01373 & 21-Sep-92 & & $10045-97-3$ & CS-137 \\
\hline 362 & & & SW & & & B01374 & 21-Sep-92 & & $10045-97-3$ & CS-137 \\
\hline 363 & & & SW & & & B076P7 & 21-Sep-92 & & $10045-97-3$ & CS-137 \\
\hline 364 & & & SW & & & B01372 & 21-Sep-92 & & 10098-97-2 & SR-90 \\
\hline 365 & & & SW & & & B01373 & 21-Sep-92 & & $10098-97-2$ & SR-90 \\
\hline 366 & & & SW & & & B01374 & 21-Sep-92 & & $10098-97-2$ & SR-90 \\
\hline 367 & & & SW & & & B076P7 & 21-Sep-92 & & $10098-97-2$ & SR-90 \\
\hline 368 & & & SW & & & B01372 & 21-Sep-92 & & $14133-76-7$ & TC-99 \\
\hline 369 & & & SW & & & B01373 & 21-Sep-92 & & 14133-76-7 & TC-99 \\
\hline 370 & & & SW & & & B01374 & 21-Sep-92 & & 14133-76-7 & TC-99 \\
\hline 371 & & & SW & & & B076P7 & 21-Sep-92 & & $14133-76-7$ & TC-99 \\
\hline 372 & & & SW & & & B01372 & 21-Sep-92 & & $10028-17-8$ & TRITIUM \\
\hline 373 & & & SW & & & B01373 & 21-Sep-92 & & $10028-17-8$ & TRITIUM \\
\hline 374 & & & SW & & & B01374 & 21-Sep-92 & & $10028-17-8$ & TRITIUM \\
\hline 375 & & & SW & & & B076P7 & 21-Sep-92 & & $10028-17-8$ & TRITIUM \\
\hline 376 & & & SW & & & B01372 & 21-Sep-92 & & 15117-96-1 & U-235 \\
\hline 377 & & & SW & & & B01373 & 21-Sep-92 & & 15117-96-1 & U-235 \\
\hline 378 & & & SW & & & B01374 & 21-Sep-92 & & 15117-96-1 & U-235 \\
\hline 379 & & & SW & & & B076P7 & 21-Sep-92 & & $15117-96-1$ & U-235 \\
\hline 380 & & & SW & & & B01372 & 21-Sep-92 & & U-238 & U-238 \\
\hline 381 & & & SW & & & B01373 & 21-Sep-92 & & $\mathrm{U}-238$ & $\mathrm{U}-238$ \\
\hline
\end{tabular}




\begin{tabular}{|c|c|c|c|c|c|c|c|c|c|c|}
\hline & $\mathrm{A}$ & $\mathrm{B}$ & $C$ & $\mathrm{D}$ & $E$ & $\mathrm{~F}$ & $\mathrm{G}$ & $\mathrm{H}$ & $\mathrm{I}$ & $\mathrm{J}$ \\
\hline 1 & SAMPLE_NUM_ASSIGNED_TO & SAMPLER & MEDIA & BIOTA_MEDIA & TAG_ID & SAMP_NUM & SAMP_DATE_TIME & SAMP_DATE_TIME_ON & CON_ID & CON_SHORT_NAME \\
\hline 382 & & & SW & & & B01374 & 21-Sep-92 & & U-238 & U-238 \\
\hline 383 & & & SW & & & B076P7 & 21-Sep-92 & & U-238 & U-238 \\
\hline 384 & & & SW & & & B01375 & 21-Sep-92 & & $56-23-5$ & CARBTET \\
\hline 385 & & & SW & & & B01376 & 21-Sep-92 & & $56-23-5$ & CARBTET \\
\hline 386 & & & SW & & & B01377 & 21-Sep-92 & & $56-23-5$ & CARBTET \\
\hline 387 & & & SW & & & B01375 & 21-Sep-92 & & $7440-47-3$ & CR \\
\hline 388 & & & SW & & & B01376 & 21-Sep-92 & & $7440-47-3$ & $\mathrm{CR}$ \\
\hline 389 & & & SW & & & B01377 & 21-Sep-92 & & $7440-47-3$ & CR \\
\hline 390 & & & SW & & & B076J3 & 21-Sep-92 & & $7440-47-3$ & CR \\
\hline 391 & & & SW & & & B076J5 & 21-Sep-92 & & $7440-47-3$ & CR \\
\hline 392 & & & SW & & & B076J7 & 21-Sep-92 & & $7440-47-3$ & CR \\
\hline 393 & & & SW & & & B01375 & 21-Sep-92 & & $10045-97-3$ & CS-137 \\
\hline 394 & & & SW & & & B01376 & 21-Sep-92 & & $10045-97-3$ & CS-137 \\
\hline 395 & & & SW & & & B01377 & 21-Sep-92 & & $10045-97-3$ & CS-137 \\
\hline 396 & & & SW & & & B01375 & 21-Sep-92 & & $10098-97-2$ & SR-90 \\
\hline 397 & & & SW & & & B01376 & 21-Sep-92 & & $10098-97-2$ & SR-90 \\
\hline 398 & & & SW & & & B01377 & 21-Sep-92 & & $10098-97-2$ & SR-90 \\
\hline 399 & & & SW & & & B01375 & 21-Sep-92 & & 14133-76-7 & TC-99 \\
\hline 400 & & & SW & & & B01376 & 21-Sep-92 & & 14133-76-7 & TC-99 \\
\hline 401 & & & SW & & & B01377 & 21-Sep-92 & & $14133-76-7$ & TC-99 \\
\hline 402 & & & SW & & & B01375 & 21-Sep-92 & & $10028-17-8$ & TRITIUM \\
\hline 403 & & & SW & & & B01376 & 21-Sep-92 & & $10028-17-8$ & TRITIUM \\
\hline 404 & & & SW & & & B01377 & 21-Sep-92 & & $10028-17-8$ & TRITIUM \\
\hline 405 & & & SW & & & B01375 & 21-Sep-92 & & 15117-96-1 & U-235 \\
\hline 406 & & & SW & & & B01376 & 21-Sep-92 & & 15117-96-1 & U-235 \\
\hline 407 & & & SW & & & B01377 & 21-Sep-92 & & 15117-96-1 & U-235 \\
\hline 408 & & & SW & & & B01375 & 21-Sep-92 & & U-238 & U-238 \\
\hline 409 & & & SW & & & B01376 & 21-Sep-92 & & U-238 & $\mathrm{U}-238$ \\
\hline 410 & & & SW & & & B01377 & 21-Sep-92 & & U-238 & U-238 \\
\hline 411 & PDMS & & SW & & & E121557 & 21-Sep-92 & & $10045-97-3$ & CS-137 \\
\hline 412 & PDMS & & SW & & & E121557 & 21-Sep-92 & & 15046-84-1 & I-129 \\
\hline 413 & PDMS & & SW & & & E121557 & 21-Sep-92 & & 14133-76-7 & TC-99 \\
\hline 414 & PDMS & & SW & & & E121557 & 21-Sep-92 & & $10028-17-8$ & TRITIUM \\
\hline 415 & PDMS & & SW & & & E122018 & 31-Dec-92 & & $10045-97-3$ & CS-137 \\
\hline 416 & PDMS & & SW & & & E122018 & 31-Dec-92 & & $10098-97-2$ & SR-90 \\
\hline 417 & PDMS & & SW & & & E122018 & 31-Dec-92 & & $14133-76-7$ & TC-99 \\
\hline 418 & PDMS & & SW & & & E122018 & 31-Dec-92 & & $10028-17-8$ & TRITIUM \\
\hline 419 & PDMS & & SW & & & E124513 & 23-Aug-93 & & $10045-97-3$ & CS-137 \\
\hline
\end{tabular}




\begin{tabular}{|c|c|c|c|c|c|c|c|c|c|c|}
\hline & $\mathrm{A}$ & $\mathrm{B}$ & $C$ & $\mathrm{D}$ & $E$ & $\mathrm{~F}$ & $\mathrm{G}$ & $\mathrm{H}$ & $\mathrm{I}$ & $\mathrm{J}$ \\
\hline 1 & SAMPLE_NUM_ASSIGNED_TO & SAMPLER & MEDIA & BIOTA_MEDIA & TAG_ID & SAMP_NUM & SAMP_DATE_TIME & SAMP_DATE_TIME_ON & CON_ID & CON_SHORT_NAME \\
\hline 420 & PDMS & & SW & & & E124513 & 23-Aug-93 & & $10098-97-2$ & SR-90 \\
\hline 421 & PDMS & & SW & & & E124513 & 23-Aug-93 & & $14133-76-7$ & TC-99 \\
\hline 422 & PDMS & & SW & & & E124513 & 23-Aug-93 & & $10028-17-8$ & TRITIUM \\
\hline 423 & PDMS & & SW & & & E124513 & 23-Aug-93 & & $13966-29-5$ & U-234 \\
\hline 424 & PDMS & & SW & & & E124513 & 23-Aug-93 & & 15117-96-1 & U-235 \\
\hline 425 & PDMS & & SW & & & E124513 & 23-Aug-93 & & U-238 & $\mathrm{U}-238$ \\
\hline 426 & PDMS & & SW & & & E124514 & 23-Aug-93 & & $10045-97-3$ & CS-137 \\
\hline 427 & PDMS & & SW & & & E124514 & 23-Aug-93 & & $10098-97-2$ & SR-90 \\
\hline 428 & PDMS & & SW & & & E124514 & 23-Aug-93 & & $14133-76-7$ & TC-99 \\
\hline 429 & PDMS & & SW & & & E124514 & 23-Aug-93 & & $10028-17-8$ & TRITIUM \\
\hline 430 & PDMS & & SW & & & E124514 & 23-Aug-93 & & $13966-29-5$ & U-234 \\
\hline 431 & PDMS & & SW & & & E124514 & 23-Aug-93 & & 15117-96-1 & U-235 \\
\hline 432 & PDMS & & SW & & & E124514 & 23-Aug-93 & & U-238 & $\mathrm{U}-238$ \\
\hline 433 & & LD WALKER & SW & & & B091L1 & 15-Sep-93 & & $7440-47-3$ & CR \\
\hline 434 & & LD WALKER & SW & & & B091L2 & 15-Sep-93 & & $7440-47-3$ & CR \\
\hline 435 & & LD WALKER & SW & & & B091L1 & 15-Sep-93 & & $10098-97-2$ & SR-90 \\
\hline 436 & & LD WALKER & SW & & & B091L1 & 15-Sep-93 & & $10028-17-8$ & TRITIUM \\
\hline 437 & & LD WALKER & SW & & & B091M3 & 15-Sep-93 & & $7440-47-3$ & CR \\
\hline 438 & & LD WALKER & SW & & & B091M4 & 15-Sep-93 & & $7440-47-3$ & CR \\
\hline 439 & & LD WALKER & SW & & & B091M3 & 15-Sep-93 & & $10098-97-2$ & SR-90 \\
\hline 440 & & LD WALKER & SW & & & B091M3 & 15-Sep-93 & & $10028-17-8$ & TRITIUM \\
\hline 441 & PDMS & & SW & & & E124664 & 20-Sep-93 & & $10045-97-3$ & CS-137 \\
\hline 442 & PDMS & & SW & & & E124664 & 20-Sep-93 & & $10098-97-2$ & SR-90 \\
\hline 443 & PDMS & & SW & & & E124664 & 20-Sep-93 & & $14133-76-7$ & TC-99 \\
\hline 444 & PDMS & & SW & & & E124664 & 20-Sep-93 & & $10028-17-8$ & TRITIUM \\
\hline 445 & PDMS & & SW & & & E124664 & 20-Sep-93 & & 13966-29-5 & U-234 \\
\hline 446 & PDMS & & SW & & & E124664 & 20-Sep-93 & & 15117-96-1 & U-235 \\
\hline 447 & PDMS & & SW & & & E124664 & 20-Sep-93 & & U-238 & U-238 \\
\hline 448 & PDMS & & SW & & & E124663 & 20-Sep-93 & & $10045-97-3$ & CS-137 \\
\hline 449 & PDMS & & SW & & & E124663 & 20-Sep-93 & & 15046-84-1 & I-129 \\
\hline 450 & PDMS & & SW & & & E124663 & 20-Sep-93 & & $10098-97-2$ & SR-90 \\
\hline 451 & PDMS & & SW & & & E124663 & 20-Sep-93 & & $14133-76-7$ & TC-99 \\
\hline 452 & PDMS & & SW & & & E124663 & 20-Sep-93 & & $10028-17-8$ & TRITIUM \\
\hline 453 & PDMS & & SW & & & E124663 & 20-Sep-93 & & 13966-29-5 & U-234 \\
\hline 454 & PDMS & & SW & & & E124663 & 20-Sep-93 & & 15117-96-1 & U-235 \\
\hline 455 & PDMS & & SW & & & E124663 & 20-Sep-93 & & U-238 & $U-238$ \\
\hline 456 & PDMS & & SW & & & E124669 & 20-Sep-93 & & $10045-97-3$ & CS-137 \\
\hline 457 & PDMS & & SW & & & E124669 & 20-Sep-93 & & 15046-84-1 & $1-129$ \\
\hline
\end{tabular}




\begin{tabular}{|c|c|c|c|c|c|c|c|c|c|c|}
\hline & $\mathrm{A}$ & $\mathrm{B}$ & $C$ & $\mathrm{D}$ & $E$ & $\mathrm{~F}$ & $\mathrm{G}$ & $\mathrm{H}$ & $\mathrm{I}$ & $\mathrm{J}$ \\
\hline 1 & SAMPLE_NUM_ASSIGNED_TO & SAMPLER & MEDIA & BIOTA_MEDIA & TAG_ID & SAMP_NUM & SAMP_DATE_TIME & SAMP_DATE_TIME_ON & CON_ID & CON_SHORT_NAME \\
\hline 458 & PDMS & & SW & & & E124669 & 20-Sep-93 & & $10098-97-2$ & SR-90 \\
\hline 459 & PDMS & & SW & & & E124669 & 20-Sep-93 & & $14133-76-7$ & TC-99 \\
\hline 460 & PDMS & & SW & & & E124669 & 20-Sep-93 & & $10028-17-8$ & TRITIUM \\
\hline 461 & PDMS & & SW & & & E124669 & 20-Sep-93 & & $13966-29-5$ & U-234 \\
\hline 462 & PDMS & & SW & & & E124669 & 20-Sep-93 & & 15117-96-1 & U-235 \\
\hline 463 & PDMS & & SW & & & E124669 & 20-Sep-93 & & U-238 & $\mathrm{U}-238$ \\
\hline 464 & & LD WALKER & SW & & & B091L5 & 20-Sep-93 & & $7440-47-3$ & CR \\
\hline 465 & & LD WALKER & SW & & & B091L6 & 20-Sep-93 & & $7440-47-3$ & CR \\
\hline 466 & & LD WALKER & SW & & & B091L5 & 20-Sep-93 & & $10098-97-2$ & SR-90 \\
\hline 467 & & LD WALKER & SW & & & B091L5 & 20-Sep-93 & & $10028-17-8$ & TRITIUM \\
\hline 468 & & LD WALKER & SW & & & B091M1 & 22-Sep-93 & & $7440-47-3$ & CR \\
\hline 469 & & LD WALKER & SW & & & B091M2 & 22-Sep-93 & & $7440-47-3$ & CR \\
\hline 470 & & LD WALKER & SW & & & B091M1 & 22-Sep-93 & & $10098-97-2$ & SR-90 \\
\hline 471 & & LD WALKER & SW & & & B091M1 & 22-Sep-93 & & $10028-17-8$ & TRITIUM \\
\hline 472 & & LD WALKER & SW & & & B091L3 & 22-Sep-93 & & $7440-47-3$ & CR \\
\hline 473 & & LD WALKER & SW & & & B091L4 & 22-Sep-93 & & $7440-47-3$ & CR \\
\hline 474 & & LD WALKER & SW & & & B091L3 & 22-Sep-93 & & $10098-97-2$ & SR-90 \\
\hline 475 & & LD WALKER & SW & & & B091L3 & 22-Sep-93 & & $10028-17-8$ & TRITIUM \\
\hline 476 & PDMS & & SW & & & E124668 & 26-Sep-93 & & $10045-97-3$ & CS-137 \\
\hline 477 & PDMS & & SW & & & E124668 & 26-Sep-93 & & $10098-97-2$ & SR-90 \\
\hline 478 & PDMS & & SW & & & E124668 & 26-Sep-93 & & $14133-76-7$ & TC-99 \\
\hline 479 & PDMS & & SW & & & E124668 & 26-Sep-93 & & $10028-17-8$ & TRITIUM \\
\hline 480 & PDMS & & SW & & & E124668 & 26-Sep-93 & & 13966-29-5 & U-234 \\
\hline 481 & PDMS & & SW & & & E124668 & 26-Sep-93 & & 15117-96-1 & U-235 \\
\hline 482 & PDMS & & SW & & & E124668 & 26-Sep-93 & & U-238 & $\mathrm{U}-238$ \\
\hline 483 & PDMS & & SW & & & E124666 & 26-Sep-93 & & $10045-97-3$ & CS-137 \\
\hline 484 & PDMS & & SW & & & E124666 & 26-Sep-93 & & $10098-97-2$ & SR-90 \\
\hline 485 & PDMS & & SW & & & E124666 & 26-Sep-93 & & $14133-76-7$ & TC-99 \\
\hline 486 & PDMS & & SW & & & E124666 & 26-Sep-93 & & $10028-17-8$ & TRITIUM \\
\hline 487 & PDMS & & SW & & & E124666 & 26-Sep-93 & & 13966-29-5 & U-234 \\
\hline 488 & PDMS & & SW & & & E124666 & 26-Sep-93 & & 15117-96-1 & U-235 \\
\hline 489 & PDMS & & SW & & & E124666 & 26-Sep-93 & & U-238 & U-238 \\
\hline 490 & PDMS & & SW & & & E124667 & 26-Sep-93 & & $10045-97-3$ & CS-137 \\
\hline 491 & PDMS & & SW & & & E124667 & 26-Sep-93 & & $10098-97-2$ & SR-90 \\
\hline 492 & PDMS & & SW & & & E124667 & 26-Sep-93 & & $14133-76-7$ & TC-99 \\
\hline 493 & PDMS & & SW & & & E124667 & 26-Sep-93 & & $10028-17-8$ & TRITIUM \\
\hline 494 & PDMS & & SW & & & E124667 & 26-Sep-93 & & 13966-29-5 & U-234 \\
\hline 495 & PDMS & & SW & & & E124667 & 26-Sep-93 & & $15117-96-1$ & $\mathrm{U}-235$ \\
\hline
\end{tabular}




\begin{tabular}{|c|c|c|c|c|c|c|c|c|c|c|}
\hline & A & $\mathrm{B}$ & $\mathrm{C}$ & $\mathrm{D}$ & $\mathrm{E}$ & $\mathrm{F}$ & $\mathrm{G}$ & $\mathrm{H}$ & $\mathrm{I}$ & $\mathrm{J}$ \\
\hline 1 & SAMPLE_NUM_ASSIGNED_TO & SAMPLER & MEDIA & BIOTA_MEDIA & TAG_ID & SAMP_NUM & SAMP_DATE_TIME & SAMP_DATE_TIME_ON & CON_ID & CON_SHORT_NAME \\
\hline 496 & PDMS & & SW & & & E124667 & 26-Sep-93 & & U-238 & U-238 \\
\hline 497 & PDMS & & SW & & & E124672 & 26-Sep-93 & & $10045-97-3$ & CS-137 \\
\hline 498 & PDMS & & SW & & & E124665 & 26-Sep-93 & & $10045-97-3$ & CS-137 \\
\hline 499 & PDMS & & SW & & & E124672 & 26-Sep-93 & & $10098-97-2$ & SR-90 \\
\hline 500 & PDMS & & SW & & & E124665 & 26-Sep-93 & & $10098-97-2$ & SR-90 \\
\hline 501 & PDMS & & SW & & & E124672 & 26-Sep-93 & & $14133-76-7$ & TC-99 \\
\hline 502 & PDMS & & SW & & & E124665 & 26-Sep-93 & & $14133-76-7$ & TC-99 \\
\hline 503 & PDMS & & SW & & & E124672 & 26-Sep-93 & & $10028-17-8$ & TRITIUM \\
\hline 504 & PDMS & & SW & & & E124665 & 26-Sep-93 & & $10028-17-8$ & TRITIUM \\
\hline 505 & PDMS & & SW & & & E124672 & 26-Sep-93 & & $13966-29-5$ & U-234 \\
\hline 506 & PDMS & & SW & & & E124665 & 26-Sep-93 & & $13966-29-5$ & U-234 \\
\hline 507 & PDMS & & SW & & & E124672 & 26-Sep-93 & & $15117-96-1$ & U-235 \\
\hline 508 & PDMS & & SW & & & E124665 & 26-Sep-93 & & $15117-96-1$ & U-235 \\
\hline 509 & PDMS & & SW & & & E124672 & 26-Sep-93 & & U-238 & $\mathrm{U}-238$ \\
\hline 510 & PDMS & & SW & & & E124665 & 26-Sep-93 & & U-238 & U-238 \\
\hline 511 & PDMS & & SW & & & E124697 & 26-Sep-93 & & $10045-97-3$ & CS-137 \\
\hline 512 & PDMS & & SW & & & E124770 & 26-Sep-93 & & $10045-97-3$ & CS-137 \\
\hline 513 & PDMS & & SW & & & E124697 & 26-Sep-93 & & $10098-97-2$ & SR-90 \\
\hline 514 & PDMS & & SW & & & E124770 & 26-Sep-93 & & $10098-97-2$ & SR-90 \\
\hline 515 & PDMS & & SW & & & E124697 & 26-Sep-93 & & $14133-76-7$ & TC-99 \\
\hline 516 & PDMS & & SW & & & E124770 & 26-Sep-93 & & $14133-76-7$ & TC-99 \\
\hline 517 & PDMS & & SW & & & E124697 & 26-Sep-93 & & $10028-17-8$ & TRITIUM \\
\hline 518 & PDMS & & SW & & & E124770 & 26-Sep-93 & & $10028-17-8$ & TRITIUM \\
\hline 519 & PDMS & & SW & & & E124697 & 26-Sep-93 & & $13966-29-5$ & U-234 \\
\hline 520 & PDMS & & SW & & & E124770 & 26-Sep-93 & & $13966-29-5$ & U-234 \\
\hline 521 & PDMS & & SW & & & E124697 & 26-Sep-93 & & $15117-96-1$ & U-235 \\
\hline 522 & PDMS & & SW & & & E124770 & 26-Sep-93 & & 15117-96-1 & U-235 \\
\hline 523 & PDMS & & SW & & & E124697 & 26-Sep-93 & & U-238 & U-238 \\
\hline 524 & PDMS & & SW & & & E124770 & 26-Sep-93 & & U-238 & U-238 \\
\hline 525 & PDMS & & SW & & & E124698 & 26-Sep-93 & & $10045-97-3$ & CS-137 \\
\hline 526 & PDMS & & SW & & & E124698 & 26-Sep-93 & & $10098-97-2$ & SR-90 \\
\hline 527 & PDMS & & SW & & & E124698 & 26-Sep-93 & & $14133-76-7$ & TC-99 \\
\hline 528 & PDMS & & SW & & & E124698 & 26-Sep-93 & & $10028-17-8$ & TRITIUM \\
\hline 529 & PDMS & & SW & & & E124698 & 26-Sep-93 & & $13966-29-5$ & U-234 \\
\hline 530 & PDMS & & SW & & & E124698 & 26-Sep-93 & & $15117-96-1$ & U-235 \\
\hline 531 & PDMS & & SW & & & E124698 & 26-Sep-93 & & U-238 & $\mathrm{U}-238$ \\
\hline 532 & & LD WALKER & SW & & & B091L9 & 28-Sep-93 & & $7440-47-3$ & $\mathrm{CR}$ \\
\hline 533 & & LD WALKER & SW & & & B091M0 & 28-Sep-93 & & $7440-47-3$ & CR \\
\hline
\end{tabular}




\begin{tabular}{|c|c|c|c|c|c|c|c|c|c|c|}
\hline & $\mathrm{A}$ & $\mathrm{B}$ & $C$ & $\mathrm{D}$ & $E$ & $\mathrm{~F}$ & $\mathrm{G}$ & $\mathrm{H}$ & $\mathrm{I}$ & $\mathrm{J}$ \\
\hline 1 & SAMPLE_NUM_ASSIGNED_TO & SAMPLER & MEDIA & BIOTA_MEDIA & TAG_ID & SAMP_NUM & SAMP_DATE_TIME & SAMP_DATE_TIME_ON & CON_ID & CON_SHORT_NAME \\
\hline 534 & & LD WALKER & SW & & & B091L9 & 28-Sep-93 & & $10098-97-2$ & SR-90 \\
\hline 535 & & LD WALKER & SW & & & B091L9 & 28-Sep-93 & & $10028-17-8$ & TRITIUM \\
\hline 536 & & LD WALKER & SW & & & B091L7 & 28-Sep-93 & & $7440-47-3$ & CR \\
\hline 537 & & LD WALKER & SW & & & B091L8 & 28-Sep-93 & & $7440-47-3$ & CR \\
\hline 538 & & LD WALKER & SW & & & B091L7 & 28-Sep-93 & & $10098-97-2$ & SR-90 \\
\hline 539 & & LD WALKER & SW & & & B091L7 & 28-Sep-93 & & $10028-17-8$ & TRITIUM \\
\hline 540 & PDMS & & SW & & & E124782 & 30-Sep-93 & & $10045-97-3$ & CS-137 \\
\hline 541 & PDMS & & SW & & & E124782 & 30-Sep-93 & & $10098-97-2$ & SR-90 \\
\hline 542 & PDMS & & SW & & & E124782 & 30-Sep-93 & & $14133-76-7$ & TC-99 \\
\hline 543 & PDMS & & SW & & & E124782 & 30-Sep-93 & & $10028-17-8$ & TRITIUM \\
\hline 544 & PDMS & & SW & & & E124782 & 30-Sep-93 & & $13966-29-5$ & U-234 \\
\hline 545 & PDMS & & SW & & & E124782 & 30-Sep-93 & & 15117-96-1 & U-235 \\
\hline 546 & PDMS & & SW & & & E124782 & 30-Sep-93 & & U-238 & $\mathrm{U}-238$ \\
\hline 547 & & GG HAMILTON & SW & & & B09BB2 & 04-Oct-93 & & $10098-97-2$ & SR-90 \\
\hline 548 & & GG HAMILTON & SW & & & B09BB3 & 04-Oct-93 & & $10098-97-2$ & SR-90 \\
\hline 549 & & LD WALKER & SW & & & B0BMJ6 & 31-Mar-94 & & $7440-47-3$ & CR \\
\hline 550 & & LD WALKER & SW & & & B0BMJ7 & 31-Mar-94 & & $7440-47-3$ & CR \\
\hline 551 & & LD WALKER & SW & & & B0BMJ8 & 31-Mar-94 & & $18540-29-9$ & $\mathrm{CR}+6$ \\
\hline 552 & & LD WALKER & SW & & & B0BMJ8 & 31-Mar-94 & & $18540-29-9$ & $\mathrm{CR}+6$ \\
\hline 553 & & LD WALKER & SW & & & B0BMJ9 & 01-Apr-94 & & $7440-47-3$ & CR \\
\hline 554 & & LD WALKER & SW & & & BOBMKO & 01-Apr-94 & & $7440-47-3$ & CR \\
\hline 555 & & LD WALKER & SW & & & B0BMK1 & 01-Apr-94 & & $18540-29-9$ & $\mathrm{CR}+6$ \\
\hline 556 & & LD WALKER & SW & & & B0BMK1 & 01-Apr-94 & & $18540-29-9$ & $\mathrm{CR}+6$ \\
\hline 557 & & LD WALKER & SW & & & BOBMK2 & 01-Apr-94 & & $7440-47-3$ & CR \\
\hline 558 & & LD WALKER & SW & & & BOBMK3 & 01-Apr-94 & & $7440-47-3$ & $\mathrm{CR}$ \\
\hline 559 & & LD WALKER & SW & & & B0BMK4 & 01-Apr-94 & & $18540-29-9$ & $\mathrm{CR}+6$ \\
\hline 560 & & LD WALKER & SW & & & B0BMK4 & 01-Apr-94 & & $18540-29-9$ & $\mathrm{CR}+6$ \\
\hline 561 & & LD WALKER & SW & & & B0BMK5 & 01-Apr-94 & & $7440-47-3$ & CR \\
\hline 562 & & LD WALKER & SW & & & B0BMK6 & 01-Apr-94 & & $7440-47-3$ & CR \\
\hline 563 & & LD WALKER & SW & & & B0BMK7 & 01-Apr-94 & & $18540-29-9$ & $\mathrm{CR}+6$ \\
\hline 564 & & LD WALKER & SW & & & B0BMK7 & 01-Apr-94 & & $18540-29-9$ & $\mathrm{CR}+6$ \\
\hline 565 & PS HENRY/LE BISPING & & SW & & & B0CSD9 & 22-Aug-94 & & $56-23-5$ & CARBTET \\
\hline 566 & PS HENRY/LE BISPING & & SW & & & B0CSD9 & 22-Aug-94 & & $7440-47-3$ & CR \\
\hline 567 & SESPSCH & & SW & & & B0C5D7 & 22-Aug-94 & & $10045-97-3$ & CS-137 \\
\hline 568 & SESPSCH & & SW & & & B0C5D7 & 22-Aug-94 & & $10098-97-2$ & SR-90 \\
\hline 569 & SESPSCH & & SW & & & B0C5D7 & 22-Aug-94 & & $14133-76-7$ & TC-99 \\
\hline 570 & SESPSCH & & SW & & & B0C5D7 & 22-Aug-94 & & $10028-17-8$ & TRITIUM \\
\hline 571 & SESPSCH & & SW & & & B0C5D7 & 22-Aug-94 & & 13966-29-5 & $\mathrm{U}-234$ \\
\hline
\end{tabular}




\begin{tabular}{|c|c|c|c|c|c|c|c|c|c|c|}
\hline & A & $B$ & $\mathrm{C}$ & $\mathrm{D}$ & $\mathrm{E}$ & $\mathrm{F}$ & G & $\mathrm{H}$ & $\mathrm{I}$ & $\mathrm{J}$ \\
\hline 1 & SAMPLE_NUM_ASSIGNED_TO & SAMPLER & MEDIA & BIOTA_MEDIA & TAG_ID & SAMP_NUM & SAMP_DATE_TIME & SAMP_DATE_TIME_ON & CON_ID & CON_SHORT_NAME \\
\hline 572 & SESPSCH & & SW & & & B0C5D7 & 22-Aug-94 & & 15117-96-1 & U-235 \\
\hline 573 & SESPSCH & & SW & & & B0C5D7 & 22-Aug-94 & & U-238 & $\mathrm{U}-238$ \\
\hline 574 & PS HENRY/LE BISPING & & SW & & & B0CSF5 & 22-Aug-94 & & $56-23-5$ & CARBTET \\
\hline 575 & PS HENRY/LE BISPING & & SW & & & B0CSF5 & 22-Aug-94 & & $7440-47-3$ & CR \\
\hline 576 & SESPSCH & & SW & & & B0C5F5 & 22-Aug-94 & & $10045-97-3$ & CS-137 \\
\hline 577 & SESPSCH & & SW & & & B0C5F5 & 22-Aug-94 & & $10098-97-2$ & SR-90 \\
\hline 578 & SESPSCH & & SW & & & B0C5F5 & 22-Aug-94 & & 14133-76-7 & TC-99 \\
\hline 579 & SESPSCH & & SW & & & B0C5F5 & 22-Aug-94 & & $10028-17-8$ & TRITIUM \\
\hline 580 & SESPSCH & & SW & & & B0C5F5 & 22-Aug-94 & & 13966-29-5 & U-234 \\
\hline 581 & SESPSCH & & SW & & & B0C5F5 & 22-Aug-94 & & 15117-96-1 & U-235 \\
\hline 582 & SESPSCH & & SW & & & B0C5F5 & 22-Aug-94 & & U-238 & $\mathrm{U}-238$ \\
\hline 583 & PS HENRY/LE BISPING & & SW & & & B0CSF7 & 25-Aug-94 & & $56-23-5$ & CARBTET \\
\hline 584 & PS HENRY/LE BISPING & & SW & & & B0CSF7 & 25-Aug-94 & & $7440-47-3$ & $\mathrm{CR}$ \\
\hline 585 & SESPSCH & & SW & & & B0C5F7 & 25-Aug-94 & & $10045-97-3$ & CS-137 \\
\hline 586 & SESPSCH & & SW & & & B0C5F7 & 25-Aug-94 & & $10098-97-2$ & SR-90 \\
\hline 587 & SESPSCH & & SW & & & B0C5F7 & 25-Aug-94 & & 14133-76-7 & TC-99 \\
\hline 588 & SESPSCH & & SW & & & B0C5F7 & 25-Aug-94 & & $10028-17-8$ & TRITIUM \\
\hline 589 & SESPSCH & & SW & & & B0C5F7 & 25-Aug-94 & & 13966-29-5 & $\mathrm{U}-234$ \\
\hline 590 & SESPSCH & & SW & & & B0C5F7 & 25-Aug-94 & & 15117-96-1 & U-235 \\
\hline 591 & SESPSCH & & SW & & & B0C5F7 & 25-Aug-94 & & U-238 & $\mathrm{U}-238$ \\
\hline 592 & PS HENRY/LE BISPING & & SW & & & B0CSG3 & 29-Aug-94 & & $56-23-5$ & CARBTET \\
\hline 593 & PS HENRY/LE BISPING & & SW & & & B0CSG3 & 29-Aug-94 & & $7440-47-3$ & CR \\
\hline 594 & SESPSCH & & SW & & & B0C5G4 & 29-Aug-94 & & $10045-97-3$ & CS-137 \\
\hline 595 & SESPSCH & & SW & & & B0C5G5 & 29-Aug-94 & & 15046-84-1 & I-129 \\
\hline 596 & SESPSCH & & SW & & & B0C5G4 & 29-Aug-94 & & 10098-97-2 & SR-90 \\
\hline 597 & SESPSCH & & SW & & & B0C5G4 & 29-Aug-94 & & $14133-76-7$ & TC-99 \\
\hline 598 & SESPSCH & & SW & & & B0C5G4 & 29-Aug-94 & & $10028-17-8$ & TRITIUM \\
\hline 599 & SESPSCH & & SW & & & B0C5G4 & 29-Aug-94 & & 13966-29-5 & $\mathrm{U}-234$ \\
\hline 600 & SESPSCH & & SW & & & B0C5G4 & 29-Aug-94 & & 15117-96-1 & U-235 \\
\hline 601 & SESPSCH & & SW & & & B0C5G4 & 29-Aug-94 & & U-238 & $\mathrm{U}-238$ \\
\hline 602 & PS HENRY/LE BISPING & & SW & & & B0CSG1 & 02-Sep-94 & & $56-23-5$ & CARBTET \\
\hline 603 & PS HENRY/LE BISPING & & SW & & & B0CSG1 & 02-Sep-94 & & $7440-47-3$ & $\mathrm{CR}$ \\
\hline 604 & SESPSCH & & SW & & & B0C5G1 & 02-Sep-94 & & $10045-97-3$ & CS-137 \\
\hline 605 & SESPSCH & & SW & & & B0C5G2 & 02-Sep-94 & & 15046-84-1 & I-129 \\
\hline 606 & SESPSCH & & SW & & & B0C5G1 & 02-Sep-94 & & $10098-97-2$ & SR-90 \\
\hline 607 & SESPSCH & & SW & & & B0C5G1 & 02-Sep-94 & & 14133-76-7 & TC-99 \\
\hline 608 & SESPSCH & & SW & & & B0C5G1 & 02-Sep-94 & & $10028-17-8$ & TRITIUM \\
\hline 609 & SESPSCH & & SW & & & B0C5G1 & 02-Sep-94 & & 13966-29-5 & U-234 \\
\hline
\end{tabular}




\begin{tabular}{|c|c|c|c|c|c|c|c|c|c|c|}
\hline & A & $B$ & $\mathrm{C}$ & $\mathrm{D}$ & $\mathrm{E}$ & $\mathrm{F}$ & G & $\mathrm{H}$ & $\mathrm{I}$ & $\mathrm{J}$ \\
\hline 1 & SAMPLE_NUM_ASSIGNED_TO & SAMPLER & MEDIA & BIOTA_MEDIA & TAG_ID & SAMP_NUM & SAMP_DATE_TIME & SAMP_DATE_TIME_ON & CON_ID & CON_SHORT_NAME \\
\hline 610 & SESPSCH & & SW & & & B0C5G1 & 02-Sep-94 & & $15117-96-1$ & U-235 \\
\hline 611 & SESPSCH & & SW & & & B0C5G1 & 02-Sep-94 & & U-238 & $\mathrm{U}-238$ \\
\hline 612 & PS HENRY/LE BISPING & & SW & & & BOCSFO & 12-Sep-94 & & $56-23-5$ & CARBTET \\
\hline 613 & PS HENRY/LE BISPING & & SW & & & BOCSFO & 12-Sep-94 & & $7440-47-3$ & CR \\
\hline 614 & SESPSCH & & SW & & & B0C5D8 & 12-Sep-94 & & $10045-97-3$ & CS-137 \\
\hline 615 & SESPSCH & & SW & & & B0C5D8 & 12-Sep-94 & & $10098-97-2$ & SR-90 \\
\hline 616 & SESPSCH & & SW & & & B0C5D8 & 12-Sep-94 & & 14133-76-7 & TC-99 \\
\hline 617 & SESPSCH & & SW & & & B0C5D8 & 12-Sep-94 & & $10028-17-8$ & TRITIUM \\
\hline 618 & SESPSCH & & SW & & & B0C5D8 & 12-Sep-94 & & 13966-29-5 & U-234 \\
\hline 619 & SESPSCH & & SW & & & B0C5D8 & 12-Sep-94 & & 15117-96-1 & U-235 \\
\hline 620 & SESPSCH & & SW & & & B0C5D8 & 12-Sep-94 & & U-238 & $\mathrm{U}-238$ \\
\hline 621 & PS HENRY/LE BISPING & & SW & & & B0CSG2 & 12-Sep-94 & & $56-23-5$ & CARBTET \\
\hline 622 & PS HENRY/LE BISPING & & SW & & & B0CSG2 & 12-Sep-94 & & $7440-47-3$ & $\mathrm{CR}$ \\
\hline 623 & SESPSCH & & SW & & & B0C5G3 & 12-Sep-94 & & $10045-97-3$ & CS-137 \\
\hline 624 & SESPSCH & & SW & & & B0C5G3 & 12-Sep-94 & & $10098-97-2$ & SR-90 \\
\hline 625 & SESPSCH & & SW & & & B0C5G3 & 12-Sep-94 & & 14133-76-7 & TC-99 \\
\hline 626 & SESPSCH & & SW & & & B0C5G3 & 12-Sep-94 & & $10028-17-8$ & TRITIUM \\
\hline 627 & SESPSCH & & SW & & & B0C5G3 & 12-Sep-94 & & 13966-29-5 & $\mathrm{U}-234$ \\
\hline 628 & SESPSCH & & SW & & & B0C5G3 & 12-Sep-94 & & 15117-96-1 & U-235 \\
\hline 629 & SESPSCH & & SW & & & B0C5G3 & 12-Sep-94 & & U-238 & $\mathrm{U}-238$ \\
\hline 630 & SESPSCH & & SW & & & B0C5G6 & 25-Sep-94 & & $10045-97-3$ & CS-137 \\
\hline 631 & SESPSCH & & SW & & & B0C5G6 & 25-Sep-94 & & $10098-97-2$ & SR-90 \\
\hline 632 & SESPSCH & & SW & & & B0C5G6 & 25-Sep-94 & & 14133-76-7 & TC-99 \\
\hline 633 & SESPSCH & & SW & & & B0C5G6 & 25-Sep-94 & & $10028-17-8$ & TRITIUM \\
\hline 634 & SESPSCH & & SW & & & B0C5G6 & 25-Sep-94 & & 13966-29-5 & $\mathrm{U}-234$ \\
\hline 635 & SESPSCH & & SW & & & B0C5G6 & 25-Sep-94 & & 15117-96-1 & U-235 \\
\hline 636 & SESPSCH & & SW & & & B0C5G6 & 25-Sep-94 & & U-238 & $\mathrm{U}-238$ \\
\hline 637 & PS HENRY/LE BISPING & & SW & & & B0CSF6 & 26-Sep-94 & & $56-23-5$ & CARBTET \\
\hline 638 & PS HENRY/LE BISPING & & SW & & & B0CSF6 & 26-Sep-94 & & $7440-47-3$ & CR \\
\hline 639 & SESPSCH & & SW & & & B0C5F6 & 26-Sep-94 & & $10045-97-3$ & CS-137 \\
\hline 640 & SESPSCH & & SW & & & B0C5F6 & 26-Sep-94 & & $10098-97-2$ & SR-90 \\
\hline 641 & SESPSCH & & SW & & & B0C5F6 & 26-Sep-94 & & 14133-76-7 & TC-99 \\
\hline 642 & SESPSCH & & SW & & & B0C5F6 & 26-Sep-94 & & $10028-17-8$ & TRITIUM \\
\hline 643 & SESPSCH & & SW & & & B0C5F6 & 26-Sep-94 & & 13966-29-5 & U-234 \\
\hline 644 & SESPSCH & & SW & & & B0C5F6 & 26-Sep-94 & & 15117-96-1 & $\mathrm{U}-235$ \\
\hline 645 & SESPSCH & & SW & & & B0C5F6 & 26-Sep-94 & & U-238 & $\mathrm{U}-238$ \\
\hline 646 & PS HENRY/LE BISPING & & SW & & & BOCSG0 & 26-Sep-94 & & $56-23-5$ & CARBTET \\
\hline 647 & PS HENRY/LE BISPING & & SW & & & BOCSG0 & 26-Sep-94 & & $7440-47-3$ & $\mathrm{CR}$ \\
\hline
\end{tabular}




\begin{tabular}{|c|c|c|c|c|c|c|c|c|c|c|}
\hline & A & $\mathrm{B}$ & $\mathrm{C}$ & $\mathrm{D}$ & $\mathrm{E}$ & $\mathrm{F}$ & $\mathrm{G}$ & $\mathrm{H}$ & $\mathrm{I}$ & $\mathrm{J}$ \\
\hline 1 & SAMPLE_NUM_ASSIGNED_TO & SAMPLER & MEDIA & BIOTA_MEDIA & TAG_ID & SAMP_NUM & SAMP_DATE_TIME & SAMP_DATE_TIME_ON & CON_ID & CON_SHORT_NAME \\
\hline 648 & SESPSCH & & SW & & & B0C5G0 & 26-Sep-94 & & $10045-97-3$ & CS-137 \\
\hline 649 & SESPSCH & & SW & & & B0C5G0 & 26-Sep-94 & & $10098-97-2$ & SR-90 \\
\hline 650 & SESPSCH & & SW & & & B0C5G0 & 26-Sep-94 & & 14133-76-7 & TC-99 \\
\hline 651 & SESPSCH & & SW & & & B0C5G0 & 26-Sep-94 & & $10028-17-8$ & TRITIUM \\
\hline 652 & SESPSCH & & SW & & & B0C5G0 & 26-Sep-94 & & $13966-29-5$ & $\mathrm{U}-234$ \\
\hline 653 & SESPSCH & & SW & & & B0C5G0 & 26-Sep-94 & & 15117-96-1 & U-235 \\
\hline 654 & SESPSCH & & SW & & & B0C5G0 & 26-Sep-94 & & U-238 & $\mathrm{U}-238$ \\
\hline 655 & PS HENRY/LE BISPING & & SW & & & BOCSF8 & 26-Sep-94 & & $56-23-5$ & CARBTET \\
\hline 656 & PS HENRY/LE BISPING & & SW & & & BOCSF8 & 26-Sep-94 & & $7440-47-3$ & $\mathrm{CR}$ \\
\hline 657 & SESPSCH & & SW & & & B0C5F8 & 26-Sep-94 & & $10045-97-3$ & CS-137 \\
\hline 658 & SESPSCH & & SW & & & B0C5F8 & 26-Sep-94 & & $10098-97-2$ & SR-90 \\
\hline 659 & SESPSCH & & SW & & & B0C5F8 & 26-Sep-94 & & $14133-76-7$ & TC-99 \\
\hline 660 & SESPSCH & & SW & & & B0C5F8 & 26-Sep-94 & & $10028-17-8$ & TRITIUM \\
\hline 661 & SESPSCH & & SW & & & B0C5F8 & 26-Sep-94 & & $13966-29-5$ & U-234 \\
\hline 662 & SESPSCH & & SW & & & B0C5F8 & 26-Sep-94 & & $15117-96-1$ & U-235 \\
\hline 663 & SESPSCH & & SW & & & B0C5F8 & 26-Sep-94 & & U-238 & U-238 \\
\hline 664 & PS HENRY/LE BISPING & & SW & & & B0CSF3 & 26-Sep-94 & & $56-23-5$ & CARBTET \\
\hline 665 & PS HENRY/LE BISPING & & SW & & & BOCSF3 & 26-Sep-94 & & $7440-47-3$ & $\mathrm{CR}$ \\
\hline 666 & SESPSCH & & SW & & & B0C5F2 & 26-Sep-94 & & $10045-97-3$ & CS-137 \\
\hline 667 & SESPSCH & & SW & & & B0C5F2 & 26-Sep-94 & & $10098-97-2$ & SR-90 \\
\hline 668 & SESPSCH & & SW & & & B0C5F2 & 26-Sep-94 & & $14133-76-7$ & TC-99 \\
\hline 669 & SESPSCH & & SW & & & B0C5F2 & 26-Sep-94 & & $10028-17-8$ & TRITIUM \\
\hline 670 & SESPSCH & & SW & & & B0C5F2 & 26-Sep-94 & & $13966-29-5$ & U-234 \\
\hline 671 & SESPSCH & & SW & & & B0C5F2 & 26-Sep-94 & & $15117-96-1$ & $\mathrm{U}-235$ \\
\hline 672 & SESPSCH & & SW & & & B0C5F2 & 26-Sep-94 & & U-238 & U-238 \\
\hline 673 & & DOUG BOWERS & SW & & & BOGBJ2 & 03-Aug-95 & & $10098-97-2$ & SR-90 \\
\hline 674 & & DOUG BOWERS & SW & & & B0GBH5 & 03-Aug-95 & & $10098-97-2$ & SR-90 \\
\hline 675 & & DOUG BOWERS & SW & & & B0GBH6 & 03-Aug-95 & & $10098-97-2$ & SR-90 \\
\hline 676 & & DOUG BOWERS & SW & & & B0GBH7 & 03-Aug-95 & & $10098-97-2$ & SR-90 \\
\hline 677 & & DOUG BOWERS & SW & & & B0GBH8 & 03-Aug-95 & & $10098-97-2$ & SR-90 \\
\hline 678 & & DOUG BOWERS & SW & & & BOGBH9 & 03-Aug-95 & & $10098-97-2$ & SR-90 \\
\hline 679 & & DOUG BOWERS & SW & & & BOGBJO & 03-Aug-95 & & $10098-97-2$ & SR-90 \\
\hline 680 & & DOUG BOWERS & SW & & & B0GBJ1 & 03-Aug-95 & & $10098-97-2$ & SR-90 \\
\hline 681 & & DOUG BOWERS & SW & & & B0GBJ3 & 03-Aug-95 & & 10098-97-2 & SR-90 \\
\hline 682 & & DOUG BOWERS & SW & & & B0GBJ4 & 04-Aug-95 & & $10098-97-2$ & SR-90 \\
\hline 683 & & DOUG BOWERS & SW & & & B0GBJ5 & 04-Aug-95 & & $10098-97-2$ & SR-90 \\
\hline 684 & & DOUG BOWERS & SW & & & B0GBJ6 & 04-Aug-95 & & $10098-97-2$ & SR-90 \\
\hline 685 & & DOUG BOWERS & SW & & & B0GBJ7 & 04-Aug-95 & & $10098-97-2$ & SR-90 \\
\hline
\end{tabular}




\begin{tabular}{|c|c|c|c|c|c|c|c|c|c|c|}
\hline & A & $\mathrm{B}$ & $\mathrm{C}$ & $\mathrm{D}$ & $\mathrm{E}$ & $\mathrm{F}$ & $\mathrm{G}$ & $\mathrm{H}$ & $\mathrm{I}$ & $\mathrm{J}$ \\
\hline 1 & SAMPLE_NUM_ASSIGNED_TO & SAMPLER & MEDIA & BIOTA_MEDIA & TAG_ID & SAMP_NUM & SAMP_DATE_TIME & SAMP_DATE_TIME_ON & CON_ID & CON_SHORT_NAME \\
\hline 686 & & DOUG BOWERS & SW & & & B0GBJ9 & 04-Aug-95 & & $10098-97-2$ & SR-90 \\
\hline 687 & & DOUG BOWERS & SW & & & BOGBK2 & 04-Aug-95 & & $10098-97-2$ & SR-90 \\
\hline 688 & PS HENRY/LE BISPING & & SW & & & B0G0Q1 & 28-Aug-95 & & $56-23-5$ & CARBTET \\
\hline 689 & PS HENRY/LE BISPING & & SW & & & B0G0Q1 & 28-Aug-95 & & $7440-47-3$ & CR \\
\hline 690 & SESPSCH & & SW & & & B0G8B3 & 28-Aug-95 & & $10045-97-3$ & CS-137 \\
\hline 691 & SESPSCH & & SW & & & B0G8B3 & 28-Aug-95 & & $10098-97-2$ & SR-90 \\
\hline 692 & SESPSCH & & SW & & & B0G8B3 & 28-Aug-95 & & $14133-76-7$ & TC-99 \\
\hline 693 & SESPSCH & & SW & & & B0G8B3 & 28-Aug-95 & & $10028-17-8$ & TRITIUM \\
\hline 694 & SESPSCH & & SW & & & B0G8B3 & 28-Aug-95 & & $13966-29-5$ & U-234 \\
\hline 695 & SESPSCH & & SW & & & B0G8B3 & 28-Aug-95 & & $15117-96-1$ & $\mathrm{U}-235$ \\
\hline 696 & SESPSCH & & SW & & & B0G8B3 & 28-Aug-95 & & U-238 & $\mathrm{U}-238$ \\
\hline 697 & PS HENRY/LE BISPING & & SW & & & B0G0Q4 & 29-Aug-95 & & $56-23-5$ & CARBTET \\
\hline 698 & PS HENRY/LE BISPING & & SW & & & B0G0Q4 & 29-Aug-95 & & $7440-47-3$ & $\mathrm{CR}$ \\
\hline 699 & SESPSCH & & SW & & & B0G8B7 & 29-Aug-95 & & $10045-97-3$ & CS-137 \\
\hline 700 & SESPSCH & & SW & & & B0G8B7 & 29-Aug-95 & & $10098-97-2$ & SR-90 \\
\hline 701 & SESPSCH & & SW & & & B0G8B7 & 29-Aug-95 & & $14133-76-7$ & TC-99 \\
\hline 702 & SESPSCH & & SW & & & B0G8B7 & 29-Aug-95 & & $10028-17-8$ & TRITIUM \\
\hline 703 & SESPSCH & & SW & & & B0G8B7 & 29-Aug-95 & & $13966-29-5$ & U-234 \\
\hline 704 & SESPSCH & & SW & & & B0G8B7 & 29-Aug-95 & & $15117-96-1$ & U-235 \\
\hline 705 & SESPSCH & & SW & & & B0G8B7 & 29-Aug-95 & & U-238 & $\mathrm{U}-238$ \\
\hline 706 & PS HENRY/LE BISPING & & SW & & & B0G0Q3 & 29-Aug-95 & & $56-23-5$ & CARBTET \\
\hline 707 & PS HENRY/LE BISPING & & SW & & & B0G0Q3 & 29-Aug-95 & & $7440-47-3$ & CR \\
\hline 708 & SESPSCH & & SW & & & B0G8B5 & 29-Aug-95 & & $10045-97-3$ & CS-137 \\
\hline 709 & SESPSCH & & SW & & & B0G8B5 & 29-Aug-95 & & $10098-97-2$ & SR-90 \\
\hline 710 & SESPSCH & & SW & & & B0G8B5 & 29-Aug-95 & & $14133-76-7$ & TC-99 \\
\hline 711 & SESPSCH & & SW & & & B0G8B5 & 29-Aug-95 & & $10028-17-8$ & TRITIUM \\
\hline 712 & SESPSCH & & SW & & & B0G8B5 & 29-Aug-95 & & $13966-29-5$ & $U-234$ \\
\hline 713 & SESPSCH & & SW & & & B0G8B5 & 29-Aug-95 & & $15117-96-1$ & U-235 \\
\hline 714 & SESPSCH & & SW & & & B0G8B5 & 29-Aug-95 & & U-238 & $\mathrm{U}-238$ \\
\hline 715 & PS HENRY/LE BISPING & & SW & & & B0G0Q5 & 05-Sep-95 & & $56-23-5$ & CARBTET \\
\hline 716 & PS HENRY/LE BISPING & & SW & & & B0G0Q5 & 05-Sep-95 & & $7440-47-3$ & CR \\
\hline 717 & SESPSCH & & SW & & & B0G8B8 & 05-Sep-95 & & $10045-97-3$ & CS-137 \\
\hline 718 & SESPSCH & & SW & & & B0G8B8 & 05-Sep-95 & & $10098-97-2$ & SR-90 \\
\hline 719 & SESPSCH & & SW & & & B0G8B8 & 05-Sep-95 & & $14133-76-7$ & TC-99 \\
\hline 720 & SESPSCH & & SW & & & B0G8B8 & 05-Sep-95 & & $10028-17-8$ & TRITIUM \\
\hline 721 & SESPSCH & & SW & & & B0G8B8 & 05-Sep-95 & & $13966-29-5$ & $\mathrm{U}-234$ \\
\hline 722 & SESPSCH & & SW & & & B0G8B8 & 05-Sep-95 & & $15117-96-1$ & U-235 \\
\hline 723 & SESPSCH & & SW & & & B0G8B8 & 05-Sep-95 & & U-238 & U-238 \\
\hline
\end{tabular}




\begin{tabular}{|c|c|c|c|c|c|c|c|c|c|c|}
\hline & A & $\mathrm{B}$ & $\mathrm{C}$ & $\mathrm{D}$ & $\mathrm{E}$ & $\mathrm{F}$ & $\mathrm{G}$ & $\mathrm{H}$ & $\mathrm{I}$ & $\mathrm{J}$ \\
\hline 1 & SAMPLE_NUM_ASSIGNED_TO & SAMPLER & MEDIA & BIOTA_MEDIA & TAG_ID & SAMP_NUM & SAMP_DATE_TIME & SAMP_DATE_TIME_ON & CON_ID & CON_SHORT_NAME \\
\hline 724 & PS HENRY/LE BISPING & & SW & & & B0G0Q2 & 05-Sep-95 & & $56-23-5$ & CARBTET \\
\hline 725 & PS HENRY/LE BISPING & & SW & & & B0G0Q2 & 05-Sep-95 & & $7440-47-3$ & CR \\
\hline 726 & SESPSCH & & SW & & & B0G8B4 & 05-Sep-95 & & $10045-97-3$ & CS-137 \\
\hline 727 & SESPSCH & & SW & & & B0G8B4 & 05-Sep-95 & & $10098-97-2$ & SR-90 \\
\hline 728 & SESPSCH & & SW & & & B0G8B4 & 05-Sep-95 & & 14133-76-7 & TC-99 \\
\hline 729 & SESPSCH & & SW & & & B0G8B4 & 05-Sep-95 & & $10028-17-8$ & TRITIUM \\
\hline 730 & SESPSCH & & SW & & & B0G8B4 & 05-Sep-95 & & $13966-29-5$ & U-234 \\
\hline 731 & SESPSCH & & SW & & & B0G8B4 & 05-Sep-95 & & 15117-96-1 & U-235 \\
\hline 732 & SESPSCH & & SW & & & B0G8B4 & 05-Sep-95 & & U-238 & $\mathrm{U}-238$ \\
\hline 733 & PS HENRY/LE BISPING & & SW & & & B0G0Q8 & 05-Sep-95 & & $56-23-5$ & CARBTET \\
\hline 734 & PS HENRY/LE BISPING & & SW & & & B0G0Q8 & 05-Sep-95 & & $7440-47-3$ & $\mathrm{CR}$ \\
\hline 735 & SESPSCH & & SW & & & B0G8C1 & 05-Sep-95 & & $10045-97-3$ & CS-137 \\
\hline 736 & SESPSCH & & SW & & & B0G8F2 & 05-Sep-95 & & $15046-84-1$ & I-129 \\
\hline 737 & SESPSCH & & SW & & & B0G8C1 & 05-Sep-95 & & $10098-97-2$ & SR-90 \\
\hline 738 & SESPSCH & & SW & & & B0G8C1 & 05-Sep-95 & & 14133-76-7 & TC-99 \\
\hline 739 & SESPSCH & & SW & & & B0G8C1 & 05-Sep-95 & & $10028-17-8$ & TRITIUM \\
\hline 740 & SESPSCH & & SW & & & B0G8C1 & 05-Sep-95 & & $13966-29-5$ & U-234 \\
\hline 741 & SESPSCH & & SW & & & B0G8C1 & 05-Sep-95 & & 15117-96-1 & U-235 \\
\hline 742 & SESPSCH & & SW & & & B0G8C1 & 05-Sep-95 & & U-238 & U-238 \\
\hline 743 & PS HENRY/LE BISPING & & SW & & & B0G0Q7 & 05-Sep-95 & & $56-23-5$ & CARBTET \\
\hline 744 & PS HENRY/LE BISPING & & SW & & & B0G0Q7 & 05-Sep-95 & & $7440-47-3$ & $\mathrm{CR}$ \\
\hline 745 & SESPSCH & & SW & & & BOG8C0 & 05-Sep-95 & & $10045-97-3$ & CS-137 \\
\hline 746 & SESPSCH & & SW & & & B0G8F1 & 05-Sep-95 & & $15046-84-1$ & I-129 \\
\hline 747 & SESPSCH & & SW & & & B0G8C0 & 05-Sep-95 & & $10098-97-2$ & SR-90 \\
\hline 748 & SESPSCH & & SW & & & B0G8C0 & 05-Sep-95 & & 14133-76-7 & TC-99 \\
\hline 749 & SESPSCH & & SW & & & B0G8C0 & 05-Sep-95 & & $10028-17-8$ & TRITIUM \\
\hline 750 & SESPSCH & & SW & & & B0G8C0 & 05-Sep-95 & & $13966-29-5$ & U-234 \\
\hline 751 & SESPSCH & & SW & & & B0G8C0 & 05-Sep-95 & & $15117-96-1$ & U-235 \\
\hline 752 & SESPSCH & & SW & & & B0G8C0 & 05-Sep-95 & & U-238 & $\mathrm{U}-238$ \\
\hline 753 & PS HENRY/LE BISPING & & SW & & & B0G0Y1 & 11-Sep-95 & & $56-23-5$ & CARBTET \\
\hline 754 & PS HENRY/LE BISPING & & SW & & & B0G0Y1 & 11-Sep-95 & & $7440-47-3$ & CR \\
\hline 755 & SESPSCH & & SW & & & B0GKD5 & 11-Sep-95 & & $10045-97-3$ & CS-137 \\
\hline 756 & SESPSCH & & SW & & & B0GKD5 & 11-Sep-95 & & $10098-97-2$ & SR-90 \\
\hline 757 & SESPSCH & & SW & & & B0GKD5 & 11-Sep-95 & & $14133-76-7$ & TC-99 \\
\hline 758 & SESPSCH & & SW & & & B0GKD5 & 11-Sep-95 & & $10028-17-8$ & TRITIUM \\
\hline 759 & SESPSCH & & SW & & & B0GKD5 & 11-Sep-95 & & $13966-29-5$ & $\mathrm{U}-234$ \\
\hline 760 & SESPSCH & & SW & & & B0GKD5 & 11-Sep-95 & & $15117-96-1$ & U-235 \\
\hline 761 & SESPSCH & & SW & & & B0GKD5 & 11-Sep-95 & & U-238 & U-238 \\
\hline
\end{tabular}

Page 20 


\begin{tabular}{|c|c|c|c|c|c|c|c|c|c|c|}
\hline & A & $\mathrm{B}$ & $\mathrm{C}$ & $\mathrm{D}$ & $\mathrm{E}$ & $\mathrm{F}$ & $\mathrm{G}$ & $\mathrm{H}$ & $\mathrm{I}$ & $\mathrm{J}$ \\
\hline 1 & SAMPLE_NUM_ASSIGNED_TO & SAMPLER & MEDIA & BIOTA_MEDIA & TAG_ID & SAMP_NUM & SAMP_DATE_TIME & SAMP_DATE_TIME_ON & CON_ID & CON_SHORT_NAME \\
\hline 762 & PS HENRY/LE BISPING & & SW & & & B0G0Q6 & 12-Sep-95 & & $56-23-5$ & CARBTET \\
\hline 763 & PS HENRY/LE BISPING & & SW & & & B0G0Q6 & 12-Sep-95 & & $7440-47-3$ & CR \\
\hline 764 & SESPSCH & & SW & & & B0G8B9 & 12-Sep-95 & & $10045-97-3$ & CS-137 \\
\hline 765 & SESPSCH & & SW & & & B0G8B9 & 12-Sep-95 & & $10098-97-2$ & SR-90 \\
\hline 766 & SESPSCH & & SW & & & B0G8B9 & 12-Sep-95 & & 14133-76-7 & TC-99 \\
\hline 767 & SESPSCH & & SW & & & B0G8B9 & 12-Sep-95 & & $10028-17-8$ & TRITIUM \\
\hline 768 & SESPSCH & & SW & & & B0G8B9 & 12-Sep-95 & & $13966-29-5$ & U-234 \\
\hline 769 & SESPSCH & & SW & & & B0G8B9 & 12-Sep-95 & & 15117-96-1 & U-235 \\
\hline 770 & SESPSCH & & SW & & & B0G8B9 & 12-Sep-95 & & U-238 & $\mathrm{U}-238$ \\
\hline 771 & TRAPP, KF & BOWERS/MEHLHORN/JOHN & SW & & & BOGYX4 & 09-Jan-96 & & $10098-97-2$ & SR-90 \\
\hline 772 & TRAPP, KF & BOWERS/MEHLHORN/JOHN & SW & & & BOGYZO & 09-Jan-96 & & $10098-97-2$ & SR-90 \\
\hline 773 & TRAPP, KF & BOWERS/MEHLHORN/JOHN & SW & & & B0GYX5 & 09-Jan-96 & & $10098-97-2$ & SR-90 \\
\hline 774 & TRAPP, KF & BOWERS/MEHLHORN/JOHN & SW & & & BOGYX6 & 09-Jan-96 & & $10098-97-2$ & SR-90 \\
\hline 775 & TRAPP, KF & BOWERS/MEHLHORN/JOHN & SW & & & BOGYX7 & 10-Jan-96 & & $10098-97-2$ & SR-90 \\
\hline 776 & TRAPP, KF & BOWERS/MEHLHORN/JOHN & SW & & & BOGYX8 & 10-Jan-96 & & $10098-97-2$ & SR-90 \\
\hline 777 & TRAPP, KF & BOWERS/MEHLHORN/JOHN & SW & & & BOGYX9 & 10-Jan-96 & & $10098-97-2$ & SR-90 \\
\hline 778 & TRAPP, KF & BOWERS/MEHLHORN/JOHN & SW & & & BOGYYO & 10-Jan-96 & & $10098-97-2$ & SR-90 \\
\hline 779 & TRAPP, KF & BOWERS/MEHLHORN/JOHN & SW & & & B0GYY1 & 10-Jan-96 & & $10098-97-2$ & SR-90 \\
\hline 780 & TRAPP, KF & BOWERS/MEHLHORN/JOHN & SW & & & BOGYY2 & 10-Jan-96 & & $10098-97-2$ & SR-90 \\
\hline 781 & TRAPP, KF & BOWERS/MEHLHORN/JOHN & SW & & & BOGYY4 & 10-Jan-96 & & $10098-97-2$ & SR-90 \\
\hline 782 & TRAPP, KF & BOWERS/MEHLHORN/JOHN & SW & & & B0GYY5 & 10-Jan-96 & & $10098-97-2$ & SR-90 \\
\hline 783 & BRYANT, DL & BRYANT,DL;ELLINGSON, & SW & & & B0J263 & 19-Sep-96 & & $10045-97-3$ & CS-137 \\
\hline 784 & BRYANT, DL & BRYANT,DL;ELLINGSON, & SW & & & B0J263 & 19-Sep-96 & & $10098-97-2$ & SR-90 \\
\hline 785 & BRYANT, DL & BRYANT,DL;ELLINGSON, & SW & & & BOJ263 & 19-Sep-96 & & $10028-17-8$ & TRITIUM \\
\hline 786 & BRYANT, DL & BRYANT,DL;ELLINGSON, & SW & & & B0J264 & 19-Sep-96 & & $10045-97-3$ & CS-137 \\
\hline 787 & BRYANT, DL & BRYANT,DL;ELLINGSON, & SW & & & B0J264 & 19-Sep-96 & & $10098-97-2$ & SR-90 \\
\hline 788 & BRYANT, DL & BRYANT,DL;ELLINGSON, & SW & & & B0J264 & 19-Sep-96 & & $10028-17-8$ & TRITIUM \\
\hline 789 & BRYANT, DL & BRYANT,DL;ELLINGSON, & SW & & & B0J265 & 19-Sep-96 & & $10045-97-3$ & CS-137 \\
\hline 790 & BRYANT, DL & BRYANT,DL;ELLINGSON, & SW & & & B0J265 & 19-Sep-96 & & $10098-97-2$ & SR-90 \\
\hline 791 & BRYANT, DL & BRYANT,DL;ELLINGSON, & SW & & & B0J265 & 19-Sep-96 & & $10028-17-8$ & TRITIUM \\
\hline 792 & BRYANT, DL & BRYANT,DL;ELLINGSON, & SW & & & B0J266 & 19-Sep-96 & & $10045-97-3$ & CS-137 \\
\hline 793 & BRYANT, DL & BRYANT,DL;ELLINGSON, & SW & & & B0J266 & 19-Sep-96 & & $10098-97-2$ & SR-90 \\
\hline 794 & BRYANT, DL & BRYANT,DL;ELLINGSON, & SW & & & B0J266 & 19-Sep-96 & & $10028-17-8$ & TRITIUM \\
\hline 795 & BRYANT, DL & BRYANT,DL;ELLINGSON, & SW & & & BOJ267 & 19-Sep-96 & & $10045-97-3$ & CS-137 \\
\hline 796 & BRYANT, DL & BRYANT,DL;ELLINGSON, & SW & & & B0J267 & 19-Sep-96 & & $10098-97-2$ & SR-90 \\
\hline 797 & BRYANT, DL & BRYANT,DL;ELLINGSON, & SW & & & B0J267 & 19-Sep-96 & & $10028-17-8$ & TRITIUM \\
\hline 798 & BRYANT, DL & BRYANT,DL;ELLINGSON, & SW & & & B0J268 & 19-Sep-96 & & $10045-97-3$ & CS-137 \\
\hline 799 & BRYANT, DL & BRYANT,DL;ELLINGSON, & SW & & & B0J268 & 19-Sep-96 & & $10098-97-2$ & SR-90 \\
\hline
\end{tabular}




\begin{tabular}{|c|c|c|c|c|c|c|c|c|c|c|}
\hline & A & $\mathrm{B}$ & $\mathrm{C}$ & $\mathrm{D}$ & $\mathrm{E}$ & $\mathrm{F}$ & $\mathrm{G}$ & $\mathrm{H}$ & $\mathrm{I}$ & $\mathrm{J}$ \\
\hline 1 & SAMPLE_NUM_ASSIGNED_TO & SAMPLER & MEDIA & BIOTA_MEDIA & TAG_ID & SAMP_NUM & SAMP_DATE_TIME & SAMP_DATE_TIME_ON & CON_ID & CON_SHORT_NAME \\
\hline 800 & BRYANT, DL & BRYANT,DL;ELLINGSON, & SW & & & B0J268 & 19-Sep-96 & & $10028-17-8$ & TRITIUM \\
\hline 801 & BRYANT, DL & BRYANT,DL;ELLINGSON, & SW & & & B0J269 & 19-Sep-96 & & $10045-97-3$ & CS-137 \\
\hline 802 & BRYANT, DL & BRYANT,DL;ELLINGSON, & SW & & & B0J269 & 19-Sep-96 & & $10098-97-2$ & SR-90 \\
\hline 803 & BRYANT, DL & BRYANT,DL;ELLINGSON, & SW & & & B0J269 & 19-Sep-96 & & $10028-17-8$ & TRITIUM \\
\hline 804 & BRYANT, DL & BRYANT,DL;ELLINGSON, & SW & & & B0J270 & 19-Sep-96 & & $10045-97-3$ & CS-137 \\
\hline 805 & BRYANT, DL & BRYANT,DL;ELLINGSON, & SW & & & B0J270 & 19-Sep-96 & & $10098-97-2$ & SR-90 \\
\hline 806 & BRYANT, DL & BRYANT,DL;ELLINGSON, & SW & & & B0J270 & 19-Sep-96 & & $10028-17-8$ & TRITIUM \\
\hline 807 & BRYANT, DL & BRYANT,DL;ELLINGSON, & SW & & & B0J271 & 19-Sep-96 & & $10045-97-3$ & CS-137 \\
\hline 808 & BRYANT, DL & BRYANT,DL;ELLINGSON, & SW & & & B0J271 & 19-Sep-96 & & $10098-97-2$ & SR-90 \\
\hline 809 & BRYANT, DL & BRYANT,DL;ELLINGSON, & SW & & & B0J271 & 19-Sep-96 & & $10028-17-8$ & TRITIUM \\
\hline 810 & TRAPP, KF & BOWERS & SW & & & B0JJW2 & $28-$ Oct-96 & & $7440-47-3$ & CR \\
\hline 811 & TRAPP, KF & BOWERS & SW & & & B0JJW4 & 28-Oct-96 & & $7440-47-3$ & CR \\
\hline 812 & TRAPP, KF & BOWERS & SW & & & BOJKJO & $29-$ Oct-96 & & $7440-47-3$ & CR \\
\hline 813 & TRAPP, KF & BOWERS & SW & & & B0JK47 & 29-Oct-96 & & $7440-47-3$ & CR \\
\hline 814 & TRAPP, KF & BOWERS & SW & & & B0JJY3 & 30-Oct-96 & & $7440-47-3$ & CR \\
\hline 815 & TRAPP, KF & BOWERS & SW & & & B0JJZ5 & $31-O c t-96$ & & $7440-47-3$ & CR \\
\hline 816 & TRAPP, KF & BOWERS & SW & & & B0JJZ2 & 31-Oct-96 & & $7440-47-3$ & CR \\
\hline 817 & TRAPP, KF & BOWERS & SW & & & B0JJZ8 & 31-Oct-96 & & $7440-47-3$ & CR \\
\hline 818 & PS HENRY & & SW & & & B0G1B0 & 04-Nov-96 & & $56-23-5$ & CARBTET \\
\hline 819 & PS HENRY & & SW & & & B0G1B0 & 04-Nov-96 & & $7440-47-3$ & CR \\
\hline 820 & SESPSCH & & SW & & & B0JRS1 & 04-Nov-96 & & $18540-29-9$ & $\mathrm{CR}+6$ \\
\hline 821 & SESPSCH & & SW & & & B0J5B8 & 04-Nov-96 & & $10045-97-3$ & CS-137 \\
\hline 822 & SESPSCH & & SW & & & B0J5B8 & 04-Nov-96 & & $10098-97-2$ & SR-90 \\
\hline 823 & SESPSCH & & SW & & & B0J5B8 & 04-Nov-96 & & $14133-76-7$ & TC-99 \\
\hline 824 & SESPSCH & & SW & & & B0J5B8 & 04-Nov-96 & & $10028-17-8$ & TRITIUM \\
\hline 825 & PS HENRY & & SW & & & B0G1B2 & 04-Nov-96 & & $56-23-5$ & CARBTET \\
\hline 826 & PS HENRY & & SW & & & B0G1B2 & 04-Nov-96 & & $7440-47-3$ & CR \\
\hline 827 & SESPSCH & & SW & & & B0JRS4 & 04-Nov-96 & & $18540-29-9$ & $\mathrm{CR}+6$ \\
\hline 828 & SESPSCH & & SW & & & B0JRS5 & 04-Nov-96 & & $18540-29-9$ & $\mathrm{CR}+6$ \\
\hline 829 & SESPSCH & & SW & & & B0J5C4 & 04-Nov-96 & & $10045-97-3$ & CS-137 \\
\hline 830 & SESPSCH & & SW & & & B0J5C4 & 04-Nov-96 & & $10098-97-2$ & SR-90 \\
\hline 831 & SESPSCH & & SW & & & B0J5C4 & 04-Nov-96 & & $10028-17-8$ & TRITIUM \\
\hline 832 & PS HENRY & & SW & & & B0G1D8 & 04-Nov-96 & & $56-23-5$ & CARBTET \\
\hline 833 & PS HENRY & & SW & & & B0G1D8 & 04-Nov-96 & & $7440-47-3$ & CR \\
\hline 834 & SESPSCH & & SW & & & B0JRS2 & 04-Nov-96 & & $18540-29-9$ & $\mathrm{CR}+6$ \\
\hline 835 & SESPSCH & & SW & & & B0JRS3 & 04-Nov-96 & & $18540-29-9$ & $\mathrm{CR}+6$ \\
\hline 836 & SESPSCH & & SW & & & B0J5C2 & 04-Nov-96 & & $10045-97-3$ & CS-137 \\
\hline 837 & SESPSCH & & SW & & & B0J5C2 & 04-Nov-96 & & $10098-97-2$ & SR-90 \\
\hline
\end{tabular}




\begin{tabular}{|c|c|c|c|c|c|c|c|c|c|c|}
\hline & A & $\mathrm{B}$ & $\mathrm{C}$ & $\mathrm{D}$ & $\mathrm{E}$ & $\mathrm{F}$ & $\mathrm{G}$ & $\mathrm{H}$ & $\mathrm{I}$ & $\mathrm{J}$ \\
\hline 1 & SAMPLE_NUM_ASSIGNED_TO & SAMPLER & MEDIA & BIOTA_MEDIA & TAG_ID & SAMP_NUM & SAMP_DATE_TIME & SAMP_DATE_TIME_ON & CON_ID & CON_SHORT_NAME \\
\hline 838 & SESPSCH & & SW & & & B0J5C2 & 04-Nov-96 & & $10028-17-8$ & TRITIUM \\
\hline 839 & TRAPP, KF & BOWERS & SW & & & BOJJY6 & 04-Nov-96 & & $7440-47-3$ & CR \\
\hline 840 & TRAPP, KF & BOWERS & SW & & & BOJJY9 & 04-Nov-96 & & $7440-47-3$ & CR \\
\hline 841 & TRAPP, KF & BOWERS & SW & & & B0JK01 & 04-Nov-96 & & $7440-47-3$ & CR \\
\hline 842 & TRAPP, KF & BOWERS & SW & & & B0JK04 & 04-Nov-96 & & $7440-47-3$ & CR \\
\hline 843 & TRAPP, KF & BOWERS & SW & & & B0JKH7 & 06-Nov-96 & & $7440-47-3$ & CR \\
\hline 844 & PS HENRY & & SW & & & B0G1C3 & 11-Nov-96 & & $56-23-5$ & CARBTET \\
\hline 845 & PS HENRY & & SW & & & B0G1C3 & 11-Nov-96 & & $7440-47-3$ & $\mathrm{CR}$ \\
\hline 846 & SESPSCH & & SW & & & B0JRS8 & 11-Nov-96 & & $18540-29-9$ & $\mathrm{CR}+6$ \\
\hline 847 & SESPSCH & & SW & & & B0JRS9 & $11-N o v-96$ & & $18540-29-9$ & $\mathrm{CR}+6$ \\
\hline 848 & SESPSCH & & SW & & & B0J5C6 & 11-Nov-96 & & $10045-97-3$ & CS-137 \\
\hline 849 & SESPSCH & & SW & & & B0J5C6 & 11-Nov-96 & & $10098-97-2$ & SR-90 \\
\hline 850 & SESPSCH & & SW & & & B0J5C6 & 11-Nov-96 & & $10028-17-8$ & TRITIUM \\
\hline 851 & SESPSCH & & SW & & & B0J5C6 & 11-Nov-96 & & 13966-29-5 & U-234 \\
\hline 852 & SESPSCH & & SW & & & B0J5C6 & 11-Nov-96 & & $15117-96-1$ & U-235 \\
\hline 853 & SESPSCH & & SW & & & B0J5C6 & 11-Nov-96 & & U-238 & U-238 \\
\hline 854 & PS HENRY & & SW & & & B0G1H3 & 11-Nov-96 & & $56-23-5$ & CARBTET \\
\hline 855 & PS HENRY & & SW & & & B0G1H3 & 11-Nov-96 & & $7440-47-3$ & CR \\
\hline 856 & SESPSCH & & SW & & & B0JRS6 & 11-Nov-96 & & $18540-29-9$ & $\mathrm{CR}+6$ \\
\hline 857 & SESPSCH & & SW & & & B0JRS7 & 11-Nov-96 & & $18540-29-9$ & $\mathrm{CR}+6$ \\
\hline 858 & SESPSCH & & SW & & & B0J5C8 & 11-Nov-96 & & $10045-97-3$ & CS-137 \\
\hline 859 & SESPSCH & & SW & & & B0J5H4 & 11-Nov-96 & & 15046-84-1 & I-129 \\
\hline 860 & SESPSCH & & SW & & & B0J5C8 & 11-Nov-96 & & $14133-76-7$ & TC-99 \\
\hline 861 & SESPSCH & & SW & & & B0J5C8 & 11-Nov-96 & & $10028-17-8$ & TRITIUM \\
\hline 862 & SESPSCH & & SW & & & B0J5C8 & 11-Nov-96 & & 13966-29-5 & U-234 \\
\hline 863 & SESPSCH & & SW & & & B0J5C8 & $11-N o v-96$ & & $15117-96-1$ & U-235 \\
\hline 864 & SESPSCH & & SW & & & B0J5C8 & 11-Nov-96 & & U-238 & U-238 \\
\hline 865 & PS HENRY & & SW & & & B0G1G0 & 21-Nov-96 & & $7440-47-3$ & CR \\
\hline 866 & SESPSCH & & SW & & & B0JRR9 & 21-Nov-96 & & $18540-29-9$ & $\mathrm{CR}+6$ \\
\hline 867 & SESPSCH & & SW & & & BOJRSO & 21-Nov-96 & & $18540-29-9$ & $\mathrm{CR}+6$ \\
\hline 868 & SESPSCH & & SW & & & B0J5D0 & 21-Nov-96 & & $10045-97-3$ & CS-137 \\
\hline 869 & SESPSCH & & SW & & & B0J5H5 & 21-Nov-96 & & 15046-84-1 & I-129 \\
\hline 870 & SESPSCH & & SW & & & B0J5D0 & 21-Nov-96 & & $14133-76-7$ & TC-99 \\
\hline 871 & SESPSCH & & SW & & & B0J5D0 & 21-Nov-96 & & $10028-17-8$ & TRITIUM \\
\hline 872 & SESPSCH & & SW & & & B0J5D0 & 21-Nov-96 & & $13966-29-5$ & U-234 \\
\hline 873 & SESPSCH & & SW & & & B0J5D0 & 21-Nov-96 & & $15117-96-1$ & $\mathrm{U}-235$ \\
\hline 874 & SESPSCH & & SW & & & B0J5D0 & 21-Nov-96 & & $\mathrm{U}-238$ & $\mathrm{U}-238$ \\
\hline 875 & DEAYRES & BOWERS, DL & SW & & & B0M228 & 26-Sep-97 & & $7440-47-3$ & $\mathrm{CR}$ \\
\hline
\end{tabular}




\begin{tabular}{|c|c|c|c|c|c|c|c|c|c|c|}
\hline & $\mathrm{A}$ & $\mathrm{B}$ & $\mathrm{C}$ & $\mathrm{D}$ & $E$ & $\mathrm{~F}$ & $\mathrm{G}$ & $\mathrm{H}$ & $\mathrm{I}$ & $\mathrm{J}$ \\
\hline 1 & SAMPLE_NUM_ASSIGNED_TO & SAMPLER & MEDIA & BIOTA_MEDIA & TAG_ID & SAMP_NUM & SAMP_DATE_TIME & SAMP_DATE_TIME_ON & CON_ID & CON_SHORT_NAME \\
\hline 876 & PSHENRY & STEFFLER, RZ & SW & & & B0M6W1 & 20-Oct-97 & & $7440-47-3$ & CR \\
\hline 877 & PSHENRY & STEFFLER, RZ & SW & & & B0M6W2 & 20-Oct-97 & & $7440-47-3$ & CR \\
\hline 878 & PSHENRY & STEFFLER, RZ & SW & & & B0M6W1 & 20-Oct-97 & & $10098-97-2$ & SR-90 \\
\hline 879 & PSHENRY & STEFFLER, RZ & SW & & & B0M6W1 & 20-Oct-97 & & $10028-17-8$ & TRITIUM \\
\hline 880 & PSHENRY & STEFFLER, RZ & SW & & & B0M6W3 & 20-Oct-97 & & $7440-47-3$ & CR \\
\hline 881 & PSHENRY & STEFFLER, RZ & SW & & & B0M6W4 & $20-$ Oct-97 & & $7440-47-3$ & CR \\
\hline 882 & PSHENRY & STEFFLER, RZ & SW & & & B0M6W3 & 20-Oct-97 & & $10098-97-2$ & SR-90 \\
\hline 883 & PSHENRY & STEFFLER, RZ & SW & & & B0M6W3 & 20-Oct-97 & & $10028-17-8$ & TRITIUM \\
\hline 884 & PSHENRY & STEFFLER, RZ & SW & & & B0M351 & 21-Oct-97 & & $7440-47-3$ & CR \\
\hline 885 & PSHENRY & STEFFLER, RZ & SW & & & B0M352 & 21-Oct-97 & & $10098-97-2$ & SR-90 \\
\hline 886 & PSHENRY & STEFFLER, RZ & SW & & & B0M338 & 21-Oct-97 & & $7440-47-3$ & CR \\
\hline 887 & PSHENRY & STEFFLER, RZ & SW & & & B0M340 & 21-Oct-97 & & $7440-47-3$ & CR \\
\hline 888 & PSHENRY & STEFFLER, RZ & SW & & & B0M339 & 21-Oct-97 & & $10098-97-2$ & SR-90 \\
\hline 889 & PSHENRY & STEFFLER, RZ & SW & & & B0M341 & 21-Oct-97 & & $10098-97-2$ & SR-90 \\
\hline 890 & PSHENRY & STEFFLER, RZ & SW & & & B0M343 & 21-Oct-97 & & $7440-47-3$ & CR \\
\hline 891 & PSHENRY & STEFFLER, RZ & SW & & & B0M344 & 21-Oct-97 & & $10098-97-2$ & SR-90 \\
\hline 892 & PSHENRY & STEFFLER, RZ & SW & & & B0M373 & 22-Oct-97 & & $7440-47-3$ & CR \\
\hline 893 & PSHENRY & STEFFLER, RZ & SW & & & B0M374 & 22-Oct-97 & & $10028-17-8$ & TRITIUM \\
\hline 894 & SESP Scheduling & & SW & & & B0M7V4 & 23-Oct-97 & & $10045-97-3$ & CS-137 \\
\hline 895 & SESP Scheduling & & SW & & & B0M7V4 & 23-Oct-97 & & $10098-97-2$ & SR-90 \\
\hline 896 & SESP Scheduling & & SW & & & B0M7V4 & 23-Oct-97 & & 14133-76-7 & TC-99 \\
\hline 897 & SESP Scheduling & & SW & & & B0M7V4 & 23-Oct-97 & & $10028-17-8$ & TRITIUM \\
\hline 898 & SESP Scheduling & & SW & & & B0M7W1 & 23-Oct-97 & & $10045-97-3$ & CS-137 \\
\hline 899 & SESP Scheduling & & SW & & & B0M7W1 & 23-Oct-97 & & $10098-97-2$ & SR-90 \\
\hline 900 & SESP Scheduling & & SW & & & B0M7W1 & 23-Oct-97 & & $10028-17-8$ & TRITIUM \\
\hline 901 & SESP Scheduling & & SW & & & B0M7W1 & 23-Oct-97 & & $13966-29-5$ & U-234 \\
\hline 902 & SESP Scheduling & & SW & & & B0M7W1 & 23-Oct-97 & & 15117-96-1 & U-235 \\
\hline 903 & SESP Scheduling & & SW & & & B0M7W1 & 23-Oct-97 & & $\mathrm{U}-238$ & $\mathrm{U}-238$ \\
\hline 904 & PSHENRY & STEFFLER, RZ & SW & & & B0M7M3 & 23-Oct-97 & & $7440-47-3$ & CR \\
\hline 905 & PSHENRY & STEFFLER, RZ & SW & & & B0M7M4 & 23-Oct-97 & & $7440-47-3$ & CR \\
\hline 906 & PSHENRY & STEFFLER, RZ & SW & & & B0M7M3 & 23-Oct-97 & & $10045-97-3$ & CS-137 \\
\hline 907 & PSHENRY & STEFFLER, RZ & SW & & & B0M7M3 & 23-Oct-97 & & $10028-17-8$ & TRITIUM \\
\hline 908 & PSHENRY & STEFFLER, RZ & SW & & & B0M7M7 & 23-Oct-97 & & $7440-47-3$ & CR \\
\hline 909 & PSHENRY & STEFFLER, RZ & SW & & & B0M7M8 & 23-Oct-97 & & $7440-47-3$ & CR \\
\hline 910 & PSHENRY & STEFFLER, RZ & SW & & & B0M7M7 & 23-Oct-97 & & $10045-97-3$ & CS-137 \\
\hline 911 & PSHENRY & STEFFLER, RZ & SW & & & B0M7M7 & 23-Oct-97 & & $10028-17-8$ & TRITIUM \\
\hline 912 & SESP Scheduling & & SW & & & B0M7V7 & $27-$ Oct-97 & & $10045-97-3$ & CS-137 \\
\hline 913 & SESP Scheduling & & SW & & & B0M7V7 & 27-Oct-97 & & $10098-97-2$ & SR-90 \\
\hline
\end{tabular}




\begin{tabular}{|c|c|c|c|c|c|c|c|c|c|c|}
\hline & A & $\mathrm{B}$ & $\mathrm{C}$ & $\mathrm{D}$ & $\mathrm{E}$ & $\mathrm{F}$ & $\mathrm{G}$ & $\mathrm{H}$ & $\mathrm{I}$ & $\mathrm{J}$ \\
\hline 1 & SAMPLE_NUM_ASSIGNED_TO & SAMPLER & MEDIA & BIOTA_MEDIA & TAG_ID & SAMP_NUM & SAMP_DATE_TIME & SAMP_DATE_TIME_ON & CON_ID & CON_SHORT_NAME \\
\hline 914 & SESP Scheduling & & SW & & & B0M7V7 & $27-O c t-97$ & & $10028-17-8$ & TRITIUM \\
\hline 915 & SESP Scheduling & & SW & & & B0M7V9 & $27-$ Oct-97 & & $10045-97-3$ & CS-137 \\
\hline 916 & SESP Scheduling & & SW & & & B0M7V9 & $27-$ Oct-97 & & $10098-97-2$ & SR-90 \\
\hline 917 & SESP Scheduling & & SW & & & B0M7V9 & 27-Oct-97 & & 14133-76-7 & TC-99 \\
\hline 918 & SESP Scheduling & & SW & & & B0M7V9 & 27-Oct-97 & & $10028-17-8$ & TRITIUM \\
\hline 919 & SESP Scheduling & & SW & & & B0M7V9 & 27-Oct-97 & & $13966-29-5$ & U-234 \\
\hline 920 & SESP Scheduling & & SW & & & B0M7V9 & 27-Oct-97 & & 15117-96-1 & U-235 \\
\hline 921 & SESP Scheduling & & SW & & & B0M7V9 & 27-Oct-97 & & U-238 & $\mathrm{U}-238$ \\
\hline 922 & SESP Scheduling & & SW & & & B0M7W3 & 27-Oct-97 & & $10045-97-3$ & CS-137 \\
\hline 923 & SESP Scheduling & & SW & & & BOM7V0 & $27-$ Oct-97 & & $15046-84-1$ & $1-129$ \\
\hline 924 & SESP Scheduling & & SW & & & B0M7W3 & 27-Oct-97 & & $14133-76-7$ & TC-99 \\
\hline 925 & SESP Scheduling & & SW & & & B0M7W3 & 27-Oct-97 & & $10028-17-8$ & TRITIUM \\
\hline 926 & SESP Scheduling & & SW & & & B0M7W3 & 27-Oct-97 & & $13966-29-5$ & U-234 \\
\hline 927 & SESP Scheduling & & SW & & & B0M7W3 & 27-Oct-97 & & 15117-96-1 & U-235 \\
\hline 928 & SESP Scheduling & & SW & & & B0M7W3 & 27-Oct-97 & & U-238 & U-238 \\
\hline 929 & SESP Scheduling & & SW & & & B0M7W2 & 27-Oct-97 & & $10045-97-3$ & CS-137 \\
\hline 930 & SESP Scheduling & & SW & & & B0M7T9 & $27-$ Oct- 97 & & 15046-84-1 & I-129 \\
\hline 931 & SESP Scheduling & & SW & & & B0M7W2 & 27-Oct-97 & & $14133-76-7$ & TC-99 \\
\hline 932 & SESP Scheduling & & SW & & & B0M7W2 & 27-Oct-97 & & $10028-17-8$ & TRITIUM \\
\hline 933 & SESP Scheduling & & SW & & & B0M7W2 & 27-Oct-97 & & $13966-29-5$ & U-234 \\
\hline 934 & SESP Scheduling & & SW & & & B0M7W2 & 27-Oct-97 & & $15117-96-1$ & $\mathrm{U}-235$ \\
\hline 935 & SESP Scheduling & & SW & & & B0M7W2 & 27-Oct-97 & & U-238 & $\mathrm{U}-238$ \\
\hline 936 & PSHENRY & STEFFLER, RZ & SW & & & B0M355 & 28-Oct-97 & & $7440-47-3$ & $\mathrm{CR}$ \\
\hline 937 & PSHENRY & STEFFLER, RZ & SW & & & B0M357 & 28-Oct-97 & & $7440-47-3$ & CR \\
\hline 938 & PSHENRY & STEFFLER, RZ & SW & & & B0M356 & 28-Oct-97 & & $10028-17-8$ & TRITIUM \\
\hline 939 & PSHENRY & STEFFLER, RZ & SW & & & B0M358 & 28-Oct-97 & & $10028-17-8$ & TRITIUM \\
\hline 940 & PSHENRY & STEFFLER, RZ & SW & & & В0М369 & 28-Oct-97 & & $7440-47-3$ & CR \\
\hline 941 & PSHENRY & STEFFLER, RZ & SW & & & B0M370 & 28-Oct-97 & & $10028-17-8$ & TRITIUM \\
\hline 942 & PSHENRY & STEFFLER, RZ & SW & & & B0M347 & 29-Oct-97 & & $7440-47-3$ & CR \\
\hline 943 & PSHENRY & STEFFLER, RZ & SW & & & В0М348 & 29-Oct-97 & & $10098-97-2$ & SR-90 \\
\hline 944 & PSHENRY & STEFFLER, RZ & SW & & & B0M7M5 & 29-Oct-97 & & $7440-47-3$ & CR \\
\hline 945 & PSHENRY & STEFFLER, RZ & SW & & & B0M7M6 & 29-Oct-97 & & $7440-47-3$ & $\mathrm{CR}$ \\
\hline 946 & PSHENRY & STEFFLER, RZ & SW & & & B0M7M5 & 29-Oct-97 & & $10045-97-3$ & CS-137 \\
\hline 947 & PSHENRY & STEFFLER, RZ & SW & & & B0M7M5 & 29-Oct-97 & & $10028-17-8$ & TRITIUM \\
\hline 948 & SESP SCHEDULING & & SW & & & B0MD34 & 03-Nov-97 & & $10045-97-3$ & CS-137 \\
\hline 949 & SESP SCHEDULING & & SW & & & B0MD34 & 03-Nov-97 & & $10098-97-2$ & SR-90 \\
\hline 950 & SESP SCHEDULING & & SW & & & B0MD34 & 03-Nov-97 & & $10028-17-8$ & TRITIUM \\
\hline 951 & SESP Scheduling & & SW & & & B0M7V5 & 03-Nov-97 & & $10045-97-3$ & CS-137 \\
\hline
\end{tabular}




\begin{tabular}{|c|c|c|c|c|c|c|c|c|c|c|}
\hline & A & $B$ & $C$ & $\mathrm{D}$ & $\mathrm{E}$ & $\mathrm{F}$ & G & $\mathrm{H}$ & $\mathrm{I}$ & $\mathrm{J}$ \\
\hline 1 & SAMPLE_NUM_ASSIGNED_TO & SAMPLER & MEDIA & BIOTA_MEDIA & TAG_ID & SAMP_NUM & SAMP_DATE_TIME & SAMP_DATE_TIME_ON & CON_ID & CON_SHORT_NAME \\
\hline 952 & SESP Scheduling & & SW & & & B0M7V5 & 03-Nov-97 & & $10098-97-2$ & SR-90 \\
\hline 953 & SESP Scheduling & & SW & & & B0M7V5 & 03-Nov-97 & & $10028-17-8$ & TRITIUM \\
\hline 954 & SESP Scheduling & & SW & & & B0M7V6 & 03-Nov-97 & & $10045-97-3$ & CS-137 \\
\hline 955 & SESP Scheduling & & SW & & & B0M7V6 & 03-Nov-97 & & 10098-97-2 & SR-90 \\
\hline 956 & SESP Scheduling & & SW & & & B0M7V6 & 03-Nov-97 & & 10028-17-8 & TRITIUM \\
\hline 957 & SESP SCHEDULING & & SW & & & B0PXB3 & 22-Sep-98 & & $10045-97-3$ & CS-137 \\
\hline 958 & SESP SCHEDULING & & SW & & & B0PX66 & 22-Sep-98 & & 15046-84-1 & $\mathrm{I}-129$ \\
\hline 959 & SESP SCHEDULING & & SW & & & B0PXB3 & 22-Sep-98 & & 14133-76-7 & TC-99 \\
\hline 960 & SESP SCHEDULING & & SW & & & B0PXB3 & 22-Sep-98 & & 10028-17-8 & TRITIUM \\
\hline 961 & SESP SCHEDULING & & SW & & & B0PXB3 & 22-Sep-98 & & 13966-29-5 & $\mathrm{U}-234$ \\
\hline 962 & SESP SCHEDULING & & SW & & & B0PXB3 & 22-Sep-98 & & 15117-96-1 & U-235 \\
\hline 963 & SESP SCHEDULING & & SW & & & B0РXВ3 & 22-Sep-98 & & U-238 & U-238 \\
\hline 964 & SESP SCHEDULING & & SW & & & B0PXB1 & 22-Sep-98 & & $10045-97-3$ & CS-137 \\
\hline 965 & SESP SCHEDULING & & SW & & & B0P 665 & 22-Sep-98 & & 15046-84-1 & I-129 \\
\hline 966 & SESP SCHEDULING & & SW & & & B0PXB1 & 22-Sep-98 & & 14133-76-7 & TC-99 \\
\hline 967 & SESP SCHEDULING & & SW & & & B0PXB1 & 22-Sep-98 & & $10028-17-8$ & TRITIUM \\
\hline 968 & SESP SCHEDULING & & SW & & & B0PXB1 & 22-Sep-98 & & 13966-29-5 & U-234 \\
\hline 969 & SESP SCHEDULING & & SW & & & B0PXB1 & 22-Sep-98 & & 15117-96-1 & U-235 \\
\hline 970 & SESP SCHEDULING & & SW & & & B0PXB1 & 22-Sep-98 & & U-238 & $\mathrm{U}-238$ \\
\hline 971 & SESP SCHEDULING & & SW & & & B0P X97 & 23-Sep-98 & & $10045-97-3$ & CS-137 \\
\hline 972 & SESP SCHEDULING & & SW & & & B0P $\times 97$ & 23-Sep-98 & & $10098-97-2$ & SR-90 \\
\hline 973 & SESP SCHEDULING & & SW & & & B0P X97 & 23-Sep-98 & & $10028-17-8$ & TRITIUM \\
\hline 974 & SESP SCHEDULING & & SW & & & B0PX98 & 23-Sep-98 & & $10045-97-3$ & CS-137 \\
\hline 975 & SESP SCHEDULING & & SW & & & B0P X98 & 23-Sep-98 & & $10098-97-2$ & SR-90 \\
\hline 976 & SESP SCHEDULING & & SW & & & B0РX98 & 23-Sep-98 & & 14133-76-7 & TC-99 \\
\hline 977 & SESP SCHEDULING & & SW & & & B0PX98 & 23-Sep-98 & & $10028-17-8$ & TRITIUM \\
\hline 978 & SESP SCHEDULING & & SW & & & B0P X98 & 23-Sep-98 & & 13966-29-5 & U-234 \\
\hline 979 & SESP SCHEDULING & & SW & & & B0PX98 & 23-Sep-98 & & 15117-96-1 & U-235 \\
\hline 980 & SESP SCHEDULING & & SW & & & B0P X98 & 23-Sep-98 & & U-238 & $\mathrm{U}-238$ \\
\hline 981 & SESP SCHEDULING & & SW & & & B0P $\times 92$ & 24-Sep-98 & & $10045-97-3$ & CS-137 \\
\hline 982 & SESP SCHEDULING & & SW & & & B0P X92 & 24-Sep-98 & & $10098-97-2$ & SR-90 \\
\hline 983 & SESP SCHEDULING & & SW & & & B0PX92 & 24-Sep-98 & & 14133-76-7 & TC-99 \\
\hline 984 & SESP SCHEDULING & & SW & & & B0P $\times 92$ & 24-Sep-98 & & $10028-17-8$ & TRITIUM \\
\hline 985 & SESP SCHEDULING & & SW & & & B0PX99 & 20-Oct-98 & & $10045-97-3$ & CS-137 \\
\hline 986 & SESP SCHEDULING & & SW & & & B0PX99 & 20-Oct-98 & & $10098-97-2$ & SR-90 \\
\hline 987 & SESP SCHEDULING & & SW & & & B0PX99 & 20-Oct-98 & & $10028-17-8$ & TRITIUM \\
\hline 988 & SESP SCHEDULING & & SW & & & B0P X99 & 20-Oct-98 & & 13966-29-5 & U-234 \\
\hline 989 & SESP SCHEDULING & & SW & & & B0P $\times 99$ & 20-Oct-98 & & 15117-96-1 & U-235 \\
\hline
\end{tabular}




\begin{tabular}{|c|c|c|c|c|c|c|c|c|c|c|}
\hline & A & $B$ & $C$ & $\mathrm{D}$ & $\mathrm{E}$ & $\mathrm{F}$ & G & $\mathrm{H}$ & $\mathrm{I}$ & $\mathrm{J}$ \\
\hline 1 & SAMPLE_NUM_ASSIGNED_TO & SAMPLER & MEDIA & BIOTA_MEDIA & TAG_ID & SAMP_NUM & SAMP_DATE_TIME & SAMP_DATE_TIME_ON & CON_ID & CON_SHORT_NAME \\
\hline 990 & SESP SCHEDULING & & SW & & & B0PX99 & $20-O c t-98$ & & $\mathrm{U}-238$ & U-238 \\
\hline 991 & SESP SCHEDULING & & SW & & & B0P $\times 93$ & 26-Oct-98 & & $10045-97-3$ & CS-137 \\
\hline 992 & SESP SCHEDULING & & SW & & & B0P X93 & $26-$ Oct-98 & & $10098-97-2$ & SR-90 \\
\hline 993 & SESP SCHEDULING & & SW & & & B0P X93 & 26-Oct-98 & & $10028-17-8$ & TRITIUM \\
\hline 994 & SESP SCHEDULING & & SW & & & B0PX95 & 26-Oct-98 & & $10045-97-3$ & CS-137 \\
\hline 995 & SESP SCHEDULING & & SW & & & B0PX95 & 26-Oct-98 & & $10098-97-2$ & SR-90 \\
\hline 996 & SESP SCHEDULING & & SW & & & B0PX95 & 26-Oct-98 & & 10028-17-8 & TRITIUM \\
\hline 997 & SESP SCHEDULING & & SW & & & B0WP11 & 25-Oct-99 & & $10045-97-3$ & CS-137 \\
\hline 998 & SESP SCHEDULING & & SW & & & B0WP11 & 25-Oct-99 & & $10098-97-2$ & SR-90 \\
\hline 999 & SESP SCHEDULING & & SW & & & B0WP11 & 25-Oct-99 & & $14133-76-7$ & TC-99 \\
\hline 1000 & SESP SCHEDULING & & SW & & & B0WP11 & 25-Oct-99 & & $10028-17-8$ & TRITIUM \\
\hline 1001 & SESP SCHEDULING & & SW & & & B0WP12 & 25-Oct-99 & & $10045-97-3$ & CS-137 \\
\hline 1002 & SESP SCHEDULING & & SW & & & B0WP12 & 25-Oct-99 & & $10098-97-2$ & SR-90 \\
\hline 1003 & SESP SCHEDULING & & SW & & & B0WP12 & 25-Oct-99 & & 14133-76-7 & TC-99 \\
\hline 1004 & SESP SCHEDULING & & SW & & & B0WP12 & 25-Oct-99 & & $10028-17-8$ & TRITIUM \\
\hline 1005 & SESP SCHEDULING & & SW & & & B0WP13 & 25-Oct-99 & & $10045-97-3$ & CS-137 \\
\hline 1006 & SESP SCHEDULING & & SW & & & B0WP13 & 25-Oct-99 & & $10098-97-2$ & SR-90 \\
\hline 1007 & SESP SCHEDULING & & SW & & & B0WP13 & 25-Oct-99 & & $10028-17-8$ & TRITIUM \\
\hline 1008 & SESP SCHEDULING & & SW & & & BOWNX9 & 25-Oct-99 & & $10045-97-3$ & CS-137 \\
\hline 1009 & SESP SCHEDULING & & SW & & & BOWNX9 & 25-Oct-99 & & $10098-97-2$ & SR-90 \\
\hline 1010 & SESP SCHEDULING & & SW & & & BOWNX9 & 25-Oct-99 & & $10028-17-8$ & TRITIUM \\
\hline 1011 & PSHENRY & BELT, LL & SW & & & B0WMT1 & 25-Oct-99 & & $56-23-5$ & CARBTET \\
\hline 1012 & SESP SCHEDULING & & SW & & & B0WP18 & 27-Oct-99 & & $10045-97-3$ & CS-137 \\
\hline 1013 & SESP SCHEDULING & & SW & & & B0WP18 & 27-Oct-99 & & $10098-97-2$ & SR-90 \\
\hline 1014 & SESP SCHEDULING & & SW & & & B0WP18 & 27-Oct-99 & & 10028-17-8 & TRITIUM \\
\hline 1015 & SESP SCHEDULING & & SW & & & B0WP17 & 27-Oct-99 & & $10045-97-3$ & CS-137 \\
\hline 1016 & SESP SCHEDULING & & SW & & & B0WP17 & 27-Oct-99 & & $10098-97-2$ & SR-90 \\
\hline 1017 & SESP SCHEDULING & & SW & & & B0WP17 & 27-Oct-99 & & $10028-17-8$ & TRITIUM \\
\hline 1018 & SESP SCHEDULING & & SW & & & B0WP21 & 27-Oct-99 & & $10045-97-3$ & CS-137 \\
\hline 1019 & SESP SCHEDULING & & SW & & & B0WP21 & 27-Oct-99 & & $10098-97-2$ & SR-90 \\
\hline 1020 & SESP SCHEDULING & & SW & & & B0WP21 & 27-Oct-99 & & 14133-76-7 & TC-99 \\
\hline 1021 & SESP SCHEDULING & & SW & & & B0WP21 & 27-Oct-99 & & $10028-17-8$ & TRITIUM \\
\hline 1022 & SESP SCHEDULING & & SW & & & B0WP21 & 27-Oct-99 & & 13966-29-5 & $\mathrm{U}-234$ \\
\hline 1023 & SESP SCHEDULING & & SW & & & B0WP21 & 27-Oct-99 & & 15117-96-1 & U-235 \\
\hline 1024 & SESP SCHEDULING & & SW & & & BOWP21 & 27-Oct-99 & & $\mathrm{U}-238$ & $\mathrm{U}-238$ \\
\hline 1025 & SESP SCHEDULING & & SW & & & B0WP19 & 27-Oct-99 & & $10045-97-3$ & CS-137 \\
\hline 1026 & SESP SCHEDULING & & SW & & & B0WP19 & 27-Oct-99 & & 10098-97-2 & SR-90 \\
\hline 1027 & SESP SCHEDULING & & SW & & & B0WP19 & 27-Oct-99 & & 14133-76-7 & TC-99 \\
\hline
\end{tabular}




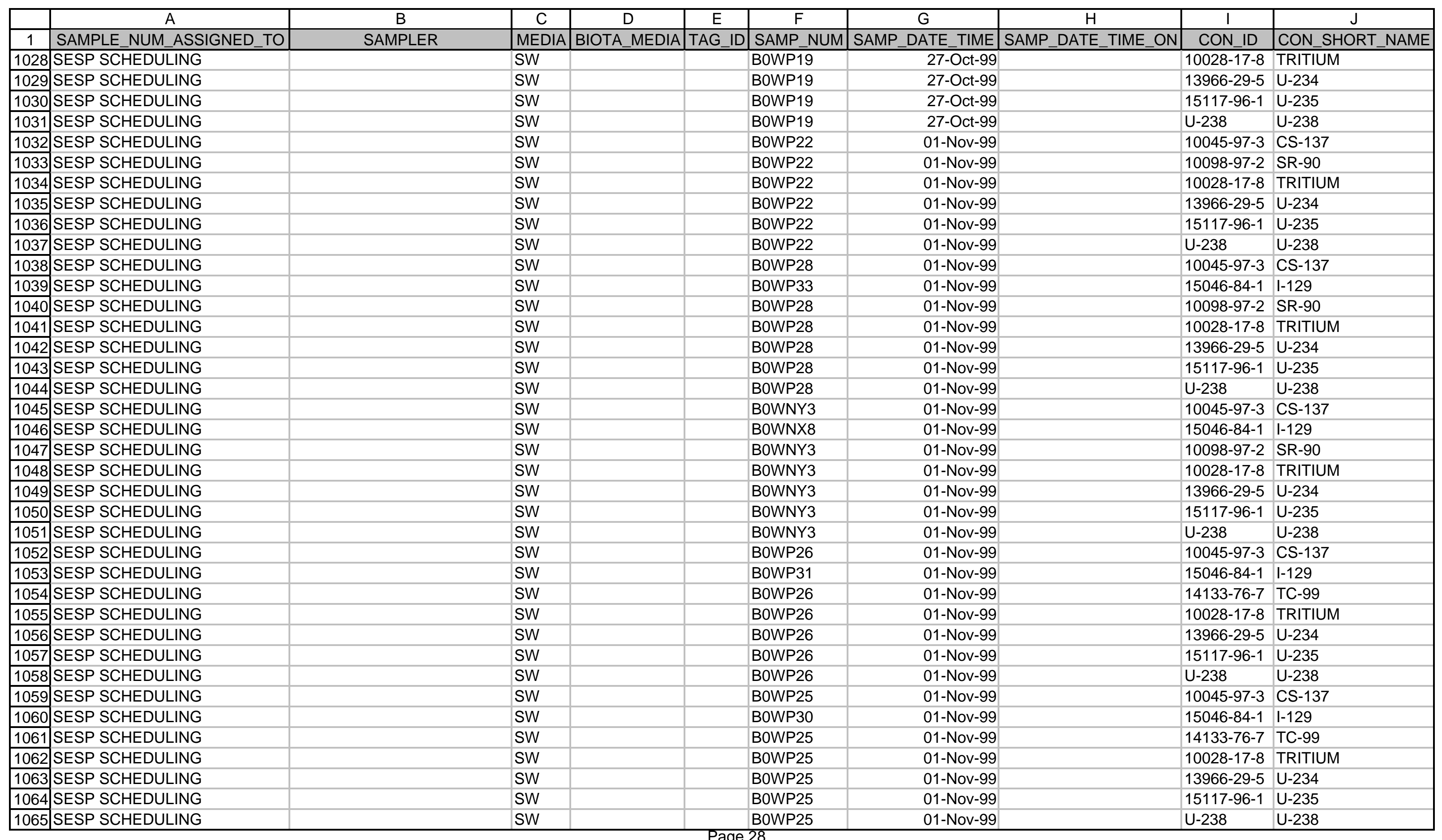




\begin{tabular}{|c|c|c|c|c|c|c|c|c|c|c|}
\hline & $A$ & $\mathrm{~B}$ & $C$ & $\mathrm{D}$ & $\mathrm{E}$ & $\mathrm{F}$ & $\mathrm{G}$ & $\mathrm{H}$ & $\mathrm{I}$ & $\mathrm{J}$ \\
\hline 1 & SAMPLE_NUM_ASSIGNED_TO & SAMPLER & MEDIA & BIOTA_MEDIA & TAG_ID & SAMP_NUM & SAMP_DATE_TIME & SAMP_DATE_TIME_ON & CON_ID & CON_SHORT_NAME \\
\hline 1066 & SESP SCHEDULING & & SW & & & BOWNY1 & 01-Nov-99 & & $10045-97-3$ & CS-137 \\
\hline 1067 & SESP SCHEDULING & & SW & & & BOWNX7 & 01-Nov-99 & & $15046-84-1$ & I-129 \\
\hline 1068 & SESP SCHEDULING & & SW & & & BOWNY1 & 01-Nov-99 & & $14133-76-7$ & TC-99 \\
\hline 1069 & SESP SCHEDULING & & SW & & & BOWNY1 & 01-Nov-99 & & $10028-17-8$ & TRITIUM \\
\hline 1070 & SESP SCHEDULING & & SW & & & BOWNY1 & 01-Nov-99 & & $13966-29-5$ & U-234 \\
\hline 1071 & SESP SCHEDULING & & SW & & & BOWNY1 & 01-Nov-99 & & 15117-96-1 & $\mathrm{U}-235$ \\
\hline 1072 & SESP SCHEDULING & & SW & & & BOWNY1 & 01-Nov-99 & & U-238 & $\mathrm{U}-238$ \\
\hline 1073 & PSHENRY & BELT, LL & SW & & & BOWMT9 & 01-Nov-99 & & $56-23-5$ & CARBTET \\
\hline 1074 & PSHENRY & BELT, LL & SW & & & BOWMV3 & 01-Nov-99 & & $56-23-5$ & CARBTET \\
\hline 1075 & PSHENRY & BELT, LL & SW & & & BOWMV4 & 01-Nov-99 & & $56-23-5$ & CARBTET \\
\hline 1076 & SESP SCHEDULING & & SW & & & B0YRB8 & 12-Jun-00 & & $10045-97-3$ & CS-137 \\
\hline 1077 & SESP SCHEDULING & & SW & & & B0YRB8 & 12-Jun-00 & & $13966-29-5$ & $\mathrm{U}-234$ \\
\hline 1078 & SESP SCHEDULING & & SW & & & B0YRB8 & 12-Jun-00 & & $15117-96-1$ & U-235 \\
\hline 1079 & SESP SCHEDULING & & SW & & & B0YRB8 & 12-Jun-00 & & U-238 & $\mathrm{U}-238$ \\
\hline
\end{tabular}




\begin{tabular}{|c|c|c|c|c|c|c|c|c|c|}
\hline & $\mathrm{K}$ & $\mathrm{L}$ & $\mathrm{M}$ & $\mathrm{N}$ & $\mathrm{O}$ & $\mathrm{P}$ & $\mathrm{Q}$ & $\mathrm{R}$ & $\mathrm{S}$ \\
\hline 1 & METHOD NAME & LAB CODE & VALUE RPTD & ANAL UNITS RPTD & COUNTING ERROR & TOTAL ANAL ERROR & MIN DETECTABLE ACTIVITY & PCNT MOISTURE & LAB QUALIFIER \\
\hline 2 & CLP_METALS_ICP & SKINER & $36 . c$ & $u g / L$ & & & & & $\mathrm{~J}$ \\
\hline 3 & CLP_METALS_ICP & SKINER & 40.6 & ug/L & & & & & \\
\hline 4 & GAMMA_GS & TMANC & 10.16 & $\mathrm{pCi} / \mathrm{L}$ & & & 10.16 & & UJ \\
\hline 5 & SAS_COUNT_LSC & TMANC & 0.96 & $\mathrm{pCi} / \mathrm{L}$ & 0.29 & & & & $\mathrm{~J}$ \\
\hline 6 & SAS_COUNT_LSC & TMANC & 20600 & $\mathrm{pCi} / \mathrm{L}$ & 900 & & & & $\mathrm{~J}$ \\
\hline 7 & CLP_METALS_ICP & SKINER & & ug/L & & & & & UJ \\
\hline 8 & CLP METALS ICP & SKINER & & $\mathrm{ug} / \mathrm{L}$ & & & & & UJ \\
\hline 9 & GAMMA_GS & TMANC & 15.25 & $\mathrm{pCi} / \mathrm{L}$ & & & 15.25 & & UJ \\
\hline 11 & SAS_COUNT_LSC & TMANC & 300 & $\mathrm{pCi} / \mathrm{L}$ & 200 & & & & $\mathrm{~J}$ \\
\hline 12 & CLP_METALS_ICP & SKINER & 27.7 & $\mathrm{ug} / \mathrm{L}$ & & & & & $\mathrm{J}$ \\
\hline 13 & CLP_METALS_ICP & SKINER & 54.1 & $\mathrm{ug} / \mathrm{L}$ & & & & & \\
\hline 14 & GAMMA_GS & TMANC & 11.7 & $\mathrm{pCi} / \mathrm{L}$ & & & 11.7 & & UJ \\
\hline 15 & SAS_COUNT_LSC & TMANC & 0.4 & $\mathrm{pCi} / \mathrm{L}$ & & & 0.4 & & UJ \\
\hline 16 & SAS_COUNT_LSC & TMANC & 13000 & $\mathrm{pCi} / \mathrm{L}$ & 700 & & & & $\mathrm{~J}$ \\
\hline 17 & CLP_METALS_ICP & SKINER & 13.7 & $\mathrm{ug} / \mathrm{L}$ & & & & & \\
\hline 18 & CLP METALS ICP & SKINER & 16.9 & $\mathrm{ug} / \mathrm{L}$ & & & & & \\
\hline 19 & GAMMA_GS & TMANC & 12.27 & $7 \mathrm{pCi} / \mathrm{L}$ & & & 12.27 & & $U$ \\
\hline 21 & SAS_COUNT_LSC & TMANC & 3100 & $\mathrm{pCi} / \mathrm{L}$ & 200 & & & & \\
\hline 22 & CLP_METALS_ICP & SKINER & 75.8 & $3 \mathrm{ug} / \mathrm{L}$ & & & & & \\
\hline 23 & CLP_METALS_ICP & SKINER & 71.7 & $7 \mathrm{ug} / \mathrm{L}$ & & & & & \\
\hline 24 & GAMMA_GS & TMANC & 11.06 & $\mathrm{pCi} / \mathrm{L}$ & & & 11.06 & & U \\
\hline 25 & SAS COUNT LSC & TMANC & 4.5 & $5 \mathrm{pCi} / \mathrm{L}$ & 0.3 & & & & \\
\hline 26 & SAS_COUNT_LSC & TMANC & & $3 \mathrm{pCi} / \mathrm{L}$ & 1 & & & & $U$ \\
\hline 27 & SAS_COUNT_LSC & TMANC & 1200 & $\mathrm{pCi} / \mathrm{L}$ & 200 & & & & \\
\hline 28 & CLP_METALS_ICP & SKINER & 46.3 & $3 \mathrm{ug} / \mathrm{L}$ & & & & & \\
\hline 29 & CLP_METALS_ICP & SKINER & 43.3 & $3 \mathrm{ug} / \mathrm{L}$ & & & & & \\
\hline 30 & CLP_METALS_ICP & SKINER & & $\mathrm{ug} / \mathrm{L}$ & & & & & $U$ \\
\hline 31 & CLP_METALS_ICP & SKINER & & $\mathrm{ug} / \mathrm{L}$ & & & & & $U$ \\
\hline 32 & GAMMA_GS & TMANC & 7.522 & $2 \mathrm{pCi} / \mathrm{L}$ & & & & & $U$ \\
\hline 33 & GAMMA_GS & TMANC & 13.8 & $4 \mathrm{pCi} / \mathrm{L}$ & & & & & $U$ \\
\hline 34 & SAS COUNT LSC & TMANC & 0.4 & $4 \mathrm{pCi} / \mathrm{L}$ & & & & & \\
\hline 35 & SAS_COUNT_LSC & TMANC & 0.01 & $\mathrm{pCi} / \mathrm{L}$ & & & & & U \\
\hline 36 & SAS_COUNT_LSC & TMANC & & $\mathrm{pCi} / \mathrm{L}$ & & & & & U \\
\hline 37 & SAS_COUNT_LSC & TMANC & & $3 \mathrm{pCi} / \mathrm{L}$ & & & & & $U$ \\
\hline 38 & SAS_COUNT_LSC & TMANC & 2900 & $\mathrm{pCi} / \mathrm{L}$ & & & & & \\
\hline
\end{tabular}




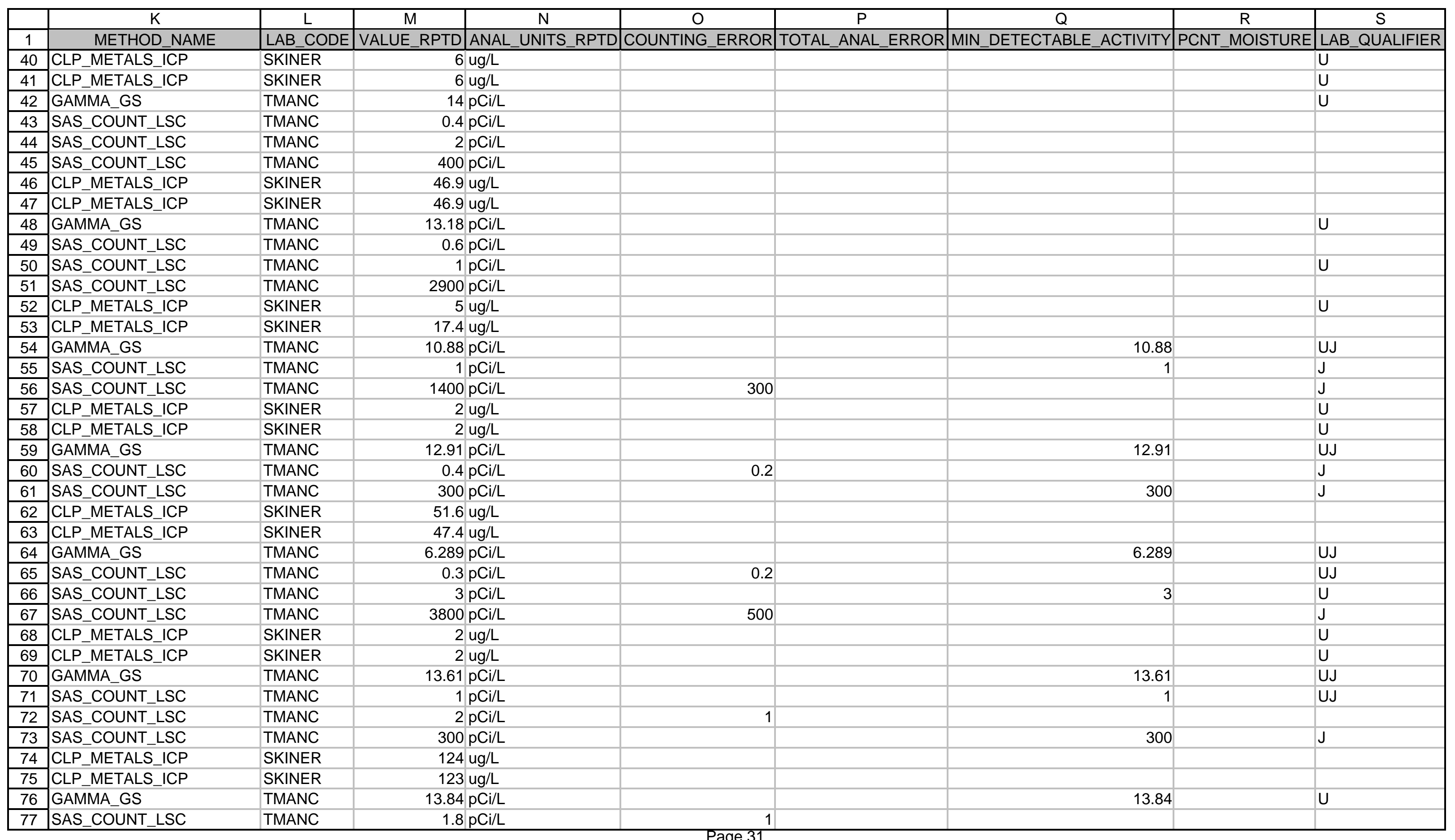




\begin{tabular}{|c|c|c|c|c|c|c|c|c|c|}
\hline & $\mathrm{K}$ & $\mathrm{L}$ & $\mathrm{M}$ & $\mathrm{N}$ & $\mathrm{O}$ & $\mathrm{P}$ & $\mathrm{Q}$ & $\mathrm{R}$ & $\mathrm{S}$ \\
\hline 1 & METHOD NAME & LAB CODE & VALUE RPTD & ANAL UNITS RPTD & COUNTING ERROR & TOTAL ANAL ERROR & MIN DETECTABLE ACTIVITY & PCNT MOISTURE & LAB QUALIFIER \\
\hline 78 & SAS_COUNT_LSC & TMANC & 4.9 & $\mathrm{pCi} / \mathrm{L}$ & 1.1 & & & & \\
\hline 79 & SAS_COUNT_LSC & TMANC & 3100 & $\mathrm{pCi} / \mathrm{L}$ & 400 & & & & \\
\hline 80 & CLP_METALS_ICP & SKINER & 8.8 & ug/L & & & & & $\mathrm{U}$ \\
\hline 81 & CLP_METALS_ICP & SKINER & 2.4 & $u g / L$ & & & & & B \\
\hline 82 & GAMMA_GS & TMANC & 12.91 & $\mathrm{pCi} / \mathrm{L}$ & & & 12.91 & & U \\
\hline 83 & SAS_COUNT_LSC & TMANC & 1 & $\mathrm{pCi} / \mathrm{L}$ & & & 1 & & $U$ \\
\hline 84 & SAS COUNT ${ }^{-}$LSC & TMANC & 7 & $\mathrm{pCi} / \mathrm{L}$ & & & 7 & & $\mathrm{U}$ \\
\hline 85 & SAS_COUNT_LSC & TMANC & 400 & $\mathrm{pCi} / \mathrm{L}$ & & & 400 & & $U$ \\
\hline 87 & CLP_METALS_ICP & SKINER & 21.2 & $\mathrm{ug} / \mathrm{L}$ & & & & & \\
\hline 88 & GAMMA_GS & TMANC & 15.47 & $\mathrm{pCi} / \mathrm{L}$ & & & 15.47 & & U \\
\hline 89 & SAS_COUNT_LSC & TMANC & & $\mathrm{pCi} / \mathrm{L}$ & & & 1 & & $U$ \\
\hline 90 & SAS_COUNT_LSC & TMANC & & $\mathrm{pCi} / \mathrm{L}$ & & & 6 & & $\mathrm{U}$ \\
\hline 91 & SAS_COUNT_LSC & TMANC & 1100 & $\mathrm{pCi} / \mathrm{L}$ & 300 & & & & \\
\hline 92 & CLP_METALS_ICP & SKINER & & $u g / L$ & & & & & $\mathrm{U}$ \\
\hline 93 & CLP_METALS_ICP & SKINER & & $\mathrm{ug} / \mathrm{L}$ & & & & & $\mathrm{U}$ \\
\hline 94 & GAMMA_GS & TMANC & 11.76 & $\mathrm{pCi} / \mathrm{L}$ & & & 11.76 & & $\mathrm{U}$ \\
\hline 95 & SAS_COUNT_LSC & TMANC & 1 & $\mathrm{pCi} / \mathrm{L}$ & & & 1 & & $U$ \\
\hline 97 & SAS_COUNT_LSC & TMANC & 400 & $\mathrm{pCi} / \mathrm{L}$ & & & 400 & & $\mathrm{U}$ \\
\hline 98 & CLP_METALS_ICP & SKINER & & ug/L & & & & & $U$ \\
\hline 99 & CLP_METALS_ICP & SKINER & & $\mathrm{ug} / \mathrm{L}$ & & & & & $\mathrm{U}$ \\
\hline 100 & GAMMA_GS & TMANC & 15.35 & $\mathrm{pCi} / \mathrm{L}$ & & & 15.35 & & U \\
\hline 101 & SAS_COUNT_LSC & TMANC & 2.5 & $\mathrm{pCi} / \mathrm{L}$ & 0.4 & & & & \\
\hline 102 & SAS_COUNT_LSC & TMANC & 400 & $\mathrm{pCi} / \mathrm{L}$ & & & 400 & & $U$ \\
\hline 103 & CLP_METALS_ICP & SKINER & 2.2 & $u g / L$ & & & & & U \\
\hline 104 & CLP_METALS_ICP & SKINER & & $\mathrm{ug} / \mathrm{L}$ & & & & & $U$ \\
\hline 105 & GAMMA_GS & TMANC & 10.92 & $\mathrm{pCi} / \mathrm{L}$ & & & 10.92 & & $\mathrm{U}$ \\
\hline 106 & SAS_COUNT_LSC & TMANC & 1 & $\mathrm{pCi} / \mathrm{L}$ & & & 1 & & $\mathrm{U}$ \\
\hline 107 & SAS_COUNT_LSC & TMANC & 400 & $\mathrm{pCi} / \mathrm{L}$ & & & 400 & & $U$ \\
\hline 108 & CLP_METALS_ICP & SKINER & 7.4 & ug/L & & & & & U \\
\hline 109 & CLP_METALS_ICP & SKINER & 2.7 & $\mathrm{ug} / \mathrm{L}$ & & & & & B \\
\hline 110 & GAMMA GS & TMANC & 11.74 & $\mathrm{pCi} / \mathrm{L}$ & & & 11.74 & & $\mathrm{U}$ \\
\hline 111 & SAS_COUNT_LSC & TMANC & 0.6 & $\mathrm{pCi} / \mathrm{L}$ & & & 0.6 & & $\mathrm{U}$ \\
\hline 112 & SAS_COUNT_LSC & TMANC & 400 & $\mathrm{pCi} / \mathrm{L}$ & & & 400 & & $\mathrm{U}$ \\
\hline 113 & CLP_METALS_ICP & SKINER & 6.3 & $\mathrm{ug} / \mathrm{L}$ & & & & & U \\
\hline 114 & CLP_METALS_ICP & SKINER & & ug/L & & & & & $U$ \\
\hline
\end{tabular}




\begin{tabular}{|c|c|c|c|c|c|c|c|c|c|}
\hline & $\mathrm{K}$ & $\mathrm{L}$ & $\mathrm{M}$ & $\mathrm{N}$ & $\mathrm{O}$ & $\mathrm{P}$ & $\mathrm{Q}$ & $\mathrm{R}$ & $\mathrm{S}$ \\
\hline 1 & METHOD NAME & LAB CODE & VALUE RPTD & ANAL UNITS RPTD & COUNTING ERROR & TOTAL ANAL ERROR & MIN DETECTABLE ACTIVITY & PCNT MOISTURE & LAB QUALIFIER \\
\hline 116 & SAS_COUNT_LSC & TMANC & & $\mathrm{pCi} / \mathrm{L}$ & & & 1 & & $\mathrm{U}$ \\
\hline 117 & SAS_COUNT_LSC & TMANC & 400 & $\mathrm{pCi} / \mathrm{L}$ & & & 400 & & $\mathrm{U}$ \\
\hline 118 & CLP_METALS_ICP & SKINER & & $\mathrm{ug} / \mathrm{L}$ & & & & & $B$ \\
\hline 119 & CLP_METALS_ICP & SKINER & 32.1 & $u g / L$ & & & & & \\
\hline 120 & GAMMA_GS & TMANC & 13.78 & $\mathrm{pCi} / \mathrm{L}$ & & & 13.78 & & U \\
\hline 121 & SAS_COUNT_LSC & TMANC & & $\mathrm{pCi} / \mathrm{L}$ & & & 1 & & $U$ \\
\hline 122 & SAS COUNT ${ }^{-}$LSC & TMANC & $40 c$ & $\mathrm{pCi} / \mathrm{L}$ & & & 400 & & $\mathrm{U}$ \\
\hline 123 & CLP_METALS_ICP & SKINER & 11.9 & $\mathrm{ug} / \mathrm{L}$ & & & & & $U$ \\
\hline 125 & GAMMA_GS & TMANC & 12.51 & $\mathrm{pCi} / \mathrm{L}$ & & & 12.51 & & $\mathrm{U}$ \\
\hline 126 & SAS_COUNT_LSC & TMANC & 0.4 & $\mathrm{pCi} / \mathrm{L}$ & & & 0.4 & & U \\
\hline 127 & SAS_COUNT_LSC & TMANC & 400 & $\mathrm{pCi} / \mathrm{L}$ & & & 400 & & $U$ \\
\hline 128 & CLP_METALS_ICP & SKINER & 6.9 & $\mathrm{ug} / \mathrm{L}$ & & & & & $\mathrm{U}$ \\
\hline 129 & CLP_METALS_ICP & SKINER & 2.4 & $\mathrm{ug} / \mathrm{L}$ & & & & & B \\
\hline 130 & GAMMA_GS & TMANC & 14.77 & $\mathrm{pCi} / \mathrm{L}$ & & & 14.77 & & $U$ \\
\hline 131 & SAS_COUNT_LSC & TMANC & 0.4 & $\mathrm{pCi} / \mathrm{L}$ & & & 0.4 & & $\mathrm{U}$ \\
\hline 132 & SAS_COUNT_LSC & TMANC & 400 & $\mathrm{pCi} / \mathrm{L}$ & & & 400 & & $\mathrm{U}$ \\
\hline 133 & CLP_METALS_ICP & SKINER & 11.3 & $3 \mathrm{ug} / \mathrm{L}$ & & & & & $U$ \\
\hline 135 & GAMMA_GS & TMANC & 10.71 & $\mathrm{pCi} / \mathrm{L}$ & & & 10.71 & & $\mathrm{U}$ \\
\hline 136 & SAS COUNT LSC & TMANC & & $\mathrm{pCi} / \mathrm{L}$ & & & 1 & & $\mathrm{U}$ \\
\hline 137 & SAS_COUNT_LSC & TMANC & 400 & $\mathrm{pCi} / \mathrm{L}$ & & & 400 & & $\mathrm{U}$ \\
\hline 138 & CLP_METALS_ICP & SKINER & 5.2 & $2 \mathrm{ug} / \mathrm{L}$ & & & & & U \\
\hline 139 & CLP_METALS_ICP & SKINER & & $2 \mathrm{ug} / \mathrm{L}$ & & & & & U \\
\hline 140 & CLP_METALS_ICP & SKINER & 4.2 & $2 \mathrm{ug} / \mathrm{L}$ & & & & & U \\
\hline 141 & GAMMA_GS & TMANC & 12.47 & $7 \mathrm{pCi} / \mathrm{L}$ & & & 12.47 & & U \\
\hline 142 & GAMMA_GS & TMANC & 13.97 & $7 \mathrm{pCi} / \mathrm{L}$ & & & 13.97 & & $U$ \\
\hline 143 & SAS_COUNT_LSC & TMANC & & $\mathrm{pCi} / \mathrm{L}$ & & & 1 & & $\mathrm{U}$ \\
\hline 144 & SAS_COUNT_LSC & TMANC & & $\mathrm{pCi} / \mathrm{L}$ & & & 1 & & $\mathrm{U}$ \\
\hline 145 & SAS_COUNT_LSC & TMANC & 400 & $\mathrm{pCi} / \mathrm{L}$ & & & 400 & & $U$ \\
\hline 146 & SAS_COUNT_LSC & TMANC & 400 & $\mathrm{pCi} / \mathrm{L}$ & & & 400 & & U \\
\hline 147 & CLP_METALS_ICP & SKINER & & $2 \mathrm{ug} / \mathrm{L}$ & & & & & $\mathrm{U}$ \\
\hline 148 & CLP METALS ICP & SKINER & 2.2 & $2 \mathrm{ug} / \mathrm{L}$ & & & & & $\mathrm{U}$ \\
\hline 149 & CLP_METALS_ICP & SKINER & & $2 \mathrm{ug} / \mathrm{L}$ & & & & & $\mathrm{U}$ \\
\hline 150 & GAMMA_GS & TMANC & 11.39 & $9 \mathrm{pCi} / \mathrm{L}$ & & & 11.39 & & U \\
\hline 151 & SAS_COUNT_LSC & TMANC & & $\mathrm{pCi} / \mathrm{L}$ & & & 1 & & U \\
\hline 152 & SAS_COUNT_LSC & TMANC & 400 & $\mathrm{pCi} / \mathrm{L}$ & & & 400 & & $U$ \\
\hline
\end{tabular}




\begin{tabular}{|c|c|c|c|c|c|c|c|c|c|}
\hline & $\mathrm{K}$ & $\mathrm{L}$ & $\mathrm{M}$ & $\mathrm{N}$ & $\mathrm{O}$ & $\mathrm{P}$ & $\mathrm{Q}$ & $\mathrm{R}$ & $\mathrm{S}$ \\
\hline 1 & METHOD NAME & LAB CODE & VALUE RPTD & ANAL UNITS RPTD & COUNTING ERROR & TOTAL ANAL ERROR & MIN DETECTABLE ACTIVITY & PCNT MOISTURE & LAB QUALIFIER \\
\hline 154 & CLP_METALS_ICP & SKINER & & ug/L & & & & & $\mathrm{U}$ \\
\hline 155 & GAMMA_GS & TMANC & 12.99 & $\mathrm{pCi} / \mathrm{L}$ & & & & & U \\
\hline 156 & SAS_COUNT_LSC & TMANC & 0.1 & $\mathrm{pCi} / \mathrm{L}$ & 0.2 & & & & $\mathrm{U}$ \\
\hline 157 & SAS_COUNT_LSC & TMANC & -46 & $\mathrm{pCi} / \mathrm{L}$ & 126 & & & & $\mathrm{U}$ \\
\hline 158 & CLP_METALS_ICP & SKINER & 9.6 & ug/L & & & & & B \\
\hline 159 & CLP_METALS_ICP & SKINER & & ug/L & & & & & B \\
\hline 160 & GAMMA GS & TMANC & 11.8 & $\mathrm{pCi} / \mathrm{L}$ & & & 11.8 & & $\mathrm{U}$ \\
\hline 161 & SAS_COUNT_LSC & TMANC & 0.24 & $\mathrm{pCi} / \mathrm{L}$ & 0.07 & & & & \\
\hline 163 & CLP_METALS_ICP & SKINER & & ug/L & & & & & $\mathrm{U}$ \\
\hline 164 & CLP_METALS_ICP & SKINER & & $\mathrm{ug} / \mathrm{L}$ & & & & & U \\
\hline 165 & GAMMA_GS & TMANC & 10.87 & $\mathrm{pCi} / \mathrm{L}$ & & & 10.87 & & $U$ \\
\hline 166 & SAS_COUNT_LSC & TMANC & -0.4 & $\mathrm{pCi} / \mathrm{L}$ & 0.4 & & & & $\mathrm{U}$ \\
\hline 167 & SAS_COUNT_LSC & TMANC & 110 & $\mathrm{pCi} / \mathrm{L}$ & 130 & & & & $U$ \\
\hline 168 & CLP_METALS_ICP & SKINER & & ug/L & & & & & $U$ \\
\hline 169 & CLP_METALS_ICP & SKINER & & $\mathrm{ug} / \mathrm{L}$ & & & & & $\mathrm{U}$ \\
\hline 170 & GAMMA_GS & TMANC & 13.26 & $\mathrm{pCi} / \mathrm{L}$ & & & 13.26 & & U \\
\hline 171 & SAS_COUNT_LSC & TMANC & 0.4 & $\mathrm{pCi} / \mathrm{L}$ & & & 0.4 & & $U$ \\
\hline 173 & CLP_METALS_ICP & SKINER & & $\mathrm{ug} / \mathrm{L}$ & & & & & U \\
\hline 174 & CLP_METALS_ICP & SKINER & & $\mathrm{ug} / \mathrm{L}$ & & & & & $U$ \\
\hline 175 & GAMMA_GS & TMANC & & $\mathrm{pCi} / \mathrm{L}$ & & & 10 & & $\mathrm{U}$ \\
\hline 176 & SAS_COUNT_LSC & TMANC & 0.8 & $\mathrm{pCi} / \mathrm{L}$ & & & 0.8 & & U \\
\hline 177 & SAS_COUNT_LSC & TMANC & 170 & $\mathrm{pCi} / \mathrm{L}$ & & & 170 & & U \\
\hline 178 & CLP_METALS_ICP & SKINER & & ug/L & & & & & U \\
\hline 179 & CLP_METALS_ICP & SKINER & & $\mathrm{ug} / \mathrm{L}$ & & & & & $\mathrm{U}$ \\
\hline 180 & GAMMA_GS & TMANC & 14.03 & $\mathrm{pCi} / \mathrm{L}$ & & & 14.03 & & $U$ \\
\hline 181 & SAS_COUNT_LSC & TMANC & 0.3 & $\mathrm{pCi} / \mathrm{L}$ & & & 0.3 & & $\mathrm{U}$ \\
\hline 182 & SAS_COUNT_LSC & TMANC & 240 & $\mathrm{pCi} / \mathrm{L}$ & & & 240 & & $\mathrm{U}$ \\
\hline 183 & CLP_METALS_ICP & SKINER & & ug/L & & & & & $U$ \\
\hline 184 & CLP_METALS_ICP & SKINER & & $\mathrm{ug} / \mathrm{L}$ & & & & & U \\
\hline 185 & GAMMA_GS & TMANC & 14.43 & $\mathrm{pCi} / \mathrm{L}$ & & & 14.43 & & $\mathrm{U}$ \\
\hline 186 & SAS COUNT LSC & TMANC & 0.4 & $\mathrm{pCi} / \mathrm{L}$ & & & 0.4 & & $\mathrm{U}$ \\
\hline 187 & SAS_COUNT_LSC & TMANC & 190 & $\mathrm{pCi} / \mathrm{L}$ & & & 190 & & $\mathrm{U}$ \\
\hline 188 & CLP_METALS_ICP & SKINER & & $\mathrm{ug} / \mathrm{L}$ & & & & & U \\
\hline 189 & CLP_METALS_ICP & SKINER & & $\mathrm{ug} / \mathrm{L}$ & & & & & U \\
\hline 190 & CLP_METALS_ICP & SKINER & & ug/L & & & & & $U$ \\
\hline
\end{tabular}

Page 34 


\begin{tabular}{|c|c|c|c|c|c|c|c|c|c|}
\hline & $\mathrm{K}$ & $\mathrm{L}$ & $\mathrm{M}$ & $\mathrm{N}$ & $\mathrm{O}$ & $\mathrm{P}$ & $\mathrm{Q}$ & $\mathrm{R}$ & $\mathrm{S}$ \\
\hline 1 & METHOD NAME & LAB CODE & VALUE RPTD & ANAL UNITS RPTD & COUNTING ERROR & TOTAL ANAL ERROR & MIN DETECTABLE ACTIVITY & PCNT MOISTURE & LAB QUALIFIER \\
\hline 192 & GAMMA_GS & TMANC & 14.13 & $\mathrm{pCi} / \mathrm{L}$ & & & 14.13 & & $U$ \\
\hline 193 & GAMMA_GS & TMANC & 13.02 & $\mathrm{pCi} / \mathrm{L}$ & & & 13.02 & & $U$ \\
\hline 194 & SAS_COUNT_LSC & TMANC & & $\mathrm{pCi} / \mathrm{L}$ & & & 1 & & $\mathrm{U}$ \\
\hline 195 & SAS_COUNT_LSC & TMANC & 0.4 & $\mathrm{pCi} / \mathrm{L}$ & 0.2 & & & & \\
\hline 196 & SAS_COUNT_LSC & TMANC & 270 & $\mathrm{pCi} / \mathrm{L}$ & & & 270 & & $U$ \\
\hline 197 & SAS_COUNT_LSC & TMANC & 170 & $\mathrm{pCi} / \mathrm{L}$ & & & 170 & & U \\
\hline 198 & CLP METALS̄ ICP & SKINER & 2 & $u g / L$ & & & & & $\mathrm{U}$ \\
\hline 199 & GAMMA_GS & TMANC & 13.82 & $\mathrm{pCi} / \mathrm{L}$ & & & 13.82 & & $U$ \\
\hline 201 & SAS_COUNT_LSC & TMANC & 2.4 & $\mathrm{pCi} / \mathrm{L}$ & & & 2.4 & & $U$ \\
\hline 202 & SAS_COUNT_LSC & TMANC & 300 & $\mathrm{pCi} / \mathrm{L}$ & 100 & & & & \\
\hline 203 & CLP_METALS_ICP & SKINER & & ug/L & & & & & $U$ \\
\hline 204 & CLP_METALS_ICP & SKINER & 2.3 & $\mathrm{ug} / \mathrm{L}$ & & & & & 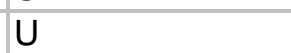 \\
\hline 205 & GAMMA_GS & TMANC & 11.79 & $\mathrm{pCi} / \mathrm{L}$ & & & 11.79 & & $U$ \\
\hline 206 & SAS_COUNT_LSC & TMANC & 3210 & $\mathrm{pCi} / \mathrm{L}$ & 70 & & & & \\
\hline 207 & SAS_COUNT_LSC & TMANC & 2.5 & $\mathrm{pCi} / \mathrm{L}$ & 2.2 & & & & \\
\hline 208 & SAS_COUNT_LSC & TMANC & 15900 & $\mathrm{pCi} / \mathrm{L}$ & 800 & & & & \\
\hline 209 & CLP_METALS_ICP & SKINER & 2 & ug/L & & & & & $U$ \\
\hline 211 & GAMMA_GS & TMANC & 15.06 & $\mathrm{pCi} / \mathrm{L}$ & & & 15.06 & & $\mathrm{U}$ \\
\hline 212 & SAS_COUNT_LSC & TMANC & 3 & $\mathrm{pCi} / \mathrm{L}$ & 1 & & & & $\mathrm{~J}$ \\
\hline 213 & SAS_COUNT_LSC & TMANC & 3.9 & $\mathrm{pCi} / \mathrm{L}$ & 1.6 & & & & \\
\hline 214 & SAS_COUNT_LSC & TMANC & 200 & $\mathrm{pCi} / \mathrm{L}$ & 100 & & & & \\
\hline 215 & CLP_METALS_ICP & SKINER & 2.6 & $\mathrm{ug} / \mathrm{L}$ & & & & & $u$ \\
\hline 216 & CLP_METALS_ICP & SKINER & 2 & $\mathrm{ug} / \mathrm{L}$ & & & & & 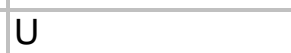 \\
\hline 217 & GAMMA_GS & TMANC & 12.25 & $\mathrm{pCi} / \mathrm{L}$ & & & 12.25 & & U \\
\hline 218 & SAS_COUNT_LSC & TMANC & 395 & $\mathrm{pCi} / \mathrm{L}$ & 12 & & & & \\
\hline 219 & SAS_COUNT_LSC & TMANC & 3.6 & $\mathrm{pCi} / \mathrm{L}$ & 1.3 & & & & \\
\hline 220 & SAS_COUNT_LSC & TMANC & 3400 & $\mathrm{pCi} / \mathrm{L}$ & 200 & & & & \\
\hline 221 & CLP_METALS_ICP & SKINER & 6 & $\mathrm{ug} / \mathrm{L}$ & & & & & $U$ \\
\hline 222 & CLP_METALS_ICP & SKINER & 2 & $\mathrm{ug} / \mathrm{L}$ & & & & & $U$ \\
\hline 223 & GAMMA_GS & TMANC & 11.54 & $\mathrm{pCi} / \mathrm{L}$ & & & 11.54 & & $U$ \\
\hline 224 & SAS_COUNT_LSC & TMANC & 0.7 & $\mathrm{pCi} / \mathrm{L}$ & 0.2 & & & & $\mathrm{~J}$ \\
\hline 225 & SAS_COUNT_LSC & TMANC & 2 & $\mathrm{pCi} / \mathrm{L}$ & 1 & & & & \\
\hline 226 & SAS_COUNT_LSC & TMANC & 200 & $\mathrm{pCi} / \mathrm{L}$ & & & 200 & & $U$ \\
\hline 227 & CLP_METALS_ICP & SKINER & 68.7 & $\mathrm{ug} / \mathrm{L}$ & & & & & \\
\hline 228 & CLP_METALS_ICP & SKINER & 64.5 & $\mathrm{ug} / \mathrm{L}$ & & & & & \\
\hline
\end{tabular}




\begin{tabular}{|c|c|c|c|c|c|c|c|c|c|}
\hline & $\mathrm{K}$ & $\mathrm{L}$ & $\mathrm{M}$ & $\mathrm{N}$ & $\mathrm{O}$ & $\mathrm{P}$ & $\mathrm{Q}$ & $\mathrm{R}$ & $\mathrm{S}$ \\
\hline 1 & METHOD NAME & LAB CODE & VALUE RPTD & ANAL UNITS RPTD & COUNTING ERROR & TOTAL ANAL ERROR & MIN DETECTABLE ACTIVITY & PCNT MOISTURE & LAB QUALIFIER \\
\hline 230 & SAS_COUNT_LSC & TMANC & 8.8 & $\mathrm{pCi} / \mathrm{L}$ & 0.6 & & & & \\
\hline 231 & SAS_COUNT_LSC & TMANC & 5.2 & $\mathrm{pCi} / \mathrm{L}$ & 1.6 & & & & \\
\hline 232 & SAS_COUNT_LSC & TMANC & 400 & $\mathrm{pCi} / \mathrm{L}$ & 100 & & & & \\
\hline 233 & CLP_METALS_ICP & SKINER & & ug/L & & & & & $\mathrm{U}$ \\
\hline 234 & CLP_METALS_ICP & SKINER & & ug/L & & & & & U \\
\hline 235 & GAMMA_GS & TMANC & 11.9 & $\mathrm{pCi} / \mathrm{L}$ & & & 11.9 & & $U$ \\
\hline 236 & SAS COUNT LSC & TMANC & 0.9 & $\mathrm{pCi} / \mathrm{L}$ & 0.8 & & & & $\mathrm{~J}$ \\
\hline 237 & SAS_COUNT_LSC & TMANC & 1.7 & $\mathrm{pCi} / \mathrm{L}$ & 1.2 & & & & \\
\hline 239 & CLP_METALS_ICP & SKINER & & $\mathrm{ug} / \mathrm{L}$ & & & & & $\mathrm{U}$ \\
\hline 240 & CLP_METALS_ICP & SKINER & & $\mathrm{ug} / \mathrm{L}$ & & & & & U \\
\hline 241 & GAMMA_GS & TMANC & 12.67 & $\mathrm{pCi} / \mathrm{L}$ & & & 12.67 & & $U$ \\
\hline 242 & SAS_COUNT_LSC & TMANC & & $\mathrm{pCi} / \mathrm{L}$ & & & 1 & & $\mathrm{U}$ \\
\hline 243 & SAS_COUNT_LSC & TMANC & & $\mathrm{pCi} / \mathrm{L}$ & 3 & & & & \\
\hline 244 & SAS_COUNT_LSC & TMANC & 20300 & $\mathrm{pCi} / \mathrm{L}$ & 1000 & & & & \\
\hline 245 & CLP_METALS_ICP & SKINER & & $u g / L$ & & & & & $\mathrm{U}$ \\
\hline 246 & CLP_METALS_ICP & SKINER & & $\mathrm{ug} / \mathrm{L}$ & & & & & $\mathrm{U}$ \\
\hline 247 & GAMMA_GS & TMANC & 9.389 & $\mathrm{pCi} / \mathrm{L}$ & & & 9.389 & & $U$ \\
\hline 249 & SAS_COUNT_LSC & TMANC & & $\mathrm{pCi} / \mathrm{L}$ & & & 5 & & $\mathrm{U}$ \\
\hline 250 & SAS_COUNT_LSC & TMANC & 800 & $\mathrm{pCi} / \mathrm{L}$ & 100 & & & & \\
\hline 251 & CLP_METALS_ICP & SKINER & & ug/L & & & & & U \\
\hline 252 & CLP_METALS_ICP & SKINER & 3.1 & $\mathrm{ug} / \mathrm{L}$ & & & & & U \\
\hline 253 & GAMMA_GS & TMANC & 14.95 & $\mathrm{pCi} / \mathrm{L}$ & & & 14.95 & & U \\
\hline 254 & SAS_COUNT_LSC & TMANC & 0.2 & $\mathrm{pCi} / \mathrm{L}$ & & & 0.2 & & U \\
\hline 255 & SAS_COUNT_LSC & TMANC & 6.2 & $\mathrm{pCi} / \mathrm{L}$ & 3 & & & & \\
\hline 256 & SAS_COUNT_LSC & TMANC & 23900 & $\mathrm{pCi} / \mathrm{L}$ & 1200 & & & & \\
\hline 257 & CLP_METALS_ICP & SKINER & & ug/L & & & & & U \\
\hline 258 & CLP_METALS_ICP & SKINER & & ug/L & & & & & $\mathrm{U}$ \\
\hline 259 & GAMMA_GS & TMANC & 11.68 & $\mathrm{pCi} / \mathrm{L}$ & & & 11.68 & & $U$ \\
\hline 260 & SAS_COUNT_LSC & TMANC & 0.4 & $\mathrm{pCi} / \mathrm{L}$ & & & 0.4 & & U \\
\hline 261 & SAS_COUNT_LSC & TMANC & 3 & $\mathrm{pCi} / \mathrm{L}$ & & & 3 & & $\mathrm{U}$ \\
\hline 262 & SAS COUNT LSC & TMANC & 200 & $\mathrm{pCi} / \mathrm{L}$ & & & 200 & & $\mathrm{U}$ \\
\hline 263 & CLP_METALS_ICP & SKINER & & ug/L & & & & & U \\
\hline 264 & CLP_METALS_ICP & SKINER & 2.4 & $\mathrm{ug} / \mathrm{L}$ & & & & & U \\
\hline 265 & GAMMA_GS & TMANC & 12.26 & $\mathrm{pCi} / \mathrm{L}$ & & & 12.26 & & U \\
\hline 266 & SAS_COUNT_LSC & TMANC & & $\mathrm{pCi} / \mathrm{L}$ & & & 3 & & $U$ \\
\hline
\end{tabular}




\begin{tabular}{|c|c|c|c|c|c|c|c|c|c|}
\hline & $\mathrm{K}$ & $\mathrm{L}$ & $\mathrm{M}$ & $\mathrm{N}$ & $\mathrm{O}$ & $\mathrm{P}$ & $\mathrm{Q}$ & $\mathrm{R}$ & $\mathrm{S}$ \\
\hline 1 & METHOD_NAME & LAB_CODE & VALUE_RPTD & ANAL_UNITS_RPTD & |COUNTING_ERROR & TOTAL_ANAL_ERROR & MIN_DETECTABLE_ACTIVITY & PCNT_MOISTURE & LAB_QUALIFIER \\
\hline 268 & SAS_COUNT_LSC & TMANC & 24300 & $\mathrm{pCi} / \mathrm{L}$ & 1200 & & & & \\
\hline 269 & CLP_METALS_ICP & SKINER & & ug/L & & & & & $\mathrm{U}$ \\
\hline 270 & CLP_METALS_ICP & SKINER & 2.6 & $\mathrm{ug} / \mathrm{L}$ & & & & & $U$ \\
\hline 271 & GAMMA_GS & TMANC & 8.608 & $\mathrm{pCi} / \mathrm{L}$ & & & 8.608 & & $U$ \\
\hline 272 & SAS_COUNT_LSC & TMANC & 0.4 & $\mathrm{pCi} / \mathrm{L}$ & & & 0.4 & & $U$ \\
\hline 273 & SAS_COUNT_LSC & TMANC & 3 & $\mathrm{pCi} / \mathrm{L}$ & & & 3 & & $U$ \\
\hline 274 & SAS_COUNT_LSC & TMANC & 200 & $\mathrm{pCi} / \mathrm{L}$ & & & 200 & & $U$ \\
\hline 275 & CLP_METALS_ICP & SKINER & 10.5 & $\mathrm{ug} / \mathrm{L}$ & & & & & \\
\hline 277 & GAMMA_GS & TMANC & 14.44 & $\mathrm{pCi} / \mathrm{L}$ & & & 14.44 & & $U$ \\
\hline 278 & SAS_COUNT_LSC & TMANC & 0.5 & $\mathrm{pCi} / \mathrm{L}$ & & & 0.5 & & $U$ \\
\hline 279 & SAS_COUNT_LSC & TMANC & & $\mathrm{pCi} / \mathrm{L}$ & & & 2 & & $U$ \\
\hline 280 & SAS_COUNT_LSC & TMANC & 8900 & $\mathrm{pCi} / \mathrm{L}$ & 500 & & & & \\
\hline 281 & CLP_METALS_ICP & SKINER & 15.7 & ug/L & & & & & \\
\hline 282 & CLP_METALS_ICP & SKINER & & ug/L & & & & & $U$ \\
\hline 283 & CLP_METALS_ICP & SKINER & & ug/L & & & & & $\mathrm{U}$ \\
\hline 284 & CLP_METALS_ICP & SKINER & 20.9 & $\mathrm{ug} / \mathrm{L}$ & & & & & \\
\hline 285 & GAMMA_GS & TMANC & 8.863 & $\mathrm{pCi} / \mathrm{L}$ & & & 8.863 & & $U$ \\
\hline 287 & SAS_COUNT_LSC & TMANC & 0.7 & $\mathrm{pCi} / \mathrm{L}$ & 0.2 & & & & $\mathrm{~J}$ \\
\hline 288 & SAS_COUNT_LSC & TMANC & 12.7 & $\mathrm{pCi} / \mathrm{L}$ & 1.4 & & & & \\
\hline 289 & SAS_COUNT_LSC & TMANC & 2.6 & $\mathrm{pCi} / \mathrm{L}$ & 1.4 & & & & \\
\hline 290 & SAS_COUNT_LSC & TMANC & 12 & $\mathrm{pCi} / \mathrm{L}$ & 2 & & & & \\
\hline 291 & SAS_COUNT_LSC & TMANC & 200 & $\mathrm{pCi} / \mathrm{L}$ & & & 200 & & $U$ \\
\hline 292 & SAS_COUNT_LSC & TMANC & 400 & $\mathrm{pCi} / \mathrm{L}$ & 100 & & & & \\
\hline 293 & GAMMA_GS & ITASRL & -0.149 & $\mathrm{pCi} / \mathrm{L}$ & 1.15 & 1.15 & & & $U$ \\
\hline 294 & TC99_SEP_LSC & ITASRL & 124 & $\mathrm{pCi} / \mathrm{L}$ & 2.6 & 15.5 & & & \\
\hline 295 & TRITIUM_DIST_LSC & ITASRL & 138000 & $\mathrm{pCi} / \mathrm{L}$ & 1190 & 10300 & & & \\
\hline 296 & GAMMA_GS & ITASRL & -0.834 & $\mathrm{pCi} / \mathrm{L}$ & 5.46 & 5.46 & & & $U$ \\
\hline 297 & SRISO_SEP_PRECIP_GPC & ITASRL & 5110 & $\mathrm{pCi} / \mathrm{L}$ & 244 & 1000 & & & \\
\hline 298 & TC99_SEP_LSC & ITASRL & 2.44 & $\mathrm{pCi} / \mathrm{L}$ & 0.134 & 0.685 & & & \\
\hline 299 & TRITIUM_DIST_LSC & ITASRL & 11300 & $\mathrm{pCi} / \mathrm{L}$ & 370 & 1040 & & & \\
\hline 300 & GAMMA_GS & ITASRL & 1.09 & $\mathrm{pCi} / \mathrm{L}$ & 1.27 & 1.27 & & & $U$ \\
\hline 301 & TC99_SËP_LSC & ITASRL & 2.07 & $\mathrm{pCi} / \mathrm{L}$ & 0.132 & 0.642 & & & \\
\hline 302 & TRITIŪM_DIST_LSC & ITASRL & 6470 & $\mathrm{pCi} / \mathrm{L}$ & 294 & 699 & & & \\
\hline 303 & UISO_AEA & ITASRL & 28.6 & $\mathrm{pCi} / \mathrm{L}$ & 0.467 & 2.74 & & & \\
\hline 304 & UISO_AEA & ITASRL & 1.53 & $\mathrm{pCi} / \mathrm{L}$ & 0.108 & 0.18 & & & \\
\hline
\end{tabular}




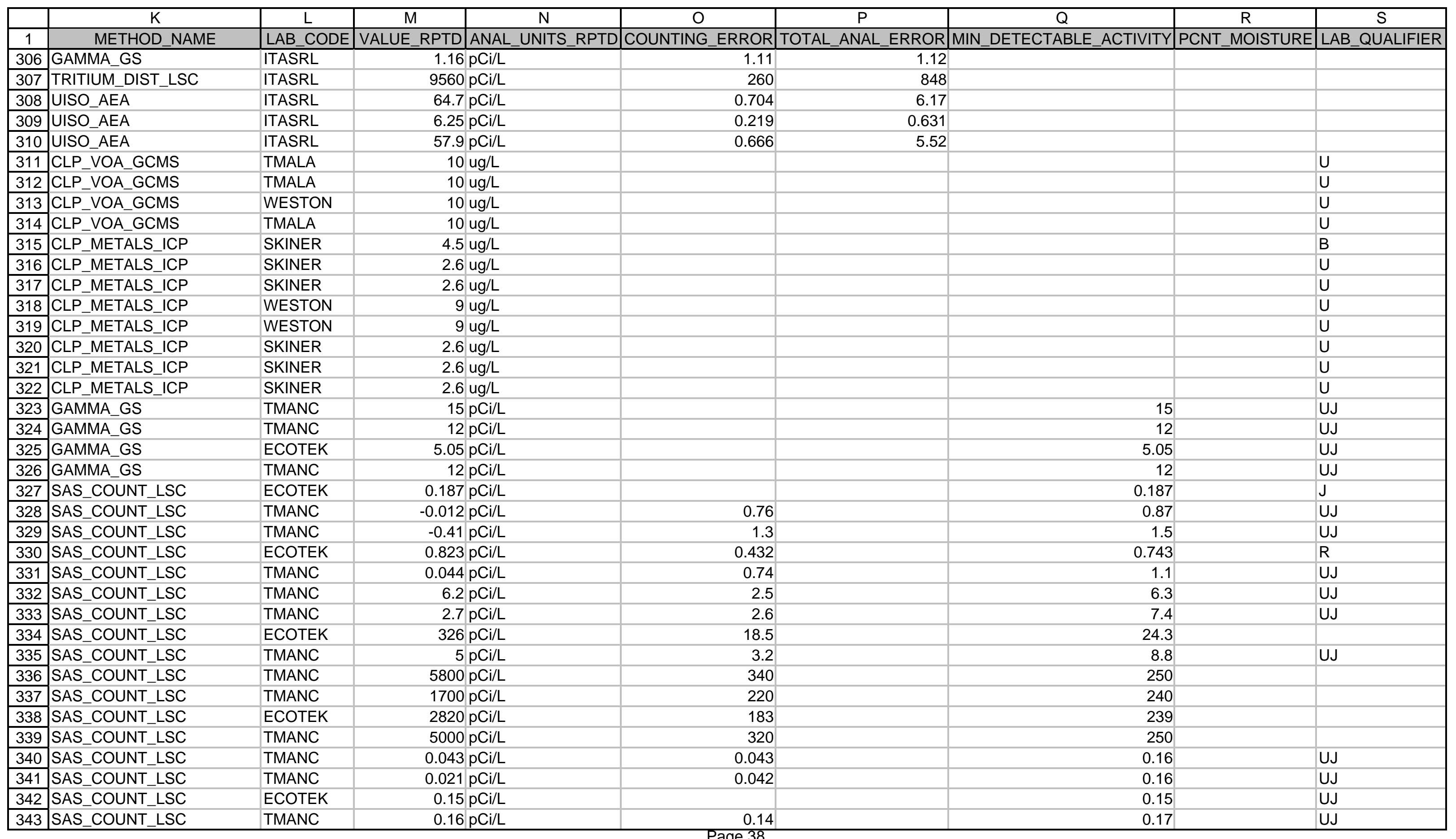




\begin{tabular}{|c|c|c|c|c|c|c|c|c|c|}
\hline & $\mathrm{K}$ & $\mathrm{L}$ & $\mathrm{M}$ & $\mathrm{N}$ & $\mathrm{O}$ & $P$ & $\mathrm{Q}$ & $\mathrm{R}$ & $\mathrm{S}$ \\
\hline 1 & METHOD_NAME & LAB_CODE & VALUE_RPTD & ANAL_UNITS_RPTD & COUNTING_ERROR & TOTAL_ANAL_ERROR & MIN_DETECTABLE_ACTIVITY & PCNT_MOISTURE & LAB_QUALIFIER \\
\hline 344 & SAS_COUNT_LSC & TMANC & 1.1 & $\mathrm{pCi} / \mathrm{L}$ & 0.32 & & 0.14 & & \\
\hline 345 & SAS_COUNT_LSC & TMANC & 0.47 & $\mathrm{pCi} / \mathrm{L}$ & 0.18 & & 0.13 & & \\
\hline 346 & SAS_COUNT_LSC & ECOTEK & 1.44 & $\mathrm{pCi} / \mathrm{L}$ & 0.607 & & 0.149 & & $\mathrm{~J}$ \\
\hline 347 & SAS_COUNT_LSC & TMANC & 1.1 & $\mathrm{pCi} / \mathrm{L}$ & 0.33 & & 0.14 & & \\
\hline 348 & CLP_VOA_GCMS & TMALA & 10 & $\mathrm{ug} / \mathrm{L}$ & & & & & $U$ \\
\hline 349 & CLP_VOA_GCMS & TMALA & 10 & ug/L & & & & & $U$ \\
\hline 350 & CLP_VOA_GCMS & TMALA & 10 & $\mathrm{ug} / \mathrm{L}$ & & & & & $\mathrm{U}$ \\
\hline 351 & CLP_VOA_GCMS & TMALA & 10 & $\mathrm{ug} / \mathrm{L}$ & & & & & $U$ \\
\hline 353 & CLP_METALS_ICP & SKINER & 2.6 & ug/L & & & & & $\mathrm{U}$ \\
\hline 354 & CLP_METALS_ICP & SKINER & 2.6 & ug/L & & & & & $\mathrm{U}$ \\
\hline 355 & CLP_METALS_ICP & SKINER & 5.2 & ug/L & & & & & B \\
\hline 356 & CLP_METALS_ICP & SKINER & 2.6 & ug/L & & & & & U \\
\hline 357 & CLP_METALS_ICP & SKINER & 2.6 & $\mathrm{ug} / \mathrm{L}$ & & & & & $U$ \\
\hline 358 & CLP_METALS_ICP & SKINER & 2.6 & ug/L & & & & & $U$ \\
\hline 359 & CLP_METALS_ICP & SKINER & 2.6 & $\mathrm{ug} / \mathrm{L}$ & & & & & $\mathrm{U}$ \\
\hline 360 & GAMMA_GS & TMANC & 15 & $\mathrm{pCi} / \mathrm{L}$ & & & 15 & & UJ \\
\hline 361 & GAMMA_GS & TMANC & 11 & $\mathrm{pCi} / \mathrm{L}$ & & & 11 & & UJ \\
\hline 363 & GAMMA_GS & TMANC & 13 & $\mathrm{pCi} / \mathrm{L}$ & & & 13 & & $\mathrm{U}$ \\
\hline 364 & SAS_COUNT_LSC & TMANC & 0.43 & $\mathrm{pCi} / \mathrm{L}$ & 0.36 & & 0.73 & & UJ \\
\hline 365 & SAS_COUNT_LSC & TMANC & 0.57 & $\mathrm{pCi} / \mathrm{L}$ & 0.69 & & 1.5 & & UJ \\
\hline 366 & SAS_COUNT_LSC & TMANC & 0.32 & pCi/L & 0.4 & & 0.86 & & UJ \\
\hline 367 & SAS_COUNT_LSC & TMANC & -0.07 & $\mathrm{pCi} / \mathrm{L}$ & 0.84 & & 0.81 & & $\mathrm{U}$ \\
\hline 368 & SAS_COUNT_LSC & TMANC & 0.53 & $\mathrm{pCi} / \mathrm{L}$ & 2.6 & & 5 & & UJ \\
\hline 369 & SAS_COUNT_LSC & TMANC & 5 & pCi/L & 3.3 & & 9.2 & & UJ \\
\hline 370 & SAS_COUNT_LSC & TMANC & 1.2 & $\mathrm{pCi} / \mathrm{L}$ & 2 & & 6.2 & & UJ \\
\hline 371 & SAS_COUNT_LSC & TMANC & 1.7 & $\mathrm{pCi} / \mathrm{L}$ & 1.5 & & 3.5 & & $\mathrm{U}$ \\
\hline 372 & SAS_COUNT_LSC & TMANC & 1900 & $\mathrm{pCi} / \mathrm{L}$ & 230 & & 250 & & \\
\hline 373 & SAS_COUNT_LSC & TMANC & 1800 & $\mathrm{pCi} / \mathrm{L}$ & 220 & & 240 & & \\
\hline 374 & SAS_COUNT_LSC & TMANC & 750 & $\mathrm{pCi} / \mathrm{L}$ & 180 & & 240 & & \\
\hline 375 & SAS_COUNT_LSC & TMANC & 1900 & $\mathrm{pCi} / \mathrm{L}$ & 220 & & 240 & & \\
\hline 376 & SAS_COUNT_LSC & TMANC & 0.79 & $\mathrm{pCi} / \mathrm{L}$ & 0.25 & & 0.15 & & $\mathrm{~J}$ \\
\hline 377 & SAS_COUNT_LSC & TMANC & 0.56 & $\mathrm{pCi} / \mathrm{L}$ & 0.21 & & 0.15 & & UJ \\
\hline 378 & SAS_COUNT_LSC & TMANC & 0.12 & $\mathrm{pCi} / \mathrm{L}$ & 0.12 & & 0.23 & & UJ \\
\hline 379 & SAS_COUNT_LSC & TMANC & 0.94 & $\mathrm{pCi} / \mathrm{L}$ & 0.29 & & 0.15 & & \\
\hline 380 & SAS_COUNT_LSC & TMANC & 7.5 & $\mathrm{pCi} / \mathrm{L}$ & 1.1 & & 0.2 & & \\
\hline
\end{tabular}

Page 39 


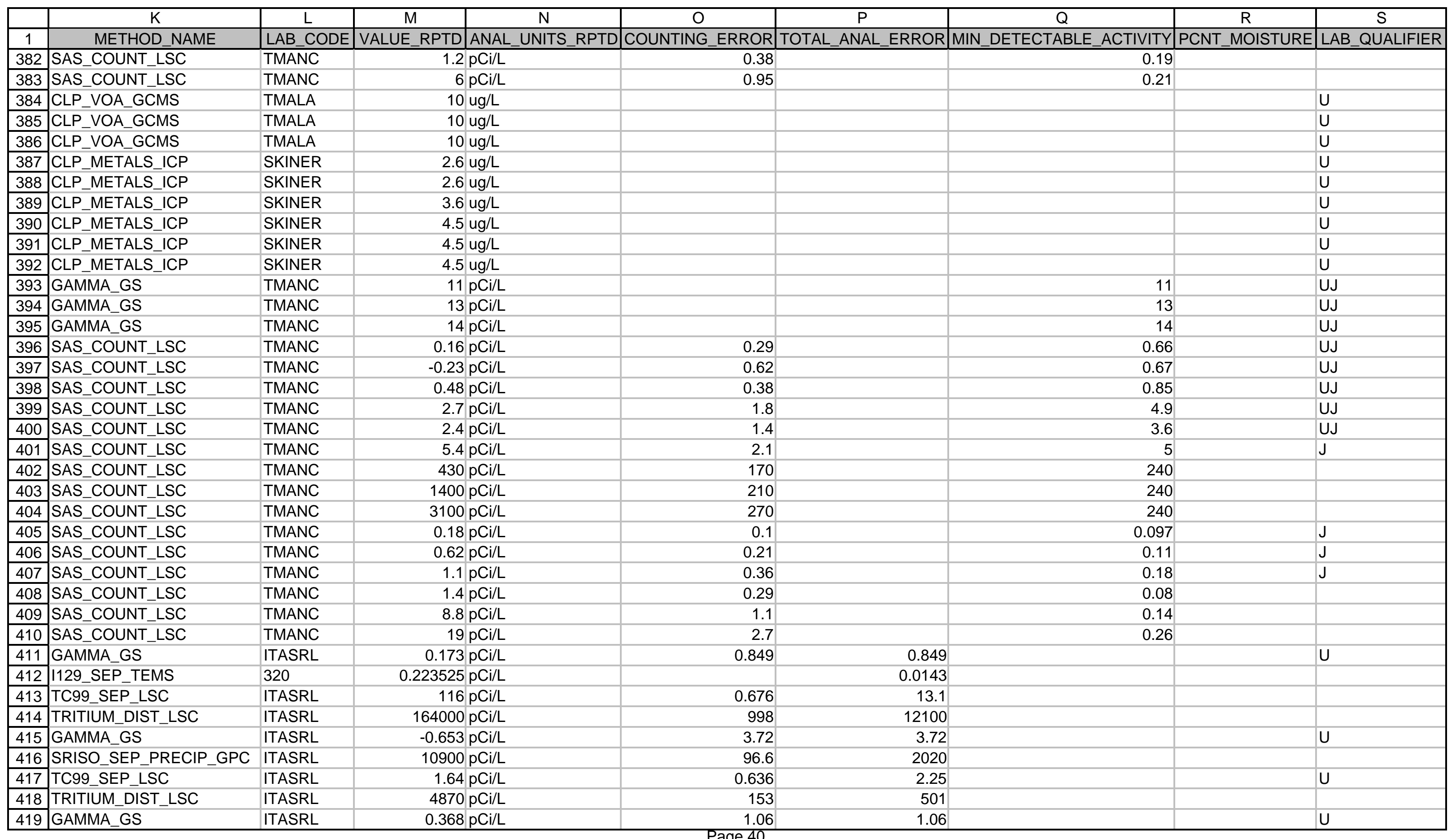

Page 40 


\begin{tabular}{|c|c|c|c|c|c|c|c|c|c|}
\hline & $\mathrm{K}$ & L & $\mathrm{M}$ & $\mathrm{N}$ & $\mathrm{O}$ & $\mathrm{P}$ & $\mathrm{Q}$ & $\mathrm{R}$ & $\mathrm{S}$ \\
\hline 1 & METHOD NAME & LAB CODE & VALUE RPTD & ANAL UNITS RPTD & COUNTING ERROR & TOTAL ANAL ERROR & MIN DETECTABLE ACTIVITY & PCNT MOISTURE & LAB QUALIFIER \\
\hline 420 & SRISO_SEP_PRECIP_GPC & ITASRL & 0.0029 & $\mathrm{pCi} / \mathrm{L}$ & 0.0913 & 0.0924 & & & $U$ \\
\hline 421 & TC99_SEP_LSC & ITASRL & 23.5 & $\mathrm{pCi} / \mathrm{L}$ & 0.646 & 4.18 & & & \\
\hline 422 & TRITIUM_DIST_LSC & ITASRL & 12900 & $\mathrm{pCi} / \mathrm{L}$ & 233 & 1070 & & & \\
\hline 423 & UISO_AEA & ITASRL & 1.05 & $\mathrm{pCi} / \mathrm{L}$ & 0.0943 & 0.139 & & & \\
\hline 424 & UISO_AEA & ITASRL & 0.044 & $\mathrm{pCi} / \mathrm{L}$ & 0.0201 & 0.0206 & & & \\
\hline 425 & UISO_AEA & ITASRL & 0.885 & $\mathrm{pCi} / \mathrm{L}$ & 0.0864 & 0.122 & & & \\
\hline 426 & GAMMĀGS & ITASRL & -0.77 & $\mathrm{pCi} / \mathrm{L}$ & 1.17 & 1.17 & & & $\mathrm{U}$ \\
\hline 427 & SRISO_SEP_PRECIP_GPC & ITASRL & 4.44 & $\mathrm{pCi} / \mathrm{L}$ & 0.317 & 0.868 & & & \\
\hline 429 & TRITIUM_DIST_LSC & ITASRL & 6520 & $\mathrm{pCi} / \mathrm{L}$ & 175 & 618 & & & \\
\hline 430 & UISO_AEA & ITASRL & 0.635 & $\mathrm{pCi} / \mathrm{L}$ & 0.0734 & 0.096 & & & \\
\hline 431 & UISO_AEA & ITASRL & 0.0184 & $\mathrm{pCi} / \mathrm{L}$ & 0.0147 & 0.0149 & & & \\
\hline 432 & UISO_AEA & ITASRL & 0.495 & $\mathrm{pCi} / \mathrm{L}$ & 0.0646 & 0.0805 & & & \\
\hline 433 & CLP_METALS_ICP & SKINER & 20.5 & ug/L & & & & & \\
\hline 434 & CLP_METALS_ICP & SKINER & 20.5 & $u g / L$ & & & & & \\
\hline 435 & SAS_COUNT_LSC & TMANC & 12 & $\mathrm{pCi} / \mathrm{L}$ & 2.4 & & 0.8 & & \\
\hline 436 & SAS_COUNT_LSC & TMANC & 380 & $\mathrm{pCi} / \mathrm{L}$ & 170 & & 300 & & $\mathrm{~J}$ \\
\hline 437 & CLP_METALS_ICP & SKINER & 176 & $\mathrm{ug} / \mathrm{L}$ & & & & & \\
\hline 439 & SAS_COUNT_LSC & TMANC & 3.7 & $\mathrm{pCi} / \mathrm{L}$ & 1.5 & & 0.8 & & \\
\hline 440 & SAS_COUNT_LSC & TMANC & 5300 & $\mathrm{pCi} / \mathrm{L}$ & 320 & & 200 & & \\
\hline 441 & GAMMA_GS & ITASRL & 0.0295 & $\mathrm{pCi} / \mathrm{L}$ & 0.895 & 0.895 & & & $u$ \\
\hline 442 & SRISO_SEP_PRECIP_GPC & ITASRL & 18.6 & $\mathrm{pCi} / \mathrm{L}$ & 0.586 & 4.52 & & & \\
\hline 443 & TC99_SEP_LSC & ITASRL & 133 & $\mathrm{pCi} / \mathrm{L}$ & 0.63 & 14.9 & & & \\
\hline 444 & TRITIŪM_DIST_LSC & ITASRL & 1190 & $\mathrm{pCi} / \mathrm{L}$ & 97.7 & 236 & & & \\
\hline 445 & UISO_AEA & ITASRL & 3.57 & $\mathrm{pCi} / \mathrm{L}$ & 0.181 & 0.391 & & & \\
\hline 446 & UISO_AEA & ITASRL & 0.224 & $\mathrm{pCi} / \mathrm{L}$ & 0.046 & 0.0509 & & & \\
\hline 447 & UISO_AEA & ITASRL & 2.79 & $\mathrm{pCi} / \mathrm{L}$ & 0.16 & 0.314 & & & \\
\hline 448 & GAMMA_GS & ITASRL & -0.226 & $\mathrm{pCi} / \mathrm{L}$ & 0.856 & 0.856 & & & $U$ \\
\hline 449 & I129_SEP_TEMS & 320 & 0.0018705 & $\mathrm{pCi} / \mathrm{L}$ & & 0.000202 & & & \\
\hline 450 & SRISO_SËP_PRECIP_GPC & ITASRL & 0.149 & $\mathrm{pCi} / \mathrm{L}$ & 0.169 & 0.175 & & & $u$ \\
\hline 451 & TC99_SEP_LSC & ITASRL & 9.25 & $\mathrm{pCi} / \mathrm{L}$ & 0.191 & 1.41 & & & \\
\hline 452 & TRITIŪM_DIST_LSC & ITASRL & 9850 & $\mathrm{pCi} / \mathrm{L}$ & 200 & 838 & & & \\
\hline 453 & UISO_AËA & ITASRL & 57.8 & $\mathrm{pCi} / \mathrm{L}$ & 0.764 & 5.69 & & & \\
\hline 454 & UISO_AEA & ITASRL & 0.585 & $\mathrm{pCi} / \mathrm{L}$ & 0.077 & 0.0959 & & & \\
\hline 455 & UISO_AEA & ITASRL & 45.6 & $\mathrm{pCi} / \mathrm{L}$ & 0.679 & 4.5 & & & \\
\hline 456 & GAMM̄A_GS & ITASRL & -2.14 & $\mathrm{pCi} / \mathrm{L}$ & 9.19 & 9.19 & & & $u$ \\
\hline
\end{tabular}




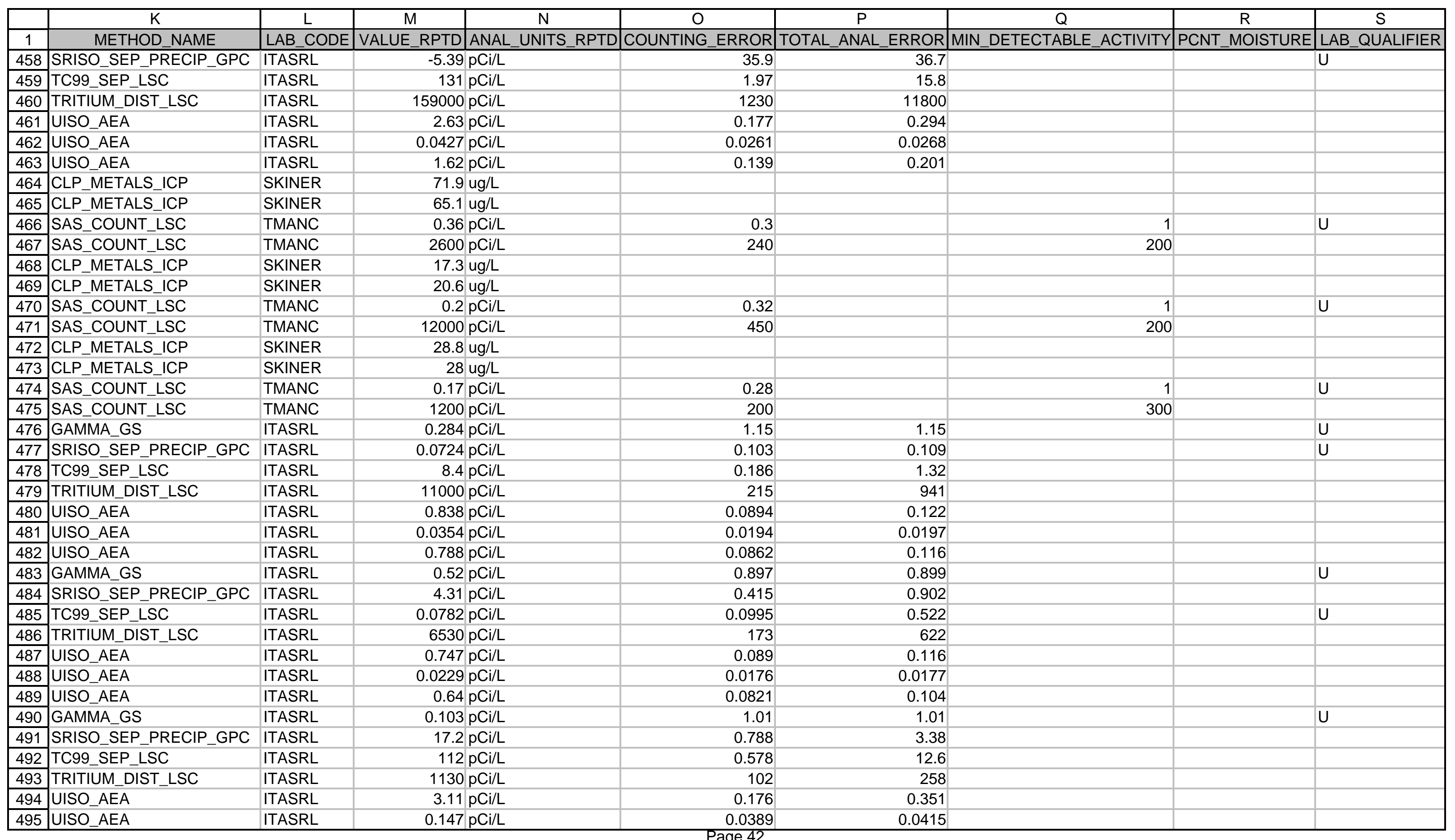




\begin{tabular}{|c|c|c|c|c|c|c|c|c|c|}
\hline & $\mathrm{K}$ & $\mathrm{L}$ & $\mathrm{M}$ & $\mathrm{N}$ & $\mathrm{O}$ & $P$ & $\mathrm{Q}$ & $\mathrm{R}$ & $\mathrm{S}$ \\
\hline 1 & METHOD_NAME & LAB_CODE & VALUE_RPTD & ANAL_UNITS_RPTD & COUNTING_ERROR & TOTAL_ANAL_ERROR & MIN_DETECTABLE_ACTIVITY & PCNT_MOISTURE & LAB_QUALIFIER \\
\hline 496 & UISO_AEA & ITASRL & 2.39 & $\mathrm{pCi} / \mathrm{L}$ & 0.154 & 0.28 & & & \\
\hline 497 & GAMMA_GS & ITASRL & 0.993 & $\mathrm{pCi} / \mathrm{L}$ & 0.992 & 0.997 & & & $\mathrm{U}$ \\
\hline 498 & GAMMA_GS & ITASRL & 0.159 & $\mathrm{pCi} / \mathrm{L}$ & 1.01 & 1.01 & & & $\mathrm{U}$ \\
\hline 499 & SRISO_SEP_PRECIP_GPC & ITASRL & -0.031 & $\mathrm{pCi} / \mathrm{L}$ & 0.103 & 0.105 & & & $U$ \\
\hline 500 & SRISO_SEP_PRECIP_GPC & ITASRL & 0.106 & $\mathrm{pCi} / \mathrm{L}$ & 0.124 & 0.129 & & & $\mathrm{U}$ \\
\hline 501 & TC99_SEP_LSC & ITASRL & 0.805 & $\mathrm{pCi} / \mathrm{L}$ & 0.108 & 0.579 & & & \\
\hline 502 & TC99_SEP_LSC & ITASRL & 0.451 & $\mathrm{pCi} / \mathrm{L}$ & 0.105 & 0.551 & & & $U$ \\
\hline 503 & TRITIUM_DIST_LSC & ITASRL & 18300 & $\mathrm{pCi} / \mathrm{L}$ & 270 & 1470 & & & \\
\hline 505 & UISO_AËA & ITASRL & 1.21 & $\mathrm{pCi} / \mathrm{L}$ & 0.105 & 0.158 & & & \\
\hline 506 & UISO_AEA & ITASRL & 1.22 & pCi/L & 0.107 & 0.16 & & & \\
\hline 507 & UISO_AEA & ITASRL & 0.0358 & $\mathrm{pCi} / \mathrm{L}$ & 0.0195 & 0.0199 & & & \\
\hline 508 & UISO_AEA & ITASRL & 0.0388 & $\mathrm{pCi} / \mathrm{L}$ & 0.02 & 0.0204 & & & \\
\hline 509 & UISO_AEA & ITASRL & 0.948 & $\mathrm{pCi} / \mathrm{L}$ & 0.0933 & 0.131 & & & \\
\hline 510 & UISO_AEA & ITASRL & 1.02 & $\mathrm{pCi} / \mathrm{L}$ & 0.0983 & 0.14 & & & \\
\hline 511 & GAMMĀA_GS & ITASRL & 0.252 & $\mathrm{pCi} / \mathrm{L}$ & 3.66 & 3.66 & & & $U$ \\
\hline 512 & GAMMA_GS & ITASRL & -5.54 & $\mathrm{pCi} / \mathrm{L}$ & 4.88 & 4.91 & & & $U$ \\
\hline 513 & SRISO_SEP_PRECIP_GPC & ITASRL & -0.0104 & $\mathrm{pCi} / \mathrm{L}$ & 0.218 & 0.221 & & & $\mathrm{U}$ \\
\hline 515 & TC99_SEEP_LSC & ITASRL & 2.09 & $\mathrm{pCi} / \mathrm{L}$ & 0.643 & 2.31 & & & $\mathrm{U}$ \\
\hline 516 & TC99_SEP_LSC & ITASRL & 1.93 & $\mathrm{pCi} / \mathrm{L}$ & 0.634 & 2.29 & & & $\mathrm{U}$ \\
\hline 517 & TRITIUM_DIST_LSC & ITASRL & 28900 & $\mathrm{pCi} / \mathrm{L}$ & 472 & 2260 & & & \\
\hline 518 & TRITIUM_DIST_LSC & ITASRL & 28500 & $\mathrm{pCi} / \mathrm{L}$ & 469 & 2220 & & & \\
\hline 519 & UISO_AEA & ITASRL & 0.368 & $\mathrm{pCi} / \mathrm{L}$ & 0.0594 & 0.0705 & & & \\
\hline 520 & UISO_AEA & ITASRL & 0.435 & $\mathrm{pCi} / \mathrm{L}$ & 0.0661 & 0.0798 & & & \\
\hline 521 & UISO_AEA & ITASRL & 0.00305 & pCi/L & 0.0112 & 0.0119 & & & $\mathrm{U}$ \\
\hline 522 & UISO_AEA & ITASRL & 0.0137 & $\mathrm{pCi} / \mathrm{L}$ & 0.0161 & 0.0167 & & & $U$ \\
\hline 523 & UISO_AEA & ITASRL & 0.283 & $\mathrm{pCi} / \mathrm{L}$ & 0.0524 & 0.0606 & & & \\
\hline 524 & UISO_AEA & ITASRL & 0.322 & $\mathrm{pCi} / \mathrm{L}$ & 0.0571 & 0.0666 & & & \\
\hline 525 & GAMM̄A_GS & ITASRL & 1.15 & $\mathrm{pCi} / \mathrm{L}$ & 3.69 & 3.69 & & & $U$ \\
\hline 526 & SRISO_SEP_PRECIP_GPC & ITASRL & -0.165 & $\mathrm{pCi} / \mathrm{L}$ & 0.195 & 0.198 & & & $\mathrm{U}$ \\
\hline 527 & TC99_SEEP_LEC & ITASRL & 111 & $\mathrm{pCi} / \mathrm{L}$ & 1.82 & 13.6 & & & \\
\hline 528 & TRITIUM_DIST_LSC & ITASRL & 125000 & $\mathrm{pCi} / \mathrm{L}$ & 963 & 9260 & & & \\
\hline 529 & UISO_AEA & ITASRL & 1.62 & $\mathrm{pCi} / \mathrm{L}$ & 0.121 & 0.199 & & & \\
\hline 530 & UISO_AEA & ITASRL & 0.0416 & $\mathrm{pCi} / \mathrm{L}$ & 0.0214 & 0.0222 & & & \\
\hline 531 & UISO_AEA & ITASRL & 1.07 & $\mathrm{pCi} / \mathrm{L}$ & 0.0984 & 0.144 & & & \\
\hline 532 & CLP_METALS_ICP & SKINER & 36.4 & ug/L & & & & & \\
\hline
\end{tabular}




\begin{tabular}{|c|c|c|c|c|c|c|c|c|c|}
\hline & $\mathrm{K}$ & $L$ & $\mathrm{M}$ & $\mathrm{N}$ & $\mathrm{O}$ & $\mathrm{P}$ & $\mathrm{Q}$ & $\mathrm{R}$ & $\mathrm{S}$ \\
\hline 1 & METHOD_NAME & LAB_CODE & VALUE_RPTD & ANAL_UNITS_RPTD & |COUNTING_ERROR & TOTAL_ANAL_ERROR & MIN_DETECTABLE_ACTIVITY & PCNT_MOISTURE & LAB_QUALIFIER \\
\hline 534 & SAS_COUNT_LSC & TMANC & 3.9 & $\mathrm{pCi} / \mathrm{L}$ & 0.99 & & 0.2 & & \\
\hline 535 & SAS_COUNT_LSC & TMANC & 140 & $\mathrm{pCi} / \mathrm{L}$ & 150 & & 300 & & $\mathrm{U}$ \\
\hline 536 & CLP_METALS_ICP & SKINER & 2.6 & ug/L & & & & & B \\
\hline 537 & CLP_METALS_ICP & SKINER & 2.1 & ug/L & & & & & $\mathrm{U}$ \\
\hline 538 & SAS_COUNT_LSC & TMANC & 0.35 & $\mathrm{pCi} / \mathrm{L}$ & 0.62 & & 0.2 & & $U$ \\
\hline 539 & SAS_COUNT_LSC & TMANC & 29 & $\mathrm{pCi} / \mathrm{L}$ & 150 & & 300 & & $\mathrm{U}$ \\
\hline 540 & GAMMA_GS & ITASRL & -0.416 & $\mathrm{pCi} / \mathrm{L}$ & 0.892 & 0.893 & & & $U$ \\
\hline 541 & SRISO_SEP_PRECIP_GPC & ITASRL & 0.116 & $\mathrm{pCi} / \mathrm{L}$ & 0.0793 & 0.0847 & & & \\
\hline 543 & TRITIUM_DIST_LSC & ITASRL & 1260 & $\mathrm{pCi} / \mathrm{L}$ & 105 & 264 & & & \\
\hline 544 & UISO_AEA & ITASRL & 12.8 & $\mathrm{pCi} / \mathrm{L}$ & 0.397 & 1.35 & & & \\
\hline 545 & UISO_AEA & ITASRL & 0.516 & $\mathrm{pCi} / \mathrm{L}$ & 0.0798 & 0.0951 & & & \\
\hline 546 & UISO_AEA & ITASRL & 10.9 & $\mathrm{pCi} / \mathrm{L}$ & 0.366 & 1.15 & & & \\
\hline 547 & SAS_COUNT_LSC & TMANC & 22 & $\mathrm{pCi} / \mathrm{L}$ & 1.8 & & 0.2 & & $\mathrm{R}$ \\
\hline 548 & SAS_COUNT_LSC & TMANC & 16 & $\mathrm{pCi} / \mathrm{L}$ & 0.59 & & 0.2 & & $\mathrm{R}$ \\
\hline 549 & CLP_METALS_ICP & WESTON & 26.4 & ug/L & & & & & $\mathrm{E}$ \\
\hline 550 & CLP_METALS_ICP & WESTON & 24.8 & ug/L & & & & & E \\
\hline 551 & 7196_CR6 & TMANC & 0.05 & $\mathrm{mg} / \mathrm{L}$ & & & & & $U$ \\
\hline 553 & CLP_METALS_ICP & WESTON & 24.6 & $\mathrm{ug} / \mathrm{L}$ & & & & & E \\
\hline 554 & CLP_METALS_ICP & WESTON & 16 & $\mathrm{ug} / \mathrm{L}$ & & & & & $\mathrm{E}$ \\
\hline 555 & 7196_CR6 & TMANC & 0.05 & $\mathrm{mg} / \mathrm{L}$ & & & & & $U$ \\
\hline 556 & 7197_CR6 & TMANC & 10 & ug/L & & & & & $\mathrm{U}$ \\
\hline 557 & CLP_METALS_ICP & WESTON & 12700 & ug/L & & & & & $\mathrm{E}$ \\
\hline 558 & CLP_METALS_ICP & WESTON & 21.7 & $\mathrm{ug} / \mathrm{L}$ & & & & & $\mathrm{E}$ \\
\hline 559 & 7196_CR6 & TMANC & 0.05 & $\mathrm{mg} / \mathrm{L}$ & & & & & U \\
\hline 560 & 7197_CR6 & TMANC & 10 & ug/L & & & & & U \\
\hline 561 & CLP_METALS_ICP & WESTON & 19.8 & $\mathrm{ug} / \mathrm{L}$ & & & & & $\mathrm{E}$ \\
\hline 562 & CLP_METALS_ICP & WESTON & 20.8 & $\mathrm{ug} / \mathrm{L}$ & & & & & $\mathrm{E}$ \\
\hline 563 & 7196_CR6 & TMANC & 0.05 & $\mathrm{mg} / \mathrm{L}$ & & & & & $\mathrm{U}$ \\
\hline 564 & 7197_CR6 & TMANC & 10 & $\mathrm{ug} / \mathrm{L}$ & & & & & U \\
\hline 565 & 8240_VOA_GCMS & DATACH & & & & & & & $\mathrm{U}$ \\
\hline 566 & 6010_METALS_ICP & DATACH & 21 & ug/L & & 10.1 & & & \\
\hline 567 & GAMMA_GS & ITASRL & 0.399 & $\mathrm{pCi} / \mathrm{L}$ & 1.05 & 1.05 & & & $\mathrm{U}$ \\
\hline 568 & SRISO_SEP_PRECIP_GPC & ITASRL & -0.111 & $\mathrm{pCi} / \mathrm{L}$ & 0.124 & 0.126 & & & $\mathrm{U}$ \\
\hline 569 & TC99_SEP_LSC & ITASRL & 10 & $\mathrm{pCi} / \mathrm{L}$ & 0.2 & 1.5 & & & \\
\hline 570 & TRITIUM_DIST_LSC & ITASRL & 13900 & $\mathrm{pCi} / \mathrm{L}$ & 239 & 1140 & & & \\
\hline
\end{tabular}




\begin{tabular}{|c|c|c|c|c|c|c|c|c|c|}
\hline & $\mathrm{K}$ & $\mathrm{L}$ & $\mathrm{M}$ & $\mathrm{N}$ & $\mathrm{O}$ & $\mathrm{P}$ & $\mathrm{Q}$ & $\mathrm{R}$ & $\mathrm{S}$ \\
\hline 1 & METHOD_NAME & LAB_CODE & VALUE_RPTD & ANAL_UNITS_RPTD & COUNTING_ERROR| & TOTAL_ANAL_ERROR & MIN_DETECTABLE_ACTIVITY & PCNT_MOISTURE & LAB_QUALIFIER \\
\hline 572 & UISO_AEA & ITASRL & 0.0247 & $\mathrm{pCi} / \mathrm{L}$ & 0.0166 & 0.0168 & & & \\
\hline 573 & UISO_AEA & ITASRL & 0.78 & $\mathrm{pCi} / \mathrm{L}$ & 0.0829 & 0.113 & & & \\
\hline 574 & 8240_VOA_GCMS & DATACH & & & & & & & U \\
\hline 575 & 6010_METALS_ICP & DATACH & 350 & ug/L & & 168 & & & \\
\hline 576 & GAMMA_GS & ITASRL & 1.18 & $\mathrm{pCi} / \mathrm{L}$ & 1.03 & 1.04 & & & \\
\hline 577 & SRISO_SEP_PRECIP_GPC & ITASRL & 7 & $\mathrm{pCi} / \mathrm{L}$ & 0.596 & 1.41 & & & \\
\hline 578 & TC99_SEP_LSC & ITASRL & 0.0299 & $\mathrm{pCi} / \mathrm{L}$ & 0.102 & 0.527 & & & $U$ \\
\hline 579 & TRITIŪM_DIST_LSC & ITASRL & 8010 & $\mathrm{pCi} / \mathrm{L}$ & 189 & 718 & & & \\
\hline 581 & UISO_AEA & ITASRL & 0.028 & $\mathrm{pCi} / \mathrm{L}$ & 0.0168 & 0.017 & & & \\
\hline 582 & UISO_AEA & ITASRL & 0.614 & $\mathrm{pCi} / \mathrm{L}$ & 0.0745 & 0.0957 & & & \\
\hline 583 & 8240_VOA_GCMS & DATACH & & & & & & & $U$ \\
\hline 584 & 6010_METALS_ICP & DATACH & 18 & ug/L & & 8.64 & & & LB \\
\hline 585 & GAMMA_GS & ITASRL & 1.88 & $\mathrm{pCi} / \mathrm{L}$ & 1.34 & 1.35 & & & \\
\hline 586 & SRISO_SEP_PRECIP_GPC & ITASRL & 12.5 & $\mathrm{pCi} / \mathrm{L}$ & 0.782 & 2.47 & & & \\
\hline 587 & TC99_SEP_LSC & ITASRL & 43.7 & $\mathrm{pCi} / \mathrm{L}$ & 0.373 & 5.14 & & & \\
\hline 588 & TRITIUM_DIST_LSC & ITASRL & 691 & $\mathrm{pCi} / \mathrm{L}$ & 92.1 & 221 & & & \\
\hline 589 & UISO_AEA & ITASRL & 2.92 & $\mathrm{pCi} / \mathrm{L}$ & 0.164 & 0.328 & & & \\
\hline 591 & UISO_AEA & ITASRL & 2.21 & $\mathrm{pCi} / \mathrm{L}$ & 0.143 & 0.258 & & & \\
\hline 592 & 8240_VOA_GCMS & DATACH & & & & & & & $U$ \\
\hline 593 & 6010_METÁLS_ICP & DATACH & & & & & & & $U$ \\
\hline 594 & GAMMA_GS & ITASRL & -1.78 & $\mathrm{pCi} / \mathrm{L}$ & 3.32 & 3.32 & & & U \\
\hline 595 & I129_SEP_TEMS & 320 & 0.004393 & $\mathrm{pCi} / \mathrm{L}$ & & 0.0004217 & & & \\
\hline 596 & SRISO_SEP_PRECIP_GPC & ITASRL & 0.0136 & $\mathrm{pCi} / \mathrm{L}$ & 0.486 & 0.496 & & & $U$ \\
\hline 597 & TC99_SEP_LSC & ITASRL & 1.3 & $\mathrm{pCi} / \mathrm{L}$ & 0.119 & 0.609 & & & \\
\hline 598 & TRITIUM_DIST_LSC & ITASRL & 10200 & $\mathrm{pCi} / \mathrm{L}$ & 209 & 878 & & & \\
\hline 599 & UISO_AEA & ITASRL & 58.6 & $\mathrm{pCi} / \mathrm{L}$ & 1.22 & 6.8 & & & \\
\hline 600 & UISO_AEA & ITASRL & 3.88 & $\mathrm{pCi} / \mathrm{L}$ & 0.315 & 0.543 & & & \\
\hline 601 & UISO_AEA & ITASRL & 50.9 & $\mathrm{pCi} / \mathrm{L}$ & 1.14 & 5.92 & & & \\
\hline 602 & 8240_VOA_GCMS & DATACH & & & & & & & $U$ \\
\hline 603 & 6010_METALS_ICP & DATACH & & & & & & & U \\
\hline 604 & GAMMA_GS & ITASRL & -3.62 & $\mathrm{pCi} / \mathrm{L}$ & 4.38 & 4.4 & & & U \\
\hline 605 & I129_SEP_LEPS_GS & ITASRL & 0.0435 & $\mathrm{pCi} / \mathrm{L}$ & 0.347 & 0.347 & & & U \\
\hline 606 & SRISO_SEP_PRECIP_GPC & ITASRL & -0.324 & $\mathrm{pCi} / \mathrm{L}$ & 0.34 & 0.347 & & & U \\
\hline 607 & TC99_SEP_LSC & ITASRL & 2.04 & $\mathrm{pCi} / \mathrm{L}$ & 0.124 & 0.674 & & & \\
\hline 608 & TRITIUM_DIST_LSC & ITASRL & 6340 & pCi/L & 170 & 599 & & & \\
\hline
\end{tabular}




\begin{tabular}{|c|c|c|c|c|c|c|c|c|c|}
\hline & $\mathrm{K}$ & L & $\mathrm{M}$ & $\mathrm{N}$ & $\mathrm{O}$ & $\mathrm{P}$ & $\mathrm{Q}$ & $\mathrm{R}$ & $\mathrm{S}$ \\
\hline 1 & METHOD NAME & LAB CODE & VALUE RPTD & ANAL UNITS RPTD & COUNTING ERROR & TOTAL ANAL ERROR & MIN DETECTABLE ACTIVITY & PCNT MOISTURE & LAB QUALIFIER \\
\hline 610 & UISO_AEA & ITASRL & 0.0605 & $5 \mathrm{pCi} / \mathrm{L}$ & 0.0403 & 0.0409 & & & \\
\hline 611 & UISO_AEA & ITASRL & 1.10 & $\mathrm{pCi} / \mathrm{L}$ & 0.171 & 0.218 & & & \\
\hline 612 & 8240_VOA_GCMS & DATACH & & & & & & & $U$ \\
\hline 613 & 6010_METALS_ICP & DATACH & 25 & $5 \mathrm{ug} / \mathrm{L}$ & & 10.5 & & & \\
\hline 614 & GAMMĀG_GS & ITASRL & -1.45 & $5 \mathrm{pCi} / \mathrm{L}$ & 1.22 & 1.23 & & & $U$ \\
\hline 615 & SRISO_SEP_PRECIP_GPC & ITASRL & 0.027 & $7 \mathrm{pCi} / \mathrm{L}$ & 0.11 & 0.112 & & & 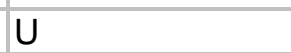 \\
\hline 616 & TC99 SEP LSC & ITASRL & 10.2 & $2 \mathrm{pCi} / \mathrm{L}$ & 0.202 & 1.5 & & & \\
\hline 617 & TRITIŪM_DIST_LSC & ITASRL & 14300 & $\mathrm{pCi} / \mathrm{L}$ & 246 & 1190 & & & \\
\hline 619 & UISO_AEA & ITASRL & 0.0388 & $\mathrm{pCi} / \mathrm{L}$ & 0.0208 & 0.0213 & & & \\
\hline 620 & UISO_AEA & ITASRL & $0.75 \mathrm{~s}$ & $\mathrm{pCi} / \mathrm{L}$ & 0.0882 & 0.122 & & & \\
\hline 621 & 8240_VOA_GCMS & DATACH & & & & & & & $u$ \\
\hline 622 & 6010_METALS_ICP & DATACH & & & & & & & $u$ \\
\hline 623 & GAMMA_GS & ITASRL & 0.281 & $\mathrm{pCi} / \mathrm{L}$ & 1.2 & 1.2 & & & $U$ \\
\hline 624 & SRISO_SEP_PRECIP_GPC & ITASRL & 0.123 & $3 \mathrm{pCi} / \mathrm{L}$ & 0.16 & 0.167 & & & $u$ \\
\hline 625 & TC99_SEP_LSC & ITASRL & 54.4 & $4 \mathrm{pCi} / \mathrm{L}$ & 0.412 & 6.29 & & & \\
\hline 626 & TRITIUM_DIST_LSC & ITASRL & 173000 & $\mathrm{pCi} / \mathrm{L}$ & 810 & 12700 & & & \\
\hline 627 & UISO_AËA & ITASRL & 2.52 & $2 \mathrm{pCi} / \mathrm{L}$ & 0.179 & 0.341 & & & \\
\hline 629 & UISO_AEA & ITASRL & 1.47 & $7 \mathrm{pCi} / \mathrm{L}$ & 0.136 & 0.217 & & & \\
\hline 630 & GAMM̄A_GS & ITASRL & -0.487 & $7 \mathrm{pCi} / \mathrm{L}$ & 1.85 & 1.85 & & & $u$ \\
\hline 631 & SRISO_SEP_PRECIP_GPC & ITASRL & 0.198 & $3 \mathrm{pCi} / \mathrm{L}$ & 0.0957 & 0.107 & & & \\
\hline 632 & TC99_SEP_LSC & ITASRL & 12.7 & $7 \mathrm{pCi} / \mathrm{L}$ & 0.291 & 2.04 & & & \\
\hline 633 & TRITIUM_DIST_LSC & ITASRL & 11300 & $\mathrm{pCi} / \mathrm{L}$ & 219 & 954 & & & \\
\hline 634 & UISO_AEA & ITASRL & 33.1 & $\mathrm{pCi} / \mathrm{L}$ & 1.73 & 3.97 & & & \\
\hline 635 & UISO_AEA & ITASRL & 1.87 & $7 \mathrm{pCi} / \mathrm{L}$ & 0.419 & 0.466 & & & \\
\hline 636 & UISO_AEA & ITASRL & 25.8 & $\mathrm{pCi} / \mathrm{L}$ & 1.52 & 3.17 & & & \\
\hline 637 & 8240_VOA_GCMS & DATACH & & & & & & & 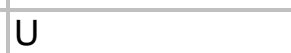 \\
\hline 638 & 6010_METALS_ICP & DATACH & 400 & $u g / L$ & & 168 & & & B \\
\hline 639 & GAMMA_GS & ITASRL & & $\mathrm{pCi} / \mathrm{L}$ & 2.12 & 2.12 & & & $U$ \\
\hline 640 & SRISO_SEP_PRECIP_GPC & ITASRL & 9.41 & $\mathrm{pCi} / \mathrm{L}$ & 0.506 & 1.78 & & & \\
\hline 641 & TC99_SEP_LSC & ITASRL & -0.0435 & $5 \mathrm{pCi} / \mathrm{L}$ & 0.0992 & 0.51 & & & $u$ \\
\hline 642 & TRITIUM_DIST_LSC & ITASRL & 12500 & $\mathrm{pCi} / \mathrm{L}$ & 228 & 1040 & & & \\
\hline 643 & UISO_AËA & ITASRL & 1.03 & $3 \mathrm{pCi} / \mathrm{L}$ & 0.0927 & 0.144 & & & \\
\hline 644 & UISO_AEA & ITASRL & 0.0317 & $7 \mathrm{pCi} / \mathrm{L}$ & 0.0168 & 0.0172 & & & \\
\hline 645 & UISO_AEA & ITASRL & $0.85 \varepsilon$ & $3 \mathrm{pCi} / \mathrm{L}$ & 0.0841 & 0.124 & & & \\
\hline 646 & 8240_VOA_GCMS & DATACH & & & & & & & $U$ \\
\hline
\end{tabular}




\begin{tabular}{|c|c|c|c|c|c|c|c|c|c|}
\hline & $\mathrm{K}$ & $\mathrm{L}$ & $\mathrm{M}$ & $\mathrm{N}$ & $\mathrm{O}$ & $\mathrm{P}$ & $\mathrm{Q}$ & $\mathrm{R}$ & $\mathrm{S}$ \\
\hline 1 & METHOD_NAME & LAB_CODE & VALUE_RPTD & ANAL_UNITS_RPTD & COUNTING_ERROR & TOTAL_ANAL_ERROR & MIN_DETECTABLE_ACTIVITY & PCNT_MOISTURE & LAB_QUALIFIER \\
\hline 648 & GAMMA_GS & ITASRL & 0.631 & $\mathrm{pCi} / \mathrm{L}$ & 1.77 & 1.77 & & & U \\
\hline 649 & ]SRISO_SEP_PRECIP_GPC & ITASRL & 0.0986 & $\mathrm{pCi} / \mathrm{L}$ & 0.0849 & 0.0906 & & & \\
\hline 650 & TC99_SEP_LSC & ITASRL & -0.0303 & $\mathrm{pCi} / \mathrm{L}$ & 0.121 & 0.629 & & & $\mathrm{U}$ \\
\hline 651 & TRITIUM_DIST_LSC & ITASRL & 623 & $\mathrm{pCi} / \mathrm{L}$ & 90.7 & 215 & & & \\
\hline 652 & UISO_AËA & ITASRL & 2.57 & $\mathrm{pCi} / \mathrm{L}$ & 0.214 & 0.347 & & & \\
\hline 653 & UISO_AEA & ITASRL & 0.0733 & pCi/L & 0.0385 & 0.0395 & & & \\
\hline 654 & UISO_AEA & ITASRL & 1.98 & $\mathrm{pCi} / \mathrm{L}$ & 0.188 & 0.281 & & & \\
\hline 655 & 8240_VOA_GCMS & DATACH & & & & & & & U \\
\hline 657 & GAMMA_GS & ITASRL & 2.14 & $\mathrm{pCi} / \mathrm{L}$ & 2.79 & 2.8 & & & $\mathrm{U}$ \\
\hline 658 & SRISO_SEP_PRECIP_GPC & ITASRL & 25.2 & $\mathrm{pCi} / \mathrm{L}$ & 0.811 & 4.5 & & & \\
\hline 659 & TC99_SEP_LSC & ITASRL & 62.3 & pCi/L & 0.44 & 7.15 & & & \\
\hline 660 & TRITIUM_DIST_LSC & ITASRL & 1150 & $\mathrm{pCi} / \mathrm{L}$ & 102 & 251 & & & \\
\hline 661 & ]UISO_AEA & ITASRL & 4.14 & $\mathrm{pCi} / \mathrm{L}$ & 0.27 & 0.587 & & & \\
\hline 662 & UISO_AEA & ITASRL & 0.17 & $\mathrm{pCi} / \mathrm{L}$ & 0.0561 & 0.0601 & & & \\
\hline 663 & UISO_AEA & ITASRL & 4.04 & $\mathrm{pCi} / \mathrm{L}$ & 0.266 & 0.573 & & & \\
\hline 664 & 8240_VOA_GCMS & DATACH & & & & & & & 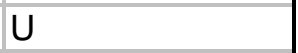 \\
\hline 665 & 6010_METALS_ICP & DATACH & 45 & ug/L & & 18.9 & & & B \\
\hline 667 & SRISO_SEP_PRECIP_GPC & ITASRL & 0.129 & $\mathrm{pCi} / \mathrm{L}$ & 0.1 & 0.107 & & & \\
\hline 668 & TC99_SEEP_LSC & ITASRL & 2.42 & $\mathrm{pCi} / \mathrm{L}$ & 0.13 & 0.72 & & & \\
\hline 669 & TRITIŪM_DIST_LSC & ITASRL & 30900 & $\mathrm{pCi} / \mathrm{L}$ & 351 & 2380 & & & \\
\hline 670 & UISO_AEA & ITASRL & 1.35 & $\mathrm{pCi} / \mathrm{L}$ & 0.117 & 0.189 & & & \\
\hline 671 & UISO_AEA & ITASRL & 0.0405 & $\mathrm{pCi} / \mathrm{L}$ & 0.0215 & 0.022 & & & \\
\hline 672 & UISO_AEA & ITASRL & 1.08 & $\mathrm{pCi} / \mathrm{L}$ & 0.105 & 0.158 & & & \\
\hline 673 & ISRTOT_SEP_PRECIP_GPC & QTESRL & 34.8 & $\mathrm{pCi} / \mathrm{L}$ & 1.52 & 9.1 & 0.786 & & \\
\hline 674 & SRTOT_SEP_PRECIP_GPC & QTESRL & 50.3 & $\mathrm{pCi} / \mathrm{L}$ & 1.81 & 13.2 & 0.749 & & \\
\hline 675 & SRTOT_SEP_PRECIP_GPC & QTESRL & 222 & $\mathrm{pCi} / \mathrm{L}$ & 3.76 & 57.9 & 0.718 & & \\
\hline 676 & SRTOT_SEP_PRECIP_GPC & QTESRL & 2000 & $\mathrm{pCi} / \mathrm{L}$ & 11.3 & 545 & 0.789 & & \\
\hline 677 & SRTOT_SEP_PRECIP_GPC & QTESRL & 624 & $\mathrm{pCi} / \mathrm{L}$ & 6.47 & 158 & 0.758 & & \\
\hline 678 & SRTOT_SEP_PRECIP_GPC & QTESRL & 53.6 & $\mathrm{pCi} / \mathrm{L}$ & 1.88 & 13.9 & 0.744 & & \\
\hline 679 & SRTOT_SEP_PRECIP_GPC & QTESRL & 23.9 & $\mathrm{pCi} / \mathrm{L}$ & 1.27 & 6.27 & 0.759 & & \\
\hline 680 & SRTOT_SEP_PRECIP_GPC & QTESRL & 6.73 & $\mathrm{pCi} / \mathrm{L}$ & 0.69 & 1.92 & 0.718 & & \\
\hline 681 & SRTOT_SEP_PRECIP_GPC & QTESRL & 26.6 & $\mathrm{pCi} / \mathrm{L}$ & 1.32 & 7.13 & 0.717 & & \\
\hline 682 & SRTOT_SEP_PRECIP_GPC & QTESRL & 37 & $\mathrm{pCi} / \mathrm{L}$ & 1.5 & 9.65 & 0.784 & & \\
\hline 683 & SRTOT_SEP_PRECIP_GPC & QTESRL & 3.8 & $\mathrm{pCi} / \mathrm{L}$ & 0.518 & 1.11 & 0.756 & & \\
\hline 684 & SRTOT_SEP_PRECIP_GPC & QTESRL & 213 & $\mathrm{pCi} / \mathrm{L}$ & 3.49 & 55.7 & 0.796 & & \\
\hline
\end{tabular}




\begin{tabular}{|c|c|c|c|c|c|c|c|c|c|}
\hline & $\mathrm{K}$ & $L$ & $\mathrm{M}$ & $\mathrm{N}$ & $\mathrm{O}$ & $\mathrm{P}$ & $\mathrm{Q}$ & $\mathrm{R}$ & $\mathrm{S}$ \\
\hline 1 & METHOD_NAME & LAB_CODE & VALUE_RPTD & ANAL_UNITS_RPTD & COUNTING_ERROR & TOTAL_ANAL_ERROR & MIN_DETECTABLE_ACTIVITY & PCNT_MOISTURE & LAB_QUALIFIER \\
\hline 686 & SRTOT_SEP_PRECIP_GPC & QTESRL & 4.95 & $\mathrm{pCi} / \mathrm{L}$ & 0.562 & 1.42 & 0.743 & & \\
\hline 687 & SRTOT_SEP_PRECIP_GPC & QTESRL & 5.91 & $\mathrm{pCi} / \mathrm{L}$ & 0.618 & 1.63 & 0.72 & & \\
\hline 688 & 8260_VOA_GCMS & DATACH & & & & & & & $\mathrm{U}$ \\
\hline 689 & 6010_METALS_ICP & DATACH & & ug/L & & 9.24 & & & \\
\hline 690 & GAMMA_GS & ITASRL & 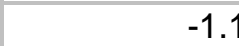 & $\mathrm{pCi} / \mathrm{L}$ & 2.21 & 2.21 & & & $U$ \\
\hline 691 & SRISO_SEP_PRECIP_GPC & ITASRL & 0.0198 & $\mathrm{pCi} / \mathrm{L}$ & 0.147 & 0.149 & & & $\mathrm{U}$ \\
\hline 692 & TC99_SEP_LSC & ITASRL & 25.3 & $\mathrm{pCi} / \mathrm{L}$ & 0.291 & 3.16 & & & \\
\hline 693 & TRITIUM_DIST_LSC & ITASRL & 22500 & $\mathrm{pCi} / \mathrm{L}$ & 365 & 1730 & & & \\
\hline 695 & UISO_AEA & ITASRL & 0.0456 & $\mathrm{pCi} / \mathrm{L}$ & 0.0194 & 0.02 & & & \\
\hline 696 & UISO_AEA & ITASRL & 1.4 & $\mathrm{pCi} / \mathrm{L}$ & 0.102 & 0.176 & & & \\
\hline 697 & 8260_VOA_GCMS & DATACH & & & & & & & $\mathrm{U}$ \\
\hline 698 & 6010_METALS_ICP & DATACH & & & & & & & $\mathrm{U}$ \\
\hline 699 & GAMMA_GS & ITASRL & 0.167 & $\mathrm{pCi} / \mathrm{L}$ & 2.08 & 2.08 & & & U \\
\hline 700 & SRISO_SEP_PRECIP_GPC & ITASRL & 0.069 & $\mathrm{pCi} / \mathrm{L}$ & 0.0911 & 0.0957 & & & $U$ \\
\hline 701 & TC99_SEP_LSC & ITASRL & -0.117 & $\mathrm{pCi} / \mathrm{L}$ & 0.101 & 0.542 & & & $\mathrm{U}$ \\
\hline 702 & TRITIUM_DIST_LSC & ITASRL & 86.5 & $\mathrm{pCi} / \mathrm{L}$ & 73.9 & 133 & & & $\mathrm{U}$ \\
\hline 703 & UISO_AEA & ITASRL & $0.15 s$ & $\mathrm{pCi} / \mathrm{L}$ & 0.0362 & 0.0405 & & & \\
\hline 705 & UISO_AEA & ITASRL & 0.125 & $\mathrm{pCi} / \mathrm{L}$ & 0.0316 & 0.0345 & & & \\
\hline 706 & 8260_VOA_GCMS & DATACH & & & & & & & $U$ \\
\hline 707 & 6010_METALS_ICP & DATACH & & & & & & & $U$ \\
\hline 708 & GAMMA_GS & ITASRL & $-0.11 \varepsilon$ & $\mathrm{pCi} / \mathrm{L}$ & 1.36 & 1.36 & & & $\mathrm{U}$ \\
\hline 709 & SRISO_SEP_PRECIP_GPC & ITASRL & 0.079 & $\mathrm{pCi} / \mathrm{L}$ & 0.0994 & 0.104 & & & $U$ \\
\hline 710 & TC99_SEP_LSC & ITASRL & 0.842 & $\mathrm{pCi} / \mathrm{L}$ & 0.113 & 0.617 & & & \\
\hline 711 & TRITIUM_DIST_LSC & ITASRL & $1200 c$ & $\mathrm{pCi} / \mathrm{L}$ & 271 & 969 & & & \\
\hline 712 & UISO_AEA & ITASRL & 0.125 & $\mathrm{pCi} / \mathrm{L}$ & 0.0344 & 0.0377 & & & \\
\hline 713 & UISO_AEA & ITASRL & -0.000526 & $\mathrm{pCi} / \mathrm{L}$ & 0.00608 & 0.00622 & & & $\mathrm{U}$ \\
\hline 714 & UISO_AEA & ITASRL & 0.111 & $\mathrm{pCi} / \mathrm{L}$ & 0.0315 & 0.034 & & & \\
\hline 715 & 8260_VOA_GCMS & DATACH & & & & & & & $U$ \\
\hline 716 & 6010_METALS_ICP & DATACH & & $\mathrm{ug} / \mathrm{L}$ & & 18.5 & & & \\
\hline 717 & GAMMA_GS & ITASRL & 3.02 & $\mathrm{pCi} / \mathrm{L}$ & 4.15 & 4.16 & & & $\mathrm{U}$ \\
\hline 718 & SRISO_SEP_PRECIP_GPC & ITASRL & 12.4 & $\mathrm{pCi} / \mathrm{L}$ & 0.626 & 2.42 & & & \\
\hline 719 & TC99_SEP_LSC & ITASRL & 136 & $\mathrm{pCi} / \mathrm{L}$ & 0.646 & 15.2 & & & \\
\hline 720 & TRITIUM_DIST_LSC & ITASRL & 1100 & $\mathrm{pCi} / \mathrm{L}$ & 105 & 194 & & & \\
\hline 721 & UISO_AËA - & ITASRL & 4.35 & $\mathrm{pCi} / \mathrm{L}$ & 0.219 & 0.533 & & & \\
\hline 722 & UISO_AEA & ITASRL & 0.201 & $\mathrm{pCi} / \mathrm{L}$ & 0.0483 & 0.0534 & & & \\
\hline
\end{tabular}




\begin{tabular}{|c|c|c|c|c|c|c|c|c|c|}
\hline & $\mathrm{K}$ & $L$ & $\mathrm{M}$ & $\mathrm{N}$ & $\mathrm{O}$ & $\mathrm{P}$ & $\mathrm{Q}$ & $\mathrm{R}$ & $\mathrm{S}$ \\
\hline 1 & METHOD_NAME & LAB_CODE & VALUE_RPTD & ANAL_UNITS_RPTD & |COUNTING_ERROR & TOTAL_ANAL_ERROR & MIN_DETECTABLE_ACTIVITY & PCNT_MOISTURE & LAB_QUALIFIER \\
\hline 724 & 8260_VOA_GCMS & DATACH & & & & & & & $U$ \\
\hline 725 & 6010_METALS_ICP & DATACH & 66 & ug/L & & 27.7 & & & \\
\hline 726 & GAMMA_GS & ITASRL & 3.23 & $\mathrm{pCi} / \mathrm{L}$ & 2.23 & 2.25 & & & \\
\hline 727 & SRISO_SEP_PRECIP_GPC & ITASRL & -0.0244 & $\mathrm{pCi} / \mathrm{L}$ & 0.115 & 0.116 & & & $U$ \\
\hline 728 & TC99_SEP_LSC & ITASRL & -0.0211 & $\mathrm{pCi} / \mathrm{L}$ & 0.0988 & 0.512 & & & $U$ \\
\hline 729 & TRITIUM_DIST_LSC & ITASRL & 19700 & $\mathrm{pCi} / \mathrm{L}$ & 342 & 1530 & & & \\
\hline 730 & UISO_AEA & ITASRL & 0.697 & $\mathrm{pCi} / \mathrm{L}$ & 0.0781 & 0.109 & & & \\
\hline 731 & UISO_AEA & ITASRL & 0.0367 & $\mathrm{pCi} / \mathrm{L}$ & 0.0191 & 0.0196 & & & \\
\hline 733 & 8260_VOA_GCMS & DATACH & & & & & & & $U$ \\
\hline 734 & 6010_METALS_ICP & DATACH & 4.4 & $u g / L$ & & 1.85 & & & $L$ \\
\hline 735 & GAMMA_GS & ITASRL & $0.21<$ & $\mathrm{pCi} / \mathrm{L}$ & 1.65 & 1.65 & & & $\mathrm{U}$ \\
\hline 736 & I129_SEP_TEMS & 320 & 0.004923 & $\mathrm{pCi} / \mathrm{L}$ & & 0.0006302 & & & \\
\hline 737 & SRISO_SEP_PRECIP_GPC & ITASRL & 0.195 & $\mathrm{pCi} / \mathrm{L}$ & 0.101 & 0.11 & & & \\
\hline 738 & TC99_SEP_LSC & ITASRL & 13.5 & $\mathrm{pCi} / \mathrm{L}$ & 0.22 & 1.86 & & & \\
\hline 739 & TRITIUM_DIST_LSC & ITASRL & 11600 & $\mathrm{pCi} / \mathrm{L}$ & 266 & 940 & & & \\
\hline 740 & UISO_AEA & ITASRL & 47 & $\mathrm{pCi} / \mathrm{L}$ & 0.605 & 4.96 & & & \\
\hline 741 & UISO_AEA & ITASRL & & & & & & & \\
\hline 743 & 8260_VOA_GCMS & DATACH & & & & & & & $U$ \\
\hline 744 & 6010_METALS_ICP & DATACH & & & & & & & U \\
\hline 745 & GAMMA_GS & ITASRL & -0.112 & $\mathrm{pCi} / \mathrm{L}$ & 1.29 & 1.29 & & & U \\
\hline 746 & I129_SEP_TEMS & 320 & 0.06381 & $\mathrm{pCi} / \mathrm{L}$ & & 0.005743 & & & \\
\hline 747 & SRISO_SEP_PRECIP_GPC & ITASRL & 0.0823 & $\mathrm{pCi} / \mathrm{L}$ & 0.11 & 0.115 & & & $U$ \\
\hline 748 & TC99_SEP_LSC & ITASRL & 6.11 & $\mathrm{pCi} / \mathrm{L}$ & 0.169 & 1.09 & & & \\
\hline 749 & TRITIUM_DIST_LSC & ITASRL & 22200 & $\mathrm{pCi} / \mathrm{L}$ & 361 & 1710 & & & \\
\hline 750 & UISO_AEA & ITASRL & 1.31 & $\mathrm{pCi} / \mathrm{L}$ & 0.111 & 0.181 & & & \\
\hline 751 & UISO_AEA & ITASRL & 0.0291 & $\mathrm{pCi} / \mathrm{L}$ & 0.0183 & 0.0186 & & & \\
\hline 752 & UISO_AEA & ITASRL & 0.979 & $\mathrm{pCi} / \mathrm{L}$ & 0.0962 & 0.143 & & & \\
\hline 753 & 8260_VOA_GCMS & DATACH & & & & & & & $U$ \\
\hline 754 & 6010_METALS_ICP & DATACH & 240 & $\mathrm{ug} / \mathrm{L}$ & & 101 & & & \\
\hline 755 & GAMMA_GS & ITASRL & 0.26 & $\mathrm{pCi} / \mathrm{L}$ & 1.71 & 1.71 & & & $U$ \\
\hline 756 & SRISO_SEP_PRECIP_GPC & ITASRL & 3.96 & $\mathrm{pCi} / \mathrm{L}$ & 0.311 & 0.871 & & & \\
\hline 757 & TC99_SEP_LSC & ITASRL & -0.182 & $\mathrm{pCi} / \mathrm{L}$ & 0.195 & 1.01 & & & U \\
\hline 758 & TRITIUM_DIST_LSC & ITASRL & 5870 & $\mathrm{pCi} / \mathrm{L}$ & 196 & 531 & & & \\
\hline 759 & UISO_AEA & ITASRL & 0.666 & $\mathrm{pCi} / \mathrm{L}$ & 0.0749 & 0.104 & & & \\
\hline 760 & UISO_AEA & ITASRL & 0.0171 & $\mathrm{pCi} / \mathrm{L}$ & 0.013 & 0.0132 & & & \\
\hline
\end{tabular}




\begin{tabular}{|c|c|c|c|c|c|c|c|c|c|}
\hline & $\mathrm{K}$ & L & $\mathrm{M}$ & $\mathrm{N}$ & $\mathrm{O}$ & $\mathrm{P}$ & $\mathrm{Q}$ & $\mathrm{R}$ & $\mathrm{S}$ \\
\hline 1 & METHOD NAME & LAB CODE & VALUE RPTD & ANAL UNITS RPTD & COUNTING ERROR & TOTAL ANAL ERROR & MIN DETECTABLE ACTIVITY & PCNT MOISTURE & LAB QUALIFIER \\
\hline 762 & 8260_VOA_GCMS & DATACH & & & & & & & $\mathrm{U}$ \\
\hline 763 & 6010_METALS_ICP & DATACH & 37 & ug/L & & 15.5 & & & \\
\hline 764 & GAMMA_GS & ITASRL & 1.12 & $\mathrm{pCi} / \mathrm{L}$ & 1.97 & 1.97 & & & $\mathrm{U}$ \\
\hline 765 & SRISO_SEP_PRECIP_GPC & ITASRL & -0.0303 & $\mathrm{pCi} / \mathrm{L}$ & 0.0422 & 0.0427 & & & $\mathrm{U}$ \\
\hline 766 & TC99_SEP_LSC & ITASRL & & & & & & & \\
\hline 767 & TRITIŪM_DIST_LSC & ITASRL & 1620 & $\mathrm{pCi} / \mathrm{L}$ & 120 & 233 & & & \\
\hline 768 & UISO AEA & ITASRL & 1.78 & $\mathrm{pCi} / \mathrm{L}$ & 0.128 & 0.23 & & & \\
\hline 769 & UISO_AEA & ITASRL & 0.0563 & $\mathrm{pCi} / \mathrm{L}$ & 0.0235 & 0.0244 & & & \\
\hline 771 & SRTOT_SEP_PRECIP_GPC & QTESRL & 0.834 & $\mathrm{pCi} / \mathrm{L}$ & 0.268 & 0.366 & 0.82 & & $\mathrm{~J}$ \\
\hline 772 & SRTOT_SEP_PRECIP_GPC & QTESRL & 0.79 & $\mathrm{pCi} / \mathrm{L}$ & 0.256 & 0.351 & 0.778 & & $\mathrm{~J}$ \\
\hline 773 & SRTOT_SEP_PRECIP_GPC & QTESRL & 9.15 & $\mathrm{pCi} / \mathrm{L}$ & 0.684 & 2.82 & 0.8 & & \\
\hline 774 & SRTOT_SEP_PRECIP_GPC & QTESRL & 30.7 & $\mathrm{pCi} / \mathrm{L}$ & 1.22 & 9.31 & 0.787 & & \\
\hline 775 & SRTOT_SEP_PRECIP_GPC & QTESRL & 84 & $\mathrm{pCi} / \mathrm{L}$ & 2.05 & 24.9 & 0.824 & & \\
\hline 776 & SRTOT_SEP_PRECIP_GPC & QTESRL & 37.4 & $\mathrm{pCi} / \mathrm{L}$ & 1.34 & 11.3 & 0.791 & & \\
\hline 777 & SRTOT_SEP_PRECIP_GPC & QTESRL & 0.625 & $\mathrm{pCi} / \mathrm{L}$ & 0.251 & 0.312 & 0.824 & & $U$ \\
\hline 778 & SRTOT_SEP_PRECIP_GPC & QTESRL & 0.898 & $\mathrm{pCi} / \mathrm{L}$ & 0.277 & 0.385 & 0.836 & & $\mathrm{~J}$ \\
\hline 779 & SRTOT_SEP_PRECIP_GPC & QTESRL & 1.77 & $\mathrm{pCi} / \mathrm{L}$ & 0.341 & 0.626 & 0.812 & & $\mathrm{~J}$ \\
\hline 781 & SRTOT_SEP_PRECIP_GPC & QTESRL & 0.031 & $\mathrm{pCi} / \mathrm{L}$ & 0.184 & 0.184 & 0.824 & & U \\
\hline 782 & SRTOT_SEP_PRECIP_GPC & QTESRL & 18.6 & $\mathrm{pCi} / \mathrm{L}$ & 0.994 & 5.52 & 0.851 & & \\
\hline 783 & GAMMA_GS & QTESRL & 1.91 & $\mathrm{pCi} / \mathrm{L}$ & 3.72 & 3.72 & 7.49 & & $U$ \\
\hline 784 & SRISO_SEP_PRECIP_GPC & QTESRL & 57.8 & $\mathrm{pCi} / \mathrm{L}$ & 1.84 & 15.3 & 0.535 & & \\
\hline 785 & TRITIUM_DIST_LSC & QTESRL & 251 & $\mathrm{pCi} / \mathrm{L}$ & 142 & 213 & 301 & & U \\
\hline 786 & GAMMA_GS & QTESRL & -2.82 & $\mathrm{pCi} / \mathrm{L}$ & 4.85 & 4.86 & 8.13 & & $U$ \\
\hline 787 & SRISO_SEP_PRECIP_GPC & QTESRL & 258 & $\mathrm{pCi} / \mathrm{L}$ & 3.8 & 63.9 & 0.493 & & \\
\hline 788 & TRITIUMM_DISTT_LSC & QTESRL & 854 & $\mathrm{pCi} / \mathrm{L}$ & 175 & 253 & 301 & & \\
\hline 789 & GAMMA_GS & QTESRL & -1.96 & $\mathrm{pCi} / \mathrm{L}$ & 4.11 & 4.12 & 7.21 & & $U$ \\
\hline 790 & SRISO_SEP_PRECIP_GPC & QTESRL & 5780 & $\mathrm{pCi} / \mathrm{L}$ & 126 & 1580 & 26 & & \\
\hline 791 & TRITIUMM_DISTT_LSC & QTESRL & 16100 & $\mathrm{pCi} / \mathrm{L}$ & 544 & 1350 & 301 & & \\
\hline 792 & GAMMA_GS & QTESRL & 0.184 & $\mathrm{pCi} / \mathrm{L}$ & 4.25 & 4.25 & 7.72 & & $U$ \\
\hline 793 & SRISO_SEP_PRECIP_GPC & QTESRL & 953 & $\mathrm{pCi} / \mathrm{L}$ & 23.9 & 264 & 5.61 & & \\
\hline 794 & TRITIUM_DIST_LSC & QTESRL & 4200 & $\mathrm{pCi} / \mathrm{L}$ & 300 & 489 & 301 & & \\
\hline 795 & GAMMA_GS & QTESRL & 3.35 & $\mathrm{pCi} / \mathrm{L}$ & 4.3 & 4.31 & 8.57 & & $U$ \\
\hline 796 & SRISO_SEP_PRECIP_GPC & QTESRL & 37.2 & $\mathrm{pCi} / \mathrm{L}$ & 1.53 & 10.1 & 0.569 & & \\
\hline 797 & TRITIUM_DIST_LSC & QTESRL & 155 & $\mathrm{pCi} / \mathrm{L}$ & 138 & 207 & 301 & & U \\
\hline 798 & GAMMA_GS & QTESRL & -0.0613 & $\mathrm{pCi} / \mathrm{L}$ & 4.66 & 4.66 & 8.4 & & $U$ \\
\hline
\end{tabular}




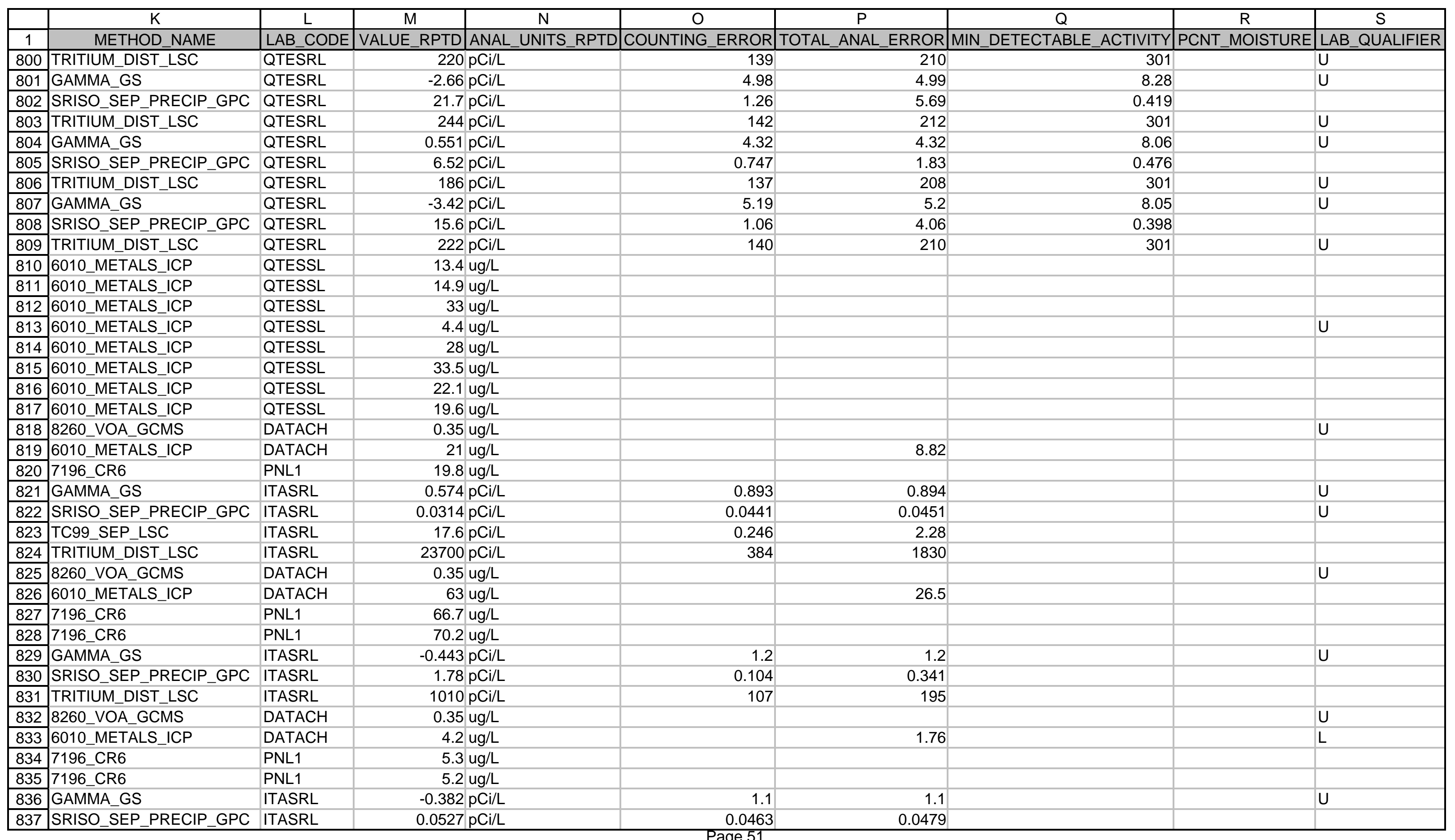




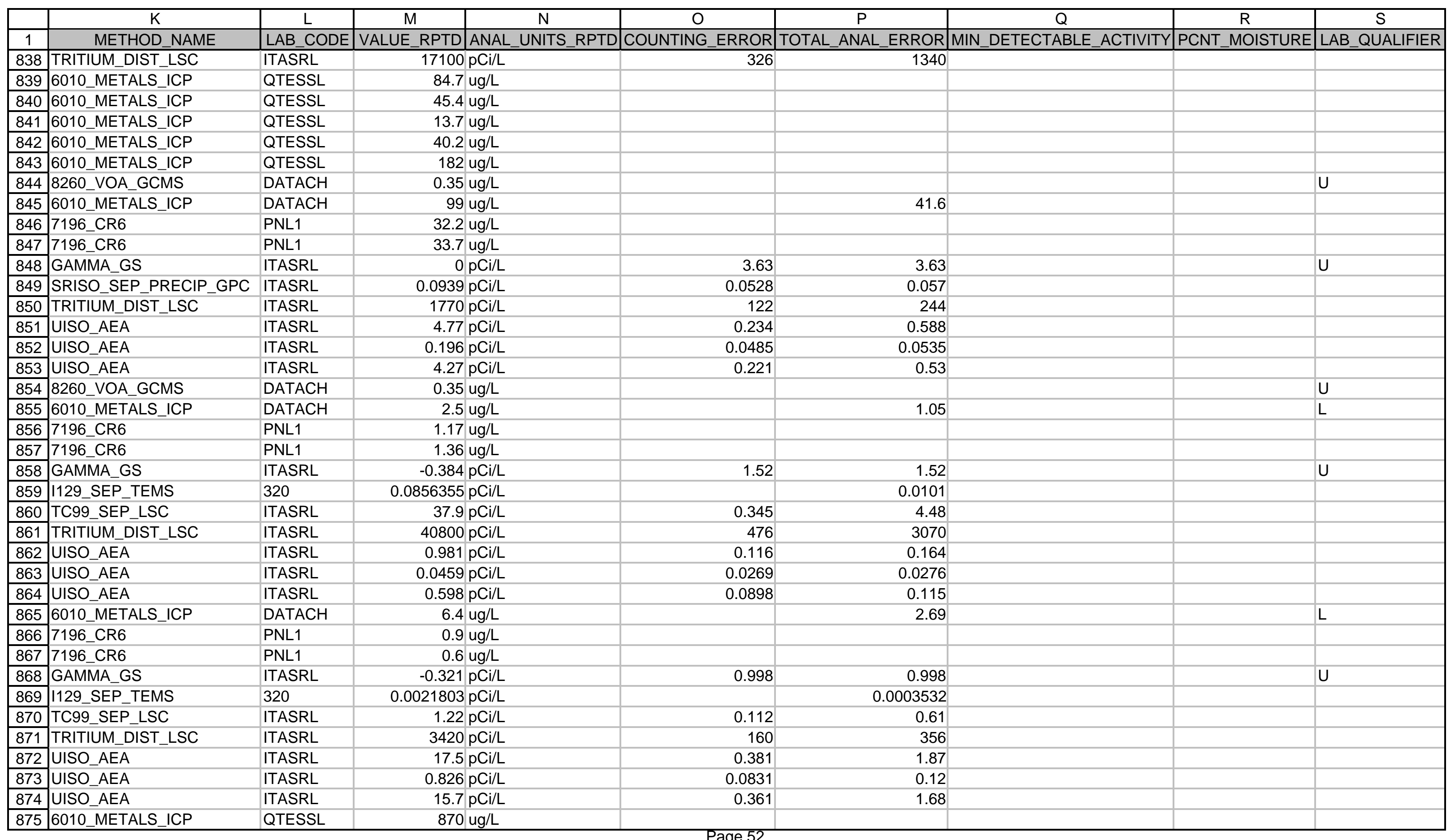




\begin{tabular}{|c|c|c|c|c|c|c|c|c|c|}
\hline & $\mathrm{K}$ & $\mathrm{L}$ & $\mathrm{M}$ & $\mathrm{N}$ & $\mathrm{O}$ & $\mathrm{P}$ & $\mathrm{Q}$ & $\mathrm{R}$ & $\mathrm{S}$ \\
\hline 1 & METHOD_NAME & LAB_CODE & VALUE_RPTD & ANAL_UNITS_RPTD & COUNTING_ERROR & TOTAL_ANAL_ERROR & MIN_DETECTABLE_ACTIVITY & PCNT_MOISTURE & LAB_QUALIFIER \\
\hline 876 & 6010_METALS_ICP & QTESSL & 18.8 & $3 \mathrm{ug} / \mathrm{L}$ & & & & & \\
\hline 877 & 6010_METALS_ICP & QTESSL & 11.5 & $5 \mathrm{ug} / \mathrm{L}$ & & & & & \\
\hline 878 & SRISO_SEP_PRECIP_GPC & QTESRL & 0.0295 & $5 \mathrm{pCi} / \mathrm{L}$ & 0.263 & 0.263 & 0.6 & & $U$ \\
\hline 879 & TRITIUM_DIST_LSC & QTESRL & 11700 & $\mathrm{pCi} / \mathrm{L}$ & 481 & 1040 & 315 & & \\
\hline 880 & 6010_METALS_ICP & QTESSL & 14 & $4 \mathrm{ug} / \mathrm{L}$ & & & & & \\
\hline 881 & 6010_METALS_ICP & QTESSL & 13.9 & ug/L & & & & & \\
\hline 882 & SRISO_SEP_PRECIP_GPC & QTESRL & 7.41 & $\mathrm{pCi} / \mathrm{L}$ & 0.844 & 1.58 & 0.525 & & \\
\hline 883 & TRITIUM_DIST_LSC & QTESRL & 2690 & $\mathrm{pCi} / \mathrm{L}$ & 258 & 389 & 315 & & \\
\hline 885 & SRISO_SEP_PRECIP_GPC & QTESRL & 0.45 & $5 \mathrm{pCi} / \mathrm{L}$ & 0.291 & 0.302 & 0.492 & & $U$ \\
\hline 886 & 6010_METALS_ICP & QTESSL & 215 & $5 \mathrm{ug} / \mathrm{L}$ & & & & & \\
\hline 887 & 6010_METALS_ICP & RFWLVL & 236 & $\mathrm{ug} / \mathrm{L}$ & & & & & \\
\hline 888 & SRISO_SEP_PRECIP_GPC & QTESRL & 0.336 & $\mathrm{pCi} / \mathrm{L}$ & 0.344 & 0.361 & 0.681 & & $\mathrm{U}$ \\
\hline 889 & SRISO_SEP_PRECIP_GPC & TMANC & 0.026 & $\mathrm{pCi} / \mathrm{L}$ & 0.1 & 0.1 & 0.27 & & $U$ \\
\hline 890 & 6010_METALS_ICP & QTESSL & 55.3 & $3 \mathrm{ug} / \mathrm{L}$ & & & & & \\
\hline 891 & SRISO_SEP_PRECIP_GPC & QTESRL & 1.88 & $3 \mathrm{pCi} / \mathrm{L}$ & 0.447 & 0.636 & 0.623 & & $\mathrm{~J}$ \\
\hline 892 & 6010_METALS_ICP & QTESSL & 17.4 & $4 \mathrm{ug} / \mathrm{L}$ & & & & & \\
\hline 893 & TRITIUM_DIST_LSC & QTESRL & 140 & $\mathrm{pCi} / \mathrm{L}$ & 154 & 235 & 355 & & $U$ \\
\hline 895 & SRISO_SEP_PRECIP_GPC & ITASRL & 0.0242 & $2 \mathrm{pCi} / \mathrm{L}$ & 0.0291 & 0.0304 & & & $U$ \\
\hline 896 & TC99_SEP_LSC & ITASRL & 5.83 & $3 \mathrm{pCi} / \mathrm{L}$ & 0.162 & 1.06 & & & \\
\hline 897 & TRITIUM_DIST_LSC & ITASRL & 11100 & $\mathrm{pCi} / \mathrm{L}$ & 274 & 912 & & & \\
\hline 898 & GAMMA_GS & ITASRL & -1.06 & $\mathrm{pCi} / \mathrm{L}$ & 1.21 & 1.22 & & & $U$ \\
\hline 899 & SRISO_SEP_PRECIP_GPC & ITASRL & 0.0342 & $2 \mathrm{pCi} / \mathrm{L}$ & 0.0313 & 0.0329 & & & \\
\hline 900 & TRITIUM_DIST_LSC & ITASRL & 1140 & $\mathrm{pCi} / \mathrm{L}$ & 114 & 210 & & & \\
\hline 901 & UISO_AEA & ITASRL & 2.44 & $4 \mathrm{pCi} / \mathrm{L}$ & 0.147 & 0.3 & & & \\
\hline 902 & UISO_AEA & ITASRL & 0.13 & $4 \mathrm{pCi} / \mathrm{L}$ & 0.0348 & 0.0378 & & & \\
\hline 903 & JUISO_AEA & ITASRL & $1.9 \mathrm{~s}$ & $\mathrm{pCi} / \mathrm{L}$ & 0.133 & 0.251 & & & \\
\hline 904 & 6010_METALS_ICP & QTESSL & 5.5 & $5 \mathrm{ug} / \mathrm{L}$ & & & & & B \\
\hline 905 & 6010_METALS_ICP & QTESSL & & $4 \mathrm{ug} / \mathrm{L}$ & & & & & B \\
\hline 906 & GAMMA_GS & QTESRL & -0.817 & $7 \mathrm{pCi} / \mathrm{L}$ & 4.87 & 4.87 & 8.36 & & $U$ \\
\hline 907 & TRITIUM_DIST_LSC & QTESRL & 1570 & $\mathrm{pCi} / \mathrm{L}$ & 224 & 319 & 329 & & \\
\hline 908 & 6010_METALS_ICP & QTESSL & 41.9 & ug/L & & & & & \\
\hline 909 & 6010_METALS_ICP & QTESSL & 39.5 & $5 \mathrm{ug} / \mathrm{L}$ & & & & & \\
\hline 910 & GAMMA_GS & QTESRL & 1.28 & $3 \mathrm{pCi} / \mathrm{L}$ & 4.42 & 4.43 & 8.42 & & $U$ \\
\hline 911 & TRITIUM_DIST_LSC & QTESRL & 4400 & $\mathrm{pCi} / \mathrm{L}$ & 327 & 522 & 329 & & \\
\hline 912 & GAMMA_GS & ITASRL & -0.535 & $5 \mathrm{pCi} / \mathrm{L}$ & 1.19 & 1.19 & & & $U$ \\
\hline
\end{tabular}




\begin{tabular}{|c|c|c|c|c|c|c|c|c|c|}
\hline & $\mathrm{K}$ & $\mathrm{L}$ & $\mathrm{M}$ & $\mathrm{N}$ & $\mathrm{O}$ & $\mathrm{P}$ & $\mathrm{Q}$ & $\mathrm{R}$ & $\mathrm{S}$ \\
\hline 1 & METHOD_NAME & LAB_CODE & VALUE_RPTD & ANAL_UNITS_RPTD & COUNTING_ERROR| & TOTAL_ANAL_ERROR| & MIN_DETECTABLE_ACTIVITY & PCNT_MOISTURE & LAB_QUALIFIER \\
\hline 914 & TRITIUM_DIST_LSC & ITASRL & 356 & $\mathrm{pCi} / \mathrm{L}$ & 91 & 162 & & & \\
\hline 915 & GAMMA_GS & ITASRL & 0.0312 & $\mathrm{pCi} / \mathrm{L}$ & 0.948 & 0.948 & & & U \\
\hline 916 & SRISO_SEP_PRECIP_GPC & ITASRL & 17.4 & $\mathrm{pCi} / \mathrm{L}$ & 0.346 & 3.07 & & & \\
\hline 917 & TC99_SEP_LSC & ITASRL & 18.3 & $\mathrm{pCi} / \mathrm{L}$ & 0.249 & 2.38 & & & \\
\hline 918 & TRITIUMM_DIST_LSC & ITASRL & 428 & $\mathrm{pCi} / \mathrm{L}$ & 93.8 & 167 & & & \\
\hline 919 & UISO_AEA & ITASRL & 0.868 & $\mathrm{pCi} / \mathrm{L}$ & 0.0792 & 0.12 & & & \\
\hline 920 & UISO_AEA & ITASRL & 0.0378 & $\mathrm{pCi} / \mathrm{L}$ & 0.0171 & 0.0177 & & & \\
\hline 921 & UISO_AEA & ITASRL & 0.759 & $\mathrm{pCi} / \mathrm{L}$ & 0.074 & 0.108 & & & \\
\hline 923 & I129_SEP_TEMS & 320 & 0.005471 & $\mathrm{pCi} / \mathrm{L}$ & & 0.0005799 & & & \\
\hline 924 & TC99_SEP_LSC & ITASRL & 8.76 & $\mathrm{pCi} / \mathrm{L}$ & 0.186 & 1.36 & & & \\
\hline 925 & TRITIŪM_DIST_LSC & ITASRL & 7880 & pCi/L & 234 & 682 & & & \\
\hline 926 & UISO_AEA & ITASRL & 26.9 & $\mathrm{pCi} / \mathrm{L}$ & 0.451 & 2.83 & & & \\
\hline 927 & ]UISO_AEA & ITASRL & 1.28 & $\mathrm{pCi} / \mathrm{L}$ & 0.0985 & 0.165 & & & \\
\hline 928 & UISO_AEA & ITASRL & 24.6 & $\mathrm{pCi} / \mathrm{L}$ & 0.432 & 2.59 & & & \\
\hline 929 & GAMMA_GS & ITASRL & -0.0612 & $\mathrm{pCi} / \mathrm{L}$ & 0.983 & 0.983 & & & U \\
\hline 930 & I129_SEP_TEMS & 320 & 0.1351058 & $\mathrm{pCi} / \mathrm{L}$ & & 0.008106 & & & \\
\hline 931 & TC99_SEP_LSC & ITASRL & 43.4 & $\mathrm{pCi} / \mathrm{L}$ & 0.366 & 5.09 & & & \\
\hline 933 & UISO_AEA & ITASRL & 1.23 & $\mathrm{pCi} / \mathrm{L}$ & 0.106 & 0.17 & & & \\
\hline 934 & UISO_AEA & ITASRL & 0.0295 & $\mathrm{pCi} / \mathrm{L}$ & 0.0175 & 0.0179 & & & \\
\hline 935 & UISO_AEA & ITASRL & 0.773 & $\mathrm{pCi} / \mathrm{L}$ & 0.0838 & 0.118 & & & \\
\hline 936 & 6010_METALS_ICP & QTESSL & 39.4 & ug/L & & & & & \\
\hline 937 & 6010_METALS_ICP & QTESSL & 41.6 & $\mathrm{ug} / \mathrm{L}$ & & & & & \\
\hline 938 & TRITIUM_DIST_LSC & QTESRL & 1680 & $\mathrm{pCi} / \mathrm{L}$ & 233 & 339 & 355 & & \\
\hline 939 & TRITIUM_DIST_LSC & QTESRL & 1840 & $\mathrm{pCi} / \mathrm{L}$ & 238 & 349 & 355 & & \\
\hline 940 & 6010_METALS_ICP & QTESSL & 5.2 & ug/L & & & & & B \\
\hline 941 & TRITIUM_DIST_LSC & QTESRL & 222 & $\mathrm{pCi} / \mathrm{L}$ & 159 & 241 & 355 & & $\mathrm{U}$ \\
\hline 942 & 6010_METALS_ICP & QTESSL & 40.4 & ug/L & & & & & \\
\hline 943 & SRISO_SEP_PRECIP_GPC & QTESRL & 0.932 & $\mathrm{pCi} / \mathrm{L}$ & 0.328 & 0.375 & 0.443 & & $\mathrm{~J}$ \\
\hline 944 & 6010_METALS_ICP & QTESSL & 23.7 & $\mathrm{ug} / \mathrm{L}$ & & & & & \\
\hline 945 & 6010_METALS_ICP & QTESSL & 25.7 & ug/L & & & & & \\
\hline 946 & GAMMA_GS & QTESRL & -5.35 & $\mathrm{pCi} / \mathrm{L}$ & 4.72 & 4.75 & 6.68 & & $\mathrm{U}$ \\
\hline 947 & TRITIUM_DIST_LSC & QTESRL & 300 & pCi/L & 153 & 231 & 324 & & $\mathrm{U}$ \\
\hline 948 & GAMMA_GS & ITASRL & 0.352 & $\mathrm{pCi} / \mathrm{L}$ & 1.49 & 1.49 & & & $\mathrm{U}$ \\
\hline 949 & SRISO_SEP_PRECIP_GPC & ITASRL & 9870 & $\mathrm{pCi} / \mathrm{L}$ & 98.2 & 1850 & & & \\
\hline 950 & TRITIUM_DIST_LSC & ITASRL & 13700 & pCi/L & 302 & 1100 & & & \\
\hline
\end{tabular}




\begin{tabular}{|c|c|c|c|c|c|c|c|c|c|}
\hline & $\mathrm{K}$ & $L$ & $\mathrm{M}$ & $\mathrm{N}$ & $\mathrm{O}$ & $\mathrm{P}$ & $\mathrm{Q}$ & $\mathrm{R}$ & $\mathrm{S}$ \\
\hline 1 & METHOD_NAME & LAB_CODE & VALUE_RPTD & ANAL_UNITS_RPTD & |COUNTING_ERROR & TOTAL_ANAL_ERROR & MIN_DETECTABLE_ACTIVITY & PCNT_MOISTURE & LAB_QUALIFIER \\
\hline 952 & SRISO_SEP_PRECIP_GPC & ITASRL & 0.593 & $\mathrm{pCi} / \mathrm{L}$ & 0.0625 & 0.129 & & & \\
\hline 953 & TRITIUM_DIST_LSC & ITASRL & 108 & $\mathrm{pCi} / \mathrm{L}$ & 79.2 & 141 & & & $\mathrm{U}$ \\
\hline 954 & GAMMA_GS & ITASRL & 0.718 & $\mathrm{pCi} / \mathrm{L}$ & 0.845 & 0.848 & & & $\mathrm{U}$ \\
\hline 955 & SRISO_SEP_PRECIP_GPC & ITASRL & 0.591 & $\mathrm{pCi} / \mathrm{L}$ & 0.067 & 0.134 & & & \\
\hline 956 & TRITIUM_DIST_LSC & ITASRL & 18900 & $\mathrm{pCi} / \mathrm{L}$ & 354 & 1480 & & & \\
\hline 957 & GAMMA_GS & ITASRL & -0.0888 & $\mathrm{pCi} / \mathrm{L}$ & 1.13 & 1.13 & & & $U$ \\
\hline 958 & I129_SEP_TEMS & 320 & 0.004723 & $\mathrm{pCi} / \mathrm{L}$ & & 0.0003967 & & & \\
\hline 959 & TC99_SEP_LSC & ITASRL & 12.6 & $\mathrm{pCi} / \mathrm{L}$ & 0.212 & 1.74 & & & \\
\hline 961 & UISO_IE_PLATE_AEA & ITASRL & 30.9 & $\mathrm{pCi} / \mathrm{L}$ & 0.485 & 3.24 & & & \\
\hline 962 & UISO_IE_PLATE_AEA & ITASRL & 0.567 & $\mathrm{pCi} / \mathrm{L}$ & 0.0658 & 0.0885 & & & \\
\hline 963 & UISO_IE_PLATE_AEA & ITASRL & 26.2 & $\mathrm{pCi} / \mathrm{L}$ & 0.446 & 2.75 & & & \\
\hline 964 & GAMMA_GS & ITASRL & 0.391 & $\mathrm{pCi} / \mathrm{L}$ & 1.09 & 1.09 & & & $\mathrm{U}$ \\
\hline 965 & I129_SEP_TEMS & 320 & 0.2184497 & $\mathrm{pCi} / \mathrm{L}$ & & 0.03 & & & \\
\hline 966 & TC99_SEP_LSC & ITASRL & 105 & $\mathrm{pCi} / \mathrm{L}$ & 0.557 & 11.8 & & & \\
\hline 967 & TRITIUM_DIST_LSC & ITASRL & 120000 & $\mathrm{pCi} / \mathrm{L}$ & 845 & 8850 & & & \\
\hline 968 & UISO_IE_PLATE_AEA & ITASRL & 2.1 & $\mathrm{pCi} / \mathrm{L}$ & 0.121 & 0.247 & & & \\
\hline 969 & UISO_IE_PLATE_AEA & ITASRL & 0.0427 & $\mathrm{pCi} / \mathrm{L}$ & 0.018 & 0.0187 & & & \\
\hline 971 & GAMMA_GS & ITASRL & -0.106 & $\mathrm{pCi} / \mathrm{L}$ & 1.07 & 1.07 & & & $U$ \\
\hline 972 & SRISO_SEP_PRECIP_GPC & ITASRL & 5.28 & $\mathrm{pCi} / \mathrm{L}$ & 0.219 & 1.2 & & & \\
\hline 973 & TRITIUM_DIST_LSC & ITASRL & 4850 & $\mathrm{pCi} / \mathrm{L}$ & 181 & 453 & & & \\
\hline 974 & GAMMA_GS & ITASRL & 0.0692 & $\mathrm{pCi} / \mathrm{L}$ & 1.34 & 1.34 & & & $\mathrm{U}$ \\
\hline 975 & SRISO_SEP_PRECIP_GPC & ITASRL & & & & & & & \\
\hline 976 & TC99_SEP_LSC & ITASRL & 76.7 & $\mathrm{pCi} / \mathrm{L}$ & 0.477 & 8.69 & & & \\
\hline 977 & TRITIUM_DIST_LSC & ITASRL & 2280 & $\mathrm{pCi} / \mathrm{L}$ & 135 & 273 & & & \\
\hline 978 & UISO_IE_PLATE_AEA & ITASRL & 4.99 & $\mathrm{pCi} / \mathrm{L}$ & 0.19 & 0.549 & & & \\
\hline 979 & UISO_IE_PLATE_AEA & ITASRL & 0.359 & $\mathrm{pCi} / \mathrm{L}$ & 0.0514 & 0.0636 & & & \\
\hline 980 & UISO_IE_PLATE_AEA & ITASRL & 3.91 & $\mathrm{pCi} / \mathrm{L}$ & 0.168 & 0.437 & & & \\
\hline 981 & GAMM̄A_GS - & ITASRL & 1.02 & $\mathrm{pCi} / \mathrm{L}$ & 1.14 & 1.14 & & & $U$ \\
\hline 982 & SRISO_SEP_PRECIP_GPC & ITASRL & -0.0219 & $\mathrm{pCi} / \mathrm{L}$ & 0.0671 & 0.0697 & & & $U$ \\
\hline 983 & TC99_SEP_LSC & ITASRL & 10 & $\mathrm{pCi} / \mathrm{L}$ & 0.193 & 1.45 & & & \\
\hline 984 & TRITIUM_DIST_LSC & ITASRL & 14300 & $\mathrm{pCi} / \mathrm{L}$ & 295 & 1140 & & & \\
\hline 985 & GAMMA_GS & QTESRL & -0.132 & $\mathrm{pCi} / \mathrm{L}$ & 1.1 & 1.1 & 2.02 & & $\mathrm{U}$ \\
\hline 986 & SRISO_SEP_PRECIP_GPC & QTESRL & 0.0123 & $\mathrm{pCi} / \mathrm{L}$ & 0.024 & 0.024 & 0.042 & & $\mathrm{U}$ \\
\hline 987 & TRITIUM_DIST_LSC & QTESRL & 738 & $\mathrm{pCi} / \mathrm{L}$ & 98 & 170 & 173 & & \\
\hline 988 & UISO_IE_PLATE_AEA & QTESRL & 1.63 & $\mathrm{pCi} / \mathrm{L}$ & 0.11 & 0.2 & 0.0161 & & \\
\hline
\end{tabular}




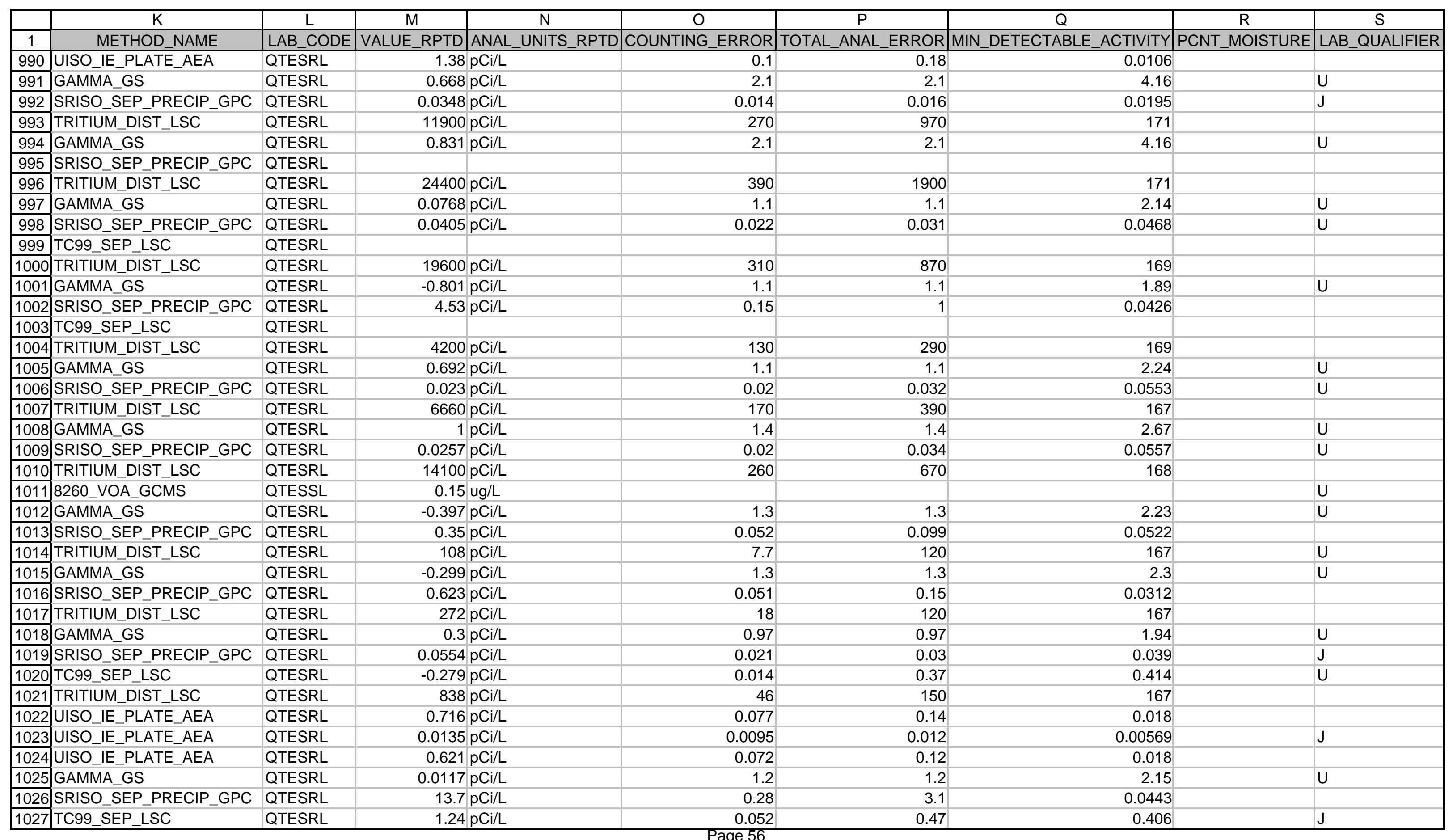




\begin{tabular}{|c|c|c|c|c|c|c|c|c|c|}
\hline & $\mathrm{K}$ & $\mathrm{L}$ & $\mathrm{M}$ & $\mathrm{N}$ & $\mathrm{O}$ & $\mathrm{P}$ & $\mathrm{Q}$ & $\mathrm{R}$ & $\mathrm{S}$ \\
\hline 1 & METHOD_NAME & LAB_CODE & VALUE_RPTD & ANAL_UNITS_RPTD & COUNTING_ERROR & TOTAL_ANAL_ERROR & MIN_DETECTABLE_ACTIVITY & PCNT_MOISTURE & LAB_QUALIFIER \\
\hline 1028 & TRITIUM_DIST_LSC & QTESRL & 525 & $\mathrm{pCi} / \mathrm{L}$ & 32 & 140 & 168 & & \\
\hline 1029 & UISO_IE_PLATE_AEA & QTESRL & 0.655 & $\mathrm{pCi} / \mathrm{L}$ & 0.073 & 0.13 & 0.0254 & & \\
\hline 1030 & UISO_IE_PLATE_AEA & QTESRL & 0.0142 & $\mathrm{pCi} / \mathrm{L}$ & 0.011 & 0.014 & 0.0202 & & U \\
\hline 1031 & UISO_IE_PLATE_AEA & QTESRL & 0.455 & $\mathrm{pCi} / \mathrm{L}$ & 0.062 & 0.095 & 0.0271 & & \\
\hline 1032 & GAMMA_G_GS & QTESRL & 0.488 & $\mathrm{pCi} / \mathrm{L}$ & 1.2 & 1.2 & 2.34 & & U \\
\hline 1033 & SRISO_SEP_PRECIP_GPC & QTESRL & 0.00358 & $\mathrm{pCi} / \mathrm{L}$ & 0.0069 & 0.027 & 0.0508 & & $U$ \\
\hline 1034 & TRITIUM_DIST_LSC & QTESRL & 1450 & $\mathrm{pCi} / \mathrm{L}$ & 69 & 180 & 171 & & \\
\hline 1035 & UISO_IE_PLATE_AEA & QTESRL & 2.57 & $\mathrm{pCi} / \mathrm{L}$ & 0.13 & 0.42 & 0.0195 & & \\
\hline 1036 & UISO_IE_PLATE_AEA & QTESRL & 0.0926 & $\mathrm{pCi} / \mathrm{L}$ & 0.025 & 0.03 & 0.0122 & & \\
\hline 1037 & UISO_IE_PLATE_AEA & QTESRL & 2.09 & $\mathrm{pCi} / \mathrm{L}$ & 0.12 & 0.35 & 0.0138 & & \\
\hline 1038 & GAMMA_GS & QTESRL & -0.702 & $\mathrm{pCi} / \mathrm{L}$ & 1.3 & 1.3 & 2.12 & & U \\
\hline 1039 & I129_SEP_TEMS & 320 & 0.006197 & $\mathrm{pCi} / \mathrm{L}$ & & 0.000558 & & & \\
\hline 1040 & SRISŌSEETP_PRECIP_GPC & QTESRL & 0.279 & $\mathrm{pCi} / \mathrm{L}$ & 0.037 & 0.076 & 0.0325 & & \\
\hline 1041 & TRITIUMM_DIST_LSC & QTESRL & 11300 & $\mathrm{pCi} / \mathrm{L}$ & 230 & 570 & 169 & & \\
\hline 1042 & UISO_IE_PLATE_AEA & QTESRL & 111 & $\mathrm{pCi} / \mathrm{L}$ & 8.6 & 19 & 1.31 & & \\
\hline 1043 & UISO_IE_PLATE_AEA & QTESRL & 2.65 & $\mathrm{pCi} / \mathrm{L}$ & 1.3 & 1.6 & 1.31 & & \\
\hline 1044 & UISO_IE_PLATE_AEA & QTESRL & 99.3 & $\mathrm{pCi} / \mathrm{L}$ & 8.1 & 17 & 0.947 & & \\
\hline 1045 & GAMMA_GS & QTESRL & 0.209 & $\mathrm{pCi} / \mathrm{L}$ & 1.4 & 1.4 & 2.53 & & $U$ \\
\hline 1047 & SRISO_SEEPP_PRECIP_GPC & QTESRL & 0.212 & $\mathrm{pCi} / \mathrm{L}$ & 0.036 & 0.063 & 0.0392 & & \\
\hline 1048 & TRITIUM_DIST_LSC & QTESRL & 10300 & $\mathrm{pCi} / \mathrm{L}$ & 220 & 530 & 169 & & \\
\hline 1049 & UISO_IE_PLATE_AEA & QTESRL & 41.6 & $\mathrm{pCi} / \mathrm{L}$ & 0.62 & 6.5 & 0.0269 & & \\
\hline 1050 & UISO_IE_PLATE_AEA & QTESRL & 2.06 & $\mathrm{pCi} / \mathrm{L}$ & 0.14 & 0.35 & 0.0182 & & \\
\hline 1051 & UISO_IE_PLATE_AEA & QTESRL & 37.6 & $\mathrm{pCi} / \mathrm{L}$ & 0.59 & 5.9 & 0.0345 & & \\
\hline 1052 & GAMMA_GS & QTESRL & -0.381 & $\mathrm{pCi} / \mathrm{L}$ & 1 & 1 & 1.86 & & U \\
\hline 1053 & I129_SEP_TEMS & 320 & 0.1696 & $\mathrm{pCi} / \mathrm{L}$ & & 0.0129 & & & \\
\hline 1054 & TC99_SEP_LSC & QTESRL & 88 & $\mathrm{pCi} / \mathrm{L}$ & 0.49 & 6 & 0.223 & & \\
\hline 1055 & TRITIUM_DIST_LSC & QTESRL & 86100 & $\mathrm{pCi} / \mathrm{L}$ & 670 & 3300 & 168 & & \\
\hline 1056 & UISO_IE_PLATE_AEA & QTESRL & 2.82 & $\mathrm{pCi} / \mathrm{L}$ & 0.16 & 0.47 & 0.0189 & & \\
\hline 1057 & UISO_IE_PLATE_AEA & QTESRL & 0.063 & $\mathrm{pCi} / \mathrm{L}$ & 0.023 & 0.027 & 0.0151 & & \\
\hline 1058 & UISO_IE_PLATE_AEA & QTESRL & 2.08 & $\mathrm{pCi} / \mathrm{L}$ & 0.14 & 0.35 & 0.0242 & & \\
\hline 1059 & GAMMA_GS & QTESRL & 0.789 & $\mathrm{pCi} / \mathrm{L}$ & 1.3 & 1.3 & 2.61 & & $U$ \\
\hline 1060 & I129_SEP_TEMS & 320 & 0.4056 & $\mathrm{pCi} / \mathrm{L}$ & & 0.0243 & & & \\
\hline 1061 & TC99_SEP_LSC & QTESRL & 57.4 & $\mathrm{pCi} / \mathrm{L}$ & 0.39 & 4 & 0.222 & & \\
\hline 1062 & TRITIUM_DIST_LSC & QTESRL & 75000 & $\mathrm{pCi} / \mathrm{L}$ & 620 & 2900 & 170 & & \\
\hline 1063 & UISO_IE_PLATE_AEA & QTESRL & 1.94 & $\mathrm{pCi} / \mathrm{L}$ & 0.12 & 0.33 & 0.0235 & & \\
\hline 1064 & UISO_IE_PLATE_AEA & QTESRL & 0.0358 & $\mathrm{pCi} / \mathrm{L}$ & 0.017 & 0.02 & 0.0182 & & $\mathrm{~J}$ \\
\hline
\end{tabular}




\begin{tabular}{|c|c|c|c|c|c|c|c|c|c|}
\hline & $\mathrm{K}$ & $L$ & $\mathrm{M}$ & $\mathrm{N}$ & $\mathrm{O}$ & $\mathrm{P}$ & $\mathrm{Q}$ & $\mathrm{R}$ & $\mathrm{S}$ \\
\hline 1 & METHOD_NAME & LAB_CODE & VALUE_RPTD & ANAL_UNITS_RPTD & COUNTING_ERROR & TOTAL_ANAL_ERROR & MIN_DETECTABLE_ACTIVITY & PCNT_MOISTURE & LAB_QUALIFIER \\
\hline 1066 & GAMMA_GS & QTESRL & 0.943 & $\mathrm{pCi} / \mathrm{L}$ & 1.3 & 1.3 & 2.55 & & $U$ \\
\hline 1067 & I129_SEP_TEMS & 320 & 0.2271 & $\mathrm{pCi} / \mathrm{L}$ & & 0.0163 & & & \\
\hline 1068 & TC99_SEP_LSC & QTESRL & 120 & $\mathrm{pCi} / \mathrm{L}$ & 0.58 & 8 & 0.222 & & \\
\hline 1069 & TRITIUM_DIST_LSC & QTESRL & 106000 & $\mathrm{pCi} / \mathrm{L}$ & 740 & 4100 & 168 & & \\
\hline 1070 & UISO_IE_PLATE_AEA & QTESRL & 5.06 & $\mathrm{pCi} / \mathrm{L}$ & 0.25 & 0.84 & 0.0385 & & \\
\hline 1071 & UISO_IE_PLATE_AEA & QTESRL & 0.135 & $\mathrm{pCi} / \mathrm{L}$ & 0.041 & 0.047 & 0.00869 & & \\
\hline 1072 & UISO_IE_PLATE_AEA & QTESRL & 3.42 & $\mathrm{pCi} / \mathrm{L}$ & 0.21 & 0.58 & 0.0276 & & \\
\hline 1073 & 8260_VOA_GCMS & QTESSL & 0.15 & $\mathrm{ug} / \mathrm{L}$ & & & & & U \\
\hline 1075 & 8260_VOA_GCMS & QTESSL & 0.15 & ug/L & & & & & $U$ \\
\hline 1076 & GAMMA_GS & STLRL & -1.05 & $\mathrm{pCi} / \mathrm{L}$ & 4.1 & 4.1 & 7.45 & & U \\
\hline 1077 & |UISO_IE_PLATE_AEA & STLRL & 29.1 & $\mathrm{pCi} / \mathrm{L}$ & 2 & 5.5 & 0.42 & & \\
\hline 1078 & UISO_IE_PLATE_AEA & STLRL & 0.952 & $\mathrm{pCi} / \mathrm{L}$ & 0.35 & 0.42 & 0.232 & & \\
\hline 1079 & UISO_IE_PLATE_AEA & STLRL & 27.7 & $\mathrm{pCi} / \mathrm{L}$ & 1.9 & 5.2 & 0.389 & & \\
\hline
\end{tabular}




\begin{tabular}{|c|c|c|c|c|c|c|c|c|}
\hline & $\mathrm{T}$ & $U$ & $\mathrm{~V}$ & $\mathrm{~W}$ & $x$ & $Y$ & $Z$ & $\mathrm{AA}$ \\
\hline 1 & REVIEW_QUALIFIER & SAMP_FROM & SAMP_ITEM & SAMP_MTHD & COLL_MTHD & SAMP_SITE_ID & SAMP_SITE_NAME & WELL_NAME \\
\hline 2 & & SEEP & WATER & & & 2217 & 7 SEEP 038-3 & \\
\hline 3 & & SEEP & WATER & & & 2217 & 7 SEEP 038-3 & \\
\hline 4 & & SEEP & WATER & & & 2217 & 7 SEEP 038-3 & \\
\hline 5 & & SEEP & WATER & & & 2217 & 7 SEEP 038-3 & \\
\hline 6 & & SEEP & WATER & & & 2217 & 7 SEEP 038-3 & \\
\hline 7 & & SEEP & WATER & & & 2216 & 6 SEEP 037-1 & \\
\hline 8 & & SEEP & WATER & & & 2216 & 6 SEEP 037-1 & \\
\hline 9 & & SEEP & WATER & & & 2216 & 6 SEEP 037-1 & \\
\hline 11 & & SEEP & WATER & & & 2216 & 6 SEEP 037-1 & \\
\hline 12 & & SEEP & WATER & & & 2216 & 5 SEEP 037-1 & \\
\hline 13 & & SEEP & WATER & & & 2216 & 6 SEEP 037-1 & \\
\hline 14 & & SEEP & WATER & & & 2216 & 6 SEEP 037-1 & \\
\hline 15 & & SEEP & WATER & & & 2216 & o SEEP 037-1 & \\
\hline 16 & & SEEP & WATER & & & 2216 & 6 SEEP 037-1 & \\
\hline 17 & & SEEP & WATER & & & 2218 & 3 SEEP 039-2 & \\
\hline 18 & & SEEP & WATER & & & 2218 & 3 SEEP 039-2 & \\
\hline 19 & & SEEP & WATER & & & 2218 & 3 SEEP 039-2 & \\
\hline 21 & & SEEP & WATER & & & 2218 & 3 SEEP 039-2 & \\
\hline 22 & & SEEP & WATER & & & 2228 & 3 SEEP 110-1 & \\
\hline 23 & & SEEP & WATER & & & 2228 & 3 SEEP 110-1 & \\
\hline 24 & & SEEP & WATER & & & 2228 & 3 SEEP 110-1 & \\
\hline 25 & & SEEP & WATER & & & 2228 & 3 SEEP $110-1$ & \\
\hline 26 & & SEEP & WATER & & & 2228 & 3 SEEP 110-1 & \\
\hline 27 & & SEEP & WATER & & & 2228 & 3 SEEP $110-1$ & \\
\hline 28 & & SEEP & WATER & & & 2231 & 1 SEEP 143-1 & \\
\hline 29 & & SEEP & WATER & & & 2231 & 1 SEEP 143-1 & \\
\hline 30 & & SEEP & WATER & & & 2231 & 1 SEEP 143-1 & \\
\hline 31 & & SEEP & WATER & & & 2231 & 1 SEEP 143-1 & \\
\hline 32 & & SEEP & WATER & & & 2231 & 1 SEEP 143-1 & \\
\hline 33 & & SEEP & WATER & & & 2231 & 1 SEEP 143-1 & \\
\hline 34 & & SEEP & WATER & & & 2231 & 1 SEEP 143-1 & \\
\hline 35 & & SEEP & WATER & & & 2231 & 1 SEEP 143-1 & \\
\hline 36 & & SEEP & WATER & & & 2231 & 1 SEEP 143-1 & \\
\hline 37 & & SEEP & WATER & & & 2231 & 1 SEEP $143-1$ & \\
\hline 38 & & SEEP & WATER & & & 2231 & 1 SEEP 143-1 & \\
\hline
\end{tabular}




\begin{tabular}{|c|c|c|c|c|c|c|c|c|}
\hline & $T$ & $U$ & $\mathrm{~V}$ & $\mathrm{~W}$ & $x$ & $Y$ & $Z$ & $\mathrm{AA}$ \\
\hline 1 & REVIEW_QUALIFIER & SAMP_FROM & SAMP_ITEM & SAMP_MTHD & COLL_MTHD & SAMP_SITE_ID & SAMP_SITE_NAME & WELL_NAME \\
\hline 40 & & SEEP & WATER & & & 2232 & SEEP 144-1 & \\
\hline 41 & & SEEP & WATER & & & 2232 & SEEP 144-1 & \\
\hline 42 & & SEEP & WATER & & & 2232 & SEEP 144-1 & \\
\hline 43 & & SEEP & WATER & & & 2232 & SEEP 144-1 & \\
\hline 44 & & SEEP & WATER & & & 2232 & SEEP 144-1 & \\
\hline 45 & & SEEP & WATER & & & 2232 & SEEP 144-1 & \\
\hline 46 & & SEEP & WATER & & & 2232 & SEEP 144-1 & \\
\hline 47 & & SEEP & WATER & & & 2232 & SEEP 144-1 & \\
\hline 49 & & SEEP & WATER & & & 2232 & SEEP 144-1 & \\
\hline 50 & & SEEP & WATER & & & 2232 & SEEP 144-1 & \\
\hline 51 & & SEEP & WATER & & & 2232 & SEEP 144-1 & \\
\hline 52 & & SEEP & WATER & & & 2220 & SEEP 056-3 & \\
\hline 53 & & SEEP & WATER & & & 2220 & SEEP 056-3 & \\
\hline 54 & & SEEP & WATER & & & 2220 & SEEP 056-3 & \\
\hline 55 & & SEEP & WATER & & & 2220 & SEEP 056-3 & \\
\hline 56 & & SEEP & WATER & & & 2220 & SEEP 056-3 & \\
\hline 57 & & SEEP & WATER & & & 2220 & SEEP 056-3 & \\
\hline 59 & & SEEP & WATER & & & 2220 & SEEP 056-3 & \\
\hline 60 & & SEEP & WATER & & & 2220 & SEEP 056-3 & \\
\hline 61 & & SEEP & WATER & & & 2220 & SEEP 056-3 & \\
\hline 62 & & SEEP & WATER & & & 2233 & SEEP 146-1 & \\
\hline 63 & & SEEP & WATER & & & 2233 & SEEP 146-1 & \\
\hline 64 & & SEEP & WATER & & & 2233 & SEEP 146-1 & \\
\hline 65 & & SEEP & WATER & & & 2233 & SEEP 146-1 & \\
\hline 66 & & SEEP & WATER & & & 2233 & SEEP 146-1 & \\
\hline 67 & & SEEP & WATER & & & 2233 & SEEP 146-1 & \\
\hline 68 & & SEEP & WATER & & & 2233 & SEEP 146-1 & \\
\hline 69 & & SEEP & WATER & & & 2233 & SEEP 146-1 & \\
\hline 70 & & SEEP & WATER & & & 2233 & SEEP 146-1 & \\
\hline 71 & & SEEP & WATER & & & 2233 & SEEP 146-1 & \\
\hline 72 & & SEEP & WATER & & & 2233 & SEEP 146-1 & \\
\hline 73 & & SEEP & WATER & & & 2233 & SEEP 146-1 & \\
\hline 74 & & SEEP & WATER & & & 2229 & SEEP $110-2$ & \\
\hline 75 & & SEEP & WATER & & & 2229 & SEEP $110-2$ & \\
\hline 76 & & SEEP & WATER & & & 2229 & SEEP $110-2$ & \\
\hline
\end{tabular}




\begin{tabular}{|c|c|c|c|c|c|c|c|c|}
\hline & $T$ & $U$ & $\mathrm{~V}$ & $\mathrm{~W}$ & $x$ & $Y$ & $Z$ & $\mathrm{AA}$ \\
\hline 1 & REVIEW_QUALIFIER & SAMP_FROM & SAMP_ITEM & SAMP_MTHD & COLL_MTHD & SAMP_SITE_ID & SAMP_SITE_NAME & WELL_NAME \\
\hline 78 & & SEEP & WATER & & & 2229 & SEEP $110-2$ & \\
\hline 79 & & SEEP & WATER & & & 2229 & SEEP 110-2 & \\
\hline 80 & & SEEP & WATER & & & 2229 & SEEP $110-2$ & \\
\hline 81 & & SEEP & WATER & & & 2229 & SEEP $110-2$ & \\
\hline 82 & & SEEP & WATER & & & 2229 & SEEP 110-2 & \\
\hline 83 & & SEEP & WATER & & & 2229 & SEEP 110-2 & \\
\hline 84 & & SEEP & WATER & & & 2229 & SEEP $110-2$ & \\
\hline 85 & & SEEP & WATER & & & 2229 & SEEP 110-2 & \\
\hline 87 & & SEEP & WATER & & & 2234 & 4 SEEP 149-1 & \\
\hline 88 & & SEEP & WATER & & & 2234 & SEEP $149-1$ & \\
\hline 89 & & SEEP & WATER & & & 2234 & 4 SEEP 149-1 & \\
\hline 90 & & SEEP & WATER & & & 2234 & SEEP 149-1 & \\
\hline 91 & & SEEP & WATER & & & 2234 & SEEP $149-1$ & \\
\hline 92 & & SEEP & WATER & & & 2234 & SEEP 149-1 & \\
\hline 93 & & SEEP & WATER & & & 2234 & 4 SEEP 149-1 & \\
\hline 94 & & SEEP & WATER & & & 2234 & SEEP 149-1 & \\
\hline 95 & & SEEP & WATER & & & 2234 & SEEP 149-1 & \\
\hline 97 & & SEEP & WATER & & & 2234 & 4 SEEP 149-1 & \\
\hline 98 & & SEEP & WATER & & & 2237 & SEEP 190-4 & \\
\hline 99 & & SEEP & WATER & & & 2237 & SEEP 190-4 & \\
\hline 100 & & SEEP & WATER & & & 2237 & SEEP 190-4 & \\
\hline 101 & & SEEP & WATER & & & 2237 & SEEP 190-4 & \\
\hline 102 & & SEEP & WATER & & & 2237 & SEEP 190-4 & \\
\hline 103 & & SEEP & WATER & & & 2237 & SEEP 190-4 & \\
\hline 104 & & SEEP & WATER & & & 2237 & SEEP 190-4 & \\
\hline 105 & & SEEP & WATER & & & 2237 & SEEP 190-4 & \\
\hline 106 & & SEEP & WATER & & & 2237 & SEEP 190-4 & \\
\hline 107 & & SEEP & WATER & & & 2237 & SEEP 190-4 & \\
\hline 108 & & SEEP & WATER & & & 2236 & SEEP 187-1 & \\
\hline 109 & & SEEP & WATER & & & 2236 & SEEP 187-1 & \\
\hline 110 & & SEEP & WATER & & & 2236 & SEEP 187-1 & \\
\hline 111 & & SEEP & WATER & & & 2236 & SEEP 187-1 & \\
\hline 112 & & SEEP & WATER & & & 2236 & SEEP 187-1 & \\
\hline 113 & & SEEP & WATER & & & 2236 & SEEP $187-1$ & \\
\hline 114 & & SEEP & WATER & & & 2236 & SEEP 187-1 & \\
\hline
\end{tabular}




\begin{tabular}{|c|c|c|c|c|c|c|c|c|}
\hline & $\mathrm{T}$ & $U$ & $\mathrm{~V}$ & $\mathrm{~W}$ & $x$ & $Y$ & $Z$ & $\mathrm{AA}$ \\
\hline 1 & REVIEW_QUALIFIER & SAMP_FROM & SAMP_ITEM & SAMP_MTHD & COLL_MTHD & SAMP_SITE_ID & SAMP_SITE_NAME & WELL_NAME \\
\hline 116 & & SEEP & WATER & & & 2236 & SEEP 187-1 & \\
\hline 117 & & SEEP & WATER & & & 2236 & SEEP 187-1 & \\
\hline 118 & & SEEP & WATER & & & 2241 & SEEP 221-1 & \\
\hline 119 & & SEEP & WATER & & & 2241 & SEEP 221-1 & \\
\hline 120 & & SEEP & WATER & & & 2241 & SEEP 221-1 & \\
\hline 121 & & SEEP & WATER & & & 2241 & SEEP 221-1 & \\
\hline 122 & & SEEP & WATER & & & 2241 & SEEP 221-1 & \\
\hline 123 & & SEEP & WATER & & & 2241 & SEEP 221-1 & \\
\hline 125 & & SEEP & WATER & & & 2241 & SEEP 221-1 & \\
\hline 126 & & SEEP & WATER & & & 2241 & SEEP 221-1 & \\
\hline 127 & & SEEP & WATER & & & 2241 & SEEP 221-1 & \\
\hline 128 & & SEEP & WATER & & & 2240 & SEEP 218-1 & \\
\hline 129 & & SEEP & WATER & & & 2240 & SEEP 218-1 & \\
\hline 130 & & SEEP & WATER & & & 2240 & SEEP 218-1 & \\
\hline 131 & & SEEP & WATER & & & 2240 & SEEP 218-1 & \\
\hline 132 & & SEEP & WATER & & & 2240 & SEEP 218-1 & \\
\hline 133 & & SEEP & WATER & & & 2240 & SEEP $218-1$ & \\
\hline 135 & & SEEP & WATER & & & 2240 & SEEP 218-1 & \\
\hline 136 & & SEEP & WATER & & & 2240 & SEEP 218-1 & \\
\hline 137 & & SEEP & WATER & & & 2240 & SEEP 218-1 & \\
\hline 138 & & SEEP & WATER & & & 2239 & SEEP 216-1 & \\
\hline 139 & & SEEP & WATER & & & 2239 & SEEP 216-1 & \\
\hline 140 & & SEEP & WATER & & & 2239 & SEEP 216-1 & \\
\hline 141 & & SEEP & WATER & & & 2239 & SEEP $216-1$ & \\
\hline 142 & & SEEP & WATER & & & 2239 & SEEP 216-1 & \\
\hline 143 & & SEEP & WATER & & & 2239 & SEEP 216-1 & \\
\hline 144 & & SEEP & WATER & & & 2239 & SEEP 216-1 & \\
\hline 145 & & SEEP & WATER & & & 2239 & SEEP 216-1 & \\
\hline 146 & & SEEP & WATER & & & 2239 & SEEP 216-1 & \\
\hline 147 & & SEEP & WATER & & & 2239 & SEEP 216-1 & \\
\hline 148 & & SEEP & WATER & & & 2239 & SEEP 216-1 & \\
\hline 149 & & SEEP & WATER & & & 2239 & SEEP 216-1 & \\
\hline 150 & & SEEP & WATER & & & 2239 & SEEP 216-1 & \\
\hline 151 & & SEEP & WATER & & & 2239 & SEEP 216-1 & \\
\hline 152 & & SEEP & WATER & & & 2239 & SEEP 216-1 & \\
\hline
\end{tabular}




\begin{tabular}{|c|c|c|c|c|c|c|c|c|}
\hline & $T$ & $U$ & $\mathrm{~V}$ & $\mathrm{~W}$ & $x$ & $Y$ & $Z$ & $\mathrm{AA}$ \\
\hline 1 & REVIEW_QUALIFIER & SAMP_FROM & SAMP_ITEM & SAMP_MTHD & COLL_MTHD & SAMP_SITE_ID & SAMP_SITE_NAME & WELL_NAME \\
\hline 154 & & SEEP & WATER & & & 2239 & SEEP 216-1 & \\
\hline 155 & & SEEP & WATER & & & 2239 & SEEP 216-1 & \\
\hline 156 & & SEEP & WATER & & & 2239 & SEEP 216-1 & \\
\hline 157 & & SEEP & WATER & & & 2239 & SEEP 216-1 & \\
\hline 158 & & SEEP & WATER & & & 2238 & SEEP 208-1 & \\
\hline 159 & & SEEP & WATER & & & 2238 & SEEP 208-1 & \\
\hline 160 & & SEEP & WATER & & & 2238 & SEEP 208-1 & \\
\hline 161 & & SEEP & WATER & & & 2238 & SEEP 208-1 & \\
\hline 163 & & SEEP & WATER & & & 2238 & SEEP 208-1 & \\
\hline 164 & & SEEP & WATER & & & 2238 & SEEP 208-1 & \\
\hline 165 & & SEEP & WATER & & & 2238 & SEEP 208-1 & \\
\hline 166 & & SEEP & WATER & & & 2238 & SEEP 208-1 & \\
\hline 167 & & SEEP & WATER & & & 2238 & SEEP 208-1 & \\
\hline 168 & & SEEP & WATER & & & 2243 & SEEP 252-1 & \\
\hline 169 & & SEEP & WATER & & & 2243 & SEEP 252-1 & \\
\hline 170 & & SEEP & WATER & & & 2243 & SEEP 252-1 & \\
\hline 171 & & SEEP & WATER & & & 2243 & SEEP 252-1 & \\
\hline 173 & & SEEP & WATER & & & 2243 & SEEP 252-1 & \\
\hline 174 & & SEEP & WATER & & & 2243 & SEEP 252-1 & \\
\hline 175 & & SEEP & WATER & & & 2243 & SEEP 252-1 & \\
\hline 176 & & SEEP & WATER & & & 2243 & SEEP 252-1 & \\
\hline 177 & & SEEP & WATER & & & 2243 & SEEP 252-1 & \\
\hline 178 & & SEEP & WATER & & & 2242 & SEEP $246-1$ & \\
\hline 179 & & SEEP & WATER & & & 2242 & SEEP $246-1$ & \\
\hline 180 & & SEEP & WATER & & & 2242 & SEEP $246-1$ & \\
\hline 181 & & SEEP & WATER & & & 2242 & SEEP $246-1$ & \\
\hline 182 & & SEEP & WATER & & & 2242 & SEEP $246-1$ & \\
\hline 183 & & SEEP & WATER & & & 2242 & SEEP 246-1 & \\
\hline 184 & & SEEP & WATER & & & 2242 & SEEP $246-1$ & \\
\hline 185 & & SEEP & WATER & & & 2242 & SEEP 246-1 & \\
\hline 186 & & SEEP & WATER & & & 2242 & SEEP 246-1 & \\
\hline 187 & & SEEP & WATER & & & 2242 & SEEP $246-1$ & \\
\hline 188 & & SEEP & WATER & & & 2242 & SEEP $246-1$ & \\
\hline 189 & & SEEP & WATER & & & 2242 & SEEP $246-1$ & \\
\hline 190 & & SEEP & WATER & & & 2242 & SEEP $246-1$ & \\
\hline
\end{tabular}




\begin{tabular}{|c|c|c|c|c|c|c|c|c|}
\hline & $T$ & $\mathrm{U}$ & $\mathrm{V}$ & $\mathrm{W}$ & $\mathrm{X}$ & $\mathrm{Y}$ & $Z$ & AA \\
\hline 1 & REVIEW_QUALIFIER & SAMP_FROM & SAMP_ITEM & SAMP_MTHD & COLL_MTHD & SAMP_SITE_ID & SAMP_SITE_NAME & WELL_NAME \\
\hline 192 & & SEEP & WATER & & & 2242 & SEEP $246-1$ & \\
\hline 193 & & SEEP & WATER & & & 2242 & SEEP $246-1$ & \\
\hline 194 & & SEEP & WATER & & & 2242 & SEEP $246-1$ & \\
\hline 195 & & SEEP & WATER & & & 2242 & SEEP $246-1$ & \\
\hline 196 & & SEEP & WATER & & & 2242 & SEEP $246-1$ & \\
\hline 197 & & SEEP & WATER & & & 2242 & SEEP $246-1$ & \\
\hline 198 & & SEEP & WATER & & & 2223 & SEEP 090-1 & \\
\hline 199 & & SEEP & WATER & & & 2223 & SEEP 090-1 & \\
\hline 201 & & SEEP & WATER & & & 2223 & SEEP 090-1 & \\
\hline 202 & & SEEP & WATER & & & 2223 & SEEP 090-1 & \\
\hline 203 & & SEEP & WATER & & & 2223 & SEEP 090-1 & \\
\hline 204 & & SEEP & WATER & & & 2223 & SEEP 090-1 & \\
\hline 205 & & SEEP & WATER & & & 2223 & SEEP 090-1 & \\
\hline 206 & & SEEP & WATER & & & 2223 & SEEP 090-1 & \\
\hline 207 & & SEEP & WATER & & & 2223 & SEEP 090-1 & \\
\hline 208 & & SEEP & WATER & & & 2223 & SEEP 090-1 & \\
\hline 209 & & SEEP & WATER & & & 2224 & SEEP 091-2 & \\
\hline 211 & & SEEP & WATER & & & 2224 & SEEP 091-2 & \\
\hline 212 & & SEEP & WATER & & & 2224 & SEEP 091-2 & \\
\hline 213 & & SEEP & WATER & & & 2224 & SEEP 091-2 & \\
\hline 214 & & SEEP & WATER & & & 2224 & SEEP 091-2 & \\
\hline 215 & & SEEP & WATER & & & 2224 & SEEP 091-2 & \\
\hline 216 & & SEEP & WATER & & & 2224 & SEEP 091-2 & \\
\hline 217 & & SEEP & WATER & & & 2224 & SEEP 091-2 & \\
\hline 218 & & SEEP & WATER & & & 2224 & SEEP 091-2 & \\
\hline 219 & & SEEP & WATER & & & 2224 & SEEP 091-2 & \\
\hline 220 & & SEEP & WATER & & & 2224 & SEEP 091-2 & \\
\hline 221 & & SEEP & WATER & & & 2221 & SEEP 074-1 & \\
\hline 222 & & SEEP & WATER & & & 2221 & SEEP 074-1 & \\
\hline 223 & & SEEP & WATER & & & 2221 & SEEP 074-1 & \\
\hline 224 & & SEEP & WATER & & & 2221 & SEEP 074-1 & \\
\hline 225 & & SEEP & WATER & & & 2221 & SEEP 074-1 & \\
\hline 226 & & SEEP & WATER & & & 2221 & SEEP 074-1 & \\
\hline 227 & & SEEP & WATER & & & 2221 & SEEP 074-1 & \\
\hline 228 & & SEEP & WATER & & & 2221 & SEEP 074-1 & \\
\hline
\end{tabular}




\begin{tabular}{|c|c|c|c|c|c|c|c|c|}
\hline & $T$ & $\mathrm{U}$ & $\mathrm{V}$ & $\mathrm{W}$ & $\mathrm{X}$ & $\mathrm{Y}$ & $Z$ & AA \\
\hline 1 & REVIEW_QUALIFIER & SAMP_FROM & SAMP_ITEM & SAMP_MTHD & COLL_MTHD & SAMP_SITE_ID & SAMP_SITE_NAME & WELL_NAME \\
\hline 230 & & SEEP & WATER & & & 2221 & 1 SEEP 074-1 & \\
\hline 231 & & SEEP & WATER & & & 2221 & 1 SEEP 074-1 & \\
\hline 232 & & SEEP & WATER & & & 2221 & 1 SEEP 074-1 & \\
\hline 233 & & SEEP & WATER & & & 2227 & 7 SEEP 094-1 & \\
\hline 234 & & SEEP & WATER & & & 2227 & 7 SEEP 094-1 & \\
\hline 235 & & SEEP & WATER & & & 2227 & 7 SEEP 094-1 & \\
\hline 236 & & SEEP & WATER & & & 2227 & 7 SEEP 094-1 & \\
\hline 237 & & SEEP & WATER & & & 2227 & 7 SEEP 094-1 & \\
\hline 239 & & SEEP & WATER & & & 2227 & 7 SEEP 094-1 & \\
\hline 240 & & SEEP & WATER & & & 2227 & 7 SEEP 094-1 & \\
\hline 241 & & SEEP & WATER & & & 2227 & 7 SEEP 094-1 & \\
\hline 242 & & SEEP & WATER & & & 2227 & 7 SEEP 094-1 & \\
\hline 243 & & SEEP & WATER & & & 2227 & 7 SEEP 094-1 & \\
\hline 244 & & SEEP & WATER & & & 2227 & 7 SEEP 094-1 & \\
\hline 245 & & SEEP & WATER & & & 2226 & 6 SEEP 092-2 & \\
\hline 246 & & SEEP & WATER & & & 2226 & 6 SEEP 092-2 & \\
\hline 247 & & SEEP & WATER & & & 2226 & 6 SEEP 092-2 & \\
\hline 249 & & SEEP & WATER & & & 2226 & 6 SEEP 092-2 & \\
\hline 250 & & SEEP & WATER & & & 2226 & 6 SEEP 092-2 & \\
\hline 251 & & SEEP & WATER & & & 2226 & 6 SEEP 092-2 & \\
\hline 252 & & SEEP & WATER & & & 2226 & 6 SEEP 092-2 & \\
\hline 253 & & SEEP & WATER & & & 2226 & SEEP 092-2 & \\
\hline 254 & & SEEP & WATER & & & 2226 & 6 SEEP 092-2 & \\
\hline 255 & & SEEP & WATER & & & 2226 & 6 SEEP 092-2 & \\
\hline 256 & & SEEP & WATER & & & 2226 & 6 SEEP 092-2 & \\
\hline 257 & & SEEP & WATER & & & 2225 & 5 SEEP 092-1 & \\
\hline 258 & & SEEP & WATER & & & 2225 & 5 SEEP 092-1 & \\
\hline 259 & & SEEP & WATER & & & 2225 & SEEP 092-1 & \\
\hline 260 & & SEEP & WATER & & & 2225 & 5 SEEP 092-1 & \\
\hline 261 & & SEEP & WATER & & & 2225 & 5 SEEP 092-1 & \\
\hline 262 & & SEEP & WATER & & & 2225 & 5 SEEP 092-1 & \\
\hline 263 & & SEEP & WATER & & & 2225 & 5 SEEP 092-1 & \\
\hline 264 & & SEEP & WATER & & & 2225 & 5 SEEP 092-1 & \\
\hline 265 & & SEEP & WATER & & & 2225 & 5 SEEP 092-1 & \\
\hline 266 & & SEEP & WATER & & & 2225 & 5 SEEP 092-1 & \\
\hline
\end{tabular}




\begin{tabular}{|c|c|c|c|c|c|c|c|c|}
\hline & $\mathrm{T}$ & $U$ & $\mathrm{~V}$ & $\mathrm{~W}$ & $x$ & $Y$ & $Z$ & $\mathrm{AA}$ \\
\hline 1 & REVIEW_QUALIFIER & SAMP_FROM & SAMP_ITEM & SAMP_MTHD & COLL_MTHD & SAMP_SITE_ID & SAMP_SITE_NAME & WELL_NAME \\
\hline 268 & & SEEP & WATER & & & 2225 & 5 SEEP 092-1 & \\
\hline 269 & & SEEP & WATER & & & 2222 & 2 SEEP 080-2 & \\
\hline 270 & & SEEP & WATER & & & 2222 & 2 SEEP 080-2 & \\
\hline 271 & & SEEP & WATER & & & 2222 & 2 SEEP 080-2 & \\
\hline 272 & & SEEP & WATER & & & 2222 & 2 SEEP 080-2 & \\
\hline 273 & & SEEP & WATER & & & 2222 & 2 SEEP 080-2 & \\
\hline 274 & & SEEP & WATER & & & 2222 & 2 SEEP 080-2 & \\
\hline 275 & & SEEP & WATER & & & 2222 & 2 SEEP 080-2 & \\
\hline 277 & & SEEP & WATER & & & 2222 & 2 SEEP 080-2 & \\
\hline 278 & & SEEP & WATER & & & 2222 & 2 SEEP 080-2 & \\
\hline 279 & & SEEP & WATER & & & 2222 & 2 SEEP 080-2 & \\
\hline 280 & & SEEP & WATER & & & 2222 & 2 SEEP 080-2 & \\
\hline 281 & & SEEP & WATER & & & 2235 & 5 SEEP 154-1 & \\
\hline 282 & & SEEP & WATER & & & 2235 & 5 SEEP 154-1 & \\
\hline 283 & & SEEP & WATER & & & 2235 & 5 SEEP 154-1 & \\
\hline 284 & & SEEP & WATER & & & 2235 & 5 SEEP 154-1 & \\
\hline 285 & & SEEP & WATER & & & 2235 & 5 SEEP 154-1 & \\
\hline 287 & & SEEP & WATER & & & 2235 & 5 SEEP 154-1 & \\
\hline 288 & & SEEP & WATER & & & 2235 & 5 SEEP 154-1 & \\
\hline 289 & & SEEP & WATER & & & 2235 & 5 SEEP 154-1 & \\
\hline 290 & & SEEP & WATER & & & 2235 & 5 SEEP 154-1 & \\
\hline 291 & & SEEP & WATER & & & 2235 & 5 SEEP 154-1 & \\
\hline 292 & & SEEP & WATER & & & 2235 & 5 SEEP 154-1 & \\
\hline 293 & & SEEP & & RAW GRAB & UNFILTERED & 151 & 1 HANFORD SPRING 28-2 & \\
\hline 294 & & SEEP & & RAW GRAB & UNFILTERED & 151 & 1 HANFORD SPRING 28-2 & \\
\hline 295 & & SEEP & & RAW GRAB & UNFILTERED & 151 & 1 HANFORD SPRING 28-2 & \\
\hline 296 & & SEEP & & RAW GRAB & UNFILTERED & 202 & 2 100-N SPRING 8-13 & \\
\hline 297 & & SEEP & & RAW GRAB & UNFILTERED & 202 & 2 100-N SPRING 8-13 & \\
\hline 298 & & SEEP & & RAW GRAB & UNFILTERED & 202 & 2 100-N SPRING 8-13 & \\
\hline 299 & & SEEP & & RAW GRAB & UNFILTERED & 202 & 2 100-N SPRING 8-13 & \\
\hline 300 & & SEEP & & RAW GRAB & UNFILTERED & 488 & 3300 AREA SPRING 42-2 & \\
\hline 301 & & SEEP & & RAW GRAB & UNFILTERED & 488 & 300 AREA SPRING 42-2 & \\
\hline 302 & & SEEP & & RAW GRAB & UNFILTERED & 488 & 3300 AREA SPRING 42-2 & \\
\hline 303 & & SEEP & & RAW GRAB & UNFILTERED & 488 & 3300 AREA SPRING 42-2 & \\
\hline 304 & & SEEP & & RAW GRAB & UNFILTERED & 488 & 300 AREA SPRING 42-2 & \\
\hline
\end{tabular}

488300 AREA SPRING 42-2 


\begin{tabular}{|c|c|c|c|c|c|c|c|c|}
\hline & $\mathrm{T}$ & $U$ & $\mathrm{~V}$ & $\mathrm{~W}$ & $\mathrm{X}$ & $Y$ & $Z$ & $\mathrm{AA}$ \\
\hline 1 & REVIEW_QUALIFIER & SAMP_FROM & SAMP_ITEM & SAMP_MTHD & COLL_MTHD & SAMP_SITE_ID & SAMP_SITE_NAME & WELL_NAME \\
\hline 306 & & SEEP & & RAW GRAB & UNFILTERED & 488 & 3 300 AREA SPRING 42-2 & \\
\hline 307 & & SEEP & & RAW GRAB & UNFILTERED & 488 & 3300 AREA SPRING 42-2 & \\
\hline 308 & & SEEP & & RAW GRAB & UNFILTERED & 488 & 3300 AREA SPRING 42-2 & \\
\hline 309 & & SEEP & & RAW GRAB & UNFILTERED & 488 & 3300 AREA SPRING 42-2 & \\
\hline 310 & & SEEP & & RAW GRAB & UNFILTERED & 488 & 3300 AREA SPRING 42-2 & \\
\hline 311 & & SEEP & WATER & & & 5241 & 1 300-FF-5 RI|B01311 & \\
\hline 312 & & SEEP & WATER & & & 5241 & 1 300-FF-5 RI|B01311 & \\
\hline 313 & & SEEP & WATER & & & 5241 & 1 300-FF-5 RI|B01311 & \\
\hline 315 & & SEEP & WATER & & & 5241 & 1 300-FF-5 RI|B01311 & \\
\hline 316 & & SEEP & WATER & & & 5241 & 1 300-FF-5 RI|B01311 & \\
\hline 317 & & SEEP & WATER & & & 5241 & 1 300-FF-5 RI|B01311 & \\
\hline 318 & & SEEP & WATER & & & 5241 & 1 300-FF-5 RI|B01311 & \\
\hline 319 & & SEEP & WATER & & & 5241 & 1 300-FF-5 RI|B01311 & \\
\hline 320 & & SEEP & WATER & & & 5241 & 1 300-FF-5 RI|B01311 & \\
\hline 321 & & SEEP & WATER & & & 5241 & 1 300-FF-5 RI|B01311 & \\
\hline 322 & & SEEP & WATER & & & 5241 & 1 300-FF-5 RI|B01311 & \\
\hline 323 & & SEEP & WATER & & & 5241 & 1 300-FF-5 RI|B01311 & \\
\hline 325 & & SEEP & WATER & & & 5241 & 1 300-FF-5 RI|B01311 & \\
\hline 326 & & SEEP & WATER & & & 5241 & 1 300-FF-5 RI|B01311 & \\
\hline 327 & & SEEP & WATER & & & 5241 & 1 300-FF-5 RI|B01311 & \\
\hline 328 & & SEEP & WATER & & & 5241 & 1 300-FF-5 RI|B01311 & \\
\hline 329 & & SEEP & WATER & & & 5241 & 1 300-FF-5 RI|B01311 & \\
\hline 330 & & SEEP & WATER & & & 5241 & 1 300-FF-5 RI|B01311 & \\
\hline 331 & & SEEP & WATER & & & 5241 & 1 300-FF-5 RI|B01311 & \\
\hline 332 & & SEEP & WATER & & & 5241 & 1 300-FF-5 RI|B01311 & \\
\hline 333 & & SEEP & WATER & & & 5241 & 1 300-FF-5 RI|B01311 & \\
\hline 334 & & SEEP & WATER & & & 5241 & 1 300-FF-5 RI|B01311 & \\
\hline 335 & & SEEP & WATER & & & 5241 & 1 300-FF-5 RI|B01311 & \\
\hline 336 & & SEEP & WATER & & & 5241 & 1 300-FF-5 RI|B01311 & \\
\hline 337 & & SEEP & WATER & & & 5241 & 1 300-FF-5 RI|B01311 & \\
\hline 338 & & SEEP & WATER & & & 5241 & 1 300-FF-5 RI|B01311 & \\
\hline 339 & & SEEP & WATER & & & 5241 & 1 300-FF-5 RI|B01311 & \\
\hline 340 & & SEEP & WATER & & & 5241 & 1 300-FF-5 RI|B01311 & \\
\hline 341 & & SEEP & WATER & & & 5241 & $1300-F F-5$ RI|B01311 & \\
\hline 342 & & SEEP & WATER & & & 5241 & 1 300-FF-5 RI|B01311 & \\
\hline
\end{tabular}




\begin{tabular}{|c|c|c|c|c|c|c|c|c|}
\hline & $\mathrm{T}$ & $U$ & $\mathrm{~V}$ & $\mathrm{~W}$ & $\mathrm{X}$ & $Y$ & $Z$ & $\mathrm{AA}$ \\
\hline 1 & REVIEW_QUALIFIER & SAMP_FROM & SAMP_ITEM & SAMP_MTHD & COLL_MTHD & SAMP_SITE_ID & SAMP_SITE_NAME & WELL_NAME \\
\hline 344 & & SEEP & WATER & & & 5241 & 300-FF-5 RI|B01311 & \\
\hline 345 & & SEEP & WATER & & & 5241 & 300-FF-5 RI|B01311 & \\
\hline 346 & & SEEP & WATER & & & 5241 & 300-FF-5 RI|B01311 & \\
\hline 347 & & SEEP & WATER & & & 5241 & 300-FF-5 RI|B01311 & \\
\hline 348 & & SEEP & WATER & & & 5226 & 300-FF-5 RI|B01318 & \\
\hline 349 & & SEEP & WATER & & & 5226 & 300-FF-5 RI|B01318 & \\
\hline 350 & & SEEP & WATER & & & 5226 & 300-FF-5 RI|B01318 & \\
\hline 351 & & SEEP & WATER & & & 5226 & 300-FF-5 RI|B01318 & \\
\hline 353 & & SEEP & WATER & & & 5226 & 300-FF-5 RI|B01318 & \\
\hline 354 & & SEEP & WATER & & & 5226 & 300-FF-5 RI|B01318 & \\
\hline 355 & & SEEP & WATER & & & 5226 & 300-FF-5 RI|B01318 & \\
\hline 356 & & SEEP & WATER & & & 5226 & 300-FF-5 RI|B01318 & \\
\hline 357 & & SEEP & WATER & & & 5226 & 300-FF-5 RI|B01318 & \\
\hline 358 & & SEEP & WATER & & & 5226 & 300-FF-5 RI|B01318 & \\
\hline 359 & & SEEP & WATER & & & 5226 & 300-FF-5 RI|B01318 & \\
\hline 360 & & SEEP & WATER & & & 5226 & 300-FF-5 RI|B01318 & \\
\hline 361 & & SEEP & WATER & & & 5226 & 300-FF-5 RI|B01318 & \\
\hline 363 & & SEEP & WATER & & & 5226 & 300-FF-5 RI|B01318 & \\
\hline 364 & & SEEP & WATER & & & 5226 & 300-FF-5 RI|B01318 & \\
\hline 365 & & SEEP & WATER & & & 5226 & 300-FF-5 RI|B01318 & \\
\hline 366 & & SEEP & WATER & & & 5226 & 300-FF-5 RI|B01318 & \\
\hline 367 & & SEEP & WATER & & & 5226 & 300-FF-5 RI|B01318 & \\
\hline 368 & & SEEP & WATER & & & 5226 & 300-FF-5 RI|B01318 & \\
\hline 369 & & SEEP & WATER & & & 5226 & 300-FF-5 RI|B01318 & \\
\hline 370 & & SEEP & WATER & & & 5226 & 300-FF-5 RI|B01318 & \\
\hline 371 & & SEEP & WATER & & & 5226 & 300-FF-5 RI|B01318 & \\
\hline 372 & & SEEP & WATER & & & 5226 & 300-FF-5 RI|B01318 & \\
\hline 373 & & SEEP & WATER & & & 5226 & 300-FF-5 RI|B01318 & \\
\hline 374 & & SEEP & WATER & & & 5226 & 300-FF-5 RI|B01318 & \\
\hline 375 & & SEEP & WATER & & & 5226 & 300-FF-5 RI|B01318 & \\
\hline 376 & & SEEP & WATER & & & 5226 & 300-FF-5 RI|B01318 & \\
\hline 377 & & SEEP & WATER & & & 5226 & 300-FF-5 RI|B01318 & \\
\hline 378 & & SEEP & WATER & & & 5226 & 300-FF-5 RI|B01318 & \\
\hline 379 & & SEEP & WATER & & & 5226 & 300-FF-5 RI|B01318 & \\
\hline 380 & & SEEP & WATER & & & 5226 & 300-FF-5 RI|B01318 & \\
\hline
\end{tabular}




\begin{tabular}{|c|c|c|c|c|c|c|c|c|}
\hline & $\mathrm{T}$ & $U$ & $\mathrm{~V}$ & $\mathrm{~W}$ & $\mathrm{X}$ & $Y$ & $Z$ & $\mathrm{AA}$ \\
\hline 1 & REVIEW_QUALIFIER & SAMP_FROM & SAMP_ITEM & SAMP_MTHD & COLL_MTHD & SAMP_SITE_ID & SAMP_SITE_NAME & WELL_NAME \\
\hline 382 & & SEEP & WATER & & & 5226 & 300-FF-5 RI|B01318 & \\
\hline 383 & & SEEP & WATER & & & 5226 & 300-FF-5 RI|B01318 & \\
\hline 384 & & SEEP & WATER & & & 5228 & 300-FF-5 RI|B01322 & \\
\hline 385 & & SEEP & WATER & & & 5228 & 300-FF-5 RI|B01322 & \\
\hline 386 & & SEEP & WATER & & & 5228 & 300-FF-5 RI|B01322 & \\
\hline 387 & & SEEP & WATER & & & 5228 & 300-FF-5 RI|B01322 & \\
\hline 388 & & SEEP & WATER & & & 5228 & 300-FF-5 RI|B01322 & \\
\hline 389 & & SEEP & WATER & & & 5228 & 300-FF-5 RI|B01322 & \\
\hline 391 & & SEEP & WATER & & & 5228 & 300-FF-5 RI|B01322 & \\
\hline 392 & & SEEP & WATER & & & 5228 & 300-FF-5 RI|B01322 & \\
\hline 393 & & SEEP & WATER & & & 5228 & 300-FF-5 RI|B01322 & \\
\hline 394 & & SEEP & WATER & & & 5228 & 300-FF-5 RI|B01322 & \\
\hline 395 & & SEEP & WATER & & & 5228 & 300-FF-5 RI|B01322 & \\
\hline 396 & & SEEP & WATER & & & 5228 & 300-FF-5 RI|B01322 & \\
\hline 397 & & SEEP & WATER & & & 5228 & 300-FF-5 RI|B01322 & \\
\hline 398 & & SEEP & WATER & & & 5228 & 300-FF-5 RI|B01322 & \\
\hline 399 & & SEEP & WATER & & & 5228 & 300-FF-5 RI|B01322 & \\
\hline 401 & & SEEP & WATER & & & 5228 & 300-FF-5 RI|B01322 & \\
\hline 402 & & SEEP & WATER & & & 5228 & 300-FF-5 RI|B01322 & \\
\hline 403 & & SEEP & WATER & & & 5228 & 300-FF-5 RI|B01322 & \\
\hline 404 & & SEEP & WATER & & & 5228 & 300-FF-5 RI|B01322 & \\
\hline 405 & & SEEP & WATER & & & 5228 & 300-FF-5 RI|B01322 & \\
\hline 406 & & SEEP & WATER & & & 5228 & 300-FF-5 RI|B01322 & \\
\hline 407 & & SEEP & WATER & & & 5228 & 300-FF-5 RI|B01322 & \\
\hline 408 & & SEEP & WATER & & & 5228 & 300-FF-5 RI|B01322 & \\
\hline 409 & & SEEP & WATER & & & 5228 & 300-FF-5 RI|B01322 & \\
\hline 410 & & SEEP & WATER & & & 5228 & 300-FF-5 RI|B01322 & \\
\hline 411 & & SEEP & & RAW GRAB & UNFILTERED & 151 & HANFORD SPRING 28-2 & \\
\hline 412 & & SEEP & & RAW GRAB & UNFILTERED & 151 & HANFORD SPRING 28-2 & \\
\hline 413 & & SEEP & & RAW GRAB & UNFILTERED & 151 & HANFORD SPRING 28-2 & \\
\hline 414 & & SEEP & & RAW GRAB & UNFILTERED & 151 & HANFORD SPRING 28-2 & \\
\hline 415 & & SEEP & & RAW GRAB & UNFILTERED & 202 & 100-N SPRING 8-13 & \\
\hline 416 & & SEEP & & RAW GRAB & UNFILTERED & 202 & $100-N$ SPRING 8-13 & \\
\hline 417 & & SEEP & & RAW GRAB & UNFILTERED & 202 & $100-N$ SPRING 8-13 & \\
\hline 418 & & SEEP & & RAW GRAB & UNFILTERED & 202 & $100-N$ SPRING 8-13 & \\
\hline
\end{tabular}

52 100-B SPRING 


\begin{tabular}{|c|c|c|c|c|c|c|c|c|}
\hline & $T$ & $U$ & $\mathrm{~V}$ & $\mathrm{~W}$ & $x$ & $Y$ & $Z$ & $\mathrm{AA}$ \\
\hline 1 & REVIEW_QUALIFIER & SAMP_FROM & SAMP_ITEM & SAMP_MTHD & COLL_MTHD & SAMP_SITE_ID & SAMP_SITE_NAME & WELL_NAME \\
\hline 420 & & SEEP & & RAW GRAB & UNFILTERED & & 100-B SPRING & \\
\hline 421 & & SEEP & & RAW GRAB & UNFILTERED & 52 & 100-B SPRING & \\
\hline 422 & & SEEP & & RAW GRAB & UNFILTERED & 52 & 100-B SPRING & \\
\hline 423 & & SEEP & & RAW GRAB & UNFILTERED & 52 & 100-B SPRING & \\
\hline 424 & & SEEP & & RAW GRAB & UNFILTERED & 52 & 100-B SPRING & \\
\hline 425 & & SEEP & & RAW GRAB & UNFILTERED & 52 & 100-B SPRING & \\
\hline 426 & & SEEP & & RAW GRAB & UNFILTERED & 193 & 100-D SPRING & \\
\hline 427 & & SEEP & & RAW GRAB & UNFILTERED & 193 & 100-D SPRING & \\
\hline 429 & & SEEP & & RAW GRAB & UNFILTERED & 193 & 100-D SPRING & \\
\hline 430 & & SEEP & & RAW GRAB & UNFILTERED & 193 & 100-D SPRING & \\
\hline 431 & & SEEP & & RAW GRAB & UNFILTERED & 193 & 100-D SPRING & \\
\hline 432 & & SEEP & & RAW GRAB & UNFILTERED & 193 & 100-D SPRING & \\
\hline 433 & & SEEP & WATER & & & 2235 & SEEP 154-1 & \\
\hline 434 & & SEEP & WATER & & & 2235 & SEEP 154-1 & \\
\hline 435 & & SEEP & WATER & & & 2235 & SEEP 154-1 & \\
\hline 436 & & SEEP & WATER & & & 2235 & SEEP 154-1 & \\
\hline 437 & & SEEP & WATER & & & 2228 & SEEP 110-1 & \\
\hline 439 & & SEEP & WATER & & & 2228 & SEEP 110-1 & \\
\hline 440 & & SEEP & WATER & & & 2228 & SEEP 110-1 & \\
\hline 441 & & SEEP & & RAW GRAB & UNFILTERED & 199 & 100-H SPRING & \\
\hline 442 & & SEEP & & RAW GRAB & UNFILTERED & 199 & 100-H SPRING & \\
\hline 443 & & SEEP & & RAW GRAB & UNFILTERED & 199 & 100-H SPRING & \\
\hline 444 & & SEEP & & RAW GRAB & UNFILTERED & 199 & 100-H SPRING & \\
\hline 445 & & SEEP & & RAW GRAB & UNFILTERED & 199 & 100-H SPRING & \\
\hline 446 & & SEEP & & RAW GRAB & UNFILTERED & 199 & 100-H SPRING & \\
\hline 447 & & SEEP & & RAW GRAB & UNFILTERED & 199 & 100-H SPRING & \\
\hline 448 & & SEEP & & RAW GRAB & UNFILTERED & 488 & 300 AREA SPRING 42-2 & \\
\hline 449 & & SEEP & & RAW GRAB & UNFILTERED & 488 & 300 AREA SPRING 42-2 & \\
\hline 450 & & SEEP & & RAW GRAB & UNFILTERED & 488 & 300 AREA SPRING 42-2 & \\
\hline 451 & & SEEP & & RAW GRAB & UNFILTERED & 488 & 300 AREA SPRING 42-2 & \\
\hline 452 & & SEEP & & RAW GRAB & UNFILTERED & 488 & 300 AREA SPRING 42-2 & \\
\hline 453 & & SEEP & & RAW GRAB & UNFILTERED & 488 & 300 AREA SPRING 42-2 & \\
\hline 454 & & SEEP & & RAW GRAB & UNFILTERED & 488 & 300 AREA SPRING 42-2 & \\
\hline 455 & & SEEP & & RAW GRAB & UNFILTERED & 488 & 300 AREA SPRING 42-2 & \\
\hline 456 & & SEEP & & RAW GRAB & UNFILTERED & 151 & HANFORD SPRING 28-2 & \\
\hline
\end{tabular}




\begin{tabular}{|c|c|c|c|c|c|c|c|c|}
\hline & $\mathrm{T}$ & $\mathrm{U}$ & $\mathrm{V}$ & $\mathrm{W}$ & $\mathrm{X}$ & $\mathrm{Y}$ & $Z$ & $\mathrm{AA}$ \\
\hline 1 & REVIEW_QUALIFIER & SAMP_FROM & SAMP_ITEM & SAMP_MTHD & COLL_MTHD & SAMP_SITE_ID & SAMP_SITE_NAME & WELL_NAME \\
\hline 458 & & SEEP & & RAW GRAB & UNFILTERED & 151 & 1 HANFORD SPRING 28-2 & \\
\hline 459 & & SEEP & & RAW GRAB & UNFILTERED & 151 & 1 HANFORD SPRING 28-2 & \\
\hline 460 & & SEEP & & RAW GRAB & UNFILTERED & 151 & 1 HANFORD SPRING 28-2 & \\
\hline 461 & & SEEP & & RAW GRAB & UNFILTERED & 151 & 1 HANFORD SPRING 28-2 & \\
\hline 462 & & SEEP & & RAW GRAB & UNFILTERED & 151 & 1 HANFORD SPRING 28-2 & \\
\hline 463 & & SEEP & & RAW GRAB & UNFILTERED & 151 & 1 HANFORD SPRING 28-2 & \\
\hline 464 & & SEEP & WATER & & & 2233 & 3 SEEP 146-1 & \\
\hline 465 & & SEEP & WATER & & & 2233 & 3 SEEP 146-1 & \\
\hline 467 & & SEEP & WATER & & & 2233 & 3 SEEP 146-1 & \\
\hline 468 & & SEEP & WATER & & & 2216 & 6 SEEP 037-1 & \\
\hline 469 & & SEEP & WATER & & & 2216 & 6 SEEP 037-1 & \\
\hline 470 & & SEEP & WATER & & & 2216 & 6 SEEP 037-1 & \\
\hline 471 & & SEEP & WATER & & & 2216 & o SEEP 037-1 & \\
\hline 472 & & SEEP & WATER & & & 2247 & 7 SEEP 205-1 & \\
\hline 473 & & SEEP & WATER & & & 2247 & 7 SEEP 205-1 & \\
\hline 474 & & SEEP & WATER & & & 2247 & 7 SEEP 205-1 & \\
\hline 475 & & SEEP & WATER & & & 2247 & 7 SEEP 205-1 & \\
\hline 477 & & SEEP & & RAW GRAB & UNFILTERED & 52 & 2 100-B SPRING & \\
\hline 478 & & SEEP & & RAW GRAB & UNFILTERED & 52 & 2 100-B SPRING & \\
\hline 479 & & SEEP & & RAW GRAB & UNFILTERED & 52 & 2 100-B SPRING & \\
\hline 480 & & SEEP & & RAW GRAB & UNFILTERED & 52 & 2 100-B SPRING & \\
\hline 481 & & SEEP & & RAW GRAB & UNFILTERED & 52 & 2 100-B SPRING & \\
\hline 482 & & SEEP & & RAW GRAB & UNFILTERED & 52 & 2 100-B SPRING & \\
\hline 483 & & SEEP & & RAW GRAB & UNFILTERED & 193 & 3 100-D SPRING & \\
\hline 484 & & SEEP & & RAW GRAB & UNFILTERED & 193 & 3 100-D SPRING & \\
\hline 485 & & SEEP & & RAW GRAB & UNFILTERED & 193 & 3 100-D SPRING & \\
\hline 486 & & SEEP & & RAW GRAB & UNFILTERED & 193 & 3 100-D SPRING & \\
\hline 487 & & SEEP & & RAW GRAB & UNFILTERED & 193 & 3 100-D SPRING & \\
\hline 488 & & SEEP & & RAW GRAB & UNFILTERED & 193 & 3 100-D SPRING & \\
\hline 489 & & SEEP & & RAW GRAB & UNFILTERED & 193 & 3 100-D SPRING & \\
\hline 490 & & SEEP & & RAW GRAB & UNFILTERED & 199 & 9 100-H SPRING & \\
\hline 491 & & SEEP & & RAW GRAB & UNFILTERED & 199 & 9 100-H SPRING & \\
\hline 492 & & SEEP & & RAW GRAB & UNFILTERED & 199 & 9 100-H SPRING & \\
\hline 493 & & SEEP & & RAW GRAB & UNFILTERED & 199 & $9100-H$ SPRING & \\
\hline 494 & & SEEP & & RAW GRAB & UNFILTERED & 199 & 9 100-H SPRING & \\
\hline
\end{tabular}




\begin{tabular}{|c|c|c|c|c|c|c|c|c|}
\hline & $\mathrm{T}$ & $\mathrm{U}$ & $\mathrm{V}$ & $\mathrm{W}$ & $x$ & $\mathrm{Y}$ & $Z$ & AA \\
\hline 1 & REVIEW_QUALIFIER & SAMP_FROM & SAMP_ITEM & SAMP_MTHD & COLL_MTHD & SAMP_SITE_ID & SAMP_SITE_NAME & WELL_NAME \\
\hline 496 & & SEEP & & RAW GRAB & UNFILTERED & 1991 & 100-H SPRING & \\
\hline 497 & & SEEP & & RAW GRAB & UNFILTERED & 5331 & 100-K SPRING & \\
\hline 498 & & SEEP & & RAW GRAB & UNFILTERED & 5331 & 100-K SPRING & \\
\hline 499 & & SEEP & & RAW GRAB & UNFILTERED & 5331 & 100-K SPRING & \\
\hline 500 & & SEEP & & RAW GRAB & UNFILTERED & 5331 & 100-K SPRING & \\
\hline 501 & & SEEP & & RAW GRAB & UNFILTERED & 5331 & 100-K SPRING & \\
\hline 502 & & SEEP & & RAW GRAB & UNFILTERED & 5331 & 100-K SPRING & \\
\hline 503 & & SEEP & & RAW GRAB & UNFILTERED & 5331 & 100-K SPRING & \\
\hline 505 & & SEEP & & RAW GRAB & UNFILTERED & 5331 & 100-K SPRING & \\
\hline 506 & & SEEP & & RAW GRAB & UNFILTERED & 5331 & 100-K SPRING & \\
\hline 507 & & SEEP & & RAW GRAB & UNFILTERED & 5331 & 100-K SPRING & \\
\hline 508 & & SEEP & & RAW GRAB & UNFILTERED & 533 & 100-K SPRING & \\
\hline 509 & & SEEP & & RAW GRAB & UNFILTERED & 5331 & 100-K SPRING & \\
\hline 510 & & SEEP & & RAW GRAB & UNFILTERED & 5331 & 100-K SPRING & \\
\hline 511 & & SEEP & & RAW GRAB & UNFILTERED & 2021 & 100-N SPRING 8-13 & \\
\hline 512 & & SEEP & & RAW GRAB & UNFILTERED & 2021 & 100-N SPRING 8-13 & \\
\hline 513 & & SEEP & & RAW GRAB & UNFILTERED & 2021 & 100-N SPRING 8-13 & \\
\hline 515 & & SEEP & & RAW GRAB & UNFILTERED & 2021 & 100-N SPRING 8-13 & \\
\hline 516 & & SEEP & & RAW GRAB & UNFILTERED & 2021 & 100-N SPRING 8-13 & \\
\hline 517 & & SEEP & & RAW GRAB & UNFILTERED & 2021 & 100-N SPRING 8-13 & \\
\hline 518 & & SEEP & & RAW GRAB & UNFILTERED & 2021 & 100-N SPRING 8-13 & \\
\hline 519 & & SEEP & & RAW GRAB & UNFILTERED & 2021 & 100-N SPRING 8-13 & \\
\hline 520 & & SEEP & & RAW GRAB & UNFILTERED & 2021 & 100-N SPRING 8-13 & \\
\hline 521 & & SEEP & & RAW GRAB & UNFILTERED & 2021 & 100-N SPRING 8-13 & \\
\hline 522 & & SEEP & & RAW GRAB & UNFILTERED & 2021 & 100-N SPRING 8-13 & \\
\hline 523 & & SEEP & & RAW GRAB & UNFILTERED & 2021 & 100-N SPRING 8-13 & \\
\hline 524 & & SEEP & & RAW GRAB & UNFILTERED & 2021 & 100-N SPRING 8-13 & \\
\hline 525 & & SEEP & & RAW GRAB & UNFILTERED & 151 & HANFORD SPRING 28-2 & \\
\hline 526 & & SEEP & & RAW GRAB & UNFILTERED & $151 \vdash$ & HANFORD SPRING 28-2 & \\
\hline 527 & & SEEP & & RAW GRAB & UNFILTERED & 151 & HANFORD SPRING 28-2 & \\
\hline 528 & & SEEP & & RAW GRAB & UNFILTERED & 151 & HANFORD SPRING 28-2 & \\
\hline 529 & & SEEP & & RAW GRAB & UNFILTERED & $151 \vdash$ & HANFORD SPRING 28-2 & \\
\hline 530 & & SEEP & & RAW GRAB & UNFILTERED & 151 & HANFORD SPRING 28-2 & \\
\hline 531 & & SEEP & & RAW GRAB & UNFILTERED & $151 \vdash$ & HANFORD SPRING 28-2 & \\
\hline 532 & & SEEP & WATER & & & 2221 & SEEP 074-1 & \\
\hline
\end{tabular}




\begin{tabular}{|c|c|c|c|c|c|c|c|c|}
\hline & $\mathrm{T}$ & $\mathrm{U}$ & $\mathrm{V}$ & $\mathrm{W}$ & $\mathrm{X}$ & $\mathrm{Y}$ & $Z$ & $\mathrm{AA}$ \\
\hline 1 & REVIEW_QUALIFIER & SAMP_FROM & SAMP_ITEM & SAMP_MTHD & COLL_MTHD & SAMP_SITE_ID & SAMP_SITE_NAME & WELL_NAME \\
\hline 534 & & SEEP & WATER & & & 2221 & SEEP 074-1 & \\
\hline 535 & & SEEP & WATER & & & 2221 & SEEP 074-1 & \\
\hline 536 & & SEEP & WATER & & & 2248 & SEEP 189-1 & \\
\hline 537 & & SEEP & WATER & & & 2248 & SEEP 189-1 & \\
\hline 538 & & SEEP & WATER & & & 2248 & SEEP 189-1 & \\
\hline 539 & & SEEP & WATER & & & 2248 & SEEP 189-1 & \\
\hline 540 & & SEEP & & RAW GRAB & UNFILTERED & 488 & 300 AREA SPRING 42-2 & \\
\hline 541 & & SEEP & & RAW GRAB & UNFILTERED & 488 & 300 AREA SPRING 42-2 & \\
\hline 543 & & SEEP & & RAW GRAB & UNFILTERED & 488 & 300 AREA SPRING 42-2 & \\
\hline 544 & & SEEP & & RAW GRAB & UNFILTERED & 488 & 300 AREA SPRING 42-2 & \\
\hline 545 & & SEEP & & RAW GRAB & UNFILTERED & 488 & 300 AREA SPRING 42-2 & \\
\hline 546 & & SEEP & & RAW GRAB & UNFILTERED & 488 & 300 AREA SPRING 42-2 & \\
\hline 547 & & SEEP & WATER & & & 2245 & SEEP 152-2 & \\
\hline 548 & & SEEP & WATER & & & 2246 & SEEP 152-3 & \\
\hline 549 & & SEEP & WATER & & & 2228 & SEEP 110-1 & \\
\hline 550 & & SEEP & WATER & & & 2228 & SEEP 110-1 & \\
\hline 551 & & SEEP & WATER & & & 2228 & SEEP 110-1 & \\
\hline 553 & & SEEP & WATER & & & 2228 & SEEP 110-1 & \\
\hline 554 & & SEEP & WATER & & & 2228 & SEEP 110-1 & \\
\hline 555 & & SEEP & WATER & & & 2228 & SEEP 110-1 & \\
\hline 556 & & SEEP & WATER & & & 2228 & SEEP 110-1 & \\
\hline 557 & & SEEP & WATER & & & 2228 & SEEP $110-1$ & \\
\hline 558 & & SEEP & WATER & & & 2228 & SEEP 110-1 & \\
\hline 559 & & SEEP & WATER & & & 2228 & SEEP $110-1$ & \\
\hline 560 & & SEEP & WATER & & & 2228 & SEEP $110-1$ & \\
\hline 561 & & SEEP & WATER & & & 2228 & SEEP 110-1 & \\
\hline 562 & & SEEP & WATER & & & 2228 & SEEP 110-1 & \\
\hline 563 & & SEEP & WATER & & & 2228 & SEEP 110-1 & \\
\hline 564 & & SEEP & WATER & & & 2228 & SEEP 110-1 & \\
\hline 565 & & SEEP & & RAW GRAB & UNFILTERED & 52 & 100-B SPRING & \\
\hline 566 & & SEEP & & RAW GRAB & UNFILTERED & 52 & 100-B SPRING & \\
\hline 567 & & SEEP & & RAW GRAB & UNFILTERED & 52 & 100-B SPRING & \\
\hline 568 & $F$ & SEEP & & RAW GRAB & UNFILTERED & 52 & 100-B SPRING & \\
\hline 569 & & SEEP & & RAW GRAB & UNFILTERED & 52 & 100-B SPRING & \\
\hline 570 & $F$ & SEEP & & RAW GRAB & UNFILTERED & 52 & 100-B SPRING & \\
\hline
\end{tabular}




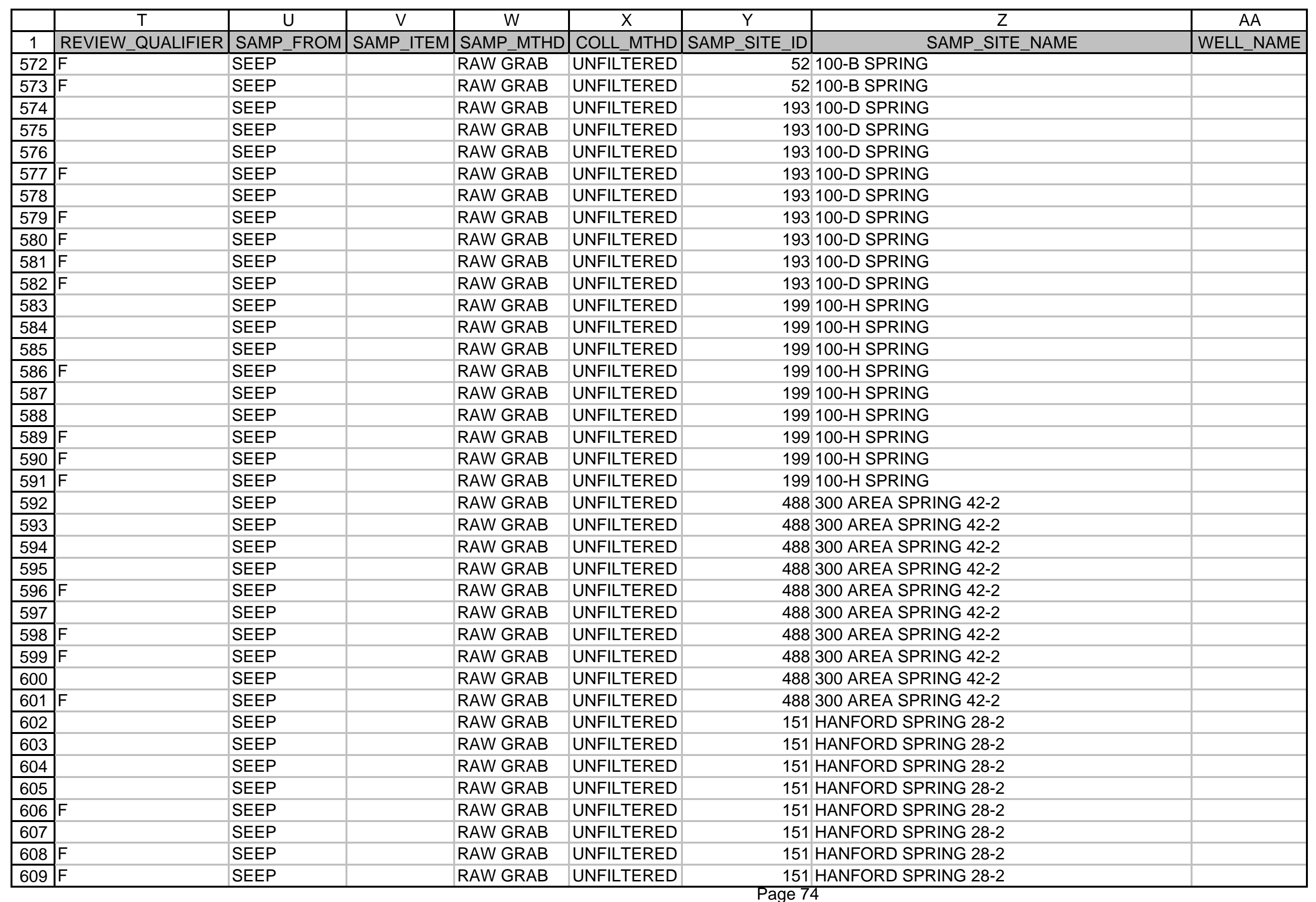




\begin{tabular}{|c|c|c|c|c|c|c|c|c|}
\hline & $\mathrm{T}$ & $\mathrm{U}$ & $\mathrm{V}$ & $\mathrm{W}$ & $\mathrm{X}$ & $\mathrm{Y}$ & $Z$ & $\mathrm{AA}$ \\
\hline 1 & REVIEW_QUALIFIER & SAMP_FROM & SAMP_ITEM & SAMP_MTHD & COLL_MTHD & SAMP_SITE_ID & SAMP_SITE_NAME & WELL_NAME \\
\hline 610 & $\mathrm{~F}$ & SEEP & & RAW GRAB & UNFILTERED & 151 & 1 HANFORD SPRING 28-2 & \\
\hline 611 & $\mathrm{~F}$ & SEEP & & RAW GRAB & UNFILTERED & 151 & 1 HANFORD SPRING 28-2 & \\
\hline 612 & & SEEP & & RAW GRAB & UNFILTERED & 52 & 2 100-B SPRING & \\
\hline 613 & & SEEP & & RAW GRAB & UNFILTERED & 52 & 2 100-B SPRING & \\
\hline 614 & & SEEP & & RAW GRAB & UNFILTERED & 52 & 2 100-B SPRING & \\
\hline 615 & $F$ & SEEP & & RAW GRAB & UNFILTERED & 52 & 2 100-B SPRING & \\
\hline 616 & & SEEP & & RAW GRAB & UNFILTERED & 52 & 2 100-B SPRING & \\
\hline 617 & $F$ & SEEP & & RAW GRAB & UNFILTERED & 52 & 2 100-B SPRING & \\
\hline 619 & $F$ & SEEP & & RAW GRAB & UNFILTERED & 52 & 2 100-B SPRING & \\
\hline 620 & $F$ & SEEP & & RAW GRAB & UNFILTERED & 52 & 2 100-B SPRING & \\
\hline 621 & & SEEP & & RAW GRAB & UNFILTERED & 151 & 1 HANFORD SPRING 28-2 & \\
\hline 622 & & SEEP & & RAW GRAB & UNFILTERED & 151 & 1 HANFORD SPRING 28-2 & \\
\hline 623 & & SEEP & & RAW GRAB & UNFILTERED & 151 & 1 HANFORD SPRING 28-2 & \\
\hline 624 & $\mathrm{~F}$ & SEEP & & RAW GRAB & UNFILTERED & 151 & 1 HANFORD SPRING 28-2 & \\
\hline 625 & & SEEP & & RAW GRAB & UNFILTERED & 151 & 1 HANFORD SPRING 28-2 & \\
\hline 626 & $F$ & SEEP & & RAW GRAB & UNFILTERED & 151 & 1 HANFORD SPRING 28-2 & \\
\hline 627 & $F$ & SEEP & & RAW GRAB & UNFILTERED & 151 & 1 HANFORD SPRING 28-2 & \\
\hline 629 & $F$ & SEEP & & RAW GRAB & UNFILTERED & 151 & 1 HANFORD SPRING 28-2 & \\
\hline 630 & & SEEP & & RAW GRAB & UNFILTERED & 488 & 3300 AREA SPRING 42-2 & \\
\hline 631 & $F$ & SEEP & & RAW GRAB & UNFILTERED & 488 & 300 AREA SPRING 42-2 & \\
\hline 632 & & SEEP & & RAW GRAB & UNFILTERED & 488 & 3300 AREA SPRING 42-2 & \\
\hline 633 & $F$ & SEEP & & RAW GRAB & UNFILTERED & 488 & 3300 AREA SPRING 42-2 & \\
\hline 634 & $\mathrm{~F}$ & SEEP & & RAW GRAB & UNFILTERED & 488 & 3300 AREA SPRING 42-2 & \\
\hline 635 & & SEEP & & RAW GRAB & UNFILTERED & 488 & 3300 AREA SPRING 42-2 & \\
\hline 636 & $F$ & SEEP & & RAW GRAB & UNFILTERED & 488 & 300 AREA SPRING 42-2 & \\
\hline 637 & & SEEP & & RAW GRAB & UNFILTERED & 193 & 3 100-D SPRING & \\
\hline 638 & & SEEP & & RAW GRAB & UNFILTERED & 193 & 3 100-D SPRING & \\
\hline 639 & & SEEP & & RAW GRAB & UNFILTERED & 193 & 3 100-D SPRING & \\
\hline 640 & $F$ & SEEP & & RAW GRAB & UNFILTERED & 193 & 3 100-D SPRING & \\
\hline 641 & & SEEP & & RAW GRAB & UNFILTERED & 193 & 3 100-D SPRING & \\
\hline 642 & $F$ & SEEP & & RAW GRAB & UNFILTERED & 193 & 3 100-D SPRING & \\
\hline 643 & $F$ & SEEP & & RAW GRAB & UNFILTERED & 193 & 3 100-D SPRING & \\
\hline 644 & $F$ & SEEP & & RAW GRAB & UNFILTERED & 193 & 3 100-D SPRING & \\
\hline 645 & $F$ & SEEP & & RAW GRAB & UNFILTERED & 193 & 3 100-D SPRING & \\
\hline 646 & & SEEP & & RAW GRAB & UNFILTERED & 196 & 6 100-F SPRING & \\
\hline
\end{tabular}




\begin{tabular}{|c|c|c|c|c|c|c|c|c|}
\hline & $\mathrm{T}$ & $U$ & $\mathrm{~V}$ & $\mathrm{~W}$ & $\mathrm{X}$ & $Y$ & $Z$ & $\mathrm{AA}$ \\
\hline 1 & REVIEW_QUALIFIER & SAMP_FROM & SAMP_ITEM & SAMP_MTHD & COLL_MTHD & SAMP_SITE_ID & SAMP_SITE_NAME & WELL_NAME \\
\hline 648 & & SEEP & & RAW GRAB & UNFILTERED & 196 & 6 100-F SPRING & \\
\hline 649 & $\mathrm{~F}$ & SEEP & & RAW GRAB & UNFILTERED & 196 & 5 100-F SPRING & \\
\hline 650 & & SEEP & & RAW GRAB & UNFILTERED & 196 & 6 100-F SPRING & \\
\hline 651 & & SEEP & & RAW GRAB & UNFILTERED & 196 & $5100-F$ SPRING & \\
\hline 652 & $\mathrm{~F}$ & SEEP & & RAW GRAB & UNFILTERED & 196 & $6100-F$ SPRING & \\
\hline 653 & $F$ & SEEP & & RAW GRAB & UNFILTERED & 196 & 100-F SPRING & \\
\hline 654 & $F$ & SEEP & & RAW GRAB & UNFILTERED & 196 & $100-F$ SPRING & \\
\hline 655 & & SEEP & & RAW GRAB & UNFILTERED & 199 & 9 100-H SPRING & \\
\hline 657 & & SEEP & & RAW GRAB & UNFILTERED & 199 & 9 100-H SPRING & \\
\hline 658 & $F$ & SEEP & & RAW GRAB & UNFILTERED & 199 & 9 100-H SPRING & \\
\hline 659 & & SEEP & & RAW GRAB & UNFILTERED & 199 & 9 100-H SPRING & \\
\hline 660 & $\mathrm{~F}$ & SEEP & & RAW GRAB & UNFILTERED & 199 & 9 100-H SPRING & \\
\hline 661 & $\mathrm{~F}$ & SEEP & & RAW GRAB & UNFILTERED & 199 & 9 100-H SPRING & \\
\hline 662 & $F$ & SEEP & & RAW GRAB & UNFILTERED & 199 & 9 100-H SPRING & \\
\hline 663 & $F$ & SEEP & & RAW GRAB & UNFILTERED & 199 & 9 100-H SPRING & \\
\hline 664 & & SEEP & & RAW GRAB & UNFILTERED & 202 & 2 100-N SPRING 8-13 & \\
\hline 665 & & SEEP & & RAW GRAB & UNFILTERED & 202 & 2 100-N SPRING 8-13 & \\
\hline 667 & $F$ & SEEP & & RAW GRAB & UNFILTERED & 202 & 2 100-N SPRING 8-13 & \\
\hline 668 & & SEEP & & RAW GRAB & UNFILTERED & 202 & 2 100-N SPRING 8-13 & \\
\hline 669 & $F$ & SEEP & & RAW GRAB & UNFILTERED & 202 & 2 100-N SPRING 8-13 & \\
\hline 670 & $F$ & SEEP & & RAW GRAB & UNFILTERED & 202 & 2 100-N SPRING 8-13 & \\
\hline 671 & $F$ & SEEP & & RAW GRAB & UNFILTERED & 202 & 2 100-N SPRING 8-13 & \\
\hline 672 & $F$ & SEEP & & RAW GRAB & UNFILTERED & 202 & 2 100-N SPRING 8-13 & \\
\hline 673 & & SEEP & WATER & & & 2343 & 3 NS-8A SEEP & \\
\hline 674 & & SEEP & WATER & & & 2351 & 1 NS-1 SEEP & \\
\hline 675 & & SEEP & WATER & & & 2350 & NS-2 SEEP & \\
\hline 676 & & SEEP & WATER & & & 2349 & 9 NS-3 SEEP & \\
\hline 677 & & SEEP & WATER & & & 2348 & 3 NS-4 SEEP & \\
\hline 678 & & SEEP & WATER & & & 2347 & 7 NS-5 SEEP & \\
\hline 679 & & SEEP & WATER & & & 2346 & 6 NS-6 SEEP & \\
\hline 680 & & SEEP & WATER & & & 2345 & 5 NS-7 SEEP & \\
\hline 681 & & SEEP & WATER & & & 2344 & 4 NS-8B SEEP & \\
\hline 682 & & SEEP & WATER & & & 2342 & 2 NS-9 SEEP & \\
\hline 683 & & SEEP & WATER & & & 2341 & 1 NS-10 SEEP & \\
\hline 684 & & SEEP & WATER & & & 2340 & NS-11 SEEP & \\
\hline
\end{tabular}




\begin{tabular}{|c|c|c|c|c|c|c|c|c|}
\hline & $\mathrm{T}$ & $U$ & $\mathrm{~V}$ & $\mathrm{~W}$ & $\mathrm{X}$ & $Y$ & $Z$ & $\mathrm{AA}$ \\
\hline 1 & REVIEW_QUALIFIER & SAMP_FROM & SAMP_ITEM & SAMP_MTHD & COLL_MTHD & SAMP_SITE_ID & SAMP_SITE_NAME & WELL_NAME \\
\hline 686 & & SEEP & WATER & & & 2337 & 7 NS-13 SEEP & \\
\hline 687 & & SEEP & WATER & & & 2337 & 7 NS-13 SEEP & \\
\hline 688 & & SEEP & & RAW GRAB & UNFILTERED & 52 & 2 100-B SPRING & \\
\hline 689 & & SEEP & & RAW GRAB & UNFILTERED & 52 & 2 100-B SPRING & \\
\hline 690 & & SEEP & & RAW GRAB & UNFILTERED & 52 & 2 100-B SPRING & \\
\hline 691 & $F$ & SEEP & & RAW GRAB & UNFILTERED & 52 & 2 100-B SPRING & \\
\hline 692 & & SEEP & & RAW GRAB & UNFILTERED & 52 & 2 100-B SPRING & \\
\hline 693 & $F$ & SEEP & & RAW GRAB & UNFILTERED & 52 & 2 100-B SPRING & \\
\hline 695 & $F$ & SEEP & & RAW GRAB & UNFILTERED & 52 & 2 100-B SPRING & \\
\hline 696 & $F$ & SEEP & & RAW GRAB & UNFILTERED & 52 & 2 100-B SPRING & \\
\hline 697 & & SEEP & & RAW GRAB & UNFILTERED & 193 & 3 100-D SPRING & \\
\hline 698 & & SEEP & & RAW GRAB & UNFILTERED & 193 & 3 100-D SPRING & \\
\hline 699 & & SEEP & & RAW GRAB & UNFILTERED & 193 & 3 100-D SPRING & \\
\hline 700 & $F$ & SEEP & & RAW GRAB & UNFILTERED & 193 & 3 100-D SPRING & \\
\hline 701 & & SEEP & & RAW GRAB & UNFILTERED & 193 & 3 100-D SPRING & \\
\hline 702 & $F$ & SEEP & & RAW GRAB & UNFILTERED & 193 & 3 100-D SPRING & \\
\hline 703 & $F$ & SEEP & & RAW GRAB & UNFILTERED & 193 & 3 100-D SPRING & \\
\hline 705 & $F$ & SEEP & & RAW GRAB & UNFILTERED & 193 & 3 100-D SPRING & \\
\hline 706 & & SEEP & & RAW GRAB & UNFILTERED & 202 & 2 100-N SPRING 8-13 & \\
\hline 707 & & SEEP & & RAW GRAB & UNFILTERED & 202 & 2 100-N SPRING 8-13 & \\
\hline 708 & & SEEP & & RAW GRAB & UNFILTERED & 202 & 2 100-N SPRING 8-13 & \\
\hline 709 & $F$ & SEEP & & RAW GRAB & UNFILTERED & 202 & 2 100-N SPRING 8-13 & \\
\hline 710 & & SEEP & & RAW GRAB & UNFILTERED & 202 & 2 100-N SPRING 8-13 & \\
\hline 711 & $\mathrm{~F}$ & SEEP & & RAW GRAB & UNFILTERED & 202 & 2 100-N SPRING 8-13 & \\
\hline 712 & $\mathrm{~F}$ & SEEP & & RAW GRAB & UNFILTERED & 202 & $2100-N$ SPRING 8-13 & \\
\hline 713 & & SEEP & & RAW GRAB & UNFILTERED & 202 & 2 100-N SPRING 8-13 & \\
\hline 714 & $F$ & SEEP & & RAW GRAB & UNFILTERED & 202 & 2 100-N SPRING 8-13 & \\
\hline 715 & & SEEP & & RAW GRAB & UNFILTERED & 199 & 9 100-H SPRING & \\
\hline 716 & & SEEP & & RAW GRAB & UNFILTERED & 199 & 9 100-H SPRING & \\
\hline 717 & & SEEP & & RAW GRAB & UNFILTERED & 199 & 9 100-H SPRING & \\
\hline 718 & $F$ & SEEP & & RAW GRAB & UNFILTERED & 199 & 9 100-H SPRING & \\
\hline 719 & & SEEP & & RAW GRAB & UNFILTERED & 199 & 9 100-H SPRING & \\
\hline 720 & $F$ & SEEP & & RAW GRAB & UNFILTERED & 199 & 9 100-H SPRING & \\
\hline 721 & $F$ & SEEP & & RAW GRAB & UNFILTERED & 199 & 9 100-H SPRING & \\
\hline 722 & $\mathrm{~F}$ & SEEP & & RAW GRAB & UNFILTERED & 199 & 9 100-H SPRING & \\
\hline
\end{tabular}




\begin{tabular}{|c|c|c|c|c|c|c|c|c|}
\hline & $\mathrm{T}$ & $\mathrm{U}$ & $\mathrm{V}$ & $\mathrm{W}$ & $\mathrm{X}$ & $\mathrm{Y}$ & $Z$ & $\mathrm{AA}$ \\
\hline 1 & REVIEW_QUALIFIER & SAMP_FROM & SAMP_ITEM & SAMP_MTHD & COLL_MTHD & SAMP_SITE_ID & SAMP_SITE_NAME & WELL_NAME \\
\hline 724 & & SEEP & & RAW GRAB & UNFILTERED & 533 & 3 100-K SPRING & \\
\hline 725 & & SEEP & & RAW GRAB & UNFILTERED & 533 & 3 100-K SPRING & \\
\hline 726 & & SEEP & & RAW GRAB & UNFILTERED & 533 & 3 100-K SPRING & \\
\hline 727 & $\mathrm{~F}$ & SEEP & & RAW GRAB & UNFILTERED & 533 & 3 100-K SPRING & \\
\hline 728 & & SEEP & & RAW GRAB & UNFILTERED & 533 & 3 100-K SPRING & \\
\hline 729 & $F$ & SEEP & & RAW GRAB & UNFILTERED & 533 & 3 100-K SPRING & \\
\hline 730 & $F$ & SEEP & & RAW GRAB & UNFILTERED & 533 & 3 100-K SPRING & \\
\hline 731 & $\mathrm{~F}$ & SEEP & & RAW GRAB & UNFILTERED & 533 & 3 100-K SPRING & \\
\hline 733 & & SEEP & & RAW GRAB & UNFILTERED & 488 & 3300 AREA SPRING 42-2 & \\
\hline 734 & & SEEP & & RAW GRAB & UNFILTERED & 488 & 3300 AREA SPRING 42-2 & \\
\hline 735 & & SEEP & & RAW GRAB & UNFILTERED & 488 & 8300 AREA SPRING 42-2 & \\
\hline 736 & & SEEP & & RAW GRAB & UNFILTERED & 488 & 3300 AREA SPRING 42-2 & \\
\hline 737 & $\mathrm{~F}$ & SEEP & & RAW GRAB & UNFILTERED & 488 & 3300 AREA SPRING 42-2 & \\
\hline 738 & & SEEP & & RAW GRAB & UNFILTERED & 488 & 3300 AREA SPRING 42-2 & \\
\hline 739 & $F$ & SEEP & & RAW GRAB & UNFILTERED & 488 & 3300 AREA SPRING 42-2 & \\
\hline 740 & $F$ & SEEP & & RAW GRAB & UNFILTERED & 488 & 3300 AREA SPRING 42-2 & \\
\hline 741 & & SEEP & & RAW GRAB & UNFILTERED & 488 & 300 AREA SPRING 42-2 & \\
\hline 743 & & SEEP & & RAW GRAB & UNFILTERED & 151 & 1 HANFORD SPRING 28-2 & \\
\hline 744 & & SEEP & & RAW GRAB & UNFILTERED & 151 & 1 HANFORD SPRING 28-2 & \\
\hline 745 & & SEEP & & RAW GRAB & UNFILTERED & 151 & 1 HANFORD SPRING 28-2 & \\
\hline 746 & & SEEP & & RAW GRAB & UNFILTERED & 151 & 1 HANFORD SPRING 28-2 & \\
\hline 747 & $F$ & SEEP & & RAW GRAB & UNFILTERED & 151 & 1 HANFORD SPRING 28-2 & \\
\hline 748 & & SEEP & & RAW GRAB & UNFILTERED & 151 & 1 HANFORD SPRING 28-2 & \\
\hline 749 & $\mathrm{~F}$ & SEEP & & RAW GRAB & UNFILTERED & 151 & 1 HANFORD SPRING 28-2 & \\
\hline 750 & $\mathrm{~F}$ & SEEP & & RAW GRAB & UNFILTERED & 151 & 1 HANFORD SPRING 28-2 & \\
\hline 751 & $F$ & SEEP & & RAW GRAB & UNFILTERED & 151 & 1 HANFORD SPRING 28-2 & \\
\hline 752 & $F$ & SEEP & & RAW GRAB & UNFILTERED & 151 & 1 HANFORD SPRING 28-2 & \\
\hline 753 & & SEEP & & RAW GRAB & UNFILTERED & 193 & 3 100-D SPRING & \\
\hline 754 & & SEEP & & RAW GRAB & UNFILTERED & 193 & 3 100-D SPRING & \\
\hline 755 & & SEEP & & RAW GRAB & UNFILTERED & 193 & 3 100-D SPRING & \\
\hline 756 & & SEEP & & RAW GRAB & UNFILTERED & 193 & 3 100-D SPRING & \\
\hline 757 & & SEEP & & RAW GRAB & UNFILTERED & 193 & 3 100-D SPRING & \\
\hline 758 & $F$ & SEEP & & RAW GRAB & UNFILTERED & 193 & 3 100-D SPRING & \\
\hline 759 & $F$ & SEEP & & RAW GRAB & UNFILTERED & 193 & 3 100-D SPRING & \\
\hline 760 & $\mathrm{~F}$ & SEEP & & RAW GRAB & UNFILTERED & 193 & 3 100-D SPRING & \\
\hline
\end{tabular}




\begin{tabular}{|c|c|c|c|c|c|c|c|c|}
\hline & $T$ & $U$ & $\mathrm{~V}$ & $\mathrm{~W}$ & $\bar{x}$ & $\bar{Y}$ & $Z$ & $\mathrm{AA}$ \\
\hline 1 & REVIEW QUALIFIER & SAMP FROM & SAMP ITEM & SAMP MTHD & COLL MTHD & SAMP SITE ID & SAMP SITE NAME & WELL NAME \\
\hline 762 & & SEEP & & RAW GRAB & UNFILTERED & 196 & 100-F SPRING & \\
\hline 763 & & SEEP & & RAW GRAB & UNFILTERED & 196 & 100-F SPRING & \\
\hline 764 & & SEEP & & RAW GRAB & UNFILTERED & 196 & 100-F SPRING & \\
\hline 765 & & SEEP & & RAW GRAB & UNFILTERED & 196 & $100-F$ SPRING & \\
\hline 766 & & SEEP & & RAW GRAB & UNFILTERED & 196 & 6 100-F SPRING & \\
\hline 767 & $F$ & SEEP & & RAW GRAB & UNFILTERED & 196 & 6 100-F SPRING & \\
\hline 768 & $\mathrm{~F}$ & SEEP & & RAW GRAB & UNFILTERED & 196 & $100-F$ SPRING & \\
\hline 769 & $F$ & SEEP & & RAW GRAB & UNFILTERED & 196 & 6 100-F SPRING & \\
\hline 771 & & SEEP & WATER & & & 2351 & 1 NS-1 SEEP & \\
\hline 772 & & SEEP & WATER & & & 2351 & 1 NS-1 SEEP & \\
\hline 773 & & SEEP & WATER & & & 2350 & NS-2 SEEP & \\
\hline 774 & & SEEP & WATER & & & 2349 & 9 NS-3 SEEP & \\
\hline 775 & & SEEP & WATER & & & 2348 & 3 NS-4 SEEP & \\
\hline 776 & & SEEP & WATER & & & 2347 & 7 NS-5 SEEP & \\
\hline 777 & & SEEP & WATER & & & 2346 & DS-6 SEEP & \\
\hline 778 & & SEEP & WATER & & & 2345 & 5 NS-7 SEEP & \\
\hline 779 & & SEEP & WATER & & & 2343 & 3 NS-8A SEEP & \\
\hline 781 & & SEEP & WATER & & & 2341 & 1 NS-10 SEEP & \\
\hline 782 & & SEEP & WATER & & & 2340 & NS-11 SEEP & \\
\hline 783 & & SEEP & WATER & & & 5498 & 3 N SPRINGS SEEPAGE SAMPLE LOCATION \#1 & \\
\hline 784 & & SEEP & WATER & & & 5498 & 3 N SPRINGS SEEPAGE SAMPLE LOCATION \#1 & \\
\hline 785 & & SEEP & WATER & & & 5498 & 3 N SPRINGS SEEPAGE SAMPLE LOCATION \#1 & \\
\hline 786 & & SEEP & WATER & & & 5499 & 9 N SPRINGS SEEPAGE SAMPLE LOCATION \#2 & \\
\hline 787 & & SEEP & WATER & & & 5499 & 9 N SPRINGS SEEPAGE SAMPLE LOCATION \#2 & \\
\hline 788 & & SEEP & WATER & & & 5499 & 9 N SPRINGS SEEPAGE SAMPLE LOCATION \#2 & \\
\hline 789 & & SEEP & WATER & & & 5500 & N SPRINGS SEEPAGE SAMPLE LOCATION \#3 & \\
\hline 790 & & SEEP & WATER & & & 5500 & N SPRINGS SEEPAGE SAMPLE LOCATION \#3 & \\
\hline 791 & & SEEP & WATER & & & 5500 & N SPRINGS SEEPAGE SAMPLE LOCATION \#3 & \\
\hline 792 & & SEEP & WATER & & & 5501 & 1 N SPRINGS SEEPAGE SAMPLE LOCATION \#4 & \\
\hline 793 & & SEEP & WATER & & & 5501 & 1 N SPRINGS SEEPAGE SAMPLE LOCATION \#4 & \\
\hline 794 & & SEEP & WATER & & & 5501 & 1 N SPRINGS SEEPAGE SAMPLE LOCATION \#4 & \\
\hline 795 & & SEEP & WATER & & & 5502 & 2 N SPRINGS SEEPAGE SAMPLE LOCATION \#5 & \\
\hline 796 & & SEEP & WATER & & & 5502 & 2 N SPRINGS SEEPAGE SAMPLE LOCATION \#5 & \\
\hline 797 & & SEEP & WATER & & & 5502 & 2 N SPRINGS SEEPAGE SAMPLE LOCATION \#5 & \\
\hline 798 & & SEEP & WATER & & & 5503 & 3 N SPRINGS SEEPAGE SAMPLE LOCATION \#6 & \\
\hline
\end{tabular}




\begin{tabular}{|c|c|c|c|c|c|c|c|c|}
\hline & $\mathrm{T}$ & $\mathrm{U}$ & $\mathrm{V}$ & $\mathrm{W}$ & $\mathrm{X}$ & $\mathrm{Y}$ & Z & $\mathrm{AA}$ \\
\hline 1 & REVIEW_QUALIFIER & SAMP_FROM & SAMP_ITEM & SAMP_MTHD & COLL_MTHD & SAMP_SITE_ID & SAMP_SITE_NAME & WELL_NAME \\
\hline 800 & & SEEP & WATER & & & 5503 & 3 N SPRINGS SEEPAGE SAMPLE LOCATION \#6 & \\
\hline 801 & & SEEP & WATER & & & 5504 & 4 N SPRINGS SEEPAGE SAMPLE LOCATION \#7 & \\
\hline 802 & & SEEP & WATER & & & 5504 & 4 N SPRINGS SEEPAGE SAMPLE LOCATION \#7 & \\
\hline 803 & & SEEP & WATER & & & 5504 & 4 N SPRINGS SEEPAGE SAMPLE LOCATION \#7 & \\
\hline 804 & & SEEP & WATER & & & 5505 & $5 \mathrm{~N}$ SPRINGS SEEPAGE SAMPLE LOCATION \#8 & \\
\hline 805 & & SEEP & WATER & & & 5505 & 5 N SPRINGS SEEPAGE SAMPLE LOCATION \#8 & \\
\hline 806 & & SEEP & WATER & & & 5505 & 5 N SPRINGS SEEPAGE SAMPLE LOCATION \#8 & \\
\hline 807 & & SEEP & WATER & & & 5506 & 6 N SPRINGS SEEPAGE SAMPLE LOCATION \#9 & \\
\hline 809 & & SEEP & WATER & & & 5506 & o N SPRINGS SEEPAGE SAMPLE LOCATION \#9 & \\
\hline 810 & & SEEP & WATER & & & 2216 & 6 SEEP 037-1 & \\
\hline 811 & & SEEP & WATER & & & 2218 & 3 SEEP 039-2 & \\
\hline 812 & & SEEP & WATER & & & 5304 & 4 SEEP 082-2 & \\
\hline 813 & & SEEP & WATER & & & 5303 & 3 SEEP 057-3 & \\
\hline 814 & & SEEP & WATER & & & 4721 & SD-098-1 & \\
\hline 815 & & SEEP & WATER & & & 5306 & 6 SEEP $145-2$ & \\
\hline 816 & & SEEP & WATER & & & 2232 & 2 SEEP 144-1 & \\
\hline 817 & & SEEP & WATER & & & 5307 & 7 SEEP $150-3$ & \\
\hline 819 & & SEEP & & RAW GRAB & UNFILTERED & 52 & 2 100-B SPRING & \\
\hline 820 & & SEEP & & RAW GRAB & UNFILTERED & 52 & 2 100-B SPRING & \\
\hline 821 & & SEEP & & RAW GRAB & UNFILTERED & 52 & 2 100-B SPRING & \\
\hline 822 & & SEEP & & RAW GRAB & UNFILTERED & 52 & 2 100-B SPRING & \\
\hline 823 & & SEEP & & RAW GRAB & UNFILTERED & 52 & 2 100-B SPRING & \\
\hline 824 & $F$ & SEEP & & RAW GRAB & UNFILTERED & 52 & 2 100-B SPRING & \\
\hline 825 & & SEEP & & RAW GRAB & UNFILTERED & 193 & 3 100-D SPRING & \\
\hline 826 & & SEEP & & RAW GRAB & UNFILTERED & 193 & 3 100-D SPRING & \\
\hline 827 & & SEEP & & RAW GRAB & UNFILTERED & 193 & 3 100-D SPRING & \\
\hline 828 & & SEEP & & RAW GRAB & UNFILTERED & 193 & 3 100-D SPRING & \\
\hline 829 & & SEEP & & RAW GRAB & UNFILTERED & 193 & 3 100-D SPRING & \\
\hline 830 & & SEEP & & RAW GRAB & UNFILTERED & 193 & 3 100-D SPRING & \\
\hline 831 & $F$ & SEEP & & RAW GRAB & UNFILTERED & 193 & 3 100-D SPRING & \\
\hline 832 & & SEEP & & RAW GRAB & UNFILTERED & 202 & 2 100-N SPRING 8-13 & \\
\hline 833 & & SEEP & & RAW GRAB & UNFILTERED & 202 & 2 100-N SPRING 8-13 & \\
\hline 834 & & SEEP & & RAW GRAB & UNFILTERED & 202 & 2 100-N SPRING 8-13 & \\
\hline 835 & & SEEP & & RAW GRAB & UNFILTERED & 202 & 2 100-N SPRING 8-13 & \\
\hline 836 & & SEEP & & RAW GRAB & UNFILTERED & 202 & 2 100-N SPRING 8-13 & \\
\hline
\end{tabular}




\begin{tabular}{|c|c|c|c|c|c|c|c|c|}
\hline & $\mathrm{T}$ & $\mathrm{U}$ & $\mathrm{V}$ & $\mathrm{W}$ & $\mathrm{X}$ & $\mathrm{Y}$ & $Z$ & $\mathrm{AA}$ \\
\hline 1 & REVIEW_QUALIFIER & SAMP_FROM & SAMP_ITEM & SAMP_MTHD & COLL_MTHD & SAMP_SITE_ID & SAMP_SITE_NAME & WELL_NAME \\
\hline 838 & $\mathrm{~F}$ & SEEP & & RAW GRAB & UNFILTERED & 202 & $2100-N$ SPRING 8-13 & \\
\hline 839 & & SEEP & WATER & & & 2228 & 3 SEEP $110-1$ & \\
\hline 840 & & SEEP & WATER & & & 2229 & 9 SEEP $110-2$ & \\
\hline 841 & & SEEP & WATER & & & 2245 & 5 SEEP $152-2$ & \\
\hline 842 & & SEEP & WATER & & & 5325 & 5 SEEP 153-1 & \\
\hline 843 & & SEEP & WATER & & & 5326 & 6 SEEP 102-1 & \\
\hline 844 & & SEEP & & RAW GRAB & UNFILTERED & 196 & $100-F$ SPRING & \\
\hline 845 & & SEEP & & RAW GRAB & UNFILTERED & 196 & 100-F SPRING & \\
\hline 847 & & SEEP & & RAW GRAB & UNFILTERED & 196 & $100-F$ SPRING & \\
\hline 848 & & SEEP & & RAW GRAB & UNFILTERED & 196 & 100-F SPRING & \\
\hline 849 & $F$ & SEEP & & RAW GRAB & UNFILTERED & 196 & 6 100-F SPRING & \\
\hline 850 & $\mathrm{~F}$ & SEEP & & RAW GRAB & UNFILTERED & 196 & 6 100-F SPRING & \\
\hline 851 & $\mathrm{~F}$ & SEEP & & RAW GRAB & UNFILTERED & 196 & 6 100-F SPRING & \\
\hline 852 & $F$ & SEEP & & RAW GRAB & UNFILTERED & 196 & 100-F SPRING & \\
\hline 853 & $F$ & SEEP & & RAW GRAB & UNFILTERED & 196 & $100-F$ SPRING & \\
\hline 854 & & SEEP & & RAW GRAB & UNFILTERED & 151 & 1 HANFORD SPRING 28-2 & \\
\hline 855 & & SEEP & & RAW GRAB & UNFILTERED & 151 & 1 HANFORD SPRING 28-2 & \\
\hline 857 & & SEEP & & RAW GRAB & UNFILTERED & 151 & 1 HANFORD SPRING 28-2 & \\
\hline 858 & & SEEP & & RAW GRAB & UNFILTERED & 151 & 1 HANFORD SPRING 28-2 & \\
\hline 859 & & SEEP & & RAW GRAB & UNFILTERED & 151 & 1 HANFORD SPRING 28-2 & \\
\hline 860 & & SEEP & & RAW GRAB & UNFILTERED & 151 & 1 HANFORD SPRING 28-2 & \\
\hline 861 & $F$ & SEEP & & RAW GRAB & UNFILTERED & 151 & 1 HANFORD SPRING 28-2 & \\
\hline 862 & $\mathrm{~F}$ & SEEP & & RAW GRAB & UNFILTERED & 151 & 1 HANFORD SPRING 28-2 & \\
\hline 863 & $\mathrm{~F}$ & SEEP & & RAW GRAB & UNFILTERED & 151 & 1 HANFORD SPRING 28-2 & \\
\hline 864 & $\mathrm{~F}$ & SEEP & & RAW GRAB & UNFILTERED & 151 & 1 HANFORD SPRING 28-2 & \\
\hline 865 & & SEEP & & RAW GRAB & UNFILTERED & 488 & 300 AREA SPRING 42-2 & \\
\hline 866 & & SEEP & & RAW GRAB & UNFILTERED & 488 & 3300 AREA SPRING 42-2 & \\
\hline 867 & & SEEP & & RAW GRAB & UNFILTERED & 488 & 3300 AREA SPRING 42-2 & \\
\hline 868 & & SEEP & & RAW GRAB & UNFILTERED & 488 & 300 AREA SPRING 42-2 & \\
\hline 869 & & SEEP & & RAW GRAB & UNFILTERED & 488 & 3300 AREA SPRING 42-2 & \\
\hline 870 & & SEEP & & RAW GRAB & UNFILTERED & 488 & 3300 AREA SPRING 42-2 & \\
\hline 871 & $F$ & SEEP & & RAW GRAB & UNFILTERED & 488 & 300 AREA SPRING 42-2 & \\
\hline 872 & $F$ & SEEP & & RAW GRAB & UNFILTERED & 488 & 3300 AREA SPRING 42-2 & \\
\hline 873 & $F$ & SEEP & & RAW GRAB & UNFILTERED & 488 & 3300 AREA SPRING 42-2 & \\
\hline 874 & $F$ & SEEP & & RAW GRAB & UNFILTERED & 488 & 300 AREA SPRING 42-2 & \\
\hline
\end{tabular}




\begin{tabular}{|c|c|c|c|c|c|c|c|c|}
\hline & $\mathrm{T}$ & $U$ & $\mathrm{~V}$ & $\mathrm{~W}$ & $x$ & $Y$ & $Z$ & $\mathrm{AA}$ \\
\hline 1 & REVIEW_QUALIFIER & SAMP_FROM & SAMP_ITEM & SAMP_MTHD & COLL_MTHD & SAMP_SITE_ID & SAMP_SITE_NAME & WELL_NAME \\
\hline 876 & & SEEP & WATER & & & 2216 & 5 SEEP 037-1 & \\
\hline 877 & & SEEP & WATER & & & 2216 & 6 SEEP 037-1 & \\
\hline 878 & & SEEP & WATER & & & 2216 & 6 SEEP 037-1 & \\
\hline 879 & & SEEP & WATER & & & 2216 & 6 SEEP 037-1 & \\
\hline 880 & & SEEP & WATER & & & 2218 & 3 SEEP 039-2 & \\
\hline 881 & & SEEP & WATER & & & 2218 & 3 SEEP 039-2 & \\
\hline 882 & & SEEP & WATER & & & 2218 & 3 SEEP 039-2 & \\
\hline 883 & & SEEP & WATER & & & 2218 & 3 SEEP 039-2 & \\
\hline 885 & & SEEP & WATER & & & 4721 & 1 SD-098-1 & \\
\hline 886 & & SEEP & WATER & & & 5326 & 5 SEEP 102-1 & \\
\hline 887 & & SEEP & WATER & & & 5326 & 6 SEEP 102-1 & \\
\hline 888 & & SEEP & WATER & & & 5326 & 6 SEEP 102-1 & \\
\hline 889 & & SEEP & WATER & & & 5326 & o SEEP 102-1 & \\
\hline 890 & & SEEP & WATER & & & 2228 & 3 SEEP 110-1 & \\
\hline 891 & & SEEP & WATER & & & 2228 & 3 SEEP 110-1 & \\
\hline 892 & & SEEP & WATER & & & 5325 & 5 SEEP 153-1 & \\
\hline 893 & & SEEP & WATER & & & 5325 & 5 SEEP 153-1 & \\
\hline 895 & & SEEP & & RAW GRAB & UNFILTERED & 52 & 2 100-B SPRING & \\
\hline 896 & & SEEP & & RAW GRAB & UNFILTERED & 52 & 2 100-B SPRING & \\
\hline 897 & $F$ & SEEP & & RAW GRAB & UNFILTERED & 52 & 2 100-B SPRING & \\
\hline 898 & & SEEP & & RAW GRAB & UNFILTERED & 196 & 6 100-F SPRING & \\
\hline 899 & $F$ & SEEP & & RAW GRAB & UNFILTERED & 196 & $100-F$ SPRING & \\
\hline 900 & $F$ & SEEP & & RAW GRAB & UNFILTERED & 196 & 6 100-F SPRING & \\
\hline 901 & $\mathrm{~F}$ & SEEP & & RAW GRAB & UNFILTERED & 196 & 0 100-F SPRING & \\
\hline 902 & $F$ & SEEP & & RAW GRAB & UNFILTERED & 196 & 6 100-F SPRING & \\
\hline 903 & $F$ & SEEP & & RAW GRAB & UNFILTERED & 196 & 6 100-F SPRING & \\
\hline 904 & & SEEP & WATER & & & 5303 & 3 SEEP 057-3 & \\
\hline 905 & & SEEP & WATER & & & 5303 & 3 SEEP 057-3 & \\
\hline 906 & & SEEP & WATER & & & 5303 & 3 SEEP 057-3 & \\
\hline 907 & & SEEP & WATER & & & 5303 & 3 SEEP 057-3 & \\
\hline 908 & & SEEP & WATER & & & 5304 & 4 SEEP 082-2 & \\
\hline 909 & & SEEP & WATER & & & 5304 & 4 SEEP 082-2 & \\
\hline 910 & & SEEP & WATER & & & 5304 & 4 SEEP 082-2 & \\
\hline 911 & & SEEP & WATER & & & 5304 & 4 SEEP 082-2 & \\
\hline 912 & & SEEP & & RAW GRAB & UNFILTERED & 193 & 3 100-D SPRING & \\
\hline
\end{tabular}




\begin{tabular}{|c|c|c|c|c|c|c|c|c|}
\hline & $\mathrm{T}$ & $\mathrm{U}$ & $\mathrm{V}$ & $\mathrm{W}$ & $\mathrm{X}$ & $\mathrm{Y}$ & $Z$ & $\mathrm{AA}$ \\
\hline 1 & REVIEW_QUALIFIER & SAMP_FROM & SAMP_ITEM & SAMP_MTHD & COLL_MTHD & SAMP_SITE_ID & SAMP_SITE_NAME & WELL_NAME \\
\hline 914 & & SEEP & & RAW GRAB & UNFILTERED & 193 & 3 100-D SPRING & \\
\hline 915 & & SEEP & & RAW GRAB & UNFILTERED & 199 & 9 100-H SPRING & \\
\hline 916 & $F$ & SEEP & & RAW GRAB & UNFILTERED & 199 & 9 100-H SPRING & \\
\hline 917 & & SEEP & & RAW GRAB & UNFILTERED & 199 & 9 100-H SPRING & \\
\hline 918 & & SEEP & & RAW GRAB & UNFILTERED & 199 & 9 100-H SPRING & \\
\hline 919 & $F$ & SEEP & & RAW GRAB & UNFILTERED & 199 & 9 100-H SPRING & \\
\hline 920 & $F$ & SEEP & & RAW GRAB & UNFILTERED & 199 & 9 100-H SPRING & \\
\hline 921 & $F$ & SEEP & & RAW GRAB & UNFILTERED & 199 & 9 100-H SPRING & \\
\hline 923 & & SEEP & & RAW GRAB & UNFILTERED & 488 & 3300 AREA SPRING 42-2 & \\
\hline 924 & & SEEP & & RAW GRAB & UNFILTERED & 488 & 3300 AREA SPRING 42-2 & \\
\hline 925 & $F$ & SEEP & & RAW GRAB & UNFILTERED & 488 & 8300 AREA SPRING 42-2 & \\
\hline 926 & $\mathrm{~F}$ & SEEP & & RAW GRAB & UNFILTERED & 488 & 3300 AREA SPRING 42-2 & \\
\hline 927 & & SEEP & & RAW GRAB & UNFILTERED & 488 & 3300 AREA SPRING 42-2 & \\
\hline 928 & $F$ & SEEP & & RAW GRAB & UNFILTERED & 488 & 300 AREA SPRING 42-2 & \\
\hline 929 & & SEEP & & RAW GRAB & UNFILTERED & 151 & 1 HANFORD SPRING 28-2 & \\
\hline 930 & & SEEP & & RAW GRAB & UNFILTERED & 151 & 1 HANFORD SPRING 28-2 & \\
\hline 931 & & SEEP & & RAW GRAB & UNFILTERED & 151 & 1 HANFORD SPRING 28-2 & \\
\hline 933 & $F$ & SEEP & & RAW GRAB & UNFILTERED & 151 & 1 HANFORD SPRING 28-2 & \\
\hline 934 & $F$ & SEEP & & RAW GRAB & UNFILTERED & 151 & 1 HANFORD SPRING 28-2 & \\
\hline 935 & $F$ & SEEP & & RAW GRAB & UNFILTERED & 151 & 1 HANFORD SPRING 28-2 & \\
\hline 936 & & SEEP & WATER & & & 2232 & 2 SEEP $144-1$ & \\
\hline 937 & & SEEP & WATER & & & 2232 & 2 SEEP 144-1 & \\
\hline 938 & & SEEP & WATER & & & 2232 & 2 SEEP $144-1$ & \\
\hline 939 & & SEEP & WATER & & & 2232 & 2 SEEP $144-1$ & \\
\hline 940 & & SEEP & WATER & & & 2245 & 5 SEEP 152-2 & \\
\hline 941 & & SEEP & WATER & & & 2245 & 5 SEEP 152-2 & \\
\hline 942 & & SEEP & WATER & & & 2229 & 9 SEEP $110-2$ & \\
\hline 943 & & SEEP & WATER & & & 2229 & 9 SEEP $110-2$ & \\
\hline 944 & & SEEP & WATER & & & 5324 & 4 SEEP 077-1 & \\
\hline 945 & & SEEP & WATER & & & 5324 & 4 SEEP 077-1 & \\
\hline 946 & & SEEP & WATER & & & 5324 & 4 SEEP 077-1 & \\
\hline 947 & & SEEP & WATER & & & 5324 & 4 SEEP 077-1 & \\
\hline 948 & & SEEP & & RAW GRAB & UNFILTERED & 211 & 100 N SPRING & \\
\hline 949 & $F$ & SEEP & & RAW GRAB & UNFILTERED & 211 & 100 N SPRING & \\
\hline 950 & $F$ & SEEP & & RAW GRAB & UNFILTERED & 211 & 100 N SPRING & \\
\hline
\end{tabular}




\begin{tabular}{|c|c|c|c|c|c|c|c|c|}
\hline & $\mathrm{T}$ & $U$ & $\mathrm{~V}$ & $\mathrm{~W}$ & $\mathrm{X}$ & $\mathrm{Y}$ & $Z$ & $\mathrm{AA}$ \\
\hline 1 & REVIEW_QUALIFIER & SAMP_FROM & SAMP_ITEM & SAMP_MTHD & COLL_MTHD & SAMP_SITE_ID & SAMP_SITE_NAME & WELL_NAME \\
\hline 952 & & SEEP & & RAW GRAB & UNFILTERED & 533 & 3 100-K SPRING & \\
\hline 953 & $\mathrm{~F}$ & SEEP & & RAW GRAB & UNFILTERED & 533 & 3 100-K SPRING & \\
\hline 954 & & SEEP & & RAW GRAB & UNFILTERED & 202 & 2 100-N SPRING 8-13 & \\
\hline 955 & & SEEP & & RAW GRAB & UNFILTERED & 202 & 2 100-N SPRING 8-13 & \\
\hline 956 & $F$ & SEEP & & RAW GRAB & UNFILTERED & 202 & 2 100-N SPRING 8-13 & \\
\hline 957 & & SEEP & & RAW GRAB & UNFILTERED & 488 & 300 AREA SPRING 42-2 & \\
\hline 958 & & SEEP & & RAW GRAB & UNFILTERED & 488 & 300 AREA SPRING 42-2 & \\
\hline 959 & & SEEP & & RAW GRAB & UNFILTERED & 488 & 3300 AREA SPRING 42-2 & \\
\hline 961 & $F$ & SEEP & & RAW GRAB & UNFILTERED & 488 & 3300 AREA SPRING 42-2 & \\
\hline 962 & $F$ & SEEP & & RAW GRAB & UNFILTERED & 488 & 3300 AREA SPRING 42-2 & \\
\hline 963 & $\mathrm{~F}$ & SEEP & & RAW GRAB & UNFILTERED & 488 & 3300 AREA SPRING 42-2 & \\
\hline 964 & & SEEP & & RAW GRAB & UNFILTERED & 151 & 1 HANFORD SPRING 28-2 & \\
\hline 965 & & SEEP & & RAW GRAB & UNFILTERED & 151 & 1 HANFORD SPRING 28-2 & \\
\hline 966 & & SEEP & & RAW GRAB & UNFILTERED & 151 & 1 HANFORD SPRING 28-2 & \\
\hline 967 & $F$ & SEEP & & RAW GRAB & UNFILTERED & 151 & 1 HANFORD SPRING 28-2 & \\
\hline 968 & $F$ & SEEP & & RAW GRAB & UNFILTERED & 151 & 1 HANFORD SPRING $28-2$ & \\
\hline 969 & $F$ & SEEP & & RAW GRAB & UNFILTERED & 151 & 1 HANFORD SPRING 28-2 & \\
\hline 971 & & SEEP & & RAW GRAB & UNFILTERED & 193 & 3 100-D SPRING & \\
\hline 972 & $F$ & SEEP & & RAW GRAB & UNFILTERED & 193 & 3 100-D SPRING & \\
\hline 973 & $F$ & SEEP & & RAW GRAB & UNFILTERED & 193 & 3 100-D SPRING & \\
\hline 974 & & SEEP & & RAW GRAB & UNFILTERED & 199 & 9 100-H SPRING & \\
\hline 975 & & SEEP & & RAW GRAB & UNFILTERED & 199 & 9 100-H SPRING & \\
\hline 976 & & SEEP & & RAW GRAB & UNFILTERED & 199 & 9 100-H SPRING & \\
\hline 977 & $F$ & SEEP & & RAW GRAB & UNFILTERED & 199 & 9 100-H SPRING & \\
\hline 978 & $F$ & SEEP & & RAW GRAB & UNFILTERED & 199 & 9 100-H SPRING & \\
\hline 979 & $F$ & SEEP & & RAW GRAB & UNFILTERED & 199 & 9 100-H SPRING & \\
\hline 980 & $F$ & SEEP & & RAW GRAB & UNFILTERED & 199 & 9 100-H SPRING & \\
\hline 981 & & SEEP & & RAW GRAB & UNFILTERED & 52 & 2 100-B SPRING & \\
\hline 982 & $F$ & SEEP & & RAW GRAB & UNFILTERED & 52 & 2 100-B SPRING & \\
\hline 983 & & SEEP & & RAW GRAB & UNFILTERED & 52 & 2 100-B SPRING & \\
\hline 984 & $F$ & SEEP & & RAW GRAB & UNFILTERED & 52 & 2 100-B SPRING & \\
\hline 985 & & SEEP & & RAW GRAB & UNFILTERED & 196 & $100-F$ SPRING & \\
\hline 986 & & SEEP & & RAW GRAB & UNFILTERED & 196 & 6 100-F SPRING & \\
\hline 987 & & SEEP & & RAW GRAB & UNFILTERED & 196 & 6 100-F SPRING & \\
\hline 988 & $\mathrm{~F}$ & SEEP & & RAW GRAB & UNFILTERED & 196 & 6 100-F SPRING & \\
\hline
\end{tabular}




\begin{tabular}{|c|c|c|c|c|c|c|c|c|}
\hline & $T$ & $U$ & $\mathrm{~V}$ & $\mathrm{~W}$ & $\mathrm{X}$ & $\bar{Y}$ & Z & $\mathrm{AA}$ \\
\hline 1 & REVIEW QUALIFIER & SAMP FROM & SAMP ITEM & SAMP MTHD & COLL MTHD & SAMP SITE ID & SAMP SITE NAME & WELL NAME \\
\hline 990 & $F$ & SEEP & & RAW GRAB & UNFILTERED & 196 & $6100-F$ SPRING & \\
\hline 991 & & SEEP & & RAW GRAB & UNFILTERED & 533 & 3 100-K SPRING & \\
\hline 992 & $F$ & SEEP & & RAW GRAB & UNFILTERED & 533 & 3 100-K SPRING & \\
\hline 993 & $F$ & SEEP & & RAW GRAB & UNFILTERED & 533 & 3 100-K SPRING & \\
\hline 994 & & SEEP & & RAW GRAB & UNFILTERED & 202 & 2 100-N SPRING 8-13 & \\
\hline 995 & & SEEP & & RAW GRAB & UNFILTERED & 202 & 2 100-N SPRING 8-13 & \\
\hline \begin{tabular}{|l|}
996 \\
\end{tabular} & $\mathrm{~F}$ & SEEP & & RAW GRAB & UNFILTERED & 202 & 2 100-N SPRING 8-13 & \\
\hline 997 & & SEEP & & RAW GRAB & UNFILTERED & 8140 & 100-B SPRING 38-3 & \\
\hline 999 & & SEEP & & RAW GRAB & UNFILTERED & 8140 & 100-B SPRING 38-3 & \\
\hline 1000 & $F$ & SEEP & & RAW GRAB & UNFILTERED & 8140 & 100-B SPRING 38-3 & \\
\hline 1001 & & SEEP & & RAW GRAB & UNFILTERED & 8141 & 1 100-B SPRING 39-2 & \\
\hline 1002 & & SEEP & & RAW GRAB & UNFILTERED & 8141 & 1 100-B SPRING 39-2 & \\
\hline 1003 & & SEEP & & RAW GRAB & UNFILTERED & 8141 & 1 100-B SPRING 39-2 & \\
\hline 1004 & $F$ & SEEP & & RAW GRAB & UNFILTERED & 8141 & 1 100-B SPRING 39-2 & \\
\hline 1005 & & SEEP & & RAW GRAB & UNFILTERED & 8145 & 5 100-K SPRING 63-1 & \\
\hline 1006 & $F$ & SEEP & & RAW GRAB & UNFILTERED & 8145 & 5 100-K SPRING 63-1 & \\
\hline 1007 & $F$ & SEEP & & RAW GRAB & UNFILTERED & 8145 & 5 100-K SPRING 63-1 & \\
\hline 1009 & $F$ & SEEP & & RAW GRAB & UNFILTERED & 202 & 2 100-N SPRING 8-13 & \\
\hline 1010 & $F$ & SEEP & & RAW GRAB & UNFILTERED & 202 & 2 100-N SPRING 8-13 & \\
\hline 1011 & & SEEP & & RAW GRAB & UNFILTERED & 8145 & 5 100-K SPRING 63-1 & \\
\hline 1012 & & SEEP & & RAW GRAB & UNFILTERED & 8163 & 3 100-D SPRING 102-1 & \\
\hline 1013 & $F$ & SEEP & & RAW GRAB & UNFILTERED & 8163 & 3 100-D SPRING 102-1 & \\
\hline 1014 & $F$ & SEEP & & RAW GRAB & UNFILTERED & 8163 & 3 100-D SPRING 102-1 & \\
\hline 1015 & & SEEP & & RAW GRAB & UNFILTERED & 8162 & 2 100-D SPRING 110-1 & \\
\hline 1016 & & SEEP & & RAW GRAB & UNFILTERED & 8162 & 2 100-D SPRING 110-1 & \\
\hline 1017 & & SEEP & & RAW GRAB & UNFILTERED & 8162 & 2 100-D SPRING 110-1 & \\
\hline 1018 & & SEEP & & RAW GRAB & UNFILTERED & 8165 & 5 100-H SPRING 145-1 & \\
\hline 1019 & $F$ & SEEP & & RAW GRAB & UNFILTERED & 8165 & 5 100-H SPRING 145-1 & \\
\hline 1020 & & SEEP & & RAW GRAB & UNFILTERED & 8165 & 5 100-H SPRING 145-1 & \\
\hline 1021 & & SEEP & & RAW GRAB & UNFILTERED & 8165 & 5 100-H SPRING 145-1 & \\
\hline 1022 & $F$ & SEEP & & RAW GRAB & UNFILTERED & 8165 & 5 100-H SPRING 145-1 & \\
\hline 1023 & $F$ & SEEP & & RAW GRAB & UNFILTERED & 8165 & 5 100-H SPRING 145-1 & \\
\hline 1024 & $F$ & SEEP & & RAW GRAB & UNFILTERED & 8165 & 5 100-H SPRING 145-1 & \\
\hline 1025 & & SEEP & & RAW GRAB & UNFILTERED & 8164 & 4 100-H SPRING 153-1 & \\
\hline 1026 & $F$ & SEEP & & RAW GRAB & UNFILTERED & 8164 & 4 100-H SPRING 153-1 & \\
\hline
\end{tabular}




\begin{tabular}{|c|c|c|c|c|c|c|c|c|}
\hline & $T$ & $\bar{U}$ & $\mathrm{~V}$ & $\mathrm{~W}$ & $\bar{X}$ & $\bar{Y}$ & $\bar{Z}$ & AA \\
\hline 1 & REVIEW_QUALIFIER & SAMP_FROM & SAMP_ITEM & SAMP_MTHD & COLL_MTHD & SAMP_SITE_ID & SAMP_SITE_NAME & WELL_NAME \\
\hline 1028 & & SEEP & & RAW GRAB & UNFILTERED & 8164 & 4 100-H SPRING 153-1 & \\
\hline 1029 & $F$ & SEEP & & RAW GRAB & UNFILTERED & 8164 & 4 100-H SPRING 153-1 & \\
\hline 1030 & $\mathrm{~F}$ & SEEP & & RAW GRAB & UNFILTERED & 8164 & 4 100-H SPRING 153-1 & \\
\hline 1031 & $F$ & SEEP & & RAW GRAB & UNFILTERED & 8164 & 4 100-H SPRING 153-1 & \\
\hline 1032 & & SEEP & & RAW GRAB & UNFILTERED & 8147 & 7 100-F SPRING 207-1 & \\
\hline 1033 & & SEEP & & RAW GRAB & UNFILTERED & 8147 & 7 100-F SPRING 207-1 & \\
\hline 1034 & $F$ & SEEP & & RAW GRAB & UNFILTERED & 8147 & 7 100-F SPRING 207-1 & \\
\hline 1035 & $F$ & SEEP & & RAW GRAB & UNFILTERED & 8147 & 7 100-F SPRING 207-1 & \\
\hline 1037 & $F$ & SEEP & & RAW GRAB & UNFILTERED & 8147 & 7 100-F SPRING 207-1 & \\
\hline 1038 & & SEEP & & RAW GRAB & UNFILTERED & 8144 & 4300 AREA SPR DR 42-2 & \\
\hline 1039 & & SEEP & & RAW GRAB & UNFILTERED & 8144 & 4300 AREA SPR DR 42-2 & \\
\hline 1040 & $\mathrm{~F}$ & SEEP & & RAW GRAB & UNFILTERED & 8144 & 4300 AREA SPR DR 42-2 & \\
\hline 1041 & $F$ & SEEP & & RAW GRAB & UNFILTERED & 8144 & 4300 AREA SPR DR 42-2 & \\
\hline 1042 & & SEEP & & RAW GRAB & UNFILTERED & 8144 & 4300 AREA SPR DR 42-2 & \\
\hline 1043 & & SEEP & & RAW GRAB & UNFILTERED & 8144 & 4300 AREA SPR DR 42-2 & \\
\hline 1044 & & SEEP & & RAW GRAB & UNFILTERED & 8144 & 4300 AREA SPR DR 42-2 & \\
\hline 1045 & & SEEP & & RAW GRAB & UNFILTERED & 488 & 300 AREA SPRING 42-2 & \\
\hline 1047 & $F$ & SEEP & & RAW GRAB & UNFILTERED & 488 & 3300 AREA SPRING 42-2 & \\
\hline 1048 & $F$ & SEEP & & RAW GRAB & UNFILTERED & 488 & 300 AREA SPRING 42-2 & \\
\hline 1049 & $F$ & SEEP & & RAW GRAB & UNFILTERED & 488 & 300 AREA SPRING 42-2 & \\
\hline 1050 & & SEEP & & RAW GRAB & UNFILTERED & 488 & 300 AREA SPRING 42-2 & \\
\hline 1051 & $\mathrm{~F}$ & SEEP & & RAW GRAB & UNFILTERED & 488 & 3300 AREA SPRING 42-2 & \\
\hline 1052 & & SEEP & & RAW GRAB & UNFILTERED & 8143 & 3 HANFORD SPR DR 28-2 & \\
\hline 1053 & & SEEP & & RAW GRAB & UNFILTERED & 8143 & 3 HANFORD SPR DR 28-2 & \\
\hline 1054 & & SEEP & & RAW GRAB & UNFILTERED & 8143 & 3 HANFORD SPR DR 28-2 & \\
\hline 1055 & $F$ & SEEP & & RAW GRAB & UNFILTERED & 8143 & 3 HANFORD SPR DR 28-2 & \\
\hline 1056 & $F$ & SEEP & & RAW GRAB & UNFILTERED & 8143 & 3 HANFORD SPR DR 28-2 & \\
\hline 1057 & $F$ & SEEP & & RAW GRAB & UNFILTERED & 8143 & 3 HANFORD SPR DR 28-2 & \\
\hline 1058 & $F$ & SEEP & & RAW GRAB & UNFILTERED & 8143 & 3 HANFORD SPR DR 28-2 & \\
\hline 1059 & & SEEP & & RAW GRAB & UNFILTERED & 8142 & 2 HANFORD SPR UR 28-2 & \\
\hline 1060 & & SEEP & & RAW GRAB & UNFILTERED & 8142 & 2 HANFORD SPR UR 28-2 & \\
\hline 1061 & & SEEP & & RAW GRAB & UNFILTERED & 8142 & 2 HANFORD SPR UR 28-2 & \\
\hline 1062 & $F$ & SEEP & & RAW GRAB & UNFILTERED & 8142 & 2 HANFORD SPR UR 28-2 & \\
\hline 1063 & $\mathrm{~F}$ & SEEP & & RAW GRAB & UNFILTERED & 8142 & 2 HANFORD SPR UR 28-2 & \\
\hline 1064 & $\mathrm{~F}$ & SEEP & & RAW GRAB & UNFILTERED & 8142 & 2 HANFORD SPR UR 28-2 & \\
\hline
\end{tabular}




\begin{tabular}{|c|c|c|c|c|c|c|c|c|}
\hline & $T$ & $\bar{U}$ & $\mathrm{~V}$ & $\mathrm{~W}$ & $\bar{x}$ & $\bar{Y}$ & $\bar{Z}$ & $\overline{A A}$ \\
\hline 1 & REVIEW_QUALIFIER & SAMP_FROM & SAMP_ITEM & SAMP_MTHD & COLL_MTHD & SAMP_SITE_ID & SAMP_SITE_NAME & WELL_NAME \\
\hline 1066 & & SEEP & & RAW GRAB & UNFILTERED & 151 & HANFORD SPRING $28-2$ & \\
\hline 1067 & & SEEP & & RAW GRAB & UNFILTERED & 151 & HANFORD SPRING 28-2 & \\
\hline 1068 & & SEEP & & RAW GRAB & UNFILTERED & 151 & HANFORD SPRING 28-2 & \\
\hline 1069 & $\mathrm{~F}$ & SEEP & & RAW GRAB & UNFILTERED & 151 & HANFORD SPRING $28-2$ & \\
\hline $\mid 1070$ & $\mathrm{~F}$ & SEEP & & RAW GRAB & UNFILTERED & 151 & HANFORD SPRING $28-2$ & \\
\hline 1071 & $\mathrm{~F}$ & SEEP & & RAW GRAB & UNFILTERED & 151 & HANFORD SPRING 28-2 & \\
\hline \begin{tabular}{|l|}
1072 \\
\end{tabular} & $\mathrm{~F}$ & SEEP & & RAW GRAB & UNFILTERED & 151 & HANFORD SPRING $28-2$ & \\
\hline 1073 & & SEEP & & RAW GRAB & UNFILTERED & 8147 & 100-F SPRING 207-1 & \\
\hline 1075 & & SEEP & & RAW GRAB & UNFILTERED & 8144 & 300 AREA SPR DR 42-2 & \\
\hline 1076 & & SEEP & & RAW GRAB & & 8626 & WEST LAKE SPRING & \\
\hline 1077 & $\mathrm{~F}$ & SEEP & & RAW GRAB & & 8626 & WEST LAKE SPRING & \\
\hline 1078 & $\mathrm{~F}$ & SEEP & & RAW GRAB & & 8626 & WEST LAKE SPRING & \\
\hline 1079 & $\mathrm{~F}$ & SEEP & & RAW GRAB & & 8626 & WEST LAKE SPRING & \\
\hline
\end{tabular}




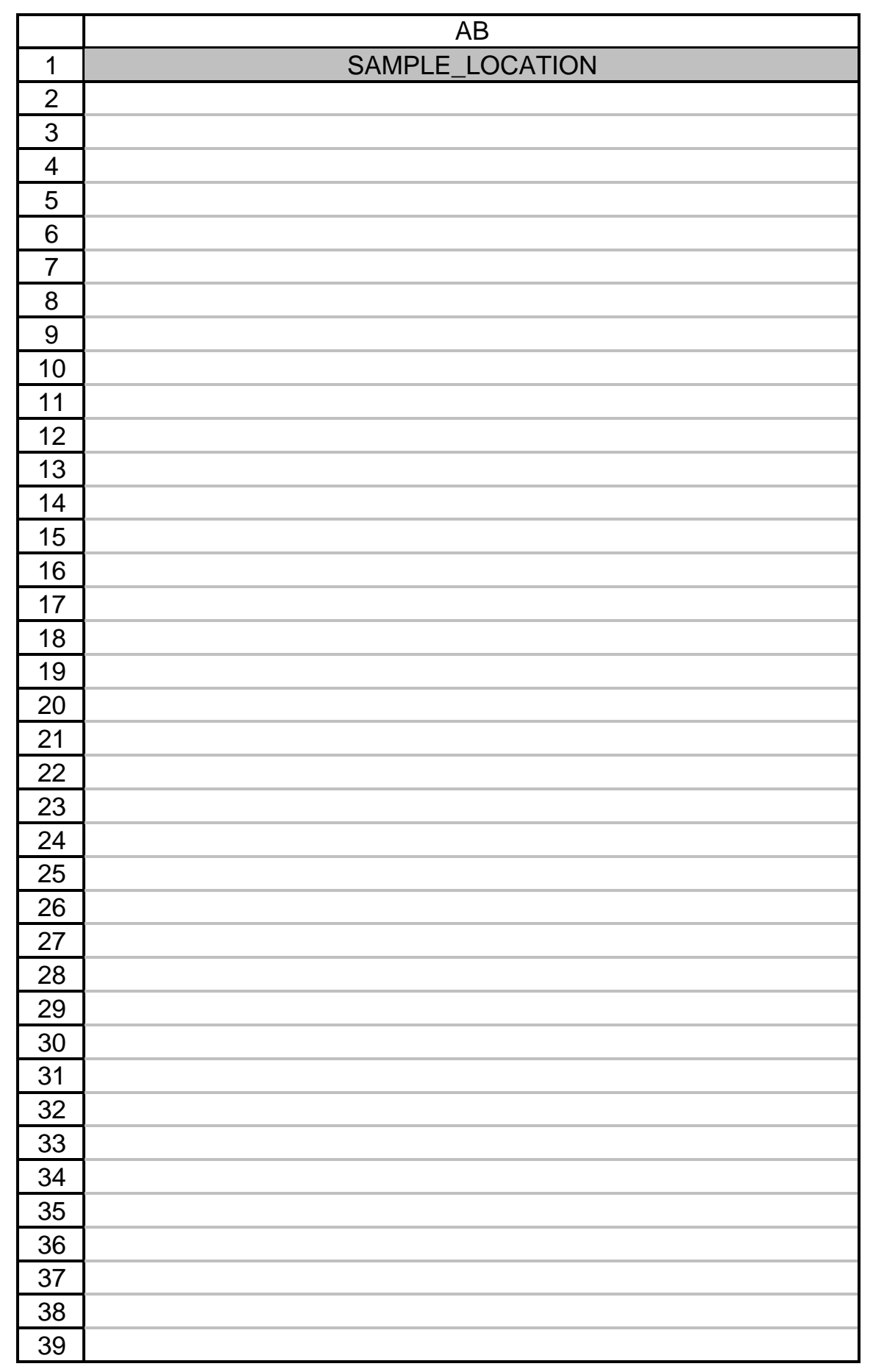

Page 88 


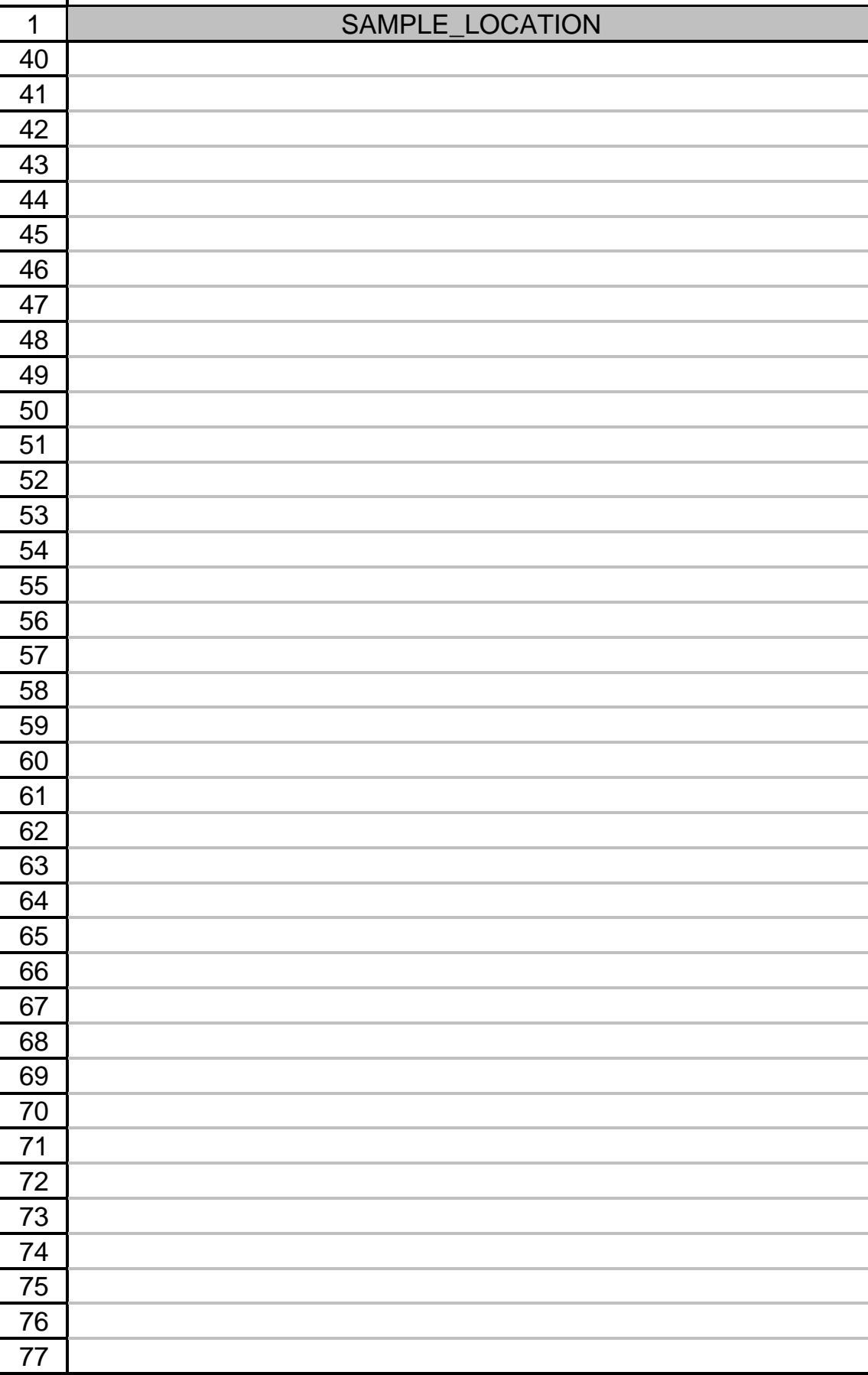

Page 89 
AB

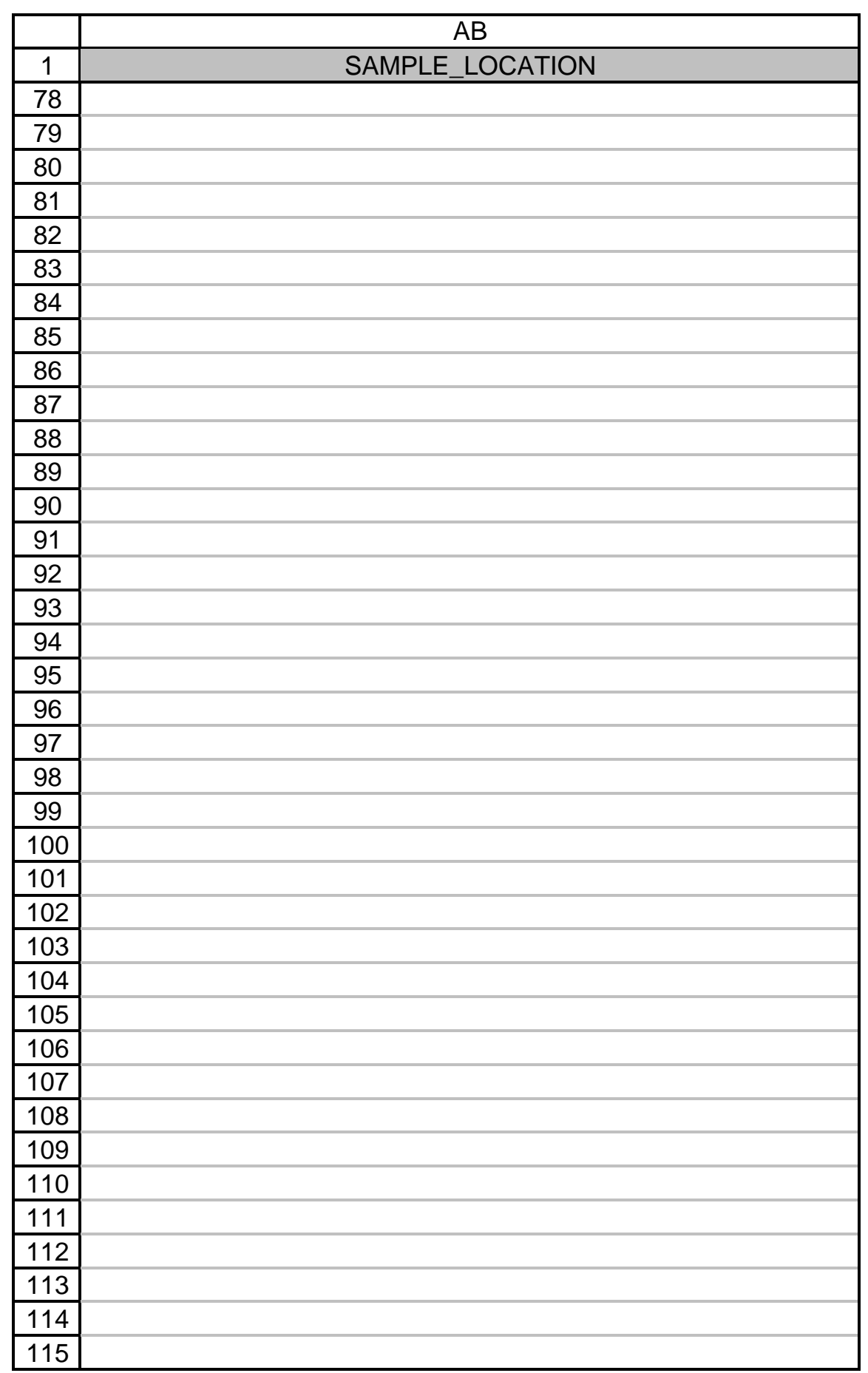

Page 90 
AB

\begin{tabular}{|c|c|}
\hline & AB \\
\hline 1 & \\
\hline 116 & \\
\hline 117 & \\
\hline 118 & \\
\hline 119 & \\
\hline 120 & \\
\hline 121 & \\
\hline 122 & \\
\hline 123 & \\
\hline 124 & \\
\hline 125 \\
\hline 126 \\
\hline 127 \\
\hline 128 \\
\hline 129 \\
\hline 130 \\
\hline 131 \\
\hline 132 \\
\hline 133 \\
\hline 134 \\
\hline 135 \\
\hline 136 \\
\hline 137 \\
\hline 138 \\
\hline 139 \\
\hline 140 \\
\hline 141 \\
\hline 142 \\
\hline 143 \\
\hline 144 \\
\hline 145 \\
\hline 146 \\
\hline 147 \\
\hline 148 \\
\hline 149 \\
\hline 150 \\
\hline 151 \\
\hline 152 \\
\hline 153 & \\
\hline
\end{tabular}

Page 91 
AB

\begin{tabular}{|c|c|}
\hline 1 & AB \\
\hline 154 & \\
\hline 155 & \\
\hline 156 \\
\hline 157 \\
\hline 158 \\
\hline 159 \\
\hline 160 \\
\hline 161 \\
\hline 162 \\
\hline 163 \\
\hline 164 \\
\hline 165 \\
\hline 166 \\
\hline 167 \\
\hline 168 \\
\hline 169 \\
\hline 170 \\
\hline 171 \\
\hline 172 \\
\hline 173 \\
\hline 174 \\
\hline 175 \\
\hline 176 \\
\hline 177 \\
\hline 178 \\
\hline 179 \\
\hline 180 \\
\hline 181 \\
\hline 182 \\
\hline 183 \\
\hline 184 \\
\hline 185 \\
\hline 186 \\
\hline 187 \\
\hline 188 \\
\hline 189 \\
\hline 190 \\
\hline 191 & \\
\hline
\end{tabular}


AB

\begin{tabular}{|c|c|}
\hline & AB \\
\hline 1 & \\
\hline 192 & \\
\hline 193 & \\
\hline 194 \\
\hline 195 \\
\hline 196 \\
\hline 197 \\
\hline 198 \\
\hline 199 \\
\hline 200 \\
\hline 201 \\
\hline 202 \\
\hline 203 \\
\hline 204 \\
\hline 205 \\
\hline 206 \\
\hline 207 \\
\hline 208 \\
\hline 209 \\
\hline 210 \\
\hline 211 \\
\hline 212 \\
\hline 213 \\
\hline 214 \\
\hline 215 \\
\hline 216 \\
\hline 217 \\
\hline 218 \\
\hline 219 \\
\hline 220 \\
\hline 221 \\
\hline 222 \\
\hline 223 \\
\hline 224 \\
\hline 225 \\
\hline 226 \\
\hline 227 \\
\hline 228 \\
\hline 229 & \\
\hline
\end{tabular}

Page 93 
AB

\begin{tabular}{|r|r|}
\hline & AB \\
\hline 1 & \\
\hline 230 & \\
\hline 231 & \\
\hline 232 & \\
\hline 233 & \\
\hline 234 & \\
\hline 235 & \\
\hline 236 & \\
\hline 237 & \\
\hline 238 & \\
\hline 239 & \\
\hline 240 & \\
\hline 241 & \\
\hline 242 & \\
\hline 243 & \\
\hline 244 & \\
\hline 245 & \\
\hline 246 & \\
\hline 247 & \\
\hline 248 & \\
\hline 249 & \\
\hline 250 & \\
\hline 251 & \\
\hline 252 & \\
\hline 253 & \\
\hline 254 & \\
\hline 255 & \\
\hline 256 & \\
\hline 257 & \\
\hline 258 & \\
\hline 259 & \\
\hline 260 & \\
\hline 261 & \\
\hline 262 & \\
\hline 263 & \\
\hline 264 \\
\hline 265 \\
\hline 266 \\
\hline 267 & \\
\hline
\end{tabular}

Page 94 
$A B$

\begin{tabular}{|c|c|}
\hline & \multicolumn{1}{|c|}{ AB } \\
\hline 1 & SAMPLE_LOCATION \\
\hline 268 & \\
\hline 269 & \\
\hline 270 & \\
\hline 271 & \\
\hline 272 & \\
\hline 273 & \\
\hline 274 & \\
\hline 275 & \\
\hline 276 & \\
\hline 277 & \\
\hline 278 & \\
\hline 279 & \\
\hline 280 & \\
\hline 281 & \\
\hline 282 & \\
\hline 283 & \\
\hline 284 & \\
\hline 285 & \\
\hline 286 & \\
\hline 287 & \\
\hline 288 & \\
\hline 289 & \\
\hline 290 & \\
\hline 291 & \\
\hline 292 & \\
\hline 293 & \\
\hline 294 & \\
\hline 295 & \\
\hline 296 & COLLECTED FROM WELL \#N-46 \\
\hline 297 & COLLECTED FROM WELL \#N-46 \\
\hline 298 & COLLECTED FROM WELL \#N-46 \\
\hline 299 & COLLECTED FROM WELL \#N-46 \\
\hline 300 & \\
\hline 301 & \\
\hline 302 & \\
\hline 303 & \\
\hline 304 & \\
\hline 305 & \\
\hline
\end{tabular}


AB

\begin{tabular}{|c|c|}
\hline & AB \\
\hline 1 & \\
\hline 306 & \\
\hline 307 & \\
\hline 308 & \\
\hline 309 & \\
\hline 310 & \\
\hline 311 & \\
\hline 312 & \\
\hline 313 & \\
\hline 314 & \\
\hline 315 \\
\hline 316 \\
\hline 317 \\
\hline 318 \\
\hline 319 \\
\hline 320 \\
\hline 321 \\
\hline 322 \\
\hline 323 \\
\hline 324 \\
\hline 325 \\
\hline 326 \\
\hline 327 \\
\hline 328 \\
\hline 329 \\
\hline 330 \\
\hline 331 \\
\hline 332 \\
\hline 333 \\
\hline 334 \\
\hline 335 \\
\hline 336 \\
\hline 337 \\
\hline 338 \\
\hline 339 \\
\hline 340 \\
\hline 341 \\
\hline 342 \\
\hline 343 & \\
\hline
\end{tabular}

Page 96 
AB

\begin{tabular}{|c|c|}
\hline & \multicolumn{1}{|c|}{ AB } \\
\hline 1 & SAMPLE_LOCATION \\
\hline 344 & \\
\hline 345 & \\
\hline 346 \\
\hline 347 \\
\hline 348 \\
\hline 349 \\
\hline 350 \\
\hline 351 \\
\hline 352 \\
\hline 353 \\
\hline 354 \\
\hline 355 \\
\hline 356 \\
\hline 357 \\
\hline 358 \\
\hline 359 \\
\hline 360 \\
\hline 361 \\
\hline 362 \\
\hline 363 \\
\hline 364 \\
\hline 365 \\
\hline 366 \\
\hline 367 \\
\hline 368 \\
\hline 369 \\
\hline 370 \\
\hline 371 \\
\hline 372 \\
\hline 373 \\
\hline 374 \\
\hline 375 \\
\hline 376 \\
\hline 377 \\
\hline 378 \\
\hline 379 \\
\hline 380 \\
\hline 381 & \\
\hline
\end{tabular}

Page 97 
AB

\begin{tabular}{|l|l|}
\hline & AB \\
\hline 1 & SAMPLE_LOCATION \\
\hline 382 & \\
\hline 383 & \\
\hline 384 & \\
\hline 385 & \\
\hline 386 & \\
\hline 387 & \\
\hline 388 & \\
\hline 389 \\
\hline 390 \\
\hline 391 \\
\hline 392 \\
\hline 393 \\
\hline 394 \\
\hline 395 \\
\hline 396 \\
\hline 397 \\
\hline 398 \\
\hline 399 \\
\hline 400 \\
\hline 401 \\
\hline 402 \\
\hline 403 \\
\hline 404 \\
\hline 405 \\
\hline 406 \\
\hline 407 \\
\hline 408 \\
\hline 409 \\
\hline 410 \\
\hline 411 \\
\hline 412 \\
\hline 413 \\
\hline 414 \\
\hline 415 \\
\hline 416 \\
\hline 417 \\
\hline 418 \\
\hline 419 & \\
\hline
\end{tabular}

Page 98 
AB

\begin{tabular}{|r|r|}
\hline & AB \\
\hline 1 & SAMPLE_LOCATION \\
\hline 420 & \\
\hline 421 & \\
\hline 422 & \\
\hline 423 & \\
\hline 424 & \\
\hline 425 & \\
\hline 426 & \\
\hline 427 & \\
\hline 428 & \\
\hline 429 & \\
\hline 430 & \\
\hline 431 & \\
\hline 432 & \\
\hline 433 & \\
\hline 434 & \\
\hline 435 & \\
\hline 436 & \\
\hline 437 & \\
\hline 438 & \\
\hline 439 & \\
\hline 440 & \\
\hline 441 & \\
\hline 442 & \\
\hline 443 & \\
\hline 444 & \\
\hline 445 & \\
\hline 446 & \\
\hline 447 & \\
\hline 448 & \\
\hline 449 & \\
\hline 450 & \\
\hline 451 & \\
\hline 452 & \\
\hline 453 & \\
\hline 454 & \\
\hline 455 & \\
\hline 456 & \\
\hline 457 & \\
\hline
\end{tabular}

Page 99 
AB

\begin{tabular}{|l|l|}
\hline & AB \\
\hline 1 & \\
\hline 458 & \\
\hline 459 & \\
\hline 460 \\
\hline 461 \\
\hline 462 \\
\hline 463 \\
\hline 464 \\
\hline 465 \\
\hline 466 \\
\hline 467 \\
\hline 468 \\
\hline 469 \\
\hline 470 \\
\hline 471 \\
\hline 472 \\
\hline 473 \\
\hline 474 \\
\hline 475 \\
\hline 476 \\
\hline 477 \\
\hline 478 \\
\hline 479 \\
\hline 480 \\
\hline 481 \\
\hline 482 \\
\hline 483 \\
\hline 484 \\
\hline 485 \\
\hline 486 \\
\hline 487 \\
\hline 488 \\
\hline 489 \\
\hline 490 \\
\hline 491 \\
\hline 492 \\
\hline 493 \\
\hline 494 \\
\hline 495 \\
\hline
\end{tabular}


AB

\begin{tabular}{|c|c|}
\hline & AB \\
\hline 1 & \\
\hline 496 & \\
\hline 497 & \\
\hline 498 \\
\hline 499 \\
\hline 500 \\
\hline 501 \\
\hline 502 \\
\hline 503 \\
\hline 504 \\
\hline 505 \\
\hline 506 \\
\hline 507 \\
\hline 508 \\
\hline 509 \\
\hline 510 \\
\hline 511 \\
\hline 512 \\
\hline 513 \\
\hline 514 \\
\hline 515 \\
\hline 516 \\
\hline 517 \\
\hline 518 \\
\hline 519 \\
\hline 520 \\
\hline 521 \\
\hline 522 \\
\hline 523 \\
\hline 524 \\
\hline 525 \\
\hline 526 \\
\hline 527 \\
\hline 528 \\
\hline 529 \\
\hline 530 \\
\hline 531 \\
\hline 532 \\
\hline 533 & \\
\hline
\end{tabular}




\begin{tabular}{|l|l|}
\hline & \multicolumn{1}{|c|}{ AB } \\
\hline 1 & \\
\hline 534 & \\
\hline 535 & SAMPLE_LOCATION \\
\hline 536 \\
\hline 537 \\
\hline 538 \\
\hline 539 \\
\hline 540 \\
\hline 541 \\
\hline 542 \\
\hline 543 \\
\hline 544 \\
\hline 545 \\
\hline 546 \\
\hline 547 \\
\hline 548 \\
\hline 549 \\
\hline 550 \\
\hline 551 \\
\hline 552 \\
\hline 553 \\
\hline 554 \\
\hline 555 \\
\hline 556 \\
\hline 557 \\
\hline 558 \\
\hline 559 \\
\hline 560 \\
\hline 561 \\
\hline 562 \\
\hline 563 \\
\hline 564 \\
\hline 565 \\
\hline 566 \\
\hline 567 \\
\hline 568 \\
\hline 569 \\
\hline 570 \\
\hline 571 \\
\hline
\end{tabular}




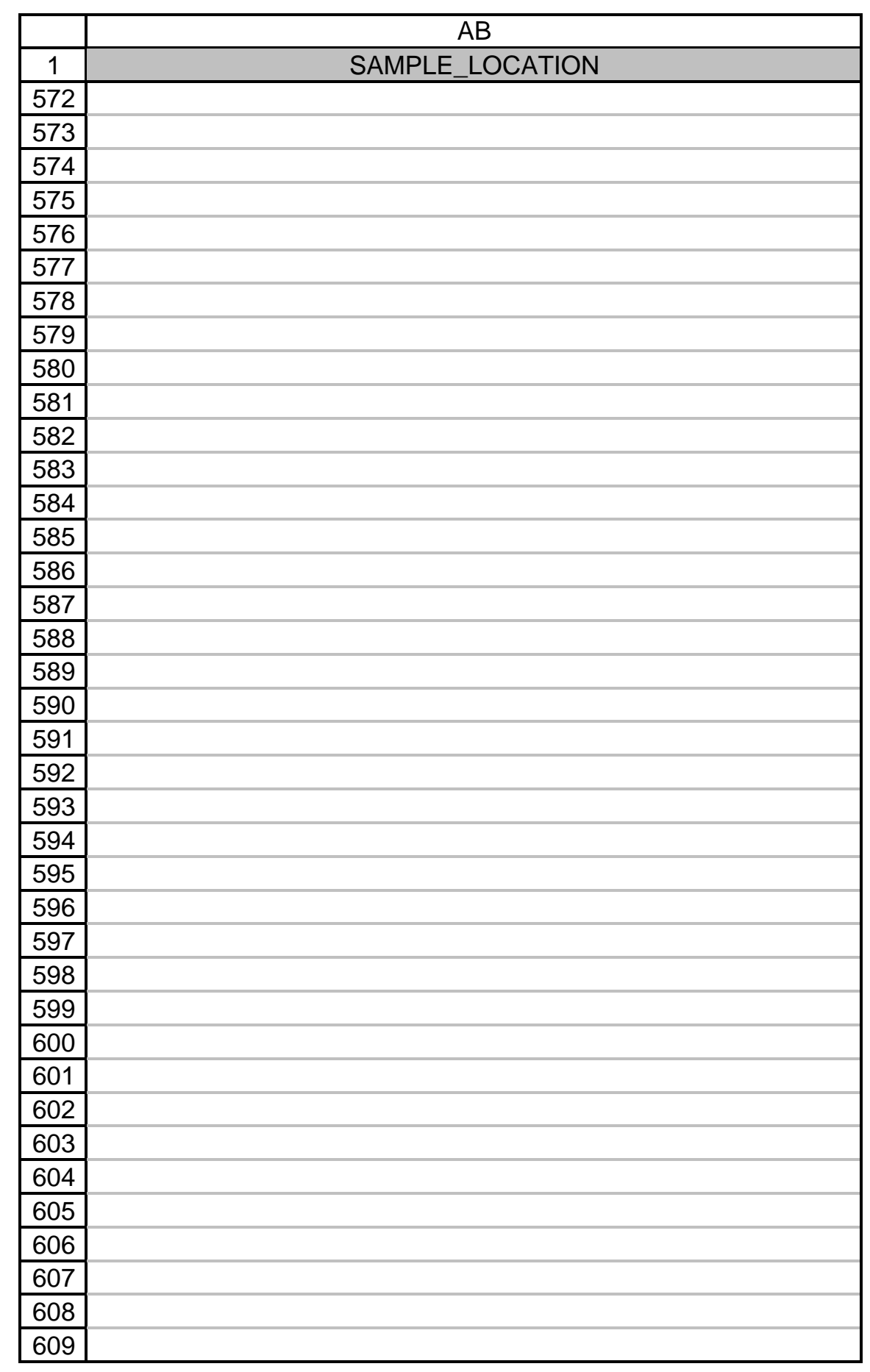


AB

\begin{tabular}{|l|l|}
\hline & \multicolumn{1}{|c|}{ AB } \\
\hline 1 & \\
\hline 610 & \\
\hline 611 & \\
\hline 612 & \\
\hline 613 & \\
\hline 614 & \\
\hline 615 & \\
\hline 616 & \\
\hline 617 & \\
\hline 618 & \\
\hline 619 & \\
\hline 620 & \\
\hline 621 & \\
\hline 622 & \\
\hline 623 & \\
\hline 624 & \\
\hline 625 & \\
\hline 626 & \\
\hline 627 & \\
\hline 628 & \\
\hline 629 & \\
\hline 630 & \\
\hline 631 & \\
\hline 632 \\
\hline 633 \\
\hline 634 \\
\hline 635 \\
\hline 636 \\
\hline 637 \\
\hline 638 \\
\hline 639 \\
\hline 640 \\
\hline 641 \\
\hline 642 \\
\hline 643 \\
\hline 644 \\
\hline 645 \\
\hline 646 & \\
\hline 647 & \\
\hline
\end{tabular}


AB

\begin{tabular}{|l|l|}
\hline & \multicolumn{1}{|c|}{ AB } \\
\hline 1 & SAMPLE_LOCATION \\
\hline 648 & \\
\hline 649 & \\
\hline 650 & \\
\hline 651 & \\
\hline 652 & \\
\hline 653 & \\
\hline 654 & \\
\hline 655 & \\
\hline 656 & \\
\hline 657 & \\
\hline 658 & \\
\hline 659 & \\
\hline 660 & \\
\hline 661 & \\
\hline 662 & \\
\hline 663 & \\
\hline 664 & \\
\hline 665 & \\
\hline 666 & \\
\hline 667 & \\
\hline 668 \\
\hline 669 \\
\hline 670 \\
\hline 671 \\
\hline 672 \\
\hline 673 \\
\hline 674 \\
\hline 675 \\
\hline 676 \\
\hline 677 \\
\hline 678 \\
\hline 679 \\
\hline 680 \\
\hline 681 \\
\hline 682 \\
\hline 683 \\
\hline 684 \\
\hline 685 & \\
\hline
\end{tabular}


AB

\begin{tabular}{|l|l|}
\hline & AB \\
\hline 1 & \\
\hline 686 & \\
\hline 687 & \\
\hline 688 & \\
\hline 689 \\
\hline 690 \\
\hline 691 \\
\hline 692 \\
\hline 693 \\
\hline 694 \\
\hline 695 \\
\hline 696 \\
\hline 697 \\
\hline 698 \\
\hline 699 \\
\hline 700 \\
\hline 701 \\
\hline 702 \\
\hline 703 \\
\hline 704 \\
\hline 705 \\
\hline 706 \\
\hline 707 \\
\hline 708 \\
\hline 709 \\
\hline 710 \\
\hline 711 \\
\hline 712 \\
\hline 713 \\
\hline 714 \\
\hline 715 \\
\hline 716 \\
\hline 717 \\
\hline 718 \\
\hline 719 \\
\hline 720 \\
\hline 721 \\
\hline 722 \\
\hline 723 & \\
\hline
\end{tabular}


AB

\begin{tabular}{|l|l|}
\hline & AB \\
\hline 1 & \\
\hline 724 & \\
\hline 725 \\
\hline 726 \\
\hline 727 \\
\hline 728 \\
\hline 729 \\
\hline 730 \\
\hline 731 \\
\hline 732 \\
\hline 733 \\
\hline 734 \\
\hline 735 \\
\hline 736 \\
\hline 737 \\
\hline 738 \\
\hline 739 \\
\hline 740 \\
\hline 741 \\
\hline 742 \\
\hline 743 \\
\hline 744 \\
\hline 745 \\
\hline 746 \\
\hline 747 \\
\hline 748 \\
\hline 749 \\
\hline 750 \\
\hline 751 \\
\hline 752 \\
\hline 753 \\
\hline 754 \\
\hline 755 \\
\hline 756 \\
\hline 757 \\
\hline 758 \\
\hline 759 \\
\hline 760 \\
\hline 761 \\
\hline
\end{tabular}


AB

\begin{tabular}{|l|l|}
\hline & \multicolumn{1}{|c|}{ AB } \\
\hline 1 & \\
\hline 762 & \\
\hline 763 & \\
\hline 764 & \\
\hline 765 & \\
\hline 766 & \\
\hline 767 & \\
\hline 768 & \\
\hline 769 & \\
\hline 770 & \\
\hline 771 & NS-1 \\
\hline 772 & NS-1 \\
\hline 773 & NS-2 \\
\hline 774 & NS-3 \\
\hline 775 & NS-4 \\
\hline 776 & NS-5 \\
\hline 777 & NS-6 \\
\hline 778 & NS-7 \\
\hline 779 & NS-8A \\
\hline 780 & NS-8B \\
\hline 781 & NS-10 \\
\hline 782 & NS-11 \\
\hline 783 & \\
\hline 784 & \\
\hline 785 & \\
\hline 786 & \\
\hline 787 & \\
\hline 788 & \\
\hline 789 & \\
\hline 790 & \\
\hline 791 & \\
\hline 792 & \\
\hline 793 & \\
\hline 794 & \\
\hline 795 & \\
\hline 796 & \\
\hline 797 & \\
\hline 798 & \\
\hline 799 & \\
\hline
\end{tabular}


AB

\begin{tabular}{|l|l|}
\hline & \multicolumn{1}{|c|}{ AB } \\
\hline 1 & \\
\hline 800 & \\
\hline 801 & \\
\hline 802 & \\
\hline 803 & \\
\hline 804 & \\
\hline 805 & \\
\hline 806 & \\
\hline 807 & \\
\hline 808 & \\
\hline 809 & \\
\hline 810 & $037-1$ \\
\hline 811 & $039-2$ \\
\hline 812 & $082-2$ \\
\hline 813 & $057-3$ \\
\hline 814 & $098-1$ \\
\hline 815 & $145-2$ \\
\hline 816 & $144-1$ \\
\hline 817 & $150-3$ \\
\hline 818 & \\
\hline 819 & \\
\hline 820 & \\
\hline 821 & \\
\hline 822 & \\
\hline 823 & \\
\hline 824 & \\
\hline 825 & \\
\hline 826 & \\
\hline 827 & \\
\hline 828 & \\
\hline 829 & \\
\hline 830 & \\
\hline 831 & \\
\hline 832 & \\
\hline 833 & \\
\hline 834 & \\
\hline 835 & \\
\hline 836 & \\
\hline 837 & \\
\hline
\end{tabular}


$A B$

\begin{tabular}{|r|r|}
\hline & \multicolumn{2}{|c|}{ AB } \\
\hline 1 & \multicolumn{2}{|c|}{ SAMPLE_LOCATION } \\
\hline 838 & \\
\hline 839 & $110-1$ \\
\hline 840 & $110-2$ \\
\hline 841 & $152-2$ \\
\hline 842 & $153-1$ \\
\hline 843 & $102-1$ \\
\hline 844 & \\
\hline 845 & \\
\hline 846 & \\
\hline 847 & \\
\hline 848 & \\
\hline 849 & \\
\hline 850 & \\
\hline 851 & \\
\hline 852 & \\
\hline 853 & \\
\hline 854 & \\
\hline 855 & \\
\hline 856 & \\
\hline 857 & \\
\hline 858 & \\
\hline 859 & \\
\hline 860 & \\
\hline 861 & \\
\hline 862 & \\
\hline 863 & \\
\hline 864 & \\
\hline 865 & \\
\hline 866 & \\
\hline 867 & \\
\hline 868 & \\
\hline 869 & \\
\hline 870 & \\
\hline 871 & \\
\hline 872 & \\
\hline 873 & \\
\hline 874 & \\
\hline 875 & $190-D$ \\
\hline
\end{tabular}


$A B$

\begin{tabular}{|c|l|}
\hline & \multicolumn{1}{|c|}{ AB } \\
\hline 1 & \\
\hline 876 & \\
\hline 877 & \\
\hline 878 & \\
\hline 879 & \\
\hline 880 & \\
\hline 881 & \\
\hline 882 & \\
\hline 883 & \\
\hline 884 & SD-98-1 \\
\hline 885 & SD-98-1 \\
\hline 886 & SD-102-1 \\
\hline 887 & SD-102-1 \\
\hline 888 & SD-102-1 \\
\hline 889 & SD-102-1 \\
\hline 890 & SD-110-1 \\
\hline 891 & SD-110-1 \\
\hline 892 & SH-153-1 \\
\hline 893 & SH-153-1 \\
\hline 894 & \\
\hline 895 & \\
\hline 896 & \\
\hline 897 & \\
\hline 898 & \\
\hline 899 & \\
\hline 900 & \\
\hline 901 & \\
\hline 902 & \\
\hline 903 & \\
\hline 904 & SK-057-3 \\
\hline 905 & SK-057-3 \\
\hline 906 & SK-057-3 \\
\hline 907 & SK-057-3 \\
\hline 908 & SK-082-2 \\
\hline 909 & SK-082-2 \\
\hline 910 & SK-082-2 \\
\hline 911 & SK-082-2 \\
\hline 912 & \\
\hline 913 & \\
\hline
\end{tabular}


$A B$

\begin{tabular}{|c|l|}
\hline & \multicolumn{1}{|c|}{ AB } \\
\hline 1 & SAMPLE_LOCATION \\
\hline 914 & \\
\hline 915 & \\
\hline 916 & \\
\hline 917 & \\
\hline 918 & \\
\hline 919 & \\
\hline 920 & \\
\hline 921 & \\
\hline 922 & \\
\hline 923 & \\
\hline 924 & \\
\hline 925 & \\
\hline 926 & \\
\hline 927 & \\
\hline 928 & \\
\hline 929 & \\
\hline 930 & \\
\hline 931 & \\
\hline 932 & \\
\hline 933 & \\
\hline 934 & \\
\hline 935 & \\
\hline 936 & SH-144-1 \\
\hline 937 & SH-144-1 \\
\hline 938 & SH-144-1 \\
\hline 939 & SH-144-1 \\
\hline 940 & SH-152-2 \\
\hline 941 & SH-152-2 \\
\hline 942 & SD-110-2 \\
\hline 943 & SD-110-2 \\
\hline 944 & SK-077-1 \\
\hline 945 & SK-077-1 \\
\hline 946 & SK-077-1 \\
\hline 947 & SK-077-1 \\
\hline 948 & COLLECTED BELOW WELL BLDG. NEAR 199-8T. \\
\hline 949 & COLLECTED BELOW WELL BLDG. NEAR 199-8T. \\
\hline 950 & COLLECTED BELOW WELL BLDG. NEAR 199-8T. \\
\hline 951 & \\
\hline
\end{tabular}


$A B$

\begin{tabular}{|c|c|}
\hline & AB \\
\hline 1 & SAMPLE_LOCATION \\
\hline 952 & \\
\hline 953 & \\
\hline 954 & SAMPLE COLLECTED 60 YARDS UPRIVER FROM RAD ZONE. \\
\hline 955 & SAMPLE COLLECTED 60 YARDS UPRIVER FROM RAD ZONE. \\
\hline 956 & SAMPLE COLLECTED 60 YARDS UPRIVER FROM RAD ZONE. \\
\hline 957 & \\
\hline 958 & \\
\hline 959 & \\
\hline 960 & \\
\hline 961 & \\
\hline 962 & \\
\hline 963 & \\
\hline 964 & \\
\hline 965 & \\
\hline 966 & \\
\hline 967 & \\
\hline 968 & \\
\hline 969 & \\
\hline 970 & \\
\hline 971 & \\
\hline 972 & \\
\hline 973 & \\
\hline 974 & \\
\hline 975 & \\
\hline 976 & \\
\hline 977 & \\
\hline 978 & \\
\hline 979 & \\
\hline 980 & \\
\hline 981 & \\
\hline 982 & \\
\hline 983 & \\
\hline 984 & \\
\hline 985 & \\
\hline 986 & \\
\hline 987 & \\
\hline 988 & \\
\hline 989 & \\
\hline
\end{tabular}




\begin{tabular}{|c|c|}
\hline & AB \\
\hline 1 & \\
\hline 990 & \\
\hline 991 & \\
\hline 992 & \\
\hline 993 & \\
\hline 994 & \\
\hline 995 & \\
\hline 996 & \\
\hline 997 & \\
\hline 998 & \\
\hline 999 & \\
\hline 1000 & \\
\hline 1001 & \\
\hline 1002 & \\
\hline 1003 & \\
\hline 1004 & \\
\hline 1005 & \\
\hline 1006 & \\
\hline 1007 & \\
\hline 1008 & \\
\hline 1009 & \\
\hline 1010 & \\
\hline 1011 & \\
\hline 1012 & \\
\hline 1013 & \\
\hline 1014 & \\
\hline 1015 & \\
\hline 1016 & \\
\hline 1017 & \\
\hline 1018 & \\
\hline 1019 & \\
\hline 1020 & \\
\hline 1021 & \\
\hline 1022 & \\
\hline 1023 & \\
\hline 1024 & \\
\hline 1025 & \\
\hline 1026 & \\
\hline 1027 & \\
\hline
\end{tabular}




\begin{tabular}{|c|c|}
\hline & \multicolumn{1}{|c|}{ AB } \\
\hline 1 & \\
\hline 1028 & \\
\hline 1029 & SAMPLE_LOCATION \\
\hline 1030 & \\
\hline 1031 & \\
\hline 1032 & \\
\hline 1033 & \\
\hline 1034 & \\
\hline 1035 & \\
\hline 1036 & \\
\hline 1037 & \\
\hline 1038 & \\
\hline 1039 & \\
\hline 1040 & \\
\hline 1041 & \\
\hline 1042 & \\
\hline 1043 & \\
\hline 1044 & \\
\hline 1045 & \\
\hline 1046 & \\
\hline 1047 & \\
\hline 1048 & \\
\hline 1049 & \\
\hline 1050 & \\
\hline 1051 & \\
\hline 1052 & \\
\hline 1053 & \\
\hline 1054 & \\
\hline 1055 & \\
\hline 1056 & \\
\hline 1057 & \\
\hline 1058 & \\
\hline 1059 & \\
\hline 1060 & \\
\hline 1061 & \\
\hline 1062 & \\
\hline 1063 & \\
\hline 1064 & \\
\hline 1065 & \\
\hline
\end{tabular}




\begin{tabular}{|c|c|}
\hline 1 & AB \\
\hline 1066 & SAMPLE_LOCATION \\
\hline 1067 & \\
\hline 1068 & \\
\hline 1069 & \\
\hline 1070 & \\
\hline 1071 & \\
\hline 1072 & \\
\hline 1073 & \\
\hline 1074 & \\
\hline 1075 & \\
\hline 1077 & \\
\hline 1078 & \\
\hline 1079 & \\
\hline
\end{tabular}


AC

\begin{tabular}{|c|c|}
\hline & $\mathrm{AC}$ \\
\hline 1 & SAMP_COMMENT \\
\hline 2 & River Mile 3.8 - spring water 100B intake \\
\hline 3 & River Mile 3.8 - spring water 100B intake \\
\hline 4 & River Mile 3.8 - spring water 100B intake \\
\hline 5 & River Mile 3.8 - spring water 100B intake \\
\hline 6 & River Mile 3.8 - spring water 100B intake \\
\hline 7 & River Mile 3.7 - river water \\
\hline 8 & River Mile 3.7 - river water \\
\hline 9 & River Mile 3.7 - river water \\
\hline 10 & River Mile 3.7 - river water \\
\hline 11 & River Mile 3.7 - river water \\
\hline 12 & \\
\hline 13 & \\
\hline 14 & \\
\hline 15 & \\
\hline 16 & \\
\hline 17 & River Mile 3.9 - spring water $\sim 100$ ' downstream of old concrete slabs from 100B/C intake structure \\
\hline 18 & River Mile 3.9 - spring water $\sim 100$ downstream of old concrete slabs from 100B/C intake structure \\
\hline 19 & River Mile 3.9 - spring water $\sim 100$ downstream of old concrete slabs from 100B/C intake structure \\
\hline 20 & River Mile 3.9 - spring water $\sim 100$ downstream of old concrete slabs from 100B/C intake structure \\
\hline 21 & River Mile 3.9 - spring water $\sim 100$ downstream of old concrete slabs from 100B/C intake structure \\
\hline 22 & River Mile 11.0 - spring water \\
\hline 23 & River Mile 11.0 - spring water \\
\hline 24 & River Mile 11.0 - spring water \\
\hline 25 & River Mile 11.0 - spring water \\
\hline 26 & River Mile 11.0 - spring water \\
\hline 27 & River Mile 11.0 - spring water \\
\hline 28 & River Mile 14.3 - spring water \\
\hline 29 & River Mile 14.3 - spring water \\
\hline 30 & River Mile 14.3 - river water \\
\hline 31 & River Mile 14.3 - river water \\
\hline 32 & River Mile 14.3 - spring water \\
\hline 33 & River Mile 14.3 - river water \\
\hline 34 & River Mile 14.3 - spring water \\
\hline 35 & River Mile 14.3 - river water \\
\hline 36 & River Mile 14.3 - spring water \\
\hline 37 & River Mile 14.3 - river water \\
\hline 38 & River Mile 14.3 - spring water \\
\hline 39 & River Mile 14.3 - river water \\
\hline
\end{tabular}


AC

\begin{tabular}{|c|c|}
\hline & 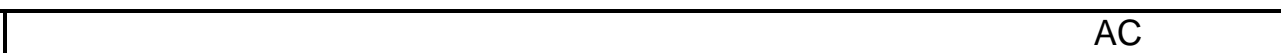 \\
\hline 1 & SAMP_COMMENT \\
\hline 40 & River Mile 14.4 - river water \\
\hline 41 & River Mile 14.4 - river water \\
\hline 42 & River Mile 14.4 - river water \\
\hline 43 & River Mile 14.4 - river water \\
\hline 44 & River Mile 14.4 - river water \\
\hline 45 & River Mile 14.4 - river water \\
\hline 46 & River Mile 14.4 - spring water \\
\hline 47 & River Mile 14.4 - spring water \\
\hline 48 & River Mile 14.4 - spring water \\
\hline 49 & River Mile 14.4 - spring water \\
\hline 50 & River Mile 14.4 - spring water \\
\hline 51 & River Mile 14.4 - spring water \\
\hline 52 & River Mile 5.6 - spring water \\
\hline 53 & River Mile 5.6 - spring water \\
\hline 54 & River Mile 5.6 - spring water \\
\hline 55 & River Mile 5.6 - spring water \\
\hline 56 & River Mile 5.6 - spring water \\
\hline 57 & River Mile 5.6 - river water \\
\hline 58 & River Mile 5.6 - river water \\
\hline 59 & River Mile 5.6 - river water \\
\hline 60 & River Mile 5.6 - river water \\
\hline 61 & River Mile 5.6 - river water \\
\hline 62 & River Mile 14.5 - spring water \\
\hline 63 & River Mile 14.5 - spring water \\
\hline 64 & River Mile 14.5 - spring water \\
\hline 65 & River Mile 14.5 - spring water \\
\hline 66 & River Mile 14.5 - spring water \\
\hline 67 & River Mile 14.5 - spring water \\
\hline 68 & River Mile 14.5 - river water - water is turbid \\
\hline 69 & River Mile 14.5 - river water - water is turbid \\
\hline 70 & River Mile 14.5 - river water - water is turbid \\
\hline 71 & River Mile 14.5 - river water - water is turbid \\
\hline 72 & River Mile 14.5 - river water - water is turbid \\
\hline 73 & River Mile 14.5 - river water - water is turbid \\
\hline 74 & River Mile 11.0 - spring water \\
\hline 75 & River Mile 11.0 - spring water \\
\hline 76 & River Mile 11.0 - spring water \\
\hline 77 & River Mile 11.0 - spring water \\
\hline
\end{tabular}


AC

\begin{tabular}{|c|c|}
\hline & 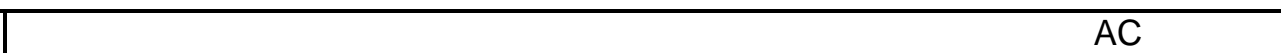 \\
\hline 1 & $\begin{array}{l}\text { SAMP_COMMENT } \\
\end{array}$ \\
\hline 78 & River Mile 11.0 - spring water \\
\hline 79 & River Mile 11.0 - spring water \\
\hline 80 & River Mile 11.0 - river water \\
\hline 81 & River Mile 11.0 - river water \\
\hline 82 & River Mile 11.0 - river water \\
\hline 83 & River Mile 11.0 - river water \\
\hline 84 & River Mile 11.0 - river water \\
\hline 85 & River Mile 11.0 - river water \\
\hline 86 & River Mile 14.9 - spring water \\
\hline 87 & River Mile 14.9 - spring water \\
\hline 88 & River Mile 14.9 - spring water \\
\hline 89 & River Mile 14.9 - spring water \\
\hline 90 & River Mile 14.9 - spring water \\
\hline 91 & River Mile 14.9 - spring water \\
\hline 92 & River Mile 14.9 - river water \\
\hline 93 & River Mile 14.9 - river water \\
\hline 94 & River Mile 14.9 - river water \\
\hline 95 & River Mile 14.9 - river water \\
\hline 96 & River Mile 14.9 - river water \\
\hline 97 & River Mile 14.9 - river water \\
\hline 98 & River Mile 19.0 - spring water \\
\hline 99 & River Mile 19.0 - spring water \\
\hline 100 & River Mile 19.0 - spring water \\
\hline 101 & River Mile 19.0 - spring water \\
\hline 102 & River Mile 19.0 - spring water \\
\hline 103 & River Mile 19.0 - river water \\
\hline 104 & River Mile 19.0 - river water \\
\hline 105 & River Mile 19.0 - river water \\
\hline 106 & River Mile 19.0 - river water \\
\hline 107 & River Mile 19.0 - river water \\
\hline 108 & River Mile 18.7 - spring water \\
\hline 109 & River Mile 18.7 - spring water \\
\hline 110 & River Mile 18.7 - spring water \\
\hline 111 & River Mile 18.7 - spring water \\
\hline 112 & River Mile 18.7 - spring water \\
\hline 113 & River Mile 18.7 - river water \\
\hline 114 & River Mile 18.7 - river water \\
\hline 115 & River Mile 18.7 - river water \\
\hline
\end{tabular}


AC

\begin{tabular}{|c|c|}
\hline 1 & SAMP_COMMENT \\
\hline 116 & River Mile 18.7 - river water \\
\hline 117 & River Mile 18.7 - river water \\
\hline 118 & River Mile 22.1 - spring water \\
\hline 119 & River Mile 22.1 - spring water \\
\hline 120 & River Mile 22.1 - spring water \\
\hline 121 & River Mile 22.1 - spring water \\
\hline 122 & River Mile 22.1 - spring water \\
\hline 123 & River Mile 22.1 - river water \\
\hline 124 & River Mile 22.1 - river water \\
\hline 125 & River Mile 22.1 - river water \\
\hline 126 & River Mile 22.1 - river water \\
\hline 127 & River Mile 22.1 - river water \\
\hline 128 & River Mile 21.8 - spring water \\
\hline 129 & River Mile 21.8 - spring water \\
\hline 130 & River Mile 21.8 - spring water \\
\hline 131 & River Mile 21.8 - spring water \\
\hline 132 & River Mile 21.8 - spring water \\
\hline 133 & River Mile 21.8 - river water \\
\hline 134 & River Mile 21.8 - river water \\
\hline 135 & River Mile 21.8 - river water \\
\hline 136 & River Mile 21.8 - river water \\
\hline 137 & River Mile 21.8 - river water \\
\hline 138 & River Mile 21.6 - spring water \\
\hline 139 & River Mile 21.6 - spring water \\
\hline 140 & River Mile 21.6 - spring water \\
\hline 141 & River Mile 21.6 - spring water \\
\hline 142 & River Mile 21.6 - spring water \\
\hline 143 & River Mile 21.6 - spring water \\
\hline 144 & River Mile 21.6 - spring water \\
\hline 145 & River Mile 21.6 - spring water \\
\hline 146 & River Mile 21.6 - spring water \\
\hline 147 & River Mile 21.6 - spring water \\
\hline 148 & River Mile 21.6 - river water \\
\hline 149 & River Mile 21.6 - river water \\
\hline 150 & River Mile 21.6 - river water \\
\hline 151 & River Mile 21.6 - river water \\
\hline 152 & River Mile 21.6 - river water \\
\hline 153 & River Mile 21.6 - river water \\
\hline
\end{tabular}


$\mathrm{AC}$

\begin{tabular}{|c|c|}
\hline 1 & SAMP_COMMENT \\
\hline 154 & River Mile 21.6 - spring water \\
\hline 155 & River Mile 21.6 - river water \\
\hline 156 & River Mile 21.6 - river water \\
\hline 157 & River Mile 21.6 - river water \\
\hline 158 & River Mile 20.8 - spring water \\
\hline 159 & River Mile 20.8 - spring water \\
\hline 160 & River Mile 20.8 - spring water \\
\hline 161 & River Mile 20.8 - spring water \\
\hline 162 & River Mile 20.8 - spring water \\
\hline 163 & River Mile 20.8 - river water - $11 "$ to $12 "$ \\
\hline 164 & River Mile 20.8 - river water - $11 "$ to $12 "$ \\
\hline 165 & River Mile 20.8 - river water - 11" to $12 "$ \\
\hline 166 & River Mile 20.8 - river water - 11" to $12 "$ \\
\hline 167 & River Mile 20.8 - river water - 11" to $12 "$ \\
\hline 168 & River Mile 25.2 - spring water \\
\hline 169 & River Mile 25.2 - spring water \\
\hline 170 & River Mile 25.2 - spring water \\
\hline 171 & River Mile 25.2 - spring water \\
\hline 172 & River Mile 25.2 - spring water \\
\hline 173 & River Mile 25.2 - river water \\
\hline 174 & River Mile 25.2 - river water \\
\hline 175 & River Mile 25.2 - river water \\
\hline 176 & River Mile 25.2 - river water \\
\hline 177 & River Mile 25.2 - river water \\
\hline 178 & River Mile 24.6 - spring water \\
\hline 179 & River Mile 24.6 - spring water \\
\hline 180 & River Mile 24.6 - spring water \\
\hline 181 & River Mile 24.6 - spring water \\
\hline 182 & River Mile 24.6 - spring water \\
\hline 183 & River Mile 24.6 - spring water \\
\hline 184 & River Mile 24.6 - spring water \\
\hline 185 & River Mile 24.6 - spring water \\
\hline 186 & River Mile 24.6 - spring water \\
\hline 187 & River Mile 24.6 - spring water \\
\hline 188 & River Mile 24.6 - river water \\
\hline 189 & River Mile 24.6 - river water \\
\hline 190 & River Mile 24.6 - river water \\
\hline
\end{tabular}

191 River Mile 24.6 - river water 
AC

\begin{tabular}{|c|c|}
\hline 1 & $\begin{array}{l}\text { SAMP_COMMENT } \\
\end{array}$ \\
\hline 192 & River Mile 24.6 - river water \\
\hline 193 & River Mile 24.6 - river water \\
\hline 194 & River Mile 24.6 - river water \\
\hline 195 & River Mile 24.6 - river water \\
\hline 196 & River Mile 24.6 - river water \\
\hline 197 & River Mile 24.6 - river water \\
\hline 198 & River Mile 9.0 - river water \\
\hline 199 & River Mile 9.0 - river water \\
\hline 200 & River Mile 9.0 - river water \\
\hline 201 & River Mile 9.0 - river water \\
\hline 202 & River Mile 9.0 - river water \\
\hline 203 & River Mile 9.0 - spring water \\
\hline 204 & River Mile 9.0 - spring water \\
\hline 205 & River Mile 9.0 - spring water \\
\hline 206 & River Mile 9.0 - spring water \\
\hline 207 & River Mile 9.0 - spring water \\
\hline 208 & River Mile 9.0 - spring water \\
\hline 209 & River Mile 9.1 - river water \\
\hline 210 & River Mile 9.1 - river water \\
\hline 211 & River Mile 9.1 - river water \\
\hline 212 & River Mile 9.1 - river water \\
\hline 213 & River Mile 9.1 - river water \\
\hline 214 & River Mile 9.1 - river water \\
\hline 215 & River Mile 9.1 - spring water \\
\hline 216 & River Mile 9.1 - spring water \\
\hline 217 & River Mile 9.1 - spring water \\
\hline 218 & River Mile 9.1 - spring water \\
\hline 219 & River Mile 9.1 - spring water \\
\hline 220 & River Mile 9.1 - spring water \\
\hline 221 & River Mile 7.4 - river water \\
\hline 222 & River Mile 7.4 - river water \\
\hline 223 & River Mile 7.4 - river water \\
\hline 224 & River Mile 7.4 - river water \\
\hline 225 & River Mile 7.4 - river water \\
\hline 226 & River Mile 7.4 - river water \\
\hline 227 & River Mile 7.4 - spring water \\
\hline 228 & River Mile 7.4 - spring water \\
\hline 229 & River Mile 7.4 - spring water \\
\hline
\end{tabular}


AC

\begin{tabular}{|c|c|}
\hline 1 & $\begin{array}{l}\text { SAMP_COMMENT } \\
\end{array}$ \\
\hline 230 & River Mile 7.4 - spring water \\
\hline 231 & River Mile 7.4 - spring water \\
\hline 232 & River Mile 7.4 - spring water \\
\hline 233 & River Mile 9.4 - river water \\
\hline 234 & River Mile 9.4 - river water \\
\hline 235 & River Mile 9.4 - river water \\
\hline 236 & River Mile 9.4 - river water \\
\hline 237 & River Mile 9.4 - river water \\
\hline 238 & River Mile 9.4 - river water \\
\hline 239 & River Mile 9.4 - spring water \\
\hline 240 & River Mile 9.4 - spring water \\
\hline 241 & River Mile 9.4 - spring water \\
\hline 242 & River Mile 9.4 - spring water \\
\hline 243 & River Mile 9.4 - spring water \\
\hline 244 & River Mile 9.4 - spring water \\
\hline 245 & River Mile 9.2 - river water \\
\hline 246 & River Mile 9.2 - river water \\
\hline 247 & River Mile 9.2 - river water \\
\hline 248 & River Mile 9.2 - river water \\
\hline 249 & River Mile 9.2 - river water \\
\hline 250 & River Mile 9.2 - river water \\
\hline 251 & River Mile 9.2 - spring water \\
\hline 252 & River Mile 9.2 - spring water \\
\hline 253 & River Mile 9.2 - spring water \\
\hline 254 & River Mile 9.2 - spring water \\
\hline 255 & River Mile 9.2 - spring water \\
\hline 256 & River Mile 9.2 - spring water \\
\hline 257 & River Mile 9.2 - river water \\
\hline 258 & River Mile 9.2 - river water \\
\hline 259 & River Mile 9.2 - river water \\
\hline 260 & River Mile 9.2 - river water \\
\hline 261 & River Mile 9.2 - river water \\
\hline 262 & River Mile 9.2 - river water \\
\hline 263 & River Mile 9.2 - spring water \\
\hline 264 & River Mile 9.2 - spring water \\
\hline 265 & River Mile 9.2 - spring water \\
\hline 266 & River Mile 9.2 - spring water \\
\hline 267 & River Mile 9.2 - spring water \\
\hline
\end{tabular}


$\mathrm{AC}$

\begin{tabular}{|c|c|}
\hline & ${ }_{2}$ \\
\hline 1 & $\begin{array}{r}\text { SAMP_COMMENT } \\
\end{array}$ \\
\hline 268 & River Mile 9.2 - spring water \\
\hline 269 & River Mile 8.1 - river water \\
\hline 270 & River Mile 8.1 - river water \\
\hline 271 & River Mile 8.1 - river water \\
\hline 272 & River Mile 8.1 - river water \\
\hline 273 & River Mile 8.1 - river water \\
\hline 274 & River Mile 8.1 - river water \\
\hline 275 & River Mile 8.1 - spring water \\
\hline 276 & River Mile 8.1 - spring water \\
\hline 277 & River Mile 8.1 - spring water \\
\hline 278 & River Mile 8.1 - spring water \\
\hline 279 & River Mile 8.1 - spring water \\
\hline 280 & River Mile 8.1 - spring water \\
\hline 281 & River Mile 15.3 - spring water \\
\hline 282 & River Mile 15.3 - river water \\
\hline 283 & River Mile 15.3 - river water \\
\hline 284 & River Mile 15.3 - spring water \\
\hline 285 & River Mile 15.3 - river water \\
\hline 286 & River Mile 15.3 - spring water \\
\hline 287 & River Mile 15.3 - river water \\
\hline 288 & River Mile 15.3 - spring water \\
\hline 289 & River Mile 15.3 - river water \\
\hline 290 & River Mile 15.3 - spring water \\
\hline 291 & River Mile 15.3 - river water \\
\hline 292 & River Mile 15.3 - spring water \\
\hline 293 & \\
\hline 294 & \\
\hline 295 & \\
\hline 296 & \\
\hline 297 & \\
\hline 298 & \\
\hline 299 & \\
\hline 300 & \\
\hline 301 & \\
\hline 302 & \\
\hline 303 & \\
\hline 304 & \\
\hline 305 & \\
\hline
\end{tabular}




\begin{tabular}{|c|c|}
\hline 1 & SAMP_COMMENT \\
\hline 306 & FILTERED SAMPLE. \\
\hline 307 & FILTERED SAMPLE. \\
\hline 308 & FILTERED SAMPLE. \\
\hline 309 & FILTERED SAMPLE. \\
\hline 310 & FILTERED SAMPLE. \\
\hline 311 & Notice: Sample taken three (3) feet from the shore \\
\hline 312 & Notice: Sample taken twenty (20) feet from the shore \\
\hline 313 & NOTICE: B076Q3 IS A SPLIT OF B01321 (20ft. from the shore) \\
\hline 314 & Notice: Sample taken ten (10) feet from the shore \\
\hline 315 & Notice: Sample taken three (3) feet from the shore \\
\hline 316 & Notice: Sample taken twenty (20) feet from the shore \\
\hline 317 & Notice: Sample taken twenty (20) feet from the shore \\
\hline 318 & NOTICE: B076Q3 IS A SPLIT OF B01321 (20ft. from the shore) \\
\hline 319 & NOTICE: B076Q4 IS A SPLIT OF B076H5 (20ft. from the shore) \\
\hline 320 & Notice: Sample taken three (3) feet from the shore \\
\hline 321 & Notice: Sample taken ten (10) feet from the shore \\
\hline 322 & \\
\hline 323 & Notice: Sample taken three (3) feet from the shore \\
\hline 324 & Notice: Sample taken twenty (20) feet from the shore \\
\hline 325 & NOTICE: B076Q3 IS A SPLIT OF B01321 (20ft. from the shore) \\
\hline 326 & Notice: Sample taken ten (10) feet from the shore \\
\hline 327 & NOTICE: B076Q3 IS A SPLIT OF B01321 (20ft. from the shore) \\
\hline 328 & Notice: Sample taken three (3) feet from the shore \\
\hline 329 & Notice: Sample taken twenty (20) feet from the shore \\
\hline 330 & NOTICE: B076Q3 IS A SPLIT OF B01321 (20ft. from the shore) \\
\hline 331 & Notice: Sample taken ten (10) feet from the shore \\
\hline 332 & Notice: Sample taken three (3) feet from the shore \\
\hline 333 & Notice: Sample taken twenty (20) feet from the shore \\
\hline 334 & NOTICE: B076Q3 IS A SPLIT OF B01321 (20ft. from the shore) \\
\hline 335 & Notice: Sample taken ten (10) feet from the shore \\
\hline 336 & Notice: Sample taken three (3) feet from the shore \\
\hline 337 & Notice: Sample taken twenty (20) feet from the shore \\
\hline 338 & NOTICE: B076Q3 IS A SPLIT OF B01321 (20ft. from the shore) \\
\hline 339 & Notice: Sample taken ten (10) feet from the shore \\
\hline 340 & Notice: Sample taken three (3) feet from the shore \\
\hline 341 & Notice: Sample taken twenty (20) feet from the shore \\
\hline 342 & NOTICE: B076Q3 IS A SPLIT OF B01321 (20ft. from the shore) \\
\hline 343 & Notice: Sample taken ten (10) feet from the shore \\
\hline
\end{tabular}

$\begin{array}{ll}343 & \text { Notice: Sample taken ten (10) feet from the shore }\end{array}$ 
344 Notice: Sample taken three (3) feet from the shore

345 Notice: Sample taken twenty (20) feet from the shore

346 NOTICE: B076Q3 IS A SPLIT OF B01321 (20ft. from the shore)

347 Notice: Sample taken ten (10) feet from the shore

$\begin{array}{lll}348 & \text { Notice: Sample was taken three (3) feet from the shore }\end{array}$

349 Notice: Sample was taken ten (10) feet from shore

350 Notice: Sample was taken twenty (20) feet from the shore

351 NOTICE: B076P7 IS A REPLICATE OF B01372 (3ft. from shore)

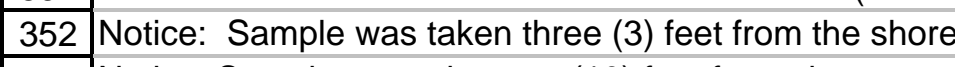

353 Notice: Sample was taken ten (10) feet from shore

354 Notice: Sample was taken twenty (20) feet from the shore

355 Notice: Sample taken three (3) feet from the shore

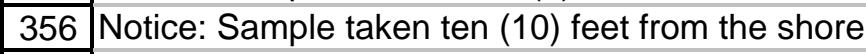

357 Notice: Sample taken twenty (20) feet from the shore

358 NOTICE: B076P7 IS A REPLICATE OF B01372 (3ft. from shore)

359 NOTICE: B076P8 IS A REPLICATE OF B076H7 (3ft. from shore)

360 Notice: Sample was taken three (3) feet from the shore

361 Notice: Sample was taken ten (10) feet from shore

362 Notice: Sample was taken twenty (20) feet from the shore

363 NOTICE: B076P7 IS A REPLICATE OF B01372 (3ft. from shore)

364 Notice: Sample was taken three (3) feet from the shore

365 Notice: Sample was taken ten (10) feet from shore

366 Notice: Sample was taken twenty (20) feet from the shore

367 NOTICE: B076P7 IS A REPLICATE OF B01372 (3ft. from shore)

368 Notice: Sample was taken three (3) feet from the shore

369 Notice: Sample was taken ten (10) feet from shore

370 Notice: Sample was taken twenty (20) feet from the shore

371 NOTICE: B076P7 IS A REPLICATE OF B01372 (3ft. from shore)

372 Notice: Sample was taken three (3) feet from the shore

\begin{tabular}{l|l}
373 & Notice: Sample was taken ten (10) feet from shore
\end{tabular}

374 Notice: Sample was taken twenty (20) feet from the shore

375 NOTICE: B076P7 IS A REPLICATE OF B01372 (3ft. from shore)

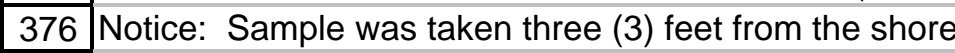

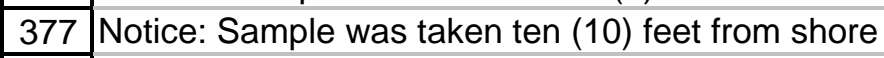

378 Notice: Sample was taken twenty (20) feet from the shore

379 NOTICE: B076P7 IS A REPLICATE OF B01372 (3ft. from shore)

380 Notice: Sample was taken three (3) feet from the shore

$\begin{array}{lll}381 & \text { Notice: Sample was taken ten (10) feet from shore }\end{array}$ 


\begin{tabular}{|c|c|}
\hline 1 & SAMP_COMMENT \\
\hline 382 & Notice: Sample was taken twenty (20) feet from the shore \\
\hline 383 & NOTICE: B076P7 IS A REPLICATE OF B01372 (3ft. from shore) \\
\hline 384 & Notice: Sample was taken twenty (20) feet from the shore \\
\hline 385 & Notice: Sample was taken ten (10) feet from the shore \\
\hline 386 & Notice: Sample was taken three (3) feet from the shore \\
\hline 387 & Notice: Sample was taken twenty (20) feet from the shore \\
\hline 388 & Notice: Sample was taken ten (10) feet from the shore \\
\hline 389 & Notice: Sample was taken three (3) feet from the shore \\
\hline 390 & Notice: Sample taken twenty (20) feet from the shore \\
\hline 391 & Notice: Sample taken ten (10) feet from the shore \\
\hline 392 & Notice: Sample taken three (3) feet from the shore \\
\hline 393 & Notice: Sample was taken twenty (20) feet from the shore \\
\hline 394 & Notice: Sample was taken ten (10) feet from the shore \\
\hline 395 & Notice: Sample was taken three (3) feet from the shore \\
\hline 396 & Notice: Sample was taken twenty (20) feet from the shore \\
\hline 397 & Notice: Sample was taken ten (10) feet from the shore \\
\hline 398 & Notice: Sample was taken three (3) feet from the shore \\
\hline 399 & Notice: Sample was taken twenty (20) feet from the shore \\
\hline 400 & Notice: Sample was taken ten (10) feet from the shore \\
\hline 401 & Notice: Sample was taken three (3) feet from the shore \\
\hline 402 & Notice: Sample was taken twenty (20) feet from the shore \\
\hline 403 & Notice: Sample was taken ten (10) feet from the shore \\
\hline 404 & Notice: Sample was taken three (3) feet from the shore \\
\hline 405 & Notice: Sample was taken twenty (20) feet from the shore \\
\hline 406 & Notice: Sample was taken ten (10) feet from the shore \\
\hline 407 & Notice: Sample was taken three (3) feet from the shore \\
\hline 408 & Notice: Sample was taken twenty (20) feet from the shore \\
\hline 409 & Notice: Sample was taken ten (10) feet from the shore \\
\hline 410 & Notice: Sample was taken three (3) feet from the shore \\
\hline 411 & \\
\hline 412 & \\
\hline 413 & \\
\hline 414 & \\
\hline 415 & REFER TO FOLLOW-UP REQUESTS \#W008 \& W008-1 REGARDING BETA AND SR-90. \\
\hline 416 & REFER TO FOLLOW-UP REQUESTS \#W008 \& W008-1 REGARDING BETA AND SR-90. \\
\hline 417 & REFER TO FOLLOW-UP REQUESTS \#W008 \& W008-1 REGARDING BETA AND SR-90. \\
\hline 418 & REFER TO FOLLOW-UP REQUESTS \#W008 \& W008-1 REGARDING BETA AND SR-90. \\
\hline
\end{tabular}


$A C$

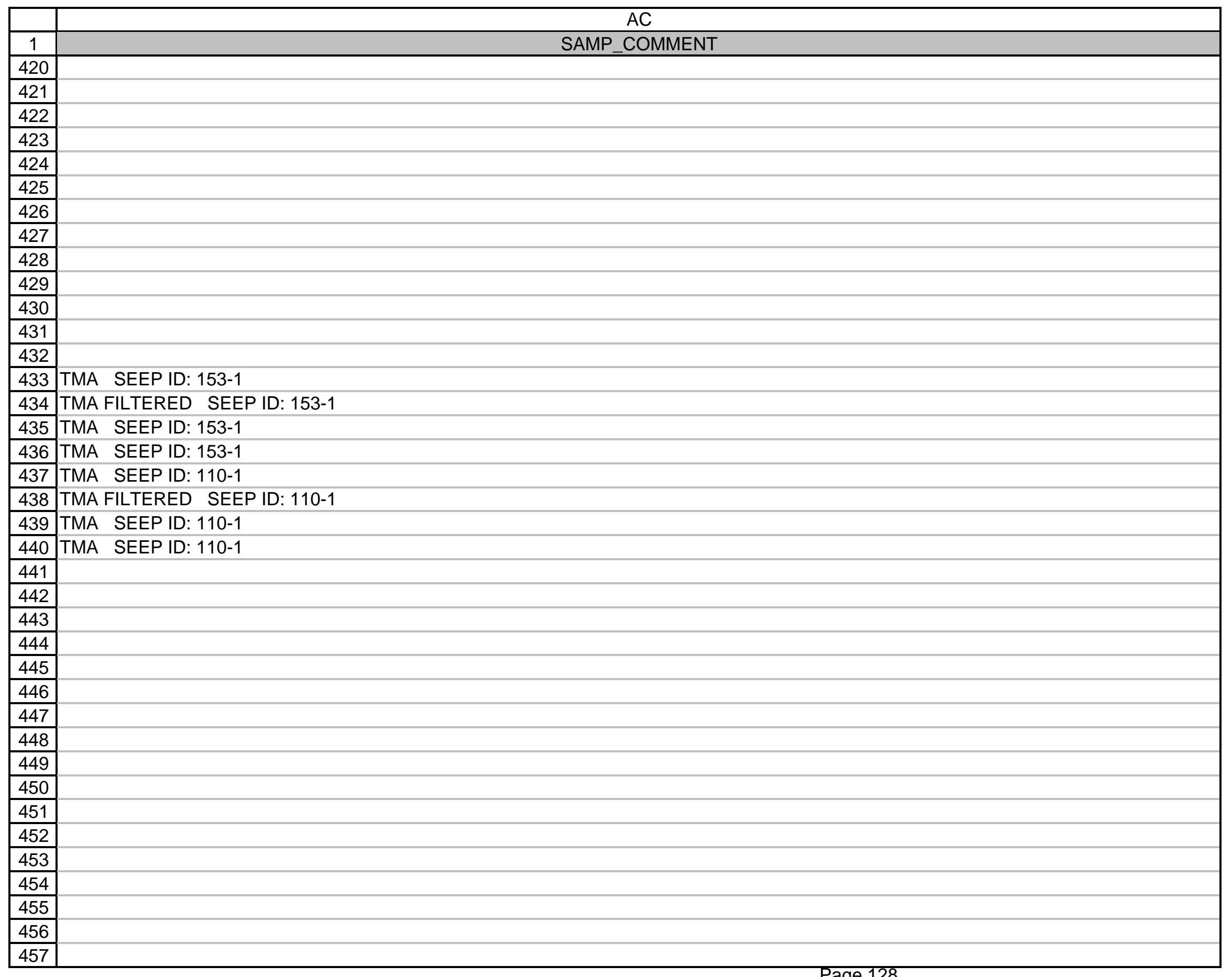


AC

\begin{tabular}{|c|c|}
\hline & $\mathrm{AC}$ \\
\hline 1 & SAMP_COMMENT \\
\hline 458 & \\
\hline 459 & \\
\hline 460 & \\
\hline 461 & \\
\hline 462 & \\
\hline 463 & \\
\hline 464 & TMA SEEP ID: $145-2$ \\
\hline 465 & TMA FILTERED SEEP ID: $145-2$ \\
\hline 466 & TMA SEEP ID: $145-2$ \\
\hline 467 & TMA SEEP ID: $145-2$ \\
\hline 468 & TMA SEEP ID: 037-1 \\
\hline 469 & TMA FILTERED SEEP ID: 037-1 \\
\hline 470 & TMA SEEP ID: 037-1 \\
\hline 471 & TMA SEEP ID: 037-1 \\
\hline 472 & TMA SEEP ID: 205-1 \\
\hline 473 & TMA FILTERED SEEP ID: 205-1 \\
\hline 474 & TMA SEEP ID: 205-1 \\
\hline 475 & TMA SEEP ID: 205-1 \\
\hline 476 & \\
\hline 477 & \\
\hline 478 & \\
\hline 479 & \\
\hline 480 & \\
\hline 481 & \\
\hline 482 & \\
\hline 483 & \\
\hline 484 & \\
\hline 485 & \\
\hline 486 & \\
\hline 487 & \\
\hline 488 & \\
\hline 489 & \\
\hline 490 & REFER TO INCIDENT REPORT 11/11/93 REGARDING INCORRECT ALPHA SPEC DETECTOR BACKGROUND USED FOR U-ISOTOPIC. \\
\hline 491 & REFER TO INCIDENT REPORT 11/11/93 REGARDING INCORRECT ALPHA SPEC DETECTOR BACKGROUND USED FOR U-ISOTOPIC. \\
\hline 492 & REFER TO INCIDENT REPORT 11/11/93 REGARDING INCORRECT ALPHA SPEC DETECTOR BACKGROUND USED FOR U-ISOTOPIC. \\
\hline 493 & REFER TO INCIDENT REPORT 11/11/93 REGARDING INCORRECT ALPHA SPEC DETECTOR BACKGROUND USED FOR U-ISOTOPIC. \\
\hline 494 & REFER TO INCIDENT REPORT 11/11/93 REGARDING INCORRECT ALPHA SPEC DETECTOR BACKGROUND USED FOR U-ISOTOPIC. \\
\hline 495 & REFER TO INCIDENT REPORT 11/11/93 REGARDING INCORRECT ALPHA SPEC DETECTOR BACKGROUND USED FOR U-ISOTOPIC. \\
\hline
\end{tabular}


496 REFER TO INCIDENT REPORT 11/11/93 REGARDING INCORRECT ALPHA SPEC DETECTOR BACKGROUND USED FOR U-ISOTOPIC. 497

498

499

500

501

502

503

504

\begin{tabular}{|l|}
504 \\
\hline 505 \\
\hline 506 \\
\hline 5
\end{tabular}

507

508

509

510

511

512

513

514

515

516

517

518

519

520

521

522

523

524

525

531

532 TMA SEEP ID: 077-1

\begin{tabular}{|l|l}
533 & TMA FILTERED SEEP ID: 077-1 \\
\hline
\end{tabular} 
AC

\begin{tabular}{|c|c|}
\hline 1 & SAMP_COMMENT \\
\hline 534 & TMA SEEP ID: 077-1 \\
\hline 535 & TMA SEEP ID: 077-1 \\
\hline 536 & TMA SEEP ID: $189-1$ \\
\hline 537 & TMA-FILTERED SEEP ID: $189-1$ \\
\hline 538 & TMA SEEP ID: $189-1$ \\
\hline 539 & TMA SEEP ID: $189-1$ \\
\hline 540 & \\
\hline 541 & \\
\hline 542 & \\
\hline 543 & \\
\hline 544 & \\
\hline 545 & \\
\hline 546 & \\
\hline 547 & TMA SEEP ID: 152-2 \\
\hline 548 & TMA SEEP ID: $152-3$ \\
\hline 549 & \\
\hline 550 & \\
\hline 551 & \\
\hline 552 & \\
\hline 553 & \\
\hline 554 & \\
\hline 555 & \\
\hline 556 & \\
\hline 557 & \\
\hline 558 & \\
\hline 559 & \\
\hline 560 & \\
\hline 561 & Duplicate of B0BMJ9 \\
\hline 562 & Duplicate of BOBMKO \\
\hline 563 & Duplicate of BOBMK1 \\
\hline 564 & Duplicate of BOBMK1 \\
\hline 565 & \\
\hline 566 & \\
\hline 567 & \\
\hline 568 & \\
\hline 569 & \\
\hline 570 & \\
\hline 571 & \\
\hline
\end{tabular}




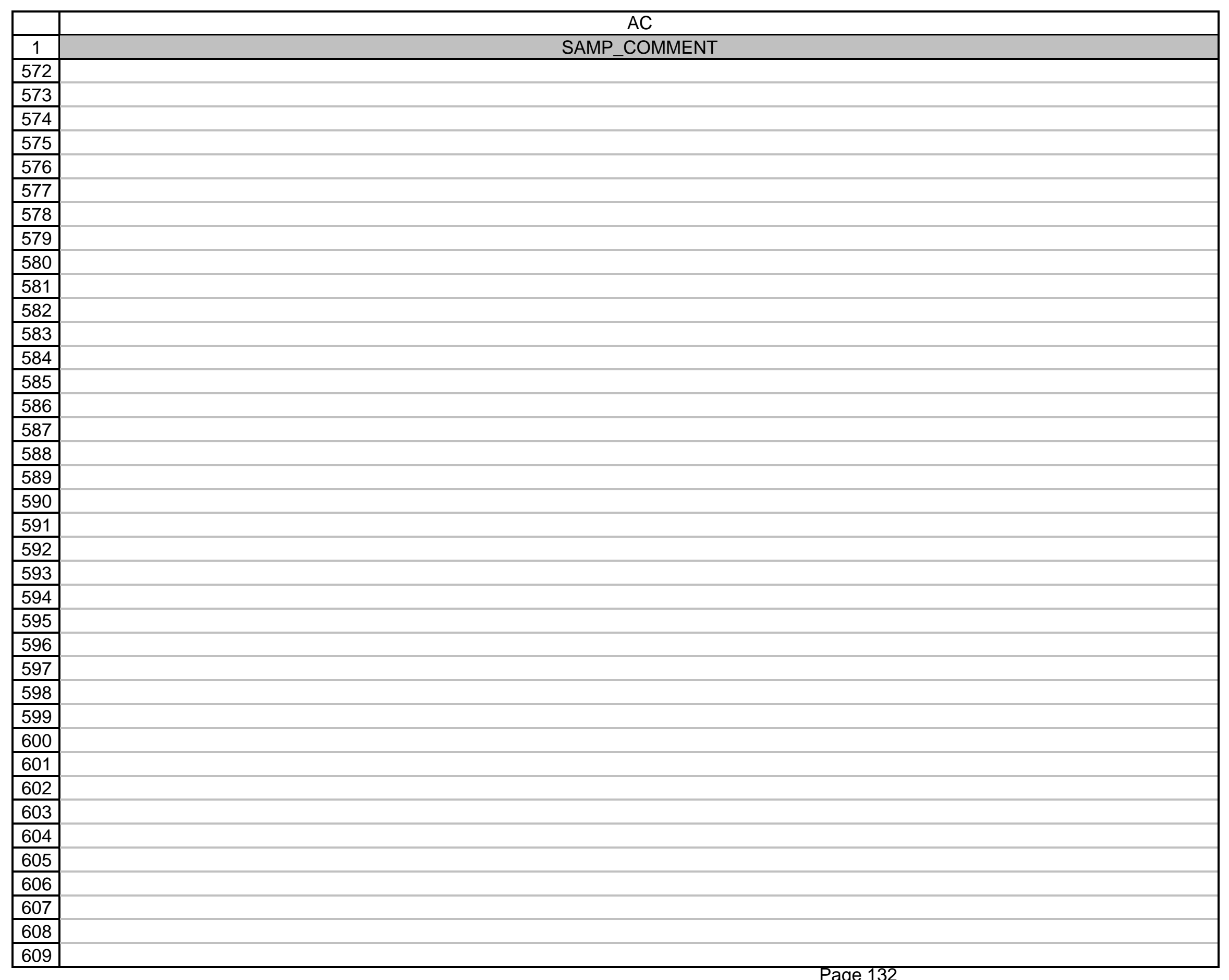




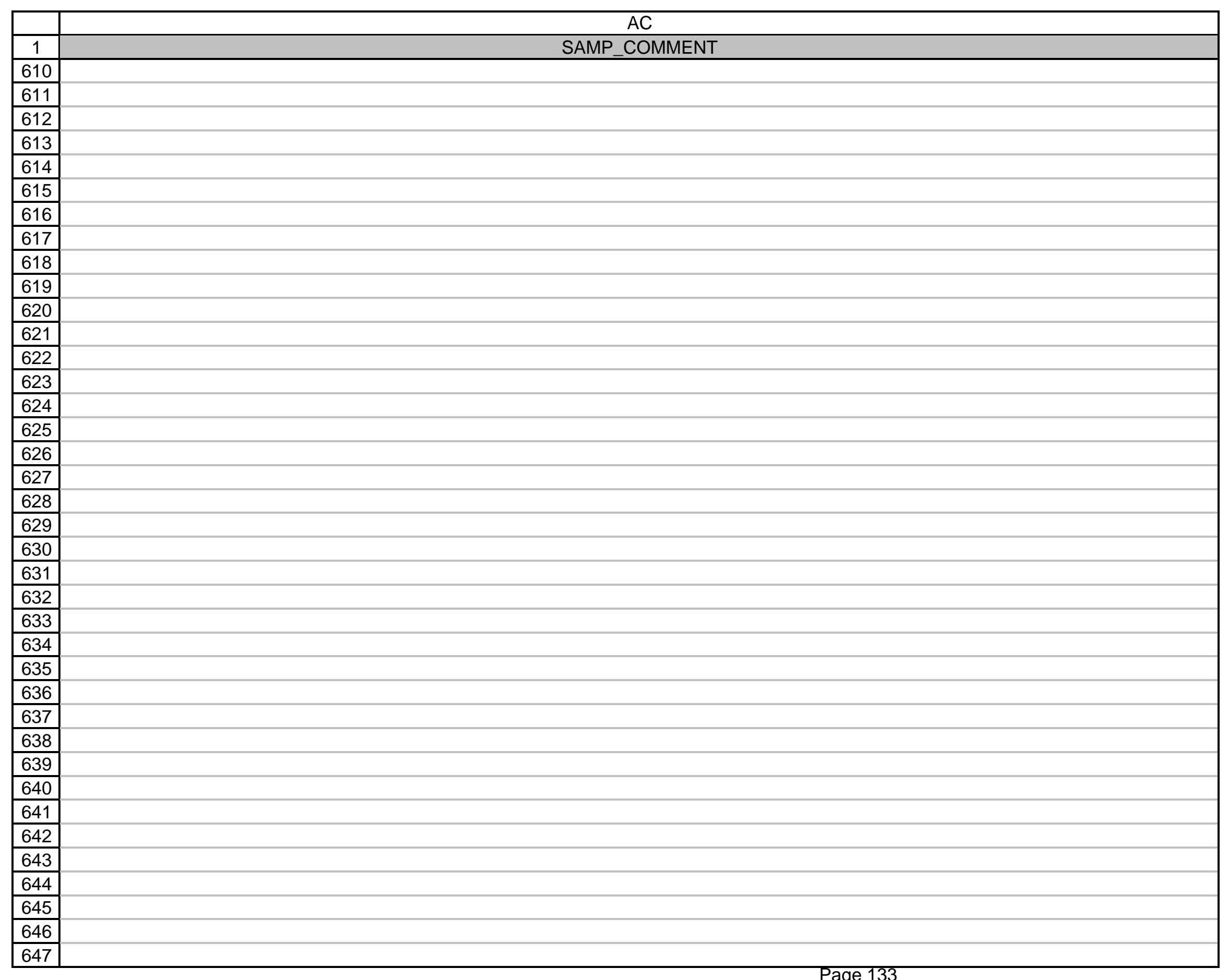




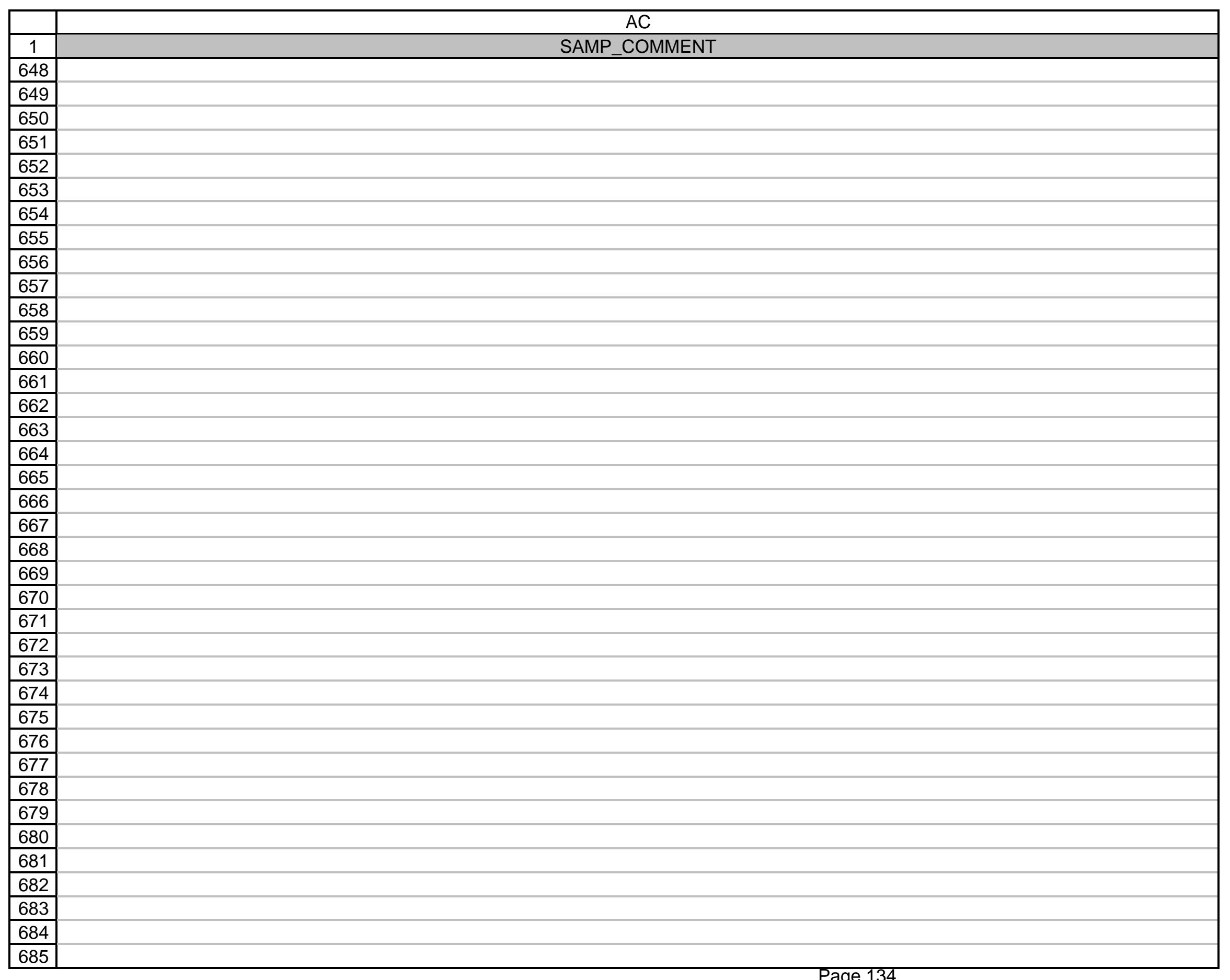


$A C$

\begin{tabular}{|c|c|}
\hline & $A C$ \\
\hline 1 & SAMP_COMMENT \\
\hline 686 & \\
\hline 687 & Duplicate sample of B0GBJ9 \\
\hline 688 & \\
\hline 689 & \\
\hline 690 & HIGH AMOUNT OF SUSPENDED SEDIMENT IN THE SAMPLE. \\
\hline 691 & HIGH AMOUNT OF SUSPENDED SEDIMENT IN THE SAMPLE. \\
\hline 692 & HIGH AMOUNT OF SUSPENDED SEDIMENT IN THE SAMPLE. \\
\hline 693 & HIGH AMOUNT OF SUSPENDED SEDIMENT IN THE SAMPLE. \\
\hline 694 & HIGH AMOUNT OF SUSPENDED SEDIMENT IN THE SAMPLE. \\
\hline 695 & HIGH AMOUNT OF SUSPENDED SEDIMENT IN THE SAMPLE. \\
\hline 696 & HIGH AMOUNT OF SUSPENDED SEDIMENT IN THE SAMPLE. \\
\hline 697 & \\
\hline 698 & \\
\hline 699 & \\
\hline 700 & \\
\hline 701 & \\
\hline 702 & \\
\hline 703 & \\
\hline 704 & \\
\hline 705 & \\
\hline 706 & \\
\hline 707 & \\
\hline 708 & \\
\hline 709 & \\
\hline 710 & \\
\hline 711 & \\
\hline 712 & \\
\hline 713 & \\
\hline 714 & \\
\hline 715 & \\
\hline 716 & \\
\hline 717 & \\
\hline 718 & \\
\hline 719 & \\
\hline 720 & \\
\hline 721 & \\
\hline 722 & \\
\hline 723 & \\
\hline
\end{tabular}




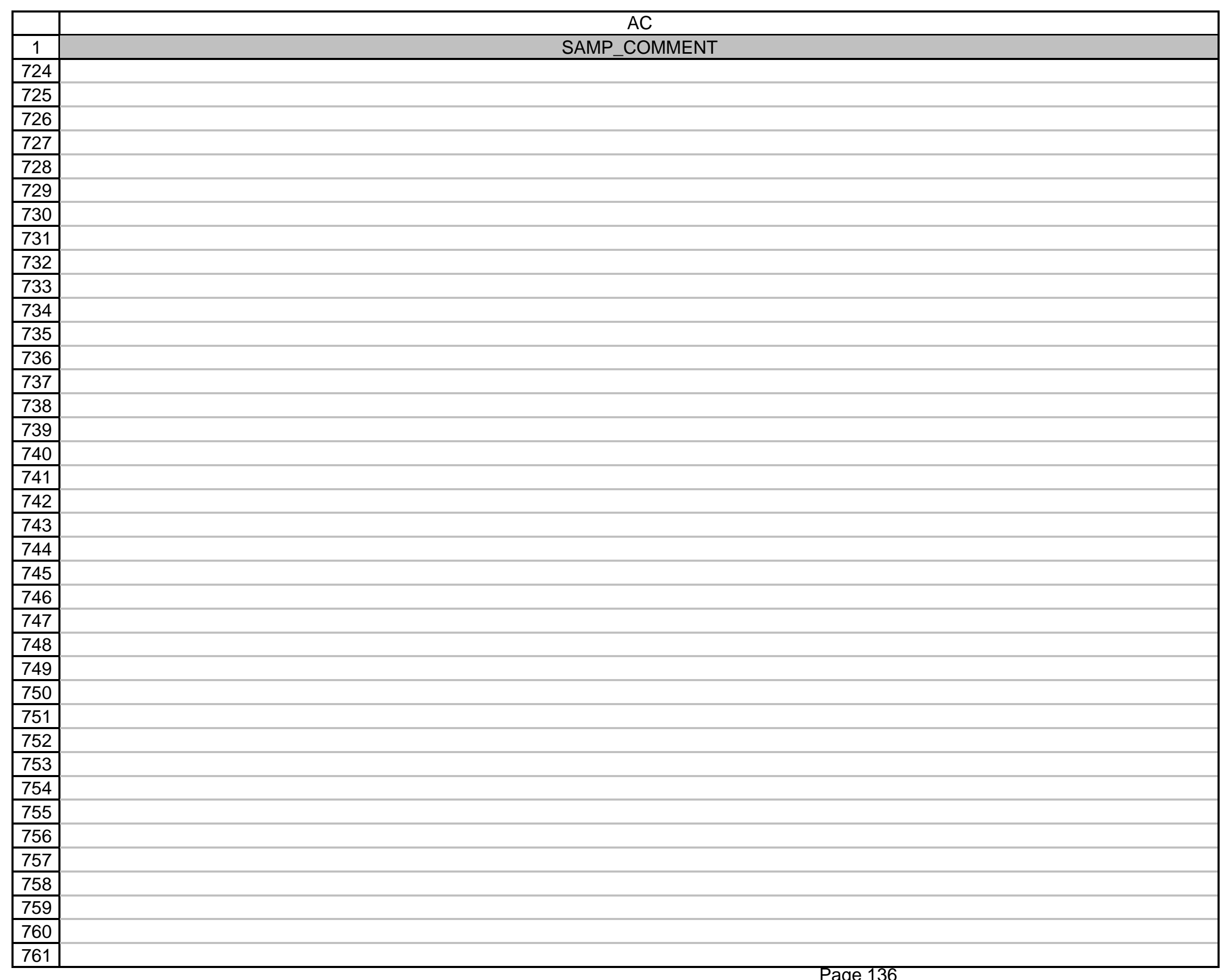


AC

\begin{tabular}{|c|c|}
\hline & $\mathrm{AC}$ \\
\hline 1 & SAMP_COMMENT \\
\hline 762 & \\
\hline 763 & \\
\hline 764 & \\
\hline 765 & \\
\hline 766 & \\
\hline 767 & \\
\hline 768 & \\
\hline 769 & \\
\hline 770 & \\
\hline 771 & Analysis by Quanterra/ Seep \# NS-1 \\
\hline 772 & Analysis by Quanterra/ Seep \# NS-1 / Duplicate of B0GYX4 \\
\hline 773 & Analysis by Quanterra/ Seep \# NS-2 \\
\hline 774 & Analysis by Quanterra/ Seep \# NS-3 \\
\hline 775 & Analysis by Quanterra/ Seep \# NS-4 \\
\hline 776 & Analysis by Quanterra/ Seep \# NS-5 \\
\hline 777 & Analysis by Quanterra/ Seep \# NS-6 \\
\hline 778 & Analysis by Quanterra/ Seep \# NS-7 \\
\hline 779 & Analysis by Quanterra/ Seep \# NS-8A \\
\hline 780 & Analysis by Quanterra/ Seep \# NS-8B \\
\hline 781 & Analysis by Quanterra/ Seep \# NS-10 \\
\hline 782 & Analysis by Quanterra/ Seep \# NS-11 \\
\hline 783 & Sampling Points Documented in WHC-SP-0098-8 \\
\hline 784 & Sampling Points Documented in WHC-SP-0098-8 \\
\hline 785 & Sampling Points Documented in WHC-SP-0098-8 \\
\hline 786 & Sampling Points Documented in WHC-SP-0098-8 \\
\hline 787 & Sampling Points Documented in WHC-SP-0098-8 \\
\hline 788 & Sampling Points Documented in WHC-SP-0098-8 \\
\hline 789 & Sampling Points Documented in WHC-SP-0098-8 \\
\hline 790 & Sampling Points Documented in WHC-SP-0098-8 \\
\hline 791 & Sampling Points Documented in WHC-SP-0098-8 \\
\hline 792 & Sampling Points Documented in WHC-SP-0098-8 \\
\hline 793 & Sampling Points Documented in WHC-SP-0098-8 \\
\hline 794 & Sampling Points Documented in WHC-SP-0098-8 \\
\hline 795 & Sampling Points Documented in WHC-SP-0098-8 \\
\hline 796 & Sampling Points Documented in WHC-SP-0098-8 \\
\hline 797 & Sampling Points Documented in WHC-SP-0098-8 \\
\hline 798 & Sampling Points Documented in WHC-SP-0098-8 \\
\hline 799 & Sampling Points Documented in WHC-SP-0098-8 \\
\hline
\end{tabular}


AC

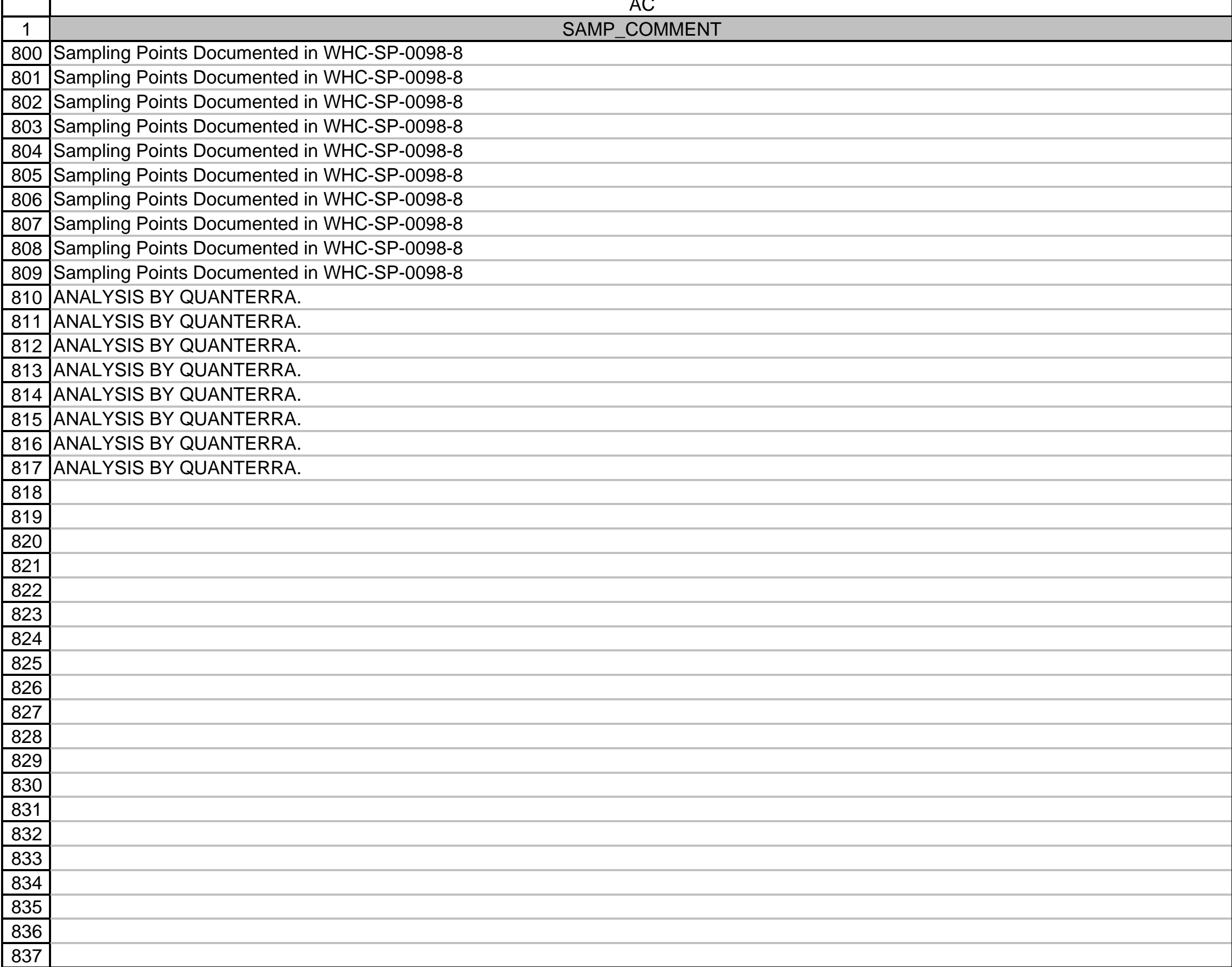


$A C$

\begin{tabular}{|c|c|}
\hline & $A C$ \\
\hline 1 & SAMP_COMMENT \\
\hline 838 & \\
\hline 839 & ANALYSIS BY QUANTERRA. \\
\hline 840 & ANALYSIS BY QUANTERRA. \\
\hline 841 & ANALYSIS BY QUANTERRA. \\
\hline 842 & ANALYSIS BY QUANTERRA. \\
\hline 843 & ANALYSIS BY QUANTERRA. \\
\hline 844 & \\
\hline 845 & \\
\hline 846 & \\
\hline 847 & \\
\hline 848 & \\
\hline 849 & \\
\hline 850 & \\
\hline 851 & \\
\hline 852 & \\
\hline 853 & \\
\hline 854 & \\
\hline 855 & \\
\hline 856 & \\
\hline 857 & \\
\hline 858 & \\
\hline 859 & \\
\hline 860 & \\
\hline 861 & \\
\hline 862 & \\
\hline 863 & \\
\hline 864 & \\
\hline 865 & \\
\hline 866 & \\
\hline 867 & \\
\hline 868 & \\
\hline 869 & \\
\hline 870 & \\
\hline 871 & \\
\hline 872 & \\
\hline 873 & \\
\hline 874 & \\
\hline 875 & \\
\hline
\end{tabular}




\begin{tabular}{|c|c|}
\hline & 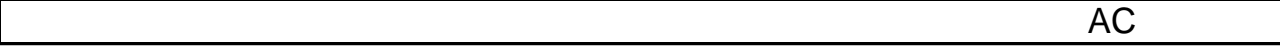 \\
\hline 1 & SAMP_COMMENT \\
\hline 876 & \\
\hline 877 & \\
\hline 878 & \\
\hline 879 & \\
\hline 880 & \\
\hline 881 & \\
\hline 882 & \\
\hline 883 & \\
\hline 884 & \\
\hline 885 & \\
\hline 886 & \\
\hline 887 & \\
\hline 888 & \\
\hline 889 & \\
\hline 890 & \\
\hline 891 & \\
\hline 892 & No sample date \& time on the Chain of Custody. \\
\hline 893 & No sample date \& time on the Chain of Custody. \\
\hline 894 & \\
\hline 895 & \\
\hline 896 & \\
\hline 897 & \\
\hline 898 & \\
\hline 899 & \\
\hline 900 & \\
\hline 901 & \\
\hline 902 & \\
\hline 903 & \\
\hline 904 & \\
\hline 905 & \\
\hline 906 & \\
\hline 907 & \\
\hline 908 & \\
\hline 909 & \\
\hline 910 & \\
\hline 911 & \\
\hline 912 & \\
\hline 913 & \\
\hline
\end{tabular}




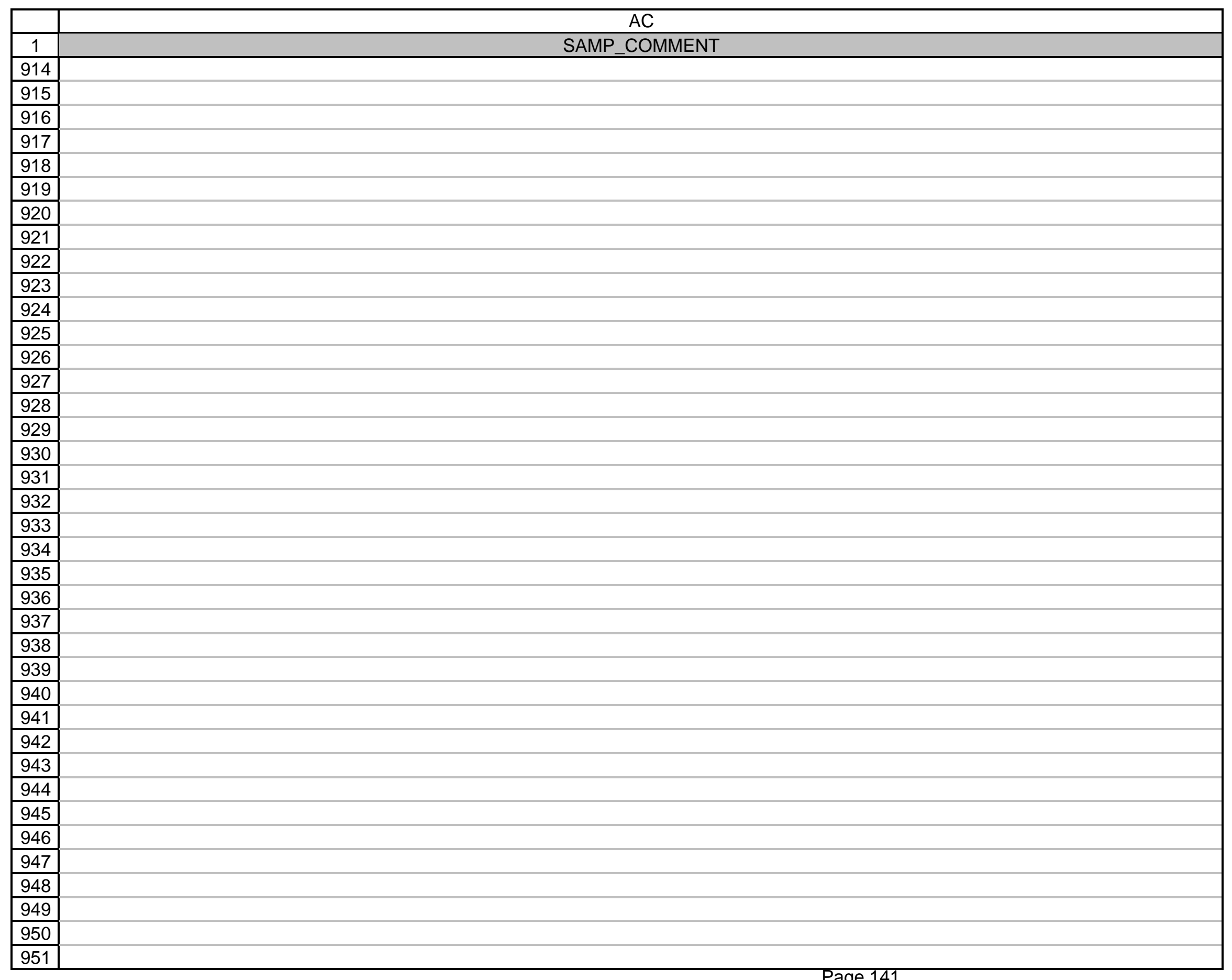




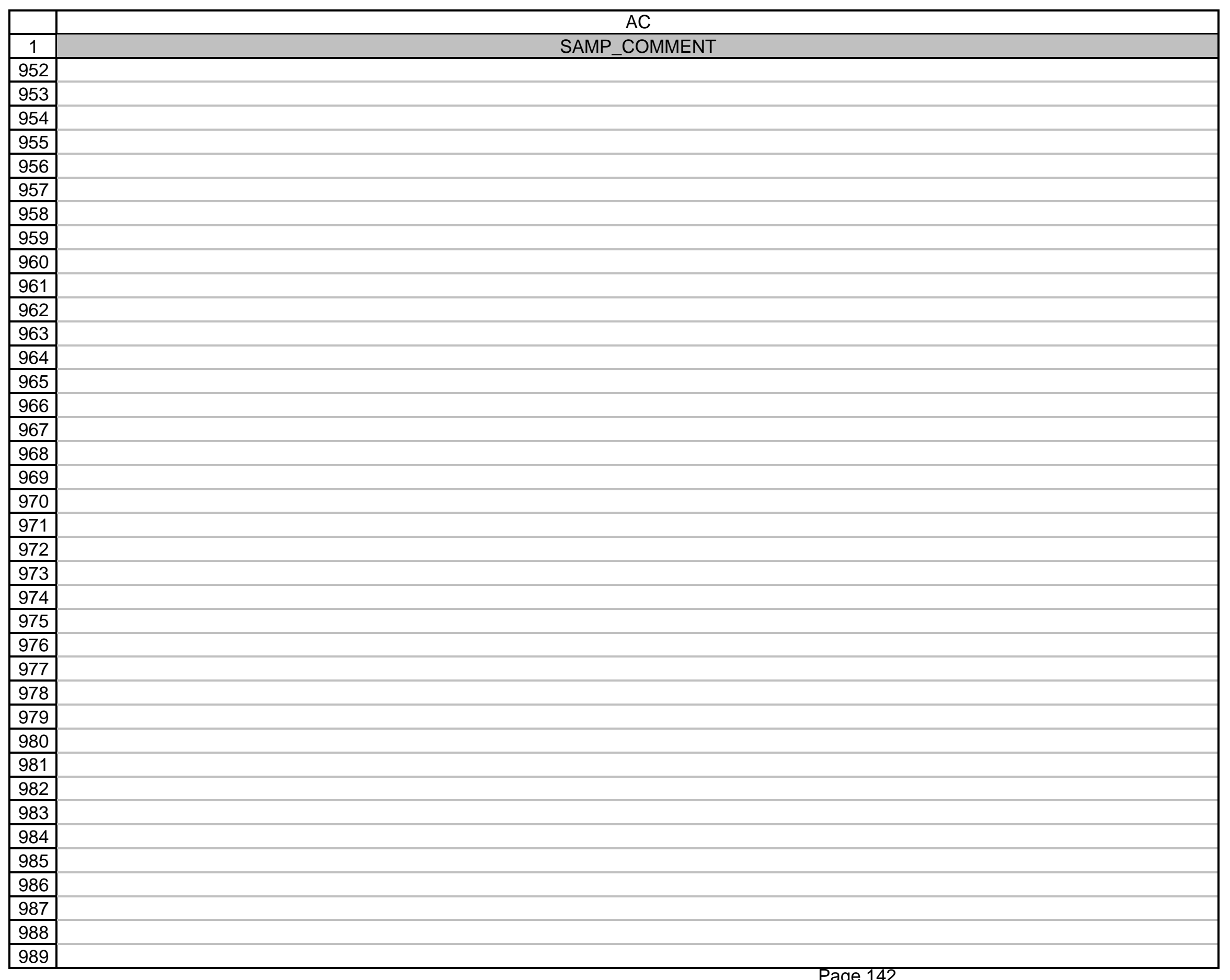




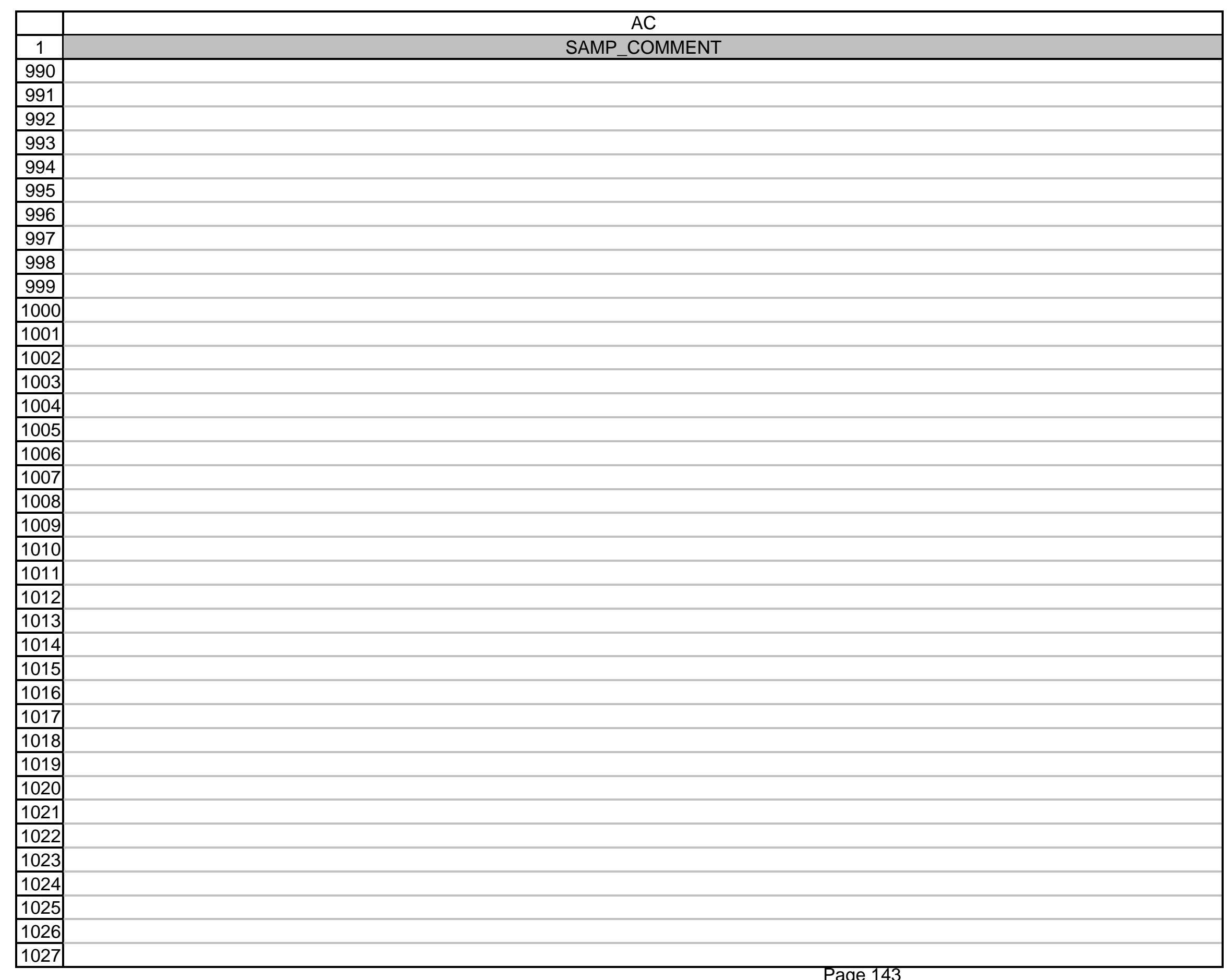




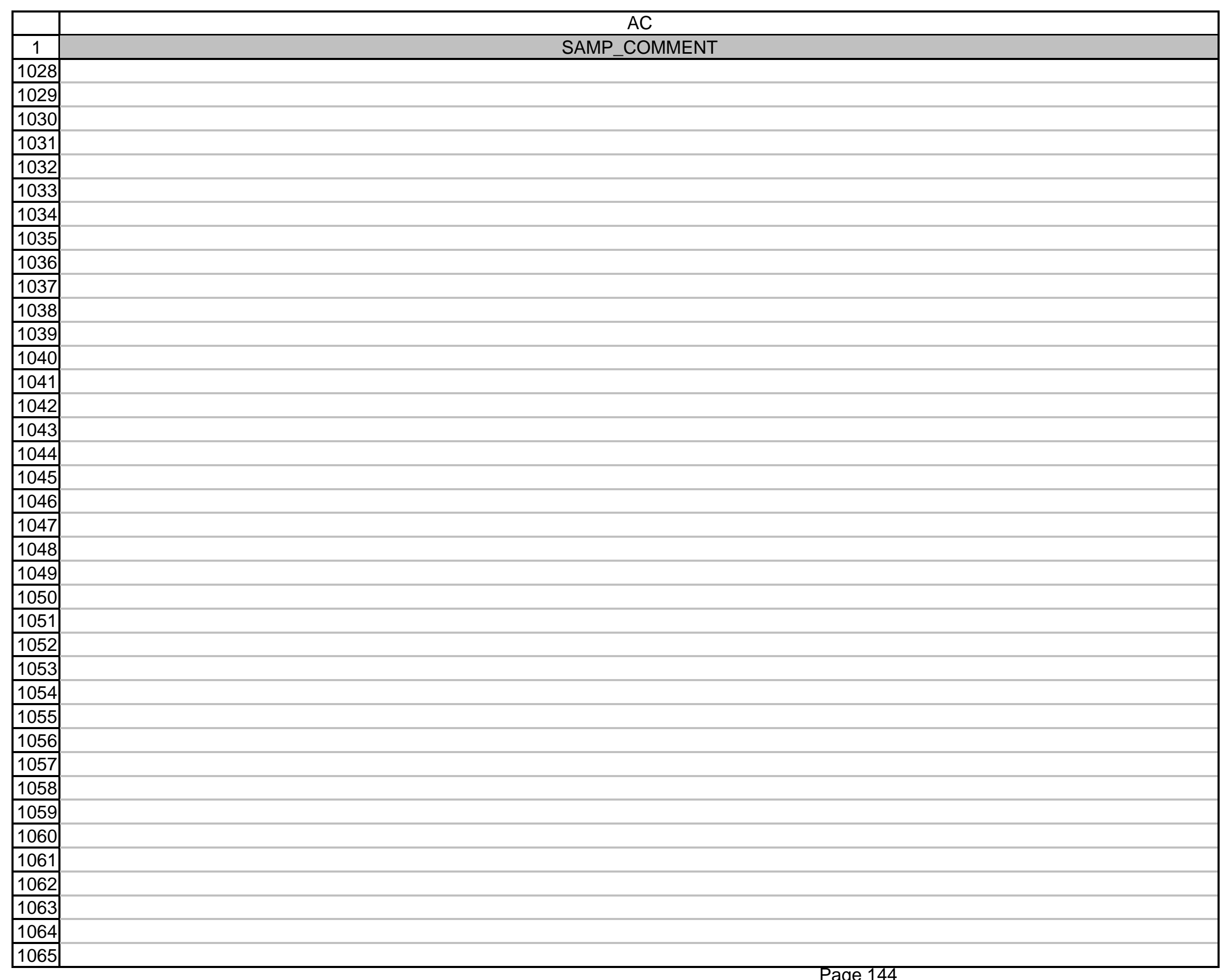




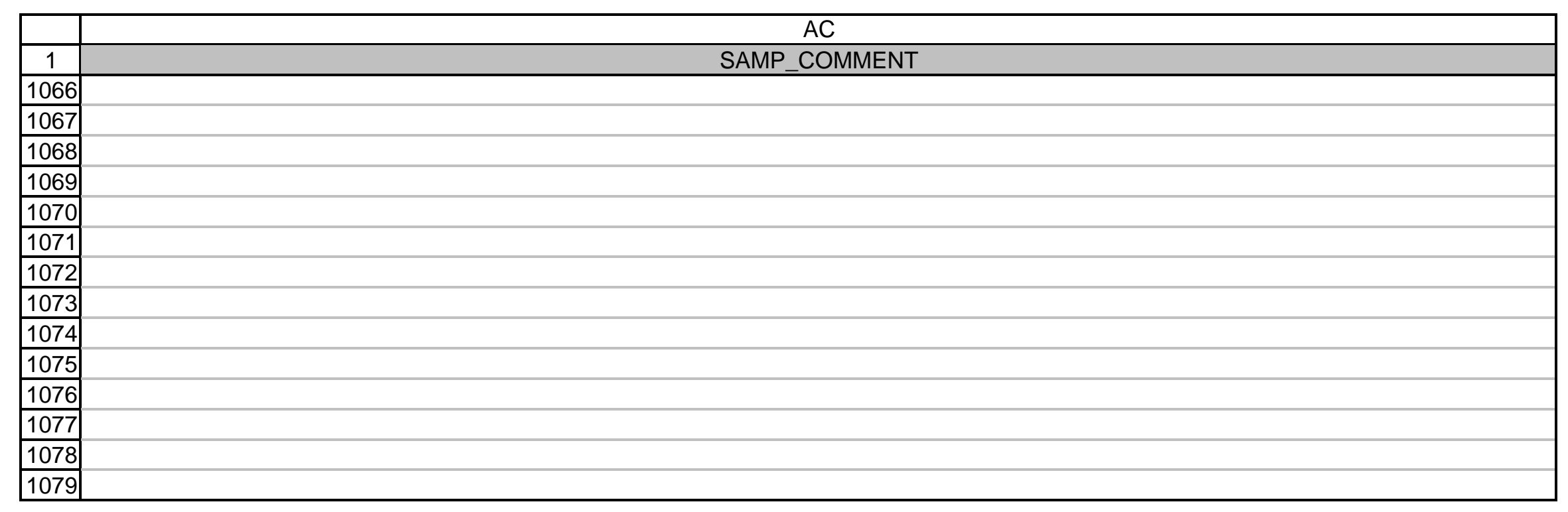

Page 145 


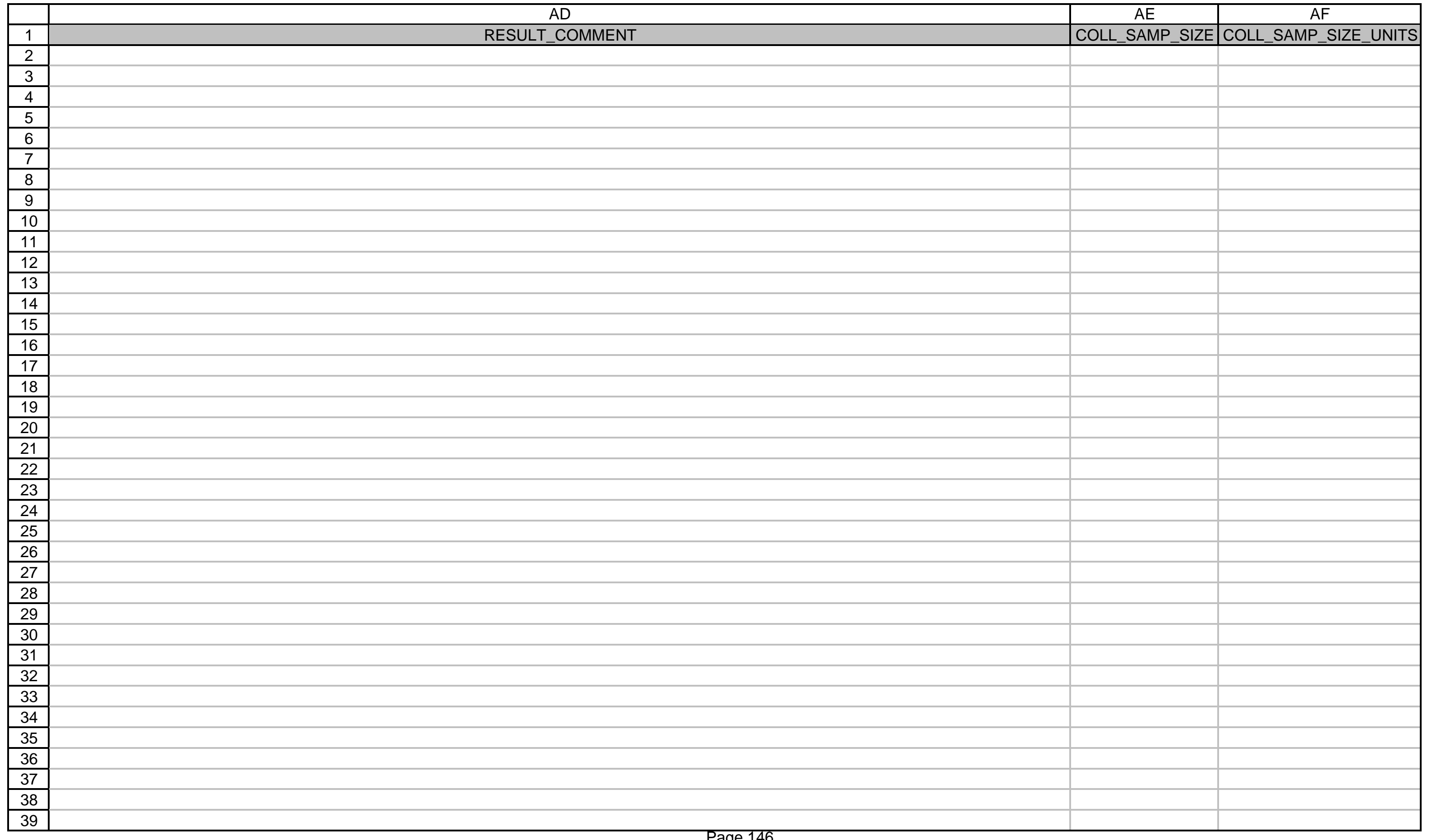




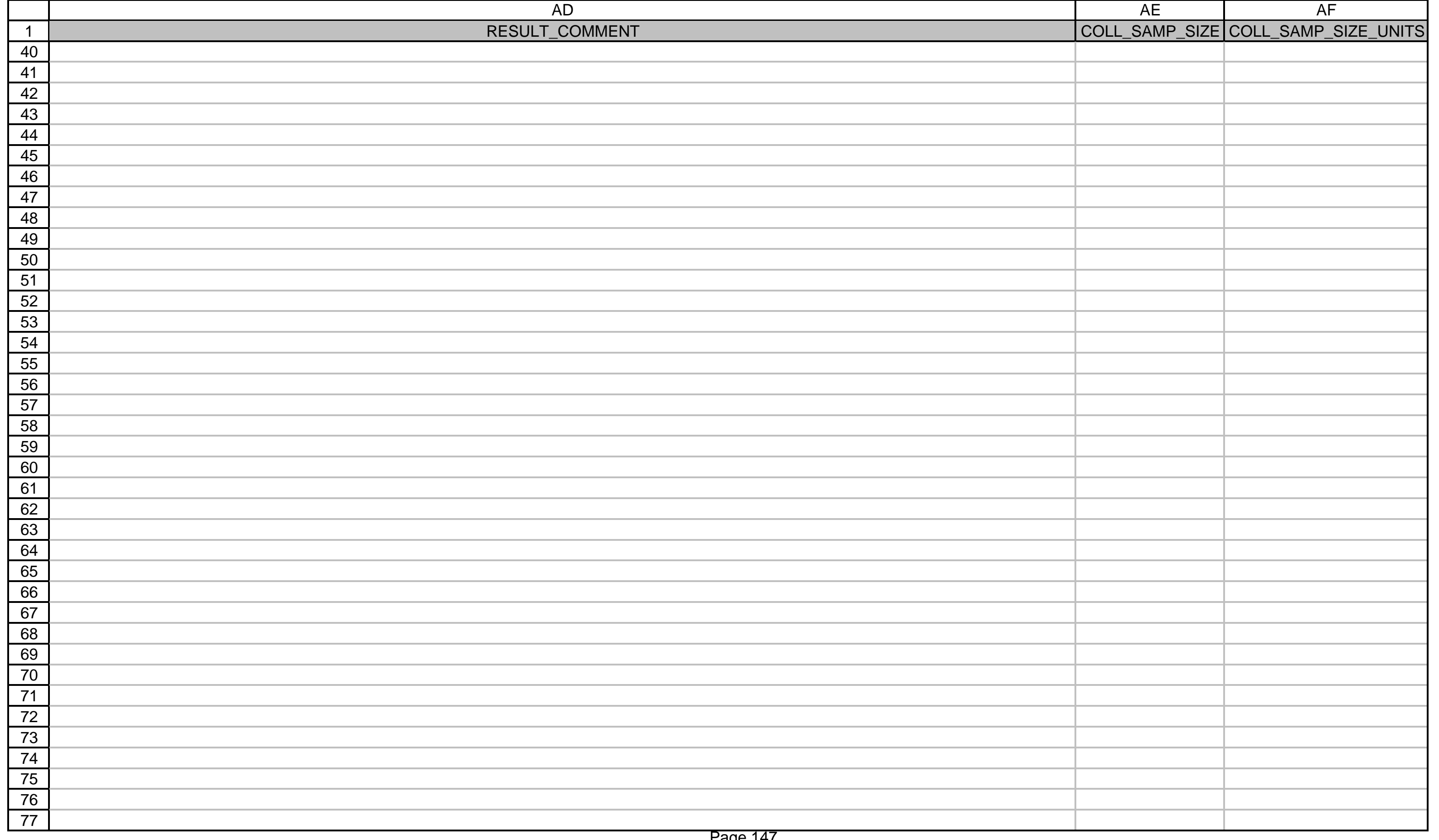




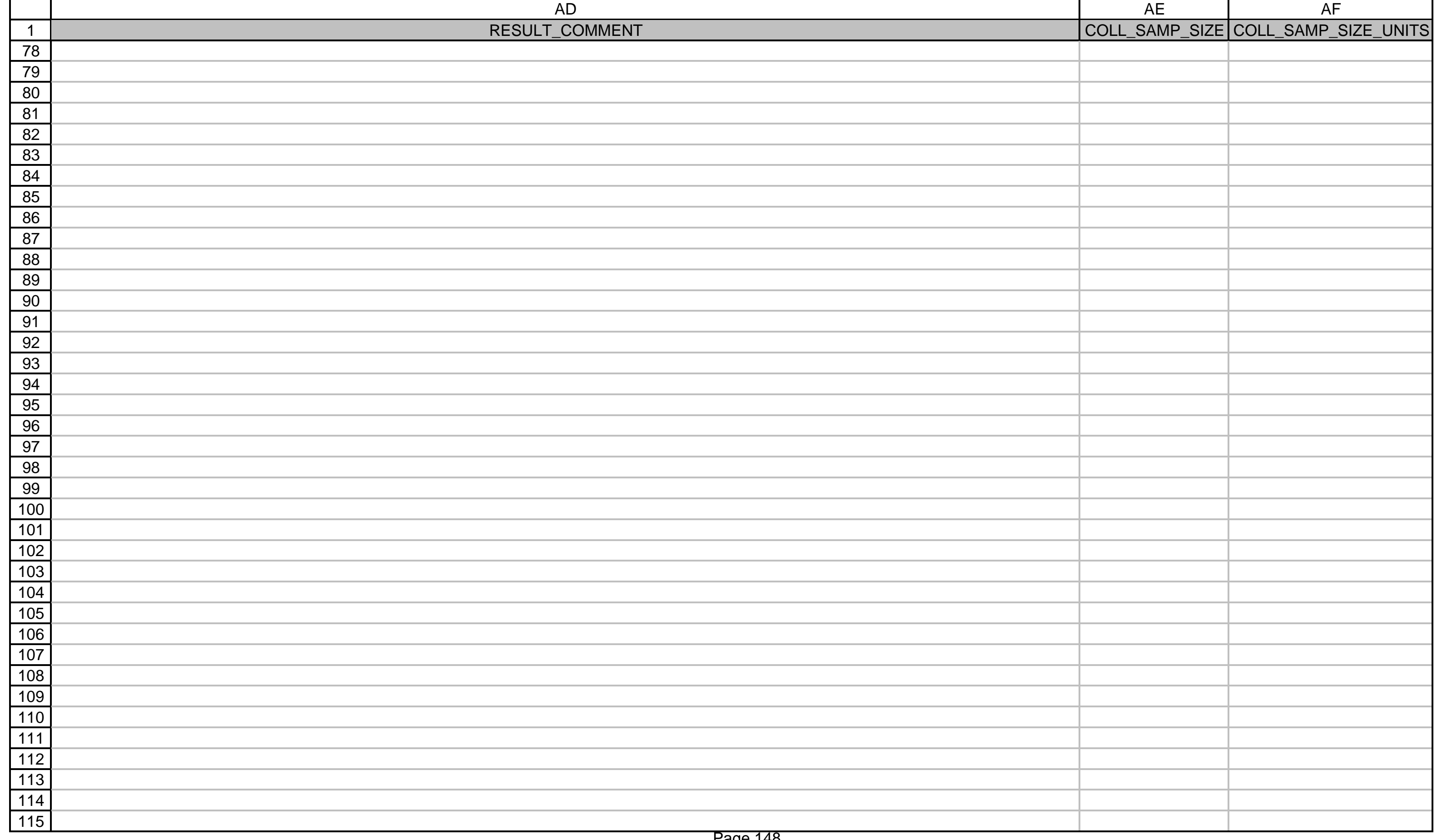




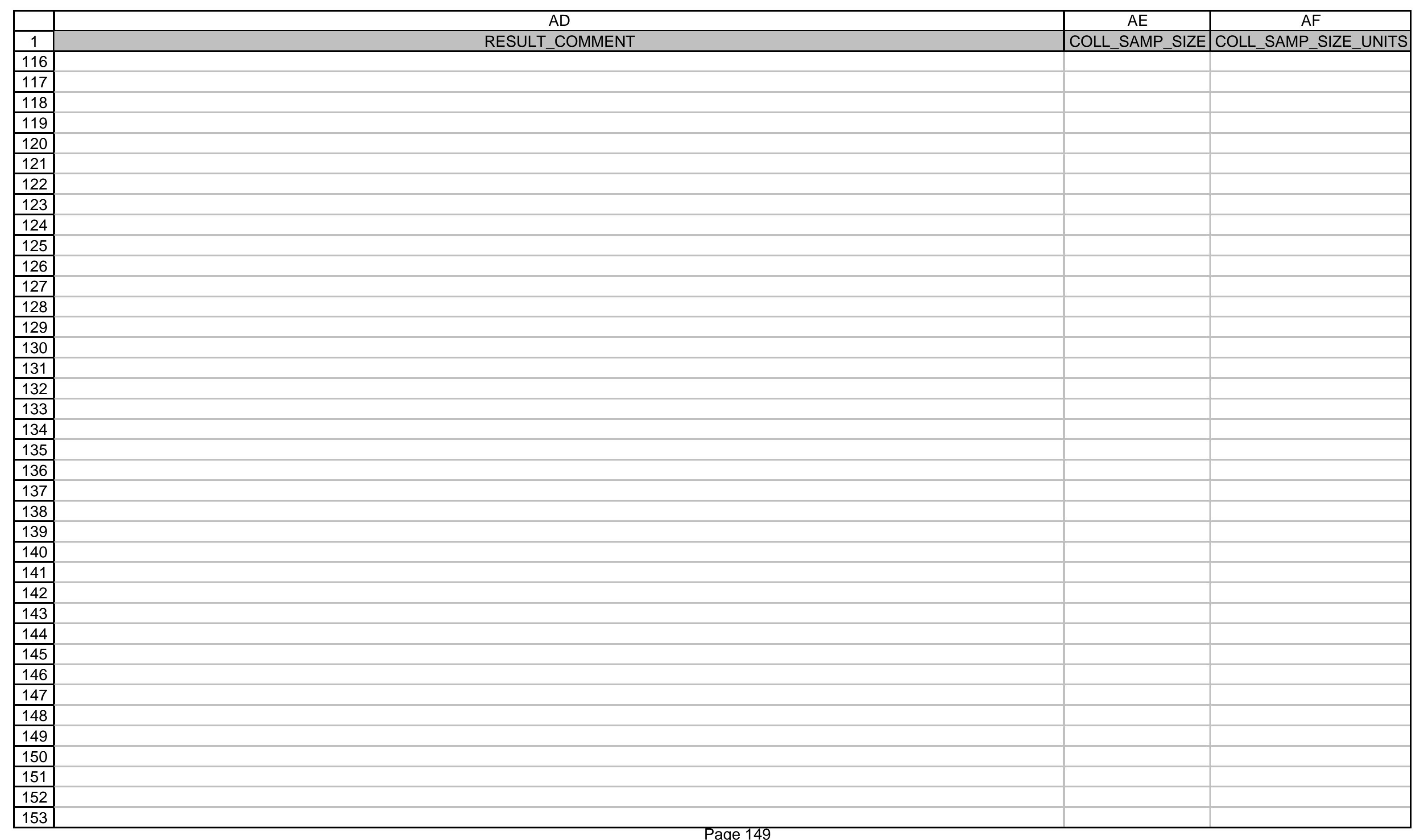




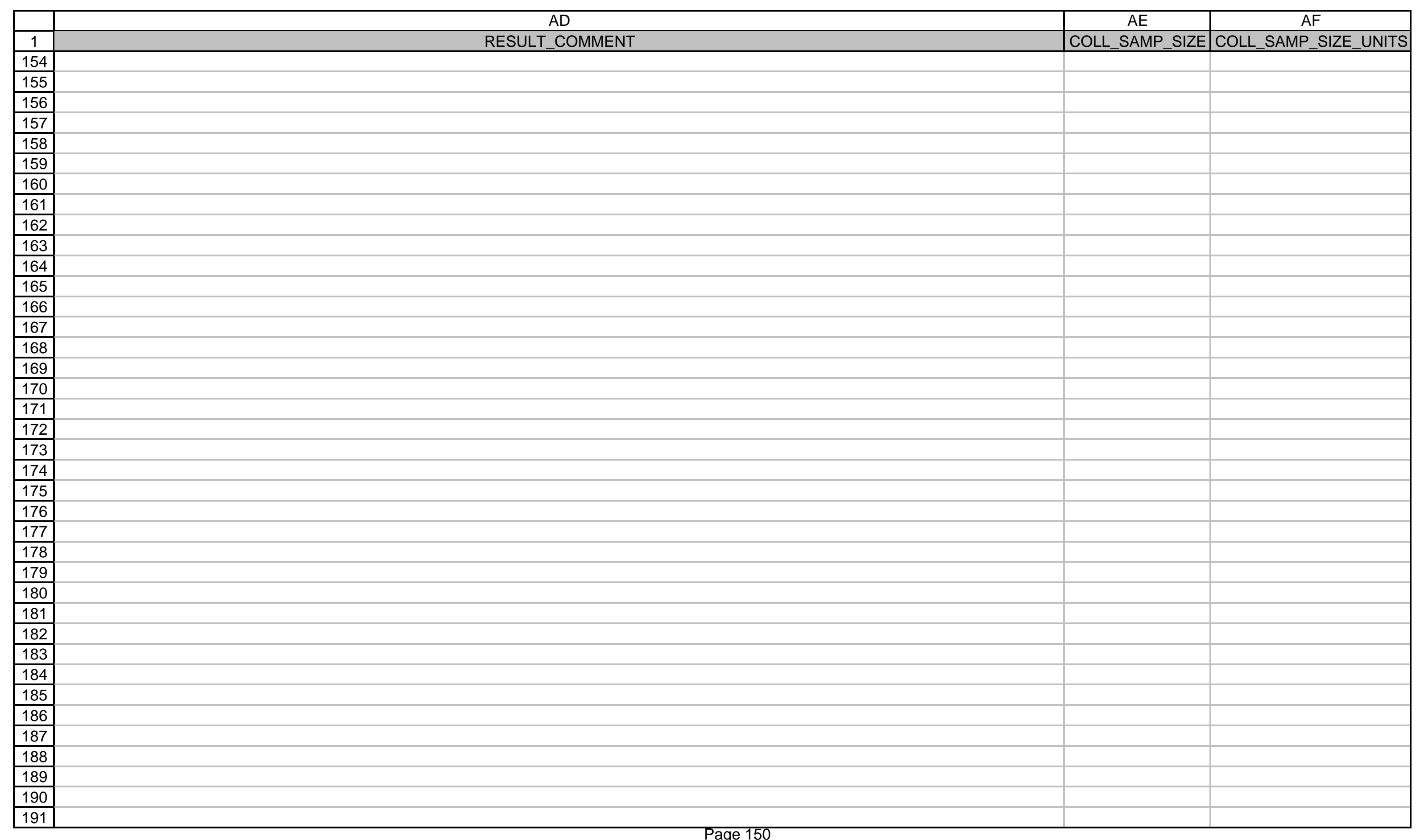




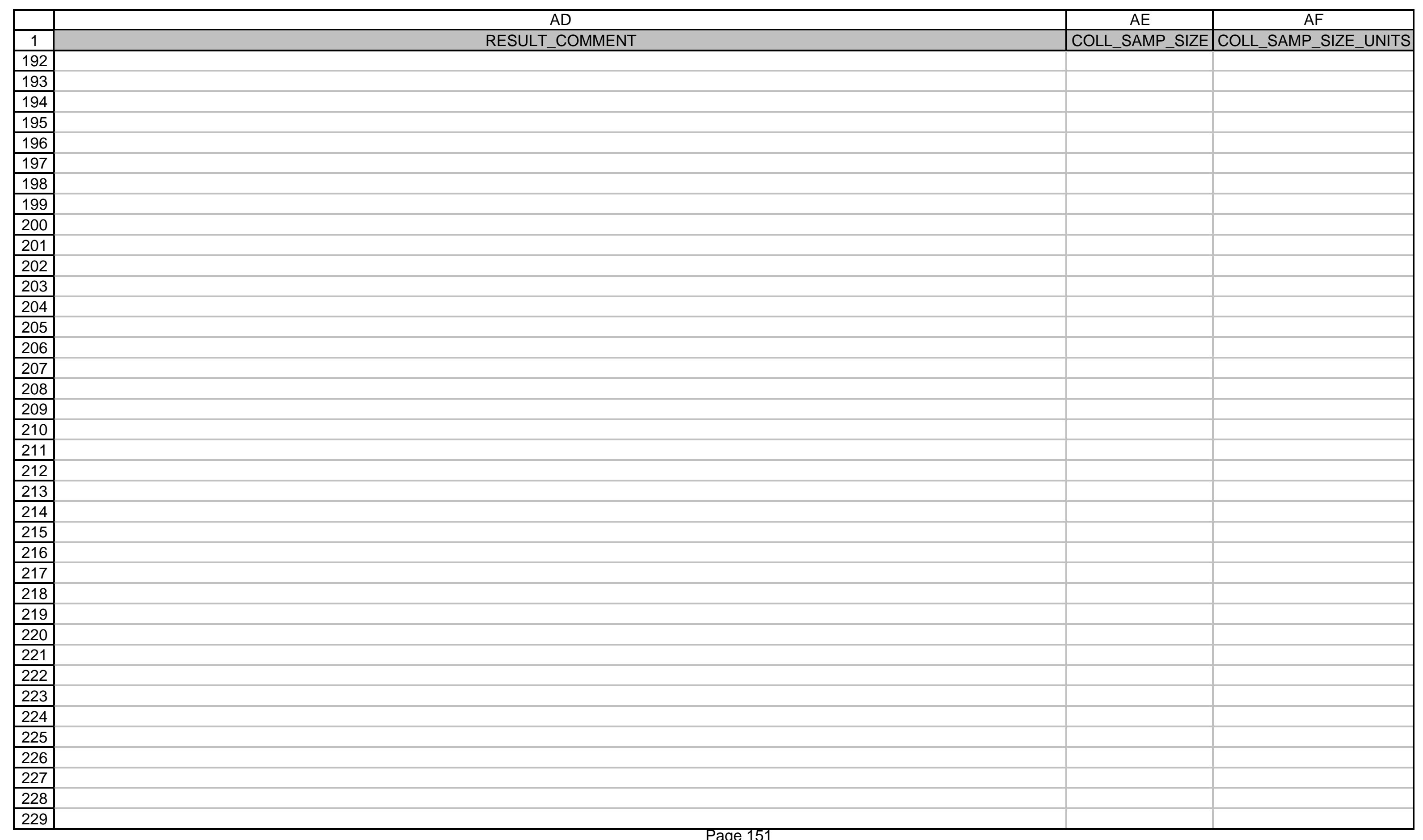




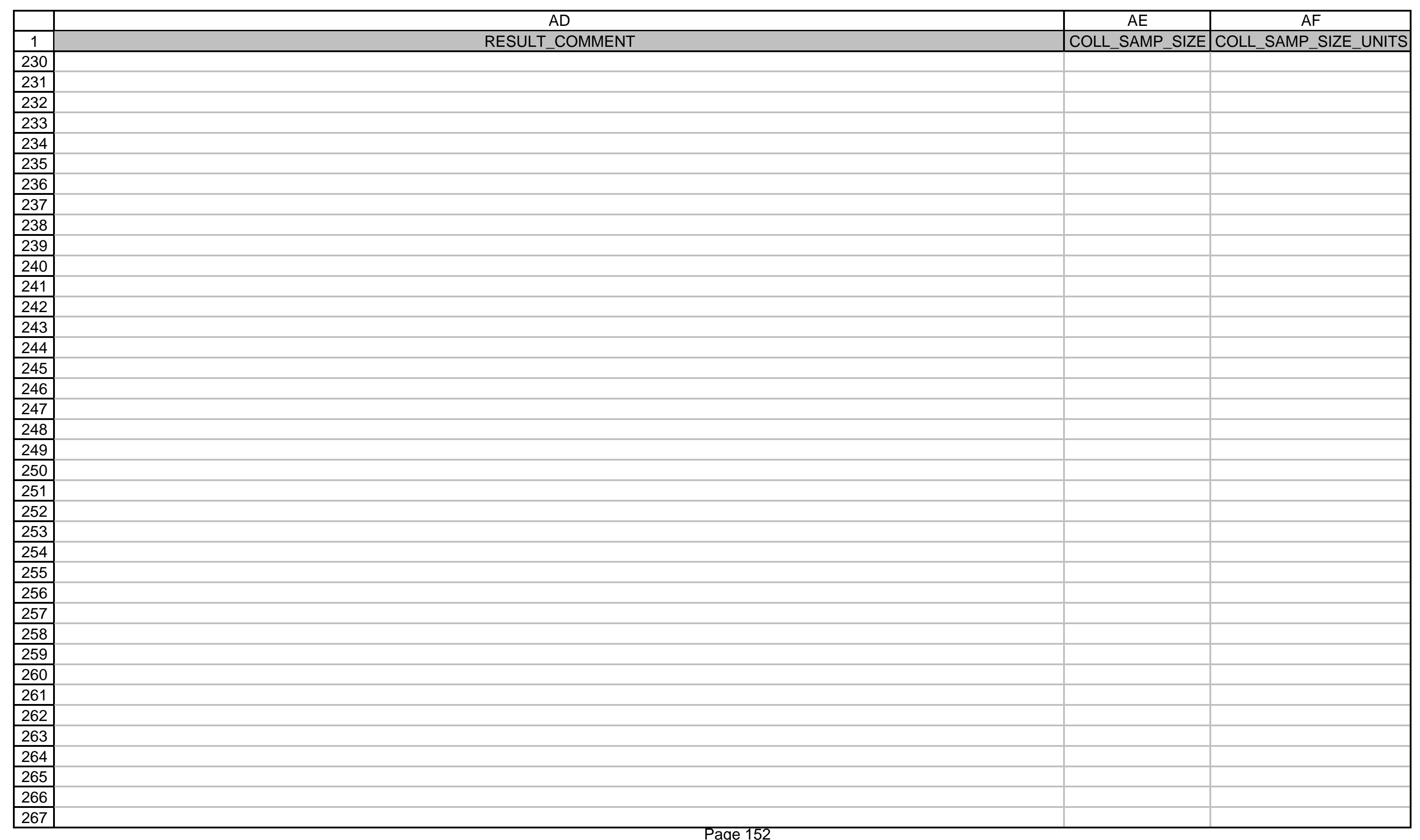




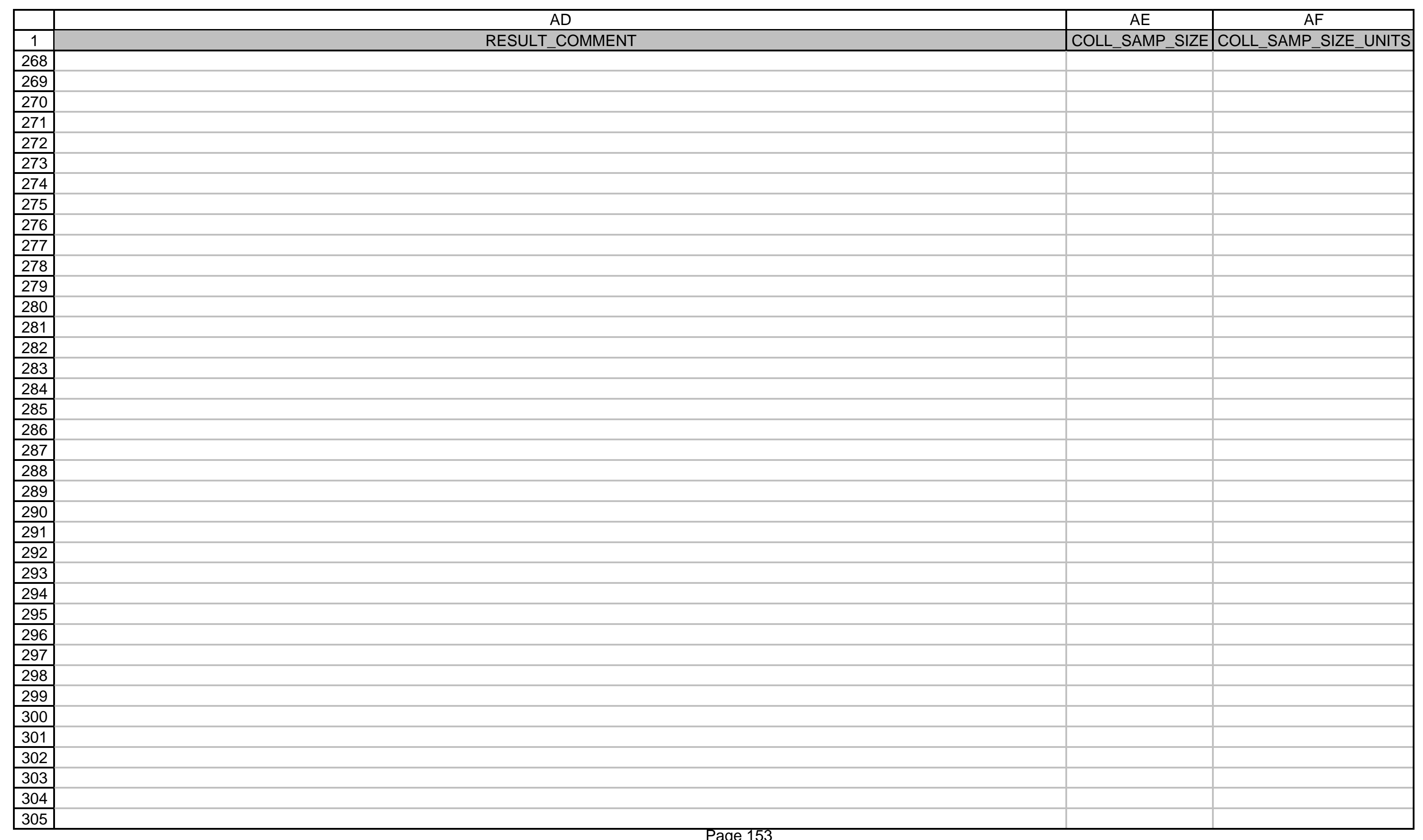


307 See sample record for comment.

308 See sample record for comment.

309 See sample record for comment.

\begin{tabular}{|l|l|}
310 & See sample record for comment.
\end{tabular}

311

\begin{tabular}{|l|}
\hline 311 \\
\hline 312 \\
\hline 313 \\
\hline 311
\end{tabular}

313

314

315

316

317

318

319

320

321 


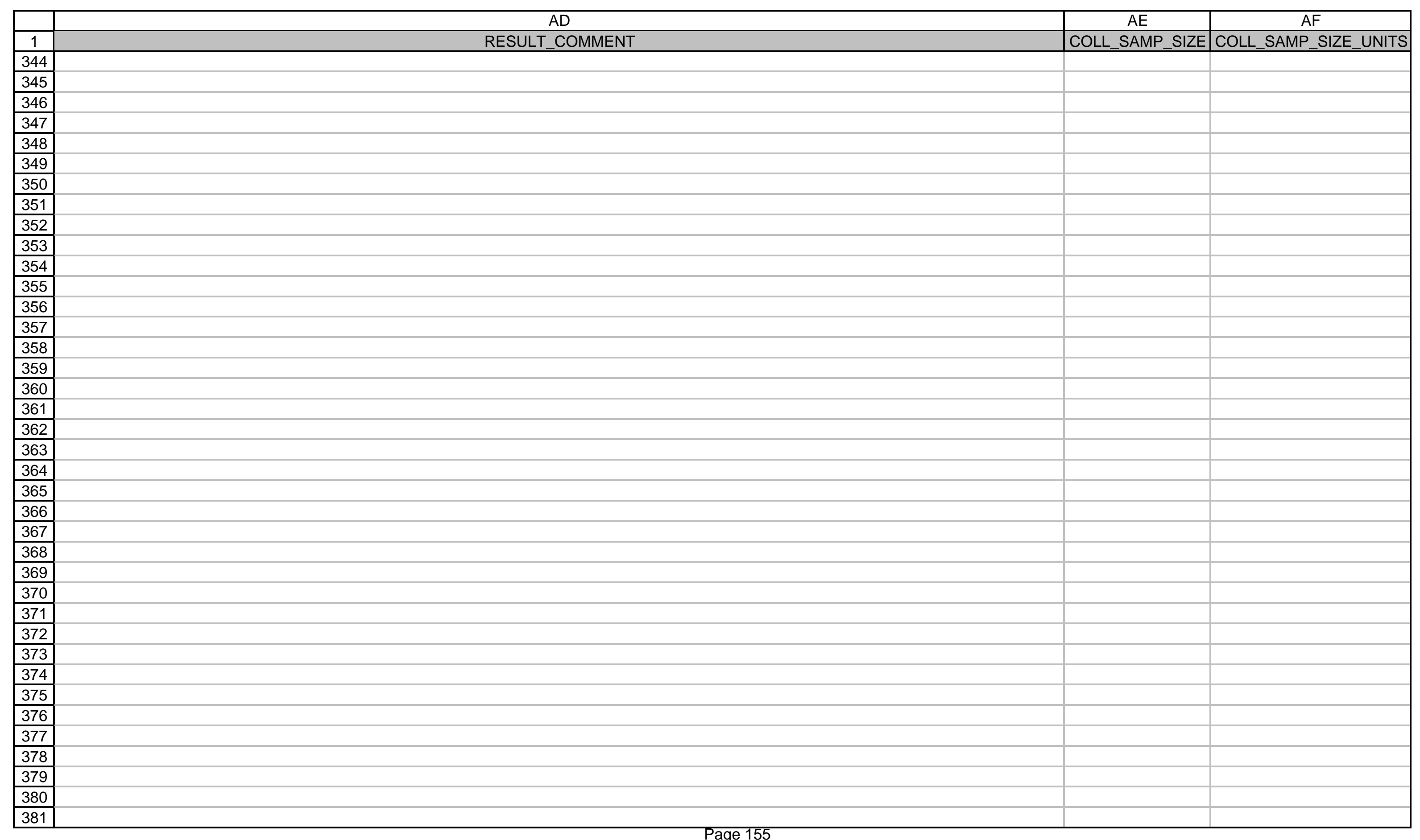




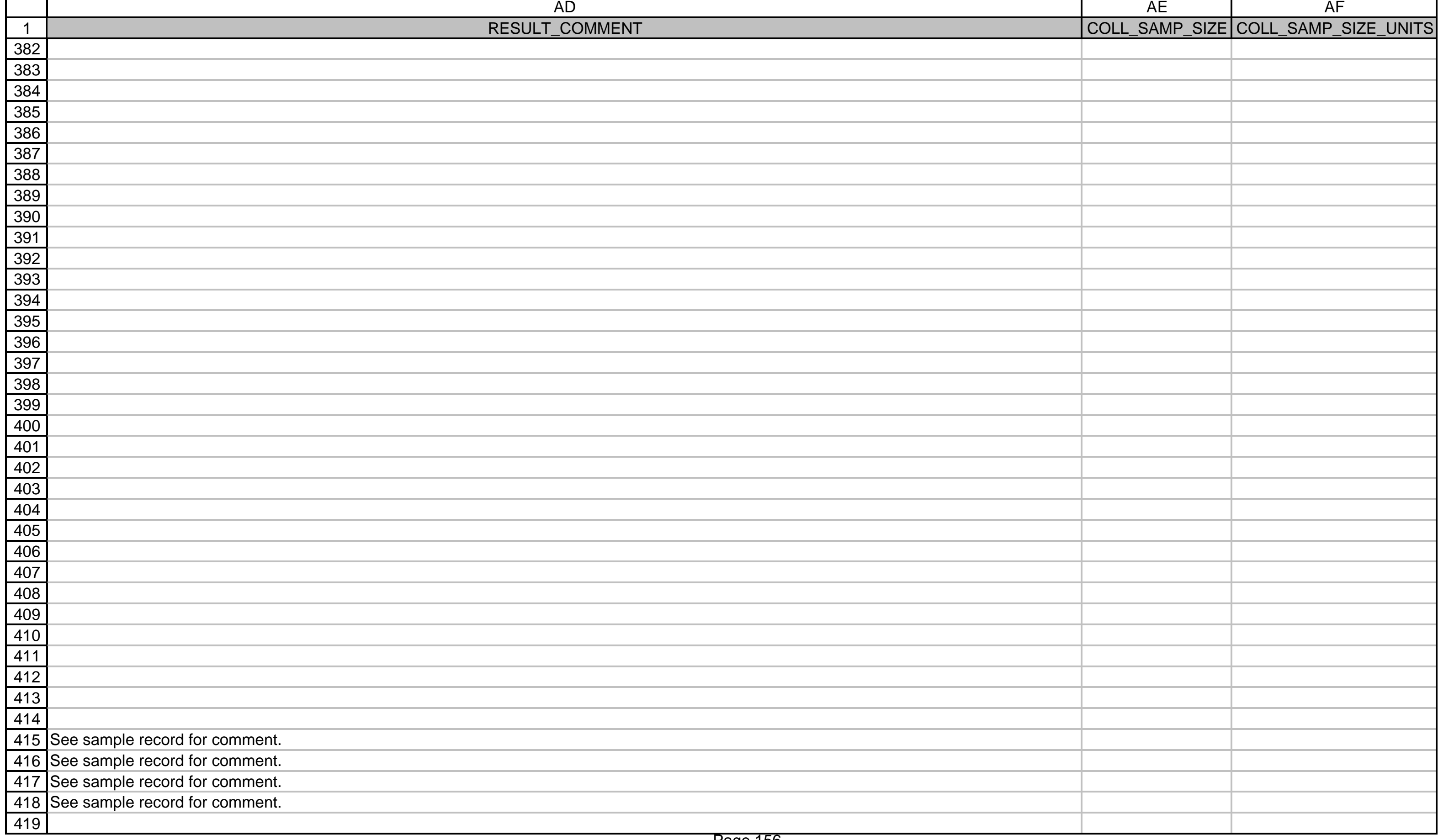




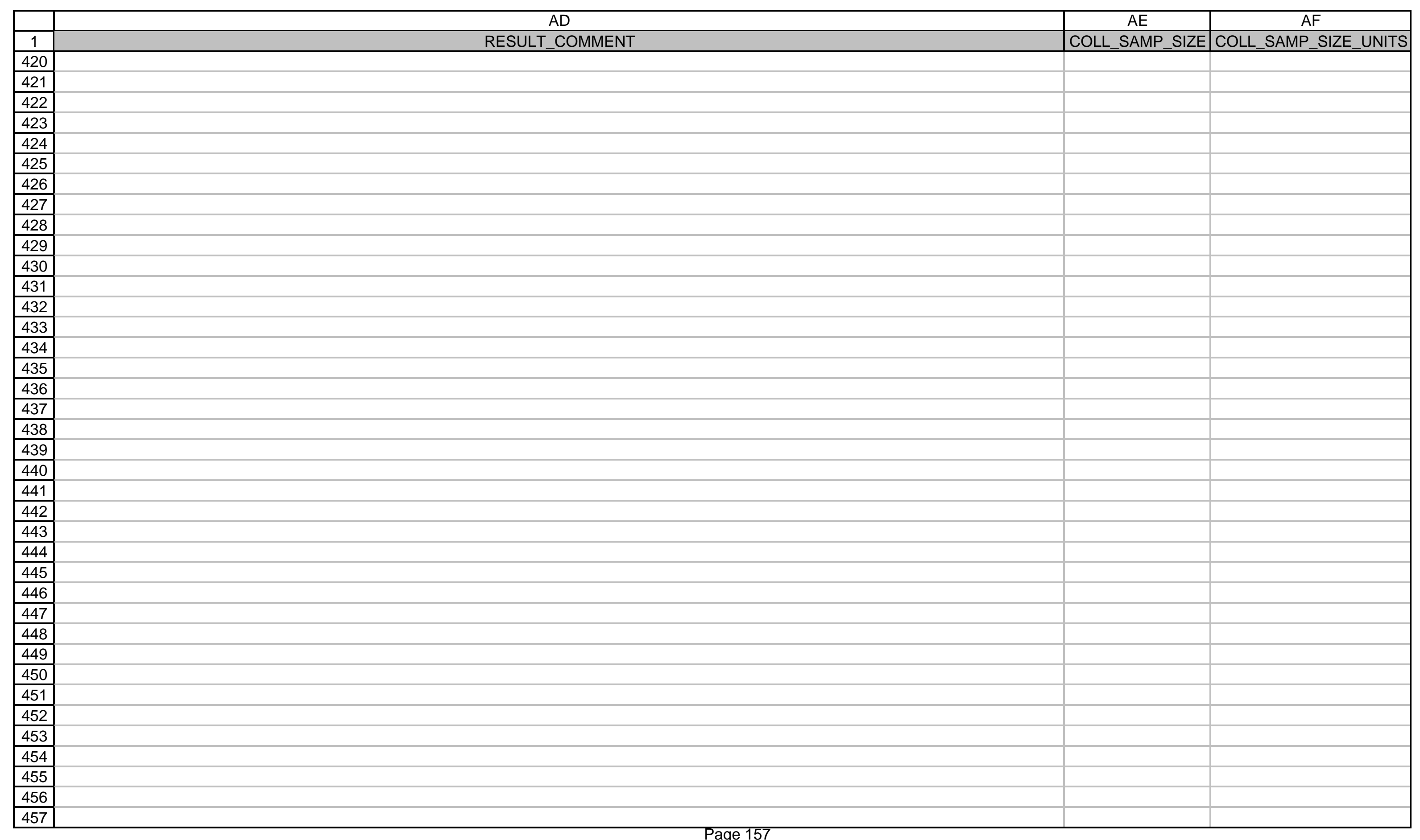




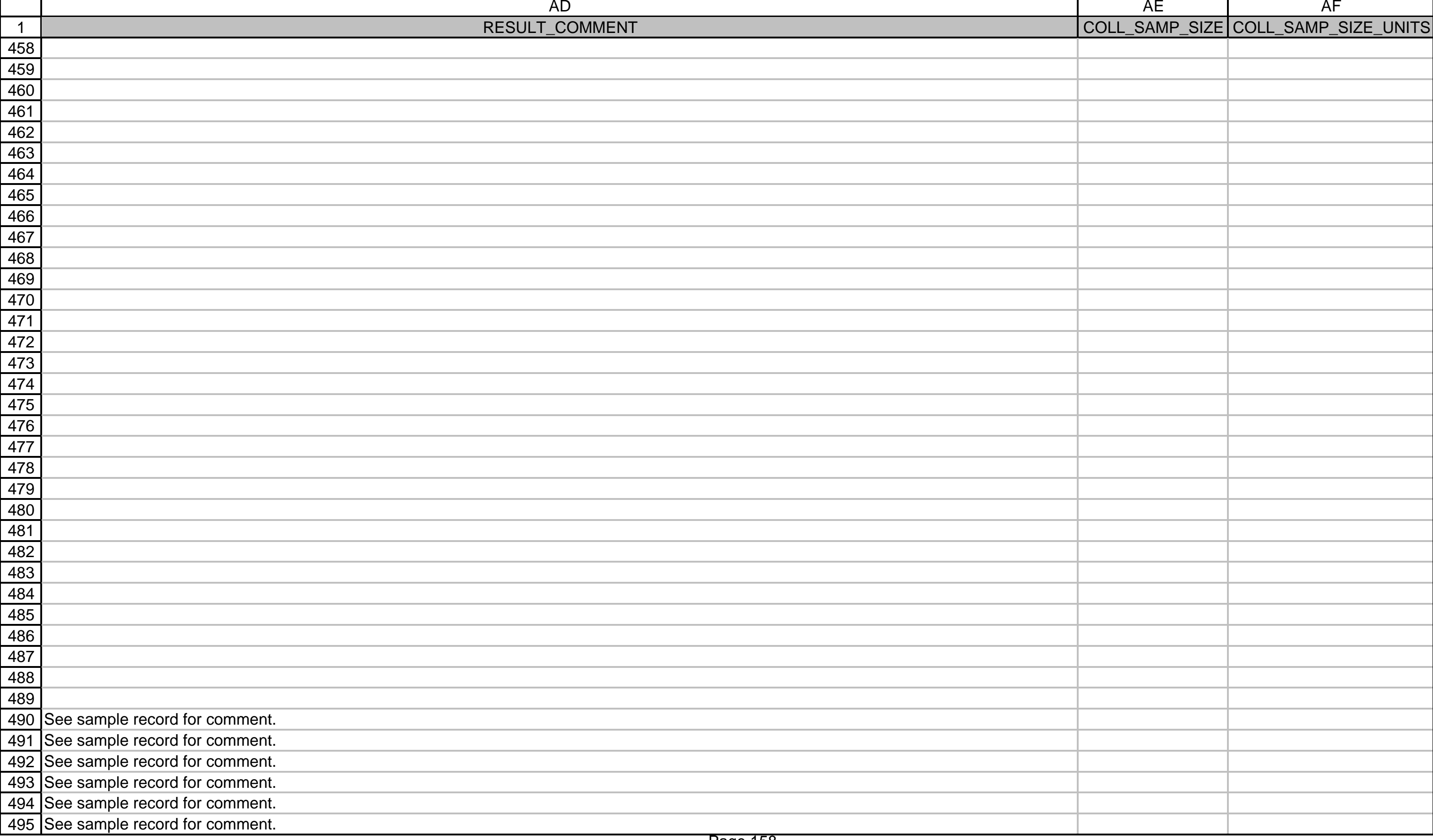

\begin{tabular}{|l|l}
494 & See sample record for comment. \\
495 & See sample record for comment.
\end{tabular} 


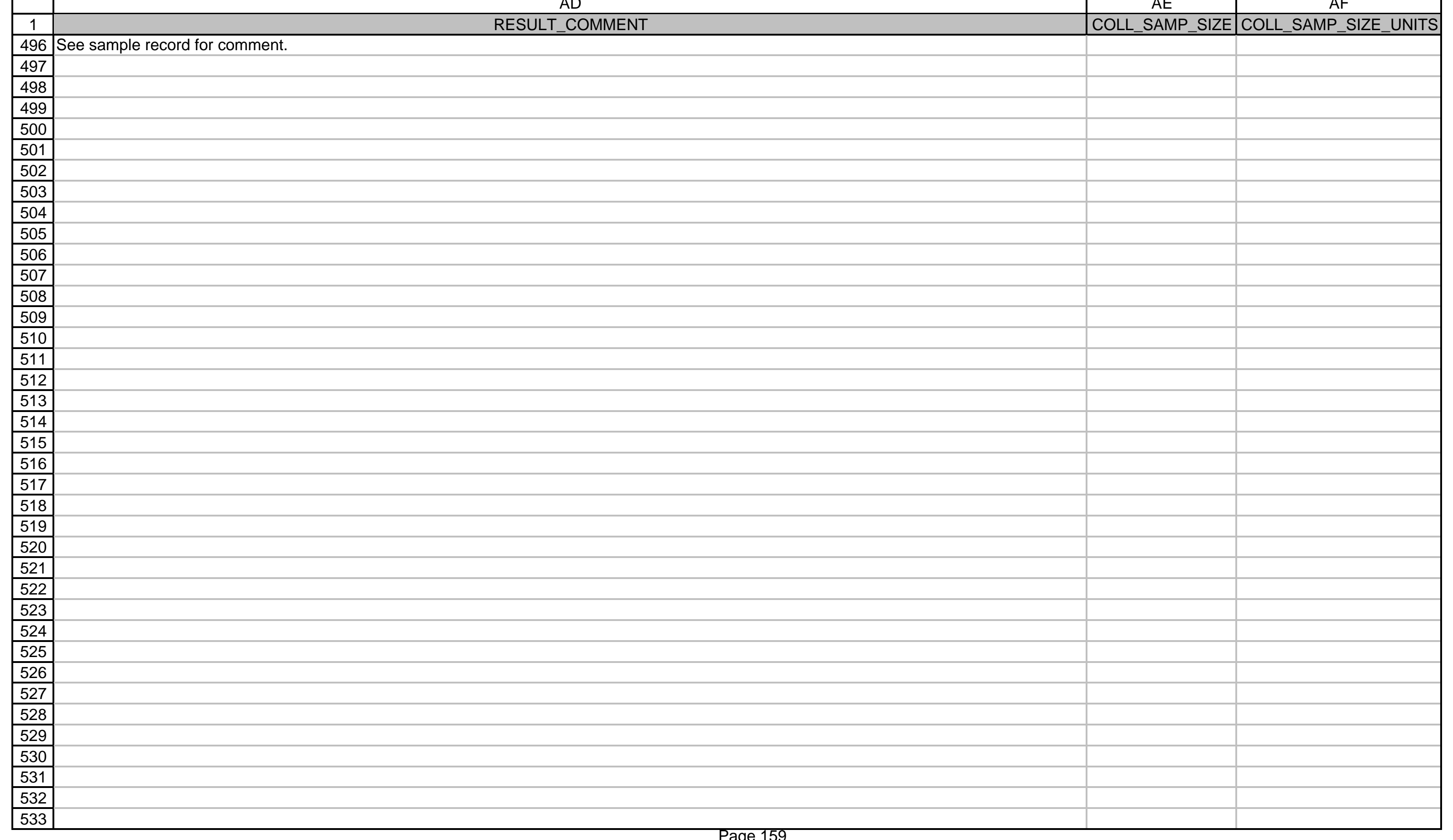




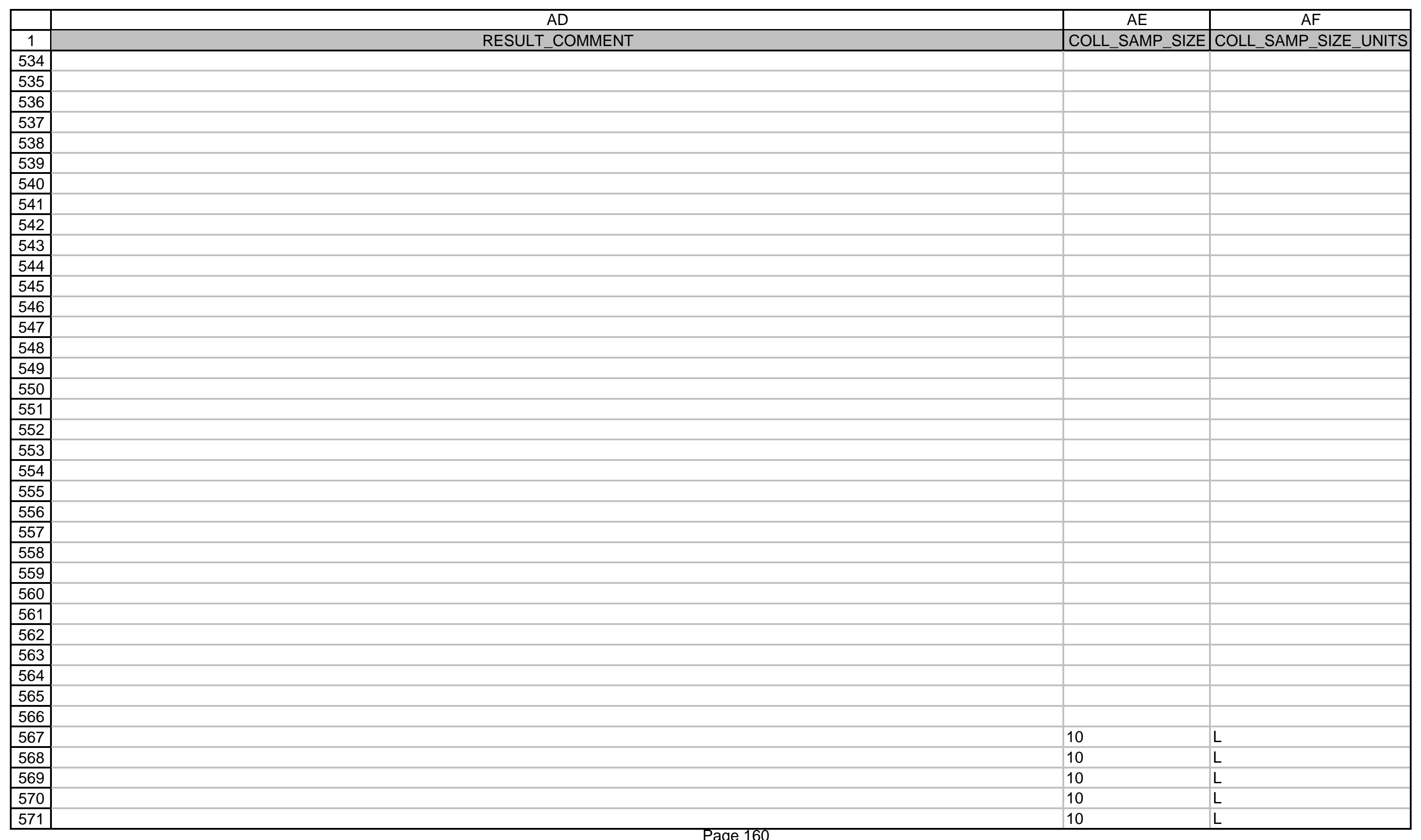




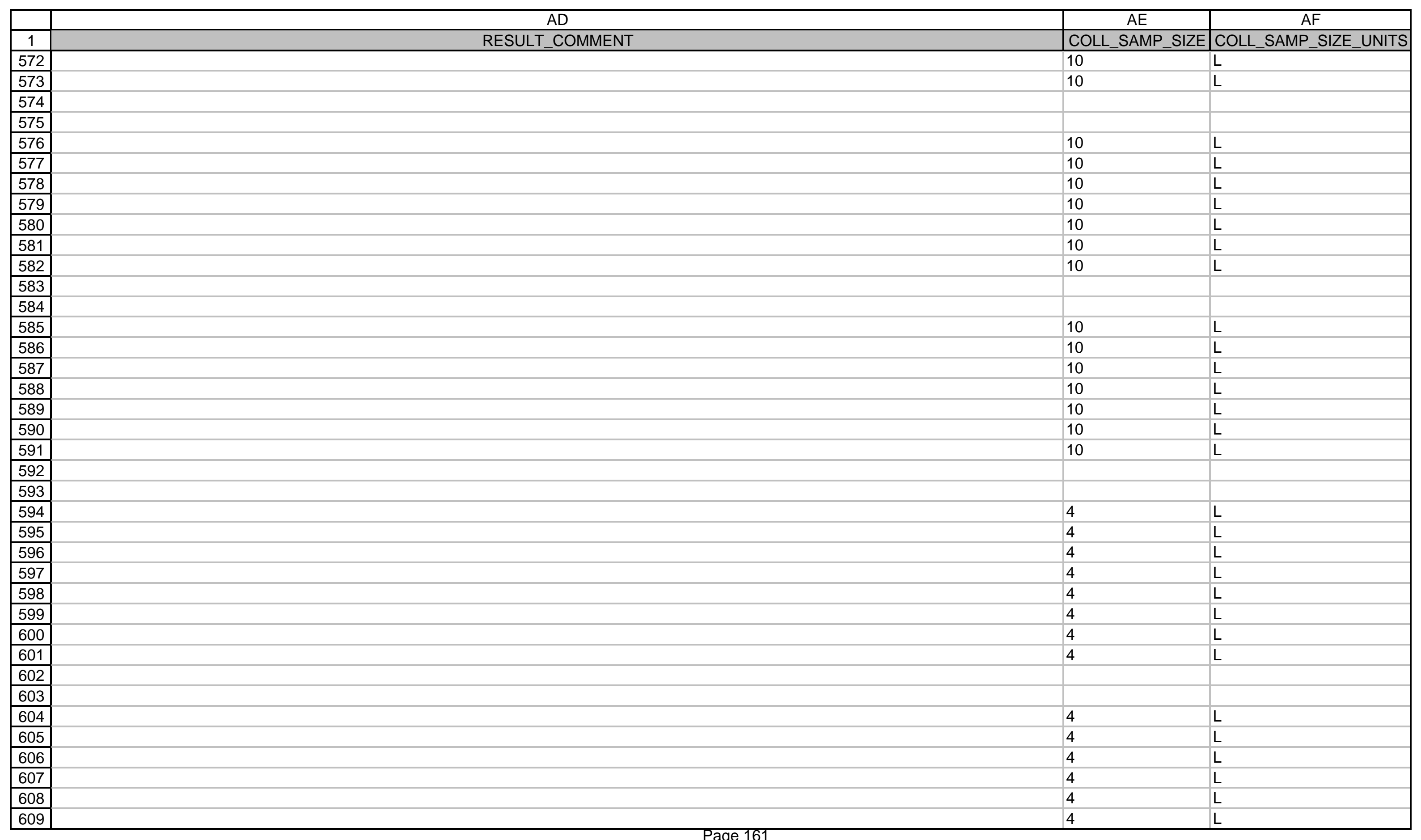




\begin{tabular}{|c|c|c|}
\hline & $A D$ & $\mathrm{AF}$ \\
\hline 1 & RESULT_COMMENT & COLL_SAMP_SIZE_UNITS \\
\hline 610 & & $\mathrm{~L}$ \\
\hline 611 & & $\mathrm{~L}$ \\
\hline \multicolumn{3}{|l|}{612} \\
\hline \multicolumn{3}{|l|}{613} \\
\hline 614 & & L \\
\hline 615 & & L \\
\hline 616 & 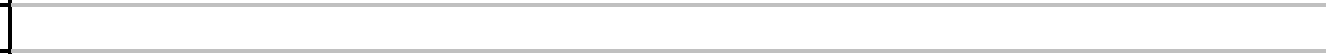 & L \\
\hline 617 & 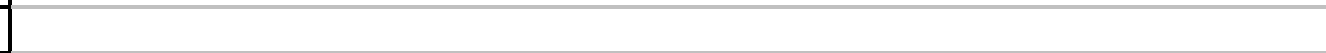 & L \\
\hline 618 & 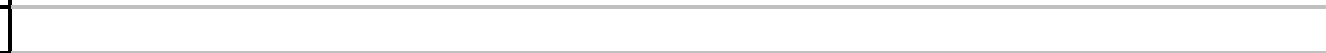 & L \\
\hline 619 & 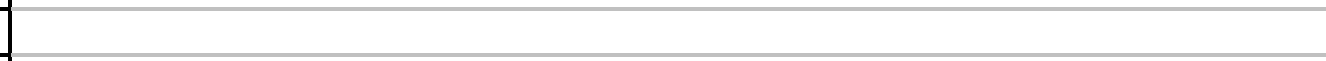 & L \\
\hline 620 & 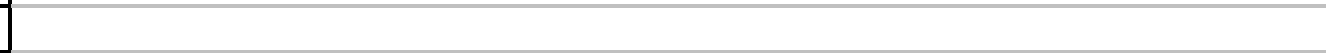 & L \\
\hline \multicolumn{3}{|l|}{621} \\
\hline \multicolumn{3}{|l|}{622} \\
\hline 623 & & $\mathrm{~L}$ \\
\hline 624 & & L \\
\hline 625 & & L \\
\hline 626 & & L \\
\hline 627 & & L \\
\hline 628 & & $\bar{L}$ \\
\hline 629 & & L \\
\hline 630 & & L \\
\hline 631 & & L \\
\hline 632 & & $\mathrm{~L}$ \\
\hline 633 & & $\mathrm{~L}$ \\
\hline 634 & & $\mathrm{~L}$ \\
\hline 635 & & L \\
\hline 636 & & L \\
\hline \multicolumn{3}{|l|}{637} \\
\hline \multicolumn{3}{|l|}{638} \\
\hline 639 & & L \\
\hline 640 & & L \\
\hline 641 & & $\bar{L}$ \\
\hline 642 & & L \\
\hline 643 & & L \\
\hline 644 & & L \\
\hline 645 & & L \\
\hline \multicolumn{3}{|l|}{646} \\
\hline 647 & $x_{2}^{2}$ & \\
\hline
\end{tabular}




\begin{tabular}{|c|c|c|}
\hline & $A D$ & $\mathrm{AF}$ \\
\hline 1 & RESULT_COMMENT & COLL_SAMP_SIZE_UNITS \\
\hline 648 & & $\mathrm{~L}$ \\
\hline 649 & & $\mathrm{~L}$ \\
\hline 650 & & $\mathrm{~L}$ \\
\hline 651 & & $\mathrm{~L}$ \\
\hline 652 & & $\mathrm{~L}$ \\
\hline 653 & & $L$ \\
\hline 654 & & L \\
\hline \multicolumn{3}{|l|}{655} \\
\hline \multicolumn{3}{|l|}{656} \\
\hline 657 & & L \\
\hline 658 & - & L \\
\hline 659 & & $\mathrm{~L}$ \\
\hline 660 & & L \\
\hline 661 & & $\mathrm{~L}$ \\
\hline 662 & & $\mathrm{~L}$ \\
\hline 663 & & $\mathrm{~L}$ \\
\hline \multicolumn{3}{|l|}{664} \\
\hline \multicolumn{3}{|l|}{665} \\
\hline 666 & & L \\
\hline 667 & & L \\
\hline 668 & & L \\
\hline 669 & & L \\
\hline 670 & & L \\
\hline 671 & & L \\
\hline 672 & & L \\
\hline \multicolumn{3}{|l|}{673} \\
\hline \multicolumn{3}{|l|}{674} \\
\hline \multicolumn{3}{|l|}{675} \\
\hline \multicolumn{3}{|l|}{676} \\
\hline \multicolumn{3}{|l|}{677} \\
\hline \multicolumn{3}{|l|}{678} \\
\hline \multicolumn{3}{|l|}{679} \\
\hline \multicolumn{3}{|l|}{680} \\
\hline \multicolumn{3}{|l|}{681} \\
\hline \multicolumn{3}{|l|}{682} \\
\hline \multicolumn{3}{|l|}{683} \\
\hline \multicolumn{3}{|l|}{684} \\
\hline 685 & 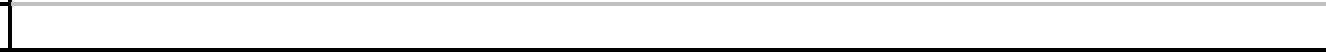 & \\
\hline
\end{tabular}




\begin{tabular}{|c|c|c|c|}
\hline & $A D$ & $\mathrm{AE}$ & $\mathrm{AF}$ \\
\hline 1 & RESULT_COMMENT & COLL_SAMP_SIZE & COLL_SAMP_SIZE_UNITS \\
\hline \multicolumn{4}{|c|}{ 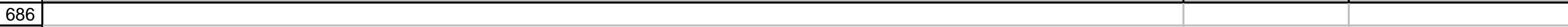 } \\
\hline \multicolumn{4}{|l|}{687} \\
\hline \multicolumn{4}{|l|}{688} \\
\hline \multicolumn{4}{|l|}{689} \\
\hline 690 & & 10 & L \\
\hline 691 & & 10 & L \\
\hline 692 & 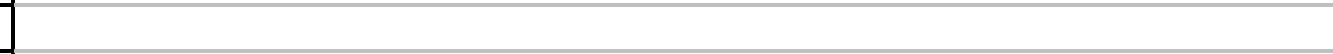 & 10 & L \\
\hline 693 & 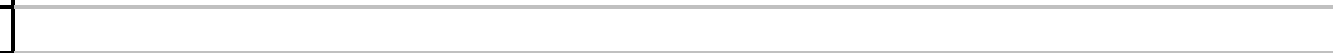 & 10 & L \\
\hline 694 & & 10 & L \\
\hline 695 & 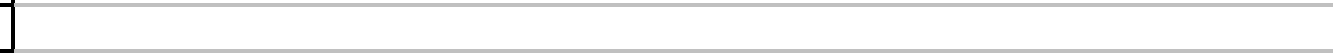 & 10 & L \\
\hline 696 & & 10 & L \\
\hline \multicolumn{4}{|l|}{697} \\
\hline \multicolumn{4}{|l|}{698} \\
\hline 699 & & 10 & $\mathrm{~L}$ \\
\hline 700 & & 10 & $\mathrm{~L}$ \\
\hline 701 & & 10 & $\mathrm{~L}$ \\
\hline 702 & & 10 & L \\
\hline 703 & & 10 & L \\
\hline 704 & & 10 & $\mathrm{~L}$ \\
\hline 705 & & 10 & L \\
\hline \multicolumn{4}{|l|}{706} \\
\hline \multicolumn{4}{|l|}{707} \\
\hline 708 & & 10 & L \\
\hline 709 & & 10 & L \\
\hline 710 & & 10 & L \\
\hline 711 & & 10 & L \\
\hline 712 & & 10 & L \\
\hline 713 & & 10 & L \\
\hline 714 & & 10 & L \\
\hline \multicolumn{4}{|l|}{715} \\
\hline \multicolumn{4}{|l|}{716} \\
\hline 717 & & 10 & L \\
\hline 718 & & 10 & L \\
\hline 719 & & 10 & $\mathrm{~L}$ \\
\hline 720 & & 10 & L \\
\hline 721 & & 10 & L \\
\hline 722 & & 10 & $\mathrm{~L}$ \\
\hline 723 & $x_{1}^{2}$ & 10 & L \\
\hline
\end{tabular}




\begin{tabular}{|c|c|c|c|}
\hline & $A D$ & $\mathrm{AE}$ & $\mathrm{AF}$ \\
\hline 1 & RESULT_COMMENT & COLL_SAMP_SIZE & COLL_SAMP_SIZE_UNITS \\
\hline \multicolumn{4}{|l|}{724} \\
\hline \multicolumn{4}{|l|}{725} \\
\hline 726 & & 10 & L \\
\hline 727 & & 10 & $\mathrm{~L}$ \\
\hline 728 & & 10 & $\mathrm{~L}$ \\
\hline 729 & & 10 & $\mathrm{~L}$ \\
\hline 730 & & 10 & L \\
\hline 731 & & 10 & $\mathrm{~L}$ \\
\hline 732 & & 10 & L \\
\hline \multicolumn{4}{|l|}{733} \\
\hline \multicolumn{4}{|l|}{734} \\
\hline 735 & & 10 & L \\
\hline 736 & & 4 & $\mathrm{~L}$ \\
\hline 737 & & 10 & $\mathrm{~L}$ \\
\hline 738 & & 10 & L \\
\hline 739 & & 10 & $\mathrm{~L}$ \\
\hline 740 & & 10 & $\mathrm{~L}$ \\
\hline 741 & REFER TO 3/12/96 INCIDENT REPORT, U-235 ACTIVITY COULD NOT BE CALCULATED. & 10 & $\mathrm{~L}$ \\
\hline 742 & & 10 & $\mathrm{~L}$ \\
\hline \multicolumn{4}{|l|}{743} \\
\hline \multicolumn{4}{|l|}{744} \\
\hline 745 & & 10 & L \\
\hline 746 & & 4 & L \\
\hline 747 & & 10 & L \\
\hline 748 & & 10 & L \\
\hline 749 & & 10 & L \\
\hline 750 & & 10 & L \\
\hline 751 & & 10 & $\mathrm{~L}$ \\
\hline 752 & & 10 & L \\
\hline \multicolumn{4}{|l|}{753} \\
\hline \multicolumn{4}{|l|}{754} \\
\hline 755 & & 10 & L \\
\hline 756 & & 10 & L \\
\hline 757 & & 10 & $\mathrm{~L}$ \\
\hline 758 & & 10 & L \\
\hline 759 & & 10 & L \\
\hline 760 & & 10 & L \\
\hline 761 & & 10 & L \\
\hline
\end{tabular}




\begin{tabular}{|c|c|c|c|}
\hline & AD & $\mathrm{AE}$ & $\mathrm{AF}$ \\
\hline 1 & RESULT_COMMENT & COLL_SAMP_SIZE & COLL_SAMP_SIZE_UNITS \\
\hline \multicolumn{4}{|l|}{762} \\
\hline \multicolumn{4}{|l|}{763} \\
\hline 764 & & 10 & L \\
\hline 765 & & 10 & L \\
\hline 766 & REFER TO 3/12/96 AND 4/12/96 INCIDENT REPORTS. REQUESTED TC-99 FROM GAMMA FRACTION, BUT LAB UNABLE TO PERFORM. & 10 & L \\
\hline 767 & & 10 & $\mathrm{~L}$ \\
\hline 768 & & 10 & $\mathrm{~L}$ \\
\hline 769 & & 10 & $\mathrm{~L}$ \\
\hline 770 & & 10 & L \\
\hline \multicolumn{4}{|l|}{771} \\
\hline \multicolumn{4}{|c|}{772} \\
\hline \multicolumn{4}{|l|}{773} \\
\hline \multicolumn{4}{|l|}{774} \\
\hline \multicolumn{4}{|l|}{775} \\
\hline \multicolumn{4}{|l|}{776} \\
\hline \multicolumn{4}{|l|}{777} \\
\hline \multicolumn{4}{|l|}{778} \\
\hline \multicolumn{4}{|l|}{779} \\
\hline \multicolumn{4}{|l|}{780} \\
\hline \multicolumn{4}{|l|}{781} \\
\hline \multicolumn{4}{|l|}{782} \\
\hline \multicolumn{4}{|l|}{783} \\
\hline \multicolumn{4}{|l|}{784} \\
\hline \multicolumn{4}{|l|}{785} \\
\hline \multicolumn{4}{|l|}{786} \\
\hline \multicolumn{4}{|l|}{787} \\
\hline \multicolumn{4}{|l|}{788} \\
\hline \multicolumn{4}{|c|}{789} \\
\hline \multicolumn{4}{|l|}{790} \\
\hline \multicolumn{4}{|l|}{791} \\
\hline \multicolumn{4}{|l|}{792} \\
\hline \multicolumn{4}{|l|}{793} \\
\hline \multicolumn{4}{|l|}{794} \\
\hline \multicolumn{4}{|l|}{795} \\
\hline 796 & & & \\
\hline 797 & & & \\
\hline 798 & & & \\
\hline 799 & & & \\
\hline
\end{tabular}




\begin{tabular}{|c|c|c|c|}
\hline & (2) & $\mathrm{AE}$ & $\mathrm{AF}$ \\
\hline 1 & 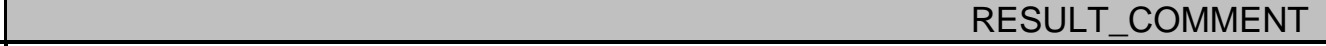 & COLL_SAMP_SIZE & COLL_SAMP_SIZE_UNITS \\
\hline \multicolumn{4}{|c|}{ 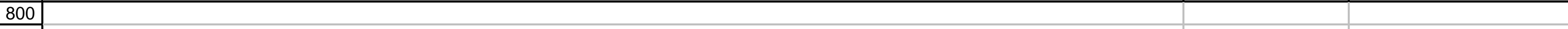 } \\
\hline \multicolumn{4}{|l|}{801} \\
\hline \multicolumn{4}{|l|}{802} \\
\hline \multicolumn{4}{|l|}{803} \\
\hline \multicolumn{4}{|l|}{804} \\
\hline \multicolumn{4}{|l|}{805} \\
\hline \multicolumn{4}{|l|}{806} \\
\hline \multicolumn{4}{|l|}{807} \\
\hline \multicolumn{4}{|l|}{808} \\
\hline \multicolumn{4}{|l|}{809} \\
\hline \multicolumn{4}{|l|}{810} \\
\hline \multicolumn{4}{|l|}{811} \\
\hline \multicolumn{4}{|l|}{812} \\
\hline \multicolumn{4}{|l|}{813} \\
\hline \multicolumn{4}{|l|}{814} \\
\hline \multicolumn{4}{|l|}{815} \\
\hline \multicolumn{4}{|l|}{816} \\
\hline \multicolumn{4}{|l|}{817} \\
\hline \multicolumn{4}{|l|}{818} \\
\hline \multicolumn{4}{|l|}{819} \\
\hline 820 & ( & 125 & $\mathrm{~mL}$ \\
\hline 821 & & 20 & $\mathrm{~L}$ \\
\hline 822 & & 20 & L \\
\hline 823 & & 20 & $\mathrm{~L}$ \\
\hline 824 & & 20 & L \\
\hline \multicolumn{4}{|l|}{825} \\
\hline \multicolumn{4}{|l|}{826} \\
\hline 827 & & 125 & $\mathrm{~mL}$ \\
\hline 828 & & 125 & $\mathrm{~mL}$ \\
\hline 829 & & 20 & $\mathrm{~L}$ \\
\hline 830 & & 20 & $\mathrm{~L}$ \\
\hline 831 & 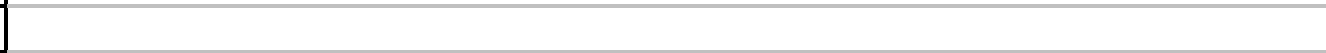 & 20 & L \\
\hline \multicolumn{4}{|l|}{832} \\
\hline \multicolumn{4}{|l|}{833} \\
\hline 834 & & 125 & $\mathrm{~mL}$ \\
\hline 835 & & 125 & $\mathrm{~mL}$ \\
\hline 836 & & 20 & $\mathrm{~L}$ \\
\hline 837 & 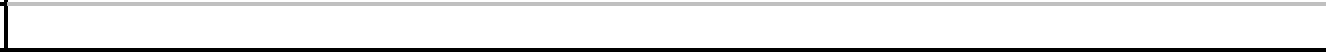 & 20 & $\mathrm{~L}$ \\
\hline
\end{tabular}




\begin{tabular}{|c|c|c|c|}
\hline & 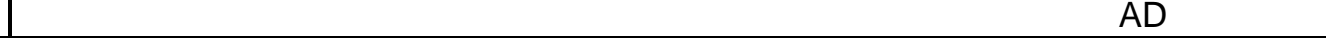 & $\mathrm{AE}$ & AF \\
\hline 1 & 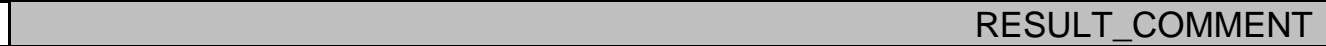 & COLL_SAMP_SIZE & COLL_SAMP_SIZE_UNITS \\
\hline 838 & & 20 & $\mathrm{~L}$ \\
\hline \multicolumn{4}{|l|}{839} \\
\hline \multicolumn{4}{|l|}{840} \\
\hline \multicolumn{4}{|l|}{841} \\
\hline \multicolumn{4}{|l|}{842} \\
\hline \multicolumn{4}{|l|}{843} \\
\hline \multicolumn{4}{|l|}{844} \\
\hline \multicolumn{4}{|l|}{845} \\
\hline 846 & & 125 & $\mathrm{~mL}$ \\
\hline 847 & & 125 & $\mathrm{~mL}$ \\
\hline 848 & & 20 & $\mathrm{~L}$ \\
\hline 849 & & 20 & $\mathrm{~L}$ \\
\hline 850 & & 20 & $\mathrm{~L}$ \\
\hline 851 & & 20 & $\mathrm{~L}$ \\
\hline 852 & & 20 & $\mathrm{~L}$ \\
\hline 853 & & 20 & $\mathrm{~L}$ \\
\hline \multicolumn{4}{|l|}{854} \\
\hline \multicolumn{4}{|l|}{855} \\
\hline 856 & 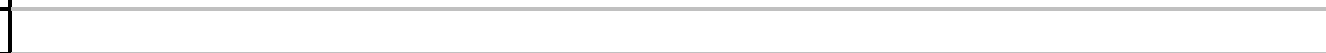 & 125 & $\mathrm{~mL}$ \\
\hline 857 & & 125 & $\mathrm{~mL}$ \\
\hline 858 & & 10 & $\mathrm{~L}$ \\
\hline 859 & & 4 & $\mathrm{~L}$ \\
\hline 860 & & 10 & $\mathrm{~L}$ \\
\hline 861 & & 10 & $\mathrm{~L}$ \\
\hline 862 & & 10 & $\mathrm{~L}$ \\
\hline 863 & & 10 & $\mathrm{~L}$ \\
\hline 864 & & 10 & $\mathrm{~L}$ \\
\hline \multicolumn{4}{|l|}{865} \\
\hline 866 & & 125 & $\mathrm{~mL}$ \\
\hline 867 & & 125 & $\mathrm{~mL}$ \\
\hline 868 & & 10 & $\mathrm{~L}$ \\
\hline 869 & & 4 & L \\
\hline 870 & & 10 & $\mathrm{~L}$ \\
\hline 871 & & 10 & $L$ \\
\hline 872 & & 10 & $\mathrm{~L}$ \\
\hline 873 & & 10 & $\mathrm{~L}$ \\
\hline 874 & & 10 & $\mathrm{~L}$ \\
\hline 875 & 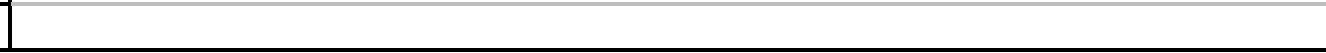 & & \\
\hline
\end{tabular}




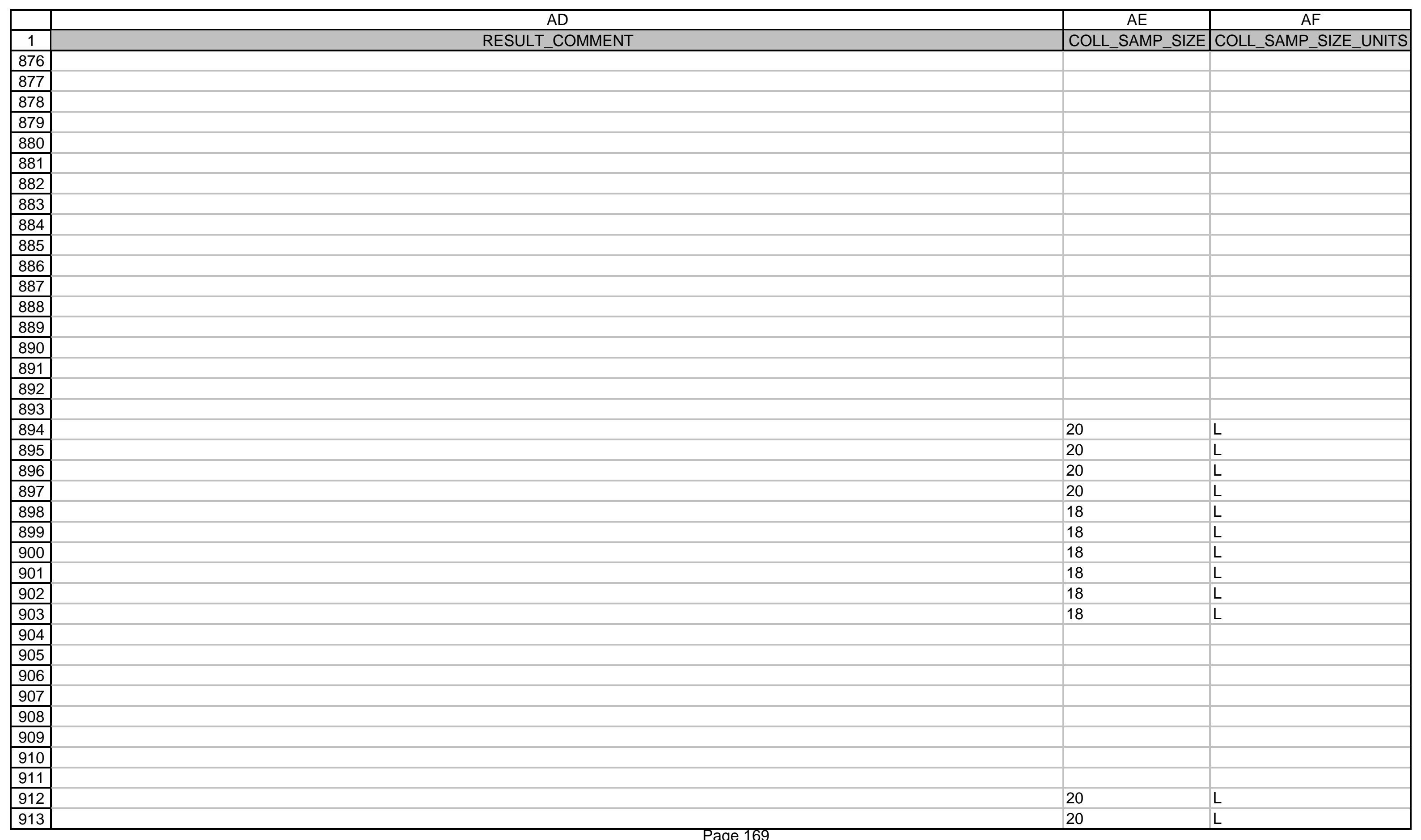




\begin{tabular}{|c|c|c|}
\hline & 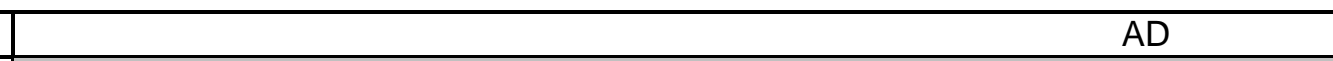 & $\mathrm{AF}$ \\
\hline 1 & 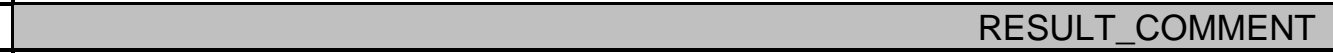 & COLL_SAMP_SIZE_UNITS \\
\hline 914 & & $\mathrm{~L}$ \\
\hline 915 & & L \\
\hline 916 & & L \\
\hline 917 & & L \\
\hline 918 & & L \\
\hline 919 & & $\mathrm{~L}$ \\
\hline 920 & & L \\
\hline 921 & trat & $\mathrm{L}$ \\
\hline 922 & & $\mathrm{~L}$ \\
\hline 923 & - & $\mathrm{L}$ \\
\hline 924 & & L \\
\hline 925 & & L \\
\hline 926 & & L \\
\hline 927 & & $\mathrm{~L}$ \\
\hline 928 & & $\mathrm{~L}$ \\
\hline 929 & & L \\
\hline 930 & & L \\
\hline 931 & & $\mathrm{~L}$ \\
\hline 932 & & $\mathrm{~L}$ \\
\hline 933 & & L \\
\hline 934 & & $\mathrm{~L}$ \\
\hline 935 & & L \\
\hline \multicolumn{3}{|l|}{936} \\
\hline \multicolumn{3}{|l|}{937} \\
\hline \multicolumn{3}{|l|}{938} \\
\hline \multicolumn{3}{|l|}{939} \\
\hline \multicolumn{3}{|l|}{940} \\
\hline \multicolumn{3}{|l|}{941} \\
\hline \multicolumn{3}{|l|}{942} \\
\hline \multicolumn{3}{|l|}{943} \\
\hline \multicolumn{3}{|l|}{944} \\
\hline \multicolumn{3}{|l|}{945} \\
\hline \multicolumn{3}{|l|}{946} \\
\hline \multicolumn{3}{|l|}{947} \\
\hline 948 & & $\mathrm{~L}$ \\
\hline 949 & & $\mathrm{~L}$ \\
\hline 950 & & L \\
\hline 951 & $x_{1}^{2}$ & L \\
\hline
\end{tabular}




\begin{tabular}{|c|c|c|c|}
\hline & 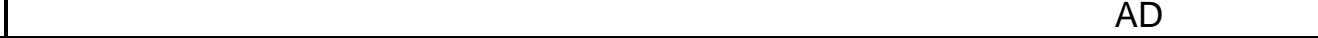 & $\mathrm{AE}$ & $\mathrm{AF}$ \\
\hline 1 & RESULT_COMMENT & COLL_SAMP_SIZE & COLL_SAMP_SIZE_UNITS \\
\hline 952 & & 20 & $\mathrm{~L}$ \\
\hline 953 & & 20 & L \\
\hline 954 & & 20 & L \\
\hline 955 & & 20 & L \\
\hline 956 & & 20 & L \\
\hline 957 & & 20 & L \\
\hline 958 & & 4 & L \\
\hline 959 & & 20 & L \\
\hline 960 & & 20 & $\mathrm{~L}$ \\
\hline 961 & & 20 & L \\
\hline 962 & & 20 & L \\
\hline 963 & & 20 & L \\
\hline 964 & & 20 & L \\
\hline 965 & & 4 & L \\
\hline 966 & & 20 & L \\
\hline 967 & & 20 & L \\
\hline 968 & & 20 & $\mathrm{~L}$ \\
\hline 969 & & 20 & L \\
\hline 970 & & 20 & L \\
\hline 971 & & 20 & L \\
\hline 972 & & 20 & L \\
\hline 973 & & 20 & L \\
\hline 974 & & 20 & L \\
\hline 975 & REFER TO 3/29/99 INCIDENT REPORT, LOST IN LAB. & 20 & L \\
\hline 976 & & 20 & L \\
\hline 977 & & 20 & L \\
\hline 978 & & 20 & L \\
\hline 979 & & 20 & L \\
\hline 980 & & 20 & L \\
\hline 981 & & 20 & L \\
\hline 982 & & 20 & L \\
\hline 983 & & 20 & L \\
\hline 984 & & 20 & $\mathrm{~L}$ \\
\hline 985 & & 20 & $\mathrm{~L}$ \\
\hline 986 & & 20 & L \\
\hline 987 & & 20 & L \\
\hline 988 & & 20 & $\mathrm{~L}$ \\
\hline 989 & & 20 & $\mathrm{~L}$ \\
\hline
\end{tabular}




\begin{tabular}{|c|c|c|c|}
\hline & AD & $\mathrm{AE}$ & AF \\
\hline 1 & RESULT_COMMENT & COLL_SAMP_SIZE & COLL_SAMP_SIZE_UNITS \\
\hline 990 & & 20 & $\mathrm{~L}$ \\
\hline 991 & & 20 & L \\
\hline 992 & & 20 & L \\
\hline 993 & & 20 & L \\
\hline 994 & & 20 & L \\
\hline \begin{tabular}{|l|l|}
995 \\
909
\end{tabular} & REFER TO 12/09/98 INCIDENT REPORT, SR-90 LOST. & 20 & $\mathrm{~L}$ \\
\hline 996 & & 20 & L \\
\hline 997 & & 20 & L \\
\hline 998 & & 20 & $\mathrm{~L}$ \\
\hline 999 & IR-0031 FAILED INSUFFICIENT VOLUME & 20 & L \\
\hline 1000 & & 20 & L \\
\hline 1001 & & 20 & L \\
\hline 1002 & & 20 & L \\
\hline 1003 & IR-0031 FAILED INSUFFICIENT VOLUME & 20 & L \\
\hline 1004 & & 20 & L \\
\hline 1005 & & 20 & L \\
\hline 1006 & & 20 & L \\
\hline 1007 & & 20 & L \\
\hline 1008 & & 20 & L \\
\hline 1009 & & 20 & L \\
\hline 1010 & & 20 & L \\
\hline 1011 & & & \\
\hline 1012 & & 14 & L \\
\hline 1013 & & 14 & L \\
\hline 1014 & & 14 & L \\
\hline 1015 & & 20 & L \\
\hline 1016 & & 20 & L \\
\hline 1017 & & 20 & L \\
\hline 1018 & & 20 & L \\
\hline \begin{tabular}{|l|}
1019 \\
\end{tabular} & & 20 & L \\
\hline 1020 & & 20 & L \\
\hline \begin{tabular}{|l|}
1021 \\
\end{tabular} & & 20 & L \\
\hline 1022 & & 20 & L \\
\hline \begin{tabular}{|c|}
1023 \\
\end{tabular} & & 20 & $\mathrm{~L}$ \\
\hline 1024 & & 20 & $\mathrm{~L}$ \\
\hline \begin{tabular}{|l|}
1025 \\
\end{tabular} & & 20 & L \\
\hline 1026 & & 20 & $\mathrm{~L}$ \\
\hline \begin{tabular}{|l|}
1027 \\
\end{tabular} & 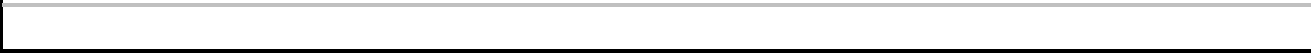 & 20 & $\mathrm{~L}$ \\
\hline
\end{tabular}




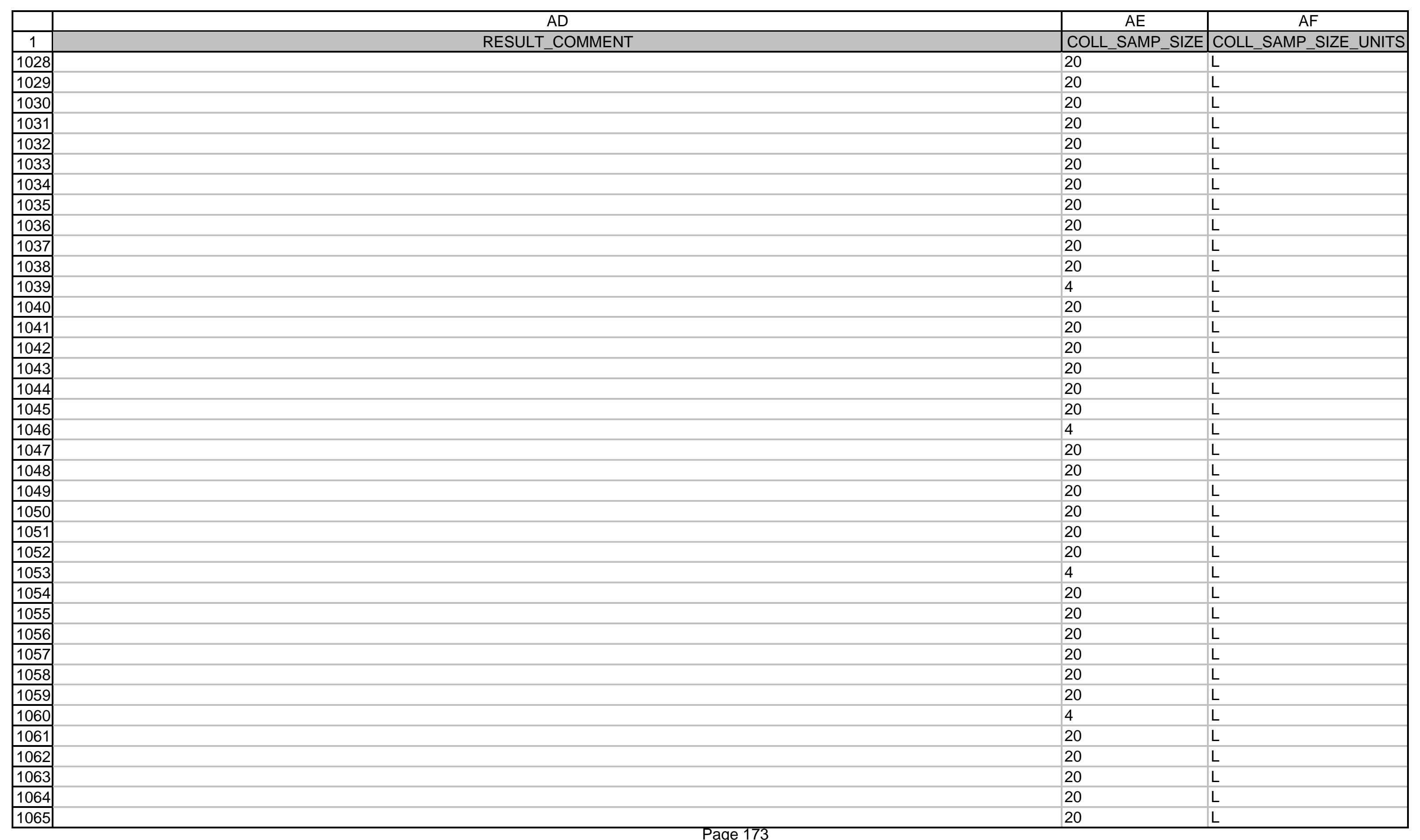


AD

$\mathrm{AE}$

AF

\begin{tabular}{|c|c|c|c|}
\hline & $A D$ & $\mathrm{AE}$ & $\mathrm{AF}$ \\
\hline 1 & RESULT_COMMENT & COLL_SAMP_SIZE & COLL_SAMP_SIZE_UNITS \\
\hline 1066 & & 20 & $\mathrm{~L}$ \\
\hline 1067 & & 4 & L \\
\hline 1068 & & 20 & L \\
\hline 1069 & & 20 & $\mathrm{~L}$ \\
\hline 1070 & & 20 & L \\
\hline 1071 & & 20 & $\mathrm{~L}$ \\
\hline 1072 & & 20 & L \\
\hline \multicolumn{4}{|c|}{1073} \\
\hline \multicolumn{4}{|c|}{1074} \\
\hline \multicolumn{4}{|c|}{\begin{tabular}{|l|}
1075 \\
\end{tabular}} \\
\hline 1076 & & 4 & L \\
\hline 1077 & \#IR-0062 MDA > CRDL & 4 & L \\
\hline 1078 & \#IR-0062 MDA > CRDL & 4 & $\mathrm{~L}$ \\
\hline 1079 & \#IR-0062 MDA > CRDL & 4 & $\mathrm{~L}$ \\
\hline
\end{tabular}




\begin{tabular}{|c|c|c|c|}
\hline & $A G$ & $\overline{\mathrm{AH}}$ & $\mathrm{Al}$ \\
\hline 1 & DIST_CLASS & OWNER_ID & COLLECTION_PURPOSE \\
\hline 2 & & HEISPROD & \\
\hline 3 & & HEISPROD & \\
\hline 4 & & HEISPROD & \\
\hline 5 & & HEISPROD & \\
\hline 6 & & HEISPROD & \\
\hline 7 & & HEISPROD & \\
\hline 8 & & HEISPROD & \\
\hline 9 & & HEISPROD & \\
\hline 10 & & HEISPROD & \\
\hline 11 & & HEISPROD & \\
\hline 12 & & HEISPROD & \\
\hline 13 & & HEISPROD & \\
\hline 14 & & HEISPROD & \\
\hline 15 & & HEISPROD & \\
\hline 16 & & HEISPROD & \\
\hline 17 & & HEISPROD & \\
\hline 18 & & HEISPROD & \\
\hline 19 & & HEISPROD & \\
\hline 20 & & HEISPROD & \\
\hline 21 & & HEISPROD & \\
\hline 22 & & HEISPROD & \\
\hline 23 & & HEISPROD & \\
\hline 24 & & HEISPROD & \\
\hline 25 & & HEISPROD & \\
\hline 26 & & HEISPROD & \\
\hline 27 & & HEISPROD & \\
\hline 28 & & HEISPROD & \\
\hline 29 & & HEISPROD & \\
\hline 30 & & HEISPROD & \\
\hline 31 & & HEISPROD & \\
\hline 32 & & HEISPROD & \\
\hline 33 & & HEISPROD & \\
\hline 34 & & HEISPROD & \\
\hline 35 & & HEISPROD & \\
\hline 36 & & HEISPROD & \\
\hline 37 & & HEISPROD & \\
\hline 38 & & HEISPROD & \\
\hline 39 & & HEISPROD & \\
\hline
\end{tabular}




\begin{tabular}{|c|c|c|c|}
\hline & AG & $\mathrm{AH}$ & $\mathrm{Al}$ \\
\hline 1 & DIST_CLASS & OWNER_ID & COLLECTION_PURPOSE \\
\hline 40 & & HEISPROD & \\
\hline 41 & & HEISPROD & \\
\hline 42 & & HEISPROD & \\
\hline 43 & & HEISPROD & \\
\hline 44 & & HEISPROD & \\
\hline 45 & & HEISPROD & \\
\hline 46 & & HEISPROD & \\
\hline 47 & & HEISPROD & \\
\hline 48 & & HEISPROD & \\
\hline 49 & & HEISPROD & \\
\hline 50 & & HEISPROD & \\
\hline 51 & & HEISPROD & \\
\hline 52 & & HEISPROD & \\
\hline 53 & & HEISPROD & \\
\hline 54 & & HEISPROD & \\
\hline 55 & & HEISPROD & \\
\hline 56 & & HEISPROD & \\
\hline 57 & & HEISPROD & \\
\hline 58 & & HEISPROD & \\
\hline 59 & & HEISPROD & \\
\hline 60 & & HEISPROD & \\
\hline 61 & & HEISPROD & \\
\hline 62 & & HEISPROD & \\
\hline 63 & & HEISPROD & \\
\hline 64 & & HEISPROD & \\
\hline 65 & & HEISPROD & \\
\hline 66 & & HEISPROD & \\
\hline 67 & & HEISPROD & \\
\hline 68 & & HEISPROD & \\
\hline 69 & & HEISPROD & \\
\hline 70 & & HEISPROD & \\
\hline 71 & & HEISPROD & \\
\hline 72 & & HEISPROD & \\
\hline 73 & & HEISPROD & \\
\hline 74 & & HEISPROD & \\
\hline 75 & & HEISPROD & \\
\hline 76 & & HEISPROD & \\
\hline 77 & & HEISPROD & \\
\hline
\end{tabular}




\begin{tabular}{|c|c|c|c|}
\hline & $A G$ & $\mathrm{AH}$ & Al \\
\hline 1 & DIST_CLASS & OWNER_ID & COLLECTION_PURPOSE \\
\hline 78 & & HEISPROD & \\
\hline 79 & & HEISPROD & \\
\hline 80 & & HEISPROD & \\
\hline 81 & & HEISPROD & \\
\hline 82 & & HEISPROD & \\
\hline 83 & & HEISPROD & \\
\hline 84 & & HEISPROD & \\
\hline 85 & & HEISPROD & \\
\hline 86 & & HEISPROD & \\
\hline 87 & & HEISPROD & \\
\hline 88 & & HEISPROD & \\
\hline 89 & & HEISPROD & \\
\hline 90 & & HEISPROD & \\
\hline 91 & & HEISPROD & \\
\hline 92 & & HEISPROD & \\
\hline 93 & & HEISPROD & \\
\hline 94 & & HEISPROD & \\
\hline 95 & & HEISPROD & \\
\hline 96 & & HEISPROD & \\
\hline 97 & & HEISPROD & \\
\hline 98 & & HEISPROD & \\
\hline 99 & & HEISPROD & \\
\hline 100 & & HEISPROD & \\
\hline 101 & & HEISPROD & \\
\hline 102 & & HEISPROD & \\
\hline 103 & & HEISPROD & \\
\hline 104 & & HEISPROD & \\
\hline 105 & & HEISPROD & \\
\hline 106 & & HEISPROD & \\
\hline 107 & & HEISPROD & \\
\hline 108 & & HEISPROD & \\
\hline 109 & & HEISPROD & \\
\hline 110 & & HEISPROD & \\
\hline 111 & & HEISPROD & \\
\hline 112 & & HEISPROD & \\
\hline 113 & & HEISPROD & \\
\hline 114 & & HEISPROD & \\
\hline 115 & & HEISPROD & \\
\hline
\end{tabular}




\begin{tabular}{|c|c|c|c|}
\hline & $A G$ & $\overline{\mathrm{AH}}$ & $\mathrm{Al}$ \\
\hline 1 & DIST_CLASS & OWNER_ID & COLLECTION_PURPOSE \\
\hline 116 & & HEISPROD & \\
\hline 117 & & HEISPROD & \\
\hline 118 & & HEISPROD & \\
\hline 119 & & HEISPROD & \\
\hline 120 & & HEISPROD & \\
\hline 121 & & HEISPROD & \\
\hline 122 & & HEISPROD & \\
\hline 123 & & HEISPROD & \\
\hline 124 & & HEISPROD & \\
\hline 125 & & HEISPROD & \\
\hline 126 & & HEISPROD & \\
\hline 127 & & HEISPROD & \\
\hline 128 & & HEISPROD & \\
\hline 129 & & HEISPROD & \\
\hline 130 & & HEISPROD & \\
\hline 131 & & HEISPROD & \\
\hline 132 & & HEISPROD & \\
\hline 133 & & HEISPROD & \\
\hline 134 & & HEISPROD & \\
\hline 135 & & HEISPROD & \\
\hline 136 & & HEISPROD & \\
\hline 137 & & HEISPROD & \\
\hline 138 & & HEISPROD & \\
\hline 139 & & HEISPROD & \\
\hline 140 & & HEISPROD & \\
\hline 141 & & HEISPROD & \\
\hline 142 & & HEISPROD & \\
\hline 143 & & HEISPROD & \\
\hline 144 & & HEISPROD & \\
\hline 145 & & HEISPROD & \\
\hline 146 & & HEISPROD & \\
\hline 147 & & HEISPROD & \\
\hline 148 & & HEISPROD & \\
\hline 149 & & HEISPROD & \\
\hline 150 & & HEISPROD & \\
\hline 151 & & HEISPROD & \\
\hline 152 & & HEISPROD & \\
\hline 153 & & HEISPROD & \\
\hline
\end{tabular}




\begin{tabular}{|c|c|c|c|}
\hline & $A G$ & $\mathrm{AH}$ & $\mathrm{Al}$ \\
\hline 1 & DIST_CLASS & OWNER_ID & COLLECTION_PURPOSE \\
\hline 154 & & HEISPROD & \\
\hline 155 & & HEISPROD & \\
\hline 156 & & HEISPROD & \\
\hline 157 & & HEISPROD & \\
\hline 158 & & HEISPROD & \\
\hline 159 & & HEISPROD & \\
\hline 160 & & HEISPROD & \\
\hline 161 & & HEISPROD & \\
\hline 162 & & HEISPROD & \\
\hline 163 & & HEISPROD & \\
\hline 164 & & HEISPROD & \\
\hline 165 & & HEISPROD & \\
\hline 166 & & HEISPROD & \\
\hline 167 & & HEISPROD & \\
\hline 168 & & HEISPROD & \\
\hline 169 & & HEISPROD & \\
\hline 170 & & HEISPROD & \\
\hline 171 & & HEISPROD & \\
\hline 172 & & HEISPROD & \\
\hline 173 & & HEISPROD & \\
\hline 174 & & HEISPROD & \\
\hline 175 & & HEISPROD & \\
\hline 176 & & HEISPROD & \\
\hline 177 & & HEISPROD & \\
\hline 178 & & HEISPROD & \\
\hline 179 & & HEISPROD & \\
\hline 180 & & HEISPROD & \\
\hline 181 & & HEISPROD & \\
\hline 182 & & HEISPROD & \\
\hline 183 & & HEISPROD & \\
\hline 184 & & HEISPROD & \\
\hline 185 & & HEISPROD & \\
\hline 186 & & HEISPROD & \\
\hline 187 & & HEISPROD & \\
\hline 188 & & HEISPROD & \\
\hline 189 & & HEISPROD & \\
\hline 190 & & HEISPROD & \\
\hline 191 & & HEISPROD & \\
\hline
\end{tabular}




\begin{tabular}{|c|c|c|c|}
\hline & $A G$ & $\mathrm{AH}$ & Al \\
\hline 1 & DIST_CLASS & OWNER_ID & COLLECTION_PURPOSE \\
\hline 192 & & HEISPROD & \\
\hline 193 & & HEISPROD & \\
\hline 194 & & HEISPROD & \\
\hline 195 & & HEISPROD & \\
\hline 196 & & HEISPROD & \\
\hline 197 & & HEISPROD & \\
\hline 198 & & HEISPROD & \\
\hline 199 & & HEISPROD & \\
\hline 200 & & HEISPROD & \\
\hline 201 & & HEISPROD & \\
\hline 202 & & HEISPROD & \\
\hline 203 & & HEISPROD & \\
\hline 204 & & HEISPROD & \\
\hline 205 & & HEISPROD & \\
\hline 206 & & HEISPROD & \\
\hline 207 & & HEISPROD & \\
\hline 208 & & HEISPROD & \\
\hline 209 & & HEISPROD & \\
\hline 210 & & HEISPROD & \\
\hline 211 & & HEISPROD & \\
\hline 212 & & HEISPROD & \\
\hline 213 & & HEISPROD & \\
\hline 214 & & HEISPROD & \\
\hline 215 & & HEISPROD & \\
\hline 216 & & HEISPROD & \\
\hline 217 & & HEISPROD & \\
\hline 218 & & HEISPROD & \\
\hline 219 & & HEISPROD & \\
\hline 220 & & HEISPROD & \\
\hline 221 & & HEISPROD & \\
\hline 222 & & HEISPROD & \\
\hline 223 & & HEISPROD & \\
\hline 224 & & HEISPROD & \\
\hline 225 & & HEISPROD & \\
\hline 226 & & HEISPROD & \\
\hline 227 & & HEISPROD & \\
\hline 228 & & HEISPROD & \\
\hline 229 & & HEISPROD & \\
\hline
\end{tabular}




\begin{tabular}{|c|c|c|c|}
\hline & $A G$ & $\mathrm{AH}$ & Al \\
\hline 1 & DIST_CLASS & OWNER_ID & COLLECTION_PURPOSE \\
\hline 230 & & HEISPROD & \\
\hline 231 & & HEISPROD & \\
\hline 232 & & HEISPROD & \\
\hline 233 & & HEISPROD & \\
\hline 234 & & HEISPROD & \\
\hline 235 & & HEISPROD & \\
\hline 236 & & HEISPROD & \\
\hline 237 & & HEISPROD & \\
\hline 238 & & HEISPROD & \\
\hline 239 & & HEISPROD & \\
\hline 240 & & HEISPROD & \\
\hline 241 & & HEISPROD & \\
\hline 242 & & HEISPROD & \\
\hline 243 & & HEISPROD & \\
\hline 244 & & HEISPROD & \\
\hline 245 & & HEISPROD & \\
\hline 246 & & HEISPROD & \\
\hline 247 & & HEISPROD & \\
\hline 248 & & HEISPROD & \\
\hline 249 & & HEISPROD & \\
\hline 250 & & HEISPROD & \\
\hline 251 & & HEISPROD & \\
\hline 252 & & HEISPROD & \\
\hline 253 & & HEISPROD & \\
\hline 254 & & HEISPROD & \\
\hline 255 & & HEISPROD & \\
\hline 256 & & HEISPROD & \\
\hline 257 & & HEISPROD & \\
\hline 258 & & HEISPROD & \\
\hline 259 & & HEISPROD & \\
\hline 260 & & HEISPROD & \\
\hline 261 & & HEISPROD & \\
\hline 262 & & HEISPROD & \\
\hline 263 & & HEISPROD & \\
\hline 264 & & HEISPROD & \\
\hline 265 & & HEISPROD & \\
\hline 266 & & HEISPROD & \\
\hline 267 & & HEISPROD & \\
\hline
\end{tabular}




\begin{tabular}{|c|c|c|c|}
\hline & $A G$ & $\overline{\mathrm{AH}}$ & $\mathrm{Al}$ \\
\hline 1 & DIST_CLASS & OWNER_ID & COLLECTION_PURPOSE \\
\hline 268 & & HEISPROD & \\
\hline 269 & & HEISPROD & \\
\hline 270 & & HEISPROD & \\
\hline 271 & & HEISPROD & \\
\hline 272 & & HEISPROD & \\
\hline 273 & & HEISPROD & \\
\hline 274 & & HEISPROD & \\
\hline 275 & & HEISPROD & \\
\hline 276 & & HEISPROD & \\
\hline 277 & & HEISPROD & \\
\hline 278 & & HEISPROD & \\
\hline 279 & & HEISPROD & \\
\hline 280 & & HEISPROD & \\
\hline 281 & & HEISPROD & \\
\hline 282 & & HEISPROD & \\
\hline 283 & & HEISPROD & \\
\hline 284 & & HEISPROD & \\
\hline 285 & & HEISPROD & \\
\hline 286 & & HEISPROD & \\
\hline 287 & & HEISPROD & \\
\hline 288 & & HEISPROD & \\
\hline 289 & & HEISPROD & \\
\hline 290 & & HEISPROD & \\
\hline 291 & & HEISPROD & \\
\hline 292 & & HEISPROD & \\
\hline 293 & ONSITE & SESPMNT & $\mathrm{R}$ \\
\hline 294 & ONSITE & SESPMNT & $\mathrm{R}$ \\
\hline 295 & ONSITE & SESPMNT & $\mathrm{R}$ \\
\hline 296 & ONSITE & SESPMNT & $\mathrm{R}$ \\
\hline 297 & ONSITE & SESPMNT & $\mathrm{R}$ \\
\hline 298 & ONSITE & SESPMNT & $\mathrm{R}$ \\
\hline 299 & ONSITE & SESPMNT & $\mathrm{R}$ \\
\hline 300 & ONSITE & SESPMNT & $\mathrm{R}$ \\
\hline 301 & ONSITE & SESPMNT & $\mathrm{R}$ \\
\hline 302 & ONSITE & SESPMNT & $\mathrm{R}$ \\
\hline 303 & ONSITE & SESPMNT & $\mathrm{R}$ \\
\hline 304 & ONSITE & SESPMNT & $\mathrm{R}$ \\
\hline 305 & ONSITE & SESPMNT & $\mathrm{R}$ \\
\hline
\end{tabular}




\begin{tabular}{|c|c|c|c|}
\hline & $A G$ & $\mathrm{AH}$ & Al \\
\hline 1 & DIST_CLASS & OWNER_ID & COLLECTION_PURPOSE \\
\hline 306 & ONSITE & SESPMNT & $\mathrm{R}$ \\
\hline 307 & ONSITE & SESPMNT & $\mathrm{R}$ \\
\hline 308 & ONSITE & SESPMNT & $\mathrm{R}$ \\
\hline 309 & ONSITE & SESPMNT & $\mathrm{R}$ \\
\hline 310 & ONSITE & SESPMNT & $\mathrm{R}$ \\
\hline 311 & & HEISPROD & \\
\hline 312 & & HEISPROD & \\
\hline 313 & & HEISPROD & \\
\hline 314 & & HEISPROD & \\
\hline 315 & & HEISPROD & \\
\hline 316 & & HEISPROD & \\
\hline 317 & & HEISPROD & \\
\hline 318 & & HEISPROD & \\
\hline 319 & & HEISPROD & \\
\hline 320 & & HEISPROD & \\
\hline 321 & & HEISPROD & \\
\hline 322 & & HEISPROD & \\
\hline 323 & & HEISPROD & \\
\hline 324 & & HEISPROD & \\
\hline 325 & & HEISPROD & \\
\hline 326 & & HEISPROD & \\
\hline 327 & & HEISPROD & \\
\hline 328 & & HEISPROD & \\
\hline 329 & & HEISPROD & \\
\hline 330 & & HEISPROD & \\
\hline 331 & & HEISPROD & \\
\hline 332 & & HEISPROD & \\
\hline 333 & & HEISPROD & \\
\hline 334 & & HEISPROD & \\
\hline 335 & & HEISPROD & \\
\hline 336 & & HEISPROD & \\
\hline 337 & & HEISPROD & \\
\hline 338 & & HEISPROD & \\
\hline 339 & & HEISPROD & \\
\hline 340 & & HEISPROD & \\
\hline 341 & & HEISPROD & \\
\hline 342 & & HEISPROD & \\
\hline 343 & & HEISPROD & \\
\hline
\end{tabular}




\begin{tabular}{|c|c|c|c|}
\hline & $A G$ & $\mathrm{AH}$ & Al \\
\hline 1 & DIST_CLASS & OWNER_ID & COLLECTION_PURPOSE \\
\hline 344 & & HEISPROD & \\
\hline 345 & & HEISPROD & \\
\hline 346 & & HEISPROD & \\
\hline 347 & & HEISPROD & \\
\hline 348 & & HEISPROD & \\
\hline 349 & & HEISPROD & \\
\hline 350 & & HEISPROD & \\
\hline 351 & & HEISPROD & \\
\hline 352 & & HEISPROD & \\
\hline 353 & & HEISPROD & \\
\hline 354 & & HEISPROD & \\
\hline 355 & & HEISPROD & \\
\hline 356 & & HEISPROD & \\
\hline 357 & & HEISPROD & \\
\hline 358 & & HEISPROD & \\
\hline 359 & & HEISPROD & \\
\hline 360 & & HEISPROD & \\
\hline 361 & & HEISPROD & \\
\hline 362 & & HEISPROD & \\
\hline 363 & & HEISPROD & \\
\hline 364 & & HEISPROD & \\
\hline 365 & & HEISPROD & \\
\hline 366 & & HEISPROD & \\
\hline 367 & & HEISPROD & \\
\hline 368 & & HEISPROD & \\
\hline 369 & & HEISPROD & \\
\hline 370 & & HEISPROD & \\
\hline 371 & & HEISPROD & \\
\hline 372 & & HEISPROD & \\
\hline 373 & & HEISPROD & \\
\hline 374 & & HEISPROD & \\
\hline 375 & & HEISPROD & \\
\hline 376 & & HEISPROD & \\
\hline 377 & & HEISPROD & \\
\hline 378 & & HEISPROD & \\
\hline 379 & & HEISPROD & \\
\hline 380 & & HEISPROD & \\
\hline 381 & & HEISPROD & \\
\hline
\end{tabular}




\begin{tabular}{|c|c|c|c|}
\hline & $A G$ & $\mathrm{AH}$ & Al \\
\hline 1 & DIST_CLASS & OWNER_ID & COLLECTION_PURPOSE \\
\hline 382 & & HEISPROD & \\
\hline 383 & & HEISPROD & \\
\hline 384 & & HEISPROD & \\
\hline 385 & & HEISPROD & \\
\hline 386 & & HEISPROD & \\
\hline 387 & & HEISPROD & \\
\hline 388 & & HEISPROD & \\
\hline 389 & & HEISPROD & \\
\hline 390 & & HEISPROD & \\
\hline 391 & & HEISPROD & \\
\hline 392 & & HEISPROD & \\
\hline 393 & & HEISPROD & \\
\hline 394 & & HEISPROD & \\
\hline 395 & & HEISPROD & \\
\hline 396 & & HEISPROD & \\
\hline 397 & & HEISPROD & \\
\hline 398 & & HEISPROD & \\
\hline 399 & & HEISPROD & \\
\hline 400 & & HEISPROD & \\
\hline 401 & & HEISPROD & \\
\hline 402 & & HEISPROD & \\
\hline 403 & & HEISPROD & \\
\hline 404 & & HEISPROD & \\
\hline 405 & & HEISPROD & \\
\hline 406 & & HEISPROD & \\
\hline 407 & & HEISPROD & \\
\hline 408 & & HEISPROD & \\
\hline 409 & & HEISPROD & \\
\hline 410 & & HEISPROD & \\
\hline 411 & ONSITE & SESPMNT & $\mathrm{R}$ \\
\hline 412 & ONSITE & SESPMNT & $\mathrm{R}$ \\
\hline 413 & ONSITE & SESPMNT & $\mathrm{R}$ \\
\hline 414 & ONSITE & SESPMNT & $\mathrm{R}$ \\
\hline 415 & ONSITE & SESPMNT & $\mathrm{R}$ \\
\hline 416 & ONSITE & SESPMNT & $\mathrm{R}$ \\
\hline 417 & ONSITE & SESPMNT & $\mathrm{R}$ \\
\hline 418 & ONSITE & SESPMNT & $\mathrm{R}$ \\
\hline 419 & ONSITE & SESPMNT & $\mathrm{R}$ \\
\hline
\end{tabular}




\begin{tabular}{|c|c|c|c|}
\hline & $A G$ & $\mathrm{AH}$ & Al \\
\hline 1 & DIST_CLASS & OWNER_ID & COLLECTION_PURPOSE \\
\hline 420 & ONSITE & SESPMNT & $\mathrm{R}$ \\
\hline 421 & ONSITE & SESPMNT & $\mathrm{R}$ \\
\hline 422 & ONSITE & SESPMNT & $\mathrm{R}$ \\
\hline 423 & ONSITE & SESPMNT & $\mathrm{R}$ \\
\hline 424 & ONSITE & SESPMNT & $\mathrm{R}$ \\
\hline 425 & ONSITE & SESPMNT & $\mathrm{R}$ \\
\hline 426 & ONSITE & SESPMNT & $\mathrm{R}$ \\
\hline 427 & ONSITE & SESPMNT & $\mathrm{R}$ \\
\hline 428 & ONSITE & SESPMNT & $\mathrm{R}$ \\
\hline 429 & ONSITE & SESPMNT & $\mathrm{R}$ \\
\hline 430 & ONSITE & SESPMNT & $\mathrm{R}$ \\
\hline 431 & ONSITE & SESPMNT & $\mathrm{R}$ \\
\hline 432 & ONSITE & SESPMNT & $\mathrm{R}$ \\
\hline 433 & & HEISPROD & \\
\hline 434 & & HEISPROD & \\
\hline 435 & & HEISPROD & \\
\hline 436 & & HEISPROD & \\
\hline 437 & & HEISPROD & \\
\hline 438 & & HEISPROD & \\
\hline 439 & & HEISPROD & \\
\hline 440 & & HEISPROD & \\
\hline 441 & ONSITE & SESPMNT & $\mathrm{R}$ \\
\hline 442 & ONSITE & SESPMNT & $\mathrm{R}$ \\
\hline 443 & ONSITE & SESPMNT & $\mathrm{R}$ \\
\hline 444 & ONSITE & SESPMNT & $\mathrm{R}$ \\
\hline 445 & ONSITE & SESPMNT & $\mathrm{R}$ \\
\hline 446 & ONSITE & SESPMNT & $\mathrm{R}$ \\
\hline 447 & ONSITE & SESPMNT & $\mathrm{R}$ \\
\hline 448 & ONSITE & SESPMNT & $\mathrm{R}$ \\
\hline 449 & ONSITE & SESPMNT & $\mathrm{R}$ \\
\hline 450 & ONSITE & SESPMNT & $\mathrm{R}$ \\
\hline 451 & ONSITE & SESPMNT & $\mathrm{R}$ \\
\hline 452 & ONSITE & SESPMNT & $\mathrm{R}$ \\
\hline 453 & ONSITE & SESPMNT & $\mathrm{R}$ \\
\hline 454 & ONSITE & SESPMNT & $\mathrm{R}$ \\
\hline 455 & ONSITE & SESPMNT & $\mathrm{R}$ \\
\hline 456 & ONSITE & SESPMNT & $\mathrm{R}$ \\
\hline 457 & ONSITE & SESPMNT & $\mathrm{R}$ \\
\hline
\end{tabular}




\begin{tabular}{|c|c|c|c|}
\hline & $A G$ & $\overline{\mathrm{AH}}$ & $\mathrm{Al}$ \\
\hline 1 & DIST_CLASS & OWNER_ID & COLLECTION_PURPOSE \\
\hline 458 & ONSITE & SESPMNT & $\mathrm{R}$ \\
\hline 459 & ONSITE & SESPMNT & $\mathrm{R}$ \\
\hline 460 & ONSITE & SESPMNT & $\mathrm{R}$ \\
\hline 461 & ONSITE & SESPMNT & $\mathrm{R}$ \\
\hline 462 & ONSITE & SESPMNT & $\mathrm{R}$ \\
\hline 463 & ONSITE & SESPMNT & $\mathrm{R}$ \\
\hline 464 & & HEISPROD & \\
\hline 465 & & HEISPROD & \\
\hline 466 & & HEISPROD & \\
\hline 467 & & HEISPROD & \\
\hline 468 & & HEISPROD & \\
\hline 469 & & HEISPROD & \\
\hline 470 & & HEISPROD & \\
\hline 471 & & HEISPROD & \\
\hline 472 & & HEISPROD & \\
\hline 473 & & HEISPROD & \\
\hline 474 & & HEISPROD & \\
\hline 475 & & HEISPROD & \\
\hline 476 & ONSITE & SESPMNT & $\mathrm{R}$ \\
\hline 477 & ONSITE & SESPMNT & $\mathrm{R}$ \\
\hline 478 & ONSITE & SESPMNT & $\mathrm{R}$ \\
\hline 479 & ONSITE & SESPMNT & $\mathrm{R}$ \\
\hline 480 & ONSITE & SESPMNT & $\mathrm{R}$ \\
\hline 481 & ONSITE & SESPMNT & $\mathrm{R}$ \\
\hline 482 & ONSITE & SESPMNT & $\mathrm{R}$ \\
\hline 483 & ONSITE & SESPMNT & $\mathrm{R}$ \\
\hline 484 & ONSITE & SESPMNT & $\mathrm{R}$ \\
\hline 485 & ONSITE & SESPMNT & $\mathrm{R}$ \\
\hline 486 & ONSITE & SESPMNT & $\mathrm{R}$ \\
\hline 487 & ONSITE & SESPMNT & $\mathrm{R}$ \\
\hline 488 & ONSITE & SESPMNT & $\mathrm{R}$ \\
\hline 489 & ONSITE & SESPMNT & $\mathrm{R}$ \\
\hline 490 & ONSITE & SESPMNT & $\mathrm{R}$ \\
\hline 491 & ONSITE & SESPMNT & $\mathrm{R}$ \\
\hline 492 & ONSITE & SESPMNT & $\mathrm{R}$ \\
\hline 493 & ONSITE & SESPMNT & $\mathrm{R}$ \\
\hline 494 & ONSITE & SESPMNT & $\mathrm{R}$ \\
\hline 495 & ONSITE & SESPMNT & $\mathrm{R}$ \\
\hline
\end{tabular}




\begin{tabular}{|c|c|c|c|}
\hline & $A G$ & $\mathrm{AH}$ & Al \\
\hline 1 & DIST_CLASS & OWNER_ID & COLLECTION_PURPOSE \\
\hline 496 & ONSITE & SESPMNT & $\mathrm{R}$ \\
\hline 497 & ONSITE & SESPMNT & $\mathrm{R}$ \\
\hline 498 & ONSITE & SESPMNT & $\mathrm{R}$ \\
\hline 499 & ONSITE & SESPMNT & $\mathrm{R}$ \\
\hline 500 & ONSITE & SESPMNT & $\mathrm{R}$ \\
\hline 501 & ONSITE & SESPMNT & $\mathrm{R}$ \\
\hline 502 & ONSITE & SESPMNT & $\mathrm{R}$ \\
\hline 503 & ONSITE & SESPMNT & $\mathrm{R}$ \\
\hline 504 & ONSITE & SESPMNT & $\mathrm{R}$ \\
\hline 505 & ONSITE & SESPMNT & $\mathrm{R}$ \\
\hline 506 & ONSITE & SESPMNT & $\mathrm{R}$ \\
\hline 507 & ONSITE & SESPMNT & $\mathrm{R}$ \\
\hline 508 & ONSITE & SESPMNT & $\mathrm{R}$ \\
\hline 509 & ONSITE & SESPMNT & $\mathrm{R}$ \\
\hline 510 & ONSITE & SESPMNT & $\mathrm{R}$ \\
\hline 511 & ONSITE & SESPMNT & $\mathrm{R}$ \\
\hline 512 & ONSITE & SESPMNT & $\mathrm{R}$ \\
\hline 513 & ONSITE & SESPMNT & $\mathrm{R}$ \\
\hline 514 & ONSITE & SESPMNT & $\mathrm{R}$ \\
\hline 515 & ONSITE & SESPMNT & $\mathrm{R}$ \\
\hline 516 & ONSITE & SESPMNT & $\mathrm{R}$ \\
\hline 517 & ONSITE & SESPMNT & $\mathrm{R}$ \\
\hline 518 & ONSITE & SESPMNT & $\mathrm{R}$ \\
\hline 519 & ONSITE & SESPMNT & $\mathrm{R}$ \\
\hline 520 & ONSITE & SESPMNT & $\mathrm{R}$ \\
\hline 521 & ONSITE & SESPMNT & $\mathrm{R}$ \\
\hline 522 & ONSITE & SESPMNT & $\mathrm{R}$ \\
\hline 523 & ONSITE & SESPMNT & $\mathrm{R}$ \\
\hline 524 & ONSITE & SESPMNT & $\mathrm{R}$ \\
\hline 525 & ONSITE & SESPMNT & $\mathrm{R}$ \\
\hline 526 & ONSITE & SESPMNT & $\mathrm{R}$ \\
\hline 527 & ONSITE & SESPMNT & $\mathrm{R}$ \\
\hline 528 & ONSITE & SESPMNT & $\mathrm{R}$ \\
\hline 529 & ONSITE & SESPMNT & $\mathrm{R}$ \\
\hline 530 & ONSITE & SESPMNT & $\mathrm{R}$ \\
\hline 531 & ONSITE & SESPMNT & $\mathrm{R}$ \\
\hline 532 & & HEISPROD & \\
\hline 533 & & HEISPROD & \\
\hline
\end{tabular}




\begin{tabular}{|c|c|c|c|}
\hline & $A G$ & $\mathrm{AH}$ & Al \\
\hline 1 & DIST_CLASS & OWNER_ID & COLLECTION_PURPOSE \\
\hline 534 & & HEISPROD & \\
\hline 535 & & HEISPROD & \\
\hline 536 & & HEISPROD & \\
\hline 537 & & HEISPROD & \\
\hline 538 & & HEISPROD & \\
\hline 539 & & HEISPROD & \\
\hline 540 & ONSITE & SESPMNT & $\mathrm{R}$ \\
\hline 541 & ONSITE & SESPMNT & $\mathrm{R}$ \\
\hline 542 & ONSITE & SESPMNT & $\mathrm{R}$ \\
\hline 543 & ONSITE & SESPMNT & $\mathrm{R}$ \\
\hline 544 & ONSITE & SESPMNT & $\mathrm{R}$ \\
\hline 545 & ONSITE & SESPMNT & $\mathrm{R}$ \\
\hline 546 & ONSITE & SESPMNT & $\mathrm{R}$ \\
\hline 547 & & HEISPROD & \\
\hline 548 & & HEISPROD & \\
\hline 549 & & HEISPROD & \\
\hline 550 & & HEISPROD & \\
\hline 551 & & HEISPROD & \\
\hline 552 & & HEISPROD & \\
\hline 553 & & HEISPROD & \\
\hline 554 & & HEISPROD & \\
\hline 555 & & HEISPROD & \\
\hline 556 & & HEISPROD & \\
\hline 557 & & HEISPROD & \\
\hline 558 & & HEISPROD & \\
\hline 559 & & HEISPROD & \\
\hline 560 & & HEISPROD & \\
\hline 561 & & HEISPROD & \\
\hline 562 & & HEISPROD & \\
\hline 563 & & HEISPROD & \\
\hline 564 & & HEISPROD & \\
\hline 565 & ONSITE & SESPMNT & $\mathrm{R}$ \\
\hline 566 & ONSITE & SESPMNT & $\mathrm{R}$ \\
\hline 567 & ONSITE & SESPMNT & $\mathrm{R}$ \\
\hline 568 & ONSITE & SESPMNT & $\mathrm{R}$ \\
\hline 569 & ONSITE & SESPMNT & $\mathrm{R}$ \\
\hline 570 & ONSITE & SESPMNT & $\mathrm{R}$ \\
\hline 571 & ONSITE & SESPMNT & $\mathrm{R}$ \\
\hline
\end{tabular}




\begin{tabular}{|c|c|c|c|}
\hline & $A G$ & $\mathrm{AH}$ & Al \\
\hline 1 & DIST_CLASS & OWNER_ID & COLLECTION_PURPOSE \\
\hline 572 & ONSITE & SESPMNT & $\mathrm{R}$ \\
\hline 573 & ONSITE & SESPMNT & $\mathrm{R}$ \\
\hline 574 & ONSITE & SESPMNT & $\mathrm{R}$ \\
\hline 575 & ONSITE & SESPMNT & $\mathrm{R}$ \\
\hline 576 & ONSITE & SESPMNT & $\mathrm{R}$ \\
\hline 577 & ONSITE & SESPMNT & $\mathrm{R}$ \\
\hline 578 & ONSITE & SESPMNT & $\mathrm{R}$ \\
\hline 579 & ONSITE & SESPMNT & $\mathrm{R}$ \\
\hline 580 & ONSITE & SESPMNT & $\mathrm{R}$ \\
\hline 581 & ONSITE & SESPMNT & $\mathrm{R}$ \\
\hline 582 & ONSITE & SESPMNT & $\mathrm{R}$ \\
\hline 583 & ONSITE & SESPMNT & $\mathrm{R}$ \\
\hline 584 & ONSITE & SESPMNT & $\mathrm{R}$ \\
\hline 585 & ONSITE & SESPMNT & $\mathrm{R}$ \\
\hline 586 & ONSITE & SESPMNT & $\mathrm{R}$ \\
\hline 587 & ONSITE & SESPMNT & $\mathrm{R}$ \\
\hline 588 & ONSITE & SESPMNT & $\mathrm{R}$ \\
\hline 589 & ONSITE & SESPMNT & $\mathrm{R}$ \\
\hline 590 & ONSITE & SESPMNT & $\mathrm{R}$ \\
\hline 591 & ONSITE & SESPMNT & $\mathrm{R}$ \\
\hline 592 & ONSITE & SESPMNT & $\mathrm{R}$ \\
\hline 593 & ONSITE & SESPMNT & $\mathrm{R}$ \\
\hline 594 & ONSITE & SESPMNT & $\mathrm{R}$ \\
\hline 595 & ONSITE & SESPMNT & $\mathrm{R}$ \\
\hline 596 & ONSITE & SESPMNT & $\mathrm{R}$ \\
\hline 597 & ONSITE & SESPMNT & $\mathrm{R}$ \\
\hline 598 & ONSITE & SESPMNT & $\mathrm{R}$ \\
\hline 599 & ONSITE & SESPMNT & $\mathrm{R}$ \\
\hline 600 & ONSITE & SESPMNT & $\mathrm{R}$ \\
\hline 601 & ONSITE & SESPMNT & $\mathrm{R}$ \\
\hline 602 & ONSITE & SESPMNT & $\mathrm{R}$ \\
\hline 603 & ONSITE & SESPMNT & $\mathrm{R}$ \\
\hline 604 & ONSITE & SESPMNT & $\mathrm{R}$ \\
\hline 605 & ONSITE & SESPMNT & $\mathrm{R}$ \\
\hline 606 & ONSITE & SESPMNT & $\mathrm{R}$ \\
\hline 607 & ONSITE & SESPMNT & $\mathrm{R}$ \\
\hline 608 & ONSITE & SESPMNT & $\mathrm{R}$ \\
\hline 609 & ONSITE & SESPMNT & $\mathrm{R}$ \\
\hline
\end{tabular}




\begin{tabular}{|c|c|c|c|}
\hline & $A G$ & $\mathrm{AH}$ & Al \\
\hline 1 & DIST_CLASS & OWNER_ID & COLLECTION_PURPOSE \\
\hline 610 & ONSITE & SESPMNT & $\mathrm{R}$ \\
\hline 611 & ONSITE & SESPMNT & $\mathrm{R}$ \\
\hline 612 & ONSITE & SESPMNT & $\mathrm{R}$ \\
\hline 613 & ONSITE & SESPMNT & $\mathrm{R}$ \\
\hline 614 & ONSITE & SESPMNT & $\mathrm{R}$ \\
\hline 615 & ONSITE & SESPMNT & $\mathrm{R}$ \\
\hline 616 & ONSITE & SESPMNT & $\mathrm{R}$ \\
\hline 617 & ONSITE & SESPMNT & $\mathrm{R}$ \\
\hline 618 & ONSITE & SESPMNT & $\mathrm{R}$ \\
\hline 619 & ONSITE & SESPMNT & $\mathrm{R}$ \\
\hline 620 & ONSITE & SESPMNT & $\mathrm{R}$ \\
\hline 621 & ONSITE & SESPMNT & $\mathrm{R}$ \\
\hline 622 & ONSITE & SESPMNT & $\mathrm{R}$ \\
\hline 623 & ONSITE & SESPMNT & $\mathrm{R}$ \\
\hline 624 & ONSITE & SESPMNT & $\mathrm{R}$ \\
\hline 625 & ONSITE & SESPMNT & $\mathrm{R}$ \\
\hline 626 & ONSITE & SESPMNT & $\mathrm{R}$ \\
\hline 627 & ONSITE & SESPMNT & $\mathrm{R}$ \\
\hline 628 & ONSITE & SESPMNT & $\mathrm{R}$ \\
\hline 629 & ONSITE & SESPMNT & $\mathrm{R}$ \\
\hline 630 & ONSITE & SESPMNT & $\mathrm{R}$ \\
\hline 631 & ONSITE & SESPMNT & $\mathrm{R}$ \\
\hline 632 & ONSITE & SESPMNT & $\mathrm{R}$ \\
\hline 633 & ONSITE & SESPMNT & $\mathrm{R}$ \\
\hline 634 & ONSITE & SESPMNT & $\mathrm{R}$ \\
\hline 635 & ONSITE & SESPMNT & $\mathrm{R}$ \\
\hline 636 & ONSITE & SESPMNT & $\mathrm{R}$ \\
\hline 637 & ONSITE & SESPMNT & $\mathrm{R}$ \\
\hline 638 & ONSITE & SESPMNT & $\mathrm{R}$ \\
\hline 639 & ONSITE & SESPMNT & $\mathrm{R}$ \\
\hline 640 & ONSITE & SESPMNT & $\mathrm{R}$ \\
\hline 641 & ONSITE & SESPMNT & $\mathrm{R}$ \\
\hline 642 & ONSITE & SESPMNT & $\mathrm{R}$ \\
\hline 643 & ONSITE & SESPMNT & $\mathrm{R}$ \\
\hline 644 & ONSITE & SESPMNT & $\mathrm{R}$ \\
\hline 645 & ONSITE & SESPMNT & $\mathrm{R}$ \\
\hline 646 & ONSITE & SESPMNT & $\mathrm{R}$ \\
\hline 647 & ONSITE & SESPMNT & $\mathrm{R}$ \\
\hline
\end{tabular}




\begin{tabular}{|c|c|c|c|}
\hline & $A G$ & $\mathrm{AH}$ & Al \\
\hline 1 & DIST_CLASS & OWNER_ID & COLLECTION_PURPOSE \\
\hline 648 & ONSITE & SESPMNT & $\mathrm{R}$ \\
\hline 649 & ONSITE & SESPMNT & $\mathrm{R}$ \\
\hline 650 & ONSITE & SESPMNT & $\mathrm{R}$ \\
\hline 651 & ONSITE & SESPMNT & $\mathrm{R}$ \\
\hline 652 & ONSITE & SESPMNT & $\mathrm{R}$ \\
\hline 653 & ONSITE & SESPMNT & $\mathrm{R}$ \\
\hline 654 & ONSITE & SESPMNT & $\mathrm{R}$ \\
\hline 655 & ONSITE & SESPMNT & $\mathrm{R}$ \\
\hline 656 & ONSITE & SESPMNT & $\mathrm{R}$ \\
\hline 657 & ONSITE & SESPMNT & $\mathrm{R}$ \\
\hline 658 & ONSITE & SESPMNT & $\mathrm{R}$ \\
\hline 659 & ONSITE & SESPMNT & $\mathrm{R}$ \\
\hline 660 & ONSITE & SESPMNT & $\mathrm{R}$ \\
\hline 661 & ONSITE & SESPMNT & $\mathrm{R}$ \\
\hline 662 & ONSITE & SESPMNT & $\mathrm{R}$ \\
\hline 663 & ONSITE & SESPMNT & $\mathrm{R}$ \\
\hline 664 & ONSITE & SESPMNT & $\mathrm{R}$ \\
\hline 665 & ONSITE & SESPMNT & $\mathrm{R}$ \\
\hline 666 & ONSITE & SESPMNT & $\mathrm{R}$ \\
\hline 667 & ONSITE & SESPMNT & $\mathrm{R}$ \\
\hline 668 & ONSITE & SESPMNT & $\mathrm{R}$ \\
\hline 669 & ONSITE & SESPMNT & $\mathrm{R}$ \\
\hline 670 & ONSITE & SESPMNT & $\mathrm{R}$ \\
\hline 671 & ONSITE & SESPMNT & $\mathrm{R}$ \\
\hline 672 & ONSITE & SESPMNT & $\mathrm{R}$ \\
\hline 673 & & HEISPROD & \\
\hline 674 & & HEISPROD & \\
\hline 675 & & HEISPROD & \\
\hline 676 & & HEISPROD & \\
\hline 677 & & HEISPROD & \\
\hline 678 & & HEISPROD & \\
\hline 679 & & HEISPROD & \\
\hline 680 & & HEISPROD & \\
\hline 681 & & HEISPROD & \\
\hline 682 & & HEISPROD & \\
\hline 683 & & HEISPROD & \\
\hline 684 & & HEISPROD & \\
\hline 685 & & HEISPROD & \\
\hline
\end{tabular}




\begin{tabular}{|c|c|c|c|}
\hline & $A G$ & $\mathrm{AH}$ & Al \\
\hline 1 & DIST_CLASS & OWNER_ID & COLLECTION_PURPOSE \\
\hline 686 & & HEISPROD & \\
\hline 687 & & HEISPROD & \\
\hline 688 & ONSITE & SESPMNT & $\mathrm{R}$ \\
\hline 689 & ONSITE & SESPMNT & $\mathrm{R}$ \\
\hline 690 & ONSITE & SESPMNT & $\mathrm{R}$ \\
\hline 691 & ONSITE & SESPMNT & $\mathrm{R}$ \\
\hline 692 & ONSITE & SESPMNT & $\mathrm{R}$ \\
\hline 693 & ONSITE & SESPMNT & $\mathrm{R}$ \\
\hline 694 & ONSITE & SESPMNT & $\mathrm{R}$ \\
\hline 695 & ONSITE & SESPMNT & $\mathrm{R}$ \\
\hline 696 & ONSITE & SESPMNT & $\mathrm{R}$ \\
\hline 697 & ONSITE & SESPMNT & $\mathrm{R}$ \\
\hline 698 & ONSITE & SESPMNT & $\mathrm{R}$ \\
\hline 699 & ONSITE & SESPMNT & $\mathrm{R}$ \\
\hline 700 & ONSITE & SESPMNT & $\mathrm{R}$ \\
\hline 701 & ONSITE & SESPMNT & $\mathrm{R}$ \\
\hline 702 & ONSITE & SESPMNT & $\mathrm{R}$ \\
\hline 703 & ONSITE & SESPMNT & $\mathrm{R}$ \\
\hline 704 & ONSITE & SESPMNT & $\mathrm{R}$ \\
\hline 705 & ONSITE & SESPMNT & $\mathrm{R}$ \\
\hline 706 & ONSITE & SESPMNT & $\mathrm{R}$ \\
\hline 707 & ONSITE & SESPMNT & $\mathrm{R}$ \\
\hline 708 & ONSITE & SESPMNT & $\mathrm{R}$ \\
\hline 709 & ONSITE & SESPMNT & $\mathrm{R}$ \\
\hline 710 & ONSITE & SESPMNT & $\mathrm{R}$ \\
\hline 711 & ONSITE & SESPMNT & $\mathrm{R}$ \\
\hline 712 & ONSITE & SESPMNT & $\mathrm{R}$ \\
\hline 713 & ONSITE & SESPMNT & $\mathrm{R}$ \\
\hline 714 & ONSITE & SESPMNT & $\mathrm{R}$ \\
\hline 715 & ONSITE & SESPMNT & $\mathrm{R}$ \\
\hline 716 & ONSITE & SESPMNT & $\mathrm{R}$ \\
\hline 717 & ONSITE & SESPMNT & $\mathrm{R}$ \\
\hline 718 & ONSITE & SESPMNT & $\mathrm{R}$ \\
\hline 719 & ONSITE & SESPMNT & $\mathrm{R}$ \\
\hline 720 & ONSITE & SESPMNT & $\mathrm{R}$ \\
\hline 721 & ONSITE & SESPMNT & $\mathrm{R}$ \\
\hline 722 & ONSITE & SESPMNT & $\mathrm{R}$ \\
\hline 723 & ONSITE & SESPMNT & $\mathrm{R}$ \\
\hline
\end{tabular}


AG

\begin{tabular}{|c|l|l|l|}
\hline & \multicolumn{1}{|c|}{ AG } & AH & \\
\hline 1 & DIST_CLASS & OWNER_ID & COLLECTION_PURPOSE \\
\hline 724 & ONSITE & SESPMNT & $\mathrm{R}$ \\
\hline 725 & ONSITE & SESPMNT & $\mathrm{R}$ \\
\hline 726 & ONSITE & SESPMNT & $\mathrm{R}$ \\
\hline 727 & ONSITE & SESPMNT & $\mathrm{R}$ \\
\hline 728 & ONSITE & SESPMNT & $\mathrm{R}$ \\
\hline 729 & ONSITE & SESPMNT & $\mathrm{R}$ \\
\hline 730 & ONSITE & SESPMNT & $\mathrm{R}$ \\
\hline 731 & ONSITE & SESPMNT & $\mathrm{R}$ \\
\hline 732 & ONSITE & SESPMNT & $\mathrm{R}$ \\
\hline 733 & ONSITE & SESPMNT & $\mathrm{R}$ \\
\hline 734 & ONSITE & SESPMNT & $\mathrm{R}$ \\
\hline 735 & ONSITE & SESPMNT & $\mathrm{R}$ \\
\hline 736 & ONSITE & SESPMNT & $\mathrm{R}$ \\
\hline 737 & ONSITE & SESPMNT & $\mathrm{R}$ \\
\hline 738 & ONSITE & SESPMNT & $\mathrm{R}$ \\
\hline 739 & ONSITE & SESPMNT & $\mathrm{R}$ \\
\hline 740 & ONSITE & SESPMNT & $\mathrm{R}$ \\
\hline 741 & ONSITE & SESPMNT & $\mathrm{R}$ \\
\hline 742 & ONSITE & SESPMNT & $\mathrm{R}$ \\
\hline 743 & ONSITE & SESPMNT & $\mathrm{R}$ \\
\hline 744 & ONSITE & SESPMNT & $\mathrm{R}$ \\
\hline 745 & ONSITE & SESPMNT & $\mathrm{R}$ \\
\hline 746 & ONSITE & SESPMNT & $\mathrm{R}$ \\
\hline 747 & ONSITE & SESPMNT & $\mathrm{R}$ \\
\hline 748 & ONSITE & SESPMNT & $\mathrm{R}$ \\
\hline 749 & ONSITE & SESPMNT & $\mathrm{R}$ \\
\hline 750 & ONSITE & SESPMNT & $\mathrm{R}$ \\
\hline 751 & ONSITE & SESPMNT & $\mathrm{R}$ \\
\hline 752 & ONSITE & SESPMNT & $\mathrm{R}$ \\
\hline 753 & ONSITE & SESPMNT & $\mathrm{R}$ \\
\hline 754 & ONSITE & SESPMNT & $\mathrm{R}$ \\
\hline 755 & ONSITE & SESPMNT & $\mathrm{R}$ \\
\hline 756 & ONSITE & SESPMNT & $\mathrm{R}$ \\
\hline 757 & ONSITE & SESPMNT & $\mathrm{R}$ \\
\hline 758 & ONSITE & SESPMNT & $\mathrm{R}$ \\
\hline 759 & ONSITE & SESPMNT & $\mathrm{R}$ \\
\hline 760 & ONSITE & SESPMNT & $\mathrm{R}$ \\
\hline 761 & ONSITE & SESPMNT & $\mathrm{R}$ \\
\hline & & & \\
\hline
\end{tabular}


AG

\begin{tabular}{|c|c|c|c|}
\hline & AG & $\mathrm{AH}$ & $\mathrm{Al}$ \\
\hline 1 & DIST_CLASS & OWNER_ID & COLLECTION_PURPOSE \\
\hline 762 & ONSITE & SESPMNT & $\mathrm{R}$ \\
\hline 763 & ONSITE & SESPMNT & $\mathrm{R}$ \\
\hline 764 & ONSITE & SESPMNT & $\mathrm{R}$ \\
\hline 765 & ONSITE & SESPMNT & $\mathrm{R}$ \\
\hline 766 & ONSITE & SESPMNT & $\mathrm{R}$ \\
\hline 767 & ONSITE & SESPMNT & $\mathrm{R}$ \\
\hline 768 & ONSITE & SESPMNT & $\mathrm{R}$ \\
\hline 769 & ONSITE & SESPMNT & $\mathrm{R}$ \\
\hline 770 & ONSITE & SESPMNT & $\mathrm{R}$ \\
\hline 771 & & HEISPROD & \\
\hline 772 & & HEISPROD & \\
\hline 773 & & HEISPROD & \\
\hline 774 & & HEISPROD & \\
\hline 775 & & HEISPROD & \\
\hline 776 & & HEISPROD & \\
\hline 777 & & HEISPROD & \\
\hline 778 & & HEISPROD & \\
\hline 779 & & HEISPROD & \\
\hline 780 & & HEISPROD & \\
\hline 781 & & HEISPROD & \\
\hline 782 & & HEISPROD & \\
\hline 783 & RIVER_SHORELINE & HEISPROD & \\
\hline 784 & RIVER_SHORELINE & HEISPROD & \\
\hline 785 & RIVER_SHORELINE & HEISPROD & \\
\hline 786 & RIVER_SHORELINE & HEISPROD & \\
\hline 787 & RIVER_SHORELINE & HEISPROD & \\
\hline 788 & RIVER_SHORELINE & HEISPROD & \\
\hline 789 & RIVER_SHORELINE & HEISPROD & \\
\hline 790 & RIVER SHORELINE & HEISPROD & \\
\hline 791 & RIVER_SHORELINE & HEISPROD & \\
\hline 792 & RIVER_SHORELINE & HEISPROD & \\
\hline 793 & RIVER_SHORELINE & HEISPROD & \\
\hline 794 & RIVER_SHORELINE & HEISPROD & \\
\hline 795 & RIVER_SHORELINE & HEISPROD & \\
\hline 796 & RIVER_SHORELINE & HEISPROD & \\
\hline 797 & RIVER_SHORELINE & HEISPROD & \\
\hline 798 & RIVER_SHORELINE & HEISPROD & \\
\hline 799 & RIVER_SHORELINE & HEISPROD & \\
\hline
\end{tabular}




\begin{tabular}{|c|c|c|c|}
\hline & & & \\
\hline 1 & DIST_CLASS & OWNER_ID & COLLECTION_PURPOSE \\
\hline 800 & RIVER_SHORELINE & HEISPROD & \\
\hline 801 & RIVER_SHORELINE & HEISPROD & \\
\hline 802 & RIVER_SHORELINE & HEISPROD & \\
\hline 803 & RIVER_SHORELINE & HEISPROD & \\
\hline 804 & RIVER_SHORELINE & HEISPROD & \\
\hline 805 & RIVER_SHORELINE & HEISPROD & \\
\hline 806 & RIVER_SHORELINE & HEISPROD & \\
\hline 807 & RIVER SHORELINE & HEISPROD & \\
\hline 808 & RIVER_SHORELINE & HEISPROD & \\
\hline 809 & RIVER_SHORELINE & HEISPROD & \\
\hline 810 & & HEISPROD & \\
\hline 811 & & HEISPROD & \\
\hline 812 & & HEISPROD & \\
\hline 813 & & HEISPROD & \\
\hline 814 & & HEISPROD & \\
\hline 815 & & HEISPROD & \\
\hline 816 & & HEISPROD & \\
\hline 817 & & HEISPROD & \\
\hline 818 & ONSITE & SESPMNT & $\mathrm{R}$ \\
\hline 819 & ONSITE & SESPMNT & $\mathrm{R}$ \\
\hline 820 & ONSITE & SESPMNT & $\mathrm{R}$ \\
\hline 821 & ONSITE & SESPMNT & $\mathrm{R}$ \\
\hline 822 & ONSITE & SESPMNT & $\mathrm{R}$ \\
\hline 823 & ONSITE & SESPMNT & $\mathrm{R}$ \\
\hline 824 & ONSITE & SESPMNT & $\mathrm{R}$ \\
\hline 825 & ONSITE & SESPMNT & $\mathrm{R}$ \\
\hline 826 & ONSITE & SESPMNT & $\mathrm{R}$ \\
\hline 827 & ONSITE & SESPMNT & $\mathrm{R}$ \\
\hline 828 & ONSITE & SESPMNT & $\mathrm{R}$ \\
\hline 829 & ONSITE & SESPMNT & $\mathrm{R}$ \\
\hline 830 & ONSITE & SESPMNT & $\mathrm{R}$ \\
\hline 831 & ONSITE & SESPMNT & $\mathrm{R}$ \\
\hline 832 & ONSITE & SESPMNT & $\mathrm{R}$ \\
\hline 833 & ONSITE & SESPMNT & $\mathrm{R}$ \\
\hline 834 & ONSITE & SESPMNT & $\mathrm{R}$ \\
\hline 835 & ONSITE & SESPMNT & $\mathrm{R}$ \\
\hline 836 & ONSITE & SESPMNT & $\mathrm{R}$ \\
\hline 837 & ONSITE & SESPMNT & $\mathrm{R}$ \\
\hline
\end{tabular}




\begin{tabular}{|c|c|c|c|}
\hline & $A G$ & $\mathrm{AH}$ & Al \\
\hline 1 & DIST_CLASS & OWNER_ID & COLLECTION_PURPOSE \\
\hline 838 & ONSITE & SESPMNT & $\mathrm{R}$ \\
\hline 839 & & HEISPROD & \\
\hline 840 & & HEISPROD & \\
\hline 841 & & HEISPROD & \\
\hline 842 & & HEISPROD & \\
\hline 843 & & HEISPROD & \\
\hline 844 & ONSITE & SESPMNT & $\mathrm{R}$ \\
\hline 845 & ONSITE & SESPMNT & $\mathrm{R}$ \\
\hline 846 & ONSITE & SESPMNT & $\mathrm{R}$ \\
\hline 847 & ONSITE & SESPMNT & $\mathrm{R}$ \\
\hline 848 & ONSITE & SESPMNT & $\mathrm{R}$ \\
\hline 849 & ONSITE & SESPMNT & $\mathrm{R}$ \\
\hline 850 & ONSITE & SESPMNT & $\mathrm{R}$ \\
\hline 851 & ONSITE & SESPMNT & $\mathrm{R}$ \\
\hline 852 & ONSITE & SESPMNT & $\mathrm{R}$ \\
\hline 853 & ONSITE & SESPMNT & $\mathrm{R}$ \\
\hline 854 & ONSITE & SESPMNT & $\mathrm{R}$ \\
\hline 855 & ONSITE & SESPMNT & $\mathrm{R}$ \\
\hline 856 & ONSITE & SESPMNT & $\mathrm{R}$ \\
\hline 857 & ONSITE & SESPMNT & $\mathrm{R}$ \\
\hline 858 & ONSITE & SESPMNT & $\mathrm{R}$ \\
\hline 859 & ONSITE & SESPMNT & $\mathrm{R}$ \\
\hline 860 & ONSITE & SESPMNT & $\mathrm{R}$ \\
\hline 861 & ONSITE & SESPMNT & $\mathrm{R}$ \\
\hline 862 & ONSITE & SESPMNT & $\mathrm{R}$ \\
\hline 863 & ONSITE & SESPMNT & $\mathrm{R}$ \\
\hline 864 & ONSITE & SESPMNT & $\mathrm{R}$ \\
\hline 865 & ONSITE & SESPMNT & $\mathrm{R}$ \\
\hline 866 & ONSITE & SESPMNT & $\mathrm{R}$ \\
\hline 867 & ONSITE & SESPMNT & $\mathrm{R}$ \\
\hline 868 & ONSITE & SESPMNT & $\mathrm{R}$ \\
\hline 869 & ONSITE & SESPMNT & $\mathrm{R}$ \\
\hline 870 & ONSITE & SESPMNT & $\mathrm{R}$ \\
\hline 871 & ONSITE & SESPMNT & $\mathrm{R}$ \\
\hline 872 & ONSITE & SESPMNT & $\mathrm{R}$ \\
\hline 873 & ONSITE & SESPMNT & $\mathrm{R}$ \\
\hline 874 & ONSITE & SESPMNT & $\mathrm{R}$ \\
\hline 875 & & HEISPROD & \\
\hline
\end{tabular}




\begin{tabular}{|c|c|c|c|}
\hline & $A G$ & $\mathrm{AH}$ & Al \\
\hline 1 & DIST_CLASS & OWNER_ID & COLLECTION_PURPOSE \\
\hline 876 & & PNLGW & \\
\hline 877 & & PNLGW & \\
\hline 878 & & PNLGW & \\
\hline 879 & & PNLGW & \\
\hline 880 & & PNLGW & \\
\hline 881 & & PNLGW & \\
\hline 882 & & PNLGW & \\
\hline 883 & & PNLGW & \\
\hline 884 & & HEISPROD & \\
\hline 885 & & HEISPROD & \\
\hline 886 & & HEISPROD & \\
\hline 887 & & HEISPROD & \\
\hline 888 & & HEISPROD & \\
\hline 889 & & HEISPROD & \\
\hline 890 & & HEISPROD & \\
\hline 891 & & HEISPROD & \\
\hline 892 & & HEISPROD & \\
\hline 893 & & HEISPROD & \\
\hline 894 & ONSITE & SESPMNT & $\mathrm{R}$ \\
\hline 895 & ONSITE & SESPMNT & $\mathrm{R}$ \\
\hline 896 & ONSITE & SESPMNT & $\mathrm{R}$ \\
\hline 897 & ONSITE & SESPMNT & $\mathrm{R}$ \\
\hline 898 & ONSITE & SESPMNT & $\mathrm{R}$ \\
\hline 899 & ONSITE & SESPMNT & $\mathrm{R}$ \\
\hline 900 & ONSITE & SESPMNT & $\mathrm{R}$ \\
\hline 901 & ONSITE & SESPMNT & $\mathrm{R}$ \\
\hline 902 & ONSITE & SESPMNT & $\mathrm{R}$ \\
\hline 903 & ONSITE & SESPMNT & $\mathrm{R}$ \\
\hline 904 & & HEISPROD & \\
\hline 905 & & HEISPROD & \\
\hline 906 & & HEISPROD & \\
\hline 907 & & HEISPROD & \\
\hline 908 & & HEISPROD & \\
\hline 909 & & HEISPROD & \\
\hline 910 & & HEISPROD & \\
\hline 911 & & HEISPROD & \\
\hline 912 & ONSITE & SESPMNT & $\mathrm{R}$ \\
\hline 913 & ONSITE & SESPMNT & $\mathrm{R}$ \\
\hline
\end{tabular}




\begin{tabular}{|c|c|c|c|}
\hline & $A G$ & $\mathrm{AH}$ & Al \\
\hline 1 & DIST_CLASS & OWNER_ID & COLLECTION_PURPOSE \\
\hline 914 & ONSITE & SESPMNT & $\mathrm{R}$ \\
\hline 915 & ONSITE & SESPMNT & $\mathrm{R}$ \\
\hline 916 & ONSITE & SESPMNT & $\mathrm{R}$ \\
\hline 917 & ONSITE & SESPMNT & $\mathrm{R}$ \\
\hline 918 & ONSITE & SESPMNT & $\mathrm{R}$ \\
\hline 919 & ONSITE & SESPMNT & $\mathrm{R}$ \\
\hline 920 & ONSITE & SESPMNT & $\mathrm{R}$ \\
\hline 921 & ONSITE & SESPMNT & $\mathrm{R}$ \\
\hline 922 & ONSITE & SESPMNT & $\mathrm{R}$ \\
\hline 923 & ONSITE & SESPMNT & $\mathrm{R}$ \\
\hline 924 & ONSITE & SESPMNT & $\mathrm{R}$ \\
\hline 925 & ONSITE & SESPMNT & $\mathrm{R}$ \\
\hline 926 & ONSITE & SESPMNT & $\mathrm{R}$ \\
\hline 927 & ONSITE & SESPMNT & $\mathrm{R}$ \\
\hline 928 & ONSITE & SESPMNT & $\mathrm{R}$ \\
\hline 929 & ONSITE & SESPMNT & $\mathrm{R}$ \\
\hline 930 & ONSITE & SESPMNT & $\mathrm{R}$ \\
\hline 931 & ONSITE & SESPMNT & $\mathrm{R}$ \\
\hline 932 & ONSITE & SESPMNT & $\mathrm{R}$ \\
\hline 933 & ONSITE & SESPMNT & $\mathrm{R}$ \\
\hline 934 & ONSITE & SESPMNT & $\mathrm{R}$ \\
\hline 935 & ONSITE & SESPMNT & $\mathrm{R}$ \\
\hline 936 & & HEISPROD & \\
\hline 937 & & HEISPROD & \\
\hline 938 & & HEISPROD & \\
\hline 939 & & HEISPROD & \\
\hline 940 & & HEISPROD & \\
\hline 941 & & HEISPROD & \\
\hline 942 & & HEISPROD & \\
\hline 943 & & HEISPROD & \\
\hline 944 & & HEISPROD & \\
\hline 945 & & HEISPROD & \\
\hline 946 & & HEISPROD & \\
\hline 947 & & HEISPROD & \\
\hline 948 & ONSITE & SESPSPEC & S \\
\hline 949 & ONSITE & SESPSPEC & S \\
\hline 950 & ONSITE & SESPSPEC & $\mathrm{S}$ \\
\hline 951 & ONSITE & SESPMNT & $\mathrm{R}$ \\
\hline
\end{tabular}




\begin{tabular}{|c|c|c|c|}
\hline & $A G$ & $\mathrm{AH}$ & Al \\
\hline 1 & DIST_CLASS & OWNER_ID & COLLECTION_PURPOSE \\
\hline 952 & ONSITE & SESPMNT & $\mathrm{R}$ \\
\hline 953 & ONSITE & SESPMNT & $\mathrm{R}$ \\
\hline 954 & ONSITE & SESPMNT & $\mathrm{R}$ \\
\hline 955 & ONSITE & SESPMNT & $\mathrm{R}$ \\
\hline 956 & ONSITE & SESPMNT & $\mathrm{R}$ \\
\hline 957 & ONSITE & SESPMNT & $\mathrm{R}$ \\
\hline 958 & ONSITE & SESPMNT & $\mathrm{R}$ \\
\hline 959 & ONSITE & SESPMNT & $\mathrm{R}$ \\
\hline 960 & ONSITE & SESPMNT & $\mathrm{R}$ \\
\hline 961 & ONSITE & SESPMNT & $\mathrm{R}$ \\
\hline 962 & ONSITE & SESPMNT & $\mathrm{R}$ \\
\hline 963 & ONSITE & SESPMNT & $\mathrm{R}$ \\
\hline 964 & ONSITE & SESPMNT & $\mathrm{R}$ \\
\hline 965 & ONSITE & SESPMNT & $\mathrm{R}$ \\
\hline 966 & ONSITE & SESPMNT & $\mathrm{R}$ \\
\hline 967 & ONSITE & SESPMNT & $\mathrm{R}$ \\
\hline 968 & ONSITE & SESPMNT & $\mathrm{R}$ \\
\hline 969 & ONSITE & SESPMNT & $\mathrm{R}$ \\
\hline 970 & ONSITE & SESPMNT & $\mathrm{R}$ \\
\hline 971 & ONSITE & SESPMNT & $\mathrm{R}$ \\
\hline 972 & ONSITE & SESPMNT & $\mathrm{R}$ \\
\hline 973 & ONSITE & SESPMNT & $\mathrm{R}$ \\
\hline 974 & ONSITE & SESPMNT & $\mathrm{R}$ \\
\hline 975 & ONSITE & SESPMNT & $\mathrm{R}$ \\
\hline 976 & ONSITE & SESPMNT & $\mathrm{R}$ \\
\hline 977 & ONSITE & SESPMNT & $\mathrm{R}$ \\
\hline 978 & ONSITE & SESPMNT & $\mathrm{R}$ \\
\hline 979 & ONSITE & SESPMNT & $\mathrm{R}$ \\
\hline 980 & ONSITE & SESPMNT & $\mathrm{R}$ \\
\hline 981 & ONSITE & SESPMNT & $\mathrm{R}$ \\
\hline 982 & ONSITE & SESPMNT & $\mathrm{R}$ \\
\hline 983 & ONSITE & SESPMNT & $\mathrm{R}$ \\
\hline 984 & ONSITE & SESPMNT & $\mathrm{R}$ \\
\hline 985 & ONSITE & SESPMNT & $\mathrm{R}$ \\
\hline 986 & ONSITE & SESPMNT & $\mathrm{R}$ \\
\hline 987 & ONSITE & SESPMNT & $\mathrm{R}$ \\
\hline 988 & ONSITE & SESPMNT & $\mathrm{R}$ \\
\hline 989 & ONSITE & SESPMNT & $\mathrm{R}$ \\
\hline
\end{tabular}




\begin{tabular}{|c|c|c|c|}
\hline & $A G$ & $\mathrm{AH}$ & Al \\
\hline 1 & DIST_CLASS & OWNER_ID & COLLECTION_PURPOSE \\
\hline 990 & ONSITE & SESPMNT & $\mathrm{R}$ \\
\hline 991 & ONSITE & SESPMNT & $\mathrm{R}$ \\
\hline 992 & ONSITE & SESPMNT & $\mathrm{R}$ \\
\hline 993 & ONSITE & SESPMNT & $\mathrm{R}$ \\
\hline 994 & ONSITE & SESPMNT & $\mathrm{R}$ \\
\hline 995 & ONSITE & SESPMNT & $\mathrm{R}$ \\
\hline 996 & ONSITE & SESPMNT & $\mathrm{R}$ \\
\hline 997 & ONSITE & SESPMNT & $\mathrm{R}$ \\
\hline 998 & ONSITE & SESPMNT & $\mathrm{R}$ \\
\hline 999 & ONSITE & SESPMNT & $\mathrm{R}$ \\
\hline 1000 & ONSITE & SESPMNT & $\mathrm{R}$ \\
\hline 1001 & ONSITE & SESPMNT & $\mathrm{R}$ \\
\hline 1002 & ONSITE & SESPMNT & $\mathrm{R}$ \\
\hline 1003 & ONSITE & SESPMNT & $\mathrm{R}$ \\
\hline 1004 & ONSITE & SESPMNT & $\mathrm{R}$ \\
\hline 1005 & ONSITE & SESPMNT & $\mathrm{R}$ \\
\hline 1006 & ONSITE & SESPMNT & $\mathrm{R}$ \\
\hline 1007 & ONSITE & SESPMNT & $\mathrm{R}$ \\
\hline 1008 & ONSITE & SESPMNT & $\mathrm{R}$ \\
\hline 1009 & ONSITE & SESPMNT & $\mathrm{R}$ \\
\hline 1010 & ONSITE & SESPMNT & $\mathrm{R}$ \\
\hline 1011 & ONSITE & SESPMNT & \\
\hline 1012 & ONSITE & SESPMNT & $\mathrm{R}$ \\
\hline 1013 & ONSITE & SESPMNT & $\mathrm{R}$ \\
\hline 1014 & ONSITE & SESPMNT & $\mathrm{R}$ \\
\hline 1015 & ONSITE & SESPMNT & $\mathrm{R}$ \\
\hline 1016 & ONSITE & SESPMNT & $\mathrm{R}$ \\
\hline 1017 & ONSITE & SESPMNT & $\mathrm{R}$ \\
\hline 1018 & ONSITE & SESPMNT & $\mathrm{R}$ \\
\hline 1019 & ONSITE & SESPMNT & $\mathrm{R}$ \\
\hline 1020 & ONSITE & SESPMNT & $\mathrm{R}$ \\
\hline 1021 & ONSITE & SESPMNT & $\mathrm{R}$ \\
\hline 1022 & ONSITE & SESPMNT & $\mathrm{R}$ \\
\hline 1023 & ONSITE & SESPMNT & $\mathrm{R}$ \\
\hline 1024 & ONSITE & SESPMNT & $\mathrm{R}$ \\
\hline 1025 & ONSITE & SESPMNT & $\mathrm{R}$ \\
\hline 1026 & ONSITE & SESPMNT & $\mathrm{R}$ \\
\hline 1027 & ONSITE & SESPMNT & $\mathrm{R}$ \\
\hline
\end{tabular}




\begin{tabular}{|c|l|l|l|}
\hline & AG & AH & \\
\hline 1 & DIST_CLASS & OWNER_ID & COLLECTION_PURPOSE \\
\hline 1028 & ONSITE & SESPMNT & $\mathrm{R}$ \\
\hline 1029 & ONSITE & SESPMNT & $\mathrm{R}$ \\
\hline 1030 & ONSITE & SESPMNT & $\mathrm{R}$ \\
\hline 1031 & ONSITE & SESPMNT & $\mathrm{R}$ \\
\hline 1032 & ONSITE & SESPMNT & $\mathrm{R}$ \\
\hline 1033 & ONSITE & SESPMNT & $\mathrm{R}$ \\
\hline 1034 & ONSITE & SESPMNT & $\mathrm{R}$ \\
\hline 1035 & ONSITE & SESPMNT & $\mathrm{R}$ \\
\hline 1036 & ONSITE & SESPMNT & $\mathrm{R}$ \\
\hline 1037 & ONSITE & SESPMNT & $\mathrm{R}$ \\
\hline 1038 & ONSITE & SESPMNT & $\mathrm{R}$ \\
\hline 1039 & ONSITE & SESPMNT & $\mathrm{R}$ \\
\hline 1040 & ONSITE & SESPMNT & $\mathrm{R}$ \\
\hline 1041 & ONSITE & SESPMNT & $\mathrm{R}$ \\
\hline 1042 & ONSITE & SESPMNT & $\mathrm{R}$ \\
\hline 1043 & ONSITE & SESPMNT & $\mathrm{R}$ \\
\hline 1044 & ONSITE & SESPMNT & $\mathrm{R}$ \\
\hline 1045 & ONSITE & SESPMNT & $\mathrm{R}$ \\
\hline 1046 & ONSITE & SESPMNT & $\mathrm{R}$ \\
\hline 1047 & ONSITE & SESPMNT & $\mathrm{R}$ \\
\hline 1048 & ONSITE & SESPMNT & $\mathrm{R}$ \\
\hline 1049 & ONSITE & SESPMNT & $\mathrm{R}$ \\
\hline 1050 & ONSITE & SESPMNT & $\mathrm{R}$ \\
\hline 1051 & ONSITE & SESPMNT & $\mathrm{R}$ \\
\hline 1052 & ONSITE & SESPMNT & $\mathrm{R}$ \\
\hline 1053 & ONSITE & SESPMNT & $\mathrm{R}$ \\
\hline 1054 & ONSITE & SESPMNT & $\mathrm{R}$ \\
\hline 1055 & ONSITE & SESPMNT & $\mathrm{R}$ \\
\hline 1056 & ONSITE & SESPMNT & $\mathrm{R}$ \\
\hline 1057 & ONSITE & SESPMNT & $\mathrm{R}$ \\
\hline 1058 & ONSITE & SESPMNT & $\mathrm{R}$ \\
\hline 1059 & ONSITE & SESPMNT & $\mathrm{R}$ \\
\hline 1060 & ONSITE & SESPMNT & $\mathrm{R}$ \\
\hline 1061 & ONSITE & SESPMNT & $\mathrm{R}$ \\
\hline 1062 & ONSITE & SESPMNT & $\mathrm{R}$ \\
\hline 1063 & ONSITE & SESPMNT & $\mathrm{R}$ \\
\hline 1064 & ONSITE & SESPMNT & $\mathrm{R}$ \\
\hline 1065 & ONSITE & SESPMNT & $\mathrm{R}$ \\
\hline & & \\
\hline
\end{tabular}


$\mathrm{AH} \quad \mathrm{Al}$

\begin{tabular}{|c|c|c|c|}
\hline & & & \\
\hline 1 & DIST_CLASS & OWNER_ID & COLLECTION PURPOSE \\
\hline 1066 & ONSITE & SESPMNT & $\mathrm{R}$ \\
\hline 1067 & ONSITE & SESPMNT & $\mathrm{R}$ \\
\hline 1068 & ONSITE & SESPMNT & $\mathrm{R}$ \\
\hline 1069 & ONSITE & SESPMNT & $\mathrm{R}$ \\
\hline 1070 & ONSITE & SESPMNT & $\mathrm{R}$ \\
\hline 1071 & ONSITE & SESPMNT & $\mathrm{R}$ \\
\hline 1072 & ONSITE & SESPMNT & $\mathrm{R}$ \\
\hline 1073 & ONSITE & SESPMNT & \\
\hline 1074 & ONSITE & SESPMNT & \\
\hline 1075 & ONSITE & SESPMNT & \\
\hline 1076 & ONSITE & SESPSPEC & $\mathrm{S}$ \\
\hline 1077 & ONSITE & SESPSPEC & S \\
\hline 1078 & ONSITE & SESPSPEC & $\mathrm{S}$ \\
\hline 1079 & ONSITE & SESPSPEC & $\mathrm{S}$ \\
\hline
\end{tabular}




\section{0 - 00 Transect}




\begin{tabular}{|c|c|c|c|c|c|c|c|c|c|c|}
\hline & $\mathrm{A}$ & $B$ & $\mathrm{C}$ & $\mathrm{D}$ & $E$ & $\mathrm{~F}$ & $G$ & $\mathrm{H}$ & $I$ & $\mathrm{~J}$ \\
\hline 1 & SAMPLE NUM ASSIGNED TO & SAMPLER & MEDIA & BIOTA MEDIA & TAG ID & SAMP NUM & SAMP DATE TIME & SAMP DATE TIME ON & CON ID & CON SHORT NAME \\
\hline 2 & PDMS & & SW & & & E117334 & 07-Aug-91 & & $10098-97-2$ & SR-90 \\
\hline 3 & PDMS & & SW & & & E117334 & 07-Aug-91 & & 10028-17-8 & TRITIUM \\
\hline 4 & PDMS & & SW & & & E117334 & 07-Aug-91 & & 13966-29-5 & U-234 \\
\hline 5 & PDMS & & SW & & & E117334 & 07-Aug-91 & & 15117-96-1 & U-235 \\
\hline 6 & PDMS & & SW & & & E117334 & 07-Aug-91 & & U-238 & U-238 \\
\hline 7 & PDMS & & SW & & & E117335 & 07-Aug-91 & & $10098-97-2$ & SR-90 \\
\hline 8 & PDMS & & SW & & & E117335 & 07-Aug-91 & & $10028-17-8$ & TRITIUM \\
\hline 9 & PDMS & & SW & & & E117335 & 07-Aug-91 & & 13966-29-5 & U-234 \\
\hline 10 & PDMS & & SW & & & E117335 & 07-Aug-91 & & $15117-96-1$ & U-235 \\
\hline 11 & PDMS & & SW & & & E117335 & 07-Aug-91 & & U-238 & U-238 \\
\hline 12 & PDMS & & SW & & & E117336 & 07-Aug-91 & & $10098-97-2$ & SR-90 \\
\hline 13 & PDMS & & SW & & & E117336 & 07-Aug-91 & & $10028-17-8$ & TRITIUM \\
\hline 14 & PDMS & & SW & & & E117336 & 07-Aug-91 & & $13966-29-5$ & $\mathrm{U}-234$ \\
\hline 15 & PDMS & & SW & & & E117336 & 07-Aug-91 & & 15117-96-1 & U-235 \\
\hline 16 & PDMS & & SW & & & E117336 & 07-Aug-91 & & U-238 & U-238 \\
\hline 17 & PDMS & & SW & & & E117337 & 07-Aug-91 & & $10098-97-2$ & SR-90 \\
\hline 18 & PDMS & & SW & & & E117337 & 07-Aug-91 & & 10028-17-8 & TRITIUM \\
\hline 19 & PDMS & & SW & & & E117337 & 07-Aug-91 & & 13966-29-5 & U-234 \\
\hline 20 & PDMS & & SW & & & E117337 & 07-Aug-91 & & $15117-96-1$ & U-235 \\
\hline 21 & PDMS & & SW & & & E117337 & 07-Aug-91 & & U-238 & U-238 \\
\hline 22 & PDMS & & SW & & & E117338 & 08-Aug-91 & & $10098-97-2$ & SR-90 \\
\hline 23 & PDMS & & SW & & & E117338 & 08-Aug-91 & & $10028-17-8$ & TRITIUM \\
\hline 24 & PDMS & & SW & & & E117338 & 08-Aug-91 & & 13966-29-5 & U-234 \\
\hline 25 & PDMS & & SW & & & E117338 & 08-Aug-91 & & $15117-96-1$ & U-235 \\
\hline 26 & PDMS & & SW & & & E117338 & 08-Aug-91 & & U-238 & U-238 \\
\hline 27 & PDMS & & SW & & & E117339 & $08-A u g-91$ & & $10098-97-2$ & SR-90 \\
\hline 28 & PDMS & & SW & & & E117339 & 08-Aug-91 & & $10028-17-8$ & TRITIUM \\
\hline 29 & PDMS & & SW & & & E117339 & 08-Aug-91 & & 13966-29-5 & U-234 \\
\hline 30 & PDMS & & SW & & & E117339 & 08-Aug-91 & & $15117-96-1$ & U-235 \\
\hline 31 & PDMS & & SW & & & E117339 & 08-Aug-91 & & U-238 & U-238 \\
\hline 32 & PDMS & & SW & & & E117340 & 08-Aug-91 & & $10098-97-2$ & SR-90 \\
\hline 33 & PDMS & & SW & & & E117340 & 08-Aug-91 & & $10028-17-8$ & TRITIUM \\
\hline 34 & PDMS & & SW & & & E117340 & 08-Aug-91 & & $13966-29-5$ & U-234 \\
\hline 35 & PDMS & & SW & & & E117340 & 08-Aug-91 & & $15117-96-1$ & U-235 \\
\hline 36 & PDMS & & SW & & & E117340 & 08-Aug-91 & & U-238 & U-238 \\
\hline 37 & PDMS & & SW & & & E117341 & 08-Aug-91 & & $10098-97-2$ & SR-90 \\
\hline 38 & PDMS & & SW & & & E117341 & 08-Aug-91 & & $10028-17-8$ & TRITIUM \\
\hline 39 & PDMS & & SW & & & E117341 & 08-Aug-91 & & 13966-29-5 & U-234 \\
\hline
\end{tabular}




\begin{tabular}{|c|c|c|c|c|c|c|c|c|c|c|}
\hline & $\mathrm{A}$ & $\mathrm{B}$ & $\mathrm{C}$ & $\mathrm{D}$ & $\mathrm{E}$ & $\mathrm{F}$ & $\mathrm{G}$ & $\mathrm{H}$ & $\mathrm{I}$ & $\mathrm{J}$ \\
\hline 1 & SAMPLE_NUM_ASSIGNED_TO & SAMPLER & MEDIA & BIOTA_MEDIA & TAG_ID & SAMP_NUM & SAMP_DATE_TIME & SAMP_DATE_TIME_ON & CON_ID & CON_SHORT_NAME \\
\hline 40 & PDMS & & SW & & & E117341 & 08-Aug-91 & & 15117-96-1 & U-235 \\
\hline 41 & PDMS & & SW & & & E117341 & 08-Aug-91 & & U-238 & U-238 \\
\hline 42 & PDMS & & SW & & & E118570 & 24-Sep-91 & & $10098-97-2$ & SR-90 \\
\hline 43 & PDMS & & SW & & & E118570 & 24-Sep-91 & & $10028-17-8$ & TRITIUM \\
\hline 44 & PDMS & & SW & & & E118570 & 24-Sep-91 & & 13966-29-5 & U-234 \\
\hline 45 & PDMS & & SW & & & E118570 & 24-Sep-91 & & 15117-96-1 & U-235 \\
\hline 46 & PDMS & & SW & & & E118570 & 24-Sep-91 & & U-238 & U-238 \\
\hline 47 & PDMS & & SW & & & E118571 & 24-Sep-91 & & $10098-97-2$ & SR-90 \\
\hline 48 & PDMS & & SW & & & E118571 & 24-Sep-91 & & $10028-17-8$ & TRITIUM \\
\hline 49 & PDMS & & SW & & & E118571 & 24-Sep-91 & & 13966-29-5 & U-234 \\
\hline 50 & PDMS & & SW & & & E118571 & 24-Sep-91 & & 15117-96-1 & U-235 \\
\hline 51 & PDMS & & SW & & & E118571 & 24-Sep-91 & & $\mathrm{U}-238$ & U-238 \\
\hline 52 & PDMS & & SW & & & E118572 & 24-Sep-91 & & $10098-97-2$ & SR-90 \\
\hline 53 & PDMS & & SW & & & E118572 & 24-Sep-91 & & 10028-17-8 & TRITIUM \\
\hline 54 & PDMS & & SW & & & E118572 & 24-Sep-91 & & $13966-29-5$ & U-234 \\
\hline 55 & PDMS & & SW & & & E118572 & 24-Sep-91 & & 15117-96-1 & U-235 \\
\hline 56 & PDMS & & SW & & & E118572 & 24-Sep-91 & & U-238 & U-238 \\
\hline 57 & PDMS & & SW & & & E118573 & 24-Sep-91 & & $10098-97-2$ & SR-90 \\
\hline 58 & PDMS & & SW & & & E118573 & 24-Sep-91 & & $10028-17-8$ & TRITIUM \\
\hline 59 & PDMS & & SW & & & E118573 & 24-Sep-91 & & 13966-29-5 & U-234 \\
\hline 60 & PDMS & & SW & & & E118573 & 24-Sep-91 & & 15117-96-1 & U-235 \\
\hline 61 & PDMS & & SW & & & E118573 & 24-Sep-91 & & U-238 & U-238 \\
\hline 62 & PDMS & & SW & & & E117946 & 25-Sep-91 & & $10098-97-2$ & SR-90 \\
\hline 63 & PDMS & & SW & & & E117946 & 25-Sep-91 & & $10028-17-8$ & TRITIUM \\
\hline 64 & PDMS & & SW & & & E117946 & 25-Sep-91 & & 13966-29-5 & U-234 \\
\hline 65 & PDMS & & SW & & & E117946 & 25-Sep-91 & & 15117-96-1 & U-235 \\
\hline 66 & PDMS & & SW & & & E117946 & 25-Sep-91 & & U-238 & U-238 \\
\hline 67 & PDMS & & SW & & & E117947 & 25-Sep-91 & & $10098-97-2$ & SR-90 \\
\hline 68 & PDMS & & SW & & & E117947 & 25-Sep-91 & & $10028-17-8$ & TRITIUM \\
\hline 69 & PDMS & & SW & & & E117947 & 25-Sep-91 & & 13966-29-5 & U-234 \\
\hline 70 & PDMS & & SW & & & E117947 & 25-Sep-91 & & 15117-96-1 & U-235 \\
\hline 71 & PDMS & & SW & & & E117947 & 25-Sep-91 & & $\mathrm{U}-238$ & U-238 \\
\hline 72 & PDMS & & SW & & & E117948 & 25-Sep-91 & & $10098-97-2$ & SR-90 \\
\hline 73 & PDMS & & SW & & & E117948 & 25-Sep-91 & & $10028-17-8$ & TRITIUM \\
\hline 74 & PDMS & & SW & & & E117948 & 25-Sep-91 & & 13966-29-5 & U-234 \\
\hline 75 & PDMS & & SW & & & E117948 & 25-Sep-91 & & 15117-96-1 & U-235 \\
\hline 76 & PDMS & & SW & & & E117948 & 25-Sep-91 & & U-238 & U-238 \\
\hline 77 & PDMS & & SW & & & E117949 & 25-Sep-91 & & 10098-97-2 & SR-90 \\
\hline
\end{tabular}




\begin{tabular}{|c|c|c|c|c|c|c|c|c|c|c|}
\hline & $\mathrm{A}$ & $B$ & $\mathrm{C}$ & $\mathrm{D}$ & $E$ & $\mathrm{~F}$ & $G$ & $\mathrm{H}$ & $I$ & $\mathrm{~J}$ \\
\hline 1 & SAMPLE NUM ASSIGNED TO & SAMPLER & MEDIA & BIOTA MEDIA & TAG ID & SAMP NUM & SAMP DATE TIME & SAMP DATE TIME ON & CON ID & CON SHORT NAME \\
\hline 78 & PDMS & & SW & & & E117949 & 25-Sep-91 & & $10028-17-8$ & TRITIUM \\
\hline 79 & PDMS & & SW & & & E117949 & 25-Sep-91 & & $13966-29-5$ & U-234 \\
\hline 80 & PDMS & & SW & & & E117949 & 25-Sep-91 & & 15117-96-1 & U-235 \\
\hline 81 & PDMS & & SW & & & E117949 & 25-Sep-91 & & U-238 & U-238 \\
\hline 82 & PDMS & & SW & & & E118587 & 17-Oct-91 & & $10098-97-2$ & SR-90 \\
\hline 83 & PDMS & & SW & & & E118587 & 17-Oct-91 & & $10028-17-8$ & TRITIUM \\
\hline 84 & PDMS & & SW & & & E118587 & 17-Oct-91 & & 13966-29-5 & U-234 \\
\hline 85 & PDMS & & SW & & & E118587 & 17-Oct-91 & & 15117-96-1 & U-235 \\
\hline 86 & PDMS & & SW & & & E118587 & 17-Oct-91 & & U-238 & U-238 \\
\hline 87 & PDMS & & SW & & & E118588 & 17-Oct-91 & & $10098-97-2$ & SR-90 \\
\hline 88 & PDMS & & SW & & & E118588 & 17-Oct-91 & & $10028-17-8$ & TRITIUM \\
\hline 89 & PDMS & & SW & & & E118588 & 17-Oct-91 & & $13966-29-5$ & U-234 \\
\hline 90 & PDMS & & SW & & & E118588 & 17-Oct-91 & & $15117-96-1$ & U-235 \\
\hline 91 & PDMS & & SW & & & E118588 & 17-Oct-91 & & U-238 & U-238 \\
\hline 92 & PDMS & & SW & & & E118589 & 17-Oct-91 & & $10098-97-2$ & SR-90 \\
\hline 93 & PDMS & & SW & & & E118589 & 17-Oct-91 & & 10028-17-8 & TRITIUM \\
\hline 94 & PDMS & & SW & & & E118589 & 17-Oct-91 & & 13966-29-5 & U-234 \\
\hline 95 & PDMS & & SW & & & E118589 & 17-Oct-91 & & 15117-96-1 & U-235 \\
\hline 96 & PDMS & & SW & & & E118589 & 17-Oct-91 & & U-238 & U-238 \\
\hline 97 & PDMS & & SW & & & E118590 & 17-Oct-91 & & $10098-97-2$ & SR-90 \\
\hline 98 & PDMS & & SW & & & E118590 & 17-Oct-91 & & $10028-17-8$ & TRITIUM \\
\hline 99 & PDMS & & SW & & & E118590 & 17-Oct-91 & & 13966-29-5 & U-234 \\
\hline 100 & PDMS & & SW & & & E118590 & 17-Oct-91 & & $15117-96-1$ & U-235 \\
\hline 101 & PDMS & & SW & & & E118590 & 17-Oct-91 & & $U-238$ & U-238 \\
\hline 102 & PDMS & & SW & & & E118579 & 17-Oct-91 & & $10098-97-2$ & SR-90 \\
\hline 103 & PDMS & & SW & & & E118579 & 17-Oct-91 & & $10028-17-8$ & TRITIUM \\
\hline 104 & PDMS & & SW & & & E118579 & 17-Oct-91 & & 13966-29-5 & U-234 \\
\hline 105 & PDMS & & SW & & & E118579 & 17-Oct-91 & & $15117-96-1$ & U-235 \\
\hline 106 & PDMS & & SW & & & E118579 & 17-Oct-91 & & U-238 & U-238 \\
\hline 107 & PDMS & & SW & & & E118580 & 17-Oct-91 & & $10098-97-2$ & SR-90 \\
\hline 108 & PDMS & & SW & & & E118580 & 17-Oct-91 & & $10028-17-8$ & TRITIUM \\
\hline 109 & PDMS & & SW & & & E118580 & 17-Oct-91 & & 13966-29-5 & U-234 \\
\hline 110 & PDMS & & SW & & & E118580 & 17-Oct-91 & & $15117-96-1$ & U-235 \\
\hline$\frac{110}{111}$ & PDMS & & SW & & & E118580 & 17-Oct-91 & & $\mathrm{U}-238$ & $\mathrm{U}-238$ \\
\hline 112 & PDMS & & SW & & & E118581 & 17-Oct-91 & & $10098-97-2$ & SR-90 \\
\hline 113 & PDMS & & SW & & & E118581 & 17-Oct-91 & & $10028-17-8$ & TRITIUM \\
\hline 114 & PDMS & & SW & & & E118581 & 17-Oct-91 & & 13966-29-5 & U-234 \\
\hline 115 & PDMS & & SW & & & E118581 & 17-Oct-91 & & $15117-96-1$ & U-235 \\
\hline
\end{tabular}

Page 3 


\begin{tabular}{|c|c|c|c|c|c|c|c|c|c|c|}
\hline & $\mathrm{A}$ & $B$ & $\mathrm{C}$ & $\mathrm{D}$ & $E$ & $\mathrm{~F}$ & $G$ & $\mathrm{H}$ & $I$ & $\mathrm{~J}$ \\
\hline 1 & SAMPLE NUM ASSIGNED TO & SAMPLER & MEDIA & BIOTA MEDIA & TAG ID & SAMP NUM & SAMP DATE TIME & SAMP DATE TIME ON & CON ID & CON SHORT NAME \\
\hline 116 & PDMS & & SW & & & E118581 & 17-Oct-91 & & U-238 & U-238 \\
\hline 117 & PDMS & & SW & & & E118582 & 17-Oct-91 & & $10098-97-2$ & SR-90 \\
\hline 118 & PDMS & & SW & & & E118582 & 17-Oct-91 & & $10028-17-8$ & TRITIUM \\
\hline 119 & PDMS & & SW & & & E118582 & 17-Oct-91 & & $13966-29-5$ & U-234 \\
\hline 120 & PDMS & & SW & & & E118582 & 17-Oct-91 & & 15117-96-1 & U-235 \\
\hline 121 & PDMS & & SW & & & E118582 & 17-Oct-91 & & U-238 & U-238 \\
\hline 122 & PDMS & & SW & & & E118278 & 25-Nov-91 & & $10098-97-2$ & SR-90 \\
\hline 123 & PDMS & & SW & & & E118278 & 25-Nov-91 & & $10028-17-8$ & TRITIUM \\
\hline 124 & PDMS & & SW & & & E118278 & 25-Nov-91 & & 13966-29-5 & U-234 \\
\hline 125 & PDMS & & SW & & & E118278 & 25-Nov-91 & & $15117-96-1$ & U-235 \\
\hline 126 & PDMS & & SW & & & E118278 & 25-Nov-91 & & U-238 & U-238 \\
\hline 127 & PDMS & & SW & & & E118279 & 25-Nov-91 & & $10098-97-2$ & SR-90 \\
\hline 128 & PDMS & & SW & & & E118279 & 25-Nov-91 & & $10028-17-8$ & TRITIUM \\
\hline 129 & PDMS & & SW & & & E118279 & 25-Nov-91 & & 13966-29-5 & U-234 \\
\hline 130 & PDMS & & SW & & & E118279 & 25-Nov-91 & & 15117-96-1 & U-235 \\
\hline 131 & PDMS & & SW & & & E118279 & 25-Nov-91 & & U-238 & U-238 \\
\hline 132 & PDMS & & SW & & & E118280 & 25-Nov-91 & & 10098-97-2 & SR-90 \\
\hline 133 & PDMS & & SW & & & E118280 & 25-Nov-91 & & $10028-17-8$ & TRITIUM \\
\hline 134 & PDMS & & SW & & & E118280 & 25-Nov-91 & & $13966-29-5$ & U-234 \\
\hline 135 & PDMS & & SW & & & E118280 & 25-Nov-91 & & $15117-96-1$ & U-235 \\
\hline 136 & PDMS & & SW & & & E118280 & 25-Nov-91 & & U-238 & U-238 \\
\hline 137 & PDMS & & SW & & & E118281 & 25-Nov-91 & & $10098-97-2$ & SR-90 \\
\hline 138 & PDMS & & SW & & & E118281 & 25-Nov-91 & & $10028-17-8$ & TRITIUM \\
\hline 139 & PDMS & & SW & & & E118281 & 25-Nov-91 & & 13966-29-5 & U-234 \\
\hline 140 & PDMS & & SW & & & E118281 & 25-Nov-91 & & 15117-96-1 & U-235 \\
\hline 141 & PDMS & & SW & & & E118281 & $25-$ Nov-91 & & $\mathrm{U}-238$ & U-238 \\
\hline 142 & PDMS & & SW & & & E118274 & 27-Nov-91 & & 10098-97-2 & SR-90 \\
\hline 143 & PDMS & & SW & & & E118274 & 27-Nov-91 & & $10028-17-8$ & TRITIUM \\
\hline 144 & PDMS & & SW & & & E118274 & 27-Nov-91 & & $13966-29-5$ & U-234 \\
\hline 145 & PDMS & & SW & & & E118274 & 27-Nov-91 & & 15117-96-1 & U-235 \\
\hline 146 & PDMS & & SW & & & E118274 & 27-Nov-91 & & U-238 & U-238 \\
\hline 147 & PDMS & & SW & & & E118275 & 27-Nov-91 & & $10098-97-2$ & SR-90 \\
\hline 148 & PDMS & & SW & & & E118275 & 27-Nov-91 & & $10028-17-8$ & TRITIUM \\
\hline 149 & PDMS & & SW & & & E118275 & 27-Nov-91 & & 13966-29-5 & U-234 \\
\hline 150 & PDMS & & SW & & & E118275 & 27-Nov-91 & & $15117-96-1$ & U-235 \\
\hline 151 & PDMS & & SW & & & E118275 & 27-Nov-91 & & U-238 & $\mathrm{U}-238$ \\
\hline 152 & PDMS & & SW & & & E118276 & 27-Nov-91 & & $10098-97-2$ & SR-90 \\
\hline 153 & PDMS & & SW & & & E118276 & 27-Nov-91 & & $10028-17-8$ & TRITIUM \\
\hline
\end{tabular}




\begin{tabular}{|c|c|c|c|c|c|c|c|c|c|c|}
\hline & $\mathrm{A}$ & $B$ & $\mathrm{C}$ & $\mathrm{D}$ & $E$ & $\mathrm{~F}$ & $G$ & $\mathrm{H}$ & $I$ & $\mathrm{~J}$ \\
\hline 1 & SAMPLE NUM ASSIGNED TO & SAMPLER & MEDIA & BIOTA MEDIA & TAG ID & SAMP NUM & SAMP DATE TIME & SAMP DATE TIME ON & CON ID & CON SHORT NAME \\
\hline 154 & PDMS & & SW & & & E118276 & 27-Nov-91 & & $13966-29-5$ & U-234 \\
\hline 155 & PDMS & & SW & & & E118276 & 27-Nov-91 & & 15117-96-1 & U-235 \\
\hline 156 & PDMS & & SW & & & E118276 & 27-Nov-91 & & U-238 & U-238 \\
\hline 157 & PDMS & & SW & & & E118277 & 27-Nov-91 & & 10098-97-2 & SR-90 \\
\hline 158 & PDMS & & SW & & & E118277 & 27-Nov-91 & & 10028-17-8 & TRITIUM \\
\hline 159 & PDMS & & SW & & & E118277 & 27-Nov-91 & & $13966-29-5$ & U-234 \\
\hline 160 & PDMS & & SW & & & E118277 & 27-Nov-91 & & $15117-96-1$ & U-235 \\
\hline 161 & PDMS & & SW & & & E118277 & 27-Nov-91 & & U-238 & U-238 \\
\hline 162 & PDMS & & SW & & & E118380 & 17-Dec-91 & & $10098-97-2$ & SR-90 \\
\hline 163 & PDMS & & SW & & & E118380 & 17-Dec-91 & & $10028-17-8$ & TRITIUM \\
\hline 164 & PDMS & & SW & & & E118380 & 17-Dec-91 & & $13966-29-5$ & U-234 \\
\hline 165 & PDMS & & SW & & & E118380 & 17-Dec-91 & & $15117-96-1$ & U-235 \\
\hline 166 & PDMS & & SW & & & E118380 & 17-Dec-91 & & U-238 & U-238 \\
\hline 167 & PDMS & & SW & & & E118381 & 17-Dec-91 & & $10098-97-2$ & SR-90 \\
\hline 168 & PDMS & & SW & & & E118381 & 17-Dec-91 & & 10028-17-8 & TRITIUM \\
\hline 169 & PDMS & & SW & & & E118381 & 17-Dec-91 & & 13966-29-5 & U-234 \\
\hline 170 & PDMS & & SW & & & E118381 & 17-Dec-91 & & 15117-96-1 & U-235 \\
\hline 171 & PDMS & & SW & & & E118381 & 17-Dec-91 & & U-238 & U-238 \\
\hline 172 & PDMS & & SW & & & E118382 & 17-Dec-91 & & $10098-97-2$ & SR-90 \\
\hline 173 & PDMS & & SW & & & E118382 & 17-Dec-91 & & $10028-17-8$ & TRITIUM \\
\hline 174 & PDMS & & SW & & & E118382 & 17-Dec-91 & & $13966-29-5$ & U-234 \\
\hline 175 & PDMS & & SW & & & E118382 & 17-Dec-91 & & $15117-96-1$ & U-235 \\
\hline 176 & PDMS & & SW & & & E118382 & 17-Dec-91 & & U-238 & U-238 \\
\hline 177 & PDMS & & SW & & & E118383 & 17-Dec-91 & & $10098-97-2$ & SR-90 \\
\hline 178 & PDMS & & SW & & & E118383 & 17-Dec-91 & & $10028-17-8$ & TRITIUM \\
\hline 179 & PDMS & & SW & & & E118383 & 17-Dec-91 & & $13966-29-5$ & U-234 \\
\hline 180 & PDMS & & SW & & & E118383 & 17-Dec-91 & & 15117-96-1 & U-235 \\
\hline 181 & PDMS & & SW & & & E118383 & 17-Dec-91 & & U-238 & U-238 \\
\hline 182 & PDMS & & SW & & & E118803 & 18-Dec-91 & & 10098-97-2 & SR-90 \\
\hline 183 & PDMS & & SW & & & E118803 & 18-Dec-91 & & $10028-17-8$ & TRITIUM \\
\hline 184 & PDMS & & SW & & & E118803 & 18-Dec-91 & & 13966-29-5 & U-234 \\
\hline 185 & PDMS & & SW & & & E118803 & 18-Dec-91 & & $15117-96-1$ & U-235 \\
\hline 186 & PDMS & & SW & & & E118803 & 18-Dec-91 & & $\mathrm{U}-238$ & U-238 \\
\hline \begin{tabular}{|l|}
187 \\
187
\end{tabular} & PDMS & & SW & & & E118804 & 18-Dec-91 & & $10098-97-2$ & SR-90 \\
\hline 188 & PDMS & & SW & & & E118804 & 18-Dec-91 & & $10028-17-8$ & TRITIUM \\
\hline 189 & PDMS & & SW & & & E118804 & 18-Dec-91 & & $13966-29-5$ & U-234 \\
\hline 190 & PDMS & & SW & & & E118804 & 18-Dec-91 & & $15117-96-1$ & U-235 \\
\hline 191 & PDMS & & SW & & & E118804 & 18-Dec-91 & & U-238 & U-238 \\
\hline
\end{tabular}




\begin{tabular}{|c|c|c|c|c|c|c|c|c|c|c|}
\hline & $\mathrm{A}$ & $B$ & $\mathrm{C}$ & $\mathrm{D}$ & $E$ & $\mathrm{~F}$ & $G$ & $\mathrm{H}$ & $\mathrm{I}$ & $\mathrm{J}$ \\
\hline 1 & SAMPLE_NUM_ASSIGNED_TO & SAMPLER & MEDIA & BIOTA_MEDIA & TAG_ID & SAMP_NUM & SAMP_DATE_TIME| & SAMP_DATE_TIME_ON & CON_ID & CON_SHORT_NAME \\
\hline 192 & PDMS & & SW & & & E118805 & 18-Dec-91 & & $10098-97-2$ & SR-90 \\
\hline 193 & PDMS & & SW & & & E118805 & 18-Dec-91 & & $10028-17-8$ & TRITIUM \\
\hline 194 & PDMS & & SW & & & E118805 & 18-Dec-91 & & 13966-29-5 & U-234 \\
\hline 195 & PDMS & & SW & & & E118805 & 18-Dec-91 & & 15117-96-1 & U-235 \\
\hline 196 & PDMS & & SW & & & E118805 & 18-Dec-91 & & U-238 & U-238 \\
\hline 197 & PDMS & & SW & & & E118806 & 18-Dec-91 & & 10098-97-2 & SR-90 \\
\hline 198 & PDMS & & SW & & & E118806 & 18-Dec-91 & & $10028-17-8$ & TRITIUM \\
\hline 199 & PDMS & & SW & & & E118806 & 18-Dec-91 & & 13966-29-5 & U-234 \\
\hline 200 & PDMS & & SW & & & E118806 & 18-Dec-91 & & 15117-96-1 & U-235 \\
\hline 201 & PDMS & & SW & & & E118806 & 18-Dec-91 & & U-238 & U-238 \\
\hline 202 & PDMS & & SW & & & E120423 & 25-Mar-92 & & $10098-97-2$ & SR-90 \\
\hline 203 & PDMS & & SW & & & E120423 & 25-Mar-92 & & $10028-17-8$ & TRITIUM \\
\hline 204 & PDMS & & SW & & & E120423 & 25-Mar-92 & & 10028-17-8 & TRITIUM \\
\hline 205 & PDMS & & SW & & & E120423 & 25-Mar-92 & & 13966-29-5 & U-234 \\
\hline 206 & PDMS & & SW & & & E120423 & 25-Mar-92 & & 15117-96-1 & U-235 \\
\hline 207 & PDMS & & SW & & & E120423 & 25-Mar-92 & & U-238 & U-238 \\
\hline 208 & PDMS & & SW & & & E120424 & 25-Mar-92 & & $10098-97-2$ & SR-90 \\
\hline 209 & PDMS & & SW & & & E120424 & 25-Mar-92 & & $10028-17-8$ & TRITIUM \\
\hline 210 & PDMS & & SW & & & E120424 & 25-Mar-92 & & $10028-17-8$ & TRITIUM \\
\hline 211 & PDMS & & SW & & & E120424 & 25-Mar-92 & & 13966-29-5 & U-234 \\
\hline 212 & PDMS & & SW & & & E120424 & 25-Mar-92 & & 15117-96-1 & U-235 \\
\hline 213 & PDMS & & SW & & & E120424 & 25-Mar-92 & & U-238 & U-238 \\
\hline 214 & PDMS & & SW & & & E120425 & 25-Mar-92 & & $10098-97-2$ & SR-90 \\
\hline 215 & PDMS & & SW & & & E120425 & 25-Mar-92 & & $10028-17-8$ & TRITIUM \\
\hline 216 & PDMS & & SW & & & E120425 & 25-Mar-92 & & $10028-17-8$ & TRITIUM \\
\hline 217 & PDMS & & SW & & & E120425 & 25-Mar-92 & & 13966-29-5 & U-234 \\
\hline 218 & PDMS & & SW & & & E120425 & 25-Mar-92 & & 15117-96-1 & U-235 \\
\hline 219 & PDMS & & SW & & & E120425 & 25-Mar-92 & & U-238 & U-238 \\
\hline 220 & PDMS & & SW & & & E120426 & 25-Mar-92 & & $10098-97-2$ & SR-90 \\
\hline 221 & PDMS & & SW & & & E120426 & 25-Mar-92 & & $10028-17-8$ & TRITIUM \\
\hline 222 & PDMS & & SW & & & E120426 & 25-Mar-92 & & $10028-17-8$ & TRITIUM \\
\hline 223 & PDMS & & SW & & & E120426 & 25-Mar-92 & & 13966-29-5 & U-234 \\
\hline 224 & PDMS & & SW & & & E120426 & 25-Mar-92 & & 15117-96-1 & U-235 \\
\hline 225 & PDMS & & SW & & & E120426 & 25-Mar-92 & & U-238 & U-238 \\
\hline 226 & PDMS & & SW & & & E120344 & 30-Mar-92 & & $10098-97-2$ & SR-90 \\
\hline 227 & PDMS & & SW & & & E120344 & 30-Mar-92 & & $10028-17-8$ & TRITIUM \\
\hline 228 & PDMS & & SW & & & E120344 & 30-Mar-92 & & 13966-29-5 & U-234 \\
\hline 229 & PDMS & & SW & & & E120344 & 30-Mar-92 & & 15117-96-1 & U-235 \\
\hline
\end{tabular}




\begin{tabular}{|c|c|c|c|c|c|c|c|c|c|c|}
\hline & $\mathrm{A}$ & $B$ & $\mathrm{C}$ & $\mathrm{D}$ & $E$ & $\mathrm{~F}$ & $G$ & $\mathrm{H}$ & $I$ & $\mathrm{~J}$ \\
\hline 1 & SAMPLE NUM ASSIGNED TO & SAMPLER & MEDIA & BIOTA MEDIA & TAG ID & SAMP NUM & SAMP DATE TIME & SAMP DATE TIME ON & CON ID & CON SHORT NAME \\
\hline 230 & PDMS & & SW & & & E120344 & 30-Mar-92 & & U-238 & U-238 \\
\hline 231 & PDMS & & SW & & & E120353 & 30-Mar-92 & & $10098-97-2$ & SR-90 \\
\hline 232 & PDMS & & SW & & & E120353 & 30-Mar-92 & & $10028-17-8$ & TRITIUM \\
\hline 233 & PDMS & & SW & & & E120353 & 30-Mar-92 & & $13966-29-5$ & U-234 \\
\hline 234 & PDMS & & SW & & & E120353 & 30-Mar-92 & & 15117-96-1 & U-235 \\
\hline 235 & PDMS & & SW & & & E120353 & 30-Mar-92 & & U-238 & U-238 \\
\hline 236 & PDMS & & SW & & & E120345 & 30-Mar-92 & & $10098-97-2$ & SR-90 \\
\hline 237 & PDMS & & SW & & & E120345 & 30-Mar-92 & & $10028-17-8$ & TRITIUM \\
\hline 238 & PDMS & & SW & & & E120345 & 30-Mar-92 & & 13966-29-5 & U-234 \\
\hline 239 & PDMS & & SW & & & E120345 & 30-Mar-92 & & $15117-96-1$ & U-235 \\
\hline 240 & PDMS & & SW & & & E120345 & 30-Mar-92 & & U-238 & U-238 \\
\hline 241 & PDMS & & SW & & & E120346 & 30-Mar-92 & & $10098-97-2$ & SR-90 \\
\hline 242 & PDMS & & SW & & & E120346 & 30-Mar-92 & & $10028-17-8$ & TRITIUM \\
\hline 243 & PDMS & & SW & & & E120346 & 30-Mar-92 & & 13966-29-5 & U-234 \\
\hline 244 & PDMS & & SW & & & E120346 & 30-Mar-92 & & 15117-96-1 & U-235 \\
\hline 245 & PDMS & & SW & & & E120346 & 30-Mar-92 & & U-238 & U-238 \\
\hline 246 & PDMS & & SW & & & E120347 & 30-Mar-92 & & 10098-97-2 & SR-90 \\
\hline 247 & PDMS & & SW & & & E120347 & 30-Mar-92 & & $10028-17-8$ & TRITIUM \\
\hline 248 & PDMS & & SW & & & E120347 & 30-Mar-92 & & $13966-29-5$ & U-234 \\
\hline 249 & PDMS & & SW & & & E120347 & 30-Mar-92 & & $15117-96-1$ & U-235 \\
\hline 250 & PDMS & & SW & & & E120347 & 30-Mar-92 & & U-238 & U-238 \\
\hline 251 & PDMS & & SW & & & E120348 & 30-Mar-92 & & $10098-97-2$ & SR-90 \\
\hline 252 & PDMS & & SW & & & E120348 & 30-Mar-92 & & $10028-17-8$ & TRITIUM \\
\hline 253 & PDMS & & SW & & & E120348 & 30-Mar-92 & & 13966-29-5 & U-234 \\
\hline 254 & PDMS & & SW & & & E120348 & 30-Mar-92 & & 15117-96-1 & U-235 \\
\hline 255 & PDMS & & SW & & & E120348 & 30-Mar-92 & & U-238 & U-238 \\
\hline 256 & PDMS & & SW & & & E120349 & 30-Mar-92 & & 10098-97-2 & SR-90 \\
\hline 257 & PDMS & & SW & & & E120349 & 30-Mar-92 & & $10028-17-8$ & TRITIUM \\
\hline 258 & PDMS & & SW & & & E120349 & 30-Mar-92 & & $13966-29-5$ & U-234 \\
\hline 259 & PDMS & & SW & & & E120349 & 30-Mar-92 & & 15117-96-1 & U-235 \\
\hline 260 & PDMS & & SW & & & E120349 & 30-Mar-92 & & U-238 & U-238 \\
\hline 261 & PDMS & & SW & & & E120350 & 30-Mar-92 & & $10098-97-2$ & SR-90 \\
\hline 262 & PDMS & & SW & & & E120350 & 30-Mar-92 & & $10028-17-8$ & TRITIUM \\
\hline 263 & PDMS & & SW & & & E120350 & 30-Mar-92 & & 13966-29-5 & U-234 \\
\hline 264 & PDMS & & SW & & & E120350 & 30-Mar-92 & & $15117-96-1$ & U-235 \\
\hline 265 & PDMS & & SW & & & E120350 & 30-Mar-92 & & U-238 & U-238 \\
\hline 266 & PDMS & & SW & & & E120351 & 30-Mar-92 & & $10098-97-2$ & SR-90 \\
\hline 267 & PDMS & & SW & & & E120351 & 30-Mar-92 & & $10028-17-8$ & TRITIUM \\
\hline
\end{tabular}




\begin{tabular}{|c|c|c|c|c|c|c|c|c|c|c|}
\hline & $\mathrm{A}$ & $B$ & $\mathrm{C}$ & $\mathrm{D}$ & $E$ & $\mathrm{~F}$ & $G$ & $\mathrm{H}$ & $I$ & $\mathrm{~J}$ \\
\hline 1 & SAMPLE NUM ASSIGNED TO & SAMPLER & MEDIA & BIOTA MEDIA & TAG ID & SAMP NUM & SAMP DATE TIME & SAMP DATE TIME ON & CON ID & CON SHORT NAME \\
\hline 268 & PDMS & & SW & & & E120351 & 30-Mar-92 & & $13966-29-5$ & U-234 \\
\hline 269 & PDMS & & SW & & & E120351 & 30-Mar-92 & & 15117-96-1 & U-235 \\
\hline 270 & PDMS & & SW & & & E120351 & 30-Mar-92 & & U-238 & U-238 \\
\hline 271 & PDMS & & SW & & & E120352 & 30-Mar-92 & & 10098-97-2 & SR-90 \\
\hline 272 & PDMS & & SW & & & E120352 & 30-Mar-92 & & 10028-17-8 & TRITIUM \\
\hline 273 & PDMS & & SW & & & E120352 & 30-Mar-92 & & $13966-29-5$ & U-234 \\
\hline 274 & PDMS & & SW & & & E120352 & 30-Mar-92 & & 15117-96-1 & U-235 \\
\hline 275 & PDMS & & SW & & & E120352 & 30-Mar-92 & & U-238 & U-238 \\
\hline 276 & PDMS & & SW & & & E120954 & 11-Jun-92 & & $10098-97-2$ & SR-90 \\
\hline 277 & PDMS & & SW & & & E120954 & 11-Jun-92 & & $10028-17-8$ & TRITIUM \\
\hline 278 & PDMS & & SW & & & E120954 & 11-Jun-92 & & 13966-29-5 & U-234 \\
\hline 279 & PDMS & & SW & & & E120954 & 11-Jun-92 & & $15117-96-1$ & U-235 \\
\hline 280 & PDMS & & SW & & & E120954 & 11-Jun-92 & & U-238 & U-238 \\
\hline 281 & PDMS & & SW & & & E120955 & 11-Jun-92 & & $10098-97-2$ & SR-90 \\
\hline 282 & PDMS & & SW & & & E120955 & 11-Jun-92 & & 10028-17-8 & TRITIUM \\
\hline 283 & PDMS & & SW & & & E120955 & 11-Jun-92 & & 13966-29-5 & U-234 \\
\hline 284 & PDMS & & SW & & & E120955 & 11-Jun-92 & & 15117-96-1 & U-235 \\
\hline 285 & PDMS & & SW & & & E120955 & 11-Jun-92 & & U-238 & U-238 \\
\hline 286 & PDMS & & SW & & & E120956 & 11-Jun-92 & & $10098-97-2$ & SR-90 \\
\hline 287 & PDMS & & SW & & & E120956 & 11-Jun-92 & & $10028-17-8$ & TRITIUM \\
\hline 288 & PDMS & & SW & & & E120956 & 11-Jun-92 & & $13966-29-5$ & U-234 \\
\hline 289 & PDMS & & SW & & & E120956 & 11-Jun-92 & & $15117-96-1$ & U-235 \\
\hline 290 & PDMS & & SW & & & E120956 & 11-Jun-92 & & U-238 & U-238 \\
\hline 291 & PDMS & & SW & & & E120957 & 11-Jun-92 & & $10098-97-2$ & SR-90 \\
\hline 292 & PDMS & & SW & & & E120957 & 11-Jun-92 & & $10028-17-8$ & TRITIUM \\
\hline 293 & PDMS & & SW & & & E120957 & 11-Jun-92 & & $13966-29-5$ & U-234 \\
\hline 294 & PDMS & & SW & & & E120957 & 11-Jun-92 & & 15117-96-1 & U-235 \\
\hline 295 & PDMS & & SW & & & E120957 & 11-Jun-92 & & U-238 & U-238 \\
\hline 296 & PDMS & & SW & & & E120890 & 12-Jun-92 & & 10098-97-2 & SR-90 \\
\hline 297 & PDMS & & SW & & & E120890 & 12-Jun-92 & & $10028-17-8$ & TRITIUM \\
\hline 298 & PDMS & & SW & & & E120890 & 12-Jun-92 & & 13966-29-5 & U-234 \\
\hline 299 & PDMS & & SW & & & E120890 & 12-Jun-92 & & $15117-96-1$ & U-235 \\
\hline 300 & PDMS & & SW & & & E120890 & 12-Jun-92 & & $\mathrm{U}-238$ & U-238 \\
\hline 301 & PDMS & & SW & & & E120899 & 12-Jun-92 & & $10098-97-2$ & SR-90 \\
\hline 302 & PDMS & & SW & & & E120899 & 12-Jun-92 & & $10028-17-8$ & TRITIUM \\
\hline 303 & PDMS & & SW & & & E120899 & 12-Jun-92 & & 13966-29-5 & U-234 \\
\hline 304 & PDMS & & SW & & & E120899 & 12-Jun-92 & & $15117-96-1$ & U-235 \\
\hline 305 & PDMS & & SW & & & E120899 & 12-Jun-92 & & U-238 & U-238 \\
\hline
\end{tabular}




\begin{tabular}{|c|c|c|c|c|c|c|c|c|c|c|}
\hline & $\mathrm{A}$ & $B$ & $\mathrm{C}$ & $\mathrm{D}$ & $E$ & $\mathrm{~F}$ & $G$ & $\mathrm{H}$ & $\mathrm{I}$ & $\mathrm{J}$ \\
\hline 1 & SAMPLE_NUM_ASSIGNED_TO & SAMPLER & MEDIA & BIOTA_MEDIA & TAG_ID & SAMP_NUM & SAMP_DATE_TIME| & SAMP_DATE_TIME_ON & CON_ID & CON_SHORT_NAME \\
\hline 306 & PDMS & & SW & & & E120891 & 12-Jun-92 & & $10098-97-2$ & SR-90 \\
\hline 307 & PDMS & & SW & & & E120891 & 12-Jun-92 & & $10028-17-8$ & TRITIUM \\
\hline 308 & PDMS & & SW & & & E120891 & 12-Jun-92 & & 13966-29-5 & U-234 \\
\hline 309 & PDMS & & SW & & & E120891 & 12-Jun-92 & & 15117-96-1 & U-235 \\
\hline 310 & PDMS & & SW & & & E120891 & 12-Jun-92 & & U-238 & U-238 \\
\hline 311 & PDMS & & SW & & & E120892 & 12-Jun-92 & & 10098-97-2 & SR-90 \\
\hline 312 & PDMS & & SW & & & E120892 & 12-Jun-92 & & $10028-17-8$ & TRITIUM \\
\hline 313 & PDMS & & SW & & & E120892 & 12-Jun-92 & & 13966-29-5 & U-234 \\
\hline 314 & PDMS & & SW & & & E120892 & 12-Jun-92 & & 15117-96-1 & U-235 \\
\hline 315 & PDMS & & SW & & & E120892 & 12-Jun-92 & & U-238 & U-238 \\
\hline 316 & PDMS & & SW & & & E120893 & 12-Jun-92 & & $10098-97-2$ & SR-90 \\
\hline 317 & PDMS & & SW & & & E120893 & 12-Jun-92 & & $10028-17-8$ & TRITIUM \\
\hline 318 & PDMS & & SW & & & E120893 & 12-Jun-92 & & 13966-29-5 & U-234 \\
\hline 319 & PDMS & & SW & & & E120893 & 12-Jun-92 & & 15117-96-1 & U-235 \\
\hline 320 & PDMS & & SW & & & E120893 & 12-Jun-92 & & U-238 & U-238 \\
\hline 321 & PDMS & & SW & & & E120894 & 12-Jun-92 & & $10098-97-2$ & SR-90 \\
\hline 322 & PDMS & & SW & & & E120894 & 12-Jun-92 & & $10028-17-8$ & TRITIUM \\
\hline 323 & PDMS & & SW & & & E120894 & 12-Jun-92 & & 13966-29-5 & U-234 \\
\hline 324 & PDMS & & SW & & & E120894 & 12-Jun-92 & & 15117-96-1 & U-235 \\
\hline 325 & PDMS & & SW & & & E120894 & 12-Jun-92 & & U-238 & U-238 \\
\hline 326 & PDMS & & SW & & & E120895 & 12-Jun-92 & & $10098-97-2$ & SR-90 \\
\hline 327 & PDMS & & SW & & & E120895 & 12-Jun-92 & & $10028-17-8$ & TRITIUM \\
\hline 328 & PDMS & & SW & & & E120895 & 12-Jun-92 & & 13966-29-5 & U-234 \\
\hline 329 & PDMS & & SW & & & E120895 & 12-Jun-92 & & 15117-96-1 & U-235 \\
\hline 330 & PDMS & & SW & & & E120895 & 12-Jun-92 & & U-238 & U-238 \\
\hline 331 & PDMS & & SW & & & E120896 & 12-Jun-92 & & $10098-97-2$ & SR-90 \\
\hline 332 & PDMS & & SW & & & E120896 & 12-Jun-92 & & $10028-17-8$ & TRITIUM \\
\hline 333 & PDMS & & SW & & & E120896 & 12-Jun-92 & & 13966-29-5 & U-234 \\
\hline 334 & PDMS & & SW & & & E120896 & 12-Jun-92 & & 15117-96-1 & U-235 \\
\hline 335 & PDMS & & SW & & & E120896 & 12-Jun-92 & & U-238 & U-238 \\
\hline 336 & PDMS & & SW & & & E120897 & 12-Jun-92 & & $10098-97-2$ & SR-90 \\
\hline 337 & PDMS & & SW & & & E120897 & 12-Jun-92 & & $10028-17-8$ & TRITIUM \\
\hline 338 & PDMS & & SW & & & E120897 & 12-Jun-92 & & 13966-29-5 & U-234 \\
\hline 339 & PDMS & & SW & & & E120897 & 12-Jun-92 & & 15117-96-1 & U-235 \\
\hline 340 & PDMS & & SW & & & E120897 & 12-Jun-92 & & U-238 & U-238 \\
\hline 341 & PDMS & & SW & & & E120898 & 12-Jun-92 & & $10098-97-2$ & SR-90 \\
\hline 342 & PDMS & & SW & & & E120898 & 12-Jun-92 & & $10028-17-8$ & TRITIUM \\
\hline 343 & PDMS & & SW & & & E120898 & 12-Jun-92 & & 13966-29-5 & U-234 \\
\hline
\end{tabular}




\begin{tabular}{|c|c|c|c|c|c|c|c|c|c|c|}
\hline & $\mathrm{A}$ & $B$ & $\mathrm{C}$ & $\mathrm{D}$ & $E$ & $\mathrm{~F}$ & $G$ & $\mathrm{H}$ & $I$ & $\mathrm{~J}$ \\
\hline 1 & SAMPLE NUM ASSIGNED TO & SAMPLER & MEDIA & BIOTA MEDIA & TAG ID & SAMP NUM & SAMP DATE TIME & SAMP DATE TIME ON & CON ID & CON SHORT NAME \\
\hline 344 & PDMS & & SW & & & E120898 & 12-Jun-92 & & 15117-96-1 & U-235 \\
\hline 345 & PDMS & & SW & & & E120898 & 12-Jun-92 & & U-238 & U-238 \\
\hline 346 & PDMS & & SW & & & E121756 & 30-Sep-92 & & $10098-97-2$ & SR-90 \\
\hline 347 & PDMS & & SW & & & E121756 & 30-Sep-92 & & $10028-17-8$ & TRITIUM \\
\hline 348 & PDMS & & SW & & & E121756 & 30-Sep-92 & & 13966-29-5 & U-234 \\
\hline 349 & PDMS & & SW & & & E121756 & 30-Sep-92 & & 15117-96-1 & U-235 \\
\hline 350 & PDMS & & SW & & & E121756 & 30-Sep-92 & & U-238 & $\mathrm{U}-238$ \\
\hline 351 & PDMS & & SW & & & E121757 & 30-Sep-92 & & $10098-97-2$ & SR-90 \\
\hline 352 & PDMS & & SW & & & E121757 & 30-Sep-92 & & $10028-17-8$ & TRITIUM \\
\hline 353 & PDMS & & SW & & & E121757 & 30-Sep-92 & & $13966-29-5$ & U-234 \\
\hline 354 & PDMS & & SW & & & E121757 & 30-Sep-92 & & 15117-96-1 & U-235 \\
\hline 355 & PDMS & & SW & & & E121757 & 30-Sep-92 & & U-238 & U-238 \\
\hline 356 & PDMS & & SW & & & E121758 & 30-Sep-92 & & $10098-97-2$ & SR-90 \\
\hline 357 & PDMS & & SW & & & E121758 & 30-Sep-92 & & $10028-17-8$ & TRITIUM \\
\hline 358 & PDMS & & SW & & & E121758 & 30-Sep-92 & & $13966-29-5$ & U-234 \\
\hline 359 & PDMS & & SW & & & E121758 & 30-Sep-92 & & 15117-96-1 & U-235 \\
\hline 360 & PDMS & & SW & & & E121758 & 30-Sep-92 & & U-238 & $\mathrm{U}-238$ \\
\hline 361 & PDMS & & SW & & & E121759 & 30-Sep-92 & & 10098-97-2 & SR-90 \\
\hline 362 & PDMS & & SW & & & E121759 & 30-Sep-92 & & $10028-17-8$ & TRITIUM \\
\hline 363 & PDMS & & SW & & & E121759 & 30-Sep-92 & & $13966-29-5$ & U-234 \\
\hline 364 & PDMS & & SW & & & E121759 & 30-Sep-92 & & 15117-96-1 & U-235 \\
\hline 365 & PDMS & & SW & & & E121759 & 30-Sep-92 & & U-238 & U-238 \\
\hline 366 & PDMS & & SW & & & E121794 & 02-Oct-92 & & $10098-97-2$ & SR-90 \\
\hline 367 & PDMS & & SW & & & E121794 & 02-Oct-92 & & $10028-17-8$ & TRITIUM \\
\hline 368 & PDMS & & SW & & & E121794 & 02-Oct-92 & & 13966-29-5 & U-234 \\
\hline 369 & PDMS & & SW & & & E121794 & 02-Oct-92 & & $15117-96-1$ & U-235 \\
\hline 370 & PDMS & & SW & & & E121794 & 02-Oct-92 & & U-238 & U-238 \\
\hline 371 & PDMS & & SW & & & E121803 & 02-Oct-92 & & 10098-97-2 & SR-90 \\
\hline 372 & PDMS & & SW & & & E121803 & 02-Oct-92 & & $10028-17-8$ & TRITIUM \\
\hline 373 & PDMS & & SW & & & E121803 & 02-Oct-92 & & 13966-29-5 & U-234 \\
\hline 374 & PDMS & & SW & & & E121803 & 02-Oct-92 & & 15117-96-1 & U-235 \\
\hline 375 & PDMS & & SW & & & E121803 & 02-Oct-92 & & U-238 & U-238 \\
\hline 376 & PDMS & & SW & & & E121795 & 02-Oct-92 & & $10098-97-2$ & SR-90 \\
\hline 377 & PDMS & & SW & & & E121795 & 02-Oct-92 & & $10028-17-8$ & TRITIUM \\
\hline 378 & PDMS & & SW & & & E121795 & 02-Oct-92 & & $13966-29-5$ & U-234 \\
\hline 379 & PDMS & & SW & & & E121795 & 02-Oct-92 & & $15117-96-1$ & U-235 \\
\hline 380 & PDMS & & SW & & & E121795 & 02-Oct-92 & & U-238 & U-238 \\
\hline 381 & PDMS & & SW & & & E121796 & 02-Oct-92 & & $10098-97-2$ & SR-90 \\
\hline
\end{tabular}




\begin{tabular}{|c|c|c|c|c|c|c|c|c|c|c|}
\hline & $\mathrm{A}$ & $B$ & $\mathrm{C}$ & $\mathrm{D}$ & $E$ & $\mathrm{~F}$ & $G$ & $\mathrm{H}$ & $I$ & $\mathrm{~J}$ \\
\hline 1 & SAMPLE NUM ASSIGNED TO & SAMPLER & MEDIA & BIOTA MEDIA & TAG ID & SAMP NUM & SAMP DATE TIME & SAMP DATE TIME ON & CON ID & CON SHORT NAME \\
\hline 382 & PDMS & & SW & & & E121796 & 02-Oct-92 & & $10028-17-8$ & TRITIUM \\
\hline 383 & PDMS & & SW & & & E121796 & 02-Oct-92 & & $13966-29-5$ & U-234 \\
\hline 384 & PDMS & & SW & & & E121796 & 02-Oct-92 & & 15117-96-1 & U-235 \\
\hline 385 & PDMS & & SW & & & E121796 & 02-Oct-92 & & U-238 & U-238 \\
\hline 386 & PDMS & & SW & & & E121797 & 02-Oct-92 & & $10098-97-2$ & SR-90 \\
\hline 387 & PDMS & & SW & & & E121797 & 02-Oct-92 & & $10028-17-8$ & TRITIUM \\
\hline 388 & PDMS & & SW & & & E121797 & 02-Oct-92 & & $13966-29-5$ & $\mathrm{U}-234$ \\
\hline 389 & PDMS & & SW & & & E121797 & 02-Oct-92 & & 15117-96-1 & U-235 \\
\hline 390 & PDMS & & SW & & & E121797 & 02-Oct-92 & & U-238 & U-238 \\
\hline 391 & PDMS & & SW & & & E121798 & 02-Oct-92 & & $10098-97-2$ & SR-90 \\
\hline 392 & PDMS & & SW & & & E121798 & 02-Oct-92 & & $10028-17-8$ & TRITIUM \\
\hline 393 & PDMS & & SW & & & E121798 & 02-Oct-92 & & $13966-29-5$ & U-234 \\
\hline 394 & PDMS & & SW & & & E121798 & 02-Oct-92 & & $15117-96-1$ & U-235 \\
\hline 395 & PDMS & & SW & & & E121798 & 02-Oct-92 & & U-238 & U-238 \\
\hline 396 & PDMS & & SW & & & E121799 & 02-Oct-92 & & $10098-97-2$ & SR-90 \\
\hline 397 & PDMS & & SW & & & E121799 & $02-O c t-92$ & & 10028-17-8 & TRITIUM \\
\hline 398 & PDMS & & SW & & & E121799 & 02-Oct-92 & & 13966-29-5 & U-234 \\
\hline 399 & PDMS & & SW & & & E121799 & 02-Oct-92 & & 15117-96-1 & U-235 \\
\hline 400 & PDMS & & SW & & & E121799 & 02-Oct-92 & & U-238 & U-238 \\
\hline 401 & PDMS & & SW & & & E121800 & 02-Oct-92 & & $10098-97-2$ & SR-90 \\
\hline 402 & PDMS & & SW & & & E121800 & 02-Oct-92 & & $10028-17-8$ & TRITIUM \\
\hline 403 & PDMS & & SW & & & E121800 & 02-Oct-92 & & 13966-29-5 & U-234 \\
\hline 404 & PDMS & & SW & & & E121800 & 02-Oct-92 & & $15117-96-1$ & U-235 \\
\hline 405 & PDMS & & SW & & & E121800 & 02-Oct-92 & & U-238 & U-238 \\
\hline 406 & PDMS & & SW & & & E121801 & 02-Oct-92 & & $10098-97-2$ & SR-90 \\
\hline 407 & PDMS & & SW & & & E121801 & $02-$ Oct-92 & & $10028-17-8$ & TRITIUM \\
\hline 408 & PDMS & & SW & & & E121801 & 02-Oct-92 & & 13966-29-5 & U-234 \\
\hline 409 & PDMS & & SW & & & E121801 & 02-Oct-92 & & $15117-96-1$ & U-235 \\
\hline 410 & PDMS & & SW & & & E121801 & 02-Oct-92 & & U-238 & U-238 \\
\hline 411 & PDMS & & SW & & & E121802 & 02-Oct-92 & & $10098-97-2$ & SR-90 \\
\hline 412 & PDMS & & SW & & & E121802 & 02-Oct-92 & & $10028-17-8$ & TRITIUM \\
\hline 413 & PDMS & & SW & & & E121802 & 02-Oct-92 & & 13966-29-5 & U-234 \\
\hline 414 & PDMS & & SW & & & E121802 & 02-Oct-92 & & $15117-96-1$ & U-235 \\
\hline 415 & PDMS & & SW & & & E121802 & 02-Oct-92 & & U-238 & U-238 \\
\hline 416 & PDMS & & SW & & & E119997 & 10-Dec-92 & & $10098-97-2$ & SR-90 \\
\hline 417 & PDMS & & SW & & & E119997 & 10-Dec-92 & & $10028-17-8$ & TRITIUM \\
\hline 418 & PDMS & & SW & & & E119997 & 10-Dec-92 & & 13966-29-5 & U-234 \\
\hline 419 & PDMS & & SW & & & E119997 & 10-Dec-92 & & $15117-96-1$ & U-235 \\
\hline
\end{tabular}




\begin{tabular}{|c|c|c|c|c|c|c|c|c|c|c|}
\hline & $\mathrm{A}$ & $\mathrm{B}$ & $\mathrm{C}$ & $\mathrm{D}$ & $E$ & $\mathrm{~F}$ & $G$ & $\mathrm{H}$ & $\mathrm{I}$ & $\mathrm{J}$ \\
\hline 1 & SAMPLE_NUM_ASSIGNED_TO & SAMPLER & MEDIA & BIOTA_MEDIA & TAG_ID & SAMP_NUM & SAMP_DATE_TIME & SAMP_DATE_TIME_ON & CON_ID & CON_SHORT_NAME \\
\hline 420 & PDMS & & SW & & & E119997 & 10-Dec-92 & & U-238 & U-238 \\
\hline 421 & PDMS & & SW & & & E120006 & 10-Dec-92 & & $10098-97-2$ & SR-90 \\
\hline 422 & PDMS & & SW & & & E120006 & 10-Dec-92 & & 10028-17-8 & TRITIUM \\
\hline 423 & PDMS & & SW & & & E120006 & 10-Dec-92 & & 13966-29-5 & U-234 \\
\hline 424 & PDMS & & SW & & & E120006 & 10-Dec-92 & & 15117-96-1 & U-235 \\
\hline 425 & PDMS & & SW & & & E120006 & 10-Dec-92 & & U-238 & U-238 \\
\hline 426 & PDMS & & SW & & & E119998 & 10-Dec-92 & & $10098-97-2$ & SR-90 \\
\hline 427 & PDMS & & SW & & & E119998 & 10-Dec-92 & & 10028-17-8 & TRITIUM \\
\hline 428 & PDMS & & SW & & & E119998 & 10-Dec-92 & & 13966-29-5 & U-234 \\
\hline 429 & PDMS & & SW & & & E119998 & 10-Dec-92 & & $15117-96-1$ & U-235 \\
\hline 430 & PDMS & & SW & & & E119998 & 10-Dec-92 & & U-238 & U-238 \\
\hline 431 & PDMS & & SW & & & E119999 & 10-Dec-92 & & $10098-97-2$ & SR-90 \\
\hline 432 & PDMS & & SW & & & E119999 & 10-Dec-92 & & $10028-17-8$ & TRITIUM \\
\hline 433 & PDMS & & SW & & & E119999 & 10-Dec-92 & & 13966-29-5 & U-234 \\
\hline 434 & PDMS & & SW & & & E119999 & 10-Dec-92 & & 15117-96-1 & U-235 \\
\hline 435 & PDMS & & SW & & & E119999 & 10-Dec-92 & & U-238 & U-238 \\
\hline 436 & PDMS & & SW & & & E120000 & 10-Dec-92 & & $10098-97-2$ & SR-90 \\
\hline 437 & PDMS & & SW & & & E120000 & 10-Dec-92 & & $10028-17-8$ & TRITIUM \\
\hline 438 & PDMS & & SW & & & E120000 & 10-Dec-92 & & 13966-29-5 & U-234 \\
\hline 439 & PDMS & & SW & & & E120000 & 10-Dec-92 & & $15117-96-1$ & U-235 \\
\hline 440 & PDMS & & SW & & & E120000 & 10-Dec-92 & & U-238 & U-238 \\
\hline 441 & PDMS & & SW & & & E120001 & 10-Dec-92 & & $10098-97-2$ & SR-90 \\
\hline 442 & PDMS & & SW & & & E120001 & 10-Dec-92 & & $10028-17-8$ & TRITIUM \\
\hline 443 & PDMS & & SW & & & E120001 & 10-Dec-92 & & 13966-29-5 & U-234 \\
\hline 444 & PDMS & & SW & & & E120001 & 10-Dec-92 & & $15117-96-1$ & U-235 \\
\hline 445 & PDMS & & SW & & & E120001 & 10-Dec-92 & & $\mathrm{U}-238$ & U-238 \\
\hline 446 & PDMS & & SW & & & E120002 & 10-Dec-92 & & $10098-97-2$ & SR-90 \\
\hline 447 & PDMS & & SW & & & E120002 & 10-Dec-92 & & $10028-17-8$ & TRITIUM \\
\hline 448 & PDMS & & SW & & & E120002 & 10-Dec-92 & & 13966-29-5 & U-234 \\
\hline 449 & PDMS & & SW & & & E120002 & 10-Dec-92 & & 15117-96-1 & U-235 \\
\hline 450 & PDMS & & SW & & & E120002 & 10-Dec-92 & & U-238 & U-238 \\
\hline 451 & PDMS & & SW & & & E120003 & 10-Dec-92 & & $10098-97-2$ & SR-90 \\
\hline 452 & PDMS & & SW & & & E120003 & 10-Dec-92 & & $10028-17-8$ & TRITIUM \\
\hline 453 & PDMS & & SW & & & E120003 & 10-Dec-92 & & $13966-29-5$ & $\mathrm{U}-234$ \\
\hline 454 & PDMS & & SW & & & E120003 & 10-Dec-92 & & $15117-96-1$ & U-235 \\
\hline 455 & PDMS & & SW & & & E120003 & 10-Dec-92 & & U-238 & U-238 \\
\hline 456 & PDMS & & SW & & & E120004 & 10-Dec-92 & & $10098-97-2$ & SR-90 \\
\hline 457 & PDMS & & SW & & & E120004 & 10-Dec-92 & & $10028-17-8$ & TRITIUM \\
\hline
\end{tabular}




\begin{tabular}{|c|c|c|c|c|c|c|c|c|c|c|}
\hline & $\mathrm{A}$ & $\mathrm{B}$ & $\mathrm{C}$ & $\mathrm{D}$ & $E$ & $\mathrm{~F}$ & $G$ & $\mathrm{H}$ & $\mathrm{I}$ & $\mathrm{J}$ \\
\hline 1 & SAMPLE_NUM_ASSIGNED_TO & SAMPLER & MEDIA & BIOTA_MEDIA & TAG_ID & SAMP_NUM & SAMP_DATE_TIME & SAMP_DATE_TIME_ON & CON_ID & CON_SHORT_NAME \\
\hline 458 & PDMS & & SW & & & E120004 & 10-Dec-92 & & $13966-29-5$ & U-234 \\
\hline 459 & PDMS & & SW & & & E120004 & 10-Dec-92 & & 15117-96-1 & U-235 \\
\hline 460 & PDMS & & SW & & & E120004 & 10-Dec-92 & & U-238 & U-238 \\
\hline 461 & PDMS & & SW & & & E120005 & 10-Dec-92 & & 10098-97-2 & SR-90 \\
\hline 462 & PDMS & & SW & & & E120005 & 10-Dec-92 & & 10028-17-8 & TRITIUM \\
\hline 463 & PDMS & & SW & & & E120005 & 10-Dec-92 & & 13966-29-5 & U-234 \\
\hline 464 & PDMS & & SW & & & E120005 & 10-Dec-92 & & $15117-96-1$ & U-235 \\
\hline 465 & PDMS & & SW & & & E120005 & 10-Dec-92 & & U-238 & U-238 \\
\hline 466 & PDMS & & SW & & & E120007 & 11-Dec-92 & & $10098-97-2$ & SR-90 \\
\hline 467 & PDMS & & SW & & & E120007 & 11-Dec-92 & & $10028-17-8$ & TRITIUM \\
\hline 468 & PDMS & & SW & & & E120007 & 11-Dec-92 & & $13966-29-5$ & U-234 \\
\hline 469 & PDMS & & SW & & & E120007 & 11-Dec-92 & & $15117-96-1$ & U-235 \\
\hline 470 & PDMS & & SW & & & E120007 & 11-Dec-92 & & U-238 & U-238 \\
\hline 471 & PDMS & & SW & & & E120008 & 11-Dec-92 & & $10098-97-2$ & SR-90 \\
\hline 472 & PDMS & & SW & & & E120008 & 11-Dec-92 & & 10028-17-8 & TRITIUM \\
\hline 473 & PDMS & & SW & & & E120008 & 11-Dec-92 & & 13966-29-5 & U-234 \\
\hline 474 & PDMS & & SW & & & E120008 & 11-Dec-92 & & 15117-96-1 & U-235 \\
\hline 475 & PDMS & & SW & & & E120008 & 11-Dec-92 & & U-238 & U-238 \\
\hline 476 & PDMS & & SW & & & E120009 & 11-Dec-92 & & $10098-97-2$ & SR-90 \\
\hline 477 & PDMS & & SW & & & E120009 & 11-Dec-92 & & $10028-17-8$ & TRITIUM \\
\hline 478 & PDMS & & SW & & & E120009 & 11-Dec-92 & & $13966-29-5$ & U-234 \\
\hline 479 & PDMS & & SW & & & E120009 & 11-Dec-92 & & $15117-96-1$ & U-235 \\
\hline 480 & PDMS & & SW & & & E120009 & 11-Dec-92 & & U-238 & U-238 \\
\hline 481 & PDMS & & SW & & & E120010 & 11-Dec-92 & & $10098-97-2$ & SR-90 \\
\hline 482 & PDMS & & SW & & & E120010 & 11-Dec-92 & & $10028-17-8$ & TRITIUM \\
\hline 483 & PDMS & & SW & & & E120010 & 11-Dec-92 & & $13966-29-5$ & U-234 \\
\hline 484 & PDMS & & SW & & & E120010 & 11-Dec-92 & & 15117-96-1 & U-235 \\
\hline 485 & PDMS & & SW & & & E120010 & 11-Dec-92 & & U-238 & U-238 \\
\hline 486 & PDMS & & SW & & & E123569 & 25-Mar-93 & & 10098-97-2 & SR-90 \\
\hline 487 & PDMS & & SW & & & E123569 & 25-Mar-93 & & $10028-17-8$ & TRITIUM \\
\hline 488 & PDMS & & SW & & & E123569 & 25-Mar-93 & & 13966-29-5 & U-234 \\
\hline 489 & PDMS & & SW & & & E123569 & 25-Mar-93 & & $15117-96-1$ & U-235 \\
\hline 490 & PDMS & & SW & & & E123569 & 25-Mar-93 & & $\mathrm{U}-238$ & U-238 \\
\hline 491 & PDMS & & SW & & & E123570 & 25-Mar-93 & & $10098-97-2$ & SR-90 \\
\hline 492 & PDMS & & SW & & & E123570 & 25-Mar-93 & & $10028-17-8$ & TRITIUM \\
\hline 493 & PDMS & & SW & & & E123570 & 25-Mar-93 & & $13966-29-5$ & U-234 \\
\hline 494 & PDMS & & SW & & & E123570 & 25-Mar-93 & & $15117-96-1$ & U-235 \\
\hline 495 & PDMS & & SW & & & E123570 & 25-Mar-93 & & U-238 & U-238 \\
\hline
\end{tabular}




\begin{tabular}{|c|c|c|c|c|c|c|c|c|c|c|}
\hline & $\mathrm{A}$ & $B$ & $\mathrm{C}$ & $\mathrm{D}$ & $E$ & $\mathrm{~F}$ & $G$ & $\mathrm{H}$ & $I$ & $\mathrm{~J}$ \\
\hline 1 & SAMPLE NUM ASSIGNED TO & SAMPLER & MEDIA & BIOTA MEDIA & TAG ID & SAMP NUM & SAMP DATE TIME & SAMP DATE TIME ON & CON ID & CON SHORT NAME \\
\hline 496 & PDMS & & SW & & & E123571 & 25-Mar-93 & & $10098-97-2$ & SR-90 \\
\hline 497 & PDMS & & SW & & & E123571 & 25-Mar-93 & & 10028-17-8 & TRITIUM \\
\hline 498 & PDMS & & SW & & & E123571 & 25-Mar-93 & & 13966-29-5 & U-234 \\
\hline 499 & PDMS & & SW & & & E123571 & 25-Mar-93 & & 15117-96-1 & U-235 \\
\hline 500 & PDMS & & SW & & & E123571 & 25-Mar-93 & & U-238 & U-238 \\
\hline 501 & PDMS & & SW & & & E123572 & 25-Mar-93 & & $10098-97-2$ & SR-90 \\
\hline 502 & PDMS & & SW & & & E123572 & 25-Mar-93 & & $10028-17-8$ & TRITIUM \\
\hline 503 & PDMS & & SW & & & E123572 & 25-Mar-93 & & $13966-29-5$ & $\mathrm{U}-234$ \\
\hline 504 & PDMS & & SW & & & E123572 & 25-Mar-93 & & $15117-96-1$ & U-235 \\
\hline 505 & PDMS & & SW & & & E123572 & 25-Mar-93 & & U-238 & U-238 \\
\hline 506 & PDMS & & SW & & & E123598 & 26-Mar-93 & & $10098-97-2$ & SR-90 \\
\hline 507 & PDMS & & SW & & & E123599 & 26-Mar-93 & & $10098-97-2$ & SR-90 \\
\hline 508 & PDMS & & SW & & & E123598 & 26-Mar-93 & & $10028-17-8$ & TRITIUM \\
\hline 509 & PDMS & & SW & & & E123599 & 26-Mar-93 & & $10028-17-8$ & TRITIUM \\
\hline 510 & PDMS & & SW & & & E123598 & 26-Mar-93 & & 13966-29-5 & U-234 \\
\hline 511 & PDMS & & SW & & & E123598 & 26-Mar-93 & & 15117-96-1 & U-235 \\
\hline 512 & PDMS & & SW & & & E123598 & 26-Mar-93 & & U-238 & $\mathrm{U}-238$ \\
\hline 513 & PDMS & & SW & & & E123599 & 26-Mar-93 & & 13966-29-5 & U-234 \\
\hline 514 & PDMS & & SW & & & E123599 & 26-Mar-93 & & $15117-96-1$ & U-235 \\
\hline 515 & PDMS & & SW & & & E123599 & 26-Mar-93 & & U-238 & U-238 \\
\hline 516 & PDMS & & SW & & & E123588 & 26-Mar-93 & & $10098-97-2$ & SR-90 \\
\hline 517 & PDMS & & SW & & & E123588 & 26-Mar-93 & & $10028-17-8$ & TRITIUM \\
\hline 518 & PDMS & & SW & & & E123588 & 26-Mar-93 & & 13966-29-5 & U-234 \\
\hline 519 & PDMS & & SW & & & E123588 & 26-Mar-93 & & 15117-96-1 & U-235 \\
\hline 520 & PDMS & & SW & & & E123588 & 26-Mar-93 & & U-238 & U-238 \\
\hline 521 & PDMS & & SW & & & E123597 & 26-Mar-93 & & $10098-97-2$ & SR-90 \\
\hline 522 & PDMS & & SW & & & E123597 & 26-Mar-93 & & $10028-17-8$ & TRITIUM \\
\hline 523 & PDMS & & SW & & & E123597 & 26-Mar-93 & & 13966-29-5 & U-234 \\
\hline 524 & PDMS & & SW & & & E123597 & 26-Mar-93 & & 15117-96-1 & U-235 \\
\hline 525 & PDMS & & SW & & & E123597 & 26-Mar-93 & & U-238 & U-238 \\
\hline 526 & PDMS & & SW & & & E123589 & 26-Mar-93 & & $10098-97-2$ & SR-90 \\
\hline 527 & PDMS & & SW & & & E123589 & 26-Mar-93 & & $10028-17-8$ & TRITIUM \\
\hline 528 & PDMS & & SW & & & E123589 & 26-Mar-93 & & $13966-29-5$ & U-234 \\
\hline 529 & PDMS & & SW & & & E123589 & 26-Mar-93 & & $15117-96-1$ & U-235 \\
\hline 530 & PDMS & & SW & & & E123589 & 26-Mar-93 & & U-238 & U-238 \\
\hline 531 & PDMS & & SW & & & E123590 & 26-Mar-93 & & $10098-97-2$ & SR-90 \\
\hline 532 & PDMS & & SW & & & E123590 & 26-Mar-93 & & $10028-17-8$ & TRITIUM \\
\hline 533 & PDMS & & SW & & & E123590 & 26-Mar-93 & & 13966-29-5 & U-234 \\
\hline
\end{tabular}




\begin{tabular}{|c|c|c|c|c|c|c|c|c|c|c|}
\hline & $\mathrm{A}$ & $B$ & $\mathrm{C}$ & $\mathrm{D}$ & $E$ & $\mathrm{~F}$ & $G$ & $\mathrm{H}$ & $I$ & $\mathrm{~J}$ \\
\hline 1 & SAMPLE NUM ASSIGNED TO & SAMPLER & MEDIA & BIOTA MEDIA & TAG ID & SAMP NUM & SAMP DATE TIME & SAMP DATE TIME ON & CON ID & CON SHORT NAME \\
\hline 534 & PDMS & & SW & & & E123590 & 26-Mar-93 & & 15117-96-1 & U-235 \\
\hline 535 & PDMS & & SW & & & E123590 & 26-Mar-93 & & U-238 & U-238 \\
\hline 536 & PDMS & & SW & & & E123591 & 26-Mar-93 & & $10098-97-2$ & SR-90 \\
\hline 537 & PDMS & & SW & & & E123591 & 26-Mar-93 & & $10028-17-8$ & TRITIUM \\
\hline 538 & PDMS & & SW & & & E123591 & 26-Mar-93 & & 13966-29-5 & U-234 \\
\hline 539 & PDMS & & SW & & & E123591 & 26-Mar-93 & & 15117-96-1 & U-235 \\
\hline 540 & PDMS & & SW & & & E123591 & 26-Mar-93 & & U-238 & $\mathrm{U}-238$ \\
\hline 541 & PDMS & & SW & & & E123592 & 26-Mar-93 & & $10098-97-2$ & SR-90 \\
\hline 542 & PDMS & & SW & & & E123592 & 26-Mar-93 & & $10028-17-8$ & TRITIUM \\
\hline 543 & PDMS & & SW & & & E123592 & 26-Mar-93 & & $13966-29-5$ & U-234 \\
\hline 544 & PDMS & & SW & & & E123592 & 26-Mar-93 & & $15117-96-1$ & U-235 \\
\hline 545 & PDMS & & SW & & & E123592 & 26-Mar-93 & & U-238 & U-238 \\
\hline 546 & PDMS & & SW & & & E123593 & 26-Mar-93 & & $10098-97-2$ & SR-90 \\
\hline 547 & PDMS & & SW & & & E123593 & 26-Mar-93 & & $10028-17-8$ & TRITIUM \\
\hline 548 & PDMS & & SW & & & E123593 & 26-Mar-93 & & $13966-29-5$ & U-234 \\
\hline 549 & PDMS & & SW & & & E123593 & 26-Mar-93 & & 15117-96-1 & U-235 \\
\hline 550 & PDMS & & SW & & & E123593 & 26-Mar-93 & & U-238 & $\mathrm{U}-238$ \\
\hline 551 & PDMS & & SW & & & E123594 & 26-Mar-93 & & 10098-97-2 & SR-90 \\
\hline 552 & PDMS & & SW & & & E123594 & 26-Mar-93 & & $10028-17-8$ & TRITIUM \\
\hline 553 & PDMS & & SW & & & E123594 & 26-Mar-93 & & $13966-29-5$ & U-234 \\
\hline 554 & PDMS & & SW & & & E123594 & 26-Mar-93 & & 15117-96-1 & U-235 \\
\hline 555 & PDMS & & SW & & & E123594 & 26-Mar-93 & & U-238 & U-238 \\
\hline 556 & PDMS & & SW & & & E123595 & 26-Mar-93 & & $10098-97-2$ & SR-90 \\
\hline 557 & PDMS & & SW & & & E123595 & 26-Mar-93 & & $10028-17-8$ & TRITIUM \\
\hline 558 & PDMS & & SW & & & E123595 & 26-Mar-93 & & 13966-29-5 & U-234 \\
\hline 559 & PDMS & & SW & & & E123595 & 26-Mar-93 & & $15117-96-1$ & U-235 \\
\hline 560 & PDMS & & SW & & & E123595 & 26-Mar-93 & & U-238 & U-238 \\
\hline 561 & PDMS & & SW & & & E123596 & 26-Mar-93 & & $10098-97-2$ & SR-90 \\
\hline 562 & PDMS & & SW & & & E123596 & 26-Mar-93 & & $10028-17-8$ & TRITIUM \\
\hline 563 & PDMS & & SW & & & E123596 & 26-Mar-93 & & $13966-29-5$ & U-234 \\
\hline 564 & PDMS & & SW & & & E123596 & 26-Mar-93 & & 15117-96-1 & U-235 \\
\hline 565 & PDMS & & SW & & & E123596 & 26-Mar-93 & & U-238 & U-238 \\
\hline 566 & PDMS & & SW & & & E124100 & 01-Jul-93 & & $10098-97-2$ & SR-90 \\
\hline 567 & PDMS & & SW & & & E124100 & 01-Jul-93 & & $10028-17-8$ & TRITIUM \\
\hline 568 & PDMS & & SW & & & E124100 & 01-Jul-93 & & $13966-29-5$ & U-234 \\
\hline 569 & PDMS & & SW & & & E124100 & 01-Jul-93 & & $15117-96-1$ & U-235 \\
\hline 570 & PDMS & & SW & & & E124100 & 01-Jul-93 & & U-238 & U-238 \\
\hline 571 & PDMS & & SW & & & E124101 & 01-Jul-93 & & $10098-97-2$ & SR-90 \\
\hline
\end{tabular}




\begin{tabular}{|c|c|c|c|c|c|c|c|c|c|c|}
\hline & $\mathrm{A}$ & $\mathrm{B}$ & $\mathrm{C}$ & $\mathrm{D}$ & $E$ & $\mathrm{~F}$ & $G$ & $\mathrm{H}$ & $\mathrm{I}$ & $\mathrm{J}$ \\
\hline 1 & SAMPLE_NUM_ASSIGNED_TO & SAMPLER & MEDIA & BIOTA_MEDIA & TAG_ID & SAMP_NUM & SAMP_DATE_TIME & SAMP_DATE_TIME_ON & CON_ID & CON_SHORT_NAME \\
\hline 572 & PDMS & & SW & & & E124101 & 01-Jul-93 & & $10028-17-8$ & TRITIUM \\
\hline 573 & PDMS & & SW & & & E124101 & 01-Jul-93 & & $13966-29-5$ & U-234 \\
\hline 574 & PDMS & & SW & & & E124101 & 01-Jul-93 & & 15117-96-1 & U-235 \\
\hline 575 & PDMS & & SW & & & E124101 & 01-Jul-93 & & U-238 & U-238 \\
\hline 576 & PDMS & & SW & & & E124102 & 01-Jul-93 & & $10098-97-2$ & SR-90 \\
\hline 577 & PDMS & & SW & & & E124102 & 01-Jul-93 & & $10028-17-8$ & TRITIUM \\
\hline 578 & PDMS & & SW & & & E124102 & 01-Jul-93 & & $13966-29-5$ & $\mathrm{U}-234$ \\
\hline 579 & PDMS & & SW & & & E124102 & 01-Jul-93 & & 15117-96-1 & U-235 \\
\hline 580 & PDMS & & SW & & & E124102 & 01-Jul-93 & & U-238 & U-238 \\
\hline 581 & PDMS & & SW & & & E124103 & 01-Jul-93 & & $10098-97-2$ & SR-90 \\
\hline 582 & PDMS & & SW & & & E124103 & 01-Jul-93 & & $10028-17-8$ & TRITIUM \\
\hline 583 & PDMS & & SW & & & E124103 & 01-Jul-93 & & 13966-29-5 & U-234 \\
\hline 584 & PDMS & & SW & & & E124103 & 01-Jul-93 & & $15117-96-1$ & U-235 \\
\hline 585 & PDMS & & SW & & & E124103 & 01-Jul-93 & & U-238 & U-238 \\
\hline 586 & PDMS & & SW & & & E124158 & 02-Jul-93 & & $10098-97-2$ & SR-90 \\
\hline 587 & PDMS & & SW & & & E124158 & 02-Jul-93 & & 10028-17-8 & TRITIUM \\
\hline 588 & PDMS & & SW & & & E124158 & 02-Jul-93 & & 13966-29-5 & U-234 \\
\hline 589 & PDMS & & SW & & & E124158 & 02-Jul-93 & & 15117-96-1 & U-235 \\
\hline 590 & PDMS & & SW & & & E124158 & 02-Jul-93 & & U-238 & U-238 \\
\hline 591 & PDMS & & SW & & & E124167 & 02-Jul-93 & & $10098-97-2$ & SR-90 \\
\hline 592 & PDMS & & SW & & & E124167 & 02-Jul-93 & & $10028-17-8$ & TRITIUM \\
\hline 593 & PDMS & & SW & & & E124167 & 02-Jul-93 & & 13966-29-5 & U-234 \\
\hline 594 & PDMS & & SW & & & E124167 & 02-Jul-93 & & $15117-96-1$ & U-235 \\
\hline 595 & PDMS & & SW & & & E124167 & 02-Jul-93 & & U-238 & U-238 \\
\hline 596 & PDMS & & SW & & & E124159 & 02-Jul-93 & & $10098-97-2$ & SR-90 \\
\hline 597 & PDMS & & SW & & & E124159 & 02-Jul-93 & & $10028-17-8$ & TRITIUM \\
\hline 598 & PDMS & & SW & & & E124159 & 02-Jul-93 & & 13966-29-5 & U-234 \\
\hline 599 & PDMS & & SW & & & E124159 & 02-Jul-93 & & $15117-96-1$ & U-235 \\
\hline 600 & PDMS & & SW & & & E124159 & 02-Jul-93 & & U-238 & U-238 \\
\hline 601 & PDMS & & SW & & & E124160 & 02-Jul-93 & & $10098-97-2$ & SR-90 \\
\hline 602 & PDMS & & SW & & & E124160 & 02-Jul-93 & & $10028-17-8$ & TRITIUM \\
\hline 603 & PDMS & & SW & & & E124160 & 02-Jul-93 & & 13966-29-5 & U-234 \\
\hline 604 & PDMS & & SW & & & E124160 & 02-Jul-93 & & $15117-96-1$ & U-235 \\
\hline 605 & PDMS & & SW & & & E124160 & 02-Jul-93 & & U-238 & U-238 \\
\hline 606 & PDMS & & SW & & & E124161 & 02-Jul-93 & & $10098-97-2$ & SR-90 \\
\hline 607 & PDMS & & SW & & & E124161 & 02-Jul-93 & & $10028-17-8$ & TRITIUM \\
\hline 608 & PDMS & & SW & & & E124161 & 02-Jul-93 & & 13966-29-5 & U-234 \\
\hline 609 & PDMS & & SW & & & E124161 & 02-Jul-93 & & $15117-96-1$ & U-235 \\
\hline
\end{tabular}




\begin{tabular}{|c|c|c|c|c|c|c|c|c|c|c|}
\hline & $\mathrm{A}$ & $B$ & $\mathrm{C}$ & $\mathrm{D}$ & $E$ & $\mathrm{~F}$ & $G$ & $\mathrm{H}$ & $\mathrm{I}$ & $\mathrm{J}$ \\
\hline 1 & SAMPLE_NUM_ASSIGNED_TO & SAMPLER & MEDIA & BIOTA_MEDIA & TAG_ID & SAMP_NUM & SAMP_DATE_TIME| & SAMP_DATE_TIME_ON & CON_ID & CON_SHORT_NAME \\
\hline 610 & PDMS & & SW & & & E124161 & 02-Jul-93 & & $\mathrm{U}-238$ & U-238 \\
\hline 611 & PDMS & & SW & & & E124162 & 02-Jul-93 & & 10098-97-2 & SR-90 \\
\hline 612 & PDMS & & SW & & & E124162 & 02-Jul-93 & & 10028-17-8 & TRITIUM \\
\hline 613 & PDMS & & SW & & & E124162 & 02-Jul-93 & & 13966-29-5 & U-234 \\
\hline 614 & PDMS & & SW & & & E124162 & 02-Jul-93 & & 15117-96-1 & U-235 \\
\hline 615 & PDMS & & SW & & & E124162 & 02-Jul-93 & & U-238 & $\mathrm{U}-238$ \\
\hline 616 & PDMS & & SW & & & E124163 & 02-Jul-93 & & $10098-97-2$ & SR-90 \\
\hline 617 & PDMS & & SW & & & E124163 & 02-Jul-93 & & $10028-17-8$ & TRITIUM \\
\hline 618 & PDMS & & SW & & & E124163 & 02-Jul-93 & & 13966-29-5 & U-234 \\
\hline 619 & PDMS & & SW & & & E124163 & 02-Jul-93 & & 15117-96-1 & U-235 \\
\hline 620 & PDMS & & SW & & & E124163 & 02-Jul-93 & & U-238 & U-238 \\
\hline 621 & PDMS & & SW & & & E124164 & 02-Jul-93 & & $10098-97-2$ & SR-90 \\
\hline 622 & PDMS & & SW & & & E124164 & 02-Jul-93 & & 10028-17-8 & TRITIUM \\
\hline 623 & PDMS & & SW & & & E124164 & 02-Jul-93 & & 13966-29-5 & U-234 \\
\hline 624 & PDMS & & SW & & & E124164 & 02-Jul-93 & & 15117-96-1 & U-235 \\
\hline 625 & PDMS & & SW & & & E124164 & 02-Jul-93 & & U-238 & U-238 \\
\hline 626 & PDMS & & SW & & & E124165 & 02-Jul-93 & & $10098-97-2$ & SR-90 \\
\hline 627 & PDMS & & SW & & & E124165 & 02-Jul-93 & & $10028-17-8$ & TRITIUM \\
\hline 628 & PDMS & & SW & & & E124165 & 02-Jul-93 & & 13966-29-5 & U-234 \\
\hline 629 & PDMS & & SW & & & E124165 & 02-Jul-93 & & 15117-96-1 & U-235 \\
\hline 630 & PDMS & & SW & & & E124165 & 02-Jul-93 & & U-238 & U-238 \\
\hline 631 & PDMS & & SW & & & E124166 & 02-Jul-93 & & $10098-97-2$ & SR-90 \\
\hline 632 & PDMS & & SW & & & E124166 & 02-Jul-93 & & $10028-17-8$ & TRITIUM \\
\hline 633 & PDMS & & SW & & & E124166 & 02-Jul-93 & & 13966-29-5 & U-234 \\
\hline 634 & PDMS & & SW & & & E124166 & 02-Jul-93 & & 15117-96-1 & U-235 \\
\hline 635 & PDMS & & SW & & & E124166 & 02-Jul-93 & & U-238 & U-238 \\
\hline 636 & PDMS & & SW & & & E124774 & 16-Sep-93 & & $10098-97-2$ & SR-90 \\
\hline 637 & PDMS & & SW & & & E124774 & 16-Sep-93 & & 14133-76-7 & TC-99 \\
\hline 638 & PDMS & & SW & & & E124774 & 16-Sep-93 & & $10028-17-8$ & TRITIUM \\
\hline 639 & PDMS & & SW & & & E124774 & 16-Sep-93 & & 13966-29-5 & U-234 \\
\hline 640 & PDMS & & SW & & & E124774 & 16-Sep-93 & & 15117-96-1 & U-235 \\
\hline 641 & PDMS & & SW & & & E124774 & 16-Sep-93 & & U-238 & U-238 \\
\hline 642 & PDMS & & SW & & & E124775 & 16-Sep-93 & & $10098-97-2$ & SR-90 \\
\hline 643 & PDMS & & SW & & & E124775 & 16-Sep-93 & & 14133-76-7 & TC-99 \\
\hline 644 & PDMS & & SW & & & E124775 & 16-Sep-93 & & $10028-17-8$ & TRITIUM \\
\hline 645 & PDMS & & SW & & & E124775 & 16-Sep-93 & & 13966-29-5 & U-234 \\
\hline 646 & PDMS & & SW & & & E124775 & 16-Sep-93 & & 15117-96-1 & U-235 \\
\hline 647 & PDMS & & SW & & & E124775 & 16-Sep-93 & & U-238 & U-238 \\
\hline
\end{tabular}




\begin{tabular}{|c|c|c|c|c|c|c|c|c|c|c|}
\hline & $\mathrm{A}$ & $B$ & $\mathrm{C}$ & $\mathrm{D}$ & $E$ & $\mathrm{~F}$ & $G$ & $\mathrm{H}$ & $I$ & $\mathrm{~J}$ \\
\hline 1 & SAMPLE NUM ASSIGNED TO & SAMPLER & MEDIA & BIOTA MEDIA & TAG ID & SAMP NUM & SAMP DATE TIME & SAMP DATE TIME ON & CON ID & CON SHORT NAME \\
\hline 648 & PDMS & & SW & & & E124776 & 16-Sep-93 & & $10098-97-2$ & SR-90 \\
\hline 649 & PDMS & & SW & & & E124776 & 16-Sep-93 & & 14133-76-7 & TC-99 \\
\hline 650 & PDMS & & SW & & & E124776 & 16-Sep-93 & & $10028-17-8$ & TRITIUM \\
\hline 651 & PDMS & & SW & & & E124776 & 16-Sep-93 & & $13966-29-5$ & U-234 \\
\hline 652 & PDMS & & SW & & & E124776 & 16-Sep-93 & & 15117-96-1 & U-235 \\
\hline 653 & PDMS & & SW & & & E124776 & 16-Sep-93 & & U-238 & U-238 \\
\hline 654 & PDMS & & SW & & & E124777 & 16-Sep-93 & & $10098-97-2$ & SR-90 \\
\hline 655 & PDMS & & SW & & & E124777 & 16-Sep-93 & & 14133-76-7 & TC-99 \\
\hline 656 & PDMS & & SW & & & E124777 & 16-Sep-93 & & $10028-17-8$ & TRITIUM \\
\hline 657 & PDMS & & SW & & & E124777 & 16-Sep-93 & & $13966-29-5$ & U-234 \\
\hline 658 & PDMS & & SW & & & E124777 & 16-Sep-93 & & 15117-96-1 & U-235 \\
\hline 659 & PDMS & & SW & & & E124777 & 16-Sep-93 & & U-238 & U-238 \\
\hline 660 & PDMS & & SW & & & E124683 & 17-Sep-93 & & $10098-97-2$ & SR-90 \\
\hline 661 & PDMS & & SW & & & E124683 & 17-Sep-93 & & $10028-17-8$ & TRITIUM \\
\hline 662 & PDMS & & SW & & & E124683 & 17-Sep-93 & & $13966-29-5$ & U-234 \\
\hline 663 & PDMS & & SW & & & E124683 & 17-Sep-93 & & $15117-96-1$ & U-235 \\
\hline 664 & PDMS & & SW & & & E124683 & 17-Sep-93 & & U-238 & $\mathrm{U}-238$ \\
\hline 665 & PDMS & & SW & & & E124684 & 17-Sep-93 & & 10098-97-2 & SR-90 \\
\hline 666 & PDMS & & SW & & & E124684 & 17-Sep-93 & & $10028-17-8$ & TRITIUM \\
\hline 667 & PDMS & & SW & & & E124684 & 17-Sep-93 & & $13966-29-5$ & U-234 \\
\hline 668 & PDMS & & SW & & & E124684 & 17-Sep-93 & & 15117-96-1 & U-235 \\
\hline 669 & PDMS & & SW & & & E124684 & 17-Sep-93 & & U-238 & U-238 \\
\hline 670 & PDMS & & SW & & & E124685 & 17-Sep-93 & & $10098-97-2$ & SR-90 \\
\hline 671 & PDMS & & SW & & & E124685 & 17-Sep-93 & & $10028-17-8$ & TRITIUM \\
\hline 672 & PDMS & & SW & & & E124685 & 17-Sep-93 & & 13966-29-5 & U-234 \\
\hline 673 & PDMS & & SW & & & E124685 & 17-Sep-93 & & $15117-96-1$ & U-235 \\
\hline 674 & PDMS & & SW & & & E124685 & 17-Sep-93 & & U-238 & U-238 \\
\hline 675 & PDMS & & SW & & & E124686 & 17-Sep-93 & & $10098-97-2$ & SR-90 \\
\hline 676 & PDMS & & SW & & & E124686 & 17-Sep-93 & & $10028-17-8$ & TRITIUM \\
\hline 677 & PDMS & & SW & & & E124686 & 17-Sep-93 & & 13966-29-5 & U-234 \\
\hline 678 & PDMS & & SW & & & E124686 & 17-Sep-93 & & 15117-96-1 & U-235 \\
\hline 679 & PDMS & & SW & & & E124686 & 17-Sep-93 & & U-238 & U-238 \\
\hline 680 & PDMS & & SW & & & E124687 & 17-Sep-93 & & $10098-97-2$ & SR-90 \\
\hline 681 & PDMS & & SW & & & E124687 & 17-Sep-93 & & $10028-17-8$ & TRITIUM \\
\hline 682 & PDMS & & SW & & & E124687 & 17-Sep-93 & & $13966-29-5$ & U-234 \\
\hline 683 & PDMS & & SW & & & E124687 & 17-Sep-93 & & $15117-96-1$ & U-235 \\
\hline 684 & PDMS & & SW & & & E124687 & 17-Sep-93 & & U-238 & U-238 \\
\hline 685 & PDMS & & SW & & & E124688 & 17-Sep-93 & & $10098-97-2$ & SR-90 \\
\hline
\end{tabular}




\begin{tabular}{|c|c|c|c|c|c|c|c|c|c|c|}
\hline & $\mathrm{A}$ & $\mathrm{B}$ & $\mathrm{C}$ & $\mathrm{D}$ & $\mathrm{E}$ & $\mathrm{F}$ & G & $\mathrm{H}$ & $\mathrm{I}$ & $\mathrm{J}$ \\
\hline 1 & SAMPLE_NUM_ASSIGNED_TO & SAMPLER & MEDIA & BIOTA_MEDIA & TAG_ID & SAMP_NUM & SAMP_DATE_TIME & SAMP_DATE_TIME_ON & CON_ID & CON_SHORT_NAME \\
\hline 686 & PDMS & & SW & & & E124688 & 17-Sep-93 & & $10028-17-8$ & TRITIUM \\
\hline 687 & PDMS & & SW & & & E124688 & 17-Sep-93 & & $13966-29-5$ & U-234 \\
\hline 688 & PDMS & & SW & & & E124688 & 17-Sep-93 & & 15117-96-1 & U-235 \\
\hline 689 & PDMS & & SW & & & E124688 & 17-Sep-93 & & U-238 & $\mathrm{U}-238$ \\
\hline 690 & PDMS & & SW & & & E124689 & 17-Sep-93 & & 10098-97-2 & SR-90 \\
\hline 691 & PDMS & & SW & & & E124689 & 17-Sep-93 & & $10028-17-8$ & TRITIUM \\
\hline 692 & PDMS & & SW & & & E124689 & 17-Sep-93 & & 13966-29-5 & U-234 \\
\hline 693 & PDMS & & SW & & & E124689 & 17-Sep-93 & & 15117-96-1 & U-235 \\
\hline 694 & PDMS & & SW & & & E124689 & 17-Sep-93 & & U-238 & U-238 \\
\hline 695 & PDMS & & SW & & & E124690 & 17-Sep-93 & & $10098-97-2$ & SR-90 \\
\hline 696 & PDMS & & SW & & & E124690 & 17-Sep-93 & & $10028-17-8$ & TRITIUM \\
\hline 697 & PDMS & & SW & & & E124690 & 17-Sep-93 & & $13966-29-5$ & U-234 \\
\hline 698 & PDMS & & SW & & & E124690 & 17-Sep-93 & & 15117-96-1 & U-235 \\
\hline 699 & PDMS & & SW & & & E124690 & 17-Sep-93 & & U-238 & U-238 \\
\hline 700 & PDMS & & SW & & & E124691 & 17-Sep-93 & & $10098-97-2$ & SR-90 \\
\hline 701 & PDMS & & SW & & & E124691 & 17-Sep-93 & & $10028-17-8$ & TRITIUM \\
\hline 702 & PDMS & & SW & & & E124691 & 17-Sep-93 & & 13966-29-5 & U-234 \\
\hline 703 & PDMS & & SW & & & E124691 & 17-Sep-93 & & 15117-96-1 & U-235 \\
\hline 704 & PDMS & & SW & & & E124691 & 17-Sep-93 & & U-238 & U-238 \\
\hline 705 & PDMS & & SW & & & E124692 & 17-Sep-93 & & $10098-97-2$ & SR-90 \\
\hline 706 & PDMS & & SW & & & E124692 & 17-Sep-93 & & $10028-17-8$ & TRITIUM \\
\hline 707 & PDMS & & SW & & & E124692 & 17-Sep-93 & & 13966-29-5 & U-234 \\
\hline 708 & PDMS & & SW & & & E124692 & 17-Sep-93 & & $15117-96-1$ & U-235 \\
\hline 709 & PDMS & & SW & & & E124692 & 17-Sep-93 & & U-238 & U-238 \\
\hline 710 & PDMS & & SW & & & E124693 & 27-Sep-93 & & $10045-97-3$ & CS-137 \\
\hline 711 & PDMS & & SW & & & E124693 & 27-Sep-93 & & $10098-97-2$ & SR-90 \\
\hline 712 & PDMS & & SW & & & E124693 & 27-Sep-93 & & 14133-76-7 & TC-99 \\
\hline 713 & PDMS & & SW & & & E124693 & 27-Sep-93 & & $10028-17-8$ & TRITIUM \\
\hline 714 & PDMS & & SW & & & E124693 & 27-Sep-93 & & 13966-29-5 & U-234 \\
\hline 715 & PDMS & & SW & & & E124693 & 27-Sep-93 & & 15117-96-1 & U-235 \\
\hline 716 & PDMS & & SW & & & E124693 & 27-Sep-93 & & U-238 & U-238 \\
\hline 717 & PDMS & & SW & & & E124950 & 27-Sep-93 & & $10098-97-2$ & SR-90 \\
\hline 718 & PDMS & & SW & & & E124950 & 27-Sep-93 & & $10028-17-8$ & TRITIUM \\
\hline 719 & PDMS & & SW & & & E124950 & 27-Sep-93 & & 13966-29-5 & U-234 \\
\hline 720 & PDMS & & SW & & & E124950 & 27-Sep-93 & & 15117-96-1 & U-235 \\
\hline 721 & PDMS & & SW & & & E124950 & 27-Sep-93 & & U-238 & U-238 \\
\hline 722 & PDMS & & SW & & & E124959 & 27-Sep-93 & & $10098-97-2$ & SR-90 \\
\hline 723 & PDMS & & SW & & & E124959 & 27-Sep-93 & & $10028-17-8$ & TRITIUM \\
\hline
\end{tabular}




\begin{tabular}{|c|c|c|c|c|c|c|c|c|c|c|}
\hline & $\mathrm{A}$ & $B$ & $\mathrm{C}$ & $\mathrm{D}$ & $E$ & $\mathrm{~F}$ & $G$ & $\mathrm{H}$ & $\mathrm{I}$ & $\mathrm{J}$ \\
\hline 1 & SAMPLE_NUM_ASSIGNED_TO & SAMPLER & MEDIA & BIOTA_MEDIA & TAG_ID & SAMP_NUM & SAMP_DATE_TIME| & SAMP_DATE_TIME_ON & CON_ID & CON_SHORT_NAME \\
\hline 724 & PDMS & & SW & & & E124959 & 27-Sep-93 & & 13966-29-5 & U-234 \\
\hline 725 & PDMS & & SW & & & E124959 & 27-Sep-93 & & 15117-96-1 & U-235 \\
\hline 726 & PDMS & & SW & & & E124959 & 27-Sep-93 & & U-238 & U-238 \\
\hline 727 & PDMS & & SW & & & E124694 & 27-Sep-93 & & $10045-97-3$ & CS-137 \\
\hline 728 & PDMS & & SW & & & E124694 & 27-Sep-93 & & $10098-97-2$ & SR-90 \\
\hline 729 & PDMS & & SW & & & E124694 & 27-Sep-93 & & 14133-76-7 & TC-99 \\
\hline 730 & PDMS & & SW & & & E124694 & 27-Sep-93 & & $10028-17-8$ & TRITIUM \\
\hline 731 & PDMS & & SW & & & E124694 & 27-Sep-93 & & 13966-29-5 & U-234 \\
\hline 732 & PDMS & & SW & & & E124694 & 27-Sep-93 & & 15117-96-1 & U-235 \\
\hline 733 & PDMS & & SW & & & E124694 & 27-Sep-93 & & U-238 & U-238 \\
\hline 734 & PDMS & & SW & & & E124951 & 27-Sep-93 & & $10098-97-2$ & SR-90 \\
\hline 735 & PDMS & & SW & & & E124951 & 27-Sep-93 & & $10028-17-8$ & TRITIUM \\
\hline 736 & PDMS & & SW & & & E124951 & 27-Sep-93 & & 13966-29-5 & U-234 \\
\hline 737 & PDMS & & SW & & & E124951 & 27-Sep-93 & & 15117-96-1 & U-235 \\
\hline 738 & PDMS & & SW & & & E124951 & 27-Sep-93 & & U-238 & U-238 \\
\hline 739 & PDMS & & SW & & & E124952 & 27-Sep-93 & & $10098-97-2$ & SR-90 \\
\hline 740 & PDMS & & SW & & & E124952 & 27-Sep-93 & & $10028-17-8$ & TRITIUM \\
\hline 741 & PDMS & & SW & & & E124952 & 27-Sep-93 & & 13966-29-5 & U-234 \\
\hline 742 & PDMS & & SW & & & E124952 & 27-Sep-93 & & 15117-96-1 & U-235 \\
\hline 743 & PDMS & & SW & & & E124952 & 27-Sep-93 & & U-238 & U-238 \\
\hline 744 & PDMS & & SW & & & E124953 & 27-Sep-93 & & $10098-97-2$ & SR-90 \\
\hline 745 & PDMS & & SW & & & E124953 & 27-Sep-93 & & $10028-17-8$ & TRITIUM \\
\hline 746 & PDMS & & SW & & & E124953 & 27-Sep-93 & & 13966-29-5 & U-234 \\
\hline 747 & PDMS & & SW & & & E124953 & 27-Sep-93 & & 15117-96-1 & U-235 \\
\hline 748 & PDMS & & SW & & & E124953 & 27-Sep-93 & & U-238 & U-238 \\
\hline 749 & PDMS & & SW & & & E124954 & 27-Sep-93 & & $10098-97-2$ & SR-90 \\
\hline 750 & PDMS & & SW & & & E124954 & 27-Sep-93 & & $10028-17-8$ & TRITIUM \\
\hline 751 & PDMS & & SW & & & E124954 & 27-Sep-93 & & $13966-29-5$ & U-234 \\
\hline 752 & PDMS & & SW & & & E124954 & 27-Sep-93 & & 15117-96-1 & U-235 \\
\hline 753 & PDMS & & SW & & & E124954 & 27-Sep-93 & & U-238 & U-238 \\
\hline 754 & PDMS & & SW & & & E124955 & 27-Sep-93 & & $10098-97-2$ & SR-90 \\
\hline 755 & PDMS & & SW & & & E124955 & 27-Sep-93 & & $10028-17-8$ & TRITIUM \\
\hline 756 & PDMS & & SW & & & E124955 & 27-Sep-93 & & 13966-29-5 & U-234 \\
\hline 757 & PDMS & & SW & & & E124955 & 27-Sep-93 & & 15117-96-1 & U-235 \\
\hline 758 & PDMS & & SW & & & E124955 & 27-Sep-93 & & U-238 & U-238 \\
\hline 759 & PDMS & & SW & & & E124956 & 27-Sep-93 & & $10098-97-2$ & SR-90 \\
\hline 760 & PDMS & & SW & & & E124956 & 27-Sep-93 & & $10028-17-8$ & TRITIUM \\
\hline 761 & PDMS & & SW & & & E124956 & 27-Sep-93 & & 13966-29-5 & U-234 \\
\hline
\end{tabular}

Page 20 


\begin{tabular}{|c|c|c|c|c|c|c|c|c|c|c|}
\hline & $\mathrm{A}$ & $B$ & $\mathrm{C}$ & $\mathrm{D}$ & $E$ & $\mathrm{~F}$ & $G$ & $\mathrm{H}$ & $\mathrm{I}$ & $\mathrm{J}$ \\
\hline 1 & SAMPLE_NUM_ASSIGNED_TO & SAMPLER & MEDIA & BIOTA_MEDIA & TAG_ID & SAMP_NUM & SAMP_DATE_TIME| & SAMP_DATE_TIME_ON & CON_ID & CON_SHORT_NAME \\
\hline 762 & PDMS & & SW & & & E124956 & 27-Sep-93 & & 15117-96-1 & U-235 \\
\hline 763 & PDMS & & SW & & & E124956 & 27-Sep-93 & & U-238 & U-238 \\
\hline 764 & PDMS & & SW & & & E124957 & 27-Sep-93 & & $10098-97-2$ & SR-90 \\
\hline 765 & PDMS & & SW & & & E124957 & 27-Sep-93 & & $10028-17-8$ & TRITIUM \\
\hline 766 & PDMS & & SW & & & E124957 & 27-Sep-93 & & 13966-29-5 & U-234 \\
\hline 767 & PDMS & & SW & & & E124957 & 27-Sep-93 & & 15117-96-1 & U-235 \\
\hline 768 & PDMS & & SW & & & E124957 & 27-Sep-93 & & U-238 & U-238 \\
\hline 769 & PDMS & & SW & & & E124958 & 27-Sep-93 & & $10098-97-2$ & SR-90 \\
\hline 770 & PDMS & & SW & & & E124958 & 27-Sep-93 & & $10028-17-8$ & TRITIUM \\
\hline 771 & PDMS & & SW & & & E124958 & 27-Sep-93 & & $13966-29-5$ & U-234 \\
\hline 772 & PDMS & & SW & & & E124958 & 27-Sep-93 & & 15117-96-1 & U-235 \\
\hline 773 & PDMS & & SW & & & E124958 & 27-Sep-93 & & U-238 & U-238 \\
\hline 774 & PDMS & & SW & & & E124695 & 27-Sep-93 & & $10045-97-3$ & CS-137 \\
\hline 775 & PDMS & & SW & & & E124695 & 27-Sep-93 & & 14133-76-7 & TC-99 \\
\hline 776 & PDMS & & SW & & & E124695 & 27-Sep-93 & & $10028-17-8$ & TRITIUM \\
\hline 777 & PDMS & & SW & & & E124695 & 27-Sep-93 & & $13966-29-5$ & U-234 \\
\hline 778 & PDMS & & SW & & & E124695 & 27-Sep-93 & & 15117-96-1 & U-235 \\
\hline 779 & PDMS & & SW & & & E124695 & 27-Sep-93 & & U-238 & U-238 \\
\hline 780 & PDMS & & SW & & & E124582 & 27-Sep-93 & & $10045-97-3$ & CS-137 \\
\hline 781 & PDMS & & SW & & & E124582 & 27-Sep-93 & & $14133-76-7$ & TC-99 \\
\hline 782 & PDMS & & SW & & & E124582 & 27-Sep-93 & & $10028-17-8$ & TRITIUM \\
\hline 783 & PDMS & & SW & & & E124582 & 27-Sep-93 & & 13966-29-5 & U-234 \\
\hline 784 & PDMS & & SW & & & E124582 & 27-Sep-93 & & 15117-96-1 & U-235 \\
\hline 785 & PDMS & & SW & & & E124582 & 27-Sep-93 & & U-238 & U-238 \\
\hline 786 & PDMS & & SW & & & E124696 & 27-Sep-93 & & $10045-97-3$ & CS-137 \\
\hline 787 & PDMS & & SW & & & E124696 & 27-Sep-93 & & 14133-76-7 & TC-99 \\
\hline 788 & PDMS & & SW & & & E124696 & 27-Sep-93 & & 10028-17-8 & TRITIUM \\
\hline 789 & PDMS & & SW & & & E124696 & 27-Sep-93 & & $13966-29-5$ & U-234 \\
\hline 790 & PDMS & & SW & & & E124696 & 27-Sep-93 & & 15117-96-1 & U-235 \\
\hline 791 & PDMS & & SW & & & E124696 & 27-Sep-93 & & U-238 & U-238 \\
\hline 792 & PDMS & & SW & & & E124573 & 27-Sep-93 & & $10045-97-3$ & CS-137 \\
\hline 793 & PDMS & & SW & & & E124573 & 27-Sep-93 & & 14133-76-7 & TC-99 \\
\hline 794 & PDMS & & SW & & & E124573 & 27-Sep-93 & & $10028-17-8$ & TRITIUM \\
\hline 795 & PDMS & & SW & & & E124573 & 27-Sep-93 & & 13966-29-5 & U-234 \\
\hline 796 & PDMS & & SW & & & E124573 & 27-Sep-93 & & 15117-96-1 & U-235 \\
\hline 797 & PDMS & & SW & & & E124573 & 27-Sep-93 & & U-238 & U-238 \\
\hline 798 & PDMS & & SW & & & E124575 & 27-Sep-93 & & $10045-97-3$ & CS-137 \\
\hline 799 & PDMS & & SW & & & E124575 & 27-Sep-93 & & 14133-76-7 & TC-99 \\
\hline
\end{tabular}




\begin{tabular}{|c|c|c|c|c|c|c|c|c|c|c|}
\hline & $\mathrm{A}$ & $B$ & $\mathrm{C}$ & $\mathrm{D}$ & $E$ & $\mathrm{~F}$ & $G$ & $\mathrm{H}$ & $\mathrm{I}$ & $\mathrm{J}$ \\
\hline 1 & SAMPLE_NUM_ASSIGNED_TO & SAMPLER & MEDIA & BIOTA_MEDIA & TAG_ID & SAMP_NUM & SAMP_DATE_TIME| & SAMP_DATE_TIME_ON & CON_ID & CON_SHORT_NAME \\
\hline 800 & PDMS & & SW & & & E124575 & 27-Sep-93 & & 10028-17-8 & TRITIUM \\
\hline 801 & PDMS & & SW & & & E124575 & 27-Sep-93 & & $13966-29-5$ & U-234 \\
\hline 802 & PDMS & & SW & & & E124575 & 27-Sep-93 & & 15117-96-1 & U-235 \\
\hline 803 & PDMS & & SW & & & E124575 & 27-Sep-93 & & U-238 & U-238 \\
\hline 804 & PDMS & & SW & & & E124574 & 27-Sep-93 & & $10045-97-3$ & CS-137 \\
\hline 805 & PDMS & & SW & & & E124574 & 27-Sep-93 & & 14133-76-7 & TC-99 \\
\hline 806 & PDMS & & SW & & & E124574 & 27-Sep-93 & & $10028-17-8$ & TRITIUM \\
\hline 807 & PDMS & & SW & & & E124574 & 27-Sep-93 & & 13966-29-5 & U-234 \\
\hline 808 & PDMS & & SW & & & E124574 & 27-Sep-93 & & 15117-96-1 & U-235 \\
\hline 809 & PDMS & & SW & & & E124574 & 27-Sep-93 & & U-238 & U-238 \\
\hline 810 & PDMS & & SW & & & E124576 & 27-Sep-93 & & $10045-97-3$ & CS-137 \\
\hline 811 & PDMS & & SW & & & E124576 & 27-Sep-93 & & 14133-76-7 & TC-99 \\
\hline 812 & PDMS & & SW & & & E124576 & 27-Sep-93 & & 10028-17-8 & TRITIUM \\
\hline 813 & PDMS & & SW & & & E124576 & 27-Sep-93 & & $13966-29-5$ & U-234 \\
\hline 814 & PDMS & & SW & & & E124576 & 27-Sep-93 & & 15117-96-1 & U-235 \\
\hline 815 & PDMS & & SW & & & E124576 & 27-Sep-93 & & U-238 & U-238 \\
\hline 816 & PDMS & & SW & & & E124577 & 27-Sep-93 & & $10045-97-3$ & CS-137 \\
\hline 817 & PDMS & & SW & & & E124577 & 27-Sep-93 & & 14133-76-7 & TC-99 \\
\hline 818 & PDMS & & SW & & & E124577 & 27-Sep-93 & & $10028-17-8$ & TRITIUM \\
\hline 819 & PDMS & & SW & & & E124577 & 27-Sep-93 & & 13966-29-5 & U-234 \\
\hline 820 & PDMS & & SW & & & E124577 & 27-Sep-93 & & 15117-96-1 & U-235 \\
\hline 821 & PDMS & & SW & & & E124577 & 27-Sep-93 & & U-238 & U-238 \\
\hline 822 & PDMS & & SW & & & E124578 & 27-Sep-93 & & $10045-97-3$ & CS-137 \\
\hline 823 & PDMS & & SW & & & E124578 & 27-Sep-93 & & 14133-76-7 & TC-99 \\
\hline 824 & PDMS & & SW & & & E124578 & 27-Sep-93 & & $10028-17-8$ & TRITIUM \\
\hline 825 & PDMS & & SW & & & E124578 & 27-Sep-93 & & $13966-29-5$ & U-234 \\
\hline 826 & PDMS & & SW & & & E124578 & 27-Sep-93 & & 15117-96-1 & U-235 \\
\hline 827 & PDMS & & SW & & & E124578 & 27-Sep-93 & & U-238 & U-238 \\
\hline 828 & PDMS & & SW & & & E124579 & 27-Sep-93 & & $10045-97-3$ & CS-137 \\
\hline 829 & PDMS & & SW & & & E124579 & 27-Sep-93 & & 14133-76-7 & TC-99 \\
\hline 830 & PDMS & & SW & & & E124579 & 27-Sep-93 & & $10028-17-8$ & TRITIUM \\
\hline 831 & PDMS & & SW & & & E124579 & 27-Sep-93 & & 13966-29-5 & U-234 \\
\hline 832 & PDMS & & SW & & & E124579 & 27-Sep-93 & & 15117-96-1 & U-235 \\
\hline 833 & PDMS & & SW & & & E124579 & 27-Sep-93 & & U-238 & U-238 \\
\hline 834 & PDMS & & SW & & & E124580 & 27-Sep-93 & & $10045-97-3$ & CS-137 \\
\hline 835 & PDMS & & SW & & & E124580 & 27-Sep-93 & & 14133-76-7 & TC-99 \\
\hline 836 & PDMS & & SW & & & E124580 & 27-Sep-93 & & $10028-17-8$ & TRITIUM \\
\hline 837 & PDMS & & SW & & & E124580 & 27-Sep-93 & & 13966-29-5 & U-234 \\
\hline
\end{tabular}

Page 22 


\begin{tabular}{|c|c|c|c|c|c|c|c|c|c|c|}
\hline & $\mathrm{A}$ & $B$ & $\mathrm{C}$ & $\mathrm{D}$ & $E$ & $\mathrm{~F}$ & $G$ & $\mathrm{H}$ & $I$ & $\mathrm{~J}$ \\
\hline 1 & SAMPLE NUM ASSIGNED TO & SAMPLER & MEDIA & BIOTA MEDIA & TAG ID & SAMP NUM & SAMP DATE TIME & SAMP DATE TIME ON & CON ID & CON SHORT NAME \\
\hline 838 & PDMS & & SW & & & E124580 & 27-Sep-93 & & $15117-96-1$ & U-235 \\
\hline 839 & PDMS & & SW & & & E124580 & 27-Sep-93 & & U-238 & U-238 \\
\hline 840 & PDMS & & SW & & & E124581 & 27-Sep-93 & & $10045-97-3$ & CS-137 \\
\hline 841 & PDMS & & SW & & & E124581 & 27-Sep-93 & & 14133-76-7 & TC-99 \\
\hline 842 & PDMS & & SW & & & E124581 & 27-Sep-93 & & 10028-17-8 & TRITIUM \\
\hline 843 & PDMS & & SW & & & E124581 & 27-Sep-93 & & $13966-29-5$ & U-234 \\
\hline 844 & PDMS & & SW & & & E124581 & 27-Sep-93 & & 15117-96-1 & U-235 \\
\hline 845 & PDMS & & SW & & & E124581 & 27-Sep-93 & & U-238 & U-238 \\
\hline 846 & PDMS & & SW & & & E124940 & 27-Sep-93 & & $10098-97-2$ & SR-90 \\
\hline 847 & PDMS & & SW & & & E124940 & 27-Sep-93 & & $10028-17-8$ & TRITIUM \\
\hline 848 & PDMS & & SW & & & E124940 & 27-Sep-93 & & 13966-29-5 & U-234 \\
\hline 849 & PDMS & & SW & & & E124940 & 27-Sep-93 & & $15117-96-1$ & U-235 \\
\hline 850 & PDMS & & SW & & & E124940 & 27-Sep-93 & & U-238 & U-238 \\
\hline 851 & PDMS & & SW & & & E124949 & 27-Sep-93 & & $10098-97-2$ & SR-90 \\
\hline 852 & PDMS & & SW & & & E124949 & 27-Sep-93 & & 10028-17-8 & TRITIUM \\
\hline 853 & PDMS & & SW & & & E124949 & 27-Sep-93 & & 13966-29-5 & U-234 \\
\hline 854 & PDMS & & SW & & & E124949 & 27-Sep-93 & & 15117-96-1 & U-235 \\
\hline 855 & PDMS & & SW & & & E124949 & 27-Sep-93 & & U-238 & U-238 \\
\hline 856 & PDMS & & SW & & & E124941 & 27-Sep-93 & & $10098-97-2$ & SR-90 \\
\hline 857 & PDMS & & SW & & & E124941 & 27-Sep-93 & & $10028-17-8$ & TRITIUM \\
\hline 858 & PDMS & & SW & & & E124941 & 27-Sep-93 & & $13966-29-5$ & U-234 \\
\hline 859 & PDMS & & SW & & & E124941 & 27-Sep-93 & & $15117-96-1$ & U-235 \\
\hline 860 & PDMS & & SW & & & E124941 & 27-Sep-93 & & U-238 & U-238 \\
\hline 861 & PDMS & & SW & & & E124942 & 27-Sep-93 & & $10098-97-2$ & SR-90 \\
\hline 862 & PDMS & & SW & & & E124942 & 27-Sep-93 & & $10028-17-8$ & TRITIUM \\
\hline 863 & PDMS & & SW & & & E124942 & 27-Sep-93 & & $13966-29-5$ & U-234 \\
\hline 864 & PDMS & & SW & & & E124942 & 27-Sep-93 & & 15117-96-1 & U-235 \\
\hline 865 & PDMS & & SW & & & E124942 & 27-Sep-93 & & U-238 & U-238 \\
\hline 866 & PDMS & & SW & & & E124943 & 27-Sep-93 & & 10098-97-2 & SR-90 \\
\hline 867 & PDMS & & SW & & & E124943 & 27-Sep-93 & & $10028-17-8$ & TRITIUM \\
\hline 868 & PDMS & & SW & & & E124943 & 27-Sep-93 & & 13966-29-5 & U-234 \\
\hline 869 & PDMS & & SW & & & E124943 & 27-Sep-93 & & $15117-96-1$ & U-235 \\
\hline 870 & PDMS & & SW & & & E124943 & 27-Sep-93 & & $\mathrm{U}-238$ & U-238 \\
\hline 871 & PDMS & & SW & & & E124944 & 27-Sep-93 & & $10098-97-2$ & SR-90 \\
\hline 872 & PDMS & & SW & & & E124944 & 27-Sep-93 & & $10028-17-8$ & TRITIUM \\
\hline 873 & PDMS & & SW & & & E124944 & 27-Sep-93 & & $13966-29-5$ & U-234 \\
\hline 874 & PDMS & & SW & & & E124944 & 27-Sep-93 & & $15117-96-1$ & U-235 \\
\hline 875 & PDMS & & SW & & & E124944 & 27-Sep-93 & & U-238 & U-238 \\
\hline
\end{tabular}

Page 23 


\begin{tabular}{|c|c|c|c|c|c|c|c|c|c|c|}
\hline & $\mathrm{A}$ & $B$ & $\mathrm{C}$ & $\mathrm{D}$ & $E$ & $\mathrm{~F}$ & $G$ & $\mathrm{H}$ & $I$ & $\mathrm{~J}$ \\
\hline 1 & SAMPLE NUM ASSIGNED TO & SAMPLER & MEDIA & BIOTA MEDIA & TAG ID & SAMP NUM & SAMP DATE TIME & SAMP DATE TIME ON & CON ID & CON SHORT NAME \\
\hline 876 & PDMS & & SW & & & E124945 & 27-Sep-93 & & $10098-97-2$ & SR-90 \\
\hline 877 & PDMS & & SW & & & E124945 & 27-Sep-93 & & $10028-17-8$ & TRITIUM \\
\hline 878 & PDMS & & SW & & & E124945 & 27-Sep-93 & & $13966-29-5$ & U-234 \\
\hline 879 & PDMS & & SW & & & E124945 & 27-Sep-93 & & 15117-96-1 & U-235 \\
\hline 880 & PDMS & & SW & & & E124945 & 27-Sep-93 & & U-238 & U-238 \\
\hline 881 & PDMS & & SW & & & E124946 & 27-Sep-93 & & 10098-97-2 & SR-90 \\
\hline 882 & PDMS & & SW & & & E124946 & 27-Sep-93 & & $10028-17-8$ & TRITIUM \\
\hline 883 & PDMS & & SW & & & E124946 & 27-Sep-93 & & 13966-29-5 & U-234 \\
\hline 884 & PDMS & & SW & & & E124946 & 27-Sep-93 & & 15117-96-1 & U-235 \\
\hline 885 & PDMS & & SW & & & E124946 & 27-Sep-93 & & U-238 & U-238 \\
\hline 886 & PDMS & & SW & & & E124947 & 27-Sep-93 & & 10098-97-2 & SR-90 \\
\hline 887 & PDMS & & SW & & & E124947 & 27-Sep-93 & & $10028-17-8$ & TRITIUM \\
\hline 888 & PDMS & & SW & & & E124947 & 27-Sep-93 & & $13966-29-5$ & U-234 \\
\hline 889 & PDMS & & SW & & & E124947 & 27-Sep-93 & & 15117-96-1 & U-235 \\
\hline 890 & PDMS & & SW & & & E124947 & 27-Sep-93 & & U-238 & U-238 \\
\hline 891 & PDMS & & SW & & & E124948 & 27-Sep-93 & & $10098-97-2$ & SR-90 \\
\hline 892 & PDMS & & SW & & & E124948 & 27-Sep-93 & & $10028-17-8$ & TRITIUM \\
\hline 893 & PDMS & & SW & & & E124948 & 27-Sep-93 & & 13966-29-5 & U-234 \\
\hline 894 & PDMS & & SW & & & E124948 & 27-Sep-93 & & $15117-96-1$ & U-235 \\
\hline 895 & PDMS & & SW & & & E124948 & 27-Sep-93 & & U-238 & U-238 \\
\hline 896 & PDMS & & SW & & & E124589 & 30-Sep-93 & & $10045-97-3$ & CS-137 \\
\hline 897 & PDMS & & SW & & & E124589 & 30-Sep-93 & & $10098-97-2$ & SR-90 \\
\hline 898 & PDMS & & SW & & & E124589 & 30-Sep-93 & & $14133-76-7$ & TC-99 \\
\hline 899 & PDMS & & SW & & & E124589 & 30-Sep-93 & & $10028-17-8$ & TRITIUM \\
\hline 900 & PDMS & & SW & & & E124589 & 30-Sep-93 & & 13966-29-5 & U-234 \\
\hline 901 & PDMS & & SW & & & E124589 & 30-Sep-93 & & $15117-96-1$ & U-235 \\
\hline 902 & PDMS & & SW & & & E124589 & 30-Sep-93 & & U-238 & U-238 \\
\hline 903 & PDMS & & SW & & & E124583 & 30-Sep-93 & & $10045-97-3$ & CS-137 \\
\hline 904 & PDMS & & SW & & & E124583 & 30-Sep-93 & & $10098-97-2$ & SR-90 \\
\hline 905 & PDMS & & SW & & & E124583 & 30-Sep-93 & & $14133-76-7$ & TC-99 \\
\hline 906 & PDMS & & SW & & & E124583 & 30-Sep-93 & & $10028-17-8$ & TRITIUM \\
\hline 907 & PDMS & & SW & & & E124583 & 30-Sep-93 & & $13966-29-5$ & U-234 \\
\hline 908 & PDMS & & SW & & & E124583 & 30-Sep-93 & & $15117-96-1$ & U-235 \\
\hline 909 & PDMS & & SW & & & E124583 & 30-Sep-93 & & U-238 & U-238 \\
\hline 910 & PDMS & & SW & & & E124584 & 30-Sep-93 & & $10045-97-3$ & CS-137 \\
\hline 911 & PDMS & & SW & & & E124584 & 30-Sep-93 & & $10098-97-2$ & SR-90 \\
\hline 912 & PDMS & & SW & & & E124584 & 30-Sep-93 & & $14133-76-7$ & TC-99 \\
\hline 913 & PDMS & & SW & & & E124584 & 30-Sep-93 & & $10028-17-8$ & TRITIUM \\
\hline
\end{tabular}

Page 24 


\begin{tabular}{|c|c|c|c|c|c|c|c|c|c|c|}
\hline & $\mathrm{A}$ & $B$ & $\mathrm{C}$ & $\mathrm{D}$ & $E$ & $\mathrm{~F}$ & $G$ & $\mathrm{H}$ & $I$ & $\mathrm{~J}$ \\
\hline 1 & SAMPLE NUM ASSIGNED TO & SAMPLER & MEDIA & BIOTA MEDIA & TAG ID & SAMP NUM & SAMP DATE TIME & SAMP DATE TIME ON & CON ID & CON SHORT NAME \\
\hline 914 & PDMS & & SW & & & E124584 & 30-Sep-93 & & $13966-29-5$ & U-234 \\
\hline 915 & PDMS & & SW & & & E124584 & 30-Sep-93 & & 15117-96-1 & U-235 \\
\hline 916 & PDMS & & SW & & & E124584 & 30-Sep-93 & & U-238 & U-238 \\
\hline 917 & PDMS & & SW & & & E124585 & 30-Sep-93 & & $10045-97-3$ & CS-137 \\
\hline 918 & PDMS & & SW & & & E124585 & 30-Sep-93 & & $10098-97-2$ & SR-90 \\
\hline 919 & PDMS & & SW & & & E124585 & 30-Sep-93 & & $14133-76-7$ & TC-99 \\
\hline 920 & PDMS & & SW & & & E124585 & 30-Sep-93 & & $10028-17-8$ & TRITIUM \\
\hline 921 & PDMS & & SW & & & E124585 & 30-Sep-93 & & $13966-29-5$ & $\mathrm{U}-234$ \\
\hline 922 & PDMS & & SW & & & E124585 & 30-Sep-93 & & $15117-96-1$ & U-235 \\
\hline 923 & PDMS & & SW & & & E124585 & 30-Sep-93 & & U-238 & U-238 \\
\hline 924 & PDMS & & SW & & & E124586 & 30-Sep-93 & & $10045-97-3$ & CS-137 \\
\hline 925 & PDMS & & SW & & & E124586 & 30-Sep-93 & & $10098-97-2$ & SR-90 \\
\hline 926 & PDMS & & SW & & & E124586 & 30-Sep-93 & & 14133-76-7 & TC-99 \\
\hline 927 & PDMS & & SW & & & E124586 & 30-Sep-93 & & $10028-17-8$ & TRITIUM \\
\hline 928 & PDMS & & SW & & & E124586 & 30-Sep-93 & & 13966-29-5 & U-234 \\
\hline 929 & PDMS & & SW & & & E124586 & 30-Sep-93 & & $15117-96-1$ & U-235 \\
\hline 930 & PDMS & & SW & & & E124586 & 30-Sep-93 & & U-238 & U-238 \\
\hline 931 & PDMS & & SW & & & E124587 & 30-Sep-93 & & $10045-97-3$ & CS-137 \\
\hline 932 & PDMS & & SW & & & E124587 & 30-Sep-93 & & $10098-97-2$ & SR-90 \\
\hline 933 & PDMS & & SW & & & E124587 & 30-Sep-93 & & $14133-76-7$ & TC-99 \\
\hline 934 & PDMS & & SW & & & E124587 & 30-Sep-93 & & $10028-17-8$ & TRITIUM \\
\hline 935 & PDMS & & SW & & & E124587 & 30-Sep-93 & & 13966-29-5 & U-234 \\
\hline 936 & PDMS & & SW & & & E124587 & 30-Sep-93 & & $15117-96-1$ & U-235 \\
\hline 937 & PDMS & & SW & & & E124587 & 30-Sep-93 & & $U-238$ & U-238 \\
\hline 938 & PDMS & & SW & & & E124588 & 30-Sep-93 & & $10045-97-3$ & CS-137 \\
\hline 939 & PDMS & & SW & & & E124588 & 30-Sep-93 & & $10098-97-2$ & SR-90 \\
\hline 940 & PDMS & & SW & & & E124588 & 30-Sep-93 & & 14133-76-7 & TC-99 \\
\hline 941 & PDMS & & SW & & & E124588 & 30-Sep-93 & & $10028-17-8$ & TRITIUM \\
\hline 942 & PDMS & & SW & & & E124588 & 30-Sep-93 & & 13966-29-5 & U-234 \\
\hline 943 & PDMS & & SW & & & E124588 & 30-Sep-93 & & 15117-96-1 & U-235 \\
\hline 944 & PDMS & & SW & & & E124588 & 30-Sep-93 & & U-238 & U-238 \\
\hline 945 & PDMS & & SW & & & E124785 & 30-Sep-93 & & $10045-97-3$ & CS-137 \\
\hline 946 & PDMS & & SW & & & E124785 & 30-Sep-93 & & $10098-97-2$ & SR-90 \\
\hline 947 & PDMS & & SW & & & E124785 & 30-Sep-93 & & $14133-76-7$ & TC-99 \\
\hline 948 & PDMS & & SW & & & E124785 & 30-Sep-93 & & $10028-17-8$ & TRITIUM \\
\hline 949 & PDMS & & SW & & & E124785 & 30-Sep-93 & & $13966-29-5$ & U-234 \\
\hline 950 & PDMS & & SW & & & E124785 & 30-Sep-93 & & $15117-96-1$ & U-235 \\
\hline 951 & PDMS & & SW & & & E124785 & 30-Sep-93 & & U-238 & U-238 \\
\hline
\end{tabular}




\begin{tabular}{|c|c|c|c|c|c|c|c|c|c|c|}
\hline & $\mathrm{A}$ & $B$ & $\mathrm{C}$ & $\mathrm{D}$ & $E$ & $\mathrm{~F}$ & $G$ & $\mathrm{H}$ & $\mathrm{I}$ & $\mathrm{J}$ \\
\hline 1 & SAMPLE_NUM_ASSIGNED_TO & SAMPLER & MEDIA & BIOTA_MEDIA & TAG_ID & SAMP_NUM & SAMP_DATE_TIME| & SAMP_DATE_TIME_ON & CON_ID & CON_SHORT_NAME \\
\hline 952 & PDMS & & SW & & & E124783 & 30-Sep-93 & & $10045-97-3$ & CS-137 \\
\hline 953 & PDMS & & SW & & & E124783 & 30-Sep-93 & & 10098-97-2 & SR-90 \\
\hline 954 & PDMS & & SW & & & E124783 & 30-Sep-93 & & 14133-76-7 & TC-99 \\
\hline 955 & PDMS & & SW & & & E124783 & 30-Sep-93 & & 10028-17-8 & TRITIUM \\
\hline 956 & PDMS & & SW & & & E124783 & 30-Sep-93 & & 13966-29-5 & U-234 \\
\hline 957 & PDMS & & SW & & & E124783 & 30-Sep-93 & & 15117-96-1 & U-235 \\
\hline 958 & PDMS & & SW & & & E124783 & 30-Sep-93 & & U-238 & U-238 \\
\hline 959 & PDMS & & SW & & & E124784 & 30-Sep-93 & & $10045-97-3$ & CS-137 \\
\hline 960 & PDMS & & SW & & & E124784 & 30-Sep-93 & & $10098-97-2$ & SR-90 \\
\hline 961 & PDMS & & SW & & & E124784 & 30-Sep-93 & & 14133-76-7 & TC-99 \\
\hline 962 & PDMS & & SW & & & E124784 & 30-Sep-93 & & 10028-17-8 & TRITIUM \\
\hline 963 & PDMS & & SW & & & E124784 & 30-Sep-93 & & 13966-29-5 & U-234 \\
\hline 964 & PDMS & & SW & & & E124784 & 30-Sep-93 & & 15117-96-1 & U-235 \\
\hline 965 & PDMS & & SW & & & E124784 & 30-Sep-93 & & U-238 & U-238 \\
\hline 966 & PDMS & & SW & & & E124874 & 30-Sep-93 & & $10098-97-2$ & SR-90 \\
\hline 967 & PDMS & & SW & & & E124874 & 30-Sep-93 & & $10028-17-8$ & TRITIUM \\
\hline 968 & PDMS & & SW & & & E124874 & 30-Sep-93 & & 13966-29-5 & U-234 \\
\hline 969 & PDMS & & SW & & & E124874 & 30-Sep-93 & & 15117-96-1 & U-235 \\
\hline 970 & PDMS & & SW & & & E124874 & 30-Sep-93 & & U-238 & U-238 \\
\hline 971 & PDMS & & SW & & & E124883 & 30-Sep-93 & & $10098-97-2$ & SR-90 \\
\hline 972 & PDMS & & SW & & & E124883 & 30-Sep-93 & & $10028-17-8$ & TRITIUM \\
\hline 973 & PDMS & & SW & & & E124883 & 30-Sep-93 & & 13966-29-5 & U-234 \\
\hline 974 & PDMS & & SW & & & E124883 & 30-Sep-93 & & 15117-96-1 & U-235 \\
\hline 975 & PDMS & & SW & & & E124883 & 30-Sep-93 & & U-238 & U-238 \\
\hline 976 & PDMS & & SW & & & E124875 & 30-Sep-93 & & $10098-97-2$ & SR-90 \\
\hline 977 & PDMS & & SW & & & E124875 & 30-Sep-93 & & 10028-17-8 & TRITIUM \\
\hline 978 & PDMS & & SW & & & E124875 & 30-Sep-93 & & $13966-29-5$ & U-234 \\
\hline 979 & PDMS & & SW & & & E124875 & 30-Sep-93 & & 15117-96-1 & U-235 \\
\hline 980 & PDMS & & SW & & & E124875 & 30-Sep-93 & & U-238 & U-238 \\
\hline 981 & PDMS & & SW & & & E124876 & 30-Sep-93 & & $10098-97-2$ & SR-90 \\
\hline 982 & PDMS & & SW & & & E124876 & 30-Sep-93 & & $10028-17-8$ & TRITIUM \\
\hline 983 & PDMS & & SW & & & E124876 & 30-Sep-93 & & 13966-29-5 & U-234 \\
\hline 984 & PDMS & & SW & & & E124876 & 30-Sep-93 & & 15117-96-1 & U-235 \\
\hline 985 & PDMS & & SW & & & E124876 & 30-Sep-93 & & U-238 & U-238 \\
\hline 986 & PDMS & & SW & & & E124877 & 30-Sep-93 & & $10098-97-2$ & SR-90 \\
\hline 987 & PDMS & & SW & & & E124877 & 30-Sep-93 & & $10028-17-8$ & TRITIUM \\
\hline 988 & PDMS & & SW & & & E124877 & 30-Sep-93 & & 13966-29-5 & U-234 \\
\hline 989 & PDMS & & SW & & & E124877 & 30-Sep-93 & & 15117-96-1 & U-235 \\
\hline
\end{tabular}




\begin{tabular}{|c|c|c|c|c|c|c|c|c|c|c|}
\hline & A & $\mathrm{B}$ & C & $\mathrm{D}$ & $E$ & $\mathrm{~F}$ & G & $\mathrm{H}$ & $\mathrm{I}$ & $\mathrm{J}$ \\
\hline 1 & SAMPLE_NUM_ASSIGNED_TO & SAMPLER & MEDIA & BIOTA_MEDIA & TAG_ID & SAMP_NUM & SAMP_DATE_TIME & SAMP_DATE_TIME_ON & CON_ID & CON_SHORT_NAME \\
\hline 990 & PDMS & & SW & & & E124877 & 30-Sep-93 & & $\mathrm{U}-238$ & $\mathrm{U}-238$ \\
\hline \begin{tabular}{|l|}
991 \\
\end{tabular} & PDMS & & SW & & & E124878 & 30-Sep-93 & & $10098-97-2$ & SR-90 \\
\hline \begin{tabular}{|l|}
992 \\
\end{tabular} & PDMS & & SW & & & E124878 & 30-Sep-93 & & $10028-17-8$ & TRITIUM \\
\hline \begin{tabular}{|l|}
993 \\
\end{tabular} & PDMS & & SW & & & E124878 & 30-Sep-93 & & 13966-29-5 & U-234 \\
\hline \begin{tabular}{|l|}
994 \\
\end{tabular} & PDMS & & SW & & & E124878 & 30-Sep-93 & & $15117-96-1$ & U-235 \\
\hline 995 & PDMS & & SW & & & E124878 & 30-Sep-93 & & $\mathrm{U}-238$ & U-238 \\
\hline 996 & PDMS & & SW & & & E124879 & 30-Sep-93 & & $10098-97-2$ & SR-90 \\
\hline 997 & PDMS & & SW & & & E124879 & 30-Sep-93 & & $10028-17-8$ & TRITIUM \\
\hline 998 & PDMS & & SW & & & E124879 & 30-Sep-93 & & $13966-29-5$ & U-234 \\
\hline 999 & PDMS & & SW & & & E124879 & 30-Sep-93 & & $15117-96-1$ & U-235 \\
\hline 1000 & PDMS & & SW & & & E124879 & 30-Sep-93 & & $\mathrm{U}-238$ & $\mathrm{U}-238$ \\
\hline 1001 & PDMS & & SW & & & E124880 & 30-Sep-93 & & $10098-97-2$ & SR-90 \\
\hline 1002 & PDMS & & SW & & & E124880 & 30-Sep-93 & & 10028-17-8 & TRITIUM \\
\hline 1003 & PDMS & & SW & & & E124880 & 30-Sep-93 & & $13966-29-5$ & U-234 \\
\hline 1004 & PDMS & & SW & & & E124880 & 30-Sep-93 & & $15117-96-1$ & U-235 \\
\hline \begin{tabular}{|l|}
1005 \\
\end{tabular} & PDMS & & SW & & & E124880 & 30-Sep-93 & & U-238 & U-238 \\
\hline \begin{tabular}{|l|}
1006 \\
\end{tabular} & PDMS & & SW & & & E124881 & 30-Sep-93 & & $10098-97-2$ & SR-90 \\
\hline \begin{tabular}{|l|}
1007 \\
100
\end{tabular} & PDMS & & SW & & & E124881 & 30-Sep-93 & & $10028-17-8$ & TRITIUM \\
\hline \begin{tabular}{|l|}
1008 \\
\end{tabular} & PDMS & & SW & & & E124881 & 30-Sep-93 & & $13966-29-5$ & U-234 \\
\hline 1009 & PDMS & & SW & & & E124881 & 30-Sep-93 & & $15117-96-1$ & $\mathrm{U}-235$ \\
\hline 1010 & PDMS & & SW & & & E124881 & 30-Sep-93 & & U-238 & U-238 \\
\hline \begin{tabular}{|l|}
1011 \\
\end{tabular} & PDMS & & SW & & & E124882 & 30-Sep-93 & & $10098-97-2$ & SR-90 \\
\hline \begin{tabular}{|l|}
1012 \\
\end{tabular} & PDMS & & SW & & & E124882 & 30-Sep-93 & & $10028-17-8$ & TRITIUM \\
\hline 1013 & PDMS & & SW & & & E124882 & 30-Sep-93 & & $13966-29-5$ & U-234 \\
\hline \begin{tabular}{|l|}
1014 \\
\end{tabular} & PDMS & & SW & & & E124882 & 30-Sep-93 & & $15117-96-1$ & U-235 \\
\hline \begin{tabular}{|l|}
1015 \\
\end{tabular} & PDMS & & SW & & & E124882 & 30-Sep-93 & & U-238 & U-238 \\
\hline 1016 & PDMS & & SW & & & E125158 & 22-Nov-93 & & $10098-97-2$ & SR-90 \\
\hline \begin{tabular}{|l|}
1017 \\
\end{tabular} & PDMS & & SW & & & E125158 & 22-Nov-93 & & $10028-17-8$ & TRITIUM \\
\hline \begin{tabular}{|l|}
1018 \\
\end{tabular} & PDMS & & SW & & & E125158 & 22-Nov-93 & & $13966-29-5$ & U-234 \\
\hline \begin{tabular}{|l|}
1019 \\
\end{tabular} & PDMS & & SW & & & E125158 & 22-Nov-93 & & $15117-96-1$ & U-235 \\
\hline \begin{tabular}{|l|}
1020 \\
1020
\end{tabular} & PDMS & & SW & & & E125158 & 22-Nov-93 & & $\mathrm{U}-238$ & $\mathrm{U}-238$ \\
\hline \begin{tabular}{|l|}
1021 \\
\end{tabular} & PDMS & & SW & & & E125167 & 22-Nov-93 & & $10098-97-2$ & SR-90 \\
\hline \begin{tabular}{|l|}
1022 \\
\end{tabular} & PDMS & & SW & & & E125167 & 22-Nov-93 & & $10028-17-8$ & TRITIUM \\
\hline \begin{tabular}{|l|}
1023 \\
\end{tabular} & PDMS & & SW & & & E125167 & 22-Nov-93 & & $13966-29-5$ & U-234 \\
\hline \begin{tabular}{|l|}
1024 \\
\end{tabular} & PDMS & & SW & & & E125167 & 22-Nov-93 & & $15117-96-1$ & U-235 \\
\hline \begin{tabular}{|l|}
1025 \\
\end{tabular} & PDMS & & SW & & & E125167 & 22-Nov-93 & & $\mathrm{U}-238$ & U-238 \\
\hline 1026 & PDMS & & SW & & & E125159 & 22-Nov-93 & & $10098-97-2$ & SR-90 \\
\hline \begin{tabular}{|l|}
1027 \\
\end{tabular} & PDMS & & SW & & & E125159 & 22-Nov-93 & & $10028-17-8$ & TRITIUM \\
\hline
\end{tabular}




\begin{tabular}{|c|c|c|c|c|c|c|c|c|c|c|}
\hline & A & $\mathrm{B}$ & C & $\mathrm{D}$ & $E$ & $\mathrm{~F}$ & G & $\mathrm{H}$ & $\mathrm{I}$ & $\mathrm{J}$ \\
\hline 1 & SAMPLE_NUM_ASSIGNED_TO & SAMPLER & MEDIA & BIOTA_MEDIA & TAG_ID & SAMP_NUM & SAMP_DATE_TIME & SAMP_DATE_TIME_ON & CON_ID & CON_SHORT_NAME \\
\hline 1028 & PDMS & & SW & & & E125159 & 22-Nov-93 & & $13966-29-5$ & $\mathrm{U}-234$ \\
\hline 1029 & PDMS & & SW & & & E125159 & 22-Nov-93 & & $15117-96-1$ & U-235 \\
\hline 1030 & PDMS & & SW & & & E125159 & 22-Nov-93 & & U-238 & U-238 \\
\hline \begin{tabular}{|l|}
1031 \\
\end{tabular} & PDMS & & SW & & & E125160 & 22-Nov-93 & & $10098-97-2$ & SR-90 \\
\hline \begin{tabular}{|l|}
1032 \\
\end{tabular} & PDMS & & SW & & & E125160 & 22-Nov-93 & & $10028-17-8$ & TRITIUM \\
\hline 1033 & PDMS & & SW & & & E125160 & 22-Nov-93 & & $13966-29-5$ & U-234 \\
\hline \begin{tabular}{|l|l|}
1034 \\
\end{tabular} & PDMS & & SW & & & E125160 & 22-Nov-93 & & $15117-96-1$ & U-235 \\
\hline 1035 & PDMS & & SW & & & E125160 & 22-Nov-93 & & $U-238$ & $\mathrm{U}-238$ \\
\hline 1036 & PDMS & & SW & & & E125161 & 22-Nov-93 & & $10098-97-2$ & SR-90 \\
\hline 1037 & PDMS & & SW & & & E125161 & 22-Nov-93 & & $10028-17-8$ & TRITIUM \\
\hline 1038 & PDMS & & SW & & & E125161 & 22-Nov-93 & & 13966-29-5 & U-234 \\
\hline 1039 & PDMS & & SW & & & E125161 & 22-Nov-93 & & $15117-96-1$ & U-235 \\
\hline 1040 & PDMS & & SW & & & E125161 & 22-Nov-93 & & U-238 & U-238 \\
\hline 1041 & PDMS & & SW & & & E125162 & 22-Nov-93 & & $10098-97-2$ & SR-90 \\
\hline 1042 & PDMS & & SW & & & E125162 & 22-Nov-93 & & $10028-17-8$ & TRITIUM \\
\hline \begin{tabular}{|l|}
1043 \\
\end{tabular} & PDMS & & SW & & & E125162 & 22-Nov-93 & & $13966-29-5$ & U-234 \\
\hline \begin{tabular}{|l|}
1044 \\
\end{tabular} & PDMS & & SW & & & E125162 & 22-Nov-93 & & $15117-96-1$ & U-235 \\
\hline 1045 & PDMS & & SW & & & E125162 & 22-Nov-93 & & U-238 & U-238 \\
\hline 1046 & PDMS & & SW & & & E125163 & 22-Nov-93 & & $10098-97-2$ & SR-90 \\
\hline \begin{tabular}{|l|}
1047 \\
\end{tabular} & PDMS & & SW & & & E125163 & 22-Nov-93 & & $10028-17-8$ & TRITIUM \\
\hline 1048 & PDMS & & SW & & & E125163 & 22-Nov-93 & & $13966-29-5$ & U-234 \\
\hline 1049 & PDMS & & SW & & & E125163 & 22-Nov-93 & & $15117-96-1$ & U-235 \\
\hline 1050 & PDMS & & SW & & & E125163 & 22-Nov-93 & & U-238 & U-238 \\
\hline 1051 & PDMS & & SW & & & E125164 & 22-Nov-93 & & $10098-97-2$ & SR-90 \\
\hline 1052 & PDMS & & SW & & & E125164 & 22-Nov-93 & & $10028-17-8$ & TRITIUM \\
\hline \begin{tabular}{|l|}
1053 \\
\end{tabular} & PDMS & & SW & & & E125164 & 22-Nov-93 & & $13966-29-5$ & U-234 \\
\hline 1054 & PDMS & & SW & & & E125164 & 22-Nov-93 & & $15117-96-1$ & U-235 \\
\hline \begin{tabular}{|l|}
1055 \\
\end{tabular} & PDMS & & SW & & & E125164 & 22-Nov-93 & & U-238 & U-238 \\
\hline \begin{tabular}{|l|}
1056 \\
\end{tabular} & PDMS & & SW & & & E125165 & 22-Nov-93 & & $10098-97-2$ & SR-90 \\
\hline \begin{tabular}{|l|}
1057 \\
100
\end{tabular} & PDMS & & SW & & & E125165 & 22-Nov-93 & & $10028-17-8$ & TRITIUM \\
\hline \begin{tabular}{|l|}
1058 \\
\end{tabular} & PDMS & & SW & & & E125165 & 22-Nov-93 & & $13966-29-5$ & U-234 \\
\hline \begin{tabular}{|l|}
1059 \\
\end{tabular} & PDMS & & SW & & & E125165 & 22-Nov-93 & & $15117-96-1$ & U-235 \\
\hline \begin{tabular}{|l|}
1060 \\
\end{tabular} & PDMS & & SW & & & E125165 & 22-Nov-93 & & $U-238$ & $\mathrm{U}-238$ \\
\hline \begin{tabular}{|l|}
1061 \\
\end{tabular} & PDMS & & SW & & & E125166 & 22-Nov-93 & & $10098-97-2$ & SR-90 \\
\hline \begin{tabular}{|l|}
1062 \\
\end{tabular} & PDMS & & SW & & & E125166 & 22-Nov-93 & & $10028-17-8$ & TRITIUM \\
\hline \begin{tabular}{|l|}
1063 \\
\end{tabular} & PDMS & & SW & & & E125166 & 22-Nov-93 & & $13966-29-5$ & U-234 \\
\hline \begin{tabular}{|l|}
1064 \\
\end{tabular} & PDMS & & SW & & & E125166 & 22-Nov-93 & & $15117-96-1$ & U-235 \\
\hline 1065 & PDMS & & SW & & & E125166 & 22-Nov-93 & & U-238 & U-238 \\
\hline
\end{tabular}




\begin{tabular}{|c|c|c|c|c|c|c|c|c|c|c|}
\hline & A & $\mathrm{B}$ & C & $\mathrm{D}$ & $E$ & $\mathrm{~F}$ & G & $\mathrm{H}$ & $\mathrm{I}$ & $\mathrm{J}$ \\
\hline 1 & SAMPLE_NUM_ASSIGNED_TO & SAMPLER & MEDIA & BIOTA_MEDIA & TAG_ID & SAMP_NUM & SAMP_DATE_TIME & SAMP_DATE_TIME_ON & CON_ID & CON_SHORT_NAME \\
\hline 1066 & PDMS & & SW & & & E125267 & 16-Dec-93 & & $10098-97-2$ & SR-90 \\
\hline 1067 & PDMS & & SW & & & E125267 & 16-Dec-93 & & $10028-17-8$ & TRITIUM \\
\hline \begin{tabular}{|l|}
1068 \\
\end{tabular} & PDMS & & SW & & & E125267 & 16-Dec-93 & & 13966-29-5 & U-234 \\
\hline 1069 & PDMS & & SW & & & E125267 & 16-Dec-93 & & $15117-96-1$ & U-235 \\
\hline \begin{tabular}{|l|}
1070 \\
\end{tabular} & PDMS & & SW & & & E125267 & 16-Dec-93 & & U-238 & U-238 \\
\hline 1071 & PDMS & & SW & & & E125268 & 16-Dec-93 & & $10098-97-2$ & SR-90 \\
\hline 1072 & PDMS & & SW & & & E125268 & 16-Dec-93 & & $10028-17-8$ & TRITIUM \\
\hline 1073 & PDMS & & SW & & & E125268 & 16-Dec-93 & & $13966-29-5$ & U-234 \\
\hline 1074 & PDMS & & SW & & & E125268 & 16-Dec-93 & & $15117-96-1$ & U-235 \\
\hline 1075 & PDMS & & SW & & & E125268 & 16-Dec-93 & & U-238 & U-238 \\
\hline 1076 & PDMS & & SW & & & E125269 & 16-Dec-93 & & $10098-97-2$ & SR-90 \\
\hline 1077 & PDMS & & SW & & & E125269 & 16-Dec-93 & & $10028-17-8$ & TRITIUM \\
\hline \begin{tabular}{|l|}
1078 \\
\end{tabular} & PDMS & & SW & & & E125269 & 16-Dec-93 & & $13966-29-5$ & U-234 \\
\hline 1079 & PDMS & & SW & & & E125269 & 16-Dec-93 & & $15117-96-1$ & U-235 \\
\hline 1080 & PDMS & & SW & & & E125269 & 16-Dec-93 & & U-238 & U-238 \\
\hline \begin{tabular}{|l|}
1081 \\
\end{tabular} & PDMS & & SW & & & E125270 & 16-Dec-93 & & $10098-97-2$ & SR-90 \\
\hline \begin{tabular}{|l|}
1082 \\
\end{tabular} & PDMS & & SW & & & E125270 & 16-Dec-93 & & $10028-17-8$ & TRITIUM \\
\hline 1083 & PDMS & & SW & & & E125270 & 16-Dec-93 & & 13966-29-5 & U-234 \\
\hline \begin{tabular}{|l|}
1084 \\
\end{tabular} & PDMS & & SW & & & E125270 & 16-Dec-93 & & $15117-96-1$ & U-235 \\
\hline \begin{tabular}{|l|}
1085 \\
\end{tabular} & PDMS & & SW & & & E125270 & 16-Dec-93 & & U-238 & U-238 \\
\hline 1086 & PDMS & & SW & & & E125331 & 17-Dec-93 & & $10098-97-2$ & SR-90 \\
\hline \begin{tabular}{|l|}
1087 \\
\end{tabular} & PDMS & & SW & & & E125331 & 17-Dec-93 & & $10028-17-8$ & TRITIUM \\
\hline 1088 & PDMS & & SW & & & E125331 & 17-Dec-93 & & $13966-29-5$ & U-234 \\
\hline 1089 & PDMS & & SW & & & E125331 & 17-Dec-93 & & $15117-96-1$ & U-235 \\
\hline 1090 & PDMS & & SW & & & E125331 & 17-Dec-93 & & U-238 & U-238 \\
\hline \begin{tabular}{|l|}
1091 \\
\end{tabular} & PDMS & & SW & & & E125315 & 17-Dec-93 & & 10098-97-2 & SR-90 \\
\hline 1092 & PDMS & & SW & & & E125315 & 17-Dec-93 & & $10028-17-8$ & TRITIUM \\
\hline 1093 & PDMS & & SW & & & E125315 & 17-Dec-93 & & 13966-29-5 & U-234 \\
\hline \begin{tabular}{|l|}
1094 \\
\end{tabular} & PDMS & & SW & & & E125315 & 17-Dec-93 & & $15117-96-1$ & U-235 \\
\hline \begin{tabular}{|l|}
1095 \\
\end{tabular} & PDMS & & SW & & & E125315 & 17-Dec-93 & & $\mathrm{U}-238$ & U-238 \\
\hline 1096 & PDMS & & SW & & & E125324 & 17-Dec-93 & & $10098-97-2$ & SR-90 \\
\hline \begin{tabular}{|l|}
1097 \\
\end{tabular} & PDMS & & SW & & & E125324 & 17-Dec-93 & & $10028-17-8$ & TRITIUM \\
\hline \begin{tabular}{|l|}
1098 \\
\end{tabular} & PDMS & & SW & & & E125324 & 17-Dec-93 & & $13966-29-5$ & U-234 \\
\hline 1099 & PDMS & & SW & & & E125324 & 17-Dec-93 & & $15117-96-1$ & U-235 \\
\hline 1100 & PDMS & & SW & & & E125324 & 17-Dec-93 & & U-238 & U-238 \\
\hline 1101 & PDMS & & SW & & & E125316 & 17-Dec-93 & & $10098-97-2$ & SR-90 \\
\hline \begin{tabular}{|l|}
1102 \\
\end{tabular} & PDMS & & SW & & & E125316 & 17-Dec-93 & & $10028-17-8$ & TRITIUM \\
\hline 1103 & PDMS & & SW & & & E125316 & 17-Dec-93 & & $13966-29-5$ & U-234 \\
\hline
\end{tabular}




\begin{tabular}{|c|c|c|c|c|c|c|c|c|c|c|}
\hline & A & $\mathrm{B}$ & C & $\mathrm{D}$ & $E$ & $\mathrm{~F}$ & G & $\mathrm{H}$ & $\mathrm{I}$ & $\mathrm{J}$ \\
\hline 1 & SAMPLE_NUM_ASSIGNED_TO & SAMPLER & MEDIA & BIOTA_MEDIA & TAG_ID & SAMP_NUM & SAMP_DATE_TIME & SAMP_DATE_TIME_ON & CON_ID & CON_SHORT_NAME \\
\hline 1104 & PDMS & & SW & & & E125316 & 17-Dec-93 & & $15117-96-1$ & $\mathrm{U}-235$ \\
\hline 1105 & PDMS & & SW & & & E125316 & 17-Dec-93 & & $U-238$ & $\mathrm{U}-238$ \\
\hline 1106 & PDMS & & SW & & & E125317 & 17-Dec-93 & & $10098-97-2$ & SR-90 \\
\hline \begin{tabular}{|l|}
1107 \\
\end{tabular} & PDMS & & SW & & & E125317 & 17-Dec-93 & & $10028-17-8$ & TRITIUM \\
\hline \begin{tabular}{|l|}
1108 \\
\end{tabular} & PDMS & & SW & & & E125317 & 17-Dec-93 & & 13966-29-5 & U-234 \\
\hline 1109 & PDMS & & SW & & & E125317 & 17-Dec-93 & & $15117-96-1$ & U-235 \\
\hline 1110 & PDMS & & SW & & & E125317 & 17-Dec-93 & & U-238 & $\mathrm{U}-238$ \\
\hline 1111 & PDMS & & SW & & & E125318 & 17-Dec-93 & & $10098-97-2$ & SR-90 \\
\hline 1112 & PDMS & & SW & & & E125318 & 17-Dec-93 & & $10028-17-8$ & TRITIUM \\
\hline 1113 & PDMS & & SW & & & E125318 & 17-Dec-93 & & $13966-29-5$ & U-234 \\
\hline 1114 & PDMS & & SW & & & E125318 & 17-Dec-93 & & $15117-96-1$ & U-235 \\
\hline 1115 & PDMS & & SW & & & E125318 & 17-Dec-93 & & U-238 & U-238 \\
\hline 1116 & PDMS & & SW & & & E125319 & 17-Dec-93 & & $10098-97-2$ & SR-90 \\
\hline \begin{tabular}{|l|}
1117 \\
\end{tabular} & PDMS & & SW & & & E125319 & 17-Dec-93 & & $10028-17-8$ & TRITIUM \\
\hline 1118 & PDMS & & SW & & & E125319 & 17-Dec-93 & & $13966-29-5$ & U-234 \\
\hline \begin{tabular}{|l|}
1119 \\
\end{tabular} & PDMS & & SW & & & E125319 & 17-Dec-93 & & $15117-96-1$ & U-235 \\
\hline \begin{tabular}{|l}
1120 \\
\end{tabular} & PDMS & & SW & & & E125319 & 17-Dec-93 & & U-238 & U-238 \\
\hline \begin{tabular}{|l|}
1121 \\
\end{tabular} & PDMS & & SW & & & E125320 & 17-Dec-93 & & $10098-97-2$ & SR-90 \\
\hline 1122 & PDMS & & SW & & & E125320 & 17-Dec-93 & & $10028-17-8$ & TRITIUM \\
\hline \begin{tabular}{|l}
1123 \\
\end{tabular} & PDMS & & SW & & & E125320 & 17-Dec-93 & & $13966-29-5$ & U-234 \\
\hline \begin{tabular}{|l|}
1124 \\
\end{tabular} & PDMS & & SW & & & E125320 & 17-Dec-93 & & $15117-96-1$ & U-235 \\
\hline \begin{tabular}{|l|}
1125 \\
\end{tabular} & PDMS & & SW & & & E125320 & 17-Dec-93 & & U-238 & U-238 \\
\hline 1126 & PDMS & & SW & & & E125321 & 17-Dec-93 & & $10098-97-2$ & SR-90 \\
\hline \begin{tabular}{|l|}
1127 \\
\end{tabular} & PDMS & & SW & & & E125321 & 17-Dec-93 & & $10028-17-8$ & TRITIUM \\
\hline 1128 & PDMS & & SW & & & E125321 & 17-Dec-93 & & $13966-29-5$ & U-234 \\
\hline 1129 & PDMS & & SW & & & E125321 & 17-Dec-93 & & 15117-96-1 & U-235 \\
\hline 1130 & PDMS & & SW & & & E125321 & 17-Dec-93 & & U-238 & U-238 \\
\hline \begin{tabular}{|l|}
1131 \\
\end{tabular} & PDMS & & SW & & & E125322 & 17-Dec-93 & & $10098-97-2$ & SR-90 \\
\hline 1132 & PDMS & & SW & & & E125322 & 17-Dec-93 & & $10028-17-8$ & TRITIUM \\
\hline \begin{tabular}{|l}
1133 \\
\end{tabular} & PDMS & & SW & & & E125322 & 17-Dec-93 & & $13966-29-5$ & U-234 \\
\hline \begin{tabular}{|l|}
1130 \\
1134 \\
\end{tabular} & PDMS & & SW & & & E125322 & 17-Dec-93 & & $15117-96-1$ & U-235 \\
\hline \begin{tabular}{|l|}
1135 \\
\end{tabular} & PDMS & & SW & & & E125322 & 17-Dec-93 & & U-238 & U-238 \\
\hline \begin{tabular}{|l|}
1136 \\
\end{tabular} & PDMS & & SW & & & E125323 & 17-Dec-93 & & $10098-97-2$ & SR-90 \\
\hline \begin{tabular}{|l|}
1137 \\
\end{tabular} & PDMS & & SW & & & E125323 & 17-Dec-93 & & $10028-17-8$ & TRITIUM \\
\hline 1138 & PDMS & & SW & & & E125323 & 17-Dec-93 & & $13966-29-5$ & U-234 \\
\hline \begin{tabular}{|l|}
1139 \\
\end{tabular} & PDMS & & SW & & & E125323 & 17-Dec-93 & & $15117-96-1$ & U-235 \\
\hline 1140 & PDMS & & SW & & & E125323 & 17-Dec-93 & & U-238 & U-238 \\
\hline 1141 & SESPSCH & & SW & & & B0BG75 & 17-Mar-94 & & $10098-97-2$ & SR-90 \\
\hline
\end{tabular}




\begin{tabular}{|c|c|c|c|c|c|c|c|c|c|c|}
\hline & A & $\mathrm{B}$ & $\mathrm{C}$ & $\mathrm{D}$ & $E$ & $\mathrm{~F}$ & G & $\mathrm{H}$ & $\mathrm{I}$ & $\mathrm{J}$ \\
\hline 1 & SAMPLE_NUM_ASSIGNED_TO & SAMPLER & MEDIA & BIOTA_MEDIA & TAG_ID & SAMP_NUM & SAMP_DATE_TIME & SAMP_DATE_TIME_ON & CON_ID & CON_SHORT_NAME \\
\hline 1142 & SESPSCH & & SW & & & B0BG75 & 17-Mar-94 & & $10028-17-8$ & TRITIUM \\
\hline 1143 & SESPSCH & & SW & & & BOBG75 & 17-Mar-94 & & 13966-29-5 & U-234 \\
\hline \begin{tabular}{|l|}
1144 \\
\end{tabular} & SESPSCH & & SW & & & BOBG75 & 17-Mar-94 & & $15117-96-1$ & U-235 \\
\hline \begin{tabular}{|l|}
1145 \\
\end{tabular} & SESPSCH & & SW & & & BOBG75 & 17-Mar-94 & & U-238 & U-238 \\
\hline \begin{tabular}{|l|}
1146 \\
\end{tabular} & SESPSCH & & SW & & & BOBG76 & 17-Mar-94 & & $10098-97-2$ & SR-90 \\
\hline 1147 & SESPSCH & & SW & & & B0BG76 & 17-Mar-94 & & $10028-17-8$ & TRITIUM \\
\hline 1148 & SESPSCH & & SW & & & B0BG76 & 17-Mar-94 & & $13966-29-5$ & U-234 \\
\hline 1149 & SESPSCH & & SW & & & B0BG76 & 17-Mar-94 & & $15117-96-1$ & U-235 \\
\hline 1150 & SESPSCH & & SW & & & B0BG76 & 17-Mar-94 & & $\mathrm{U}-238$ & $\mathrm{U}-238$ \\
\hline 1151 & SESPSCH & & SW & & & B0BG79 & 31-Mar-94 & & $10098-97-2$ & SR-90 \\
\hline 1152 & SESPSCH & & SW & & & B0BG79 & 31-Mar-94 & & $10028-17-8$ & TRITIUM \\
\hline 1153 & SESPSCH & & SW & & & BOBG79 & 31-Mar-94 & & $13966-29-5$ & U-234 \\
\hline 1154 & SESPSCH & & SW & & & BOBG79 & 31-Mar-94 & & 15117-96-1 & U-235 \\
\hline 1155 & SESPSCH & & SW & & & B0BG79 & 31-Mar-94 & & U-238 & U-238 \\
\hline 1156 & SESPSCH & & SW & & & B0BG88 & 31-Mar-94 & & $10098-97-2$ & SR-90 \\
\hline \begin{tabular}{|l}
1157 \\
\end{tabular} & SESPSCH & & SW & & & B0BG88 & 31-Mar-94 & & $10028-17-8$ & TRITIUM \\
\hline \begin{tabular}{|l|}
1158 \\
\end{tabular} & SESPSCH & & SW & & & B0BG88 & 31-Mar-94 & & $13966-29-5$ & U-234 \\
\hline 1159 & SESPSCH & & SW & & & B0BG88 & 31-Mar-94 & & $15117-96-1$ & U-235 \\
\hline 1160 & SESPSCH & & SW & & & B0BG88 & 31-Mar-94 & & U-238 & U-238 \\
\hline \begin{tabular}{|l|}
1161 \\
\end{tabular} & SESPSCH & & SW & & & BOBG80 & 31-Mar-94 & & $10098-97-2$ & SR-90 \\
\hline \begin{tabular}{|l|}
1162 \\
\end{tabular} & SESPSCH & & SW & & & B0BG80 & 31-Mar-94 & & $10028-17-8$ & TRITIUM \\
\hline \begin{tabular}{|l|}
1163 \\
\end{tabular} & SESPSCH & & SW & & & B0BG80 & 31-Mar-94 & & $13966-29-5$ & U-234 \\
\hline \begin{tabular}{|l|}
1164 \\
\end{tabular} & SESPSCH & & SW & & & B0BG80 & 31-Mar-94 & & $15117-96-1$ & U-235 \\
\hline 1165 & SESPSCH & & SW & & & B0BG80 & 31-Mar-94 & & U-238 & U-238 \\
\hline 1166 & SESPSCH & & SW & & & B0BG81 & 31-Mar-94 & & $10098-97-2$ & SR-90 \\
\hline 1167 & SESPSCH & & SW & & & B0BG81 & 31-Mar-94 & & $10028-17-8$ & TRITIUM \\
\hline 1168 & SESPSCH & & SW & & & B0BG81 & 31-Mar-94 & & 13966-29-5 & U-234 \\
\hline 1169 & SESPSCH & & SW & & & B0BG81 & 31-Mar-94 & & $15117-96-1$ & U-235 \\
\hline 1170 & SESPSCH & & SW & & & B0BG81 & 31-Mar-94 & & U-238 & U-238 \\
\hline \begin{tabular}{|l|}
1171 \\
\end{tabular} & SESPSCH & & SW & & & B0BG82 & 31-Mar-94 & & $10098-97-2$ & SR-90 \\
\hline \begin{tabular}{|l|}
1172 \\
\end{tabular} & SESPSCH & & SW & & & B0BG82 & 31-Mar-94 & & $10028-17-8$ & TRITIUM \\
\hline \begin{tabular}{|l}
1173 \\
\end{tabular} & SESPSCH & & SW & & & B0BG82 & 31-Mar-94 & & $13966-29-5$ & U-234 \\
\hline \begin{tabular}{|l|}
1174 \\
\end{tabular} & SESPSCH & & SW & & & B0BG82 & 31-Mar-94 & & 15117-96-1 & U-235 \\
\hline \begin{tabular}{|l|}
1175 \\
\end{tabular} & SESPSCH & & SW & & & B0BG82 & 31-Mar-94 & & U-238 & U-238 \\
\hline 1176 & SESPSCH & & SW & & & B0BG83 & 31-Mar-94 & & $10098-97-2$ & SR-90 \\
\hline \begin{tabular}{|l|}
1177 \\
\end{tabular} & SESPSCH & & SW & & & B0BG83 & 31-Mar-94 & & $10028-17-8$ & TRITIUM \\
\hline 1178 & SESPSCH & & SW & & & B0BG83 & 31-Mar-94 & & $13966-29-5$ & U-234 \\
\hline 1179 & SESPSCH & & SW & & & B0BG83 & 31-Mar-94 & & $15117-96-1$ & U-235 \\
\hline
\end{tabular}




\begin{tabular}{|c|c|c|c|c|c|c|c|c|c|c|}
\hline & A & $\mathrm{B}$ & $\mathrm{C}$ & $\mathrm{D}$ & $E$ & $\mathrm{~F}$ & G & $\mathrm{H}$ & $\mathrm{I}$ & $\mathrm{J}$ \\
\hline 1 & SAMPLE_NUM_ASSIGNED_TO & SAMPLER & MEDIA & BIOTA_MEDIA & TAG_ID & SAMP_NUM & SAMP_DATE_TIME & SAMP_DATE_TIME_ON & CON_ID & CON_SHORT_NAME \\
\hline 1180 & SESPSCH & & SW & & & B0BG83 & 31-Mar-94 & & U-238 & U-238 \\
\hline 1181 & SESPSCH & & SW & & & B0BG84 & 31-Mar-94 & & 10098-97-2 & SR-90 \\
\hline \begin{tabular}{|l|}
1182 \\
\end{tabular} & SESPSCH & & SW & & & B0BG84 & 31-Mar-94 & & $10028-17-8$ & TRITIUM \\
\hline 1183 & SESPSCH & & SW & & & B0BG84 & 31-Mar-94 & & 13966-29-5 & U-234 \\
\hline \begin{tabular}{|l|}
1184 \\
\end{tabular} & SESPSCH & & SW & & & B0BG84 & 31-Mar-94 & & $15117-96-1$ & U-235 \\
\hline 1185 & SESPSCH & & SW & & & B0BG84 & 31-Mar-94 & & U-238 & U-238 \\
\hline 1186 & SESPSCH & & SW & & & B0BG85 & 31-Mar-94 & & $10098-97-2$ & SR-90 \\
\hline 1187 & SESPSCH & & SW & & & B0BG85 & 31-Mar-94 & & $10028-17-8$ & TRITIUM \\
\hline 1188 & SESPSCH & & SW & & & B0BG85 & 31-Mar-94 & & $13966-29-5$ & U-234 \\
\hline 1189 & SESPSCH & & SW & & & B0BG85 & 31-Mar-94 & & $15117-96-1$ & U-235 \\
\hline 1190 & SESPSCH & & SW & & & B0BG85 & 31-Mar-94 & & U-238 & U-238 \\
\hline 1191 & SESPSCH & & SW & & & B0BG86 & 31-Mar-94 & & $10098-97-2$ & SR-90 \\
\hline 1192 & SESPSCH & & SW & & & B0BG86 & 31-Mar-94 & & 10028-17-8 & TRITIUM \\
\hline 1193 & SESPSCH & & SW & & & B0BG86 & 31-Mar-94 & & $13966-29-5$ & U-234 \\
\hline 1194 & SESPSCH & & SW & & & B0BG86 & 31-Mar-94 & & $15117-96-1$ & U-235 \\
\hline \begin{tabular}{|l|}
1195 \\
\end{tabular} & SESPSCH & & SW & & & B0BG86 & 31-Mar-94 & & U-238 & U-238 \\
\hline \begin{tabular}{|l|}
1196 \\
\end{tabular} & SESPSCH & & SW & & & B0BG87 & 31-Mar-94 & & $10098-97-2$ & SR-90 \\
\hline 1197 & SESPSCH & & SW & & & B0BG87 & 31-Mar-94 & & $10028-17-8$ & TRITIUM \\
\hline \begin{tabular}{|l|}
1198 \\
\end{tabular} & SESPSCH & & SW & & & B0BG87 & 31-Mar-94 & & $13966-29-5$ & U-234 \\
\hline \begin{tabular}{|l|}
1199 \\
\end{tabular} & SESPSCH & & SW & & & B0BG87 & 31-Mar-94 & & $15117-96-1$ & U-235 \\
\hline 1200 & SESPSCH & & SW & & & B0BG87 & 31-Mar-94 & & U-238 & U-238 \\
\hline \begin{tabular}{|l|}
1201 \\
\end{tabular} & SESPSCH & & SW & & & B0BQM7 & 30-Jun-94 & & $10098-97-2$ & SR-90 \\
\hline \begin{tabular}{|l|}
1202 \\
\end{tabular} & SESPSCH & & SW & & & BOBQM7 & 30-Jun-94 & & $10028-17-8$ & TRITIUM \\
\hline 1203 & SESPSCH & & SW & & & BOBQM7 & 30-Jun-94 & & $13966-29-5$ & U-234 \\
\hline 1204 & SESPSCH & & SW & & & B0BQM7 & 30-Jun-94 & & 15117-96-1 & U-235 \\
\hline 1205 & SESPSCH & & SW & & & B0BQM7 & 30-Jun-94 & & $\mathrm{U}-238$ & U-238 \\
\hline 1206 & SESPSCH & & SW & & & BOBQM8 & 30-Jun-94 & & $10098-97-2$ & SR-90 \\
\hline \begin{tabular}{|l|}
1207 \\
\end{tabular} & SESPSCH & & SW & & & BOBQM8 & 30-Jun-94 & & $10028-17-8$ & TRITIUM \\
\hline \begin{tabular}{|l|}
1208 \\
\end{tabular} & SESPSCH & & SW & & & BOBQM8 & 30-Jun-94 & & $13966-29-5$ & U-234 \\
\hline \begin{tabular}{|l|}
1209 \\
\end{tabular} & SESPSCH & & SW & & & B0BQM8 & 30-Jun-94 & & $15117-96-1$ & U-235 \\
\hline 1210 & SESPSCH & & SW & & & BOBQM8 & 30-Jun-94 & & U-238 & U-238 \\
\hline \begin{tabular}{|l|}
1211 \\
\end{tabular} & SESPSCH & & SW & & & B0BQM9 & 30-Jun-94 & & $10098-97-2$ & SR-90 \\
\hline \begin{tabular}{|l|}
1212 \\
\end{tabular} & SESPSCH & & SW & & & B0BQM9 & 30-Jun-94 & & $10028-17-8$ & TRITIUM \\
\hline \begin{tabular}{|l|}
1213 \\
\end{tabular} & SESPSCH & & SW & & & BOBQM9 & 30-Jun-94 & & $13966-29-5$ & U-234 \\
\hline \begin{tabular}{|l|}
1214 \\
\end{tabular} & SESPSCH & & SW & & & BOBQM9 & 30-Jun-94 & & $15117-96-1$ & U-235 \\
\hline \begin{tabular}{|l|}
1215 \\
\end{tabular} & SESPSCH & & SW & & & B0BQM9 & 30-Jun-94 & & U-238 & U-238 \\
\hline 1216 & SESPSCH & & SW & & & BOBQNO & 30-Jun-94 & & $10098-97-2$ & SR-90 \\
\hline \begin{tabular}{|l|}
1217 \\
\end{tabular} & SESPSCH & & SW & & & BOBQNO & 30-Jun-94 & & 10028-17-8 & TRITIUM \\
\hline
\end{tabular}




\begin{tabular}{|c|c|c|c|c|c|c|c|c|c|c|}
\hline & A & $\mathrm{B}$ & $\mathrm{C}$ & $\mathrm{D}$ & $E$ & $\mathrm{~F}$ & G & $\mathrm{H}$ & $\mathrm{I}$ & $\mathrm{J}$ \\
\hline 1 & SAMPLE_NUM_ASSIGNED_TO & SAMPLER & MEDIA & BIOTA_MEDIA & TAG_ID & SAMP_NUM & SAMP_DATE_TIME & SAMP_DATE_TIME_ON & CON_ID & CON_SHORT_NAME \\
\hline 1218 & SESPSCH & & SW & & & BOBQNO & 30-Jun-94 & & $13966-29-5$ & $\mathrm{U}-234$ \\
\hline \begin{tabular}{|l|}
1219 \\
\end{tabular} & SESPSCH & & SW & & & BOBQNO & 30-Jun-94 & & 15117-96-1 & U-235 \\
\hline 1220 & SESPSCH & & SW & & & BOBQNO & 30-Jun-94 & & U-238 & U-238 \\
\hline 1221 & SESPSCH & & SW & & & B0BQN1 & 01-Jul-94 & & $10098-97-2$ & SR-90 \\
\hline 1222 & SESPSCH & & SW & & & B0BQN1 & 01-Jul-94 & & $10028-17-8$ & TRITIUM \\
\hline 1223 & SESPSCH & & SW & & & B0BQN1 & 01-Jul-94 & & 13966-29-5 & U-234 \\
\hline 1224 & SESPSCH & & SW & & & B0BQN1 & 01-Jul-94 & & 15117-96-1 & U-235 \\
\hline 1225 & SESPSCH & & SW & & & B0BQN1 & 01-Jul-94 & & U-238 & U-238 \\
\hline 1226 & SESPSCH & & SW & & & BOBQPO & 01-Jul-94 & & $10098-97-2$ & SR-90 \\
\hline 1227 & SESPSCH & & SW & & & BOBQP0 & 01-Jul-94 & & $10028-17-8$ & TRITIUM \\
\hline 1228 & SESPSCH & & SW & & & BOBQP0 & 01-Jul-94 & & $13966-29-5$ & U-234 \\
\hline 1229 & SESPSCH & & SW & & & BOBQPO & 01-Jul-94 & & 15117-96-1 & U-235 \\
\hline 1230 & SESPSCH & & SW & & & BOBQPO & 01-Jul-94 & & U-238 & U-238 \\
\hline \begin{tabular}{|l|}
1231 \\
\end{tabular} & SESPSCH & & SW & & & B0BQN2 & 01-Jul-94 & & $10098-97-2$ & SR-90 \\
\hline \begin{tabular}{|l|}
1232 \\
\end{tabular} & SESPSCH & & SW & & & BOBQN2 & 01-Jul-94 & & $10028-17-8$ & TRITIUM \\
\hline \begin{tabular}{|l|}
1233 \\
\end{tabular} & SESPSCH & & SW & & & BOBQN2 & 01-Jul-94 & & $13966-29-5$ & U-234 \\
\hline 1234 & SESPSCH & & SW & & & BOBQN2 & 01-Jul-94 & & $15117-96-1$ & U-235 \\
\hline 1235 & SESPSCH & & SW & & & BOBQN2 & 01-Jul-94 & & U-238 & U-238 \\
\hline \begin{tabular}{|l|}
1236 \\
\end{tabular} & SESPSCH & & SW & & & BOBQN3 & 01-Jul-94 & & $10098-97-2$ & SR-90 \\
\hline \begin{tabular}{|l|}
1237 \\
\end{tabular} & SESPSCH & & SW & & & B0BQN3 & 01-Jul-94 & & $10028-17-8$ & TRITIUM \\
\hline \begin{tabular}{|l|}
1238 \\
\end{tabular} & SESPSCH & & SW & & & BOBQN3 & 01-Jul-94 & & $13966-29-5$ & $\mathrm{U}-234$ \\
\hline 1239 & SESPSCH & & SW & & & B0BQN3 & 01-Jul-94 & & $15117-96-1$ & U-235 \\
\hline 1240 & SESPSCH & & SW & & & B0BQN3 & 01-Jul-94 & & U-238 & U-238 \\
\hline 1241 & SESPSCH & & SW & & & BOBQN4 & 01-Jul-94 & & $10098-97-2$ & SR-90 \\
\hline 1242 & SESPSCH & & SW & & & BOBQN4 & 01-Jul-94 & & $10028-17-8$ & TRITIUM \\
\hline 1243 & SESPSCH & & SW & & & BOBQN4 & 01-Jul-94 & & $13966-29-5$ & U-234 \\
\hline \begin{tabular}{|l|}
1244 \\
\end{tabular} & SESPSCH & & SW & & & BOBQN4 & 01-Jul-94 & & $15117-96-1$ & U-235 \\
\hline \begin{tabular}{|l|}
1245 \\
\end{tabular} & SESPSCH & & SW & & & BOBQN4 & 01-Jul-94 & & U-238 & U-238 \\
\hline 1246 & SESPSCH & & SW & & & B0BQN5 & 01-Jul-94 & & $10098-97-2$ & SR-90 \\
\hline \begin{tabular}{|l|}
1247 \\
\end{tabular} & SESPSCH & & SW & & & B0BQN5 & 01-Jul-94 & & $10028-17-8$ & TRITIUM \\
\hline \begin{tabular}{|l|}
1248 \\
\end{tabular} & SESPSCH & & SW & & & B0BQN5 & 01-Jul-94 & & $13966-29-5$ & U-234 \\
\hline 1249 & SESPSCH & & SW & & & B0BQN5 & 01-Jul-94 & & $15117-96-1$ & U-235 \\
\hline 1250 & SESPSCH & & SW & & & B0BQN5 & 01-Jul-94 & & U-238 & U-238 \\
\hline 1251 & SESPSCH & & SW & & & B0BQN6 & 01-Jul-94 & & $10098-97-2$ & SR-90 \\
\hline 1252 & SESPSCH & & SW & & & B0BQN6 & 01-Jul-94 & & $10028-17-8$ & TRITIUM \\
\hline 1253 & SESPSCH & & SW & & & B0BQN6 & 01-Jul-94 & & $13966-29-5$ & U-234 \\
\hline 1254 & SESPSCH & & SW & & & B0BQN6 & 01-Jul-94 & & 15117-96-1 & U-235 \\
\hline 1255 & SESPSCH & & SW & & & B0BQN6 & 01-Jul-94 & & $\mathrm{U}-238$ & $\mathrm{U}-238$ \\
\hline
\end{tabular}




\begin{tabular}{|c|c|c|c|c|c|c|c|c|c|c|}
\hline & A & $\mathrm{B}$ & $C$ & $\mathrm{D}$ & $E$ & $\mathrm{~F}$ & $G$ & $\mathrm{H}$ & $\mathrm{I}$ & $\mathrm{J}$ \\
\hline 1 & SAMPLE_NUM_ASSIGNED_TO & SAMPLER & MEDIA & BIOTA_MEDIA & TAG_ID & SAMP_NUM & SAMP_DATE_TIME| & SAMP_DATE_TIME_ON & CON_ID & CON_SHORT_NAME \\
\hline 1256 & SESPSCH & & SW & & & BOBQN7 & 01-Jul-94 & & $10098-97-2$ & SR-90 \\
\hline 1257 & SESPSCH & & SW & & & BOBQN7 & 01-Jul-94 & & $10028-17-8$ & TRITIUM \\
\hline 1258 & SESPSCH & & SW & & & BOBQN7 & 01-Jul-94 & & $13966-29-5$ & U-234 \\
\hline 1259 & SESPSCH & & SW & & & BOBQN7 & 01-Jul-94 & & 15117-96-1 & U-235 \\
\hline 1260 & SESPSCH & & SW & & & BOBQN7 & 01-Jul-94 & & $\mathrm{U}-238$ & $\mathrm{U}-238$ \\
\hline 1261 & SESPSCH & & SW & & & BOBQN8 & 01-Jul-94 & & $10098-97-2$ & SR-90 \\
\hline 1262 & SESPSCH & & SW & & & B0BQN8 & 01-Jul-94 & & $10028-17-8$ & TRITIUM \\
\hline 1263 & SESPSCH & & SW & & & BOBQN8 & 01-Jul-94 & & $13966-29-5$ & U-234 \\
\hline 1264 & SESPSCH & & SW & & & B0BQN8 & 01-Jul-94 & & 15117-96-1 & U-235 \\
\hline 1265 & SESPSCH & & SW & & & B0BQN8 & 01-Jul-94 & & $\mathrm{U}-238$ & U-238 \\
\hline 1266 & SESPSCH & & SW & & & BOBQN9 & 01-Jul-94 & & $10098-97-2$ & SR-90 \\
\hline 1267 & SESPSCH & & SW & & & BOBQN9 & 01-Jul-94 & & $10028-17-8$ & TRITIUM \\
\hline 1268 & SESPSCH & & SW & & & BOBQN9 & 01-Jul-94 & & $13966-29-5$ & U-234 \\
\hline 1269 & SESPSCH & & SW & & & BOBQN9 & 01-Jul-94 & & $15117-96-1$ & U-235 \\
\hline 1270 & SESPSCH & & SW & & & BOBQN9 & 01-Jul-94 & & U-238 & U-238 \\
\hline 1271 & JL JULYA/LE BISPING & & SW & & & B09J78 & 25-Aug-94 & & $7440-47-3$ & $\mathrm{CR}$ \\
\hline 1272 & JL JULYA/LE BISPING & & SW & & & B09J78 & 25-Aug-94 & & $56-23-5$ & CARBTET \\
\hline 1273 & SESPSCH & & SW & & & B0C588 & 25-Aug-94 & & $10098-97-2$ & SR-90 \\
\hline 1274 & SESPSCH & & SW & & & B0C588 & 25-Aug-94 & & $10028-17-8$ & TRITIUM \\
\hline 1275 & SESPSCH & & SW & & & B0C588 & 25-Aug-94 & & 13966-29-5 & U-234 \\
\hline 1276 & SESPSCH & & SW & & & B0C588 & 25-Aug-94 & & $15117-96-1$ & U-235 \\
\hline 1277 & SESPSCH & & SW & & & B0C588 & 25-Aug-94 & & $\mathrm{U}-238$ & $\mathrm{U}-238$ \\
\hline 1278 & JL JULYA/LE BISPING & & SW & & & B09J87 & 25-Aug-94 & & $7440-47-3$ & CR \\
\hline 1279 & JL JULYA/LE BISPING & & SW & & & B09J87 & 25-Aug-94 & & $56-23-5$ & CARBTET \\
\hline 1280 & SESPSCH & & SW & & & B0C598 & 25-Aug-94 & & $10098-97-2$ & SR-90 \\
\hline 1281 & SESPSCH & & SW & & & B0C598 & 25-Aug-94 & & $10028-17-8$ & TRITIUM \\
\hline 1282 & SESPSCH & & SW & & & B0C598 & 25-Aug-94 & & $13966-29-5$ & U-234 \\
\hline 1283 & SESPSCH & & SW & & & B0C598 & 25-Aug-94 & & 15117-96-1 & U-235 \\
\hline 1284 & SESPSCH & & SW & & & B0C598 & 25-Aug-94 & & $\mathrm{U}-238$ & $\mathrm{U}-238$ \\
\hline 1285 & JL JULYA/LE BISPING & & SW & & & B09J79 & 25-Aug-94 & & $7440-47-3$ & CR \\
\hline 1286 & JL JULYA/LE BISPING & & SW & & & B09J79 & 25-Aug-94 & & $56-23-5$ & CARBTET \\
\hline 1287 & SESPSCH & & SW & & & B0C592 & 25-Aug-94 & & $10098-97-2$ & SR-90 \\
\hline 1288 & SESPSCH & & SW & & & B0C592 & 25-Aug-94 & & $10028-17-8$ & TRITIUM \\
\hline 1289 & SESPSCH & & SW & & & B0C592 & 25-Aug-94 & & $13966-29-5$ & U-234 \\
\hline 1290 & SESPSCH & & SW & & & B0C592 & 25-Aug-94 & & 15117-96-1 & U-235 \\
\hline 1291 & SESPSCH & & SW & & & B0C592 & 25-Aug-94 & & $\mathrm{U}-238$ & $\mathrm{U}-238$ \\
\hline 1292 & JL JULYA/LE BISPING & & SW & & & B09J80 & 25-Aug-94 & & $7440-47-3$ & CR \\
\hline 1293 & JL JULYA/LE BISPING & & SW & & & B09J80 & 25-Aug-94 & & $56-23-5$ & CARBTET \\
\hline
\end{tabular}




\begin{tabular}{|c|c|c|c|c|c|c|c|c|c|c|}
\hline & A & $\mathrm{B}$ & $\mathrm{C}$ & $\mathrm{D}$ & $E$ & $\mathrm{~F}$ & G & $\mathrm{H}$ & $\mathrm{I}$ & $\mathrm{J}$ \\
\hline 1 & SAMPLE_NUM_ASSIGNED_TO & SAMPLER & MEDIA & BIOTA_MEDIA & TAG_ID & SAMP_NUM & SAMP_DATE_TIME & SAMP_DATE_TIME_ON & CON_ID & CON_SHORT_NAME \\
\hline 1294 & SESPSCH & & SW & & & B0C589 & 25-Aug-94 & & $10098-97-2$ & SR-90 \\
\hline 1295 & SESPSCH & & SW & & & B0C589 & 25-Aug-94 & & $10028-17-8$ & TRITIUM \\
\hline 1296 & SESPSCH & & SW & & & B0C589 & 25-Aug-94 & & $13966-29-5$ & U-234 \\
\hline 1297 & SESPSCH & & SW & & & B0C589 & 25-Aug-94 & & $15117-96-1$ & U-235 \\
\hline 1298 & SESPSCH & & SW & & & B0C589 & 25-Aug-94 & & $\mathrm{U}-238$ & U-238 \\
\hline 1299 & JL JULYA/LE BISPING & & SW & & & B09J81 & 25-Aug-94 & & $7440-47-3$ & CR \\
\hline 1300 & JL JULYA/LE BISPING & & SW & & & B09J81 & 25-Aug-94 & & $56-23-5$ & CARBTET \\
\hline 1301 & SESPSCH & & SW & & & B0C593 & 25-Aug-94 & & $10098-97-2$ & SR-90 \\
\hline 1302 & SESPSCH & & SW & & & B0C593 & 25-Aug-94 & & $10028-17-8$ & TRITIUM \\
\hline 1303 & SESPSCH & & SW & & & B0C593 & 25-Aug-94 & & $13966-29-5$ & $\mathrm{U}-234$ \\
\hline 1304 & SESPSCH & & SW & & & B0C593 & 25-Aug-94 & & $15117-96-1$ & U-235 \\
\hline 1305 & SESPSCH & & SW & & & B0C593 & 25-Aug-94 & & $\mathrm{U}-238$ & $\mathrm{U}-238$ \\
\hline 1306 & JL JULYA/LE BISPING & & SW & & & B09J82 & 25-Aug-94 & & $7440-47-3$ & CR \\
\hline 1307 & JL JULYA/LE BISPING & & SW & & & B09J82 & 25-Aug-94 & & $56-23-5$ & CARBTET \\
\hline 1308 & SESPSCH & & SW & & & B0C594 & 25-Aug-94 & & $10098-97-2$ & SR-90 \\
\hline 1309 & SESPSCH & & SW & & & B0C594 & 25-Aug-94 & & $10028-17-8$ & TRITIUM \\
\hline 1310 & SESPSCH & & SW & & & B0C594 & 25-Aug-94 & & 13966-29-5 & U-234 \\
\hline 1311 & SESPSCH & & SW & & & B0C594 & 25-Aug-94 & & 15117-96-1 & U-235 \\
\hline 1312 & SESPSCH & & SW & & & B0C594 & 25-Aug-94 & & $\mathrm{U}-238$ & $\mathrm{U}-238$ \\
\hline 1313 & JL JULYA/LE BISPING & & SW & & & B09J83 & 25-Aug-94 & & $7440-47-3$ & $\mathrm{CR}$ \\
\hline 1314 & JL JULYA/LE BISPING & & SW & & & B09J83 & 25-Aug-94 & & $56-23-5$ & CARBTET \\
\hline 1315 & SESPSCH & & SW & & & B0C595 & 25-Aug-94 & & $10098-97-2$ & SR-90 \\
\hline 1316 & SESPSCH & & SW & & & B0C595 & 25-Aug-94 & & $10028-17-8$ & TRITIUM \\
\hline 1317 & SESPSCH & & SW & & & B0C595 & 25-Aug-94 & & $13966-29-5$ & U-234 \\
\hline 1318 & SESPSCH & & SW & & & B0C595 & 25-Aug-94 & & $15117-96-1$ & U-235 \\
\hline 1319 & SESPSCH & & SW & & & B0C595 & 25-Aug-94 & & $\mathrm{U}-238$ & $\mathrm{U}-238$ \\
\hline 1320 & JL JULYA/LE BISPING & & SW & & & B09J84 & 25-Aug-94 & & $7440-47-3$ & CR \\
\hline 1321 & JL JULYA/LE BISPING & & SW & & & B09J84 & 25-Aug-94 & & $56-23-5$ & CARBTET \\
\hline 1322 & SESPSCH & & SW & & & B0C596 & 25-Aug-94 & & $10098-97-2$ & SR-90 \\
\hline 1323 & SESPSCH & & SW & & & B0C596 & 25-Aug-94 & & $10028-17-8$ & TRITIUM \\
\hline 1324 & SESPSCH & & SW & & & B0C596 & 25-Aug-94 & & 13966-29-5 & U-234 \\
\hline 1325 & SESPSCH & & SW & & & B0C596 & 25-Aug-94 & & 15117-96-1 & U-235 \\
\hline 1326 & SESPSCH & & SW & & & B0C596 & 25-Aug-94 & & U-238 & U-238 \\
\hline 1327 & JL JULYA/LE BISPING & & SW & & & B09J85 & 25-Aug-94 & & $7440-47-3$ & $\mathrm{CR}$ \\
\hline 1328 & JL JULYA/LE BISPING & & SW & & & B09J85 & 25-Aug-94 & & $56-23-5$ & CARBTET \\
\hline 1329 & SESPSCH & & SW & & & B0C597 & 25-Aug-94 & & $10098-97-2$ & SR-90 \\
\hline 1330 & SESPSCH & & SW & & & B0C597 & 25-Aug-94 & & $10028-17-8$ & TRITIUM \\
\hline 1331 & SESPSCH & & SW & & & B0C597 & 25-Aug-94 & & 13966-29-5 & $\mathrm{U}-234$ \\
\hline
\end{tabular}




\begin{tabular}{|c|c|c|c|c|c|c|c|c|c|c|}
\hline & A & $\mathrm{B}$ & $C$ & $\mathrm{D}$ & $E$ & $\mathrm{~F}$ & $G$ & $\mathrm{H}$ & $\mathrm{I}$ & $\mathrm{J}$ \\
\hline 1 & SAMPLE_NUM_ASSIGNED_TO & SAMPLER & MEDIA & BIOTA_MEDIA & TAG_ID & SAMP_NUM & SAMP_DATE_TIME| & SAMP_DATE_TIME_ON & CON_ID & CON_SHORT_NAME \\
\hline 1332 & SESPSCH & & SW & & & B0C597 & 25-Aug-94 & & $15117-96-1$ & U-235 \\
\hline 1333 & SESPSCH & & SW & & & B0C597 & 25-Aug-94 & & $\mathrm{U}-238$ & $\mathrm{U}-238$ \\
\hline 1334 & JL JULYA/LE BISPING & & SW & & & B09J86 & 25-Aug-94 & & $7440-47-3$ & $\mathrm{CR}$ \\
\hline 1335 & JL JULYA/LE BISPING & & SW & & & B09J86 & 25-Aug-94 & & $56-23-5$ & CARBTET \\
\hline 1336 & SESPSCH & & SW & & & B0C599 & 25-Aug-94 & & $10098-97-2$ & SR-90 \\
\hline 1337 & SESPSCH & & SW & & & B0C599 & 25-Aug-94 & & $10028-17-8$ & TRITIUM \\
\hline 1338 & SESPSCH & & SW & & & B0C599 & 25-Aug-94 & & $13966-29-5$ & U-234 \\
\hline 1339 & SESPSCH & & SW & & & B0C599 & 25-Aug-94 & & 15117-96-1 & U-235 \\
\hline 1340 & SESPSCH & & SW & & & B0C599 & 25-Aug-94 & & U-238 & U-238 \\
\hline 1341 & JL JULYA/LE BISPING & & SW & & & B09J68 & 25-Aug-94 & & $7440-47-3$ & $\mathrm{CR}$ \\
\hline 1342 & JL JULYA/LE BISPING & & SW & & & B09J68 & 25-Aug-94 & & $56-23-5$ & CARBTET \\
\hline 1343 & SESPSCH & & SW & & & B0C584 & 25-Aug-94 & & $10045-97-3$ & CS-137 \\
\hline 1344 & SESPSCH & & SW & & & B0C584 & 25-Aug-94 & & $10098-97-2$ & SR-90 \\
\hline 1345 & SESPSCH & & SW & & & B0C584 & 25-Aug-94 & & 14133-76-7 & TC-99 \\
\hline 1346 & SESPSCH & & SW & & & B0C584 & 25-Aug-94 & & $10028-17-8$ & TRITIUM \\
\hline 1347 & SESPSCH & & SW & & & B0C584 & 25-Aug-94 & & $13966-29-5$ & U-234 \\
\hline 1348 & SESPSCH & & SW & & & B0C584 & 25-Aug-94 & & 15117-96-1 & U-235 \\
\hline 1349 & SESPSCH & & SW & & & B0C584 & 25-Aug-94 & & $\mathrm{U}-238$ & $\mathrm{U}-238$ \\
\hline 1350 & JL JULYA/LE BISPING & & SW & & & B09J77 & 25-Aug-94 & & $7440-47-3$ & $\mathrm{CR}$ \\
\hline 1351 & JL JULYA/LE BISPING & & SW & & & B09J77 & 25-Aug-94 & & $56-23-5$ & CARBTET \\
\hline 1352 & SESPSCH & & SW & & & $\mathrm{BOC} 5 \mathrm{H} 2$ & 25-Aug-94 & & $10045-97-3$ & CS-137 \\
\hline 1353 & SESPSCH & & SW & & & $\mathrm{B} 0 \mathrm{C} 5 \mathrm{H} 2$ & 25-Aug-94 & & $10098-97-2$ & SR-90 \\
\hline 1354 & SESPSCH & & SW & & & $\mathrm{B} 0 \mathrm{C} 5 \mathrm{H} 2$ & 25-Aug-94 & & 14133-76-7 & TC-99 \\
\hline 1355 & SESPSCH & & SW & & & $\mathrm{B} 0 \mathrm{C} 5 \mathrm{H} 2$ & 25-Aug-94 & & $10028-17-8$ & TRITIUM \\
\hline 1356 & SESPSCH & & SW & & & $\mathrm{BOC} 5 \mathrm{H} 2$ & 25-Aug-94 & & $13966-29-5$ & U-234 \\
\hline 1357 & SESPSCH & & SW & & & $\mathrm{B} 0 \mathrm{C} 5 \mathrm{H} 2$ & 25-Aug-94 & & $15117-96-1$ & U-235 \\
\hline 1358 & SESPSCH & & SW & & & $\mathrm{B} 0 \mathrm{C} 5 \mathrm{H} 2$ & 25-Aug-94 & & U-238 & U-238 \\
\hline 1359 & JL JULYA/LE BISPING & & SW & & & B09J69 & 25-Aug-94 & & $7440-47-3$ & $\mathrm{CR}$ \\
\hline 1360 & JL JULYA/LE BISPING & & SW & & & B09J69 & 25-Aug-94 & & $56-23-5$ & CARBTET \\
\hline 1361 & SESPSCH & & SW & & & B0C585 & 25-Aug-94 & & $10045-97-3$ & CS-137 \\
\hline 1362 & SESPSCH & & SW & & & B0C585 & 25-Aug-94 & & $10098-97-2$ & SR-90 \\
\hline 1363 & SESPSCH & & SW & & & B0C585 & 25-Aug-94 & & 14133-76-7 & TC-99 \\
\hline 1364 & SESPSCH & & SW & & & B0C585 & 25-Aug-94 & & $10028-17-8$ & TRITIUM \\
\hline 1365 & SESPSCH & & SW & & & B0C585 & 25-Aug-94 & & $13966-29-5$ & U-234 \\
\hline 1366 & SESPSCH & & SW & & & B0C585 & 25-Aug-94 & & 15117-96-1 & U-235 \\
\hline 1367 & SESPSCH & & SW & & & B0C585 & 25-Aug-94 & & $\mathrm{U}-238$ & $\mathrm{U}-238$ \\
\hline 1368 & JL JULYA/LE BISPING & & SW & & & B09J70 & 25-Aug-94 & & $7440-47-3$ & CR \\
\hline 1369 & JL JULYA/LE BISPING & & SW & & & B09J70 & 25-Aug-94 & & $56-23-5$ & CARBTET \\
\hline
\end{tabular}




\begin{tabular}{|c|c|c|c|c|c|c|c|c|c|c|}
\hline & $\mathrm{A}$ & $\mathrm{B}$ & $\mathrm{C}$ & $\mathrm{D}$ & $\mathrm{E}$ & $\mathrm{F}$ & $G$ & $\mathrm{H}$ & I & $\mathrm{J}$ \\
\hline 1 & SAMPLE_NUM_ASSIGNED_TO & SAMPLER & MEDIA & BIOTA_MEDIA & TAG_ID & SAMP_NUM & SAMP_DATE_TIME & SAMP_DATE_TIME_ON & CON_ID & CON_SHORT_NAME \\
\hline 1370 & SESPSCH & & SW & & & B0C5H5 & 25-Aug-94 & & $10045-97-3$ & CS-137 \\
\hline 1371 & SESPSCH & & SW & & & B0C5H5 & 25-Aug-94 & & $10098-97-2$ & SR-90 \\
\hline 1372 & SESPSCH & & SW & & & B0C5H5 & 25-Aug-94 & & 14133-76-7 & TC-99 \\
\hline 1373 & SESPSCH & & SW & & & B0C5H5 & 25-Aug-94 & & $10028-17-8$ & TRITIUM \\
\hline 1374 & SESPSCH & & SW & & & B0C5H5 & 25-Aug-94 & & $13966-29-5$ & $\mathrm{U}-234$ \\
\hline 1375 & SESPSCH & & SW & & & B0C5H5 & 25-Aug-94 & & $15117-96-1$ & U-235 \\
\hline 1376 & SESPSCH & & SW & & & B0C5H5 & 25-Aug-94 & & $\mathrm{U}-238$ & $\mathrm{U}-238$ \\
\hline 1377 & JL JULYA/LE BISPING & & SW & & & B09J71 & 25-Aug-94 & & $7440-47-3$ & $\mathrm{CR}$ \\
\hline 1378 & JL JULYA/LE BISPING & & SW & & & B09J71 & 25-Aug-94 & & $56-23-5$ & CARBTET \\
\hline 1379 & SESPSCH & & SW & & & B0C5H6 & 25-Aug-94 & & $10045-97-3$ & CS-137 \\
\hline 1380 & SESPSCH & & SW & & & B0C5H6 & 25-Aug-94 & & $10098-97-2$ & SR-90 \\
\hline 1381 & SESPSCH & & SW & & & B0C5H6 & 25-Aug-94 & & 14133-76-7 & TC-99 \\
\hline 1382 & SESPSCH & & SW & & & B0C5H6 & 25-Aug-94 & & $10028-17-8$ & TRITIUM \\
\hline 1383 & SESPSCH & & SW & & & B0C5H6 & 25-Aug-94 & & $13966-29-5$ & U-234 \\
\hline 1384 & SESPSCH & & SW & & & B0C5H6 & 25-Aug-94 & & 15117-96-1 & U-235 \\
\hline 1385 & SESPSCH & & SW & & & B0C5H6 & 25-Aug-94 & & $\mathrm{U}-238$ & U-238 \\
\hline 1386 & JL JULYA/LE BISPING & & SW & & & B09J72 & 25-Aug-94 & & $7440-47-3$ & $\mathrm{CR}$ \\
\hline 1387 & JL JULYA/LE BISPING & & SW & & & B09J72 & 25-Aug-94 & & $56-23-5$ & CARBTET \\
\hline 1388 & SESPSCH & & SW & & & B0C587 & 25-Aug-94 & & $10045-97-3$ & CS-137 \\
\hline 1389 & SESPSCH & & SW & & & B0C587 & 25-Aug-94 & & $10098-97-2$ & SR-90 \\
\hline 1390 & SESPSCH & & SW & & & B0C587 & 25-Aug-94 & & 14133-76-7 & TC-99 \\
\hline 1391 & SESPSCH & & SW & & & B0C587 & 25-Aug-94 & & $10028-17-8$ & TRITIUM \\
\hline 1392 & SESPSCH & & SW & & & B0C587 & 25-Aug-94 & & $13966-29-5$ & U-234 \\
\hline 1393 & SESPSCH & & SW & & & B0C587 & 25-Aug-94 & & $15117-96-1$ & U-235 \\
\hline 1394 & SESPSCH & & SW & & & B0C587 & 25-Aug-94 & & $\mathrm{U}-238$ & $\mathrm{U}-238$ \\
\hline 1395 & JL JULYA/LE BISPING & & SW & & & B09J73 & 25-Aug-94 & & $7440-47-3$ & $\mathrm{CR}$ \\
\hline 1396 & JL JULYA/LE BISPING & & SW & & & B09J73 & 25-Aug-94 & & $56-23-5$ & CARBTET \\
\hline 1397 & SESPSCH & & SW & & & B0C5H8 & 25-Aug-94 & & $10045-97-3$ & CS-137 \\
\hline 1398 & SESPSCH & & SW & & & $\mathrm{B} 0 \mathrm{C} 5 \mathrm{H} 8$ & 25-Aug-94 & & $10098-97-2$ & SR-90 \\
\hline 1399 & SESPSCH & & SW & & & $\mathrm{B} 0 \mathrm{C} 5 \mathrm{H} 8$ & 25-Aug-94 & & 14133-76-7 & TC-99 \\
\hline 1400 & SESPSCH & & SW & & & B0C5H8 & 25-Aug-94 & & $10028-17-8$ & TRITIUM \\
\hline 1401 & SESPSCH & & SW & & & B0C5H8 & 25-Aug-94 & & $13966-29-5$ & U-234 \\
\hline 1402 & SESPSCH & & SW & & & B0C5H8 & 25-Aug-94 & & 15117-96-1 & U-235 \\
\hline 1403 & SESPSCH & & SW & & & B0C5H8 & 25-Aug-94 & & $\mathrm{U}-238$ & $\mathrm{U}-238$ \\
\hline 1404 & JL JULYA/LE BISPING & & SW & & & B09J74 & 25-Aug-94 & & $7440-47-3$ & $\mathrm{CR}$ \\
\hline 1405 & JL JULYA/LE BISPING & & SW & & & B09J74 & 25-Aug-94 & & $56-23-5$ & CARBTET \\
\hline 1406 & SESPSCH & & SW & & & B0C5H7 & 25-Aug-94 & & $10045-97-3$ & CS-137 \\
\hline 1407 & SESPSCH & & SW & & & B0C5H7 & 25-Aug-94 & & $10098-97-2$ & SR-90 \\
\hline
\end{tabular}




\begin{tabular}{|c|c|c|c|c|c|c|c|c|c|c|}
\hline & A & $\mathrm{B}$ & $C$ & $\mathrm{D}$ & $E$ & $\mathrm{~F}$ & $G$ & $\mathrm{H}$ & $\mathrm{I}$ & $\mathrm{J}$ \\
\hline 1 & SAMPLE_NUM_ASSIGNED_TO & SAMPLER & MEDIA & BIOTA_MEDIA & TAG_ID & SAMP_NUM & SAMP_DATE_TIME| & SAMP_DATE_TIME_ON & CON_ID & CON_SHORT_NAME \\
\hline 1408 & SESPSCH & & SW & & & B0C5H7 & 25-Aug-94 & & 14133-76-7 & TC-99 \\
\hline 1409 & SESPSCH & & SW & & & $\mathrm{B} 0 \mathrm{C} 5 \mathrm{H} 7$ & 25-Aug-94 & & $10028-17-8$ & TRITIUM \\
\hline 1410 & SESPSCH & & SW & & & B0C5H7 & 25-Aug-94 & & $13966-29-5$ & U-234 \\
\hline 1411 & SESPSCH & & SW & & & B0C5H7 & 25-Aug-94 & & 15117-96-1 & U-235 \\
\hline 1412 & SESPSCH & & SW & & & B0C5H7 & 25-Aug-94 & & $\mathrm{U}-238$ & $\mathrm{U}-238$ \\
\hline 1413 & JL JULYA/LE BISPING & & SW & & & B09J75 & 25-Aug-94 & & $7440-47-3$ & CR \\
\hline 1414 & JL JULYA/LE BISPING & & SW & & & B09J75 & 25-Aug-94 & & $56-23-5$ & CARBTET \\
\hline 1415 & SESPSCH & & SW & & & B0С $5 \mathrm{H} 9$ & 25-Aug-94 & & $10045-97-3$ & CS-137 \\
\hline 1416 & SESPSCH & & SW & & & B0C5H9 & 25-Aug-94 & & $10098-97-2$ & SR-90 \\
\hline 1417 & SESPSCH & & SW & & & B0С $5 \mathrm{H} 9$ & 25-Aug-94 & & 14133-76-7 & TC-99 \\
\hline 1418 & SESPSCH & & SW & & & B0С5H9 & 25-Aug-94 & & $10028-17-8$ & TRITIUM \\
\hline 1419 & SESPSCH & & SW & & & B0C5H9 & 25-Aug-94 & & $13966-29-5$ & U-234 \\
\hline 1420 & SESPSCH & & SW & & & B0С $5 \mathrm{H} 9$ & 25-Aug-94 & & $15117-96-1$ & U-235 \\
\hline 1421 & SESPSCH & & SW & & & B0C5H9 & 25-Aug-94 & & U-238 & U-238 \\
\hline 1422 & JL JULYA/LE BISPING & & SW & & & B09J76 & 25-Aug-94 & & $7440-47-3$ & CR \\
\hline 1423 & JL JULYA/LE BISPING & & SW & & & B09J76 & 25-Aug-94 & & $56-23-5$ & CARBTET \\
\hline 1424 & SESPSCH & & SW & & & B0C5J0 & 25-Aug-94 & & $10045-97-3$ & CS-137 \\
\hline 1425 & SESPSCH & & SW & & & B0C5J0 & 25-Aug-94 & & $10098-97-2$ & SR-90 \\
\hline 1426 & SESPSCH & & SW & & & B0C5J0 & 25-Aug-94 & & 14133-76-7 & TC-99 \\
\hline 1427 & SESPSCH & & SW & & & B0C5J0 & 25-Aug-94 & & $10028-17-8$ & TRITIUM \\
\hline 1428 & SESPSCH & & SW & & & B0C5J0 & 25-Aug-94 & & $13966-29-5$ & U-234 \\
\hline 1429 & SESPSCH & & SW & & & B0C5J0 & 25-Aug-94 & & 15117-96-1 & U-235 \\
\hline 1430 & SESPSCH & & SW & & & B0C5J0 & 25-Aug-94 & & $\mathrm{U}-238$ & $\mathrm{U}-238$ \\
\hline 1431 & JL JULYA/LE BISPING & & SW & & & B09J64 & 25-Aug-94 & & $7440-47-3$ & CR \\
\hline 1432 & JL JULYA/LE BISPING & & SW & & & B09J64 & 25-Aug-94 & & $56-23-5$ & CARBTET \\
\hline 1433 & SESPSCH & & SW & & & B0C580 & 25-Aug-94 & & $10098-97-2$ & SR-90 \\
\hline 1434 & SESPSCH & & SW & & & B0C580 & 25-Aug-94 & & $10028-17-8$ & TRITIUM \\
\hline 1435 & SESPSCH & & SW & & & B0C580 & 25-Aug-94 & & $13966-29-5$ & U-234 \\
\hline 1436 & SESPSCH & & SW & & & B0C580 & 25-Aug-94 & & 15117-96-1 & U-235 \\
\hline 1437 & SESPSCH & & SW & & & B0C580 & 25-Aug-94 & & $\mathrm{U}-238$ & $\mathrm{U}-238$ \\
\hline 1438 & JL JULYA/LE BISPING & & SW & & & B09J65 & 25-Aug-94 & & $7440-47-3$ & CR \\
\hline 1439 & JL JULYA/LE BISPING & & SW & & & B09J65 & 25-Aug-94 & & $56-23-5$ & CARBTET \\
\hline 1440 & SESPSCH & & SW & & & B0C581 & 25-Aug-94 & & $10098-97-2$ & SR-90 \\
\hline 1441 & SESPSCH & & SW & & & B0C581 & 25-Aug-94 & & $10028-17-8$ & TRITIUM \\
\hline 1442 & SESPSCH & & SW & & & B0C581 & 25-Aug-94 & & 13966-29-5 & U-234 \\
\hline 1443 & SESPSCH & & SW & & & B0C581 & 25-Aug-94 & & 15117-96-1 & U-235 \\
\hline 1444 & SESPSCH & & SW & & & B0C581 & 25-Aug-94 & & U-238 & U-238 \\
\hline 1445 & JL JULYA/LE BISPING & & SW & & & B09J66 & 25-Aug-94 & & $7440-47-3$ & CR \\
\hline
\end{tabular}




\begin{tabular}{|c|c|c|c|c|c|c|c|c|c|c|}
\hline & A & $\mathrm{B}$ & $C$ & $\mathrm{D}$ & $E$ & $\mathrm{~F}$ & $G$ & $\mathrm{H}$ & $\mathrm{I}$ & $\mathrm{J}$ \\
\hline 1 & SAMPLE_NUM_ASSIGNED_TO & SAMPLER & MEDIA & BIOTA_MEDIA & TAG_ID & SAMP_NUM & SAMP_DATE_TIME| & SAMP_DATE_TIME_ON & CON_ID & CON_SHORT_NAME \\
\hline 1446 & JL JULYA/LE BISPING & & SW & & & B09J66 & 25-Aug-94 & & $56-23-5$ & CARBTET \\
\hline 1447 & SESPSCH & & SW & & & B0C582 & 25-Aug-94 & & $10098-97-2$ & SR-90 \\
\hline 1448 & SESPSCH & & SW & & & B0C582 & 25-Aug-94 & & $10028-17-8$ & TRITIUM \\
\hline 1449 & SESPSCH & & SW & & & B0C582 & 25-Aug-94 & & $13966-29-5$ & U-234 \\
\hline 1450 & SESPSCH & & SW & & & B0C582 & 25-Aug-94 & & $15117-96-1$ & U-235 \\
\hline 1451 & SESPSCH & & SW & & & B0C582 & 25-Aug-94 & & $\mathrm{U}-238$ & $\mathrm{U}-238$ \\
\hline 1452 & JL JULYA/LE BISPING & & SW & & & B09J67 & 25-Aug-94 & & $7440-47-3$ & CR \\
\hline 1453 & JL JULYA/LE BISPING & & SW & & & B09J67 & 25-Aug-94 & & $56-23-5$ & CARBTET \\
\hline 1454 & SESPSCH & & SW & & & B0C583 & 25-Aug-94 & & $10098-97-2$ & SR-90 \\
\hline 1455 & SESPSCH & & SW & & & B0C583 & 25-Aug-94 & & $10028-17-8$ & TRITIUM \\
\hline 1456 & SESPSCH & & SW & & & B0C583 & 25-Aug-94 & & $13966-29-5$ & U-234 \\
\hline 1457 & SESPSCH & & SW & & & B0C583 & 25-Aug-94 & & 15117-96-1 & U-235 \\
\hline 1458 & SESPSCH & & SW & & & B0C583 & 25-Aug-94 & & U-238 & U-238 \\
\hline 1459 & JL JULYA/LE BISPING & & SW & & & B09J98 & 26-Aug-94 & & $7440-47-3$ & CR \\
\hline 1460 & JL JULYA/LE BISPING & & SW & & & B09J98 & 26-Aug-94 & & $56-23-5$ & CARBTET \\
\hline 1461 & SESPSCH & & SW & & & B0C5B7 & 26-Aug-94 & & $10098-97-2$ & SR-90 \\
\hline 1462 & SESPSCH & & SW & & & B0C5B7 & 26-Aug-94 & & $10028-17-8$ & TRITIUM \\
\hline 1463 & SESPSCH & & SW & & & B0C5B7 & 26-Aug-94 & & 13966-29-5 & U-234 \\
\hline 1464 & SESPSCH & & SW & & & B0C5B7 & 26-Aug-94 & & 15117-96-1 & U-235 \\
\hline 1465 & SESPSCH & & SW & & & B0C5B7 & 26-Aug-94 & & $\mathrm{U}-238$ & $\mathrm{U}-238$ \\
\hline 1466 & JL JULYA/LE BISPING & & SW & & & B09J99 & 26-Aug-94 & & $7440-47-3$ & CR \\
\hline 1467 & JL JULYA/LE BISPING & & SW & & & B09J99 & 26-Aug-94 & & $56-23-5$ & CARBTET \\
\hline 1468 & SESPSCH & & SW & & & B0C5B8 & 26-Aug-94 & & $10098-97-2$ & SR-90 \\
\hline 1469 & SESPSCH & & SW & & & B0C5B8 & 26-Aug-94 & & $10028-17-8$ & TRITIUM \\
\hline 1470 & SESPSCH & & SW & & & B0C5B8 & 26-Aug-94 & & $13966-29-5$ & U-234 \\
\hline 1471 & SESPSCH & & SW & & & B0С5B8 & 26-Aug-94 & & $15117-96-1$ & U-235 \\
\hline 1472 & SESPSCH & & SW & & & В0С5B8 & 26-Aug-94 & & U-238 & U-238 \\
\hline 1473 & JL JULYA/LE BISPING & & SW & & & B09JB0 & 26-Aug-94 & & $7440-47-3$ & $\mathrm{CR}$ \\
\hline 1474 & JL JULYA/LE BISPING & & SW & & & B09JB0 & 26-Aug-94 & & $56-23-5$ & CARBTET \\
\hline 1475 & SESPSCH & & SW & & & B0С5B9 & 26-Aug-94 & & $10098-97-2$ & SR-90 \\
\hline 1476 & SESPSCH & & SW & & & B0С5B9 & 26-Aug-94 & & $10028-17-8$ & TRITIUM \\
\hline 1477 & SESPSCH & & SW & & & B0С5B9 & 26-Aug-94 & & 13966-29-5 & U-234 \\
\hline 1478 & SESPSCH & & SW & & & B0С5B9 & 26-Aug-94 & & 15117-96-1 & U-235 \\
\hline 1479 & SESPSCH & & SW & & & В0С5B9 & 26-Aug-94 & & U-238 & U-238 \\
\hline 1480 & JL JULYA/LE BISPING & & SW & & & B09J60 & 26-Aug-94 & & $7440-47-3$ & $\mathrm{CR}$ \\
\hline 1481 & JL JULYA/LE BISPING & & SW & & & B09J60 & 26-Aug-94 & & $56-23-5$ & CARBTET \\
\hline 1482 & SESPSCH & & SW & & & $\mathrm{B} 0 \mathrm{C} 5 \mathrm{CO}$ & 26-Aug-94 & & $10098-97-2$ & SR-90 \\
\hline 1483 & SESPSCH & & SW & & & B0C5C0 & 26-Aug-94 & & $10028-17-8$ & TRITIUM \\
\hline
\end{tabular}




\begin{tabular}{|c|c|c|c|c|c|c|c|c|c|c|}
\hline & A & $\mathrm{B}$ & $C$ & $\mathrm{D}$ & $E$ & $\mathrm{~F}$ & $G$ & $\mathrm{H}$ & $\mathrm{I}$ & $\mathrm{J}$ \\
\hline 1 & SAMPLE_NUM_ASSIGNED_TO & SAMPLER & MEDIA & BIOTA_MEDIA & TAG_ID & SAMP_NUM & SAMP_DATE_TIME| & SAMP_DATE_TIME_ON & CON_ID & CON_SHORT_NAME \\
\hline 1484 & SESPSCH & & SW & & & $\mathrm{B} 0 \mathrm{C} 5 \mathrm{CO}$ & 26-Aug-94 & & 13966-29-5 & U-234 \\
\hline 1485 & SESPSCH & & SW & & & B0C5C0 & 26-Aug-94 & & 15117-96-1 & U-235 \\
\hline 1486 & SESPSCH & & SW & & & B0C5C0 & 26-Aug-94 & & U-238 & U-238 \\
\hline 1487 & PS HENRY/LE BISPING & & SW & & & B0CSC3 & 26-Aug-94 & & $7440-47-3$ & CR \\
\hline 1488 & PS HENRY/LE BISPING & & SW & & & B0CSC3 & 26-Aug-94 & & $56-23-5$ & CARBTET \\
\hline 1489 & SESPSCH & & SW & & & B0C5C1 & 26-Aug-94 & & $10098-97-2$ & SR-90 \\
\hline 1490 & SESPSCH & & SW & & & B0C5C1 & 26-Aug-94 & & $10028-17-8$ & TRITIUM \\
\hline 1491 & SESPSCH & & SW & & & B0C5C1 & 26-Aug-94 & & $13966-29-5$ & $\mathrm{U}-234$ \\
\hline 1492 & SESPSCH & & SW & & & B0C5C1 & 26-Aug-94 & & 15117-96-1 & U-235 \\
\hline 1493 & SESPSCH & & SW & & & B0C5C1 & 26-Aug-94 & & U-238 & U-238 \\
\hline 1494 & PS HENRY/LE BISPING & & SW & & & B0CSC4 & 26-Aug-94 & & $7440-47-3$ & $\mathrm{CR}$ \\
\hline 1495 & PS HENRY/LE BISPING & & SW & & & BoCSC4 & 26-Aug-94 & & $56-23-5$ & CARBTET \\
\hline 1496 & SESPSCH & & SW & & & B0C5C2 & 26-Aug-94 & & $10098-97-2$ & SR-90 \\
\hline 1497 & SESPSCH & & SW & & & B0C5C2 & 26-Aug-94 & & 10028-17-8 & TRITIUM \\
\hline 1498 & SESPSCH & & SW & & & B0C5C2 & 26-Aug-94 & & 13966-29-5 & U-234 \\
\hline 1499 & SESPSCH & & SW & & & B0C5C2 & 26-Aug-94 & & 15117-96-1 & U-235 \\
\hline 1500 & SESPSCH & & SW & & & B0C5C2 & 26-Aug-94 & & U-238 & $\mathrm{U}-238$ \\
\hline 1501 & PS HENRY/LE BISPING & & SW & & & B0CSC5 & 26-Aug-94 & & $7440-47-3$ & $\mathrm{CR}$ \\
\hline 1502 & PS HENRY/LE BISPING & & SW & & & B0CSC5 & 26-Aug-94 & & $56-23-5$ & CARBTET \\
\hline 1503 & SESPSCH & & SW & & & B0C5C3 & 26-Aug-94 & & $10098-97-2$ & SR-90 \\
\hline 1504 & SESPSCH & & SW & & & B0C5C3 & 26-Aug-94 & & 10028-17-8 & TRITIUM \\
\hline 1505 & SESPSCH & & SW & & & B0C5C3 & 26-Aug-94 & & 13966-29-5 & U-234 \\
\hline 1506 & SESPSCH & & SW & & & B0C5C3 & 26-Aug-94 & & $15117-96-1$ & U-235 \\
\hline 1507 & SESPSCH & & SW & & & $\mathrm{B} 0 \mathrm{C} 5 \mathrm{C} 3$ & 26-Aug-94 & & $U-238$ & $\mathrm{U}-238$ \\
\hline 1508 & PS HENRY/LE BISPING & & SW & & & B0CSC6 & 26-Aug-94 & & $7440-47-3$ & $\mathrm{CR}$ \\
\hline 1509 & PS HENRY/LE BISPING & & SW & & & B0CSC6 & 26-Aug-94 & & $56-23-5$ & CARBTET \\
\hline 1510 & SESPSCH & & SW & & & B0C5C4 & 26-Aug-94 & & $10098-97-2$ & SR-90 \\
\hline 1511 & SESPSCH & & SW & & & B0C5C4 & 26-Aug-94 & & $10028-17-8$ & TRITIUM \\
\hline 1512 & SESPSCH & & SW & & & B0C5C4 & 26-Aug-94 & & $13966-29-5$ & U-234 \\
\hline 1513 & SESPSCH & & SW & & & B0C5C4 & 26-Aug-94 & & $15117-96-1$ & U-235 \\
\hline 1514 & SESPSCH & & SW & & & B0C5C4 & 26-Aug-94 & & U-238 & $\mathrm{U}-238$ \\
\hline 1515 & PS HENRY/LE BISPING & & SW & & & B0CSC7 & 26-Aug-94 & & $7440-47-3$ & CR \\
\hline 1516 & PS HENRY/LE BISPING & & SW & & & B0CSC7 & 26-Aug-94 & & $56-23-5$ & CARBTET \\
\hline 1517 & SESPSCH & & SW & & & B0C5C5 & 26-Aug-94 & & $10098-97-2$ & SR-90 \\
\hline 1518 & SESPSCH & & SW & & & B0C5C5 & 26-Aug-94 & & $10028-17-8$ & TRITIUM \\
\hline 1519 & SESPSCH & & SW & & & B0C5C5 & 26-Aug-94 & & 13966-29-5 & U-234 \\
\hline 1520 & SESPSCH & & SW & & & B0C5C5 & 26-Aug-94 & & $15117-96-1$ & U-235 \\
\hline 1521 & SESPSCH & & SW & & & B0C5C5 & 26-Aug-94 & & U-238 & U-238 \\
\hline
\end{tabular}




\begin{tabular}{|c|c|c|}
\hline & A & B \\
\hline 1 & SAMPLE_NUM_ASSIGNED_TO & SAMPLER \\
\hline
\end{tabular}

\begin{tabular}{c|c|c|c|}
\hline C & D & E \\
\hline & MEDIA & BIOTA MEDIA & TAG ID \\
\hline
\end{tabular} 1522 PS HENRY/LE BISPING

\begin{tabular}{|l|l|}
1523 & PS HENRY/LE BISPING \\
\hline 1524 & SESPSCH \\
\hline
\end{tabular}

1524 SESPSCH

1525 SESPSCH

1526 SESPSCH

\begin{tabular}{|l|l|l|}
\hline 1527 & SESPSCH \\
\hline 1528 & SESPSCH \\
\hline
\end{tabular}

\begin{tabular}{|l|l}
1528 & SESPSCH \\
\hline 1529 & PS HENRYILE
\end{tabular}

1529 PS HENRY/LE BISPING

\begin{tabular}{|l|l}
1530 & PS HENRY/LE BISPING \\
\hline
\end{tabular}

1531 SESPSCH

1532 SESPSCH

\begin{tabular}{|l|l|l}
1533 & SESPSCH \\
\hline 1534 & SESPSCH \\
\hline
\end{tabular}

1534 SESPSCH

1535 SESPSCH

1536 PS HENRY/LE BISPING

1537 PS HENRY/LE BISPING

1538 SESPSCH

1539 SESPSCH

\begin{tabular}{|l|l|}
1540 & SESPSCH \\
\hline
\end{tabular}

1541 SESPSCH

1542 SESPSCH

\begin{tabular}{|l|l|}
1543 & PS HENRY/LE BISPING \\
\hline 1544 & PS HENRY/LE BISPING \\
\hline
\end{tabular}

\begin{tabular}{|l|l|}
1544 & PS HENRY/LE BISPING \\
\hline
\end{tabular}

1545 SESPSCH

1546 SESPSCH

\begin{tabular}{|l|l|}
1547 & SESPSCH \\
\hline 1548 & SESPSCH \\
\hline
\end{tabular}

\begin{tabular}{|l|l|}
1548 & SESPSCH \\
\hline 1549 & SESPSCH \\
\hline
\end{tabular}

1549 SESPSCH

1550 PS HENRY/LE BISPING

\begin{tabular}{|l|l}
1551 & PS HENRY/LE BISPING \\
\hline
\end{tabular}

\begin{tabular}{|l|l|}
1552 & SESPSCH \\
\hline 1553 & SESPSCH
\end{tabular}

1553 SESPSCH

\begin{tabular}{rl|l}
1554 & SESPSCH \\
\hline 1555 & SESPSCH
\end{tabular}

\begin{tabular}{|l|l|}
1555 & SESPSCH \\
\hline 1556 & SESPSCH
\end{tabular}

1556 SESPSCH

1557 PS HENRY/LE BISPING

\begin{tabular}{|l|l|}
1558 & PS HENRY/LE BISPING \\
\hline 1559 & SESPSCH \\
\hline
\end{tabular}

\begin{tabular}{|l|l|}
\hline 1559 & SESPSCH \\
\hline
\end{tabular}

\begin{tabular}{|c|c|c|c|c|c|c|c|}
\hline MEDIA & BIOTA_MEDIA & TAG_ID & SAMP_NUM & SAMP_DATE_TIME & SAMP_DATE_TIME_ON & CON_ID & CON_SHORT_NAME \\
\hline SW & & & B0CSC8 & 26-Aug-94 & & $7440-47-3$ & CR \\
\hline SW & & & B0CSC8 & 26-Aug-94 & & $56-23-5$ & CARBTET \\
\hline SW & & & B0C5C6 & 26-Aug-94 & & $10098-97-2$ & SR-90 \\
\hline SW & & & B0C5C6 & 26-Aug-94 & & $10028-17-8$ & TRITIUM \\
\hline SW & & & B0C5C6 & 26-Aug-94 & & 13966-29-5 & U-234 \\
\hline SW & & & B0C5C6 & 26-Aug-94 & & 15117-96-1 & U-235 \\
\hline SW & & & B0C5C6 & 26-Aug-94 & & $\mathrm{U}-238$ & U-238 \\
\hline SW & & & B0CSC9 & 26-Aug-94 & & $7440-47-3$ & CR \\
\hline SW & & & B0CSC9 & 26-Aug-94 & & $56-23-5$ & CARBTET \\
\hline SW & & & B0C5C7 & 26-Aug-94 & & $10098-97-2$ & SR-90 \\
\hline SW & & & B0C5C7 & 26-Aug-94 & & $10028-17-8$ & TRITIUM \\
\hline SW & & & B0C5C7 & 26-Aug-94 & & $13966-29-5$ & $\mathrm{U}-234$ \\
\hline SW & & & B0C5C7 & 26-Aug-94 & & 15117-96-1 & U-235 \\
\hline SW & & & B0C5C7 & 26-Aug-94 & & $\mathrm{U}-238$ & U-238 \\
\hline SW & & & B0CSD8 & 26-Aug-94 & & $7440-47-3$ & CR \\
\hline SW & & & B0CSD8 & 26-Aug-94 & & $56-23-5$ & CARBTET \\
\hline SW & & & B0C5D6 & 26-Aug-94 & & $10098-97-2$ & SR-90 \\
\hline SW & & & B0C5D6 & 26-Aug-94 & & $10028-17-8$ & TRITIUM \\
\hline SW & & & B0C5D6 & 26-Aug-94 & & $13966-29-5$ & U-234 \\
\hline SW & & & B0C5D6 & 26-Aug-94 & & 15117-96-1 & U-235 \\
\hline SW & & & B0C5D6 & 26-Aug-94 & & U-238 & U-238 \\
\hline SW & & & BOCSD0 & 26-Aug-94 & & $7440-47-3$ & CR \\
\hline SW & & & BOCSDO & 26-Aug-94 & & $56-23-5$ & CARBTET \\
\hline SW & & & B0C5C8 & 26-Aug-94 & & $10098-97-2$ & SR-90 \\
\hline SW & & & B0C5C8 & 26-Aug-94 & & $10028-17-8$ & TRITIUM \\
\hline SW & & & B0C5C8 & 26-Aug-94 & & $13966-29-5$ & U-234 \\
\hline SW & & & B0C5C8 & 26-Aug-94 & & 15117-96-1 & U-235 \\
\hline SW & & & B0C5C8 & 26-Aug-94 & & U-238 & U-238 \\
\hline SW & & & B0CSD1 & 26-Aug-94 & & $7440-47-3$ & CR \\
\hline SW & & & B0CSD1 & 26-Aug-94 & & $56-23-5$ & CARBTET \\
\hline SW & & & B0C5C9 & 26-Aug-94 & & $10098-97-2$ & SR-90 \\
\hline SW & & & B0C5C9 & 26-Aug-94 & & $10028-17-8$ & TRITIUM \\
\hline SW & & & B0C5C9 & 26-Aug-94 & & $13966-29-5$ & U-234 \\
\hline SW & & & B0C5C9 & 26-Aug-94 & & 15117-96-1 & U-235 \\
\hline SW & & & В0С5C9 & 26-Aug-94 & & U-238 & U-238 \\
\hline SW & & & B0CSD2 & 26-Aug-94 & & $7440-47-3$ & CR \\
\hline SW & & & B0CSD2 & 26-Aug-94 & & $56-23-5$ & CARBTET \\
\hline SW & & & B0C5D0 & 26-Aug-94 & & $10098-97-2$ & SR-90 \\
\hline
\end{tabular}

Page 41 


\begin{tabular}{|c|c|c|c|c|c|c|c|c|c|c|}
\hline & A & $\mathrm{B}$ & $C$ & $\mathrm{D}$ & $E$ & $\mathrm{~F}$ & $G$ & $\mathrm{H}$ & $\mathrm{I}$ & $\mathrm{J}$ \\
\hline 1 & SAMPLE_NUM_ASSIGNED_TO & SAMPLER & MEDIA & BIOTA_MEDIA & TAG_ID & SAMP_NUM & SAMP_DATE_TIME| & SAMP_DATE_TIME_ON & CON_ID & CON_SHORT_NAME \\
\hline 1560 & SESPSCH & & SW & & & B0C5D0 & 26-Aug-94 & & 10028-17-8 & TRITIUM \\
\hline 1561 & SESPSCH & & SW & & & B0C5D0 & 26-Aug-94 & & 13966-29-5 & $\mathrm{U}-234$ \\
\hline 1562 & SESPSCH & & SW & & & B0C5D0 & 26-Aug-94 & & 15117-96-1 & U-235 \\
\hline 1563 & SESPSCH & & SW & & & B0C5D0 & 26-Aug-94 & & $\mathrm{U}-238$ & $\mathrm{U}-238$ \\
\hline 1564 & PS HENRY/LE BISPING & & SW & & & B0CSD3 & 26-Aug-94 & & $7440-47-3$ & CR \\
\hline 1565 & PS HENRY/LE BISPING & & SW & & & B0CSD3 & 26-Aug-94 & & $56-23-5$ & CARBTET \\
\hline 1566 & SESPSCH & & SW & & & B0C5D1 & 26-Aug-94 & & $10098-97-2$ & SR-90 \\
\hline 1567 & SESPSCH & & SW & & & B0C5D1 & 26-Aug-94 & & 10028-17-8 & TRITIUM \\
\hline 1568 & SESPSCH & & SW & & & B0C5D1 & 26-Aug-94 & & 13966-29-5 & U-234 \\
\hline 1569 & SESPSCH & & SW & & & B0C5D1 & 26-Aug-94 & & 15117-96-1 & U-235 \\
\hline 1570 & SESPSCH & & SW & & & B0C5D1 & 26-Aug-94 & & U-238 & $\mathrm{U}-238$ \\
\hline 1571 & PS HENRY/LE BISPING & & SW & & & B0CSD4 & 26-Aug-94 & & $7440-47-3$ & CR \\
\hline 1572 & PS HENRY/LE BISPING & & SW & & & B0CSD4 & 26-Aug-94 & & $56-23-5$ & CARBTET \\
\hline 1573 & SESPSCH & & SW & & & B0C5D2 & 26-Aug-94 & & $10098-97-2$ & SR-90 \\
\hline 1574 & SESPSCH & & SW & & & B0C5D2 & 26-Aug-94 & & 10028-17-8 & TRITIUM \\
\hline 1575 & SESPSCH & & SW & & & B0C5D2 & 26-Aug-94 & & $13966-29-5$ & U-234 \\
\hline 1576 & SESPSCH & & SW & & & B0C5D2 & 26-Aug-94 & & $15117-96-1$ & U-235 \\
\hline 1577 & SESPSCH & & SW & & & B0C5D2 & 26-Aug-94 & & U-238 & $\mathrm{U}-238$ \\
\hline 1578 & PS HENRY/LE BISPING & & SW & & & B0CSD5 & 26-Aug-94 & & $7440-47-3$ & $\mathrm{CR}$ \\
\hline 1579 & PS HENRY/LE BISPING & & SW & & & B0CSD5 & 26-Aug-94 & & $56-23-5$ & CARBTET \\
\hline 1580 & SESPSCH & & SW & & & B0C5D3 & 26-Aug-94 & & $10098-97-2$ & SR-90 \\
\hline 1581 & SESPSCH & & SW & & & B0C5D3 & 26-Aug-94 & & $10028-17-8$ & TRITIUM \\
\hline 1582 & SESPSCH & & SW & & & B0C5D3 & 26-Aug-94 & & $13966-29-5$ & U-234 \\
\hline 1583 & SESPSCH & & SW & & & B0C5D3 & 26-Aug-94 & & $15117-96-1$ & $\mathrm{U}-235$ \\
\hline 1584 & SESPSCH & & SW & & & B0C5D3 & 26-Aug-94 & & U-238 & $\mathrm{U}-238$ \\
\hline 1585 & PS HENRY/LE BISPING & & SW & & & B0CSD6 & 26-Aug-94 & & $7440-47-3$ & CR \\
\hline 1586 & PS HENRY/LE BISPING & & SW & & & B0CSD6 & 26-Aug-94 & & $56-23-5$ & CARBTET \\
\hline 1587 & SESPSCH & & SW & & & B0C5D4 & 26-Aug-94 & & $10098-97-2$ & SR-90 \\
\hline 1588 & SESPSCH & & SW & & & B0C5D4 & 26-Aug-94 & & $10028-17-8$ & TRITIUM \\
\hline 1589 & SESPSCH & & SW & & & B0C5D4 & 26-Aug-94 & & $13966-29-5$ & U-234 \\
\hline 1590 & SESPSCH & & SW & & & B0C5D4 & 26-Aug-94 & & $15117-96-1$ & U-235 \\
\hline 1591 & SESPSCH & & SW & & & B0C5D4 & 26-Aug-94 & & U-238 & U-238 \\
\hline 1592 & PS HENRY/LE BISPING & & SW & & & B0CSD7 & 26-Aug-94 & & $7440-47-3$ & $\mathrm{CR}$ \\
\hline 1593 & PS HENRY/LE BISPING & & SW & & & B0CSD7 & 26-Aug-94 & & $56-23-5$ & CARBTET \\
\hline 1594 & SESPSCH & & SW & & & B0C5D5 & 26-Aug-94 & & $10098-97-2$ & SR-90 \\
\hline 1595 & SESPSCH & & SW & & & B0C5D5 & 26-Aug-94 & & $10028-17-8$ & TRITIUM \\
\hline 1596 & SESPSCH & & SW & & & B0C5D5 & 26-Aug-94 & & $13966-29-5$ & U-234 \\
\hline 1597 & SESPSCH & & SW & & & B0C5D5 & 26-Aug-94 & & $15117-96-1$ & U-235 \\
\hline
\end{tabular}




\begin{tabular}{|c|c|c|c|c|c|c|c|c|c|c|}
\hline & A & $\mathrm{B}$ & $C$ & $\mathrm{D}$ & $E$ & $\mathrm{~F}$ & $G$ & $\mathrm{H}$ & $\mathrm{I}$ & $\mathrm{J}$ \\
\hline 1 & SAMPLE_NUM_ASSIGNED_TO & SAMPLER & MEDIA & BIOTA_MEDIA & TAG_ID & SAMP_NUM & SAMP_DATE_TIME| & SAMP_DATE_TIME_ON & CON_ID & CON_SHORT_NAME \\
\hline 1598 & SESPSCH & & SW & & & B0C5D5 & 26-Aug-94 & & $\mathrm{U}-238$ & U-238 \\
\hline 1599 & JL JULYA/LE BISPING & & SW & & & B09J88 & 02-Sep-94 & & $7440-47-3$ & $\mathrm{CR}$ \\
\hline 1600 & JL JULYA/LE BISPING & & SW & & & B09J88 & 02-Sep-94 & & $56-23-5$ & CARBTET \\
\hline 1601 & SESPSCH & & SW & & & B0C5B0 & 02-Sep-94 & & $10098-97-2$ & SR-90 \\
\hline 1602 & SESPSCH & & SW & & & B0С5B0 & 02-Sep-94 & & 14133-76-7 & TC-99 \\
\hline 1603 & SESPSCH & & SW & & & B0C5B0 & 02-Sep-94 & & $10028-17-8$ & TRITIUM \\
\hline 1604 & SESPSCH & & SW & & & B0C5B0 & 02-Sep-94 & & $13966-29-5$ & U-234 \\
\hline 1605 & SESPSCH & & SW & & & B0С5B0 & 02-Sep-94 & & 15117-96-1 & U-235 \\
\hline 1606 & SESPSCH & & SW & & & B0C5B0 & 02-Sep-94 & & $\mathrm{U}-238$ & $\mathrm{U}-238$ \\
\hline 1607 & JL JULYA/LE BISPING & & SW & & & B09J97 & 02-Sep-94 & & $7440-47-3$ & $\mathrm{CR}$ \\
\hline 1608 & JL JULYA/LE BISPING & & SW & & & B09J97 & 02-Sep-94 & & $56-23-5$ & CARBTET \\
\hline 1609 & SESPSCH & & SW & & & B0C5B5 & 02-Sep-94 & & $10098-97-2$ & SR-90 \\
\hline 1610 & SESPSCH & & SW & & & B0C5B5 & 02-Sep-94 & & 14133-76-7 & TC-99 \\
\hline 1611 & SESPSCH & & SW & & & B0C5B5 & 02-Sep-94 & & $10028-17-8$ & TRITIUM \\
\hline 1612 & SESPSCH & & SW & & & B0C5B5 & 02-Sep-94 & & $13966-29-5$ & U-234 \\
\hline 1613 & SESPSCH & & SW & & & B0C5B5 & 02-Sep-94 & & 15117-96-1 & U-235 \\
\hline 1614 & SESPSCH & & SW & & & B0C5B5 & 02-Sep-94 & & $\mathrm{U}-238$ & $\mathrm{U}-238$ \\
\hline 1615 & JL JULYA/LE BISPING & & SW & & & B09J89 & 02-Sep-94 & & $7440-47-3$ & $\mathrm{CR}$ \\
\hline 1616 & JL JULYA/LE BISPING & & SW & & & B09J89 & 02-Sep-94 & & $56-23-5$ & CARBTET \\
\hline 1617 & SESPSCH & & SW & & & B0C5B1 & 02-Sep-94 & & $10098-97-2$ & SR-90 \\
\hline 1618 & SESPSCH & & SW & & & B0C5B1 & 02-Sep-94 & & 14133-76-7 & TC-99 \\
\hline 1619 & SESPSCH & & SW & & & B0C5B1 & 02-Sep-94 & & $10028-17-8$ & TRITIUM \\
\hline 1620 & SESPSCH & & SW & & & B0C5B1 & 02-Sep-94 & & $13966-29-5$ & U-234 \\
\hline 1621 & SESPSCH & & SW & & & B0C5B1 & 02-Sep-94 & & $15117-96-1$ & U-235 \\
\hline 1622 & SESPSCH & & SW & & & B0C5B1 & 02-Sep-94 & & $\mathrm{U}-238$ & $\mathrm{U}-238$ \\
\hline 1623 & JL JULYA/LE BISPING & & SW & & & B09J90 & 02-Sep-94 & & $7440-47-3$ & CR \\
\hline 1624 & JL JULYA/LE BISPING & & SW & & & B09J90 & 02-Sep-94 & & $56-23-5$ & CARBTET \\
\hline 1625 & SESPSCH & & SW & & & B0C5B6 & 02-Sep-94 & & $10098-97-2$ & SR-90 \\
\hline 1626 & SESPSCH & & SW & & & B0C5B6 & 02-Sep-94 & & 14133-76-7 & TC-99 \\
\hline 1627 & SESPSCH & & SW & & & B0C5B6 & 02-Sep-94 & & $10028-17-8$ & TRITIUM \\
\hline 1628 & SESPSCH & & SW & & & B0C5B6 & 02-Sep-94 & & $13966-29-5$ & $\mathrm{U}-234$ \\
\hline 1629 & SESPSCH & & SW & & & B0C5B6 & 02-Sep-94 & & 15117-96-1 & U-235 \\
\hline 1630 & SESPSCH & & SW & & & B0C5B6 & 02-Sep-94 & & $\mathrm{U}-238$ & $\mathrm{U}-238$ \\
\hline 1631 & JL JULYA/LE BISPING & & SW & & & B09J91 & 02-Sep-94 & & $7440-47-3$ & CR \\
\hline 1632 & JL JULYA/LE BISPING & & SW & & & B09J91 & 02-Sep-94 & & $56-23-5$ & CARBTET \\
\hline 1633 & SESPSCH & & SW & & & B0C586 & 02-Sep-94 & & $10098-97-2$ & SR-90 \\
\hline 1634 & SESPSCH & & SW & & & B0C586 & 02-Sep-94 & & $14133-76-7$ & TC-99 \\
\hline 1635 & SESPSCH & & SW & & & B0C586 & 02-Sep-94 & & $10028-17-8$ & TRITIUM \\
\hline
\end{tabular}




\begin{tabular}{|c|c|c|c|c|c|c|c|c|c|c|}
\hline & A & $\mathrm{B}$ & $\mathrm{C}$ & $\mathrm{D}$ & $E$ & $\mathrm{~F}$ & G & $\mathrm{H}$ & $\mathrm{I}$ & $\mathrm{J}$ \\
\hline 1 & SAMPLE_NUM_ASSIGNED_TO & SAMPLER & MEDIA & BIOTA_MEDIA & TAG_ID & SAMP_NUM & SAMP_DATE_TIME & SAMP_DATE_TIME_ON & CON_ID & CON_SHORT_NAME \\
\hline 1636 & SESPSCH & & SW & & & B0C586 & 02-Sep-94 & & $13966-29-5$ & $\mathrm{U}-234$ \\
\hline 1637 & SESPSCH & & SW & & & B0C586 & 02-Sep-94 & & 15117-96-1 & U-235 \\
\hline 1638 & SESPSCH & & SW & & & B0C586 & 02-Sep-94 & & U-238 & U-238 \\
\hline 1639 & JL JULYA/LE BISPING & & SW & & & B09J92 & 02-Sep-94 & & $7440-47-3$ & $\mathrm{CR}$ \\
\hline 1640 & JL JULYA/LE BISPING & & SW & & & B09J92 & 02-Sep-94 & & $56-23-5$ & CARBTET \\
\hline 1641 & SESPSCH & & SW & & & B0C590 & 02-Sep-94 & & $10098-97-2$ & SR-90 \\
\hline \begin{tabular}{|l|l|}
1642 \\
\end{tabular} & SESPSCH & & SW & & & B0C590 & 02-Sep-94 & & 14133-76-7 & TC-99 \\
\hline 1643 & SESPSCH & & SW & & & B0C590 & 02-Sep-94 & & $10028-17-8$ & TRITIUM \\
\hline 1644 & SESPSCH & & SW & & & B0C590 & 02-Sep-94 & & $13966-29-5$ & U-234 \\
\hline 1645 & SESPSCH & & SW & & & B0C590 & 02-Sep-94 & & $15117-96-1$ & U-235 \\
\hline 1646 & SESPSCH & & SW & & & B0C590 & 02-Sep-94 & & U-238 & U-238 \\
\hline 1647 & JL JULYA/LE BISPING & & SW & & & B09J93 & 02-Sep-94 & & $7440-47-3$ & CR \\
\hline 1648 & JL JULYA/LE BISPING & & SW & & & B09J93 & 02-Sep-94 & & $56-23-5$ & CARBTET \\
\hline 1649 & SESPSCH & & SW & & & B0C591 & 02-Sep-94 & & $10098-97-2$ & SR-90 \\
\hline 1650 & SESPSCH & & SW & & & B0C591 & 02-Sep-94 & & 14133-76-7 & TC-99 \\
\hline 1651 & SESPSCH & & SW & & & B0C591 & 02-Sep-94 & & $10028-17-8$ & TRITIUM \\
\hline \begin{tabular}{|l|}
1652 \\
165
\end{tabular} & SESPSCH & & SW & & & B0C591 & 02-Sep-94 & & $13966-29-5$ & U-234 \\
\hline 1653 & SESPSCH & & SW & & & B0C591 & 02-Sep-94 & & 15117-96-1 & U-235 \\
\hline 1654 & SESPSCH & & SW & & & B0C591 & 02-Sep-94 & & U-238 & U-238 \\
\hline \begin{tabular}{|l|}
1655 \\
\end{tabular} & JL JULYA/LE BISPING & & SW & & & B09J94 & 02-Sep-94 & & $7440-47-3$ & $\mathrm{CR}$ \\
\hline 1656 & JL JULYA/LE BISPING & & SW & & & B09J94 & 02-Sep-94 & & $56-23-5$ & CARBTET \\
\hline 1657 & SESPSCH & & SW & & & B0C5B2 & 02-Sep-94 & & $10098-97-2$ & SR-90 \\
\hline 1658 & SESPSCH & & SW & & & B0C5B2 & 02-Sep-94 & & 14133-76-7 & TC-99 \\
\hline 1659 & SESPSCH & & SW & & & B0C5B2 & 02-Sep-94 & & $10028-17-8$ & TRITIUM \\
\hline 1660 & SESPSCH & & SW & & & B0C5B2 & 02-Sep-94 & & $13966-29-5$ & U-234 \\
\hline 1661 & SESPSCH & & SW & & & B0C5B2 & 02-Sep-94 & & 15117-96-1 & U-235 \\
\hline 1662 & SESPSCH & & SW & & & B0C5B2 & 02-Sep-94 & & U-238 & U-238 \\
\hline 1663 & JL JULYA/LE BISPING & & SW & & & B09J95 & 02-Sep-94 & & $7440-47-3$ & $\mathrm{CR}$ \\
\hline 1664 & JL JULYA/LE BISPING & & SW & & & B09J95 & 02-Sep-94 & & $56-23-5$ & CARBTET \\
\hline \begin{tabular}{|l|l|}
1665 \\
\end{tabular} & SESPSCH & & SW & & & B0C5B3 & 02-Sep-94 & & $10098-97-2$ & SR-90 \\
\hline 1666 & SESPSCH & & SW & & & B0C5B3 & 02-Sep-94 & & 14133-76-7 & TC-99 \\
\hline \begin{tabular}{|l|}
1667 \\
\end{tabular} & SESPSCH & & SW & & & B0C5B3 & 02-Sep-94 & & $10028-17-8$ & TRITIUM \\
\hline 1668 & SESPSCH & & SW & & & B0C5B3 & 02-Sep-94 & & $13966-29-5$ & U-234 \\
\hline 1669 & SESPSCH & & SW & & & B0C5B3 & 02-Sep-94 & & 15117-96-1 & U-235 \\
\hline 1670 & SESPSCH & & SW & & & B0C5B3 & 02-Sep-94 & & U-238 & U-238 \\
\hline \begin{tabular}{|l|}
1671 \\
\end{tabular} & JL JULYA/LE BISPING & & SW & & & B09J96 & 02-Sep-94 & & $7440-47-3$ & $\mathrm{CR}$ \\
\hline 1672 & JL JULYA/LE BISPING & & SW & & & B09J96 & 02-Sep-94 & & $56-23-5$ & CARBTET \\
\hline 1673 & SESPSCH & & SW & & & B0C5B4 & 02-Sep-94 & & $10098-97-2$ & SR-90 \\
\hline
\end{tabular}




\begin{tabular}{|c|c|c|c|c|c|c|c|c|c|c|}
\hline & A & $\mathrm{B}$ & $C$ & $\mathrm{D}$ & $E$ & $\mathrm{~F}$ & $G$ & $\mathrm{H}$ & $\mathrm{I}$ & $\mathrm{J}$ \\
\hline 1 & SAMPLE_NUM_ASSIGNED_TO & SAMPLER & MEDIA & BIOTA_MEDIA & TAG_ID & SAMP_NUM & SAMP_DATE_TIME| & SAMP_DATE_TIME_ON & CON_ID & CON_SHORT_NAME \\
\hline 1674 & SESPSCH & & SW & & & B0C5B4 & 02-Sep-94 & & 14133-76-7 & TC-99 \\
\hline 1675 & SESPSCH & & SW & & & B0C5B4 & 02-Sep-94 & & $10028-17-8$ & TRITIUM \\
\hline 1676 & SESPSCH & & SW & & & B0C5B4 & 02-Sep-94 & & $13966-29-5$ & U-234 \\
\hline 1677 & SESPSCH & & SW & & & B0C5B4 & 02-Sep-94 & & 15117-96-1 & U-235 \\
\hline 1678 & SESPSCH & & SW & & & B0C5B4 & 02-Sep-94 & & $\mathrm{U}-238$ & $\mathrm{U}-238$ \\
\hline 1679 & PS HENRY/LE BISPING & & SW & & & BOCV80 & 15-Dec-94 & & $7440-47-3$ & CR \\
\hline 1680 & PS HENRY/LE BISPING & & SW & & & B0CV80 & 15-Dec-94 & & $56-23-5$ & CARBTET \\
\hline 1681 & SESPSCH & & SW & & & BOD1Y0 & 15-Dec-94 & & $10098-97-2$ & SR-90 \\
\hline 1682 & SESPSCH & & SW & & & B0D1Y0 & 15-Dec-94 & & $10028-17-8$ & TRITIUM \\
\hline 1683 & SESPSCH & & SW & & & B0D1Y0 & 15-Dec-94 & & $13966-29-5$ & U-234 \\
\hline 1684 & SESPSCH & & SW & & & BOD1Y0 & 15-Dec-94 & & $15117-96-1$ & U-235 \\
\hline 1685 & SESPSCH & & SW & & & B0D1Y0 & 15-Dec-94 & & $\mathrm{U}-238$ & $\mathrm{U}-238$ \\
\hline 1686 & PS HENRY/LE BISPING & & SW & & & B0CV81 & 15-Dec-94 & & $7440-47-3$ & CR \\
\hline 1687 & PS HENRY/LE BISPING & & SW & & & B0CV81 & 15-Dec-94 & & $56-23-5$ & CARBTET \\
\hline 1688 & SESPSCH & & SW & & & B0D1Y1 & 15-Dec-94 & & $10098-97-2$ & SR-90 \\
\hline 1689 & SESPSCH & & SW & & & B0D1Y1 & 15-Dec-94 & & $10028-17-8$ & TRITIUM \\
\hline 1690 & SESPSCH & & SW & & & B0D1Y1 & 15-Dec-94 & & 13966-29-5 & U-234 \\
\hline 1691 & SESPSCH & & SW & & & B0D1Y1 & 15-Dec-94 & & $15117-96-1$ & U-235 \\
\hline 1692 & SESPSCH & & SW & & & B0D1Y1 & 15-Dec-94 & & $\mathrm{U}-238$ & $\mathrm{U}-238$ \\
\hline 1693 & PS HENRY/LE BISPING & & SW & & & B0CV82 & 15-Dec-94 & & $7440-47-3$ & CR \\
\hline 1694 & PS HENRY/LE BISPING & & SW & & & B0CV82 & 15-Dec-94 & & $56-23-5$ & CARBTET \\
\hline 1695 & SESPSCH & & SW & & & B0D1Y2 & 15-Dec-94 & & $10098-97-2$ & SR-90 \\
\hline 1696 & SESPSCH & & SW & & & BOD1Y2 & 15-Dec-94 & & $10028-17-8$ & TRITIUM \\
\hline 1697 & SESPSCH & & SW & & & BOD1Y2 & 15-Dec-94 & & 13966-29-5 & U-234 \\
\hline 1698 & SESPSCH & & SW & & & B0D1Y2 & 15-Dec-94 & & 15117-96-1 & U-235 \\
\hline 1699 & SESPSCH & & SW & & & B0D1Y2 & 15-Dec-94 & & U-238 & U-238 \\
\hline 1700 & PS HENRY/LE BISPING & & SW & & & B0CV83 & 15-Dec-94 & & $7440-47-3$ & CR \\
\hline 1701 & PS HENRY/LE BISPING & & SW & & & B0CV83 & 15-Dec-94 & & $56-23-5$ & CARBTET \\
\hline 1702 & SESPSCH & & SW & & & B0D1Y3 & 15-Dec-94 & & $10098-97-2$ & SR-90 \\
\hline 1703 & SESPSCH & & SW & & & B0D1Y3 & 15-Dec-94 & & $10028-17-8$ & TRITIUM \\
\hline 1704 & SESPSCH & & SW & & & B0D1Y3 & 15-Dec-94 & & $13966-29-5$ & $\mathrm{U}-234$ \\
\hline 1705 & SESPSCH & & SW & & & B0D1Y3 & 15-Dec-94 & & 15117-96-1 & U-235 \\
\hline 1706 & SESPSCH & & SW & & & B0D1Y3 & 15-Dec-94 & & $\mathrm{U}-238$ & $\mathrm{U}-238$ \\
\hline 1707 & PS HENRY/LE BISPING & & SW & & & B0CV84 & 21-Dec-94 & & $7440-47-3$ & CR \\
\hline 1708 & PS HENRY/LE BISPING & & SW & & & B0CV84 & 21-Dec-94 & & $56-23-5$ & CARBTET \\
\hline 1709 & PS HENRY/LE BISPING & & SW & & & B0CV93 & 21-Dec-94 & & $7440-47-3$ & CR \\
\hline 1710 & PS HENRY/LE BISPING & & SW & & & B0CV93 & 21-Dec-94 & & $56-23-5$ & CARBTET \\
\hline 1711 & PS HENRY/LE BISPING & & SW & & & B0CV85 & 21-Dec-94 & & $7440-47-3$ & CR \\
\hline
\end{tabular}


\begin{tabular}{l|l}
$D$ & $E$
\end{tabular}

G

$\mathrm{H}$

\begin{tabular}{|l|l|}
\hline & \\
\hline & \\
& \\
&
\end{tabular}

MEDIA 1 BIOTA_MEDIA TAG_ID

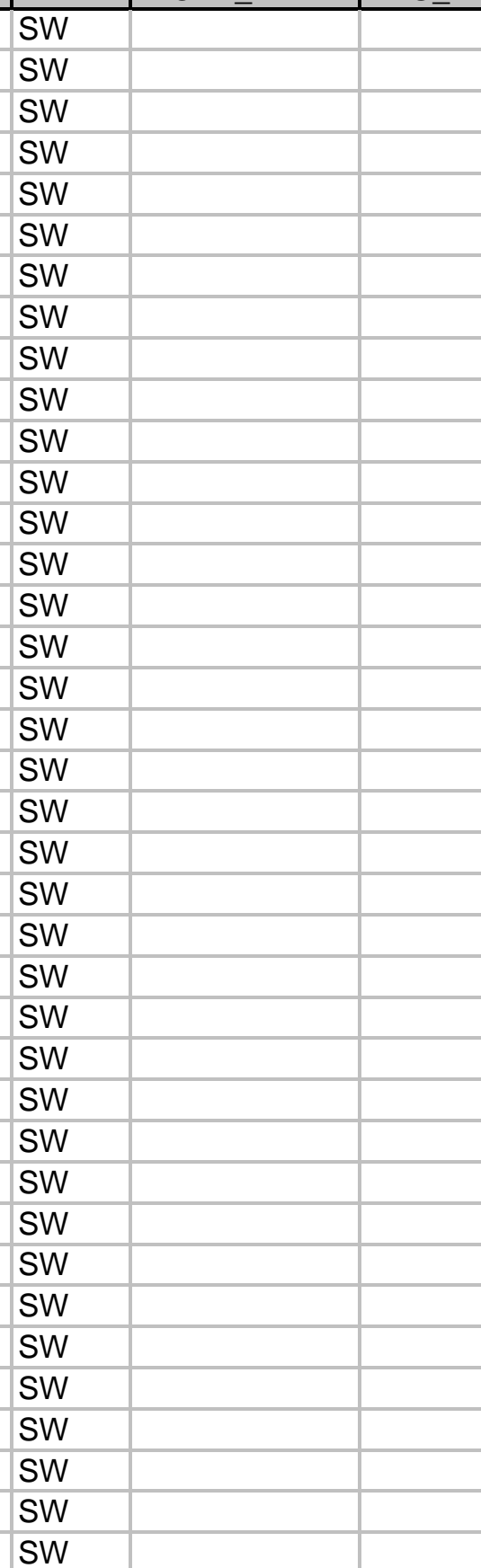

\begin{tabular}{l|l} 
SAMP NUM & SAMP DAT \\
\hline
\end{tabular}

B0CV85

B0CV86

B0CV86

B0CV87

B0CV87

B0CV88

B0CV88

B0CV89

B0CV89

B0CV90

B0CV90

B0CV91

B0CV91

B0CV92

B0CV92

B0DG81

B0DG81

B0DG81

BODG81

B0DG81

B0D1Y4

B0D1Y4

BOD1Y4

BOD1Y4

B0D1Y4

B0D1Y5

B0D1Y5

B0D1Y5

B0D1Y5

B0D1Y5

B0D1Y6

B0D1Y6

B0D1Y6

BOD1Y6

BOD1Y6

B0D1Y7

B0D1Y7

B0D1Y7

$\mathrm{H}$

21-Dec-94

21-Dec-94

21-Dec-94

21-Dec-94

21-Dec-94

21-Dec-94

21-Dec-94

21-Dec-94

21-Dec-94

21-Dec-94

21-Dec-94

21-Dec-94

21-Dec-94

21-Dec-94

21-Dec-94

21-Dec-94

21-Dec-94

21-Dec-94

21-Dec-94

21-Dec-94

21-Dec-94

21-Dec-94

21-Dec-94

21-Dec-94

21-Dec-94

21-Dec-94

21-Dec-94

21-Dec-94

21-Dec-94

21-Dec-94

21-Dec-94

21-Dec-94

21-Dec-94

21-Dec-94

21-Dec-94

21-Dec-94

21-Dec-94

21-Dec-94

I

CON_ID CON_SHORT_NAME

56-23-5 CARBTET

7440-47-3 CR

56-23-5 CARBTET

7440-47-3 CR

56-23-5 CARBTET

7440-47-3 CR

56-23-5 CARBTET

7440-47-3 CR

56-23-5 CARBTET

7440-47-3 CR

56-23-5 CARBTET

7440-47-3 CR

56-23-5 CARBTET

7440-47-3 CR

56-23-5 CARBTET

10098-97-2 SR-90

10028-17-8 TRITIUM

13966-29-5 U-234

15117-96-1 U-235

\begin{tabular}{l|l} 
U-238 U-238 &
\end{tabular}

10098-97-2 SR-90

10028-17-8 TRITIUM

13966-29-5 U-234

15117-96-1 U-235

U-238 U-238

10098-97-2 SR-90

10028-17-8 TRITIUM

13966-29-5 U-234

15117-96-1 U-235

U-238 U-238

10098-97-2 SR-90

10028-17-8 TRITIUM

13966-29-5 U-234

15117-96-1 U-235

U-238 U-238

10098-97-2 SR-90

10028-17-8 TRITIUM

Page 46 


\begin{tabular}{|c|c|c|c|c|c|c|c|c|c|c|}
\hline & A & $\mathrm{B}$ & $\mathrm{C}$ & $\mathrm{D}$ & $E$ & $\mathrm{~F}$ & G & $\mathrm{H}$ & $\mathrm{I}$ & $\mathrm{J}$ \\
\hline 1 & SAMPLE_NUM_ASSIGNED_TO & SAMPLER & MEDIA & BIOTA_MEDIA & TAG_ID & SAMP_NUM & SAMP_DATE_TIME & SAMP_DATE_TIME_ON & CON_ID & CON_SHORT_NAME \\
\hline 1750 & SESPSCH & & SW & & & B0D1Y7 & 21-Dec-94 & & $15117-96-1$ & $\mathrm{U}-235$ \\
\hline 1751 & SESPSCH & & SW & & & B0D1Y7 & 21-Dec-94 & & U-238 & U-238 \\
\hline \begin{tabular}{|l|}
1752 \\
\end{tabular} & SESPSCH & & SW & & & B0D1Y8 & 21-Dec-94 & & $10098-97-2$ & SR-90 \\
\hline 1753 & SESPSCH & & SW & & & B0D1Y8 & 21-Dec-94 & & $10028-17-8$ & TRITIUM \\
\hline \begin{tabular}{|l|}
1754 \\
\end{tabular} & SESPSCH & & SW & & & B0D1Y8 & 21-Dec-94 & & 13966-29-5 & U-234 \\
\hline 1755 & SESPSCH & & SW & & & B0D1Y8 & 21-Dec-94 & & $15117-96-1$ & U-235 \\
\hline 1756 & SESPSCH & & SW & & & B0D1Y8 & 21-Dec-94 & & $\mathrm{U}-238$ & $\mathrm{U}-238$ \\
\hline 1757 & SESPSCH & & SW & & & B0D1Y9 & 21-Dec-94 & & $10098-97-2$ & SR-90 \\
\hline 1758 & SESPSCH & & SW & & & BOD1Y9 & 21-Dec-94 & & $10028-17-8$ & TRITIUM \\
\hline 1759 & SESPSCH & & SW & & & BOD1Y9 & 21-Dec-94 & & $13966-29-5$ & U-234 \\
\hline 1760 & SESPSCH & & SW & & & B0D1Y9 & 21-Dec-94 & & $15117-96-1$ & U-235 \\
\hline 1761 & SESPSCH & & SW & & & B0D1Y9 & 21-Dec-94 & & U-238 & U-238 \\
\hline 1762 & SESPSCH & & SW & & & BOD1Z0 & 21-Dec-94 & & 10098-97-2 & SR-90 \\
\hline 1763 & SESPSCH & & SW & & & B0D1Z0 & 21-Dec-94 & & $10028-17-8$ & TRITIUM \\
\hline \begin{tabular}{|l|}
1764 \\
\end{tabular} & SESPSCH & & SW & & & BOD1Z0 & 21-Dec-94 & & 13966-29-5 & U-234 \\
\hline \begin{tabular}{|l|}
1765 \\
\end{tabular} & SESPSCH & & SW & & & BOD1Z0 & 21-Dec-94 & & $15117-96-1$ & U-235 \\
\hline \begin{tabular}{|l|}
1766 \\
\end{tabular} & SESPSCH & & SW & & & BOD1Z0 & 21-Dec-94 & & U-238 & U-238 \\
\hline \begin{tabular}{|l|}
1767 \\
\end{tabular} & SESPSCH & & SW & & & B0D1Z1 & 21-Dec-94 & & $10098-97-2$ & SR-90 \\
\hline \begin{tabular}{|l|}
1768 \\
\end{tabular} & SESPSCH & & SW & & & B0D1Z1 & 21-Dec-94 & & $10028-17-8$ & TRITIUM \\
\hline \begin{tabular}{|l|}
1769 \\
\end{tabular} & SESPSCH & & SW & & & B0D1Z1 & 21-Dec-94 & & $13966-29-5$ & U-234 \\
\hline 1770 & SESPSCH & & SW & & & B0D1Z1 & 21-Dec-94 & & $15117-96-1$ & U-235 \\
\hline \begin{tabular}{|l|}
1771 \\
\end{tabular} & SESPSCH & & SW & & & B0D1Z1 & 21-Dec-94 & & U-238 & U-238 \\
\hline \begin{tabular}{|l|}
1772 \\
\end{tabular} & SESPSCH & & SW & & & B0D1Z2 & 21-Dec-94 & & $10098-97-2$ & SR-90 \\
\hline 1773 & SESPSCH & & SW & & & BOD1Z2 & 21-Dec-94 & & $10028-17-8$ & TRITIUM \\
\hline 1774 & SESPSCH & & SW & & & B0D1Z2 & 21-Dec-94 & & $13966-29-5$ & U-234 \\
\hline 1775 & SESPSCH & & SW & & & B0D1Z2 & 21-Dec-94 & & 15117-96-1 & U-235 \\
\hline 1776 & SESPSCH & & SW & & & BoD1Z2 & 21-Dec-94 & & U-238 & U-238 \\
\hline \begin{tabular}{|l|}
1777 \\
\end{tabular} & SESPSCH & & SW & & & BOD1Z3 & 21-Dec-94 & & $10098-97-2$ & SR-90 \\
\hline \begin{tabular}{|l|}
1778 \\
\end{tabular} & SESPSCH & & SW & & & B0D1Z3 & 21-Dec-94 & & $10028-17-8$ & TRITIUM \\
\hline \begin{tabular}{|l|}
1779 \\
\end{tabular} & SESPSCH & & SW & & & BoD1Z3 & 21-Dec-94 & & $13966-29-5$ & U-234 \\
\hline 1780 & SESPSCH & & SW & & & B0D1Z3 & 21-Dec-94 & & $15117-96-1$ & U-235 \\
\hline \begin{tabular}{|l|}
1781 \\
\end{tabular} & SESPSCH & & SW & & & B0D1Z3 & 21-Dec-94 & & U-238 & U-238 \\
\hline \begin{tabular}{|l|}
1782 \\
\end{tabular} & PS HENRY/LE BISPING & & SW & & & B0CVY1 & 16-Mar-95 & & $7440-47-3$ & $\mathrm{CR}$ \\
\hline 1783 & PS HENRY/LE BISPING & & SW & & & B0CVY1 & 16-Mar-95 & & $56-23-5$ & CARBTET \\
\hline 1784 & SESPSCH & & SW & & & B0DX17 & 16-Mar-95 & & $10098-97-2$ & SR-90 \\
\hline 1785 & SESPSCH & & SW & & & B0DX17 & 16-Mar-95 & & $10028-17-8$ & TRITIUM \\
\hline 1786 & SESPSCH & & SW & & & B0DX17 & 16-Mar-95 & & $13966-29-5$ & U-234 \\
\hline \begin{tabular}{|l|}
1787 \\
\end{tabular} & SESPSCH & & SW & & & B0DX17 & 16-Mar-95 & & 15117-96-1 & U-235 \\
\hline
\end{tabular}




\begin{tabular}{|c|c|c|c|c|c|c|c|c|c|c|}
\hline & A & $\mathrm{B}$ & $C$ & $\mathrm{D}$ & $E$ & $\mathrm{~F}$ & $G$ & $\mathrm{H}$ & $\mathrm{I}$ & $\mathrm{J}$ \\
\hline 1 & SAMPLE_NUM_ASSIGNED_TO & SAMPLER & MEDIA & BIOTA_MEDIA & TAG_ID & SAMP_NUM & SAMP_DATE_TIME| & SAMP_DATE_TIME_ON & CON_ID & CON_SHORT_NAME \\
\hline 1788 & SESPSCH & & SW & & & B0DX17 & 16-Mar-95 & & $\mathrm{U}-238$ & U-238 \\
\hline 1789 & PS HENRY/LE BISPING & & SW & & & B0CVY2 & 16-Mar-95 & & $7440-47-3$ & $\mathrm{CR}$ \\
\hline 1790 & PS HENRY/LE BISPING & & SW & & & BOCVY2 & 16-Mar-95 & & $56-23-5$ & CARBTET \\
\hline 1791 & SESPSCH & & SW & & & B0DX18 & 16-Mar-95 & & $10098-97-2$ & SR-90 \\
\hline 1792 & SESPSCH & & SW & & & B0DX18 & 16-Mar-95 & & $10028-17-8$ & TRITIUM \\
\hline 1793 & SESPSCH & & SW & & & B0DX18 & 16-Mar-95 & & $13966-29-5$ & U-234 \\
\hline 1794 & SESPSCH & & SW & & & B0DX18 & 16-Mar-95 & & 15117-96-1 & U-235 \\
\hline 1795 & SESPSCH & & SW & & & B0DX18 & 16-Mar-95 & & $\mathrm{U}-238$ & $\mathrm{U}-238$ \\
\hline 1796 & PS HENRY/LE BISPING & & SW & & & B0CVY3 & 16-Mar-95 & & $7440-47-3$ & $\mathrm{CR}$ \\
\hline 1797 & PS HENRY/LE BISPING & & SW & & & B0CVY3 & 16-Mar-95 & & $56-23-5$ & CARBTET \\
\hline 1798 & SESPSCH & & SW & & & B0DX19 & 16-Mar-95 & & $10098-97-2$ & SR-90 \\
\hline 1799 & SESPSCH & & SW & & & B0DX19 & 16-Mar-95 & & $10028-17-8$ & TRITIUM \\
\hline 1800 & SESPSCH & & SW & & & B0DX19 & 16-Mar-95 & & $13966-29-5$ & U-234 \\
\hline 1801 & SESPSCH & & SW & & & B0DX19 & 16-Mar-95 & & $15117-96-1$ & U-235 \\
\hline 1802 & SESPSCH & & SW & & & B0DX19 & 16-Mar-95 & & U-238 & U-238 \\
\hline 1803 & PS HENRY/LE BISPING & & SW & & & B0CVY4 & 16-Mar-95 & & $7440-47-3$ & $\mathrm{CR}$ \\
\hline 1804 & PS HENRY/LE BISPING & & SW & & & B0CVY4 & 16-Mar-95 & & $56-23-5$ & CARBTET \\
\hline 1805 & SESPSCH & & SW & & & BOD $\times 20$ & 16-Mar-95 & & $10098-97-2$ & SR-90 \\
\hline 1806 & SESPSCH & & SW & & & B0D $\times 20$ & 16-Mar-95 & & $10028-17-8$ & TRITIUM \\
\hline 1807 & SESPSCH & & SW & & & BODX20 & 16-Mar-95 & & 13966-29-5 & U-234 \\
\hline 1808 & SESPSCH & & SW & & & B0DX20 & 16-Mar-95 & & $15117-96-1$ & U-235 \\
\hline 1809 & SESPSCH & & SW & & & B0DX20 & 16-Mar-95 & & $\mathrm{U}-238$ & $\mathrm{U}-238$ \\
\hline 1810 & PS HENRY/LE BISPING & & SW & & & B0CVY5 & 17-Mar-95 & & $7440-47-3$ & CR \\
\hline 1811 & PS HENRY/LE BISPING & & SW & & & B0CVY5 & 17-Mar-95 & & $56-23-5$ & CARBTET \\
\hline 1812 & SESPSCH & & SW & & & B0DX24 & 17-Mar-95 & & $10098-97-2$ & SR-90 \\
\hline 1813 & SESPSCH & & SW & & & B0DX24 & 17-Mar-95 & & $10028-17-8$ & TRITIUM \\
\hline 1814 & SESPSCH & & SW & & & B0DX24 & 17-Mar-95 & & $13966-29-5$ & U-234 \\
\hline 1815 & SESPSCH & & SW & & & B0DX24 & 17-Mar-95 & & 15117-96-1 & U-235 \\
\hline 1816 & SESPSCH & & SW & & & B0DX24 & 17-Mar-95 & & $\mathrm{U}-238$ & $\mathrm{U}-238$ \\
\hline 1817 & PS HENRY/LE BISPING & & SW & & & B0CVZ4 & 17-Mar-95 & & $7440-47-3$ & CR \\
\hline 1818 & PS HENRY/LE BISPING & & SW & & & BOCVZ4 & 17-Mar-95 & & $56-23-5$ & CARBTET \\
\hline 1819 & SESPSCH & & SW & & & B0DX33 & 17-Mar-95 & & $10098-97-2$ & SR-90 \\
\hline 1820 & SESPSCH & & SW & & & B0DX33 & 17-Mar-95 & & $10028-17-8$ & TRITIUM \\
\hline 1821 & SESPSCH & & SW & & & B0DX33 & 17-Mar-95 & & $13966-29-5$ & U-234 \\
\hline 1822 & SESPSCH & & SW & & & B0DX33 & 17-Mar-95 & & 15117-96-1 & U-235 \\
\hline 1823 & SESPSCH & & SW & & & B0DX33 & 17-Mar-95 & & $\mathrm{U}-238$ & $\mathrm{U}-238$ \\
\hline 1824 & PS HENRY/LE BISPING & & SW & & & BOCVY6 & 17-Mar-95 & & $7440-47-3$ & CR \\
\hline 1825 & PS HENRY/LE BISPING & & SW & & & B0CVY6 & 17-Mar-95 & & $56-23-5$ & CARBTET \\
\hline
\end{tabular}




\begin{tabular}{|c|c|c|c|c|c|c|c|c|c|c|}
\hline & A & $\mathrm{B}$ & $C$ & $\mathrm{D}$ & $E$ & $\mathrm{~F}$ & $G$ & $\mathrm{H}$ & $\mathrm{I}$ & $\mathrm{J}$ \\
\hline 1 & SAMPLE_NUM_ASSIGNED_TO & SAMPLER & MEDIA & BIOTA_MEDIA & TAG_ID & SAMP_NUM & SAMP_DATE_TIME| & SAMP_DATE_TIME_ON & CON_ID & CON_SHORT_NAME \\
\hline 1826 & SESPSCH & & SW & & & B0DX25 & 17-Mar-95 & & $10098-97-2$ & SR-90 \\
\hline 1827 & SESPSCH & & SW & & & B0DX25 & 17-Mar-95 & & $10028-17-8$ & TRITIUM \\
\hline 1828 & SESPSCH & & SW & & & B0D $\times 25$ & 17-Mar-95 & & $13966-29-5$ & U-234 \\
\hline 1829 & SESPSCH & & SW & & & B0DX25 & 17-Mar-95 & & 15117-96-1 & U-235 \\
\hline 1830 & SESPSCH & & SW & & & B0DX25 & 17-Mar-95 & & U-238 & $\mathrm{U}-238$ \\
\hline 1831 & PS HENRY/LE BISPING & & SW & & & B0CVY7 & 17-Mar-95 & & $7440-47-3$ & CR \\
\hline 1832 & PS HENRY/LE BISPING & & SW & & & B0CVY7 & 17-Mar-95 & & $56-23-5$ & CARBTET \\
\hline 1833 & SESPSCH & & SW & & & B0D $\times 26$ & 17-Mar-95 & & $10098-97-2$ & SR-90 \\
\hline 1834 & SESPSCH & & SW & & & B0DX26 & 17-Mar-95 & & $10028-17-8$ & TRITIUM \\
\hline 1835 & SESPSCH & & SW & & & B0D $\times 26$ & 17-Mar-95 & & $13966-29-5$ & U-234 \\
\hline 1836 & SESPSCH & & SW & & & B0D $\times 26$ & 17-Mar-95 & & $15117-96-1$ & U-235 \\
\hline 1837 & SESPSCH & & SW & & & B0DX26 & 17-Mar-95 & & U-238 & $\mathrm{U}-238$ \\
\hline 1838 & PS HENRY/LE BISPING & & SW & & & BOCVY8 & 17-Mar-95 & & $7440-47-3$ & CR \\
\hline 1839 & PS HENRY/LE BISPING & & SW & & & B0CVY8 & 17-Mar-95 & & $56-23-5$ & CARBTET \\
\hline 1840 & SESPSCH & & SW & & & B0DX27 & 17-Mar-95 & & 10098-97-2 & SR-90 \\
\hline 1841 & SESPSCH & & SW & & & B0DX27 & 17-Mar-95 & & $10028-17-8$ & TRITIUM \\
\hline 1842 & SESPSCH & & SW & & & BODX27 & 17-Mar-95 & & $13966-29-5$ & U-234 \\
\hline 1843 & SESPSCH & & SW & & & BOD $\times 27$ & 17-Mar-95 & & $15117-96-1$ & U-235 \\
\hline 1844 & SESPSCH & & SW & & & B0DX27 & 17-Mar-95 & & $\mathrm{U}-238$ & $\mathrm{U}-238$ \\
\hline 1845 & PS HENRY/LE BISPING & & SW & & & BOCVY9 & 17-Mar-95 & & $7440-47-3$ & CR \\
\hline 1846 & PS HENRY/LE BISPING & & SW & & & BOCVY9 & 17-Mar-95 & & $56-23-5$ & CARBTET \\
\hline 1847 & SESPSCH & & SW & & & B0DX28 & 17-Mar-95 & & $10098-97-2$ & SR-90 \\
\hline 1848 & SESPSCH & & SW & & & B0DX28 & 17-Mar-95 & & $10028-17-8$ & TRITIUM \\
\hline 1849 & SESPSCH & & SW & & & BODX28 & 17-Mar-95 & & $13966-29-5$ & $\mathrm{U}-234$ \\
\hline 1850 & SESPSCH & & SW & & & B0DX28 & 17-Mar-95 & & 15117-96-1 & U-235 \\
\hline 1851 & SESPSCH & & SW & & & B0DX28 & 17-Mar-95 & & U-238 & U-238 \\
\hline 1852 & PS HENRY/LE BISPING & & SW & & & BOCVZO & 17-Mar-95 & & $7440-47-3$ & CR \\
\hline 1853 & PS HENRY/LE BISPING & & SW & & & BOCVZO & 17-Mar-95 & & $56-23-5$ & CARBTET \\
\hline 1854 & SESPSCH & & SW & & & B0DX29 & 17-Mar-95 & & $10098-97-2$ & SR-90 \\
\hline 1855 & SESPSCH & & SW & & & BOD $\times 29$ & 17-Mar-95 & & $10028-17-8$ & TRITIUM \\
\hline 1856 & SESPSCH & & SW & & & B0DX29 & 17-Mar-95 & & $13966-29-5$ & $\mathrm{U}-234$ \\
\hline 1857 & SESPSCH & & SW & & & B0DX29 & 17-Mar-95 & & $15117-96-1$ & U-235 \\
\hline 1858 & SESPSCH & & SW & & & BODX29 & 17-Mar-95 & & U-238 & $\mathrm{U}-238$ \\
\hline 1859 & PS HENRY/LE BISPING & & SW & & & B0CVZ1 & 17-Mar-95 & & $7440-47-3$ & CR \\
\hline 1860 & PS HENRY/LE BISPING & & SW & & & B0CVZ1 & 17-Mar-95 & & $56-23-5$ & CARBTET \\
\hline 1861 & SESPSCH & & SW & & & B0DX30 & 17-Mar-95 & & $10098-97-2$ & SR-90 \\
\hline 1862 & SESPSCH & & SW & & & B0DX30 & 17-Mar-95 & & $10028-17-8$ & TRITIUM \\
\hline 1863 & SESPSCH & & SW & & & B0DX30 & 17-Mar-95 & & $13966-29-5$ & U-234 \\
\hline
\end{tabular}




\begin{tabular}{|c|c|c|c|c|c|c|c|c|c|c|}
\hline & A & $\mathrm{B}$ & $C$ & $\mathrm{D}$ & $E$ & $\mathrm{~F}$ & $G$ & $\mathrm{H}$ & $\mathrm{I}$ & $\mathrm{J}$ \\
\hline 1 & SAMPLE_NUM_ASSIGNED_TO & SAMPLER & MEDIA & BIOTA_MEDIA & TAG_ID & SAMP_NUM & SAMP_DATE_TIME| & SAMP_DATE_TIME_ON & CON_ID & CON_SHORT_NAME \\
\hline 1864 & SESPSCH & & SW & & & B0DX30 & 17-Mar-95 & & 15117-96-1 & U-235 \\
\hline 1865 & SESPSCH & & SW & & & BODX30 & 17-Mar-95 & & U-238 & $\mathrm{U}-238$ \\
\hline 1866 & PS HENRY/LE BISPING & & SW & & & B0CVZ2 & 17-Mar-95 & & $7440-47-3$ & $\mathrm{CR}$ \\
\hline 1867 & PS HENRY/LE BISPING & & SW & & & B0CVZ2 & 17-Mar-95 & & $56-23-5$ & CARBTET \\
\hline 1868 & SESPSCH & & SW & & & B0DX31 & 17-Mar-95 & & $10098-97-2$ & SR-90 \\
\hline 1869 & SESPSCH & & SW & & & B0DX31 & 17-Mar-95 & & 10028-17-8 & TRITIUM \\
\hline 1870 & SESPSCH & & SW & & & B0DX31 & 17-Mar-95 & & $13966-29-5$ & $\mathrm{U}-234$ \\
\hline 1871 & SESPSCH & & SW & & & B0DX31 & 17-Mar-95 & & $15117-96-1$ & U-235 \\
\hline 1872 & SESPSCH & & SW & & & B0DX31 & 17-Mar-95 & & U-238 & $\mathrm{U}-238$ \\
\hline 1873 & PS HENRY/LE BISPING & & SW & & & B0CVZ3 & 17-Mar-95 & & $7440-47-3$ & $\mathrm{CR}$ \\
\hline 1874 & PS HENRY/LE BISPING & & SW & & & B0CVZ3 & 17-Mar-95 & & $56-23-5$ & CARBTET \\
\hline 1875 & SESPSCH & & SW & & & B0DX32 & 17-Mar-95 & & $10098-97-2$ & SR-90 \\
\hline 1876 & SESPSCH & & SW & & & B0DX32 & 17-Mar-95 & & 10028-17-8 & TRITIUM \\
\hline 1877 & SESPSCH & & SW & & & B0DX32 & 17-Mar-95 & & 13966-29-5 & U-234 \\
\hline 1878 & SESPSCH & & SW & & & B0DX32 & 17-Mar-95 & & 15117-96-1 & U-235 \\
\hline 1879 & SESPSCH & & SW & & & B0DX32 & 17-Mar-95 & & U-238 & $\mathrm{U}-238$ \\
\hline 1880 & PS HENRY/LE BISPING & & SW & & & B0CW59 & 15-Jun-95 & & $7440-47-3$ & $\mathrm{CR}$ \\
\hline 1881 & PS HENRY/LE BISPING & & SW & & & B0CW59 & 15-Jun-95 & & $56-23-5$ & CARBTET \\
\hline 1882 & SESPSCH & & SW & & & B0FL62 & 15-Jun-95 & & $10098-97-2$ & SR-90 \\
\hline 1883 & SESPSCH & & SW & & & BOFL62 & 15-Jun-95 & & $10028-17-8$ & TRITIUM \\
\hline 1884 & SESPSCH & & SW & & & BOFL62 & 15-Jun-95 & & 13966-29-5 & U-234 \\
\hline 1885 & SESPSCH & & SW & & & B0FL62 & 15-Jun-95 & & 15117-96-1 & U-235 \\
\hline 1886 & SESPSCH & & SW & & & B0FL62 & 15-Jun-95 & & U-238 & $\mathrm{U}-238$ \\
\hline 1887 & PS HENRY/LE BISPING & & SW & & & B0CW60 & 15-Jun-95 & & $7440-47-3$ & CR \\
\hline 1888 & PS HENRY/LE BISPING & & SW & & & B0CW60 & 15-Jun-95 & & $56-23-5$ & CARBTET \\
\hline 1889 & SESPSCH & & SW & & & B0FL63 & 15-Jun-95 & & $10098-97-2$ & SR-90 \\
\hline 1890 & SESPSCH & & SW & & & B0FL63 & 15-Jun-95 & & $10028-17-8$ & TRITIUM \\
\hline 1891 & SESPSCH & & SW & & & B0FL63 & 15-Jun-95 & & $13966-29-5$ & U-234 \\
\hline 1892 & SESPSCH & & SW & & & B0FL63 & 15-Jun-95 & & 15117-96-1 & U-235 \\
\hline 1893 & SESPSCH & & SW & & & B0FL63 & 15-Jun-95 & & U-238 & U-238 \\
\hline 1894 & PS HENRY/LE BISPING & & SW & & & B0CW61 & 15-Jun-95 & & $7440-47-3$ & CR \\
\hline 1895 & PS HENRY/LE BISPING & & SW & & & B0CW61 & 15-Jun-95 & & $56-23-5$ & CARBTET \\
\hline 1896 & SESPSCH & & SW & & & B0FL64 & 15-Jun-95 & & $10098-97-2$ & SR-90 \\
\hline 1897 & SESPSCH & & SW & & & B0FL64 & 15-Jun-95 & & $10028-17-8$ & TRITIUM \\
\hline 1898 & SESPSCH & & SW & & & B0FL64 & 15-Jun-95 & & $13966-29-5$ & U-234 \\
\hline 1899 & SESPSCH & & SW & & & BOFL64 & 15-Jun-95 & & 15117-96-1 & U-235 \\
\hline 1900 & SESPSCH & & SW & & & B0FL64 & 15-Jun-95 & & U-238 & U-238 \\
\hline 1901 & PS HENRY/LE BISPING & & SW & & & B0CW62 & 15-Jun-95 & & $7440-47-3$ & $\mathrm{CR}$ \\
\hline
\end{tabular}




\begin{tabular}{|c|c|c|c|c|c|c|c|c|c|c|}
\hline & A & $\mathrm{B}$ & $C$ & $\mathrm{D}$ & $E$ & $\mathrm{~F}$ & $G$ & $\mathrm{H}$ & $\mathrm{I}$ & $\mathrm{J}$ \\
\hline 1 & SAMPLE_NUM_ASSIGNED_TO & SAMPLER & MEDIA & BIOTA_MEDIA & TAG_ID & SAMP_NUM & SAMP_DATE_TIME| & SAMP_DATE_TIME_ON & CON_ID & CON_SHORT_NAME \\
\hline 1902 & PS HENRY/LE BISPING & & SW & & & B0CW62 & 15-Jun-95 & & $56-23-5$ & CARBTET \\
\hline 1903 & SESPSCH & & SW & & & B0FL65 & 15-Jun-95 & & $10098-97-2$ & SR-90 \\
\hline 1904 & SESPSCH & & SW & & & B0FL65 & 15-Jun-95 & & 10028-17-8 & TRITIUM \\
\hline 1905 & SESPSCH & & SW & & & B0FL65 & 15-Jun-95 & & $13966-29-5$ & U-234 \\
\hline 1906 & SESPSCH & & SW & & & B0FL65 & 15-Jun-95 & & $15117-96-1$ & U-235 \\
\hline 1907 & SESPSCH & & SW & & & B0FL65 & 15-Jun-95 & & U-238 & $\mathrm{U}-238$ \\
\hline 1908 & PS HENRY/LE BISPING & & SW & & & B0CW63 & 16-Jun-95 & & $7440-47-3$ & CR \\
\hline 1909 & PS HENRY/LE BISPING & & SW & & & B0CW63 & 16-Jun-95 & & $56-23-5$ & CARBTET \\
\hline 1910 & SESPSCH & & SW & & & B0FL66 & 16-Jun-95 & & $10098-97-2$ & SR-90 \\
\hline 1911 & SESPSCH & & SW & & & B0FL66 & 16-Jun-95 & & $10028-17-8$ & TRITIUM \\
\hline 1912 & SESPSCH & & SW & & & B0FL66 & 16-Jun-95 & & $13966-29-5$ & U-234 \\
\hline 1913 & SESPSCH & & SW & & & B0FL66 & 16-Jun-95 & & $15117-96-1$ & U-235 \\
\hline 1914 & SESPSCH & & SW & & & BOFL66 & 16-Jun-95 & & U-238 & U-238 \\
\hline 1915 & PS HENRY/LE BISPING & & SW & & & B0CW72 & 16-Jun-95 & & $7440-47-3$ & CR \\
\hline 1916 & PS HENRY/LE BISPING & & SW & & & B0CW72 & 16-Jun-95 & & $56-23-5$ & CARBTET \\
\hline 1917 & SESPSCH & & SW & & & B0FL75 & 16-Jun-95 & & $10098-97-2$ & SR-90 \\
\hline 1918 & SESPSCH & & SW & & & BOFL75 & 16-Jun-95 & & $10028-17-8$ & TRITIUM \\
\hline 1919 & SESPSCH & & SW & & & B0FL75 & 16-Jun-95 & & $13966-29-5$ & U-234 \\
\hline 1920 & SESPSCH & & SW & & & B0FL75 & 16-Jun-95 & & 15117-96-1 & U-235 \\
\hline 1921 & SESPSCH & & SW & & & B0FL75 & 16-Jun-95 & & U-238 & $\mathrm{U}-238$ \\
\hline 1922 & PS HENRY/LE BISPING & & SW & & & B0CW64 & 16-Jun-95 & & $7440-47-3$ & CR \\
\hline 1923 & PS HENRY/LE BISPING & & SW & & & B0CW64 & 16-Jun-95 & & $56-23-5$ & CARBTET \\
\hline 1924 & SESPSCH & & SW & & & BOFL67 & 16-Jun-95 & & $10098-97-2$ & SR-90 \\
\hline 1925 & SESPSCH & & SW & & & BOFL67 & 16-Jun-95 & & $10028-17-8$ & TRITIUM \\
\hline 1926 & SESPSCH & & SW & & & B0FL67 & 16-Jun-95 & & $13966-29-5$ & U-234 \\
\hline 1927 & SESPSCH & & SW & & & B0FL67 & 16-Jun-95 & & 15117-96-1 & U-235 \\
\hline 1928 & SESPSCH & & SW & & & BOFL67 & 16-Jun-95 & & U-238 & U-238 \\
\hline 1929 & PS HENRY/LE BISPING & & SW & & & B0CW65 & 16-Jun-95 & & $7440-47-3$ & $\mathrm{CR}$ \\
\hline 1930 & PS HENRY/LE BISPING & & SW & & & B0CW65 & 16-Jun-95 & & $56-23-5$ & CARBTET \\
\hline 1931 & SESPSCH & & SW & & & BOFL68 & 16-Jun-95 & & $10098-97-2$ & SR-90 \\
\hline 1932 & SESPSCH & & SW & & & B0FL68 & 16-Jun-95 & & $10028-17-8$ & TRITIUM \\
\hline 1933 & SESPSCH & & SW & & & BOFL68 & 16-Jun-95 & & $13966-29-5$ & U-234 \\
\hline 1934 & SESPSCH & & SW & & & B0FL68 & 16-Jun-95 & & $15117-96-1$ & U-235 \\
\hline 1935 & SESPSCH & & SW & & & B0FL68 & 16-Jun-95 & & U-238 & U-238 \\
\hline 1936 & PS HENRY/LE BISPING & & SW & & & B0CW66 & 16-Jun-95 & & $7440-47-3$ & $\mathrm{CR}$ \\
\hline 1937 & PS HENRY/LE BISPING & & SW & & & B0CW66 & 16-Jun-95 & & $56-23-5$ & CARBTET \\
\hline 1938 & SESPSCH & & SW & & & BOFL69 & 16-Jun-95 & & $10098-97-2$ & SR-90 \\
\hline 1939 & SESPSCH & & SW & & & B0FL69 & 16-Jun-95 & & $10028-17-8$ & TRITIUM \\
\hline
\end{tabular}




\begin{tabular}{|c|c|c|c|c|c|c|c|c|c|c|}
\hline & $\mathrm{A}$ & $\mathrm{B}$ & $\mathrm{C}$ & $\mathrm{D}$ & $\mathrm{E}$ & $\mathrm{F}$ & $G$ & $\mathrm{H}$ & I & $\mathrm{J}$ \\
\hline 1 & SAMPLE_NUM_ASSIGNED_TO & SAMPLER & MEDIA & BIOTA_MEDIA & TAG_ID & SAMP_NUM & SAMP_DATE_TIME & SAMP_DATE_TIME_ON & CON_ID & CON_SHORT_NAME \\
\hline 1940 & SESPSCH & & SW & & & B0FL69 & 16-Jun-95 & & $13966-29-5$ & $\mathrm{U}-234$ \\
\hline 1941 & SESPSCH & & SW & & & BOFL69 & 16-Jun-95 & & $15117-96-1$ & U-235 \\
\hline 1942 & SESPSCH & & SW & & & BOFL69 & 16-Jun-95 & & $\mathrm{U}-238$ & U-238 \\
\hline 1943 & PS HENRY/LE BISPING & & SW & & & B0CW67 & 16-Jun-95 & & $7440-47-3$ & CR \\
\hline 1944 & PS HENRY/LE BISPING & & SW & & & B0CW67 & 16-Jun-95 & & $56-23-5$ & CARBTET \\
\hline 1945 & SESPSCH & & SW & & & B0FL70 & 16-Jun-95 & & $10098-97-2$ & SR-90 \\
\hline 1946 & SESPSCH & & SW & & & B0FL70 & 16-Jun-95 & & $10028-17-8$ & TRITIUM \\
\hline 1947 & SESPSCH & & SW & & & BOFL70 & 16-Jun-95 & & $13966-29-5$ & $\mathrm{U}-234$ \\
\hline 1948 & SESPSCH & & SW & & & BOFL70 & 16-Jun-95 & & $15117-96-1$ & U-235 \\
\hline 1949 & SESPSCH & & SW & & & BOFL70 & 16-Jun-95 & & $\mathrm{U}-238$ & $\mathrm{U}-238$ \\
\hline 1950 & PS HENRY/LE BISPING & & SW & & & B0CW68 & 16-Jun-95 & & $7440-47-3$ & $\mathrm{CR}$ \\
\hline 1951 & PS HENRY/LE BISPING & & SW & & & B0CW68 & 16-Jun-95 & & $56-23-5$ & CARBTET \\
\hline 1952 & SESPSCH & & SW & & & B0FL71 & 16-Jun-95 & & $10098-97-2$ & SR-90 \\
\hline 1953 & SESPSCH & & SW & & & B0FL71 & 16-Jun-95 & & $10028-17-8$ & TRITIUM \\
\hline 1954 & SESPSCH & & SW & & & B0FL71 & 16-Jun-95 & & $13966-29-5$ & U-234 \\
\hline 1955 & SESPSCH & & SW & & & B0FL71 & 16-Jun-95 & & $15117-96-1$ & U-235 \\
\hline 1956 & SESPSCH & & SW & & & B0FL71 & 16-Jun-95 & & $\mathrm{U}-238$ & $\mathrm{U}-238$ \\
\hline 1957 & PS HENRY/LE BISPING & & SW & & & B0CW69 & 16-Jun-95 & & $7440-47-3$ & CR \\
\hline 1958 & PS HENRY/LE BISPING & & SW & & & BoCW69 & 16-Jun-95 & & $56-23-5$ & CARBTET \\
\hline 1959 & SESPSCH & & SW & & & B0FL72 & 16-Jun-95 & & $10098-97-2$ & SR-90 \\
\hline 1960 & SESPSCH & & SW & & & BOFL72 & 16-Jun-95 & & $10028-17-8$ & TRITIUM \\
\hline 1961 & SESPSCH & & SW & & & B0FL72 & 16-Jun-95 & & $13966-29-5$ & $\mathrm{U}-234$ \\
\hline 1962 & SESPSCH & & SW & & & B0FL72 & 16-Jun-95 & & 15117-96-1 & $\mathrm{U}-235$ \\
\hline 1963 & SESPSCH & & SW & & & BOFL72 & 16-Jun-95 & & $\mathrm{U}-238$ & $\mathrm{U}-238$ \\
\hline 1964 & PS HENRY/LE BISPING & & SW & & & B0CW70 & 16-Jun-95 & & $7440-47-3$ & $\mathrm{CR}$ \\
\hline 1965 & PS HENRY/LE BISPING & & SW & & & B0CW70 & 16-Jun-95 & & $56-23-5$ & CARBTET \\
\hline 1966 & SESPSCH & & SW & & & B0FL73 & 16-Jun-95 & & $10098-97-2$ & SR-90 \\
\hline 1967 & SESPSCH & & SW & & & B0FL73 & 16-Jun-95 & & $10028-17-8$ & TRITIUM \\
\hline 1968 & SESPSCH & & SW & & & B0FL73 & 16-Jun-95 & & $13966-29-5$ & U-234 \\
\hline 1969 & SESPSCH & & SW & & & B0FL73 & 16-Jun-95 & & $15117-96-1$ & $\mathrm{U}-235$ \\
\hline 1970 & SESPSCH & & SW & & & B0FL73 & 16-Jun-95 & & $\mathrm{U}-238$ & $\mathrm{U}-238$ \\
\hline 1971 & PS HENRY/LE BISPING & & SW & & & B0CW71 & 16-Jun-95 & & $7440-47-3$ & $\mathrm{CR}$ \\
\hline 1972 & PS HENRY/LE BISPING & & SW & & & B0CW71 & 16-Jun-95 & & $56-23-5$ & CARBTET \\
\hline 1973 & SESPSCH & & SW & & & B0FL74 & 16-Jun-95 & & $10098-97-2$ & SR-90 \\
\hline 1974 & SESPSCH & & SW & & & B0FL74 & 16-Jun-95 & & $10028-17-8$ & TRITIUM \\
\hline 1975 & SESPSCH & & SW & & & B0FL74 & 16-Jun-95 & & $13966-29-5$ & U-234 \\
\hline 1976 & SESPSCH & & SW & & & B0FL74 & 16-Jun-95 & & $15117-96-1$ & $\mathrm{U}-235$ \\
\hline 1977 & SESPSCH & & SW & & & B0FL74 & 16-Jun-95 & & $\mathrm{U}-238$ & U-238 \\
\hline
\end{tabular}




\begin{tabular}{|c|c|c|c|c|c|c|c|c|c|c|}
\hline & A & $\mathrm{B}$ & $C$ & $\mathrm{D}$ & $E$ & $\mathrm{~F}$ & $G$ & $\mathrm{H}$ & $\mathrm{I}$ & $\mathrm{J}$ \\
\hline 1 & SAMPLE_NUM_ASSIGNED_TO & SAMPLER & MEDIA & BIOTA_MEDIA & TAG_ID & SAMP_NUM & SAMP_DATE_TIME| & SAMP_DATE_TIME_ON & CON_ID & CON_SHORT_NAME \\
\hline 1978 & PS HENRY/LE BISPING & & SW & & & BOG0S3 & 11-Sep-95 & & $7440-47-3$ & CR \\
\hline 1979 & PS HENRY/LE BISPING & & SW & & & B0G0S3 & 11-Sep-95 & & $56-23-5$ & CARBTET \\
\hline 1980 & SESPSCH & & SW & & & B0G868 & 11-Sep-95 & & $10045-97-3$ & CS-137 \\
\hline 1981 & SESPSCH & & SW & & & B0G868 & 11-Sep-95 & & $10098-97-2$ & SR-90 \\
\hline 1982 & SESPSCH & & SW & & & B0G868 & 11-Sep-95 & & 14133-76-7 & TC-99 \\
\hline 1983 & SESPSCH & & SW & & & B0G868 & 11-Sep-95 & & $10028-17-8$ & TRITIUM \\
\hline 1984 & SESPSCH & & SW & & & B0G868 & 11-Sep-95 & & $13966-29-5$ & $\mathrm{U}-234$ \\
\hline 1985 & SESPSCH & & SW & & & B0G868 & 11-Sep-95 & & 15117-96-1 & U-235 \\
\hline 1986 & SESPSCH & & SW & & & B0G868 & 11-Sep-95 & & $\mathrm{U}-238$ & $\mathrm{U}-238$ \\
\hline 1987 & PS HENRY/LE BISPING & & SW & & & B0G0T2 & 11-Sep-95 & & $7440-47-3$ & $\mathrm{CR}$ \\
\hline 1988 & PS HENRY/LE BISPING & & SW & & & B0G0T2 & 11-Sep-95 & & $56-23-5$ & CARBTET \\
\hline 1989 & SESPSCH & & SW & & & B0G8C4 & 11-Sep-95 & & $10045-97-3$ & CS-137 \\
\hline 1990 & SESPSCH & & SW & & & B0G8C4 & 11-Sep-95 & & $10098-97-2$ & SR-90 \\
\hline 1991 & SESPSCH & & SW & & & B0G8C4 & 11-Sep-95 & & 14133-76-7 & TC-99 \\
\hline 1992 & SESPSCH & & SW & & & B0G8C4 & 11-Sep-95 & & $10028-17-8$ & TRITIUM \\
\hline 1993 & SESPSCH & & SW & & & B0G8C4 & 11-Sep-95 & & $\mathrm{U}-238$ & $\mathrm{U}-238$ \\
\hline 1994 & SESPSCH & & SW & & & B0G8C4 & 11-Sep-95 & & 15117-96-1 & U-235 \\
\hline 1995 & SESPSCH & & SW & & & B0G8C4 & 11-Sep-95 & & 13966-29-5 & $\mathrm{U}-234$ \\
\hline 1996 & PS HENRY/LE BISPING & & SW & & & B0G0S4 & 11-Sep-95 & & $7440-47-3$ & $\mathrm{CR}$ \\
\hline 1997 & PS HENRY/LE BISPING & & SW & & & B0G0S4 & 11-Sep-95 & & $56-23-5$ & CARBTET \\
\hline 1998 & SESPSCH & & SW & & & B0G870 & 11-Sep-95 & & $10045-97-3$ & CS-137 \\
\hline 1999 & SESPSCH & & SW & & & B0G870 & 11-Sep-95 & & $10098-97-2$ & SR-90 \\
\hline 2000 & SESPSCH & & SW & & & B0G870 & 11-Sep-95 & & 14133-76-7 & TC-99 \\
\hline 2001 & SESPSCH & & SW & & & B0G870 & 11-Sep-95 & & $10028-17-8$ & TRITIUM \\
\hline 2002 & SESPSCH & & SW & & & B0G870 & 11-Sep-95 & & $13966-29-5$ & U-234 \\
\hline 2003 & SESPSCH & & SW & & & B0G870 & 11-Sep-95 & & $15117-96-1$ & U-235 \\
\hline 2004 & SESPSCH & & SW & & & B0G870 & 11-Sep-95 & & U-238 & U-238 \\
\hline 2005 & PS HENRY/LE BISPING & & SW & & & B0G0S5 & 11-Sep-95 & & $7440-47-3$ & $\mathrm{CR}$ \\
\hline 2006 & PS HENRY/LE BISPING & & SW & & & B0G0S5 & 11-Sep-95 & & $56-23-5$ & CARBTET \\
\hline 2007 & SESPSCH & & SW & & & B0G8C5 & 11-Sep-95 & & $10045-97-3$ & CS-137 \\
\hline 2008 & SESPSCH & & SW & & & B0G8C5 & 11-Sep-95 & & $10098-97-2$ & SR-90 \\
\hline 2009 & SESPSCH & & SW & & & B0G8C5 & 11-Sep-95 & & 14133-76-7 & TC-99 \\
\hline 2010 & SESPSCH & & SW & & & B0G8C5 & 11-Sep-95 & & $10028-17-8$ & TRITIUM \\
\hline 2011 & SESPSCH & & SW & & & B0G8C5 & 11-Sep-95 & & $\mathrm{U}-238$ & U-238 \\
\hline 2012 & SESPSCH & & SW & & & B0G8C5 & 11-Sep-95 & & 15117-96-1 & U-235 \\
\hline 2013 & SESPSCH & & SW & & & B0G8C5 & 11-Sep-95 & & 13966-29-5 & U-234 \\
\hline 2014 & PS HENRY/LE BISPING & & SW & & & B0G0S6 & 11-Sep-95 & & $7440-47-3$ & $\mathrm{CR}$ \\
\hline 2015 & PS HENRY/LE BISPING & & SW & & & B0G0S6 & 11-Sep-95 & & $56-23-5$ & CARBTET \\
\hline
\end{tabular}




\begin{tabular}{|c|c|c|c|c|c|c|c|c|c|c|}
\hline & A & $\mathrm{B}$ & $\mathrm{C}$ & $\mathrm{D}$ & $E$ & $\mathrm{~F}$ & G & $\mathrm{H}$ & $\mathrm{I}$ & $\mathrm{J}$ \\
\hline 1 & SAMPLE_NUM_ASSIGNED_TO & SAMPLER & MEDIA & BIOTA_MEDIA & TAG_ID & SAMP_NUM & SAMP_DATE_TIME & SAMP_DATE_TIME_ON & CON_ID & CON_SHORT_NAME \\
\hline 2016 & SESPSCH & & SW & & & B0G8C6 & 11-Sep-95 & & $10045-97-3$ & CS-137 \\
\hline 2017 & SESPSCH & & SW & & & B0G8C6 & 11-Sep-95 & & 10098-97-2 & SR-90 \\
\hline \begin{tabular}{|l|}
2018 \\
\end{tabular} & SESPSCH & & SW & & & B0G8C6 & 11-Sep-95 & & $14133-76-7$ & TC-99 \\
\hline 2019 & SESPSCH & & SW & & & B0G8C6 & 11-Sep-95 & & $10028-17-8$ & TRITIUM \\
\hline 2020 & SESPSCH & & SW & & & B0G8C6 & 11-Sep-95 & & U-238 & U-238 \\
\hline 2021 & SESPSCH & & SW & & & B0G8C6 & 11-Sep-95 & & $15117-96-1$ & U-235 \\
\hline 2022 & SESPSCH & & SW & & & B0G8C6 & 11-Sep-95 & & $13966-29-5$ & U-234 \\
\hline 2023 & PS HENRY/LE BISPING & & SW & & & B0G0S7 & 11-Sep-95 & & $7440-47-3$ & CR \\
\hline 2024 & PS HENRY/LE BISPING & & SW & & & BOG0S7 & 11-Sep-95 & & $56-23-5$ & CARBTET \\
\hline 2025 & SESPSCH & & SW & & & B0G873 & 11-Sep-95 & & $10045-97-3$ & CS-137 \\
\hline 2026 & SESPSCH & & SW & & & B0G873 & 11-Sep-95 & & $10098-97-2$ & SR-90 \\
\hline 2027 & SESPSCH & & SW & & & B0G873 & 11-Sep-95 & & $14133-76-7$ & TC-99 \\
\hline 2028 & SESPSCH & & SW & & & B0G873 & 11-Sep-95 & & $10028-17-8$ & TRITIUM \\
\hline 2029 & SESPSCH & & SW & & & B0G873 & 11-Sep-95 & & $13966-29-5$ & U-234 \\
\hline 2030 & SESPSCH & & SW & & & B0G873 & 11-Sep-95 & & $15117-96-1$ & U-235 \\
\hline 2031 & SESPSCH & & SW & & & B0G873 & 11-Sep-95 & & $\mathrm{U}-238$ & $\mathrm{U}-238$ \\
\hline \begin{tabular}{|l|}
2032 \\
203
\end{tabular} & PS HENRY/LE BISPING & & SW & & & B0G0S8 & 11-Sep-95 & & $7440-47-3$ & $\mathrm{CR}$ \\
\hline 2033 & PS HENRY/LE BISPING & & SW & & & B0G0S8 & 11-Sep-95 & & $56-23-5$ & CARBTET \\
\hline 2034 & SESPSCH & & SW & & & B0G8C9 & 11-Sep-95 & & $10045-97-3$ & CS-137 \\
\hline \begin{tabular}{|l|}
2035 \\
\end{tabular} & SESPSCH & & SW & & & B0G8C9 & 11-Sep-95 & & $10098-97-2$ & SR-90 \\
\hline 2036 & SESPSCH & & SW & & & B0G8C9 & 11-Sep-95 & & 14133-76-7 & TC-99 \\
\hline 2037 & SESPSCH & & SW & & & B0G8C9 & 11-Sep-95 & & $10028-17-8$ & TRITIUM \\
\hline 2038 & SESPSCH & & SW & & & B0G8C9 & 11-Sep-95 & & $\mathrm{U}-238$ & U-238 \\
\hline 2039 & SESPSCH & & SW & & & B0G8C9 & 11-Sep-95 & & $15117-96-1$ & U-235 \\
\hline 2040 & SESPSCH & & SW & & & B0G8C9 & 11-Sep-95 & & $13966-29-5$ & U-234 \\
\hline 2041 & PS HENRY/LE BISPING & & SW & & & B0G0S9 & 11-Sep-95 & & $7440-47-3$ & CR \\
\hline 2042 & PS HENRY/LE BISPING & & SW & & & B0G0S9 & 11-Sep-95 & & $56-23-5$ & CARBTET \\
\hline 2043 & SESPSCH & & SW & & & B0G8C8 & 11-Sep-95 & & $10045-97-3$ & CS-137 \\
\hline 2044 & SESPSCH & & SW & & & B0G8C8 & 11-Sep-95 & & $10098-97-2$ & SR-90 \\
\hline \begin{tabular}{|l|}
2045 \\
\end{tabular} & SESPSCH & & SW & & & B0G8C8 & 11-Sep-95 & & $14133-76-7$ & TC-99 \\
\hline 2046 & SESPSCH & & SW & & & B0G8C8 & 11-Sep-95 & & $10028-17-8$ & TRITIUM \\
\hline \begin{tabular}{|l|}
2047 \\
\end{tabular} & SESPSCH & & SW & & & B0G8C8 & 11-Sep-95 & & $\mathrm{U}-238$ & U-238 \\
\hline 2048 & SESPSCH & & SW & & & B0G8C8 & 11-Sep-95 & & $15117-96-1$ & $\mathrm{U}-235$ \\
\hline 2049 & SESPSCH & & SW & & & B0G8C8 & 11-Sep-95 & & $13966-29-5$ & U-234 \\
\hline 2050 & PS HENRY/LE BISPING & & SW & & & BOGOTO & 11-Sep-95 & & $7440-47-3$ & $\mathrm{CR}$ \\
\hline 2051 & PS HENRY/LE BISPING & & SW & & & BOGOTO & 11-Sep-95 & & $56-23-5$ & CARBTET \\
\hline 2052 & SESPSCH & & SW & & & B0G8D0 & 11-Sep-95 & & $10045-97-3$ & CS-137 \\
\hline 2053 & SESPSCH & & SW & & & B0G8D0 & 11-Sep-95 & & $10098-97-2$ & SR-90 \\
\hline
\end{tabular}




\begin{tabular}{|c|c|c|c|c|c|c|c|c|c|c|}
\hline & $\mathrm{A}$ & $\mathrm{B}$ & $\mathrm{C}$ & $\mathrm{D}$ & $E$ & $\mathrm{~F}$ & $G$ & $\mathrm{H}$ & $\mathrm{I}$ & $\mathrm{J}$ \\
\hline 1 & SAMPLE NUM ASSIGNED TO & SAMPLER & MEDIA & BIOTA MEDIA & TAG ID & SAMP NUM & SAMP DATE TIME & SAMP DATE TIME ON & CON ID & CON SHORT NAME \\
\hline 2054 & SESPSCH & & SW & & & B0G8D0 & 11-Sep-95 & & 14133-76-7 & TC-99 \\
\hline 2055 & SESPSCH & & SW & & & B0G8D0 & 11-Sep-95 & & $10028-17-8$ & TRITIUM \\
\hline 2056 & SESPSCH & & SW & & & B0G8D0 & 11-Sep-95 & & $13966-29-5$ & U-234 \\
\hline 2057 & SESPSCH & & SW & & & B0G8D0 & 11-Sep-95 & & 15117-96-1 & U-235 \\
\hline 2058 & SESPSCH & & SW & & & B0G8D0 & 11-Sep-95 & & U-238 & U-238 \\
\hline 2059 & PS HENRY/LE BISPING & & SW & & & B0G0T1 & 11-Sep-95 & & $7440-47-3$ & $\mathrm{CR}$ \\
\hline 2060 & PS HENRY/LE BISPING & & SW & & & B0G0T1 & 11-Sep-95 & & $56-23-5$ & CARBTET \\
\hline 2061 & SESPSCH & & SW & & & B0G8D1 & 11-Sep-95 & & $10045-97-3$ & CS-137 \\
\hline 2062 & SESPSCH & & SW & & & B0G8D1 & 11-Sep-95 & & $10098-97-2$ & SR-90 \\
\hline 2063 & SESPSCH & & SW & & & B0G8D1 & 11-Sep-95 & & 14133-76-7 & TC-99 \\
\hline 2064 & SESPSCH & & SW & & & B0G8D1 & 11-Sep-95 & & $10028-17-8$ & TRITIUM \\
\hline 2065 & SESPSCH & & SW & & & B0G8D1 & 11-Sep-95 & & $13966-29-5$ & U-234 \\
\hline 2066 & SESPSCH & & SW & & & B0G8D1 & 11-Sep-95 & & 15117-96-1 & U-235 \\
\hline 2067 & SESPSCH & & SW & & & B0G8D1 & 11-Sep-95 & & U-238 & U-238 \\
\hline 2068 & PS HENRY/LE BISPING & & SW & & & B0G0Q9 & 11-Sep-95 & & $7440-47-3$ & CR \\
\hline 2069 & PS HENRY/LE BISPING & & SW & & & B0G0Q9 & 11-Sep-95 & & $56-23-5$ & CARBTET \\
\hline 2070 & SESPSCH & & SW & & & B0GBM4 & 11-Sep-95 & & 10098-97-2 & SR-90 \\
\hline 2071 & SESPSCH & & SW & & & B0GBM4 & 11-Sep-95 & & $10028-17-8$ & TRITIUM \\
\hline 2072 & SESPSCH & & SW & & & B0GBM4 & 11-Sep-95 & & $13966-29-5$ & U-234 \\
\hline 2073 & SESPSCH & & SW & & & B0GBM4 & 11-Sep-95 & & $15117-96-1$ & U-235 \\
\hline 2074 & SESPSCH & & SW & & & B0GBM4 & 11-Sep-95 & & U-238 & U-238 \\
\hline 2075 & PS HENRY/LE BISPING & & SW & & & BOGORO & 11-Sep-95 & & $7440-47-3$ & $\mathrm{CR}$ \\
\hline 2076 & PS HENRY/LE BISPING & & SW & & & BOGORO & 11-Sep-95 & & $56-23-5$ & CARBTET \\
\hline 2077 & SESPSCH & & SW & & & B0GBM5 & 11-Sep-95 & & 10098-97-2 & SR-90 \\
\hline 2078 & SESPSCH & & SW & & & B0GBM5 & 11-Sep-95 & & $10028-17-8$ & TRITIUM \\
\hline 2079 & SESPSCH & & SW & & & B0GBM5 & 11-Sep-95 & & $13966-29-5$ & U-234 \\
\hline 2080 & SESPSCH & & SW & & & B0GBM5 & 11-Sep-95 & & 15117-96-1 & U-235 \\
\hline 2081 & SESPSCH & & SW & & & B0GBM5 & 11-Sep-95 & & U-238 & U-238 \\
\hline 2082 & PS HENRY/LE BISPING & & SW & & & B0G0R1 & 11-Sep-95 & & $7440-47-3$ & CR \\
\hline 2083 & PS HENRY/LE BISPING & & SW & & & B0G0R1 & 11-Sep-95 & & $56-23-5$ & CARBTET \\
\hline 2084 & SESPSCH & & SW & & & B0GBM6 & 11-Sep-95 & & $10098-97-2$ & SR-90 \\
\hline 2085 & SESPSCH & & SW & & & B0GBM6 & 11-Sep-95 & & $10028-17-8$ & TRITIUM \\
\hline 2086 & SESPSCH & & SW & & & B0GBM6 & 11-Sep-95 & & 13966-29-5 & U-234 \\
\hline 2087 & SESPSCH & & SW & & & B0GBM6 & 11-Sep-95 & & 15117-96-1 & U-235 \\
\hline 2088 & SESPSCH & & SW & & & B0GBM6 & 11-Sep-95 & & U-238 & U-238 \\
\hline 2089 & PS HENRY/LE BISPING & & SW & & & B0G0R2 & 11-Sep-95 & & $7440-47-3$ & CR \\
\hline 2090 & PS HENRY/LE BISPING & & SW & & & BOG0R2 & 11-Sep-95 & & $56-23-5$ & CARBTET \\
\hline 2091 & SESPSCH & & SW & & & B0GBM7 & 11-Sep-95 & & $10098-97-2$ & SR-90 \\
\hline
\end{tabular}




\begin{tabular}{|c|c|c|c|c|c|c|c|c|c|c|}
\hline & A & $\mathrm{B}$ & $C$ & $\mathrm{D}$ & $E$ & $\mathrm{~F}$ & $G$ & $\mathrm{H}$ & $\mathrm{I}$ & $\mathrm{J}$ \\
\hline 1 & SAMPLE_NUM_ASSIGNED_TO & SAMPLER & MEDIA & BIOTA_MEDIA & TAG_ID & SAMP_NUM & SAMP_DATE_TIME| & SAMP_DATE_TIME_ON & CON_ID & CON_SHORT_NAME \\
\hline 2092 & SESPSCH & & SW & & & B0GBM7 & 11-Sep-95 & & $10028-17-8$ & TRITIUM \\
\hline 2093 & SESPSCH & & SW & & & B0GBM7 & 11-Sep-95 & & $13966-29-5$ & $\mathrm{U}-234$ \\
\hline 2094 & SESPSCH & & SW & & & B0GBM7 & 11-Sep-95 & & 15117-96-1 & U-235 \\
\hline 2095 & SESPSCH & & SW & & & B0GBM7 & 11-Sep-95 & & U-238 & $\mathrm{U}-238$ \\
\hline 2096 & PS HENRY/LE BISPING & & SW & & & B0G0T3 & 12-Sep-95 & & $7440-47-3$ & CR \\
\hline 2097 & PS HENRY/LE BISPING & & SW & & & B0G0T3 & 12-Sep-95 & & $56-23-5$ & CARBTET \\
\hline 2098 & SESPSCH & & SW & & & B0G874 & 12-Sep-95 & & $10098-97-2$ & SR-90 \\
\hline 2099 & SESPSCH & & SW & & & B0G874 & 12-Sep-95 & & $10028-17-8$ & TRITIUM \\
\hline 2100 & SESPSCH & & SW & & & B0G874 & 12-Sep-95 & & $13966-29-5$ & U-234 \\
\hline 2101 & SESPSCH & & SW & & & B0G874 & 12-Sep-95 & & 15117-96-1 & U-235 \\
\hline 2102 & SESPSCH & & SW & & & B0G874 & 12-Sep-95 & & U-238 & $\mathrm{U}-238$ \\
\hline 2103 & PS HENRY/LE BISPING & & SW & & & B0G0V2 & 12-Sep-95 & & $7440-47-3$ & CR \\
\hline 2104 & PS HENRY/LE BISPING & & SW & & & BOGOV2 & 12-Sep-95 & & $56-23-5$ & CARBTET \\
\hline 2105 & SESPSCH & & SW & & & B0G884 & 12-Sep-95 & & $10098-97-2$ & SR-90 \\
\hline 2106 & SESPSCH & & SW & & & B0G884 & 12-Sep-95 & & $10028-17-8$ & TRITIUM \\
\hline 2107 & SESPSCH & & SW & & & B0G884 & 12-Sep-95 & & U-238 & $\mathrm{U}-238$ \\
\hline 2108 & SESPSCH & & SW & & & B0G884 & 12-Sep-95 & & 15117-96-1 & U-235 \\
\hline 2109 & SESPSCH & & SW & & & B0G884 & 12-Sep-95 & & 13966-29-5 & $\mathrm{U}-234$ \\
\hline 2110 & PS HENRY/LE BISPING & & SW & & & B0G0T4 & 12-Sep-95 & & $7440-47-3$ & $\mathrm{CR}$ \\
\hline 2111 & PS HENRY/LE BISPING & & SW & & & BOG0T4 & 12-Sep-95 & & $56-23-5$ & CARBTET \\
\hline 2112 & SESPSCH & & SW & & & B0G878 & 12-Sep-95 & & $10098-97-2$ & SR-90 \\
\hline 2113 & SESPSCH & & SW & & & B0G878 & 12-Sep-95 & & $10028-17-8$ & TRITIUM \\
\hline 2114 & SESPSCH & & SW & & & B0G878 & 12-Sep-95 & & U-238 & $\mathrm{U}-238$ \\
\hline 2115 & SESPSCH & & SW & & & B0G878 & 12-Sep-95 & & 15117-96-1 & U-235 \\
\hline 2116 & SESPSCH & & SW & & & B0G878 & 12-Sep-95 & & $13966-29-5$ & U-234 \\
\hline 2117 & PS HENRY/LE BISPING & & SW & & & B0G0T5 & 12-Sep-95 & & $7440-47-3$ & CR \\
\hline 2118 & PS HENRY/LE BISPING & & SW & & & B0G0T5 & 12-Sep-95 & & $56-23-5$ & CARBTET \\
\hline 2119 & SESPSCH & & SW & & & B0G875 & 12-Sep-95 & & $10098-97-2$ & SR-90 \\
\hline 2120 & SESPSCH & & SW & & & B0G875 & 12-Sep-95 & & $10028-17-8$ & TRITIUM \\
\hline 2121 & SESPSCH & & SW & & & B0G875 & 12-Sep-95 & & 13966-29-5 & U-234 \\
\hline 2122 & SESPSCH & & SW & & & B0G875 & 12-Sep-95 & & 15117-96-1 & U-235 \\
\hline 2123 & SESPSCH & & SW & & & B0G875 & 12-Sep-95 & & $\mathrm{U}-238$ & U-238 \\
\hline 2124 & PS HENRY/LE BISPING & & SW & & & B0G0T6 & 12-Sep-95 & & $7440-47-3$ & CR \\
\hline 2125 & PS HENRY/LE BISPING & & SW & & & B0G0T6 & 12-Sep-95 & & $56-23-5$ & CARBTET \\
\hline 2126 & SESPSCH & & SW & & & B0G879 & 12-Sep-95 & & $10098-97-2$ & SR-90 \\
\hline 2127 & SESPSCH & & SW & & & B0G879 & 12-Sep-95 & & $10028-17-8$ & TRITIUM \\
\hline 2128 & SESPSCH & & SW & & & B0G879 & 12-Sep-95 & & U-238 & U-238 \\
\hline 2129 & SESPSCH & & SW & & & B0G879 & 12-Sep-95 & & $15117-96-1$ & U-235 \\
\hline
\end{tabular}




\begin{tabular}{|c|c|c|c|c|c|c|c|c|c|c|}
\hline & A & $\mathrm{B}$ & $C$ & $\mathrm{D}$ & $E$ & $\mathrm{~F}$ & $G$ & $\mathrm{H}$ & $\mathrm{I}$ & $\mathrm{J}$ \\
\hline 1 & SAMPLE_NUM_ASSIGNED_TO & SAMPLER & MEDIA & BIOTA_MEDIA & TAG_ID & SAMP_NUM & SAMP_DATE_TIME| & SAMP_DATE_TIME_ON & CON_ID & CON_SHORT_NAME \\
\hline 2130 & SESPSCH & & SW & & & B0G879 & 12-Sep-95 & & $13966-29-5$ & U-234 \\
\hline 2131 & PS HENRY/LE BISPING & & SW & & & B0G0T7 & 12-Sep-95 & & $7440-47-3$ & $\mathrm{CR}$ \\
\hline 2132 & PS HENRY/LE BISPING & & SW & & & B0G0T7 & 12-Sep-95 & & $56-23-5$ & CARBTET \\
\hline 2133 & SESPSCH & & SW & & & B0G880 & 12-Sep-95 & & $10098-97-2$ & SR-90 \\
\hline 2134 & SESPSCH & & SW & & & B0G880 & 12-Sep-95 & & $10028-17-8$ & TRITIUM \\
\hline 2135 & SESPSCH & & SW & & & B0G880 & 12-Sep-95 & & $\mathrm{U}-238$ & $\mathrm{U}-238$ \\
\hline 2136 & SESPSCH & & SW & & & B0G880 & 12-Sep-95 & & 15117-96-1 & U-235 \\
\hline 2137 & SESPSCH & & SW & & & B0G880 & 12-Sep-95 & & 13966-29-5 & $\mathrm{U}-234$ \\
\hline 2138 & PS HENRY/LE BISPING & & SW & & & B0G0T8 & 12-Sep-95 & & $7440-47-3$ & $\mathrm{CR}$ \\
\hline 2139 & PS HENRY/LE BISPING & & SW & & & B0G0T8 & 12-Sep-95 & & $56-23-5$ & CARBTET \\
\hline 2140 & SESPSCH & & SW & & & B0G881 & 12-Sep-95 & & $10098-97-2$ & SR-90 \\
\hline 2141 & SESPSCH & & SW & & & B0G881 & 12-Sep-95 & & $10028-17-8$ & TRITIUM \\
\hline 2142 & SESPSCH & & SW & & & B0G881 & 12-Sep-95 & & U-238 & U-238 \\
\hline 2143 & SESPSCH & & SW & & & B0G881 & 12-Sep-95 & & $15117-96-1$ & U-235 \\
\hline 2144 & SESPSCH & & SW & & & B0G881 & 12-Sep-95 & & $13966-29-5$ & U-234 \\
\hline 2145 & PS HENRY/LE BISPING & & SW & & & B0G0T9 & 12-Sep-95 & & $7440-47-3$ & $\mathrm{CR}$ \\
\hline 2146 & PS HENRY/LE BISPING & & SW & & & BOG0T9 & 12-Sep-95 & & $56-23-5$ & CARBTET \\
\hline 2147 & SESPSCH & & SW & & & B0G882 & 12-Sep-95 & & $10098-97-2$ & SR-90 \\
\hline 2148 & SESPSCH & & SW & & & B0G882 & 12-Sep-95 & & $10028-17-8$ & TRITIUM \\
\hline 2149 & SESPSCH & & SW & & & B0G882 & 12-Sep-95 & & $\mathrm{U}-238$ & $\mathrm{U}-238$ \\
\hline 2150 & SESPSCH & & SW & & & B0G882 & 12-Sep-95 & & $15117-96-1$ & U-235 \\
\hline 2151 & SESPSCH & & SW & & & B0G882 & 12-Sep-95 & & $13966-29-5$ & U-234 \\
\hline 2152 & PS HENRY/LE BISPING & & SW & & & BOGOV0 & 12-Sep-95 & & $7440-47-3$ & CR \\
\hline 2153 & PS HENRY/LE BISPING & & SW & & & BOGOV0 & 12-Sep-95 & & $56-23-5$ & CARBTET \\
\hline 2154 & SESPSCH & & SW & & & B0G883 & 12-Sep-95 & & $10098-97-2$ & SR-90 \\
\hline 2155 & SESPSCH & & SW & & & B0G883 & 12-Sep-95 & & $10028-17-8$ & TRITIUM \\
\hline 2156 & SESPSCH & & SW & & & B0G883 & 12-Sep-95 & & U-238 & U-238 \\
\hline 2157 & SESPSCH & & SW & & & B0G883 & 12-Sep-95 & & 15117-96-1 & U-235 \\
\hline 2158 & SESPSCH & & SW & & & B0G883 & 12-Sep-95 & & $13966-29-5$ & U-234 \\
\hline 2159 & PS HENRY/LE BISPING & & SW & & & B0G0V1 & 12-Sep-95 & & $7440-47-3$ & CR \\
\hline 2160 & PS HENRY/LE BISPING & & SW & & & B0G0V1 & 12-Sep-95 & & $56-23-5$ & CARBTET \\
\hline 2161 & SESPSCH & & SW & & & B0G885 & 12-Sep-95 & & $10098-97-2$ & SR-90 \\
\hline 2162 & SESPSCH & & SW & & & B0G885 & 12-Sep-95 & & $10028-17-8$ & TRITIUM \\
\hline 2163 & SESPSCH & & SW & & & B0G885 & 12-Sep-95 & & U-238 & U-238 \\
\hline 2164 & SESPSCH & & SW & & & B0G885 & 12-Sep-95 & & 15117-96-1 & U-235 \\
\hline 2165 & SESPSCH & & SW & & & B0G885 & 12-Sep-95 & & 13966-29-5 & U-234 \\
\hline 2166 & PS HENRY/LE BISPING & & SW & & & BOGOV3 & 12-Sep-95 & & $7440-47-3$ & $\mathrm{CR}$ \\
\hline 2167 & PS HENRY/LE BISPING & & SW & & & BOGOV3 & 12-Sep-95 & & $56-23-5$ & CARBTET \\
\hline
\end{tabular}




\begin{tabular}{|c|c|c|c|c|c|c|c|c|c|c|}
\hline & A & $\mathrm{B}$ & $\mathrm{C}$ & $\mathrm{D}$ & $E$ & $\mathrm{~F}$ & G & $\mathrm{H}$ & $\mathrm{I}$ & $\mathrm{J}$ \\
\hline 1 & SAMPLE_NUM_ASSIGNED_TO & SAMPLER & MEDIA & BIOTA_MEDIA & TAG_ID & SAMP_NUM & SAMP_DATE_TIME & SAMP_DATE_TIME_ON & CON_ID & CON_SHORT_NAME \\
\hline 2168 & SESPSCH & & SW & & & B0G886 & 12-Sep-95 & & $10098-97-2$ & SR-90 \\
\hline 2169 & SESPSCH & & SW & & & B0G886 & 12-Sep-95 & & $14133-76-7$ & TC-99 \\
\hline 2170 & SESPSCH & & SW & & & B0G886 & 12-Sep-95 & & $10028-17-8$ & TRITIUM \\
\hline \begin{tabular}{|l|}
2171 \\
\end{tabular} & SESPSCH & & SW & & & B0G886 & 12-Sep-95 & & U-238 & U-238 \\
\hline $2 \mid$ & SESPSCH & & SW & & & B0G886 & 12-Sep-95 & & $15117-96-1$ & U-235 \\
\hline 2173 & SESPSCH & & SW & & & B0G886 & 12-Sep-95 & & $13966-29-5$ & U-234 \\
\hline 2174 & PS HENRY/LE BISPING & & SW & & & BOGOW2 & 12-Sep-95 & & $7440-47-3$ & $\mathrm{CR}$ \\
\hline 2175 & PS HENRY/LE BISPING & & SW & & & B0G0W2 & 12-Sep-95 & & $56-23-5$ & CARBTET \\
\hline 2176 & SESPSCH & & SW & & & B0G891 & 12-Sep-95 & & $10098-97-2$ & SR-90 \\
\hline 2177 & SESPSCH & & SW & & & B0G891 & 12-Sep-95 & & $14133-76-7$ & TC-99 \\
\hline 2178 & SESPSCH & & SW & & & B0G891 & 12-Sep-95 & & $10028-17-8$ & TRITIUM \\
\hline 2179 & SESPSCH & & SW & & & B0G891 & 12-Sep-95 & & U-238 & U-238 \\
\hline 2180 & SESPSCH & & SW & & & B0G891 & 12-Sep-95 & & $15117-96-1$ & U-235 \\
\hline 2181 & SESPSCH & & SW & & & B0G891 & 12-Sep-95 & & $13966-29-5$ & U-234 \\
\hline 2182 & PS HENRY/LE BISPING & & SW & & & B0G0V4 & 12-Sep-95 & & $7440-47-3$ & $\mathrm{CR}$ \\
\hline 2183 & PS HENRY/LE BISPING & & SW & & & B0G0V4 & 12-Sep-95 & & $56-23-5$ & CARBTET \\
\hline 2184 & SESPSCH & & SW & & & B0G887 & 12-Sep-95 & & $10098-97-2$ & SR-90 \\
\hline 2185 & SESPSCH & & SW & & & B0G887 & 12-Sep-95 & & 14133-76-7 & TC-99 \\
\hline 2186 & SESPSCH & & SW & & & B0G887 & 12-Sep-95 & & $10028-17-8$ & TRITIUM \\
\hline \begin{tabular}{|l|}
2187 \\
\end{tabular} & SESPSCH & & SW & & & B0G887 & 12-Sep-95 & & $\mathrm{U}-238$ & U-238 \\
\hline 2188 & SESPSCH & & SW & & & B0G887 & 12-Sep-95 & & $15117-96-1$ & U-235 \\
\hline 2189 & SESPSCH & & SW & & & B0G887 & 12-Sep-95 & & $13966-29-5$ & U-234 \\
\hline 2190 & PS HENRY/LE BISPING & & SW & & & B0G0V5 & 12-Sep-95 & & $7440-47-3$ & $\mathrm{CR}$ \\
\hline 2191 & PS HENRY/LE BISPING & & SW & & & B0G0V5 & 12-Sep-95 & & $56-23-5$ & CARBTET \\
\hline 2192 & SESPSCH & & SW & & & B0G892 & 12-Sep-95 & & $10098-97-2$ & SR-90 \\
\hline 2193 & SESPSCH & & SW & & & B0G892 & 12-Sep-95 & & 14133-76-7 & TC-99 \\
\hline 2194 & SESPSCH & & SW & & & B0G892 & 12-Sep-95 & & $10028-17-8$ & TRITIUM \\
\hline 2195 & SESPSCH & & SW & & & B0G892 & 12-Sep-95 & & U-238 & U-238 \\
\hline 2196 & SESPSCH & & SW & & & B0G892 & 12-Sep-95 & & $15117-96-1$ & U-235 \\
\hline \begin{tabular}{|l|}
2197 \\
2197
\end{tabular} & SESPSCH & & SW & & & B0G892 & 12-Sep-95 & & $13966-29-5$ & $\mathrm{U}-234$ \\
\hline 2198 & PS HENRY/LE BISPING & & SW & & & B0G0V6 & 12-Sep-95 & & $7440-47-3$ & CR \\
\hline 2199 & PS HENRY/LE BISPING & & SW & & & B0G0V6 & 12-Sep-95 & & $56-23-5$ & CARBTET \\
\hline \begin{tabular}{|l|}
2200 \\
\end{tabular} & SESPSCH & & SW & & & B0G872 & 12-Sep-95 & & $10098-97-2$ & SR-90 \\
\hline 2201 & SESPSCH & & SW & & & B0G872 & 12-Sep-95 & & $14133-76-7$ & TC-99 \\
\hline 2202 & SESPSCH & & SW & & & B0G872 & 12-Sep-95 & & $10028-17-8$ & TRITIUM \\
\hline 2203 & SESPSCH & & SW & & & B0G872 & 12-Sep-95 & & $13966-29-5$ & U-234 \\
\hline 2204 & SESPSCH & & SW & & & B0G872 & 12-Sep-95 & & $15117-96-1$ & U-235 \\
\hline 2205 & SESPSCH & & SW & & & B0G872 & 12-Sep-95 & & U-238 & U-238 \\
\hline
\end{tabular}




\begin{tabular}{|c|c|c|c|c|c|c|c|c|c|c|}
\hline & A & $\mathrm{B}$ & $C$ & $\mathrm{D}$ & $E$ & $\mathrm{~F}$ & $G$ & $\mathrm{H}$ & $\mathrm{I}$ & $\mathrm{J}$ \\
\hline 1 & SAMPLE_NUM_ASSIGNED_TO & SAMPLER & MEDIA & BIOTA_MEDIA & TAG_ID & SAMP_NUM & SAMP_DATE_TIME| & SAMP_DATE_TIME_ON & CON_ID & CON_SHORT_NAME \\
\hline 2206 & PS HENRY/LE BISPING & & SW & & & B0G0V7 & 12-Sep-95 & & $7440-47-3$ & CR \\
\hline 2207 & PS HENRY/LE BISPING & & SW & & & B0G0V7 & 12-Sep-95 & & $56-23-5$ & CARBTET \\
\hline 2208 & SESPSCH & & SW & & & B0G876 & 12-Sep-95 & & $10098-97-2$ & SR-90 \\
\hline 2209 & SESPSCH & & SW & & & B0G876 & 12-Sep-95 & & 14133-76-7 & TC-99 \\
\hline 2210 & SESPSCH & & SW & & & B0G876 & 12-Sep-95 & & $10028-17-8$ & TRITIUM \\
\hline 2211 & SESPSCH & & SW & & & B0G876 & 12-Sep-95 & & $13966-29-5$ & $\mathrm{U}-234$ \\
\hline 2212 & SESPSCH & & SW & & & B0G876 & 12-Sep-95 & & 15117-96-1 & U-235 \\
\hline 2213 & SESPSCH & & SW & & & B0G876 & 12-Sep-95 & & U-238 & U-238 \\
\hline 2214 & PS HENRY/LE BISPING & & SW & & & B0G0V8 & 12-Sep-95 & & $7440-47-3$ & $\mathrm{CR}$ \\
\hline 2215 & PS HENRY/LE BISPING & & SW & & & B0G0V8 & 12-Sep-95 & & $56-23-5$ & CARBTET \\
\hline 2216 & SESPSCH & & SW & & & B0G877 & 12-Sep-95 & & $10098-97-2$ & SR-90 \\
\hline 2217 & SESPSCH & & SW & & & B0G877 & 12-Sep-95 & & 14133-76-7 & TC-99 \\
\hline 2218 & SESPSCH & & SW & & & B0G877 & 12-Sep-95 & & $10028-17-8$ & TRITIUM \\
\hline 2219 & SESPSCH & & SW & & & B0G877 & 12-Sep-95 & & $13966-29-5$ & U-234 \\
\hline 2220 & SESPSCH & & SW & & & B0G877 & 12-Sep-95 & & $\mathrm{U}-238$ & $\mathrm{U}-238$ \\
\hline 2221 & SESPSCH & & SW & & & B0G877 & 12-Sep-95 & & 15117-96-1 & U-235 \\
\hline 2222 & PS HENRY/LE BISPING & & SW & & & B0G0V9 & 12-Sep-95 & & $7440-47-3$ & $\mathrm{CR}$ \\
\hline 2223 & PS HENRY/LE BISPING & & SW & & & B0G0V9 & 12-Sep-95 & & $56-23-5$ & CARBTET \\
\hline 2224 & SESPSCH & & SW & & & B0G888 & 12-Sep-95 & & $10098-97-2$ & SR-90 \\
\hline 2225 & SESPSCH & & SW & & & B0G888 & 12-Sep-95 & & 14133-76-7 & TC-99 \\
\hline 2226 & SESPSCH & & SW & & & B0G888 & 12-Sep-95 & & $10028-17-8$ & TRITIUM \\
\hline 2227 & SESPSCH & & SW & & & B0G888 & 12-Sep-95 & & $\mathrm{U}-238$ & $\mathrm{U}-238$ \\
\hline 2228 & SESPSCH & & SW & & & B0G888 & 12-Sep-95 & & 15117-96-1 & U-235 \\
\hline 2229 & SESPSCH & & SW & & & B0G888 & 12-Sep-95 & & 13966-29-5 & $\mathrm{U}-234$ \\
\hline 2230 & PS HENRY/LE BISPING & & SW & & & BOGOWO & 12-Sep-95 & & $7440-47-3$ & $\mathrm{CR}$ \\
\hline 2231 & PS HENRY/LE BISPING & & SW & & & BOGOWO & 12-Sep-95 & & $56-23-5$ & CARBTET \\
\hline 2232 & SESPSCH & & SW & & & B0G889 & 12-Sep-95 & & $10098-97-2$ & SR-90 \\
\hline 2233 & SESPSCH & & SW & & & B0G889 & 12-Sep-95 & & $14133-76-7$ & TC-99 \\
\hline 2234 & SESPSCH & & SW & & & B0G889 & 12-Sep-95 & & $10028-17-8$ & TRITIUM \\
\hline 2235 & SESPSCH & & SW & & & B0G889 & 12-Sep-95 & & $\mathrm{U}-238$ & U-238 \\
\hline 2236 & SESPSCH & & SW & & & B0G889 & 12-Sep-95 & & $15117-96-1$ & U-235 \\
\hline 2237 & SESPSCH & & SW & & & B0G889 & 12-Sep-95 & & 13966-29-5 & U-234 \\
\hline 2238 & PS HENRY/LE BISPING & & SW & & & B0G0W1 & 12-Sep-95 & & $7440-47-3$ & $\mathrm{CR}$ \\
\hline 2239 & PS HENRY/LE BISPING & & SW & & & B0G0W1 & 12-Sep-95 & & $56-23-5$ & CARBTET \\
\hline 2240 & SESPSCH & & SW & & & B0G890 & 12-Sep-95 & & $10098-97-2$ & SR-90 \\
\hline 2241 & SESPSCH & & SW & & & B0G890 & 12-Sep-95 & & 14133-76-7 & TC-99 \\
\hline 2242 & SESPSCH & & SW & & & B0G890 & 12-Sep-95 & & $10028-17-8$ & TRITIUM \\
\hline 2243 & SESPSCH & & SW & & & B0G890 & 12-Sep-95 & & $\mathrm{U}-238$ & U-238 \\
\hline
\end{tabular}




\begin{tabular}{|c|c|c|c|c|c|c|c|c|c|c|}
\hline & A & $\mathrm{B}$ & $\mathrm{C}$ & $\mathrm{D}$ & $E$ & $\mathrm{~F}$ & G & $\mathrm{H}$ & $\mathrm{I}$ & $\mathrm{J}$ \\
\hline 1 & SAMPLE_NUM_ASSIGNED_TO & SAMPLER & MEDIA & BIOTA_MEDIA & TAG_ID & SAMP_NUM & SAMP_DATE_TIME & SAMP_DATE_TIME_ON & CON_ID & CON_SHORT_NAME \\
\hline 2244 & SESPSCH & & SW & & & B0G890 & 12-Sep-95 & & 15117-96-1 & U-235 \\
\hline 2245 & SESPSCH & & SW & & & B0G890 & 12-Sep-95 & & 13966-29-5 & $\mathrm{U}-234$ \\
\hline 2246 & PS HENRY/LE BISPING & & SW & & & B0G0W3 & 18-Sep-95 & & $7440-47-3$ & CR \\
\hline 2247 & PS HENRY/LE BISPING & & SW & & & B0G0W3 & 18-Sep-95 & & $56-23-5$ & CARBTET \\
\hline 2248 & SESPSCH & & SW & & & B0G893 & 18-Sep-95 & & $10098-97-2$ & SR-90 \\
\hline 2249 & SESPSCH & & SW & & & B0G893 & 18-Sep-95 & & $10028-17-8$ & TRITIUM \\
\hline 2250 & SESPSCH & & SW & & & B0G893 & 18-Sep-95 & & $13966-29-5$ & $\mathrm{U}-234$ \\
\hline 2251 & SESPSCH & & SW & & & B0G893 & 18-Sep-95 & & U-238 & $\mathrm{U}-238$ \\
\hline 2252 & SESPSCH & & SW & & & B0G893 & 18-Sep-95 & & 15117-96-1 & U-235 \\
\hline 2253 & PS HENRY/LE BISPING & & SW & & & B0G0W4 & 18-Sep-95 & & $7440-47-3$ & $\mathrm{CR}$ \\
\hline 2254 & PS HENRY/LE BISPING & & SW & & & B0G0W4 & 18-Sep-95 & & $56-23-5$ & CARBTET \\
\hline 2255 & SESPSCH & & SW & & & B0G894 & 18-Sep-95 & & $10098-97-2$ & SR-90 \\
\hline 2256 & SESPSCH & & SW & & & B0G894 & 18-Sep-95 & & $10028-17-8$ & TRITIUM \\
\hline 2257 & SESPSCH & & SW & & & B0G894 & 18-Sep-95 & & U-238 & $\mathrm{U}-238$ \\
\hline 2258 & SESPSCH & & SW & & & B0G894 & 18-Sep-95 & & 15117-96-1 & U-235 \\
\hline 2259 & SESPSCH & & SW & & & B0G894 & 18-Sep-95 & & $13966-29-5$ & U-234 \\
\hline 2260 & PS HENRY/LE BISPING & & SW & & & B0G0W5 & 18-Sep-95 & & $7440-47-3$ & $\mathrm{CR}$ \\
\hline 2261 & PS HENRY/LE BISPING & & SW & & & B0G0W5 & 18-Sep-95 & & $56-23-5$ & CARBTET \\
\hline 2262 & SESPSCH & & SW & & & B0G895 & 18-Sep-95 & & $10098-97-2$ & SR-90 \\
\hline 2263 & SESPSCH & & SW & & & B0G895 & 18-Sep-95 & & $10028-17-8$ & TRITIUM \\
\hline 2264 & SESPSCH & & SW & & & B0G895 & 18-Sep-95 & & U-238 & $\mathrm{U}-238$ \\
\hline 2265 & SESPSCH & & SW & & & B0G895 & 18-Sep-95 & & 15117-96-1 & U-235 \\
\hline 2266 & SESPSCH & & SW & & & B0G895 & 18-Sep-95 & & $13966-29-5$ & $\mathrm{U}-234$ \\
\hline 2267 & PS HENRY/LE BISPING & & SW & & & B0G0W6 & 18-Sep-95 & & $7440-47-3$ & $\mathrm{CR}$ \\
\hline 2268 & PS HENRY/LE BISPING & & SW & & & B0G0W6 & 18-Sep-95 & & $56-23-5$ & CARBTET \\
\hline 2269 & SESPSCH & & SW & & & B0G896 & 18-Sep-95 & & $10098-97-2$ & SR-90 \\
\hline 2270 & SESPSCH & & SW & & & B0G896 & 18-Sep-95 & & $10028-17-8$ & TRITIUM \\
\hline 2271 & SESPSCH & & SW & & & B0G896 & 18-Sep-95 & & U-238 & $\mathrm{U}-238$ \\
\hline 2272 & SESPSCH & & SW & & & B0G896 & 18-Sep-95 & & 15117-96-1 & U-235 \\
\hline 2273 & SESPSCH & & SW & & & B0G896 & 18-Sep-95 & & $13966-29-5$ & $\mathrm{U}-234$ \\
\hline 2274 & PS HENRY/LE BISPING & & SW & & & B0G0W7 & 18-Sep-95 & & $7440-47-3$ & CR \\
\hline 2275 & PS HENRY/LE BISPING & & SW & & & B0G0W7 & 18-Sep-95 & & $56-23-5$ & CARBTET \\
\hline 2276 & SESPSCH & & SW & & & B0G897 & 18-Sep-95 & & 10098-97-2 & SR-90 \\
\hline 2277 & SESPSCH & & SW & & & B0G897 & 18-Sep-95 & & $10028-17-8$ & TRITIUM \\
\hline 2278 & SESPSCH & & SW & & & B0G897 & 18-Sep-95 & & U-238 & $\mathrm{U}-238$ \\
\hline 2279 & SESPSCH & & SW & & & B0G897 & 18-Sep-95 & & $15117-96-1$ & $\mathrm{U}-235$ \\
\hline 2280 & SESPSCH & & SW & & & B0G897 & 18-Sep-95 & & 13966-29-5 & $\mathrm{U}-234$ \\
\hline 2281 & PS HENRY/LE BISPING & & SW & & & B0G0W8 & 18-Sep-95 & & $7440-47-3$ & CR \\
\hline
\end{tabular}




\begin{tabular}{|c|c|c|c|c|c|c|c|c|c|c|}
\hline & A & $\mathrm{B}$ & $C$ & $\mathrm{D}$ & $E$ & $\mathrm{~F}$ & $G$ & $\mathrm{H}$ & $\mathrm{I}$ & $\mathrm{J}$ \\
\hline 1 & SAMPLE_NUM_ASSIGNED_TO & SAMPLER & MEDIA & BIOTA_MEDIA & TAG_ID & SAMP_NUM & SAMP_DATE_TIME| & SAMP_DATE_TIME_ON & CON_ID & CON_SHORT_NAME \\
\hline 2282 & PS HENRY/LE BISPING & & SW & & & B0G0W8 & 18-Sep-95 & & $56-23-5$ & CARBTET \\
\hline 2283 & SESPSCH & & SW & & & B0G898 & 18-Sep-95 & & $10098-97-2$ & SR-90 \\
\hline 2284 & SESPSCH & & SW & & & B0G898 & 18-Sep-95 & & 10028-17-8 & TRITIUM \\
\hline 2285 & SESPSCH & & SW & & & B0G898 & 18-Sep-95 & & $\mathrm{U}-238$ & U-238 \\
\hline 2286 & SESPSCH & & SW & & & B0G898 & 18-Sep-95 & & $15117-96-1$ & U-235 \\
\hline 2287 & SESPSCH & & SW & & & B0G898 & 18-Sep-95 & & $13966-29-5$ & U-234 \\
\hline 2288 & PS HENRY/LE BISPING & & SW & & & B0G0W9 & 18-Sep-95 & & $7440-47-3$ & CR \\
\hline 2289 & PS HENRY/LE BISPING & & SW & & & B0G0W9 & 18-Sep-95 & & $56-23-5$ & CARBTET \\
\hline 2290 & SESPSCH & & SW & & & B0G899 & 18-Sep-95 & & $10098-97-2$ & SR-90 \\
\hline 2291 & SESPSCH & & SW & & & B0G899 & 18-Sep-95 & & $10028-17-8$ & TRITIUM \\
\hline 2292 & SESPSCH & & SW & & & B0G899 & 18-Sep-95 & & U-238 & $\mathrm{U}-238$ \\
\hline 2293 & SESPSCH & & SW & & & B0G899 & 18-Sep-95 & & 15117-96-1 & U-235 \\
\hline 2294 & SESPSCH & & SW & & & B0G899 & 18-Sep-95 & & $13966-29-5$ & U-234 \\
\hline 2295 & PS HENRY/LE BISPING & & SW & & & BOGOX0 & 18-Sep-95 & & $7440-47-3$ & CR \\
\hline 2296 & PS HENRY/LE BISPING & & SW & & & BOG0X0 & 18-Sep-95 & & $56-23-5$ & CARBTET \\
\hline 2297 & SESPSCH & & SW & & & B0G8B0 & 18-Sep-95 & & $10098-97-2$ & SR-90 \\
\hline 2298 & SESPSCH & & SW & & & B0G8B0 & 18-Sep-95 & & $10028-17-8$ & TRITIUM \\
\hline 2299 & SESPSCH & & SW & & & B0G8B0 & 18-Sep-95 & & U-238 & $\mathrm{U}-238$ \\
\hline 2300 & SESPSCH & & SW & & & B0G8B0 & 18-Sep-95 & & 15117-96-1 & U-235 \\
\hline 2301 & SESPSCH & & SW & & & B0G8B0 & 18-Sep-95 & & $13966-29-5$ & U-234 \\
\hline 2302 & PS HENRY/LE BISPING & & SW & & & B0G0X1 & 18-Sep-95 & & $7440-47-3$ & CR \\
\hline 2303 & PS HENRY/LE BISPING & & SW & & & B0G0X1 & 18-Sep-95 & & $56-23-5$ & CARBTET \\
\hline 2304 & SESPSCH & & SW & & & B0G8B1 & 18-Sep-95 & & $10098-97-2$ & SR-90 \\
\hline 2305 & SESPSCH & & SW & & & B0G8B1 & 18-Sep-95 & & $10028-17-8$ & TRITIUM \\
\hline 2306 & SESPSCH & & SW & & & B0G8B1 & 18-Sep-95 & & U-238 & $\mathrm{U}-238$ \\
\hline 2307 & SESPSCH & & SW & & & B0G8B1 & 18-Sep-95 & & 15117-96-1 & U-235 \\
\hline 2308 & SESPSCH & & SW & & & B0G8B1 & 18-Sep-95 & & 13966-29-5 & U-234 \\
\hline 2309 & PS HENRY/LE BISPING & & SW & & & B0G0X2 & 18-Sep-95 & & $7440-47-3$ & $\mathrm{CR}$ \\
\hline 2310 & PS HENRY/LE BISPING & & SW & & & B0G0X2 & 18-Sep-95 & & $56-23-5$ & CARBTET \\
\hline 2311 & SESPSCH & & SW & & & B0G8B2 & 18-Sep-95 & & $10098-97-2$ & SR-90 \\
\hline 2312 & SESPSCH & & SW & & & B0G8B2 & 18-Sep-95 & & $10028-17-8$ & TRITIUM \\
\hline 2313 & SESPSCH & & SW & & & B0G8B2 & 18-Sep-95 & & U-238 & $\mathrm{U}-238$ \\
\hline 2314 & SESPSCH & & SW & & & B0G8B2 & 18-Sep-95 & & $15117-96-1$ & U-235 \\
\hline 2315 & SESPSCH & & SW & & & B0G8B2 & 18-Sep-95 & & 13966-29-5 & U-234 \\
\hline 2316 & PS HENRY/LE BISPING & & SW & & & B0G0R3 & 18-Sep-95 & & $7440-47-3$ & $\mathrm{CR}$ \\
\hline 2317 & PS HENRY/LE BISPING & & SW & & & B0G0R3 & 18-Sep-95 & & $56-23-5$ & CARBTET \\
\hline 2318 & SESPSCH & & SW & & & B0GBM8 & 18-Sep-95 & & $10098-97-2$ & SR-90 \\
\hline 2319 & SESPSCH & & SW & & & B0GBM8 & 18-Sep-95 & & $10028-17-8$ & TRITIUM \\
\hline
\end{tabular}




\begin{tabular}{|c|c|c|c|c|c|c|c|c|c|c|}
\hline & A & $\mathrm{B}$ & $\mathrm{C}$ & $\mathrm{D}$ & $E$ & $\mathrm{~F}$ & G & $\mathrm{H}$ & $\mathrm{I}$ & $\mathrm{J}$ \\
\hline 1 & SAMPLE_NUM_ASSIGNED_TO & SAMPLER & MEDIA & BIOTA_MEDIA & TAG_ID & SAMP_NUM & SAMP_DATE_TIME & SAMP_DATE_TIME_ON & CON_ID & CON_SHORT_NAME \\
\hline 2320 & SESPSCH & & SW & & & B0GBM8 & 18-Sep-95 & & $13966-29-5$ & U-234 \\
\hline 2321 & SESPSCH & & SW & & & B0GBM8 & 18-Sep-95 & & $15117-96-1$ & U-235 \\
\hline 2322 & SESPSCH & & SW & & & B0GBM8 & 18-Sep-95 & & U-238 & U-238 \\
\hline 2323 & PS HENRY/LE BISPING & & SW & & & BOG0S2 & 18-Sep-95 & & $7440-47-3$ & $\mathrm{CR}$ \\
\hline 2324 & PS HENRY/LE BISPING & & SW & & & BOG0S2 & 18-Sep-95 & & $56-23-5$ & CARBTET \\
\hline 2325 & SESPSCH & & SW & & & B0GBN7 & 18-Sep-95 & & $10098-97-2$ & SR-90 \\
\hline \begin{tabular}{|l|l|}
2326 \\
\end{tabular} & SESPSCH & & SW & & & B0GBN7 & 18-Sep-95 & & $10028-17-8$ & TRITIUM \\
\hline 2327 & SESPSCH & & SW & & & B0GBN7 & 18-Sep-95 & & $13966-29-5$ & U-234 \\
\hline 2328 & SESPSCH & & SW & & & B0GBN7 & 18-Sep-95 & & $15117-96-1$ & U-235 \\
\hline 2329 & SESPSCH & & SW & & & B0GBN7 & 18-Sep-95 & & $\mathrm{U}-238$ & $\mathrm{U}-238$ \\
\hline 2330 & PS HENRY/LE BISPING & & SW & & & B0G0R4 & 18-Sep-95 & & $7440-47-3$ & $\mathrm{CR}$ \\
\hline 2331 & PS HENRY/LE BISPING & & SW & & & B0G0R4 & 18-Sep-95 & & $56-23-5$ & CARBTET \\
\hline 2332 & SESPSCH & & SW & & & B0GBM9 & 18-Sep-95 & & $10098-97-2$ & SR-90 \\
\hline 2333 & SESPSCH & & SW & & & B0GBM9 & 18-Sep-95 & & $10028-17-8$ & TRITIUM \\
\hline 2334 & SESPSCH & & SW & & & BOGBM9 & 18-Sep-95 & & $13966-29-5$ & U-234 \\
\hline 2335 & SESPSCH & & SW & & & BOGBM9 & 18-Sep-95 & & $15117-96-1$ & U-235 \\
\hline \begin{tabular}{|l|}
2336 \\
\end{tabular} & SESPSCH & & SW & & & B0GBM9 & 18-Sep-95 & & $\mathrm{U}-238$ & $\mathrm{U}-238$ \\
\hline 2337 & PS HENRY/LE BISPING & & SW & & & B0G0R5 & 18-Sep-95 & & $7440-47-3$ & CR \\
\hline 2338 & PS HENRY/LE BISPING & & SW & & & B0G0R5 & 18-Sep-95 & & $56-23-5$ & CARBTET \\
\hline \begin{tabular}{|l|}
2339 \\
\end{tabular} & SESPSCH & & SW & & & BOGBNO & 18-Sep-95 & & $10098-97-2$ & SR-90 \\
\hline 2340 & SESPSCH & & SW & & & BOGBNO & 18-Sep-95 & & $10028-17-8$ & TRITIUM \\
\hline 2341 & SESPSCH & & SW & & & BOGBNO & 18-Sep-95 & & $13966-29-5$ & U-234 \\
\hline 2342 & SESPSCH & & SW & & & BOGBNO & 18-Sep-95 & & $15117-96-1$ & U-235 \\
\hline 2343 & SESPSCH & & SW & & & BOGBNO & 18-Sep-95 & & $\mathrm{U}-238$ & $\mathrm{U}-238$ \\
\hline 2344 & PS HENRY/LE BISPING & & SW & & & B0G0R6 & 18-Sep-95 & & $7440-47-3$ & CR \\
\hline 2345 & PS HENRY/LE BISPING & & SW & & & B0G0R6 & 18-Sep-95 & & $56-23-5$ & CARBTET \\
\hline 2346 & SESPSCH & & SW & & & B0GBN1 & 18-Sep-95 & & $10098-97-2$ & SR-90 \\
\hline 2347 & SESPSCH & & SW & & & B0GBN1 & 18-Sep-95 & & $10028-17-8$ & TRITIUM \\
\hline 2348 & SESPSCH & & SW & & & B0GBN1 & 18-Sep-95 & & $13966-29-5$ & U-234 \\
\hline \begin{tabular}{|l|}
2349 \\
\end{tabular} & SESPSCH & & SW & & & B0GBN1 & 18-Sep-95 & & $15117-96-1$ & $\mathrm{U}-235$ \\
\hline 2350 & SESPSCH & & SW & & & B0GBN1 & 18-Sep-95 & & $\mathrm{U}-238$ & U-238 \\
\hline \begin{tabular}{|l|}
2351 \\
\end{tabular} & PS HENRY/LE BISPING & & SW & & & B0G0R7 & 18-Sep-95 & & $7440-47-3$ & $\mathrm{CR}$ \\
\hline 2352 & PS HENRY/LE BISPING & & SW & & & B0G0R7 & 18-Sep-95 & & $56-23-5$ & CARBTET \\
\hline 2353 & SESPSCH & & SW & & & B0GBN2 & 18-Sep-95 & & $10098-97-2$ & SR-90 \\
\hline 2354 & SESPSCH & & SW & & & B0GBN2 & 18-Sep-95 & & $10028-17-8$ & TRITIUM \\
\hline 2355 & SESPSCH & & SW & & & BOGBN2 & 18-Sep-95 & & $13966-29-5$ & U-234 \\
\hline 2356 & SESPSCH & & SW & & & B0GBN2 & 18-Sep-95 & & $15117-96-1$ & U-235 \\
\hline 2357 & SESPSCH & & SW & & & B0GBN2 & 18-Sep-95 & & U-238 & U-238 \\
\hline
\end{tabular}


\begin{tabular}{l|l}
$D$ & $E$
\end{tabular}

G

\begin{tabular}{|c|c|c|c|c|c|c|c|c|c|c|}
\hline 1 & SAMPLE NUM ASSIGNED TO & SAMPLER & MEDIA & BIOTA MEDIA & TAG ID & SAMP_NUM & SAMP_DATE_TIME & SAMP_DATE_TIME_ON & CON_ID & CON_SHORT_NAME \\
\hline 2358 & PS HENRY/LE BISPING & & SW & & & B0G0R8 & 18-Sep-95 & & $7440-47-3$ & $\mathrm{CR}$ \\
\hline 2359 & PS HENRY/LE BISPING & & SW & & & B0G0R8 & 18-Sep-95 & & $56-23-5$ & CARBTET \\
\hline 2360 & SESPSCH & & SW & & & BOGBN3 & 18-Sep-95 & & $10098-97-2$ & SR-90 \\
\hline 2361 & SESPSCH & & SW & & & BOGBN3 & 18-Sep-95 & & $10028-17-8$ & TRITIUM \\
\hline 2362 & SESPSCH & & SW & & & B0GBN3 & 18-Sep-95 & & $13966-29-5$ & U-234 \\
\hline 2363 & SESPSCH & & SW & & & B0GBN3 & 18-Sep-95 & & 15117-96-1 & U-235 \\
\hline 2364 & SESPSCH & & SW & & & B0GBN3 & 18-Sep-95 & & U-238 & U-238 \\
\hline 2365 & PS HENRY/LE BISPING & & SW & & & B0G0R9 & 18-Sep-95 & & $7440-47-3$ & CR \\
\hline 2366 & PS HENRY/LE BISPING & & SW & & & B0G0R9 & 18-Sep-95 & & $56-23-5$ & CARBTET \\
\hline 2367 & SESPSCH & & SW & & & B0GBN4 & 18-Sep-95 & & $10098-97-2$ & SR-90 \\
\hline 2368 & SESPSCH & & SW & & & B0GBN4 & 18-Sep-95 & & $10028-17-8$ & TRITIUM \\
\hline 2369 & SESPSCH & & SW & & & B0GBN4 & 18-Sep-95 & & 13966-29-5 & U-234 \\
\hline 2370 & SESPSCH & & SW & & & B0GBN4 & 18-Sep-95 & & 15117-96-1 & U-235 \\
\hline 2371 & SESPSCH & & SW & & & B0GBN4 & 18-Sep-95 & & $\mathrm{U}-238$ & U-238 \\
\hline 2372 & PS HENRY/LE BISPING & & SW & & & BOGOSO & 18-Sep-95 & & 7440-47-3 & CR \\
\hline 2373 & PS HENRY/LE BISPING & & SW & & & BOGOSO & 18-Sep-95 & & $56-23-5$ & CARBTET \\
\hline 2374 & SESPSCH & & SW & & & B0GBN5 & 18-Sep-95 & & $10098-97-2$ & SR-90 \\
\hline 2375 & SESPSCH & & SW & & & B0GBN5 & 18-Sep-95 & & $10028-17-8$ & TRITIUM \\
\hline 2376 & SESPSCH & & SW & & & B0GBN5 & 18-Sep-95 & & $13966-29-5$ & U-234 \\
\hline 2377 & SESPSCH & & SW & & & B0GBN5 & 18-Sep-95 & & 15117-96-1 & U-235 \\
\hline 2378 & SESPSCH & & SW & & & B0GBN5 & 18-Sep-95 & & $\mathrm{U}-238$ & U-238 \\
\hline 2379 & PS HENRY/LE BISPING & & SW & & & B0G0S1 & 18-Sep-95 & & $7440-47-3$ & CR \\
\hline 2380 & PS HENRY/LE BISPING & & SW & & & B0G0S1 & 18-Sep-95 & & $56-23-5$ & CARBTET \\
\hline 2381 & SESPSCH & & SW & & & B0GBN6 & 18-Sep-95 & & $10098-97-2$ & SR-90 \\
\hline 2382 & SESPSCH & & SW & & & B0GBN6 & 18-Sep-95 & & $10028-17-8$ & TRITIUM \\
\hline 2383 & SESPSCH & & SW & & & B0GBN6 & 18-Sep-95 & & $13966-29-5$ & U-234 \\
\hline 2384 & SESPSCH & & SW & & & B0GBN6 & 18-Sep-95 & & 15117-96-1 & U-235 \\
\hline 2385 & SESPSCH & & SW & & & B0GBN6 & 18-Sep-95 & & $\mathrm{U}-238$ & U-238 \\
\hline 2386 & PS HENRY/LE BISPING & & SW & & & B0G132 & 07-Dec-95 & & $7440-47-3$ & $\mathrm{CR}$ \\
\hline 2387 & PS HENRY/LE BISPING & & SW & & & B0G132 & 07-Dec-95 & & $56-23-5$ & CARBTET \\
\hline 2388 & SESPSCH & & SW & & & B0GVY1 & 07-Dec-95 & & $10098-97-2$ & SR-90 \\
\hline 2389 & SESPSCH & & SW & & & B0GVY1 & 07-Dec-95 & & $10028-17-8$ & TRITIUM \\
\hline 2390 & SESPSCH & & SW & & & B0GVY1 & 07-Dec-95 & & 13966-29-5 & U-234 \\
\hline 2391 & SESPSCH & & SW & & & B0GVY1 & 07-Dec-95 & & 15117-96-1 & U-235 \\
\hline 2392 & SESPSCH & & SW & & & B0GVY1 & 07-Dec-95 & & $\mathrm{U}-238$ & $\mathrm{U}-238$ \\
\hline 2393 & PS HENRY/LE BISPING & & SW & & & B0G145 & 07-Dec-95 & & $7440-47-3$ & $\mathrm{CR}$ \\
\hline 2394 & PS HENRY/LE BISPING & & SW & & & B0G145 & 07-Dec-95 & & $56-23-5$ & CARBTET \\
\hline 2395 & SESPSCH & & SW & & & BOGVZ0 & 07-Dec-95 & & $10098-97-2$ & SR-90 \\
\hline
\end{tabular}




\begin{tabular}{|c|c|c|c|c|c|c|c|c|c|c|}
\hline & $\mathrm{A}$ & $\mathrm{B}$ & $\mathrm{C}$ & $\mathrm{D}$ & $\mathrm{E}$ & $\mathrm{F}$ & $G$ & $\mathrm{H}$ & I & $\mathrm{J}$ \\
\hline 1 & SAMPLE_NUM_ASSIGNED_TO & SAMPLER & MEDIA & BIOTA_MEDIA & TAG_ID & SAMP_NUM & SAMP_DATE_TIME & SAMP_DATE_TIME_ON & CON_ID & CON_SHORT_NAME \\
\hline 2396 & SESPSCH & & SW & & & BOGVZO & 07-Dec-95 & & $10028-17-8$ & TRITIUM \\
\hline 2397 & SESPSCH & & SW & & & BOGVZ0 & 07-Dec-95 & & 13966-29-5 & $\mathrm{U}-234$ \\
\hline 2398 & SESPSCH & & SW & & & BOGVZ0 & 07-Dec-95 & & 15117-96-1 & U-235 \\
\hline 2399 & SESPSCH & & SW & & & B0GVZ0 & 07-Dec-95 & & $\mathrm{U}-238$ & $\mathrm{U}-238$ \\
\hline 2400 & PS HENRY/LE BISPING & & SW & & & B0G133 & 07-Dec-95 & & $7440-47-3$ & $\mathrm{CR}$ \\
\hline 2401 & PS HENRY/LE BISPING & & SW & & & B0G133 & 07-Dec-95 & & $56-23-5$ & CARBTET \\
\hline 2402 & SESPSCH & & SW & & & B0GVY2 & 07-Dec-95 & & $10098-97-2$ & SR-90 \\
\hline 2403 & SESPSCH & & SW & & & B0GVY2 & 07-Dec-95 & & $10028-17-8$ & TRITIUM \\
\hline 2404 & SESPSCH & & SW & & & B0GVY2 & 07-Dec-95 & & 13966-29-5 & U-234 \\
\hline 2405 & SESPSCH & & SW & & & B0GVY2 & 07-Dec-95 & & $15117-96-1$ & U-235 \\
\hline 2406 & SESPSCH & & SW & & & B0GVY2 & 07-Dec-95 & & $\mathrm{U}-238$ & $\mathrm{U}-238$ \\
\hline 2407 & PS HENRY/LE BISPING & & SW & & & B0G134 & 07-Dec-95 & & $7440-47-3$ & CR \\
\hline 2408 & PS HENRY/LE BISPING & & SW & & & B0G134 & 07-Dec-95 & & $56-23-5$ & CARBTET \\
\hline 2409 & SESPSCH & & SW & & & B0GVY3 & 07-Dec-95 & & $10098-97-2$ & SR-90 \\
\hline 2410 & SESPSCH & & SW & & & B0GVY3 & 07-Dec-95 & & $10028-17-8$ & TRITIUM \\
\hline 2411 & SESPSCH & & SW & & & B0GVY3 & 07-Dec-95 & & $13966-29-5$ & $\mathrm{U}-234$ \\
\hline 2412 & SESPSCH & & SW & & & B0GVY3 & 07-Dec-95 & & 15117-96-1 & U-235 \\
\hline 2413 & SESPSCH & & SW & & & BOGVY3 & 07-Dec-95 & & $\mathrm{U}-238$ & $\mathrm{U}-238$ \\
\hline 2414 & PS HENRY/LE BISPING & & SW & & & B0G135 & 07-Dec-95 & & $7440-47-3$ & $\mathrm{CR}$ \\
\hline 2415 & PS HENRY/LE BISPING & & SW & & & B0G135 & 07-Dec-95 & & $56-23-5$ & CARBTET \\
\hline 2416 & SESPSCH & & SW & & & BOGVY4 & 07-Dec-95 & & $10098-97-2$ & SR-90 \\
\hline 2417 & SESPSCH & & SW & & & B0GVY4 & 07-Dec-95 & & $10028-17-8$ & TRITIUM \\
\hline 2418 & SESPSCH & & SW & & & B0GVY4 & 07-Dec-95 & & $13966-29-5$ & $\mathrm{U}-234$ \\
\hline 2419 & SESPSCH & & SW & & & B0GVY4 & 07-Dec-95 & & $15117-96-1$ & U-235 \\
\hline 2420 & SESPSCH & & SW & & & BOGVY4 & 07-Dec-95 & & $\mathrm{U}-238$ & $\mathrm{U}-238$ \\
\hline 2421 & PS HENRY/LE BISPING & & SW & & & B0G140 & 07-Dec-95 & & $7440-47-3$ & $\mathrm{CR}$ \\
\hline 2422 & PS HENRY/LE BISPING & & SW & & & B0G140 & 07-Dec-95 & & $56-23-5$ & CARBTET \\
\hline 2423 & SESPSCH & & SW & & & B0GVY5 & 07-Dec-95 & & $10098-97-2$ & SR-90 \\
\hline 2424 & SESPSCH & & SW & & & B0GVY5 & 07-Dec-95 & & $10028-17-8$ & TRITIUM \\
\hline 2425 & SESPSCH & & SW & & & B0GVY5 & 07-Dec-95 & & $13966-29-5$ & $\mathrm{U}-234$ \\
\hline 2426 & SESPSCH & & SW & & & B0GVY5 & 07-Dec-95 & & $15117-96-1$ & $\mathrm{U}-235$ \\
\hline 2427 & SESPSCH & & SW & & & B0GVY5 & 07-Dec-95 & & $\mathrm{U}-238$ & $\mathrm{U}-238$ \\
\hline 2428 & PS HENRY/LE BISPING & & SW & & & B0G141 & 07-Dec-95 & & $7440-47-3$ & CR \\
\hline 2429 & PS HENRY/LE BISPING & & SW & & & B0G141 & 07-Dec-95 & & $56-23-5$ & CARBTET \\
\hline 2430 & SESPSCH & & SW & & & B0GVY6 & 07-Dec-95 & & $10098-97-2$ & SR-90 \\
\hline 2431 & SESPSCH & & SW & & & B0GVY6 & 07-Dec-95 & & $10028-17-8$ & TRITIUM \\
\hline 2432 & SESPSCH & & SW & & & B0GVY6 & 07-Dec-95 & & $13966-29-5$ & U-234 \\
\hline 2433 & SESPSCH & & SW & & & B0GVY6 & 07-Dec-95 & & 15117-96-1 & $\mathrm{U}-235$ \\
\hline
\end{tabular}




\begin{tabular}{|c|c|c|c|c|c|c|c|c|c|c|}
\hline & A & $\mathrm{B}$ & $C$ & $\mathrm{D}$ & $E$ & $\mathrm{~F}$ & $G$ & $\mathrm{H}$ & $\mathrm{I}$ & $\mathrm{J}$ \\
\hline 1 & SAMPLE_NUM_ASSIGNED_TO & SAMPLER & MEDIA & BIOTA_MEDIA & TAG_ID & SAMP_NUM & SAMP_DATE_TIME| & SAMP_DATE_TIME_ON & CON_ID & CON_SHORT_NAME \\
\hline 2434 & SESPSCH & & SW & & & B0GVY6 & 07-Dec-95 & & $\mathrm{U}-238$ & U-238 \\
\hline 2435 & PS HENRY/LE BISPING & & SW & & & B0G142 & 07-Dec-95 & & $7440-47-3$ & $\mathrm{CR}$ \\
\hline 2436 & PS HENRY/LE BISPING & & SW & & & B0G142 & 07-Dec-95 & & $56-23-5$ & CARBTET \\
\hline 2437 & SESPSCH & & SW & & & B0GVY7 & 07-Dec-95 & & $10098-97-2$ & SR-90 \\
\hline 2438 & SESPSCH & & SW & & & B0GVY7 & 07-Dec-95 & & $10028-17-8$ & TRITIUM \\
\hline 2439 & SESPSCH & & SW & & & B0GVY7 & 07-Dec-95 & & $13966-29-5$ & U-234 \\
\hline 2440 & SESPSCH & & SW & & & B0GVY7 & 07-Dec-95 & & 15117-96-1 & U-235 \\
\hline 2441 & SESPSCH & & SW & & & B0GVY7 & 07-Dec-95 & & $\mathrm{U}-238$ & U-238 \\
\hline 2442 & PS HENRY/LE BISPING & & SW & & & B0G143 & 07-Dec-95 & & $7440-47-3$ & $\mathrm{CR}$ \\
\hline 2443 & PS HENRY/LE BISPING & & SW & & & B0G143 & 07-Dec-95 & & $56-23-5$ & CARBTET \\
\hline 2444 & SESPSCH & & SW & & & B0GVY8 & 07-Dec-95 & & $10098-97-2$ & SR-90 \\
\hline 2445 & SESPSCH & & SW & & & B0GVY8 & 07-Dec-95 & & $10028-17-8$ & TRITIUM \\
\hline 2446 & SESPSCH & & SW & & & BOGVY8 & 07-Dec-95 & & $13966-29-5$ & U-234 \\
\hline 2447 & SESPSCH & & SW & & & B0GVY8 & 07-Dec-95 & & $15117-96-1$ & U-235 \\
\hline 2448 & SESPSCH & & SW & & & B0GVY8 & 07-Dec-95 & & $\mathrm{U}-238$ & $\mathrm{U}-238$ \\
\hline 2449 & PS HENRY/LE BISPING & & SW & & & B0G144 & 07-Dec-95 & & $7440-47-3$ & $\mathrm{CR}$ \\
\hline 2450 & PS HENRY/LE BISPING & & SW & & & B0G144 & 07-Dec-95 & & $56-23-5$ & CARBTET \\
\hline 2451 & SESPSCH & & SW & & & B0GVY9 & 07-Dec-95 & & $10098-97-2$ & SR-90 \\
\hline 2452 & SESPSCH & & SW & & & B0GVY9 & 07-Dec-95 & & $10028-17-8$ & TRITIUM \\
\hline 2453 & SESPSCH & & SW & & & B0GVY9 & 07-Dec-95 & & $13966-29-5$ & U-234 \\
\hline 2454 & SESPSCH & & SW & & & BOGVY9 & 07-Dec-95 & & 15117-96-1 & U-235 \\
\hline 2455 & SESPSCH & & SW & & & B0GVY9 & 07-Dec-95 & & $\mathrm{U}-238$ & $\mathrm{U}-238$ \\
\hline 2456 & PS HENRY/LE BISPING & & SW & & & B0G136 & 08-Dec-95 & & $7440-47-3$ & CR \\
\hline 2457 & PS HENRY/LE BISPING & & SW & & & B0G136 & 08-Dec-95 & & $56-23-5$ & CARBTET \\
\hline 2458 & SESPSCH & & SW & & & B0GVX7 & 08-Dec-95 & & $10098-97-2$ & SR-90 \\
\hline 2459 & SESPSCH & & SW & & & B0GVX7 & 08-Dec-95 & & $10028-17-8$ & TRITIUM \\
\hline 2460 & SESPSCH & & SW & & & B0GVX7 & 08-Dec-95 & & $13966-29-5$ & U-234 \\
\hline 2461 & SESPSCH & & SW & & & B0GVX7 & 08-Dec-95 & & 15117-96-1 & U-235 \\
\hline 2462 & SESPSCH & & SW & & & B0GVX7 & 08-Dec-95 & & $\mathrm{U}-238$ & $\mathrm{U}-238$ \\
\hline 2463 & PS HENRY/LE BISPING & & SW & & & B0G137 & 08-Dec-95 & & $7440-47-3$ & CR \\
\hline 2464 & PS HENRY/LE BISPING & & SW & & & B0G137 & 08-Dec-95 & & $56-23-5$ & CARBTET \\
\hline 2465 & SESPSCH & & SW & & & B0GVX8 & 08-Dec-95 & & $10098-97-2$ & SR-90 \\
\hline 2466 & SESPSCH & & SW & & & B0GVX8 & 08-Dec-95 & & $10028-17-8$ & TRITIUM \\
\hline 2467 & SESPSCH & & SW & & & B0GVX8 & 08-Dec-95 & & $13966-29-5$ & U-234 \\
\hline 2468 & SESPSCH & & SW & & & B0GVX8 & 08-Dec-95 & & 15117-96-1 & U-235 \\
\hline 2469 & SESPSCH & & SW & & & B0GVX8 & 08-Dec-95 & & $\mathrm{U}-238$ & $\mathrm{U}-238$ \\
\hline 2470 & PS HENRY/LE BISPING & & SW & & & B0G138 & 08-Dec-95 & & $7440-47-3$ & $\mathrm{CR}$ \\
\hline 2471 & PS HENRY/LE BISPING & & SW & & & B0G138 & 08-Dec-95 & & $56-23-5$ & CARBTET \\
\hline
\end{tabular}




\begin{tabular}{|c|c|c|c|c|c|c|c|c|c|c|}
\hline & A & $\mathrm{B}$ & $C$ & $\mathrm{D}$ & $E$ & $\mathrm{~F}$ & $G$ & $\mathrm{H}$ & $\mathrm{I}$ & $\mathrm{J}$ \\
\hline 1 & SAMPLE_NUM_ASSIGNED_TO & SAMPLER & MEDIA & BIOTA_MEDIA & TAG_ID & SAMP_NUM & SAMP_DATE_TIME| & SAMP_DATE_TIME_ON & CON_ID & CON_SHORT_NAME \\
\hline 2472 & SESPSCH & & SW & & & BOGVX9 & 08-Dec-95 & & $10098-97-2$ & SR-90 \\
\hline 2473 & SESPSCH & & SW & & & B0GVX9 & 08-Dec-95 & & $10028-17-8$ & TRITIUM \\
\hline 2474 & SESPSCH & & SW & & & BOGVX9 & 08-Dec-95 & & $13966-29-5$ & U-234 \\
\hline 2475 & SESPSCH & & SW & & & BOGVX9 & 08-Dec-95 & & 15117-96-1 & U-235 \\
\hline 2476 & SESPSCH & & SW & & & B0GVX9 & 08-Dec-95 & & U-238 & U-238 \\
\hline 2477 & PS HENRY/LE BISPING & & SW & & & B0G139 & 08-Dec-95 & & $7440-47-3$ & $\mathrm{CR}$ \\
\hline 2478 & PS HENRY/LE BISPING & & SW & & & B0G139 & 08-Dec-95 & & $56-23-5$ & CARBTET \\
\hline 2479 & SESPSCH & & SW & & & BOGVYO & 08-Dec-95 & & $10098-97-2$ & SR-90 \\
\hline 2480 & SESPSCH & & SW & & & BOGVYO & 08-Dec-95 & & $10028-17-8$ & TRITIUM \\
\hline 2481 & SESPSCH & & SW & & & BOGVYO & 08-Dec-95 & & $13966-29-5$ & U-234 \\
\hline 2482 & SESPSCH & & SW & & & BOGVYO & 08-Dec-95 & & $15117-96-1$ & U-235 \\
\hline 2483 & SESPSCH & & SW & & & BOGVYO & 08-Dec-95 & & U-238 & $\mathrm{U}-238$ \\
\hline 2484 & PS HENRY & & SW & & & B0G161 & 14-Mar-96 & & $7440-47-3$ & CR \\
\hline 2485 & PS HENRY & & SW & & & B0G161 & 14-Mar-96 & & $56-23-5$ & CARBTET \\
\hline 2486 & SESPSCH & & SW & & & В0H9K9 & 14-Mar-96 & & $10098-97-2$ & SR-90 \\
\hline 2487 & SESPSCH & & SW & & & ВОН9K9 & 14-Mar-96 & & $10028-17-8$ & TRITIUM \\
\hline 2488 & SESPSCH & & SW & & & ВОН9K9 & 14-Mar-96 & & $13966-29-5$ & U-234 \\
\hline 2489 & SESPSCH & & SW & & & ВоH9K9 & 14-Mar-96 & & $15117-96-1$ & U-235 \\
\hline 2490 & SESPSCH & & SW & & & ВОН9К9 & 14-Mar-96 & & U-238 & U-238 \\
\hline 2491 & PS HENRY & & SW & & & B0G162 & 14-Mar-96 & & $7440-47-3$ & CR \\
\hline 2492 & PS HENRY & & SW & & & B0G162 & 14-Mar-96 & & $56-23-5$ & CARBTET \\
\hline 2493 & SESPSCH & & SW & & & BOH9LO & 14-Mar-96 & & $10098-97-2$ & SR-90 \\
\hline 2494 & SESPSCH & & SW & & & BOH9LO & 14-Mar-96 & & $10028-17-8$ & TRITIUM \\
\hline 2495 & SESPSCH & & SW & & & BOH9LO & 14-Mar-96 & & $13966-29-5$ & U-234 \\
\hline 2496 & SESPSCH & & SW & & & BOH9LO & 14-Mar-96 & & 15117-96-1 & U-235 \\
\hline 2497 & SESPSCH & & SW & & & BOH9LO & 14-Mar-96 & & U-238 & U-238 \\
\hline 2498 & PS HENRY & & SW & & & B0G163 & 14-Mar-96 & & $7440-47-3$ & CR \\
\hline 2499 & PS HENRY & & SW & & & B0G163 & 14-Mar-96 & & $56-23-5$ & CARBTET \\
\hline 2500 & SESPSCH & & SW & & & B0H9L1 & 14-Mar-96 & & $10098-97-2$ & SR-90 \\
\hline 2501 & SESPSCH & & SW & & & B0H9L1 & 14-Mar-96 & & $10028-17-8$ & TRITIUM \\
\hline 2502 & SESPSCH & & SW & & & B0H9L1 & 14-Mar-96 & & $13966-29-5$ & U-234 \\
\hline 2503 & SESPSCH & & SW & & & B0H9L1 & 14-Mar-96 & & $15117-96-1$ & U-235 \\
\hline 2504 & SESPSCH & & SW & & & B0H9L1 & 14-Mar-96 & & U-238 & U-238 \\
\hline 2505 & PS HENRY & & SW & & & B0G164 & 14-Mar-96 & & $7440-47-3$ & CR \\
\hline 2506 & PS HENRY & & SW & & & B0G164 & 14-Mar-96 & & $56-23-5$ & CARBTET \\
\hline 2507 & SESPSCH & & SW & & & BOH9L2 & 14-Mar-96 & & $10098-97-2$ & SR-90 \\
\hline 2508 & SESPSCH & & SW & & & BOH9L2 & 14-Mar-96 & & $10028-17-8$ & TRITIUM \\
\hline 2509 & SESPSCH & & SW & & & BOH9L2 & 14-Mar-96 & & $13966-29-5$ & U-234 \\
\hline
\end{tabular}




\begin{tabular}{|c|c|c|c|c|c|c|c|c|c|c|}
\hline & A & $\mathrm{B}$ & $C$ & $\mathrm{D}$ & $E$ & $\mathrm{~F}$ & $G$ & $\mathrm{H}$ & 1 & $\mathrm{~J}$ \\
\hline 1 & SAMPLE_NUM_ASSIGNED_TO & SAMPLER & MEDIA & BIOTA_MEDIA & TAG_ID & SAMP_NUM & SAMP_DATE_TIME| & SAMP_DATE_TIME_ON & CON_ID & CON_SHORT_NAME \\
\hline 2510 & SESPSCH & & SW & & & BOH9L2 & 14-Mar-96 & & 15117-96-1 & U-235 \\
\hline 2511 & SESPSCH & & SW & & & BOH9L2 & 14-Mar-96 & & U-238 & U-238 \\
\hline 2512 & PS HENRY & & SW & & & B0G150 & 18-Mar-96 & & $7440-47-3$ & $\mathrm{CR}$ \\
\hline 2513 & PS HENRY & & SW & & & B0G151 & 18-Mar-96 & & $7440-47-3$ & CR \\
\hline 2514 & PS HENRY & & SW & & & B0G150 & 18-Mar-96 & & $56-23-5$ & CARBTET \\
\hline 2515 & PS HENRY & & SW & & & B0G151 & 18-Mar-96 & & $56-23-5$ & CARBTET \\
\hline 2516 & SESPSCH & & SW & & & BOH9L3 & 18-Mar-96 & & $10098-97-2$ & SR-90 \\
\hline 2517 & SESPSCH & & SW & & & BOHL07 & 18-Mar-96 & & $10098-97-2$ & SR-90 \\
\hline 2518 & SESPSCH & & SW & & & BOH9L3 & 18-Mar-96 & & $10028-17-8$ & TRITIUM \\
\hline 2519 & SESPSCH & & SW & & & BOHL07 & 18-Mar-96 & & $10028-17-8$ & TRITIUM \\
\hline 2520 & SESPSCH & & SW & & & BOH9L3 & 18-Mar-96 & & 13966-29-5 & U-234 \\
\hline 2521 & SESPSCH & & SW & & & BOH9L3 & 18-Mar-96 & & $15117-96-1$ & U-235 \\
\hline 2522 & SESPSCH & & SW & & & BOH9L3 & 18-Mar-96 & & U-238 & U-238 \\
\hline 2523 & SESPSCH & & SW & & & BOHLO7 & 18-Mar-96 & & 13966-29-5 & U-234 \\
\hline 2524 & SESPSCH & & SW & & & BOHL07 & 18-Mar-96 & & 15117-96-1 & U-235 \\
\hline 2525 & SESPSCH & & SW & & & BOHL07 & 18-Mar-96 & & U-238 & U-238 \\
\hline 2526 & PS HENRY & & SW & & & B0G160 & 18-Mar-96 & & $7440-47-3$ & CR \\
\hline 2527 & PS HENRY & & SW & & & B0G160 & 18-Mar-96 & & $56-23-5$ & CARBTET \\
\hline 2528 & SESPSCH & & SW & & & BOH9M2 & 18-Mar-96 & & $10098-97-2$ & SR-90 \\
\hline 2529 & SESPSCH & & SW & & & BOH9M2 & 18-Mar-96 & & $10028-17-8$ & TRITIUM \\
\hline 2530 & SESPSCH & & SW & & & BOH9M2 & 18-Mar-96 & & 13966-29-5 & U-234 \\
\hline 2531 & SESPSCH & & SW & & & BOH9M2 & 18-Mar-96 & & 15117-96-1 & U-235 \\
\hline 2532 & SESPSCH & & SW & & & BOH9M2 & 18-Mar-96 & & U-238 & U-238 \\
\hline 2533 & PS HENRY & & SW & & & B0G152 & 18-Mar-96 & & $7440-47-3$ & CR \\
\hline 2534 & PS HENRY & & SW & & & B0G152 & 18-Mar-96 & & $56-23-5$ & CARBTET \\
\hline 2535 & SESPSCH & & SW & & & BOH9L4 & 18-Mar-96 & & 10098-97-2 & SR-90 \\
\hline 2536 & SESPSCH & & SW & & & BOH9L4 & 18-Mar-96 & & $10028-17-8$ & TRITIUM \\
\hline 2537 & SESPSCH & & SW & & & BOH9L4 & 18-Mar-96 & & 13966-29-5 & U-234 \\
\hline 2538 & SESPSCH & & SW & & & BOH9L4 & 18-Mar-96 & & 15117-96-1 & U-235 \\
\hline 2539 & SESPSCH & & SW & & & BOH9L4 & 18-Mar-96 & & U-238 & U-238 \\
\hline 2540 & PS HENRY & & SW & & & B0G153 & 18-Mar-96 & & $7440-47-3$ & CR \\
\hline 2541 & PS HENRY & & SW & & & B0G153 & 18-Mar-96 & & $56-23-5$ & CARBTET \\
\hline 2542 & SESPSCH & & SW & & & BOH9L5 & 18-Mar-96 & & $10098-97-2$ & SR-90 \\
\hline 2543 & SESPSCH & & SW & & & BOH9L5 & 18-Mar-96 & & $10028-17-8$ & TRITIUM \\
\hline 2544 & SESPSCH & & SW & & & B0H9L5 & 18-Mar-96 & & 13966-29-5 & U-234 \\
\hline 2545 & SESPSCH & & SW & & & B0H9L5 & 18-Mar-96 & & $15117-96-1$ & U-235 \\
\hline 2546 & SESPSCH & & SW & & & BOH9L5 & 18-Mar-96 & & U-238 & U-238 \\
\hline 2547 & PS HENRY & & SW & & & B0G154 & 18-Mar-96 & & $7440-47-3$ & CR \\
\hline
\end{tabular}




\begin{tabular}{|c|c|c|c|c|c|c|c|c|c|c|}
\hline & $\mathrm{A}$ & $B$ & $\mathrm{C}$ & $\mathrm{D}$ & $E$ & $\mathrm{~F}$ & $G$ & $\mathrm{H}$ & $I$ & $\mathrm{~J}$ \\
\hline 1 & SAMPLE NUM ASSIGNED TO & SAMPLER & MEDIA & BIOTA MEDIA & TAG ID & SAMP NUM & SAMP DATE TIME & SAMP DATE TIME ON & CON ID & CON SHORT NAME \\
\hline 2548 & PS HENRY & & SW & & & B0G154 & 18-Mar-96 & & $56-23-5$ & CARBTET \\
\hline 2549 & SESPSCH & & SW & & & BOH9L6 & 18-Mar-96 & & $10098-97-2$ & SR-90 \\
\hline 2550 & SESPSCH & & SW & & & BOH9L6 & 18-Mar-96 & & $10028-17-8$ & TRITIUM \\
\hline 2551 & SESPSCH & & SW & & & BOH9L6 & 18-Mar-96 & & 13966-29-5 & U-234 \\
\hline 2552 & SESPSCH & & SW & & & BOH9L6 & 18-Mar-96 & & 15117-96-1 & U-235 \\
\hline 2553 & SESPSCH & & SW & & & BOH9L6 & 18-Mar-96 & & U-238 & U-238 \\
\hline 2554 & PS HENRY & & SW & & & B0G155 & 18-Mar-96 & & $7440-47-3$ & $\mathrm{CR}$ \\
\hline 2555 & PS HENRY & & SW & & & B0G155 & 18-Mar-96 & & $56-23-5$ & CARBTET \\
\hline 2556 & SESPSCH & & SW & & & BOH9L7 & 18-Mar-96 & & $10098-97-2$ & SR-90 \\
\hline 2557 & SESPSCH & & SW & & & BOH9L7 & 18-Mar-96 & & $10028-17-8$ & TRITIUM \\
\hline 2558 & SESPSCH & & SW & & & BOH9L7 & 18-Mar-96 & & 13966-29-5 & U-234 \\
\hline 2559 & SESPSCH & & SW & & & BOH9L7 & 18-Mar-96 & & 15117-96-1 & U-235 \\
\hline 2560 & SESPSCH & & SW & & & BOH9L7 & 18-Mar-96 & & $\mathrm{U}-238$ & U-238 \\
\hline 2561 & PS HENRY & & SW & & & B0G156 & 18-Mar-96 & & $7440-47-3$ & CR \\
\hline 2562 & PS HENRY & & SW & & & B0G156 & 18-Mar-96 & & $56-23-5$ & CARBTET \\
\hline 2563 & SESPSCH & & SW & & & BOH9L8 & 18-Mar-96 & & $10098-97-2$ & SR-90 \\
\hline 2564 & SESPSCH & & SW & & & BOH9L8 & 18-Mar-96 & & $10028-17-8$ & TRITIUM \\
\hline 2565 & SESPSCH & & SW & & & BOH9L8 & 18-Mar-96 & & 13966-29-5 & U-234 \\
\hline 2566 & SESPSCH & & SW & & & BOH9L8 & 18-Mar-96 & & $15117-96-1$ & U-235 \\
\hline 2567 & SESPSCH & & SW & & & BOH9L8 & 18-Mar-96 & & U-238 & U-238 \\
\hline 2568 & PS HENRY & & SW & & & B0G157 & 18-Mar-96 & & $7440-47-3$ & $\mathrm{CR}$ \\
\hline 2569 & PS HENRY & & SW & & & B0G157 & 18-Mar-96 & & $56-23-5$ & CARBTET \\
\hline 2570 & SESPSCH & & SW & & & BOH9L9 & 18-Mar-96 & & $10098-97-2$ & SR-90 \\
\hline 2571 & SESPSCH & & SW & & & BOH9L9 & 18-Mar-96 & & $10028-17-8$ & TRITIUM \\
\hline 2572 & SESPSCH & & SW & & & BOH9L9 & 18-Mar-96 & & 13966-29-5 & U-234 \\
\hline 2573 & SESPSCH & & SW & & & BOH9L9 & 18-Mar-96 & & 15117-96-1 & U-235 \\
\hline 2574 & SESPSCH & & SW & & & BOH9L9 & 18-Mar-96 & & U-238 & U-238 \\
\hline 2575 & PS HENRY & & SW & & & B0G158 & 18-Mar-96 & & $7440-47-3$ & CR \\
\hline 2576 & PS HENRY & & SW & & & B0G158 & 18-Mar-96 & & $56-23-5$ & CARBTET \\
\hline 2577 & SESPSCH & & SW & & & В0Н9MO & 18-Mar-96 & & 10098-97-2 & SR-90 \\
\hline 2578 & SESPSCH & & SW & & & ВОН9M0 & 18-Mar-96 & & $10028-17-8$ & TRITIUM \\
\hline 2579 & SESPSCH & & SW & & & ВОН9MO & 18-Mar-96 & & $13966-29-5$ & U-234 \\
\hline 2580 & SESPSCH & & SW & & & В0Н9MO & 18-Mar-96 & & 15117-96-1 & U-235 \\
\hline 2581 & SESPSCH & & SW & & & В0H9MO & 18-Mar-96 & & U-238 & U-238 \\
\hline 2582 & PS HENRY & & SW & & & B0G159 & 18-Mar-96 & & $7440-47-3$ & $\mathrm{CR}$ \\
\hline 2583 & PS HENRY & & SW & & & B0G159 & 18-Mar-96 & & $56-23-5$ & CARBTET \\
\hline 2584 & SESPSCH & & SW & & & B0H9M1 & 18-Mar-96 & & $10098-97-2$ & SR-90 \\
\hline 2585 & SESPSCH & & SW & & & В0H9M1 & 18-Mar-96 & & $10028-17-8$ & TRITIUM \\
\hline
\end{tabular}




\begin{tabular}{|c|c|c|c|c|c|c|c|c|c|c|}
\hline & A & $\mathrm{B}$ & C & $\mathrm{D}$ & $E$ & $\mathrm{~F}$ & $G$ & $\mathrm{H}$ & $\mathrm{I}$ & $\mathrm{J}$ \\
\hline 1 & SAMPLE_NUM_ASSIGNED_TO & \begin{tabular}{|l|} 
SAMPLER \\
\end{tabular} & MEDIA & BIOTA_MEDIA & TAG_ID & SAMP_NUM & SAMP_DATE_TIME & SAMP_DATE_TIME_ON & CON_ID & CON_SHORT_NAME \\
\hline 2586 & SESPSCH & & SW & & & BOH9M1 & 18-Mar-96 & & $13966-29-5$ & $\mathrm{U}-234$ \\
\hline 2587 & SESPSCH & & SW & & & B0H9M1 & 18-Mar-96 & & 15117-96-1 & U-235 \\
\hline 2588 & SESPSCH & & SW & & & B0H9M1 & 18-Mar-96 & & U-238 & U-238 \\
\hline 2589 & PS HENRY & & SW & & & B0G180 & 06-Jun-96 & & $7440-47-3$ & CR \\
\hline 2590 & PS HENRY & & SW & & & B0G180 & 06-Jun-96 & & $56-23-5$ & CARBTET \\
\hline 2591 & SESPSCH & & SW & & & B0HTG1 & 06-Jun-96 & & $10098-97-2$ & SR-90 \\
\hline 2592 & SESPSCH & & SW & & & B0HTG1 & 06-Jun-96 & & $10028-17-8$ & TRITIUM \\
\hline 2593 & SESPSCH & & SW & & & BOHTG1 & 06-Jun-96 & & $13966-29-5$ & $\mathrm{U}-234$ \\
\hline 2594 & SESPSCH & & SW & & & B0HTG1 & 06-Jun-96 & & 15117-96-1 & U-235 \\
\hline 2595 & SESPSCH & & SW & & & B0HTG1 & 06-Jun-96 & & U-238 & U-238 \\
\hline 2596 & PS HENRY & & SW & & & B0G181 & 06-Jun-96 & & $7440-47-3$ & CR \\
\hline 2597 & PS HENRY & & SW & & & B0G181 & 06-Jun-96 & & $56-23-5$ & CARBTET \\
\hline 2598 & SESPSCH & & SW & & & BOHTG2 & 06-Jun-96 & & $10098-97-2$ & SR-90 \\
\hline 2599 & SESPSCH & & SW & & & BOHTG2 & 06-Jun-96 & & $10028-17-8$ & TRITIUM \\
\hline 2600 & SESPSCH & & SW & & & BOHTG2 & 06-Jun-96 & & $13966-29-5$ & U-234 \\
\hline 2601 & SESPSCH & & SW & & & BOHTG2 & 06-Jun-96 & & 15117-96-1 & U-235 \\
\hline 2602 & SESPSCH & & SW & & & BOHTG2 & 06-Jun-96 & & $\mathrm{U}-238$ & U-238 \\
\hline 2603 & PS HENRY & & SW & & & B0G182 & 06-Jun-96 & & $7440-47-3$ & $\mathrm{CR}$ \\
\hline 2604 & PS HENRY & & SW & & & B0G182 & 06-Jun-96 & & $56-23-5$ & CARBTET \\
\hline 2605 & SESPSCH & & SW & & & BOHTG3 & 06-Jun-96 & & $10098-97-2$ & SR-90 \\
\hline 2606 & SESPSCH & & SW & & & BOHTG3 & 06-Jun-96 & & $10028-17-8$ & TRITIUM \\
\hline 2607 & SESPSCH & & SW & & & B0HTG3 & 06-Jun-96 & & $13966-29-5$ & U-234 \\
\hline 2608 & SESPSCH & & SW & & & BOHTG3 & 06-Jun-96 & & 15117-96-1 & U-235 \\
\hline 2609 & SESPSCH & & SW & & & B0HTG3 & 06-Jun-96 & & U-238 & U-238 \\
\hline 2610 & PS HENRY & & SW & & & B0G183 & 06-Jun-96 & & $7440-47-3$ & CR \\
\hline 2611 & PS HENRY & & SW & & & B0G183 & 06-Jun-96 & & $56-23-5$ & CARBTET \\
\hline 2612 & SESPSCH & & SW & & & BOHTG4 & 06-Jun-96 & & 10098-97-2 & SR-90 \\
\hline 2613 & SESPSCH & & SW & & & BOHTG4 & 06-Jun-96 & & $10028-17-8$ & TRITIUM \\
\hline 2614 & SESPSCH & & SW & & & BOHTG4 & 06-Jun-96 & & $13966-29-5$ & U-234 \\
\hline 2615 & SESPSCH & & SW & & & BOHTG4 & 06-Jun-96 & & 15117-96-1 & U-235 \\
\hline 2616 & SESPSCH & & SW & & & BOHTG4 & 06-Jun-96 & & U-238 & U-238 \\
\hline 2617 & PS HENRY & & SW & & & B0G170 & 07-Jun-96 & & $7440-47-3$ & $\mathrm{CR}$ \\
\hline 2618 & PS HENRY & & SW & & & B0G170 & 07-Jun-96 & & $56-23-5$ & CARBTET \\
\hline 2619 & SESPSCH & & SW & & & BOHTH4 & 07-Jun-96 & & $10098-97-2$ & SR-90 \\
\hline 2620 & SESPSCH & & SW & & & BOHTH4 & 07-Jun-96 & & $10028-17-8$ & TRITIUM \\
\hline 2621 & SESPSCH & & SW & & & B0HTH4 & 07-Jun-96 & & 13966-29-5 & U-234 \\
\hline 2622 & SESPSCH & & SW & & & B0HTH4 & 07-Jun-96 & & 15117-96-1 & U-235 \\
\hline 2623 & SESPSCH & & SW & & & BOHTH4 & 07-Jun-96 & & $\mathrm{U}-238$ & U-238 \\
\hline
\end{tabular}




\begin{tabular}{|c|c|c|c|c|c|c|c|c|c|c|}
\hline & $\mathrm{A}$ & $B$ & $C$ & $\mathrm{D}$ & $E$ & $\mathrm{~F}$ & $G$ & $\mathrm{H}$ & $I$ & $\mathrm{~J}$ \\
\hline 1 & SAMPLE_NUM_ASSIGNED_TO & SAMPLER & MEDIA & BIOTA_MEDIA & TAG_ID & SAMP_NUM & SAMP_DATE_TIME| & SAMP_DATE_TIME_ON & CON_ID & CON_SHORT_NAME \\
\hline 2624 & PS HENRY & & SW & & & B0G179 & 07-Jun-96 & & 7440-47-3 & CR \\
\hline 2625 & PS HENRY & & SW & & & B0G179 & 07-Jun-96 & & $56-23-5$ & CARBTET \\
\hline 2626 & SESPSCH & & SW & & & BOHTG5 & 07-Jun-96 & & $10098-97-2$ & SR-90 \\
\hline 2627 & SESPSCH & & SW & & & BOHTG5 & 07-Jun-96 & & $10028-17-8$ & TRITIUM \\
\hline 2628 & SESPSCH & & SW & & & B0HTG5 & 07-Jun-96 & & 13966-29-5 & U-234 \\
\hline 2629 & SESPSCH & & SW & & & B0HTG5 & 07-Jun-96 & & 15117-96-1 & U-235 \\
\hline 2630 & SESPSCH & & SW & & & B0HTG5 & 07-Jun-96 & & U-238 & U-238 \\
\hline 2631 & PS HENRY & & SW & & & B0G171 & 07-Jun-96 & & $7440-47-3$ & CR \\
\hline 2632 & PS HENRY & & SW & & & B0G171 & 07-Jun-96 & & $56-23-5$ & CARBTET \\
\hline 2633 & SESPSCH & & SW & & & ВОНТНЗ & 07-Jun-96 & & $10098-97-2$ & SR-90 \\
\hline 2634 & SESPSCH & & SW & & & ВОНТНЗ & 07-Jun-96 & & $10028-17-8$ & TRITIUM \\
\hline 2635 & SESPSCH & & SW & & & BOHTH3 & 07-Jun-96 & & $13966-29-5$ & U-234 \\
\hline 2636 & SESPSCH & & SW & & & BOHTH3 & 07-Jun-96 & & 15117-96-1 & U-235 \\
\hline 2637 & SESPSCH & & SW & & & В0HTH3 & 07-Jun-96 & & U-238 & U-238 \\
\hline 2638 & PS HENRY & & SW & & & B0G172 & 07-Jun-96 & & $7440-47-3$ & CR \\
\hline 2639 & PS HENRY & & SW & & & B0G172 & 07-Jun-96 & & $56-23-5$ & CARBTET \\
\hline 2640 & SESPSCH & & SW & & & BOHTH2 & 07-Jun-96 & & $10098-97-2$ & SR-90 \\
\hline 2641 & SESPSCH & & SW & & & BOHTH2 & 07-Jun-96 & & $10028-17-8$ & TRITIUM \\
\hline 2642 & SESPSCH & & SW & & & В0HTH2 & 07-Jun-96 & & $13966-29-5$ & U-234 \\
\hline 2643 & SESPSCH & & SW & & & BOHTH2 & 07-Jun-96 & & 15117-96-1 & U-235 \\
\hline 2644 & SESPSCH & & SW & & & BOHTH2 & 07-Jun-96 & & U-238 & U-238 \\
\hline 2645 & PS HENRY & & SW & & & B0G173 & 07-Jun-96 & & $7440-47-3$ & CR \\
\hline 2646 & PS HENRY & & SW & & & B0G173 & 07-Jun-96 & & $56-23-5$ & CARBTET \\
\hline 2647 & SESPSCH & & SW & & & BOHTH1 & 07-Jun-96 & & $10098-97-2$ & SR-90 \\
\hline 2648 & SESPSCH & & SW & & & BOHTH1 & 07-Jun-96 & & 10028-17-8 & TRITIUM \\
\hline 2649 & SESPSCH & & SW & & & BOHTH1 & 07-Jun-96 & & $13966-29-5$ & U-234 \\
\hline 2650 & SESPSCH & & SW & & & B0HTH1 & 07-Jun-96 & & $15117-96-1$ & U-235 \\
\hline 2651 & SESPSCH & & SW & & & B0HTH1 & 07-Jun-96 & & U-238 & U-238 \\
\hline 2652 & PS HENRY & & SW & & & B0G174 & 07-Jun-96 & & $7440-47-3$ & CR \\
\hline 2653 & PS HENRY & & SW & & & B0G174 & 07-Jun-96 & & $56-23-5$ & CARBTET \\
\hline 2654 & SESPSCH & & SW & & & BOHTHO & 07-Jun-96 & & $10098-97-2$ & SR-90 \\
\hline 2655 & SESPSCH & & SW & & & ВОНТНО & 07-Jun-96 & & $10028-17-8$ & TRITIUM \\
\hline 2656 & SESPSCH & & SW & & & ВOHTHO & 07-Jun-96 & & 13966-29-5 & U-234 \\
\hline 2657 & SESPSCH & & SW & & & ВОНТНО & 07-Jun-96 & & $15117-96-1$ & U-235 \\
\hline 2658 & SESPSCH & & SW & & & ВОНТНО & 07-Jun-96 & & U-238 & U-238 \\
\hline 2659 & PS HENRY & & SW & & & B0G175 & 07-Jun-96 & & $7440-47-3$ & CR \\
\hline 2660 & PS HENRY & & SW & & & B0G175 & 07-Jun-96 & & $56-23-5$ & CARBTET \\
\hline 2661 & SESPSCH & & SW & & & B0HTG9 & 07-Jun-96 & & 10098-97-2 & SR-90 \\
\hline
\end{tabular}




\begin{tabular}{|c|c|c|c|c|c|c|c|c|c|c|}
\hline & $\mathrm{A}$ & $B$ & $\mathrm{C}$ & $\mathrm{D}$ & $E$ & $\mathrm{~F}$ & $G$ & $\mathrm{H}$ & $I$ & $\mathrm{~J}$ \\
\hline 1 & SAMPLE NUM ASSIGNED TO & SAMPLER & MEDIA & BIOTA MEDIA & TAG ID & SAMP NUM & SAMP DATE TIME & SAMP DATE TIME ON & CON ID & CON SHORT NAME \\
\hline 2662 & SESPSCH & & SW & & & BOHTG9 & 07-Jun-96 & & $10028-17-8$ & TRITIUM \\
\hline 2663 & SESPSCH & & SW & & & BOHTG9 & 07-Jun-96 & & $13966-29-5$ & U-234 \\
\hline 2664 & SESPSCH & & SW & & & BOHTG9 & 07-Jun-96 & & 15117-96-1 & U-235 \\
\hline 2665 & SESPSCH & & SW & & & B0HTG9 & 07-Jun-96 & & U-238 & U-238 \\
\hline 2666 & PS HENRY & & SW & & & B0G176 & 07-Jun-96 & & $7440-47-3$ & CR \\
\hline 2667 & PS HENRY & & SW & & & B0G176 & 07-Jun-96 & & $56-23-5$ & CARBTET \\
\hline 2668 & SESPSCH & & SW & & & BOHTG8 & 07-Jun-96 & & $10098-97-2$ & SR-90 \\
\hline 2669 & SESPSCH & & SW & & & B0HTG8 & 07-Jun-96 & & $10028-17-8$ & TRITIUM \\
\hline 2670 & SESPSCH & & SW & & & BOHTG8 & 07-Jun-96 & & $13966-29-5$ & U-234 \\
\hline 2671 & SESPSCH & & SW & & & BOHTG8 & 07-Jun-96 & & 15117-96-1 & U-235 \\
\hline 2672 & SESPSCH & & SW & & & BOHTG8 & 07-Jun-96 & & U-238 & U-238 \\
\hline 2673 & PS HENRY & & SW & & & B0G177 & 07-Jun-96 & & $7440-47-3$ & $\mathrm{CR}$ \\
\hline 2674 & PS HENRY & & SW & & & B0G177 & 07-Jun-96 & & 56-23-5 & CARBTET \\
\hline 2675 & SESPSCH & & SW & & & BOHTG7 & 07-Jun-96 & & $10098-97-2$ & SR-90 \\
\hline 2676 & SESPSCH & & SW & & & BOHTG7 & 07-Jun-96 & & 10028-17-8 & TRITIUM \\
\hline 2677 & SESPSCH & & SW & & & BOHTG7 & 07-Jun-96 & & 13966-29-5 & U-234 \\
\hline 2678 & SESPSCH & & SW & & & B0HTG7 & 07-Jun-96 & & 15117-96-1 & U-235 \\
\hline 2679 & SESPSCH & & SW & & & BOHTG7 & 07-Jun-96 & & U-238 & U-238 \\
\hline 2680 & PS HENRY & & SW & & & B0G178 & 07-Jun-96 & & $7440-47-3$ & $\mathrm{CR}$ \\
\hline 2681 & PS HENRY & & SW & & & B0G178 & 07-Jun-96 & & $56-23-5$ & CARBTET \\
\hline 2682 & SESPSCH & & SW & & & BOHTG6 & 07-Jun-96 & & $10098-97-2$ & SR-90 \\
\hline 2683 & SESPSCH & & SW & & & BOHTG6 & 07-Jun-96 & & $10028-17-8$ & TRITIUM \\
\hline 2684 & SESPSCH & & SW & & & BOHTG6 & 07-Jun-96 & & 13966-29-5 & U-234 \\
\hline 2685 & SESPSCH & & SW & & & B0HTG6 & 07-Jun-96 & & 15117-96-1 & U-235 \\
\hline 2686 & SESPSCH & & SW & & & B0HTG6 & 07-Jun-96 & & U-238 & U-238 \\
\hline 2687 & PS HENRY & & SW & & & B0G1J4 & 19-Sep-96 & & $7440-47-3$ & CR \\
\hline 2688 & PS HENRY & & SW & & & B0G1J4 & 19-Sep-96 & & $56-23-5$ & CARBTET \\
\hline 2689 & SESPSCH & & SW & & & B0J8S7 & 19-Sep-96 & & 10098-97-2 & SR-90 \\
\hline 2690 & SESPSCH & & SW & & & B0J8S7 & 19-Sep-96 & & $10028-17-8$ & TRITIUM \\
\hline 2691 & SESPSCH & & SW & & & B0J8S7 & 19-Sep-96 & & 13966-29-5 & U-234 \\
\hline 2692 & SESPSCH & & SW & & & B0J8S7 & 19-Sep-96 & & $15117-96-1$ & U-235 \\
\hline 2693 & SESPSCH & & SW & & & B0J8S7 & 19-Sep-96 & & U-238 & U-238 \\
\hline 2694 & PS HENRY & & SW & & & B0G1J5 & 19-Sep-96 & & $7440-47-3$ & CR \\
\hline 2695 & PS HENRY & & SW & & & B0G1J5 & 19-Sep-96 & & $56-23-5$ & CARBTET \\
\hline 2696 & SESPSCH & & SW & & & B0J8S8 & 19-Sep-96 & & $10098-97-2$ & SR-90 \\
\hline 2697 & SESPSCH & & SW & & & B0J8S8 & 19-Sep-96 & & $10028-17-8$ & TRITIUM \\
\hline 2698 & SESPSCH & & SW & & & B0J8S8 & 19-Sep-96 & & 13966-29-5 & U-234 \\
\hline 2699 & SESPSCH & & SW & & & B0J8S8 & 19-Sep-96 & & $15117-96-1$ & U-235 \\
\hline
\end{tabular}




\begin{tabular}{|c|c|c|c|c|c|c|c|c|c|c|}
\hline & $\mathrm{A}$ & $B$ & $\mathrm{C}$ & $\mathrm{D}$ & $E$ & $\mathrm{~F}$ & $G$ & $\mathrm{H}$ & $I$ & $\mathrm{~J}$ \\
\hline 1 & SAMPLE NUM ASSIGNED TO & SAMPLER & MEDIA & BIOTA MEDIA & TAG ID & SAMP NUM & SAMP DATE TIME & SAMP DATE TIME ON & CON ID & CON SHORT NAME \\
\hline 2700 & SESPSCH & & SW & & & B0J8S8 & 19-Sep-96 & & U-238 & U-238 \\
\hline 2701 & PS HENRY & & SW & & & B0G1J6 & 19-Sep-96 & & $7440-47-3$ & CR \\
\hline 2702 & PS HENRY & & SW & & & B0G1J6 & 19-Sep-96 & & $56-23-5$ & CARBTET \\
\hline 2703 & SESPSCH & & SW & & & B0J8S9 & 19-Sep-96 & & $10098-97-2$ & SR-90 \\
\hline 2704 & SESPSCH & & SW & & & B0J8S9 & 19-Sep-96 & & 10028-17-8 & TRITIUM \\
\hline 2705 & SESPSCH & & SW & & & B0J8S9 & 19-Sep-96 & & $13966-29-5$ & U-234 \\
\hline 2706 & SESPSCH & & SW & & & B0J8S9 & 19-Sep-96 & & 15117-96-1 & U-235 \\
\hline 2707 & SESPSCH & & SW & & & B0J8S9 & 19-Sep-96 & & U-238 & U-238 \\
\hline 2708 & PS HENRY & & SW & & & B0G1J7 & 19-Sep-96 & & $7440-47-3$ & $\mathrm{CR}$ \\
\hline 2709 & PS HENRY & & SW & & & B0G1J7 & 19-Sep-96 & & $56-23-5$ & CARBTET \\
\hline 2710 & SESPSCH & & SW & & & B0J8T0 & 19-Sep-96 & & $10098-97-2$ & SR-90 \\
\hline 2711 & SESPSCH & & SW & & & B0J8T0 & 19-Sep-96 & & $10028-17-8$ & TRITIUM \\
\hline 2712 & SESPSCH & & SW & & & B0J8T0 & 19-Sep-96 & & 13966-29-5 & U-234 \\
\hline 2713 & SESPSCH & & SW & & & BOJ8T0 & 19-Sep-96 & & 15117-96-1 & U-235 \\
\hline 2714 & SESPSCH & & SW & & & B0J8T0 & 19-Sep-96 & & U-238 & U-238 \\
\hline 2715 & PS HENRY & & SW & & & B0G1F0 & 20-Sep-96 & & $7440-47-3$ & CR \\
\hline 2716 & PS HENRY & & SW & & & B0G1F0 & 20-Sep-96 & & $56-23-5$ & CARBTET \\
\hline 2717 & SESPSCH & & SW & & & B0J8X2 & 20-Sep-96 & & $10098-97-2$ & SR-90 \\
\hline 2718 & SESPSCH & & SW & & & B0J8X2 & 20-Sep-96 & & $10028-17-8$ & TRITIUM \\
\hline 2719 & SESPSCH & & SW & & & B0J8X2 & 20-Sep-96 & & 13966-29-5 & U-234 \\
\hline 2720 & SESPSCH & & SW & & & B0J8X2 & 20-Sep-96 & & $15117-96-1$ & U-235 \\
\hline 2721 & SESPSCH & & SW & & & B0J8X2 & 20-Sep-96 & & U-238 & U-238 \\
\hline 2722 & PS HENRY & & SW & & & B0G1F1 & 20-Sep-96 & & $7440-47-3$ & CR \\
\hline 2723 & PS HENRY & & SW & & & B0G1F1 & 20-Sep-96 & & $56-23-5$ & CARBTET \\
\hline 2724 & SESPSCH & & SW & & & B0J8X4 & 20-Sep-96 & & $10098-97-2$ & SR-90 \\
\hline 2725 & SESPSCH & & SW & & & B0J8X4 & 20-Sep-96 & & $10028-17-8$ & TRITIUM \\
\hline 2726 & SESPSCH & & SW & & & B0J8X4 & 20-Sep-96 & & $13966-29-5$ & U-234 \\
\hline 2727 & SESPSCH & & SW & & & B0J8X4 & 20-Sep-96 & & 15117-96-1 & U-235 \\
\hline 2728 & SESPSCH & & SW & & & B0J8X4 & 20-Sep-96 & & U-238 & U-238 \\
\hline 2729 & PS HENRY & & SW & & & B0G1F2 & 20-Sep-96 & & $7440-47-3$ & CR \\
\hline 2730 & PS HENRY & & SW & & & B0G1F2 & 20-Sep-96 & & $56-23-5$ & CARBTET \\
\hline 2731 & SESPSCH & & SW & & & B0J8X6 & 20-Sep-96 & & $10098-97-2$ & SR-90 \\
\hline 2732 & SESPSCH & & SW & & & B0J8X6 & 20-Sep-96 & & $10028-17-8$ & TRITIUM \\
\hline 2733 & SESPSCH & & SW & & & B0J8X6 & 20-Sep-96 & & 13966-29-5 & U-234 \\
\hline 2734 & SESPSCH & & SW & & & B0J8X6 & 20-Sep-96 & & $15117-96-1$ & U-235 \\
\hline 2735 & SESPSCH & & SW & & & B0J8X6 & 20-Sep-96 & & U-238 & U-238 \\
\hline 2736 & PS HENRY & & SW & & & B0G1F3 & 20-Sep-96 & & $7440-47-3$ & $\mathrm{CR}$ \\
\hline 2737 & PS HENRY & & SW & & & B0G1F3 & 20-Sep-96 & & $56-23-5$ & CARBTET \\
\hline
\end{tabular}

Page 72 


\begin{tabular}{|c|c|c|c|c|c|c|c|c|c|c|}
\hline & A & $B$ & $\mathrm{C}$ & $\mathrm{D}$ & $\mathrm{E}$ & $\mathrm{F}$ & $G$ & $\mathrm{H}$ & $I$ & $\mathrm{~J}$ \\
\hline 1 & SAMPLE_NUM_ASSIGNED_TO & SAMPLER & MEDIA & BIOTA_MEDIA & TAG_ID & SAMP_NUM & SAMP_DATE_TIME| & SAMP_DATE_TIME_ON & CON_ID & CON_SHORT_NAME \\
\hline 2738 & SESPSCH & & SW & & & B0J8X8 & 20-Sep-96 & & $10098-97-2$ & SR-90 \\
\hline 2739 & SESPSCH & & SW & & & B0J8X8 & 20-Sep-96 & & $10028-17-8$ & TRITIUM \\
\hline 2740 & SESPSCH & & SW & & & B0J8X8 & 20-Sep-96 & & $13966-29-5$ & U-234 \\
\hline 2741 & SESPSCH & & SW & & & B0J8X8 & 20-Sep-96 & & 15117-96-1 & U-235 \\
\hline 2742 & SESPSCH & & SW & & & B0J8X8 & 20-Sep-96 & & U-238 & U-238 \\
\hline 2743 & PS HENRY & & SW & & & B0G1F4 & 20-Sep-96 & & $7440-47-3$ & CR \\
\hline 2744 & PS HENRY & & SW & & & B0G1F4 & 20-Sep-96 & & $56-23-5$ & CARBTET \\
\hline 2745 & SESPSCH & & SW & & & BOJ8YO & 20-Sep-96 & & $10098-97-2$ & SR-90 \\
\hline 2746 & SESPSCH & & SW & & & BOJ8Y0 & 20-Sep-96 & & $10028-17-8$ & TRITIUM \\
\hline 2747 & SESPSCH & & SW & & & BOJ8Y0 & 20-Sep-96 & & 13966-29-5 & U-234 \\
\hline 2748 & SESPSCH & & SW & & & BOJ8YO & 20-Sep-96 & & 15117-96-1 & U-235 \\
\hline 2749 & SESPSCH & & SW & & & BOJ8YO & 20-Sep-96 & & U-238 & U-238 \\
\hline 2750 & PS HENRY & & SW & & & B0G1F5 & 20-Sep-96 & & $7440-47-3$ & $\mathrm{CR}$ \\
\hline 2751 & PS HENRY & & SW & & & B0G1F5 & 20-Sep-96 & & $56-23-5$ & CARBTET \\
\hline 2752 & SESPSCH & & SW & & & B0J8Y2 & 20-Sep-96 & & $10098-97-2$ & SR-90 \\
\hline 2753 & SESPSCH & & SW & & & B0J8Y2 & 20-Sep-96 & & $10028-17-8$ & TRITIUM \\
\hline 2754 & SESPSCH & & SW & & & B0J8Y2 & 20-Sep-96 & & 13966-29-5 & U-234 \\
\hline 2755 & SESPSCH & & SW & & & B0J8Y2 & 20-Sep-96 & & 15117-96-1 & U-235 \\
\hline 2756 & SESPSCH & & SW & & & B0J8Y2 & 20-Sep-96 & & U-238 & U-238 \\
\hline 2757 & PS HENRY & & SW & & & B0G1F6 & 20-Sep-96 & & $7440-47-3$ & CR \\
\hline 2758 & PS HENRY & & SW & & & B0G1F6 & 20-Sep-96 & & $56-23-5$ & CARBTET \\
\hline 2759 & SESPSCH & & SW & & & B0J8Y3 & 20-Sep-96 & & $10098-97-2$ & SR-90 \\
\hline 2760 & SESPSCH & & SW & & & B0J8Y3 & 20-Sep-96 & & $10028-17-8$ & TRITIUM \\
\hline 2761 & SESPSCH & & SW & & & B0J8Y3 & 20-Sep-96 & & 13966-29-5 & U-234 \\
\hline 2762 & SESPSCH & & SW & & & B0J8Y3 & 20-Sep-96 & & 15117-96-1 & U-235 \\
\hline 2763 & SESPSCH & & SW & & & B0J8Y3 & 20-Sep-96 & & U-238 & U-238 \\
\hline 2764 & PS HENRY & & SW & & & B0G1F7 & 20-Sep-96 & & $7440-47-3$ & CR \\
\hline 2765 & PS HENRY & & SW & & & B0G1F7 & 20-Sep-96 & & $56-23-5$ & CARBTET \\
\hline 2766 & SESPSCH & & SW & & & B0J8Y4 & 20-Sep-96 & & $10098-97-2$ & SR-90 \\
\hline 2767 & SESPSCH & & SW & & & B0J8Y4 & 20-Sep-96 & & $10028-17-8$ & TRITIUM \\
\hline 2768 & SESPSCH & & SW & & & B0J8Y4 & 20-Sep-96 & & 13966-29-5 & U-234 \\
\hline 2769 & SESPSCH & & SW & & & B0J8Y4 & 20-Sep-96 & & 15117-96-1 & U-235 \\
\hline 2770 & SESPSCH & & SW & & & B0J8Y4 & 20-Sep-96 & & U-238 & U-238 \\
\hline 2771 & PS HENRY & & SW & & & B0G1F8 & 20-Sep-96 & & $7440-47-3$ & CR \\
\hline 2772 & PS HENRY & & SW & & & B0G1F8 & 20-Sep-96 & & $56-23-5$ & CARBTET \\
\hline 2773 & SESPSCH & & SW & & & B0J8Y5 & 20-Sep-96 & & $10098-97-2$ & SR-90 \\
\hline 2774 & SESPSCH & & SW & & & B0J8Y5 & 20-Sep-96 & & $10028-17-8$ & TRITIUM \\
\hline 2775 & SESPSCH & & SW & & & B0J8Y5 & 20-Sep-96 & & $13966-29-5$ & U-234 \\
\hline
\end{tabular}




\begin{tabular}{|c|c|c|c|c|c|c|c|c|c|c|}
\hline & A & $\mathrm{B}$ & $C$ & $\mathrm{D}$ & $E$ & $\mathrm{~F}$ & $G$ & $\mathrm{H}$ & 1 & $\mathrm{~J}$ \\
\hline 1 & SAMPLE_NUM_ASSIGNED_TO & SAMPLER & MEDIA & BIOTA_MEDIA & TAG_ID & SAMP_NUM & SAMP_DATE_TIME| & SAMP_DATE_TIME_ON & CON_ID & CON_SHORT_NAME \\
\hline 2776 & SESPSCH & & SW & & & B0J8Y5 & 20-Sep-96 & & 15117-96-1 & U-235 \\
\hline 2777 & SESPSCH & & SW & & & B0J8Y5 & 20-Sep-96 & & U-238 & U-238 \\
\hline 2778 & PS HENRY & & SW & & & B0G1F9 & 20-Sep-96 & & $7440-47-3$ & $\mathrm{CR}$ \\
\hline 2779 & PS HENRY & & SW & & & B0G1F9 & 20-Sep-96 & & $56-23-5$ & CARBTET \\
\hline 2780 & SESPSCH & & SW & & & B0J8Y6 & 20-Sep-96 & & $10098-97-2$ & SR-90 \\
\hline 2781 & SESPSCH & & SW & & & B0J8Y6 & 20-Sep-96 & & 10028-17-8 & TRITIUM \\
\hline 2782 & SESPSCH & & SW & & & B0J8Y6 & 20-Sep-96 & & 13966-29-5 & U-234 \\
\hline 2783 & SESPSCH & & SW & & & B0J8Y6 & 20-Sep-96 & & $15117-96-1$ & U-235 \\
\hline 2784 & SESPSCH & & SW & & & B0J8Y6 & 20-Sep-96 & & U-238 & U-238 \\
\hline 2785 & PS HENRY & & SW & & & B0G1H4 & 20-Sep-96 & & $7440-47-3$ & CR \\
\hline 2786 & PS HENRY & & SW & & & B0G1H4 & 20-Sep-96 & & $56-23-5$ & CARBTET \\
\hline 2787 & SESPSCH & & SW & & & B0J8Y7 & 20-Sep-96 & & $10098-97-2$ & SR-90 \\
\hline 2788 & SESPSCH & & SW & & & B0J8Y7 & 20-Sep-96 & & 10028-17-8 & TRITIUM \\
\hline 2789 & SESPSCH & & SW & & & B0J8Y7 & 20-Sep-96 & & 13966-29-5 & U-234 \\
\hline 2790 & SESPSCH & & SW & & & B0J8Y7 & 20-Sep-96 & & 15117-96-1 & U-235 \\
\hline 2791 & SESPSCH & & SW & & & B0J8Y7 & 20-Sep-96 & & U-238 & U-238 \\
\hline 2792 & PS HENRY & & SW & & & B0G1J3 & 20-Sep-96 & & $7440-47-3$ & CR \\
\hline 2793 & PS HENRY & & SW & & & B0G1J3 & 20-Sep-96 & & $56-23-5$ & CARBTET \\
\hline 2794 & SESPSCH & & SW & & & B0J8Z6 & 20-Sep-96 & & $10098-97-2$ & SR-90 \\
\hline 2795 & SESPSCH & & SW & & & B0J8Z6 & 20-Sep-96 & & $10028-17-8$ & TRITIUM \\
\hline 2796 & SESPSCH & & SW & & & B0J8Z6 & 20-Sep-96 & & $13966-29-5$ & U-234 \\
\hline 2797 & SESPSCH & & SW & & & B0J8Z6 & 20-Sep-96 & & 15117-96-1 & U-235 \\
\hline 2798 & SESPSCH & & SW & & & B0J8Z6 & 20-Sep-96 & & U-238 & U-238 \\
\hline 2799 & PS HENRY & & SW & & & B0G1H5 & 20-Sep-96 & & $7440-47-3$ & CR \\
\hline 2800 & PS HENRY & & SW & & & B0G1H5 & 20-Sep-96 & & $56-23-5$ & CARBTET \\
\hline 2801 & SESPSCH & & SW & & & B0J8Y8 & 20-Sep-96 & & 10098-97-2 & SR-90 \\
\hline 2802 & SESPSCH & & SW & & & B0J8Y8 & 20-Sep-96 & & 10028-17-8 & TRITIUM \\
\hline 2803 & SESPSCH & & SW & & & B0J8Y8 & 20-Sep-96 & & 13966-29-5 & U-234 \\
\hline 2804 & SESPSCH & & SW & & & B0J8Y8 & 20-Sep-96 & & 15117-96-1 & U-235 \\
\hline 2805 & SESPSCH & & SW & & & B0J8Y8 & 20-Sep-96 & & U-238 & U-238 \\
\hline 2806 & PS HENRY & & SW & & & B0G1H6 & 20-Sep-96 & & $7440-47-3$ & CR \\
\hline 2807 & PS HENRY & & SW & & & B0G1H6 & 20-Sep-96 & & $56-23-5$ & CARBTET \\
\hline 2808 & SESPSCH & & SW & & & B0J8Y9 & 20-Sep-96 & & $10098-97-2$ & SR-90 \\
\hline 2809 & SESPSCH & & SW & & & B0J8Y9 & 20-Sep-96 & & $10028-17-8$ & TRITIUM \\
\hline 2810 & SESPSCH & & SW & & & B0J8Y9 & 20-Sep-96 & & 13966-29-5 & U-234 \\
\hline 2811 & SESPSCH & & SW & & & B0J8Y9 & 20-Sep-96 & & $15117-96-1$ & U-235 \\
\hline 2812 & SESPSCH & & SW & & & B0J8Y9 & 20-Sep-96 & & U-238 & U-238 \\
\hline 2813 & PS HENRY & & SW & & & B0G1H7 & 20-Sep-96 & & $7440-47-3$ & CR \\
\hline
\end{tabular}




\begin{tabular}{|c|c|c|c|c|c|c|c|c|c|c|}
\hline & $\mathrm{A}$ & $B$ & $\mathrm{C}$ & $\mathrm{D}$ & $E$ & $\mathrm{~F}$ & $G$ & $\mathrm{H}$ & $I$ & $\mathrm{~J}$ \\
\hline 1 & SAMPLE NUM ASSIGNED TO & SAMPLER & MEDIA & BIOTA MEDIA & TAG ID & SAMP NUM & SAMP DATE TIME & SAMP DATE TIME ON & CON ID & CON SHORT NAME \\
\hline 2814 & PS HENRY & & SW & & & B0G1H7 & 20-Sep-96 & & $56-23-5$ & CARBTET \\
\hline 2815 & SESPSCH & & SW & & & BOJ8Z0 & 20-Sep-96 & & $10098-97-2$ & SR-90 \\
\hline 2816 & SESPSCH & & SW & & & B0J8Z0 & 20-Sep-96 & & $10028-17-8$ & TRITIUM \\
\hline 2817 & SESPSCH & & SW & & & B0J8Z0 & 20-Sep-96 & & 13966-29-5 & U-234 \\
\hline 2818 & SESPSCH & & SW & & & B0J8ZO & 20-Sep-96 & & 15117-96-1 & U-235 \\
\hline 2819 & SESPSCH & & SW & & & B0J8ZO & 20-Sep-96 & & U-238 & U-238 \\
\hline 2820 & PS HENRY & & SW & & & B0G1H8 & 20-Sep-96 & & $7440-47-3$ & $\mathrm{CR}$ \\
\hline 2821 & PS HENRY & & SW & & & B0G1H8 & 20-Sep-96 & & $56-23-5$ & CARBTET \\
\hline 2822 & SESPSCH & & SW & & & B0J8Z1 & 20-Sep-96 & & $10098-97-2$ & SR-90 \\
\hline 2823 & SESPSCH & & SW & & & B0J8Z1 & 20-Sep-96 & & $10028-17-8$ & TRITIUM \\
\hline 2824 & SESPSCH & & SW & & & B0J8Z1 & 20-Sep-96 & & 13966-29-5 & U-234 \\
\hline 2825 & SESPSCH & & SW & & & B0J8Z1 & 20-Sep-96 & & $15117-96-1$ & U-235 \\
\hline 2826 & SESPSCH & & SW & & & B0J8Z1 & 20-Sep-96 & & $\mathrm{U}-238$ & U-238 \\
\hline 2827 & PS HENRY & & SW & & & B0G1H9 & 20-Sep-96 & & $7440-47-3$ & CR \\
\hline 2828 & PS HENRY & & SW & & & B0G1H9 & 20-Sep-96 & & $56-23-5$ & CARBTET \\
\hline 2829 & SESPSCH & & SW & & & B0J8Z2 & 20-Sep-96 & & $10098-97-2$ & SR-90 \\
\hline 2830 & SESPSCH & & SW & & & B0J8Z2 & 20-Sep-96 & & $10028-17-8$ & TRITIUM \\
\hline 2831 & SESPSCH & & SW & & & B0J8Z2 & 20-Sep-96 & & 13966-29-5 & U-234 \\
\hline 2832 & SESPSCH & & SW & & & B0J8Z2 & 20-Sep-96 & & $15117-96-1$ & U-235 \\
\hline 2833 & SESPSCH & & SW & & & B0J8Z2 & 20-Sep-96 & & U-238 & U-238 \\
\hline 2834 & PS HENRY & & SW & & & B0G1J0 & 20-Sep-96 & & $7440-47-3$ & $\mathrm{CR}$ \\
\hline 2835 & PS HENRY & & SW & & & B0G1J0 & 20-Sep-96 & & $56-23-5$ & CARBTET \\
\hline 2836 & SESPSCH & & SW & & & B0J8Z3 & 20-Sep-96 & & 10098-97-2 & SR-90 \\
\hline 2837 & SESPSCH & & SW & & & B0J8Z3 & 20-Sep-96 & & $10028-17-8$ & TRITIUM \\
\hline 2838 & SESPSCH & & SW & & & B0J8Z3 & 20-Sep-96 & & 13966-29-5 & U-234 \\
\hline 2839 & SESPSCH & & SW & & & B0J8Z3 & 20-Sep-96 & & 15117-96-1 & U-235 \\
\hline 2840 & SESPSCH & & SW & & & B0J8Z3 & 20-Sep-96 & & U-238 & U-238 \\
\hline 2841 & PS HENRY & & SW & & & B0G1J1 & 20-Sep-96 & & $7440-47-3$ & CR \\
\hline 2842 & PS HENRY & & SW & & & B0G1J1 & 20-Sep-96 & & $56-23-5$ & CARBTET \\
\hline 2843 & SESPSCH & & SW & & & B0J8Z4 & 20-Sep-96 & & 10098-97-2 & SR-90 \\
\hline 2844 & SESPSCH & & SW & & & B0J8Z4 & 20-Sep-96 & & $10028-17-8$ & TRITIUM \\
\hline 2845 & SESPSCH & & SW & & & B0J8Z4 & 20-Sep-96 & & $13966-29-5$ & U-234 \\
\hline 2846 & SESPSCH & & SW & & & B0J8Z4 & 20-Sep-96 & & 15117-96-1 & U-235 \\
\hline 2847 & SESPSCH & & SW & & & B0J8Z4 & 20-Sep-96 & & U-238 & U-238 \\
\hline 2848 & PS HENRY & & SW & & & B0G1J2 & 20-Sep-96 & & $7440-47-3$ & $\mathrm{CR}$ \\
\hline 2849 & PS HENRY & & SW & & & B0G1J2 & 20-Sep-96 & & $56-23-5$ & CARBTET \\
\hline 2850 & SESPSCH & & SW & & & B0J8Z5 & 20-Sep-96 & & $10098-97-2$ & SR-90 \\
\hline 2851 & SESPSCH & & SW & & & B0J8Z5 & 20-Sep-96 & & $10028-17-8$ & TRITIUM \\
\hline
\end{tabular}




\begin{tabular}{|c|c|c|c|c|c|c|c|c|c|c|}
\hline & $\mathrm{A}$ & $B$ & $\mathrm{C}$ & $\mathrm{D}$ & $E$ & $\mathrm{~F}$ & $G$ & $\mathrm{H}$ & $I$ & $\mathrm{~J}$ \\
\hline 1 & SAMPLE NUM ASSIGNED TO & SAMPLER & MEDIA & BIOTA MEDIA & TAG ID & SAMP NUM & SAMP DATE TIME & SAMP DATE TIME ON & CON ID & CON SHORT NAME \\
\hline 2852 & SESPSCH & & SW & & & B0J8Z5 & 20-Sep-96 & & $13966-29-5$ & U-234 \\
\hline 2853 & SESPSCH & & SW & & & B0J8Z5 & 20-Sep-96 & & 15117-96-1 & U-235 \\
\hline 2854 & SESPSCH & & SW & & & B0J8Z5 & 20-Sep-96 & & U-238 & U-238 \\
\hline 2855 & PS HENRY & & SW & & & B0G1C8 & 25-Sep-96 & & $7440-47-3$ & CR \\
\hline 2856 & PS HENRY & & SW & & & B0G1C8 & 25-Sep-96 & & $56-23-5$ & CARBTET \\
\hline 2857 & SESPSCH & & SW & & & B0J8T1 & 25-Sep-96 & & 10098-97-2 & SR-90 \\
\hline 2858 & SESPSCH & & SW & & & B0J907 & 25-Sep-96 & & $10098-97-2$ & SR-90 \\
\hline 2859 & SESPSCH & & SW & & & B0J8T1 & 25-Sep-96 & & $10028-17-8$ & TRITIUM \\
\hline 2860 & SESPSCH & & SW & & & B0J907 & 25-Sep-96 & & $10028-17-8$ & TRITIUM \\
\hline 2861 & SESPSCH & & SW & & & B0J8T1 & 25-Sep-96 & & $13966-29-5$ & U-234 \\
\hline 2862 & SESPSCH & & SW & & & B0J8T1 & 25-Sep-96 & & 15117-96-1 & U-235 \\
\hline 2863 & SESPSCH & & SW & & & B0J8T1 & 25-Sep-96 & & U-238 & U-238 \\
\hline 2864 & SESPSCH & & SW & & & B0J907 & 25-Sep-96 & & $13966-29-5$ & U-234 \\
\hline 2865 & SESPSCH & & SW & & & B0J907 & 25-Sep-96 & & 15117-96-1 & U-235 \\
\hline 2866 & SESPSCH & & SW & & & B0J907 & 25-Sep-96 & & U-238 & U-238 \\
\hline 2867 & PS HENRY & & SW & & & B0G1D7 & 25-Sep-96 & & $7440-47-3$ & CR \\
\hline 2868 & PS HENRY & & SW & & & B0G1D7 & 25-Sep-96 & & $56-23-5$ & CARBTET \\
\hline 2869 & SESPSCH & & SW & & & B0J8Z8 & 25-Sep-96 & & 10098-97-2 & SR-90 \\
\hline 2870 & SESPSCH & & SW & & & B0J8Z8 & 25-Sep-96 & & $10028-17-8$ & TRITIUM \\
\hline 2871 & SESPSCH & & SW & & & B0J8Z8 & 25-Sep-96 & & 13966-29-5 & U-234 \\
\hline 2872 & SESPSCH & & SW & & & B0J8Z8 & 25-Sep-96 & & 15117-96-1 & U-235 \\
\hline 2873 & SESPSCH & & SW & & & B0J8Z8 & 25-Sep-96 & & U-238 & U-238 \\
\hline 2874 & PS HENRY & & SW & & & B0G1C9 & 25-Sep-96 & & $7440-47-3$ & CR \\
\hline 2875 & PS HENRY & & SW & & & B0G1C9 & 25-Sep-96 & & $56-23-5$ & CARBTET \\
\hline 2876 & SESPSCH & & SW & & & B0J8T3 & 25-Sep-96 & & $10098-97-2$ & SR-90 \\
\hline 2877 & SESPSCH & & SW & & & B0J8T3 & 25-Sep-96 & & $10028-17-8$ & TRITIUM \\
\hline 2878 & SESPSCH & & SW & & & B0J8T3 & 25-Sep-96 & & $13966-29-5$ & U-234 \\
\hline 2879 & SESPSCH & & SW & & & B0J8T3 & 25-Sep-96 & & 15117-96-1 & U-235 \\
\hline 2880 & SESPSCH & & SW & & & B0J8T3 & 25-Sep-96 & & U-238 & U-238 \\
\hline 2881 & PS HENRY & & SW & & & B0G1D0 & 25-Sep-96 & & $7440-47-3$ & CR \\
\hline 2882 & PS HENRY & & SW & & & B0G1D0 & 25-Sep-96 & & $56-23-5$ & CARBTET \\
\hline 2883 & SESPSCH & & SW & & & B0J8Z9 & 25-Sep-96 & & $10098-97-2$ & SR-90 \\
\hline 2884 & SESPSCH & & SW & & & B0J8Z9 & 25-Sep-96 & & $10028-17-8$ & TRITIUM \\
\hline 2885 & SESPSCH & & SW & & & B0J8Z9 & 25-Sep-96 & & 13966-29-5 & U-234 \\
\hline 2886 & SESPSCH & & SW & & & B0J8Z9 & 25-Sep-96 & & $15117-96-1$ & U-235 \\
\hline 2887 & SESPSCH & & SW & & & B0J8Z9 & 25-Sep-96 & & U-238 & U-238 \\
\hline 2888 & PS HENRY & & SW & & & B0G1D1 & 25-Sep-96 & & $7440-47-3$ & $\mathrm{CR}$ \\
\hline 2889 & PS HENRY & & SW & & & B0G1D1 & 25-Sep-96 & & $56-23-5$ & CARBTET \\
\hline
\end{tabular}




\begin{tabular}{|c|c|c|c|c|c|c|c|c|c|c|}
\hline & A & $B$ & $\mathrm{C}$ & $\mathrm{D}$ & $E$ & $\mathrm{~F}$ & G & $\mathrm{H}$ & $\mathrm{I}$ & $\mathrm{J}$ \\
\hline 1 & SAMPLE_NUM_ASSIGNED_TO & SAMPLER & MEDIA & BIOTA_MEDIA & TAG_ID & SAMP_NUM & SAMP_DATE_TIME & SAMP_DATE_TIME_ON & CON_ID & CON_SHORT_NAME \\
\hline 2890 & SESPSCH & & SW & & & B0J901 & 25-Sep-96 & & $10098-97-2$ & SR-90 \\
\hline 2891 & SESPSCH & & SW & & & B0J901 & 25-Sep-96 & & 10028-17-8 & TRITIUM \\
\hline 2892 & SESPSCH & & SW & & & B0J901 & 25-Sep-96 & & $13966-29-5$ & U-234 \\
\hline 2893 & SESPSCH & & SW & & & B0J901 & 25-Sep-96 & & 15117-96-1 & U-235 \\
\hline 2894 & SESPSCH & & SW & & & B0J901 & 25-Sep-96 & & U-238 & U-238 \\
\hline 2895 & PS HENRY & & SW & & & B0G1D2 & 25-Sep-96 & & $7440-47-3$ & $\mathrm{CR}$ \\
\hline 2896 & PS HENRY & & SW & & & B0G1D2 & 25-Sep-96 & & $56-23-5$ & CARBTET \\
\hline 2897 & SESPSCH & & SW & & & B0J8T6 & 25-Sep-96 & & 10098-97-2 & SR-90 \\
\hline 2898 & SESPSCH & & SW & & & B0J8T6 & 25-Sep-96 & & $10028-17-8$ & TRITIUM \\
\hline 2899 & SESPSCH & & SW & & & B0J8T6 & 25-Sep-96 & & $13966-29-5$ & U-234 \\
\hline 2900 & SESPSCH & & SW & & & B0J8T6 & 25-Sep-96 & & 15117-96-1 & U-235 \\
\hline 2901 & SESPSCH & & SW & & & B0J8T6 & 25-Sep-96 & & U-238 & U-238 \\
\hline 2902 & PS HENRY & & SW & & & B0G1D3 & 25-Sep-96 & & $7440-47-3$ & CR \\
\hline 2903 & PS HENRY & & SW & & & B0G1D3 & 25-Sep-96 & & $56-23-5$ & CARBTET \\
\hline 2904 & SESPSCH & & SW & & & B0J904 & 25-Sep-96 & & $10098-97-2$ & SR-90 \\
\hline 2905 & SESPSCH & & SW & & & B0J904 & 25-Sep-96 & & $10028-17-8$ & TRITIUM \\
\hline 2906 & SESPSCH & & SW & & & B0J904 & 25-Sep-96 & & $13966-29-5$ & U-234 \\
\hline 2907 & SESPSCH & & SW & & & B0J904 & 25-Sep-96 & & $15117-96-1$ & U-235 \\
\hline 2908 & SESPSCH & & SW & & & B0J904 & 25-Sep-96 & & $\mathrm{U}-238$ & $\mathrm{U}-238$ \\
\hline 2909 & PS HENRY & & SW & & & B0G1D4 & 25-Sep-96 & & $7440-47-3$ & $\mathrm{CR}$ \\
\hline 2910 & PS HENRY & & SW & & & B0G1D4 & 25-Sep-96 & & $56-23-5$ & CARBTET \\
\hline 2911 & SESPSCH & & SW & & & B0J903 & 25-Sep-96 & & $10098-97-2$ & SR-90 \\
\hline 2912 & SESPSCH & & SW & & & B0J903 & 25-Sep-96 & & 10028-17-8 & TRITIUM \\
\hline 2913 & SESPSCH & & SW & & & B0J903 & 25-Sep-96 & & $13966-29-5$ & U-234 \\
\hline 2914 & SESPSCH & & SW & & & B0J903 & 25-Sep-96 & & 15117-96-1 & U-235 \\
\hline 2915 & SESPSCH & & SW & & & B0J903 & 25-Sep-96 & & U-238 & U-238 \\
\hline 2916 & PS HENRY & & SW & & & B0G1D5 & 25-Sep-96 & & $7440-47-3$ & $\mathrm{CR}$ \\
\hline 2917 & PS HENRY & & SW & & & B0G1D5 & 25-Sep-96 & & $56-23-5$ & CARBTET \\
\hline 2918 & SESPSCH & & SW & & & B0J905 & 25-Sep-96 & & $10098-97-2$ & SR-90 \\
\hline 2919 & SESPSCH & & SW & & & B0J905 & 25-Sep-96 & & $10028-17-8$ & TRITIUM \\
\hline 2920 & SESPSCH & & SW & & & B0J905 & 25-Sep-96 & & 13966-29-5 & U-234 \\
\hline 2921 & SESPSCH & & SW & & & B0J905 & 25-Sep-96 & & 15117-96-1 & U-235 \\
\hline 2922 & SESPSCH & & SW & & & B0J905 & 25-Sep-96 & & U-238 & U-238 \\
\hline 2923 & PS HENRY & & SW & & & B0G1D6 & 25-Sep-96 & & $7440-47-3$ & $\mathrm{CR}$ \\
\hline 2924 & PS HENRY & & SW & & & B0G1D6 & 25-Sep-96 & & $56-23-5$ & CARBTET \\
\hline 2925 & SESPSCH & & SW & & & B0J906 & 25-Sep-96 & & 10098-97-2 & SR-90 \\
\hline 2926 & SESPSCH & & SW & & & B0J906 & 25-Sep-96 & & $10028-17-8$ & TRITIUM \\
\hline 2927 & SESPSCH & & SW & & & B0J906 & 25-Sep-96 & & 13966-29-5 & U-234 \\
\hline
\end{tabular}




\begin{tabular}{|c|c|c|c|c|c|c|c|c|c|c|}
\hline & $\mathrm{A}$ & $B$ & $\mathrm{C}$ & $\mathrm{D}$ & $E$ & $\mathrm{~F}$ & $G$ & $\mathrm{H}$ & $I$ & $\mathrm{~J}$ \\
\hline 1 & SAMPLE NUM ASSIGNED TO & SAMPLER & MEDIA & BIOTA MEDIA & TAG ID & SAMP NUM & SAMP DATE TIME & SAMP DATE TIME ON & CON ID & CON SHORT NAME \\
\hline 2928 & SESPSCH & & SW & & & B0J906 & 25-Sep-96 & & 15117-96-1 & U-235 \\
\hline 2929 & SESPSCH & & SW & & & B0J906 & 25-Sep-96 & & U-238 & U-238 \\
\hline 2930 & PS HENRY & & SW & & & B0G1B3 & 30-Sep-96 & & $7440-47-3$ & CR \\
\hline 2931 & PS HENRY & & SW & & & B0G1B3 & 30-Sep-96 & & $56-23-5$ & CARBTET \\
\hline 2932 & SESPSCH & & SW & & & B0J8T8 & 30-Sep-96 & & 10098-97-2 & SR-90 \\
\hline 2933 & SESPSCH & & SW & & & B0J8T8 & 30-Sep-96 & & $10028-17-8$ & TRITIUM \\
\hline 2934 & SESPSCH & & SW & & & B0J8T8 & 30-Sep-96 & & $13966-29-5$ & $\mathrm{U}-234$ \\
\hline 2935 & SESPSCH & & SW & & & B0J8T8 & 30-Sep-96 & & 15117-96-1 & U-235 \\
\hline 2936 & SESPSCH & & SW & & & B0J8T8 & 30-Sep-96 & & U-238 & U-238 \\
\hline 2937 & PS HENRY & & SW & & & B0G1C2 & 30-Sep-96 & & $7440-47-3$ & CR \\
\hline 2938 & PS HENRY & & SW & & & B0G1C2 & 30-Sep-96 & & $56-23-5$ & CARBTET \\
\hline 2939 & SESPSCH & & SW & & & B0J8W3 & 30-Sep-96 & & $10098-97-2$ & SR-90 \\
\hline 2940 & SESPSCH & & SW & & & B0J8W3 & 30-Sep-96 & & $10028-17-8$ & TRITIUM \\
\hline 2941 & SESPSCH & & SW & & & B0J8W3 & 30-Sep-96 & & $13966-29-5$ & U-234 \\
\hline 2942 & SESPSCH & & SW & & & B0J8W3 & 30-Sep-96 & & 15117-96-1 & U-235 \\
\hline 2943 & SESPSCH & & SW & & & B0J8W3 & 30-Sep-96 & & U-238 & U-238 \\
\hline 2944 & PS HENRY & & SW & & & B0G1B4 & 30-Sep-96 & & $7440-47-3$ & CR \\
\hline 2945 & PS HENRY & & SW & & & B0G1B4 & 30-Sep-96 & & $56-23-5$ & CARBTET \\
\hline 2946 & SESPSCH & & SW & & & B0J8V4 & 30-Sep-96 & & $10098-97-2$ & SR-90 \\
\hline 2947 & SESPSCH & & SW & & & B0J8V4 & 30-Sep-96 & & $10028-17-8$ & TRITIUM \\
\hline 2948 & SESPSCH & & SW & & & B0J8V4 & 30-Sep-96 & & 13966-29-5 & U-234 \\
\hline 2949 & SESPSCH & & SW & & & B0J8V4 & 30-Sep-96 & & $15117-96-1$ & U-235 \\
\hline 2950 & SESPSCH & & SW & & & B0J8V4 & 30-Sep-96 & & U-238 & U-238 \\
\hline 2951 & PS HENRY & & SW & & & B0G1B5 & 30-Sep-96 & & $7440-47-3$ & CR \\
\hline 2952 & PS HENRY & & SW & & & B0G1B5 & 30-Sep-96 & & $56-23-5$ & CARBTET \\
\hline 2953 & SESPSCH & & SW & & & BOJ8V0 & 30-Sep-96 & & 10098-97-2 & SR-90 \\
\hline 2954 & SESPSCH & & SW & & & BOJ8V0 & 30-Sep-96 & & $10028-17-8$ & TRITIUM \\
\hline 2955 & SESPSCH & & SW & & & BOJ8V0 & 30-Sep-96 & & $13966-29-5$ & U-234 \\
\hline 2956 & SESPSCH & & SW & & & B0J8V0 & 30-Sep-96 & & 15117-96-1 & U-235 \\
\hline 2957 & SESPSCH & & SW & & & B0J8V0 & 30-Sep-96 & & U-238 & U-238 \\
\hline 2958 & PS HENRY & & SW & & & B0G1B6 & 30-Sep-96 & & $7440-47-3$ & $\mathrm{CR}$ \\
\hline 2959 & PS HENRY & & SW & & & B0G1B6 & 30-Sep-96 & & $56-23-5$ & CARBTET \\
\hline 2960 & SESPSCH & & SW & & & B0J8V6 & 30-Sep-96 & & 10098-97-2 & SR-90 \\
\hline 2961 & SESPSCH & & SW & & & B0J8V6 & 30-Sep-96 & & $10028-17-8$ & TRITIUM \\
\hline 2962 & SESPSCH & & SW & & & B0J8V6 & 30-Sep-96 & & $13966-29-5$ & U-234 \\
\hline 2963 & SESPSCH & & SW & & & B0J8V6 & 30-Sep-96 & & 15117-96-1 & U-235 \\
\hline 2964 & SESPSCH & & SW & & & B0J8V6 & 30-Sep-96 & & U-238 & U-238 \\
\hline 2965 & PS HENRY & & SW & & & B0G1B7 & 30-Sep-96 & & $7440-47-3$ & $\mathrm{CR}$ \\
\hline
\end{tabular}




\begin{tabular}{|c|c|c|c|c|c|c|c|c|c|c|}
\hline & $\mathrm{A}$ & $B$ & $\mathrm{C}$ & $\mathrm{D}$ & $E$ & $\mathrm{~F}$ & $G$ & $\mathrm{H}$ & $I$ & $\mathrm{~J}$ \\
\hline 1 & SAMPLE NUM ASSIGNED TO & SAMPLER & MEDIA & BIOTA MEDIA & TAG ID & SAMP NUM & SAMP DATE TIME & SAMP DATE TIME ON & CON ID & CON SHORT NAME \\
\hline 2966 & PS HENRY & & SW & & & B0G1B7 & 30-Sep-96 & & $56-23-5$ & CARBTET \\
\hline 2967 & SESPSCH & & SW & & & B0J8V8 & 30-Sep-96 & & $10098-97-2$ & SR-90 \\
\hline 2968 & SESPSCH & & SW & & & B0J8V8 & 30-Sep-96 & & $10028-17-8$ & TRITIUM \\
\hline 2969 & SESPSCH & & SW & & & B0J8V8 & 30-Sep-96 & & 13966-29-5 & U-234 \\
\hline 2970 & SESPSCH & & SW & & & B0J8V8 & 30-Sep-96 & & 15117-96-1 & U-235 \\
\hline 2971 & SESPSCH & & SW & & & B0J8V8 & 30-Sep-96 & & U-238 & U-238 \\
\hline 2972 & PS HENRY & & SW & & & B0G1B8 & 30-Sep-96 & & $7440-47-3$ & $\mathrm{CR}$ \\
\hline 2973 & PS HENRY & & SW & & & B0G1B8 & 30-Sep-96 & & $56-23-5$ & CARBTET \\
\hline 2974 & SESPSCH & & SW & & & B0J8W0 & 30-Sep-96 & & $10098-97-2$ & SR-90 \\
\hline 2975 & SESPSCH & & SW & & & B0J8W0 & 30-Sep-96 & & $10028-17-8$ & TRITIUM \\
\hline 2976 & SESPSCH & & SW & & & B0J8W0 & 30-Sep-96 & & 13966-29-5 & U-234 \\
\hline 2977 & SESPSCH & & SW & & & B0J8W0 & 30-Sep-96 & & $15117-96-1$ & U-235 \\
\hline 2978 & SESPSCH & & SW & & & B0J8W0 & 30-Sep-96 & & $\mathrm{U}-238$ & $\mathrm{U}-238$ \\
\hline 2979 & PS HENRY & & SW & & & B0G1B9 & 30-Sep-96 & & $7440-47-3$ & CR \\
\hline 2980 & PS HENRY & & SW & & & B0G1B9 & 30-Sep-96 & & $56-23-5$ & CARBTET \\
\hline 2981 & SESPSCH & & SW & & & B0J8W1 & 30-Sep-96 & & $10098-97-2$ & SR-90 \\
\hline 2982 & SESPSCH & & SW & & & B0J8W1 & 30-Sep-96 & & $10028-17-8$ & TRITIUM \\
\hline 2983 & SESPSCH & & SW & & & B0J8W1 & 30-Sep-96 & & 13966-29-5 & U-234 \\
\hline 2984 & SESPSCH & & SW & & & B0J8W1 & 30-Sep-96 & & $15117-96-1$ & U-235 \\
\hline 2985 & SESPSCH & & SW & & & B0J8W1 & 30-Sep-96 & & U-238 & U-238 \\
\hline 2986 & PS HENRY & & SW & & & B0G1C0 & 30-Sep-96 & & $7440-47-3$ & $\mathrm{CR}$ \\
\hline 2987 & PS HENRY & & SW & & & B0G1C0 & 30-Sep-96 & & $56-23-5$ & CARBTET \\
\hline 2988 & SESPSCH & & SW & & & B0J8W2 & 30-Sep-96 & & $10098-97-2$ & SR-90 \\
\hline 2989 & SESPSCH & & SW & & & B0J8W2 & 30-Sep-96 & & $10028-17-8$ & TRITIUM \\
\hline 2990 & SESPSCH & & SW & & & B0J8W2 & 30-Sep-96 & & 13966-29-5 & U-234 \\
\hline 2991 & SESPSCH & & SW & & & B0J8W2 & 30-Sep-96 & & 15117-96-1 & U-235 \\
\hline 2992 & SESPSCH & & SW & & & B0J8W2 & 30-Sep-96 & & U-238 & U-238 \\
\hline 2993 & PS HENRY & & SW & & & B0G1C1 & 30-Sep-96 & & $7440-47-3$ & CR \\
\hline 2994 & PS HENRY & & SW & & & B0G1C1 & 30-Sep-96 & & $56-23-5$ & CARBTET \\
\hline 2995 & SESPSCH & & SW & & & B0J8W4 & 30-Sep-96 & & 10098-97-2 & SR-90 \\
\hline 2996 & SESPSCH & & SW & & & B0J8W4 & 30-Sep-96 & & $10028-17-8$ & TRITIUM \\
\hline 2997 & SESPSCH & & SW & & & B0J8W4 & 30-Sep-96 & & $13966-29-5$ & U-234 \\
\hline 2998 & SESPSCH & & SW & & & B0J8W4 & 30-Sep-96 & & 15117-96-1 & U-235 \\
\hline 2999 & SESPSCH & & SW & & & B0J8W4 & 30-Sep-96 & & U-238 & U-238 \\
\hline 3000 & PS HENRY & & SW & & & B0G1G3 & 30-Sep-96 & & $7440-47-3$ & $\mathrm{CR}$ \\
\hline 3001 & PS HENRY & & SW & & & B0G1G3 & 30-Sep-96 & & $56-23-5$ & CARBTET \\
\hline 3002 & SESPSCH & & SW & & & B0J8W5 & 30-Sep-96 & & $10098-97-2$ & SR-90 \\
\hline 3003 & SESPSCH & & SW & & & B0J8W5 & 30-Sep-96 & & $10028-17-8$ & TRITIUM \\
\hline
\end{tabular}




\begin{tabular}{|c|c|c|c|c|c|c|c|c|c|c|}
\hline & $\mathrm{A}$ & $B$ & $\mathrm{C}$ & $\mathrm{D}$ & $E$ & $\mathrm{~F}$ & $G$ & $\mathrm{H}$ & $I$ & $\mathrm{~J}$ \\
\hline 1 & SAMPLE NUM ASSIGNED TO & SAMPLER & MEDIA & BIOTA MEDIA & TAG ID & SAMP NUM & SAMP DATE TIME & SAMP DATE TIME ON & CON ID & CON SHORT NAME \\
\hline 3004 & SESPSCH & & SW & & & B0J8W5 & 30-Sep-96 & & $13966-29-5$ & U-234 \\
\hline 3005 & SESPSCH & & SW & & & B0J8W5 & 30-Sep-96 & & 15117-96-1 & U-235 \\
\hline 3006 & SESPSCH & & SW & & & B0J8W5 & 30-Sep-96 & & U-238 & U-238 \\
\hline 3007 & PS HENRY & & SW & & & B0G1H2 & 30-Sep-96 & & $7440-47-3$ & CR \\
\hline 3008 & PS HENRY & & SW & & & B0G1H2 & 30-Sep-96 & & $56-23-5$ & CARBTET \\
\hline 3009 & SESPSCH & & SW & & & B0J8X0 & 30-Sep-96 & & 10098-97-2 & SR-90 \\
\hline 3010 & SESPSCH & & SW & & & B0J8X0 & 30-Sep-96 & & $10028-17-8$ & TRITIUM \\
\hline 3011 & SESPSCH & & SW & & & B0J8X0 & 30-Sep-96 & & 13966-29-5 & U-234 \\
\hline 3012 & SESPSCH & & SW & & & BOJ8X0 & 30-Sep-96 & & 15117-96-1 & U-235 \\
\hline 3013 & SESPSCH & & SW & & & B0J8X0 & 30-Sep-96 & & U-238 & $\mathrm{U}-238$ \\
\hline 3014 & PS HENRY & & SW & & & B0G1G4 & 30-Sep-96 & & $7440-47-3$ & CR \\
\hline 3015 & PS HENRY & & SW & & & B0G1G4 & 30-Sep-96 & & $56-23-5$ & CARBTET \\
\hline 3016 & SESPSCH & & SW & & & B0J8W6 & 30-Sep-96 & & $10098-97-2$ & SR-90 \\
\hline 3017 & SESPSCH & & SW & & & B0J8W6 & 30-Sep-96 & & $10028-17-8$ & TRITIUM \\
\hline 3018 & SESPSCH & & SW & & & B0J8W6 & 30-Sep-96 & & $13966-29-5$ & U-234 \\
\hline 3019 & SESPSCH & & SW & & & B0J8W6 & 30-Sep-96 & & 15117-96-1 & U-235 \\
\hline 3020 & SESPSCH & & SW & & & B0J8W6 & 30-Sep-96 & & U-238 & U-238 \\
\hline 3021 & PS HENRY & & SW & & & B0G1G5 & 30-Sep-96 & & $7440-47-3$ & CR \\
\hline 3022 & PS HENRY & & SW & & & B0G1G5 & 30-Sep-96 & & $56-23-5$ & CARBTET \\
\hline 3023 & SESPSCH & & SW & & & B0J8X1 & 30-Sep-96 & & $10098-97-2$ & SR-90 \\
\hline 3024 & SESPSCH & & SW & & & B0J8X1 & 30-Sep-96 & & $10028-17-8$ & TRITIUM \\
\hline 3025 & SESPSCH & & SW & & & B0J8X1 & 30-Sep-96 & & $13966-29-5$ & U-234 \\
\hline 3026 & SESPSCH & & SW & & & B0J8X1 & 30-Sep-96 & & 15117-96-1 & U-235 \\
\hline 3027 & SESPSCH & & SW & & & B0J8X1 & 30-Sep-96 & & U-238 & U-238 \\
\hline 3028 & PS HENRY & & SW & & & B0G1G6 & 30-Sep-96 & & $7440-47-3$ & $\mathrm{CR}$ \\
\hline 3029 & PS HENRY & & SW & & & B0G1G6 & 30-Sep-96 & & $56-23-5$ & CARBTET \\
\hline 3030 & SESPSCH & & SW & & & B0J8T5 & 30-Sep-96 & & $10098-97-2$ & SR-90 \\
\hline 3031 & SESPSCH & & SW & & & B0J8T5 & 30-Sep-96 & & $10028-17-8$ & TRITIUM \\
\hline 3032 & SESPSCH & & SW & & & B0J8T5 & 30-Sep-96 & & 13966-29-5 & U-234 \\
\hline 3033 & SESPSCH & & SW & & & B0J8T5 & 30-Sep-96 & & 15117-96-1 & U-235 \\
\hline 3034 & SESPSCH & & SW & & & B0J8T5 & 30-Sep-96 & & U-238 & U-238 \\
\hline 3035 & PS HENRY & & SW & & & B0G1G7 & 30-Sep-96 & & $7440-47-3$ & $\mathrm{CR}$ \\
\hline 3036 & PS HENRY & & SW & & & B0G1G7 & 30-Sep-96 & & $56-23-5$ & CARBTET \\
\hline 3037 & SESPSCH & & SW & & & B0J8V2 & 30-Sep-96 & & 10098-97-2 & SR-90 \\
\hline 3038 & SESPSCH & & SW & & & B0J8V2 & 30-Sep-96 & & $10028-17-8$ & TRITIUM \\
\hline 3039 & SESPSCH & & SW & & & B0J8V2 & 30-Sep-96 & & 13966-29-5 & U-234 \\
\hline 3040 & SESPSCH & & SW & & & B0J8V2 & 30-Sep-96 & & $15117-96-1$ & U-235 \\
\hline 3041 & SESPSCH & & SW & & & B0J8V2 & 30-Sep-96 & & U-238 & U-238 \\
\hline
\end{tabular}

Page 80 


\begin{tabular}{|c|c|c|c|c|c|c|c|c|c|c|}
\hline & $\mathrm{A}$ & $B$ & $\mathrm{C}$ & $\mathrm{D}$ & $E$ & $\mathrm{~F}$ & $G$ & $\mathrm{H}$ & $\mathrm{I}$ & $\mathrm{J}$ \\
\hline 1 & SAMPLE_NUM_ASSIGNED_TO & SAMPLER & MEDIA & BIOTA_MEDIA & TAG_ID & SAMP_NUM & SAMP_DATE_TIME| & SAMP_DATE_TIME_ON & CON_ID & CON_SHORT_NAME \\
\hline 3042 & PS HENRY & & SW & & & B0G1G8 & 30-Sep-96 & & 7440-47-3 & CR \\
\hline 3043 & PS HENRY & & SW & & & B0G1G8 & 30-Sep-96 & & $56-23-5$ & CARBTET \\
\hline 3044 & SESPSCH & & SW & & & B0J8V3 & 30-Sep-96 & & $10098-97-2$ & SR-90 \\
\hline 3045 & SESPSCH & & SW & & & B0J8V3 & $30-$ Sep-96 & & $10028-17-8$ & TRITIUM \\
\hline 3046 & SESPSCH & & SW & & & B0J8V3 & 30-Sep-96 & & 13966-29-5 & U-234 \\
\hline 3047 & SESPSCH & & SW & & & B0J8V3 & 30-Sep-96 & & 15117-96-1 & U-235 \\
\hline 3048 & SESPSCH & & SW & & & B0J8V3 & 30-Sep-96 & & U-238 & U-238 \\
\hline 3049 & PS HENRY & & SW & & & B0G1G9 & 30-Sep-96 & & $7440-47-3$ & CR \\
\hline 3050 & PS HENRY & & SW & & & B0G1G9 & 30-Sep-96 & & $56-23-5$ & CARBTET \\
\hline 3051 & SESPSCH & & SW & & & B0J8W7 & 30-Sep-96 & & $10098-97-2$ & SR-90 \\
\hline 3052 & SESPSCH & & SW & & & B0J8W7 & 30-Sep-96 & & $10028-17-8$ & TRITIUM \\
\hline 3053 & SESPSCH & & SW & & & B0J8W7 & 30-Sep-96 & & 13966-29-5 & U-234 \\
\hline 3054 & SESPSCH & & SW & & & B0J8W7 & 30-Sep-96 & & 15117-96-1 & U-235 \\
\hline 3055 & SESPSCH & & SW & & & B0J8W7 & 30-Sep-96 & & U-238 & U-238 \\
\hline 3056 & PS HENRY & & SW & & & B0G1H0 & 30-Sep-96 & & $7440-47-3$ & CR \\
\hline 3057 & PS HENRY & & SW & & & B0G1H0 & 30-Sep-96 & & $56-23-5$ & CARBTET \\
\hline 3058 & SESPSCH & & SW & & & B0J8W8 & 30-Sep-96 & & $10098-97-2$ & SR-90 \\
\hline 3059 & SESPSCH & & SW & & & B0J8W8 & 30-Sep-96 & & $10028-17-8$ & TRITIUM \\
\hline 3060 & SESPSCH & & SW & & & B0J8W8 & 30-Sep-96 & & $13966-29-5$ & U-234 \\
\hline 3061 & SESPSCH & & SW & & & B0J8W8 & 30-Sep-96 & & 15117-96-1 & U-235 \\
\hline 3062 & SESPSCH & & SW & & & B0J8W8 & 30-Sep-96 & & U-238 & U-238 \\
\hline 3063 & PS HENRY & & SW & & & B0G1H1 & 30-Sep-96 & & $7440-47-3$ & CR \\
\hline 3064 & PS HENRY & & SW & & & B0G1H1 & 30-Sep-96 & & $56-23-5$ & CARBTET \\
\hline 3065 & SESPSCH & & SW & & & B0J8W9 & 30-Sep-96 & & $10098-97-2$ & SR-90 \\
\hline 3066 & SESPSCH & & SW & & & B0J8W9 & 30-Sep-96 & & 10028-17-8 & TRITIUM \\
\hline 3067 & SESPSCH & & SW & & & B0J8W9 & 30-Sep-96 & & $13966-29-5$ & U-234 \\
\hline 3068 & SESPSCH & & SW & & & B0J8W9 & 30-Sep-96 & & 15117-96-1 & U-235 \\
\hline 3069 & SESPSCH & & SW & & & B0J8W9 & 30-Sep-96 & & U-238 & U-238 \\
\hline 3070 & PS HENRY & & SW & & & B0G1L0 & 06-Dec-96 & & $7440-47-3$ & CR \\
\hline 3071 & PS HENRY & & SW & & & B0G1L0 & 06-Dec-96 & & $56-23-5$ & CARBTET \\
\hline 3072 & SESPSCH & & SW & & & BOJNO9 & 06-Dec-96 & & $10098-97-2$ & SR-90 \\
\hline 3073 & SESPSCH & & SW & & & B0JN09 & 06-Dec-96 & & $10028-17-8$ & TRITIUM \\
\hline 3074 & SESPSCH & & SW & & & B0JN09 & 06-Dec-96 & & 13966-29-5 & U-234 \\
\hline 3075 & SESPSCH & & SW & & & BOJNO9 & 06-Dec-96 & & $15117-96-1$ & U-235 \\
\hline 3076 & SESPSCH & & SW & & & BOJNO9 & 06-Dec-96 & & U-238 & U-238 \\
\hline 3077 & PS HENRY & & SW & & & B0G1L1 & 06-Dec-96 & & $7440-47-3$ & CR \\
\hline 3078 & PS HENRY & & SW & & & B0G1L1 & 06-Dec-96 & & $56-23-5$ & CARBTET \\
\hline 3079 & SESPSCH & & SW & & & B0JN10 & 06-Dec-96 & & 10098-97-2 & SR-90 \\
\hline
\end{tabular}




\begin{tabular}{|c|c|c|c|c|c|c|c|c|c|c|}
\hline & $\mathrm{A}$ & $\mathrm{B}$ & $\mathrm{C}$ & $\mathrm{D}$ & $\mathrm{E}$ & $\mathrm{F}$ & G & $\mathrm{H}$ & $\mathrm{I}$ & $\mathrm{J}$ \\
\hline 1 & SAMPLE_NUM_ASSIGNED_TO & SAMPLER & MEDIA & BIOTA_MEDIA & TAG_ID & SAMP_NUM & SAMP_DATE_TIME & SAMP_DATE_TIME_ON & CON_ID & CON_SHORT_NAME \\
\hline 3080 & SESPSCH & & SW & & & B0JN10 & 06-Dec-96 & & $10028-17-8$ & TRITIUM \\
\hline 3081 & SESPSCH & & SW & & & B0JN10 & 06-Dec-96 & & 13966-29-5 & U-234 \\
\hline 3082 & SESPSCH & & SW & & & B0JN10 & 06-Dec-96 & & 15117-96-1 & U-235 \\
\hline 3083 & SESPSCH & & SW & & & B0JN10 & 06-Dec-96 & & U-238 & U-238 \\
\hline 3084 & PS HENRY & & SW & & & B0G1L2 & 06-Dec-96 & & $7440-47-3$ & CR \\
\hline 3085 & PS HENRY & & SW & & & B0G1L2 & 06-Dec-96 & & $56-23-5$ & CARBTET \\
\hline 3086 & SESPSCH & & SW & & & B0JN11 & 06-Dec-96 & & $10098-97-2$ & SR-90 \\
\hline 3087 & SESPSCH & & SW & & & B0JN11 & 06-Dec-96 & & $10028-17-8$ & TRITIUM \\
\hline 3088 & SESPSCH & & SW & & & B0JN11 & 06-Dec-96 & & 13966-29-5 & U-234 \\
\hline 3089 & SESPSCH & & SW & & & B0JN11 & 06-Dec-96 & & 15117-96-1 & U-235 \\
\hline 3090 & SESPSCH & & SW & & & B0JN11 & 06-Dec-96 & & U-238 & U-238 \\
\hline 3091 & PS HENRY & & SW & & & B0G1L3 & 06-Dec-96 & & $7440-47-3$ & CR \\
\hline 3092 & PS HENRY & & SW & & & B0G1L3 & 06-Dec-96 & & $56-23-5$ & CARBTET \\
\hline 3093 & SESPSCH & & SW & & & B0JN12 & 06-Dec-96 & & $10098-97-2$ & SR-90 \\
\hline 3094 & SESPSCH & & SW & & & B0JN12 & 06-Dec-96 & & $10028-17-8$ & TRITIUM \\
\hline 3095 & SESPSCH & & SW & & & B0JN12 & 06-Dec-96 & & 13966-29-5 & U-234 \\
\hline 3096 & SESPSCH & & SW & & & B0JN12 & 06-Dec-96 & & 15117-96-1 & U-235 \\
\hline 3097 & SESPSCH & & SW & & & B0JN12 & 06-Dec-96 & & U-238 & U-238 \\
\hline 3098 & PS HENRY & & SW & & & B0G1K0 & 09-Dec-96 & & $7440-47-3$ & CR \\
\hline 3099 & PS HENRY & & SW & & & B0G1K0 & 09-Dec-96 & & $56-23-5$ & CARBTET \\
\hline 3100 & SESPSCH & & SW & & & B0JN13 & 09-Dec-96 & & $10098-97-2$ & SR-90 \\
\hline 3101 & SESPSCH & & SW & & & B0JN33 & 09-Dec-96 & & $10098-97-2$ & SR-90 \\
\hline 3102 & SESPSCH & & SW & & & B0JN13 & 09-Dec-96 & & $10028-17-8$ & TRITIUM \\
\hline 3103 & SESPSCH & & SW & & & B0JN33 & 09-Dec-96 & & $10028-17-8$ & TRITIUM \\
\hline 3104 & SESPSCH & & SW & & & B0JN13 & 09-Dec-96 & & 13966-29-5 & U-234 \\
\hline 3105 & SESPSCH & & SW & & & B0JN13 & 09-Dec-96 & & 15117-96-1 & U-235 \\
\hline 3106 & SESPSCH & & SW & & & B0JN13 & 09-Dec-96 & & U-238 & U-238 \\
\hline 3107 & SESPSCH & & SW & & & B0JN33 & 09-Dec-96 & & 13966-29-5 & U-234 \\
\hline 3108 & SESPSCH & & SW & & & B0JN33 & 09-Dec-96 & & 15117-96-1 & U-235 \\
\hline 3109 & SESPSCH & & SW & & & B0JN33 & 09-Dec-96 & & U-238 & U-238 \\
\hline 3110 & PS HENRY & & SW & & & B0G1K9 & 09-Dec-96 & & $7440-47-3$ & CR \\
\hline 3111 & PS HENRY & & SW & & & B0G1K9 & 09-Dec-96 & & $56-23-5$ & CARBTET \\
\hline 3112 & SESPSCH & & SW & & & B0JN22 & 09-Dec-96 & & $10098-97-2$ & SR-90 \\
\hline 3113 & SESPSCH & & SW & & & B0JN22 & 09-Dec-96 & & $10028-17-8$ & TRITIUM \\
\hline 3114 & SESPSCH & & SW & & & B0JN22 & 09-Dec-96 & & 13966-29-5 & U-234 \\
\hline 3115 & SESPSCH & & SW & & & B0JN22 & 09-Dec-96 & & 15117-96-1 & U-235 \\
\hline 3116 & SESPSCH & & SW & & & B0JN22 & 09-Dec-96 & & U-238 & U-238 \\
\hline 3117 & PS HENRY & & SW & & & B0G1K1 & 09-Dec-96 & & $7440-47-3$ & CR \\
\hline
\end{tabular}




\begin{tabular}{|c|c|c|c|c|c|c|c|c|c|c|}
\hline & A & $\mathrm{B}$ & C & $\mathrm{D}$ & $E$ & $\mathrm{~F}$ & G & $\mathrm{H}$ & $\mathrm{I}$ & $\mathrm{J}$ \\
\hline 1 & SAMPLE_NUM_ASSIGNED_TO & SAMPLER & MEDIA & BIOTA_MEDIA & TAG_ID & SAMP_NUM & SAMP_DATE_TIME & SAMP_DATE_TIME_ON & CON_ID & CON_SHORT_NAME \\
\hline 3118 & PS HENRY & & SW & & & B0G1K1 & 09-Dec-96 & & $56-23-5$ & CARBTET \\
\hline 3119 & SESPSCH & & SW & & & B0JN14 & 09-Dec-96 & & 10098-97-2 & SR-90 \\
\hline 3120 & SESPSCH & & SW & & & B0JN14 & 09-Dec-96 & & $10028-17-8$ & TRITIUM \\
\hline \begin{tabular}{|l|}
3121 \\
\end{tabular} & SESPSCH & & SW & & & B0JN14 & 09-Dec-96 & & 13966-29-5 & U-234 \\
\hline \begin{tabular}{|l|}
3122 \\
\end{tabular} & SESPSCH & & SW & & & B0JN14 & 09-Dec-96 & & $15117-96-1$ & U-235 \\
\hline 3123 & SESPSCH & & SW & & & B0JN14 & 09-Dec-96 & & U-238 & U-238 \\
\hline 3124 & PS HENRY & & SW & & & B0G1K2 & 09-Dec-96 & & $7440-47-3$ & $\mathrm{CR}$ \\
\hline 3125 & PS HENRY & & SW & & & B0G1K2 & 09-Dec-96 & & $56-23-5$ & CARBTET \\
\hline 3126 & SESPSCH & & SW & & & B0JN15 & 09-Dec-96 & & $10098-97-2$ & SR-90 \\
\hline 3127 & SESPSCH & & SW & & & B0JN15 & 09-Dec-96 & & $10028-17-8$ & TRITIUM \\
\hline 3128 & SESPSCH & & SW & & & B0JN15 & 09-Dec-96 & & $13966-29-5$ & U-234 \\
\hline 3129 & SESPSCH & & SW & & & B0JN15 & 09-Dec-96 & & 15117-96-1 & U-235 \\
\hline 3130 & SESPSCH & & SW & & & B0JN15 & 09-Dec-96 & & U-238 & U-238 \\
\hline 3131 & PS HENRY & & SW & & & B0G1K3 & 09-Dec-96 & & $7440-47-3$ & CR \\
\hline 3132 & PS HENRY & & SW & & & B0G1K3 & 09-Dec-96 & & $56-23-5$ & CARBTET \\
\hline \begin{tabular}{|l|}
3133 \\
\end{tabular} & SESPSCH & & SW & & & B0JN16 & 09-Dec-96 & & $10098-97-2$ & SR-90 \\
\hline \begin{tabular}{|l|}
3134 \\
\end{tabular} & SESPSCH & & SW & & & B0JN16 & 09-Dec-96 & & $10028-17-8$ & TRITIUM \\
\hline 3135 & SESPSCH & & SW & & & B0JN16 & 09-Dec-96 & & 13966-29-5 & U-234 \\
\hline 3136 & SESPSCH & & SW & & & B0JN16 & 09-Dec-96 & & $15117-96-1$ & U-235 \\
\hline \begin{tabular}{|l|}
3137 \\
\end{tabular} & SESPSCH & & SW & & & B0JN16 & 09-Dec-96 & & U-238 & U-238 \\
\hline 3138 & PS HENRY & & SW & & & B0G1K4 & 09-Dec-96 & & $7440-47-3$ & CR \\
\hline 3139 & PS HENRY & & SW & & & B0G1K4 & 09-Dec-96 & & $56-23-5$ & CARBTET \\
\hline 3140 & SESPSCH & & SW & & & B0JN17 & 09-Dec-96 & & $10098-97-2$ & SR-90 \\
\hline \begin{tabular}{|l|}
3141 \\
\end{tabular} & SESPSCH & & SW & & & B0JN17 & 09-Dec-96 & & $10028-17-8$ & TRITIUM \\
\hline 3142 & SESPSCH & & SW & & & B0JN17 & 09-Dec-96 & & $13966-29-5$ & U-234 \\
\hline 3143 & SESPSCH & & SW & & & B0JN17 & 09-Dec-96 & & 15117-96-1 & U-235 \\
\hline 3144 & SESPSCH & & SW & & & B0JN17 & 09-Dec-96 & & U-238 & U-238 \\
\hline 3145 & PS HENRY & & SW & & & B0G1K5 & 09-Dec-96 & & $7440-47-3$ & CR \\
\hline 3146 & PS HENRY & & SW & & & B0G1K5 & 09-Dec-96 & & $56-23-5$ & CARBTET \\
\hline \begin{tabular}{|l|}
3147 \\
\end{tabular} & SESPSCH & & SW & & & B0JN18 & 09-Dec-96 & & $10098-97-2$ & SR-90 \\
\hline 3148 & SESPSCH & & SW & & & B0JN18 & 09-Dec-96 & & $10028-17-8$ & TRITIUM \\
\hline \begin{tabular}{|l|}
3149 \\
\end{tabular} & SESPSCH & & SW & & & B0JN18 & 09-Dec-96 & & $13966-29-5$ & U-234 \\
\hline \begin{tabular}{|l|}
3150 \\
\end{tabular} & SESPSCH & & SW & & & B0JN18 & 09-Dec-96 & & $15117-96-1$ & U-235 \\
\hline 3151 & SESPSCH & & SW & & & B0JN18 & 09-Dec-96 & & U-238 & U-238 \\
\hline 3152 & PS HENRY & & SW & & & B0G1K6 & 09-Dec-96 & & $7440-47-3$ & CR \\
\hline \begin{tabular}{|l|}
3153 \\
\end{tabular} & PS HENRY & & SW & & & B0G1K6 & 09-Dec-96 & & $56-23-5$ & CARBTET \\
\hline 3154 & SESPSCH & & SW & & & B0JN19 & 09-Dec-96 & & $10098-97-2$ & SR-90 \\
\hline 3155 & SESPSCH & & SW & & & B0JN19 & 09-Dec-96 & & $10028-17-8$ & TRITIUM \\
\hline
\end{tabular}




\begin{tabular}{|c|c|c|c|c|c|c|c|c|c|c|}
\hline & $\mathrm{A}$ & $\mathrm{B}$ & $\mathrm{C}$ & $\mathrm{D}$ & $\mathrm{E}$ & $\mathrm{F}$ & G & $\mathrm{H}$ & $\mathrm{I}$ & $\mathrm{J}$ \\
\hline 1 & SAMPLE_NUM_ASSIGNED_TO & SAMPLER & MEDIA & BIOTA_MEDIA & TAG_ID & SAMP_NUM & SAMP_DATE_TIME & SAMP_DATE_TIME_ON & CON_ID & CON_SHORT_NAME \\
\hline 3156 & SESPSCH & & SW & & & B0JN19 & 09-Dec-96 & & $13966-29-5$ & U-234 \\
\hline 3157 & SESPSCH & & SW & & & B0JN19 & 09-Dec-96 & & 15117-96-1 & U-235 \\
\hline 3158 & SESPSCH & & SW & & & B0JN19 & 09-Dec-96 & & U-238 & U-238 \\
\hline 3159 & PS HENRY & & SW & & & B0G1K7 & 09-Dec-96 & & $7440-47-3$ & CR \\
\hline 3160 & PS HENRY & & SW & & & B0G1K7 & 09-Dec-96 & & $56-23-5$ & CARBTET \\
\hline 3161 & SESPSCH & & SW & & & BOJN20 & 09-Dec-96 & & $10098-97-2$ & SR-90 \\
\hline 3162 & SESPSCH & & SW & & & B0JN20 & 09-Dec-96 & & $10028-17-8$ & TRITIUM \\
\hline 3163 & SESPSCH & & SW & & & BOJN20 & 09-Dec-96 & & $13966-29-5$ & U-234 \\
\hline 3164 & SESPSCH & & SW & & & BOJN20 & 09-Dec-96 & & 15117-96-1 & U-235 \\
\hline 3165 & SESPSCH & & SW & & & B0JN20 & 09-Dec-96 & & U-238 & U-238 \\
\hline 3166 & PS HENRY & & SW & & & B0G1K8 & 09-Dec-96 & & $7440-47-3$ & CR \\
\hline 3167 & PS HENRY & & SW & & & B0G1K8 & 09-Dec-96 & & 56-23-5 & CARBTET \\
\hline 3168 & SESPSCH & & SW & & & B0JN21 & 09-Dec-96 & & 10098-97-2 & SR-90 \\
\hline 3169 & SESPSCH & & SW & & & B0JN21 & 09-Dec-96 & & $10028-17-8$ & TRITIUM \\
\hline 3170 & SESPSCH & & SW & & & B0JN21 & 09-Dec-96 & & 13966-29-5 & U-234 \\
\hline 3171 & SESPSCH & & SW & & & B0JN21 & 09-Dec-96 & & 15117-96-1 & U-235 \\
\hline 3172 & SESPSCH & & SW & & & B0JN21 & 09-Dec-96 & & U-238 & U-238 \\
\hline 3173 & SESPSCH & & SW & & & B0K261 & 31-Mar-97 & & $10098-97-2$ & SR-90 \\
\hline 3174 & SESPSCH & & SW & & & B0K261 & 31-Mar-97 & & $10028-17-8$ & TRITIUM \\
\hline 3175 & SESPSCH & & SW & & & B0K261 & 31-Mar-97 & & $13966-29-5$ & U-234 \\
\hline 3176 & SESPSCH & & SW & & & B0K261 & 31-Mar-97 & & 15117-96-1 & U-235 \\
\hline 3177 & SESPSCH & & SW & & & B0K261 & 31-Mar-97 & & $\mathrm{U}-238$ & U-238 \\
\hline 3178 & SESPSCH & & SW & & & B0K262 & 31-Mar-97 & & 10098-97-2 & SR-90 \\
\hline 3179 & SESPSCH & & SW & & & B0K262 & 31-Mar-97 & & $10028-17-8$ & TRITIUM \\
\hline 3180 & SESPSCH & & SW & & & B0K262 & 31-Mar-97 & & $13966-29-5$ & U-234 \\
\hline 3181 & SESPSCH & & SW & & & B0K262 & 31-Mar-97 & & $15117-96-1$ & U-235 \\
\hline 3182 & SESPSCH & & SW & & & B0K262 & 31-Mar-97 & & U-238 & U-238 \\
\hline 3183 & SESPSCH & & SW & & & B0K263 & 31-Mar-97 & & $10098-97-2$ & SR-90 \\
\hline 3184 & SESPSCH & & SW & & & B0K263 & 31-Mar-97 & & $10028-17-8$ & TRITIUM \\
\hline 3185 & SESPSCH & & SW & & & B0K263 & 31-Mar-97 & & $13966-29-5$ & U-234 \\
\hline 3186 & SESPSCH & & SW & & & B0K263 & 31-Mar-97 & & 15117-96-1 & U-235 \\
\hline 3187 & SESPSCH & & SW & & & B0K263 & 31-Mar-97 & & U-238 & U-238 \\
\hline 3188 & SESPSCH & & SW & & & B0K264 & 31-Mar-97 & & $10098-97-2$ & SR-90 \\
\hline 3189 & SESPSCH & & SW & & & B0K264 & 31-Mar-97 & & $10028-17-8$ & TRITIUM \\
\hline 3190 & SESPSCH & & SW & & & B0K264 & 31-Mar-97 & & 13966-29-5 & U-234 \\
\hline 3191 & SESPSCH & & SW & & & B0K264 & 31-Mar-97 & & 15117-96-1 & U-235 \\
\hline 3192 & SESPSCH & & SW & & & B0K264 & 31-Mar-97 & & U-238 & U-238 \\
\hline 3193 & SESPSCH & & SW & & & B0K265 & 01-Apr-97 & & 10098-97-2 & SR-90 \\
\hline
\end{tabular}

Page 84 


\begin{tabular}{|c|c|c|c|c|c|c|c|c|c|c|}
\hline & $\mathrm{A}$ & $\mathrm{B}$ & $\mathrm{C}$ & $\mathrm{D}$ & $\mathrm{E}$ & $\mathrm{F}$ & G & $\mathrm{H}$ & $\mathrm{I}$ & $\mathrm{J}$ \\
\hline 1 & SAMPLE_NUM_ASSIGNED_TO & SAMPLER & MEDIA & BIOTA_MEDIA & TAG_ID & SAMP_NUM & SAMP_DATE_TIME & SAMP_DATE_TIME_ON & CON_ID & CON_SHORT_NAME \\
\hline 3194 & SESPSCH & & SW & & & BOK265 & 01-Apr-97 & & $10028-17-8$ & TRITIUM \\
\hline 3195 & SESPSCH & & SW & & & B0K265 & 01-Apr-97 & & $13966-29-5$ & U-234 \\
\hline 3196 & SESPSCH & & SW & & & B0K265 & 01-Apr-97 & & 15117-96-1 & U-235 \\
\hline 3197 & SESPSCH & & SW & & & B0K265 & 01-Apr-97 & & U-238 & U-238 \\
\hline 3198 & SESPSCH & & SW & & & B0K273 & 01-Apr-97 & & $10098-97-2$ & SR-90 \\
\hline 3199 & SESPSCH & & SW & & & B0K273 & 01-Apr-97 & & $10028-17-8$ & TRITIUM \\
\hline 3200 & SESPSCH & & SW & & & B0K273 & 01-Apr-97 & & $13966-29-5$ & U-234 \\
\hline 3201 & SESPSCH & & SW & & & B0K273 & 01-Apr-97 & & 15117-96-1 & U-235 \\
\hline 3202 & SESPSCH & & SW & & & B0K273 & 01-Apr-97 & & U-238 & U-238 \\
\hline 3203 & SESPSCH & & SW & & & B0K285 & 01-Apr-97 & & $10098-97-2$ & SR-90 \\
\hline 3204 & SESPSCH & & SW & & & B0K285 & 01-Apr-97 & & $10028-17-8$ & TRITIUM \\
\hline 3205 & SESPSCH & & SW & & & B0K285 & 01-Apr-97 & & $13966-29-5$ & U-234 \\
\hline 3206 & SESPSCH & & SW & & & B0K285 & 01-Apr-97 & & $15117-96-1$ & U-235 \\
\hline 3207 & SESPSCH & & SW & & & B0K285 & 01-Apr-97 & & U-238 & U-238 \\
\hline 3208 & SESPSCH & & SW & & & B0K266 & 01-Apr-97 & & $10098-97-2$ & SR-90 \\
\hline 3209 & SESPSCH & & SW & & & B0K266 & 01-Apr-97 & & $10028-17-8$ & TRITIUM \\
\hline 3210 & SESPSCH & & SW & & & B0K266 & 01-Apr-97 & & 13966-29-5 & U-234 \\
\hline 3211 & SESPSCH & & SW & & & B0K266 & 01-Apr-97 & & 15117-96-1 & U-235 \\
\hline 3212 & SESPSCH & & SW & & & B0K266 & 01-Apr-97 & & U-238 & U-238 \\
\hline 3213 & SESPSCH & & SW & & & B0K267 & 01-Apr-97 & & $10098-97-2$ & SR-90 \\
\hline 3214 & SESPSCH & & SW & & & B0K267 & 01-Apr-97 & & $10028-17-8$ & TRITIUM \\
\hline 3215 & SESPSCH & & SW & & & B0K267 & 01-Apr-97 & & 13966-29-5 & U-234 \\
\hline 3216 & SESPSCH & & SW & & & B0K267 & 01-Apr-97 & & $15117-96-1$ & U-235 \\
\hline 3217 & SESPSCH & & SW & & & B0K267 & 01-Apr-97 & & U-238 & U-238 \\
\hline 3218 & SESPSCH & & SW & & & B0K268 & 01-Apr-97 & & $10098-97-2$ & SR-90 \\
\hline 3219 & SESPSCH & & SW & & & B0K268 & 01-Apr-97 & & $10028-17-8$ & TRITIUM \\
\hline 3220 & SESPSCH & & SW & & & B0K268 & 01-Apr-97 & & 13966-29-5 & U-234 \\
\hline 3221 & SESPSCH & & SW & & & B0K268 & 01-Apr-97 & & 15117-96-1 & U-235 \\
\hline 3222 & SESPSCH & & SW & & & B0K268 & 01-Apr-97 & & U-238 & U-238 \\
\hline 3223 & SESPSCH & & SW & & & B0K269 & 01-Apr-97 & & $10098-97-2$ & SR-90 \\
\hline 3224 & SESPSCH & & SW & & & B0K269 & 01-Apr-97 & & $10028-17-8$ & TRITIUM \\
\hline 3225 & SESPSCH & & SW & & & BOK269 & 01-Apr-97 & & 13966-29-5 & U-234 \\
\hline 3226 & SESPSCH & & SW & & & BOK269 & 01-Apr-97 & & 15117-96-1 & U-235 \\
\hline 3227 & SESPSCH & & SW & & & B0K269 & 01-Apr-97 & & U-238 & U-238 \\
\hline 3228 & SESPSCH & & SW & & & BOK270 & 01-Apr-97 & & $10098-97-2$ & SR-90 \\
\hline 3229 & SESPSCH & & SW & & & B0K270 & 01-Apr-97 & & $10028-17-8$ & TRITIUM \\
\hline 3230 & SESPSCH & & SW & & & B0K270 & 01-Apr-97 & & $13966-29-5$ & U-234 \\
\hline 3231 & SESPSCH & & SW & & & B0K270 & 01-Apr-97 & & 15117-96-1 & U-235 \\
\hline
\end{tabular}




\begin{tabular}{|c|c|c|c|c|c|c|c|c|c|c|}
\hline & $\mathrm{A}$ & $B$ & $C$ & $\mathrm{D}$ & $E$ & $\mathrm{~F}$ & $G$ & $\mathrm{H}$ & $I$ & $\mathrm{~J}$ \\
\hline 1 & SAMPLE_NUM_ASSIGNED_TO & SAMPLER & MEDIA & BIOTA_MEDIA & TAG_ID & SAMP_NUM & SAMP_DATE_TIME| & SAMP_DATE_TIME_ON & CON_ID & CON_SHORT_NAME \\
\hline 3232 & SESPSCH & & SW & & & BOK270 & 01-Apr-97 & & $\mathrm{U}-238$ & $\mathrm{U}-238$ \\
\hline 3233 & SESPSCH & & SW & & & B0K271 & 01-Apr-97 & & $10098-97-2$ & SR-90 \\
\hline 3234 & SESPSCH & & SW & & & B0K271 & 01-Apr-97 & & $10028-17-8$ & TRITIUM \\
\hline 3235 & SESPSCH & & SW & & & B0K271 & 01-Apr-97 & & $13966-29-5$ & U-234 \\
\hline 3236 & SESPSCH & & SW & & & B0K271 & 01-Apr-97 & & 15117-96-1 & U-235 \\
\hline 3237 & SESPSCH & & SW & & & B0K271 & 01-Apr-97 & & U-238 & $\mathrm{U}-238$ \\
\hline 3238 & SESPSCH & & SW & & & B0K272 & 01-Apr-97 & & $10098-97-2$ & SR-90 \\
\hline 3239 & SESPSCH & & SW & & & B0K272 & 01-Apr-97 & & $10028-17-8$ & TRITIUM \\
\hline 3240 & SESPSCH & & SW & & & B0K272 & 01-Apr-97 & & 13966-29-5 & U-234 \\
\hline 3241 & SESPSCH & & SW & & & B0K272 & 01-Apr-97 & & 15117-96-1 & U-235 \\
\hline 3242 & SESPSCH & & SW & & & B0K272 & 01-Apr-97 & & U-238 & U-238 \\
\hline 3243 & SESPSCH & & SW & & & B0LVS1 & 22-Aug-97 & & 10098-97-2 & SR-90 \\
\hline 3244 & SESPSCH & & SW & & & BOLVY6 & 22-Aug-97 & & $10098-97-2$ & SR-90 \\
\hline 3245 & SESPSCH & & SW & & & BOLVS1 & 22-Aug-97 & & $10028-17-8$ & TRITIUM \\
\hline 3246 & SESPSCH & & SW & & & BOLVY6 & 22-Aug-97 & & $10028-17-8$ & TRITIUM \\
\hline 3247 & SESPSCH & & SW & & & B0LVS1 & 22-Aug-97 & & $13966-29-5$ & U-234 \\
\hline 3248 & SESPSCH & & SW & & & B0LVS1 & 22-Aug-97 & & 15117-96-1 & U-235 \\
\hline 3249 & SESPSCH & & SW & & & B0LVS1 & 22-Aug-97 & & U-238 & U-238 \\
\hline 3250 & SESPSCH & & SW & & & B0LVY6 & 22-Aug-97 & & $13966-29-5$ & U-234 \\
\hline 3251 & SESPSCH & & SW & & & BOLVY6 & 22-Aug-97 & & 15117-96-1 & U-235 \\
\hline 3252 & SESPSCH & & SW & & & BOLVY6 & 22-Aug-97 & & U-238 & U-238 \\
\hline 3253 & SESPSCH & & SW & & & B0LVS3 & 22-Aug-97 & & 10098-97-2 & SR-90 \\
\hline 3254 & SESPSCH & & SW & & & BOLVS3 & 22-Aug-97 & & $10028-17-8$ & TRITIUM \\
\hline 3255 & SESPSCH & & SW & & & BOLVS3 & 22-Aug-97 & & $13966-29-5$ & U-234 \\
\hline 3256 & SESPSCH & & SW & & & BOLVS3 & 22-Aug-97 & & 15117-96-1 & U-235 \\
\hline 3257 & SESPSCH & & SW & & & BOLVS3 & 22-Aug-97 & & U-238 & U-238 \\
\hline 3258 & SESPSCH & & SW & & & SOLVX8 & 22-Aug-97 & & $10098-97-2$ & SR-90 \\
\hline 3259 & SESPSCH & & SW & & & SOLVX8 & 22-Aug-97 & & $10028-17-8$ & TRITIUM \\
\hline 3260 & SESPSCH & & SW & & & SOLVX8 & 22-Aug-97 & & $13966-29-5$ & U-234 \\
\hline 3261 & SESPSCH & & SW & & & SOLVX8 & 22-Aug-97 & & 15117-96-1 & U-235 \\
\hline 3262 & SESPSCH & & SW & & & SOLVX8 & 22-Aug-97 & & U-238 & U-238 \\
\hline 3263 & SESPSCH & & SW & & & SOLVYO & 22-Aug-97 & & $10098-97-2$ & SR-90 \\
\hline 3264 & SESPSCH & & SW & & & SOLVYO & 22-Aug-97 & & $10028-17-8$ & TRITIUM \\
\hline 3265 & SESPSCH & & SW & & & SOLVYO & 22-Aug-97 & & 13966-29-5 & U-234 \\
\hline 3266 & SESPSCH & & SW & & & SOLVYO & 22-Aug-97 & & 15117-96-1 & U-235 \\
\hline 3267 & SESPSCH & & SW & & & SOLVYO & 22-Aug-97 & & U-238 & U-238 \\
\hline 3268 & SESPSCH & & SW & & & B0LVR7 & 22-Aug-97 & & 10098-97-2 & SR-90 \\
\hline 3269 & SESPSCH & & SW & & & BOLVR7 & 22-Aug-97 & & $10028-17-8$ & TRITIUM \\
\hline
\end{tabular}




\begin{tabular}{|c|c|c|c|c|c|c|c|c|c|c|}
\hline & A & $\mathrm{B}$ & $\mathrm{C}$ & $\mathrm{D}$ & $E$ & $\mathrm{~F}$ & G & $\mathrm{H}$ & $\mathrm{I}$ & $\mathrm{J}$ \\
\hline 1 & SAMPLE_NUM_ASSIGNED_TO & SAMPLER & MEDIA & BIOTA_MEDIA & TAG_ID & SAMP_NUM & SAMP_DATE_TIME & SAMP_DATE_TIME_ON & CON_ID & CON_SHORT_NAME \\
\hline 3270 & SESPSCH & & SW & & & BOLVR7 & 22-Aug-97 & & 13966-29-5 & U-234 \\
\hline 3271 & SESPSCH & & SW & & & BOLVR7 & 22-Aug-97 & & 15117-96-1 & U-235 \\
\hline \begin{tabular}{|l|}
3272 \\
\end{tabular} & SESPSCH & & SW & & & BOLVR7 & 22-Aug-97 & & U-238 & U-238 \\
\hline \begin{tabular}{|l|}
3273 \\
\end{tabular} & SESPSCH & & SW & & & BOLVR8 & 22-Aug-97 & & $10098-97-2$ & SR-90 \\
\hline 3274 & SESPSCH & & SW & & & BOLVR8 & 22-Aug-97 & & $10028-17-8$ & TRITIUM \\
\hline 3275 & SESPSCH & & SW & & & BOLVR8 & 22-Aug-97 & & 13966-29-5 & U-234 \\
\hline 3276 & SESPSCH & & SW & & & BOLVR8 & 22-Aug-97 & & $15117-96-1$ & $\mathrm{U}-235$ \\
\hline 3277 & SESPSCH & & SW & & & BOLVR8 & 22-Aug-97 & & $U-238$ & $\mathrm{U}-238$ \\
\hline 3278 & SESPSCH & & SW & & & BOLVR9 & 22-Aug-97 & & $10098-97-2$ & SR-90 \\
\hline 3279 & SESPSCH & & SW & & & BOLVR9 & 22-Aug-97 & & $10028-17-8$ & TRITIUM \\
\hline 3280 & SESPSCH & & SW & & & BOLVR9 & 22-Aug-97 & & $13966-29-5$ & U-234 \\
\hline 3281 & SESPSCH & & SW & & & BOLVR9 & 22-Aug-97 & & 15117-96-1 & U-235 \\
\hline 3282 & SESPSCH & & SW & & & BOLVR9 & 22-Aug-97 & & U-238 & U-238 \\
\hline 3283 & SESPSCH & & SW & & & BOLVSO & 22-Aug-97 & & $10098-97-2$ & SR-90 \\
\hline 3284 & SESPSCH & & SW & & & BOLVSO & 22-Aug-97 & & $10028-17-8$ & TRITIUM \\
\hline \begin{tabular}{|l}
3285 \\
\end{tabular} & SESPSCH & & SW & & & BOLVSO & 22-Aug-97 & & $13966-29-5$ & U-234 \\
\hline \begin{tabular}{|l|}
3286 \\
\end{tabular} & SESPSCH & & SW & & & BOLVSO & 22-Aug-97 & & $15117-96-1$ & U-235 \\
\hline 3287 & SESPSCH & & SW & & & BOLVSO & 22-Aug-97 & & U-238 & U-238 \\
\hline \begin{tabular}{|l|}
3288 \\
\end{tabular} & SESPSCH & & SW & & & BOLVS8 & 23-Aug-97 & & $10098-97-2$ & SR-90 \\
\hline \begin{tabular}{|l|}
3289 \\
\end{tabular} & SESPSCH & & SW & & & BOLVS8 & 23-Aug-97 & & $10028-17-8$ & TRITIUM \\
\hline 3290 & SESPSCH & & SW & & & BOLVS8 & 23-Aug-97 & & 13966-29-5 & U-234 \\
\hline 3291 & SESPSCH & & SW & & & BOLVS8 & 23-Aug-97 & & $15117-96-1$ & U-235 \\
\hline \begin{tabular}{|l|}
3292 \\
\end{tabular} & SESPSCH & & SW & & & BOLVS8 & 23-Aug-97 & & $\mathrm{U}-238$ & U-238 \\
\hline 3293 & SESPSCH & & SW & & & BOLVT8 & 23-Aug-97 & & $10098-97-2$ & SR-90 \\
\hline 3294 & SESPSCH & & SW & & & BOLVT8 & 23-Aug-97 & & $10028-17-8$ & TRITIUM \\
\hline 3295 & SESPSCH & & SW & & & BOLVT8 & 23-Aug-97 & & $13966-29-5$ & U-234 \\
\hline 3296 & SESPSCH & & SW & & & BOLVT8 & 23-Aug-97 & & $15117-96-1$ & U-235 \\
\hline 3297 & SESPSCH & & SW & & & BOLVT8 & 23-Aug-97 & & U-238 & U-238 \\
\hline \begin{tabular}{|l|}
3298 \\
\end{tabular} & SESPSCH & & SW & & & BOLVT2 & 23-Aug-97 & & $10098-97-2$ & SR-90 \\
\hline \begin{tabular}{|l|}
3299 \\
\end{tabular} & SESPSCH & & SW & & & BOLVT2 & 23-Aug-97 & & $10028-17-8$ & TRITIUM \\
\hline 3300 & SESPSCH & & SW & & & BOLVT2 & 23-Aug-97 & & $13966-29-5$ & U-234 \\
\hline \begin{tabular}{|l|}
3301 \\
\end{tabular} & SESPSCH & & SW & & & BOLVT2 & 23-Aug-97 & & $15117-96-1$ & U-235 \\
\hline \begin{tabular}{|l|}
3302 \\
\end{tabular} & SESPSCH & & SW & & & BOLVT2 & 23-Aug-97 & & U-238 & U-238 \\
\hline 3303 & SESPSCH & & SW & & & BOLVS9 & 23-Aug-97 & & $10098-97-2$ & SR-90 \\
\hline \begin{tabular}{|l|}
3304 \\
\end{tabular} & SESPSCH & & SW & & & BOLVS9 & 23-Aug-97 & & $10028-17-8$ & TRITIUM \\
\hline \begin{tabular}{|l|}
3305 \\
\end{tabular} & SESPSCH & & SW & & & BOLVS9 & 23-Aug-97 & & $13966-29-5$ & U-234 \\
\hline 3306 & SESPSCH & & SW & & & BOLVS9 & 23-Aug-97 & & $15117-96-1$ & U-235 \\
\hline \begin{tabular}{|l|}
3307 \\
\end{tabular} & SESPSCH & & SW & & & BOLVS9 & 23-Aug-97 & & U-238 & U-238 \\
\hline
\end{tabular}




\begin{tabular}{|c|c|c|c|c|c|c|c|c|c|c|}
\hline & $\mathrm{A}$ & $B$ & $C$ & $\mathrm{D}$ & $E$ & $\mathrm{~F}$ & $G$ & $\mathrm{H}$ & $I$ & $\mathrm{~J}$ \\
\hline 1 & SAMPLE_NUM_ASSIGNED_TO & SAMPLER & MEDIA & BIOTA_MEDIA & TAG_ID & SAMP_NUM & SAMP_DATE_TIME| & SAMP_DATE_TIME_ON & CON_ID & CON_SHORT_NAME \\
\hline 3308 & SESPSCH & & SW & & & BOLVT3 & 23-Aug-97 & & $10098-97-2$ & SR-90 \\
\hline 3309 & SESPSCH & & SW & & & BOLVT3 & 23-Aug-97 & & $10028-17-8$ & TRITIUM \\
\hline 3310 & SESPSCH & & SW & & & BOLVT3 & 23-Aug-97 & & $13966-29-5$ & U-234 \\
\hline 3311 & SESPSCH & & SW & & & BOLVT3 & 23-Aug-97 & & 15117-96-1 & U-235 \\
\hline 3312 & SESPSCH & & SW & & & BOLVT3 & 23-Aug-97 & & U-238 & U-238 \\
\hline 3313 & SESPSCH & & SW & & & BOLVT4 & 23-Aug-97 & & 10098-97-2 & SR-90 \\
\hline 3314 & SESPSCH & & SW & & & BOLVT4 & 23-Aug-97 & & $10028-17-8$ & TRITIUM \\
\hline 3315 & SESPSCH & & SW & & & BOLVT4 & 23-Aug-97 & & 13966-29-5 & U-234 \\
\hline 3316 & SESPSCH & & SW & & & BOLVT4 & 23-Aug-97 & & 15117-96-1 & U-235 \\
\hline 3317 & SESPSCH & & SW & & & BOLVT4 & 23-Aug-97 & & U-238 & U-238 \\
\hline 3318 & SESPSCH & & SW & & & B0LVT5 & 23-Aug-97 & & $10098-97-2$ & SR-90 \\
\hline 3319 & SESPSCH & & SW & & & BOLVT5 & 23-Aug-97 & & $10028-17-8$ & TRITIUM \\
\hline 3320 & SESPSCH & & SW & & & BOLVT5 & 23-Aug-97 & & 13966-29-5 & U-234 \\
\hline 3321 & SESPSCH & & SW & & & B0LVT5 & 23-Aug-97 & & 15117-96-1 & U-235 \\
\hline 3322 & SESPSCH & & SW & & & B0LVT5 & 23-Aug-97 & & U-238 & U-238 \\
\hline 3323 & SESPSCH & & SW & & & BOLVT6 & 23-Aug-97 & & $10098-97-2$ & SR-90 \\
\hline 3324 & SESPSCH & & SW & & & BOLVT6 & 23-Aug-97 & & $10028-17-8$ & TRITIUM \\
\hline 3325 & SESPSCH & & SW & & & BOLVT6 & 23-Aug-97 & & 13966-29-5 & U-234 \\
\hline 3326 & SESPSCH & & SW & & & BOLVT6 & 23-Aug-97 & & 15117-96-1 & U-235 \\
\hline 3327 & SESPSCH & & SW & & & BOLVT6 & 23-Aug-97 & & U-238 & U-238 \\
\hline 3328 & SESPSCH & & SW & & & BOLVT7 & 23-Aug-97 & & $10098-97-2$ & SR-90 \\
\hline 3329 & SESPSCH & & SW & & & BOLVT7 & 23-Aug-97 & & $10028-17-8$ & TRITIUM \\
\hline 3330 & SESPSCH & & SW & & & BOLVT7 & 23-Aug-97 & & 13966-29-5 & U-234 \\
\hline 3331 & SESPSCH & & SW & & & BOLVT7 & 23-Aug-97 & & $15117-96-1$ & U-235 \\
\hline 3332 & SESPSCH & & SW & & & BOLVT7 & 23-Aug-97 & & $\mathrm{U}-238$ & U-238 \\
\hline 3333 & SESPSCH & & SW & & & BOLVT9 & 23-Aug-97 & & $10098-97-2$ & SR-90 \\
\hline 3334 & SESPSCH & & SW & & & BOLVT9 & 23-Aug-97 & & $10028-17-8$ & TRITIUM \\
\hline 3335 & SESPSCH & & SW & & & BOLVT9 & 23-Aug-97 & & 13966-29-5 & U-234 \\
\hline 3336 & SESPSCH & & SW & & & BOLVT9 & 23-Aug-97 & & 15117-96-1 & U-235 \\
\hline 3337 & SESPSCH & & SW & & & BOLVT9 & 23-Aug-97 & & U-238 & U-238 \\
\hline 3338 & SESPSCH & & SW & & & BOLVVO & 23-Aug-97 & & 10098-97-2 & SR-90 \\
\hline 3339 & SESPSCH & & SW & & & BOLVVO & 23-Aug-97 & & $10028-17-8$ & TRITIUM \\
\hline 3340 & SESPSCH & & SW & & & BOLVVO & 23-Aug-97 & & 13966-29-5 & U-234 \\
\hline 3341 & SESPSCH & & SW & & & BOLVV0 & 23-Aug-97 & & 15117-96-1 & U-235 \\
\hline 3342 & SESPSCH & & SW & & & BOLVV0 & 23-Aug-97 & & U-238 & U-238 \\
\hline 3343 & SESPSCH & & SW & & & B0LVV5 & 23-Aug-97 & & $10098-97-2$ & SR-90 \\
\hline 3344 & SESPSCH & & SW & & & B0LVV5 & 23-Aug-97 & & $10028-17-8$ & TRITIUM \\
\hline 3345 & SESPSCH & & SW & & & B0LVV5 & 23-Aug-97 & & 13966-29-5 & U-234 \\
\hline
\end{tabular}




\begin{tabular}{|c|c|c|c|c|c|c|c|c|c|c|}
\hline & $\mathrm{A}$ & $B$ & $\mathrm{C}$ & $\mathrm{D}$ & $E$ & $\mathrm{~F}$ & $G$ & $\mathrm{H}$ & $I$ & $\mathrm{~J}$ \\
\hline 1 & SAMPLE NUM ASSIGNED TO & SAMPLER & MEDIA & BIOTA MEDIA & TAG ID & SAMP NUM & SAMP DATE TIME & SAMP DATE TIME ON & CON ID & CON SHORT NAME \\
\hline 3346 & SESPSCH & & SW & & & B0LVV5 & 23-Aug-97 & & 15117-96-1 & U-235 \\
\hline 3347 & SESPSCH & & SW & & & BOLVV5 & 23-Aug-97 & & U-238 & U-238 \\
\hline 3348 & SESPSCH & & SW & & & BOLVV1 & 23-Aug-97 & & $10098-97-2$ & SR-90 \\
\hline 3349 & SESPSCH & & SW & & & BOLVV1 & 23-Aug-97 & & $10028-17-8$ & TRITIUM \\
\hline 3350 & SESPSCH & & SW & & & BOLVV1 & 23-Aug-97 & & 13966-29-5 & U-234 \\
\hline 3351 & SESPSCH & & SW & & & BoLVV1 & 23-Aug-97 & & 15117-96-1 & U-235 \\
\hline 3352 & SESPSCH & & SW & & & BOLVV1 & 23-Aug-97 & & U-238 & $\mathrm{U}-238$ \\
\hline 3353 & SESPSCH & & SW & & & B0LVV6 & 23-Aug-97 & & 10098-97-2 & SR-90 \\
\hline 3354 & SESPSCH & & SW & & & BOLVV6 & 23-Aug-97 & & $10028-17-8$ & TRITIUM \\
\hline 3355 & SESPSCH & & SW & & & BOLVV6 & 23-Aug-97 & & $13966-29-5$ & U-234 \\
\hline 3356 & SESPSCH & & SW & & & B0LVV6 & 23-Aug-97 & & 15117-96-1 & U-235 \\
\hline 3357 & SESPSCH & & SW & & & BOLVV6 & 23-Aug-97 & & U-238 & U-238 \\
\hline 3358 & SESPSCH & & SW & & & B0LVS5 & 23-Aug-97 & & $10098-97-2$ & SR-90 \\
\hline 3359 & SESPSCH & & SW & & & B0LVS5 & 23-Aug-97 & & $10028-17-8$ & TRITIUM \\
\hline 3360 & SESPSCH & & SW & & & B0LVS5 & 23-Aug-97 & & $13966-29-5$ & U-234 \\
\hline 3361 & SESPSCH & & SW & & & B0LVS5 & 23-Aug-97 & & 15117-96-1 & U-235 \\
\hline 3362 & SESPSCH & & SW & & & B0LVS5 & 23-Aug-97 & & U-238 & U-238 \\
\hline 3363 & SESPSCH & & SW & & & BOLVTO & 23-Aug-97 & & 10098-97-2 & SR-90 \\
\hline 3364 & SESPSCH & & SW & & & BOLVTO & 23-Aug-97 & & $10028-17-8$ & TRITIUM \\
\hline 3365 & SESPSCH & & SW & & & BOLVTO & 23-Aug-97 & & 13966-29-5 & U-234 \\
\hline 3366 & SESPSCH & & SW & & & BOLVTO & 23-Aug-97 & & 15117-96-1 & U-235 \\
\hline 3367 & SESPSCH & & SW & & & BOLVTO & 23-Aug-97 & & U-238 & U-238 \\
\hline 3368 & SESPSCH & & SW & & & B0LVT1 & 23-Aug-97 & & 10098-97-2 & SR-90 \\
\hline 3369 & SESPSCH & & SW & & & B0LVT1 & 23-Aug-97 & & $10028-17-8$ & TRITIUM \\
\hline 3370 & SESPSCH & & SW & & & BOLVT1 & 23-Aug-97 & & 13966-29-5 & U-234 \\
\hline 3371 & SESPSCH & & SW & & & B0LVT1 & 23-Aug-97 & & 15117-96-1 & U-235 \\
\hline 3372 & SESPSCH & & SW & & & BOLVT1 & 23-Aug-97 & & U-238 & U-238 \\
\hline 3373 & SESPSCH & & SW & & & BOLVV2 & 23-Aug-97 & & 10098-97-2 & SR-90 \\
\hline 3374 & SESPSCH & & SW & & & BOLVV2 & 23-Aug-97 & & $10028-17-8$ & TRITIUM \\
\hline 3375 & SESPSCH & & SW & & & BOLVV2 & 23-Aug-97 & & 13966-29-5 & U-234 \\
\hline 3376 & SESPSCH & & SW & & & BOLVV2 & 23-Aug-97 & & 15117-96-1 & U-235 \\
\hline 3377 & SESPSCH & & SW & & & BOLVV2 & 23-Aug-97 & & U-238 & U-238 \\
\hline 3378 & SESPSCH & & SW & & & B0LVV3 & 23-Aug-97 & & 10098-97-2 & SR-90 \\
\hline 3379 & SESPSCH & & SW & & & B0LVV3 & 23-Aug-97 & & $10028-17-8$ & TRITIUM \\
\hline 3380 & SESPSCH & & SW & & & BOLVV3 & 23-Aug-97 & & $13966-29-5$ & U-234 \\
\hline 3381 & SESPSCH & & SW & & & B0LVV3 & 23-Aug-97 & & 15117-96-1 & U-235 \\
\hline 3382 & SESPSCH & & SW & & & B0LVV3 & 23-Aug-97 & & U-238 & U-238 \\
\hline 3383 & SESPSCH & & SW & & & BOLVV4 & 23-Aug-97 & & $10098-97-2$ & SR-90 \\
\hline
\end{tabular}

Page 89 


\begin{tabular}{|c|c|c|c|c|c|c|c|c|c|c|}
\hline & $\mathrm{A}$ & $B$ & $\mathrm{C}$ & $\mathrm{D}$ & $E$ & $\mathrm{~F}$ & $G$ & $\mathrm{H}$ & $\mathrm{I}$ & $\mathrm{J}$ \\
\hline 1 & SAMPLE_NUM_ASSIGNED_TO & SAMPLER & MEDIA & BIOTA_MEDIA & TAG_ID & SAMP_NUM & SAMP_DATE_TIME| & SAMP_DATE_TIME_ON & CON_ID & CON_SHORT_NAME \\
\hline 3384 & SESPSCH & & SW & & & BOLVV4 & 23-Aug-97 & & 10028-17-8 & TRITIUM \\
\hline 3385 & SESPSCH & & SW & & & BOLVV4 & 23-Aug-97 & & 13966-29-5 & U-234 \\
\hline 3386 & SESPSCH & & SW & & & B0LVV4 & 23-Aug-97 & & 15117-96-1 & U-235 \\
\hline 3387 & SESPSCH & & SW & & & BOLVV4 & 23-Aug-97 & & U-238 & U-238 \\
\hline 3388 & SESPSCH & & SW & & & BOLVV7 & 25-Aug-97 & & $10098-97-2$ & SR-90 \\
\hline 3389 & SESPSCH & & SW & & & BOLVV7 & 25-Aug-97 & & 10028-17-8 & TRITIUM \\
\hline 3390 & SESPSCH & & SW & & & B0LVV7 & 25-Aug-97 & & 13966-29-5 & U-234 \\
\hline 3391 & SESPSCH & & SW & & & BOLVV7 & 25-Aug-97 & & 15117-96-1 & U-235 \\
\hline 3392 & SESPSCH & & SW & & & BOLVV7 & 25-Aug-97 & & U-238 & U-238 \\
\hline 3393 & SESPSCH & & SW & & & B0LVV8 & 25-Aug-97 & & $10098-97-2$ & SR-90 \\
\hline 3394 & SESPSCH & & SW & & & B0LVV8 & 25-Aug-97 & & 10028-17-8 & TRITIUM \\
\hline 3395 & SESPSCH & & SW & & & B0LVV8 & 25-Aug-97 & & 13966-29-5 & U-234 \\
\hline 3396 & SESPSCH & & SW & & & BOLVV8 & 25-Aug-97 & & 15117-96-1 & U-235 \\
\hline 3397 & SESPSCH & & SW & & & BOLVV8 & 25-Aug-97 & & U-238 & U-238 \\
\hline 3398 & SESPSCH & & SW & & & BOLVV9 & 25-Aug-97 & & $10098-97-2$ & SR-90 \\
\hline 3399 & SESPSCH & & SW & & & B0LVV9 & 25-Aug-97 & & $10028-17-8$ & TRITIUM \\
\hline 3400 & SESPSCH & & SW & & & BOLVV9 & 25-Aug-97 & & 13966-29-5 & U-234 \\
\hline 3401 & SESPSCH & & SW & & & BOLVV9 & 25-Aug-97 & & 15117-96-1 & U-235 \\
\hline 3402 & SESPSCH & & SW & & & B0LVV9 & 25-Aug-97 & & U-238 & U-238 \\
\hline 3403 & SESPSCH & & SW & & & BOLVWO & 25-Aug-97 & & $10098-97-2$ & SR-90 \\
\hline 3404 & SESPSCH & & SW & & & BOLVWO & 25-Aug-97 & & $10028-17-8$ & TRITIUM \\
\hline 3405 & SESPSCH & & SW & & & BOLVWO & 25-Aug-97 & & 13966-29-5 & U-234 \\
\hline 3406 & SESPSCH & & SW & & & BOLVWO & 25-Aug-97 & & 15117-96-1 & U-235 \\
\hline 3407 & SESPSCH & & SW & & & BOLVWO & 25-Aug-97 & & U-238 & U-238 \\
\hline 3408 & SESPSCH & & SW & & & B0LVW1 & 25-Aug-97 & & $10098-97-2$ & SR-90 \\
\hline 3409 & SESPSCH & & SW & & & BOLVW1 & 25-Aug-97 & & 10028-17-8 & TRITIUM \\
\hline 3410 & SESPSCH & & SW & & & BOLVW1 & 25-Aug-97 & & $13966-29-5$ & U-234 \\
\hline 3411 & SESPSCH & & SW & & & B0LVW1 & 25-Aug-97 & & 15117-96-1 & U-235 \\
\hline 3412 & SESPSCH & & SW & & & B0LVW1 & 25-Aug-97 & & U-238 & U-238 \\
\hline 3413 & SESPSCH & & SW & & & B0LVW2 & 25-Aug-97 & & $10098-97-2$ & SR-90 \\
\hline 3414 & SESPSCH & & SW & & & B0LVW2 & 25-Aug-97 & & $10028-17-8$ & TRITIUM \\
\hline 3415 & SESPSCH & & SW & & & B0LVW2 & 25-Aug-97 & & 13966-29-5 & U-234 \\
\hline 3416 & SESPSCH & & SW & & & B0LVW2 & 25-Aug-97 & & 15117-96-1 & U-235 \\
\hline 3417 & SESPSCH & & SW & & & B0LVW2 & 25-Aug-97 & & U-238 & U-238 \\
\hline 3418 & SESPSCH & & SW & & & B0LVW3 & 25-Aug-97 & & $10098-97-2$ & SR-90 \\
\hline 3419 & SESPSCH & & SW & & & B0LVW3 & 25-Aug-97 & & $10028-17-8$ & TRITIUM \\
\hline 3420 & SESPSCH & & SW & & & BOLVW3 & 25-Aug-97 & & $13966-29-5$ & U-234 \\
\hline 3421 & SESPSCH & & SW & & & B0LVW3 & 25-Aug-97 & & 15117-96-1 & U-235 \\
\hline
\end{tabular}

Page 90 


\begin{tabular}{|c|c|c|c|c|c|c|c|c|c|c|}
\hline & $\mathrm{A}$ & $B$ & $\mathrm{C}$ & $\mathrm{D}$ & $E$ & $\mathrm{~F}$ & $G$ & $\mathrm{H}$ & $\mathrm{I}$ & $\mathrm{J}$ \\
\hline 1 & SAMPLE_NUM_ASSIGNED_TO & SAMPLER & MEDIA & BIOTA_MEDIA & TAG_ID & SAMP_NUM & SAMP_DATE_TIME| & SAMP_DATE_TIME_ON & CON_ID & CON_SHORT_NAME \\
\hline 3422 & SESPSCH & & SW & & & B0LVW3 & 25-Aug-97 & & $\mathrm{U}-238$ & $\mathrm{U}-238$ \\
\hline 3423 & SESPSCH & & SW & & & BOLVW4 & 25-Aug-97 & & $10098-97-2$ & SR-90 \\
\hline 3424 & SESPSCH & & SW & & & B0LVW4 & 25-Aug-97 & & $10028-17-8$ & TRITIUM \\
\hline 3425 & SESPSCH & & SW & & & BOLVW4 & 25-Aug-97 & & $13966-29-5$ & U-234 \\
\hline 3426 & SESPSCH & & SW & & & B0LVW4 & 25-Aug-97 & & 15117-96-1 & U-235 \\
\hline 3427 & SESPSCH & & SW & & & BOLVW4 & 25-Aug-97 & & U-238 & $\mathrm{U}-238$ \\
\hline 3428 & SESPSCH & & SW & & & B0LVW5 & 25-Aug-97 & & $10098-97-2$ & SR-90 \\
\hline 3429 & SESPSCH & & SW & & & B0LVW5 & 25-Aug-97 & & $10028-17-8$ & TRITIUM \\
\hline 3430 & SESPSCH & & SW & & & B0LVW5 & 25-Aug-97 & & 13966-29-5 & U-234 \\
\hline 3431 & SESPSCH & & SW & & & B0LVW5 & 25-Aug-97 & & 15117-96-1 & U-235 \\
\hline 3432 & SESPSCH & & SW & & & B0LVW5 & 25-Aug-97 & & U-238 & U-238 \\
\hline 3433 & SESPSCH & & SW & & & B0LVW6 & 25-Aug-97 & & $10098-97-2$ & SR-90 \\
\hline 3434 & SESPSCH & & SW & & & BOLVW6 & 25-Aug-97 & & 10028-17-8 & TRITIUM \\
\hline 3435 & SESPSCH & & SW & & & B0LVW6 & 25-Aug-97 & & $13966-29-5$ & U-234 \\
\hline 3436 & SESPSCH & & SW & & & B0LVW6 & 25-Aug-97 & & $15117-96-1$ & U-235 \\
\hline 3437 & SESPSCH & & SW & & & B0LVW6 & 25-Aug-97 & & U-238 & U-238 \\
\hline 3438 & SESPSCH & & SW & & & SOLVW7 & 25-Aug-97 & & $10098-97-2$ & SR-90 \\
\hline 3439 & SESPSCH & & SW & & & SOLVW7 & 25-Aug-97 & & $10028-17-8$ & TRITIUM \\
\hline 3440 & SESPSCH & & SW & & & SOLVW7 & 25-Aug-97 & & $13966-29-5$ & U-234 \\
\hline 3441 & SESPSCH & & SW & & & SOLVW7 & 25-Aug-97 & & 15117-96-1 & U-235 \\
\hline 3442 & SESPSCH & & SW & & & SOLVW7 & 25-Aug-97 & & U-238 & U-238 \\
\hline 3443 & SESPSCH & & SW & & & B0LVX6 & 25-Aug-97 & & 10098-97-2 & SR-90 \\
\hline 3444 & SESPSCH & & SW & & & BOLVX6 & 25-Aug-97 & & $10028-17-8$ & TRITIUM \\
\hline 3445 & SESPSCH & & SW & & & BOLVX6 & 25-Aug-97 & & 13966-29-5 & U-234 \\
\hline 3446 & SESPSCH & & SW & & & BOLVX6 & 25-Aug-97 & & 15117-96-1 & U-235 \\
\hline 3447 & SESPSCH & & SW & & & BOLVX6 & 25-Aug-97 & & U-238 & U-238 \\
\hline 3448 & SESPSCH & & SW & & & SOLVW8 & 25-Aug-97 & & $10098-97-2$ & SR-90 \\
\hline 3449 & SESPSCH & & SW & & & SOLVW8 & 25-Aug-97 & & $10028-17-8$ & TRITIUM \\
\hline 3450 & SESPSCH & & SW & & & SOLVW8 & 25-Aug-97 & & $13966-29-5$ & U-234 \\
\hline 3451 & SESPSCH & & SW & & & SOLVW8 & 25-Aug-97 & & 15117-96-1 & U-235 \\
\hline 3452 & SESPSCH & & SW & & & SOLVW8 & 25-Aug-97 & & U-238 & U-238 \\
\hline 3453 & SESPSCH & & SW & & & SOLVW9 & 25-Aug-97 & & $10098-97-2$ & SR-90 \\
\hline 3454 & SESPSCH & & SW & & & SOLVW9 & 25-Aug-97 & & $10028-17-8$ & TRITIUM \\
\hline 3455 & SESPSCH & & SW & & & SOLVW9 & 25-Aug-97 & & 13966-29-5 & U-234 \\
\hline 3456 & SESPSCH & & SW & & & SOLVW9 & 25-Aug-97 & & 15117-96-1 & U-235 \\
\hline 3457 & SESPSCH & & SW & & & SOLVW9 & 25-Aug-97 & & U-238 & U-238 \\
\hline 3458 & SESPSCH & & SW & & & SOLVXO & 25-Aug-97 & & 10098-97-2 & SR-90 \\
\hline 3459 & SESPSCH & & SW & & & SOLVXO & 25-Aug-97 & & $10028-17-8$ & TRITIUM \\
\hline
\end{tabular}




\begin{tabular}{|c|c|c|c|c|c|c|c|c|c|c|}
\hline & $\mathrm{A}$ & $B$ & $\mathrm{C}$ & $\mathrm{D}$ & $E$ & $\mathrm{~F}$ & $G$ & $\mathrm{H}$ & $\mathrm{I}$ & $\mathrm{J}$ \\
\hline 1 & SAMPLE_NUM_ASSIGNED_TO & SAMPLER & MEDIA & BIOTA_MEDIA & TAG_ID & SAMP_NUM & SAMP_DATE_TIME| & SAMP_DATE_TIME_ON & CON_ID & CON_SHORT_NAME \\
\hline 3460 & SESPSCH & & SW & & & SOLVXO & 25-Aug-97 & & 13966-29-5 & U-234 \\
\hline 3461 & SESPSCH & & SW & & & SOLVXO & 25-Aug-97 & & 15117-96-1 & U-235 \\
\hline 3462 & SESPSCH & & SW & & & SOLVXO & 25-Aug-97 & & U-238 & U-238 \\
\hline 3463 & SESPSCH & & SW & & & B0LVX1 & 25-Aug-97 & & $10098-97-2$ & SR-90 \\
\hline 3464 & SESPSCH & & SW & & & BOLVX1 & 25-Aug-97 & & $10028-17-8$ & TRITIUM \\
\hline 3465 & SESPSCH & & SW & & & B0LVX1 & 25-Aug-97 & & 13966-29-5 & U-234 \\
\hline 3466 & SESPSCH & & SW & & & B0LVX1 & 25-Aug-97 & & 15117-96-1 & U-235 \\
\hline 3467 & SESPSCH & & SW & & & BOLVX1 & 25-Aug-97 & & U-238 & U-238 \\
\hline 3468 & SESPSCH & & SW & & & BOLVX2 & 25-Aug-97 & & $10098-97-2$ & SR-90 \\
\hline 3469 & SESPSCH & & SW & & & BOLVX2 & 25-Aug-97 & & $10028-17-8$ & TRITIUM \\
\hline 3470 & SESPSCH & & SW & & & BOLVX2 & 25-Aug-97 & & 13966-29-5 & U-234 \\
\hline 3471 & SESPSCH & & SW & & & BOLVX2 & 25-Aug-97 & & 15117-96-1 & U-235 \\
\hline 3472 & SESPSCH & & SW & & & BOLVX2 & 25-Aug-97 & & U-238 & U-238 \\
\hline 3473 & SESPSCH & & SW & & & BOLVX3 & 25-Aug-97 & & 10098-97-2 & SR-90 \\
\hline 3474 & SESPSCH & & SW & & & B0LVX3 & 25-Aug-97 & & $10028-17-8$ & TRITIUM \\
\hline 3475 & SESPSCH & & SW & & & B0LVX3 & 25-Aug-97 & & 13966-29-5 & U-234 \\
\hline 3476 & SESPSCH & & SW & & & BOLVX3 & 25-Aug-97 & & 15117-96-1 & U-235 \\
\hline 3477 & SESPSCH & & SW & & & B0LVX3 & 25-Aug-97 & & U-238 & U-238 \\
\hline 3478 & SESPSCH & & SW & & & B0LVX4 & 25-Aug-97 & & $10098-97-2$ & SR-90 \\
\hline 3479 & SESPSCH & & SW & & & BOLVX4 & 25-Aug-97 & & $10028-17-8$ & TRITIUM \\
\hline 3480 & SESPSCH & & SW & & & BOLVX4 & 25-Aug-97 & & 13966-29-5 & U-234 \\
\hline 3481 & SESPSCH & & SW & & & BOLVX4 & 25-Aug-97 & & 15117-96-1 & U-235 \\
\hline 3482 & SESPSCH & & SW & & & BOLVX4 & 25-Aug-97 & & U-238 & U-238 \\
\hline 3483 & SESPSCH & & SW & & & B0LVX5 & 25-Aug-97 & & $10098-97-2$ & SR-90 \\
\hline 3484 & SESPSCH & & SW & & & B0LVX5 & 25-Aug-97 & & $10028-17-8$ & TRITIUM \\
\hline 3485 & SESPSCH & & SW & & & B0LVX5 & 25-Aug-97 & & 13966-29-5 & U-234 \\
\hline 3486 & SESPSCH & & SW & & & B0LVX5 & 25-Aug-97 & & 15117-96-1 & U-235 \\
\hline 3487 & SESPSCH & & SW & & & B0LVX5 & 25-Aug-97 & & U-238 & U-238 \\
\hline 3488 & SESPSCH & & SW & & & SOLW98 & 08-Sep-97 & & $10045-97-3$ & CS-137 \\
\hline 3489 & SESPSCH & & SW & & & SOLW98 & 08-Sep-97 & & $10098-97-2$ & SR-90 \\
\hline 3490 & SESPSCH & & SW & & & SOLW98 & 08-Sep-97 & & $10028-17-8$ & TRITIUM \\
\hline 3491 & SESPSCH & & SW & & & SOLWBO & 08-Sep-97 & & $10045-97-3$ & CS-137 \\
\hline 3492 & SESPSCH & & SW & & & SOLWBO & 08-Sep-97 & & $10098-97-2$ & SR-90 \\
\hline 3493 & SESPSCH & & SW & & & SOLWBO & 08-Sep-97 & & $10028-17-8$ & TRITIUM \\
\hline 3494 & SESPSCH & & SW & & & SOLWB2 & 08-Sep-97 & & $10045-97-3$ & CS-137 \\
\hline 3495 & SESPSCH & & SW & & & SOLWB2 & 08-Sep-97 & & $10098-97-2$ & SR-90 \\
\hline 3496 & SESPSCH & & SW & & & SOLWB2 & 08-Sep-97 & & $10028-17-8$ & TRITIUM \\
\hline 3497 & SESPSCH & & SW & & & B0LWB4 & 08-Sep-97 & & $10045-97-3$ & CS-137 \\
\hline
\end{tabular}




\begin{tabular}{|c|c|c|c|c|c|c|c|c|c|c|}
\hline & A & $\mathrm{B}$ & $\mathrm{C}$ & $\mathrm{D}$ & $E$ & $\mathrm{~F}$ & G & $\mathrm{H}$ & $\mathrm{I}$ & $\mathrm{J}$ \\
\hline 1 & SAMPLE_NUM_ASSIGNED_TO & SAMPLER & MEDIA & BIOTA_MEDIA & TAG_ID & SAMP_NUM & SAMP_DATE_TIME & SAMP_DATE_TIME_ON & CON_ID & CON_SHORT_NAME \\
\hline 3498 & SESPSCH & & SW & & & BOLWB4 & 08-Sep-97 & & $10098-97-2$ & SR-90 \\
\hline 3499 & SESPSCH & & SW & & & BOLWB4 & 08-Sep-97 & & $10028-17-8$ & TRITIUM \\
\hline 3500 & SESPSCH & & SW & & & SOLWD2 & 08-Sep-97 & & $10045-97-3$ & CS-137 \\
\hline 3501 & SESPSCH & & SW & & & SOLWD2 & 08-Sep-97 & & $10098-97-2$ & SR-90 \\
\hline 3502 & SESPSCH & & SW & & & SOLWD2 & 08-Sep-97 & & $10028-17-8$ & TRITIUM \\
\hline 3503 & SESPSCH & & SW & & & B0LWB6 & 08-Sep-97 & & $10045-97-3$ & CS-137 \\
\hline 3504 & SESPSCH & & SW & & & B0LWB6 & 08-Sep-97 & & $10098-97-2$ & SR-90 \\
\hline 3505 & SESPSCH & & SW & & & B0LWB6 & 08-Sep-97 & & $10028-17-8$ & TRITIUM \\
\hline 3506 & SESPSCH & & SW & & & SOLWB8 & 08-Sep-97 & & $10045-97-3$ & CS-137 \\
\hline 3507 & SESPSCH & & SW & & & SOLWB8 & 08-Sep-97 & & $10098-97-2$ & SR-90 \\
\hline 3508 & SESPSCH & & SW & & & SOLWB8 & 08-Sep-97 & & 10028-17-8 & TRITIUM \\
\hline 3509 & SESPSCH & & SW & & & BOLWCO & 08-Sep-97 & & $10045-97-3$ & CS-137 \\
\hline 3510 & SESPSCH & & SW & & & BOLWCO & 08-Sep-97 & & $10098-97-2$ & SR-90 \\
\hline 3511 & SESPSCH & & SW & & & BOLWCO & 08-Sep-97 & & $10028-17-8$ & TRITIUM \\
\hline 3512 & SESPSCH & & SW & & & SOLWC2 & 08-Sep-97 & & $10045-97-3$ & CS-137 \\
\hline 3513 & SESPSCH & & SW & & & SOLWC2 & 08-Sep-97 & & $10098-97-2$ & SR-90 \\
\hline 3514 & SESPSCH & & SW & & & SOLWC2 & 08-Sep-97 & & $10028-17-8$ & TRITIUM \\
\hline 3515 & SESPSCH & & SW & & & SOLWC4 & 08-Sep-97 & & $10045-97-3$ & CS-137 \\
\hline 3516 & SESPSCH & & SW & & & SOLWC4 & 08-Sep-97 & & $10098-97-2$ & SR-90 \\
\hline 3517 & SESPSCH & & SW & & & SOLWC4 & 08-Sep-97 & & $10028-17-8$ & TRITIUM \\
\hline 3518 & SESPSCH & & SW & & & SOLWC6 & 08-Sep-97 & & $10028-17-8$ & TRITIUM \\
\hline 3519 & SESPSCH & & SW & & & SOLWC8 & 08-Sep-97 & & $10028-17-8$ & TRITIUM \\
\hline 3520 & SESPSCH & & SW & & & SOLWDO & 08-Sep-97 & & $10028-17-8$ & TRITIUM \\
\hline 3521 & SESPSCH & & SW & & & SOLWD4 & 08-Sep-97 & & $10045-97-3$ & CS-137 \\
\hline 3522 & SESPSCH & & SW & & & SOLWD4 & 08-Sep-97 & & $10098-97-2$ & SR-90 \\
\hline 3523 & SESPSCH & & SW & & & SOLWD4 & 08-Sep-97 & & $10028-17-8$ & TRITIUM \\
\hline 3524 & SESP SCHEDULING & & SW & & & BOMLC2 & 18-Dec-97 & & $10098-97-2$ & SR-90 \\
\hline 3525 & SESP SCHEDULING & & SW & & & BOMLC2 & 18-Dec-97 & & $10028-17-8$ & TRITIUM \\
\hline 3526 & SESP SCHEDULING & & SW & & & BOMLC2 & 18-Dec-97 & & $13966-29-5$ & U-234 \\
\hline \begin{tabular}{|l|}
3527 \\
35
\end{tabular} & SESP SCHEDULING & & SW & & & BOMLC2 & 18-Dec-97 & & 15117-96-1 & $\mathrm{U}-235$ \\
\hline 3528 & SESP SCHEDULING & & SW & & & BOMLC2 & 18-Dec-97 & & $\mathrm{U}-238$ & U-238 \\
\hline \begin{tabular}{|l|}
3529 \\
\end{tabular} & SESP SCHEDULING & & SW & & & BOMLC1 & 18-Dec-97 & & $10098-97-2$ & SR-90 \\
\hline 3530 & SESP SCHEDULING & & SW & & & B0MLC1 & 18-Dec-97 & & $10028-17-8$ & TRITIUM \\
\hline 3531 & SESP SCHEDULING & & SW & & & B0MLC1 & 18-Dec-97 & & $13966-29-5$ & U-234 \\
\hline 3532 & SESP SCHEDULING & & SW & & & BOMLC1 & 18-Dec-97 & & 15117-96-1 & U-235 \\
\hline 3533 & SESP SCHEDULING & & SW & & & BOMLC1 & 18-Dec-97 & & $\mathrm{U}-238$ & $\mathrm{U}-238$ \\
\hline 3534 & SESP SCHEDULING & & SW & & & BOMLCO & 18-Dec-97 & & $10098-97-2$ & SR-90 \\
\hline 3535 & SESP SCHEDULING & & SW & & & BOMLCO & 18-Dec-97 & & $10028-17-8$ & TRITIUM \\
\hline
\end{tabular}




\begin{tabular}{|c|c|c|c|c|c|c|c|c|c|c|}
\hline & A & $\mathrm{B}$ & $\mathrm{C}$ & $\mathrm{D}$ & $E$ & $\mathrm{~F}$ & $G$ & $\mathrm{H}$ & $\mathrm{I}$ & $\mathrm{J}$ \\
\hline 1 & SAMPLE_NUM_ASSIGNED_TO & SAMPLER & MEDIA & BIOTA_MEDIA & TAG_ID & SAMP_NUM & SAMP_DATE_TIME & SAMP_DATE_TIME_ON & CON_ID & CON_SHORT_NAME \\
\hline 3536 & SESP SCHEDULING & & SW & & & BOMLCO & 18-Dec-97 & & $13966-29-5$ & U-234 \\
\hline 3537 & SESP SCHEDULING & & SW & & & BOMLCO & 18-Dec-97 & & $15117-96-1$ & U-235 \\
\hline 3538 & SESP SCHEDULING & & SW & & & BOMLCO & 18-Dec-97 & & U-238 & U-238 \\
\hline 3539 & SESP SCHEDULING & & SW & & & BOMLB9 & 18-Dec-97 & & $10098-97-2$ & SR-90 \\
\hline 3540 & SESP SCHEDULING & & SW & & & B0MLB9 & 18-Dec-97 & & 10028-17-8 & TRITIUM \\
\hline 3541 & SESP SCHEDULING & & SW & & & BOMLB9 & 18-Dec-97 & & $13966-29-5$ & U-234 \\
\hline 3542 & SESP SCHEDULING & & SW & & & BOMLB9 & 18-Dec-97 & & $15117-96-1$ & U-235 \\
\hline 3543 & SESP SCHEDULING & & SW & & & B0MLB9 & 18-Dec-97 & & U-238 & $\mathrm{U}-238$ \\
\hline 3544 & SESP SCHEDULING & & SW & & & BOMLD2 & 19-Dec-97 & & $10098-97-2$ & SR-90 \\
\hline 3545 & SESP SCHEDULING & & SW & & & B0MLD2 & 19-Dec-97 & & $10028-17-8$ & TRITIUM \\
\hline 3546 & SESP SCHEDULING & & SW & & & B0MLD2 & 19-Dec-97 & & 13966-29-5 & $\mathrm{U}-234$ \\
\hline 3547 & SESP SCHEDULING & & SW & & & BOMLD2 & 19-Dec-97 & & 15117-96-1 & U-235 \\
\hline 3548 & SESP SCHEDULING & & SW & & & BOMLD2 & 19-Dec-97 & & $\mathrm{U}-238$ & $\mathrm{U}-238$ \\
\hline 3549 & SESP SCHEDULING & & SW & & & B0MLD1 & 19-Dec-97 & & $10098-97-2$ & SR-90 \\
\hline 3550 & SESP SCHEDULING & & SW & & & B0MLD1 & 19-Dec-97 & & $10028-17-8$ & TRITIUM \\
\hline 3551 & SESP SCHEDULING & & SW & & & B0MLD1 & 19-Dec-97 & & $13966-29-5$ & U-234 \\
\hline 3552 & SESP SCHEDULING & & SW & & & B0MLD1 & 19-Dec-97 & & 15117-96-1 & U-235 \\
\hline 3553 & SESP SCHEDULING & & SW & & & B0MLD1 & 19-Dec-97 & & U-238 & $\mathrm{U}-238$ \\
\hline 3554 & SESP SCHEDULING & & SW & & & BOMLDO & 19-Dec-97 & & $10098-97-2$ & SR-90 \\
\hline 3555 & SESP SCHEDULING & & SW & & & BOMLDO & 19-Dec-97 & & $10028-17-8$ & TRITIUM \\
\hline 3556 & SESP SCHEDULING & & SW & & & BOMLDO & 19-Dec-97 & & 13966-29-5 & U-234 \\
\hline 3557 & SESP SCHEDULING & & SW & & & BOMLDO & 19-Dec-97 & & 15117-96-1 & U-235 \\
\hline 3558 & SESP SCHEDULING & & SW & & & BOMLDO & 19-Dec-97 & & U-238 & U-238 \\
\hline 3559 & SESP SCHEDULING & & SW & & & BOMLC9 & 19-Dec-97 & & 10098-97-2 & SR-90 \\
\hline 3560 & SESP SCHEDULING & & SW & & & BOMLC9 & 19-Dec-97 & & $10028-17-8$ & TRITIUM \\
\hline 3561 & SESP SCHEDULING & & SW & & & BOMLC9 & 19-Dec-97 & & 13966-29-5 & $\mathrm{U}-234$ \\
\hline 3562 & SESP SCHEDULING & & SW & & & BOMLC9 & 19-Dec-97 & & 15117-96-1 & U-235 \\
\hline 3563 & SESP SCHEDULING & & SW & & & BOMLC9 & 19-Dec-97 & & U-238 & U-238 \\
\hline 3564 & SESP SCHEDULING & & SW & & & BOMLC8 & 19-Dec-97 & & $10098-97-2$ & SR-90 \\
\hline 3565 & SESP SCHEDULING & & SW & & & BOMLC8 & 19-Dec-97 & & $10028-17-8$ & TRITIUM \\
\hline 3566 & SESP SCHEDULING & & SW & & & BOMLC8 & 19-Dec-97 & & 13966-29-5 & U-234 \\
\hline 3567 & SESP SCHEDULING & & SW & & & BOMLC8 & 19-Dec-97 & & $15117-96-1$ & U-235 \\
\hline 3568 & SESP SCHEDULING & & SW & & & BOMLC8 & 19-Dec-97 & & $\mathrm{U}-238$ & U-238 \\
\hline 3569 & SESP SCHEDULING & & SW & & & BOMLC7 & 19-Dec-97 & & 10098-97-2 & SR-90 \\
\hline 3570 & SESP SCHEDULING & & SW & & & BOMLC7 & 19-Dec-97 & & $10028-17-8$ & TRITIUM \\
\hline 3571 & SESP SCHEDULING & & SW & & & BOMLC7 & 19-Dec-97 & & 13966-29-5 & $\mathrm{U}-234$ \\
\hline 3572 & SESP SCHEDULING & & SW & & & BOMLC7 & 19-Dec-97 & & 15117-96-1 & U-235 \\
\hline 3573 & SESP SCHEDULING & & SW & & & BOMLC7 & 19-Dec-97 & & U-238 & U-238 \\
\hline
\end{tabular}




\begin{tabular}{|c|c|c|c|c|c|c|c|c|c|c|}
\hline & A & $\mathrm{B}$ & $C$ & $\mathrm{D}$ & $E$ & $\mathrm{~F}$ & $G$ & $\mathrm{H}$ & $\mathrm{I}$ & $\mathrm{J}$ \\
\hline 1 & SAMPLE_NUM_ASSIGNED_TO & SAMPLER & MEDIA & BIOTA_MEDIA & TAG_ID & SAMP_NUM & SAMP_DATE_TIME| & SAMP_DATE_TIME_ON & CON_ID & CON_SHORT_NAME \\
\hline 3574 & SESP SCHEDULING & & SW & & & B0MLC6 & 19-Dec-97 & & $10098-97-2$ & SR-90 \\
\hline 3575 & SESP SCHEDULING & & SW & & & B0MLC6 & 19-Dec-97 & & $10028-17-8$ & TRITIUM \\
\hline 3576 & SESP SCHEDULING & & SW & & & B0MLC6 & 19-Dec-97 & & $13966-29-5$ & U-234 \\
\hline 3577 & SESP SCHEDULING & & SW & & & B0MLC6 & 19-Dec-97 & & $15117-96-1$ & U-235 \\
\hline 3578 & SESP SCHEDULING & & SW & & & B0MLC6 & 19-Dec-97 & & $\mathrm{U}-238$ & U-238 \\
\hline 3579 & SESP SCHEDULING & & SW & & & B0MLC5 & 19-Dec-97 & & $10098-97-2$ & SR-90 \\
\hline 3580 & SESP SCHEDULING & & SW & & & B0MLC5 & 19-Dec-97 & & $10028-17-8$ & TRITIUM \\
\hline 3581 & SESP SCHEDULING & & SW & & & B0MLC5 & 19-Dec-97 & & 13966-29-5 & $\mathrm{U}-234$ \\
\hline 3582 & SESP SCHEDULING & & SW & & & B0MLC5 & 19-Dec-97 & & 15117-96-1 & U-235 \\
\hline 3583 & SESP SCHEDULING & & SW & & & B0MLC5 & 19-Dec-97 & & $\mathrm{U}-238$ & U-238 \\
\hline 3584 & SESP SCHEDULING & & SW & & & BOMLC4 & 19-Dec-97 & & $10098-97-2$ & SR-90 \\
\hline 3585 & SESP SCHEDULING & & SW & & & BOMLC4 & 19-Dec-97 & & $10028-17-8$ & TRITIUM \\
\hline 3586 & SESP SCHEDULING & & SW & & & BOMLC4 & 19-Dec-97 & & $13966-29-5$ & U-234 \\
\hline 3587 & SESP SCHEDULING & & SW & & & BOMLC4 & 19-Dec-97 & & $15117-96-1$ & U-235 \\
\hline 3588 & SESP SCHEDULING & & SW & & & BOMLC4 & 19-Dec-97 & & $\mathrm{U}-238$ & U-238 \\
\hline 3589 & SESP SCHEDULING & & SW & & & B0MLB7 & 19-Dec-97 & & $10098-97-2$ & SR-90 \\
\hline 3590 & SESP SCHEDULING & & SW & & & BOMLC3 & 19-Dec-97 & & $10098-97-2$ & SR-90 \\
\hline 3591 & SESP SCHEDULING & & SW & & & B0MLB7 & 19-Dec-97 & & $10028-17-8$ & TRITIUM \\
\hline 3592 & SESP SCHEDULING & & SW & & & B0MLC3 & 19-Dec-97 & & $10028-17-8$ & TRITIUM \\
\hline 3593 & SESP SCHEDULING & & SW & & & B0MLB7 & 19-Dec-97 & & 13966-29-5 & U-234 \\
\hline 3594 & SESP SCHEDULING & & SW & & & B0MLB7 & 19-Dec-97 & & $15117-96-1$ & U-235 \\
\hline 3595 & SESP SCHEDULING & & SW & & & B0MLB7 & 19-Dec-97 & & $\mathrm{U}-238$ & $\mathrm{U}-238$ \\
\hline 3596 & SESP SCHEDULING & & SW & & & BOMLC3 & 19-Dec-97 & & 13966-29-5 & U-234 \\
\hline 3597 & SESP SCHEDULING & & SW & & & BOMLC3 & 19-Dec-97 & & $15117-96-1$ & U-235 \\
\hline 3598 & SESP SCHEDULING & & SW & & & B0MLC3 & 19-Dec-97 & & $\mathrm{U}-238$ & U-238 \\
\hline 3599 & SESP SCHEDULING & & SW & & & B0N6Y5 & 23-Mar-98 & & $10098-97-2$ & SR-90 \\
\hline 3600 & SESP SCHEDULING & & SW & & & B0N6Y5 & 23-Mar-98 & & $10028-17-8$ & TRITIUM \\
\hline 3601 & SESP SCHEDULING & & SW & & & B0N6Y5 & 23-Mar-98 & & $13966-29-5$ & U-234 \\
\hline 3602 & SESP SCHEDULING & & SW & & & B0N6Y5 & 23-Mar-98 & & 15117-96-1 & U-235 \\
\hline 3603 & SESP SCHEDULING & & SW & & & B0N6Y5 & 23-Mar-98 & & $\mathrm{U}-238$ & U-238 \\
\hline 3604 & SESP SCHEDULING & & SW & & & B0N6Y6 & 23-Mar-98 & & $10098-97-2$ & SR-90 \\
\hline 3605 & SESP SCHEDULING & & SW & & & B0N6Y6 & 23-Mar-98 & & $10028-17-8$ & TRITIUM \\
\hline 3606 & SESP SCHEDULING & & SW & & & BON6Y6 & 23-Mar-98 & & 13966-29-5 & U-234 \\
\hline 3607 & SESP SCHEDULING & & SW & & & B0N6Y6 & 23-Mar-98 & & $15117-96-1$ & $\mathrm{U}-235$ \\
\hline 3608 & SESP SCHEDULING & & SW & & & B0N6Y6 & 23-Mar-98 & & $\mathrm{U}-238$ & U-238 \\
\hline 3609 & SESP SCHEDULING & & SW & & & B0N6Y7 & 23-Mar-98 & & $10098-97-2$ & SR-90 \\
\hline 3610 & SESP SCHEDULING & & SW & & & B0N6Y7 & 23-Mar-98 & & $10028-17-8$ & TRITIUM \\
\hline 3611 & SESP SCHEDULING & & SW & & & B0N6Y7 & 23-Mar-98 & & $13966-29-5$ & U-234 \\
\hline
\end{tabular}




\begin{tabular}{|c|c|c|c|c|c|c|c|c|c|c|}
\hline & A & $\mathrm{B}$ & $C$ & $\mathrm{D}$ & $E$ & $\mathrm{~F}$ & $G$ & $\mathrm{H}$ & $\mathrm{I}$ & $\mathrm{J}$ \\
\hline 1 & SAMPLE_NUM_ASSIGNED_TO & SAMPLER & MEDIA & BIOTA_MEDIA & TAG_ID & SAMP_NUM & SAMP_DATE_TIME| & SAMP_DATE_TIME_ON & CON_ID & CON_SHORT_NAME \\
\hline 3612 & SESP SCHEDULING & & SW & & & B0N6Y7 & 23-Mar-98 & & 15117-96-1 & U-235 \\
\hline 3613 & SESP SCHEDULING & & SW & & & B0N6Y7 & 23-Mar-98 & & U-238 & $\mathrm{U}-238$ \\
\hline 3614 & SESP SCHEDULING & & SW & & & B0N6Y8 & 23-Mar-98 & & 10098-97-2 & SR-90 \\
\hline 3615 & SESP SCHEDULING & & SW & & & B0N6Y8 & 23-Mar-98 & & 10028-17-8 & TRITIUM \\
\hline 3616 & SESP SCHEDULING & & SW & & & B0N6Y8 & 23-Mar-98 & & 13966-29-5 & U-234 \\
\hline 3617 & SESP SCHEDULING & & SW & & & B0N6Y8 & 23-Mar-98 & & 15117-96-1 & U-235 \\
\hline 3618 & SESP SCHEDULING & & SW & & & B0N6Y8 & 23-Mar-98 & & U-238 & $\mathrm{U}-238$ \\
\hline 3619 & SESP SCHEDULING & & SW & & & BON6Y4 & 24-Mar-98 & & $10098-97-2$ & SR-90 \\
\hline 3620 & SESP SCHEDULING & & SW & & & BON6Y9 & 24-Mar-98 & & $10098-97-2$ & SR-90 \\
\hline 3621 & SESP SCHEDULING & & SW & & & B0N6Y4 & 24-Mar-98 & & $10028-17-8$ & TRITIUM \\
\hline 3622 & SESP SCHEDULING & & SW & & & BON6Y9 & 24-Mar-98 & & $10028-17-8$ & TRITIUM \\
\hline 3623 & SESP SCHEDULING & & SW & & & B0N6Y4 & 24-Mar-98 & & $13966-29-5$ & U-234 \\
\hline 3624 & SESP SCHEDULING & & SW & & & BON6Y4 & 24-Mar-98 & & 15117-96-1 & U-235 \\
\hline 3625 & SESP SCHEDULING & & SW & & & B0N6Y4 & 24-Mar-98 & & U-238 & U-238 \\
\hline 3626 & SESP SCHEDULING & & SW & & & B0N6Y9 & 24-Mar-98 & & 13966-29-5 & U-234 \\
\hline 3627 & SESP SCHEDULING & & SW & & & B0N6Y9 & 24-Mar-98 & & 15117-96-1 & U-235 \\
\hline 3628 & SESP SCHEDULING & & SW & & & BON6Y9 & 24-Mar-98 & & U-238 & U-238 \\
\hline 3629 & SESP SCHEDULING & & SW & & & BON708 & 24-Mar-98 & & $10098-97-2$ & SR-90 \\
\hline 3630 & SESP SCHEDULING & & SW & & & B0N708 & 24-Mar-98 & & $10028-17-8$ & TRITIUM \\
\hline 3631 & SESP SCHEDULING & & SW & & & BON708 & 24-Mar-98 & & $13966-29-5$ & U-234 \\
\hline 3632 & SESP SCHEDULING & & SW & & & B0N708 & 24-Mar-98 & & 15117-96-1 & U-235 \\
\hline 3633 & SESP SCHEDULING & & SW & & & B0N708 & 24-Mar-98 & & U-238 & $\mathrm{U}-238$ \\
\hline 3634 & SESP SCHEDULING & & SW & & & BON700 & 24-Mar-98 & & $10098-97-2$ & SR-90 \\
\hline 3635 & SESP SCHEDULING & & SW & & & B0N700 & 24-Mar-98 & & $10028-17-8$ & TRITIUM \\
\hline 3636 & SESP SCHEDULING & & SW & & & BON700 & 24-Mar-98 & & $13966-29-5$ & U-234 \\
\hline 3637 & SESP SCHEDULING & & SW & & & BON700 & 24-Mar-98 & & 15117-96-1 & U-235 \\
\hline 3638 & SESP SCHEDULING & & SW & & & BON700 & 24-Mar-98 & & U-238 & U-238 \\
\hline 3639 & SESP SCHEDULING & & SW & & & B0N701 & 24-Mar-98 & & $10098-97-2$ & SR-90 \\
\hline 3640 & SESP SCHEDULING & & SW & & & B0N701 & 24-Mar-98 & & $10028-17-8$ & TRITIUM \\
\hline 3641 & SESP SCHEDULING & & SW & & & B0N701 & 24-Mar-98 & & $13966-29-5$ & U-234 \\
\hline 3642 & SESP SCHEDULING & & SW & & & B0N701 & 24-Mar-98 & & $15117-96-1$ & U-235 \\
\hline 3643 & SESP SCHEDULING & & SW & & & B0N701 & 24-Mar-98 & & U-238 & U-238 \\
\hline 3644 & SESP SCHEDULING & & SW & & & BON702 & 24-Mar-98 & & $10098-97-2$ & SR-90 \\
\hline 3645 & SESP SCHEDULING & & SW & & & B0N702 & 24-Mar-98 & & 10028-17-8 & TRITIUM \\
\hline 3646 & SESP SCHEDULING & & SW & & & BON702 & 24-Mar-98 & & $13966-29-5$ & U-234 \\
\hline 3647 & SESP SCHEDULING & & SW & & & BON702 & 24-Mar-98 & & 15117-96-1 & $\mathrm{U}-235$ \\
\hline 3648 & SESP SCHEDULING & & SW & & & BON702 & 24-Mar-98 & & U-238 & U-238 \\
\hline 3649 & SESP SCHEDULING & & SW & & & B0N703 & 24-Mar-98 & & $10098-97-2$ & SR-90 \\
\hline
\end{tabular}




\begin{tabular}{|c|c|c|c|c|c|c|c|c|c|c|}
\hline & A & $\mathrm{B}$ & $C$ & $\mathrm{D}$ & $E$ & $\mathrm{~F}$ & $G$ & $\mathrm{H}$ & $\mathrm{I}$ & $\mathrm{J}$ \\
\hline 1 & SAMPLE_NUM_ASSIGNED_TO & SAMPLER & MEDIA & BIOTA_MEDIA & TAG_ID & SAMP_NUM & SAMP_DATE_TIME| & SAMP_DATE_TIME_ON & CON_ID & CON_SHORT_NAME \\
\hline 3650 & SESP SCHEDULING & & SW & & & B0N703 & 24-Mar-98 & & 10028-17-8 & TRITIUM \\
\hline 3651 & SESP SCHEDULING & & SW & & & B0N703 & 24-Mar-98 & & 13966-29-5 & U-234 \\
\hline 3652 & SESP SCHEDULING & & SW & & & B0N703 & 24-Mar-98 & & 15117-96-1 & U-235 \\
\hline 3653 & SESP SCHEDULING & & SW & & & B0N703 & 24-Mar-98 & & $\mathrm{U}-238$ & U-238 \\
\hline 3654 & SESP SCHEDULING & & SW & & & BON704 & 24-Mar-98 & & $10098-97-2$ & SR-90 \\
\hline 3655 & SESP SCHEDULING & & SW & & & B0N704 & 24-Mar-98 & & 10028-17-8 & TRITIUM \\
\hline 3656 & SESP SCHEDULING & & SW & & & BON704 & 24-Mar-98 & & $13966-29-5$ & U-234 \\
\hline 3657 & SESP SCHEDULING & & SW & & & B0N704 & 24-Mar-98 & & $15117-96-1$ & U-235 \\
\hline 3658 & SESP SCHEDULING & & SW & & & B0N704 & 24-Mar-98 & & U-238 & U-238 \\
\hline 3659 & SESP SCHEDULING & & SW & & & B0N705 & 24-Mar-98 & & $10098-97-2$ & SR-90 \\
\hline 3660 & SESP SCHEDULING & & SW & & & B0N705 & 24-Mar-98 & & $10028-17-8$ & TRITIUM \\
\hline 3661 & SESP SCHEDULING & & SW & & & B0N705 & 24-Mar-98 & & 13966-29-5 & U-234 \\
\hline 3662 & SESP SCHEDULING & & SW & & & B0N705 & 24-Mar-98 & & 15117-96-1 & U-235 \\
\hline 3663 & SESP SCHEDULING & & SW & & & B0N705 & 24-Mar-98 & & U-238 & U-238 \\
\hline 3664 & SESP SCHEDULING & & SW & & & B0N706 & 24-Mar-98 & & $10098-97-2$ & SR-90 \\
\hline 3665 & SESP SCHEDULING & & SW & & & B0N706 & 24-Mar-98 & & $10028-17-8$ & TRITIUM \\
\hline 3666 & SESP SCHEDULING & & SW & & & B0N706 & 24-Mar-98 & & $13966-29-5$ & U-234 \\
\hline 3667 & SESP SCHEDULING & & SW & & & B0N706 & 24-Mar-98 & & $15117-96-1$ & U-235 \\
\hline 3668 & SESP SCHEDULING & & SW & & & B0N706 & 24-Mar-98 & & U-238 & U-238 \\
\hline 3669 & SESP SCHEDULING & & SW & & & B0N707 & 24-Mar-98 & & $10098-97-2$ & SR-90 \\
\hline 3670 & SESP SCHEDULING & & SW & & & BON707 & 24-Mar-98 & & 10028-17-8 & TRITIUM \\
\hline 3671 & SESP SCHEDULING & & SW & & & B0N707 & 24-Mar-98 & & 13966-29-5 & U-234 \\
\hline 3672 & SESP SCHEDULING & & SW & & & B0N707 & 24-Mar-98 & & $15117-96-1$ & U-235 \\
\hline 3673 & SESP SCHEDULING & & SW & & & B0N707 & 24-Mar-98 & & $U-238$ & $\mathrm{U}-238$ \\
\hline 3674 & SESP SCHEDULING & & SW & & & B0P327 & 22-Jun-98 & & $10098-97-2$ & SR-90 \\
\hline 3675 & SESP SCHEDULING & & SW & & & B0P327 & 22-Jun-98 & & 10028-17-8 & TRITIUM \\
\hline 3676 & SESP SCHEDULING & & SW & & & B0P327 & 22-Jun-98 & & 13966-29-5 & U-234 \\
\hline 3677 & SESP SCHEDULING & & SW & & & B0P327 & 22-Jun-98 & & 15117-96-1 & U-235 \\
\hline 3678 & SESP SCHEDULING & & SW & & & B0P327 & 22-Jun-98 & & U-238 & U-238 \\
\hline 3679 & SESP SCHEDULING & & SW & & & B0P328 & 22-Jun-98 & & $10098-97-2$ & SR-90 \\
\hline 3680 & SESP SCHEDULING & & SW & & & B0P328 & 22-Jun-98 & & $10028-17-8$ & TRITIUM \\
\hline 3681 & SESP SCHEDULING & & SW & & & B0P328 & 22-Jun-98 & & $13966-29-5$ & U-234 \\
\hline 3682 & SESP SCHEDULING & & SW & & & B0P328 & 22-Jun-98 & & $15117-96-1$ & U-235 \\
\hline 3683 & SESP SCHEDULING & & SW & & & B0P328 & 22-Jun-98 & & U-238 & U-238 \\
\hline 3684 & SESP SCHEDULING & & SW & & & B0P329 & 22-Jun-98 & & $10098-97-2$ & SR-90 \\
\hline 3685 & SESP SCHEDULING & & SW & & & B0P329 & 22-Jun-98 & & $10028-17-8$ & TRITIUM \\
\hline 3686 & SESP SCHEDULING & & SW & & & B0P329 & 22-Jun-98 & & $13966-29-5$ & $\mathrm{U}-234$ \\
\hline 3687 & SESP SCHEDULING & & SW & & & B0P329 & 22-Jun-98 & & $15117-96-1$ & U-235 \\
\hline
\end{tabular}




\begin{tabular}{|c|c|c|c|c|c|c|c|c|c|c|}
\hline & A & $\mathrm{B}$ & $C$ & $\mathrm{D}$ & $E$ & $\mathrm{~F}$ & $G$ & $\mathrm{H}$ & $\mathrm{I}$ & $\mathrm{J}$ \\
\hline 1 & SAMPLE_NUM_ASSIGNED_TO & SAMPLER & MEDIA & BIOTA_MEDIA & TAG_ID & SAMP_NUM & SAMP_DATE_TIME| & SAMP_DATE_TIME_ON & CON_ID & CON_SHORT_NAME \\
\hline 3688 & SESP SCHEDULING & & SW & & & B0P329 & 22-Jun-98 & & $\mathrm{U}-238$ & U-238 \\
\hline 3689 & SESP SCHEDULING & & SW & & & B0P330 & 22-Jun-98 & & $10098-97-2$ & SR-90 \\
\hline 3690 & SESP SCHEDULING & & SW & & & B0P330 & 22-Jun-98 & & $10028-17-8$ & TRITIUM \\
\hline 3691 & SESP SCHEDULING & & SW & & & B0P330 & 22-Jun-98 & & $13966-29-5$ & U-234 \\
\hline 3692 & SESP SCHEDULING & & SW & & & B0P330 & 22-Jun-98 & & 15117-96-1 & U-235 \\
\hline 3693 & SESP SCHEDULING & & SW & & & B0P330 & 22-Jun-98 & & $\mathrm{U}-238$ & $\mathrm{U}-238$ \\
\hline 3694 & SESP SCHEDULING & & SW & & & B0P331 & 23-Jun-98 & & $10098-97-2$ & SR-90 \\
\hline 3695 & SESP SCHEDULING & & SW & & & B0P331 & 23-Jun-98 & & $10028-17-8$ & TRITIUM \\
\hline 3696 & SESP SCHEDULING & & SW & & & B0P331 & 23-Jun-98 & & 13966-29-5 & U-234 \\
\hline 3697 & SESP SCHEDULING & & SW & & & B0P331 & 23-Jun-98 & & 15117-96-1 & U-235 \\
\hline 3698 & SESP SCHEDULING & & SW & & & B0P331 & 23-Jun-98 & & $\mathrm{U}-238$ & U-238 \\
\hline 3699 & SESP SCHEDULING & & SW & & & B0P340 & 23-Jun-98 & & $10098-97-2$ & SR-90 \\
\hline 3700 & SESP SCHEDULING & & SW & & & B0P340 & 23-Jun-98 & & $10028-17-8$ & TRITIUM \\
\hline 3701 & SESP SCHEDULING & & SW & & & B0P340 & 23-Jun-98 & & $13966-29-5$ & U-234 \\
\hline 3702 & SESP SCHEDULING & & SW & & & B0P340 & 23-Jun-98 & & 15117-96-1 & U-235 \\
\hline 3703 & SESP SCHEDULING & & SW & & & B0P340 & 23-Jun-98 & & $\mathrm{U}-238$ & $\mathrm{U}-238$ \\
\hline 3704 & SESP SCHEDULING & & SW & & & B0P332 & 23-Jun-98 & & $10098-97-2$ & SR-90 \\
\hline 3705 & SESP SCHEDULING & & SW & & & B0P332 & 23-Jun-98 & & $10028-17-8$ & TRITIUM \\
\hline 3706 & SESP SCHEDULING & & SW & & & B0P332 & 23-Jun-98 & & $13966-29-5$ & U-234 \\
\hline 3707 & SESP SCHEDULING & & SW & & & B0P332 & 23-Jun-98 & & 15117-96-1 & U-235 \\
\hline 3708 & SESP SCHEDULING & & SW & & & B0P332 & 23-Jun-98 & & U-238 & U-238 \\
\hline 3709 & SESP SCHEDULING & & SW & & & B0P333 & 23-Jun-98 & & $10098-97-2$ & SR-90 \\
\hline 3710 & SESP SCHEDULING & & SW & & & B0P333 & 23-Jun-98 & & $10028-17-8$ & TRITIUM \\
\hline 3711 & SESP SCHEDULING & & SW & & & B0P333 & 23-Jun-98 & & $13966-29-5$ & $\mathrm{U}-234$ \\
\hline 3712 & SESP SCHEDULING & & SW & & & B0P333 & 23-Jun-98 & & 15117-96-1 & U-235 \\
\hline 3713 & SESP SCHEDULING & & SW & & & B0P333 & 23-Jun-98 & & U-238 & U-238 \\
\hline 3714 & SESP SCHEDULING & & SW & & & B0P334 & 23-Jun-98 & & $10098-97-2$ & SR-90 \\
\hline 3715 & SESP SCHEDULING & & SW & & & B0P334 & 23-Jun-98 & & $10028-17-8$ & TRITIUM \\
\hline 3716 & SESP SCHEDULING & & SW & & & B0P334 & 23-Jun-98 & & $13966-29-5$ & U-234 \\
\hline 3717 & SESP SCHEDULING & & SW & & & B0P334 & 23-Jun-98 & & 15117-96-1 & U-235 \\
\hline 3718 & SESP SCHEDULING & & SW & & & B0P334 & 23-Jun-98 & & $\mathrm{U}-238$ & $\mathrm{U}-238$ \\
\hline 3719 & SESP SCHEDULING & & SW & & & B0P335 & 23-Jun-98 & & $10098-97-2$ & SR-90 \\
\hline 3720 & SESP SCHEDULING & & SW & & & B0P335 & 23-Jun-98 & & $10028-17-8$ & TRITIUM \\
\hline 3721 & SESP SCHEDULING & & SW & & & B0P335 & 23-Jun-98 & & 13966-29-5 & U-234 \\
\hline 3722 & SESP SCHEDULING & & SW & & & B0P335 & 23-Jun-98 & & 15117-96-1 & U-235 \\
\hline 3723 & SESP SCHEDULING & & SW & & & B0P335 & 23-Jun-98 & & $\mathrm{U}-238$ & U-238 \\
\hline 3724 & SESP SCHEDULING & & SW & & & B0P336 & 23-Jun-98 & & $10098-97-2$ & SR-90 \\
\hline 3725 & SESP SCHEDULING & & SW & & & B0P336 & 23-Jun-98 & & $10028-17-8$ & TRITIUM \\
\hline
\end{tabular}




\begin{tabular}{|c|c|c|c|c|c|c|c|c|c|c|}
\hline & A & $\mathrm{B}$ & $\mathrm{C}$ & $\mathrm{D}$ & $E$ & $\mathrm{~F}$ & G & $\mathrm{H}$ & $\mathrm{I}$ & $\mathrm{J}$ \\
\hline 1 & SAMPLE_NUM_ASSIGNED_TO & SAMPLER & MEDIA & BIOTA_MEDIA & TAG_ID & SAMP_NUM & SAMP_DATE_TIME & SAMP_DATE_TIME_ON & CON_ID & CON_SHORT_NAME \\
\hline 3726 & SESP SCHEDULING & & SW & & & B0P336 & 23-Jun-98 & & $13966-29-5$ & U-234 \\
\hline 3727 & SESP SCHEDULING & & SW & & & B0P336 & 23-Jun-98 & & $15117-96-1$ & U-235 \\
\hline 3728 & SESP SCHEDULING & & SW & & & B0P336 & 23-Jun-98 & & U-238 & U-238 \\
\hline 3729 & SESP SCHEDULING & & SW & & & B0P337 & 23-Jun-98 & & $10098-97-2$ & SR-90 \\
\hline 3730 & SESP SCHEDULING & & SW & & & B0P337 & 23-Jun-98 & & $10028-17-8$ & TRITIUM \\
\hline 3731 & SESP SCHEDULING & & SW & & & B0P337 & 23-Jun-98 & & $13966-29-5$ & $\mathrm{U}-234$ \\
\hline 3732 & SESP SCHEDULING & & SW & & & B0P337 & 23-Jun-98 & & $15117-96-1$ & U-235 \\
\hline 3733 & SESP SCHEDULING & & SW & & & B0P337 & 23-Jun-98 & & $\mathrm{U}-238$ & $\mathrm{U}-238$ \\
\hline 3734 & SESP SCHEDULING & & SW & & & B0P338 & 23-Jun-98 & & $10098-97-2$ & SR-90 \\
\hline 3735 & SESP SCHEDULING & & SW & & & B0P338 & 23-Jun-98 & & $10028-17-8$ & TRITIUM \\
\hline 3736 & SESP SCHEDULING & & SW & & & B0P338 & 23-Jun-98 & & $13966-29-5$ & U-234 \\
\hline 3737 & SESP SCHEDULING & & SW & & & B0P338 & 23-Jun-98 & & $15117-96-1$ & U-235 \\
\hline 3738 & SESP SCHEDULING & & SW & & & B0P338 & 23-Jun-98 & & U-238 & U-238 \\
\hline 3739 & SESP SCHEDULING & & SW & & & B0P339 & 23-Jun-98 & & $10098-97-2$ & SR-90 \\
\hline 3740 & SESP SCHEDULING & & SW & & & B0P339 & 23-Jun-98 & & $10028-17-8$ & TRITIUM \\
\hline 3741 & SESP SCHEDULING & & SW & & & B0P339 & 23-Jun-98 & & 13966-29-5 & $\mathrm{U}-234$ \\
\hline 3742 & SESP SCHEDULING & & SW & & & B0P339 & 23-Jun-98 & & 15117-96-1 & U-235 \\
\hline 3743 & SESP SCHEDULING & & SW & & & B0P339 & 23-Jun-98 & & $\mathrm{U}-238$ & $\mathrm{U}-238$ \\
\hline 3744 & SESP SCHEDULING & & SW & & & BOPVN8 & 14-Sep-98 & & $10098-97-2$ & SR-90 \\
\hline 3745 & SESP SCHEDULING & & SW & & & BOPVN8 & 14-Sep-98 & & $10028-17-8$ & TRITIUM \\
\hline 3746 & SESP SCHEDULING & & SW & & & BOPVN8 & 14-Sep-98 & & 13966-29-5 & U-234 \\
\hline 3747 & SESP SCHEDULING & & SW & & & BOPVN8 & 14-Sep-98 & & 15117-96-1 & U-235 \\
\hline 3748 & SESP SCHEDULING & & SW & & & BOPVN8 & 14-Sep-98 & & $\mathrm{U}-238$ & $\mathrm{U}-238$ \\
\hline 3749 & SESP SCHEDULING & & SW & & & BOPVT4 & 14-Sep-98 & & $10098-97-2$ & SR-90 \\
\hline 3750 & SESP SCHEDULING & & SW & & & BOPVT4 & 14-Sep-98 & & $10028-17-8$ & TRITIUM \\
\hline 3751 & SESP SCHEDULING & & SW & & & BOPVT4 & 14-Sep-98 & & $13966-29-5$ & U-234 \\
\hline 3752 & SESP SCHEDULING & & SW & & & B0PVT4 & 14-Sep-98 & & 15117-96-1 & U-235 \\
\hline 3753 & SESP SCHEDULING & & SW & & & B0PVT4 & 14-Sep-98 & & $\mathrm{U}-238$ & $\mathrm{U}-238$ \\
\hline 3754 & SESP SCHEDULING & & SW & & & BOPVPO & 14-Sep-98 & & $10098-97-2$ & SR-90 \\
\hline 3755 & SESP SCHEDULING & & SW & & & BOPVPO & 14-Sep-98 & & $10028-17-8$ & TRITIUM \\
\hline 3756 & SESP SCHEDULING & & SW & & & BOPVPO & 14-Sep-98 & & 13966-29-5 & U-234 \\
\hline 3757 & SESP SCHEDULING & & SW & & & BOPVPO & 14-Sep-98 & & 15117-96-1 & U-235 \\
\hline 3758 & SESP SCHEDULING & & SW & & & BOPVPO & 14-Sep-98 & & U-238 & U-238 \\
\hline 3759 & SESP SCHEDULING & & SW & & & B0PVT5 & 14-Sep-98 & & $10098-97-2$ & SR-90 \\
\hline 3760 & SESP SCHEDULING & & SW & & & B0PVT5 & 14-Sep-98 & & $10028-17-8$ & TRITIUM \\
\hline 3761 & SESP SCHEDULING & & SW & & & BOPVT5 & 14-Sep-98 & & $13966-29-5$ & U-234 \\
\hline 3762 & SESP SCHEDULING & & SW & & & B0PVT5 & 14-Sep-98 & & 15117-96-1 & U-235 \\
\hline 3763 & SESP SCHEDULING & & SW & & & BOPVT5 & 14-Sep-98 & & U-238 & U-238 \\
\hline
\end{tabular}




\begin{tabular}{|c|c|c|c|c|c|c|c|c|c|c|}
\hline & A & $\mathrm{B}$ & $C$ & $\mathrm{D}$ & $E$ & $\mathrm{~F}$ & $G$ & $\mathrm{H}$ & $\mathrm{I}$ & $\mathrm{J}$ \\
\hline 1 & SAMPLE_NUM_ASSIGNED_TO & SAMPLER & MEDIA & BIOTA_MEDIA & TAG_ID & SAMP_NUM & SAMP_DATE_TIME| & SAMP_DATE_TIME_ON & CON_ID & CON_SHORT_NAME \\
\hline 3764 & SESP SCHEDULING & & SW & & & BOPVT7 & 14-Sep-98 & & $10098-97-2$ & SR-90 \\
\hline 3765 & SESP SCHEDULING & & SW & & & BOPVT7 & 14-Sep-98 & & $10028-17-8$ & TRITIUM \\
\hline 3766 & SESP SCHEDULING & & SW & & & BOPVT7 & 14-Sep-98 & & $13966-29-5$ & U-234 \\
\hline 3767 & SESP SCHEDULING & & SW & & & BOPVT7 & 14-Sep-98 & & 15117-96-1 & U-235 \\
\hline 3768 & SESP SCHEDULING & & SW & & & B0PVT7 & 14-Sep-98 & & U-238 & U-238 \\
\hline 3769 & SESP SCHEDULING & & SW & & & BOPVP2 & 14-Sep-98 & & $10098-97-2$ & SR-90 \\
\hline 3770 & SESP SCHEDULING & & SW & & & B0PVP2 & 14-Sep-98 & & $10028-17-8$ & TRITIUM \\
\hline 3771 & SESP SCHEDULING & & SW & & & B0PVP2 & 14-Sep-98 & & $13966-29-5$ & $\mathrm{U}-234$ \\
\hline 3772 & SESP SCHEDULING & & SW & & & B0PVP2 & 14-Sep-98 & & 15117-96-1 & U-235 \\
\hline 3773 & SESP SCHEDULING & & SW & & & B0PVP2 & 14-Sep-98 & & U-238 & U-238 \\
\hline 3774 & SESP SCHEDULING & & SW & & & BOPVVO & 14-Sep-98 & & $10098-97-2$ & SR-90 \\
\hline 3775 & SESP SCHEDULING & & SW & & & BOPVV0 & 14-Sep-98 & & $10028-17-8$ & TRITIUM \\
\hline 3776 & SESP SCHEDULING & & SW & & & BOPVVO & 14-Sep-98 & & $13966-29-5$ & U-234 \\
\hline 3777 & SESP SCHEDULING & & SW & & & BOPVVO & 14-Sep-98 & & 15117-96-1 & U-235 \\
\hline 3778 & SESP SCHEDULING & & SW & & & BOPVV0 & 14-Sep-98 & & U-238 & U-238 \\
\hline 3779 & SESP SCHEDULING & & SW & & & BOPVT9 & 14-Sep-98 & & $10098-97-2$ & SR-90 \\
\hline 3780 & SESP SCHEDULING & & SW & & & BOPVT9 & 14-Sep-98 & & $10028-17-8$ & TRITIUM \\
\hline 3781 & SESP SCHEDULING & & SW & & & BOPVT9 & 14-Sep-98 & & $13966-29-5$ & U-234 \\
\hline 3782 & SESP SCHEDULING & & SW & & & BOPVT9 & 14-Sep-98 & & 15117-96-1 & U-235 \\
\hline 3783 & SESP SCHEDULING & & SW & & & BOPVT9 & 14-Sep-98 & & U-238 & U-238 \\
\hline 3784 & SESP SCHEDULING & & SW & & & BOPVV1 & 14-Sep-98 & & 10098-97-2 & SR-90 \\
\hline 3785 & SESP SCHEDULING & & SW & & & B0PVV1 & 14-Sep-98 & & $10028-17-8$ & TRITIUM \\
\hline 3786 & SESP SCHEDULING & & SW & & & B0PVV1 & 14-Sep-98 & & $13966-29-5$ & U-234 \\
\hline 3787 & SESP SCHEDULING & & SW & & & BOPVV1 & 14-Sep-98 & & $15117-96-1$ & U-235 \\
\hline 3788 & SESP SCHEDULING & & SW & & & B0PVV1 & 14-Sep-98 & & U-238 & U-238 \\
\hline 3789 & SESP SCHEDULING & & SW & & & BOPVV2 & 14-Sep-98 & & 10098-97-2 & SR-90 \\
\hline 3790 & SESP SCHEDULING & & SW & & & BOPVV2 & 14-Sep-98 & & 10028-17-8 & TRITIUM \\
\hline 3791 & SESP SCHEDULING & & SW & & & B0PVV2 & 14-Sep-98 & & 13966-29-5 & U-234 \\
\hline 3792 & SESP SCHEDULING & & SW & & & B0PVV2 & 14-Sep-98 & & 15117-96-1 & U-235 \\
\hline 3793 & SESP SCHEDULING & & SW & & & B0PVV2 & 14-Sep-98 & & U-238 & U-238 \\
\hline 3794 & SESP SCHEDULING & & SW & & & BOPVN4 & 14-Sep-98 & & $10098-97-2$ & SR-90 \\
\hline 3795 & SESP SCHEDULING & & SW & & & BOPVN4 & 14-Sep-98 & & $10028-17-8$ & TRITIUM \\
\hline 3796 & SESP SCHEDULING & & SW & & & BOPVN4 & 14-Sep-98 & & $13966-29-5$ & U-234 \\
\hline 3797 & SESP SCHEDULING & & SW & & & BOPVN4 & 14-Sep-98 & & 15117-96-1 & $\mathrm{U}-235$ \\
\hline 3798 & SESP SCHEDULING & & SW & & & BOPVN4 & 14-Sep-98 & & U-238 & U-238 \\
\hline 3799 & SESP SCHEDULING & & SW & & & B0PVN5 & 14-Sep-98 & & $10098-97-2$ & SR-90 \\
\hline 3800 & SESP SCHEDULING & & SW & & & B0PVN5 & 14-Sep-98 & & $10028-17-8$ & TRITIUM \\
\hline 3801 & SESP SCHEDULING & & SW & & & BOPVN5 & 14-Sep-98 & & $13966-29-5$ & U-234 \\
\hline
\end{tabular}




\begin{tabular}{|c|c|c|c|c|c|c|c|c|c|c|}
\hline & A & $\mathrm{B}$ & $\mathrm{C}$ & $\mathrm{D}$ & $E$ & $\mathrm{~F}$ & $G$ & $\mathrm{H}$ & $\mathrm{I}$ & $\mathrm{J}$ \\
\hline 1 & SAMPLE_NUM_ASSIGNED_TO & SAMPLER & MEDIA & BIOTA_MEDIA & TAG_ID & SAMP_NUM & SAMP_DATE_TIME & SAMP_DATE_TIME_ON & CON_ID & CON_SHORT_NAME \\
\hline 3802 & SESP SCHEDULING & & SW & & & B0PVN5 & 14-Sep-98 & & 15117-96-1 & U-235 \\
\hline 3803 & SESP SCHEDULING & & SW & & & BOPVN5 & 14-Sep-98 & & U-238 & U-238 \\
\hline 3804 & SESP SCHEDULING & & SW & & & BOPVN6 & 14-Sep-98 & & $10098-97-2$ & SR-90 \\
\hline 3805 & SESP SCHEDULING & & SW & & & BOPVN6 & 14-Sep-98 & & $10028-17-8$ & TRITIUM \\
\hline 3806 & SESP SCHEDULING & & SW & & & BOPVN6 & 14-Sep-98 & & 13966-29-5 & $\mathrm{U}-234$ \\
\hline 3807 & SESP SCHEDULING & & SW & & & BOPVN6 & 14-Sep-98 & & 15117-96-1 & U-235 \\
\hline 3808 & SESP SCHEDULING & & SW & & & BOPVN6 & 14-Sep-98 & & $\mathrm{U}-238$ & U-238 \\
\hline 3809 & SESP SCHEDULING & & SW & & & BOPVN7 & 14-Sep-98 & & 10098-97-2 & SR-90 \\
\hline 3810 & SESP SCHEDULING & & SW & & & BOPVN7 & 14-Sep-98 & & $10028-17-8$ & TRITIUM \\
\hline 3811 & SESP SCHEDULING & & SW & & & BOPVN7 & 14-Sep-98 & & $13966-29-5$ & U-234 \\
\hline 3812 & SESP SCHEDULING & & SW & & & BOPVN7 & 14-Sep-98 & & 15117-96-1 & U-235 \\
\hline 3813 & SESP SCHEDULING & & SW & & & BOPVN7 & 14-Sep-98 & & U-238 & U-238 \\
\hline 3814 & SESP SCHEDULING & & SW & & & BOPVP4 & 15-Sep-98 & & 10098-97-2 & SR-90 \\
\hline 3815 & SESP SCHEDULING & & SW & & & BOPVP4 & 15-Sep-98 & & $10028-17-8$ & TRITIUM \\
\hline 3816 & SESP SCHEDULING & & SW & & & BOPVP4 & 15-Sep-98 & & $13966-29-5$ & U-234 \\
\hline 3817 & SESP SCHEDULING & & SW & & & BOPVP4 & 15-Sep-98 & & $15117-96-1$ & U-235 \\
\hline 3818 & SESP SCHEDULING & & SW & & & BOPVP4 & 15-Sep-98 & & U-238 & $\mathrm{U}-238$ \\
\hline 3819 & SESP SCHEDULING & & SW & & & B0PVP5 & 15-Sep-98 & & 10098-97-2 & SR-90 \\
\hline 3820 & SESP SCHEDULING & & SW & & & B0PVP5 & 15-Sep-98 & & $10028-17-8$ & TRITIUM \\
\hline 3821 & SESP SCHEDULING & & SW & & & B0PVP5 & 15-Sep-98 & & $13966-29-5$ & U-234 \\
\hline 3822 & SESP SCHEDULING & & SW & & & B0PVP5 & 15-Sep-98 & & 15117-96-1 & U-235 \\
\hline 3823 & SESP SCHEDULING & & SW & & & B0PVP5 & 15-Sep-98 & & U-238 & U-238 \\
\hline 3824 & SESP SCHEDULING & & SW & & & B0PVP6 & 15-Sep-98 & & 10098-97-2 & SR-90 \\
\hline 3825 & SESP SCHEDULING & & SW & & & BOPVP6 & 15-Sep-98 & & $10028-17-8$ & TRITIUM \\
\hline 3826 & SESP SCHEDULING & & SW & & & BOPVP6 & 15-Sep-98 & & $13966-29-5$ & U-234 \\
\hline 3827 & SESP SCHEDULING & & SW & & & B0PVP6 & 15-Sep-98 & & 15117-96-1 & U-235 \\
\hline 3828 & SESP SCHEDULING & & SW & & & BOPVP6 & 15-Sep-98 & & U-238 & U-238 \\
\hline 3829 & SESP SCHEDULING & & SW & & & BOPVP7 & 15-Sep-98 & & $10098-97-2$ & SR-90 \\
\hline 3830 & SESP SCHEDULING & & SW & & & BOPVP7 & 15-Sep-98 & & $10028-17-8$ & TRITIUM \\
\hline 3831 & SESP SCHEDULING & & SW & & & BOPVP7 & 15-Sep-98 & & 13966-29-5 & U-234 \\
\hline 3832 & SESP SCHEDULING & & SW & & & BOPVP7 & 15-Sep-98 & & 15117-96-1 & U-235 \\
\hline 3833 & SESP SCHEDULING & & SW & & & BOPVP7 & 15-Sep-98 & & U-238 & U-238 \\
\hline 3834 & SESP SCHEDULING & & SW & & & BOPVP8 & 15-Sep-98 & & 10098-97-2 & SR-90 \\
\hline 3835 & SESP SCHEDULING & & SW & & & BOPVP8 & 15-Sep-98 & & $10028-17-8$ & TRITIUM \\
\hline 3836 & SESP SCHEDULING & & SW & & & BOPVP8 & 15-Sep-98 & & 13966-29-5 & U-234 \\
\hline 3837 & SESP SCHEDULING & & SW & & & B0PVP8 & 15-Sep-98 & & 15117-96-1 & $\mathrm{U}-235$ \\
\hline 3838 & SESP SCHEDULING & & SW & & & BOPVP8 & 15-Sep-98 & & U-238 & U-238 \\
\hline 3839 & SESP SCHEDULING & & SW & & & BOPVP9 & 15-Sep-98 & & $10098-97-2$ & SR-90 \\
\hline
\end{tabular}




\begin{tabular}{|c|c|c|c|c|c|c|c|c|c|c|}
\hline & A & $\mathrm{B}$ & $C$ & $\mathrm{D}$ & $E$ & $\mathrm{~F}$ & $G$ & $\mathrm{H}$ & $\mathrm{I}$ & $\mathrm{J}$ \\
\hline 1 & SAMPLE_NUM_ASSIGNED_TO & SAMPLER & MEDIA & BIOTA_MEDIA & TAG_ID & SAMP_NUM & SAMP_DATE_TIME| & SAMP_DATE_TIME_ON & CON_ID & CON_SHORT_NAME \\
\hline 3840 & SESP SCHEDULING & & SW & & & BOPVP9 & 15-Sep-98 & & 10028-17-8 & TRITIUM \\
\hline 3841 & SESP SCHEDULING & & SW & & & B0PVP9 & 15-Sep-98 & & 13966-29-5 & U-234 \\
\hline 3842 & SESP SCHEDULING & & SW & & & BOPVP9 & 15-Sep-98 & & 15117-96-1 & U-235 \\
\hline 3843 & SESP SCHEDULING & & SW & & & BOPVP9 & 15-Sep-98 & & U-238 & U-238 \\
\hline 3844 & SESP SCHEDULING & & SW & & & BOPVRO & 15-Sep-98 & & $10098-97-2$ & SR-90 \\
\hline 3845 & SESP SCHEDULING & & SW & & & BOPVRO & 15-Sep-98 & & 10028-17-8 & TRITIUM \\
\hline 3846 & SESP SCHEDULING & & SW & & & BOPVRO & 15-Sep-98 & & $13966-29-5$ & U-234 \\
\hline 3847 & SESP SCHEDULING & & SW & & & BOPVRO & 15-Sep-98 & & $15117-96-1$ & U-235 \\
\hline 3848 & SESP SCHEDULING & & SW & & & BOPVRO & 15-Sep-98 & & U-238 & U-238 \\
\hline 3849 & SESP SCHEDULING & & SW & & & B0PVR1 & 15-Sep-98 & & $10098-97-2$ & SR-90 \\
\hline 3850 & SESP SCHEDULING & & SW & & & B0PVR1 & 15-Sep-98 & & $10028-17-8$ & TRITIUM \\
\hline 3851 & SESP SCHEDULING & & SW & & & B0PVR1 & 15-Sep-98 & & 13966-29-5 & U-234 \\
\hline 3852 & SESP SCHEDULING & & SW & & & BOPVR1 & 15-Sep-98 & & 15117-96-1 & U-235 \\
\hline 3853 & SESP SCHEDULING & & SW & & & BOPVR1 & 15-Sep-98 & & U-238 & U-238 \\
\hline 3854 & SESP SCHEDULING & & SW & & & B0PVR2 & 15-Sep-98 & & $10098-97-2$ & SR-90 \\
\hline 3855 & SESP SCHEDULING & & SW & & & B0PVR2 & 15-Sep-98 & & $10028-17-8$ & TRITIUM \\
\hline 3856 & SESP SCHEDULING & & SW & & & B0PVR2 & 15-Sep-98 & & $13966-29-5$ & U-234 \\
\hline 3857 & SESP SCHEDULING & & SW & & & BOPVR2 & 15-Sep-98 & & $15117-96-1$ & U-235 \\
\hline 3858 & SESP SCHEDULING & & SW & & & B0PVR2 & 15-Sep-98 & & U-238 & U-238 \\
\hline 3859 & SESP SCHEDULING & & SW & & & B0PVR3 & 15-Sep-98 & & $10098-97-2$ & SR-90 \\
\hline 3860 & SESP SCHEDULING & & SW & & & BOPVR3 & 15-Sep-98 & & 10028-17-8 & TRITIUM \\
\hline 3861 & SESP SCHEDULING & & SW & & & B0PVR3 & 15-Sep-98 & & 13966-29-5 & U-234 \\
\hline 3862 & SESP SCHEDULING & & SW & & & B0PVR3 & 15-Sep-98 & & $15117-96-1$ & U-235 \\
\hline 3863 & SESP SCHEDULING & & SW & & & BOPVR3 & 15-Sep-98 & & $U-238$ & $\mathrm{U}-238$ \\
\hline 3864 & SESP SCHEDULING & & SW & & & BOPVR4 & 15-Sep-98 & & $10098-97-2$ & SR-90 \\
\hline 3865 & SESP SCHEDULING & & SW & & & BOPVR4 & 15-Sep-98 & & 10028-17-8 & TRITIUM \\
\hline 3866 & SESP SCHEDULING & & SW & & & BOPVR4 & 15-Sep-98 & & 13966-29-5 & U-234 \\
\hline 3867 & SESP SCHEDULING & & SW & & & B0PVR4 & 15-Sep-98 & & 15117-96-1 & U-235 \\
\hline 3868 & SESP SCHEDULING & & SW & & & B0PVR4 & 15-Sep-98 & & U-238 & U-238 \\
\hline 3869 & SESP SCHEDULING & & SW & & & B0PVT3 & 15-Sep-98 & & $10098-97-2$ & SR-90 \\
\hline 3870 & SESP SCHEDULING & & SW & & & BOPVT3 & 15-Sep-98 & & $10028-17-8$ & TRITIUM \\
\hline 3871 & SESP SCHEDULING & & SW & & & B0PVT3 & 15-Sep-98 & & $13966-29-5$ & U-234 \\
\hline 3872 & SESP SCHEDULING & & SW & & & BOPVT3 & 15-Sep-98 & & 15117-96-1 & U-235 \\
\hline 3873 & SESP SCHEDULING & & SW & & & BOPVT3 & 15-Sep-98 & & U-238 & U-238 \\
\hline 3874 & SESP SCHEDULING & & SW & & & B0PVR5 & 15-Sep-98 & & $10098-97-2$ & SR-90 \\
\hline 3875 & SESP SCHEDULING & & SW & & & B0PVR5 & 15-Sep-98 & & $10028-17-8$ & TRITIUM \\
\hline 3876 & SESP SCHEDULING & & SW & & & B0PVR5 & 15-Sep-98 & & 13966-29-5 & U-234 \\
\hline 3877 & SESP SCHEDULING & & SW & & & BOPVR5 & 15-Sep-98 & & $15117-96-1$ & U-235 \\
\hline
\end{tabular}




\begin{tabular}{|c|c|c|c|c|c|c|c|c|c|c|}
\hline & A & $\mathrm{B}$ & $\mathrm{C}$ & $\mathrm{D}$ & $\mathrm{E}$ & $\mathrm{F}$ & $G$ & $\mathrm{H}$ & I & $\mathrm{J}$ \\
\hline 1 & SAMPLE_NUM_ASSIGNED_TO & SAMPLER & MEDIA & BIOTA_MEDIA & TAG_ID & SAMP_NUM & SAMP_DATE_TIME & SAMP_DATE_TIME_ON & CON_ID & CON_SHORT_NAME \\
\hline 3878 & SESP SCHEDULING & & SW & & & B0PVR5 & 15-Sep-98 & & $\mathrm{U}-238$ & $\mathrm{U}-238$ \\
\hline 3879 & SESP SCHEDULING & & SW & & & BOPVR6 & 15-Sep-98 & & $10098-97-2$ & SR-90 \\
\hline 3880 & SESP SCHEDULING & & SW & & & BOPVR6 & 15-Sep-98 & & $10028-17-8$ & TRITIUM \\
\hline 3881 & SESP SCHEDULING & & SW & & & BOPVR6 & 15-Sep-98 & & $13966-29-5$ & U-234 \\
\hline 3882 & SESP SCHEDULING & & SW & & & BOPVR6 & 15-Sep-98 & & $15117-96-1$ & U-235 \\
\hline 3883 & SESP SCHEDULING & & SW & & & B0PVR6 & 15-Sep-98 & & $\mathrm{U}-238$ & $\mathrm{U}-238$ \\
\hline 3884 & SESP SCHEDULING & & SW & & & B0PVR7 & 15-Sep-98 & & $10098-97-2$ & SR-90 \\
\hline 3885 & SESP SCHEDULING & & SW & & & B0PVR7 & 15-Sep-98 & & $10028-17-8$ & TRITIUM \\
\hline 3886 & SESP SCHEDULING & & SW & & & B0PVR7 & 15-Sep-98 & & 13966-29-5 & U-234 \\
\hline 3887 & SESP SCHEDULING & & SW & & & BOPVR7 & 15-Sep-98 & & 15117-96-1 & $\mathrm{U}-235$ \\
\hline 3888 & SESP SCHEDULING & & SW & & & BOPVR7 & 15-Sep-98 & & $\mathrm{U}-238$ & U-238 \\
\hline 3889 & SESP SCHEDULING & & SW & & & BOPVR8 & 15-Sep-98 & & $10098-97-2$ & SR-90 \\
\hline 3890 & SESP SCHEDULING & & SW & & & B0PVR8 & 15-Sep-98 & & $10028-17-8$ & TRITIUM \\
\hline 3891 & SESP SCHEDULING & & SW & & & B0PVR8 & 15-Sep-98 & & 13966-29-5 & U-234 \\
\hline 3892 & SESP SCHEDULING & & SW & & & B0PVR8 & 15-Sep-98 & & 15117-96-1 & U-235 \\
\hline 3893 & SESP SCHEDULING & & SW & & & B0PVR8 & 15-Sep-98 & & $\mathrm{U}-238$ & $\mathrm{U}-238$ \\
\hline 3894 & SESP SCHEDULING & & SW & & & BOPVR9 & 15-Sep-98 & & $10098-97-2$ & SR-90 \\
\hline 3895 & SESP SCHEDULING & & SW & & & B0PVR9 & 15-Sep-98 & & $10028-17-8$ & TRITIUM \\
\hline 3896 & SESP SCHEDULING & & SW & & & B0PVR9 & 15-Sep-98 & & 13966-29-5 & $\mathrm{U}-234$ \\
\hline 3897 & SESP SCHEDULING & & SW & & & BOPVR9 & 15-Sep-98 & & 15117-96-1 & U-235 \\
\hline 3898 & SESP SCHEDULING & & SW & & & B0PVR9 & 15-Sep-98 & & $\mathrm{U}-238$ & U-238 \\
\hline 3899 & SESP SCHEDULING & & SW & & & BOPVTO & 15-Sep-98 & & $10098-97-2$ & SR-90 \\
\hline 3900 & SESP SCHEDULING & & SW & & & BOPVTO & 15-Sep-98 & & $10028-17-8$ & TRITIUM \\
\hline 3901 & SESP SCHEDULING & & SW & & & BOPVTO & 15-Sep-98 & & $13966-29-5$ & U-234 \\
\hline 3902 & SESP SCHEDULING & & SW & & & BOPVTO & 15-Sep-98 & & $15117-96-1$ & U-235 \\
\hline 3903 & SESP SCHEDULING & & SW & & & BOPVTO & 15-Sep-98 & & $\mathrm{U}-238$ & U-238 \\
\hline 3904 & SESP SCHEDULING & & SW & & & BOPVT1 & 15-Sep-98 & & $10098-97-2$ & SR-90 \\
\hline 3905 & SESP SCHEDULING & & SW & & & B0PVT1 & 15-Sep-98 & & $10028-17-8$ & TRITIUM \\
\hline 3906 & SESP SCHEDULING & & SW & & & B0PVT1 & 15-Sep-98 & & 13966-29-5 & U-234 \\
\hline 3907 & SESP SCHEDULING & & SW & & & B0PVT1 & 15-Sep-98 & & 15117-96-1 & U-235 \\
\hline 3908 & SESP SCHEDULING & & SW & & & BOPVT1 & 15-Sep-98 & & $\mathrm{U}-238$ & U-238 \\
\hline 3909 & SESP SCHEDULING & & SW & & & BOPVT2 & 15-Sep-98 & & $10098-97-2$ & SR-90 \\
\hline 3910 & SESP SCHEDULING & & SW & & & BOPVT2 & 15-Sep-98 & & $10028-17-8$ & TRITIUM \\
\hline 3911 & SESP SCHEDULING & & SW & & & BOPVT2 & 15-Sep-98 & & $13966-29-5$ & U-234 \\
\hline 3912 & SESP SCHEDULING & & SW & & & BOPVT2 & 15-Sep-98 & & 15117-96-1 & U-235 \\
\hline 3913 & SESP SCHEDULING & & SW & & & BOPVT2 & 15-Sep-98 & & $\mathrm{U}-238$ & U-238 \\
\hline 3914 & SESP SCHEDULING & & SW & & & B0PX68 & 21-Sep-98 & & $10098-97-2$ & SR-90 \\
\hline 3915 & SESP SCHEDULING & & SW & & & B0PX68 & 21-Sep-98 & & $10028-17-8$ & TRITIUM \\
\hline
\end{tabular}


\begin{tabular}{c|c|c}
$F$ & $G$
\end{tabular}

$\mathrm{H}$

$\mathrm{J}$

\begin{tabular}{|c|c|}
1 & SAMPLE_NUM_ASSIC \\
\hline 3916 & SESP SCHEDULING
\end{tabular}

\begin{tabular}{l|l} 
& GNED_TO \\
\hline
\end{tabular} 3917 SESP SCHEDULING 3918 SESP SCHEDULING 3919 SESP SCHEDULING 3920 SESP SCHEDULING \begin{tabular}{|l|l}
3921 & SESP SCHEDULING \\
\hline 3922 & SESP SCHEDULING
\end{tabular} 3922 SESP SCHEDULING 3923 SESP SCHEDULING 3924 SESP SCHEDULING 3925 SESP SCHEDULING 3926 SESP SCHEDULING 3927 SESP SCHEDULING 3928 SESP SCHEDULING 3929 SESP SCHEDULING 3930 SESP SCHEDULING 3931 SESP SCHEDULING 3932 SESP SCHEDULING 3933 SESP SCHEDULING 3934 SESP SCHEDULING 3935 SESP SCHEDULING 3936 SESP SCHEDULING 3937 SESP SCHEDULING 3938 SESP SCHEDULING 3939 SESP SCHEDULING 3940 SESP SCHEDULING 3941 SESP SCHEDULING 3942 SESP SCHEDULING 3943 SESP SCHEDULING 3944 SESP SCHEDULING 3945 SESP SCHEDULING 3946 SESP SCHEDULING 3947 SESP SCHEDULING 3948 SESP SCHEDULING 3949 SESP SCHEDULING 3950 SESP SCHEDULING 3951 SESP SCHEDULING

\begin{tabular}{|c|c|c|c|c|c|}
\hline MEDIA & BIOTA_MEDIA & \begin{tabular}{|l|l|l|} 
TAG_ID & SAMP_NUM \\
\end{tabular} & SAMP_DATE_TIME SAMP_DATE_TIME_ON & CON_ID & CON_SHORT_NAME \\
\hline SW & & B0PX68 & 21-Sep-98 & $13966-29-5$ & U-234 \\
\hline SW & & B0PX68 & 21-Sep-98 & 15117-96-1 & U-235 \\
\hline SW & & B0PX68 & 21-Sep-98 & U-238 & U-238 \\
\hline SW & & B0PX83 & 21-Sep-98 & $10098-97-2$ & SR-90 \\
\hline SW & & B0PX83 & 21-Sep-98 & $10028-17-8$ & TRITIUM \\
\hline SW & & B0PX83 & 21-Sep-98 & $13966-29-5$ & U-234 \\
\hline SW & & B0PX83 & 21-Sep-98 & 15117-96-1 & U-235 \\
\hline SW & & B0PX83 & 21-Sep-98 & U-238 & U-238 \\
\hline SW & & B0PX74 & 21-Sep-98 & $10098-97-2$ & SR-90 \\
\hline SW & & B0PX74 & 21-Sep-98 & $10028-17-8$ & TRITIUM \\
\hline SW & & B0PX74 & 21-Sep-98 & $13966-29-5$ & U-234 \\
\hline SW & & B0PX74 & 21-Sep-98 & 15117-96-1 & U-235 \\
\hline SW & & BOPX74 & 21-Sep-98 & $\mathrm{U}-238$ & U-238 \\
\hline SW & & BOPX70 & 21-Sep-98 & 10098-97-2 & SR-90 \\
\hline SW & & B0PX70 & 21-Sep-98 & $10028-17-8$ & TRITIUM \\
\hline SW & & BOPX70 & 21-Sep-98 & $13966-29-5$ & U-234 \\
\hline SW & & B0PX70 & 21-Sep-98 & 15117-96-1 & U-235 \\
\hline SW & & B0PX70 & 21-Sep-98 & U-238 & U-238 \\
\hline SW & & B0PX76 & 21-Sep-98 & $10098-97-2$ & SR-90 \\
\hline SW & & B0PX76 & 21-Sep-98 & 10028-17-8 & TRITIUM \\
\hline SW & & B0PX76 & 21-Sep-98 & $13966-29-5$ & U-234 \\
\hline SW & & BOPX76 & 21-Sep-98 & 15117-96-1 & U-235 \\
\hline SW & & BOPX76 & 21-Sep-98 & U-238 & U-238 \\
\hline SW & & B0PX78 & 21-Sep-98 & $10098-97-2$ & SR-90 \\
\hline SW & & B0PX78 & 21-Sep-98 & $10028-17-8$ & TRITIUM \\
\hline SW & & B0PX78 & 21-Sep-98 & $13966-29-5$ & U-234 \\
\hline SW & & B0PX78 & 21-Sep-98 & 15117-96-1 & U-235 \\
\hline SW & & B0PX78 & 21-Sep-98 & U-238 & U-238 \\
\hline SW & & B0PX80 & 21-Sep-98 & $10098-97-2$ & SR-90 \\
\hline SW & & B0PX80 & 21-Sep-98 & $10028-17-8$ & TRITIUM \\
\hline SW & & B0PX80 & 21-Sep-98 & $13966-29-5$ & U-234 \\
\hline SW & & B0PX80 & 21-Sep-98 & 15117-96-1 & U-235 \\
\hline SW & & B0PX80 & 21-Sep-98 & U-238 & U-238 \\
\hline SW & & B0PX81 & 21-Sep-98 & 10098-97-2 & SR-90 \\
\hline SW & & B0PX81 & 21-Sep-98 & $10028-17-8$ & TRITIUM \\
\hline SW & & B0PX81 & 21-Sep-98 & $13966-29-5$ & U-234 \\
\hline SW & & B0PX81 & 21-Sep-98 & 15117-96-1 & U-235 \\
\hline SW & & B0PX81 & 21-Sep-98 & $\mathrm{U}-238$ & U-238 \\
\hline
\end{tabular}

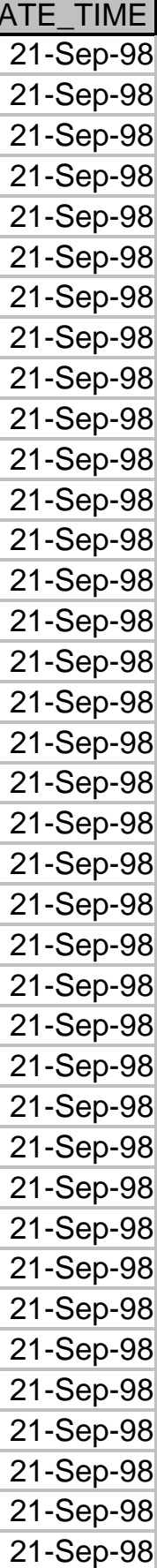




\begin{tabular}{|c|c|c|c|c|c|c|c|c|c|c|}
\hline & A & $\mathrm{B}$ & $C$ & $\mathrm{D}$ & $E$ & $\mathrm{~F}$ & $G$ & $\mathrm{H}$ & $\mathrm{I}$ & $\mathrm{J}$ \\
\hline 1 & SAMPLE_NUM_ASSIGNED_TO & SAMPLER & MEDIA & BIOTA_MEDIA & TAG_ID & SAMP_NUM & SAMP_DATE_TIME| & SAMP_DATE_TIME_ON & CON_ID & CON_SHORT_NAME \\
\hline 3954 & SESP SCHEDULING & & SW & & & B0PX82 & 21-Sep-98 & & $10098-97-2$ & SR-90 \\
\hline 3955 & SESP SCHEDULING & & SW & & & B0PX82 & 21-Sep-98 & & $10028-17-8$ & TRITIUM \\
\hline 3956 & SESP SCHEDULING & & SW & & & B0PX82 & 21-Sep-98 & & $13966-29-5$ & U-234 \\
\hline 3957 & SESP SCHEDULING & & SW & & & B0PX82 & 21-Sep-98 & & 15117-96-1 & U-235 \\
\hline 3958 & SESP SCHEDULING & & SW & & & B0PX82 & 21-Sep-98 & & U-238 & U-238 \\
\hline 3959 & SESP SCHEDULING & & SW & & & B0PX84 & 21-Sep-98 & & $10098-97-2$ & SR-90 \\
\hline 3960 & SESP SCHEDULING & & SW & & & B0PX84 & 21-Sep-98 & & $10028-17-8$ & TRITIUM \\
\hline 3961 & SESP SCHEDULING & & SW & & & B0PX84 & 21-Sep-98 & & $13966-29-5$ & $\mathrm{U}-234$ \\
\hline 3962 & SESP SCHEDULING & & SW & & & B0PX84 & 21-Sep-98 & & 15117-96-1 & U-235 \\
\hline 3963 & SESP SCHEDULING & & SW & & & B0PX84 & 21-Sep-98 & & U-238 & U-238 \\
\hline 3964 & SESP SCHEDULING & & SW & & & B0PX85 & 21-Sep-98 & & $10098-97-2$ & SR-90 \\
\hline 3965 & SESP SCHEDULING & & SW & & & B0PX85 & 21-Sep-98 & & $10028-17-8$ & TRITIUM \\
\hline 3966 & SESP SCHEDULING & & SW & & & B0PX85 & 21-Sep-98 & & $13966-29-5$ & U-234 \\
\hline 3967 & SESP SCHEDULING & & SW & & & B0PX85 & 21-Sep-98 & & 15117-96-1 & U-235 \\
\hline 3968 & SESP SCHEDULING & & SW & & & B0PX85 & 21-Sep-98 & & U-238 & U-238 \\
\hline 3969 & SESP SCHEDULING & & SW & & & B0PX90 & 21-Sep-98 & & $10098-97-2$ & SR-90 \\
\hline 3970 & SESP SCHEDULING & & SW & & & B0PX90 & 21-Sep-98 & & $10028-17-8$ & TRITIUM \\
\hline 3971 & SESP SCHEDULING & & SW & & & B0PX90 & 21-Sep-98 & & $13966-29-5$ & U-234 \\
\hline 3972 & SESP SCHEDULING & & SW & & & B0PX90 & 21-Sep-98 & & 15117-96-1 & U-235 \\
\hline 3973 & SESP SCHEDULING & & SW & & & B0PX90 & 21-Sep-98 & & U-238 & U-238 \\
\hline 3974 & SESP SCHEDULING & & SW & & & B0PX86 & 21-Sep-98 & & $10098-97-2$ & SR-90 \\
\hline 3975 & SESP SCHEDULING & & SW & & & B0PX86 & 21-Sep-98 & & $10028-17-8$ & TRITIUM \\
\hline 3976 & SESP SCHEDULING & & SW & & & B0PX86 & 21-Sep-98 & & $13966-29-5$ & U-234 \\
\hline 3977 & SESP SCHEDULING & & SW & & & B0PX86 & 21-Sep-98 & & $15117-96-1$ & U-235 \\
\hline 3978 & SESP SCHEDULING & & SW & & & B0PX86 & 21-Sep-98 & & U-238 & U-238 \\
\hline 3979 & SESP SCHEDULING & & SW & & & B0PX91 & 21-Sep-98 & & 10098-97-2 & SR-90 \\
\hline 3980 & SESP SCHEDULING & & SW & & & B0PX91 & 21-Sep-98 & & 10028-17-8 & TRITIUM \\
\hline 3981 & SESP SCHEDULING & & SW & & & B0PX91 & 21-Sep-98 & & 13966-29-5 & U-234 \\
\hline 3982 & SESP SCHEDULING & & SW & & & B0PX91 & 21-Sep-98 & & 15117-96-1 & U-235 \\
\hline 3983 & SESP SCHEDULING & & SW & & & B0PX91 & 21-Sep-98 & & U-238 & U-238 \\
\hline 3984 & SESP SCHEDULING & & SW & & & B0PX67 & 21-Sep-98 & & $10098-97-2$ & SR-90 \\
\hline 3985 & SESP SCHEDULING & & SW & & & B0PX67 & 21-Sep-98 & & $10028-17-8$ & TRITIUM \\
\hline 3986 & SESP SCHEDULING & & SW & & & B0PX67 & 21-Sep-98 & & $13966-29-5$ & U-234 \\
\hline 3987 & SESP SCHEDULING & & SW & & & B0PX67 & 21-Sep-98 & & 15117-96-1 & $\mathrm{U}-235$ \\
\hline 3988 & SESP SCHEDULING & & SW & & & B0PX67 & 21-Sep-98 & & U-238 & U-238 \\
\hline 3989 & SESP SCHEDULING & & SW & & & B0PX72 & 21-Sep-98 & & $10098-97-2$ & SR-90 \\
\hline 3990 & SESP SCHEDULING & & SW & & & B0PX72 & 21-Sep-98 & & $10028-17-8$ & TRITIUM \\
\hline 3991 & SESP SCHEDULING & & SW & & & B0PX72 & 21-Sep-98 & & $13966-29-5$ & U-234 \\
\hline
\end{tabular}




\begin{tabular}{|c|c|c|c|c|c|c|c|c|c|c|}
\hline & A & $\mathrm{B}$ & $\mathrm{C}$ & $\mathrm{D}$ & $E$ & $\mathrm{~F}$ & $G$ & $\mathrm{H}$ & $\mathrm{I}$ & $\mathrm{J}$ \\
\hline 1 & SAMPLE_NUM_ASSIGNED_TO & SAMPLER & MEDIA & BIOTA_MEDIA & TAG_ID & SAMP_NUM & SAMP_DATE_TIME & SAMP_DATE_TIME_ON & CON_ID & CON_SHORT_NAME \\
\hline 3992 & SESP SCHEDULING & & SW & & & B0PX72 & 21-Sep-98 & & 15117-96-1 & U-235 \\
\hline 3993 & SESP SCHEDULING & & SW & & & BOPX72 & 21-Sep-98 & & U-238 & U-238 \\
\hline 3994 & SESP SCHEDULING & & SW & & & BOPX73 & 21-Sep-98 & & $10098-97-2$ & SR-90 \\
\hline 3995 & SESP SCHEDULING & & SW & & & B0PX73 & 21-Sep-98 & & $10028-17-8$ & TRITIUM \\
\hline 3996 & SESP SCHEDULING & & SW & & & B0PX73 & 21-Sep-98 & & 13966-29-5 & $\mathrm{U}-234$ \\
\hline 3997 & SESP SCHEDULING & & SW & & & BOPX73 & 21-Sep-98 & & 15117-96-1 & U-235 \\
\hline 3998 & SESP SCHEDULING & & SW & & & B0PX73 & 21-Sep-98 & & $\mathrm{U}-238$ & U-238 \\
\hline 3999 & SESP SCHEDULING & & SW & & & B0PX87 & 21-Sep-98 & & 10098-97-2 & SR-90 \\
\hline 4000 & SESP SCHEDULING & & SW & & & B0P $\times 87$ & 21-Sep-98 & & $10028-17-8$ & TRITIUM \\
\hline 4001 & SESP SCHEDULING & & SW & & & B0PX87 & 21-Sep-98 & & $13966-29-5$ & U-234 \\
\hline 4002 & SESP SCHEDULING & & SW & & & B0PX87 & 21-Sep-98 & & 15117-96-1 & U-235 \\
\hline 4003 & SESP SCHEDULING & & SW & & & B0PX87 & 21-Sep-98 & & U-238 & U-238 \\
\hline 4004 & SESP SCHEDULING & & SW & & & B0PX88 & 21-Sep-98 & & 10098-97-2 & SR-90 \\
\hline 4005 & SESP SCHEDULING & & SW & & & B0PX88 & 21-Sep-98 & & $10028-17-8$ & TRITIUM \\
\hline 4006 & SESP SCHEDULING & & SW & & & B0PX88 & 21-Sep-98 & & $13966-29-5$ & U-234 \\
\hline 4007 & SESP SCHEDULING & & SW & & & B0PX88 & 21-Sep-98 & & $15117-96-1$ & U-235 \\
\hline 4008 & SESP SCHEDULING & & SW & & & B0PX88 & 21-Sep-98 & & U-238 & $\mathrm{U}-238$ \\
\hline 4009 & SESP SCHEDULING & & SW & & & B0PX89 & 21-Sep-98 & & 10098-97-2 & SR-90 \\
\hline 4010 & SESP SCHEDULING & & SW & & & B0PX89 & 21-Sep-98 & & $10028-17-8$ & TRITIUM \\
\hline 4011 & SESP SCHEDULING & & SW & & & B0PX89 & 21-Sep-98 & & $13966-29-5$ & U-234 \\
\hline 4012 & SESP SCHEDULING & & SW & & & B0PX89 & 21-Sep-98 & & 15117-96-1 & U-235 \\
\hline 4013 & SESP SCHEDULING & & SW & & & B0PX89 & 21-Sep-98 & & U-238 & U-238 \\
\hline 4014 & SESP SCHEDULING & & SW & & & B0T7R6 & 14-Dec-98 & & 10098-97-2 & SR-90 \\
\hline 4015 & SESP SCHEDULING & & SW & & & B0T7R6 & 14-Dec-98 & & $10028-17-8$ & TRITIUM \\
\hline 4016 & SESP SCHEDULING & & SW & & & B0T7R6 & 14-Dec-98 & & $13966-29-5$ & U-234 \\
\hline 4017 & SESP SCHEDULING & & SW & & & B0T7R6 & 14-Dec-98 & & 15117-96-1 & U-235 \\
\hline 4018 & SESP SCHEDULING & & SW & & & B0T7R6 & 14-Dec-98 & & U-238 & U-238 \\
\hline 4019 & SESP SCHEDULING & & SW & & & B0T7R7 & 14-Dec-98 & & $10098-97-2$ & SR-90 \\
\hline 4020 & SESP SCHEDULING & & SW & & & B0T7R7 & 14-Dec-98 & & $10028-17-8$ & TRITIUM \\
\hline 4021 & SESP SCHEDULING & & SW & & & B0T7R7 & 14-Dec-98 & & 13966-29-5 & U-234 \\
\hline 4022 & SESP SCHEDULING & & SW & & & B0T7R7 & 14-Dec-98 & & 15117-96-1 & U-235 \\
\hline 4023 & SESP SCHEDULING & & SW & & & BOT7R7 & 14-Dec-98 & & U-238 & U-238 \\
\hline 4024 & SESP SCHEDULING & & SW & & & B0T7R8 & 14-Dec-98 & & 10098-97-2 & SR-90 \\
\hline 4025 & SESP SCHEDULING & & SW & & & B0T7R8 & 14-Dec-98 & & $10028-17-8$ & TRITIUM \\
\hline 4026 & SESP SCHEDULING & & SW & & & B0T7R8 & 14-Dec-98 & & $13966-29-5$ & U-234 \\
\hline 4027 & SESP SCHEDULING & & SW & & & B0T7R8 & 14-Dec-98 & & 15117-96-1 & $\mathrm{U}-235$ \\
\hline 4028 & SESP SCHEDULING & & SW & & & B0T7R8 & 14-Dec-98 & & U-238 & U-238 \\
\hline 4029 & SESP SCHEDULING & & SW & & & BOT7R9 & 14-Dec-98 & & $10098-97-2$ & SR-90 \\
\hline
\end{tabular}




\begin{tabular}{|c|c|c|c|c|c|c|c|c|c|c|}
\hline & A & $\mathrm{B}$ & $C$ & $\mathrm{D}$ & $E$ & $\mathrm{~F}$ & $G$ & $\mathrm{H}$ & $\mathrm{I}$ & $\mathrm{J}$ \\
\hline 1 & SAMPLE_NUM_ASSIGNED_TO & SAMPLER & MEDIA & BIOTA_MEDIA & TAG_ID & SAMP_NUM & SAMP_DATE_TIME| & SAMP_DATE_TIME_ON & CON_ID & CON_SHORT_NAME \\
\hline 4030 & SESP SCHEDULING & & SW & & & B0T7R9 & 14-Dec-98 & & 10028-17-8 & TRITIUM \\
\hline 4031 & SESP SCHEDULING & & SW & & & B0T7R9 & 14-Dec-98 & & 13966-29-5 & U-234 \\
\hline 4032 & SESP SCHEDULING & & SW & & & B0T7R9 & 14-Dec-98 & & 15117-96-1 & U-235 \\
\hline 4033 & SESP SCHEDULING & & SW & & & B0T7R9 & 14-Dec-98 & & U-238 & U-238 \\
\hline 4034 & SESP SCHEDULING & & SW & & & B0T7R5 & 15-Dec-98 & & $10098-97-2$ & SR-90 \\
\hline 4035 & SESP SCHEDULING & & SW & & & B0T7T0 & 15-Dec-98 & & $10098-97-2$ & SR-90 \\
\hline 4036 & SESP SCHEDULING & & SW & & & B0T7R5 & 15-Dec-98 & & $10028-17-8$ & TRITIUM \\
\hline 4037 & SESP SCHEDULING & & SW & & & В0T7T0 & 15-Dec-98 & & $10028-17-8$ & TRITIUM \\
\hline 4038 & SESP SCHEDULING & & SW & & & B0T7R5 & 15-Dec-98 & & 13966-29-5 & U-234 \\
\hline 4039 & SESP SCHEDULING & & SW & & & B0T7R5 & 15-Dec-98 & & 15117-96-1 & U-235 \\
\hline 4040 & SESP SCHEDULING & & SW & & & B0T7R5 & 15-Dec-98 & & U-238 & U-238 \\
\hline 4041 & SESP SCHEDULING & & SW & & & B0T7T0 & 15-Dec-98 & & 13966-29-5 & U-234 \\
\hline 4042 & SESP SCHEDULING & & SW & & & B0T7T0 & 15-Dec-98 & & 15117-96-1 & U-235 \\
\hline 4043 & SESP SCHEDULING & & SW & & & В0T7T0 & 15-Dec-98 & & U-238 & U-238 \\
\hline 4044 & SESP SCHEDULING & & SW & & & B0T7T9 & 15-Dec-98 & & 10098-97-2 & SR-90 \\
\hline 4045 & SESP SCHEDULING & & SW & & & В0T7T9 & 15-Dec-98 & & $10028-17-8$ & TRITIUM \\
\hline 4046 & SESP SCHEDULING & & SW & & & В0T7T9 & 15-Dec-98 & & $13966-29-5$ & U-234 \\
\hline 4047 & SESP SCHEDULING & & SW & & & В0T7T9 & 15-Dec-98 & & $15117-96-1$ & U-235 \\
\hline 4048 & SESP SCHEDULING & & SW & & & В0T7T9 & 15-Dec-98 & & U-238 & U-238 \\
\hline 4049 & SESP SCHEDULING & & SW & & & B0T7T1 & 15-Dec-98 & & $10098-97-2$ & SR-90 \\
\hline 4050 & SESP SCHEDULING & & SW & & & B0T7T1 & 15-Dec-98 & & 10028-17-8 & TRITIUM \\
\hline 4051 & SESP SCHEDULING & & SW & & & B0T7T1 & 15-Dec-98 & & 13966-29-5 & U-234 \\
\hline 4052 & SESP SCHEDULING & & SW & & & B0T7T1 & 15-Dec-98 & & $15117-96-1$ & U-235 \\
\hline 4053 & SESP SCHEDULING & & SW & & & B0T7T1 & 15-Dec-98 & & U-238 & $\mathrm{U}-238$ \\
\hline 4054 & SESP SCHEDULING & & SW & & & В0T7T2 & 15-Dec-98 & & $10098-97-2$ & SR-90 \\
\hline 4055 & SESP SCHEDULING & & SW & & & B0T7T2 & 15-Dec-98 & & 10028-17-8 & TRITIUM \\
\hline 4056 & SESP SCHEDULING & & SW & & & B0T7T2 & 15-Dec-98 & & 13966-29-5 & U-234 \\
\hline 4057 & SESP SCHEDULING & & SW & & & B0T7T2 & 15-Dec-98 & & 15117-96-1 & U-235 \\
\hline 4058 & SESP SCHEDULING & & SW & & & В0T7T2 & 15-Dec-98 & & U-238 & U-238 \\
\hline 4059 & SESP SCHEDULING & & SW & & & В0T7Т3 & 15-Dec-98 & & $10098-97-2$ & SR-90 \\
\hline 4060 & SESP SCHEDULING & & SW & & & В0T7T3 & 15-Dec-98 & & $10028-17-8$ & TRITIUM \\
\hline 4061 & SESP SCHEDULING & & SW & & & В0T7Т3 & 15-Dec-98 & & $13966-29-5$ & U-234 \\
\hline 4062 & SESP SCHEDULING & & SW & & & В0T7T3 & 15-Dec-98 & & $15117-96-1$ & U-235 \\
\hline 4063 & SESP SCHEDULING & & SW & & & B0T7T3 & 15-Dec-98 & & U-238 & U-238 \\
\hline 4064 & SESP SCHEDULING & & SW & & & B0T7T4 & 15-Dec-98 & & $10098-97-2$ & SR-90 \\
\hline 4065 & SESP SCHEDULING & & SW & & & B0T7T4 & 15-Dec-98 & & $10028-17-8$ & TRITIUM \\
\hline 4066 & SESP SCHEDULING & & SW & & & B0T7T4 & 15-Dec-98 & & 13966-29-5 & U-234 \\
\hline 4067 & SESP SCHEDULING & & SW & & & B0T7T4 & 15-Dec-98 & & $15117-96-1$ & U-235 \\
\hline
\end{tabular}




\begin{tabular}{|c|c|c|c|c|c|c|c|c|c|c|}
\hline & A & $\mathrm{B}$ & $\mathrm{C}$ & $\mathrm{D}$ & $E$ & $\mathrm{~F}$ & $G$ & $\mathrm{H}$ & $\mathrm{I}$ & $\mathrm{J}$ \\
\hline 1 & SAMPLE_NUM_ASSIGNED_TO & SAMPLER & MEDIA & BIOTA_MEDIA & TAG_ID & SAMP_NUM & SAMP_DATE_TIME & SAMP_DATE_TIME_ON & CON_ID & CON_SHORT_NAME \\
\hline 4068 & SESP SCHEDULING & & SW & & & B0T7T4 & 15-Dec-98 & & U-238 & U-238 \\
\hline 4069 & SESP SCHEDULING & & SW & & & B0T7T5 & 15-Dec-98 & & $10098-97-2$ & SR-90 \\
\hline 4070 & SESP SCHEDULING & & SW & & & B0T7T5 & 15-Dec-98 & & $10028-17-8$ & TRITIUM \\
\hline 4071 & SESP SCHEDULING & & SW & & & B0T7T5 & 15-Dec-98 & & 13966-29-5 & $\mathrm{U}-234$ \\
\hline 4072 & SESP SCHEDULING & & SW & & & B0T7T5 & 15-Dec-98 & & $15117-96-1$ & U-235 \\
\hline 4073 & SESP SCHEDULING & & SW & & & B0T7T5 & 15-Dec-98 & & U-238 & U-238 \\
\hline 4074 & SESP SCHEDULING & & SW & & & B0T7T6 & 15-Dec-98 & & $10098-97-2$ & SR-90 \\
\hline 4075 & SESP SCHEDULING & & SW & & & B0T7T6 & 15-Dec-98 & & $10028-17-8$ & TRITIUM \\
\hline 4076 & SESP SCHEDULING & & SW & & & B0T7T6 & 15-Dec-98 & & 13966-29-5 & U-234 \\
\hline 4077 & SESP SCHEDULING & & SW & & & B0T7T6 & 15-Dec-98 & & 15117-96-1 & U-235 \\
\hline 4078 & SESP SCHEDULING & & SW & & & B0T7T6 & 15-Dec-98 & & $\mathrm{U}-238$ & $\mathrm{U}-238$ \\
\hline 4079 & SESP SCHEDULING & & SW & & & B0T7T7 & 15-Dec-98 & & $10098-97-2$ & SR-90 \\
\hline 4080 & SESP SCHEDULING & & SW & & & B0T7T7 & 15-Dec-98 & & $10028-17-8$ & TRITIUM \\
\hline 4081 & SESP SCHEDULING & & SW & & & B0T7T7 & 15-Dec-98 & & $13966-29-5$ & U-234 \\
\hline 4082 & SESP SCHEDULING & & SW & & & B0T7T7 & 15-Dec-98 & & 15117-96-1 & U-235 \\
\hline 4083 & SESP SCHEDULING & & SW & & & B0T7T7 & 15-Dec-98 & & U-238 & U-238 \\
\hline 4084 & SESP SCHEDULING & & SW & & & В0T7T8 & 15-Dec-98 & & $10098-97-2$ & SR-90 \\
\hline 4085 & SESP SCHEDULING & & SW & & & В0Т7Т8 & 15-Dec-98 & & $10028-17-8$ & TRITIUM \\
\hline 4086 & SESP SCHEDULING & & SW & & & В0T7T8 & 15-Dec-98 & & $13966-29-5$ & U-234 \\
\hline 4087 & SESP SCHEDULING & & SW & & & B0T7T8 & 15-Dec-98 & & $15117-96-1$ & U-235 \\
\hline 4088 & SESP SCHEDULING & & SW & & & В0T7T8 & 15-Dec-98 & & U-238 & U-238 \\
\hline 4089 & SESP SCHEDULING & & SW & & & B0V231 & 11-Jun-99 & & $10098-97-2$ & SR-90 \\
\hline 4090 & SESP SCHEDULING & & SW & & & B0V231 & 11-Jun-99 & & $10028-17-8$ & TRITIUM \\
\hline 4091 & SESP SCHEDULING & & SW & & & B0V231 & 11-Jun-99 & & $13966-29-5$ & U-234 \\
\hline 4092 & SESP SCHEDULING & & SW & & & B0V231 & 11-Jun-99 & & $15117-96-1$ & U-235 \\
\hline 4093 & SESP SCHEDULING & & SW & & & B0V231 & 11-Jun-99 & & $\mathrm{U}-238$ & $\mathrm{U}-238$ \\
\hline 4094 & SESP SCHEDULING & & SW & & & B0V232 & 11-Jun-99 & & $10098-97-2$ & SR-90 \\
\hline 4095 & SESP SCHEDULING & & SW & & & BOV232 & 11-Jun-99 & & $10028-17-8$ & TRITIUM \\
\hline 4096 & SESP SCHEDULING & & SW & & & B0V232 & 11-Jun-99 & & 13966-29-5 & U-234 \\
\hline 4097 & SESP SCHEDULING & & SW & & & B0V232 & 11-Jun-99 & & $15117-96-1$ & U-235 \\
\hline 4098 & SESP SCHEDULING & & SW & & & B0V232 & 11-Jun-99 & & U-238 & U-238 \\
\hline 4099 & SESP SCHEDULING & & SW & & & B0V233 & 11-Jun-99 & & $10098-97-2$ & SR-90 \\
\hline 4100 & SESP SCHEDULING & & SW & & & B0V233 & 11-Jun-99 & & $10028-17-8$ & TRITIUM \\
\hline 4101 & SESP SCHEDULING & & SW & & & B0V233 & 11-Jun-99 & & $13966-29-5$ & U-234 \\
\hline 4102 & SESP SCHEDULING & & SW & & & B0V233 & 11-Jun-99 & & $15117-96-1$ & U-235 \\
\hline 4103 & SESP SCHEDULING & & SW & & & B0V233 & 11-Jun-99 & & $\mathrm{U}-238$ & $\mathrm{U}-238$ \\
\hline 4104 & SESP SCHEDULING & & SW & & & B0V234 & 11-Jun-99 & & $10098-97-2$ & SR-90 \\
\hline 4105 & SESP SCHEDULING & & SW & & & B0V234 & 11-Jun-99 & & $10028-17-8$ & TRITIUM \\
\hline
\end{tabular}


\begin{tabular}{c|c|c|c}
\hline & D & D & E \\
\hline
\end{tabular}

\begin{tabular}{|c|c}
$F$ & $G$ \\
\hline
\end{tabular}

\begin{tabular}{l|l}
$\mathrm{G}$ & $\mathrm{H}$
\end{tabular}

\begin{tabular}{|l|l|l}
$\mathrm{H}$ & $\mathrm{I}$ & $\mathrm{J}$
\end{tabular}

\begin{tabular}{|c|c|c|c|c|c|c|c|c|c|c|}
\hline & & & & & & & & & & \\
\hline 1 & SAMPLE_NUM_ASSIGNED_TO & SAMPLER & MEDIA & BIOTA_MEDIA & TAG_ID & SAMP_NUM & SAMP_DATE_TIME & SAMP_DATE_TIME_ON & CON_ID & CON_SHORT_NAME \\
\hline 4106 & SESP SCHEDULING & & SW & & & B0V234 & 11-Jun-99 & & $13966-29-5$ & U-234 \\
\hline 4107 & SESP SCHEDULING & & SW & & & B0V234 & 11-Jun-99 & & 15117-96-1 & U-235 \\
\hline 4108 & SESP SCHEDULING & & SW & & & B0V234 & 11-Jun-99 & & U-238 & U-238 \\
\hline 4109 & SESP SCHEDULING & & SW & & & B0V230 & 14-Jun-99 & & $10098-97-2$ & SR-90 \\
\hline 4110 & SESP SCHEDULING & & SW & & & B0V235 & 14-Jun-99 & & $10098-97-2$ & SR-90 \\
\hline 4111 & SESP SCHEDULING & & SW & & & B0V230 & 14-Jun-99 & & $10028-17-8$ & TRITIUM \\
\hline 4112 & SESP SCHEDULING & & SW & & & B0V235 & 14-Jun-99 & & $10028-17-8$ & TRITIUM \\
\hline 4113 & SESP SCHEDULING & & SW & & & B0V230 & 14-Jun-99 & & 13966-29-5 & U-234 \\
\hline 4114 & SESP SCHEDULING & & SW & & & Bov230 & 14-Jun-99 & & 15117-96-1 & U-235 \\
\hline 4115 & SESP SCHEDULING & & SW & & & B0V230 & 14-Jun-99 & & $\mathrm{U}-238$ & $\mathrm{U}-238$ \\
\hline 4116 & SESP SCHEDULING & & SW & & & B0V235 & 14-Jun-99 & & $13966-29-5$ & $\mathrm{U}-234$ \\
\hline 4117 & SESP SCHEDULING & & SW & & & B0V235 & 14-Jun-99 & & 15117-96-1 & U-235 \\
\hline 4118 & SESP SCHEDULING & & SW & & & B0V235 & 14-Jun-99 & & $\mathrm{U}-238$ & U-238 \\
\hline 4119 & SESP SCHEDULING & & SW & & & B0V244 & 14-Jun-99 & & $10098-97-2$ & SR-90 \\
\hline 4120 & SESP SCHEDULING & & SW & & & B0V244 & 14-Jun-99 & & $10028-17-8$ & TRITIUM \\
\hline 4121 & SESP SCHEDULING & & SW & & & BoV244 & 14-Jun-99 & & $13966-29-5$ & U-234 \\
\hline 4122 & SESP SCHEDULING & & SW & & & B0V244 & 14-Jun-99 & & 15117-96-1 & U-235 \\
\hline 4123 & SESP SCHEDULING & & SW & & & B0V244 & 14-Jun-99 & & $\mathrm{U}-238$ & U-238 \\
\hline 4124 & SESP SCHEDULING & & SW & & & B0V236 & 14-Jun-99 & & $10098-97-2$ & SR-90 \\
\hline 4125 & SESP SCHEDULING & & SW & & & B0V236 & 14-Jun-99 & & $10028-17-8$ & TRITIUM \\
\hline 4126 & SESP SCHEDULING & & SW & & & B0V236 & 14-Jun-99 & & $13966-29-5$ & $U-234$ \\
\hline 4127 & SESP SCHEDULING & & SW & & & B0V236 & 14-Jun-99 & & 15117-96-1 & U-235 \\
\hline 4128 & SESP SCHEDULING & & SW & & & BoV236 & 14-Jun-99 & & $\mathrm{U}-238$ & $\mathrm{U}-238$ \\
\hline 4129 & SESP SCHEDULING & & SW & & & B0V237 & 14-Jun-99 & & $10098-97-2$ & SR-90 \\
\hline 4130 & SESP SCHEDULING & & SW & & & B0V237 & 14-Jun-99 & & $10028-17-8$ & TRITIUM \\
\hline 4131 & SESP SCHEDULING & & SW & & & B0V237 & 14-Jun-99 & & $13966-29-5$ & $\mathrm{U}-234$ \\
\hline 4132 & SESP SCHEDULING & & SW & & & B0V237 & 14-Jun-99 & & 15117-96-1 & U-235 \\
\hline 4133 & SESP SCHEDULING & & SW & & & B0V237 & 14-Jun-99 & & $\mathrm{U}-238$ & U-238 \\
\hline 4134 & SESP SCHEDULING & & SW & & & B0V238 & 14-Jun-99 & & $10098-97-2$ & SR-90 \\
\hline 4135 & SESP SCHEDULING & & SW & & & B0V238 & 14-Jun-99 & & $10028-17-8$ & TRITIUM \\
\hline 4136 & SESP SCHEDULING & & SW & & & B0V238 & 14-Jun-99 & & $13966-29-5$ & $\mathrm{U}-234$ \\
\hline 4137 & SESP SCHEDULING & & SW & & & B0V238 & 14-Jun-99 & & 15117-96-1 & U-235 \\
\hline 4138 & SESP SCHEDULING & & SW & & & B0V238 & 14-Jun-99 & & $\mathrm{U}-238$ & $\mathrm{U}-238$ \\
\hline 4139 & SESP SCHEDULING & & SW & & & B0V239 & 14-Jun-99 & & $10098-97-2$ & SR-90 \\
\hline 4140 & SESP SCHEDULING & & SW & & & BoV239 & 14-Jun-99 & & $10028-17-8$ & TRITIUM \\
\hline 4141 & SESP SCHEDULING & & SW & & & B0V239 & 14-Jun-99 & & $13966-29-5$ & $\mathrm{U}-234$ \\
\hline 4142 & SESP SCHEDULING & & SW & & & B0V239 & 14-Jun-99 & & 15117-96-1 & $\mathrm{U}-235$ \\
\hline 4143 & SESP SCHEDULING & & SW & & & BoV239 & 14-Jun-99 & & U-238 & $\mathrm{U}-238$ \\
\hline
\end{tabular}

\begin{tabular}{l|l|l}
\hline 4142 & SESP SCHEDULING \\
\hline 4143 & SESP SCHEDULING \\
\hline
\end{tabular} 


\begin{tabular}{|c|c|c|c|c|c|c|c|c|c|c|}
\hline & A & $\mathrm{B}$ & $\mathrm{C}$ & $\mathrm{D}$ & $\mathrm{E}$ & $\mathrm{F}$ & $G$ & $\mathrm{H}$ & I & $\mathrm{J}$ \\
\hline 1 & SAMPLE_NUM_ASSIGNED_TO & SAMPLER & MEDIA & BIOTA_MEDIA & TAG_ID & SAMP_NUM & SAMP_DATE_TIME & SAMP_DATE_TIME_ON & CON_ID & CON_SHORT_NAME \\
\hline 4144 & SESP SCHEDULING & & SW & & & BOV240 & 14-Jun-99 & & $10098-97-2$ & SR-90 \\
\hline 4145 & SESP SCHEDULING & & SW & & & BOV240 & 14-Jun-99 & & $10028-17-8$ & TRITIUM \\
\hline 4146 & SESP SCHEDULING & & SW & & & BOV240 & 14-Jun-99 & & $13966-29-5$ & U-234 \\
\hline 4147 & SESP SCHEDULING & & SW & & & BOV240 & 14-Jun-99 & & 15117-96-1 & U-235 \\
\hline 4148 & SESP SCHEDULING & & SW & & & BOV240 & 14-Jun-99 & & $\mathrm{U}-238$ & U-238 \\
\hline 4149 & SESP SCHEDULING & & SW & & & B0V241 & 14-Jun-99 & & $10098-97-2$ & SR-90 \\
\hline 4150 & SESP SCHEDULING & & SW & & & B0V241 & 14-Jun-99 & & $10028-17-8$ & TRITIUM \\
\hline 4151 & SESP SCHEDULING & & SW & & & B0V241 & 14-Jun-99 & & $13966-29-5$ & $\mathrm{U}-234$ \\
\hline 4152 & SESP SCHEDULING & & SW & & & B0V241 & 14-Jun-99 & & 15117-96-1 & U-235 \\
\hline 4153 & SESP SCHEDULING & & SW & & & B0V241 & 14-Jun-99 & & $\mathrm{U}-238$ & $\mathrm{U}-238$ \\
\hline 4154 & SESP SCHEDULING & & SW & & & B0V242 & 14-Jun-99 & & $10098-97-2$ & SR-90 \\
\hline 4155 & SESP SCHEDULING & & SW & & & BOV242 & 14-Jun-99 & & $10028-17-8$ & TRITIUM \\
\hline 4156 & SESP SCHEDULING & & SW & & & BoV242 & 14-Jun-99 & & 13966-29-5 & U-234 \\
\hline 4157 & SESP SCHEDULING & & SW & & & B0V242 & 14-Jun-99 & & 15117-96-1 & U-235 \\
\hline 4158 & SESP SCHEDULING & & SW & & & B0V242 & 14-Jun-99 & & $\mathrm{U}-238$ & U-238 \\
\hline 4159 & SESP SCHEDULING & & SW & & & B0V243 & 14-Jun-99 & & $10098-97-2$ & SR-90 \\
\hline 4160 & SESP SCHEDULING & & SW & & & BOV243 & 14-Jun-99 & & $10028-17-8$ & TRITIUM \\
\hline 4161 & SESP SCHEDULING & & SW & & & B0V243 & 14-Jun-99 & & 13966-29-5 & U-234 \\
\hline 4162 & SESP SCHEDULING & & SW & & & B0V243 & 14-Jun-99 & & 15117-96-1 & U-235 \\
\hline 4163 & SESP SCHEDULING & & SW & & & B0V243 & 14-Jun-99 & & $\mathrm{U}-238$ & U-238 \\
\hline 4164 & SESP SCHEDULING & & SW & & & B0W930 & 10-Sep-99 & & $10098-97-2$ & SR-90 \\
\hline 4165 & SESP SCHEDULING & & SW & & & BoW936 & 10-Sep-99 & & $10098-97-2$ & SR-90 \\
\hline 4166 & SESP SCHEDULING & & SW & & & BoW930 & 10-Sep-99 & & $10028-17-8$ & TRITIUM \\
\hline 4167 & SESP SCHEDULING & & SW & & & B0W936 & 10-Sep-99 & & $10028-17-8$ & TRITIUM \\
\hline 4168 & SESP SCHEDULING & & SW & & & B0W930 & 10-Sep-99 & & $13966-29-5$ & U-234 \\
\hline 4169 & SESP SCHEDULING & & SW & & & BoW930 & 10-Sep-99 & & 15117-96-1 & $\mathrm{U}-235$ \\
\hline 4170 & SESP SCHEDULING & & SW & & & B0W930 & 10-Sep-99 & & $\mathrm{U}-238$ & $\mathrm{U}-238$ \\
\hline 4171 & SESP SCHEDULING & & SW & & & B0W936 & 10-Sep-99 & & 13966-29-5 & U-234 \\
\hline 4172 & SESP SCHEDULING & & SW & & & Bow936 & 10-Sep-99 & & 15117-96-1 & U-235 \\
\hline 4173 & SESP SCHEDULING & & SW & & & BoW936 & 10-Sep-99 & & $\mathrm{U}-238$ & $\mathrm{U}-238$ \\
\hline 4174 & SESP SCHEDULING & & SW & & & BoW942 & 10-Sep-99 & & $10098-97-2$ & SR-90 \\
\hline 4175 & SESP SCHEDULING & & SW & & & BoW942 & 10-Sep-99 & & $10028-17-8$ & TRITIUM \\
\hline 4176 & SESP SCHEDULING & & SW & & & BoW942 & 10-Sep-99 & & 13966-29-5 & U-234 \\
\hline 4177 & SESP SCHEDULING & & SW & & & BoW942 & 10-Sep-99 & & 15117-96-1 & U-235 \\
\hline 4178 & SESP SCHEDULING & & SW & & & Bow942 & 10-Sep-99 & & $\mathrm{U}-238$ & $\mathrm{U}-238$ \\
\hline 4179 & SESP SCHEDULING & & SW & & & B0W938 & 10-Sep-99 & & $10098-97-2$ & SR-90 \\
\hline 4180 & SESP SCHEDULING & & SW & & & B0W938 & 10-Sep-99 & & $10028-17-8$ & TRITIUM \\
\hline 4181 & SESP SCHEDULING & & SW & & & BoW938 & 10-Sep-99 & & 13966-29-5 & U-234 \\
\hline
\end{tabular}




\begin{tabular}{|c|c|c|c|c|c|c|c|c|c|c|}
\hline & A & $\mathrm{B}$ & $\mathrm{C}$ & $\mathrm{D}$ & $E$ & $\mathrm{~F}$ & $G$ & $\mathrm{H}$ & $\mathrm{I}$ & $\mathrm{J}$ \\
\hline 1 & SAMPLE_NUM_ASSIGNED_TO & SAMPLER & MEDIA & BIOTA_MEDIA & TAG_ID & SAMP_NUM & SAMP_DATE_TIME & SAMP_DATE_TIME_ON & CON_ID & CON_SHORT_NAME \\
\hline 4182 & SESP SCHEDULING & & SW & & & B0W938 & 10-Sep-99 & & 15117-96-1 & U-235 \\
\hline 4183 & SESP SCHEDULING & & SW & & & B0W938 & 10-Sep-99 & & U-238 & U-238 \\
\hline 4184 & SESP SCHEDULING & & SW & & & B0W943 & 10-Sep-99 & & $10098-97-2$ & SR-90 \\
\hline 4185 & SESP SCHEDULING & & SW & & & B0W943 & 10-Sep-99 & & $10028-17-8$ & TRITIUM \\
\hline 4186 & SESP SCHEDULING & & SW & & & Bow943 & 10-Sep-99 & & $13966-29-5$ & $\mathrm{U}-234$ \\
\hline 4187 & SESP SCHEDULING & & SW & & & Bow943 & 10-Sep-99 & & $15117-96-1$ & U-235 \\
\hline 4188 & SESP SCHEDULING & & SW & & & BoW943 & 10-Sep-99 & & U-238 & U-238 \\
\hline 4189 & SESP SCHEDULING & & SW & & & Bow940 & 10-Sep-99 & & $10098-97-2$ & SR-90 \\
\hline 4190 & SESP SCHEDULING & & SW & & & Bow940 & 10-Sep-99 & & $10028-17-8$ & TRITIUM \\
\hline 4191 & SESP SCHEDULING & & SW & & & BoW940 & 10-Sep-99 & & $13966-29-5$ & U-234 \\
\hline 4192 & SESP SCHEDULING & & SW & & & Bow940 & 10-Sep-99 & & 15117-96-1 & U-235 \\
\hline 4193 & SESP SCHEDULING & & SW & & & Bow940 & 10-Sep-99 & & U-238 & U-238 \\
\hline 4194 & SESP SCHEDULING & & SW & & & Bow945 & 10-Sep-99 & & $10098-97-2$ & SR-90 \\
\hline 4195 & SESP SCHEDULING & & SW & & & B0W945 & 10-Sep-99 & & $10028-17-8$ & TRITIUM \\
\hline 4196 & SESP SCHEDULING & & SW & & & B0W945 & 10-Sep-99 & & $13966-29-5$ & U-234 \\
\hline 4197 & SESP SCHEDULING & & SW & & & B0W945 & 10-Sep-99 & & 15117-96-1 & U-235 \\
\hline 4198 & SESP SCHEDULING & & SW & & & Bow945 & 10-Sep-99 & & $\mathrm{U}-238$ & $\mathrm{U}-238$ \\
\hline 4199 & SESP SCHEDULING & & SW & & & B0W957 & 10-Sep-99 & & $10098-97-2$ & SR-90 \\
\hline 4200 & SESP SCHEDULING & & SW & & & B0W957 & 10-Sep-99 & & $10028-17-8$ & TRITIUM \\
\hline 4201 & SESP SCHEDULING & & SW & & & B0W957 & 10-Sep-99 & & $13966-29-5$ & U-234 \\
\hline 4202 & SESP SCHEDULING & & SW & & & BoW957 & 10-Sep-99 & & $15117-96-1$ & U-235 \\
\hline 4203 & SESP SCHEDULING & & SW & & & BoW957 & 10-Sep-99 & & U-238 & U-238 \\
\hline 4204 & SESP SCHEDULING & & SW & & & B0W958 & 10-Sep-99 & & $10098-97-2$ & SR-90 \\
\hline 4205 & SESP SCHEDULING & & SW & & & B0W958 & 10-Sep-99 & & $10028-17-8$ & TRITIUM \\
\hline 4206 & SESP SCHEDULING & & SW & & & B0W958 & 10-Sep-99 & & $13966-29-5$ & U-234 \\
\hline 4207 & SESP SCHEDULING & & SW & & & B0W958 & 10-Sep-99 & & $15117-96-1$ & U-235 \\
\hline 4208 & SESP SCHEDULING & & SW & & & B0W958 & 10-Sep-99 & & U-238 & U-238 \\
\hline 4209 & SESP SCHEDULING & & SW & & & B0W959 & 10-Sep-99 & & $10098-97-2$ & SR-90 \\
\hline 4210 & SESP SCHEDULING & & SW & & & B0W959 & 10-Sep-99 & & $10028-17-8$ & TRITIUM \\
\hline 4211 & SESP SCHEDULING & & SW & & & BoW959 & 10-Sep-99 & & $13966-29-5$ & U-234 \\
\hline 4212 & SESP SCHEDULING & & SW & & & Bow959 & 10-Sep-99 & & $15117-96-1$ & U-235 \\
\hline 4213 & SESP SCHEDULING & & SW & & & B0W959 & 10-Sep-99 & & U-238 & U-238 \\
\hline 4214 & SESP SCHEDULING & & SW & & & Bow960 & 10-Sep-99 & & $10098-97-2$ & SR-90 \\
\hline 4215 & SESP SCHEDULING & & SW & & & BoW960 & 10-Sep-99 & & $10028-17-8$ & TRITIUM \\
\hline 4216 & SESP SCHEDULING & & SW & & & B0W960 & 10-Sep-99 & & $13966-29-5$ & U-234 \\
\hline 4217 & SESP SCHEDULING & & SW & & & BoW960 & 10-Sep-99 & & $15117-96-1$ & $\mathrm{U}-235$ \\
\hline 4218 & SESP SCHEDULING & & SW & & & Bow960 & 10-Sep-99 & & $U-238$ & U-238 \\
\hline 4219 & SESP SCHEDULING & & SW & & & B0W932 & 10-Sep-99 & & $10098-97-2$ & SR-90 \\
\hline
\end{tabular}




\begin{tabular}{|c|c|c|c|c|c|c|c|c|c|c|}
\hline & A & $\mathrm{B}$ & $\mathrm{C}$ & $\mathrm{D}$ & $E$ & $\mathrm{~F}$ & $G$ & $\mathrm{H}$ & $\mathrm{I}$ & $\mathrm{J}$ \\
\hline 1 & SAMPLE_NUM_ASSIGNED_TO & SAMPLER & MEDIA & BIOTA_MEDIA & TAG_ID & SAMP_NUM & SAMP_DATE_TIME & SAMP_DATE_TIME_ON & CON_ID & CON_SHORT_NAME \\
\hline 4220 & SESP SCHEDULING & & SW & & & B0W932 & 10-Sep-99 & & $10028-17-8$ & TRITIUM \\
\hline 4221 & SESP SCHEDULING & & SW & & & B0W932 & 10-Sep-99 & & $13966-29-5$ & U-234 \\
\hline 4222 & SESP SCHEDULING & & SW & & & B0W932 & 10-Sep-99 & & 15117-96-1 & U-235 \\
\hline 4223 & SESP SCHEDULING & & SW & & & B0W932 & 10-Sep-99 & & $\mathrm{U}-238$ & $\mathrm{U}-238$ \\
\hline 4224 & SESP SCHEDULING & & SW & & & B0W933 & 10-Sep-99 & & $10098-97-2$ & SR-90 \\
\hline 4225 & SESP SCHEDULING & & SW & & & Bow933 & 10-Sep-99 & & $10028-17-8$ & TRITIUM \\
\hline 4226 & SESP SCHEDULING & & SW & & & B0W933 & 10-Sep-99 & & $13966-29-5$ & U-234 \\
\hline 4227 & SESP SCHEDULING & & SW & & & Bow933 & 10-Sep-99 & & $15117-96-1$ & U-235 \\
\hline 4228 & SESP SCHEDULING & & SW & & & Bow933 & 10-Sep-99 & & $\mathrm{U}-238$ & U-238 \\
\hline 4229 & SESP SCHEDULING & & SW & & & B0W934 & 10-Sep-99 & & $10098-97-2$ & SR-90 \\
\hline 4230 & SESP SCHEDULING & & SW & & & B0W934 & 10-Sep-99 & & $10028-17-8$ & TRITIUM \\
\hline 4231 & SESP SCHEDULING & & SW & & & Bow934 & 10-Sep-99 & & $13966-29-5$ & U-234 \\
\hline 4232 & SESP SCHEDULING & & SW & & & BoW934 & 10-Sep-99 & & $15117-96-1$ & U-235 \\
\hline 4233 & SESP SCHEDULING & & SW & & & B0W934 & 10-Sep-99 & & $\mathrm{U}-238$ & $\mathrm{U}-238$ \\
\hline 4234 & SESP SCHEDULING & & SW & & & B0W935 & 10-Sep-99 & & $10098-97-2$ & SR-90 \\
\hline 4235 & SESP SCHEDULING & & SW & & & B0W935 & 10-Sep-99 & & $10028-17-8$ & TRITIUM \\
\hline 4236 & SESP SCHEDULING & & SW & & & B0W935 & 10-Sep-99 & & $13966-29-5$ & $\mathrm{U}-234$ \\
\hline 4237 & SESP SCHEDULING & & SW & & & BoW935 & 10-Sep-99 & & $15117-96-1$ & U-235 \\
\hline 4238 & SESP SCHEDULING & & SW & & & BoW935 & 10-Sep-99 & & U-238 & U-238 \\
\hline 4239 & SESP SCHEDULING & & SW & & & B0WB23 & 16-Sep-99 & & $10098-97-2$ & SR-90 \\
\hline 4240 & SESP SCHEDULING & & SW & & & BoWB23 & 16-Sep-99 & & $10028-17-8$ & TRITIUM \\
\hline 4241 & SESP SCHEDULING & & SW & & & B0WB23 & 16-Sep-99 & & $13966-29-5$ & U-234 \\
\hline 4242 & SESP SCHEDULING & & SW & & & B0WB23 & 16-Sep-99 & & $15117-96-1$ & U-235 \\
\hline 4243 & SESP SCHEDULING & & SW & & & B0WB23 & 16-Sep-99 & & $U-238$ & U-238 \\
\hline 4244 & SESP SCHEDULING & & SW & & & BoWB24 & 16-Sep-99 & & $10098-97-2$ & SR-90 \\
\hline 4245 & SESP SCHEDULING & & SW & & & BOWB24 & 16-Sep-99 & & $10028-17-8$ & TRITIUM \\
\hline 4246 & SESP SCHEDULING & & SW & & & BOWB24 & 16-Sep-99 & & $13966-29-5$ & U-234 \\
\hline 4247 & SESP SCHEDULING & & SW & & & BOWB24 & 16-Sep-99 & & 15117-96-1 & U-235 \\
\hline 4248 & SESP SCHEDULING & & SW & & & BOWB24 & 16-Sep-99 & & U-238 & U-238 \\
\hline 4249 & SESP SCHEDULING & & SW & & & B0WB25 & 16-Sep-99 & & $10098-97-2$ & SR-90 \\
\hline 4250 & SESP SCHEDULING & & SW & & & B0WB25 & 16-Sep-99 & & $10028-17-8$ & TRITIUM \\
\hline 4251 & SESP SCHEDULING & & SW & & & B0WB25 & 16-Sep-99 & & $13966-29-5$ & U-234 \\
\hline 4252 & SESP SCHEDULING & & SW & & & B0WB25 & 16-Sep-99 & & $15117-96-1$ & $\mathrm{U}-235$ \\
\hline 4253 & SESP SCHEDULING & & SW & & & B0WB25 & 16-Sep-99 & & $\mathrm{U}-238$ & $\mathrm{U}-238$ \\
\hline 4254 & SESP SCHEDULING & & SW & & & BoWB26 & 16-Sep-99 & & $10098-97-2$ & SR-90 \\
\hline 4255 & SESP SCHEDULING & & SW & & & B0WB26 & 16-Sep-99 & & $10028-17-8$ & TRITIUM \\
\hline 4256 & SESP SCHEDULING & & SW & & & BoWB26 & 16-Sep-99 & & $13966-29-5$ & U-234 \\
\hline 4257 & SESP SCHEDULING & & SW & & & BoWB26 & 16-Sep-99 & & $15117-96-1$ & U-235 \\
\hline
\end{tabular}




\begin{tabular}{|c|c|c|c|c|c|c|c|c|c|c|}
\hline & A & $\mathrm{B}$ & $\mathrm{C}$ & $\mathrm{D}$ & $E$ & $\mathrm{~F}$ & $G$ & $\mathrm{H}$ & $\mathrm{I}$ & $\mathrm{J}$ \\
\hline 1 & SAMPLE_NUM_ASSIGNED_TO & SAMPLER & MEDIA & BIOTA_MEDIA & TAG_ID & SAMP_NUM & SAMP_DATE_TIME & SAMP_DATE_TIME_ON & CON_ID & CON_SHORT_NAME \\
\hline 4258 & SESP SCHEDULING & & SW & & & B0WB26 & 16-Sep-99 & & U-238 & U-238 \\
\hline 4259 & SESP SCHEDULING & & SW & & & BOWB27 & 16-Sep-99 & & $10098-97-2$ & SR-90 \\
\hline 4260 & SESP SCHEDULING & & SW & & & B0WB27 & 16-Sep-99 & & $10028-17-8$ & TRITIUM \\
\hline 4261 & SESP SCHEDULING & & SW & & & B0WB27 & 16-Sep-99 & & 13966-29-5 & $\mathrm{U}-234$ \\
\hline 4262 & SESP SCHEDULING & & SW & & & B0WB27 & 16-Sep-99 & & $15117-96-1$ & U-235 \\
\hline 4263 & SESP SCHEDULING & & SW & & & B0WB27 & 16-Sep-99 & & U-238 & U-238 \\
\hline 4264 & SESP SCHEDULING & & SW & & & B0WB53 & 16-Sep-99 & & $10098-97-2$ & SR-90 \\
\hline 4265 & SESP SCHEDULING & & SW & & & B0WB53 & 16-Sep-99 & & $10028-17-8$ & TRITIUM \\
\hline 4266 & SESP SCHEDULING & & SW & & & B0WB53 & 16-Sep-99 & & 13966-29-5 & U-234 \\
\hline 4267 & SESP SCHEDULING & & SW & & & B0WB53 & 16-Sep-99 & & $15117-96-1$ & U-235 \\
\hline 4268 & SESP SCHEDULING & & SW & & & B0WB53 & 16-Sep-99 & & $\mathrm{U}-238$ & $\mathrm{U}-238$ \\
\hline 4269 & SESP SCHEDULING & & SW & & & B0WB54 & 16-Sep-99 & & $10098-97-2$ & SR-90 \\
\hline 4270 & SESP SCHEDULING & & SW & & & B0WB54 & 16-Sep-99 & & $10028-17-8$ & TRITIUM \\
\hline 4271 & SESP SCHEDULING & & SW & & & B0WB54 & 16-Sep-99 & & $13966-29-5$ & U-234 \\
\hline 4272 & SESP SCHEDULING & & SW & & & B0WB54 & 16-Sep-99 & & 15117-96-1 & U-235 \\
\hline 4273 & SESP SCHEDULING & & SW & & & B0WB54 & 16-Sep-99 & & U-238 & U-238 \\
\hline 4274 & SESP SCHEDULING & & SW & & & B0WB55 & 16-Sep-99 & & $10098-97-2$ & SR-90 \\
\hline 4275 & SESP SCHEDULING & & SW & & & B0WB55 & 16-Sep-99 & & $10028-17-8$ & TRITIUM \\
\hline 4276 & SESP SCHEDULING & & SW & & & B0WB55 & 16-Sep-99 & & $13966-29-5$ & U-234 \\
\hline 4277 & SESP SCHEDULING & & SW & & & B0WB55 & 16-Sep-99 & & $15117-96-1$ & U-235 \\
\hline 4278 & SESP SCHEDULING & & SW & & & B0WB55 & 16-Sep-99 & & U-238 & U-238 \\
\hline 4279 & SESP SCHEDULING & & SW & & & B0WB56 & 16-Sep-99 & & $10098-97-2$ & SR-90 \\
\hline 4280 & SESP SCHEDULING & & SW & & & B0WB56 & 16-Sep-99 & & $10028-17-8$ & TRITIUM \\
\hline 4281 & SESP SCHEDULING & & SW & & & B0WB56 & 16-Sep-99 & & $13966-29-5$ & U-234 \\
\hline 4282 & SESP SCHEDULING & & SW & & & B0WB56 & 16-Sep-99 & & $15117-96-1$ & U-235 \\
\hline 4283 & SESP SCHEDULING & & SW & & & B0WB56 & 16-Sep-99 & & $\mathrm{U}-238$ & $\mathrm{U}-238$ \\
\hline 4284 & SESP SCHEDULING & & SW & & & BOWB28 & 16-Sep-99 & & $10098-97-2$ & SR-90 \\
\hline 4285 & SESP SCHEDULING & & SW & & & BOWB28 & 16-Sep-99 & & $10028-17-8$ & TRITIUM \\
\hline 4286 & SESP SCHEDULING & & SW & & & BOWB28 & 16-Sep-99 & & $13966-29-5$ & U-234 \\
\hline 4287 & SESP SCHEDULING & & SW & & & B0WB28 & 16-Sep-99 & & $15117-96-1$ & U-235 \\
\hline 4288 & SESP SCHEDULING & & SW & & & B0WB28 & 16-Sep-99 & & U-238 & U-238 \\
\hline 4289 & SESP SCHEDULING & & SW & & & BOWBB8 & 16-Sep-99 & & $10098-97-2$ & SR-90 \\
\hline 4290 & SESP SCHEDULING & & SW & & & B0WBB8 & 16-Sep-99 & & $10028-17-8$ & TRITIUM \\
\hline 4291 & SESP SCHEDULING & & SW & & & B0WBB8 & 16-Sep-99 & & $13966-29-5$ & U-234 \\
\hline 4292 & SESP SCHEDULING & & SW & & & BOWBB8 & 16-Sep-99 & & $15117-96-1$ & U-235 \\
\hline 4293 & SESP SCHEDULING & & SW & & & B0WBB8 & 16-Sep-99 & & $\mathrm{U}-238$ & U-238 \\
\hline 4294 & SESP SCHEDULING & & SW & & & BOWBB7 & 16-Sep-99 & & $10098-97-2$ & SR-90 \\
\hline 4295 & SESP SCHEDULING & & SW & & & B0WBB7 & 16-Sep-99 & & $10028-17-8$ & TRITIUM \\
\hline
\end{tabular}




\begin{tabular}{|c|c|c|c|c|c|c|c|c|c|c|}
\hline & A & $\mathrm{B}$ & $\mathrm{C}$ & $\mathrm{D}$ & $\mathrm{E}$ & $\mathrm{F}$ & $G$ & $\mathrm{H}$ & I & $\mathrm{J}$ \\
\hline 1 & SAMPLE_NUM_ASSIGNED_TO & SAMPLER & MEDIA & BIOTA_MEDIA & TAG_ID & SAMP_NUM & SAMP_DATE_TIME & SAMP_DATE_TIME_ON & CON_ID & CON_SHORT_NAME \\
\hline 4296 & SESP SCHEDULING & & SW & & & B0WBB7 & 16-Sep-99 & & $13966-29-5$ & $\mathrm{U}-234$ \\
\hline 4297 & SESP SCHEDULING & & SW & & & B0WBB7 & 16-Sep-99 & & 15117-96-1 & U-235 \\
\hline 4298 & SESP SCHEDULING & & SW & & & B0WBB7 & 16-Sep-99 & & U-238 & U-238 \\
\hline 4299 & SESP SCHEDULING & & SW & & & B0WBB6 & 16-Sep-99 & & $10098-97-2$ & SR-90 \\
\hline 4300 & SESP SCHEDULING & & SW & & & B0WBB6 & 16-Sep-99 & & $10028-17-8$ & TRITIUM \\
\hline 4301 & SESP SCHEDULING & & SW & & & B0WBB6 & 16-Sep-99 & & $13966-29-5$ & U-234 \\
\hline 4302 & SESP SCHEDULING & & SW & & & B0WBB6 & 16-Sep-99 & & 15117-96-1 & U-235 \\
\hline 4303 & SESP SCHEDULING & & SW & & & B0WBB6 & 16-Sep-99 & & U-238 & $\mathrm{U}-238$ \\
\hline 4304 & SESP SCHEDULING & & SW & & & B0WBB5 & 16-Sep-99 & & $10098-97-2$ & SR-90 \\
\hline 4305 & SESP SCHEDULING & & SW & & & B0WBB5 & 16-Sep-99 & & $10028-17-8$ & TRITIUM \\
\hline 4306 & SESP SCHEDULING & & SW & & & B0WBB5 & 16-Sep-99 & & $13966-29-5$ & U-234 \\
\hline 4307 & SESP SCHEDULING & & SW & & & B0WBB5 & 16-Sep-99 & & $15117-96-1$ & U-235 \\
\hline 4308 & SESP SCHEDULING & & SW & & & B0WBB5 & 16-Sep-99 & & U-238 & U-238 \\
\hline 4309 & SESP SCHEDULING & & SW & & & B0WB29 & 16-Sep-99 & & $10098-97-2$ & SR-90 \\
\hline 4310 & SESP SCHEDULING & & SW & & & B0WB29 & 16-Sep-99 & & $10028-17-8$ & TRITIUM \\
\hline 4311 & SESP SCHEDULING & & SW & & & B0WB29 & 16-Sep-99 & & $13966-29-5$ & U-234 \\
\hline 4312 & SESP SCHEDULING & & SW & & & B0WB29 & 16-Sep-99 & & 15117-96-1 & U-235 \\
\hline 4313 & SESP SCHEDULING & & SW & & & B0WB29 & 16-Sep-99 & & U-238 & $\mathrm{U}-238$ \\
\hline 4314 & SESP SCHEDULING & & SW & & & B0WB34 & 16-Sep-99 & & $10098-97-2$ & SR-90 \\
\hline 4315 & SESP SCHEDULING & & SW & & & B0WB34 & 16-Sep-99 & & $10028-17-8$ & TRITIUM \\
\hline 4316 & SESP SCHEDULING & & SW & & & B0WB34 & 16-Sep-99 & & $13966-29-5$ & U-234 \\
\hline 4317 & SESP SCHEDULING & & SW & & & BOWB34 & 16-Sep-99 & & $15117-96-1$ & U-235 \\
\hline 4318 & SESP SCHEDULING & & SW & & & B0WB34 & 16-Sep-99 & & U-238 & $\mathrm{U}-238$ \\
\hline 4319 & SESP SCHEDULING & & SW & & & BOWB30 & 16-Sep-99 & & $10098-97-2$ & SR-90 \\
\hline 4320 & SESP SCHEDULING & & SW & & & B0WB30 & 16-Sep-99 & & 10028-17-8 & TRITIUM \\
\hline 4321 & SESP SCHEDULING & & SW & & & B0WB30 & 16-Sep-99 & & 13966-29-5 & U-234 \\
\hline 4322 & SESP SCHEDULING & & SW & & & B0WB30 & 16-Sep-99 & & 15117-96-1 & U-235 \\
\hline 4323 & SESP SCHEDULING & & SW & & & B0WB30 & 16-Sep-99 & & U-238 & U-238 \\
\hline 4324 & SESP SCHEDULING & & SW & & & B0WB31 & 16-Sep-99 & & $10098-97-2$ & SR-90 \\
\hline 4325 & SESP SCHEDULING & & SW & & & B0WB31 & 16-Sep-99 & & $10028-17-8$ & TRITIUM \\
\hline 4326 & SESP SCHEDULING & & SW & & & B0WB31 & 16-Sep-99 & & $13966-29-5$ & U-234 \\
\hline 4327 & SESP SCHEDULING & & SW & & & B0WB31 & 16-Sep-99 & & 15117-96-1 & U-235 \\
\hline 4328 & SESP SCHEDULING & & SW & & & B0WB31 & 16-Sep-99 & & U-238 & U-238 \\
\hline 4329 & SESP SCHEDULING & & SW & & & B0WB32 & 16-Sep-99 & & $10098-97-2$ & SR-90 \\
\hline 4330 & SESP SCHEDULING & & SW & & & BOWB32 & 16-Sep-99 & & $10028-17-8$ & TRITIUM \\
\hline 4331 & SESP SCHEDULING & & SW & & & BOWB32 & 16-Sep-99 & & $13966-29-5$ & U-234 \\
\hline 4332 & SESP SCHEDULING & & SW & & & B0WB32 & 16-Sep-99 & & 15117-96-1 & U-235 \\
\hline 4333 & SESP SCHEDULING & & SW & & & B0WB32 & 16-Sep-99 & & U-238 & $\mathrm{U}-238$ \\
\hline
\end{tabular}




\begin{tabular}{|c|c|c|c|c|c|c|c|c|c|c|}
\hline & A & $\mathrm{B}$ & $C$ & $\mathrm{D}$ & $E$ & $\mathrm{~F}$ & $G$ & $\mathrm{H}$ & $\mathrm{I}$ & $\mathrm{J}$ \\
\hline 1 & SAMPLE_NUM_ASSIGNED_TO & SAMPLER & MEDIA & BIOTA_MEDIA & TAG_ID & SAMP_NUM & SAMP_DATE_TIME| & SAMP_DATE_TIME_ON & CON_ID & CON_SHORT_NAME \\
\hline 4334 & SESP SCHEDULING & & SW & & & B0WB33 & 16-Sep-99 & & $10098-97-2$ & SR-90 \\
\hline 4335 & SESP SCHEDULING & & SW & & & B0WB33 & 16-Sep-99 & & $10028-17-8$ & TRITIUM \\
\hline 4336 & SESP SCHEDULING & & SW & & & B0WB33 & 16-Sep-99 & & $13966-29-5$ & U-234 \\
\hline 4337 & SESP SCHEDULING & & SW & & & B0WB33 & 16-Sep-99 & & 15117-96-1 & U-235 \\
\hline 4338 & SESP SCHEDULING & & SW & & & B0WB33 & 16-Sep-99 & & U-238 & U-238 \\
\hline 4339 & SESP SCHEDULING & & SW & & & BOWB02 & 21-Sep-99 & & $10098-97-2$ & SR-90 \\
\hline 4340 & SESP SCHEDULING & & SW & & & B0WB02 & 21-Sep-99 & & $10028-17-8$ & TRITIUM \\
\hline 4341 & SESP SCHEDULING & & SW & & & B0WB02 & 21-Sep-99 & & $13966-29-5$ & $\mathrm{U}-234$ \\
\hline 4342 & SESP SCHEDULING & & SW & & & B0WB02 & 21-Sep-99 & & 15117-96-1 & U-235 \\
\hline 4343 & SESP SCHEDULING & & SW & & & B0WB02 & 21-Sep-99 & & U-238 & U-238 \\
\hline 4344 & SESP SCHEDULING & & SW & & & BoWB12 & 21-Sep-99 & & $10098-97-2$ & SR-90 \\
\hline 4345 & SESP SCHEDULING & & SW & & & B0WB12 & 21-Sep-99 & & $10028-17-8$ & TRITIUM \\
\hline 4346 & SESP SCHEDULING & & SW & & & B0WB12 & 21-Sep-99 & & $13966-29-5$ & U-234 \\
\hline 4347 & SESP SCHEDULING & & SW & & & B0WB12 & 21-Sep-99 & & 15117-96-1 & U-235 \\
\hline 4348 & SESP SCHEDULING & & SW & & & B0WB12 & 21-Sep-99 & & U-238 & U-238 \\
\hline 4349 & SESP SCHEDULING & & SW & & & B0WB06 & 21-Sep-99 & & $10098-97-2$ & SR-90 \\
\hline 4350 & SESP SCHEDULING & & SW & & & B0WB06 & 21-Sep-99 & & $10028-17-8$ & TRITIUM \\
\hline 4351 & SESP SCHEDULING & & SW & & & B0WB06 & 21-Sep-99 & & $13966-29-5$ & U-234 \\
\hline 4352 & SESP SCHEDULING & & SW & & & B0WB06 & 21-Sep-99 & & 15117-96-1 & U-235 \\
\hline 4353 & SESP SCHEDULING & & SW & & & B0WB06 & 21-Sep-99 & & U-238 & $\mathrm{U}-238$ \\
\hline 4354 & SESP SCHEDULING & & SW & & & B0WB03 & 21-Sep-99 & & 10098-97-2 & SR-90 \\
\hline 4355 & SESP SCHEDULING & & SW & & & B0WB03 & 21-Sep-99 & & $10028-17-8$ & TRITIUM \\
\hline 4356 & SESP SCHEDULING & & SW & & & B0WB03 & 21-Sep-99 & & $13966-29-5$ & U-234 \\
\hline 4357 & SESP SCHEDULING & & SW & & & B0WB03 & 21-Sep-99 & & $15117-96-1$ & U-235 \\
\hline 4358 & SESP SCHEDULING & & SW & & & B0WB03 & 21-Sep-99 & & U-238 & U-238 \\
\hline 4359 & SESP SCHEDULING & & SW & & & BOWB07 & 21-Sep-99 & & 10098-97-2 & SR-90 \\
\hline 4360 & SESP SCHEDULING & & SW & & & BOWB07 & 21-Sep-99 & & 10028-17-8 & TRITIUM \\
\hline 4361 & SESP SCHEDULING & & SW & & & B0WB07 & 21-Sep-99 & & 13966-29-5 & U-234 \\
\hline 4362 & SESP SCHEDULING & & SW & & & B0WB07 & 21-Sep-99 & & 15117-96-1 & U-235 \\
\hline 4363 & SESP SCHEDULING & & SW & & & B0WB07 & 21-Sep-99 & & U-238 & U-238 \\
\hline 4364 & SESP SCHEDULING & & SW & & & B0WB08 & 21-Sep-99 & & $10098-97-2$ & SR-90 \\
\hline 4365 & SESP SCHEDULING & & SW & & & B0WB08 & 21-Sep-99 & & $10028-17-8$ & TRITIUM \\
\hline 4366 & SESP SCHEDULING & & SW & & & B0WB08 & 21-Sep-99 & & $13966-29-5$ & U-234 \\
\hline 4367 & SESP SCHEDULING & & SW & & & B0WB08 & 21-Sep-99 & & 15117-96-1 & $\mathrm{U}-235$ \\
\hline 4368 & SESP SCHEDULING & & SW & & & B0WB08 & 21-Sep-99 & & U-238 & U-238 \\
\hline 4369 & SESP SCHEDULING & & SW & & & B0WB09 & 21-Sep-99 & & $10098-97-2$ & SR-90 \\
\hline 4370 & SESP SCHEDULING & & SW & & & B0WB09 & 21-Sep-99 & & $10028-17-8$ & TRITIUM \\
\hline 4371 & SESP SCHEDULING & & SW & & & B0WB09 & 21-Sep-99 & & $13966-29-5$ & U-234 \\
\hline
\end{tabular}




\begin{tabular}{|c|c|c|c|c|c|c|c|c|c|c|}
\hline & A & $\mathrm{B}$ & $\mathrm{C}$ & $\mathrm{D}$ & $E$ & $\mathrm{~F}$ & $G$ & $\mathrm{H}$ & $\mathrm{I}$ & $\mathrm{J}$ \\
\hline 1 & SAMPLE_NUM_ASSIGNED_TO & SAMPLER & MEDIA & BIOTA_MEDIA & TAG_ID & SAMP_NUM & SAMP_DATE_TIME & SAMP_DATE_TIME_ON & CON_ID & CON_SHORT_NAME \\
\hline 4372 & SESP SCHEDULING & & SW & & & B0WB09 & 21-Sep-99 & & 15117-96-1 & U-235 \\
\hline 4373 & SESP SCHEDULING & & SW & & & BOWB09 & 21-Sep-99 & & U-238 & U-238 \\
\hline 4374 & SESP SCHEDULING & & SW & & & B0WB10 & 21-Sep-99 & & $10098-97-2$ & SR-90 \\
\hline 4375 & SESP SCHEDULING & & SW & & & B0WB10 & 21-Sep-99 & & $10028-17-8$ & TRITIUM \\
\hline 4376 & SESP SCHEDULING & & SW & & & B0WB10 & 21-Sep-99 & & 13966-29-5 & $\mathrm{U}-234$ \\
\hline 4377 & SESP SCHEDULING & & SW & & & BoWB10 & 21-Sep-99 & & 15117-96-1 & U-235 \\
\hline 4378 & SESP SCHEDULING & & SW & & & BOWB10 & 21-Sep-99 & & $\mathrm{U}-238$ & U-238 \\
\hline 4379 & SESP SCHEDULING & & SW & & & BoWB11 & 21-Sep-99 & & 10098-97-2 & SR-90 \\
\hline 4380 & SESP SCHEDULING & & SW & & & B0WB11 & 21-Sep-99 & & $10028-17-8$ & TRITIUM \\
\hline 4381 & SESP SCHEDULING & & SW & & & B0WB11 & 21-Sep-99 & & $13966-29-5$ & U-234 \\
\hline 4382 & SESP SCHEDULING & & SW & & & B0WB11 & 21-Sep-99 & & 15117-96-1 & U-235 \\
\hline 4383 & SESP SCHEDULING & & SW & & & BoWB11 & 21-Sep-99 & & U-238 & U-238 \\
\hline 4384 & SESP SCHEDULING & & SW & & & B0WB13 & 21-Sep-99 & & $10098-97-2$ & SR-90 \\
\hline 4385 & SESP SCHEDULING & & SW & & & B0WB13 & 21-Sep-99 & & $10028-17-8$ & TRITIUM \\
\hline 4386 & SESP SCHEDULING & & SW & & & B0WB13 & 21-Sep-99 & & $13966-29-5$ & U-234 \\
\hline 4387 & SESP SCHEDULING & & SW & & & B0WB13 & 21-Sep-99 & & $15117-96-1$ & U-235 \\
\hline 4388 & SESP SCHEDULING & & SW & & & BoWB13 & 21-Sep-99 & & U-238 & $\mathrm{U}-238$ \\
\hline 4389 & SESP SCHEDULING & & SW & & & BoWB16 & 21-Sep-99 & & 10098-97-2 & SR-90 \\
\hline 4390 & SESP SCHEDULING & & SW & & & BoWB16 & 21-Sep-99 & & $10028-17-8$ & TRITIUM \\
\hline 4391 & SESP SCHEDULING & & SW & & & BoWB16 & 21-Sep-99 & & $13966-29-5$ & U-234 \\
\hline 4392 & SESP SCHEDULING & & SW & & & BoWB16 & 21-Sep-99 & & 15117-96-1 & U-235 \\
\hline 4393 & SESP SCHEDULING & & SW & & & BoWB16 & 21-Sep-99 & & U-238 & U-238 \\
\hline 4394 & SESP SCHEDULING & & SW & & & BOWB20 & 21-Sep-99 & & $10098-97-2$ & SR-90 \\
\hline 4395 & SESP SCHEDULING & & SW & & & BOWB20 & 21-Sep-99 & & $10028-17-8$ & TRITIUM \\
\hline 4396 & SESP SCHEDULING & & SW & & & BOWB20 & 21-Sep-99 & & $13966-29-5$ & U-234 \\
\hline 4397 & SESP SCHEDULING & & SW & & & BOWB20 & 21-Sep-99 & & 15117-96-1 & U-235 \\
\hline 4398 & SESP SCHEDULING & & SW & & & BOWB20 & 21-Sep-99 & & U-238 & U-238 \\
\hline 4399 & SESP SCHEDULING & & SW & & & B0WB14 & 21-Sep-99 & & $10098-97-2$ & SR-90 \\
\hline 4400 & SESP SCHEDULING & & SW & & & B0WB14 & 21-Sep-99 & & $10028-17-8$ & TRITIUM \\
\hline 4401 & SESP SCHEDULING & & SW & & & BoWB14 & 21-Sep-99 & & 13966-29-5 & U-234 \\
\hline 4402 & SESP SCHEDULING & & SW & & & BoWB14 & 21-Sep-99 & & 15117-96-1 & U-235 \\
\hline 4403 & SESP SCHEDULING & & SW & & & BoWB14 & 21-Sep-99 & & U-238 & U-238 \\
\hline 4404 & SESP SCHEDULING & & SW & & & BoWB21 & 21-Sep-99 & & $10098-97-2$ & SR-90 \\
\hline 4405 & SESP SCHEDULING & & SW & & & BoWB21 & 21-Sep-99 & & $10028-17-8$ & TRITIUM \\
\hline 4406 & SESP SCHEDULING & & SW & & & BOWB21 & 21-Sep-99 & & $13966-29-5$ & U-234 \\
\hline 4407 & SESP SCHEDULING & & SW & & & BoWB21 & 21-Sep-99 & & 15117-96-1 & $\mathrm{U}-235$ \\
\hline 4408 & SESP SCHEDULING & & SW & & & BoWB21 & 21-Sep-99 & & U-238 & U-238 \\
\hline 4409 & SESP SCHEDULING & & SW & & & BOWB04 & 21-Sep-99 & & $10098-97-2$ & SR-90 \\
\hline
\end{tabular}


\begin{tabular}{c|c|c}
$F$ & $G$
\end{tabular}

$\mathrm{H}$

\begin{tabular}{c|l}
1 & SAMPLE_NUM_ASSIC \\
4410 & SESP SCHEDULING
\end{tabular}

\begin{tabular}{|l|l}
4411 & SESP SCHEDULING \\
\hline 4412 & SESP SCHEDULING \\
\hline
\end{tabular}

4412 SESP SCHEDULING

4413 SESP SCHEDULING

4414 SESP SCHEDULING

\begin{tabular}{|l|l|}
\hline 4415 & SESP SCHEDULING \\
\hline 4416 & SESP SCHEDULING
\end{tabular}

\begin{tabular}{|l|l}
4416 & SESP SCHEDULING \\
\hline
\end{tabular}

4417 SESP SCHEDULING

\begin{tabular}{|l|l}
4418 & SESP SCHEDULING \\
\hline 4419 & SESP SCHEDULING
\end{tabular}

\begin{tabular}{|l|l|}
4419 & SESP SCHEDULING \\
\hline 4420 &
\end{tabular}

4420 SESP SCHEDULING

4421 SESP SCHEDULING

\begin{tabular}{l|l}
4422 & SESP SCHEDULING \\
\hline 4423 & SESP SCHEDULING
\end{tabular}

4423 SESP SCHEDULING

4424 SESP SCHEDULING

\begin{tabular}{l|l}
4425 & SESP SCHEDULING \\
\hline 4426 & SESP SCHEDULING
\end{tabular}

4426 SESP SCHEDULING

4427 SESP SCHEDULING

4428 SESP SCHEDULING

\begin{tabular}{l|l}
4429 & SESP SCHEDULING \\
\hline 4430 & SESP SCHEDULING
\end{tabular}

4430 SESP SCHEDULING

4431 SESP SCHEDULING

4432 SESP SCHEDULING

\begin{tabular}{|l|l}
4433 & SESP SCHEDULING \\
\hline
\end{tabular}

4434 SESP SCHEDULING

4435 SESP SCHEDULING

4436 SESP SCHEDULING

4437 SESP SCHEDULING

4438 SESP SCHEDULING

4439 SESP SCHEDULING

4440 SESP SCHEDULING

4441 SESP SCHEDULING

4442 SESP SCHEDULING

\begin{tabular}{|l|l|}
44443 & SESP SCHEDULING \\
\hline 4444 & SESP SCHEDULING \\
\hline
\end{tabular}

4444 SESP SCHEDULING

4445 SESP SCHEDULING

4446 SESP SCHEDULING

\begin{tabular}{|l|l|}
\hline 4447 & SESP SCHEDULING \\
\hline
\end{tabular}

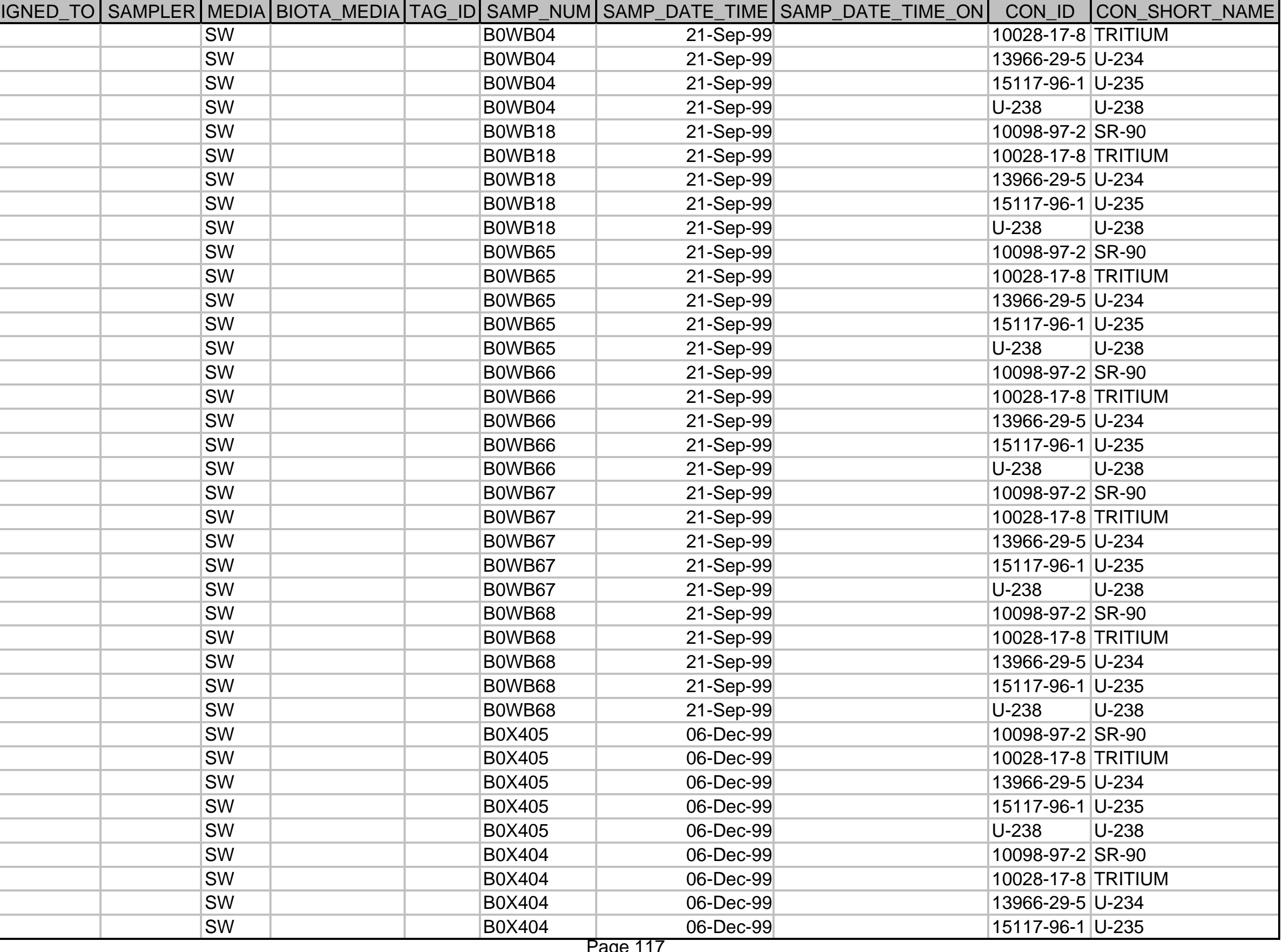

Page 117 


\begin{tabular}{|c|c|c|c|c|c|c|c|c|c|c|}
\hline & A & $\mathrm{B}$ & $\mathrm{C}$ & $\mathrm{D}$ & $\mathrm{E}$ & $\mathrm{F}$ & $G$ & $\mathrm{H}$ & I & $\mathrm{J}$ \\
\hline 1 & SAMPLE_NUM_ASSIGNED_TO & SAMPLER & MEDIA & BIOTA_MEDIA & TAG_ID & SAMP_NUM & SAMP_DATE_TIME & SAMP_DATE_TIME_ON & CON_ID & CON_SHORT_NAME \\
\hline 4448 & SESP SCHEDULING & & SW & & & B0X404 & 06-Dec-99 & & $\mathrm{U}-238$ & $\mathrm{U}-238$ \\
\hline 4449 & SESP SCHEDULING & & SW & & & B0X403 & 06-Dec-99 & & $10098-97-2$ & SR-90 \\
\hline 4450 & SESP SCHEDULING & & SW & & & B0X403 & 06-Dec-99 & & $10028-17-8$ & TRITIUM \\
\hline 4451 & SESP SCHEDULING & & SW & & & B0X403 & 06-Dec-99 & & $13966-29-5$ & U-234 \\
\hline 4452 & SESP SCHEDULING & & SW & & & B0X403 & 06-Dec-99 & & 15117-96-1 & U-235 \\
\hline 4453 & SESP SCHEDULING & & SW & & & B0X403 & 06-Dec-99 & & $\mathrm{U}-238$ & $\mathrm{U}-238$ \\
\hline 4454 & SESP SCHEDULING & & SW & & & B0X402 & 06-Dec-99 & & $10098-97-2$ & SR-90 \\
\hline 4455 & SESP SCHEDULING & & SW & & & B0X402 & 06-Dec-99 & & $10028-17-8$ & TRITIUM \\
\hline 4456 & SESP SCHEDULING & & SW & & & B0X402 & 06-Dec-99 & & 13966-29-5 & U-234 \\
\hline 4457 & SESP SCHEDULING & & SW & & & B0X402 & 06-Dec-99 & & $15117-96-1$ & U-235 \\
\hline 4458 & SESP SCHEDULING & & SW & & & B0X402 & 06-Dec-99 & & $\mathrm{U}-238$ & U-238 \\
\hline 4459 & SESP SCHEDULING & & SW & & & B0X3X1 & 06-Dec-99 & & $10098-97-2$ & SR-90 \\
\hline 4460 & SESP SCHEDULING & & SW & & & B0X3X6 & 06-Dec-99 & & $10098-97-2$ & SR-90 \\
\hline 4461 & SESP SCHEDULING & & SW & & & B0X3X1 & 06-Dec-99 & & $10028-17-8$ & TRITIUM \\
\hline 4462 & SESP SCHEDULING & & SW & & & B0X3X6 & 06-Dec-99 & & $10028-17-8$ & TRITIUM \\
\hline 4463 & SESP SCHEDULING & & SW & & & B0X3X1 & 06-Dec-99 & & 13966-29-5 & U-234 \\
\hline 4464 & SESP SCHEDULING & & SW & & & B0X3X1 & 06-Dec-99 & & 15117-96-1 & U-235 \\
\hline 4465 & SESP SCHEDULING & & SW & & & B0X3X1 & 06-Dec-99 & & $\mathrm{U}-238$ & U-238 \\
\hline 4466 & SESP SCHEDULING & & SW & & & B0X3X6 & 06-Dec-99 & & 13966-29-5 & $\mathrm{U}-234$ \\
\hline 4467 & SESP SCHEDULING & & SW & & & B0X3X6 & 06-Dec-99 & & 15117-96-1 & U-235 \\
\hline 4468 & SESP SCHEDULING & & SW & & & B0X3X6 & 06-Dec-99 & & $\mathrm{U}-238$ & U-238 \\
\hline 4469 & SESP SCHEDULING & & SW & & & B0X3Y1 & 06-Dec-99 & & $10098-97-2$ & SR-90 \\
\hline 4470 & SESP SCHEDULING & & SW & & & B0X3Y1 & 06-Dec-99 & & $10028-17-8$ & TRITIUM \\
\hline 4471 & SESP SCHEDULING & & SW & & & B0X3Y1 & 06-Dec-99 & & $13966-29-5$ & U-234 \\
\hline 4472 & SESP SCHEDULING & & SW & & & B0X3Y1 & 06-Dec-99 & & $15117-96-1$ & U-235 \\
\hline 4473 & SESP SCHEDULING & & SW & & & B0X3Y1 & 06-Dec-99 & & $\mathrm{U}-238$ & U-238 \\
\hline 4474 & SESP SCHEDULING & & SW & & & B0X3X7 & 06-Dec-99 & & $10098-97-2$ & SR-90 \\
\hline 4475 & SESP SCHEDULING & & SW & & & B0X3X7 & 06-Dec-99 & & $10028-17-8$ & TRITIUM \\
\hline 4476 & SESP SCHEDULING & & SW & & & B0X3X7 & 06-Dec-99 & & 13966-29-5 & U-234 \\
\hline 4477 & SESP SCHEDULING & & SW & & & B0X3X7 & 06-Dec-99 & & 15117-96-1 & U-235 \\
\hline 4478 & SESP SCHEDULING & & SW & & & B0X3X7 & 06-Dec-99 & & $\mathrm{U}-238$ & $\mathrm{U}-238$ \\
\hline 4479 & SESP SCHEDULING & & SW & & & B0X3X8 & 06-Dec-99 & & $10098-97-2$ & SR-90 \\
\hline 4480 & SESP SCHEDULING & & SW & & & B0X3X8 & 06-Dec-99 & & $10028-17-8$ & TRITIUM \\
\hline 4481 & SESP SCHEDULING & & SW & & & B0X3X8 & 06-Dec-99 & & 13966-29-5 & U-234 \\
\hline 4482 & SESP SCHEDULING & & SW & & & B0X3X8 & 06-Dec-99 & & $15117-96-1$ & $\mathrm{U}-235$ \\
\hline 4483 & SESP SCHEDULING & & SW & & & B0X3X8 & 06-Dec-99 & & U-238 & U-238 \\
\hline 4484 & SESP SCHEDULING & & SW & & & B0X3X9 & 06-Dec-99 & & $10098-97-2$ & SR-90 \\
\hline 4485 & SESP SCHEDULING & & SW & & & B0X3X9 & 06-Dec-99 & & $10028-17-8$ & TRITIUM \\
\hline
\end{tabular}




\begin{tabular}{|c|c|c|c|c|c|c|c|c|c|c|}
\hline & A & $\mathrm{B}$ & $\mathrm{C}$ & $\mathrm{D}$ & $E$ & $\mathrm{~F}$ & $G$ & $\mathrm{H}$ & $\mathrm{I}$ & $\mathrm{J}$ \\
\hline 1 & SAMPLE_NUM_ASSIGNED_TO & SAMPLER & MEDIA & BIOTA_MEDIA & TAG_ID & SAMP_NUM & SAMP_DATE_TIME & SAMP_DATE_TIME_ON & CON_ID & CON_SHORT_NAME \\
\hline 4486 & SESP SCHEDULING & & SW & & & B0X3X9 & 06-Dec-99 & & $13966-29-5$ & U-234 \\
\hline 4487 & SESP SCHEDULING & & SW & & & B0X3X9 & 06-Dec-99 & & 15117-96-1 & U-235 \\
\hline 4488 & SESP SCHEDULING & & SW & & & B0X3X9 & 06-Dec-99 & & U-238 & U-238 \\
\hline 4489 & SESP SCHEDULING & & SW & & & BOX3Y0 & 06-Dec-99 & & $10098-97-2$ & SR-90 \\
\hline 4490 & SESP SCHEDULING & & SW & & & BOX3Y0 & 06-Dec-99 & & $10028-17-8$ & TRITIUM \\
\hline 4491 & SESP SCHEDULING & & SW & & & BOX3Y0 & 06-Dec-99 & & 13966-29-5 & U-234 \\
\hline 4492 & SESP SCHEDULING & & SW & & & B0X3Y0 & 06-Dec-99 & & $15117-96-1$ & U-235 \\
\hline 4493 & SESP SCHEDULING & & SW & & & BOX3Y0 & 06-Dec-99 & & $U-238$ & $\mathrm{U}-238$ \\
\hline 4494 & PSHENRY & BELT, LL & SW & & & B0X450 & 06-Dec-99 & & $56-23-5$ & CARBTET \\
\hline 4495 & PSHENRY & BELT, LL & SW & & & B0X449 & 06-Dec-99 & & $56-23-5$ & CARBTET \\
\hline 4496 & PSHENRY & BELT, LL & SW & & & B0X448 & 06-Dec-99 & & $56-23-5$ & CARBTET \\
\hline 4497 & PSHENRY & BELT, LL & SW & & & B0X447 & 06-Dec-99 & & $56-23-5$ & CARBTET \\
\hline 4498 & PSHENRY & BELT, LL & SW & & & B0X446 & 06-Dec-99 & & $56-23-5$ & CARBTET \\
\hline 4499 & PSHENRY & BELT, LL & SW & & & B0X445 & 06-Dec-99 & & $56-23-5$ & CARBTET \\
\hline 4500 & PSHENRY & BELT, LL & SW & & & B0X451 & 06-Dec-99 & & $56-23-5$ & CARBTET \\
\hline 4501 & PSHENRY & BELT, LL & SW & & & B0X452 & 06-Dec-99 & & $56-23-5$ & CARBTET \\
\hline 4502 & PSHENRY & BELT, LL & SW & & & B0X453 & 06-Dec-99 & & $56-23-5$ & CARBTET \\
\hline 4503 & PSHENRY & BELT, LL & SW & & & B0X454 & 06-Dec-99 & & $56-23-5$ & CARBTET \\
\hline 4504 & SESP SCHEDULING & & SW & & & B0X3X2 & 07-Dec-99 & & $10098-97-2$ & SR-90 \\
\hline 4505 & SESP SCHEDULING & & SW & & & B0X3X2 & 07-Dec-99 & & $10028-17-8$ & TRITIUM \\
\hline 4506 & SESP SCHEDULING & & SW & & & B0X3X2 & 07-Dec-99 & & 13966-29-5 & U-234 \\
\hline 4507 & SESP SCHEDULING & & SW & & & B0X3X2 & 07-Dec-99 & & $15117-96-1$ & U-235 \\
\hline 4508 & SESP SCHEDULING & & SW & & & B0X3X2 & 07-Dec-99 & & U-238 & U-238 \\
\hline 4509 & SESP SCHEDULING & & SW & & & B0X3X3 & 07-Dec-99 & & $10098-97-2$ & SR-90 \\
\hline 4510 & SESP SCHEDULING & & SW & & & B0X3X3 & 07-Dec-99 & & $10028-17-8$ & TRITIUM \\
\hline 4511 & SESP SCHEDULING & & SW & & & B0X3X3 & 07-Dec-99 & & $13966-29-5$ & U-234 \\
\hline 4512 & SESP SCHEDULING & & SW & & & B0X3X3 & 07-Dec-99 & & 15117-96-1 & U-235 \\
\hline 4513 & SESP SCHEDULING & & SW & & & B0X3X3 & 07-Dec-99 & & U-238 & U-238 \\
\hline 4514 & SESP SCHEDULING & & SW & & & B0X3X4 & 07-Dec-99 & & $10098-97-2$ & SR-90 \\
\hline 4515 & SESP SCHEDULING & & SW & & & B0X3X4 & 07-Dec-99 & & $10028-17-8$ & TRITIUM \\
\hline 4516 & SESP SCHEDULING & & SW & & & B0X3X4 & 07-Dec-99 & & $13966-29-5$ & U-234 \\
\hline 4517 & SESP SCHEDULING & & SW & & & B0X3X4 & 07-Dec-99 & & $15117-96-1$ & U-235 \\
\hline 4518 & SESP SCHEDULING & & SW & & & B0X3X4 & 07-Dec-99 & & $\mathrm{U}-238$ & $\mathrm{U}-238$ \\
\hline 4519 & SESP SCHEDULING & & SW & & & B0X3X5 & 07-Dec-99 & & $10098-97-2$ & SR-90 \\
\hline 4520 & SESP SCHEDULING & & SW & & & B0X3X5 & 07-Dec-99 & & $10028-17-8$ & TRITIUM \\
\hline 4521 & SESP SCHEDULING & & SW & & & B0X3X5 & 07-Dec-99 & & $13966-29-5$ & $\mathrm{U}-234$ \\
\hline 4522 & SESP SCHEDULING & & SW & & & B0X3X5 & 07-Dec-99 & & $15117-96-1$ & U-235 \\
\hline 4523 & SESP SCHEDULING & & SW & & & B0X3X5 & 07-Dec-99 & & U-238 & U-238 \\
\hline
\end{tabular}




\begin{tabular}{|c|c|c|c|c|c|c|c|c|c|c|}
\hline & A & $\mathrm{B}$ & $\mathrm{C}$ & $\mathrm{D}$ & $E$ & $\mathrm{~F}$ & $G$ & $\mathrm{H}$ & $\mathrm{I}$ & $\mathrm{J}$ \\
\hline 1 & SAMPLE_NUM_ASSIGNED_TO & SAMPLER & MEDIA & BIOTA_MEDIA & TAG_ID & SAMP_NUM & SAMP_DATE_TIME & SAMP_DATE_TIME_ON & CON_ID & CON_SHORT_NAME \\
\hline 4524 & PSHENRY & BELT, LL & SW & & & B0X458 & 07-Dec-99 & & $56-23-5$ & CARBTET \\
\hline 4525 & PSHENRY & BELT, LL & SW & & & B0X457 & 07-Dec-99 & & $56-23-5$ & CARBTET \\
\hline 4526 & PSHENRY & BELT, LL & SW & & & B0X456 & 07-Dec-99 & & $56-23-5$ & CARBTET \\
\hline 4527 & PSHENRY & BELT, LL & SW & & & B0X455 & 07-Dec-99 & & $56-23-5$ & CARBTET \\
\hline 4528 & SESP SCHEDULING & & SW & & & BOXRK2 & 27-Mar-00 & & $10098-97-2$ & SR-90 \\
\hline 4529 & SESP SCHEDULING & & SW & & & BOXRK2 & 27-Mar-00 & & $10028-17-8$ & TRITIUM \\
\hline 4530 & SESP SCHEDULING & & SW & & & BOXRK2 & 27-Mar-00 & & 13966-29-5 & U-234 \\
\hline 4531 & SESP SCHEDULING & & SW & & & B0XRK2 & 27-Mar-00 & & $15117-96-1$ & U-235 \\
\hline 4532 & SESP SCHEDULING & & SW & & & BOXRK2 & 27-Mar-00 & & U-238 & U-238 \\
\hline 4533 & SESP SCHEDULING & & SW & & & B0XRK3 & 27-Mar-00 & & $10098-97-2$ & SR-90 \\
\hline 4534 & SESP SCHEDULING & & SW & & & BOXRK3 & 27-Mar-00 & & $10028-17-8$ & TRITIUM \\
\hline 4535 & SESP SCHEDULING & & SW & & & B0XRK3 & 27-Mar-00 & & $13966-29-5$ & $\mathrm{U}-234$ \\
\hline 4536 & SESP SCHEDULING & & SW & & & BOXRK3 & 27-Mar-00 & & 15117-96-1 & U-235 \\
\hline 4537 & SESP SCHEDULING & & SW & & & B0XRK3 & 27-Mar-00 & & U-238 & U-238 \\
\hline 4538 & SESP SCHEDULING & & SW & & & B0XRK4 & 27-Mar-00 & & $10098-97-2$ & SR-90 \\
\hline 4539 & SESP SCHEDULING & & SW & & & B0XRK4 & 27-Mar-00 & & $10028-17-8$ & TRITIUM \\
\hline 4540 & SESP SCHEDULING & & SW & & & B0XRK4 & 27-Mar-00 & & 13966-29-5 & U-234 \\
\hline 4541 & SESP SCHEDULING & & SW & & & BOXRK4 & 27-Mar-00 & & 15117-96-1 & U-235 \\
\hline 4542 & SESP SCHEDULING & & SW & & & B0XRK4 & 27-Mar-00 & & U-238 & U-238 \\
\hline 4543 & SESP SCHEDULING & & SW & & & B0XRK5 & 27-Mar-00 & & $10098-97-2$ & SR-90 \\
\hline 4544 & SESP SCHEDULING & & SW & & & B0XRK5 & 27-Mar-00 & & $10028-17-8$ & TRITIUM \\
\hline 4545 & SESP SCHEDULING & & SW & & & B0XRK5 & 27-Mar-00 & & $13966-29-5$ & U-234 \\
\hline 4546 & SESP SCHEDULING & & SW & & & B0XRK5 & 27-Mar-00 & & 15117-96-1 & U-235 \\
\hline 4547 & SESP SCHEDULING & & SW & & & B0XRK5 & 27-Mar-00 & & U-238 & U-238 \\
\hline 4548 & SESP SCHEDULING & & SW & & & B0XRP1 & 28-Mar-00 & & $10098-97-2$ & SR-90 \\
\hline 4549 & SESP SCHEDULING & & SW & & & B0XRP1 & 28-Mar-00 & & $10028-17-8$ & TRITIUM \\
\hline 4550 & SESP SCHEDULING & & SW & & & B0XRP1 & 28-Mar-00 & & 13966-29-5 & U-234 \\
\hline 4551 & SESP SCHEDULING & & SW & & & B0XRP1 & 28-Mar-00 & & 15117-96-1 & U-235 \\
\hline 4552 & SESP SCHEDULING & & SW & & & B0XRP1 & 28-Mar-00 & & U-238 & U-238 \\
\hline 4553 & SESP SCHEDULING & & SW & & & BOXRPO & 28-Mar-00 & & $10098-97-2$ & SR-90 \\
\hline 4554 & SESP SCHEDULING & & SW & & & BOXRPO & 28-Mar-00 & & $10028-17-8$ & TRITIUM \\
\hline 4555 & SESP SCHEDULING & & SW & & & BOXRPO & 28-Mar-00 & & 13966-29-5 & U-234 \\
\hline 4556 & SESP SCHEDULING & & SW & & & BOXRPO & 28-Mar-00 & & $15117-96-1$ & U-235 \\
\hline 4557 & SESP SCHEDULING & & SW & & & BOXRPO & 28-Mar-00 & & U-238 & U-238 \\
\hline 4558 & SESP SCHEDULING & & SW & & & BOXRN9 & 28-Mar-00 & & $10098-97-2$ & SR-90 \\
\hline 4559 & SESP SCHEDULING & & SW & & & BOXRN9 & 28-Mar-00 & & $10028-17-8$ & TRITIUM \\
\hline 4560 & SESP SCHEDULING & & SW & & & BOXRN9 & 28-Mar-00 & & $13966-29-5$ & U-234 \\
\hline 4561 & SESP SCHEDULING & & SW & & & BOXRN9 & 28-Mar-00 & & 15117-96-1 & $\mathrm{U}-235$ \\
\hline
\end{tabular}




\begin{tabular}{|c|c|c|c|c|c|c|c|c|c|c|}
\hline & A & $\mathrm{B}$ & $\mathrm{C}$ & $\mathrm{D}$ & $E$ & $\mathrm{~F}$ & $G$ & $\mathrm{H}$ & $\mathrm{I}$ & $\mathrm{J}$ \\
\hline 1 & SAMPLE_NUM_ASSIGNED_TO & SAMPLER & MEDIA & BIOTA_MEDIA & TAG_ID & SAMP_NUM & SAMP_DATE_TIME & SAMP_DATE_TIME_ON & CON_ID & CON_SHORT_NAME \\
\hline 4562 & SESP SCHEDULING & & SW & & & BOXRN9 & 28-Mar-00 & & U-238 & U-238 \\
\hline 4563 & SESP SCHEDULING & & SW & & & BOXRN8 & 28-Mar-00 & & $10098-97-2$ & SR-90 \\
\hline 4564 & SESP SCHEDULING & & SW & & & BOXRN8 & 28-Mar-00 & & $10028-17-8$ & TRITIUM \\
\hline 4565 & SESP SCHEDULING & & SW & & & BOXRN8 & 28-Mar-00 & & $13966-29-5$ & U-234 \\
\hline 4566 & SESP SCHEDULING & & SW & & & BOXRN8 & 28-Mar-00 & & 15117-96-1 & U-235 \\
\hline 4567 & SESP SCHEDULING & & SW & & & BOXRN8 & 28-Mar-00 & & $U-238$ & U-238 \\
\hline 4568 & SESP SCHEDULING & & SW & & & BOXRKO & 28-Mar-00 & & $10098-97-2$ & SR-90 \\
\hline 4569 & SESP SCHEDULING & & SW & & & B0XRK6 & 28-Mar-00 & & $10098-97-2$ & SR-90 \\
\hline 4570 & SESP SCHEDULING & & SW & & & BOXRKO & 28-Mar-00 & & $10028-17-8$ & TRITIUM \\
\hline 4571 & SESP SCHEDULING & & SW & & & B0XRK6 & 28-Mar-00 & & $10028-17-8$ & TRITIUM \\
\hline 4572 & SESP SCHEDULING & & SW & & & BOXRKO & 28-Mar-00 & & $13966-29-5$ & $\mathrm{U}-234$ \\
\hline 4573 & SESP SCHEDULING & & SW & & & BOXRKO & 28-Mar-00 & & $15117-96-1$ & U-235 \\
\hline 4574 & SESP SCHEDULING & & SW & & & BOXRKO & 28-Mar-00 & & U-238 & U-238 \\
\hline 4575 & SESP SCHEDULING & & SW & & & B0XRK6 & 28-Mar-00 & & 13966-29-5 & U-234 \\
\hline 4576 & SESP SCHEDULING & & SW & & & B0XRK6 & 28-Mar-00 & & 15117-96-1 & U-235 \\
\hline 4577 & SESP SCHEDULING & & SW & & & B0XRK6 & 28-Mar-00 & & $\mathrm{U}-238$ & U-238 \\
\hline 4578 & SESP SCHEDULING & & SW & & & B0XRL1 & 28-Mar-00 & & $10098-97-2$ & SR-90 \\
\hline 4579 & SESP SCHEDULING & & SW & & & B0XRL1 & 28-Mar-00 & & $10028-17-8$ & TRITIUM \\
\hline 4580 & SESP SCHEDULING & & SW & & & B0XRL1 & 28-Mar-00 & & $13966-29-5$ & U-234 \\
\hline 4581 & SESP SCHEDULING & & SW & & & B0XRL1 & 28-Mar-00 & & $15117-96-1$ & U-235 \\
\hline 4582 & SESP SCHEDULING & & SW & & & B0XRL1 & 28-Mar-00 & & $U-238$ & U-238 \\
\hline 4583 & SESP SCHEDULING & & SW & & & BOXRK7 & 28-Mar-00 & & $10098-97-2$ & SR-90 \\
\hline 4584 & SESP SCHEDULING & & SW & & & B0XRK7 & 28-Mar-00 & & $10028-17-8$ & TRITIUM \\
\hline 4585 & SESP SCHEDULING & & SW & & & BOXRK7 & 28-Mar-00 & & $13966-29-5$ & U-234 \\
\hline 4586 & SESP SCHEDULING & & SW & & & BOXRK7 & 28-Mar-00 & & $15117-96-1$ & U-235 \\
\hline 4587 & SESP SCHEDULING & & SW & & & B0XRK7 & 28-Mar-00 & & U-238 & $\mathrm{U}-238$ \\
\hline 4588 & SESP SCHEDULING & & SW & & & BOXRK8 & 28-Mar-00 & & $10098-97-2$ & SR-90 \\
\hline 4589 & SESP SCHEDULING & & SW & & & BOXRK8 & 28-Mar-00 & & $10028-17-8$ & TRITIUM \\
\hline 4590 & SESP SCHEDULING & & SW & & & B0XRK8 & 28-Mar-00 & & $13966-29-5$ & U-234 \\
\hline 4591 & SESP SCHEDULING & & SW & & & BOXRK8 & 28-Mar-00 & & $15117-96-1$ & U-235 \\
\hline 4592 & SESP SCHEDULING & & SW & & & BOXRK8 & 28-Mar-00 & & $U-238$ & U-238 \\
\hline 4593 & SESP SCHEDULING & & SW & & & BOXRK9 & 28-Mar-00 & & $10098-97-2$ & SR-90 \\
\hline 4594 & SESP SCHEDULING & & SW & & & BOXRK9 & 28-Mar-00 & & $10028-17-8$ & TRITIUM \\
\hline 4595 & SESP SCHEDULING & & SW & & & BOXRK9 & 28-Mar-00 & & $13966-29-5$ & U-234 \\
\hline 4596 & SESP SCHEDULING & & SW & & & BOXRK9 & 28-Mar-00 & & $15117-96-1$ & U-235 \\
\hline 4597 & SESP SCHEDULING & & SW & & & B0XRK9 & 28-Mar-00 & & U-238 & $\mathrm{U}-238$ \\
\hline 4598 & SESP SCHEDULING & & SW & & & BOXRLO & 28-Mar-00 & & $10098-97-2$ & SR-90 \\
\hline 4599 & SESP SCHEDULING & & SW & & & BOXRLO & 28-Mar-00 & & $10028-17-8$ & TRITIUM \\
\hline
\end{tabular}




\begin{tabular}{|c|c|c|c|c|c|c|c|c|c|c|}
\hline & A & $\mathrm{B}$ & $\mathrm{C}$ & $\mathrm{D}$ & $E$ & $\mathrm{~F}$ & $G$ & $\mathrm{H}$ & $\mathrm{I}$ & $\mathrm{J}$ \\
\hline 1 & SAMPLE_NUM_ASSIGNED_TO & SAMPLER & MEDIA & BIOTA_MEDIA & TAG_ID & SAMP_NUM & SAMP_DATE_TIME & SAMP_DATE_TIME_ON & CON_ID & CON_SHORT_NAME \\
\hline 4600 & SESP SCHEDULING & & SW & & & BOXRL0 & 28-Mar-00 & & $13966-29-5$ & U-234 \\
\hline 4601 & SESP SCHEDULING & & SW & & & BOXRLO & 28-Mar-00 & & 15117-96-1 & U-235 \\
\hline 4602 & SESP SCHEDULING & & SW & & & BOXRLO & 28-Mar-00 & & U-238 & U-238 \\
\hline 4603 & SESP SCHEDULING & & SW & & & B0YCD7 & 19-Jun-00 & & $10098-97-2$ & SR-90 \\
\hline 4604 & SESP SCHEDULING & & SW & & & B0YCD7 & 19-Jun-00 & & $10028-17-8$ & TRITIUM \\
\hline 4605 & SESP SCHEDULING & & SW & & & B0YCD7 & 19-Jun-00 & & 13966-29-5 & U-234 \\
\hline 4606 & SESP SCHEDULING & & SW & & & BOYCD7 & 19-Jun-00 & & $15117-96-1$ & U-235 \\
\hline 4607 & SESP SCHEDULING & & SW & & & B0YCD7 & 19-Jun-00 & & $U-238$ & $\mathrm{U}-238$ \\
\hline 4608 & SESP SCHEDULING & & SW & & & BOYCD8 & 19-Jun-00 & & $10098-97-2$ & SR-90 \\
\hline 4609 & SESP SCHEDULING & & SW & & & BOYCD8 & 19-Jun-00 & & $10028-17-8$ & TRITIUM \\
\hline 4610 & SESP SCHEDULING & & SW & & & B0YCD8 & 19-Jun-00 & & $13966-29-5$ & $\mathrm{U}-234$ \\
\hline 4611 & SESP SCHEDULING & & SW & & & B0YCD8 & 19-Jun-00 & & $15117-96-1$ & U-235 \\
\hline 4612 & SESP SCHEDULING & & SW & & & B0YCD8 & 19-Jun-00 & & U-238 & U-238 \\
\hline 4613 & SESP SCHEDULING & & SW & & & BOYCD9 & 19-Jun-00 & & $10098-97-2$ & SR-90 \\
\hline 4614 & SESP SCHEDULING & & SW & & & BOYCD9 & 19-Jun-00 & & $10028-17-8$ & TRITIUM \\
\hline 4615 & SESP SCHEDULING & & SW & & & BOYCD9 & 19-Jun-00 & & $13966-29-5$ & U-234 \\
\hline 4616 & SESP SCHEDULING & & SW & & & B0YCD9 & 19-Jun-00 & & 15117-96-1 & U-235 \\
\hline 4617 & SESP SCHEDULING & & SW & & & B0YCD9 & 19-Jun-00 & & $\mathrm{U}-238$ & $\mathrm{U}-238$ \\
\hline 4618 & SESP SCHEDULING & & SW & & & BOYCFO & 19-Jun-00 & & $10098-97-2$ & SR-90 \\
\hline 4619 & SESP SCHEDULING & & SW & & & BOYCFO & 19-Jun-00 & & $10028-17-8$ & TRITIUM \\
\hline 4620 & SESP SCHEDULING & & SW & & & BOYCFO & 19-Jun-00 & & 13966-29-5 & U-234 \\
\hline 4621 & SESP SCHEDULING & & SW & & & BOYCFO & 19-Jun-00 & & $15117-96-1$ & U-235 \\
\hline 4622 & SESP SCHEDULING & & SW & & & BOYCFO & 19-Jun-00 & & U-238 & U-238 \\
\hline 4623 & SESP SCHEDULING & & SW & & & B0YCK6 & 20-Jun-00 & & $10098-97-2$ & SR-90 \\
\hline 4624 & SESP SCHEDULING & & SW & & & B0YCK6 & 20-Jun-00 & & $10028-17-8$ & TRITIUM \\
\hline 4625 & SESP SCHEDULING & & SW & & & BOYCK6 & 20-Jun-00 & & $13966-29-5$ & U-234 \\
\hline 4626 & SESP SCHEDULING & & SW & & & BOYCK6 & 20-Jun-00 & & 15117-96-1 & U-235 \\
\hline 4627 & SESP SCHEDULING & & SW & & & BOYCK6 & 20-Jun-00 & & U-238 & U-238 \\
\hline 4628 & SESP SCHEDULING & & SW & & & B0YCK5 & 20-Jun-00 & & $10098-97-2$ & SR-90 \\
\hline 4629 & SESP SCHEDULING & & SW & & & B0YCK5 & 20-Jun-00 & & $10028-17-8$ & TRITIUM \\
\hline 4630 & SESP SCHEDULING & & SW & & & B0YCK5 & 20-Jun-00 & & $13966-29-5$ & U-234 \\
\hline 4631 & SESP SCHEDULING & & SW & & & B0YCK5 & 20-Jun-00 & & $15117-96-1$ & U-235 \\
\hline 4632 & SESP SCHEDULING & & SW & & & BOYCK5 & 20-Jun-00 & & $\mathrm{U}-238$ & $\mathrm{U}-238$ \\
\hline 4633 & SESP SCHEDULING & & SW & & & BOYCK4 & 20-Jun-00 & & $10098-97-2$ & SR-90 \\
\hline 4634 & SESP SCHEDULING & & SW & & & BOYCK4 & 20-Jun-00 & & $10028-17-8$ & TRITIUM \\
\hline 4635 & SESP SCHEDULING & & SW & & & BOYCK4 & 20-Jun-00 & & $13966-29-5$ & U-234 \\
\hline 4636 & SESP SCHEDULING & & SW & & & BOYCK4 & 20-Jun-00 & & $15117-96-1$ & U-235 \\
\hline 4637 & SESP SCHEDULING & & SW & & & BOYCK4 & 20-Jun-00 & & U-238 & U-238 \\
\hline
\end{tabular}




\begin{tabular}{|c|c|c|c|c|c|c|c|c|c|c|}
\hline & 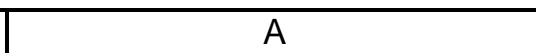 & $\mathrm{B}$ & $\mathrm{C}$ & $\mathrm{D}$ & $E$ & $\mathrm{~F}$ & G & $\mathrm{H}$ & $\mathrm{I}$ & $\mathrm{J}$ \\
\hline 1 & SAMPLE_NUM_ASSIGNED_TO & SAMPLER & MEDIA & BIOTA_MEDIA & $\overline{T A G \_I D}$ & SAMP_NUM & SAMP_DATE_TIME & SAMP_DATE_TIME_ON & CON_ID & CON_SHORT_NAME \\
\hline 4638 & SESP SCHEDULING & & SW & & & B0YCK3 & 20-Jun-00 & & $10098-97-2$ & SR-90 \\
\hline 4639 & SESP SCHEDULING & & SW & & & BOYCK3 & 20-Jun-00 & & 10028-17-8 & TRITIUM \\
\hline 4640 & SESP SCHEDULING & & SW & & & BOYCK3 & 20-Jun-00 & & $13966-29-5$ & U-234 \\
\hline 4641 & SESP SCHEDULING & & SW & & & BOYCK3 & 20-Jun-00 & & $15117-96-1$ & U-235 \\
\hline 4642 & SESP SCHEDULING & & SW & & & BOYCK3 & 20-Jun-00 & & $\mathrm{U}-238$ & $\mathrm{U}-238$ \\
\hline 4643 & SESP SCHEDULING & & SW & & & BOYCF1 & 20-Jun-00 & & $10098-97-2$ & SR-90 \\
\hline 4644 & SESP SCHEDULING & & SW & & & BOYCF1 & 20-Jun-00 & & $10028-17-8$ & TRITIUM \\
\hline 4645 & SESP SCHEDULING & & SW & & & BOYCF1 & 20-Jun-00 & & $13966-29-5$ & U-234 \\
\hline 4646 & SESP SCHEDULING & & SW & & & BOYCF1 & 20-Jun-00 & & $15117-96-1$ & U-235 \\
\hline 4647 & SESP SCHEDULING & & SW & & & BOYCF1 & 20-Jun-00 & & $\mathrm{U}-238$ & $\mathrm{U}-238$ \\
\hline 4648 & SESP SCHEDULING & & SW & & & BOYCF6 & 20-Jun-00 & & $10098-97-2$ & SR-90 \\
\hline 4649 & SESP SCHEDULING & & SW & & & BOYCF6 & 20-Jun-00 & & $10028-17-8$ & TRITIUM \\
\hline 4650 & SESP SCHEDULING & & SW & & & BOYCF6 & 20-Jun-00 & & $13966-29-5$ & U-234 \\
\hline 4651 & SESP SCHEDULING & & SW & & & B0YCF6 & 20-Jun-00 & & 15117-96-1 & U-235 \\
\hline 4652 & SESP SCHEDULING & & SW & & & BOYCF6 & 20-Jun-00 & & $U-238$ & U-238 \\
\hline 4653 & SESP SCHEDULING & & SW & & & BOYCF2 & 20-Jun-00 & & $10098-97-2$ & SR-90 \\
\hline 4654 & SESP SCHEDULING & & SW & & & BOYCF2 & 20-Jun-00 & & $10028-17-8$ & TRITIUM \\
\hline 4655 & SESP SCHEDULING & & SW & & & BOYCF2 & 20-Jun-00 & & $13966-29-5$ & U-234 \\
\hline 4656 & SESP SCHEDULING & & SW & & & BOYCF2 & 20-Jun-00 & & $15117-96-1$ & U-235 \\
\hline 4657 & SESP SCHEDULING & & SW & & & BOYCF2 & 20-Jun-00 & & $\mathrm{U}-238$ & $\mathrm{U}-238$ \\
\hline 4658 & SESP SCHEDULING & & SW & & & BOYCF3 & 20-Jun-00 & & $10098-97-2$ & SR-90 \\
\hline 4659 & SESP SCHEDULING & & SW & & & BOYCF3 & 20-Jun-00 & & $10028-17-8$ & TRITIUM \\
\hline 4660 & SESP SCHEDULING & & SW & & & BOYCF3 & 20-Jun-00 & & $13966-29-5$ & U-234 \\
\hline 4661 & SESP SCHEDULING & & SW & & & BOYCF3 & 20-Jun-00 & & 15117-96-1 & U-235 \\
\hline 4662 & SESP SCHEDULING & & SW & & & BOYCF3 & 20-Jun-00 & & U-238 & U-238 \\
\hline 4663 & SESP SCHEDULING & & SW & & & BOYCF4 & 20-Jun-00 & & $10098-97-2$ & SR-90 \\
\hline 4664 & SESP SCHEDULING & & SW & & & BOYCF4 & 20-Jun-00 & & $10028-17-8$ & TRITIUM \\
\hline 4665 & SESP SCHEDULING & & SW & & & BOYCF4 & 20-Jun-00 & & $13966-29-5$ & U-234 \\
\hline 4666 & SESP SCHEDULING & & SW & & & BOYCF4 & 20-Jun-00 & & $15117-96-1$ & U-235 \\
\hline 4667 & SESP SCHEDULING & & SW & & & BOYCF4 & 20-Jun-00 & & $\mathrm{U}-238$ & $\mathrm{U}-238$ \\
\hline 4668 & SESP SCHEDULING & & SW & & & BOYCF5 & 20-Jun-00 & & $10098-97-2$ & SR-90 \\
\hline 4669 & SESP SCHEDULING & & SW & & & B0YCF5 & 20-Jun-00 & & $10028-17-8$ & TRITIUM \\
\hline 4670 & SESP SCHEDULING & & SW & & & BOYCF5 & 20-Jun-00 & & $13966-29-5$ & U-234 \\
\hline 4671 & SESP SCHEDULING & & SW & & & BOYCF5 & 20-Jun-00 & & 15117-96-1 & U-235 \\
\hline 4672 & SESP SCHEDULING & & SW & & & BOYCF5 & 20-Jun-00 & & U-238 & U-238 \\
\hline
\end{tabular}




\begin{tabular}{|c|c|c|c|c|c|c|c|c|c|}
\hline & $\mathrm{K}$ & $\mathrm{L}$ & $\bar{M}$ & $\mathrm{~N}$ & $\mathrm{O}$ & $\mathrm{P}$ & $\mathrm{Q}$ & $\mathrm{R}$ & $\mathrm{S}$ \\
\hline 1 & METHOD NAME & LAB CODE & VALUE RPTD & ANAL UNITS RPTD & COUNTING ERROR & TOTAL ANAL ERROR & MIN DETECTABLE ACTIVITY & PCNT MOISTURE & LAB QUALIFIER \\
\hline 2 & SRISO_SEP_PRECIP_GPC & ITASRL & -0.0164 & $\mathrm{pCi} / \mathrm{L}$ & 0.0358 & 0.0379 & & & $U$ \\
\hline 3 & TRITIUM_DIST_LSC & ITASRL & 45.3 & $\mathrm{pCi} / \mathrm{L}$ & 104 & 200 & & & $\mathrm{U}$ \\
\hline 4 & UISO_AEA & ITASRL & 0.192 & $\mathrm{pCi} / \mathrm{L}$ & 0.0542 & 0.058 & & & \\
\hline 5 & UISO_AEA & ITASRL & 0.00713 & $\mathrm{pCi} / \mathrm{L}$ & 0.0151 & 0.0152 & & & $U$ \\
\hline 6 & UISO_AEA & ITASRL & 0.144 & $\mathrm{pCi} / \mathrm{L}$ & 0.0469 & 0.0493 & & & \\
\hline 7 & SRISŌSEP_PRECIP_GPC & ITASRL & 0.0608 & $\mathrm{pCi} / \mathrm{L}$ & 0.0279 & 0.032 & & & \\
\hline 8 & TRITIUM_DIST_LSC & ITASRL & 108 & $\mathrm{pCi} / \mathrm{L}$ & 106 & 203 & & & 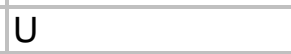 \\
\hline 9 & UISO_AEA & ITASRL & 0.194 & $\mathrm{pCi} / \mathrm{L}$ & 0.0507 & 0.0546 & & & \\
\hline 11 & UISO_AEA & ITASRL & 0.142 & $\mathrm{pCi} / \mathrm{L}$ & 0.0431 & 0.0455 & & & \\
\hline 12 & SRISO_SEP_PRECIP_GPC & ITASRL & 0.0585 & $\mathrm{pCi} / \mathrm{L}$ & 0.0247 & 0.0285 & & & \\
\hline 13 & TRITIUMM_DIST_LSC & ITASRL & 98.4 & $\mathrm{pCi} / \mathrm{L}$ & 106 & 202 & & & $U$ \\
\hline 14 & UISO_AEA & ITASRL & 0.196 & $\mathrm{pCi} / \mathrm{L}$ & 0.0476 & 0.0517 & & & \\
\hline 15 & UISO_AEA & ITASRL & 0.00711 & $\mathrm{pCi} / \mathrm{L}$ & 0.0109 & 0.011 & & & $\mathrm{U}$ \\
\hline 16 & UISO_AEA & ITASRL & 0.154 & $\mathrm{pCi} / \mathrm{L}$ & 0.0411 & 0.044 & & & \\
\hline 17 & SRISO_SEP_PRECIP_GPC & ITASRL & 0.0652 & $\mathrm{pCi} / \mathrm{L}$ & 0.0312 & 0.0349 & & & \\
\hline 18 & TRITIUM_DIST_LSC & ITASRL & 70.4 & $\mathrm{pCi} / \mathrm{L}$ & 103 & 200 & & & $U$ \\
\hline 19 & UISO_AËA & ITASRL & 0.23 & $\mathrm{pCi} / \mathrm{L}$ & 0.0639 & 0.0687 & & & \\
\hline 21 & UISO_AEA & ITASRL & 0.125 & $\mathrm{pCi} / \mathrm{L}$ & 0.0466 & 0.0486 & & & \\
\hline 22 & SRISŌSEP_PRECIP_GPC & ITASRL & 0.0728 & $\mathrm{pCi} / \mathrm{L}$ & 0.0404 & 0.046 & & & \\
\hline 23 & TRITIUM_DIST_LSC & ITASRL & 55 & $\mathrm{pCi} / \mathrm{L}$ & 105 & 200 & & & $U$ \\
\hline 24 & UISO_AEA & ITASRL & 0.138 & $\mathrm{pCi} / \mathrm{L}$ & 0.0417 & 0.0443 & & & \\
\hline 25 & UISO_AEA & ITASRL & 0.00745 & $\mathrm{pCi} / \mathrm{L}$ & 0.0101 & 0.0102 & & & $U$ \\
\hline 26 & UISO_AEA & ITASRL & 0.136 & $\mathrm{pCi} / \mathrm{L}$ & 0.0409 & 0.0433 & & & \\
\hline 27 & SRISO_SEP_PRECIP_GPC & ITASRL & 0.0847 & $\mathrm{pCi} / \mathrm{L}$ & 0.0327 & 0.0408 & & & \\
\hline 28 & TRITIUM_DISTT_LSC & ITASRL & 59.8 & $\mathrm{pCi} / \mathrm{L}$ & 104 & 200 & & & $U$ \\
\hline 29 & UISO_AEA & ITASRL & 0.206 & $\mathrm{pCi} / \mathrm{L}$ & 0.0541 & 0.0584 & & & \\
\hline 30 & UISO_AEA & ITASRL & 0.00209 & $\mathrm{pCi} / \mathrm{L}$ & 0.00676 & 0.00682 & & & $U$ \\
\hline 31 & UISO_AEA & ITASRL & 0.16 & $\mathrm{pCi} / \mathrm{L}$ & 0.0474 & 0.0502 & & & \\
\hline 32 & SRISŌSEP_PRECIP_GPC & ITASRL & 0.0847 & $\mathrm{pCi} / \mathrm{L}$ & 0.0327 & 0.0408 & & & \\
\hline 33 & TRITIUM_DIST_LSC & ITASRL & 20.2 & $\mathrm{pCi} / \mathrm{L}$ & 104 & 199 & & & $U$ \\
\hline 34 & UISO_AEA & ITASRL & 0.206 & $\mathrm{pCi} / \mathrm{L}$ & 0.0541 & 0.0584 & & & \\
\hline 35 & UISO_AEA & ITASRL & 0.00209 & $\mathrm{pCi} / \mathrm{L}$ & 0.00676 & 0.00682 & & & $U$ \\
\hline 36 & UISO_AEA & ITASRL & 0.16 & $\mathrm{pCi} / \mathrm{L}$ & 0.0474 & 0.0502 & & & \\
\hline 37 & SRISO_SEP_PRECIP_GPC & ITASRL & 0.0829 & $\mathrm{pCi} / \mathrm{L}$ & 0.0479 & 0.0515 & & & \\
\hline 38 & TRITIUM_DISTT_LSC & ITASRL & 5.79 & $\mathrm{pCi} / \mathrm{L}$ & 103 & 198 & & & $U$ \\
\hline
\end{tabular}




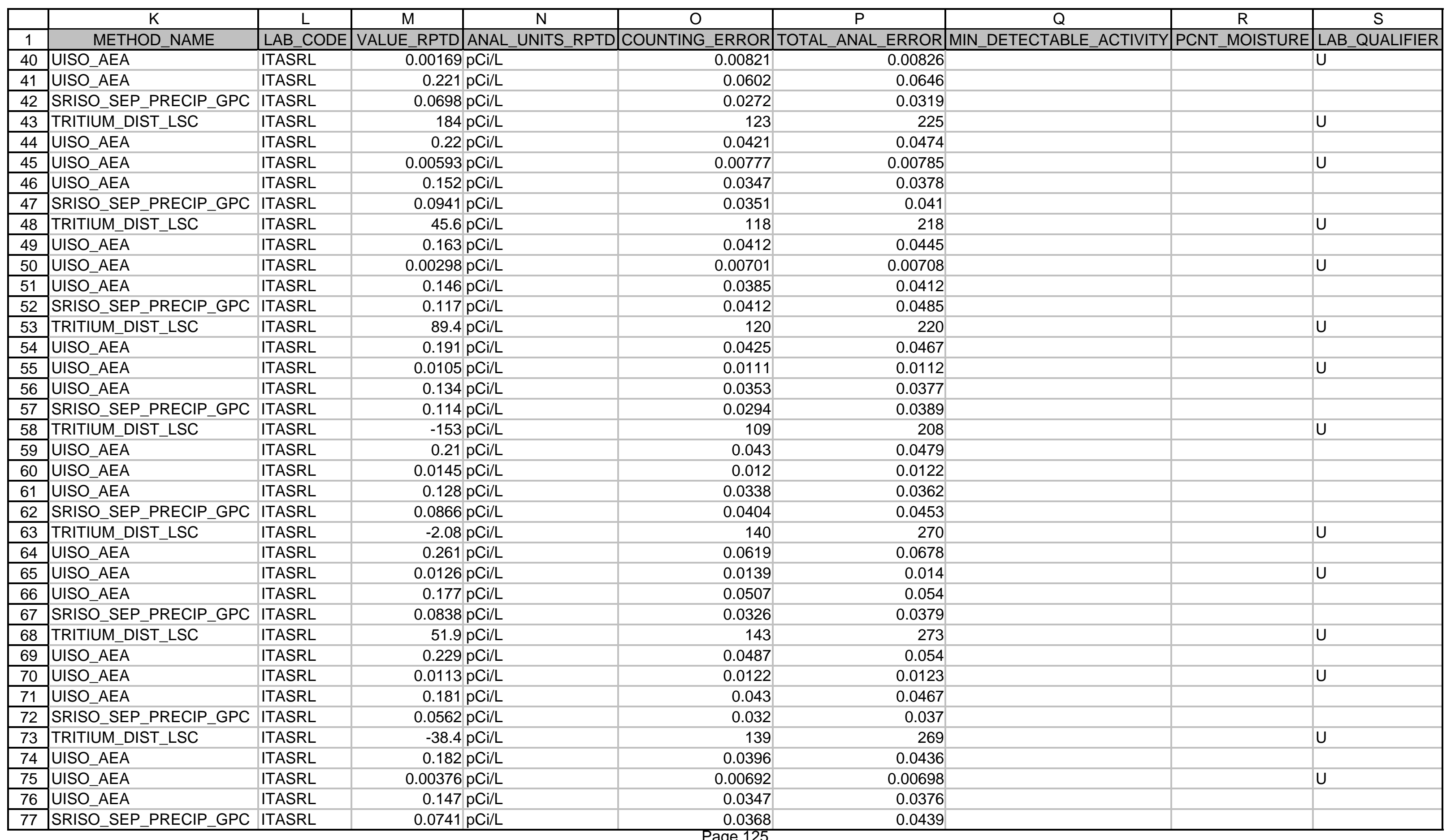




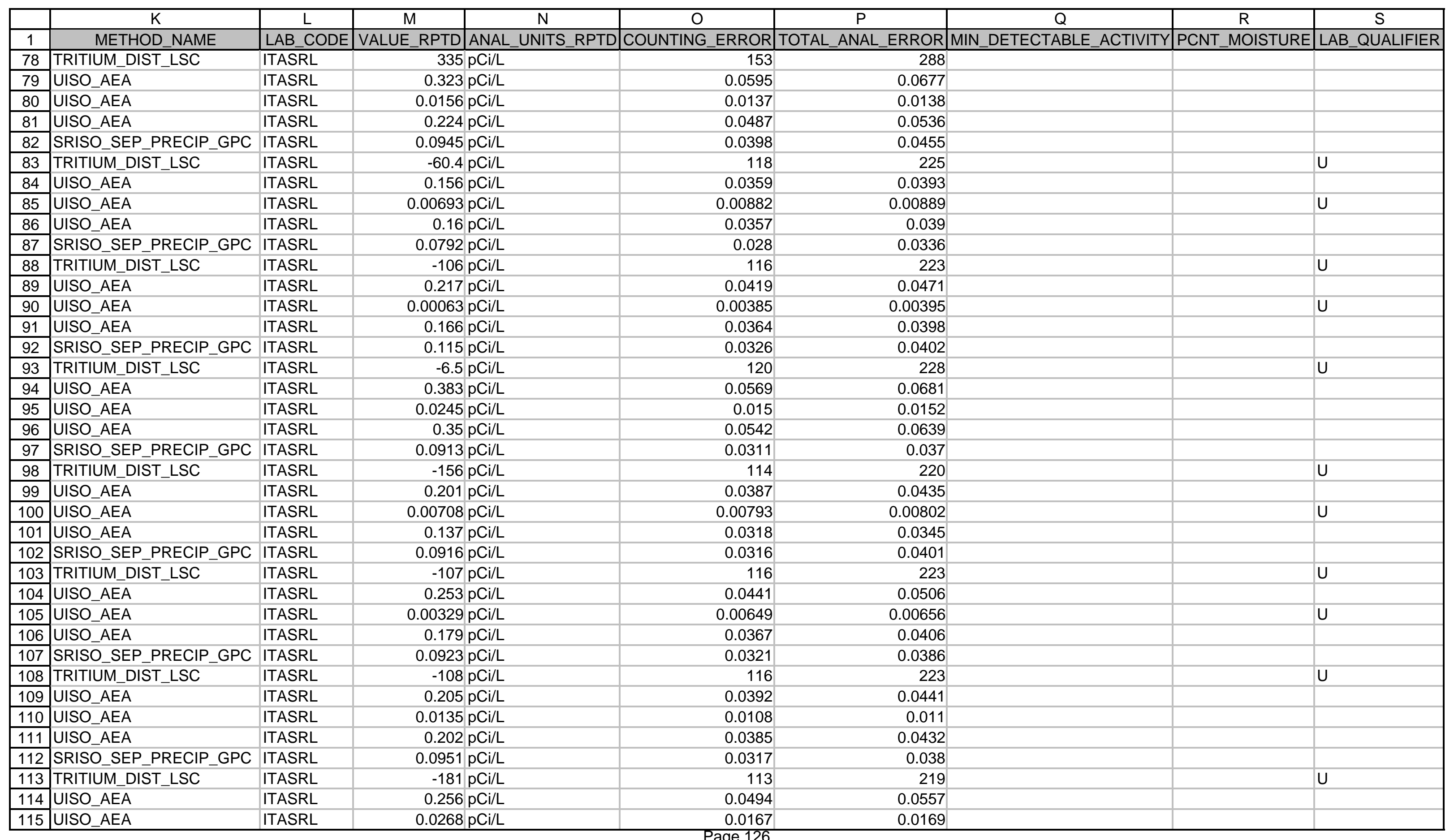




\begin{tabular}{|c|c|c|c|c|c|c|c|c|c|}
\hline & $\mathrm{K}$ & $\mathrm{L}$ & $\mathrm{M}$ & $\mathrm{N}$ & $\mathrm{O}$ & $\mathrm{P}$ & $\mathrm{Q}$ & $\mathrm{R}$ & $\mathrm{S}$ \\
\hline 1 & METHOD NAME & LAB CODE & VALUE RPTD & ANAL UNITS RPTD & COUNTING ERROR & TOTAL ANAL ERROR & MIN DETECTABLE ACTIVITY & PCNT MOISTURE & LAB QUALIFIER \\
\hline 116 & UISO_AEA & ITASRL & 0.188 & $\mathrm{pCi} / \mathrm{L}$ & 0.0421 & 0.046 & & & \\
\hline 117 & SRISO_SEP_PRECIP_GPC & ITASRL & 0.136 & $\mathrm{pCi} / \mathrm{L}$ & 0.0409 & 0.0497 & & & \\
\hline 118 & TRITIUM_DIST_LSC & ITASRL & -22.3 & $\mathrm{pCi} / \mathrm{L}$ & 119 & 227 & & & $\mathrm{U}$ \\
\hline 119 & UISO_AEA & ITASRL & 3.65 & $\mathrm{pCi} / \mathrm{L}$ & 0.181 & 0.395 & & & \\
\hline 120 & UISO_AEA & ITASRL & 0.675 & $\mathrm{pCi} / \mathrm{L}$ & 0.0777 & 0.101 & & & \\
\hline 121 & UISO_AEA & ITASRL & 2.87 & $\mathrm{pCi} / \mathrm{L}$ & 0.16 & 0.319 & & & \\
\hline 122 & SRISO_SEP_PRECIP_GPC & ITASRL & 0.0383 & $\mathrm{pCi} / \mathrm{L}$ & 0.0413 & 0.0438 & & & 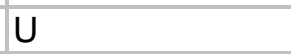 \\
\hline 123 & TRITIUM_DISTT_LSC & ITASRL & -37 & $\mathrm{pCi} / \mathrm{L}$ & 112 & 210 & & & $U$ \\
\hline 125 & UISO_AEA & ITASRL & 0.0139 & $\mathrm{pCi} / \mathrm{L}$ & 0.0316 & 0.0316 & & & $U$ \\
\hline 126 & UISO_AEA & ITASRL & 0.243 & $\mathrm{pCi} / \mathrm{L}$ & 0.0932 & 0.0979 & & & \\
\hline 127 & SRISŌSEP_PRECIP_GPC & ITASRL & 0.0637 & $\mathrm{pCi} / \mathrm{L}$ & 0.0265 & 0.0336 & & & \\
\hline 128 & TRITIUM_DIST_LSC & ITASRL & -76.7 & $\mathrm{pCi} / \mathrm{L}$ & 111 & 208 & & & $U$ \\
\hline 129 & UISO_AEA & ITASRL & 0.23 & $\mathrm{pCi} / \mathrm{L}$ & 0.044 & 0.0496 & & & \\
\hline 130 & UISO_AEA & ITASRL & 0.00222 & $\mathrm{pCi} / \mathrm{L}$ & 0.00576 & 0.00584 & & & $U$ \\
\hline 131 & UISO_AEA & ITASRL & 0.165 & $\mathrm{pCi} / \mathrm{L}$ & 0.037 & 0.0404 & & & \\
\hline 132 & SRISO_SEP_PRECIP_GPC & ITASRL & 0.0469 & $\mathrm{pCi} / \mathrm{L}$ & 0.0383 & 0.0418 & & & \\
\hline 133 & TRITIUM_DISTT_LSC & ITASRL & 20.3 & $\mathrm{pCi} / \mathrm{L}$ & 115 & 213 & & & $U$ \\
\hline 135 & UISO_AEA & ITASRL & 0.014 & $\mathrm{pCi} / \mathrm{L}$ & 0.0164 & 0.0165 & & & $U$ \\
\hline 136 & UISO_AEA & ITASRL & 0.179 & $\mathrm{pCi} / \mathrm{L}$ & 0.0555 & 0.0587 & & & \\
\hline 137 & SRISŌSEP_PRECIP_GPC & ITASRL & 0.0763 & $\mathrm{pCi} / \mathrm{L}$ & 0.0325 & 0.0404 & & & \\
\hline 138 & TRITIUM_DIST_LSC & ITASRL & -53.6 & $\mathrm{pCi} / \mathrm{L}$ & 112 & 209 & & & U \\
\hline 139 & UISO_AËA & ITASRL & 0.267 & $\mathrm{pCi} / \mathrm{L}$ & 0.0533 & 0.0597 & & & \\
\hline 140 & UISO_AEA & ITASRL & 0.00571 & $\mathrm{pCi} / \mathrm{L}$ & 0.0106 & 0.0106 & & & $U$ \\
\hline 141 & UISO_AEA & ITASRL & 0.172 & $\mathrm{pCi} / \mathrm{L}$ & 0.0431 & 0.0464 & & & \\
\hline 142 & SRISŌSEP_PRECIP_GPC & ITASRL & 0.0627 & $\mathrm{pCi} / \mathrm{L}$ & 0.0243 & 0.0318 & & & \\
\hline 143 & TRITIUM_DIST_LSC & ITASRL & 40.2 & $\mathrm{pCi} / \mathrm{L}$ & 143 & 277 & & & $\mathrm{U}$ \\
\hline 144 & UISO_AEA & ITASRL & 0.274 & $\mathrm{pCi} / \mathrm{L}$ & 0.0462 & 0.0534 & & & \\
\hline 145 & UISO_AEA & ITASRL & 0.0136 & $\mathrm{pCi} / \mathrm{L}$ & 0.0105 & 0.0106 & & & \\
\hline 146 & UISO_AEA & ITASRL & 0.207 & $\mathrm{pCi} / \mathrm{L}$ & 0.0397 & 0.0445 & & & \\
\hline 147 & SRISO_SEP_PRECIP_GPC & ITASRL & 0.0637 & $\mathrm{pCi} / \mathrm{L}$ & 0.0243 & 0.032 & & & \\
\hline 148 & TRITIUM_DISTT_LSC & ITASRL & -47.4 & $\mathrm{pCi} / \mathrm{L}$ & 140 & 273 & & & $U$ \\
\hline 149 & UISO_AËA & ITASRL & 0.182 & $\mathrm{pCi} / \mathrm{L}$ & 0.0656 & 0.069 & & & \\
\hline 150 & UISO_AEA & ITASRL & 0.00202 & $\mathrm{pCi} / \mathrm{L}$ & 0.0119 & 0.012 & & & U \\
\hline 151 & UISO_AEA & ITASRL & 0.141 & $\mathrm{pCi} / \mathrm{L}$ & 0.0578 & 0.06 & & & \\
\hline 152 & SRISŌSEP_PRECIP_GPC & ITASRL & 0.0697 & $\mathrm{pCi} / \mathrm{L}$ & 0.0255 & 0.0338 & & & \\
\hline
\end{tabular}




\begin{tabular}{|c|c|c|c|c|c|c|c|c|c|}
\hline & $\mathrm{K}$ & $\mathrm{L}$ & $\mathrm{M}$ & $\mathrm{N}$ & $\mathrm{O}$ & $\mathrm{P}$ & $\mathrm{Q}$ & $\mathrm{R}$ & $\mathrm{S}$ \\
\hline 1 & METHOD NAME & LAB CODE & VALUE RPTD & ANAL UNITS RPTD & COUNTING ERROR & TOTAL ANAL ERROR & MIN DETECTABLE ACTIVITY & PCNT MOISTURE & LAB QUALIFIER \\
\hline 154 & UISO_AEA & ITASRL & 0.238 & $\mathrm{pCi} / \mathrm{L}$ & 0.0445 & 0.0504 & & & \\
\hline 155 & UISO_AEA & ITASRL & 0.00666 & $\mathrm{pCi} / \mathrm{L}$ & 0.00796 & 0.00804 & & & $\mathrm{U}$ \\
\hline 156 & UISO_AEA & ITASRL & 0.196 & $\mathrm{pCi} / \mathrm{L}$ & 0.04 & 0.0443 & & & \\
\hline 157 & SRISO_SEP_PRECIP_GPC & ITASRL & 0.0479 & $\mathrm{pCi} / \mathrm{L}$ & 0.029 & 0.0339 & & & \\
\hline 158 & TRITIUM_DISTT_LSC & ITASRL & 37.1 & $\mathrm{pCi} / \mathrm{L}$ & 143 & 277 & & & $U$ \\
\hline 159 & UISO_AEA & ITASRL & 0.252 & $\mathrm{pCi} / \mathrm{L}$ & 0.0646 & 0.0701 & & & \\
\hline 160 & UISO AEA & ITASRL & 0.0143 & $\mathrm{pCi} / \mathrm{L}$ & 0.0156 & 0.0157 & & & $\mathrm{U}$ \\
\hline 161 & UISO_AEA & ITASRL & 0.241 & $\mathrm{pCi} / \mathrm{L}$ & 0.0617 & 0.0668 & & & \\
\hline 163 & TRITIUM_DIST_LSC & ITASRL & 14.4 & $\mathrm{pCi} / \mathrm{L}$ & 151 & 302 & & & $U$ \\
\hline 164 & UISO_AËA & ITASRL & 0.712 & $\mathrm{pCi} / \mathrm{L}$ & 0.0853 & 0.11 & & & \\
\hline 165 & UISO_AEA & ITASRL & 0.0479 & $\mathrm{pCi} / \mathrm{L}$ & 0.0231 & 0.0236 & & & \\
\hline 166 & UISO_AEA & ITASRL & 0.298 & $\mathrm{pCi} / \mathrm{L}$ & 0.0554 & 0.0628 & & & \\
\hline 167 & SRISŌSEP_PRECIP_GPC & ITASRL & 0.0724 & $\mathrm{pCi} / \mathrm{L}$ & 0.0294 & 0.0343 & & & \\
\hline 168 & TRITIUM_DIS̄T_LSC & ITASRL & -115 & $\mathrm{pCi} / \mathrm{L}$ & 147 & 296 & & & $U$ \\
\hline 169 & UISO_AEA & ITASRL & 0.251 & $\mathrm{pCi} / \mathrm{L}$ & 0.0456 & 0.052 & & & \\
\hline 170 & UISO_AEA & ITASRL & 0.00707 & $\mathrm{pCi} / \mathrm{L}$ & 0.00919 & 0.00926 & & & $U$ \\
\hline 171 & UISO_AEA & ITASRL & 0.135 & $\mathrm{pCi} / \mathrm{L}$ & 0.0337 & 0.0362 & & & \\
\hline 173 & TRITIUM_DIST_LSC & ITASRL & -12.4 & $\mathrm{pCi} / \mathrm{L}$ & 151 & 301 & & & U \\
\hline 174 & UISO_AEA & ITASRL & 0.25 & $\mathrm{pCi} / \mathrm{L}$ & 0.0439 & 0.0503 & & & \\
\hline 175 & UISO_AEA & ITASRL & 0.00914 & $\mathrm{pCi} / \mathrm{L}$ & 0.00925 & 0.00934 & & & $U$ \\
\hline 176 & UISO_AEA & ITASRL & 0.174 & $\mathrm{pCi} / \mathrm{L}$ & 0.0368 & 0.0405 & & & \\
\hline 177 & SRISŌSEP_PRECIP_GPC & ITASRL & 0.122 & $\mathrm{pCi} / \mathrm{L}$ & 0.0735 & 0.0785 & & & \\
\hline 178 & TRITIUM_DISTT_LSC & ITASRL & 4.13 & $\mathrm{pCi} / \mathrm{L}$ & 151 & 302 & & & $U$ \\
\hline 179 & UISO_AËA & ITASRL & 0.24 & $\mathrm{pCi} / \mathrm{L}$ & 0.0466 & 0.0524 & & & \\
\hline 180 & UISO_AEA & ITASRL & 0.00652 & $\mathrm{pCi} / \mathrm{L}$ & 0.00886 & 0.00893 & & & $U$ \\
\hline 181 & UISO_AEA & ITASRL & 0.166 & $\mathrm{pCi} / \mathrm{L}$ & 0.0386 & 0.042 & & & \\
\hline 182 & SRISO_SEP_PRECIP_GPC & ITASRL & 0.0679 & $\mathrm{pCi} / \mathrm{L}$ & 0.0374 & 0.0408 & & & \\
\hline 183 & TRITIUM_DISTT_LSC & ITASRL & -156 & $\mathrm{pCi} / \mathrm{L}$ & 147 & 295 & & & $U$ \\
\hline 184 & UISO_AEA & ITASRL & 0.259 & $\mathrm{pCi} / \mathrm{L}$ & 0.0509 & 0.0573 & & & \\
\hline 185 & UISO_AEA & ITASRL & 0.00159 & $\mathrm{pCi} / \mathrm{L}$ & 0.00733 & 0.00738 & & & $U$ \\
\hline 186 & UISO_AEA & ITASRL & 0.207 & $\mathrm{pCi} / \mathrm{L}$ & 0.0455 & 0.0499 & & & \\
\hline 187 & SRISŌSEP_PRECIP_GPC & ITASRL & 0.0942 & $\mathrm{pCi} / \mathrm{L}$ & 0.0356 & 0.0418 & & & \\
\hline 188 & TRITIUM_DIST_LSC & ITASRL & -150 & $\mathrm{pCi} / \mathrm{L}$ & 146 & 294 & & & U \\
\hline 189 & UISO_AEA & ITASRL & 0.279 & $\mathrm{pCi} / \mathrm{L}$ & 0.0489 & 0.0561 & & & \\
\hline 190 & UISO_AEA & ITASRL & 0.00407 & $\mathrm{pCi} / \mathrm{L}$ & 0.00734 & 0.00741 & & & U \\
\hline
\end{tabular}


N

O

P

Q

R

S

\begin{tabular}{|c|c|c|c|c|c|c|c|c|c|}
\hline & & & & & & & & & \\
\hline 1 & METHOD_NAME & LAB_CODE & VALUE_RPTD & ANAL_UNITS_RPTD & COUNTING_ERROR & TOTAL_ANAL_ERROR & MIN_DETECTABLE_ACTIVITY & PCNT_MOISTURE & LAB_QUALIFIER \\
\hline 192 & SRISO_SEP_PRECIP_GPC & ITASRL & 0.0849 & $\mathrm{pCi} / \mathrm{L}$ & 0.0334 & 0.039 & & & \\
\hline 193 & TRITIUM_DIST_LSC & ITASRL & 400 & $\mathrm{pCi} / \mathrm{L}$ & 173 & 327 & & & \\
\hline 194 & UISO_AËA & ITASRL & 0.289 & $\mathrm{pCi} / \mathrm{L}$ & 0.0484 & 0.0561 & & & \\
\hline 195 & UISO_AEA & ITASRL & 0.00183 & $\mathrm{pCi} / \mathrm{L}$ & 0.00575 & 0.00582 & & & $\mathrm{U}$ \\
\hline 196 & UISO_AEA & ITASRL & 0.233 & $\mathrm{pCi} / \mathrm{L}$ & 0.0432 & 0.0488 & & & \\
\hline 197 & SRISO-SEP_PRECIP_GPC & ITASRL & 0.0716 & $\mathrm{pCi} / \mathrm{L}$ & 0.0305 & 0.0351 & & & \\
\hline 198 & TRITIUMM_DISTT_LSC & ITASRL & 34 & $\mathrm{pCi} / \mathrm{L}$ & 155 & 305 & & & $U$ \\
\hline 199 & UISO AEA & ITASRL & 0.277 & $\mathrm{pCi} / \mathrm{L}$ & 0.0504 & 0.0574 & & & \\
\hline 200 & UISO_AEA & ITASRL & 0.0255 & $\mathrm{pCi} / \mathrm{L}$ & 0.016 & 0.0163 & & & \\
\hline 201 & UISO_AEA & ITASRL & 0.225 & $\mathrm{pCi} / \mathrm{L}$ & 0.0457 & 0.0507 & & & \\
\hline 202 & SRISO-̄SEP_PRECIP_GPC & ITASRL & 0.0914 & $\mathrm{pCi} / \mathrm{L}$ & 0.0261 & 0.0376 & & & \\
\hline 203 & TRITIUM_DIST_LSC & ITASRL & 102 & $\mathrm{pCi} / \mathrm{L}$ & 103 & 213 & & & $\mathrm{U}$ \\
\hline 204 & TRITIUM_ELECT_LSC & ITASRL & 25.7 & $\mathrm{pCi} / \mathrm{L}$ & 8.39 & 28.4 & & & $U$ \\
\hline 205 & UISO_AEA & ITASRL & 0.238 & $\mathrm{pCi} / \mathrm{L}$ & 0.044 & 0.0499 & & & \\
\hline 206 & UISO_AEA & ITASRL & 0.00642 & $\mathrm{pCi} / \mathrm{L}$ & 0.00772 & 0.0078 & & & U \\
\hline 207 & UISO_AEA & ITASRL & 0.165 & $\mathrm{pCi} / \mathrm{L}$ & 0.0374 & 0.0407 & & & \\
\hline 208 & SRISO_SEP_PRECIP_GPC & ITASRL & 0.139 & $\mathrm{pCi} / \mathrm{L}$ & 0.0345 & 0.0506 & & & \\
\hline 209 & TRITIUM_DIST_LSC & ITASRL & & $\mathrm{pCi} / \mathrm{L}$ & 100 & 208 & & & U \\
\hline 212 & UISO_AEA & ITASRL & 0.00796 & $\mathrm{pCi} / \mathrm{L}$ & 0.00828 & 0.00837 & & & U \\
\hline 213 & UISO_AEA & ITASRL & 0.175 & $\mathrm{pCi} / \mathrm{L}$ & 0.0372 & 0.0409 & & & \\
\hline 214 & SRISŌ_SEP_PRECIP_GPC & ITASRL & 0.119 & $\mathrm{pCi} / \mathrm{L}$ & 0.0411 & 0.0495 & & & \\
\hline 215 & TRITIŪM_DISTT_LSC & ITASRL & -67.4 & $\mathrm{pCi} / \mathrm{L}$ & 97.9 & 204 & & & U \\
\hline 216 & TRITIUM_ELECTT_LSC & ITASRL & & $\mathrm{pCi} / \mathrm{L}$ & 5.87 & 20 & & & \\
\hline 217 & UISO_AEA & ITASRL & 0.239 & $\mathrm{pCi} / \mathrm{L}$ & 0.0437 & 0.0497 & & & \\
\hline 218 & UISO_AEA & ITASRL & 0.0139 & $\mathrm{pCi} / \mathrm{L}$ & 0.012 & 0.0121 & & & \\
\hline 219 & UISO_AEA & ITASRL & 0.195 & $\mathrm{pCi} / \mathrm{L}$ & 0.0388 & 0.0432 & & & \\
\hline 220 & SRISŌ_SEP_PRECIP_GPC & ITASRL & 0.108 & $\mathrm{pCi} / \mathrm{L}$ & 0.0293 & 0.0408 & & & \\
\hline 221 & TRITIUM_DIST_LSC & ITASRL & 39.5 & $\mathrm{pCi} / \mathrm{L}$ & 102 & 210 & & & $\mathrm{U}$ \\
\hline 222 & TRITIUM_ELECTT_LSC & ITASRL & 34.2 & $\mathrm{pCi} / \mathrm{L}$ & 6.09 & 20.6 & & & \\
\hline 223 & UISO_AEA & ITASRL & 0.271 & $\mathrm{pCi} / \mathrm{L}$ & 0.0487 & 0.0556 & & & \\
\hline 224 & UISO_AEA & ITASRL & 0.0158 & $\mathrm{pCi} / \mathrm{L}$ & 0.0127 & 0.0129 & & & \\
\hline 225 & UISO_AEA & ITASRL & 0.164 & $\mathrm{pCi} / \mathrm{L}$ & 0.0379 & 0.0411 & & & \\
\hline 226 & SRISO_SEP_PRECIP_GPC & ITASRL & 0.101 & $\mathrm{pCi} / \mathrm{L}$ & 0.0367 & 0.0426 & & & \\
\hline 227 & TRITIŪM_ELEECT_LSC̄ & ITASRL & 126 & $\mathrm{pCi} / \mathrm{L}$ & 3.81 & 16.1 & & & \\
\hline 228 & UISO_AEA & ITASRL & 0.25 & $\mathrm{pCi} / \mathrm{L}$ & 0.0515 & 0.0574 & & & \\
\hline 229 & UISO_AEA & ITASRL & 0.0138 & $\mathrm{pCi} / \mathrm{L}$ & 0.0159 & 0.016 & & & $U$ \\
\hline
\end{tabular}




\begin{tabular}{|c|c|c|c|c|c|c|c|c|c|}
\hline & $\mathrm{K}$ & $\mathrm{L}$ & $\mathrm{M}$ & $\mathrm{N}$ & $\mathrm{O}$ & $\mathrm{P}$ & $\mathrm{Q}$ & $\mathrm{R}$ & $\mathrm{S}$ \\
\hline 1 & METHOD_NAME & LAB_CODE & VALUE_RPTD & ANAL_UNITS_RPTD & COUNTING_ERROR & TOTAL_ANAL_ERROR & MIN_DETECTABLE_ACTIVITY & PCNT_MOISTURE & LAB_QUALIFIER \\
\hline 230 & UISO_AEA & ITASRL & 0.227 & $7 \mathrm{pCi} / \mathrm{L}$ & 0.0482 & 0.0533 & & & \\
\hline 231 & SRISO_SEP_PRECIP_GPC & ITASRL & 0.0966 & $\mathrm{pCi} / \mathrm{L}$ & 0.0301 & 0.0416 & & & \\
\hline 232 & TRITIUM_ELECT_LSC & ITASRL & 52.5 & $5 \mathrm{pCi} / \mathrm{L}$ & 2.96 & 10.4 & & & \\
\hline 233 & UISO_AEA & ITASRL & 0.373 & $3 \mathrm{pCi} / \mathrm{L}$ & 0.0539 & 0.0648 & & & \\
\hline 234 & UISO_AEA & ITASRL & 0.025 & $5 \mathrm{pCi} / \mathrm{L}$ & 0.015 & 0.0153 & & & \\
\hline 235 & UISO_AEA & ITASRL & 0.336 & $\mathrm{pCi} / \mathrm{L}$ & 0.0509 & 0.0602 & & & \\
\hline 236 & SRISO_SEP_PRECIP_GPC & ITASRL & 0.0736 & $\mathrm{pCi} / \mathrm{L}$ & 0.0306 & 0.0351 & & & \\
\hline 237 & TRITIUM_ELECT_LSC & ITASRL & 118 & $\mathrm{pCi} / \mathrm{L}$ & 3.71 & 15.4 & & & \\
\hline 239 & UISO_AEA & ITASRL & 0.0154 & $4 \mathrm{pCi} / \mathrm{L}$ & 0.0134 & 0.0135 & & & \\
\hline 240 & UISO_AEA & ITASRL & 0.167 & $7 \mathrm{pCi} / \mathrm{L}$ & 0.0364 & 0.0399 & & & \\
\hline 241 & SRISO_SEP_PRECIP_GPC & ITASRL & 0.0897 & $7 \mathrm{pCi} / \mathrm{L}$ & 0.0342 & 0.0394 & & & \\
\hline 242 & TRITIUM_ELECT_LSC & ITASRL & 86.8 & $3 \mathrm{pCi} / \mathrm{L}$ & 3.38 & 13 & & & \\
\hline 243 & ]UISO_AEA & ITASRL & 0.249 & $\mathrm{pCi} / \mathrm{L}$ & 0.0489 & 0.0549 & & & \\
\hline 244 & UISO_AEA & ITASRL & 0.0152 & $2 \mathrm{pCi} / \mathrm{L}$ & 0.0132 & 0.0133 & & & \\
\hline 245 & UISO_AEA & ITASRL & 0.233 & $3 \mathrm{pCi} / \mathrm{L}$ & 0.047 & 0.0522 & & & \\
\hline 246 & SRISO_SEP_PRECIP_GPC & ITASRL & 0.0884 & $4 \mathrm{pCi} / \mathrm{L}$ & 0.0319 & 0.0372 & & & \\
\hline 247 & TRITIUM_ELECET_LSC & ITASRL & 83.1 & $\mathrm{pCi} / \mathrm{L}$ & 3.35 & 12.8 & & & \\
\hline 249 & UISO_AEA & ITASRL & 0.0144 & $4 \mathrm{pCi} / \mathrm{L}$ & 0.0126 & 0.0127 & & & \\
\hline 250 & UISO_AEA & ITASRL & 0.189 & $\mathrm{pCi} / \mathrm{L}$ & 0.0413 & 0.0453 & & & \\
\hline 251 & SRISO_SEP_PRECIP_GPC & ITASRL & 0.11 & $\mathrm{pCi} / \mathrm{L}$ & 0.0363 & 0.0438 & & & \\
\hline 252 & TRITIUM_ELECT_LSC & ITASRL & 70.4 & $4 \mathrm{pCi} / \mathrm{L}$ & 3.17 & 11.7 & & & \\
\hline 253 & UISO_AEA & ITASRL & 0.304 & $4 \mathrm{pCi} / \mathrm{L}$ & 0.0499 & 0.0581 & & & \\
\hline 254 & UISO_AEA & ITASRL & 0.00986 & $\mathrm{pCi} / \mathrm{L}$ & 0.00989 & 0.00998 & & & $U$ \\
\hline 255 & ]UISO_AEA & ITASRL & 0.252 & $2 \mathrm{pCi} / \mathrm{L}$ & 0.0455 & 0.0516 & & & \\
\hline 256 & SRISO_SEP_PRECIP_GPC & ITASRL & 0.107 & $7 \mathrm{pCi} / \mathrm{L}$ & 0.0259 & 0.035 & & & \\
\hline 257 & TRITIUM_ELECT_LSC & ITASRL & 64.6 & $\mathrm{pCi} / \mathrm{L}$ & 3.1 & 11.2 & & & \\
\hline 258 & UISO_AEA & ITASRL & 0.256 & $\mathrm{pCi} / \mathrm{L}$ & 0.0485 & 0.0547 & & & \\
\hline 259 & UISO_AEA & ITASRL & 0.00999 & $9 \mathrm{pCi} / \mathrm{L}$ & 0.0109 & 0.011 & & & $\mathrm{U}$ \\
\hline 260 & UISO_AEA & ITASRL & 0.218 & $\mathrm{pCi} / \mathrm{L}$ & 0.045 & 0.0498 & & & \\
\hline 261 & SRISO_SEP_PRECIP_GPC & ITASRL & 0.103 & $3 \mathrm{pCi} / \mathrm{L}$ & 0.0268 & 0.0354 & & & \\
\hline 262 & TRITIUM_ELEECT_LSC & ITASRL & 68.7 & $7 \mathrm{pCi} / \mathrm{L}$ & 3.29 & 12 & & & \\
\hline 263 & UISO_AEA & ITASRL & 0.228 & $3 \mathrm{pCi} / \mathrm{L}$ & 0.0435 & 0.049 & & & \\
\hline 264 & UISO_AEA & ITASRL & 0.00897 & $7 \mathrm{pCi} / \mathrm{L}$ & 0.00993 & 0.01 & & & $U$ \\
\hline 265 & UISO_AEA & ITASRL & 0.211 & $\mathrm{pCi} / \mathrm{L}$ & 0.0415 & 0.0462 & & & \\
\hline 266 & SRISO_SEP_PRECIP_GPC & ITASRL & 0.0886 & $\mathrm{pCi} / \mathrm{L}$ & 0.0288 & 0.0357 & & & \\
\hline
\end{tabular}




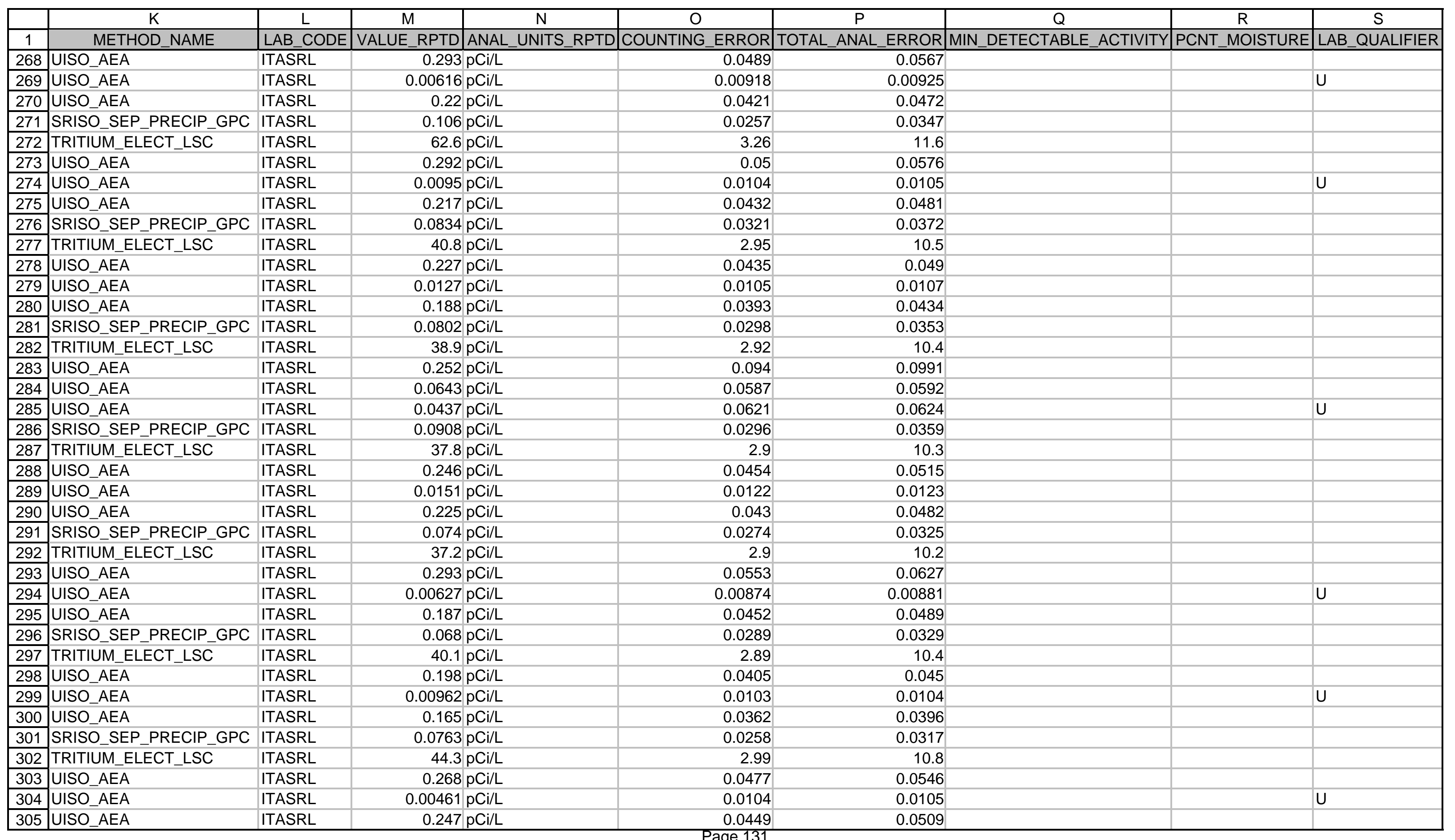




\begin{tabular}{|c|c|c|c|c|c|c|c|c|c|}
\hline & $\mathrm{K}$ & $\mathrm{L}$ & $\mathrm{M}$ & $\mathrm{N}$ & $\mathrm{O}$ & $P$ & $\mathrm{Q}$ & $\mathrm{R}$ & $S$ \\
\hline 1 & METHOD_NAME & LAB_CODE & VALUE_RPTD & ANAL_UNITS_RPTD & COUNTING_ERROR & TOTAL_ANAL_ERROR & MIN_DETECTABLE_ACTIVITY & PCNT_MOISTURE & LAB_QUALIFIER \\
\hline 306 & SRISO_SEP_PRECIP_GPC & ITASRL & 0.0803 & $\mathrm{pCi} / \mathrm{L}$ & 0.0298 & 0.0348 & & & \\
\hline 307 & TRITIUM_ELECT_LSC & ITASRL & 38.2 & $\mathrm{pCi} / \mathrm{L}$ & 2.9 & 10.3 & & & \\
\hline 308 & UISO_AEA & ITASRL & 0.219 & $\mathrm{pCi} / \mathrm{L}$ & 0.0417 & 0.047 & & & \\
\hline 309 & UISO_AEA & ITASRL & 0.0119 & $\mathrm{pCi} / \mathrm{L}$ & 0.0118 & 0.0119 & & & \\
\hline 310 & UISO_AEA & ITASRL & 0.164 & $\mathrm{pCi} / \mathrm{L}$ & 0.0358 & 0.0392 & & & \\
\hline 311 & SRISO_SEP_PRECIP_GPC & ITASRL & 0.0882 & $\mathrm{pCi} / \mathrm{L}$ & 0.0313 & 0.0367 & & & \\
\hline 312 & TRITIUM_ELECT_LSC & ITASRL & 35.4 & $\mathrm{pCi} / \mathrm{L}$ & 2.88 & 10.2 & & & \\
\hline 313 & UISO_AEA & ITASRL & 0.224 & $\mathrm{pCi} / \mathrm{L}$ & 0.0411 & 0.0467 & & & \\
\hline 315 & UISO_AEA & ITASRL & 0.197 & $\mathrm{pCi} / \mathrm{L}$ & 0.0382 & 0.0426 & & & \\
\hline 316 & SRISO_SEP_PRECIP_GPC & ITASRL & 0.0822 & $\mathrm{pCi} / \mathrm{L}$ & 0.0298 & 0.0353 & & & \\
\hline 317 & TRITIUM_ELECT_LSC & ITASRL & 37.8 & $\mathrm{pCi} / \mathrm{L}$ & 2.85 & 10.2 & & & \\
\hline 318 & UISO_AEA & ITASRL & 0.234 & $\mathrm{pCi} / \mathrm{L}$ & 0.0424 & 0.0482 & & & \\
\hline 319 & ]UISO_AEA & ITASRL & 0.0116 & $\mathrm{pCi} / \mathrm{L}$ & 0.00975 & 0.00987 & & & \\
\hline 320 & UISO_AEA & ITASRL & 0.201 & $\mathrm{pCi} / \mathrm{L}$ & 0.039 & 0.0436 & & & \\
\hline 321 & SRISO_SEP_PRECIP_GPC & ITASRL & 0.0755 & $\mathrm{pCi} / \mathrm{L}$ & 0.0229 & 0.0289 & & & \\
\hline 322 & TRITIUM_ELECT_LSC & ITASRL & 36.5 & $\mathrm{pCi} / \mathrm{L}$ & 2.85 & 10.2 & & & \\
\hline 323 & UISO_AEA & ITASRL & 0.238 & $\mathrm{pCi} / \mathrm{L}$ & 0.0419 & 0.048 & & & \\
\hline 325 & UISO_AEA & ITASRL & 0.176 & $\mathrm{pCi} / \mathrm{L}$ & 0.0362 & 0.04 & & & \\
\hline 326 & SRISŌ_SEP_PRECIP_GPC & ITASRL & 0.0733 & $\mathrm{pCi} / \mathrm{L}$ & 0.0259 & 0.0316 & & & \\
\hline 327 & TRITIUMM_ELEECT_LSC̄ & ITASRL & 39.2 & $\mathrm{pCi} / \mathrm{L}$ & 2.91 & 10.4 & & & \\
\hline 328 & UISO_AEA & ITASRL & 0.25 & $\mathrm{pCi} / \mathrm{L}$ & 0.0441 & 0.0505 & & & \\
\hline 329 & UISO_AEA & ITASRL & 0.00466 & $\mathrm{pCi} / \mathrm{L}$ & 0.00774 & 0.0078 & & & U \\
\hline 330 & UISO_AEA & ITASRL & 0.22 & $\mathrm{pCi} / \mathrm{L}$ & 0.041 & 0.0461 & & & \\
\hline 331 & SRISO_SEP_PRECIP_GPC & ITASRL & 0.0931 & $\mathrm{pCi} / \mathrm{L}$ & 0.0309 & 0.0372 & & & \\
\hline 332 & TRITIUM_ELECT_LSC & ITASRL & 38.4 & $\mathrm{pCi} / \mathrm{L}$ & 2.91 & 10.3 & & & \\
\hline 333 & UISO_AEA & ITASRL & 0.22 & $\mathrm{pCi} / \mathrm{L}$ & 0.0456 & 0.0507 & & & \\
\hline 334 & UISO_AEA & ITASRL & 0.00772 & $\mathrm{pCi} / \mathrm{L}$ & 0.00902 & 0.0091 & & & $\mathrm{U}$ \\
\hline 335 & UISO_AEA & ITASRL & 0.187 & $\mathrm{pCi} / \mathrm{L}$ & 0.0426 & 0.0464 & & & \\
\hline 336 & SRISO_SEP_PRECIP_GPC & ITASRL & 0.0488 & $\mathrm{pCi} / \mathrm{L}$ & 0.0278 & 0.0311 & & & \\
\hline 337 & TRITIUM_ELECT_LSC & ITASRL & 36.8 & $\mathrm{pCi} / \mathrm{L}$ & 2.88 & 10.2 & & & \\
\hline 338 & UISO_AEA & ITASRL & 0.261 & $\mathrm{pCi} / \mathrm{L}$ & 0.0465 & 0.0531 & & & \\
\hline 339 & UISO_AEA & ITASRL & 0.0099 & $\mathrm{pCi} / \mathrm{L}$ & 0.00993 & 0.01 & & & $\mathrm{U}$ \\
\hline 340 & UISO_AEA & ITASRL & 0.191 & $\mathrm{pCi} / \mathrm{L}$ & 0.0399 & 0.044 & & & \\
\hline 341 & SRISO_SEP_PRECIP_GPC & ITASRL & 0.09 & $\mathrm{pCi} / \mathrm{L}$ & 0.0247 & 0.0326 & & & \\
\hline 342 & TRITIUM_ELEECT_LSC & ITASRL & 39.3 & $\mathrm{pCi} / \mathrm{L}$ & 2.91 & 10.4 & & & \\
\hline
\end{tabular}




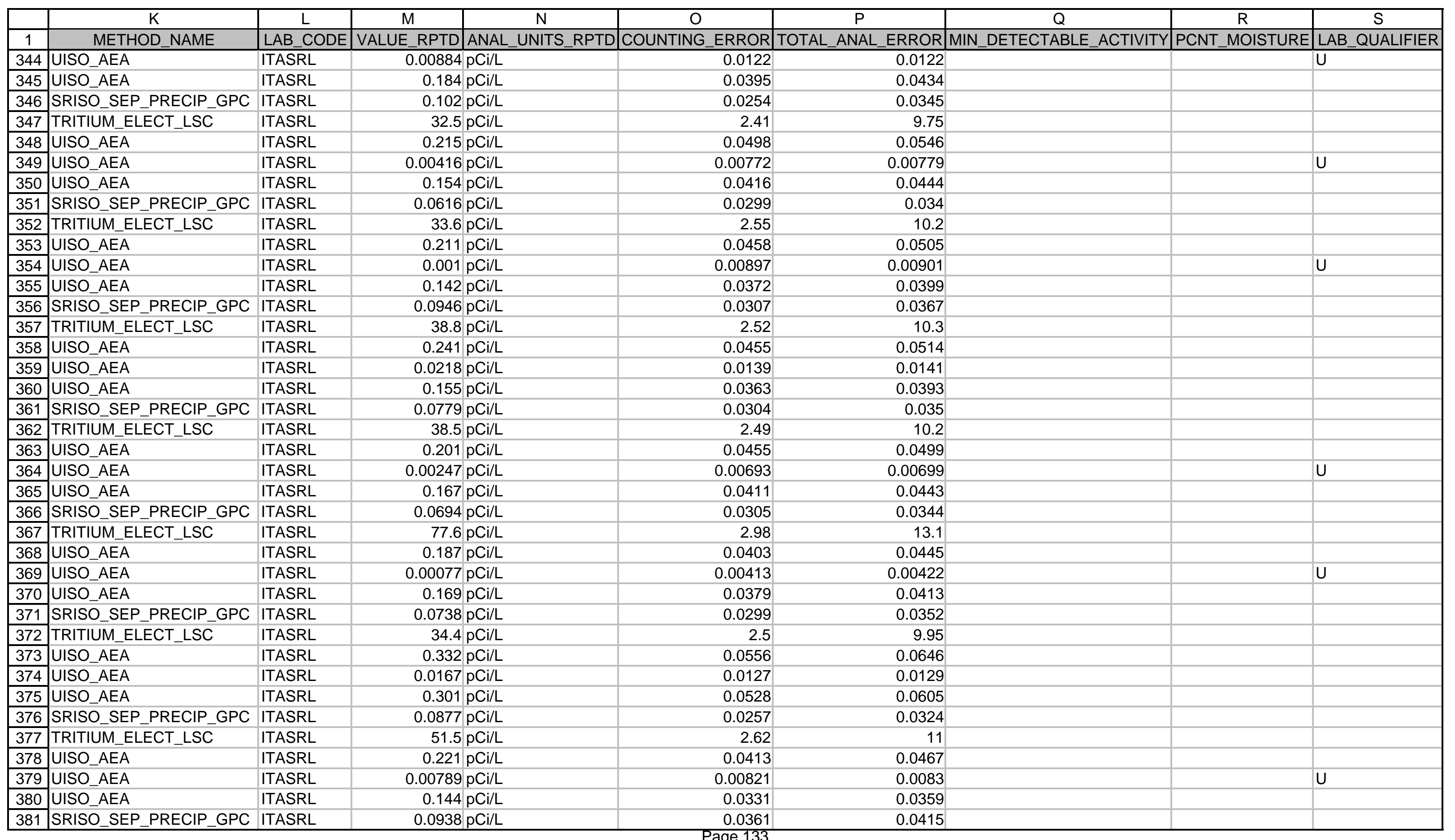




\begin{tabular}{|c|c|c|c|c|c|c|c|c|c|}
\hline & $\mathrm{K}$ & $\mathrm{L}$ & $\mathrm{M}$ & $\mathrm{N}$ & $\mathrm{O}$ & $\mathrm{P}$ & $\mathrm{Q}$ & $\mathrm{R}$ & $\mathrm{S}$ \\
\hline 1 & METHOD_NAME & LAB_CODE & VALUE_RPTD & ANAL_UNITS_RPTD & COUNTING_ERROR & TOTAL_ANAL_ERROR & MIN_DETECTABLE_ACTIVITY & PCNT_MOISTURE & LAB_QUALIFIER \\
\hline 382 & TRITIUM_ELECT_LSC & ITASRL & 42.3 & $\mathrm{pCi} / \mathrm{L}$ & 2.7 & 10.9 & & & \\
\hline 383 & UISO_AEA & ITASRL & 0.185 & $\mathrm{pCi} / \mathrm{L}$ & 0.0447 & 0.0486 & & & \\
\hline 384 & UISO_AEA & ITASRL & 0.00521 & $\mathrm{pCi} / \mathrm{L}$ & 0.0089 & 0.00896 & & & $\mathrm{U}$ \\
\hline 385 & UISO_AEA & ITASRL & 0.16 & $\mathrm{pCi} / \mathrm{L}$ & 0.0407 & 0.0438 & & & \\
\hline 386 & SRISŌSEP_PRECIP_GPC & ITASRL & 0.0985 & $\mathrm{pCi} / \mathrm{L}$ & 0.0275 & 0.0351 & & & \\
\hline 387 & TRITIUM_ELEECT_LSC & ITASRL & 36 & $\mathrm{pCi} / \mathrm{L}$ & 2.46 & 9.98 & & & \\
\hline 388 & UISO AEA & ITASRL & 0.195 & $\mathrm{pCi} / \mathrm{L}$ & 0.0408 & 0.0452 & & & \\
\hline 389 & UISO_AEA & ITASRL & -0.0013 & $\mathrm{pCi} / \mathrm{L}$ & 0.0066 & 0.00665 & & & $U$ \\
\hline 391 & SRISO_SEP_PRECIP_GPC & ITASRL & 0.0907 & $\mathrm{pCi} / \mathrm{L}$ & 0.0309 & 0.0366 & & & \\
\hline 392 & TRITIUM_ELECT_LSC & ITASRL & 34.3 & $\mathrm{pCi} / \mathrm{L}$ & 2.45 & 9.89 & & & \\
\hline 393 & UISO_AEA & ITASRL & 0.242 & $\mathrm{pCi} / \mathrm{L}$ & 0.0491 & 0.0548 & & & \\
\hline 394 & UISO_AEA & ITASRL & 0.00288 & $\mathrm{pCi} / \mathrm{L}$ & 0.00865 & 0.0087 & & & U \\
\hline 395 & UISO_AEA & ITASRL & 0.191 & $\mathrm{pCi} / \mathrm{L}$ & 0.0428 & 0.0468 & & & \\
\hline 396 & SRISO_SEP_PRECIP_GPC & ITASRL & 0.0923 & $\mathrm{pCi} / \mathrm{L}$ & 0.026 & 0.0338 & & & \\
\hline 397 & TRITIUM_ELECT_LSC & ITASRL & 35.7 & $\mathrm{pCi} / \mathrm{L}$ & 2.52 & 10.1 & & & \\
\hline 398 & UISO_AEA & ITASRL & 0.219 & $\mathrm{pCi} / \mathrm{L}$ & 0.0498 & 0.0547 & & & \\
\hline 399 & UISO_AEA & ITASRL & 0.0104 & $\mathrm{pCi} / \mathrm{L}$ & 0.0137 & 0.0138 & & & $U$ \\
\hline 401 & SRISO_SEP_PRECIP_GPC & ITASRL & 0.0604 & $\mathrm{pCi} / \mathrm{L}$ & 0.0326 & 0.0362 & & & \\
\hline 402 & TRITIUM_ELECTI_LSC & ITASRL & 36.1 & $\mathrm{pCi} / \mathrm{L}$ & 2.46 & 10 & & & \\
\hline 403 & UISO_AEA & ITASRL & 0.221 & $\mathrm{pCi} / \mathrm{L}$ & 0.0426 & 0.0479 & & & \\
\hline 404 & UISO_AEA & ITASRL & 0.0156 & $\mathrm{pCi} / \mathrm{L}$ & 0.0119 & 0.0121 & & & \\
\hline 405 & UISO_AEA & ITASRL & 0.168 & $\mathrm{pCi} / \mathrm{L}$ & 0.0375 & 0.041 & & & \\
\hline 406 & SRISŌ_SEP_PRECIP_GPC & ITASRL & 0.0927 & $\mathrm{pCi} / \mathrm{L}$ & 0.035 & 0.041 & & & \\
\hline 407 & TRITIUM_ELEECT_LSC & ITASRL & 34.8 & $\mathrm{pCi} / \mathrm{L}$ & 2.5 & 10 & & & \\
\hline 408 & UISO_AEA & ITASRL & 0.24 & $\mathrm{pCi} / \mathrm{L}$ & 0.0463 & 0.0521 & & & \\
\hline 409 & UISO_AEA & ITASRL & 0.00505 & $\mathrm{pCi} / \mathrm{L}$ & 0.00733 & 0.0074 & & & $U$ \\
\hline 410 & UISO_AEA & ITASRL & 0.181 & $\mathrm{pCi} / \mathrm{L}$ & 0.04 & 0.0438 & & & \\
\hline 411 & SRISŌSEP_PRECIP_GPC & ITASRL & 0.06 & $\mathrm{pCi} / \mathrm{L}$ & 0.0246 & 0.0289 & & & \\
\hline 412 & TRITIUM_ELECTILLSC & ITASRL & & & & & & & \\
\hline 413 & UISO_AEA & ITASRL & 0.249 & $\mathrm{pCi} / \mathrm{L}$ & 0.0463 & 0.0524 & & & \\
\hline 414 & UISO_AEA & ITASRL & 0.00116 & $\mathrm{pCi} / \mathrm{L}$ & 0.00624 & 0.0063 & & & $U$ \\
\hline 415 & UISO_AEA & ITASRL & 0.195 & $\mathrm{pCi} / \mathrm{L}$ & 0.0409 & 0.0451 & & & \\
\hline 416 & SRISO_SEP_PRECIP_GPC & ITASRL & -0.00784 & $\mathrm{pCi} / \mathrm{L}$ & 0.0247 & 0.0253 & & & U \\
\hline 417 & TRITIUM_ELECT_LSC & ITASRL & 38.9 & $\mathrm{pCi} / \mathrm{L}$ & 2.5 & 10 & & & \\
\hline 418 & UISO_AEA & ITASRL & 0.243 & $\mathrm{pCi} / \mathrm{L}$ & 0.0468 & 0.0527 & & & \\
\hline
\end{tabular}




\begin{tabular}{|c|c|c|c|c|c|c|c|c|c|}
\hline & $\mathrm{K}$ & $\mathrm{L}$ & $\mathrm{M}$ & $\mathrm{N}$ & $\mathrm{O}$ & $\mathrm{P}$ & $\mathrm{Q}$ & $\mathrm{R}$ & $\mathrm{S}$ \\
\hline 1 & METHOD_NAME & LAB_CODE & VALUE_RPTD & ANAL_UNITS_RPTD & COUNTING_ERROR & TOTAL_ANAL_ERROR & MIN_DETECTABLE_ACTIVITY & PCNT_MOISTURE & LAB_QUALIFIER \\
\hline 420 & UISO_AEA & ITASRL & 0.206 & $\mathrm{pCi} / \mathrm{L}$ & 0.0433 & 0.0478 & & & \\
\hline 421 & SRISO_SEP_PRECIP_GPC & ITASRL & 0.0716 & $\mathrm{pCi} / \mathrm{L}$ & 0.0266 & 0.0346 & & & \\
\hline 422 & TRITIUM_ELECT_LSC & ITASRL & 41.6 & $\mathrm{pCi} / \mathrm{L}$ & 2.52 & 10.2 & & & \\
\hline 423 & UISO_AEA & ITASRL & 0.309 & $\mathrm{pCi} / \mathrm{L}$ & 0.0547 & 0.0628 & & & \\
\hline 424 & UISO_AEA & ITASRL & 0.0284 & $\mathrm{pCi} / \mathrm{L}$ & 0.0176 & 0.0179 & & & \\
\hline 425 & UISO_AEA & ITASRL & 0.248 & $\mathrm{pCi} / \mathrm{L}$ & 0.0493 & 0.055 & & & \\
\hline 426 & SRISO_SEP_PRECIP_GPC & ITASRL & 0.0866 & $\mathrm{pCi} / \mathrm{L}$ & 0.0332 & 0.0382 & & & \\
\hline 427 & TRITIUM_ELECET_LSC & ITASRL & 38.7 & $\mathrm{pCi} / \mathrm{L}$ & 2.48 & 9.95 & & & \\
\hline 429 & UISO_AEA & ITASRL & 0.0064 & $\mathrm{pCi} / \mathrm{L}$ & 0.00948 & 0.00954 & & & $\mathrm{U}$ \\
\hline 430 & UISO_AEA & ITASRL & 0.182 & $\mathrm{pCi} / \mathrm{L}$ & 0.0391 & 0.043 & & & \\
\hline 431 & SRISO_SEP_PRECIP_GPC & ITASRL & 0.0765 & $\mathrm{pCi} / \mathrm{L}$ & 0.0285 & 0.0337 & & & \\
\hline 432 & TRITIUM_ELECT_LSC & ITASRL & 39.6 & $\mathrm{pCi} / \mathrm{L}$ & 2.52 & 10.1 & & & \\
\hline 433 & ]UISO_AEA & ITASRL & 0.276 & $\mathrm{pCi} / \mathrm{L}$ & 0.0492 & 0.0563 & & & \\
\hline 434 & UISO_AEA & ITASRL & 0.00584 & $\mathrm{pCi} / \mathrm{L}$ & 0.00998 & 0.01 & & & $\mathrm{U}$ \\
\hline 435 & UISO_AEA & ITASRL & 0.227 & $\mathrm{pCi} / \mathrm{L}$ & 0.0451 & 0.0503 & & & \\
\hline 436 & SRISO_SEP_PRECIP_GPC & ITASRL & 0.0665 & $\mathrm{pCi} / \mathrm{L}$ & 0.0309 & 0.0348 & & & \\
\hline 437 & TRITIUM_ELECET_LSC & ITASRL & 38.6 & $\mathrm{pCi} / \mathrm{L}$ & 2.52 & 10.1 & & & \\
\hline 439 & UISO_AEA & ITASRL & 0.00404 & $\mathrm{pCi} / \mathrm{L}$ & 0.00884 & 0.0089 & & & $\mathrm{U}$ \\
\hline 440 & UISO_AEA & ITASRL & 0.201 & $\mathrm{pCi} / \mathrm{L}$ & 0.0448 & 0.0491 & & & \\
\hline 441 & SRISŌSEP_PRECIP_GPC & ITASRL & 0.0731 & $\mathrm{pCi} / \mathrm{L}$ & 0.0324 & 0.0363 & & & \\
\hline 442 & TRITIUM_ELECT_LSC & ITASRL & 35.3 & $\mathrm{pCi} / \mathrm{L}$ & 2.45 & 9.75 & & & \\
\hline 443 & UISO_AEA & ITASRL & 0.222 & $\mathrm{pCi} / \mathrm{L}$ & 0.0489 & 0.0539 & & & \\
\hline 444 & UISO_AEA & ITASRL & 0.0017 & $\mathrm{pCi} / \mathrm{L}$ & 0.0076 & 0.00765 & & & $U$ \\
\hline 445 & UISO_AEA & ITASRL & 0.228 & $\mathrm{pCi} / \mathrm{L}$ & 0.0485 & 0.0535 & & & \\
\hline 446 & SRISO_SEP_PRECIP_GPC & ITASRL & 0.0582 & $\mathrm{pCi} / \mathrm{L}$ & 0.0474 & 0.0498 & & & \\
\hline 447 & TRITIUM_ELECT_LSC & ITASRL & 40 & $\mathrm{pCi} / \mathrm{L}$ & 2.51 & 10.1 & & & \\
\hline 448 & UISO_AEA & ITASRL & 0.223 & $\mathrm{pCi} / \mathrm{L}$ & 0.0534 & 0.0583 & & & \\
\hline 449 & UISO_AEA & ITASRL & 0.00402 & $\mathrm{pCi} / \mathrm{L}$ & 0.011 & 0.0111 & & & $\mathrm{U}$ \\
\hline 450 & UISO_AEA & ITASRL & 0.184 & $\mathrm{pCi} / \mathrm{L}$ & 0.0478 & 0.0513 & & & \\
\hline 451 & SRISO_SEP_PRECIP_GPC & ITASRL & 0.0723 & $\mathrm{pCi} / \mathrm{L}$ & 0.0393 & 0.0442 & & & \\
\hline 452 & TRITIUM_ELEECT_LSC & ITASRL & 37.6 & $\mathrm{pCi} / \mathrm{L}$ & 2.47 & 9.83 & & & \\
\hline 453 & UISO_AËA & ITASRL & 0.308 & $\mathrm{pCi} / \mathrm{L}$ & 0.0558 & 0.0637 & & & \\
\hline 454 & UISO_AEA & ITASRL & 0.0108 & $\mathrm{pCi} / \mathrm{L}$ & 0.0108 & 0.0109 & & & U \\
\hline 455 & UISO_AEA & ITASRL & 0.197 & $\mathrm{pCi} / \mathrm{L}$ & 0.0445 & 0.0486 & & & \\
\hline 456 & SRISO_SEP_PRECIP_GPC & ITASRL & 0.0782 & $\mathrm{pCi} / \mathrm{L}$ & 0.032 & 0.0364 & & & \\
\hline
\end{tabular}




\begin{tabular}{|c|c|c|c|c|c|c|c|c|c|}
\hline & $\mathrm{K}$ & $\mathrm{L}$ & $\mathrm{M}$ & $\mathrm{N}$ & $\mathrm{O}$ & $\mathrm{P}$ & $\mathrm{Q}$ & $\mathrm{R}$ & $\mathrm{S}$ \\
\hline 1 & METHOD_NAME & LAB_CODE & VALUE_RPTD & ANAL_UNITS_RPTD & |COUNTING_ERROR| & TOTAL_ANAL_ERROR & MIN_DETECTABLE_ACTIVITY & PCNT_MOISTURE & LAB_QUALIFIER \\
\hline 458 & UISO_AEA & ITASRL & 0.257 & $\mathrm{pCi} / \mathrm{L}$ & 0.0667 & 0.0723 & & & \\
\hline 459 & UISO_AEA & ITASRL & 0.00908 & $\mathrm{pCi} / \mathrm{L}$ & 0.0142 & 0.0142 & & & U \\
\hline 460 & UISO_AEA & ITASRL & 0.212 & $\mathrm{pCi} / \mathrm{L}$ & 0.0593 & 0.0635 & & & \\
\hline 461 & SRISO_SEP_PRECIP_GPC & ITASRL & 0.129 & $\mathrm{pCi} / \mathrm{L}$ & 0.0363 & 0.0457 & & & \\
\hline 462 & TRITIUM_ELECET_LSC & ITASRL & 37.9 & $\mathrm{pCi} / \mathrm{L}$ & 2.49 & 9.95 & & & \\
\hline 463 & UISO_AEA & ITASRL & 0.31 & $\mathrm{pCi} / \mathrm{L}$ & 0.0607 & 0.0685 & & & \\
\hline 464 & UISO_AEA & ITASRL & 0.00261 & $\mathrm{pCi} / \mathrm{L}$ & 0.0107 & 0.0107 & & & $\mathrm{U}$ \\
\hline 465 & UISO_AEA & ITASRL & 0.211 & $\mathrm{pCi} / \mathrm{L}$ & 0.0503 & 0.0547 & & & \\
\hline 467 & TRITIUM_ELECT_LSC & ITASRL & 39.8 & $\mathrm{pCi} / \mathrm{L}$ & 2.52 & 10.1 & & & \\
\hline 468 & UISO_AEA & ITASRL & 0.298 & $\mathrm{pCi} / \mathrm{L}$ & 0.0508 & 0.0587 & & & \\
\hline 469 & UISO_AEA & ITASRL & 0.00367 & $\mathrm{pCi} / \mathrm{L}$ & 0.00892 & 0.00897 & & & $U$ \\
\hline 470 & UISO_AEA & ITASRL & 0.205 & $\mathrm{pCi} / \mathrm{L}$ & 0.0427 & 0.0472 & & & \\
\hline 471 & SRISO_SEP_PRECIP_GPC & ITASRL & 0.0629 & $\mathrm{pCi} / \mathrm{L}$ & 0.0288 & 0.0329 & & & \\
\hline 472 & TRITIUM_ELECT_LSC & ITASRL & 36.9 & $\mathrm{pCi} / \mathrm{L}$ & 2.5 & 9.9 & & & \\
\hline 473 & UISO_AEA & ITASRL & 0.255 & $\mathrm{pCi} / \mathrm{L}$ & 0.0483 & 0.0546 & & & \\
\hline 474 & UISO_AEA & ITASRL & 0.00783 & $\mathrm{pCi} / \mathrm{L}$ & 0.01 & 0.0101 & & & $\mathrm{U}$ \\
\hline 475 & UISO_AEA & ITASRL & 0.223 & $\mathrm{pCi} / \mathrm{L}$ & 0.0445 & 0.0496 & & & \\
\hline 477 & TRITIUM_ELECT_LSC & ITASRL & 39 & $\mathrm{pCi} / \mathrm{L}$ & 2.53 & 10.1 & & & \\
\hline 478 & UISO_AËA & ITASRL & 0.228 & $\mathrm{pCi} / \mathrm{L}$ & 0.0458 & 0.0512 & & & \\
\hline 479 & UISO_AEA & ITASRL & 0.0141 & $\mathrm{pCi} / \mathrm{L}$ & 0.0123 & 0.0124 & & & \\
\hline 480 & UISO_AEA & ITASRL & 0.203 & $\mathrm{pCi} / \mathrm{L}$ & 0.0423 & 0.0468 & & & \\
\hline 481 & SRISO_SEP_PRECIP_GPC & ITASRL & 0.0809 & $\mathrm{pCi} / \mathrm{L}$ & 0.0348 & 0.0425 & & & \\
\hline 482 & TRITIUM_ELECT_LSC & ITASRL & 39.7 & $\mathrm{pCi} / \mathrm{L}$ & 2.51 & 10 & & & \\
\hline 483 & UISO_AEA & ITASRL & 0.232 & $\mathrm{pCi} / \mathrm{L}$ & 0.0472 & 0.0527 & & & \\
\hline 484 & UISO_AEA & ITASRL & 0.00718 & $\mathrm{pCi} / \mathrm{L}$ & 0.0104 & 0.0105 & & & U \\
\hline 485 & JUISO_AEA & ITASRL & 0.208 & $\mathrm{pCi} / \mathrm{L}$ & 0.0441 & 0.0486 & & & \\
\hline 486 & SRISO_SEP_PRECIP_GPC & ITASRL & 0.0922 & $\mathrm{pCi} / \mathrm{L}$ & 0.034 & 0.0402 & & & \\
\hline 487 & TRITIUM_ELECT_LSC & ITASRL & 99.7 & $\mathrm{pCi} / \mathrm{L}$ & 3.27 & 15.2 & & & \\
\hline 488 & UISO_AEA & ITASRL & 0.304 & $\mathrm{pCi} / \mathrm{L}$ & 0.0532 & 0.0611 & & & \\
\hline 489 & UISO_AEA & ITASRL & 0.00656 & $\mathrm{pCi} / \mathrm{L}$ & 0.0115 & 0.0116 & & & $\mathrm{U}$ \\
\hline 490 & UISO_AEA & ITASRL & 0.218 & $\mathrm{pCi} / \mathrm{L}$ & 0.045 & 0.0498 & & & \\
\hline 491 & SRISO_SEP_PRECIP_GPC & ITASRL & 0.104 & $\mathrm{pCi} / \mathrm{L}$ & 0.0272 & 0.0348 & & & \\
\hline 492 & TRITIUM_ELECT_LSC & ITASRL & 45.3 & $\mathrm{pCi} / \mathrm{L}$ & 2.72 & 11.3 & & & \\
\hline 493 & UISO_AEA & ITASRL & 0.28 & $\mathrm{pCi} / \mathrm{L}$ & 0.0571 & 0.0638 & & & \\
\hline 494 & UISO_AEA & ITASRL & 0.0241 & $\mathrm{pCi} / \mathrm{L}$ & 0.0178 & 0.018 & & & \\
\hline
\end{tabular}




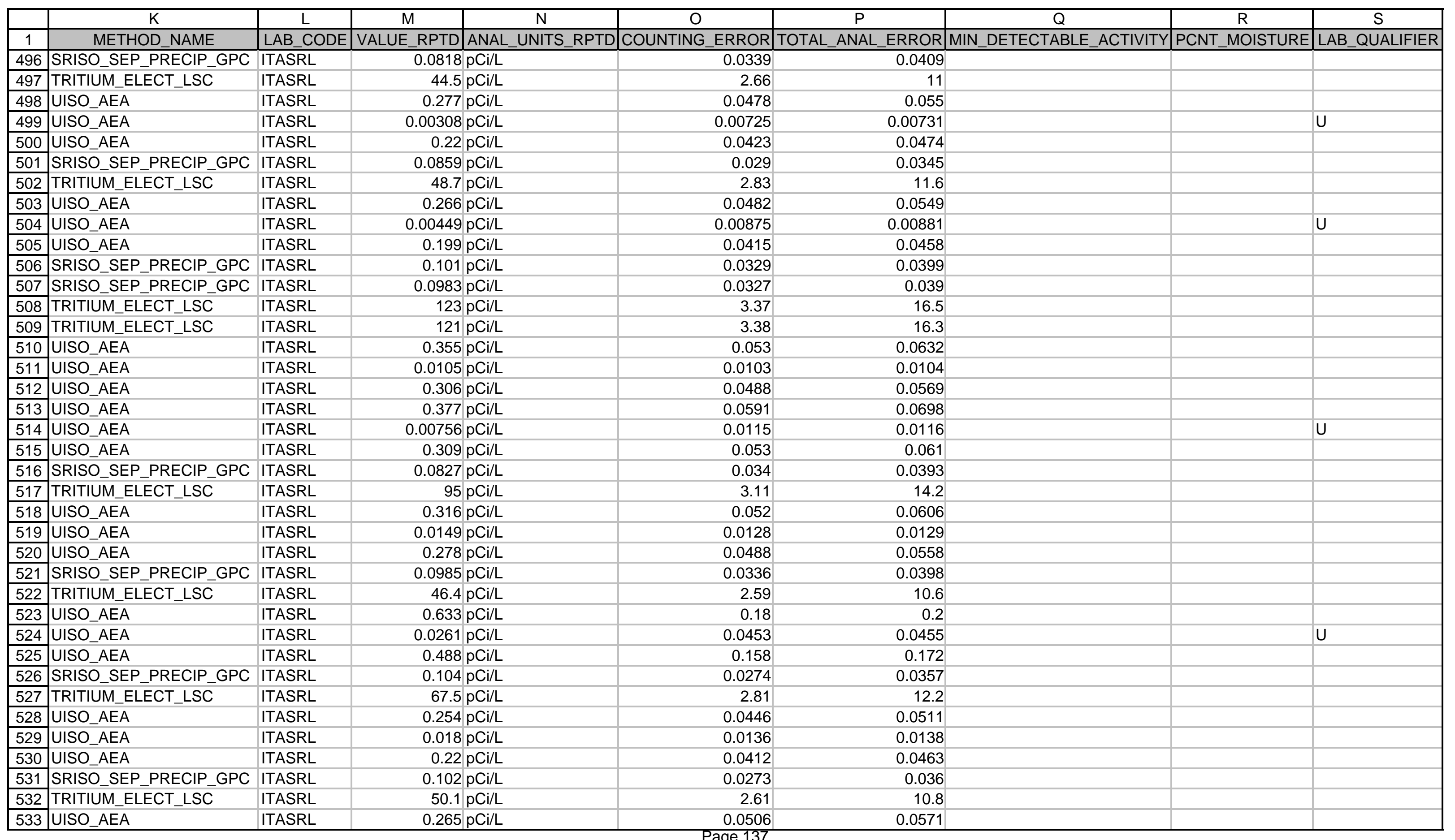




\begin{tabular}{|c|c|c|c|c|c|c|c|c|c|}
\hline & $\mathrm{K}$ & $\mathrm{L}$ & $\mathrm{M}$ & $\mathrm{N}$ & $\mathrm{O}$ & $\mathrm{P}$ & $\mathrm{Q}$ & $\mathrm{R}$ & $\mathrm{S}$ \\
\hline 1 & METHOD_NAME & LAB_CODE & VALUE_RPTD & ANAL_UNITS_RPTD & |COUNTING_ERROR| & TOTAL_ANAL_ERROR & MIN_DETECTABLE_ACTIVITY & PCNT_MOISTURE & LAB_QUALIFIER \\
\hline 534 & UISO_AEA & ITASRL & 0.0343 & $3 \mathrm{pCi} / \mathrm{L}$ & 0.0184 & 0.0187 & & & \\
\hline 535 & UISO_AEA & ITASRL & 0.202 & $2 \mathrm{pCi} / \mathrm{L}$ & 0.0443 & 0.0486 & & & \\
\hline 536 & SRISO_SEP_PRECIP_GPC & ITASRL & 0.104 & $4 \mathrm{pCi} / \mathrm{L}$ & 0.0353 & 0.0416 & & & \\
\hline 537 & TRITIUM_ELECT_LSC & ITASRL & 41.8 & $\mathrm{pCi} / \mathrm{L}$ & 2.52 & 10.3 & & & \\
\hline 538 & UISO_AEA & ITASRL & 0.275 & $5 \mathrm{pCi} / \mathrm{L}$ & 0.0482 & 0.0554 & & & \\
\hline 539 & UISO_AEA & ITASRL & 0.00522 & $2 \mathrm{pCi} / \mathrm{L}$ & 0.00847 & 0.00853 & & & $U$ \\
\hline 540 & UISO_AEA & ITASRL & 0.232 & $2 \mathrm{pCi} / \mathrm{L}$ & 0.0441 & 0.0496 & & & \\
\hline 541 & SRISO_SEP_PRECIP_GPC & ITASRL & 0.0922 & $2 \mathrm{pCi} / \mathrm{L}$ & 0.0301 & 0.0406 & & & \\
\hline 543 & UISO_AEA & ITASRL & 0.316 & $\mathrm{pCi} / \mathrm{L}$ & 0.0507 & 0.0594 & & & \\
\hline 544 & UISO_AEA & ITASRL & 0.00688 & $3 \mathrm{pCi} / \mathrm{L}$ & 0.00898 & 0.00905 & & & $\mathrm{U}$ \\
\hline 545 & UISO_AEA & ITASRL & 0.259 & $\mathrm{pCi} / \mathrm{L}$ & 0.0459 & 0.0523 & & & \\
\hline 546 & SRISO_SEP_PRECIP_GPC & ITASRL & 0.101 & $\mathrm{LCCi} / \mathrm{L}$ & 0.0417 & 0.0506 & & & \\
\hline 547 & TRITIUM_ELECT_LSC & ITASRL & 44 & $4 \mathrm{pCi} / \mathrm{L}$ & 2.56 & 10.4 & & & \\
\hline 548 & UISO_AEA & ITASRL & 0.296 & $\mathrm{pCi} / \mathrm{L}$ & 0.0514 & 0.0592 & & & \\
\hline 549 & UISO_AEA & ITASRL & 0.00252 & $2 \mathrm{pCi} / \mathrm{L}$ & 0.00791 & 0.00797 & & & $\mathrm{U}$ \\
\hline 550 & UISO_AEA & ITASRL & 0.185 & $5 \mathrm{pCi} / \mathrm{L}$ & 0.0406 & 0.0445 & & & \\
\hline 551 & SRISO_SEP_PRECIP_GPC & ITASRL & 0.0814 & $4 \mathrm{pCi} / \mathrm{L}$ & 0.0327 & 0.0375 & & & \\
\hline 553 & UISO_AEA & ITASRL & 0.294 & $4 \mathrm{pCi} / \mathrm{L}$ & 0.0506 & 0.0584 & & & \\
\hline 554 & UISO_AEA & ITASRL & 0.00958 & $\mathrm{pCi} / \mathrm{L}$ & 0.0105 & 0.0106 & & & $\mathrm{U}$ \\
\hline 555 & UISO_AEA & ITASRL & 0.235 & $\mathrm{pCi} / \mathrm{L}$ & 0.0449 & 0.0504 & & & \\
\hline 556 & SRISO_SEP_PRECIP_GPC & ITASRL & 0.197 & $7 \mathrm{pCi} / \mathrm{L}$ & 0.0318 & 0.0575 & & & \\
\hline 557 & TRITIUM_ELEECT_LSC & ITASRL & 43.6 & $\mathrm{pCi} / \mathrm{L}$ & 2.55 & 10.4 & & & \\
\hline 558 & UISO_AEA & ITASRL & 0.279 & $\mathrm{pCi} / \mathrm{L}$ & 0.0493 & 0.0565 & & & \\
\hline 559 & ]UISO_AEA & ITASRL & 0.0127 & $7 \mathrm{pCi} / \mathrm{L}$ & 0.012 & 0.0121 & & & \\
\hline 560 & UISO_AEA & ITASRL & 0.247 & $7 \mathrm{pCi} / \mathrm{L}$ & 0.0458 & 0.0517 & & & \\
\hline 561 & SRISO_SEP_PRECIP_GPC & ITASRL & 0.0901 & $\mathrm{p} \mathrm{pCi} / \mathrm{L}$ & 0.0325 & 0.0378 & & & \\
\hline 562 & TRITIUM_ELECT_LSC & ITASRL & 44.6 & $\mathrm{pCi} / \mathrm{L}$ & 2.57 & 10.5 & & & \\
\hline 563 & UISO_AEA & ITASRL & 0.26 & $\mathrm{pCi} / \mathrm{L}$ & 0.0551 & 0.0612 & & & \\
\hline 564 & UISO_AEA & ITASRL & 0.0141 & $\mathrm{pCi} / \mathrm{L}$ & 0.0136 & 0.0137 & & & \\
\hline 565 & UISO_AEA & ITASRL & 0.201 & $\mathrm{pCi} / \mathrm{L}$ & 0.048 & 0.0521 & & & \\
\hline 566 & SRISO_SEP_PRECIP_GPC & ITASRL & 0.0725 & $5 \mathrm{pCi} / \mathrm{L}$ & 0.0266 & 0.031 & & & \\
\hline 567 & TRITIUM_ELEECT_LSC & ITASRL & 35.6 & $\mathrm{pCi} / \mathrm{L}$ & 2.43 & 9.63 & & & \\
\hline 568 & UISO_AEA & ITASRL & 0.252 & $2 \mathrm{pCi} / \mathrm{L}$ & 0.0514 & 0.0575 & & & \\
\hline 569 & UISO_AEA & ITASRL & 0.0175 & $5 \mathrm{pCi} / \mathrm{L}$ & 0.0148 & 0.015 & & & \\
\hline 570 & UISO_AEA & ITASRL & 0.19 & $\mathrm{pCi} / \mathrm{L}$ & 0.0446 & 0.0486 & & & \\
\hline
\end{tabular}




\begin{tabular}{|c|c|c|c|c|c|c|c|c|c|}
\hline & $\mathrm{K}$ & $\mathrm{L}$ & $\bar{M}$ & $\mathrm{~N}$ & $\mathrm{O}$ & $\mathrm{P}$ & $\mathrm{Q}$ & $\mathrm{R}$ & $\mathrm{S}$ \\
\hline 1 & METHOD NAME & LAB CODE & VALUE RPTD & ANAL UNITS RPTD & COUNTING ERROR & TOTAL ANAL ERROR & MIN DETECTABLE ACTIVITY & PCNT MOISTURE & LAB QUALIFIER \\
\hline 572 & TRITIUM_ELECT_LSC & ITASRL & 37.4 & $\mathrm{pCi} / \mathrm{L}$ & 2.51 & 9.92 & & & \\
\hline 573 & UISO_AEA & ITASRL & 0.217 & $\mathrm{pCi} / \mathrm{L}$ & 0.0504 & 0.0553 & & & \\
\hline 574 & UISO_AEA & ITASRL & 0.0257 & $\mathrm{pCi} / \mathrm{L}$ & 0.019 & 0.0192 & & & \\
\hline 575 & UISO_AEA & ITASRL & 0.212 & $\mathrm{pCi} / \mathrm{L}$ & 0.0479 & 0.0526 & & & \\
\hline 576 & SRISŌSEP_PRECIP_GPC & ITASRL & 0.0791 & $\mathrm{pCi} / \mathrm{L}$ & 0.0228 & 0.0286 & & & \\
\hline 577 & TRITIUM_ELEECT_LSC & ITASRL & 39.4 & $\mathrm{pCi} / \mathrm{L}$ & 2.55 & 10.1 & & & \\
\hline 578 & UISO_AE- AE & ITASRL & 0.255 & $\mathrm{pCi} / \mathrm{L}$ & 0.0551 & 0.0612 & & & \\
\hline 579 & UISO_AEA & ITASRL & 0.000912 & $\mathrm{pCi} / \mathrm{L}$ & 0.00869 & 0.00874 & & & $U$ \\
\hline 581 & SRISO_SEP_PRECIP_GPC & ITASRL & 0.0855 & $\mathrm{pCi} / \mathrm{L}$ & 0.0219 & 0.0284 & & & \\
\hline 582 & TRITIUM_ELECTILSC & ITASRL & 38.9 & $\mathrm{pCi} / \mathrm{L}$ & 2.52 & 10 & & & \\
\hline 583 & UISO_AEA & ITASRL & 0.256 & $\mathrm{pCi} / \mathrm{L}$ & 0.0543 & 0.0605 & & & \\
\hline 584 & UISO_AEA & ITASRL & 0.00353 & $\mathrm{pCi} / \mathrm{L}$ & 0.01 & 0.0101 & & & U \\
\hline 585 & UISO_AEA & ITASRL & 0.156 & $\mathrm{pCi} / \mathrm{L}$ & 0.0427 & 0.0457 & & & \\
\hline 586 & SRISO_SEP_PRECIP_GPC & ITASRL & 0.0761 & $\mathrm{pCi} / \mathrm{L}$ & 0.024 & 0.0289 & & & \\
\hline 587 & TRITIUM_ELECT_LSC & ITASRL & 37.6 & $\mathrm{pCi} / \mathrm{L}$ & 2.44 & 9.76 & & & \\
\hline 588 & UISO_AEA & ITASRL & 0.219 & $\mathrm{pCi} / \mathrm{L}$ & 0.0491 & 0.0541 & & & \\
\hline 589 & UISO_AEA & ITASRL & 0.00156 & $\mathrm{pCi} / \mathrm{L}$ & 0.0134 & 0.0134 & & & $U$ \\
\hline 591 & SRISO_SEP_PRECIP_GPC & ITASRL & 0.0435 & $\mathrm{pCi} / \mathrm{L}$ & 0.0275 & 0.0303 & & & \\
\hline 592 & TRITIUM_ELECTI_LSC & ITASRL & 39.4 & $\mathrm{pCi} / \mathrm{L}$ & 2.49 & 9.85 & & & \\
\hline 593 & UISO_AEA & ITASRL & 0.309 & $\mathrm{pCi} / \mathrm{L}$ & 0.059 & 0.0671 & & & \\
\hline 594 & UISO_AEA & ITASRL & 0.0131 & $\mathrm{pCi} / \mathrm{L}$ & 0.0147 & 0.0148 & & & $U$ \\
\hline 595 & UISO_AEA & ITASRL & 0.237 & $\mathrm{pCi} / \mathrm{L}$ & 0.0513 & 0.0567 & & & \\
\hline 596 & SRISŌ_SEP_PRECIP_GPC & ITASRL & 0.0789 & $\mathrm{pCi} / \mathrm{L}$ & 0.0301 & 0.0345 & & & \\
\hline 597 & TRITIUM_ELEECT_LSC & ITASRL & 39.4 & $\mathrm{pCi} / \mathrm{L}$ & 2.49 & 9.92 & & & \\
\hline 598 & UISO_AEA & ITASRL & 0.216 & $\mathrm{pCi} / \mathrm{L}$ & 0.0492 & 0.0541 & & & \\
\hline 599 & UISO_AEA & ITASRL & 0.00924 & $\mathrm{pCi} / \mathrm{L}$ & 0.0116 & 0.0116 & & & $U$ \\
\hline 600 & UISO_AEA & ITASRL & 0.179 & $\mathrm{pCi} / \mathrm{L}$ & 0.0442 & 0.0479 & & & \\
\hline 601 & SRISŌSEP_PRECIP_GPC & ITASRL & 0.0701 & $\mathrm{pCi} / \mathrm{L}$ & 0.0258 & 0.0301 & & & \\
\hline 602 & TRITIUM_ELECTILLSC & ITASRL & 38.1 & $\mathrm{pCi} / \mathrm{L}$ & 2.47 & 9.8 & & & \\
\hline 603 & UISO_AEA & ITASRL & 0.248 & $\mathrm{pCi} / \mathrm{L}$ & 0.0474 & 0.0537 & & & \\
\hline 604 & UISO_AEA & ITASRL & -0.000449 & $\mathrm{pCi} / \mathrm{L}$ & 0.00688 & 0.00694 & & & $U$ \\
\hline 605 & UISO_AEA & ITASRL & 0.173 & $\mathrm{pCi} / \mathrm{L}$ & 0.0398 & 0.0436 & & & \\
\hline 606 & SRISO_SEP_PRECIP_GPC & ITASRL & 0.0564 & $\mathrm{pCi} / \mathrm{L}$ & 0.0289 & 0.0314 & & & \\
\hline 607 & TRITIUMM_ELECT_LSC & ITASRL & 42.1 & $\mathrm{pCi} / \mathrm{L}$ & 2.52 & 10 & & & \\
\hline 608 & UISO_AEA & ITASRL & 0.248 & $\mathrm{pCi} / \mathrm{L}$ & 0.0496 & 0.0557 & & & \\
\hline
\end{tabular}




\begin{tabular}{|c|c|c|c|c|c|c|c|c|c|}
\hline & $\mathrm{K}$ & $\mathrm{L}$ & $\mathrm{M}$ & $\mathrm{N}$ & $\mathrm{O}$ & $\mathrm{P}$ & $\mathrm{Q}$ & $\mathrm{R}$ & $\mathrm{S}$ \\
\hline 1 & METHOD_NAME & LAB_CODE & VALUE_RPTD & ANAL_UNITS_RPTD & COUNTING_ERROR & TOTAL_ANAL_ERROR & MIN_DETECTABLE_ACTIVITY & PCNT_MOISTURE & LAB_QUALIFIER \\
\hline 610 & UISO_AEA & ITASRL & 0.197 & $7 \mathrm{pCi} / \mathrm{L}$ & 0.044 & 0.0484 & & & \\
\hline 611 & SRISO_SEP_PRECIP_GPC & ITASRL & 0.0753 & $3 \mathrm{pCi} / \mathrm{L}$ & 0.0245 & 0.0296 & & & \\
\hline 612 & TRITIUM_ELECT_LSC & ITASRL & 40.7 & $7 \mathrm{pCi} / \mathrm{L}$ & 2.49 & 10 & & & \\
\hline 613 & UISO_AEA & ITASRL & 0.2 & $\mathrm{pCi} / \mathrm{L}$ & 0.0737 & 0.0775 & & & \\
\hline 614 & UISO_AEA & ITASRL & 0.0296 & $\mathrm{pCi} / \mathrm{L}$ & 0.033 & 0.0332 & & & $\mathrm{U}$ \\
\hline 615 & UISO_AEA & ITASRL & 0.235 & $5 \mathrm{pCi} / \mathrm{L}$ & 0.0741 & 0.079 & & & \\
\hline 616 & SRISO_SEP_PRECIP_GPC & ITASRL & 0.0904 & $4 \mathrm{pCi} / \mathrm{L}$ & 0.055 & 0.0592 & & & \\
\hline 617 & TRITIUM_ELECT_LSC & ITASRL & 39.5 & $5 \mathrm{pCi} / \mathrm{L}$ & 2.47 & 9.91 & & & \\
\hline 619 & UISO_AEA & ITASRL & -0.00183 & $3 \mathrm{pCi} / \mathrm{L}$ & 0.00654 & 0.0066 & & & $\mathrm{U}$ \\
\hline 620 & UISO_AEA & ITASRL & 0.163 & $3 \mathrm{pCi} / \mathrm{L}$ & 0.0434 & 0.0466 & & & \\
\hline 621 & SRISO_SEP_PRECIP_GPC & ITASRL & 0.0587 & $7 \mathrm{pCi} / \mathrm{L}$ & 0.0284 & 0.0332 & & & \\
\hline 622 & TRITIUM_ELECT_LSC & ITASRL & 40.1 & $\mathrm{pCi} / \mathrm{L}$ & 2.49 & 9.94 & & & \\
\hline 623 & UISO_AEA & ITASRL & 0.24 & $4 \mathrm{pCi} / \mathrm{L}$ & 0.0494 & 0.0552 & & & \\
\hline 624 & UISO_AEA & ITASRL & 0.0189 & $\mathrm{pCi} / \mathrm{L}$ & 0.0149 & 0.015 & & & \\
\hline 625 & UISO_AEA & ITASRL & 0.132 & $2 \mathrm{pCi} / \mathrm{L}$ & 0.0375 & 0.0399 & & & \\
\hline 626 & SRISO_SEP_PRECIP_GPC & ITASRL & 0.0947 & $7 \mathrm{pCi} / \mathrm{L}$ & 0.0362 & 0.044 & & & \\
\hline 627 & TRITIUM_ELECT_LSC & ITASRL & 38.5 & $5 \mathrm{pCi} / \mathrm{L}$ & 2.46 & 9.86 & & & \\
\hline 629 & UISO_AEA & ITASRL & 0.0103 & $3 \mathrm{pCi} / \mathrm{L}$ & 0.0202 & 0.0202 & & & $U$ \\
\hline 630 & UISO_AEA & ITASRL & 0.17 & $7 \mathrm{pCi} / \mathrm{L}$ & 0.0507 & 0.0539 & & & \\
\hline 631 & SRISO_SEP_PRECIP_GPC & ITASRL & 0.0639 & $9 \mathrm{pCi} / \mathrm{L}$ & 0.0289 & 0.0322 & & & \\
\hline 632 & TRITIUM_ELECT_LSC & ITASRL & 35.6 & $\mathrm{pCi} / \mathrm{L}$ & 2.42 & 9.59 & & & \\
\hline 633 & UISO_AEA & ITASRL & 0.253 & $3 \mathrm{pCi} / \mathrm{L}$ & 0.0584 & 0.0643 & & & \\
\hline 634 & ]UISO_AEA & ITASRL & 0.00491 & $1 \mathrm{pCi} / \mathrm{L}$ & 0.00877 & 0.00884 & & & U \\
\hline 635 & UISO_AEA & ITASRL & 0.223 & $3 \mathrm{pCi} / \mathrm{L}$ & 0.0547 & 0.0595 & & & \\
\hline 636 & SRISO_SEP_PRECIP_GPC & ITASRL & 0.0806 & $\mathrm{pCi} / \mathrm{L}$ & 0.0622 & 0.0663 & & & \\
\hline 637 & TC99_SEP_LSC & ITASRL & 0.0924 & $4 \mathrm{pCi} / \mathrm{L}$ & 0.0976 & 0.516 & & & $\mathrm{U}$ \\
\hline 638 & TRITIUM_ELECT_LSC & ITASRL & 37.3 & $\mathrm{pCi} / \mathrm{L}$ & 2.37 & 9.06 & & & \\
\hline 639 & UISO_AEA & ITASRL & 0.245 & $5 \mathrm{pCi} / \mathrm{L}$ & 0.0475 & 0.0536 & & & \\
\hline 640 & UISO_AEA & ITASRL & 0.00768 & $3 \mathrm{pCi} / \mathrm{L}$ & 0.00986 & 0.00993 & & & $\mathrm{U}$ \\
\hline 641 & UISO_AEA & ITASRL & 0.228 & $\mathrm{pCi} / \mathrm{L}$ & 0.0456 & 0.0509 & & & \\
\hline 642 & SRISO_SEP_PRECIP_GPC & ITASRL & 0.0945 & $5 \mathrm{pCi} / \mathrm{L}$ & 0.0477 & 0.0538 & & & \\
\hline 643 & TC99_SEP_LSC & ITASRL & 0.212 & $\mathrm{pCi} / \mathrm{L}$ & 0.0993 & 0.525 & & & $\mathrm{U}$ \\
\hline 644 & TRITIUMM_ELECT_LSC & ITASRL & 40.1 & $\mathrm{pCi} / \mathrm{L}$ & 2.42 & 9.24 & & & \\
\hline 645 & UISO_AEA & ITASRL & & & & & & & $U$ \\
\hline 646 & UISO_AEA & ITASRL & & & & & & & $U$ \\
\hline
\end{tabular}




\begin{tabular}{|c|c|c|c|c|c|c|c|c|c|}
\hline & $\mathrm{K}$ & $\mathrm{L}$ & $\mathrm{M}$ & $\mathrm{N}$ & $\mathrm{O}$ & $P$ & $\mathrm{Q}$ & $\mathrm{R}$ & $S$ \\
\hline 1 & METHOD_NAME & LAB_CODE & VALUE_RPTD & ANAL_UNITS_RPTD & COUNTING_ERROR & TOTAL_ANAL_ERROR|I & MIN_DETECTABLE_ACTIVITY & PCNT_MOISTURE & LAB_QUALIFIER \\
\hline 648 & SRISO_SEP_PRECIP_GPC & ITASRL & & & & & & & $\mathrm{U}$ \\
\hline 649 & TC99_SEP_LSC & ITASRL & 0.327 & $\mathrm{pCi} / \mathrm{L}$ & 0.102 & 0.534 & & & $\mathrm{U}$ \\
\hline 650 & TRITIUM_ELECT_LSC & ITASRL & 38.6 & $\mathrm{pCi} / \mathrm{L}$ & 2.39 & 9.13 & & & \\
\hline 651 & UISO_AEA & ITASRL & 0.238 & $\mathrm{pCi} / \mathrm{L}$ & 0.0527 & 0.0583 & & & \\
\hline 652 & UISO_AEA & ITASRL & 0.00825 & $\mathrm{pCi} / \mathrm{L}$ & 0.0108 & 0.0109 & & & U \\
\hline 653 & UISO_AEA & ITASRL & 0.149 & $\mathrm{pCi} / \mathrm{L}$ & 0.0415 & 0.0444 & & & \\
\hline 654 & SRISO_SEP_PRECIP_GPC & ITASRL & 0.0751 & $\mathrm{pCi} / \mathrm{L}$ & 0.0479 & 0.0532 & & & \\
\hline 655 & TC99_SEP_LSC & ITASRL & 0.295 & $\mathrm{pCi} / \mathrm{L}$ & 0.102 & 0.532 & & & $U$ \\
\hline 657 & UISO_AEA & ITASRL & 0.226 & $\mathrm{pCi} / \mathrm{L}$ & 0.0477 & 0.0531 & & & \\
\hline 658 & UISO_AEA & ITASRL & 0.00337 & $\mathrm{pCi} / \mathrm{L}$ & 0.0102 & 0.0103 & & & U \\
\hline 659 & UISO_AEA & ITASRL & 0.177 & $\mathrm{pCi} / \mathrm{L}$ & 0.0424 & 0.0462 & & & \\
\hline 660 & SRISO_SEP_PRECIP_GPC & ITASRL & 0.188 & $\mathrm{pCi} / \mathrm{L}$ & 0.0538 & 0.065 & & & \\
\hline 661 & TRITIUM_ELECT_LSC & ITASRL & 64.5 & $\mathrm{pCi} / \mathrm{L}$ & 2.68 & 11 & & & \\
\hline 662 & UISO_AEA & ITASRL & 0.407 & $\mathrm{pCi} / \mathrm{L}$ & 0.0597 & 0.0721 & & & \\
\hline 663 & UISO_AEA & ITASRL & 0.0107 & $\mathrm{pCi} / \mathrm{L}$ & 0.0114 & 0.0114 & & & $\mathrm{U}$ \\
\hline 664 & UISO_AEA & ITASRL & 0.371 & $\mathrm{pCi} / \mathrm{L}$ & 0.0566 & 0.0673 & & & \\
\hline 665 & SRISO_SEP_PRECIP_GPC & ITASRL & 0.247 & $\mathrm{pCi} / \mathrm{L}$ & 0.0614 & 0.0773 & & & \\
\hline 667 & UISO_AEA & ITASRL & 0.268 & $\mathrm{pCi} / \mathrm{L}$ & 0.0502 & 0.0571 & & & \\
\hline 668 & UISO_AEA & ITASRL & 0.00669 & $\mathrm{pCi} / \mathrm{L}$ & 0.0117 & 0.0118 & & & U \\
\hline 669 & UISO_AEA & ITASRL & 0.19 & $\mathrm{pCi} / \mathrm{L}$ & 0.0427 & 0.0468 & & & \\
\hline 670 & SRISO_SEP_PRECIP_GPC & ITASRL & 0.114 & $\mathrm{pCi} / \mathrm{L}$ & 0.0353 & 0.0424 & & & \\
\hline 671 & TRITIUM_ELEECT_LSC & ITASRL & 53.2 & $\mathrm{pCi} / \mathrm{L}$ & 2.58 & 10.2 & & & \\
\hline 672 & UISO_AEA & ITASRL & 0.306 & $\mathrm{pCi} / \mathrm{L}$ & 0.0579 & 0.0658 & & & \\
\hline 673 & UISO_AEA & ITASRL & 0.0129 & $\mathrm{pCi} / \mathrm{L}$ & 0.0151 & 0.0152 & & & U \\
\hline 674 & UISO_AEA & ITASRL & 0.201 & $\mathrm{pCi} / \mathrm{L}$ & 0.0467 & 0.0511 & & & \\
\hline 675 & SRISO_SEP_PRECIP_GPC & ITASRL & 0.112 & $\mathrm{pCi} / \mathrm{L}$ & 0.039 & 0.0455 & & & \\
\hline 676 & TRITIUM_ELECT_LSC & ITASRL & 44.8 & $\mathrm{pCi} / \mathrm{L}$ & 2.45 & 9.57 & & & \\
\hline 677 & UISO_AEA & ITASRL & 0.247 & $\mathrm{pCi} / \mathrm{L}$ & 0.047 & 0.0533 & & & \\
\hline 678 & UISO_AEA & ITASRL & 0.0136 & $\mathrm{pCi} / \mathrm{L}$ & 0.0119 & 0.012 & & & \\
\hline 679 & UISO_AEA & ITASRL & 0.16 & $\mathrm{pCi} / \mathrm{L}$ & 0.038 & 0.0413 & & & \\
\hline 680 & SRISO_SEP_PRECIP_GPC & ITASRL & 0.144 & $\mathrm{pCi} / \mathrm{L}$ & 0.0416 & 0.0514 & & & \\
\hline 681 & TRITIUMM_ELEECT_LSC̄ & ITASRL & 40.1 & $\mathrm{pCi} / \mathrm{L}$ & 2.42 & 9.28 & & & \\
\hline 682 & UISO_AEA & ITASRL & 0.284 & $\mathrm{pCi} / \mathrm{L}$ & 0.0539 & 0.0612 & & & \\
\hline 683 & UISO_AEA & ITASRL & 0.0121 & $\mathrm{pCi} / \mathrm{L}$ & 0.0118 & 0.0119 & & & \\
\hline 684 & UISO_AEA & ITASRL & 0.175 & $\mathrm{pCi} / \mathrm{L}$ & 0.0426 & 0.0462 & & & \\
\hline
\end{tabular}




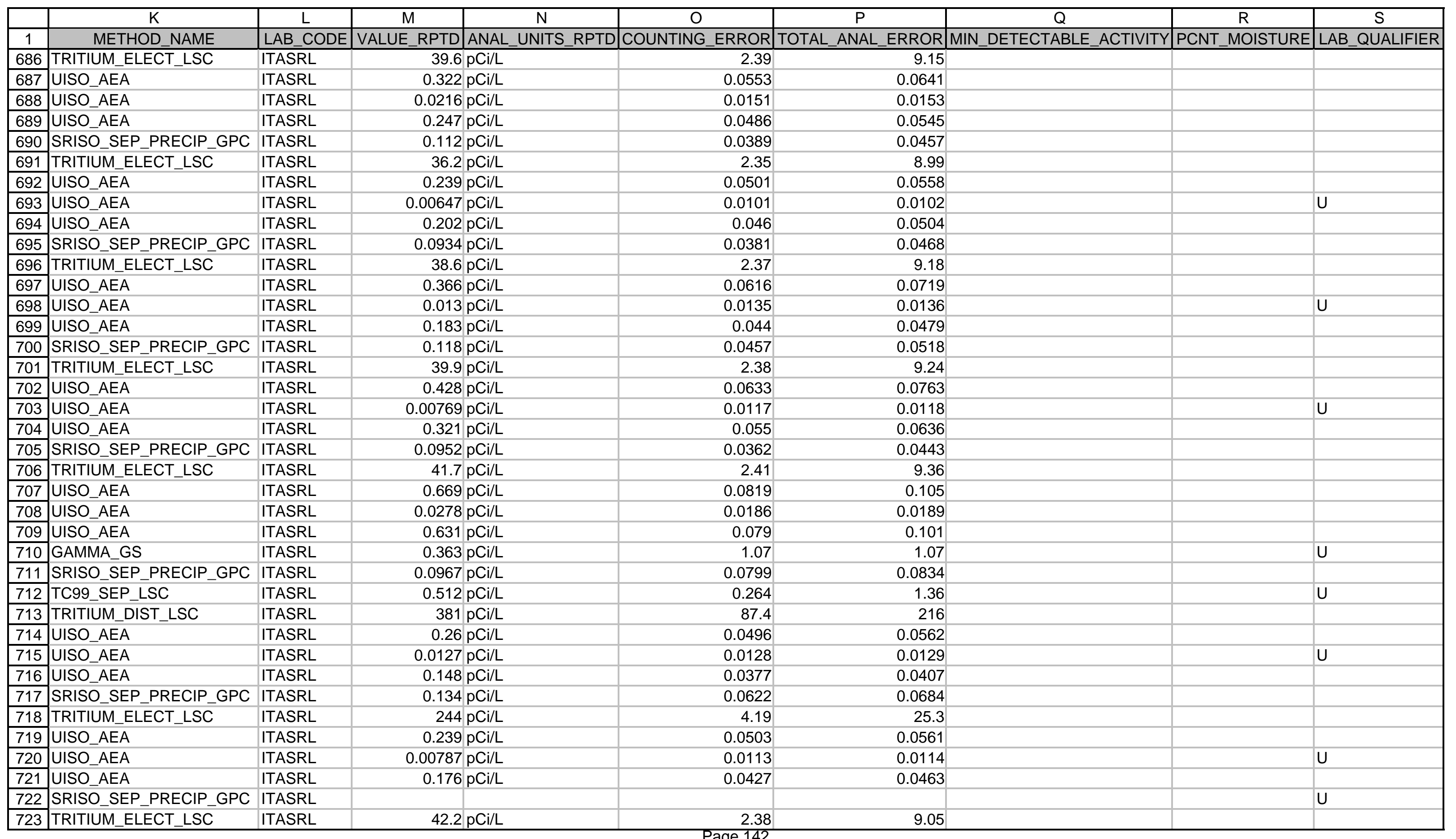




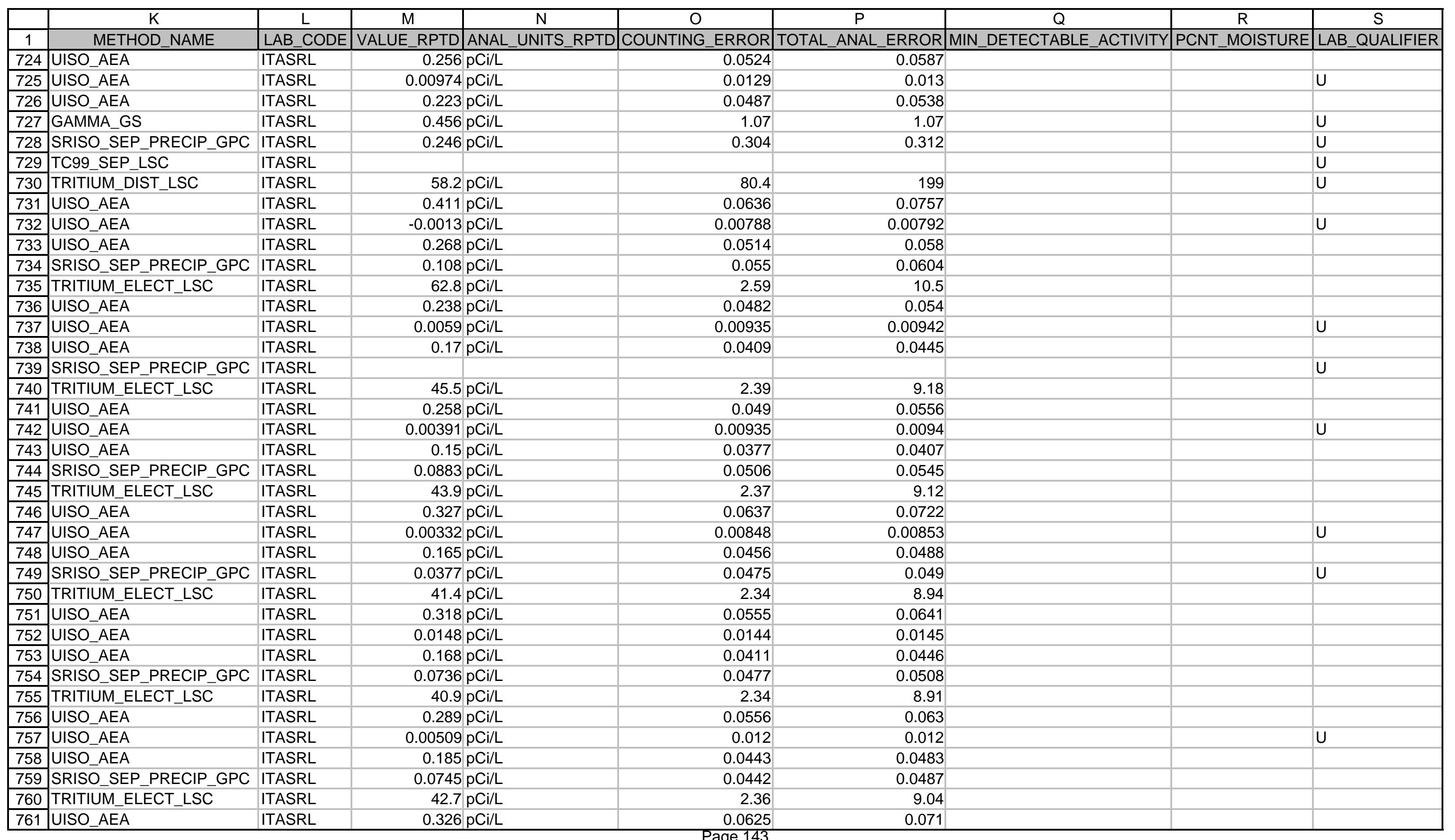




\begin{tabular}{|c|c|c|c|c|c|c|c|c|c|}
\hline & $\mathrm{K}$ & $\mathrm{L}$ & $\mathrm{M}$ & $\mathrm{N}$ & $\mathrm{O}$ & $\mathrm{P}$ & $Q$ & $\mathrm{R}$ & $\mathrm{S}$ \\
\hline 1 & METHOD_NAME & LAB_CODE & VALUE_RPTD & ANAL_UNITS_RPTD & COUNTING_ERROR & TOTAL_ANAL_ERROR & MIN_DETECTABLE_ACTIVITY & PCNT_MOISTURE & LAB_QUALIFIER \\
\hline 762 & UISO_AEA & ITASRL & 0.01 & $\mathrm{pCi} / \mathrm{L}$ & 0.0113 & 0.0114 & & & $U$ \\
\hline 763 & UISO_AEA & ITASRL & 0.204 & $\mathrm{pCi} / \mathrm{L}$ & 0.0497 & 0.054 & & & \\
\hline 764 & SRISO_SEP_PRECIP_GPC & ITASRL & 0.0625 & $\mathrm{pCi} / \mathrm{L}$ & 0.0404 & 0.044 & & & \\
\hline 765 & TRITIUM_ELECTILSC & ITASRL & 41.2 & $\mathrm{pCi} / \mathrm{L}$ & 2.35 & 8.93 & & & \\
\hline 766 & UISO_AEA & ITASRL & 0.36 & $\mathrm{pCi} / \mathrm{L}$ & 0.0569 & 0.0673 & & & \\
\hline 767 & UISO AEA & ITASRL & 0.0133 & $\mathrm{pCi} / \mathrm{L}$ & 0.0125 & 0.0126 & & & \\
\hline 768 & UISO_AEA & ITASRL & 0.184 & $\mathrm{pCi} / \mathrm{L}$ & 0.0414 & 0.0454 & & & \\
\hline 769 & SRISO_SEP_PRECIP_GPC & ITASRL & 0.084 & $\mathrm{pCi} / \mathrm{L}$ & 0.0534 & 0.0569 & & & \\
\hline 771 & UISO_AEA & ITASRL & 0.304 & $\mathrm{pCi} / \mathrm{L}$ & 0.0583 & 0.0662 & & & \\
\hline 772 & UISO_AEA & ITASRL & 0.00494 & $\mathrm{pCi} / \mathrm{L}$ & 0.0112 & 0.0113 & & & $\mathrm{U}$ \\
\hline 773 & UISO_AEA & ITASRL & 0.201 & $\mathrm{pCi} / \mathrm{L}$ & 0.0472 & 0.0515 & & & \\
\hline 774 & GAMMA_GS & ITASRL & 0.425 & $\mathrm{pCi} / \mathrm{L}$ & 0.882 & 0.883 & & & U \\
\hline 775 & TC99_SEP_LSC & ITASRL & 12.9 & $\mathrm{pCi} / \mathrm{L}$ & 0.216 & 1.78 & & & \\
\hline 776 & TRITIUM_DIST_LSC & ITASRL & 17600 & $\mathrm{pCi} / \mathrm{L}$ & 265 & 1420 & & & \\
\hline 777 & UISO_AEA & ITASRL & 0.505 & $\mathrm{pCi} / \mathrm{L}$ & 0.0748 & 0.0904 & & & \\
\hline 778 & UISO_AEA & ITASRL & 0.0128 & $\mathrm{pCi} / \mathrm{L}$ & 0.0144 & 0.0145 & & & $\mathrm{U}$ \\
\hline 779 & UISO_AEA & ITASRL & 0.328 & $\mathrm{pCi} / \mathrm{L}$ & 0.0596 & 0.0682 & & & \\
\hline 781 & TC99_SEP_LSC & ITASRL & & & & & & & \\
\hline 782 & TRITIŪM_DIST_LSC & ITASRL & & & & & & & \\
\hline 783 & UISO_AEA & ITASRL & & & & & & & \\
\hline 784 & UISO_AEA & ITASRL & & & & & & & \\
\hline 785 & UISO_AEA & ITASRL & & & & & & & \\
\hline 786 & GAMMA_GS & ITASRL & -1.01 & $\mathrm{pCi} / \mathrm{L}$ & 1.11 & 1.12 & & & $\mathrm{U}$ \\
\hline 787 & TC99_SEP_LSC & ITASRL & 0.187 & $\mathrm{pCi} / \mathrm{L}$ & 0.0992 & 0.511 & & & $\mathrm{U}$ \\
\hline 788 & TRITIUM_DIST_LSC & ITASRL & 65.6 & $\mathrm{pCi} / \mathrm{L}$ & 80.7 & 200 & & & $\mathrm{U}$ \\
\hline 789 & UISO_AEA & ITASRL & 0.315 & $\mathrm{pCi} / \mathrm{L}$ & 0.0554 & 0.0638 & & & \\
\hline 790 & UISO_AEA & ITASRL & 0.00823 & $\mathrm{pCi} / \mathrm{L}$ & 0.0105 & 0.0105 & & & $\mathrm{U}$ \\
\hline 791 & UISO_AEA & ITASRL & 0.23 & $\mathrm{pCi} / \mathrm{L}$ & 0.047 & 0.0524 & & & \\
\hline 792 & GAMMA_GS & ITASRL & & & & & & & \\
\hline 793 & TC99_SEP_LSC & ITASRL & & & & & & & \\
\hline 794 & TRITIŪM_DIST_LSC & ITASRL & & & & & & & \\
\hline 795 & UISO_AĒA & ITASRL & & & & & & & \\
\hline 796 & UISO_AEA & ITASRL & & & & & & & \\
\hline 797 & UISO_AEA & ITASRL & & & & & & & \\
\hline 798 & GAMMA_GS & ITASRL & & & & & & & \\
\hline
\end{tabular}




\begin{tabular}{|c|c|c|c|c|c|c|c|c|c|}
\hline & $\mathrm{K}$ & $\mathrm{L}$ & $\mathrm{M}$ & $\mathrm{N}$ & $\mathrm{O}$ & $\mathrm{P}$ & $\mathrm{Q}$ & $\mathrm{R}$ & $\mathrm{S}$ \\
\hline 1 & METHOD_NAME & LAB_CODE & VALUE_RPTD & ANAL_UNITS_RPTD & $\mid$ COUNTING_ERROR & TOTAL_ANAL_ERROR & MIN_DETECTABLE_ACTIVITY & PCNT_MOISTURE & LAB_QUALIFIER \\
\hline 800 & TRITIUM_DIST_LSC & ITASRL & & & & & & & \\
\hline 801 & UISO_AËA & ITASRL & & & & & & & \\
\hline 802 & UISO_AEA & ITASRL & & & & & & & \\
\hline 803 & UISO_AEA & ITASRL & & & & & & & \\
\hline 804 & GAMMA_A_GS & ITASRL & & & & & & & \\
\hline 805 & TC99_SËP_LSC & ITASRL & & & & & & & \\
\hline 806 & TRITIUM_DIST_LSC & ITASRL & & & & & & & \\
\hline 807 & UISO_AEA & ITASRL & & & & & & & \\
\hline 808 & UISO_AEA & ITASRL & & & & & & & \\
\hline 809 & UISO_AEA & ITASRL & & & & & & & \\
\hline 810 & GAMMA_GS & ITASRL & & & & & & & \\
\hline 811 & TC99_SEP_LSC & ITASRL & & & & & & & \\
\hline 812 & TRITIUMM_DIST_LSC & ITASRL & & & & & & & \\
\hline 813 & UISO_AËA - & ITASRL & & & & & & & \\
\hline 814 & UISO_AEA & ITASRL & & & & & & & \\
\hline 815 & UISO_AEA & ITASRL & & & & & & & \\
\hline 816 & GAMMĀA_GS & ITASRL & & & & & & & \\
\hline 817 & TC99_SËP_LSC & ITASRL & & & & & & & \\
\hline 819 & UISO_AEA & ITASRL & & & & & & & \\
\hline 820 & UISO_AEA & ITASRL & & & & & & & \\
\hline 821 & UISO_AEA & ITASRL & & & & & & & \\
\hline 822 & GAMMA_GS & ITASRL & & & & & & & \\
\hline 823 & TC99_SEP_LSC & ITASRL & & & & & & & \\
\hline 824 & TRITIUM_DIST_LSC & ITASRL & & & & & & & \\
\hline 825 & UISO_AEA & ITASRL & & & & & & & \\
\hline 826 & UISO_AEA & ITASRL & & & & & & & \\
\hline 827 & UISO_AEA & ITASRL & & & & & & & \\
\hline 828 & GAMMA_GS & ITASRL & & & & & & & \\
\hline 829 & TC99_SËP_LSC & ITASRL & & & & & & & \\
\hline 830 & TRITIUM_DIST_LSC & ITASRL & & & & & & & \\
\hline 831 & UISO_AEA & ITASRL & & & & & & & \\
\hline 832 & UISO_AEA & ITASRL & & & & & & & \\
\hline 833 & UISO_AEA & ITASRL & & & & & & & \\
\hline 834 & GAMMA_GS & ITASRL & & & & & & & \\
\hline 835 & TC99_SEP_LSC & ITASRL & & & & & & & \\
\hline 836 & TRITIUM_DIST_LSC & ITASRL & & & & & & & \\
\hline
\end{tabular}




\begin{tabular}{|c|c|c|c|c|c|c|c|c|c|}
\hline & $\mathrm{K}$ & $\mathrm{L}$ & $\mathrm{M}$ & $\mathrm{N}$ & $\mathrm{O}$ & $\mathrm{P}$ & $\mathrm{Q}$ & $\mathrm{R}$ & $\mathrm{S}$ \\
\hline 1 & METHOD_NAME & LAB_CODE & VALUE_RPTD & ANAL_UNITS_RPTD & COUNTING_ERROR & TOTAL_ANAL_ERROR & MIN_DETECTABLE_ACTIVITY & PCNT_MOISTURE & LAB_QUALIFIER \\
\hline 838 & UISO_AEA & ITASRL & & & & & & & \\
\hline 839 & UISO_AEA & ITASRL & & & & & & & \\
\hline 840 & GAMMA_GS & ITASRL & & & & & & & \\
\hline 841 & TC99_SEP_LSC & ITASRL & & & & & & & \\
\hline 842 & TRITIUMM_DIST_LSC & ITASRL & & & & & & & \\
\hline 843 & UISO_AEA & ITASRL & & & & & & & \\
\hline 844 & UISO_AEA & ITASRL & & & & & & & \\
\hline 845 & UISO_AEA & ITASRL & & & & & & & \\
\hline 847 & TRITIUM_ELECT_LSC & ITASRL & & $\mathrm{pCi} / \mathrm{L}$ & 3.03 & 13.3 & & & \\
\hline 848 & UISO_AEA & ITASRL & 0.227 & $7 \mathrm{pCi} / \mathrm{L}$ & 0.049 & 0.0544 & & & \\
\hline 849 & UISO_AEA & ITASRL & 0.00409 & $9 \mathrm{pCi} / \mathrm{L}$ & 0.00891 & 0.00897 & & & $U$ \\
\hline 850 & UISO_AEA & ITASRL & 0.196 & $\mathrm{pCi} / \mathrm{L}$ & 0.0455 & 0.0498 & & & \\
\hline 851 & SRISO_SEP_PRECIP_GPC & ITASRL & 0.121 & $\mathrm{l} \mathrm{pCi} / \mathrm{L}$ & 0.0544 & 0.0604 & & & \\
\hline 852 & TRITIUM_ELECT_LSC & ITASRL & 42.9 & $\mathrm{pCi} / \mathrm{L}$ & 2.61 & 10.5 & & & \\
\hline 853 & UISO_AEA & ITASRL & 0.264 & $4 \mathrm{pCi} / \mathrm{L}$ & 0.0884 & 0.094 & & & \\
\hline 854 & UISO_AEA & ITASRL & 0.01 & $\mathrm{pCi} / \mathrm{L}$ & 0.0235 & 0.0235 & & & $\mathrm{U}$ \\
\hline 855 & UISO_AEA & ITASRL & 0.223 & $3 \mathrm{pCi} / \mathrm{L}$ & 0.0779 & 0.0823 & & & \\
\hline 857 & TRITIUM_ELECT_LSC & ITASRL & 55.2 & $2 \mathrm{pCi} / \mathrm{L}$ & 2.73 & 11.3 & & & \\
\hline 858 & UISO_AËA & ITASRL & 0.273 & $3 \mathrm{pCi} / \mathrm{L}$ & 0.0493 & 0.0565 & & & \\
\hline 859 & UISO_AEA & ITASRL & 0.00873 & $3 \mathrm{pCi} / \mathrm{L}$ & 0.0106 & 0.0107 & & & $U$ \\
\hline 860 & UISO_AEA & ITASRL & 0.19 & $\mathrm{pCi} / \mathrm{L}$ & 0.0412 & 0.0455 & & & \\
\hline 861 & SRISO_SEP_PRECIP_GPC & ITASRL & 0.0958 & $3 \mathrm{pCi} / \mathrm{L}$ & 0.0472 & 0.0534 & & & \\
\hline 862 & TRITIUM_ELECT_LSC & ITASRL & 50 & $\mathrm{pCi} / \mathrm{L}$ & 2.68 & 10.9 & & & \\
\hline 863 & UISO_AEA & ITASRL & 0.269 & $\mathrm{pCi} / \mathrm{L}$ & 0.0501 & 0.057 & & & \\
\hline 864 & UISO_AEA & ITASRL & 0.0154 & $4 \mathrm{pCi} / \mathrm{L}$ & 0.0132 & 0.0133 & & & \\
\hline 865 & JUISO_AEA & ITASRL & 0.189 & $\mathrm{pCi} / \mathrm{L}$ & 0.0418 & 0.046 & & & \\
\hline 866 & SRISO_SEP_PRECIP_GPC & ITASRL & 0.104 & $4 \mathrm{pCi} / \mathrm{L}$ & 0.0583 & 0.0629 & & & \\
\hline 867 & TRITIUM_ELECT_LSC & ITASRL & 47.5 & $\mathrm{pCi} / \mathrm{L}$ & 2.64 & 10.7 & & & \\
\hline 868 & UISO_AEA & ITASRL & 0.283 & $3 \mathrm{pCi} / \mathrm{L}$ & 0.0489 & 0.0565 & & & \\
\hline 869 & UISO_AEA & ITASRL & 0.00871 & $\mathrm{pCi} / \mathrm{L}$ & 0.0112 & 0.0113 & & & $\mathrm{U}$ \\
\hline 870 & UISO_AEA & ITASRL & 0.166 & $\mathrm{pCi} / \mathrm{L}$ & 0.0381 & 0.0417 & & & \\
\hline 871 & SRISO_SEP_PRECIP_GPC & ITASRL & 0.0941 & $1 \mathrm{pCi} / \mathrm{L}$ & 0.0479 & 0.0525 & & & \\
\hline 872 & TRITIUMM_ELECT_LSC & ITASRL & 41.9 & $\mathrm{pCi} / \mathrm{L}$ & 2.58 & 10.3 & & & \\
\hline 873 & UISO_AEA & ITASRL & 0.286 & $\mathrm{pCi} / \mathrm{L}$ & 0.0524 & 0.0599 & & & \\
\hline 874 & UISO_AEA & ITASRL & 0.0179 & $9 \mathrm{pCi} / \mathrm{L}$ & 0.0154 & 0.0156 & & & \\
\hline
\end{tabular}




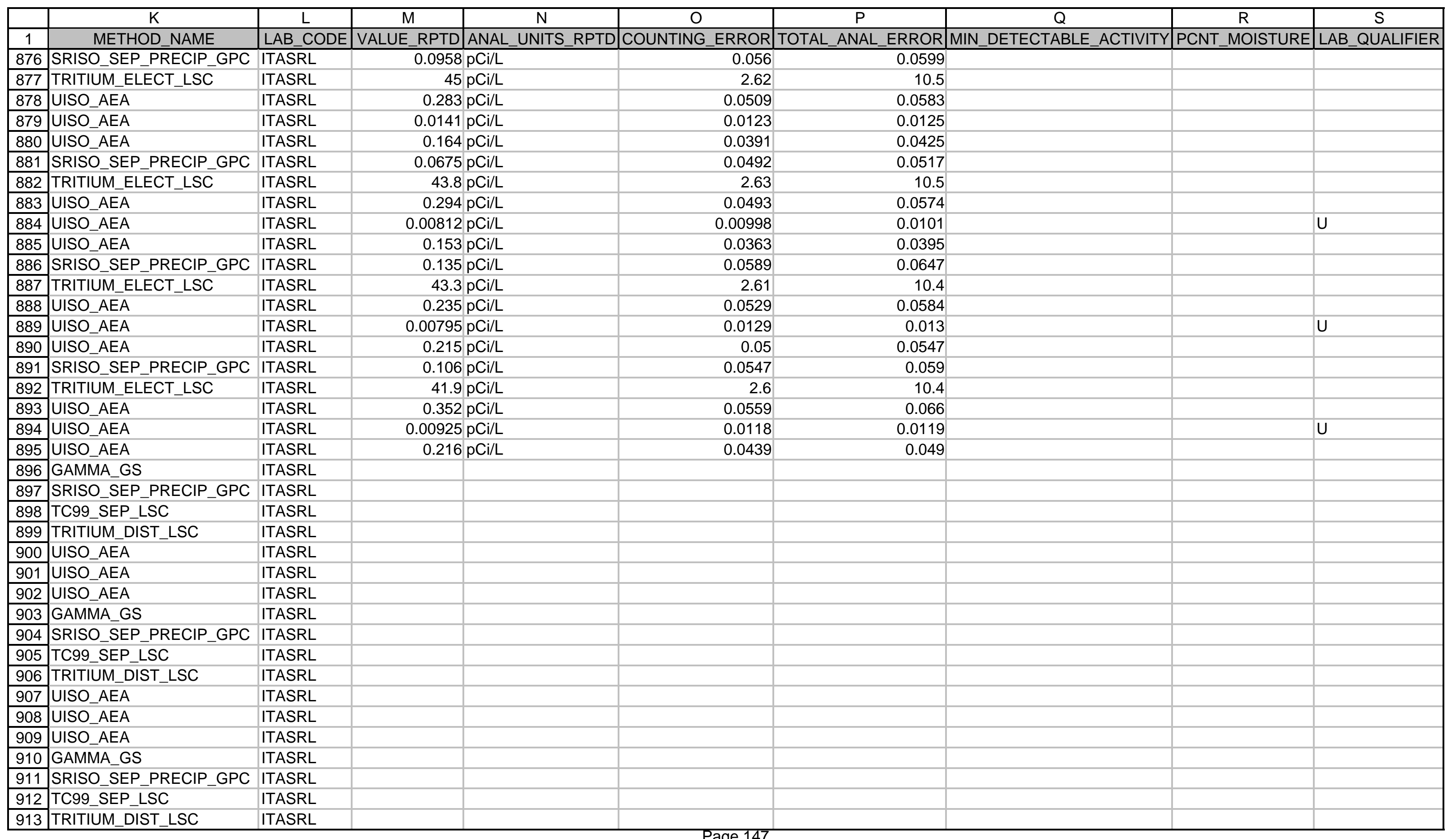




\begin{tabular}{|c|c|c|c|c|c|c|c|c|c|}
\hline & $\mathrm{K}$ & $\mathrm{L}$ & $\mathrm{M}$ & $\mathrm{N}$ & $\mathrm{O}$ & $P$ & $\mathrm{Q}$ & $\mathrm{R}$ & $\mathrm{S}$ \\
\hline 1 & METHOD NAME & LAB CODE & VALUE RPTD & ANAL UNITS RPTD & COUNTING ERROR & TOTAL ANAL ERROR & MIN DETECTABLE ACTIVITY & PCNT MOISTURE & LAB QUALIFIER \\
\hline 914 & UISO_AEA & ITASRL & & & & & & & \\
\hline 915 & UISO_AEA & ITASRL & & & & & & & \\
\hline 916 & UISO_AEA & ITASRL & & & & & & & \\
\hline 917 & GAMMA_GS & ITASRL & & & & & & & \\
\hline 918 & SRISO_SEP_PRECIP_GPC & ITASRL & & & & & & & \\
\hline 919 & TC99_SEPP_LSC & ITASRL & & & & & & & \\
\hline 920 & TRITIŪM_DIST_LSC & ITASRL & & & & & & & \\
\hline 921 & UISO_AËA & ITASRL & & & & & & & \\
\hline 923 & UISO_AEA & ITASRL & & & & & & & \\
\hline 924 & GAMMĀA_GS & ITASRL & & & & & & & \\
\hline 925 & SRISO_SEPP_PRECIP_GPC & ITASRL & & & & & & & \\
\hline 926 & TC99_SEP_LSC & ITASRL & & & & & & & \\
\hline 927 & TRITIUM_DIST_LSC & ITASRL & & & & & & & \\
\hline 928 & UISO_AEA & ITASRL & & & & & & & \\
\hline 929 & UISO_AEA & ITASRL & & & & & & & \\
\hline 930 & UISO_AEA & ITASRL & & & & & & & \\
\hline 931 & GAMM̄A_GS & ITASRL & & & & & & & \\
\hline 933 & TC99_SEPP_LSC & ITASRL & & & & & & & \\
\hline 934 & TRITIŪM_DIST_LSC & ITASRL & & & & & & & \\
\hline 935 & UISO_AEA & ITASRL & & & & & & & \\
\hline 936 & UISO_AEA & ITASRL & & & & & & & \\
\hline 937 & UISO_AEA & ITASRL & & & & & & & \\
\hline 938 & GAMM̄A_GS & ITASRL & & & & & & & \\
\hline 939 & SRISO_SEP_PRECIP_GPC & ITASRL & & & & & & & \\
\hline 940 & TC99_SEPP_LSC & ITASRL & & & & & & & \\
\hline 941 & TRITIUM_DIST_LSC & ITASRL & & & & & & & \\
\hline 942 & UISO_AEA & ITASRL & & & & & & & \\
\hline 943 & UISO_AEA & ITASRL & & & & & & & \\
\hline 944 & UISO_AEA & ITASRL & & & & & & & \\
\hline 945 & GAMMA_GS & ITASRL & 0.884 & $\mathrm{pCi} / \mathrm{L}$ & 0.861 & 0.866 & & & \\
\hline 946 & SRISO_SEP_PRECIP_GPC & ITASRL & 0.0319 & $\mathrm{pCi} / \mathrm{L}$ & 0.0738 & 0.0757 & & & $U$ \\
\hline 947 & TC99_SEP_LEC & ITASRL & 8.76 & $\mathrm{pCi} / \mathrm{L}$ & 0.376 & 2.24 & & & \\
\hline 948 & TRITIUM_DIST_LSC & ITASRL & 8530 & $\mathrm{pCi} / \mathrm{L}$ & 195 & 763 & & & \\
\hline 949 & UISO_AEA & ITASRL & 2.21 & $\mathrm{pCi} / \mathrm{L}$ & 0.171 & 0.283 & & & \\
\hline 950 & UISO_AEA & ITASRL & 0.083 & $\mathrm{pCi} / \mathrm{L}$ & 0.0342 & 0.0353 & & & \\
\hline
\end{tabular}




\begin{tabular}{|c|c|c|c|c|c|c|c|c|c|}
\hline & $\mathrm{K}$ & $\mathrm{L}$ & $\bar{M}$ & $\mathrm{~N}$ & $\mathrm{O}$ & $\mathrm{P}$ & $\mathrm{Q}$ & $\mathrm{R}$ & $\mathrm{S}$ \\
\hline 1 & METHOD_NAME & LAB_CODE & VALUE_RPTD & ANAL_UNITS_RPTD & COUNTING_ERROR & TOTAL_ANAL_ERROR & MIN_DETECTABLE_ACTIVITY & PCNT_MOISTURE & LAB_QUALIFIER \\
\hline 952 & GAMMA_GS & ITASRL & 0.402 & $\mathrm{pCi} / \mathrm{L}$ & 0.717 & 0.718 & & & $U$ \\
\hline 953 & SRISO_SEP_PRECIP_GPC & ITASRL & 0.126 & $\mathrm{pCi} / \mathrm{L}$ & 0.0814 & 0.0871 & & & \\
\hline 954 & TC99_SEP_LSC & ITASRL & -0.596 & $\mathrm{pCi} / \mathrm{L}$ & 0.973 & 5.28 & & & $\mathrm{U}$ \\
\hline 955 & TRITIUM_DIST_LSC & ITASRL & 57.6 & $\mathrm{pCi} / \mathrm{L}$ & 80.9 & 197 & & & $U$ \\
\hline 956 & UISO_AEA & ITASRL & 0.251 & $\mathrm{pCi} / \mathrm{L}$ & 0.056 & 0.0619 & & & \\
\hline 957 & UISO_AEA & ITASRL & 0.00672 & $\mathrm{pCi} / \mathrm{L}$ & 0.0121 & 0.0122 & & & $U$ \\
\hline 958 & UISO AEA & ITASRL & 0.2 & $\mathrm{pCi} / \mathrm{L}$ & 0.0493 & 0.0535 & & & \\
\hline 959 & GAMMĀA_GS & ITASRL & -0.239 & $\mathrm{pCi} / \mathrm{L}$ & 0.944 & 0.944 & & & $U$ \\
\hline 961 & TC99_SEP_LSC & ITASRL & 10.7 & $\mathrm{pCi} / \mathrm{L}$ & 0.516 & 2.99 & & & \\
\hline 962 & TRITIUM_DIST_LSC & ITASRL & 118 & $\mathrm{pCi} / \mathrm{L}$ & 82.2 & 200 & & & $\mathrm{U}$ \\
\hline 963 & UISO_AEA & ITASRL & 0.464 & $\mathrm{pCi} / \mathrm{L}$ & 0.0684 & 0.0827 & & & \\
\hline 964 & UISO_AEA & ITASRL & -0.00178 & $\mathrm{pCi} / \mathrm{L}$ & 0.0101 & 0.0101 & & & U \\
\hline 965 & UISO_AEA & ITASRL & 0.345 & $\mathrm{pCi} / \mathrm{L}$ & 0.0592 & 0.0685 & & & \\
\hline 966 & SRISŌSEP_PRECIP_GPC & ITASRL & 0.139 & $\mathrm{pCi} / \mathrm{L}$ & 0.0607 & 0.067 & & & \\
\hline 967 & TRITIUM_ELECT_LSC & ITASRL & 55.3 & $\mathrm{pCi} / \mathrm{L}$ & 2.68 & 11 & & & \\
\hline 968 & UISO_AEA & ITASRL & 0.291 & $\mathrm{pCi} / \mathrm{L}$ & 0.0595 & 0.0668 & & & \\
\hline 969 & UISO_AEA & ITASRL & 0.0021 & $\mathrm{pCi} / \mathrm{L}$ & 0.00862 & 0.00867 & & & $U$ \\
\hline 971 & SRISO_SEP_PRECIP_GPC & ITASRL & 0.0792 & $\mathrm{pCi} / \mathrm{L}$ & 0.0541 & 0.0573 & & & \\
\hline 972 & TRITIUM_ELECTI_LSC & ITASRL & 46.3 & $\mathrm{pCi} / \mathrm{L}$ & 2.7 & 10.7 & & & \\
\hline 973 & UISO_AEA & ITASRL & 0.462 & $\mathrm{pCi} / \mathrm{L}$ & 0.0673 & 0.0816 & & & \\
\hline 974 & UISO_AEA & ITASRL & 0.00531 & $\mathrm{pCi} / \mathrm{L}$ & 0.00999 & 0.01 & & & $U$ \\
\hline 975 & UISO_AEA & ITASRL & 0.246 & $\mathrm{pCi} / \mathrm{L}$ & 0.0496 & 0.0555 & & & \\
\hline 976 & SRISŌ_SEP_PRECIP_GPC & ITASRL & 0.106 & $\mathrm{pCi} / \mathrm{L}$ & 0.0563 & 0.0608 & & & \\
\hline 977 & TRITIUM_ELEECT_LSC & ITASRL & 48.3 & $\mathrm{pCi} / \mathrm{L}$ & 2.6 & 10.5 & & & \\
\hline 978 & UISO_AEA & ITASRL & 0.288 & $\mathrm{pCi} / \mathrm{L}$ & 0.0579 & 0.0652 & & & \\
\hline 979 & UISO_AEA & ITASRL & 0.00635 & $\mathrm{pCi} / \mathrm{L}$ & 0.0116 & 0.0116 & & & $U$ \\
\hline 980 & UISO_AEA & ITASRL & 0.184 & $\mathrm{pCi} / \mathrm{L}$ & 0.0465 & 0.0503 & & & \\
\hline 981 & SRISŌSEP_PRECIP_GPC & ITASRL & 0.0787 & $\mathrm{pCi} / \mathrm{L}$ & 0.0569 & 0.0598 & & & \\
\hline 982 & TRITIUM_ELECTILLSC & ITASRL & 43.4 & $\mathrm{pCi} / \mathrm{L}$ & 2.54 & 10.1 & & & \\
\hline 983 & UISO_AEA & ITASRL & 0.231 & $\mathrm{pCi} / \mathrm{L}$ & 0.051 & 0.0564 & & & \\
\hline 984 & UISO_AEA & ITASRL & 0.000243 & $\mathrm{pCi} / \mathrm{L}$ & 0.00553 & 0.00559 & & & U \\
\hline 985 & UISO_AEA & ITASRL & 0.159 & $\mathrm{pCi} / \mathrm{L}$ & 0.0421 & 0.0453 & & & \\
\hline 986 & SRISO_SEP_PRECIP_GPC & ITASRL & 0.128 & $\mathrm{pCi} / \mathrm{L}$ & 0.0586 & 0.0649 & & & \\
\hline 987 & TRITIUM_ELECT_LSC & ITASRL & 41.7 & $\mathrm{pCi} / \mathrm{L}$ & 2.53 & 10 & & & \\
\hline 988 & UISO_AEA & ITASRL & 0.222 & $\mathrm{pCi} / \mathrm{L}$ & 0.0463 & 0.0516 & & & \\
\hline
\end{tabular}




\begin{tabular}{|c|c|c|c|c|c|c|c|c|c|}
\hline & $\mathrm{K}$ & $\mathrm{L}$ & $\mathrm{M}$ & $\mathrm{N}$ & $\mathrm{O}$ & $\mathrm{P}$ & $\mathrm{Q}$ & $\mathrm{R}$ & $\mathrm{S}$ \\
\hline 1 & METHOD_NAME & LAB_CODE & VALUE_RPTD & ANAL_UNITS_RPTD & COUNTING_ERROR & TOTAL_ANAL_ERROR & MIN_DETECTABLE_ACTIVITY & PCNT_MOISTURE & LAB_QUALIFIER \\
\hline 990 & UISO_AEA & ITASRL & 0.196 & $\mathrm{pCi} / \mathrm{L}$ & 0.0437 & 0.048 & & & \\
\hline 991 & SRISO_SEP_PRECIP_GPC & ITASRL & 0.113 & $\mathrm{pCi} / \mathrm{L}$ & 0.0613 & 0.066 & & & \\
\hline 992 & TRITIUM_ELECT_LSC & ITASRL & 38.7 & $\mathrm{pCi} / \mathrm{L}$ & 2.48 & 9.77 & & & \\
\hline 993 & UISO_AEA & ITASRL & 0.302 & $\mathrm{pCi} / \mathrm{L}$ & 0.0536 & 0.0617 & & & \\
\hline 994 & UISO_AEA & ITASRL & -0.00085 & $\mathrm{pCi} / \mathrm{L}$ & 0.00904 & 0.00908 & & & $\mathrm{U}$ \\
\hline 995 & UISO_AEA & ITASRL & 0.19 & $\mathrm{pCi} / \mathrm{L}$ & 0.0426 & 0.0468 & & & \\
\hline 996 & SRISO_SEP_PRECIP_GPC & ITASRL & 0.128 & $\mathrm{pCi} / \mathrm{L}$ & 0.0574 & 0.0639 & & & \\
\hline 997 & TRITIUM_ELECT_LSC & ITASRL & 38.7 & $\mathrm{pCi} / \mathrm{L}$ & 2.49 & 9.84 & & & \\
\hline 999 & UISO_AEA & ITASRL & 0.0237 & $\mathrm{pCi} / \mathrm{L}$ & 0.0165 & 0.0167 & & & \\
\hline 1000 & UISO_AEA & ITASRL & 0.195 & $\mathrm{pCi} / \mathrm{L}$ & 0.0453 & 0.0495 & & & \\
\hline 1001 & SRISO_SEP_PRECIP_GPC & ITASRL & 0.138 & $\mathrm{pCi} / \mathrm{L}$ & 0.0777 & 0.0837 & & & \\
\hline 1002 & TRITIUM_ELECT_LSC & ITASRL & 41.5 & $\mathrm{pCi} / \mathrm{L}$ & 2.52 & 9.99 & & & \\
\hline 1003 & UISO_AEA & ITASRL & 0.234 & $\mathrm{pCi} / \mathrm{L}$ & 0.0539 & 0.0593 & & & \\
\hline 1004 & UISO_AEA & ITASRL & 0.00554 & $\mathrm{pCi} / \mathrm{L}$ & 0.0123 & 0.0123 & & & $U$ \\
\hline 1005 & UISO_AEA & ITASRL & 0.193 & $\mathrm{pCi} / \mathrm{L}$ & 0.0484 & 0.0524 & & & \\
\hline 1006 & SRISO_SEP_PRECIP_GPC & ITASRL & 0.115 & $\mathrm{pCi} / \mathrm{L}$ & 0.0654 & 0.07 & & & \\
\hline 1007 & TRITIUM_ELECT_LSC & ITASRL & 37.5 & $\mathrm{pCi} / \mathrm{L}$ & 2.47 & 9.7 & & & \\
\hline 1009 & UISO_AEA & ITASRL & 0.00843 & $\mathrm{pCi} / \mathrm{L}$ & 0.00961 & 0.00969 & & & $\mathrm{U}$ \\
\hline 1010 & UISO_AEA & ITASRL & 0.18 & $\mathrm{pCi} / \mathrm{L}$ & 0.0404 & 0.0444 & & & \\
\hline 1011 & ISRISO_SEP_PRECIP_GPC & ITASRL & 0.0762 & $\mathrm{pCi} / \mathrm{L}$ & 0.0534 & 0.0565 & & & \\
\hline 1012 & TRITIUM_ELECT_LSC & ITASRL & 39.2 & $\mathrm{pCi} / \mathrm{L}$ & 2.5 & 9.87 & & & \\
\hline 1013 & UISO_AEA & ITASRL & 0.25 & $\mathrm{pCi} / \mathrm{L}$ & 0.047 & 0.0534 & & & \\
\hline 1014 & ]UISO_AEA & ITASRL & 0.00946 & $\mathrm{pCi} / \mathrm{L}$ & 0.0104 & 0.0105 & & & U \\
\hline 1015 & JUISO_AEA & ITASRL & 0.214 & $\mathrm{pCi} / \mathrm{L}$ & 0.0435 & 0.0485 & & & \\
\hline 1016 & SRISO_SEP_PRECIP_GPC & ITASRL & 0.0824 & $\mathrm{pCi} / \mathrm{L}$ & 0.0377 & 0.0419 & & & \\
\hline 1017 & TRITIUM_ELECT_LSC & ITASRL & 39.2 & $\mathrm{pCi} / \mathrm{L}$ & 2.56 & 10.2 & & & \\
\hline 1018 & UISO_AEA & ITASRL & 0.274 & $\mathrm{pCi} / \mathrm{L}$ & 0.0609 & 0.0675 & & & \\
\hline 1019 & UISO_AEA & ITASRL & 0.00737 & $\mathrm{pCi} / \mathrm{L}$ & 0.0131 & 0.0132 & & & $\mathrm{U}$ \\
\hline 1020 & UISO_AEA & ITASRL & 0.191 & $\mathrm{pCi} / \mathrm{L}$ & 0.0504 & 0.0543 & & & \\
\hline 1021 & SRISO_SEP_PRECIP_GPC & ITASRL & 0.157 & $\mathrm{pCi} / \mathrm{L}$ & 0.0485 & 0.0578 & & & \\
\hline 1022 & TRITIUM_ELECT_LSC & ITASRL & 40.5 & $\mathrm{pCi} / \mathrm{L}$ & 2.59 & 10.3 & & & \\
\hline 1023 & UISO_AEA & ITASRL & 0.234 & $\mathrm{pCi} / \mathrm{L}$ & 0.0558 & 0.0611 & & & \\
\hline 1024 & UISO_AEA & ITASRL & 0.0159 & $\mathrm{pCi} / \mathrm{L}$ & 0.0153 & 0.0154 & & & \\
\hline 1025 & UISO_AEA & ITASRL & 0.184 & $\mathrm{pCi} / \mathrm{L}$ & 0.0493 & 0.053 & & & \\
\hline 1026 & SRISO_SEP_PRECIP_GPC & ITASRL & 0.115 & $\mathrm{pCi} / \mathrm{L}$ & 0.0447 & 0.0505 & & & \\
\hline
\end{tabular}




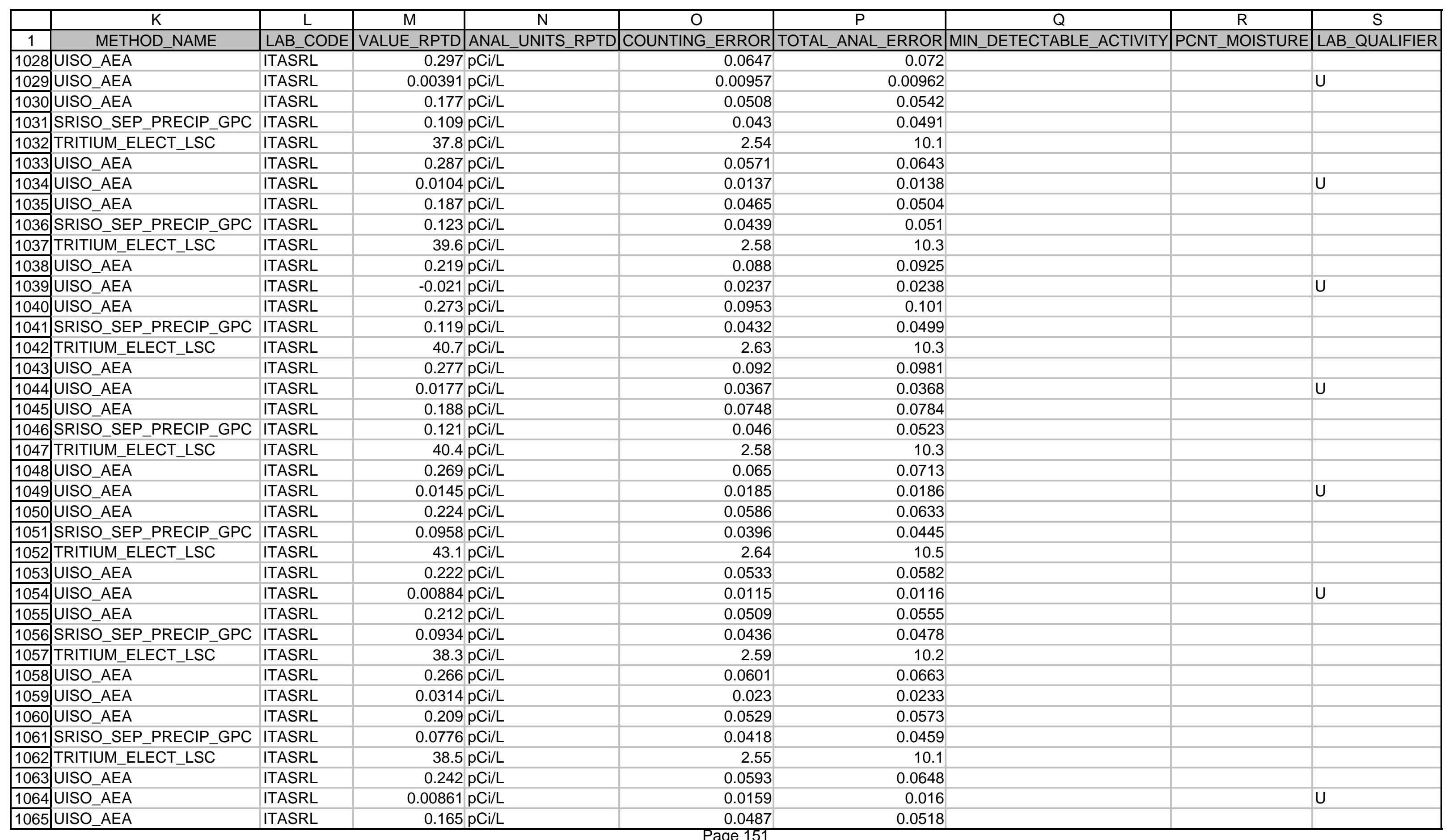




\begin{tabular}{|c|c|}
\hline 1 & METHOD_NAME \\
\hline 1066 & SRISO_SEP_PRECIP_GPC \\
\hline 1067 & TRITIUM_ELECT_LSC \\
\hline 1068
\end{tabular}

\begin{tabular}{c|c}
$\mathrm{N}$ & $\mathrm{O}$ \\
\hline
\end{tabular}

$\mathrm{P}$

Q $\mathrm{R}$ $\mathrm{S}$ 1067 TRITIUM_ELECT_LSC1068 UISO_AEA

1069 UISO_AEA \begin{tabular}{l|l}
\hline ITASRL & $0.0975 \mathrm{pCi} / \mathrm{L}$ \\
\hline
\end{tabular} 1070 UISO_AEA $33.6 \mathrm{pCi} / \mathrm{L}$ \begin{tabular}{l|l} 
ITASRL & $0.262 \mathrm{pCi} / \mathrm{L}$
\end{tabular} \begin{tabular}{l|r}
\hline ITASRL & $0.0177 \mathrm{pCi} / \mathrm{L}$
\end{tabular} \begin{tabular}{|l|r}
\hline ITASRL & $0.274 \mathrm{pCi} / \mathrm{L}$ \\
\hline ITASRL & $0.144 \mathrm{pCi} / \mathrm{L}$
\end{tabular}

1071 SRISO_SEP_PRECIP_GPC ITASRL 1072 TRITIUM_ELECT_LSC

$39.1 \mathrm{pCi} / \mathrm{L}$ \begin{tabular}{|l|r}
\hline ITASRL & $0.29 \mathrm{pCi} / \mathrm{L}$ \\
\hline ITASRL & $0.00917 \mathrm{pCi} / \mathrm{L}$ \\
\hline
\end{tabular}

1074 UISO_AEA

$0.00917 \mathrm{pCi} / \mathrm{L}$

1075 UISO_AEA

$0.0971 \mathrm{pCi} / \mathrm{L}$ $37.7 \mathrm{pCi} / \mathrm{L}$ $0.233 \mathrm{pCi} / \mathrm{L}$ $0.0229 \mathrm{pCi} / \mathrm{L}$ $0.218 \mathrm{pCi} / \mathrm{L}$

$0.124 \mathrm{pCi} / \mathrm{L}$

$39.6 \mathrm{pCi} / \mathrm{L}$

$0.274 \mathrm{pCi} / \mathrm{L}$

$0.0153 \mathrm{pCi} / \mathrm{L}$

$0.187 \mathrm{pCi} / \mathrm{L}$

$0.111 \mathrm{pCi} / \mathrm{L}$

$38 \mathrm{pCi} / \mathrm{L}$ $0.245 \mathrm{pCi} / \mathrm{L}$

$0.00536 \mathrm{pCi} / \mathrm{L}$

$0.231 \mathrm{pCi} / \mathrm{L}$

$0.0804 \mathrm{pCi} / \mathrm{L}$

$48.9 \mathrm{pCi} / \mathrm{L}$

$0.247 \mathrm{pCi} / \mathrm{L}$

$0.00788 \mathrm{pCi} / \mathrm{L}$

$0.221 \mathrm{pCi} / \mathrm{L}$

$0.0817 \mathrm{pCi} / \mathrm{L}$

$33.4 \mathrm{pCi} / \mathrm{L}$

$0.411 \mathrm{pCi} / \mathrm{L}$

$0.017 \mathrm{pCi} / \mathrm{L}$

$0.362 \mathrm{pCi} / \mathrm{L}$

$0.0982 \mathrm{pCi} / \mathrm{L}$

$46 \mathrm{pCi} / \mathrm{L}$

$0.241 \mathrm{pCi} / \mathrm{L}$

\begin{tabular}{|c|c|c|c|c|c|}
\hline UNITS_RPTD & COUNTING_ERROR & TOTAL_ANAL_ERROR & MIN_DETECTABLE_ACTIVITY & PCNT_MOISTURE & LAB_QUALIFIER \\
\hline & 0.0412 & 0.0483 & & & \\
\hline & 2.38 & 9.02 & & & \\
\hline & 0.0527 & 0.0592 & & & \\
\hline & 0.015 & 0.0151 & & & \\
\hline & 0.0528 & 0.0595 & & & \\
\hline & 0.049 & 0.0625 & & & \\
\hline & 2.45 & 9.41 & & & \\
\hline & 0.0573 & 0.0646 & & & \\
\hline & 0.0105 & 0.0105 & & & $U$ \\
\hline & 0.0469 & 0.0511 & & & \\
\hline & 0.0453 & 0.0499 & & & \\
\hline & 2.46 & 9.35 & & & \\
\hline & 0.0464 & 0.0522 & & & \\
\hline & 0.0145 & 0.0147 & & & \\
\hline & 0.0442 & 0.0493 & & & \\
\hline & 0.0489 & 0.0593 & & & \\
\hline & 2.46 & 9.43 & & & \\
\hline & 0.048 & 0.0553 & & & \\
\hline & 0.0134 & 0.0135 & & & \\
\hline & 0.0395 & 0.0438 & & & \\
\hline & 0.0453 & 0.0511 & & & \\
\hline & 2.41 & 9.21 & & & \\
\hline & 0.0446 & 0.0509 & & & \\
\hline & 0.00755 & 0.00762 & & & $U$ \\
\hline & 0.0426 & 0.0483 & & & \\
\hline & 0.0437 & 0.0467 & & & \\
\hline & 2.58 & 10.1 & & & \\
\hline & 0.0499 & 0.0559 & & & \\
\hline & 0.012 & 0.012 & & & $U$ \\
\hline & 0.0464 & 0.0515 & & & \\
\hline & 0.0401 & 0.0469 & & & \\
\hline & 2.35 & 8.87 & & & \\
\hline & 0.0595 & 0.072 & & & \\
\hline & 0.0122 & 0.0124 & & & \\
\hline & 0.0553 & 0.0657 & & & \\
\hline & 0.0442 & 0.0486 & & & \\
\hline & 2.58 & 9.93 & & & \\
\hline & 0.0471 & 0.0531 & & & \\
\hline
\end{tabular}

\begin{tabular}{|l|l|}
11102 & TRITIUM_ELECT_LSC \\
\hline
\end{tabular}

\begin{tabular}{l|l}
1102 & UISO_AEA \\
\hline 1103
\end{tabular}

ITASRL

ITASRL

P.0471 


\begin{tabular}{|c|c|c|c|c|c|c|c|c|c|}
\hline & $\mathrm{K}$ & $L$ & $\mathrm{M}$ & $\mathrm{N}$ & $\mathrm{O}$ & $\mathrm{P}$ & $\mathrm{Q}$ & $\mathrm{R}$ & $\mathrm{S}$ \\
\hline 1 & METHOD_NAME & LAB_CODE & VALUE_RPTD & ANAL_UNITS_RPTD & COUNTING_ERROR & \begin{tabular}{|l||} 
TOTAL_ANAL_ERROR \\
\end{tabular} & MIN_DETECTABLE_ACTIVITY & PCNT_MOISTURE & LAB_QUALIFIER \\
\hline 1104 & UISO_AEA & ITASRL & 0.019 & $\mathrm{pCi} / \mathrm{L}$ & 0.0152 & 0.0154 & & & \\
\hline 1105 & UISO_AEA & ITASRL & 0.197 & $\mathrm{pCi} / \mathrm{L}$ & 0.0422 & 0.0466 & & & \\
\hline 1106 & SRISO_SEP_PRECIP_GPC & ITASRL & 0.117 & $\mathrm{pCi} / \mathrm{L}$ & 0.0457 & 0.0511 & & & \\
\hline 1107 & TRITIUM_E_ELECT_LSC & ITASRL & 39.6 & $\mathrm{pCi} / \mathrm{L}$ & 2.5 & 9.45 & & & \\
\hline 1108 & UISO_AEA & ITASRL & 0.307 & $\mathrm{pCi} / \mathrm{L}$ & 0.0518 & 0.0602 & & & \\
\hline 1109 & UISO_AEA & ITASRL & 0.0129 & $\mathrm{pCi} / \mathrm{L}$ & 0.0108 & 0.0109 & & & \\
\hline 1110 & UISO_AEA & ITASRL & 0.215 & $\mathrm{pCi} / \mathrm{L}$ & 0.0429 & 0.0479 & & & \\
\hline 1111 & SRISO_SEP_PRECIP_GPC & ITASRL & 0.12 & $\mathrm{pCi} / \mathrm{L}$ & 0.0469 & 0.0526 & & & \\
\hline 1112 & TRITIUM_ELECT_LSC & ITASRL & 39.1 & $\mathrm{pCi} / \mathrm{L}$ & 2.43 & 9.31 & & & \\
\hline 1113 & UISO_AEA & ITASRL & 0.239 & $\mathrm{pCi} / \mathrm{L}$ & 0.0442 & 0.0503 & & & \\
\hline 1114 & UISO_AEA & ITASRL & 0.00322 & $\mathrm{pCi} / \mathrm{L}$ & 0.00811 & 0.00817 & & & $\mathrm{U}$ \\
\hline 1115 & UISO_AEA & ITASRL & 0.219 & $\mathrm{pCi} / \mathrm{L}$ & 0.0418 & 0.0471 & & & \\
\hline 1116 & SRISO_SEP_PRECIP_GPC & ITASRL & 0.116 & $\mathrm{pCi} / \mathrm{L}$ & 0.0482 & 0.0543 & & & \\
\hline 1117 & TRITIUM_ELECT_LSC & ITASRL & 37.9 & $\mathrm{pCi} / \mathrm{L}$ & 2.43 & 9.23 & & & \\
\hline 1118 & UISO_AEA & ITASRL & 0.316 & $\mathrm{pCi} / \mathrm{L}$ & 0.0537 & 0.0623 & & & \\
\hline 1119 & UISO_AEA & ITASRL & 0.0177 & $\mathrm{pCi} / \mathrm{L}$ & 0.0127 & 0.0128 & & & \\
\hline 1120 & UISO_AEA & ITASRL & 0.192 & $\mathrm{pCi} / \mathrm{L}$ & 0.0417 & 0.046 & & & \\
\hline 1121 & SRISO_SEP_PRECIP_GPC & ITASRL & 0.114 & $\mathrm{pCi} / \mathrm{L}$ & 0.0467 & 0.0521 & & & \\
\hline 1123 & UISO_AEA & ITASRL & 0.24 & $\mathrm{pCi} / \mathrm{L}$ & 0.0451 & 0.0512 & & & \\
\hline 1124 & UISO_AEA & ITASRL & 0.00582 & $\mathrm{pCi} / \mathrm{L}$ & 0.00806 & 0.00813 & & & U \\
\hline 1125 & UISO_AEA & ITASRL & 0.24 & $\mathrm{pCi} / \mathrm{L}$ & 0.045 & 0.0509 & & & \\
\hline 1126 & SRISO_SEP_PRECIP_GPC & ITASRL & 0.106 & $\mathrm{pCi} / \mathrm{L}$ & 0.0449 & 0.0504 & & & \\
\hline 1127 & TRITIUM_ELECT_LSC & ITASRL & 37.6 & $\mathrm{pCi} / \mathrm{L}$ & 2.45 & 9.26 & & & \\
\hline 1128 & UISO_AEA & ITASRL & 0.291 & $\mathrm{pCi} / \mathrm{L}$ & 0.0497 & 0.0576 & & & \\
\hline 1129 & UISO_AEA & ITASRL & 0.00545 & $\mathrm{pCi} / \mathrm{L}$ & 0.0115 & 0.0116 & & & U \\
\hline 1130 & UISO_AEA & ITASRL & 0.197 & $\mathrm{pCi} / \mathrm{L}$ & 0.041 & 0.0455 & & & \\
\hline 1131 & SRISO_SEP_PRECIP_GPC & ITASRL & 0.0778 & $\mathrm{pCi} / \mathrm{L}$ & 0.0374 & 0.0427 & & & \\
\hline 1132 & TRITIUM̄E_ELECT_LSC̄ & ITASRL & 28.9 & $\mathrm{pCi} / \mathrm{L}$ & 2.29 & 8.59 & & & \\
\hline 1133 & UISO_AEA & ITASRL & 0.248 & $\mathrm{pCi} / \mathrm{L}$ & 0.0484 & 0.0546 & & & \\
\hline 1134 & UISO_AEA & ITASRL & 0.0135 & $\mathrm{pCi} / \mathrm{L}$ & 0.0146 & 0.0147 & & & $\mathrm{U}$ \\
\hline 1135 & UISO_AEA & ITASRL & 0.228 & $\mathrm{pCi} / \mathrm{L}$ & 0.0457 & 0.0511 & & & \\
\hline 1136 & SRISO_SEP_PRECIP_GPC & ITASRL & 0.0752 & $\mathrm{pCi} / \mathrm{L}$ & 0.0412 & 0.0446 & & & \\
\hline 1137 & TRITIUM_ELECT_LSC & ITASRL & 36.7 & $\mathrm{pCi} / \mathrm{L}$ & 2.46 & 9.32 & & & \\
\hline 1138 & UISO_AEA & ITASRL & 0.296 & $\mathrm{pCi} / \mathrm{L}$ & 0.0513 & 0.0593 & & & \\
\hline 1139 & UISO_AEA & ITASRL & 0.0119 & $\mathrm{pCi} / \mathrm{L}$ & 0.0122 & 0.0123 & & & $\mathrm{U}$ \\
\hline 1140 & UISO_AEA & ITASRL & 0.186 & $\mathrm{pCi} / \mathrm{L}$ & 0.0403 & 0.0445 & & & \\
\hline
\end{tabular}


$\mathrm{N}$

$\mathrm{O}$

$P$

Q \begin{tabular}{l|r} 
ITASRL & $42.2 \mathrm{pCi} / \mathrm{L}$
\end{tabular} \begin{tabular}{r|r}
$42.2 \mathrm{pCi} / \mathrm{L}$ & 2.57 \\
0.287 & 0.055
\end{tabular}

1143 UISO_AEA

1144 UISO_AEA

1145 UISO_AEA

\begin{tabular}{|l|r}
\hline ITASRL & $0.287 \mathrm{pCi} / \mathrm{L}$ \\
\hline ITASRL & $0.0183 \mathrm{pCi} / \mathrm{L}$
\end{tabular}

\begin{tabular}{l|c} 
ITASRL & $0.0183 \mathrm{pCi} / \mathrm{L}$ \\
\hline
\end{tabular}

$0.173 \mathrm{pCi} / \mathrm{L}$

$0.11 \mathrm{pCi} / \mathrm{L}$

$42.5 \mathrm{pCi} / \mathrm{L}$

1147 TRITIUM_ELECT_LSC

1148 UISO_AEA

1149 UISO AEA

$0.262 \mathrm{pCi} / \mathrm{L}$

ITASRL

ITASRL

ITASRL

$0.0165 \mathrm{pCi} / \mathrm{L}$

$0.216 \mathrm{pCi} / \mathrm{L}$

1151 SRISO SEP PRECIP GPC ITASRL

1152 TRITIUM_ELECT_LSC

1153 UISO_AEA

1154 UISO_AEA

1155 UISO AEA

$0.0958 \mathrm{pCi} / \mathrm{L}$

$51 \mathrm{pCi} / \mathrm{L}$

\begin{tabular}{l|l} 
ITASRL & $0.264 \mathrm{pCi} / \mathrm{L}$
\end{tabular}

$0.1 \mathrm{pCi} / \mathrm{L}$

ITASRL

$0.141 \mathrm{pCi} / \mathrm{L}$

1156 SRISO_SEP_PRECIP_GPC ITASRL

\begin{tabular}{|l|l|l}
1157 & TRITIUM_ELECT_LSC \\
\hline 1158 & UISO_AEA
\end{tabular}

1158 UISO_AEA

1159 UISO AEA

\begin{tabular}{l|r}
\hline ITASRL & $0.0671 \mathrm{pCi} / \mathrm{L}$ \\
\hline ITASRL & $39.4 \mathrm{pCi} / \mathrm{L}$
\end{tabular}

$39.4 \mathrm{pCi} / \mathrm{L}$

\begin{tabular}{l|r}
\hline ITASRL & $0.414 \mathrm{pCi} / \mathrm{L}$ \\
\hline ITASRL & $0.0917 \mathrm{pCi} / \mathrm{L}$
\end{tabular}

\begin{tabular}{|l|r}
\hline ITASRL & $0.276 \mathrm{pCi} / \mathrm{L}$ \\
\hline ITASRL & $0.0828 \mathrm{pCi} / \mathrm{L}$
\end{tabular}

$43.6 \mathrm{pCi} / \mathrm{L}$

1161 SRISO_SEP_PRECIP_GPC ITASRL

1162 TRITIUM_ELECT_LSC

ITASRL

ITASRL

$0.261 \mathrm{pCi} / \mathrm{L}$

1163 UISO_AEA

\begin{tabular}{|l|l|l|}
\hline 1164 & UISO_AEA \\
\hline 1165 & UISO_AEA \\
\hline 1166 & SRISO SEP
\end{tabular}

\begin{tabular}{l|l} 
ITASRL & $0.0103 \mathrm{pCi} / \mathrm{L}$
\end{tabular}

$0.216 \mathrm{pCi} / \mathrm{L}$

$0.0944 \mathrm{pCi} / \mathrm{L}$

$42.5 \mathrm{pCi} / \mathrm{L}$

$0.236 \mathrm{pCi} / \mathrm{L}$

$0.0498 \mathrm{pCi} / \mathrm{L}$

$0.186 \mathrm{pCi} / \mathrm{L}$

$0.103 \mathrm{pCi} / \mathrm{L}$

$38.4 \mathrm{pCi} / \mathrm{L}$

$0.244 \mathrm{pCi} / \mathrm{L}$

$0.0175 \mathrm{pCi} / \mathrm{L}$

$0.201 \mathrm{pCi} / \mathrm{L}$

$0.873 \mathrm{pCi} / \mathrm{L}$

$39.3 \mathrm{pCi} / \mathrm{L}$

$0.263 \mathrm{pCi} / \mathrm{L}$

$0.0215 \mathrm{pCi} / \mathrm{L}$

\begin{tabular}{|r|r|r|}
\hline 2.57 & 10.4 \\
\hline 0.055 & 0.0624 \\
\hline 0.0139 & 0.014 \\
\hline 0.0333 & 0.0469 \\
\hline 2.58 & 0.0451 \\
\hline 0.0515 & 10.4 \\
\hline 0.0155 & 0.0581 \\
\hline 0.0469 & 0.0156 \\
\hline 0.0389 & 0.0518 \\
\hline 2.5 & 0.0433 \\
\hline 0.0451 & 9.52 \\
\hline 0.0273 & 0.0522 \\
\hline 0.0329 & 0.029 \\
\hline 0.034 & 0.0359 \\
\hline 2.33 & 0.0371 \\
\hline 0.0566 & 8.68 \\
\hline 0.027 & 0.0697 \\
\hline 0.0461 & 0.0284 \\
\hline 0.0358 & 0.0535 \\
\hline 2.4 & 0.0398 \\
\hline 0.0444 & 8.99 \\
\hline 0.00937 & 0.0515 \\
\hline 0.0404 & 0.00947 \\
\hline 0.0371 & 0.0457 \\
\hline 2.38 & 0.0416 \\
\hline 0.0431 & 8.91 \\
\hline 0.0196 & 0.0492 \\
\hline 0.0378 & 0.0202 \\
\hline 0.0378 & 0.0421 \\
\hline 2.3 & 0.0428 \\
\hline 0.0428 & 8.53 \\
\hline 0.0123 & 0.0493 \\
\hline 0.0387 & 0.0124 \\
\hline 0.0884 & 0.0435 \\
\hline 2.32 & 0.187 \\
\hline 0.0451 & 8.64 \\
\hline 0.0136 & 0.0522 \\
\hline 9154 & 0.0138 \\
\hline
\end{tabular}

\begin{tabular}{|l|l|l|} 
& \\
\hline
\end{tabular}

0.0624

$\begin{array}{r}0.0624 \\ 0.014 \\ \hline 0.0469\end{array}$

10.4

0.0156

0.0433

9.52

0.029

0.0371
8.68

0.0697

0.0284

0.0535

8.99

0.0515

0.0515
0.0457

0.0416

8.91
0.0492

0.0202

0.0421

8.53

0.0493

0.0124

0.187

ITASRL

ITASRL

0.0136

0.0138

\begin{tabular}{|l|l|l|}
\hline 1178 & UISO_AEA \\
\hline 1179 & UISO AEA \\
\hline
\end{tabular} 


\begin{tabular}{|c|c|c|c|c|c|c|c|c|c|}
\hline & $\mathrm{K}$ & $\mathrm{L}$ & $\mathrm{M}$ & $\mathrm{N}$ & $\mathrm{O}$ & $\mathrm{P}$ & $\mathrm{Q}$ & $\mathrm{R}$ & $\mathrm{S}$ \\
\hline 1 & METHOD_NAME & LAB_CODE & VALUE_RPTD & ANAL_UNITS_RPTD & COUNTING_ERROR & TOTAL_ANAL_ERROR & MIN_DETECTABLE_ACTIVITY & PCNT_MOISTURE & LAB_QUALIFIER \\
\hline 1180 & UISO_AEA & ITASRL & 0.188 & $\mathrm{pCi} / \mathrm{L}$ & 0.038 & 0.0424 & & & \\
\hline 1181 & SRISO_SEP_PRECIP_GPC & ITASRL & 0.111 & $\mathrm{pCi} / \mathrm{L}$ & 0.0377 & 0.0438 & & & \\
\hline 1182 & TRITIUM_ELECT_LSC & ITASRL & 40.7 & $\mathrm{pCi} / \mathrm{L}$ & 2.32 & 8.69 & & & \\
\hline 1183 & UISO_AEA & ITASRL & 0.248 & $\mathrm{pCi} / \mathrm{L}$ & 0.0461 & 0.0524 & & & \\
\hline 1184 & UISO_AEA & ITASRL & 0.0179 & $\mathrm{pCi} / \mathrm{L}$ & 0.0133 & 0.0134 & & & \\
\hline 1185 & UISO_AEA & ITASRL & 0.264 & $\mathrm{pCi} / \mathrm{L}$ & 0.0466 & 0.0534 & & & \\
\hline 1186 & SRISO_SEP_PRECIP_GPC & ITASRL & 0.0962 & $\mathrm{pCi} / \mathrm{L}$ & 0.0374 & 0.0429 & & & \\
\hline 1187 & TRITIUM_ELECT_LSC & ITASRL & 48.9 & $\mathrm{pCi} / \mathrm{L}$ & 2.43 & 9.35 & & & \\
\hline 1188 & UISO_AEA & ITASRL & 0.275 & $\mathrm{pCi} / \mathrm{L}$ & 0.0459 & 0.0534 & & & \\
\hline 1189 & UISO_AEA & ITASRL & 0.0729 & $\mathrm{pCi} / \mathrm{L}$ & 0.0234 & 0.0244 & & & \\
\hline 1190 & UISO_AEA & ITASRL & 0.143 & $\mathrm{pCi} / \mathrm{L}$ & 0.0337 & 0.0367 & & & \\
\hline 1191 & SRISO_SEP_PRECIP_GPC & ITASRL & 0.0775 & $\mathrm{pCi} / \mathrm{L}$ & 0.0336 & 0.0376 & & & \\
\hline 1192 & TRITIUM_ELECT_LSC & ITASRL & 40.6 & $\mathrm{pCi} / \mathrm{L}$ & 2.35 & 8.74 & & & \\
\hline 1193 & UISO_AEA & ITASRL & 0.263 & $\mathrm{pCi} / \mathrm{L}$ & 0.0439 & 0.0511 & & & \\
\hline 1194 & UISO_AEA & ITASRL & 0.00652 & $\mathrm{pCi} / \mathrm{L}$ & 0.00772 & 0.0078 & & & $U$ \\
\hline 1195 & UISO_AEA & ITASRL & 0.171 & $\mathrm{pCi} / \mathrm{L}$ & 0.0356 & 0.0395 & & & \\
\hline 1196 & SRISO_SEP_PRECIP_GPC & ITASRL & 0.0913 & $\mathrm{pCi} / \mathrm{L}$ & 0.0391 & 0.0434 & & & \\
\hline 1199 & UISO_AEA & ITASRL & 0.0399 & $\mathrm{pCi} / \mathrm{L}$ & 0.0171 & 0.0176 & & & \\
\hline 1200 & UISO_AEA & ITASRL & 0.183 & $\mathrm{pCi} / \mathrm{L}$ & 0.0366 & 0.0409 & & & \\
\hline 1201 & SRISO_SEP_PRECIP_GPC & ITASRL & 0.0945 & $\mathrm{pCi} / \mathrm{L}$ & 0.0386 & 0.0434 & & & \\
\hline 1202 & TRITIUM_ELECT_LSC & ITASRL & 32.3 & $\mathrm{pCi} / \mathrm{L}$ & 2.28 & 8.32 & & & \\
\hline 1203 & UISO_AEA & ITASRL & 0.199 & $\mathrm{pCi} / \mathrm{L}$ & 0.0479 & 0.0523 & & & \\
\hline 1204 & UISO_AEA & ITASRL & 0.00919 & $\mathrm{pCi} / \mathrm{L}$ & 0.012 & 0.0121 & & & U \\
\hline 1205 & UISO_AEA & ITASRL & 0.157 & $\mathrm{pCi} / \mathrm{L}$ & 0.0424 & 0.0455 & & & \\
\hline 1206 & SRISO_SEP_PRECIP_GPC & ITASRL & 0.0986 & $\mathrm{pCi} / \mathrm{L}$ & 0.0401 & 0.0448 & & & \\
\hline 1207 & TRITIUM̄ELEEECT_LSC̄ & ITASRL & 34.5 & $\mathrm{pCi} / \mathrm{L}$ & 2.3 & 8.46 & & & \\
\hline 1208 & UISO_AEA & ITASRL & 0.277 & $\mathrm{pCi} / \mathrm{L}$ & 0.0632 & 0.0698 & & & \\
\hline 1209 & UISO_AEA & ITASRL & 0.00463 & $\mathrm{pCi} / \mathrm{L}$ & 0.00972 & 0.00978 & & & $U$ \\
\hline 1210 & UISO_AEA & ITASRL & 0.152 & $\mathrm{pCi} / \mathrm{L}$ & 0.0469 & 0.0497 & & & \\
\hline 1211 & SRISO_SEP_PRECIP_GPC & ITASRL & 0.0926 & $\mathrm{pCi} / \mathrm{L}$ & 0.0394 & 0.0444 & & & \\
\hline 1212 & TRITIUM_ELECT_LSC & ITASRL & 33.6 & $\mathrm{pCi} / \mathrm{L}$ & 2.3 & 8.37 & & & \\
\hline 1213 & UISO_AEA & ITASRL & 0.203 & $\mathrm{pCi} / \mathrm{L}$ & 0.0485 & 0.0531 & & & \\
\hline 1214 & UISO_AEA & ITASRL & 0.00674 & $\mathrm{pCi} / \mathrm{L}$ & 0.00928 & 0.00935 & & & $U$ \\
\hline 1215 & UISO_AEA & ITASRL & 0.198 & $\mathrm{pCi} / \mathrm{L}$ & 0.0477 & 0.0519 & & & \\
\hline 1216 & SRISŌ_SEP_PRECIP_GPC & ITASRL & 0.091 & $\mathrm{pCi} / \mathrm{L}$ & 0.0361 & 0.0411 & & & \\
\hline
\end{tabular}




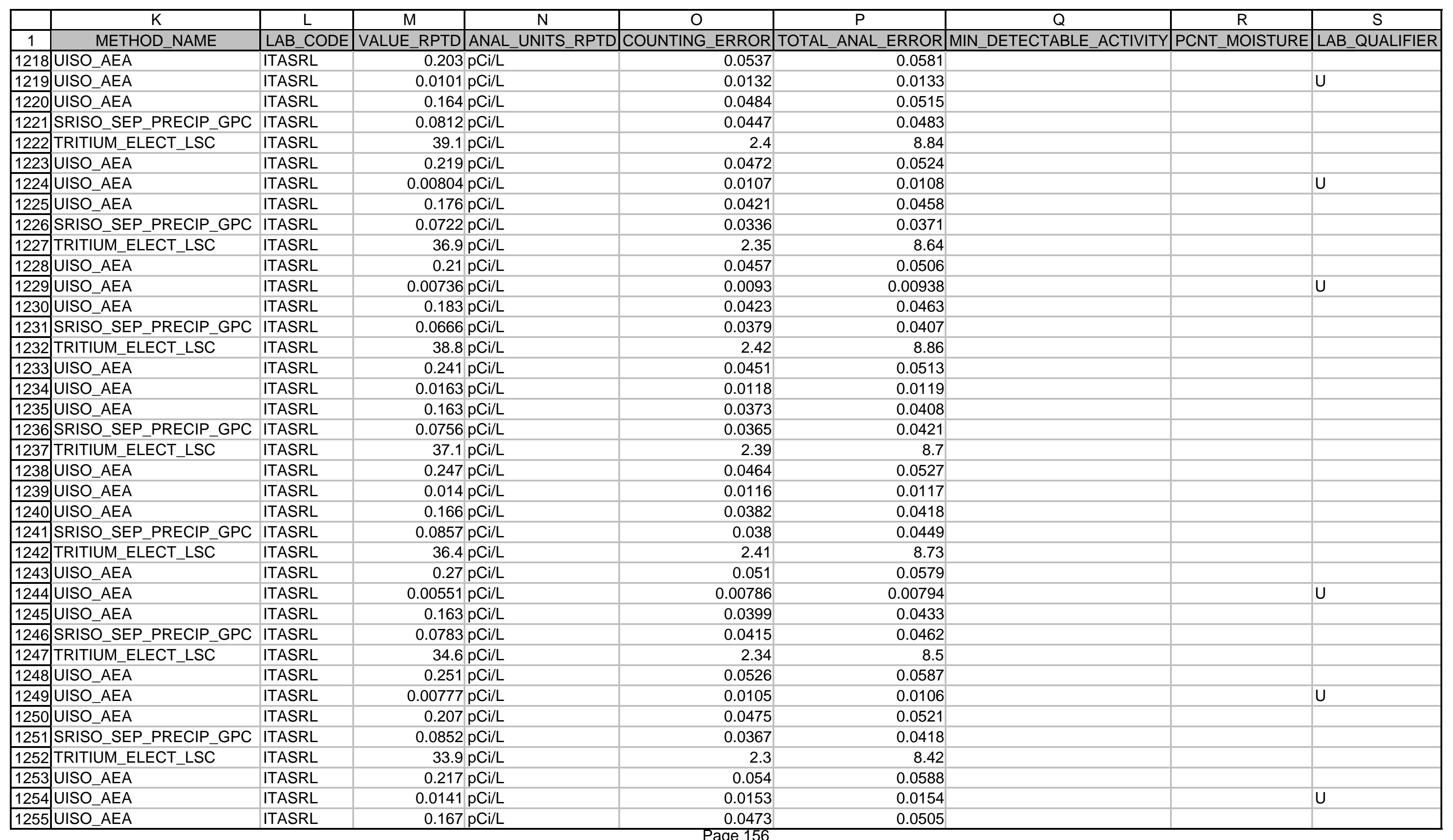


1260 UISO_AEA \begin{tabular}{|l|r}
\hline ITASRL & $34.7 \mathrm{pCi} / \mathrm{L}$ \\
\hline ITASRL & $0.238 \mathrm{pCi} / \mathrm{L}$
\end{tabular} ITASRL $0.0105 \mathrm{pCi} / \mathrm{L}$ \begin{tabular}{l|l} 
ITASRL & $0.202 \mathrm{pCi} / \mathrm{L}$
\end{tabular}

1261 SRISO SEP PRECIP_GPC ITASRL 1262 TRITIUM_ELECT_LSC $0.0573 \mathrm{pCi} / \mathrm{L}$ \begin{tabular}{l|l}
\hline ITASRL & $36.1 \mathrm{pCi} / \mathrm{L}$ \\
\hline ITASRL & $0.25 \mathrm{pCi} / \mathrm{L}$
\end{tabular}

1263 UISO_AEA

1264 UISO_AEA

$0.25 \mathrm{pCi} / \mathrm{L}$

\begin{tabular}{|l|r|}
\hline ITASRL & $0.0093 \mathrm{pCi} / \mathrm{L}$ \\
\hline ITASRL & $0.169 \mathrm{pCi} / \mathrm{L}$
\end{tabular}

\begin{tabular}{|l|l|l|l}
\hline 1266 & SRISO SEP PRECIP GPC & ITASRL & $0.0863 \mathrm{pCi} / \mathrm{L}$
\end{tabular}

1267 TRITIUM_ELECT_LSC

1268 UISO_AEA

1269 UISO_AEA

1270 UISO AEA

1271 6010_METALS_ICP

1272 8240_VOA_GCMS

$34.3 \mathrm{pCi} / \mathrm{L}$

\begin{tabular}{l|r}
\hline ITASRL & $34.3 \mathrm{pCi} / \mathrm{L}$ \\
\hline ITASRL & $0.257 \mathrm{pCi} / \mathrm{L}$ \\
\hline
\end{tabular}

\begin{tabular}{l|l} 
ITASRL & $0.0144 \mathrm{pCi} / \mathrm{L}$
\end{tabular}

ITASRL $\quad 0.213 \mathrm{pCi} / \mathrm{L}$

$\frac{0.213 \mathrm{pCi} / \mathrm{L}}{17 \mathrm{ug} / \mathrm{L}}$

1273 SRISO SEP_PRECIP_GPC ITASRL $\quad 0.0914 \mathrm{pCi} / \mathrm{L}$

1274 TRITIUM_ELECT_LSC

1275 UISO_AEA

1276 UISO_AEA

1277 UISO_AEA

1278 6010_METALS_ICP

1279 8240_VOA_GCMS

\begin{tabular}{l|r} 
& $0.0914 \mathrm{pCi} / \mathrm{L}$ \\
\hline ITASRL & $40 \mathrm{pCi} / \mathrm{L}$
\end{tabular}

$0.201 \mathrm{pCi} / \mathrm{L}$

ITASRL

ITASRL

ITASRL

SRISO SEP PRECIP GPC ITASRL

1281 TRITIUM_ELECT_LSC

1282 UISO_AEA

1283 UISO_AEA

1284 UISO_AEA

1285 6010_METALS_ICP

1286 8240_VOA_GCMS

1288 TRITIUM_ELECT_LSC

1289 UISO_AEA

1290 UISO_AEA

1291 UISO_AEA

1292 6010_METALS_ICP

\begin{tabular}{|l|l|}
1293 & $8240 \_V O A \_G C M S$ \\
\hline
\end{tabular}

\begin{tabular}{l|l} 
& $0.113 \mathrm{pCi} / \mathrm{L}$
\end{tabular}

$32.9 \mathrm{pCi} / \mathrm{L}$

$0.216 \mathrm{pCi} / \mathrm{L}$

$0.0111 \mathrm{pCi} / \mathrm{L}$

\begin{tabular}{|l|r|}
\hline ITASRL & $0.0111 \mathrm{pCi} / \mathrm{L}$ \\
\hline ITASRL & $0.161 \mathrm{pCi} / \mathrm{L}$ \\
\hline DATACH & $18 \mathrm{ug} / \mathrm{L}$ \\
\hline
\end{tabular}

DATACH

$107 \mathrm{pCi} / \mathrm{L}$

$35.6 \mathrm{pCi} / \mathrm{L}$

ITASRL

ITASRL

ITASRL

$0.246 \mathrm{pCi} / \mathrm{L}$

$0.0153 \mathrm{pCi} / \mathrm{L}$

$0.152 \mathrm{pCi} / \mathrm{L}$

DATACH

$18 \mathrm{ug} / \mathrm{L}$

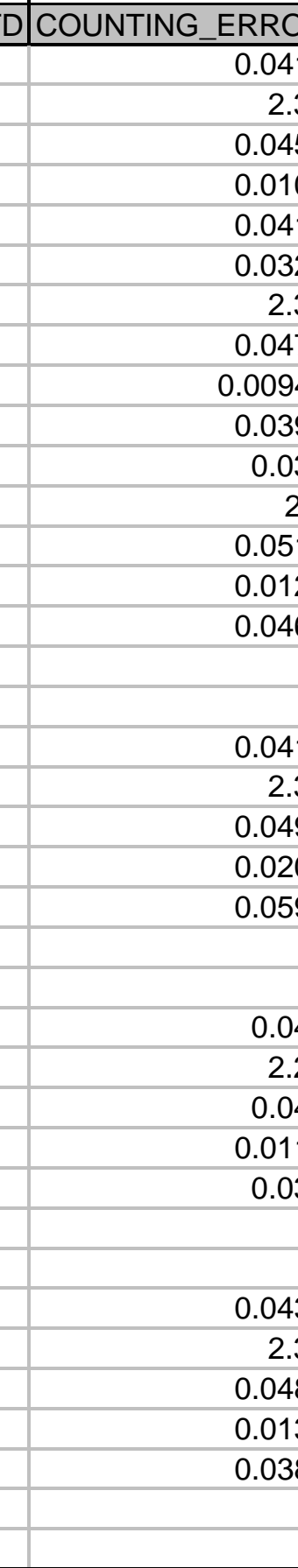

\subsection{9}

0.0497

$0.0456 \quad 0.051$

0.0102

$0.0418 \quad 0.0465$

$0.0324 \quad 0.035$

8.55

$0.0476 \quad 0.054$

\begin{tabular}{r|r|}
0.00948 & 0.00957 \\
\hline 0.0394 & 0.043 \\
\hline
\end{tabular}

$0.037 \quad 0.0415$

\begin{tabular}{rr}
2.3 & 8.41 \\
\hline
\end{tabular}

$0.0517 \quad 0.058$

0.013

$0.0467 \quad 0.0515$

8.16

0.0459

8.69

0.0534

0.0203

\begin{tabular}{|r|r|r|}
\hline 0.0202 & 0.0605 & \\
\hline 27.8 & $B$ \\
\hline
\end{tabular}

$0.041 \quad 0.0476$

8.19

0.0493

0.0112

0.0414

8.64

0.0489

2.31

0.0488

0.0139

0.0387

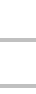

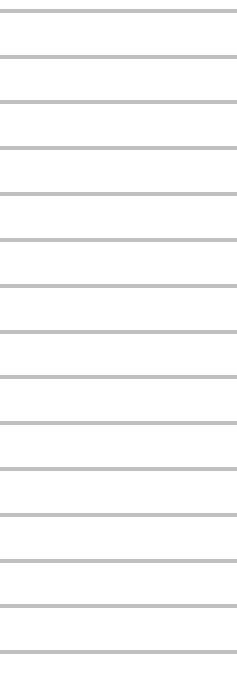




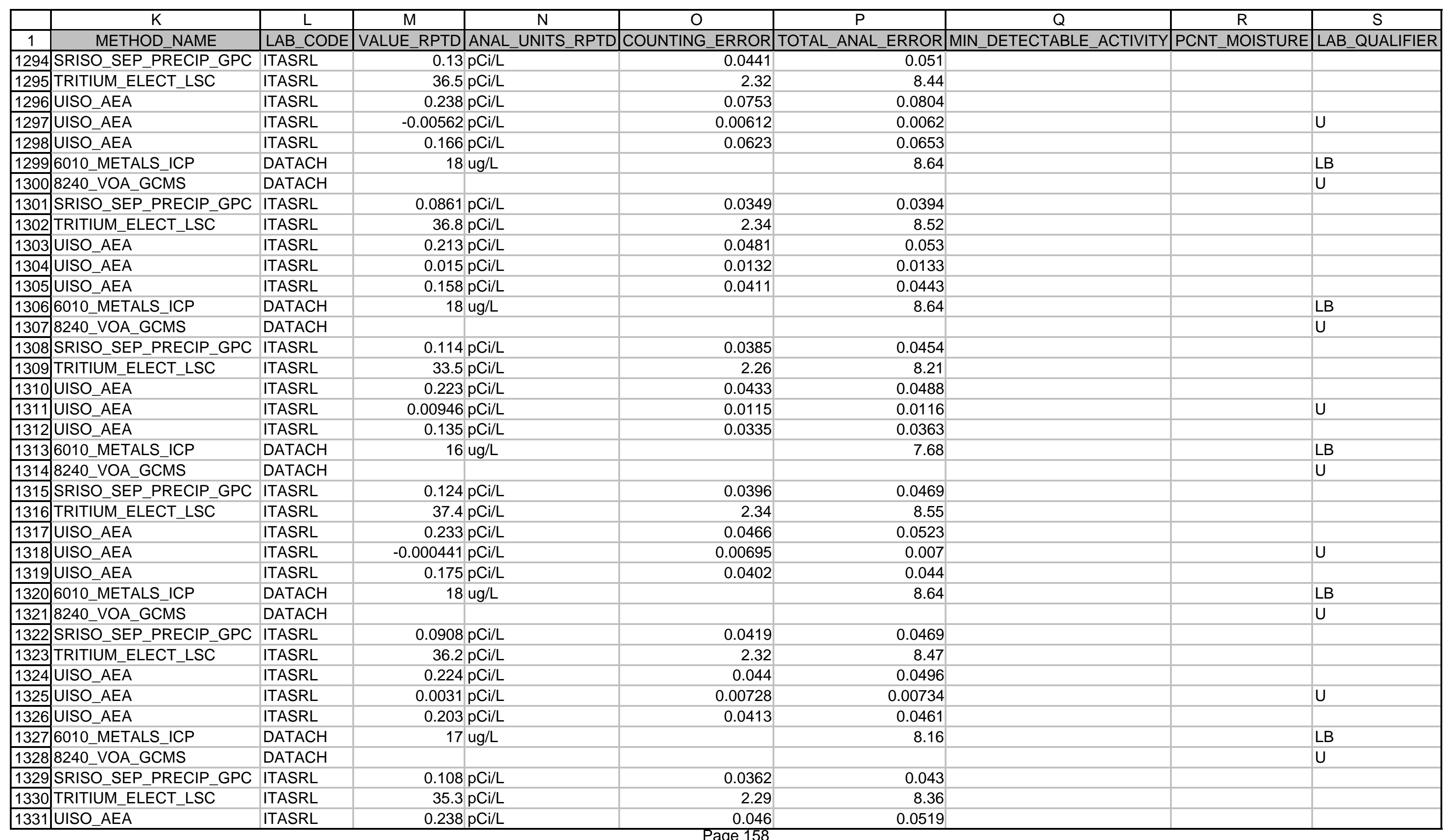




\begin{tabular}{|c|c|c|c|c|c|c|c|c|c|}
\hline & $\mathrm{K}$ & $\mathrm{L}$ & $\mathrm{M}$ & $\mathrm{N}$ & $\mathrm{O}$ & $\mathrm{P}$ & $\mathrm{Q}$ & $\mathrm{R}$ & $\mathrm{S}$ \\
\hline 1 & METHOD_NAME & LAB_CODE & VALUE_RPTD & ANAL_UNITS_RPTD & COUNTING_ERROR & $\mid$ TOTAL_ANAL_ERROR| & MIN_DETECTABLE_ACTIVITY & PCNT_MOISTURE & LAB_QUALIFIER \\
\hline 1332 & UISO_AEA & ITASRL & 0.00703 & $\mathrm{pCi} / \mathrm{L}$ & 0.00832 & 0.0084 & & & $U$ \\
\hline 1333 & UISO AEA & ITASRL & 0.145 & $\mathrm{pCi} / \mathrm{L}$ & 0.036 & 0.039 & & & \\
\hline 1334 & 6010_METALS_ICP & DATACH & 17 & ug/L & & 8.16 & & & LB \\
\hline 1335 & 8240_VOA_GCMS & DATACH & & & & & & & $U$ \\
\hline 1336 & SRISO_SEPP_PRECIP_GPC & ITASRL & 0.0833 & $\mathrm{pCi} / \mathrm{L}$ & 0.035 & 0.0397 & & & \\
\hline 1337 & TRITIUM_ELECT_LSC & ITASRL & 40.3 & $\mathrm{pCi} / \mathrm{L}$ & 2.39 & 8.79 & & & \\
\hline 1338 & UISO_AËA & ITASRL & 0.209 & $\mathrm{pCi} / \mathrm{L}$ & 0.0417 & 0.0468 & & & \\
\hline 1339 & UISO AEA & ITASRL & 0.00209 & $\mathrm{pCi} / \mathrm{L}$ & 0.00556 & 0.00564 & & & $\mathrm{U}$ \\
\hline 1340 & UISO_AEA & ITASRL & 0.154 & $\mathrm{pCi} / \mathrm{L}$ & 0.0357 & 0.039 & & & \\
\hline 1341 & 6010_METALS_ICP & DATACH & & & & & & & U \\
\hline 1342 & 8240_VOA_GCMS & DATACH & & & & & & & U \\
\hline 1343 & GAMMA_GS & ITASRL & -0.343 & $\mathrm{pCi} / \mathrm{L}$ & 2.05 & 2.05 & & & U \\
\hline 1344 & SRISO_SEP_PRECIP_GPC & ITASRL & 1.25 & $\mathrm{pCi} / \mathrm{L}$ & 0.201 & 0.321 & & & \\
\hline 1345 & TC99_SEPP_LSC & ITASRL & 0.0524 & $\mathrm{pCi} / \mathrm{L}$ & 0.102 & 0.528 & & & $U$ \\
\hline 1346 & TRITIÜM ELECT LSC & ITASRL & 50.2 & $\mathrm{pCi} / \mathrm{L}$ & 2.57 & 9.55 & & & \\
\hline 1347 & UISO_AEA & ITASRL & 0.207 & $\mathrm{pCi} / \mathrm{L}$ & 0.0506 & 0.0551 & & & \\
\hline 1348 & UISO_AEA & ITASRL & 0.011 & $\mathrm{pCi} / \mathrm{L}$ & 0.0151 & 0.0151 & & & $U$ \\
\hline 1349 & UISO_AEA & ITASRL & 0.152 & $\mathrm{pCi} / \mathrm{L}$ & 0.0422 & 0.0451 & & & \\
\hline 1351 & 8240_VOA_GCMS & DATACH & & & & & & & $U$ \\
\hline 1352 & GAMMA_GS & ITASRL & -0.063 & $\mathrm{pCi} / \mathrm{L}$ & 1.23 & 1.24 & & & $\mathrm{U}$ \\
\hline 1353 & SRISO_SEP_PRECIP_GPC & ITASRL & 0.068 & $\mathrm{pCi} / \mathrm{L}$ & 0.139 & 0.142 & & & $\mathrm{U}$ \\
\hline 1354 & TC99_SEP_LSC & ITASRL & -0.00092 & $\mathrm{pCi} / \mathrm{L}$ & 0.101 & 0.524 & & & U \\
\hline 1355 & TRITIUM_ELECT_LSC & ITASRL & 35.1 & $\mathrm{pCi} / \mathrm{L}$ & 2.3 & 8.36 & & & \\
\hline 1356 & UISO_AEA & ITASRL & 0.267 & $\mathrm{pCi} / \mathrm{L}$ & 0.0483 & 0.0553 & & & \\
\hline 1357 & UISO_AEA & ITASRL & 0.00482 & $\mathrm{pCi} / \mathrm{L}$ & 0.0084 & 0.00846 & & & $U$ \\
\hline 1358 & UISO_AEA & ITASRL & 0.193 & $\mathrm{pCi} / \mathrm{L}$ & 0.041 & 0.0454 & & & \\
\hline 1359 & 6010_METALS_ICP & DATACH & & & & & & & $U$ \\
\hline 1360 & 8240_VOA_GCMS & DATACH & & & & & & & $U$ \\
\hline 1361 & GAMMA_GS & ITASRL & -0.756 & $\mathrm{pCi} / \mathrm{L}$ & 1.25 & 1.26 & & & U \\
\hline 1362 & SRISO_SEP_PRECIP_GPC & ITASRL & 0.000673 & $\mathrm{pCi} / \mathrm{L}$ & 0.13 & 0.132 & & & $U$ \\
\hline 1363 & TC99_SEP_LSC & ITASRL & -0.0495 & $\mathrm{pCi} / \mathrm{L}$ & 0.0997 & 0.52 & & & $U$ \\
\hline 1364 & TRITIŪM_ĒELET_LSC & ITASRL & 37.6 & $\mathrm{pCi} / \mathrm{L}$ & 2.34 & 8.54 & & & \\
\hline 1365 & UISO_AEA & ITASRL & 0.221 & $\mathrm{pCi} / \mathrm{L}$ & 0.0473 & 0.0526 & & & \\
\hline 1366 & UISO_AEA & ITASRL & 0.000943 & $\mathrm{pCi} / \mathrm{L}$ & 0.00873 & 0.00877 & & & U \\
\hline 1367 & UISO_AEA & ITASRL & 0.146 & $\mathrm{pCi} / \mathrm{L}$ & 0.0379 & 0.0408 & & & \\
\hline 1368 & 6010_METALS_ICP & DATACH & & & & & & & $U$ \\
\hline
\end{tabular}




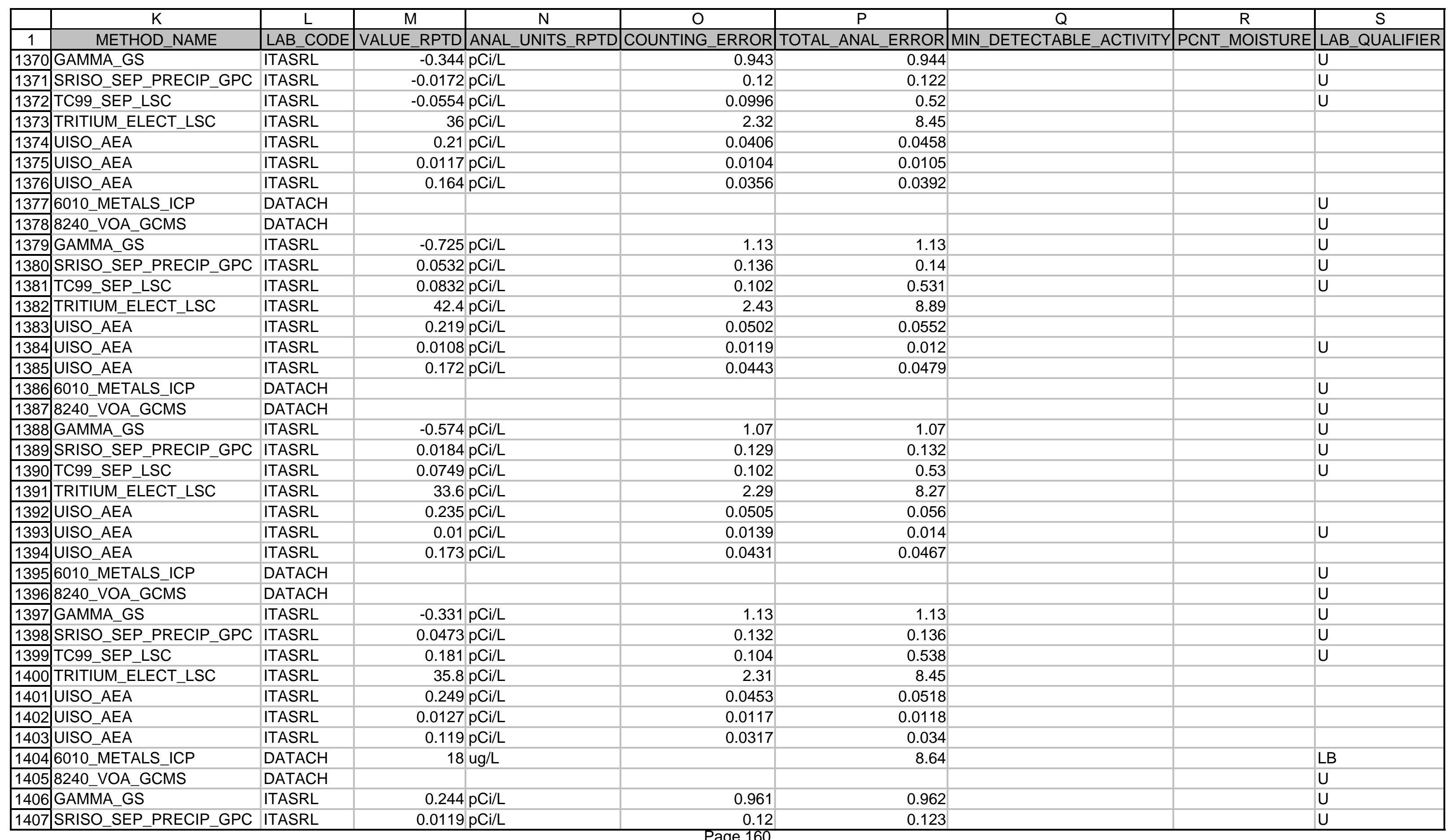




\begin{tabular}{|c|c|c|c|c|c|c|c|c|c|}
\hline & $\mathrm{K}$ & $\mathrm{L}$ & $\mathrm{M}$ & $\mathrm{N}$ & $\mathrm{O}$ & $\mathrm{P}$ & $\mathrm{Q}$ & $\mathrm{R}$ & $\mathrm{S}$ \\
\hline 1 & METHOD_NAME & LAB_CODE & VALUE_RPTD & ANAL_UNITS_RPTD & COUNTING_ERROR & TOTAL_ANAL_ERROR I & MIN_DETECTABLE_ACTIVITY & PCNT_MOISTURE & LAB_QUALIFIER \\
\hline 1408 & TC99_SEP_LSC & ITASRL & 0.166 & $\mathrm{pCi} / \mathrm{L}$ & 0.103 & 0.537 & & & $U$ \\
\hline 1409 & TRITIUM ELECT_LSC & ITASRL & 33.9 & $\mathrm{pCi} / \mathrm{L}$ & 2.31 & 8.35 & & & \\
\hline 1410 & UISO_AEA & ITASRL & 0.199 & $\mathrm{pCi} / \mathrm{L}$ & 0.0639 & 0.068 & & & \\
\hline 1411 & UISO_AEA & ITASRL & 0.00893 & $\mathrm{pCi} / \mathrm{L}$ & 0.0169 & 0.017 & & & $U$ \\
\hline 1412 & UISO_AEA & ITASRL & 0.187 & $\mathrm{pCi} / \mathrm{L}$ & 0.0611 & 0.0647 & & & \\
\hline 1413 & 6010_METALS_ICP & DATACH & 18 & ug/L & & 8.64 & & & LB \\
\hline 1414 & 8240_VOA_GCMS & DATACH & & & & & & & $\mathrm{U}$ \\
\hline 1415 & GAMMA_GS & ITASRL & 1.1 & $\mathrm{pCi} / \mathrm{L}$ & 1.29 & 1.29 & & & $U$ \\
\hline 1416 & SRISO_SEP_PRECIP_GPC & ITASRL & 0.00135 & $\mathrm{pCi} / \mathrm{L}$ & 0.14 & 0.143 & & & U \\
\hline 1417 & TC99_SEP_LSC & ITASRL & -0.131 & $\mathrm{pCi} / \mathrm{L}$ & 0.0993 & 0.515 & & & U \\
\hline 1418 & TRITIUM_ELECT_LSC & ITASRL & 31.4 & $\mathrm{pCi} / \mathrm{L}$ & 2.15 & 7.81 & & & \\
\hline 1419 & UISO_AEA & ITASRL & 0.18 & $\mathrm{pCi} / \mathrm{L}$ & 0.041 & 0.0451 & & & \\
\hline 1420 & UISO_AEA & ITASRL & 0.00515 & $\mathrm{pCi} / \mathrm{L}$ & 0.00744 & 0.00751 & & & $U$ \\
\hline 1421 & UISO AEA & ITASRL & 0.171 & $\mathrm{pCi} / \mathrm{L}$ & 0.0396 & 0.0433 & & & \\
\hline 1422 & 6010_METALS_ICP & DATACH & 19 & ug/L & & 9.12 & & & LB \\
\hline 1423 & 8240_VOA_GCMS & DATACH & & & & & & & $U$ \\
\hline 1424 & GAMMA_GS & ITASRL & 0.158 & $\mathrm{pCi} / \mathrm{L}$ & 1.14 & 1.14 & & & U \\
\hline 1425 & SRISO_SEP_PRECIP_GPC & ITASRL & 0.109 & $\mathrm{pCi} / \mathrm{L}$ & 0.149 & 0.155 & & & $U$ \\
\hline 1427 & TRITIUMM_ELECT_LSC & ITASRL & 34 & $\mathrm{pCi} / \mathrm{L}$ & 2.29 & 8.33 & & & \\
\hline 1428 & UISO_AEA & ITASRL & 0.185 & $\mathrm{pCi} / \mathrm{L}$ & 0.0424 & 0.0466 & & & \\
\hline 1429 & UISO_AEA & ITASRL & -0.000172 & $\mathrm{pCi} / \mathrm{L}$ & 0.00477 & 0.00485 & & & U \\
\hline 1430 & UISO_AEA & ITASRL & 0.185 & $\mathrm{pCi} / \mathrm{L}$ & 0.0419 & 0.046 & & & \\
\hline 1431 & 6010_METALS_ICP & DATACH & & & & & & & $U$ \\
\hline 1432 & 8240_VOA_GCMS & DATACH & & & & & & & $U$ \\
\hline 1433 & SRISO_SEPP_PRECIP_GPC & ITASRL & 0.0727 & $\mathrm{pCi} / \mathrm{L}$ & 0.035 & 0.0386 & & & \\
\hline 1434 & TRITIUM ELECT LSC & ITASRL & 32.8 & $\mathrm{pCi} / \mathrm{L}$ & 2.27 & 8.25 & & & \\
\hline 1435 & UISO_AEA & ITASRL & 0.223 & $\mathrm{pCi} / \mathrm{L}$ & 0.0422 & 0.0478 & & & \\
\hline 1436 & UISO_AEA & ITASRL & 0.00567 & $\mathrm{pCi} / \mathrm{L}$ & 0.00748 & 0.00756 & & & $\mathrm{U}$ \\
\hline 1437 & UISO_AEA & ITASRL & 0.168 & $\mathrm{pCi} / \mathrm{L}$ & 0.0364 & 0.0401 & & & \\
\hline 1438 & 6010_METALS_ICP & DATACH & & & & & & & $U$ \\
\hline 1439 & 8240_VOA_GCMS & DATACH & & & & & & & $\mathrm{U}$ \\
\hline 1440 & SRISO_SĒP_PRECIP_GPC & ITASRL & 0.0794 & $\mathrm{pCi} / \mathrm{L}$ & 0.0358 & 0.04 & & & \\
\hline 1441 & TRITIUM_ELECT_LSC & ITASRL & 37.6 & $\mathrm{pCi} / \mathrm{L}$ & 2.34 & 8.6 & & & \\
\hline 1442 & UISO_AEA & ITASRL & 0.163 & $\mathrm{pCi} / \mathrm{L}$ & 0.0654 & 0.0684 & & & \\
\hline 1443 & UISO_AEA & ITASRL & 0.0217 & $\mathrm{pCi} / \mathrm{L}$ & 0.023 & 0.0232 & & & $U$ \\
\hline 1444 & UISO_AEA & ITASRL & 0.109 & $\mathrm{pCi} / \mathrm{L}$ & 0.0544 & 0.056 & & & \\
\hline
\end{tabular}




\begin{tabular}{|c|c|c|c|c|c|c|c|c|c|}
\hline & $\mathrm{K}$ & $\mathrm{L}$ & $\mathrm{M}$ & $\mathrm{N}$ & $\mathrm{O}$ & $\mathrm{P}$ & $\mathrm{Q}$ & $\mathrm{R}$ & $\mathrm{S}$ \\
\hline 1 & METHOD_NAME & LAB_CODE & VALUE_RPTD & ANAL_UNITS_RPTD & COUNTING_ERROR & $\mid$ TOTAL_ANAL_ERROR| & MIN_DETECTABLE_ACTIVITY & PCNT_MOISTURE & LAB_QUALIFIER \\
\hline 1446 & 8240 VOA_GCMS & DATACH & & & & & & & $\mathrm{U}$ \\
\hline 1447 & SRISO_SEPP_PRECIP_GPC & ITASRL & 0.0814 & $\mathrm{pCi} / \mathrm{L}$ & 0.0366 & 0.0404 & & & \\
\hline 1448 & TRITIUMM ELECT LSC̄ & ITASRL & 37.6 & $\mathrm{pCi} / \mathrm{L}$ & 2.34 & 8.6 & & & \\
\hline 1449 & UISO AËA & ITASRL & 0.224 & $\mathrm{pCi} / \mathrm{L}$ & 0.076 & 0.0806 & & & \\
\hline 1450 & UISO_AEA & ITASRL & 0.0109 & $\mathrm{pCi} / \mathrm{L}$ & 0.0274 & 0.0274 & & & $U$ \\
\hline 1451 & UISO_AEA & ITASRL & 0.223 & $\mathrm{pCi} / \mathrm{L}$ & 0.0729 & 0.0774 & & & \\
\hline 1452 & 6010_METALS_ICP & DATACH & & & & & & & $U$ \\
\hline 1453 & 8240_VOA_GCMS & DATACH & & & & & & & $U$ \\
\hline 1454 & SRISO_SEP_PRECIP_GPC & ITASRL & 0.098 & $\mathrm{pCi} / \mathrm{L}$ & 0.0378 & 0.0432 & & & \\
\hline 1455 & TRITIUMM_ELEECT_LSC & ITASRL & 36 & $\mathrm{pCi} / \mathrm{L}$ & 2.3 & 8.4 & & & \\
\hline 1456 & UISO_AEA & ITASRL & 0.205 & $\mathrm{pCi} / \mathrm{L}$ & 0.0406 & 0.0456 & & & \\
\hline 1457 & UISO_AEA & ITASRL & 0.00718 & $\mathrm{pCi} / \mathrm{L}$ & 0.00837 & 0.00845 & & & U \\
\hline 1458 & UISO_AEA & ITASRL & 0.182 & $\mathrm{pCi} / \mathrm{L}$ & 0.038 & 0.0422 & & & \\
\hline 1459 & 6010_METALS_ICP & DATACH & & & & & & & $U$ \\
\hline 1460 & 8240_VOA_GCMS & DATACH & & & & & & & $U$ \\
\hline 1461 & SRISO_SEP_PRECIP_GPC & ITASRL & 0.081 & $\mathrm{pCi} / \mathrm{L}$ & 0.0488 & 0.0545 & & & \\
\hline 1462 & TRITIŪM ELEECT LSC̄ & ITASRL & 66.6 & $\mathrm{pCi} / \mathrm{L}$ & 2.69 & 11.1 & & & \\
\hline 1463 & UISO_AËA & ITASRL & 0.317 & $\mathrm{pCi} / \mathrm{L}$ & 0.141 & 0.147 & & & \\
\hline 1465 & UISO_AEA & ITASRL & 0.156 & $\mathrm{pCi} / \mathrm{L}$ & 0.14 & 0.142 & & & \\
\hline 1466 & 6010_METALS_ICP & DATACH & & & & & & & $U$ \\
\hline 1467 & 8240_VOA_GCMS & DATACH & & & & & & & $U$ \\
\hline 1468 & SRISO_SEPP_PRECIP_GPC & ITASRL & 0.0863 & $\mathrm{pCi} / \mathrm{L}$ & 0.0453 & 0.0518 & & & \\
\hline 1469 & TRITIUM_ELECT_LSC & ITASRL & 52.7 & $\mathrm{pCi} / \mathrm{L}$ & 2.54 & 10 & & & \\
\hline 1470 & UISO_AEA & ITASRL & 0.112 & $\mathrm{pCi} / \mathrm{L}$ & 0.0788 & 0.0806 & & & \\
\hline 1471 & UISO_AEA & ITASRL & 0.00282 & $\mathrm{pCi} / \mathrm{L}$ & 0.0335 & 0.0335 & & & $U$ \\
\hline 1472 & UISO_AEA & ITASRL & 0.152 & $\mathrm{pCi} / \mathrm{L}$ & 0.0861 & 0.0888 & & & \\
\hline 1473 & 6010_METALS_ICP & DATACH & & & & & & & $U$ \\
\hline \begin{tabular}{|l|l|}
1474 \\
\end{tabular} & 8240_VOA_GCMS & DATACH & & & & & & & $U$ \\
\hline 1475 & SRISO_SEPP_PRECIP_GPC & ITASRL & 0.087 & $\mathrm{pCi} / \mathrm{L}$ & 0.0376 & 0.0436 & & & \\
\hline 1476 & TRITIUMM_ELECCT_LSC̄ & ITASRL & 41.6 & $\mathrm{pCi} / \mathrm{L}$ & 2.48 & 9.32 & & & \\
\hline 1477 & UISO_AEA & ITASRL & 0.265 & $\mathrm{pCi} / \mathrm{L}$ & 0.201 & 0.209 & & & \\
\hline 1478 & UISO_AEA & ITASRL & 0.117 & $\mathrm{pCi} / \mathrm{L}$ & 0.129 & 0.132 & & & $U$ \\
\hline 1479 & UISO_AEA & ITASRL & 0.287 & $\mathrm{pCi} / \mathrm{L}$ & 0.188 & 0.197 & & & \\
\hline 1480 & 6010_METALS_ICP & DATACH & & & & & & & $U$ \\
\hline 1481 & 8240_VOA_GCMS & DATACH & & & & & & & U \\
\hline 1482 & SRISO_SEP_PRECIP_GPC & ITASRL & 0.0747 & $\mathrm{pCi} / \mathrm{L}$ & 0.0384 & 0.0435 & & & \\
\hline
\end{tabular}




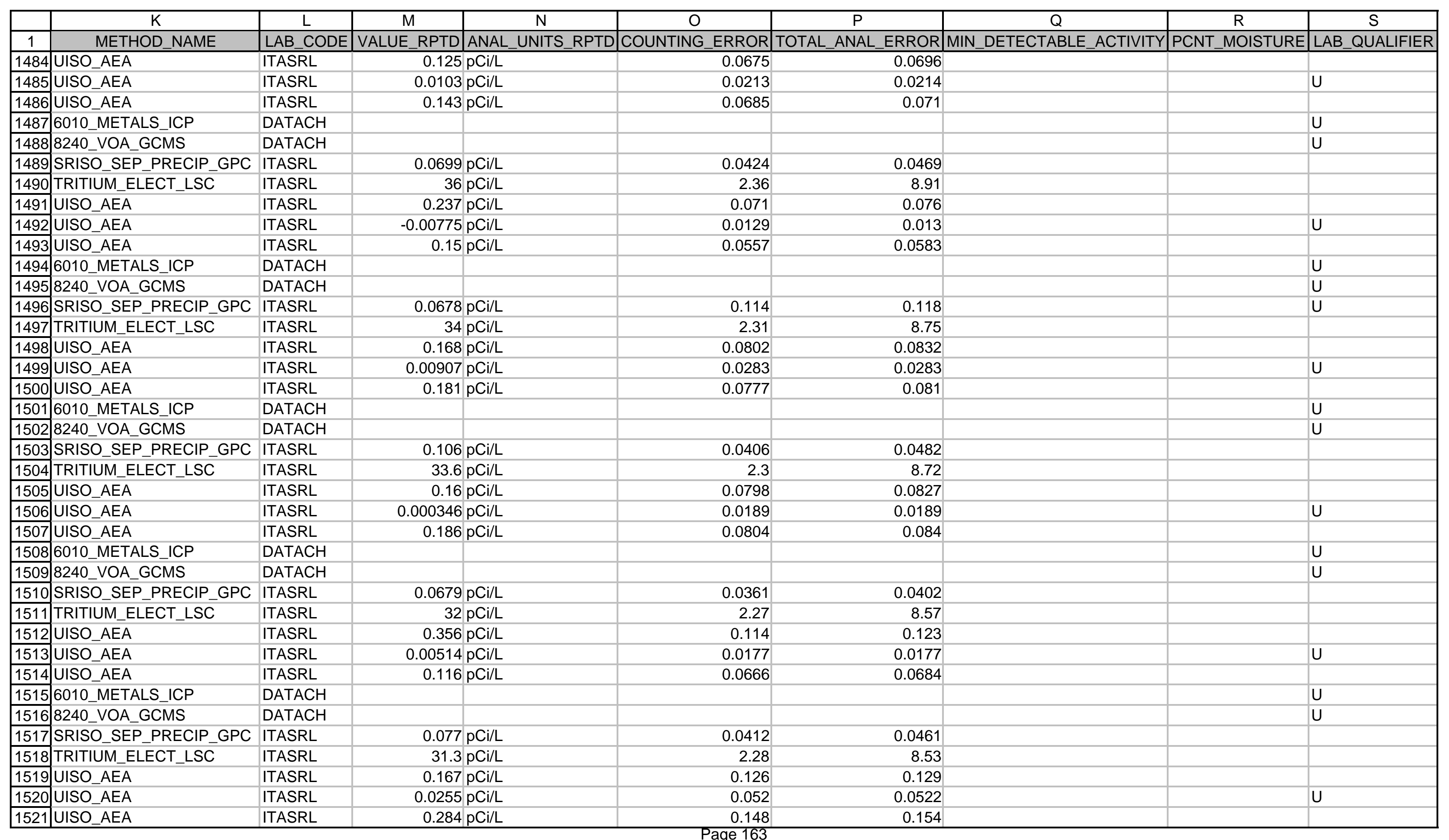




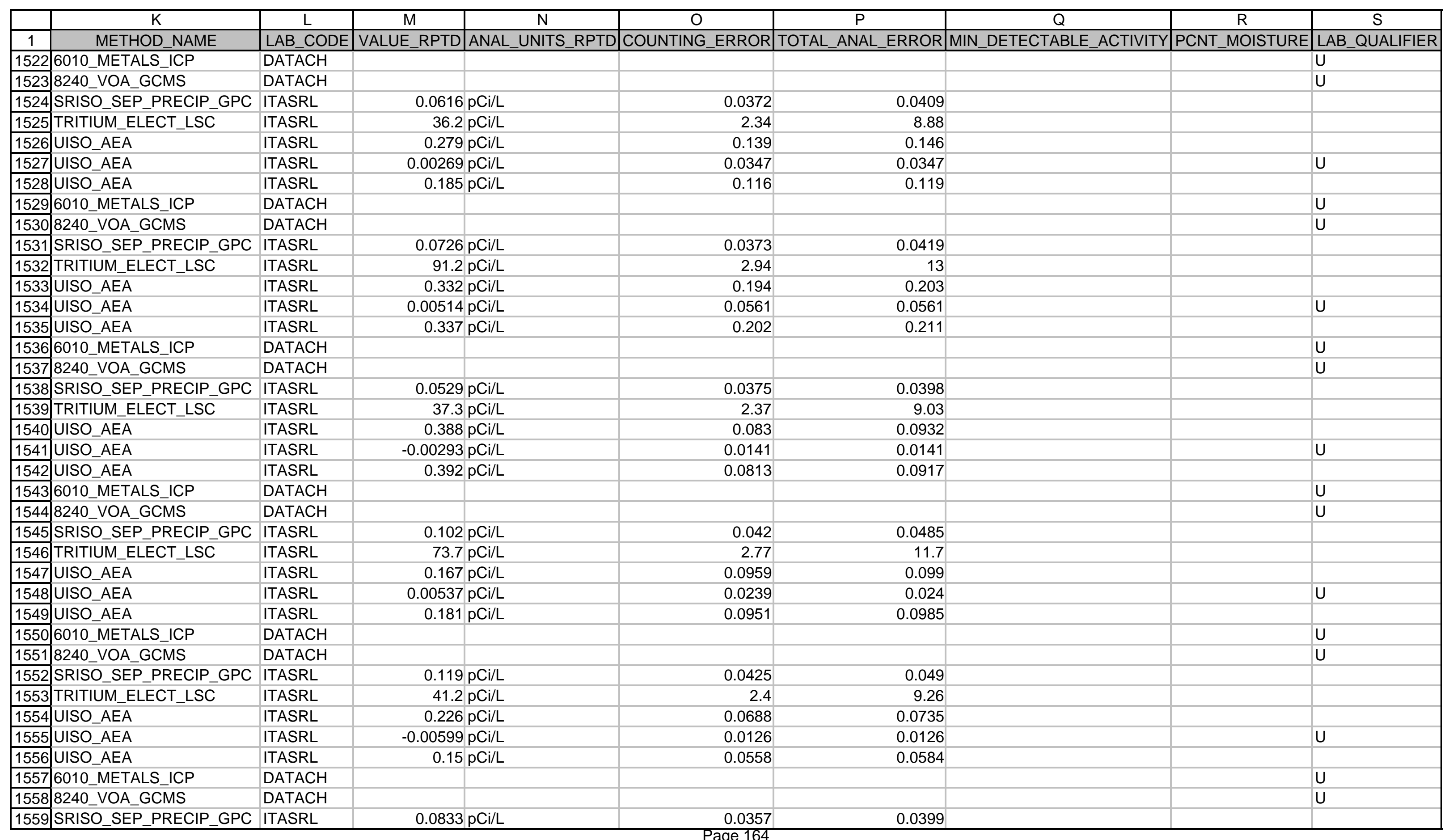


$\mathrm{N}$

O

P

Q

$\mathrm{R}$

$\mathrm{S}$

\begin{tabular}{|c|c|c|c|c|c|c|c|c|c|}
\hline & & & & & & & & & \\
\hline 1 & METHOD_NAME & LAB_CODE & VALUE_RPTD & ANAL_UNITS_RPTD & COUNTING_ERROR & TOTAL_ANAL_ERROR & MIN_DETECTABLE_ACTIVITY & PCNT_MOISTURE & LAB_QUALIFIER \\
\hline 1560 & TRITIUM_ELECT_LSC & ITASRL & 34 & $\mathrm{pCi} / \mathrm{L}$ & 2.3 & 8.71 & & & \\
\hline 1561 & UISO_AEA & ITASRL & 0.231 & $\mathrm{pCi} / \mathrm{L}$ & 0.102 & 0.105 & & & \\
\hline 1562 & UISO_AEA & ITASRL & -0.00245 & $\mathrm{pCi} / \mathrm{L}$ & 0.0562 & 0.0562 & & & U \\
\hline 1563 & UISO_AEA & ITASRL & 0.121 & $\mathrm{pCi} / \mathrm{L}$ & 0.0995 & 0.101 & & & \\
\hline 1564 & 6010_METALS_ICP & DATACH & & & & & & & U \\
\hline 1565 & 8240_VOA_GCMS & DATACH & & & & & & & U \\
\hline 1566 & SRISO_SEP_PRECIP_GPC & ITASRL & 0.108 & $3 \mathrm{pCi} / \mathrm{L}$ & 0.045 & 0.0502 & & & \\
\hline 1567 & TRITIUM_ELECT_LSC & ITASRL & 35.6 & $\mathrm{pCi} / \mathrm{L}$ & 2.33 & 8.88 & & & \\
\hline 1568 & UISO_AEA & ITASRL & 0.248 & $3 \mathrm{pCi} / \mathrm{L}$ & 0.0839 & 0.089 & & & \\
\hline 1569 & UISO_AEA & ITASRL & 0.0103 & $3 \mathrm{pCi} / \mathrm{L}$ & 0.0255 & 0.0256 & & & $U$ \\
\hline 1570 & UISO_AEA & ITASRL & 0.122 & $\mathrm{pCi} / \mathrm{L}$ & 0.0576 & 0.0596 & & & \\
\hline 1571 & 6010_METALS_ICP & DATACH & & & & & & & U \\
\hline 1572 & 8240_VOA_GCMS & DATACH & & & & & & & U \\
\hline 1573 & SRISO_SEP_PRECIP_GPC & ITASRL & 0.086 & $\mathrm{pCi} / \mathrm{L}$ & 0.0407 & 0.0447 & & & \\
\hline 1574 & TRITIUM_ELECT_LSC & ITASRL & 31.4 & $4 \mathrm{pCi} / \mathrm{L}$ & 2.27 & 8.53 & & & \\
\hline 1575 & UISO_AEA & ITASRL & 0.211 & $\mathrm{pCi} / \mathrm{L}$ & 0.0714 & 0.0757 & & & \\
\hline 1576 & UISO_AEA & ITASRL & 0.00925 & $5 \mathrm{pCi} / \mathrm{L}$ & 0.0231 & 0.0232 & & & $U$ \\
\hline 1577 & UISO_AEA & ITASRL & 0.151 & $\mathrm{pCi} / \mathrm{L}$ & 0.0615 & 0.064 & & & \\
\hline 1580 & SRISO_SEP_PRECIP_GPC & ITASRL & 0.112 & $2 \mathrm{pCi} / \mathrm{L}$ & 0.0419 & 0.0478 & & & \\
\hline 1581 & TRITIUM_ELECT_LSC & ITASRL & 35.2 & $\mathrm{pCi} / \mathrm{L}$ & 2.33 & 8.84 & & & \\
\hline 1582 & UISO_AEA & ITASRL & 0.223 & $3 \mathrm{pCi} / \mathrm{L}$ & 0.0732 & 0.0777 & & & \\
\hline 1583 & UISO_AEA & ITASRL & -0.00618 & $3 \mathrm{pCi} / \mathrm{L}$ & 0.0131 & 0.0132 & & & $U$ \\
\hline 1584 & UISO_AEA & ITASRL & 0.197 & $7 \mathrm{pCi} / \mathrm{L}$ & 0.0653 & 0.0691 & & & \\
\hline 1585 & 6010_METALS_ICP & DATACH & & & & & & & $U$ \\
\hline 1586 & 8240_VOA_GCMS & DATACH & & & & & & & U \\
\hline 1587. & SRISO_SEP_PRECIP_GPC & ITASRL & 0.0779 & $9 \mathrm{pCi} / \mathrm{L}$ & 0.0393 & 0.0429 & & & \\
\hline 1588 & TRITIUM_ELECT_LSC & ITASRL & 29.1 & $\mathrm{pCi} / \mathrm{L}$ & 2.24 & 8.43 & & & \\
\hline 1589 & UISO_AEA & ITASRL & 0.217 & $7 \mathrm{pCi} / \mathrm{L}$ & 0.0649 & 0.0695 & & & \\
\hline 1590 & UISO_AEA & ITASRL & 0.00828 & $3 \mathrm{pCi} / \mathrm{L}$ & 0.0159 & 0.0159 & & & $U$ \\
\hline 1591 & UISO_AEA & ITASRL & 0.17 & $7 \mathrm{pCi} / \mathrm{L}$ & 0.0565 & 0.0597 & & & \\
\hline 1592 & 6010_METALS_ICP & DATACH & & & & & & & U \\
\hline 1593 & 8240_VOA_GCMS & DATACH & & & & & & & U \\
\hline 1594 & SRISO_SEP_PRECIP_GPC & ITASRL & 0.0724 & $4 \mathrm{pCi} / \mathrm{L}$ & 0.0384 & 0.0418 & & & \\
\hline 1595 & TRITIUM_ELECT_LSC & ITASRL & 30 & $\mathrm{pCi} / \mathrm{L}$ & 2.32 & 8.68 & & & \\
\hline 1596 & UISO_AEA & ITASRL & 0.272 & $2 \mathrm{pCi} / \mathrm{L}$ & 0.0818 & 0.0879 & & & \\
\hline 1597 & UISO_AEA & ITASRL & 0.0132 & $\mathrm{pCi} / \mathrm{L}$ & 0.0199 & 0.0199 & & & U \\
\hline
\end{tabular}

DATACH

ITASRL

DATACH

ITASRL

DATACH

ITASRL

ITASRL

ITASRL

DATACH

ITASRL

ITASRL

ITASRL

DATACH

ITASRL

ITASRL

$0.0132 \mathrm{pCi} / \mathrm{L}$

Page 165 


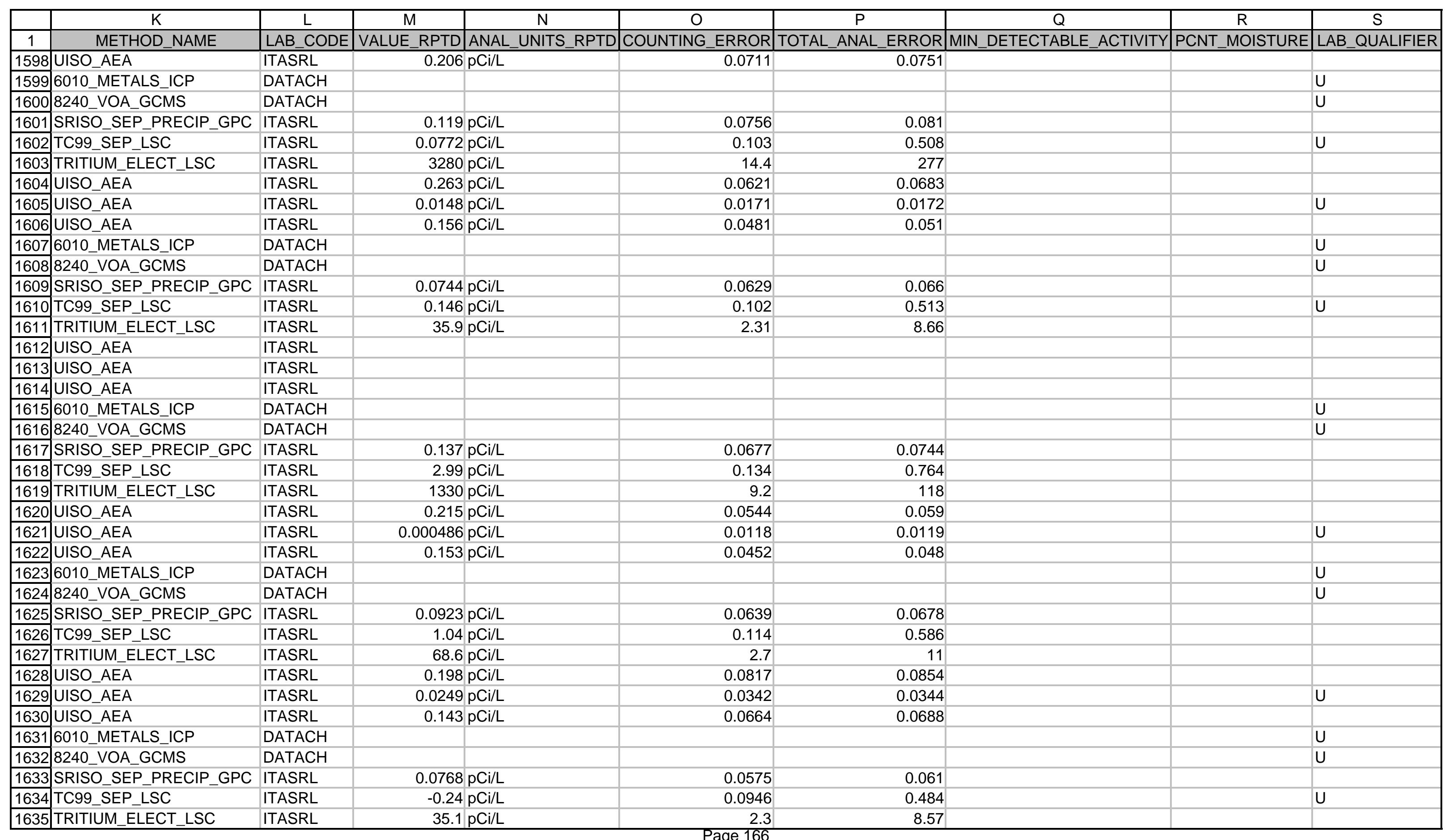




\begin{tabular}{|c|c|c|c|c|c|c|c|c|c|}
\hline & $\mathrm{K}$ & $\mathrm{L}$ & $\mathrm{M}$ & $\mathrm{N}$ & $\mathrm{O}$ & $\mathrm{P}$ & $\mathrm{Q}$ & $\mathrm{R}$ & $\mathrm{S}$ \\
\hline 1 & METHOD_NAME & LAB_CODE & VALUE_RPTD & ANAL_UNITS_RPTD & COUNTING_ERROR & $\mid$ TOTAL_ANAL_ERROR| & MIN_DETECTABLE_ACTIVITY & PCNT_MOISTURE & LAB_QUALIFIER \\
\hline 1636 & UISO_AEA & ITASRL & 0.225 & $\mathrm{pCi} / \mathrm{L}$ & 0.0596 & 0.0645 & & & \\
\hline 1637 & UISO_AEA & ITASRL & 0.01 & $\mathrm{pCi} / \mathrm{L}$ & 0.0147 & 0.0148 & & & $\mathrm{U}$ \\
\hline 1638 & UISO_AEA & ITASRL & 0.191 & $\mathrm{pCi} / \mathrm{L}$ & 0.0534 & 0.0573 & & & \\
\hline 1639 & 6010 METALS ICP & DATACH & & & & & & & $\mathrm{U}$ \\
\hline 1640 & 8240_VOA_GCMS & DATACH & & & & & & & $U$ \\
\hline 1641 & SRISO-SĒP_PRECIP_GPC & ITASRL & 0.11 & $\mathrm{pCi} / \mathrm{L}$ & 0.0638 & 0.0695 & & & \\
\hline 1642 & TC99_SEP_LSC & ITASRL & 0.207 & $\mathrm{pCi} / \mathrm{L}$ & 0.104 & 0.518 & & & $U$ \\
\hline 1643 & TRITIŪM_ĒELET_LSC & ITASRL & 29.8 & $\mathrm{pCi} / \mathrm{L}$ & 2.24 & 8.22 & & & \\
\hline 1645 & UISO_AEA & ITASRL & 0.00689 & $\mathrm{pCi} / \mathrm{L}$ & 0.0116 & 0.0116 & & & $U$ \\
\hline 1646 & UISO_AEA & ITASRL & 0.133 & $\mathrm{pCi} / \mathrm{L}$ & 0.0441 & 0.0464 & & & \\
\hline 1647 & 6010_METALS_ICP & DATACH & & & & & & & $\mathrm{U}$ \\
\hline 1648 & 8240_VOA_GCMS & DATACH & & & & & & & U \\
\hline 1649 & SRISO_SEPP_PRECIP_GPC & ITASRL & 0.108 & $\mathrm{pCi} / \mathrm{L}$ & 0.0634 & 0.0687 & & & \\
\hline 1650 & TC99_SEP_LESC & ITASRL & -0.111 & $\mathrm{pCi} / \mathrm{L}$ & 0.0985 & 0.494 & & & $U$ \\
\hline 1651 & TRITIUM_ELECT_LSC & ITASRL & 39.1 & $\mathrm{pCi} / \mathrm{L}$ & 2.36 & 8.87 & & & \\
\hline 1652 & UISO AEA & ITASRL & 0.167 & $\mathrm{pCi} / \mathrm{L}$ & 0.0514 & 0.0547 & & & \\
\hline 1653 & UISO_AEA & ITASRL & 0.00493 & $\mathrm{pCi} / \mathrm{L}$ & 0.0102 & 0.0103 & & & $U$ \\
\hline 1655 & 6010_METALS_ICP & DATACH & & & & & & & $U$ \\
\hline 1656 & 8240_VOA_GCMS & DATACH & & & & & & & $U$ \\
\hline 1657 & SRISO_SEP_PRECIP_GPC & ITASRL & 0.0849 & $\mathrm{pCi} / \mathrm{L}$ & 0.0608 & 0.065 & & & \\
\hline 1658 & TC99_SEP_LSC & ITASRL & -0.258 & $\mathrm{pCi} / \mathrm{L}$ & 0.0945 & 0.483 & & & U \\
\hline 1659 & TRITIŪM_ĒELET_LSC & ITASRL & 38.9 & $\mathrm{pCi} / \mathrm{L}$ & 2.36 & 8.86 & & & \\
\hline 1660 & UISO_AEA & ITASRL & 0.201 & $\mathrm{pCi} / \mathrm{L}$ & 0.0517 & 0.0561 & & & \\
\hline 1661 & UISO_AEA & ITASRL & 0.0153 & $\mathrm{pCi} / \mathrm{L}$ & 0.015 & 0.0151 & & & \\
\hline 1662 & UISO_AEA & ITASRL & 0.184 & $\mathrm{pCi} / \mathrm{L}$ & 0.0488 & 0.0525 & & & \\
\hline 1663 & 6010_METALS_ICP & DATACH & & & & & & & $U$ \\
\hline 1664 & 8240_VOA_GCMS & DATACH & & & & & & & U \\
\hline \begin{tabular}{|l|l|}
1665 \\
160
\end{tabular} & SRISO SEP PRECIP GPC & ITASRL & 0.0845 & $\mathrm{pCi} / \mathrm{L}$ & 0.0595 & 0.0633 & & & \\
\hline 1666 & TC99_SEP_LEC & ITASRL & 0.194 & $\mathrm{pCi} / \mathrm{L}$ & 0.104 & 0.517 & & & $U$ \\
\hline \begin{tabular}{|l|l|}
1667 \\
\end{tabular} & TRITIUM_ELECT_LSC & ITASRL & 37.7 & $\mathrm{pCi} / \mathrm{L}$ & 2.33 & 8.74 & & & \\
\hline 1668 & UISO_AEA & ITASRL & 0.13 & $\mathrm{pCi} / \mathrm{L}$ & 0.0353 & 0.0382 & & & \\
\hline 1669 & UISO_AEA & ITASRL & 0.0056 & $\mathrm{pCi} / \mathrm{L}$ & 0.00869 & 0.00876 & & & $U$ \\
\hline 1670 & UISO_AEA & ITASRL & 0.107 & $\mathrm{pCi} / \mathrm{L}$ & 0.0318 & 0.0339 & & & \\
\hline 1671 & 6010_METALS_ICP & DATACH & & & & & & & $U$ \\
\hline 1672 & 8240_VOA_GCMS & DATACH & & & & & & & $\mathrm{U}$ \\
\hline
\end{tabular}




\begin{tabular}{|c|c|c|c|c|c|c|c|c|c|}
\hline & $\mathrm{K}$ & $\mathrm{L}$ & $\mathrm{M}$ & $\mathrm{N}$ & $\mathrm{O}$ & $\mathrm{P}$ & $\mathrm{Q}$ & $\mathrm{R}$ & $\mathrm{S}$ \\
\hline 1 & METHOD_NAME & LAB_CODE & VALUE_RPTD & ANAL_UNITS_RPTD & COUNTING_ERROR & $\mid$ TOTAL_ANAL_ERROR| & MIN_DETECTABLE_ACTIVITY & PCNT_MOISTURE & LAB_QUALIFIER \\
\hline 1674 & TC99_SEP_LSC & ITASRL & -0.0708 & $\mathrm{pCi} / \mathrm{L}$ & 0.0972 & 0.496 & & & $U$ \\
\hline 1675 & TRITIUM ELECT_LSC & ITASRL & 38.1 & $\mathrm{pCi} / \mathrm{L}$ & 2.33 & 8.79 & & & \\
\hline 1676 & UISO_AEA & ITASRL & 0.154 & $\mathrm{pCi} / \mathrm{L}$ & 0.0345 & 0.0383 & & & \\
\hline 1677 & UISO_AEA & ITASRL & 0.00784 & $\mathrm{pCi} / \mathrm{L}$ & 0.00866 & 0.00874 & & & $U$ \\
\hline 1678 & UISO_AEA & ITASRL & 0.113 & $\mathrm{pCi} / \mathrm{L}$ & 0.0296 & 0.032 & & & \\
\hline 1679 & 6010_METALS_ICP & DATACH & & & & & & & $U$ \\
\hline 1680 & 8240_VOA_GCMS & DATACH & & & & & & & $U$ \\
\hline 1681 & SRISO_SEP_PRECIP_GPC & ITASRL & 0.0982 & $\mathrm{pCi} / \mathrm{L}$ & 0.0383 & 0.0432 & & & \\
\hline 1682 & |TRITIUM_ELECT_LSC & ITASRL & 40.6 & $\mathrm{pCi} / \mathrm{L}$ & 2.39 & 8.81 & & & \\
\hline 1683 & UISO_AEA & ITASRL & 0.206 & $\mathrm{pCi} / \mathrm{L}$ & 0.0405 & 0.0466 & & & \\
\hline 1684 & UISO_AEA & ITASRL & 0.0156 & $\mathrm{pCi} / \mathrm{L}$ & 0.0143 & 0.0144 & & & \\
\hline 1685 & UISO_AEA & ITASRL & 0.165 & $\mathrm{pCi} / \mathrm{L}$ & 0.0351 & 0.0394 & & & \\
\hline 1686 & 6010_METALS_ICP & DATACH & 4.5 & ug/L & & 1.89 & & & $\mathrm{~L}$ \\
\hline 1687 & 8240_VOA_GCMS & DATACH & & & & & & & $U$ \\
\hline 1688 & SRISO SEP PRECIP GPC & ITASRL & 0.0914 & $\mathrm{pCi} / \mathrm{L}$ & 0.0352 & 0.0402 & & & \\
\hline 1689 & TRITIUMM_ELEECT_LSC & ITASRL & 40.3 & $\mathrm{pCi} / \mathrm{L}$ & 2.38 & 8.76 & & & \\
\hline 1690 & UISO_AËA & ITASRL & 0.296 & $\mathrm{pCi} / \mathrm{L}$ & 0.0506 & 0.0603 & & & \\
\hline 1691 & UISO_AEA & ITASRL & 0.0504 & $\mathrm{pCi} / \mathrm{L}$ & 0.022 & 0.0227 & & & \\
\hline 1693 & 6010_METALS_ICP & DATACH & & & & & & & $U$ \\
\hline 1694 & 8240_VOA_GCMS & DATACH & & & & & & & 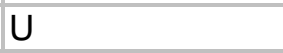 \\
\hline 1695 & SRISO_SEP_PRECIP_GPC & ITASRL & 0.0734 & $\mathrm{pCi} / \mathrm{L}$ & 0.0402 & 0.0434 & & & \\
\hline 1696 & |TRITIUM_ELECT_LSC & ITASRL & 39.8 & $\mathrm{pCi} / \mathrm{L}$ & 2.38 & 8.75 & & & \\
\hline 1697 & UISO_AEA & ITASRL & 0.186 & $\mathrm{pCi} / \mathrm{L}$ & 0.0383 & 0.0437 & & & \\
\hline 1698 & UISO_AEA & ITASRL & 0.00299 & $\mathrm{pCi} / \mathrm{L}$ & 0.00826 & 0.00838 & & & $U$ \\
\hline 1699 & UISO_AEA & ITASRL & 0.193 & $\mathrm{pCi} / \mathrm{L}$ & 0.0379 & 0.0432 & & & \\
\hline 1700 & 6010_METALS_ICP & DATACH & & & & & & & $U$ \\
\hline 1701 & 8240_VOA_GCMS & DATACH & & & & & & & $U$ \\
\hline 1702 & SRISO_SEPP_PRECIP_GPC & ITASRL & 0.0955 & $\mathrm{pCi} / \mathrm{L}$ & 0.0405 & 0.0458 & & & \\
\hline 1703 & TRITIŪM_ELECCT_LSC̄ & ITASRL & 37.3 & $\mathrm{pCi} / \mathrm{L}$ & 2.33 & 8.58 & & & \\
\hline 1704 & UISO_AEA & ITASRL & 0.225 & $\mathrm{pCi} / \mathrm{L}$ & 0.0412 & 0.0481 & & & \\
\hline 1705 & UISO_AEA & ITASRL & 0.00725 & $\mathrm{pCi} / \mathrm{L}$ & 0.0116 & 0.0117 & & & $U$ \\
\hline 1706 & UISO_AEA & ITASRL & 0.166 & $\mathrm{pCi} / \mathrm{L}$ & 0.0353 & 0.0396 & & & \\
\hline 1707 & 6010_METALS_ICP & DATACH & 5.3 & ug/L & & 2.23 & & & L \\
\hline 1708 & 8240_VOA_GCMS & DATACH & & & & & & & U \\
\hline 1709 & 6010_METALS_ICP & DATACH & & & & & & & U \\
\hline 1710 & 8240_VOA_GCMS & DATACH & & & & & & & U \\
\hline
\end{tabular}




\begin{tabular}{|c|c|c|c|c|c|c|c|c|c|}
\hline & $\mathrm{K}$ & $\mathrm{L}$ & $\mathrm{M}$ & $\mathrm{N}$ & $\mathrm{O}$ & $\mathrm{P}$ & $\mathrm{Q}$ & $\mathrm{R}$ & $\mathrm{S}$ \\
\hline 1 & METHOD_NAME & LAB_CODE & VALUE_RPTD & ANAL_UNITS_RPTD & COUNTING_ERROR & $\mid$ TOTAL_ANAL_ERROR| & MIN_DETECTABLE_ACTIVITY & PCNT_MOISTURE & LAB_QUALIFIER \\
\hline 1712 & 8240_VOA_GCMS & DATACH & & & & & & & $U$ \\
\hline 1713 & 6010_METALSS_ICP & DATACH & 4.7 & ug/L & & 1.97 & & & L \\
\hline 1714 & 8240 VOA GCMS & DATACH & & & & & & & $\mathrm{U}$ \\
\hline 1715 & 6010_METALS_ICP & DATACH & & & & & & & U \\
\hline 1716 & $8240^{-}$VOA GCMS & DATACH & & & & & & & $\mathrm{U}$ \\
\hline 1717 & 6010_METÁLS_ICP & DATACH & & ug/L & & 2.1 & & & L \\
\hline 1718 & 8240_VOA_GCMS & DATACH & & & & & & & U \\
\hline 1719 & 6010_METÁ_S_ICP & DATACH & & ug/L & & 2.1 & & & L \\
\hline 1721 & 6010_METALS_ICP & DATACH & 4.7 & ug/L & & 1.97 & & & L \\
\hline 1722 & 8240_VOA_GCMS & DATACH & & & & & & & $U$ \\
\hline 1723 & 6010_METALS_ICP & DATACH & & & & & & & U \\
\hline 1724 & 8240_VOA_GCMS & DATACH & & & & & & & U \\
\hline 1725 & 6010_METÁ_S_ICP & DATACH & & & & & & & $U$ \\
\hline 1726 & 8240_VOA_GCMS & DATACH & & & & & & & $U$ \\
\hline 1727 & SRISO_SEP_PRECIP_GPC & ITASRL & 0.0868 & $\mathrm{pCi} / \mathrm{L}$ & 0.0369 & 0.0419 & & & \\
\hline \begin{tabular}{|l|l|}
1728 \\
\end{tabular} & TRITIUM_ELECT_LSC & ITASRL & 165 & $\mathrm{pCi} / \mathrm{L}$ & 3.69 & 18.7 & & & \\
\hline 1729 & UISO_AEA & ITASRL & & & & & & & \\
\hline 1731 & UISO_AEA & ITASRL & & & & & & & \\
\hline 1732 & SRISŌ_SEP_PRECIP_GPC & ITASRL & 0.059 & $\mathrm{pCi} / \mathrm{L}$ & 0.0426 & 0.0458 & & & \\
\hline 1733 & TRITIUMM_ELECT_LSC- & ITASRL & 166 & $\mathrm{pCi} / \mathrm{L}$ & 3.66 & 18.5 & & & \\
\hline \begin{tabular}{|c|}
1734 \\
\end{tabular} & UISO_AEA & ITASRL & 0.369 & $\mathrm{pCi} / \mathrm{L}$ & 0.134 & 0.143 & & & \\
\hline 1735 & UISO_AEA & ITASRL & 0.00619 & $\mathrm{pCi} / \mathrm{L}$ & 0.045 & 0.0454 & & & $U$ \\
\hline 1736 & UISO_AEA & ITASRL & 0.329 & $\mathrm{pCi} / \mathrm{L}$ & 0.12 & 0.127 & & & \\
\hline 1737 & SRISO_SEP_PRECIP_GPC & ITASRL & 0.0733 & $\mathrm{pCi} / \mathrm{L}$ & 0.0418 & 0.047 & & & \\
\hline 1738 & TRITIUMM_ELECCT_LSC̄ & ITASRL & 139 & $\mathrm{pCi} / \mathrm{L}$ & 3.48 & 16.6 & & & \\
\hline 1739 & UISO_AEA & ITASRL & 0.27 & $\mathrm{pCi} / \mathrm{L}$ & 0.0756 & 0.0824 & & & \\
\hline 1740 & UISO_AEA & ITASRL & 0.00581 & $\mathrm{pCi} / \mathrm{L}$ & 0.0212 & 0.0215 & & & $U$ \\
\hline 1741 & UISO_AEA & ITASRL & 0.182 & $\mathrm{pCi} / \mathrm{L}$ & 0.0629 & 0.0667 & & & \\
\hline 1742 & SRISŌ_SEP_PRECIP_GPC & ITASRL & 0.0986 & $\mathrm{pCi} / \mathrm{L}$ & 0.0421 & 0.0469 & & & \\
\hline 1743 & TRITIUMM_ELECT_LSC & ITASRL & 82 & $\mathrm{pCi} / \mathrm{L}$ & 2.87 & 11.9 & & & \\
\hline 1744 & UISO_AEA & ITASRL & 0.239 & $\mathrm{pCi} / \mathrm{L}$ & 0.0724 & 0.0783 & & & \\
\hline 1745 & UISO_AEA & ITASRL & -0.00271 & $\mathrm{pCi} / \mathrm{L}$ & 0.0177 & 0.018 & & & $U$ \\
\hline \begin{tabular}{|c|c|}
1746 \\
\end{tabular} & UISO_AEA & ITASRL & 0.194 & $\mathrm{pCi} / \mathrm{L}$ & 0.0638 & 0.0679 & & & \\
\hline \begin{tabular}{|l|l|}
+1747 \\
1747 \\
\end{tabular} & SRISO_SEP_PRECIP_GPC & ITASRL & 0.0879 & $\mathrm{pCi} / \mathrm{L}$ & 0.0424 & 0.0463 & & & \\
\hline 1748 & TRITIUMM_ELECCT_LSC̄ & ITASRL & 66.2 & $\mathrm{pCi} / \mathrm{L}$ & 2.67 & 10.7 & & & \\
\hline
\end{tabular}




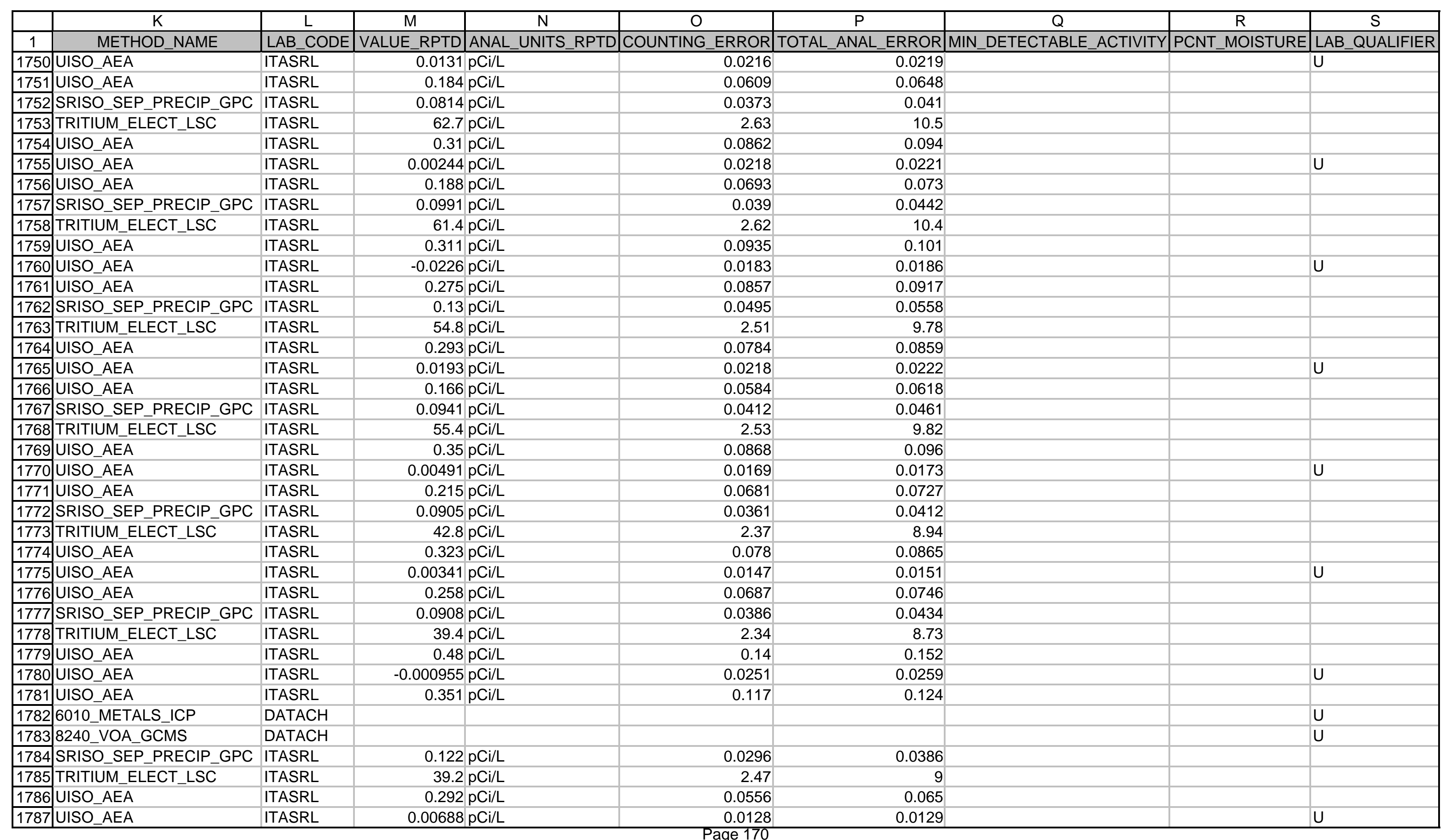




\begin{tabular}{|c|c|c|c|c|c|c|c|c|c|}
\hline & $\mathrm{K}$ & $\mathrm{L}$ & $\mathrm{M}$ & $\mathrm{N}$ & $\mathrm{O}$ & $\mathrm{P}$ & $\mathrm{Q}$ & $\mathrm{R}$ & $\mathrm{S}$ \\
\hline 1 & METHOD_NAME & LAB_CODE & VALUE_RPTD & ANAL_UNITS_RPTD & COUNTING_ERROR & $\mid$ TOTAL_ANAL_ERROR| & MIN_DETECTABLE_ACTIVITY & PCNT_MOISTURE & LAB_QUALIFIER \\
\hline 1788 & UISO_AEA & ITASRL & 0.231 & $\mathrm{pCi} / \mathrm{L}$ & 0.0495 & 0.056 & & & \\
\hline 1789 & 6010_METALS_ICP & DATACH & & & & & & & $U$ \\
\hline 1790 & 8240_VOA_GCMS & DATACH & & & & & & & 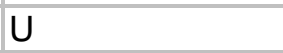 \\
\hline 1791 & SRISO_SEPP_PRECIP_GPC & ITASRL & 0.085 & $\mathrm{pCi} / \mathrm{L}$ & 0.026 & 0.032 & & & \\
\hline 1792 & TRITIUMM_ELECT_LSC & ITASRL & 37.6 & $\mathrm{pCi} / \mathrm{L}$ & 2.44 & 8.85 & & & \\
\hline 1793 & UISO_AEA & ITASRL & 0.315 & $\mathrm{pCi} / \mathrm{L}$ & 0.0563 & 0.0667 & & & \\
\hline 1794 & UISO_AEA & ITASRL & 0.0018 & $\mathrm{pCi} / \mathrm{L}$ & 0.0103 & 0.0104 & & & $U$ \\
\hline 1795 & UISO_AEA & ITASRL & 0.227 & $\mathrm{pCi} / \mathrm{L}$ & 0.0474 & 0.0539 & & & \\
\hline 1796 & 6010_METALS_ICP & DATACH & & & & & & & U \\
\hline 1797 & 8240_VOA_GCMS & DATACH & & & & & & & U \\
\hline 1798 & SRISO_SEP_PRECIP_GPC & ITASRL & 0.108 & $\mathrm{pCi} / \mathrm{L}$ & 0.0301 & 0.0373 & & & \\
\hline 1799 & TRITIUM_ELECT_LSC & ITASRL & 36.4 & $\mathrm{pCi} / \mathrm{L}$ & 2.4 & 8.78 & & & \\
\hline 1800 & UISO_AEA & ITASRL & 0.284 & $\mathrm{pCi} / \mathrm{L}$ & 0.0488 & 0.0581 & & & \\
\hline 1801 & UISO_AEA & ITASRL & 0.00644 & $\mathrm{pCi} / \mathrm{L}$ & 0.00993 & 0.0101 & & & $U$ \\
\hline 1802 & UISO AEA & ITASRL & 0.215 & $\mathrm{pCi} / \mathrm{L}$ & 0.0423 & 0.0484 & & & \\
\hline 1803 & 6010 METALS ICP & DATACH & & & & & & & $\mathrm{U}$ \\
\hline 1804 & 8240_VOA_GCMS & DATACH & & & & & & & $U$ \\
\hline 1805 & SRISO_SEP_PRECIP_GPC & ITASRL & 0.0643 & $\mathrm{pCi} / \mathrm{L}$ & 0.0238 & 0.0284 & & & \\
\hline 1807 & UISO_AEA & ITASRL & 0.285 & $\mathrm{pCi} / \mathrm{L}$ & 0.0531 & 0.0622 & & & \\
\hline 1808 & UISO_AEA & ITASRL & 0.00714 & $\mathrm{pCi} / \mathrm{L}$ & 0.0118 & 0.0119 & & & $U$ \\
\hline 1809 & UISO_AEA & ITASRL & 0.289 & $\mathrm{pCi} / \mathrm{L}$ & 0.0529 & 0.0619 & & & \\
\hline 1810 & 6010_METALS_ICP & DATACH & & & & & & & U \\
\hline 1811 & 8240_VOA_GCMS & DATACH & & & & & & & $\mathrm{U}$ \\
\hline 1812 & SRISO_SEP_PRECIP_GPC & ITASRL & 0.0818 & $\mathrm{pCi} / \mathrm{L}$ & 0.0309 & 0.0356 & & & \\
\hline 1813 & TRITIUMM_ELECCT_LSC̄ & ITASRL & 224 & $\mathrm{pCi} / \mathrm{L}$ & 4.23 & 23.3 & & & \\
\hline 1814 & UISO AEA & ITASRL & 0.203 & $\mathrm{pCi} / \mathrm{L}$ & 0.0378 & 0.0438 & & & \\
\hline 1815 & UISO_AEA & ITASRL & 0.00627 & $\mathrm{pCi} / \mathrm{L}$ & 0.00787 & 0.00803 & & & $U$ \\
\hline 1816 & UISO_AEA & ITASRL & 0.164 & $\mathrm{pCi} / \mathrm{L}$ & 0.0336 & 0.0379 & & & \\
\hline 1817 & 6010_METALS_ICP & DATACH & & & & & & & $U$ \\
\hline 1818 & 8240_VOA_GCMS & DATACH & & & & & & & $U$ \\
\hline 1819 & SRISO_SEPP_PRECIP_GPC & ITASRL & 0.106 & $\mathrm{pCi} / \mathrm{L}$ & 0.0336 & 0.0406 & & & \\
\hline 1820 & TRITIŪM_ELEECT_LSC̄ & ITASRL & 42.1 & $\mathrm{pCi} / \mathrm{L}$ & 2.51 & 9.15 & & & \\
\hline 1821 & UISO_AEA- & ITASRL & 0.399 & $\mathrm{pCi} / \mathrm{L}$ & 0.0584 & 0.0729 & & & \\
\hline 1822 & UISO_AEA & ITASRL & 0.00887 & $\mathrm{pCi} / \mathrm{L}$ & 0.0111 & 0.0113 & & & U \\
\hline 1823 & UISO_AEA & ITASRL & 0.316 & $\mathrm{pCi} / \mathrm{L}$ & 0.0518 & 0.0621 & & & \\
\hline 1824 & 6010_METALS_ICP & DATACH & & & & & & & 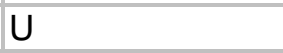 \\
\hline
\end{tabular}


N

O

P

Q

\begin{tabular}{|c|c|c|c|c|c|c|c|c|c|}
\hline & & & & & & & & & \\
\hline 1 & METHOD_NAME & LAB_CODE & VALUE_RPTD & ANAL_UNITS_RPTD & $\mid$ COUNTING_ERROR & TOTAL_ANAL_ERROR & MIN_DETECTABLE_ACTIVITY & PCNT_MOISTURE & LAB_QUALIFIER \\
\hline 1826 & SRISO_SEP_PRECIP_GPC & ITASRL & 0.0933 & $\mathrm{pCi} / \mathrm{L}$ & 0.0327 & 0.0381 & & & \\
\hline 1827 & TRITIUM_ELECT_LSC & ITASRL & 167 & $\mathrm{pCi} / \mathrm{L}$ & 3.76 & 19.1 & & & \\
\hline 1828 & UISO_AEA & ITASRL & 0.246 & $\mathrm{pCi} / \mathrm{L}$ & 0.0445 & 0.0521 & & & \\
\hline 1829 & UISO_AEA & ITASRL & 0.0172 & $\mathrm{pCi} / \mathrm{L}$ & 0.0125 & 0.0127 & & & \\
\hline 1830 & UISO_AEA & ITASRL & 0.177 & $\mathrm{pCi} / \mathrm{L}$ & 0.0375 & 0.0421 & & & \\
\hline 1831 & 6010_METALS_ICP & DATACH & & & & & & & U \\
\hline 1832 & 8240_VOA_GCMS & DATACH & & & & & & & U \\
\hline 1833 & SRISO_SEP_PRECIP_GPC & ITASRL & 0.476 & $\mathrm{pCi} / \mathrm{L}$ & 0.0512 & 0.1 & & & \\
\hline 1834 & TRITIUM_ELECT_LSC & ITASRL & 117 & $\mathrm{pCi} / \mathrm{L}$ & 3.29 & 15 & & & \\
\hline 1835 & UISO_AEA & ITASRL & 0.197 & $\mathrm{pCi} / \mathrm{L}$ & 0.04 & 0.0457 & & & \\
\hline 1836 & UISO AEA & ITASRL & 0.00626 & $\mathrm{pCi} / \mathrm{L}$ & 0.00821 & 0.00837 & & & $\mathrm{U}$ \\
\hline 1837 & UISO_AEA & ITASRL & 0.203 & $\mathrm{pCi} / \mathrm{L}$ & 0.0396 & 0.0452 & & & \\
\hline 1838 & 6010_METALS_ICP & DATACH & & & & & & & $U$ \\
\hline 1839 & 8240_VOA_GCMS & DATACH & & & & & & & U \\
\hline 1840 & SRISO_SEP_PRECIP_GPC & ITASRL & 0.403 & $\mathrm{pCi} / \mathrm{L}$ & 0.0455 & 0.0904 & & & \\
\hline 1841 & TRITIUM_ELECT_LSC & ITASRL & 59.6 & $\mathrm{pCi} / \mathrm{L}$ & 2.71 & 10.5 & & & \\
\hline 1842 & UISO_AEA & ITASRL & 0.215 & $\mathrm{pCi} / \mathrm{L}$ & 0.0399 & 0.0464 & & & \\
\hline 1843 & UISO_AEA & ITASRL & 0.00514 & $\mathrm{pCi} / \mathrm{L}$ & 0.00764 & 0.00779 & & & U \\
\hline 1846 & 8240_VOA_GCMS & DATACH & & & & & & & $\mathrm{U}$ \\
\hline 1847 & SRISO_SEP_PRECIP_GPC & ITASRL & 0.109 & $\mathrm{pCi} / \mathrm{L}$ & 0.029 & 0.0367 & & & \\
\hline 1848 & TRITIUM_ELECT_LSC & ITASRL & 47.4 & $\mathrm{pCi} / \mathrm{L}$ & 2.55 & 9.58 & & & \\
\hline 1849 & UISO_AEA & ITASRL & 0.28 & $\mathrm{pCi} / \mathrm{L}$ & 0.0519 & 0.0607 & & & \\
\hline 1850 & UISO_AEA & ITASRL & 0.00382 & $\mathrm{pCi} / \mathrm{L}$ & 0.00778 & 0.00792 & & & $U$ \\
\hline 1851 & UISO_AEA & ITASRL & 0.194 & $\mathrm{pCi} / \mathrm{L}$ & 0.0429 & 0.0481 & & & \\
\hline 1852 & 6010_METALS_ICP & DATACH & & & & & & & $U$ \\
\hline 1853 & 8240_VOA_GCMS & DATACH & & & & & & & U \\
\hline 1854 & SRISO_SEP_PRECIP_GPC & ITASRL & 0.0822 & $\mathrm{pCi} / \mathrm{L}$ & 0.0266 & 0.0327 & & & \\
\hline 1855 & TRITIUM_ELECT_LSC & ITASRL & 40.8 & $\mathrm{pCi} / \mathrm{L}$ & 2.46 & 9.05 & & & \\
\hline 1856 & UISO_AEA & ITASRL & 0.201 & $\mathrm{pCi} / \mathrm{L}$ & 0.0437 & 0.0494 & & & \\
\hline 1857 & UISO_AEA & ITASRL & 0.01 & $\mathrm{pCi} / \mathrm{L}$ & 0.0106 & 0.0107 & & & $U$ \\
\hline 1858 & UISO_AEA & ITASRL & 0.169 & $\mathrm{pCi} / \mathrm{L}$ & 0.0393 & 0.0436 & & & \\
\hline 1859 & 6010_METALS_ICP & DATACH & & & & & & & $U$ \\
\hline 1860 & 8240_VOA_GCMS & DATACH & & & & & & & $\mathrm{U}$ \\
\hline 1861 & SRISO_SEP_PRECIP_GPC & ITASRL & 0.0934 & $\mathrm{pCi} / \mathrm{L}$ & 0.0292 & 0.0357 & & & \\
\hline 1862 & TRITIUM_ELEECT_LSC & ITASRL & 42.4 & $\mathrm{pCi} / \mathrm{L}$ & 2.47 & 9.18 & & & \\
\hline 1863 & UISO_AEA & ITASRL & 0.279 & $\mathrm{pCi} / \mathrm{L}$ & 0.0455 & 0.0545 & & & \\
\hline
\end{tabular}




\begin{tabular}{|c|c|c|c|c|c|c|c|c|c|}
\hline & $\mathrm{K}$ & $\mathrm{L}$ & $\mathrm{M}$ & $\mathrm{N}$ & $\mathrm{O}$ & $\mathrm{P}$ & $\mathrm{Q}$ & $\mathrm{R}$ & $\mathrm{S}$ \\
\hline 1 & METHOD_NAME & LAB_CODE & VALUE_RPTD & ANAL_UNITS_RPTD & COUNTING_ERROR & TOTAL_ANAL_ERROR I & MIN_DETECTABLE_ACTIVITY & PCNT_MOISTURE & LAB_QUALIFIER \\
\hline 1864 & UISO_AEA & ITASRL & 0.0197 & $\mathrm{pCi} / \mathrm{L}$ & 0.0126 & 0.0128 & & & \\
\hline 1865 & UISO AEA & ITASRL & 0.198 & $\mathrm{pCi} / \mathrm{L}$ & 0.0382 & 0.0437 & & & \\
\hline 1866 & 6010_METALS_ICP & DATACH & & & & & & & $U$ \\
\hline 1867 & 8240_VOA_GCMS & DATACH & & & & & & & $U$ \\
\hline 1868 & SRISO_SEPP_PRECIP_GPC & ITASRL & 0.0753 & $\mathrm{pCi} / \mathrm{L}$ & 0.0291 & 0.0339 & & & \\
\hline 1869 & TRITIUM_ELECT_LSC & ITASRL & 42.8 & $\mathrm{pCi} / \mathrm{L}$ & 2.49 & 9.19 & & & \\
\hline 1870 & UISO_AËA & ITASRL & 0.234 & $\mathrm{pCi} / \mathrm{L}$ & 0.0486 & 0.0556 & & & \\
\hline 1871 & UISO_AEA & ITASRL & 0.00316 & $\mathrm{pCi} / \mathrm{L}$ & 0.00831 & 0.00844 & & & $U$ \\
\hline 1872 & UISO_AEA & ITASRL & 0.221 & $\mathrm{pCi} / \mathrm{L}$ & 0.0465 & 0.0527 & & & \\
\hline 1873 & 6010_METALS_ICP & DATACH & & & & & & & U \\
\hline 1874 & 8240_VOA_GCMS & DATACH & & & & & & & U \\
\hline 1875 & SRISO_SEP_PRECIP_GPC & ITASRL & 0.0974 & $\mathrm{pCi} / \mathrm{L}$ & 0.028 & 0.035 & & & \\
\hline 1876 & TRITIŪM_ELEECT_LSC̄ & ITASRL & 41.7 & $\mathrm{pCi} / \mathrm{L}$ & 2.48 & 9.14 & & & \\
\hline 1877 & UISO_AËA & ITASRL & 0.393 & $\mathrm{pCi} / \mathrm{L}$ & 0.0678 & 0.0815 & & & \\
\hline 1878 & UISO AEA & ITASRL & 0.0126 & $\mathrm{pCi} / \mathrm{L}$ & 0.0138 & 0.0139 & & & $\mathrm{U}$ \\
\hline 1879 & UISO_AEA & ITASRL & 0.345 & $\mathrm{pCi} / \mathrm{L}$ & 0.0634 & 0.0745 & & & \\
\hline 1880 & 6010_METALS_ICP & DATACH & & & & & & & $U$ \\
\hline 1881 & 8260_VOA_GCMS & DATACH & & & & & & & $U$ \\
\hline 1883 & TRITIUM_ELECT_LSC & ITASRL & 33 & $\mathrm{pCi} / \mathrm{L}$ & 2.37 & 8.4 & & & \\
\hline 1884 & UISO_AEA & ITASRL & 0.237 & $\mathrm{pCi} / \mathrm{L}$ & 0.0438 & 0.0511 & & & \\
\hline 1885 & UISO_AEA & ITASRL & 0.0139 & $\mathrm{pCi} / \mathrm{L}$ & 0.0112 & 0.0114 & & & \\
\hline 1886 & UISO_AEA & ITASRL & 0.143 & $\mathrm{pCi} / \mathrm{L}$ & 0.0338 & 0.0374 & & & \\
\hline 1887 & 6010_METALS_ICP & DATACH & & & & & & & $U$ \\
\hline 1888 & 8260_VOA_GCMS & DATACH & & & & & & & $U$ \\
\hline 1889 & SRISO_SEPP_PRECIP_GPC & ITASRL & 0.0398 & $\mathrm{pCi} / \mathrm{L}$ & 0.0371 & 0.0394 & & & \\
\hline 1890 & TRITIUMM_ELECTT_LSC̄ & ITASRL & 33 & $\mathrm{pCi} / \mathrm{L}$ & 2.35 & 8.41 & & & \\
\hline 1891 & UISO_AEA & ITASRL & 0.23 & $\mathrm{pCi} / \mathrm{L}$ & 0.0435 & 0.0505 & & & \\
\hline 1892 & UISO_AEA & ITASRL & 0.00966 & $\mathrm{pCi} / \mathrm{L}$ & 0.0102 & 0.0104 & & & $U$ \\
\hline 1893 & UISO_AEA & ITASRL & 0.155 & $\mathrm{pCi} / \mathrm{L}$ & 0.0356 & 0.0395 & & & \\
\hline 1894 & 6010_METALS_ICP & DATACH & & & & & & & $U$ \\
\hline 1895 & 8260_VOA_GCMS & DATACH & & & & & & & $U$ \\
\hline 1896 & SRISO_SEPP_PRECIP_GPC & ITASRL & 0.0763 & $\mathrm{pCi} / \mathrm{L}$ & 0.038 & 0.0419 & & & \\
\hline 1897 & TRITIUM_ELECTI_LSC & ITASRL & 33.9 & $\mathrm{pCi} / \mathrm{L}$ & 2.39 & 8.53 & & & \\
\hline 1898 & UISO_AEA & ITASRL & 0.232 & $\mathrm{pCi} / \mathrm{L}$ & 0.0429 & 0.05 & & & \\
\hline 1899 & UISO_AEA & ITASRL & 0.0086 & $\mathrm{pCi} / \mathrm{L}$ & 0.0106 & 0.0107 & & & U \\
\hline 1900 & UISO_AEA & ITASRL & 0.173 & $\mathrm{pCi} / \mathrm{L}$ & 0.0366 & 0.0412 & & & \\
\hline
\end{tabular}




\begin{tabular}{|c|c|c|c|c|c|c|c|c|c|}
\hline & $\mathrm{K}$ & $\mathrm{L}$ & $\mathrm{M}$ & $\mathrm{N}$ & $\mathrm{O}$ & $\mathrm{P}$ & $\mathrm{Q}$ & $\mathrm{R}$ & $\mathrm{S}$ \\
\hline 1 & METHOD_NAME & LAB_CODE & VALUE_RPTD & ANAL_UNITS_RPTD & COUNTING_ERROR & TOTAL_ANAL_ERROR & MIN_DETECTABLE_ACTIVITY & PCNT_MOISTURE & LAB_QUALIFIER \\
\hline 1902 & 8260_VOA_GCMS & DATACH & & & & & & & $\mathrm{U}$ \\
\hline 1903 & SRISO_SEP_PRECIP_GPC & ITASRL & 0.0535 & $\mathrm{pCi} / \mathrm{L}$ & 0.0343 & 0.0367 & & & \\
\hline 1904 & TRITIUM_ELECT_LSC & ITASRL & 33.5 & $\mathrm{pCi} / \mathrm{L}$ & 2.38 & 8.47 & & & \\
\hline 1905 & UISO_AEA & ITASRL & 0.279 & $\mathrm{pCi} / \mathrm{L}$ & 0.048 & 0.0571 & & & \\
\hline 1906 & UISO_AEA & ITASRL & -0.00139 & $\mathrm{pCi} / \mathrm{L}$ & 0.00604 & 0.00618 & & & U \\
\hline 1907 & UISO_AEA & ITASRL & 0.178 & $\mathrm{pCi} / \mathrm{L}$ & 0.0385 & 0.0432 & & & \\
\hline 1908 & 6010_METALS_ICP & DATACH & & & & & & & U \\
\hline 1909 \& & 8260_VOA_GCMS & DATACH & & & & & & & U \\
\hline 1910 & SRISO_SEP_PRECIP_GPC & ITASRL & 0.0756 & $\mathrm{pCi} / \mathrm{L}$ & 0.0407 & 0.0444 & & & \\
\hline 1911 & TRITIUM_ELECT_LSC & ITASRL & 33.3 & $\mathrm{pCi} / \mathrm{L}$ & 2.35 & 8.43 & & & \\
\hline 1912 & UISO_AEA & ITASRL & 0.197 & $\mathrm{pCi} / \mathrm{L}$ & 0.0399 & 0.0456 & & & \\
\hline 1913 & UISO_AEA & ITASRL & 0.00899 & $\mathrm{pCi} / \mathrm{L}$ & 0.0109 & 0.0111 & & & $U$ \\
\hline 1914 & UISO_AEA & ITASRL & 0.202 & $\mathrm{pCi} / \mathrm{L}$ & 0.04 & 0.0456 & & & \\
\hline 1915 & 6010_METALS_ICP & DATACH & & & & & & & $U$ \\
\hline 1916 & 8260_VOA_GCMS & DATACH & & & & & & & U \\
\hline 1917 & SRISO_SEP_PRECIP_GPC & ITASRL & 0.369 & $\mathrm{pCi} / \mathrm{L}$ & 0.0703 & 0.0991 & & & \\
\hline 1918 & TRITIUM_ELECT_LSC & ITASRL & 33.4 & $\mathrm{pCi} / \mathrm{L}$ & 2.37 & 8.42 & & & \\
\hline 1921 & UISO_AEA & ITASRL & 0.281 & $\mathrm{pCi} / \mathrm{L}$ & 0.0479 & 0.0567 & & & \\
\hline 1922 & 6010_METALS_ICP & DATACH & & & & & & & U \\
\hline 1923 & 8260_VOA_GCMS & DATACH & & & & & & & $U$ \\
\hline 1924 & SRISO_SEP_PRECIP_GPC & ITASRL & 0.105 & $\mathrm{pCi} / \mathrm{L}$ & 0.0468 & 0.0518 & & & \\
\hline 1925 & TRITIUM_ELECTI_LSC & ITASRL & 32.4 & $\mathrm{pCi} / \mathrm{L}$ & 2.35 & 8.4 & & & \\
\hline \begin{tabular}{|l|l|}
1926 \\
\end{tabular} & UISO_AĒA & ITASRL & 0.227 & $\mathrm{pCi} / \mathrm{L}$ & 0.0467 & 0.0535 & & & \\
\hline 1927 & UISO_AEA & ITASRL & 0.00441 & $\mathrm{pCi} / \mathrm{L}$ & 0.0102 & 0.0104 & & & U \\
\hline 1928 & UISO_AEA & ITASRL & 0.154 & $\mathrm{pCi} / \mathrm{L}$ & 0.0379 & 0.0418 & & & \\
\hline 1929 & 6010_METALS_ICP & DATACH & & & & & & & U \\
\hline 1930 & 8260_VOA_GCMS & DATACH & & & & & & & U \\
\hline 1931 & SRISO_SEP_PRECIP_GPC & ITASRL & 0.0943 & $\mathrm{pCi} / \mathrm{L}$ & 0.0509 & 0.0554 & & & \\
\hline 1932 & TRITIUM_ELECT_LSC & ITASRL & 33.9 & $\mathrm{pCi} / \mathrm{L}$ & 2.39 & 8.53 & & & \\
\hline 1933 & UISO_AEA & ITASRL & 0.271 & $\mathrm{pCi} / \mathrm{L}$ & 0.0495 & 0.0582 & & & \\
\hline 1934 & UISO_AEA & ITASRL & 0.00543 & $\mathrm{pCi} / \mathrm{L}$ & 0.0109 & 0.011 & & & U \\
\hline 1935 & UISO_AEA & ITASRL & 0.202 & $\mathrm{pCi} / \mathrm{L}$ & 0.0425 & 0.0481 & & & \\
\hline 1936 & 6010_METALS_ICP & DATACH & & & & & & & U \\
\hline 1937 & 8260_VOA_GCMS & DATACH & & & & & & & U \\
\hline 1938 & SRISO_SEPP_PRECIP_GPC & ITASRL & 0.0715 & $\mathrm{pCi} / \mathrm{L}$ & 0.038 & 0.0415 & & & \\
\hline
\end{tabular}




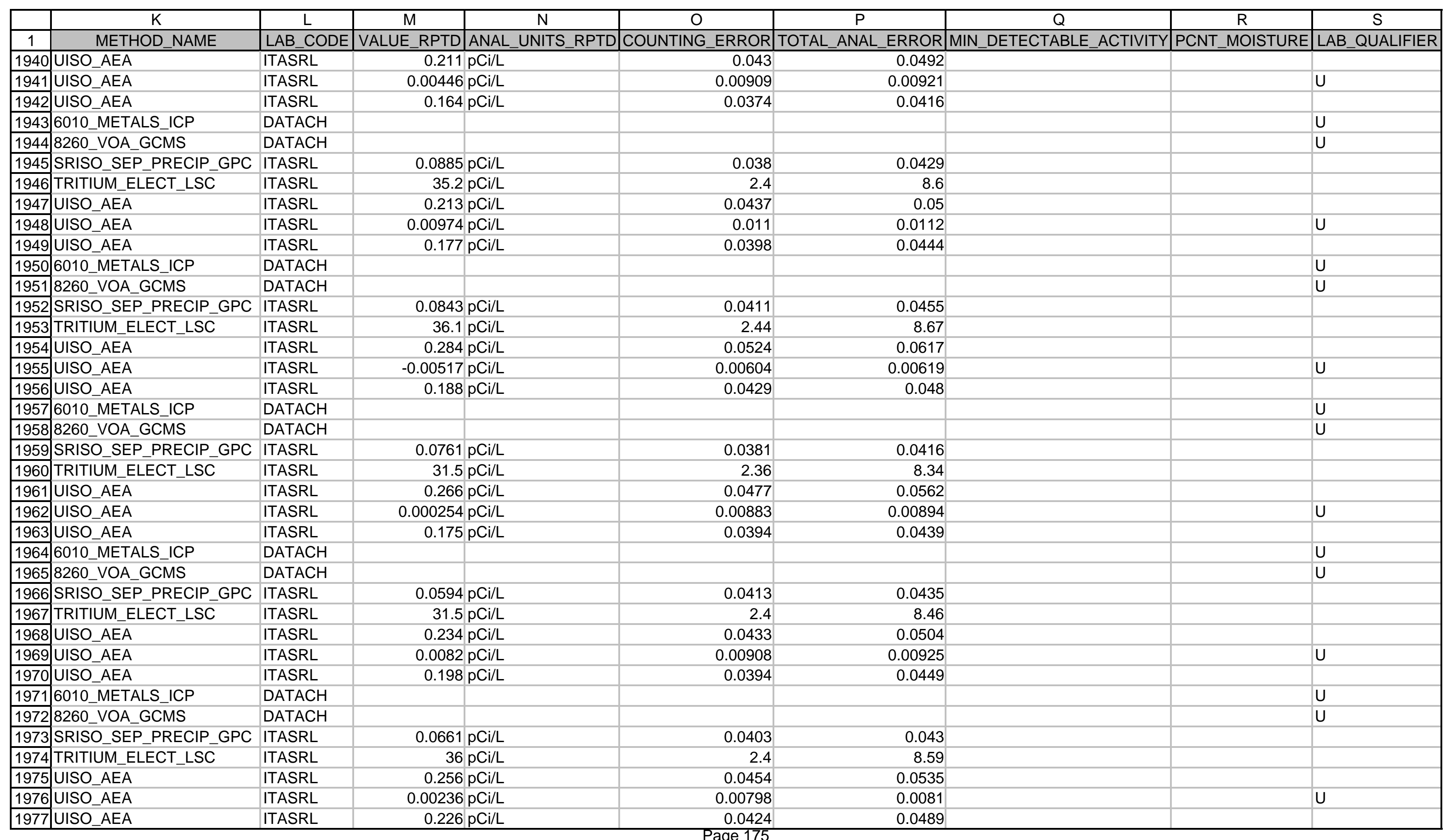




\begin{tabular}{|c|c|c|c|c|c|c|c|c|c|}
\hline & $\mathrm{K}$ & $\mathrm{L}$ & $\mathrm{M}$ & $\mathrm{N}$ & $\mathrm{O}$ & $\mathrm{P}$ & $\mathrm{Q}$ & $\mathrm{R}$ & $\mathrm{S}$ \\
\hline 1 & METHOD_NAME & LAB_CODE & VALUE_RPTD & ANAL_UNITS_RPTD & COUNTING_ERROR & TOTAL_ANAL_ERROR & MIN_DETECTABLE_ACTIVITY & PCNT_MOISTURE & LAB_QUALIFIER \\
\hline 1978 & 6010_METALS_ICP & DATACH & & & & & & & $\mathrm{U}$ \\
\hline 1979 & 8260 VOA GCMS & DATACH & & & & & & & $\mathrm{U}$ \\
\hline 1980 & GAMMA_GS & ITASRL & -0.395 & $\mathrm{pCi} / \mathrm{L}$ & 1.6 & 1.6 & & & $U$ \\
\hline 1981 & SRISO_SEP_PRECIP_GPC & ITASRL & 0.168 & $\mathrm{pCi} / \mathrm{L}$ & 0.106 & 0.115 & & & \\
\hline 1982 & TC99_SEP_LSC & ITASRL & -0.284 & $\mathrm{pCi} / \mathrm{L}$ & 0.196 & 1.01 & & & U \\
\hline 1983 & TRITIUMM_ELECT_LSC & ITASRL & 70 & $\mathrm{pCi} / \mathrm{L}$ & 2.86 & 11.4 & & & \\
\hline 1984 & UISO_AËA & ITASRL & 0.246 & $\mathrm{pCi} / \mathrm{L}$ & 0.036 & 0.0446 & & & \\
\hline 1985 & UISO AEA & ITASRL & 0.00634 & $\mathrm{pCi} / \mathrm{L}$ & 0.00672 & 0.00683 & & & $\mathrm{U}$ \\
\hline 1986 & UISO_AEA & ITASRL & 0.173 & $\mathrm{pCi} / \mathrm{L}$ & 0.0302 & 0.0354 & & & \\
\hline 1987 & 6010_METALS_ICP & DATACH & 4.4 & $u g / L$ & & 1.85 & & & $\mathrm{~L}$ \\
\hline 1988 & 8260_VOA_GCMS & DATACH & & & & & & & $\mathrm{U}$ \\
\hline 1989 & GAMMA_GS & ITASRL & 0.879 & $\mathrm{pCi} / \mathrm{L}$ & 2.6 & 2.6 & & & U \\
\hline 1990 & SRISO_SEP_PRECIP_GPC & ITASRL & 0.131 & $\mathrm{pCi} / \mathrm{L}$ & 0.103 & 0.109 & & & \\
\hline 1991 & TC99_SEPP_LSC & ITASRL & -0.378 & $\mathrm{pCi} / \mathrm{L}$ & 0.194 & 0.999 & & & $U$ \\
\hline 1992 & TRITIÜM ELECT LSC & ITASRL & 34 & $\mathrm{pCi} / \mathrm{L}$ & 2.42 & 8.77 & & & \\
\hline 1993 & UISO AËA & ITASRL & 0.182 & $\mathrm{pCi} / \mathrm{L}$ & 0.0382 & 0.0431 & & & \\
\hline \begin{tabular}{|l|}
1994 \\
\end{tabular} & UISO AEA & ITASRL & 0.00421 & $\mathrm{pCi} / \mathrm{L}$ & 0.0107 & 0.0108 & & & U \\
\hline 1995 & UISO_AEA & ITASRL & 0.298 & $\mathrm{pCi} / \mathrm{L}$ & 0.0492 & 0.0591 & & & \\
\hline 1997 & 8260_VOA_GCMS & DATACH & & & & & & & U \\
\hline 1998 & GAMMA_GS & ITASRL & 1.35 & $\mathrm{pCi} / \mathrm{L}$ & 1.65 & 1.66 & & & U \\
\hline 1999 & SRISO_SEP_PRECIP_GPC & ITASRL & 0.122 & $\mathrm{pCi} / \mathrm{L}$ & 0.0938 & 0.0996 & & & \\
\hline 2000 & TC99_SEP_LSC & ITASRL & -0.213 & $\mathrm{pCi} / \mathrm{L}$ & 0.195 & 1.01 & & & $\mathrm{U}$ \\
\hline 2001 & TRITIUM_ELECT_LSC & ITASRL & 37.4 & $\mathrm{pCi} / \mathrm{L}$ & 2.48 & 9.02 & & & \\
\hline 2002 & UISO_AEA & ITASRL & 0.248 & $\mathrm{pCi} / \mathrm{L}$ & 0.0482 & 0.0558 & & & \\
\hline 2003 & UISO_AEA & ITASRL & 0.0122 & $\mathrm{pCi} / \mathrm{L}$ & 0.0114 & 0.0116 & & & \\
\hline 2004 & UISO AEA & ITASRL & 0.14 & $\mathrm{pCi} / \mathrm{L}$ & 0.0362 & 0.0395 & & & \\
\hline 2005 & 6010_METALS_ICP & DATACH & & & & & & & U \\
\hline 2006 & 8260_VOA_GCMS & DATACH & & & & & & & $\mathrm{U}$ \\
\hline 2007 & GAMMA_GS & ITASRL & 0.612 & $\mathrm{pCi} / \mathrm{L}$ & 1.85 & 1.85 & & & U \\
\hline 2008 & SRISO_SEP_PRECIP_GPC & ITASRL & 0.0792 & $\mathrm{pCi} / \mathrm{L}$ & 0.0844 & 0.0883 & & & $\mathrm{U}$ \\
\hline 2009 & TC99_SEP_LSC & ITASRL & 0.156 & $\mathrm{pCi} / \mathrm{L}$ & 0.132 & 0.732 & & & $U$ \\
\hline 2010 & TRITIUM_ELECT_LSC & ITASRL & 37.1 & $\mathrm{pCi} / \mathrm{L}$ & 2.45 & 8.86 & & & \\
\hline 2011 & UISO_AEA & ITASRL & 0.183 & $\mathrm{pCi} / \mathrm{L}$ & 0.0402 & 0.0451 & & & \\
\hline 2012 & UISO_AEA & ITASRL & -0.000403 & $\mathrm{pCi} / \mathrm{L}$ & 0.00631 & 0.00645 & & & $\mathrm{U}$ \\
\hline 2013 & UISO_AEA & ITASRL & 0.21 & $\mathrm{pCi} / \mathrm{L}$ & 0.0438 & 0.0498 & & & \\
\hline 2014 & 6010_METALS_ICP & DATACH & & & & & & & $U$ \\
\hline
\end{tabular}




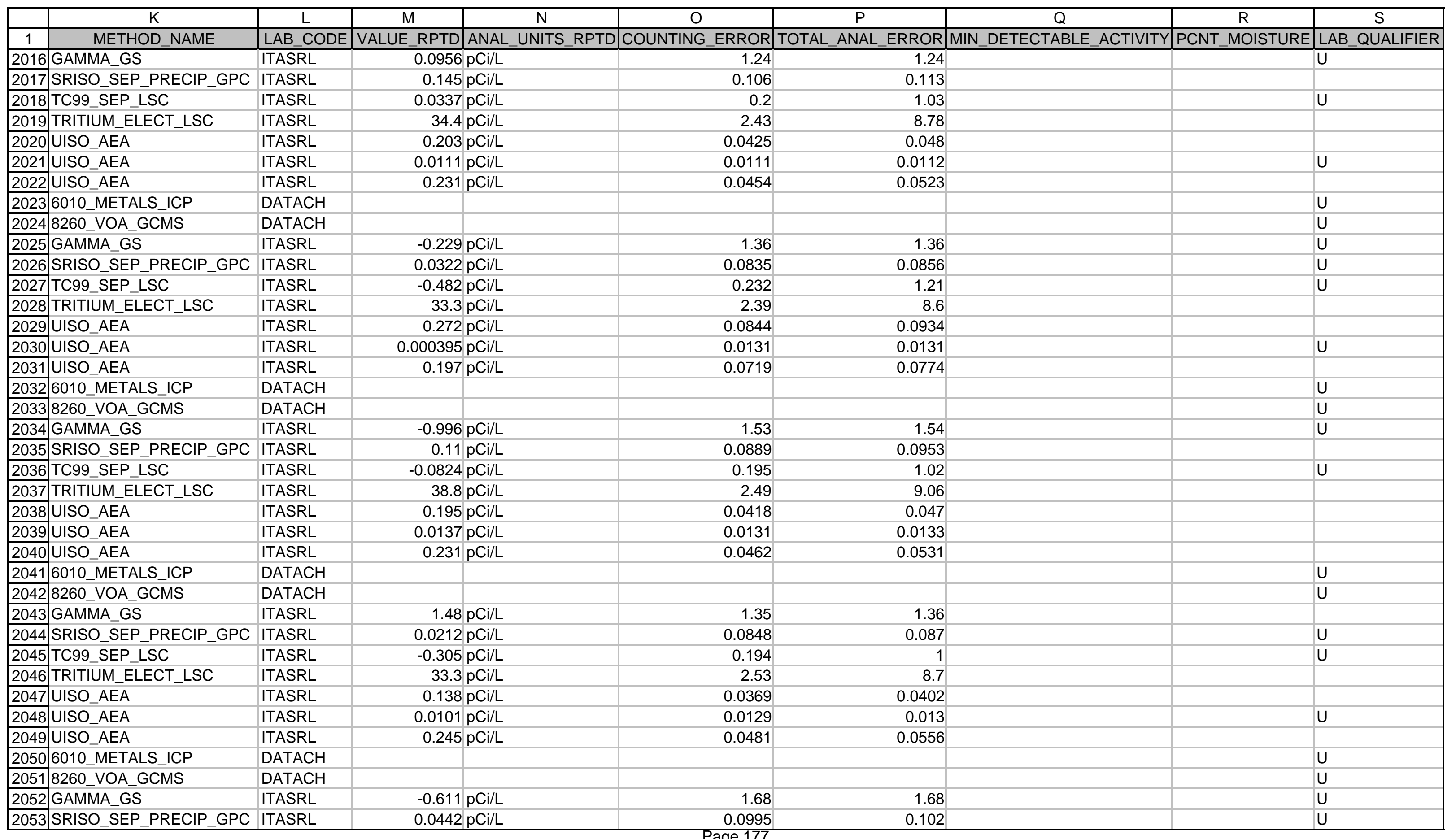




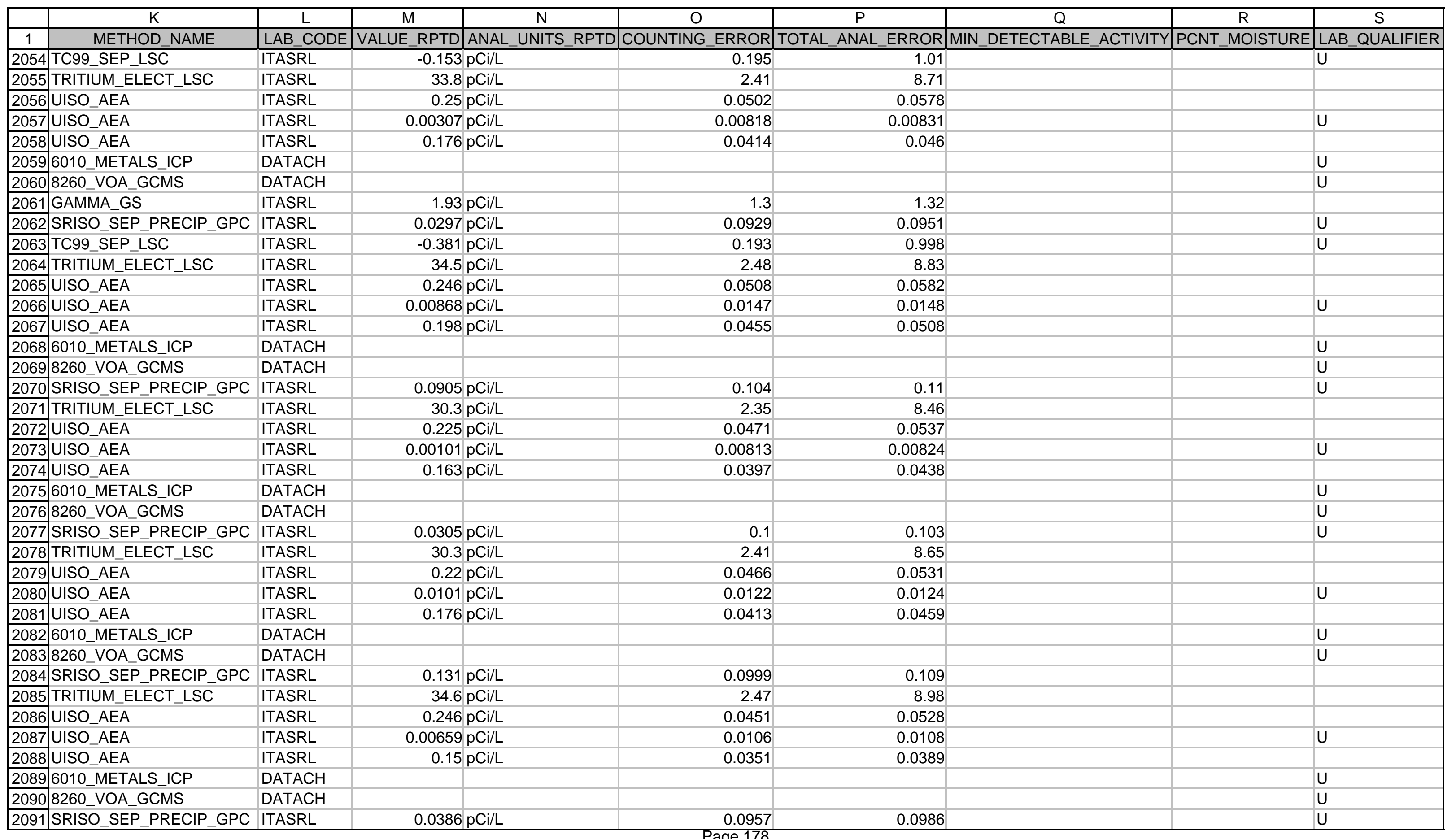




\begin{tabular}{|c|c|c|c|c|c|c|c|c|c|}
\hline & $\mathrm{K}$ & $\mathrm{L}$ & $\mathrm{M}$ & $\mathrm{N}$ & $\mathrm{O}$ & $\mathrm{P}$ & $\mathrm{Q}$ & $\mathrm{R}$ & $\mathrm{S}$ \\
\hline 1 & METHOD_NAME & LAB_CODE & VALUE_RPTD & ANAL_UNITS_RPTD & COUNTING_ERROR & TOTAL_ANAL_ERROR & MIN_DETECTABLE_ACTIVITY & PCNT_MOISTURE & LAB_QUALIFIER \\
\hline 2092 & TRITIUM_ELECT_LSC & ITASRL & 30 & $\mathrm{pCi} / \mathrm{L}$ & 2.36 & 8.46 & & & \\
\hline 2093 & UISO_AEA & ITASRL & 0.195 & $5 \mathrm{pCi} / \mathrm{L}$ & 0.0395 & 0.0452 & & & \\
\hline 2094 & UISO_AEA & ITASRL & -0.00277 & $7 \mathrm{pCi} / \mathrm{L}$ & 0.00598 & 0.00611 & & & $U$ \\
\hline 2095 & UISO_AEA & ITASRL & 0.161 & $\mathrm{pCi} / \mathrm{L}$ & 0.0356 & 0.0397 & & & \\
\hline 2096 & 6010_METALS_ICP & DATACH & & & & & & & $U$ \\
\hline 2097 & 8260_VOA_GCMS & DATACH & & & & & & & $U$ \\
\hline 2098 & SRISO_SEP_PRECIP_GPC & ITASRL & 0.0597 & $7 \mathrm{pCi} / \mathrm{L}$ & 0.0377 & 0.0405 & & & \\
\hline 2099 & TRITIUM_ELECT_LSC & ITASRL & 36 & $\mathrm{pCi} / \mathrm{L}$ & 2.46 & 8.93 & & & \\
\hline 2101 & UISO_AEA & ITASRL & 0.011 & $\mathrm{pCi} / \mathrm{L}$ & 0.0133 & 0.0135 & & & $\mathrm{U}$ \\
\hline 2102 & UISO_AEA & ITASRL & 0.203 & $3 \mathrm{pCi} / \mathrm{L}$ & 0.0428 & 0.0484 & & & \\
\hline 2103 & 6010_METALS_ICP & DATACH & & & & & & & $\mathrm{U}$ \\
\hline 2104 & 8260_VOA_GCMS & DATACH & & & & & & & U \\
\hline 2105 & SRISO_SEP_PRECIP_GPC & ITASRL & 0.0719 & $9 \mathrm{pCi} / \mathrm{L}$ & 0.0762 & 0.081 & & & $U$ \\
\hline 2106 & TRITIUM_ELECT_LSC & ITASRL & 36.1 & $\mathrm{pCi} / \mathrm{L}$ & 2.36 & 8.61 & & & \\
\hline 2107 & UISO_AEA & ITASRL & 0.173 & $3 \mathrm{pCi} / \mathrm{L}$ & 0.04 & 0.0445 & & & \\
\hline 2108 & UISO_AEA & ITASRL & 0.00619 & $9 \mathrm{pCi} / \mathrm{L}$ & 0.01 & 0.0101 & & & $\mathrm{U}$ \\
\hline 2109 & UISO_AEA & ITASRL & 0.248 & $3 \mathrm{pCi} / \mathrm{L}$ & 0.0485 & 0.056 & & & \\
\hline 2111 & 8260_VOA_GCMS & DATACH & & & & & & & $\mathrm{U}$ \\
\hline 2112 & SRISO_SEP_PRECIP_GPC & ITASRL & 0.0806 & $5 \mathrm{pCi} / \mathrm{L}$ & 0.0508 & 0.0562 & & & \\
\hline 2113 & TRITIUM_ELECT_LSC & ITASRL & 37.9 & $\mathrm{pCi} / \mathrm{L}$ & 2.47 & 8.98 & & & \\
\hline 2114 & UISO_AEA & ITASRL & 0.193 & $3 \mathrm{pCi} / \mathrm{L}$ & 0.0433 & 0.0484 & & & \\
\hline 2115 & UISO_AEA & ITASRL & 0.00767 & $7 \mathrm{pCi} / \mathrm{L}$ & 0.0112 & 0.0114 & & & $U$ \\
\hline 2116 & UISO_AEA & ITASRL & 0.225 & $5 \mathrm{pCi} / \mathrm{L}$ & 0.0475 & 0.0541 & & & \\
\hline 2117 & 6010_METALS_ICP & DATACH & & & & & & & U \\
\hline 2118 & 8260_VOA_GCMS & DATACH & & & & & & & $U$ \\
\hline 2119 & SRISO_SEP_PRECIP_GPC & ITASRL & 0.0745 & $\mathrm{pCi} / \mathrm{L}$ & 0.046 & 0.0514 & & & \\
\hline 2120 & TRITIUM_ELECT_LSC & ITASRL & 36.3 & $\mathrm{pCi} / \mathrm{L}$ & 2.45 & 8.9 & & & \\
\hline 2121 & UISO_AEA & ITASRL & 0.209 & $\mathrm{pCi} / \mathrm{L}$ & 0.0486 & 0.0546 & & & \\
\hline 2122 & UISO_AEA & ITASRL & 0.00934 & $4 \mathrm{pCi} / \mathrm{L}$ & 0.013 & 0.0131 & & & $U$ \\
\hline 2123 & UISO_AEA & ITASRL & 0.18 & $3 \mathrm{pCi} / \mathrm{L}$ & 0.0447 & 0.0493 & & & \\
\hline 2124 & 6010_METALS_ICP & DATACH & & & & & & & $U$ \\
\hline 2125 & 8260_VOA_GCMS & DATACH & & & & & & & $U$ \\
\hline 2126 & SRISO_SEP_PRECIP_GPC & ITASRL & 0.0907 & $7 \mathrm{pCi} / \mathrm{L}$ & 0.0526 & 0.0584 & & & \\
\hline 2127 & TRITIUM_ELECT_LSC & ITASRL & 36.2 & $\mathrm{pCi} / \mathrm{L}$ & 2.43 & 8.88 & & & \\
\hline 2128 & UISO_AEA & ITASRL & 0.176 & $\mathrm{pCi} / \mathrm{L}$ & 0.0418 & 0.0464 & & & \\
\hline
\end{tabular}




\begin{tabular}{|c|c|c|c|c|c|c|c|c|c|}
\hline & $\mathrm{K}$ & $\mathrm{L}$ & $\mathrm{M}$ & $\mathrm{N}$ & $\mathrm{O}$ & $\mathrm{P}$ & $\mathrm{Q}$ & $\mathrm{R}$ & $\mathrm{S}$ \\
\hline 1 & METHOD_NAME & LAB_CODE & VALUE_RPTD & ANAL_UNITS_RPTD & COUNTING_ERROR & TOTAL_ANAL_ERROR I & MIN_DETECTABLE_ACTIVITY & PCNT_MOISTURE & LAB_QUALIFIER \\
\hline 2130 & UISO_AEA & ITASRL & 0.287 & $\mathrm{pCi} / \mathrm{L}$ & 0.0537 & 0.0629 & & & \\
\hline 2131 & 6010 METALS ICP & DATACH & & & & & & & U \\
\hline 2132 & $8260^{\circ}$ VOA GCMS & DATACH & & & & & & & $\mathrm{U}$ \\
\hline 2133 & SRISO_SEPP_PRECIP_GPC & ITASRL & 0.0621 & $\mathrm{pCi} / \mathrm{L}$ & 0.0512 & 0.0544 & & & \\
\hline 2134 & TRITIUMM_ELECT_LSC & ITASRL & 36.4 & $\mathrm{pCi} / \mathrm{L}$ & 2.46 & 8.93 & & & \\
\hline 2135 & UISO_AEA & ITASRL & 0.213 & $\mathrm{pCi} / \mathrm{L}$ & 0.0441 & 0.0501 & & & \\
\hline 2136 & UISO_AEA & ITASRL & 0.00558 & $\mathrm{pCi} / \mathrm{L}$ & 0.0111 & 0.0112 & & & $U$ \\
\hline 2137 & UISO_AEA & ITASRL & 0.217 & $\mathrm{pCi} / \mathrm{L}$ & 0.0454 & 0.0518 & & & \\
\hline 2138 & 6010_METALS_ICP & DATACH & & & & & & & $\mathrm{U}$ \\
\hline 2139 & 8260_VOA_GCMS & DATACH & & & & & & & U \\
\hline 2140 & SRISO_SEP_PRECIP_GPC & ITASRL & 0.0868 & $\mathrm{pCi} / \mathrm{L}$ & 0.0455 & 0.0495 & & & \\
\hline 2141 & TRITIUM_ELECT_LSC & ITASRL & 35.5 & $\mathrm{pCi} / \mathrm{L}$ & 2.42 & 8.81 & & & \\
\hline 2142 & UISO_AEA & ITASRL & 0.187 & $\mathrm{pCi} / \mathrm{L}$ & 0.0502 & 0.055 & & & \\
\hline 2143 & UISO_AEA & ITASRL & 0.00498 & $\mathrm{pCi} / \mathrm{L}$ & 0.0112 & 0.0113 & & & $U$ \\
\hline 2144 & UISO_AEA & ITASRL & 0.305 & $\mathrm{pCi} / \mathrm{L}$ & 0.0638 & 0.0736 & & & \\
\hline 2145 & 6010_METALS_ICP & DATACH & & & & & & & $\mathrm{U}$ \\
\hline 2146 & 8260_VOA_GCMS & DATACH & & & & & & & $U$ \\
\hline 2147 & SRISO-SĒP_PRECIP_GPC & ITASRL & 0.0897 & $\mathrm{pCi} / \mathrm{L}$ & 0.0423 & 0.0469 & & & \\
\hline 2149 & UISO_AEA & ITASRL & 0.193 & $\mathrm{pCi} / \mathrm{L}$ & 0.0423 & 0.0475 & & & \\
\hline 2150 & UISO_AEA & ITASRL & 0.0119 & $\mathrm{pCi} / \mathrm{L}$ & 0.0117 & 0.0118 & & & \\
\hline 2151 & UISO_AEA & ITASRL & 0.258 & $\mathrm{pCi} / \mathrm{L}$ & 0.0493 & 0.0572 & & & \\
\hline 2152 & 6010_METALS_ICP & DATACH & & & & & & & $U$ \\
\hline 2153 & 8260_VOA_GCMS & DATACH & & & & & & & $\mathrm{U}$ \\
\hline 2154 & SRISO_SEP_PRECIP_GPC & ITASRL & 0.0274 & $\mathrm{pCi} / \mathrm{L}$ & 0.0468 & 0.0477 & & & $\mathrm{U}$ \\
\hline 2155 & TRITIUMM_ELECCT_LSC̄ & ITASRL & 36 & $\mathrm{pCi} / \mathrm{L}$ & 2.44 & 8.81 & & & \\
\hline 2156 & UISO_AEA & ITASRL & 0.168 & $\mathrm{pCi} / \mathrm{L}$ & 0.0382 & 0.0426 & & & \\
\hline \begin{tabular}{|l|l|}
2157 \\
\end{tabular} & UISO_AEA & ITASRL & 0.00563 & $\mathrm{pCi} / \mathrm{L}$ & 0.00939 & 0.00953 & & & U \\
\hline 2158 & UISO_AEA & ITASRL & 0.25 & $\mathrm{pCi} / \mathrm{L}$ & 0.0466 & 0.0543 & & & \\
\hline 2159 & 6010_METALS_ICP & DATACH & & & & & & & $U$ \\
\hline 2160 & 8260_VOA_GCMS & DATACH & & & & & & & $U$ \\
\hline 2161 & SRISO_SEPP_PRECIP_GPC & ITASRL & 0.0636 & $\mathrm{pCi} / \mathrm{L}$ & 0.049 & 0.0533 & & & \\
\hline 2162 & TRITIŪM_ELECCT_LSC̄ & ITASRL & 36.4 & $\mathrm{pCi} / \mathrm{L}$ & 2.45 & 8.92 & & & \\
\hline 2163 & UISO_AEA- & ITASRL & 0.173 & $\mathrm{pCi} / \mathrm{L}$ & 0.0405 & 0.045 & & & \\
\hline 2164 & UISO_AEA & ITASRL & 0.00468 & $\mathrm{pCi} / \mathrm{L}$ & 0.0106 & 0.0107 & & & $\mathrm{U}$ \\
\hline 2165 & UISO_AEA & ITASRL & 0.18 & $\mathrm{pCi} / \mathrm{L}$ & 0.0423 & 0.0472 & & & \\
\hline 2166 & 6010_METALS_ICP & DATACH & & & & & & & U \\
\hline
\end{tabular}


$\mathrm{N}$

O

P

Q

$\mathrm{R}$

$\mathrm{S}$

\begin{tabular}{|c|c|c|c|c|c|c|c|c|c|}
\hline 1 & METHOD_NAME & LAB_CODE & VALUE_RPTD & ANAL_UNITS_RPTD & COUNTING_ERROR & TOTAL_ANAL_ERROR & MIN_DETECTABLE_ACTIVITY & PCNT_MOISTURE & LAB_QUALIFIER \\
\hline 2168 & SRISO_SEP_PRECIP_GPC & ITASRL & 0.0624 & $\mathrm{pCi} / \mathrm{L}$ & 0.0431 & 0.0472 & & & \\
\hline 2169 & TC99_SEP_LSC & ITASRL & -0.539 & $\mathrm{pCi} / \mathrm{L}$ & 0.214 & 1.14 & & & U \\
\hline 2170 & TRITIUM_ELECT_LSC & ITASRL & 190 & $\mathrm{pCi} / \mathrm{L}$ & 4.01 & 21.1 & & & \\
\hline 2171 & UISO_AEA & ITASRL & 0.204 & $\mathrm{pCi} / \mathrm{L}$ & 0.0415 & 0.0472 & & & \\
\hline 2173 & UISO_AEA & ITASRL & 0.238 & $\mathrm{pCi} / \mathrm{L}$ & 0.0455 & 0.0527 & & & \\
\hline 2174 & 6010_METALS_ICP & DATACH & & & & & & & $\mathrm{U}$ \\
\hline 2175 & 8260_VOA_GCMS & DATACH & & & & & & & $\mathrm{U}$ \\
\hline 2176 & SRISO_SEP_PRECIP_GPC & ITASRL & 0.0819 & $\mathrm{pCi} / \mathrm{L}$ & 0.0846 & 0.0877 & & & $U$ \\
\hline 2178 & TRITIŪM_ĒELECT_LSC & ITASRL & 34.4 & $\mathrm{pCi} / \mathrm{L}$ & 2.44 & 8.83 & & & \\
\hline 2179 & UISO_AEA & ITASRL & 0.158 & $\mathrm{pCi} / \mathrm{L}$ & 0.0365 & 0.0406 & & & \\
\hline 2180 & UISO_AEA & ITASRL & 0.0158 & $\mathrm{pCi} / \mathrm{L}$ & 0.0141 & 0.0143 & & & \\
\hline 2181 & UISO_AEA & ITASRL & 0.189 & $\mathrm{pCi} / \mathrm{L}$ & 0.041 & 0.0464 & & & \\
\hline 2182 & 6010_METALS_ICP & DATACH & & & & & & & $\mathrm{U}$ \\
\hline 2183 & 8260_VOA_GCMS & DATACH & & & & & & & $U$ \\
\hline 2184 & SRISO_SEP_PRECIP_GPC & ITASRL & 0.0857 & $\mathrm{pCi} / \mathrm{L}$ & 0.0427 & 0.0467 & & & \\
\hline 2185 & TC99_SEP_LSC & ITASRL & -0.342 & $\mathrm{pCi} / \mathrm{L}$ & 0.217 & 1.16 & & & $U$ \\
\hline 2186 & TRITIUM_ELECT_LSC & ITASRL & 50.4 & $\mathrm{pCi} / \mathrm{L}$ & 2.64 & 9.95 & & & \\
\hline 2188 & UISO_AEA & ITASRL & 0.00413 & $\mathrm{pCi} / \mathrm{L}$ & 0.00916 & 0.00929 & & & $\mathrm{U}$ \\
\hline 2189 & UISO_AEA & ITASRL & 0.213 & $\mathrm{pCi} / \mathrm{L}$ & 0.0455 & 0.0517 & & & \\
\hline 2190 & 6010_METALS_ICP & DATACH & & & & & & & $\mathrm{U}$ \\
\hline 2191 & 8260_VOA_GCMS & DATACH & & & & & & & U \\
\hline 2192 & SRISO_SEP_PRECIP_GPC & ITASRL & 0.0869 & $\mathrm{pCi} / \mathrm{L}$ & 0.0459 & 0.0498 & & & \\
\hline 2193 & TC99_SEP_LSC & ITASRL & -0.837 & $\mathrm{pCi} / \mathrm{L}$ & 0.238 & 1.27 & & & U \\
\hline 2194 & TRITIUM_ELECT_LSC & ITASRL & 39.1 & $\mathrm{pCi} / \mathrm{L}$ & 2.5 & 9.11 & & & \\
\hline 2195 & UISO_AEA & ITASRL & 0.172 & $\mathrm{pCi} / \mathrm{L}$ & 0.0411 & 0.0454 & & & \\
\hline 2196 & UISO_AEA & ITASRL & 0.00359 & $\mathrm{pCi} / \mathrm{L}$ & 0.0105 & 0.0106 & & & $U$ \\
\hline 2197 & UISO_AEA & ITASRL & 0.224 & $\mathrm{pCi} / \mathrm{L}$ & 0.0463 & 0.0529 & & & \\
\hline 2198 & 6010_METALS_ICP & DATACH & & & & & & & $U$ \\
\hline 2199 & 8260_VOA_GCMS & DATACH & & & & & & & $U$ \\
\hline 2200 & SRISO_SEP_PRECIP_GPC & ITASRL & 0.0843 & $\mathrm{pCi} / \mathrm{L}$ & 0.0445 & 0.0489 & & & \\
\hline 2201 & TC99_SEP_LSC & ITASRL & -0.587 & $\mathrm{pCi} / \mathrm{L}$ & 0.192 & 1.02 & & & $\mathrm{U}$ \\
\hline 2202 & TRITIUM_ELECT_LSC & ITASRL & 34.9 & $\mathrm{pCi} / \mathrm{L}$ & 2.43 & 8.79 & & & \\
\hline 2203 & UISO_AEA & ITASRL & 0.202 & $\mathrm{pCi} / \mathrm{L}$ & 0.0428 & 0.0487 & & & \\
\hline 2204 & UISO_AEA & ITASRL & 0.00444 & $\mathrm{pCi} / \mathrm{L}$ & 0.00834 & 0.00848 & & & $U$ \\
\hline 2205 & UISO_AEA & ITASRL & 0.154 & $\mathrm{pCi} / \mathrm{L}$ & 0.0366 & 0.0404 & & & \\
\hline
\end{tabular}

ITASRL 


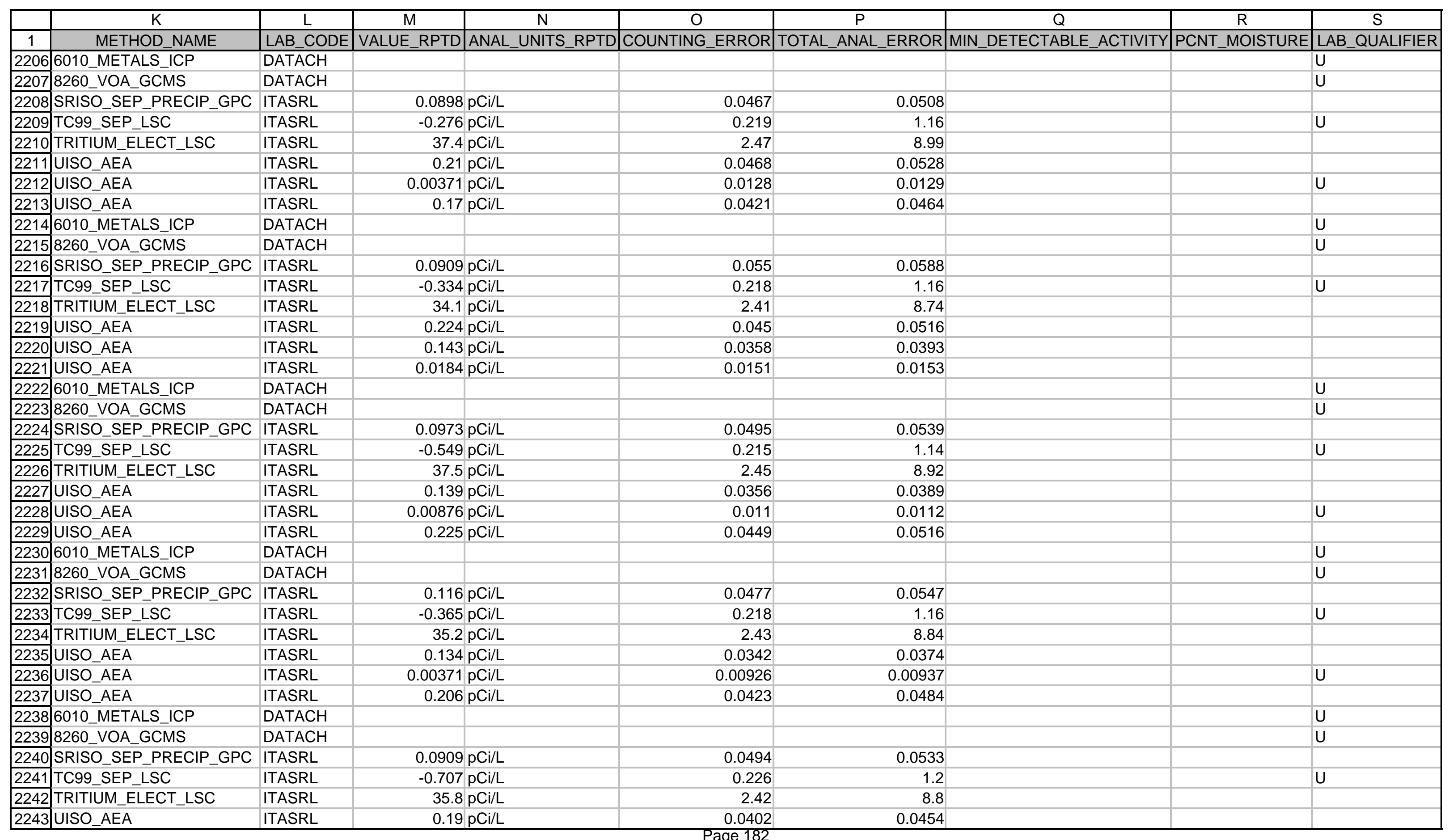




\begin{tabular}{|c|c|c|c|c|c|c|c|c|c|}
\hline & $\mathrm{K}$ & $\mathrm{L}$ & $\mathrm{M}$ & $\mathrm{N}$ & $\mathrm{O}$ & $\mathrm{P}$ & $\mathrm{Q}$ & $\mathrm{R}$ & $\mathrm{S}$ \\
\hline 1 & METHOD_NAME & LAB_CODE & VALUE_RPTD & ANAL_UNITS_RPTD & COUNTING_ERROR & $\mid$ TOTAL_ANAL_ERROR| & MIN_DETECTABLE_ACTIVITY & PCNT_MOISTURE & LAB_QUALIFIER \\
\hline 2244 & UISO_AEA & ITASRL & 0.0131 & $\mathrm{pCi} / \mathrm{L}$ & 0.0131 & 0.0133 & & & $U$ \\
\hline 2245 & UISO_AEA & ITASRL & 0.21 & $\mathrm{pCi} / \mathrm{L}$ & 0.043 & 0.0492 & & & \\
\hline 2246 & 6010 METALS ICP & DATACH & & & & & & & $\mathrm{U}$ \\
\hline 2247 & $8260^{\circ}$ VOA GCMS & DATACH & & & & & & & $\mathrm{U}$ \\
\hline 2248 & SRISO_SEPP_PRECIP_GPC & ITASRL & 0.0834 & $\mathrm{pCi} / \mathrm{L}$ & 0.0465 & 0.0505 & & & \\
\hline 2249 & TRITIUMM_ELECTT_LSC̄ & ITASRL & 128 & $\mathrm{pCi} / \mathrm{L}$ & 3.42 & 15.9 & & & \\
\hline 2250 & UISO_AËA & ITASRL & 0.299 & $\mathrm{pCi} / \mathrm{L}$ & 0.0513 & 0.0611 & & & \\
\hline 2251 & UISO_AEA & ITASRL & 0.246 & $\mathrm{pCi} / \mathrm{L}$ & 0.0467 & 0.0539 & & & \\
\hline 2253 & 6010_METALS_ICP & DATACH & & & & & & & $\mathrm{U}$ \\
\hline 2254 & 8260_VOA_GCMS & DATACH & & & & & & & $U$ \\
\hline 2255 & SRISO_SEP_PRECIP_GPC & ITASRL & 0.118 & $\mathrm{pCi} / \mathrm{L}$ & 0.066 & 0.074 & & & \\
\hline 2256 & TRITIUM_ELECT_LSC & ITASRL & 79.2 & $\mathrm{pCi} / \mathrm{L}$ & 2.95 & 12.1 & & & \\
\hline 2257 & UISO_AEA & ITASRL & 0.167 & $\mathrm{pCi} / \mathrm{L}$ & 0.0449 & 0.0491 & & & \\
\hline 2258 & UISO_AEA & ITASRL & 0.00484 & $\mathrm{pCi} / \mathrm{L}$ & 0.015 & 0.015 & & & $U$ \\
\hline 2259 & UISO_AEA & ITASRL & 0.215 & $\mathrm{pCi} / \mathrm{L}$ & 0.0521 & 0.0583 & & & \\
\hline 2260 & 6010_METALS_ICP & DATACH & & & & & & & $U$ \\
\hline 2261 & 8260_VOA_GCMS & DATACH & & & & & & & $U$ \\
\hline 2263 & TRITIŪM_ELEECT_LSC & ITASRL & 40 & $\mathrm{pCi} / \mathrm{L}$ & 2.46 & 9.07 & & & \\
\hline 2264 & UISO_AËA & ITASRL & 0.139 & $\mathrm{pCi} / \mathrm{L}$ & 0.0352 & 0.0385 & & & \\
\hline 2265 & UISO_AEA & ITASRL & 0.00775 & $\mathrm{pCi} / \mathrm{L}$ & 0.0109 & 0.0111 & & & $U$ \\
\hline 2266 & UISO_AEA & ITASRL & 0.233 & $\mathrm{pCi} / \mathrm{L}$ & 0.0456 & 0.0525 & & & \\
\hline 2267 & 6010_METALS_ICP & DATACH & & & & & & & $\mathrm{U}$ \\
\hline 2268 & 8260_VOA_GCMS & DATACH & & & & & & & U \\
\hline 2269 & SRISO_SEPP_PRECIP_GPC & ITASRL & 0.0875 & $\mathrm{pCi} / \mathrm{L}$ & 0.0459 & 0.0521 & & & \\
\hline 2270 & TRITIUMM_ELECCT_LSC̄ & ITASRL & 38 & $\mathrm{pCi} / \mathrm{L}$ & 2.45 & 9 & & & \\
\hline 2271 & UISO_AEA & ITASRL & 0.174 & $\mathrm{pCi} / \mathrm{L}$ & 0.0394 & 0.0439 & & & \\
\hline 2272 & UISO_AEA & ITASRL & 0.000782 & $\mathrm{pCi} / \mathrm{L}$ & 0.00767 & 0.00779 & & & $U$ \\
\hline 2273 & UISO_AEA & ITASRL & 0.211 & $\mathrm{pCi} / \mathrm{L}$ & 0.0439 & 0.0501 & & & \\
\hline 2274 & 6010_METALS_ICP & DATACH & & & & & & & $U$ \\
\hline 2275 & 8260 VOA_GCMS & DATACH & & & & & & & U \\
\hline 2276 & SRISO_SEPP_PRECIP_GPC & ITASRL & 0.0763 & $\mathrm{pCi} / \mathrm{L}$ & 0.0503 & 0.0548 & & & \\
\hline 2277 & TRITIUM_ELEECT_LSC- & ITASRL & 37.1 & $\mathrm{pCi} / \mathrm{L}$ & 2.44 & 8.92 & & & \\
\hline 2278 & UISO_AEA & ITASRL & 0.19 & $\mathrm{pCi} / \mathrm{L}$ & 0.0479 & 0.0528 & & & \\
\hline 2279 & UISO_AEA & ITASRL & 0.00783 & $\mathrm{pCi} / \mathrm{L}$ & 0.014 & 0.0141 & & & $U$ \\
\hline 2280 & UISO_AEA & ITASRL & 0.221 & $\mathrm{pCi} / \mathrm{L}$ & 0.0519 & 0.0583 & & & \\
\hline
\end{tabular}




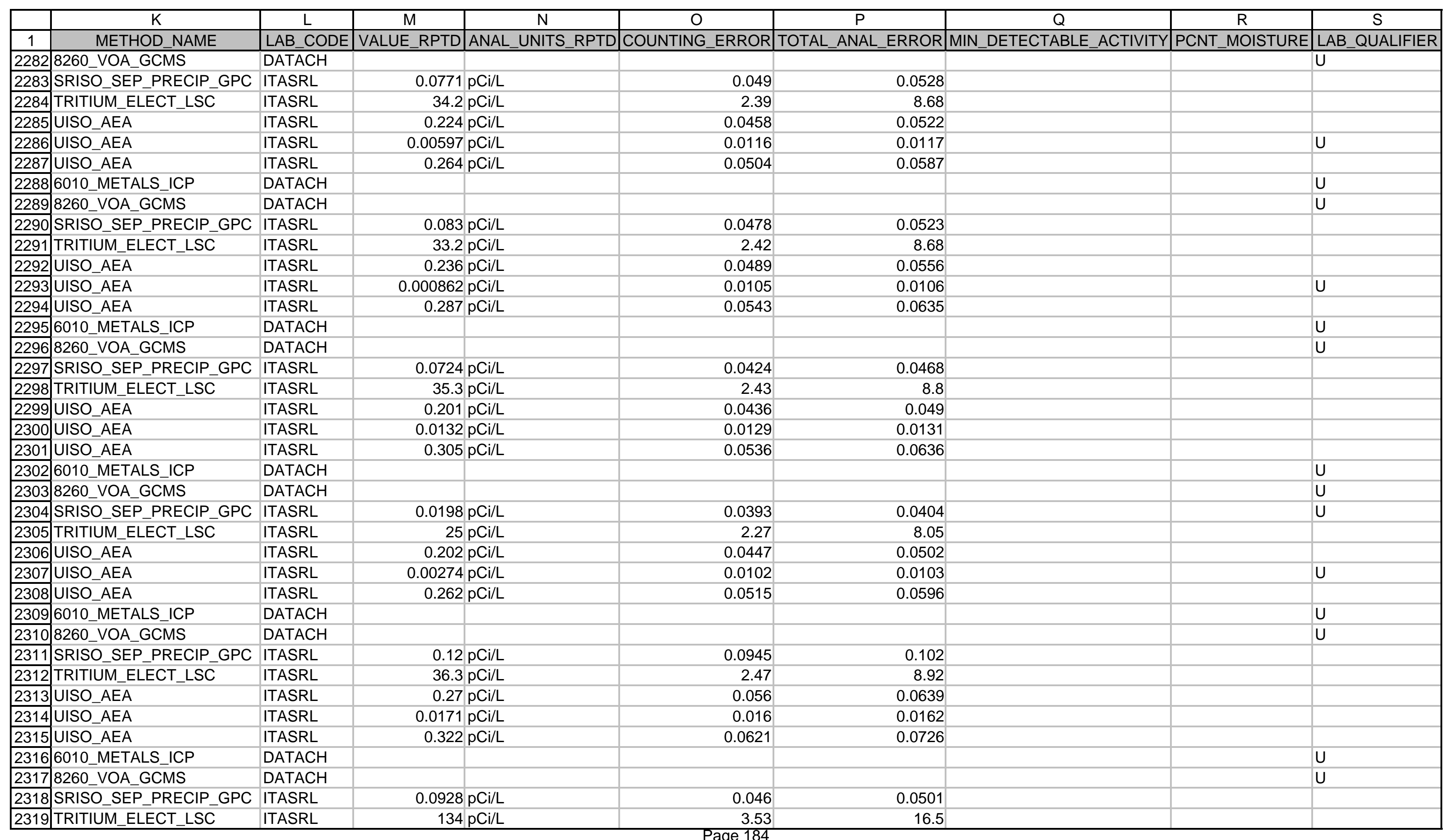




\begin{tabular}{|c|c|c|c|c|c|c|c|c|c|}
\hline & $\mathrm{K}$ & $\mathrm{L}$ & $\mathrm{M}$ & $\mathrm{N}$ & $\mathrm{O}$ & $P$ & $\mathrm{Q}$ & $\mathrm{R}$ & $\mathrm{S}$ \\
\hline 1 & METHOD_NAME & LAB_CODE & VALUE_RPTD & ANAL_UNITS_RPTD & COUNTING_ERROR| & TOTAL_ANAL_ERROR & MIN_DETECTABLE_ACTIVITY & PCNT_MOISTURE & LAB_QUALIFIER \\
\hline 2320 & UISO_AEA & ITASRL & 0.258 & $\mathrm{pCi} / \mathrm{L}$ & 0.0516 & 0.0595 & & & \\
\hline 2321 & UISO_AEA & ITASRL & 0.00664 & $\mathrm{pCi} / \mathrm{L}$ & 0.00956 & 0.0097 & & & $U$ \\
\hline 2322 & UISO_AEA & ITASRL & 0.156 & $\mathrm{pCi} / \mathrm{L}$ & 0.04 & 0.0439 & & & \\
\hline 2323 & 6010_METALS_ICP & DATACH & & & & & & & U \\
\hline 2324 & 8260_VOA_GCMS & DATACH & & & & & & & U \\
\hline 2325 & SRISO_SEP_PRECIP_GPC & ITASRL & 0.108 & $\mathrm{pCi} / \mathrm{L}$ & 0.076 & 0.081 & & & \\
\hline 2326 & TRITIUM_ELECT_LSC & ITASRL & 47.4 & $\mathrm{pCi} / \mathrm{L}$ & 2.61 & 9.65 & & & \\
\hline 2327 & UISO_AEA & ITASRL & 0.325 & $\mathrm{pCi} / \mathrm{L}$ & 0.059 & 0.0697 & & & \\
\hline 2329 & UISO_AEA & ITASRL & 0.235 & $\mathrm{pCi} / \mathrm{L}$ & 0.0501 & 0.0568 & & & \\
\hline 2330 & 6010_METALS_ICP & DATACH & & & & & & & $\mathrm{U}$ \\
\hline 2331 & 8260_VOA_GCMS & DATACH & & & & & & & $\mathrm{U}$ \\
\hline 2332 & SRISO_SEP_PRECIP_GPC & ITASRL & 0.0613 & $\mathrm{pCi} / \mathrm{L}$ & 0.0479 & 0.0504 & & & \\
\hline 2333 & TRITIUM_ELECT_LSC & ITASRL & 57.4 & $\mathrm{pCi} / \mathrm{L}$ & 2.71 & 10.4 & & & \\
\hline 2334 & UISO_AEA & ITASRL & 0.265 & $\mathrm{pCi} / \mathrm{L}$ & 0.0512 & 0.0594 & & & \\
\hline 2335 & UISO_AEA & ITASRL & 0.00211 & $\mathrm{pCi} / \mathrm{L}$ & 0.00833 & 0.00845 & & & $\mathrm{U}$ \\
\hline 2336 & UISO_AEA & ITASRL & 0.189 & $\mathrm{pCi} / \mathrm{L}$ & 0.043 & 0.048 & & & \\
\hline 2337 & 6010_METALS_ICP & DATACH & & & & & & & $\mathrm{U}$ \\
\hline 2339 & SRISO_SEP_PRECIP_GPC & ITASRL & 0.0477 & pCi/L & 0.0421 & 0.044 & & & \\
\hline 2340 & TRITIUM_ELECT_LSC & ITASRL & 54.8 & $\mathrm{pCi} / \mathrm{L}$ & 2.67 & 10.1 & & & \\
\hline 2341 & UISO_AEA & ITASRL & 0.23 & $\mathrm{pCi} / \mathrm{L}$ & 0.0514 & 0.0581 & & & \\
\hline 2342 & UISO_AEA & ITASRL & 0.00689 & $\mathrm{pCi} / \mathrm{L}$ & 0.0121 & 0.0122 & & & $U$ \\
\hline 2343 & UISO_AEA & ITASRL & 0.223 & $\mathrm{pCi} / \mathrm{L}$ & 0.0497 & 0.056 & & & \\
\hline 2344 & 6010_METALS_ICP & DATACH & & & & & & & $\mathrm{U}$ \\
\hline 2345 & 8260_VOA_GCMS & DATACH & & & & & & & $\mathrm{U}$ \\
\hline 2346 & SRISO_SEP_PRECIP_GPC & ITASRL & 0.0834 & $\mathrm{pCi} / \mathrm{L}$ & 0.0463 & 0.05 & & & \\
\hline 2347 & TRITIUM_ELECT_LSC & ITASRL & 48.5 & $\mathrm{pCi} / \mathrm{L}$ & 2.61 & 9.68 & & & \\
\hline 2348 & UISO_AEA & ITASRL & 0.279 & $\mathrm{pCi} / \mathrm{L}$ & 0.0664 & 0.0751 & & & \\
\hline 2349 & UISO_AEA & ITASRL & 0.0113 & $\mathrm{pCi} / \mathrm{L}$ & 0.0185 & 0.0187 & & & $U$ \\
\hline 2350 & UISO_AEA & ITASRL & 0.216 & $\mathrm{pCi} / \mathrm{L}$ & 0.0582 & 0.0641 & & & \\
\hline 2351 & 6010_METALS_ICP & DATACH & 4.3 & ug/L & & 1.81 & & & $\mathrm{~L}$ \\
\hline 2352 & 8260_VOA_GCMS & DATACH & & & & & & & $\mathrm{U}$ \\
\hline 2353 & SRISO_SEP_PRECIP_GPC & ITASRL & 0.106 & $\mathrm{pCi} / \mathrm{L}$ & 0.0487 & 0.054 & & & \\
\hline 2354 & TRITIUM_ELECT_LSC & ITASRL & 53.9 & $\mathrm{pCi} / \mathrm{L}$ & 2.67 & 10.1 & & & \\
\hline 2355 & UISO_AEA & ITASRL & 0.192 & $\mathrm{pCi} / \mathrm{L}$ & 0.046 & 0.0514 & & & \\
\hline 2356 & UISO_AEA & ITASRL & 0.00953 & $\mathrm{pCi} / \mathrm{L}$ & 0.0139 & 0.014 & & & $\mathrm{U}$ \\
\hline
\end{tabular}




\begin{tabular}{|c|c|c|c|c|c|c|c|c|c|}
\hline & $\mathrm{K}$ & $\mathrm{L}$ & $\mathrm{M}$ & $\mathrm{N}$ & $\mathrm{O}$ & $\mathrm{P}$ & $\mathrm{Q}$ & $\mathrm{R}$ & $\mathrm{S}$ \\
\hline 1 & METHOD NAME & LAB CODE & VALUE RPTD & ANAL UNITS RPTD & COUNTING ERROR & TOTAL ANAL ERROR & MIN DETECTABLE ACTIVITY & PCNT MOISTURE & LAB QUALIFIER \\
\hline 2358 & 6010_METALS_ICP & DATACH & & & & & & & $U$ \\
\hline 2359 & 8260_VOA_GCMS & DATACH & & & & & & & $\mathrm{U}$ \\
\hline 2360 & SRISO_SEP_PRECIP_GPC & ITASRL & 0.0627 & $\mathrm{pCi} / \mathrm{L}$ & 0.0426 & 0.0452 & & & \\
\hline 2361 & TRITIUM_ELEECT_LSC & ITASRL & 51.2 & $\mathrm{pCi} / \mathrm{L}$ & 2.64 & 9.95 & & & \\
\hline 2362 & UISO_AEA & ITASRL & 0.194 & $\mathrm{pCi} / \mathrm{L}$ & 0.0443 & 0.0498 & & & \\
\hline 2363 & UISO_AEA & ITASRL & 0.00416 & $\mathrm{pCi} / \mathrm{L}$ & 0.0118 & 0.0119 & & & $U$ \\
\hline 2364 & UISO_AEA & ITASRL & 0.165 & $\mathrm{pCi} / \mathrm{L}$ & 0.04 & 0.0442 & & & \\
\hline 2365 & 6010_METALS_ICP & DATACH & & & & & & & $U$ \\
\hline 2367 & SRISO_SEP_PRECIP_GPC & ITASRL & 0.0863 & $\mathrm{pCi} / \mathrm{L}$ & 0.05 & 0.0537 & & & \\
\hline 2368 & TRITIUM_ELEECT_LSC & ITASRL & 52.6 & $\mathrm{pCi} / \mathrm{L}$ & 2.66 & 10.1 & & & \\
\hline 2369 & UISO_AEA & ITASRL & 0.219 & $\mathrm{pCi} / \mathrm{L}$ & 0.0471 & 0.0535 & & & \\
\hline 2370 & UISO_AEA & ITASRL & -0.00245 & $\mathrm{pCi} / \mathrm{L}$ & 0.00541 & 0.00557 & & & $\mathrm{U}$ \\
\hline 2371 & UISO_AEA & ITASRL & 0.147 & $\mathrm{pCi} / \mathrm{L}$ & 0.0386 & 0.0421 & & & \\
\hline 2372 & 6010_METALS_ICP & DATACH & 3.7 & $\mathrm{ug} / \mathrm{L}$ & & 1.55 & & & $\mathrm{~L}$ \\
\hline 2373 & 8260_VOA_GCMS & DATACH & & & & & & & $U$ \\
\hline 2374 & SRISO_SEP_PRECIP_GPC & ITASRL & 0.106 & $\mathrm{pCi} / \mathrm{L}$ & 0.0449 & 0.0504 & & & \\
\hline 2375 & TRITIUM_ELEECT_LSC & ITASRL & 53.3 & $\mathrm{pCi} / \mathrm{L}$ & 2.69 & 10.1 & & & \\
\hline 2377 & UISO_AEA & ITASRL & 0.00533 & $\mathrm{pCi} / \mathrm{L}$ & 0.0148 & 0.0149 & & & $U$ \\
\hline 2378 & UISO_AEA & ITASRL & 0.176 & $\mathrm{pCi} / \mathrm{L}$ & 0.0505 & 0.0549 & & & \\
\hline 2379 & 6010_METALS_ICP & DATACH & & & & & & & $U$ \\
\hline 2380 & 8260_VOA_GCMS & DATACH & & & & & & & $U$ \\
\hline 2381 & SRISO_SEP_PRECIP_GPC & ITASRL & 0.0715 & $\mathrm{pCi} / \mathrm{L}$ & 0.0453 & 0.0483 & & & \\
\hline \begin{tabular}{|l|l|}
2382 \\
230 \\
\end{tabular} & TRITIUM_ELECT_LSC & ITASRL & 49.6 & $\mathrm{pCi} / \mathrm{L}$ & 2.64 & 9.86 & & & \\
\hline 2383 & UISO_AEA & ITASRL & 0.248 & $\mathrm{pCi} / \mathrm{L}$ & 0.0603 & 0.0676 & & & \\
\hline 2384 & UISO_AEA & ITASRL & 0.011 & $\mathrm{pCi} / \mathrm{L}$ & 0.0153 & 0.0154 & & & $\mathrm{U}$ \\
\hline 2385 & UISO_AEA & ITASRL & 0.287 & $\mathrm{pCi} / \mathrm{L}$ & 0.0629 & 0.0716 & & & \\
\hline 2386 & 6010_METALS_ICP & DATACH & 3.7 & ug/L & & & & & $\mathrm{U}$ \\
\hline 2387 & 8260_VOA_GCMS & DATACH & 0.22 & $\mathrm{ug} / \mathrm{L}$ & & & & & $U$ \\
\hline 2388 & SRISO_SĒP_PRECIP_GPC & ITASRL & 0.115 & $\mathrm{pCi} / \mathrm{L}$ & 0.144 & 0.146 & & & $U$ \\
\hline 2389 & TRITIUM_ELEECT_LSC & ITASRL & 36.1 & $\mathrm{pCi} / \mathrm{L}$ & 2.43 & 8.83 & & & \\
\hline 2390 & UISO_AEA & ITASRL & 0.182 & $\mathrm{pCi} / \mathrm{L}$ & 0.0454 & 0.0501 & & & \\
\hline 2391 & UISO_AEA & ITASRL & 0.0084 & $\mathrm{pCi} / \mathrm{L}$ & 0.0116 & 0.0118 & & & $\mathrm{U}$ \\
\hline 2392 & UISO_AEA & ITASRL & 0.156 & $\mathrm{pCi} / \mathrm{L}$ & 0.041 & 0.0446 & & & \\
\hline 2393 & 6010_METALS_ICP & DATACH & 3.7 & ug/L & & & & & $U$ \\
\hline 2394 & 8260_VOA_GCMS & DATACH & 0.22 & $\mathrm{ug} / \mathrm{L}$ & & & & & $U$ \\
\hline
\end{tabular}




\begin{tabular}{|c|c|c|c|c|c|c|c|c|c|}
\hline & $\mathrm{K}$ & $\mathrm{L}$ & $\mathrm{M}$ & $\mathrm{N}$ & $\mathrm{O}$ & $\mathrm{P}$ & $\mathrm{Q}$ & $\mathrm{R}$ & $\mathrm{S}$ \\
\hline 1 & METHOD_NAME & LAB_CODE & VALUE_RPTD & ANAL_UNITS_RPTD & COUNTING_ERROR & TOTAL_ANAL_ERROR & MIN_DETECTABLE_ACTIVITY & PCNT_MOISTURE & LAB_QUALIFIER \\
\hline 2396 & TRITIUM_ELECT_LSC & ITASRL & 28.7 & $\mathrm{pCi} / \mathrm{L}$ & 2.36 & 8.38 & & & \\
\hline 2397 & UISO_AEA & ITASRL & 0.376 & $\mathrm{pCi} / \mathrm{L}$ & 0.0661 & 0.0788 & & & \\
\hline 2398 & UISO AEA & ITASRL & 0.00316 & $\mathrm{pCi} / \mathrm{L}$ & 0.0117 & 0.0118 & & & $\mathrm{U}$ \\
\hline 2399 & UISO AEA & ITASRL & 0.283 & $\mathrm{pCi} / \mathrm{L}$ & 0.0565 & 0.0649 & & & \\
\hline 2400 & 6010_METALS_ICP & DATACH & 3.7 & ug/L & & & & & $U$ \\
\hline 2401 & 8260_VOA_GCMS & DATACH & 0.22 & ug/L & & & & & $U$ \\
\hline 2402 & SRISO_SEPP_PRECIP_GPC & ITASRL & 0.0305 & $\mathrm{pCi} / \mathrm{L}$ & 0.107 & 0.107 & & & $U$ \\
\hline 2403 & TRITIŪM_ELECCT_LSC̄ & ITASRL & 34.1 & $\mathrm{pCi} / \mathrm{L}$ & 2.4 & 8.67 & & & \\
\hline \begin{tabular}{|l|l|}
2405 \\
\end{tabular} & UISO_AEA & ITASRL & 0.00885 & $\mathrm{pCi} / \mathrm{L}$ & 0.012 & 0.0122 & & & U \\
\hline 2406 & UISO_AEA & ITASRL & 0.157 & $\mathrm{pCi} / \mathrm{L}$ & 0.0418 & 0.0455 & & & \\
\hline 2407 & 6010_METALS_ICP & DATACH & 3.7 & ug/L & & & & & U \\
\hline 2408 & 8260_VOA_GCMS & DATACH & 0.22 & ug/L & & & & & $\mathrm{U}$ \\
\hline 2409 & SRISO_SEPP_PRECIP_GPC & ITASRL & 0.0646 & $\mathrm{pCi} / \mathrm{L}$ & 0.0543 & 0.0566 & & & \\
\hline 2410 & TRITIUMM_ELECTT_LSC & ITASRL & 31.8 & $\mathrm{pCi} / \mathrm{L}$ & 2.4 & 8.59 & & & \\
\hline 2411 & UISO_AEA & ITASRL & 0.196 & $\mathrm{pCi} / \mathrm{L}$ & 0.0483 & 0.0536 & & & \\
\hline 2412 & UISO AEA & ITASRL & 0.00653 & $\mathrm{pCi} / \mathrm{L}$ & 0.0112 & 0.0114 & & & $U$ \\
\hline 2413 & UISO_AEA & ITASRL & 0.129 & $\mathrm{pCi} / \mathrm{L}$ & 0.0389 & 0.0417 & & & \\
\hline 2415 & 8260_VOA_GCMS & DATACH & 0.22 & $\mathrm{ug} / \mathrm{L}$ & & & & & $\mathrm{U}$ \\
\hline 2416 & SRISO-SĒP_PRECIP_GPC & ITASRL & & & & & & & \\
\hline 2417 & TRITIUMM_ELECT_LSC- & ITASRL & 32.2 & $\mathrm{pCi} / \mathrm{L}$ & 2.39 & 8.54 & & & \\
\hline 2418 & UISO_AEA & ITASRL & 0.173 & $\mathrm{pCi} / \mathrm{L}$ & 0.0374 & 0.042 & & & \\
\hline 2419 & UISO_AEA & ITASRL & -0.00034 & $\mathrm{pCi} / \mathrm{L}$ & 0.00551 & 0.00581 & & & $\mathrm{U}$ \\
\hline 2420 & UISO_AEA & ITASRL & 0.124 & $\mathrm{pCi} / \mathrm{L}$ & 0.0315 & 0.0342 & & & \\
\hline 2421 & 6010_METALS_ICP & DATACH & 3.7 & ug/L & & & & & $U$ \\
\hline 2422 & 8260_VOA_GCMS & DATACH & 0.22 & ug/L & & & & & $U$ \\
\hline 2423 & SRISO_SEP_PRECIP_GPC & ITASRL & 0.0778 & $\mathrm{pCi} / \mathrm{L}$ & 0.0807 & 0.0859 & & & $U$ \\
\hline 2424 & TRITIUM_ELEECT_LSC & ITASRL & 31.4 & $\mathrm{pCi} / \mathrm{L}$ & 2.41 & 8.55 & & & \\
\hline 2425 & UISO_AEA & ITASRL & 0.173 & $\mathrm{pCi} / \mathrm{L}$ & 0.0376 & 0.0422 & & & \\
\hline 2426 & UISO_AEA & ITASRL & 0.00462 & $\mathrm{pCi} / \mathrm{L}$ & 0.00875 & 0.00897 & & & $U$ \\
\hline \begin{tabular}{|l|}
2427 \\
\end{tabular} & UISO AEA & ITASRL & 0.143 & $\mathrm{pCi} / \mathrm{L}$ & 0.0338 & 0.0371 & & & \\
\hline 2428 & 6010_METALS_ICP & DATACH & 3.7 & ug/L & & & & & $U$ \\
\hline 2429 & 8260_VOA_GCMS & DATACH & 0.22 & ug/L & & & & & $U$ \\
\hline 2430 & SRISO_SEP_PRECIP_GPC & ITASRL & 0.074 & $\mathrm{pCi} / \mathrm{L}$ & 0.05 & 0.0549 & & & \\
\hline 2431 & TRITIUM_ELECT_LSC & ITASRL & 25.6 & $\mathrm{pCi} / \mathrm{L}$ & 2.37 & 8.27 & & & \\
\hline 2432 & UISO_AEA & ITASRL & 0.163 & $\mathrm{pCi} / \mathrm{L}$ & 0.0419 & 0.046 & & & \\
\hline
\end{tabular}




\begin{tabular}{|c|c|c|c|c|c|c|c|c|c|}
\hline & $\mathrm{K}$ & $\mathrm{L}$ & $\mathrm{M}$ & $\mathrm{N}$ & $\mathrm{O}$ & $\mathrm{P}$ & $\mathrm{Q}$ & $\mathrm{R}$ & $\mathrm{S}$ \\
\hline 1 & METHOD NAME & LAB CODE & VALUE RPTD & ANAL UNITS RPTD & COUNTING ERROR & TOTAL ANAL ERROR & MIN DETECTABLE ACTIVITY & PCNT MOISTURE & LAB QUALIFIER \\
\hline 2434 & UISO_AEA & ITASRL & 0.167 & $\mathrm{pCi} / \mathrm{L}$ & 0.0416 & 0.0456 & & & \\
\hline 2435 & 6010_METALS_ICP & DATACH & 3.7 & ug/L & & & & & $\mathrm{U}$ \\
\hline 2436 & 8260_VOA_GCMS & DATACH & 0.22 & ug/L & & & & & $\mathrm{U}$ \\
\hline 2437 & SRISO_SEP_PRECIP_GPC & ITASRL & 0.455 & $\mathrm{pCi} / \mathrm{L}$ & 0.0812 & 0.121 & & & \\
\hline 2438 & TRITIUM_ELEECT_LSC & ITASRL & 31.7 & $\mathrm{pCi} / \mathrm{L}$ & 2.39 & 8.51 & & & \\
\hline 2439 & UISO_AEA & ITASRL & 0.191 & $\mathrm{pCi} / \mathrm{L}$ & 0.0404 & 0.0456 & & & \\
\hline 2440 & UISO_AEA & ITASRL & 0.00579 & $\mathrm{pCi} / \mathrm{L}$ & 0.00902 & 0.00924 & & & 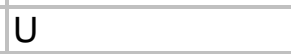 \\
\hline 2441 & UISO_AEA & ITASRL & 0.126 & $\mathrm{pCi} / \mathrm{L}$ & 0.0329 & 0.0356 & & & \\
\hline 2443 & 8260_VOA_GCMS & DATACH & 0.22 & ug/L & & & & & $\mathrm{U}$ \\
\hline 2444 & SRISO_SEP_PRECIP_GPC & ITASRL & 0.0526 & $\mathrm{pCi} / \mathrm{L}$ & 0.0445 & 0.048 & & & \\
\hline \begin{tabular}{|l|l|}
2445 \\
\end{tabular} & TRITIUM_ELECT_LSC & ITASRL & 27.5 & $\mathrm{pCi} / \mathrm{L}$ & 2.34 & 8.22 & & & \\
\hline 2446 & UISO_AEA & ITASRL & 0.213 & $\mathrm{pCi} / \mathrm{L}$ & 0.0451 & 0.051 & & & \\
\hline 2447 & UISO_AEA & ITASRL & 0.0114 & $\mathrm{pCi} / \mathrm{L}$ & 0.012 & 0.0122 & & & $\mathrm{U}$ \\
\hline 2448 & UISO_AEA & ITASRL & 0.123 & $\mathrm{pCi} / \mathrm{L}$ & 0.0342 & 0.0368 & & & \\
\hline 2449 & 6010_METALS_ICP & DATACH & 3.7 & ug/L & & & & & $\mathrm{U}$ \\
\hline 2450 & 8260_VOA_GCMS & DATACH & 0.22 & ug/L & & & & & $U$ \\
\hline 2451 & SRISO_SEP_PRECIP_GPC & ITASRL & 0.0777 & $\mathrm{pCi} / \mathrm{L}$ & 0.0458 & 0.0495 & & & \\
\hline 2453 & UISO_AEA & ITASRL & 0.169 & $\mathrm{pCi} / \mathrm{L}$ & 0.0442 & 0.0484 & & & \\
\hline 2454 & UISO_AEA & ITASRL & 0.0168 & $\mathrm{pCi} / \mathrm{L}$ & 0.0151 & 0.0154 & & & \\
\hline 2455 & UISO_AEA & ITASRL & 0.197 & $\mathrm{pCi} / \mathrm{L}$ & 0.047 & 0.052 & & & \\
\hline 2456 & 6010_METALS_ICP & DATACH & 3.7 & ug/L & & & & & $U$ \\
\hline 2457 & 8260_VOA_GCMS & DATACH & 0.22 & ug/L & & & & & $U$ \\
\hline \begin{tabular}{|l|l|}
2458 \\
\end{tabular} & SRISO SEP PRECIP GPC & ITASRL & 0.0676 & $\mathrm{pCi} / \mathrm{L}$ & 0.047 & 0.0495 & & & \\
\hline 2459 & TRITIUM_ELECT_LSC & ITASRL & 29.1 & $\mathrm{pCi} / \mathrm{L}$ & 2.35 & 8.32 & & & \\
\hline 2460 & UISO_AEA & ITASRL & 0.218 & $\mathrm{pCi} / \mathrm{L}$ & 0.0441 & 0.0503 & & & \\
\hline 2461 & UISO_AEA & ITASRL & 0.00114 & $\mathrm{pCi} / \mathrm{L}$ & 0.00742 & 0.00765 & & & $\mathrm{U}$ \\
\hline 2462 & UISO_AEA & ITASRL & 0.159 & $\mathrm{pCi} / \mathrm{L}$ & 0.0373 & 0.0411 & & & \\
\hline 2463 & 6010_METALS_ICP & DATACH & 3.7 & ug/L & & & & & $U$ \\
\hline 2464 & 8260_VOA_GCMS & DATACH & 0.22 & ug/L & & & & & $U$ \\
\hline 2465 & SRISO_SEP_PRECIP_GPC & ITASRL & 0.115 & $\mathrm{pCi} / \mathrm{L}$ & 0.078 & 0.0818 & & & \\
\hline 2466 & TRITIUM_ELEECT_LSC & ITASRL & 33.1 & $\mathrm{pCi} / \mathrm{L}$ & 2.4 & 8.55 & & & \\
\hline 2467 & UISO_AEA & ITASRL & 0.205 & $\mathrm{pCi} / \mathrm{L}$ & 0.0453 & 0.0509 & & & \\
\hline 2468 & UISO_AEA & ITASRL & 0.0209 & $\mathrm{pCi} / \mathrm{L}$ & 0.0159 & 0.0162 & & & \\
\hline 2469 & UISO_AEA & ITASRL & 0.15 & $\mathrm{pCi} / \mathrm{L}$ & 0.0388 & 0.0423 & & & \\
\hline 2470 & 6010_METALS_ICP & DATACH & 4.6 & ug/L & & 1.93 & & & $\mathrm{~L}$ \\
\hline
\end{tabular}

Page 188 
$\mathrm{N}$

O

$\mathrm{P}$

Q

R

$\mathrm{S}$

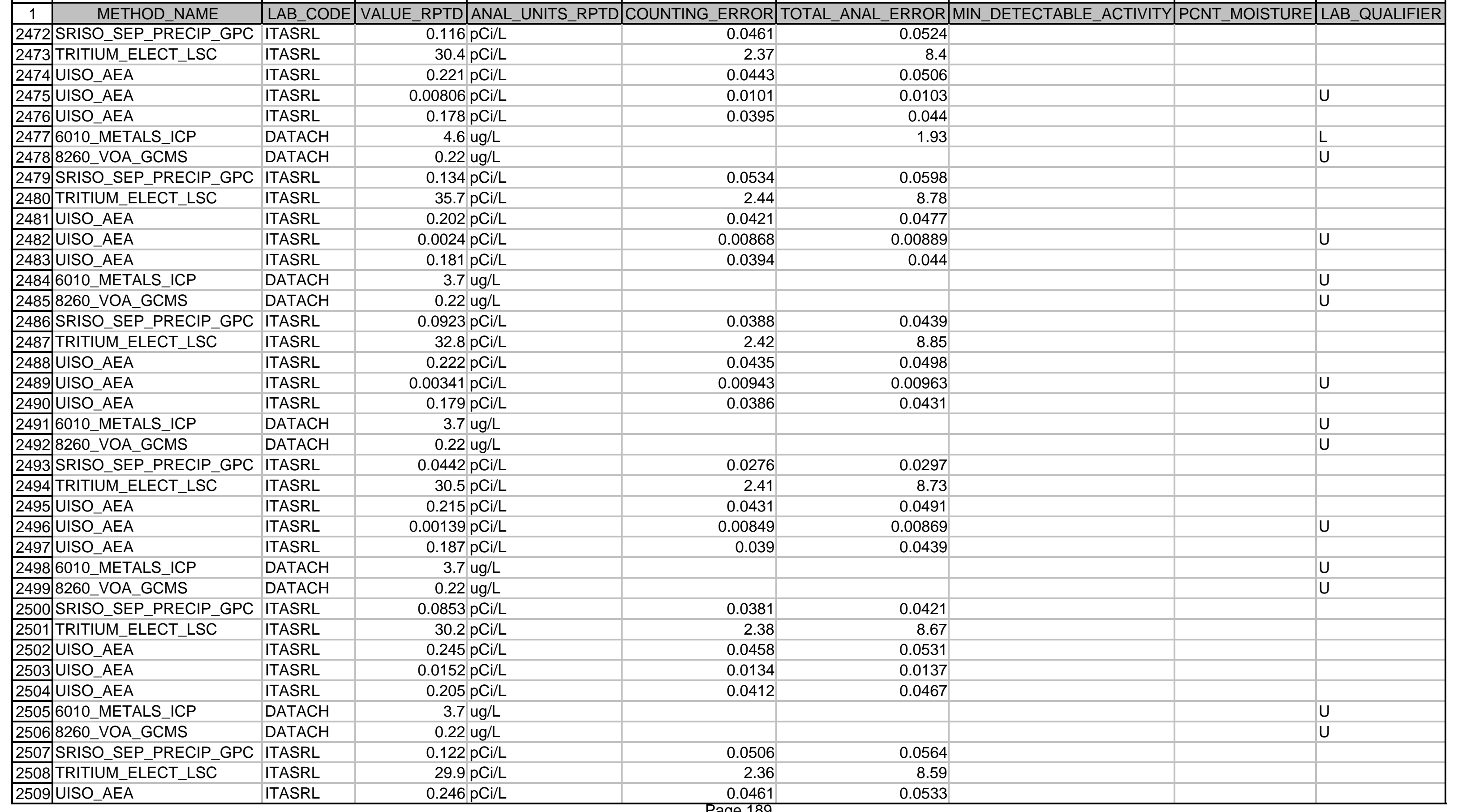

\begin{tabular}{|l|l|}
\hline 2508 & UISO_AEA \\
\hline
\end{tabular}

$0.246 \mathrm{pCi} / \mathrm{L}$

0.0461
Page 189 


\begin{tabular}{|c|c|c|c|c|c|c|c|c|c|}
\hline & $\mathrm{K}$ & $\mathrm{L}$ & $M$ & $\mathrm{~N}$ & $\mathrm{O}$ & $\mathrm{P}$ & $\mathrm{Q}$ & $\mathrm{R}$ & $\mathrm{S}$ \\
\hline 1 & METHOD_NAME & LAB_CODE & VALUE_RPTD & ANAL_UNITS_RPTD & COUNTING_ERROR & TOTAL_ANAL_ERROR & MIN_DETECTABLE_ACTIVITY & PCNT_MOISTURE & LAB_QUALIFIER \\
\hline 2510 & UISO_AEA & ITASRL & 0.00336 & $\mathrm{pCi} / \mathrm{L}$ & 0.00935 & 0.00955 & & & $\mathrm{U}$ \\
\hline 2511 & UISO_AEA & ITASRL & 0.198 & $\mathrm{pCi} / \mathrm{L}$ & 0.0405 & 0.0457 & & & \\
\hline 2512 & 6010_METALS_ICP & DATACH & 3.7 & ug/L & & & & & $\mathrm{U}$ \\
\hline 2513 & 6010_METALS_ICP & DATACH & 3.7 & ug/L & & & & & $\mathrm{U}$ \\
\hline 2514 & 8260_VOA_GCMS & DATACH & 0.22 & ug/L & & & & & $\mathrm{U}$ \\
\hline 2515 & 8260_VOA_GCMS & DATACH & 0.22 & ug/L & & & & & U \\
\hline 2516 & SRISO_SEP_PRECIP_GPC & ITASRL & 0.0486 & pCi/L & 0.042 & 0.0439 & & & \\
\hline 2517 & SRISO_SEP_PRECIP_GPC & ITASRL & 0.0861 & $\mathrm{pCi} / \mathrm{L}$ & 0.0471 & 0.0508 & & & \\
\hline 2519 & TRITIUM_ELECT_LSC & ITASRL & 43 & $\mathrm{pCi} / \mathrm{L}$ & 2.55 & 9.54 & & & \\
\hline 2520 & UISO_AEA & ITASRL & 0.17 & $\mathrm{pCi} / \mathrm{L}$ & 0.0397 & 0.044 & & & \\
\hline 2521 & UISO_AEA & ITASRL & 0.0118 & $\mathrm{pCi} / \mathrm{L}$ & 0.0127 & 0.013 & & & $\mathrm{U}$ \\
\hline 2522 & UISO_AEA & ITASRL & 0.154 & $\mathrm{pCi} / \mathrm{L}$ & 0.0362 & 0.0399 & & & \\
\hline 2523 & UISO_AEA & ITASRL & 0.226 & $\mathrm{pCi} / \mathrm{L}$ & 0.0513 & 0.0577 & & & \\
\hline 2524 & UISO_AEA & ITASRL & 0.0112 & $\mathrm{pCi} / \mathrm{L}$ & 0.0149 & 0.0151 & & & U \\
\hline 2525 & UISO_AEA & ITASRL & 0.207 & $\mathrm{pCi} / \mathrm{L}$ & 0.0483 & 0.0537 & & & \\
\hline 2526 & 6010_METALS_ICP & DATACH & 3.7 & ug/L & & & & & $\mathrm{U}$ \\
\hline 2527 & 8260_VOA_GCMS & DATACH & 0.22 & ug/L & & & & & U \\
\hline 2529 & TRITIUMM_ELECTT_LSC & ITASRL & 32.1 & $\mathrm{pCi} / \mathrm{L}$ & 2.42 & 8.8 & & & \\
\hline 2530 & UISO_AEA & ITASRL & 0.256 & $\mathrm{pCi} / \mathrm{L}$ & 0.0501 & 0.0577 & & & \\
\hline 2531 & UISO_AEA & ITASRL & 0.00769 & $\mathrm{pCi} / \mathrm{L}$ & 0.0117 & 0.0119 & & & U \\
\hline 2532 & UISO_AEA & ITASRL & 0.207 & $\mathrm{pCi} / \mathrm{L}$ & 0.0443 & 0.0499 & & & \\
\hline 2533 & 6010_METALS_ICP & DATACH & 3.7 & ug/L & & & & & $\mathrm{U}$ \\
\hline 2534 & 8260_VOA_GCMS & DATACH & 0.22 & ug/L & & & & & $U$ \\
\hline 2535 & SRISO_SEP_PRECIP_GPC & ITASRL & 0.0819 & pCi/L & 0.0449 & 0.0491 & & & \\
\hline 2536 & TRITIUM_ELECT_LSC & ITASRL & 33.9 & $\mathrm{pCi} / \mathrm{L}$ & 2.46 & 8.91 & & & \\
\hline 2537 & UISO_AEEA & ITASRL & 0.22 & $\mathrm{pCi} / \mathrm{L}$ & 0.0456 & 0.0519 & & & \\
\hline 2538 & UISO_AEA & ITASRL & -0.000327 & pCi/L & 0.00821 & 0.00842 & & & $\mathrm{U}$ \\
\hline 2539 & UISO_AEA & ITASRL & 0.179 & $\mathrm{pCi} / \mathrm{L}$ & 0.0406 & 0.0451 & & & \\
\hline 2540 & 6010_METALS_ICP & DATACH & 3.7 & $\mathrm{ug} / \mathrm{L}$ & & & & & $U$ \\
\hline 2541 & 8260_VOA_GCMS & DATACH & 0.22 & ug/L & & & & & $\mathrm{U}$ \\
\hline 2542 & SRISO_SEP_PRECIP_GPC & ITASRL & 0.0907 & $\mathrm{pCi} / \mathrm{L}$ & 0.0497 & 0.0535 & & & \\
\hline 2543 & TRITIUM_ELEECT_LSC & ITASRL & 32.5 & $\mathrm{pCi} / \mathrm{L}$ & 2.44 & 8.82 & & & \\
\hline 2544 & UISO_AEA & ITASRL & 0.244 & $\mathrm{pCi} / \mathrm{L}$ & 0.0463 & 0.0534 & & & \\
\hline 2545 & UISO_AEA & ITASRL & 0.00346 & $\mathrm{pCi} / \mathrm{L}$ & 0.0095 & 0.00969 & & & $\mathrm{U}$ \\
\hline 2546 & UISO_AEA & ITASRL & 0.181 & $\mathrm{pCi} / \mathrm{L}$ & 0.0391 & 0.0437 & & & \\
\hline
\end{tabular}




\begin{tabular}{|c|c|c|c|c|c|c|c|c|c|}
\hline & $\mathrm{K}$ & $\mathrm{L}$ & $\mathrm{M}$ & $\mathrm{N}$ & $\mathrm{O}$ & $\mathrm{P}$ & $\mathrm{Q}$ & $\mathrm{R}$ & $\mathrm{S}$ \\
\hline 1 & METHOD_NAME & LAB_CODE & VALUE_RPTD & ANAL_UNITS_RPTD & COUNTING_ERROR & TOTAL_ANAL_ERROR & MIN_DETECTABLE_ACTIVITY & PCNT_MOISTURE & LAB_QUALIFIER \\
\hline 2548 & 8260 VOA_GCMS & DATACH & 0.22 & $u g / L$ & & & & & $U$ \\
\hline 2549 & SRISO_SEP_PRECIP_GPC & ITASRL & 0.0757 & $\mathrm{pCi} / \mathrm{L}$ & 0.0563 & 0.0589 & & & \\
\hline 2550 & TRITIUMM ELECT LSC̄ & ITASRL & 31.3 & $\mathrm{pCi} / \mathrm{L}$ & 2.4 & 8.75 & & & \\
\hline 2551 & UISO AËA & ITASRL & 0.241 & $\mathrm{pCi} / \mathrm{L}$ & 0.0455 & 0.0526 & & & \\
\hline 2552 & UISO_AEA & ITASRL & 0.0136 & $\mathrm{pCi} / \mathrm{L}$ & 0.0119 & 0.0122 & & & \\
\hline 2553 & UISO_AEA & ITASRL & 0.176 & $\mathrm{pCi} / \mathrm{L}$ & 0.038 & 0.0425 & & & \\
\hline 2554 & 6010_METALS_ICP & DATACH & 3.7 & ug/L & & & & & $U$ \\
\hline 2555 & 8260_VOA_GCMS & DATACH & 0.22 & ug/L & & & & & $U$ \\
\hline 2556 & SRISO SEP PRECIP GPC & ITASRL & 0.0751 & $\mathrm{pCi} / \mathrm{L}$ & 0.0552 & 0.0583 & & & \\
\hline 2557 & TRITIUM_ELECT_LSC & ITASRL & 29.2 & $\mathrm{pCi} / \mathrm{L}$ & 2.37 & 8.6 & & & \\
\hline 2558 & UISO_AEA & ITASRL & 0.252 & $\mathrm{pCi} / \mathrm{L}$ & 0.0461 & 0.0536 & & & \\
\hline 2559 & UISO_AEA & ITASRL & 0.00219 & $\mathrm{pCi} / \mathrm{L}$ & 0.00837 & 0.00858 & & & $\mathrm{U}$ \\
\hline 2560 & UISO_AEA & ITASRL & 0.215 & $\mathrm{pCi} / \mathrm{L}$ & 0.0419 & 0.0477 & & & \\
\hline 2561 & 6010_METALS_ICP & DATACH & 3.7 & ug/L & & & & & $U$ \\
\hline 2562 & 8260_VOA_GCMS & DATACH & 0.22 & ug/L & & & & & $U$ \\
\hline 2563 & SRISO_SEP_PRECIP_GPC & ITASRL & 0.0751 & $\mathrm{pCi} / \mathrm{L}$ & 0.0538 & 0.0569 & & & \\
\hline 2564 & TRITIŪM_ELEECT_LSC & ITASRL & 31.5 & $\mathrm{pCi} / \mathrm{L}$ & 2.4 & 8.73 & & & \\
\hline 2565 & UISO_AËA & ITASRL & 0.23 & $\mathrm{pCi} / \mathrm{L}$ & 0.0496 & 0.0561 & & & \\
\hline 2567 & UISO_AEA & ITASRL & 0.197 & $\mathrm{pCi} / \mathrm{L}$ & 0.0445 & 0.0496 & & & \\
\hline 2568 & 6010_METALS_ICP & DATACH & 3.7 & ug/L & & & & & $U$ \\
\hline 2569 & 8260_VOA_GCMS & DATACH & 0.22 & $\mathrm{ug} / \mathrm{L}$ & & & & & $\mathrm{U}$ \\
\hline 2570 & SRISO_SEP_PRECIP_GPC & ITASRL & 0.0694 & $\mathrm{pCi} / \mathrm{L}$ & 0.125 & 0.126 & & & U \\
\hline 2571 & TRITIUM_ELECT_LSC & ITASRL & 32 & $\mathrm{pCi} / \mathrm{L}$ & 2.43 & 8.84 & & & \\
\hline 2572 & UISO_AEA & ITASRL & 0.26 & $\mathrm{pCi} / \mathrm{L}$ & 0.0477 & 0.0556 & & & \\
\hline 2573 & UISO_AEA & ITASRL & 0.0118 & $\mathrm{pCi} / \mathrm{L}$ & 0.0127 & 0.0129 & & & $U$ \\
\hline 2574 & UISO_AEA & ITASRL & 0.182 & $\mathrm{pCi} / \mathrm{L}$ & 0.0396 & 0.0442 & & & \\
\hline 2575 & 6010_METALS_ICP & DATACH & 4.1 & ug/L & & 1.72 & & & L \\
\hline 2576 & $8260^{\circ}$ VOA GCMS & DATACH & 0.22 & $\mathrm{ug} / \mathrm{L}$ & & & & & $\mathrm{U}$ \\
\hline 2577 & SRISO_SEPP_PRECIP_GPC & ITASRL & 0.0624 & $\mathrm{pCi} / \mathrm{L}$ & 0.0389 & 0.0419 & & & \\
\hline 2578 & TRITIUMM_ELECCT_LSC̄ & ITASRL & 29.4 & $\mathrm{pCi} / \mathrm{L}$ & 2.39 & 8.66 & & & \\
\hline 2579 & UISO_AEA & ITASRL & 0.292 & $\mathrm{pCi} / \mathrm{L}$ & 0.0485 & 0.0578 & & & \\
\hline 2580 & UISO_AEA & ITASRL & 0.0163 & $\mathrm{pCi} / \mathrm{L}$ & 0.0134 & 0.0137 & & & \\
\hline 2581 & UISO AEA & ITASRL & 0.215 & $\mathrm{pCi} / \mathrm{L}$ & 0.041 & 0.0469 & & & \\
\hline 2582 & 6010 METALS ICP & DATACH & 3.7 & $\mathrm{ug} / \mathrm{L}$ & & & & & $\mathrm{U}$ \\
\hline 2583 & 8260_VOA_GCMS & DATACH & 0.22 & ug/L & & & & & U \\
\hline 2584 & SRISO_SEP_PRECIP_GPC & ITASRL & 0.104 & $\mathrm{pCi} / \mathrm{L}$ & 0.0532 & 0.0579 & & & \\
\hline
\end{tabular}




\begin{tabular}{|c|c|c|c|c|c|c|c|c|c|}
\hline & $\mathrm{K}$ & $\mathrm{L}$ & $\mathrm{M}$ & $\mathrm{N}$ & $\mathrm{O}$ & $\mathrm{P}$ & $\mathrm{Q}$ & $\mathrm{R}$ & $\mathrm{S}$ \\
\hline 1 & METHOD_NAME & LAB_CODE & VALUE_RPTD & ANAL_UNITS_RPTD & COUNTING_ERROR & TOTAL_ANAL_ERROR & MIN_DETECTABLE_ACTIVITY & PCNT_MOISTURE & LAB_QUALIFIER \\
\hline 2586 & UISO_AEA & ITASRL & 0.235 & $\mathrm{pCi} / \mathrm{L}$ & 0.0458 & 0.0526 & & & \\
\hline 2587 & UISO_AEA & ITASRL & 0.002 & $2 \mathrm{pCi} / \mathrm{L}$ & 0.0073 & 0.00754 & & & $U$ \\
\hline 2588 & UISO_AEA & ITASRL & 0.184 & $4 \mathrm{pCi} / \mathrm{L}$ & 0.0397 & 0.0444 & & & \\
\hline 2589 & 6010_METALS_ICP & DATACH & 1.8 & ug/L & & & & & $U$ \\
\hline 2590 & 8260_VOA_GCMS & DATACH & 0.35 & $\mathrm{ug} / \mathrm{L}$ & & & & & $\mathrm{U}$ \\
\hline 2591 & SRISO_SEP_PRECIP_GPC & ITASRL & 0.0936 & $\mathrm{pCi} / \mathrm{L}$ & 0.0417 & 0.0461 & & & \\
\hline 2592 & TRITIUM_ELECT_LSC & ITASRL & 25.7 & $7 \mathrm{pCi} / \mathrm{L}$ & 2.36 & 8.39 & & & \\
\hline 2593 & UISO_AEA & ITASRL & 0.264 & $4 \mathrm{pCi} / \mathrm{L}$ & 0.0464 & 0.0545 & & & \\
\hline 2595 & UISO_AEA & ITASRL & 0.208 & $3 \mathrm{pCi} / \mathrm{L}$ & 0.0408 & 0.0464 & & & \\
\hline 2596 & 6010_METALS_ICP & DATACH & 1.8 & $3 \mathrm{ug} / \mathrm{L}$ & & & & & $\mathrm{U}$ \\
\hline 2597 & 8260_VOA_GCMS & DATACH & 0.35 & $5 \mathrm{ug} / \mathrm{L}$ & & & & & $U$ \\
\hline 2598 & SRISO_SEP_PRECIP_GPC & ITASRL & 0.129 & $\mathrm{pCi} / \mathrm{L}$ & 0.0481 & 0.0551 & & & \\
\hline 2599 & TRITIUM_ELECT_LSC & ITASRL & 27.4 & $4 \mathrm{pCi} / \mathrm{L}$ & 2.41 & 8.56 & & & \\
\hline 2600 & UISO_AEA & ITASRL & 0.238 & $3 \mathrm{pCi} / \mathrm{L}$ & 0.0414 & 0.0486 & & & \\
\hline 2601 & UISO_AEA & ITASRL & 0.0277 & $7 \mathrm{pCi} / \mathrm{L}$ & 0.0146 & 0.015 & & & \\
\hline 2602 & UISO_AEA & ITASRL & 0.182 & $2 \mathrm{pCi} / \mathrm{L}$ & 0.0361 & 0.0408 & & & \\
\hline 2603 & 6010_METALS_ICP & DATACH & 1.8 & $3 \mathrm{ug} / \mathrm{L}$ & & & & & $\mathrm{U}$ \\
\hline 2605 & SRISO_SEP_PRECIP_GPC & ITASRL & 0.0686 & $\mathrm{pCi} / \mathrm{L}$ & 0.0409 & 0.0441 & & & \\
\hline 2606 & TRITIUM_ELECT_LSC & ITASRL & 27.1 & $\mathrm{pCi} / \mathrm{L}$ & 2.43 & 8.59 & & & \\
\hline 2607 & UISO_AEA & ITASRL & 0.256 & $\mathrm{pCi} / \mathrm{L}$ & 0.0449 & 0.0527 & & & \\
\hline 2608 & UISO_AEA & ITASRL & 0.00619 & $9 \mathrm{pCi} / \mathrm{L}$ & 0.00845 & 0.00869 & & & $U$ \\
\hline 2609 & UISO_AEA & ITASRL & 0.172 & $2 \mathrm{pCi} / \mathrm{L}$ & 0.0367 & 0.041 & & & \\
\hline 2610 & 6010_METALS_ICP & DATACH & 1.8 & ug/L & & & & & $U$ \\
\hline 2611 & 8260_VOA_GCMS & DATACH & 0.35 & $\mathrm{ug} / \mathrm{L}$ & & & & & $U$ \\
\hline 2612 & SRISO_SEP_PRECIP_GPC & ITASRL & 0.0799 & $\mathrm{pCi} / \mathrm{L}$ & 0.0413 & 0.0451 & & & \\
\hline 2613 & TRITIUM_ELECTI_LSC & ITASRL & 26.2 & $\mathrm{pCi} / \mathrm{L}$ & 2.37 & 8.39 & & & \\
\hline 2614 & UISO_AEA & ITASRL & 0.252 & $2 \mathrm{pCi} / \mathrm{L}$ & 0.0479 & 0.0554 & & & \\
\hline 2615 & UISO_AEA & ITASRL & 0.00459 & $9 \mathrm{pCi} / \mathrm{L}$ & 0.00891 & 0.00912 & & & $U$ \\
\hline 2616 & UISO_AEA & ITASRL & 0.259 & $\mathrm{pCi} / \mathrm{L}$ & 0.0481 & 0.0556 & & & \\
\hline 2617 & 6010_METALS_ICP & DATACH & 2.2 & ug/L & & 0.84 & & & $\mathrm{BL}$ \\
\hline 2618 & 8260_VOA_GCMS & DATACH & 0.35 & $\mathrm{ug} / \mathrm{L}$ & & & & & $U$ \\
\hline 2619 & SRISO_SEP_PRECIP_GPC & ITASRL & 0.0941 & $\mathrm{pCi} / \mathrm{L}$ & 0.0527 & 0.0568 & & & \\
\hline 2620 & TRITIUM_ELECT_LSC & ITASRL & 53.8 & $\mathrm{pCi} / \mathrm{L}$ & 2.99 & 11.1 & & & \\
\hline 2621 & UISO_AEA & ITASRL & 0.267 & $7 \mathrm{pCi} / \mathrm{L}$ & 0.0448 & 0.0531 & & & \\
\hline 2622 & UISO_AEA & ITASRL & 0.00142 & $2 \mathrm{pCi} / \mathrm{L}$ & 0.00644 & 0.0067 & & & $U$ \\
\hline
\end{tabular}




\begin{tabular}{|c|c|c|c|c|c|c|c|c|c|}
\hline & $\mathrm{K}$ & $\mathrm{L}$ & $\mathrm{M}$ & $\mathrm{N}$ & $\mathrm{O}$ & $\mathrm{P}$ & $\mathrm{Q}$ & $\mathrm{R}$ & $\mathrm{S}$ \\
\hline 1 & METHOD_NAME & LAB_CODE & VALUE_RPTD & ANAL_UNITS_RPTD & COUNTING_ERROR & TOTAL_ANAL_ERROR & MIN_DETECTABLE_ACTIVITY & PCNT_MOISTURE & LAB_QUALIFIER \\
\hline 2624 & 6010_METALS_ICP & DATACH & & ug/L & & & & & $U$ \\
\hline 2625 & 8260_VOA_GCMS & DATACH & 0.35 & ug/L & & & & & $U$ \\
\hline 2626 & SRISO_SEP_PRECIP_GPC & ITASRL & 0.0901 & $\mathrm{pCi} / \mathrm{L}$ & 0.0477 & 0.052 & & & \\
\hline 2627 & TRITIUM_ELEECT_LSC & ITASRL & 30.2 & $\mathrm{pCi} / \mathrm{L}$ & 2.44 & 8.75 & & & \\
\hline 2628 & UISO_AEA & ITASRL & 0.272 & $\mathrm{pCi} / \mathrm{L}$ & 0.0505 & 0.0588 & & & \\
\hline 2629 & UISO_AEA & ITASRL & 0.00346 & $\mathrm{pCi} / \mathrm{L}$ & 0.00782 & 0.00805 & & & $U$ \\
\hline 2630 & UISO_AEA & ITASRL & 0.245 & $\mathrm{pCi} / \mathrm{L}$ & 0.0476 & 0.0545 & & & \\
\hline 2631 & 6010_METALS_ICP & DATACH & 1.8 & ug/L & & & & & $U$ \\
\hline 2633 & SRISO_SEP_PRECIP_GPC & ITASRL & 0.081 & $\mathrm{pCi} / \mathrm{L}$ & 0.0431 & 0.0469 & & & \\
\hline 2634 & TRITIUM_ELEECT_LSC & ITASRL & 34.6 & $\mathrm{pCi} / \mathrm{L}$ & 2.5 & 9.02 & & & \\
\hline 2635 & UISO_AEA & ITASRL & 0.25 & $\mathrm{pCi} / \mathrm{L}$ & 0.0458 & 0.0533 & & & \\
\hline 2636 & UISO_AEA & ITASRL & 0.0107 & $\mathrm{pCi} / \mathrm{L}$ & 0.0106 & 0.0109 & & & $\mathrm{U}$ \\
\hline 2637 & UISO_AEA & ITASRL & 0.214 & $\mathrm{pCi} / \mathrm{L}$ & 0.0421 & 0.0479 & & & \\
\hline 2638 & 6010_METALS_ICP & DATACH & 1.8 & ug/L & & & & & $U$ \\
\hline 2639 & 8260_VOA_GCMS & DATACH & 0.35 & ug/L & & & & & $\mathrm{U}$ \\
\hline 2640 & SRISO_SEP_PRECIP_GPC & ITASRL & 0.105 & $\mathrm{pCi} / \mathrm{L}$ & 0.0422 & 0.0474 & & & \\
\hline 2641 & TRITIUM_ELEECT_LSC & ITASRL & 30.6 & $\mathrm{pCi} / \mathrm{L}$ & 2.44 & 8.74 & & & \\
\hline 2643 & UISO_AEA & ITASRL & 0.0149 & $\mathrm{pCi} / \mathrm{L}$ & 0.0121 & 0.0124 & & & \\
\hline 2644 & UISO_AEA & ITASRL & 0.205 & $\mathrm{pCi} / \mathrm{L}$ & 0.0414 & 0.0469 & & & \\
\hline 2645 & 6010_METALS_ICP & DATACH & 1.8 & ug/L & & & & & $\mathrm{U}$ \\
\hline 2646 & 8260_VOA_GCMS & DATACH & 0.35 & ug/L & & & & & $U$ \\
\hline 2647 & SRISO_SEP_PRECIP_GPC & ITASRL & 0.0632 & $\mathrm{pCi} / \mathrm{L}$ & 0.0446 & 0.0471 & & & \\
\hline 2648 & TRITIUM_ELEECT_LSC & ITASRL & 30 & $\mathrm{pCi} / \mathrm{L}$ & 2.41 & 8.64 & & & \\
\hline 2649 & UISO_AEA & ITASRL & 0.247 & $\mathrm{pCi} / \mathrm{L}$ & 0.0427 & 0.0502 & & & \\
\hline 2650 & UISO_AEA & ITASRL & 0.00476 & $\mathrm{pCi} / \mathrm{L}$ & 0.00799 & 0.00823 & & & $U$ \\
\hline 2651 & UISO_AEA & ITASRL & 0.174 & $\mathrm{pCi} / \mathrm{L}$ & 0.0355 & 0.04 & & & \\
\hline 2652 & 6010_METALS_ICP & DATACH & 2.2 & ug/L & & 0.84 & & & $\mathrm{BL}$ \\
\hline 2653 & 8260_VOA_GCMS & DATACH & 0.35 & ug/L & & & & & $U$ \\
\hline 2654 & SRISO_SĒP_PRECIP_GPC & ITASRL & 0.086 & $\mathrm{pCi} / \mathrm{L}$ & 0.0462 & 0.0502 & & & \\
\hline 2655 & TRITIUM_ELEECT_LSC & ITASRL & 28.1 & $\mathrm{pCi} / \mathrm{L}$ & 2.31 & 8.28 & & & \\
\hline 2656 & UISO_AEA & ITASRL & 0.226 & $\mathrm{pCi} / \mathrm{L}$ & 0.0417 & 0.0483 & & & \\
\hline 2657 & UISO_AEA & ITASRL & 0.0125 & $\mathrm{pCi} / \mathrm{L}$ & 0.0111 & 0.0114 & & & \\
\hline 2658 & UISO_AEA & ITASRL & 0.186 & $\mathrm{pCi} / \mathrm{L}$ & 0.0374 & 0.0423 & & & \\
\hline 2659 & 6010_METALS_ICP & DATACH & 1.8 & ug/L & & & & & $U$ \\
\hline 2660 & 8260 VOA GCMS & DATACH & 0.35 & ug/L & & & & & $U$ \\
\hline
\end{tabular}


N

O

P

Q

$\mathrm{R}$

$S$

\begin{tabular}{|c|c|c|c|c|c|c|c|c|c|}
\hline & & & & & & & & & \\
\hline 1 & METHOD_NAME & LAB_CODE & VALUE_RPTD & ANAL_UNITS_RPTD & COUNTING_ERROR & TOTAL_ANAL_ERROR & MIN_DETECTABLE_ACTIVITY & PCNT_MOISTURE & LAB_QUALIFIER \\
\hline 2662 & $\begin{array}{l}2 \text { TRITIUM_ELECT_LSC } \\
\end{array}$ & ITASRL & 31.8 & $8 \mathrm{pCi} / \mathrm{L}$ & 2.44 & 8.76 & & & \\
\hline 2663 & UISO_AEA & ITASRL & 0.265 & $5 \mathrm{pCi} / \mathrm{L}$ & 0.0439 & 0.0521 & & & \\
\hline 2664 & $\begin{array}{l}4 \text { UISO_AEA } \\
\text { UISO_A }\end{array}$ & ITASRL & 0.00891 & $1 \mathrm{pCi} / \mathrm{L}$ & 0.00924 & 0.00949 & & & $U$ \\
\hline 2665 & DISO_AEA & ITASRL & 0.171 & $1 \mathrm{pCi} / \mathrm{L}$ & 0.0351 & 0.0394 & & & \\
\hline 2666 & 6010_METALS_ICP & DATACH & 1.8 & $8 \mathrm{ug} / \mathrm{L}$ & & & & & $\mathrm{U}$ \\
\hline 2667 & 7 8260_VOA_GCMS & DATACH & 0.35 & $5 \mathrm{ug} / \mathrm{L}$ & & & & & $\mathrm{U}$ \\
\hline 2668 & SRISO_SEP_PRECIP_GPC & ITASRL & 0.106 & $6 \mathrm{pCi} / \mathrm{L}$ & 0.0413 & 0.0472 & & & \\
\hline 2669 & 9 & ITASRL & 26.6 & $6 \mathrm{pCi} / \mathrm{L}$ & 2.35 & 8.39 & & & \\
\hline 2670 & UISO_AEA & ITASRL & 0.292 & $2 \mathrm{pCi} / \mathrm{L}$ & 0.0504 & 0.0597 & & & \\
\hline 2671 & UISO_AEA & ITASRL & 0.00847 & $7 \mathrm{pCi} / \mathrm{L}$ & 0.0103 & 0.0106 & & & $\mathrm{U}$ \\
\hline 2672 & 2 UISO_AEA & ITASRL & 0.204 & $4 \mathrm{pCi} / \mathrm{L}$ & 0.0419 & 0.0474 & & & \\
\hline 2673 & 6010_METALS_ICP & DATACH & 1.8 & $8 \mathrm{ug} / \mathrm{L}$ & & & & & $\mathrm{U}$ \\
\hline 2674 & 8260_VOA_GCMS & DATACH & 0.35 & $5 \mathrm{ug} / \mathrm{L}$ & & & & & $\mathrm{U}$ \\
\hline 2675 & DSRISO_SEP_PRECIP_GPC & ITASRL & 0.0941 & $1 \mathrm{pCi} / \mathrm{L}$ & 0.0381 & 0.0434 & & & \\
\hline 2676 & TRITIUM_ELECT_LSC & ITASRL & 30.6 & $6 \mathrm{pCi} / \mathrm{L}$ & 2.42 & 8.75 & & & \\
\hline 2677 & 7]UISO_AEA & ITASRL & 0.224 & $4 \mathrm{pCi} / \mathrm{L}$ & 0.0434 & 0.0498 & & & \\
\hline 2678 & UISO_AEA & ITASRL & 0.00779 & $9 \mathrm{pCi} / \mathrm{L}$ & 0.00985 & 0.0101 & & & $\mathrm{U}$ \\
\hline 2679 & UISO_AEA & ITASRL & 0.201 & $1 \mathrm{pCi} / \mathrm{L}$ & 0.0405 & 0.0458 & & & \\
\hline 2682 & SRISO_SEP_PRECIP_GPC & ITASRL & 0.206 & $6 \mathrm{pCi} / \mathrm{L}$ & 0.0514 & 0.0663 & & & \\
\hline 2683 & \begin{tabular}{|l|l} 
TRITIUM_ELECT_LSC \\
\end{tabular} & ITASRL & 29 & $9 \mathrm{pCi} / \mathrm{L}$ & 2.41 & 8.57 & & & \\
\hline 2684 & UISO_AEA & ITASRL & 0.262 & $2 \mathrm{pCi} / \mathrm{L}$ & 0.0469 & 0.0548 & & & \\
\hline 2685 & UISO_AEA & ITASRL & 0.0107 & $7 \mathrm{pCi} / \mathrm{L}$ & 0.0106 & 0.0108 & & & $\mathrm{U}$ \\
\hline 2686 & UISO_AEA & ITASRL & 0.207 & $7 \mathrm{pCi} / \mathrm{L}$ & 0.0412 & 0.0467 & & & \\
\hline 2687 & 7 6010_METALS_ICP & DATACH & 2.2 & $2 \mathrm{ug} / \mathrm{L}$ & & 0.924 & & & $\mathrm{BL}$ \\
\hline 2688 & 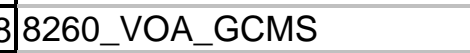 & DATACH & 0.35 & $5 \mathrm{ug} / \mathrm{L}$ & & & & & U \\
\hline 2689 & 9RISO_SEP_PRECIP_GPC & ITASRL & 0.0868 & $8 \mathrm{pCi} / \mathrm{L}$ & 0.036 & 0.0438 & & & \\
\hline 2690 & TRITIUM_ELECT_LSC & ITASRL & 32.2 & $2 \mathrm{pCi} / \mathrm{L}$ & 2.42 & 8.56 & & & \\
\hline 2691 & UISO_AEA & ITASRL & 0.23 & $3 \mathrm{pCi} / \mathrm{L}$ & 0.0465 & 0.0531 & & & \\
\hline 2692 & UISO_AEA & ITASRL & 0.0011 & $1 \mathrm{pCi} / \mathrm{L}$ & 0.00626 & 0.00653 & & & $\mathrm{U}$ \\
\hline 2693 & UISO_AEA & ITASRL & 0.171 & $1 \mathrm{pCi} / \mathrm{L}$ & 0.0396 & 0.0438 & & & \\
\hline 2694 & 46010_METALS_ICP & DATACH & 4.4 & $4 \mathrm{ug} / \mathrm{L}$ & & 1.85 & & & $\mathrm{BL}$ \\
\hline 2695 & D260_VOA_GCMS & DATACH & 0.35 & $5 \mathrm{ug} / \mathrm{L}$ & & & & & $\mathrm{U}$ \\
\hline 2696 & SRISO_SEP_PRECIP_GPC & ITASRL & 0.0671 & $1 \mathrm{pCi} / \mathrm{L}$ & 0.0335 & 0.0391 & & & \\
\hline 2697 & 7) TRITIUM_ELECT_LSC & ITASRL & 33 & $3 \mathrm{pCi} / \mathrm{L}$ & 2.43 & 8.61 & & & \\
\hline 2698 & UISO_AEA & ITASRL & 0.184 & $4 \mathrm{pCi} / \mathrm{L}$ & 0.0372 & 0.0423 & & & \\
\hline 2699 & UISO_AEA & ITASRL & 0.00747 & $7 \mathrm{pCi} / \mathrm{L}$ & 0.00872 & 0.00896 & & & $\mathrm{U}$ \\
\hline
\end{tabular}




\begin{tabular}{|c|c|c|c|c|c|c|c|c|c|}
\hline & $\mathrm{K}$ & $\mathrm{L}$ & $\mathrm{M}$ & $\mathrm{N}$ & $\mathrm{O}$ & $\mathrm{P}$ & $\mathrm{Q}$ & $\mathrm{R}$ & $\mathrm{S}$ \\
\hline 1 & METHOD NAME & LAB CODE & VALUE RPTD & ANAL UNITS RPTD & COUNTING ERROR & TOTAL ANAL ERROR & MIN DETECTABLE ACTIVITY & PCNT MOISTURE & LAB QUALIFIER \\
\hline 2700 & UISO_AEA & ITASRL & 0.182 & $\mathrm{pCi} / \mathrm{L}$ & 0.0365 & 0.0412 & & & \\
\hline 2701 & 6010_METALS_ICP & DATACH & 1.8 & ug/L & & & & & $\mathrm{U}$ \\
\hline 2702 & 8260_VOA_GCMS & DATACH & 0.35 & ug/L & & & & & $\mathrm{U}$ \\
\hline 2703 & SRISO_SEP_PRECIP_GPC & ITASRL & 0.0995 & $\mathrm{pCi} / \mathrm{L}$ & 0.0381 & 0.0464 & & & \\
\hline 2704 & TRITIUM_ELEECT_LSC & ITASRL & 31.7 & $\mathrm{pCi} / \mathrm{L}$ & 2.4 & 8.48 & & & \\
\hline 2705 & UISO_AEA & ITASRL & 0.262 & $\mathrm{pCi} / \mathrm{L}$ & 0.0483 & 0.0562 & & & \\
\hline 2706 & UISO_AEA & ITASRL & 0.0216 & $\mathrm{pCi} / \mathrm{L}$ & 0.0149 & 0.0152 & & & \\
\hline 2707 & UISO_AEA & ITASRL & 0.169 & $\mathrm{pCi} / \mathrm{L}$ & 0.0386 & 0.0427 & & & \\
\hline 2709 & 8260 VOA_GCMS & DATACH & 0.35 & ug/L & & & & & $\mathrm{U}$ \\
\hline 2710 & SRISO_SEP_PRECIP_GPC & ITASRL & 0.0895 & $\mathrm{pCi} / \mathrm{L}$ & 0.0356 & 0.0416 & & & \\
\hline 2711 & TRITIUM_ELEECT_LSC & ITASRL & 31.7 & $\mathrm{pCi} / \mathrm{L}$ & 2.43 & 8.51 & & & \\
\hline 2712 & UISO_AEA & ITASRL & 0.241 & $\mathrm{pCi} / \mathrm{L}$ & 0.0458 & 0.0529 & & & \\
\hline 2713 & UISO_AEA & ITASRL & 0.000189 & $\mathrm{pCi} / \mathrm{L}$ & 0.00731 & 0.00754 & & & $\mathrm{U}$ \\
\hline 2714 & UISO_AEA & ITASRL & 0.166 & $\mathrm{pCi} / \mathrm{L}$ & 0.0374 & 0.0415 & & & \\
\hline 2715 & 6010_METALS_ICP & DATACH & 3.7 & ug/L & & 1.55 & & & $\mathrm{BL}$ \\
\hline 2716 & 8260_VOA_GCMS & DATACH & 0.35 & ug/L & & & & & $U$ \\
\hline 2717 & SRISO_SEP_PRECIP_GPC & ITASRL & 0.0873 & $\mathrm{pCi} / \mathrm{L}$ & 0.035 & 0.0402 & & & \\
\hline 2719 & UISO_AEA & ITASRL & 0.591 & $\mathrm{pCi} / \mathrm{L}$ & 0.0671 & 0.0913 & & & \\
\hline 2720 & UISO_AEA & ITASRL & 0.0214 & $\mathrm{pCi} / \mathrm{L}$ & 0.0146 & 0.0149 & & & \\
\hline 2721 & UISO_AEA & ITASRL & 0.494 & $\mathrm{pCi} / \mathrm{L}$ & 0.061 & 0.0797 & & & \\
\hline 2722 & 6010_METALS_ICP & DATACH & 2.6 & ug/L & & 1.09 & & & $\mathrm{BL}$ \\
\hline 2723 & 8260_VOA_GCMS & DATACH & 0.35 & ug/L & & & & & $U$ \\
\hline \begin{tabular}{|l|l|}
2724 \\
\end{tabular} & SRISO_SEP_PRECIP_GPC & ITASRL & 0.11 & $\mathrm{pCi} / \mathrm{L}$ & 0.0376 & 0.0443 & & & \\
\hline 2725 & TRITIUM_ELECT_LSC & ITASRL & 97.9 & $\mathrm{pCi} / \mathrm{L}$ & 3.19 & 13.4 & & & \\
\hline 2726 & UISO_AEA & ITASRL & 0.325 & $\mathrm{pCi} / \mathrm{L}$ & 0.0554 & 0.066 & & & \\
\hline 2727 & UISO_AEA & ITASRL & 0.0191 & $\mathrm{pCi} / \mathrm{L}$ & 0.0142 & 0.0145 & & & \\
\hline 2728 & UISO_AEA & ITASRL & 0.304 & $\mathrm{pCi} / \mathrm{L}$ & 0.0532 & 0.0625 & & & \\
\hline 2729 & 6010_METALS_ICP & DATACH & 3.2 & ug/L & & 1.34 & & & $\mathrm{BL}$ \\
\hline 2730 & 8260_VOA_GCMS & DATACH & 0.35 & ug/L & & & & & $U$ \\
\hline 2731 & SRISO_SEP_PRECIP_GPC & ITASRL & 0.0729 & $\mathrm{pCi} / \mathrm{L}$ & 0.0395 & 0.0439 & & & \\
\hline 2732 & TRITIUM_ELEECT_LSC & ITASRL & 42.6 & $\mathrm{pCi} / \mathrm{L}$ & 2.54 & 9.28 & & & \\
\hline 2733 & UISO_AEA & ITASRL & 0.212 & $\mathrm{pCi} / \mathrm{L}$ & 0.0449 & 0.0508 & & & \\
\hline 2734 & UISO_AEA & ITASRL & -0.00334 & $\mathrm{pCi} / \mathrm{L}$ & 0.00726 & 0.00748 & & & $\mathrm{U}$ \\
\hline 2735 & UISO_AEA & ITASRL & 0.177 & $\mathrm{pCi} / \mathrm{L}$ & 0.0404 & 0.0448 & & & \\
\hline 2736 & 6010_METALS_ICP & DATACH & 1.8 & ug/L & & & & & $U$ \\
\hline
\end{tabular}

Page 195 


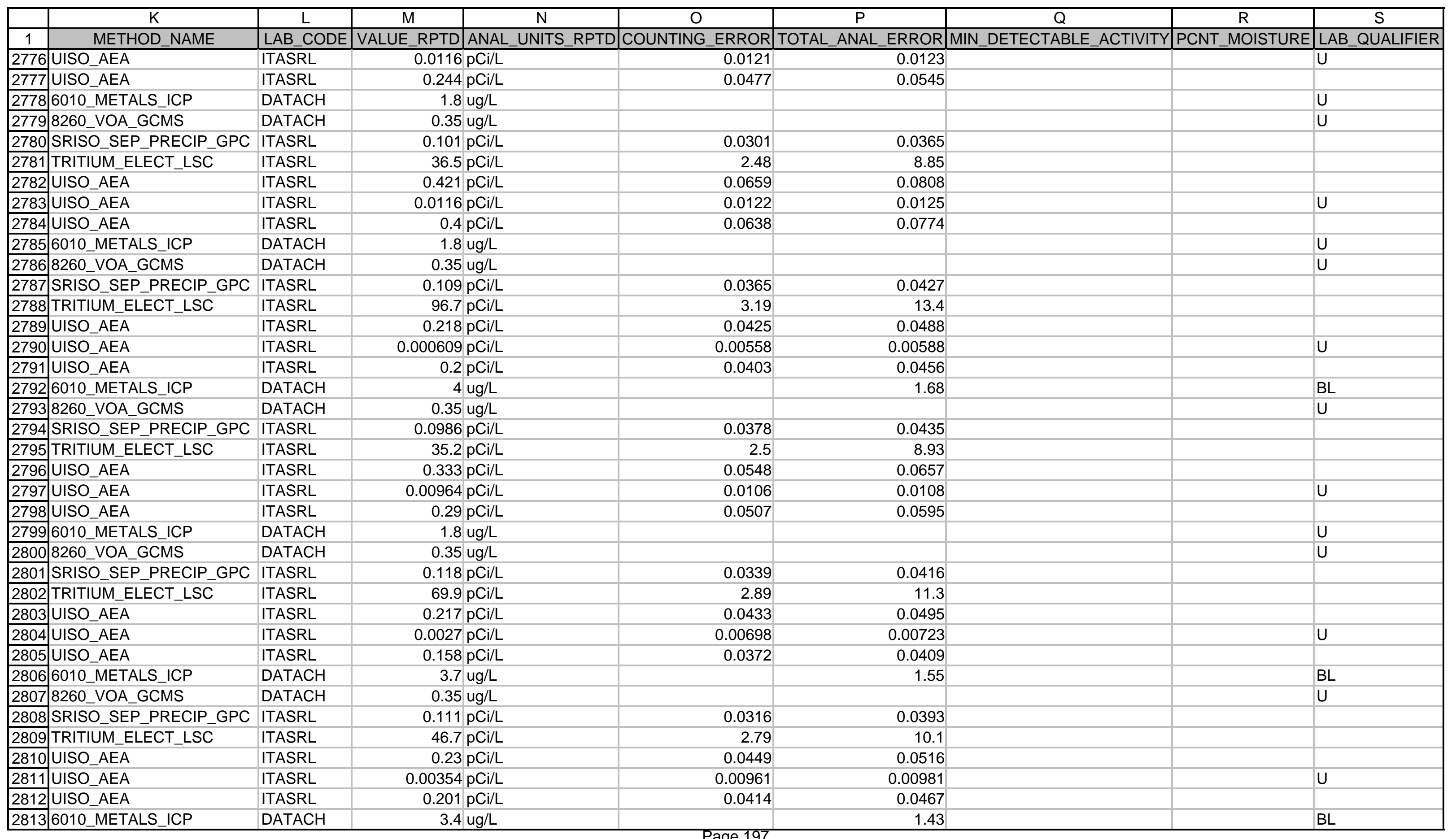




\begin{tabular}{|c|c|c|c|c|c|c|c|c|c|}
\hline & $\mathrm{K}$ & $\mathrm{L}$ & $\mathrm{M}$ & $\mathrm{N}$ & $\mathrm{O}$ & $\mathrm{P}$ & $\mathrm{Q}$ & $\mathrm{R}$ & $\mathrm{S}$ \\
\hline 1 & METHOD_NAME & LAB_CODE & VALUE_RPTD & ANAL_UNITS_RPTD & COUNTING_ERROR & $\mid$ TOTAL_ANAL_ERROR| & MIN_DETECTABLE_ACTIVITY & PCNT_MOISTURE & LAB_QUALIFIER \\
\hline 2814 & 8260_VOA_GCMS & DATACH & 0.35 & $u g / L$ & & & & & $U$ \\
\hline 2815 & SRISO_SEPP_PRECIP_GPC & ITASRL & 0.112 & $\mathrm{pCi} / \mathrm{L}$ & 0.0343 & 0.0419 & & & \\
\hline 2816 & TRITIUMM ELECT LSC̄ & ITASRL & 35.1 & $\mathrm{pCi} / \mathrm{L}$ & 2.45 & 8.71 & & & \\
\hline 2817 & UISO AËA & ITASRL & 0.217 & $\mathrm{pCi} / \mathrm{L}$ & 0.0479 & 0.054 & & & \\
\hline 2818 & UISO_AEA & ITASRL & 0.0207 & $\mathrm{pCi} / \mathrm{L}$ & 0.0185 & 0.0188 & & & \\
\hline 2819 & UISO_AEA & ITASRL & 0.134 & $\mathrm{pCi} / \mathrm{L}$ & 0.0374 & 0.0404 & & & \\
\hline 2820 & 6010_METALS_ICP & DATACH & 4.8 & ug/L & & 2.02 & & & $\mathrm{BL}$ \\
\hline 2821 & 8260_VOA_GCMS & DATACH & 0.35 & ug/L & & & & & $U$ \\
\hline \begin{tabular}{|l|l|}
2823 \\
2823 \\
\end{tabular} & TRITIUM_ELECT_LSC & ITASRL & 32.6 & $\mathrm{pCi} / \mathrm{L}$ & 2.38 & 8.44 & & & \\
\hline 2824 & UISO_AEA & ITASRL & 0.252 & $\mathrm{pCi} / \mathrm{L}$ & 0.0459 & 0.0534 & & & \\
\hline 2825 & UISO_AEA & ITASRL & 0.00408 & $\mathrm{pCi} / \mathrm{L}$ & 0.00914 & 0.00935 & & & U \\
\hline 2826 & UISO_AEA & ITASRL & 0.189 & $\mathrm{pCi} / \mathrm{L}$ & 0.0393 & 0.0443 & & & \\
\hline 2827 & 6010_METALS_ICP & DATACH & 1.8 & ug/L & & & & & $U$ \\
\hline 2828 & 8260_VOA_GCMS & DATACH & 0.35 & ug/L & & & & & $U$ \\
\hline 2829 & SRISO_SEP_PRECIP_GPC & ITASRL & 0.135 & $\mathrm{pCi} / \mathrm{L}$ & 0.0377 & 0.0481 & & & \\
\hline 2830 & TRITIŪM ELEECT LSC̄ & ITASRL & 33.2 & $\mathrm{pCi} / \mathrm{L}$ & 2.41 & 8.53 & & & \\
\hline 2831 & UISO_AEA & ITASRL & 0.241 & $\mathrm{pCi} / \mathrm{L}$ & 0.048 & 0.055 & & & \\
\hline 2833 & UISO AEA & ITASRL & 0.236 & $\mathrm{pCi} / \mathrm{L}$ & 0.0469 & 0.0535 & & & \\
\hline 2834 & 6010_METALS_ICP & DATACH & 4.2 & ug/L & & 1.76 & & & $\mathrm{BL}$ \\
\hline 2835 & 8260_VOA_GCMS & DATACH & 0.35 & $\mathrm{ug} / \mathrm{L}$ & & & & & $U$ \\
\hline 2836 & SRISO_SEP_PRECIP_GPC & ITASRL & 0.105 & $\mathrm{pCi} / \mathrm{L}$ & 0.0375 & 0.0435 & & & \\
\hline 2837 & TRITIŪM_ELECCT_LSC̄ & ITASRL & 35.3 & $\mathrm{pCi} / \mathrm{L}$ & 2.49 & 8.87 & & & \\
\hline 2838 & UISO_AEA & ITASRL & 0.198 & $\mathrm{pCi} / \mathrm{L}$ & 0.0417 & 0.0471 & & & \\
\hline 2839 & UISO_AEA & ITASRL & -0.00295 & $\mathrm{pCi} / \mathrm{L}$ & 0.00453 & 0.00488 & & & $U$ \\
\hline 2840 & UISO_AEA & ITASRL & 0.167 & $\mathrm{pCi} / \mathrm{L}$ & 0.0376 & 0.0417 & & & \\
\hline 2841 & 6010_METALS_ICP & DATACH & 3.2 & ug/L & & 1.34 & & & $\mathrm{BL}$ \\
\hline 2842 & 8260_VOA_GCMS & DATACH & 0.35 & ug/L & & & & & $U$ \\
\hline 2843 & SRISO_SEPP_PRECIP_GPC & ITASRL & 0.134 & $\mathrm{pCi} / \mathrm{L}$ & 0.0451 & 0.0527 & & & \\
\hline 2844 & TRITIUMM_ELECCT_LSC̄ & ITASRL & 31.7 & $\mathrm{pCi} / \mathrm{L}$ & 2.47 & 8.72 & & & \\
\hline 2845 & UISO_AEA & ITASRL & 0.271 & $\mathrm{pCi} / \mathrm{L}$ & 0.0496 & 0.0579 & & & \\
\hline 2846 & UISO_AEA & ITASRL & 0.00321 & $\mathrm{pCi} / \mathrm{L}$ & 0.00757 & 0.00781 & & & $U$ \\
\hline 2847 & UISO_AEA & ITASRL & 0.209 & $\mathrm{pCi} / \mathrm{L}$ & 0.0432 & 0.0488 & & & \\
\hline 2848 & 6010_METALS_ICP & DATACH & 1.8 & ug/L & & 0.756 & & & $\mathrm{BL}$ \\
\hline 2849 & 8260_VOA_GCMS & DATACH & 0.35 & ug/L & & & & & $U$ \\
\hline 2850 & SRISO_SEP_PRECIP_GPC & ITASRL & 0.112 & $\mathrm{pCi} / \mathrm{L}$ & 0.033 & 0.0406 & & & \\
\hline
\end{tabular}




\begin{tabular}{|c|c|c|c|c|c|c|c|c|c|}
\hline & $\mathrm{K}$ & $\mathrm{L}$ & $\mathrm{M}$ & $\mathrm{N}$ & $\mathrm{O}$ & $\mathrm{P}$ & $\mathrm{Q}$ & $\mathrm{R}$ & $\mathrm{S}$ \\
\hline 1 & METHOD NAME & LAB CODE & VALUE RPTD & ANAL UNITS RPTD & COUNTING ERROR & TOTAL ANAL ERROR & MIN DETECTABLE ACTIVITY & PCNT MOISTURE & LAB QUALIFIER \\
\hline 2852 & UISO_AEA & ITASRL & 0.274 & $\mathrm{pCi} / \mathrm{L}$ & 0.0486 & 0.057 & & & \\
\hline 2853 & UISO_AEA & ITASRL & 0.004 & $\mathrm{pCi} / \mathrm{L}$ & 0.00832 & 0.00855 & & & $\mathrm{U}$ \\
\hline 2854 & UISO_AEA & ITASRL & 0.196 & $\mathrm{pCi} / \mathrm{L}$ & 0.0409 & 0.046 & & & \\
\hline 2855 & 6010_METALS_ICP & DATACH & 1.8 & ug/L & & & & & $\mathrm{U}$ \\
\hline 2856 & 8260_VOA_GCMS & DATACH & 0.35 & $\mathrm{ug} / \mathrm{L}$ & & & & & $U$ \\
\hline 2857 & SRISO_SĒP_PRECIP_GPC & ITASRL & & & & & & & \\
\hline 2858 & SRISO SEP PRECIP GPC & ITASRL & 0.18 & $\mathrm{pCi} / \mathrm{L}$ & 0.0411 & 0.0535 & & & \\
\hline 2859 & TRITIUM_ELEECT_LSC & ITASRL & 42.6 & $\mathrm{pCi} / \mathrm{L}$ & 2.54 & 9.21 & & & \\
\hline \begin{tabular}{|l|}
2861 \\
\end{tabular} & UISO_AEA & ITASRL & 0.218 & $\mathrm{pCi} / \mathrm{L}$ & 0.0435 & 0.0497 & & & \\
\hline 2862 & UISO_AEA & ITASRL & 0.00682 & $\mathrm{pCi} / \mathrm{L}$ & 0.00909 & 0.00932 & & & $U$ \\
\hline 2863 & UISO_AEA & ITASRL & 0.168 & $\mathrm{pCi} / \mathrm{L}$ & 0.0377 & 0.0419 & & & \\
\hline 2864 & UISO_AEA & ITASRL & 0.2 & $\mathrm{pCi} / \mathrm{L}$ & 0.0414 & 0.047 & & & \\
\hline 2865 & UISO_AEA & ITASRL & -0.00293 & $\mathrm{pCi} / \mathrm{L}$ & 0.00469 & 0.00503 & & & $\mathrm{U}$ \\
\hline 2866 & UISO_AEA & ITASRL & 0.117 & $\mathrm{pCi} / \mathrm{L}$ & 0.0316 & 0.0341 & & & \\
\hline 2867 & 6010_METALS_ICP & DATACH & 1.8 & $\mathrm{ug} / \mathrm{L}$ & & & & & $\mathrm{U}$ \\
\hline 2868 & 8260_VOA_GCMS & DATACH & 0.35 & $\mathrm{ug} / \mathrm{L}$ & & & & & $U$ \\
\hline 2869 & SRISO_SEP_PRECIP_GPC & ITASRL & 0.0705 & $\mathrm{pCi} / \mathrm{L}$ & 0.0295 & 0.0337 & & & \\
\hline 2871 & UISO_AEA & ITASRL & 0.274 & $\mathrm{pCi} / \mathrm{L}$ & 0.0507 & 0.059 & & & \\
\hline 2872 & UISO_AEA & ITASRL & 0.00118 & $\mathrm{pCi} / \mathrm{L}$ & 0.00639 & 0.00665 & & & $U$ \\
\hline \begin{tabular}{|l|}
2873 \\
287
\end{tabular} & UISO_AEA & ITASRL & 0.205 & $\mathrm{pCi} / \mathrm{L}$ & 0.0435 & 0.0489 & & & \\
\hline 2874 & 6010_METALS_ICP & DATACH & 1.8 & ug/L & & & & & $U$ \\
\hline 2875 & 8260_VOA_GCMS & DATACH & 0.35 & $\mathrm{ug} / \mathrm{L}$ & & & & & $U$ \\
\hline \begin{tabular}{|l|l|}
2876 \\
\end{tabular} & SRISO_SEP_PRECIP_GPC & ITASRL & 0.0886 & $\mathrm{pCi} / \mathrm{L}$ & 0.0323 & 0.0373 & & & \\
\hline 2877 & TRITIUM_ELEECT_LSC & ITASRL & 45.4 & $\mathrm{pCi} / \mathrm{L}$ & 2.6 & 9.51 & & & \\
\hline 2878 & UISO_AEA & ITASRL & 0.242 & $\mathrm{pCi} / \mathrm{L}$ & 0.0455 & 0.0526 & & & \\
\hline 2879 & UISO_AEA & ITASRL & 0.00603 & $\mathrm{pCi} / \mathrm{L}$ & 0.00993 & 0.0101 & & & $\mathrm{U}$ \\
\hline 2880 & UISO_AEA & ITASRL & 0.188 & $\mathrm{pCi} / \mathrm{L}$ & 0.0396 & 0.0445 & & & \\
\hline 2881 & 6010_METALS_ICP & DATACH & 1.8 & ug/L & & & & & $U$ \\
\hline 2882 & 8260_VOA_GCMS & DATACH & 0.35 & $\mathrm{ug} / \mathrm{L}$ & & & & & $U$ \\
\hline 2883 & SRISO_SEP_PRECIP_GPC & ITASRL & 0.111 & $\mathrm{pCi} / \mathrm{L}$ & 0.0318 & 0.0396 & & & \\
\hline 2884 & TRITIUM_ELEECT_LSC & ITASRL & 33.4 & $\mathrm{pCi} / \mathrm{L}$ & 2.39 & 8.48 & & & \\
\hline 2885 & UISO_AEA & ITASRL & 0.215 & $\mathrm{pCi} / \mathrm{L}$ & 0.0442 & 0.0502 & & & \\
\hline 2886 & UISO_AEA & ITASRL & -0.0000191 & $\mathrm{pCi} / \mathrm{L}$ & 0.0061 & 0.00637 & & & $\mathrm{U}$ \\
\hline 2887 & UISO_AEA & ITASRL & 0.159 & $\mathrm{pCi} / \mathrm{L}$ & 0.0378 & 0.0416 & & & \\
\hline 2888 & 6010_METALS_ICP & DATACH & 1.8 & ug/L & & & & & $U$ \\
\hline
\end{tabular}



$0.125 \mathrm{pCi} / \mathrm{L}$

$28.4 \mathrm{pCi} / \mathrm{L}$

2893 UISO_AEA

\begin{tabular}{lr}
$0.173 \mathrm{pCi} / \mathrm{L}$ \\
\hline ITASRL
\end{tabular}

$0.00598 \mathrm{pCi} / \mathrm{L}$

2895 6010_METALS_ICP

28968260 VOA GCMS

ITASR

$0.155 \mathrm{pCi} / \mathrm{L}$

\begin{tabular}{l|l}
$\mathrm{DATACH}$ & $2.4 \mathrm{ug} / \mathrm{L}$
\end{tabular}

DATACH

2897 SRISO_SEP_PRECIP_GPC ITASRL

$0.35 \mathrm{ug} / \mathrm{L}$

2898 TRITIUM_DIST_LSC

2899 UISO_AEA

2900 UISO AEA

2901 UISO_AEA

2902 6010_METALS_ICP

29038260 VOA GCMS

\begin{tabular}{|l|r|}
\hline ITASRL & $0.0858 \mathrm{pCi} / \mathrm{L}$ \\
\hline ITASRL & $19.5 \mathrm{pCi} / \mathrm{L}$ \\
\hline ITASRL & $0.185 \mathrm{pCi} / \mathrm{L}$ \\
\hline
\end{tabular}

\begin{tabular}{l|r}
\hline ITASRL & $0.00205 \mathrm{pCi} / \mathrm{L}$ \\
\hline ITASRL & $0.149 \mathrm{pCi} / \mathrm{L}$
\end{tabular}

\begin{tabular}{l|r}
\hline ITASRL & $0.149 \mathrm{pCI} / \mathrm{L}$ \\
\hline DATACH & $1.8 \mathrm{ug} / \mathrm{L}$ \\
\hline
\end{tabular}

2904 SRISO_SEP_PRECIP_GPC ITASRL

2905 TRITIUM_ELECT_LSC

2906 UISO_AEA

2907 UISO AEA

2908 UISO_AEA

2909 6010_METALS_ICP

2910 8260_VOA_GCMS

\begin{tabular}{l|l} 
ITASRL & $0.0922 \mathrm{pCi} / \mathrm{L}$
\end{tabular}

$0.0922 \mathrm{pCi} / \mathrm{L}$
$38.9 \mathrm{pCi} / \mathrm{L}$

ITASRL $\quad 0.199 \mathrm{pCi} / \mathrm{L}$

$0.0115 \mathrm{pCi} / \mathrm{L}$

\begin{tabular}{l|r}
\hline ITASRL & $0.0115 \mathrm{pCi} / \mathrm{L}$ \\
\hline ITASRL & $0.189 \mathrm{pCi} / \mathrm{L}$
\end{tabular}

\begin{tabular}{l|r}
\hline ITASRL & $0.189 \mathrm{pC} / \mathrm{L}$ \\
\hline DATACH & $1.8 \mathrm{ug} / \mathrm{L}$ \\
\hline
\end{tabular}

\begin{tabular}{l|l} 
DATACH & $0.35 \mathrm{ug} / \mathrm{L}$
\end{tabular}

ECIP GPC ITASRL

2912 TRITIUM_ELECT_LSC

2913 UISO_AEA

2914 UISO_AEA

2915 UISO_AEA

2916 6010_METALS_ICP

29178260 VOA GCMS

\begin{tabular}{l|r}
\hline ITASRL & $0.0838 \mathrm{pCi} / \mathrm{L}$ \\
\hline ITASRL & $36.2 \mathrm{pCi} / \mathrm{L}$ \\
\hline
\end{tabular}

$36.2 \mathrm{pCi} / \mathrm{L}$
$0.226 \mathrm{pCi} / \mathrm{L}$

\begin{tabular}{l|r}
\hline ITASRL & $0.226 \mathrm{pCi} / \mathrm{L}$ \\
\hline ITASRL & $0.00585 \mathrm{pCi} / \mathrm{L}$
\end{tabular}

\begin{tabular}{|l|r}
\hline ITASRL & $0.174 \mathrm{pCi} / \mathrm{L}$ \\
\hline DATACH & $1.8 \mathrm{ug} / \mathrm{L}$
\end{tabular}

\begin{tabular}{l|l} 
& \\
\hline RROR & TOT \\
\hline 0.0328
\end{tabular}

$0.35 \mathrm{ug} / \mathrm{L}$

2918 SRISO_SEP_PRECIP_GPC ITASRL $\quad 0.0931 \mathrm{pCi} / \mathrm{L}$

\begin{tabular}{l|r}
\hline ITASRL & $33.6 \mathrm{pCi} / \mathrm{L}$ \\
\hline ITASRL & $0.204 \mathrm{pCi} / \mathrm{L}$
\end{tabular}

2919

2920 UISO_AEA

2921 UISO_AEA

$0.0162 \mathrm{pCi} / \mathrm{L}$

2922 UISO_AEA

2923 6010_METALS_ICP

29248260 VOA GCMS

$0.178 \mathrm{pCi} / \mathrm{L}$

ITASRL

ITASRL

\begin{tabular}{l|l} 
DATACH \\
\hline
\end{tabular}

SRISO SEP PRECIP GPC ITASRL

22926 TRITIUM_ELECT_LSC

2927 UISO_AEA

ITASRL

$0.35 \mathrm{ug} / \mathrm{L}$

$0.35 \mathrm{ug} / \mathrm{L}$

$0.15 \mathrm{pCi} / \mathrm{L}$

$0.245 \mathrm{pCi} / \mathrm{L}$

\begin{tabular}{|r|}
\hline 0.0328 \\
\hline 2.32 \\
\hline 0.0414 \\
\hline 0.00932 \\
\hline 0.0387 \\
\hline \\
\hline 0.0306 \\
\hline 72.1 \\
\hline 0.0407 \\
\hline 0.00736 \\
\hline 0.0359 \\
\hline \\
\hline 0.0329 \\
\hline 2.52 \\
\hline 0.0426 \\
\hline 0.0113 \\
\hline 0.0408 \\
\hline \\
\hline 0.035 \\
\hline 2.5 \\
\hline 0.0466 \\
\hline 0.00919 \\
\hline 0.0406 \\
\hline 0.0324 \\
\hline 2.47 \\
\hline 0.013 \\
\hline
\end{tabular}

ERROR

ITASRL

0.0457

\begin{tabular}{|c|c|c|}
\hline 3 & 0.0413 & \\
\hline 2 & 8.13 & \\
\hline 4 & 0.0459 & \\
\hline ? & 0.00954 & $\mathrm{U}$ \\
\hline 7 & 0.0424 & \\
\hline & 1.01 & L \\
\hline & & $\mathrm{U}$ \\
\hline 6 & 0.0364 & \\
\hline 1 & 133 & U \\
\hline 7 & 0.0456 & \\
\hline 6 & 0.0076 & $\mathrm{U}$ \\
\hline ) & 0.0394 & \\
\hline & & U \\
\hline & & $\mathrm{U}$ \\
\hline 9 & 0.0385 & \\
\hline ? & 9.01 & \\
\hline 6 & 0.0481 & \\
\hline 3 & 0.0115 & \\
\hline 8 & 0.0457 & \\
\hline & & $\mathrm{U}$ \\
\hline & & $\mathrm{U}$ \\
\hline 5 & 0.04 & \\
\hline 5 & 8.88 & \\
\hline 6 & 0.0531 & \\
\hline 9 & 0.00942 & $\mathrm{U}$ \\
\hline 5 & 0.0449 & \\
\hline & & $\mathrm{U}$ \\
\hline & & U \\
\hline 4 & 0.0384 & \\
\hline 7 & 8.69 & \\
\hline 3 & 0.0489 & \\
\hline 3 & 0.0133 & \\
\hline 1 & 0.0446 & \\
\hline & & $\mathrm{U}$ \\
\hline & & U \\
\hline & 0.0493 & \\
\hline & 8.5 & \\
\hline & 0.053 & \\
\hline
\end{tabular}




\begin{tabular}{|c|c|c|c|c|c|c|c|c|c|}
\hline & $\mathrm{K}$ & $\mathrm{L}$ & $\mathrm{M}$ & $\mathrm{N}$ & $\mathrm{O}$ & $\mathrm{P}$ & Q & $\mathrm{R}$ & $\mathrm{S}$ \\
\hline 1 & METHOD_NAME & LAB_CODE & VALUE_RPTD & ANAL_UNITS_RPTD & COUNTING_ERROR & TOTAL_ANAL_ERROR & MIN_DETECTABLE_ACTIVITY & PCNT_MOISTURE & LAB_QUALIFIER \\
\hline 2928 & UISO_AEA & ITASRL & 0.00484 & $4 \mathrm{pCi} / \mathrm{L}$ & 0.00818 & 0.00842 & & & $U$ \\
\hline 2929 & UISO_AEA & ITASRL & 0.198 & $3 \mathrm{pCi} / \mathrm{L}$ & 0.041 & 0.0462 & & & \\
\hline 2930 & 6010_METALS_ICP & DATACH & 2.9 & ug/L & & 1.22 & & & $\mathrm{BL}$ \\
\hline 2931 & 8260_VOA_GCMS & DATACH & 0.35 & $\mathrm{ug} / \mathrm{L}$ & & & & & $\mathrm{U}$ \\
\hline 2932 & SRISO_SEP_PRECIP_GPC & ITASRL & 0.108 & $3 \mathrm{pCi} / \mathrm{L}$ & 0.0343 & 0.0417 & & & \\
\hline 2933 & TRITIUM_ELECT_LSC & ITASRL & 34 & $4 \mathrm{pCi} / \mathrm{L}$ & 2.42 & 8.54 & & & \\
\hline 2934 & UISO_AEA & ITASRL & 0.249 & $\mathrm{pCi} / \mathrm{L}$ & 0.0523 & 0.0596 & & & \\
\hline 2935 & UISO_AEA & ITASRL & 0.00286 & $5 \mathrm{pCi} / \mathrm{L}$ & 0.0111 & 0.0113 & & & $U$ \\
\hline 2936 & UISO_AEA & ITASRL & 0.202 & $2 \mathrm{pCi} / \mathrm{L}$ & 0.0466 & 0.0518 & & & \\
\hline 2937 & 6010_METALS_ICP & DATACH & 3.7 & $\mathrm{ug} / \mathrm{L}$ & & 1.55 & & & $\mathrm{BL}$ \\
\hline 2938 & 8260_VOA_GCMS & DATACH & 0.35 & $5 \mathrm{ug} / \mathrm{L}$ & & & & & $U$ \\
\hline 2939 & SRISO_SEP_PRECIP_GPC & ITASRL & 0.0947 & $7 \mathrm{pCi} / \mathrm{L}$ & 0.0358 & 0.0413 & & & \\
\hline 2940 & TRITIUM_ELECT_LSC & ITASRL & 33.7 & $7 \mathrm{pCi} / \mathrm{L}$ & 2.41 & 8.52 & & & \\
\hline 2941 & UISO_AEA & ITASRL & 0.25 & $5 \mathrm{pCi} / \mathrm{L}$ & 0.0441 & 0.0517 & & & \\
\hline 2942 & UISO_AEA & ITASRL & 0.00226 & $\mathrm{pCi} / \mathrm{L}$ & 0.00646 & 0.00673 & & & $U$ \\
\hline 2943 & UISO_AEA & ITASRL & 0.179 & $9 \mathrm{pCi} / \mathrm{L}$ & 0.0371 & 0.0417 & & & \\
\hline 2944 & 6010_METALS_ICP & DATACH & 1.8 & $3 \mathrm{ug} / \mathrm{L}$ & & & & & $\mathrm{U}$ \\
\hline 2945 & 8260_VOA_GCMS & DATACH & 0.35 & $\mathrm{ug} / \mathrm{L}$ & & & & & $U$ \\
\hline 2947 & TRITIUM_ELECT_LSC & ITASRL & 36.3 & $3 \mathrm{pCi} / \mathrm{L}$ & 2.43 & 8.65 & & & \\
\hline 2948 & UISO_AEA & ITASRL & 0.219 & $\mathrm{pCi} / \mathrm{L}$ & 0.0415 & 0.0478 & & & \\
\hline 2949 & UISO_AEA & ITASRL & 0.00293 & $3 \mathrm{pCi} / \mathrm{L}$ & 0.00876 & 0.00896 & & & $U$ \\
\hline 2950 & UISO_AEA & ITASRL & 0.17 & $7 \mathrm{pCi} / \mathrm{L}$ & 0.0365 & 0.0407 & & & \\
\hline 2951 & 6010_METALS_ICP & DATACH & 4.5 & $5 \mathrm{ug} / \mathrm{L}$ & & 1.89 & & & $\mathrm{BL}$ \\
\hline 2952 & 8260_VOA_GCMS & DATACH & 0.35 & $5 \mathrm{ug} / \mathrm{L}$ & & & & & U \\
\hline 2953 & SRISO_SEP_PRECIP_GPC & ITASRL & 0.0847 & $7 \mathrm{pCi} / \mathrm{L}$ & 0.0333 & 0.0413 & & & \\
\hline 2954 & TRITIUM_ELECT_LSC & ITASRL & 33.6 & $\mathrm{pCi} / \mathrm{L}$ & 2.4 & 8.47 & & & \\
\hline 2955 & UISO_AEA & ITASRL & 0.232 & $2 \mathrm{pCi} / \mathrm{L}$ & 0.0503 & 0.0569 & & & \\
\hline 2956 & UISO_AEA & ITASRL & 0.0108 & $3 \mathrm{pCi} / \mathrm{L}$ & 0.0134 & 0.0136 & & & $\mathrm{U}$ \\
\hline 2957 & UISO_AEA & ITASRL & 0.185 & $5 \mathrm{pCi} / \mathrm{L}$ & 0.0444 & 0.049 & & & \\
\hline 2958 & 6010_METALS_ICP & DATACH & 4.2 & ug/L & & 1.76 & & & $\mathrm{BL}$ \\
\hline 2959 & 8260_VOA_GCMS & DATACH & 0.35 & ug/L & & & & & $U$ \\
\hline 2960 & SRISO_SEP_PRECIP_GPC & ITASRL & 0.108 & $3 \mathrm{pCi} / \mathrm{L}$ & 0.0307 & 0.0431 & & & \\
\hline 2961 & TRITIUM_ELECT_LSC & ITASRL & 36.4 & $4 \mathrm{pCi} / \mathrm{L}$ & 2.46 & 8.77 & & & \\
\hline 2962 & UISO_AEA & ITASRL & 0.242 & $2 \mathrm{pCi} / \mathrm{L}$ & 0.0464 & 0.0535 & & & \\
\hline 2963 & UISO_AEA & ITASRL & 0.00328 & $3 \mathrm{pCi} / \mathrm{L}$ & 0.00859 & 0.00881 & & & $U$ \\
\hline 2964 & UISO_AEA & ITASRL & 0.207 & $7 \mathrm{pCi} / \mathrm{L}$ & 0.0423 & 0.0478 & & & \\
\hline
\end{tabular}

Page 201 


\begin{tabular}{|c|c|c|c|c|c|c|c|c|c|}
\hline & $\mathrm{K}$ & $\mathrm{L}$ & $\mathrm{M}$ & $\mathrm{N}$ & $\mathrm{O}$ & $\mathrm{P}$ & $\mathrm{Q}$ & $\mathrm{R}$ & $\mathrm{S}$ \\
\hline 1 & METHOD_NAME & LAB_CODE & VALUE_RPTD & ANAL_UNITS_RPTD & COUNTING_ERROR & TOTAL_ANAL_ERROR & MIN_DETECTABLE_ACTIVITY & PCNT_MOISTURE & LAB_QUALIFIER \\
\hline 2966 & 8260_VOA_GCMS & DATACH & 0.35 & ug/L & & & & & $U$ \\
\hline 2967 & SRISO_SEP_PRECIP_GPC & ITASRL & 0.0896 & $\mathrm{pCi} / \mathrm{L}$ & 0.0342 & 0.0423 & & & \\
\hline 2968 & TRITIUM_ELECT_LSC & ITASRL & 33.2 & $\mathrm{pCi} / \mathrm{L}$ & 2.38 & 8.42 & & & \\
\hline 2969 & UISO_AEA & ITASRL & 0.186 & $\mathrm{pCi} / \mathrm{L}$ & 0.0398 & 0.0448 & & & \\
\hline 2970 & UISO_AEA & ITASRL & 0.0047 & $\mathrm{pCi} / \mathrm{L}$ & 0.00804 & 0.00828 & & & U \\
\hline 2971 & UISO_AEA & ITASRL & 0.155 & $\mathrm{pCi} / \mathrm{L}$ & 0.036 & 0.0397 & & & \\
\hline 2972 & 6010_METALS_ICP & DATACH & 2.1 & ug/L & & 0.882 & & & $\mathrm{BL}$ \\
\hline 2973 \& & 8260_VOA_GCMS & DATACH & 0.35 & ug/L & & & & & U \\
\hline 2974 & SRISO_SEP_PRECIP_GPC & ITASRL & 0.058 & $\mathrm{pCi} / \mathrm{L}$ & 0.0281 & 0.0327 & & & \\
\hline 2975 & TRITIUM_ELECT_LSC & ITASRL & 34.3 & $\mathrm{pCi} / \mathrm{L}$ & 2.41 & 8.54 & & & \\
\hline 2976 & UISO_AEA & ITASRL & 0.255 & $\mathrm{pCi} / \mathrm{L}$ & 0.0489 & 0.0564 & & & \\
\hline 2977 & UISO_AEA & ITASRL & 0.00682 & $\mathrm{pCi} / \mathrm{L}$ & 0.01 & 0.0102 & & & $U$ \\
\hline 2978 & UISO_AEA & ITASRL & 0.161 & $\mathrm{pCi} / \mathrm{L}$ & 0.0385 & 0.0424 & & & \\
\hline 2979 & 6010_METALS_ICP & DATACH & 3.2 & ug/L & & 1.34 & & & $\mathrm{BL}$ \\
\hline 2980 & 8260_VOA_GCMS & DATACH & 0.35 & ug/L & & & & & $U$ \\
\hline 2981 & SRISO_SEP_PRECIP_GPC & ITASRL & 0.0925 & $\mathrm{pCi} / \mathrm{L}$ & 0.0294 & 0.0355 & & & \\
\hline 2982 & TRITIUM_ELECT_LSC & ITASRL & 30.9 & $\mathrm{pCi} / \mathrm{L}$ & 2.37 & 8.33 & & & \\
\hline 2985 & UISO_AEA & ITASRL & 0.204 & $\mathrm{pCi} / \mathrm{L}$ & 0.0415 & 0.047 & & & \\
\hline 2986 & 6010_METALS_ICP & DATACH & 4.2 & ug/L & & 1.76 & & & $\mathrm{BL}$ \\
\hline 2987 & 8260_VOA_GCMS & DATACH & 0.35 & ug/L & & & & & $U$ \\
\hline 2988 & SRISO_SEP_PRECIP_GPC & ITASRL & 0.0728 & $\mathrm{pCi} / \mathrm{L}$ & 0.0283 & 0.0328 & & & \\
\hline 2989 & TRITIUM_ELECTI_LSC & ITASRL & 36.1 & $\mathrm{pCi} / \mathrm{L}$ & 2.47 & 8.77 & & & \\
\hline 2990 & UISO_AEA & ITASRL & 0.221 & $\mathrm{pCi} / \mathrm{L}$ & 0.0535 & 0.0595 & & & \\
\hline 2991 & UISO_AEA & ITASRL & 0.00283 & $\mathrm{pCi} / \mathrm{L}$ & 0.00873 & 0.00894 & & & U \\
\hline 2992 & UISO_AEA & ITASRL & 0.17 & $\mathrm{pCi} / \mathrm{L}$ & 0.0466 & 0.0507 & & & \\
\hline 2993 & 6010_METALS_ICP & DATACH & 3.1 & ug/L & & 1.3 & & & $\mathrm{BL}$ \\
\hline 2994 & 8260_VOA_GCMS & DATACH & 0.35 & ug/L & & & & & U \\
\hline 2995 & SRISO_SEP_PRECIP_GPC & ITASRL & 0.0789 & $\mathrm{pCi} / \mathrm{L}$ & 0.0344 & 0.039 & & & \\
\hline 2996 & TRITIUM_ELECT_LSC & ITASRL & 38.3 & $\mathrm{pCi} / \mathrm{L}$ & 2.49 & 8.88 & & & \\
\hline 2997 I & UISO_AEA & ITASRL & 0.239 & $\mathrm{pCi} / \mathrm{L}$ & 0.0447 & 0.0518 & & & \\
\hline 2998 & UISO_AEA & ITASRL & 0.00462 & $\mathrm{pCi} / \mathrm{L}$ & 0.00796 & 0.00821 & & & U \\
\hline 2999 & UISO_AEA & ITASRL & 0.163 & $\mathrm{pCi} / \mathrm{L}$ & 0.0367 & 0.0407 & & & \\
\hline 3000 & 6010_METALS_ICP & DATACH & 4.8 & ug/L & & 2.02 & & & $\mathrm{BL}$ \\
\hline 3001 & 8260_VOA_GCMS & DATACH & 0.35 & $\mathrm{ug} / \mathrm{L}$ & & & & & $\mathrm{U}$ \\
\hline 3002 & SRISO_SEP_PRECIP_GPC & ITASRL & 0.112 & $\mathrm{pCi} / \mathrm{L}$ & 0.0321 & 0.0391 & & & \\
\hline
\end{tabular}




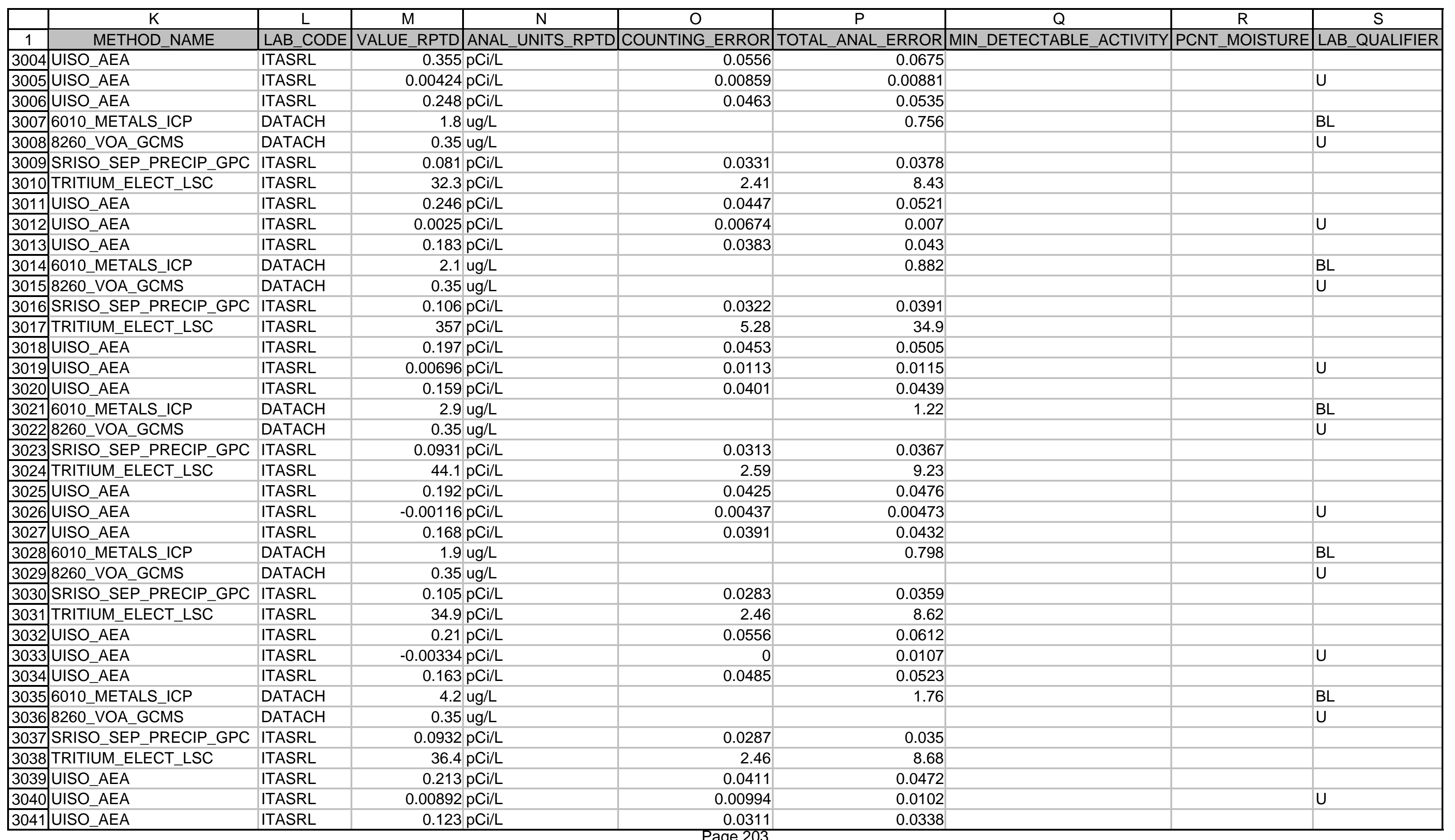




\begin{tabular}{|c|c|c|c|c|c|c|c|c|c|}
\hline & $\mathrm{K}$ & $\mathrm{L}$ & $M$ & $\mathrm{~N}$ & $\mathrm{O}$ & $\mathrm{P}$ & $\mathrm{Q}$ & $\mathrm{R}$ & $\mathrm{S}$ \\
\hline 1 & METHOD_NAME & LAB_CODE & VALUE_RPTD & ANAL_UNITS_RPTD & COUNTING_ERROR & TOTAL_ANAL_ERROR & MIN_DETECTABLE_ACTIVITY & PCNT_MOISTURE & LAB_QUALIFIER \\
\hline 3042 & 6010_METALS_ICP & DATACH & & $\mathrm{ug} / \mathrm{L}$ & & 1.68 & & & $\mathrm{BL}$ \\
\hline 3043 & 8260_VOA_GCMS & DATACH & 0.35 & $u g / L$ & & & & & $\mathrm{U}$ \\
\hline 3044 & SRISO_SEP_PRECIP_GPC & ITASRL & 0.0814 & $\mathrm{pCi} / \mathrm{L}$ & 0.0289 & 0.0343 & & & \\
\hline 3045 & TRITIUM_ELECT_LSC & ITASRL & 38.1 & $\mathrm{pCi} / \mathrm{L}$ & 2.51 & 8.84 & & & \\
\hline 3046 & UISO_AEA & ITASRL & 0.216 & $\mathrm{pCi} / \mathrm{L}$ & 0.0438 & 0.0499 & & & \\
\hline 3047 & UISO_AEA & ITASRL & 0.00291 & $\mathrm{pCi} / \mathrm{L}$ & 0.00722 & 0.00747 & & & U \\
\hline 3048 & UISO_AEA & ITASRL & 0.144 & pCi/L & 0.0354 & 0.0387 & & & \\
\hline 3049 & 6010_METALS_ICP & DATACH & 2.4 & ug/L & & 1.01 & & & $\mathrm{BL}$ \\
\hline 3051 & SRISO_SEP_PRECIP_GPC & ITASRL & 0.0982 & $\mathrm{pCi} / \mathrm{L}$ & 0.0325 & 0.0391 & & & \\
\hline 3052 & TRITIUM_ELECT_LSC & ITASRL & 32.8 & $\mathrm{pCi} / \mathrm{L}$ & 2.41 & 8.43 & & & \\
\hline 3053 & UISO_AEA & ITASRL & 0.248 & $\mathrm{pCi} / \mathrm{L}$ & 0.0474 & 0.0547 & & & \\
\hline 3054 & UISO_AEA & ITASRL & 0.00101 & $\mathrm{pCi} / \mathrm{L}$ & 0.00615 & 0.00642 & & & $\mathrm{U}$ \\
\hline 3055 & UISO_AEA & ITASRL & 0.153 & $\mathrm{pCi} / \mathrm{L}$ & 0.0371 & 0.0407 & & & \\
\hline 3056 & 6010_METALS_ICP & DATACH & & ug/L & & 2.1 & & & $\mathrm{BL}$ \\
\hline 3057 & 8260_VOA_GCMS & DATACH & 0.35 & ug/L & & & & & U \\
\hline 3058 & SRISO_SEP_PRECIP_GPC & ITASRL & 0.11 & pCi/L & 0.0301 & 0.0383 & & & \\
\hline 3059 & TRITIUM_ELECT_LSC & ITASRL & 32 & $\mathrm{pCi} / \mathrm{L}$ & 2.42 & 8.4 & & & \\
\hline 3061 & UISO_AEA & ITASRL & 0.00441 & $\mathrm{pCi} / \mathrm{L}$ & 0.00774 & 0.00799 & & & $\mathrm{U}$ \\
\hline 3062 & UISO_AEA & ITASRL & 0.166 & $\mathrm{pCi} / \mathrm{L}$ & 0.0366 & 0.0407 & & & \\
\hline 3063 & 6010_METALS_ICP & DATACH & 4.8 & $\mathrm{ug} / \mathrm{L}$ & & 2.02 & & & $\mathrm{BL}$ \\
\hline 3064 & 8260_VOA_GCMS & DATACH & 0.35 & ug/L & & & & & $\mathrm{U}$ \\
\hline 3065 & SRISO_SEP_PRECIP_GPC & ITASRL & 0.12 & $\mathrm{pCi} / \mathrm{L}$ & 0.0447 & 0.0515 & & & \\
\hline 3066 & TRITIUM_ELECT_LSC & ITASRL & 34.3 & $\mathrm{pCi} / \mathrm{L}$ & 2.43 & 8.54 & & & \\
\hline 3067 & UISO_AEA & ITASRL & 0.214 & $\mathrm{pCi} / \mathrm{L}$ & 0.0417 & 0.0478 & & & \\
\hline 3068 & UISO_AEA & ITASRL & 0.000535 & $\mathrm{pCi} / \mathrm{L}$ & 0.00548 & 0.00578 & & & U \\
\hline 3069 & UISO_AEA & ITASRL & 0.172 & $\mathrm{pCi} / \mathrm{L}$ & 0.0372 & 0.0415 & & & \\
\hline 3070 & 6010_METALS_ICP & DATACH & 1.8 & $\mathrm{ug} / \mathrm{L}$ & & & & & $\mathrm{U}$ \\
\hline 3071 & 8260_VOA_GCMS & DATACH & 0.35 & ug/L & & & & & U \\
\hline 3072 & SRISO_SEP_PRECIP_GPC & ITASRL & 0.0832 & $\mathrm{pCi} / \mathrm{L}$ & 0.0292 & 0.0346 & & & \\
\hline 3073 & TRITIUM_ELECT_LSC & ITASRL & 29.5 & $\mathrm{pCi} / \mathrm{L}$ & 2.35 & 8.13 & & & \\
\hline 3074 & UISO_AEA & ITASRL & 0.17 & $\mathrm{pCi} / \mathrm{L}$ & 0.0388 & 0.0432 & & & \\
\hline 3075 & UISO_AEA & ITASRL & 0.0239 & $\mathrm{pCi} / \mathrm{L}$ & 0.0156 & 0.016 & & & \\
\hline 3076 & UISO_AEA & ITASRL & 0.157 & $\mathrm{pCi} / \mathrm{L}$ & 0.0367 & 0.0405 & & & \\
\hline 3077 & 6010_METALS_ICP & DATACH & 1.8 & ug/L & & & & & $\mathrm{U}$ \\
\hline 3078 & 8260_VOA_GCMS & DATACH & 0.35 & ug/L & & & & & $U$ \\
\hline
\end{tabular}


$\mathrm{N}$

$\mathrm{O}$

P

Q

R

$S$

\begin{tabular}{|c|c|c|c|c|c|c|c|c|c|}
\hline & & & & & & & & & \\
\hline 1 & METHOD_NAME & LAB_CODE & VALUE_RPTD & ANAL_UNITS_RPTD & COUNTING_ERROR & TOTAL_ANAL_ERROR & MIN_DETECTABLE_ACTIVITY & PCNT_MOISTURE & LAB_QUALIFIER \\
\hline 3080 & TRITIUM_ELECT_LSC & ITASRL & 25.4 & $7 \mathrm{pCi} / \mathrm{L}$ & 2.29 & 7.84 & & & \\
\hline 3081 & UISO_AEA & ITASRL & 0.195 & $\mathrm{pCi} / \mathrm{L}$ & 0.0426 & 0.0479 & & & \\
\hline 3082 & UISO_AEA & ITASRL & 0.00233 & $3 \mathrm{pCi} / \mathrm{L}$ & 0.00775 & 0.00798 & & & $\mathrm{U}$ \\
\hline 3083 & UISO_AEA & ITASRL & 0.149 & $\mathrm{pCi} / \mathrm{L}$ & 0.0368 & 0.0402 & & & \\
\hline 3084 & 6010_METALS_ICP & DATACH & 1.8 & $3 \mathrm{ug} / \mathrm{L}$ & & & & & $\mathrm{U}$ \\
\hline 3085 & 8260_VOA_GCMS & DATACH & 0.35 & $\mathrm{ug} / \mathrm{L}$ & & & & & $\mathrm{U}$ \\
\hline 3086 & SRISO_SEP_PRECIP_GPC & ITASRL & 0.0567 & $\mathrm{pCi} / \mathrm{L}$ & 0.0348 & 0.0392 & & & \\
\hline 3087 & TRITIUM_ELECT_LSC & ITASRL & 30.9 & $\mathrm{pCi} / \mathrm{L}$ & 2.37 & 8.21 & & & \\
\hline 3088 & UISO_AËA & ITASRL & 0.224 & $\mathrm{pCi} / \mathrm{L}$ & 0.0455 & 0.0519 & & & \\
\hline 3089 & UISO_AEA & ITASRL & 0.00992 & $\mathrm{pCi} / \mathrm{L}$ & 0.0108 & 0.0111 & & & U \\
\hline 3090 & UISO_AEA & ITASRL & 0.166 & $\mathrm{pCi} / \mathrm{L}$ & 0.0391 & 0.0431 & & & \\
\hline 3091 & 6010_METALS_ICP & DATACH & 1.8 & $3 \mathrm{ug} / \mathrm{L}$ & & & & & U \\
\hline 3092 & 8260 VOA_GCMS & DATACH & 0.35 & $\mathrm{ug} / \mathrm{L}$ & & & & & $\mathrm{U}$ \\
\hline 3093 & SRISO_SEP_PRECIP_GPC & ITASRL & 0.0589 & $\mathrm{pCi} / \mathrm{L}$ & 0.0357 & 0.0384 & & & \\
\hline 3094 & TRITIUM_ELECT_LSC & ITASRL & 27.6 & $\mathrm{pCi} / \mathrm{L}$ & 2.31 & 7.97 & & & \\
\hline 3095 & UISO_AEA & ITASRL & 0.186 & $\mathrm{pCi} / \mathrm{L}$ & 0.045 & 0.0498 & & & \\
\hline 3096 & UISO_AEA & ITASRL & 0.00999 & $\mathrm{pCi} / \mathrm{L}$ & 0.0136 & 0.0138 & & & $U$ \\
\hline 3097 & UISO_AEA & ITASRL & 0.169 & $\mathrm{pCi} / \mathrm{L}$ & 0.0425 & 0.0465 & & & \\
\hline 3100 & SRISO_SEP_PRECIP_GPC & ITASRL & 0.153 & $3 \mathrm{pCi} / \mathrm{L}$ & 0.0451 & 0.0549 & & & \\
\hline 3101 & SRISO_SEP_PRECIP_GPC & ITASRL & 0.146 & $\mathrm{pCi} / \mathrm{L}$ & 0.0414 & 0.055 & & & \\
\hline 3102 & TRITIUM_ELECT_LSC & ITASRL & 109 & $\mathrm{pCi} / \mathrm{L}$ & 3.33 & 14.3 & & & \\
\hline 3103 & TRITIUM_ELECT_LSC & ITASRL & 115 & $\mathrm{pCi} / \mathrm{L}$ & 3.38 & 14.5 & & & \\
\hline 3104 & UISO_AEA & ITASRL & 0.223 & $\mathrm{pCi} / \mathrm{L}$ & 0.0467 & 0.053 & & & \\
\hline 3105 & UISO_AEA & ITASRL & -0.000659 & $\mathrm{pCi} / \mathrm{L}$ & 0.0068 & 0.00705 & & & $U$ \\
\hline 3106 & UISO_AEA & ITASRL & 0.196 & $\mathrm{pCi} / \mathrm{L}$ & 0.0427 & 0.0478 & & & \\
\hline 3107 & UISO_AEA & ITASRL & 0.239 & $\mathrm{pCi} / \mathrm{L}$ & 0.0462 & 0.0533 & & & \\
\hline 3108 & UISO_AEA & ITASRL & 0.0211 & $\mathrm{pCi} / \mathrm{L}$ & 0.0147 & 0.015 & & & \\
\hline 3109 & UISO_AEA & ITASRL & 0.209 & $\mathrm{pCi} / \mathrm{L}$ & 0.0428 & 0.0484 & & & \\
\hline 3110 & 6010_METALS_ICP & DATACH & 1.8 & $3 \mathrm{ug} / \mathrm{L}$ & & & & & $U$ \\
\hline 3111 & 8260_VOA_GCMS & DATACH & 0.35 & $\mathrm{ug} / \mathrm{L}$ & & & & & U \\
\hline 3112 & SRISO_SEP_PRECIP_GPC & ITASRL & 0.0949 & $\mathrm{pCi} / \mathrm{L}$ & 0.0308 & 0.0367 & & & \\
\hline 3113 & \begin{tabular}{|l} 
TRITIUM_ELECT_LSC \\
TEMCT
\end{tabular} & ITASRL & 30.8 & $3 \mathrm{pCi} / \mathrm{L}$ & 2.37 & 8.21 & & & \\
\hline 3114 & UISO_AEA & ITASRL & 0.309 & $\mathrm{pCi} / \mathrm{L}$ & 0.0566 & 0.0664 & & & \\
\hline 3115 & UISO_AEA & ITASRL & 0.0142 & $\mathrm{pCi} / \mathrm{L}$ & 0.0132 & 0.0135 & & & \\
\hline 3116 & UISO_AEA & ITASRL & 0.237 & $7 \mathrm{pCi} / \mathrm{L}$ & 0.0493 & 0.0558 & & & \\
\hline 3117 & 6010_METALS_ICP & DATACH & 1.8 & $3 \mathrm{ug} / \mathrm{L}$ & & & & & $U$ \\
\hline
\end{tabular}

Page 205 


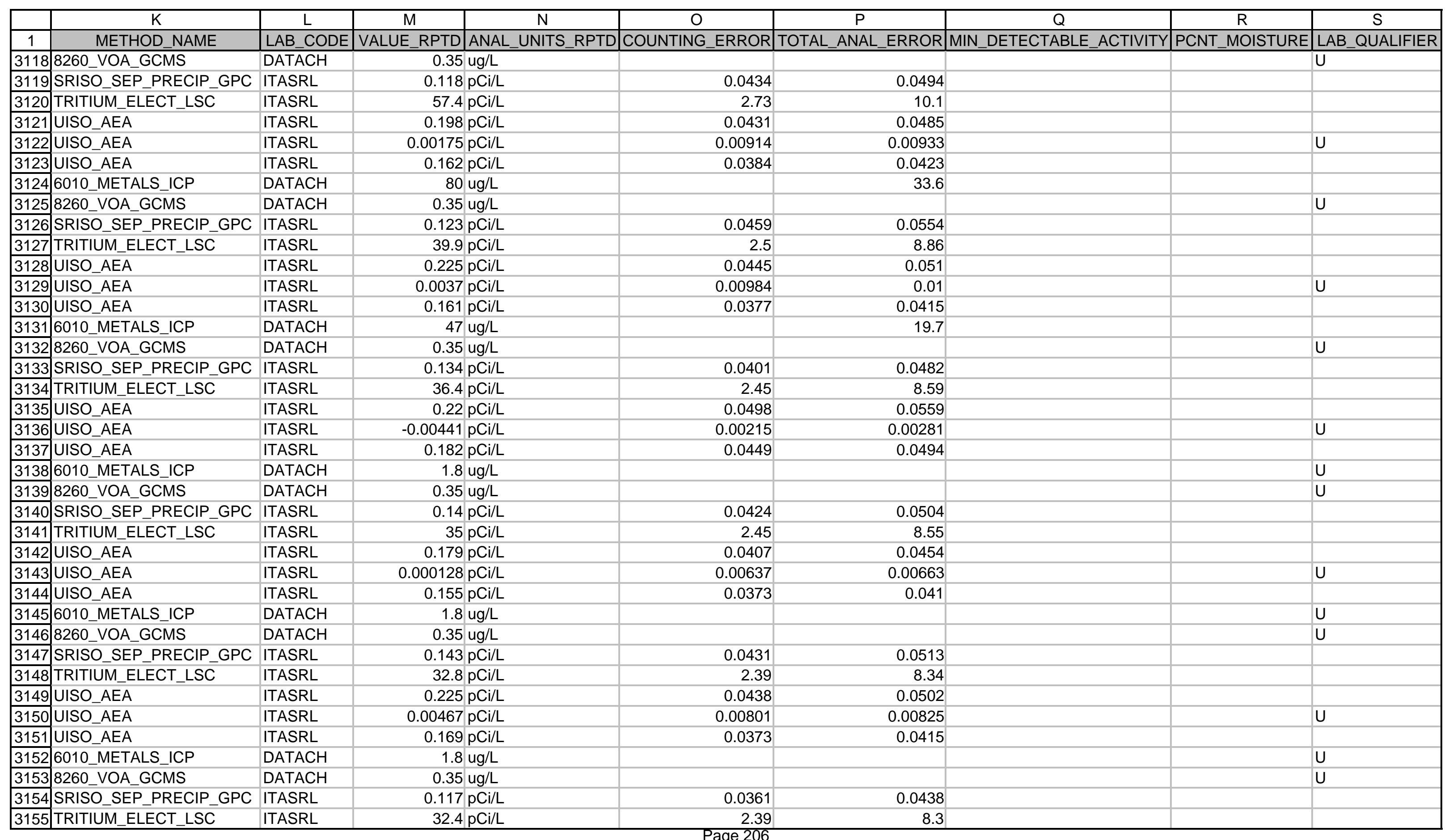




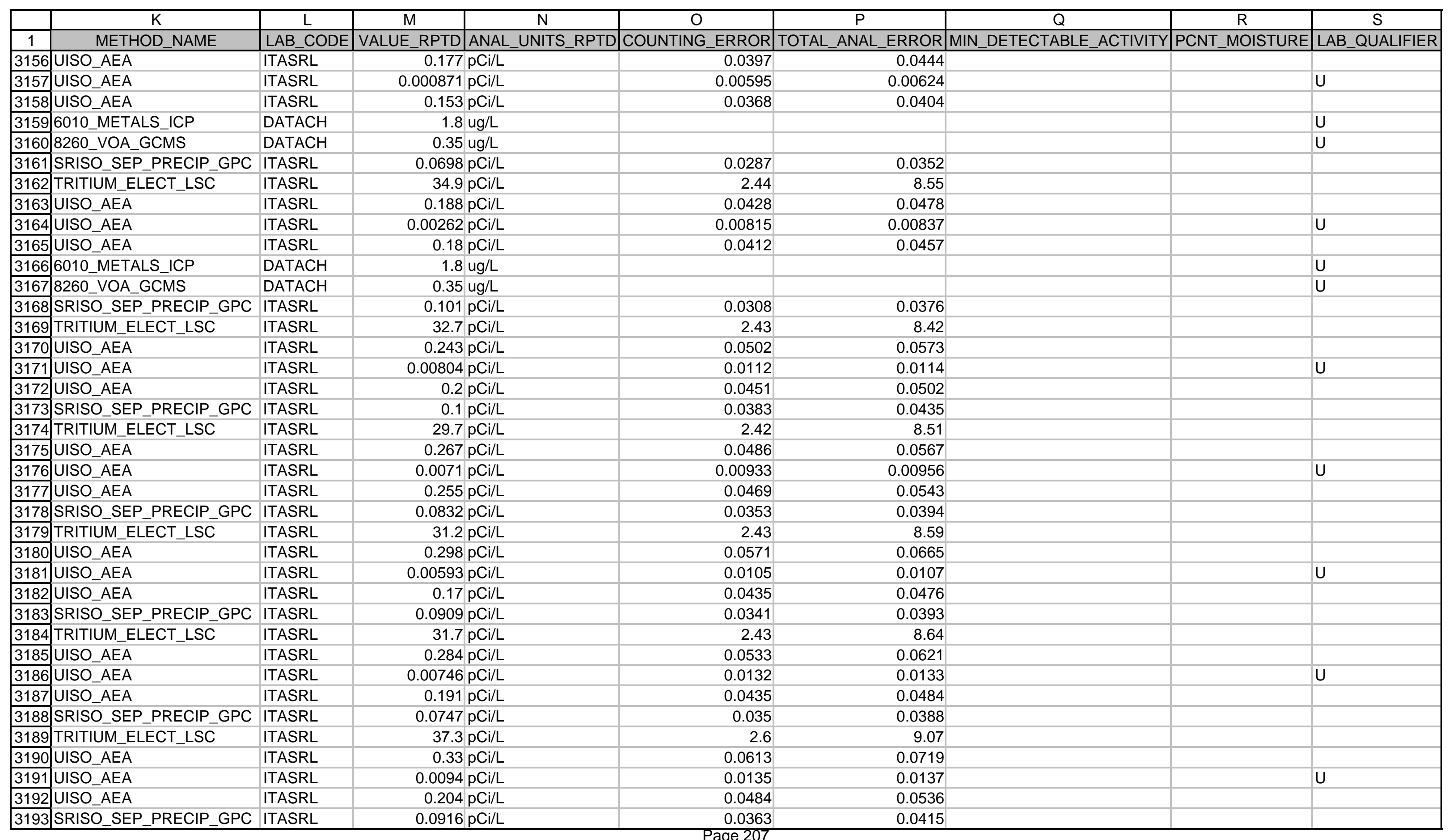


N

O

P

Q

\begin{tabular}{|c|c|c|c|c|c|c|c|c|c|}
\hline & & & & & & & & & \\
\hline 1 & METHOD_NAME & LAB_CODE & VALUE_RPTD & ANAL_UNITS_RPTD & COUNTING_ERROR & TOTAL_ANAL_ERROR & MIN_DETECTABLE_ACTIVITY & PCNT_MOISTURE & LAB_QUALIFIER \\
\hline 3194 & $\begin{array}{l}4 \mid \text { TRITIUM_ELECT_LSC } \\
\text { TIM_ET }\end{array}$ & ITASRL & 31 & $\mathrm{pCi} / \mathrm{L}$ & 2.46 & 8.69 & & & \\
\hline 3195 & UISO_AEA & ITASRL & 0.299 & $\mathrm{pCi} / \mathrm{L}$ & 0.0569 & 0.0662 & & & \\
\hline 3196 & UISO_AEA & ITASRL & -0.000702 & $\mathrm{pCi} / \mathrm{L}$ & 0.00516 & 0.00546 & & & $U$ \\
\hline 3197 & 7) UISO_AEA & ITASRL & 0.218 & $\mathrm{pCi} / \mathrm{L}$ & 0.0482 & 0.0541 & & & \\
\hline 3198 & SRISO_SEP_PRECIP_GPC & ITASRL & 0.0731 & $\mathrm{pCi} / \mathrm{L}$ & 0.0343 & 0.0379 & & & \\
\hline 3199 & ]TRITIUM_ELECT_LSC & ITASRL & 32.6 & $\mathrm{pCi} / \mathrm{L}$ & 2.48 & 8.72 & & & \\
\hline 3200 & UISO_AEA & ITASRL & 0.336 & $\mathrm{pCi} / \mathrm{L}$ & 0.061 & 0.072 & & & \\
\hline 3201 & UISO_AEA & ITASRL & 0.0101 & $\mathrm{pCi} / \mathrm{L}$ & 0.012 & 0.0122 & & & $\mathrm{U}$ \\
\hline 3202 & UISO_AEA & ITASRL & 0.26 & $\mathrm{pCi} / \mathrm{L}$ & 0.0534 & 0.0609 & & & \\
\hline 3203 & SRISŌSEP_PRECIP_GPC & ITASRL & 0.0588 & $\mathrm{pCi} / \mathrm{L}$ & 0.0374 & 0.04 & & & \\
\hline 3204 & 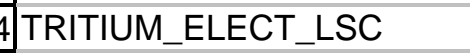 & ITASRL & 30 & $\mathrm{pCi} / \mathrm{L}$ & 2.42 & 8.54 & & & \\
\hline 3205 & UISO_AEA & ITASRL & 0.254 & $\mathrm{pCi} / \mathrm{L}$ & 0.0508 & 0.0584 & & & \\
\hline 3206 & UISO_AEA & ITASRL & -0.00422 & $\mathrm{pCi} / \mathrm{L}$ & 0.00195 & 0.00263 & & & $\mathrm{U}$ \\
\hline 3207 & 7 UISO_AEA & ITASRL & 0.215 & $\mathrm{pCi} / \mathrm{L}$ & 0.0465 & 0.0523 & & & \\
\hline 3208 & SRISO_SEP_PRECIP_GPC & ITASRL & 0.0905 & $\mathrm{pCi} / \mathrm{L}$ & 0.0389 & 0.0437 & & & \\
\hline 3209 & \begin{tabular}{|l|l} 
TRITIUM_ELECT_LSC \\
\end{tabular} & ITASRL & 29.6 & $\mathrm{pCi} / \mathrm{L}$ & 2.43 & 8.52 & & & \\
\hline 3210 & UISO_AEA & ITASRL & 0.246 & $\mathrm{pCi} / \mathrm{L}$ & 0.052 & 0.0591 & & & \\
\hline 3211 & UISO_AEA & ITASRL & 0.0046 & $\mathrm{pCi} / \mathrm{L}$ & 0.00903 & 0.00924 & & & $\mathrm{U}$ \\
\hline 3214 & 4 TRITIUM_ELECT_LSC & ITASRL & 35.9 & $\mathrm{pCi} / \mathrm{L}$ & 2.5 & 8.92 & & & \\
\hline 3215 & UISO_AEA & ITASRL & 0.341 & $\mathrm{pCi} / \mathrm{L}$ & 0.0555 & 0.0669 & & & \\
\hline 3216 & UISO_AEA & ITASRL & 0.00328 & $\mathrm{pCi} / \mathrm{L}$ & 0.00758 & 0.00781 & & & $\mathrm{U}$ \\
\hline 3217 & 7 UISO_AEA & ITASRL & 0.282 & $\mathrm{pCi} / \mathrm{L}$ & 0.0503 & 0.0588 & & & \\
\hline 3218 & SRISO_SEP_PRECIP_GPC & ITASRL & 0.0995 & $\mathrm{pCi} / \mathrm{L}$ & 0.0378 & 0.0434 & & & \\
\hline 3219 & TRITIUM_ELECT_LSC & ITASRL & 34.4 & $\mathrm{pCi} / \mathrm{L}$ & 2.63 & 9.29 & & & \\
\hline 3220 & UISO_AEA & ITASRL & 0.295 & $\mathrm{pCi} / \mathrm{L}$ & 0.0496 & 0.059 & & & \\
\hline 3221 & UISO_AEA & ITASRL & 0.0127 & $\mathrm{pCi} / \mathrm{L}$ & 0.0113 & 0.0116 & & & \\
\hline 3222 & UISO_AEA & ITASRL & 0.222 & $\mathrm{pCi} / \mathrm{L}$ & 0.0426 & 0.0488 & & & \\
\hline 3223 & SRISO_SEP_PRECIP_GPC & ITASRL & 0.0798 & $\mathrm{pCi} / \mathrm{L}$ & 0.0398 & 0.0435 & & & \\
\hline 3224 & TRITIUM_ELECT_LSC & ITASRL & 31.7 & $\mathrm{pCi} / \mathrm{L}$ & 2.47 & 8.65 & & & \\
\hline 3225 & UISO_AEA & ITASRL & 0.279 & $\mathrm{pCi} / \mathrm{L}$ & 0.0517 & 0.0603 & & & \\
\hline 3226 & UISO_AEA & ITASRL & 0.0123 & $\mathrm{pCi} / \mathrm{L}$ & 0.0134 & 0.0136 & & & $\mathrm{U}$ \\
\hline 3227 & 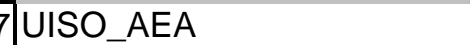 & ITASRL & 0.227 & $\mathrm{pCi} / \mathrm{L}$ & 0.0463 & 0.0525 & & & \\
\hline 3228 & SRISO_SEP_PRECIP_GPC & ITASRL & 0.0792 & $\mathrm{pCi} / \mathrm{L}$ & 0.0372 & 0.0408 & & & \\
\hline 3229 & TRITIUM_ELECT_LSC & ITASRL & 29.6 & $\mathrm{pCi} / \mathrm{L}$ & 2.41 & 8.48 & & & \\
\hline 3230 & UISO_AEA & ITASRL & 0.336 & $\mathrm{pCi} / \mathrm{L}$ & 0.0605 & 0.0716 & & & \\
\hline 3231 & UISO_AEA & ITASRL & 0.0168 & $\mathrm{pCi} / \mathrm{L}$ & 0.0151 & 0.0154 & & & \\
\hline
\end{tabular}




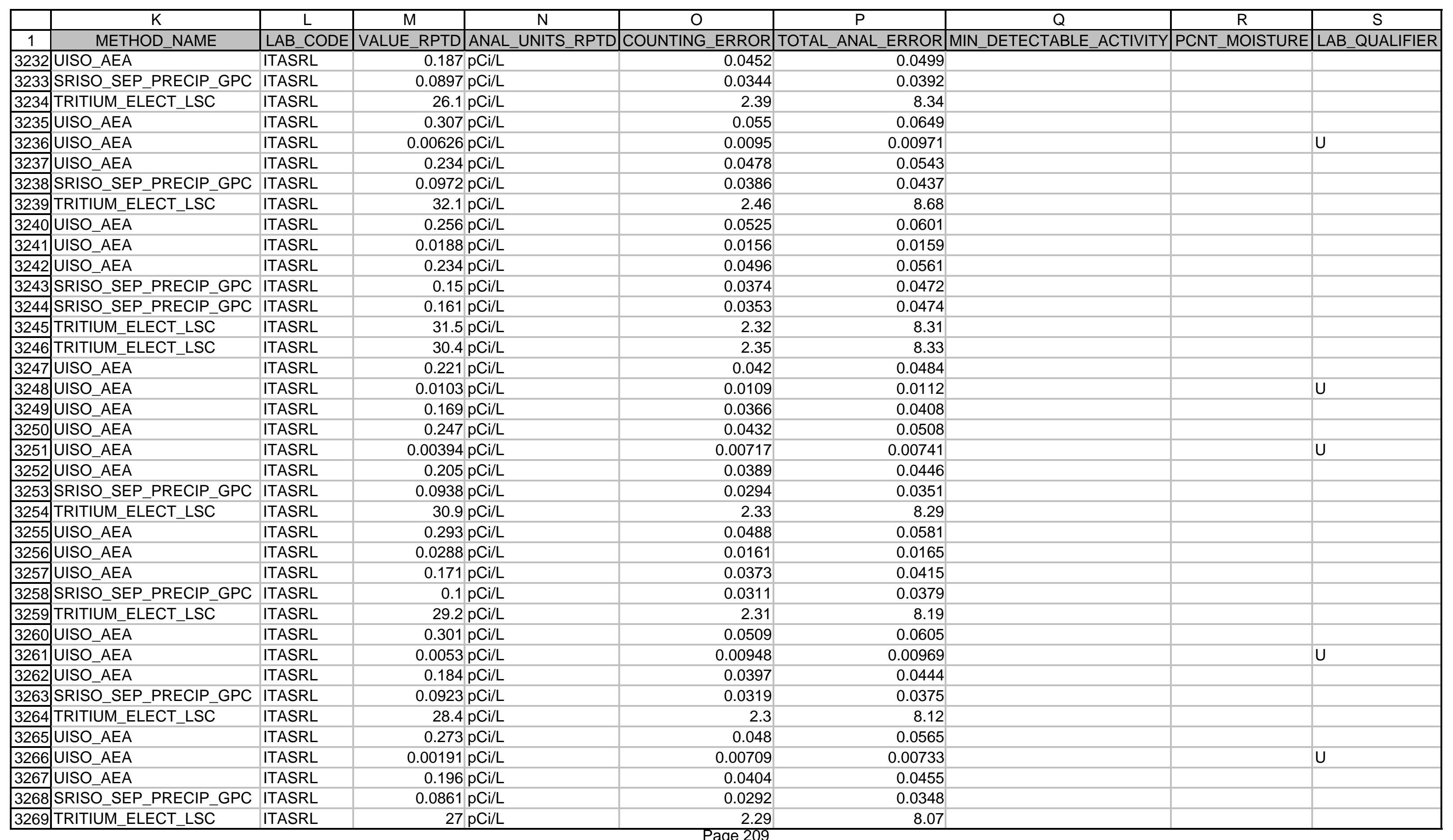




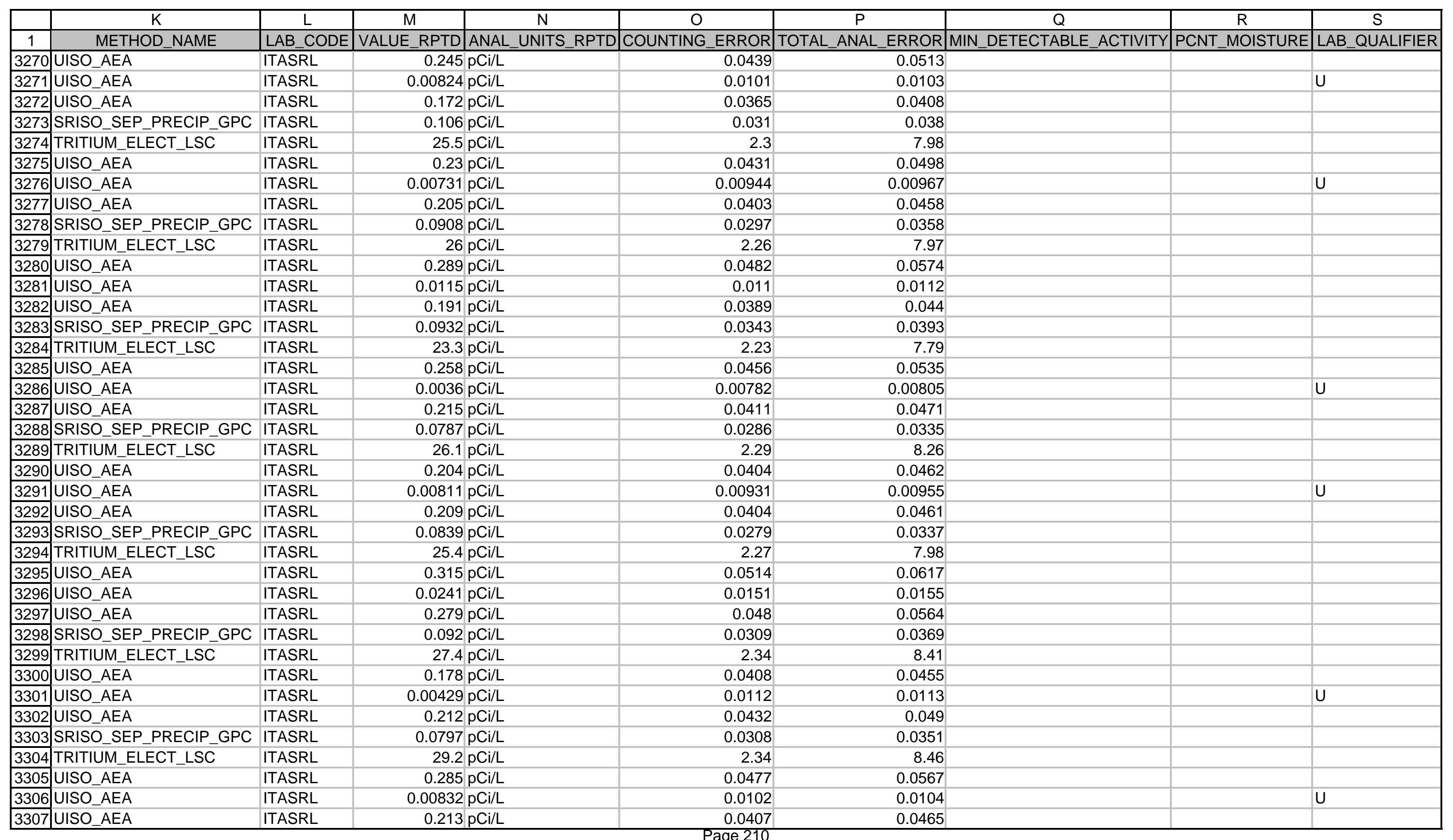


N

O

P

Q

R

$\mathrm{S}$

\begin{tabular}{|c|c|c|c|c|c|c|c|c|c|}
\hline & & & & & & & & & \\
\hline 1 & METHOD_NAME & LAB_CODE & VALUE_RPTD & ANAL_UNITS_RPTD & COUNTING_ERROR & TOTAL_ANAL_ERROR & MIN_DETECTABLE_ACTIVITY & PCNT_MOISTURE & LAB_QUALIFIER \\
\hline 3308 & SRISO_SEP_PRECIP_GPC & ITASRL & 0.0847 & $\mathrm{pCi} / \mathrm{L}$ & 0.0302 & 0.0352 & & & \\
\hline 3309 & TRITIUM_ELECT_LSC & ITASRL & 25.8 & $\mathrm{pCi} / \mathrm{L}$ & 2.28 & 8.21 & & & \\
\hline 3310 & UISO_AEA & ITASRL & 0.238 & $\mathrm{pCi} / \mathrm{L}$ & 0.046 & 0.0531 & & & \\
\hline 3311 & UISO_AEA & ITASRL & 0.00726 & $\mathrm{pCi} / \mathrm{L}$ & 0.00942 & 0.00965 & & & $\mathrm{U}$ \\
\hline 3312 & UISO AEA & ITASRL & 0.189 & $\mathrm{pCi} / \mathrm{L}$ & 0.0407 & 0.0456 & & & \\
\hline 3313 & SRISO_SEP_PRECIP_GPC & ITASRL & 0.0883 & $\mathrm{pCi} / \mathrm{L}$ & 0.0299 & 0.035 & & & \\
\hline 3314 & TRITIUM_ELECT_LSC & ITASRL & 23.6 & $\mathrm{pCi} / \mathrm{L}$ & 2.28 & 8.08 & & & \\
\hline 3315 & UISO_AEA & ITASRL & 0.198 & $\mathrm{pCi} / \mathrm{L}$ & 0.0441 & 0.0495 & & & \\
\hline 3316 & UISO_AEA & ITASRL & 0.00505 & $\mathrm{pCi} / \mathrm{L}$ & 0.00947 & 0.00968 & & & $\mathrm{U}$ \\
\hline 3317 & UISO_AEA & ITASRL & 0.192 & $\mathrm{pCi} / \mathrm{L}$ & 0.0428 & 0.0478 & & & \\
\hline 3318 & SRISO SEP PRECIP GPC & ITASRL & 0.098 & $\mathrm{pCi} / \mathrm{L}$ & 0.0272 & 0.0347 & & & \\
\hline 3319 & TRITIUM_ELEECT_LSC & ITASRL & 29.7 & $\mathrm{pCi} / \mathrm{L}$ & 2.34 & 8.26 & & & \\
\hline 3320 & UISO_AEA & ITASRL & 0.202 & $\mathrm{pCi} / \mathrm{L}$ & 0.0396 & 0.0453 & & & \\
\hline 3321 & UISO_AEA & ITASRL & 0.00507 & $\mathrm{pCi} / \mathrm{L}$ & 0.0083 & 0.00854 & & & $U$ \\
\hline 3322 & UISO_AEA & ITASRL & 0.15 & $\mathrm{pCi} / \mathrm{L}$ & 0.0337 & 0.0373 & & & \\
\hline 3323 & SRISO_SEP_PRECIP_GPC & ITASRL & 0.0852 & $\mathrm{pCi} / \mathrm{L}$ & 0.0281 & 0.0338 & & & \\
\hline 3324 & TRITIUM ELECT LSC & ITASRL & 28.1 & $\mathrm{pCi} / \mathrm{L}$ & 2.34 & 8.15 & & & \\
\hline 3325 & UISO_AEA & ITASRL & 0.237 & $\mathrm{pCi} / \mathrm{L}$ & 0.0435 & 0.0505 & & & \\
\hline 3328 & SRISO-SEP_PRECIP_GPC & ITASRL & 0.104 & $\mathrm{pCi} / \mathrm{L}$ & 0.0302 & 0.038 & & & \\
\hline 3329 & TRITIUM_ELECT_LSC & ITASRL & 28.8 & $\mathrm{pCi} / \mathrm{L}$ & 2.36 & 8.17 & & & \\
\hline 3330 & UISO_AEA & ITASRL & 0.26 & $\mathrm{pCi} / \mathrm{L}$ & 0.0454 & 0.0533 & & & \\
\hline 3331 & UISO_AEA & ITASRL & 0.00419 & $\mathrm{pCi} / \mathrm{L}$ & 0.00753 & 0.00778 & & & $U$ \\
\hline 3332 & UISO_AEA & ITASRL & 0.229 & $\mathrm{pCi} / \mathrm{L}$ & 0.0421 & 0.0486 & & & \\
\hline 3333 & SRISO_SEP_PRECIP_GPC & ITASRL & 0.0836 & $\mathrm{pCi} / \mathrm{L}$ & 0.03 & 0.0354 & & & \\
\hline 3334 & TRITIUM_ELEECT_LSC & ITASRL & 26.3 & $\mathrm{pCi} / \mathrm{L}$ & 2.31 & 8 & & & \\
\hline 3335 & UISO_AEA & ITASRL & 0.258 & $\mathrm{pCi} / \mathrm{L}$ & 0.0426 & 0.0507 & & & \\
\hline 3336 & UISO_AEA & ITASRL & 0.00478 & $\mathrm{pCi} / \mathrm{L}$ & 0.00858 & 0.0088 & & & $\mathrm{U}$ \\
\hline 3337 & UISO_AEA & ITASRL & 0.167 & $\mathrm{pCi} / \mathrm{L}$ & 0.0342 & 0.0385 & & & \\
\hline 3338 & SRISO_SEP_PRECIP_GPC & ITASRL & 0.0672 & $\mathrm{pCi} / \mathrm{L}$ & 0.0294 & 0.0329 & & & \\
\hline 3339 & TRITIUM_ELECT_LSC & ITASRL & 1550 & $\mathrm{pCi} / \mathrm{L}$ & 10.4 & 137 & & & \\
\hline 3340 & UISO_AEA & ITASRL & 0.216 & $\mathrm{pCi} / \mathrm{L}$ & 0.0426 & 0.0487 & & & \\
\hline 3341 & UISO_AEA & ITASRL & 0.0058 & $\mathrm{pCi} / \mathrm{L}$ & 0.00901 & 0.00923 & & & $\mathrm{U}$ \\
\hline 3342 & UISO_AEA & ITASRL & 0.173 & $\mathrm{pCi} / \mathrm{L}$ & 0.0376 & 0.042 & & & \\
\hline 3343 & SRISO SEP PRECIP GPC & ITASRL & 0.0839 & $\mathrm{pCi} / \mathrm{L}$ & 0.0315 & 0.0366 & & & \\
\hline 3344 & TRITIUMM_ELECT_LSC̄ & ITASRL & 30.7 & $\mathrm{pCi} / \mathrm{L}$ & 2.4 & 8.65 & & & \\
\hline 3345 & UISO_AEA & ITASRL & 0.239 & $\mathrm{pCi} / \mathrm{L}$ & 0.044 & 0.051 & & & \\
\hline
\end{tabular}




\begin{tabular}{|c|c|c|c|c|c|c|c|c|c|}
\hline & $\mathrm{K}$ & $\mathrm{L}$ & $\mathrm{M}$ & $\mathrm{N}$ & $\mathrm{O}$ & $P$ & $\mathrm{Q}$ & $\mathrm{R}$ & $S$ \\
\hline 1 & METHOD_NAME & LAB_CODE & VALUE_RPTD & ANAL_UNITS_RPTD & COUNTING_ERROR & TOTAL_ANAL_ERROR & MIN_DETECTABLE_ACTIVITY & PCNT_MOISTURE & LAB_QUALIFIER \\
\hline 3346 & UISO_AEA & ITASRL & 0.00791 & $\mathrm{pCi} / \mathrm{L}$ & 0.0127 & 0.0129 & & & $\mathrm{U}$ \\
\hline 3347 & UISO_AEA & ITASRL & 0.187 & $\mathrm{pCi} / \mathrm{L}$ & 0.0387 & 0.0435 & & & \\
\hline 3348 & SRISO_SEP_PRECIP_GPC & ITASRL & 0.0702 & $\mathrm{pCi} / \mathrm{L}$ & 0.0284 & 0.0324 & & & \\
\hline 3349 & TRITIUM_ELECT_LSC & ITASRL & 50 & $\mathrm{pCi} / \mathrm{L}$ & 2.62 & 9.93 & & & \\
\hline 3350 & UISO_AEA & ITASRL & 0.283 & $\mathrm{pCi} / \mathrm{L}$ & 0.0484 & 0.0572 & & & \\
\hline 3351 & UISO_AEA & ITASRL & 0.00104 & $\mathrm{pCi} / \mathrm{L}$ & 0.00724 & 0.00747 & & & $\mathrm{U}$ \\
\hline 3352 & UISO_AEA & ITASRL & 0.168 & $\mathrm{pCi} / \mathrm{L}$ & 0.0373 & 0.0415 & & & \\
\hline 3353 & SRISŌSEP_PRECIP_GPC & ITASRL & 0.125 & $\mathrm{pCi} / \mathrm{L}$ & 0.0326 & 0.0408 & & & \\
\hline 3355 & UISO_AEA & ITASRL & 0.276 & $\mathrm{pCi} / \mathrm{L}$ & 0.0491 & 0.0576 & & & \\
\hline 3356 & UISO_AEA & ITASRL & 0.0167 & $\mathrm{pCi} / \mathrm{L}$ & 0.0133 & 0.0136 & & & \\
\hline 3357 & UISO_AEA & ITASRL & 0.196 & $\mathrm{pCi} / \mathrm{L}$ & 0.0412 & 0.0463 & & & \\
\hline 3358 & SRISO_SEP_PRECIP_GPC & ITASRL & 0.105 & $\mathrm{pCi} / \mathrm{L}$ & 0.0332 & 0.0394 & & & \\
\hline 3359 & TRITIUM_ELECT_LSC & ITASRL & 27.5 & $\mathrm{pCi} / \mathrm{L}$ & 2.31 & 8.28 & & & \\
\hline 3360 & UISO_AEA & ITASRL & 0.247 & $\mathrm{pCi} / \mathrm{L}$ & 0.0468 & 0.0541 & & & \\
\hline 3361 & UISO_AEA & ITASRL & 0.0117 & $\mathrm{pCi} / \mathrm{L}$ & 0.0121 & 0.0123 & & & $\mathrm{U}$ \\
\hline 3362 & UISO_AEA & ITASRL & 0.193 & $\mathrm{pCi} / \mathrm{L}$ & 0.041 & 0.046 & & & \\
\hline 3363 & SRISO-SEP_PRECIP_GPC & ITASRL & 0.0766 & $\mathrm{pCi} / \mathrm{L}$ & 0.0313 & 0.0358 & & & \\
\hline 3365 & UISO_AEA & ITASRL & 0.193 & $\mathrm{pCi} / \mathrm{L}$ & 0.0392 & 0.0445 & & & \\
\hline 3366 & UISO_AEA & ITASRL & 0.0126 & $\mathrm{pCi} / \mathrm{L}$ & 0.0112 & 0.0115 & & & \\
\hline 3367 & UISO_AEA & ITASRL & 0.176 & $\mathrm{pCi} / \mathrm{L}$ & 0.037 & 0.0415 & & & \\
\hline 3368 & SRISO_SEP_PRECIP_GPC & ITASRL & 0.0934 & $\mathrm{pCi} / \mathrm{L}$ & 0.0301 & 0.0362 & & & \\
\hline 3369 & TRITIUMM_ELECT_LSC̄ & ITASRL & 25.3 & $\mathrm{pCi} / \mathrm{L}$ & 2.28 & 8.19 & & & \\
\hline 3370 & UISO_AEA & ITASRL & 0.237 & $\mathrm{pCi} / \mathrm{L}$ & 0.0445 & 0.0515 & & & \\
\hline 3371 & UISO_AEA & ITASRL & 0.00567 & $\mathrm{pCi} / \mathrm{L}$ & 0.00888 & 0.0091 & & & $\mathrm{U}$ \\
\hline 3372 & UISO_AEA & ITASRL & 0.191 & $\mathrm{pCi} / \mathrm{L}$ & 0.0396 & 0.0446 & & & \\
\hline 3373 & SRISO_SEP_PRECIP_GPC & ITASRL & 0.0979 & $\mathrm{pCi} / \mathrm{L}$ & 0.0293 & 0.0365 & & & \\
\hline 3374 & TRITIUM_ELECT_LSC & ITASRL & 27.6 & $\mathrm{pCi} / \mathrm{L}$ & 2.32 & 8.36 & & & \\
\hline 3375 & UISO_AEA & ITASRL & 0.214 & $\mathrm{pCi} / \mathrm{L}$ & 0.0424 & 0.0485 & & & \\
\hline 3376 & UISO_AEA & ITASRL & 0.00291 & $\mathrm{pCi} / \mathrm{L}$ & 0.00809 & 0.00831 & & & $\mathrm{U}$ \\
\hline 3377 & UISO_AEA & ITASRL & 0.17 & $\mathrm{pCi} / \mathrm{L}$ & 0.0369 & 0.0412 & & & \\
\hline 3378 & SRISŌSEP_PRECIP_GPC & ITASRL & 0.109 & $\mathrm{pCi} / \mathrm{L}$ & 0.0311 & 0.0391 & & & \\
\hline 3379 & TRITIUM_ELECT_LSC & ITASRL & 26 & $\mathrm{pCi} / \mathrm{L}$ & 2.29 & 8.24 & & & \\
\hline 3380 & UISO_AEA & ITASRL & 0.23 & $\mathrm{pCi} / \mathrm{L}$ & 0.0432 & 0.0499 & & & \\
\hline 3381 & UISO_AEA & ITASRL & 0.016 & $\mathrm{pCi} / \mathrm{L}$ & 0.0122 & 0.0125 & & & \\
\hline 3382 & UISO_AEA & ITASRL & 0.165 & $\mathrm{pCi} / \mathrm{L}$ & 0.0365 & 0.0405 & & & \\
\hline
\end{tabular}


$\mathrm{N}$

O

P

Q

\begin{tabular}{|c|c|c|c|c|c|c|c|c|c|}
\hline & & & & & & & & & \\
\hline 1 & METHOD_NAME & LAB_CODE & VALUE_RPTD & ANAL_UNITS_RPTD & COUNTING_ERROR & TOTAL_ANAL_ERROR & MIN_DETECTABLE_ACTIVITY & PCNT_MOISTURE & LAB_QUALIFIER \\
\hline 3384 & TRITIUM_ELECT_LSC & ITASRL & 29.4 & $\mathrm{pCi} / \mathrm{L}$ & 2.38 & 8.59 & & & \\
\hline 3385 & UISO_AEA & ITASRL & 0.216 & $\mathrm{pCi} / \mathrm{L}$ & 0.041 & 0.0473 & & & \\
\hline 3386 & UISO_AEA & ITASRL & 0.00595 & $\mathrm{pCi} / \mathrm{L}$ & 0.00827 & 0.00851 & & & $U$ \\
\hline 3387 & UISO_AEA & ITASRL & 0.171 & $\mathrm{pCi} / \mathrm{L}$ & 0.0363 & 0.0406 & & & \\
\hline 3388 & SRISO_SEP_PRECIP_GPC & ITASRL & 0.0965 & $\mathrm{pCi} / \mathrm{L}$ & 0.0289 & 0.0353 & & & \\
\hline 3389 & TRITIUM_ELECT_LSC & ITASRL & 63 & $\mathrm{pCi} / \mathrm{L}$ & 2.86 & 10.8 & & & \\
\hline 3390 & UISO_AEA & ITASRL & 0.244 & $\mathrm{pCi} / \mathrm{L}$ & 0.0436 & 0.0509 & & & \\
\hline 3391 & UISO_AEA & ITASRL & 0.00225 & $\mathrm{pCi} / \mathrm{L}$ & 0.00645 & 0.00672 & & & $U$ \\
\hline 3392 & UISO_AEA & ITASRL & 0.209 & $\mathrm{pCi} / \mathrm{L}$ & 0.0401 & 0.0458 & & & \\
\hline 3393 & SRISO_SEP_PRECIP_GPC & ITASRL & 0.0996 & $\mathrm{pCi} / \mathrm{L}$ & 0.0303 & 0.037 & & & \\
\hline 3394 & TRITIUM_ELECT_LSC & ITASRL & 41.6 & $\mathrm{pCi} / \mathrm{L}$ & 2.54 & 9.1 & & & \\
\hline 3395 & UISO_AEA & ITASRL & 0.245 & $\mathrm{pCi} / \mathrm{L}$ & 0.0456 & 0.0529 & & & \\
\hline 3396 & UISO_AEA & ITASRL & 0.00783 & $\mathrm{pCi} / \mathrm{L}$ & 0.0099 & 0.0101 & & & $\mathrm{U}$ \\
\hline 3397 & UISO_AEA & ITASRL & 0.166 & $\mathrm{pCi} / \mathrm{L}$ & 0.0373 & 0.0414 & & & \\
\hline 3398 & SRISO_SEP_PRECIP_GPC & ITASRL & 0.0839 & $\mathrm{pCi} / \mathrm{L}$ & 0.0305 & 0.0355 & & & \\
\hline 3399 & TRITIUM_ELECT_LSC & ITASRL & 33.8 & $\mathrm{pCi} / \mathrm{L}$ & 2.48 & 8.67 & & & \\
\hline 3400 & UISO_AEA & ITASRL & 0.248 & $\mathrm{pCi} / \mathrm{L}$ & 0.047 & 0.0543 & & & \\
\hline 3401 & UISO_AEA & ITASRL & 0.00301 & $\mathrm{pCi} / \mathrm{L}$ & 0.00733 & 0.00758 & & & $\mathrm{U}$ \\
\hline 3404 & TRITIUM_ELECT_LSC & ITASRL & 29.3 & $\mathrm{pCi} / \mathrm{L}$ & 2.39 & 8.25 & & & \\
\hline 3405 & UISO_AEA & ITASRL & 0.234 & $\mathrm{pCi} / \mathrm{L}$ & 0.0449 & 0.0516 & & & \\
\hline 3406 & UISO_AEA & ITASRL & 0.00866 & $\mathrm{pCi} / \mathrm{L}$ & 0.00979 & 0.01 & & & $\mathrm{U}$ \\
\hline 3407 & UISO_AEA & ITASRL & 0.187 & $\mathrm{pCi} / \mathrm{L}$ & 0.0394 & 0.0442 & & & \\
\hline 3408 & SRISO_SEP_PRECIP_GPC & ITASRL & 0.0897 & $\mathrm{pCi} / \mathrm{L}$ & 0.0279 & 0.034 & & & \\
\hline 3409 & TRITIUM_ELECT_LSC & ITASRL & 27.6 & $\mathrm{pCi} / \mathrm{L}$ & 2.36 & 8.11 & & & \\
\hline 3410 & UISO_AEA & ITASRL & 0.266 & $\mathrm{pCi} / \mathrm{L}$ & 0.0476 & 0.0555 & & & \\
\hline 3411 & UISO_AEA & ITASRL & 0.00475 & $\mathrm{pCi} / \mathrm{L}$ & 0.011 & 0.0112 & & & $\mathrm{U}$ \\
\hline 3412 & UISO_AEA & ITASRL & 0.191 & $\mathrm{pCi} / \mathrm{L}$ & 0.0402 & 0.0451 & & & \\
\hline 3413 & SRISO_SEP_PRECIP_GPC & ITASRL & 0.103 & $\mathrm{pCi} / \mathrm{L}$ & 0.0314 & 0.0385 & & & \\
\hline 3414 & TRITIUM_ELECT_LSC & ITASRL & 27.6 & $\mathrm{pCi} / \mathrm{L}$ & 2.38 & 8.09 & & & \\
\hline 3415 & UISO_AEA & ITASRL & 0.239 & $\mathrm{pCi} / \mathrm{L}$ & 0.0421 & 0.0492 & & & \\
\hline 3416 & UISO_AEA & ITASRL & 0.0108 & $\mathrm{pCi} / \mathrm{L}$ & 0.01 & 0.0103 & & & \\
\hline 3417 & UISO_AEA & ITASRL & 0.19 & $\mathrm{pCi} / \mathrm{L}$ & 0.0373 & 0.0424 & & & \\
\hline 3418 & SRISO_SEP_PRECIP_GPC & ITASRL & 0.09 & $\mathrm{pCi} / \mathrm{L}$ & 0.0287 & 0.0348 & & & \\
\hline 3419 & TRITIUM_ELECT_LSC & ITASRL & 27.4 & $\mathrm{pCi} / \mathrm{L}$ & 2.33 & 8.05 & & & \\
\hline 3420 & UISO_AEA & ITASRL & 0.277 & $\mathrm{pCi} / \mathrm{L}$ & 0.0498 & 0.0583 & & & \\
\hline 3421 & UISO_AEA & ITASRL & 0.0000148 & $\mathrm{pCi} / \mathrm{L}$ & 0.00933 & 0.00951 & & & U \\
\hline
\end{tabular}

ITASRL
$29.4 \mathrm{pCi} / \mathrm{L}$
$0.216 \mathrm{pCi} / \mathrm{L}$

$0.00595 \mathrm{pCi} / \mathrm{L}$

$0.0965 \mathrm{pCi} / \mathrm{L}$

$00225 \mathrm{pCi} / \mathrm{L}$

$.0996 \mathrm{pCi} / \mathrm{L}$

$1.6 \mathrm{pCi} / \mathrm{L}$

$0.245 \mathrm{pCi} / \mathrm{L}$

$166 \mathrm{pCi}$

$.0839 \mathrm{pCi} / \mathrm{L}$

$33.8 \mathrm{pCi} /$

$0.221 \mathrm{pCi} / \mathrm{L}$

$0.0896 \mathrm{pCi} / \mathrm{L}$

$0.234 \mathrm{pCi} / \mathrm{L}$

$.00866 \mathrm{pCi} / \mathrm{L}$

$27.6 \mathrm{pCi} / \mathrm{L}$

$0.191 \mathrm{pCi} / \mathrm{L}$

$0.103 \mathrm{pCi} / \mathrm{L}$

$0108 \mathrm{pCi} / \mathrm{L}$

$0.09 \mathrm{pCi} / \mathrm{L}$

$27.4 \mathrm{pCi} / \mathrm{L}$ 


\begin{tabular}{|c|c|c|c|c|c|c|c|c|c|}
\hline & $\mathrm{K}$ & $\mathrm{L}$ & $\mathrm{M}$ & $\mathrm{N}$ & $\mathrm{O}$ & $\mathrm{P}$ & $\mathrm{Q}$ & $\mathrm{R}$ & $\mathrm{S}$ \\
\hline 1 & METHOD_NAME & LAB_CODE & VALUE_RPTD & ANAL_UNITS_RPTD & COUNTING_ERROR & \begin{tabular}{|l||} 
TOTAL_ANAL_ERROR \\
\end{tabular} & MIN_DETECTABLE_ACTIVITY & PCNT_MOISTURE & LAB_QUALIFIER \\
\hline 3422 & UISO_AEA & ITASRL & 0.224 & $\mathrm{pCi} / \mathrm{L}$ & 0.0443 & 0.0505 & & & \\
\hline 3423 & SRISO_SEP_PRECIP_GPC & ITASRL & 0.111 & $\mathrm{pCi} / \mathrm{L}$ & 0.0308 & 0.0384 & & & \\
\hline 3424 & TRITIUM_ELEECT_LSC̄ & ITASRL & 29.4 & $\mathrm{pCi} / \mathrm{L}$ & 2.4 & 8.29 & & & \\
\hline 3425 & UISO_AEA & ITASRL & 0.298 & $\mathrm{pCi} / \mathrm{L}$ & 0.0492 & 0.0588 & & & \\
\hline 3426 & UISO_AEA & ITASRL & 0.0173 & $\mathrm{pCi} / \mathrm{L}$ & 0.0135 & 0.0138 & & & \\
\hline 3427 & UISO_AEA & ITASRL & 0.258 & $\mathrm{pCi} / \mathrm{L}$ & 0.0451 & 0.0527 & & & \\
\hline 3428 & SRISO_SEP_PRECIP_GPC & ITASRL & 0.0917 & $\mathrm{pCi} / \mathrm{L}$ & 0.0301 & 0.0362 & & & \\
\hline 3429 & TRITIUM_ELECT_LSC & ITASRL & 29.9 & $\mathrm{pCi} / \mathrm{L}$ & 2.41 & 8.37 & & & \\
\hline 3430 & UISO_AEA & ITASRL & 0.277 & $\mathrm{pCi} / \mathrm{L}$ & 0.047 & 0.0558 & & & \\
\hline 3431 & UISO_AEA & ITASRL & 0.0119 & $\mathrm{pCi} / \mathrm{L}$ & 0.0108 & 0.011 & & & \\
\hline 3432 & UISO_AEA & ITASRL & 0.203 & $\mathrm{pCi} / \mathrm{L}$ & 0.04 & 0.0455 & & & \\
\hline 3433 & SRISO_SEP_PRECIP_GPC & ITASRL & 0.122 & $\mathrm{pCi} / \mathrm{L}$ & 0.0352 & 0.043 & & & \\
\hline 3434 & TRITIUMM_ELECT_LSC & ITASRL & 33.3 & $\mathrm{pCi} / \mathrm{L}$ & 2.45 & 8.58 & & & \\
\hline 3435 & UISO_AEA & ITASRL & 0.464 & $\mathrm{pCi} / \mathrm{L}$ & 0.0618 & 0.0792 & & & \\
\hline 3436 & UISO_AEA & ITASRL & 0.0126 & $\mathrm{pCi} / \mathrm{L}$ & 0.0113 & 0.0116 & & & \\
\hline 3437 & UISO_AEA & ITASRL & 0.417 & $\mathrm{pCi} / \mathrm{L}$ & 0.0582 & 0.073 & & & \\
\hline 3438 & SRISO_SEP_PRECIP_GPC & ITASRL & 0.0949 & $\mathrm{pCi} / \mathrm{L}$ & 0.0306 & 0.0366 & & & \\
\hline 3439 & TRITIUM_ELECT_LSC & ITASRL & 71 & $\mathrm{pCi} / \mathrm{L}$ & 3.39 & 11.4 & & & \\
\hline 3441 & UISO_AEA & ITASRL & 0.00394 & $\mathrm{pCi} / \mathrm{L}$ & 0.008 & 0.00823 & & & $\mathrm{U}$ \\
\hline 3442 & UISO_AEA & ITASRL & 0.161 & $\mathrm{pCi} / \mathrm{L}$ & 0.034 & 0.038 & & & \\
\hline 3443 & SRISO_SEP_PRECIP_GPC & ITASRL & 0.088 & $\mathrm{pCi} / \mathrm{L}$ & 0.0287 & 0.0347 & & & \\
\hline 3444 & TRITIUM_ELECT_LSC & ITASRL & 31.2 & $\mathrm{pCi} / \mathrm{L}$ & 2.37 & 8.4 & & & \\
\hline 3445 & UISO_AEA & ITASRL & 0.377 & $\mathrm{pCi} / \mathrm{L}$ & 0.0528 & 0.0661 & & & \\
\hline 3446 & UISO_AEA & ITASRL & 0.0103 & $\mathrm{pCi} / \mathrm{L}$ & 0.0102 & 0.0105 & & & U \\
\hline 3447 & UISO_AEA & ITASRL & 0.274 & $\mathrm{pCi} / \mathrm{L}$ & 0.045 & 0.0533 & & & \\
\hline 3448 & SRISO_SEP_PRECIP_GPC & ITASRL & 0.0949 & $\mathrm{pCi} / \mathrm{L}$ & 0.0322 & 0.038 & & & \\
\hline 3449 & TRITIUM̄E_ELECTI_LSC̄ & ITASRL & 41.1 & $\mathrm{pCi} / \mathrm{L}$ & 2.57 & 9.07 & & & \\
\hline 3450 & UISO_AEA & ITASRL & 0.19 & $\mathrm{pCi} / \mathrm{L}$ & 0.0401 & 0.0453 & & & \\
\hline 3451 & UISO_AEA & ITASRL & 0.0116 & $\mathrm{pCi} / \mathrm{L}$ & 0.0112 & 0.0115 & & & \\
\hline 3452 & UISO_AEA & ITASRL & 0.194 & $\mathrm{pCi} / \mathrm{L}$ & 0.0397 & 0.0448 & & & \\
\hline 3453 & SRISO_SEP_PRECIP_GPC & ITASRL & 0.0989 & $\mathrm{pCi} / \mathrm{L}$ & 0.0306 & 0.037 & & & \\
\hline 3454 & TRITIUM_ELECT_LSC & ITASRL & 34.7 & $\mathrm{pCi} / \mathrm{L}$ & 2.47 & 8.64 & & & \\
\hline 3455 & UISO_AEA & ITASRL & 0.262 & $\mathrm{pCi} / \mathrm{L}$ & 0.0461 & 0.0541 & & & \\
\hline 3456 & UISO_AEA & ITASRL & 0.00555 & $\mathrm{pCi} / \mathrm{L}$ & 0.00878 & 0.00901 & & & $U$ \\
\hline 3457 & UISO_AEA & ITASRL & 0.186 & $\mathrm{pCi} / \mathrm{L}$ & 0.0387 & 0.0435 & & & \\
\hline 3458 & SRISO_SEP_PRECIP_GPC & ITASRL & 0.0881 & $\mathrm{pCi} / \mathrm{L}$ & 0.0285 & 0.0343 & & & \\
\hline
\end{tabular}




\begin{tabular}{|c|c|c|c|c|c|c|c|c|c|}
\hline & $\mathrm{K}$ & $\mathrm{L}$ & $\mathrm{M}$ & $\mathrm{N}$ & $\mathrm{O}$ & $\mathrm{P}$ & $\mathrm{Q}$ & $\mathrm{R}$ & $\mathrm{S}$ \\
\hline 1 & METHOD_NAME & LAB_CODE & VALUE_RPTD & ANAL_UNITS_RPTD & COUNTING_ERROR & TOTAL_ANAL_ERROR I & MIN_DETECTABLE_ACTIVITY & PCNT_MOISTURE & LAB_QUALIFIER \\
\hline 3460 & UISO_AEA & ITASRL & 0.261 & $\mathrm{pCi} / \mathrm{L}$ & 0.0471 & 0.055 & & & \\
\hline 3461 & UISO AEA & ITASRL & 0.00804 & $\mathrm{pCi} / \mathrm{L}$ & 0.0101 & 0.0103 & & & $\mathrm{U}$ \\
\hline 3462 & UISO_AEA & ITASRL & 0.227 & $\mathrm{pCi} / \mathrm{L}$ & 0.0436 & 0.05 & & & \\
\hline 3463 & SRISO-SEP_PRECIP_GPC & ITASRL & 0.0744 & $\mathrm{pCi} / \mathrm{L}$ & 0.027 & 0.0316 & & & \\
\hline 3464 & TRITIUM_ELECT_LSC & ITASRL & 28.4 & $\mathrm{pCi} / \mathrm{L}$ & 2.33 & 8.22 & & & \\
\hline 3465 & UISO_AEA & ITASRL & 0.269 & $\mathrm{pCi} / \mathrm{L}$ & 0.0589 & 0.0669 & & & \\
\hline 3466 & UISO_AEA & ITASRL & 0.0121 & $\mathrm{pCi} / \mathrm{L}$ & 0.0138 & 0.014 & & & $U$ \\
\hline 3467 i & UISO_AEA & ITASRL & 0.189 & $\mathrm{pCi} / \mathrm{L}$ & 0.049 & 0.0537 & & & \\
\hline 3468 & SRISO_SEP_PRECIP_GPC & ITASRL & 0.091 & $\mathrm{pCi} / \mathrm{L}$ & 0.0277 & 0.0343 & & & \\
\hline 3469 & TRITIUM_ELECT_LSC & ITASRL & 30.7 & $\mathrm{pCi} / \mathrm{L}$ & 2.37 & 8.39 & & & \\
\hline 3470 & UISO_AEA & ITASRL & 0.236 & $\mathrm{pCi} / \mathrm{L}$ & 0.0435 & 0.0505 & & & \\
\hline 3471 & UISO_AEA & ITASRL & 0.00744 & $\mathrm{pCi} / \mathrm{L}$ & 0.00955 & 0.00978 & & & $U$ \\
\hline 3472 & UISO_AEA & ITASRL & 0.181 & $\mathrm{pCi} / \mathrm{L}$ & 0.0379 & 0.0426 & & & \\
\hline 3473 & SRISŌ_SEP_PRECIP_GPC & ITASRL & 0.103 & $\mathrm{pCi} / \mathrm{L}$ & 0.0292 & 0.0364 & & & \\
\hline 3474 & TRITIUMM ELECT LSC̄ & ITASRL & 31.2 & $\mathrm{pCi} / \mathrm{L}$ & 2.37 & 8.41 & & & \\
\hline 3475 & UISO_AEA & ITASRL & 0.261 & $\mathrm{pCi} / \mathrm{L}$ & 0.0453 & 0.0533 & & & \\
\hline 3476 & UISO AEA & ITASRL & 0.0113 & $\mathrm{pCi} / \mathrm{L}$ & 0.0115 & 0.0118 & & & U \\
\hline 3477 & UISO_AEA & ITASRL & 0.181 & $\mathrm{pCi} / \mathrm{L}$ & 0.0375 & 0.0421 & & & \\
\hline 3479 & TRITIUM_ELECT_LSC & ITASRL & 30 & $\mathrm{pCi} / \mathrm{L}$ & 2.37 & 8.35 & & & \\
\hline 3480 & UISO_AEA & ITASRL & 0.234 & $\mathrm{pCi} / \mathrm{L}$ & 0.0454 & 0.0523 & & & \\
\hline 3481 & UISO_AEA & ITASRL & -0.000833 & $\mathrm{pCi} / \mathrm{L}$ & 0.00636 & 0.00662 & & & U \\
\hline 3482 & UISO_AEA & ITASRL & 0.193 & $\mathrm{pCi} / \mathrm{L}$ & 0.0407 & 0.0457 & & & \\
\hline 3483 & SRISO_SEP_PRECIP_GPC & ITASRL & 0.0717 & $\mathrm{pCi} / \mathrm{L}$ & 0.0265 & 0.0312 & & & \\
\hline 3484 & TRITIUMM_ELECTT_LSC & ITASRL & 33.2 & $\mathrm{pCi} / \mathrm{L}$ & 2.42 & 8.61 & & & \\
\hline 3485 & UISO_AEA & ITASRL & 0.279 & $\mathrm{pCi} / \mathrm{L}$ & 0.0487 & 0.0573 & & & \\
\hline 3486 & UISO_AEA & ITASRL & 0.0059 & $\mathrm{pCi} / \mathrm{L}$ & 0.00913 & 0.00935 & & & $U$ \\
\hline 3487 & UISO_AEA & ITASRL & 0.233 & $\mathrm{pCi} / \mathrm{L}$ & 0.0439 & 0.0504 & & & \\
\hline 3488 & GAMMA_A_GS & ITASRL & 0.0459 & $\mathrm{pCi} / \mathrm{L}$ & 1.06 & 1.06 & & & $U$ \\
\hline 3489 & SRISO_SEPP_PRECIP_GPC & ITASRL & 0.231 & $\mathrm{pCi} / \mathrm{L}$ & 0.0422 & 0.0602 & & & \\
\hline 3490 & TRITIUMM_ELEECT_LSC & ITASRL & 998 & $\mathrm{pCi} / \mathrm{L}$ & 8.68 & 90.2 & & & \\
\hline 3491 & GAMMA_GS & ITASRL & -0.904 & $\mathrm{pCi} / \mathrm{L}$ & 1.13 & 1.13 & & & $U$ \\
\hline 3492 & SRISO_SEP_PRECIP_GPC & ITASRL & 0.297 & $\mathrm{pCi} / \mathrm{L}$ & 0.044 & 0.0702 & & & \\
\hline 3493 & TRITIUMM_ELEECT_LSC & ITASRL & 227 & $\mathrm{pCi} / \mathrm{L}$ & 4.5 & 24.8 & & & \\
\hline 3494 & GAMMA_GS & ITASRL & 0.934 & $\mathrm{pCi} / \mathrm{L}$ & 1.04 & 1.04 & & & U \\
\hline 3495 & SRISO_SEP_PRECIP_GPC & ITASRL & 0.299 & $\mathrm{pCi} / \mathrm{L}$ & 0.0442 & 0.0733 & & & \\
\hline 3496 & TRITIUM_ELECT_LSC & ITASRL & 320 & $\mathrm{pCi} / \mathrm{L}$ & 5.17 & 32.6 & & & \\
\hline
\end{tabular}




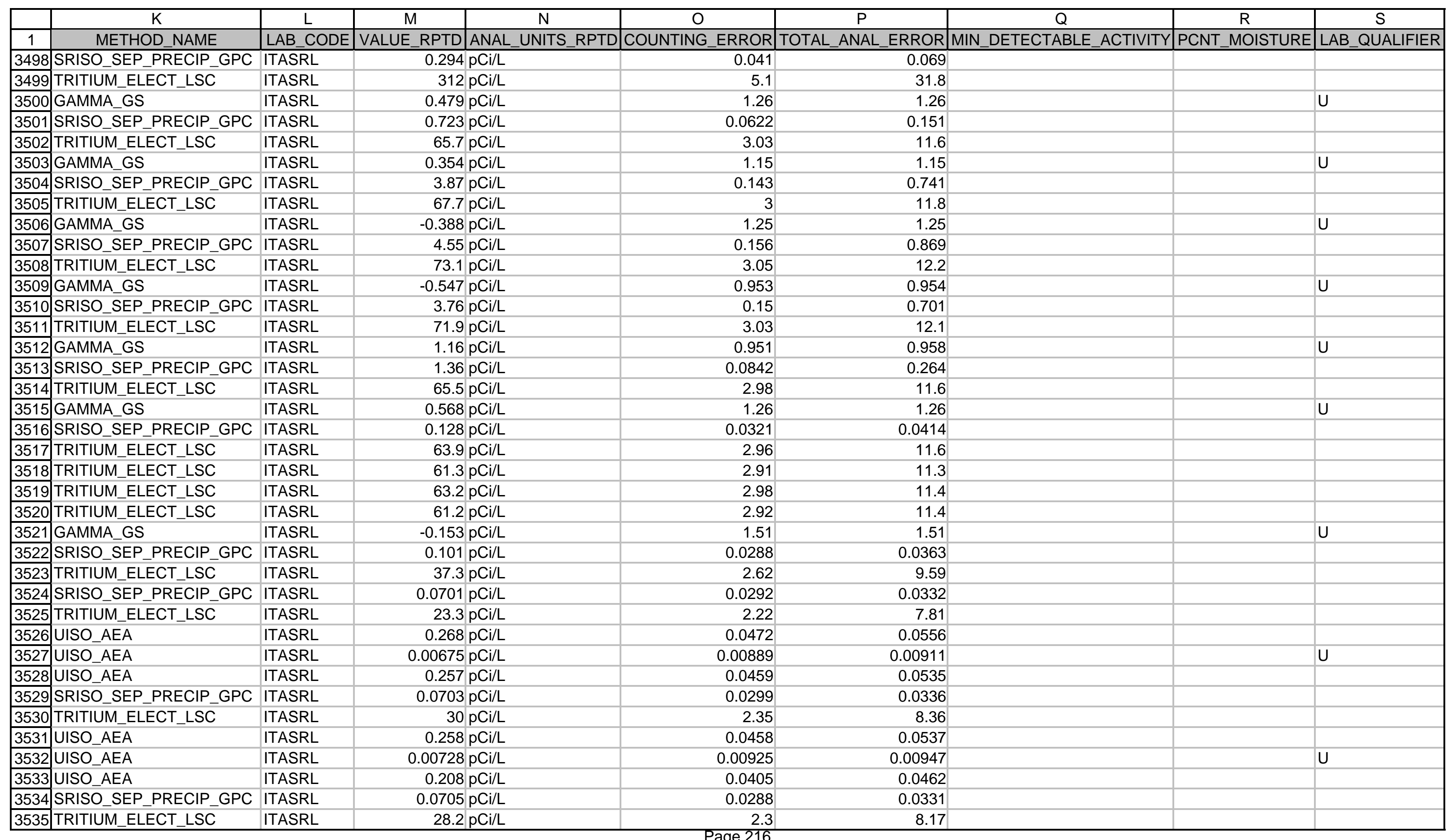




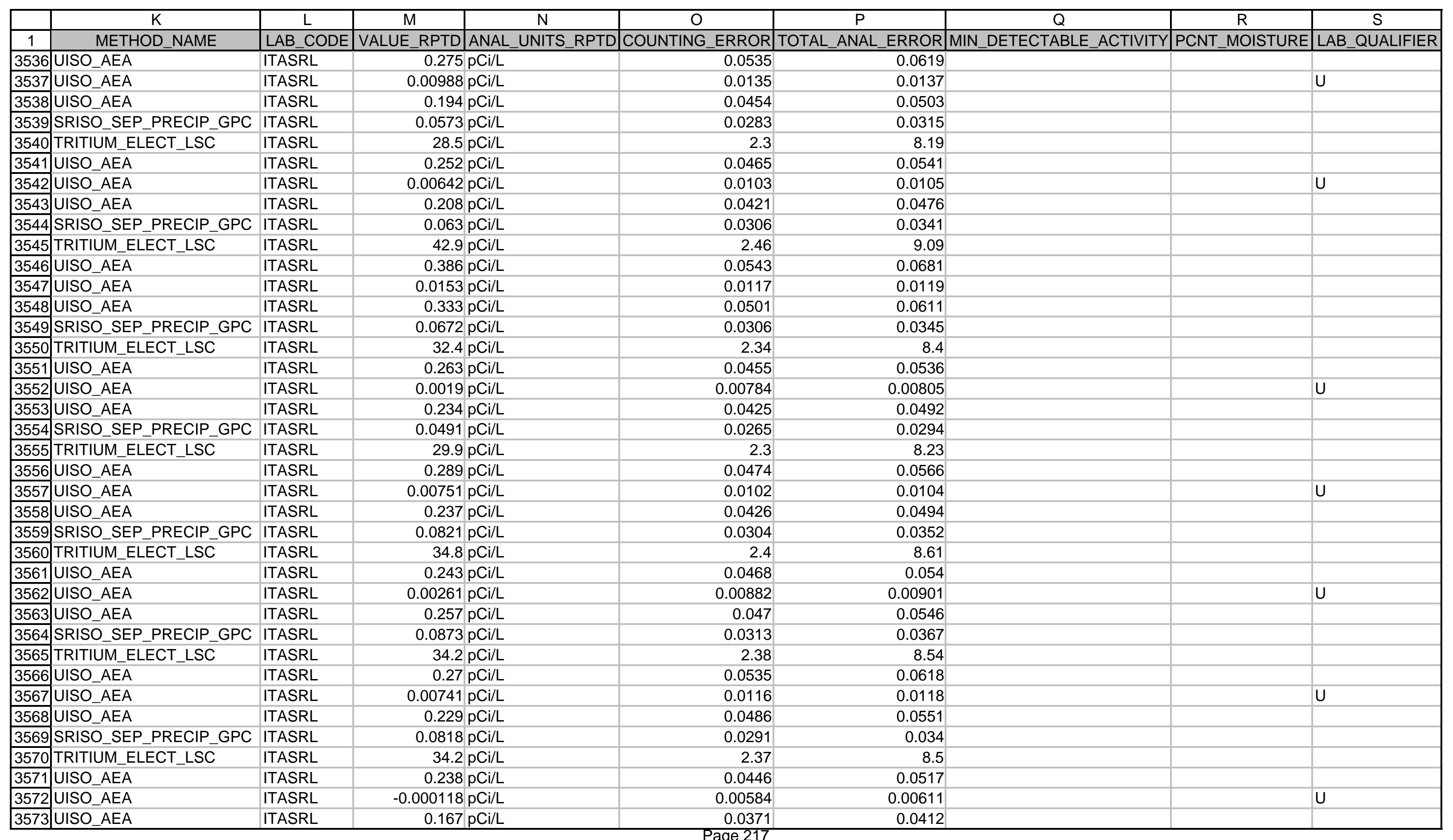


$\mathrm{N}$

O

$\mathrm{P}$

Q

R

$\mathrm{S}$

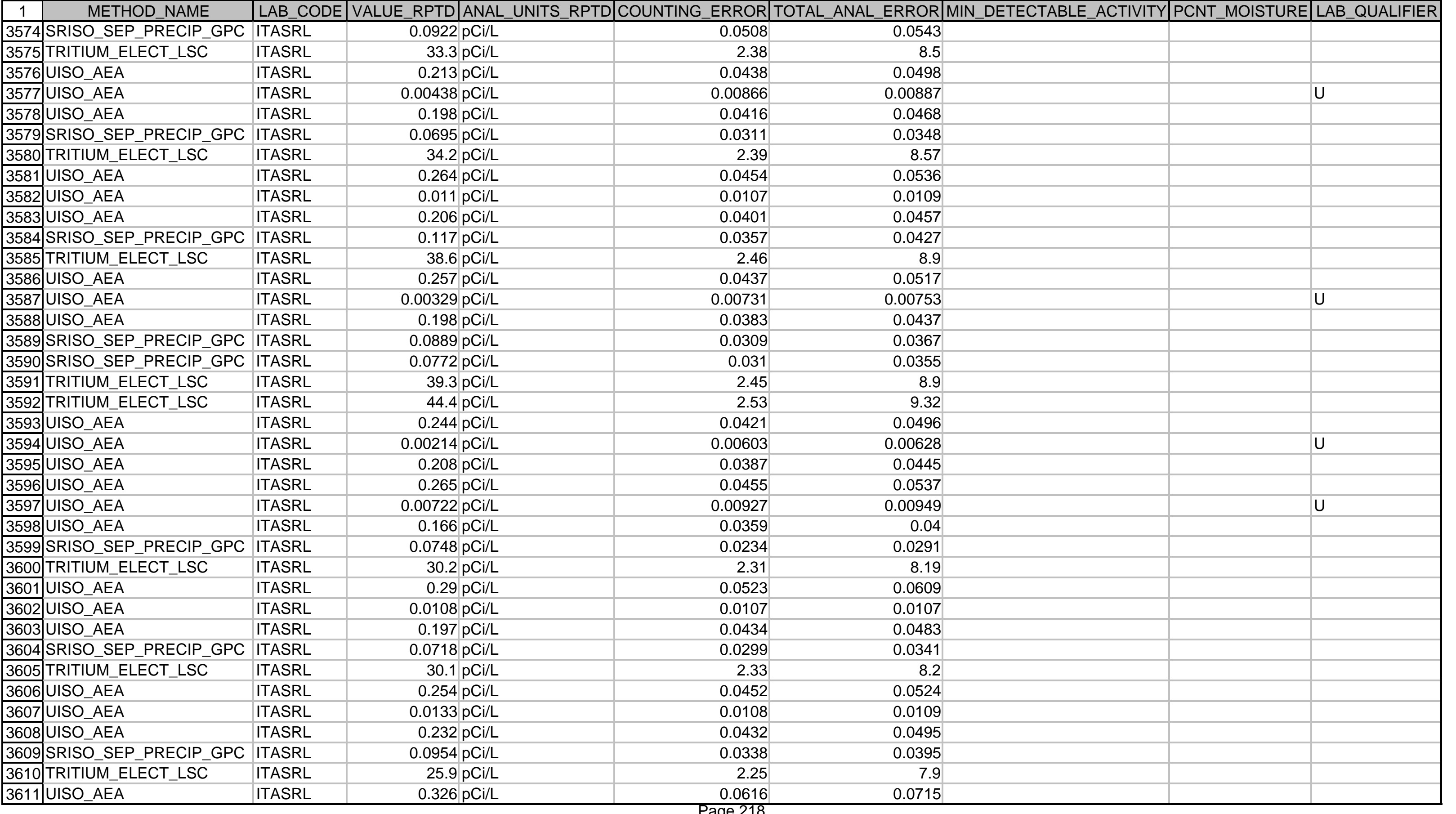

\begin{tabular}{|l|l|}
\hline 3611 & UISO_AEA \\
\hline
\end{tabular}

ITASRL

$0.326 \mathrm{pCi} / \mathrm{L}$ 


\begin{tabular}{|c|c|c|c|c|c|c|c|c|c|}
\hline & $\mathrm{K}$ & $\mathrm{L}$ & $\bar{M}$ & $\mathrm{~N}$ & $\mathrm{O}$ & $P$ & $\mathrm{Q}$ & $\mathrm{R}$ & $\mathrm{S}$ \\
\hline 1 & METHOD NAME & LAB CODE & VALUE RPTD & ANAL UNITS RPTD & COUNTING ERROR & TOTAL ANAL ERROR & MIN DETECTABLE ACTIVITY & PCNT MOISTURE & LAB QUALIFIER \\
\hline 3612 & UISO_AEA & ITASRL & 0.0112 & $\mathrm{pCi} / \mathrm{L}$ & 0.0146 & 0.0146 & & & $U$ \\
\hline 3613 & UISO_AEA & ITASRL & 0.265 & $\mathrm{pCi} / \mathrm{L}$ & 0.0549 & 0.0624 & & & \\
\hline 3614 & SRISO_SEP_PRECIP_GPC & ITASRL & 0.147 & $\mathrm{pCi} / \mathrm{L}$ & 0.0417 & 0.0517 & & & \\
\hline 3615 & TRITIUM_ELEECT_LSC & ITASRL & 27.2 & $\mathrm{pCi} / \mathrm{L}$ & 2.22 & 7.84 & & & \\
\hline 3616 & UISO_AEA & ITASRL & 0.286 & $\mathrm{pCi} / \mathrm{L}$ & 0.0532 & 0.0615 & & & \\
\hline 3617 & UISO_AEA & ITASRL & 0.0103 & $\mathrm{pCi} / \mathrm{L}$ & 0.0144 & 0.0144 & & & $U$ \\
\hline 3618 & UISO AEA & ITASRL & 0.225 & $\mathrm{pCi} / \mathrm{L}$ & 0.0469 & 0.0528 & & & \\
\hline 3619 & SRISŌSEP_PRECIP_GPC & ITASRL & 0.0745 & $\mathrm{pCi} / \mathrm{L}$ & 0.0256 & 0.0303 & & & \\
\hline 3621 & TRITIUM_ELECT_LSC & ITASRL & 39.4 & $\mathrm{pCi} / \mathrm{L}$ & 2.44 & 8.77 & & & \\
\hline 3622 & TRITIUM_ELECT_LSC & ITASRL & 37.5 & $\mathrm{pCi} / \mathrm{L}$ & 2.44 & 8.73 & & & \\
\hline 3623 & UISO_AEA & ITASRL & 0.284 & $\mathrm{pCi} / \mathrm{L}$ & 0.0474 & 0.0559 & & & \\
\hline 3624 & UISO_AEA & ITASRL & 0.00711 & $\mathrm{pCi} / \mathrm{L}$ & 0.00806 & 0.00809 & & & $\mathrm{U}$ \\
\hline 3625 & UISO_AEA & ITASRL & 0.246 & $\mathrm{pCi} / \mathrm{L}$ & 0.0442 & 0.0511 & & & \\
\hline 3626 & UISO_AEA & ITASRL & 0.28 & $\mathrm{pCi} / \mathrm{L}$ & 0.051 & 0.0591 & & & \\
\hline 3627 & UISO_AEA & ITASRL & 0.0139 & $\mathrm{pCi} / \mathrm{L}$ & 0.0114 & 0.0114 & & & \\
\hline 3628 & UISO_AEA & ITASRL & 0.273 & $\mathrm{pCi} / \mathrm{L}$ & 0.0507 & 0.0585 & & & \\
\hline 3629 & SRISŌSEP_PRECIP_GPC & ITASRL & 0.071 & $\mathrm{pCi} / \mathrm{L}$ & 0.091 & 0.0927 & & & $U$ \\
\hline 3631 & UISO_AEA & ITASRL & 0.501 & $\mathrm{pCi} / \mathrm{L}$ & 0.0612 & 0.08 & & & \\
\hline 3632 & UISO_AEA & ITASRL & 0.0172 & $\mathrm{pCi} / \mathrm{L}$ & 0.0139 & 0.014 & & & \\
\hline 3633 & UISO_AEA & ITASRL & 0.364 & $\mathrm{pCi} / \mathrm{L}$ & 0.052 & 0.0641 & & & \\
\hline 3634 & SRISO_SEP_PRECIP_GPC & ITASRL & 0.0862 & $\mathrm{pCi} / \mathrm{L}$ & 0.0306 & 0.0358 & & & \\
\hline 3635 & TRITIUM_ELEECT_LSC & ITASRL & 29.7 & $\mathrm{pCi} / \mathrm{L}$ & 2.31 & 8.16 & & & \\
\hline 3636 & UISO_AEA & ITASRL & 0.288 & $\mathrm{pCi} / \mathrm{L}$ & 0.0494 & 0.058 & & & \\
\hline 3637 & UISO_AEA & ITASRL & 0.0127 & $\mathrm{pCi} / \mathrm{L}$ & 0.0103 & 0.0104 & & & \\
\hline 3638 & UISO_AEA & ITASRL & 0.212 & $\mathrm{pCi} / \mathrm{L}$ & 0.0424 & 0.0479 & & & \\
\hline 3639 & SRISO_SEP_PRECIP_GPC & ITASRL & 0.0908 & $\mathrm{pCi} / \mathrm{L}$ & 0.0274 & 0.0332 & & & \\
\hline 3640 & TRITIUM_ELEECT_LSC & ITASRL & 26.2 & $\mathrm{pCi} / \mathrm{L}$ & 2.25 & 7.87 & & & \\
\hline 3641 & UISO_AEA & ITASRL & 0.321 & $\mathrm{pCi} / \mathrm{L}$ & 0.0526 & 0.0626 & & & \\
\hline 3642 & UISO_AEA & ITASRL & 0.0191 & $\mathrm{pCi} / \mathrm{L}$ & 0.0127 & 0.0129 & & & \\
\hline 3643 & UISO_AEA & ITASRL & 0.235 & $\mathrm{pCi} / \mathrm{L}$ & 0.0448 & 0.0511 & & & \\
\hline 3644 & SRISŌSEP_PRECIP_GPC & ITASRL & 0.083 & $\mathrm{pCi} / \mathrm{L}$ & 0.0337 & 0.0382 & & & \\
\hline 3645 & TRITIUM_ELEECT_LSC & ITASRL & 29.3 & $\mathrm{pCi} / \mathrm{L}$ & 2.3 & 8.09 & & & \\
\hline 3646 & UISO_AEA & ITASRL & 0.303 & $\mathrm{pCi} / \mathrm{L}$ & 0.0514 & 0.0606 & & & \\
\hline 3647 & UISO_AEA & ITASRL & 0.0195 & $\mathrm{pCi} / \mathrm{L}$ & 0.0136 & 0.0138 & & & \\
\hline 3648 & UISO AEA & ITASRL & 0.25 & $\mathrm{pCi} / \mathrm{L}$ & 0.0466 & 0.0536 & & & \\
\hline
\end{tabular}


$\mathrm{N}$

O

$\mathrm{P}$

Q

\begin{tabular}{|c|c|c|c|c|c|c|c|c|c|}
\hline & & & & & & & & & \\
\hline 1 & METHOD_NAME & LAB_CODE & VALUE_RPTD & ANAL_UNITS_RPTD & COUNTING_ERROR & TOTAL_ANAL_ERROR & MIN_DETECTABLE_ACTIVITY & PCNT_MOISTURE & LAB_QUALIFIER \\
\hline 3650 & TRITIUM_ELECT_LSC & ITASRL & 31.3 & $\mathrm{pCi} / \mathrm{L}$ & 2.34 & 8.26 & & & \\
\hline 3651 & UISO_AEA & ITASRL & 0.359 & $\mathrm{pCi} / \mathrm{L}$ & 0.0533 & 0.0651 & & & \\
\hline 3652 & UISO_AEA & ITASRL & 0.0128 & $3 \mathrm{pCi} / \mathrm{L}$ & 0.0103 & 0.0104 & & & \\
\hline 3653 & UISO_AEA & ITASRL & 0.254 & $+\mathrm{pCi} / \mathrm{L}$ & 0.0445 & 0.0517 & & & \\
\hline 3654 & SRISO_SEP_PRECIP_GPC & ITASRL & 0.0876 & $\mathrm{pCi} / \mathrm{L}$ & 0.038 & 0.0427 & & & \\
\hline 3655 & TRITIUM_ELECT_LSC & ITASRL & 25.4 & $\mathrm{pCi} / \mathrm{L}$ & 2.24 & 7.82 & & & \\
\hline 3656 & UISO_AEA & ITASRL & 0.324 & $+\mathrm{pCi} / \mathrm{L}$ & 0.052 & 0.0622 & & & \\
\hline 3657 & UISO_AEA & ITASRL & -0.000832 & $2 \mathrm{pCi} / \mathrm{L}$ & 0.00166 & 0.00167 & & & $U$ \\
\hline 3658 & UISO_AEA & ITASRL & 0.214 & $\mathrm{pCi} / \mathrm{L}$ & 0.0425 & 0.0481 & & & \\
\hline 3659 & SRISO_SEP_PRECIP_GPC & ITASRL & 0.0939 & $\mathrm{pCi} / \mathrm{L}$ & 0.0325 & 0.0387 & & & \\
\hline 3660 & TRITIUM_ELECT_LSC & ITASRL & 26.9 & $\mathrm{pCi} / \mathrm{L}$ & 2.27 & 7.95 & & & \\
\hline 3661 & UISO_AEA & ITASRL & 0.328 & $\mathrm{pCi} / \mathrm{L}$ & 0.0503 & 0.0607 & & & \\
\hline 3662 & UISO_AEA & ITASRL & 0.00382 & $2 \mathrm{pCi} / \mathrm{L}$ & 0.00836 & 0.00837 & & & $U$ \\
\hline 3663 & UISO_AEA & ITASRL & 0.218 & $\mathrm{pCi} / \mathrm{L}$ & 0.0413 & 0.047 & & & \\
\hline 3664 & SRISO_SEP_PRECIP_GPC & ITASRL & 0.096 & $\mathrm{pCi} / \mathrm{L}$ & 0.0328 & 0.0389 & & & \\
\hline 3665 & TRITIUM_ELECT_LSC & ITASRL & 22.9 & $\mathrm{pCi} / \mathrm{L}$ & 2.21 & 7.64 & & & \\
\hline 3666 & UISO_AEA & ITASRL & 0.244 & $\mathrm{pCi} / \mathrm{L}$ & 0.0434 & 0.0502 & & & \\
\hline 3667 & UISO_AEA & ITASRL & 0.00885 & $\mathrm{pCi} / \mathrm{L}$ & 0.00874 & 0.00879 & & & \\
\hline 3670 & TRITIUM_ELECT_LSC & ITASRL & 24.3 & $\mathrm{pCi} / \mathrm{L}$ & 2.25 & 7.83 & & & \\
\hline 3671 & UISO_AEA & ITASRL & 0.31 & $\mathrm{pCi} / \mathrm{L}$ & 0.0545 & 0.0638 & & & \\
\hline 3672 & UISO_AEA & ITASRL & 0.0229 & $\mathrm{pCi} / \mathrm{L}$ & 0.0164 & 0.0165 & & & \\
\hline 3673 & UISO_AEA & ITASRL & 0.205 & $5 \mathrm{pCi} / \mathrm{L}$ & 0.0437 & 0.049 & & & \\
\hline 3674 & SRISO_SEP_PRECIP_GPC & ITASRL & 0.0454 & $\mathrm{pCi} / \mathrm{L}$ & 0.0288 & 0.0311 & & & \\
\hline 3675 & TRITIUM_ELECT_LSC & ITASRL & 59.4 & $\mathrm{pCi} / \mathrm{L}$ & 2.92 & 11.1 & & & \\
\hline 3676 & UISO_AEA & ITASRL & 0.22 & $\mathrm{pCi} / \mathrm{L}$ & 0.0515 & 0.0575 & & & \\
\hline 3677 & UISO_AEA & ITASRL & 0.00793 & $3 \mathrm{pCi} / \mathrm{L}$ & 0.0113 & 0.0115 & & & U \\
\hline 3678 & UISO_AEA & ITASRL & 0.154 & $\mathrm{pCi} / \mathrm{L}$ & 0.0426 & 0.0461 & & & \\
\hline 3679 & SRISO_SEP_PRECIP_GPC & ITASRL & 0.0767 & $\mathrm{pCi} / \mathrm{L}$ & 0.0376 & 0.0413 & & & \\
\hline 3680 & TRITIUM_ELECT_LSC & ITASRL & 53.5 & $\mathrm{pCi} / \mathrm{L}$ & 2.82 & 10.7 & & & \\
\hline 3681 & UISO_AEA & ITASRL & 0.252 & $\mathrm{pCi} / \mathrm{L}$ & 0.0517 & 0.059 & & & \\
\hline 3682 & UISO_AEA & ITASRL & 0.0176 & $\mathrm{pCi} / \mathrm{L}$ & 0.0148 & 0.015 & & & \\
\hline 3683 & UISO_AEA & ITASRL & 0.193 & $3 \mathrm{pCi} / \mathrm{L}$ & 0.0446 & 0.0495 & & & \\
\hline 3684 & SRISO_SEP_PRECIP_GPC & ITASRL & 0.0678 & $3 \mathrm{pCi} / \mathrm{L}$ & 0.0321 & 0.0357 & & & \\
\hline 3685 & TRITIUM_ELECT_LSC & ITASRL & 85.4 & $\mathrm{pCi} / \mathrm{L}$ & 3.2 & 13.1 & & & \\
\hline 3686 & UISO_AEA & ITASRL & 0.239 & $\mathrm{pCi} / \mathrm{L}$ & 0.0445 & 0.0516 & & & \\
\hline 3687 & UISO_AEA & ITASRL & 0.0143 & $\mathrm{pCi} / \mathrm{L}$ & 0.0117 & 0.012 & & & \\
\hline
\end{tabular}

\begin{abstract}
ITASRL
\end{abstract}
page 220 


\begin{tabular}{|c|c|c|c|c|c|c|c|c|c|}
\hline & $\mathrm{K}$ & $\mathrm{L}$ & $\mathrm{M}$ & $\mathrm{N}$ & $\mathrm{O}$ & $\mathrm{P}$ & $\mathrm{Q}$ & $\mathrm{R}$ & $\mathrm{S}$ \\
\hline 1 & METHOD_NAME & LAB_CODE & VALUE_RPTD & ANAL_UNITS_RPTD & COUNTING_ERROR & $\mid$ TOTAL_ANAL_ERROR| & MIN_DETECTABLE_ACTIVITY & PCNT_MOISTURE & LAB_QUALIFIER \\
\hline 3688 & UISO_AEA & ITASRL & 0.178 & $\mathrm{pCi} / \mathrm{L}$ & 0.038 & 0.0425 & & & \\
\hline 3689 & SRISO_SEP_PRECIP_GPC & ITASRL & 0.0673 & $\mathrm{pCi} / \mathrm{L}$ & 0.0287 & 0.0323 & & & \\
\hline 3690 & TRITIUMM ELECT LSC̄ & ITASRL & 78.2 & $\mathrm{pCi} / \mathrm{L}$ & 3.17 & 12.6 & & & \\
\hline 3691 & UISO IE PLATE AEA & ITASRL & 0.237 & $\mathrm{pCi} / \mathrm{L}$ & 0.0425 & 0.0496 & & & \\
\hline 3692 & UISO_IE_PLATE_AEA & ITASRL & 0.00551 & $\mathrm{pCi} / \mathrm{L}$ & 0.00988 & 0.0101 & & & $U$ \\
\hline 3693 & UISO_IE_PLATE_AEA & ITASRL & 0.218 & $\mathrm{pCi} / \mathrm{L}$ & 0.0401 & 0.0462 & & & \\
\hline 3694 & SRISO-SEPP_PRECCIP_GPC & ITASRL & 0.052 & $\mathrm{pCi} / \mathrm{L}$ & 0.0268 & 0.0298 & & & \\
\hline 3695 & TRITIŪM_ELECCT_LSC̄ & ITASRL & 98.7 & $\mathrm{pCi} / \mathrm{L}$ & 3.09 & 13.1 & & & \\
\hline \begin{tabular}{|l|l|}
3697 \\
\end{tabular} & UISO_AEA & ITASRL & 0.0105 & $\mathrm{pCi} / \mathrm{L}$ & 0.016 & 0.0162 & & & $U$ \\
\hline 3698 & UISO_AEA & ITASRL & 0.221 & $\mathrm{pCi} / \mathrm{L}$ & 0.0494 & 0.0555 & & & \\
\hline 3699 & SRISO_SEP_PRECIP_GPC & ITASRL & 0.0499 & $\mathrm{pCi} / \mathrm{L}$ & 0.0307 & 0.0332 & & & \\
\hline 3700 & TRITIUM_ELECT_LSC & ITASRL & 65.1 & $\mathrm{pCi} / \mathrm{L}$ & 2.72 & 10.5 & & & \\
\hline 3701 & UISO_AEA & ITASRL & 0.396 & $\mathrm{pCi} / \mathrm{L}$ & 0.0847 & 0.0984 & & & \\
\hline 3702 & UISO_AEA & ITASRL & 0.0138 & $\mathrm{pCi} / \mathrm{L}$ & 0.0171 & 0.0173 & & & $U$ \\
\hline 3703 & UISO_AEA & ITASRL & 0.271 & $\mathrm{pCi} / \mathrm{L}$ & 0.0691 & 0.077 & & & \\
\hline 3704 & SRISŌ SEP PRECIP GPC & ITASRL & 0.0643 & $\mathrm{pCi} / \mathrm{L}$ & 0.0311 & 0.0343 & & & \\
\hline 3705 & TRITIUMM_ELECCT_LSC̄ & ITASRL & 61.6 & $\mathrm{pCi} / \mathrm{L}$ & 2.69 & 10.2 & & & \\
\hline 3707 & UISO_AEA & ITASRL & 0.0163 & $\mathrm{pCi} / \mathrm{L}$ & 0.0161 & 0.0163 & & & \\
\hline 3708 & UISO_AEA & ITASRL & 0.211 & $\mathrm{pCi} / \mathrm{L}$ & 0.0533 & 0.0588 & & & \\
\hline 3709 & SRISO_SEP_PRECIP_GPC & ITASRL & 0.0779 & $\mathrm{pCi} / \mathrm{L}$ & 0.0317 & 0.0361 & & & \\
\hline 3710 & TRITIŪM_ELEECT_LSC & ITASRL & 58 & $\mathrm{pCi} / \mathrm{L}$ & 2.65 & 9.97 & & & \\
\hline 3711 & UISO_AEA & ITASRL & 0.198 & $\mathrm{pCi} / \mathrm{L}$ & 0.0409 & 0.0463 & & & \\
\hline 3712 & UISO_AEA & ITASRL & 0.00792 & $\mathrm{pCi} / \mathrm{L}$ & 0.0106 & 0.0108 & & & U \\
\hline 3713 & UISO_AEA & ITASRL & 0.137 & $\mathrm{pCi} / \mathrm{L}$ & 0.0341 & 0.0372 & & & \\
\hline 3714 & SRISŌ_SEP_PRECIP_GPC & ITASRL & 0.0686 & $\mathrm{pCi} / \mathrm{L}$ & 0.0278 & 0.0316 & & & \\
\hline 3715 & TRITIUM_ELEECT_LSC & ITASRL & 59.5 & $\mathrm{pCi} / \mathrm{L}$ & 2.65 & 10 & & & \\
\hline 3716 & UISO_AEA & ITASRL & 0.22 & $\mathrm{pCi} / \mathrm{L}$ & 0.0435 & 0.0498 & & & \\
\hline 3717 & UISO_AEA & ITASRL & 0.00276 & $\mathrm{pCi} / \mathrm{L}$ & 0.00705 & 0.0073 & & & $U$ \\
\hline 3718 & UISO_AEA & ITASRL & 0.15 & $\mathrm{pCi} / \mathrm{L}$ & 0.0357 & 0.0393 & & & \\
\hline 3719 & SRISO-SEP_PRECIP_GPC & ITASRL & 0.0808 & $\mathrm{pCi} / \mathrm{L}$ & 0.0281 & 0.0336 & & & \\
\hline 3720 & TRITIUM_ELEECT_LSC & ITASRL & 76.9 & $\mathrm{pCi} / \mathrm{L}$ & 2.88 & 11.4 & & & \\
\hline 3721 & UISO_AEA & ITASRL & 0.201 & $\mathrm{pCi} / \mathrm{L}$ & 0.0429 & 0.0484 & & & \\
\hline 3722 & UISO_AEA & ITASRL & 0.00423 & $\mathrm{pCi} / \mathrm{L}$ & 0.0111 & 0.0113 & & & U \\
\hline 3723 & UISO_AEA & ITASRL & 0.181 & $\mathrm{pCi} / \mathrm{L}$ & 0.0406 & 0.0451 & & & \\
\hline 3724 & SRISO_SEP_PRECIP_GPC & ITASRL & 0.0536 & $\mathrm{pCi} / \mathrm{L}$ & 0.0252 & 0.0284 & & & \\
\hline
\end{tabular}




\begin{tabular}{|c|c|c|c|c|c|c|c|c|c|}
\hline & $\mathrm{K}$ & $\mathrm{L}$ & $\mathrm{M}$ & $\mathrm{N}$ & $\mathrm{O}$ & $\mathrm{P}$ & $\mathrm{Q}$ & $\mathrm{R}$ & $\mathrm{S}$ \\
\hline 1 & METHOD_NAME & LAB_CODE & VALUE_RPTD & ANAL_UNITS_RPTD & COUNTING_ERROR & TOTAL_ANAL_ERROR & MIN_DETECTABLE_ACTIVITY & PCNT_MOISTURE & LAB_QUALIFIER \\
\hline 3726 & UISO_AEA & ITASRL & 0.246 & $\mathrm{pCi} / \mathrm{L}$ & 0.0474 & 0.0546 & & & \\
\hline 3727 & UISO_AEA & ITASRL & 0.00632 & $\mathrm{pCi} / \mathrm{L}$ & 0.0118 & 0.012 & & & U \\
\hline 3728 & UISO_AEA & ITASRL & 0.183 & $\mathrm{pCi} / \mathrm{L}$ & 0.0404 & 0.0451 & & & \\
\hline 3729 & SRISO_SEP_PRECIP_GPC & ITASRL & 0.0599 & $\mathrm{pCi} / \mathrm{L}$ & 0.0402 & 0.0435 & & & \\
\hline 3730 & TRITIUM_ELECT_LSC & ITASRL & 58.9 & $\mathrm{pCi} / \mathrm{L}$ & 2.67 & 10 & & & \\
\hline 3731 & UISO_AEA & ITASRL & 0.214 & $\mathrm{pCi} / \mathrm{L}$ & 0.0618 & 0.0676 & & & \\
\hline 3732 & UISO_AEA & ITASRL & 0.000861 & $\mathrm{pCi} / \mathrm{L}$ & 0.0084 & 0.00861 & & & $U$ \\
\hline 3733 & UISO_AEA & ITASRL & 0.203 & $\mathrm{pCi} / \mathrm{L}$ & 0.0595 & 0.0647 & & & \\
\hline 3734 & SRISO_SEP_PRECIP_GPC & ITASRL & 0.0441 & $\mathrm{pCi} / \mathrm{L}$ & 0.0368 & 0.0396 & & & \\
\hline 3735 & TRITIUM_ELECT_LSC & ITASRL & 63.5 & $\mathrm{pCi} / \mathrm{L}$ & 2.73 & 10.4 & & & \\
\hline 3736 & UISO_AEA & ITASRL & 0.266 & $\mathrm{pCi} / \mathrm{L}$ & 0.0559 & 0.0639 & & & \\
\hline 3737 & UISO_AEA & ITASRL & -0.00000958 & $\mathrm{pCi} / \mathrm{L}$ & 0.00845 & 0.00865 & & & $U$ \\
\hline 3738 & UISO_AEA & ITASRL & 0.186 & $\mathrm{pCi} / \mathrm{L}$ & 0.047 & 0.0516 & & & \\
\hline 3739 & SRISO_SEP_PRECIP_GPC & ITASRL & 0.0688 & $\mathrm{pCi} / \mathrm{L}$ & 0.0329 & 0.0367 & & & \\
\hline 3740 & TRITIUM_ELECT_LSC & ITASRL & 55.7 & $\mathrm{pCi} / \mathrm{L}$ & 2.62 & 9.78 & & & \\
\hline 3741 & UISO_AEA & ITASRL & 0.234 & $\mathrm{pCi} / \mathrm{L}$ & 0.0527 & 0.0594 & & & \\
\hline 3742 & UISO_AEA & ITASRL & -0.000552 & $\mathrm{pCi} / \mathrm{L}$ & 0.00558 & 0.00587 & & & $U$ \\
\hline 3745 & TRITIUM_ELECT_LSC & ITASRL & 60.6 & $\mathrm{pCi} / \mathrm{L}$ & 3 & 12.2 & & & \\
\hline 3746 & UISO_IE_PLATE_AEA & ITASRL & 0.22 & $\mathrm{pCi} / \mathrm{L}$ & 0.0387 & 0.0452 & & & \\
\hline 3747 & UISO_IE_PLATE_AEA & ITASRL & -0.00301 & $\mathrm{pCi} / \mathrm{L}$ & 0.00372 & 0.00413 & & & $U$ \\
\hline 3748 & UISO_IE_PLATE_AEA & ITASRL & 0.164 & $\mathrm{pCi} / \mathrm{L}$ & 0.0331 & 0.0373 & & & \\
\hline 3749 & SRISO_SEP_PRECIP_GPC & ITASRL & 0.0651 & $\mathrm{pCi} / \mathrm{L}$ & 0.0274 & 0.0313 & & & \\
\hline 3750 & TRITIUM_ELECT_LSC & ITASRL & 43.1 & $\mathrm{pCi} / \mathrm{L}$ & 2.82 & 11.1 & & & \\
\hline 3751 & UISO_IE_PLATE_AEA & ITASRL & 0.236 & $\mathrm{pCi} / \mathrm{L}$ & 0.0437 & 0.0507 & & & \\
\hline 3752 & UISO_IE_PLATE_AEA & ITASRL & 0.00877 & $\mathrm{pCi} / \mathrm{L}$ & 0.0111 & 0.0113 & & & $\mathrm{U}$ \\
\hline 3753 & UISO_IE_PLATE_AEA & ITASRL & 0.209 & $\mathrm{pCi} / \mathrm{L}$ & 0.0409 & 0.0465 & & & \\
\hline 3754 & SRISO_SEP_PRECIP_GPC & ITASRL & 0.07 & $\mathrm{pCi} / \mathrm{L}$ & 0.0286 & 0.0327 & & & \\
\hline 3755 & TRITIUM_ELECT_LSC & ITASRL & 44.4 & $\mathrm{pCi} / \mathrm{L}$ & 2.82 & 11.1 & & & \\
\hline 3756 & UISO_IE_PLATE_AEA & ITASRL & 0.195 & $\mathrm{pCi} / \mathrm{L}$ & 0.031 & 0.0369 & & & \\
\hline 3757 & UISO_IE_PLATE_AEA & ITASRL & 0.00598 & $\mathrm{pCi} / \mathrm{L}$ & 0.00658 & 0.00688 & & & U \\
\hline 3758 & UISO_IE_PLATE_AEA & ITASRL & 0.157 & $\mathrm{pCi} / \mathrm{L}$ & 0.0275 & 0.0317 & & & \\
\hline 3759 & SRISO_SEP_PRECIP_GPC & ITASRL & 0.0742 & $\mathrm{pCi} / \mathrm{L}$ & 0.0337 & 0.0376 & & & \\
\hline 3760 & TRITIUM_ELECT_LSC & ITASRL & 49.8 & $\mathrm{pCi} / \mathrm{L}$ & 2.88 & 11.4 & & & \\
\hline 3761 & UISO_IE_PLATE_AEA & ITASRL & 0.209 & $\mathrm{pCi} / \mathrm{L}$ & 0.0387 & 0.0447 & & & \\
\hline 3762 & UISO_IE_PLATE_AEA & ITASRL & 0.00951 & $\mathrm{pCi} / \mathrm{L}$ & 0.0101 & 0.0104 & & & U \\
\hline
\end{tabular}




\begin{tabular}{|c|c|c|c|c|c|c|c|c|c|}
\hline & $\mathrm{K}$ & $L$ & $\mathrm{M}$ & $\mathrm{N}$ & $\mathrm{O}$ & $\mathrm{P}$ & $\mathrm{Q}$ & $\mathrm{R}$ & $\mathrm{S}$ \\
\hline 1 & METHOD_NAME & LAB_CODE & VALUE_RPTD & ANAL_UNITS_RPTD & COUNTING_ERROR & TOTAL_ANAL_ERROR & MIN_DETECTABLE_ACTIVITY & PCNT_MOISTURE & LAB_QUALIFIER \\
\hline 3764 & SRISO_SEP_PRECIP_GPC & ITASRL & 0.0564 & $\mathrm{pCi} / \mathrm{L}$ & 0.0268 & 0.0301 & & & \\
\hline 3765 & TRITIUM_ELECT_LSC & ITASRL & 45.7 & $\mathrm{pCi} / \mathrm{L}$ & 2.83 & 11.2 & & & \\
\hline 3766 & UISO_IE_PLATE_AEA & ITASRL & 0.184 & $\mathrm{pCi} / \mathrm{L}$ & 0.0352 & 0.0404 & & & \\
\hline 3767 & UISO_IE_PLATE_AEA & ITASRL & 0.0000955 & $\mathrm{pCi} / \mathrm{L}$ & 0.00568 & 0.00597 & & & $U$ \\
\hline 3768 & UISO_IE_PLATE_AEA & ITASRL & 0.16 & $\mathrm{pCi} / \mathrm{L}$ & 0.0323 & 0.0363 & & & \\
\hline 3769 & SRISO_SEP_PRECIP_GPC & ITASRL & 0.0713 & $\mathrm{pCi} / \mathrm{L}$ & 0.0291 & 0.0331 & & & \\
\hline 3770 & TRITIUM_ELECT_LSC & ITASRL & 32.7 & $\mathrm{pCi} / \mathrm{L}$ & 2.66 & 10.2 & & & \\
\hline 3771 & UISO_IE_PLATE_AEA & ITASRL & 0.224 & $\mathrm{pCi} / \mathrm{L}$ & 0.0382 & 0.0449 & & & \\
\hline 3772 & UISO_IE_PLATE_AEA & ITASRL & 0.00316 & $\mathrm{pCi} / \mathrm{L}$ & 0.00712 & 0.00737 & & & $U$ \\
\hline 3773 & UISO_IE_PLATE_AEA & ITASRL & 0.155 & $\mathrm{pCi} / \mathrm{L}$ & 0.0315 & 0.0354 & & & \\
\hline 3774 & SRISO_SEP_PRECIP_GPC & ITASRL & 0.0833 & $\mathrm{pCi} / \mathrm{L}$ & 0.0291 & 0.0341 & & & \\
\hline 3775 & TRITIUM_ELECT_LSC & ITASRL & 41.1 & $\mathrm{pCi} / \mathrm{L}$ & 2.81 & 10.9 & & & \\
\hline 3776 & UISO_IE_PLATE_AEA & ITASRL & 0.242 & $\mathrm{pCi} / \mathrm{L}$ & 0.0409 & 0.0483 & & & \\
\hline 3777 & UISO_IE_PLATE_AEA & ITASRL & 0.009 & $\mathrm{pCi} / \mathrm{L}$ & 0.00923 & 0.00948 & & & $\mathrm{U}$ \\
\hline 3778 & UISO_IE_PLATE_AEA & ITASRL & 0.157 & $\mathrm{pCi} / \mathrm{L}$ & 0.0332 & 0.037 & & & \\
\hline 3779 & SRISO_SËP_PRËCIP_GPC & ITASRL & 0.0768 & $\mathrm{pCi} / \mathrm{L}$ & 0.0398 & 0.0433 & & & \\
\hline 3780 & TRITIUM_ELECT_LSC & ITASRL & & & & & & & \\
\hline 3781 & UISO_IE_PLATE_AEA & ITASRL & 0.19 & $\mathrm{pCi} / \mathrm{L}$ & 0.0364 & 0.0417 & & & \\
\hline 3783 & UISO_IE_PLATE_AEA & ITASRL & 0.22 & $\mathrm{pCi} / \mathrm{L}$ & 0.039 & 0.0451 & & & \\
\hline 3784 & SRISO_SEP_PRECIP_GPC & ITASRL & 0.0533 & $\mathrm{pCi} / \mathrm{L}$ & 0.0393 & 0.0423 & & & \\
\hline 3785 & TRITIUM_ELECT_LSC & ITASRL & & & & & & & \\
\hline 3786 & UISO_IE_PLATE_AEA & ITASRL & 0.243 & $\mathrm{pCi} / \mathrm{L}$ & 0.0426 & 0.0499 & & & \\
\hline 3787 & UISO_IE_PLATE_AEA & ITASRL & 0.00233 & $\mathrm{pCi} / \mathrm{L}$ & 0.00734 & 0.00758 & & & $\mathrm{U}$ \\
\hline 3788 & UISO_IE_PLATE_AEA & ITASRL & 0.171 & $\mathrm{pCi} / \mathrm{L}$ & 0.0358 & 0.0401 & & & \\
\hline 3789 & SRISO_SEP_PRECIP_GPC & ITASRL & 0.0765 & $\mathrm{pCi} / \mathrm{L}$ & 0.0282 & 0.033 & & & \\
\hline 3790 & TRITIUM_ELECT_LSC & ITASRL & & & & & & & \\
\hline 3791 & UISO_IE_PLATE_AEA & ITASRL & 0.225 & $\mathrm{pCi} / \mathrm{L}$ & 0.0425 & 0.049 & & & \\
\hline 3792 & UISO_IE_PLATE_AEA & ITASRL & -0.00219 & $\mathrm{pCi} / \mathrm{L}$ & 0.00407 & 0.00445 & & & $U$ \\
\hline 3793 & UISO_IE_PLATE_AEA & ITASRL & 0.222 & $\mathrm{pCi} / \mathrm{L}$ & 0.0416 & 0.0478 & & & \\
\hline 3794 & SRISO_SEP_PRECIP_GPC & ITASRL & 0.0868 & $\mathrm{pCi} / \mathrm{L}$ & 0.0262 & 0.0326 & & & \\
\hline 3795 & TRITIUM_ELECT_LSC & ITASRL & 43 & $\mathrm{pCi} / \mathrm{L}$ & 2.8 & 10.9 & & & \\
\hline 3796 & UISO_IE_PLATE_AEA & ITASRL & 0.199 & $\mathrm{pCi} / \mathrm{L}$ & 0.0386 & 0.0442 & & & \\
\hline 3797 & UISO_IE_PLATE_AEA & ITASRL & 0.00514 & $\mathrm{pCi} / \mathrm{L}$ & 0.00947 & 0.00967 & & & U \\
\hline 3798 & UISO_IE_PLATE_AEA & ITASRL & 0.149 & $\mathrm{pCi} / \mathrm{L}$ & 0.0338 & 0.0373 & & & \\
\hline 3799 & SRISO_SEP_PRECIP_GPC & ITASRL & 0.075 & $\mathrm{pCi} / \mathrm{L}$ & 0.0259 & 0.0308 & & & \\
\hline 3800 & TRITIUMM_ELECTI_LSC & ITASRL & 40.5 & $\mathrm{pCi} / \mathrm{L}$ & 2.77 & 10.8 & & & \\
\hline
\end{tabular}




\begin{tabular}{|c|c|c|c|c|c|c|c|c|c|}
\hline & $\mathrm{K}$ & $\mathrm{L}$ & $\mathrm{M}$ & $\mathrm{N}$ & $\mathrm{O}$ & $P$ & $\mathrm{Q}$ & $\mathrm{R}$ & $S$ \\
\hline 1 & METHOD_NAME & LAB_CODE & VALUE_RPTD & ANAL_UNITS_RPTD & COUNTING_ERROR & TOTAL_ANAL_ERROR & MIN_DETECTABLE_ACTIVITY & PCNT_MOISTURE & LAB_QUALIFIER \\
\hline 3802 & UISO_IE_PLATE_AEA & ITASRL & -0.00108 & $\mathrm{pCi} / \mathrm{L}$ & 0.00611 & 0.00637 & & & $\mathrm{U}$ \\
\hline 3803 & UISO_IE_PLATE_AEA & ITASRL & 0.156 & $\mathrm{pCi} / \mathrm{L}$ & 0.0326 & 0.0365 & & & \\
\hline 3804 & SRISO_SEP_PRECIP_GPC & ITASRL & 0.0927 & $\mathrm{pCi} / \mathrm{L}$ & 0.0224 & 0.0305 & & & \\
\hline 3805 & TRITIUM_ELECT_LSC & ITASRL & 50.1 & $\mathrm{pCi} / \mathrm{L}$ & 2.88 & 11.4 & & & \\
\hline 3806 & UISO_IE_PLATE_AEA & ITASRL & 0.219 & $\mathrm{pCi} / \mathrm{L}$ & 0.0407 & 0.047 & & & \\
\hline 3807 & UISO_IE_PLATE_AEA & ITASRL & 0.00411 & $\mathrm{pCi} / \mathrm{L}$ & 0.00817 & 0.0084 & & & $\mathrm{U}$ \\
\hline 3808 & UISO_IE_PLATE_AEA & ITASRL & 0.152 & $\mathrm{pCi} / \mathrm{L}$ & 0.0335 & 0.0371 & & & \\
\hline 3809 & SRISO_SEP_PRECIP_GPC & ITASRL & 0.088 & $\mathrm{pCi} / \mathrm{L}$ & 0.0248 & 0.0315 & & & \\
\hline 3811 & UISO_IE_PLATE_AEA & ITASRL & 0.242 & $\mathrm{pCi} / \mathrm{L}$ & 0.0403 & 0.0477 & & & \\
\hline 3812 & UISO_IE_PLATE_AEA & ITASRL & 0.0056 & $\mathrm{pCi} / \mathrm{L}$ & 0.0079 & 0.00815 & & & $\mathrm{U}$ \\
\hline 3813 & UISO_IE_PLATE_AEA & ITASRL & 0.165 & $\mathrm{pCi} / \mathrm{L}$ & 0.0329 & 0.0371 & & & \\
\hline 3814 & SRISO_SEP_PRECIP_GPC & ITASRL & 0.0617 & $\mathrm{pCi} / \mathrm{L}$ & 0.0359 & 0.0386 & & & \\
\hline 3815 & TRITIUM_ELECT_LSC & ITASRL & 63.3 & $\mathrm{pCi} / \mathrm{L}$ & 2.82 & 10.6 & & & \\
\hline 3816 & UISO_IE_PLATE_AEA & ITASRL & 0.21 & $\mathrm{pCi} / \mathrm{L}$ & 0.0466 & 0.0524 & & & \\
\hline 3817 & UISO_IE_PLATE_AEA & ITASRL & 0.00421 & $\mathrm{pCi} / \mathrm{L}$ & 0.00977 & 0.00997 & & & $\mathrm{U}$ \\
\hline 3818 & UISO_IE_PLATE_AEA & ITASRL & 0.166 & $\mathrm{pCi} / \mathrm{L}$ & 0.0406 & 0.0446 & & & \\
\hline 3819 & SRISO_SEP_PRECIP_GPC & ITASRL & 0.0842 & $\mathrm{pCi} / \mathrm{L}$ & 0.066 & 0.0685 & & & \\
\hline 3821 & UISO_IE_PLATE_AEA & ITASRL & 0.201 & $\mathrm{pCi} / \mathrm{L}$ & 0.0509 & 0.0563 & & & \\
\hline 3822 & UISO_IE_PLATE_AEA & ITASRL & 0.00333 & $\mathrm{pCi} / \mathrm{L}$ & 0.011 & 0.0111 & & & $\mathrm{U}$ \\
\hline 3823 & UISO_IE_PLATE_AEA & ITASRL & 0.15 & $\mathrm{pCi} / \mathrm{L}$ & 0.0435 & 0.0469 & & & \\
\hline 3824 & SRISO_SEP_PRECIP_GPC & ITASRL & 0.0655 & $\mathrm{pCi} / \mathrm{L}$ & 0.0349 & 0.038 & & & \\
\hline 3825 & TRITIUMM_ELECT_LSC & ITASRL & 38.3 & $\mathrm{pCi} / \mathrm{L}$ & 2.41 & 8.73 & & & \\
\hline 3826 & UISO_IE_PLATE_AEA & ITASRL & 0.178 & $\mathrm{pCi} / \mathrm{L}$ & 0.0752 & 0.0798 & & & \\
\hline 3827 & UISO_IE_PLATE_AEA & ITASRL & -0.00844 & $\mathrm{pCi} / \mathrm{L}$ & 0.00725 & 0.00751 & & & $\mathrm{U}$ \\
\hline 3828 & UISO_IE_PLATE_AEA & ITASRL & 0.0958 & $\mathrm{pCi} / \mathrm{L}$ & 0.0577 & 0.0595 & & & \\
\hline 3829 & SRISO_SEP_PRECIP_GPC & ITASRL & 0.0851 & $\mathrm{pCi} / \mathrm{L}$ & 0.0415 & 0.0456 & & & \\
\hline 3830 & TRITIUM_ELECT_LSC & ITASRL & 38.6 & $\mathrm{pCi} / \mathrm{L}$ & 2.42 & 8.75 & & & \\
\hline 3831 & UISO_IE_PLATE_AEA & ITASRL & 0.192 & $\mathrm{pCi} / \mathrm{L}$ & 0.0448 & 0.0499 & & & \\
\hline 3832 & UISO_IE_PLATE_AEA & ITASRL & 0.00292 & $\mathrm{pCi} / \mathrm{L}$ & 0.00849 & 0.0087 & & & $\mathrm{U}$ \\
\hline 3833 & UISO_IE_PLATE_AEA & ITASRL & 0.18 & $\mathrm{pCi} / \mathrm{L}$ & 0.0426 & 0.0471 & & & \\
\hline 3834 & SRISO_SEP_PRECIP_GPC & ITASRL & 0.0634 & $\mathrm{pCi} / \mathrm{L}$ & 0.0365 & 0.0396 & & & \\
\hline 3835 & TRITIUM_ELECT_LSC & ITASRL & 33.6 & $\mathrm{pCi} / \mathrm{L}$ & 2.36 & 8.44 & & & \\
\hline 3836 & UISO_IE_PLATE_AEA & ITASRL & 0.195 & $\mathrm{pCi} / \mathrm{L}$ & 0.0442 & 0.0495 & & & \\
\hline 3837 & UISO_IE_PLATE_AEA & ITASRL & 0.00412 & $\mathrm{pCi} / \mathrm{L}$ & 0.00966 & 0.00985 & & & $\mathrm{U}$ \\
\hline 3838 & UISO_IE_PLATE_AEA & ITASRL & 0.167 & $\mathrm{pCi} / \mathrm{L}$ & 0.0407 & 0.0447 & & & \\
\hline
\end{tabular}




\begin{tabular}{|c|c|c|c|c|c|c|c|c|c|}
\hline & $\mathrm{K}$ & $\mathrm{L}$ & $\mathrm{M}$ & $\mathrm{N}$ & $\mathrm{O}$ & $\mathrm{P}$ & $\mathrm{Q}$ & $\mathrm{R}$ & $\mathrm{S}$ \\
\hline 1 & METHOD_NAME & LAB_CODE & VALUE_RPTD & ANAL_UNITS_RPTD & COUNTING_ERROR & $\mid$ TOTAL_ANAL_ERROR| & MIN_DETECTABLE_ACTIVITY & PCNT_MOISTURE & LAB_QUALIFIER \\
\hline 3840 & TRITIUM_ELECT_LSC & ITASRL & 40.4 & $\mathrm{pCi} / \mathrm{L}$ & 2.48 & 9 & & & \\
\hline 3841 & UISO_IE_PLATE_AEA & ITASRL & & & & & & & \\
\hline 3842 & UISO_IE_PLATE_AEA & ITASRL & & & & & & & \\
\hline 3843 & UISO_IE_PLATE_AEA & ITASRL & & & & & & & \\
\hline 3844 & SRISO-SEPP_PRËCIP_GPC & ITASRL & 0.0667 & $\mathrm{pCi} / \mathrm{L}$ & 0.0347 & 0.0379 & & & \\
\hline 3845 & TRITIUMM_ELECTT_LSC̄ & ITASRL & 38.1 & $\mathrm{pCi} / \mathrm{L}$ & 2.42 & 8.67 & & & \\
\hline 3846 & UISO_IE_PLATE_AEA & ITASRL & 0.347 & $\mathrm{pCi} / \mathrm{L}$ & 0.119 & 0.132 & & & \\
\hline 3847 & UISO_IE_PLATE_AEA & ITASRL & -0.0154 & $\mathrm{pCi} / \mathrm{L}$ & 0.0244 & 0.0245 & & & $U$ \\
\hline 3849 & SRISO_SEP_PRECIP_GPC & ITASRL & 0.0677 & $\mathrm{pCi} / \mathrm{L}$ & 0.0452 & 0.048 & & & \\
\hline 3850 & TRITIUM_ELEECT_LSC & ITASRL & 38.1 & $\mathrm{pCi} / \mathrm{L}$ & 2.39 & 8.64 & & & \\
\hline 3851 & UISO_IE_PLATE_AEA & ITASRL & 0.224 & $\mathrm{pCi} / \mathrm{L}$ & 0.0505 & 0.0568 & & & \\
\hline 3852 & UISO_IE_PLATE_AEA & ITASRL & -0.00333 & $\mathrm{pCi} / \mathrm{L}$ & 0.00867 & 0.00885 & & & $\mathrm{U}$ \\
\hline 3853 & UISO_IE_PLATE_AEA & ITASRL & 0.195 & $\mathrm{pCi} / \mathrm{L}$ & 0.047 & 0.052 & & & \\
\hline 3854 & SRISŌ_ĒSEP_PRËCIP_GPC & ITASRL & -0.22 & $\mathrm{pCi} / \mathrm{L}$ & 0.329 & 0.331 & & & $U$ \\
\hline 3855 & TRITIUM_ELECCT_LSC & ITASRL & 40.8 & $\mathrm{pCi} / \mathrm{L}$ & 2.47 & 8.94 & & & \\
\hline 3856 & UISO_IE_PLATE_AEA & ITASRL & & & & & & & \\
\hline 3857 & UISO_IE_PLATE_AEA & ITASRL & & & & & & & \\
\hline 3859 & SRISO-SE_SEP_PRĒCIP_GPC & ITASRL & 0.073 & $\mathrm{pCi} / \mathrm{L}$ & 0.0399 & 0.043 & & & \\
\hline 3860 & TRITIUMM_ELECCT_LSC & ITASRL & 36.3 & $\mathrm{pCi} / \mathrm{L}$ & 2.42 & 8.65 & & & \\
\hline 3861 & UISO_IE_PLATE_AEA & ITASRL & 0.451 & $\mathrm{pCi} / \mathrm{L}$ & 0.114 & 0.132 & & & \\
\hline 3862 & UISO_IE_PLATE_AEA & ITASRL & 0.000768 & $\mathrm{pCi} / \mathrm{L}$ & 0.0146 & 0.0147 & & & $U$ \\
\hline 3863 & UISO_IE_PLATE_AEA & ITASRL & 0.318 & $\mathrm{pCi} / \mathrm{L}$ & 0.0942 & 0.105 & & & \\
\hline 3864 & SRISO_SEP_PRECIP_GPC & ITASRL & 0.0964 & $\mathrm{pCi} / \mathrm{L}$ & 0.0355 & 0.0416 & & & \\
\hline 3865 & TRITIUM_ELECT_LSC & ITASRL & 52.1 & $\mathrm{pCi} / \mathrm{L}$ & 2.79 & 11.3 & & & \\
\hline 3866 & UISO_IE_PLATE_AEA & ITASRL & 0.207 & $\mathrm{pCi} / \mathrm{L}$ & 0.0383 & 0.0443 & & & \\
\hline 3867 & UISO_IE_PLATE_AEA & ITASRL & 0.00608 & $\mathrm{pCi} / \mathrm{L}$ & 0.00833 & 0.00857 & & & $U$ \\
\hline 3868 & UISO_IE_PLATE_AEA & ITASRL & 0.161 & $\mathrm{pCi} / \mathrm{L}$ & 0.0338 & 0.0378 & & & \\
\hline 3869 & SRISO-SE_SEP_PRËCIP_GPC & ITASRL & 0.0786 & $\mathrm{pCi} / \mathrm{L}$ & 0.0282 & 0.0334 & & & \\
\hline 3870 & TRITIUMM_ELECCT_LSC̄ & ITASRL & 40.5 & $\mathrm{pCi} / \mathrm{L}$ & 2.64 & 10.4 & & & \\
\hline 3871 & UISO_IE_PLATE_AEA & ITASRL & 0.372 & $\mathrm{pCi} / \mathrm{L}$ & 0.0509 & 0.064 & & & \\
\hline 3872 & UISO_IE_PLATE_AEA & ITASRL & 0.00945 & $\mathrm{pCi} / \mathrm{L}$ & 0.0096 & 0.00984 & & & $U$ \\
\hline 3873 & UISO_IE_PLATE_AEA & ITASRL & 0.29 & $\mathrm{pCi} / \mathrm{L}$ & 0.0446 & 0.0537 & & & \\
\hline 3874 & SRISO_SEP_PRECCIP_GPC & ITASRL & 0.0849 & $\mathrm{pCi} / \mathrm{L}$ & 0.0321 & 0.037 & & & \\
\hline 3875 & TRITIUMM_ELECCT_LSC & ITASRL & 70.6 & $\mathrm{pCi} / \mathrm{L}$ & 3 & 12.7 & & & \\
\hline 3876 & UISO_IE_PLATE_AEA & ITASRL & 0.27 & $\mathrm{pCi} / \mathrm{L}$ & 0.098 & 0.107 & & & \\
\hline
\end{tabular}




\begin{tabular}{|c|c|c|c|c|c|c|c|c|c|}
\hline & $\mathrm{K}$ & $\mathrm{L}$ & $\mathrm{M}$ & $\mathrm{N}$ & $\mathrm{O}$ & $\mathrm{P}$ & $\mathrm{Q}$ & $\mathrm{R}$ & $\mathrm{S}$ \\
\hline 1 & METHOD_NAME & LAB_CODE & VALUE_RPTD & ANAL_UNITS_RPTD & COUNTING_ERROR & TOTAL_ANAL_ERROR & MIN_DETECTABLE_ACTIVITY & PCNT_MOISTURE & LAB_QUALIFIER \\
\hline 3878 & UISO_IE_PLATE_AEA & ITASRL & 0.123 & $\mathrm{pCi} / \mathrm{L}$ & 0.0703 & 0.073 & & & \\
\hline 3879 & SRISO_SEP_PRECIP_GPC & ITASRL & 0.0752 & $\mathrm{pCi} / \mathrm{L}$ & 0.0269 & 0.0316 & & & \\
\hline 3880 & TRITIUM_ELECT_LSC & ITASRL & 79.8 & $\mathrm{pCi} / \mathrm{L}$ & 3.09 & 13.2 & & & \\
\hline 3881 & UISO_IE_PLATE_AEA & ITASRL & 0.234 & $\mathrm{pCi} / \mathrm{L}$ & 0.0451 & 0.0519 & & & \\
\hline 3882 & UISO_IE_PLATE_AEA & ITASRL & 0.0143 & $\mathrm{pCi} / \mathrm{L}$ & 0.0135 & 0.0137 & & & \\
\hline 3883 & UISO_IE_PLATE_AEA & ITASRL & 0.171 & $\mathrm{pCi} / \mathrm{L}$ & 0.0386 & 0.0428 & & & \\
\hline 3884 & SRISO_SEP_PRECIP_GPC & ITASRL & 0.0801 & $\mathrm{pCi} / \mathrm{L}$ & 0.0262 & 0.0313 & & & \\
\hline 3885 & TRITIUM_ELECT_LSC & ITASRL & 65.4 & $\mathrm{pCi} / \mathrm{L}$ & 2.94 & 12.2 & & & \\
\hline 3886 & UISO_IE_PLATE_AEA & ITASRL & 0.201 & $\mathrm{pCi} / \mathrm{L}$ & 0.0482 & 0.0536 & & & \\
\hline 3887 & UISO_IE_PLATE_AEA & ITASRL & 0.00557 & $\mathrm{pCi} / \mathrm{L}$ & 0.0124 & 0.0126 & & & $\mathrm{U}$ \\
\hline 3888 & UISO_IE_PLATE_AEA & ITASRL & 0.178 & $\mathrm{pCi} / \mathrm{L}$ & 0.045 & 0.0493 & & & \\
\hline 3889 & SRISO_SEP_PRECIP_GPC & ITASRL & 0.0872 & $\mathrm{pCi} / \mathrm{L}$ & 0.0271 & 0.0326 & & & \\
\hline 3890 & TRITIUM_ELECT_LSC & ITASRL & 65.6 & $\mathrm{pCi} / \mathrm{L}$ & 3.15 & 13 & & & \\
\hline 3891 & UISO_IE_PLATE_AEA & ITASRL & 0.199 & $\mathrm{pCi} / \mathrm{L}$ & 0.045 & 0.0504 & & & \\
\hline 3892 & UISO_IE_PLATE_AEA & ITASRL & 0.00756 & $\mathrm{pCi} / \mathrm{L}$ & 0.0108 & 0.011 & & & $U$ \\
\hline 3893 & UISO_IE_PLATE_AEA & ITASRL & 0.216 & $\mathrm{pCi} / \mathrm{L}$ & 0.0459 & 0.0518 & & & \\
\hline 3894 & SRISO_SEP_PRECIP_GPC & ITASRL & 0.0783 & $\mathrm{pCi} / \mathrm{L}$ & 0.0335 & 0.0379 & & & \\
\hline 3895 & TRITIUM_ELECT_LSC & ITASRL & 63.6 & $\mathrm{pCi} / \mathrm{L}$ & 2.9 & 12.1 & & & \\
\hline 3897 & UISO_IE_PLATE_AEA & ITASRL & 0.00764 & $\mathrm{pCi} / \mathrm{L}$ & 0.00976 & 0.00998 & & & U \\
\hline 3898 & UISO_IE_PLATE_AEA & ITASRL & 0.16 & $\mathrm{pCi} / \mathrm{L}$ & 0.0384 & 0.0423 & & & \\
\hline 3899 & SRISO_SEP_PRECIP_GPC & ITASRL & 0.0709 & $\mathrm{pCi} / \mathrm{L}$ & 0.026 & 0.0306 & & & \\
\hline 3900 & TRITIUM_ELECT_LSC & ITASRL & 50.3 & $\mathrm{pCi} / \mathrm{L}$ & 2.73 & 11.1 & & & \\
\hline 3901 & UISO_IE_PLATE_AEA & ITASRL & 0.191 & $\mathrm{pCi} / \mathrm{L}$ & 0.0392 & 0.0445 & & & \\
\hline 3902 & UISO_IE_PLATE_AEA & ITASRL & 0.00459 & $\mathrm{pCi} / \mathrm{L}$ & 0.00869 & 0.00891 & & & U \\
\hline 3903 & UISO_IE_PLATE_AEA & ITASRL & 0.221 & $\mathrm{pCi} / \mathrm{L}$ & 0.0418 & 0.0478 & & & \\
\hline 3904 & SRISO_SEP_PRECIP_GPC & ITASRL & 0.0521 & $\mathrm{pCi} / \mathrm{L}$ & 0.0311 & 0.0337 & & & \\
\hline 3905 & TRITIUM_ELECT_LSC & ITASRL & 43.4 & $\mathrm{pCi} / \mathrm{L}$ & 2.63 & 10.5 & & & \\
\hline 3906 & UISO_IE_PLATE_AEA & ITASRL & 0.194 & $\mathrm{pCi} / \mathrm{L}$ & 0.0767 & 0.0819 & & & \\
\hline 3907 & UISO_IE_PLATE_AEA & ITASRL & 0.00461 & $\mathrm{pCi} / \mathrm{L}$ & 0.0201 & 0.0202 & & & $U$ \\
\hline 3908 & UISO_IE_PLATE_AEA & ITASRL & 0.219 & $\mathrm{pCi} / \mathrm{L}$ & 0.0801 & 0.086 & & & \\
\hline 3909 & SRISO_SEP_PRECIP_GPC & ITASRL & 0.0581 & $\mathrm{pCi} / \mathrm{L}$ & 0.0226 & 0.0268 & & & \\
\hline 3910 & TRITIUM_ELECT_LSC & ITASRL & 44.5 & $\mathrm{pCi} / \mathrm{L}$ & 2.68 & 10.7 & & & \\
\hline 3911 & UISO_IE_PLATE_AEA & ITASRL & 0.34 & $\mathrm{pCi} / \mathrm{L}$ & 0.0544 & 0.0659 & & & \\
\hline 3912 & UISO_IE_PLATE_AEA & ITASRL & 0.008 & $\mathrm{pCi} / \mathrm{L}$ & 0.0116 & 0.0118 & & & $\mathrm{U}$ \\
\hline 3913 & UISO_IE_PLATE_AEA & ITASRL & 0.223 & $\mathrm{pCi} / \mathrm{L}$ & 0.0445 & 0.0506 & & & \\
\hline 3914 & SRISO_SEP_PRECIP_GPC & ITASRL & 0.0953 & $\mathrm{pCi} / \mathrm{L}$ & 0.0308 & 0.0369 & & & \\
\hline
\end{tabular}




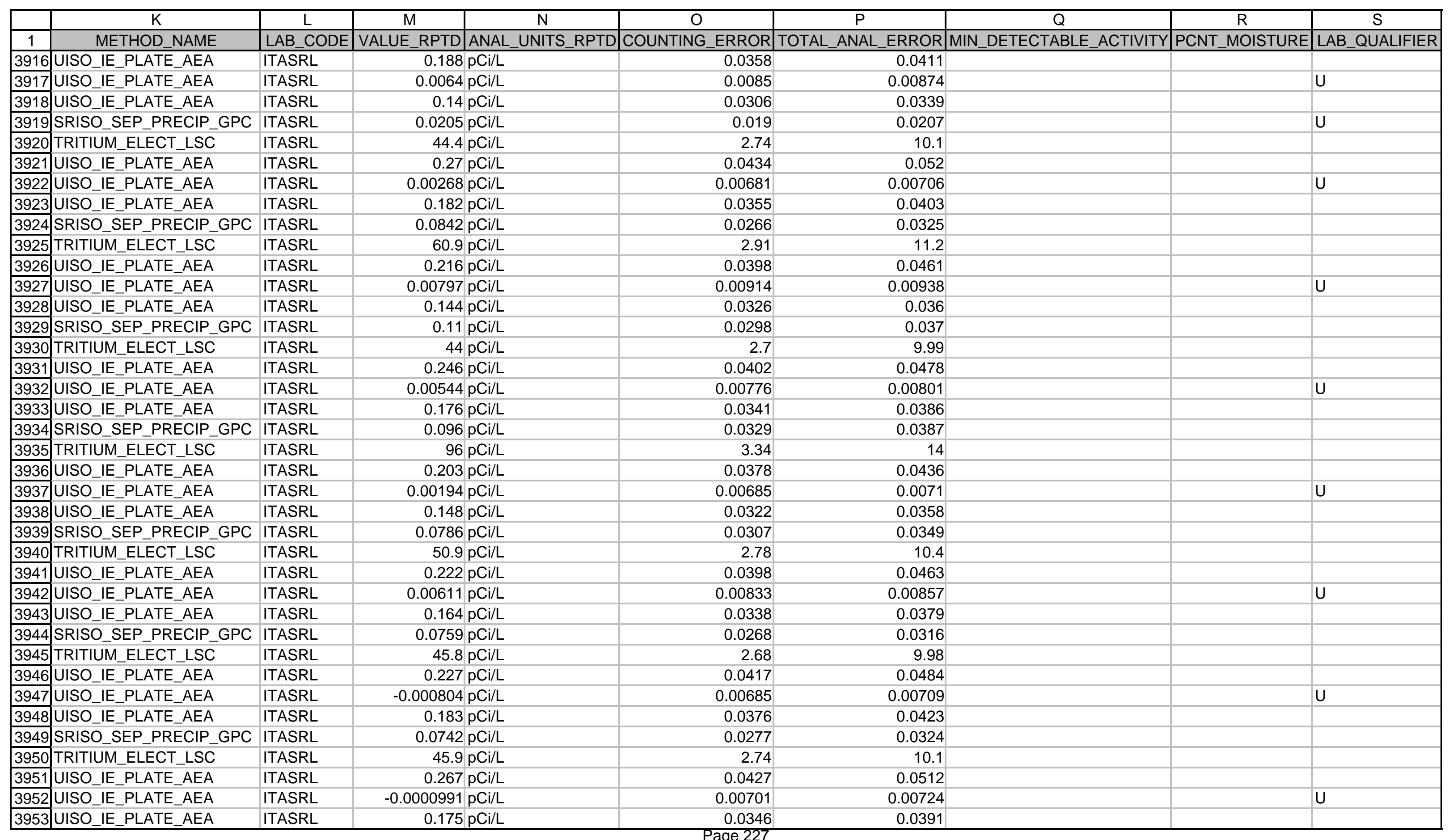




\begin{tabular}{|c|c|c|c|c|c|c|c|c|c|}
\hline & $\mathrm{K}$ & $\mathrm{L}$ & $\mathrm{M}$ & $\mathrm{N}$ & $\mathrm{O}$ & $\mathrm{P}$ & $\mathrm{Q}$ & $\mathrm{R}$ & $\mathrm{S}$ \\
\hline 1 & METHOD_NAME & LAB_CODE & VALUE_RPTD & ANAL_UNITS_RPTD & COUNTING_ERROR| & TOTAL_ANAL_ERROR|I & MIN_DETECTABLE_ACTIVITY & PCNT_MOISTURE & LAB_QUALIFIER \\
\hline 3954 & SRISO_SEP_PRECIP_GPC & ITASRL & 0.0648 & $\mathrm{pCi} / \mathrm{L}$ & 0.0241 & 0.0285 & & & \\
\hline 3955 & TRITIUM_ELECT_LSC & ITASRL & 39.4 & $\mathrm{pCi} / \mathrm{L}$ & 2.65 & 9.67 & & & \\
\hline 3956 & UISO_IE_PLATE_AEA & ITASRL & 0.249 & $\mathrm{pCi} / \mathrm{L}$ & 0.0421 & 0.0497 & & & \\
\hline 3957 & UISO_IE_PLATE_AEA & ITASRL & -0.000949 & $\mathrm{pCi} / \mathrm{L}$ & 0.00636 & 0.00661 & & & $\mathrm{U}$ \\
\hline 3958 & UISO_IE_PLATE_AEA & ITASRL & 0.161 & $\mathrm{pCi} / \mathrm{L}$ & 0.0338 & 0.0379 & & & \\
\hline 3959 & SRISO_SEP_PRECIP_GPC & ITASRL & 0.0641 & $\mathrm{pCi} / \mathrm{L}$ & 0.0246 & 0.029 & & & \\
\hline 3960 & TRITIUM_ELECT_LSC & ITASRL & 39.5 & $\mathrm{pCi} / \mathrm{L}$ & 2.66 & 9.7 & & & \\
\hline 3961 & UISO_IE_PLATE_AEA & ITASRL & 0.262 & $\mathrm{pCi} / \mathrm{L}$ & 0.0439 & 0.052 & & & \\
\hline 3962 & UISO_IE_PLATE_AEA & ITASRL & 0.00645 & $\mathrm{pCi} / \mathrm{L}$ & 0.00865 & 0.00889 & & & $\mathrm{U}$ \\
\hline 3963 & UISO_IE_PLATE_AEA & ITASRL & 0.18 & $\mathrm{pCi} / \mathrm{L}$ & 0.0361 & 0.0408 & & & \\
\hline 3964 & SRISO_SEP_PRECIP_GPC & ITASRL & 0.0863 & $\mathrm{pCi} / \mathrm{L}$ & 0.0281 & 0.0336 & & & \\
\hline 3965 & TRITIUM_ELECT_LSC & ITASRL & 4070 & $\mathrm{pCi} / \mathrm{L}$ & 16.4 & 352 & & & \\
\hline 3966 & UISO_IE_PLATE_AEA & ITASRL & 0.346 & $\mathrm{pCi} / \mathrm{L}$ & 0.051 & 0.0628 & & & \\
\hline 3967 & UISO_IE_PLATE_AEA & ITASRL & 0.00316 & $\mathrm{pCi} / \mathrm{L}$ & 0.00734 & 0.00759 & & & $U$ \\
\hline 3968 & UISO_IE_PLATE_AEA & ITASRL & 0.235 & $\mathrm{pCi} / \mathrm{L}$ & 0.0417 & 0.0484 & & & \\
\hline 3969 & SRISO_SEP_PRECIP_GPC & ITASRL & 0.0808 & $\mathrm{pCi} / \mathrm{L}$ & 0.0258 & 0.0312 & & & \\
\hline 3970 & TRITIUM_ELECT_LSC & ITASRL & 52.9 & $\mathrm{pCi} / \mathrm{L}$ & 2.68 & 10.2 & & & \\
\hline 3971 & UISO_IE_PLATE_AEA & ITASRL & 0.238 & $\mathrm{pCi} / \mathrm{L}$ & 0.069 & 0.0758 & & & \\
\hline 3973 & UISO_IE_PLATE_AEA & ITASRL & 0.222 & $\mathrm{pCi} / \mathrm{L}$ & 0.0656 & 0.0716 & & & \\
\hline 3974 & SRISO_SEP_PRECIP_GPC & ITASRL & 0.0806 & $\mathrm{pCi} / \mathrm{L}$ & 0.0281 & 0.0328 & & & \\
\hline 3975 & TRITIUM_ELECT_LSC & ITASRL & 2710 & $\mathrm{pCi} / \mathrm{L}$ & 13.5 & 235 & & & \\
\hline 3976 & UISO_IE_PLATE_AEA & ITASRL & 0.311 & $\mathrm{pCi} / \mathrm{L}$ & 0.0463 & 0.0567 & & & \\
\hline 3977 & UISO_IE_PLATE_AEA & ITASRL & 0.00525 & $\mathrm{pCi} / \mathrm{L}$ & 0.00828 & 0.00852 & & & $\mathrm{U}$ \\
\hline 3978 & UISO_IE_PLATE_AEA & ITASRL & 0.195 & $\mathrm{pCi} / \mathrm{L}$ & 0.0366 & 0.0419 & & & \\
\hline 3979 & SRISO_SEP_PRECIP_GPC & ITASRL & 0.0826 & $\mathrm{pCi} / \mathrm{L}$ & 0.0285 & 0.0334 & & & \\
\hline 3980 & TRITIUM_ELECT_LSC & ITASRL & 94.3 & $\mathrm{pCi} / \mathrm{L}$ & 3.15 & 13.4 & & & \\
\hline 3981 & UISO_IE_PLATE_AEA & ITASRL & 0.209 & $\mathrm{pCi} / \mathrm{L}$ & 0.0371 & 0.0432 & & & \\
\hline 3982 & UISO_IE_PLATE_AEA & ITASRL & 0.0116 & $\mathrm{pCi} / \mathrm{L}$ & 0.00988 & 0.0101 & & & \\
\hline 3983 & UISO_IE_PLATE_AEA & ITASRL & 0.145 & $\mathrm{pCi} / \mathrm{L}$ & 0.0312 & 0.0346 & & & \\
\hline 3984 & SRISO_SEP_PRECIP_GPC & ITASRL & 0.0632 & $\mathrm{pCi} / \mathrm{L}$ & 0.0259 & 0.03 & & & \\
\hline 3985 & TRITIUM_ELECT_LSC & ITASRL & 66.5 & $\mathrm{pCi} / \mathrm{L}$ & 2.83 & 11.1 & & & \\
\hline 3986 & UISO_IE_PLATE_AEA & ITASRL & 0.166 & $\mathrm{pCi} / \mathrm{L}$ & 0.0391 & 0.0433 & & & \\
\hline 3987 & UISO_IE_PLATE_AEA & ITASRL & -0.00374 & $\mathrm{pCi} / \mathrm{L}$ & 0.00504 & 0.00535 & & & $\mathrm{U}$ \\
\hline 3988 & UISO_IE_PLATE_AEA & ITASRL & 0.135 & $\mathrm{pCi} / \mathrm{L}$ & 0.0342 & 0.0372 & & & \\
\hline 3989 & SRISO_SEP_PRECIP_GPC & ITASRL & 0.071 & $\mathrm{pCi} / \mathrm{L}$ & 0.0247 & 0.0293 & & & \\
\hline 3990 & TRITIUM_ELECT_LSC & ITASRL & 83.2 & $\mathrm{pCi} / \mathrm{L}$ & 3.02 & 12.5 & & & \\
\hline
\end{tabular}




\begin{tabular}{|c|c|c|c|c|c|c|c|c|c|}
\hline & $\mathrm{K}$ & $\mathrm{L}$ & $\mathrm{M}$ & $\mathrm{N}$ & $\mathrm{O}$ & $\mathrm{P}$ & $\mathrm{Q}$ & $\mathrm{R}$ & $\mathrm{S}$ \\
\hline 1 & METHOD_NAME & LAB_CODE & VALUE_RPTD & ANAL_UNITS_RPTD & COUNTING_ERROR & TOTAL_ANAL_ERROR & MIN_DETECTABLE_ACTIVITY & PCNT_MOISTURE & LAB_QUALIFIER \\
\hline 3992 & UISO IE_PLATE_AEA & ITASRL & 0.0167 & $\mathrm{pCi} / \mathrm{L}$ & 0.0366 & 0.0369 & & & $U$ \\
\hline 3993 & UISO_IE_PLATE_AEA & ITASRL & 0.182 & $\mathrm{pCi} / \mathrm{L}$ & 0.0966 & 0.102 & & & \\
\hline 3994 & SRISŌ SEP PRËCIP GPC & ITASRL & 0.0597 & $\mathrm{pCi} / \mathrm{L}$ & 0.0248 & 0.0287 & & & \\
\hline 3995 & TRITIŪM ELEECT LSC̄ & ITASRL & 72.7 & $\mathrm{pCi} / \mathrm{L}$ & 2.92 & 11.7 & & & \\
\hline 3996 & UISO_IE_PLATE_AEA & ITASRL & 0.247 & $\mathrm{pCi} / \mathrm{L}$ & 0.0594 & 0.0667 & & & \\
\hline 3997 & UISO_IE_PLATE_AEA & ITASRL & 0.000818 & $\mathrm{pCi} / \mathrm{L}$ & 0.0153 & 0.0154 & & & $U$ \\
\hline 3998 & UISO_IE_PLATE_AEA & ITASRL & 0.16 & $\mathrm{pCi} / \mathrm{L}$ & 0.0486 & 0.0524 & & & \\
\hline 3999 & SRISO-SE_SEP_PRËCIP_GPC & ITASRL & 0.0623 & $\mathrm{pCi} / \mathrm{L}$ & 0.0237 & 0.0278 & & & \\
\hline \begin{tabular}{|l|}
4001 \\
\end{tabular} & UISO_IE_PLATE_AEA & ITASRL & 0.225 & $\mathrm{pCi} / \mathrm{L}$ & 0.039 & 0.0457 & & & \\
\hline 4002 & UISO_IE_PLATE_AEA & ITASRL & 0.00167 & $\mathrm{pCi} / \mathrm{L}$ & 0.00829 & 0.00849 & & & U \\
\hline 4003 & UISO_IE_PLATE_AEA & ITASRL & 0.177 & $\mathrm{pCi} / \mathrm{L}$ & 0.034 & 0.0386 & & & \\
\hline 4004 & SRISO_SEP_PRECIP_GPC & ITASRL & 0.0549 & $\mathrm{pCi} / \mathrm{L}$ & 0.0237 & 0.0274 & & & \\
\hline 4005 & TRITIUMM_ELECCT_LSC & ITASRL & 55.5 & $\mathrm{pCi} / \mathrm{L}$ & 2.72 & 10.4 & & & \\
\hline 4006 & UISO_IE_PLATE_AEA & ITASRL & 0.178 & $\mathrm{pCi} / \mathrm{L}$ & 0.0372 & 0.042 & & & \\
\hline 4007 & UISO_IE_PLATE_AEA & ITASRL & 0.0139 & $\mathrm{pCi} / \mathrm{L}$ & 0.0114 & 0.0117 & & & \\
\hline 4008 & UISO IE PLATE AEA & ITASRL & 0.195 & $\mathrm{pCi} / \mathrm{L}$ & 0.0383 & 0.0436 & & & \\
\hline 4009 & SRISŌ_ĒSEP_PRĒCIP_GPC & ITASRL & 0.0751 & $\mathrm{pCi} / \mathrm{L}$ & 0.025 & 0.0299 & & & \\
\hline 4011 & UISO_IE_PLATE_AEA & ITASRL & 0.22 & $\mathrm{pCi} / \mathrm{L}$ & 0.0399 & 0.0464 & & & \\
\hline 4012 & UISO_IE_PLATE_AEA & ITASRL & 0.00621 & $\mathrm{pCi} / \mathrm{L}$ & 0.00845 & 0.00869 & & & $U$ \\
\hline 4013 & UISO_IE_PLATE_AEA & ITASRL & 0.186 & $\mathrm{pCi} / \mathrm{L}$ & 0.0362 & 0.0411 & & & \\
\hline 4014 & SRISO_SEPP_PRECCIP_GPC & QTESRL & 0.0856 & $\mathrm{pCi} / \mathrm{L}$ & 0.028 & 0.034 & 0.0357 & & \\
\hline 4015 & TRITIUM_ELECT_LSC & QTESRL & 28.5 & $\mathrm{pCi} / \mathrm{L}$ & 2.5 & 9.7 & 4.88 & & \\
\hline 4016 & UISO_IE_PLATE_AEA & QTESRL & 0.23 & $\mathrm{pCi} / \mathrm{L}$ & 0.043 & 0.05 & 0.0243 & & $\mathrm{~J}$ \\
\hline 4017 & UISO_IE_PLATE_AEA & QTESRL & 0.00359 & $\mathrm{pCi} / \mathrm{L}$ & 0.0085 & 0.0088 & 0.0142 & & $U$ \\
\hline 4018 & UISO_IE_PLATE_AEA & QTESRL & 0.155 & $\mathrm{pCi} / \mathrm{L}$ & 0.034 & 0.038 & 0.0125 & & $\mathrm{~J}$ \\
\hline 4019 & SRISO_SEP_PRECCIP_GPC & QTESRL & 0.0755 & $\mathrm{pCi} / \mathrm{L}$ & 0.025 & 0.031 & 0.0332 & & \\
\hline 4020 & TRITIUM_ELEECT_LSC & QTESRL & 29.4 & $\mathrm{pCi} / \mathrm{L}$ & 2.6 & 9.9 & 4.88 & & \\
\hline 4021 & UISO_IE_PLATE_AEA & QTESRL & 0.263 & $\mathrm{pCi} / \mathrm{L}$ & 0.045 & 0.053 & 0.018 & & $\mathrm{~J}$ \\
\hline 4022 & UISO_IE_PLATE_AEA & QTESRL & 0.000661 & $\mathrm{pCi} / \mathrm{L}$ & 0.0066 & 0.0069 & 0.0125 & & $U$ \\
\hline 4023 & UISO_IE_PLATE_AEA & QTESRL & 0.191 & $\mathrm{pCi} / \mathrm{L}$ & 0.038 & 0.043 & 0.0125 & & $\mathrm{~J}$ \\
\hline 4024 & SRISO-SE_SEP_PRËCIP_GPC & QTESRL & 0.0703 & $\mathrm{pCi} / \mathrm{L}$ & 0.026 & 0.031 & 0.0366 & & \\
\hline 4025 & TRITIUM_ELEECT_LSC & QTESRL & 29.2 & $\mathrm{pCi} / \mathrm{L}$ & 2.6 & 9.7 & 4.88 & & \\
\hline 4026 & UISO_IE_PLATE_AEA & QTESRL & 0.239 & $\mathrm{pCi} / \mathrm{L}$ & 0.04 & 0.047 & 0.011 & & $\mathrm{~J}$ \\
\hline 4027 & UISO_IE_PLATE_AEA & QTESRL & 0.0114 & $\mathrm{pCi} / \mathrm{L}$ & 0.01 & 0.011 & 0.011 & & $\mathrm{~J}$ \\
\hline 4028 & UISO_IE_PLATE_AEA & QTESRL & 0.18 & $\mathrm{pCi} / \mathrm{L}$ & 0.035 & 0.039 & 0.00908 & & $\mathrm{~J}$ \\
\hline
\end{tabular}




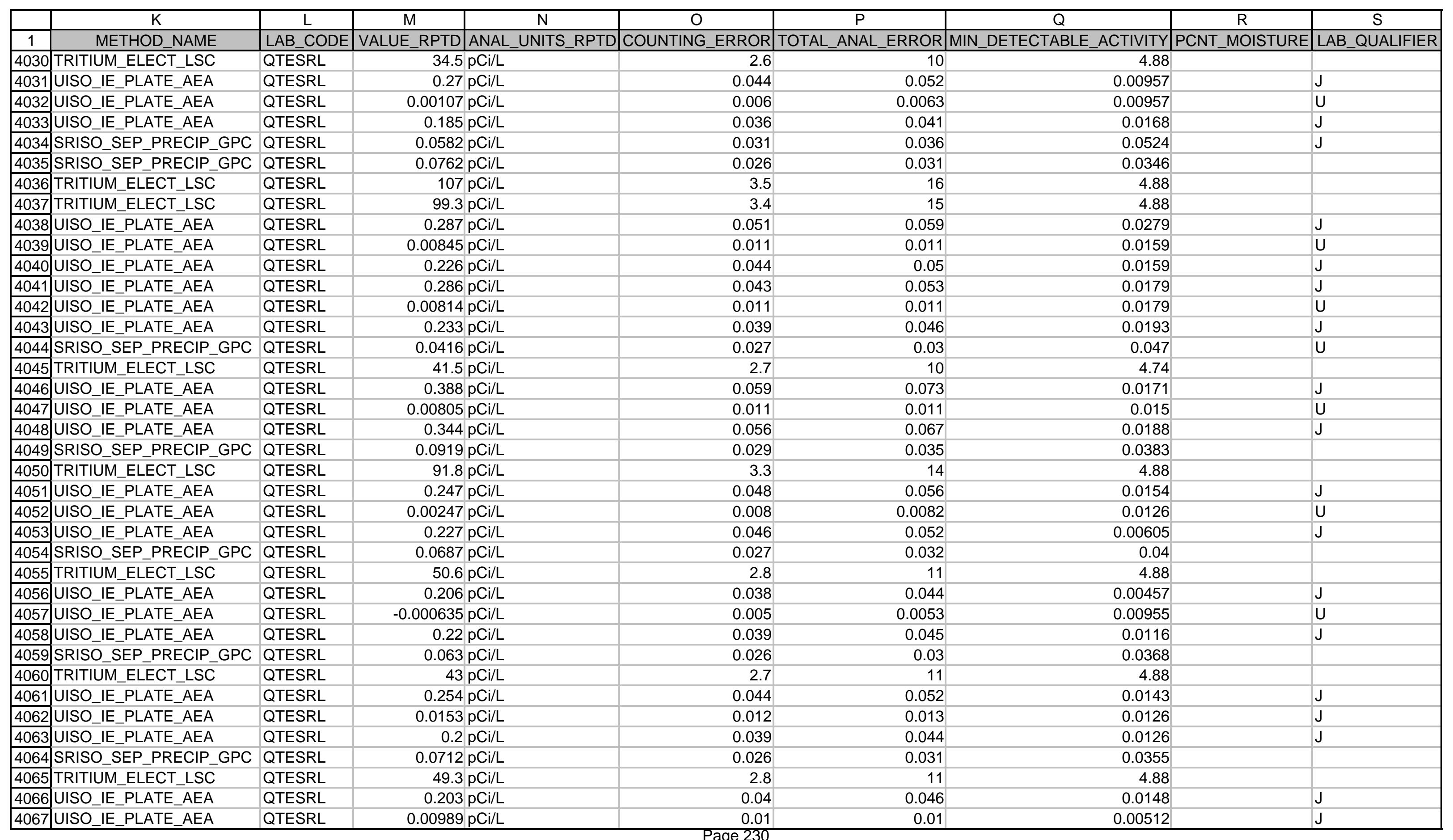




\begin{tabular}{|c|c|c|c|c|c|c|c|c|c|}
\hline & $\mathrm{K}$ & $\mathrm{L}$ & $\mathrm{M}$ & $\mathrm{N}$ & $\mathrm{O}$ & $\mathrm{P}$ & $\mathrm{Q}$ & $\mathrm{R}$ & $\mathrm{S}$ \\
\hline 1 & METHOD_NAME & LAB_CODE & VALUE_RPTD & ANAL_UNITS_RPTD & COUNTING_ERROR & TOTAL_ANAL_ERROR & MIN_DETECTABLE_ACTIVITY & PCNT_MOISTURE & LAB_QUALIFIER \\
\hline 4068 & UISO_IE_PLATE_AEA & QTESRL & 0.205 & $\mathrm{pCi} / \mathrm{L}$ & 0.04 & 0.046 & 0.013 & & $\mathrm{~J}$ \\
\hline 4069 & SRISO_SEP_PRECIP_GPC & QTESRL & 0.0581 & $\mathrm{pCi} / \mathrm{L}$ & 0.029 & 0.032 & 0.0489 & & $\mathrm{~J}$ \\
\hline 4070 & TRITIUM_ELECT_LSC & QTESRL & 44.6 & $\mathrm{pCi} / \mathrm{L}$ & 2.9 & 11 & 4.88 & & \\
\hline 4071 & UISO_IE_PLATE_AEA & QTESRL & 0.209 & $\mathrm{pCi} / \mathrm{L}$ & 0.036 & 0.042 & 0.00829 & & $\mathrm{~J}$ \\
\hline 4072 & UISO_IE_PLATE_AEA & QTESRL & 0.00429 & $\mathrm{pCi} / \mathrm{L}$ & 0.0074 & 0.0076 & 0.0101 & & U \\
\hline 4073 & UISO_IE_PLATE_AEA & QTESRL & 0.186 & $\mathrm{pCi} / \mathrm{L}$ & 0.034 & 0.039 & 0.00829 & & $\mathrm{~J}$ \\
\hline 4074 & SRISO_SEP_PRECIP_GPC & QTESRL & 0.0573 & $\mathrm{pCi} / \mathrm{L}$ & 0.029 & 0.034 & 0.0496 & & $\mathrm{~J}$ \\
\hline 4075 & TRITIUM_ELECT_LSC & QTESRL & 42.1 & $\mathrm{pCi} / \mathrm{L}$ & 2.7 & 10 & 4.78 & & \\
\hline 4076 & UISO_IE_PLATE_AEA & QTESRL & 0.241 & $\mathrm{pCi} / \mathrm{L}$ & 0.052 & 0.059 & 0.0312 & & $\mathrm{~J}$ \\
\hline 4077 & UISO_IE_PLATE_AEA & QTESRL & 0.0137 & $\mathrm{pCi} / \mathrm{L}$ & 0.015 & 0.015 & 0.0195 & & U \\
\hline 4078 & UISO_IE_PLATE_AEA & QTESRL & 0.184 & $\mathrm{pCi} / \mathrm{L}$ & 0.044 & 0.049 & 0.0248 & & $\mathrm{~J}$ \\
\hline 4079 & SRISO_SEP_PRECIP_GPC & QTESRL & 0.0787 & $\mathrm{pCi} / \mathrm{L}$ & 0.028 & 0.036 & 0.0432 & & \\
\hline 4080 & TRITIUM_ELECT_LSC & QTESRL & 45.6 & $\mathrm{pCi} / \mathrm{L}$ & 2.8 & 10 & 4.78 & & \\
\hline 4081 & UISO_IE_PLATE_AEA & QTESRL & 0.224 & $\mathrm{pCi} / \mathrm{L}$ & 0.051 & 0.057 & 0.0187 & & $\mathrm{~J}$ \\
\hline 4082 & UISO_IE_PLATE_AEA & QTESRL & 0.0103 & $\mathrm{pCi} / \mathrm{L}$ & 0.012 & 0.012 & 0.00738 & & $\mathrm{~J}$ \\
\hline 4083 & UISO_IE_PLATE_AEA & QTESRL & 0.229 & $\mathrm{pCi} / \mathrm{L}$ & 0.051 & 0.057 & 0.00738 & & $\mathrm{~J}$ \\
\hline 4084 & SRISO_SEP_PRECIP_GPC & QTESRL & 0.0687 & $\mathrm{pCi} / \mathrm{L}$ & 0.027 & 0.034 & 0.043 & & \\
\hline 4087 & UISO_IE_PLATE_AEA & QTESRL & 0.00502 & $\mathrm{pCi} / \mathrm{L}$ & 0.0083 & 0.0085 & 0.0103 & & U \\
\hline 4088 & UISO_IE_PLATE_AEA & QTESRL & 0.248 & $\mathrm{pCi} / \mathrm{L}$ & 0.043 & 0.05 & 0.0125 & & $\mathrm{~J}$ \\
\hline 4089 & SRISO_SEP_PRECIP_GPC & QTESRL & 0.0779 & $\mathrm{pCi} / \mathrm{L}$ & 0.025 & 0.036 & 0.0417 & & \\
\hline 4090 & TRITIUM_ELECT_LSC & QTESRL & 30.1 & $\mathrm{pCi} / \mathrm{L}$ & 0.83 & 5.7 & 4.93 & & \\
\hline 4091 & UISO_IE_PLATE_AEA & QTESRL & 0.243 & $\mathrm{pCi} / \mathrm{L}$ & 0.045 & 0.061 & 0.0198 & & \\
\hline 4092 & UISO_IE_PLATE_AEA & QTESRL & 0.00433 & $\mathrm{pCi} / \mathrm{L}$ & 0.0049 & 0.0089 & 0.012 & & U \\
\hline 4093 & UISO_IE_PLATE_AEA & QTESRL & 0.201 & $\mathrm{pCi} / \mathrm{L}$ & 0.041 & 0.053 & 0.0166 & & \\
\hline 4094 & SRISO_SEP_PRECCIP_GPC & QTESRL & 0.0837 & $\mathrm{pCi} / \mathrm{L}$ & 0.026 & 0.037 & 0.0405 & & \\
\hline 4095 & TRITIUM̄ELEEECT_LSC̄ & QTESRL & 32.7 & $\mathrm{pCi} / \mathrm{L}$ & 0.89 & 5.9 & 4.91 & & \\
\hline 4096 & UISO_IE_PLATE_AEA & QTESRL & 0.234 & $\mathrm{pCi} / \mathrm{L}$ & 0.043 & 0.058 & 0.0185 & & \\
\hline 4097 & UISO_IE_PLATE_AEA & QTESRL & 0.015 & $\mathrm{pCi} / \mathrm{L}$ & 0.01 & 0.013 & 0.0137 & & $\mathrm{~J}$ \\
\hline 4098 & UISO_IE_PLATE_AEA & QTESRL & 0.204 & $\mathrm{pCi} / \mathrm{L}$ & 0.04 & 0.052 & 0.0137 & & \\
\hline 4099 & SRISO_SEP_PRECIP_GPC & QTESRL & 0.0798 & $\mathrm{pCi} / \mathrm{L}$ & 0.026 & 0.037 & 0.0414 & & \\
\hline 4100 & TRITIUM_ELECT_LSC & QTESRL & 37.9 & $\mathrm{pCi} / \mathrm{L}$ & 1 & 6.2 & 4.87 & & \\
\hline 4101 & UISO_IE_PLATE_AEA & QTESRL & 0.224 & $\mathrm{pCi} / \mathrm{L}$ & 0.043 & 0.058 & 0.0219 & & \\
\hline 4102 & UISO_IE_PLATE_AEA & QTESRL & 0.0109 & $\mathrm{pCi} / \mathrm{L}$ & 0.011 & 0.014 & 0.0231 & & U \\
\hline 4103 & UISO_IE_PLATE_AEA & QTESRL & 0.207 & $\mathrm{pCi} / \mathrm{L}$ & 0.042 & 0.054 & 0.0207 & & \\
\hline 4104 & SRISŌ_ĒSEP_PRĒCIP_GPC & QTESRL & 0.062 & $\mathrm{pCi} / \mathrm{L}$ & 0.019 & 0.029 & 0.0322 & & \\
\hline
\end{tabular}




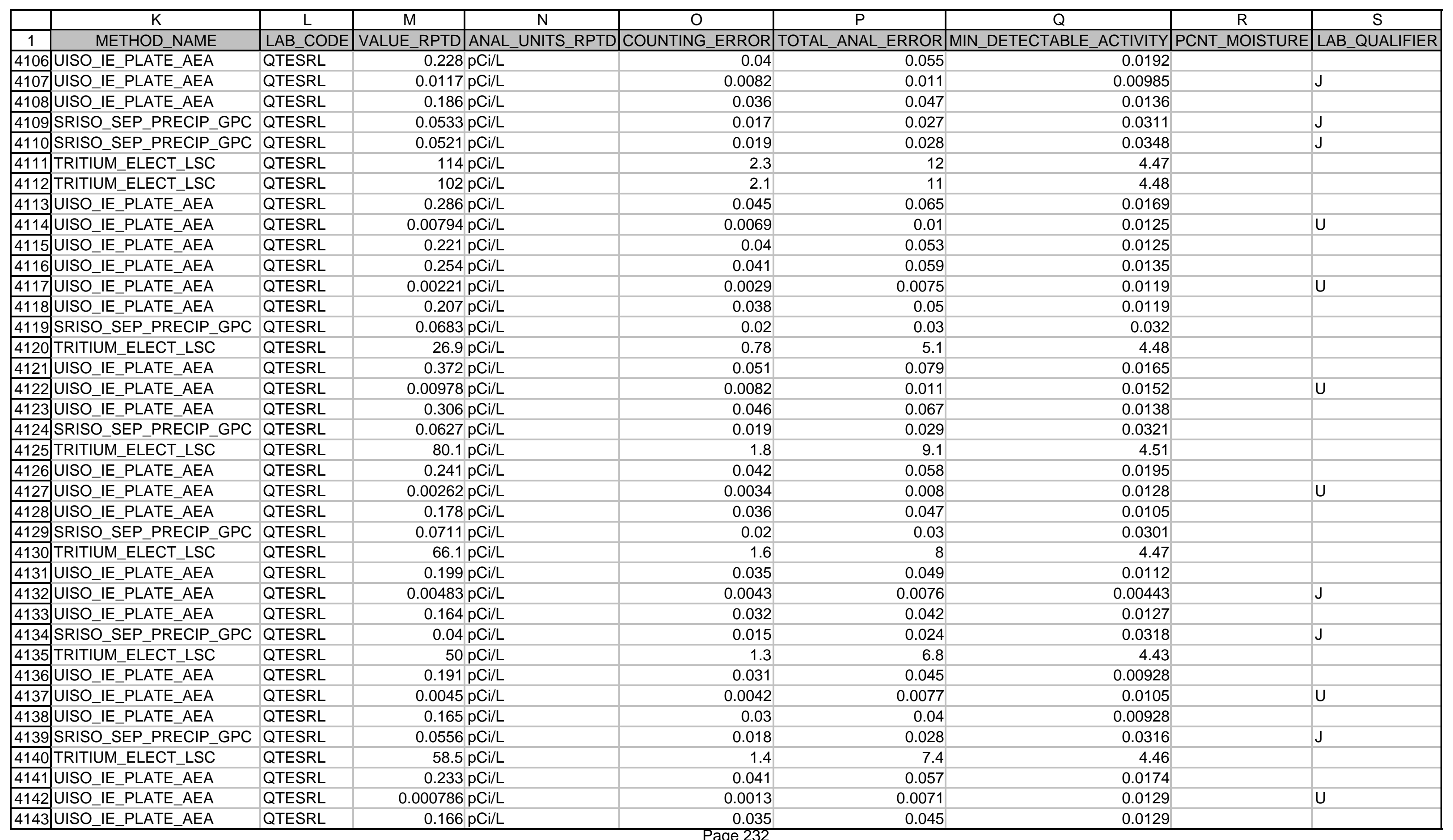




\begin{tabular}{|c|c|c|c|c|c|c|c|c|c|}
\hline & $\mathrm{K}$ & $\mathrm{L}$ & $\mathrm{M}$ & $\mathrm{N}$ & $\mathrm{O}$ & $P$ & $\mathrm{Q}$ & $\mathrm{R}$ & $\mathrm{S}$ \\
\hline 1 & METHOD_NAME & LAB_CODE & VALUE_RPTD & ANAL_UNITS_RPTD & |COUNTING_ERROR & TOTAL_ANAL_ERROR & |MIN_DETECTABLE_ACTIVITY & PCNT_MOISTURE & LAB_QUALIFIER \\
\hline 4144 & SRISO_SEP_PRECIP_GPC & QTESRL & 0.0769 & $\mathrm{pCi} / \mathrm{L}$ & 0.021 & 0.033 & 0.033 & & \\
\hline 4145 & TRITIUM_ELECT_LSC & QTESRL & 67.3 & $\mathrm{pCi} / \mathrm{L}$ & 1.6 & 8.1 & 4.44 & & \\
\hline 4146 & UISO_IE_PLATE_AEA & QTESRL & 0.21 & $\mathrm{pCi} / \mathrm{L}$ & 0.037 & 0.051 & 0.0145 & & \\
\hline 4147 & UISO_IE_PLATE_AEA & QTESRL & 0.00716 & $\mathrm{pCi} / \mathrm{L}$ & 0.0062 & 0.0094 & 0.0116 & & $\mathrm{U}$ \\
\hline 4148 & UISO_IE_PLATE_AEA & QTESRL & 0.178 & $\mathrm{pCi} / \mathrm{L}$ & 0.034 & 0.045 & 0.00957 & & \\
\hline 4149 & SRISO_SEP_PRECIP_GPC & QTESRL & 0.0629 & pCi/L & 0.02 & 0.03 & 0.0344 & & \\
\hline 4150 & TRITIUM_ELECT_LSC & QTESRL & 244 & $\mathrm{pCi} / \mathrm{L}$ & 3.7 & 23 & 4.49 & & \\
\hline 4151 & UISO_IE_PLATE_AEA & QTESRL & 0.226 & $\mathrm{pCi} / \mathrm{L}$ & 0.038 & 0.054 & 0.0117 & & \\
\hline 4152 & UISO_IE_PLATE_AEA & QTESRL & 0.00482 & $\mathrm{pCi} / \mathrm{L}$ & 0.0051 & 0.0089 & 0.0133 & & $\mathrm{U}$ \\
\hline 4153 & UISO_IE_PLATE_AEA & QTESRL & 0.163 & $\mathrm{pCi} / \mathrm{L}$ & 0.033 & 0.043 & 0.0117 & & \\
\hline 4154 & SRISO_SEP_PRECIP_GPC & QTESRL & 0.0619 & $\mathrm{pCi} / \mathrm{L}$ & 0.021 & 0.031 & 0.0364 & & \\
\hline 4155 & TRITIUM_ELECT_LSC & QTESRL & 73.7 & $\mathrm{pCi} / \mathrm{L}$ & 1.7 & 8.6 & 4.53 & & \\
\hline 4156 & UISO_IE_PLATE_AEA & QTESRL & 0.207 & $\mathrm{pCi} / \mathrm{L}$ & 0.037 & 0.051 & 0.0134 & & \\
\hline 4157 & UISO_IE_PLATE_AEA & QTESRL & 0.00559 & $\mathrm{pCi} / \mathrm{L}$ & 0.0054 & 0.0089 & 0.0118 & & U \\
\hline 4158 & UISO_IE_PLATE_AEA & QTESRL & 0.172 & $\mathrm{pCi} / \mathrm{L}$ & 0.034 & 0.044 & 0.0118 & & \\
\hline 4159 & SRISO_SEP_PRECIP_GPC & QTESRL & 0.0534 & $\mathrm{pCi} / \mathrm{L}$ & 0.017 & 0.026 & 0.0292 & & $\mathrm{~J}$ \\
\hline 4160 & TRITIUM_ELECT_LSC & QTESRL & 36.7 & $\mathrm{pCi} / \mathrm{L}$ & 1 & 5.8 & 4.46 & & \\
\hline 4161 & UISO_IE_PLATE_AEA & QTESRL & 0.287 & $\mathrm{pCi} / \mathrm{L}$ & 0.043 & 0.064 & 0.0115 & & \\
\hline 4162 & UISO_IE_PLATE_AEA & QTESRL & 0.013 & $\mathrm{pCi} / \mathrm{L}$ & 0.009 & 0.012 & 0.013 & & $\mathrm{U}$ \\
\hline 4163 & UISO_IE_PLATE_AEA & QTESRL & 0.202 & $\mathrm{pCi} / \mathrm{L}$ & 0.036 & 0.049 & 0.0115 & & \\
\hline 4164 & SRISO_SEP_PRECIP_GPC & QTESRL & 0.0935 & $\mathrm{pCi} / \mathrm{L}$ & 0.022 & 0.035 & 0.0311 & & \\
\hline 4165 & SRISO_SEP_PRECIP_GPC & QTESRL & 0.26 & pCi/L & 0.038 & 0.073 & 0.0362 & & \\
\hline 4166 & TRITIUM_ELECT_LSC & QTESRL & 69.9 & $\mathrm{pCi} / \mathrm{L}$ & 1.6 & 8.6 & 4.94 & & \\
\hline 4167 & TRITIUM_ELECT_LSC & QTESRL & 86.8 & $\mathrm{pCi} / \mathrm{L}$ & 1.8 & 10 & 4.97 & & \\
\hline 4168 & UISO_IE_PLATE_AEA & QTESRL & 0.173 & $\mathrm{pCi} / \mathrm{L}$ & 0.032 & 0.044 & 0.0136 & & \\
\hline 4169 & ]UISO_IE_PLATE_AEA & QTESRL & 0.00459 & $\mathrm{pCi} / \mathrm{L}$ & 0.0051 & 0.0091 & 0.0147 & & U \\
\hline 4170 & ] UISO_IE_PLATE_AEA & QTESRL & 0.162 & $\mathrm{pCi} / \mathrm{L}$ & 0.032 & 0.041 & 0.00893 & & \\
\hline 4171 & UISO_IE_PLATE_AEA & QTESRL & 0.24 & pCi/L & 0.04 & 0.057 & 0.0161 & & \\
\hline 4172 & UISO_IE_PLATE_AEA & QTESRL & 0.00567 & $\mathrm{pCi} / \mathrm{L}$ & 0.0066 & 0.011 & 0.0181 & & $\mathrm{U}$ \\
\hline 4173 & UISO_IE_PLATE_AEA & QTESRL & 0.213 & $\mathrm{pCi} / \mathrm{L}$ & 0.041 & 0.054 & 0.0384 & & \\
\hline 4174 & SRISO_SEP_PRECIP_GPC & QTESRL & 0.0784 & pCi/L & 0.023 & 0.034 & 0.0351 & & \\
\hline 4175 & TRITIUM_ELECT_LSC & QTESRL & 38.9 & $\mathrm{pCi} / \mathrm{L}$ & 0.99 & 6.3 & 4.87 & & \\
\hline 4176 & UISO_IE_PLATE_AEA & QTESRL & 0.303 & pCi/L & 0.084 & 0.1 & 0.0621 & & \\
\hline 4177 & UISO_IE_PLATE_AEA & QTESRL & 0.0327 & pCi/L & 0.03 & 0.034 & 0.0483 & & $\mathrm{U}$ \\
\hline 4178 & UISO_IE_PLATE_AEA & QTESRL & 0.275 & $\mathrm{pCi} / \mathrm{L}$ & 0.087 & 0.1 & 0.0946 & & \\
\hline 4179 & SRISO_SEP_PRECIP_GPC & QTESRL & 0.0818 & $\mathrm{pCi} / \mathrm{L}$ & 0.022 & 0.034 & 0.0341 & & \\
\hline 4180 & TRITIUM_ELECT_LSC & QTESRL & 40.8 & pCi/L & 1 & 6.5 & 5.05 & & \\
\hline 4181 & UISO_IE_PLATE_AEA & QTESRL & 0.192 & $\mathrm{pCi} / \mathrm{L}$ & 0.035 & 0.048 & 0.0162 & & \\
\hline
\end{tabular}




\begin{tabular}{|c|c|c|c|c|c|c|c|c|c|}
\hline & $\mathrm{K}$ & $\mathrm{L}$ & $\mathrm{M}$ & $\mathrm{N}$ & $\mathrm{O}$ & $\mathrm{P}$ & $\mathrm{Q}$ & $\mathrm{R}$ & $\mathrm{S}$ \\
\hline 1 & METHOD_NAME & LAB_CODE & VALUE_RPTD & ANAL_UNITS_RPTD & COUNTING_ERROR & TOTAL_ANAL_ERROR & MIN_DETECTABLE_ACTIVITY & PCNT_MOISTURE & LAB_QUALIFIER \\
\hline 4182 & UISO_IE_PLATE_AEA & QTESRL & 0.00518 & $\mathrm{pCi} / \mathrm{L}$ & 0.006 & 0.01 & 0.0172 & & $U$ \\
\hline 4183 & UISO_IE_PLATE_AEA & QTESRL & 0.134 & $\mathrm{pCi} / \mathrm{L}$ & 0.033 & 0.04 & 0.0345 & & \\
\hline 4184 & SRISO_SEEP_PRËEIP_GPC & QTESRL & 0.0813 & $\mathrm{pCi} / \mathrm{L}$ & 0.024 & 0.036 & 0.0378 & & \\
\hline 4185 & TRITIUM_ELECT_LSC & QTESRL & 36.5 & $\mathrm{pCi} / \mathrm{L}$ & 0.94 & 6.2 & 4.84 & & \\
\hline 4186 & UISO_IE_PLATE_AEA & QTESRL & 0.178 & $\mathrm{pCi} / \mathrm{L}$ & 0.035 & 0.047 & 0.0122 & & \\
\hline 4187 & UISO_IE_PLATE_AEA & QTESRL & 0.00413 & $\mathrm{pCi} / \mathrm{L}$ & 0.0045 & 0.0084 & 0.0122 & & U \\
\hline 4188 & UISO_IE_PLATE_AEA & QTESRL & 0.17 & $\mathrm{pCi} / \mathrm{L}$ & 0.034 & 0.044 & 0.00481 & & \\
\hline 4189 & SRISO_SEP_PRECIP_GPC & QTESRL & 0.0664 & $\mathrm{pCi} / \mathrm{L}$ & 0.021 & 0.032 & 0.0366 & & \\
\hline 4190 & |TRITIUM_ELECT_LSC & QTESRL & 43 & $\mathrm{pCi} / \mathrm{L}$ & 1.1 & 6.6 & 4.83 & & \\
\hline 4191 & UISO_IE_PLATE_AEA & QTESRL & 0.176 & $\mathrm{pCi} / \mathrm{L}$ & 0.033 & 0.045 & 0.0129 & & \\
\hline 4192 & UISO_IE_PLATE_AEA & QTESRL & 0.00758 & $\mathrm{pCi} / \mathrm{L}$ & 0.0061 & 0.0091 & 0.00934 & & U \\
\hline 4193 & UISO_IE_PLATE_AEA & QTESRL & 0.145 & $\mathrm{pCi} / \mathrm{L}$ & 0.031 & 0.039 & 0.0129 & & \\
\hline 4194 & SRISO_SEP_PRECIP_GPC & QTESRL & 0.0852 & $\mathrm{pCi} / \mathrm{L}$ & 0.023 & 0.035 & 0.0331 & & \\
\hline \begin{tabular}{|l|l|}
4195 \\
\end{tabular} & TRITIUM_ELECT_LSC & QTESRL & 42.8 & $\mathrm{pCi} / \mathrm{L}$ & 1.1 & 6.6 & 4.87 & & \\
\hline 4196 & UISO_IE_PLATE_AEA & QTESRL & 0.22 & $\mathrm{pCi} / \mathrm{L}$ & 0.038 & 0.053 & 0.0164 & & \\
\hline 4197 & UISO_IE_PLATE_AEA & QTESRL & 0.00196 & $\mathrm{pCi} / \mathrm{L}$ & 0.0025 & 0.0071 & 0.0113 & & U \\
\hline 4198 & UISO_IE_PLATE_AEA & QTESRL & 0.129 & $\mathrm{pCi} / \mathrm{L}$ & 0.029 & 0.036 & 0.0113 & & \\
\hline 4201 & UISO_IE_PLATE_AEA & QTESRL & 0.219 & $\mathrm{pCi} / \mathrm{L}$ & 0.037 & 0.052 & 0.0126 & & \\
\hline 4202 & UISO_IE_PLATE_AEA & QTESRL & 0.00992 & $\mathrm{pCi} / \mathrm{L}$ & 0.0074 & 0.01 & 0.0111 & & U \\
\hline 4203 & UISO_IE_PLATE_AEA & QTESRL & 0.155 & $\mathrm{pCi} / \mathrm{L}$ & 0.032 & 0.041 & 0.0194 & & \\
\hline 4204 & SRISO_SEP_PRECIP_GPC & QTESRL & 0.51 & $\mathrm{pCi} / \mathrm{L}$ & 0.048 & 0.13 & 0.0337 & & \\
\hline 4205 & TRITIUM_ELECTI_LSC & QTESRL & 50.2 & $\mathrm{pCi} / \mathrm{L}$ & 1.2 & 7.1 & 4.89 & & \\
\hline 4206 & UISO_IE_PLATE_AEA & QTESRL & 0.192 & $\mathrm{pCi} / \mathrm{L}$ & 0.041 & 0.053 & 0.0333 & & \\
\hline 4207 & UISO_IE_PLATE_AEA & QTESRL & 0.00531 & $\mathrm{pCi} / \mathrm{L}$ & 0.0072 & 0.012 & 0.0216 & & U \\
\hline 4208 & UISO_IE_PLATE_AEA & QTESRL & 0.17 & $\mathrm{pCi} / \mathrm{L}$ & 0.039 & 0.048 & 0.0282 & & \\
\hline 4209 & SRISO_SEEP_PRËCIP_GPC & QTESRL & 1.27 & $\mathrm{pCi} / \mathrm{L}$ & 0.077 & 0.3 & 0.0337 & & \\
\hline 4210 & TRITIUM_ELECT_LSC & QTESRL & 47.2 & $\mathrm{pCi} / \mathrm{L}$ & 1.2 & 6.9 & 4.87 & & \\
\hline 4211 & UISO_IE_PLATE_AEA & QTESRL & 0.171 & $\mathrm{pCi} / \mathrm{L}$ & 0.035 & 0.047 & 0.0131 & & \\
\hline 4212 & UISO_IE_PLATE_AEA & QTESRL & 0.00586 & $\mathrm{pCi} / \mathrm{L}$ & 0.0062 & 0.01 & 0.0149 & & U \\
\hline 4213 & UISO_IE_PLATE_AEA & QTESRL & 0.144 & $\mathrm{pCi} / \mathrm{L}$ & 0.033 & 0.041 & 0.0164 & & \\
\hline 4214 & SRISO_SEP_PRECIP_GPC & QTESRL & 0.0613 & $\mathrm{pCi} / \mathrm{L}$ & 0.021 & 0.031 & 0.0366 & & \\
\hline 4215 & TRITIUM_ELECT_LSC & QTESRL & 112 & $\mathrm{pCi} / \mathrm{L}$ & 2.2 & 12 & 4.94 & & \\
\hline 4216 & UISO_IE_PLATE_AEA & QTESRL & 0.184 & $\mathrm{pCi} / \mathrm{L}$ & 0.036 & 0.048 & 0.0192 & & \\
\hline 4217 & UISO_IE_PLATE_AEA & QTESRL & 0.00878 & $\mathrm{pCi} / \mathrm{L}$ & 0.0071 & 0.01 & 0.0104 & & U \\
\hline 4218 & UISO_IE_PLATE_AEA & QTESRL & 0.164 & $\mathrm{pCi} / \mathrm{L}$ & 0.034 & 0.044 & 0.0126 & & \\
\hline
\end{tabular}




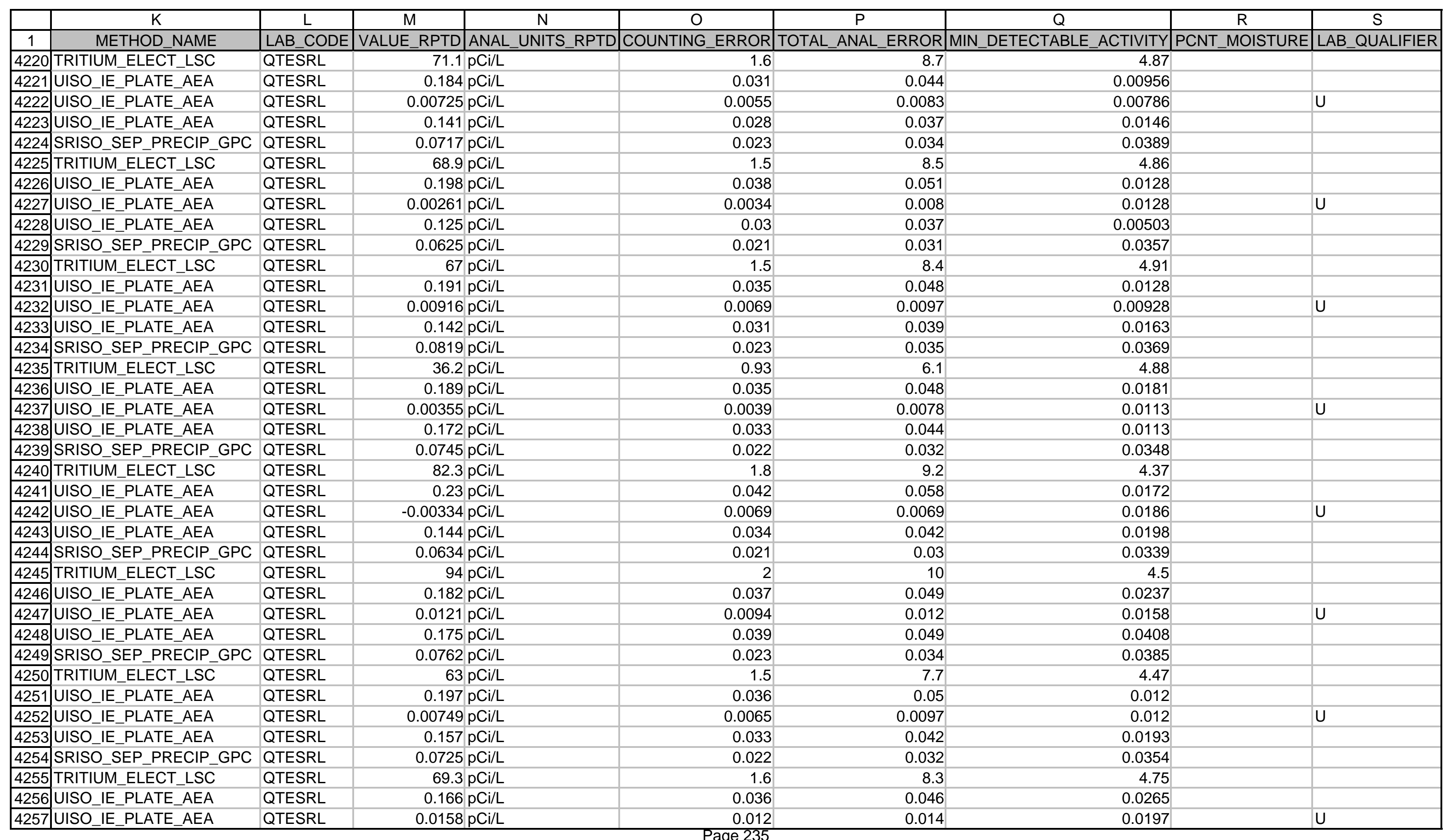




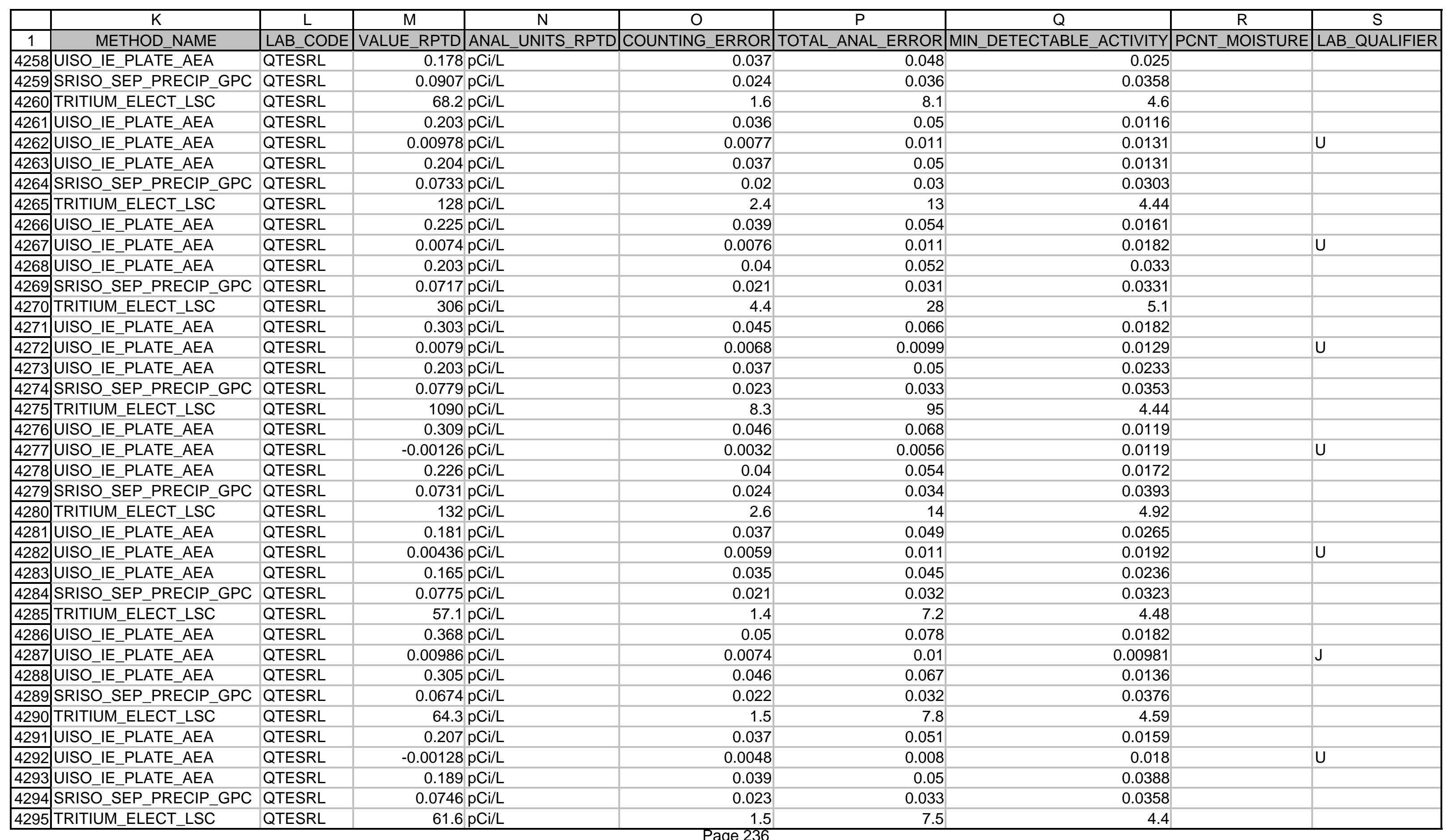




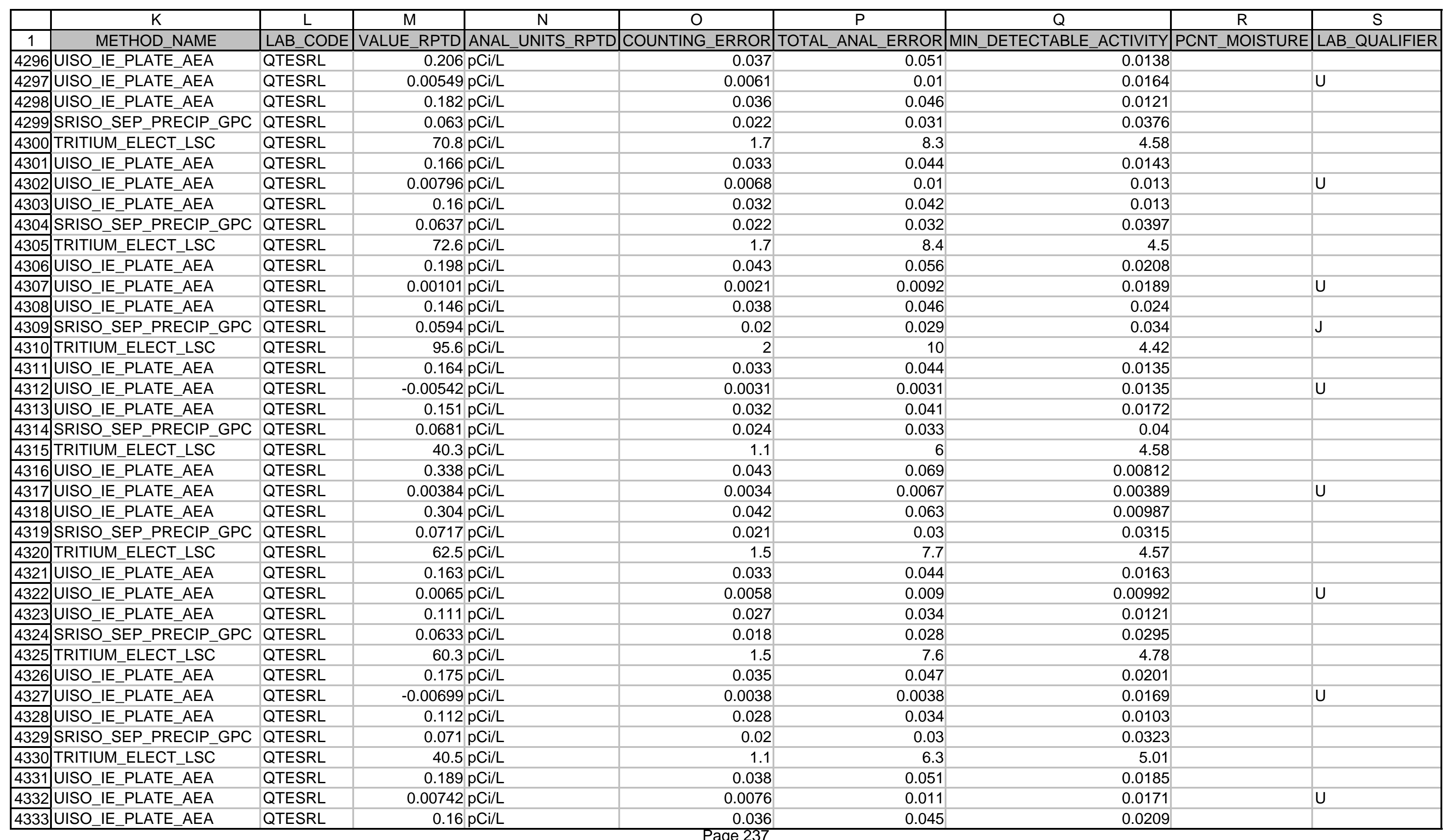




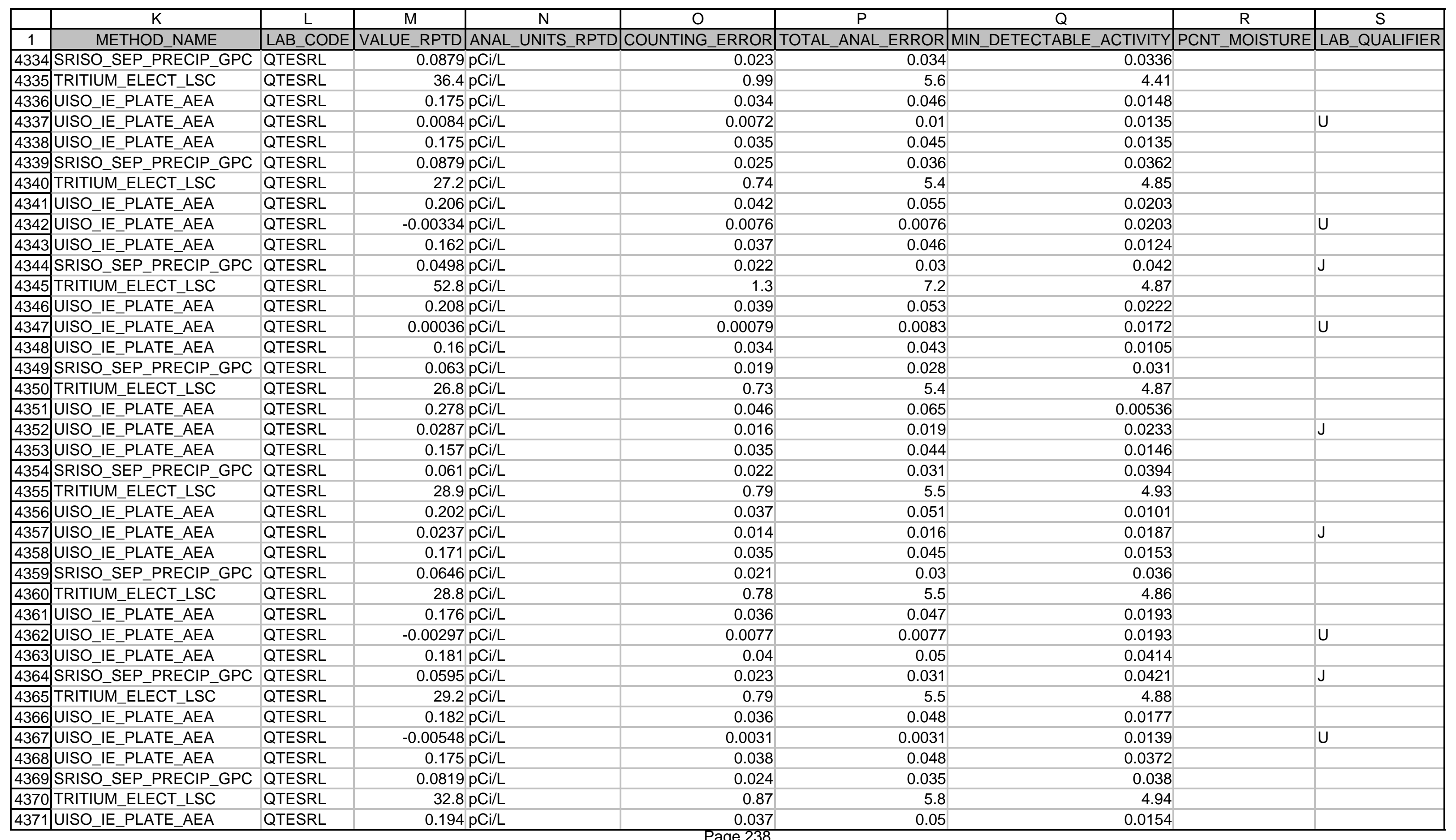




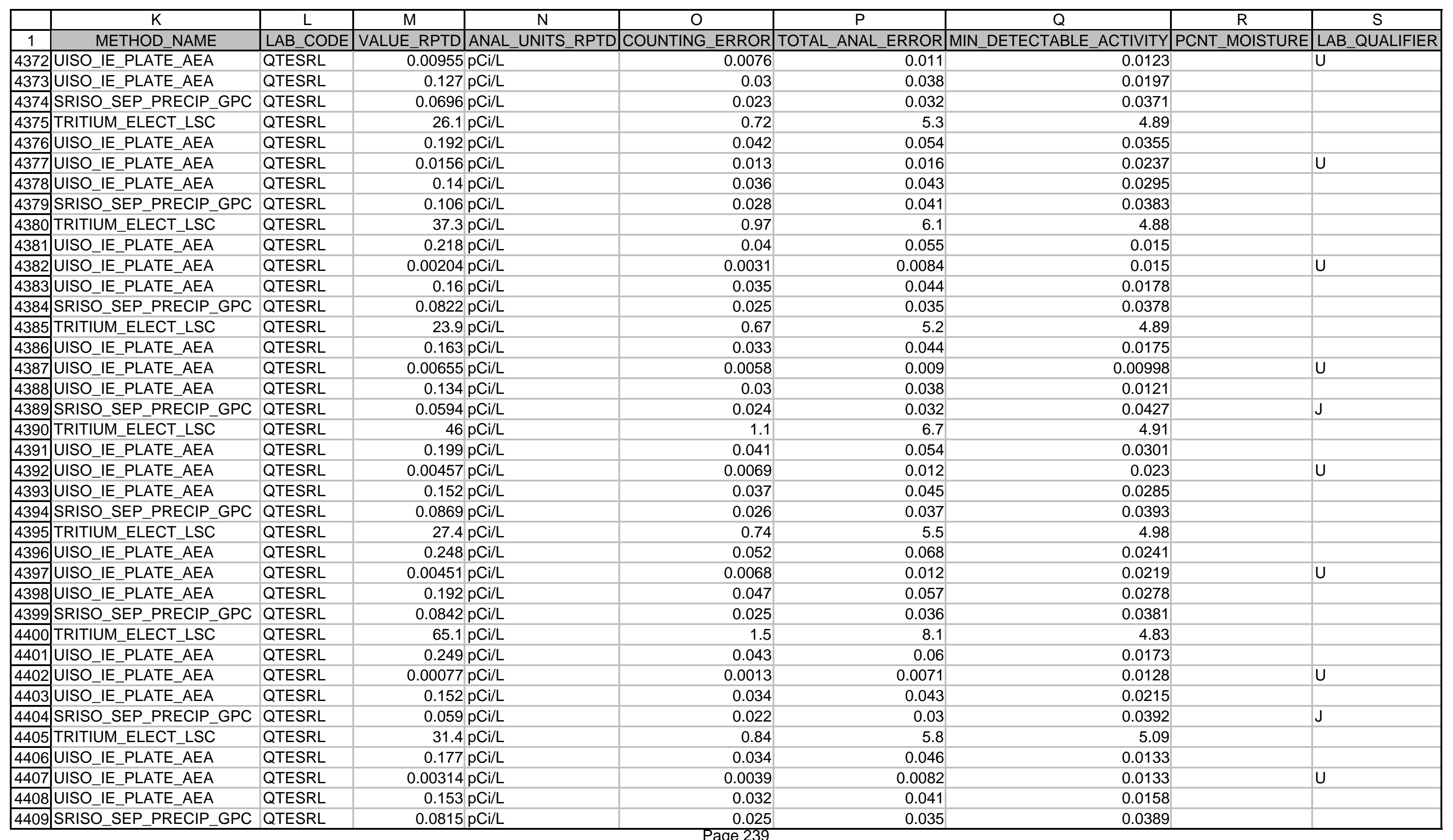




\begin{tabular}{|c|c|c|c|c|c|c|c|c|c|}
\hline & $\mathrm{K}$ & $\mathrm{L}$ & $\mathrm{M}$ & $\mathrm{N}$ & $\mathrm{O}$ & $\mathrm{P}$ & $\mathrm{Q}$ & $\mathrm{R}$ & $\mathrm{S}$ \\
\hline 1 & METHOD_NAME & LAB_CODE & VALUE_RPTD & ANAL_UNITS_RPTD & COUNTING_ERROR & TOTAL_ANAL_ERROR & MIN_DETECTABLE_ACTIVITY & PCNT_MOISTURE & LAB_QUALIFIER \\
\hline 4410 & TRITIUM ELECT LSC & QTESRL & 24.2 & $\mathrm{pCi} / \mathrm{L}$ & 0.67 & 5.3 & 5.04 & & \\
\hline 4411 & UISO_IE_PLATE_AEA & QTESRL & 0.194 & $\mathrm{pCi} / \mathrm{L}$ & 0.037 & 0.05 & 0.0179 & & \\
\hline 4412 & UISO_IE_PLATE_AEA & QTESRL & 0.00135 & $\mathrm{pCi} / \mathrm{L}$ & 0.0018 & 0.0067 & 0.0102 & & $U$ \\
\hline 4413 & UISO_IE_PLATE_AEA & QTESRL & 0.152 & $\mathrm{pCi} / \mathrm{L}$ & 0.033 & 0.042 & 0.0124 & & \\
\hline 4414 & SRISŌ_SEP_PRĒCIP_GPC & QTESRL & 0.0952 & $\mathrm{pCi} / \mathrm{L}$ & 0.027 & 0.039 & 0.0414 & & \\
\hline 4415 & TRITIUM_ELECT_LSC & QTESRL & 25.5 & $\mathrm{pCi} / \mathrm{L}$ & 0.7 & 5.4 & 5.06 & & \\
\hline 4416 & UISO_IE_PLATE_AEA & QTESRL & 0.174 & $\mathrm{pCi} / \mathrm{L}$ & 0.037 & 0.048 & 0.0195 & & \\
\hline 4417 & UISO_IE_PLATE_AEA & QTESRL & -0.00137 & $\mathrm{pCi} / \mathrm{L}$ & 0.0053 & 0.0079 & 0.0183 & & $U$ \\
\hline 4418 & UISO_IE_PLATE_AEA & QTESRL & 0.126 & $\mathrm{pCi} / \mathrm{L}$ & 0.031 & 0.038 & 0.0111 & & \\
\hline 4419 & SRISO-SE_SEP_PRĒCIP_GPC & QTESRL & 0.057 & $\mathrm{pCi} / \mathrm{L}$ & 0.024 & 0.033 & 0.0442 & & $\mathrm{~J}$ \\
\hline 4420 & TRITIUM_ELECT_LSC & QTESRL & 27.6 & $\mathrm{pCi} / \mathrm{L}$ & 0.76 & 6 & 5.64 & & \\
\hline 4421 & UISO_IE_PLATE_AEA & QTESRL & 0.232 & $\mathrm{pCi} / \mathrm{L}$ & 0.052 & 0.066 & 0.0253 & & \\
\hline 4422 & UISO_IE_PLATE_AEA & QTESRL & 0.00196 & $\mathrm{pCi} / \mathrm{L}$ & 0.0041 & 0.011 & 0.023 & & $U$ \\
\hline 4423 & UISO_IE_PLATE_AEA & QTESRL & 0.153 & $\mathrm{pCi} / \mathrm{L}$ & 0.042 & 0.05 & 0.023 & & \\
\hline 4424 & SRISO_SEP_PRECIP_GPC & QTESRL & 0.0924 & $\mathrm{pCi} / \mathrm{L}$ & 0.026 & 0.038 & 0.0406 & & \\
\hline 4425 & TRITIUMM_ELECTI_LSC & QTESRL & 30.4 & $\mathrm{pCi} / \mathrm{L}$ & 0.81 & 5.6 & 4.91 & & \\
\hline 4426 & UISO_IE_PLATE_AEA & QTESRL & 0.162 & $\mathrm{pCi} / \mathrm{L}$ & 0.029 & 0.04 & 0.00364 & & \\
\hline 4427 & UISO_IE_PLATE_AEA & QTESRL & 0.00418 & $\mathrm{pCi} / \mathrm{L}$ & 0.0037 & 0.007 & 0.00759 & & U \\
\hline 4429 & SRISO_SEP_PRECIP_GPC & QTESRL & 0.0568 & $\mathrm{pCi} / \mathrm{L}$ & 0.024 & 0.035 & 0.0475 & & J \\
\hline 4430 & TRITIUMM_ELEECT_LSC & QTESRL & 51.4 & $\mathrm{pCi} / \mathrm{L}$ & 1.2 & 7.2 & 4.93 & & \\
\hline 4431 & UISO_IE_PLATE_AEA & QTESRL & 0.201 & $\mathrm{pCi} / \mathrm{L}$ & 0.038 & 0.052 & 0.0205 & & \\
\hline 4432 & UISO_IE_PLATE_AEA & QTESRL & 0.000744 & $\mathrm{pCi} / \mathrm{L}$ & 0.0017 & 0.0094 & 0.0195 & & $U$ \\
\hline 4433 & UISO_IE_PLATE_AEA & QTESRL & 0.105 & $\mathrm{pCi} / \mathrm{L}$ & 0.031 & 0.037 & 0.0369 & & \\
\hline 4434 & SRISO-SE_SEP_PRĒCIP_GPC & QTESRL & 0.0583 & $\mathrm{pCi} / \mathrm{L}$ & 0.03 & 0.039 & 0.0588 & & $U$ \\
\hline 4435 & TRITIŪM_ELECTT_LSC̄ & QTESRL & 33.2 & $\mathrm{pCi} / \mathrm{L}$ & 0.87 & 5.8 & 4.92 & & \\
\hline 4436 & UISO_IE_PLATE_AEA & QTESRL & 0.169 & $\mathrm{pCi} / \mathrm{L}$ & 0.034 & 0.045 & 0.0179 & & \\
\hline 4437 & UISO_IE_PLATE_AEA & QTESRL & 0.00486 & $\mathrm{pCi} / \mathrm{L}$ & 0.0052 & 0.0089 & 0.0133 & & U \\
\hline 4438 & UISO_IE_PLATE_AEA & QTESRL & 0.164 & $\mathrm{pCi} / \mathrm{L}$ & 0.034 & 0.044 & 0.0241 & & \\
\hline 4439 & SRISŌ_SEP_PRĒCIP_GPC & QTESRL & 0.0678 & $\mathrm{pCi} / \mathrm{L}$ & 0.022 & 0.032 & 0.0375 & & \\
\hline 4440 & TRITIŪM_ELEECT_LSC & QTESRL & 84.6 & $\mathrm{pCi} / \mathrm{L}$ & 1.8 & 9.8 & 5.11 & & \\
\hline 4441 & UISO_IE_PLATE_AEA & QTESRL & 0.196 & $\mathrm{pCi} / \mathrm{L}$ & 0.041 & 0.053 & 0.0287 & & \\
\hline 4442 & UISO_IE_PLATE_AEA & QTESRL & 0.00764 & $\mathrm{pCi} / \mathrm{L}$ & 0.0078 & 0.011 & 0.0175 & & $U$ \\
\hline 4443 & UISO_IE_PLATE_AEA & QTESRL & 0.172 & $\mathrm{pCi} / \mathrm{L}$ & 0.038 & 0.048 & 0.0234 & & \\
\hline 4444 & SRISO-SE_SEP_PRĒCIP_GPC & QTESRL & 0.0759 & $\mathrm{pCi} / \mathrm{L}$ & 0.02 & 0.031 & 0.031 & & \\
\hline 4445 & TRITIUM_ELECT_LSC & QTESRL & 84.3 & $\mathrm{pCi} / \mathrm{L}$ & 1.8 & 10 & 5.44 & & \\
\hline 4446 & UISO_IE_PLATE_AEA & QTESRL & 0.238 & $\mathrm{pCi} / \mathrm{L}$ & 0.043 & 0.059 & 0.0243 & & \\
\hline
\end{tabular}




\begin{tabular}{|c|c|c|c|c|c|c|c|c|c|}
\hline & $\mathrm{K}$ & $\mathrm{L}$ & $\mathrm{M}$ & $\mathrm{N}$ & $\mathrm{O}$ & $\mathrm{P}$ & $\mathrm{Q}$ & $\mathrm{R}$ & $\mathrm{S}$ \\
\hline 1 & METHOD_NAME & LAB_CODE & VALUE_RPTD & ANAL_UNITS_RPTD & COUNTING_ERROR & TOTAL_ANAL_ERROR & MIN_DETECTABLE_ACTIVITY & PCNT_MOISTURE & LAB_QUALIFIER \\
\hline 4448 & UISO_IE_PLATE_AEA & QTESRL & 0.113 & $\mathrm{pCi} / \mathrm{L}$ & 0.03 & 0.036 & 0.0194 & & \\
\hline 4449 & SRISO SEP PRECCIP GPC & QTESRL & 0.0501 & $\mathrm{pCi} / \mathrm{L}$ & 0.018 & 0.027 & 0.0331 & & $\mathrm{~J}$ \\
\hline 4450 & TRITIUMM_ELECTT_LSC & QTESRL & 82.6 & $\mathrm{pCi} / \mathrm{L}$ & 1.7 & 9.7 & 5.09 & & \\
\hline 4451 & UISO_IE_PLATE_AEA & QTESRL & 0.228 & $\mathrm{pCi} / \mathrm{L}$ & 0.042 & 0.057 & 0.0206 & & \\
\hline 4452 & UISO_IE_PLATE_AEA & QTESRL & 0.00411 & $\mathrm{pCi} / \mathrm{L}$ & 0.0051 & 0.0094 & 0.0153 & & $\mathrm{U}$ \\
\hline 4453 & UISO_IE_PLATE_AEA & QTESRL & 0.157 & $\mathrm{pCi} / \mathrm{L}$ & 0.037 & 0.046 & 0.0311 & & \\
\hline 4454 & SRISO_SEP_PRECIP_GPC & QTESRL & 0.0633 & $\mathrm{pCi} / \mathrm{L}$ & 0.021 & 0.03 & 0.0358 & & \\
\hline 4455 & TRITIUM_ELECT_LSC & QTESRL & 81.4 & $\mathrm{pCi} / \mathrm{L}$ & 1.7 & 9.6 & 5.1 & & \\
\hline 4456 & UISO_IE_PLATE_AEA & QTESRL & 0.199 & $\mathrm{pCi} / \mathrm{L}$ & 0.038 & 0.051 & 0.0192 & & \\
\hline 4457 & UISO_IE_PLATE_AEA & QTESRL & 0.00398 & $\mathrm{pCi} / \mathrm{L}$ & 0.0052 & 0.0098 & 0.017 & & U \\
\hline 4458 & UISO_IE_PLATE_AEA & QTESRL & 0.153 & $\mathrm{pCi} / \mathrm{L}$ & 0.035 & 0.043 & 0.0278 & & \\
\hline 4459 & SRISO_SEP_PRECIP_GPC & QTESRL & 0.0696 & $\mathrm{pCi} / \mathrm{L}$ & 0.019 & 0.029 & 0.0288 & & \\
\hline 4460 & SRISO_SEP_PRECIP_GPC & QTESRL & 0.0821 & $\mathrm{pCi} / \mathrm{L}$ & 0.024 & 0.035 & 0.0379 & & \\
\hline 4461 & TRITIUM ELECT LSC & QTESRL & 107 & $\mathrm{pCi} / \mathrm{L}$ & 2.1 & 12 & 5.08 & & \\
\hline 44462 & TRITIUM ELECT LSC & QTESRL & 109 & $\mathrm{pCi} / \mathrm{L}$ & 2.1 & 12 & 5.13 & & \\
\hline 4463 & UISO_IE_PLATE_AEA & QTESRL & 0.15 & $\mathrm{pCi} / \mathrm{L}$ & 0.034 & 0.043 & 0.0183 & & \\
\hline 4464 & UISO_IE_PLATE_AEA & QTESRL & 0.00572 & $\mathrm{pCi} / \mathrm{L}$ & 0.0057 & 0.0092 & 0.0111 & & U \\
\hline 4465 & UISO_IE_PLATE_AEA & QTESRL & 0.192 & $\mathrm{pCi} / \mathrm{L}$ & 0.039 & 0.05 & 0.0169 & & \\
\hline 4467 & UISO_IE_PLATE_AEA & QTESRL & 0.00543 & $\mathrm{pCi} / \mathrm{L}$ & 0.0069 & 0.011 & 0.0202 & & $U$ \\
\hline 4468 & UISO_IE_PLATE_AEA & QTESRL & 0.173 & $\mathrm{pCi} / \mathrm{L}$ & 0.039 & 0.048 & 0.0381 & & \\
\hline 4469 & SRISO_SEP_PRECIP_GPC & QTESRL & 0.0695 & $\mathrm{pCi} / \mathrm{L}$ & 0.022 & 0.033 & 0.0378 & & \\
\hline 4470 & |TRITIUM_ELECT_LSC & QTESRL & 82.5 & $\mathrm{pCi} / \mathrm{L}$ & 1.8 & 9.7 & 5.17 & & \\
\hline 4471 & UISO_IE_PLATE_AEA & QTESRL & 0.303 & $\mathrm{pCi} / \mathrm{L}$ & 0.051 & 0.071 & 0.0124 & & \\
\hline 4472 & UISO_IE_PLATE_AEA & QTESRL & 0.0116 & $\mathrm{pCi} / \mathrm{L}$ & 0.0099 & 0.013 & 0.0171 & & $U$ \\
\hline 4473 & UISO_IE_PLATE_AEA & QTESRL & 0.234 & $\mathrm{pCi} / \mathrm{L}$ & 0.045 & 0.059 & 0.0151 & & \\
\hline 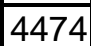 & SRISO SEP PRECCIP GPC & QTESRL & 0.0832 & $\mathrm{pCi} / \mathrm{L}$ & 0.022 & 0.033 & 0.0313 & & \\
\hline 4475 & TRITIUMM_ELEECT_LSC & QTESRL & 125 & $\mathrm{pCi} / \mathrm{L}$ & 2.3 & 13 & 5.12 & & \\
\hline 4476 & UISO_IE_PLATE_AEA & QTESRL & 0.213 & $\mathrm{pCi} / \mathrm{L}$ & 0.041 & 0.055 & 0.0256 & & \\
\hline 4477 & UISO_IE_PLATE_AEA & QTESRL & 0.00166 & $\mathrm{pCi} / \mathrm{L}$ & 0.0023 & 0.0071 & 0.0109 & & $U$ \\
\hline 4478 & UISO_IE_PLATE_AEA & QTESRL & 0.192 & $\mathrm{pCi} / \mathrm{L}$ & 0.039 & 0.05 & 0.0191 & & \\
\hline 4479 & SRISO_SEP_PRECIP_GPC & QTESRL & 0.0659 & $\mathrm{pCi} / \mathrm{L}$ & 0.019 & 0.029 & 0.0313 & & \\
\hline 4480 & TRITIUM_ELECT_LSC & QTESRL & 99.7 & $\mathrm{pCi} / \mathrm{L}$ & 2 & 11 & 5.12 & & \\
\hline 4481 & UISO_IE_PLATE_AEA & QTESRL & 0.202 & $\mathrm{pCi} / \mathrm{L}$ & 0.038 & 0.052 & 0.0174 & & \\
\hline 4482 & UISO_IE_PLATE_AEA & QTESRL & 0.00414 & $\mathrm{pCi} / \mathrm{L}$ & 0.0054 & 0.01 & 0.0174 & & U \\
\hline 4483 & UISO_IE_PLATE_AEA & QTESRL & 0.178 & $\mathrm{pCi} / \mathrm{L}$ & 0.036 & 0.047 & 0.0146 & & \\
\hline 4484 & SRISO_SEP_PRECIP_GPC & QTESRL & 0.0822 & $\mathrm{pCi} / \mathrm{L}$ & 0.022 & 0.032 & 0.0317 & & \\
\hline
\end{tabular}




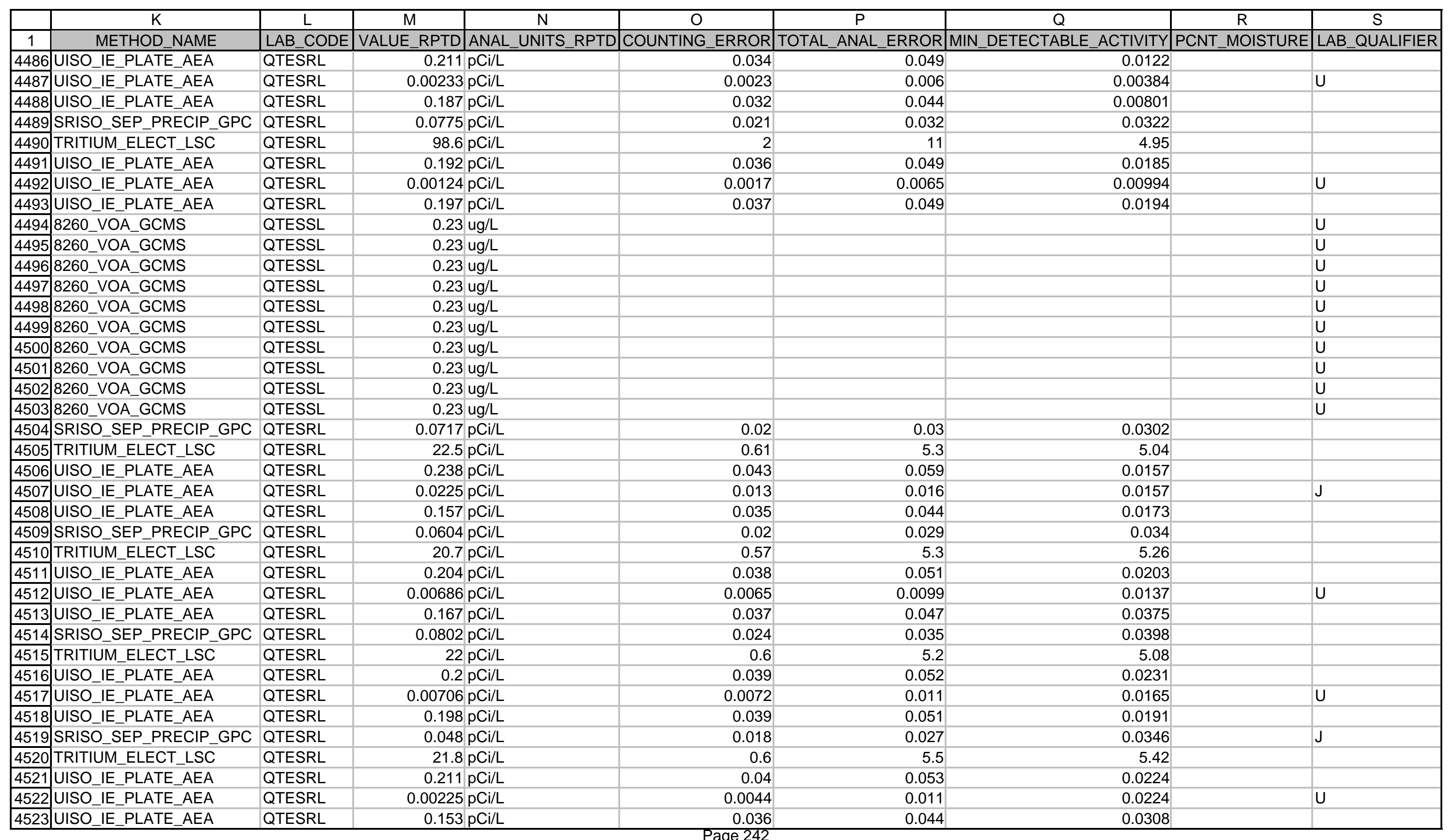




\begin{tabular}{|c|c|c|c|c|c|c|c|c|c|}
\hline & $\mathrm{K}$ & $\mathrm{L}$ & $\mathrm{M}$ & $\mathrm{N}$ & $\mathrm{O}$ & $\mathrm{P}$ & $\mathrm{Q}$ & $\mathrm{R}$ & $\mathrm{S}$ \\
\hline 1 & METHOD_NAME & LAB_CODE & VALUE_RPTD & ANAL_UNITS_RPTD & COUNTING_ERROR & TOTAL_ANAL_ERROR & MIN_DETECTABLE_ACTIVITY & PCNT_MOISTURE & LAB_QUALIFIER \\
\hline 4524 & 8260_VOA_GCMS & QTESSL & 0.23 & $u g / L$ & & & & & $U$ \\
\hline 4525 & 8260_VOA_GCMS & QTESSL & 0.23 & ug/L & & & & & $\mathrm{U}$ \\
\hline 4526 & $8260^{-}$VOA GCMS & QTESSL & 0.23 & $\mathrm{ug} / \mathrm{L}$ & & & & & $\mathrm{U}$ \\
\hline 4527 & $8260^{\circ}$ VOA GCMS & QTESSL & 0.23 & $\mathrm{ug} / \mathrm{L}$ & & & & & $\mathrm{U}$ \\
\hline 4528 & SRISO_SEPP_PRECIP_GPC & QTESRL & 0.0783 & $\mathrm{pCi} / \mathrm{L}$ & 0.024 & 0.034 & 0.0374 & & \\
\hline 4529 & TRITIUMM_ELECTT_LSC̄ & QTESRL & 29.8 & $\mathrm{pCi} / \mathrm{L}$ & 0.86 & 5.2 & 4.57 & & \\
\hline 4530 & UISO_IE_PLATE_AEA & QTESRL & 0.224 & $\mathrm{pCi} / \mathrm{L}$ & 0.044 & 0.058 & 0.0281 & & \\
\hline 4531 & UISO_IE_PLATE_AEA & QTESRL & 0.0148 & $\mathrm{pCi} / \mathrm{L}$ & 0.012 & 0.015 & 0.0209 & & $U$ \\
\hline 4533 & SRISO_SEP_PRECIP_GPC & QTESRL & 0.0709 & $\mathrm{pCi} / \mathrm{L}$ & 0.024 & 0.033 & 0.0388 & & \\
\hline 4534 & TRITIUM_ELECT_LSC & QTESRL & 28.6 & $\mathrm{pCi} / \mathrm{L}$ & 0.83 & 5 & 4.32 & & \\
\hline 4535 & UISO_IE_PLATE_AEA & QTESRL & 0.223 & $\mathrm{pCi} / \mathrm{L}$ & 0.04 & 0.055 & 0.0159 & & \\
\hline 4536 & UISO_IE_PLATE_AEA & QTESRL & 0.00962 & $\mathrm{pCi} / \mathrm{L}$ & 0.0073 & 0.01 & 0.00502 & & $\mathrm{~J}$ \\
\hline 4537 & UISO_IE_PLATE_AEA & QTESRL & 0.2 & $\mathrm{pCi} / \mathrm{L}$ & 0.038 & 0.051 & 0.0172 & & \\
\hline 4538 & SRISŌ_ĒSEP_PRËCIP_GPC & QTESRL & 0.0696 & $\mathrm{pCi} / \mathrm{L}$ & 0.022 & 0.032 & 0.0364 & & \\
\hline 4539 & TRITIUM_ELECCT_LSC & QTESRL & 29.8 & $\mathrm{pCi} / \mathrm{L}$ & 0.86 & 5 & 4.33 & & \\
\hline 4540 & UISO IE PLATE AEA & QTESRL & 0.257 & $\mathrm{pCi} / \mathrm{L}$ & 0.043 & 0.06 & 0.0187 & & \\
\hline 4541 & UISO_IE_PLATE_AEA & QTESRL & 0.012 & $\mathrm{pCi} / \mathrm{L}$ & 0.0085 & 0.011 & 0.0101 & & $\mathrm{~J}$ \\
\hline 4543 & SRISO-SE_SEP_PRĒCIP_GPC & QTESRL & 0.0852 & $\mathrm{pCi} / \mathrm{L}$ & 0.023 & 0.034 & 0.0349 & & \\
\hline 4544 & TRITIUMM_ELECCT_LSC & QTESRL & 26.6 & $\mathrm{pCi} / \mathrm{L}$ & 0.78 & 4.8 & 4.35 & & \\
\hline 4545 & UISO_IE_PLATE_AEA & QTESRL & 0.278 & $\mathrm{pCi} / \mathrm{L}$ & 0.046 & 0.064 & 0.0216 & & \\
\hline 4546 & UISO_IE_PLATE_AEA & QTESRL & 0.0281 & $\mathrm{pCi} / \mathrm{L}$ & 0.014 & 0.017 & 0.0146 & & $\mathrm{~J}$ \\
\hline 4547 & UISO_IE_PLATE_AEA & QTESRL & 0.203 & $\mathrm{pCi} / \mathrm{L}$ & 0.039 & 0.052 & 0.0216 & & \\
\hline 4548 & SRISO_SEP_PRECIP_GPC & QTESRL & 0.0873 & $\mathrm{pCi} / \mathrm{L}$ & 0.028 & 0.038 & 0.0425 & & \\
\hline 4549 & TRITIUM_ELECT_LSC & QTESRL & 70.7 & $\mathrm{pCi} / \mathrm{L}$ & 1.7 & 8.3 & 4.56 & & \\
\hline 4550 & UISO_IE_PLATE_AEA & QTESRL & 0.271 & $\mathrm{pCi} / \mathrm{L}$ & 0.037 & 0.057 & 0.0103 & & \\
\hline 4551 & UISO_IE_PLATE_AEA & QTESRL & 0.0114 & $\mathrm{pCi} / \mathrm{L}$ & 0.0072 & 0.0097 & 0.00906 & & $\mathrm{~J}$ \\
\hline 4552 & UISO_IE_PLATE_AEA & QTESRL & 0.189 & $\mathrm{pCi} / \mathrm{L}$ & 0.031 & 0.044 & 0.00745 & & \\
\hline 4553 & SRISO-SE_SEP_PRËCIP_GPC & QTESRL & 0.0805 & $\mathrm{pCi} / \mathrm{L}$ & 0.028 & 0.038 & 0.0458 & & \\
\hline 4554 & TRITIUMM_ELECCT_LSC̄ & QTESRL & 82 & $\mathrm{pCi} / \mathrm{L}$ & 1.9 & 9.2 & 4.58 & & \\
\hline 4555 & UISO_IE_PLATE_AEA & QTESRL & 0.245 & $\mathrm{pCi} / \mathrm{L}$ & 0.04 & 0.056 & 0.0128 & & \\
\hline 4556 & UISO_IE_PLATE_AEA & QTESRL & 0.00486 & $\mathrm{pCi} / \mathrm{L}$ & 0.0054 & 0.0094 & 0.0152 & & $U$ \\
\hline 4557 & UISO_IE_PLATE_AEA & QTESRL & 0.21 & $\mathrm{pCi} / \mathrm{L}$ & 0.037 & 0.05 & 0.0141 & & \\
\hline 4558 & SRISO_SEP_PRECCIP_GPC & QTESRL & 0.0817 & $\mathrm{pCi} / \mathrm{L}$ & 0.028 & 0.038 & 0.0448 & & \\
\hline 4559 & TRITIUMM_ELECCT_LSC & QTESRL & 69.9 & $\mathrm{pCi} / \mathrm{L}$ & 1.7 & 8.2 & 4.5 & & \\
\hline 4560 & UISO_IE_PLATE_AEA & QTESRL & 0.227 & $\mathrm{pCi} / \mathrm{L}$ & 0.039 & 0.054 & 0.00976 & & \\
\hline
\end{tabular}




\begin{tabular}{|c|c|c|c|c|c|c|c|c|c|}
\hline & $\mathrm{K}$ & $\mathrm{L}$ & $\mathrm{M}$ & $\mathrm{N}$ & $\mathrm{O}$ & $\mathrm{P}$ & $\mathrm{Q}$ & $\mathrm{R}$ & $\mathrm{S}$ \\
\hline 1 & METHOD_NAME & LAB_CODE & VALUE_RPTD & ANAL_UNITS_RPTD & COUNTING_ERROR & TOTAL_ANAL_ERROR & MIN_DETECTABLE_ACTIVITY & PCNT_MOISTURE & LAB_QUALIFIER \\
\hline 4562 & UISO_IE_PLATE_AEA & QTESRL & 0.228 & $\mathrm{pCi} / \mathrm{L}$ & 0.039 & 0.054 & 0.0119 & & \\
\hline \begin{tabular}{|l|}
4563 \\
\end{tabular} & SRISO SEP PRECCIP GPC & QTESRL & 0.0952 & $\mathrm{pCi} / \mathrm{L}$ & 0.031 & 0.043 & 0.0494 & & \\
\hline 4564 & TRITIUMM_ELECTT_LSC & QTESRL & 68.9 & $\mathrm{pCi} / \mathrm{L}$ & 1.6 & 8.2 & 4.54 & & \\
\hline 4565 & UISO_IE_PLATE_AEA & QTESRL & 0.228 & $\mathrm{pCi} / \mathrm{L}$ & 0.04 & 0.055 & 0.0167 & & \\
\hline 4566 & UISO_IE_PLATE_AEA & QTESRL & 0.0035 & $\mathrm{pCi} / \mathrm{L}$ & 0.0043 & 0.0087 & 0.014 & & $U$ \\
\hline 4567 & UISO_IE_PLATE_AEA & QTESRL & 0.184 & $\mathrm{pCi} / \mathrm{L}$ & 0.036 & 0.047 & 0.0167 & & \\
\hline 4568 & SRISO-SEPP_PRECCIP_GPC & QTESRL & 0.0942 & $\mathrm{pCi} / \mathrm{L}$ & 0.029 & 0.041 & 0.0468 & & \\
\hline 4569 & SRISO_SEP_PRECIP_GPC & QTESRL & 0.0943 & $\mathrm{pCi} / \mathrm{L}$ & 0.033 & 0.044 & 0.0515 & & \\
\hline 4570 & |TRITIUM_ELECT_LSC & QTESRL & 84.8 & $\mathrm{pCi} / \mathrm{L}$ & 1.9 & 9.4 & 4.43 & & \\
\hline 4571 & TRITIUM_ELECT_LSC & QTESRL & 86.8 & $\mathrm{pCi} / \mathrm{L}$ & 1.9 & 9.6 & 4.51 & & \\
\hline 4572 & UISO_IE_PLATE_AEA & QTESRL & 0.255 & $\mathrm{pCi} / \mathrm{L}$ & 0.041 & 0.058 & 0.0172 & & \\
\hline 4573 & UISO_IE_PLATE_AEA & QTESRL & 0.00421 & $\mathrm{pCi} / \mathrm{L}$ & 0.0042 & 0.0077 & 0.00927 & & $U$ \\
\hline 4574 & UISO_IE_PLATE_AEA & QTESRL & 0.222 & $\mathrm{pCi} / \mathrm{L}$ & 0.041 & 0.055 & 0.0389 & & \\
\hline 4575 & UISO_IE_PLATE_AEA & QTESRL & 0.301 & $\mathrm{pCi} / \mathrm{L}$ & 0.045 & 0.066 & 0.0169 & & \\
\hline 4576 & UISO_IE_PLATE_AEA & QTESRL & 0.0124 & $\mathrm{pCi} / \mathrm{L}$ & 0.0087 & 0.011 & 0.0117 & & $\mathrm{~J}$ \\
\hline 4577 & UISO_IE_PLATE_AEA & QTESRL & 0.212 & $\mathrm{pCi} / \mathrm{L}$ & 0.038 & 0.051 & 0.0159 & & \\
\hline 4578 & SRISO-SEPP_PRECCIP_GPC & QTESRL & 0.0637 & $\mathrm{pCi} / \mathrm{L}$ & 0.023 & 0.032 & 0.0399 & & \\
\hline 4579 & TRITIUMM_ELECCT_LSC & QTESRL & 43.4 & $\mathrm{pCi} / \mathrm{L}$ & 1.2 & 6.2 & 4.5 & & \\
\hline 4581 & UISO_IE_PLATE_AEA & QTESRL & 0.0201 & $\mathrm{pCi} / \mathrm{L}$ & 0.011 & 0.013 & 0.00454 & & $\mathrm{~J}$ \\
\hline 4582 & UISO_IE_PLATE_AEA & QTESRL & 0.39 & $\mathrm{pCi} / \mathrm{L}$ & 0.051 & 0.08 & 0.0156 & & \\
\hline 4583 & SRISO_SEP_PRECIP_GPC & QTESRL & 0.0664 & $\mathrm{pCi} / \mathrm{L}$ & 0.025 & 0.036 & 0.0462 & & \\
\hline 4584 & |TRITIUM_ELECT_LSC & QTESRL & 50.5 & $\mathrm{pCi} / \mathrm{L}$ & 1.3 & 6.7 & 4.55 & & \\
\hline 4585 & UISO_IE_PLATE_AEA & QTESRL & 0.251 & $\mathrm{pCi} / \mathrm{L}$ & 0.042 & 0.059 & 0.0154 & & \\
\hline 4586 & UISO_IE_PLATE_AEA & QTESRL & 0.00454 & $\mathrm{pCi} / \mathrm{L}$ & 0.0053 & 0.0095 & 0.0154 & & $U$ \\
\hline 4587 & UISO_IE_PLATE_AEA & QTESRL & 0.147 & $\mathrm{pCi} / \mathrm{L}$ & 0.033 & 0.041 & 0.0215 & & \\
\hline 4588 & SRISŌ_ĒSEP_PRËCIP_GPC & QTESRL & 0.0792 & $\mathrm{pCi} / \mathrm{L}$ & 0.026 & 0.037 & 0.043 & & \\
\hline 4589 & TRITIUM_ELEECT_LSC & QTESRL & 41.5 & $\mathrm{pCi} / \mathrm{L}$ & 1.1 & 6 & 4.47 & & \\
\hline 4590 & UISO_IE_PLATE_AEA & QTESRL & 0.28 & $\mathrm{pCi} / \mathrm{L}$ & 0.044 & 0.064 & 0.0166 & & \\
\hline 4591 & UISO_IE_PLATE_AEA & QTESRL & 0.00954 & $\mathrm{pCi} / \mathrm{L}$ & 0.0076 & 0.011 & 0.0123 & & $U$ \\
\hline 4592 & UISO_IE_PLATE_AEA & QTESRL & 0.213 & $\mathrm{pCi} / \mathrm{L}$ & 0.039 & 0.052 & 0.0188 & & \\
\hline 4593 & SRISO-SEPP_PRECCIP_GPC & QTESRL & 0.0699 & $\mathrm{pCi} / \mathrm{L}$ & 0.025 & 0.034 & 0.0405 & & \\
\hline 4594 & TRITIŪM_ELECCT_LSC̄ & QTESRL & 41.8 & $\mathrm{pCi} / \mathrm{L}$ & 1.1 & 6 & 4.47 & & \\
\hline 4595 & UISO_IE_PLATE_AEA & QTESRL & 0.292 & $\mathrm{pCi} / \mathrm{L}$ & 0.047 & 0.067 & 0.0249 & & \\
\hline 4596 & UISO_IE_PLATE_AEA & QTESRL & 0.012 & $\mathrm{pCi} / \mathrm{L}$ & 0.01 & 0.013 & 0.0196 & & U \\
\hline 4597 & UISO_IE_PLATE_AEA & QTESRL & 0.177 & $\mathrm{pCi} / \mathrm{L}$ & 0.036 & 0.047 & 0.0186 & & \\
\hline 4598 & SRISO_SEP_PRECIP_GPC & QTESRL & 0.0699 & $\mathrm{pCi} / \mathrm{L}$ & 0.026 & 0.035 & 0.044 & & \\
\hline
\end{tabular}




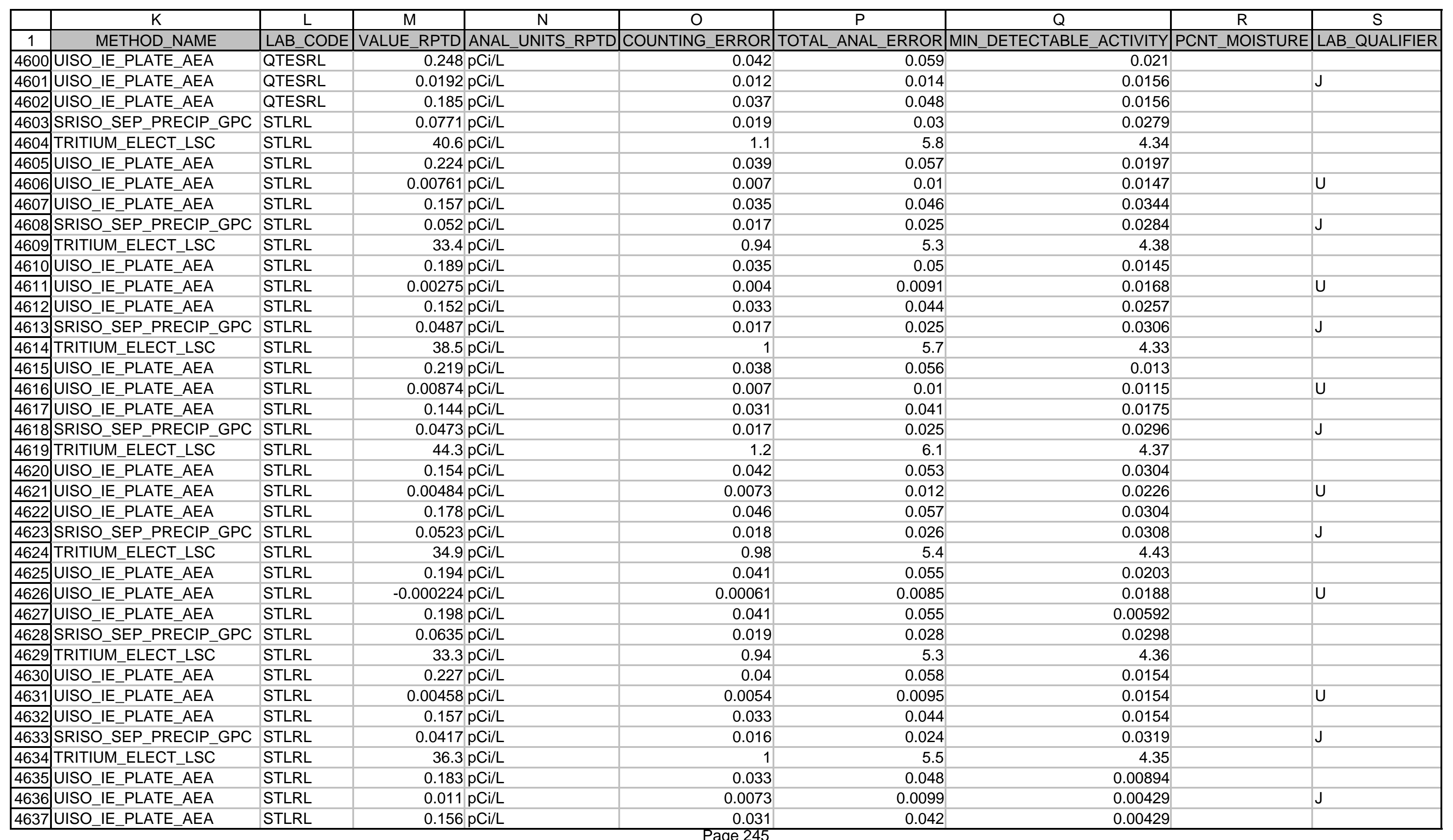




\begin{tabular}{|c|c|c|c|c|c|c|c|c|c|}
\hline & $\mathrm{K}$ & $\mathrm{L}$ & $\mathrm{M}$ & $\mathrm{N}$ & $\mathrm{O}$ & $\mathrm{P}$ & $\mathrm{Q}$ & $\mathrm{R}$ & $\mathrm{S}$ \\
\hline 1 & METHOD_NAME & LAB_CODE & VALUE_RPTD & ANAL_UNITS_RPTD & COUNTING_ERROR & TOTAL_ANAL_ERROR & MIN_DETECTABLE_ACTIVITY & PCNT_MOISTURE & LAB_QUALIFIER \\
\hline 4638 & SRISO_SEP_PRECIP_GPC & STLRL & 0.0485 & $\mathrm{pCi} / \mathrm{L}$ & 0.017 & 0.025 & 0.0309 & & $\mathrm{~J}$ \\
\hline 4639 & TRITIUM_ELECT_LSC & STLRL & 42.3 & $\mathrm{pCi} / \mathrm{L}$ & 1.1 & 6 & 4.4 & & \\
\hline 4640 & UISO_IE_PLATE_AEA & STLRL & 0.192 & $\mathrm{pCi} / \mathrm{L}$ & 0.039 & 0.053 & 0.0157 & & \\
\hline 4641 & UISO_IE_PLATE_AEA & STLRL & 0.00353 & $\mathrm{pCi} / \mathrm{L}$ & 0.006 & 0.012 & 0.0233 & & $\mathrm{U}$ \\
\hline 4642 & UISO_IE_PLATE_AEA & STLRL & 0.163 & $\mathrm{pCi} / \mathrm{L}$ & 0.036 & 0.048 & 0.02 & & \\
\hline 4643 & SRISO SEP PRECIP GPC & STLRL & 0.0644 & $\mathrm{pCi} / \mathrm{L}$ & 0.018 & 0.028 & 0.0299 & & \\
\hline 4644 & TRITIUMM_ELEECT_LSC & STLRL & 68.9 & $\mathrm{pCi} / \mathrm{L}$ & 1.6 & 8 & 4.33 & & \\
\hline 4645 & UISO_IE_PLATE_AEA & STLRL & 0.221 & $\mathrm{pCi} / \mathrm{L}$ & 0.04 & 0.057 & 0.0126 & & \\
\hline 4647 & UISO_IE_PLATE_AEA & STLRL & 0.17 & $\mathrm{pCi} / \mathrm{L}$ & 0.035 & 0.047 & 0.0126 & & \\
\hline 4648 & SRISO_SEP_PRECIP_GPC & STLRL & 0.0572 & $\mathrm{pCi} / \mathrm{L}$ & 0.019 & 0.027 & 0.0317 & & $\mathrm{~J}$ \\
\hline 4649 & |TRITIUM_ELECT_LSC & STLRL & 147 & $\mathrm{pCi} / \mathrm{L}$ & 2.7 & 14 & 4.37 & & \\
\hline 4650 & UISO_IE_PLATE_AEA & STLRL & 0.255 & $\mathrm{pCi} / \mathrm{L}$ & 0.047 & 0.067 & 0.0215 & & \\
\hline 4651 & UISO_IE_PLATE_AEA & STLRL & 0.00927 & $\mathrm{pCi} / \mathrm{L}$ & 0.0087 & 0.012 & 0.017 & & $\mathrm{U}$ \\
\hline 4652 & UISO_IE_PLATE_AEA & STLRL & 0.258 & $\mathrm{pCi} / \mathrm{L}$ & 0.047 & 0.066 & 0.0149 & & \\
\hline 4653 & SRISO_SEP_PRECIP_GPC & STLRL & 0.049 & $\mathrm{pCi} / \mathrm{L}$ & 0.016 & 0.024 & 0.0267 & & $\mathrm{~J}$ \\
\hline 4654 & TRITIUM_ELECT_LSC & STLRL & 120 & $\mathrm{pCi} / \mathrm{L}$ & 2.4 & 12 & 4.35 & & \\
\hline 4655 & UISO_IE_PLATE_AEA & STLRL & 0.221 & $\mathrm{pCi} / \mathrm{L}$ & 0.038 & 0.056 & 0.0131 & & \\
\hline 4657 & UISO_IE_PLATE_AEA & STLRL & 0.156 & $\mathrm{pCi} / \mathrm{L}$ & 0.032 & 0.043 & 0.0116 & & \\
\hline 4658 & SRISO_SEP_PRECIP_GPC & STLRL & 0.0606 & $\mathrm{pCi} / \mathrm{L}$ & 0.018 & 0.027 & 0.0277 & & \\
\hline 4659 & TRITIUM_ELECT_LSC & STLRL & 130 & $\mathrm{pCi} / \mathrm{L}$ & 2.5 & 13 & 4.36 & & \\
\hline 4660 & UISO_IE_PLATE_AEA & STLRL & 0.235 & $\mathrm{pCi} / \mathrm{L}$ & 0.036 & 0.056 & 0.0124 & & \\
\hline 4661 & UISO_IE_PLATE_AEA & STLRL & 0.0101 & $\mathrm{pCi} / \mathrm{L}$ & 0.007 & 0.0097 & 0.00995 & & $\mathrm{~J}$ \\
\hline 4662 & UISO_IE_PLATE_AEA & STLRL & 0.166 & $\mathrm{pCi} / \mathrm{L}$ & 0.031 & 0.043 & 0.00995 & & \\
\hline 4663 & SRISO_SEP_PRECIP_GPC & STLRL & 0.0465 & $\mathrm{pCi} / \mathrm{L}$ & 0.016 & 0.024 & 0.0289 & & $\mathrm{~J}$ \\
\hline 4664 & TRITIUM_ELECT_LSC & STLRL & 119 & $\mathrm{pCi} / \mathrm{L}$ & 2.3 & 12 & 4.31 & & \\
\hline 4665 & UISO_IE_PLATE_AEA & STLRL & 0.203 & $\mathrm{pCi} / \mathrm{L}$ & 0.04 & 0.056 & 0.0176 & & \\
\hline 4666 & UISO_IE_PLATE_AEA & STLRL & 0.0016 & $\mathrm{pCi} / \mathrm{L}$ & 0.0029 & 0.009 & 0.0176 & & $U$ \\
\hline 4667 & UISO_IE_PLATE_AEA & STLRL & 0.15 & $\mathrm{pCi} / \mathrm{L}$ & 0.034 & 0.045 & 0.00555 & & \\
\hline 4668 & SRISO_SEP_PRECIP_GPC & STLRL & 0.0566 & $\mathrm{pCi} / \mathrm{L}$ & 0.019 & 0.027 & 0.0314 & & $\mathrm{~J}$ \\
\hline 4669 & TRITIUM_ELECT_LSC & STLRL & 131 & $\mathrm{pCi} / \mathrm{L}$ & 2.5 & 13 & 4.35 & & \\
\hline 4670 & UISO_IE_PLATE_AEA & STLRL & 0.211 & $\mathrm{pCi} / \mathrm{L}$ & 0.04 & 0.056 & 0.0134 & & \\
\hline 4671 & UISO_IE_PLATE_AEA & STLRL & 0.00872 & $\mathrm{pCi} / \mathrm{L}$ & 0.0076 & 0.011 & 0.0134 & & U \\
\hline 4672 & UISO_IE_PLATE_AEA & STLRL & 0.152 & $\mathrm{pCi} / \mathrm{L}$ & 0.034 & 0.045 & 0.0134 & & \\
\hline
\end{tabular}




\begin{tabular}{|c|c|c|c|c|c|c|c|c|c|}
\hline & $\mathrm{T}$ & $\mathrm{U}$ & $\mathrm{V}$ & $\mathrm{W}$ & $\mathrm{X}$ & $\bar{Y}$ & $\bar{Z}$ & $\overline{A A}$ & $A B$ \\
\hline 1 & REVIEW_QUALIFIER & SAMP_FROM & SAMP_ITEM & SAMP_MTHD & COLL_MTHD & SAMP_SITE_ID & SAMP_SITE_NAME & WELL_NAME & SAMPLE_LOCATION \\
\hline 2 & & RIVER & & RAW GRAB & TRANSECT & 571 & RICH.PMPHS-1 HRM46.4 & & \\
\hline 3 & & RIVER & & RAW GRAB & TRANSECT & 571 & RICH.PMPHS-1 HRM46.4 & & \\
\hline 4 & & RIVER & & RAW GRAB & TRANSECT & 571 & RICH.PMPHS-1 HRM46.4 & & \\
\hline 5 & & RIVER & & RAW GRAB & TRANSECT & 571 & RICH.PMPHS-1 HRM46.4 & & \\
\hline 6 & & RIVER & & RAW GRAB & TRANSECT & 571 & RICH.PMPHS-1 HRM46.4 & & \\
\hline 7 & & RIVER & & RAW GRAB & TRANSECT & 321 & RICH.PMPHS-2 HRM46.4 & & \\
\hline 8 & & RIVER & & RAW GRAB & TRANSECT & 321 & RICH.PMPHS-2 HRM46.4 & & \\
\hline 9 & & RIVER & & RAW GRAB & TRANSECT & 321 & RICH.PMPHS-2 HRM46.4 & & \\
\hline 10 & & RIVER & & RAW GRAB & TRANSECT & 321 & RICH.PMPHS-2 HRM46.4 & & \\
\hline 11 & & RIVER & & RAW GRAB & TRANSECT & 321 & RICH.PMPHS-2 HRM46.4 & & \\
\hline 12 & & RIVER & & RAW GRAB & TRANSECT & 322 & RICH.PMPHS-3 HRM46.4 & & \\
\hline 13 & & RIVER & & RAW GRAB & TRANSECT & 322 & RICH.PMPHS-3 HRM46.4 & & \\
\hline 14 & & RIVER & & RAW GRAB & TRANSECT & 322 & RICH.PMPHS-3 HRM46.4 & & \\
\hline 15 & & RIVER & & RAW GRAB & TRANSECT & 322 & RICH.PMPHS-3 HRM46.4 & & \\
\hline 16 & & RIVER & & RAW GRAB & TRANSECT & 322 & RICH.PMPHS-3 HRM46.4 & & \\
\hline 17 & & RIVER & & RAW GRAB & TRANSECT & 572 & RICH.PMPHS-4 HRM46.4 & & \\
\hline 18 & & RIVER & & RAW GRAB & TRANSECT & 572 & RICH.PMPHS-4 HRM46.4 & & \\
\hline 19 & & RIVER & & RAW GRAB & TRANSECT & 572 & RICH.PMPHS-4 HRM46.4 & & \\
\hline 20 & & RIVER & & RAW GRAB & TRANSECT & 572 & RICH.PMPHS-4 HRM46.4 & & \\
\hline 21 & & RIVER & & RAW GRAB & TRANSECT & 572 & RICH.PMPHS-4 HRM46.4 & & \\
\hline 22 & & RIVER & & RAW GRAB & TRANSECT & 317 & VERNITA-1 HRM 0.3 & & \\
\hline 23 & & RIVER & & RAW GRAB & TRANSECT & 317 & VERNITA-1 HRM 0.3 & & \\
\hline 24 & & RIVER & & RAW GRAB & TRANSECT & 317 & VERNITA-1 HRM 0.3 & & \\
\hline 25 & & RIVER & & RAW GRAB & TRANSECT & 317 & VERNITA-1 HRM 0.3 & & \\
\hline 26 & & RIVER & & RAW GRAB & TRANSECT & 317 & VERNITA-1 HRM 0.3 & & \\
\hline 27 & & RIVER & & RAW GRAB & TRANSECT & 570 & VERNITA-2 HRM 0.3 & & \\
\hline 28 & & RIVER & & RAW GRAB & TRANSECT & 570 & VERNITA-2 HRM 0.3 & & \\
\hline 29 & & RIVER & & RAW GRAB & TRANSECT & 570 & VERNITA-2 HRM 0.3 & & \\
\hline 30 & & RIVER & & RAW GRAB & TRANSECT & 570 & VERNITA-2 HRM 0.3 & & \\
\hline 31 & & RIVER & & RAW GRAB & TRANSECT & 570 & VERNITA-2 HRM 0.3 & & \\
\hline 32 & & RIVER & & RAW GRAB & TRANSECT & 318 & VERNITA-3 HRM 0.3 & & \\
\hline 33 & & RIVER & & RAW GRAB & TRANSECT & 318 & VERNITA-3 HRM 0.3 & & \\
\hline 34 & & RIVER & & RAW GRAB & TRANSECT & 318 & VERNITA-3 HRM 0.3 & & \\
\hline 35 & & RIVER & & RAW GRAB & TRANSECT & 318 & VERNITA-3 HRM 0.3 & & \\
\hline 36 & & RIVER & & RAW GRAB & TRANSECT & 318 & VERNITA-3 HRM 0.3 & & \\
\hline 37 & & RIVER & & RAW GRAB & TRANSECT & 319 & VERNITA-4 HRM 0.3 & & \\
\hline 38 & & RIVER & & RAW GRAB & TRANSECT & 319 & VERNITA-4 HRM 0.3 & & \\
\hline 39 & & RIVER & & RAW GRAB & TRANSECT & 319 & VERNITA-4 HRM 0.3 & & \\
\hline
\end{tabular}




\begin{tabular}{|c|c|c|c|c|c|c|c|c|c|}
\hline & $\mathrm{T}$ & $U$ & $\mathrm{~V}$ & $\mathrm{~W}$ & $\mathrm{X}$ & $\bar{Y}$ & $Z$ & AA & $A B$ \\
\hline 1 & REVIEW_QUALIFIER & SAMP_FROM & SAMP_ITEM & SAMP_MTHD & COLL_MTHD & SAMP_SITE_ID & SAMP_SITE_NAME & WELL_NAME & SAMPLE_LOCATION \\
\hline 40 & & RIVER & & RAW GRAB & TRANSECT & 319 & VERNITA-4 HRM 0.3 & & \\
\hline 41 & & RIVER & & RAW GRAB & TRANSECT & 319 & VERNITA-4 HRM 0.3 & & \\
\hline 42 & & RIVER & & RAW GRAB & TRANSECT & 317 & VERNITA-1 HRM 0.3 & & \\
\hline 43 & & RIVER & & RAW GRAB & TRANSECT & 317 & VERNITA-1 HRM 0.3 & & \\
\hline 44 & & RIVER & & RAW GRAB & TRANSECT & 317 & VERNITA-1 HRM 0.3 & & \\
\hline 45 & & RIVER & & RAW GRAB & TRANSECT & 317 & VERNITA-1 HRM 0.3 & & \\
\hline 46 & & RIVER & & RAW GRAB & TRANSECT & 317 & VERNITA-1 HRM 0.3 & & \\
\hline 47 & & RIVER & & RAW GRAB & TRANSECT & 570 & VERNITA-2 HRM 0.3 & & \\
\hline 48 & & RIVER & & RAW GRAB & TRANSECT & 570 & VERNITA-2 HRM 0.3 & & \\
\hline 49 & & RIVER & & RAW GRAB & TRANSECT & 570 & VERNITA-2 HRM 0.3 & & \\
\hline 50 & & RIVER & & RAW GRAB & TRANSECT & 570 & VERNITA-2 HRM 0.3 & & \\
\hline 51 & & RIVER & & RAW GRAB & TRANSECT & 570 & VERNITA-2 HRM 0.3 & & \\
\hline 52 & & RIVER & & RAW GRAB & TRANSECT & 318 & VERNITA-3 HRM 0.3 & & \\
\hline 53 & & RIVER & & RAW GRAB & TRANSECT & 318 & VERNITA-3 HRM 0.3 & & \\
\hline 54 & & RIVER & & RAW GRAB & TRANSECT & 318 & VERNITA-3 HRM 0.3 & & \\
\hline 55 & & RIVER & & RAW GRAB & TRANSECT & 318 & VERNITA-3 HRM 0.3 & & \\
\hline 56 & & RIVER & & RAW GRAB & TRANSECT & 318 & VERNITA-3 HRM 0.3 & & \\
\hline 57 & & RIVER & & RAW GRAB & TRANSECT & 319 & VERNITA-4 HRM 0.3 & & \\
\hline 58 & & RIVER & & RAW GRAB & TRANSECT & 319 & VERNITA-4 HRM 0.3 & & \\
\hline 59 & & RIVER & & RAW GRAB & TRANSECT & 319 & VERNITA-4 HRM 0.3 & & \\
\hline 60 & & RIVER & & RAW GRAB & TRANSECT & 319 & VERNITA-4 HRM 0.3 & & \\
\hline 61 & & RIVER & & RAW GRAB & TRANSECT & 319 & VERNITA-4 HRM 0.3 & & \\
\hline 62 & & RIVER & & RAW GRAB & TRANSECT & 571 & RICH.PMPHS-1 HRM46.4 & & \\
\hline 63 & & RIVER & & RAW GRAB & TRANSECT & 571 & RICH.PMPHS-1 HRM46.4 & & \\
\hline 64 & & RIVER & & RAW GRAB & TRANSECT & 571 & RICH.PMPHS-1 HRM46.4 & & \\
\hline 65 & & RIVER & & RAW GRAB & TRANSECT & 571 & RICH.PMPHS-1 HRM46.4 & & \\
\hline 66 & & RIVER & & RAW GRAB & TRANSECT & 571 & RICH.PMPHS-1 HRM46.4 & & \\
\hline 67 & & RIVER & & RAW GRAB & TRANSECT & 321 & RICH.PMPHS-2 HRM46.4 & & \\
\hline 68 & & RIVER & & RAW GRAB & TRANSECT & 321 & RICH.PMPHS-2 HRM46.4 & & \\
\hline 69 & & RIVER & & RAW GRAB & TRANSECT & 321 & RICH.PMPHS-2 HRM46.4 & & \\
\hline 70 & & RIVER & & RAW GRAB & TRANSECT & 321 & RICH.PMPHS-2 HRM46.4 & & \\
\hline 71 & & RIVER & & RAW GRAB & TRANSECT & 321 & RICH.PMPHS-2 HRM46.4 & & \\
\hline 72 & & RIVER & & RAW GRAB & TRANSECT & 322 & RICH.PMPHS-3 HRM46.4 & & \\
\hline 73 & & RIVER & & RAW GRAB & TRANSECT & 322 & RICH.PMPHS-3 HRM46.4 & & \\
\hline 74 & & RIVER & & RAW GRAB & TRANSECT & 322 & RICH.PMPHS-3 HRM46.4 & & \\
\hline 75 & & RIVER & & RAW GRAB & TRANSECT & 322 & RICH.PMPHS-3 HRM46.4 & & \\
\hline 76 & & RIVER & & RAW GRAB & TRANSECT & 322 & RICH.PMPHS-3 HRM46.4 & & \\
\hline 77 & & RIVER & & RAW GRAB & TRANSECT & 572 & RICH.PMPHS-4 HRM46.4 & & \\
\hline
\end{tabular}




\begin{tabular}{|c|c|c|c|c|c|c|c|c|c|}
\hline & $\mathrm{T}$ & $U$ & $\mathrm{~V}$ & $\mathrm{~W}$ & $\mathrm{X}$ & $\mathrm{Y}$ & $Z$ & $\mathrm{AA}$ & $A B$ \\
\hline 1 & REVIEW_QUALIFIER & SAMP_FROM & SAMP_ITEM & SAMP_MTHD & COLL_MTHD & SAMP_SITE_ID & SAMP_SITE_NAME & WELL_NAME & SAMPLE_LOCATION \\
\hline 78 & & RIVER & & RAW GRAB & TRANSECT & 572 & 2 RICH.PMPHS-4 HRM46.4 & & \\
\hline 79 & & RIVER & & RAW GRAB & TRANSECT & 572 & 2 RICH.PMPHS-4 HRM46.4 & & \\
\hline 80 & & RIVER & & RAW GRAB & TRANSECT & 572 & 2 RICH.PMPHS-4 HRM46.4 & & \\
\hline 81 & & RIVER & & RAW GRAB & TRANSECT & 572 & 2 RICH.PMPHS-4 HRM46.4 & & \\
\hline 82 & & RIVER & & RAW GRAB & TRANSECT & 571 & RICH.PMPHS-1 HRM46.4 & & \\
\hline 83 & & RIVER & & RAW GRAB & TRANSECT & 571 & RICH.PMPHS-1 HRM46.4 & & \\
\hline 84 & & RIVER & & RAW GRAB & TRANSECT & 571 & RICH.PMPHS-1 HRM46.4 & & \\
\hline 85 & & RIVER & & RAW GRAB & TRANSECT & 571 & RICH.PMPHS-1 HRM46.4 & & \\
\hline 86 & & RIVER & & RAW GRAB & TRANSECT & 571 & RICH.PMPHS-1 HRM46.4 & & \\
\hline 87 & & RIVER & & RAW GRAB & TRANSECT & 321 & RICH.PMPHS-2 HRM46.4 & & \\
\hline 88 & & RIVER & & RAW GRAB & TRANSECT & 321 & RICH.PMPHS-2 HRM46.4 & & \\
\hline 89 & & RIVER & & RAW GRAB & TRANSECT & 321 & RICH.PMPHS-2 HRM46.4 & & \\
\hline 90 & & RIVER & & RAW GRAB & TRANSECT & 321 & RICH.PMPHS-2 HRM46.4 & & \\
\hline 91 & & RIVER & & RAW GRAB & TRANSECT & 321 & RICH.PMPHS-2 HRM46.4 & & \\
\hline 92 & & RIVER & & RAW GRAB & TRANSECT & 322 & RICH.PMPHS-3 HRM46.4 & & \\
\hline 93 & & RIVER & & RAW GRAB & TRANSECT & 322 & RICH.PMPHS-3 HRM46.4 & & \\
\hline 94 & & RIVER & & RAW GRAB & TRANSECT & 322 & RICH.PMPHS-3 HRM46.4 & & \\
\hline 95 & & RIVER & & RAW GRAB & TRANSECT & 322 & RICH.PMPHS-3 HRM46.4 & & \\
\hline 96 & & RIVER & & RAW GRAB & TRANSECT & 322 & RICH.PMPHS-3 HRM46.4 & & \\
\hline 97 & & RIVER & & RAW GRAB & TRANSECT & 572 & RICH.PMPHS-4 HRM46.4 & & \\
\hline 98 & & RIVER & & RAW GRAB & TRANSECT & 572 & RICH.PMPHS-4 HRM46.4 & & \\
\hline 99 & & RIVER & & RAW GRAB & TRANSECT & 572 & RICH.PMPHS-4 HRM46.4 & & \\
\hline 100 & & RIVER & & RAW GRAB & TRANSECT & 572 & RICH.PMPHS-4 HRM46.4 & & \\
\hline 101 & & RIVER & & RAW GRAB & TRANSECT & 572 & RICH.PMPHS-4 HRM46.4 & & \\
\hline 102 & & RIVER & & RAW GRAB & TRANSECT & 317 & VERNITA-1 HRM 0.3 & & \\
\hline 103 & & RIVER & & RAW GRAB & TRANSECT & 317 & VERNITA-1 HRM 0.3 & & \\
\hline 104 & & RIVER & & RAW GRAB & TRANSECT & 317 & VERNITA-1 HRM 0.3 & & \\
\hline 105 & & RIVER & & RAW GRAB & TRANSECT & 317 & VERNITA-1 HRM 0.3 & & \\
\hline 106 & & RIVER & & RAW GRAB & TRANSECT & 317 & VERNITA-1 HRM 0.3 & & \\
\hline 107 & & RIVER & & RAW GRAB & TRANSECT & 570 & VERNITA-2 HRM 0.3 & & \\
\hline 108 & & RIVER & & RAW GRAB & TRANSECT & 570 & VERNITA-2 HRM 0.3 & & \\
\hline 109 & & RIVER & & RAW GRAB & TRANSECT & 570 & VERNITA-2 HRM 0.3 & & \\
\hline 110 & & RIVER & & RAW GRAB & TRANSECT & 570 & VERNITA-2 HRM 0.3 & & \\
\hline 111 & & RIVER & & RAW GRAB & TRANSECT & 570 & VERNITA-2 HRM 0.3 & & \\
\hline 112 & & RIVER & & RAW GRAB & TRANSECT & 318 & VERNITA-3 HRM 0.3 & & \\
\hline 113 & & RIVER & & RAW GRAB & TRANSECT & 318 & VERNITA-3 HRM 0.3 & & \\
\hline 114 & & RIVER & & RAW GRAB & TRANSECT & 318 & VERNITA-3 HRM 0.3 & & \\
\hline 115 & & RIVER & & RAW GRAB & TRANSECT & 318 & VERNITA-3 HRM 0.3 & & \\
\hline
\end{tabular}




\begin{tabular}{|c|c|c|c|c|c|c|c|c|c|}
\hline & $\mathrm{T}$ & $\mathrm{U}$ & $\mathrm{V}$ & $\mathrm{W}$ & $\mathrm{X}$ & $\bar{Y}$ & $Z$ & $\overline{A A}$ & $A B$ \\
\hline 1 & REVIEW_QUALIFIER & SAMP_FROM & SAMP_ITEM & SAMP_MTHD & COLL_MTHD & SAMP_SITE_ID & SAMP_SITE_NAME & WELL_NAME & SAMPLE_LOCATION \\
\hline 116 & & RIVER & & RAW GRAB & TRANSECT & 318 & VERNITA-3 HRM 0.3 & & \\
\hline 117 & & RIVER & & RAW GRAB & TRANSECT & 319 & VERNITA-4 HRM 0.3 & & \\
\hline 118 & & RIVER & & RAW GRAB & TRANSECT & 319 & VERNITA-4 HRM 0.3 & & \\
\hline 119 & & RIVER & & RAW GRAB & TRANSECT & 319 & VERNITA-4 HRM 0.3 & & \\
\hline 120 & & RIVER & & RAW GRAB & TRANSECT & 319 & VERNITA-4 HRM 0.3 & & \\
\hline 121 & & RIVER & & RAW GRAB & TRANSECT & 319 & VERNITA-4 HRM 0.3 & & \\
\hline 122 & & RIVER & & RAW GRAB & TRANSECT & 317 & VERNITA-1 HRM 0.3 & & \\
\hline 123 & & RIVER & & RAW GRAB & TRANSECT & 317 & VERNITA-1 HRM 0.3 & & \\
\hline 124 & & RIVER & & RAW GRAB & TRANSECT & 317 & VERNITA-1 HRM 0.3 & & \\
\hline 125 & & RIVER & & RAW GRAB & TRANSECT & 317 & VERNITA-1 HRM 0.3 & & \\
\hline 126 & & RIVER & & RAW GRAB & TRANSECT & 317 & VERNITA-1 HRM 0.3 & & \\
\hline 127 & & RIVER & & RAW GRAB & TRANSECT & 570 & VERNITA-2 HRM 0.3 & & \\
\hline 128 & & RIVER & & RAW GRAB & TRANSECT & 570 & VERNITA-2 HRM 0.3 & & \\
\hline 129 & & RIVER & & RAW GRAB & TRANSECT & 570 & VERNITA-2 HRM 0.3 & & \\
\hline 130 & & RIVER & & RAW GRAB & TRANSECT & 570 & VERNITA-2 HRM 0.3 & & \\
\hline 131 & & RIVER & & RAW GRAB & TRANSECT & 570 & VERNITA-2 HRM 0.3 & & \\
\hline 132 & & RIVER & & RAW GRAB & TRANSECT & 318 & VERNITA-3 HRM 0.3 & & \\
\hline 133 & & RIVER & & RAW GRAB & TRANSECT & 318 & VERNITA-3 HRM 0.3 & & \\
\hline 134 & & RIVER & & RAW GRAB & TRANSECT & 318 & VERNITA-3 HRM 0.3 & & \\
\hline 135 & & RIVER & & RAW GRAB & TRANSECT & 318 & VERNITA-3 HRM 0.3 & & \\
\hline 136 & & RIVER & & RAW GRAB & TRANSECT & 318 & VERNITA-3 HRM 0.3 & & \\
\hline 137 & & RIVER & & RAW GRAB & TRANSECT & 319 & VERNITA-4 HRM 0.3 & & \\
\hline 138 & & RIVER & & RAW GRAB & TRANSECT & 319 & VERNITA-4 HRM 0.3 & & \\
\hline 139 & & RIVER & & RAW GRAB & TRANSECT & 319 & VERNITA-4 HRM 0.3 & & \\
\hline 140 & & RIVER & & RAW GRAB & TRANSECT & 319 & VERNITA-4 HRM 0.3 & & \\
\hline 141 & & RIVER & & RAW GRAB & TRANSECT & 319 & VERNITA-4 HRM 0.3 & & \\
\hline 142 & & RIVER & & RAW GRAB & TRANSECT & 571 & RICH.PMPHS-1 HRM46.4 & & \\
\hline 143 & & RIVER & & RAW GRAB & TRANSECT & 571 & RICH.PMPHS-1 HRM46.4 & & \\
\hline 144 & & RIVER & & RAW GRAB & TRANSECT & 571 & RICH.PMPHS-1 HRM46.4 & & \\
\hline 145 & & RIVER & & RAW GRAB & TRANSECT & 571 & RICH.PMPHS-1 HRM46.4 & & \\
\hline 146 & & RIVER & & RAW GRAB & TRANSECT & 571 & RICH.PMPHS-1 HRM46.4 & & \\
\hline 147 & & RIVER & & RAW GRAB & TRANSECT & 321 & RICH.PMPHS-2 HRM46.4 & & \\
\hline 148 & & RIVER & & RAW GRAB & TRANSECT & 321 & RICH.PMPHS-2 HRM46.4 & & \\
\hline 149 & & RIVER & & RAW GRAB & TRANSECT & 321 & RICH.PMPHS-2 HRM46.4 & & \\
\hline 150 & & RIVER & & RAW GRAB & TRANSECT & 321 & RICH.PMPHS-2 HRM46.4 & & \\
\hline 151 & & RIVER & & RAW GRAB & TRANSECT & 321 & RICH.PMPHS-2 HRM46.4 & & \\
\hline 152 & & RIVER & & RAW GRAB & TRANSECT & 322 & RICH.PMPHS-3 HRM46.4 & & \\
\hline 153 & & RIVER & & RAW GRAB & TRANSECT & 322 & RICH.PMPHS-3 HRM46.4 & & \\
\hline
\end{tabular}




\begin{tabular}{|c|c|c|c|c|c|c|c|c|c|}
\hline & $\mathrm{T}$ & $\mathrm{U}$ & $\mathrm{V}$ & $\mathrm{W}$ & $\mathrm{X}$ & $\bar{Y}$ & $\bar{Z}$ & $\overline{A A}$ & $A B$ \\
\hline 1 & REVIEW_QUALIFIER & SAMP_FROM & SAMP_ITEM & SAMP_MTHD & COLL_MTHD & SAMP_SITE_ID & SAMP_SITE_NAME & WELL_NAME & SAMPLE_LOCATION \\
\hline 154 & & RIVER & & RAW GRAB & TRANSECT & 322 & RICH.PMPHS-3 HRM46.4 & & \\
\hline 155 & & RIVER & & RAW GRAB & TRANSECT & 322 & RICH.PMPHS-3 HRM46.4 & & \\
\hline 156 & & RIVER & & RAW GRAB & TRANSECT & 322 & RICH.PMPHS-3 HRM46.4 & & \\
\hline 157 & & RIVER & & RAW GRAB & TRANSECT & 572 & RICH.PMPHS-4 HRM46.4 & & \\
\hline 158 & & RIVER & & RAW GRAB & TRANSECT & 572 & RICH.PMPHS-4 HRM46.4 & & \\
\hline 159 & & RIVER & & RAW GRAB & TRANSECT & 572 & RICH.PMPHS-4 HRM46.4 & & \\
\hline 160 & & RIVER & & RAW GRAB & TRANSECT & 572 & RICH.PMPHS-4 HRM46.4 & & \\
\hline 161 & & RIVER & & RAW GRAB & TRANSECT & 572 & RICH.PMPHS-4 HRM46.4 & & \\
\hline 162 & & RIVER & & RAW GRAB & TRANSECT & 317 & VERNITA-1 HRM 0.3 & & \\
\hline 163 & & RIVER & & RAW GRAB & TRANSECT & 317 & VERNITA-1 HRM 0.3 & & \\
\hline 164 & & RIVER & & RAW GRAB & TRANSECT & 317 & VERNITA-1 HRM 0.3 & & \\
\hline 165 & & RIVER & & RAW GRAB & TRANSECT & 317 & VERNITA-1 HRM 0.3 & & \\
\hline 166 & & RIVER & & RAW GRAB & TRANSECT & 317 & VERNITA-1 HRM 0.3 & & \\
\hline 167 & & RIVER & & RAW GRAB & TRANSECT & 570 & VERNITA-2 HRM 0.3 & & \\
\hline 168 & & RIVER & & RAW GRAB & TRANSECT & 570 & VERNITA-2 HRM 0.3 & & \\
\hline 169 & & RIVER & & RAW GRAB & TRANSECT & 570 & VERNITA-2 HRM 0.3 & & \\
\hline 170 & & RIVER & & RAW GRAB & TRANSECT & 570 & VERNITA-2 HRM 0.3 & & \\
\hline 171 & & RIVER & & RAW GRAB & TRANSECT & 570 & VERNITA-2 HRM 0.3 & & \\
\hline 172 & & RIVER & & RAW GRAB & TRANSECT & 318 & VERNITA-3 HRM 0.3 & & \\
\hline 173 & & RIVER & & RAW GRAB & TRANSECT & 318 & VERNITA-3 HRM 0.3 & & \\
\hline 174 & & RIVER & & RAW GRAB & TRANSECT & 318 & VERNITA-3 HRM 0.3 & & \\
\hline 175 & & RIVER & & RAW GRAB & TRANSECT & 318 & VERNITA-3 HRM 0.3 & & \\
\hline 176 & & RIVER & & RAW GRAB & TRANSECT & 318 & VERNITA-3 HRM 0.3 & & \\
\hline 177 & & RIVER & & RAW GRAB & TRANSECT & 319 & VERNITA-4 HRM 0.3 & & \\
\hline 178 & & RIVER & & RAW GRAB & TRANSECT & 319 & VERNITA-4 HRM 0.3 & & \\
\hline 179 & & RIVER & & RAW GRAB & TRANSECT & 319 & VERNITA-4 HRM 0.3 & & \\
\hline 180 & & RIVER & & RAW GRAB & TRANSECT & 319 & VERNITA-4 HRM 0.3 & & \\
\hline 181 & & RIVER & & RAW GRAB & TRANSECT & 319 & VERNITA-4 HRM 0.3 & & \\
\hline 182 & & RIVER & & RAW GRAB & TRANSECT & 571 & RICH.PMPHS-1 HRM46.4 & & \\
\hline 183 & & RIVER & & RAW GRAB & TRANSECT & 571 & RICH.PMPHS-1 HRM46.4 & & \\
\hline 184 & & RIVER & & RAW GRAB & TRANSECT & 571 & RICH.PMPHS-1 HRM46.4 & & \\
\hline 185 & & RIVER & & RAW GRAB & TRANSECT & 571 & RICH.PMPHS-1 HRM46.4 & & \\
\hline 186 & & RIVER & & RAW GRAB & TRANSECT & 571 & RICH.PMPHS-1 HRM46.4 & & \\
\hline 187 & & RIVER & & RAW GRAB & TRANSECT & 321 & RICH.PMPHS-2 HRM46.4 & & \\
\hline 188 & & RIVER & & RAW GRAB & TRANSECT & 321 & RICH.PMPHS-2 HRM46.4 & & \\
\hline 189 & & RIVER & & RAW GRAB & TRANSECT & 321 & RICH.PMPHS-2 HRM46.4 & & \\
\hline 190 & & RIVER & & RAW GRAB & TRANSECT & 321 & RICH.PMPHS-2 HRM46.4 & & \\
\hline 191 & & RIVER & & RAW GRAB & TRANSECT & 321 & RICH.PMPHS-2 HRM46.4 & & \\
\hline
\end{tabular}




\begin{tabular}{|c|c|c|c|c|c|c|c|c|c|}
\hline & $\mathrm{T}$ & $\mathrm{U}$ & $\mathrm{V}$ & $\mathrm{W}$ & $\mathrm{X}$ & $\bar{Y}$ & $Z$ & AA & $A B$ \\
\hline 1 & REVIEW_QUALIFIER & SAMP_FROM & SAMP_ITEM & SAMP_MTHD & COLL_MTHD & SAMP_SITE_ID & SAMP_SITE_NAME & WELL_NAME & SAMPLE_LOCATION \\
\hline 192 & & RIVER & & RAW GRAB & TRANSECT & 322 & $2 \mathrm{RICH} . \mathrm{PMPHS}-3 \mathrm{HRM} 46.4$ & & \\
\hline 193 & & RIVER & & RAW GRAB & TRANSECT & 322 & 2 RICH.PMPHS-3 HRM46.4 & & \\
\hline 194 & & RIVER & & RAW GRAB & TRANSECT & 322 & 2 RICH.PMPHS-3 HRM46.4 & & \\
\hline 195 & & RIVER & & RAW GRAB & TRANSECT & 322 & 2 RICH.PMPHS-3 HRM46.4 & & \\
\hline 196 & & RIVER & & RAW GRAB & TRANSECT & 322 & 2 RICH.PMPHS-3 HRM46.4 & & \\
\hline 197 & & RIVER & & RAW GRAB & TRANSECT & 572 & 2 RICH.PMPHS-4 HRM46.4 & & \\
\hline 198 & & RIVER & & RAW GRAB & TRANSECT & 572 & 2 RICH.PMPHS-4 HRM46.4 & & \\
\hline 199 & & RIVER & & RAW GRAB & TRANSECT & 572 & 2 RICH.PMPHS-4 HRM46.4 & & \\
\hline 200 & & RIVER & & RAW GRAB & TRANSECT & 572 & 2 RICH.PMPHS-4 HRM46.4 & & \\
\hline 201 & & RIVER & & RAW GRAB & TRANSECT & 572 & 2 RICH.PMPHS-4 HRM46.4 & & \\
\hline 202 & & RIVER & & RAW GRAB & TRANSECT & 317 & 7 VERNITA-1 HRM 0.3 & & \\
\hline 203 & & RIVER & & RAW GRAB & TRANSECT & 317 & 7 VERNITA-1 HRM 0.3 & & \\
\hline 204 & & RIVER & & RAW GRAB & TRANSECT & 317 & 7 VERNITA-1 HRM 0.3 & & \\
\hline 205 & & RIVER & & RAW GRAB & TRANSECT & 317 & 7 VERNITA-1 HRM 0.3 & & \\
\hline 206 & & RIVER & & RAW GRAB & TRANSECT & 317 & 7 VERNITA-1 HRM 0.3 & & \\
\hline 207 & & RIVER & & RAW GRAB & TRANSECT & 317 & 7 VERNITA-1 HRM 0.3 & & \\
\hline 208 & & RIVER & & RAW GRAB & TRANSECT & 570 & VERNITA-2 HRM 0.3 & & \\
\hline 209 & & RIVER & & RAW GRAB & TRANSECT & 570 & VERNITA-2 HRM 0.3 & & \\
\hline 210 & & RIVER & & RAW GRAB & TRANSECT & 570 & VERNITA-2 HRM 0.3 & & \\
\hline 211 & & RIVER & & RAW GRAB & TRANSECT & 570 & VERNITA-2 HRM 0.3 & & \\
\hline 212 & & RIVER & & RAW GRAB & TRANSECT & 570 & VERNITA-2 HRM 0.3 & & \\
\hline 213 & & RIVER & & RAW GRAB & TRANSECT & 570 & VERNITA-2 HRM 0.3 & & \\
\hline 214 & & RIVER & & RAW GRAB & TRANSECT & 318 & 3 VERNITA-3 HRM 0.3 & & \\
\hline 215 & & RIVER & & RAW GRAB & TRANSECT & 318 & 3 VERNITA-3 HRM 0.3 & & \\
\hline 216 & & RIVER & & RAW GRAB & TRANSECT & 318 & 3 VERNITA-3 HRM 0.3 & & \\
\hline 217 & & RIVER & & RAW GRAB & TRANSECT & 318 & 3 VERNITA-3 HRM 0.3 & & \\
\hline 218 & & RIVER & & RAW GRAB & TRANSECT & 318 & 3 VERNITA-3 HRM 0.3 & & \\
\hline 219 & & RIVER & & RAW GRAB & TRANSECT & 318 & 3 VERNITA-3 HRM 0.3 & & \\
\hline 220 & & RIVER & & RAW GRAB & TRANSECT & 319 & 9 VERNITA-4 HRM 0.3 & & \\
\hline 221 & & RIVER & & RAW GRAB & TRANSECT & 319 & 9 VERNITA-4 HRM 0.3 & & \\
\hline 222 & & RIVER & & RAW GRAB & TRANSECT & 319 & 9 VERNITA-4 HRM 0.3 & & \\
\hline 223 & & RIVER & & RAW GRAB & TRANSECT & 319 & 9 VERNITA-4 HRM 0.3 & & \\
\hline 224 & & RIVER & & RAW GRAB & TRANSECT & 319 & 9 VERNITA-4 HRM 0.3 & & \\
\hline 225 & & RIVER & & RAW GRAB & TRANSECT & 319 & 9 VERNITA-4 HRM 0.3 & & \\
\hline 226 & & RIVER & & RAW GRAB & TRANSECT & 571 & RICH.PMPHS-1 HRM46.4 & & \\
\hline 227 & & RIVER & & RAW GRAB & TRANSECT & 571 & RICH.PMPHS-1 HRM46.4 & & \\
\hline 228 & & RIVER & & RAW GRAB & TRANSECT & 571 & RICH.PMPHS-1 HRM46.4 & & \\
\hline 229 & & RIVER & & RAW GRAB & TRANSECT & 571 & IICH.PMPHS-1 HRM46.4 & & \\
\hline
\end{tabular}




\begin{tabular}{|c|c|c|c|c|c|c|c|c|c|}
\hline & $\mathrm{T}$ & $U$ & $\mathrm{~V}$ & $\mathrm{~W}$ & $\mathrm{X}$ & $\mathrm{Y}$ & $Z$ & $\mathrm{AA}$ & $A B$ \\
\hline 1 & REVIEW_QUALIFIER & SAMP_FROM & SAMP_ITEM & SAMP_MTHD & COLL_MTHD & SAMP_SITE_ID & SAMP_SITE_NAME & WELL_NAME & SAMPLE_LOCATION \\
\hline 230 & & RIVER & & RAW GRAB & TRANSECT & 571 & RICH.PMPHS-1 HRM46.4 & & \\
\hline 231 & & RIVER & & RAW GRAB & TRANSECT & 327 & RICH.PMPHS-10 HRM46.4 & & \\
\hline 232 & & RIVER & & RAW GRAB & TRANSECT & 327 & RICH.PMPHS-10 HRM46.4 & & \\
\hline 233 & & RIVER & & RAW GRAB & TRANSECT & 327 & RICH.PMPHS-10 HRM46.4 & & \\
\hline 234 & & RIVER & & RAW GRAB & TRANSECT & 327 & RICH.PMPHS-10 HRM46.4 & & \\
\hline 235 & & RIVER & & RAW GRAB & TRANSECT & 327 & RICH.PMPHS-10 HRM46.4 & & \\
\hline 236 & & RIVER & & RAW GRAB & TRANSECT & 321 & RICH.PMPHS-2 HRM46.4 & & \\
\hline 237 & & RIVER & & RAW GRAB & TRANSECT & 321 & RICH.PMPHS-2 HRM46.4 & & \\
\hline 238 & & RIVER & & RAW GRAB & TRANSECT & 321 & RICH.PMPHS-2 HRM46.4 & & \\
\hline 239 & & RIVER & & RAW GRAB & TRANSECT & 321 & RICH.PMPHS-2 HRM46.4 & & \\
\hline 240 & & RIVER & & RAW GRAB & TRANSECT & 321 & RICH.PMPHS-2 HRM46.4 & & \\
\hline 241 & & RIVER & & RAW GRAB & TRANSECT & 322 & RICH.PMPHS-3 HRM46.4 & & \\
\hline 242 & & RIVER & & RAW GRAB & TRANSECT & 322 & RICH.PMPHS-3 HRM46.4 & & \\
\hline 243 & & RIVER & & RAW GRAB & TRANSECT & 322 & RICH.PMPHS-3 HRM46.4 & & \\
\hline 244 & & RIVER & & RAW GRAB & TRANSECT & 322 & RICH.PMPHS-3 HRM46.4 & & \\
\hline 245 & & RIVER & & RAW GRAB & TRANSECT & 322 & RICH.PMPHS-3 HRM46.4 & & \\
\hline 246 & & RIVER & & RAW GRAB & TRANSECT & 572 & RICH.PMPHS-4 HRM46.4 & & \\
\hline 247 & & RIVER & & RAW GRAB & TRANSECT & 572 & RICH.PMPHS-4 HRM46.4 & & \\
\hline 248 & & RIVER & & RAW GRAB & TRANSECT & 572 & RICH.PMPHS-4 HRM46.4 & & \\
\hline 249 & & RIVER & & RAW GRAB & TRANSECT & 572 & RICH.PMPHS-4 HRM46.4 & & \\
\hline 250 & & RIVER & & RAW GRAB & TRANSECT & 572 & RICH.PMPHS-4 HRM46.4 & & \\
\hline 251 & & RIVER & & RAW GRAB & TRANSECT & 323 & RICH.PMPHS-5 HRM46.4 & & \\
\hline 252 & & RIVER & & RAW GRAB & TRANSECT & 323 & RICH.PMPHS-5 HRM46.4 & & \\
\hline 253 & & RIVER & & RAW GRAB & TRANSECT & 323 & RICH.PMPHS-5 HRM46.4 & & \\
\hline 254 & & RIVER & & RAW GRAB & TRANSECT & 323 & RICH.PMPHS-5 HRM46.4 & & \\
\hline 255 & & RIVER & & RAW GRAB & TRANSECT & 323 & RICH.PMPHS-5 HRM46.4 & & \\
\hline 256 & & RIVER & & RAW GRAB & TRANSECT & 324 & RICH.PMPHS-6 HRM46.4 & & \\
\hline 257 & & RIVER & & RAW GRAB & TRANSECT & 324 & RICH.PMPHS-6 HRM46.4 & & \\
\hline 258 & & RIVER & & RAW GRAB & TRANSECT & 324 & RICH.PMPHS-6 HRM46.4 & & \\
\hline 259 & & RIVER & & RAW GRAB & TRANSECT & 324 & RICH.PMPHS-6 HRM46.4 & & \\
\hline 260 & & RIVER & & RAW GRAB & TRANSECT & 324 & RICH.PMPHS-6 HRM46.4 & & \\
\hline 261 & & RIVER & & RAW GRAB & TRANSECT & 325 & RICH.PMPHS-7 HRM46.4 & & \\
\hline 262 & & RIVER & & RAW GRAB & TRANSECT & 325 & RICH.PMPHS-7 HRM46.4 & & \\
\hline 263 & & RIVER & & RAW GRAB & TRANSECT & 325 & RICH.PMPHS-7 HRM46.4 & & \\
\hline 264 & & RIVER & & RAW GRAB & TRANSECT & 325 & RICH.PMPHS-7 HRM46.4 & & \\
\hline 265 & & RIVER & & RAW GRAB & TRANSECT & 325 & RICH.PMPHS-7 HRM46.4 & & \\
\hline 266 & & RIVER & & RAW GRAB & TRANSECT & 326 & RICH.PMPHS-8 HRM46.4 & & \\
\hline 267 & & RIVER & & RAW GRAB & TRANSECT & 326 & RICH.PMPHS-8 HRM46.4 & & \\
\hline
\end{tabular}




\begin{tabular}{|c|c|c|c|c|c|c|c|c|c|}
\hline & $\mathrm{T}$ & $\mathrm{U}$ & $\mathrm{V}$ & $\mathrm{W}$ & $\mathrm{X}$ & $\bar{Y}$ & $Z$ & AA & $A B$ \\
\hline 1 & REVIEW_QUALIFIER & SAMP_FROM & SAMP_ITEM & SAMP_MTHD & COLL_MTHD & SAMP_SITE_ID & SAMP_SITE_NAME & WELL_NAME & SAMPLE_LOCATION \\
\hline 268 & & RIVER & & RAW GRAB & TRANSECT & 326 & RICH.PMPHS-8 HRM46.4 & & \\
\hline 269 & & RIVER & & RAW GRAB & TRANSECT & 326 & RICH.PMPHS-8 HRM46.4 & & \\
\hline 270 & & RIVER & & RAW GRAB & TRANSECT & 326 & RICH.PMPHS-8 HRM46.4 & & \\
\hline 271 & & RIVER & & RAW GRAB & TRANSECT & 573 & RICH.PMPHS-9 HRM46.4 & & \\
\hline 272 & & RIVER & & RAW GRAB & TRANSECT & 573 & RICH.PMPHS-9 HRM46.4 & & \\
\hline 273 & & RIVER & & RAW GRAB & TRANSECT & 573 & RICH.PMPHS-9 HRM46.4 & & \\
\hline 274 & & RIVER & & RAW GRAB & TRANSECT & 573 & RICH.PMPHS-9 HRM46.4 & & \\
\hline 275 & & RIVER & & RAW GRAB & TRANSECT & 573 & RICH.PMPHS-9 HRM46.4 & & \\
\hline 276 & & RIVER & & RAW GRAB & TRANSECT & 317 & VERNITA-1 HRM 0.3 & & \\
\hline 277 & & RIVER & & RAW GRAB & TRANSECT & 317 & VERNITA-1 HRM 0.3 & & \\
\hline 278 & & RIVER & & RAW GRAB & TRANSECT & 317 & VERNITA-1 HRM 0.3 & & \\
\hline 279 & & RIVER & & RAW GRAB & TRANSECT & 317 & VERNITA-1 HRM 0.3 & & \\
\hline 280 & & RIVER & & RAW GRAB & TRANSECT & 317 & VERNITA-1 HRM 0.3 & & \\
\hline 281 & & RIVER & & RAW GRAB & TRANSECT & 570 & VERNITA-2 HRM 0.3 & & \\
\hline 282 & & RIVER & & RAW GRAB & TRANSECT & 570 & VERNITA-2 HRM 0.3 & & \\
\hline 283 & & RIVER & & RAW GRAB & TRANSECT & 570 & VERNITA-2 HRM 0.3 & & \\
\hline 284 & & RIVER & & RAW GRAB & TRANSECT & 570 & VERNITA-2 HRM 0.3 & & \\
\hline 285 & & RIVER & & RAW GRAB & TRANSECT & 570 & VERNITA-2 HRM 0.3 & & \\
\hline 286 & & RIVER & & RAW GRAB & TRANSECT & 318 & VERNITA-3 HRM 0.3 & & \\
\hline 287 & & RIVER & & RAW GRAB & TRANSECT & 318 & VERNITA-3 HRM 0.3 & & \\
\hline 288 & & RIVER & & RAW GRAB & TRANSECT & 318 & VERNITA-3 HRM 0.3 & & \\
\hline 289 & & RIVER & & RAW GRAB & TRANSECT & 318 & VERNITA-3 HRM 0.3 & & \\
\hline 290 & & RIVER & & RAW GRAB & TRANSECT & 318 & VERNITA-3 HRM 0.3 & & \\
\hline 291 & & RIVER & & RAW GRAB & TRANSECT & 319 & VERNITA-4 HRM 0.3 & & \\
\hline 292 & & RIVER & & RAW GRAB & TRANSECT & 319 & VERNITA-4 HRM 0.3 & & \\
\hline 293 & & RIVER & & RAW GRAB & TRANSECT & 319 & VERNITA-4 HRM 0.3 & & \\
\hline 294 & & RIVER & & RAW GRAB & TRANSECT & 319 & VERNITA-4 HRM 0.3 & & \\
\hline 295 & & RIVER & & RAW GRAB & TRANSECT & 319 & VERNITA-4 HRM 0.3 & & \\
\hline 296 & & RIVER & & RAW GRAB & TRANSECT & 571 & RICH.PMPHS-1 HRM46.4 & & \\
\hline 297 & & RIVER & & RAW GRAB & TRANSECT & 571 & RICH.PMPHS-1 HRM46.4 & & \\
\hline 298 & & RIVER & & RAW GRAB & TRANSECT & 571 & RICH.PMPHS-1 HRM46.4 & & \\
\hline 299 & & RIVER & & RAW GRAB & TRANSECT & 571 & RICH.PMPHS-1 HRM46.4 & & \\
\hline 300 & & RIVER & & RAW GRAB & TRANSECT & 571 & RICH.PMPHS-1 HRM46.4 & & \\
\hline 301 & & RIVER & & RAW GRAB & TRANSECT & 327 & RICH.PMPHS-10 HRM46.4 & & \\
\hline 302 & & RIVER & & RAW GRAB & TRANSECT & 327 & RICH.PMPHS-10 HRM46.4 & & \\
\hline 303 & & RIVER & & RAW GRAB & TRANSECT & 327 & RICH.PMPHS-10 HRM46.4 & & \\
\hline 304 & & RIVER & & RAW GRAB & TRANSECT & 327 & RICH.PMPHS-10 HRM46.4 & & \\
\hline 305 & & RIVER & & RAW GRAB & TRANSECT & 327 & RICH.PMPHS-10 HRM46.4 & & \\
\hline
\end{tabular}




\begin{tabular}{|c|c|c|c|c|c|c|c|c|c|}
\hline & $\mathrm{T}$ & $U$ & $\mathrm{~V}$ & $\mathrm{~W}$ & $\mathrm{X}$ & $\mathrm{Y}$ & $Z$ & $\mathrm{AA}$ & $A B$ \\
\hline 1 & REVIEW_QUALIFIER & SAMP_FROM & SAMP_ITEM & SAMP_MTHD & COLL_MTHD & SAMP_SITE_ID & SAMP_SITE_NAME & WELL_NAME & SAMPLE_LOCATION \\
\hline 306 & & RIVER & & RAW GRAB & TRANSECT & 321 & RICH.PMPHS-2 HRM46.4 & & \\
\hline 307 & & RIVER & & RAW GRAB & TRANSECT & 321 & RICH.PMPHS-2 HRM46.4 & & \\
\hline 308 & & RIVER & & RAW GRAB & TRANSECT & 321 & RICH.PMPHS-2 HRM46.4 & & \\
\hline 309 & & RIVER & & RAW GRAB & TRANSECT & 321 & RICH.PMPHS-2 HRM46.4 & & \\
\hline 310 & & RIVER & & RAW GRAB & TRANSECT & 321 & RICH.PMPHS-2 HRM46.4 & & \\
\hline 311 & & RIVER & & RAW GRAB & TRANSECT & 322 & RICH.PMPHS-3 HRM46.4 & & \\
\hline 312 & & RIVER & & RAW GRAB & TRANSECT & 322 & RICH.PMPHS-3 HRM46.4 & & \\
\hline 313 & & RIVER & & RAW GRAB & TRANSECT & 322 & RICH.PMPHS-3 HRM46.4 & & \\
\hline 314 & & RIVER & & RAW GRAB & TRANSECT & 322 & RICH.PMPHS-3 HRM46.4 & & \\
\hline 315 & & RIVER & & RAW GRAB & TRANSECT & 322 & RICH.PMPHS-3 HRM46.4 & & \\
\hline 316 & & RIVER & & RAW GRAB & TRANSECT & 572 & RICH.PMPHS-4 HRM46.4 & & \\
\hline 317 & & RIVER & & RAW GRAB & TRANSECT & 572 & RICH.PMPHS-4 HRM46.4 & & \\
\hline 318 & & RIVER & & RAW GRAB & TRANSECT & 572 & RICH.PMPHS-4 HRM46.4 & & \\
\hline 319 & & RIVER & & RAW GRAB & TRANSECT & 572 & RICH.PMPHS-4 HRM46.4 & & \\
\hline 320 & & RIVER & & RAW GRAB & TRANSECT & 572 & RICH.PMPHS-4 HRM46.4 & & \\
\hline 321 & & RIVER & & RAW GRAB & TRANSECT & 323 & RICH.PMPHS-5 HRM46.4 & & \\
\hline 322 & & RIVER & & RAW GRAB & TRANSECT & 323 & RICH.PMPHS-5 HRM46.4 & & \\
\hline 323 & & RIVER & & RAW GRAB & TRANSECT & 323 & RICH.PMPHS-5 HRM46.4 & & \\
\hline 324 & & RIVER & & RAW GRAB & TRANSECT & 323 & RICH.PMPHS-5 HRM46.4 & & \\
\hline 325 & & RIVER & & RAW GRAB & TRANSECT & 323 & RICH.PMPHS-5 HRM46.4 & & \\
\hline 326 & & RIVER & & RAW GRAB & TRANSECT & 324 & RICH.PMPHS-6 HRM46.4 & & \\
\hline 327 & & RIVER & & RAW GRAB & TRANSECT & 324 & RICH.PMPHS-6 HRM46.4 & & \\
\hline 328 & & RIVER & & RAW GRAB & TRANSECT & 324 & RICH.PMPHS-6 HRM46.4 & & \\
\hline 329 & & RIVER & & RAW GRAB & TRANSECT & 324 & RICH.PMPHS-6 HRM46.4 & & \\
\hline 330 & & RIVER & & RAW GRAB & TRANSECT & 324 & RICH.PMPHS-6 HRM46.4 & & \\
\hline 331 & & RIVER & & RAW GRAB & TRANSECT & 325 & RICH.PMPHS-7 HRM46.4 & & \\
\hline 332 & & RIVER & & RAW GRAB & TRANSECT & 325 & RICH.PMPHS-7 HRM46.4 & & \\
\hline 333 & & RIVER & & RAW GRAB & TRANSECT & 325 & RICH.PMPHS-7 HRM46.4 & & \\
\hline 334 & & RIVER & & RAW GRAB & TRANSECT & 325 & RICH.PMPHS-7 HRM46.4 & & \\
\hline 335 & & RIVER & & RAW GRAB & TRANSECT & 325 & RICH.PMPHS-7 HRM46.4 & & \\
\hline 336 & & RIVER & & RAW GRAB & TRANSECT & 326 & RICH.PMPHS-8 HRM46.4 & & \\
\hline 337 & & RIVER & & RAW GRAB & TRANSECT & 326 & RICH.PMPHS-8 HRM46.4 & & \\
\hline 338 & & RIVER & & RAW GRAB & TRANSECT & 326 & RICH.PMPHS-8 HRM46.4 & & \\
\hline 339 & & RIVER & & RAW GRAB & TRANSECT & 326 & RICH.PMPHS-8 HRM46.4 & & \\
\hline 340 & & RIVER & & RAW GRAB & TRANSECT & 326 & RICH.PMPHS-8 HRM46.4 & & \\
\hline 341 & & RIVER & & RAW GRAB & TRANSECT & 573 & RICH.PMPHS-9 HRM46.4 & & \\
\hline 342 & & RIVER & & RAW GRAB & TRANSECT & 573 & RICH.PMPHS-9 HRM46.4 & & \\
\hline 343 & & RIVER & & RAW GRAB & TRANSECT & 573 & RICH.PMPHS-9 HRM46.4 & & \\
\hline
\end{tabular}




\begin{tabular}{|c|c|c|c|c|c|c|c|c|c|}
\hline & $\mathrm{T}$ & $\mathrm{U}$ & $\mathrm{V}$ & $\mathrm{W}$ & $\mathrm{X}$ & $\bar{Y}$ & $Z$ & AA & $A B$ \\
\hline 1 & REVIEW_QUALIFIER & SAMP_FROM & SAMP_ITEM & SAMP_MTHD & COLL_MTHD & SAMP_SITE_ID & SAMP_SITE_NAME & WELL_NAME & SAMPLE_LOCATION \\
\hline 344 & & RIVER & & RAW GRAB & TRANSECT & 573 & RICH.PMPHS-9 HRM46.4 & & \\
\hline 345 & & RIVER & & RAW GRAB & TRANSECT & 573 & RICH.PMPHS-9 HRM46.4 & & \\
\hline 346 & & RIVER & & RAW GRAB & TRANSECT & 317 & VERNITA-1 HRM 0.3 & & \\
\hline 347 & & RIVER & & RAW GRAB & TRANSECT & 317 & VERNITA-1 HRM 0.3 & & \\
\hline 348 & & RIVER & & RAW GRAB & TRANSECT & 317 & VERNITA-1 HRM 0.3 & & \\
\hline 349 & & RIVER & & RAW GRAB & TRANSECT & 317 & VERNITA-1 HRM 0.3 & & \\
\hline 350 & & RIVER & & RAW GRAB & TRANSECT & 317 & VERNITA-1 HRM 0.3 & & \\
\hline 351 & & RIVER & & RAW GRAB & TRANSECT & 570 & VERNITA-2 HRM 0.3 & & \\
\hline 352 & & RIVER & & RAW GRAB & TRANSECT & 570 & VERNITA-2 HRM 0.3 & & \\
\hline 353 & & RIVER & & RAW GRAB & TRANSECT & 570 & VERNITA-2 HRM 0.3 & & \\
\hline 354 & & RIVER & & RAW GRAB & TRANSECT & 570 & VERNITA-2 HRM 0.3 & & \\
\hline 355 & & RIVER & & RAW GRAB & TRANSECT & 570 & VERNITA-2 HRM 0.3 & & \\
\hline 356 & & RIVER & & RAW GRAB & TRANSECT & 318 & VERNITA-3 HRM 0.3 & & \\
\hline 357 & & RIVER & & RAW GRAB & TRANSECT & 318 & VERNITA-3 HRM 0.3 & & \\
\hline 358 & & RIVER & & RAW GRAB & TRANSECT & 318 & VERNITA-3 HRM 0.3 & & \\
\hline 359 & & RIVER & & RAW GRAB & TRANSECT & 318 & VERNITA-3 HRM 0.3 & & \\
\hline 360 & & RIVER & & RAW GRAB & TRANSECT & 318 & VERNITA-3 HRM 0.3 & & \\
\hline 361 & & RIVER & & RAW GRAB & TRANSECT & 319 & VERNITA-4 HRM 0.3 & & \\
\hline 362 & & RIVER & & RAW GRAB & TRANSECT & 319 & VERNITA-4 HRM 0.3 & & \\
\hline 363 & & RIVER & & RAW GRAB & TRANSECT & 319 & VERNITA-4 HRM 0.3 & & \\
\hline 364 & & RIVER & & RAW GRAB & TRANSECT & 319 & VERNITA-4 HRM 0.3 & & \\
\hline 365 & & RIVER & & RAW GRAB & TRANSECT & 319 & VERNITA-4 HRM 0.3 & & \\
\hline 366 & & RIVER & & RAW GRAB & TRANSECT & 571 & RICH.PMPHS-1 HRM46.4 & & \\
\hline 367 & & RIVER & & RAW GRAB & TRANSECT & 571 & RICH.PMPHS-1 HRM46.4 & & \\
\hline 368 & & RIVER & & RAW GRAB & TRANSECT & 571 & RICH.PMPHS-1 HRM46.4 & & \\
\hline 369 & & RIVER & & RAW GRAB & TRANSECT & 571 & RICH.PMPHS-1 HRM46.4 & & \\
\hline 370 & & RIVER & & RAW GRAB & TRANSECT & 571 & RICH.PMPHS-1 HRM46.4 & & \\
\hline 371 & & RIVER & & RAW GRAB & TRANSECT & 327 & RICH.PMPHS-10 HRM46.4 & & \\
\hline 372 & & RIVER & & RAW GRAB & TRANSECT & 327 & RICH.PMPHS-10 HRM46.4 & & \\
\hline 373 & & RIVER & & RAW GRAB & TRANSECT & 327 & RICH.PMPHS-10 HRM46.4 & & \\
\hline 374 & & RIVER & & RAW GRAB & TRANSECT & 327 & RICH.PMPHS-10 HRM46.4 & & \\
\hline 375 & & RIVER & & RAW GRAB & TRANSECT & 327 & RICH.PMPHS-10 HRM46.4 & & \\
\hline 376 & & RIVER & & RAW GRAB & TRANSECT & 321 & RICH.PMPHS-2 HRM46.4 & & \\
\hline 377 & & RIVER & & RAW GRAB & TRANSECT & 321 & RICH.PMPHS-2 HRM46.4 & & \\
\hline 378 & & RIVER & & RAW GRAB & TRANSECT & 321 & RICH.PMPHS-2 HRM46.4 & & \\
\hline 379 & & RIVER & & RAW GRAB & TRANSECT & 321 & RICH.PMPHS-2 HRM46.4 & & \\
\hline 380 & & RIVER & & RAW GRAB & TRANSECT & 321 & RICH.PMPHS-2 HRM46.4 & & \\
\hline 381 & & RIVER & & RAW GRAB & TRANSECT & 322 & RICH.PMPHS-3 HRM46.4 & & \\
\hline
\end{tabular}




\begin{tabular}{|c|c|c|c|c|c|c|c|c|c|}
\hline & $\mathrm{T}$ & $\mathrm{U}$ & $\mathrm{V}$ & $\mathrm{W}$ & $\mathrm{X}$ & $\bar{Y}$ & $\bar{Z}$ & $\overline{A A}$ & $A B$ \\
\hline 1 & REVIEW_QUALIFIER & SAMP_FROM & SAMP_ITEM & SAMP_MTHD & COLL_MTHD & SAMP_SITE_ID & SAMP_SITE_NAME & WELL_NAME & SAMPLE_LOCATION \\
\hline 382 & & RIVER & & RAW GRAB & TRANSECT & 322 & RICH.PMPHS-3 HRM46.4 & & \\
\hline 383 & & RIVER & & RAW GRAB & TRANSECT & 322 & RICH.PMPHS-3 HRM46.4 & & \\
\hline 384 & & RIVER & & RAW GRAB & TRANSECT & 322 & RICH.PMPHS-3 HRM46.4 & & \\
\hline 385 & & RIVER & & RAW GRAB & TRANSECT & 322 & RICH.PMPHS-3 HRM46.4 & & \\
\hline 386 & & RIVER & & RAW GRAB & TRANSECT & 572 & RICH.PMPHS-4 HRM46.4 & & \\
\hline 387 & & RIVER & & RAW GRAB & TRANSECT & 572 & RICH.PMPHS-4 HRM46.4 & & \\
\hline 388 & & RIVER & & RAW GRAB & TRANSECT & 572 & RICH.PMPHS-4 HRM46.4 & & \\
\hline 389 & & RIVER & & RAW GRAB & TRANSECT & 572 & RICH.PMPHS-4 HRM46.4 & & \\
\hline 390 & & RIVER & & RAW GRAB & TRANSECT & 572 & RICH.PMPHS-4 HRM46.4 & & \\
\hline 391 & & RIVER & & RAW GRAB & TRANSECT & 323 & RICH.PMPHS-5 HRM46.4 & & \\
\hline 392 & & RIVER & & RAW GRAB & TRANSECT & 323 & RICH.PMPHS-5 HRM46.4 & & \\
\hline 393 & & RIVER & & RAW GRAB & TRANSECT & 323 & RICH.PMPHS-5 HRM46.4 & & \\
\hline 394 & & RIVER & & RAW GRAB & TRANSECT & 323 & RICH.PMPHS-5 HRM46.4 & & \\
\hline 395 & & RIVER & & RAW GRAB & TRANSECT & 323 & RICH.PMPHS-5 HRM46.4 & & \\
\hline 396 & & RIVER & & RAW GRAB & TRANSECT & 324 & RICH.PMPHS-6 HRM46.4 & & \\
\hline 397 & & RIVER & & RAW GRAB & TRANSECT & 324 & RICH.PMPHS-6 HRM46.4 & & \\
\hline 398 & & RIVER & & RAW GRAB & TRANSECT & 324 & RICH.PMPHS-6 HRM46.4 & & \\
\hline 399 & & RIVER & & RAW GRAB & TRANSECT & 324 & RICH.PMPHS-6 HRM46.4 & & \\
\hline 400 & & RIVER & & RAW GRAB & TRANSECT & 324 & RICH.PMPHS-6 HRM46.4 & & \\
\hline 401 & & RIVER & & RAW GRAB & TRANSECT & 325 & RICH.PMPHS-7 HRM46.4 & & \\
\hline 402 & & RIVER & & RAW GRAB & TRANSECT & 325 & RICH.PMPHS-7 HRM46.4 & & \\
\hline 403 & & RIVER & & RAW GRAB & TRANSECT & 325 & RICH.PMPHS-7 HRM46.4 & & \\
\hline 404 & & RIVER & & RAW GRAB & TRANSECT & 325 & RICH.PMPHS-7 HRM46.4 & & \\
\hline 405 & & RIVER & & RAW GRAB & TRANSECT & 325 & RICH.PMPHS-7 HRM46.4 & & \\
\hline 406 & & RIVER & & RAW GRAB & TRANSECT & 326 & RICH.PMPHS-8 HRM46.4 & & \\
\hline 407 & & RIVER & & RAW GRAB & TRANSECT & 326 & RICH.PMPHS-8 HRM46.4 & & \\
\hline 408 & & RIVER & & RAW GRAB & TRANSECT & 326 & RICH.PMPHS-8 HRM46.4 & & \\
\hline 409 & & RIVER & & RAW GRAB & TRANSECT & 326 & RICH.PMPHS-8 HRM46.4 & & \\
\hline 410 & & RIVER & & RAW GRAB & TRANSECT & 326 & RICH.PMPHS-8 HRM46.4 & & \\
\hline 411 & & RIVER & & RAW GRAB & TRANSECT & 573 & RICH.PMPHS-9 HRM46.4 & & \\
\hline 412 & & RIVER & & RAW GRAB & TRANSECT & 573 & RICH.PMPHS-9 HRM46.4 & & \\
\hline 413 & & RIVER & & RAW GRAB & TRANSECT & 573 & RICH.PMPHS-9 HRM46.4 & & \\
\hline 414 & & RIVER & & RAW GRAB & TRANSECT & 573 & RICH.PMPHS-9 HRM46.4 & & \\
\hline 415 & & RIVER & & RAW GRAB & TRANSECT & 573 & RICH.PMPHS-9 HRM46.4 & & \\
\hline 416 & & RIVER & & RAW GRAB & TRANSECT & 571 & RICH.PMPHS-1 HRM46.4 & & \\
\hline 417 & & RIVER & & RAW GRAB & TRANSECT & 571 & RICH.PMPHS-1 HRM46.4 & & \\
\hline 418 & & RIVER & & RAW GRAB & TRANSECT & 571 & RICH.PMPHS-1 HRM46.4 & & \\
\hline 419 & & RIVER & & RAW GRAB & TRANSECT & 571 & RICH.PMPHS-1 HRM46.4 & & \\
\hline
\end{tabular}




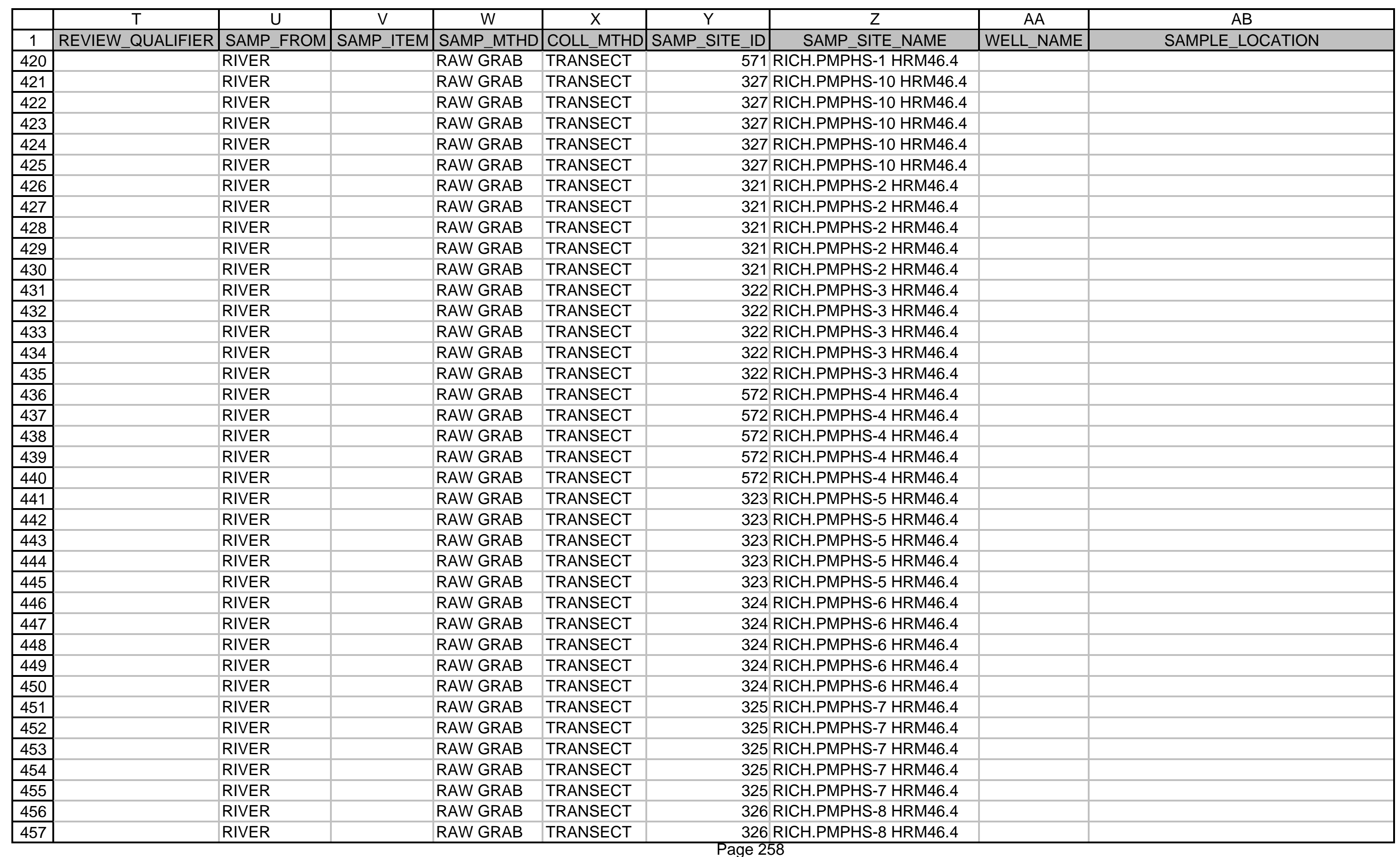




\begin{tabular}{|c|c|c|c|c|c|c|c|c|c|}
\hline & $\mathrm{T}$ & $U$ & $\mathrm{~V}$ & $\mathrm{~W}$ & $\mathrm{X}$ & $\mathrm{Y}$ & $Z$ & $\mathrm{AA}$ & $A B$ \\
\hline 1 & REVIEW_QUALIFIER & SAMP_FROM & SAMP_ITEM & SAMP_MTHD & COLL_MTHD & SAMP_SITE_ID & SAMP_SITE_NAME & WELL_NAME & SAMPLE_LOCATION \\
\hline 458 & & RIVER & & RAW GRAB & TRANSECT & 326 & 5 RICH.PMPHS-8 HRM46.4 & & \\
\hline 459 & & RIVER & & RAW GRAB & TRANSECT & 326 & 6 RICH.PMPHS-8 HRM46.4 & & \\
\hline 460 & & RIVER & & RAW GRAB & TRANSECT & 326 & 6 RICH.PMPHS-8 HRM46.4 & & \\
\hline 461 & & RIVER & & RAW GRAB & TRANSECT & 573 & 3 RICH.PMPHS-9 HRM46.4 & & \\
\hline 462 & & RIVER & & RAW GRAB & TRANSECT & 573 & 3 RICH.PMPHS-9 HRM46.4 & & \\
\hline 463 & & RIVER & & RAW GRAB & TRANSECT & 573 & 3 RICH.PMPHS-9 HRM46.4 & & \\
\hline 464 & & RIVER & & RAW GRAB & TRANSECT & 573 & 3 RICH.PMPHS-9 HRM46.4 & & \\
\hline 465 & & RIVER & & RAW GRAB & TRANSECT & 573 & 3 RICH.PMPHS-9 HRM46.4 & & \\
\hline 466 & & RIVER & & RAW GRAB & TRANSECT & 317 & 7 VERNITA-1 HRM 0.3 & & \\
\hline 467 & & RIVER & & RAW GRAB & TRANSECT & 317 & 7 VERNITA-1 HRM 0.3 & & \\
\hline 468 & & RIVER & & RAW GRAB & TRANSECT & 317 & 7 VERNITA-1 HRM 0.3 & & \\
\hline 469 & & RIVER & & RAW GRAB & TRANSECT & 317 & 7 VERNITA-1 HRM 0.3 & & \\
\hline 470 & & RIVER & & RAW GRAB & TRANSECT & 317 & 7 VERNITA-1 HRM 0.3 & & \\
\hline 471 & & RIVER & & RAW GRAB & TRANSECT & 570 & VERNITA-2 HRM 0.3 & & \\
\hline 472 & & RIVER & & RAW GRAB & TRANSECT & 570 & VERNITA-2 HRM 0.3 & & \\
\hline 473 & & RIVER & & RAW GRAB & TRANSECT & 570 & VERNITA-2 HRM 0.3 & & \\
\hline 474 & & RIVER & & RAW GRAB & TRANSECT & 570 & VERNITA-2 HRM 0.3 & & \\
\hline 475 & & RIVER & & RAW GRAB & TRANSECT & 570 & VERNITA-2 HRM 0.3 & & \\
\hline 476 & & RIVER & & RAW GRAB & TRANSECT & 318 & 3 VERNITA-3 HRM 0.3 & & \\
\hline 477 & & RIVER & & RAW GRAB & TRANSECT & 318 & 3 VERNITA-3 HRM 0.3 & & \\
\hline 478 & & RIVER & & RAW GRAB & TRANSECT & 318 & 3 VERNITA-3 HRM 0.3 & & \\
\hline 479 & & RIVER & & RAW GRAB & TRANSECT & 318 & 3 VERNITA-3 HRM 0.3 & & \\
\hline 480 & & RIVER & & RAW GRAB & TRANSECT & 318 & 3 VERNITA-3 HRM 0.3 & & \\
\hline 481 & & RIVER & & RAW GRAB & TRANSECT & 319 & 9 VERNITA-4 HRM 0.3 & & \\
\hline 482 & & RIVER & & RAW GRAB & TRANSECT & 319 & 9 VERNITA-4 HRM 0.3 & & \\
\hline 483 & & RIVER & & RAW GRAB & TRANSECT & 319 & 9 VERNITA-4 HRM 0.3 & & \\
\hline 484 & & RIVER & & RAW GRAB & TRANSECT & 319 & 9 VERNITA-4 HRM 0.3 & & \\
\hline 485 & & RIVER & & RAW GRAB & TRANSECT & 319 & 9 VERNITA-4 HRM 0.3 & & \\
\hline 486 & & RIVER & & RAW GRAB & TRANSECT & 317 & 7 VERNITA-1 HRM 0.3 & & \\
\hline 487 & & RIVER & & RAW GRAB & TRANSECT & 317 & 7 VERNITA-1 HRM 0.3 & & \\
\hline 488 & & RIVER & & RAW GRAB & TRANSECT & 317 & 7 VERNITA-1 HRM 0.3 & & \\
\hline 489 & & RIVER & & RAW GRAB & TRANSECT & 317 & 7 VERNITA-1 HRM 0.3 & & \\
\hline 490 & & RIVER & & RAW GRAB & TRANSECT & 317 & 7 VERNITA-1 HRM 0.3 & & \\
\hline 491 & & RIVER & & RAW GRAB & TRANSECT & 570 & VERNITA-2 HRM 0.3 & & \\
\hline 492 & & RIVER & & RAW GRAB & TRANSECT & 570 & VERNITA-2 HRM 0.3 & & \\
\hline 493 & & RIVER & & RAW GRAB & TRANSECT & 570 & VERNITA-2 HRM 0.3 & & \\
\hline 494 & & RIVER & & RAW GRAB & TRANSECT & 570 & VERNITA-2 HRM 0.3 & & \\
\hline 495 & & RIVER & & RAW GRAB & TRANSECT & 570 & VERNITA-2 HRM 0.3 & & \\
\hline
\end{tabular}




\begin{tabular}{|c|c|c|c|c|c|c|c|c|c|}
\hline & $\mathrm{T}$ & $U$ & $\mathrm{~V}$ & $\mathrm{~W}$ & $\mathrm{X}$ & $\mathrm{Y}$ & $Z$ & $\mathrm{AA}$ & $A B$ \\
\hline 1 & REVIEW_QUALIFIER & SAMP_FROM & SAMP_ITEM & SAMP_MTHD & COLL_MTHD & SAMP_SITE_ID & SAMP_SITE_NAME & WELL_NAME & SAMPLE_LOCATION \\
\hline 496 & & RIVER & & RAW GRAB & TRANSECT & 318 & VERNITA-3 HRM 0.3 & & \\
\hline 497 & & RIVER & & RAW GRAB & TRANSECT & 318 & VERNITA-3 HRM 0.3 & & \\
\hline 498 & & RIVER & & RAW GRAB & TRANSECT & 318 & VERNITA-3 HRM 0.3 & & \\
\hline 499 & & RIVER & & RAW GRAB & TRANSECT & 318 & VERNITA-3 HRM 0.3 & & \\
\hline 500 & & RIVER & & RAW GRAB & TRANSECT & 318 & VERNITA-3 HRM 0.3 & & \\
\hline 501 & & RIVER & & RAW GRAB & TRANSECT & 319 & VERNITA-4 HRM 0.3 & & \\
\hline 502 & & RIVER & & RAW GRAB & TRANSECT & 319 & VERNITA-4 HRM 0.3 & & \\
\hline 503 & & RIVER & & RAW GRAB & TRANSECT & 319 & VERNITA-4 HRM 0.3 & & \\
\hline 504 & & RIVER & & RAW GRAB & TRANSECT & 319 & VERNITA-4 HRM 0.3 & & \\
\hline 505 & & RIVER & & RAW GRAB & TRANSECT & 319 & VERNITA-4 HRM 0.3 & & \\
\hline 506 & & RIVER & & RAW GRAB & TRANSECT & 463 & RICH.PMPHS HRM 46.4 & & \\
\hline 507 & & RIVER & & RAW GRAB & TRANSECT & 463 & RICH.PMPHS HRM 46.4 & & \\
\hline 508 & & RIVER & & RAW GRAB & TRANSECT & 463 & RICH.PMPHS HRM 46.4 & & \\
\hline 509 & & RIVER & & RAW GRAB & TRANSECT & 463 & RICH.PMPHS HRM 46.4 & & \\
\hline 510 & & RIVER & & RAW GRAB & TRANSECT & 463 & RICH.PMPHS HRM 46.4 & & \\
\hline 511 & & RIVER & & RAW GRAB & TRANSECT & 463 & RICH.PMPHS HRM 46.4 & & \\
\hline 512 & & RIVER & & RAW GRAB & TRANSECT & 463 & RICH.PMPHS HRM 46.4 & & \\
\hline 513 & & RIVER & & RAW GRAB & TRANSECT & 463 & RICH.PMPHS HRM 46.4 & & \\
\hline 514 & & RIVER & & RAW GRAB & TRANSECT & 463 & RICH.PMPHS HRM 46.4 & & \\
\hline 515 & & RIVER & & RAW GRAB & TRANSECT & 463 & RICH.PMPHS HRM 46.4 & & \\
\hline 516 & & RIVER & & RAW GRAB & TRANSECT & 571 & RICH.PMPHS-1 HRM46.4 & & \\
\hline 517 & & RIVER & & RAW GRAB & TRANSECT & 571 & RICH.PMPHS-1 HRM46.4 & & \\
\hline 518 & & RIVER & & RAW GRAB & TRANSECT & 571 & RICH.PMPHS-1 HRM46.4 & & \\
\hline 519 & & RIVER & & RAW GRAB & TRANSECT & 571 & RICH.PMPHS-1 HRM46.4 & & \\
\hline 520 & & RIVER & & RAW GRAB & TRANSECT & 571 & RICH.PMPHS-1 HRM46.4 & & \\
\hline 521 & & RIVER & & RAW GRAB & TRANSECT & 327 & RICH.PMPHS-10 HRM46.4 & & \\
\hline 522 & & RIVER & & RAW GRAB & TRANSECT & 327 & RICH.PMPHS-10 HRM46.4 & & \\
\hline 523 & & RIVER & & RAW GRAB & TRANSECT & 327 & RICH.PMPHS-10 HRM46.4 & & \\
\hline 524 & & RIVER & & RAW GRAB & TRANSECT & 327 & RICH.PMPHS-10 HRM46.4 & & \\
\hline 525 & & RIVER & & RAW GRAB & TRANSECT & 327 & RICH.PMPHS-10 HRM46.4 & & \\
\hline 526 & & RIVER & & RAW GRAB & TRANSECT & 321 & RICH.PMPHS-2 HRM46.4 & & \\
\hline 527 & & RIVER & & RAW GRAB & TRANSECT & 321 & RICH.PMPHS-2 HRM46.4 & & \\
\hline 528 & & RIVER & & RAW GRAB & TRANSECT & 321 & RICH.PMPHS-2 HRM46.4 & & \\
\hline 529 & & RIVER & & RAW GRAB & TRANSECT & 321 & RICH.PMPHS-2 HRM46.4 & & \\
\hline 530 & & RIVER & & RAW GRAB & TRANSECT & 321 & RICH.PMPHS-2 HRM46.4 & & \\
\hline 531 & & RIVER & & RAW GRAB & TRANSECT & 322 & RICH.PMPHS-3 HRM46.4 & & \\
\hline 532 & & RIVER & & RAW GRAB & TRANSECT & 322 & RICH.PMPHS-3 HRM46.4 & & \\
\hline 533 & & RIVER & & RAW GRAB & TRANSECT & 322 & RICH.PMPHS-3 HRM46.4 & & \\
\hline
\end{tabular}




\begin{tabular}{|c|c|c|c|c|c|c|c|c|c|}
\hline & $\mathrm{T}$ & $\mathrm{U}$ & $\mathrm{V}$ & W & $\mathrm{X}$ & $\mathrm{Y}$ & Z & $\mathrm{AA}$ & $A B$ \\
\hline 1 & REVIEW_QUALIFIER & SAMP_FROM & SAMP_ITEM & SAMP_MTHD & COLL_MTHD & SAMP_SITE_ID & SAMP_SITE_NAME & WELL_NAME & SAMPLE_LOCATION \\
\hline 534 & & RIVER & & RAW GRAB & TRANSECT & 322 & RICH.PMPHS-3 HRM46.4 & & \\
\hline 535 & & RIVER & & RAW GRAB & TRANSECT & 322 & RICH.PMPHS-3 HRM46.4 & & \\
\hline 536 & & RIVER & & RAW GRAB & TRANSECT & 572 & RICH.PMPHS-4 HRM46.4 & & \\
\hline 537 & & RIVER & & RAW GRAB & TRANSECT & 572 & RICH.PMPHS-4 HRM46.4 & & \\
\hline 538 & & RIVER & & RAW GRAB & TRANSECT & 572 & RICH.PMPHS-4 HRM46.4 & & \\
\hline 539 & & RIVER & & RAW GRAB & TRANSECT & 572 & RICH.PMPHS-4 HRM46.4 & & \\
\hline 540 & & RIVER & & RAW GRAB & TRANSECT & 572 & RICH.PMPHS-4 HRM46.4 & & \\
\hline 541 & & RIVER & & RAW GRAB & TRANSECT & 323 & RICH.PMPHS-5 HRM46.4 & & \\
\hline 542 & & RIVER & & RAW GRAB & TRANSECT & 323 & RICH.PMPHS-5 HRM46.4 & & \\
\hline 543 & & RIVER & & RAW GRAB & TRANSECT & 323 & RICH.PMPHS-5 HRM46.4 & & \\
\hline 544 & & RIVER & & RAW GRAB & TRANSECT & 323 & RICH.PMPHS-5 HRM46.4 & & \\
\hline 545 & & RIVER & & RAW GRAB & TRANSECT & 323 & RICH.PMPHS-5 HRM46.4 & & \\
\hline 546 & & RIVER & & RAW GRAB & TRANSECT & 324 & RICH.PMPHS-6 HRM46.4 & & \\
\hline 547 & & RIVER & & RAW GRAB & TRANSECT & 324 & RICH.PMPHS-6 HRM46.4 & & \\
\hline 548 & & RIVER & & RAW GRAB & TRANSECT & 324 & RICH.PMPHS-6 HRM46.4 & & \\
\hline 549 & & RIVER & & RAW GRAB & TRANSECT & 324 & RICH.PMPHS-6 HRM46.4 & & \\
\hline 550 & & RIVER & & RAW GRAB & TRANSECT & 324 & RICH.PMPHS-6 HRM46.4 & & \\
\hline 551 & & RIVER & & RAW GRAB & TRANSECT & 325 & RICH.PMPHS-7 HRM46.4 & & \\
\hline 552 & & RIVER & & RAW GRAB & TRANSECT & 325 & RICH.PMPHS-7 HRM46.4 & & \\
\hline 553 & & RIVER & & RAW GRAB & TRANSECT & 325 & RICH.PMPHS-7 HRM46.4 & & \\
\hline 554 & & RIVER & & RAW GRAB & TRANSECT & 325 & RICH.PMPHS-7 HRM46.4 & & \\
\hline 555 & & RIVER & & RAW GRAB & TRANSECT & 325 & RICH.PMPHS-7 HRM46.4 & & \\
\hline 556 & & RIVER & & RAW GRAB & TRANSECT & 326 & RICH.PMPHS-8 HRM46.4 & & \\
\hline 557 & & RIVER & & RAW GRAB & TRANSECT & 326 & RICH.PMPHS-8 HRM46.4 & & \\
\hline 558 & & RIVER & & RAW GRAB & TRANSECT & 326 & RICH.PMPHS-8 HRM46.4 & & \\
\hline 559 & & RIVER & & RAW GRAB & TRANSECT & 326 & RICH.PMPHS-8 HRM46.4 & & \\
\hline 560 & & RIVER & & RAW GRAB & TRANSECT & 326 & RICH.PMPHS-8 HRM46.4 & & \\
\hline 561 & & RIVER & & RAW GRAB & TRANSECT & 573 & RICH.PMPHS-9 HRM46.4 & & \\
\hline 562 & & RIVER & & RAW GRAB & TRANSECT & 573 & RICH.PMPHS-9 HRM46.4 & & \\
\hline 563 & & RIVER & & RAW GRAB & TRANSECT & 573 & RICH.PMPHS-9 HRM46.4 & & \\
\hline 564 & & RIVER & & RAW GRAB & TRANSECT & 573 & RICH.PMPHS-9 HRM46.4 & & \\
\hline 565 & & RIVER & & RAW GRAB & TRANSECT & 573 & RICH.PMPHS-9 HRM46.4 & & \\
\hline 566 & & RIVER & & RAW GRAB & TRANSECT & 317 & VERNITA-1 HRM 0.3 & & \\
\hline 567 & & RIVER & & RAW GRAB & TRANSECT & 317 & VERNITA-1 HRM 0.3 & & \\
\hline 568 & & RIVER & & RAW GRAB & TRANSECT & 317 & VERNITA-1 HRM 0.3 & & \\
\hline 569 & & RIVER & & RAW GRAB & TRANSECT & 317 & VERNITA-1 HRM 0.3 & & \\
\hline 570 & & RIVER & & RAW GRAB & TRANSECT & 317 & VERNITA-1 HRM 0.3 & & \\
\hline 571 & & RIVER & & RAW GRAB & TRANSECT & 570 & VERNITA-2 HRM 0.3 & & \\
\hline
\end{tabular}




\begin{tabular}{|c|c|c|c|c|c|c|c|c|c|}
\hline & $\mathrm{T}$ & $U$ & $\mathrm{~V}$ & $\mathrm{~W}$ & $\mathrm{X}$ & $\mathrm{Y}$ & $Z$ & $\mathrm{AA}$ & $A B$ \\
\hline 1 & REVIEW_QUALIFIER & SAMP_FROM & SAMP_ITEM & SAMP_MTHD & COLL_MTHD & SAMP_SITE_ID & SAMP_SITE_NAME & WELL_NAME & SAMPLE_LOCATION \\
\hline 572 & & RIVER & & RAW GRAB & TRANSECT & 570 & VERNITA-2 HRM 0.3 & & \\
\hline 573 & & RIVER & & RAW GRAB & TRANSECT & 570 & VERNITA-2 HRM 0.3 & & \\
\hline 574 & & RIVER & & RAW GRAB & TRANSECT & 570 & VERNITA-2 HRM 0.3 & & \\
\hline 575 & & RIVER & & RAW GRAB & TRANSECT & 570 & VERNITA-2 HRM 0.3 & & \\
\hline 576 & & RIVER & & RAW GRAB & TRANSECT & 318 & 3 VERNITA-3 HRM 0.3 & & \\
\hline 577 & & RIVER & & RAW GRAB & TRANSECT & 318 & 3 VERNITA-3 HRM 0.3 & & \\
\hline 578 & & RIVER & & RAW GRAB & TRANSECT & 318 & 3 VERNITA-3 HRM 0.3 & & \\
\hline 579 & & RIVER & & RAW GRAB & TRANSECT & 318 & 3 VERNITA-3 HRM 0.3 & & \\
\hline 580 & & RIVER & & RAW GRAB & TRANSECT & 318 & 3 VERNITA-3 HRM 0.3 & & \\
\hline 581 & & RIVER & & RAW GRAB & TRANSECT & 319 & 9 VERNITA-4 HRM 0.3 & & \\
\hline 582 & & RIVER & & RAW GRAB & TRANSECT & 319 & 9 VERNITA-4 HRM 0.3 & & \\
\hline 583 & & RIVER & & RAW GRAB & TRANSECT & 319 & 9 VERNITA-4 HRM 0.3 & & \\
\hline 584 & & RIVER & & RAW GRAB & TRANSECT & 319 & 9 VERNITA-4 HRM 0.3 & & \\
\hline 585 & & RIVER & & RAW GRAB & TRANSECT & 319 & 9 VERNITA-4 HRM 0.3 & & \\
\hline 586 & & RIVER & & RAW GRAB & TRANSECT & 571 & RICH.PMPHS-1 HRM46.4 & & \\
\hline 587 & & RIVER & & RAW GRAB & TRANSECT & 571 & RICH.PMPHS-1 HRM46.4 & & \\
\hline 588 & & RIVER & & RAW GRAB & TRANSECT & 571 & RICH.PMPHS-1 HRM46.4 & & \\
\hline 589 & & RIVER & & RAW GRAB & TRANSECT & 571 & RICH.PMPHS-1 HRM46.4 & & \\
\hline 590 & & RIVER & & RAW GRAB & TRANSECT & 571 & RICH.PMPHS-1 HRM46.4 & & \\
\hline 591 & & RIVER & & RAW GRAB & TRANSECT & 327 & 7 RICH.PMPHS-10 HRM46.4 & & \\
\hline 592 & & RIVER & & RAW GRAB & TRANSECT & 327 & 7 RICH.PMPHS-10 HRM46.4 & & \\
\hline 593 & & RIVER & & RAW GRAB & TRANSECT & 327 & 7 RICH.PMPHS-10 HRM46.4 & & \\
\hline 594 & & RIVER & & RAW GRAB & TRANSECT & 327 & 7 RICH.PMPHS-10 HRM46.4 & & \\
\hline 595 & & RIVER & & RAW GRAB & TRANSECT & 327 & 7 RICH.PMPHS-10 HRM46.4 & & \\
\hline 596 & & RIVER & & RAW GRAB & TRANSECT & 321 & RICH.PMPHS-2 HRM46.4 & & \\
\hline 597 & & RIVER & & RAW GRAB & TRANSECT & 321 & 1 RICH.PMPHS-2 HRM46.4 & & \\
\hline 598 & & RIVER & & RAW GRAB & TRANSECT & 321 & RICH.PMPHS-2 HRM46.4 & & \\
\hline 599 & & RIVER & & RAW GRAB & TRANSECT & 321 & RICH.PMPHS-2 HRM46.4 & & \\
\hline 600 & & RIVER & & RAW GRAB & TRANSECT & 321 & RICH.PMPHS-2 HRM46.4 & & \\
\hline 601 & & RIVER & & RAW GRAB & TRANSECT & 322 & 2 RICH.PMPHS-3 HRM46.4 & & \\
\hline 602 & & RIVER & & RAW GRAB & TRANSECT & 322 & 2 RICH.PMPHS-3 HRM46.4 & & \\
\hline 603 & & RIVER & & RAW GRAB & TRANSECT & 322 & 2 RICH.PMPHS-3 HRM46.4 & & \\
\hline 604 & & RIVER & & RAW GRAB & TRANSECT & 322 & 2 RICH.PMPHS-3 HRM46.4 & & \\
\hline 605 & & RIVER & & RAW GRAB & TRANSECT & 322 & 2 RICH.PMPHS-3 HRM46.4 & & \\
\hline 606 & & RIVER & & RAW GRAB & TRANSECT & 572 & 2 RICH.PMPHS-4 HRM46.4 & & \\
\hline 607 & & RIVER & & RAW GRAB & TRANSECT & 572 & 2 RICH.PMPHS-4 HRM46.4 & & \\
\hline 608 & & RIVER & & RAW GRAB & TRANSECT & 572 & 2 RICH.PMPHS-4 HRM46.4 & & \\
\hline 609 & & RIVER & & RAW GRAB & TRANSECT & 572 & 2 RICH.PMPHS-4 HRM46.4 & & \\
\hline
\end{tabular}




\begin{tabular}{|c|c|c|c|c|c|c|c|c|c|}
\hline & $\mathrm{T}$ & $U$ & $\mathrm{~V}$ & $\mathrm{~W}$ & $\mathrm{X}$ & $\mathrm{Y}$ & $Z$ & $\mathrm{AA}$ & $A B$ \\
\hline 1 & REVIEW_QUALIFIER & SAMP_FROM & SAMP_ITEM & SAMP_MTHD & COLL_MTHD & SAMP_SITE_ID & SAMP_SITE_NAME & WELL_NAME & SAMPLE_LOCATION \\
\hline 610 & & RIVER & & RAW GRAB & TRANSECT & 572 & 2 RICH.PMPHS-4 HRM46.4 & & \\
\hline 611 & & RIVER & & RAW GRAB & TRANSECT & 323 & 3 RICH.PMPHS-5 HRM46.4 & & \\
\hline 612 & & RIVER & & RAW GRAB & TRANSECT & 323 & 3 RICH.PMPHS-5 HRM46.4 & & \\
\hline 613 & & RIVER & & RAW GRAB & TRANSECT & 323 & 3 RICH.PMPHS-5 HRM46.4 & & \\
\hline 614 & & RIVER & & RAW GRAB & TRANSECT & 323 & 3 RICH.PMPHS-5 HRM46.4 & & \\
\hline 615 & & RIVER & & RAW GRAB & TRANSECT & 323 & 3 RICH.PMPHS-5 HRM46.4 & & \\
\hline 616 & & RIVER & & RAW GRAB & TRANSECT & 324 & 4 RICH.PMPHS-6 HRM46.4 & & \\
\hline 617 & & RIVER & & RAW GRAB & TRANSECT & 324 & 4 RICH.PMPHS-6 HRM46.4 & & \\
\hline 618 & & RIVER & & RAW GRAB & TRANSECT & 324 & RICH.PMPHS-6 HRM46.4 & & \\
\hline 619 & & RIVER & & RAW GRAB & TRANSECT & 324 & RICH.PMPHS-6 HRM46.4 & & \\
\hline 620 & & RIVER & & RAW GRAB & TRANSECT & 324 & RICH.PMPHS-6 HRM46.4 & & \\
\hline 621 & & RIVER & & RAW GRAB & TRANSECT & 325 & RICH.PMPHS-7 HRM46.4 & & \\
\hline 622 & & RIVER & & RAW GRAB & TRANSECT & 325 & RICH.PMPHS-7 HRM46.4 & & \\
\hline 623 & & RIVER & & RAW GRAB & TRANSECT & 325 & RICH.PMPHS-7 HRM46.4 & & \\
\hline 624 & & RIVER & & RAW GRAB & TRANSECT & 325 & RICH.PMPHS-7 HRM46.4 & & \\
\hline 625 & & RIVER & & RAW GRAB & TRANSECT & 325 & RICH.PMPHS-7 HRM46.4 & & \\
\hline 626 & & RIVER & & RAW GRAB & TRANSECT & 326 & RICH.PMPHS-8 HRM46.4 & & \\
\hline 627 & & RIVER & & RAW GRAB & TRANSECT & 326 & RICH.PMPHS-8 HRM46.4 & & \\
\hline 628 & & RIVER & & RAW GRAB & TRANSECT & 326 & RICH.PMPHS-8 HRM46.4 & & \\
\hline 629 & & RIVER & & RAW GRAB & TRANSECT & 326 & RICH.PMPHS-8 HRM46.4 & & \\
\hline 630 & & RIVER & & RAW GRAB & TRANSECT & 326 & RICH.PMPHS-8 HRM46.4 & & \\
\hline 631 & & RIVER & & RAW GRAB & TRANSECT & 573 & RICH.PMPHS-9 HRM46.4 & & \\
\hline 632 & & RIVER & & RAW GRAB & TRANSECT & 573 & RICH.PMPHS-9 HRM46.4 & & \\
\hline 633 & & RIVER & & RAW GRAB & TRANSECT & 573 & RICH.PMPHS-9 HRM46.4 & & \\
\hline 634 & & RIVER & & RAW GRAB & TRANSECT & 573 & RICH.PMPHS-9 HRM46.4 & & \\
\hline 635 & & RIVER & & RAW GRAB & TRANSECT & 573 & RICH.PMPHS-9 HRM46.4 & & \\
\hline 636 & & RIVER & & RAW GRAB & TRANSECT & 317 & VERNITA-1 HRM 0.3 & & \\
\hline 637 & & RIVER & & RAW GRAB & TRANSECT & 317 & VERNITA-1 HRM 0.3 & & \\
\hline 638 & & RIVER & & RAW GRAB & TRANSECT & 317 & VERNITA-1 HRM 0.3 & & \\
\hline 639 & & RIVER & & RAW GRAB & TRANSECT & 317 & VERNITA-1 HRM 0.3 & & \\
\hline 640 & & RIVER & & RAW GRAB & TRANSECT & 317 & VERNITA-1 HRM 0.3 & & \\
\hline 641 & & RIVER & & RAW GRAB & TRANSECT & 317 & VERNITA-1 HRM 0.3 & & \\
\hline 642 & & RIVER & & RAW GRAB & TRANSECT & 570 & VERNITA-2 HRM 0.3 & & \\
\hline 643 & & RIVER & & RAW GRAB & TRANSECT & 570 & VERNITA-2 HRM 0.3 & & \\
\hline 644 & & RIVER & & RAW GRAB & TRANSECT & 570 & VERNITA-2 HRM 0.3 & & \\
\hline 645 & & RIVER & & RAW GRAB & TRANSECT & 570 & VERNITA-2 HRM 0.3 & & \\
\hline 646 & & RIVER & & RAW GRAB & TRANSECT & 570 & VERNITA-2 HRM 0.3 & & \\
\hline 647 & & RIVER & & RAW GRAB & TRANSECT & 570 & VERNITA-2 HRM 0.3 & & \\
\hline
\end{tabular}




\begin{tabular}{|c|c|c|c|c|c|c|c|c|c|}
\hline & $\mathrm{T}$ & $U$ & $\mathrm{~V}$ & $\mathrm{~W}$ & $\mathrm{X}$ & $\bar{Y}$ & $Z$ & AA & $A B$ \\
\hline 1 & REVIEW_QUALIFIER & SAMP_FROM & SAMP_ITEM & SAMP_MTHD & COLL_MTHD & SAMP_SITE_ID & SAMP_SITE_NAME & WELL_NAME & SAMPLE_LOCATION \\
\hline 648 & & RIVER & & RAW GRAB & TRANSECT & 318 & ERNITA-3 HRM 0.3 & & \\
\hline 649 & & RIVER & & RAW GRAB & TRANSECT & 318 & /ERNITA-3 HRM 0.3 & & \\
\hline 650 & & RIVER & & RAW GRAB & TRANSECT & 318 & ERNITA-3 HRM 0.3 & & \\
\hline 651 & & RIVER & & RAW GRAB & TRANSECT & 318 & ERNITA-3 HRM 0.3 & & \\
\hline 652 & & RIVER & & RAW GRAB & TRANSECT & 318 & ERNITA-3 HRM 0.3 & & \\
\hline 653 & & RIVER & & RAW GRAB & TRANSECT & 318 & ERNITA-3 HRM 0.3 & & \\
\hline 654 & & RIVER & & RAW GRAB & TRANSECT & 319 & ERNITA-4 HRM 0.3 & & \\
\hline 655 & & RIVER & & RAW GRAB & TRANSECT & 319 & ERNITA-4 HRM 0.3 & & \\
\hline 656 & & RIVER & & RAW GRAB & TRANSECT & 319 & ERNITA-4 HRM 0.3 & & \\
\hline 657 & & RIVER & & RAW GRAB & TRANSECT & 319 & ERNITA-4 HRM 0.3 & & \\
\hline 658 & & RIVER & & RAW GRAB & TRANSECT & 319 & ERNITA-4 HRM 0.3 & & \\
\hline 659 & & RIVER & & RAW GRAB & TRANSECT & 319 & ERNITA-4 HRM 0.3 & & \\
\hline 660 & & RIVER & & RAW GRAB & TRANSECT & 348 & 00 AREA -1 HRM 43.1 & & \\
\hline 661 & & RIVER & & RAW GRAB & TRANSECT & 348 & 00 AREA -1 HRM 43.1 & & \\
\hline 662 & & RIVER & & RAW GRAB & TRANSECT & 348 & 00 AREA -1 HRM 43.1 & & \\
\hline 663 & & RIVER & & RAW GRAB & TRANSECT & 348 & 00 AREA -1 HRM 43.1 & & \\
\hline 664 & & RIVER & & RAW GRAB & TRANSECT & 348 & 00 AREA -1 HRM 43.1 & & \\
\hline 665 & & RIVER & & RAW GRAB & TRANSECT & 470 & 00 AREA -2 HRM 43.1 & & \\
\hline 666 & & RIVER & & RAW GRAB & TRANSECT & 470 & 00 AREA -2 HRM 43.1 & & \\
\hline 667 & & RIVER & & RAW GRAB & TRANSECT & 470 & 00 AREA -2 HRM 43.1 & & \\
\hline 668 & & RIVER & & RAW GRAB & TRANSECT & 470 & 00 AREA -2 HRM 43.1 & & \\
\hline 669 & & RIVER & & RAW GRAB & TRANSECT & 470 & 00 AREA -2 HRM 43.1 & & \\
\hline 670 & & RIVER & & RAW GRAB & TRANSECT & 580 & 00 AREA -3 HRM 43.1 & & \\
\hline 671 & & RIVER & & RAW GRAB & TRANSECT & 580 & 00 AREA -3 HRM 43.1 & & \\
\hline 672 & & RIVER & & RAW GRAB & TRANSECT & 580 & 00 AREA -3 HRM 43.1 & & \\
\hline 673 & & RIVER & & RAW GRAB & TRANSECT & 580 & 00 AREA -3 HRM 43.1 & & \\
\hline 674 & & RIVER & & RAW GRAB & TRANSECT & 580 & 00 AREA -3 HRM 43.1 & & \\
\hline 675 & & RIVER & & RAW GRAB & TRANSECT & 349 & 00 AREA -4 HRM 43.1 & & \\
\hline 676 & & RIVER & & RAW GRAB & TRANSECT & 349 & 00 AREA -4 HRM 43.1 & & \\
\hline 677 & & RIVER & & RAW GRAB & TRANSECT & 349 & 00 AREA -4 HRM 43.1 & & \\
\hline 678 & & RIVER & & RAW GRAB & TRANSECT & 349 & 00 AREA -4 HRM 43.1 & & \\
\hline 679 & & RIVER & & RAW GRAB & TRANSECT & 349 & 00 AREA -4 HRM 43.1 & & \\
\hline 680 & & RIVER & & RAW GRAB & TRANSECT & 607 & 00 AREA -5 HRM 43.1 & & \\
\hline 681 & & RIVER & & RAW GRAB & TRANSECT & 607 & 00 AREA -5 HRM 43.1 & & \\
\hline 682 & & RIVER & & RAW GRAB & TRANSECT & 607 & 00 AREA -5 HRM 43.1 & & \\
\hline 683 & & RIVER & & RAW GRAB & TRANSECT & 607 & 00 AREA -5 HRM 43.1 & & \\
\hline 684 & & RIVER & & RAW GRAB & TRANSECT & 607 & 00 AREA -5 HRM 43.1 & & \\
\hline 685 & & RIVER & & RAW GRAB & TRANSECT & 350 & 00 AREA -6 HRM 43.1 & & \\
\hline
\end{tabular}




\begin{tabular}{|c|c|c|c|c|c|c|c|c|c|}
\hline & $\mathrm{T}$ & $U$ & $\mathrm{~V}$ & $\mathrm{~W}$ & $\mathrm{X}$ & $\mathrm{Y}$ & $Z$ & $\mathrm{AA}$ & $A B$ \\
\hline 1 & REVIEW_QUALIFIER & SAMP_FROM & SAMP_ITEM & SAMP_MTHD & COLL_MTHD & SAMP_SITE_ID & SAMP_SITE_NAME & WELL_NAME & SAMPLE_LOCATION \\
\hline 686 & & RIVER & & RAW GRAB & TRANSECT & 350 & 300 AREA -6 HRM 43.1 & & \\
\hline 687 & & RIVER & & RAW GRAB & TRANSECT & 350 & 300 AREA -6 HRM 43.1 & & \\
\hline 688 & & RIVER & & RAW GRAB & TRANSECT & 350 & 300 AREA -6 HRM 43.1 & & \\
\hline 689 & & RIVER & & RAW GRAB & TRANSECT & 350 & 300 AREA -6 HRM 43.1 & & \\
\hline 690 & & RIVER & & RAW GRAB & TRANSECT & 351 & 300 AREA -7 HRM 43.1 & & \\
\hline 691 & & RIVER & & RAW GRAB & TRANSECT & 351 & 300 AREA -7 HRM 43.1 & & \\
\hline 692 & & RIVER & & RAW GRAB & TRANSECT & 351 & 300 AREA -7 HRM 43.1 & & \\
\hline 693 & & RIVER & & RAW GRAB & TRANSECT & 351 & 300 AREA -7 HRM 43.1 & & \\
\hline 694 & & RIVER & & RAW GRAB & TRANSECT & 351 & 300 AREA -7 HRM 43.1 & & \\
\hline 695 & & RIVER & & RAW GRAB & TRANSECT & 581 & 300 AREA -8 HRM 43.1 & & \\
\hline 696 & & RIVER & & RAW GRAB & TRANSECT & 581 & 300 AREA -8 HRM 43.1 & & \\
\hline 697 & & RIVER & & RAW GRAB & TRANSECT & 581 & 300 AREA -8 HRM 43.1 & & \\
\hline 698 & & RIVER & & RAW GRAB & TRANSECT & 581 & 1300 AREA -8 HRM 43.1 & & \\
\hline 699 & & RIVER & & RAW GRAB & TRANSECT & 581 & 1300 AREA -8 HRM 43.1 & & \\
\hline 700 & & RIVER & & RAW GRAB & TRANSECT & 471 & 300 AREA -9 HRM 43.1 & & \\
\hline 701 & & RIVER & & RAW GRAB & TRANSECT & 471 & 1300 AREA -9 HRM 43.1 & & \\
\hline 702 & & RIVER & & RAW GRAB & TRANSECT & 471 & 300 AREA -9 HRM 43.1 & & \\
\hline 703 & & RIVER & & RAW GRAB & TRANSECT & 471 & 300 AREA -9 HRM 43.1 & & \\
\hline 704 & & RIVER & & RAW GRAB & TRANSECT & 471 & 300 AREA -9 HRM 43.1 & & \\
\hline 705 & & RIVER & & RAW GRAB & TRANSECT & 352 & 2300 AREA-10 HRM 43.1 & & \\
\hline 706 & & RIVER & & RAW GRAB & TRANSECT & 352 & 2300 AREA-10 HRM 43.1 & & \\
\hline 707 & & RIVER & & RAW GRAB & TRANSECT & 352 & 2300 AREA-10 HRM 43.1 & & \\
\hline 708 & & RIVER & & RAW GRAB & TRANSECT & 352 & 2300 AREA-10 HRM 43.1 & & \\
\hline 709 & & RIVER & & RAW GRAB & TRANSECT & 352 & 2300 AREA-10 HRM 43.1 & & \\
\hline 710 & & RIVER & & RAW GRAB & TRANSECT & 353 & $3100 \mathrm{~N}-0 \mathrm{~B}$ & & \\
\hline 711 & & RIVER & & RAW GRAB & TRANSECT & 353 & $3100 \mathrm{~N}-0 \mathrm{~B}$ & & \\
\hline 712 & & RIVER & & RAW GRAB & TRANSECT & 353 & $3100 \mathrm{~N}-0 \mathrm{~B}$ & & \\
\hline 713 & & RIVER & & RAW GRAB & TRANSECT & 353 & $3100 \mathrm{~N}-0 \mathrm{~B}$ & & \\
\hline 714 & & RIVER & & RAW GRAB & TRANSECT & 353 & $3100 \mathrm{~N}-0 \mathrm{~B}$ & & \\
\hline 715 & & RIVER & & RAW GRAB & TRANSECT & 353 & $3100 \mathrm{~N}-0 \mathrm{~B}$ & & \\
\hline 716 & & RIVER & & RAW GRAB & TRANSECT & 353 & $3100 \mathrm{~N}-0 \mathrm{~B}$ & & \\
\hline 717 & & RIVER & & RAW GRAB & TRANSECT & 328 & 3100 N -1 HRM 9.5 & & \\
\hline 718 & & RIVER & & RAW GRAB & TRANSECT & 328 & 3100 N -1 HRM 9.5 & & \\
\hline 719 & & RIVER & & RAW GRAB & TRANSECT & 328 & 3100 N -1 HRM 9.5 & & \\
\hline 720 & & RIVER & & RAW GRAB & TRANSECT & 328 & 3100 N -1 HRM 9.5 & & \\
\hline 721 & & RIVER & & RAW GRAB & TRANSECT & 328 & 8100 N -1 HRM 9.5 & & \\
\hline 722 & & RIVER & & RAW GRAB & TRANSECT & 335 & 5100 N -10 HRM 9.5 & & \\
\hline 723 & & RIVER & & RAW GRAB & TRANSECT & 335 & $5100 \mathrm{~N}-10$ HRM 9.5 & & \\
\hline
\end{tabular}




\begin{tabular}{|c|c|c|c|c|c|c|c|c|c|}
\hline & $\mathrm{T}$ & $U$ & $\mathrm{~V}$ & $\mathrm{~W}$ & $x$ & $\bar{Y}$ & $Z$ & AA & $A B$ \\
\hline 1 & REVIEW_QUALIFIER & SAMP_FROM & SAMP_ITEM & SAMP_MTHD & COLL_MTHD & SAMP_SITE_ID & SAMP_SITE_NAME & WELL_NAME & SAMPLE_LOCATION \\
\hline 724 & & RIVER & & RAW GRAB & TRANSECT & 335 & $100 \mathrm{~N}-10 \mathrm{HRM} 9.5$ & & \\
\hline 725 & & RIVER & & RAW GRAB & TRANSECT & 335 & 100 N -10 HRM 9.5 & & \\
\hline 726 & & RIVER & & RAW GRAB & TRANSECT & 335 & 100 N -10 HRM 9.5 & & \\
\hline 727 & & RIVER & & RAW GRAB & TRANSECT & 361 & $100 N-11 B$ & & \\
\hline 728 & & RIVER & & RAW GRAB & TRANSECT & 361 & $100 \mathrm{~N}-11 \mathrm{~B}$ & & \\
\hline 729 & & RIVER & & RAW GRAB & TRANSECT & 361 & $100 N-11 B$ & & \\
\hline 730 & & RIVER & & RAW GRAB & TRANSECT & 361 & $100 N-11 B$ & & \\
\hline 731 & & RIVER & & RAW GRAB & TRANSECT & 361 & $100 \mathrm{~N}-11 \mathrm{~B}$ & & \\
\hline 732 & & RIVER & & RAW GRAB & TRANSECT & 361 & $100 N-11 B$ & & \\
\hline 733 & & RIVER & & RAW GRAB & TRANSECT & 361 & $100 \mathrm{~N}-11 \mathrm{~B}$ & & \\
\hline 734 & & RIVER & & RAW GRAB & TRANSECT & 329 & 100 N -2 HRM 9.5 & & \\
\hline 735 & & RIVER & & RAW GRAB & TRANSECT & 329 & 100 N -2 HRM 9.5 & & \\
\hline 736 & & RIVER & & RAW GRAB & TRANSECT & 329 & 100 N -2 HRM 9.5 & & \\
\hline 737 & & RIVER & & RAW GRAB & TRANSECT & 329 & 100 N -2 HRM 9.5 & & \\
\hline 738 & & RIVER & & RAW GRAB & TRANSECT & 329 & 100 N -2 HRM 9.5 & & \\
\hline 739 & & RIVER & & RAW GRAB & TRANSECT & 330 & 100 N -3 HRM 9.5 & & \\
\hline 740 & & RIVER & & RAW GRAB & TRANSECT & 330 & 100 N -3 HRM 9.5 & & \\
\hline 741 & & RIVER & & RAW GRAB & TRANSECT & 330 & $100 \mathrm{~N}-3$ HRM 9.5 & & \\
\hline 742 & & RIVER & & RAW GRAB & TRANSECT & 330 & 100 N -3 HRM 9.5 & & \\
\hline 743 & & RIVER & & RAW GRAB & TRANSECT & 330 & 100 N -3 HRM 9.5 & & \\
\hline 744 & & RIVER & & RAW GRAB & TRANSECT & 574 & 100 N -4 HRM 9.5 & & \\
\hline 745 & & RIVER & & RAW GRAB & TRANSECT & 574 & 100 N -4 HRM 9.5 & & \\
\hline 746 & & RIVER & & RAW GRAB & TRANSECT & 574 & 100 N -4 HRM 9.5 & & \\
\hline 747 & & RIVER & & RAW GRAB & TRANSECT & 574 & 100 N -4 HRM 9.5 & & \\
\hline 748 & & RIVER & & RAW GRAB & TRANSECT & 574 & 100 N -4 HRM 9.5 & & \\
\hline 749 & & RIVER & & RAW GRAB & TRANSECT & 331 & 100 N -5 HRM 9.5 & & \\
\hline 750 & & RIVER & & RAW GRAB & TRANSECT & 331 & 100 N -5 HRM 9.5 & & \\
\hline 751 & & RIVER & & RAW GRAB & TRANSECT & 331 & 100 N -5 HRM 9.5 & & \\
\hline 752 & & RIVER & & RAW GRAB & TRANSECT & 331 & 100 N -5 HRM 9.5 & & \\
\hline 753 & & RIVER & & RAW GRAB & TRANSECT & 331 & 100 N -5 HRM 9.5 & & \\
\hline 754 & & RIVER & & RAW GRAB & TRANSECT & 332 & 100 N -6 HRM 9.5 & & \\
\hline 755 & & RIVER & & RAW GRAB & TRANSECT & 332 & 100 N -6 HRM 9.5 & & \\
\hline 756 & & RIVER & & RAW GRAB & TRANSECT & 332 & 100 N -6 HRM 9.5 & & \\
\hline 757 & & RIVER & & RAW GRAB & TRANSECT & 332 & 100 N -6 HRM 9.5 & & \\
\hline 758 & & RIVER & & RAW GRAB & TRANSECT & 332 & 100 N -6 HRM 9.5 & & \\
\hline 759 & & RIVER & & RAW GRAB & TRANSECT & 333 & 100 N -7 HRM 9.5 & & \\
\hline 760 & & RIVER & & RAW GRAB & TRANSECT & 333 & 100 N -7 HRM 9.5 & & \\
\hline 761 & & RIVER & & RAW GRAB & TRANSECT & 333 & $100 \mathrm{~N}-7$ HRM 9.5 & & \\
\hline
\end{tabular}




\begin{tabular}{|c|c|c|c|c|c|c|c|c|c|}
\hline & $\mathrm{T}$ & $U$ & $\mathrm{~V}$ & $\mathrm{~W}$ & $\mathrm{X}$ & $\bar{Y}$ & $Z$ & AA & $A B$ \\
\hline 1 & REVIEW_QUALIFIER & SAMP_FROM & SAMP_ITEM & SAMP_MTHD & COLL_MTHD & SAMP_SITE_ID & SAMP_SITE_NAME & WELL_NAME & SAMPLE_LOCATION \\
\hline 762 & & RIVER & & RAW GRAB & TRANSECT & 333 & 100 N -7 HRM 9.5 & & \\
\hline 763 & & RIVER & & RAW GRAB & TRANSECT & 333 & 3100 N -7 HRM 9.5 & & \\
\hline 764 & & RIVER & & RAW GRAB & TRANSECT & 575 & 100 N -8 HRM 9.5 & & \\
\hline 765 & & RIVER & & RAW GRAB & TRANSECT & 575 & 100 N -8 HRM 9.5 & & \\
\hline 766 & & RIVER & & RAW GRAB & TRANSECT & 575 & 100 N -8 HRM 9.5 & & \\
\hline 767 & & RIVER & & RAW GRAB & TRANSECT & 575 & 100 N -8 HRM 9.5 & & \\
\hline 768 & & RIVER & & RAW GRAB & TRANSECT & 575 & 100 N -8 HRM 9.5 & & \\
\hline 769 & & RIVER & & RAW GRAB & TRANSECT & 334 & 100 N -9 HRM 9.5 & & \\
\hline 770 & & RIVER & & RAW GRAB & TRANSECT & 334 & 100 N -9 HRM 9.5 & & \\
\hline 771 & & RIVER & & RAW GRAB & TRANSECT & 334 & 100 N -9 HRM 9.5 & & \\
\hline 772 & & RIVER & & RAW GRAB & TRANSECT & 334 & 100 N -9 HRM 9.5 & & \\
\hline 773 & & RIVER & & RAW GRAB & TRANSECT & 334 & 100 N -9 HRM 9.5 & & \\
\hline 774 & & RIVER & & RAW GRAB & TRANSECT & 362 & HANFORD TOWNSITE-OB & & \\
\hline 775 & & RIVER & & RAW GRAB & TRANSECT & 362 & HANFORD TOWNSITE-OB & & \\
\hline 776 & & RIVER & & RAW GRAB & TRANSECT & 362 & HANFORD TOWNSITE-OB & & \\
\hline 777 & & RIVER & & RAW GRAB & TRANSECT & 362 & HANFORD TOWNSITE-OB & & \\
\hline 778 & & RIVER & & RAW GRAB & TRANSECT & 362 & HANFORD TOWNSITE-OB & & \\
\hline 779 & & RIVER & & RAW GRAB & TRANSECT & 362 & HANFORD TOWNSITE-OB & & \\
\hline 780 & & RIVER & & RAW GRAB & TRANSECT & 587 & HANFORD TOWNSITE-10B & & \\
\hline 781 & & RIVER & & RAW GRAB & TRANSECT & 587 & HANFORD TOWNSITE-10B & & \\
\hline 782 & & RIVER & & RAW GRAB & TRANSECT & 587 & HANFORD TOWNSITE-10B & & \\
\hline 783 & & RIVER & & RAW GRAB & TRANSECT & 587 & HANFORD TOWNSITE-10B & & \\
\hline 784 & & RIVER & & RAW GRAB & TRANSECT & 587 & HANFORD TOWNSITE-10B & & \\
\hline 785 & & RIVER & & RAW GRAB & TRANSECT & 587 & HANFORD TOWNSITE-10B & & \\
\hline 786 & & RIVER & & RAW GRAB & TRANSECT & 472 & HANFORD TOWNSITE-11B & & \\
\hline 787 & & RIVER & & RAW GRAB & TRANSECT & 472 & HANFORD TOWNSITE-11B & & \\
\hline 788 & & RIVER & & RAW GRAB & TRANSECT & 472 & HANFORD TOWNSITE-11B & & \\
\hline 789 & & RIVER & & RAW GRAB & TRANSECT & 472 & HANFORD TOWNSITE-11B & & \\
\hline 790 & & RIVER & & RAW GRAB & TRANSECT & 472 & HANFORD TOWNSITE-11B & & \\
\hline 791 & & RIVER & & RAW GRAB & TRANSECT & 472 & HANFORD TOWNSITE-11B & & \\
\hline 792 & & RIVER & & RAW GRAB & TRANSECT & 585 & HANFORD TOWNSITE-1B & & \\
\hline 793 & & RIVER & & RAW GRAB & TRANSECT & 585 & HANFORD TOWNSITE-1B & & \\
\hline 794 & & RIVER & & RAW GRAB & TRANSECT & 585 & HANFORD TOWNSITE-1B & & \\
\hline 795 & & RIVER & & RAW GRAB & TRANSECT & 585 & HANFORD TOWNSITE-1B & & \\
\hline 796 & & RIVER & & RAW GRAB & TRANSECT & 585 & HANFORD TOWNSITE-1B & & \\
\hline 797 & & RIVER & & RAW GRAB & TRANSECT & 585 & HANFORD TOWNSITE-1B & & \\
\hline 798 & & RIVER & & RAW GRAB & TRANSECT & 363 & HANFORD TOWNSITE-2B & & \\
\hline 799 & & RIVER & & RAW GRAB & TRANSECT & 363 & HANFORD TOWNSITE-2B & & \\
\hline
\end{tabular}




\begin{tabular}{|c|c|c|c|c|c|c|c|c|c|}
\hline & $\mathrm{T}$ & $\mathrm{U}$ & $\mathrm{V}$ & $\mathrm{W}$ & $\mathrm{X}$ & $\bar{Y}$ & $Z$ & AA & $A B$ \\
\hline 1 & REVIEW_QUALIFIER & SAMP_FROM & SAMP_ITEM & SAMP_MTHD & COLL_MTHD & SAMP_SITE_ID & SAMP_SITE_NAME & WELL_NAME & SAMPLE_LOCATION \\
\hline 800 & & RIVER & & RAW GRAB & TRANSECT & 363 & HANFORD TOWNSITE-2B & & \\
\hline 801 & & RIVER & & RAW GRAB & TRANSECT & 363 & HANFORD TOWNSITE-2B & & \\
\hline 802 & & RIVER & & RAW GRAB & TRANSECT & 363 & HANFORD TOWNSITE-2B & & \\
\hline 803 & & RIVER & & RAW GRAB & TRANSECT & 363 & HANFORD TOWNSITE-2B & & \\
\hline 804 & & RIVER & & RAW GRAB & TRANSECT & 364 & HANFORD TOWNSITE-3B & & \\
\hline 805 & & RIVER & & RAW GRAB & TRANSECT & 364 & HANFORD TOWNSITE-3B & & \\
\hline 806 & & RIVER & & RAW GRAB & TRANSECT & 364 & HANFORD TOWNSITE-3B & & \\
\hline 807 & & RIVER & & RAW GRAB & TRANSECT & 364 & HANFORD TOWNSITE-3B & & \\
\hline 808 & & RIVER & & RAW GRAB & TRANSECT & 364 & HANFORD TOWNSITE-3B & & \\
\hline 809 & & RIVER & & RAW GRAB & TRANSECT & 364 & HANFORD TOWNSITE-3B & & \\
\hline 810 & & RIVER & & RAW GRAB & TRANSECT & 365 & HANFORD TOWNSITE-4B & & \\
\hline 811 & & RIVER & & RAW GRAB & TRANSECT & 365 & HANFORD TOWNSITE-4B & & \\
\hline 812 & & RIVER & & RAW GRAB & TRANSECT & 365 & HANFORD TOWNSITE-4B & & \\
\hline 813 & & RIVER & & RAW GRAB & TRANSECT & 365 & HANFORD TOWNSITE-4B & & \\
\hline 814 & & RIVER & & RAW GRAB & TRANSECT & 365 & HANFORD TOWNSITE-4B & & \\
\hline 815 & & RIVER & & RAW GRAB & TRANSECT & 365 & HANFORD TOWNSITE-4B & & \\
\hline 816 & & RIVER & & RAW GRAB & TRANSECT & 586 & HANFORD TOWNSITE-5B & & \\
\hline 817 & & RIVER & & RAW GRAB & TRANSECT & 586 & HANFORD TOWNSITE-5B & & \\
\hline 818 & & RIVER & & RAW GRAB & TRANSECT & 586 & HANFORD TOWNSITE-5B & & \\
\hline 819 & & RIVER & & RAW GRAB & TRANSECT & 586 & HANFORD TOWNSITE-5B & & \\
\hline 820 & & RIVER & & RAW GRAB & TRANSECT & 586 & HANFORD TOWNSITE-5B & & \\
\hline 821 & & RIVER & & RAW GRAB & TRANSECT & 586 & HANFORD TOWNSITE-5B & & \\
\hline 822 & & RIVER & & RAW GRAB & TRANSECT & 366 & HANFORD TOWNSITE-6B & & \\
\hline 823 & & RIVER & & RAW GRAB & TRANSECT & 366 & HANFORD TOWNSITE-6B & & \\
\hline 824 & & RIVER & & RAW GRAB & TRANSECT & 366 & HANFORD TOWNSITE-6B & & \\
\hline 825 & & RIVER & & RAW GRAB & TRANSECT & 366 & HANFORD TOWNSITE-6B & & \\
\hline 826 & & RIVER & & RAW GRAB & TRANSECT & 366 & HANFORD TOWNSITE-6B & & \\
\hline 827 & & RIVER & & RAW GRAB & TRANSECT & 366 & HANFORD TOWNSITE-6B & & \\
\hline 828 & & RIVER & & RAW GRAB & TRANSECT & 367 & HANFORD TOWNSITE-7B & & \\
\hline 829 & & RIVER & & RAW GRAB & TRANSECT & 367 & HANFORD TOWNSITE-7B & & \\
\hline 830 & & RIVER & & RAW GRAB & TRANSECT & 367 & HANFORD TOWNSITE-7B & & \\
\hline 831 & & RIVER & & RAW GRAB & TRANSECT & 367 & HANFORD TOWNSITE-7B & & \\
\hline 832 & & RIVER & & RAW GRAB & TRANSECT & 367 & HANFORD TOWNSITE-7B & & \\
\hline 833 & & RIVER & & RAW GRAB & TRANSECT & 367 & HANFORD TOWNSITE-7B & & \\
\hline 834 & & RIVER & & RAW GRAB & TRANSECT & 368 & HANFORD TOWNSITE-8B & & \\
\hline 835 & & RIVER & & RAW GRAB & TRANSECT & 368 & HANFORD TOWNSITE-8B & & \\
\hline 836 & & RIVER & & RAW GRAB & TRANSECT & 368 & HANFORD TOWNSITE-8B & & \\
\hline 837 & & RIVER & & RAW GRAB & TRANSECT & 368 & HANFORD TOWNSITE-8B & & \\
\hline
\end{tabular}




\begin{tabular}{|c|c|c|c|c|c|c|c|c|c|}
\hline & $\mathrm{T}$ & $U$ & $\mathrm{~V}$ & $\mathrm{~W}$ & $\mathrm{X}$ & $\mathrm{Y}$ & $Z$ & $\mathrm{AA}$ & $A B$ \\
\hline 1 & REVIEW_QUALIFIER & SAMP_FROM & SAMP_ITEM & SAMP_MTHD & COLL_MTHD & SAMP_SITE_ID & SAMP_SITE_NAME & WELL_NAME & SAMPLE_LOCATION \\
\hline 838 & & RIVER & & RAW GRAB & TRANSECT & 368 & HANFORD TOWNSITE-8B & & \\
\hline 839 & & RIVER & & RAW GRAB & TRANSECT & 368 & HANFORD TOWNSITE-8B & & \\
\hline 840 & & RIVER & & RAW GRAB & TRANSECT & 369 & HANFORD TOWNSITE-9B & & \\
\hline 841 & & RIVER & & RAW GRAB & TRANSECT & 369 & HANFORD TOWNSITE-9B & & \\
\hline 842 & & RIVER & & RAW GRAB & TRANSECT & 369 & HANFORD TOWNSITE-9B & & \\
\hline 843 & & RIVER & & RAW GRAB & TRANSECT & 369 & HANFORD TOWNSITE-9B & & \\
\hline 844 & & RIVER & & RAW GRAB & TRANSECT & 369 & HANFORD TOWNSITE-9B & & \\
\hline 845 & & RIVER & & RAW GRAB & TRANSECT & 369 & HANFORD TOWNSITE-9B & & \\
\hline 846 & & RIVER & & RAW GRAB & TRANSECT & 578 & HANFRD TS-1 HRM 28.7 & & \\
\hline 847 & & RIVER & & RAW GRAB & TRANSECT & 578 & HANFRD TS-1 HRM 28.7 & & \\
\hline 848 & & RIVER & & RAW GRAB & TRANSECT & 578 & HANFRD TS-1 HRM 28.7 & & \\
\hline 849 & & RIVER & & RAW GRAB & TRANSECT & 578 & HANFRD TS-1 HRM 28.7 & & \\
\hline 850 & & RIVER & & RAW GRAB & TRANSECT & 578 & HANFRD TS-1 HRM 28.7 & & \\
\hline 851 & & RIVER & & RAW GRAB & TRANSECT & 347 & HANFRD TS-10 HRM 28.7 & & \\
\hline 852 & & RIVER & & RAW GRAB & TRANSECT & 347 & HANFRD TS-10 HRM 28.7 & & \\
\hline 853 & & RIVER & & RAW GRAB & TRANSECT & 347 & HANFRD TS-10 HRM 28.7 & & \\
\hline 854 & & RIVER & & RAW GRAB & TRANSECT & 347 & HANFRD TS-10 HRM 28.7 & & \\
\hline 855 & & RIVER & & RAW GRAB & TRANSECT & 347 & HANFRD TS-10 HRM 28.7 & & \\
\hline 856 & & RIVER & & RAW GRAB & TRANSECT & 342 & HANFRD TS-2 HRM 28.7 & & \\
\hline 857 & & RIVER & & RAW GRAB & TRANSECT & 342 & HANFRD TS-2 HRM 28.7 & & \\
\hline 858 & & RIVER & & RAW GRAB & TRANSECT & 342 & HANFRD TS-2 HRM 28.7 & & \\
\hline 859 & & RIVER & & RAW GRAB & TRANSECT & 342 & HANFRD TS-2 HRM 28.7 & & \\
\hline 860 & & RIVER & & RAW GRAB & TRANSECT & 342 & HANFRD TS-2 HRM 28.7 & & \\
\hline 861 & & RIVER & & RAW GRAB & TRANSECT & 468 & HANFRD TS-3 HRM 28.7 & & \\
\hline 862 & & RIVER & & RAW GRAB & TRANSECT & 468 & HANFRD TS-3 HRM 28.7 & & \\
\hline 863 & & RIVER & & RAW GRAB & TRANSECT & 468 & HANFRD TS-3 HRM 28.7 & & \\
\hline 864 & & RIVER & & RAW GRAB & TRANSECT & 468 & HANFRD TS-3 HRM 28.7 & & \\
\hline 865 & & RIVER & & RAW GRAB & TRANSECT & 468 & HANFRD TS-3 HRM 28.7 & & \\
\hline 866 & & RIVER & & RAW GRAB & TRANSECT & 343 & HANFRD TS-4 HRM 28.7 & & \\
\hline 867 & & RIVER & & RAW GRAB & TRANSECT & 343 & HANFRD TS-4 HRM 28.7 & & \\
\hline 868 & & RIVER & & RAW GRAB & TRANSECT & 343 & HANFRD TS-4 HRM 28.7 & & \\
\hline 869 & & RIVER & & RAW GRAB & TRANSECT & 343 & HANFRD TS-4 HRM 28.7 & & \\
\hline 870 & & RIVER & & RAW GRAB & TRANSECT & 343 & HANFRD TS-4 HRM 28.7 & & \\
\hline 871 & & RIVER & & RAW GRAB & TRANSECT & 344 & HANFRD TS-5 HRM 28.7 & & \\
\hline 872 & & RIVER & & RAW GRAB & TRANSECT & 344 & HANFRD TS-5 HRM 28.7 & & \\
\hline 873 & & RIVER & & RAW GRAB & TRANSECT & 344 & HANFRD TS-5 HRM 28.7 & & \\
\hline 874 & & RIVER & & RAW GRAB & TRANSECT & 344 & HANFRD TS-5 HRM 28.7 & & \\
\hline 875 & & RIVER & & RAW GRAB & TRANSECT & 344 & HANFRD TS-5 HRM 28.7 & & \\
\hline
\end{tabular}




\begin{tabular}{|c|c|c|c|c|c|c|c|c|c|}
\hline & $\mathrm{T}$ & $\mathrm{U}$ & $\mathrm{V}$ & $\mathrm{W}$ & $\mathrm{x}$ & $\bar{Y}$ & $Z$ & AA & $A B$ \\
\hline 1 & REVIEW_QUALIFIER & SAMP_FROM & SAMP_ITEM & SAMP_MTHD & COLL_MTHD & SAMP_SITE_ID & SAMP_SITE_NAME & WELL_NAME & SAMPLE_LOCATION \\
\hline 876 & & RIVER & & RAW GRAB & TRANSECT & 345 & HANFRD TS-6 HRM 28.7 & & \\
\hline 877 & & RIVER & & RAW GRAB & TRANSECT & 345 & HANFRD TS-6 HRM 28.7 & & \\
\hline 878 & & RIVER & & RAW GRAB & TRANSECT & 345 & HANFRD TS-6 HRM 28.7 & & \\
\hline 879 & & RIVER & & RAW GRAB & TRANSECT & 345 & HANFRD TS-6 HRM 28.7 & & \\
\hline 880 & & RIVER & & RAW GRAB & TRANSECT & 345 & HANFRD TS-6 HRM 28.7 & & \\
\hline 881 & & RIVER & & RAW GRAB & TRANSECT & 469 & HANFRD TS-7 HRM 28.7 & & \\
\hline 882 & & RIVER & & RAW GRAB & TRANSECT & 469 & HANFRD TS-7 HRM 28.7 & & \\
\hline 883 & & RIVER & & RAW GRAB & TRANSECT & 469 & HANFRD TS-7 HRM 28.7 & & \\
\hline 884 & & RIVER & & RAW GRAB & TRANSECT & 469 & HANFRD TS-7 HRM 28.7 & & \\
\hline 885 & & RIVER & & RAW GRAB & TRANSECT & 469 & HANFRD TS-7 HRM 28.7 & & \\
\hline 886 & & RIVER & & RAW GRAB & TRANSECT & 579 & HANFRD TS-8 HRM 28.7 & & \\
\hline 887 & & RIVER & & RAW GRAB & TRANSECT & 579 & HANFRD TS-8 HRM 28.7 & & \\
\hline 888 & & RIVER & & RAW GRAB & TRANSECT & 579 & HANFRD TS-8 HRM 28.7 & & \\
\hline 889 & & RIVER & & RAW GRAB & TRANSECT & 579 & HANFRD TS-8 HRM 28.7 & & \\
\hline 890 & & RIVER & & RAW GRAB & TRANSECT & 579 & HANFRD TS-8 HRM 28.7 & & \\
\hline 891 & & RIVER & & RAW GRAB & TRANSECT & 346 & HANFRD TS-9 HRM 28.7 & & \\
\hline 892 & & RIVER & & RAW GRAB & TRANSECT & 346 & HANFRD TS-9 HRM 28.7 & & \\
\hline 893 & & RIVER & & RAW GRAB & TRANSECT & 346 & HANFRD TS-9 HRM 28.7 & & \\
\hline 894 & & RIVER & & RAW GRAB & TRANSECT & 346 & HANFRD TS-9 HRM 28.7 & & \\
\hline 895 & & RIVER & & RAW GRAB & TRANSECT & 346 & HANFRD TS-9 HRM 28.7 & & \\
\hline 896 & & RIVER & & RAW GRAB & TRANSECT & 360 & $100 \mathrm{~N}-10 \mathrm{~B}$ & & \\
\hline 897 & & RIVER & & RAW GRAB & TRANSECT & 360 & $100 \mathrm{~N}-10 \mathrm{~B}$ & & \\
\hline 898 & & RIVER & & RAW GRAB & TRANSECT & 360 & $100 N-10 B$ & & \\
\hline 899 & & RIVER & & RAW GRAB & TRANSECT & 360 & $100 \mathrm{~N}-10 \mathrm{~B}$ & & \\
\hline 900 & & RIVER & & RAW GRAB & TRANSECT & 360 & $100 \mathrm{~N}-10 \mathrm{~B}$ & & \\
\hline 901 & & RIVER & & RAW GRAB & TRANSECT & 360 & $100 \mathrm{~N}-10 \mathrm{~B}$ & & \\
\hline 902 & & RIVER & & RAW GRAB & TRANSECT & 360 & $100 \mathrm{~N}-10 \mathrm{~B}$ & & \\
\hline 903 & & RIVER & & RAW GRAB & TRANSECT & 356 & $100 N-4 B$ & & \\
\hline 904 & & RIVER & & RAW GRAB & TRANSECT & 356 & $100 N-4 B$ & & \\
\hline 905 & & RIVER & & RAW GRAB & TRANSECT & 356 & $100 N-4 B$ & & \\
\hline 906 & & RIVER & & RAW GRAB & TRANSECT & 356 & $100 N-4 B$ & & \\
\hline 907 & & RIVER & & RAW GRAB & TRANSECT & 356 & $100 N-4 B$ & & \\
\hline 908 & & RIVER & & RAW GRAB & TRANSECT & 356 & $100 N-4 B$ & & \\
\hline 909 & & RIVER & & RAW GRAB & TRANSECT & 356 & $100 N-4 B$ & & \\
\hline 910 & & RIVER & & RAW GRAB & TRANSECT & 583 & $100 N-5 B$ & & \\
\hline 911 & & RIVER & & RAW GRAB & TRANSECT & 583 & $100 N-5 B$ & & \\
\hline 912 & & RIVER & & RAW GRAB & TRANSECT & 583 & $100 N-5 B$ & & \\
\hline 913 & & RIVER & & RAW GRAB & TRANSECT & 583 & $100 N-5 B$ & & \\
\hline
\end{tabular}




\begin{tabular}{|c|c|c|c|c|c|c|c|c|c|}
\hline & $\mathrm{T}$ & $U$ & $\mathrm{~V}$ & $\mathrm{~W}$ & $x$ & $\bar{Y}$ & $Z$ & AA & $A B$ \\
\hline 1 & REVIEW_QUALIFIER & SAMP_FROM & SAMP_ITEM & SAMP_MTHD & COLL_MTHD & SAMP_SITE_ID & SAMP_SITE_NAME & WELL_NAME & SAMPLE_LOCATION \\
\hline 914 & & RIVER & & RAW GRAB & TRANSECT & 583 & $100 N-5 B$ & & \\
\hline 915 & & RIVER & & RAW GRAB & TRANSECT & 583 & $3100 N-5 B$ & & \\
\hline 916 & & RIVER & & RAW GRAB & TRANSECT & 583 & $100 N-5 B$ & & \\
\hline 917 & & RIVER & & RAW GRAB & TRANSECT & 357 & $100 N-6 B$ & & \\
\hline 918 & & RIVER & & RAW GRAB & TRANSECT & 357 & $100 N-6 B$ & & \\
\hline 919 & & RIVER & & RAW GRAB & TRANSECT & 357 & $100 N-6 B$ & & \\
\hline 920 & & RIVER & & RAW GRAB & TRANSECT & 357 & $100 N-6 B$ & & \\
\hline 921 & & RIVER & & RAW GRAB & TRANSECT & 357 & $100 N-6 B$ & & \\
\hline 922 & & RIVER & & RAW GRAB & TRANSECT & 357 & $100 N-6 B$ & & \\
\hline 923 & & RIVER & & RAW GRAB & TRANSECT & 357 & $100 N-6 B$ & & \\
\hline 924 & & RIVER & & RAW GRAB & TRANSECT & 358 & $100 \mathrm{~N}-7 \mathrm{~B}$ & & \\
\hline 925 & & RIVER & & RAW GRAB & TRANSECT & 358 & $100 \mathrm{~N}-7 \mathrm{~B}$ & & \\
\hline 926 & & RIVER & & RAW GRAB & TRANSECT & 358 & $100 \mathrm{~N}-7 \mathrm{~B}$ & & \\
\hline 927 & & RIVER & & RAW GRAB & TRANSECT & 358 & $100 N-7 B$ & & \\
\hline 928 & & RIVER & & RAW GRAB & TRANSECT & 358 & $100 \mathrm{~N}-7 \mathrm{~B}$ & & \\
\hline 929 & & RIVER & & RAW GRAB & TRANSECT & 358 & $100 \mathrm{~N}-7 \mathrm{~B}$ & & \\
\hline 930 & & RIVER & & RAW GRAB & TRANSECT & 358 & $100 \mathrm{~N}-7 \mathrm{~B}$ & & \\
\hline 931 & & RIVER & & RAW GRAB & TRANSECT & 359 & $100 N-8 B$ & & \\
\hline 932 & & RIVER & & RAW GRAB & TRANSECT & 359 & $100 \mathrm{~N}-8 \mathrm{~B}$ & & \\
\hline 933 & & RIVER & & RAW GRAB & TRANSECT & 359 & $100 N-8 B$ & & \\
\hline 934 & & RIVER & & RAW GRAB & TRANSECT & 359 & $100 N-8 B$ & & \\
\hline 935 & & RIVER & & RAW GRAB & TRANSECT & 359 & $100 N-8 B$ & & \\
\hline 936 & & RIVER & & RAW GRAB & TRANSECT & 359 & $100 \mathrm{~N}-8 \mathrm{~B}$ & & \\
\hline 937 & & RIVER & & RAW GRAB & TRANSECT & 359 & $100 N-8 B$ & & \\
\hline 938 & & RIVER & & RAW GRAB & TRANSECT & 584 & $100 \mathrm{~N}-9 \mathrm{~B}$ & & \\
\hline 939 & & RIVER & & RAW GRAB & TRANSECT & 584 & $100 \mathrm{~N}-9 \mathrm{~B}$ & & \\
\hline 940 & & RIVER & & RAW GRAB & TRANSECT & 584 & $100 \mathrm{~N}-9 \mathrm{~B}$ & & \\
\hline 941 & & RIVER & & RAW GRAB & TRANSECT & 584 & $100 \mathrm{~N}-9 \mathrm{~B}$ & & \\
\hline 942 & & RIVER & & RAW GRAB & TRANSECT & 584 & $100 \mathrm{~N}-9 \mathrm{~B}$ & & \\
\hline 943 & & RIVER & & RAW GRAB & TRANSECT & 584 & $100 \mathrm{~N}-9 \mathrm{~B}$ & & \\
\hline 944 & & RIVER & & RAW GRAB & TRANSECT & 584 & $100 N-9 B$ & & \\
\hline 945 & & RIVER & & RAW GRAB & TRANSECT & 567 & ABOVE 300 AREA SPR & & \\
\hline 946 & & RIVER & & RAW GRAB & TRANSECT & 567 & ABOVE 300 AREA SPR & & \\
\hline 947 & & RIVER & & RAW GRAB & TRANSECT & 567 & ABOVE 300 AREA SPR & & \\
\hline 948 & & RIVER & & RAW GRAB & TRANSECT & 567 & ABOVE 300 AREA SPR & & \\
\hline 949 & & RIVER & & RAW GRAB & TRANSECT & 567 & ABOVE 300 AREA SPR & & \\
\hline 950 & & RIVER & & RAW GRAB & TRANSECT & 567 & ABOVE 300 AREA SPR & & \\
\hline 951 & & RIVER & & RAW GRAB & TRANSECT & 567 & ABOVE 300 AREA SPR & & \\
\hline
\end{tabular}




\begin{tabular}{|c|c|c|c|c|c|c|c|c|c|}
\hline & $\mathrm{T}$ & $\mathrm{U}$ & $\mathrm{V}$ & $\mathrm{W}$ & $\mathrm{X}$ & $\bar{Y}$ & $Z$ & AA & $A B$ \\
\hline 1 & REVIEW_QUALIFIER & SAMP_FROM & SAMP_ITEM & SAMP_MTHD & COLL_MTHD & SAMP_SITE_ID & SAMP_SITE_NAME & WELL_NAME & SAMPLE_LOCATION \\
\hline 952 & & RIVER & & RAW GRAB & TRANSECT & 466 & RICH. PUMPHOUSE -1B & & \\
\hline 953 & & RIVER & & RAW GRAB & TRANSECT & 466 & DICH. PUMPHOUSE -1B & & \\
\hline 954 & & RIVER & & RAW GRAB & TRANSECT & 466 & SICH. PUMPHOUSE -1B & & \\
\hline 955 & & RIVER & & RAW GRAB & TRANSECT & 466 & SICH. PUMPHOUSE -1B & & \\
\hline 956 & & RIVER & & RAW GRAB & TRANSECT & 466 & RICH. PUMPHOUSE -1B & & \\
\hline 957 & & RIVER & & RAW GRAB & TRANSECT & 466 & RICH. PUMPHOUSE -1B & & \\
\hline 958 & & RIVER & & RAW GRAB & TRANSECT & 466 & RICH. PUMPHOUSE -1B & & \\
\hline 959 & & RIVER & & RAW GRAB & TRANSECT & 320 & RICH. PUMPHOUSE -2B & & \\
\hline 960 & & RIVER & & RAW GRAB & TRANSECT & 320 & RICH. PUMPHOUSE -2B & & \\
\hline 961 & & RIVER & & RAW GRAB & TRANSECT & 320 & RICH. PUMPHOUSE -2B & & \\
\hline 962 & & RIVER & & RAW GRAB & TRANSECT & 320 & RICH. PUMPHOUSE -2B & & \\
\hline 963 & & RIVER & & RAW GRAB & TRANSECT & 320 & RICH. PUMPHOUSE -2B & & \\
\hline 964 & & RIVER & & RAW GRAB & TRANSECT & 320 & RICH. PUMPHOUSE -2B & & \\
\hline 965 & & RIVER & & RAW GRAB & TRANSECT & 320 & RICH. PUMPHOUSE -2B & & \\
\hline 966 & & RIVER & & RAW GRAB & TRANSECT & 571 & RICH.PMPHS-1 HRM46.4 & & \\
\hline 967 & & RIVER & & RAW GRAB & TRANSECT & 571 & RICH.PMPHS-1 HRM46.4 & & \\
\hline 968 & & RIVER & & RAW GRAB & TRANSECT & 571 & RICH.PMPHS-1 HRM46.4 & & \\
\hline 969 & & RIVER & & RAW GRAB & TRANSECT & 571 & RICH.PMPHS-1 HRM46.4 & & \\
\hline 970 & & RIVER & & RAW GRAB & TRANSECT & 571 & RICH.PMPHS-1 HRM46.4 & & \\
\hline 971 & & RIVER & & RAW GRAB & TRANSECT & 327 & 7 RICH.PMPHS-10 HRM46.4 & & \\
\hline 972 & & RIVER & & RAW GRAB & TRANSECT & 327 & 7 RICH.PMPHS-10 HRM46.4 & & \\
\hline 973 & & RIVER & & RAW GRAB & TRANSECT & 327 & 7 RICH.PMPHS-10 HRM46.4 & & \\
\hline 974 & & RIVER & & RAW GRAB & TRANSECT & 327 & 7 RICH.PMPHS-10 HRM46.4 & & \\
\hline 975 & & RIVER & & RAW GRAB & TRANSECT & 327 & 7 RICH.PMPHS-10 HRM46.4 & & \\
\hline 976 & & RIVER & & RAW GRAB & TRANSECT & 321 & RICH.PMPHS-2 HRM46.4 & & \\
\hline 977 & & RIVER & & RAW GRAB & TRANSECT & 321 & RICH.PMPHS-2 HRM46.4 & & \\
\hline 978 & & RIVER & & RAW GRAB & TRANSECT & 321 & RICH.PMPHS-2 HRM46.4 & & \\
\hline 979 & & RIVER & & RAW GRAB & TRANSECT & 321 & RICH.PMPHS-2 HRM46.4 & & \\
\hline 980 & & RIVER & & RAW GRAB & TRANSECT & 321 & RICH.PMPHS-2 HRM46.4 & & \\
\hline 981 & & RIVER & & RAW GRAB & TRANSECT & 322 & 2 RICH.PMPHS-3 HRM46.4 & & \\
\hline 982 & & RIVER & & RAW GRAB & TRANSECT & 322 & 2 RICH.PMPHS-3 HRM46.4 & & \\
\hline 983 & & RIVER & & RAW GRAB & TRANSECT & 322 & 2 RICH.PMPHS-3 HRM46.4 & & \\
\hline 984 & & RIVER & & RAW GRAB & TRANSECT & 322 & 2 RICH.PMPHS-3 HRM46.4 & & \\
\hline 985 & & RIVER & & RAW GRAB & TRANSECT & 322 & 2 RICH.PMPHS-3 HRM46.4 & & \\
\hline 986 & & RIVER & & RAW GRAB & TRANSECT & 572 & 2 RICH.PMPHS-4 HRM46.4 & & \\
\hline 987 & & RIVER & & RAW GRAB & TRANSECT & 572 & 2 RICH.PMPHS-4 HRM46.4 & & \\
\hline 988 & & RIVER & & RAW GRAB & TRANSECT & 572 & 2 RICH.PMPHS-4 HRM46.4 & & \\
\hline 989 & & RIVER & & RAW GRAB & TRANSECT & 572 & 2 RICH.PMPHS-4 HRM46.4 & & \\
\hline
\end{tabular}




\begin{tabular}{|c|c|c|c|c|c|c|c|c|c|}
\hline & $\mathrm{T}$ & $\mathrm{U}$ & $\mathrm{V}$ & $\mathrm{W}$ & $\mathrm{X}$ & $\bar{Y}$ & Z & AA & $A B$ \\
\hline 1 & REVIEW_QUALIFIER & SAMP_FROM & SAMP_ITEM & SAMP_MTHD & COLL_MTHD & SAMP_SITE_ID & SAMP_SITE_NAME & WELL_NAME & SAMPLE_LOCATION \\
\hline 990 & & RIVER & & RAW GRAB & TRANSECT & 572 & 2 RICH.PMPHS-4 HRM46.4 & & \\
\hline 991 & & RIVER & & RAW GRAB & TRANSECT & 323 & 3 RICH.PMPHS-5 HRM46.4 & & \\
\hline 992 & & RIVER & & RAW GRAB & TRANSECT & 323 & 3 RICH.PMPHS-5 HRM46.4 & & \\
\hline 993 & & RIVER & & RAW GRAB & TRANSECT & 323 & 3 RICH.PMPHS-5 HRM46.4 & & \\
\hline 994 & & RIVER & & RAW GRAB & TRANSECT & 323 & 3 RICH.PMPHS-5 HRM46.4 & & \\
\hline 995 & & RIVER & & RAW GRAB & TRANSECT & 323 & 3 RICH.PMPHS-5 HRM46.4 & & \\
\hline 996 & & RIVER & & RAW GRAB & TRANSECT & 324 & 4 RICH.PMPHS-6 HRM46.4 & & \\
\hline 997 & & RIVER & & RAW GRAB & TRANSECT & 324 & 4 RICH.PMPHS-6 HRM46.4 & & \\
\hline 998 & & RIVER & & RAW GRAB & TRANSECT & 324 & 4 RICH.PMPHS-6 HRM46.4 & & \\
\hline 999 & & RIVER & & RAW GRAB & TRANSECT & 324 & 4 RICH.PMPHS-6 HRM46.4 & & \\
\hline 1000 & & RIVER & & RAW GRAB & TRANSECT & 324 & 4 RICH.PMPHS-6 HRM46.4 & & \\
\hline 1001 & & RIVER & & RAW GRAB & TRANSECT & 325 & 5 RICH.PMPHS-7 HRM46.4 & & \\
\hline 1002 & & RIVER & & RAW GRAB & TRANSECT & 325 & 5 RICH.PMPHS-7 HRM46.4 & & \\
\hline 1003 & & RIVER & & RAW GRAB & TRANSECT & 325 & 5 RICH.PMPHS-7 HRM46.4 & & \\
\hline 1004 & & RIVER & & RAW GRAB & TRANSECT & 325 & 5 RICH.PMPHS-7 HRM46.4 & & \\
\hline 1005 & & RIVER & & RAW GRAB & TRANSECT & 325 & 5 RICH.PMPHS-7 HRM46.4 & & \\
\hline 1006 & & RIVER & & RAW GRAB & TRANSECT & 326 & 6 RICH.PMPHS-8 HRM46.4 & & \\
\hline 1007 & & RIVER & & RAW GRAB & TRANSECT & 326 & 6 RICH.PMPHS-8 HRM46.4 & & \\
\hline 1008 & & RIVER & & RAW GRAB & TRANSECT & 326 & 6 RICH.PMPHS-8 HRM46.4 & & \\
\hline 1009 & & RIVER & & RAW GRAB & TRANSECT & 326 & 6 RICH.PMPHS-8 HRM46.4 & & \\
\hline 1010 & & RIVER & & RAW GRAB & TRANSECT & 326 & 6 RICH.PMPHS-8 HRM46.4 & & \\
\hline 1011 & & RIVER & & RAW GRAB & TRANSECT & 573 & 3 RICH.PMPHS-9 HRM46.4 & & \\
\hline 1012 & & RIVER & & RAW GRAB & TRANSECT & 573 & 3 RICH.PMPHS-9 HRM46.4 & & \\
\hline 1013 & & RIVER & & RAW GRAB & TRANSECT & 573 & 3 RICH.PMPHS-9 HRM46.4 & & \\
\hline 1014 & & RIVER & & RAW GRAB & TRANSECT & 573 & 3 RICH.PMPHS-9 HRM46.4 & & \\
\hline 1015 & & RIVER & & RAW GRAB & TRANSECT & 573 & 3 RICH.PMPHS-9 HRM46.4 & & \\
\hline 1016 & & RIVER & & RAW GRAB & TRANSECT & 336 & 100 F -1 HRM 19.0 & & \\
\hline 1017 & & RIVER & & RAW GRAB & TRANSECT & 336 & 100 F -1 HRM 19.0 & & \\
\hline 1018 & & RIVER & & RAW GRAB & TRANSECT & 336 & 100 F -1 HRM 19.0 & & \\
\hline 1019 & & RIVER & & RAW GRAB & TRANSECT & 336 & 100 F -1 HRM 19.0 & & \\
\hline 1020 & & RIVER & & RAW GRAB & TRANSECT & 336 & 100 F -1 HRM 19.0 & & \\
\hline 1021 & & RIVER & & RAW GRAB & TRANSECT & 341 & $100 \mathrm{~F}-10$ HRM 19.0 & & \\
\hline 1022 & & RIVER & & RAW GRAB & TRANSECT & 341 & 100 F -10 HRM 19.0 & & \\
\hline 1023 & & RIVER & & RAW GRAB & TRANSECT & 341 & 100 F -10 HRM 19.0 & & \\
\hline 1024 & & RIVER & & RAW GRAB & TRANSECT & 341 & 100 F -10 HRM 19.0 & & \\
\hline 1025 & & RIVER & & RAW GRAB & TRANSECT & 341 & $100 \mathrm{~F}-10$ HRM 19.0 & & \\
\hline 1026 & & RIVER & & RAW GRAB & TRANSECT & 576 & 100 F -2 HRM 19.0 & & \\
\hline 1027 & & RIVER & & RAW GRAB & TRANSECT & 576 & b 100 F -2 HRM 19.0 & & \\
\hline
\end{tabular}




\begin{tabular}{|c|c|c|c|c|c|c|c|c|c|}
\hline & $\mathrm{T}$ & 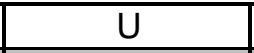 & $\mathrm{V}$ & $\mathrm{W}$ & $\mathrm{X}$ & $\mathrm{Y}$ & $Z$ & $\mathrm{AA}$ & $A B$ \\
\hline 1 & REVIEW_QUALIFIER & SAMP_FROM & SAMP_ITEM & SAMP_MTHD & COLL_MTHD & SAMP_SITE_ID & SAMP_SITE_NAME & WELL_NAME & SAMPLE_LOCATION \\
\hline 1028 & & RIVER & & RAW GRAB & TRANSECT & 576 & 100 F -2 HRM 19.0 & & \\
\hline 1029 & & RIVER & & RAW GRAB & TRANSECT & 576 & 100 F -2 HRM 19.0 & & \\
\hline 1030 & & RIVER & & RAW GRAB & TRANSECT & 576 & 100 F -2 HRM 19.0 & & \\
\hline 1031 & & RIVER & & RAW GRAB & TRANSECT & 337 & 100 F -3 HRM 19.0 & & \\
\hline 1032 & & RIVER & & RAW GRAB & TRANSECT & 337 & 100 F -3 HRM 19.0 & & \\
\hline 1033 & & RIVER & & RAW GRAB & TRANSECT & 337 & 100 F -3 HRM 19.0 & & \\
\hline 1034 & & RIVER & & RAW GRAB & TRANSECT & 337 & 100 F -3 HRM 19.0 & & \\
\hline 1035 & & RIVER & & RAW GRAB & TRANSECT & 337 & 100 F -3 HRM 19.0 & & \\
\hline 1036 & & RIVER & & RAW GRAB & TRANSECT & 338 & 100 F -4 HRM 19.0 & & \\
\hline 1037 & & RIVER & & RAW GRAB & TRANSECT & 338 & 100 F -4 HRM 19.0 & & \\
\hline 1038 & & RIVER & & RAW GRAB & TRANSECT & 338 & 100 F -4 HRM 19.0 & & \\
\hline 1039 & & RIVER & & RAW GRAB & TRANSECT & 338 & 100 F -4 HRM 19.0 & & \\
\hline 1040 & & RIVER & & RAW GRAB & TRANSECT & 338 & 100 F -4 HRM 19.0 & & \\
\hline 1041 & & RIVER & & RAW GRAB & TRANSECT & 577 & 100 F -5 HRM 19.0 & & \\
\hline 1042 & & RIVER & & RAW GRAB & TRANSECT & 577 & 100 F -5 HRM 19.0 & & \\
\hline 1043 & & RIVER & & RAW GRAB & TRANSECT & 577 & 100 F -5 HRM 19.0 & & \\
\hline 1044 & & RIVER & & RAW GRAB & TRANSECT & 577 & 100 F -5 HRM 19.0 & & \\
\hline 1045 & & RIVER & & RAW GRAB & TRANSECT & 577 & 100 F -5 HRM 19.0 & & \\
\hline 1046 & & RIVER & & RAW GRAB & TRANSECT & 606 & 100 F -6 HRM 19.0 & & \\
\hline 1047 & & RIVER & & RAW GRAB & TRANSECT & 606 & 100 F -6 HRM 19.0 & & \\
\hline 1048 & & RIVER & & RAW GRAB & TRANSECT & 606 & 100 F -6 HRM 19.0 & & \\
\hline 1049 & & RIVER & & RAW GRAB & TRANSECT & 606 & 100 F -6 HRM 19.0 & & \\
\hline 1050 & & RIVER & & RAW GRAB & TRANSECT & 606 & 100 F -6 HRM 19.0 & & \\
\hline 1051 & & RIVER & & RAW GRAB & TRANSECT & 339 & 100 F -7 HRM 19.0 & & \\
\hline 1052 & & RIVER & & RAW GRAB & TRANSECT & 339 & 100 F -7 HRM 19.0 & & \\
\hline 1053 & & RIVER & & RAW GRAB & TRANSECT & 339 & 100 F -7 HRM 19.0 & & \\
\hline 1054 & & RIVER & & RAW GRAB & TRANSECT & 339 & 100 F -7 HRM 19.0 & & \\
\hline 1055 & & RIVER & & RAW GRAB & TRANSECT & 339 & 100 F -7 HRM 19.0 & & \\
\hline 1056 & & RIVER & & RAW GRAB & TRANSECT & 340 & 100 F -8 HRM 19.0 & & \\
\hline 1057 & & RIVER & & RAW GRAB & TRANSECT & 340 & 100 F -8 HRM 19.0 & & \\
\hline 1058 & & RIVER & & RAW GRAB & TRANSECT & 340 & 100 F -8 HRM 19.0 & & \\
\hline 1059 & & RIVER & & RAW GRAB & TRANSECT & 340 & 100 F -8 HRM 19.0 & & \\
\hline 1060 & & RIVER & & RAW GRAB & TRANSECT & 340 & 100 F -8 HRM 19.0 & & \\
\hline 1061 & & RIVER & & RAW GRAB & TRANSECT & 467 & 100 F -9 HRM 19.0 & & \\
\hline 1062 & & RIVER & & RAW GRAB & TRANSECT & 467 & 100 F -9 HRM 19.0 & & \\
\hline 1063 & & RIVER & & RAW GRAB & TRANSECT & $467:$ & 100 F -9 HRM 19.0 & & \\
\hline 1064 & & RIVER & & RAW GRAB & TRANSECT & 467 & 100 F -9 HRM 19.0 & & \\
\hline 1065 & & RIVER & & RAW GRAB & TRANSECT & 467 & 100 F -9 HRM 19.0 & & \\
\hline
\end{tabular}




\begin{tabular}{|c|c|c|c|c|c|c|c|c|c|}
\hline & $\mathrm{T}$ & 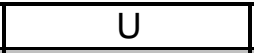 & $\mathrm{V}$ & $\mathrm{W}$ & $\mathrm{X}$ & $\mathrm{Y}$ & $Z$ & $\mathrm{AA}$ & $A B$ \\
\hline 1 & REVIEW_QUALIFIER & SAMP_FROM & SAMP_ITEM & SAMP_MTHD & COLL_MTHD & SAMP_SITE_ID & SAMP_SITE_NAME & WELL_NAME & SAMPLE_LOCATION \\
\hline 1066 & & RIVER & & RAW GRAB & TRANSECT & 317 & VERNITA-1 HRM 0.3 & & \\
\hline 1067 & & RIVER & & RAW GRAB & TRANSECT & 317 & VERNITA-1 HRM 0.3 & & \\
\hline 1068 & & RIVER & & RAW GRAB & TRANSECT & 317 & VERNITA-1 HRM 0.3 & & \\
\hline 1069 & & RIVER & & RAW GRAB & TRANSECT & 317 & VERNITA-1 HRM 0.3 & & \\
\hline 1070 & & RIVER & & RAW GRAB & TRANSECT & 317 & VERNITA-1 HRM 0.3 & & \\
\hline 1071 & & RIVER & & RAW GRAB & TRANSECT & 570 & VERNITA-2 HRM 0.3 & & \\
\hline 1072 & & RIVER & & RAW GRAB & TRANSECT & 570 & VERNITA-2 HRM 0.3 & & \\
\hline 1073 & & RIVER & & RAW GRAB & TRANSECT & 570 & VERNITA-2 HRM 0.3 & & \\
\hline 1074 & & RIVER & & RAW GRAB & TRANSECT & 570 & VERNITA-2 HRM 0.3 & & \\
\hline 1075 & & RIVER & & RAW GRAB & TRANSECT & 570 & VERNITA-2 HRM 0.3 & & \\
\hline 1076 & & RIVER & & RAW GRAB & TRANSECT & 318 & VERNITA-3 HRM 0.3 & & \\
\hline 1077 & & RIVER & & RAW GRAB & TRANSECT & 318 & VERNITA-3 HRM 0.3 & & \\
\hline 1078 & & RIVER & & RAW GRAB & TRANSECT & 318 & VERNITA-3 HRM 0.3 & & \\
\hline 1079 & & RIVER & & RAW GRAB & TRANSECT & 318 & VERNITA-3 HRM 0.3 & & \\
\hline 1080 & & RIVER & & RAW GRAB & TRANSECT & 318 & VERNITA-3 HRM 0.3 & & \\
\hline 1081 & & RIVER & & RAW GRAB & TRANSECT & 319 & VERNITA-4 HRM 0.3 & & \\
\hline 1082 & & RIVER & & RAW GRAB & TRANSECT & 319 & VERNITA-4 HRM 0.3 & & \\
\hline 1083 & & RIVER & & RAW GRAB & TRANSECT & 319 & VERNITA-4 HRM 0.3 & & \\
\hline 1084 & & RIVER & & RAW GRAB & TRANSECT & 319 & VERNITA-4 HRM 0.3 & & \\
\hline 1085 & & RIVER & & RAW GRAB & TRANSECT & 319 & VERNITA-4 HRM 0.3 & & \\
\hline 1086 & & RIVER & & RAW GRAB & TRANSECT & 463 & RICH.PMPHS HRM 46.4 & & \\
\hline 1087 & & RIVER & & RAW GRAB & TRANSECT & 463 & RICH.PMPHS HRM 46.4 & & \\
\hline 1088 & & RIVER & & RAW GRAB & TRANSECT & 463 & RICH.PMPHS HRM 46.4 & & \\
\hline 1089 & & RIVER & & RAW GRAB & TRANSECT & 463 & RICH.PMPHS HRM 46.4 & & \\
\hline 1090 & & RIVER & & RAW GRAB & TRANSECT & 463 & RICH.PMPHS HRM 46.4 & & \\
\hline 1091 & & RIVER & & RAW GRAB & TRANSECT & 571 & RICH.PMPHS-1 HRM46.4 & & \\
\hline 1092 & & RIVER & & RAW GRAB & TRANSECT & 571 & RICH.PMPHS-1 HRM46.4 & & \\
\hline 1093 & & RIVER & & RAW GRAB & TRANSECT & 571 & RICH.PMPHS-1 HRM46.4 & & \\
\hline 1094 & & RIVER & & RAW GRAB & TRANSECT & 571 & RICH.PMPHS-1 HRM46.4 & & \\
\hline 1095 & & RIVER & & RAW GRAB & TRANSECT & 571 & RICH.PMPHS-1 HRM46.4 & & \\
\hline 1096 & & RIVER & & RAW GRAB & TRANSECT & 327 & RICH.PMPHS-10 HRM46.4 & & \\
\hline 1097 & & RIVER & & RAW GRAB & TRANSECT & 327 & RICH.PMPHS-10 HRM46.4 & & \\
\hline 1098 & & RIVER & & RAW GRAB & TRANSECT & 327 & RICH.PMPHS-10 HRM46.4 & & \\
\hline 1099 & & RIVER & & RAW GRAB & TRANSECT & 327 & RICH.PMPHS-10 HRM46.4 & & \\
\hline 1100 & & RIVER & & RAW GRAB & TRANSECT & 327 & RICH.PMPHS-10 HRM46.4 & & \\
\hline 1101 & & RIVER & & RAW GRAB & TRANSECT & 321 & RICH.PMPHS-2 HRM46.4 & & \\
\hline 1102 & & RIVER & & RAW GRAB & TRANSECT & 321 & RICH.PMPHS-2 HRM46.4 & & \\
\hline 1103 & & RIVER & & RAW GRAB & TRANSECT & 321 & RICH.PMPHS-2 HRM46.4 & & \\
\hline
\end{tabular}




\begin{tabular}{|c|c|c|c|c|c|c|c|c|c|}
\hline & $\mathrm{T}$ & $\bar{U}$ & $\mathrm{~V}$ & $\mathrm{~W}$ & $\mathrm{X}$ & $\mathrm{Y}$ & $Z$ & $\mathrm{AA}$ & $\mathrm{AB}$ \\
\hline 1 & REVIEW_QUALIFIER & SAMP_FROM & SAMP_ITEM & SAMP_MTHD & COLL_MTHD & SAMP_SITE_ID & SAMP_SITE_NAME & WELL_NAME & SAMPLE_LOCATION \\
\hline 1104 & & RIVER & & RAW GRAB & TRANSECT & 321 & RICH.PMPHS-2 HRM46.4 & & \\
\hline 1105 & & RIVER & & RAW GRAB & TRANSECT & 321 & RICH.PMPHS-2 HRM46.4 & & \\
\hline 1106 & & RIVER & & RAW GRAB & TRANSECT & 322 & RICH.PMPHS-3 HRM46.4 & & \\
\hline 1107 & & RIVER & & RAW GRAB & TRANSECT & 322 & RICH.PMPHS-3 HRM46.4 & & \\
\hline 1108 & & RIVER & & RAW GRAB & TRANSECT & 322 & RICH.PMPHS-3 HRM46.4 & & \\
\hline 1109 & & RIVER & & RAW GRAB & TRANSECT & 322 & RICH.PMPHS-3 HRM46.4 & & \\
\hline 1110 & & RIVER & & RAW GRAB & TRANSECT & 322 & RICH.PMPHS-3 HRM46.4 & & \\
\hline 1111 & & RIVER & & RAW GRAB & TRANSECT & 572 & RICH.PMPHS-4 HRM46.4 & & \\
\hline 1112 & & RIVER & & RAW GRAB & TRANSECT & 572 & RICH.PMPHS-4 HRM46.4 & & \\
\hline 1113 & & RIVER & & RAW GRAB & TRANSECT & 572 & RICH.PMPHS-4 HRM46.4 & & \\
\hline 1114 & & RIVER & & RAW GRAB & TRANSECT & 572 & RICH.PMPHS-4 HRM46.4 & & \\
\hline 1115 & & RIVER & & RAW GRAB & TRANSECT & 572 & RICH.PMPHS-4 HRM46.4 & & \\
\hline 1116 & & RIVER & & RAW GRAB & TRANSECT & 323 & RICH.PMPHS-5 HRM46.4 & & \\
\hline 1117 & & RIVER & & RAW GRAB & TRANSECT & 323 & RICH.PMPHS-5 HRM46.4 & & \\
\hline 1118 & & RIVER & & RAW GRAB & TRANSECT & 323 & RICH.PMPHS-5 HRM46.4 & & \\
\hline 1119 & & RIVER & & RAW GRAB & TRANSECT & 323 & RICH.PMPHS-5 HRM46.4 & & \\
\hline 1120 & & RIVER & & RAW GRAB & TRANSECT & 323 & RICH.PMPHS-5 HRM46.4 & & \\
\hline 1121 & & RIVER & & RAW GRAB & TRANSECT & 324 & RICH.PMPHS-6 HRM46.4 & & \\
\hline 1122 & & RIVER & & RAW GRAB & TRANSECT & 324 & RICH.PMPHS-6 HRM46.4 & & \\
\hline 1123 & & RIVER & & RAW GRAB & TRANSECT & 324 & RICH.PMPHS-6 HRM46.4 & & \\
\hline 1124 & & RIVER & & RAW GRAB & TRANSECT & 324 & RICH.PMPHS-6 HRM46.4 & & \\
\hline 1125 & & RIVER & & RAW GRAB & TRANSECT & 324 & RICH.PMPHS-6 HRM46.4 & & \\
\hline 1126 & & RIVER & & RAW GRAB & TRANSECT & 325 & RICH.PMPHS-7 HRM46.4 & & \\
\hline 1127 & & RIVER & & RAW GRAB & TRANSECT & 325 & RICH.PMPHS-7 HRM46.4 & & \\
\hline 1128 & & RIVER & & RAW GRAB & TRANSECT & 325 & RICH.PMPHS-7 HRM46.4 & & \\
\hline 1129 & & RIVER & & RAW GRAB & TRANSECT & 325 & RICH.PMPHS-7 HRM46.4 & & \\
\hline 1130 & & RIVER & & RAW GRAB & TRANSECT & 325 & RICH.PMPHS-7 HRM46.4 & & \\
\hline 1131 & & RIVER & & RAW GRAB & TRANSECT & 326 & RICH.PMPHS-8 HRM46.4 & & \\
\hline 1132 & & RIVER & & RAW GRAB & TRANSECT & 326 & RICH.PMPHS-8 HRM46.4 & & \\
\hline 1133 & & RIVER & & RAW GRAB & TRANSECT & 326 & RICH.PMPHS-8 HRM46.4 & & \\
\hline 1134 & & RIVER & & RAW GRAB & TRANSECT & 326 & RICH.PMPHS-8 HRM46.4 & & \\
\hline 1135 & & RIVER & & RAW GRAB & TRANSECT & 326 & RICH.PMPHS-8 HRM46.4 & & \\
\hline 1136 & & RIVER & & RAW GRAB & TRANSECT & 573 & RICH.PMPHS-9 HRM46.4 & & \\
\hline 1137 & & RIVER & & RAW GRAB & TRANSECT & 573 & RICH.PMPHS-9 HRM46.4 & & \\
\hline 1138 & & RIVER & & RAW GRAB & TRANSECT & 573 & RICH.PMPHS-9 HRM46.4 & & \\
\hline 1139 & & RIVER & & RAW GRAB & TRANSECT & 573 & RICH.PMPHS-9 HRM46.4 & & \\
\hline 1140 & & RIVER & & RAW GRAB & TRANSECT & 573 & RICH.PMPHS-9 HRM46.4 & & \\
\hline 1141 & & RIVER & & RAW GRAB & TRANSECT & 317 & VERNITA-1 HRM 0.3 & & \\
\hline
\end{tabular}




\begin{tabular}{|c|c|c|c|c|c|c|c|c|c|}
\hline & $\mathrm{T}$ & $\bar{U}$ & $\mathrm{~V}$ & $\mathrm{~W}$ & $\mathrm{X}$ & $\mathrm{Y}$ & $Z$ & $\mathrm{AA}$ & $\mathrm{AB}$ \\
\hline 1 & REVIEW_QUALIFIER & SAMP_FROM & SAMP_ITEM & SAMP_MTHD & COLL_MTHD & SAMP_SITE_ID & SAMP_SITE_NAME & WELL_NAME & SAMPLE_LOCATION \\
\hline 1142 & $F$ & RIVER & & RAW GRAB & TRANSECT & 317 & VERNITA-1 HRM 0.3 & & \\
\hline 1143 & & RIVER & & RAW GRAB & TRANSECT & 317 & VERNITA-1 HRM 0.3 & & \\
\hline 1144 & & RIVER & & RAW GRAB & TRANSECT & 317 & VERNITA-1 HRM 0.3 & & \\
\hline 1145 & & RIVER & & RAW GRAB & TRANSECT & 317 & VERNITA-1 HRM 0.3 & & \\
\hline 1146 & & RIVER & & RAW GRAB & TRANSECT & 570 & VERNITA-2 HRM 0.3 & & \\
\hline 1147 & $F$ & RIVER & & RAW GRAB & TRANSECT & 570 & VERNITA-2 HRM 0.3 & & \\
\hline 1148 & & RIVER & & RAW GRAB & TRANSECT & 570 & VERNITA-2 HRM 0.3 & & \\
\hline 1149 & & RIVER & & RAW GRAB & TRANSECT & 570 & VERNITA-2 HRM 0.3 & & \\
\hline 1150 & & RIVER & & RAW GRAB & TRANSECT & 570 & VERNITA-2 HRM 0.3 & & \\
\hline 1151 & & RIVER & & RAW GRAB & TRANSECT & 571 & RICH.PMPHS-1 HRM46.4 & & \\
\hline 1152 & & RIVER & & RAW GRAB & TRANSECT & 571 & RICH.PMPHS-1 HRM46.4 & & \\
\hline 1153 & & RIVER & & RAW GRAB & TRANSECT & 571 & RICH.PMPHS-1 HRM46.4 & & \\
\hline 1154 & $F$ & RIVER & & RAW GRAB & TRANSECT & 571 & RICH.PMPHS-1 HRM46.4 & & \\
\hline 1155 & & RIVER & & RAW GRAB & TRANSECT & 571 & RICH.PMPHS-1 HRM46.4 & & \\
\hline 1156 & & RIVER & & RAW GRAB & TRANSECT & 327 & RICH.PMPHS-10 HRM46.4 & & \\
\hline 1157 & $F$ & RIVER & & RAW GRAB & TRANSECT & 327 & RICH.PMPHS-10 HRM46.4 & & \\
\hline 1158 & $F$ & RIVER & & RAW GRAB & TRANSECT & 327 & RICH.PMPHS-10 HRM46.4 & & \\
\hline 1159 & $F$ & RIVER & & RAW GRAB & TRANSECT & 327 & RICH.PMPHS-10 HRM46.4 & & \\
\hline 1160 & & RIVER & & RAW GRAB & TRANSECT & 327 & RICH.PMPHS-10 HRM46.4 & & \\
\hline 1161 & & RIVER & & RAW GRAB & TRANSECT & 321 & RICH.PMPHS-2 HRM46.4 & & \\
\hline 1162 & $F$ & RIVER & & RAW GRAB & TRANSECT & 321 & RICH.PMPHS-2 HRM46.4 & & \\
\hline 1163 & & RIVER & & RAW GRAB & TRANSECT & 321 & RICH.PMPHS-2 HRM46.4 & & \\
\hline 1164 & & RIVER & & RAW GRAB & TRANSECT & 321 & RICH.PMPHS-2 HRM46.4 & & \\
\hline 1165 & & RIVER & & RAW GRAB & TRANSECT & 321 & RICH.PMPHS-2 HRM46.4 & & \\
\hline 1166 & & RIVER & & RAW GRAB & TRANSECT & 322 & RICH.PMPHS-3 HRM46.4 & & \\
\hline 1167 & $F$ & RIVER & & RAW GRAB & TRANSECT & 322 & RICH.PMPHS-3 HRM46.4 & & \\
\hline 1168 & & RIVER & & RAW GRAB & TRANSECT & 322 & RICH.PMPHS-3 HRM46.4 & & \\
\hline 1169 & $F$ & RIVER & & RAW GRAB & TRANSECT & 322 & RICH.PMPHS-3 HRM46.4 & & \\
\hline 1170 & & RIVER & & RAW GRAB & TRANSECT & 322 & RICH.PMPHS-3 HRM46.4 & & \\
\hline 1171 & & RIVER & & RAW GRAB & TRANSECT & 572 & RICH.PMPHS-4 HRM46.4 & & \\
\hline 1172 & $F$ & RIVER & & RAW GRAB & TRANSECT & 572 & RICH.PMPHS-4 HRM46.4 & & \\
\hline 1173 & & RIVER & & RAW GRAB & TRANSECT & 572 & RICH.PMPHS-4 HRM46.4 & & \\
\hline 1174 & & RIVER & & RAW GRAB & TRANSECT & 572 & RICH.PMPHS-4 HRM46.4 & & \\
\hline 1175 & & RIVER & & RAW GRAB & TRANSECT & 572 & RICH.PMPHS-4 HRM46.4 & & \\
\hline 1176 & $F$ & RIVER & & RAW GRAB & TRANSECT & 323 & RICH.PMPHS-5 HRM46.4 & & \\
\hline 1177 & $F$ & RIVER & & RAW GRAB & TRANSECT & 323 & RICH.PMPHS-5 HRM46.4 & & \\
\hline 1178 & & RIVER & & RAW GRAB & TRANSECT & 323 & RICH.PMPHS-5 HRM46.4 & & \\
\hline 1179 & & RIVER & & RAW GRAB & TRANSECT & 323 & RICH.PMPHS-5 HRM46.4 & & \\
\hline
\end{tabular}




\begin{tabular}{|c|c|c|c|c|c|c|c|c|c|}
\hline & $T$ & $\bar{U}$ & $\overline{\mathrm{V}}$ & $\mathrm{W}$ & $\bar{X}$ & $\bar{Y}$ & $\bar{Z}$ & $\overline{A A}$ & $A B$ \\
\hline 1 & REVIEW_QUALIFIER & SAMP_FROM & SAMP_ITEM & SAMP_MTHD & COLL_MTHD & SAMP_SITE_ID & SAMP_SITE_NAME & WELL_NAME & SAMPLE_LOCATION \\
\hline 1180 & & RIVER & & RAW GRAB & TRANSECT & & $3 \mathrm{RICH} . \mathrm{PMPHS}-5$ HRM46.4 & & \\
\hline 1181 & & RIVER & & RAW GRAB & TRANSECT & 32 & 4 RICH.PMPHS-6 HRM46.4 & & \\
\hline 1182 & $\mathrm{~F}$ & RIVER & & RAW GRAB & TRANSECT & 32 & 4 RICH.PMPHS-6 HRM46.4 & & \\
\hline 1183 & & RIVER & & RAW GRAB & TRANSECT & 32 & 4 RICH.PMPHS-6 HRM46.4 & & \\
\hline 1184 & & RIVER & & RAW GRAB & TRANSECT & 32 & 4 RICH.PMPHS-6 HRM46.4 & & \\
\hline 1185 & & RIVER & & RAW GRAB & TRANSECT & 32 & RICH.PMPHS-6 HRM46.4 & & \\
\hline 1186 & & RIVER & & RAW GRAB & TRANSECT & 325 & 5 RICH.PMPHS-7 HRM46.4 & & \\
\hline 1187 & $\mathrm{~F}$ & RIVER & & RAW GRAB & TRANSECT & 325 & RICH.PMPHS-7 HRM46.4 & & \\
\hline 1188 & & RIVER & & RAW GRAB & TRANSECT & 325 & 5 RICH.PMPHS-7 HRM46.4 & & \\
\hline 1189 & $\mathrm{~F}$ & RIVER & & RAW GRAB & TRANSECT & 325 & 5 RICH.PMPHS-7 HRM46.4 & & \\
\hline 1190 & & RIVER & & RAW GRAB & TRANSECT & 325 & RICH.PMPHS-7 HRM46.4 & & \\
\hline 1191 & & RIVER & & RAW GRAB & TRANSECT & 326 & RICH.PMPHS-8 HRM46.4 & & \\
\hline 1192 & $\mathrm{~F}$ & RIVER & & RAW GRAB & TRANSECT & 326 & RICH.PMPHS-8 HRM46.4 & & \\
\hline 1193 & & RIVER & & RAW GRAB & TRANSECT & 326 & RICH.PMPHS-8 HRM46.4 & & \\
\hline 1194 & & RIVER & & RAW GRAB & TRANSECT & 326 & RICH.PMPHS-8 HRM46.4 & & \\
\hline 1195 & & RIVER & & RAW GRAB & TRANSECT & 326 & RICH.PMPHS-8 HRM46.4 & & \\
\hline 1196 & & RIVER & & RAW GRAB & TRANSECT & 573 & 3 RICH.PMPHS-9 HRM46.4 & & \\
\hline 1197 & $\mathrm{~F}$ & RIVER & & RAW GRAB & TRANSECT & 573 & RICH.PMPHS-9 HRM46.4 & & \\
\hline 1198 & & RIVER & & RAW GRAB & TRANSECT & 573 & 3 RICH.PMPHS-9 HRM46.4 & & \\
\hline 1199 & & RIVER & & RAW GRAB & TRANSECT & 573 & 3 RICH.PMPHS-9 HRM46.4 & & \\
\hline 1200 & & RIVER & & RAW GRAB & TRANSECT & 573 & RICH.PMPHS-9 HRM46.4 & & \\
\hline 1201 & & RIVER & & RAW GRAB & TRANSECT & 317 & 7 VERNITA-1 HRM 0.3 & & \\
\hline 1202 & $\mathrm{~F}$ & RIVER & & RAW GRAB & TRANSECT & 317 & 7 VERNITA-1 HRM 0.3 & & \\
\hline 1203 & & RIVER & & RAW GRAB & TRANSECT & 317 & 7 VERNITA-1 HRM 0.3 & & \\
\hline 1204 & & RIVER & & RAW GRAB & TRANSECT & 317 & 7 VERNITA-1 HRM 0.3 & & \\
\hline 1205 & & RIVER & & RAW GRAB & TRANSECT & 317 & 7 VERNITA-1 HRM 0.3 & & \\
\hline 1206 & & RIVER & & RAW GRAB & TRANSECT & 570 & VERNITA-2 HRM 0.3 & & \\
\hline 1207 & $\mathrm{~F}$ & RIVER & & RAW GRAB & TRANSECT & 570 & VERNITA-2 HRM 0.3 & & \\
\hline 1208 & & RIVER & & RAW GRAB & TRANSECT & 570 & VERNITA-2 HRM 0.3 & & \\
\hline 1209 & & RIVER & & RAW GRAB & TRANSECT & 570 & VERNITA-2 HRM 0.3 & & \\
\hline 1210 & & RIVER & & RAW GRAB & TRANSECT & 570 & VERNITA-2 HRM 0.3 & & \\
\hline 1211 & & RIVER & & RAW GRAB & TRANSECT & $31 \varepsilon$ & 3 VERNITA-3 HRM 0.3 & & \\
\hline 1212 & $\mathrm{~F}$ & RIVER & & RAW GRAB & TRANSECT & $31 \varepsilon$ & 3 VERNITA-3 HRM 0.3 & & \\
\hline 1213 & & RIVER & & RAW GRAB & TRANSECT & $31 \varepsilon$ & 3 VERNITA-3 HRM 0.3 & & \\
\hline 1214 & & RIVER & & RAW GRAB & TRANSECT & $31 \varepsilon$ & 3 VERNITA-3 HRM 0.3 & & \\
\hline 1215 & & RIVER & & RAW GRAB & TRANSECT & $31 \varepsilon$ & 3 VERNITA-3 HRM 0.3 & & \\
\hline 1216 & & RIVER & & RAW GRAB & TRANSECT & 31 & 9 VERNITA-4 HRM 0.3 & & \\
\hline 1217 & $F$ & RIVER & & RAW GRAB & TRANSECT & 315 & VERNITA-4 HRM 0.3 & & \\
\hline
\end{tabular}




\begin{tabular}{|c|c|c|c|c|c|c|c|c|c|}
\hline & $T$ & $\bar{U}$ & $\mathrm{~V}$ & $\mathrm{~W}$ & $\bar{x}$ & $\bar{Y}$ & $\bar{Z}$ & AA & $A B$ \\
\hline 1 & REVIEW_QUALIFIER & SAMP_FROM & SAMP_ITEM & SAMP_MTHD & COLL_MTHD & SAMP_SITE_ID & SAMP_SITE_NAME & WELL_NAME & SAMPLE_LOCATION \\
\hline 1218 & & RIVER & & RAW GRAB & TRANSECT & 319 & 9 VERNITA-4 HRM 0.3 & & \\
\hline 1219 & & RIVER & & RAW GRAB & TRANSECT & 319 & 9 VERNITA-4 HRM 0.3 & & \\
\hline 1220 & & RIVER & & RAW GRAB & TRANSECT & 319 & 9 VERNITA-4 HRM 0.3 & & \\
\hline 1221 & & RIVER & & RAW GRAB & TRANSECT & 571 & RICH.PMPHS-1 HRM46.4 & & \\
\hline \begin{tabular}{|l|l|}
1222 \\
\end{tabular} & $F$ & RIVER & & RAW GRAB & TRANSECT & 571 & IICH.PMPHS-1 HRM46.4 & & \\
\hline 1223 & & RIVER & & RAW GRAB & TRANSECT & 571 & RICH.PMPHS-1 HRM46.4 & & \\
\hline 1224 & & RIVER & & RAW GRAB & TRANSECT & 571 & 1 RICH.PMPHS-1 HRM46.4 & & \\
\hline 1225 & & RIVER & & RAW GRAB & TRANSECT & 571 & RICH.PMPHS-1 HRM46.4 & & \\
\hline 1226 & & RIVER & & RAW GRAB & TRANSECT & 327 & 7 RICH.PMPHS-10 HRM46.4 & & \\
\hline 1227 & $\mathrm{~F}$ & RIVER & & RAW GRAB & TRANSECT & 327 & 7 RICH.PMPHS-10 HRM46.4 & & \\
\hline 1228 & & RIVER & & RAW GRAB & TRANSECT & 327 & 7 RICH.PMPHS-10 HRM46.4 & & \\
\hline 1229 & & RIVER & & RAW GRAB & TRANSECT & 327 & 7 RICH.PMPHS-10 HRM46.4 & & \\
\hline 1230 & & RIVER & & RAW GRAB & TRANSECT & 327 & 7 RICH.PMPHS-10 HRM46.4 & & \\
\hline 1231 & & RIVER & & RAW GRAB & TRANSECT & 321 & RICH.PMPHS-2 HRM46.4 & & \\
\hline 1232 & $F$ & RIVER & & RAW GRAB & TRANSECT & 321 & RICH.PMPHS-2 HRM46.4 & & \\
\hline 1233 & & RIVER & & RAW GRAB & TRANSECT & 321 & IICH.PMPHS-2 HRM46.4 & & \\
\hline 1234 & & RIVER & & RAW GRAB & TRANSECT & 321 & RICH.PMPHS-2 HRM46.4 & & \\
\hline 1235 & & RIVER & & RAW GRAB & TRANSECT & 321 & RICH.PMPHS-2 HRM46.4 & & \\
\hline 1236 & & RIVER & & RAW GRAB & TRANSECT & 322 & 2 RICH.PMPHS-3 HRM46.4 & & \\
\hline 1237 & $F$ & RIVER & & RAW GRAB & TRANSECT & 322 & 2 RICH.PMPHS-3 HRM46.4 & & \\
\hline 1238 & & RIVER & & RAW GRAB & TRANSECT & 322 & 2 RICH.PMPHS-3 HRM46.4 & & \\
\hline 1239 & & RIVER & & RAW GRAB & TRANSECT & 322 & 2 RICH.PMPHS-3 HRM46.4 & & \\
\hline 1240 & & RIVER & & RAW GRAB & TRANSECT & 322 & 2 RICH.PMPHS-3 HRM46.4 & & \\
\hline 1241 & & RIVER & & RAW GRAB & TRANSECT & 572 & 2 RICH.PMPHS-4 HRM46.4 & & \\
\hline 1242 & $\mathrm{~F}$ & RIVER & & RAW GRAB & TRANSECT & 572 & 2 RICH.PMPHS-4 HRM46.4 & & \\
\hline 1243 & & RIVER & & RAW GRAB & TRANSECT & 572 & 2 RICH.PMPHS-4 HRM46.4 & & \\
\hline 1244 & & RIVER & & RAW GRAB & TRANSECT & 572 & 2 RICH.PMPHS-4 HRM46.4 & & \\
\hline 1245 & & RIVER & & RAW GRAB & TRANSECT & 572 & 2 RICH.PMPHS-4 HRM46.4 & & \\
\hline 1246 & & RIVER & & RAW GRAB & TRANSECT & 323 & 3 RICH.PMPHS-5 HRM46.4 & & \\
\hline 1247 & $F$ & RIVER & & RAW GRAB & TRANSECT & 323 & 3 RICH.PMPHS-5 HRM46.4 & & \\
\hline 1248 & & RIVER & & RAW GRAB & TRANSECT & 323 & 3 RICH.PMPHS-5 HRM46.4 & & \\
\hline 1249 & & RIVER & & RAW GRAB & TRANSECT & 323 & 3 RICH.PMPHS-5 HRM46.4 & & \\
\hline 1250 & & RIVER & & RAW GRAB & TRANSECT & 323 & 3 RICH.PMPHS-5 HRM46.4 & & \\
\hline 1251 & & RIVER & & RAW GRAB & TRANSECT & 324 & 4 RICH.PMPHS-6 HRM46.4 & & \\
\hline \begin{tabular}{|l|l|}
1252 \\
\end{tabular} & $F$ & RIVER & & RAW GRAB & TRANSECT & 324 & 4 RICH.PMPHS-6 HRM46.4 & & \\
\hline 1253 & & RIVER & & RAW GRAB & TRANSECT & 324 & 4 RICH.PMPHS-6 HRM46.4 & & \\
\hline 1254 & & RIVER & & RAW GRAB & TRANSECT & 324 & 4 RICH.PMPHS-6 HRM46.4 & & \\
\hline 1255 & & RIVER & & RAW GRAB & TRANSECT & 324 & 4 RICH.PMPHS-6 HRM46.4 & & \\
\hline
\end{tabular}




\begin{tabular}{|c|c|c|c|c|c|c|c|c|c|}
\hline & $\mathrm{T}$ & $\bar{U}$ & $\mathrm{~V}$ & $\mathrm{~W}$ & $\mathrm{X}$ & $\mathrm{Y}$ & $Z$ & $\mathrm{AA}$ & $\mathrm{AB}$ \\
\hline \begin{tabular}{l|l}
1 & 1 \\
\end{tabular} & REVIEW_QUALIFIER & SAMP_FROM & SAMP_ITEM & SAMP_MTHD & COLL_MTHD & SAMP_SITE_ID & SAMP_SITE_NAME & WELL_NAME & SAMPLE_LOCATION \\
\hline 1256 & & RIVER & & RAW GRAB & TRANSECT & 325 & RICH.PMPHS-7 HRM46.4 & & \\
\hline $1257 \mathrm{~F}$ & $\mathrm{~F}$ & RIVER & & RAW GRAB & TRANSECT & 325 & RICH.PMPHS-7 HRM46.4 & & \\
\hline 1258 & & RIVER & & RAW GRAB & TRANSECT & 325 & RICH.PMPHS-7 HRM46.4 & & \\
\hline 1259 & & RIVER & & RAW GRAB & TRANSECT & 325 & RICH.PMPHS-7 HRM46.4 & & \\
\hline 1260 & & RIVER & & RAW GRAB & TRANSECT & 325 & RICH.PMPHS-7 HRM46.4 & & \\
\hline 1261 & & RIVER & & RAW GRAB & TRANSECT & 326 & RICH.PMPHS-8 HRM46.4 & & \\
\hline $1262 \mathrm{~F}$ & $\mathrm{~F}$ & RIVER & & RAW GRAB & TRANSECT & 326 & RICH.PMPHS-8 HRM46.4 & & \\
\hline 1263 & & RIVER & & RAW GRAB & TRANSECT & 326 & RICH.PMPHS-8 HRM46.4 & & \\
\hline 1264 & & RIVER & & RAW GRAB & TRANSECT & 326 & RICH.PMPHS-8 HRM46.4 & & \\
\hline 1265 & & RIVER & & RAW GRAB & TRANSECT & 326 & RICH.PMPHS-8 HRM46.4 & & \\
\hline 1266 & & RIVER & & RAW GRAB & TRANSECT & 573 & RICH.PMPHS-9 HRM46.4 & & \\
\hline $1267 \mathrm{~F}$ & $\mathrm{~F}$ & RIVER & & RAW GRAB & TRANSECT & 573 & RICH.PMPHS-9 HRM46.4 & & \\
\hline 1268 & & RIVER & & RAW GRAB & TRANSECT & 573 & RICH.PMPHS-9 HRM46.4 & & \\
\hline 1269 & & RIVER & & RAW GRAB & TRANSECT & 573 & RICH.PMPHS-9 HRM46.4 & & \\
\hline 1270 & & RIVER & & RAW GRAB & TRANSECT & 573 & RICH.PMPHS-9 HRM46.4 & & \\
\hline 1271 & & RIVER & & RAW GRAB & TRANSECT & 336 & 100 F -1 HRM 19.0 & & \\
\hline 1272 & & RIVER & & RAW GRAB & TRANSECT & 336 & 100 F -1 HRM 19.0 & & \\
\hline 1273 & & RIVER & & RAW GRAB & TRANSECT & 336 & 100 F -1 HRM 19.0 & & \\
\hline $1274 \mathrm{~F}$ & $\mathrm{~F}$ & RIVER & & RAW GRAB & TRANSECT & 336 & 100 F -1 HRM 19.0 & & \\
\hline 1275 & & RIVER & & RAW GRAB & TRANSECT & 336 & 100 F -1 HRM 19.0 & & \\
\hline 1276 & & RIVER & & RAW GRAB & TRANSECT & 336 & 100 F -1 HRM 19.0 & & \\
\hline 1277 & & RIVER & & RAW GRAB & TRANSECT & 336 & 100 F -1 HRM 19.0 & & \\
\hline 1278 & & RIVER & & RAW GRAB & TRANSECT & 341 & $100 \mathrm{~F}-10 \mathrm{HRM} 19.0$ & & \\
\hline 1279 & & RIVER & & RAW GRAB & TRANSECT & 341 & 100 F -10 HRM 19.0 & & \\
\hline 1280 & & RIVER & & RAW GRAB & TRANSECT & 341 & $100 \mathrm{~F}-10 \mathrm{HRM} 19.0$ & & \\
\hline $1281 \mathrm{~F}$ & $\mathrm{~F}$ & RIVER & & RAW GRAB & TRANSECT & 341 & $100 \mathrm{~F}-10 \mathrm{HRM} 19.0$ & & \\
\hline 1282 & & RIVER & & RAW GRAB & TRANSECT & 341 & $100 \mathrm{~F}-10 \mathrm{HRM} 19.0$ & & \\
\hline 1283 & & RIVER & & RAW GRAB & TRANSECT & 341 & $100 \mathrm{~F}-10 \mathrm{HRM} 19.0$ & & \\
\hline 1284 & & RIVER & & RAW GRAB & TRANSECT & 341 & 100 F -10 HRM 19.0 & & \\
\hline 1285 & & RIVER & & RAW GRAB & TRANSECT & 576 & 100 F -2 HRM 19.0 & & \\
\hline 1286 & & RIVER & & RAW GRAB & TRANSECT & 576 & 100 F -2 HRM 19.0 & & \\
\hline 1287 & & RIVER & & RAW GRAB & TRANSECT & 576 & 100 F -2 HRM 19.0 & & \\
\hline $1288 \mathrm{~F}$ & $\mathrm{~F}$ & RIVER & & RAW GRAB & TRANSECT & 576 & 100 F -2 HRM 19.0 & & \\
\hline 1289 & & RIVER & & RAW GRAB & TRANSECT & 576 & 100 F -2 HRM 19.0 & & \\
\hline 1290 & & RIVER & & RAW GRAB & TRANSECT & 576 & 100 F -2 HRM 19.0 & & \\
\hline 1291 & & RIVER & & RAW GRAB & TRANSECT & 576 & 100 F -2 HRM 19.0 & & \\
\hline 1292 & & RIVER & & RAW GRAB & TRANSECT & 337 & 100 F -3 HRM 19.0 & & \\
\hline 1293 & & RIVER & & RAW GRAB & TRANSECT & 337 & 100 F -3 HRM 19.0 & & \\
\hline
\end{tabular}




\begin{tabular}{|c|c|c|c|c|c|c|c|c|c|}
\hline & $\mathrm{T}$ & 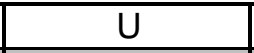 & $\mathrm{V}$ & $\mathrm{W}$ & $\mathrm{X}$ & $\mathrm{Y}$ & $Z$ & $\mathrm{AA}$ & $A B$ \\
\hline \begin{tabular}{l|l}
1 & 1 \\
\end{tabular} & REVIEW_QUALIFIER & SAMP_FROM & SAMP_ITEM & SAMP_MTHD & COLL_MTHD & SAMP_SITE_ID & SAMP_SITE_NAME & WELL_NAME & SAMPLE_LOCATION \\
\hline 1294 & & RIVER & & RAW GRAB & TRANSECT & 337 & 100 F -3 HRM 19.0 & & \\
\hline $1295 \mathrm{~F}$ & $\mathrm{~F}$ & RIVER & & RAW GRAB & TRANSECT & $337:$ & 100 F -3 HRM 19.0 & & \\
\hline 1296 & & RIVER & & RAW GRAB & TRANSECT & 337 & 100 F -3 HRM 19.0 & & \\
\hline 1297 & & RIVER & & RAW GRAB & TRANSECT & 337 & 100 F -3 HRM 19.0 & & \\
\hline 1298 & & RIVER & & RAW GRAB & TRANSECT & 337 & 100 F -3 HRM 19.0 & & \\
\hline 1299 & & RIVER & & RAW GRAB & TRANSECT & 338 & 100 F -4 HRM 19.0 & & \\
\hline 1300 & & RIVER & & RAW GRAB & TRANSECT & 338 & 100 F -4 HRM 19.0 & & \\
\hline 1301 & & RIVER & & RAW GRAB & TRANSECT & 338 & 100 F -4 HRM 19.0 & & \\
\hline $1302 \mathrm{~F}$ & $\mathrm{~F}$ & RIVER & & RAW GRAB & TRANSECT & 338 & 100 F -4 HRM 19.0 & & \\
\hline 1303 & & RIVER & & RAW GRAB & TRANSECT & 338 & 100 F -4 HRM 19.0 & & \\
\hline 1304 & & RIVER & & RAW GRAB & TRANSECT & 338 & 100 F -4 HRM 19.0 & & \\
\hline 1305 & & RIVER & & RAW GRAB & TRANSECT & 338 & 100 F -4 HRM 19.0 & & \\
\hline 1306 & & RIVER & & RAW GRAB & TRANSECT & 577 & 100 F -5 HRM 19.0 & & \\
\hline 1307 & & RIVER & & RAW GRAB & TRANSECT & 577 & 100 F -5 HRM 19.0 & & \\
\hline 1308 & & RIVER & & RAW GRAB & TRANSECT & 577 & 100 F -5 HRM 19.0 & & \\
\hline $1309 \mathrm{~F}$ & $\mathrm{~F}$ & RIVER & & RAW GRAB & TRANSECT & 577 & 100 F -5 HRM 19.0 & & \\
\hline 1310 & & RIVER & & RAW GRAB & TRANSECT & 577 & 100 F -5 HRM 19.0 & & \\
\hline 1311 & & RIVER & & RAW GRAB & TRANSECT & 577 & 100 F -5 HRM 19.0 & & \\
\hline 1312 & & RIVER & & RAW GRAB & TRANSECT & 577 & 100 F -5 HRM 19.0 & & \\
\hline 1313 & & RIVER & & RAW GRAB & TRANSECT & 606 & 100 F -6 HRM 19.0 & & \\
\hline 1314 & & RIVER & & RAW GRAB & TRANSECT & 606 & 100 F -6 HRM 19.0 & & \\
\hline 1315 & & RIVER & & RAW GRAB & TRANSECT & 606 & 100 F -6 HRM 19.0 & & \\
\hline $1316 \mathrm{~F}$ & $\mathrm{~F}$ & RIVER & & RAW GRAB & TRANSECT & 606 & 100 F -6 HRM 19.0 & & \\
\hline 1317 & & RIVER & & RAW GRAB & TRANSECT & 606 & 100 F -6 HRM 19.0 & & \\
\hline 1318 & & RIVER & & RAW GRAB & TRANSECT & 606 & 100 F -6 HRM 19.0 & & \\
\hline 1319 & & RIVER & & RAW GRAB & TRANSECT & 606 & 100 F -6 HRM 19.0 & & \\
\hline 1320 & & RIVER & & RAW GRAB & TRANSECT & 339 & 100 F -7 HRM 19.0 & & \\
\hline 1321 & & RIVER & & RAW GRAB & TRANSECT & 339 & 100 F -7 HRM 19.0 & & \\
\hline 1322 & & RIVER & & RAW GRAB & TRANSECT & 339 & 100 F -7 HRM 19.0 & & \\
\hline $1323 \mathrm{~F}$ & $\mathrm{~F}$ & RIVER & & RAW GRAB & TRANSECT & 339 & $100 \mathrm{~F}-7$ HRM 19.0 & & \\
\hline 1324 & & RIVER & & RAW GRAB & TRANSECT & 339 & 100 F -7 HRM 19.0 & & \\
\hline 1325 & & RIVER & & RAW GRAB & TRANSECT & 339 & 100 F -7 HRM 19.0 & & \\
\hline 1326 & & RIVER & & RAW GRAB & TRANSECT & 339 & 100 F -7 HRM 19.0 & & \\
\hline 1327 & & RIVER & & RAW GRAB & TRANSECT & 340 & 100 F -8 HRM 19.0 & & \\
\hline 1328 & & RIVER & & RAW GRAB & TRANSECT & 340 & 100 F -8 HRM 19.0 & & \\
\hline 1329 & & RIVER & & RAW GRAB & TRANSECT & 340 & 100 F -8 HRM 19.0 & & \\
\hline $1330 \mathrm{~F}$ & $\mathrm{~F}$ & RIVER & & RAW GRAB & TRANSECT & 340 & 100 F -8 HRM 19.0 & & \\
\hline 1331 & & RIVER & & RAW GRAB & TRANSECT & 340 & 100 F -8 HRM 19.0 & & \\
\hline
\end{tabular}




\begin{tabular}{|c|c|c|c|c|c|c|c|c|c|}
\hline & $\mathrm{T}$ & 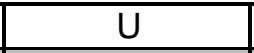 & $\mathrm{V}$ & $\mathrm{W}$ & $\mathrm{X}$ & $\mathrm{Y}$ & $Z$ & $\mathrm{AA}$ & $A B$ \\
\hline \begin{tabular}{l|l}
1 & 1 \\
\end{tabular} & REVIEW_QUALIFIER & SAMP_FROM & SAMP_ITEM & SAMP_MTHD & COLL_MTHD & SAMP_SITE_ID & SAMP_SITE_NAME & WELL_NAME & SAMPLE_LOCATION \\
\hline 1332 & & RIVER & & RAW GRAB & TRANSECT & 340 & 100 F -8 HRM 19.0 & & \\
\hline 1333 & & RIVER & & RAW GRAB & TRANSECT & 340 & 100 F -8 HRM 19.0 & & \\
\hline 1334 & & RIVER & & RAW GRAB & TRANSECT & 467 & 100 F -9 HRM 19.0 & & \\
\hline 1335 & & RIVER & & RAW GRAB & TRANSECT & 467 & 100 F -9 HRM 19.0 & & \\
\hline 1336 & & RIVER & & RAW GRAB & TRANSECT & $467:$ & 100 F -9 HRM 19.0 & & \\
\hline $1337 \mathrm{~F}$ & $\mathrm{~F}$ & RIVER & & RAW GRAB & TRANSECT & 467 & 100 F -9 HRM 19.0 & & \\
\hline 1338 & & RIVER & & RAW GRAB & TRANSECT & 467 & 100 F -9 HRM 19.0 & & \\
\hline 1339 & & RIVER & & RAW GRAB & TRANSECT & $467:$ & 100 F -9 HRM 19.0 & & \\
\hline 1340 & & RIVER & & RAW GRAB & TRANSECT & 467 & 100 F -9 HRM 19.0 & & \\
\hline 1341 & & RIVER & & RAW GRAB & TRANSECT & 328 & 100 N -1 HRM 9.5 & & \\
\hline 1342 & & RIVER & & RAW GRAB & TRANSECT & 328 & 100 N -1 HRM 9.5 & & \\
\hline 1343 & & RIVER & & RAW GRAB & TRANSECT & 328 & 100 N -1 HRM 9.5 & & \\
\hline $1344 \mathrm{~F}$ & $\mathrm{~F}$ & RIVER & & RAW GRAB & TRANSECT & 328 & 100 N -1 HRM 9.5 & & \\
\hline 1345 & & RIVER & & RAW GRAB & TRANSECT & 328 & 100 N -1 HRM 9.5 & & \\
\hline 1346 & & RIVER & & RAW GRAB & TRANSECT & 328 & 100 N -1 HRM 9.5 & & \\
\hline 1347 & & RIVER & & RAW GRAB & TRANSECT & 328 & 100 N -1 HRM 9.5 & & \\
\hline 1348 & & RIVER & & RAW GRAB & TRANSECT & 328 & 100 N -1 HRM 9.5 & & \\
\hline 1349 & & RIVER & & RAW GRAB & TRANSECT & 328 & 100 N -1 HRM 9.5 & & \\
\hline 1350 & & RIVER & & RAW GRAB & TRANSECT & 335 & $100 \mathrm{~N}-10$ HRM 9.5 & & \\
\hline 1351 & & RIVER & & RAW GRAB & TRANSECT & 335 & $100 \mathrm{~N}-10$ HRM 9.5 & & \\
\hline 1352 & & RIVER & & RAW GRAB & TRANSECT & 335 & $100 \mathrm{~N}-10$ HRM 9.5 & & \\
\hline $1353 \mathrm{~F}$ & $\mathrm{~F}$ & RIVER & & RAW GRAB & TRANSECT & 335 & $100 \mathrm{~N}-10$ HRM 9.5 & & \\
\hline 1354 & & RIVER & & RAW GRAB & TRANSECT & 335 & $100 \mathrm{~N}-10$ HRM 9.5 & & \\
\hline $1355 \mathrm{~F}$ & $\mathrm{~F}$ & RIVER & & RAW GRAB & TRANSECT & 335 & $100 \mathrm{~N}-10 \mathrm{HRM} 9.5$ & & \\
\hline 1356 & & RIVER & & RAW GRAB & TRANSECT & 335 & $100 \mathrm{~N}-10$ HRM 9.5 & & \\
\hline 1357 & & RIVER & & RAW GRAB & TRANSECT & 335 & $100 \mathrm{~N}-10$ HRM 9.5 & & \\
\hline 1358 & & RIVER & & RAW GRAB & TRANSECT & 335 & $100 \mathrm{~N}-10$ HRM 9.5 & & \\
\hline 1359 & & RIVER & & RAW GRAB & TRANSECT & 329 & 100 N -2 HRM 9.5 & & \\
\hline 1360 & & RIVER & & RAW GRAB & TRANSECT & 329 & 100 N -2 HRM 9.5 & & \\
\hline 1361 & & RIVER & & RAW GRAB & TRANSECT & 329 & 100 N -2 HRM 9.5 & & \\
\hline $1362 \mathrm{~F}$ & $\mathrm{~F}$ & RIVER & & RAW GRAB & TRANSECT & 329 & 100 N -2 HRM 9.5 & & \\
\hline 1363 & & RIVER & & RAW GRAB & TRANSECT & 329 & 100 N -2 HRM 9.5 & & \\
\hline $1364 \mathrm{~F}$ & $\mathrm{~F}$ & RIVER & & RAW GRAB & TRANSECT & 329 & 100 N -2 HRM 9.5 & & \\
\hline 1365 & & RIVER & & RAW GRAB & TRANSECT & 329 & 100 N -2 HRM 9.5 & & \\
\hline 1366 & & RIVER & & RAW GRAB & TRANSECT & 329 & 100 N -2 HRM 9.5 & & \\
\hline 1367 & & RIVER & & RAW GRAB & TRANSECT & 329 & $100 \mathrm{~N}-2$ HRM 9.5 & & \\
\hline 1368 & & RIVER & & RAW GRAB & TRANSECT & 330 & 100 N -3 HRM 9.5 & & \\
\hline 1369 & & RIVER & & RAW GRAB & TRANSECT & 330 & 100 N -3 HRM 9.5 & & \\
\hline
\end{tabular}




\begin{tabular}{|c|c|c|c|c|c|c|c|c|c|}
\hline & $\mathrm{T}$ & 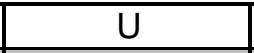 & $\mathrm{V}$ & $\mathrm{W}$ & $\mathrm{X}$ & $\mathrm{Y}$ & $Z$ & $\mathrm{AA}$ & $A B$ \\
\hline \begin{tabular}{l|l}
1 & 1 \\
\end{tabular} & REVIEW_QUALIFIER & SAMP_FROM & SAMP_ITEM & SAMP_MTHD & COLL_MTHD & SAMP_SITE_ID & SAMP_SITE_NAME & WELL_NAME & SAMPLE_LOCATION \\
\hline 1370 & & RIVER & & RAW GRAB & TRANSECT & 330 & 100 N -3 HRM 9.5 & & \\
\hline $1371 \mathrm{~F}$ & $\mathrm{~F}$ & RIVER & & RAW GRAB & TRANSECT & 330 & 100 N -3 HRM 9.5 & & \\
\hline 1372 & & RIVER & & RAW GRAB & TRANSECT & 330 & 100 N -3 HRM 9.5 & & \\
\hline $1373 \mathrm{~F}$ & $\mathrm{~F}$ & RIVER & & RAW GRAB & TRANSECT & 330 & 100 N -3 HRM 9.5 & & \\
\hline 1374 & & RIVER & & RAW GRAB & TRANSECT & 330 & 100 N -3 HRM 9.5 & & \\
\hline 1375 & & RIVER & & RAW GRAB & TRANSECT & 330 & 100 N -3 HRM 9.5 & & \\
\hline 1376 & & RIVER & & RAW GRAB & TRANSECT & 330 & 100 N -3 HRM 9.5 & & \\
\hline 1377 & & RIVER & & RAW GRAB & TRANSECT & 574 & 100 N -4 HRM 9.5 & & \\
\hline 1378 & & RIVER & & RAW GRAB & TRANSECT & 574 & 100 N -4 HRM 9.5 & & \\
\hline 1379 & & RIVER & & RAW GRAB & TRANSECT & 574 & 100 N -4 HRM 9.5 & & \\
\hline $1380 \mathrm{~F}$ & $\mathrm{~F}$ & RIVER & & RAW GRAB & TRANSECT & 574 & 100 N -4 HRM 9.5 & & \\
\hline 1381 & & RIVER & & RAW GRAB & TRANSECT & 574 & 100 N -4 HRM 9.5 & & \\
\hline $1382 \mathrm{~F}$ & $\mathrm{~F}$ & RIVER & & RAW GRAB & TRANSECT & 574 & 100 N -4 HRM 9.5 & & \\
\hline 1383 & & RIVER & & RAW GRAB & TRANSECT & $574:$ & 100 N -4 HRM 9.5 & & \\
\hline 1384 & & RIVER & & RAW GRAB & TRANSECT & 574 & 100 N -4 HRM 9.5 & & \\
\hline 1385 & & RIVER & & RAW GRAB & TRANSECT & 574 & 100 N -4 HRM 9.5 & & \\
\hline 1386 & & RIVER & & RAW GRAB & TRANSECT & 331 & 100 N -5 HRM 9.5 & & \\
\hline 1387 & & RIVER & & RAW GRAB & TRANSECT & 331 & 100 N -5 HRM 9.5 & & \\
\hline 1388 & & RIVER & & RAW GRAB & TRANSECT & 331 & 100 N -5 HRM 9.5 & & \\
\hline $1389 \mathrm{~F}$ & $\mathrm{~F}$ & RIVER & & RAW GRAB & TRANSECT & 331 & 100 N -5 HRM 9.5 & & \\
\hline 1390 & & RIVER & & RAW GRAB & TRANSECT & 331 & 100 N -5 HRM 9.5 & & \\
\hline $1391 \mathrm{~F}$ & $\mathrm{~F}$ & RIVER & & RAW GRAB & TRANSECT & 331 & 100 N -5 HRM 9.5 & & \\
\hline 1392 & & RIVER & & RAW GRAB & TRANSECT & 331 & 100 N -5 HRM 9.5 & & \\
\hline 1393 & & RIVER & & RAW GRAB & TRANSECT & 331 & 100 N -5 HRM 9.5 & & \\
\hline 1394 & & RIVER & & RAW GRAB & TRANSECT & 331 & 100 N -5 HRM 9.5 & & \\
\hline 1395 & & RIVER & & RAW GRAB & TRANSECT & 332 & 100 N -6 HRM 9.5 & & \\
\hline 1396 & & RIVER & & RAW GRAB & TRANSECT & 332 & 100 N -6 HRM 9.5 & & \\
\hline 1397 & & RIVER & & RAW GRAB & TRANSECT & 332 & 100 N -6 HRM 9.5 & & \\
\hline $1398 \mathrm{~F}$ & $\mathrm{~F}$ & RIVER & & RAW GRAB & TRANSECT & 332 & 100 N -6 HRM 9.5 & & \\
\hline 1399 & & RIVER & & RAW GRAB & TRANSECT & 332 & 100 N -6 HRM 9.5 & & \\
\hline $1400 \mathrm{~F}$ & $\mathrm{~F}$ & RIVER & & RAW GRAB & TRANSECT & 332 & 100 N -6 HRM 9.5 & & \\
\hline 1401 & & RIVER & & RAW GRAB & TRANSECT & 332 & 100 N -6 HRM 9.5 & & \\
\hline 1402 & & RIVER & & RAW GRAB & TRANSECT & 332 & 100 N -6 HRM 9.5 & & \\
\hline 1403 & & RIVER & & RAW GRAB & TRANSECT & 332 & 100 N -6 HRM 9.5 & & \\
\hline 1404 & & RIVER & & RAW GRAB & TRANSECT & 333 & 100 N -7 HRM 9.5 & & \\
\hline 1405 & & RIVER & & RAW GRAB & TRANSECT & 333 & 100 N -7 HRM 9.5 & & \\
\hline 1406 & & RIVER & & RAW GRAB & TRANSECT & 333 & 100 N -7 HRM 9.5 & & \\
\hline $1407 \mid \mathrm{F}$ & $\mathrm{F}$ & RIVER & & RAW GRAB & TRANSECT & 333 & $100 \mathrm{~N}-7$ HRM 9.5 & & \\
\hline
\end{tabular}




\begin{tabular}{|c|c|c|c|c|c|c|c|c|c|}
\hline & $\mathrm{T}$ & 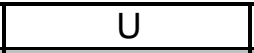 & $\mathrm{V}$ & $\mathrm{W}$ & $\mathrm{X}$ & $\mathrm{Y}$ & $Z$ & $\mathrm{AA}$ & $A B$ \\
\hline 1 & REVIEW_QUALIFIER & SAMP_FROM & SAMP_ITEM & SAMP_MTHD & COLL_MTHD & SAMP_SITE_ID & SAMP_SITE_NAME & WELL_NAME & SAMPLE_LOCATION \\
\hline 1408 & & RIVER & & RAW GRAB & TRANSECT & 333 & $00 \mathrm{~N}-7$ HRM 9.5 & & \\
\hline 1409 & $\mathrm{~F}$ & RIVER & & RAW GRAB & TRANSECT & 333 & $00 \mathrm{~N}-7$ HRM 9.5 & & \\
\hline 1410 & & RIVER & & RAW GRAB & TRANSECT & 333 & $00 \mathrm{~N}-7$ HRM 9.5 & & \\
\hline 1411 & & RIVER & & RAW GRAB & TRANSECT & 333 & $00 \mathrm{~N}-7$ HRM 9.5 & & \\
\hline 1412 & & RIVER & & RAW GRAB & TRANSECT & 333 & $00 \mathrm{~N}-7$ HRM 9.5 & & \\
\hline 1413 & & RIVER & & RAW GRAB & TRANSECT & 575 & $00 \mathrm{~N}-8$ HRM 9.5 & & \\
\hline 1414 & & RIVER & & RAW GRAB & TRANSECT & 575 & $00 \mathrm{~N}-8$ HRM 9.5 & & \\
\hline 1415 & & RIVER & & RAW GRAB & TRANSECT & 575 & $00 \mathrm{~N}-8 \mathrm{HRM} 9.5$ & & \\
\hline 1416 & $\mathrm{~F}$ & RIVER & & RAW GRAB & TRANSECT & 575 & $00 \mathrm{~N}-8 \mathrm{HRM} 9.5$ & & \\
\hline 1417 & & RIVER & & RAW GRAB & TRANSECT & 575 & $00 \mathrm{~N}-8$ HRM 9.5 & & \\
\hline 1418 & $\mathrm{~F}$ & RIVER & & RAW GRAB & TRANSECT & 575 & $00 \mathrm{~N}-8 \mathrm{HRM} 9.5$ & & \\
\hline 1419 & & RIVER & & RAW GRAB & TRANSECT & 575 & $00 \mathrm{~N}-8$ HRM 9.5 & & \\
\hline 1420 & & RIVER & & RAW GRAB & TRANSECT & 575 & $00 \mathrm{~N}-8 \mathrm{HRM} 9.5$ & & \\
\hline 1421 & & RIVER & & RAW GRAB & TRANSECT & 575 & $00 \mathrm{~N}-8 \mathrm{HRM} 9.5$ & & \\
\hline 1422 & & RIVER & & RAW GRAB & TRANSECT & 334 & $00 \mathrm{~N}-9$ HRM 9.5 & & \\
\hline 1423 & & RIVER & & RAW GRAB & TRANSECT & 334 & $00 \mathrm{~N}-9$ HRM 9.5 & & \\
\hline 1424 & & RIVER & & RAW GRAB & TRANSECT & 334 & $00 \mathrm{~N}-9$ HRM 9.5 & & \\
\hline 1425 & $\mathrm{~F}$ & RIVER & & RAW GRAB & TRANSECT & 334 & $00 \mathrm{~N}-9$ HRM 9.5 & & \\
\hline 1426 & & RIVER & & RAW GRAB & TRANSECT & 334 & $00 \mathrm{~N}-9$ HRM 9.5 & & \\
\hline 1427 & $\mathrm{~F}$ & RIVER & & RAW GRAB & TRANSECT & 334 & $00 \mathrm{~N}-9$ HRM 9.5 & & \\
\hline 1428 & & RIVER & & RAW GRAB & TRANSECT & 334 & $00 \mathrm{~N}-9$ HRM 9.5 & & \\
\hline 1429 & & RIVER & & RAW GRAB & TRANSECT & 334 & $00 \mathrm{~N}-9$ HRM 9.5 & & \\
\hline 1430 & & RIVER & & RAW GRAB & TRANSECT & 334 & $00 \mathrm{~N}-9$ HRM 9.5 & & \\
\hline 1431 & & RIVER & & RAW GRAB & TRANSECT & 317 & ERNITA-1 HRM 0.3 & & \\
\hline 1432 & & RIVER & & RAW GRAB & TRANSECT & 317 & ERNITA-1 HRM 0.3 & & \\
\hline 1433 & & RIVER & & RAW GRAB & TRANSECT & 317 & ERNITA-1 HRM 0.3 & & \\
\hline 1434 & $\mathrm{~F}$ & RIVER & & RAW GRAB & TRANSECT & 317 & ERNITA-1 HRM 0.3 & & \\
\hline 1435 & & RIVER & & RAW GRAB & TRANSECT & 317 & ERNITA-1 HRM 0.3 & & \\
\hline 1436 & & RIVER & & RAW GRAB & TRANSECT & 317 & ERNITA-1 HRM 0.3 & & \\
\hline 1437 & & RIVER & & RAW GRAB & TRANSECT & 317 & ERNITA-1 HRM 0.3 & & \\
\hline 1438 & & RIVER & & RAW GRAB & TRANSECT & 570 & ERNITA-2 HRM 0.3 & & \\
\hline 1439 & & RIVER & & RAW GRAB & TRANSECT & 570 & ERNITA-2 HRM 0.3 & & \\
\hline 1440 & & RIVER & & RAW GRAB & TRANSECT & 570 & ERNITA-2 HRM 0.3 & & \\
\hline 1441 & $\mathrm{~F}$ & RIVER & & RAW GRAB & TRANSECT & 570 & ERNITA-2 HRM 0.3 & & \\
\hline 1442 & & RIVER & & RAW GRAB & TRANSECT & 570 & ERNITA-2 HRM 0.3 & & \\
\hline 1443 & & RIVER & & RAW GRAB & TRANSECT & 570 & ERNITA-2 HRM 0.3 & & \\
\hline 1444 & & RIVER & & RAW GRAB & TRANSECT & 570 & ERNITA-2 HRM 0.3 & & \\
\hline 1445 & & RIVER & & RAW GRAB & TRANSECT & 318 & ERNITA-3 HRM 0.3 & & \\
\hline
\end{tabular}




\begin{tabular}{|c|c|c|c|c|c|c|c|c|c|}
\hline & $T$ & $\mathrm{U}$ & $\mathrm{V}$ & $\mathrm{W}$ & $\mathrm{X}$ & $\bar{Y}$ & $Z$ & $\overline{A A}$ & $A B$ \\
\hline 1 & REVIEW_QUALIFIER & SAMP_FROM & SAMP_ITEM & SAMP_MTHD & COLL_MTHD & SAMP_SITE_ID & SAMP_SITE_NAME & WELL_NAME & SAMPLE_LOCATION \\
\hline 1446 & & RIVER & & RAW GRAB & TRANSECT & 318 & VERNITA-3 HRM 0.3 & & \\
\hline 1447 & & RIVER & & RAW GRAB & TRANSECT & 318 & VERNITA-3 HRM 0.3 & & \\
\hline $1448 \mathrm{~F}$ & $\mathrm{~F}$ & RIVER & & RAW GRAB & TRANSECT & 318 & VERNITA-3 HRM 0.3 & & \\
\hline 1449 & & RIVER & & RAW GRAB & TRANSECT & 318 & VERNITA-3 HRM 0.3 & & \\
\hline 1450 & & RIVER & & RAW GRAB & TRANSECT & 318 & VERNITA-3 HRM 0.3 & & \\
\hline 1451 & & RIVER & & RAW GRAB & TRANSECT & 318 & VERNITA-3 HRM 0.3 & & \\
\hline 1452 & & RIVER & & RAW GRAB & TRANSECT & 319 & VERNITA-4 HRM 0.3 & & \\
\hline 1453 & & RIVER & & RAW GRAB & TRANSECT & 319 & VERNITA-4 HRM 0.3 & & \\
\hline 1454 & & RIVER & & RAW GRAB & TRANSECT & 319 & VERNITA-4 HRM 0.3 & & \\
\hline $1455 \mathrm{~F}$ & $\mathrm{~F}$ & RIVER & & RAW GRAB & TRANSECT & 319 & VERNITA-4 HRM 0.3 & & \\
\hline 1456 & & RIVER & & RAW GRAB & TRANSECT & 319 & VERNITA-4 HRM 0.3 & & \\
\hline 1457 & & RIVER & & RAW GRAB & TRANSECT & 319 & VERNITA-4 HRM 0.3 & & \\
\hline 1458 & & RIVER & & RAW GRAB & TRANSECT & 319 & VERNITA-4 HRM 0.3 & & \\
\hline 1459 & & RIVER & & RAW GRAB & TRANSECT & 348 & 300 AREA -1 HRM 43.1 & & \\
\hline 1460 & & RIVER & & RAW GRAB & TRANSECT & 348 & 300 AREA -1 HRM 43.1 & & \\
\hline 1461 & & RIVER & & RAW GRAB & TRANSECT & 348 & 300 AREA -1 HRM 43.1 & & \\
\hline 1462 & & RIVER & & RAW GRAB & TRANSECT & 348 & 300 AREA -1 HRM 43.1 & & \\
\hline 1463 & & RIVER & & RAW GRAB & TRANSECT & 348 & 300 AREA -1 HRM 43.1 & & \\
\hline $1464 \mathrm{~F}$ & $\mathrm{~F}$ & RIVER & & RAW GRAB & TRANSECT & 348 & 300 AREA -1 HRM 43.1 & & \\
\hline 1465 & & RIVER & & RAW GRAB & TRANSECT & 348 & 300 AREA -1 HRM 43.1 & & \\
\hline 1466 & & RIVER & & RAW GRAB & TRANSECT & 470 & 300 AREA -2 HRM 43.1 & & \\
\hline 1467 & & RIVER & & RAW GRAB & TRANSECT & 470 & 300 AREA -2 HRM 43.1 & & \\
\hline 1468 & & RIVER & & RAW GRAB & TRANSECT & 470 & 300 AREA -2 HRM 43.1 & & \\
\hline 1469 & & RIVER & & RAW GRAB & TRANSECT & 470 & 300 AREA -2 HRM 43.1 & & \\
\hline 1470 & & RIVER & & RAW GRAB & TRANSECT & 470 & 300 AREA -2 HRM 43.1 & & \\
\hline 1471 & & RIVER & & RAW GRAB & TRANSECT & 470 & 300 AREA -2 HRM 43.1 & & \\
\hline 1472 & & RIVER & & RAW GRAB & TRANSECT & 470 & 300 AREA -2 HRM 43.1 & & \\
\hline 1473 & & RIVER & & RAW GRAB & TRANSECT & 580 & 300 AREA -3 HRM 43.1 & & \\
\hline 1474 & & RIVER & & RAW GRAB & TRANSECT & 580 & 300 AREA -3 HRM 43.1 & & \\
\hline 1475 & & RIVER & & RAW GRAB & TRANSECT & 580 & 300 AREA -3 HRM 43.1 & & \\
\hline $1476 \mathrm{~F}$ & $\mathrm{~F}$ & RIVER & & RAW GRAB & TRANSECT & 580 & 300 AREA -3 HRM 43.1 & & \\
\hline 1477 & & RIVER & & RAW GRAB & TRANSECT & 580 & 300 AREA -3 HRM 43.1 & & \\
\hline $1478 \mathrm{~F}$ & $F$ & RIVER & & RAW GRAB & TRANSECT & 580 & 300 AREA -3 HRM 43.1 & & \\
\hline 1479 & & RIVER & & RAW GRAB & TRANSECT & 580 & 300 AREA -3 HRM 43.1 & & \\
\hline 1480 & & RIVER & & RAW GRAB & TRANSECT & 349 & 300 AREA -4 HRM 43.1 & & \\
\hline 1481 & & RIVER & & RAW GRAB & TRANSECT & 349 & 300 AREA -4 HRM 43.1 & & \\
\hline 1482 & & RIVER & & RAW GRAB & TRANSECT & 349 & 300 AREA -4 HRM 43.1 & & \\
\hline 1483 F & $\mathrm{F}$ & RIVER & & RAW GRAB & TRANSECT & 349 & 300 AREA -4 HRM 43.1 & & \\
\hline
\end{tabular}




\begin{tabular}{|c|c|c|c|c|c|c|c|c|c|}
\hline & $\mathrm{T}$ & $\mathrm{U}$ & $\mathrm{V}$ & $\mathrm{W}$ & $\mathrm{X}$ & $\bar{Y}$ & $Z$ & AA & $A B$ \\
\hline 1 & REVIEW_QUALIFIER & SAMP_FROM & SAMP_ITEM & SAMP_MTHD & COLL_MTHD & SAMP_SITE_ID & SAMP_SITE_NAME & WELL_NAME & SAMPLE_LOCATION \\
\hline 1484 & & RIVER & & RAW GRAB & TRANSECT & 349 & 300 AREA -4 HRM 43.1 & & \\
\hline 1485 & & RIVER & & RAW GRAB & TRANSECT & 349 & 300 AREA -4 HRM 43.1 & & \\
\hline 1486 & & RIVER & & RAW GRAB & TRANSECT & 349 & 300 AREA -4 HRM 43.1 & & \\
\hline 1487 & & RIVER & & RAW GRAB & TRANSECT & 607 & 300 AREA -5 HRM 43.1 & & \\
\hline 1488 & & RIVER & & RAW GRAB & TRANSECT & 607 & 300 AREA -5 HRM 43.1 & & \\
\hline 1489 & & RIVER & & RAW GRAB & TRANSECT & 607 & 300 AREA -5 HRM 43.1 & & \\
\hline 1490 & $F$ & RIVER & & RAW GRAB & TRANSECT & 607 & 300 AREA -5 HRM 43.1 & & \\
\hline 1491 & & RIVER & & RAW GRAB & TRANSECT & 607 & 300 AREA -5 HRM 43.1 & & \\
\hline 1492 & & RIVER & & RAW GRAB & TRANSECT & 607 & 300 AREA -5 HRM 43.1 & & \\
\hline 1493 & & RIVER & & RAW GRAB & TRANSECT & 607 & 300 AREA -5 HRM 43.1 & & \\
\hline 1494 & & RIVER & & RAW GRAB & TRANSECT & 350 & 300 AREA -6 HRM 43.1 & & \\
\hline 1495 & & RIVER & & RAW GRAB & TRANSECT & 350 & 300 AREA -6 HRM 43.1 & & \\
\hline 1496 & $\mathrm{~F}$ & RIVER & & RAW GRAB & TRANSECT & 350 & 300 AREA -6 HRM 43.1 & & \\
\hline 1497 & $F$ & RIVER & & RAW GRAB & TRANSECT & 350 & 300 AREA -6 HRM 43.1 & & \\
\hline 1498 & & RIVER & & RAW GRAB & TRANSECT & 350 & 300 AREA -6 HRM 43.1 & & \\
\hline 1499 & & RIVER & & RAW GRAB & TRANSECT & 350 & 300 AREA -6 HRM 43.1 & & \\
\hline 1500 & & RIVER & & RAW GRAB & TRANSECT & 350 & 300 AREA -6 HRM 43.1 & & \\
\hline 1501 & & RIVER & & RAW GRAB & TRANSECT & 351 & 300 AREA -7 HRM 43.1 & & \\
\hline 1502 & & RIVER & & RAW GRAB & TRANSECT & 351 & 300 AREA -7 HRM 43.1 & & \\
\hline 1503 & & RIVER & & RAW GRAB & TRANSECT & 351 & - 300 AREA -7 HRM 43.1 & & \\
\hline 1504 & $F$ & RIVER & & RAW GRAB & TRANSECT & 351 & 300 AREA -7 HRM 43.1 & & \\
\hline 1505 & & RIVER & & RAW GRAB & TRANSECT & 351 & 300 AREA -7 HRM 43.1 & & \\
\hline 1506 & & RIVER & & RAW GRAB & TRANSECT & 351 & 300 AREA -7 HRM 43.1 & & \\
\hline 1507 & & RIVER & & RAW GRAB & TRANSECT & 351 & 300 AREA -7 HRM 43.1 & & \\
\hline 1508 & & RIVER & & RAW GRAB & TRANSECT & 581 & 300 AREA -8 HRM 43.1 & & \\
\hline 1509 & & RIVER & & RAW GRAB & TRANSECT & 581 & 300 AREA -8 HRM 43.1 & & \\
\hline 1510 & & RIVER & & RAW GRAB & TRANSECT & 581 & 300 AREA -8 HRM 43.1 & & \\
\hline 1511 & $F$ & RIVER & & RAW GRAB & TRANSECT & 581 & 300 AREA -8 HRM 43.1 & & \\
\hline 1512 & & RIVER & & RAW GRAB & TRANSECT & 581 & 300 AREA -8 HRM 43.1 & & \\
\hline \begin{tabular}{|l|}
1513 \\
\end{tabular} & & RIVER & & RAW GRAB & TRANSECT & 581 & 300 AREA -8 HRM 43.1 & & \\
\hline 1514 & & RIVER & & RAW GRAB & TRANSECT & 581 & 300 AREA -8 HRM 43.1 & & \\
\hline 1515 & & RIVER & & RAW GRAB & TRANSECT & 471 & 300 AREA -9 HRM 43.1 & & \\
\hline 1516 & & RIVER & & RAW GRAB & TRANSECT & 471 & 300 AREA -9 HRM 43.1 & & \\
\hline 1517 & & RIVER & & RAW GRAB & TRANSECT & 471 & 300 AREA -9 HRM 43.1 & & \\
\hline 1518 & $F$ & RIVER & & RAW GRAB & TRANSECT & 471 & 300 AREA -9 HRM 43.1 & & \\
\hline 1519 & & RIVER & & RAW GRAB & TRANSECT & 471 & 300 AREA -9 HRM 43.1 & & \\
\hline 1520 & & RIVER & & RAW GRAB & TRANSECT & 471 & 300 AREA -9 HRM 43.1 & & \\
\hline 1521 & & RIVER & & RAW GRAB & TRANSECT & 471 & 300 AREA -9 HRM 43.1 & & \\
\hline
\end{tabular}




\begin{tabular}{|c|c|c|c|c|c|c|c|c|c|}
\hline & $\mathrm{T}$ & $\bar{U}$ & $\mathrm{~V}$ & $\mathrm{~W}$ & $\mathrm{X}$ & $\mathrm{Y}$ & $Z$ & $\mathrm{AA}$ & $\mathrm{AB}$ \\
\hline 1 & REVIEW_QUALIFIER & SAMP_FROM & SAMP_ITEM & SAMP_MTHD & COLL_MTHD & SAMP_SITE_ID & SAMP_SITE_NAME & WELL_NAME & SAMPLE_LOCATION \\
\hline 1522 & & RIVER & & RAW GRAB & TRANSECT & 352 & 300 AREA-10 HRM 43.1 & & \\
\hline 1523 & & RIVER & & RAW GRAB & TRANSECT & 352 & 300 AREA-10 HRM 43.1 & & \\
\hline 1524 & & RIVER & & RAW GRAB & TRANSECT & 352 & 300 AREA-10 HRM 43.1 & & \\
\hline 1525 & $\mathrm{~F}$ & RIVER & & RAW GRAB & TRANSECT & 352 & 300 AREA-10 HRM 43.1 & & \\
\hline 1526 & & RIVER & & RAW GRAB & TRANSECT & 352 & 300 AREA-10 HRM 43.1 & & \\
\hline 1527 & & RIVER & & RAW GRAB & TRANSECT & 352 & 300 AREA-10 HRM 43.1 & & \\
\hline 1528 & & RIVER & & RAW GRAB & TRANSECT & 352 & 300 AREA-10 HRM 43.1 & & \\
\hline 1529 & & RIVER & & RAW GRAB & TRANSECT & 571 & RICH.PMPHS-1 HRM46.4 & & \\
\hline 1530 & & RIVER & & RAW GRAB & TRANSECT & 571 & RICH.PMPHS-1 HRM46.4 & & \\
\hline 1531 & & RIVER & & RAW GRAB & TRANSECT & 571 & RICH.PMPHS-1 HRM46.4 & & \\
\hline 1532 & & RIVER & & RAW GRAB & TRANSECT & 571 & RICH.PMPHS-1 HRM46.4 & & \\
\hline 1533 & & RIVER & & RAW GRAB & TRANSECT & 571 & RICH.PMPHS-1 HRM46.4 & & \\
\hline 1534 & & RIVER & & RAW GRAB & TRANSECT & 571 & RICH.PMPHS-1 HRM46.4 & & \\
\hline 1535 & & RIVER & & RAW GRAB & TRANSECT & 571 & RICH.PMPHS-1 HRM46.4 & & \\
\hline 1536 & & RIVER & & RAW GRAB & TRANSECT & 327 & RICH.PMPHS-10 HRM46.4 & & \\
\hline 1537 & & RIVER & & RAW GRAB & TRANSECT & 327 & RICH.PMPHS-10 HRM46.4 & & \\
\hline 1538 & & RIVER & & RAW GRAB & TRANSECT & 327 & RICH.PMPHS-10 HRM46.4 & & \\
\hline 1539 & $\mathrm{~F}$ & RIVER & & RAW GRAB & TRANSECT & 327 & RICH.PMPHS-10 HRM46.4 & & \\
\hline 1540 & & RIVER & & RAW GRAB & TRANSECT & 327 & RICH.PMPHS-10 HRM46.4 & & \\
\hline 1541 & & RIVER & & RAW GRAB & TRANSECT & 327 & RICH.PMPHS-10 HRM46.4 & & \\
\hline 1542 & & RIVER & & RAW GRAB & TRANSECT & 327 & RICH.PMPHS-10 HRM46.4 & & \\
\hline 1543 & & RIVER & & RAW GRAB & TRANSECT & 321 & RICH.PMPHS-2 HRM46.4 & & \\
\hline 1544 & & RIVER & & RAW GRAB & TRANSECT & 321 & RICH.PMPHS-2 HRM46.4 & & \\
\hline 1545 & & RIVER & & RAW GRAB & TRANSECT & 321 & RICH.PMPHS-2 HRM46.4 & & \\
\hline 1546 & & RIVER & & RAW GRAB & TRANSECT & 321 & RICH.PMPHS-2 HRM46.4 & & \\
\hline 1547 & & RIVER & & RAW GRAB & TRANSECT & 321 & RICH.PMPHS-2 HRM46.4 & & \\
\hline 1548 & & RIVER & & RAW GRAB & TRANSECT & 321 & RICH.PMPHS-2 HRM46.4 & & \\
\hline 1549 & & RIVER & & RAW GRAB & TRANSECT & 321 & RICH.PMPHS-2 HRM46.4 & & \\
\hline 1550 & & RIVER & & RAW GRAB & TRANSECT & 322 & RICH.PMPHS-3 HRM46.4 & & \\
\hline 1551 & & RIVER & & RAW GRAB & TRANSECT & 322 & RICH.PMPHS-3 HRM46.4 & & \\
\hline 1552 & & RIVER & & RAW GRAB & TRANSECT & 322 & RICH.PMPHS-3 HRM46.4 & & \\
\hline 1553 & $\mathrm{~F}$ & RIVER & & RAW GRAB & TRANSECT & 322 & RICH.PMPHS-3 HRM46.4 & & \\
\hline 1554 & & RIVER & & RAW GRAB & TRANSECT & 322 & RICH.PMPHS-3 HRM46.4 & & \\
\hline 1555 & & RIVER & & RAW GRAB & TRANSECT & 322 & RICH.PMPHS-3 HRM46.4 & & \\
\hline 1556 & & RIVER & & RAW GRAB & TRANSECT & 322 & RICH.PMPHS-3 HRM46.4 & & \\
\hline 1557 & & RIVER & & RAW GRAB & TRANSECT & 572 & RICH.PMPHS-4 HRM46.4 & & \\
\hline 1558 & & RIVER & & RAW GRAB & TRANSECT & 572 & RICH.PMPHS-4 HRM46.4 & & \\
\hline 1559 & & RIVER & & RAW GRAB & TRANSECT & 572 & RICH.PMPHS-4 HRM46.4 & & \\
\hline
\end{tabular}




\begin{tabular}{|c|c|c|c|c|c|c|c|c|c|}
\hline & $\mathrm{T}$ & $\bar{U}$ & $\mathrm{~V}$ & $\mathrm{~W}$ & $\mathrm{X}$ & $\mathrm{Y}$ & $Z$ & $\mathrm{AA}$ & $\mathrm{AB}$ \\
\hline \begin{tabular}{l|l}
1 & 1 \\
\end{tabular} & REVIEW_QUALIFIER & SAMP_FROM & SAMP_ITEM & SAMP_MTHD & COLL_MTHD & SAMP_SITE_ID & SAMP_SITE_NAME & WELL_NAME & SAMPLE_LOCATION \\
\hline $1560 \mathrm{~F}$ & $\mathrm{~F}$ & RIVER & & RAW GRAB & TRANSECT & 572 & RICH.PMPHS-4 HRM46.4 & & \\
\hline 1561 & & RIVER & & RAW GRAB & TRANSECT & 572 & RICH.PMPHS-4 HRM46.4 & & \\
\hline 1562 & & RIVER & & RAW GRAB & TRANSECT & 572 & RICH.PMPHS-4 HRM46.4 & & \\
\hline 1563 & & RIVER & & RAW GRAB & TRANSECT & 572 & RICH.PMPHS-4 HRM46.4 & & \\
\hline 1564 & & RIVER & & RAW GRAB & TRANSECT & 323 & RICH.PMPHS-5 HRM46.4 & & \\
\hline 1565 & & RIVER & & RAW GRAB & TRANSECT & 323 & RICH.PMPHS-5 HRM46.4 & & \\
\hline 1566 & & RIVER & & RAW GRAB & TRANSECT & 323 & RICH.PMPHS-5 HRM46.4 & & \\
\hline $1567 \mathrm{~F}$ & $\mathrm{~F}$ & RIVER & & RAW GRAB & TRANSECT & 323 & RICH.PMPHS-5 HRM46.4 & & \\
\hline 1568 & & RIVER & & RAW GRAB & TRANSECT & 323 & RICH.PMPHS-5 HRM46.4 & & \\
\hline 1569 & & RIVER & & RAW GRAB & TRANSECT & 323 & RICH.PMPHS-5 HRM46.4 & & \\
\hline 1570 & & RIVER & & RAW GRAB & TRANSECT & 323 & RICH.PMPHS-5 HRM46.4 & & \\
\hline 1571 & & RIVER & & RAW GRAB & TRANSECT & 324 & RICH.PMPHS-6 HRM46.4 & & \\
\hline 1572 & & RIVER & & RAW GRAB & TRANSECT & 324 & RICH.PMPHS-6 HRM46.4 & & \\
\hline 1573 & & RIVER & & RAW GRAB & TRANSECT & 324 & RICH.PMPHS-6 HRM46.4 & & \\
\hline $1574 \mathrm{~F}$ & $\mathrm{~F}$ & RIVER & & RAW GRAB & TRANSECT & 324 & RICH.PMPHS-6 HRM46.4 & & \\
\hline 1575 & & RIVER & & RAW GRAB & TRANSECT & 324 & RICH.PMPHS-6 HRM46.4 & & \\
\hline 1576 & & RIVER & & RAW GRAB & TRANSECT & 324 & RICH.PMPHS-6 HRM46.4 & & \\
\hline 1577 & & RIVER & & RAW GRAB & TRANSECT & 324 & RICH.PMPHS-6 HRM46.4 & & \\
\hline 1578 & & RIVER & & RAW GRAB & TRANSECT & 325 & RICH.PMPHS-7 HRM46.4 & & \\
\hline 1579 & & RIVER & & RAW GRAB & TRANSECT & 325 & RICH.PMPHS-7 HRM46.4 & & \\
\hline 1580 & & RIVER & & RAW GRAB & TRANSECT & 325 & RICH.PMPHS-7 HRM46.4 & & \\
\hline $1581 \mathrm{~F}$ & $\mathrm{~F}$ & RIVER & & RAW GRAB & TRANSECT & 325 & RICH.PMPHS-7 HRM46.4 & & \\
\hline 1582 & & RIVER & & RAW GRAB & TRANSECT & 325 & RICH.PMPHS-7 HRM46.4 & & \\
\hline 1583 & & RIVER & & RAW GRAB & TRANSECT & 325 & RICH.PMPHS-7 HRM46.4 & & \\
\hline 1584 & & RIVER & & RAW GRAB & TRANSECT & 325 & RICH.PMPHS-7 HRM46.4 & & \\
\hline 1585 & & RIVER & & RAW GRAB & TRANSECT & 326 & RICH.PMPHS-8 HRM46.4 & & \\
\hline 1586 & & RIVER & & RAW GRAB & TRANSECT & 326 & RICH.PMPHS-8 HRM46.4 & & \\
\hline 1587 & & RIVER & & RAW GRAB & TRANSECT & 326 & RICH.PMPHS-8 HRM46.4 & & \\
\hline $1588 \mathrm{~F}$ & $\mathrm{~F}$ & RIVER & & RAW GRAB & TRANSECT & 326 & RICH.PMPHS-8 HRM46.4 & & \\
\hline 1589 & & RIVER & & RAW GRAB & TRANSECT & 326 & RICH.PMPHS-8 HRM46.4 & & \\
\hline 1590 & & RIVER & & RAW GRAB & TRANSECT & 326 & RICH.PMPHS-8 HRM46.4 & & \\
\hline 1591 & & RIVER & & RAW GRAB & TRANSECT & 326 & RICH.PMPHS-8 HRM46.4 & & \\
\hline 1592 & & RIVER & & RAW GRAB & TRANSECT & 573 & RICH.PMPHS-9 HRM46.4 & & \\
\hline 1593 & & RIVER & & RAW GRAB & TRANSECT & 573 & RICH.PMPHS-9 HRM46.4 & & \\
\hline 1594 & & RIVER & & RAW GRAB & TRANSECT & 573 & RICH.PMPHS-9 HRM46.4 & & \\
\hline 1595 & $\mathrm{~F}$ & RIVER & & RAW GRAB & TRANSECT & 573 & RICH.PMPHS-9 HRM46.4 & & \\
\hline 1596 & & RIVER & & RAW GRAB & TRANSECT & 573 & RICH.PMPHS-9 HRM46.4 & & \\
\hline 1597 & & RIVER & & RAW GRAB & TRANSECT & 573 & RICH.PMPHS-9 HRM46.4 & & \\
\hline
\end{tabular}




\begin{tabular}{|c|c|c|c|c|c|c|c|c|c|}
\hline & $\mathrm{T}$ & $\bar{U}$ & $\mathrm{~V}$ & $\mathrm{~W}$ & $\mathrm{X}$ & $\mathrm{Y}$ & $Z$ & $\mathrm{AA}$ & $\mathrm{AB}$ \\
\hline \begin{tabular}{l|l}
1 & 1 \\
\end{tabular} & REVIEW_QUALIFIER & SAMP_FROM & SAMP_ITEM & SAMP_MTHD & COLL_MTHD & SAMP_SITE_ID & SAMP_SITE_NAME & WELL_NAME & SAMPLE_LOCATION \\
\hline 1598 & & RIVER & & RAW GRAB & TRANSECT & 573 & RICH.PMPHS-9 HRM46.4 & & \\
\hline 1599 & & RIVER & & RAW GRAB & TRANSECT & 578 & HANFRD TS-1 HRM 28.7 & & \\
\hline 1600 & & RIVER & & RAW GRAB & TRANSECT & 578 & HANFRD TS-1 HRM 28.7 & & \\
\hline 1601 & & RIVER & & RAW GRAB & TRANSECT & 578 & HANFRD TS-1 HRM 28.7 & & \\
\hline 1602 & & RIVER & & RAW GRAB & TRANSECT & 578 & HANFRD TS-1 HRM 28.7 & & \\
\hline $1603 \mathrm{~F}$ & $\mathrm{~F}$ & RIVER & & RAW GRAB & TRANSECT & 578 & HANFRD TS-1 HRM 28.7 & & \\
\hline 1604 & & RIVER & & RAW GRAB & TRANSECT & 578 & HANFRD TS-1 HRM 28.7 & & \\
\hline 1605 & & RIVER & & RAW GRAB & TRANSECT & 578 & HANFRD TS-1 HRM 28.7 & & \\
\hline 1606 & & RIVER & & RAW GRAB & TRANSECT & 578 & HANFRD TS-1 HRM 28.7 & & \\
\hline 1607 & & RIVER & & RAW GRAB & TRANSECT & 347 & HANFRD TS-10 HRM 28.7 & & \\
\hline 1608 & & RIVER & & RAW GRAB & TRANSECT & 347 & HANFRD TS-10 HRM 28.7 & & \\
\hline 1609 & & RIVER & & RAW GRAB & TRANSECT & 347 & HANFRD TS-10 HRM 28.7 & & \\
\hline 1610 & & RIVER & & RAW GRAB & TRANSECT & 347 & HANFRD TS-10 HRM 28.7 & & \\
\hline $1611 \mathrm{~F}$ & $\mathrm{~F}$ & RIVER & & RAW GRAB & TRANSECT & 347 & HANFRD TS-10 HRM 28.7 & & \\
\hline 1612 & & RIVER & & RAW GRAB & TRANSECT & 347 & HANFRD TS-10 HRM 28.7 & & \\
\hline 1613 & & RIVER & & RAW GRAB & TRANSECT & 347 & HANFRD TS-10 HRM 28.7 & & \\
\hline 1614 & & RIVER & & RAW GRAB & TRANSECT & 347 & HANFRD TS-10 HRM 28.7 & & \\
\hline 1615 & & RIVER & & RAW GRAB & TRANSECT & 342 & HANFRD TS-2 HRM 28.7 & & \\
\hline 1616 & & RIVER & & RAW GRAB & TRANSECT & 342 & HANFRD TS-2 HRM 28.7 & & \\
\hline 1617 & & RIVER & & RAW GRAB & TRANSECT & 342 & HANFRD TS-2 HRM 28.7 & & \\
\hline 1618 & & RIVER & & RAW GRAB & TRANSECT & 342 & HANFRD TS-2 HRM 28.7 & & \\
\hline $1619 \mathrm{~F}$ & $\mathrm{~F}$ & RIVER & & RAW GRAB & TRANSECT & 342 & HANFRD TS-2 HRM 28.7 & & \\
\hline 1620 & & RIVER & & RAW GRAB & TRANSECT & 342 & HANFRD TS-2 HRM 28.7 & & \\
\hline 1621 & & RIVER & & RAW GRAB & TRANSECT & 342 & HANFRD TS-2 HRM 28.7 & & \\
\hline 1622 & & RIVER & & RAW GRAB & TRANSECT & 342 & HANFRD TS-2 HRM 28.7 & & \\
\hline 1623 & & RIVER & & RAW GRAB & TRANSECT & 468 & HANFRD TS-3 HRM 28.7 & & \\
\hline 1624 & & RIVER & & RAW GRAB & TRANSECT & 468 & HANFRD TS-3 HRM 28.7 & & \\
\hline 1625 & & RIVER & & RAW GRAB & TRANSECT & 468 & HANFRD TS-3 HRM 28.7 & & \\
\hline 1626 & & RIVER & & RAW GRAB & TRANSECT & 468 & HANFRD TS-3 HRM 28.7 & & \\
\hline 1627 & & RIVER & & RAW GRAB & TRANSECT & 468 & HANFRD TS-3 HRM 28.7 & & \\
\hline 1628 & & RIVER & & RAW GRAB & TRANSECT & 468 & HANFRD TS-3 HRM 28.7 & & \\
\hline 1629 & & RIVER & & RAW GRAB & TRANSECT & 468 & HANFRD TS-3 HRM 28.7 & & \\
\hline 1630 & & RIVER & & RAW GRAB & TRANSECT & 468 & HANFRD TS-3 HRM 28.7 & & \\
\hline 1631 & & RIVER & & RAW GRAB & TRANSECT & 343 & HANFRD TS-4 HRM 28.7 & & \\
\hline 1632 & & RIVER & & RAW GRAB & TRANSECT & 343 & HANFRD TS-4 HRM 28.7 & & \\
\hline 1633 & & RIVER & & RAW GRAB & TRANSECT & 343 & HANFRD TS-4 HRM 28.7 & & \\
\hline 1634 & & RIVER & & RAW GRAB & TRANSECT & 343 & HANFRD TS-4 HRM 28.7 & & \\
\hline $1635 \mathrm{~F}$ & $\mathrm{~F}$ & RIVER & & RAW GRAB & TRANSECT & 343 & HANFRD TS-4 HRM 28.7 & & \\
\hline
\end{tabular}




\begin{tabular}{|c|c|c|c|c|c|c|c|c|c|}
\hline & $\mathrm{T}$ & $\bar{U}$ & $\mathrm{~V}$ & $\mathrm{~W}$ & $\mathrm{X}$ & $\mathrm{Y}$ & $Z$ & $\mathrm{AA}$ & $\mathrm{AB}$ \\
\hline 1 & REVIEW_QUALIFIER & SAMP_FROM & SAMP_ITEM & SAMP_MTHD & COLL_MTHD & SAMP_SITE_ID & SAMP_SITE_NAME & WELL_NAME & SAMPLE_LOCATION \\
\hline 1636 & & RIVER & & RAW GRAB & TRANSECT & 343 & IANFRD TS-4 HRM 28.7 & & \\
\hline 1637 & & RIVER & & RAW GRAB & TRANSECT & 343 & IANFRD TS-4 HRM 28.7 & & \\
\hline 1638 & & RIVER & & RAW GRAB & TRANSECT & 343 & IANFRD TS-4 HRM 28.7 & & \\
\hline 1639 & & RIVER & & RAW GRAB & TRANSECT & 344 & IANFRD TS-5 HRM 28.7 & & \\
\hline 1640 & & RIVER & & RAW GRAB & TRANSECT & 344 & IANFRD TS-5 HRM 28.7 & & \\
\hline 1641 & & RIVER & & RAW GRAB & TRANSECT & 344 & IANFRD TS-5 HRM 28.7 & & \\
\hline 1642 & & RIVER & & RAW GRAB & TRANSECT & 344 & IANFRD TS-5 HRM 28.7 & & \\
\hline $1643 \mathrm{~F}$ & $\mathrm{~F}$ & RIVER & & RAW GRAB & TRANSECT & 344 & IANFRD TS-5 HRM 28.7 & & \\
\hline 1644 & & RIVER & & RAW GRAB & TRANSECT & 344 & IANFRD TS-5 HRM 28.7 & & \\
\hline 1645 & & RIVER & & RAW GRAB & TRANSECT & 344 & IANFRD TS-5 HRM 28.7 & & \\
\hline 1646 & & RIVER & & RAW GRAB & TRANSECT & 344 & IANFRD TS-5 HRM 28.7 & & \\
\hline 1647 & & RIVER & & RAW GRAB & TRANSECT & $345 \mathrm{H}$ & IANFRD TS-6 HRM 28.7 & & \\
\hline 1648 & & RIVER & & RAW GRAB & TRANSECT & 345 & IANFRD TS-6 HRM 28.7 & & \\
\hline 1649 & & RIVER & & RAW GRAB & TRANSECT & 345 & IANFRD TS-6 HRM 28.7 & & \\
\hline 1650 & & RIVER & & RAW GRAB & TRANSECT & 345 & IANFRD TS-6 HRM 28.7 & & \\
\hline $1651 \mathrm{~F}$ & $\mathrm{~F}$ & RIVER & & RAW GRAB & TRANSECT & 345 & IANFRD TS-6 HRM 28.7 & & \\
\hline 1652 & & RIVER & & RAW GRAB & TRANSECT & 345 & IANFRD TS-6 HRM 28.7 & & \\
\hline 1653 & & RIVER & & RAW GRAB & TRANSECT & 345 & IANFRD TS-6 HRM 28.7 & & \\
\hline 1654 & & RIVER & & RAW GRAB & TRANSECT & 345 & IANFRD TS-6 HRM 28.7 & & \\
\hline 1655 & & RIVER & & RAW GRAB & TRANSECT & 469 & IANFRD TS-7 HRM 28.7 & & \\
\hline 1656 & & RIVER & & RAW GRAB & TRANSECT & 469 & IANFRD TS-7 HRM 28.7 & & \\
\hline 1657 & & RIVER & & RAW GRAB & TRANSECT & 469 & IANFRD TS-7 HRM 28.7 & & \\
\hline 1658 & & RIVER & & RAW GRAB & TRANSECT & 469 & IANFRD TS-7 HRM 28.7 & & \\
\hline $1659 \mathrm{~F}$ & $\mathrm{~F}$ & RIVER & & RAW GRAB & TRANSECT & 469 & IANFRD TS-7 HRM 28.7 & & \\
\hline 1660 & & RIVER & & RAW GRAB & TRANSECT & 469 & IANFRD TS-7 HRM 28.7 & & \\
\hline 1661 & & RIVER & & RAW GRAB & TRANSECT & 469 & IANFRD TS-7 HRM 28.7 & & \\
\hline 1662 & & RIVER & & RAW GRAB & TRANSECT & 469 & IANFRD TS-7 HRM 28.7 & & \\
\hline 1663 & & RIVER & & RAW GRAB & TRANSECT & 579 & IANFRD TS-8 HRM 28.7 & & \\
\hline 1664 & & RIVER & & RAW GRAB & TRANSECT & 579 & IANFRD TS-8 HRM 28.7 & & \\
\hline 1665 & & RIVER & & RAW GRAB & TRANSECT & 579 & IANFRD TS-8 HRM 28.7 & & \\
\hline 1666 & & RIVER & & RAW GRAB & TRANSECT & 579 & IANFRD TS-8 HRM 28.7 & & \\
\hline $1667 \mathrm{~F}$ & $\mathrm{~F}$ & RIVER & & RAW GRAB & TRANSECT & 579 & IANFRD TS-8 HRM 28.7 & & \\
\hline 1668 & & RIVER & & RAW GRAB & TRANSECT & 579 & IANFRD TS-8 HRM 28.7 & & \\
\hline 1669 & & RIVER & & RAW GRAB & TRANSECT & 579 & IANFRD TS-8 HRM 28.7 & & \\
\hline 1670 & & RIVER & & RAW GRAB & TRANSECT & 579 & IANFRD TS-8 HRM 28.7 & & \\
\hline 1671 & & RIVER & & RAW GRAB & TRANSECT & 346 & IANFRD TS-9 HRM 28.7 & & \\
\hline 1672 & & RIVER & & RAW GRAB & TRANSECT & 346 & IANFRD TS-9 HRM 28.7 & & \\
\hline 1673 & & RIVER & & RAW GRAB & TRANSECT & 346 & IANFRD TS-9 HRM 28.7 & & \\
\hline
\end{tabular}




\begin{tabular}{|c|c|c|c|c|c|c|c|c|c|}
\hline & $\mathrm{T}$ & $\bar{U}$ & $\mathrm{~V}$ & $\mathrm{~W}$ & $\mathrm{X}$ & $\mathrm{Y}$ & $Z$ & $\mathrm{AA}$ & $\mathrm{AB}$ \\
\hline \begin{tabular}{l|l}
1 & 1 \\
\end{tabular} & REVIEW_QUALIFIER & SAMP_FROM & SAMP_ITEM & SAMP_MTHD & COLL_MTHD & SAMP_SITE_ID & SAMP_SITE_NAME & WELL_NAME & SAMPLE_LOCATION \\
\hline 1674 & & RIVER & & RAW GRAB & TRANSECT & 346 & HANFRD TS-9 HRM 28.7 & & \\
\hline $1675 \mathrm{~F}$ & $\mathrm{~F}$ & RIVER & & RAW GRAB & TRANSECT & 346 & HANFRD TS-9 HRM 28.7 & & \\
\hline 1676 & & RIVER & & RAW GRAB & TRANSECT & 346 & HANFRD TS-9 HRM 28.7 & & \\
\hline 1677 & & RIVER & & RAW GRAB & TRANSECT & 346 & HANFRD TS-9 HRM 28.7 & & \\
\hline 1678 & & RIVER & & RAW GRAB & TRANSECT & 346 & HANFRD TS-9 HRM 28.7 & & \\
\hline 1679 & & RIVER & & RAW GRAB & TRANSECT & 317 & VERNITA-1 HRM 0.3 & & \\
\hline 1680 & & RIVER & & RAW GRAB & TRANSECT & 317 & VERNITA-1 HRM 0.3 & & \\
\hline 1681 & & RIVER & & RAW GRAB & TRANSECT & 317 & VERNITA-1 HRM 0.3 & & \\
\hline $1682 \mathrm{~F}$ & $\mathrm{~F}$ & RIVER & & RAW GRAB & TRANSECT & 317 & VERNITA-1 HRM 0.3 & & \\
\hline 1683 & & RIVER & & RAW GRAB & TRANSECT & 317 & VERNITA-1 HRM 0.3 & & \\
\hline 1684 & & RIVER & & RAW GRAB & TRANSECT & 317 & VERNITA-1 HRM 0.3 & & \\
\hline 1685 & & RIVER & & RAW GRAB & TRANSECT & 317 & VERNITA-1 HRM 0.3 & & \\
\hline 1686 & & RIVER & & RAW GRAB & TRANSECT & 570 & VERNITA-2 HRM 0.3 & & \\
\hline 1687 & & RIVER & & RAW GRAB & TRANSECT & 570 & VERNITA-2 HRM 0.3 & & \\
\hline 1688 & & RIVER & & RAW GRAB & TRANSECT & 570 & VERNITA-2 HRM 0.3 & & \\
\hline $1689 \mathrm{~F}$ & $\mathrm{~F}$ & RIVER & & RAW GRAB & TRANSECT & 570 & VERNITA-2 HRM 0.3 & & \\
\hline 1690 & & RIVER & & RAW GRAB & TRANSECT & 570 & VERNITA-2 HRM 0.3 & & \\
\hline $1691 \mathrm{~F}$ & $\mathrm{~F}$ & RIVER & & RAW GRAB & TRANSECT & 570 & VERNITA-2 HRM 0.3 & & \\
\hline 1692 & & RIVER & & RAW GRAB & TRANSECT & 570 & VERNITA-2 HRM 0.3 & & \\
\hline 1693 & & RIVER & & RAW GRAB & TRANSECT & 318 & VERNITA-3 HRM 0.3 & & \\
\hline 1694 & & RIVER & & RAW GRAB & TRANSECT & 318 & VERNITA-3 HRM 0.3 & & \\
\hline 1695 & & RIVER & & RAW GRAB & TRANSECT & 318 & VERNITA-3 HRM 0.3 & & \\
\hline $1696 \mathrm{~F}$ & $\mathrm{~F}$ & RIVER & & RAW GRAB & TRANSECT & 318 & VERNITA-3 HRM 0.3 & & \\
\hline 1697 & & RIVER & & RAW GRAB & TRANSECT & 318 & VERNITA-3 HRM 0.3 & & \\
\hline 1698 & & RIVER & & RAW GRAB & TRANSECT & 318 & VERNITA-3 HRM 0.3 & & \\
\hline 1699 & & RIVER & & RAW GRAB & TRANSECT & 318 & VERNITA-3 HRM 0.3 & & \\
\hline 1700 & & RIVER & & RAW GRAB & TRANSECT & 319 & VERNITA-4 HRM 0.3 & & \\
\hline 1701 & & RIVER & & RAW GRAB & TRANSECT & 319 & VERNITA-4 HRM 0.3 & & \\
\hline 1702 & & RIVER & & RAW GRAB & TRANSECT & 319 & VERNITA-4 HRM 0.3 & & \\
\hline $1703 \mathrm{~F}$ & $\mathrm{~F}$ & RIVER & & RAW GRAB & TRANSECT & 319 & VERNITA-4 HRM 0.3 & & \\
\hline 1704 & & RIVER & & RAW GRAB & TRANSECT & 319 & VERNITA-4 HRM 0.3 & & \\
\hline 1705 & & RIVER & & RAW GRAB & TRANSECT & 319 & VERNITA-4 HRM 0.3 & & \\
\hline 1706 & & RIVER & & RAW GRAB & TRANSECT & 319 & VERNITA-4 HRM 0.3 & & \\
\hline 1707 & & RIVER & & RAW GRAB & TRANSECT & 571 & RICH.PMPHS-1 HRM46.4 & & \\
\hline 1708 & & RIVER & & RAW GRAB & TRANSECT & 571 & RICH.PMPHS-1 HRM46.4 & & \\
\hline 1709 & & RIVER & & RAW GRAB & TRANSECT & 327 & RICH.PMPHS-10 HRM46.4 & & \\
\hline 1710 & & RIVER & & RAW GRAB & TRANSECT & 327 & RICH.PMPHS-10 HRM46.4 & & \\
\hline 1711 & & RIVER & & RAW GRAB & TRANSECT & 321 & RICH.PMPHS-2 HRM46.4 & & \\
\hline
\end{tabular}




\begin{tabular}{|c|c|c|c|c|c|c|c|c|c|}
\hline & $\mathrm{T}$ & $\mathrm{U}$ & $\mathrm{V}$ & $\mathrm{W}$ & $\mathrm{X}$ & $\bar{Y}$ & $Z$ & AA & $A B$ \\
\hline 1 & REVIEW_QUALIFIER & SAMP_FROM & SAMP_ITEM & SAMP_MTHD & COLL_MTHD & SAMP_SITE_ID & SAMP_SITE_NAME & WELL_NAME & SAMPLE_LOCATION \\
\hline 1712 & & RIVER & & RAW GRAB & TRANSECT & 321 & RICH.PMPHS-2 HRM46.4 & & \\
\hline 1713 & & RIVER & & RAW GRAB & TRANSECT & 322 & RICH.PMPHS-3 HRM46.4 & & \\
\hline 1714 & & RIVER & & RAW GRAB & TRANSECT & 322 & RICH.PMPHS-3 HRM46.4 & & \\
\hline 1715 & & RIVER & & RAW GRAB & TRANSECT & 572 & RICH.PMPHS-4 HRM46.4 & & \\
\hline 1716 & & RIVER & & RAW GRAB & TRANSECT & 572 & RICH.PMPHS-4 HRM46.4 & & \\
\hline 1717 & & RIVER & & RAW GRAB & TRANSECT & 323 & RICH.PMPHS-5 HRM46.4 & & \\
\hline 1718 & & RIVER & & RAW GRAB & TRANSECT & 323 & RICH.PMPHS-5 HRM46.4 & & \\
\hline 1719 & & RIVER & & RAW GRAB & TRANSECT & 324 & RICH.PMPHS-6 HRM46.4 & & \\
\hline 1720 & & RIVER & & RAW GRAB & TRANSECT & 324 & RICH.PMPHS-6 HRM46.4 & & \\
\hline 1721 & & RIVER & & RAW GRAB & TRANSECT & 325 & RICH.PMPHS-7 HRM46.4 & & \\
\hline 1722 & & RIVER & & RAW GRAB & TRANSECT & 325 & RICH.PMPHS-7 HRM46.4 & & \\
\hline 1723 & & RIVER & & RAW GRAB & TRANSECT & 326 & RICH.PMPHS-8 HRM46.4 & & \\
\hline 1724 & & RIVER & & RAW GRAB & TRANSECT & 326 & RICH.PMPHS-8 HRM46.4 & & \\
\hline 1725 & & RIVER & & RAW GRAB & TRANSECT & 573 & RICH.PMPHS-9 HRM46.4 & & \\
\hline 1726 & & RIVER & & RAW GRAB & TRANSECT & 573 & RICH.PMPHS-9 HRM46.4 & & \\
\hline 1727 & & RIVER & & RAW GRAB & TRANSECT & 463 & RICH.PMPHS HRM 46.4 & & \\
\hline 1728 & & RIVER & & RAW GRAB & TRANSECT & 463 & RICH.PMPHS HRM 46.4 & & \\
\hline 1729 & & RIVER & & RAW GRAB & TRANSECT & 463 & RICH.PMPHS HRM 46.4 & & \\
\hline 1730 & & RIVER & & RAW GRAB & TRANSECT & 463 & RICH.PMPHS HRM 46.4 & & \\
\hline 1731 & & RIVER & & RAW GRAB & TRANSECT & 463 & RICH.PMPHS HRM 46.4 & & \\
\hline 1732 & & RIVER & & RAW GRAB & TRANSECT & 571 & RICH.PMPHS-1 HRM46.4 & & \\
\hline 1733 & & RIVER & & RAW GRAB & TRANSECT & 571 & RICH.PMPHS-1 HRM46.4 & & \\
\hline 1734 & & RIVER & & RAW GRAB & TRANSECT & 571 & RICH.PMPHS-1 HRM46.4 & & \\
\hline 1735 & & RIVER & & RAW GRAB & TRANSECT & 571 & RICH.PMPHS-1 HRM46.4 & & \\
\hline 1736 & & RIVER & & RAW GRAB & TRANSECT & 571 & RICH.PMPHS-1 HRM46.4 & & \\
\hline 1737 & & RIVER & & RAW GRAB & TRANSECT & 321 & RICH.PMPHS-2 HRM46.4 & & \\
\hline 1738 & & RIVER & & RAW GRAB & TRANSECT & 321 & RICH.PMPHS-2 HRM46.4 & & \\
\hline 1739 & & RIVER & & RAW GRAB & TRANSECT & 321 & RICH.PMPHS-2 HRM46.4 & & \\
\hline 1740 & & RIVER & & RAW GRAB & TRANSECT & 321 & RICH.PMPHS-2 HRM46.4 & & \\
\hline 1741 & & RIVER & & RAW GRAB & TRANSECT & 321 & RICH.PMPHS-2 HRM46.4 & & \\
\hline 1742 & & RIVER & & RAW GRAB & TRANSECT & 322 & RICH.PMPHS-3 HRM46.4 & & \\
\hline 1743 & & RIVER & & RAW GRAB & TRANSECT & 322 & RICH.PMPHS-3 HRM46.4 & & \\
\hline 1744 & & RIVER & & RAW GRAB & TRANSECT & 322 & RICH.PMPHS-3 HRM46.4 & & \\
\hline 1745 & & RIVER & & RAW GRAB & TRANSECT & 322 & RICH.PMPHS-3 HRM46.4 & & \\
\hline 1746 & & RIVER & & RAW GRAB & TRANSECT & 322 & RICH.PMPHS-3 HRM46.4 & & \\
\hline 1747 & & RIVER & & RAW GRAB & TRANSECT & 572 & RICH.PMPHS-4 HRM46.4 & & \\
\hline 1748 & & RIVER & & RAW GRAB & TRANSECT & 572 & RICH.PMPHS-4 HRM46.4 & & \\
\hline 1749 & & RIVER & & RAW GRAB & TRANSECT & 572 & RICH.PMPHS-4 HRM46.4 & & \\
\hline
\end{tabular}




\begin{tabular}{|c|c|c|c|c|c|c|c|c|c|}
\hline & $\mathrm{T}$ & $\mathrm{U}$ & $\mathrm{V}$ & $\mathrm{W}$ & $\mathrm{X}$ & $\bar{Y}$ & $\bar{Z}$ & $\overline{A A}$ & $A B$ \\
\hline 1 & REVIEW_QUALIFIER & SAMP_FROM & SAMP_ITEM & SAMP_MTHD & COLL_MTHD & SAMP_SITE_ID & SAMP_SITE_NAME & WELL_NAME & SAMPLE_LOCATION \\
\hline 1750 & & RIVER & & RAW GRAB & TRANSECT & 572 & RICH.PMPHS-4 HRM46.4 & & \\
\hline 1751 & & RIVER & & RAW GRAB & TRANSECT & 572 & RICH.PMPHS-4 HRM46.4 & & \\
\hline 1752 & & RIVER & & RAW GRAB & TRANSECT & 323 & RICH.PMPHS-5 HRM46.4 & & \\
\hline 1753 & & RIVER & & RAW GRAB & TRANSECT & 323 & RICH.PMPHS-5 HRM46.4 & & \\
\hline 1754 & & RIVER & & RAW GRAB & TRANSECT & 323 & RICH.PMPHS-5 HRM46.4 & & \\
\hline 1755 & & RIVER & & RAW GRAB & TRANSECT & 323 & RICH.PMPHS-5 HRM46.4 & & \\
\hline 1756 & & RIVER & & RAW GRAB & TRANSECT & 323 & RICH.PMPHS-5 HRM46.4 & & \\
\hline 1757 & & RIVER & & RAW GRAB & TRANSECT & 324 & RICH.PMPHS-6 HRM46.4 & & \\
\hline 1758 & & RIVER & & RAW GRAB & TRANSECT & 324 & RICH.PMPHS-6 HRM46.4 & & \\
\hline 1759 & & RIVER & & RAW GRAB & TRANSECT & 324 & RICH.PMPHS-6 HRM46.4 & & \\
\hline 1760 & & RIVER & & RAW GRAB & TRANSECT & 324 & RICH.PMPHS-6 HRM46.4 & & \\
\hline 1761 & & RIVER & & RAW GRAB & TRANSECT & 324 & RICH.PMPHS-6 HRM46.4 & & \\
\hline 1762 & & RIVER & & RAW GRAB & TRANSECT & 325 & RICH.PMPHS-7 HRM46.4 & & \\
\hline 1763 & & RIVER & & RAW GRAB & TRANSECT & 325 & RICH.PMPHS-7 HRM46.4 & & \\
\hline 1764 & & RIVER & & RAW GRAB & TRANSECT & 325 & RICH.PMPHS-7 HRM46.4 & & \\
\hline 1765 & & RIVER & & RAW GRAB & TRANSECT & 325 & RICH.PMPHS-7 HRM46.4 & & \\
\hline 1766 & & RIVER & & RAW GRAB & TRANSECT & 325 & RICH.PMPHS-7 HRM46.4 & & \\
\hline 1767 & & RIVER & & RAW GRAB & TRANSECT & 326 & RICH.PMPHS-8 HRM46.4 & & \\
\hline 1768 & & RIVER & & RAW GRAB & TRANSECT & 326 & RICH.PMPHS-8 HRM46.4 & & \\
\hline 1769 & & RIVER & & RAW GRAB & TRANSECT & 326 & RICH.PMPHS-8 HRM46.4 & & \\
\hline 1770 & & RIVER & & RAW GRAB & TRANSECT & 326 & RICH.PMPHS-8 HRM46.4 & & \\
\hline 1771 & & RIVER & & RAW GRAB & TRANSECT & 326 & RICH.PMPHS-8 HRM46.4 & & \\
\hline 1772 & & RIVER & & RAW GRAB & TRANSECT & 573 & RICH.PMPHS-9 HRM46.4 & & \\
\hline 1773 & $\mathrm{~F}$ & RIVER & & RAW GRAB & TRANSECT & 573 & RICH.PMPHS-9 HRM46.4 & & \\
\hline 1774 & & RIVER & & RAW GRAB & TRANSECT & 573 & RICH.PMPHS-9 HRM46.4 & & \\
\hline 1775 & & RIVER & & RAW GRAB & TRANSECT & 573 & RICH.PMPHS-9 HRM46.4 & & \\
\hline 1776 & & RIVER & & RAW GRAB & TRANSECT & 573 & RICH.PMPHS-9 HRM46.4 & & \\
\hline 1777 & & RIVER & & RAW GRAB & TRANSECT & 327 & RICH.PMPHS-10 HRM46.4 & & \\
\hline 1778 & $F$ & RIVER & & RAW GRAB & TRANSECT & 327 & RICH.PMPHS-10 HRM46.4 & & \\
\hline 1779 & $F$ & RIVER & & RAW GRAB & TRANSECT & 327 & RICH.PMPHS-10 HRM46.4 & & \\
\hline 1780 & & RIVER & & RAW GRAB & TRANSECT & 327 & RICH.PMPHS-10 HRM46.4 & & \\
\hline 1781 & & RIVER & & RAW GRAB & TRANSECT & 327 & RICH.PMPHS-10 HRM46.4 & & \\
\hline 1782 & & RIVER & & RAW GRAB & TRANSECT & 317 & VERNITA-1 HRM 0.3 & & \\
\hline 1783 & & RIVER & & RAW GRAB & TRANSECT & 317 & VERNITA-1 HRM 0.3 & & \\
\hline 1784 & & RIVER & & RAW GRAB & TRANSECT & 317 & VERNITA-1 HRM 0.3 & & \\
\hline 1785 & $F$ & RIVER & & RAW GRAB & TRANSECT & 317 & VERNITA-1 HRM 0.3 & & \\
\hline 1786 & & RIVER & & RAW GRAB & TRANSECT & 317 & VERNITA-1 HRM 0.3 & & \\
\hline 1787 & & RIVER & & RAW GRAB & TRANSECT & 317 & VERNITA-1 HRM 0.3 & & \\
\hline
\end{tabular}




\begin{tabular}{|c|c|c|c|c|c|c|c|c|c|}
\hline & $\mathrm{T}$ & 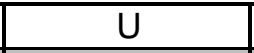 & $\mathrm{V}$ & $\mathrm{W}$ & $\mathrm{X}$ & $\mathrm{Y}$ & $Z$ & $\mathrm{AA}$ & $A B$ \\
\hline 1 & REVIEW_QUALIFIER & SAMP_FROM & SAMP_ITEM & SAMP_MTHD & COLL_MTHD & SAMP_SITE_ID & SAMP_SITE_NAME & WELL_NAME & SAMPLE_LOCATION \\
\hline 1788 & & RIVER & & RAW GRAB & TRANSECT & 317 & VERNITA-1 HRM 0.3 & & \\
\hline 1789 & & RIVER & & RAW GRAB & TRANSECT & 570 & VERNITA-2 HRM 0.3 & & \\
\hline 1790 & & RIVER & & RAW GRAB & TRANSECT & 570 & VERNITA-2 HRM 0.3 & & \\
\hline 1791 & & RIVER & & RAW GRAB & TRANSECT & 570 & VERNITA-2 HRM 0.3 & & \\
\hline 1792 & $\mathrm{~F}$ & RIVER & & RAW GRAB & TRANSECT & 570 & VERNITA-2 HRM 0.3 & & \\
\hline 1793 & & RIVER & & RAW GRAB & TRANSECT & 570 & VERNITA-2 HRM 0.3 & & \\
\hline 1794 & & RIVER & & RAW GRAB & TRANSECT & 570 & VERNITA-2 HRM 0.3 & & \\
\hline 1795 & & RIVER & & RAW GRAB & TRANSECT & 570 & VERNITA-2 HRM 0.3 & & \\
\hline 1796 & & RIVER & & RAW GRAB & TRANSECT & 318 & VERNITA-3 HRM 0.3 & & \\
\hline 1797 & & RIVER & & RAW GRAB & TRANSECT & 318 & VERNITA-3 HRM 0.3 & & \\
\hline 1798 & & RIVER & & RAW GRAB & TRANSECT & 318 & VERNITA-3 HRM 0.3 & & \\
\hline 1799 & $\mathrm{~F}$ & RIVER & & RAW GRAB & TRANSECT & 318 & VERNITA-3 HRM 0.3 & & \\
\hline 1800 & & RIVER & & RAW GRAB & TRANSECT & 318 & VERNITA-3 HRM 0.3 & & \\
\hline 1801 & & RIVER & & RAW GRAB & TRANSECT & 318 & VERNITA-3 HRM 0.3 & & \\
\hline 1802 & & RIVER & & RAW GRAB & TRANSECT & 318 & VERNITA-3 HRM 0.3 & & \\
\hline 1803 & & RIVER & & RAW GRAB & TRANSECT & 319 & VERNITA-4 HRM 0.3 & & \\
\hline 1804 & & RIVER & & RAW GRAB & TRANSECT & 319 & VERNITA-4 HRM 0.3 & & \\
\hline 1805 & & RIVER & & RAW GRAB & TRANSECT & 319 & VERNITA-4 HRM 0.3 & & \\
\hline 1806 & $\mathrm{~F}$ & RIVER & & RAW GRAB & TRANSECT & 319 & VERNITA-4 HRM 0.3 & & \\
\hline 1807 & & RIVER & & RAW GRAB & TRANSECT & 319 & VERNITA-4 HRM 0.3 & & \\
\hline 1808 & & RIVER & & RAW GRAB & TRANSECT & 319 & VERNITA-4 HRM 0.3 & & \\
\hline 1809 & & RIVER & & RAW GRAB & TRANSECT & 319 & VERNITA-4 HRM 0.3 & & \\
\hline 1810 & & RIVER & & RAW GRAB & TRANSECT & 571 & RICH.PMPHS-1 HRM46.4 & & \\
\hline 1811 & & RIVER & & RAW GRAB & TRANSECT & 571 & RICH.PMPHS-1 HRM46.4 & & \\
\hline 1812 & & RIVER & & RAW GRAB & TRANSECT & 571 & RICH.PMPHS-1 HRM46.4 & & \\
\hline 1813 & & RIVER & & RAW GRAB & TRANSECT & 571 & RICH.PMPHS-1 HRM46.4 & & \\
\hline 1814 & & RIVER & & RAW GRAB & TRANSECT & 571 & RICH.PMPHS-1 HRM46.4 & & \\
\hline 1815 & & RIVER & & RAW GRAB & TRANSECT & 571 & RICH.PMPHS-1 HRM46.4 & & \\
\hline 1816 & & RIVER & & RAW GRAB & TRANSECT & 571 & RICH.PMPHS-1 HRM46.4 & & \\
\hline 1817 & & RIVER & & RAW GRAB & TRANSECT & 327 & RICH.PMPHS-10 HRM46.4 & & \\
\hline 1818 & & RIVER & & RAW GRAB & TRANSECT & 327 & RICH.PMPHS-10 HRM46.4 & & \\
\hline 1819 & & RIVER & & RAW GRAB & TRANSECT & 327 & RICH.PMPHS-10 HRM46.4 & & \\
\hline 1820 & $\mathrm{~F}$ & RIVER & & RAW GRAB & TRANSECT & 327 & RICH.PMPHS-10 HRM46.4 & & \\
\hline 1821 & & RIVER & & RAW GRAB & TRANSECT & 327 & RICH.PMPHS-10 HRM46.4 & & \\
\hline 1822 & & RIVER & & RAW GRAB & TRANSECT & 327 & RICH.PMPHS-10 HRM46.4 & & \\
\hline 1823 & & RIVER & & RAW GRAB & TRANSECT & 327 & RICH.PMPHS-10 HRM46.4 & & \\
\hline 1824 & & RIVER & & RAW GRAB & TRANSECT & 321 & RICH.PMPHS-2 HRM46.4 & & \\
\hline 1825 & & RIVER & & RAW GRAB & TRANSECT & 321 & RICH.PMPHS-2 HRM46.4 & & \\
\hline
\end{tabular}




\begin{tabular}{|c|c|c|c|c|c|c|c|c|c|}
\hline & $\mathrm{T}$ & $\bar{U}$ & $\mathrm{~V}$ & $\mathrm{~W}$ & $\mathrm{X}$ & $\mathrm{Y}$ & $Z$ & $\mathrm{AA}$ & $\mathrm{AB}$ \\
\hline \begin{tabular}{l|l}
1 & 1 \\
\end{tabular} & REVIEW_QUALIFIER & SAMP_FROM & SAMP_ITEM & SAMP_MTHD & COLL_MTHD & SAMP_SITE_ID & SAMP_SITE_NAME & WELL_NAME & SAMPLE_LOCATION \\
\hline 1826 & & RIVER & & RAW GRAB & TRANSECT & 321 & RICH.PMPHS-2 HRM46.4 & & \\
\hline 1827 & & RIVER & & RAW GRAB & TRANSECT & 321 & RICH.PMPHS-2 HRM46.4 & & \\
\hline 1828 & & RIVER & & RAW GRAB & TRANSECT & 321 & RICH.PMPHS-2 HRM46.4 & & \\
\hline 1829 & & RIVER & & RAW GRAB & TRANSECT & 321 & RICH.PMPHS-2 HRM46.4 & & \\
\hline 1830 & & RIVER & & RAW GRAB & TRANSECT & 321 & RICH.PMPHS-2 HRM46.4 & & \\
\hline 1831 & & RIVER & & RAW GRAB & TRANSECT & 322 & RICH.PMPHS-3 HRM46.4 & & \\
\hline 1832 & & RIVER & & RAW GRAB & TRANSECT & 322 & RICH.PMPHS-3 HRM46.4 & & \\
\hline $1833 \mathrm{~F}$ & $\mathrm{~F}$ & RIVER & & RAW GRAB & TRANSECT & 322 & RICH.PMPHS-3 HRM46.4 & & \\
\hline 1834 & & RIVER & & RAW GRAB & TRANSECT & 322 & RICH.PMPHS-3 HRM46.4 & & \\
\hline 1835 & & RIVER & & RAW GRAB & TRANSECT & 322 & RICH.PMPHS-3 HRM46.4 & & \\
\hline 1836 & & RIVER & & RAW GRAB & TRANSECT & 322 & RICH.PMPHS-3 HRM46.4 & & \\
\hline 1837 & & RIVER & & RAW GRAB & TRANSECT & 322 & RICH.PMPHS-3 HRM46.4 & & \\
\hline 1838 & & RIVER & & RAW GRAB & TRANSECT & 572 & RICH.PMPHS-4 HRM46.4 & & \\
\hline 1839 & & RIVER & & RAW GRAB & TRANSECT & 572 & RICH.PMPHS-4 HRM46.4 & & \\
\hline $1840 \mathrm{~F}$ & $\mathrm{~F}$ & RIVER & & RAW GRAB & TRANSECT & 572 & RICH.PMPHS-4 HRM46.4 & & \\
\hline 1841 & & RIVER & & RAW GRAB & TRANSECT & 572 & RICH.PMPHS-4 HRM46.4 & & \\
\hline 1842 & & RIVER & & RAW GRAB & TRANSECT & 572 & RICH.PMPHS-4 HRM46.4 & & \\
\hline 1843 & & RIVER & & RAW GRAB & TRANSECT & 572 & RICH.PMPHS-4 HRM46.4 & & \\
\hline 1844 & & RIVER & & RAW GRAB & TRANSECT & 572 & RICH.PMPHS-4 HRM46.4 & & \\
\hline 1845 & & RIVER & & RAW GRAB & TRANSECT & 323 & RICH.PMPHS-5 HRM46.4 & & \\
\hline 1846 & & RIVER & & RAW GRAB & TRANSECT & 323 & RICH.PMPHS-5 HRM46.4 & & \\
\hline 1847 & & RIVER & & RAW GRAB & TRANSECT & 323 & RICH.PMPHS-5 HRM46.4 & & \\
\hline $1848 \mathrm{~F}$ & $\mathrm{~F}$ & RIVER & & RAW GRAB & TRANSECT & 323 & RICH.PMPHS-5 HRM46.4 & & \\
\hline 1849 & & RIVER & & RAW GRAB & TRANSECT & 323 & RICH.PMPHS-5 HRM46.4 & & \\
\hline 1850 & & RIVER & & RAW GRAB & TRANSECT & 323 & RICH.PMPHS-5 HRM46.4 & & \\
\hline 1851 & & RIVER & & RAW GRAB & TRANSECT & 323 & RICH.PMPHS-5 HRM46.4 & & \\
\hline 1852 & & RIVER & & RAW GRAB & TRANSECT & 324 & RICH.PMPHS-6 HRM46.4 & & \\
\hline 1853 & & RIVER & & RAW GRAB & TRANSECT & 324 & RICH.PMPHS-6 HRM46.4 & & \\
\hline 1854 & & RIVER & & RAW GRAB & TRANSECT & 324 & RICH.PMPHS-6 HRM46.4 & & \\
\hline $1855 \mathrm{~F}$ & $\mathrm{~F}$ & RIVER & & RAW GRAB & TRANSECT & 324 & RICH.PMPHS-6 HRM46.4 & & \\
\hline 1856 & & RIVER & & RAW GRAB & TRANSECT & 324 & RICH.PMPHS-6 HRM46.4 & & \\
\hline 1857 & & RIVER & & RAW GRAB & TRANSECT & 324 & RICH.PMPHS-6 HRM46.4 & & \\
\hline 1858 & & RIVER & & RAW GRAB & TRANSECT & 324 & RICH.PMPHS-6 HRM46.4 & & \\
\hline 1859 & & RIVER & & RAW GRAB & TRANSECT & 325 & RICH.PMPHS-7 HRM46.4 & & \\
\hline 1860 & & RIVER & & RAW GRAB & TRANSECT & 325 & RICH.PMPHS-7 HRM46.4 & & \\
\hline 1861 & & RIVER & & RAW GRAB & TRANSECT & 325 & RICH.PMPHS-7 HRM46.4 & & \\
\hline $1862 \mathrm{~F}$ & $\mathrm{~F}$ & RIVER & & RAW GRAB & TRANSECT & 325 & RICH.PMPHS-7 HRM46.4 & & \\
\hline 1863 & & RIVER & & RAW GRAB & TRANSECT & 325 & RICH.PMPHS-7 HRM46.4 & & \\
\hline
\end{tabular}




\begin{tabular}{|c|c|c|c|c|c|c|c|c|c|}
\hline & $T$ & $\bar{U}$ & $\mathrm{~V}$ & $\mathrm{~W}$ & $\bar{x}$ & $\bar{Y}$ & $\bar{Z}$ & AA & $A B$ \\
\hline 1 & REVIEW_QUALIFIER & SAMP_FROM & SAMP_ITEM & SAMP_MTHD & COLL_MTHD & SAMP_SITE_ID & SAMP_SITE_NAME & WELL_NAME & SAMPLE_LOCATION \\
\hline 1864 & & RIVER & & RAW GRAB & TRANSECT & 325 & 5 RICH.PMPHS-7 HRM46.4 & & \\
\hline 1865 & & RIVER & & RAW GRAB & TRANSECT & 325 & RICH.PMPHS-7 HRM46.4 & & \\
\hline 1866 & & RIVER & & RAW GRAB & TRANSECT & 326 & f RICH.PMPHS-8 HRM46.4 & & \\
\hline 1867 & & RIVER & & RAW GRAB & TRANSECT & 326 & RICH.PMPHS-8 HRM46.4 & & \\
\hline 1868 & & RIVER & & RAW GRAB & TRANSECT & 326 & f RICH.PMPHS-8 HRM46.4 & & \\
\hline 1869 & $F$ & RIVER & & RAW GRAB & TRANSECT & 326 & RICH.PMPHS-8 HRM46.4 & & \\
\hline 1870 & & RIVER & & RAW GRAB & TRANSECT & 326 & RICH.PMPHS-8 HRM46.4 & & \\
\hline 1871 & & RIVER & & RAW GRAB & TRANSECT & 326 & RICH.PMPHS-8 HRM46.4 & & \\
\hline 1872 & & RIVER & & RAW GRAB & TRANSECT & 326 & RICH.PMPHS-8 HRM46.4 & & \\
\hline 1873 & & RIVER & & RAW GRAB & TRANSECT & 573 & 3 RICH.PMPHS-9 HRM46.4 & & \\
\hline 1874 & & RIVER & & RAW GRAB & TRANSECT & 573 & 3 RICH.PMPHS-9 HRM46.4 & & \\
\hline 1875 & & RIVER & & RAW GRAB & TRANSECT & 573 & 3 RICH.PMPHS-9 HRM46.4 & & \\
\hline |1876 & $\mathrm{F}$ & RIVER & & RAW GRAB & TRANSECT & 573 & 3 RICH.PMPHS-9 HRM46.4 & & \\
\hline 1877 & & RIVER & & RAW GRAB & TRANSECT & 573 & 3 RICH.PMPHS-9 HRM46.4 & & \\
\hline 1878 & & RIVER & & RAW GRAB & TRANSECT & 573 & 3 RICH.PMPHS-9 HRM46.4 & & \\
\hline 1879 & & RIVER & & RAW GRAB & TRANSECT & 573 & 3 RICH.PMPHS-9 HRM46.4 & & \\
\hline 1880 & & RIVER & & RAW GRAB & TRANSECT & 317 & 7 VERNITA-1 HRM 0.3 & & \\
\hline 1881 & & RIVER & & RAW GRAB & TRANSECT & 317 & 7 VERNITA-1 HRM 0.3 & & \\
\hline 1882 & & RIVER & & RAW GRAB & TRANSECT & 317 & 7 VERNITA-1 HRM 0.3 & & \\
\hline 1883 & $F$ & RIVER & & RAW GRAB & TRANSECT & 317 & 7 VERNITA-1 HRM 0.3 & & \\
\hline 1884 & & RIVER & & RAW GRAB & TRANSECT & 317 & 7 VERNITA-1 HRM 0.3 & & \\
\hline 1885 & & RIVER & & RAW GRAB & TRANSECT & 317 & 7 VERNITA-1 HRM 0.3 & & \\
\hline 1886 & & RIVER & & RAW GRAB & TRANSECT & 317 & 7 VERNITA-1 HRM 0.3 & & \\
\hline 1887 & & RIVER & & RAW GRAB & TRANSECT & 570 & VERNITA-2 HRM 0.3 & & \\
\hline 1888 & & RIVER & & RAW GRAB & TRANSECT & 570 & VERNITA-2 HRM 0.3 & & \\
\hline 1889 & & RIVER & & RAW GRAB & TRANSECT & 570 & VERNITA-2 HRM 0.3 & & \\
\hline 1890 & $F$ & RIVER & & RAW GRAB & TRANSECT & 570 & VERNITA-2 HRM 0.3 & & \\
\hline 1891 & & RIVER & & RAW GRAB & TRANSECT & 570 & VERNITA-2 HRM 0.3 & & \\
\hline 1892 & & RIVER & & RAW GRAB & TRANSECT & 570 & VERNITA-2 HRM 0.3 & & \\
\hline 1893 & & RIVER & & RAW GRAB & TRANSECT & 570 & VERNITA-2 HRM 0.3 & & \\
\hline 1894 & & RIVER & & RAW GRAB & TRANSECT & 318 & 3 VERNITA-3 HRM 0.3 & & \\
\hline 1895 & & RIVER & & RAW GRAB & TRANSECT & 318 & 3 VERNITA-3 HRM 0.3 & & \\
\hline 1896 & & RIVER & & RAW GRAB & TRANSECT & 318 & 3 VERNITA-3 HRM 0.3 & & \\
\hline 1897 & $F$ & RIVER & & RAW GRAB & TRANSECT & 318 & 3 VERNITA-3 HRM 0.3 & & \\
\hline 1898 & & RIVER & & RAW GRAB & TRANSECT & 318 & 3 VERNITA-3 HRM 0.3 & & \\
\hline 1899 & & RIVER & & RAW GRAB & TRANSECT & 318 & 3 VERNITA-3 HRM 0.3 & & \\
\hline 1900 & & RIVER & & RAW GRAB & TRANSECT & 318 & 3 VERNITA-3 HRM 0.3 & & \\
\hline 1901 & & RIVER & & RAW GRAB & TRANSECT & 319 & 9 VERNITA-4 HRM 0.3 & & \\
\hline
\end{tabular}




\begin{tabular}{|c|c|c|c|c|c|c|c|c|c|}
\hline & $\mathrm{T}$ & $\bar{U}$ & $\mathrm{~V}$ & $\mathrm{~W}$ & $\mathrm{X}$ & $\mathrm{Y}$ & $Z$ & $\mathrm{AA}$ & $\mathrm{AB}$ \\
\hline 1 & REVIEW_QUALIFIER & SAMP_FROM & SAMP_ITEM & SAMP_MTHD & COLL_MTHD & SAMP_SITE_ID & SAMP_SITE_NAME & WELL_NAME & SAMPLE_LOCATION \\
\hline 1902 & & RIVER & & RAW GRAB & TRANSECT & 319 & VERNITA-4 HRM 0.3 & & \\
\hline 1903 & & RIVER & & RAW GRAB & TRANSECT & 319 & VERNITA-4 HRM 0.3 & & \\
\hline 1904 & $\mathrm{~F}$ & RIVER & & RAW GRAB & TRANSECT & 319 & VERNITA-4 HRM 0.3 & & \\
\hline 1905 & & RIVER & & RAW GRAB & TRANSECT & 319 & VERNITA-4 HRM 0.3 & & \\
\hline 1906 & & RIVER & & RAW GRAB & TRANSECT & 319 & VERNITA-4 HRM 0.3 & & \\
\hline 1907 & & RIVER & & RAW GRAB & TRANSECT & 319 & VERNITA-4 HRM 0.3 & & \\
\hline 1908 & & RIVER & & RAW GRAB & TRANSECT & 571 & RICH.PMPHS-1 HRM46.4 & & \\
\hline 1909 & & RIVER & & RAW GRAB & TRANSECT & 571 & RICH.PMPHS-1 HRM46.4 & & \\
\hline 1910 & & RIVER & & RAW GRAB & TRANSECT & 571 & RICH.PMPHS-1 HRM46.4 & & \\
\hline 1911 & $\mathrm{~F}$ & RIVER & & RAW GRAB & TRANSECT & 571 & RICH.PMPHS-1 HRM46.4 & & \\
\hline 1912 & & RIVER & & RAW GRAB & TRANSECT & 571 & RICH.PMPHS-1 HRM46.4 & & \\
\hline 1913 & & RIVER & & RAW GRAB & TRANSECT & 571 & RICH.PMPHS-1 HRM46.4 & & \\
\hline 1914 & & RIVER & & RAW GRAB & TRANSECT & 571 & RICH.PMPHS-1 HRM46.4 & & \\
\hline 1915 & & RIVER & & RAW GRAB & TRANSECT & 327 & RICH.PMPHS-10 HRM46.4 & & \\
\hline 1916 & & RIVER & & RAW GRAB & TRANSECT & 327 & RICH.PMPHS-10 HRM46.4 & & \\
\hline 1917 & $\mathrm{~F}$ & RIVER & & RAW GRAB & TRANSECT & 327 & RICH.PMPHS-10 HRM46.4 & & \\
\hline 1918 & $\mathrm{~F}$ & RIVER & & RAW GRAB & TRANSECT & 327 & RICH.PMPHS-10 HRM46.4 & & \\
\hline 1919 & & RIVER & & RAW GRAB & TRANSECT & 327 & RICH.PMPHS-10 HRM46.4 & & \\
\hline 1920 & & RIVER & & RAW GRAB & TRANSECT & 327 & RICH.PMPHS-10 HRM46.4 & & \\
\hline 1921 & & RIVER & & RAW GRAB & TRANSECT & 327 & RICH.PMPHS-10 HRM46.4 & & \\
\hline 1922 & & RIVER & & RAW GRAB & TRANSECT & 321 & RICH.PMPHS-2 HRM46.4 & & \\
\hline 1923 & & RIVER & & RAW GRAB & TRANSECT & 321 & RICH.PMPHS-2 HRM46.4 & & \\
\hline 1924 & & RIVER & & RAW GRAB & TRANSECT & 321 & RICH.PMPHS-2 HRM46.4 & & \\
\hline 1925 & $\mathrm{~F}$ & RIVER & & RAW GRAB & TRANSECT & 321 & RICH.PMPHS-2 HRM46.4 & & \\
\hline 1926 & & RIVER & & RAW GRAB & TRANSECT & 321 & RICH.PMPHS-2 HRM46.4 & & \\
\hline 1927 & & RIVER & & RAW GRAB & TRANSECT & 321 & RICH.PMPHS-2 HRM46.4 & & \\
\hline 1928 & & RIVER & & RAW GRAB & TRANSECT & 321 & RICH.PMPHS-2 HRM46.4 & & \\
\hline 1929 & & RIVER & & RAW GRAB & TRANSECT & 322 & RICH.PMPHS-3 HRM46.4 & & \\
\hline 1930 & & RIVER & & RAW GRAB & TRANSECT & 322 & RICH.PMPHS-3 HRM46.4 & & \\
\hline 1931 & & RIVER & & RAW GRAB & TRANSECT & 322 & RICH.PMPHS-3 HRM46.4 & & \\
\hline 1932 & $\mathrm{~F}$ & RIVER & & RAW GRAB & TRANSECT & 322 & RICH.PMPHS-3 HRM46.4 & & \\
\hline 1933 & & RIVER & & RAW GRAB & TRANSECT & 322 & RICH.PMPHS-3 HRM46.4 & & \\
\hline 1934 & & RIVER & & RAW GRAB & TRANSECT & 322 & RICH.PMPHS-3 HRM46.4 & & \\
\hline 1935 & & RIVER & & RAW GRAB & TRANSECT & 322 & RICH.PMPHS-3 HRM46.4 & & \\
\hline 1936 & & RIVER & & RAW GRAB & TRANSECT & 572 & RICH.PMPHS-4 HRM46.4 & & \\
\hline 1937 & & RIVER & & RAW GRAB & TRANSECT & 572 & RICH.PMPHS-4 HRM46.4 & & \\
\hline 1938 & & RIVER & & RAW GRAB & TRANSECT & 572 & RICH.PMPHS-4 HRM46.4 & & \\
\hline 1939 & $\mathrm{~F}$ & RIVER & & RAW GRAB & TRANSECT & 572 & RICH.PMPHS-4 HRM46.4 & & \\
\hline
\end{tabular}




\begin{tabular}{|c|c|c|c|c|c|c|c|c|c|}
\hline & $\mathrm{T}$ & $\mathrm{U}$ & $\mathrm{V}$ & $\mathrm{W}$ & $\mathrm{X}$ & $\bar{Y}$ & $Z$ & AA & $A B$ \\
\hline 1 & REVIEW_QUALIFIER & SAMP_FROM & SAMP_ITEM & SAMP_MTHD & COLL_MTHD & SAMP_SITE_ID & SAMP_SITE_NAME & WELL_NAME & SAMPLE_LOCATION \\
\hline 1940 & & RIVER & & RAW GRAB & TRANSECT & 572 & RICH.PMPHS-4 HRM46.4 & & \\
\hline 1941 & & RIVER & & RAW GRAB & TRANSECT & 572 & RICH.PMPHS-4 HRM46.4 & & \\
\hline 1942 & & RIVER & & RAW GRAB & TRANSECT & 572 & RICH.PMPHS-4 HRM46.4 & & \\
\hline 1943 & & RIVER & & RAW GRAB & TRANSECT & 323 & RICH.PMPHS-5 HRM46.4 & & \\
\hline 1944 & & RIVER & & RAW GRAB & TRANSECT & 323 & RICH.PMPHS-5 HRM46.4 & & \\
\hline 1945 & & RIVER & & RAW GRAB & TRANSECT & 323 & RICH.PMPHS-5 HRM46.4 & & \\
\hline 1946 & $F$ & RIVER & & RAW GRAB & TRANSECT & 323 & RICH.PMPHS-5 HRM46.4 & & \\
\hline 1947 & & RIVER & & RAW GRAB & TRANSECT & 323 & RICH.PMPHS-5 HRM46.4 & & \\
\hline 1948 & & RIVER & & RAW GRAB & TRANSECT & 323 & RICH.PMPHS-5 HRM46.4 & & \\
\hline 1949 & & RIVER & & RAW GRAB & TRANSECT & 323 & RICH.PMPHS-5 HRM46.4 & & \\
\hline 1950 & & RIVER & & RAW GRAB & TRANSECT & 324 & RICH.PMPHS-6 HRM46.4 & & \\
\hline 1951 & & RIVER & & RAW GRAB & TRANSECT & 324 & RICH.PMPHS-6 HRM46.4 & & \\
\hline 1952 & & RIVER & & RAW GRAB & TRANSECT & 324 & RICH.PMPHS-6 HRM46.4 & & \\
\hline 1953 & $F$ & RIVER & & RAW GRAB & TRANSECT & 324 & RICH.PMPHS-6 HRM46.4 & & \\
\hline 1954 & & RIVER & & RAW GRAB & TRANSECT & 324 & RICH.PMPHS-6 HRM46.4 & & \\
\hline 1955 & & RIVER & & RAW GRAB & TRANSECT & 324 & RICH.PMPHS-6 HRM46.4 & & \\
\hline 1956 & & RIVER & & RAW GRAB & TRANSECT & 324 & RICH.PMPHS-6 HRM46.4 & & \\
\hline 1957 & & RIVER & & RAW GRAB & TRANSECT & 325 & RICH.PMPHS-7 HRM46.4 & & \\
\hline 1958 & & RIVER & & RAW GRAB & TRANSECT & 325 & RICH.PMPHS-7 HRM46.4 & & \\
\hline 1959 & & RIVER & & RAW GRAB & TRANSECT & 325 & RICH.PMPHS-7 HRM46.4 & & \\
\hline 1960 & $F$ & RIVER & & RAW GRAB & TRANSECT & 325 & RICH.PMPHS-7 HRM46.4 & & \\
\hline 1961 & & RIVER & & RAW GRAB & TRANSECT & 325 & RICH.PMPHS-7 HRM46.4 & & \\
\hline 1962 & & RIVER & & RAW GRAB & TRANSECT & 325 & RICH.PMPHS-7 HRM46.4 & & \\
\hline 1963 & & RIVER & & RAW GRAB & TRANSECT & 325 & RICH.PMPHS-7 HRM46.4 & & \\
\hline 1964 & & RIVER & & RAW GRAB & TRANSECT & 326 & RICH.PMPHS-8 HRM46.4 & & \\
\hline 1965 & & RIVER & & RAW GRAB & TRANSECT & 326 & RICH.PMPHS-8 HRM46.4 & & \\
\hline 1966 & & RIVER & & RAW GRAB & TRANSECT & 326 & RICH.PMPHS-8 HRM46.4 & & \\
\hline 1967 & $F$ & RIVER & & RAW GRAB & TRANSECT & 326 & RICH.PMPHS-8 HRM46.4 & & \\
\hline 1968 & & RIVER & & RAW GRAB & TRANSECT & 326 & RICH.PMPHS-8 HRM46.4 & & \\
\hline 1969 & & RIVER & & RAW GRAB & TRANSECT & 326 & RICH.PMPHS-8 HRM46.4 & & \\
\hline 1970 & & RIVER & & RAW GRAB & TRANSECT & 326 & RICH.PMPHS-8 HRM46.4 & & \\
\hline 1971 & & RIVER & & RAW GRAB & TRANSECT & 573 & RICH.PMPHS-9 HRM46.4 & & \\
\hline 1972 & & RIVER & & RAW GRAB & TRANSECT & 573 & RICH.PMPHS-9 HRM46.4 & & \\
\hline 1973 & & RIVER & & RAW GRAB & TRANSECT & 573 & RICH.PMPHS-9 HRM46.4 & & \\
\hline \begin{tabular}{|l|l|}
1974 \\
\end{tabular} & $F$ & RIVER & & RAW GRAB & TRANSECT & 573 & RICH.PMPHS-9 HRM46.4 & & \\
\hline 1975 & & RIVER & & RAW GRAB & TRANSECT & 573 & RICH.PMPHS-9 HRM46.4 & & \\
\hline 1976 & & RIVER & & RAW GRAB & TRANSECT & 573 & RICH.PMPHS-9 HRM46.4 & & \\
\hline 1977 & & RIVER & & RAW GRAB & TRANSECT & 573 & RICH.PMPHS-9 HRM46.4 & & \\
\hline
\end{tabular}




\begin{tabular}{|c|c|c|c|c|c|c|c|c|c|}
\hline & $T$ & $\bar{U}$ & $\mathrm{~V}$ & $\mathrm{~W}$ & $\bar{x}$ & $\bar{Y}$ & $\bar{Z}$ & AA & $A B$ \\
\hline 1 & REVIEW_QUALIFIER & SAMP_FROM & SAMP_ITEM & SAMP_MTHD & COLL_MTHD & SAMP_SITE_ID & SAMP_SITE_NAME & WELL_NAME & SAMPLE_LOCATION \\
\hline 1978 & & RIVER & & RAW GRAB & TRANSECT & 328 & 3100 N -1 HRM 9.5 & & \\
\hline 1979 & & RIVER & & RAW GRAB & TRANSECT & 328 & 100 N -1 HRM 9.5 & & \\
\hline 1980 & & RIVER & & RAW GRAB & TRANSECT & 328 & 100 N -1 HRM 9.5 & & \\
\hline 1981 & & RIVER & & RAW GRAB & TRANSECT & 328 & 100 N -1 HRM 9.5 & & \\
\hline \begin{tabular}{|l|}
1982 \\
\end{tabular} & & RIVER & & RAW GRAB & TRANSECT & 328 & 100 N -1 HRM 9.5 & & \\
\hline 1983 & & RIVER & & RAW GRAB & TRANSECT & 328 & 100 N -1 HRM 9.5 & & \\
\hline 1984 & & RIVER & & RAW GRAB & TRANSECT & 328 & 100 N -1 HRM 9.5 & & \\
\hline 1985 & & RIVER & & RAW GRAB & TRANSECT & 328 & 100 N -1 HRM 9.5 & & \\
\hline 1986 & & RIVER & & RAW GRAB & TRANSECT & 328 & 100 N -1 HRM 9.5 & & \\
\hline \begin{tabular}{|l|}
1987 \\
\end{tabular} & & RIVER & & RAW GRAB & TRANSECT & 335 & 100 N -10 HRM 9.5 & & \\
\hline 1988 & & RIVER & & RAW GRAB & TRANSECT & 335 & 100 N -10 HRM 9.5 & & \\
\hline 1989 & & RIVER & & RAW GRAB & TRANSECT & 335 & 100 N -10 HRM 9.5 & & \\
\hline 1990 & & RIVER & & RAW GRAB & TRANSECT & 335 & 100 N -10 HRM 9.5 & & \\
\hline 1991 & & RIVER & & RAW GRAB & TRANSECT & 335 & $100 \mathrm{~N}-10 \mathrm{HRM} 9.5$ & & \\
\hline 1992 & $F$ & RIVER & & RAW GRAB & TRANSECT & 335 & 100 N -10 HRM 9.5 & & \\
\hline 1993 & & RIVER & & RAW GRAB & TRANSECT & 335 & $100 \mathrm{~N}-10 \mathrm{HRM} 9.5$ & & \\
\hline 1994 & & RIVER & & RAW GRAB & TRANSECT & 335 & $100 \mathrm{~N}-10 \mathrm{HRM} 9.5$ & & \\
\hline 1995 & & RIVER & & RAW GRAB & TRANSECT & 335 & 100 N -10 HRM 9.5 & & \\
\hline 1996 & & RIVER & & RAW GRAB & TRANSECT & 329 & 100 N -2 HRM 9.5 & & \\
\hline 1997 & & RIVER & & RAW GRAB & TRANSECT & 329 & 100 N -2 HRM 9.5 & & \\
\hline 1998 & & RIVER & & RAW GRAB & TRANSECT & 329 & 100 N -2 HRM 9.5 & & \\
\hline 1999 & & RIVER & & RAW GRAB & TRANSECT & 329 & 100 N -2 HRM 9.5 & & \\
\hline 2000 & & RIVER & & RAW GRAB & TRANSECT & 329 & 100 N -2 HRM 9.5 & & \\
\hline 2001 & $F$ & RIVER & & RAW GRAB & TRANSECT & 329 & $100 \mathrm{~N}-2$ HRM 9.5 & & \\
\hline 2002 & & RIVER & & RAW GRAB & TRANSECT & 329 & $100 \mathrm{~N}-2$ HRM 9.5 & & \\
\hline 2003 & & RIVER & & RAW GRAB & TRANSECT & 329 & 100 N -2 HRM 9.5 & & \\
\hline 2004 & & RIVER & & RAW GRAB & TRANSECT & 329 & 100 N -2 HRM 9.5 & & \\
\hline 2005 & & RIVER & & RAW GRAB & TRANSECT & 330 & 100 N -3 HRM 9.5 & & \\
\hline 2006 & & RIVER & & RAW GRAB & TRANSECT & 330 & 100 N -3 HRM 9.5 & & \\
\hline 2007 & & RIVER & & RAW GRAB & TRANSECT & 330 & $100 \mathrm{~N}-3$ HRM 9.5 & & \\
\hline 2008 & $F$ & RIVER & & RAW GRAB & TRANSECT & 330 & 100 N -3 HRM 9.5 & & \\
\hline 2009 & & RIVER & & RAW GRAB & TRANSECT & 330 & 100 N -3 HRM 9.5 & & \\
\hline 2010 & $F$ & RIVER & & RAW GRAB & TRANSECT & 330 & 100 N -3 HRM 9.5 & & \\
\hline 2011 & & RIVER & & RAW GRAB & TRANSECT & 330 & 100 N -3 HRM 9.5 & & \\
\hline 2012 & & RIVER & & RAW GRAB & TRANSECT & 330 & 100 N -3 HRM 9.5 & & \\
\hline 2013 & & RIVER & & RAW GRAB & TRANSECT & 330 & $100 \mathrm{~N}-3$ HRM 9.5 & & \\
\hline 2014 & & RIVER & & RAW GRAB & TRANSECT & 574 & 100 N -4 HRM 9.5 & & \\
\hline 2015 & & RIVER & & RAW GRAB & TRANSECT & 574 & 100 N -4 HRM 9.5 & & \\
\hline
\end{tabular}




\begin{tabular}{|c|c|c|c|c|c|c|c|c|c|}
\hline & $T$ & $\bar{U}$ & $\overline{\mathrm{V}}$ & $\mathrm{W}$ & $\bar{X}$ & $\bar{Y}$ & $\bar{Z}$ & $\overline{A A}$ & $A B$ \\
\hline 1 & REVIEW_QUALIFIER & SAMP_FROM & SAMP_ITEM & SAMP_MTHD & COLL_MTHD & SAMP_SITE_ID & SAMP_SITE_NAME & WELL_NAME & SAMPLE_LOCATION \\
\hline 2016 & & RIVER & & RAW GRAB & TRANSECT & 574 & $100 \mathrm{~N}-4$ HRM 9.5 & & \\
\hline 2017 & & RIVER & & RAW GRAB & TRANSECT & 574 & 100 N -4 HRM 9.5 & & \\
\hline 2018 & & RIVER & & RAW GRAB & TRANSECT & 574 & 100 N -4 HRM 9.5 & & \\
\hline 2019 & $\mathrm{~F}$ & RIVER & & RAW GRAB & TRANSECT & 574 & 100 N -4 HRM 9.5 & & \\
\hline 2020 & & RIVER & & RAW GRAB & TRANSECT & 574 & 100 N -4 HRM 9.5 & & \\
\hline 2021 & & RIVER & & RAW GRAB & TRANSECT & 574 & 100 N -4 HRM 9.5 & & \\
\hline 2022 & & RIVER & & RAW GRAB & TRANSECT & 574 & 100 N -4 HRM 9.5 & & \\
\hline 2023 & & RIVER & & RAW GRAB & TRANSECT & 331 & 100 N -5 HRM 9.5 & & \\
\hline 2024 & & RIVER & & RAW GRAB & TRANSECT & 331 & 100 N -5 HRM 9.5 & & \\
\hline 2025 & & RIVER & & RAW GRAB & TRANSECT & 331 & 100 N -5 HRM 9.5 & & \\
\hline 2026 & $\mathrm{~F}$ & RIVER & & RAW GRAB & TRANSECT & $331:$ & 100 N -5 HRM 9.5 & & \\
\hline 2027 & & RIVER & & RAW GRAB & TRANSECT & $331:$ & 100 N -5 HRM 9.5 & & \\
\hline 2028 & $\mathrm{~F}$ & RIVER & & RAW GRAB & TRANSECT & 331 & 100 N -5 HRM 9.5 & & \\
\hline 2029 & & RIVER & & RAW GRAB & TRANSECT & 331 & 100 N -5 HRM 9.5 & & \\
\hline 2030 & & RIVER & & RAW GRAB & TRANSECT & 331 & 100 N -5 HRM 9.5 & & \\
\hline 2031 & & RIVER & & RAW GRAB & TRANSECT & 331 & 100 N -5 HRM 9.5 & & \\
\hline 2032 & & RIVER & & RAW GRAB & TRANSECT & 332 & 100 N -6 HRM 9.5 & & \\
\hline 2033 & & RIVER & & RAW GRAB & TRANSECT & 332 & 100 N -6 HRM 9.5 & & \\
\hline 2034 & & RIVER & & RAW GRAB & TRANSECT & 332 & 100 N -6 HRM 9.5 & & \\
\hline 2035 & & RIVER & & RAW GRAB & TRANSECT & 332 & 100 N -6 HRM 9.5 & & \\
\hline 2036 & & RIVER & & RAW GRAB & TRANSECT & 332 & 100 N -6 HRM 9.5 & & \\
\hline 2037 & $\mathrm{~F}$ & RIVER & & RAW GRAB & TRANSECT & 332 & 100 N -6 HRM 9.5 & & \\
\hline 2038 & & RIVER & & RAW GRAB & TRANSECT & 332 & 100 N -6 HRM 9.5 & & \\
\hline 2039 & & RIVER & & RAW GRAB & TRANSECT & 332 & 100 N -6 HRM 9.5 & & \\
\hline 2040 & & RIVER & & RAW GRAB & TRANSECT & 332 & 100 N -6 HRM 9.5 & & \\
\hline 2041 & & RIVER & & RAW GRAB & TRANSECT & 333 & 100 N -7 HRM 9.5 & & \\
\hline 2042 & & RIVER & & RAW GRAB & TRANSECT & 333 & 100 N -7 HRM 9.5 & & \\
\hline 2043 & & RIVER & & RAW GRAB & TRANSECT & 3331 & 100 N -7 HRM 9.5 & & \\
\hline 2044 & $\mathrm{~F}$ & RIVER & & RAW GRAB & TRANSECT & 3331 & 100 N -7 HRM 9.5 & & \\
\hline 2045 & & RIVER & & RAW GRAB & TRANSECT & 3331 & 100 N -7 HRM 9.5 & & \\
\hline 2046 & $\mathrm{~F}$ & RIVER & & RAW GRAB & TRANSECT & 3331 & 100 N -7 HRM 9.5 & & \\
\hline 2047 & & RIVER & & RAW GRAB & TRANSECT & 3331 & 100 N -7 HRM 9.5 & & \\
\hline 2048 & & RIVER & & RAW GRAB & TRANSECT & 3331 & 100 N -7 HRM 9.5 & & \\
\hline 2049 & & RIVER & & RAW GRAB & TRANSECT & 3331 & 100 N -7 HRM 9.5 & & \\
\hline 2050 & & RIVER & & RAW GRAB & TRANSECT & 5751 & 100 N -8 HRM 9.5 & & \\
\hline 2051 & & RIVER & & RAW GRAB & TRANSECT & 5751 & 100 N -8 HRM 9.5 & & \\
\hline 2052 & & RIVER & & RAW GRAB & TRANSECT & 5751 & 100 N -8 HRM 9.5 & & \\
\hline 2053 & $F$ & RIVER & & RAW GRAB & TRANSECT & 5751 & 100 N -8 HRM 9.5 & & \\
\hline
\end{tabular}




\begin{tabular}{|c|c|c|c|c|c|c|c|c|c|}
\hline & $T$ & $\bar{U}$ & $\overline{\mathrm{V}}$ & $\mathrm{W}$ & $\bar{X}$ & $\bar{Y}$ & $\bar{Z}$ & $\overline{A A}$ & $A B$ \\
\hline 1 & REVIEW_QUALIFIER & SAMP_FROM & SAMP_ITEM & SAMP_MTHD & COLL_MTHD & SAMP_SITE_ID & SAMP_SITE_NAME & WELL_NAME & SAMPLE_LOCATION \\
\hline 2054 & & RIVER & & RAW GRAB & TRANSECT & 575 & 100 N -8 HRM 9.5 & & \\
\hline 2055 & $\mathrm{~F}$ & RIVER & & RAW GRAB & TRANSECT & 575 & 100 N -8 HRM 9.5 & & \\
\hline 2056 & & RIVER & & RAW GRAB & TRANSECT & 575 : & 100 N -8 HRM 9.5 & & \\
\hline 2057 & & RIVER & & RAW GRAB & TRANSECT & 575 & 100 N -8 HRM 9.5 & & \\
\hline 2058 & & RIVER & & RAW GRAB & TRANSECT & 575 & 100 N -8 HRM 9.5 & & \\
\hline 2059 & & RIVER & & RAW GRAB & TRANSECT & 334 & 100 N -9 HRM 9.5 & & \\
\hline 2060 & & RIVER & & RAW GRAB & TRANSECT & 334 & 100 N -9 HRM 9.5 & & \\
\hline 2061 & & RIVER & & RAW GRAB & TRANSECT & 334 & 100 N -9 HRM 9.5 & & \\
\hline 2062 & $\mathrm{~F}$ & RIVER & & RAW GRAB & TRANSECT & $334:$ & 100 N -9 HRM 9.5 & & \\
\hline 2063 & & RIVER & & RAW GRAB & TRANSECT & 334 & 100 N -9 HRM 9.5 & & \\
\hline 2064 & $\mathrm{~F}$ & RIVER & & RAW GRAB & TRANSECT & $334:$ & 100 N -9 HRM 9.5 & & \\
\hline 2065 & & RIVER & & RAW GRAB & TRANSECT & 334 & $100 \mathrm{~N}-9$ HRM 9.5 & & \\
\hline 2066 & & RIVER & & RAW GRAB & TRANSECT & 334 & 100 N -9 HRM 9.5 & & \\
\hline 2067 & & RIVER & & RAW GRAB & TRANSECT & 334 & 100 N -9 HRM 9.5 & & \\
\hline 2068 & & RIVER & & RAW GRAB & TRANSECT & 317 & VERNITA-1 HRM 0.3 & & \\
\hline 2069 & & RIVER & & RAW GRAB & TRANSECT & 317 & VERNITA-1 HRM 0.3 & & \\
\hline 2070 & $\mathrm{~F}$ & RIVER & & RAW GRAB & TRANSECT & 317 & VERNITA-1 HRM 0.3 & & \\
\hline 2071 & $\mathrm{~F}$ & RIVER & & RAW GRAB & TRANSECT & 317 & VERNITA-1 HRM 0.3 & & \\
\hline 2072 & & RIVER & & RAW GRAB & TRANSECT & 317 & VERNITA-1 HRM 0.3 & & \\
\hline 2073 & & RIVER & & RAW GRAB & TRANSECT & 317 & VERNITA-1 HRM 0.3 & & \\
\hline 2074 & & RIVER & & RAW GRAB & TRANSECT & 317 & VERNITA-1 HRM 0.3 & & \\
\hline 2075 & & RIVER & & RAW GRAB & TRANSECT & 570 & VERNITA-2 HRM 0.3 & & \\
\hline 2076 & & RIVER & & RAW GRAB & TRANSECT & 570 & VERNITA-2 HRM 0.3 & & \\
\hline 2077 & $\mathrm{~F}$ & RIVER & & RAW GRAB & TRANSECT & 570 & VERNITA-2 HRM 0.3 & & \\
\hline 2078 & $\mathrm{~F}$ & RIVER & & RAW GRAB & TRANSECT & 570 & VERNITA-2 HRM 0.3 & & \\
\hline 2079 & & RIVER & & RAW GRAB & TRANSECT & 570 & VERNITA-2 HRM 0.3 & & \\
\hline 2080 & & RIVER & & RAW GRAB & TRANSECT & 570 & VERNITA-2 HRM 0.3 & & \\
\hline 2081 & & RIVER & & RAW GRAB & TRANSECT & 570 & VERNITA-2 HRM 0.3 & & \\
\hline 2082 & & RIVER & & RAW GRAB & TRANSECT & 318 & VERNITA-3 HRM 0.3 & & \\
\hline 2083 & & RIVER & & RAW GRAB & TRANSECT & 318 & VERNITA-3 HRM 0.3 & & \\
\hline 2084 & & RIVER & & RAW GRAB & TRANSECT & 318 & VERNITA-3 HRM 0.3 & & \\
\hline 2085 & $\mathrm{~F}$ & RIVER & & RAW GRAB & TRANSECT & 318 & VERNITA-3 HRM 0.3 & & \\
\hline 2086 & & RIVER & & RAW GRAB & TRANSECT & 318 & VERNITA-3 HRM 0.3 & & \\
\hline 2087 & & RIVER & & RAW GRAB & TRANSECT & 318 & VERNITA-3 HRM 0.3 & & \\
\hline 2088 & & RIVER & & RAW GRAB & TRANSECT & 318 & VERNITA-3 HRM 0.3 & & \\
\hline 2089 & & RIVER & & RAW GRAB & TRANSECT & 319 & VERNITA-4 HRM 0.3 & & \\
\hline 2090 & & RIVER & & RAW GRAB & TRANSECT & 319 & VERNITA-4 HRM 0.3 & & \\
\hline 2091 & $F$ & RIVER & & RAW GRAB & TRANSECT & 319 & VERNITA-4 HRM 0.3 & & \\
\hline
\end{tabular}




\begin{tabular}{|c|c|c|c|c|c|c|c|c|c|}
\hline & $\mathrm{T}$ & 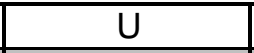 & $\mathrm{V}$ & $\mathrm{W}$ & $\mathrm{X}$ & $\mathrm{Y}$ & $Z$ & $\mathrm{AA}$ & $A B$ \\
\hline \begin{tabular}{l|l}
1 & 1 \\
\end{tabular} & REVIEW_QUALIFIER & SAMP_FROM & SAMP_ITEM & SAMP_MTHD & COLL_MTHD & SAMP_SITE_ID & SAMP_SITE_NAME & WELL_NAME & SAMPLE_LOCATION \\
\hline $2092 \mathrm{~F}$ & $\mathrm{~F}$ & RIVER & & RAW GRAB & TRANSECT & 319 & VERNITA-4 HRM 0.3 & & \\
\hline 2093 & & RIVER & & RAW GRAB & TRANSECT & 319 & VERNITA-4 HRM 0.3 & & \\
\hline 2094 & & RIVER & & RAW GRAB & TRANSECT & 319 & VERNITA-4 HRM 0.3 & & \\
\hline 2095 & & RIVER & & RAW GRAB & TRANSECT & 319 & VERNITA-4 HRM 0.3 & & \\
\hline 2096 & & RIVER & & RAW GRAB & TRANSECT & 336 & 100 F -1 HRM 19.0 & & \\
\hline 2097 & & RIVER & & RAW GRAB & TRANSECT & 336 & 100 F -1 HRM 19.0 & & \\
\hline 2098 & & RIVER & & RAW GRAB & TRANSECT & 336 & 100 F -1 HRM 19.0 & & \\
\hline $2099 \mathrm{~F}$ & $\mathrm{~F}$ & RIVER & & RAW GRAB & TRANSECT & 336 & 100 F -1 HRM 19.0 & & \\
\hline 2100 & & RIVER & & RAW GRAB & TRANSECT & 336 & 100 F -1 HRM 19.0 & & \\
\hline 2101 & & RIVER & & RAW GRAB & TRANSECT & 336 & 100 F -1 HRM 19.0 & & \\
\hline 2102 & & RIVER & & RAW GRAB & TRANSECT & 336 & 100 F -1 HRM 19.0 & & \\
\hline 2103 & & RIVER & & RAW GRAB & TRANSECT & 341 & 100 F -10 HRM 19.0 & & \\
\hline 2104 & & RIVER & & RAW GRAB & TRANSECT & 341 & 100 F -10 HRM 19.0 & & \\
\hline $2105 \mathrm{~F}$ & $\mathrm{~F}$ & RIVER & & RAW GRAB & TRANSECT & 341 & 100 F -10 HRM 19.0 & & \\
\hline $2106 \mathrm{~F}$ & $\mathrm{~F}$ & RIVER & & RAW GRAB & TRANSECT & 341 & 100 F -10 HRM 19.0 & & \\
\hline 2107 & & RIVER & & RAW GRAB & TRANSECT & 341 & 100 F -10 HRM 19.0 & & \\
\hline 2108 & & RIVER & & RAW GRAB & TRANSECT & 341 & 100 F -10 HRM 19.0 & & \\
\hline 2109 & & RIVER & & RAW GRAB & TRANSECT & 341 & 100 F -10 HRM 19.0 & & \\
\hline 2110 & & RIVER & & RAW GRAB & TRANSECT & 576 & 100 F -2 HRM 19.0 & & \\
\hline 2111 & & RIVER & & RAW GRAB & TRANSECT & 576 & 100 F -2 HRM 19.0 & & \\
\hline 2112 & & RIVER & & RAW GRAB & TRANSECT & 576 & 100 F -2 HRM 19.0 & & \\
\hline $2113 \mathrm{~F}$ & $\mathrm{~F}$ & RIVER & & RAW GRAB & TRANSECT & 576 & 100 F -2 HRM 19.0 & & \\
\hline 2114 & & RIVER & & RAW GRAB & TRANSECT & 576 & 100 F -2 HRM 19.0 & & \\
\hline 2115 & & RIVER & & RAW GRAB & TRANSECT & 576 & 100 F -2 HRM 19.0 & & \\
\hline 2116 & & RIVER & & RAW GRAB & TRANSECT & 576 & 100 F -2 HRM 19.0 & & \\
\hline 2117 & & RIVER & & RAW GRAB & TRANSECT & 337 & 100 F -3 HRM 19.0 & & \\
\hline 2118 & & RIVER & & RAW GRAB & TRANSECT & 337 & 100 F -3 HRM 19.0 & & \\
\hline 2119 & & RIVER & & RAW GRAB & TRANSECT & 337 & 100 F -3 HRM 19.0 & & \\
\hline $2120 \mathrm{~F}$ & $\mathrm{~F}$ & RIVER & & RAW GRAB & TRANSECT & 337 & 100 F -3 HRM 19.0 & & \\
\hline 2121 & & RIVER & & RAW GRAB & TRANSECT & 337 & 100 F -3 HRM 19.0 & & \\
\hline 2122 & & RIVER & & RAW GRAB & TRANSECT & 337 & 100 F -3 HRM 19.0 & & \\
\hline 2123 & & RIVER & & RAW GRAB & TRANSECT & 337 & 100 F -3 HRM 19.0 & & \\
\hline 2124 & & RIVER & & RAW GRAB & TRANSECT & 338 & 100 F -4 HRM 19.0 & & \\
\hline 2125 & & RIVER & & RAW GRAB & TRANSECT & 338 & 100 F -4 HRM 19.0 & & \\
\hline 2126 & & RIVER & & RAW GRAB & TRANSECT & 338 & 100 F -4 HRM 19.0 & & \\
\hline $2127 \mathrm{~F}$ & $\mathrm{~F}$ & RIVER & & RAW GRAB & TRANSECT & 338 & 100 F -4 HRM 19.0 & & \\
\hline 2128 & & RIVER & & RAW GRAB & TRANSECT & 338 & 100 F -4 HRM 19.0 & & \\
\hline 2129 & & RIVER & & RAW GRAB & TRANSECT & 338 & 100 F -4 HRM 19.0 & & \\
\hline
\end{tabular}




\begin{tabular}{|c|c|c|c|c|c|c|c|c|c|}
\hline & $T$ & $\bar{U}$ & $\mathrm{~V}$ & $\mathrm{~W}$ & $\bar{x}$ & $\bar{Y}$ & $\bar{Z}$ & AA & $A B$ \\
\hline 1 & REVIEW_QUALIFIER & SAMP_FROM & SAMP_ITEM & SAMP_MTHD & COLL_MTHD & SAMP_SITE_ID & SAMP_SITE_NAME & WELL_NAME & SAMPLE_LOCATION \\
\hline 2130 & & RIVER & & RAW GRAB & TRANSECT & 338 & 3100 F -4 HRM 19.0 & & \\
\hline 2131 & & RIVER & & RAW GRAB & TRANSECT & 577 & 7100 F -5 HRM 19.0 & & \\
\hline 2132 & & RIVER & & RAW GRAB & TRANSECT & 577 & 7100 F -5 HRM 19.0 & & \\
\hline 2133 & & RIVER & & RAW GRAB & TRANSECT & 577 & 7100 F -5 HRM 19.0 & & \\
\hline \begin{tabular}{|l|l|}
2134 \\
\end{tabular} & $F$ & RIVER & & RAW GRAB & TRANSECT & 577 & 7100 F -5 HRM 19.0 & & \\
\hline 2135 & & RIVER & & RAW GRAB & TRANSECT & 577 & 7 100 F -5 HRM 19.0 & & \\
\hline \begin{tabular}{|l|}
2136 \\
\end{tabular} & & RIVER & & RAW GRAB & TRANSECT & 577 & 7100 F -5 HRM 19.0 & & \\
\hline 2137 & & RIVER & & RAW GRAB & TRANSECT & 577 & 7100 F -5 HRM 19.0 & & \\
\hline 2138 & & RIVER & & RAW GRAB & TRANSECT & 606 & 100 F -6 HRM 19.0 & & \\
\hline \begin{tabular}{|l|}
2139 \\
\end{tabular} & & RIVER & & RAW GRAB & TRANSECT & 606 & 100 F -6 HRM 19.0 & & \\
\hline 2140 & & RIVER & & RAW GRAB & TRANSECT & 606 & 100 F -6 HRM 19.0 & & \\
\hline 2141 & $\mathrm{~F}$ & RIVER & & RAW GRAB & TRANSECT & 606 & 5100 F -6 HRM 19.0 & & \\
\hline 2142 & & RIVER & & RAW GRAB & TRANSECT & 606 & 6100 F -6 HRM 19.0 & & \\
\hline 2143 & & RIVER & & RAW GRAB & TRANSECT & 606 & 100 F -6 HRM 19.0 & & \\
\hline 2144 & & RIVER & & RAW GRAB & TRANSECT & 606 & 100 F -6 HRM 19.0 & & \\
\hline 2145 & & RIVER & & RAW GRAB & TRANSECT & 339 & 9100 F -7 HRM 19.0 & & \\
\hline 2146 & & RIVER & & RAW GRAB & TRANSECT & 339 & 9100 F -7 HRM 19.0 & & \\
\hline 2147 & & RIVER & & RAW GRAB & TRANSECT & 339 & 9100 F -7 HRM 19.0 & & \\
\hline 2148 & $F$ & RIVER & & RAW GRAB & TRANSECT & 339 & 9100 F -7 HRM 19.0 & & \\
\hline 2149 & & RIVER & & RAW GRAB & TRANSECT & 339 & 9100 F -7 HRM 19.0 & & \\
\hline 2150 & & RIVER & & RAW GRAB & TRANSECT & 339 & 9100 F -7 HRM 19.0 & & \\
\hline 2151 & & RIVER & & RAW GRAB & TRANSECT & 339 & 9100 F -7 HRM 19.0 & & \\
\hline 2152 & & RIVER & & RAW GRAB & TRANSECT & 340 & 100 F -8 HRM 19.0 & & \\
\hline 2153 & & RIVER & & RAW GRAB & TRANSECT & 340 & 100 F -8 HRM 19.0 & & \\
\hline 2154 & & RIVER & & RAW GRAB & TRANSECT & 340 & 100 F -8 HRM 19.0 & & \\
\hline 2155 & $F$ & RIVER & & RAW GRAB & TRANSECT & 340 & 100 F -8 HRM 19.0 & & \\
\hline 2156 & & RIVER & & RAW GRAB & TRANSECT & 340 & 100 F -8 HRM 19.0 & & \\
\hline 2157 & & RIVER & & RAW GRAB & TRANSECT & 340 & 100 F -8 HRM 19.0 & & \\
\hline 2158 & & RIVER & & RAW GRAB & TRANSECT & 340 & 100 F -8 HRM 19.0 & & \\
\hline 2159 & & RIVER & & RAW GRAB & TRANSECT & 467 & 7 100 F -9 HRM 19.0 & & \\
\hline 2160 & & RIVER & & RAW GRAB & TRANSECT & 467 & 7100 F -9 HRM 19.0 & & \\
\hline 2161 & & RIVER & & RAW GRAB & TRANSECT & 467 & 7100 F -9 HRM 19.0 & & \\
\hline \begin{tabular}{|l|l|}
2162 \\
\end{tabular} & $F$ & RIVER & & RAW GRAB & TRANSECT & 467 & 7100 F -9 HRM 19.0 & & \\
\hline 2163 & & RIVER & & RAW GRAB & TRANSECT & 467 & 7100 F -9 HRM 19.0 & & \\
\hline 2164 & & RIVER & & RAW GRAB & TRANSECT & 467 & 7 100 F -9 HRM 19.0 & & \\
\hline 2165 & & RIVER & & RAW GRAB & TRANSECT & 467 & 7 100 F -9 HRM 19.0 & & \\
\hline 2166 & & RIVER & & RAW GRAB & TRANSECT & 578 & 3 HANFRD TS-1 HRM 28.7 & & \\
\hline \begin{tabular}{|l|}
2167 \\
\end{tabular} & & RIVER & & RAW GRAB & TRANSECT & 578 & 3 HANFRD TS-1 HRM 28.7 & & \\
\hline
\end{tabular}




\begin{tabular}{|c|c|c|c|c|c|c|c|c|c|}
\hline & $\mathrm{T}$ & $U$ & $\mathrm{~V}$ & $\mathrm{~W}$ & $\mathrm{X}$ & $\mathrm{Y}$ & $Z$ & $\mathrm{AA}$ & $A B$ \\
\hline 1 & REVIEW_QUALIFIER & SAMP_FROM & SAMP_ITEM & SAMP_MTHD & COLL_MTHD & SAMP_SITE_ID & SAMP_SITE_NAME & WELL_NAME & SAMPLE_LOCATION \\
\hline 2168 & & RIVER & & RAW GRAB & TRANSECT & 578 & HANFRD TS-1 HRM 28.7 & & \\
\hline 2169 & & RIVER & & RAW GRAB & TRANSECT & 578 & HANFRD TS-1 HRM 28.7 & & \\
\hline 2170 & & RIVER & & RAW GRAB & TRANSECT & 578 & HANFRD TS-1 HRM 28.7 & & \\
\hline 2171 & & RIVER & & RAW GRAB & TRANSECT & 578 & HANFRD TS-1 HRM 28.7 & & \\
\hline 2172 & & RIVER & & RAW GRAB & TRANSECT & 578 & HANFRD TS-1 HRM 28.7 & & \\
\hline 2173 & & RIVER & & RAW GRAB & TRANSECT & 578 & HANFRD TS-1 HRM 28.7 & & \\
\hline 2174 & & RIVER & & RAW GRAB & TRANSECT & 347 & HANFRD TS-10 HRM 28.7 & & \\
\hline 2175 & & RIVER & & RAW GRAB & TRANSECT & 347 & HANFRD TS-10 HRM 28.7 & & \\
\hline 2176 & $F$ & RIVER & & RAW GRAB & TRANSECT & 347 & HANFRD TS-10 HRM 28.7 & & \\
\hline 2177 & & RIVER & & RAW GRAB & TRANSECT & 347 & HANFRD TS-10 HRM 28.7 & & \\
\hline 2178 & $F$ & RIVER & & RAW GRAB & TRANSECT & 347 & HANFRD TS-10 HRM 28.7 & & \\
\hline 2179 & & RIVER & & RAW GRAB & TRANSECT & 347 & HANFRD TS-10 HRM 28.7 & & \\
\hline 2180 & & RIVER & & RAW GRAB & TRANSECT & 347 & HANFRD TS-10 HRM 28.7 & & \\
\hline 2181 & & RIVER & & RAW GRAB & TRANSECT & 347 & HANFRD TS-10 HRM 28.7 & & \\
\hline 2182 & & RIVER & & RAW GRAB & TRANSECT & 342 & HANFRD TS-2 HRM 28.7 & & \\
\hline 2183 & & RIVER & & RAW GRAB & TRANSECT & 342 & HANFRD TS-2 HRM 28.7 & & \\
\hline 2184 & & RIVER & & RAW GRAB & TRANSECT & 342 & HANFRD TS-2 HRM 28.7 & & \\
\hline 2185 & & RIVER & & RAW GRAB & TRANSECT & 342 & HANFRD TS-2 HRM 28.7 & & \\
\hline 2186 & & RIVER & & RAW GRAB & TRANSECT & 342 & HANFRD TS-2 HRM 28.7 & & \\
\hline 2187 & & RIVER & & RAW GRAB & TRANSECT & 342 & HANFRD TS-2 HRM 28.7 & & \\
\hline 2188 & & RIVER & & RAW GRAB & TRANSECT & 342 & HANFRD TS-2 HRM 28.7 & & \\
\hline 2189 & & RIVER & & RAW GRAB & TRANSECT & 342 & HANFRD TS-2 HRM 28.7 & & \\
\hline 2190 & & RIVER & & RAW GRAB & TRANSECT & 468 & HANFRD TS-3 HRM 28.7 & & \\
\hline 2191 & & RIVER & & RAW GRAB & TRANSECT & 468 & HANFRD TS-3 HRM 28.7 & & \\
\hline 2192 & & RIVER & & RAW GRAB & TRANSECT & 468 & HANFRD TS-3 HRM 28.7 & & \\
\hline 2193 & & RIVER & & RAW GRAB & TRANSECT & 468 & HANFRD TS-3 HRM 28.7 & & \\
\hline 2194 & $F$ & RIVER & & RAW GRAB & TRANSECT & 468 & HANFRD TS-3 HRM 28.7 & & \\
\hline 2195 & & RIVER & & RAW GRAB & TRANSECT & 468 & HANFRD TS-3 HRM 28.7 & & \\
\hline 2196 & & RIVER & & RAW GRAB & TRANSECT & 468 & HANFRD TS-3 HRM 28.7 & & \\
\hline 2197 & & RIVER & & RAW GRAB & TRANSECT & 468 & HANFRD TS-3 HRM 28.7 & & \\
\hline 2198 & & RIVER & & RAW GRAB & TRANSECT & 343 & HANFRD TS-4 HRM 28.7 & & \\
\hline 2199 & & RIVER & & RAW GRAB & TRANSECT & 343 & HANFRD TS-4 HRM 28.7 & & \\
\hline 2200 & & RIVER & & RAW GRAB & TRANSECT & 343 & HANFRD TS-4 HRM 28.7 & & \\
\hline 2201 & & RIVER & & RAW GRAB & TRANSECT & 343 & HANFRD TS-4 HRM 28.7 & & \\
\hline 2202 & $F$ & RIVER & & RAW GRAB & TRANSECT & 343 & HANFRD TS-4 HRM 28.7 & & \\
\hline 2203 & & RIVER & & RAW GRAB & TRANSECT & 343 & HANFRD TS-4 HRM 28.7 & & \\
\hline 2204 & & RIVER & & RAW GRAB & TRANSECT & 343 & HANFRD TS-4 HRM 28.7 & & \\
\hline 2205 & & RIVER & & RAW GRAB & TRANSECT & 343 & HANFRD TS-4 HRM 28.7 & & \\
\hline
\end{tabular}




\begin{tabular}{|c|c|c|c|c|c|c|c|c|c|}
\hline & $\mathrm{T}$ & $\bar{U}$ & $\mathrm{~V}$ & $\mathrm{~W}$ & $\mathrm{X}$ & $\mathrm{Y}$ & $Z$ & $\mathrm{AA}$ & $\mathrm{AB}$ \\
\hline \begin{tabular}{l|l}
1 & 1 \\
\end{tabular} & REVIEW_QUALIFIER & SAMP_FROM & SAMP_ITEM & SAMP_MTHD & COLL_MTHD & SAMP_SITE_ID & SAMP_SITE_NAME & WELL_NAME & SAMPLE_LOCATION \\
\hline 2206 & & RIVER & & RAW GRAB & TRANSECT & 344 & ANFRD TS-5 HRM 28.7 & & \\
\hline 2207 & & RIVER & & RAW GRAB & TRANSECT & 344 & ANFRD TS-5 HRM 28.7 & & \\
\hline 2208 & & RIVER & & RAW GRAB & TRANSECT & 344 & ANFRD TS-5 HRM 28.7 & & \\
\hline 2209 & & RIVER & & RAW GRAB & TRANSECT & 344 & ANFRD TS-5 HRM 28.7 & & \\
\hline $2210 \mathrm{~F}$ & $\mathrm{~F}$ & RIVER & & RAW GRAB & TRANSECT & 344 & ANFRD TS-5 HRM 28.7 & & \\
\hline 2211 & & RIVER & & RAW GRAB & TRANSECT & 344 & ANFRD TS-5 HRM 28.7 & & \\
\hline 2212 & & RIVER & & RAW GRAB & TRANSECT & 344 & ANFRD TS-5 HRM 28.7 & & \\
\hline 2213 & & RIVER & & RAW GRAB & TRANSECT & 344 & ANFRD TS-5 HRM 28.7 & & \\
\hline 2214 & & RIVER & & RAW GRAB & TRANSECT & 345 & ANFRD TS-6 HRM 28.7 & & \\
\hline 2215 & & RIVER & & RAW GRAB & TRANSECT & 345 & ANFRD TS-6 HRM 28.7 & & \\
\hline 2216 & & RIVER & & RAW GRAB & TRANSECT & 345 & ANFRD TS-6 HRM 28.7 & & \\
\hline 2217 & & RIVER & & RAW GRAB & TRANSECT & 345 & ANFRD TS-6 HRM 28.7 & & \\
\hline $2218 \mathrm{~F}$ & $\mathrm{~F}$ & RIVER & & RAW GRAB & TRANSECT & 345 & ANFRD TS-6 HRM 28.7 & & \\
\hline 2219 & & RIVER & & RAW GRAB & TRANSECT & 345 & ANFRD TS-6 HRM 28.7 & & \\
\hline 2220 & & RIVER & & RAW GRAB & TRANSECT & 345 & ANFRD TS-6 HRM 28.7 & & \\
\hline 2221 & & RIVER & & RAW GRAB & TRANSECT & 345 & ANFRD TS-6 HRM 28.7 & & \\
\hline 2222 & & RIVER & & RAW GRAB & TRANSECT & 469 & ANFRD TS-7 HRM 28.7 & & \\
\hline 2223 & & RIVER & & RAW GRAB & TRANSECT & 469 & ANFRD TS-7 HRM 28.7 & & \\
\hline 2224 & & RIVER & & RAW GRAB & TRANSECT & 469 & ANFRD TS-7 HRM 28.7 & & \\
\hline 2225 & & RIVER & & RAW GRAB & TRANSECT & 469 & ANFRD TS-7 HRM 28.7 & & \\
\hline $2226 \mathrm{~F}$ & $\mathrm{~F}$ & RIVER & & RAW GRAB & TRANSECT & 469 & ANFRD TS-7 HRM 28.7 & & \\
\hline 2227 & & RIVER & & RAW GRAB & TRANSECT & 469 & ANFRD TS-7 HRM 28.7 & & \\
\hline 2228 & & RIVER & & RAW GRAB & TRANSECT & 469 & ANFRD TS-7 HRM 28.7 & & \\
\hline 2229 & & RIVER & & RAW GRAB & TRANSECT & 469 & ANFRD TS-7 HRM 28.7 & & \\
\hline 2230 & & RIVER & & RAW GRAB & TRANSECT & 579 & ANFRD TS-8 HRM 28.7 & & \\
\hline 2231 & & RIVER & & RAW GRAB & TRANSECT & 579 & ANFRD TS-8 HRM 28.7 & & \\
\hline 2232 & & RIVER & & RAW GRAB & TRANSECT & 579 & ANFRD TS-8 HRM 28.7 & & \\
\hline 2233 & & RIVER & & RAW GRAB & TRANSECT & 579 & ANFRD TS-8 HRM 28.7 & & \\
\hline $2234 \mathrm{~F}$ & $\mathrm{~F}$ & RIVER & & RAW GRAB & TRANSECT & 579 & ANFRD TS-8 HRM 28.7 & & \\
\hline 2235 & & RIVER & & RAW GRAB & TRANSECT & 579 & IANFRD TS-8 HRM 28.7 & & \\
\hline 2236 & & RIVER & & RAW GRAB & TRANSECT & 579 & ANFRD TS-8 HRM 28.7 & & \\
\hline 2237 & & RIVER & & RAW GRAB & TRANSECT & 579 & IANFRD TS-8 HRM 28.7 & & \\
\hline 2238 & & RIVER & & RAW GRAB & TRANSECT & 346 & IANFRD TS-9 HRM 28.7 & & \\
\hline 2239 & & RIVER & & RAW GRAB & TRANSECT & 346 & ANFRD TS-9 HRM 28.7 & & \\
\hline 2240 & & RIVER & & RAW GRAB & TRANSECT & 346 & IANFRD TS-9 HRM 28.7 & & \\
\hline 2241 & & RIVER & & RAW GRAB & TRANSECT & 346 & IANFRD TS-9 HRM 28.7 & & \\
\hline $2242 \mathrm{~F}$ & $\mathrm{~F}$ & RIVER & & RAW GRAB & TRANSECT & 346 & ANFRD TS-9 HRM 28.7 & & \\
\hline 2243 & & RIVER & & RAW GRAB & TRANSECT & 346 & IANFRD TS-9 HRM 28.7 & & \\
\hline
\end{tabular}




\begin{tabular}{|c|c|c|c|c|c|c|c|c|c|}
\hline & $\mathrm{T}$ & $\bar{U}$ & $\mathrm{~V}$ & $\mathrm{~W}$ & $\mathrm{X}$ & $\mathrm{Y}$ & $Z$ & $\mathrm{AA}$ & $\mathrm{AB}$ \\
\hline \begin{tabular}{l|l}
1 & 1 \\
\end{tabular} & REVIEW_QUALIFIER & SAMP_FROM & SAMP_ITEM & SAMP_MTHD & COLL_MTHD & SAMP_SITE_ID & SAMP_SITE_NAME & WELL_NAME & SAMPLE_LOCATION \\
\hline 2244 & & RIVER & & RAW GRAB & TRANSECT & 346 & HANFRD TS-9 HRM 28.7 & & \\
\hline 2245 & & RIVER & & RAW GRAB & TRANSECT & 346 & HANFRD TS-9 HRM 28.7 & & \\
\hline 2246 & & RIVER & & RAW GRAB & TRANSECT & 3483 & 300 AREA -1 HRM 43.1 & & \\
\hline 2247 & & RIVER & & RAW GRAB & TRANSECT & 3483 & 300 AREA -1 HRM 43.1 & & \\
\hline 2248 & & RIVER & & RAW GRAB & TRANSECT & 3483 & 300 AREA -1 HRM 43.1 & & \\
\hline 2249 & & RIVER & & RAW GRAB & TRANSECT & 3483 & 300 AREA -1 HRM 43.1 & & \\
\hline 2250 & & RIVER & & RAW GRAB & TRANSECT & 348 & 300 AREA -1 HRM 43.1 & & \\
\hline 2251 & & RIVER & & RAW GRAB & TRANSECT & 3483 & 300 AREA -1 HRM 43.1 & & \\
\hline 2252 & & RIVER & & RAW GRAB & TRANSECT & 348 & 300 AREA -1 HRM 43.1 & & \\
\hline 2253 & & RIVER & & RAW GRAB & TRANSECT & 4703 & 300 AREA -2 HRM 43.1 & & \\
\hline 2254 & & RIVER & & RAW GRAB & TRANSECT & 4703 & 300 AREA -2 HRM 43.1 & & \\
\hline 2255 & & RIVER & & RAW GRAB & TRANSECT & 4703 & 300 AREA -2 HRM 43.1 & & \\
\hline 2256 & & RIVER & & RAW GRAB & TRANSECT & 470 & 300 AREA -2 HRM 43.1 & & \\
\hline 2257 & & RIVER & & RAW GRAB & TRANSECT & 4703 & 300 AREA -2 HRM 43.1 & & \\
\hline 2258 & & RIVER & & RAW GRAB & TRANSECT & 4703 & 300 AREA -2 HRM 43.1 & & \\
\hline 2259 & & RIVER & & RAW GRAB & TRANSECT & 4703 & 300 AREA -2 HRM 43.1 & & \\
\hline 2260 & & RIVER & & RAW GRAB & TRANSECT & 5803 & 300 AREA -3 HRM 43.1 & & \\
\hline 2261 & & RIVER & & RAW GRAB & TRANSECT & 5803 & 300 AREA -3 HRM 43.1 & & \\
\hline 2262 & & RIVER & & RAW GRAB & TRANSECT & 5803 & 300 AREA -3 HRM 43.1 & & \\
\hline $2263 \mathrm{~F}$ & $\mathrm{~F}$ & RIVER & & RAW GRAB & TRANSECT & 5803 & 300 AREA -3 HRM 43.1 & & \\
\hline 2264 & & RIVER & & RAW GRAB & TRANSECT & 5803 & 300 AREA -3 HRM 43.1 & & \\
\hline 2265 & & RIVER & & RAW GRAB & TRANSECT & 5803 & 300 AREA -3 HRM 43.1 & & \\
\hline 2266 & & RIVER & & RAW GRAB & TRANSECT & 580 & 300 AREA -3 HRM 43.1 & & \\
\hline 2267 & & RIVER & & RAW GRAB & TRANSECT & 3493 & 300 AREA -4 HRM 43.1 & & \\
\hline 2268 & & RIVER & & RAW GRAB & TRANSECT & 3493 & 300 AREA -4 HRM 43.1 & & \\
\hline 2269 & & RIVER & & RAW GRAB & TRANSECT & 349 & 300 AREA -4 HRM 43.1 & & \\
\hline $2270 \mathrm{~F}$ & $\mathrm{~F}$ & RIVER & & RAW GRAB & TRANSECT & 349 & 300 AREA -4 HRM 43.1 & & \\
\hline 2271 & & RIVER & & RAW GRAB & TRANSECT & 3493 & 300 AREA -4 HRM 43.1 & & \\
\hline 2272 & & RIVER & & RAW GRAB & TRANSECT & 3493 & 300 AREA -4 HRM 43.1 & & \\
\hline 2273 & & RIVER & & RAW GRAB & TRANSECT & 349 & 300 AREA -4 HRM 43.1 & & \\
\hline 2274 & & RIVER & & RAW GRAB & TRANSECT & 6073 & 300 AREA -5 HRM 43.1 & & \\
\hline 2275 & & RIVER & & RAW GRAB & TRANSECT & 607 & 300 AREA -5 HRM 43.1 & & \\
\hline 2276 & & RIVER & & RAW GRAB & TRANSECT & 607 & 300 AREA -5 HRM 43.1 & & \\
\hline $2277 \mathrm{~F}$ & $\mathrm{~F}$ & RIVER & & RAW GRAB & TRANSECT & 6073 & 300 AREA -5 HRM 43.1 & & \\
\hline 2278 & & RIVER & & RAW GRAB & TRANSECT & 6073 & 300 AREA -5 HRM 43.1 & & \\
\hline 2279 & & RIVER & & RAW GRAB & TRANSECT & 607 & 300 AREA -5 HRM 43.1 & & \\
\hline 2280 & & RIVER & & RAW GRAB & TRANSECT & 6073 & 300 AREA -5 HRM 43.1 & & \\
\hline 2281 & & RIVER & & RAW GRAB & TRANSECT & $350 ?$ & 300 AREA -6 HRM 43.1 & & \\
\hline
\end{tabular}




\begin{tabular}{|c|c|c|c|c|c|c|c|c|c|}
\hline & $\mathrm{T}$ & $\bar{U}$ & $\mathrm{~V}$ & $\mathrm{~W}$ & $\mathrm{X}$ & $\mathrm{Y}$ & $Z$ & $\mathrm{AA}$ & $\mathrm{AB}$ \\
\hline \begin{tabular}{l|l}
1 & 1 \\
\end{tabular} & REVIEW_QUALIFIER & SAMP_FROM & SAMP_ITEM & SAMP_MTHD & COLL_MTHD & SAMP_SITE_ID & SAMP_SITE_NAME & WELL_NAME & SAMPLE_LOCATION \\
\hline 2282 & & RIVER & & RAW GRAB & TRANSECT & 3503 & 300 AREA -6 HRM 43.1 & & \\
\hline 2283 & & RIVER & & RAW GRAB & TRANSECT & 3503 & 300 AREA -6 HRM 43.1 & & \\
\hline $2284 \mathrm{~F}$ & $\mathrm{~F}$ & RIVER & & RAW GRAB & TRANSECT & 3503 & 300 AREA -6 HRM 43.1 & & \\
\hline 2285 & & RIVER & & RAW GRAB & TRANSECT & 3503 & 300 AREA -6 HRM 43.1 & & \\
\hline 2286 & & RIVER & & RAW GRAB & TRANSECT & 3503 & 300 AREA -6 HRM 43.1 & & \\
\hline 2287 & & RIVER & & RAW GRAB & TRANSECT & 350 & 300 AREA -6 HRM 43.1 & & \\
\hline 2288 & & RIVER & & RAW GRAB & TRANSECT & 351 & 300 AREA -7 HRM 43.1 & & \\
\hline 2289 & & RIVER & & RAW GRAB & TRANSECT & 3513 & 300 AREA -7 HRM 43.1 & & \\
\hline 2290 & & RIVER & & RAW GRAB & TRANSECT & 351 & 300 AREA -7 HRM 43.1 & & \\
\hline $2291 \mathrm{~F}$ & $\mathrm{~F}$ & RIVER & & RAW GRAB & TRANSECT & 3513 & 300 AREA -7 HRM 43.1 & & \\
\hline 2292 & & RIVER & & RAW GRAB & TRANSECT & 3513 & 300 AREA -7 HRM 43.1 & & \\
\hline 2293 & & RIVER & & RAW GRAB & TRANSECT & 3513 & 300 AREA -7 HRM 43.1 & & \\
\hline 2294 & & RIVER & & RAW GRAB & TRANSECT & 351 & 300 AREA -7 HRM 43.1 & & \\
\hline 2295 & & RIVER & & RAW GRAB & TRANSECT & 5813 & 300 AREA -8 HRM 43.1 & & \\
\hline 2296 & & RIVER & & RAW GRAB & TRANSECT & 5813 & 300 AREA -8 HRM 43.1 & & \\
\hline 2297 & & RIVER & & RAW GRAB & TRANSECT & 5813 & 300 AREA -8 HRM 43.1 & & \\
\hline $2298 \mathrm{~F}$ & $\mathrm{~F}$ & RIVER & & RAW GRAB & TRANSECT & 581 & 300 AREA -8 HRM 43.1 & & \\
\hline 2299 & & RIVER & & RAW GRAB & TRANSECT & 5813 & 300 AREA -8 HRM 43.1 & & \\
\hline 2300 & & RIVER & & RAW GRAB & TRANSECT & 5813 & 300 AREA -8 HRM 43.1 & & \\
\hline 2301 & & RIVER & & RAW GRAB & TRANSECT & 581 & 300 AREA -8 HRM 43.1 & & \\
\hline 2302 & & RIVER & & RAW GRAB & TRANSECT & 4713 & 300 AREA -9 HRM 43.1 & & \\
\hline 2303 & & RIVER & & RAW GRAB & TRANSECT & 4713 & 300 AREA -9 HRM 43.1 & & \\
\hline 2304 & & RIVER & & RAW GRAB & TRANSECT & 4713 & 300 AREA -9 HRM 43.1 & & \\
\hline $2305 \mathrm{~F}$ & $\mathrm{~F}$ & RIVER & & RAW GRAB & TRANSECT & 4713 & 300 AREA -9 HRM 43.1 & & \\
\hline 2306 & & RIVER & & RAW GRAB & TRANSECT & 4713 & 300 AREA -9 HRM 43.1 & & \\
\hline 2307 & & RIVER & & RAW GRAB & TRANSECT & 4713 & 300 AREA -9 HRM 43.1 & & \\
\hline 2308 & & RIVER & & RAW GRAB & TRANSECT & 4713 & 300 AREA -9 HRM 43.1 & & \\
\hline 2309 & & RIVER & & RAW GRAB & TRANSECT & 3523 & 300 AREA-10 HRM 43.1 & & \\
\hline 2310 & & RIVER & & RAW GRAB & TRANSECT & 3523 & 300 AREA-10 HRM 43.1 & & \\
\hline 2311 & & RIVER & & RAW GRAB & TRANSECT & 352 & 300 AREA-10 HRM 43.1 & & \\
\hline $2312 \mathrm{~F}$ & $\mathrm{~F}$ & RIVER & & RAW GRAB & TRANSECT & 3523 & 300 AREA-10 HRM 43.1 & & \\
\hline 2313 & & RIVER & & RAW GRAB & TRANSECT & 3523 & 300 AREA-10 HRM 43.1 & & \\
\hline 2314 & & RIVER & & RAW GRAB & TRANSECT & 3523 & 300 AREA-10 HRM 43.1 & & \\
\hline 2315 & & RIVER & & RAW GRAB & TRANSECT & 3523 & 300 AREA-10 HRM 43.1 & & \\
\hline 2316 & & RIVER & & RAW GRAB & TRANSECT & 571 & RICH.PMPHS-1 HRM46.4 & & \\
\hline 2317 & & RIVER & & RAW GRAB & TRANSECT & 571 & RICH.PMPHS-1 HRM46.4 & & \\
\hline 2318 & & RIVER & & RAW GRAB & TRANSECT & 571 & RICH.PMPHS-1 HRM46.4 & & \\
\hline 2319 & & RIVER & & RAW GRAB & TRANSECT & 571 & RICH.PMPHS-1 HRM46.4 & & \\
\hline
\end{tabular}




\begin{tabular}{|c|c|c|c|c|c|c|c|c|c|}
\hline & $\mathrm{T}$ & $\bar{U}$ & $\mathrm{~V}$ & $\mathrm{~W}$ & $\mathrm{X}$ & $\mathrm{Y}$ & $Z$ & $\mathrm{AA}$ & $\mathrm{AB}$ \\
\hline \begin{tabular}{l|l}
1 & 1 \\
\end{tabular} & REVIEW_QUALIFIER & SAMP_FROM & SAMP_ITEM & SAMP_MTHD & COLL_MTHD & SAMP_SITE_ID & SAMP_SITE_NAME & WELL_NAME & SAMPLE_LOCATION \\
\hline 2320 & & RIVER & & RAW GRAB & TRANSECT & 571 & RICH.PMPHS-1 HRM46.4 & & \\
\hline 2321 & & RIVER & & RAW GRAB & TRANSECT & 571 & RICH.PMPHS-1 HRM46.4 & & \\
\hline 2322 & & RIVER & & RAW GRAB & TRANSECT & 571 & RICH.PMPHS-1 HRM46.4 & & \\
\hline 2323 & & RIVER & & RAW GRAB & TRANSECT & 327 & RICH.PMPHS-10 HRM46.4 & & \\
\hline 2324 & & RIVER & & RAW GRAB & TRANSECT & 327 & RICH.PMPHS-10 HRM46.4 & & \\
\hline 2325 & & RIVER & & RAW GRAB & TRANSECT & 327 & RICH.PMPHS-10 HRM46.4 & & \\
\hline $2326 \mathrm{~F}$ & $\mathrm{~F}$ & RIVER & & RAW GRAB & TRANSECT & 327 & RICH.PMPHS-10 HRM46.4 & & \\
\hline 2327 & & RIVER & & RAW GRAB & TRANSECT & 327 & RICH.PMPHS-10 HRM46.4 & & \\
\hline 2328 & & RIVER & & RAW GRAB & TRANSECT & 327 & RICH.PMPHS-10 HRM46.4 & & \\
\hline 2329 & & RIVER & & RAW GRAB & TRANSECT & 327 & RICH.PMPHS-10 HRM46.4 & & \\
\hline 2330 & & RIVER & & RAW GRAB & TRANSECT & 321 & RICH.PMPHS-2 HRM46.4 & & \\
\hline 2331 & & RIVER & & RAW GRAB & TRANSECT & 321 & RICH.PMPHS-2 HRM46.4 & & \\
\hline 2332 & & RIVER & & RAW GRAB & TRANSECT & 321 & RICH.PMPHS-2 HRM46.4 & & \\
\hline 2333 & & RIVER & & RAW GRAB & TRANSECT & 321 & RICH.PMPHS-2 HRM46.4 & & \\
\hline 2334 & & RIVER & & RAW GRAB & TRANSECT & 321 & RICH.PMPHS-2 HRM46.4 & & \\
\hline 2335 & & RIVER & & RAW GRAB & TRANSECT & 321 & RICH.PMPHS-2 HRM46.4 & & \\
\hline 2336 & & RIVER & & RAW GRAB & TRANSECT & 321 & RICH.PMPHS-2 HRM46.4 & & \\
\hline 2337 & & RIVER & & RAW GRAB & TRANSECT & 322 & RICH.PMPHS-3 HRM46.4 & & \\
\hline 2338 & & RIVER & & RAW GRAB & TRANSECT & 322 & RICH.PMPHS-3 HRM46.4 & & \\
\hline 2339 & & RIVER & & RAW GRAB & TRANSECT & 322 & RICH.PMPHS-3 HRM46.4 & & \\
\hline 2340 & & RIVER & & RAW GRAB & TRANSECT & 322 & RICH.PMPHS-3 HRM46.4 & & \\
\hline 2341 & & RIVER & & RAW GRAB & TRANSECT & 322 & RICH.PMPHS-3 HRM46.4 & & \\
\hline 2342 & & RIVER & & RAW GRAB & TRANSECT & 322 & RICH.PMPHS-3 HRM46.4 & & \\
\hline 2343 & & RIVER & & RAW GRAB & TRANSECT & 322 & RICH.PMPHS-3 HRM46.4 & & \\
\hline 2344 & & RIVER & & RAW GRAB & TRANSECT & 572 & RICH.PMPHS-4 HRM46.4 & & \\
\hline 2345 & & RIVER & & RAW GRAB & TRANSECT & 572 & RICH.PMPHS-4 HRM46.4 & & \\
\hline 2346 & & RIVER & & RAW GRAB & TRANSECT & 572 & RICH.PMPHS-4 HRM46.4 & & \\
\hline $2347 \mathrm{~F}$ & $\mathrm{~F}$ & RIVER & & RAW GRAB & TRANSECT & 572 & RICH.PMPHS-4 HRM46.4 & & \\
\hline 2348 & & RIVER & & RAW GRAB & TRANSECT & 572 & RICH.PMPHS-4 HRM46.4 & & \\
\hline 2349 & & RIVER & & RAW GRAB & TRANSECT & 572 & RICH.PMPHS-4 HRM46.4 & & \\
\hline 2350 & & RIVER & & RAW GRAB & TRANSECT & 572 & RICH.PMPHS-4 HRM46.4 & & \\
\hline 2351 & & RIVER & & RAW GRAB & TRANSECT & 323 & RICH.PMPHS-5 HRM46.4 & & \\
\hline 2352 & & RIVER & & RAW GRAB & TRANSECT & 323 & RICH.PMPHS-5 HRM46.4 & & \\
\hline 2353 & & RIVER & & RAW GRAB & TRANSECT & 323 & RICH.PMPHS-5 HRM46.4 & & \\
\hline 2354 & & RIVER & & RAW GRAB & TRANSECT & 323 & RICH.PMPHS-5 HRM46.4 & & \\
\hline 2355 & & RIVER & & RAW GRAB & TRANSECT & 323 & RICH.PMPHS-5 HRM46.4 & & \\
\hline 2356 & & RIVER & & RAW GRAB & TRANSECT & 323 & RICH.PMPHS-5 HRM46.4 & & \\
\hline 2357 & & RIVER & & RAW GRAB & TRANSECT & 323 & RICH.PMPHS-5 HRM46.4 & & \\
\hline
\end{tabular}




\begin{tabular}{|c|c|c|c|c|c|c|c|c|c|}
\hline & $\mathrm{T}$ & $\bar{U}$ & $\mathrm{~V}$ & $\mathrm{~W}$ & $\mathrm{X}$ & $\mathrm{Y}$ & $Z$ & $\mathrm{AA}$ & $\mathrm{AB}$ \\
\hline \begin{tabular}{l|l}
1 & 1 \\
\end{tabular} & REVIEW_QUALIFIER & SAMP_FROM & SAMP_ITEM & SAMP_MTHD & COLL_MTHD & SAMP_SITE_ID & SAMP_SITE_NAME & WELL_NAME & SAMPLE_LOCATION \\
\hline 2358 & & RIVER & & RAW GRAB & TRANSECT & 324 & RICH.PMPHS-6 HRM46.4 & & \\
\hline 2359 & & RIVER & & RAW GRAB & TRANSECT & 324 & RICH.PMPHS-6 HRM46.4 & & \\
\hline 2360 & & RIVER & & RAW GRAB & TRANSECT & 324 & RICH.PMPHS-6 HRM46.4 & & \\
\hline 2361 & & RIVER & & RAW GRAB & TRANSECT & 324 & RICH.PMPHS-6 HRM46.4 & & \\
\hline 2362 & & RIVER & & RAW GRAB & TRANSECT & 324 & RICH.PMPHS-6 HRM46.4 & & \\
\hline 2363 & & RIVER & & RAW GRAB & TRANSECT & 324 & RICH.PMPHS-6 HRM46.4 & & \\
\hline 2364 & & RIVER & & RAW GRAB & TRANSECT & 324 & RICH.PMPHS-6 HRM46.4 & & \\
\hline 2365 & & RIVER & & RAW GRAB & TRANSECT & 325 & RICH.PMPHS-7 HRM46.4 & & \\
\hline 2366 & & RIVER & & RAW GRAB & TRANSECT & 325 & RICH.PMPHS-7 HRM46.4 & & \\
\hline 2367 & & RIVER & & RAW GRAB & TRANSECT & 325 & RICH.PMPHS-7 HRM46.4 & & \\
\hline 2368 & & RIVER & & RAW GRAB & TRANSECT & 325 & RICH.PMPHS-7 HRM46.4 & & \\
\hline 2369 & & RIVER & & RAW GRAB & TRANSECT & 325 & RICH.PMPHS-7 HRM46.4 & & \\
\hline 2370 & & RIVER & & RAW GRAB & TRANSECT & 325 & RICH.PMPHS-7 HRM46.4 & & \\
\hline 2371 & & RIVER & & RAW GRAB & TRANSECT & 325 & RICH.PMPHS-7 HRM46.4 & & \\
\hline 2372 & & RIVER & & RAW GRAB & TRANSECT & 326 & RICH.PMPHS-8 HRM46.4 & & \\
\hline 2373 & & RIVER & & RAW GRAB & TRANSECT & 326 & RICH.PMPHS-8 HRM46.4 & & \\
\hline 2374 & & RIVER & & RAW GRAB & TRANSECT & 326 & RICH.PMPHS-8 HRM46.4 & & \\
\hline 2375 & & RIVER & & RAW GRAB & TRANSECT & 326 & RICH.PMPHS-8 HRM46.4 & & \\
\hline 2376 & & RIVER & & RAW GRAB & TRANSECT & 326 & RICH.PMPHS-8 HRM46.4 & & \\
\hline 2377 & & RIVER & & RAW GRAB & TRANSECT & 326 & RICH.PMPHS-8 HRM46.4 & & \\
\hline 2378 & & RIVER & & RAW GRAB & TRANSECT & 326 & RICH.PMPHS-8 HRM46.4 & & \\
\hline 2379 & & RIVER & & RAW GRAB & TRANSECT & 573 & RICH.PMPHS-9 HRM46.4 & & \\
\hline 2380 & & RIVER & & RAW GRAB & TRANSECT & 573 & RICH.PMPHS-9 HRM46.4 & & \\
\hline 2381 & & RIVER & & RAW GRAB & TRANSECT & 573 & RICH.PMPHS-9 HRM46.4 & & \\
\hline $2382 \mathrm{~F}$ & $\mathrm{~F}$ & RIVER & & RAW GRAB & TRANSECT & 573 & RICH.PMPHS-9 HRM46.4 & & \\
\hline 2383 & & RIVER & & RAW GRAB & TRANSECT & 573 & RICH.PMPHS-9 HRM46.4 & & \\
\hline 2384 & & RIVER & & RAW GRAB & TRANSECT & 573 & RICH.PMPHS-9 HRM46.4 & & \\
\hline 2385 & & RIVER & & RAW GRAB & TRANSECT & 573 & RICH.PMPHS-9 HRM46.4 & & \\
\hline 2386 & & RIVER & & RAW GRAB & TRANSECT & 571 & RICH.PMPHS-1 HRM46.4 & & \\
\hline 2387 & & RIVER & & RAW GRAB & TRANSECT & 571 & RICH.PMPHS-1 HRM46.4 & & \\
\hline $2388 \mathrm{~F}$ & $\mathrm{~F}$ & RIVER & & RAW GRAB & TRANSECT & 571 & RICH.PMPHS-1 HRM46.4 & & \\
\hline $2389 \mathrm{~F}$ & $\mathrm{~F}$ & RIVER & & RAW GRAB & TRANSECT & 571 & RICH.PMPHS-1 HRM46.4 & & \\
\hline 2390 & & RIVER & & RAW GRAB & TRANSECT & 571 & RICH.PMPHS-1 HRM46.4 & & \\
\hline 2391 & & RIVER & & RAW GRAB & TRANSECT & 571 & RICH.PMPHS-1 HRM46.4 & & \\
\hline 2392 & & RIVER & & RAW GRAB & TRANSECT & 571 & RICH.PMPHS-1 HRM46.4 & & \\
\hline 2393 & & RIVER & & RAW GRAB & TRANSECT & 327 & RICH.PMPHS-10 HRM46.4 & & \\
\hline 2394 & & RIVER & & RAW GRAB & TRANSECT & 327 & RICH.PMPHS-10 HRM46.4 & & \\
\hline 2395 & & RIVER & & RAW GRAB & TRANSECT & 327 & RICH.PMPHS-10 HRM46.4 & & \\
\hline
\end{tabular}




\begin{tabular}{|c|c|c|c|c|c|c|c|c|c|}
\hline & $\mathrm{T}$ & $\mathrm{U}$ & $\mathrm{V}$ & $\mathrm{W}$ & $\mathrm{X}$ & $\bar{Y}$ & Z & AA & $A B$ \\
\hline 1 & REVIEW_QUALIFIER & SAMP_FROM & SAMP_ITEM & SAMP_MTHD & COLL_MTHD & SAMP_SITE_ID & SAMP_SITE_NAME & WELL_NAME & SAMPLE_LOCATION \\
\hline 2396 & $\mathrm{~F}$ & RIVER & & RAW GRAB & TRANSECT & 327 & RICH.PMPHS-10 HRM46.4 & & \\
\hline 2397 & & RIVER & & RAW GRAB & TRANSECT & 327 & RICH.PMPHS-10 HRM46.4 & & \\
\hline 2398 & & RIVER & & RAW GRAB & TRANSECT & 327 & RICH.PMPHS-10 HRM46.4 & & \\
\hline 2399 & & RIVER & & RAW GRAB & TRANSECT & 327 & RICH.PMPHS-10 HRM46.4 & & \\
\hline 2400 & & RIVER & & RAW GRAB & TRANSECT & 321 & RICH.PMPHS-2 HRM46.4 & & \\
\hline 2401 & & RIVER & & RAW GRAB & TRANSECT & 321 & RICH.PMPHS-2 HRM46.4 & & \\
\hline 2402 & $F$ & RIVER & & RAW GRAB & TRANSECT & 321 & RICH.PMPHS-2 HRM46.4 & & \\
\hline 2403 & $F$ & RIVER & & RAW GRAB & TRANSECT & 321 & RICH.PMPHS-2 HRM46.4 & & \\
\hline 2404 & & RIVER & & RAW GRAB & TRANSECT & 321 & RICH.PMPHS-2 HRM46.4 & & \\
\hline 2405 & & RIVER & & RAW GRAB & TRANSECT & 321 & RICH.PMPHS-2 HRM46.4 & & \\
\hline 2406 & & RIVER & & RAW GRAB & TRANSECT & 321 & RICH.PMPHS-2 HRM46.4 & & \\
\hline 2407 & & RIVER & & RAW GRAB & TRANSECT & 322 & RICH.PMPHS-3 HRM46.4 & & \\
\hline 2408 & & RIVER & & RAW GRAB & TRANSECT & 322 & RICH.PMPHS-3 HRM46.4 & & \\
\hline 2409 & & RIVER & & RAW GRAB & TRANSECT & 322 & RICH.PMPHS-3 HRM46.4 & & \\
\hline 2410 & $F$ & RIVER & & RAW GRAB & TRANSECT & 322 & RICH.PMPHS-3 HRM46.4 & & \\
\hline 2411 & & RIVER & & RAW GRAB & TRANSECT & 322 & RICH.PMPHS-3 HRM46.4 & & \\
\hline 2412 & & RIVER & & RAW GRAB & TRANSECT & 322 & RICH.PMPHS-3 HRM46.4 & & \\
\hline 2413 & & RIVER & & RAW GRAB & TRANSECT & 322 & RICH.PMPHS-3 HRM46.4 & & \\
\hline 2414 & & RIVER & & RAW GRAB & TRANSECT & 572 & RICH.PMPHS-4 HRM46.4 & & \\
\hline 2415 & & RIVER & & RAW GRAB & TRANSECT & 572 & RICH.PMPHS-4 HRM46.4 & & \\
\hline 2416 & & RIVER & & RAW GRAB & TRANSECT & 572 & RICH.PMPHS-4 HRM46.4 & & \\
\hline 2417 & $F$ & RIVER & & RAW GRAB & TRANSECT & 572 & RICH.PMPHS-4 HRM46.4 & & \\
\hline 2418 & & RIVER & & RAW GRAB & TRANSECT & 572 & RICH.PMPHS-4 HRM46.4 & & \\
\hline 2419 & & RIVER & & RAW GRAB & TRANSECT & 572 & RICH.PMPHS-4 HRM46.4 & & \\
\hline 2420 & & RIVER & & RAW GRAB & TRANSECT & 572 & RICH.PMPHS-4 HRM46.4 & & \\
\hline 2421 & & RIVER & & RAW GRAB & TRANSECT & 323 & RICH.PMPHS-5 HRM46.4 & & \\
\hline 2422 & & RIVER & & RAW GRAB & TRANSECT & 323 & RICH.PMPHS-5 HRM46.4 & & \\
\hline 2423 & $F$ & RIVER & & RAW GRAB & TRANSECT & 323 & RICH.PMPHS-5 HRM46.4 & & \\
\hline 2424 & $F$ & RIVER & & RAW GRAB & TRANSECT & 323 & RICH.PMPHS-5 HRM46.4 & & \\
\hline 2425 & & RIVER & & RAW GRAB & TRANSECT & 323 & RICH.PMPHS-5 HRM46.4 & & \\
\hline 2426 & & RIVER & & RAW GRAB & TRANSECT & 323 & RICH.PMPHS-5 HRM46.4 & & \\
\hline 2427 & & RIVER & & RAW GRAB & TRANSECT & 323 & RICH.PMPHS-5 HRM46.4 & & \\
\hline 2428 & & RIVER & & RAW GRAB & TRANSECT & 324 & RICH.PMPHS-6 HRM46.4 & & \\
\hline 2429 & & RIVER & & RAW GRAB & TRANSECT & 324 & RICH.PMPHS-6 HRM46.4 & & \\
\hline 2430 & & RIVER & & RAW GRAB & TRANSECT & 324 & RICH.PMPHS-6 HRM46.4 & & \\
\hline 2431 & $F$ & RIVER & & RAW GRAB & TRANSECT & 324 & RICH.PMPHS-6 HRM46.4 & & \\
\hline 2432 & & RIVER & & RAW GRAB & TRANSECT & 324 & RICH.PMPHS-6 HRM46.4 & & \\
\hline 2433 & & RIVER & & RAW GRAB & TRANSECT & 324 & RICH.PMPHS-6 HRM46.4 & & \\
\hline
\end{tabular}




\begin{tabular}{|c|c|c|c|c|c|c|c|c|c|}
\hline & $T$ & $\bar{U}$ & $\overline{\mathrm{V}}$ & $\mathrm{W}$ & $\bar{X}$ & $\bar{Y}$ & $\bar{Z}$ & $\overline{A A}$ & $A B$ \\
\hline 1 & REVIEW_QUALIFIER & SAMP_FROM & SAMP_ITEM & SAMP_MTHD & COLL_MTHD & SAMP_SITE_ID & SAMP_SITE_NAME & WELL_NAME & SAMPLE_LOCATION \\
\hline 2434 & & RIVER & & RAW GRAB & TRANSECT & 324 & RICH.PMPHS-6 HRM46.4 & & \\
\hline 2435 & & RIVER & & RAW GRAB & TRANSECT & 325 & RICH.PMPHS-7 HRM46.4 & & \\
\hline 2436 & & RIVER & & RAW GRAB & TRANSECT & $325 \mathrm{f}$ & RICH.PMPHS-7 HRM46.4 & & \\
\hline $2437 \mathrm{~F}$ & $\mathrm{~F}$ & RIVER & & RAW GRAB & TRANSECT & 325 & RICH.PMPHS-7 HRM46.4 & & \\
\hline $2438 \mathrm{~F}$ & $\mathrm{~F}$ & RIVER & & RAW GRAB & TRANSECT & 325 & RICH.PMPHS-7 HRM46.4 & & \\
\hline 2439 & & RIVER & & RAW GRAB & TRANSECT & 325 & RICH.PMPHS-7 HRM46.4 & & \\
\hline 2440 & & RIVER & & RAW GRAB & TRANSECT & 325 & RICH.PMPHS-7 HRM46.4 & & \\
\hline 2441 & & RIVER & & RAW GRAB & TRANSECT & 325 & RICH.PMPHS-7 HRM46.4 & & \\
\hline 2442 & & RIVER & & RAW GRAB & TRANSECT & 326 & RICH.PMPHS-8 HRM46.4 & & \\
\hline 2443 & & RIVER & & RAW GRAB & TRANSECT & 326 & RICH.PMPHS-8 HRM46.4 & & \\
\hline 2444 & & RIVER & & RAW GRAB & TRANSECT & 326 & RICH.PMPHS-8 HRM46.4 & & \\
\hline $2445 \mathrm{~F}$ & $\mathrm{~F}$ & RIVER & & RAW GRAB & TRANSECT & 326 & RICH.PMPHS-8 HRM46.4 & & \\
\hline 2446 & & RIVER & & RAW GRAB & TRANSECT & 326 & RICH.PMPHS-8 HRM46.4 & & \\
\hline 2447 & & RIVER & & RAW GRAB & TRANSECT & 326 & RICH.PMPHS-8 HRM46.4 & & \\
\hline 2448 & & RIVER & & RAW GRAB & TRANSECT & 326 & RICH.PMPHS-8 HRM46.4 & & \\
\hline 2449 & & RIVER & & RAW GRAB & TRANSECT & 573 & RICH.PMPHS-9 HRM46.4 & & \\
\hline 2450 & & RIVER & & RAW GRAB & TRANSECT & 573 & RICH.PMPHS-9 HRM46.4 & & \\
\hline 2451 & & RIVER & & RAW GRAB & TRANSECT & 573 & RICH.PMPHS-9 HRM46.4 & & \\
\hline $2452 \mathrm{~F}$ & $\mathrm{~F}$ & RIVER & & RAW GRAB & TRANSECT & 573 & RICH.PMPHS-9 HRM46.4 & & \\
\hline 2453 & & RIVER & & RAW GRAB & TRANSECT & 573 & RICH.PMPHS-9 HRM46.4 & & \\
\hline 2454 & & RIVER & & RAW GRAB & TRANSECT & 573 & RICH.PMPHS-9 HRM46.4 & & \\
\hline 2455 & & RIVER & & RAW GRAB & TRANSECT & 573 & RICH.PMPHS-9 HRM46.4 & & \\
\hline 2456 & & RIVER & & RAW GRAB & TRANSECT & 317 & VERNITA-1 HRM 0.3 & & \\
\hline 2457 & & RIVER & & RAW GRAB & TRANSECT & 317 & VERNITA-1 HRM 0.3 & & \\
\hline 2458 & & RIVER & & RAW GRAB & TRANSECT & 317 & VERNITA-1 HRM 0.3 & & \\
\hline $2459 \mathrm{~F}$ & $\mathrm{~F}$ & RIVER & & RAW GRAB & TRANSECT & 317 & VERNITA-1 HRM 0.3 & & \\
\hline 2460 & & RIVER & & RAW GRAB & TRANSECT & 317 & VERNITA-1 HRM 0.3 & & \\
\hline 2461 & & RIVER & & RAW GRAB & TRANSECT & 317 & VERNITA-1 HRM 0.3 & & \\
\hline 2462 & & RIVER & & RAW GRAB & TRANSECT & 317 & VERNITA-1 HRM 0.3 & & \\
\hline 2463 & & RIVER & & RAW GRAB & TRANSECT & 570 & VERNITA-2 HRM 0.3 & & \\
\hline 2464 & & RIVER & & RAW GRAB & TRANSECT & 570 & VERNITA-2 HRM 0.3 & & \\
\hline 2465 & & RIVER & & RAW GRAB & TRANSECT & 570 & VERNITA-2 HRM 0.3 & & \\
\hline $2466 \mathrm{~F}$ & $\mathrm{~F}$ & RIVER & & RAW GRAB & TRANSECT & 570 & VERNITA-2 HRM 0.3 & & \\
\hline 2467 & & RIVER & & RAW GRAB & TRANSECT & 570 & VERNITA-2 HRM 0.3 & & \\
\hline 2468 & & RIVER & & RAW GRAB & TRANSECT & 570 & VERNITA-2 HRM 0.3 & & \\
\hline 2469 & & RIVER & & RAW GRAB & TRANSECT & 570 & VERNITA-2 HRM 0.3 & & \\
\hline 2470 & & RIVER & & RAW GRAB & TRANSECT & 318 & VERNITA-3 HRM 0.3 & & \\
\hline 2471 & & RIVER & & RAW GRAB & TRANSECT & 318 & VERNITA-3 HRM 0.3 & & \\
\hline
\end{tabular}




\begin{tabular}{|c|c|c|c|c|c|c|c|c|c|}
\hline & $\mathrm{T}$ & 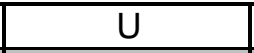 & $\mathrm{V}$ & $\mathrm{W}$ & $\mathrm{X}$ & $\mathrm{Y}$ & $Z$ & $\mathrm{AA}$ & $A B$ \\
\hline 1 & REVIEW_QUALIFIER & SAMP_FROM & SAMP_ITEM & SAMP_MTHD & COLL_MTHD & SAMP_SITE_ID & SAMP_SITE_NAME & WELL_NAME & SAMPLE_LOCATION \\
\hline 2472 & & RIVER & & RAW GRAB & TRANSECT & 318 & ERNITA-3 HRM 0.3 & & \\
\hline $2473 \mathrm{~F}$ & $\mathrm{~F}$ & RIVER & & RAW GRAB & TRANSECT & 318 & ERNITA-3 HRM 0.3 & & \\
\hline 2474 & & RIVER & & RAW GRAB & TRANSECT & 318 & ERNITA-3 HRM 0.3 & & \\
\hline 2475 & & RIVER & & RAW GRAB & TRANSECT & 318 & ERNITA-3 HRM 0.3 & & \\
\hline 2476 & & RIVER & & RAW GRAB & TRANSECT & 318 & ERNITA-3 HRM 0.3 & & \\
\hline 2477 & & RIVER & & RAW GRAB & TRANSECT & 319 & ERNITA-4 HRM 0.3 & & \\
\hline 2478 & & RIVER & & RAW GRAB & TRANSECT & 319 & ERNITA-4 HRM 0.3 & & \\
\hline 2479 & & RIVER & & RAW GRAB & TRANSECT & 319 & ERNITA-4 HRM 0.3 & & \\
\hline $2480 \mathrm{~F}$ & $\mathrm{~F}$ & RIVER & & RAW GRAB & TRANSECT & 319 & ERNITA-4 HRM 0.3 & & \\
\hline 2481 & & RIVER & & RAW GRAB & TRANSECT & 319 & ERNITA-4 HRM 0.3 & & \\
\hline 2482 & & RIVER & & RAW GRAB & TRANSECT & 319 & ERNITA-4 HRM 0.3 & & \\
\hline 2483 & & RIVER & & RAW GRAB & TRANSECT & 319 & ERNITA-4 HRM 0.3 & & \\
\hline 2484 & & RIVER & & RAW GRAB & TRANSECT & 317 & ERNITA-1 HRM 0.3 & & \\
\hline 2485 & & RIVER & & RAW GRAB & TRANSECT & 317 & ERNITA-1 HRM 0.3 & & \\
\hline 2486 & & RIVER & & RAW GRAB & TRANSECT & 317 & ERNITA-1 HRM 0.3 & & \\
\hline $2487 \mathrm{~F}$ & $\mathrm{~F}$ & RIVER & & RAW GRAB & TRANSECT & 317 & ERNITA-1 HRM 0.3 & & \\
\hline 2488 & & RIVER & & RAW GRAB & TRANSECT & 317 & ERNITA-1 HRM 0.3 & & \\
\hline 2489 & & RIVER & & RAW GRAB & TRANSECT & 317 & ERNITA-1 HRM 0.3 & & \\
\hline 2490 & & RIVER & & RAW GRAB & TRANSECT & 317 & ERNITA-1 HRM 0.3 & & \\
\hline 2491 & & RIVER & & RAW GRAB & TRANSECT & 570 & ERNITA-2 HRM 0.3 & & \\
\hline 2492 & & RIVER & & RAW GRAB & TRANSECT & 570 & ERNITA-2 HRM 0.3 & & \\
\hline 2493 & & RIVER & & RAW GRAB & TRANSECT & 570 & ERNITA-2 HRM 0.3 & & \\
\hline $2494 \mathrm{~F}$ & $\mathrm{~F}$ & RIVER & & RAW GRAB & TRANSECT & 570 & ERNITA-2 HRM 0.3 & & \\
\hline 2495 & & RIVER & & RAW GRAB & TRANSECT & 570 & ERNITA-2 HRM 0.3 & & \\
\hline 2496 & & RIVER & & RAW GRAB & TRANSECT & 570 & ERNITA-2 HRM 0.3 & & \\
\hline 2497 & & RIVER & & RAW GRAB & TRANSECT & 570 & ERNITA-2 HRM 0.3 & & \\
\hline 2498 & & RIVER & & RAW GRAB & TRANSECT & 318 & ERNITA-3 HRM 0.3 & & \\
\hline 2499 & & RIVER & & RAW GRAB & TRANSECT & 318 & ERNITA-3 HRM 0.3 & & \\
\hline 2500 & & RIVER & & RAW GRAB & TRANSECT & 318 & ERNITA-3 HRM 0.3 & & \\
\hline 2501 & $\mathrm{~F}$ & RIVER & & RAW GRAB & TRANSECT & 318 & ERNITA-3 HRM 0.3 & & \\
\hline 2502 & & RIVER & & RAW GRAB & TRANSECT & 318 & ERNITA-3 HRM 0.3 & & \\
\hline 2503 & & RIVER & & RAW GRAB & TRANSECT & 318 & ERNITA-3 HRM 0.3 & & \\
\hline 2504 & & RIVER & & RAW GRAB & TRANSECT & 318 & ERNITA-3 HRM 0.3 & & \\
\hline 2505 & & RIVER & & RAW GRAB & TRANSECT & 319 & ERNITA-4 HRM 0.3 & & \\
\hline 2506 & & RIVER & & RAW GRAB & TRANSECT & 319 & ERNITA-4 HRM 0.3 & & \\
\hline 2507 & & RIVER & & RAW GRAB & TRANSECT & 319 & ERNITA-4 HRM 0.3 & & \\
\hline $2508 \mathrm{~F}$ & $\mathrm{~F}$ & RIVER & & RAW GRAB & TRANSECT & 319 & ERNITA-4 HRM 0.3 & & \\
\hline 2509 & & RIVER & & RAW GRAB & TRANSECT & 319 & ERNITA-4 HRM 0.3 & & \\
\hline
\end{tabular}




\begin{tabular}{|c|c|c|c|c|c|c|c|c|c|}
\hline & $\mathrm{T}$ & $\bar{U}$ & $\mathrm{~V}$ & $\mathrm{~W}$ & $\mathrm{X}$ & $\mathrm{Y}$ & $Z$ & $\mathrm{AA}$ & $\mathrm{AB}$ \\
\hline 1 & REVIEW_QUALIFIER & SAMP_FROM & SAMP_ITEM & SAMP_MTHD & COLL_MTHD & SAMP_SITE_ID & SAMP_SITE_NAME & WELL_NAME & SAMPLE_LOCATION \\
\hline 2510 & & RIVER & & RAW GRAB & TRANSECT & 319 & VERNITA-4 HRM 0.3 & & \\
\hline 2511 & & RIVER & & RAW GRAB & TRANSECT & 319 & VERNITA-4 HRM 0.3 & & \\
\hline 2512 & & RIVER & & RAW GRAB & TRANSECT & 571 & RICH.PMPHS-1 HRM46.4 & & \\
\hline 2513 & & RIVER & & RAW GRAB & TRANSECT & 571 & RICH.PMPHS-1 HRM46.4 & & \\
\hline 2514 & & RIVER & & RAW GRAB & TRANSECT & 571 & RICH.PMPHS-1 HRM46.4 & & \\
\hline 2515 & & RIVER & & RAW GRAB & TRANSECT & 571 & RICH.PMPHS-1 HRM46.4 & & \\
\hline 2516 & & RIVER & & RAW GRAB & TRANSECT & 571 & RICH.PMPHS-1 HRM46.4 & & \\
\hline 2517 & & RIVER & & RAW GRAB & TRANSECT & 571 & RICH.PMPHS-1 HRM46.4 & & \\
\hline $2518 \mathrm{~F}$ & $\mathrm{~F}$ & RIVER & & RAW GRAB & TRANSECT & 571 & RICH.PMPHS-1 HRM46.4 & & \\
\hline 2519 & & RIVER & & RAW GRAB & TRANSECT & 571 & RICH.PMPHS-1 HRM46.4 & & \\
\hline 2520 & & RIVER & & RAW GRAB & TRANSECT & 571 & RICH.PMPHS-1 HRM46.4 & & \\
\hline 2521 & & RIVER & & RAW GRAB & TRANSECT & 571 & RICH.PMPHS-1 HRM46.4 & & \\
\hline 2522 & & RIVER & & RAW GRAB & TRANSECT & 571 & RICH.PMPHS-1 HRM46.4 & & \\
\hline 2523 & & RIVER & & RAW GRAB & TRANSECT & 571 & RICH.PMPHS-1 HRM46.4 & & \\
\hline 2524 & & RIVER & & RAW GRAB & TRANSECT & 571 & RICH.PMPHS-1 HRM46.4 & & \\
\hline 2525 & & RIVER & & RAW GRAB & TRANSECT & 571 & RICH.PMPHS-1 HRM46.4 & & \\
\hline 2526 & & RIVER & & RAW GRAB & TRANSECT & 327 & RICH.PMPHS-10 HRM46.4 & & \\
\hline 2527 & & RIVER & & RAW GRAB & TRANSECT & 327 & RICH.PMPHS-10 HRM46.4 & & \\
\hline 2528 & & RIVER & & RAW GRAB & TRANSECT & 327 & RICH.PMPHS-10 HRM46.4 & & \\
\hline $2529 \mathrm{~F}$ & $\mathrm{~F}$ & RIVER & & RAW GRAB & TRANSECT & 327 & RICH.PMPHS-10 HRM46.4 & & \\
\hline 2530 & & RIVER & & RAW GRAB & TRANSECT & 327 & RICH.PMPHS-10 HRM46.4 & & \\
\hline 2531 & & RIVER & & RAW GRAB & TRANSECT & 327 & RICH.PMPHS-10 HRM46.4 & & \\
\hline 2532 & & RIVER & & RAW GRAB & TRANSECT & 327 & RICH.PMPHS-10 HRM46.4 & & \\
\hline 2533 & & RIVER & & RAW GRAB & TRANSECT & 321 & RICH.PMPHS-2 HRM46.4 & & \\
\hline 2534 & & RIVER & & RAW GRAB & TRANSECT & 321 & RICH.PMPHS-2 HRM46.4 & & \\
\hline 2535 & & RIVER & & RAW GRAB & TRANSECT & 321 & RICH.PMPHS-2 HRM46.4 & & \\
\hline $2536 \mathrm{~F}$ & $\mathrm{~F}$ & RIVER & & RAW GRAB & TRANSECT & 321 & RICH.PMPHS-2 HRM46.4 & & \\
\hline 2537 & & RIVER & & RAW GRAB & TRANSECT & 321 & RICH.PMPHS-2 HRM46.4 & & \\
\hline 2538 & & RIVER & & RAW GRAB & TRANSECT & 321 & RICH.PMPHS-2 HRM46.4 & & \\
\hline 2539 & & RIVER & & RAW GRAB & TRANSECT & 321 & RICH.PMPHS-2 HRM46.4 & & \\
\hline 2540 & & RIVER & & RAW GRAB & TRANSECT & 322 & RICH.PMPHS-3 HRM46.4 & & \\
\hline 2541 & & RIVER & & RAW GRAB & TRANSECT & 322 & RICH.PMPHS-3 HRM46.4 & & \\
\hline 2542 & & RIVER & & RAW GRAB & TRANSECT & 322 & RICH.PMPHS-3 HRM46.4 & & \\
\hline $2543 \mathrm{~F}$ & $\mathrm{~F}$ & RIVER & & RAW GRAB & TRANSECT & 322 & RICH.PMPHS-3 HRM46.4 & & \\
\hline 2544 & & RIVER & & RAW GRAB & TRANSECT & 322 & RICH.PMPHS-3 HRM46.4 & & \\
\hline 2545 & & RIVER & & RAW GRAB & TRANSECT & 322 & RICH.PMPHS-3 HRM46.4 & & \\
\hline 2546 & & RIVER & & RAW GRAB & TRANSECT & 322 & RICH.PMPHS-3 HRM46.4 & & \\
\hline 2547 & & RIVER & & RAW GRAB & TRANSECT & 572 & RICH.PMPHS-4 HRM46.4 & & \\
\hline
\end{tabular}




\begin{tabular}{|c|c|c|c|c|c|c|c|c|c|}
\hline & $T$ & $\bar{U}$ & $\overline{\mathrm{V}}$ & $\mathrm{W}$ & $\bar{X}$ & $\bar{Y}$ & $\bar{Z}$ & $\overline{A A}$ & $A B$ \\
\hline 1 & REVIEW_QUALIFIER & SAMP_FROM & SAMP_ITEM & SAMP_MTHD & COLL_MTHD & SAMP_SITE_ID & SAMP_SITE_NAME & WELL_NAME & SAMPLE_LOCATION \\
\hline 2548 & & RIVER & & RAW GRAB & TRANSECT & 572 & RICH.PMPHS-4 HRM46.4 & & \\
\hline 2549 & & RIVER & & RAW GRAB & TRANSECT & 572 & RICH.PMPHS-4 HRM46.4 & & \\
\hline 2550 & $\mathrm{~F}$ & RIVER & & RAW GRAB & TRANSECT & 572 & RICH.PMPHS-4 HRM46.4 & & \\
\hline 2551 & & RIVER & & RAW GRAB & TRANSECT & 572 & RICH.PMPHS-4 HRM46.4 & & \\
\hline 2552 & & RIVER & & RAW GRAB & TRANSECT & 572 & RICH.PMPHS-4 HRM46.4 & & \\
\hline 2553 & & RIVER & & RAW GRAB & TRANSECT & 572 & RICH.PMPHS-4 HRM46.4 & & \\
\hline 2554 & & RIVER & & RAW GRAB & TRANSECT & 323 & RICH.PMPHS-5 HRM46.4 & & \\
\hline 2555 & & RIVER & & RAW GRAB & TRANSECT & 323 & RICH.PMPHS-5 HRM46.4 & & \\
\hline 2556 & & RIVER & & RAW GRAB & TRANSECT & 323 & RICH.PMPHS-5 HRM46.4 & & \\
\hline 2557 & $\mathrm{~F}$ & RIVER & & RAW GRAB & TRANSECT & 323 & RICH.PMPHS-5 HRM46.4 & & \\
\hline 2558 & & RIVER & & RAW GRAB & TRANSECT & 323 & RICH.PMPHS-5 HRM46.4 & & \\
\hline 2559 & & RIVER & & RAW GRAB & TRANSECT & 323 & RICH.PMPHS-5 HRM46.4 & & \\
\hline 2560 & & RIVER & & RAW GRAB & TRANSECT & 323 & RICH.PMPHS-5 HRM46.4 & & \\
\hline 2561 & & RIVER & & RAW GRAB & TRANSECT & 324 & RICH.PMPHS-6 HRM46.4 & & \\
\hline 2562 & & RIVER & & RAW GRAB & TRANSECT & 324 & RICH.PMPHS-6 HRM46.4 & & \\
\hline 2563 & & RIVER & & RAW GRAB & TRANSECT & 324 & RICH.PMPHS-6 HRM46.4 & & \\
\hline 2564 & $\mathrm{~F}$ & RIVER & & RAW GRAB & TRANSECT & 324 & RICH.PMPHS-6 HRM46.4 & & \\
\hline 2565 & & RIVER & & RAW GRAB & TRANSECT & 324 & RICH.PMPHS-6 HRM46.4 & & \\
\hline 2566 & & RIVER & & RAW GRAB & TRANSECT & 324 & RICH.PMPHS-6 HRM46.4 & & \\
\hline 2567 & & RIVER & & RAW GRAB & TRANSECT & 324 & RICH.PMPHS-6 HRM46.4 & & \\
\hline 2568 & & RIVER & & RAW GRAB & TRANSECT & 325 & RICH.PMPHS-7 HRM46.4 & & \\
\hline 2569 & & RIVER & & RAW GRAB & TRANSECT & 325 & RICH.PMPHS-7 HRM46.4 & & \\
\hline 2570 & $\mathrm{~F}$ & RIVER & & RAW GRAB & TRANSECT & 325 & RICH.PMPHS-7 HRM46.4 & & \\
\hline 2571 & $\mathrm{~F}$ & RIVER & & RAW GRAB & TRANSECT & 325 & RICH.PMPHS-7 HRM46.4 & & \\
\hline 2572 & & RIVER & & RAW GRAB & TRANSECT & 325 & RICH.PMPHS-7 HRM46.4 & & \\
\hline 2573 & & RIVER & & RAW GRAB & TRANSECT & 325 & RICH.PMPHS-7 HRM46.4 & & \\
\hline 2574 & & RIVER & & RAW GRAB & TRANSECT & 325 & RICH.PMPHS-7 HRM46.4 & & \\
\hline 2575 & & RIVER & & RAW GRAB & TRANSECT & 326 & RICH.PMPHS-8 HRM46.4 & & \\
\hline 2576 & & RIVER & & RAW GRAB & TRANSECT & 326 & RICH.PMPHS-8 HRM46.4 & & \\
\hline 2577 & & RIVER & & RAW GRAB & TRANSECT & 326 & RICH.PMPHS-8 HRM46.4 & & \\
\hline 2578 & $\mathrm{~F}$ & RIVER & & RAW GRAB & TRANSECT & 326 & RICH.PMPHS-8 HRM46.4 & & \\
\hline 2579 & & RIVER & & RAW GRAB & TRANSECT & 326 & RICH.PMPHS-8 HRM46.4 & & \\
\hline 2580 & & RIVER & & RAW GRAB & TRANSECT & 326 & RICH.PMPHS-8 HRM46.4 & & \\
\hline 2581 & & RIVER & & RAW GRAB & TRANSECT & 326 & RICH.PMPHS-8 HRM46.4 & & \\
\hline 2582 & & RIVER & & RAW GRAB & TRANSECT & 573 & RICH.PMPHS-9 HRM46.4 & & \\
\hline 2583 & & RIVER & & RAW GRAB & TRANSECT & 573 & RICH.PMPHS-9 HRM46.4 & & \\
\hline 2584 & & RIVER & & RAW GRAB & TRANSECT & 573 & RICH.PMPHS-9 HRM46.4 & & \\
\hline 2585 & $\mathrm{~F}$ & RIVER & & RAW GRAB & TRANSECT & 573 & RICH.PMPHS-9 HRM46.4 & & \\
\hline
\end{tabular}




\begin{tabular}{|c|c|c|c|c|c|c|c|c|c|}
\hline & $\mathrm{T}$ & $\bar{U}$ & $\mathrm{~V}$ & $\mathrm{~W}$ & $\mathrm{X}$ & $\mathrm{Y}$ & $Z$ & $\mathrm{AA}$ & $\mathrm{AB}$ \\
\hline \begin{tabular}{l|l}
1 & 1 \\
\end{tabular} & REVIEW_QUALIFIER & SAMP_FROM & SAMP_ITEM & SAMP_MTHD & COLL_MTHD & SAMP_SITE_ID & SAMP_SITE_NAME & WELL_NAME & SAMPLE_LOCATION \\
\hline 2586 & & RIVER & & RAW GRAB & TRANSECT & 573 & RICH.PMPHS-9 HRM46.4 & & \\
\hline 2587 & & RIVER & & RAW GRAB & TRANSECT & 573 & RICH.PMPHS-9 HRM46.4 & & \\
\hline 2588 & & RIVER & & RAW GRAB & TRANSECT & 573 & RICH.PMPHS-9 HRM46.4 & & \\
\hline 2589 & & RIVER & & RAW GRAB & TRANSECT & 317 & VERNITA-1 HRM 0.3 & & \\
\hline 2590 & & RIVER & & RAW GRAB & TRANSECT & 317 & VERNITA-1 HRM 0.3 & & \\
\hline 2591 & & RIVER & & RAW GRAB & TRANSECT & 317 & VERNITA-1 HRM 0.3 & & \\
\hline $2592 \mathrm{~F}$ & $\mathrm{~F}$ & RIVER & & RAW GRAB & TRANSECT & 317 & VERNITA-1 HRM 0.3 & & \\
\hline 2593 & & RIVER & & RAW GRAB & TRANSECT & 317 & VERNITA-1 HRM 0.3 & & \\
\hline 2594 & & RIVER & & RAW GRAB & TRANSECT & 317 & VERNITA-1 HRM 0.3 & & \\
\hline 2595 & & RIVER & & RAW GRAB & TRANSECT & 317 & VERNITA-1 HRM 0.3 & & \\
\hline 2596 & & RIVER & & RAW GRAB & TRANSECT & 570 & VERNITA-2 HRM 0.3 & & \\
\hline 2597 & & RIVER & & RAW GRAB & TRANSECT & 570 & VERNITA-2 HRM 0.3 & & \\
\hline 2598 & & RIVER & & RAW GRAB & TRANSECT & 570 & VERNITA-2 HRM 0.3 & & \\
\hline $2599 \mathrm{~F}$ & $\mathrm{~F}$ & RIVER & & RAW GRAB & TRANSECT & 570 & VERNITA-2 HRM 0.3 & & \\
\hline 2600 & & RIVER & & RAW GRAB & TRANSECT & 570 & VERNITA-2 HRM 0.3 & & \\
\hline 2601 & & RIVER & & RAW GRAB & TRANSECT & 570 & VERNITA-2 HRM 0.3 & & \\
\hline 2602 & & RIVER & & RAW GRAB & TRANSECT & 570 & VERNITA-2 HRM 0.3 & & \\
\hline 2603 & & RIVER & & RAW GRAB & TRANSECT & 318 & VERNITA-3 HRM 0.3 & & \\
\hline 2604 & & RIVER & & RAW GRAB & TRANSECT & 318 & VERNITA-3 HRM 0.3 & & \\
\hline 2605 & & RIVER & & RAW GRAB & TRANSECT & 318 & VERNITA-3 HRM 0.3 & & \\
\hline 2606 F & $\mathrm{F}$ & RIVER & & RAW GRAB & TRANSECT & 318 & VERNITA-3 HRM 0.3 & & \\
\hline 2607 & & RIVER & & RAW GRAB & TRANSECT & 318 & VERNITA-3 HRM 0.3 & & \\
\hline 2608 & & RIVER & & RAW GRAB & TRANSECT & 318 & VERNITA-3 HRM 0.3 & & \\
\hline 2609 & & RIVER & & RAW GRAB & TRANSECT & 318 & VERNITA-3 HRM 0.3 & & \\
\hline 2610 & & RIVER & & RAW GRAB & TRANSECT & 319 & VERNITA-4 HRM 0.3 & & \\
\hline 2611 & & RIVER & & RAW GRAB & TRANSECT & 319 & VERNITA-4 HRM 0.3 & & \\
\hline 2612 & & RIVER & & RAW GRAB & TRANSECT & 319 & VERNITA-4 HRM 0.3 & & \\
\hline $2613 \mathrm{~F}$ & $\mathrm{~F}$ & RIVER & & RAW GRAB & TRANSECT & 319 & VERNITA-4 HRM 0.3 & & \\
\hline 2614 & & RIVER & & RAW GRAB & TRANSECT & 319 & VERNITA-4 HRM 0.3 & & \\
\hline 2615 & & RIVER & & RAW GRAB & TRANSECT & 319 & VERNITA-4 HRM 0.3 & & \\
\hline 2616 & & RIVER & & RAW GRAB & TRANSECT & 319 & VERNITA-4 HRM 0.3 & & \\
\hline 2617 & & RIVER & & RAW GRAB & TRANSECT & 571 & RICH.PMPHS-1 HRM46.4 & & \\
\hline 2618 & & RIVER & & RAW GRAB & TRANSECT & 571 & RICH.PMPHS-1 HRM46.4 & & \\
\hline 2619 & & RIVER & & RAW GRAB & TRANSECT & 571 & RICH.PMPHS-1 HRM46.4 & & \\
\hline 2620 & & RIVER & & RAW GRAB & TRANSECT & 571 & RICH.PMPHS-1 HRM46.4 & & \\
\hline 2621 & & RIVER & & RAW GRAB & TRANSECT & 571 & RICH.PMPHS-1 HRM46.4 & & \\
\hline 2622 & & RIVER & & RAW GRAB & TRANSECT & 571 & RICH.PMPHS-1 HRM46.4 & & \\
\hline 2623 & & RIVER & & RAW GRAB & TRANSECT & 571 & RICH.PMPHS-1 HRM46.4 & & \\
\hline
\end{tabular}




\begin{tabular}{|c|c|c|c|c|c|c|c|c|c|}
\hline & $T$ & $\bar{U}$ & $\overline{\mathrm{V}}$ & $\mathrm{W}$ & $\bar{X}$ & $\bar{Y}$ & $\bar{Z}$ & $\overline{A A}$ & $A B$ \\
\hline 1 & REVIEW_QUALIFIER & SAMP_FROM & SAMP_ITEM & SAMP_MTHD & COLL_MTHD & SAMP_SITE_ID & SAMP_SITE_NAME & WELL_NAME & SAMPLE_LOCATION \\
\hline 2624 & & RIVER & & RAW GRAB & TRANSECT & 327 & RICH.PMPHS-10 HRM46.4 & & \\
\hline 2625 & & RIVER & & RAW GRAB & TRANSECT & 327 & RICH.PMPHS-10 HRM46.4 & & \\
\hline 2626 & & RIVER & & RAW GRAB & TRANSECT & 327 & RICH.PMPHS-10 HRM46.4 & & \\
\hline 2627 & $\mathrm{~F}$ & RIVER & & RAW GRAB & TRANSECT & 327 & RICH.PMPHS-10 HRM46.4 & & \\
\hline 2628 & & RIVER & & RAW GRAB & TRANSECT & 327 & RICH.PMPHS-10 HRM46.4 & & \\
\hline 2629 & & RIVER & & RAW GRAB & TRANSECT & 327 & RICH.PMPHS-10 HRM46.4 & & \\
\hline 2630 & & RIVER & & RAW GRAB & TRANSECT & 327 & RICH.PMPHS-10 HRM46.4 & & \\
\hline 2631 & & RIVER & & RAW GRAB & TRANSECT & 321 & RICH.PMPHS-2 HRM46.4 & & \\
\hline 2632 & & RIVER & & RAW GRAB & TRANSECT & 321 & RICH.PMPHS-2 HRM46.4 & & \\
\hline 2633 & & RIVER & & RAW GRAB & TRANSECT & 321 & RICH.PMPHS-2 HRM46.4 & & \\
\hline 2634 & $\mathrm{~F}$ & RIVER & & RAW GRAB & TRANSECT & 321 & RICH.PMPHS-2 HRM46.4 & & \\
\hline 2635 & & RIVER & & RAW GRAB & TRANSECT & 321 & RICH.PMPHS-2 HRM46.4 & & \\
\hline 2636 & & RIVER & & RAW GRAB & TRANSECT & 321 & RICH.PMPHS-2 HRM46.4 & & \\
\hline 2637 & & RIVER & & RAW GRAB & TRANSECT & 321 & RICH.PMPHS-2 HRM46.4 & & \\
\hline 2638 & & RIVER & & RAW GRAB & TRANSECT & 322 & RICH.PMPHS-3 HRM46.4 & & \\
\hline 2639 & & RIVER & & RAW GRAB & TRANSECT & 322 & RICH.PMPHS-3 HRM46.4 & & \\
\hline 2640 & & RIVER & & RAW GRAB & TRANSECT & 322 & RICH.PMPHS-3 HRM46.4 & & \\
\hline 2641 & $\mathrm{~F}$ & RIVER & & RAW GRAB & TRANSECT & 322 & RICH.PMPHS-3 HRM46.4 & & \\
\hline 2642 & & RIVER & & RAW GRAB & TRANSECT & 322 & RICH.PMPHS-3 HRM46.4 & & \\
\hline 2643 & & RIVER & & RAW GRAB & TRANSECT & 322 & RICH.PMPHS-3 HRM46.4 & & \\
\hline 2644 & & RIVER & & RAW GRAB & TRANSECT & 322 & RICH.PMPHS-3 HRM46.4 & & \\
\hline 2645 & & RIVER & & RAW GRAB & TRANSECT & 572 & RICH.PMPHS-4 HRM46.4 & & \\
\hline 2646 & & RIVER & & RAW GRAB & TRANSECT & 572 & RICH.PMPHS-4 HRM46.4 & & \\
\hline 2647 & & RIVER & & RAW GRAB & TRANSECT & 572 & RICH.PMPHS-4 HRM46.4 & & \\
\hline 2648 & $\mathrm{~F}$ & RIVER & & RAW GRAB & TRANSECT & 572 & RICH.PMPHS-4 HRM46.4 & & \\
\hline 2649 & & RIVER & & RAW GRAB & TRANSECT & 572 & RICH.PMPHS-4 HRM46.4 & & \\
\hline 2650 & & RIVER & & RAW GRAB & TRANSECT & 572 & RICH.PMPHS-4 HRM46.4 & & \\
\hline 2651 & & RIVER & & RAW GRAB & TRANSECT & 572 & RICH.PMPHS-4 HRM46.4 & & \\
\hline 2652 & & RIVER & & RAW GRAB & TRANSECT & 323 & RICH.PMPHS-5 HRM46.4 & & \\
\hline 2653 & & RIVER & & RAW GRAB & TRANSECT & 323 & RICH.PMPHS-5 HRM46.4 & & \\
\hline 2654 & & RIVER & & RAW GRAB & TRANSECT & 323 & RICH.PMPHS-5 HRM46.4 & & \\
\hline 2655 & $\mathrm{~F}$ & RIVER & & RAW GRAB & TRANSECT & 323 & RICH.PMPHS-5 HRM46.4 & & \\
\hline 2656 & & RIVER & & RAW GRAB & TRANSECT & 323 & RICH.PMPHS-5 HRM46.4 & & \\
\hline 2657 & & RIVER & & RAW GRAB & TRANSECT & 323 & RICH.PMPHS-5 HRM46.4 & & \\
\hline 2658 & & RIVER & & RAW GRAB & TRANSECT & 323 & RICH.PMPHS-5 HRM46.4 & & \\
\hline 2659 & & RIVER & & RAW GRAB & TRANSECT & 324 & RICH.PMPHS-6 HRM46.4 & & \\
\hline 2660 & & RIVER & & RAW GRAB & TRANSECT & 324 & RICH.PMPHS-6 HRM46.4 & & \\
\hline 2661 & & RIVER & & RAW GRAB & TRANSECT & 324 & RICH.PMPHS-6 HRM46.4 & & \\
\hline
\end{tabular}




\begin{tabular}{|c|c|c|c|c|c|c|c|c|c|}
\hline & $\mathrm{T}$ & $\bar{U}$ & $\mathrm{~V}$ & $\mathrm{~W}$ & $\mathrm{X}$ & $\mathrm{Y}$ & $Z$ & $\mathrm{AA}$ & $\mathrm{AB}$ \\
\hline \begin{tabular}{l|l}
1 & 1 \\
\end{tabular} & REVIEW_QUALIFIER & SAMP_FROM & SAMP_ITEM & SAMP_MTHD & COLL_MTHD & SAMP_SITE_ID & SAMP_SITE_NAME & WELL_NAME & SAMPLE_LOCATION \\
\hline $2662 \mathrm{~F}$ & $\mathrm{~F}$ & RIVER & & RAW GRAB & TRANSECT & 324 & RICH.PMPHS-6 HRM46.4 & & \\
\hline 2663 & & RIVER & & RAW GRAB & TRANSECT & 324 & RICH.PMPHS-6 HRM46.4 & & \\
\hline 2664 & & RIVER & & RAW GRAB & TRANSECT & 324 & RICH.PMPHS-6 HRM46.4 & & \\
\hline 2665 & & RIVER & & RAW GRAB & TRANSECT & 324 & RICH.PMPHS-6 HRM46.4 & & \\
\hline 2666 & & RIVER & & RAW GRAB & TRANSECT & 325 & RICH.PMPHS-7 HRM46.4 & & \\
\hline 2667 & & RIVER & & RAW GRAB & TRANSECT & 325 & RICH.PMPHS-7 HRM46.4 & & \\
\hline 2668 & & RIVER & & RAW GRAB & TRANSECT & 325 & RICH.PMPHS-7 HRM46.4 & & \\
\hline $2669 \mathrm{~F}$ & $\mathrm{~F}$ & RIVER & & RAW GRAB & TRANSECT & 325 & RICH.PMPHS-7 HRM46.4 & & \\
\hline 2670 & & RIVER & & RAW GRAB & TRANSECT & 325 & RICH.PMPHS-7 HRM46.4 & & \\
\hline 2671 & & RIVER & & RAW GRAB & TRANSECT & 325 & RICH.PMPHS-7 HRM46.4 & & \\
\hline 2672 & & RIVER & & RAW GRAB & TRANSECT & $325 \mathrm{H}$ & RICH.PMPHS-7 HRM46.4 & & \\
\hline 2673 & & RIVER & & RAW GRAB & TRANSECT & 326 & RICH.PMPHS-8 HRM46.4 & & \\
\hline 2674 & & RIVER & & RAW GRAB & TRANSECT & 326 & RICH.PMPHS-8 HRM46.4 & & \\
\hline 2675 & & RIVER & & RAW GRAB & TRANSECT & 326 & RICH.PMPHS-8 HRM46.4 & & \\
\hline $2676 \mathrm{~F}$ & $\mathrm{~F}$ & RIVER & & RAW GRAB & TRANSECT & 326 & RICH.PMPHS-8 HRM46.4 & & \\
\hline 2677 & & RIVER & & RAW GRAB & TRANSECT & 326 & RICH.PMPHS-8 HRM46.4 & & \\
\hline 2678 & & RIVER & & RAW GRAB & TRANSECT & 326 & RICH.PMPHS-8 HRM46.4 & & \\
\hline 2679 & & RIVER & & RAW GRAB & TRANSECT & 326 & RICH.PMPHS-8 HRM46.4 & & \\
\hline 2680 & & RIVER & & RAW GRAB & TRANSECT & 573 & RICH.PMPHS-9 HRM46.4 & & \\
\hline 2681 & & RIVER & & RAW GRAB & TRANSECT & 573 & RICH.PMPHS-9 HRM46.4 & & \\
\hline 2682 & & RIVER & & RAW GRAB & TRANSECT & 573 & RICH.PMPHS-9 HRM46.4 & & \\
\hline $2683 \mathrm{~F}$ & $\mathrm{~F}$ & RIVER & & RAW GRAB & TRANSECT & 573 & RICH.PMPHS-9 HRM46.4 & & \\
\hline 2684 & & RIVER & & RAW GRAB & TRANSECT & 573 & RICH.PMPHS-9 HRM46.4 & & \\
\hline 2685 & & RIVER & & RAW GRAB & TRANSECT & 573 & RICH.PMPHS-9 HRM46.4 & & \\
\hline 2686 & & RIVER & & RAW GRAB & TRANSECT & 573 & RICH.PMPHS-9 HRM46.4 & & \\
\hline 2687 & & RIVER & & RAW GRAB & TRANSECT & 317 & VERNITA-1 HRM 0.3 & & \\
\hline 2688 & & RIVER & & RAW GRAB & TRANSECT & 317 & VERNITA-1 HRM 0.3 & & \\
\hline 2689 & & RIVER & & RAW GRAB & TRANSECT & 317 & VERNITA-1 HRM 0.3 & & \\
\hline $2690 \mathrm{~F}$ & $\mathrm{~F}$ & RIVER & & RAW GRAB & TRANSECT & 317 & VERNITA-1 HRM 0.3 & & \\
\hline 2691 & & RIVER & & RAW GRAB & TRANSECT & 317 & VERNITA-1 HRM 0.3 & & \\
\hline 2692 & & RIVER & & RAW GRAB & TRANSECT & 317 & VERNITA-1 HRM 0.3 & & \\
\hline 2693 & & RIVER & & RAW GRAB & TRANSECT & 317 & VERNITA-1 HRM 0.3 & & \\
\hline 2694 & & RIVER & & RAW GRAB & TRANSECT & 570 & VERNITA-2 HRM 0.3 & & \\
\hline 2695 & & RIVER & & RAW GRAB & TRANSECT & 570 & VERNITA-2 HRM 0.3 & & \\
\hline 2696 & & RIVER & & RAW GRAB & TRANSECT & 570 & VERNITA-2 HRM 0.3 & & \\
\hline $2697 \mathrm{~F}$ & $\mathrm{~F}$ & RIVER & & RAW GRAB & TRANSECT & 570 & VERNITA-2 HRM 0.3 & & \\
\hline 2698 & & RIVER & & RAW GRAB & TRANSECT & 570 & VERNITA-2 HRM 0.3 & & \\
\hline 2699 & & RIVER & & RAW GRAB & TRANSECT & 570 & VERNITA-2 HRM 0.3 & & \\
\hline
\end{tabular}




\begin{tabular}{|c|c|c|c|c|c|c|c|c|c|}
\hline & $T$ & $\bar{U}$ & $\mathrm{~V}$ & $\mathrm{~W}$ & $\bar{x}$ & $\bar{Y}$ & $\bar{Z}$ & AA & $A B$ \\
\hline 1 & REVIEW_QUALIFIER & SAMP_FROM & SAMP_ITEM & SAMP_MTHD & COLL_MTHD & SAMP_SITE_ID & SAMP_SITE_NAME & WELL_NAME & SAMPLE_LOCATION \\
\hline 2700 & & RIVER & & RAW GRAB & TRANSECT & 570 & VERNITA-2 HRM 0.3 & & \\
\hline 2701 & & RIVER & & RAW GRAB & TRANSECT & 318 & 3 VERNITA-3 HRM 0.3 & & \\
\hline 2702 & & RIVER & & RAW GRAB & TRANSECT & 318 & 3 VERNITA-3 HRM 0.3 & & \\
\hline 2703 & & RIVER & & RAW GRAB & TRANSECT & 318 & 3 VERNITA-3 HRM 0.3 & & \\
\hline 2704 & $F$ & RIVER & & RAW GRAB & TRANSECT & 318 & 3 VERNITA-3 HRM 0.3 & & \\
\hline 2705 & & RIVER & & RAW GRAB & TRANSECT & 318 & 3 VERNITA-3 HRM 0.3 & & \\
\hline 2706 & & RIVER & & RAW GRAB & TRANSECT & 318 & 3 VERNITA-3 HRM 0.3 & & \\
\hline 2707 & & RIVER & & RAW GRAB & TRANSECT & 318 & 3 VERNITA-3 HRM 0.3 & & \\
\hline 2708 & & RIVER & & RAW GRAB & TRANSECT & 319 & 9 VERNITA-4 HRM 0.3 & & \\
\hline 2709 & & RIVER & & RAW GRAB & TRANSECT & 319 & 9 VERNITA-4 HRM 0.3 & & \\
\hline 2710 & & RIVER & & RAW GRAB & TRANSECT & 319 & 9 VERNITA-4 HRM 0.3 & & \\
\hline 2711 & $\mathrm{~F}$ & RIVER & & RAW GRAB & TRANSECT & 319 & 9 VERNITA-4 HRM 0.3 & & \\
\hline 2712 & & RIVER & & RAW GRAB & TRANSECT & 319 & 9 VERNITA-4 HRM 0.3 & & \\
\hline 2713 & & RIVER & & RAW GRAB & TRANSECT & 319 & 9 VERNITA-4 HRM 0.3 & & \\
\hline 2714 & & RIVER & & RAW GRAB & TRANSECT & 319 & 9 VERNITA-4 HRM 0.3 & & \\
\hline 2715 & & RIVER & & RAW GRAB & TRANSECT & 348 & 3300 AREA -1 HRM 43.1 & & \\
\hline 2716 & & RIVER & & RAW GRAB & TRANSECT & 348 & 3300 AREA -1 HRM 43.1 & & \\
\hline 2717 & & RIVER & & RAW GRAB & TRANSECT & 348 & 300 AREA -1 HRM 43.1 & & \\
\hline 2718 & & RIVER & & RAW GRAB & TRANSECT & 348 & 300 AREA -1 HRM 43.1 & & \\
\hline 2719 & $F$ & RIVER & & RAW GRAB & TRANSECT & 348 & 3300 AREA -1 HRM 43.1 & & \\
\hline 2720 & & RIVER & & RAW GRAB & TRANSECT & 348 & 300 AREA -1 HRM 43.1 & & \\
\hline 2721 & $F$ & RIVER & & RAW GRAB & TRANSECT & 348 & 300 AREA -1 HRM 43.1 & & \\
\hline 2722 & & RIVER & & RAW GRAB & TRANSECT & 470 & 300 AREA -2 HRM 43.1 & & \\
\hline 2723 & & RIVER & & RAW GRAB & TRANSECT & 470 & 300 AREA -2 HRM 43.1 & & \\
\hline 2724 & & RIVER & & RAW GRAB & TRANSECT & 470 & 300 AREA -2 HRM 43.1 & & \\
\hline 2725 & & RIVER & & RAW GRAB & TRANSECT & 470 & 300 AREA -2 HRM 43.1 & & \\
\hline 2726 & & RIVER & & RAW GRAB & TRANSECT & 470 & 300 AREA -2 HRM 43.1 & & \\
\hline 2727 & & RIVER & & RAW GRAB & TRANSECT & 470 & 300 AREA -2 HRM 43.1 & & \\
\hline 2728 & & RIVER & & RAW GRAB & TRANSECT & 470 & 300 AREA -2 HRM 43.1 & & \\
\hline 2729 & & RIVER & & RAW GRAB & TRANSECT & 580 & 300 AREA -3 HRM 43.1 & & \\
\hline 2730 & & RIVER & & RAW GRAB & TRANSECT & 580 & 300 AREA -3 HRM 43.1 & & \\
\hline 2731 & & RIVER & & RAW GRAB & TRANSECT & 580 & 300 AREA -3 HRM 43.1 & & \\
\hline 2732 & $F$ & RIVER & & RAW GRAB & TRANSECT & 580 & 300 AREA -3 HRM 43.1 & & \\
\hline 2733 & & RIVER & & RAW GRAB & TRANSECT & 580 & 300 AREA -3 HRM 43.1 & & \\
\hline 2734 & & RIVER & & RAW GRAB & TRANSECT & 580 & 300 AREA -3 HRM 43.1 & & \\
\hline 2735 & & RIVER & & RAW GRAB & TRANSECT & 580 & 300 AREA -3 HRM 43.1 & & \\
\hline 2736 & & RIVER & & RAW GRAB & TRANSECT & 349 & 9 300 AREA -4 HRM 43.1 & & \\
\hline 2737 & & RIVER & & RAW GRAB & TRANSECT & 349 & 9300 AREA -4 HRM 43.1 & & \\
\hline
\end{tabular}




\begin{tabular}{|c|c|c|c|c|c|c|c|c|c|}
\hline & $T$ & $\bar{U}$ & $\overline{\mathrm{V}}$ & $\mathrm{W}$ & $\bar{X}$ & $\bar{Y}$ & $\bar{Z}$ & $\overline{A A}$ & $A B$ \\
\hline 1 & REVIEW_QUALIFIER & SAMP_FROM & SAMP_ITEM & SAMP_MTHD & COLL_MTHD & SAMP_SITE_ID & SAMP_SITE_NAME & WELL_NAME & SAMPLE_LOCATION \\
\hline 2738 & & RIVER & & RAW GRAB & TRANSECT & 3493 & 300 AREA -4 HRM 43.1 & & \\
\hline 2739 & $\mathrm{~F}$ & RIVER & & RAW GRAB & TRANSECT & 349 & 300 AREA -4 HRM 43.1 & & \\
\hline 2740 & & RIVER & & RAW GRAB & TRANSECT & 3493 & 300 AREA -4 HRM 43.1 & & \\
\hline 2741 & & RIVER & & RAW GRAB & TRANSECT & 3493 & 300 AREA -4 HRM 43.1 & & \\
\hline 2742 & & RIVER & & RAW GRAB & TRANSECT & 349 & 300 AREA -4 HRM 43.1 & & \\
\hline 2743 & & RIVER & & RAW GRAB & TRANSECT & 6073 & 300 AREA -5 HRM 43.1 & & \\
\hline 2744 & & RIVER & & RAW GRAB & TRANSECT & 607 & 300 AREA -5 HRM 43.1 & & \\
\hline 2745 & & RIVER & & RAW GRAB & TRANSECT & 607 & 300 AREA -5 HRM 43.1 & & \\
\hline 2746 & $\mathrm{~F}$ & RIVER & & RAW GRAB & TRANSECT & 607 & 300 AREA -5 HRM 43.1 & & \\
\hline 2747 & & RIVER & & RAW GRAB & TRANSECT & 607 & 300 AREA -5 HRM 43.1 & & \\
\hline 2748 & & RIVER & & RAW GRAB & TRANSECT & 607 & 300 AREA -5 HRM 43.1 & & \\
\hline 2749 & & RIVER & & RAW GRAB & TRANSECT & 6073 & 300 AREA -5 HRM 43.1 & & \\
\hline 2750 & & RIVER & & RAW GRAB & TRANSECT & 350 & 300 AREA -6 HRM 43.1 & & \\
\hline 2751 & & RIVER & & RAW GRAB & TRANSECT & 350 & 300 AREA -6 HRM 43.1 & & \\
\hline 2752 & & RIVER & & RAW GRAB & TRANSECT & 3503 & 300 AREA -6 HRM 43.1 & & \\
\hline 2753 & $\mathrm{~F}$ & RIVER & & RAW GRAB & TRANSECT & 350 & 300 AREA -6 HRM 43.1 & & \\
\hline 2754 & & RIVER & & RAW GRAB & TRANSECT & 3503 & 300 AREA -6 HRM 43.1 & & \\
\hline 2755 & & RIVER & & RAW GRAB & TRANSECT & 3503 & 300 AREA -6 HRM 43.1 & & \\
\hline 2756 & & RIVER & & RAW GRAB & TRANSECT & 350 & 300 AREA -6 HRM 43.1 & & \\
\hline 2757 & & RIVER & & RAW GRAB & TRANSECT & 3513 & 300 AREA -7 HRM 43.1 & & \\
\hline 2758 & & RIVER & & RAW GRAB & TRANSECT & 3513 & 300 AREA -7 HRM 43.1 & & \\
\hline 2759 & & RIVER & & RAW GRAB & TRANSECT & 3513 & 300 AREA -7 HRM 43.1 & & \\
\hline 2760 & $\mathrm{~F}$ & RIVER & & RAW GRAB & TRANSECT & 351 & 300 AREA -7 HRM 43.1 & & \\
\hline 2761 & & RIVER & & RAW GRAB & TRANSECT & 3513 & 300 AREA -7 HRM 43.1 & & \\
\hline 2762 & & RIVER & & RAW GRAB & TRANSECT & 3513 & 300 AREA -7 HRM 43.1 & & \\
\hline 2763 & & RIVER & & RAW GRAB & TRANSECT & 3513 & 300 AREA -7 HRM 43.1 & & \\
\hline 2764 & & RIVER & & RAW GRAB & TRANSECT & 5813 & 300 AREA -8 HRM 43.1 & & \\
\hline 2765 & & RIVER & & RAW GRAB & TRANSECT & 5813 & 300 AREA -8 HRM 43.1 & & \\
\hline 2766 & & RIVER & & RAW GRAB & TRANSECT & 5813 & 300 AREA -8 HRM 43.1 & & \\
\hline 2767 & $\mathrm{~F}$ & RIVER & & RAW GRAB & TRANSECT & 5813 & 300 AREA -8 HRM 43.1 & & \\
\hline 2768 & & RIVER & & RAW GRAB & TRANSECT & 5813 & 300 AREA -8 HRM 43.1 & & \\
\hline 2769 & & RIVER & & RAW GRAB & TRANSECT & 5813 & 300 AREA -8 HRM 43.1 & & \\
\hline 2770 & & RIVER & & RAW GRAB & TRANSECT & 5813 & 300 AREA -8 HRM 43.1 & & \\
\hline 2771 & & RIVER & & RAW GRAB & TRANSECT & 4713 & 300 AREA -9 HRM 43.1 & & \\
\hline 2772 & & RIVER & & RAW GRAB & TRANSECT & 4713 & 300 AREA -9 HRM 43.1 & & \\
\hline 2773 & & RIVER & & RAW GRAB & TRANSECT & 4713 & 300 AREA -9 HRM 43.1 & & \\
\hline 2774 & $\mathrm{~F}$ & RIVER & & RAW GRAB & TRANSECT & 4713 & 300 AREA -9 HRM 43.1 & & \\
\hline 2775 & & RIVER & & RAW GRAB & TRANSECT & 4713 & 300 AREA -9 HRM 43.1 & & \\
\hline
\end{tabular}




\begin{tabular}{|c|c|c|c|c|c|c|c|c|c|}
\hline & $\mathrm{T}$ & $\bar{U}$ & $\mathrm{~V}$ & $\mathrm{~W}$ & $\mathrm{X}$ & $\mathrm{Y}$ & $Z$ & $\mathrm{AA}$ & $\mathrm{AB}$ \\
\hline \begin{tabular}{l|l}
1 & 1 \\
\end{tabular} & REVIEW_QUALIFIER & SAMP_FROM & SAMP_ITEM & SAMP_MTHD & COLL_MTHD & SAMP_SITE_ID & SAMP_SITE_NAME & WELL_NAME & SAMPLE_LOCATION \\
\hline 2776 & & RIVER & & RAW GRAB & TRANSECT & 4713 & 300 AREA -9 HRM 43.1 & & \\
\hline 2777 & & RIVER & & RAW GRAB & TRANSECT & 4713 & 300 AREA -9 HRM 43.1 & & \\
\hline 2778 & & RIVER & & RAW GRAB & TRANSECT & 3523 & 300 AREA-10 HRM 43.1 & & \\
\hline 2779 & & RIVER & & RAW GRAB & TRANSECT & 3523 & 300 AREA-10 HRM 43.1 & & \\
\hline 2780 & & RIVER & & RAW GRAB & TRANSECT & 3523 & 300 AREA-10 HRM 43.1 & & \\
\hline $2781 \mathrm{~F}$ & $\mathrm{~F}$ & RIVER & & RAW GRAB & TRANSECT & 3523 & 300 AREA-10 HRM 43.1 & & \\
\hline $2782 \mathrm{~F}$ & $\mathrm{~F}$ & RIVER & & RAW GRAB & TRANSECT & 3523 & 300 AREA-10 HRM 43.1 & & \\
\hline 2783 & & RIVER & & RAW GRAB & TRANSECT & 3523 & 300 AREA-10 HRM 43.1 & & \\
\hline 2784 & & RIVER & & RAW GRAB & TRANSECT & 352 & 300 AREA-10 HRM 43.1 & & \\
\hline 2785 & & RIVER & & RAW GRAB & TRANSECT & 571 & RICH.PMPHS-1 HRM46.4 & & \\
\hline 2786 & & RIVER & & RAW GRAB & TRANSECT & 571 & RICH.PMPHS-1 HRM46.4 & & \\
\hline 2787 & & RIVER & & RAW GRAB & TRANSECT & 571 & RICH.PMPHS-1 HRM46.4 & & \\
\hline 2788 & & RIVER & & RAW GRAB & TRANSECT & 571 & RICH.PMPHS-1 HRM46.4 & & \\
\hline 2789 & & RIVER & & RAW GRAB & TRANSECT & 571 & RICH.PMPHS-1 HRM46.4 & & \\
\hline 2790 & & RIVER & & RAW GRAB & TRANSECT & 571 & RICH.PMPHS-1 HRM46.4 & & \\
\hline 2791 & & RIVER & & RAW GRAB & TRANSECT & 571 & RICH.PMPHS-1 HRM46.4 & & \\
\hline 2792 & & RIVER & & RAW GRAB & TRANSECT & 327 & RICH.PMPHS-10 HRM46.4 & & \\
\hline 2793 & & RIVER & & RAW GRAB & TRANSECT & 327 & RICH.PMPHS-10 HRM46.4 & & \\
\hline 2794 & & RIVER & & RAW GRAB & TRANSECT & 327 & RICH.PMPHS-10 HRM46.4 & & \\
\hline 2795 F & $\mathrm{F}$ & RIVER & & RAW GRAB & TRANSECT & 327 & RICH.PMPHS-10 HRM46.4 & & \\
\hline 2796 & & RIVER & & RAW GRAB & TRANSECT & 327 & RICH.PMPHS-10 HRM46.4 & & \\
\hline 2797 & & RIVER & & RAW GRAB & TRANSECT & 327 & RICH.PMPHS-10 HRM46.4 & & \\
\hline 2798 & & RIVER & & RAW GRAB & TRANSECT & 327 & RICH.PMPHS-10 HRM46.4 & & \\
\hline 2799 & & RIVER & & RAW GRAB & TRANSECT & 321 & RICH.PMPHS-2 HRM46.4 & & \\
\hline 2800 & & RIVER & & RAW GRAB & TRANSECT & 321 & RICH.PMPHS-2 HRM46.4 & & \\
\hline 2801 & & RIVER & & RAW GRAB & TRANSECT & 321 & RICH.PMPHS-2 HRM46.4 & & \\
\hline 2802 & & RIVER & & RAW GRAB & TRANSECT & 321 & RICH.PMPHS-2 HRM46.4 & & \\
\hline 2803 & & RIVER & & RAW GRAB & TRANSECT & 321 & RICH.PMPHS-2 HRM46.4 & & \\
\hline 2804 & & RIVER & & RAW GRAB & TRANSECT & 321 & RICH.PMPHS-2 HRM46.4 & & \\
\hline 2805 & & RIVER & & RAW GRAB & TRANSECT & 321 & RICH.PMPHS-2 HRM46.4 & & \\
\hline 2806 & & RIVER & & RAW GRAB & TRANSECT & 322 & RICH.PMPHS-3 HRM46.4 & & \\
\hline 2807 & & RIVER & & RAW GRAB & TRANSECT & $322 \mathrm{~F}$ & RICH.PMPHS-3 HRM46.4 & & \\
\hline 2808 & & RIVER & & RAW GRAB & TRANSECT & 322 & RICH.PMPHS-3 HRM46.4 & & \\
\hline $2809 \mathrm{~F}$ & $\mathrm{~F}$ & RIVER & & RAW GRAB & TRANSECT & 322 & RICH.PMPHS-3 HRM46.4 & & \\
\hline 2810 & & RIVER & & RAW GRAB & TRANSECT & 322 & RICH.PMPHS-3 HRM46.4 & & \\
\hline 2811 & & RIVER & & RAW GRAB & TRANSECT & 322 & RICH.PMPHS-3 HRM46.4 & & \\
\hline 2812 & & RIVER & & RAW GRAB & TRANSECT & 322 & RICH.PMPHS-3 HRM46.4 & & \\
\hline 2813 & & RIVER & & RAW GRAB & TRANSECT & 572 & RICH.PMPHS-4 HRM46.4 & & \\
\hline
\end{tabular}




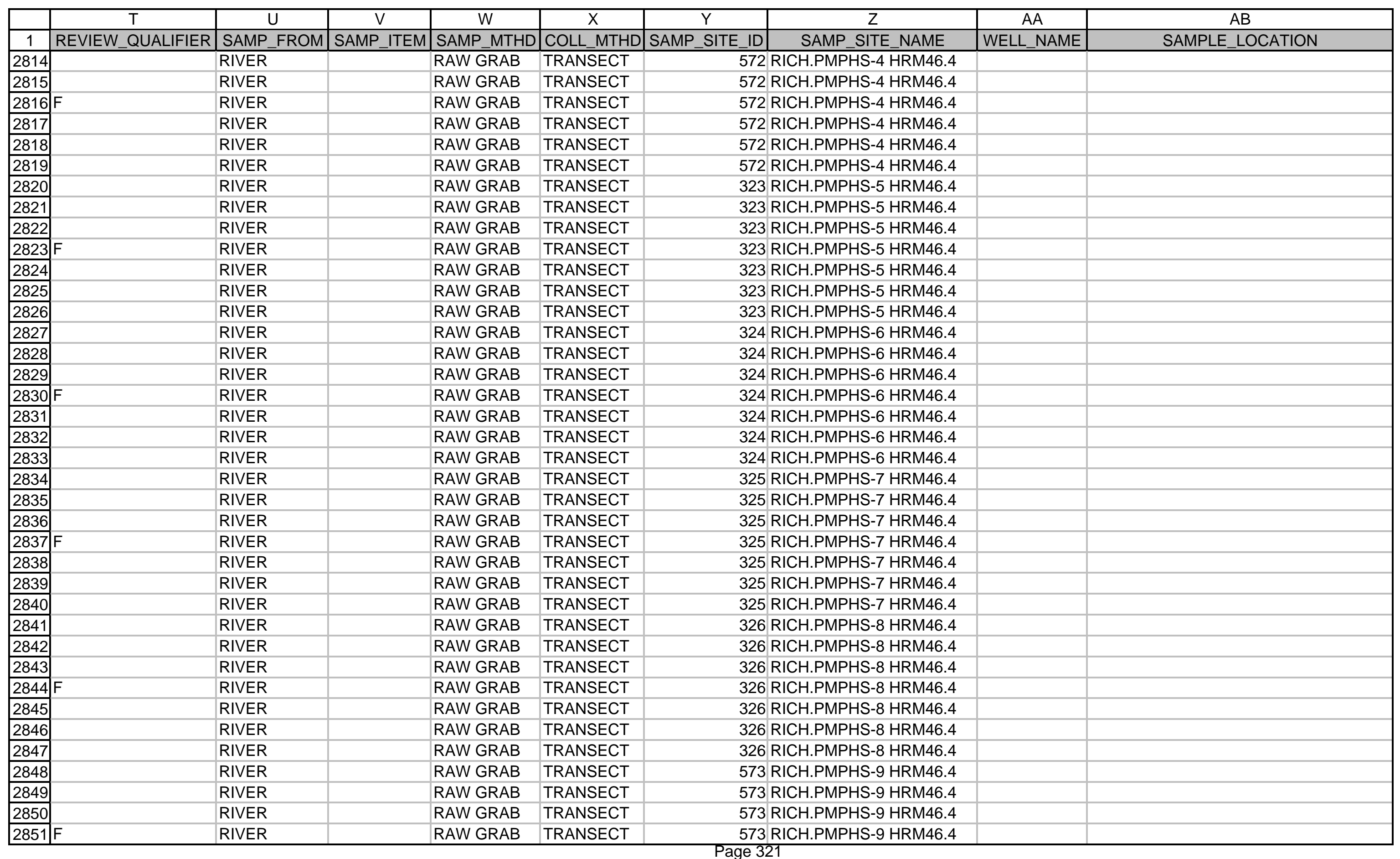




\begin{tabular}{|c|c|c|c|c|c|c|c|c|c|}
\hline & $T$ & $\bar{U}$ & $\overline{\mathrm{V}}$ & $\mathrm{W}$ & $\bar{X}$ & $\bar{Y}$ & $\bar{Z}$ & $\overline{A A}$ & $A B$ \\
\hline 1 & REVIEW_QUALIFIER & SAMP_FROM & SAMP_ITEM & SAMP_MTHD & COLL_MTHD & SAMP_SITE_ID & SAMP_SITE_NAME & WELL_NAME & SAMPLE_LOCATION \\
\hline 2852 & & RIVER & & RAW GRAB & TRANSECT & 573 & RICH.PMPHS-9 HRM46.4 & & \\
\hline 2853 & & RIVER & & RAW GRAB & TRANSECT & 573 & RICH.PMPHS-9 HRM46.4 & & \\
\hline 2854 & & RIVER & & RAW GRAB & TRANSECT & 573 & RICH.PMPHS-9 HRM46.4 & & \\
\hline 2855 & & RIVER & & RAW GRAB & TRANSECT & 328 & 100 N -1 HRM 9.5 & & \\
\hline 2856 & & RIVER & & RAW GRAB & TRANSECT & 328 & 100 N -1 HRM 9.5 & & \\
\hline 2857 & & RIVER & & RAW GRAB & TRANSECT & 328 & 100 N -1 HRM 9.5 & & \\
\hline 2858 & & RIVER & & RAW GRAB & TRANSECT & 328 & 100 N -1 HRM 9.5 & & \\
\hline 2859 & $\mathrm{~F}$ & RIVER & & RAW GRAB & TRANSECT & 328 & 100 N -1 HRM 9.5 & & \\
\hline 2860 & & RIVER & & RAW GRAB & TRANSECT & 328 & 100 N -1 HRM 9.5 & & \\
\hline 2861 & & RIVER & & RAW GRAB & TRANSECT & 328 & 100 N -1 HRM 9.5 & & \\
\hline 2862 & & RIVER & & RAW GRAB & TRANSECT & 328 & 100 N -1 HRM 9.5 & & \\
\hline 2863 & & RIVER & & RAW GRAB & TRANSECT & 328 & 100 N -1 HRM 9.5 & & \\
\hline 2864 & & RIVER & & RAW GRAB & TRANSECT & 328 & 100 N -1 HRM 9.5 & & \\
\hline 2865 & & RIVER & & RAW GRAB & TRANSECT & 328 & 100 N -1 HRM 9.5 & & \\
\hline 2866 & & RIVER & & RAW GRAB & TRANSECT & 328 & 100 N -1 HRM 9.5 & & \\
\hline 2867 & & RIVER & & RAW GRAB & TRANSECT & 335 & $100 \mathrm{~N}-10$ HRM 9.5 & & \\
\hline 2868 & & RIVER & & RAW GRAB & TRANSECT & 335 & $100 \mathrm{~N}-10$ HRM 9.5 & & \\
\hline 2869 & & RIVER & & RAW GRAB & TRANSECT & 335 & $100 \mathrm{~N}-10$ HRM 9.5 & & \\
\hline 2870 & $\mathrm{~F}$ & RIVER & & RAW GRAB & TRANSECT & 335 & $100 \mathrm{~N}-10$ HRM 9.5 & & \\
\hline 2871 & & RIVER & & RAW GRAB & TRANSECT & 335 : & $100 \mathrm{~N}-10$ HRM 9.5 & & \\
\hline 2872 & & RIVER & & RAW GRAB & TRANSECT & 335 & $100 \mathrm{~N}-10$ HRM 9.5 & & \\
\hline 2873 & & RIVER & & RAW GRAB & TRANSECT & 335 & $100 \mathrm{~N}-10$ HRM 9.5 & & \\
\hline 2874 & & RIVER & & RAW GRAB & TRANSECT & 329 & 100 N -2 HRM 9.5 & & \\
\hline 2875 & & RIVER & & RAW GRAB & TRANSECT & 329 & $100 \mathrm{~N}-2$ HRM 9.5 & & \\
\hline 2876 & & RIVER & & RAW GRAB & TRANSECT & 329 & 100 N -2 HRM 9.5 & & \\
\hline 2877 & $\mathrm{~F}$ & RIVER & & RAW GRAB & TRANSECT & 329 & 100 N -2 HRM 9.5 & & \\
\hline 2878 & & RIVER & & RAW GRAB & TRANSECT & 329 & 100 N -2 HRM 9.5 & & \\
\hline 2879 & & RIVER & & RAW GRAB & TRANSECT & 329 & 100 N -2 HRM 9.5 & & \\
\hline 2880 & & RIVER & & RAW GRAB & TRANSECT & 329 & 100 N -2 HRM 9.5 & & \\
\hline 2881 & & RIVER & & RAW GRAB & TRANSECT & 330 & 100 N -3 HRM 9.5 & & \\
\hline 2882 & & RIVER & & RAW GRAB & TRANSECT & 330 & 100 N -3 HRM 9.5 & & \\
\hline 2883 & & RIVER & & RAW GRAB & TRANSECT & 330 & 100 N -3 HRM 9.5 & & \\
\hline 2884 & $\mathrm{~F}$ & RIVER & & RAW GRAB & TRANSECT & 330 & 100 N -3 HRM 9.5 & & \\
\hline 2885 & & RIVER & & RAW GRAB & TRANSECT & 330 & 100 N -3 HRM 9.5 & & \\
\hline 2886 & & RIVER & & RAW GRAB & TRANSECT & 330 & 100 N -3 HRM 9.5 & & \\
\hline 2887 & & RIVER & & RAW GRAB & TRANSECT & 330 & 100 N -3 HRM 9.5 & & \\
\hline 2888 & & RIVER & & RAW GRAB & TRANSECT & 574 & 100 N -4 HRM 9.5 & & \\
\hline 2889 & & RIVER & & RAW GRAB & TRANSECT & $574:$ & $100 \mathrm{~N}-4$ HRM 9.5 & & \\
\hline
\end{tabular}




\begin{tabular}{|c|c|c|c|c|c|c|c|c|c|}
\hline & $T$ & $\bar{U}$ & $\overline{\mathrm{V}}$ & $\mathrm{W}$ & $\bar{X}$ & $\bar{Y}$ & $\bar{Z}$ & $\overline{A A}$ & $A B$ \\
\hline 1 & REVIEW_QUALIFIER & SAMP_FROM & SAMP_ITEM & SAMP_MTHD & COLL_MTHD & SAMP_SITE_ID & SAMP_SITE_NAME & WELL_NAME & SAMPLE_LOCATION \\
\hline 2890 & & RIVER & & RAW GRAB & TRANSECT & 574 & 100 N -4 HRM 9.5 & & \\
\hline 2891 & $\mathrm{~F}$ & RIVER & & RAW GRAB & TRANSECT & 574 ? & 100 N -4 HRM 9.5 & & \\
\hline 2892 & & RIVER & & RAW GRAB & TRANSECT & 574 : & 100 N -4 HRM 9.5 & & \\
\hline 2893 & & RIVER & & RAW GRAB & TRANSECT & 574 & 100 N -4 HRM 9.5 & & \\
\hline 2894 & & RIVER & & RAW GRAB & TRANSECT & 574 & 100 N -4 HRM 9.5 & & \\
\hline 2895 & & RIVER & & RAW GRAB & TRANSECT & 331 & 100 N -5 HRM 9.5 & & \\
\hline 2896 & & RIVER & & RAW GRAB & TRANSECT & 331 & 100 N -5 HRM 9.5 & & \\
\hline 2897 & & RIVER & & RAW GRAB & TRANSECT & 331 & 100 N -5 HRM 9.5 & & \\
\hline 2898 & & RIVER & & RAW GRAB & TRANSECT & 331 & 100 N -5 HRM 9.5 & & \\
\hline 2899 & & RIVER & & RAW GRAB & TRANSECT & 331 & 100 N -5 HRM 9.5 & & \\
\hline 2900 & & RIVER & & RAW GRAB & TRANSECT & 331 & 100 N -5 HRM 9.5 & & \\
\hline 2901 & & RIVER & & RAW GRAB & TRANSECT & $331:$ & 100 N -5 HRM 9.5 & & \\
\hline 2902 & & RIVER & & RAW GRAB & TRANSECT & 332 & 100 N -6 HRM 9.5 & & \\
\hline 2903 & & RIVER & & RAW GRAB & TRANSECT & 332 & 100 N -6 HRM 9.5 & & \\
\hline 2904 & & RIVER & & RAW GRAB & TRANSECT & 332 & 100 N -6 HRM 9.5 & & \\
\hline 2905 & $\mathrm{~F}$ & RIVER & & RAW GRAB & TRANSECT & 332 & 100 N -6 HRM 9.5 & & \\
\hline 2906 & & RIVER & & RAW GRAB & TRANSECT & 332 & 100 N -6 HRM 9.5 & & \\
\hline 2907 & & RIVER & & RAW GRAB & TRANSECT & 332 & 100 N -6 HRM 9.5 & & \\
\hline 2908 & & RIVER & & RAW GRAB & TRANSECT & 332 & 100 N -6 HRM 9.5 & & \\
\hline 2909 & & RIVER & & RAW GRAB & TRANSECT & 333 & 100 N -7 HRM 9.5 & & \\
\hline 2910 & & RIVER & & RAW GRAB & TRANSECT & 333 & 100 N -7 HRM 9.5 & & \\
\hline 2911 & & RIVER & & RAW GRAB & TRANSECT & 333 & 100 N -7 HRM 9.5 & & \\
\hline 2912 & $\mathrm{~F}$ & RIVER & & RAW GRAB & TRANSECT & 333 & $100 \mathrm{~N}-7$ HRM 9.5 & & \\
\hline 2913 & & RIVER & & RAW GRAB & TRANSECT & 333 & $100 \mathrm{~N}-7$ HRM 9.5 & & \\
\hline 2914 & & RIVER & & RAW GRAB & TRANSECT & 333 & 100 N -7 HRM 9.5 & & \\
\hline 2915 & & RIVER & & RAW GRAB & TRANSECT & 333 & 100 N -7 HRM 9.5 & & \\
\hline 2916 & & RIVER & & RAW GRAB & TRANSECT & 575 & 100 N -8 HRM 9.5 & & \\
\hline 2917 & & RIVER & & RAW GRAB & TRANSECT & 575 & 100 N -8 HRM 9.5 & & \\
\hline 2918 & & RIVER & & RAW GRAB & TRANSECT & 575 & 100 N -8 HRM 9.5 & & \\
\hline 2919 & $\mathrm{~F}$ & RIVER & & RAW GRAB & TRANSECT & 575 & 100 N -8 HRM 9.5 & & \\
\hline 2920 & & RIVER & & RAW GRAB & TRANSECT & 575 & 100 N -8 HRM 9.5 & & \\
\hline 2921 & & RIVER & & RAW GRAB & TRANSECT & 575 : & 100 N -8 HRM 9.5 & & \\
\hline 2922 & & RIVER & & RAW GRAB & TRANSECT & 575 & 100 N -8 HRM 9.5 & & \\
\hline 2923 & & RIVER & & RAW GRAB & TRANSECT & 334 & 100 N -9 HRM 9.5 & & \\
\hline 2924 & & RIVER & & RAW GRAB & TRANSECT & 334 & 100 N -9 HRM 9.5 & & \\
\hline 2925 & & RIVER & & RAW GRAB & TRANSECT & 334 & 100 N -9 HRM 9.5 & & \\
\hline 2926 & $\mathrm{~F}$ & RIVER & & RAW GRAB & TRANSECT & 334 & 100 N -9 HRM 9.5 & & \\
\hline 2927 & & RIVER & & RAW GRAB & TRANSECT & $334:$ & 100 N -9 HRM 9.5 & & \\
\hline
\end{tabular}




\begin{tabular}{|c|c|c|c|c|c|c|c|c|c|}
\hline & $\mathrm{T}$ & 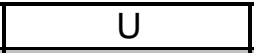 & $\mathrm{V}$ & $\mathrm{W}$ & $\mathrm{X}$ & $\mathrm{Y}$ & $Z$ & $\mathrm{AA}$ & $A B$ \\
\hline \begin{tabular}{l|l}
1 & 1 \\
\end{tabular} & REVIEW_QUALIFIER & SAMP_FROM & SAMP_ITEM & SAMP_MTHD & COLL_MTHD & SAMP_SITE_ID & SAMP_SITE_NAME & WELL_NAME & SAMPLE_LOCATION \\
\hline 2928 & & RIVER & & RAW GRAB & TRANSECT & 334 & 100 N -9 HRM 9.5 & & \\
\hline 2929 & & RIVER & & RAW GRAB & TRANSECT & 334 & $100 \mathrm{~N}-9$ HRM 9.5 & & \\
\hline 2930 & & RIVER & & RAW GRAB & TRANSECT & 336 & 100 F -1 HRM 19.0 & & \\
\hline 2931 & & RIVER & & RAW GRAB & TRANSECT & 336 & 100 F -1 HRM 19.0 & & \\
\hline 2932 & & RIVER & & RAW GRAB & TRANSECT & 336 & 100 F -1 HRM 19.0 & & \\
\hline $2933 \mathrm{~F}$ & $\mathrm{~F}$ & RIVER & & RAW GRAB & TRANSECT & 336 & 100 F -1 HRM 19.0 & & \\
\hline 2934 & & RIVER & & RAW GRAB & TRANSECT & 336 & 100 F -1 HRM 19.0 & & \\
\hline 2935 & & RIVER & & RAW GRAB & TRANSECT & 336 & 100 F -1 HRM 19.0 & & \\
\hline 2936 & & RIVER & & RAW GRAB & TRANSECT & 336 & 100 F -1 HRM 19.0 & & \\
\hline 2937 & & RIVER & & RAW GRAB & TRANSECT & 341 & 100 F -10 HRM 19.0 & & \\
\hline 2938 & & RIVER & & RAW GRAB & TRANSECT & 341 & 100 F -10 HRM 19.0 & & \\
\hline 2939 & & RIVER & & RAW GRAB & TRANSECT & 341 & 100 F -10 HRM 19.0 & & \\
\hline $2940 \mathrm{~F}$ & $\mathrm{~F}$ & RIVER & & RAW GRAB & TRANSECT & 341 & 100 F -10 HRM 19.0 & & \\
\hline 2941 & & RIVER & & RAW GRAB & TRANSECT & 341 & 100 F -10 HRM 19.0 & & \\
\hline 2942 & & RIVER & & RAW GRAB & TRANSECT & 341 & 100 F -10 HRM 19.0 & & \\
\hline 2943 & & RIVER & & RAW GRAB & TRANSECT & 341 & 100 F -10 HRM 19.0 & & \\
\hline 2944 & & RIVER & & RAW GRAB & TRANSECT & 576 & 100 F -2 HRM 19.0 & & \\
\hline 2945 & & RIVER & & RAW GRAB & TRANSECT & 576 & 100 F -2 HRM 19.0 & & \\
\hline 2946 & & RIVER & & RAW GRAB & TRANSECT & 576 & 100 F -2 HRM 19.0 & & \\
\hline $2947 \mathrm{~F}$ & $\mathrm{~F}$ & RIVER & & RAW GRAB & TRANSECT & 576 & 100 F -2 HRM 19.0 & & \\
\hline 2948 & & RIVER & & RAW GRAB & TRANSECT & 576 & 100 F -2 HRM 19.0 & & \\
\hline 2949 & & RIVER & & RAW GRAB & TRANSECT & 576 & 100 F -2 HRM 19.0 & & \\
\hline 2950 & & RIVER & & RAW GRAB & TRANSECT & 576 & 100 F -2 HRM 19.0 & & \\
\hline 2951 & & RIVER & & RAW GRAB & TRANSECT & 337 & 100 F -3 HRM 19.0 & & \\
\hline 2952 & & RIVER & & RAW GRAB & TRANSECT & 337 & 100 F -3 HRM 19.0 & & \\
\hline 2953 & & RIVER & & RAW GRAB & TRANSECT & 337 & 100 F -3 HRM 19.0 & & \\
\hline $2954 \mathrm{~F}$ & $\mathrm{~F}$ & RIVER & & RAW GRAB & TRANSECT & 337 & 100 F -3 HRM 19.0 & & \\
\hline 2955 & & RIVER & & RAW GRAB & TRANSECT & 337 & 100 F -3 HRM 19.0 & & \\
\hline 2956 & & RIVER & & RAW GRAB & TRANSECT & 337 & 100 F -3 HRM 19.0 & & \\
\hline 2957 & & RIVER & & RAW GRAB & TRANSECT & 337 & 100 F -3 HRM 19.0 & & \\
\hline 2958 & & RIVER & & RAW GRAB & TRANSECT & 338 & 100 F -4 HRM 19.0 & & \\
\hline 2959 & & RIVER & & RAW GRAB & TRANSECT & 338 & 100 F -4 HRM 19.0 & & \\
\hline 2960 & & RIVER & & RAW GRAB & TRANSECT & 338 & 100 F -4 HRM 19.0 & & \\
\hline $2961 \mathrm{~F}$ & $\mathrm{~F}$ & RIVER & & RAW GRAB & TRANSECT & 338 & 100 F -4 HRM 19.0 & & \\
\hline 2962 & & RIVER & & RAW GRAB & TRANSECT & 338 & 100 F -4 HRM 19.0 & & \\
\hline 2963 & & RIVER & & RAW GRAB & TRANSECT & 338 & 100 F -4 HRM 19.0 & & \\
\hline 2964 & & RIVER & & RAW GRAB & TRANSECT & 338 & 100 F -4 HRM 19.0 & & \\
\hline 2965 & & RIVER & & RAW GRAB & TRANSECT & $577:$ & 100 F -5 HRM 19.0 & & \\
\hline
\end{tabular}




\begin{tabular}{|c|c|c|c|c|c|c|c|c|c|}
\hline & $T$ & $\bar{U}$ & $\overline{\mathrm{V}}$ & $\mathrm{W}$ & $\bar{X}$ & $\bar{Y}$ & $\bar{Z}$ & $\overline{A A}$ & $A B$ \\
\hline 1 & REVIEW_QUALIFIER & SAMP_FROM & SAMP_ITEM & SAMP_MTHD & COLL_MTHD & SAMP_SITE_ID & SAMP_SITE_NAME & WELL_NAME & SAMPLE_LOCATION \\
\hline 2966 & & RIVER & & RAW GRAB & TRANSECT & 577 : & 100 F -5 HRM 19.0 & & \\
\hline 2967 & & RIVER & & RAW GRAB & TRANSECT & 577 & 100 F -5 HRM 19.0 & & \\
\hline 2968 & $\mathrm{~F}$ & RIVER & & RAW GRAB & TRANSECT & 577 : & 100 F -5 HRM 19.0 & & \\
\hline 2969 & & RIVER & & RAW GRAB & TRANSECT & 577 & 100 F -5 HRM 19.0 & & \\
\hline 2970 & & RIVER & & RAW GRAB & TRANSECT & 577 : & 100 F -5 HRM 19.0 & & \\
\hline 2971 & & RIVER & & RAW GRAB & TRANSECT & 577 & 100 F -5 HRM 19.0 & & \\
\hline 2972 & & RIVER & & RAW GRAB & TRANSECT & 606 & 100 F -6 HRM 19.0 & & \\
\hline 2973 & & RIVER & & RAW GRAB & TRANSECT & 606 & 100 F -6 HRM 19.0 & & \\
\hline 2974 & & RIVER & & RAW GRAB & TRANSECT & 606 & 100 F -6 HRM 19.0 & & \\
\hline 2975 & $\mathrm{~F}$ & RIVER & & RAW GRAB & TRANSECT & 606 & 100 F -6 HRM 19.0 & & \\
\hline 2976 & & RIVER & & RAW GRAB & TRANSECT & 606 & 100 F -6 HRM 19.0 & & \\
\hline 2977 & & RIVER & & RAW GRAB & TRANSECT & 606 & 100 F -6 HRM 19.0 & & \\
\hline 2978 & & RIVER & & RAW GRAB & TRANSECT & $606:$ & 100 F -6 HRM 19.0 & & \\
\hline 2979 & & RIVER & & RAW GRAB & TRANSECT & 339 & 100 F -7 HRM 19.0 & & \\
\hline 2980 & & RIVER & & RAW GRAB & TRANSECT & 339 & 100 F -7 HRM 19.0 & & \\
\hline 2981 & & RIVER & & RAW GRAB & TRANSECT & 339 & 100 F -7 HRM 19.0 & & \\
\hline 2982 & $\mathrm{~F}$ & RIVER & & RAW GRAB & TRANSECT & 339 & 100 F -7 HRM 19.0 & & \\
\hline 2983 & & RIVER & & RAW GRAB & TRANSECT & 339 & 100 F -7 HRM 19.0 & & \\
\hline 2984 & & RIVER & & RAW GRAB & TRANSECT & 339 & 100 F -7 HRM 19.0 & & \\
\hline 2985 & & RIVER & & RAW GRAB & TRANSECT & 339 & 100 F -7 HRM 19.0 & & \\
\hline 2986 & & RIVER & & RAW GRAB & TRANSECT & 340 & 100 F -8 HRM 19.0 & & \\
\hline 2987 & & RIVER & & RAW GRAB & TRANSECT & 340 & 100 F -8 HRM 19.0 & & \\
\hline 2988 & & RIVER & & RAW GRAB & TRANSECT & 340 & 100 F -8 HRM 19.0 & & \\
\hline 2989 & $\mathrm{~F}$ & RIVER & & RAW GRAB & TRANSECT & 340 & 100 F -8 HRM 19.0 & & \\
\hline 2990 & & RIVER & & RAW GRAB & TRANSECT & 340 & 100 F -8 HRM 19.0 & & \\
\hline 2991 & & RIVER & & RAW GRAB & TRANSECT & 340 & 100 F -8 HRM 19.0 & & \\
\hline 2992 & & RIVER & & RAW GRAB & TRANSECT & 340 & 100 F -8 HRM 19.0 & & \\
\hline 2993 & & RIVER & & RAW GRAB & TRANSECT & 467 & 100 F -9 HRM 19.0 & & \\
\hline 2994 & & RIVER & & RAW GRAB & TRANSECT & 467 & 100 F -9 HRM 19.0 & & \\
\hline 2995 & & RIVER & & RAW GRAB & TRANSECT & 467 & 100 F -9 HRM 19.0 & & \\
\hline 2996 & $\mathrm{~F}$ & RIVER & & RAW GRAB & TRANSECT & 467 & 100 F -9 HRM 19.0 & & \\
\hline 2997 & & RIVER & & RAW GRAB & TRANSECT & 467 & 100 F -9 HRM 19.0 & & \\
\hline 2998 & & RIVER & & RAW GRAB & TRANSECT & 467 & 100 F -9 HRM 19.0 & & \\
\hline 2999 & & RIVER & & RAW GRAB & TRANSECT & 467 & 100 F -9 HRM 19.0 & & \\
\hline 3000 & & RIVER & & RAW GRAB & TRANSECT & 578 & HANFRD TS-1 HRM 28.7 & & \\
\hline 3001 & & RIVER & & RAW GRAB & TRANSECT & 578 & HANFRD TS-1 HRM 28.7 & & \\
\hline 3002 & & RIVER & & RAW GRAB & TRANSECT & 578 & HANFRD TS-1 HRM 28.7 & & \\
\hline 3003 & $\mathrm{~F}$ & RIVER & & RAW GRAB & TRANSECT & 578 & HANFRD TS-1 HRM 28.7 & & \\
\hline
\end{tabular}




\begin{tabular}{|c|c|c|c|c|c|c|c|c|c|}
\hline & $\mathrm{T}$ & $\bar{U}$ & $\mathrm{~V}$ & $\mathrm{~W}$ & $\mathrm{X}$ & $\mathrm{Y}$ & $Z$ & $\mathrm{AA}$ & $\mathrm{AB}$ \\
\hline \begin{tabular}{l|l}
1 & 1 \\
\end{tabular} & REVIEW_QUALIFIER & SAMP_FROM & SAMP_ITEM & SAMP_MTHD & COLL_MTHD & SAMP_SITE_ID & SAMP_SITE_NAME & WELL_NAME & SAMPLE_LOCATION \\
\hline 3004 & & RIVER & & RAW GRAB & TRANSECT & 578 & HANFRD TS-1 HRM 28.7 & & \\
\hline 3005 & & RIVER & & RAW GRAB & TRANSECT & 578 & HANFRD TS-1 HRM 28.7 & & \\
\hline 3006 & & RIVER & & RAW GRAB & TRANSECT & 578 & HANFRD TS-1 HRM 28.7 & & \\
\hline 3007 & & RIVER & & RAW GRAB & TRANSECT & 347 & HANFRD TS-10 HRM 28.7 & & \\
\hline 3008 & & RIVER & & RAW GRAB & TRANSECT & 347 & HANFRD TS-10 HRM 28.7 & & \\
\hline 3009 & & RIVER & & RAW GRAB & TRANSECT & 347 & HANFRD TS-10 HRM 28.7 & & \\
\hline $3010 \mathrm{~F}$ & $\mathrm{~F}$ & RIVER & & RAW GRAB & TRANSECT & 347 & HANFRD TS-10 HRM 28.7 & & \\
\hline 3011 & & RIVER & & RAW GRAB & TRANSECT & 347 & HANFRD TS-10 HRM 28.7 & & \\
\hline 3012 & & RIVER & & RAW GRAB & TRANSECT & 347 & HANFRD TS-10 HRM 28.7 & & \\
\hline 3013 & & RIVER & & RAW GRAB & TRANSECT & 347 & HANFRD TS-10 HRM 28.7 & & \\
\hline 3014 & & RIVER & & RAW GRAB & TRANSECT & 342 & HANFRD TS-2 HRM 28.7 & & \\
\hline 3015 & & RIVER & & RAW GRAB & TRANSECT & 342 & HANFRD TS-2 HRM 28.7 & & \\
\hline 3016 & & RIVER & & RAW GRAB & TRANSECT & 342 & HANFRD TS-2 HRM 28.7 & & \\
\hline 3017 & & RIVER & & RAW GRAB & TRANSECT & 342 & HANFRD TS-2 HRM 28.7 & & \\
\hline 3018 & & RIVER & & RAW GRAB & TRANSECT & 342 & HANFRD TS-2 HRM 28.7 & & \\
\hline 3019 & & RIVER & & RAW GRAB & TRANSECT & 342 & HANFRD TS-2 HRM 28.7 & & \\
\hline 3020 & & RIVER & & RAW GRAB & TRANSECT & 342 & HANFRD TS-2 HRM 28.7 & & \\
\hline 3021 & & RIVER & & RAW GRAB & TRANSECT & 468 & HANFRD TS-3 HRM 28.7 & & \\
\hline 3022 & & RIVER & & RAW GRAB & TRANSECT & 468 & HANFRD TS-3 HRM 28.7 & & \\
\hline 3023 & & RIVER & & RAW GRAB & TRANSECT & 468 & HANFRD TS-3 HRM 28.7 & & \\
\hline $3024 \mathrm{~F}$ & $\mathrm{~F}$ & RIVER & & RAW GRAB & TRANSECT & 468 & HANFRD TS-3 HRM 28.7 & & \\
\hline 3025 & & RIVER & & RAW GRAB & TRANSECT & 468 & HANFRD TS-3 HRM 28.7 & & \\
\hline 3026 & & RIVER & & RAW GRAB & TRANSECT & 468 & HANFRD TS-3 HRM 28.7 & & \\
\hline 3027 & & RIVER & & RAW GRAB & TRANSECT & 468 & HANFRD TS-3 HRM 28.7 & & \\
\hline 3028 & & RIVER & & RAW GRAB & TRANSECT & 343 & HANFRD TS-4 HRM 28.7 & & \\
\hline 3029 & & RIVER & & RAW GRAB & TRANSECT & 343 & HANFRD TS-4 HRM 28.7 & & \\
\hline 3030 & & RIVER & & RAW GRAB & TRANSECT & 343 & HANFRD TS-4 HRM 28.7 & & \\
\hline $3031 \mathrm{~F}$ & $\mathrm{~F}$ & RIVER & & RAW GRAB & TRANSECT & 343 & HANFRD TS-4 HRM 28.7 & & \\
\hline 3032 & & RIVER & & RAW GRAB & TRANSECT & 343 & HANFRD TS-4 HRM 28.7 & & \\
\hline 3033 & & RIVER & & RAW GRAB & TRANSECT & 343 & HANFRD TS-4 HRM 28.7 & & \\
\hline 3034 & & RIVER & & RAW GRAB & TRANSECT & 343 & HANFRD TS-4 HRM 28.7 & & \\
\hline 3035 & & RIVER & & RAW GRAB & TRANSECT & 344 & HANFRD TS-5 HRM 28.7 & & \\
\hline 3036 & & RIVER & & RAW GRAB & TRANSECT & 344 & HANFRD TS-5 HRM 28.7 & & \\
\hline 3037 & & RIVER & & RAW GRAB & TRANSECT & 344 & HANFRD TS-5 HRM 28.7 & & \\
\hline $3038 \mathrm{~F}$ & $\mathrm{~F}$ & RIVER & & RAW GRAB & TRANSECT & 344 & HANFRD TS-5 HRM 28.7 & & \\
\hline 3039 & & RIVER & & RAW GRAB & TRANSECT & 344 & HANFRD TS-5 HRM 28.7 & & \\
\hline 3040 & & RIVER & & RAW GRAB & TRANSECT & 344 & HANFRD TS-5 HRM 28.7 & & \\
\hline 3041 & & RIVER & & RAW GRAB & TRANSECT & 344 & HANFRD TS-5 HRM 28.7 & & \\
\hline
\end{tabular}




\begin{tabular}{|c|c|c|c|c|c|c|c|c|c|}
\hline & $\mathrm{T}$ & $\bar{U}$ & $\mathrm{~V}$ & $\mathrm{~W}$ & $\mathrm{X}$ & $\mathrm{Y}$ & $Z$ & $\mathrm{AA}$ & $\mathrm{AB}$ \\
\hline \begin{tabular}{l|l}
1 & 1 \\
\end{tabular} & REVIEW_QUALIFIER & SAMP_FROM & SAMP_ITEM & SAMP_MTHD & COLL_MTHD & SAMP_SITE_ID & SAMP_SITE_NAME & WELL_NAME & SAMPLE_LOCATION \\
\hline 3042 & & RIVER & & RAW GRAB & TRANSECT & 345 & HANFRD TS-6 HRM 28.7 & & \\
\hline 3043 & & RIVER & & RAW GRAB & TRANSECT & 345 & HANFRD TS-6 HRM 28.7 & & \\
\hline 3044 & & RIVER & & RAW GRAB & TRANSECT & 345 & HANFRD TS-6 HRM 28.7 & & \\
\hline $3045 \mathrm{~F}$ & $\mathrm{~F}$ & RIVER & & RAW GRAB & TRANSECT & 345 & HANFRD TS-6 HRM 28.7 & & \\
\hline 3046 & & RIVER & & RAW GRAB & TRANSECT & 345 & HANFRD TS-6 HRM 28.7 & & \\
\hline 3047 & & RIVER & & RAW GRAB & TRANSECT & 345 & HANFRD TS-6 HRM 28.7 & & \\
\hline 3048 & & RIVER & & RAW GRAB & TRANSECT & 345 & HANFRD TS-6 HRM 28.7 & & \\
\hline 3049 & & RIVER & & RAW GRAB & TRANSECT & 469 & HANFRD TS-7 HRM 28.7 & & \\
\hline 3050 & & RIVER & & RAW GRAB & TRANSECT & 469 & HANFRD TS-7 HRM 28.7 & & \\
\hline 3051 & & RIVER & & RAW GRAB & TRANSECT & 469 & HANFRD TS-7 HRM 28.7 & & \\
\hline $3052 \mathrm{~F}$ & $\mathrm{~F}$ & RIVER & & RAW GRAB & TRANSECT & 469 & HANFRD TS-7 HRM 28.7 & & \\
\hline 3053 & & RIVER & & RAW GRAB & TRANSECT & 469 & HANFRD TS-7 HRM 28.7 & & \\
\hline 3054 & & RIVER & & RAW GRAB & TRANSECT & 469 & HANFRD TS-7 HRM 28.7 & & \\
\hline 3055 & & RIVER & & RAW GRAB & TRANSECT & 469 & HANFRD TS-7 HRM 28.7 & & \\
\hline 3056 & & RIVER & & RAW GRAB & TRANSECT & 579 & HANFRD TS-8 HRM 28.7 & & \\
\hline 3057 & & RIVER & & RAW GRAB & TRANSECT & 579 & HANFRD TS-8 HRM 28.7 & & \\
\hline 3058 & & RIVER & & RAW GRAB & TRANSECT & 579 & HANFRD TS-8 HRM 28.7 & & \\
\hline $3059 \mathrm{~F}$ & $\mathrm{~F}$ & RIVER & & RAW GRAB & TRANSECT & 579 & HANFRD TS-8 HRM 28.7 & & \\
\hline 3060 & & RIVER & & RAW GRAB & TRANSECT & 579 & HANFRD TS-8 HRM 28.7 & & \\
\hline 3061 & & RIVER & & RAW GRAB & TRANSECT & 579 & HANFRD TS-8 HRM 28.7 & & \\
\hline 3062 & & RIVER & & RAW GRAB & TRANSECT & 579 & HANFRD TS-8 HRM 28.7 & & \\
\hline 3063 & & RIVER & & RAW GRAB & TRANSECT & 346 & HANFRD TS-9 HRM 28.7 & & \\
\hline 3064 & & RIVER & & RAW GRAB & TRANSECT & 346 & HANFRD TS-9 HRM 28.7 & & \\
\hline 3065 & & RIVER & & RAW GRAB & TRANSECT & 346 & HANFRD TS-9 HRM 28.7 & & \\
\hline $3066 \mathrm{~F}$ & $\mathrm{~F}$ & RIVER & & RAW GRAB & TRANSECT & 346 & HANFRD TS-9 HRM 28.7 & & \\
\hline 3067 & & RIVER & & RAW GRAB & TRANSECT & 346 & HANFRD TS-9 HRM 28.7 & & \\
\hline 3068 & & RIVER & & RAW GRAB & TRANSECT & 346 & HANFRD TS-9 HRM 28.7 & & \\
\hline 3069 & & RIVER & & RAW GRAB & TRANSECT & 346 & HANFRD TS-9 HRM 28.7 & & \\
\hline 3070 & & RIVER & & RAW GRAB & TRANSECT & 317 & VERNITA-1 HRM 0.3 & & \\
\hline 3071 & & RIVER & & RAW GRAB & TRANSECT & 317 & VERNITA-1 HRM 0.3 & & \\
\hline 3072 & & RIVER & & RAW GRAB & TRANSECT & 317 & VERNITA-1 HRM 0.3 & & \\
\hline $3073 \mathrm{~F}$ & $\mathrm{~F}$ & RIVER & & RAW GRAB & TRANSECT & 317 & VERNITA-1 HRM 0.3 & & \\
\hline 3074 & & RIVER & & RAW GRAB & TRANSECT & 317 & VERNITA-1 HRM 0.3 & & \\
\hline 3075 & & RIVER & & RAW GRAB & TRANSECT & 317 & VERNITA-1 HRM 0.3 & & \\
\hline 3076 & & RIVER & & RAW GRAB & TRANSECT & 317 & VERNITA-1 HRM 0.3 & & \\
\hline 3077 & & RIVER & & RAW GRAB & TRANSECT & 570 & VERNITA-2 HRM 0.3 & & \\
\hline 3078 & & RIVER & & RAW GRAB & TRANSECT & 570 & VERNITA-2 HRM 0.3 & & \\
\hline 3079 & & RIVER & & RAW GRAB & TRANSECT & 570 & VERNITA-2 HRM 0.3 & & \\
\hline
\end{tabular}




\begin{tabular}{|c|c|c|c|c|c|c|c|c|c|}
\hline & $\mathrm{T}$ & 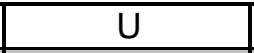 & $\mathrm{V}$ & $\mathrm{W}$ & $\mathrm{X}$ & $\mathrm{Y}$ & $Z$ & $\mathrm{AA}$ & $A B$ \\
\hline \begin{tabular}{l|l}
1 & 1 \\
\end{tabular} & REVIEW_QUALIFIER & SAMP_FROM & SAMP_ITEM & SAMP_MTHD & COLL_MTHD & SAMP_SITE_ID & SAMP_SITE_NAME & WELL_NAME & SAMPLE_LOCATION \\
\hline $3080 \mathrm{~F}$ & $\mathrm{~F}$ & RIVER & & RAW GRAB & TRANSECT & 570 & VERNITA-2 HRM 0.3 & & \\
\hline 3081 & & RIVER & & RAW GRAB & TRANSECT & 570 & VERNITA-2 HRM 0.3 & & \\
\hline 3082 & & RIVER & & RAW GRAB & TRANSECT & 570 & VERNITA-2 HRM 0.3 & & \\
\hline 3083 & & RIVER & & RAW GRAB & TRANSECT & 570 & VERNITA-2 HRM 0.3 & & \\
\hline 3084 & & RIVER & & RAW GRAB & TRANSECT & 318 & VERNITA-3 HRM 0.3 & & \\
\hline 3085 & & RIVER & & RAW GRAB & TRANSECT & 318 & VERNITA-3 HRM 0.3 & & \\
\hline 3086 & & RIVER & & RAW GRAB & TRANSECT & 318 & VERNITA-3 HRM 0.3 & & \\
\hline $3087 \mathrm{~F}$ & $\mathrm{~F}$ & RIVER & & RAW GRAB & TRANSECT & 318 & VERNITA-3 HRM 0.3 & & \\
\hline 3088 & & RIVER & & RAW GRAB & TRANSECT & 318 & VERNITA-3 HRM 0.3 & & \\
\hline 3089 & & RIVER & & RAW GRAB & TRANSECT & 318 & VERNITA-3 HRM 0.3 & & \\
\hline 3090 & & RIVER & & RAW GRAB & TRANSECT & 318 & VERNITA-3 HRM 0.3 & & \\
\hline 3091 & & RIVER & & RAW GRAB & TRANSECT & 319 & VERNITA-4 HRM 0.3 & & \\
\hline 3092 & & RIVER & & RAW GRAB & TRANSECT & 319 & VERNITA-4 HRM 0.3 & & \\
\hline 3093 & & RIVER & & RAW GRAB & TRANSECT & 319 & VERNITA-4 HRM 0.3 & & \\
\hline $3094 \mathrm{~F}$ & $\mathrm{~F}$ & RIVER & & RAW GRAB & TRANSECT & 319 & VERNITA-4 HRM 0.3 & & \\
\hline 3095 & & RIVER & & RAW GRAB & TRANSECT & 319 & VERNITA-4 HRM 0.3 & & \\
\hline 3096 & & RIVER & & RAW GRAB & TRANSECT & 319 & VERNITA-4 HRM 0.3 & & \\
\hline 3097 & & RIVER & & RAW GRAB & TRANSECT & 319 & VERNITA-4 HRM 0.3 & & \\
\hline 3098 & & RIVER & & RAW GRAB & TRANSECT & 571 & RICH.PMPHS-1 HRM46.4 & & \\
\hline 3099 & & RIVER & & RAW GRAB & TRANSECT & 571 & RICH.PMPHS-1 HRM46.4 & & \\
\hline 3100 & & RIVER & & RAW GRAB & TRANSECT & 571 & RICH.PMPHS-1 HRM46.4 & & \\
\hline 3101 & & RIVER & & RAW GRAB & TRANSECT & 571 & RICH.PMPHS-1 HRM46.4 & & \\
\hline 3102 & & RIVER & & RAW GRAB & TRANSECT & 571 & RICH.PMPHS-1 HRM46.4 & & \\
\hline 3103 & & RIVER & & RAW GRAB & TRANSECT & 571 & RICH.PMPHS-1 HRM46.4 & & \\
\hline 3104 & & RIVER & & RAW GRAB & TRANSECT & 571 & RICH.PMPHS-1 HRM46.4 & & \\
\hline 3105 & & RIVER & & RAW GRAB & TRANSECT & 571 & RICH.PMPHS-1 HRM46.4 & & \\
\hline 3106 & & RIVER & & RAW GRAB & TRANSECT & 571 & RICH.PMPHS-1 HRM46.4 & & \\
\hline 3107 & & RIVER & & RAW GRAB & TRANSECT & 571 & RICH.PMPHS-1 HRM46.4 & & \\
\hline 3108 & & RIVER & & RAW GRAB & TRANSECT & 571 & RICH.PMPHS-1 HRM46.4 & & \\
\hline 3109 & & RIVER & & RAW GRAB & TRANSECT & 571 & RICH.PMPHS-1 HRM46.4 & & \\
\hline 3110 & & RIVER & & RAW GRAB & TRANSECT & 327 & RICH.PMPHS-10 HRM46.4 & & \\
\hline 3111 & & RIVER & & RAW GRAB & TRANSECT & 327 & RICH.PMPHS-10 HRM46.4 & & \\
\hline 3112 & & RIVER & & RAW GRAB & TRANSECT & 327 & RICH.PMPHS-10 HRM46.4 & & \\
\hline $3113 \mathrm{~F}$ & $\mathrm{~F}$ & RIVER & & RAW GRAB & TRANSECT & 327 & RICH.PMPHS-10 HRM46.4 & & \\
\hline 3114 & & RIVER & & RAW GRAB & TRANSECT & 327 & RICH.PMPHS-10 HRM46.4 & & \\
\hline 3115 & & RIVER & & RAW GRAB & TRANSECT & 327 & RICH.PMPHS-10 HRM46.4 & & \\
\hline 3116 & & RIVER & & RAW GRAB & TRANSECT & 327 & RICH.PMPHS-10 HRM46.4 & & \\
\hline 3117 & & RIVER & & RAW GRAB & TRANSECT & 321 & RICH.PMPHS-2 HRM46.4 & & \\
\hline
\end{tabular}




\begin{tabular}{|c|c|c|c|c|c|c|c|c|c|}
\hline & $\mathrm{T}$ & $\bar{U}$ & $\mathrm{~V}$ & $\mathrm{~W}$ & $\mathrm{X}$ & $\mathrm{Y}$ & $Z$ & $\mathrm{AA}$ & $\mathrm{AB}$ \\
\hline \begin{tabular}{l|l}
1 & 1 \\
\end{tabular} & REVIEW_QUALIFIER & SAMP_FROM & SAMP_ITEM & SAMP_MTHD & COLL_MTHD & SAMP_SITE_ID & SAMP_SITE_NAME & WELL_NAME & SAMPLE_LOCATION \\
\hline 3118 & & RIVER & & RAW GRAB & TRANSECT & 321 & RICH.PMPHS-2 HRM46.4 & & \\
\hline 3119 & & RIVER & & RAW GRAB & TRANSECT & 321 & RICH.PMPHS-2 HRM46.4 & & \\
\hline 3120 & & RIVER & & RAW GRAB & TRANSECT & 321 & RICH.PMPHS-2 HRM46.4 & & \\
\hline 3121 & & RIVER & & RAW GRAB & TRANSECT & 321 & RICH.PMPHS-2 HRM46.4 & & \\
\hline 3122 & & RIVER & & RAW GRAB & TRANSECT & 321 & RICH.PMPHS-2 HRM46.4 & & \\
\hline 3123 & & RIVER & & RAW GRAB & TRANSECT & 321 & RICH.PMPHS-2 HRM46.4 & & \\
\hline 3124 & & RIVER & & RAW GRAB & TRANSECT & 322 & RICH.PMPHS-3 HRM46.4 & & \\
\hline 3125 & & RIVER & & RAW GRAB & TRANSECT & 322 & RICH.PMPHS-3 HRM46.4 & & \\
\hline 3126 & & RIVER & & RAW GRAB & TRANSECT & 322 & RICH.PMPHS-3 HRM46.4 & & \\
\hline $3127 \mathrm{~F}$ & $\mathrm{~F}$ & RIVER & & RAW GRAB & TRANSECT & 322 & RICH.PMPHS-3 HRM46.4 & & \\
\hline 3128 & & RIVER & & RAW GRAB & TRANSECT & 322 & RICH.PMPHS-3 HRM46.4 & & \\
\hline 3129 & & RIVER & & RAW GRAB & TRANSECT & 322 & RICH.PMPHS-3 HRM46.4 & & \\
\hline 3130 & & RIVER & & RAW GRAB & TRANSECT & 322 & RICH.PMPHS-3 HRM46.4 & & \\
\hline 3131 & & RIVER & & RAW GRAB & TRANSECT & 572 & RICH.PMPHS-4 HRM46.4 & & \\
\hline 3132 & & RIVER & & RAW GRAB & TRANSECT & 572 & RICH.PMPHS-4 HRM46.4 & & \\
\hline 3133 & & RIVER & & RAW GRAB & TRANSECT & 572 & RICH.PMPHS-4 HRM46.4 & & \\
\hline $3134 \mathrm{~F}$ & $\mathrm{~F}$ & RIVER & & RAW GRAB & TRANSECT & 572 & RICH.PMPHS-4 HRM46.4 & & \\
\hline 3135 & & RIVER & & RAW GRAB & TRANSECT & 572 & RICH.PMPHS-4 HRM46.4 & & \\
\hline 3136 & & RIVER & & RAW GRAB & TRANSECT & 572 & RICH.PMPHS-4 HRM46.4 & & \\
\hline 3137 & & RIVER & & RAW GRAB & TRANSECT & 572 & RICH.PMPHS-4 HRM46.4 & & \\
\hline 3138 & & RIVER & & RAW GRAB & TRANSECT & 323 & RICH.PMPHS-5 HRM46.4 & & \\
\hline 3139 & & RIVER & & RAW GRAB & TRANSECT & 323 & RICH.PMPHS-5 HRM46.4 & & \\
\hline 3140 & & RIVER & & RAW GRAB & TRANSECT & 323 & RICH.PMPHS-5 HRM46.4 & & \\
\hline $3141 \mathrm{~F}$ & $\mathrm{~F}$ & RIVER & & RAW GRAB & TRANSECT & 323 & RICH.PMPHS-5 HRM46.4 & & \\
\hline 3142 & & RIVER & & RAW GRAB & TRANSECT & 323 & RICH.PMPHS-5 HRM46.4 & & \\
\hline 3143 & & RIVER & & RAW GRAB & TRANSECT & 323 & RICH.PMPHS-5 HRM46.4 & & \\
\hline 3144 & & RIVER & & RAW GRAB & TRANSECT & 323 & RICH.PMPHS-5 HRM46.4 & & \\
\hline 3145 & & RIVER & & RAW GRAB & TRANSECT & 324 & RICH.PMPHS-6 HRM46.4 & & \\
\hline 3146 & & RIVER & & RAW GRAB & TRANSECT & 324 & RICH.PMPHS-6 HRM46.4 & & \\
\hline 3147 & & RIVER & & RAW GRAB & TRANSECT & 324 & RICH.PMPHS-6 HRM46.4 & & \\
\hline $3148 \mathrm{~F}$ & $\mathrm{~F}$ & RIVER & & RAW GRAB & TRANSECT & 324 & RICH.PMPHS-6 HRM46.4 & & \\
\hline 3149 & & RIVER & & RAW GRAB & TRANSECT & 324 & RICH.PMPHS-6 HRM46.4 & & \\
\hline 3150 & & RIVER & & RAW GRAB & TRANSECT & 324 & RICH.PMPHS-6 HRM46.4 & & \\
\hline 3151 & & RIVER & & RAW GRAB & TRANSECT & 324 & RICH.PMPHS-6 HRM46.4 & & \\
\hline 3152 & & RIVER & & RAW GRAB & TRANSECT & 325 & RICH.PMPHS-7 HRM46.4 & & \\
\hline 3153 & & RIVER & & RAW GRAB & TRANSECT & 325 & RICH.PMPHS-7 HRM46.4 & & \\
\hline 3154 & & RIVER & & RAW GRAB & TRANSECT & 325 & RICH.PMPHS-7 HRM46.4 & & \\
\hline 3155 F & $\mathrm{F}$ & RIVER & & RAW GRAB & TRANSECT & 325 & RICH.PMPHS-7 HRM46.4 & & \\
\hline
\end{tabular}




\begin{tabular}{|c|c|c|c|c|c|c|c|c|c|}
\hline & $T$ & $\bar{U}$ & $\overline{\mathrm{V}}$ & $\mathrm{W}$ & $\bar{X}$ & $\bar{Y}$ & $\bar{Z}$ & $\overline{A A}$ & $A B$ \\
\hline 1 & REVIEW_QUALIFIER & SAMP_FROM & SAMP_ITEM & SAMP_MTHD & COLL_MTHD & SAMP_SITE_ID & SAMP_SITE_NAME & WELL_NAME & SAMPLE_LOCATION \\
\hline 3156 & & RIVER & & RAW GRAB & TRANSECT & 325 & RICH.PMPHS-7 HRM46.4 & & \\
\hline 3157 & & RIVER & & RAW GRAB & TRANSECT & 325 & RICH.PMPHS-7 HRM46.4 & & \\
\hline 3158 & & RIVER & & RAW GRAB & TRANSECT & $325 \mathrm{f}$ & RICH.PMPHS-7 HRM46.4 & & \\
\hline 3159 & & RIVER & & RAW GRAB & TRANSECT & 326 & RICH.PMPHS-8 HRM46.4 & & \\
\hline 3160 & & RIVER & & RAW GRAB & TRANSECT & 326 & RICH.PMPHS-8 HRM46.4 & & \\
\hline 3161 & & RIVER & & RAW GRAB & TRANSECT & 326 & RICH.PMPHS-8 HRM46.4 & & \\
\hline $3162 \mathrm{~F}$ & $\mathrm{~F}$ & RIVER & & RAW GRAB & TRANSECT & 326 & RICH.PMPHS-8 HRM46.4 & & \\
\hline 3163 & & RIVER & & RAW GRAB & TRANSECT & 326 & RICH.PMPHS-8 HRM46.4 & & \\
\hline 3164 & & RIVER & & RAW GRAB & TRANSECT & 326 & RICH.PMPHS-8 HRM46.4 & & \\
\hline 3165 & & RIVER & & RAW GRAB & TRANSECT & 326 & RICH.PMPHS-8 HRM46.4 & & \\
\hline 3166 & & RIVER & & RAW GRAB & TRANSECT & 573 & RICH.PMPHS-9 HRM46.4 & & \\
\hline 3167 & & RIVER & & RAW GRAB & TRANSECT & 573 & RICH.PMPHS-9 HRM46.4 & & \\
\hline 3168 & & RIVER & & RAW GRAB & TRANSECT & 573 & RICH.PMPHS-9 HRM46.4 & & \\
\hline $3169 \mathrm{~F}$ & $\mathrm{~F}$ & RIVER & & RAW GRAB & TRANSECT & 573 & RICH.PMPHS-9 HRM46.4 & & \\
\hline 3170 & & RIVER & & RAW GRAB & TRANSECT & 573 & RICH.PMPHS-9 HRM46.4 & & \\
\hline 3171 & & RIVER & & RAW GRAB & TRANSECT & 573 & RICH.PMPHS-9 HRM46.4 & & \\
\hline 3172 & & RIVER & & RAW GRAB & TRANSECT & 573 & RICH.PMPHS-9 HRM46.4 & & \\
\hline 3173 & & RIVER & & RAW GRAB & TRANSECT & 317 & VERNITA-1 HRM 0.3 & & \\
\hline $3174 \mathrm{~F}$ & $\mathrm{~F}$ & RIVER & & RAW GRAB & TRANSECT & 317 & VERNITA-1 HRM 0.3 & & \\
\hline 3175 & & RIVER & & RAW GRAB & TRANSECT & 317 & VERNITA-1 HRM 0.3 & & \\
\hline 3176 & & RIVER & & RAW GRAB & TRANSECT & 317 & VERNITA-1 HRM 0.3 & & \\
\hline 3177 & & RIVER & & RAW GRAB & TRANSECT & 317 & VERNITA-1 HRM 0.3 & & \\
\hline 3178 & & RIVER & & RAW GRAB & TRANSECT & 570 & VERNITA-2 HRM 0.3 & & \\
\hline $3179 \mathrm{~F}$ & $\mathrm{~F}$ & RIVER & & RAW GRAB & TRANSECT & 570 & VERNITA-2 HRM 0.3 & & \\
\hline 3180 & & RIVER & & RAW GRAB & TRANSECT & 570 & VERNITA-2 HRM 0.3 & & \\
\hline 3181 & & RIVER & & RAW GRAB & TRANSECT & 570 & VERNITA-2 HRM 0.3 & & \\
\hline 3182 & & RIVER & & RAW GRAB & TRANSECT & 570 & VERNITA-2 HRM 0.3 & & \\
\hline 3183 & & RIVER & & RAW GRAB & TRANSECT & 318 & VERNITA-3 HRM 0.3 & & \\
\hline $3184 \mathrm{~F}$ & $\mathrm{~F}$ & RIVER & & RAW GRAB & TRANSECT & 318 & VERNITA-3 HRM 0.3 & & \\
\hline 3185 & & RIVER & & RAW GRAB & TRANSECT & 318 & VERNITA-3 HRM 0.3 & & \\
\hline 3186 & & RIVER & & RAW GRAB & TRANSECT & 318 & VERNITA-3 HRM 0.3 & & \\
\hline 3187 & & RIVER & & RAW GRAB & TRANSECT & 318 & VERNITA-3 HRM 0.3 & & \\
\hline 3188 & & RIVER & & RAW GRAB & TRANSECT & 319 & VERNITA-4 HRM 0.3 & & \\
\hline $3189 \mathrm{~F}$ & $\mathrm{~F}$ & RIVER & & RAW GRAB & TRANSECT & 319 & VERNITA-4 HRM 0.3 & & \\
\hline 3190 & & RIVER & & RAW GRAB & TRANSECT & 319 & VERNITA-4 HRM 0.3 & & \\
\hline 3191 & & RIVER & & RAW GRAB & TRANSECT & 319 & VERNITA-4 HRM 0.3 & & \\
\hline 3192 & & RIVER & & RAW GRAB & TRANSECT & 319 & VERNITA-4 HRM 0.3 & & \\
\hline 3193 & & RIVER & & RAW GRAB & TRANSECT & 571 & RICH.PMPHS-1 HRM46.4 & & \\
\hline
\end{tabular}




\begin{tabular}{|c|c|c|c|c|c|c|c|c|c|}
\hline & $\mathrm{T}$ & $\bar{U}$ & $\mathrm{~V}$ & $\mathrm{~W}$ & $\mathrm{X}$ & $\mathrm{Y}$ & $Z$ & $\mathrm{AA}$ & $\mathrm{AB}$ \\
\hline 1 & REVIEW_QUALIFIER & SAMP_FROM & SAMP_ITEM & SAMP_MTHD & COLL_MTHD & SAMP_SITE_ID & SAMP_SITE_NAME & WELL_NAME & SAMPLE_LOCATION \\
\hline 3194 & $F$ & RIVER & & RAW GRAB & TRANSECT & 571 & RICH.PMPHS-1 HRM46.4 & & \\
\hline 3195 & & RIVER & & RAW GRAB & TRANSECT & 571 & RICH.PMPHS-1 HRM46.4 & & \\
\hline 3196 & & RIVER & & RAW GRAB & TRANSECT & 571 & RICH.PMPHS-1 HRM46.4 & & \\
\hline 3197 & & RIVER & & RAW GRAB & TRANSECT & 571 & RICH.PMPHS-1 HRM46.4 & & \\
\hline 3198 & & RIVER & & RAW GRAB & TRANSECT & 327 & RICH.PMPHS-10 HRM46.4 & & \\
\hline 3199 & $F$ & RIVER & & RAW GRAB & TRANSECT & 327 & RICH.PMPHS-10 HRM46.4 & & \\
\hline 3200 & & RIVER & & RAW GRAB & TRANSECT & 327 & RICH.PMPHS-10 HRM46.4 & & \\
\hline 3201 & & RIVER & & RAW GRAB & TRANSECT & 327 & RICH.PMPHS-10 HRM46.4 & & \\
\hline 3202 & & RIVER & & RAW GRAB & TRANSECT & 327 & RICH.PMPHS-10 HRM46.4 & & \\
\hline 3203 & & RIVER & & RAW GRAB & TRANSECT & 321 & RICH.PMPHS-2 HRM46.4 & & \\
\hline 3204 & $F$ & RIVER & & RAW GRAB & TRANSECT & 321 & RICH.PMPHS-2 HRM46.4 & & \\
\hline 3205 & & RIVER & & RAW GRAB & TRANSECT & 321 & RICH.PMPHS-2 HRM46.4 & & \\
\hline 3206 & & RIVER & & RAW GRAB & TRANSECT & 321 & RICH.PMPHS-2 HRM46.4 & & \\
\hline 3207 & & RIVER & & RAW GRAB & TRANSECT & 321 & RICH.PMPHS-2 HRM46.4 & & \\
\hline 3208 & & RIVER & & RAW GRAB & TRANSECT & 322 & RICH.PMPHS-3 HRM46.4 & & \\
\hline 3209 & $F$ & RIVER & & RAW GRAB & TRANSECT & 322 & RICH.PMPHS-3 HRM46.4 & & \\
\hline 3210 & & RIVER & & RAW GRAB & TRANSECT & 322 & RICH.PMPHS-3 HRM46.4 & & \\
\hline 3211 & & RIVER & & RAW GRAB & TRANSECT & 322 & RICH.PMPHS-3 HRM46.4 & & \\
\hline 3212 & & RIVER & & RAW GRAB & TRANSECT & 322 & RICH.PMPHS-3 HRM46.4 & & \\
\hline 3213 & & RIVER & & RAW GRAB & TRANSECT & 572 & RICH.PMPHS-4 HRM46.4 & & \\
\hline 3214 & $F$ & RIVER & & RAW GRAB & TRANSECT & 572 & RICH.PMPHS-4 HRM46.4 & & \\
\hline 3215 & & RIVER & & RAW GRAB & TRANSECT & 572 & RICH.PMPHS-4 HRM46.4 & & \\
\hline 3216 & & RIVER & & RAW GRAB & TRANSECT & 572 & RICH.PMPHS-4 HRM46.4 & & \\
\hline 3217 & & RIVER & & RAW GRAB & TRANSECT & 572 & RICH.PMPHS-4 HRM46.4 & & \\
\hline 3218 & & RIVER & & RAW GRAB & TRANSECT & 323 & RICH.PMPHS-5 HRM46.4 & & \\
\hline 3219 & $F$ & RIVER & & RAW GRAB & TRANSECT & 323 & RICH.PMPHS-5 HRM46.4 & & \\
\hline 3220 & & RIVER & & RAW GRAB & TRANSECT & 323 & RICH.PMPHS-5 HRM46.4 & & \\
\hline 3221 & & RIVER & & RAW GRAB & TRANSECT & 323 & RICH.PMPHS-5 HRM46.4 & & \\
\hline 3222 & & RIVER & & RAW GRAB & TRANSECT & 323 & RICH.PMPHS-5 HRM46.4 & & \\
\hline 3223 & & RIVER & & RAW GRAB & TRANSECT & 324 & RICH.PMPHS-6 HRM46.4 & & \\
\hline 3224 & $F$ & RIVER & & RAW GRAB & TRANSECT & 324 & RICH.PMPHS-6 HRM46.4 & & \\
\hline 3225 & & RIVER & & RAW GRAB & TRANSECT & 324 & RICH.PMPHS-6 HRM46.4 & & \\
\hline 3226 & & RIVER & & RAW GRAB & TRANSECT & 324 & RICH.PMPHS-6 HRM46.4 & & \\
\hline 3227 & & RIVER & & RAW GRAB & TRANSECT & 324 & RICH.PMPHS-6 HRM46.4 & & \\
\hline 3228 & & RIVER & & RAW GRAB & TRANSECT & 325 & RICH.PMPHS-7 HRM46.4 & & \\
\hline 3229 & $F$ & RIVER & & RAW GRAB & TRANSECT & 325 & RICH.PMPHS-7 HRM46.4 & & \\
\hline 3230 & & RIVER & & RAW GRAB & TRANSECT & 325 & RICH.PMPHS-7 HRM46.4 & & \\
\hline 3231 & & RIVER & & RAW GRAB & TRANSECT & 325 & RICH.PMPHS-7 HRM46.4 & & \\
\hline
\end{tabular}




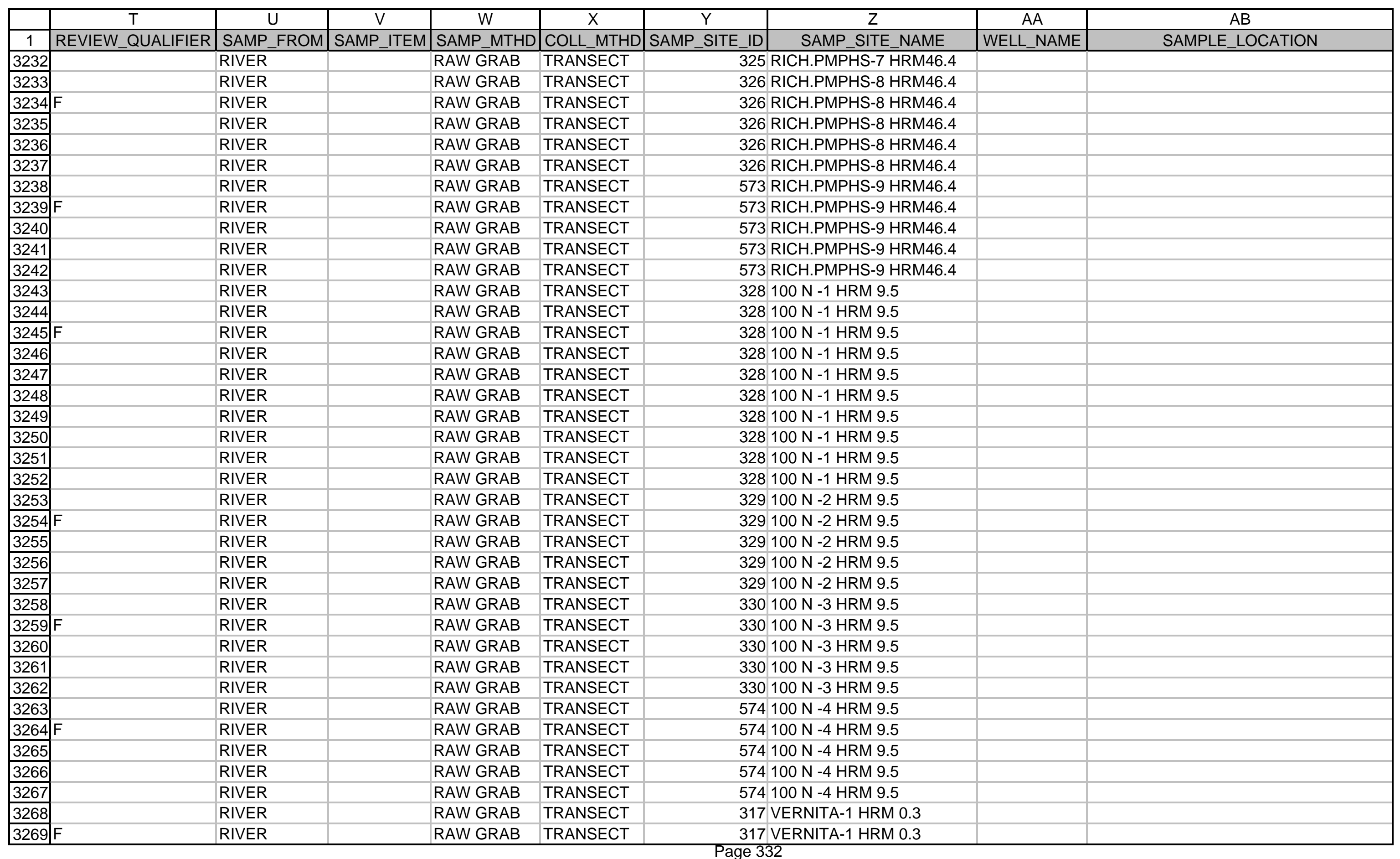




\begin{tabular}{|c|c|c|c|c|c|c|c|c|c|}
\hline & $\mathrm{T}$ & 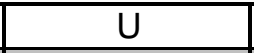 & $\mathrm{V}$ & $\mathrm{W}$ & $\mathrm{X}$ & $\mathrm{Y}$ & $Z$ & $\mathrm{AA}$ & $A B$ \\
\hline 1 & REVIEW_QUALIFIER & SAMP_FROM & SAMP_ITEM & SAMP_MTHD & COLL_MTHD & SAMP_SITE_ID & SAMP_SITE_NAME & WELL_NAME & SAMPLE_LOCATION \\
\hline 3270 & & RIVER & & RAW GRAB & TRANSECT & 317 & VERNITA-1 HRM 0.3 & & \\
\hline 3271 & & RIVER & & RAW GRAB & TRANSECT & 317 & VERNITA-1 HRM 0.3 & & \\
\hline 3272 & & RIVER & & RAW GRAB & TRANSECT & 317 & VERNITA-1 HRM 0.3 & & \\
\hline 3273 & & RIVER & & RAW GRAB & TRANSECT & 570 & VERNITA-2 HRM 0.3 & & \\
\hline 3274 & $\mathrm{~F}$ & RIVER & & RAW GRAB & TRANSECT & 570 & VERNITA-2 HRM 0.3 & & \\
\hline 3275 & & RIVER & & RAW GRAB & TRANSECT & 570 & VERNITA-2 HRM 0.3 & & \\
\hline 3276 & & RIVER & & RAW GRAB & TRANSECT & 570 & VERNITA-2 HRM 0.3 & & \\
\hline 3277 & & RIVER & & RAW GRAB & TRANSECT & 570 & VERNITA-2 HRM 0.3 & & \\
\hline 3278 & & RIVER & & RAW GRAB & TRANSECT & 318 & VERNITA-3 HRM 0.3 & & \\
\hline 3279 & $\mathrm{~F}$ & RIVER & & RAW GRAB & TRANSECT & 318 & VERNITA-3 HRM 0.3 & & \\
\hline 3280 & & RIVER & & RAW GRAB & TRANSECT & 318 & VERNITA-3 HRM 0.3 & & \\
\hline 3281 & & RIVER & & RAW GRAB & TRANSECT & 318 & VERNITA-3 HRM 0.3 & & \\
\hline 3282 & & RIVER & & RAW GRAB & TRANSECT & 318 & VERNITA-3 HRM 0.3 & & \\
\hline 3283 & & RIVER & & RAW GRAB & TRANSECT & 319 & VERNITA-4 HRM 0.3 & & \\
\hline 3284 & $\mathrm{~F}$ & RIVER & & RAW GRAB & TRANSECT & 319 & VERNITA-4 HRM 0.3 & & \\
\hline 3285 & & RIVER & & RAW GRAB & TRANSECT & 319 & VERNITA-4 HRM 0.3 & & \\
\hline 3286 & & RIVER & & RAW GRAB & TRANSECT & 319 & VERNITA-4 HRM 0.3 & & \\
\hline 3287 & & RIVER & & RAW GRAB & TRANSECT & 319 & VERNITA-4 HRM 0.3 & & \\
\hline 3288 & & RIVER & & RAW GRAB & TRANSECT & 336 & 100 F -1 HRM 19.0 & & \\
\hline 3289 & $\mathrm{~F}$ & RIVER & & RAW GRAB & TRANSECT & 336 & 100 F -1 HRM 19.0 & & \\
\hline 3290 & & RIVER & & RAW GRAB & TRANSECT & 336 & 100 F -1 HRM 19.0 & & \\
\hline 3291 & & RIVER & & RAW GRAB & TRANSECT & 336 & 100 F -1 HRM 19.0 & & \\
\hline 3292 & & RIVER & & RAW GRAB & TRANSECT & 336 & 100 F -1 HRM 19.0 & & \\
\hline 3293 & & RIVER & & RAW GRAB & TRANSECT & 341 & 100 F -10 HRM 19.0 & & \\
\hline 3294 & $\mathrm{~F}$ & RIVER & & RAW GRAB & TRANSECT & 341 & 100 F -10 HRM 19.0 & & \\
\hline 3295 & & RIVER & & RAW GRAB & TRANSECT & 341 & 100 F -10 HRM 19.0 & & \\
\hline 3296 & & RIVER & & RAW GRAB & TRANSECT & 341 & $100 \mathrm{~F}-10 \mathrm{HRM} 19.0$ & & \\
\hline 3297 & & RIVER & & RAW GRAB & TRANSECT & 341 & $100 \mathrm{~F}-10 \mathrm{HRM} 19.0$ & & \\
\hline 3298 & & RIVER & & RAW GRAB & TRANSECT & 576 & 100 F -2 HRM 19.0 & & \\
\hline 3299 & $\mathrm{~F}$ & RIVER & & RAW GRAB & TRANSECT & 576 & 100 F -2 HRM 19.0 & & \\
\hline 3300 & & RIVER & & RAW GRAB & TRANSECT & 576 & 100 F -2 HRM 19.0 & & \\
\hline 3301 & & RIVER & & RAW GRAB & TRANSECT & 576 & 100 F -2 HRM 19.0 & & \\
\hline 3302 & & RIVER & & RAW GRAB & TRANSECT & 576 & 100 F -2 HRM 19.0 & & \\
\hline 3303 & & RIVER & & RAW GRAB & TRANSECT & 337 & 100 F -3 HRM 19.0 & & \\
\hline 3304 & $\mathrm{~F}$ & RIVER & & RAW GRAB & TRANSECT & 337 & 100 F -3 HRM 19.0 & & \\
\hline 3305 & & RIVER & & RAW GRAB & TRANSECT & 337 & 100 F -3 HRM 19.0 & & \\
\hline 3306 & & RIVER & & RAW GRAB & TRANSECT & 337 & 100 F -3 HRM 19.0 & & \\
\hline 3307 & & RIVER & & RAW GRAB & TRANSECT & 337 & 100 F -3 HRM 19.0 & & \\
\hline
\end{tabular}




\begin{tabular}{|c|c|c|c|c|c|c|c|c|c|}
\hline & $\mathrm{T}$ & 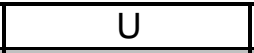 & $\mathrm{V}$ & $\mathrm{W}$ & $\mathrm{X}$ & $\mathrm{Y}$ & $Z$ & $\mathrm{AA}$ & $A B$ \\
\hline \begin{tabular}{l|l}
1 & 1 \\
\end{tabular} & REVIEW_QUALIFIER & SAMP_FROM & SAMP_ITEM & SAMP_MTHD & COLL_MTHD & SAMP_SITE_ID & SAMP_SITE_NAME & WELL_NAME & SAMPLE_LOCATION \\
\hline 3308 & & RIVER & & RAW GRAB & TRANSECT & 338 & 100 F -4 HRM 19.0 & & \\
\hline $3309 \mathrm{~F}$ & $\mathrm{~F}$ & RIVER & & RAW GRAB & TRANSECT & 338 & 100 F -4 HRM 19.0 & & \\
\hline 3310 & & RIVER & & RAW GRAB & TRANSECT & 338 & 100 F -4 HRM 19.0 & & \\
\hline 3311 & & RIVER & & RAW GRAB & TRANSECT & 338 & 100 F -4 HRM 19.0 & & \\
\hline 3312 & & RIVER & & RAW GRAB & TRANSECT & 338 & 100 F -4 HRM 19.0 & & \\
\hline 3313 & & RIVER & & RAW GRAB & TRANSECT & 577 & 100 F -5 HRM 19.0 & & \\
\hline $3314 \mathrm{~F}$ & $\mathrm{~F}$ & RIVER & & RAW GRAB & TRANSECT & 577 & 100 F -5 HRM 19.0 & & \\
\hline 3315 & & RIVER & & RAW GRAB & TRANSECT & $577:$ & 100 F -5 HRM 19.0 & & \\
\hline 3316 & & RIVER & & RAW GRAB & TRANSECT & 577 & 100 F -5 HRM 19.0 & & \\
\hline 3317 & & RIVER & & RAW GRAB & TRANSECT & $577:$ & 100 F -5 HRM 19.0 & & \\
\hline 3318 & & RIVER & & RAW GRAB & TRANSECT & 606 & 100 F -6 HRM 19.0 & & \\
\hline $3319 \mathrm{~F}$ & $\mathrm{~F}$ & RIVER & & RAW GRAB & TRANSECT & 606 & 100 F -6 HRM 19.0 & & \\
\hline 3320 & & RIVER & & RAW GRAB & TRANSECT & 606 & 100 F -6 HRM 19.0 & & \\
\hline 3321 & & RIVER & & RAW GRAB & TRANSECT & 606 & 100 F -6 HRM 19.0 & & \\
\hline 3322 & & RIVER & & RAW GRAB & TRANSECT & 606 & 100 F -6 HRM 19.0 & & \\
\hline 3323 & & RIVER & & RAW GRAB & TRANSECT & 339 & 100 F -7 HRM 19.0 & & \\
\hline $3324 \mathrm{~F}$ & $\mathrm{~F}$ & RIVER & & RAW GRAB & TRANSECT & 339 & 100 F -7 HRM 19.0 & & \\
\hline 3325 & & RIVER & & RAW GRAB & TRANSECT & 339 & 100 F -7 HRM 19.0 & & \\
\hline 3326 & & RIVER & & RAW GRAB & TRANSECT & 339 & 100 F -7 HRM 19.0 & & \\
\hline 3327 & & RIVER & & RAW GRAB & TRANSECT & 339 & 100 F -7 HRM 19.0 & & \\
\hline 3328 & & RIVER & & RAW GRAB & TRANSECT & 340 & 100 F -8 HRM 19.0 & & \\
\hline $3329 \mathrm{~F}$ & $\mathrm{~F}$ & RIVER & & RAW GRAB & TRANSECT & 340 & 100 F -8 HRM 19.0 & & \\
\hline 3330 & & RIVER & & RAW GRAB & TRANSECT & 340 & 100 F -8 HRM 19.0 & & \\
\hline 3331 & & RIVER & & RAW GRAB & TRANSECT & 340 & 100 F -8 HRM 19.0 & & \\
\hline 3332 & & RIVER & & RAW GRAB & TRANSECT & 340 & 100 F -8 HRM 19.0 & & \\
\hline 3333 & & RIVER & & RAW GRAB & TRANSECT & 467 & 100 F -9 HRM 19.0 & & \\
\hline $3334 \mathrm{~F}$ & $\mathrm{~F}$ & RIVER & & RAW GRAB & TRANSECT & 467 & 100 F -9 HRM 19.0 & & \\
\hline 3335 & & RIVER & & RAW GRAB & TRANSECT & 467 & 100 F -9 HRM 19.0 & & \\
\hline 3336 & & RIVER & & RAW GRAB & TRANSECT & 467 & 100 F -9 HRM 19.0 & & \\
\hline 3337 & & RIVER & & RAW GRAB & TRANSECT & $467:$ & 100 F -9 HRM 19.0 & & \\
\hline 3338 & & RIVER & & RAW GRAB & TRANSECT & 578 & HANFRD TS-1 HRM 28.7 & & \\
\hline $3339 \mathrm{~F}$ & $\mathrm{~F}$ & RIVER & & RAW GRAB & TRANSECT & 578 & HANFRD TS-1 HRM 28.7 & & \\
\hline 3340 & & RIVER & & RAW GRAB & TRANSECT & 578 & HANFRD TS-1 HRM 28.7 & & \\
\hline 3341 & & RIVER & & RAW GRAB & TRANSECT & 578 & HANFRD TS-1 HRM 28.7 & & \\
\hline 3342 & & RIVER & & RAW GRAB & TRANSECT & 578 & HANFRD TS-1 HRM 28.7 & & \\
\hline 3343 & & RIVER & & RAW GRAB & TRANSECT & 347 & HANFRD TS-10 HRM 28.7 & & \\
\hline $3344 \mathrm{~F}$ & $\mathrm{~F}$ & RIVER & & RAW GRAB & TRANSECT & 347 & HANFRD TS-10 HRM 28.7 & & \\
\hline 3345 & & RIVER & & RAW GRAB & TRANSECT & 347 & HANFRD TS-10 HRM 28.7 & & \\
\hline
\end{tabular}




\begin{tabular}{|c|c|c|c|c|c|c|c|c|c|}
\hline & $\mathrm{T}$ & $\bar{U}$ & $\mathrm{~V}$ & $\mathrm{~W}$ & $\mathrm{X}$ & $\mathrm{Y}$ & $Z$ & $\mathrm{AA}$ & $\mathrm{AB}$ \\
\hline \begin{tabular}{l|l}
1 & 1 \\
\end{tabular} & REVIEW_QUALIFIER & SAMP_FROM & SAMP_ITEM & SAMP_MTHD & COLL_MTHD & SAMP_SITE_ID & SAMP_SITE_NAME & WELL_NAME & SAMPLE_LOCATION \\
\hline 3346 & & RIVER & & RAW GRAB & TRANSECT & 347 & HANFRD TS-10 HRM 28.7 & & \\
\hline 3347 & & RIVER & & RAW GRAB & TRANSECT & 347 & HANFRD TS-10 HRM 28.7 & & \\
\hline 3348 & & RIVER & & RAW GRAB & TRANSECT & 342 & HANFRD TS-2 HRM 28.7 & & \\
\hline 3349 & & RIVER & & RAW GRAB & TRANSECT & 342 & HANFRD TS-2 HRM 28.7 & & \\
\hline 3350 & & RIVER & & RAW GRAB & TRANSECT & 342 & HANFRD TS-2 HRM 28.7 & & \\
\hline 3351 & & RIVER & & RAW GRAB & TRANSECT & 342 & HANFRD TS-2 HRM 28.7 & & \\
\hline 3352 & & RIVER & & RAW GRAB & TRANSECT & 342 & HANFRD TS-2 HRM 28.7 & & \\
\hline 3353 & & RIVER & & RAW GRAB & TRANSECT & 468 & HANFRD TS-3 HRM 28.7 & & \\
\hline $3354 \mathrm{~F}$ & $\mathrm{~F}$ & RIVER & & RAW GRAB & TRANSECT & 468 & HANFRD TS-3 HRM 28.7 & & \\
\hline 3355 & & RIVER & & RAW GRAB & TRANSECT & 468 & HANFRD TS-3 HRM 28.7 & & \\
\hline 3356 & & RIVER & & RAW GRAB & TRANSECT & 468 & 3 HANFRD TS-3 HRM 28.7 & & \\
\hline 3357 & & RIVER & & RAW GRAB & TRANSECT & 468 & HANFRD TS-3 HRM 28.7 & & \\
\hline 3358 & & RIVER & & RAW GRAB & TRANSECT & 343 & HANFRD TS-4 HRM 28.7 & & \\
\hline $3359 \mathrm{~F}$ & $\mathrm{~F}$ & RIVER & & RAW GRAB & TRANSECT & 343 & HANFRD TS-4 HRM 28.7 & & \\
\hline 3360 & & RIVER & & RAW GRAB & TRANSECT & 343 & HANFRD TS-4 HRM 28.7 & & \\
\hline 3361 & & RIVER & & RAW GRAB & TRANSECT & 343 & HANFRD TS-4 HRM 28.7 & & \\
\hline 3362 & & RIVER & & RAW GRAB & TRANSECT & 343 & HANFRD TS-4 HRM 28.7 & & \\
\hline 3363 & & RIVER & & RAW GRAB & TRANSECT & 344 & HANFRD TS-5 HRM 28.7 & & \\
\hline $3364 \mathrm{~F}$ & $\mathrm{~F}$ & RIVER & & RAW GRAB & TRANSECT & 344 & HANFRD TS-5 HRM 28.7 & & \\
\hline 3365 & & RIVER & & RAW GRAB & TRANSECT & 344 & HANFRD TS-5 HRM 28.7 & & \\
\hline 3366 & & RIVER & & RAW GRAB & TRANSECT & 344 & HANFRD TS-5 HRM 28.7 & & \\
\hline 3367 & & RIVER & & RAW GRAB & TRANSECT & 344 & HANFRD TS-5 HRM 28.7 & & \\
\hline 3368 & & RIVER & & RAW GRAB & TRANSECT & 345 & HANFRD TS-6 HRM 28.7 & & \\
\hline $3369 \mathrm{~F}$ & $\mathrm{~F}$ & RIVER & & RAW GRAB & TRANSECT & 345 & HANFRD TS-6 HRM 28.7 & & \\
\hline 3370 & & RIVER & & RAW GRAB & TRANSECT & 345 & HANFRD TS-6 HRM 28.7 & & \\
\hline 3371 & & RIVER & & RAW GRAB & TRANSECT & 345 & HANFRD TS-6 HRM 28.7 & & \\
\hline 3372 & & RIVER & & RAW GRAB & TRANSECT & 345 & HANFRD TS-6 HRM 28.7 & & \\
\hline 3373 & & RIVER & & RAW GRAB & TRANSECT & 469 & HANFRD TS-7 HRM 28.7 & & \\
\hline $3374 \mathrm{~F}$ & $\mathrm{~F}$ & RIVER & & RAW GRAB & TRANSECT & 469 & HANFRD TS-7 HRM 28.7 & & \\
\hline 3375 & & RIVER & & RAW GRAB & TRANSECT & 469 & HANFRD TS-7 HRM 28.7 & & \\
\hline 3376 & & RIVER & & RAW GRAB & TRANSECT & 469 & HANFRD TS-7 HRM 28.7 & & \\
\hline 3377 & & RIVER & & RAW GRAB & TRANSECT & 469 & HANFRD TS-7 HRM 28.7 & & \\
\hline 3378 & & RIVER & & RAW GRAB & TRANSECT & 579 & HANFRD TS-8 HRM 28.7 & & \\
\hline $3379 \mathrm{~F}$ & $\mathrm{~F}$ & RIVER & & RAW GRAB & TRANSECT & 579 & HANFRD TS-8 HRM 28.7 & & \\
\hline 3380 & & RIVER & & RAW GRAB & TRANSECT & 579 & HANFRD TS-8 HRM 28.7 & & \\
\hline 3381 & & RIVER & & RAW GRAB & TRANSECT & 579 & HANFRD TS-8 HRM 28.7 & & \\
\hline 3382 & & RIVER & & RAW GRAB & TRANSECT & 579 & HANFRD TS-8 HRM 28.7 & & \\
\hline 3383 & & RIVER & & RAW GRAB & TRANSECT & 346 & HANFRD TS-9 HRM 28.7 & & \\
\hline
\end{tabular}




\begin{tabular}{|c|c|c|c|c|c|c|c|c|c|}
\hline & $\mathrm{T}$ & $\bar{U}$ & $\mathrm{~V}$ & $\mathrm{~W}$ & $\mathrm{X}$ & $\mathrm{Y}$ & $Z$ & $\mathrm{AA}$ & $\mathrm{AB}$ \\
\hline 1 & REVIEW_QUALIFIER & SAMP_FROM & SAMP_ITEM & SAMP_MTHD & COLL_MTHD & SAMP_SITE_ID & SAMP_SITE_NAME & WELL_NAME & SAMPLE_LOCATION \\
\hline 3384 & $F$ & RIVER & & RAW GRAB & TRANSECT & 346 & HANFRD TS-9 HRM 28.7 & & \\
\hline 3385 & & RIVER & & RAW GRAB & TRANSECT & 346 & HANFRD TS-9 HRM 28.7 & & \\
\hline 3386 & & RIVER & & RAW GRAB & TRANSECT & 346 & HANFRD TS-9 HRM 28.7 & & \\
\hline 3387 & & RIVER & & RAW GRAB & TRANSECT & 346 & HANFRD TS-9 HRM 28.7 & & \\
\hline 3388 & & RIVER & & RAW GRAB & TRANSECT & 3483 & 300 AREA -1 HRM 43.1 & & \\
\hline 3389 & & RIVER & & RAW GRAB & TRANSECT & 3483 & 300 AREA -1 HRM 43.1 & & \\
\hline 3390 & & RIVER & & RAW GRAB & TRANSECT & 348 & 300 AREA -1 HRM 43.1 & & \\
\hline 3391 & & RIVER & & RAW GRAB & TRANSECT & 3483 & 300 AREA -1 HRM 43.1 & & \\
\hline 3392 & & RIVER & & RAW GRAB & TRANSECT & 348 & 300 AREA -1 HRM 43.1 & & \\
\hline 3393 & & RIVER & & RAW GRAB & TRANSECT & 4703 & 300 AREA -2 HRM 43.1 & & \\
\hline 3394 & $F$ & RIVER & & RAW GRAB & TRANSECT & 4703 & 300 AREA -2 HRM 43.1 & & \\
\hline 3395 & & RIVER & & RAW GRAB & TRANSECT & 4703 & 300 AREA -2 HRM 43.1 & & \\
\hline 3396 & & RIVER & & RAW GRAB & TRANSECT & 470 & 300 AREA -2 HRM 43.1 & & \\
\hline 3397 & & RIVER & & RAW GRAB & TRANSECT & 4703 & 300 AREA -2 HRM 43.1 & & \\
\hline 3398 & & RIVER & & RAW GRAB & TRANSECT & 5803 & 300 AREA -3 HRM 43.1 & & \\
\hline 3399 & $F$ & RIVER & & RAW GRAB & TRANSECT & 5803 & 300 AREA -3 HRM 43.1 & & \\
\hline 3400 & & RIVER & & RAW GRAB & TRANSECT & 5803 & 300 AREA -3 HRM 43.1 & & \\
\hline 3401 & & RIVER & & RAW GRAB & TRANSECT & 5803 & 300 AREA -3 HRM 43.1 & & \\
\hline 3402 & & RIVER & & RAW GRAB & TRANSECT & 5803 & 300 AREA -3 HRM 43.1 & & \\
\hline 3403 & & RIVER & & RAW GRAB & TRANSECT & 3493 & 300 AREA -4 HRM 43.1 & & \\
\hline 3404 & $F$ & RIVER & & RAW GRAB & TRANSECT & 3493 & 300 AREA -4 HRM 43.1 & & \\
\hline 3405 & & RIVER & & RAW GRAB & TRANSECT & 3493 & 300 AREA -4 HRM 43.1 & & \\
\hline 3406 & & RIVER & & RAW GRAB & TRANSECT & 349 & 300 AREA -4 HRM 43.1 & & \\
\hline 3407 & & RIVER & & RAW GRAB & TRANSECT & 3493 & 300 AREA -4 HRM 43.1 & & \\
\hline 3408 & & RIVER & & RAW GRAB & TRANSECT & 607 & 300 AREA -5 HRM 43.1 & & \\
\hline 3409 & $F$ & RIVER & & RAW GRAB & TRANSECT & 607 & 300 AREA -5 HRM 43.1 & & \\
\hline 3410 & & RIVER & & RAW GRAB & TRANSECT & 607 & 300 AREA -5 HRM 43.1 & & \\
\hline 3411 & & RIVER & & RAW GRAB & TRANSECT & 607 & 300 AREA -5 HRM 43.1 & & \\
\hline 3412 & & RIVER & & RAW GRAB & TRANSECT & 607 & 300 AREA -5 HRM 43.1 & & \\
\hline 3413 & & RIVER & & RAW GRAB & TRANSECT & 350 & 300 AREA -6 HRM 43.1 & & \\
\hline 3414 & $F$ & RIVER & & RAW GRAB & TRANSECT & 350 & 300 AREA -6 HRM 43.1 & & \\
\hline 3415 & & RIVER & & RAW GRAB & TRANSECT & 3503 & 300 AREA -6 HRM 43.1 & & \\
\hline 3416 & & RIVER & & RAW GRAB & TRANSECT & 350 & 300 AREA -6 HRM 43.1 & & \\
\hline 3417 & & RIVER & & RAW GRAB & TRANSECT & 350 & 300 AREA -6 HRM 43.1 & & \\
\hline 3418 & & RIVER & & RAW GRAB & TRANSECT & 3513 & 300 AREA -7 HRM 43.1 & & \\
\hline 3419 & $F$ & RIVER & & RAW GRAB & TRANSECT & 351 & 300 AREA -7 HRM 43.1 & & \\
\hline 3420 & & RIVER & & RAW GRAB & TRANSECT & 3513 & 300 AREA -7 HRM 43.1 & & \\
\hline 3421 & & RIVER & & RAW GRAB & TRANSECT & 3513 & 300 AREA -7 HRM 43.1 & & \\
\hline
\end{tabular}




\begin{tabular}{|c|c|c|c|c|c|c|c|c|c|}
\hline & $\mathrm{T}$ & $\bar{U}$ & $\mathrm{~V}$ & $\mathrm{~W}$ & $\mathrm{X}$ & $\mathrm{Y}$ & $Z$ & $\mathrm{AA}$ & $\mathrm{AB}$ \\
\hline \begin{tabular}{l|l}
1 & 1 \\
\end{tabular} & REVIEW_QUALIFIER & SAMP_FROM & SAMP_ITEM & SAMP_MTHD & COLL_MTHD & SAMP_SITE_ID & SAMP_SITE_NAME & WELL_NAME & SAMPLE_LOCATION \\
\hline 3422 & & RIVER & & RAW GRAB & TRANSECT & 351 & 300 AREA -7 HRM 43.1 & & \\
\hline 3423 & & RIVER & & RAW GRAB & TRANSECT & 581 & 300 AREA -8 HRM 43.1 & & \\
\hline $3424 \mathrm{~F}$ & $\mathrm{~F}$ & RIVER & & RAW GRAB & TRANSECT & 581 & 300 AREA -8 HRM 43.1 & & \\
\hline 3425 & & RIVER & & RAW GRAB & TRANSECT & 581 & 300 AREA -8 HRM 43.1 & & \\
\hline 3426 & & RIVER & & RAW GRAB & TRANSECT & 581 & 300 AREA -8 HRM 43.1 & & \\
\hline 3427 & & RIVER & & RAW GRAB & TRANSECT & 581 & 300 AREA -8 HRM 43.1 & & \\
\hline 3428 & & RIVER & & RAW GRAB & TRANSECT & 471 & 300 AREA -9 HRM 43.1 & & \\
\hline $3429 \mathrm{~F}$ & $\mathrm{~F}$ & RIVER & & RAW GRAB & TRANSECT & 471 & 300 AREA -9 HRM 43.1 & & \\
\hline 3430 & & RIVER & & RAW GRAB & TRANSECT & 471 & 300 AREA -9 HRM 43.1 & & \\
\hline 3431 & & RIVER & & RAW GRAB & TRANSECT & 471 & 300 AREA -9 HRM 43.1 & & \\
\hline 3432 & & RIVER & & RAW GRAB & TRANSECT & 471 & 300 AREA -9 HRM 43.1 & & \\
\hline 3433 & & RIVER & & RAW GRAB & TRANSECT & 352 & 300 AREA-10 HRM 43.1 & & \\
\hline $3434 \mathrm{~F}$ & $\mathrm{~F}$ & RIVER & & RAW GRAB & TRANSECT & 352 & 300 AREA-10 HRM 43.1 & & \\
\hline $3435 \mathrm{~F}$ & $\mathrm{~F}$ & RIVER & & RAW GRAB & TRANSECT & 352 & 300 AREA-10 HRM 43.1 & & \\
\hline 3436 & & RIVER & & RAW GRAB & TRANSECT & 352 & 300 AREA-10 HRM 43.1 & & \\
\hline $3437 \mathrm{~F}$ & $\mathrm{~F}$ & RIVER & & RAW GRAB & TRANSECT & 352 & 300 AREA-10 HRM 43.1 & & \\
\hline 3438 & & RIVER & & RAW GRAB & TRANSECT & 571 & RICH.PMPHS-1 HRM46.4 & & \\
\hline 3439 & & RIVER & & RAW GRAB & TRANSECT & 571 & RICH.PMPHS-1 HRM46.4 & & \\
\hline 3440 & & RIVER & & RAW GRAB & TRANSECT & 571 & RICH.PMPHS-1 HRM46.4 & & \\
\hline 3441 & & RIVER & & RAW GRAB & TRANSECT & 571 & RICH.PMPHS-1 HRM46.4 & & \\
\hline 3442 & & RIVER & & RAW GRAB & TRANSECT & 571 & RICH.PMPHS-1 HRM46.4 & & \\
\hline 3443 & & RIVER & & RAW GRAB & TRANSECT & 327 & RICH.PMPHS-10 HRM46.4 & & \\
\hline $3444 \mathrm{~F}$ & $\mathrm{~F}$ & RIVER & & RAW GRAB & TRANSECT & 327 & RICH.PMPHS-10 HRM46.4 & & \\
\hline 3445 & & RIVER & & RAW GRAB & TRANSECT & 327 & RICH.PMPHS-10 HRM46.4 & & \\
\hline 3446 & & RIVER & & RAW GRAB & TRANSECT & 327 & RICH.PMPHS-10 HRM46.4 & & \\
\hline 3447 & & RIVER & & RAW GRAB & TRANSECT & 327 & RICH.PMPHS-10 HRM46.4 & & \\
\hline 3448 & & RIVER & & RAW GRAB & TRANSECT & 321 & RICH.PMPHS-2 HRM46.4 & & \\
\hline $3449 \mathrm{~F}$ & $\mathrm{~F}$ & RIVER & & RAW GRAB & TRANSECT & 321 & RICH.PMPHS-2 HRM46.4 & & \\
\hline 3450 & & RIVER & & RAW GRAB & TRANSECT & 321 & RICH.PMPHS-2 HRM46.4 & & \\
\hline 3451 & & RIVER & & RAW GRAB & TRANSECT & 321 & RICH.PMPHS-2 HRM46.4 & & \\
\hline 3452 & & RIVER & & RAW GRAB & TRANSECT & 321 & RICH.PMPHS-2 HRM46.4 & & \\
\hline 3453 & & RIVER & & RAW GRAB & TRANSECT & 322 & RICH.PMPHS-3 HRM46.4 & & \\
\hline $3454 \mathrm{~F}$ & $\mathrm{~F}$ & RIVER & & RAW GRAB & TRANSECT & 322 & RICH.PMPHS-3 HRM46.4 & & \\
\hline 3455 & & RIVER & & RAW GRAB & TRANSECT & 322 & RICH.PMPHS-3 HRM46.4 & & \\
\hline 3456 & & RIVER & & RAW GRAB & TRANSECT & 322 & RICH.PMPHS-3 HRM46.4 & & \\
\hline 3457 & & RIVER & & RAW GRAB & TRANSECT & 322 & RICH.PMPHS-3 HRM46.4 & & \\
\hline 3458 & & RIVER & & RAW GRAB & TRANSECT & 572 & RICH.PMPHS-4 HRM46.4 & & \\
\hline $3459 \mathrm{~F}$ & $\mathrm{~F}$ & RIVER & & RAW GRAB & TRANSECT & 572 & RICH.PMPHS-4 HRM46.4 & & \\
\hline
\end{tabular}




\begin{tabular}{|c|c|c|c|c|c|c|c|c|c|}
\hline & $\mathrm{T}$ & $\bar{U}$ & $\mathrm{~V}$ & $\mathrm{~W}$ & $\mathrm{X}$ & $\mathrm{Y}$ & $Z$ & $\mathrm{AA}$ & $\mathrm{AB}$ \\
\hline \begin{tabular}{l|l}
1 & 1 \\
\end{tabular} & REVIEW_QUALIFIER & SAMP_FROM & SAMP_ITEM & SAMP_MTHD & COLL_MTHD & SAMP_SITE_ID & SAMP_SITE_NAME & WELL_NAME & SAMPLE_LOCATION \\
\hline 3460 & & RIVER & & RAW GRAB & TRANSECT & 572 & RICH.PMPHS-4 HRM46.4 & & \\
\hline 3461 & & RIVER & & RAW GRAB & TRANSECT & 572 & RICH.PMPHS-4 HRM46.4 & & \\
\hline 3462 & & RIVER & & RAW GRAB & TRANSECT & 572 & RICH.PMPHS-4 HRM46.4 & & \\
\hline 3463 & & RIVER & & RAW GRAB & TRANSECT & 323 & RICH.PMPHS-5 HRM46.4 & & \\
\hline $3464 \mathrm{~F}$ & $\mathrm{~F}$ & RIVER & & RAW GRAB & TRANSECT & 323 & RICH.PMPHS-5 HRM46.4 & & \\
\hline 3465 & & RIVER & & RAW GRAB & TRANSECT & 323 & RICH.PMPHS-5 HRM46.4 & & \\
\hline 3466 & & RIVER & & RAW GRAB & TRANSECT & 323 & RICH.PMPHS-5 HRM46.4 & & \\
\hline 3467 & & RIVER & & RAW GRAB & TRANSECT & 323 & RICH.PMPHS-5 HRM46.4 & & \\
\hline 3468 & & RIVER & & RAW GRAB & TRANSECT & 324 & RICH.PMPHS-6 HRM46.4 & & \\
\hline $3469 \mathrm{~F}$ & $\mathrm{~F}$ & RIVER & & RAW GRAB & TRANSECT & 324 & RICH.PMPHS-6 HRM46.4 & & \\
\hline 3470 & & RIVER & & RAW GRAB & TRANSECT & 324 & RICH.PMPHS-6 HRM46.4 & & \\
\hline 3471 & & RIVER & & RAW GRAB & TRANSECT & 324 & RICH.PMPHS-6 HRM46.4 & & \\
\hline 3472 & & RIVER & & RAW GRAB & TRANSECT & 324 & RICH.PMPHS-6 HRM46.4 & & \\
\hline 3473 & & RIVER & & RAW GRAB & TRANSECT & 325 & RICH.PMPHS-7 HRM46.4 & & \\
\hline $3474 \mathrm{~F}$ & $\mathrm{~F}$ & RIVER & & RAW GRAB & TRANSECT & 325 & RICH.PMPHS-7 HRM46.4 & & \\
\hline 3475 & & RIVER & & RAW GRAB & TRANSECT & 325 & RICH.PMPHS-7 HRM46.4 & & \\
\hline 3476 & & RIVER & & RAW GRAB & TRANSECT & 325 & RICH.PMPHS-7 HRM46.4 & & \\
\hline 3477 & & RIVER & & RAW GRAB & TRANSECT & 325 & RICH.PMPHS-7 HRM46.4 & & \\
\hline 3478 & & RIVER & & RAW GRAB & TRANSECT & 326 & RICH.PMPHS-8 HRM46.4 & & \\
\hline $3479 \mathrm{~F}$ & $\mathrm{~F}$ & RIVER & & RAW GRAB & TRANSECT & 326 & RICH.PMPHS-8 HRM46.4 & & \\
\hline 3480 & & RIVER & & RAW GRAB & TRANSECT & 326 & RICH.PMPHS-8 HRM46.4 & & \\
\hline 3481 & & RIVER & & RAW GRAB & TRANSECT & 326 & RICH.PMPHS-8 HRM46.4 & & \\
\hline 3482 & & RIVER & & RAW GRAB & TRANSECT & 326 & RICH.PMPHS-8 HRM46.4 & & \\
\hline 3483 & & RIVER & & RAW GRAB & TRANSECT & 573 & RICH.PMPHS-9 HRM46.4 & & \\
\hline $3484 \mathrm{~F}$ & $\mathrm{~F}$ & RIVER & & RAW GRAB & TRANSECT & 573 & RICH.PMPHS-9 HRM46.4 & & \\
\hline 3485 & & RIVER & & RAW GRAB & TRANSECT & 573 & RICH.PMPHS-9 HRM46.4 & & \\
\hline 3486 & & RIVER & & RAW GRAB & TRANSECT & 573 & RICH.PMPHS-9 HRM46.4 & & \\
\hline 3487 & & RIVER & & RAW GRAB & TRANSECT & 573 & RICH.PMPHS-9 HRM46.4 & & \\
\hline 3488 & & RIVER & & RAW GRAB & TRANSECT & 5527 & 100 N SPRING-1A & & \\
\hline 3489 & & RIVER & & RAW GRAB & TRANSECT & 5527 & 100 N SPRING-1A & & \\
\hline 3490 & & RIVER & & RAW GRAB & TRANSECT & 5527 & 100 N SPRING-1A & & \\
\hline 3491 & & RIVER & & RAW GRAB & TRANSECT & 5528 & 100 N SPRING-1B & & \\
\hline 3492 & & RIVER & & RAW GRAB & TRANSECT & 5528 & 100 N SPRING-1B & & \\
\hline 3493 & & RIVER & & RAW GRAB & TRANSECT & 5528 & 100 N SPRING-1B & & \\
\hline 3494 & & RIVER & & RAW GRAB & TRANSECT & 5529 & 100 N SPRING-1C & & \\
\hline 3495 & & RIVER & & RAW GRAB & TRANSECT & 5529 & 100 N SPRING-1C & & \\
\hline 3496 & & RIVER & & RAW GRAB & TRANSECT & 5529 & 100 N SPRING-1C & & \\
\hline 3497 & & RIVER & & RAW GRAB & TRANSECT & 5530 & 100 N SPRING-1D & & \\
\hline
\end{tabular}




\begin{tabular}{|c|c|c|c|c|c|c|c|c|c|}
\hline & $T$ & $\bar{U}$ & $\overline{\mathrm{V}}$ & $\mathrm{W}$ & $\bar{X}$ & $\bar{Y}$ & $\bar{Z}$ & $\overline{A A}$ & $A B$ \\
\hline 1 & REVIEW_QUALIFIER & SAMP_FROM & SAMP_ITEM & SAMP_MTHD & COLL_MTHD & SAMP_SITE_ID & SAMP_SITE_NAME & WELL_NAME & SAMPLE_LOCATION \\
\hline 3498 & & RIVER & & RAW GRAB & TRANSECT & 5530 & 100 N SPRING-1D & & \\
\hline 3499 & & RIVER & & RAW GRAB & TRANSECT & 5530 & 100 N SPRING-1D & & \\
\hline 3500 & & RIVER & & RAW GRAB & TRANSECT & 5524 & 100 N SPRING-2 & & \\
\hline 3501 & & RIVER & & RAW GRAB & TRANSECT & 5524 & 100 N SPRING-2 & & \\
\hline 3502 & & RIVER & & RAW GRAB & TRANSECT & $5524:$ & 100 N SPRING-2 & & \\
\hline 3503 & & RIVER & & RAW GRAB & TRANSECT & 5531 & 100 N SPRING-3A & & \\
\hline 3504 & & RIVER & & RAW GRAB & TRANSECT & 5531 & 100 N SPRING-3A & & \\
\hline 3505 & & RIVER & & RAW GRAB & TRANSECT & 5531 & 100 N SPRING-3A & & \\
\hline 3506 & & RIVER & & RAW GRAB & TRANSECT & 5532 & 100 N SPRING-3B & & \\
\hline 3507 & & RIVER & & RAW GRAB & TRANSECT & 5532 & 100 N SPRING-3B & & \\
\hline 3508 & & RIVER & & RAW GRAB & TRANSECT & 5532 & 100 N SPRING-3B & & \\
\hline 3509 & & RIVER & & RAW GRAB & TRANSECT & 5533 & 100 N SPRING-3C & & \\
\hline 3510 & & RIVER & & RAW GRAB & TRANSECT & 5533 & 100 N SPRING-3C & & \\
\hline 3511 & & RIVER & & RAW GRAB & TRANSECT & 5533 & 100 N SPRING-3C & & \\
\hline 3512 & & RIVER & & RAW GRAB & TRANSECT & 5534 & 100 N SPRING-3D & & \\
\hline 3513 & & RIVER & & RAW GRAB & TRANSECT & 5534 & 100 N SPRING-3D & & \\
\hline 3514 & & RIVER & & RAW GRAB & TRANSECT & 5534 & 100 N SPRING-3D & & \\
\hline 3515 & & RIVER & & RAW GRAB & TRANSECT & 5535 & 100 N SPRING-4A & & \\
\hline 3516 & & RIVER & & RAW GRAB & TRANSECT & 5535 & 100 N SPRING-4A & & \\
\hline 3517 & & RIVER & & RAW GRAB & TRANSECT & 5535 & 100 N SPRING-4A & & \\
\hline 3518 & & RIVER & & RAW GRAB & TRANSECT & 5536 & 100 N SPRING-4B & & \\
\hline 3519 & & RIVER & & RAW GRAB & TRANSECT & 5537 & 100 N SPRING-4C & & \\
\hline 3520 & & RIVER & & RAW GRAB & TRANSECT & 5538 & 100 N SPRING-4D & & \\
\hline 3521 & & RIVER & & RAW GRAB & TRANSECT & 5539 & 100 N SPRING-5 & & \\
\hline 3522 & & RIVER & & RAW GRAB & TRANSECT & 5539 & 100 N SPRING-5 & & \\
\hline 3523 & & RIVER & & RAW GRAB & TRANSECT & 5539 & 100 N SPRING-5 & & \\
\hline 3524 & & RIVER & & RAW GRAB & TRANSECT & 319 & VERNITA-4 HRM 0.3 & & \\
\hline $3525 \mathrm{~F}$ & $\mathrm{~F}$ & RIVER & & RAW GRAB & TRANSECT & 319 & VERNITA-4 HRM 0.3 & & \\
\hline 3526 & & RIVER & & RAW GRAB & TRANSECT & 319 & VERNITA-4 HRM 0.3 & & \\
\hline 3527 & & RIVER & & RAW GRAB & TRANSECT & 319 & VERNITA-4 HRM 0.3 & & \\
\hline 3528 & & RIVER & & RAW GRAB & TRANSECT & 319 & VERNITA-4 HRM 0.3 & & \\
\hline 3529 & & RIVER & & RAW GRAB & TRANSECT & 318 & VERNITA-3 HRM 0.3 & & \\
\hline 3530 & & RIVER & & RAW GRAB & TRANSECT & 318 & VERNITA-3 HRM 0.3 & & \\
\hline 3531 & & RIVER & & RAW GRAB & TRANSECT & 318 & VERNITA-3 HRM 0.3 & & \\
\hline 3532 & & RIVER & & RAW GRAB & TRANSECT & 318 & VERNITA-3 HRM 0.3 & & \\
\hline 3533 & & RIVER & & RAW GRAB & TRANSECT & 318 & VERNITA-3 HRM 0.3 & & \\
\hline 3534 & & RIVER & & RAW GRAB & TRANSECT & 570 & VERNITA-2 HRM 0.3 & & \\
\hline 3535 & & RIVER & & RAW GRAB & TRANSECT & 570 & VERNITA-2 HRM 0.3 & & \\
\hline
\end{tabular}




\begin{tabular}{|c|c|c|c|c|c|c|c|c|c|}
\hline & $\mathrm{T}$ & $\bar{U}$ & $\mathrm{~V}$ & $\mathrm{~W}$ & $\mathrm{X}$ & $\mathrm{Y}$ & $Z$ & $\mathrm{AA}$ & $\mathrm{AB}$ \\
\hline 1 & REVIEW_QUALIFIER & SAMP_FROM & SAMP_ITEM & SAMP_MTHD & COLL_MTHD & SAMP_SITE_ID & SAMP_SITE_NAME & WELL_NAME & SAMPLE_LOCATION \\
\hline 3536 & & RIVER & & RAW GRAB & TRANSECT & 570 & VERNITA-2 HRM 0.3 & & \\
\hline 3537 & & RIVER & & RAW GRAB & TRANSECT & 570 & VERNITA-2 HRM 0.3 & & \\
\hline 3538 & & RIVER & & RAW GRAB & TRANSECT & 570 & VERNITA-2 HRM 0.3 & & \\
\hline 3539 & & RIVER & & RAW GRAB & TRANSECT & 317 & VERNITA-1 HRM 0.3 & & \\
\hline 3540 & & RIVER & & RAW GRAB & TRANSECT & 317 & VERNITA-1 HRM 0.3 & & \\
\hline 3541 & & RIVER & & RAW GRAB & TRANSECT & 317 & VERNITA-1 HRM 0.3 & & \\
\hline 3542 & & RIVER & & RAW GRAB & TRANSECT & 317 & VERNITA-1 HRM 0.3 & & \\
\hline 3543 & & RIVER & & RAW GRAB & TRANSECT & 317 & VERNITA-1 HRM 0.3 & & \\
\hline 3544 & & RIVER & & RAW GRAB & TRANSECT & 327 & RICH.PMPHS-10 HRM46.4 & & \\
\hline 3545 & & RIVER & & RAW GRAB & TRANSECT & 327 & RICH.PMPHS-10 HRM46.4 & & \\
\hline 3546 & & RIVER & & RAW GRAB & TRANSECT & 327 & RICH.PMPHS-10 HRM46.4 & & \\
\hline 3547 & & RIVER & & RAW GRAB & TRANSECT & 327 & RICH.PMPHS-10 HRM46.4 & & \\
\hline 3548 & & RIVER & & RAW GRAB & TRANSECT & 327 & RICH.PMPHS-10 HRM46.4 & & \\
\hline 3549 & & RIVER & & RAW GRAB & TRANSECT & 573 & RICH.PMPHS-9 HRM46.4 & & \\
\hline 3550 & & RIVER & & RAW GRAB & TRANSECT & 573 & RICH.PMPHS-9 HRM46.4 & & \\
\hline 3551 & & RIVER & & RAW GRAB & TRANSECT & 573 & RICH.PMPHS-9 HRM46.4 & & \\
\hline 3552 & & RIVER & & RAW GRAB & TRANSECT & 573 & RICH.PMPHS-9 HRM46.4 & & \\
\hline 3553 & & RIVER & & RAW GRAB & TRANSECT & 573 & RICH.PMPHS-9 HRM46.4 & & \\
\hline 3554 & & RIVER & & RAW GRAB & TRANSECT & 326 & RICH.PMPHS-8 HRM46.4 & & \\
\hline 3555 & & RIVER & & RAW GRAB & TRANSECT & 326 & RICH.PMPHS-8 HRM46.4 & & \\
\hline 3556 & & RIVER & & RAW GRAB & TRANSECT & 326 & RICH.PMPHS-8 HRM46.4 & & \\
\hline 3557 & & RIVER & & RAW GRAB & TRANSECT & 326 & RICH.PMPHS-8 HRM46.4 & & \\
\hline 3558 & & RIVER & & RAW GRAB & TRANSECT & 326 & RICH.PMPHS-8 HRM46.4 & & \\
\hline 3559 & & RIVER & & RAW GRAB & TRANSECT & 325 & RICH.PMPHS-7 HRM46.4 & & \\
\hline 3560 & & RIVER & & RAW GRAB & TRANSECT & 325 & RICH.PMPHS-7 HRM46.4 & & \\
\hline 3561 & & RIVER & & RAW GRAB & TRANSECT & 325 & RICH.PMPHS-7 HRM46.4 & & \\
\hline 3562 & & RIVER & & RAW GRAB & TRANSECT & 325 & RICH.PMPHS-7 HRM46.4 & & \\
\hline 3563 & & RIVER & & RAW GRAB & TRANSECT & 325 & RICH.PMPHS-7 HRM46.4 & & \\
\hline 3564 & & RIVER & & RAW GRAB & TRANSECT & 324 & RICH.PMPHS-6 HRM46.4 & & \\
\hline 3565 & & RIVER & & RAW GRAB & TRANSECT & 324 & RICH.PMPHS-6 HRM46.4 & & \\
\hline 3566 & & RIVER & & RAW GRAB & TRANSECT & 324 & RICH.PMPHS-6 HRM46.4 & & \\
\hline 3567 & & RIVER & & RAW GRAB & TRANSECT & 324 & RICH.PMPHS-6 HRM46.4 & & \\
\hline 3568 & & RIVER & & RAW GRAB & TRANSECT & 324 & RICH.PMPHS-6 HRM46.4 & & \\
\hline 3569 & & RIVER & & RAW GRAB & TRANSECT & 323 & RICH.PMPHS-5 HRM46.4 & & \\
\hline 3570 & & RIVER & & RAW GRAB & TRANSECT & 323 & RICH.PMPHS-5 HRM46.4 & & \\
\hline 3571 & & RIVER & & RAW GRAB & TRANSECT & 323 & RICH.PMPHS-5 HRM46.4 & & \\
\hline 3572 & & RIVER & & RAW GRAB & TRANSECT & 323 & RICH.PMPHS-5 HRM46.4 & & \\
\hline 3573 & & RIVER & & RAW GRAB & TRANSECT & 323 & RICH.PMPHS-5 HRM46.4 & & \\
\hline
\end{tabular}




\begin{tabular}{|c|c|c|c|c|c|c|c|c|c|}
\hline & $\mathrm{T}$ & $\bar{U}$ & $\mathrm{~V}$ & $\mathrm{~W}$ & $\mathrm{X}$ & $\mathrm{Y}$ & $Z$ & $\mathrm{AA}$ & $\mathrm{AB}$ \\
\hline 1 & REVIEW_QUALIFIER & SAMP_FROM & SAMP_ITEM & SAMP_MTHD & COLL_MTHD & SAMP_SITE_ID & SAMP_SITE_NAME & WELL_NAME & SAMPLE_LOCATION \\
\hline 3574 & & RIVER & & RAW GRAB & TRANSECT & 572 & RICH.PMPHS-4 HRM46.4 & & \\
\hline 3575 & & RIVER & & RAW GRAB & TRANSECT & 572 & RICH.PMPHS-4 HRM46.4 & & \\
\hline 3576 & & RIVER & & RAW GRAB & TRANSECT & 572 & RICH.PMPHS-4 HRM46.4 & & \\
\hline 3577 & & RIVER & & RAW GRAB & TRANSECT & 572 & RICH.PMPHS-4 HRM46.4 & & \\
\hline 3578 & & RIVER & & RAW GRAB & TRANSECT & 572 & RICH.PMPHS-4 HRM46.4 & & \\
\hline 3579 & & RIVER & & RAW GRAB & TRANSECT & 322 & RICH.PMPHS-3 HRM46.4 & & \\
\hline 3580 & & RIVER & & RAW GRAB & TRANSECT & 322 & RICH.PMPHS-3 HRM46.4 & & \\
\hline 3581 & & RIVER & & RAW GRAB & TRANSECT & 322 & RICH.PMPHS-3 HRM46.4 & & \\
\hline 3582 & & RIVER & & RAW GRAB & TRANSECT & 322 & RICH.PMPHS-3 HRM46.4 & & \\
\hline 3583 & & RIVER & & RAW GRAB & TRANSECT & 322 & RICH.PMPHS-3 HRM46.4 & & \\
\hline 3584 & & RIVER & & RAW GRAB & TRANSECT & 321 & RICH.PMPHS-2 HRM46.4 & & \\
\hline 3585 & & RIVER & & RAW GRAB & TRANSECT & 321 & RICH.PMPHS-2 HRM46.4 & & \\
\hline 3586 & & RIVER & & RAW GRAB & TRANSECT & 321 & RICH.PMPHS-2 HRM46.4 & & \\
\hline 3587 & & RIVER & & RAW GRAB & TRANSECT & 321 & RICH.PMPHS-2 HRM46.4 & & \\
\hline 3588 & & RIVER & & RAW GRAB & TRANSECT & 321 & RICH.PMPHS-2 HRM46.4 & & \\
\hline 3589 & & RIVER & & RAW GRAB & TRANSECT & 571 & RICH.PMPHS-1 HRM46.4 & & \\
\hline 3590 & & RIVER & & RAW GRAB & TRANSECT & 571 & RICH.PMPHS-1 HRM46.4 & & \\
\hline 3591 & & RIVER & & RAW GRAB & TRANSECT & 571 & RICH.PMPHS-1 HRM46.4 & & \\
\hline 3592 & & RIVER & & RAW GRAB & TRANSECT & 571 & RICH.PMPHS-1 HRM46.4 & & \\
\hline 3593 & & RIVER & & RAW GRAB & TRANSECT & 571 & RICH.PMPHS-1 HRM46.4 & & \\
\hline 3594 & & RIVER & & RAW GRAB & TRANSECT & 571 & RICH.PMPHS-1 HRM46.4 & & \\
\hline 3595 & & RIVER & & RAW GRAB & TRANSECT & 571 & RICH.PMPHS-1 HRM46.4 & & \\
\hline 3596 & & RIVER & & RAW GRAB & TRANSECT & 571 & RICH.PMPHS-1 HRM46.4 & & \\
\hline 3597 & & RIVER & & RAW GRAB & TRANSECT & 571 & RICH.PMPHS-1 HRM46.4 & & \\
\hline 3598 & & RIVER & & RAW GRAB & TRANSECT & 571 & RICH.PMPHS-1 HRM46.4 & & \\
\hline 3599 & & RIVER & & RAW GRAB & TRANSECT & 317 & VERNITA-1 HRM 0.3 & & \\
\hline 3600 & & RIVER & & RAW GRAB & TRANSECT & 317 & VERNITA-1 HRM 0.3 & & \\
\hline 3601 & & RIVER & & RAW GRAB & TRANSECT & 317 & VERNITA-1 HRM 0.3 & & \\
\hline 3602 & & RIVER & & RAW GRAB & TRANSECT & 317 & VERNITA-1 HRM 0.3 & & \\
\hline 3603 & & RIVER & & RAW GRAB & TRANSECT & 317 & VERNITA-1 HRM 0.3 & & \\
\hline 3604 & & RIVER & & RAW GRAB & TRANSECT & 570 & VERNITA-2 HRM 0.3 & & \\
\hline 3605 & & RIVER & & RAW GRAB & TRANSECT & 570 & VERNITA-2 HRM 0.3 & & \\
\hline 3606 & & RIVER & & RAW GRAB & TRANSECT & 570 & VERNITA-2 HRM 0.3 & & \\
\hline 3607 & & RIVER & & RAW GRAB & TRANSECT & 570 & VERNITA-2 HRM 0.3 & & \\
\hline 3608 & & RIVER & & RAW GRAB & TRANSECT & 570 & VERNITA-2 HRM 0.3 & & \\
\hline 3609 & & RIVER & & RAW GRAB & TRANSECT & 318 & VERNITA-3 HRM 0.3 & & \\
\hline 3610 & & RIVER & & RAW GRAB & TRANSECT & 318 & VERNITA-3 HRM 0.3 & & \\
\hline 3611 & & RIVER & & RAW GRAB & TRANSECT & 318 & VERNITA-3 HRM 0.3 & & \\
\hline
\end{tabular}




\begin{tabular}{|c|c|c|c|c|c|c|c|c|c|}
\hline & $\mathrm{T}$ & $\bar{U}$ & $\mathrm{~V}$ & $\mathrm{~W}$ & $\mathrm{X}$ & $\mathrm{Y}$ & $Z$ & $\mathrm{AA}$ & $\mathrm{AB}$ \\
\hline \begin{tabular}{l|l}
1 & 1 \\
\end{tabular} & REVIEW_QUALIFIER & SAMP_FROM & SAMP_ITEM & SAMP_MTHD & COLL_MTHD & SAMP_SITE_ID & SAMP_SITE_NAME & WELL_NAME & SAMPLE_LOCATION \\
\hline 3612 & & RIVER & & RAW GRAB & TRANSECT & 318 & VERNITA-3 HRM 0.3 & & \\
\hline 3613 & & RIVER & & RAW GRAB & TRANSECT & 318 & VERNITA-3 HRM 0.3 & & \\
\hline 3614 & & RIVER & & RAW GRAB & TRANSECT & 319 & VERNITA-4 HRM 0.3 & & \\
\hline 3615 & & RIVER & & RAW GRAB & TRANSECT & 319 & VERNITA-4 HRM 0.3 & & \\
\hline 3616 & & RIVER & & RAW GRAB & TRANSECT & 319 & VERNITA-4 HRM 0.3 & & \\
\hline 3617 & & RIVER & & RAW GRAB & TRANSECT & 319 & VERNITA-4 HRM 0.3 & & \\
\hline 3618 & & RIVER & & RAW GRAB & TRANSECT & 319 & VERNITA-4 HRM 0.3 & & \\
\hline 3619 & & RIVER & & RAW GRAB & TRANSECT & 571 & RICH.PMPHS-1 HRM46.4 & & \\
\hline 3620 & & RIVER & & RAW GRAB & TRANSECT & 571 & RICH.PMPHS-1 HRM46.4 & & \\
\hline 3621 & & RIVER & & RAW GRAB & TRANSECT & 571 & RICH.PMPHS-1 HRM46.4 & & \\
\hline 3622 & & RIVER & & RAW GRAB & TRANSECT & 571 & RICH.PMPHS-1 HRM46.4 & & \\
\hline 3623 & & RIVER & & RAW GRAB & TRANSECT & 571 & RICH.PMPHS-1 HRM46.4 & & \\
\hline 3624 & & RIVER & & RAW GRAB & TRANSECT & 571 & RICH.PMPHS-1 HRM46.4 & & \\
\hline 3625 & & RIVER & & RAW GRAB & TRANSECT & 571 & RICH.PMPHS-1 HRM46.4 & & \\
\hline 3626 & & RIVER & & RAW GRAB & TRANSECT & 571 & RICH.PMPHS-1 HRM46.4 & & \\
\hline 3627 & & RIVER & & RAW GRAB & TRANSECT & 571 & RICH.PMPHS-1 HRM46.4 & & \\
\hline 3628 & & RIVER & & RAW GRAB & TRANSECT & 571 & RICH.PMPHS-1 HRM46.4 & & \\
\hline $3629 \mathrm{~F}$ & $\mathrm{~F}$ & RIVER & & RAW GRAB & TRANSECT & 327 & RICH.PMPHS-10 HRM46.4 & & \\
\hline 3630 & & RIVER & & RAW GRAB & TRANSECT & 327 & RICH.PMPHS-10 HRM46.4 & & \\
\hline $3631 \mathrm{~F}$ & $\mathrm{~F}$ & RIVER & & RAW GRAB & TRANSECT & 327 & RICH.PMPHS-10 HRM46.4 & & \\
\hline 3632 & & RIVER & & RAW GRAB & TRANSECT & 327 & RICH.PMPHS-10 HRM46.4 & & \\
\hline 3633 & & RIVER & & RAW GRAB & TRANSECT & 327 & RICH.PMPHS-10 HRM46.4 & & \\
\hline 3634 & & RIVER & & RAW GRAB & TRANSECT & 321 & RICH.PMPHS-2 HRM46.4 & & \\
\hline 3635 & & RIVER & & RAW GRAB & TRANSECT & 321 & RICH.PMPHS-2 HRM46.4 & & \\
\hline 3636 & & RIVER & & RAW GRAB & TRANSECT & 321 & RICH.PMPHS-2 HRM46.4 & & \\
\hline 3637 & & RIVER & & RAW GRAB & TRANSECT & 321 & RICH.PMPHS-2 HRM46.4 & & \\
\hline 3638 & & RIVER & & RAW GRAB & TRANSECT & 321 & RICH.PMPHS-2 HRM46.4 & & \\
\hline 3639 & & RIVER & & RAW GRAB & TRANSECT & 322 & RICH.PMPHS-3 HRM46.4 & & \\
\hline 3640 & & RIVER & & RAW GRAB & TRANSECT & 322 & RICH.PMPHS-3 HRM46.4 & & \\
\hline 3641 & & RIVER & & RAW GRAB & TRANSECT & 322 & RICH.PMPHS-3 HRM46.4 & & \\
\hline 3642 & & RIVER & & RAW GRAB & TRANSECT & 322 & RICH.PMPHS-3 HRM46.4 & & \\
\hline 3643 & & RIVER & & RAW GRAB & TRANSECT & 322 & RICH.PMPHS-3 HRM46.4 & & \\
\hline 3644 & & RIVER & & RAW GRAB & TRANSECT & 572 & RICH.PMPHS-4 HRM46.4 & & \\
\hline 3645 & & RIVER & & RAW GRAB & TRANSECT & 572 & RICH.PMPHS-4 HRM46.4 & & \\
\hline 3646 & & RIVER & & RAW GRAB & TRANSECT & 572 & RICH.PMPHS-4 HRM46.4 & & \\
\hline 3647 & & RIVER & & RAW GRAB & TRANSECT & 572 & RICH.PMPHS-4 HRM46.4 & & \\
\hline 3648 & & RIVER & & RAW GRAB & TRANSECT & 572 & RICH.PMPHS-4 HRM46.4 & & \\
\hline 3649 & & RIVER & & RAW GRAB & TRANSECT & 323 & RICH.PMPHS-5 HRM46.4 & & \\
\hline
\end{tabular}




\begin{tabular}{|c|c|c|c|c|c|c|c|c|c|}
\hline & $\mathrm{T}$ & $\bar{U}$ & $\mathrm{~V}$ & $\mathrm{~W}$ & $\mathrm{X}$ & $\mathrm{Y}$ & $Z$ & $\mathrm{AA}$ & $\mathrm{AB}$ \\
\hline \begin{tabular}{l|l}
1 & 1 \\
\end{tabular} & REVIEW_QUALIFIER & SAMP_FROM & SAMP_ITEM & SAMP_MTHD & COLL_MTHD & SAMP_SITE_ID & SAMP_SITE_NAME & WELL_NAME & SAMPLE_LOCATION \\
\hline 3650 & & RIVER & & RAW GRAB & TRANSECT & 323 & RICH.PMPHS-5 HRM46.4 & & \\
\hline 3651 & & RIVER & & RAW GRAB & TRANSECT & 323 & RICH.PMPHS-5 HRM46.4 & & \\
\hline 3652 & & RIVER & & RAW GRAB & TRANSECT & 323 & RICH.PMPHS-5 HRM46.4 & & \\
\hline 3653 & & RIVER & & RAW GRAB & TRANSECT & 323 & RICH.PMPHS-5 HRM46.4 & & \\
\hline 3654 & & RIVER & & RAW GRAB & TRANSECT & 324 & RICH.PMPHS-6 HRM46.4 & & \\
\hline 3655 & & RIVER & & RAW GRAB & TRANSECT & 324 & RICH.PMPHS-6 HRM46.4 & & \\
\hline 3656 & & RIVER & & RAW GRAB & TRANSECT & 324 & RICH.PMPHS-6 HRM46.4 & & \\
\hline 3657 & & RIVER & & RAW GRAB & TRANSECT & 324 & RICH.PMPHS-6 HRM46.4 & & \\
\hline 3658 & & RIVER & & RAW GRAB & TRANSECT & 324 & RICH.PMPHS-6 HRM46.4 & & \\
\hline 3659 & & RIVER & & RAW GRAB & TRANSECT & 325 & RICH.PMPHS-7 HRM46.4 & & \\
\hline 3660 & & RIVER & & RAW GRAB & TRANSECT & $325 \mathrm{H}$ & RICH.PMPHS-7 HRM46.4 & & \\
\hline 3661 & & RIVER & & RAW GRAB & TRANSECT & 325 & RICH.PMPHS-7 HRM46.4 & & \\
\hline 3662 & & RIVER & & RAW GRAB & TRANSECT & 325 & RICH.PMPHS-7 HRM46.4 & & \\
\hline 3663 & & RIVER & & RAW GRAB & TRANSECT & 325 & RICH.PMPHS-7 HRM46.4 & & \\
\hline 3664 & & RIVER & & RAW GRAB & TRANSECT & 326 & RICH.PMPHS-8 HRM46.4 & & \\
\hline $3665 \mathrm{~F}$ & $\mathrm{~F}$ & RIVER & & RAW GRAB & TRANSECT & 326 & RICH.PMPHS-8 HRM46.4 & & \\
\hline 3666 & & RIVER & & RAW GRAB & TRANSECT & 326 & RICH.PMPHS-8 HRM46.4 & & \\
\hline $3667 \mathrm{~F}$ & $\mathrm{~F}$ & RIVER & & RAW GRAB & TRANSECT & 326 & RICH.PMPHS-8 HRM46.4 & & \\
\hline 3668 & & RIVER & & RAW GRAB & TRANSECT & 326 & RICH.PMPHS-8 HRM46.4 & & \\
\hline 3669 & & RIVER & & RAW GRAB & TRANSECT & 573 & RICH.PMPHS-9 HRM46.4 & & \\
\hline $3670 \mathrm{~F}$ & $\mathrm{~F}$ & RIVER & & RAW GRAB & TRANSECT & 573 & RICH.PMPHS-9 HRM46.4 & & \\
\hline 3671 & & RIVER & & RAW GRAB & TRANSECT & 573 & RICH.PMPHS-9 HRM46.4 & & \\
\hline 3672 & & RIVER & & RAW GRAB & TRANSECT & 573 & RICH.PMPHS-9 HRM46.4 & & \\
\hline 3673 & & RIVER & & RAW GRAB & TRANSECT & 573 & RICH.PMPHS-9 HRM46.4 & & \\
\hline 3674 & & RIVER & & RAW GRAB & TRANSECT & 317 & VERNITA-1 HRM 0.3 & & \\
\hline 3675 & & RIVER & & RAW GRAB & TRANSECT & $317 \mid$ & VERNITA-1 HRM 0.3 & & \\
\hline 3676 & & RIVER & & RAW GRAB & TRANSECT & 317 & VERNITA-1 HRM 0.3 & & \\
\hline 3677 & & RIVER & & RAW GRAB & TRANSECT & 317 & VERNITA-1 HRM 0.3 & & \\
\hline 3678 & & RIVER & & RAW GRAB & TRANSECT & 317 & VERNITA-1 HRM 0.3 & & \\
\hline 3679 & & RIVER & & RAW GRAB & TRANSECT & 570 & VERNITA-2 HRM 0.3 & & \\
\hline 3680 & & RIVER & & RAW GRAB & TRANSECT & 570 & VERNITA-2 HRM 0.3 & & \\
\hline 3681 & & RIVER & & RAW GRAB & TRANSECT & 570 & VERNITA-2 HRM 0.3 & & \\
\hline 3682 & & RIVER & & RAW GRAB & TRANSECT & 570 & VERNITA-2 HRM 0.3 & & \\
\hline 3683 & & RIVER & & RAW GRAB & TRANSECT & 570 & VERNITA-2 HRM 0.3 & & \\
\hline 3684 & & RIVER & & RAW GRAB & TRANSECT & 318 & VERNITA-3 HRM 0.3 & & \\
\hline 3685 & & RIVER & & RAW GRAB & TRANSECT & 318 & VERNITA-3 HRM 0.3 & & \\
\hline 3686 & & RIVER & & RAW GRAB & TRANSECT & 318 & VERNITA-3 HRM 0.3 & & \\
\hline 3687 & & RIVER & & RAW GRAB & TRANSECT & 318 & VERNITA-3 HRM 0.3 & & \\
\hline
\end{tabular}




\begin{tabular}{|c|c|c|c|c|c|c|c|c|c|}
\hline & $\mathrm{T}$ & $\bar{U}$ & $\mathrm{~V}$ & $\mathrm{~W}$ & $\mathrm{X}$ & $\mathrm{Y}$ & $Z$ & $\mathrm{AA}$ & $\mathrm{AB}$ \\
\hline 1 & REVIEW_QUALIFIER & SAMP_FROM & SAMP_ITEM & SAMP_MTHD & COLL_MTHD & SAMP_SITE_ID & SAMP_SITE_NAME & WELL_NAME & SAMPLE_LOCATION \\
\hline 3688 & & RIVER & & RAW GRAB & TRANSECT & 318 & VERNITA-3 HRM 0.3 & & \\
\hline 3689 & & RIVER & & RAW GRAB & TRANSECT & 319 & VERNITA-4 HRM 0.3 & & \\
\hline 3690 & & RIVER & & RAW GRAB & TRANSECT & 319 & VERNITA-4 HRM 0.3 & & \\
\hline 3691 & & RIVER & & RAW GRAB & TRANSECT & 319 & VERNITA-4 HRM 0.3 & & \\
\hline 3692 & & RIVER & & RAW GRAB & TRANSECT & 319 & VERNITA-4 HRM 0.3 & & \\
\hline 3693 & & RIVER & & RAW GRAB & TRANSECT & 319 & VERNITA-4 HRM 0.3 & & \\
\hline 3694 & & RIVER & & RAW GRAB & TRANSECT & 571 & RICH.PMPHS-1 HRM46.4 & & \\
\hline 3695 & & RIVER & & RAW GRAB & TRANSECT & 571 & RICH.PMPHS-1 HRM46.4 & & \\
\hline 3696 & & RIVER & & RAW GRAB & TRANSECT & 571 & RICH.PMPHS-1 HRM46.4 & & \\
\hline 3697 & & RIVER & & RAW GRAB & TRANSECT & 571 & RICH.PMPHS-1 HRM46.4 & & \\
\hline 3698 & & RIVER & & RAW GRAB & TRANSECT & 571 & RICH.PMPHS-1 HRM46.4 & & \\
\hline 3699 & & RIVER & & RAW GRAB & TRANSECT & 327 & RICH.PMPHS-10 HRM46.4 & & \\
\hline 3700 & & RIVER & & RAW GRAB & TRANSECT & 327 & RICH.PMPHS-10 HRM46.4 & & \\
\hline 3701 & & RIVER & & RAW GRAB & TRANSECT & 327 & RICH.PMPHS-10 HRM46.4 & & \\
\hline 3702 & & RIVER & & RAW GRAB & TRANSECT & 327 & RICH.PMPHS-10 HRM46.4 & & \\
\hline 3703 & & RIVER & & RAW GRAB & TRANSECT & 327 & RICH.PMPHS-10 HRM46.4 & & \\
\hline 3704 & & RIVER & & RAW GRAB & TRANSECT & 321 & RICH.PMPHS-2 HRM46.4 & & \\
\hline 3705 & & RIVER & & RAW GRAB & TRANSECT & 321 & RICH.PMPHS-2 HRM46.4 & & \\
\hline 3706 & & RIVER & & RAW GRAB & TRANSECT & 321 & RICH.PMPHS-2 HRM46.4 & & \\
\hline 3707 & & RIVER & & RAW GRAB & TRANSECT & 321 & RICH.PMPHS-2 HRM46.4 & & \\
\hline 3708 & & RIVER & & RAW GRAB & TRANSECT & 321 & RICH.PMPHS-2 HRM46.4 & & \\
\hline 3709 & & RIVER & & RAW GRAB & TRANSECT & 322 & RICH.PMPHS-3 HRM46.4 & & \\
\hline 3710 & & RIVER & & RAW GRAB & TRANSECT & 322 & RICH.PMPHS-3 HRM46.4 & & \\
\hline 3711 & & RIVER & & RAW GRAB & TRANSECT & 322 & RICH.PMPHS-3 HRM46.4 & & \\
\hline 3712 & & RIVER & & RAW GRAB & TRANSECT & 322 & RICH.PMPHS-3 HRM46.4 & & \\
\hline 3713 & & RIVER & & RAW GRAB & TRANSECT & 322 & RICH.PMPHS-3 HRM46.4 & & \\
\hline 3714 & & RIVER & & RAW GRAB & TRANSECT & 572 & RICH.PMPHS-4 HRM46.4 & & \\
\hline 3715 & & RIVER & & RAW GRAB & TRANSECT & 572 & RICH.PMPHS-4 HRM46.4 & & \\
\hline 3716 & & RIVER & & RAW GRAB & TRANSECT & 572 & RICH.PMPHS-4 HRM46.4 & & \\
\hline 3717 & & RIVER & & RAW GRAB & TRANSECT & 572 & RICH.PMPHS-4 HRM46.4 & & \\
\hline 3718 & & RIVER & & RAW GRAB & TRANSECT & 572 & RICH.PMPHS-4 HRM46.4 & & \\
\hline 3719 & & RIVER & & RAW GRAB & TRANSECT & 323 & RICH.PMPHS-5 HRM46.4 & & \\
\hline 3720 & & RIVER & & RAW GRAB & TRANSECT & 323 & RICH.PMPHS-5 HRM46.4 & & \\
\hline 3721 & & RIVER & & RAW GRAB & TRANSECT & 323 & RICH.PMPHS-5 HRM46.4 & & \\
\hline 3722 & & RIVER & & RAW GRAB & TRANSECT & 323 & RICH.PMPHS-5 HRM46.4 & & \\
\hline 3723 & & RIVER & & RAW GRAB & TRANSECT & 323 & RICH.PMPHS-5 HRM46.4 & & \\
\hline 3724 & & RIVER & & RAW GRAB & TRANSECT & 324 & RICH.PMPHS-6 HRM46.4 & & \\
\hline 3725 & & RIVER & & RAW GRAB & TRANSECT & 324 & RICH.PMPHS-6 HRM46.4 & & \\
\hline
\end{tabular}




\begin{tabular}{|c|c|c|c|c|c|c|c|c|c|}
\hline & $\mathrm{T}$ & $\bar{U}$ & $\mathrm{~V}$ & $\mathrm{~W}$ & $\mathrm{X}$ & $\mathrm{Y}$ & $Z$ & $\mathrm{AA}$ & $\mathrm{AB}$ \\
\hline 1 & REVIEW_QUALIFIER & SAMP_FROM & SAMP_ITEM & SAMP_MTHD & COLL_MTHD & SAMP_SITE_ID & SAMP_SITE_NAME & WELL_NAME & SAMPLE_LOCATION \\
\hline 3726 & & RIVER & & RAW GRAB & TRANSECT & 324 & RICH.PMPHS-6 HRM46.4 & & \\
\hline 3727 & & RIVER & & RAW GRAB & TRANSECT & 324 & RICH.PMPHS-6 HRM46.4 & & \\
\hline 3728 & & RIVER & & RAW GRAB & TRANSECT & 324 & RICH.PMPHS-6 HRM46.4 & & \\
\hline 3729 & & RIVER & & RAW GRAB & TRANSECT & 325 & RICH.PMPHS-7 HRM46.4 & & \\
\hline 3730 & & RIVER & & RAW GRAB & TRANSECT & 325 & RICH.PMPHS-7 HRM46.4 & & \\
\hline 3731 & & RIVER & & RAW GRAB & TRANSECT & 325 & RICH.PMPHS-7 HRM46.4 & & \\
\hline 3732 & & RIVER & & RAW GRAB & TRANSECT & 325 & RICH.PMPHS-7 HRM46.4 & & \\
\hline 3733 & & RIVER & & RAW GRAB & TRANSECT & 325 & RICH.PMPHS-7 HRM46.4 & & \\
\hline 3734 & & RIVER & & RAW GRAB & TRANSECT & 326 & RICH.PMPHS-8 HRM46.4 & & \\
\hline 3735 & & RIVER & & RAW GRAB & TRANSECT & 326 & RICH.PMPHS-8 HRM46.4 & & \\
\hline 3736 & & RIVER & & RAW GRAB & TRANSECT & 326 & RICH.PMPHS-8 HRM46.4 & & \\
\hline 3737 & & RIVER & & RAW GRAB & TRANSECT & 326 & RICH.PMPHS-8 HRM46.4 & & \\
\hline 3738 & & RIVER & & RAW GRAB & TRANSECT & 326 & RICH.PMPHS-8 HRM46.4 & & \\
\hline 3739 & & RIVER & & RAW GRAB & TRANSECT & 573 & RICH.PMPHS-9 HRM46.4 & & \\
\hline 3740 & & RIVER & & RAW GRAB & TRANSECT & 573 & RICH.PMPHS-9 HRM46.4 & & \\
\hline 3741 & & RIVER & & RAW GRAB & TRANSECT & 573 & RICH.PMPHS-9 HRM46.4 & & \\
\hline 3742 & & RIVER & & RAW GRAB & TRANSECT & 573 & RICH.PMPHS-9 HRM46.4 & & \\
\hline 3743 & & RIVER & & RAW GRAB & TRANSECT & 573 & RICH.PMPHS-9 HRM46.4 & & \\
\hline 3744 & & RIVER & & RAW GRAB & TRANSECT & 328 & 100 N -1 HRM 9.5 & & \\
\hline 3745 & & RIVER & & RAW GRAB & TRANSECT & 328 & 100 N -1 HRM 9.5 & & \\
\hline 3746 & & RIVER & & RAW GRAB & TRANSECT & 328 & $100 \mathrm{~N}-1$ HRM 9.5 & & \\
\hline 3747 & & RIVER & & RAW GRAB & TRANSECT & 328 & $100 \mathrm{~N}-1$ HRM 9.5 & & \\
\hline 3748 & & RIVER & & RAW GRAB & TRANSECT & 328 & 100 N -1 HRM 9.5 & & \\
\hline 3749 & & RIVER & & RAW GRAB & TRANSECT & 335 & $100 \mathrm{~N}-10 \mathrm{HRM} 9.5$ & & \\
\hline 3750 & & RIVER & & RAW GRAB & TRANSECT & 335 & $100 \mathrm{~N}-10$ HRM 9.5 & & \\
\hline 3751 & & RIVER & & RAW GRAB & TRANSECT & 335 & $100 \mathrm{~N}-10$ HRM 9.5 & & \\
\hline 3752 & & RIVER & & RAW GRAB & TRANSECT & 335 & $100 \mathrm{~N}-10$ HRM 9.5 & & \\
\hline 3753 & & RIVER & & RAW GRAB & TRANSECT & 335 & $100 \mathrm{~N}-10 \mathrm{HRM} 9.5$ & & \\
\hline 3754 & & RIVER & & RAW GRAB & TRANSECT & 329 & 100 N -2 HRM 9.5 & & \\
\hline 3755 & & RIVER & & RAW GRAB & TRANSECT & 329 & 100 N -2 HRM 9.5 & & \\
\hline 3756 & & RIVER & & RAW GRAB & TRANSECT & 329 & 100 N -2 HRM 9.5 & & \\
\hline 3757 & & RIVER & & RAW GRAB & TRANSECT & 329 & 100 N -2 HRM 9.5 & & \\
\hline 3758 & & RIVER & & RAW GRAB & TRANSECT & 329 & 100 N -2 HRM 9.5 & & \\
\hline 3759 & & RIVER & & RAW GRAB & TRANSECT & 330 & 100 N -3 HRM 9.5 & & \\
\hline 3760 & & RIVER & & RAW GRAB & TRANSECT & 330 & 100 N -3 HRM 9.5 & & \\
\hline 3761 & & RIVER & & RAW GRAB & TRANSECT & 330 & $100 \mathrm{~N}-3$ HRM 9.5 & & \\
\hline 3762 & & RIVER & & RAW GRAB & TRANSECT & 330 & 100 N -3 HRM 9.5 & & \\
\hline 3763 & & RIVER & & RAW GRAB & TRANSECT & 330 & 100 N -3 HRM 9.5 & & \\
\hline
\end{tabular}




\begin{tabular}{|c|c|c|c|c|c|c|c|c|c|}
\hline & $\mathrm{T}$ & 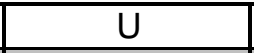 & $\mathrm{V}$ & $\mathrm{W}$ & $\mathrm{X}$ & $\mathrm{Y}$ & $Z$ & $\mathrm{AA}$ & $A B$ \\
\hline 1 & REVIEW_QUALIFIER & SAMP_FROM & SAMP_ITEM & SAMP_MTHD & COLL_MTHD & SAMP_SITE_ID & SAMP_SITE_NAME & WELL_NAME & SAMPLE_LOCATION \\
\hline 3764 & & RIVER & & RAW GRAB & TRANSECT & 574 & $100 \mathrm{~N}-4$ HRM 9.5 & & \\
\hline 3765 & & RIVER & & RAW GRAB & TRANSECT & 574 & $100 \mathrm{~N}-4$ HRM 9.5 & & \\
\hline 3766 & & RIVER & & RAW GRAB & TRANSECT & 574 & 100 N -4 HRM 9.5 & & \\
\hline 3767 & & RIVER & & RAW GRAB & TRANSECT & 574 & 100 N -4 HRM 9.5 & & \\
\hline 3768 & & RIVER & & RAW GRAB & TRANSECT & 574 & $100 \mathrm{~N}-4$ HRM 9.5 & & \\
\hline 3769 & & RIVER & & RAW GRAB & TRANSECT & 331 & 100 N -5 HRM 9.5 & & \\
\hline 3770 & & RIVER & & RAW GRAB & TRANSECT & 331 & 100 N -5 HRM 9.5 & & \\
\hline 3771 & & RIVER & & RAW GRAB & TRANSECT & 331 & 100 N -5 HRM 9.5 & & \\
\hline 3772 & & RIVER & & RAW GRAB & TRANSECT & 331 & 100 N -5 HRM 9.5 & & \\
\hline 3773 & & RIVER & & RAW GRAB & TRANSECT & $331:$ & 100 N -5 HRM 9.5 & & \\
\hline 3774 & & RIVER & & RAW GRAB & TRANSECT & 332 & 100 N -6 HRM 9.5 & & \\
\hline 3775 & & RIVER & & RAW GRAB & TRANSECT & 332 & 100 N -6 HRM 9.5 & & \\
\hline 3776 & & RIVER & & RAW GRAB & TRANSECT & 332 & 100 N -6 HRM 9.5 & & \\
\hline 3777 & & RIVER & & RAW GRAB & TRANSECT & 332 & 100 N -6 HRM 9.5 & & \\
\hline 3778 & & RIVER & & RAW GRAB & TRANSECT & 332 & 100 N -6 HRM 9.5 & & \\
\hline 3779 & & RIVER & & RAW GRAB & TRANSECT & 333 & 100 N -7 HRM 9.5 & & \\
\hline 3780 & & RIVER & & RAW GRAB & TRANSECT & 333 & 100 N -7 HRM 9.5 & & \\
\hline 3781 & & RIVER & & RAW GRAB & TRANSECT & 333 & $100 \mathrm{~N}-7$ HRM 9.5 & & \\
\hline 3782 & & RIVER & & RAW GRAB & TRANSECT & 333 & 100 N -7 HRM 9.5 & & \\
\hline 3783 & & RIVER & & RAW GRAB & TRANSECT & 333 & 100 N -7 HRM 9.5 & & \\
\hline 3784 & & RIVER & & RAW GRAB & TRANSECT & 575 & 100 N -8 HRM 9.5 & & \\
\hline 3785 & & RIVER & & RAW GRAB & TRANSECT & 575 & 100 N -8 HRM 9.5 & & \\
\hline 3786 & & RIVER & & RAW GRAB & TRANSECT & 575 & 100 N -8 HRM 9.5 & & \\
\hline 3787 & & RIVER & & RAW GRAB & TRANSECT & 575 & 100 N -8 HRM 9.5 & & \\
\hline 3788 & & RIVER & & RAW GRAB & TRANSECT & 575 & 100 N -8 HRM 9.5 & & \\
\hline 3789 & & RIVER & & RAW GRAB & TRANSECT & 334 & 100 N -9 HRM 9.5 & & \\
\hline 3790 & & RIVER & & RAW GRAB & TRANSECT & 334 & 100 N -9 HRM 9.5 & & \\
\hline 3791 & & RIVER & & RAW GRAB & TRANSECT & 334 & 100 N -9 HRM 9.5 & & \\
\hline 3792 & & RIVER & & RAW GRAB & TRANSECT & 334 & 100 N -9 HRM 9.5 & & \\
\hline 3793 & & RIVER & & RAW GRAB & TRANSECT & $334:$ & 100 N -9 HRM 9.5 & & \\
\hline 3794 & & RIVER & & RAW GRAB & TRANSECT & 317 & VERNITA-1 HRM 0.3 & & \\
\hline 3795 & & RIVER & & RAW GRAB & TRANSECT & 317 & VERNITA-1 HRM 0.3 & & \\
\hline 3796 & & RIVER & & RAW GRAB & TRANSECT & 317 & VERNITA-1 HRM 0.3 & & \\
\hline 3797 & & RIVER & & RAW GRAB & TRANSECT & 317 & VERNITA-1 HRM 0.3 & & \\
\hline 3798 & & RIVER & & RAW GRAB & TRANSECT & 317 & VERNITA-1 HRM 0.3 & & \\
\hline 3799 & & RIVER & & RAW GRAB & TRANSECT & 570 & VERNITA-2 HRM 0.3 & & \\
\hline 3800 & & RIVER & & RAW GRAB & TRANSECT & 570 & VERNITA-2 HRM 0.3 & & \\
\hline 3801 & & RIVER & & RAW GRAB & TRANSECT & 570 & VERNITA-2 HRM 0.3 & & \\
\hline
\end{tabular}




\begin{tabular}{|c|c|c|c|c|c|c|c|c|c|}
\hline & $\mathrm{T}$ & 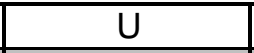 & $\mathrm{V}$ & $\mathrm{W}$ & $\mathrm{X}$ & $\mathrm{Y}$ & $Z$ & $\mathrm{AA}$ & $A B$ \\
\hline \begin{tabular}{l|l}
1 & 1 \\
\end{tabular} & REVIEW_QUALIFIER & SAMP_FROM & SAMP_ITEM & SAMP_MTHD & COLL_MTHD & SAMP_SITE_ID & SAMP_SITE_NAME & WELL_NAME & SAMPLE_LOCATION \\
\hline 3802 & & RIVER & & RAW GRAB & TRANSECT & 570 & JERNITA-2 HRM 0.3 & & \\
\hline 3803 & & RIVER & & RAW GRAB & TRANSECT & 570 & JERNITA-2 HRM 0.3 & & \\
\hline 3804 & & RIVER & & RAW GRAB & TRANSECT & 318 & JERNITA-3 HRM 0.3 & & \\
\hline 3805 & & RIVER & & RAW GRAB & TRANSECT & 318 & JERNITA-3 HRM 0.3 & & \\
\hline 3806 & & RIVER & & RAW GRAB & TRANSECT & 318 & JERNITA-3 HRM 0.3 & & \\
\hline 3807 & & RIVER & & RAW GRAB & TRANSECT & 318 & JERNITA-3 HRM 0.3 & & \\
\hline 3808 & & RIVER & & RAW GRAB & TRANSECT & 318 & JERNITA-3 HRM 0.3 & & \\
\hline 3809 & & RIVER & & RAW GRAB & TRANSECT & 319 & JERNITA-4 HRM 0.3 & & \\
\hline 3810 & & RIVER & & RAW GRAB & TRANSECT & 319 & JERNITA-4 HRM 0.3 & & \\
\hline 3811 & & RIVER & & RAW GRAB & TRANSECT & 319 & JERNITA-4 HRM 0.3 & & \\
\hline 3812 & & RIVER & & RAW GRAB & TRANSECT & 319 & VERNITA-4 HRM 0.3 & & \\
\hline 3813 & & RIVER & & RAW GRAB & TRANSECT & 319 & JERNITA-4 HRM 0.3 & & \\
\hline 3814 & & RIVER & & RAW GRAB & TRANSECT & 348 & 300 AREA -1 HRM 43.1 & & \\
\hline 3815 & & RIVER & & RAW GRAB & TRANSECT & 3483 & 300 AREA -1 HRM 43.1 & & \\
\hline 3816 & & RIVER & & RAW GRAB & TRANSECT & 348 & 300 AREA -1 HRM 43.1 & & \\
\hline 3817 & & RIVER & & RAW GRAB & TRANSECT & 348 & 300 AREA -1 HRM 43.1 & & \\
\hline 3818 & & RIVER & & RAW GRAB & TRANSECT & 348 & 300 AREA -1 HRM 43.1 & & \\
\hline 3819 & & RIVER & & RAW GRAB & TRANSECT & 4703 & 300 AREA -2 HRM 43.1 & & \\
\hline 3820 & & RIVER & & RAW GRAB & TRANSECT & 4703 & 300 AREA -2 HRM 43.1 & & \\
\hline 3821 & & RIVER & & RAW GRAB & TRANSECT & 4703 & 300 AREA -2 HRM 43.1 & & \\
\hline 3822 & & RIVER & & RAW GRAB & TRANSECT & 4703 & 300 AREA -2 HRM 43.1 & & \\
\hline 3823 & & RIVER & & RAW GRAB & TRANSECT & 4703 & 300 AREA -2 HRM 43.1 & & \\
\hline 3824 & & RIVER & & RAW GRAB & TRANSECT & 580 & 300 AREA -3 HRM 43.1 & & \\
\hline 3825 & & RIVER & & RAW GRAB & TRANSECT & 5803 & 300 AREA -3 HRM 43.1 & & \\
\hline 3826 & & RIVER & & RAW GRAB & TRANSECT & 5803 & 300 AREA -3 HRM 43.1 & & \\
\hline 3827 & & RIVER & & RAW GRAB & TRANSECT & 5803 & 300 AREA -3 HRM 43.1 & & \\
\hline $3828 \mathrm{~F}$ & $\mathrm{~F}$ & RIVER & & RAW GRAB & TRANSECT & 5803 & 300 AREA -3 HRM 43.1 & & \\
\hline 3829 & & RIVER & & RAW GRAB & TRANSECT & 3493 & 300 AREA -4 HRM 43.1 & & \\
\hline 3830 & & RIVER & & RAW GRAB & TRANSECT & 3493 & 300 AREA -4 HRM 43.1 & & \\
\hline 3831 & & RIVER & & RAW GRAB & TRANSECT & 349 & 300 AREA -4 HRM 43.1 & & \\
\hline 3832 & & RIVER & & RAW GRAB & TRANSECT & 3493 & 300 AREA -4 HRM 43.1 & & \\
\hline 3833 & & RIVER & & RAW GRAB & TRANSECT & 3493 & 300 AREA -4 HRM 43.1 & & \\
\hline 3834 & & RIVER & & RAW GRAB & TRANSECT & 607 & 300 AREA -5 HRM 43.1 & & \\
\hline 3835 & & RIVER & & RAW GRAB & TRANSECT & 6073 & 300 AREA -5 HRM 43.1 & & \\
\hline 3836 & & RIVER & & RAW GRAB & TRANSECT & 6073 & 300 AREA -5 HRM 43.1 & & \\
\hline 3837 & & RIVER & & RAW GRAB & TRANSECT & 607 & 300 AREA -5 HRM 43.1 & & \\
\hline 3838 & & RIVER & & RAW GRAB & TRANSECT & 6073 & 300 AREA -5 HRM 43.1 & & \\
\hline 3839 & & RIVER & & RAW GRAB & TRANSECT & 350 & 300 AREA -6 HRM 43.1 & & \\
\hline
\end{tabular}




\begin{tabular}{|c|c|c|c|c|c|c|c|c|c|}
\hline & $\mathrm{T}$ & $\bar{U}$ & $\mathrm{~V}$ & $\mathrm{~W}$ & $\mathrm{X}$ & $\mathrm{Y}$ & $Z$ & $\mathrm{AA}$ & $\mathrm{AB}$ \\
\hline \begin{tabular}{l|l}
1 & 1 \\
\end{tabular} & REVIEW_QUALIFIER & SAMP_FROM & SAMP_ITEM & SAMP_MTHD & COLL_MTHD & SAMP_SITE_ID & SAMP_SITE_NAME & WELL_NAME & SAMPLE_LOCATION \\
\hline 3840 & & RIVER & & RAW GRAB & TRANSECT & 3503 & 300 AREA -6 HRM 43.1 & & \\
\hline 3841 & & RIVER & & RAW GRAB & TRANSECT & 3503 & 300 AREA -6 HRM 43.1 & & \\
\hline 3842 & & RIVER & & RAW GRAB & TRANSECT & 3503 & 300 AREA -6 HRM 43.1 & & \\
\hline 3843 & & RIVER & & RAW GRAB & TRANSECT & 3503 & 300 AREA -6 HRM 43.1 & & \\
\hline 3844 & & RIVER & & RAW GRAB & TRANSECT & 3513 & 300 AREA -7 HRM 43.1 & & \\
\hline 3845 & & RIVER & & RAW GRAB & TRANSECT & 3513 & 300 AREA -7 HRM 43.1 & & \\
\hline 3846 & & RIVER & & RAW GRAB & TRANSECT & 351 & 300 AREA -7 HRM 43.1 & & \\
\hline 3847 & & RIVER & & RAW GRAB & TRANSECT & 3513 & 300 AREA -7 HRM 43.1 & & \\
\hline 3848 & & RIVER & & RAW GRAB & TRANSECT & 351 & 300 AREA -7 HRM 43.1 & & \\
\hline 3849 & & RIVER & & RAW GRAB & TRANSECT & 5813 & 300 AREA -8 HRM 43.1 & & \\
\hline 3850 & & RIVER & & RAW GRAB & TRANSECT & 5813 & 300 AREA -8 HRM 43.1 & & \\
\hline 3851 & & RIVER & & RAW GRAB & TRANSECT & 5813 & 300 AREA -8 HRM 43.1 & & \\
\hline 3852 & & RIVER & & RAW GRAB & TRANSECT & 581 & 300 AREA -8 HRM 43.1 & & \\
\hline 3853 & & RIVER & & RAW GRAB & TRANSECT & 5813 & 300 AREA -8 HRM 43.1 & & \\
\hline $3854 \mathrm{~F}$ & $\mathrm{~F}$ & RIVER & & RAW GRAB & TRANSECT & 4713 & 300 AREA -9 HRM 43.1 & & \\
\hline 3855 & & RIVER & & RAW GRAB & TRANSECT & 4713 & 300 AREA -9 HRM 43.1 & & \\
\hline 3856 & & RIVER & & RAW GRAB & TRANSECT & 4713 & 300 AREA -9 HRM 43.1 & & \\
\hline 3857 & & RIVER & & RAW GRAB & TRANSECT & 4713 & 300 AREA -9 HRM 43.1 & & \\
\hline 3858 & & RIVER & & RAW GRAB & TRANSECT & 4713 & 300 AREA -9 HRM 43.1 & & \\
\hline 3859 & & RIVER & & RAW GRAB & TRANSECT & 3523 & 300 AREA-10 HRM 43.1 & & \\
\hline 3860 & & RIVER & & RAW GRAB & TRANSECT & 3523 & 300 AREA-10 HRM 43.1 & & \\
\hline $3861 \mathrm{~F}$ & $\mathrm{~F}$ & RIVER & & RAW GRAB & TRANSECT & 3523 & 300 AREA-10 HRM 43.1 & & \\
\hline 3862 & & RIVER & & RAW GRAB & TRANSECT & 352 & 300 AREA-10 HRM 43.1 & & \\
\hline 3863 & & RIVER & & RAW GRAB & TRANSECT & 3523 & 300 AREA-10 HRM 43.1 & & \\
\hline 3864 & & RIVER & & RAW GRAB & TRANSECT & 571 & RICH.PMPHS-1 HRM46.4 & & \\
\hline 3865 & & RIVER & & RAW GRAB & TRANSECT & 571 & RICH.PMPHS-1 HRM46.4 & & \\
\hline 3866 & & RIVER & & RAW GRAB & TRANSECT & 571 & RICH.PMPHS-1 HRM46.4 & & \\
\hline 3867 & & RIVER & & RAW GRAB & TRANSECT & 571 & RICH.PMPHS-1 HRM46.4 & & \\
\hline 3868 & & RIVER & & RAW GRAB & TRANSECT & 571 & RICH.PMPHS-1 HRM46.4 & & \\
\hline 3869 & & RIVER & & RAW GRAB & TRANSECT & 327 & RICH.PMPHS-10 HRM46.4 & & \\
\hline 3870 & & RIVER & & RAW GRAB & TRANSECT & 327 & RICH.PMPHS-10 HRM46.4 & & \\
\hline 3871 & & RIVER & & RAW GRAB & TRANSECT & 327 & RICH.PMPHS-10 HRM46.4 & & \\
\hline 3872 & & RIVER & & RAW GRAB & TRANSECT & 327 & RICH.PMPHS-10 HRM46.4 & & \\
\hline 3873 & & RIVER & & RAW GRAB & TRANSECT & 327 & RICH.PMPHS-10 HRM46.4 & & \\
\hline 3874 & & RIVER & & RAW GRAB & TRANSECT & 321 & RICH.PMPHS-2 HRM46.4 & & \\
\hline 3875 & & RIVER & & RAW GRAB & TRANSECT & 321 & RICH.PMPHS-2 HRM46.4 & & \\
\hline 3876 & & RIVER & & RAW GRAB & TRANSECT & 321 & RICH.PMPHS-2 HRM46.4 & & \\
\hline 3877 & & RIVER & & RAW GRAB & TRANSECT & 321 & RICH.PMPHS-2 HRM46.4 & & \\
\hline
\end{tabular}




\begin{tabular}{|c|c|c|c|c|c|c|c|c|c|}
\hline & $\mathrm{T}$ & $\bar{U}$ & $\mathrm{~V}$ & $\mathrm{~W}$ & $\mathrm{X}$ & $\mathrm{Y}$ & $Z$ & $\mathrm{AA}$ & $\mathrm{AB}$ \\
\hline 1 & REVIEW_QUALIFIER & SAMP_FROM & SAMP_ITEM & SAMP_MTHD & COLL_MTHD & SAMP_SITE_ID & SAMP_SITE_NAME & WELL_NAME & SAMPLE_LOCATION \\
\hline 3878 & & RIVER & & RAW GRAB & TRANSECT & 321 & RICH.PMPHS-2 HRM46.4 & & \\
\hline 3879 & & RIVER & & RAW GRAB & TRANSECT & 322 & RICH.PMPHS-3 HRM46.4 & & \\
\hline 3880 & & RIVER & & RAW GRAB & TRANSECT & 322 & RICH.PMPHS-3 HRM46.4 & & \\
\hline 3881 & & RIVER & & RAW GRAB & TRANSECT & 322 & RICH.PMPHS-3 HRM46.4 & & \\
\hline 3882 & & RIVER & & RAW GRAB & TRANSECT & 322 & RICH.PMPHS-3 HRM46.4 & & \\
\hline 3883 & & RIVER & & RAW GRAB & TRANSECT & 322 & RICH.PMPHS-3 HRM46.4 & & \\
\hline 3884 & & RIVER & & RAW GRAB & TRANSECT & 572 & RICH.PMPHS-4 HRM46.4 & & \\
\hline 3885 & & RIVER & & RAW GRAB & TRANSECT & 572 & RICH.PMPHS-4 HRM46.4 & & \\
\hline 3886 & & RIVER & & RAW GRAB & TRANSECT & 572 & RICH.PMPHS-4 HRM46.4 & & \\
\hline 3887 & & RIVER & & RAW GRAB & TRANSECT & 572 & RICH.PMPHS-4 HRM46.4 & & \\
\hline 3888 & & RIVER & & RAW GRAB & TRANSECT & 572 & RICH.PMPHS-4 HRM46.4 & & \\
\hline 3889 & & RIVER & & RAW GRAB & TRANSECT & 323 & RICH.PMPHS-5 HRM46.4 & & \\
\hline 3890 & & RIVER & & RAW GRAB & TRANSECT & 323 & RICH.PMPHS-5 HRM46.4 & & \\
\hline 3891 & & RIVER & & RAW GRAB & TRANSECT & 323 & RICH.PMPHS-5 HRM46.4 & & \\
\hline 3892 & & RIVER & & RAW GRAB & TRANSECT & 323 & RICH.PMPHS-5 HRM46.4 & & \\
\hline 3893 & & RIVER & & RAW GRAB & TRANSECT & 323 & RICH.PMPHS-5 HRM46.4 & & \\
\hline 3894 & & RIVER & & RAW GRAB & TRANSECT & 324 & RICH.PMPHS-6 HRM46.4 & & \\
\hline 3895 & & RIVER & & RAW GRAB & TRANSECT & 324 & RICH.PMPHS-6 HRM46.4 & & \\
\hline 3896 & & RIVER & & RAW GRAB & TRANSECT & 324 & RICH.PMPHS-6 HRM46.4 & & \\
\hline 3897 & & RIVER & & RAW GRAB & TRANSECT & 324 & RICH.PMPHS-6 HRM46.4 & & \\
\hline 3898 & & RIVER & & RAW GRAB & TRANSECT & 324 & RICH.PMPHS-6 HRM46.4 & & \\
\hline 3899 & & RIVER & & RAW GRAB & TRANSECT & 325 & RICH.PMPHS-7 HRM46.4 & & \\
\hline 3900 & & RIVER & & RAW GRAB & TRANSECT & 325 & RICH.PMPHS-7 HRM46.4 & & \\
\hline 3901 & & RIVER & & RAW GRAB & TRANSECT & 325 & RICH.PMPHS-7 HRM46.4 & & \\
\hline 3902 & & RIVER & & RAW GRAB & TRANSECT & 325 & RICH.PMPHS-7 HRM46.4 & & \\
\hline 3903 & & RIVER & & RAW GRAB & TRANSECT & 325 & RICH.PMPHS-7 HRM46.4 & & \\
\hline 3904 & & RIVER & & RAW GRAB & TRANSECT & 326 & RICH.PMPHS-8 HRM46.4 & & \\
\hline 3905 & & RIVER & & RAW GRAB & TRANSECT & 326 & RICH.PMPHS-8 HRM46.4 & & \\
\hline 3906 & & RIVER & & RAW GRAB & TRANSECT & 326 & RICH.PMPHS-8 HRM46.4 & & \\
\hline 3907 & & RIVER & & RAW GRAB & TRANSECT & 326 & RICH.PMPHS-8 HRM46.4 & & \\
\hline 3908 & & RIVER & & RAW GRAB & TRANSECT & 326 & RICH.PMPHS-8 HRM46.4 & & \\
\hline 3909 & & RIVER & & RAW GRAB & TRANSECT & 573 & RICH.PMPHS-9 HRM46.4 & & \\
\hline 3910 & & RIVER & & RAW GRAB & TRANSECT & 573 & RICH.PMPHS-9 HRM46.4 & & \\
\hline 3911 & & RIVER & & RAW GRAB & TRANSECT & 573 & RICH.PMPHS-9 HRM46.4 & & \\
\hline 3912 & & RIVER & & RAW GRAB & TRANSECT & 573 & RICH.PMPHS-9 HRM46.4 & & \\
\hline 3913 & & RIVER & & RAW GRAB & TRANSECT & 573 & RICH.PMPHS-9 HRM46.4 & & \\
\hline 3914 & & RIVER & & RAW GRAB & TRANSECT & 336 & 100 F -1 HRM 19.0 & & \\
\hline 3915 & & RIVER & & RAW GRAB & TRANSECT & 336 & 100 F -1 HRM 19.0 & & \\
\hline
\end{tabular}




\begin{tabular}{|c|c|c|c|c|c|c|c|c|c|}
\hline & $\mathrm{T}$ & $U$ & $\mathrm{~V}$ & $\mathrm{~W}$ & $\mathrm{X}$ & $\bar{Y}$ & $Z$ & AA & $A B$ \\
\hline 1 & REVIEW_QUALIFIER & SAMP_FROM & SAMP_ITEM & SAMP_MTHD & COLL_MTHD & SAMP_SITE_ID & SAMP_SITE_NAME & WELL_NAME & SAMPLE_LOCATION \\
\hline 3916 & & RIVER & & RAW GRAB & TRANSECT & 336 & $100 \mathrm{~F}-1$ HRM 19.0 & & \\
\hline 3917 & & RIVER & & RAW GRAB & TRANSECT & 336 & 100 F -1 HRM 19.0 & & \\
\hline 3918 & & RIVER & & RAW GRAB & TRANSECT & 336 & 100 F -1 HRM 19.0 & & \\
\hline 3919 & & RIVER & & RAW GRAB & TRANSECT & 341 & 100 F -10 HRM 19.0 & & \\
\hline 3920 & & RIVER & & RAW GRAB & TRANSECT & 341 & $100 \mathrm{~F}-10 \mathrm{HRM} 19.0$ & & \\
\hline 3921 & & RIVER & & RAW GRAB & TRANSECT & 341 & $100 \mathrm{~F}-10 \mathrm{HRM} 19.0$ & & \\
\hline 3922 & & RIVER & & RAW GRAB & TRANSECT & 341 & $100 \mathrm{~F}-10 \mathrm{HRM} 19.0$ & & \\
\hline 3923 & & RIVER & & RAW GRAB & TRANSECT & 341 & $100 \mathrm{~F}-10 \mathrm{HRM} 19.0$ & & \\
\hline 3924 & & RIVER & & RAW GRAB & TRANSECT & 576 & 100 F -2 HRM 19.0 & & \\
\hline 3925 & & RIVER & & RAW GRAB & TRANSECT & 576 & 100 F -2 HRM 19.0 & & \\
\hline 3926 & & RIVER & & RAW GRAB & TRANSECT & 576 & 100 F -2 HRM 19.0 & & \\
\hline 3927 & & RIVER & & RAW GRAB & TRANSECT & 576 & 100 F -2 HRM 19.0 & & \\
\hline 3928 & & RIVER & & RAW GRAB & TRANSECT & 576 & 100 F -2 HRM 19.0 & & \\
\hline 3929 & & RIVER & & RAW GRAB & TRANSECT & 337 & 100 F -3 HRM 19.0 & & \\
\hline 3930 & & RIVER & & RAW GRAB & TRANSECT & 337 & 100 F -3 HRM 19.0 & & \\
\hline 3931 & & RIVER & & RAW GRAB & TRANSECT & 337 & 100 F -3 HRM 19.0 & & \\
\hline 3932 & & RIVER & & RAW GRAB & TRANSECT & 337 & 100 F -3 HRM 19.0 & & \\
\hline 3933 & & RIVER & & RAW GRAB & TRANSECT & 337 & 100 F -3 HRM 19.0 & & \\
\hline 3934 & & RIVER & & RAW GRAB & TRANSECT & 338 & 100 F -4 HRM 19.0 & & \\
\hline 3935 & & RIVER & & RAW GRAB & TRANSECT & 338 & 100 F -4 HRM 19.0 & & \\
\hline 3936 & & RIVER & & RAW GRAB & TRANSECT & 338 & 100 F -4 HRM 19.0 & & \\
\hline 3937 & & RIVER & & RAW GRAB & TRANSECT & 338 & 100 F -4 HRM 19.0 & & \\
\hline 3938 & & RIVER & & RAW GRAB & TRANSECT & 338 & 100 F -4 HRM 19.0 & & \\
\hline 3939 & & RIVER & & RAW GRAB & TRANSECT & 577 & 100 F -5 HRM 19.0 & & \\
\hline 3940 & & RIVER & & RAW GRAB & TRANSECT & 577 & 100 F -5 HRM 19.0 & & \\
\hline 3941 & & RIVER & & RAW GRAB & TRANSECT & 577 & 100 F -5 HRM 19.0 & & \\
\hline 3942 & & RIVER & & RAW GRAB & TRANSECT & 577 & 100 F -5 HRM 19.0 & & \\
\hline 3943 & & RIVER & & RAW GRAB & TRANSECT & 577 & 100 F -5 HRM 19.0 & & \\
\hline 3944 & & RIVER & & RAW GRAB & TRANSECT & 606 & 100 F -6 HRM 19.0 & & \\
\hline 3945 & & RIVER & & RAW GRAB & TRANSECT & 606 & 100 F -6 HRM 19.0 & & \\
\hline 3946 & & RIVER & & RAW GRAB & TRANSECT & 606 & 100 F -6 HRM 19.0 & & \\
\hline 3947 & & RIVER & & RAW GRAB & TRANSECT & 606 & 100 F -6 HRM 19.0 & & \\
\hline 3948 & & RIVER & & RAW GRAB & TRANSECT & 606 & 100 F -6 HRM 19.0 & & \\
\hline 3949 & & RIVER & & RAW GRAB & TRANSECT & 339 & 100 F -7 HRM 19.0 & & \\
\hline 3950 & & RIVER & & RAW GRAB & TRANSECT & 339 & 100 F -7 HRM 19.0 & & \\
\hline 3951 & & RIVER & & RAW GRAB & TRANSECT & 339 & 100 F -7 HRM 19.0 & & \\
\hline 3952 & & RIVER & & RAW GRAB & TRANSECT & 339 & 100 F -7 HRM 19.0 & & \\
\hline 3953 & & RIVER & & RAW GRAB & TRANSECT & 339 & 100 F -7 HRM 19.0 & & \\
\hline
\end{tabular}




\begin{tabular}{|c|c|c|c|c|c|c|c|c|c|}
\hline & $\mathrm{T}$ & $U$ & $\mathrm{~V}$ & $\mathrm{~W}$ & $\mathrm{X}$ & $\mathrm{Y}$ & $Z$ & $\mathrm{AA}$ & $A B$ \\
\hline 1 & REVIEW_QUALIFIER & SAMP_FROM & SAMP_ITEM & SAMP_MTHD & COLL_MTHD & SAMP_SITE_ID & SAMP_SITE_NAME & WELL_NAME & SAMPLE_LOCATION \\
\hline 3954 & & RIVER & & RAW GRAB & TRANSECT & 340 & 100 F -8 HRM 19.0 & & \\
\hline 3955 & & RIVER & & RAW GRAB & TRANSECT & 340 & 100 F -8 HRM 19.0 & & \\
\hline 3956 & & RIVER & & RAW GRAB & TRANSECT & 340 & 100 F -8 HRM 19.0 & & \\
\hline 3957 & & RIVER & & RAW GRAB & TRANSECT & 340 & 100 F -8 HRM 19.0 & & \\
\hline \begin{tabular}{|l|}
3958 \\
\end{tabular} & & RIVER & & RAW GRAB & TRANSECT & 340 & 100 F -8 HRM 19.0 & & \\
\hline 3959 & & RIVER & & RAW GRAB & TRANSECT & 467 & 7 100 F -9 HRM 19.0 & & \\
\hline 3960 & & RIVER & & RAW GRAB & TRANSECT & 467 & 7 100 F -9 HRM 19.0 & & \\
\hline 3961 & & RIVER & & RAW GRAB & TRANSECT & 467 & 7100 F -9 HRM 19.0 & & \\
\hline 3962 & & RIVER & & RAW GRAB & TRANSECT & 467 & 7100 F -9 HRM 19.0 & & \\
\hline 3963 & & RIVER & & RAW GRAB & TRANSECT & 467 & 7100 F -9 HRM 19.0 & & \\
\hline 3964 & & RIVER & & RAW GRAB & TRANSECT & 578 & 3 HANFRD TS-1 HRM 28.7 & & \\
\hline 3965 & $F$ & RIVER & & RAW GRAB & TRANSECT & 578 & 3 HANFRD TS-1 HRM 28.7 & & \\
\hline 3966 & & RIVER & & RAW GRAB & TRANSECT & 578 & 3 HANFRD TS-1 HRM 28.7 & & \\
\hline 3967 & & RIVER & & RAW GRAB & TRANSECT & 578 & 3 HANFRD TS-1 HRM 28.7 & & \\
\hline 3968 & & RIVER & & RAW GRAB & TRANSECT & 578 & 3 HANFRD TS-1 HRM 28.7 & & \\
\hline 3969 & & RIVER & & RAW GRAB & TRANSECT & 347 & 7 HANFRD TS-10 HRM 28.7 & & \\
\hline 3970 & & RIVER & & RAW GRAB & TRANSECT & 347 & 7 HANFRD TS-10 HRM 28.7 & & \\
\hline 3971 & & RIVER & & RAW GRAB & TRANSECT & 347 & 7 HANFRD TS-10 HRM 28.7 & & \\
\hline 3972 & & RIVER & & RAW GRAB & TRANSECT & 347 & 7 HANFRD TS-10 HRM 28.7 & & \\
\hline 3973 & & RIVER & & RAW GRAB & TRANSECT & 347 & 7 HANFRD TS-10 HRM 28.7 & & \\
\hline 3974 & & RIVER & & RAW GRAB & TRANSECT & 342 & 2 HANFRD TS-2 HRM 28.7 & & \\
\hline 3975 & $F$ & RIVER & & RAW GRAB & TRANSECT & 342 & 2 HANFRD TS-2 HRM 28.7 & & \\
\hline 3976 & & RIVER & & RAW GRAB & TRANSECT & 342 & 2 HANFRD TS-2 HRM 28.7 & & \\
\hline 3977 & & RIVER & & RAW GRAB & TRANSECT & 342 & 2 HANFRD TS-2 HRM 28.7 & & \\
\hline 3978 & & RIVER & & RAW GRAB & TRANSECT & 342 & 2 HANFRD TS-2 HRM 28.7 & & \\
\hline 3979 & & RIVER & & RAW GRAB & TRANSECT & 468 & 3 HANFRD TS-3 HRM 28.7 & & \\
\hline 3980 & & RIVER & & RAW GRAB & TRANSECT & 468 & 3 HANFRD TS-3 HRM 28.7 & & \\
\hline 3981 & & RIVER & & RAW GRAB & TRANSECT & 468 & 3 HANFRD TS-3 HRM 28.7 & & \\
\hline 3982 & & RIVER & & RAW GRAB & TRANSECT & 468 & 3 HANFRD TS-3 HRM 28.7 & & \\
\hline 3983 & & RIVER & & RAW GRAB & TRANSECT & 468 & 3 HANFRD TS-3 HRM 28.7 & & \\
\hline 3984 & & RIVER & & RAW GRAB & TRANSECT & 343 & 3 HANFRD TS-4 HRM 28.7 & & \\
\hline 3985 & & RIVER & & RAW GRAB & TRANSECT & 343 & 3 HANFRD TS-4 HRM 28.7 & & \\
\hline 3986 & & RIVER & & RAW GRAB & TRANSECT & 343 & 3 HANFRD TS-4 HRM 28.7 & & \\
\hline 3987 & & RIVER & & RAW GRAB & TRANSECT & 343 & 3 HANFRD TS-4 HRM 28.7 & & \\
\hline 3988 & & RIVER & & RAW GRAB & TRANSECT & 343 & 3 HANFRD TS-4 HRM 28.7 & & \\
\hline 3989 & & RIVER & & RAW GRAB & TRANSECT & 344 & 4 HANFRD TS-5 HRM 28.7 & & \\
\hline 3990 & & RIVER & & RAW GRAB & TRANSECT & 344 & 4 HANFRD TS-5 HRM 28.7 & & \\
\hline 3991 & & RIVER & & RAW GRAB & TRANSECT & 344 & 4 HANFRD TS-5 HRM 28.7 & & \\
\hline
\end{tabular}




\begin{tabular}{|c|c|c|c|c|c|c|c|c|c|}
\hline & $\mathrm{T}$ & $\bar{U}$ & $\mathrm{~V}$ & $\mathrm{~W}$ & $\mathrm{X}$ & $\mathrm{Y}$ & $Z$ & $\mathrm{AA}$ & $\mathrm{AB}$ \\
\hline 1 & REVIEW_QUALIFIER & SAMP_FROM & SAMP_ITEM & SAMP_MTHD & COLL_MTHD & SAMP_SITE_ID & SAMP_SITE_NAME & WELL_NAME & SAMPLE_LOCATION \\
\hline 3992 & & RIVER & & RAW GRAB & TRANSECT & 344 & IANFRD TS-5 HRM 28.7 & & \\
\hline 3993 & & RIVER & & RAW GRAB & TRANSECT & 344 & IANFRD TS-5 HRM 28.7 & & \\
\hline 3994 & & RIVER & & RAW GRAB & TRANSECT & 345 & IANFRD TS-6 HRM 28.7 & & \\
\hline 3995 & & RIVER & & RAW GRAB & TRANSECT & 345 & IANFRD TS-6 HRM 28.7 & & \\
\hline 3996 & & RIVER & & RAW GRAB & TRANSECT & 345 & IANFRD TS-6 HRM 28.7 & & \\
\hline 3997 & & RIVER & & RAW GRAB & TRANSECT & 345 & IANFRD TS-6 HRM 28.7 & & \\
\hline 3998 & & RIVER & & RAW GRAB & TRANSECT & 345 & IANFRD TS-6 HRM 28.7 & & \\
\hline 3999 & & RIVER & & RAW GRAB & TRANSECT & 469 & IANFRD TS-7 HRM 28.7 & & \\
\hline 4000 & & RIVER & & RAW GRAB & TRANSECT & 469 & IANFRD TS-7 HRM 28.7 & & \\
\hline 4001 & & RIVER & & RAW GRAB & TRANSECT & 469 & IANFRD TS-7 HRM 28.7 & & \\
\hline 4002 & & RIVER & & RAW GRAB & TRANSECT & 469 & IANFRD TS-7 HRM 28.7 & & \\
\hline 4003 & & RIVER & & RAW GRAB & TRANSECT & 469 & IANFRD TS-7 HRM 28.7 & & \\
\hline 4004 & & RIVER & & RAW GRAB & TRANSECT & 579 & IANFRD TS-8 HRM 28.7 & & \\
\hline 4005 & & RIVER & & RAW GRAB & TRANSECT & 579 & IANFRD TS-8 HRM 28.7 & & \\
\hline 4006 & & RIVER & & RAW GRAB & TRANSECT & 579 & IANFRD TS-8 HRM 28.7 & & \\
\hline 4007 & & RIVER & & RAW GRAB & TRANSECT & 579 & IANFRD TS-8 HRM 28.7 & & \\
\hline 4008 & & RIVER & & RAW GRAB & TRANSECT & 579 & IANFRD TS-8 HRM 28.7 & & \\
\hline 4009 & & RIVER & & RAW GRAB & TRANSECT & 346 & IANFRD TS-9 HRM 28.7 & & \\
\hline 4010 & & RIVER & & RAW GRAB & TRANSECT & 346 & IANFRD TS-9 HRM 28.7 & & \\
\hline 4011 & & RIVER & & RAW GRAB & TRANSECT & 346 & IANFRD TS-9 HRM 28.7 & & \\
\hline 4012 & & RIVER & & RAW GRAB & TRANSECT & 346 & IANFRD TS-9 HRM 28.7 & & \\
\hline 4013 & & RIVER & & RAW GRAB & TRANSECT & 346 & IANFRD TS-9 HRM 28.7 & & \\
\hline 4014 & & RIVER & & RAW GRAB & TRANSECT & 317 & ERNITA-1 HRM 0.3 & & \\
\hline 4015 & & RIVER & & RAW GRAB & TRANSECT & 317 & ERNITA-1 HRM 0.3 & & \\
\hline 4016 & & RIVER & & RAW GRAB & TRANSECT & 317 & ERNITA-1 HRM 0.3 & & \\
\hline 4017 & & RIVER & & RAW GRAB & TRANSECT & 317 & ERNITA-1 HRM 0.3 & & \\
\hline 4018 & & RIVER & & RAW GRAB & TRANSECT & 317 & ERNITA-1 HRM 0.3 & & \\
\hline 4019 & & RIVER & & RAW GRAB & TRANSECT & 570 & ERNITA-2 HRM 0.3 & & \\
\hline 4020 & & RIVER & & RAW GRAB & TRANSECT & 570 & ERNITA-2 HRM 0.3 & & \\
\hline 4021 & & RIVER & & RAW GRAB & TRANSECT & 570 & ERNITA-2 HRM 0.3 & & \\
\hline 4022 & & RIVER & & RAW GRAB & TRANSECT & 570 & ERNITA-2 HRM 0.3 & & \\
\hline 4023 & & RIVER & & RAW GRAB & TRANSECT & 570 & ERNITA-2 HRM 0.3 & & \\
\hline 4024 & & RIVER & & RAW GRAB & TRANSECT & 318 & ERNITA-3 HRM 0.3 & & \\
\hline 4025 & & RIVER & & RAW GRAB & TRANSECT & 318 & ERNITA-3 HRM 0.3 & & \\
\hline 4026 & & RIVER & & RAW GRAB & TRANSECT & 318 & ERNITA-3 HRM 0.3 & & \\
\hline 4027 & & RIVER & & RAW GRAB & TRANSECT & 318 & ERNITA-3 HRM 0.3 & & \\
\hline 4028 & & RIVER & & RAW GRAB & TRANSECT & 318 & ERNITA-3 HRM 0.3 & & \\
\hline 4029 & & RIVER & & RAW GRAB & TRANSECT & 319 & ERNITA-4 HRM 0.3 & & \\
\hline
\end{tabular}




\begin{tabular}{|c|c|c|c|c|c|c|c|c|c|}
\hline & $\mathrm{T}$ & $\bar{U}$ & $\mathrm{~V}$ & $\mathrm{~W}$ & $\mathrm{X}$ & $\mathrm{Y}$ & $Z$ & $\mathrm{AA}$ & $\mathrm{AB}$ \\
\hline 1 & REVIEW_QUALIFIER & SAMP_FROM & SAMP_ITEM & SAMP_MTHD & COLL_MTHD & SAMP_SITE_ID & SAMP_SITE_NAME & WELL_NAME & SAMPLE_LOCATION \\
\hline 4030 & & RIVER & & RAW GRAB & TRANSECT & 319 & VERNITA-4 HRM 0.3 & & \\
\hline 4031 & & RIVER & & RAW GRAB & TRANSECT & 319 & VERNITA-4 HRM 0.3 & & \\
\hline 4032 & & RIVER & & RAW GRAB & TRANSECT & 319 & VERNITA-4 HRM 0.3 & & \\
\hline 4033 & & RIVER & & RAW GRAB & TRANSECT & 319 & VERNITA-4 HRM 0.3 & & \\
\hline 4034 & & RIVER & & RAW GRAB & TRANSECT & 571 & RICH.PMPHS-1 HRM46.4 & & \\
\hline 4035 & & RIVER & & RAW GRAB & TRANSECT & 571 & RICH.PMPHS-1 HRM46.4 & & \\
\hline 4036 & & RIVER & & RAW GRAB & TRANSECT & 571 & RICH.PMPHS-1 HRM46.4 & & \\
\hline 4037 & & RIVER & & RAW GRAB & TRANSECT & 571 & RICH.PMPHS-1 HRM46.4 & & \\
\hline 4038 & & RIVER & & RAW GRAB & TRANSECT & 571 & RICH.PMPHS-1 HRM46.4 & & \\
\hline 4039 & & RIVER & & RAW GRAB & TRANSECT & 571 & RICH.PMPHS-1 HRM46.4 & & \\
\hline 4040 & & RIVER & & RAW GRAB & TRANSECT & 571 & RICH.PMPHS-1 HRM46.4 & & \\
\hline 4041 & & RIVER & & RAW GRAB & TRANSECT & 571 & RICH.PMPHS-1 HRM46.4 & & \\
\hline 4042 & & RIVER & & RAW GRAB & TRANSECT & 571 & RICH.PMPHS-1 HRM46.4 & & \\
\hline 4043 & & RIVER & & RAW GRAB & TRANSECT & 571 & RICH.PMPHS-1 HRM46.4 & & \\
\hline 4044 & & RIVER & & RAW GRAB & TRANSECT & 327 & RICH.PMPHS-10 HRM46.4 & & \\
\hline 4045 & & RIVER & & RAW GRAB & TRANSECT & 327 & RICH.PMPHS-10 HRM46.4 & & \\
\hline 4046 & & RIVER & & RAW GRAB & TRANSECT & 327 & RICH.PMPHS-10 HRM46.4 & & \\
\hline 4047 & & RIVER & & RAW GRAB & TRANSECT & 327 & RICH.PMPHS-10 HRM46.4 & & \\
\hline 4048 & & RIVER & & RAW GRAB & TRANSECT & 327 & RICH.PMPHS-10 HRM46.4 & & \\
\hline 4049 & & RIVER & & RAW GRAB & TRANSECT & 321 & RICH.PMPHS-2 HRM46.4 & & \\
\hline 4050 & & RIVER & & RAW GRAB & TRANSECT & 321 & RICH.PMPHS-2 HRM46.4 & & \\
\hline 4051 & & RIVER & & RAW GRAB & TRANSECT & 321 & RICH.PMPHS-2 HRM46.4 & & \\
\hline 4052 & & RIVER & & RAW GRAB & TRANSECT & 321 & RICH.PMPHS-2 HRM46.4 & & \\
\hline 4053 & & RIVER & & RAW GRAB & TRANSECT & 321 & RICH.PMPHS-2 HRM46.4 & & \\
\hline 4054 & & RIVER & & RAW GRAB & TRANSECT & 322 & RICH.PMPHS-3 HRM46.4 & & \\
\hline 4055 & & RIVER & & RAW GRAB & TRANSECT & 322 & RICH.PMPHS-3 HRM46.4 & & \\
\hline 4056 & & RIVER & & RAW GRAB & TRANSECT & 322 & RICH.PMPHS-3 HRM46.4 & & \\
\hline 4057 & & RIVER & & RAW GRAB & TRANSECT & 322 & RICH.PMPHS-3 HRM46.4 & & \\
\hline 4058 & & RIVER & & RAW GRAB & TRANSECT & 322 & RICH.PMPHS-3 HRM46.4 & & \\
\hline 4059 & & RIVER & & RAW GRAB & TRANSECT & 572 & RICH.PMPHS-4 HRM46.4 & & \\
\hline 4060 & & RIVER & & RAW GRAB & TRANSECT & 572 & RICH.PMPHS-4 HRM46.4 & & \\
\hline 4061 & & RIVER & & RAW GRAB & TRANSECT & 572 & RICH.PMPHS-4 HRM46.4 & & \\
\hline 4062 & & RIVER & & RAW GRAB & TRANSECT & 572 & RICH.PMPHS-4 HRM46.4 & & \\
\hline 4063 & & RIVER & & RAW GRAB & TRANSECT & 572 & RICH.PMPHS-4 HRM46.4 & & \\
\hline 4064 & & RIVER & & RAW GRAB & TRANSECT & 323 & RICH.PMPHS-5 HRM46.4 & & \\
\hline 4065 & & RIVER & & RAW GRAB & TRANSECT & 323 & RICH.PMPHS-5 HRM46.4 & & \\
\hline 4066 & & RIVER & & RAW GRAB & TRANSECT & 323 & RICH.PMPHS-5 HRM46.4 & & \\
\hline 4067 & & RIVER & & RAW GRAB & TRANSECT & 323 & RICH.PMPHS-5 HRM46.4 & & \\
\hline
\end{tabular}




\begin{tabular}{|c|c|c|c|c|c|c|c|c|c|}
\hline & $\mathrm{T}$ & $\bar{U}$ & $\mathrm{~V}$ & $\mathrm{~W}$ & $\mathrm{X}$ & $\mathrm{Y}$ & $Z$ & $\mathrm{AA}$ & $\mathrm{AB}$ \\
\hline 1 & REVIEW_QUALIFIER & SAMP_FROM & SAMP_ITEM & SAMP_MTHD & COLL_MTHD & SAMP_SITE_ID & SAMP_SITE_NAME & WELL_NAME & SAMPLE_LOCATION \\
\hline 4068 & & RIVER & & RAW GRAB & TRANSECT & 323 & RICH.PMPHS-5 HRM46.4 & & \\
\hline 4069 & & RIVER & & RAW GRAB & TRANSECT & 324 & RICH.PMPHS-6 HRM46.4 & & \\
\hline 4070 & & RIVER & & RAW GRAB & TRANSECT & 324 & RICH.PMPHS-6 HRM46.4 & & \\
\hline 4071 & & RIVER & & RAW GRAB & TRANSECT & 324 & RICH.PMPHS-6 HRM46.4 & & \\
\hline 4072 & & RIVER & & RAW GRAB & TRANSECT & 324 & RICH.PMPHS-6 HRM46.4 & & \\
\hline 4073 & & RIVER & & RAW GRAB & TRANSECT & 324 & RICH.PMPHS-6 HRM46.4 & & \\
\hline 4074 & & RIVER & & RAW GRAB & TRANSECT & 325 & RICH.PMPHS-7 HRM46.4 & & \\
\hline 4075 & & RIVER & & RAW GRAB & TRANSECT & 325 & RICH.PMPHS-7 HRM46.4 & & \\
\hline 4076 & & RIVER & & RAW GRAB & TRANSECT & 325 & RICH.PMPHS-7 HRM46.4 & & \\
\hline 4077 & & RIVER & & RAW GRAB & TRANSECT & 325 & RICH.PMPHS-7 HRM46.4 & & \\
\hline 4078 & & RIVER & & RAW GRAB & TRANSECT & 325 & RICH.PMPHS-7 HRM46.4 & & \\
\hline 4079 & & RIVER & & RAW GRAB & TRANSECT & 326 & RICH.PMPHS-8 HRM46.4 & & \\
\hline 4080 & & RIVER & & RAW GRAB & TRANSECT & 326 & RICH.PMPHS-8 HRM46.4 & & \\
\hline 4081 & & RIVER & & RAW GRAB & TRANSECT & 326 & RICH.PMPHS-8 HRM46.4 & & \\
\hline 4082 & & RIVER & & RAW GRAB & TRANSECT & 326 & RICH.PMPHS-8 HRM46.4 & & \\
\hline 4083 & & RIVER & & RAW GRAB & TRANSECT & 326 & RICH.PMPHS-8 HRM46.4 & & \\
\hline 4084 & & RIVER & & RAW GRAB & TRANSECT & 573 & RICH.PMPHS-9 HRM46.4 & & \\
\hline 4085 & & RIVER & & RAW GRAB & TRANSECT & 573 & RICH.PMPHS-9 HRM46.4 & & \\
\hline 4086 & & RIVER & & RAW GRAB & TRANSECT & 573 & RICH.PMPHS-9 HRM46.4 & & \\
\hline 4087 & & RIVER & & RAW GRAB & TRANSECT & 573 & RICH.PMPHS-9 HRM46.4 & & \\
\hline 4088 & & RIVER & & RAW GRAB & TRANSECT & 573 & RICH.PMPHS-9 HRM46.4 & & \\
\hline 4089 & & RIVER & & RAW GRAB & TRANSECT & 317 & VERNITA-1 HRM 0.3 & & \\
\hline 4090 & & RIVER & & RAW GRAB & TRANSECT & 317 & VERNITA-1 HRM 0.3 & & \\
\hline 4091 & & RIVER & & RAW GRAB & TRANSECT & 317 & VERNITA-1 HRM 0.3 & & \\
\hline 4092 & & RIVER & & RAW GRAB & TRANSECT & 317 & VERNITA-1 HRM 0.3 & & \\
\hline 4093 & & RIVER & & RAW GRAB & TRANSECT & 317 & VERNITA-1 HRM 0.3 & & \\
\hline 4094 & & RIVER & & RAW GRAB & TRANSECT & 570 & VERNITA-2 HRM 0.3 & & \\
\hline 4095 & & RIVER & & RAW GRAB & TRANSECT & 570 & VERNITA-2 HRM 0.3 & & \\
\hline 4096 & & RIVER & & RAW GRAB & TRANSECT & 570 & VERNITA-2 HRM 0.3 & & \\
\hline 4097 & & RIVER & & RAW GRAB & TRANSECT & 570 & VERNITA-2 HRM 0.3 & & \\
\hline 4098 & & RIVER & & RAW GRAB & TRANSECT & 570 & VERNITA-2 HRM 0.3 & & \\
\hline 4099 & & RIVER & & RAW GRAB & TRANSECT & 318 & VERNITA-3 HRM 0.3 & & \\
\hline 4100 & & RIVER & & RAW GRAB & TRANSECT & 318 & VERNITA-3 HRM 0.3 & & \\
\hline 4101 & & RIVER & & RAW GRAB & TRANSECT & 318 & VERNITA-3 HRM 0.3 & & \\
\hline 4102 & & RIVER & & RAW GRAB & TRANSECT & 318 & VERNITA-3 HRM 0.3 & & \\
\hline 4103 & & RIVER & & RAW GRAB & TRANSECT & 318 & VERNITA-3 HRM 0.3 & & \\
\hline 4104 & & RIVER & & RAW GRAB & TRANSECT & 319 & VERNITA-4 HRM 0.3 & & \\
\hline 4105 & & RIVER & & RAW GRAB & TRANSECT & 319 & VERNITA-4 HRM 0.3 & & \\
\hline
\end{tabular}




\begin{tabular}{|c|c|c|c|c|c|c|c|c|c|}
\hline & $\mathrm{T}$ & $\bar{U}$ & $\mathrm{~V}$ & $\mathrm{~W}$ & $\mathrm{X}$ & $\mathrm{Y}$ & $Z$ & $\mathrm{AA}$ & $\mathrm{AB}$ \\
\hline 1 & REVIEW_QUALIFIER & SAMP_FROM & SAMP_ITEM & SAMP_MTHD & COLL_MTHD & SAMP_SITE_ID & SAMP_SITE_NAME & WELL_NAME & SAMPLE_LOCATION \\
\hline 4106 & & RIVER & & RAW GRAB & TRANSECT & 319 & VERNITA-4 HRM 0.3 & & \\
\hline 4107 & & RIVER & & RAW GRAB & TRANSECT & 319 & VERNITA-4 HRM 0.3 & & \\
\hline 4108 & & RIVER & & RAW GRAB & TRANSECT & 319 & VERNITA-4 HRM 0.3 & & \\
\hline 4109 & & RIVER & & RAW GRAB & TRANSECT & 571 & RICH.PMPHS-1 HRM46.4 & & \\
\hline 4110 & & RIVER & & RAW GRAB & TRANSECT & 571 & RICH.PMPHS-1 HRM46.4 & & \\
\hline 4111 & & RIVER & & RAW GRAB & TRANSECT & 571 & RICH.PMPHS-1 HRM46.4 & & \\
\hline 4112 & & RIVER & & RAW GRAB & TRANSECT & 571 & RICH.PMPHS-1 HRM46.4 & & \\
\hline 4113 & & RIVER & & RAW GRAB & TRANSECT & 571 & RICH.PMPHS-1 HRM46.4 & & \\
\hline 4114 & & RIVER & & RAW GRAB & TRANSECT & 571 & RICH.PMPHS-1 HRM46.4 & & \\
\hline 4115 & & RIVER & & RAW GRAB & TRANSECT & 571 & RICH.PMPHS-1 HRM46.4 & & \\
\hline 4116 & & RIVER & & RAW GRAB & TRANSECT & 571 & RICH.PMPHS-1 HRM46.4 & & \\
\hline 4117 & & RIVER & & RAW GRAB & TRANSECT & 571 & RICH.PMPHS-1 HRM46.4 & & \\
\hline 4118 & & RIVER & & RAW GRAB & TRANSECT & 571 & RICH.PMPHS-1 HRM46.4 & & \\
\hline 4119 & & RIVER & & RAW GRAB & TRANSECT & 327 & RICH.PMPHS-10 HRM46.4 & & \\
\hline 4120 & & RIVER & & RAW GRAB & TRANSECT & 327 & RICH.PMPHS-10 HRM46.4 & & \\
\hline 4121 & & RIVER & & RAW GRAB & TRANSECT & 327 & RICH.PMPHS-10 HRM46.4 & & \\
\hline 4122 & $\mathrm{~F}$ & RIVER & & RAW GRAB & TRANSECT & 327 & RICH.PMPHS-10 HRM46.4 & & \\
\hline 4123 & & RIVER & & RAW GRAB & TRANSECT & 327 & RICH.PMPHS-10 HRM46.4 & & \\
\hline 4124 & & RIVER & & RAW GRAB & TRANSECT & 321 & RICH.PMPHS-2 HRM46.4 & & \\
\hline 4125 & & RIVER & & RAW GRAB & TRANSECT & 321 & RICH.PMPHS-2 HRM46.4 & & \\
\hline 4126 & & RIVER & & RAW GRAB & TRANSECT & 321 & RICH.PMPHS-2 HRM46.4 & & \\
\hline 4127 & & RIVER & & RAW GRAB & TRANSECT & 321 & RICH.PMPHS-2 HRM46.4 & & \\
\hline 4128 & & RIVER & & RAW GRAB & TRANSECT & 321 & RICH.PMPHS-2 HRM46.4 & & \\
\hline 4129 & & RIVER & & RAW GRAB & TRANSECT & 322 & RICH.PMPHS-3 HRM46.4 & & \\
\hline 4130 & & RIVER & & RAW GRAB & TRANSECT & 322 & RICH.PMPHS-3 HRM46.4 & & \\
\hline 4131 & & RIVER & & RAW GRAB & TRANSECT & 322 & RICH.PMPHS-3 HRM46.4 & & \\
\hline 4132 & $\mathrm{~F}$ & RIVER & & RAW GRAB & TRANSECT & 322 & RICH.PMPHS-3 HRM46.4 & & \\
\hline 4133 & & RIVER & & RAW GRAB & TRANSECT & 322 & RICH.PMPHS-3 HRM46.4 & & \\
\hline 4134 & & RIVER & & RAW GRAB & TRANSECT & 572 & RICH.PMPHS-4 HRM46.4 & & \\
\hline 4135 & & RIVER & & RAW GRAB & TRANSECT & 572 & RICH.PMPHS-4 HRM46.4 & & \\
\hline 4136 & & RIVER & & RAW GRAB & TRANSECT & 572 & RICH.PMPHS-4 HRM46.4 & & \\
\hline 4137 & $\mathrm{~F}$ & RIVER & & RAW GRAB & TRANSECT & 572 & RICH.PMPHS-4 HRM46.4 & & \\
\hline 4138 & & RIVER & & RAW GRAB & TRANSECT & 572 & RICH.PMPHS-4 HRM46.4 & & \\
\hline 4139 & & RIVER & & RAW GRAB & TRANSECT & 323 & RICH.PMPHS-5 HRM46.4 & & \\
\hline 4140 & & RIVER & & RAW GRAB & TRANSECT & 323 & RICH.PMPHS-5 HRM46.4 & & \\
\hline 4141 & & RIVER & & RAW GRAB & TRANSECT & 323 & RICH.PMPHS-5 HRM46.4 & & \\
\hline 4142 & & RIVER & & RAW GRAB & TRANSECT & 323 & RICH.PMPHS-5 HRM46.4 & & \\
\hline 4143 & & RIVER & & RAW GRAB & TRANSECT & 323 & RICH.PMPHS-5 HRM46.4 & & \\
\hline
\end{tabular}




\begin{tabular}{|c|c|c|c|c|c|c|c|c|c|}
\hline & $\mathrm{T}$ & $\bar{U}$ & $\mathrm{~V}$ & $\mathrm{~W}$ & $\mathrm{X}$ & $\mathrm{Y}$ & $Z$ & $\mathrm{AA}$ & $\mathrm{AB}$ \\
\hline \begin{tabular}{l|l}
1 & 1 \\
\end{tabular} & REVIEW_QUALIFIER & SAMP_FROM & SAMP_ITEM & SAMP_MTHD & COLL_MTHD & SAMP_SITE_ID & SAMP_SITE_NAME & WELL_NAME & SAMPLE_LOCATION \\
\hline 4144 & & RIVER & & RAW GRAB & TRANSECT & 324 & RICH.PMPHS-6 HRM46.4 & & \\
\hline 4145 & & RIVER & & RAW GRAB & TRANSECT & 324 & RICH.PMPHS-6 HRM46.4 & & \\
\hline 4146 & & RIVER & & RAW GRAB & TRANSECT & 324 & RICH.PMPHS-6 HRM46.4 & & \\
\hline $4147 \mathrm{~F}$ & $\mathrm{~F}$ & RIVER & & RAW GRAB & TRANSECT & 324 & RICH.PMPHS-6 HRM46.4 & & \\
\hline 4148 & & RIVER & & RAW GRAB & TRANSECT & 324 & RICH.PMPHS-6 HRM46.4 & & \\
\hline 4149 & & RIVER & & RAW GRAB & TRANSECT & 325 & RICH.PMPHS-7 HRM46.4 & & \\
\hline 4150 & & RIVER & & RAW GRAB & TRANSECT & 325 & RICH.PMPHS-7 HRM46.4 & & \\
\hline 4151 & & RIVER & & RAW GRAB & TRANSECT & 325 & RICH.PMPHS-7 HRM46.4 & & \\
\hline 4152 & & RIVER & & RAW GRAB & TRANSECT & 325 & RICH.PMPHS-7 HRM46.4 & & \\
\hline 4153 & & RIVER & & RAW GRAB & TRANSECT & 325 & RICH.PMPHS-7 HRM46.4 & & \\
\hline 4154 & & RIVER & & RAW GRAB & TRANSECT & 326 & RICH.PMPHS-8 HRM46.4 & & \\
\hline 4155 & & RIVER & & RAW GRAB & TRANSECT & 326 & RICH.PMPHS-8 HRM46.4 & & \\
\hline 4156 & & RIVER & & RAW GRAB & TRANSECT & 326 & RICH.PMPHS-8 HRM46.4 & & \\
\hline $4157 \mathrm{~F}$ & $\mathrm{~F}$ & RIVER & & RAW GRAB & TRANSECT & 326 & RICH.PMPHS-8 HRM46.4 & & \\
\hline 4158 & & RIVER & & RAW GRAB & TRANSECT & 326 & RICH.PMPHS-8 HRM46.4 & & \\
\hline 4159 & & RIVER & & RAW GRAB & TRANSECT & 573 & RICH.PMPHS-9 HRM46.4 & & \\
\hline 4160 & & RIVER & & RAW GRAB & TRANSECT & 573 & RICH.PMPHS-9 HRM46.4 & & \\
\hline 4161 & & RIVER & & RAW GRAB & TRANSECT & 573 & RICH.PMPHS-9 HRM46.4 & & \\
\hline 4162 & & RIVER & & RAW GRAB & TRANSECT & 573 & RICH.PMPHS-9 HRM46.4 & & \\
\hline 4163 & & RIVER & & RAW GRAB & TRANSECT & 573 & RICH.PMPHS-9 HRM46.4 & & \\
\hline 4164 & & RIVER & & RAW GRAB & TRANSECT & 328 & 100 N -1 HRM 9.5 & & \\
\hline 4165 & & RIVER & & RAW GRAB & TRANSECT & 328 & $100 \mathrm{~N}-1$ HRM 9.5 & & \\
\hline 4166 & & RIVER & & RAW GRAB & TRANSECT & 328 & 100 N -1 HRM 9.5 & & \\
\hline 4167 & & RIVER & & RAW GRAB & TRANSECT & 328 & 100 N -1 HRM 9.5 & & \\
\hline 4168 & & RIVER & & RAW GRAB & TRANSECT & 328 & 100 N -1 HRM 9.5 & & \\
\hline 4169 & & RIVER & & RAW GRAB & TRANSECT & 328 & 100 N -1 HRM 9.5 & & \\
\hline 4170 & & RIVER & & RAW GRAB & TRANSECT & 328 & $100 \mathrm{~N}-1$ HRM 9.5 & & \\
\hline 4171 & & RIVER & & RAW GRAB & TRANSECT & 328 & $100 \mathrm{~N}-1$ HRM 9.5 & & \\
\hline 4172 & & RIVER & & RAW GRAB & TRANSECT & 328 & 100 N -1 HRM 9.5 & & \\
\hline 4173 & & RIVER & & RAW GRAB & TRANSECT & 328 & 100 N -1 HRM 9.5 & & \\
\hline 4174 & & RIVER & & RAW GRAB & TRANSECT & 335 & $100 \mathrm{~N}-10$ HRM 9.5 & & \\
\hline 4175 & & RIVER & & RAW GRAB & TRANSECT & 335 & $100 \mathrm{~N}-10$ HRM 9.5 & & \\
\hline 4176 & & RIVER & & RAW GRAB & TRANSECT & 335 & $100 \mathrm{~N}-10$ HRM 9.5 & & \\
\hline 4177 & & RIVER & & RAW GRAB & TRANSECT & 335 & $100 \mathrm{~N}-10$ HRM 9.5 & & \\
\hline 4178 & & RIVER & & RAW GRAB & TRANSECT & 335 & $100 \mathrm{~N}-10$ HRM 9.5 & & \\
\hline 4179 & & RIVER & & RAW GRAB & TRANSECT & 329 & 100 N -2 HRM 9.5 & & \\
\hline 4180 & & RIVER & & RAW GRAB & TRANSECT & 329 & 100 N -2 HRM 9.5 & & \\
\hline 4181 & & RIVER & & RAW GRAB & TRANSECT & 329 & 100 N -2 HRM 9.5 & & \\
\hline
\end{tabular}




\begin{tabular}{|c|c|c|c|c|c|c|c|c|c|}
\hline & $\mathrm{T}$ & 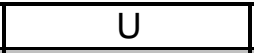 & $\mathrm{V}$ & $\mathrm{W}$ & $\mathrm{X}$ & $\mathrm{Y}$ & $Z$ & $\mathrm{AA}$ & $A B$ \\
\hline \begin{tabular}{l|l}
1 & 1 \\
\end{tabular} & REVIEW_QUALIFIER & SAMP_FROM & SAMP_ITEM & SAMP_MTHD & COLL_MTHD & SAMP_SITE_ID & SAMP_SITE_NAME & WELL_NAME & SAMPLE_LOCATION \\
\hline 4182 & & RIVER & & RAW GRAB & TRANSECT & 329 & 100 N -2 HRM 9.5 & & \\
\hline 4183 & & RIVER & & RAW GRAB & TRANSECT & 329 & 100 N -2 HRM 9.5 & & \\
\hline 4184 & & RIVER & & RAW GRAB & TRANSECT & 330 & 100 N -3 HRM 9.5 & & \\
\hline 4185 & & RIVER & & RAW GRAB & TRANSECT & 330 & 100 N -3 HRM 9.5 & & \\
\hline 4186 & & RIVER & & RAW GRAB & TRANSECT & 330 & 100 N -3 HRM 9.5 & & \\
\hline 4187 & & RIVER & & RAW GRAB & TRANSECT & 330 & 100 N -3 HRM 9.5 & & \\
\hline 4188 & & RIVER & & RAW GRAB & TRANSECT & 330 & 100 N -3 HRM 9.5 & & \\
\hline 4189 & & RIVER & & RAW GRAB & TRANSECT & 331 & 100 N -5 HRM 9.5 & & \\
\hline 4190 & & RIVER & & RAW GRAB & TRANSECT & 331 & 100 N -5 HRM 9.5 & & \\
\hline 4191 & & RIVER & & RAW GRAB & TRANSECT & $331:$ & 100 N -5 HRM 9.5 & & \\
\hline $4192 \mathrm{~F}$ & $\mathrm{~F}$ & RIVER & & RAW GRAB & TRANSECT & 331 & 100 N -5 HRM 9.5 & & \\
\hline 4193 & & RIVER & & RAW GRAB & TRANSECT & 331 & 100 N -5 HRM 9.5 & & \\
\hline 4194 & & RIVER & & RAW GRAB & TRANSECT & 333 & $100 \mathrm{~N}-7$ HRM 9.5 & & \\
\hline 4195 & & RIVER & & RAW GRAB & TRANSECT & 333 & $100 \mathrm{~N}-7$ HRM 9.5 & & \\
\hline 4196 & & RIVER & & RAW GRAB & TRANSECT & 333 & $100 \mathrm{~N}-7$ HRM 9.5 & & \\
\hline 4197 & & RIVER & & RAW GRAB & TRANSECT & 333 & $100 \mathrm{~N}-7$ HRM 9.5 & & \\
\hline 4198 & & RIVER & & RAW GRAB & TRANSECT & 333 & $100 \mathrm{~N}-7$ HRM 9.5 & & \\
\hline 4199 & & RIVER & & RAW GRAB & TRANSECT & 8244 & 100 N SHORE HRM 8.4 & & 300 METERS DOWNRIVER OF $100 \mathrm{~N}-1$. \\
\hline 4200 & & RIVER & & RAW GRAB & TRANSECT & 8244 & 100 N SHORE HRM 8.4 & & 300 METERS DOWNRIVER OF 100 N-1. \\
\hline 4201 & & RIVER & & RAW GRAB & TRANSECT & 8244 & 100 N SHORE HRM 8.4 & & 300 METERS DOWNRIVER OF 100 N-1. \\
\hline $4202 \mathrm{~F}$ & $\mathrm{~F}$ & RIVER & & RAW GRAB & TRANSECT & 8244 & 100 N SHORE HRM 8.4 & & 300 METERS DOWNRIVER OF 100 N-1. \\
\hline 4203 & & RIVER & & RAW GRAB & TRANSECT & 8244 & 100 N SHORE HRM 8.4 & & 300 METERS DOWNRIVER OF 100 N-1. \\
\hline $4204 \mathrm{~F}$ & $\mathrm{~F}$ & RIVER & & RAW GRAB & TRANSECT & 8245 & 100 N SHORE HRM 8.9 & & 300 METERS UPRIVER 100 N-1. \\
\hline 4205 & & RIVER & & RAW GRAB & TRANSECT & 8245 & 100 N SHORE HRM 8.9 & & 300 METERS UPRIVER 100 N-1. \\
\hline 4206 & & RIVER & & RAW GRAB & TRANSECT & 8245 & 100 N SHORE HRM 8.9 & & 300 METERS UPRIVER 100 N-1. \\
\hline 4207 & & RIVER & & RAW GRAB & TRANSECT & 8245 & 100 N SHORE HRM 8.9 & & 300 METERS UPRIVER 100 N-1. \\
\hline 4208 & & RIVER & & RAW GRAB & TRANSECT & 8245 & 100 N SHORE HRM 8.9 & & 300 METERS UPRIVER 100 N-1. \\
\hline $4209 \mathrm{~F}$ & $\mathrm{~F}$ & RIVER & & RAW GRAB & TRANSECT & 8246 & 100 N SHORE HRM 9.2 & & AT CASSION 1-N-8T. \\
\hline 4210 & & RIVER & & RAW GRAB & TRANSECT & 8246 & 100 N SHORE HRM 9.2 & & AT CASSION 1-N-8T. \\
\hline 4211 & & RIVER & & RAW GRAB & TRANSECT & 8246 & 100 N SHORE HRM 9.2 & & AT CASSION 1-N-8T. \\
\hline 4212 & & RIVER & & RAW GRAB & TRANSECT & 8246 & 100 N SHORE HRM 9.2 & & AT CASSION 1-N-8T. \\
\hline 4213 & & RIVER & & RAW GRAB & TRANSECT & 8246 & 100 N SHORE HRM 9.2 & & AT CASSION 1-N-8T. \\
\hline 4214 & & RIVER & & RAW GRAB & TRANSECT & 8247: & 100 N SHORE HRM 9.8 & & 2ND POWERLINE UPRIVER 100N. \\
\hline 4215 & & RIVER & & RAW GRAB & TRANSECT & 8247 & 100 N SHORE HRM 9.8 & & 2ND POWERLINE UPRIVER 100N. \\
\hline 4216 & & RIVER & & RAW GRAB & TRANSECT & 8247 & 100 N SHORE HRM 9.8 & & 2ND POWERLINE UPRIVER 100N. \\
\hline $4217 \mathrm{~F}$ & $\mathrm{~F}$ & RIVER & & RAW GRAB & TRANSECT & $8247:$ & 100 N SHORE HRM 9.8 & & 2ND POWERLINE UPRIVER 100N. \\
\hline 4218 & & RIVER & & RAW GRAB & TRANSECT & 8247 & 100 N SHORE HRM 9.8 & & 2ND POWERLINE UPRIVER 100N. \\
\hline 4219 & & RIVER & & RAW GRAB & TRANSECT & 317 & VERNITA-1 HRM 0.3 & & \\
\hline
\end{tabular}




\begin{tabular}{|c|c|c|c|c|c|c|c|c|c|}
\hline & $\mathrm{T}$ & 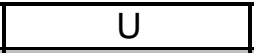 & $\mathrm{V}$ & $\mathrm{W}$ & $\mathrm{X}$ & $\mathrm{Y}$ & $Z$ & $\mathrm{AA}$ & $A B$ \\
\hline \begin{tabular}{l|l}
1 & 1 \\
\end{tabular} & REVIEW_QUALIFIER & SAMP_FROM & SAMP_ITEM & SAMP_MTHD & COLL_MTHD & SAMP_SITE_ID & SAMP_SITE_NAME & WELL_NAME & SAMPLE_LOCATION \\
\hline 4220 & & RIVER & & RAW GRAB & TRANSECT & 317 & VERNITA-1 HRM 0.3 & & \\
\hline 4221 & & RIVER & & RAW GRAB & TRANSECT & 317 & VERNITA-1 HRM 0.3 & & \\
\hline $4222 \mathrm{~F}$ & $\mathrm{~F}$ & RIVER & & RAW GRAB & TRANSECT & 317 & VERNITA-1 HRM 0.3 & & \\
\hline 4223 & & RIVER & & RAW GRAB & TRANSECT & 317 & VERNITA-1 HRM 0.3 & & \\
\hline 4224 & & RIVER & & RAW GRAB & TRANSECT & 570 & VERNITA-2 HRM 0.3 & & \\
\hline 4225 & & RIVER & & RAW GRAB & TRANSECT & 570 & VERNITA-2 HRM 0.3 & & \\
\hline 4226 & & RIVER & & RAW GRAB & TRANSECT & 570 & VERNITA-2 HRM 0.3 & & \\
\hline 4227 & & RIVER & & RAW GRAB & TRANSECT & 570 & VERNITA-2 HRM 0.3 & & \\
\hline 4228 & & RIVER & & RAW GRAB & TRANSECT & 570 & VERNITA-2 HRM 0.3 & & \\
\hline 4229 & & RIVER & & RAW GRAB & TRANSECT & 318 & VERNITA-3 HRM 0.3 & & \\
\hline 4230 & & RIVER & & RAW GRAB & TRANSECT & 318 & VERNITA-3 HRM 0.3 & & \\
\hline 4231 & & RIVER & & RAW GRAB & TRANSECT & 318 & VERNITA-3 HRM 0.3 & & \\
\hline $4232 \mathrm{~F}$ & $\mathrm{~F}$ & RIVER & & RAW GRAB & TRANSECT & 318 & VERNITA-3 HRM 0.3 & & \\
\hline 4233 & & RIVER & & RAW GRAB & TRANSECT & 318 & VERNITA-3 HRM 0.3 & & \\
\hline 4234 & & RIVER & & RAW GRAB & TRANSECT & 319 & VERNITA-4 HRM 0.3 & & \\
\hline 4235 & & RIVER & & RAW GRAB & TRANSECT & 319 & VERNITA-4 HRM 0.3 & & \\
\hline 4236 & & RIVER & & RAW GRAB & TRANSECT & 319 & VERNITA-4 HRM 0.3 & & \\
\hline 4237 & & RIVER & & RAW GRAB & TRANSECT & 319 & VERNITA-4 HRM 0.3 & & \\
\hline 4238 & & RIVER & & RAW GRAB & TRANSECT & 319 & VERNITA-4 HRM 0.3 & & \\
\hline 4239 & & RIVER & & RAW GRAB & TRANSECT & 348 & 300 AREA -1 HRM 43.1 & & \\
\hline 4240 & & RIVER & & RAW GRAB & TRANSECT & 3483 & 300 AREA -1 HRM 43.1 & & \\
\hline 4241 & & RIVER & & RAW GRAB & TRANSECT & 3483 & 300 AREA -1 HRM 43.1 & & \\
\hline 4242 & & RIVER & & RAW GRAB & TRANSECT & 348 & 300 AREA -1 HRM 43.1 & & \\
\hline 4243 & & RIVER & & RAW GRAB & TRANSECT & 3483 & 300 AREA -1 HRM 43.1 & & \\
\hline 4244 & & RIVER & & RAW GRAB & TRANSECT & 4703 & 300 AREA -2 HRM 43.1 & & \\
\hline 4245 & & RIVER & & RAW GRAB & TRANSECT & 4703 & 300 AREA -2 HRM 43.1 & & \\
\hline 4246 & & RIVER & & RAW GRAB & TRANSECT & 4703 & 300 AREA -2 HRM 43.1 & & \\
\hline 4247 & & RIVER & & RAW GRAB & TRANSECT & 4703 & 300 AREA -2 HRM 43.1 & & \\
\hline 4248 & & RIVER & & RAW GRAB & TRANSECT & 4703 & 300 AREA -2 HRM 43.1 & & \\
\hline 4249 & & RIVER & & RAW GRAB & TRANSECT & 580 & 300 AREA -3 HRM 43.1 & & \\
\hline 4250 & & RIVER & & RAW GRAB & TRANSECT & 5803 & 300 AREA -3 HRM 43.1 & & \\
\hline 4251 & & RIVER & & RAW GRAB & TRANSECT & 5803 & 300 AREA -3 HRM 43.1 & & \\
\hline $4252 \mathrm{~F}$ & $\mathrm{~F}$ & RIVER & & RAW GRAB & TRANSECT & 5803 & 300 AREA -3 HRM 43.1 & & \\
\hline 4253 & & RIVER & & RAW GRAB & TRANSECT & 5803 & 300 AREA -3 HRM 43.1 & & \\
\hline 4254 & & RIVER & & RAW GRAB & TRANSECT & 6073 & 300 AREA -5 HRM 43.1 & & \\
\hline 4255 & & RIVER & & RAW GRAB & TRANSECT & 607 & 300 AREA -5 HRM 43.1 & & \\
\hline 4256 & & RIVER & & RAW GRAB & TRANSECT & 6073 & 300 AREA -5 HRM 43.1 & & \\
\hline 4257 & & RIVER & & RAW GRAB & TRANSECT & 607 & 300 AREA -5 HRM 43.1 & & \\
\hline
\end{tabular}




\begin{tabular}{|c|c|c|c|c|c|c|c|c|c|}
\hline & $T$ & $\bar{U}$ & $\overline{\mathrm{V}}$ & $\mathrm{W}$ & $\bar{X}$ & $\bar{Y}$ & $\bar{Z}$ & $\overline{A A}$ & $A B$ \\
\hline 1 & REVIEW_QUALIFIER & SAMP_FROM & SAMP_ITEM & SAMP_MTHD & COLL_MTHD & SAMP_SITE_ID & SAMP_SITE_NAME & WELL_NAME & SAMPLE_LOCATION \\
\hline 4258 & & RIVER & & RAW GRAB & TRANSECT & 6073 & 300 AREA -5 HRM 43.1 & & \\
\hline 4259 & & RIVER & & RAW GRAB & TRANSECT & 3513 & 300 AREA -7 HRM 43.1 & & \\
\hline 4260 & & RIVER & & RAW GRAB & TRANSECT & 3513 & 300 AREA -7 HRM 43.1 & & \\
\hline 4261 & & RIVER & & RAW GRAB & TRANSECT & 3513 & 300 AREA -7 HRM 43.1 & & \\
\hline 4262 & $\mathrm{~F}$ & RIVER & & RAW GRAB & TRANSECT & 3513 & 300 AREA -7 HRM 43.1 & & \\
\hline 4263 & & RIVER & & RAW GRAB & TRANSECT & 3513 & 300 AREA -7 HRM 43.1 & & \\
\hline 4264 & & RIVER & & RAW GRAB & TRANSECT & 82403 & 300 AREA SHR HRM41.5 & & \\
\hline 4265 & & RIVER & & RAW GRAB & TRANSECT & 82403 & 300 AREA SHR HRM41.5 & & \\
\hline 4266 & & RIVER & & RAW GRAB & TRANSECT & 82403 & 300 AREA SHR HRM41.5 & & \\
\hline 4267 & & RIVER & & RAW GRAB & TRANSECT & 82403 & 300 AREA SHR HRM41.5 & & \\
\hline 4268 & & RIVER & & RAW GRAB & TRANSECT & 82403 & 300 AREA SHR HRM41.5 & & \\
\hline 4269 & & RIVER & & RAW GRAB & TRANSECT & 82413 & 300 AREA SHR HRM42.1 & & \\
\hline 4270 & & RIVER & & RAW GRAB & TRANSECT & 82413 & 300 AREA SHR HRM42.1 & & \\
\hline 4271 & & RIVER & & RAW GRAB & TRANSECT & 82413 & 300 AREA SHR HRM42.1 & & \\
\hline 4272 & $\mathrm{~F}$ & RIVER & & RAW GRAB & TRANSECT & 82413 & 300 AREA SHR HRM42.1 & & \\
\hline 4273 & & RIVER & & RAW GRAB & TRANSECT & 8241 & 300 AREA SHR HRM42.1 & & \\
\hline 4274 & & RIVER & & RAW GRAB & TRANSECT & 82423 & 300 AREA SHR HRM42.5 & & \\
\hline 4275 & $\mathrm{~F}$ & RIVER & & RAW GRAB & TRANSECT & 82423 & 300 AREA SHR HRM42.5 & & \\
\hline 4276 & & RIVER & & RAW GRAB & TRANSECT & 82423 & 300 AREA SHR HRM42.5 & & \\
\hline 4277 & & RIVER & & RAW GRAB & TRANSECT & 82423 & 300 AREA SHR HRM42.5 & & \\
\hline 4278 & & RIVER & & RAW GRAB & TRANSECT & 82423 & 300 AREA SHR HRM42.5 & & \\
\hline 4279 & & RIVER & & RAW GRAB & TRANSECT & 8243 & 300 AREA SHR HRM42.9 & & \\
\hline 4280 & & RIVER & & RAW GRAB & TRANSECT & 8243 & 300 AREA SHR HRM42.9 & & \\
\hline 4281 & & RIVER & & RAW GRAB & TRANSECT & 8243 & 300 AREA SHR HRM42.9 & & \\
\hline 4282 & & RIVER & & RAW GRAB & TRANSECT & 8243 & 300 AREA SHR HRM42.9 & & \\
\hline 4283 & & RIVER & & RAW GRAB & TRANSECT & 8243 & 300 AREA SHR HRM42.9 & & \\
\hline 4284 & & RIVER & & RAW GRAB & TRANSECT & 3523 & 300 AREA-10 HRM 43.1 & & \\
\hline 4285 & & RIVER & & RAW GRAB & TRANSECT & 3523 & 300 AREA-10 HRM 43.1 & & \\
\hline 4286 & & RIVER & & RAW GRAB & TRANSECT & 3523 & 300 AREA-10 HRM 43.1 & & \\
\hline 4287 & $\mathrm{~F}$ & RIVER & & RAW GRAB & TRANSECT & 3523 & 300 AREA-10 HRM 43.1 & & \\
\hline 4288 & & RIVER & & RAW GRAB & TRANSECT & 3523 & 300 AREA-10 HRM 43.1 & & \\
\hline 4289 & & RIVER & & RAW GRAB & TRANSECT & 8223 & RICH.PMPHS HRM 43.5 & & \\
\hline 4290 & & RIVER & & RAW GRAB & TRANSECT & 8223 & RICH.PMPHS HRM 43.5 & & \\
\hline 4291 & & RIVER & & RAW GRAB & TRANSECT & 8223 & RICH.PMPHS HRM 43.5 & & \\
\hline 4292 & & RIVER & & RAW GRAB & TRANSECT & 8223 & RICH.PMPHS HRM 43.5 & & \\
\hline 4293 & & RIVER & & RAW GRAB & TRANSECT & 8223 & RICH.PMPHS HRM 43.5 & & \\
\hline 4294 & & RIVER & & RAW GRAB & TRANSECT & 8222 & RICH.PMPHS HRM 43.9 & & \\
\hline 4295 & & RIVER & & RAW GRAB & TRANSECT & 8222 & RICH.PMPHS HRM 43.9 & & \\
\hline
\end{tabular}




\begin{tabular}{|c|c|c|c|c|c|c|c|c|c|}
\hline & $\mathrm{T}$ & $\bar{U}$ & $\mathrm{~V}$ & $\mathrm{~W}$ & $\mathrm{X}$ & $\mathrm{Y}$ & $Z$ & $\mathrm{AA}$ & $\mathrm{AB}$ \\
\hline \begin{tabular}{l|l}
1 & 1 \\
\end{tabular} & REVIEW_QUALIFIER & SAMP_FROM & SAMP_ITEM & SAMP_MTHD & COLL_MTHD & SAMP_SITE_ID & SAMP_SITE_NAME & WELL_NAME & SAMPLE_LOCATION \\
\hline 4296 & & RIVER & & RAW GRAB & TRANSECT & 8222 & RICH.PMPHS HRM 43.9 & & \\
\hline 4297 & & RIVER & & RAW GRAB & TRANSECT & 8222 & RICH.PMPHS HRM 43.9 & & \\
\hline 4298 & & RIVER & & RAW GRAB & TRANSECT & 8222 & RICH.PMPHS HRM 43.9 & & \\
\hline 4299 & & RIVER & & RAW GRAB & TRANSECT & 8221 & RICH.PMPHS HRM 45.0 & & \\
\hline 4300 & & RIVER & & RAW GRAB & TRANSECT & 8221 & RICH.PMPHS HRM 45.0 & & \\
\hline 4301 & & RIVER & & RAW GRAB & TRANSECT & 8221 & RICH.PMPHS HRM 45.0 & & \\
\hline $4302 \mathrm{~F}$ & $\mathrm{~F}$ & RIVER & & RAW GRAB & TRANSECT & 8221 & RICH.PMPHS HRM 45.0 & & \\
\hline 4303 & & RIVER & & RAW GRAB & TRANSECT & 8221 & RICH.PMPHS HRM 45.0 & & \\
\hline 4304 & & RIVER & & RAW GRAB & TRANSECT & 8220 & RICH.PMPHS HRM 45.8 & & \\
\hline 4305 & & RIVER & & RAW GRAB & TRANSECT & 8220 & RICH.PMPHS HRM 45.8 & & \\
\hline 4306 & & RIVER & & RAW GRAB & TRANSECT & 8220 & RICH.PMPHS HRM 45.8 & & \\
\hline 4307 & & RIVER & & RAW GRAB & TRANSECT & 8220 & RICH.PMPHS HRM 45.8 & & \\
\hline 4308 & & RIVER & & RAW GRAB & TRANSECT & 8220 & RICH.PMPHS HRM 45.8 & & \\
\hline 4309 & & RIVER & & RAW GRAB & TRANSECT & 571 & RICH.PMPHS-1 HRM46.4 & & \\
\hline 4310 & & RIVER & & RAW GRAB & TRANSECT & 571 & RICH.PMPHS-1 HRM46.4 & & \\
\hline 4311 & & RIVER & & RAW GRAB & TRANSECT & 571 & RICH.PMPHS-1 HRM46.4 & & \\
\hline 4312 & & RIVER & & RAW GRAB & TRANSECT & 571 & RICH.PMPHS-1 HRM46.4 & & \\
\hline 4313 & & RIVER & & RAW GRAB & TRANSECT & 571 & RICH.PMPHS-1 HRM46.4 & & \\
\hline 4314 & & RIVER & & RAW GRAB & TRANSECT & 327 & RICH.PMPHS-10 HRM46.4 & & \\
\hline 4315 & & RIVER & & RAW GRAB & TRANSECT & 327 & RICH.PMPHS-10 HRM46.4 & & \\
\hline 4316 & & RIVER & & RAW GRAB & TRANSECT & 327 & RICH.PMPHS-10 HRM46.4 & & \\
\hline $4317 \mathrm{~F}$ & $\mathrm{~F}$ & RIVER & & RAW GRAB & TRANSECT & 327 & RICH.PMPHS-10 HRM46.4 & & \\
\hline 4318 & & RIVER & & RAW GRAB & TRANSECT & 327 & RICH.PMPHS-10 HRM46.4 & & \\
\hline 4319 & & RIVER & & RAW GRAB & TRANSECT & 321 & RICH.PMPHS-2 HRM46.4 & & \\
\hline 4320 & & RIVER & & RAW GRAB & TRANSECT & 321 & RICH.PMPHS-2 HRM46.4 & & \\
\hline 4321 & & RIVER & & RAW GRAB & TRANSECT & 321 & RICH.PMPHS-2 HRM46.4 & & \\
\hline $4322 \mathrm{~F}$ & $\mathrm{~F}$ & RIVER & & RAW GRAB & TRANSECT & 321 & RICH.PMPHS-2 HRM46.4 & & \\
\hline 4323 & & RIVER & & RAW GRAB & TRANSECT & 321 & RICH.PMPHS-2 HRM46.4 & & \\
\hline 4324 & & RIVER & & RAW GRAB & TRANSECT & 322 & RICH.PMPHS-3 HRM46.4 & & \\
\hline 4325 & & RIVER & & RAW GRAB & TRANSECT & 322 & RICH.PMPHS-3 HRM46.4 & & \\
\hline 4326 & & RIVER & & RAW GRAB & TRANSECT & 322 & RICH.PMPHS-3 HRM46.4 & & \\
\hline 4327 & & RIVER & & RAW GRAB & TRANSECT & 322 & RICH.PMPHS-3 HRM46.4 & & \\
\hline 4328 & & RIVER & & RAW GRAB & TRANSECT & 322 & RICH.PMPHS-3 HRM46.4 & & \\
\hline 4329 & & RIVER & & RAW GRAB & TRANSECT & 323 & RICH.PMPHS-5 HRM46.4 & & \\
\hline 4330 & & RIVER & & RAW GRAB & TRANSECT & 323 & RICH.PMPHS-5 HRM46.4 & & \\
\hline 4331 & & RIVER & & RAW GRAB & TRANSECT & 323 & RICH.PMPHS-5 HRM46.4 & & \\
\hline 4332 & & RIVER & & RAW GRAB & TRANSECT & 323 & RICH.PMPHS-5 HRM46.4 & & \\
\hline 4333 & & RIVER & & RAW GRAB & TRANSECT & 323 & RICH.PMPHS-5 HRM46.4 & & \\
\hline
\end{tabular}




\begin{tabular}{|c|c|c|c|c|c|c|c|c|c|}
\hline & $\mathrm{T}$ & $\bar{U}$ & $\mathrm{~V}$ & $\mathrm{~W}$ & $\mathrm{X}$ & $\mathrm{Y}$ & $Z$ & $\mathrm{AA}$ & $\mathrm{AB}$ \\
\hline 1 & REVIEW_QUALIFIER & SAMP_FROM & SAMP_ITEM & SAMP_MTHD & COLL_MTHD & SAMP_SITE_ID & SAMP_SITE_NAME & WELL_NAME & SAMPLE_LOCATION \\
\hline 4334 & & RIVER & & RAW GRAB & TRANSECT & 325 & RICH.PMPHS-7 HRM46.4 & & \\
\hline 4335 & & RIVER & & RAW GRAB & TRANSECT & 325 & RICH.PMPHS-7 HRM46.4 & & \\
\hline 4336 & & RIVER & & RAW GRAB & TRANSECT & 325 & RICH.PMPHS-7 HRM46.4 & & \\
\hline 4337 & $\mathrm{~F}$ & RIVER & & RAW GRAB & TRANSECT & 325 & RICH.PMPHS-7 HRM46.4 & & \\
\hline 4338 & & RIVER & & RAW GRAB & TRANSECT & 325 & RICH.PMPHS-7 HRM46.4 & & \\
\hline 4339 & & RIVER & & RAW GRAB & TRANSECT & 336 & 100 F -1 HRM 19.0 & & \\
\hline 4340 & & RIVER & & RAW GRAB & TRANSECT & 336 & 100 F -1 HRM 19.0 & & \\
\hline 4341 & & RIVER & & RAW GRAB & TRANSECT & 336 & 100 F -1 HRM 19.0 & & \\
\hline 4342 & & RIVER & & RAW GRAB & TRANSECT & 336 & 100 F -1 HRM 19.0 & & \\
\hline 4343 & & RIVER & & RAW GRAB & TRANSECT & 336 & 100 F -1 HRM 19.0 & & \\
\hline 4344 & & RIVER & & RAW GRAB & TRANSECT & 341 & $100 \mathrm{~F}-10 \mathrm{HRM} 19.0$ & & \\
\hline 4345 & & RIVER & & RAW GRAB & TRANSECT & 341 & $100 \mathrm{~F}-10 \mathrm{HRM} 19.0$ & & \\
\hline 4346 & & RIVER & & RAW GRAB & TRANSECT & 341 & $100 \mathrm{~F}-10 \mathrm{HRM} 19.0$ & & \\
\hline 4347 & & RIVER & & RAW GRAB & TRANSECT & 341 & $100 \mathrm{~F}-10 \mathrm{HRM} 19.0$ & & \\
\hline 4348 & & RIVER & & RAW GRAB & TRANSECT & 341 & $100 \mathrm{~F}-10 \mathrm{HRM} 19.0$ & & \\
\hline 4349 & & RIVER & & RAW GRAB & TRANSECT & 576 & 100 F -2 HRM 19.0 & & \\
\hline 4350 & & RIVER & & RAW GRAB & TRANSECT & 576 & 100 F -2 HRM 19.0 & & \\
\hline 4351 & & RIVER & & RAW GRAB & TRANSECT & 576 & 100 F -2 HRM 19.0 & & \\
\hline 4352 & & RIVER & & RAW GRAB & TRANSECT & 576 & 100 F -2 HRM 19.0 & & \\
\hline 4353 & & RIVER & & RAW GRAB & TRANSECT & 576 & 100 F -2 HRM 19.0 & & \\
\hline 4354 & & RIVER & & RAW GRAB & TRANSECT & 337 & 100 F -3 HRM 19.0 & & \\
\hline 4355 & & RIVER & & RAW GRAB & TRANSECT & 337 & 100 F -3 HRM 19.0 & & \\
\hline 4356 & & RIVER & & RAW GRAB & TRANSECT & 337 & 100 F -3 HRM 19.0 & & \\
\hline 4357 & & RIVER & & RAW GRAB & TRANSECT & 337 & 100 F -3 HRM 19.0 & & \\
\hline 4358 & & RIVER & & RAW GRAB & TRANSECT & 337 & 100 F -3 HRM 19.0 & & \\
\hline 4359 & & RIVER & & RAW GRAB & TRANSECT & 338 & 100 F -4 HRM 19.0 & & \\
\hline 4360 & & RIVER & & RAW GRAB & TRANSECT & 338 & 100 F -4 HRM 19.0 & & \\
\hline 4361 & & RIVER & & RAW GRAB & TRANSECT & 338 & 100 F -4 HRM 19.0 & & \\
\hline 4362 & & RIVER & & RAW GRAB & TRANSECT & 338 & 100 F -4 HRM 19.0 & & \\
\hline 4363 & & RIVER & & RAW GRAB & TRANSECT & 338 & 100 F -4 HRM 19.0 & & \\
\hline 4364 & & RIVER & & RAW GRAB & TRANSECT & 577 & 100 F -5 HRM 19.0 & & \\
\hline 4365 & & RIVER & & RAW GRAB & TRANSECT & 577 & 100 F -5 HRM 19.0 & & \\
\hline 4366 & & RIVER & & RAW GRAB & TRANSECT & 577 & 100 F -5 HRM 19.0 & & \\
\hline 4367 & & RIVER & & RAW GRAB & TRANSECT & 577 & 100 F -5 HRM 19.0 & & \\
\hline 4368 & & RIVER & & RAW GRAB & TRANSECT & 577 & 100 F -5 HRM 19.0 & & \\
\hline 4369 & & RIVER & & RAW GRAB & TRANSECT & 606 & 100 F -6 HRM 19.0 & & \\
\hline 4370 & & RIVER & & RAW GRAB & TRANSECT & 606 & 100 F -6 HRM 19.0 & & \\
\hline 4371 & & RIVER & & RAW GRAB & TRANSECT & 606 & 100 F -6 HRM 19.0 & & \\
\hline
\end{tabular}




\begin{tabular}{|c|c|c|c|c|c|c|c|c|c|}
\hline & $\mathrm{T}$ & 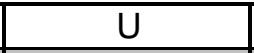 & $\mathrm{V}$ & $\mathrm{W}$ & $\mathrm{X}$ & $\mathrm{Y}$ & $Z$ & $\mathrm{AA}$ & $A B$ \\
\hline \begin{tabular}{l|l}
1 & 1 \\
\end{tabular} & REVIEW_QUALIFIER & SAMP_FROM & SAMP_ITEM & SAMP_MTHD & COLL_MTHD & SAMP_SITE_ID & SAMP_SITE_NAME & WELL_NAME & SAMPLE_LOCATION \\
\hline $4372 \mathrm{~F}$ & $\mathrm{~F}$ & RIVER & & RAW GRAB & TRANSECT & 606 & 100 F -6 HRM 19.0 & & \\
\hline 4373 & & RIVER & & RAW GRAB & TRANSECT & 606 & 100 F -6 HRM 19.0 & & \\
\hline 4374 & & RIVER & & RAW GRAB & TRANSECT & 339 & 100 F -7 HRM 19.0 & & \\
\hline 4375 & & RIVER & & RAW GRAB & TRANSECT & 339 & 100 F -7 HRM 19.0 & & \\
\hline 4376 & & RIVER & & RAW GRAB & TRANSECT & 339 & 100 F -7 HRM 19.0 & & \\
\hline 4377 & & RIVER & & RAW GRAB & TRANSECT & 339 & 100 F -7 HRM 19.0 & & \\
\hline 4378 & & RIVER & & RAW GRAB & TRANSECT & 339 & 100 F -7 HRM 19.0 & & \\
\hline 4379 & & RIVER & & RAW GRAB & TRANSECT & 340 & 100 F -8 HRM 19.0 & & \\
\hline 4380 & & RIVER & & RAW GRAB & TRANSECT & 340 & 100 F -8 HRM 19.0 & & \\
\hline 4381 & & RIVER & & RAW GRAB & TRANSECT & 340 & 100 F -8 HRM 19.0 & & \\
\hline 4382 & & RIVER & & RAW GRAB & TRANSECT & 340 & 100 F -8 HRM 19.0 & & \\
\hline 4383 & & RIVER & & RAW GRAB & TRANSECT & 340 & 100 F -8 HRM 19.0 & & \\
\hline 4384 & & RIVER & & RAW GRAB & TRANSECT & 467 & 100 F -9 HRM 19.0 & & \\
\hline $4385 \mathrm{~F}$ & $\mathrm{~F}$ & RIVER & & RAW GRAB & TRANSECT & $467:$ & 100 F -9 HRM 19.0 & & \\
\hline 4386 & & RIVER & & RAW GRAB & TRANSECT & 467 & 100 F -9 HRM 19.0 & & \\
\hline $4387 \mathrm{~F}$ & $\mathrm{~F}$ & RIVER & & RAW GRAB & TRANSECT & 467 & 100 F -9 HRM 19.0 & & \\
\hline 4388 & & RIVER & & RAW GRAB & TRANSECT & 467 & 100 F -9 HRM 19.0 & & \\
\hline 4389 & & RIVER & & RAW GRAB & TRANSECT & 578 & HANFRD TS-1 HRM 28.7 & & \\
\hline 4390 & & RIVER & & RAW GRAB & TRANSECT & 578 & HANFRD TS-1 HRM 28.7 & & \\
\hline 4391 & & RIVER & & RAW GRAB & TRANSECT & 578 & HANFRD TS-1 HRM 28.7 & & \\
\hline 4392 & & RIVER & & RAW GRAB & TRANSECT & 578 & HANFRD TS-1 HRM 28.7 & & \\
\hline 4393 & & RIVER & & RAW GRAB & TRANSECT & 578 & HANFRD TS-1 HRM 28.7 & & \\
\hline 4394 & & RIVER & & RAW GRAB & TRANSECT & $347 !$ & HANFRD TS-10 HRM 28.7 & & \\
\hline 4395 & & RIVER & & RAW GRAB & TRANSECT & $347 !$ & HANFRD TS-10 HRM 28.7 & & \\
\hline 4396 & & RIVER & & RAW GRAB & TRANSECT & $347 \vdash$ & HANFRD TS-10 HRM 28.7 & & \\
\hline 4397 & & RIVER & & RAW GRAB & TRANSECT & $347 !$ & HANFRD TS-10 HRM 28.7 & & \\
\hline 4398 & & RIVER & & RAW GRAB & TRANSECT & 347 & HANFRD TS-10 HRM 28.7 & & \\
\hline 4399 & & RIVER & & RAW GRAB & TRANSECT & 342 & HANFRD TS-2 HRM 28.7 & & \\
\hline 4400 & & RIVER & & RAW GRAB & TRANSECT & 342 & HANFRD TS-2 HRM 28.7 & & \\
\hline 4401 & & RIVER & & RAW GRAB & TRANSECT & 342 & HANFRD TS-2 HRM 28.7 & & \\
\hline 4402 & & RIVER & & RAW GRAB & TRANSECT & 342 & HANFRD TS-2 HRM 28.7 & & \\
\hline 4403 & & RIVER & & RAW GRAB & TRANSECT & 342 & HANFRD TS-2 HRM 28.7 & & \\
\hline 4404 & & RIVER & & RAW GRAB & TRANSECT & 468 & HANFRD TS-3 HRM 28.7 & & \\
\hline 4405 & & RIVER & & RAW GRAB & TRANSECT & 468 & HANFRD TS-3 HRM 28.7 & & \\
\hline 4406 & & RIVER & & RAW GRAB & TRANSECT & 468 & HANFRD TS-3 HRM 28.7 & & \\
\hline 4407 & & RIVER & & RAW GRAB & TRANSECT & 468 & HANFRD TS-3 HRM 28.7 & & \\
\hline 4408 & & RIVER & & RAW GRAB & TRANSECT & 468 & HANFRD TS-3 HRM 28.7 & & \\
\hline 4409 & & RIVER & & RAW GRAB & TRANSECT & 3441 & HANFRD TS-5 HRM 28.7 & & \\
\hline
\end{tabular}




\begin{tabular}{|c|c|c|c|c|c|c|c|c|c|}
\hline & $\mathrm{T}$ & $\bar{U}$ & $\mathrm{~V}$ & $\mathrm{~W}$ & $\mathrm{X}$ & $\mathrm{Y}$ & $Z$ & $\mathrm{AA}$ & $\mathrm{AB}$ \\
\hline 1 & REVIEW_QUALIFIER & SAMP_FROM & SAMP_ITEM & SAMP_MTHD & COLL_MTHD & SAMP_SITE_ID & SAMP_SITE_NAME & WELL_NAME & SAMPLE_LOCATION \\
\hline 4410 & $\mathrm{~F}$ & RIVER & & RAW GRAB & TRANSECT & 344 & HANFRD TS-5 HRM 28.7 & & \\
\hline 4411 & & RIVER & & RAW GRAB & TRANSECT & 344 & HANFRD TS-5 HRM 28.7 & & \\
\hline 4412 & & RIVER & & RAW GRAB & TRANSECT & 344 & HANFRD TS-5 HRM 28.7 & & \\
\hline 4413 & & RIVER & & RAW GRAB & TRANSECT & 344 & HANFRD TS-5 HRM 28.7 & & \\
\hline 4414 & & RIVER & & RAW GRAB & TRANSECT & 469 & HANFRD TS-7 HRM 28.7 & & \\
\hline 4415 & & RIVER & & RAW GRAB & TRANSECT & 469 & HANFRD TS-7 HRM 28.7 & & \\
\hline 4416 & & RIVER & & RAW GRAB & TRANSECT & 469 & HANFRD TS-7 HRM 28.7 & & \\
\hline 4417 & & RIVER & & RAW GRAB & TRANSECT & 469 & HANFRD TS-7 HRM 28.7 & & \\
\hline 4418 & & RIVER & & RAW GRAB & TRANSECT & 469 & HANFRD TS-7 HRM 28.7 & & \\
\hline 4419 & & RIVER & & RAW GRAB & TRANSECT & 8100 & HANFRD TWNSITE HRM26 & & \\
\hline 4420 & & RIVER & & RAW GRAB & TRANSECT & 8100 & HANFRD TWNSITE HRM26 & & \\
\hline 4421 & & RIVER & & RAW GRAB & TRANSECT & 8100 & HANFRD TWNSITE HRM26 & & \\
\hline 4422 & & RIVER & & RAW GRAB & TRANSECT & 8100 & HANFRD TWNSITE HRM26 & & \\
\hline 4423 & & RIVER & & RAW GRAB & TRANSECT & 8100 & HANFRD TWNSITE HRM26 & & \\
\hline 4424 & & RIVER & & RAW GRAB & TRANSECT & 8101 & HANFRD TWNSITE HRM27 & & \\
\hline 4425 & & RIVER & & RAW GRAB & TRANSECT & 8101 & HANFRD TWNSITE HRM27 & & \\
\hline 4426 & & RIVER & & RAW GRAB & TRANSECT & 8101 & HANFRD TWNSITE HRM27 & & \\
\hline 4427 & $\mathrm{~F}$ & RIVER & & RAW GRAB & TRANSECT & 8101 & HANFRD TWNSITE HRM27 & & \\
\hline 4428 & & RIVER & & RAW GRAB & TRANSECT & 8101 & HANFRD TWNSITE HRM27 & & \\
\hline 4429 & & RIVER & & RAW GRAB & TRANSECT & 8102 & HANFRD TWNSITE HRM29 & & \\
\hline 4430 & & RIVER & & RAW GRAB & TRANSECT & 8102 & HANFRD TWNSITE HRM29 & & \\
\hline 4431 & & RIVER & & RAW GRAB & TRANSECT & 8102 & HANFRD TWNSITE HRM29 & & \\
\hline 4432 & & RIVER & & RAW GRAB & TRANSECT & 8102 & HANFRD TWNSITE HRM29 & & \\
\hline 4433 & & RIVER & & RAW GRAB & TRANSECT & 8102 & HANFRD TWNSITE HRM29 & & \\
\hline 4434 & & RIVER & & RAW GRAB & TRANSECT & 8103 & HANFRD TWNSITE HRM30 & & \\
\hline 4435 & & RIVER & & RAW GRAB & TRANSECT & 8103 & HANFRD TWNSITE HRM30 & & \\
\hline 4436 & & RIVER & & RAW GRAB & TRANSECT & 8103 & HANFRD TWNSITE HRM30 & & \\
\hline 4437 & & RIVER & & RAW GRAB & TRANSECT & 8103 & HANFRD TWNSITE HRM30 & & \\
\hline 4438 & & RIVER & & RAW GRAB & TRANSECT & 8103 & HANFRD TWNSITE HRM30 & & \\
\hline 4439 & & RIVER & & RAW GRAB & TRANSECT & 8223 & RICH.PMPHS HRM 43.5 & & \\
\hline 4440 & & RIVER & & RAW GRAB & TRANSECT & 8223 & RICH.PMPHS HRM 43.5 & & \\
\hline 4441 & & RIVER & & RAW GRAB & TRANSECT & 8223 & RICH.PMPHS HRM 43.5 & & \\
\hline 4442 & & RIVER & & RAW GRAB & TRANSECT & 8223 & RICH.PMPHS HRM 43.5 & & \\
\hline 4443 & & RIVER & & RAW GRAB & TRANSECT & 8223 & RICH.PMPHS HRM 43.5 & & \\
\hline 4444 & & RIVER & & RAW GRAB & TRANSECT & 8222 & RICH.PMPHS HRM 43.9 & & \\
\hline 4445 & & RIVER & & RAW GRAB & TRANSECT & 8222 & RICH.PMPHS HRM 43.9 & & \\
\hline 4446 & & RIVER & & RAW GRAB & TRANSECT & 8222 & RICH.PMPHS HRM 43.9 & & \\
\hline 4447 & & RIVER & & RAW GRAB & TRANSECT & 8222 & RICH.PMPHS HRM 43.9 & & \\
\hline
\end{tabular}




\begin{tabular}{|c|c|c|c|c|c|c|c|c|c|}
\hline & $\mathrm{T}$ & $\bar{U}$ & $\mathrm{~V}$ & $\mathrm{~W}$ & $\mathrm{X}$ & $\mathrm{Y}$ & $Z$ & $\mathrm{AA}$ & $\mathrm{AB}$ \\
\hline 1 & REVIEW_QUALIFIER & SAMP_FROM & SAMP_ITEM & SAMP_MTHD & COLL_MTHD & SAMP_SITE_ID & SAMP_SITE_NAME & WELL_NAME & SAMPLE_LOCATION \\
\hline 4448 & & RIVER & & RAW GRAB & TRANSECT & 8222 & RICH.PMPHS HRM 43.9 & & \\
\hline 4449 & & RIVER & & RAW GRAB & TRANSECT & 8221 & RICH.PMPHS HRM 45.0 & & \\
\hline 4450 & & RIVER & & RAW GRAB & TRANSECT & 8221 & RICH.PMPHS HRM 45.0 & & \\
\hline 4451 & & RIVER & & RAW GRAB & TRANSECT & 8221 & RICH.PMPHS HRM 45.0 & & \\
\hline 4452 & & RIVER & & RAW GRAB & TRANSECT & 8221 & RICH.PMPHS HRM 45.0 & & \\
\hline 4453 & & RIVER & & RAW GRAB & TRANSECT & 8221 & RICH.PMPHS HRM 45.0 & & \\
\hline 4454 & & RIVER & & RAW GRAB & TRANSECT & 8220 & RICH.PMPHS HRM 45.8 & & \\
\hline 4455 & & RIVER & & RAW GRAB & TRANSECT & 8220 & RICH.PMPHS HRM 45.8 & & \\
\hline 4456 & & RIVER & & RAW GRAB & TRANSECT & 8220 & RICH.PMPHS HRM 45.8 & & \\
\hline 4457 & & RIVER & & RAW GRAB & TRANSECT & 8220 & RICH.PMPHS HRM 45.8 & & \\
\hline 4458 & & RIVER & & RAW GRAB & TRANSECT & 8220 & RICH.PMPHS HRM 45.8 & & \\
\hline 4459 & & RIVER & & RAW GRAB & TRANSECT & 571 & RICH.PMPHS-1 HRM46.4 & & \\
\hline 4460 & & RIVER & & RAW GRAB & TRANSECT & 571 & RICH.PMPHS-1 HRM46.4 & & \\
\hline 4461 & & RIVER & & RAW GRAB & TRANSECT & 571 & RICH.PMPHS-1 HRM46.4 & & \\
\hline 4462 & & RIVER & & RAW GRAB & TRANSECT & 571 & RICH.PMPHS-1 HRM46.4 & & \\
\hline 4463 & & RIVER & & RAW GRAB & TRANSECT & 571 & RICH.PMPHS-1 HRM46.4 & & \\
\hline 4464 & & RIVER & & RAW GRAB & TRANSECT & 571 & RICH.PMPHS-1 HRM46.4 & & \\
\hline 4465 & & RIVER & & RAW GRAB & TRANSECT & 571 & RICH.PMPHS-1 HRM46.4 & & \\
\hline 4466 & & RIVER & & RAW GRAB & TRANSECT & 571 & RICH.PMPHS-1 HRM46.4 & & \\
\hline 4467 & & RIVER & & RAW GRAB & TRANSECT & 571 & RICH.PMPHS-1 HRM46.4 & & \\
\hline 4468 & & RIVER & & RAW GRAB & TRANSECT & 571 & RICH.PMPHS-1 HRM46.4 & & \\
\hline 4469 & & RIVER & & RAW GRAB & TRANSECT & 327 & RICH.PMPHS-10 HRM46.4 & & \\
\hline 4470 & & RIVER & & RAW GRAB & TRANSECT & 327 & RICH.PMPHS-10 HRM46.4 & & \\
\hline 4471 & & RIVER & & RAW GRAB & TRANSECT & 327 & RICH.PMPHS-10 HRM46.4 & & \\
\hline 4472 & & RIVER & & RAW GRAB & TRANSECT & 327 & RICH.PMPHS-10 HRM46.4 & & \\
\hline 4473 & & RIVER & & RAW GRAB & TRANSECT & 327 & RICH.PMPHS-10 HRM46.4 & & \\
\hline 4474 & & RIVER & & RAW GRAB & TRANSECT & 321 & RICH.PMPHS-2 HRM46.4 & & \\
\hline 4475 & & RIVER & & RAW GRAB & TRANSECT & 321 & RICH.PMPHS-2 HRM46.4 & & \\
\hline 4476 & & RIVER & & RAW GRAB & TRANSECT & 321 & RICH.PMPHS-2 HRM46.4 & & \\
\hline 4477 & & RIVER & & RAW GRAB & TRANSECT & 321 & RICH.PMPHS-2 HRM46.4 & & \\
\hline 4478 & & RIVER & & RAW GRAB & TRANSECT & 321 & RICH.PMPHS-2 HRM46.4 & & \\
\hline 4479 & & RIVER & & RAW GRAB & TRANSECT & 322 & RICH.PMPHS-3 HRM46.4 & & \\
\hline 4480 & & RIVER & & RAW GRAB & TRANSECT & 322 & RICH.PMPHS-3 HRM46.4 & & \\
\hline 4481 & & RIVER & & RAW GRAB & TRANSECT & 322 & RICH.PMPHS-3 HRM46.4 & & \\
\hline 4482 & & RIVER & & RAW GRAB & TRANSECT & 322 & RICH.PMPHS-3 HRM46.4 & & \\
\hline 4483 & & RIVER & & RAW GRAB & TRANSECT & 322 & RICH.PMPHS-3 HRM46.4 & & \\
\hline 4484 & & RIVER & & RAW GRAB & TRANSECT & 323 & RICH.PMPHS-5 HRM46.4 & & \\
\hline 4485 & & RIVER & & RAW GRAB & TRANSECT & 323 & RICH.PMPHS-5 HRM46.4 & & \\
\hline
\end{tabular}




\begin{tabular}{|c|c|c|c|c|c|c|c|c|c|}
\hline & $\mathrm{T}$ & $\bar{U}$ & $\mathrm{~V}$ & $\mathrm{~W}$ & $\mathrm{X}$ & $\mathrm{Y}$ & $Z$ & $\mathrm{AA}$ & $\mathrm{AB}$ \\
\hline \begin{tabular}{l|l}
1 & 1 \\
\end{tabular} & REVIEW_QUALIFIER & SAMP_FROM & SAMP_ITEM & SAMP_MTHD & COLL_MTHD & SAMP_SITE_ID & SAMP_SITE_NAME & WELL_NAME & SAMPLE_LOCATION \\
\hline 4486 & & RIVER & & RAW GRAB & TRANSECT & 323 & RICH.PMPHS-5 HRM46.4 & & \\
\hline $4487 \mathrm{~F}$ & $\mathrm{~F}$ & RIVER & & RAW GRAB & TRANSECT & 323 & RICH.PMPHS-5 HRM46.4 & & \\
\hline 4488 & & RIVER & & RAW GRAB & TRANSECT & 323 & RICH.PMPHS-5 HRM46.4 & & \\
\hline 4489 & & RIVER & & RAW GRAB & TRANSECT & 325 & RICH.PMPHS-7 HRM46.4 & & \\
\hline 4490 & & RIVER & & RAW GRAB & TRANSECT & 325 & RICH.PMPHS-7 HRM46.4 & & \\
\hline 4491 & & RIVER & & RAW GRAB & TRANSECT & 325 & RICH.PMPHS-7 HRM46.4 & & \\
\hline 4492 & & RIVER & & RAW GRAB & TRANSECT & 325 & RICH.PMPHS-7 HRM46.4 & & \\
\hline 4493 & & RIVER & & RAW GRAB & TRANSECT & 325 & RICH.PMPHS-7 HRM46.4 & & \\
\hline 4494 & & RIVER & & RAW GRAB & TRANSECT & 327 & RICH.PMPHS-10 HRM46.4 & & \\
\hline 4495 & & RIVER & & RAW GRAB & TRANSECT & 325 & RICH.PMPHS-7 HRM46.4 & & \\
\hline 4496 & & RIVER & & RAW GRAB & TRANSECT & 323 & RICH.PMPHS-5 HRM46.4 & & \\
\hline 4497 & & RIVER & & RAW GRAB & TRANSECT & 322 & RICH.PMPHS-3 HRM46.4 & & \\
\hline 4498 & & RIVER & & RAW GRAB & TRANSECT & 321 & RICH.PMPHS-2 HRM46.4 & & \\
\hline 4499 & & RIVER & & RAW GRAB & TRANSECT & 571 & RICH.PMPHS-1 HRM46.4 & & \\
\hline 4500 & & RIVER & & RAW GRAB & TRANSECT & 8220 & RICH.PMPHS HRM 45.8 & & \\
\hline 4501 & & RIVER & & RAW GRAB & TRANSECT & 8221 & RICH.PMPHS HRM 45.0 & & \\
\hline 4502 & & RIVER & & RAW GRAB & TRANSECT & 8222 & RICH.PMPHS HRM 43.9 & & \\
\hline 4503 & & RIVER & & RAW GRAB & TRANSECT & 8223 & RICH.PMPHS HRM 43.5 & & \\
\hline 4504 & & RIVER & & RAW GRAB & TRANSECT & 317 & VERNITA-1 HRM 0.3 & & \\
\hline $4505 \mathrm{~F}$ & $\mathrm{~F}$ & RIVER & & RAW GRAB & TRANSECT & 317 & VERNITA-1 HRM 0.3 & & \\
\hline 4506 & & RIVER & & RAW GRAB & TRANSECT & 317 & VERNITA-1 HRM 0.3 & & \\
\hline 4507 & & RIVER & & RAW GRAB & TRANSECT & 317 & VERNITA-1 HRM 0.3 & & \\
\hline 4508 & & RIVER & & RAW GRAB & TRANSECT & 317 & VERNITA-1 HRM 0.3 & & \\
\hline 4509 & & RIVER & & RAW GRAB & TRANSECT & 570 & VERNITA-2 HRM 0.3 & & \\
\hline $4510 \mathrm{~F}$ & $\mathrm{~F}$ & RIVER & & RAW GRAB & TRANSECT & 570 & VERNITA-2 HRM 0.3 & & \\
\hline 4511 & & RIVER & & RAW GRAB & TRANSECT & 570 & VERNITA-2 HRM 0.3 & & \\
\hline $4512 \mathrm{~F}$ & $\mathrm{~F}$ & RIVER & & RAW GRAB & TRANSECT & 570 & VERNITA-2 HRM 0.3 & & \\
\hline 4513 & & RIVER & & RAW GRAB & TRANSECT & 570 & VERNITA-2 HRM 0.3 & & \\
\hline 4514 & & RIVER & & RAW GRAB & TRANSECT & 318 & VERNITA-3 HRM 0.3 & & \\
\hline $4515 \mathrm{~F}$ & $\mathrm{~F}$ & RIVER & & RAW GRAB & TRANSECT & 318 & VERNITA-3 HRM 0.3 & & \\
\hline 4516 & & RIVER & & RAW GRAB & TRANSECT & 318 & VERNITA-3 HRM 0.3 & & \\
\hline 4517 & & RIVER & & RAW GRAB & TRANSECT & 318 & VERNITA-3 HRM 0.3 & & \\
\hline 4518 & & RIVER & & RAW GRAB & TRANSECT & 318 & VERNITA-3 HRM 0.3 & & \\
\hline 4519 & & RIVER & & RAW GRAB & TRANSECT & 319 & VERNITA-4 HRM 0.3 & & \\
\hline $4520 \mathrm{~F}$ & $\mathrm{~F}$ & RIVER & & RAW GRAB & TRANSECT & 319 & VERNITA-4 HRM 0.3 & & \\
\hline 4521 & & RIVER & & RAW GRAB & TRANSECT & 319 & VERNITA-4 HRM 0.3 & & \\
\hline 4522 & & RIVER & & RAW GRAB & TRANSECT & 319 & VERNITA-4 HRM 0.3 & & \\
\hline 4523 & & RIVER & & RAW GRAB & TRANSECT & 319 & VERNITA-4 HRM 0.3 & & \\
\hline
\end{tabular}




\begin{tabular}{|c|c|c|c|c|c|c|c|c|c|}
\hline & $\mathrm{T}$ & 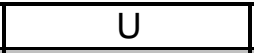 & $\mathrm{V}$ & $\mathrm{W}$ & $\mathrm{X}$ & $\mathrm{Y}$ & $Z$ & $\mathrm{AA}$ & $A B$ \\
\hline \begin{tabular}{l|l}
1 & 1 \\
\end{tabular} & REVIEW_QUALIFIER & SAMP_FROM & SAMP_ITEM & SAMP_MTHD & COLL_MTHD & SAMP_SITE_ID & SAMP_SITE_NAME & WELL_NAME & SAMPLE_LOCATION \\
\hline 4524 & & RIVER & & RAW GRAB & TRANSECT & 319 & VERNITA-4 HRM 0.3 & & \\
\hline 4525 & & RIVER & & RAW GRAB & TRANSECT & 318 & VERNITA-3 HRM 0.3 & & \\
\hline 4526 & & RIVER & & RAW GRAB & TRANSECT & 570 & VERNITA-2 HRM 0.3 & & \\
\hline 4527 & & RIVER & & RAW GRAB & TRANSECT & 317 & VERNITA-1 HRM 0.3 & & \\
\hline 4528 & & RIVER & & RAW GRAB & TRANSECT & 317 & VERNITA-1 HRM 0.3 & & \\
\hline 4529 & & RIVER & & RAW GRAB & TRANSECT & 317 & VERNITA-1 HRM 0.3 & & \\
\hline 4530 & & RIVER & & RAW GRAB & TRANSECT & 317 & VERNITA-1 HRM 0.3 & & \\
\hline 4531 & & RIVER & & RAW GRAB & TRANSECT & 317 & VERNITA-1 HRM 0.3 & & \\
\hline 4532 & & RIVER & & RAW GRAB & TRANSECT & 317 & VERNITA-1 HRM 0.3 & & \\
\hline 4533 & & RIVER & & RAW GRAB & TRANSECT & 570 & VERNITA-2 HRM 0.3 & & \\
\hline 4534 & & RIVER & & RAW GRAB & TRANSECT & 570 & VERNITA-2 HRM 0.3 & & \\
\hline 4535 & & RIVER & & RAW GRAB & TRANSECT & 570 & VERNITA-2 HRM 0.3 & & \\
\hline $4536 \mathrm{~F}$ & $\mathrm{~F}$ & RIVER & & RAW GRAB & TRANSECT & 570 & VERNITA-2 HRM 0.3 & & \\
\hline 4537 & & RIVER & & RAW GRAB & TRANSECT & 570 & VERNITA-2 HRM 0.3 & & \\
\hline 4538 & & RIVER & & RAW GRAB & TRANSECT & 318 & VERNITA-3 HRM 0.3 & & \\
\hline 4539 & & RIVER & & RAW GRAB & TRANSECT & 318 & VERNITA-3 HRM 0.3 & & \\
\hline 4540 & & RIVER & & RAW GRAB & TRANSECT & 318 & VERNITA-3 HRM 0.3 & & \\
\hline 4541 & & RIVER & & RAW GRAB & TRANSECT & 318 & VERNITA-3 HRM 0.3 & & \\
\hline 4542 & & RIVER & & RAW GRAB & TRANSECT & 318 & VERNITA-3 HRM 0.3 & & \\
\hline 4543 & & RIVER & & RAW GRAB & TRANSECT & 319 & VERNITA-4 HRM 0.3 & & \\
\hline 4544 & & RIVER & & RAW GRAB & TRANSECT & 319 & VERNITA-4 HRM 0.3 & & \\
\hline 4545 & & RIVER & & RAW GRAB & TRANSECT & 319 & VERNITA-4 HRM 0.3 & & \\
\hline 4546 & & RIVER & & RAW GRAB & TRANSECT & 319 & VERNITA-4 HRM 0.3 & & \\
\hline 4547 & & RIVER & & RAW GRAB & TRANSECT & 319 & VERNITA-4 HRM 0.3 & & \\
\hline 4548 & & RIVER & & RAW GRAB & TRANSECT & 8223 & RICH.PMPHS HRM 43.5 & & \\
\hline 4549 & & RIVER & & RAW GRAB & TRANSECT & 8223 & RICH.PMPHS HRM 43.5 & & \\
\hline 4550 & & RIVER & & RAW GRAB & TRANSECT & 8223 & RICH.PMPHS HRM 43.5 & & \\
\hline 4551 & & RIVER & & RAW GRAB & TRANSECT & 8223 & RICH.PMPHS HRM 43.5 & & \\
\hline 4552 & & RIVER & & RAW GRAB & TRANSECT & 8223 & RICH.PMPHS HRM 43.5 & & \\
\hline 4553 & & RIVER & & RAW GRAB & TRANSECT & 8222 & RICH.PMPHS HRM 43.9 & & \\
\hline 4554 & & RIVER & & RAW GRAB & TRANSECT & 8222 & RICH.PMPHS HRM 43.9 & & \\
\hline 4555 & & RIVER & & RAW GRAB & TRANSECT & 8222 & RICH.PMPHS HRM 43.9 & & \\
\hline 4556 & & RIVER & & RAW GRAB & TRANSECT & 8222 & RICH.PMPHS HRM 43.9 & & \\
\hline 4557 & & RIVER & & RAW GRAB & TRANSECT & 8222 & RICH.PMPHS HRM 43.9 & & \\
\hline 4558 & & RIVER & & RAW GRAB & TRANSECT & 8221 & RICH.PMPHS HRM 45.0 & & \\
\hline 4559 & & RIVER & & RAW GRAB & TRANSECT & 8221 & RICH.PMPHS HRM 45.0 & & \\
\hline 4560 & & RIVER & & RAW GRAB & TRANSECT & 8221 & RICH.PMPHS HRM 45.0 & & \\
\hline 4561 & & RIVER & & RAW GRAB & TRANSECT & 8221 & RICH.PMPHS HRM 45.0 & & \\
\hline
\end{tabular}




\begin{tabular}{|c|c|c|c|c|c|c|c|c|c|}
\hline & $\mathrm{T}$ & $\bar{U}$ & $\mathrm{~V}$ & $\mathrm{~W}$ & $\mathrm{X}$ & $\mathrm{Y}$ & $Z$ & $\mathrm{AA}$ & $\mathrm{AB}$ \\
\hline \begin{tabular}{l|l}
1 & 1 \\
\end{tabular} & REVIEW_QUALIFIER & SAMP_FROM & SAMP_ITEM & SAMP_MTHD & COLL_MTHD & SAMP_SITE_ID & SAMP_SITE_NAME & WELL_NAME & SAMPLE_LOCATION \\
\hline 4562 & & RIVER & & RAW GRAB & TRANSECT & 8221 & RICH.PMPHS HRM 45.0 & & \\
\hline 4563 & & RIVER & & RAW GRAB & TRANSECT & 8220 & RICH.PMPHS HRM 45.8 & & \\
\hline 4564 & & RIVER & & RAW GRAB & TRANSECT & 8220 & RICH.PMPHS HRM 45.8 & & \\
\hline 4565 & & RIVER & & RAW GRAB & TRANSECT & 8220 & RICH.PMPHS HRM 45.8 & & \\
\hline 4566 & & RIVER & & RAW GRAB & TRANSECT & 8220 & RICH.PMPHS HRM 45.8 & & \\
\hline 4567 & & RIVER & & RAW GRAB & TRANSECT & 8220 & RICH.PMPHS HRM 45.8 & & \\
\hline 4568 & & RIVER & & RAW GRAB & TRANSECT & 571 & RICH.PMPHS-1 HRM46.4 & & \\
\hline 4569 & & RIVER & & RAW GRAB & TRANSECT & 571 & RICH.PMPHS-1 HRM46.4 & & \\
\hline 4570 & & RIVER & & RAW GRAB & TRANSECT & 571 & RICH.PMPHS-1 HRM46.4 & & \\
\hline 4571 & & RIVER & & RAW GRAB & TRANSECT & 571 & RICH.PMPHS-1 HRM46.4 & & \\
\hline 4572 & & RIVER & & RAW GRAB & TRANSECT & 571 & RICH.PMPHS-1 HRM46.4 & & \\
\hline $4573 \mathrm{~F}$ & $\mathrm{~F}$ & RIVER & & RAW GRAB & TRANSECT & 571 & RICH.PMPHS-1 HRM46.4 & & \\
\hline 4574 & & RIVER & & RAW GRAB & TRANSECT & 571 & RICH.PMPHS-1 HRM46.4 & & \\
\hline 4575 & & RIVER & & RAW GRAB & TRANSECT & 571 & RICH.PMPHS-1 HRM46.4 & & \\
\hline 4576 & & RIVER & & RAW GRAB & TRANSECT & 571 & RICH.PMPHS-1 HRM46.4 & & \\
\hline 4577 & & RIVER & & RAW GRAB & TRANSECT & 571 & RICH.PMPHS-1 HRM46.4 & & \\
\hline 4578 & & RIVER & & RAW GRAB & TRANSECT & 327 & RICH.PMPHS-10 HRM46.4 & & \\
\hline 4579 & & RIVER & & RAW GRAB & TRANSECT & 327 & RICH.PMPHS-10 HRM46.4 & & \\
\hline $4580 \mathrm{~F}$ & $\mathrm{~F}$ & RIVER & & RAW GRAB & TRANSECT & 327 & RICH.PMPHS-10 HRM46.4 & & \\
\hline 4581 & & RIVER & & RAW GRAB & TRANSECT & 327 & RICH.PMPHS-10 HRM46.4 & & \\
\hline 4582 & & RIVER & & RAW GRAB & TRANSECT & 327 & RICH.PMPHS-10 HRM46.4 & & \\
\hline 4583 & & RIVER & & RAW GRAB & TRANSECT & 321 & RICH.PMPHS-2 HRM46.4 & & \\
\hline 4584 & & RIVER & & RAW GRAB & TRANSECT & 321 & RICH.PMPHS-2 HRM46.4 & & \\
\hline 4585 & & RIVER & & RAW GRAB & TRANSECT & 321 & RICH.PMPHS-2 HRM46.4 & & \\
\hline 4586 & & RIVER & & RAW GRAB & TRANSECT & 321 & RICH.PMPHS-2 HRM46.4 & & \\
\hline 4587 & & RIVER & & RAW GRAB & TRANSECT & 321 & RICH.PMPHS-2 HRM46.4 & & \\
\hline 4588 & & RIVER & & RAW GRAB & TRANSECT & 322 & RICH.PMPHS-3 HRM46.4 & & \\
\hline 4589 & & RIVER & & RAW GRAB & TRANSECT & 322 & RICH.PMPHS-3 HRM46.4 & & \\
\hline 4590 & & RIVER & & RAW GRAB & TRANSECT & 322 & RICH.PMPHS-3 HRM46.4 & & \\
\hline 4591 F & $\mathrm{F}$ & RIVER & & RAW GRAB & TRANSECT & 322 & RICH.PMPHS-3 HRM46.4 & & \\
\hline 4592 & & RIVER & & RAW GRAB & TRANSECT & 322 & RICH.PMPHS-3 HRM46.4 & & \\
\hline 4593 & & RIVER & & RAW GRAB & TRANSECT & 323 & RICH.PMPHS-5 HRM46.4 & & \\
\hline 4594 & & RIVER & & RAW GRAB & TRANSECT & 323 & RICH.PMPHS-5 HRM46.4 & & \\
\hline 4595 & & RIVER & & RAW GRAB & TRANSECT & 323 & RICH.PMPHS-5 HRM46.4 & & \\
\hline 4596 & & RIVER & & RAW GRAB & TRANSECT & 323 & RICH.PMPHS-5 HRM46.4 & & \\
\hline 4597 & & RIVER & & RAW GRAB & TRANSECT & 323 & RICH.PMPHS-5 HRM46.4 & & \\
\hline 4598 & & RIVER & & RAW GRAB & TRANSECT & 325 & RICH.PMPHS-7 HRM46.4 & & \\
\hline 4599 & & RIVER & & RAW GRAB & TRANSECT & 325 & RICH.PMPHS-7 HRM46.4 & & \\
\hline
\end{tabular}




\begin{tabular}{|c|c|c|c|c|c|c|c|c|c|}
\hline & $\mathrm{T}$ & $\bar{U}$ & $\mathrm{~V}$ & $\mathrm{~W}$ & $\mathrm{X}$ & $\mathrm{Y}$ & $Z$ & $\mathrm{AA}$ & $\mathrm{AB}$ \\
\hline \begin{tabular}{l|l}
1 & 1 \\
\end{tabular} & REVIEW_QUALIFIER & SAMP_FROM & SAMP_ITEM & SAMP_MTHD & COLL_MTHD & SAMP_SITE_ID & SAMP_SITE_NAME & WELL_NAME & SAMPLE_LOCATION \\
\hline 4600 & & RIVER & & RAW GRAB & TRANSECT & 325 & RICH.PMPHS-7 HRM46.4 & & \\
\hline 4601 & & RIVER & & RAW GRAB & TRANSECT & 325 & RICH.PMPHS-7 HRM46.4 & & \\
\hline 4602 & & RIVER & & RAW GRAB & TRANSECT & 325 & RICH.PMPHS-7 HRM46.4 & & \\
\hline 4603 & & RIVER & & RAW GRAB & TRANSECT & 317 & VERNITA-1 HRM 0.3 & & \\
\hline 4604 & & RIVER & & RAW GRAB & TRANSECT & 317 & VERNITA-1 HRM 0.3 & & \\
\hline 4605 & & RIVER & & RAW GRAB & TRANSECT & 317 & VERNITA-1 HRM 0.3 & & \\
\hline $4606 \mathrm{~F}$ & $\mathrm{~F}$ & RIVER & & RAW GRAB & TRANSECT & 317 & VERNITA-1 HRM 0.3 & & \\
\hline 4607 & & RIVER & & RAW GRAB & TRANSECT & 317 & VERNITA-1 HRM 0.3 & & \\
\hline 4608 & & RIVER & & RAW GRAB & TRANSECT & 570 & VERNITA-2 HRM 0.3 & & \\
\hline 4609 & & RIVER & & RAW GRAB & TRANSECT & 570 & VERNITA-2 HRM 0.3 & & \\
\hline 4610 & & RIVER & & RAW GRAB & TRANSECT & 570 & VERNITA-2 HRM 0.3 & & \\
\hline 4611 & & RIVER & & RAW GRAB & TRANSECT & 570 & VERNITA-2 HRM 0.3 & & \\
\hline 4612 & & RIVER & & RAW GRAB & TRANSECT & 570 & VERNITA-2 HRM 0.3 & & \\
\hline 4613 & & RIVER & & RAW GRAB & TRANSECT & 318 & VERNITA-3 HRM 0.3 & & \\
\hline 4614 & & RIVER & & RAW GRAB & TRANSECT & 318 & VERNITA-3 HRM 0.3 & & \\
\hline 4615 & & RIVER & & RAW GRAB & TRANSECT & 318 & VERNITA-3 HRM 0.3 & & \\
\hline 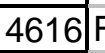 & $\mathrm{F}$ & RIVER & & RAW GRAB & TRANSECT & 318 & VERNITA-3 HRM 0.3 & & \\
\hline 4617 & & RIVER & & RAW GRAB & TRANSECT & 318 & VERNITA-3 HRM 0.3 & & \\
\hline 4618 & & RIVER & & RAW GRAB & TRANSECT & 319 & VERNITA-4 HRM 0.3 & & \\
\hline 4619 & & RIVER & & RAW GRAB & TRANSECT & 319 & VERNITA-4 HRM 0.3 & & \\
\hline 4620 & & RIVER & & RAW GRAB & TRANSECT & 319 & VERNITA-4 HRM 0.3 & & \\
\hline 4621 & & RIVER & & RAW GRAB & TRANSECT & 319 & VERNITA-4 HRM 0.3 & & \\
\hline 4622 & & RIVER & & RAW GRAB & TRANSECT & 319 & VERNITA-4 HRM 0.3 & & \\
\hline 4623 & & RIVER & & RAW GRAB & TRANSECT & 8223 & RICH.PMPHS HRM 43.5 & & \\
\hline 4624 & & RIVER & & RAW GRAB & TRANSECT & 8223 & RICH.PMPHS HRM 43.5 & & \\
\hline 4625 & & RIVER & & RAW GRAB & TRANSECT & 8223 & RICH.PMPHS HRM 43.5 & & \\
\hline 4626 & & RIVER & & RAW GRAB & TRANSECT & 8223 & RICH.PMPHS HRM 43.5 & & \\
\hline 4627 & & RIVER & & RAW GRAB & TRANSECT & 8223 & RICH.PMPHS HRM 43.5 & & \\
\hline 4628 & & RIVER & & RAW GRAB & TRANSECT & 8222 & RICH.PMPHS HRM 43.9 & & \\
\hline 4629 & & RIVER & & RAW GRAB & TRANSECT & 8222 & RICH.PMPHS HRM 43.9 & & \\
\hline 4630 & & RIVER & & RAW GRAB & TRANSECT & 8222 & RICH.PMPHS HRM 43.9 & & \\
\hline 4631 & & RIVER & & RAW GRAB & TRANSECT & 8222 & RICH.PMPHS HRM 43.9 & & \\
\hline 4632 & & RIVER & & RAW GRAB & TRANSECT & 8222 & RICH.PMPHS HRM 43.9 & & \\
\hline 4633 & & RIVER & & RAW GRAB & TRANSECT & 8221 & RICH.PMPHS HRM 45.0 & & \\
\hline 4634 & & RIVER & & RAW GRAB & TRANSECT & 8221 & RICH.PMPHS HRM 45.0 & & \\
\hline 4635 & & RIVER & & RAW GRAB & TRANSECT & 8221 & RICH.PMPHS HRM 45.0 & & \\
\hline 4636 & & RIVER & & RAW GRAB & TRANSECT & 8221 & RICH.PMPHS HRM 45.0 & & \\
\hline 4637 & & RIVER & & RAW GRAB & TRANSECT & 8221 & RICH.PMPHS HRM 45.0 & & \\
\hline
\end{tabular}




\begin{tabular}{|c|c|c|c|c|c|c|c|c|c|}
\hline & $T$ & $U$ & $\mathrm{~V}$ & $\mathrm{~W}$ & $\mathrm{X}$ & $\mathrm{Y}$ & $Z$ & $\overline{A A}$ & $A B$ \\
\hline 1 & REVIEW_QUALIFIER & SAMP_FROM & SAMP_ITEM & SAMP_MTHD & COLL_MTHD & SAMP_SITE_ID & SAMP_SITE_NAME & WELL_NAME & SAMPLE_LOCATION \\
\hline 4638 & & RIVER & & RAW GRAB & TRANSECT & 8220 & RICH.PMPHS HRM 45.8 & & \\
\hline 4639 & & RIVER & & RAW GRAB & TRANSECT & 8220 & RICH.PMPHS HRM 45.8 & & \\
\hline 4640 & & RIVER & & RAW GRAB & TRANSECT & 8220 & RICH.PMPHS HRM 45.8 & & \\
\hline 4641 & & RIVER & & RAW GRAB & TRANSECT & 8220 & RICH.PMPHS HRM 45.8 & & \\
\hline 4642 & & RIVER & & RAW GRAB & TRANSECT & 8220 & RICH.PMPHS HRM 45.8 & & \\
\hline 4643 & & RIVER & & RAW GRAB & TRANSECT & 571 & RICH.PMPHS-1 HRM46.4 & & \\
\hline 4644 & & RIVER & & RAW GRAB & TRANSECT & 571 & RICH.PMPHS-1 HRM46.4 & & \\
\hline 4645 & & RIVER & & RAW GRAB & TRANSECT & 571 & RICH.PMPHS-1 HRM46.4 & & \\
\hline 4646 & & RIVER & & RAW GRAB & TRANSECT & 571 & RICH.PMPHS-1 HRM46.4 & & \\
\hline 4647 & & RIVER & & RAW GRAB & TRANSECT & 571 & RICH.PMPHS-1 HRM46.4 & & \\
\hline 4648 & & RIVER & & RAW GRAB & TRANSECT & 327 & RICH.PMPHS-10 HRM46.4 & & \\
\hline 4649 & & RIVER & & RAW GRAB & TRANSECT & 327 & RICH.PMPHS-10 HRM46.4 & & \\
\hline 4650 & & RIVER & & RAW GRAB & TRANSECT & 327 & RICH.PMPHS-10 HRM46.4 & & \\
\hline $4651 \mathrm{~F}$ & $\mathrm{~F}$ & RIVER & & RAW GRAB & TRANSECT & 327 & RICH.PMPHS-10 HRM46.4 & & \\
\hline 4652 & & RIVER & & RAW GRAB & TRANSECT & 327 & RICH.PMPHS-10 HRM46.4 & & \\
\hline 4653 & & RIVER & & RAW GRAB & TRANSECT & 321 & RICH.PMPHS-2 HRM46.4 & & \\
\hline 4654 & & RIVER & & RAW GRAB & TRANSECT & 321 & RICH.PMPHS-2 HRM46.4 & & \\
\hline 4655 & & RIVER & & RAW GRAB & TRANSECT & 321 & RICH.PMPHS-2 HRM46.4 & & \\
\hline 4656 & & RIVER & & RAW GRAB & TRANSECT & 321 & RICH.PMPHS-2 HRM46.4 & & \\
\hline 4657 & & RIVER & & RAW GRAB & TRANSECT & 321 & RICH.PMPHS-2 HRM46.4 & & \\
\hline 4658 & & RIVER & & RAW GRAB & TRANSECT & 322 & RICH.PMPHS-3 HRM46.4 & & \\
\hline 4659 & & RIVER & & RAW GRAB & TRANSECT & 322 & RICH.PMPHS-3 HRM46.4 & & \\
\hline 4660 & & RIVER & & RAW GRAB & TRANSECT & 322 & RICH.PMPHS-3 HRM46.4 & & \\
\hline 4661 & & RIVER & & RAW GRAB & TRANSECT & 322 & RICH.PMPHS-3 HRM46.4 & & \\
\hline 4662 & & RIVER & & RAW GRAB & TRANSECT & 322 & RICH.PMPHS-3 HRM46.4 & & \\
\hline 4663 & & RIVER & & RAW GRAB & TRANSECT & 323 & RICH.PMPHS-5 HRM46.4 & & \\
\hline 4664 & & RIVER & & RAW GRAB & TRANSECT & 323 & RICH.PMPHS-5 HRM46.4 & & \\
\hline 4665 & & RIVER & & RAW GRAB & TRANSECT & 323 & RICH.PMPHS-5 HRM46.4 & & \\
\hline 4666 & & RIVER & & RAW GRAB & TRANSECT & 323 & RICH.PMPHS-5 HRM46.4 & & \\
\hline \begin{tabular}{l|l|}
4667 \\
\end{tabular} & & RIVER & & RAW GRAB & TRANSECT & 323 & RICH.PMPHS-5 HRM46.4 & & \\
\hline 4668 & & RIVER & & RAW GRAB & TRANSECT & 325 & RICH.PMPHS-7 HRM46.4 & & \\
\hline 4669 & & RIVER & & RAW GRAB & TRANSECT & 325 & RICH.PMPHS-7 HRM46.4 & & \\
\hline 4670 & & RIVER & & RAW GRAB & TRANSECT & 325 & RICH.PMPHS-7 HRM46.4 & & \\
\hline $4671 \mathrm{~F}$ & $\mathrm{~F}$ & RIVER & & RAW GRAB & TRANSECT & 325 & RICH.PMPHS-7 HRM46.4 & & \\
\hline 4672 & & RIVER & & RAW GRAB & TRANSECT & 325 & RICH.PMPHS-7 HRM46.4 & & \\
\hline
\end{tabular}




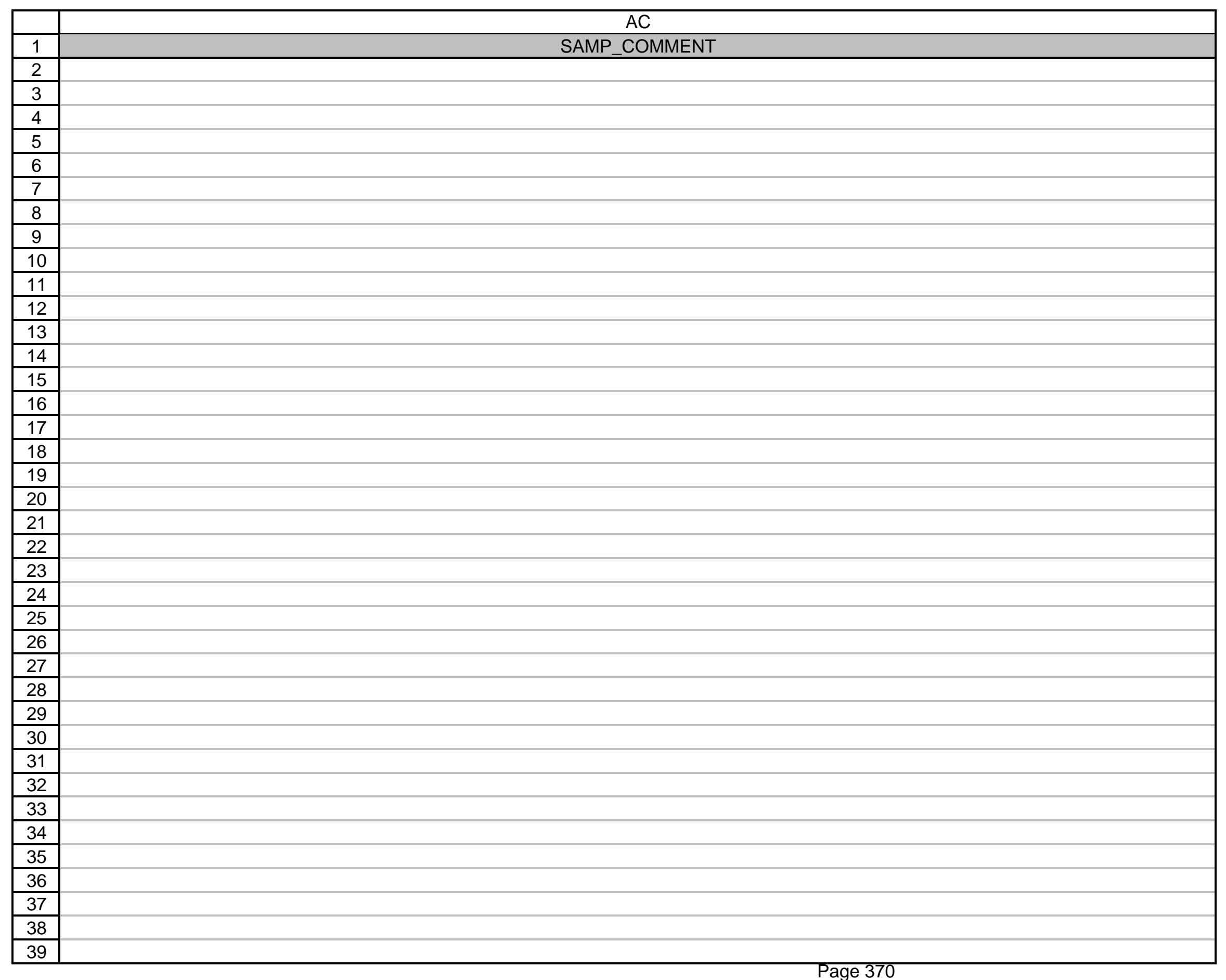




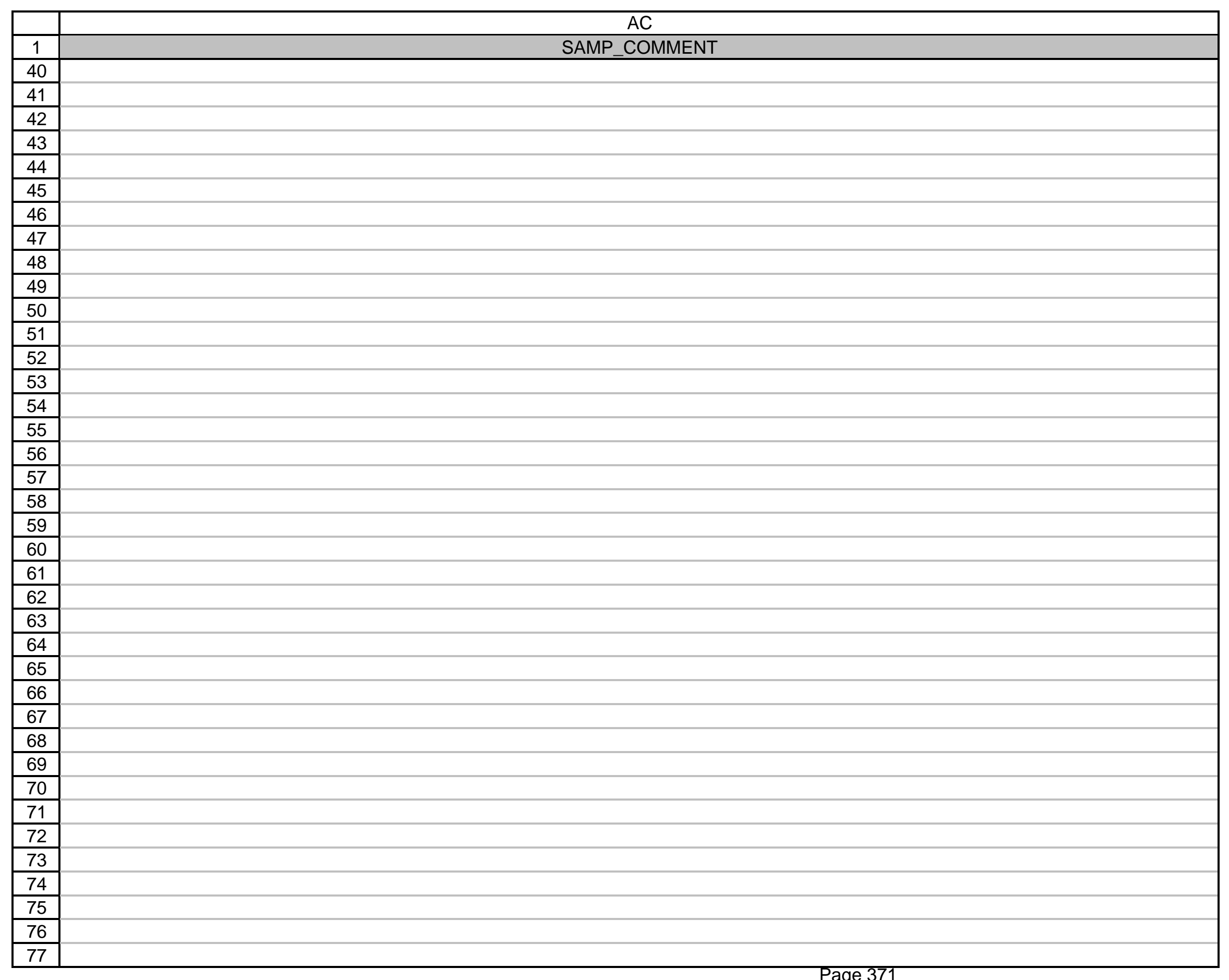




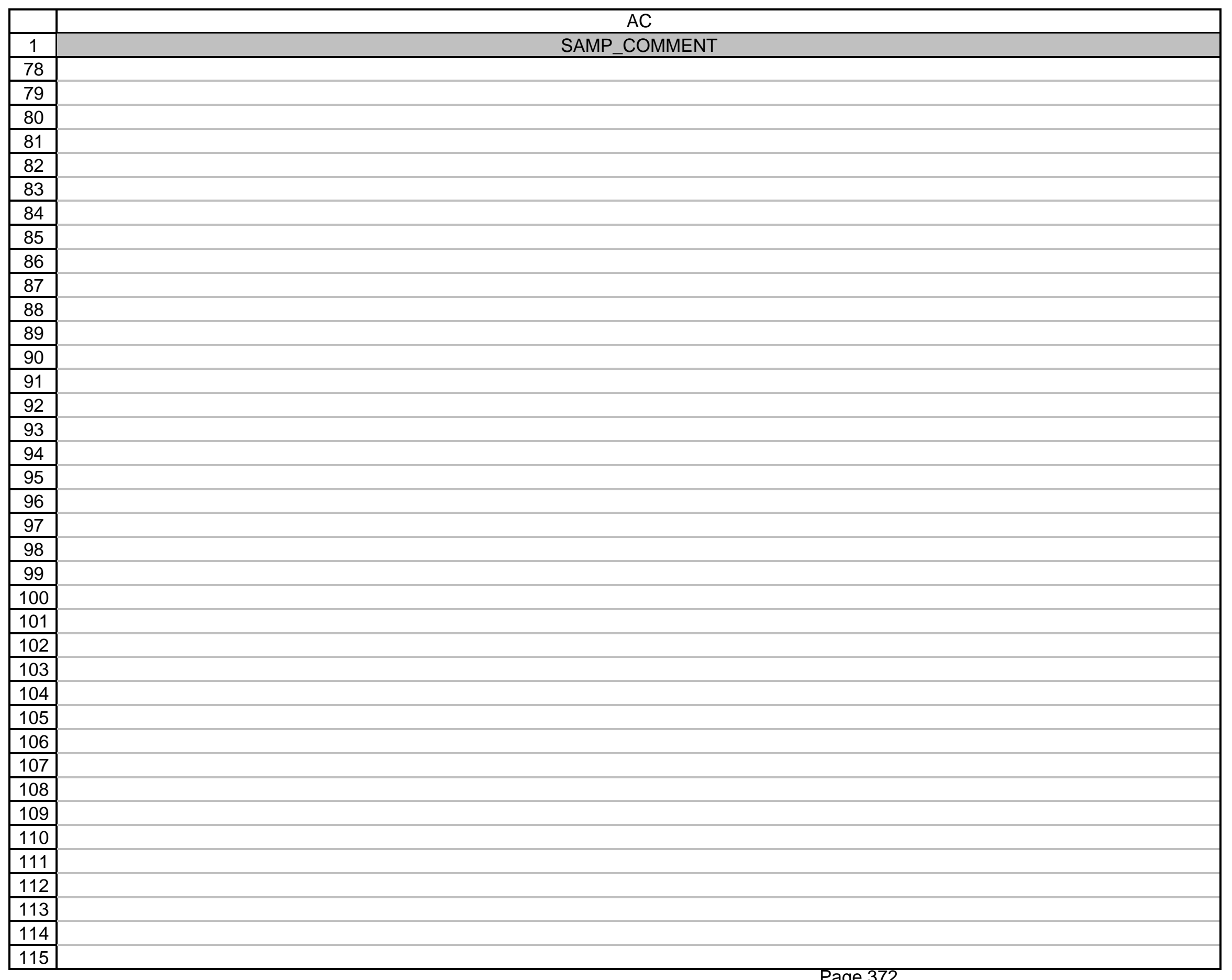




\begin{tabular}{|c|c|}
\hline 1 & SAMP COMMENT \\
\hline 116 & \\
\hline 117 & REFER TO FOLLOW-UP ANALYSIS REQUEST \#W004 REGARDING U-ISO, UNRESOLVED ANOMALOUS REPORT. \\
\hline 118 & REFER TO FOLLOW-UP ANALYSIS REQUEST \#W004 REGARDING U-ISO, UNRESOLVED ANOMALOUS REPORT. \\
\hline 119 & REFER TO FOLLOW-UP ANALYSIS REQUEST \#W004 REGARDING U-ISO, UNRESOLVED ANOMALOUS REPORT. \\
\hline 120 & REFER TO FOLLOW-UP ANALYSIS REQUEST \#W004 REGARDING U-ISO, UNRESOLVED ANOMALOUS REPORT. \\
\hline 121 & REFER TO FOLLOW-UP ANALYSIS REQUEST \#W004 REGARDING U-ISO, UNRESOLVED ANOMALOUS REPORT. \\
\hline 122 & \\
\hline 123 & \\
\hline 124 & \\
\hline 125 & \\
\hline 126 & \\
\hline 127 & \\
\hline 128 & \\
\hline 129 & \\
\hline 130 & \\
\hline 131 & \\
\hline 132 & \\
\hline 133 & \\
\hline 134 & \\
\hline 135 & \\
\hline 136 & \\
\hline \begin{tabular}{|l|}
137 \\
\end{tabular} & \\
\hline 138 & \\
\hline 139 & \\
\hline 140 & \\
\hline 141 & \\
\hline 142 & \\
\hline 143 & \\
\hline \begin{tabular}{|l|}
144 \\
\end{tabular} & \\
\hline 145 & \\
\hline \begin{tabular}{|l|}
146 \\
\end{tabular} & \\
\hline 147 & \\
\hline 148 & \\
\hline \begin{tabular}{|l|}
149 \\
\end{tabular} & \\
\hline 150 & \\
\hline 151 & \\
\hline 152 & \\
\hline 153 & \\
\hline
\end{tabular}


$A C$

\begin{tabular}{|c|c|}
\hline & AC \\
\hline 1 & SAMP_COMMENT \\
\hline 154 & \\
\hline 155 & \\
\hline 156 & \\
\hline 157 & \\
\hline 158 & \\
\hline 159 & \\
\hline 160 & \\
\hline 161 & \\
\hline 162 & \\
\hline 163 & \\
\hline 164 & \\
\hline 165 & \\
\hline 166 & \\
\hline 167 & \\
\hline 168 & \\
\hline 169 & \\
\hline 170 & \\
\hline 171 & \\
\hline 172 & \\
\hline 173 & \\
\hline 174 & \\
\hline 175 & \\
\hline 176 & \\
\hline 177 & REFER TO INCIDENT REPORT 2/14/92 REGARDING SR-90 DETECTION LIMIT. \\
\hline 178 & REFER TO INCIDENT REPORT 2/14/92 REGARDING SR-90 DETECTION LIMIT. \\
\hline 179 & REFER TO INCIDENT REPORT 2/14/92 REGARDING SR-90 DETECTION LIMIT. \\
\hline 180 & REFER TO INCIDENT REPORT 2/14/92 REGARDING SR-90 DETECTION LIMIT. \\
\hline 181 & REFER TO INCIDENT REPORT 2/14/92 REGARDING SR-90 DETECTION LIMIT. \\
\hline 182 & \\
\hline 183 & \\
\hline 184 & \\
\hline 185 & \\
\hline 186 & \\
\hline 187 & \\
\hline 188 & \\
\hline 189 & \\
\hline 190 & \\
\hline 191 & \\
\hline
\end{tabular}




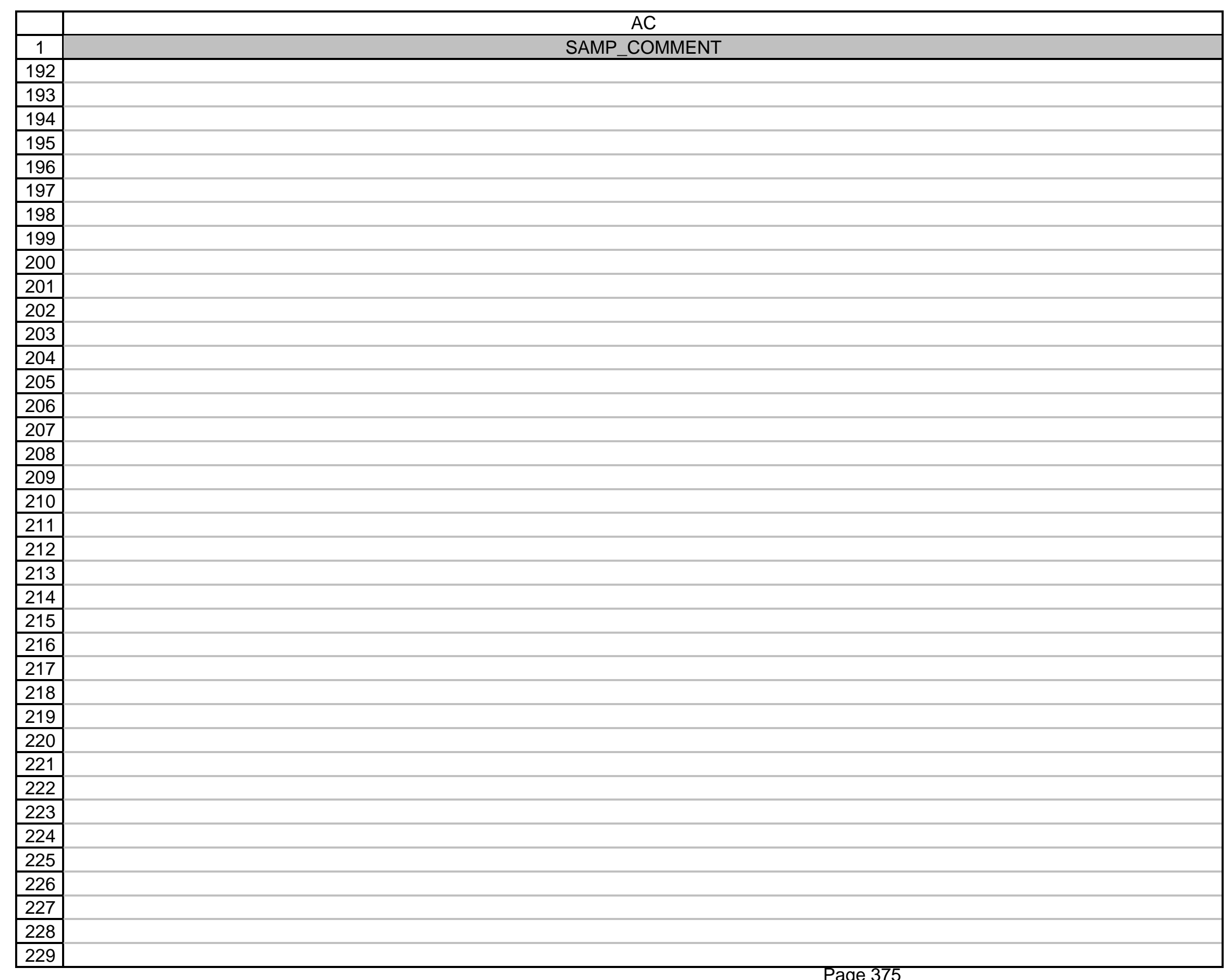




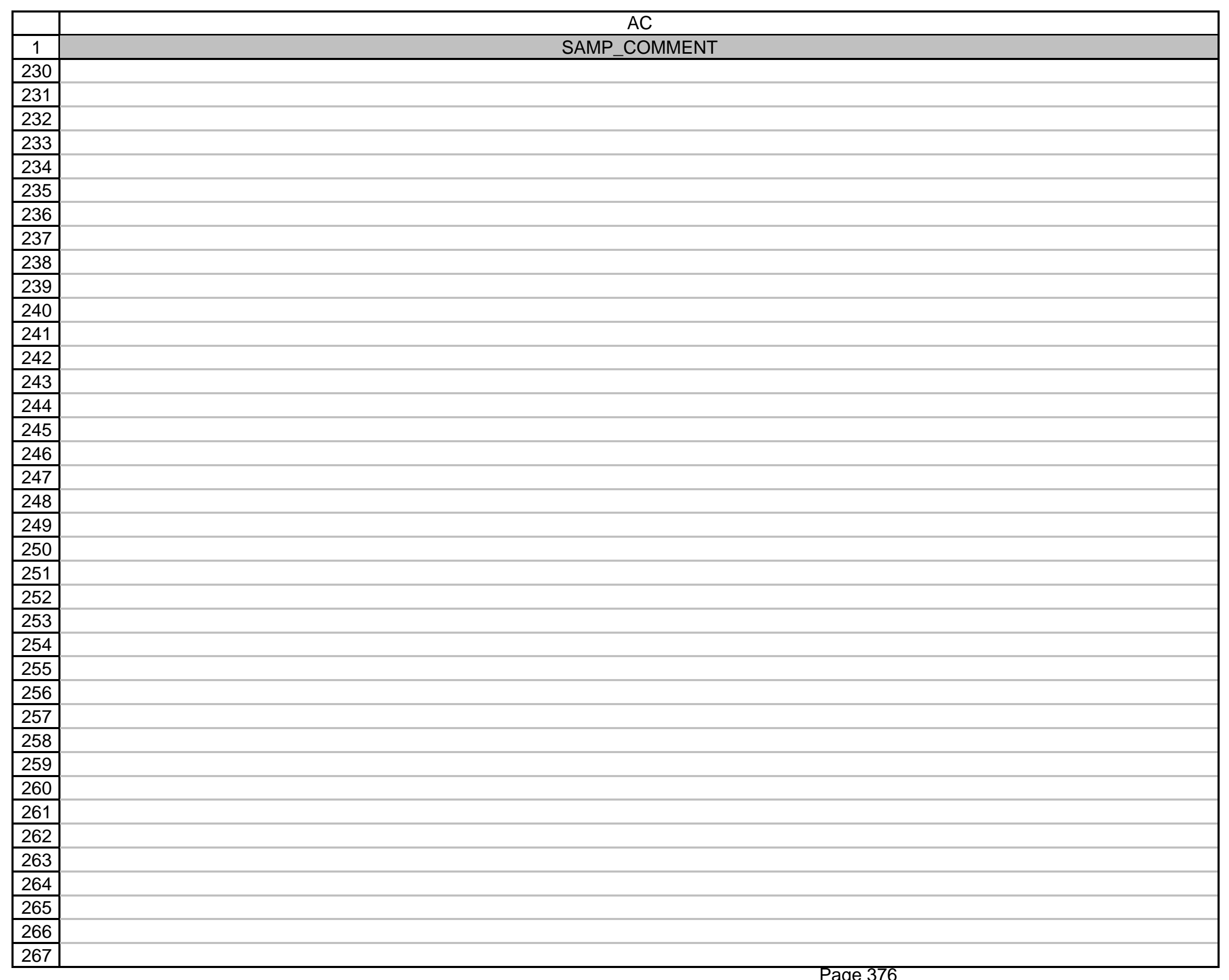


$A C$

\begin{tabular}{|c|c|}
\hline & 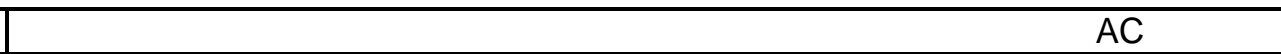 \\
\hline 1 & SAMP_COMMENT \\
\hline 268 & \\
\hline 269 & \\
\hline 270 & \\
\hline 271 & \\
\hline$\overline{272}$ & \\
\hline 273 & \\
\hline 274 & \\
\hline 275 & \\
\hline 276 & \\
\hline 277 & \\
\hline$\overline{278}$ & \\
\hline 279 & \\
\hline 280 & \\
\hline 281 & REFER TO INCIDENT REPORT 7/23/92 REGARDING ISO. URANIUM MDC. \\
\hline 282 & REFER TO INCIDENT REPORT 7/23/92 REGARDING ISO. URANIUM MDC. \\
\hline 283 & REFER TO INCIDENT REPORT 7/23/92 REGARDING ISO. URANIUM MDC. \\
\hline 284 & REFER TO INCIDENT REPORT 7/23/92 REGARDING ISO. URANIUM MDC. \\
\hline 285 & REFER TO INCIDENT REPORT 7/23/92 REGARDING ISO. URANIUM MDC. \\
\hline 286 & \\
\hline 287 & \\
\hline 288 & \\
\hline 289 & \\
\hline 290 & \\
\hline 291 & \\
\hline 292 & \\
\hline 293 & \\
\hline 294 & \\
\hline 295 & \\
\hline 296 & \\
\hline 297 & \\
\hline 298 & \\
\hline 299 & \\
\hline 300 & \\
\hline 301 & \\
\hline 302 & \\
\hline 303 & \\
\hline 304 & \\
\hline 305 & \\
\hline
\end{tabular}




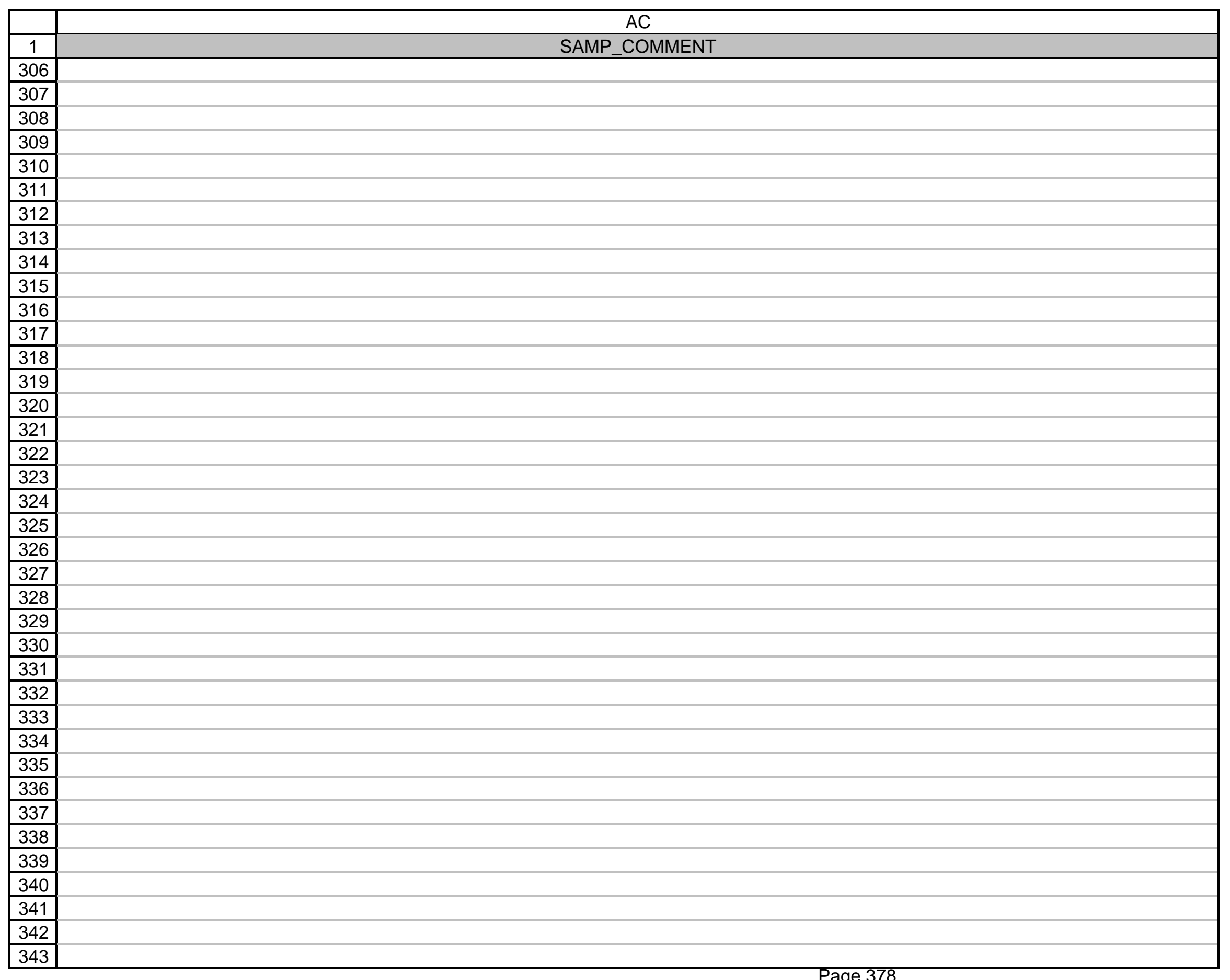




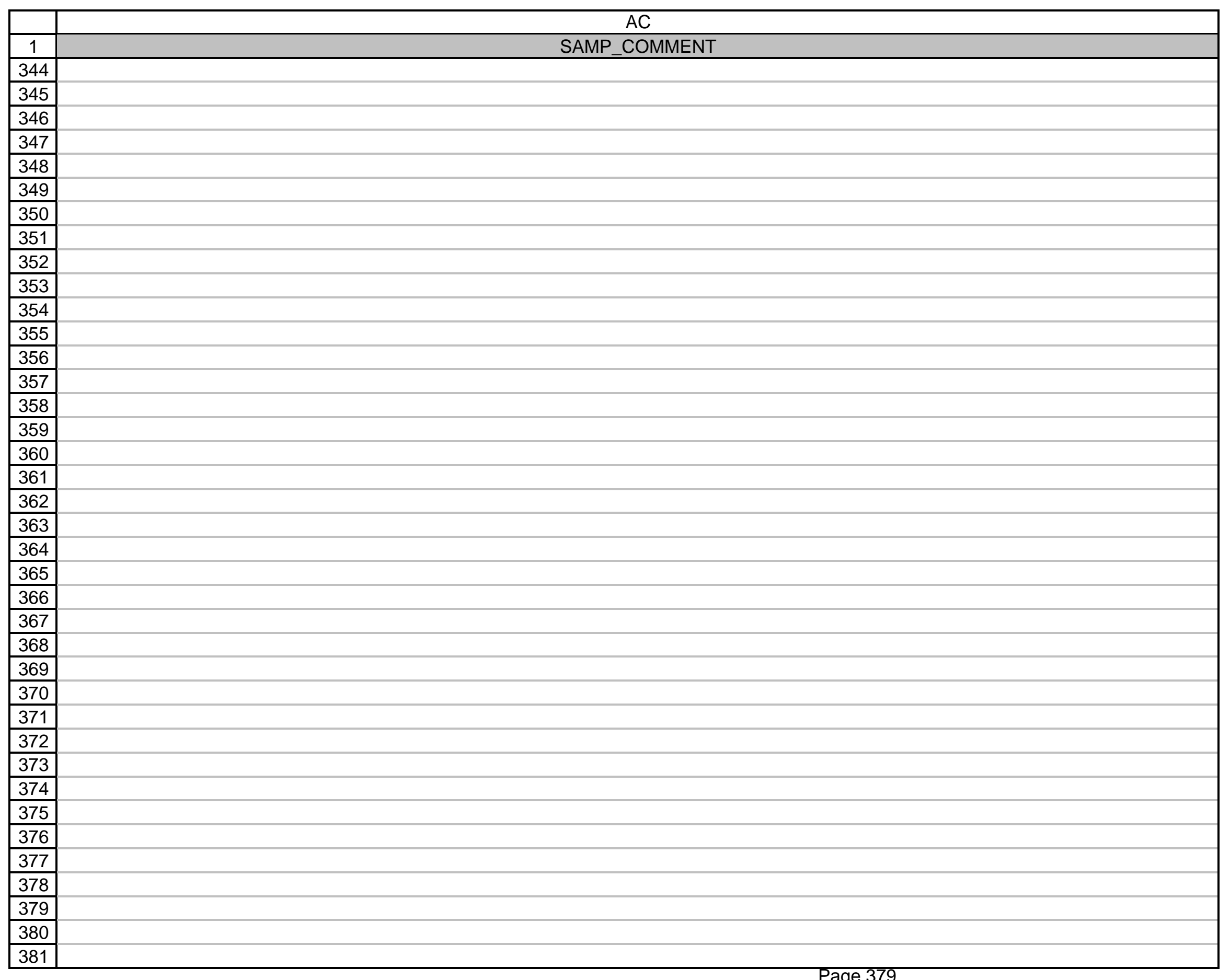


$A C$

\begin{tabular}{|c|c|}
\hline & $\begin{array}{c}\mathrm{AC} \\
\end{array}$ \\
\hline 1 & $\begin{array}{ll}\text { SAMP_COMMENT } \\
\end{array}$ \\
\hline 382 & \\
\hline 383 & \\
\hline 384 & \\
\hline 385 & \\
\hline 386 & \\
\hline 387 & \\
\hline 388 & \\
\hline 389 & \\
\hline 390 & \\
\hline 391 & \\
\hline$\overline{392}$ & \\
\hline 393 & \\
\hline 394 & \\
\hline 395 & \\
\hline 396 & \\
\hline 397 & \\
\hline 398 & \\
\hline 399 & \\
\hline 400 & \\
\hline 401 & \\
\hline 402 & \\
\hline 403 & \\
\hline 404 & \\
\hline 405 & \\
\hline 406 & \\
\hline 407 & \\
\hline 408 & \\
\hline 409 & \\
\hline 410 & \\
\hline 411 & REFER TO 10/29/92 INCIDENT REPORT REGARDING LOST LO H-3. \\
\hline 412 & REFER TO 10/29/92 INCIDENT REPORT REGARDING LOST LO H-3. \\
\hline 413 & REFER TO 10/29/92 INCIDENT REPORT REGARDING LOST LO H-3. \\
\hline 414 & REFER TO 10/29/92 INCIDENT REPORT REGARDING LOST LO H-3. \\
\hline 415 & REFER TO 10/29/92 INCIDENT REPORT REGARDING LOST LO H-3. \\
\hline 416 & \\
\hline 417 & \\
\hline 418 & \\
\hline 419 & \\
\hline
\end{tabular}


$A C$

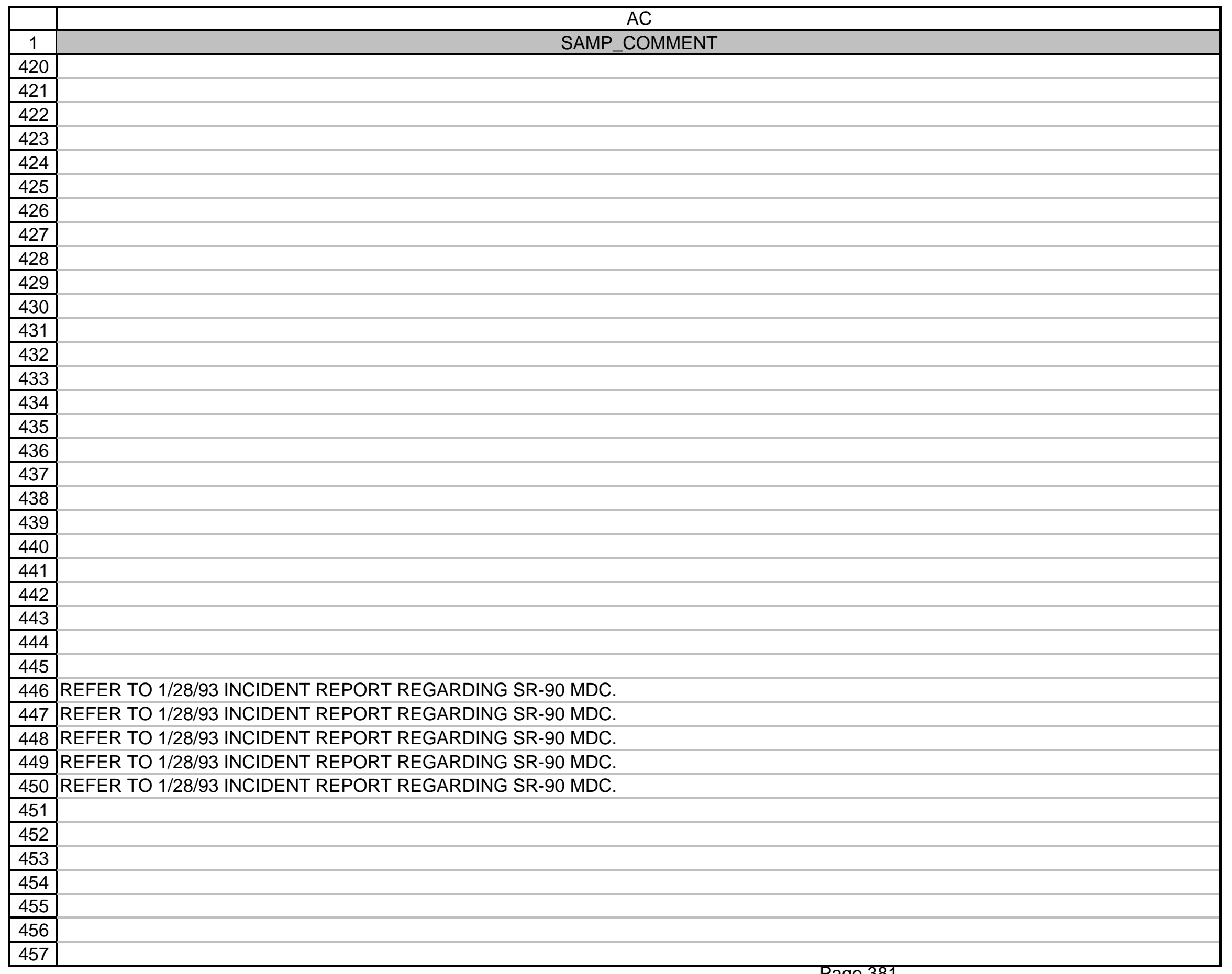




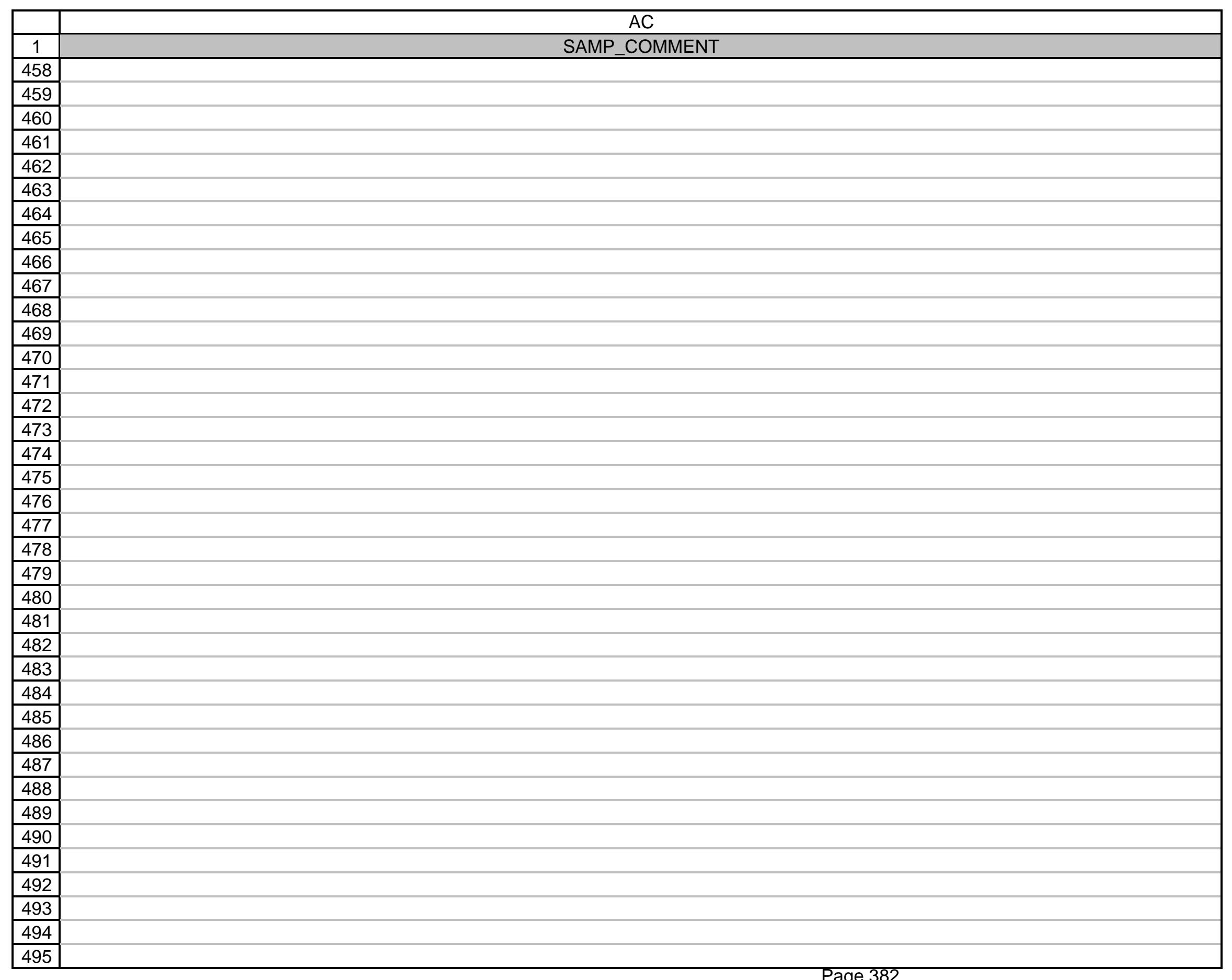


$A C$

\begin{tabular}{|c|c|}
\hline & $\begin{array}{c}\mathrm{AC} \\
\end{array}$ \\
\hline 1 & $\begin{array}{ll}\text { SAMP_COMMENT } \\
\end{array}$ \\
\hline 496 & \\
\hline 497 & \\
\hline 498 & \\
\hline 499 & \\
\hline 500 & \\
\hline 501 & \\
\hline 502 & \\
\hline 503 & \\
\hline 504 & \\
\hline 505 & \\
\hline$\overline{506}$ & \\
\hline 507 & \\
\hline 508 & \\
\hline 509 & \\
\hline 510 & \\
\hline 511 & \\
\hline 512 & \\
\hline 513 & \\
\hline 514 & \\
\hline 515 & \\
\hline$\overline{516}$ & \\
\hline 517 & \\
\hline 518 & \\
\hline 519 & \\
\hline 520 & \\
\hline 521 & REFER TO INCIDENT REPORT 4/29/93 REGARDING ISOTOPIC URANIUM MDC. \\
\hline 522 & REFER TO INCIDENT REPORT 4/29/93 REGARDING ISOTOPIC URANIUM MDC. \\
\hline 523 & REFER TO INCIDENT REPORT 4/29/93 REGARDING ISOTOPIC URANIUM MDC. \\
\hline 524 & REFER TO INCIDENT REPORT 4/29/93 REGARDING ISOTOPIC URANIUM MDC. \\
\hline 525 & REFER TO INCIDENT REPORT 4/29/93 REGARDING ISOTOPIC URANIUM MDC. \\
\hline 526 & \\
\hline 527 & \\
\hline 528 & \\
\hline 529 & \\
\hline 530 & \\
\hline 531 & \\
\hline 532 & \\
\hline 533 & \\
\hline
\end{tabular}




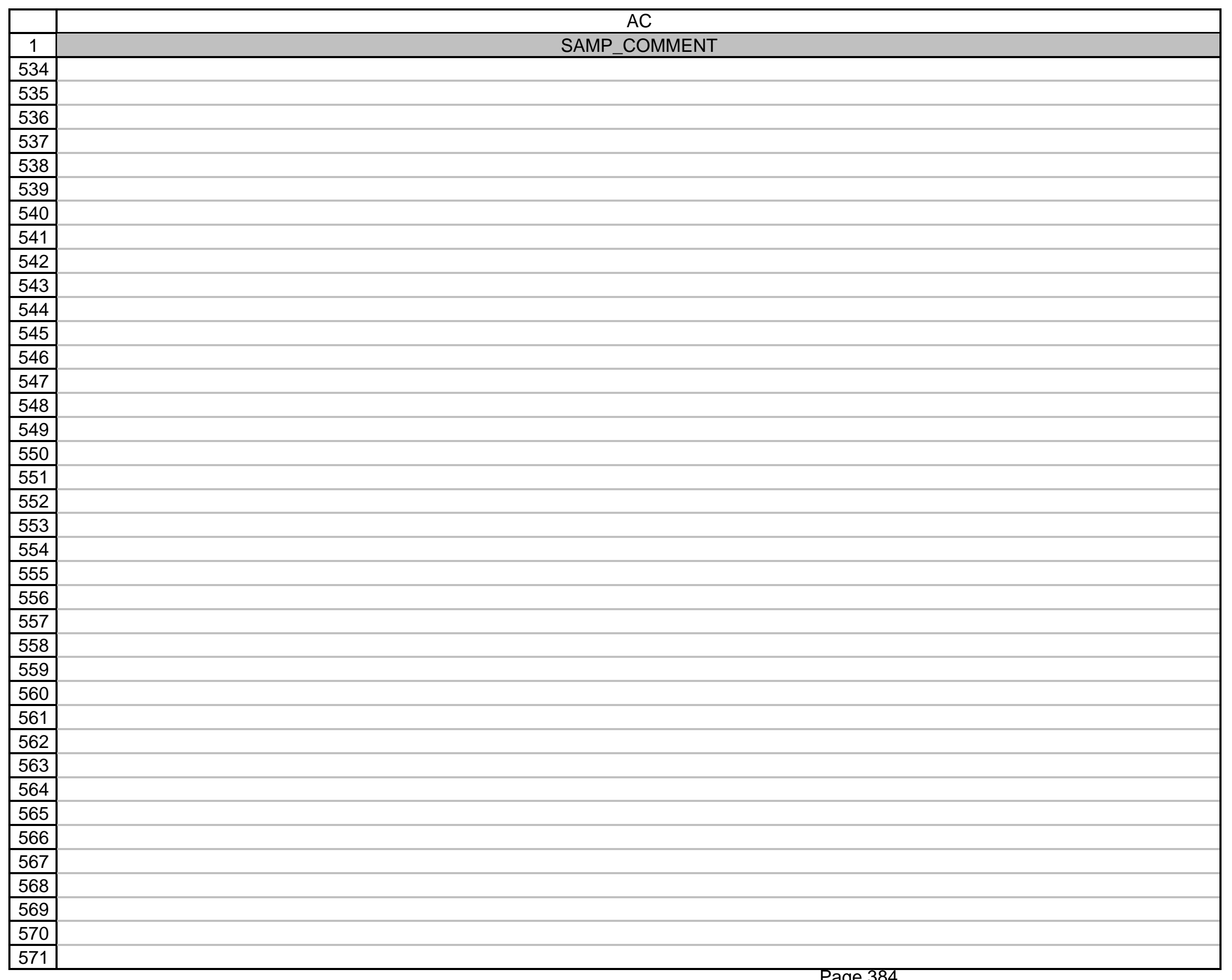




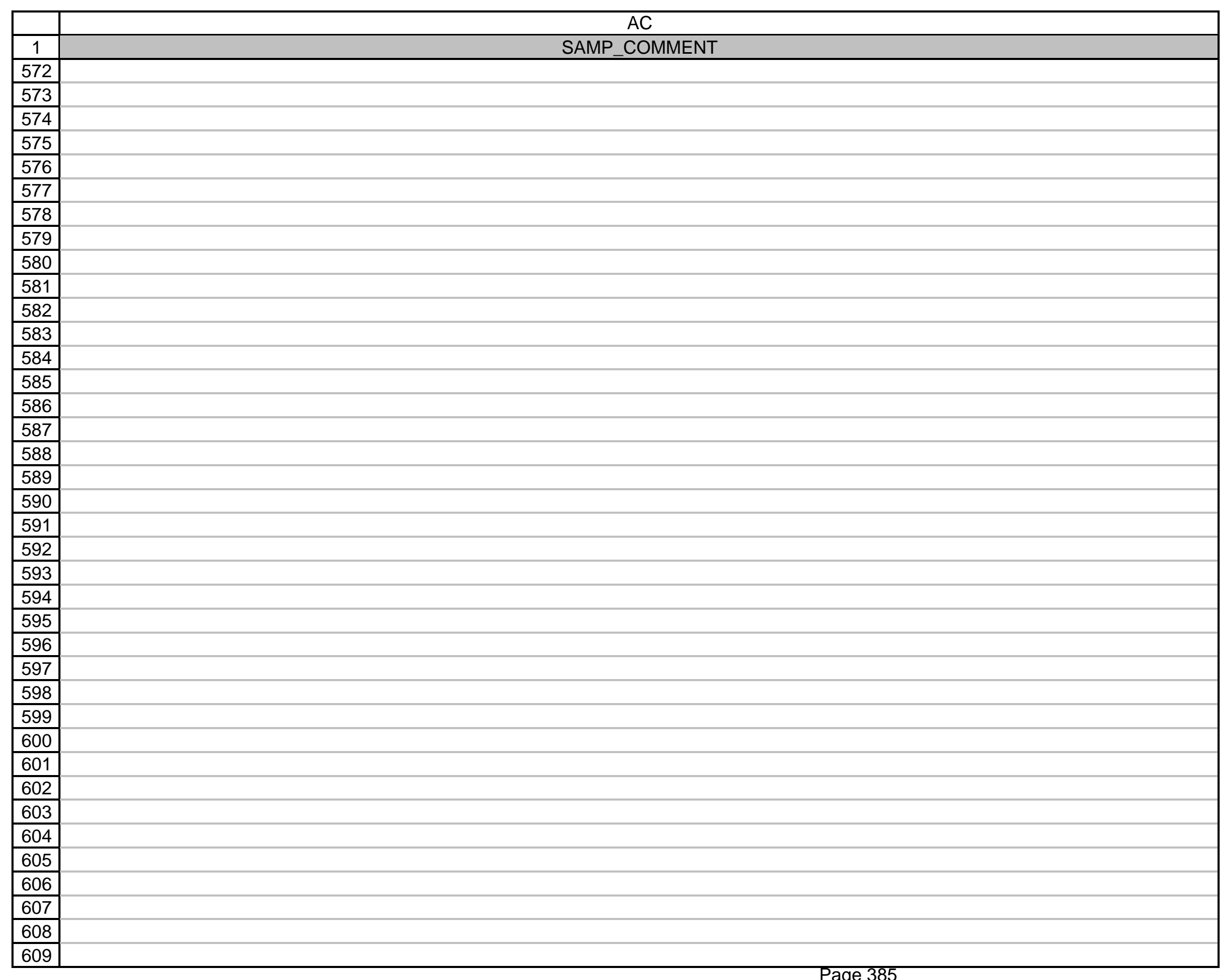


AC

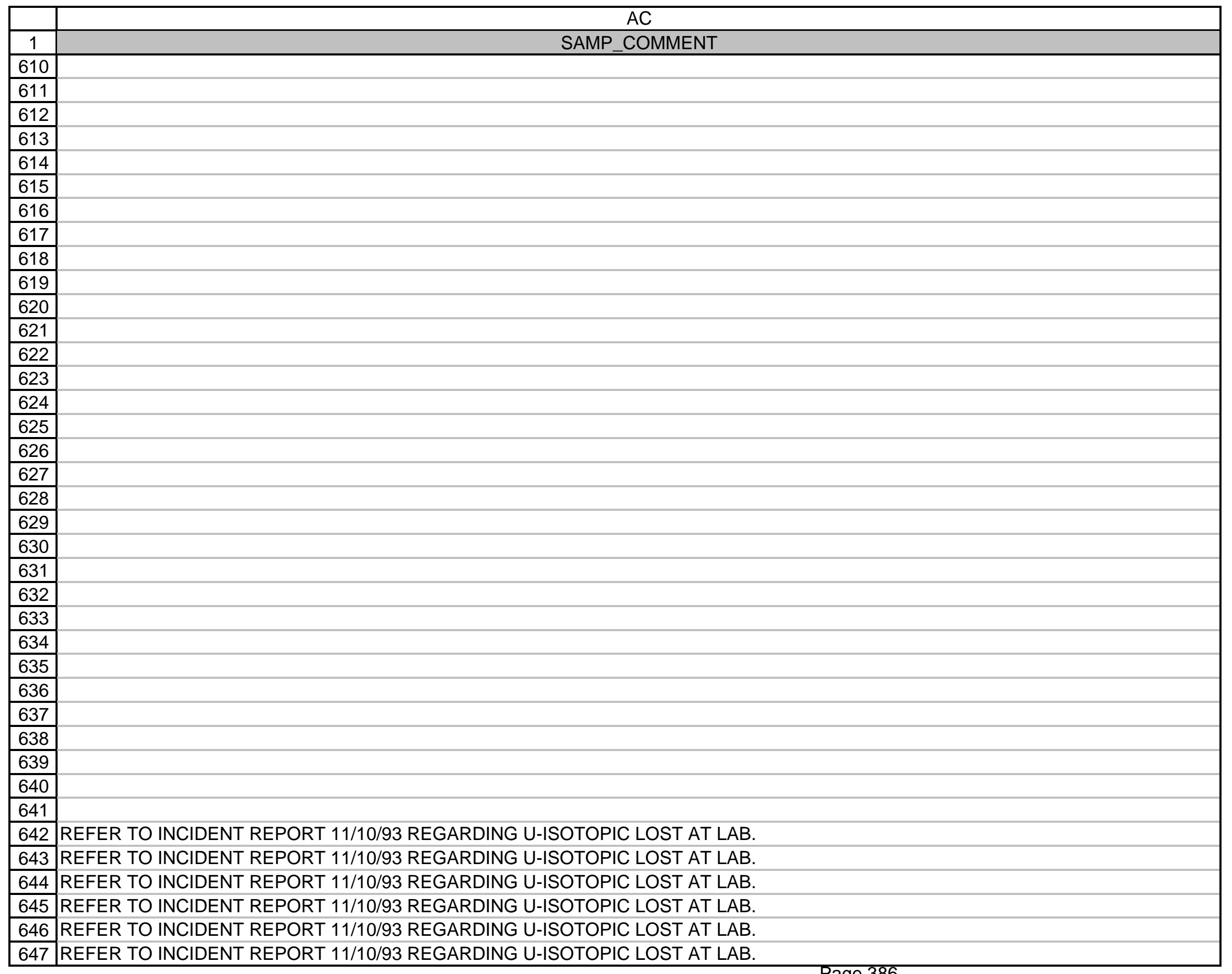


AC

\begin{tabular}{|c|c|}
\hline 1 & SAMP COMMENT \\
\hline 648 & REFER TO 11/3/93 INCIDENT REPORT REGARDING LOST SR-90. \\
\hline 649 & REFER TO 11/3/93 INCIDENT REPORT REGARDING LOST SR-90. \\
\hline 650 & REFER TO 11/3/93 INCIDENT REPORT REGARDING LOST SR-90. \\
\hline 651 & REFER TO 11/3/93 INCIDENT REPORT REGARDING LOST SR-90. \\
\hline 652 & REFER TO 11/3/93 INCIDENT REPORT REGARDING LOST SR-90. \\
\hline 653 & REFER TO 11/3/93 INCIDENT REPORT REGARDING LOST SR-90. \\
\hline 654 & \\
\hline 655 & \\
\hline 656 & \\
\hline 657 & \\
\hline 658 & \\
\hline 659 & \\
\hline 660 & \\
\hline 661 & \\
\hline 662 & \\
\hline 663 & \\
\hline 664 & \\
\hline 665 & \\
\hline 666 & \\
\hline 667 & \\
\hline 668 & \\
\hline 669 & \\
\hline 670 & \\
\hline 671 & \\
\hline 672 & \\
\hline 673 & \\
\hline 674 & \\
\hline 675 & \\
\hline 676 & \\
\hline 677 & \\
\hline 678 & \\
\hline 679 & \\
\hline 680 & \\
\hline 681 & \\
\hline 682 & \\
\hline 683 & \\
\hline 684 & \\
\hline 685 & \\
\hline
\end{tabular}


AC

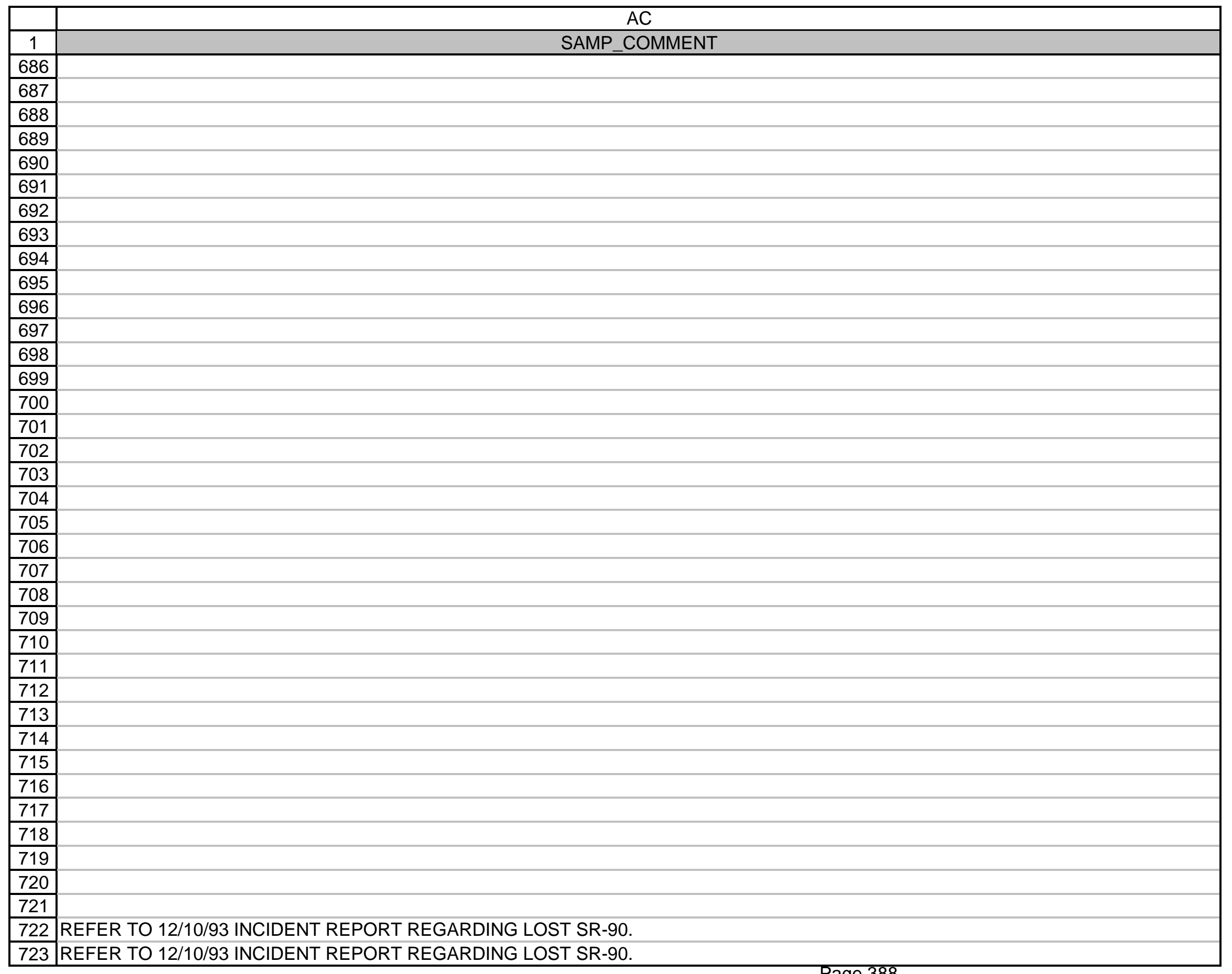




\begin{tabular}{|c|c|}
\hline 1 & SAMP_COMMENT \\
\hline 724 & REFER TO 12/10/93 INCIDENT REPORT REGARDING LOST SR-90. \\
\hline 725 & REFER TO 12/10/93 INCIDENT REPORT REGARDING LOST SR-90. \\
\hline 726 & REFER TO 12/10/93 INCIDENT REPORT REGARDING LOST SR-90. \\
\hline 727 & REFER TO 12/10/93 INCIDENT REPORT REGARDING LOST SR-90. \\
\hline 728 & REFER TO 12/10/93 INCIDENT REPORT REGARDING LOST SR-90. \\
\hline 729 & REFER TO 12/10/93 INCIDENT REPORT REGARDING LOST SR-90. \\
\hline 730 & REFER TO 12/10/93 INCIDENT REPORT REGARDING LOST SR-90. \\
\hline 731 & REFER TO 12/10/93 INCIDENT REPORT REGARDING LOST SR-90. \\
\hline 732 & REFER TO 12/10/93 INCIDENT REPORT REGARDING LOST SR-90. \\
\hline 733 & REFER TO 12/10/93 INCIDENT REPORT REGARDING LOST SR-90. \\
\hline 734 & \\
\hline 735 & \\
\hline 736 & \\
\hline 737 & \\
\hline 738 & \\
\hline 739 & REFER TO 12/10/93 INCIDENT REPORT REGARDING LOST SR-90. \\
\hline 740 & REFER TO 12/10/93 INCIDENT REPORT REGARDING LOST SR-90. \\
\hline 741 & REFER TO 12/10/93 INCIDENT REPORT REGARDING LOST SR-90. \\
\hline 742 & REFER TO 12/10/93 INCIDENT REPORT REGARDING LOST SR-90. \\
\hline 743 & REFER TO 12/10/93 INCIDENT REPORT REGARDING LOST SR-90. \\
\hline 744 & \\
\hline 745 & \\
\hline 746 & \\
\hline 747 & \\
\hline 748 & \\
\hline 749 & \\
\hline 750 & \\
\hline 751 & \\
\hline 752 & \\
\hline 753 & \\
\hline 754 & \\
\hline 755 & \\
\hline 756 & \\
\hline 757 & \\
\hline 758 & \\
\hline 759 & \\
\hline 760 & \\
\hline 761 & \\
\hline
\end{tabular}


AC

\begin{tabular}{|c|c|}
\hline & AC \\
\hline 1 & SAMP_COMMENT \\
\hline 762 & \\
\hline 763 & \\
\hline 764 & \\
\hline 765 & \\
\hline 766 & \\
\hline 767 & \\
\hline 768 & \\
\hline 769 & \\
\hline 770 & \\
\hline 771 & \\
\hline 772 & \\
\hline 773 & \\
\hline 774 & REFER TO INCIDENT REPORT 11/11/93 REGARDING INCORRECT ALPHA SPEC DETECTOR BACKGROUND USED FOR U-ISOTOPIC. \\
\hline 775 & REFER TO INCIDENT REPORT 11/11/93 REGARDING INCORRECT ALPHA SPEC DETECTOR BACKGROUND USED FOR U-ISOTOPIC. \\
\hline 776 & REFER TO INCIDENT REPORT 11/11/93 REGARDING INCORRECT ALPHA SPEC DETECTOR BACKGROUND USED FOR U-ISOTOPIC. \\
\hline 777 & REFER TO INCIDENT REPORT 11/11/93 REGARDING INCORRECT ALPHA SPEC DETECTOR BACKGROUND USED FOR U-ISOTOPIC. \\
\hline 778 & REFER TO INCIDENT REPORT 11/11/93 REGARDING INCORRECT ALPHA SPEC DETECTOR BACKGROUND USED FOR U-ISOTOPIC. \\
\hline 779 & REFER TO INCIDENT REPORT 11/11/93 REGARDING INCORRECT ALPHA SPEC DETECTOR BACKGROUND USED FOR U-ISOTOPIC. \\
\hline 780 & NO SAMPLE. \\
\hline 781 & NO SAMPLE. \\
\hline 782 & NO SAMPLE. \\
\hline 783 & NO SAMPLE. \\
\hline 784 & NO SAMPLE. \\
\hline 785 & NO SAMPLE. \\
\hline 786 & \\
\hline 787 & \\
\hline 788 & \\
\hline 789 & \\
\hline 790 & \\
\hline 791 & \\
\hline 792 & JNO SAMPLE. \\
\hline 793 & NO SAMPLE. \\
\hline 794 & NO SAMPLE. \\
\hline 795 & NO SAMPLE. \\
\hline 796 & NO SAMPLE. \\
\hline 797 & NO SAMPLE. \\
\hline 798 & NO SAMPLE. \\
\hline 799 & NO SAMPLE. \\
\hline
\end{tabular}


$A C$

\begin{tabular}{|c|c|}
\hline & 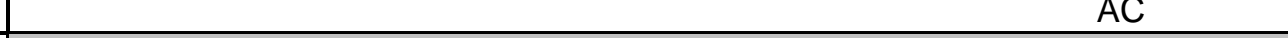 \\
\hline 1 & SAMP_COMMENT \\
\hline 800 & NO SAMPLE. \\
\hline 801 & NO SAMPLE. \\
\hline 802 & NO SAMPLE. \\
\hline 803 & NO SAMPLE. \\
\hline 804 & NO SAMPLE. \\
\hline 805 & NO SAMPLE. \\
\hline 806 & NO SAMPLE. \\
\hline 807 & NO SAMPLE. \\
\hline 808 & NO SAMPLE. \\
\hline 809 & NO SAMPLE. \\
\hline 810 & NO SAMPLE. \\
\hline 811 & NO SAMPLE. \\
\hline 812 & NO SAMPLE. \\
\hline 813 & NO SAMPLE. \\
\hline 814 & NO SAMPLE. \\
\hline 815 & NO SAMPLE. \\
\hline 816 & NO SAMPLE. \\
\hline 817 & NO SAMPLE. \\
\hline 818 & NO SAMPLE. \\
\hline 819 & NO SAMPLE. \\
\hline \begin{tabular}{|l|}
820 \\
\end{tabular} & NO SAMPLE. \\
\hline 821 & NO SAMPLE. \\
\hline 822 & NO SAMPLE. \\
\hline 823 & NO SAMPLE. \\
\hline \begin{tabular}{|l|}
824 \\
\end{tabular} & NO SAMPLE. \\
\hline 825 & NO SAMPLE. \\
\hline 826 & NO SAMPLE. \\
\hline 827 & NO SAMPLE. \\
\hline 828 & NO SAMPLE. \\
\hline 829 & NO SAMPLE. \\
\hline 830 & NO SAMPLE. \\
\hline 831 & NO SAMPLE. \\
\hline \begin{tabular}{|l|}
832 \\
\end{tabular} & NO SAMPLE. \\
\hline 833 & NO SAMPLE. \\
\hline 834 & NO SAMPLE. \\
\hline 835 & NO SAMPLE. \\
\hline 836 & NO SAMPLE. \\
\hline \begin{tabular}{|l|}
837 \\
\end{tabular} & NO SAMPLE. \\
\hline
\end{tabular}


AC

\begin{tabular}{|c|c|}
\hline 1 & SAMP_COMMENT \\
\hline 838 & NO SAMPLE. \\
\hline 839 & NO SAMPLE. \\
\hline 840 & NO SAMPLE. \\
\hline 841 & NO SAMPLE. \\
\hline 842 & NO SAMPLE. \\
\hline 843 & NO SAMPLE. \\
\hline 844 & NO SAMPLE. \\
\hline 845 & NO SAMPLE. \\
\hline 846 & \\
\hline 847 & \\
\hline 848 & \\
\hline 849 & \\
\hline 850 & \\
\hline 851 & REFER TO INCIDENT REPORT 11/11/93 REGARDING INCORRECT ALPHA SPEC DETECTOR BACKGROUND USED FOR U-ISOTOPIC. \\
\hline 852 & REFER TO INCIDENT REPORT 11/11/93 REGARDING INCORRECT ALPHA SPEC DETECTOR BACKGROUND USED FOR U-ISOTOPIC. \\
\hline 853 & REFER TO INCIDENT REPORT 11/11/93 REGARDING INCORRECT ALPHA SPEC DETECTOR BACKGROUND USED FOR U-ISOTOPIC. \\
\hline 854 & REFER TO INCIDENT REPORT 11/11/93 REGARDING INCORRECT ALPHA SPEC DETECTOR BACKGROUND USED FOR U-ISOTOPIC. \\
\hline 855 & REFER TO INCIDENT REPORT 11/11/93 REGARDING INCORRECT ALPHA SPEC DETECTOR BACKGROUND USED FOR U-ISOTOPIC. \\
\hline 856 & REFER TO 12/10/93 INCIDENT REPORT REGARDING LOST SR-90. \\
\hline 857 & REFER TO 12/10/93 INCIDENT REPORT REGARDING LOST SR-90. \\
\hline 858 & REFER TO 12/10/93 INCIDENT REPORT REGARDING LOST SR-90. \\
\hline 859 & REFER TO 12/10/93 INCIDENT REPORT REGARDING LOST SR-90. \\
\hline 860 & REFER TO 12/10/93 INCIDENT REPORT REGARDING LOST SR-90. \\
\hline 861 & \\
\hline 862 & \\
\hline 863 & \\
\hline 864 & \\
\hline 865 & \\
\hline 866 & \\
\hline 867 & \\
\hline 868 & \\
\hline 869 & \\
\hline 870 & \\
\hline 871 & \\
\hline \begin{tabular}{|l|}
872 \\
\end{tabular} & \\
\hline 873 & \\
\hline 874 & \\
\hline \begin{tabular}{|l|}
875 \\
\end{tabular} & \\
\hline
\end{tabular}


AC

\begin{tabular}{|c|c|}
\hline & $\pi$ \\
\hline 1 & $\begin{array}{r}\text { SAMP_COMMENT } \\
\end{array}$ \\
\hline 876 & \\
\hline 877 & \\
\hline 878 & \\
\hline 879 & \\
\hline 880 & \\
\hline 881 & \\
\hline 882 & \\
\hline 883 & \\
\hline 884 & \\
\hline 885 & \\
\hline 886 & \\
\hline 887 & \\
\hline 888 & \\
\hline 889 & \\
\hline 890 & \\
\hline 891 & \\
\hline 892 & \\
\hline 893 & \\
\hline 894 & \\
\hline 895 & \\
\hline 896 & NO SAMPLE. \\
\hline 897 & NO SAMPLE. \\
\hline 898 & NO SAMPLE. \\
\hline 899 & NO SAMPLE. \\
\hline 900 & NO SAMPLE. \\
\hline 901 & NO SAMPLE. \\
\hline 902 & NO SAMPLE. \\
\hline 903 & NO SAMPLE. \\
\hline 904 & NO SAMPLE. \\
\hline 905 & NO SAMPLE. \\
\hline 906 & NO SAMPLE. \\
\hline 907 & NO SAMPLE. \\
\hline 908 & NO SAMPLE. \\
\hline 909 & NO SAMPLE. \\
\hline 910 & NO SAMPLE. \\
\hline 911 & NO SAMPLE. \\
\hline 912 & NO SAMPLE. \\
\hline 913 & NO SAMPLE. \\
\hline
\end{tabular}


$A C$

\begin{tabular}{|c|c|}
\hline & \\
\hline 1 & $\begin{array}{r}\text { SAMP_COMMENT } \\
\end{array}$ \\
\hline 914 & NO SAMPLE. \\
\hline 915 & NO SAMPLE. \\
\hline 916 & NO SAMPLE. \\
\hline 917 & NO SAMPLE. \\
\hline 918 & NO SAMPLE. \\
\hline 919 & NO SAMPLE. \\
\hline 920 & NO SAMPLE. \\
\hline 921 & NO SAMPLE. \\
\hline 922 & NO SAMPLE. \\
\hline 923 & NO SAMPLE. \\
\hline 924 & NO SAMPLE. \\
\hline 925 & NO SAMPLE. \\
\hline 926 & NO SAMPLE. \\
\hline 927 & NO SAMPLE. \\
\hline 928 & NO SAMPLE. \\
\hline 929 & NO SAMPLE. \\
\hline 930 & NO SAMPLE. \\
\hline 931 & NO SAMPLE. \\
\hline 932 & NO SAMPLE. \\
\hline 933 & NO SAMPLE. \\
\hline 934 & NO SAMPLE. \\
\hline 935 & NO SAMPLE. \\
\hline 936 & NO SAMPLE. \\
\hline 937 & NO SAMPLE. \\
\hline 938 & NO SAMPLE. \\
\hline 939 & NO SAMPLE. \\
\hline 940 & NO SAMPLE. \\
\hline 941 & NO SAMPLE. \\
\hline 942 & NO SAMPLE. \\
\hline 943 & NO SAMPLE. \\
\hline 944 & NO SAMPLE. \\
\hline 945 & \\
\hline 946 & \\
\hline 947 & \\
\hline 948 & \\
\hline 949 & \\
\hline 950 & \\
\hline 951 & \\
\hline
\end{tabular}




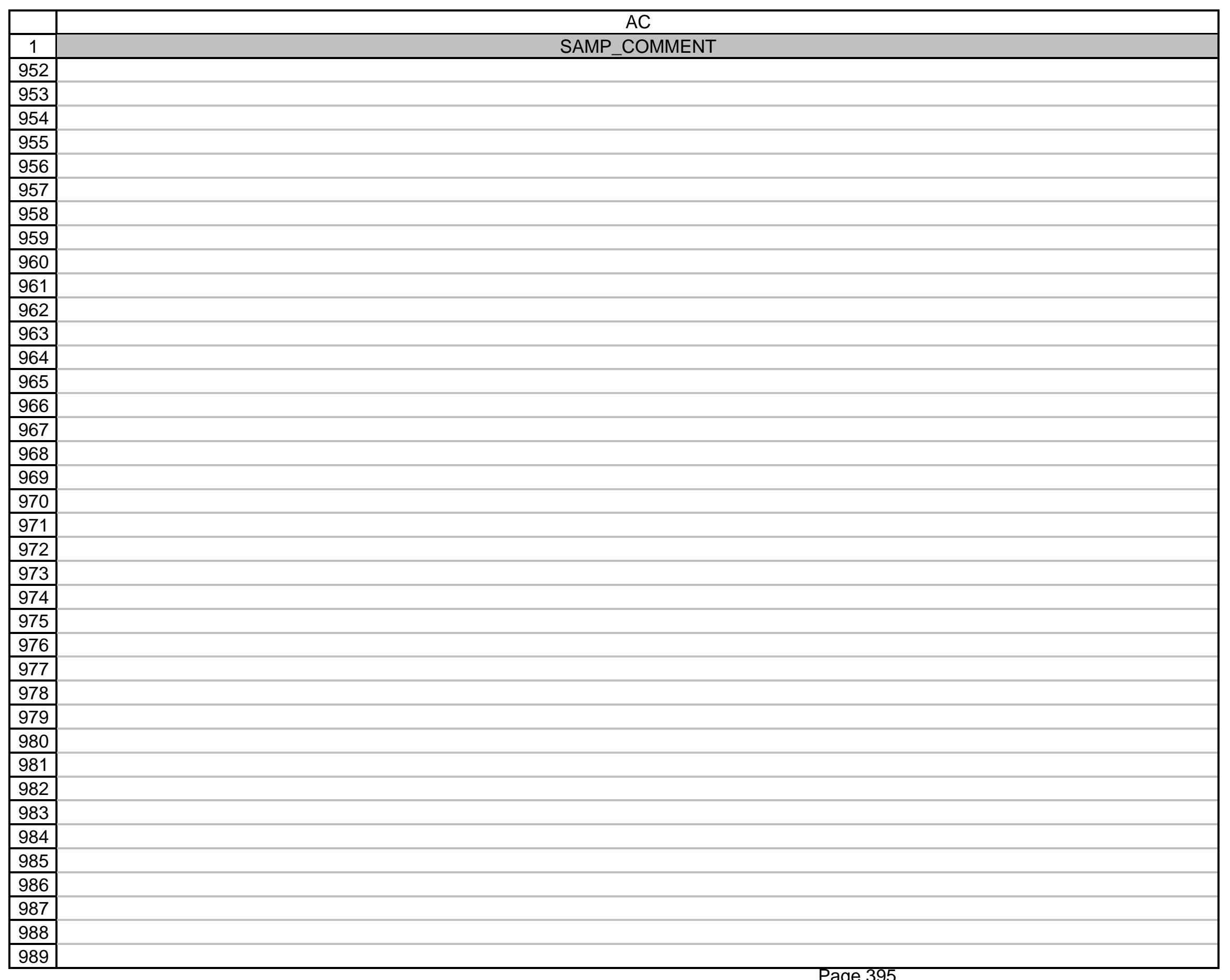




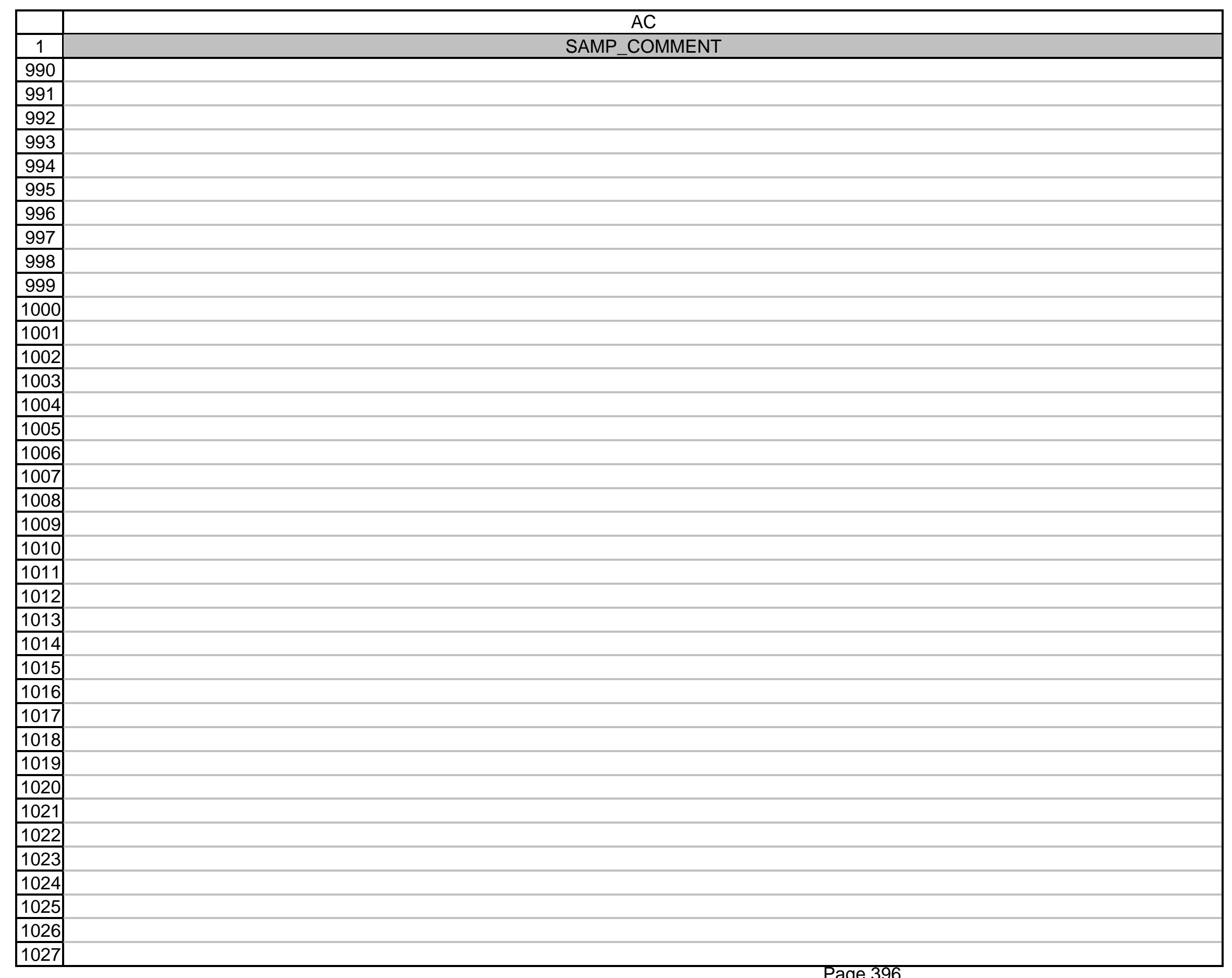




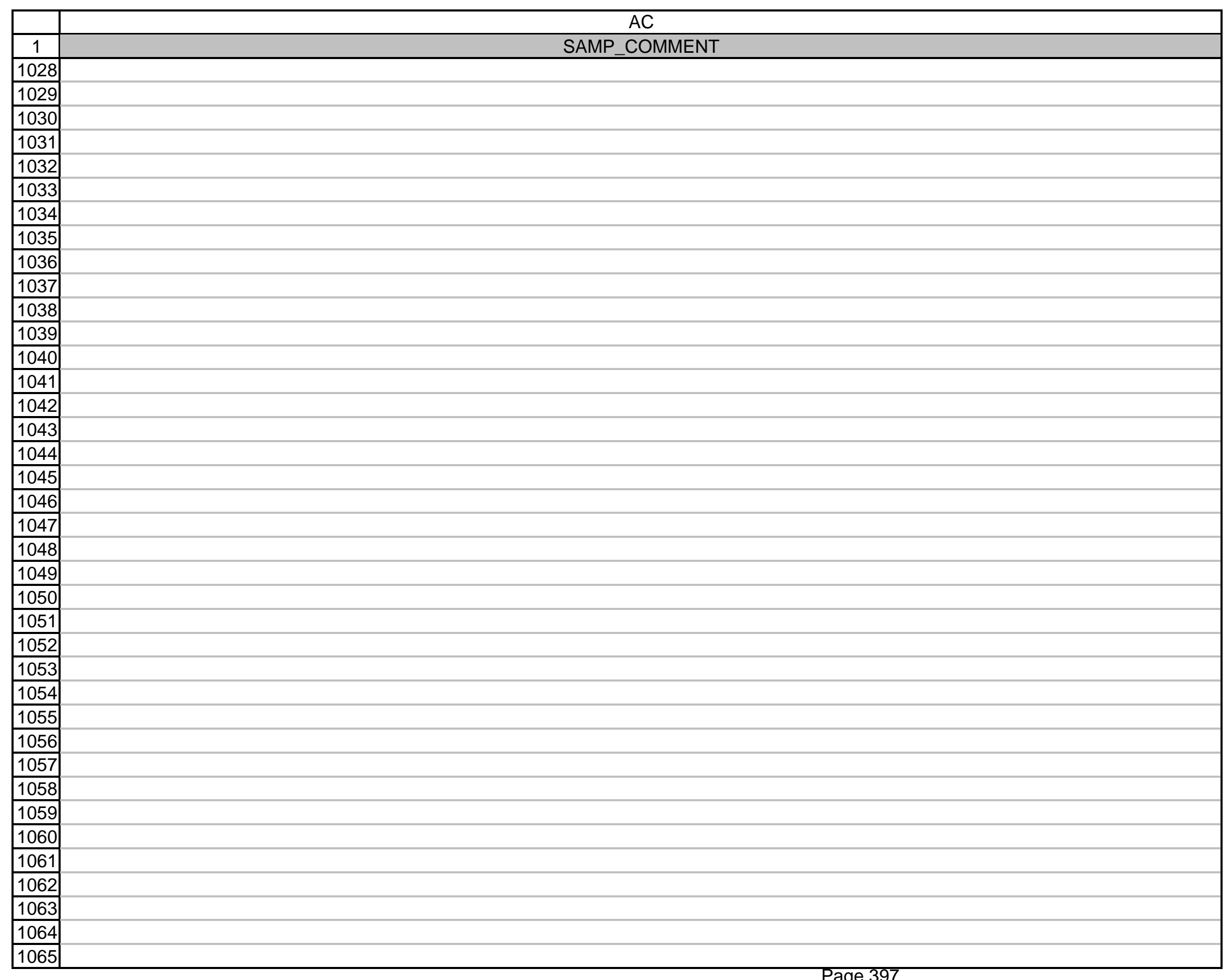




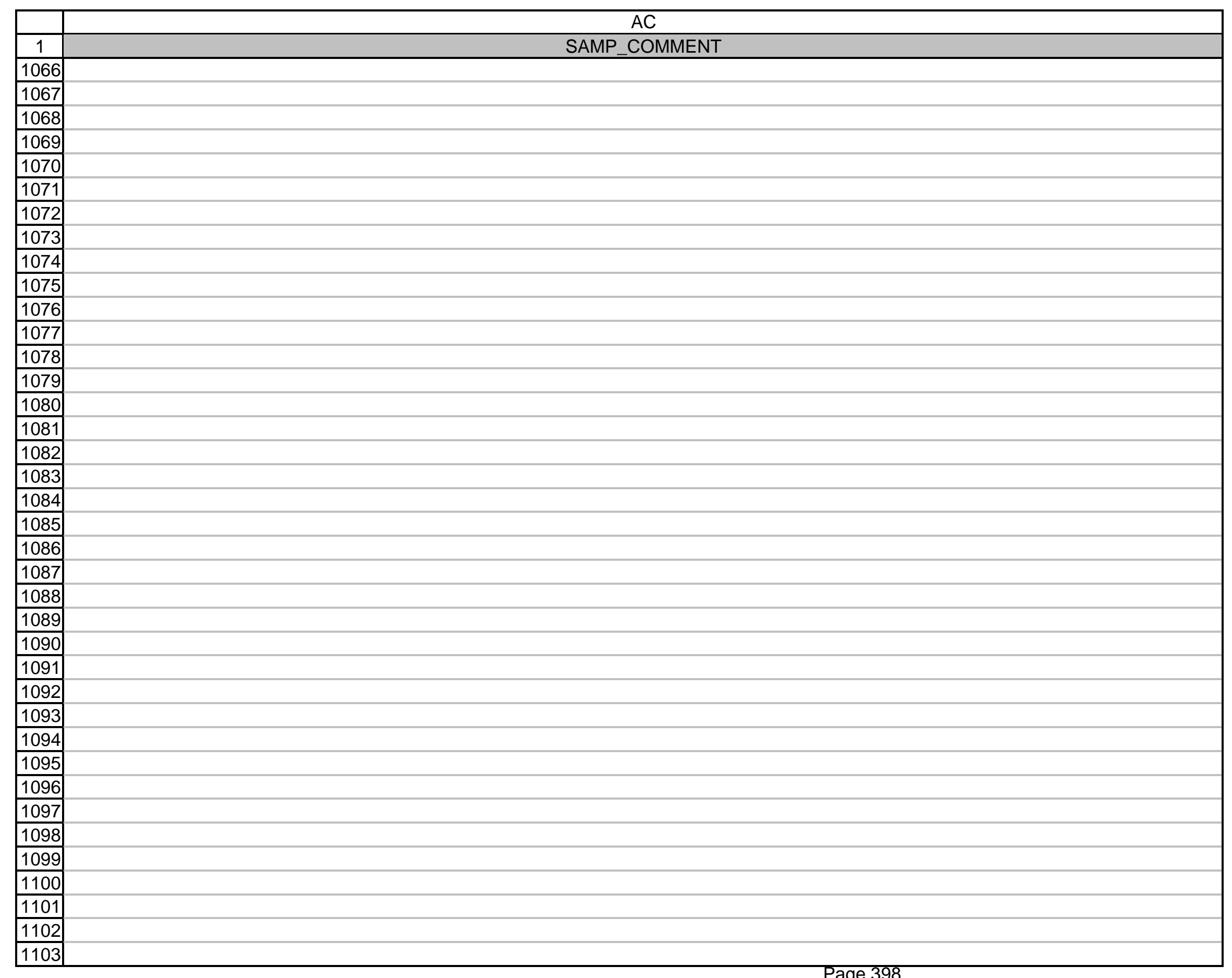




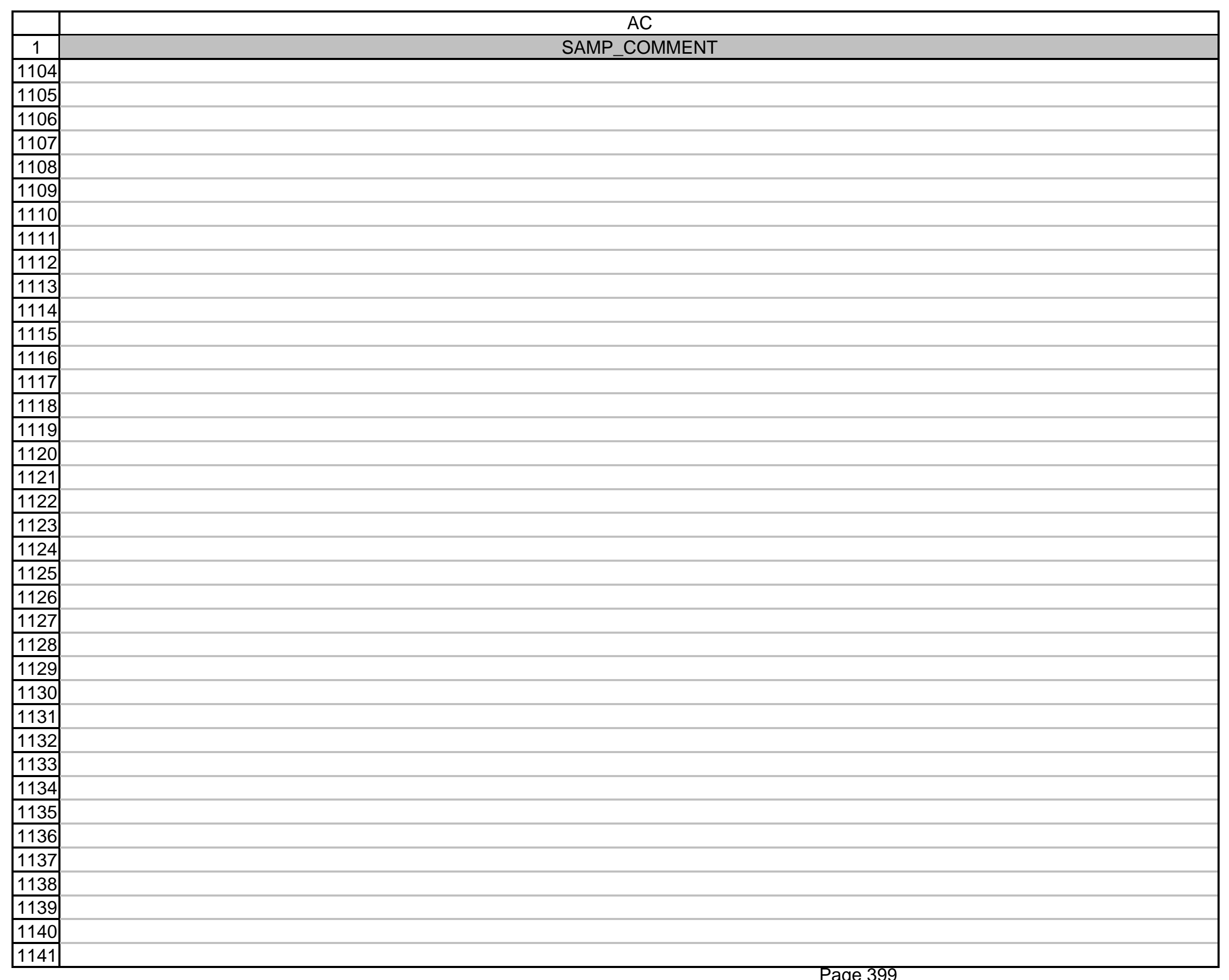




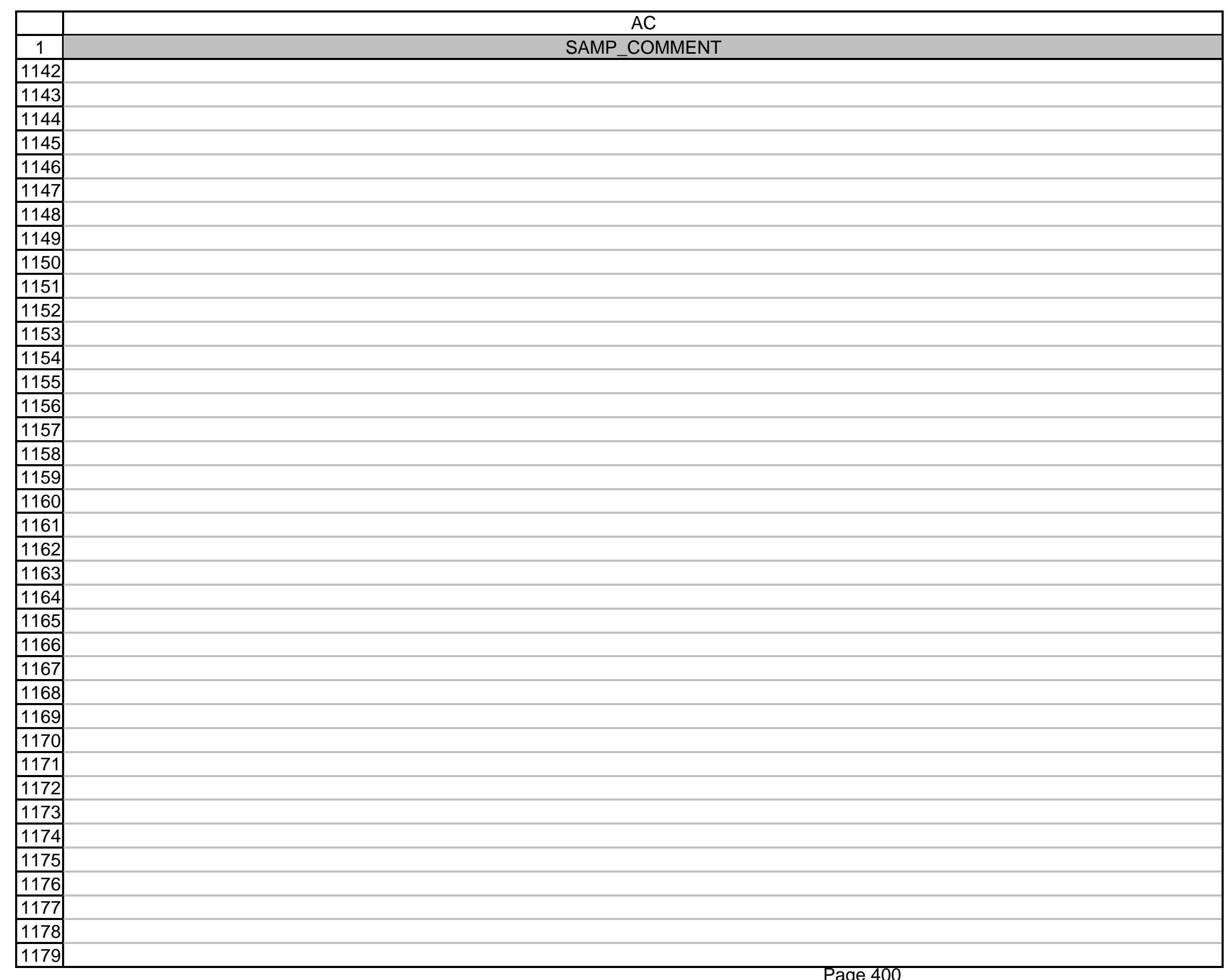




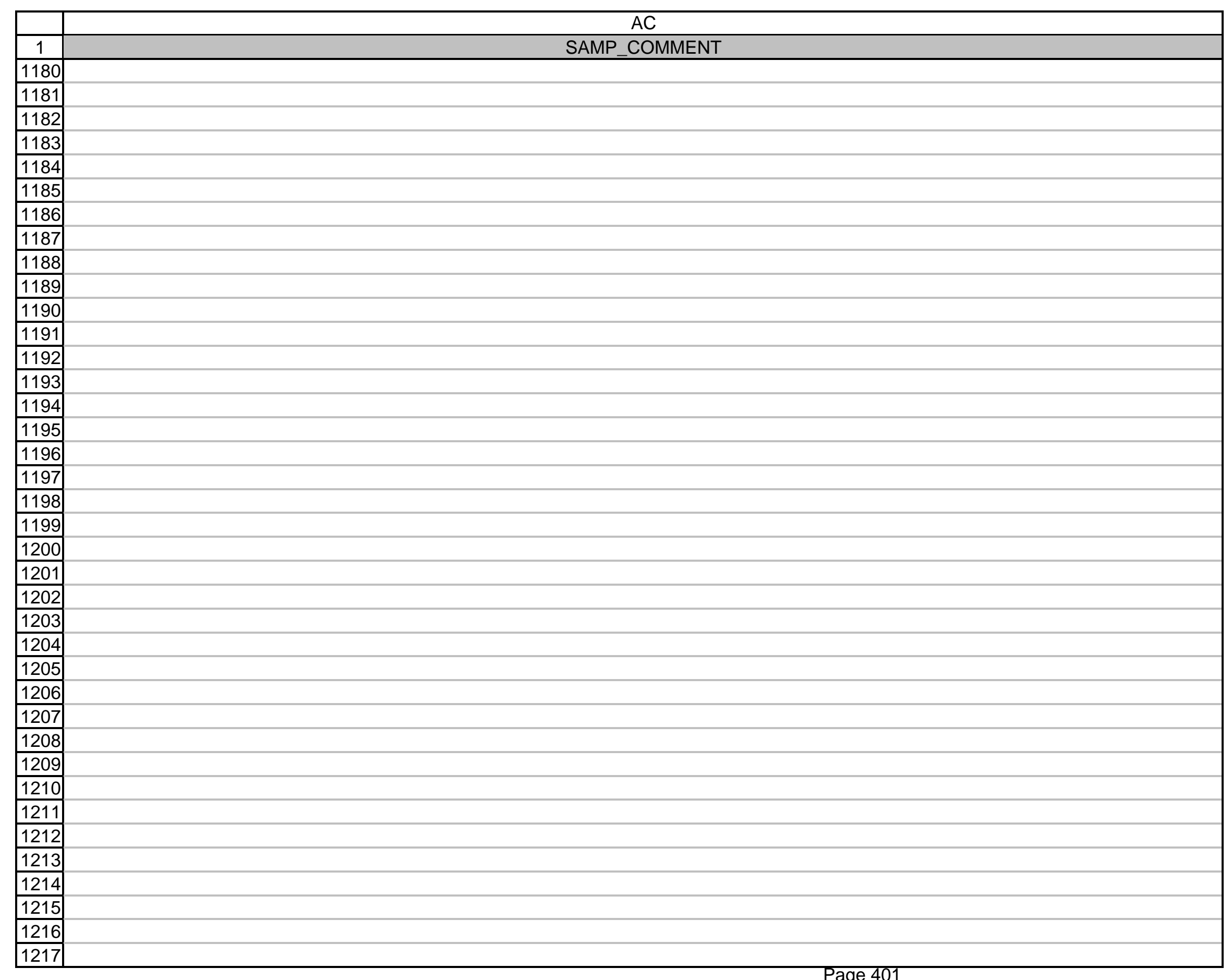




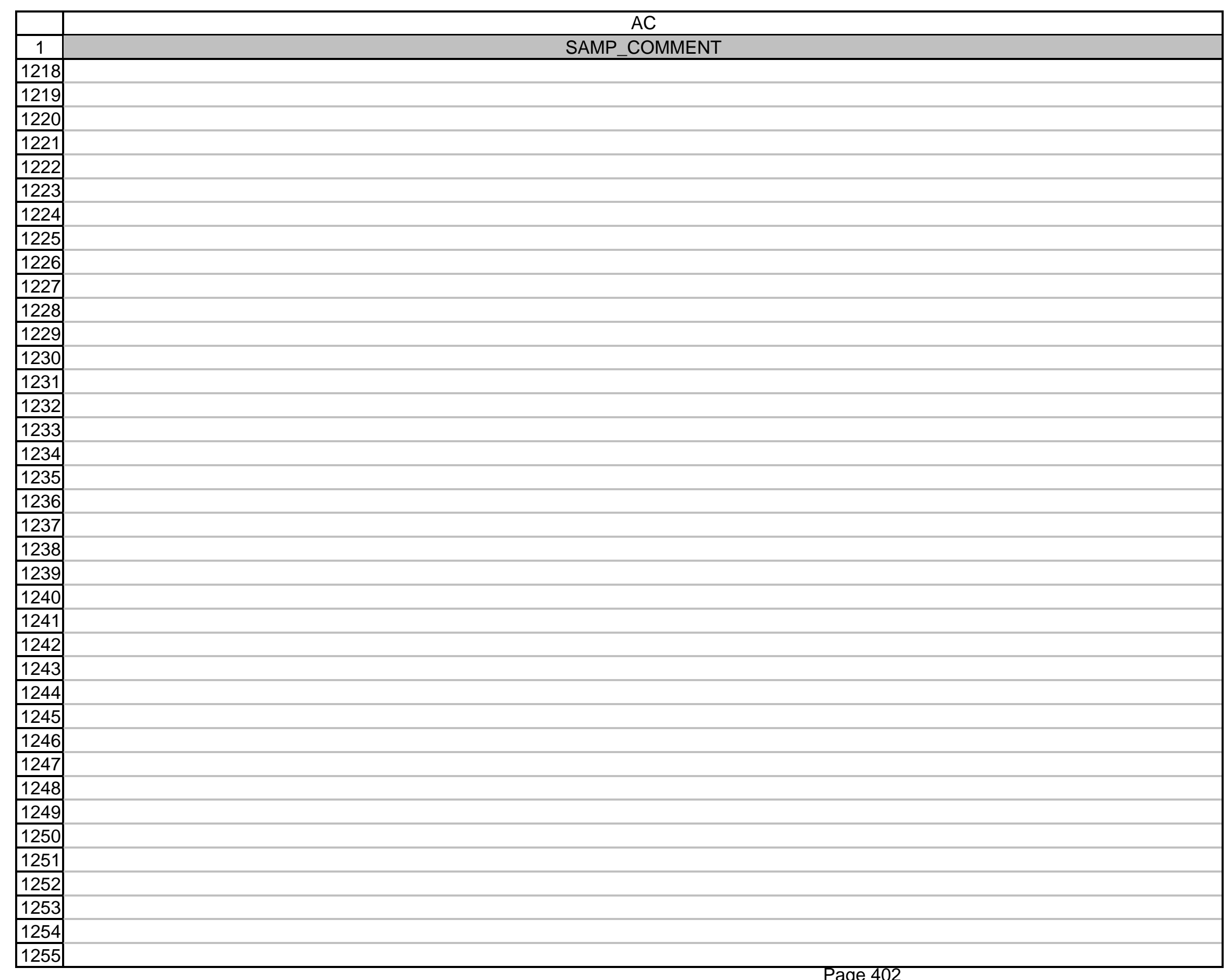




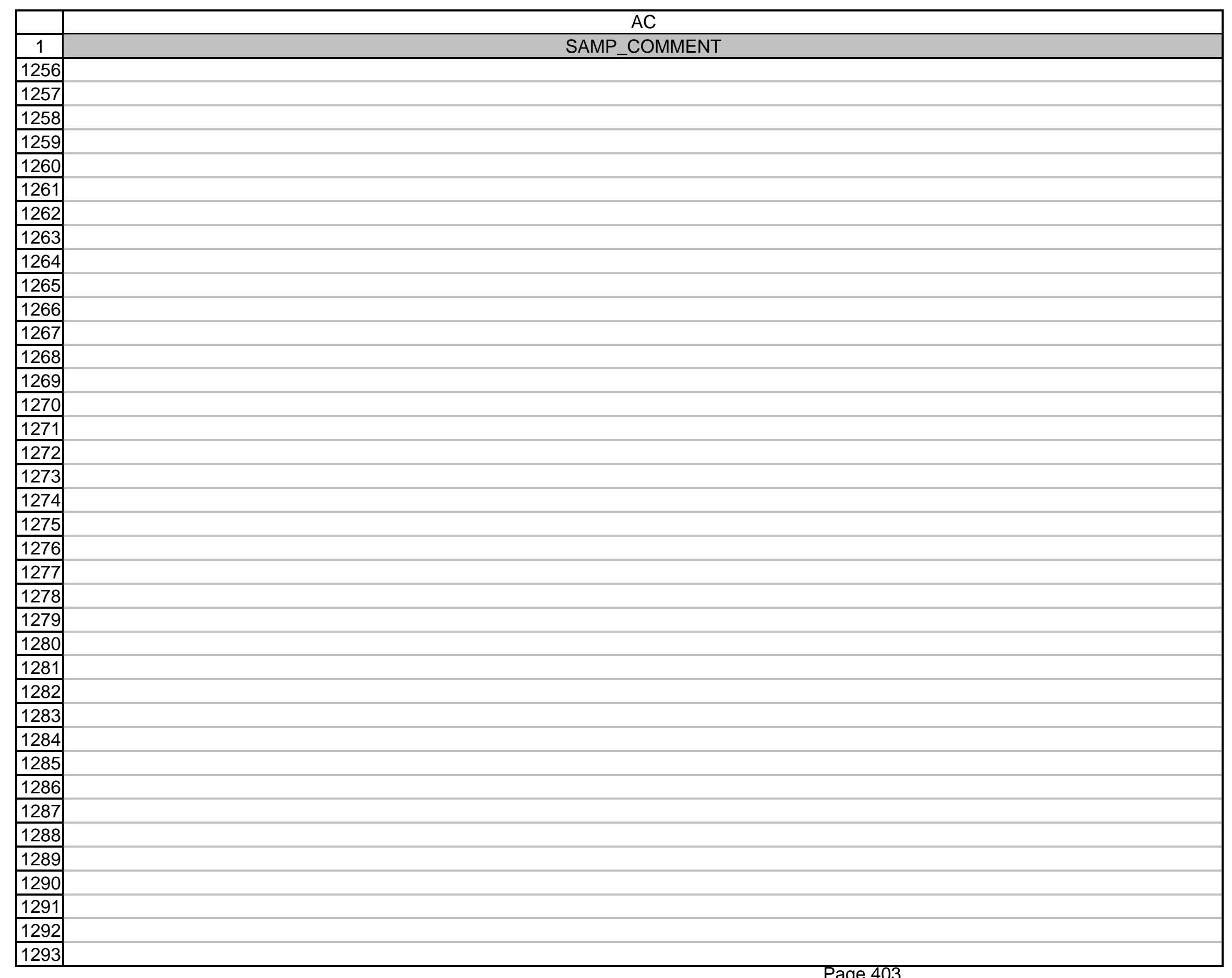




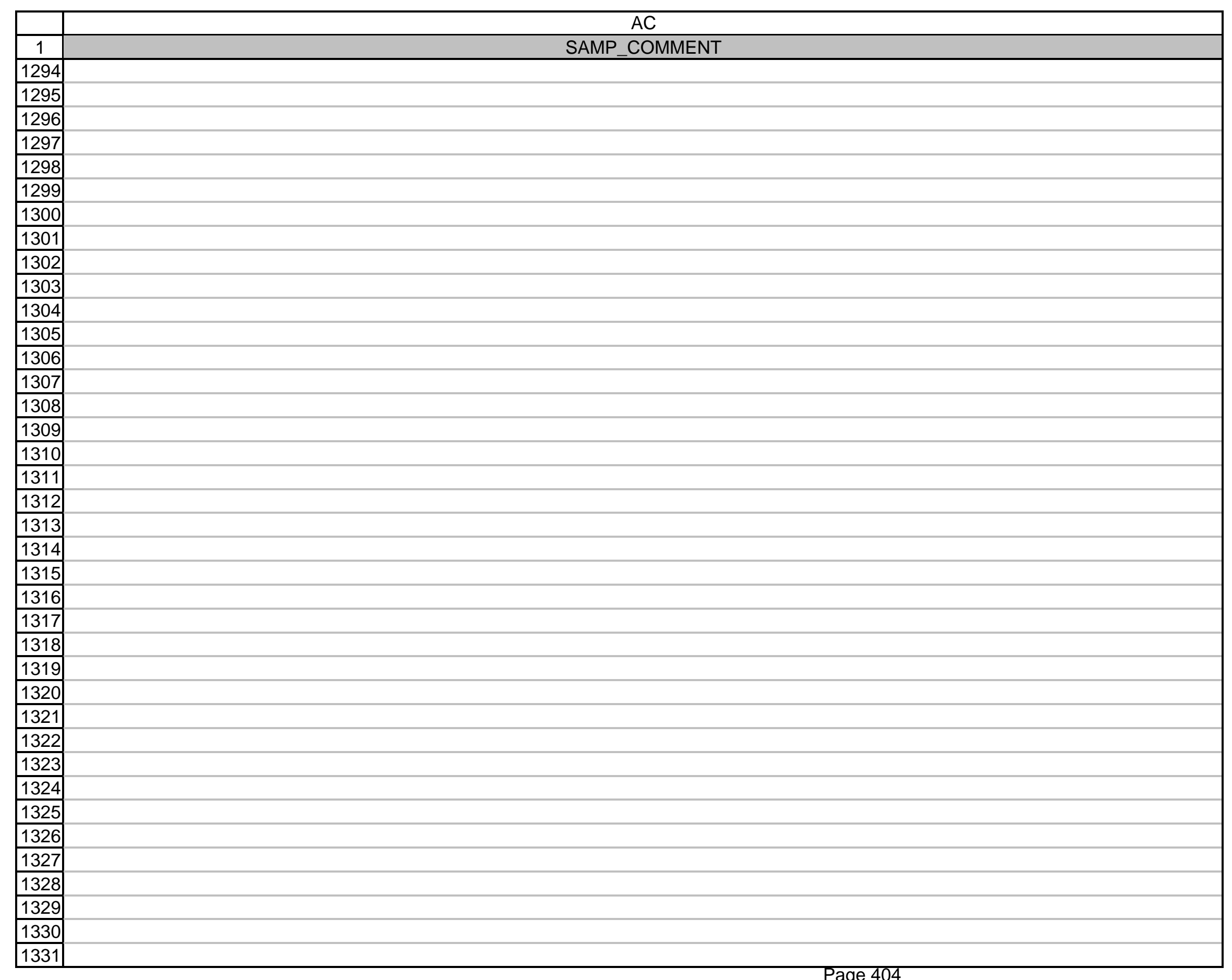




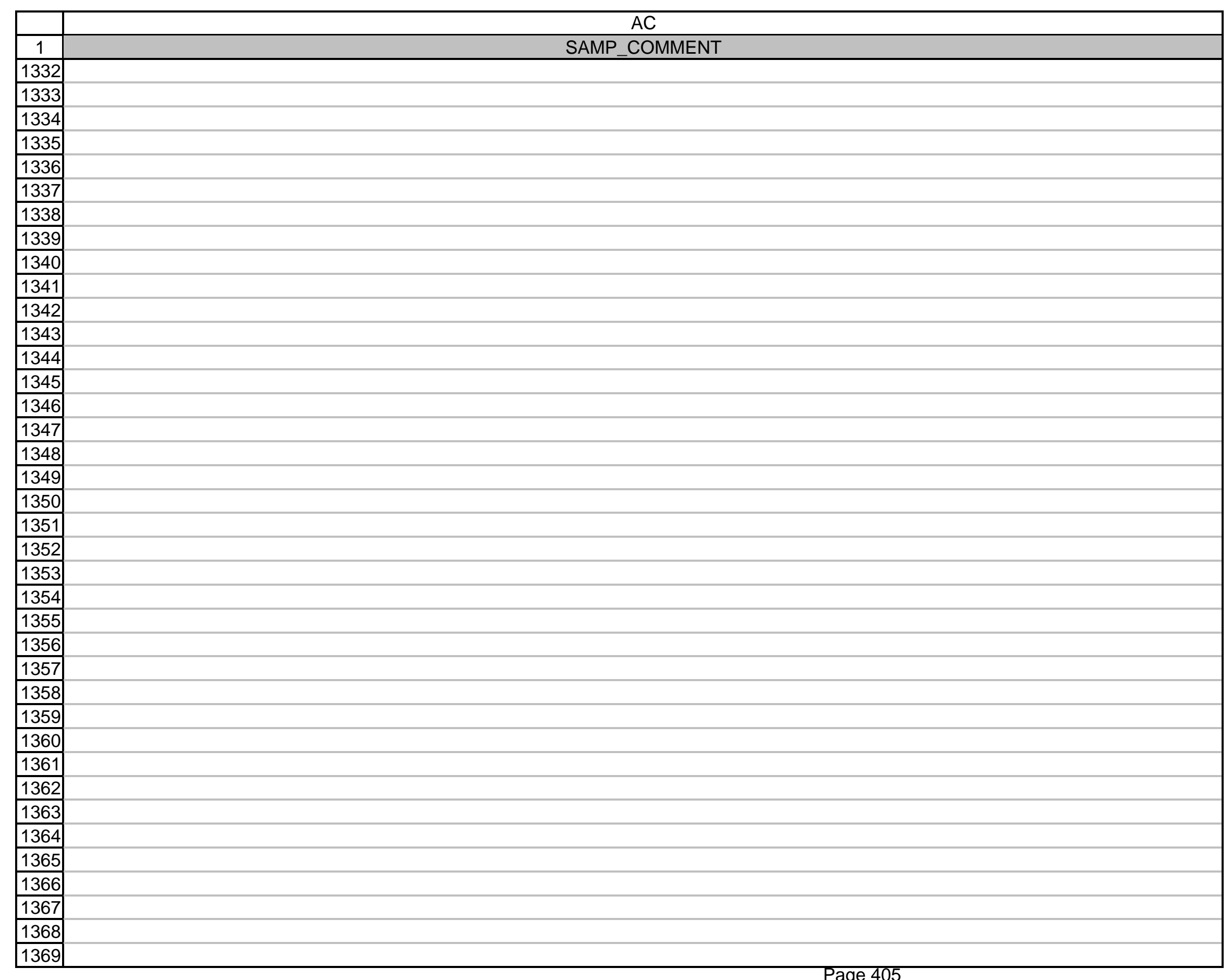




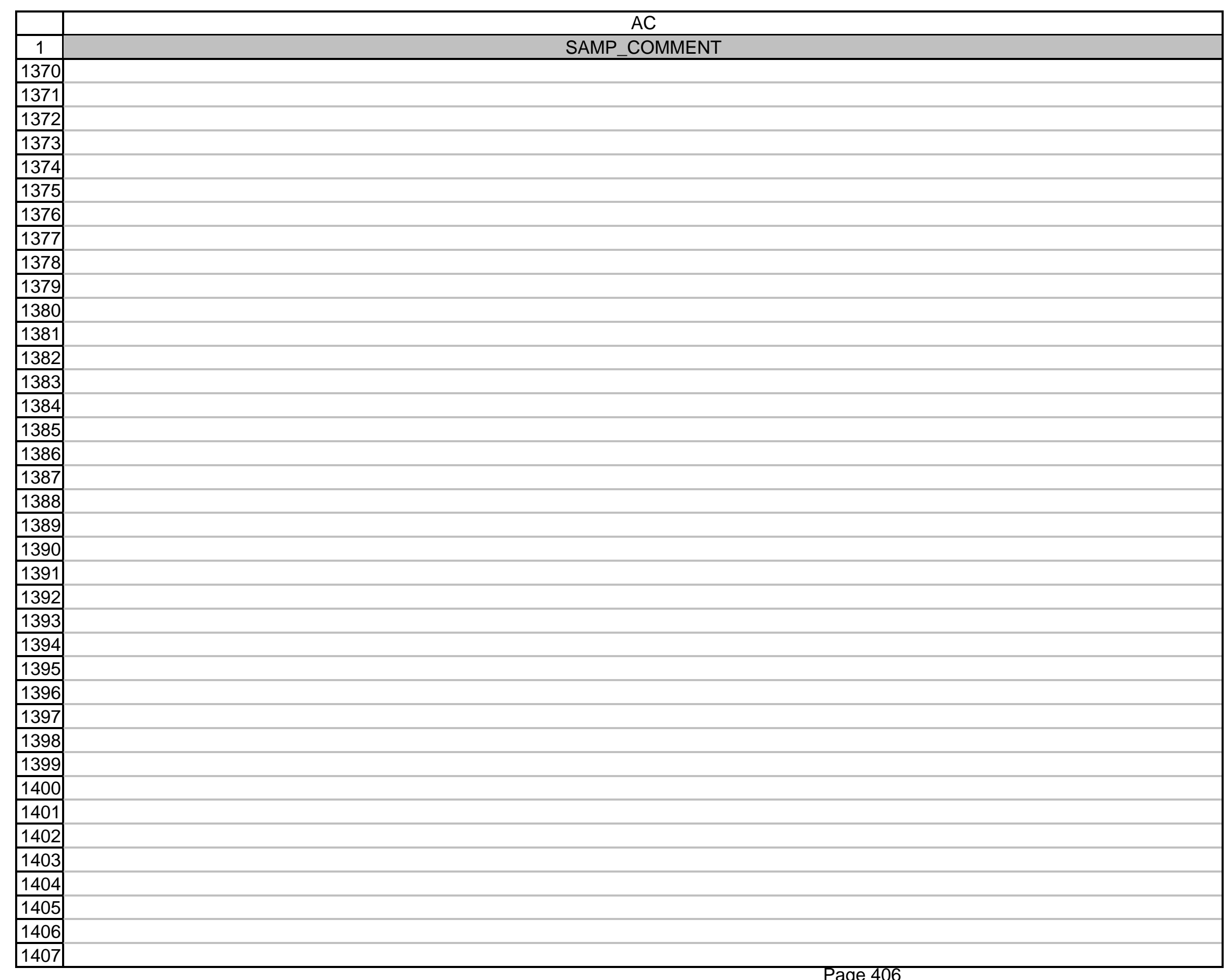




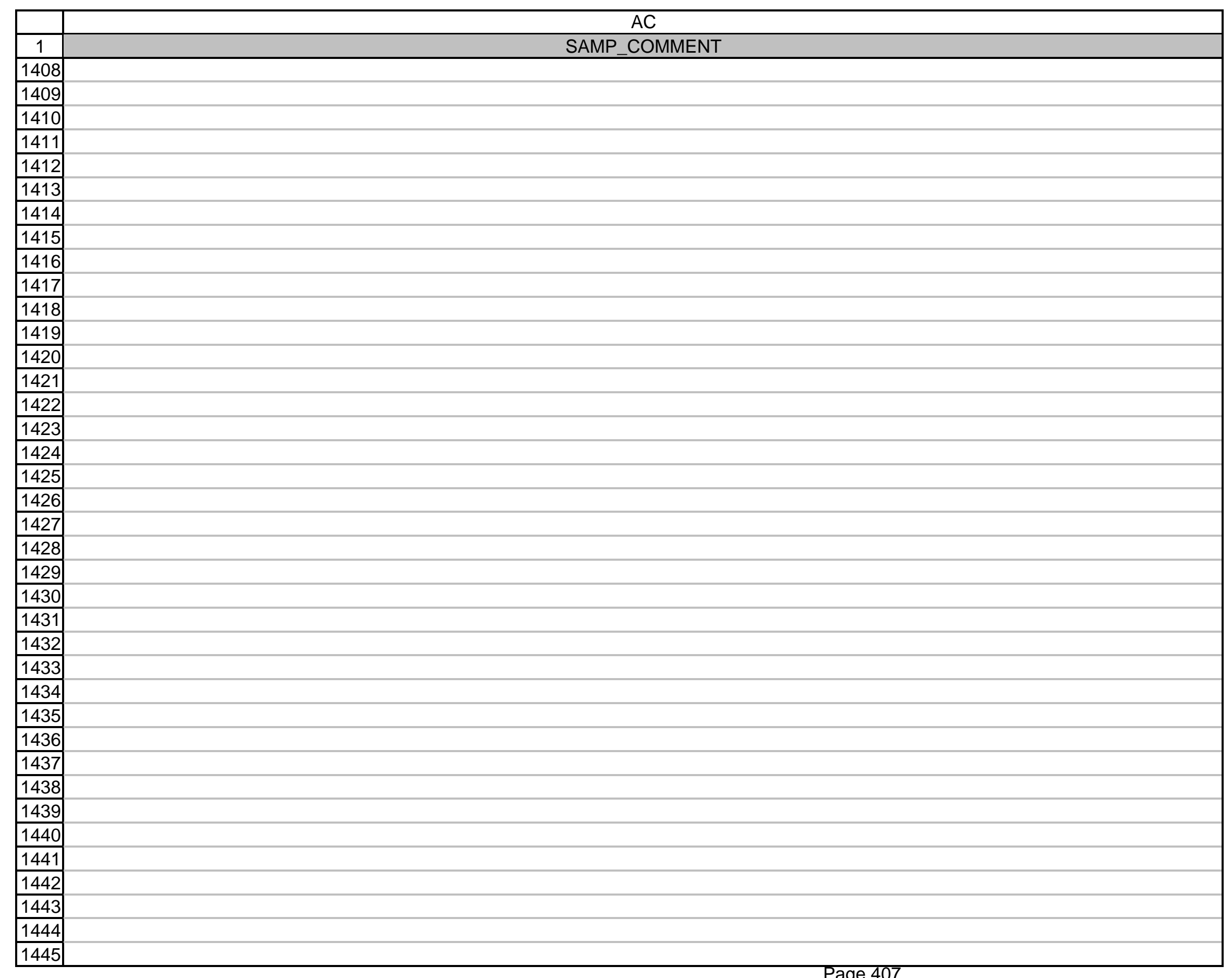




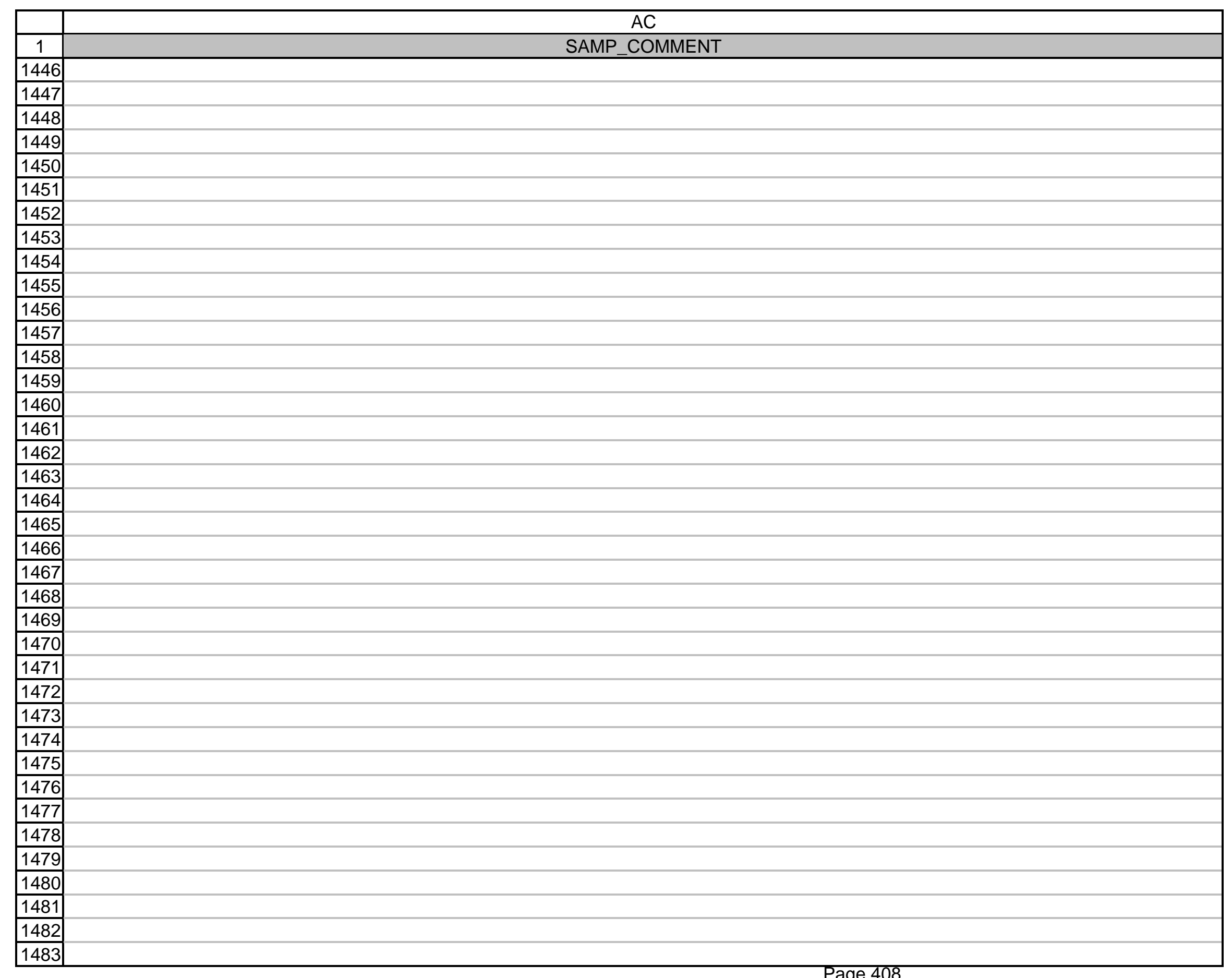




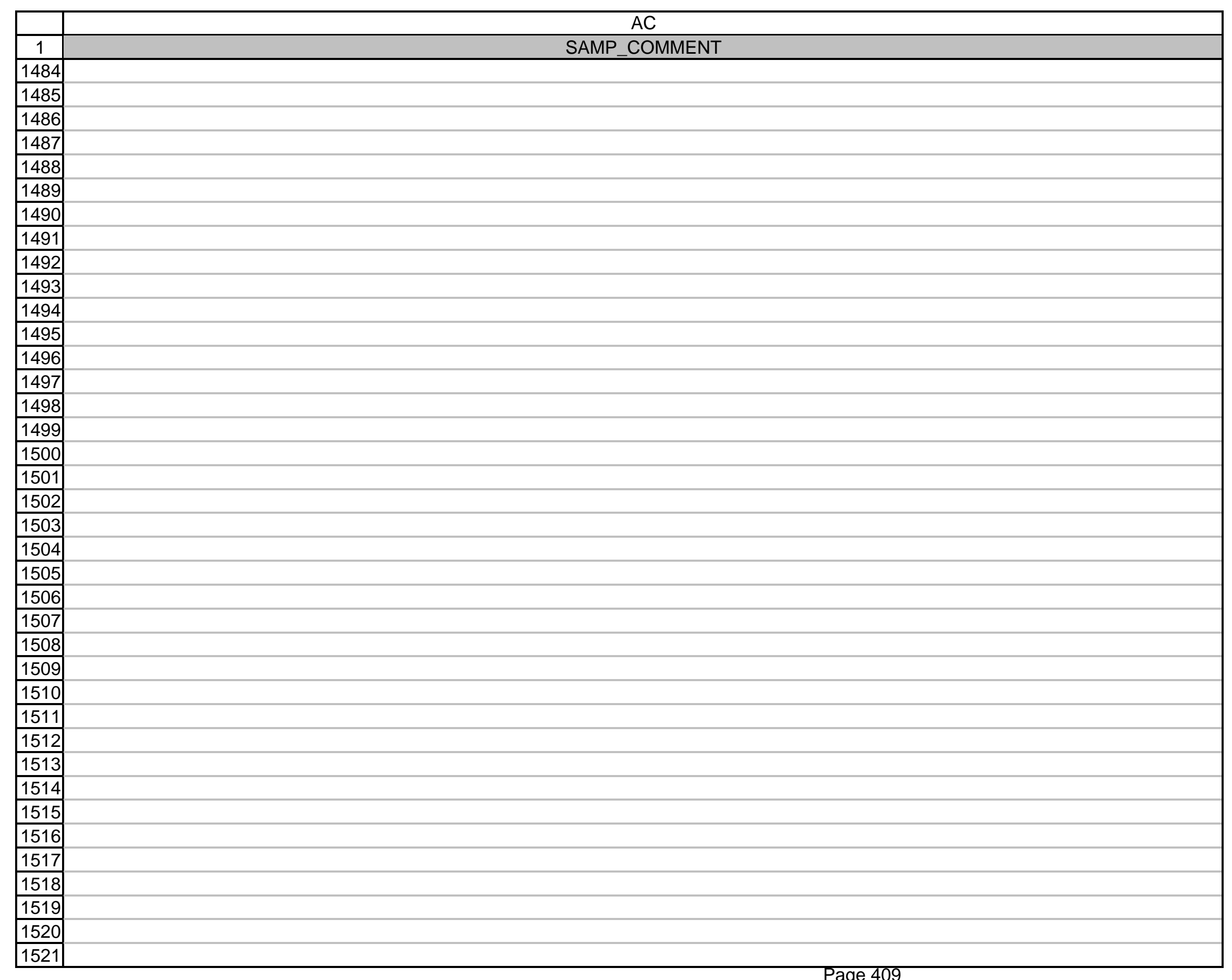




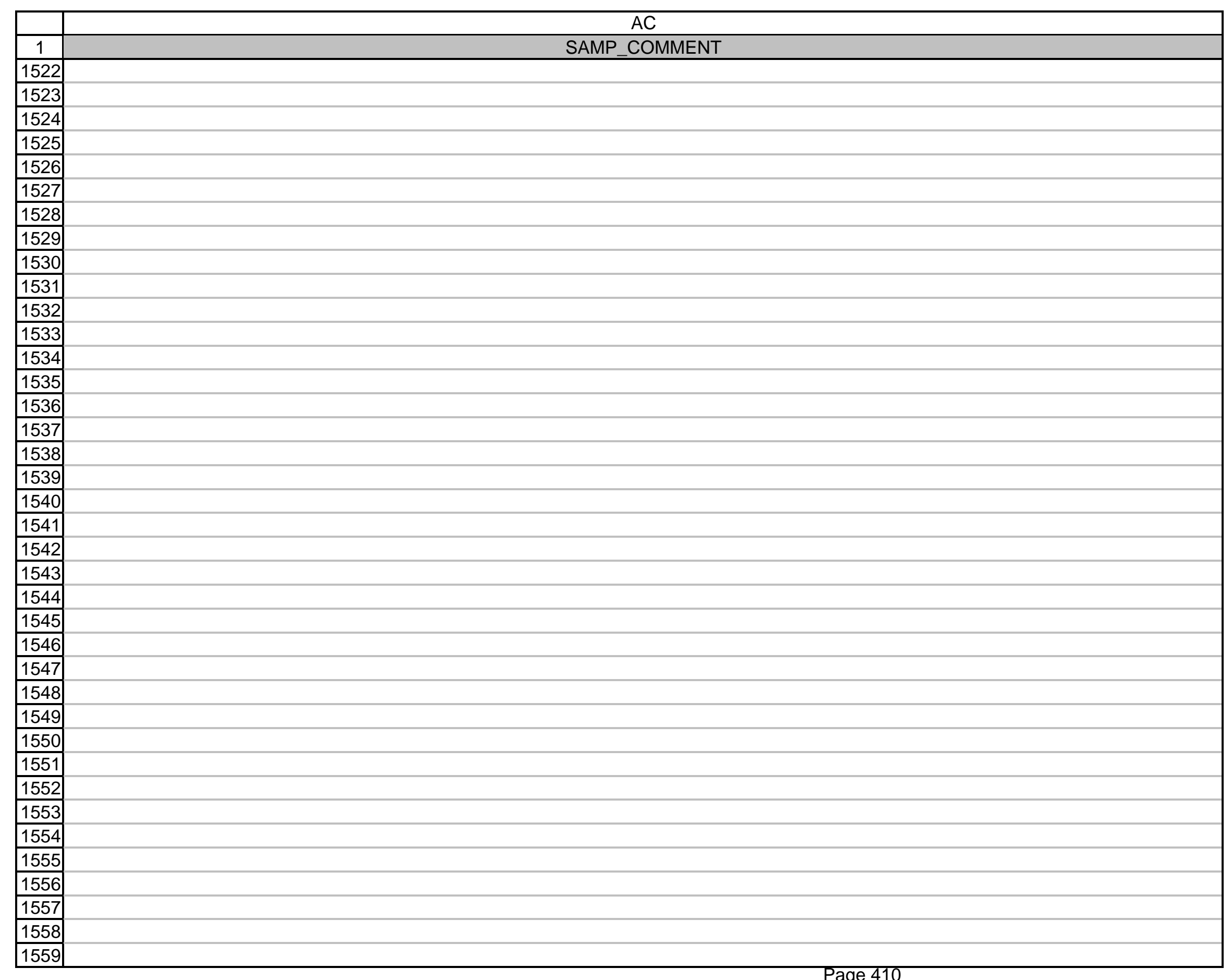




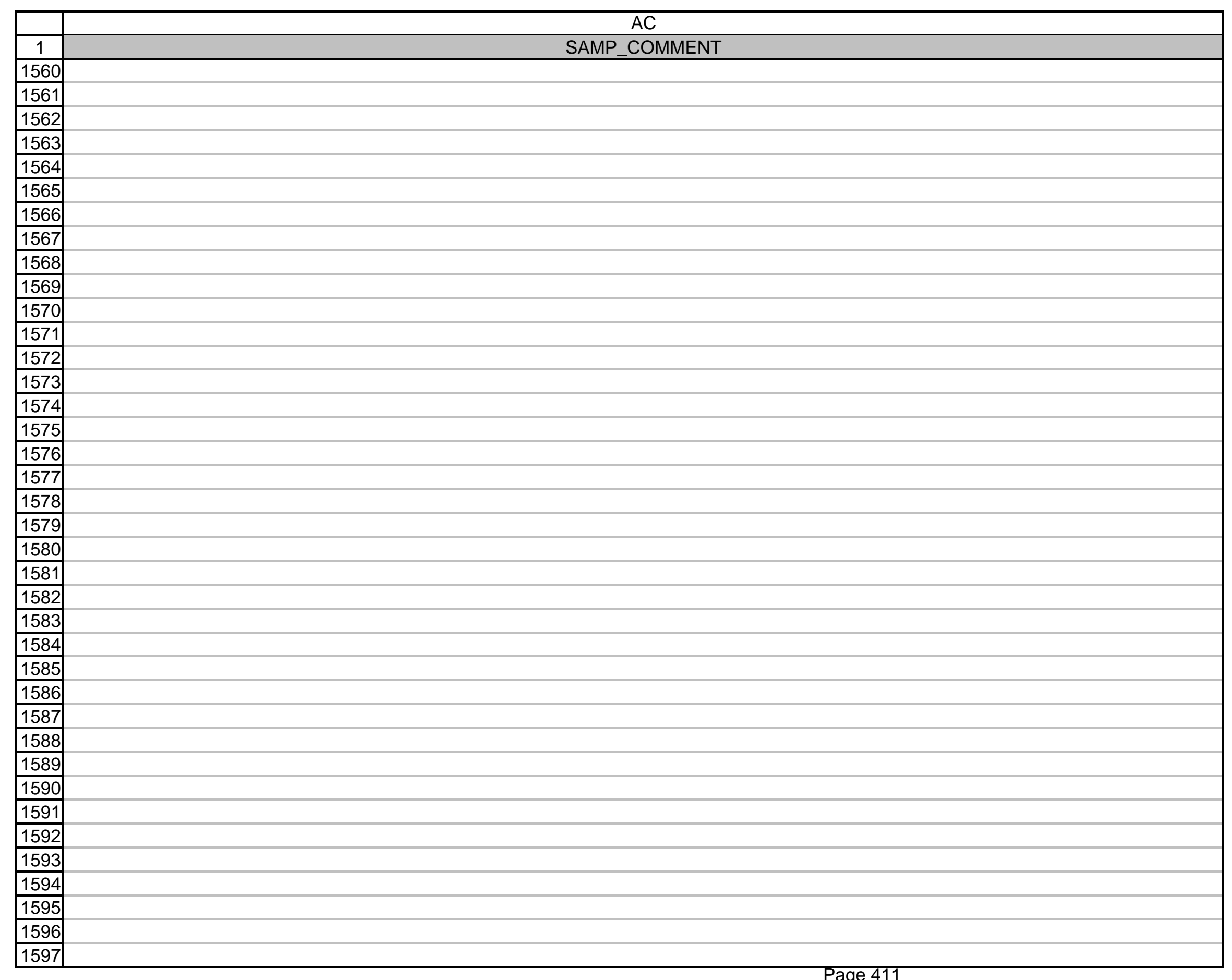




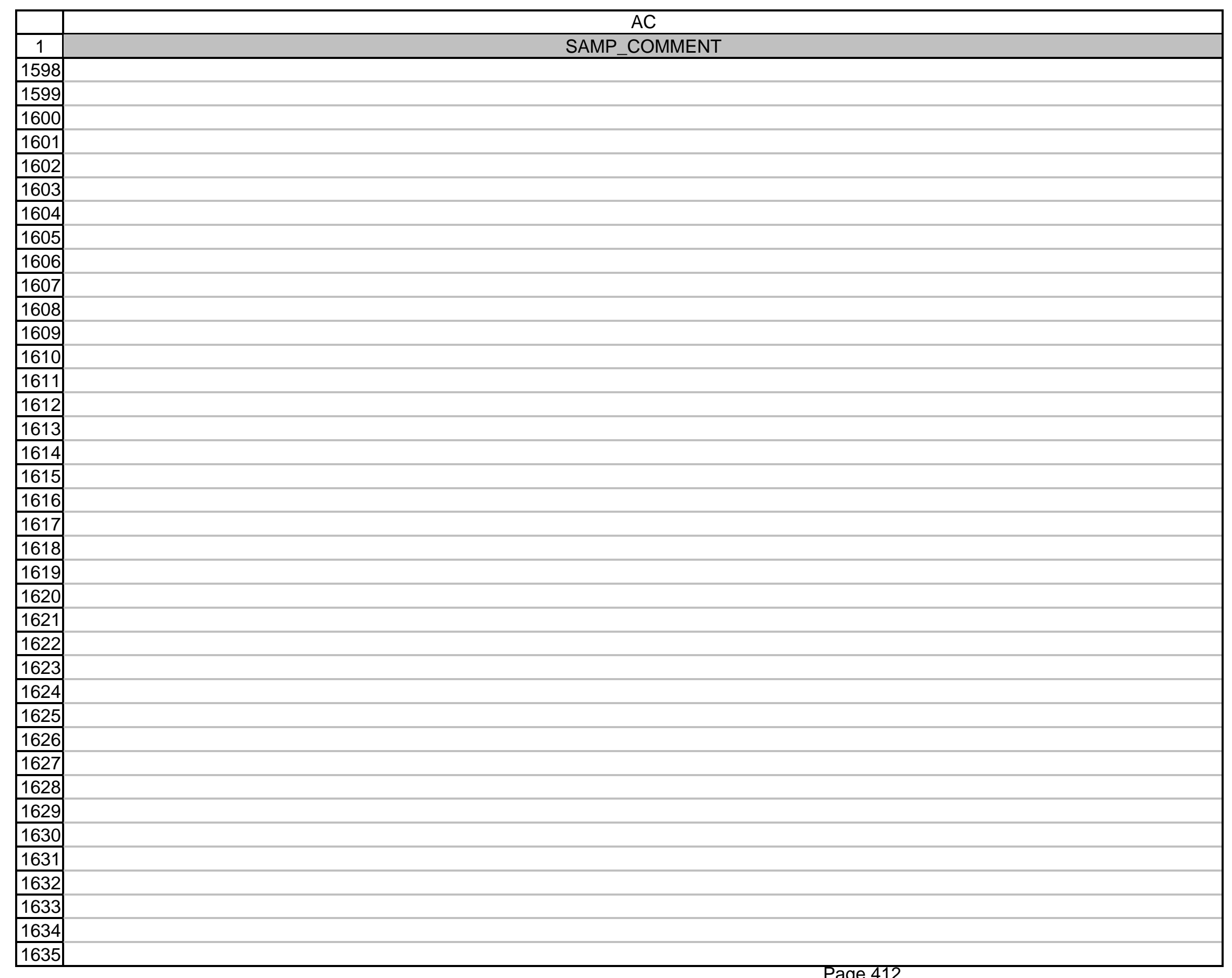




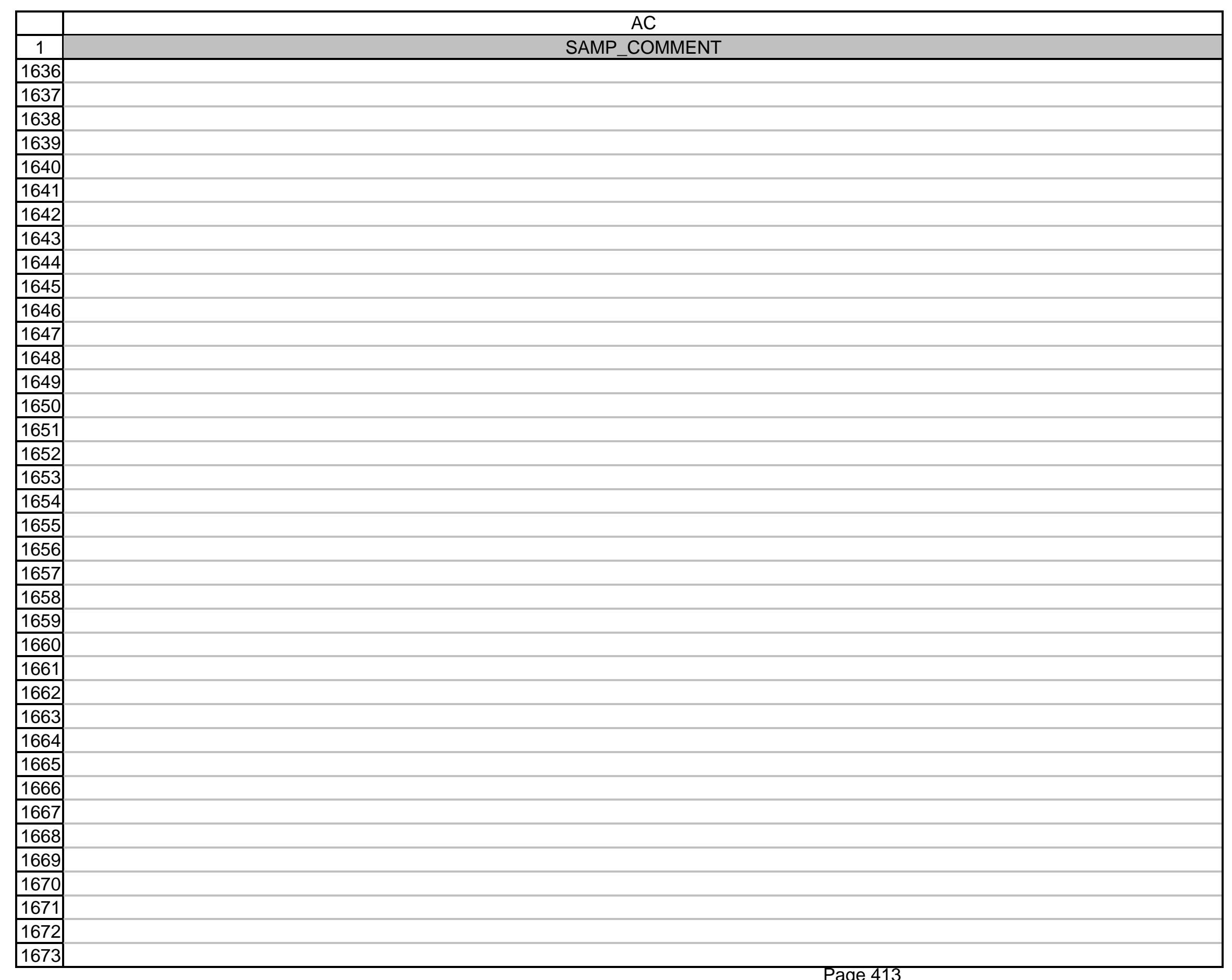




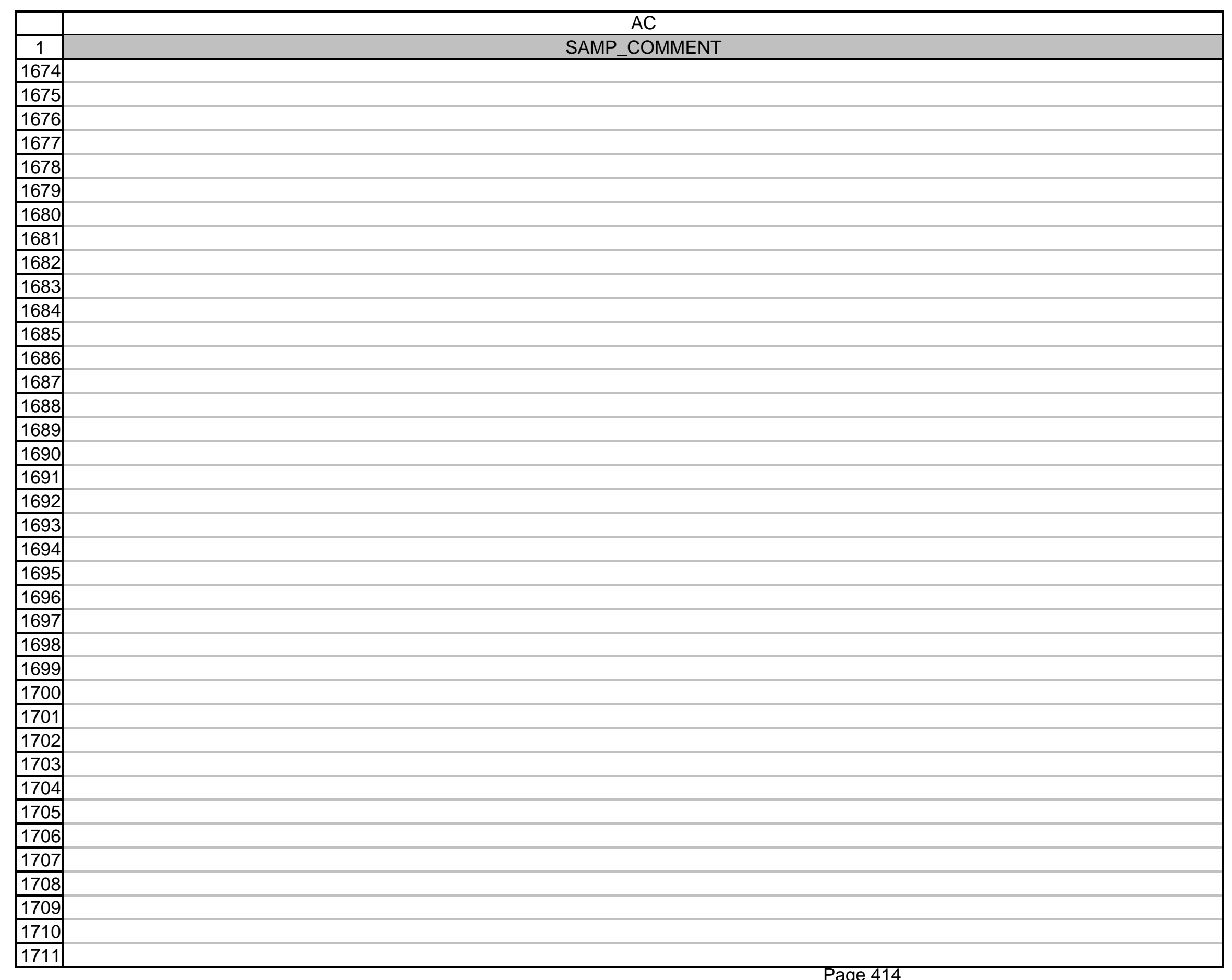




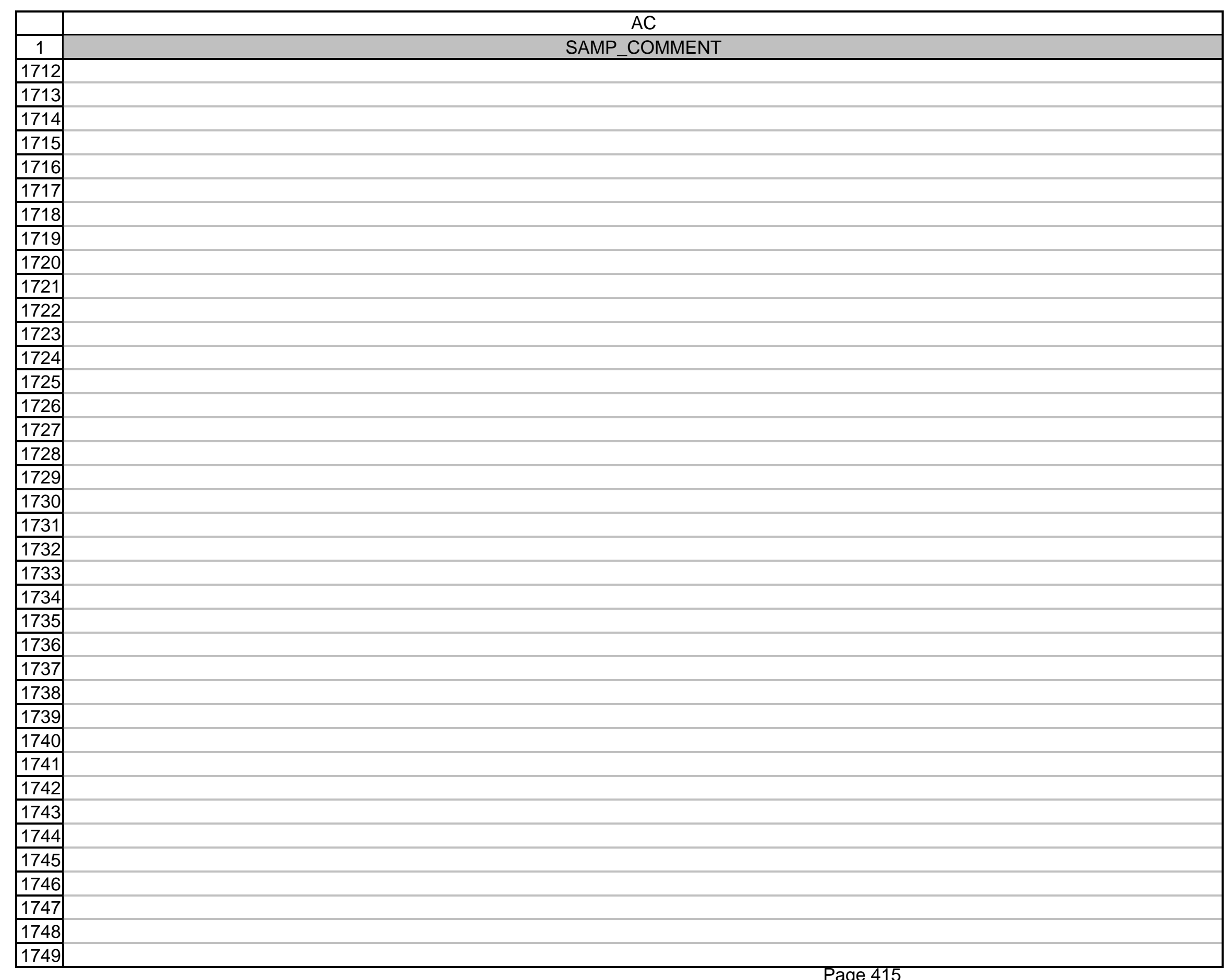




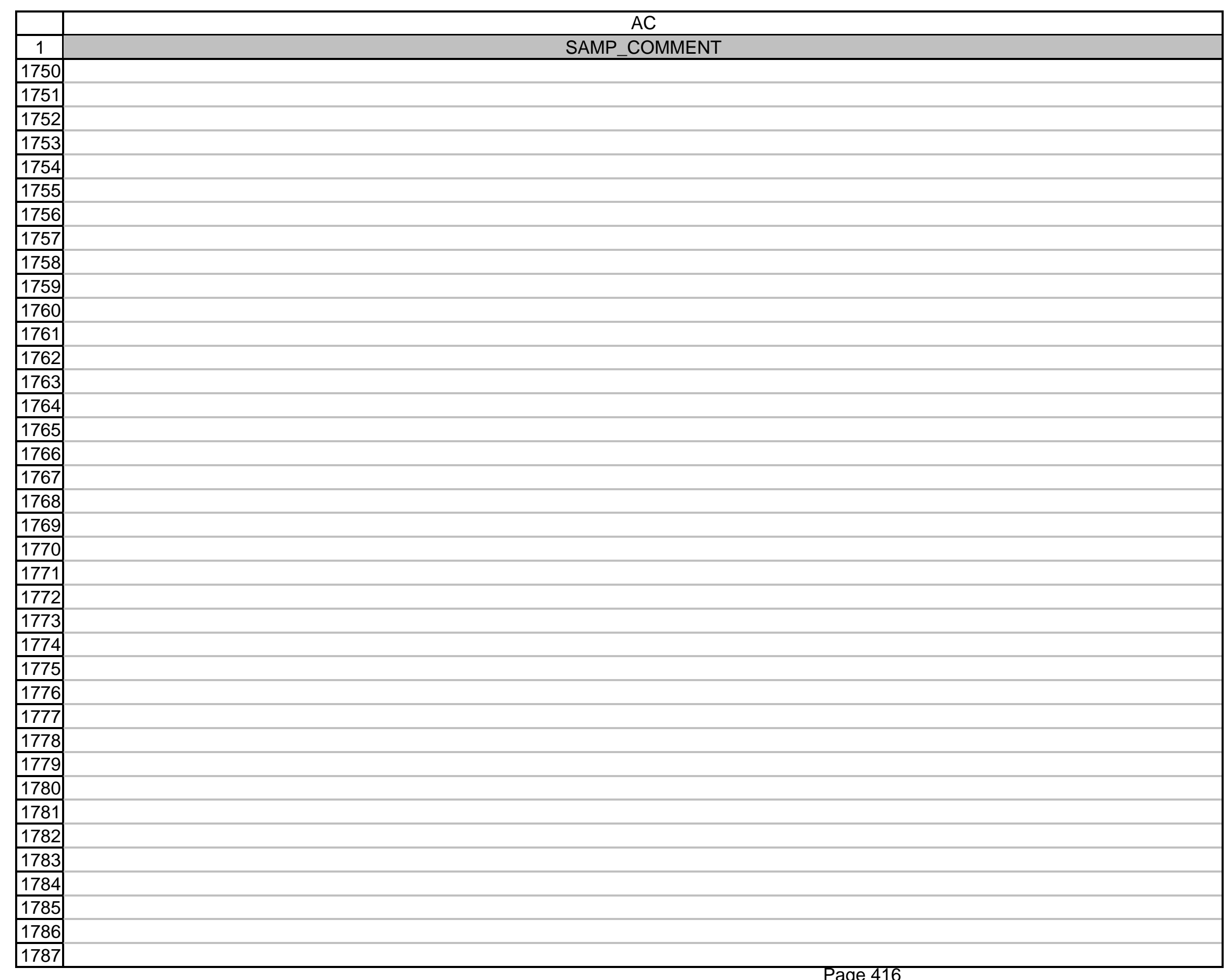




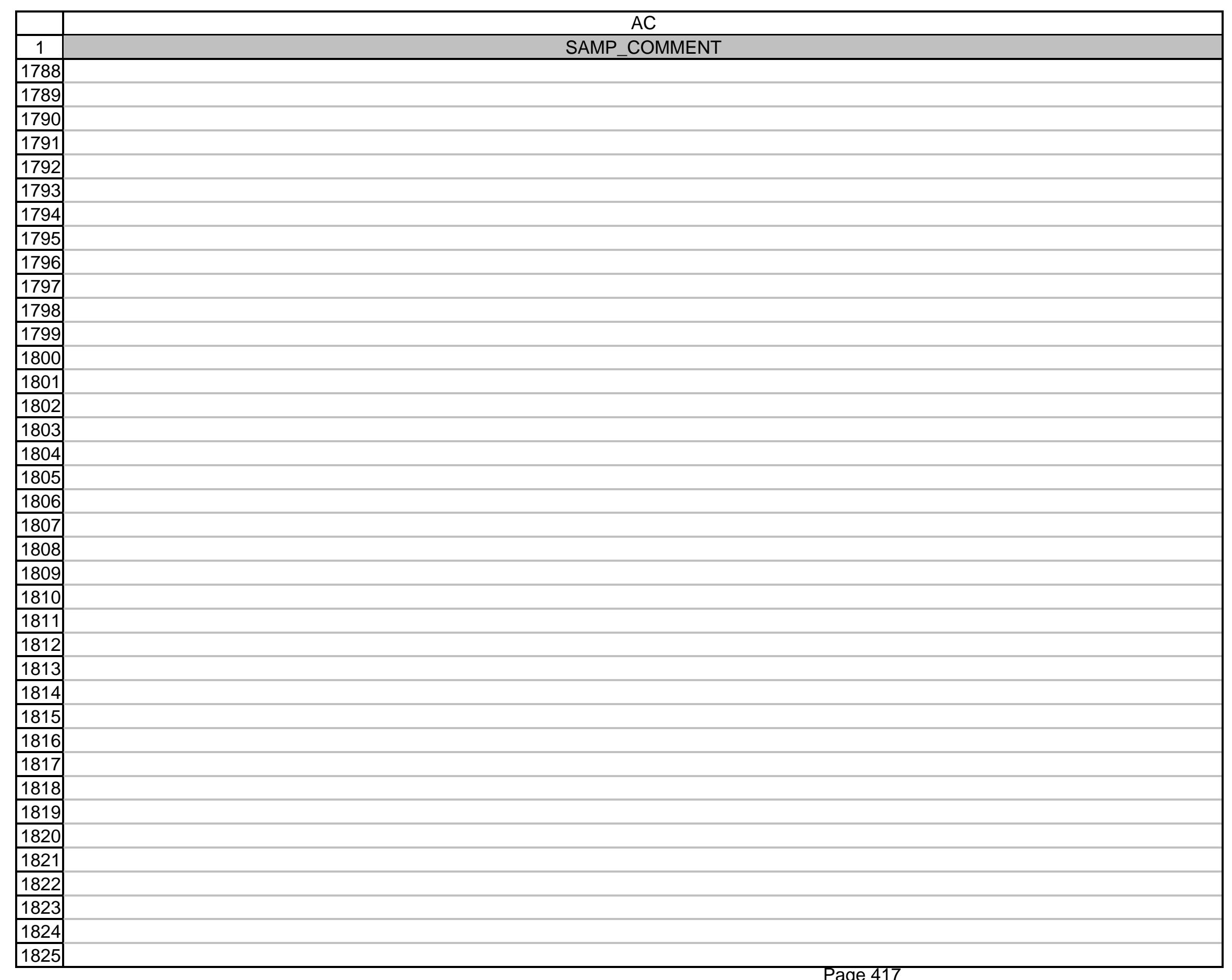




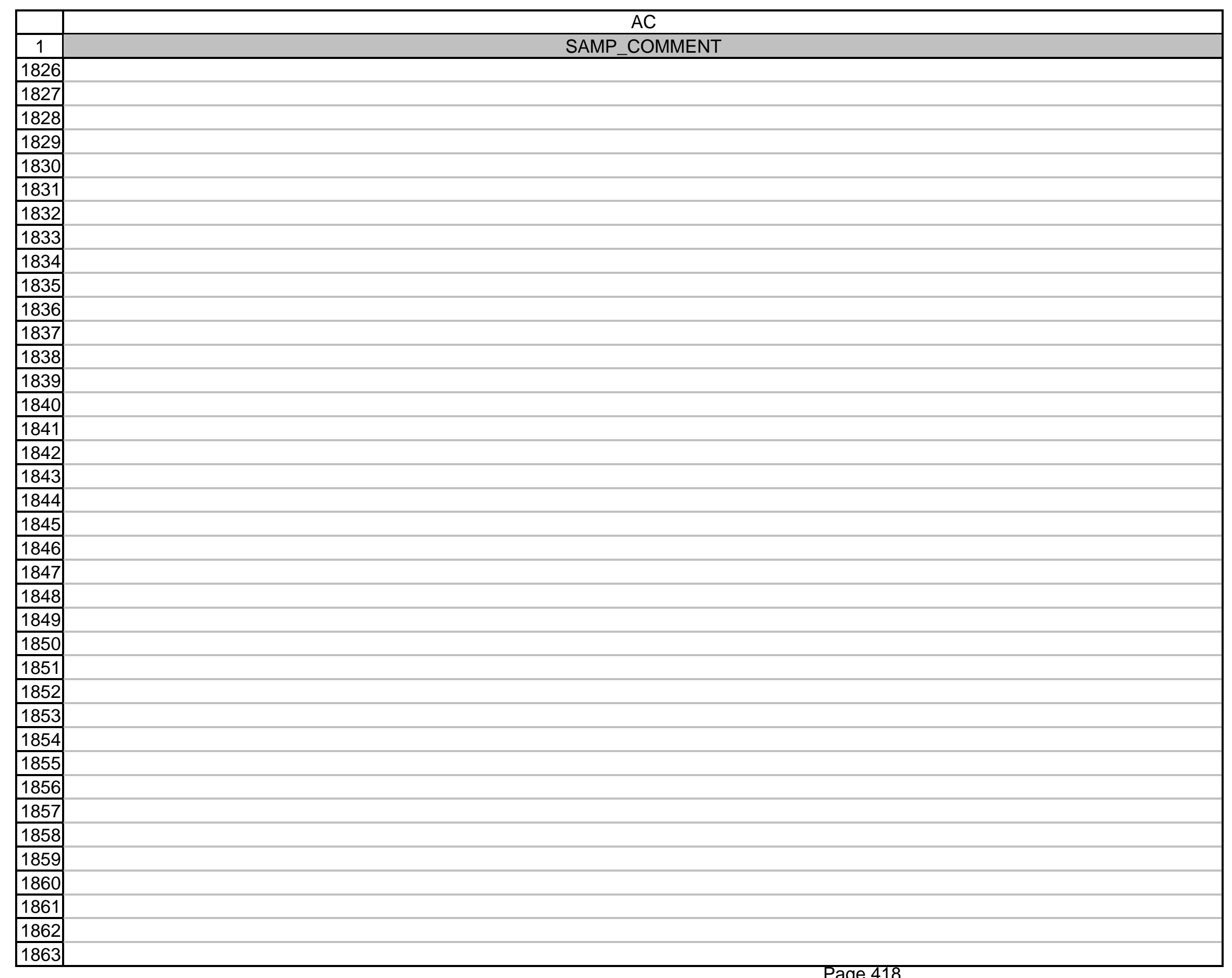




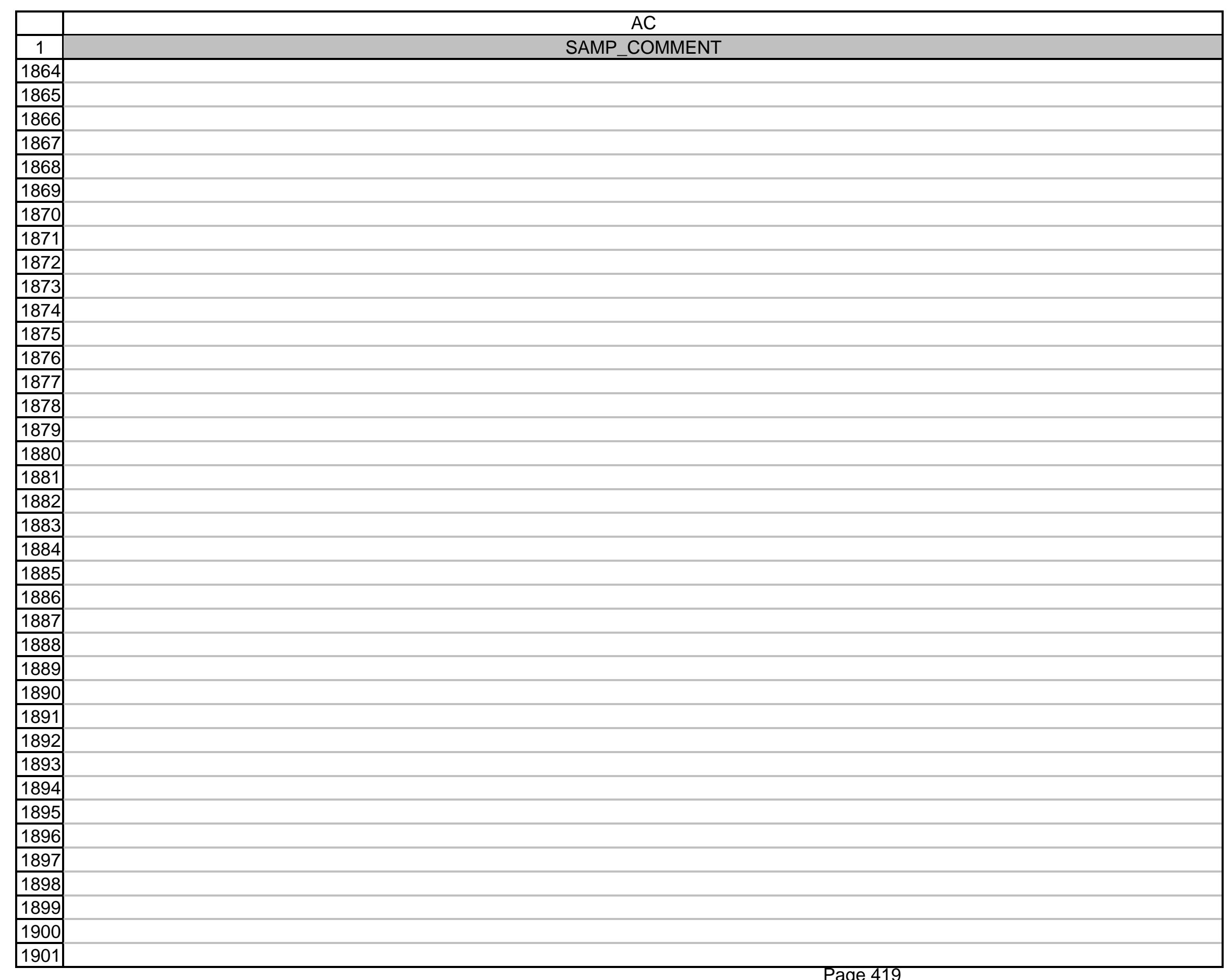




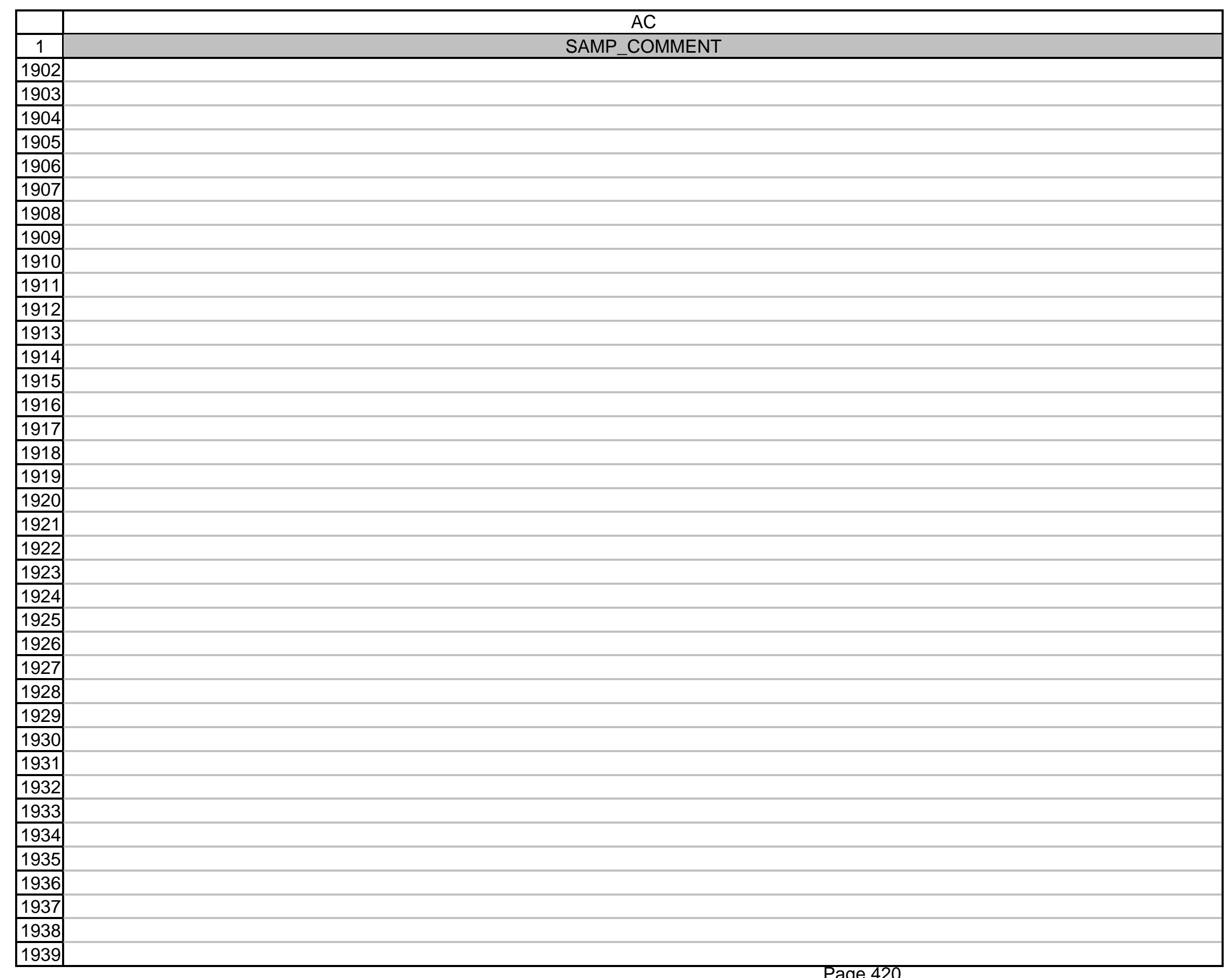




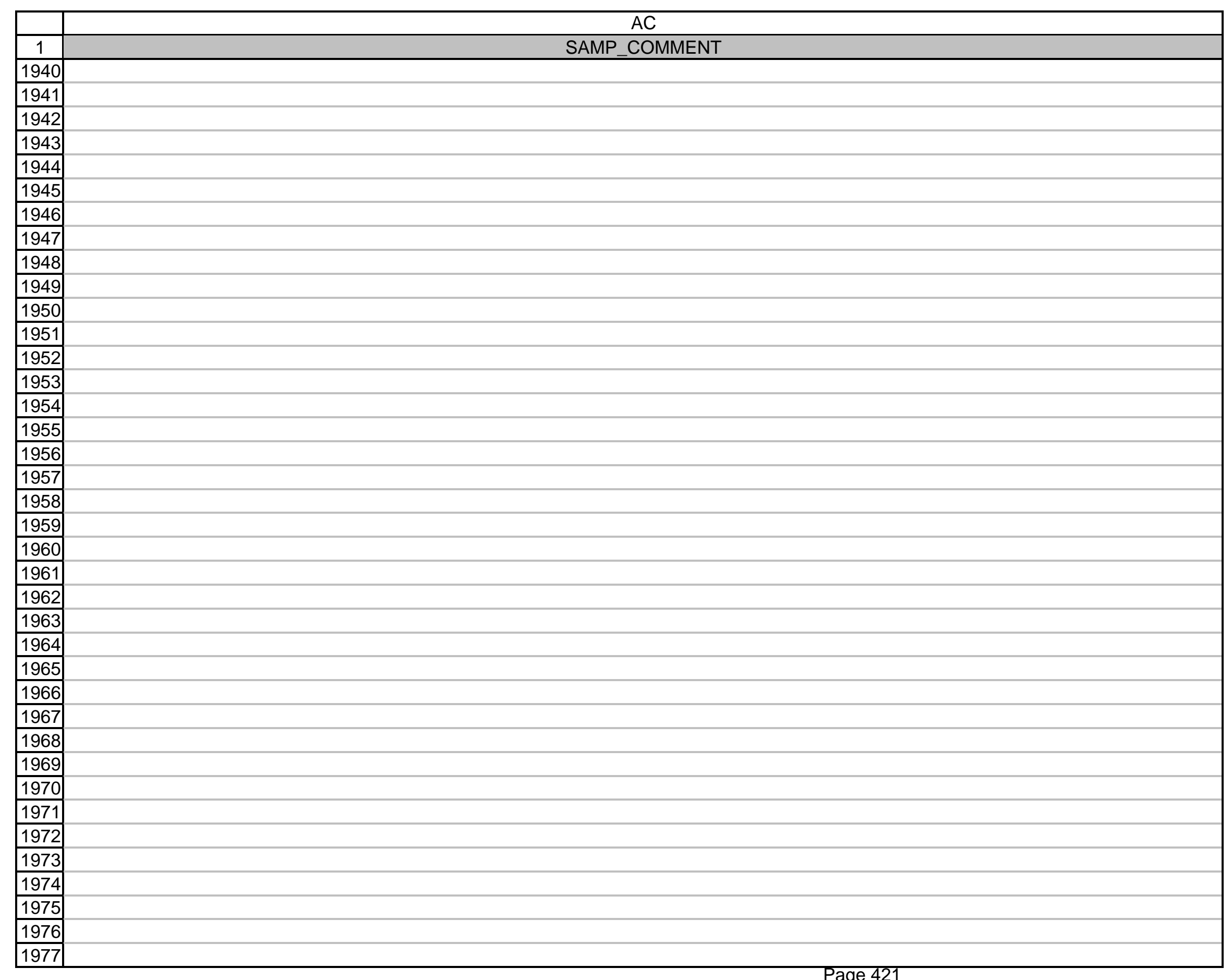




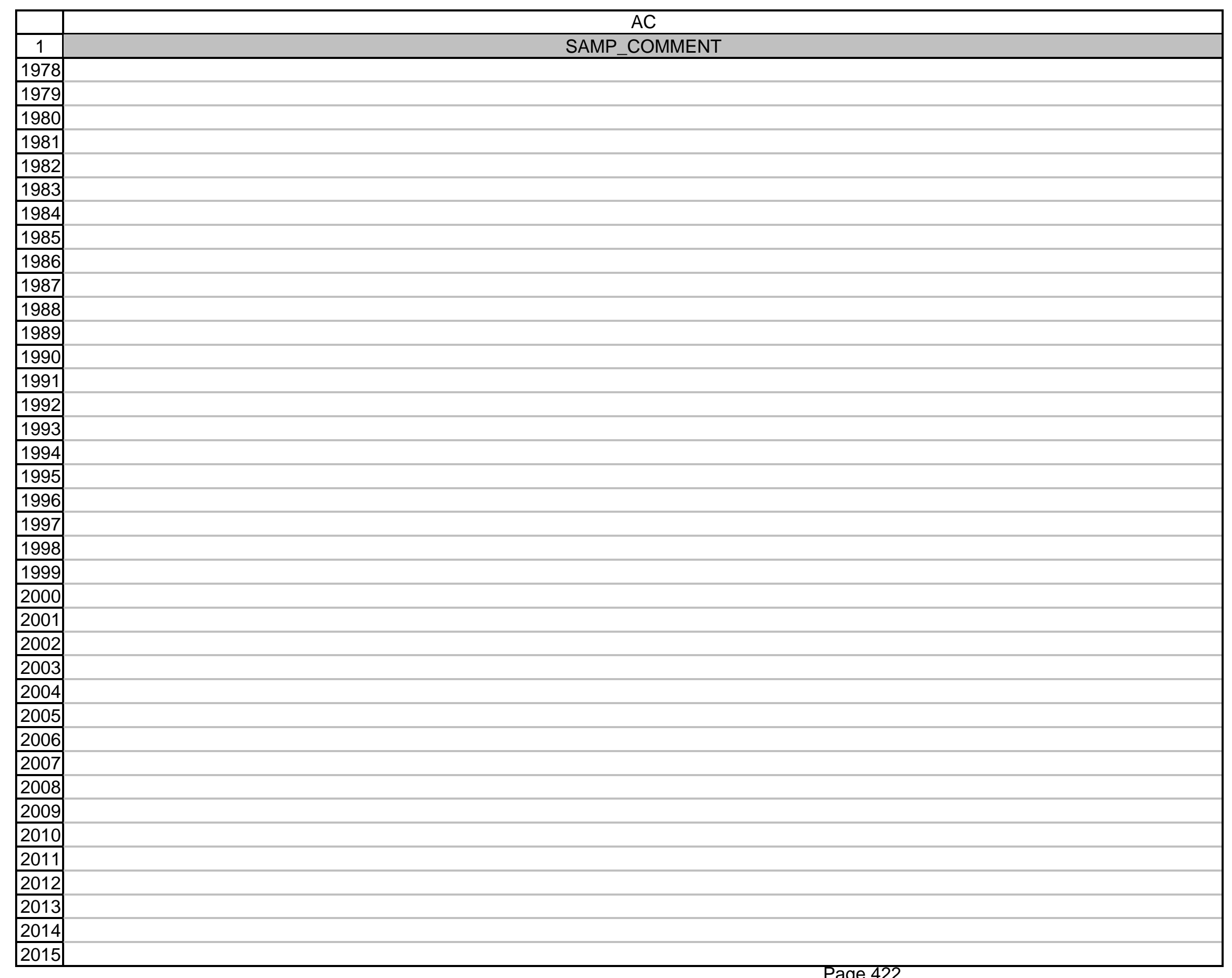




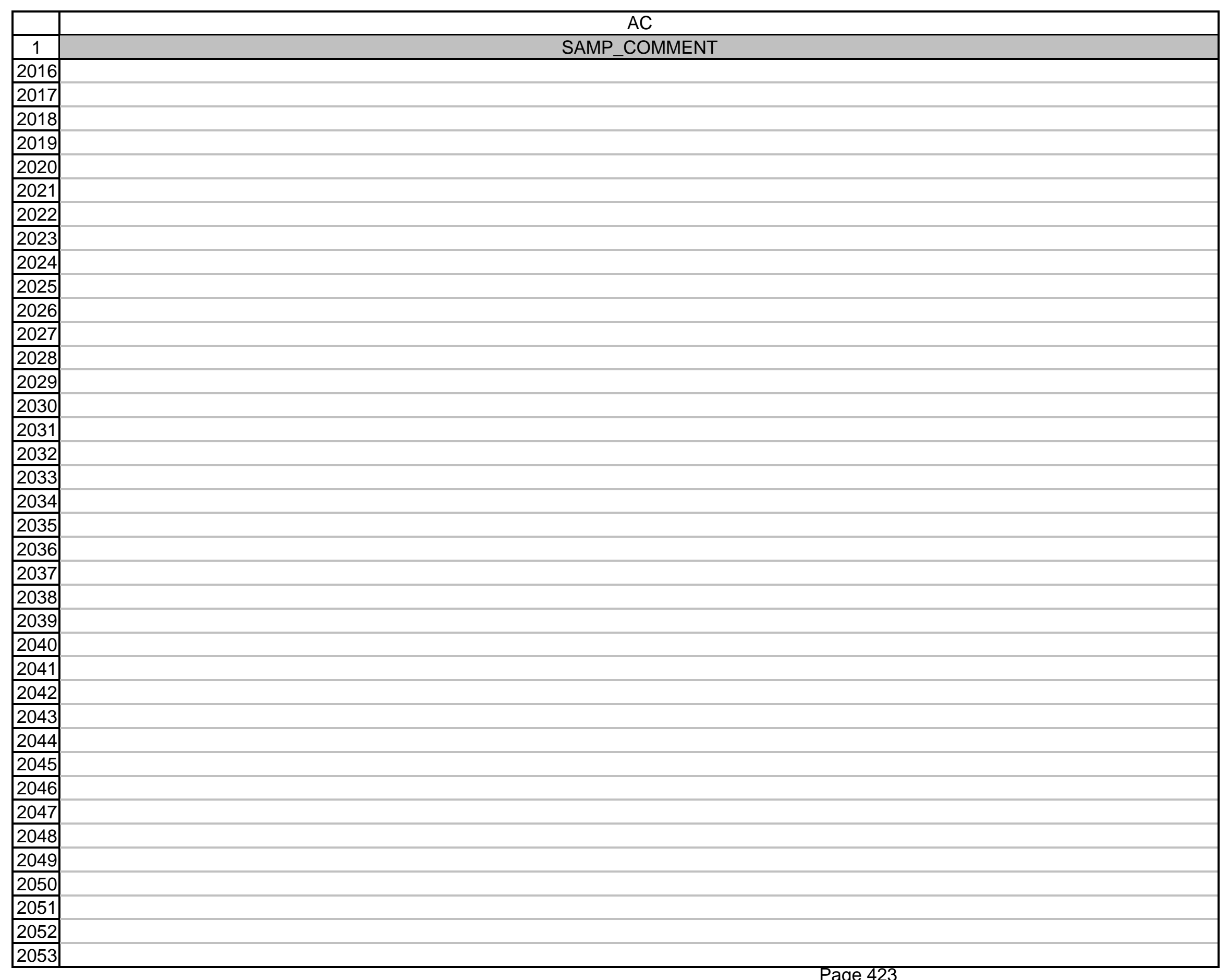




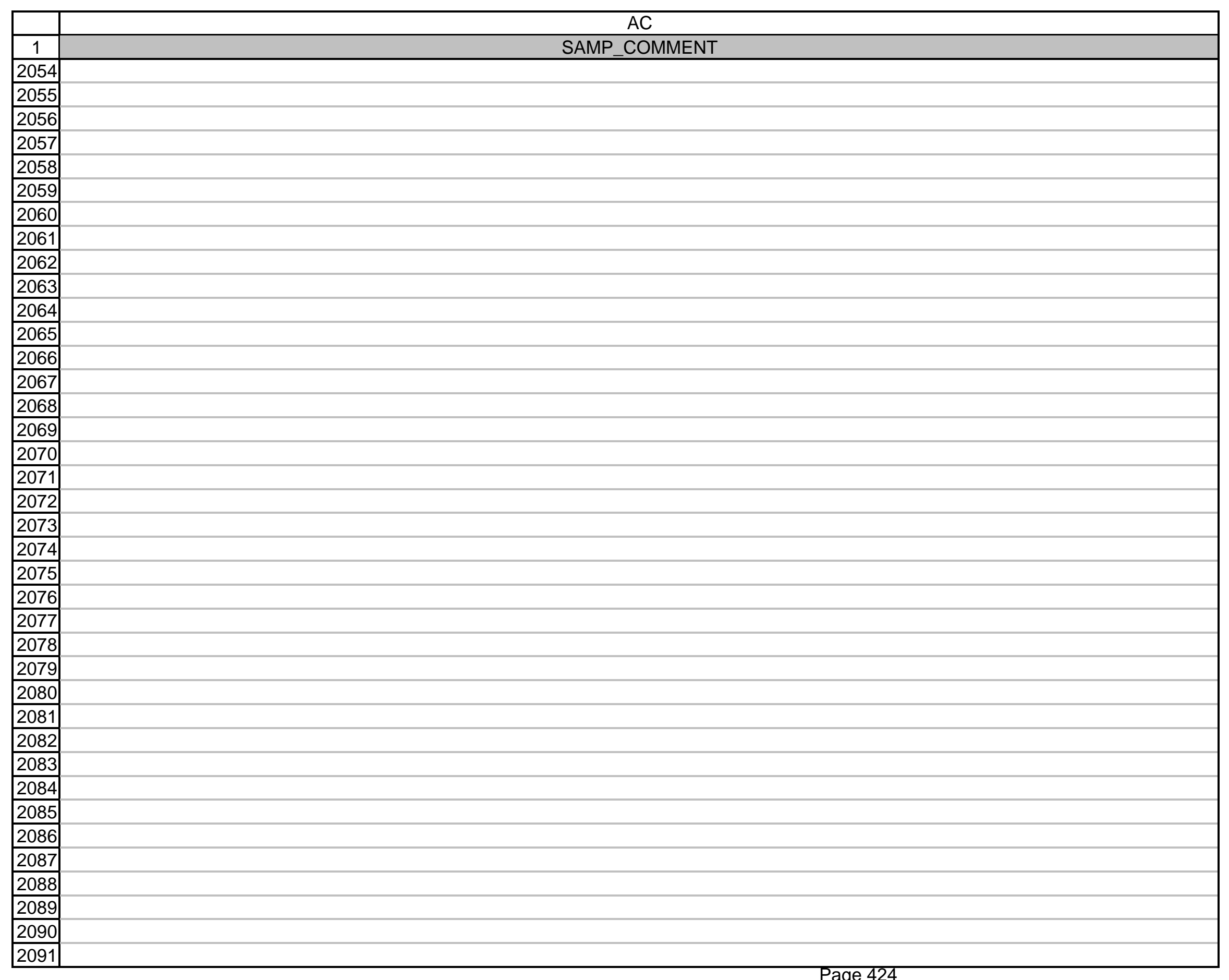




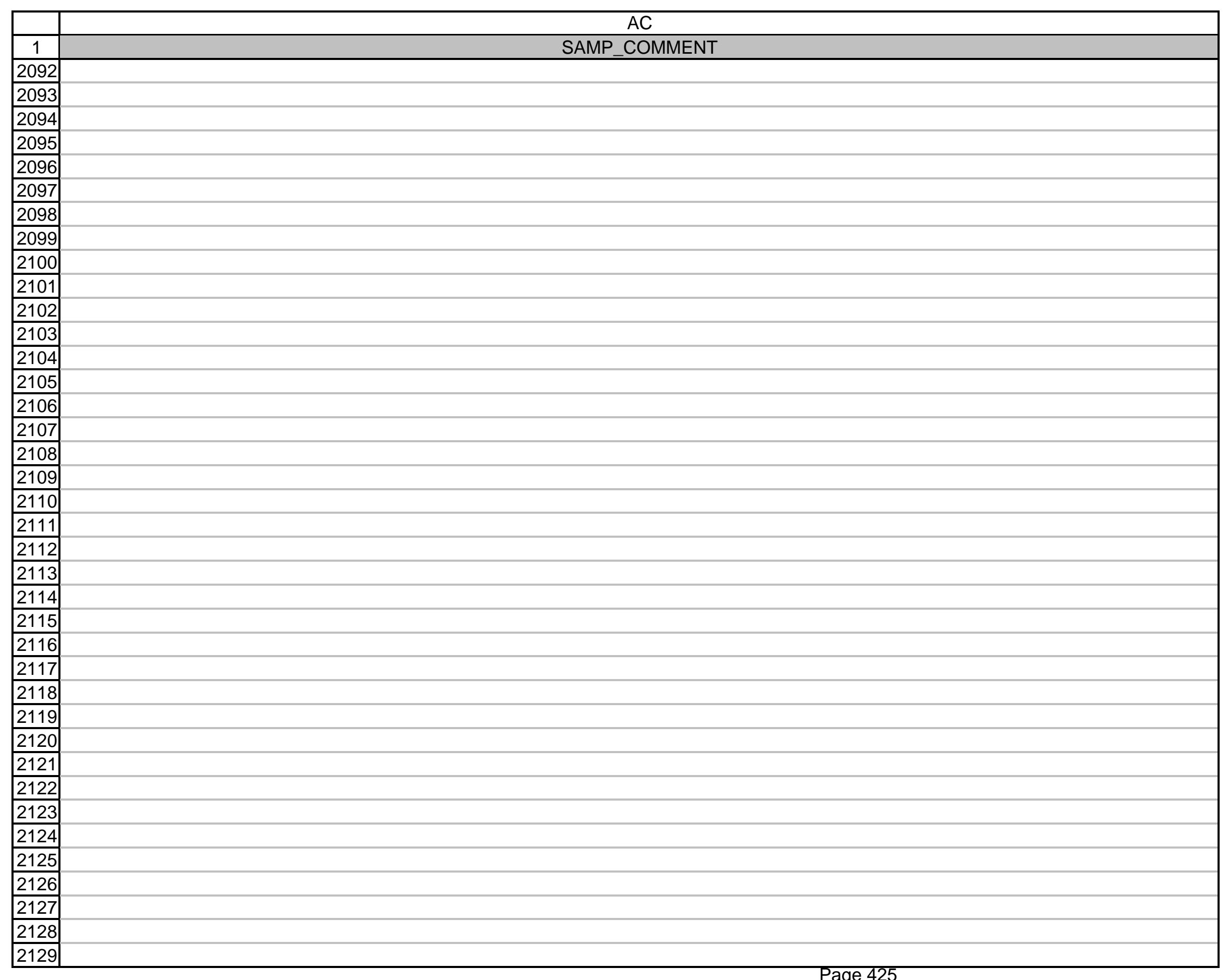




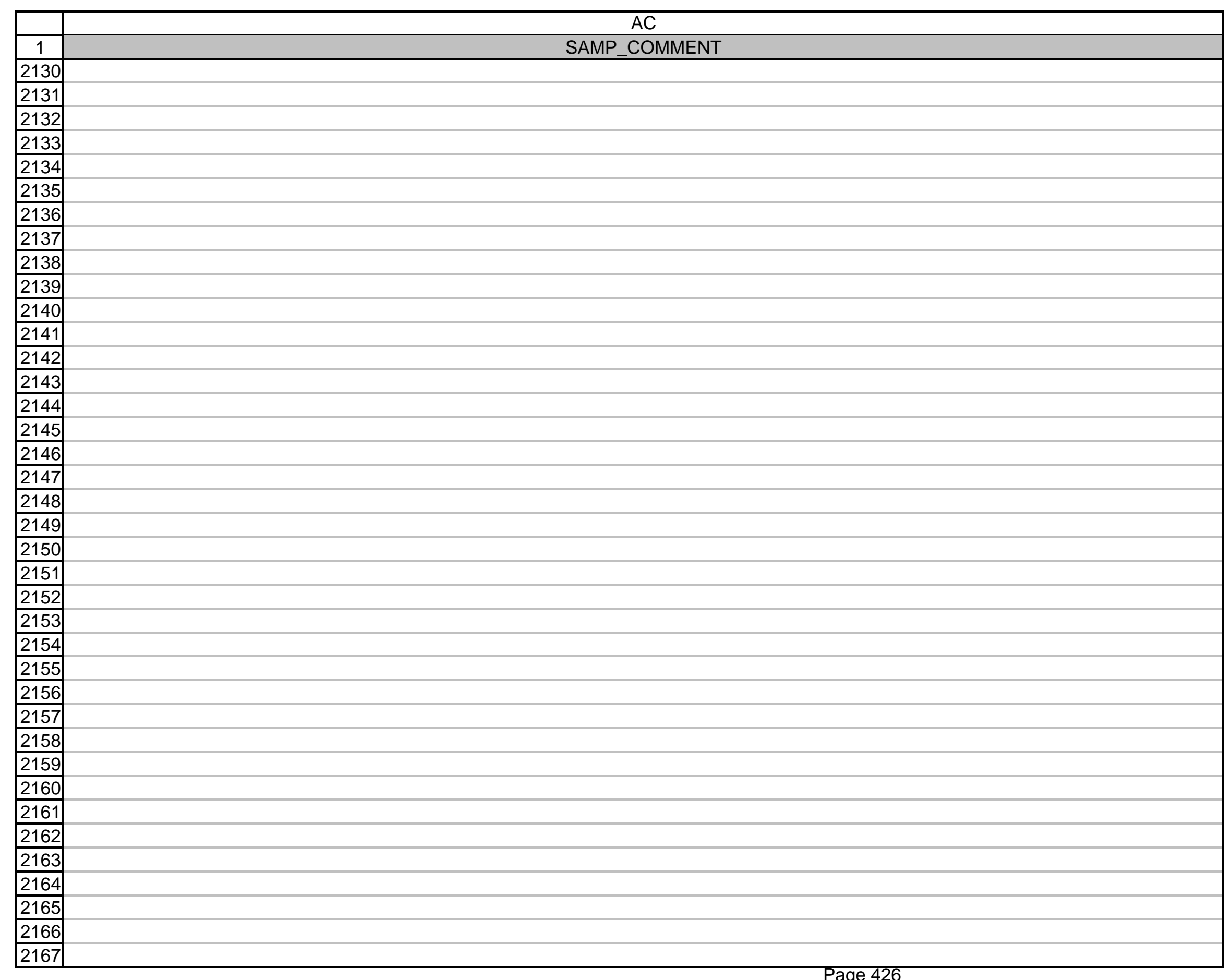




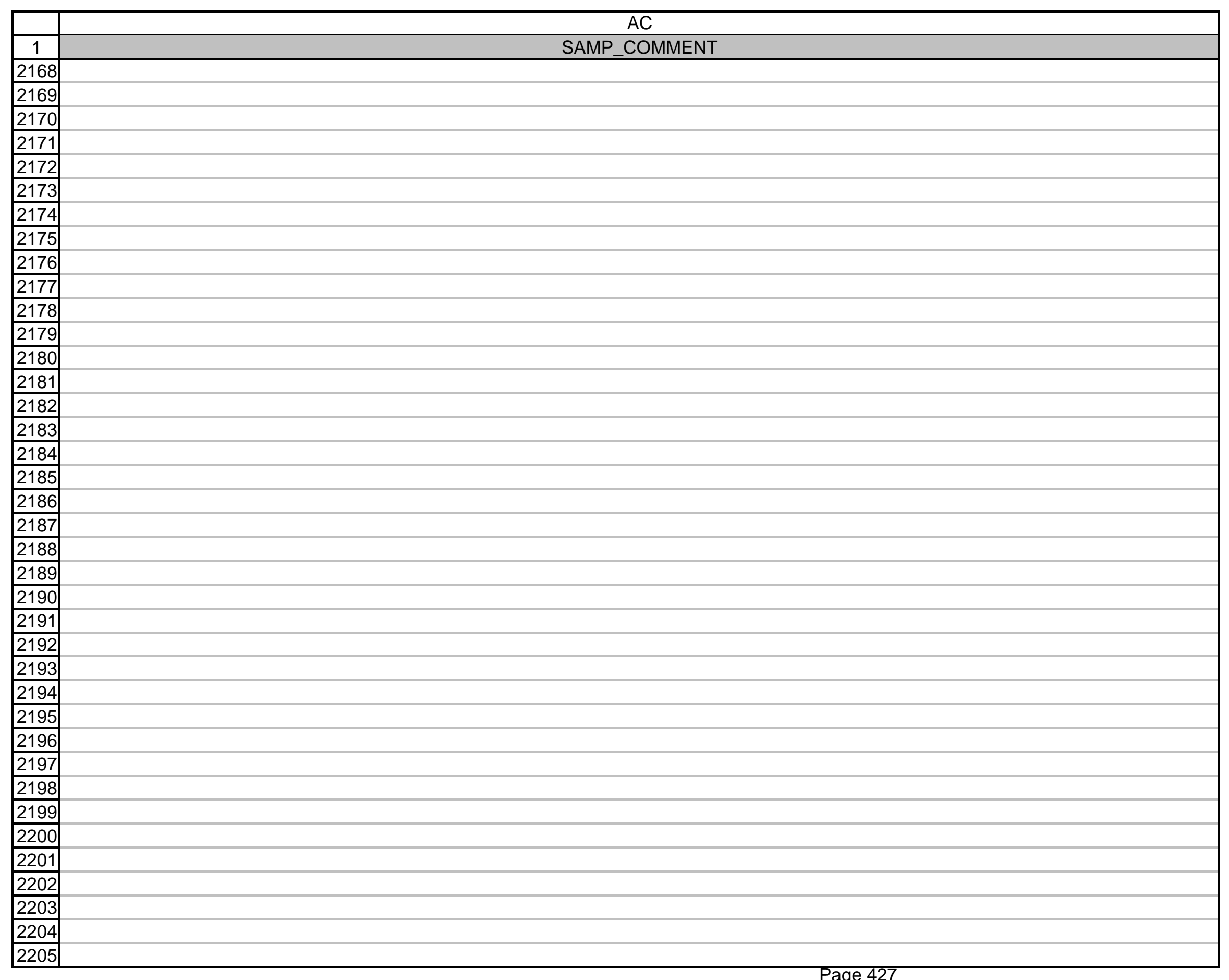




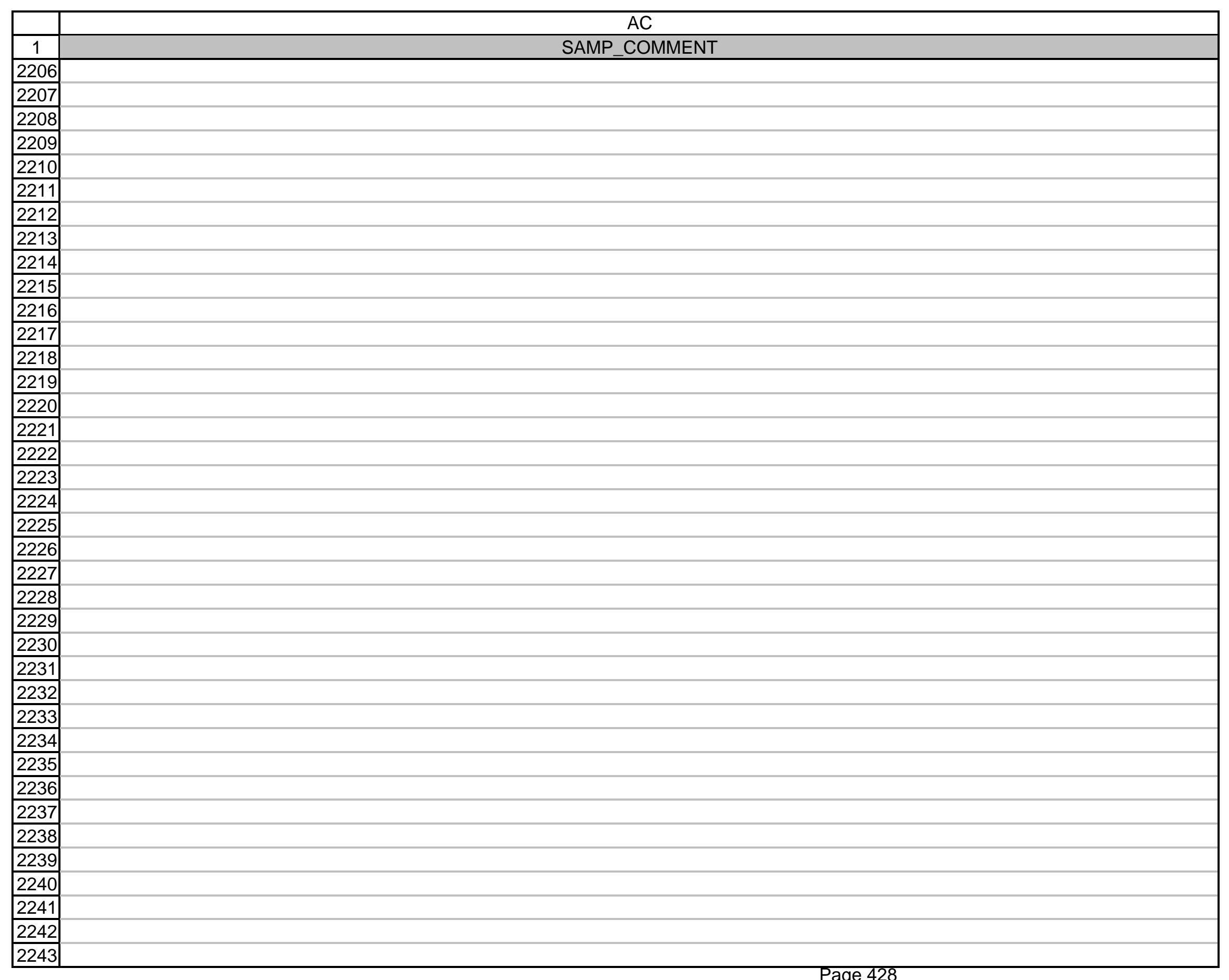




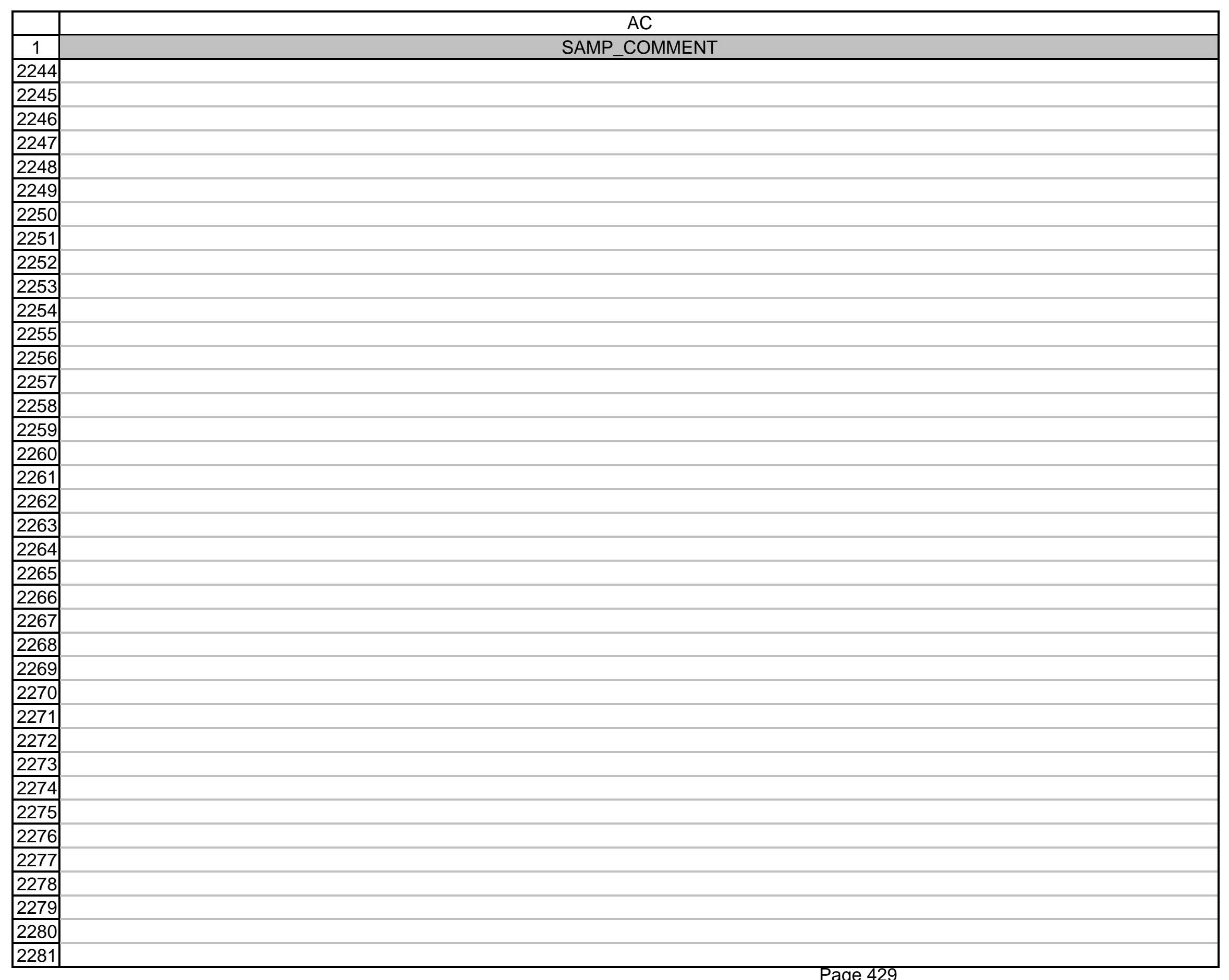




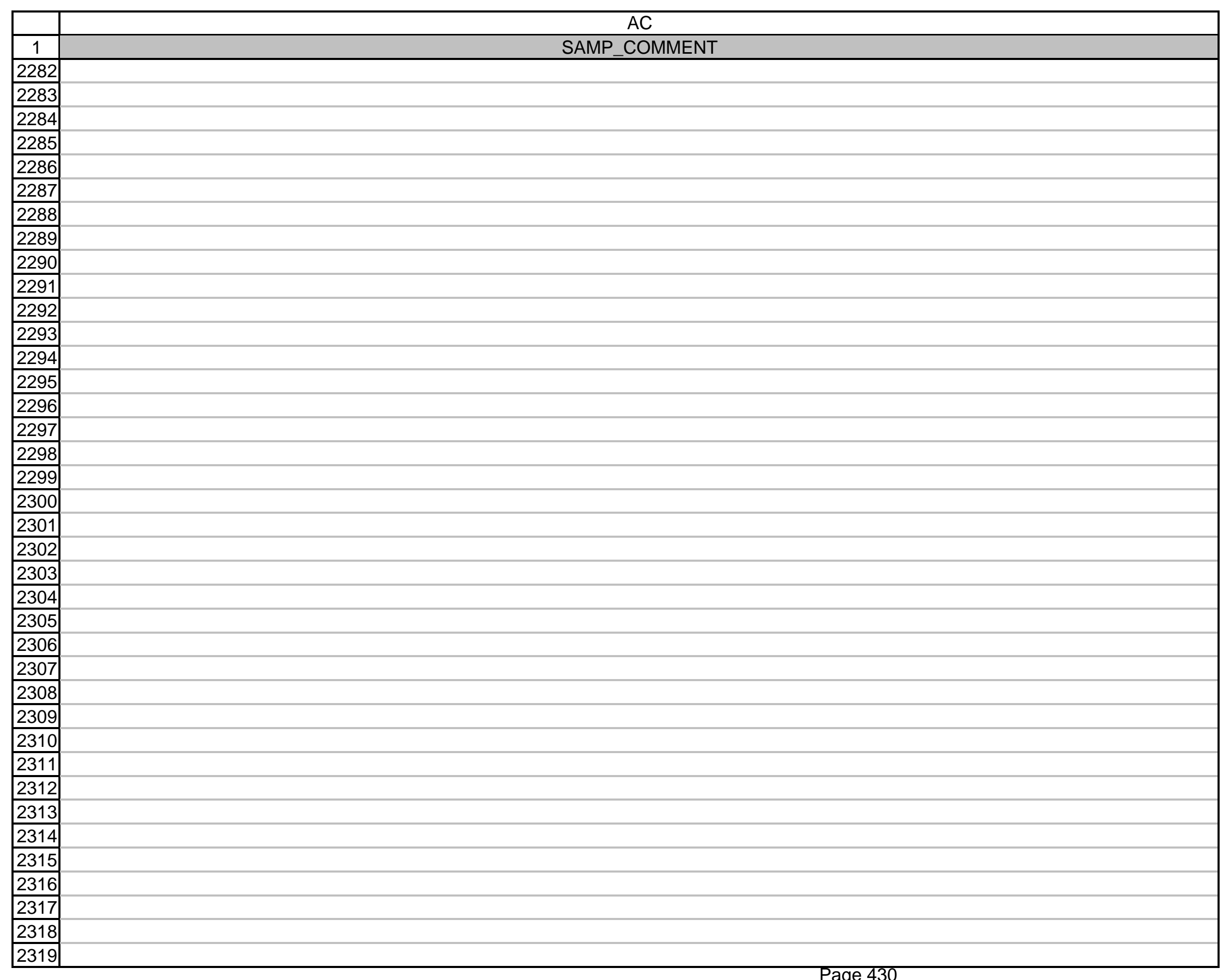




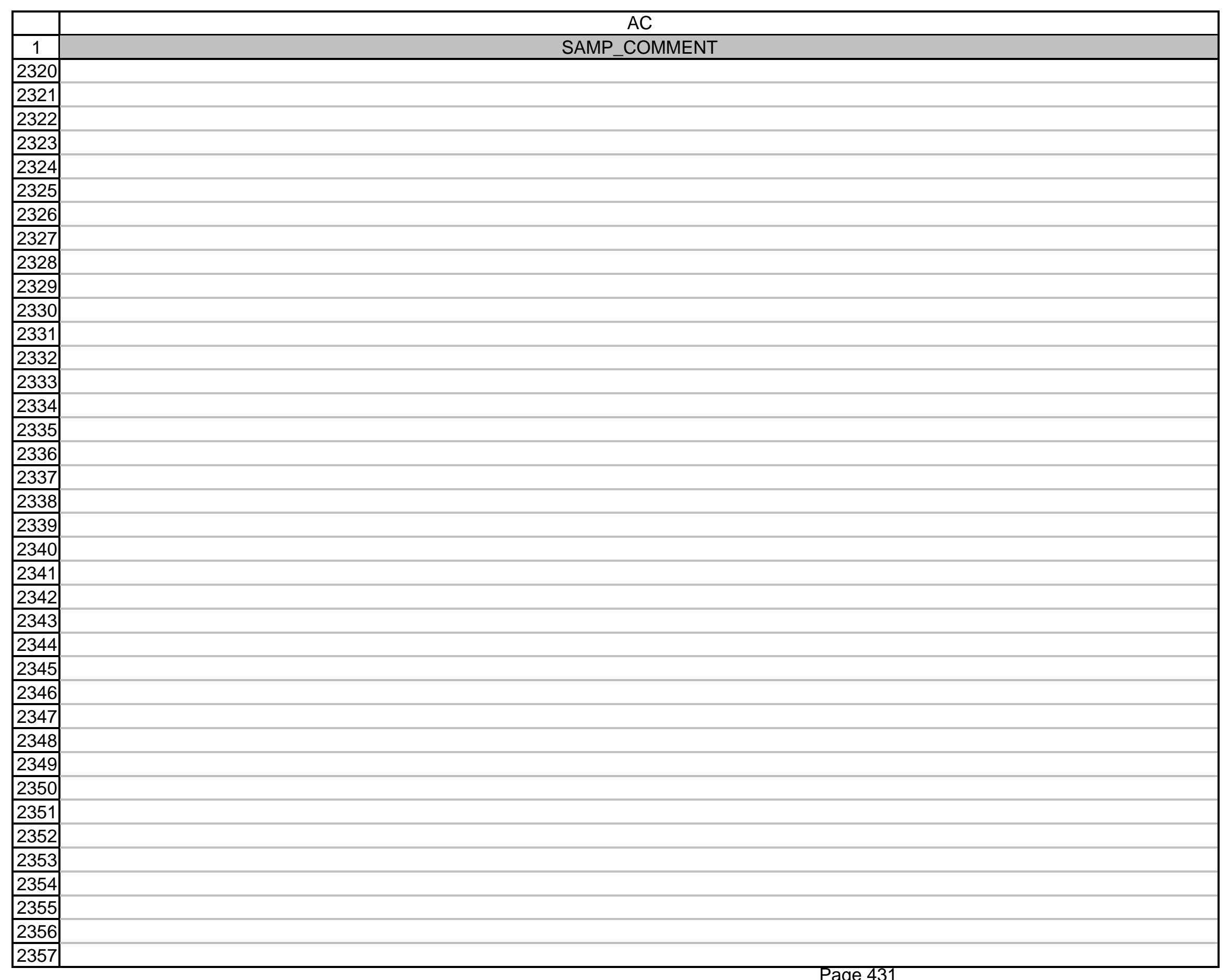




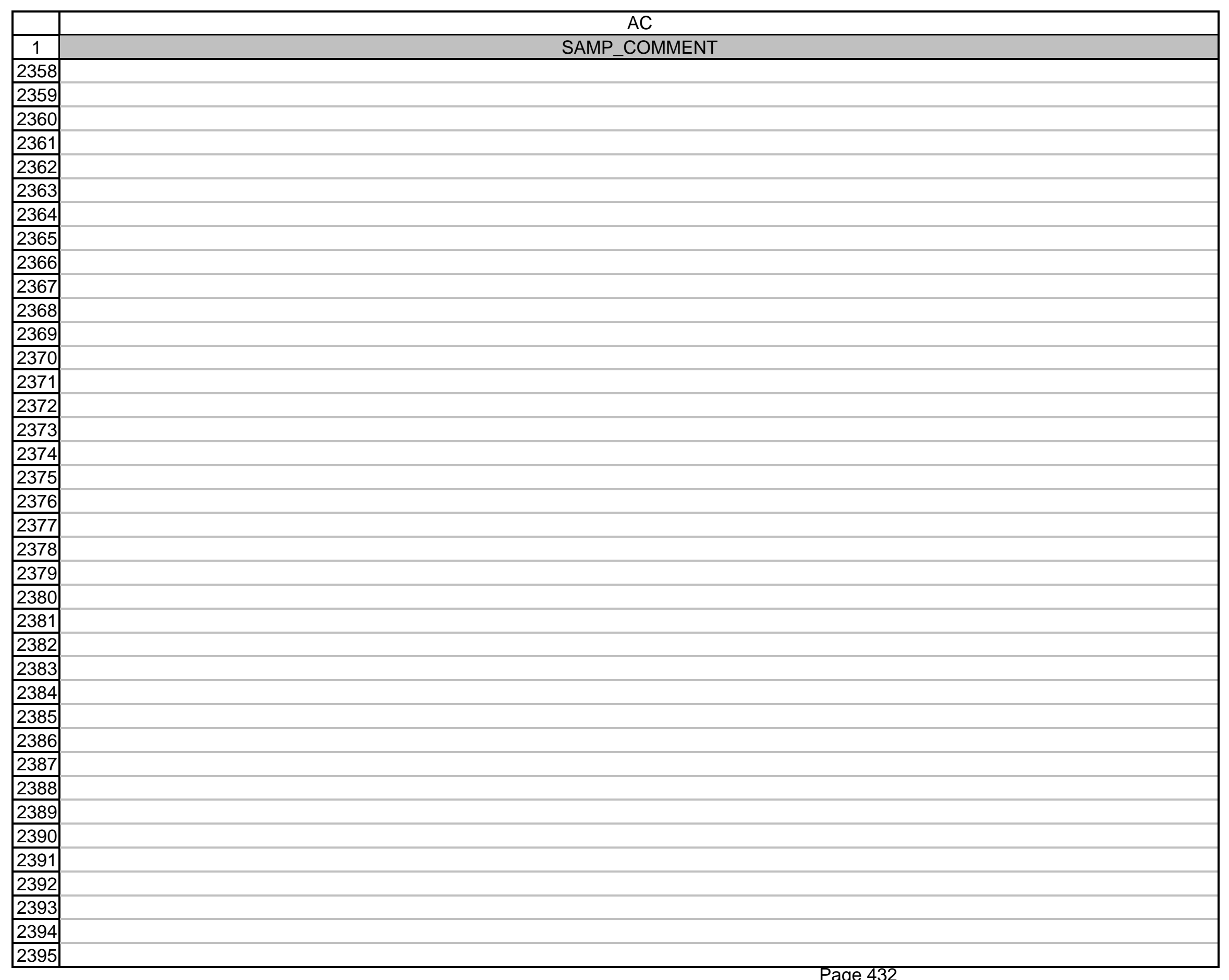




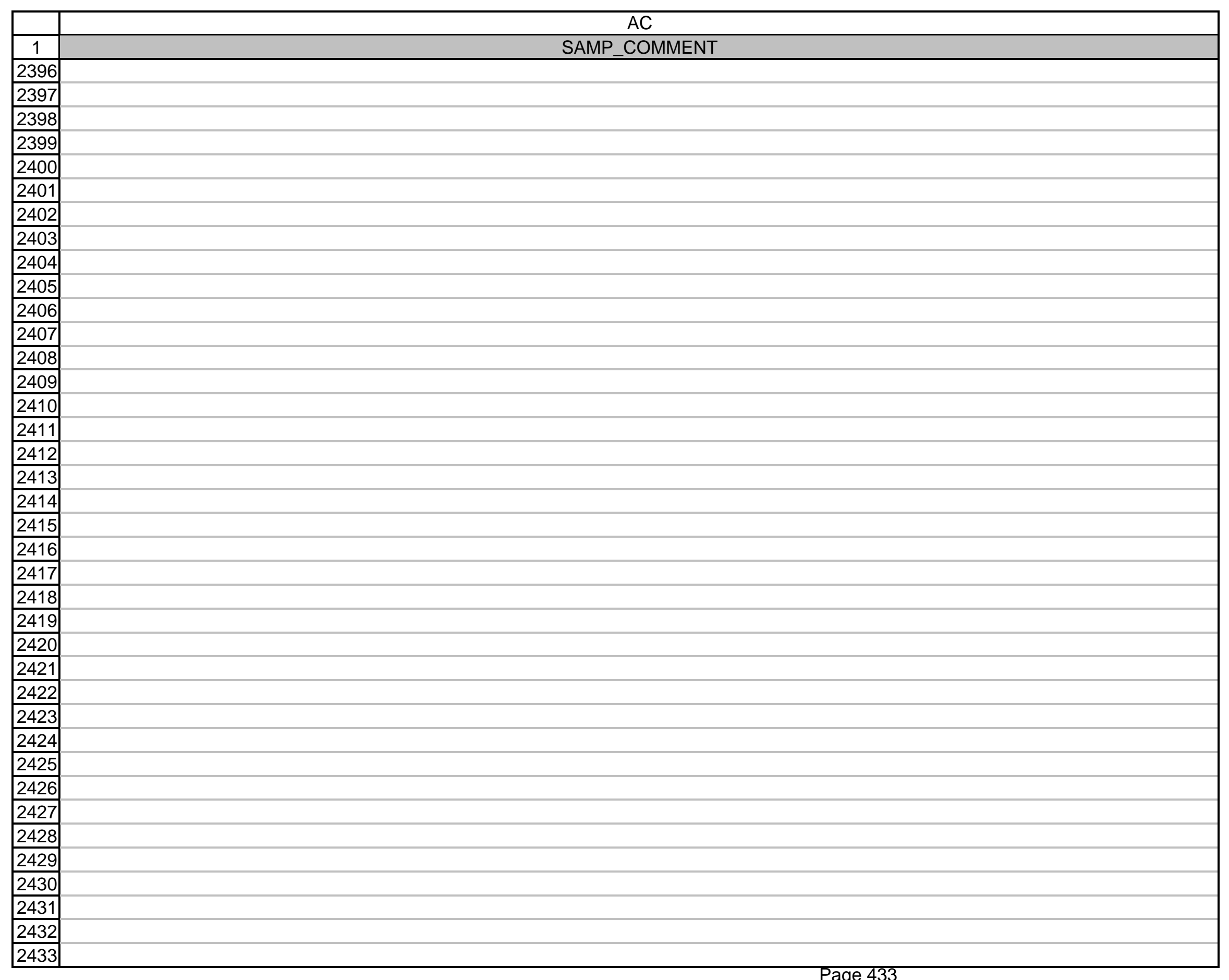




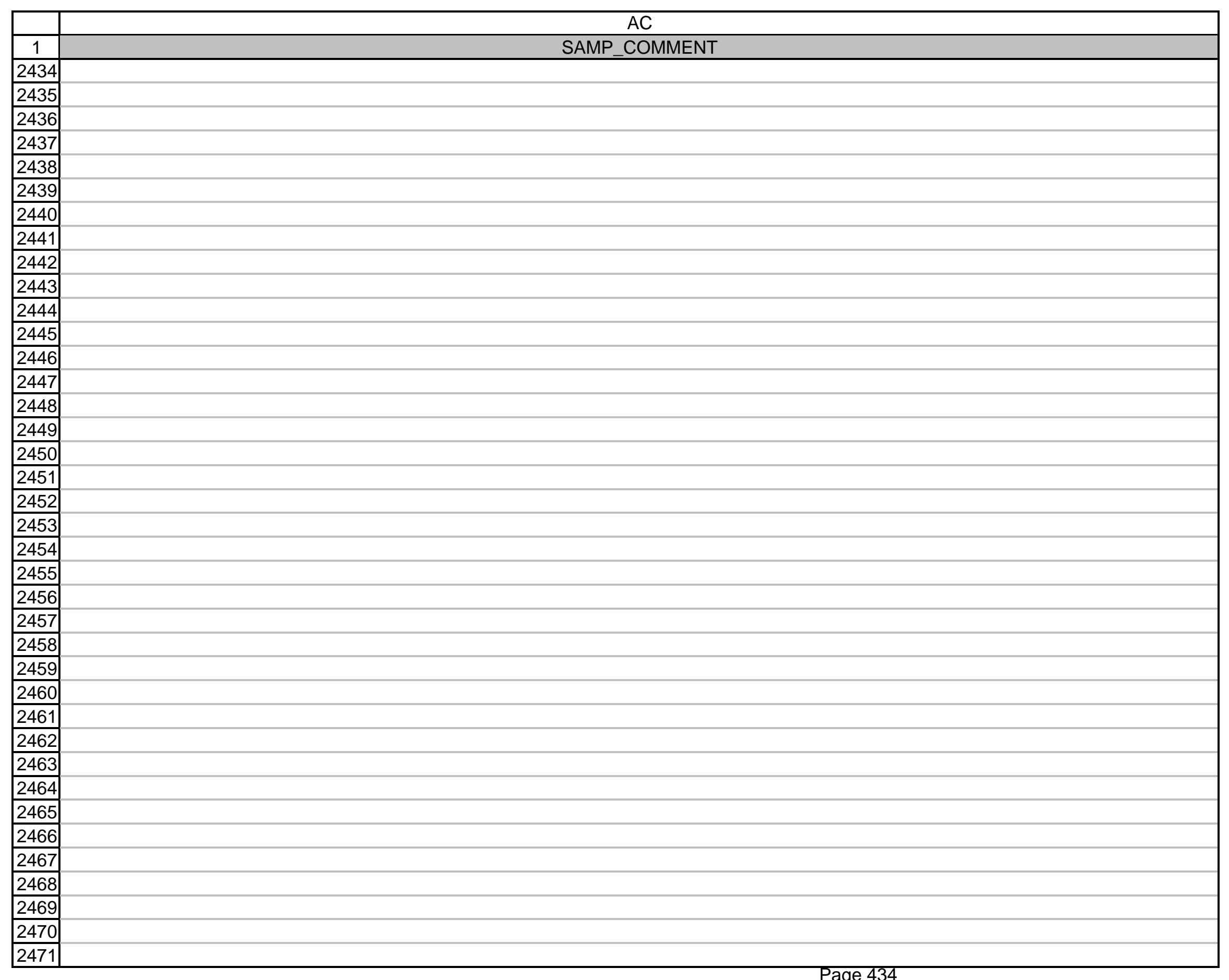


\begin{tabular}{l|l}
\hline 2472 & BOAT ENGINE LEFT RUNNING WHEN SAMPLES WERE COLLECTED.
\end{tabular}

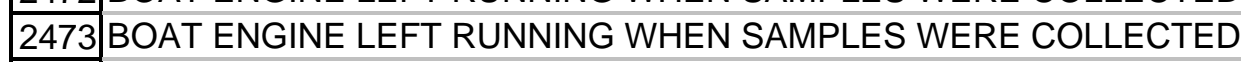

2474 BOAT ENGINE LEFT RUNNING WHEN SAMPLES WERE COLLECTED.

2475 BOAT ENGINE LEFT RUNNING WHEN SAMPLES WERE COLLECTED.

2476 BOAT ENGINE LEFT RUNNING WHEN SAMPLES WERE COLLECTED.

2477

2478

2479 BOAT ENGINE LEFT RUNNING WHEN SAMPLES WERE COLLECTED.

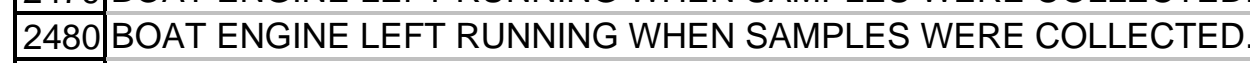

2481 BOAT ENGINE LEFT RUNNING WHEN SAMPLES WERE COLLECTED.

2482 BOAT ENGINE LEFT RUNNING WHEN SAMPLES WERE COLLECTED.

2483 BOAT ENGINE LEFT RUNNING WHEN SAMPLES WERE COLLECTED.

\section{4}

2485

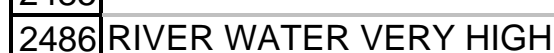

\begin{tabular}{|l|l|}
\hline 2487 & RIVER WATER VERY HIGH \\
\hline 2488 & RIVER WATER VERY HIGH. \\
\hline
\end{tabular}

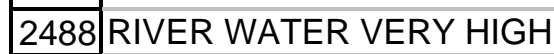

2489 RIVER WATER VERY HIGH

2490 RIVER WATER VERY HIGH.

2491

2492

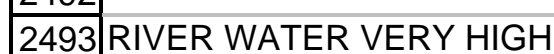

2494 RIVER WATER VERY HIGH.

2495 RIVER WATER VERY HIGH

2496 RIVER WATER VERY HIGH

2497 RIVER WATER VERY HIGH.

2498

2499

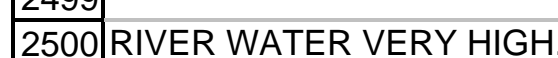

\begin{tabular}{l|l}
2501 & RIVER WATER VERY HIGH. \\
\hline
\end{tabular}

\begin{tabular}{l|l}
2502 & RIVER WATER VERY HIGH \\
\hline
\end{tabular}

2503 RIVER WATER VERY HIGH

2504 RIVER WATER VERY HIGH.

2505

2506

\begin{tabular}{|l|l}
2507 & RIVER WATER VERY HIGH \\
\hline 2508
\end{tabular}

2508 RIVER WATER VERY HIGH

\begin{tabular}{ll}
2509 & RIVER WATER VERY HIGH \\
\hline
\end{tabular} 
AC

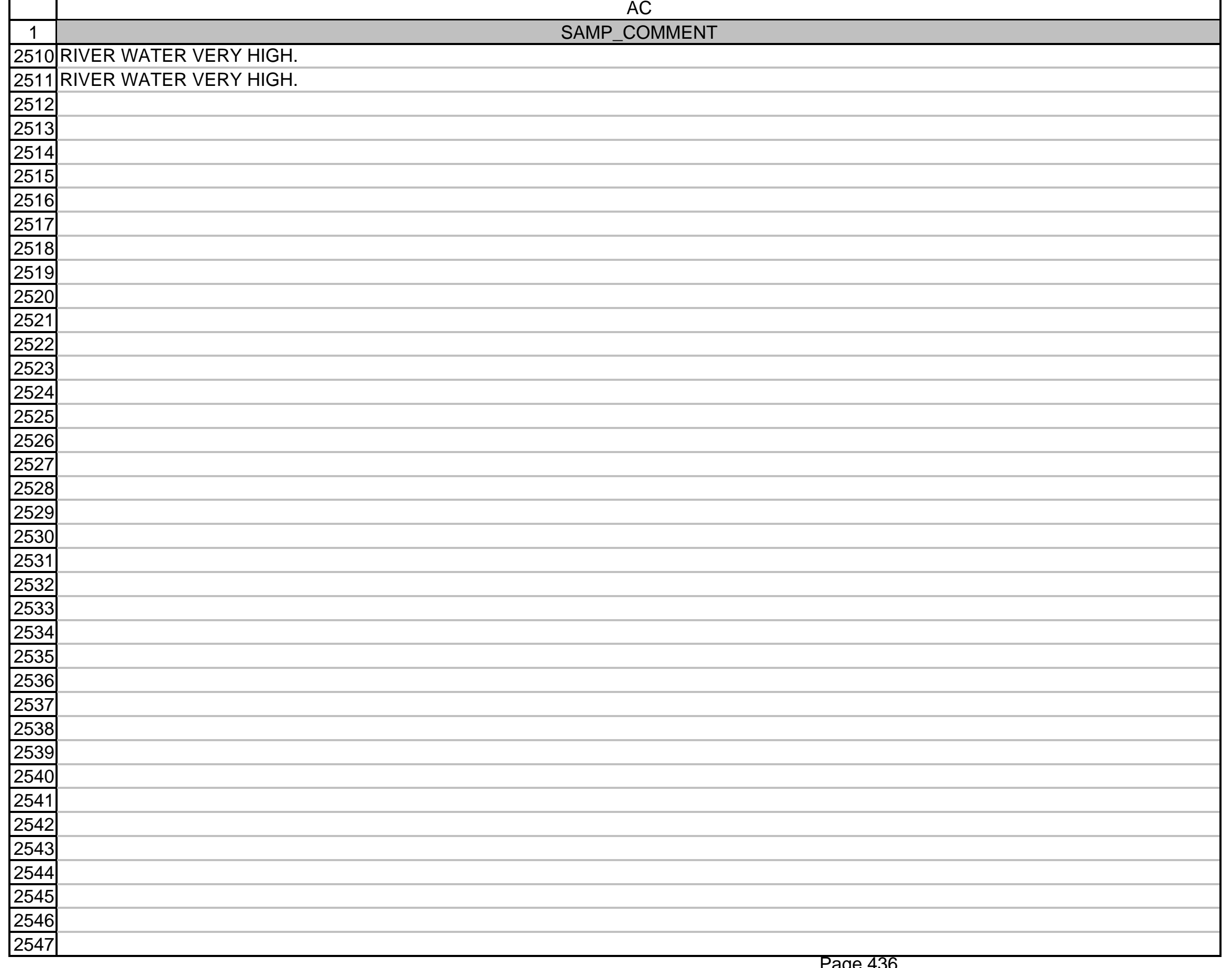




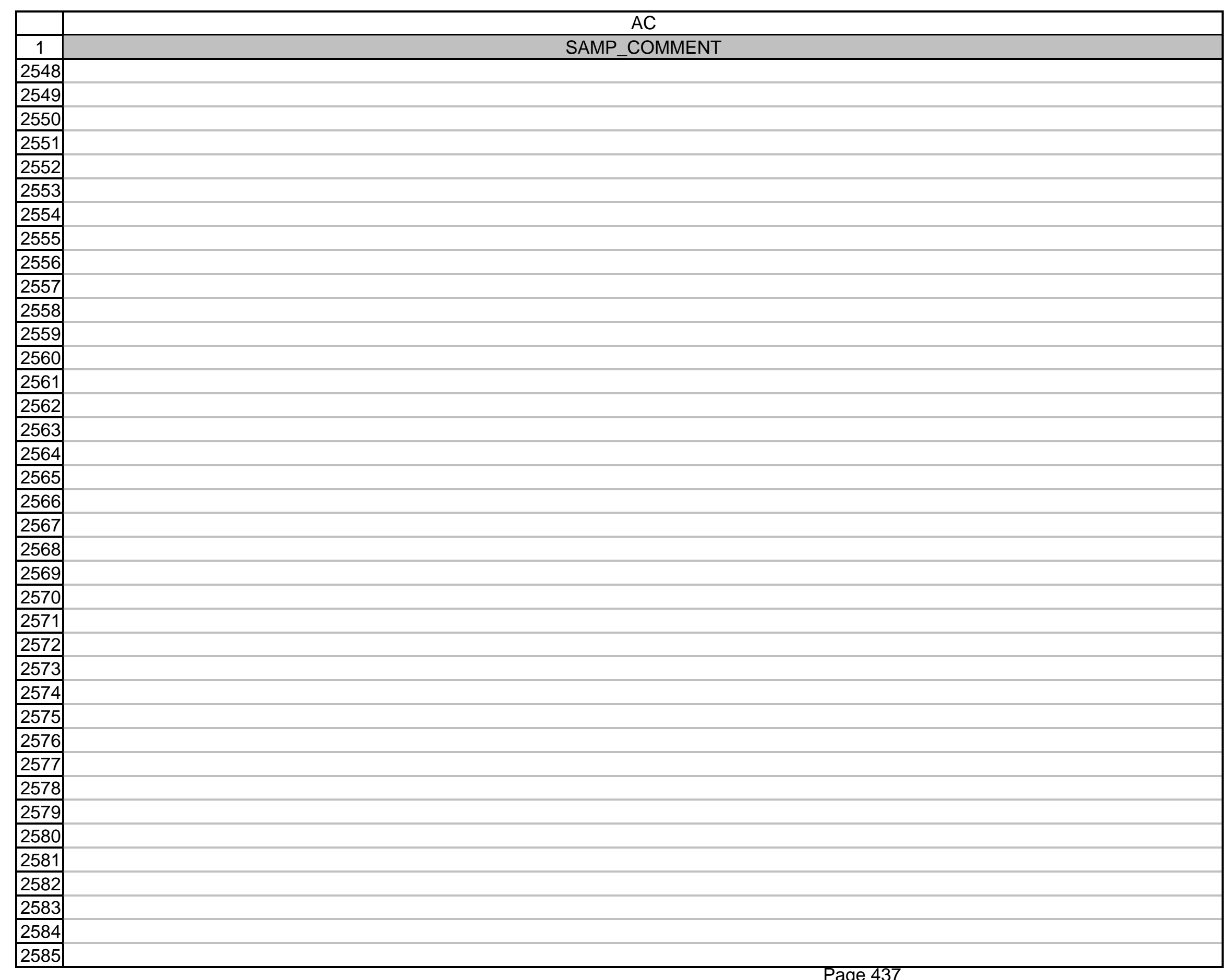


AC

\begin{tabular}{|c|c|}
\hline & $\frac{A C}{2}$ \\
\hline 1 & SAMP_COMMENT \\
\hline 2586 & \\
\hline 2587 & \\
\hline 2588 & \\
\hline 2589 & \\
\hline 2590 & \\
\hline 2591 & \\
\hline 2592 & \\
\hline 2593 & \\
\hline 2594 & \\
\hline 2595 & \\
\hline 2596 & \\
\hline 2597 & \\
\hline 2598 & \\
\hline 2599 & \\
\hline 2600 & \\
\hline 2601 & \\
\hline 2602 & \\
\hline 2603 & \\
\hline 2604 & \\
\hline 2605 & \\
\hline 2606 & \\
\hline 2607 & \\
\hline 2608 & \\
\hline 2609 & \\
\hline 2610 & \\
\hline 2611 & \\
\hline 2612 & \\
\hline 2613 & \\
\hline 2614 & \\
\hline 2615 & \\
\hline 2616 & \\
\hline 2617 & \\
\hline 2618 & \\
\hline 2619 & WATER LEVEL HIGH. \\
\hline 2620 & WATER LEVEL HIGH. \\
\hline 2621 & WATER LEVEL HIGH. \\
\hline 2622 & WATER LEVEL HIGH. \\
\hline 2623 & WATER LEVEL HIGH. \\
\hline
\end{tabular}


$\mathrm{AC}$

\begin{tabular}{|c|c|}
\hline & 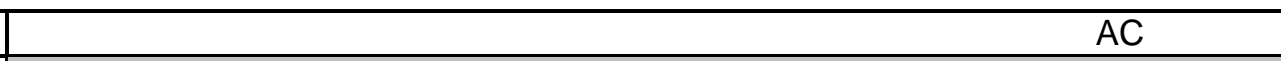 \\
\hline 1 & SAMP_COMMENT \\
\hline 2624 & \\
\hline 2625 & \\
\hline 2626 & WATER LEVEL HIGH. \\
\hline 2627 & WATER LEVEL HIGH. \\
\hline 2628 & WATER LEVEL HIGH. \\
\hline 2629 & WATER LEVEL HIGH. \\
\hline 2630 & WATER LEVEL HIGH. \\
\hline 2631 & \\
\hline 2632 & \\
\hline 2633 & WATER LEVEL HIGH. \\
\hline 2634 & WATER LEVEL HIGH. \\
\hline 2635 & WATER LEVEL HIGH. \\
\hline 2636 & WATER LEVEL HIGH. \\
\hline 2637 & WATER LEVEL HIGH. \\
\hline 2638 & \\
\hline 2639 & \\
\hline 2640 & WATER LEVEL HIGH. \\
\hline 2641 & WATER LEVEL HIGH. \\
\hline 2642 & WATER LEVEL HIGH. \\
\hline 2643 & WATER LEVEL HIGH. \\
\hline 2644 & WATER LEVEL HIGH. \\
\hline 2645 & \\
\hline 2646 & \\
\hline 2647 & WATER LEVEL HIGH. \\
\hline 2648 & WATER LEVEL HIGH. \\
\hline 2649 & WATER LEVEL HIGH. \\
\hline 2650 & WATER LEVEL HIGH. \\
\hline 2651 & WATER LEVEL HIGH. \\
\hline 2652 & \\
\hline 2653 & \\
\hline 2654 & WATER LEVEL HIGH. \\
\hline 2655 & WATER LEVEL HIGH. \\
\hline 2656 & WATER LEVEL HIGH. \\
\hline 2657 & WATER LEVEL HIGH. \\
\hline 2658 & WATER LEVEL HIGH. \\
\hline 2659 & \\
\hline 2660 & 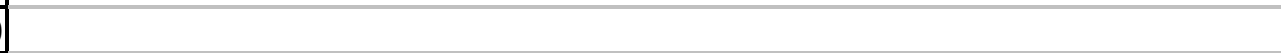 \\
\hline 2661 & WATER LEVEL HIGH. \\
\hline
\end{tabular}


$A C$

2662 WATER LEVEL HIGH

2663 WATER LEVEL HIGH.

2664 WATER LEVEL HIGH

2665 WATER LEVEL HIGH.

2666

2667

2668 WATER LEVEL HIGH

2669 WATER LEVEL HIGH.

2670 WATER LEVEL HIGH.

2671 WATER LEVEL HIGH.

2672 WATER LEVEL HIGH.

2673

2674

2675 WATER LEVEL HIGH.

2676 WATER LEVEL HIGH.

\begin{tabular}{|l|l}
2677 & WATER LEVEL HIGH. \\
\hline 2678 & WATER LEVEL HIGH.
\end{tabular}

2678 WATER LEVEL HIGH.

2679 WATER LEVEL HIGH

2680

2681

2682 WATER LEVEL HIGH.

2683 WATER LEVEL HIGH

2684 WATER LEVEL HIGH.

2685 WATER LEVEL HIGH

2686 WATER LEVEL HIGH.

2687

2688

2689

2690

2690

2692

2693

\begin{tabular}{|l|}
\hline 2693 \\
\hline 2695 \\
\hline 2696 \\
\hline
\end{tabular}

2696 


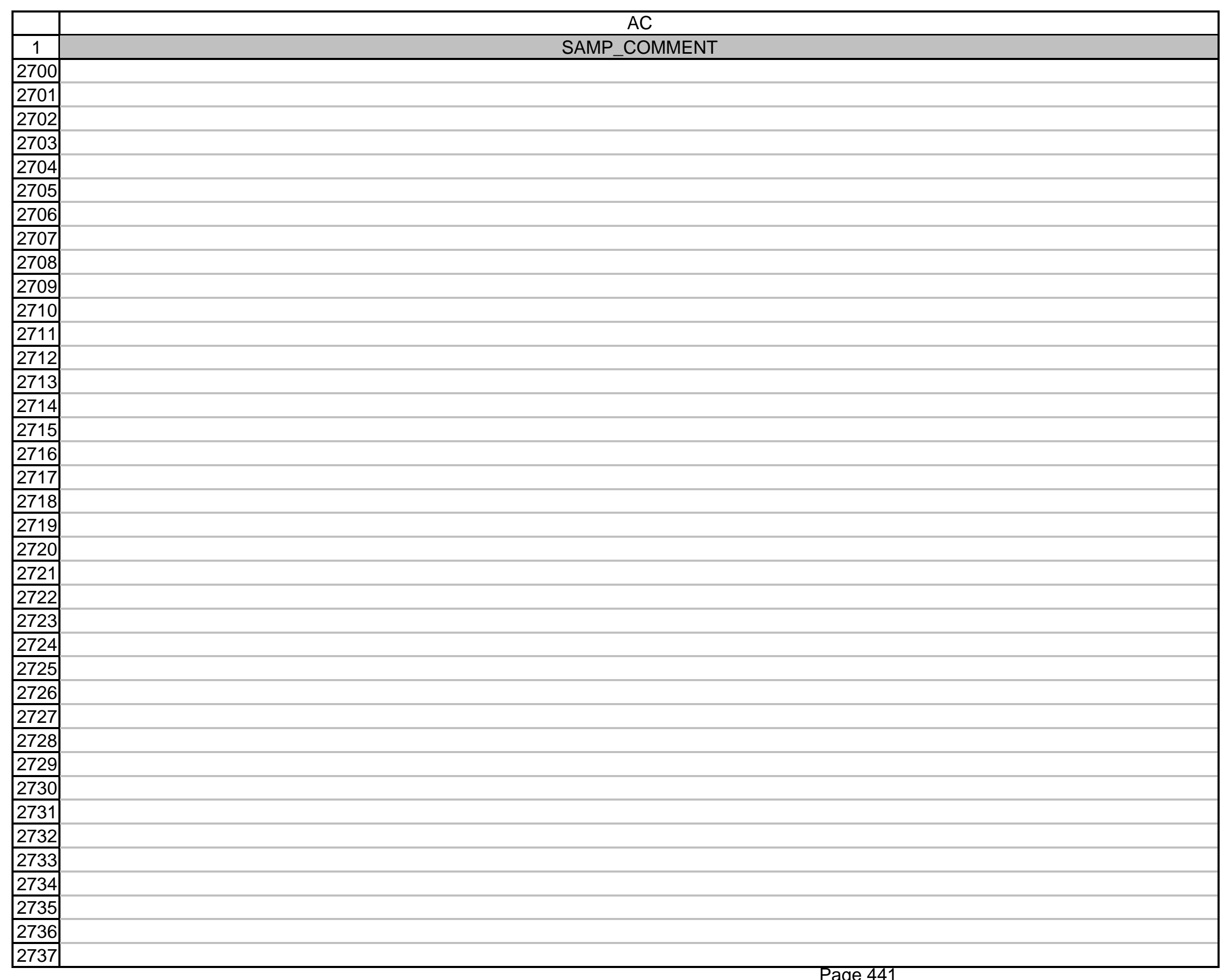




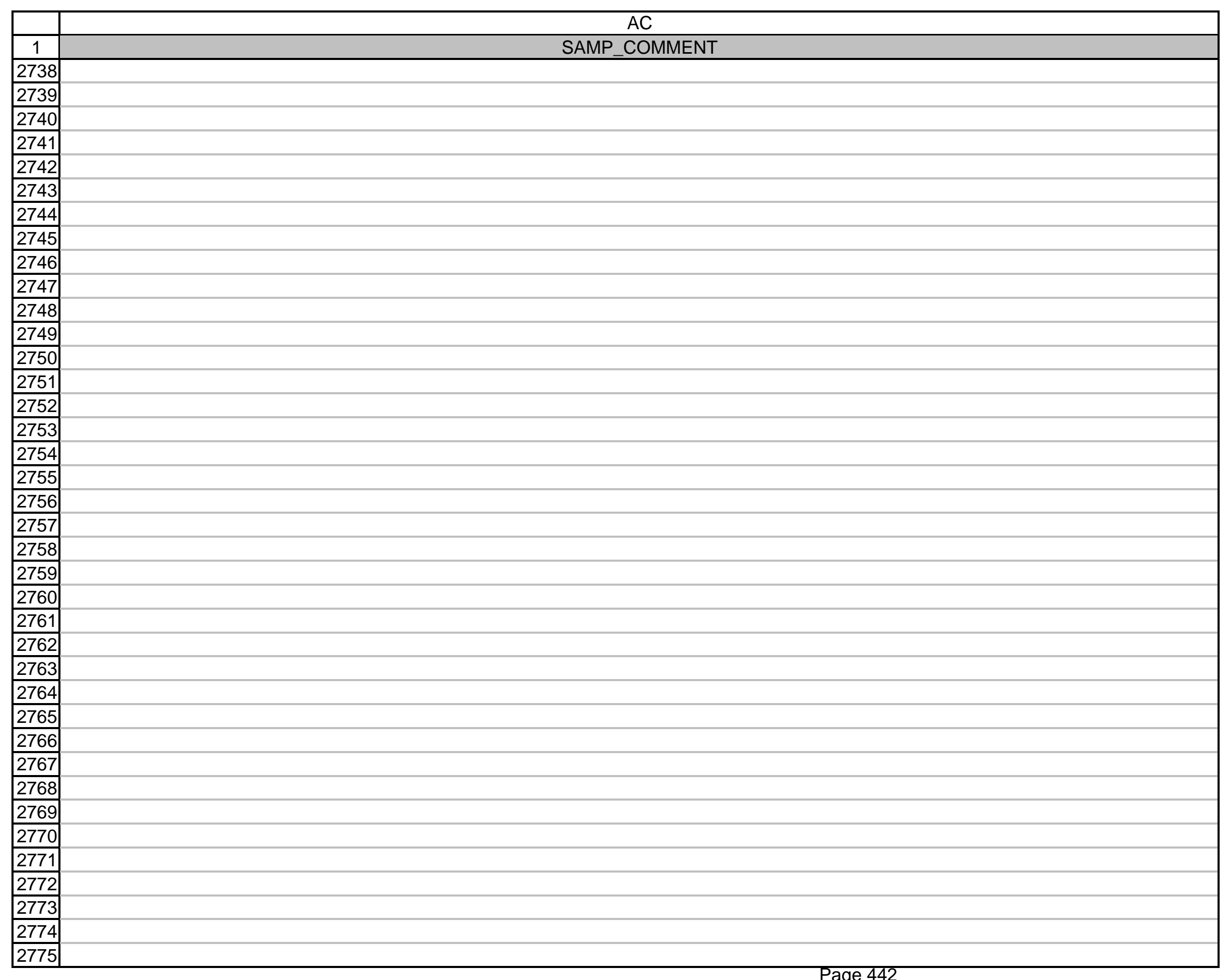




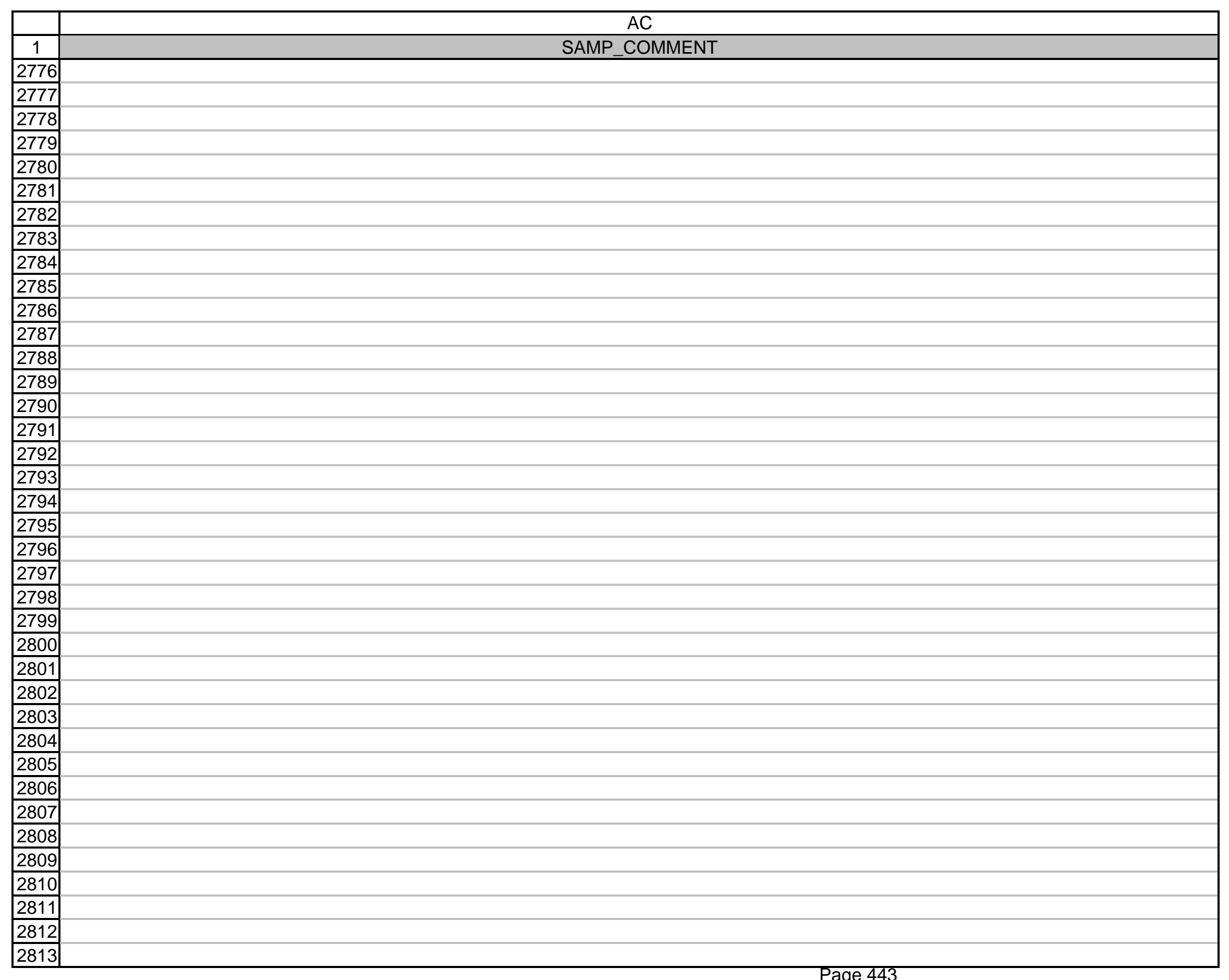




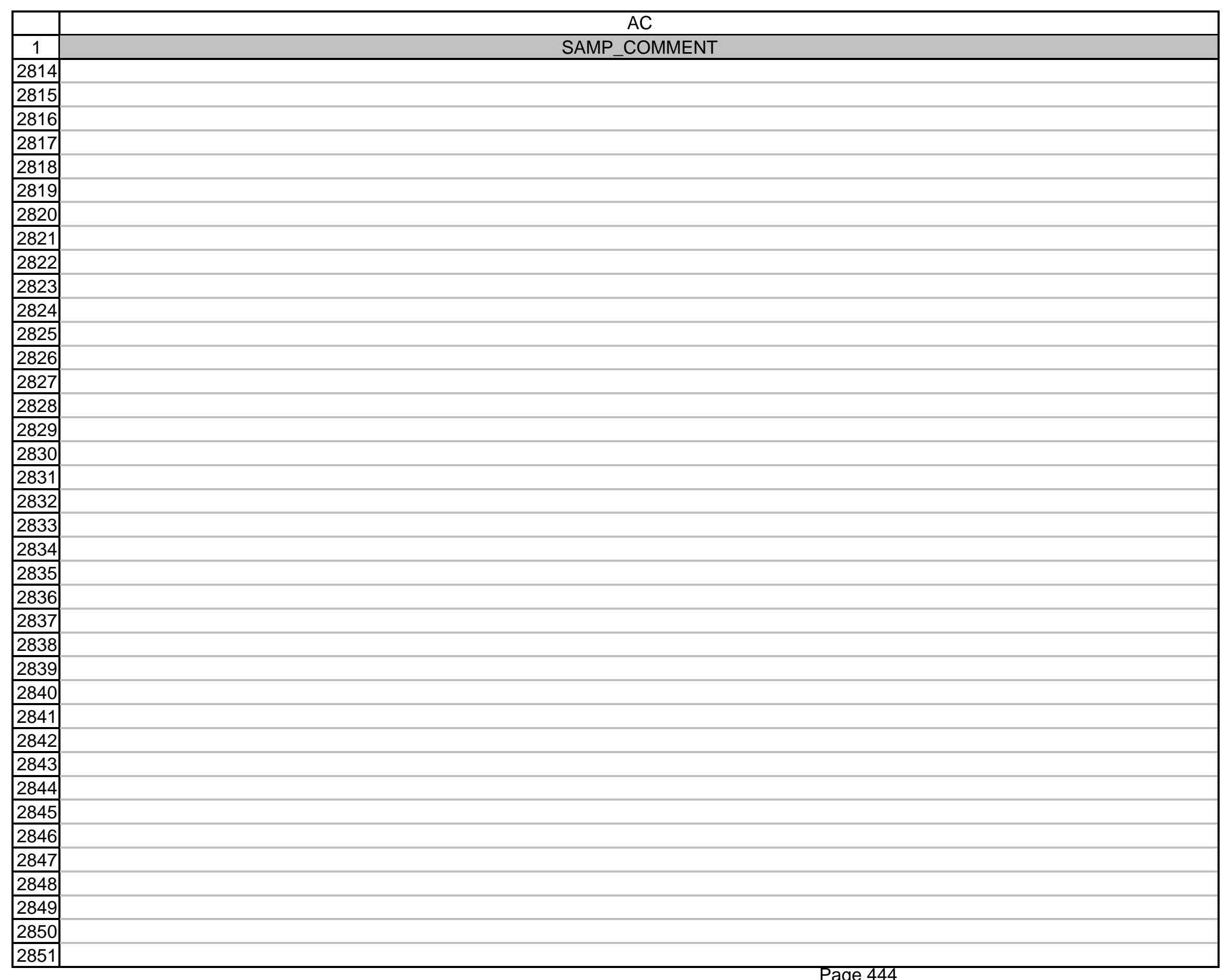




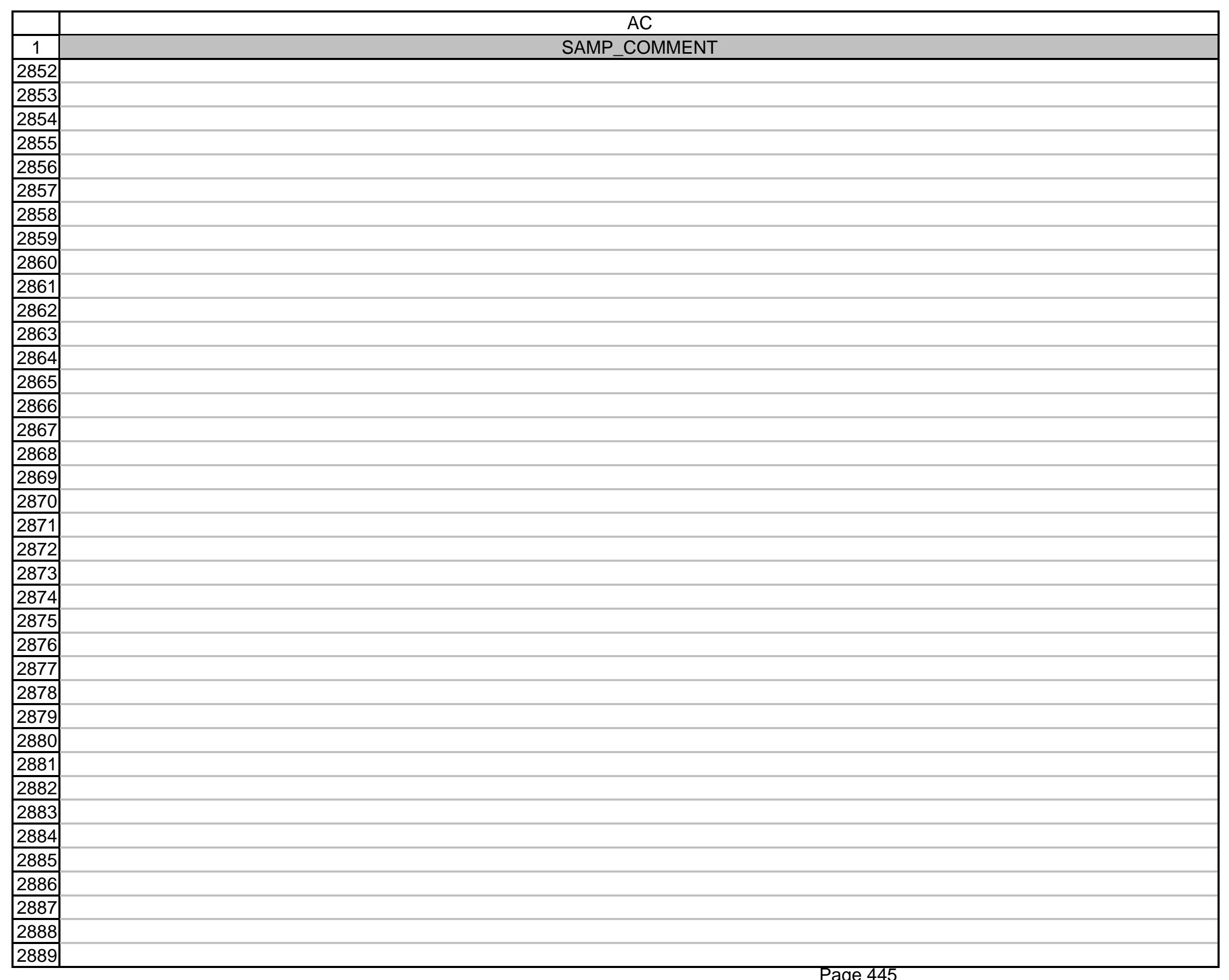




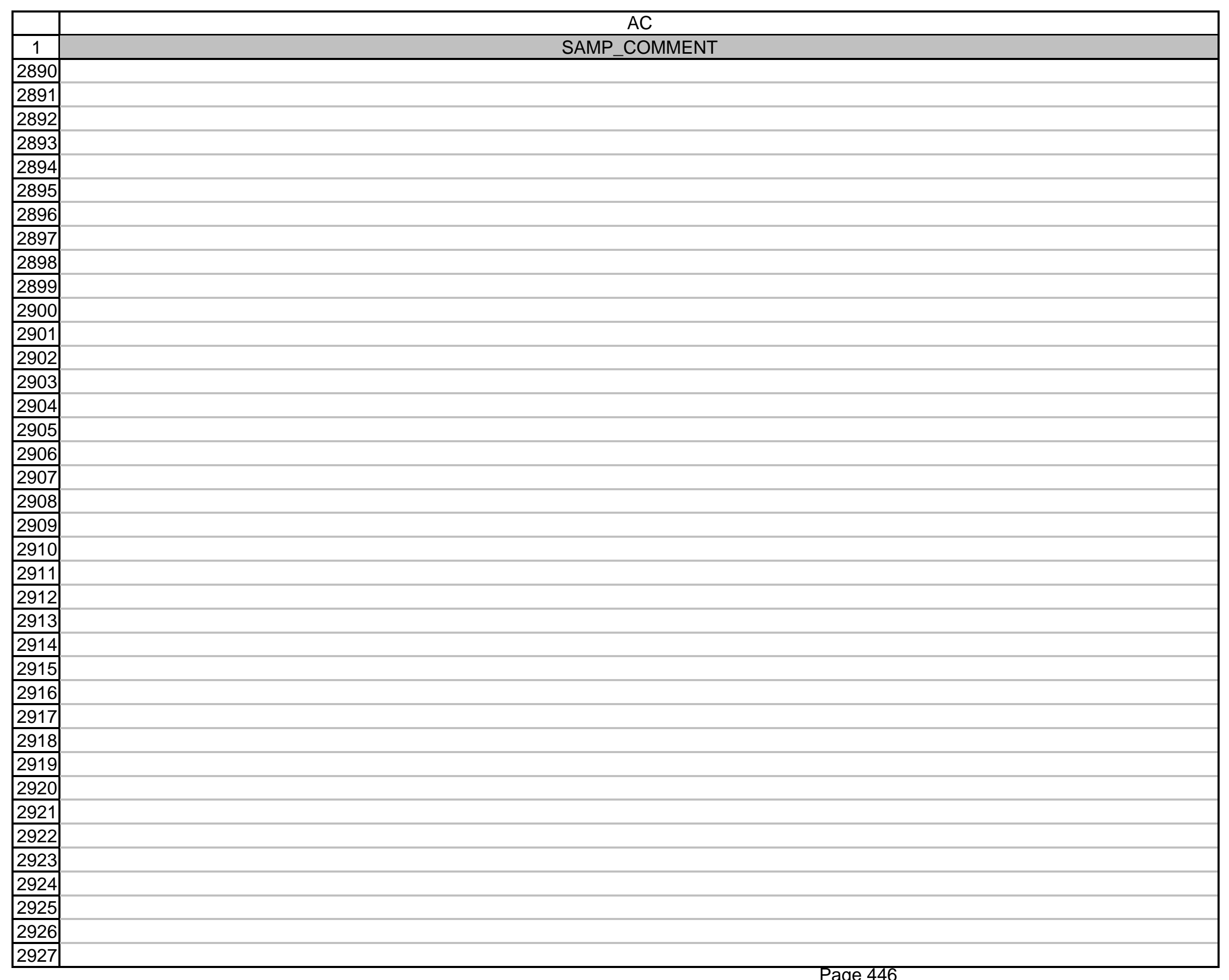


AC

\begin{tabular}{|c|c|}
\hline & 20 \\
\hline 1 & SAMP_COMMENT \\
\hline 2928 & \\
\hline 2929 & \\
\hline 2930 & \\
\hline 2931 & \\
\hline 2932 & SAMPLED AT 3 FT. OF WATER. \\
\hline 2933 & SAMPLED AT 3 FT. OF WATER. \\
\hline 2934 & SAMPLED AT 3 FT. OF WATER. \\
\hline 2935 & SAMPLED AT 3 FT. OF WATER. \\
\hline 2936 & SAMPLED AT 3 FT. OF WATER. \\
\hline 2937 & \\
\hline 2938 & \\
\hline 2939 & SAMPLED AT 2 FT. OF WATER. \\
\hline 2940 & SAMPLED AT 2 FT. OF WATER. \\
\hline 2941 & SAMPLED AT 2 FT. OF WATER. \\
\hline 2942 & SAMPLED AT 2 FT. OF WATER. \\
\hline 2943 & SAMPLED AT 2 FT. OF WATER. \\
\hline 2944 & \\
\hline 2945 & \\
\hline 2946 & SAMPLED AT 5 FT. OF WATER. \\
\hline 2947 & SAMPLED AT 5 FT. OF WATER. \\
\hline 2948 & SAMPLED AT 5 FT. OF WATER. \\
\hline 2949 & SAMPLED AT 5 FT. OF WATER. \\
\hline 2950 & SAMPLED AT 5 FT. OF WATER. \\
\hline 2951 & \\
\hline 2952 & \\
\hline 2953 & SAMPLED AT 5 FT. OF WATER. \\
\hline 2954 & SAMPLED AT 5 FT. OF WATER. \\
\hline 2955 & SAMPLED AT 5 FT. OF WATER. \\
\hline 2956 & SAMPLED AT 5 FT. OF WATER. \\
\hline 2957 & SAMPLED AT 5 FT. OF WATER. \\
\hline 2958 & \\
\hline 2959 & \\
\hline 2960 & SAMPLED AT 5.5 FT. OF WATER. \\
\hline 2961 & SAMPLED AT 5.5 FT. OF WATER. \\
\hline 2962 & SAMPLED AT 5.5 FT. OF WATER. \\
\hline 2963 & SAMPLED AT 5.5 FT. OF WATER. \\
\hline 2964 & SAMPLED AT 5.5 FT. OF WATER. \\
\hline 2965 & \\
\hline
\end{tabular}




\begin{tabular}{|c|c|}
\hline \multirow{2}{*}{\begin{tabular}{|c|}
1 \\
2966 \\
\end{tabular}} & SAMP_COMMENT \\
\hline & \\
\hline \multirow{2}{*}{\begin{tabular}{|l|}
2967 \\
2968 \\
\end{tabular}} & SAMPLED AT 5.2 FT. OF WATER. \\
\hline & SAMPLED AT 5.2 FT. OF WATER. \\
\hline 2969 & SAMPLED AT 5.2 FT. OF WATER. \\
\hline \multirow{2}{*}{\begin{tabular}{|l|}
2970 \\
2971 \\
\end{tabular}} & SAMPLED AT 5.2 FT. OF WATER. \\
\hline & SAMPLED AT 5.2 FT. OF WATER. \\
\hline 2972 & \\
\hline \begin{tabular}{|l|}
2973 \\
2071
\end{tabular} & \\
\hline \multirow{2}{*}{\begin{tabular}{|l|}
2974 \\
2975 \\
\end{tabular}} & SAMPLED AT 4 FT. OF WATER. \\
\hline & SAMPLED AT 4 FT. OF WATER. \\
\hline 2976 & SAMPLED AT 4 FT. OF WATER. \\
\hline \multirow{2}{*}{\begin{tabular}{|l|}
2977 \\
2978
\end{tabular}} & SAMPLED AT 4 FT. OF WATER. \\
\hline & SAMPLED AT 4 FT. OF WATER. \\
\hline 2979 & \\
\hline \multirow{2}{*}{\begin{tabular}{|l|}
2980 \\
2981 \\
\end{tabular}} & \\
\hline & SAMPLED AT 4 FT. OF WATER. \\
\hline 2982 & SAMPLED AT 4 FT. OF WATER. \\
\hline \multirow{2}{*}{\begin{tabular}{|l|}
2983 \\
2984
\end{tabular}} & SAMPLED AT 4 FT. OF WATER. \\
\hline & SAMPLED AT 4 FT. OF WATER. \\
\hline 2985 & SAMPLED AT 4 FT. OF WATER. \\
\hline \begin{tabular}{|l|}
2986 \\
2987 \\
\end{tabular} & \\
\hline \multirow{2}{*}{\begin{tabular}{|l|}
2987 \\
2988
\end{tabular}} & \\
\hline & SAMPLED AT 2.3 FT. OF WATER. \\
\hline 2989 & SAMPLED AT 2.3 FT. OF WATER. \\
\hline 2990 & SAMPLED AT 2.3 FT. OF WATER. \\
\hline \multirow{2}{*}{\begin{tabular}{|l|}
2991 \\
2992 \\
\end{tabular}} & SAMPLED AT 2.3 FT. OF WATER. \\
\hline & SAMPLED AT 2.3 FT. OF WATER. \\
\hline \multirow{2}{*}{\begin{tabular}{|l|}
2993 \\
2994 \\
\end{tabular}} & \\
\hline & \\
\hline 2995 & SAMPLED AT 1.5 FT. OF WATER. \\
\hline 2996 & SAMPLED AT 1.5 FT. OF WATER. \\
\hline 2997 & SAMPLED AT 1.5 FT. OF WATER. \\
\hline \begin{tabular}{|l|l|}
2998 \\
\end{tabular} & SAMPLED AT 1.5 FT. OF WATER. \\
\hline 2999 & SAMPLED AT 1.5 FT. OF WATER. \\
\hline 3000 & \\
\hline \multirow{2}{*}{\begin{tabular}{|l|}
3001 \\
3002 \\
\end{tabular}} & \\
\hline & SAMPLED AT 2 FT. OF WATER. \\
\hline & SAMPLED AT 2 FT. OF WATER. \\
\hline
\end{tabular}

\begin{tabular}{|l|l}
3003 & SAMPLED AT 2 FT. OF WATER. \\
\hline
\end{tabular} 
$A C$

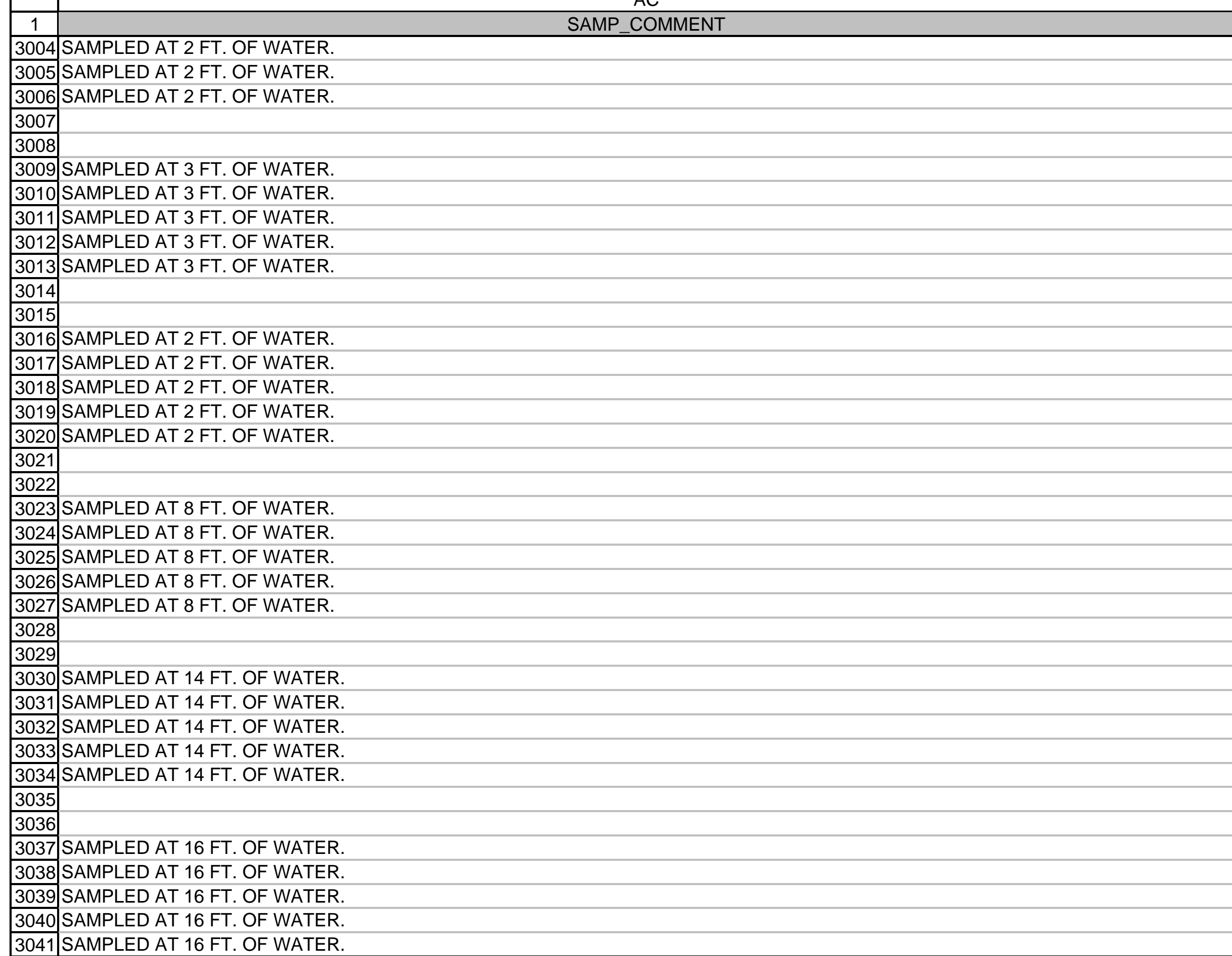

\begin{tabular}{|l|l}
3041 & SAMPLED AT 16 FT. OF WATER. \\
\hline
\end{tabular} 
$A C$

\begin{tabular}{|c|c|}
\hline 1 & SAMP_COMMENT \\
\hline 3042 & \\
\hline 3043 & \\
\hline 3044 & SAMPLED AT 16 FT. OF WATER. \\
\hline 3045 & SAMPLED AT 16 FT. OF WATER. \\
\hline 3046 & SAMPLED AT 16 FT. OF WATER. \\
\hline 3047 & SAMPLED AT 16 FT. OF WATER. \\
\hline 3048 & SAMPLED AT 16 FT. OF WATER. \\
\hline 3049 & \\
\hline 3050 & \\
\hline 3051 & SAMPLED AT 22 FT. OF WATER. \\
\hline 3052 & SAMPLED AT 22 FT. OF WATER. \\
\hline 3053 & SAMPLED AT 22 FT. OF WATER. \\
\hline 3054 & SAMPLED AT 22 FT. OF WATER. \\
\hline 3055 & SAMPLED AT 22 FT. OF WATER. \\
\hline 3056 & \\
\hline 3057 & \\
\hline 3058 & SAMPLED AT $11 \mathrm{FT}$. OF WATER. \\
\hline 3059 & SAMPLED AT 11 FT. OF WATER. \\
\hline 3060 & SAMPLED AT 11 FT. OF WATER. \\
\hline 3061 & SAMPLED AT 11 FT. OF WATER. \\
\hline 3062 & SAMPLED AT $11 \mathrm{FT}$. OF WATER. \\
\hline 3063 & \\
\hline 3064 & \\
\hline 3065 & SAMPLED AT 6 FT. OF WATER. \\
\hline 3066 & SAMPLED AT 6 FT. OF WATER. \\
\hline 3067 & SAMPLED AT 6 FT. OF WATER. \\
\hline 3068 & SAMPLED AT 6 FT. OF WATER. \\
\hline 3069 & SAMPLED AT 6 FT. OF WATER. \\
\hline 3070 & \\
\hline 3071 & \\
\hline 3072 & SAMPLE DEPTH 8 FT., HANFORD SHORE. \\
\hline 3073 & SAMPLE DEPTH 8 FT., HANFORD SHORE. \\
\hline 3074 & SAMPLE DEPTH 8 FT., HANFORD SHORE. \\
\hline 3075 & SAMPLE DEPTH 8 FT., HANFORD SHORE. \\
\hline 3076 & SAMPLE DEPTH 8 FT., HANFORD SHORE. \\
\hline 3077 & \\
\hline 3078 & \\
\hline 3079 & SAMPLE DEPTH 11 FT. WITH BOAT ENGINE RUNNING. \\
\hline
\end{tabular}

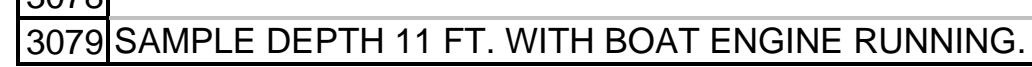




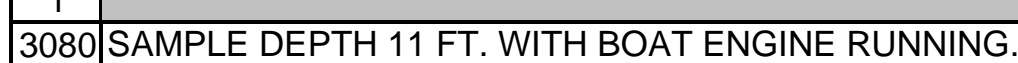

3081 SAMPLE DEPTH 11 FT. WITH BOAT ENGINE RUNNING.

3082 SAMPLE DEPTH 11 FT. WITH BOAT ENGINE RUNNING.

3083 SAMPLE DEPTH 11 FT. WITH BOAT ENGINE RUNNING.

3084

3085

3086 SAMPLE DEPTH 12 FT. WITH BOAT ENGINE RUNNING.

3087 SAMPLE DEPTH 12 FT. WITH BOAT ENGINE RUNNING.

3088 SAMPLE DEPTH 12 FT. WITH BOAT ENGINE RUNNING.

3089 SAMPLE DEPTH 12 FT. WITH BOAT ENGINE RUNNING.

3090 SAMPLE DEPTH 12 FT. WITH BOAT ENGINE RUNNING.

3091

3092

3093 SAMPLE DEPTH 6 FT. WITH BOAT ENGINE RUNNING.

3094 SAMPLE DEPTH 6 FT. WITH BOAT ENGINE RUNNING.

3095 SAMPLE DEPTH 6 FT. WITH BOAT ENGINE RUNNING.

3096 SAMPLE DEPTH 6 FT. WITH BOAT ENGINE RUNNING.

3097 SAMPLE DEPTH 6 FT. WITH BOAT ENGINE RUNNING.

3100 SAMPLE DEPTH 5.5 FT. WITH BOAT ENGINE RUNNING.

3101 SAMPLE DEPTH 5.5 FT. WITH BOAT ENGINE RUNNING.

3102 SAMPLE DEPTH 5.5 FT. WITH BOAT ENGINE RUNNING.

3103 SAMPLE DEPTH 5.5 FT. WITH BOAT ENGINE RUNNING.

3104 SAMPLE DEPTH 5.5 FT. WITH BOAT ENGINE RUNNING.

3105 SAMPLE DEPTH 5.5 FT. WITH BOAT ENGINE RUNNING.

3106 SAMPLE DEPTH 5.5 FT. WITH BOAT ENGINE RUNNING.

3107 SAMPLE DEPTH 5.5 FT. WITH BOAT ENGINE RUNNING.

3108 SAMPLE DEPTH 5.5 FT. WITH BOAT ENGINE RUNNING.

3109 SAMPLE DEPTH 5.5 FT. WITH BOAT ENGINE RUNNING.

3110

3111

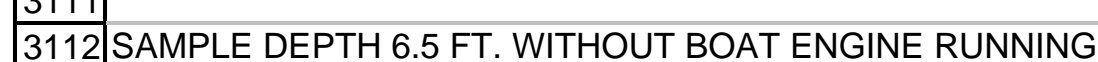

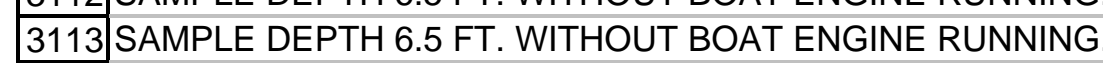

3114 SAMPLE DEPTH 6.5 FT. WITHOUT BOAT ENGINE RUNNING.

3115 SAMPLE DEPTH 6.5 FT. WITHOUT BOAT ENGINE RUNNING.

\begin{tabular}{|l|l|}
3116 & SAMPLE DEPTH 6.5 FT. WITHOUT BOAT ENGINE RUNNING \\
\hline 3117 &
\end{tabular}

(20)

(2)

(n)

(n)

(19)

G.

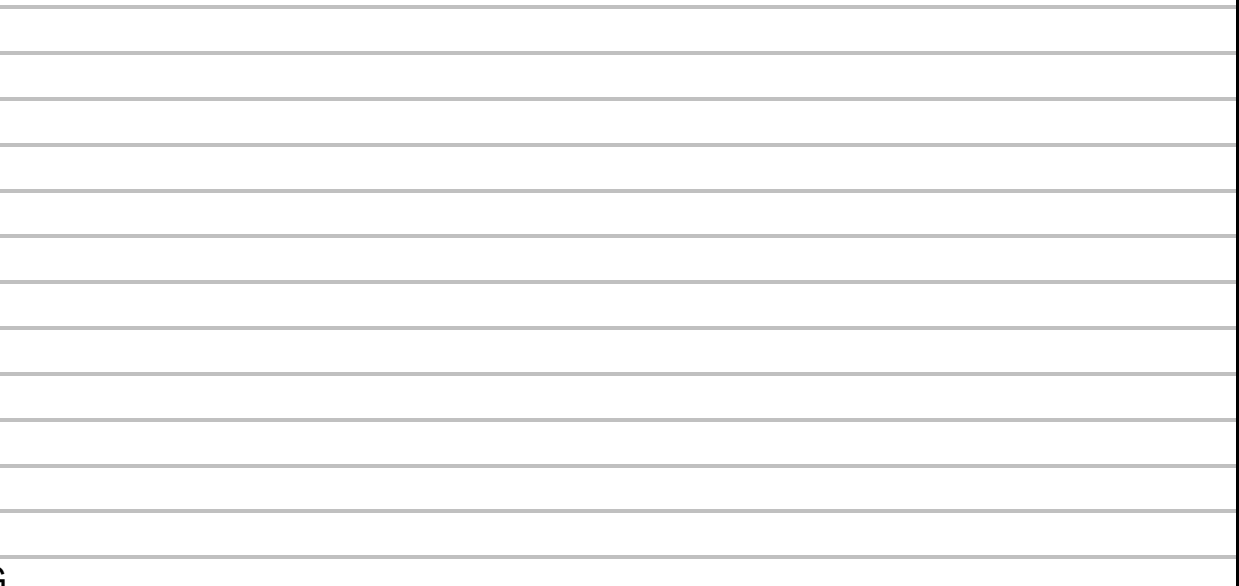

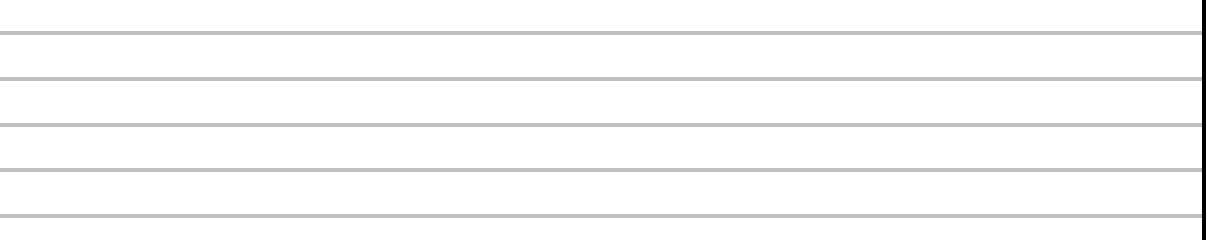

Page 451 


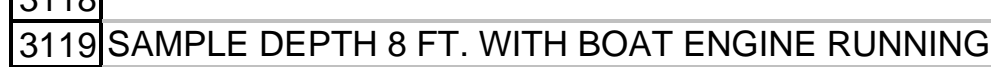
3120 SAMPLE DEPTH 8 FT. WITH BOAT ENGINE RUNNING.

3121 SAMPLE DEPTH 8 FT. WITH BOAT ENGINE RUNNING.

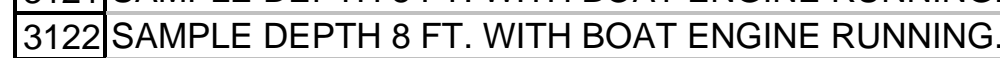

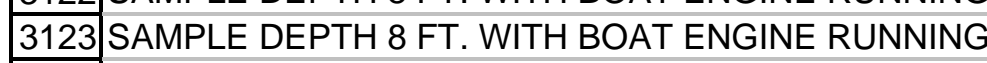

3124

3125

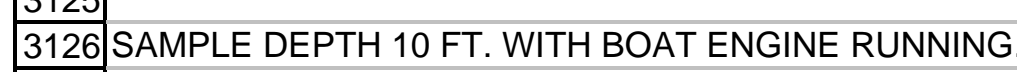
3127 SAMPLE DEPTH 10 FT. WITH BOAT ENGINE RUNNING.

3128 SAMPLE DEPTH $10 \mathrm{FT}$. WITH BOAT ENGINE RUNNING.

3129 SAMPLE DEPTH 10 FT. WITH BOAT ENGINE RUNNING.

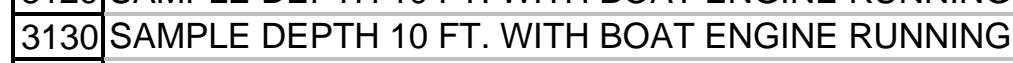

3131

3131

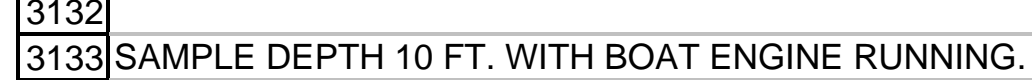

3134 SAMPLE DEPTH 10 FT. WITH BOAT ENGINE RUNNING.

3135 SAMPLE DEPTH $10 \mathrm{FT}$. WITH BOAT ENGINE RUNNING.

3136 SAMPLE DEPTH 10 FT. WITH BOAT ENGINE RUNNING.

3137 SAMPLE DEPTH 10 FT. WITH BOAT ENGINE RUNNING.

3138

3139

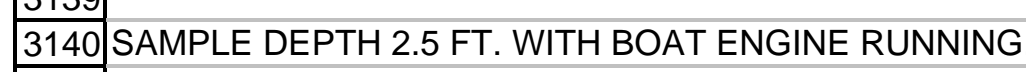

3141 SAMPLE DEPTH 2.5 FT. WITH BOAT ENGINE RUNNING.

3142 SAMPLE DEPTH 2.5 FT. WITH BOAT ENGINE RUNNING.

3143 SAMPLE DEPTH 2.5 FT. WITH BOAT ENGINE RUNNING.

3144 SAMPLE DEPTH 2.5 FT. WITH BOAT ENGINE RUNNING.

3145

3146

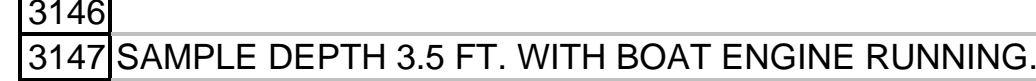

3148 SAMPLE DEPTH 3.5 FT. WITH BOAT ENGINE RUNNING.

3149 SAMPLE DEPTH 3.5 FT. WITH BOAT ENGINE RUNNING.

3150 SAMPLE DEPTH 3.5 FT. WITH BOAT ENGINE RUNNING.

\begin{tabular}{l|l}
3150 & SAMPLE DEPTH 3.5 FT. WITH BOAT ENGINE RUNNING. \\
\hline 3151 & SAMPLE DEPTH 3.5 FT. WITH BOAT ENGINE RUNNING.
\end{tabular}

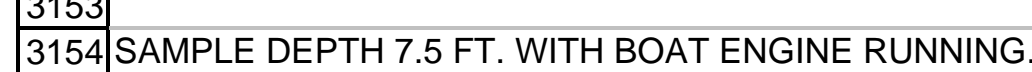

\begin{tabular}{l|l|l}
3155 & SAMPLE DEPTH 7.5 FT. WITH BOAT ENGINE RUNNING. \\
\hline
\end{tabular}

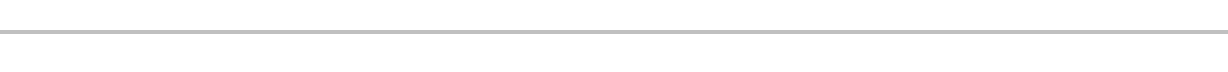

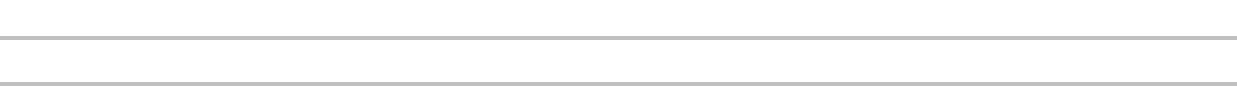

(20)

.

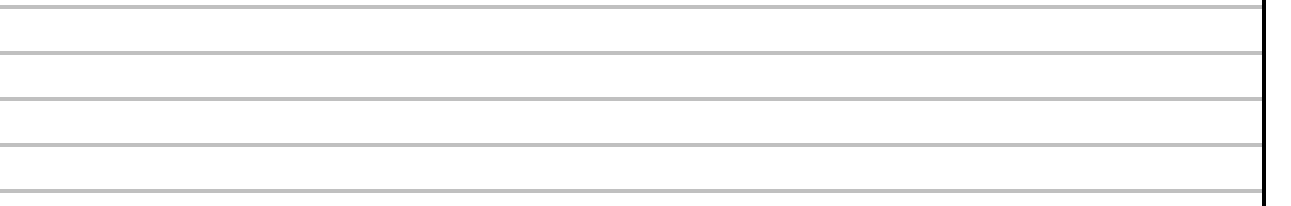

(2)

(20)

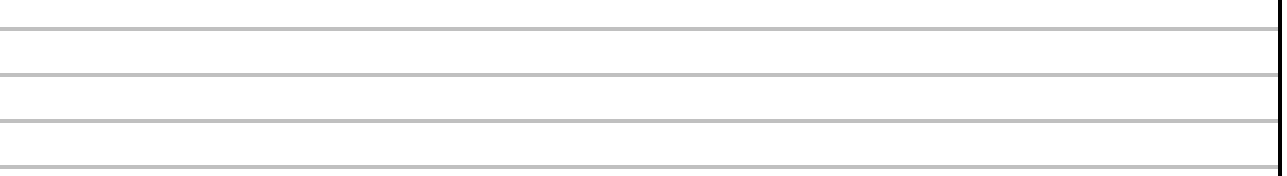

(20)

(20)

(2)

(n)

(n)

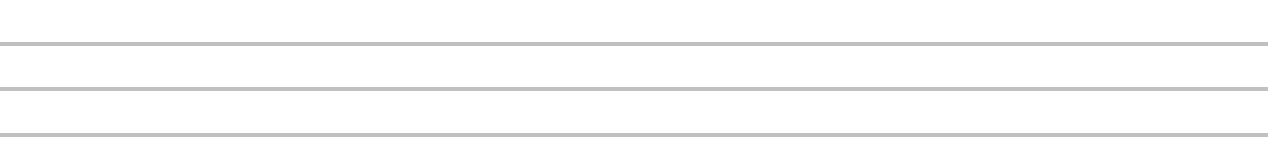

(2)

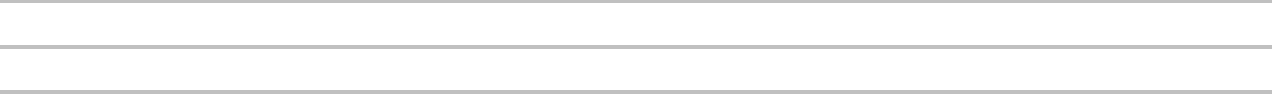


$A C$

\begin{tabular}{|c|c|}
\hline 1 & SAMP_COMMENT \\
\hline 3156 & SAMPLE DEPTH 7.5 FT. WITH BOAT ENGINE RUNNING. \\
\hline 3157 s & SAMPLE DEPTH 7.5 FT. WITH BOAT ENGINE RUNNING. \\
\hline 3158 & SAMPLE DEPTH 7.5 FT. WITH BOAT ENGINE RUNNING. \\
\hline 3159 & \\
\hline 3160 & \\
\hline 3161 & SAMPLE DEPTH 11 FT. WITH BOAT ENGINE RUNNING. \\
\hline 3162 & SAMPLE DEPTH 11 FT. WITH BOAT ENGINE RUNNING. \\
\hline 3163 & SAMPLE DEPTH 11 FT. WITH BOAT ENGINE RUNNING. \\
\hline 3164 & SAMPLE DEPTH 11 FT. WITH BOAT ENGINE RUNNING. \\
\hline 3165 & SAMPLE DEPTH 11 FT. WITH BOAT ENGINE RUNNING. \\
\hline 3166 & \\
\hline 3167 & \\
\hline 3168 & SAMPLE DEPTH 10 FT. WITH BOAT ENGINE RUNNING. \\
\hline 3169 & SAMPLE DEPTH 10 FT. WITH BOAT ENGINE RUNNING. \\
\hline 3170 & SAMPLE DEPTH 10 FT. WITH BOAT ENGINE RUNNING. \\
\hline 3171 & SAMPLE DEPTH 10 FT. WITH BOAT ENGINE RUNNING. \\
\hline 3172 & SAMPLE DEPTH 10 FT. WITH BOAT ENGINE RUNNING. \\
\hline 3173 & \\
\hline 3174 & \\
\hline 3175 & \\
\hline 3176 & \\
\hline 3177 & \\
\hline 3178 & \\
\hline 3179 & \\
\hline 3180 & \\
\hline 3181 & \\
\hline 3182 & \\
\hline 3183 & \\
\hline 3184 & \\
\hline 3185 & \\
\hline 3186 & \\
\hline 3187 & \\
\hline \begin{tabular}{|l|}
3188 \\
\end{tabular} & \\
\hline \begin{tabular}{|l|}
3189 \\
\end{tabular} & \\
\hline 3190 & \\
\hline 3191 & \\
\hline \begin{tabular}{|l|}
3192 \\
\end{tabular} & \\
\hline 3193 & \\
\hline
\end{tabular}




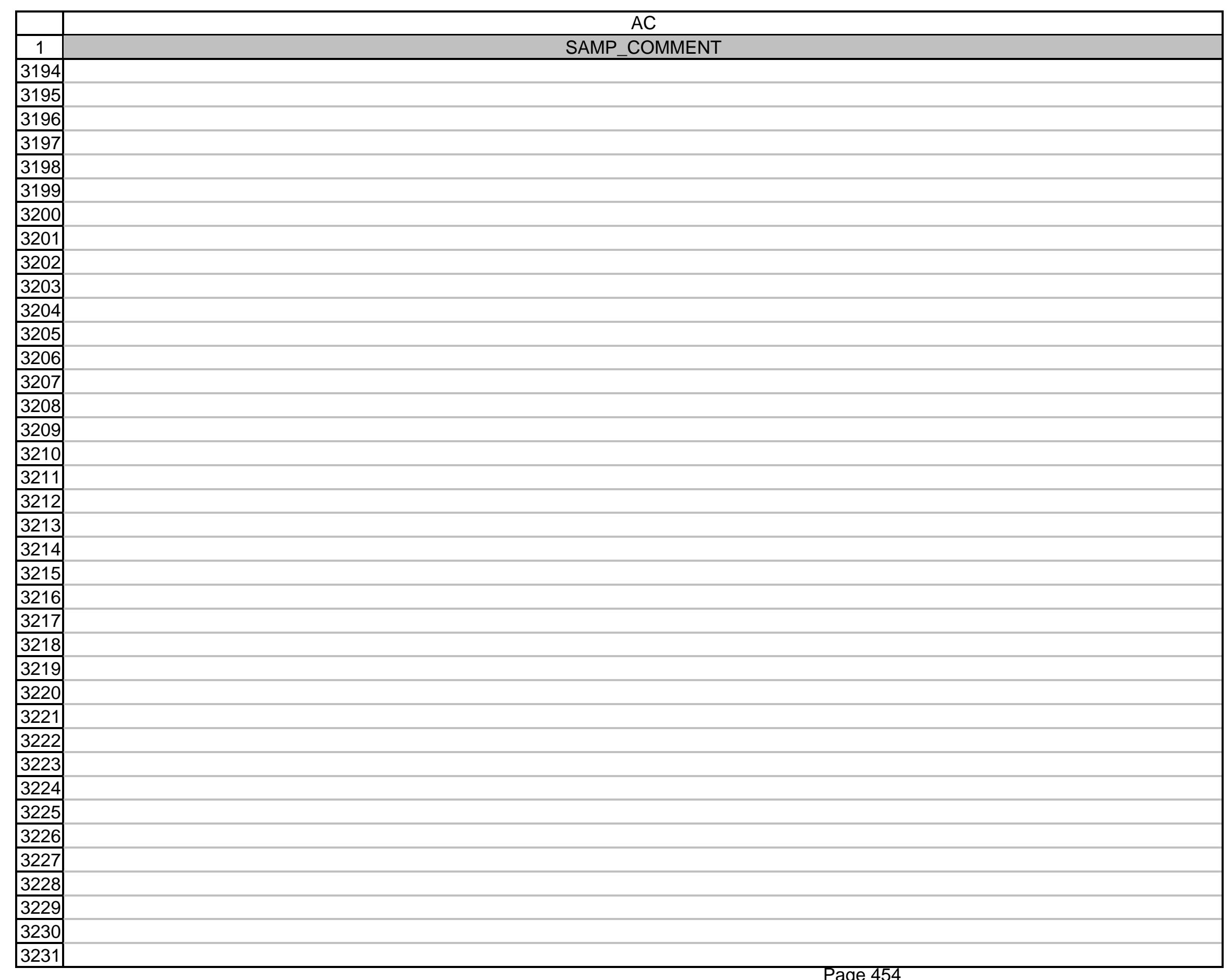


AC

\begin{tabular}{|c|c|}
\hline & 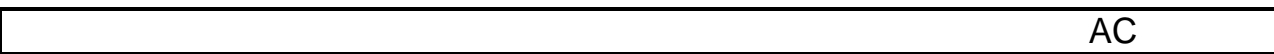 \\
\hline 1 & SAMP_COMMENT \\
\hline 3232 & \\
\hline 3233 & \\
\hline 3234 & \\
\hline 3235 & \\
\hline 3236 & \\
\hline 3237 & \\
\hline 3238 & \\
\hline 3239 & \\
\hline 3240 & \\
\hline 3241 & \\
\hline 3242 & \\
\hline 3243 & 10 FT. RIVER DEPTH. \\
\hline 3244 & 10 FT. RIVER DEPTH. \\
\hline 3245 & 10 FT. RIVER DEPTH. \\
\hline 3246 & 10 FT. RIVER DEPTH. \\
\hline 3247 & 10 FT. RIVER DEPTH. \\
\hline 3248 & 10 FT. RIVER DEPTH. \\
\hline 3249 & 10 FT. RIVER DEPTH. \\
\hline 3250 & 10 FT. RIVER DEPTH. \\
\hline 3251 & 10 FT. RIVER DEPTH. \\
\hline 3252 & 10 FT. RIVER DEPTH. \\
\hline 3253 & 20 FT. RIVER DEPTH. \\
\hline 3254 & 20 FT. RIVER DEPTH. \\
\hline 3255 & 20 FT. RIVER DEPTH. \\
\hline 3256 & 20 FT. RIVER DEPTH. \\
\hline 3257 & 20 FT. RIVER DEPTH. \\
\hline 3258 & 31 FT. RIVER DEPTH, NO ANCHOR. \\
\hline 3259 & 31 FT. RIVER DEPTH, NO ANCHOR. \\
\hline 3260 & 31 FT. RIVER DEPTH, NO ANCHOR. \\
\hline 3261 & 31 FT. RIVER DEPTH, NO ANCHOR. \\
\hline 3262 & 31 FT. RIVER DEPTH, NO ANCHOR. \\
\hline 3263 & 31 FT. RIVER DEPTH, NO ANCHOR. \\
\hline 3264 & 31 FT. RIVER DEPTH, NO ANCHOR. \\
\hline 3265 & 31 FT. RIVER DEPTH, NO ANCHOR. \\
\hline 3266 & 31 FT. RIVER DEPTH, NO ANCHOR. \\
\hline 3267 & 31 FT. RIVER DEPTH, NO ANCHOR. \\
\hline 3268 & 9 FT. SAMPLE DEPTH. \\
\hline 3269 & 9 FT. SAMPLE DEPTH. \\
\hline
\end{tabular}


$A C$

\begin{tabular}{|c|c|}
\hline 1 & 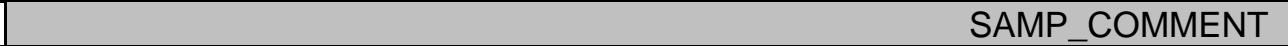 \\
\hline 3270 & 9 FT. SAMPLE DEPTH. \\
\hline 3271 & 9 FT. SAMPLE DEPTH. \\
\hline 3272 & 9 FT. SAMPLE DEPTH. \\
\hline 3273 & 20 FT. SAMPLE DEPTH. \\
\hline 3274 & 20 FT. SAMPLE DEPTH. \\
\hline 3275 & 20 FT. SAMPLE DEPTH. \\
\hline 3276 & 20 FT. SAMPLE DEPTH. \\
\hline 3277 & 20 FT. SAMPLE DEPTH. \\
\hline 3278 & 12 FT. SAMPLE DEPTH. \\
\hline 3279 & 12 FT. SAMPLE DEPTH. \\
\hline 3280 & 12 FT. SAMPLE DEPTH. \\
\hline 3281 & 12 FT. SAMPLE DEPTH. \\
\hline 3282 & 12 FT. SAMPLE DEPTH. \\
\hline 3283 & 8 FT. SAMPLE DEPTH. \\
\hline 3284 & 8 FT. SAMPLE DEPTH. \\
\hline 3285 & 8 FT. SAMPLE DEPTH. \\
\hline 3286 & 8 FT. SAMPLE DEPTH. \\
\hline 3287 & 8 FT. SAMPLE DEPTH. \\
\hline 3288 & 15 FT. RIVER DEPTH. \\
\hline 3289 & 15 FT. RIVER DEPTH. \\
\hline 3290 & 15 FT. RIVER DEPTH. \\
\hline 3291 & 15 FT. RIVER DEPTH. \\
\hline 3292 & 15 FT. RIVER DEPTH. \\
\hline 3293 & 3 FT. RIVER DEPTH. \\
\hline 3294 & 3 FT. RIVER DEPTH. \\
\hline 3295 & 3 FT. RIVER DEPTH. \\
\hline 3296 & 3 FT. RIVER DEPTH. \\
\hline 3297 & 3 FT. RIVER DEPTH. \\
\hline 3298 & 18 FT. RIVER DEPTH. \\
\hline 3299 & 18 FT. RIVER DEPTH. \\
\hline 3300 & 18 FT. RIVER DEPTH. \\
\hline 3301 & 18 FT. RIVER DEPTH. \\
\hline 3302 & 18 FT. RIVER DEPTH. \\
\hline 3303 & 18 FT. RIVER DEPTH. \\
\hline 3304 & 18 FT. RIVER DEPTH. \\
\hline 3305 & 18 FT. RIVER DEPTH. \\
\hline 3306 & 18 FT. RIVER DEPTH. \\
\hline 3307 & 18 FT. RIVER DEPTH. \\
\hline
\end{tabular}

\begin{tabular}{|l|l}
3307 & 18 FT. RIVER DEPTH. \\
\hline
\end{tabular} 
$\mathrm{AC}$

3308 16 FT. RIVER DEPTH

\begin{tabular}{|l|l|}
\hline 3309 & 16 FT. RIVER DEPTH \\
\hline 3310 & 16 FT. RIVER DEPTH \\
\hline
\end{tabular}

331016 FT. RIVER DEPTH

331116 FT. RIVER DEPTH

\begin{tabular}{l|l}
3312 & 16 FT. RIVER DEPTH
\end{tabular}

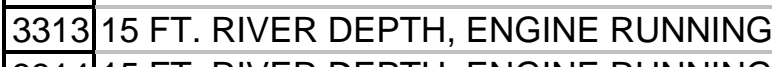

331415 FT. RIVER DEPTH, ENGINE RUNNING.

331515 FT. RIVER DEPTH, ENGINE RUNNING.

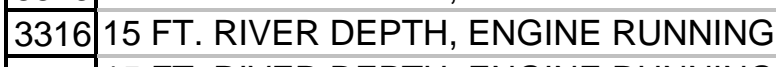

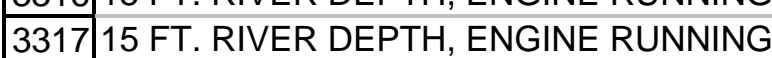

3318 14 FT. RIVER DEPTH, ENGINE RUNNING.

331914 FT. RIVER DEPTH, ENGINE RUNNING.

3320 14 FT. RIVER DEPTH, ENGINE RUNNING.

332114 FT. RIVER DEPTH, ENGINE RUNNING.

$332214 \mathrm{FT}$. RIVER DEPTH, ENGINE RUNNING.

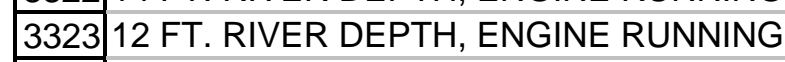

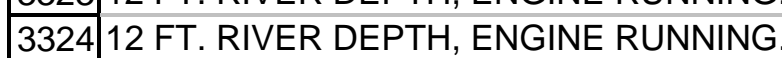

332512 FT. RIVER DEPTH, ENGINE RUNNING.

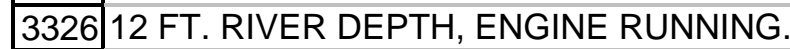

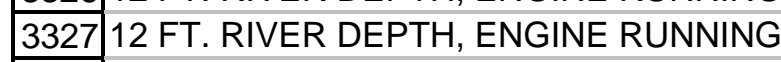

3328 8 FT. RIVER DEPTH, ENGINE RUNNING.

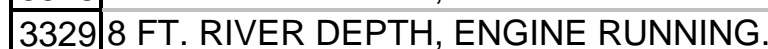

\begin{tabular}{|l|l}
3330 & 8 FT. RIVER DEPTH, ENGINE RUNNING.
\end{tabular}

3331 8 FT. RIVER DEPTH, ENGINE RUNNING.

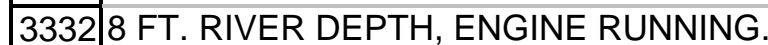

333311 FT. RIVER DEPTH.

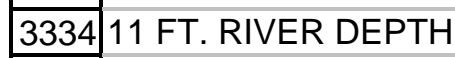

\begin{tabular}{l|l}
3335 & 11 FT. RIVER DEPTH. \\
\hline 3336
\end{tabular}

3336 11 FT. RIVER DEPTH.

\begin{tabular}{|l|l}
\hline 3337 & 11 FT. RIVER DEPTH. \\
\hline 3338 & FT. RIVER DEPTH.
\end{tabular}

33386 FT. RIVER DEPTH.

33396 FT. RIVER DEPTH.

\begin{tabular}{|l|l}
3340 & 6 FT. RIVER DEPTH.
\end{tabular}

\begin{tabular}{l|l}
3341 & 6 FT. RIVER DEPTH.
\end{tabular}

33426 FT. RIVER DEPTH.

\begin{tabular}{l|l}
3343 & 5 FT. RIVER DEPTH.
\end{tabular}

33445 FT. RIVER DEPTH.

\begin{tabular}{|l|l}
33345 & 5 FT. RIVER DEPTH. \\
\hline
\end{tabular}

SAMP COMMEN 
AC

\begin{tabular}{|c|c|}
\hline 1 & SAMP_COMMENT \\
\hline 3346 & 5 FT. RIVER DEPTH. \\
\hline 3347 & 5 FT. RIVER DEPTH. \\
\hline 3348 & 20 FT. RIVER DEPTH. \\
\hline 3349 & 20 FT. RIVER DEPTH. \\
\hline 3350 & 20 FT. RIVER DEPTH. \\
\hline 3351 & 20 FT. RIVER DEPTH. \\
\hline 3352 & 20 FT. RIVER DEPTH. \\
\hline 3353 & 18 FT. RIVER DEPTH. \\
\hline 3354 & 18 FT. RIVER DEPTH. \\
\hline 3355 & 18 FT. RIVER DEPTH. \\
\hline 3356 & 18 FT. RIVER DEPTH. \\
\hline 3357 & 18 FT. RIVER DEPTH. \\
\hline 3358 & 36 FT. RIVER DEPTH, ENGINE RUNNING. \\
\hline 3359 & 36 FT. RIVER DEPTH, ENGINE RUNNING. \\
\hline 3360 & 36 FT. RIVER DEPTH, ENGINE RUNNING. \\
\hline 3361 & 36 FT. RIVER DEPTH, ENGINE RUNNING. \\
\hline 3362 & 36 FT. RIVER DEPTH, ENGINE RUNNING. \\
\hline 3363 & 38 FT. RIVER DEPTH, ENGINE RUNNING. \\
\hline 3364 & 38 FT. RIVER DEPTH, ENGINE RUNNING. \\
\hline 3365 & 38 FT. RIVER DEPTH, ENGINE RUNNING. \\
\hline 3366 & 38 FT. RIVER DEPTH, ENGINE RUNNING. \\
\hline 3367 & 38 FT. RIVER DEPTH, ENGINE RUNNING. \\
\hline 3368 & 41 FT. RIVER DEPTH, ENGINE RUNNING. \\
\hline 3369 & 41 FT. RIVER DEPTH, ENGINE RUNNING. \\
\hline 3370 & 41 FT. RIVER DEPTH, ENGINE RUNNING. \\
\hline 3371 & 41 FT. RIVER DEPTH, ENGINE RUNNING. \\
\hline 3372 & 41 FT. RIVER DEPTH, ENGINE RUNNING. \\
\hline 3373 & 33 FT. RIVER DEPTH, ENGINE RUNNING. \\
\hline 3374 & 33 FT. RIVER DEPTH, ENGINE RUNNING. \\
\hline 3375 & 33 FT. RIVER DEPTH, ENGINE RUNNING. \\
\hline 3376 & 33 FT. RIVER DEPTH, ENGINE RUNNING. \\
\hline 3377 & 33 FT. RIVER DEPTH, ENGINE RUNNING. \\
\hline 3378 & 24 FT. RIVER DEPTH, ENGINE RUNNING. \\
\hline 3379 & 24 FT. RIVER DEPTH, ENGINE RUNNING. \\
\hline 3380 & 24 FT. RIVER DEPTH, ENGINE RUNNING. \\
\hline 3381 & 24 FT. RIVER DEPTH, ENGINE RUNNING. \\
\hline 3382 & 24 FT. RIVER DEPTH, ENGINE RUNNING. \\
\hline
\end{tabular}

\begin{tabular}{|l|l}
33383 & 14 FT. RIVER DEPTH, ENGINE RUNNING. \\
\hline
\end{tabular} 
$\mathrm{AC}$

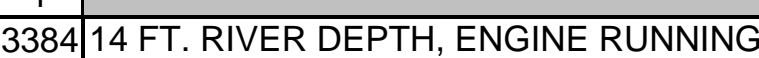

SAMP COMMENT

338514 FT. RIVER DEPTH, ENGINE RUNNING.

$338614 \mathrm{FT}$. RIVER DEPTH, ENGINE RUNNING.

338714 FT. RIVER DEPTH, ENGINE RUNNING.

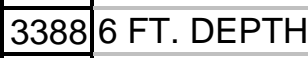

33896 FT. DEPTH.

33906 FT. DEPTH.

33916 FT. DEPTH.

\begin{tabular}{|l|l}
\hline 3392 & 6 FT. DEPTH. \\
\hline 3393 & 28 FT. DEPTH.
\end{tabular}

339328 FT. DEPTH.

339428 FT. DEPTH.

339528 FT. DEPTH.

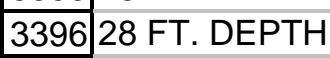

$339728 \mathrm{FT}$. DEPTH.

3398 40 FT. DEPTH.

\begin{tabular}{|l|l|}
33399 & 40 FT. DEPTH \\
\hline 3400 & 40 FT. DEPTH.
\end{tabular}

340040 FT. DEPTH.

340140 FT. DEPTH.

340240 FT. DEPTH.

\begin{tabular}{|l|l}
3402 & 40 FT. DEPTH. \\
\hline $340 \mathrm{FT}$. DEPTH.
\end{tabular}

340437 FT. DEPTH.

340537 FT. DEPTH.

\begin{tabular}{|l|l|l}
3406 & 37 FT. DEPTH. \\
\hline 3407 & 37 FT. DEPTH.
\end{tabular}

340737 FT. DEPTH.

34086 FT. DEPTH.

34096 FT. DEPTH.

\begin{tabular}{l|l}
\hline 3410 & 6 FT. DEPTH. \\
\hline 3411 & 6 FT. DEPTH.
\end{tabular}

34116 FT. DEPTH.

34126 FT. DEPTH.

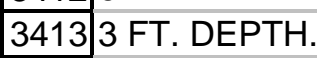

34143 FT. DEPTH.

34153 FT. DEPTH.

\begin{tabular}{l|l|l}
3416 & 3 FT. DEPTH.
\end{tabular}

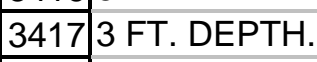

$34188 \mathrm{FT}$. DEPTH.

3419 8 FT. DEPTH.

34208 FT. DEPTH

\begin{tabular}{|l|l|}
\hline 3421 & 8 FT. DEPTH \\
\hline
\end{tabular} 
$A C$

\begin{tabular}{|c|c|}
\hline \multirow{2}{*}{\begin{tabular}{|c|}
1 \\
3422
\end{tabular}} & \multirow[t]{2}{*}{ SAMP_COMMENT } \\
\hline & \\
\hline 3423 & 8 FT. DEPTH. \\
\hline 3424 & 8 FT. DEPTH. \\
\hline 3425 & 8 FT. DEPTH. \\
\hline 3426 & 8 FT. DEPTH. \\
\hline 3427 & 8 FT. DEPTH. \\
\hline 3428 & 14 FT. DEPTH. \\
\hline 3429 & 14 FT. DEPTH. \\
\hline 3430 & 14 FT. DEPTH. \\
\hline 3431 & 14 FT. DEPTH. \\
\hline 3432 & 14 FT. DEPTH. \\
\hline 3433 & 3 FT. DEPTH. \\
\hline 3434 & 3 FT. DEPTH. \\
\hline 3435 & 3 FT. DEPTH. \\
\hline 3436 & 3 FT. DEPTH. \\
\hline 3437 & 3 FT. DEPTH. \\
\hline 3438 & 8 FT. DEPTH. \\
\hline 3439 & 8 FT. DEPTH. \\
\hline 3440 & 8 FT. DEPTH. \\
\hline 3441 & 8 FT. DEPTH. \\
\hline 3442 & 8 FT. DEPTH. \\
\hline 3443 & 6 FT. DEPTH. \\
\hline 3444 & 6 FT. DEPTH. \\
\hline 3445 & 6 FT. DEPTH. \\
\hline 3446 & 6 FT. DEPTH. \\
\hline 3447 & 6 FT. DEPTH. \\
\hline 3448 & 18 FT. DEPTH. \\
\hline 3449 & 18 FT. DEPTH. \\
\hline 3450 & 18 FT. DEPTH. \\
\hline 3451 & 18 FT. DEPTH. \\
\hline 3452 & 18 FT. DEPTH. \\
\hline 3453 & 20 FT. DEPTH. \\
\hline 3454 & 20 FT. DEPTH. \\
\hline 3455 & 20 FT. DEPTH. \\
\hline 3456 & 20 FT. DEPTH. \\
\hline 3457 & 20 FT. DEPTH. \\
\hline 3458 & 27 FT. DEPTH. \\
\hline 3459 & 27 FT. DEPTH. \\
\hline
\end{tabular}


$A C$

346027 FT. DEPTH

346127 FT. DEPTH.

3462 27 FT. DEPTH.

34636 FT. DEPTH.

\begin{tabular}{l|l|l}
3464 & 6 FT. DEPTH.
\end{tabular}

\begin{tabular}{l|l}
3465 & 6 FT. DEPTH. \\
\hline
\end{tabular}

34666 FT. DEPTH.

34676 FT. DEPTH.

\begin{tabular}{|l|l|}
\hline 3468 & 3 FT. DEPTH \\
\hline 3469 & .
\end{tabular}

34693 FT. DEPTH.

34703 FT. DEPTH.

34713 FT. DEPTH.

\begin{tabular}{|l|l|}
\hline 3472 & 3 FT. DEPTH. \\
\hline
\end{tabular}

347318 FT. DEPTH.

347418 FT. DEPTH.

\begin{tabular}{l|l}
3475 & 18 FT. DEPTH \\
\hline
\end{tabular}

347618 FT. DEPTH.

347718 FT. DEPTH.

3478 24 FT. DEPTH.

\begin{tabular}{l|l}
3479 & 24 FT. DEPTH. \\
\hline 348 & 24 FT. DEPTH.
\end{tabular}

348024 FT. DEPTH.

348124 FT. DEPTH.

\begin{tabular}{ll}
3482 & 24 FT. DEPTH \\
\hline
\end{tabular}

348321 FT. DEPTH.

348421 FT. DEPTH.

348521 FT. DEPTH

\begin{tabular}{|l|l|}
\hline 3486 & 21 FT. DEPTH \\
\hline 3487 & 21 FT. DEPTH.
\end{tabular}

$348721 \mathrm{FT}$. DEPTH.

$34888 \mathrm{INCH}$ DEPTH

34898 INCH DEPTH.

34908 INCH DEPTH.

34913 FT. DEPTH.

34923 FT. DEPTH.

\begin{tabular}{|l|l|}
\hline 3493 & 3 FT. DEPTH. \\
\hline 349 & 5 FT. DEPTH.
\end{tabular}

34945 FT. DEPTH.

34955 FT. DEPTH.

\begin{tabular}{l|l}
3496 & $5 \mathrm{FT}$. DEPTH.
\end{tabular}

\begin{tabular}{|l|l|}
\hline 3497 & 6 FT. DEPTH. \\
\hline
\end{tabular}

SAMP COMMENT 
$\mathrm{AC}$

\begin{tabular}{|c|c|}
\hline 1 & $\begin{array}{ll}\text { SAMP COMMENT } \\
\end{array}$ \\
\hline 3498 & 6 FT. DEPTH. \\
\hline 3499 & 6 FT. DEPTH. \\
\hline 3500 & $6 \mathrm{INCH}$ DEPTH. \\
\hline 3501 & $6 \mathrm{INCH}$ DEPTH. \\
\hline 3502 & 6 INCH DEPTH. \\
\hline 3503 & 6 INCH DEPTH. \\
\hline 3504 & 6 INCH DEPTH. \\
\hline 3505 & 6 INCH DEPTH. \\
\hline 3506 & 2 FT. DEPTH. \\
\hline 3507 & 2 FT. DEPTH. \\
\hline 3508 & 2 FT. DEPTH. \\
\hline 3509 & 4.5 FT. DEPTH. \\
\hline 3510 & 4.5 FT. DEPTH. \\
\hline 3511 & 4.5 FT. DEPTH. \\
\hline 3512 & 6 FT. DEPTH. \\
\hline 3513 & 6 FT. DEPTH. \\
\hline 3514 & 6 FT. DEPTH. \\
\hline 3515 & 6 INCH DEPTH. \\
\hline 3516 & 6 INCH DEPTH. \\
\hline 3517 & 6 INCH DEPTH. \\
\hline 3518 & 2 FT. DEPTH. \\
\hline 3519 & 3.5 FT. DEPTH. \\
\hline 3520 & 5 FT. DEPTH. \\
\hline 3521 & 6 INCH DEPTH. \\
\hline 3522 & 6 INCH DEPTH. \\
\hline 3523 & 6 INCH DEPTH. \\
\hline 3524 & 9 FT. RIVER DEPTH. \\
\hline 3525 & 9 FT. RIVER DEPTH. \\
\hline 3526 & 9 FT. RIVER DEPTH. \\
\hline 3527 & 9 FT. RIVER DEPTH. \\
\hline 3528 & 9 FT. RIVER DEPTH. \\
\hline 3529 & 8 FT. RIVER DEPTH. \\
\hline 3530 & 8 FT. RIVER DEPTH. \\
\hline 3531 & 8 FT. RIVER DEPTH. \\
\hline 3532 & 8 FT. RIVER DEPTH. \\
\hline 3533 & 8 FT. RIVER DEPTH. \\
\hline 3534 & 13 FT. RIVER DEPTH. \\
\hline 3535 & 13 FT. RIVER DEPTH. \\
\hline
\end{tabular}

353513 FT. RIVER DEPTH. 
AC

\begin{tabular}{|c|c|}
\hline 1 & 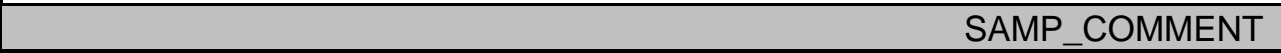 \\
\hline 3536 & 13 FT. RIVER DEPTH. \\
\hline 3537 & 13 FT. RIVER DEPTH. \\
\hline 3538 & 13 FT. RIVER DEPTH. \\
\hline 3539 & 7 FT. RIVER DEPTH. \\
\hline 3540 & 7 FT. RIVER DEPTH. \\
\hline 3541 & 7 FT. RIVER DEPTH. \\
\hline 3542 & 7 FT. RIVER DEPTH. \\
\hline 3543 & 7 FT. RIVER DEPTH. \\
\hline 3544 & 2 FT. RIVER DEPTH. \\
\hline 3545 & 2 FT. RIVER DEPTH. \\
\hline 3546 & 2 FT. RIVER DEPTH. \\
\hline 3547 & 2 FT. RIVER DEPTH. \\
\hline 3548 & 2 FT. RIVER DEPTH. \\
\hline 3549 & 4 FT. RIVER DEPTH. \\
\hline 3550 & 4 FT. RIVER DEPTH. \\
\hline 3551 & 4 FT. RIVER DEPTH. \\
\hline 3552 & 4 FT. RIVER DEPTH. \\
\hline 3553 & 4 FT. RIVER DEPTH. \\
\hline 3554 & 12 FT. RIVER DEPTH. \\
\hline 3555 & 12 FT. RIVER DEPTH. \\
\hline 3556 & 12 FT. RIVER DEPTH. \\
\hline 3557 & 12 FT. RIVER DEPTH. \\
\hline 3558 & 12 FT. RIVER DEPTH. \\
\hline 3559 & 8 FT. RIVER DEPTH. \\
\hline 3560 & 8 FT. RIVER DEPTH. \\
\hline 3561 & 8 FT. RIVER DEPTH. \\
\hline 3562 & 8 FT. RIVER DEPTH. \\
\hline 3563 & 8 FT. RIVER DEPTH. \\
\hline 3564 & 2 FT. RIVER DEPTH. \\
\hline 3565 & 2 FT. RIVER DEPTH. \\
\hline 3566 & 2 FT. RIVER DEPTH. \\
\hline 3567 & 2 FT. RIVER DEPTH. \\
\hline 3568 & 2 FT. RIVER DEPTH. \\
\hline 3569 & 2 FT. RIVER DEPTH. \\
\hline 3570 & 2 FT. RIVER DEPTH. \\
\hline 3571 & 2 FT. RIVER DEPTH. \\
\hline 3572 & 2 FT. RIVER DEPTH. \\
\hline 3573 & 2 FT. RIVER DEPTH. \\
\hline
\end{tabular}


$\mathrm{AC}$

3574 11 FT. RIVER DEPTH.

357511 FT. RIVER DEPTH.

357611 FT. RIVER DEPTH.

357711 FT. RIVER DEPTH.

\begin{tabular}{l|l}
3578 & 11 FT. RIVER DEPTH.
\end{tabular}

\begin{tabular}{|l|l}
3579 & 10 FT. RIVER DEPTH \\
\hline 3580 &
\end{tabular}

358010 FT. RIVER DEPTH.

358110 FT. RIVER DEPTH.

\begin{tabular}{|l|l}
3582 & 10 FT. RIVER DEPTH \\
\hline 3583 & 10 F. RIVER DEPTH.
\end{tabular}

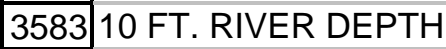

35848 FT. RIVER DEPTH.

\begin{tabular}{|l|l|l}
3585 & 8 FT. RIVER DEPTH.
\end{tabular}

\begin{tabular}{|l|l}
3588 & 8 FT. RIVER DEPTH.
\end{tabular}

35878 FT. RIVER DEPTH.

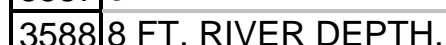

3589 3 FT. RIVER DEPTH.

35903 FT. RIVER DEPTH.

35913 FT. RIVER DEPTH.

3592 3 FT. RIVER DEPTH.

\begin{tabular}{l|l}
3593 & 3 FT. RIVER DEPTH. \\
\hline 3594 & 3 FT. RIVER DEPTH.
\end{tabular}

35943 FT. RIVER DEPTH.

$\begin{array}{ll}3595 & 3 \text { FT. RIVER DEPTH. }\end{array}$

\begin{tabular}{l|l}
3596 & 3 FT. RIVER DEPTH. \\
\hline
\end{tabular}

3597 3 FT. RIVER DEPTH.

3598 3 FT. RIVER DEPTH.

35997 FT. RIVER DEPTH.

\begin{tabular}{ll}
3600 & 7 FT. RIVER DEPTH. \\
\hline 3601 & 7 FT. RIVER DEPTH.
\end{tabular}

36017 FT. RIVER DEPTH.

36027 FT. RIVER DEPTH.

3603 7 FT. RIVER DEPTH.

360410 FT. RIVER DEPTH.

360510 FT. RIVER DEPTH.

360610 FT. RIVER DEPTH.

\begin{tabular}{l|l}
3607 & 10 FT. RIVER DEPTH \\
\hline 3608 & 10 FT. RIVER DEPTH.
\end{tabular}

3608 10 FT. RIVER DEPTH.

3609 15 FT. RIVER DEPTH.

3610 15 FT. RIVER DEPTH.

\begin{tabular}{|l|l}
3611 & 15 FT. RIVER DEPTH \\
\hline
\end{tabular}

SAMP COMMENT 
$A C$

\begin{tabular}{|c|c}
1 & \\
\hline 3612 & 15 FT. RIVER DEPTH. \\
\hline
\end{tabular}

\begin{tabular}{l|l}
3613 & 15 FT. RIVER DEPTH.
\end{tabular}

36146 FT. RIVER DEPTH.

36156 FT. RIVER DEPTH.

\begin{tabular}{|l|l}
3616 & 6 FT. RIVER DEPTH. \\
\hline 3617 & 6 FT. RIVER DEPTH.
\end{tabular}

3617 6 FT. RIVER DEPTH.

\begin{tabular}{l|l}
3618 & 6 FT. RIVER DEPTH.
\end{tabular}

\begin{tabular}{|l|l}
3619 & 4 FT. RIVER DEPTH.
\end{tabular}

3620 4 FT. RIVER DEPTH.

3621 4 FT. RIVER DEPTH.

36224 FT. RIVER DEPTH.

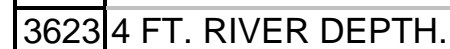

\begin{tabular}{l|l}
3624 & 4 FT. RIVER RIVER DEPTH. \\
\hline 3624 & 4 FT.
\end{tabular}

36254 FT. RIVER DEPTH.

3626 4 FT. RIVER DEPTH.

3627 4 FT. RIVER DEPTH.

3628 4 FT. RIVER DEPTH.

3629 3.5 FT. RIVER DEPTH.

3630 3.5 FT. RIVER DEPTH.

\begin{tabular}{|l|l}
3631 & 3.5 FT. RIVER DEPTH. \\
\hline 3632 & 3.5 FT. RIVER DEPTH.
\end{tabular}

3632 3.5 FT. RIVER DEPTH.

3633 3.5 FT. RIVER DEPTH.

3634 14 FT. RIVER DEPTH.

363514 FT. RIVER DEPTH.

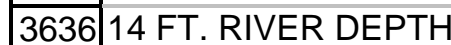

3637 14 FT. RIVER DEPTH.

\begin{tabular}{l|l}
3638 & 14 FT. RIVER DEPTH \\
\hline 3639 & 18 FT. RIVER DEPTH.
\end{tabular}

363918 FT. RIVER DEPTH.

364018 FT. RIVER DEPTH.

3641 18 FT. RIVER DEPTH.

\begin{tabular}{l|l}
3642 & 18 FT. RIVER DEPTH.
\end{tabular}

364318 FT. RIVER DEPTH.

\begin{tabular}{|l|l}
3644 & 22 FT. RIVER DEPTH. \\
\hline
\end{tabular}

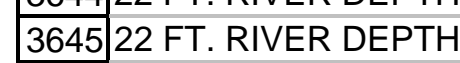

364622 FT. RIVER DEPTH.

3647 22 FT. RIVER DEPTH.

\begin{tabular}{ll}
3648 & 22 FT. RIVER DEPTH \\
\hline 3649 & FT. RIVER DEPTH.
\end{tabular}

\begin{tabular}{|l|l|}
\hline 3649 & 2 FT. RIVER DEPTH. \\
\hline
\end{tabular}

SAMP COMMENT 
$A C$

\begin{tabular}{|c|c|}
\hline \multirow{2}{*}{\begin{tabular}{|c|}
1 \\
3650 \\
\end{tabular}} & \multirow{2}{*}{\begin{tabular}{|lc} 
& SAMP_COMMENT \\
\end{tabular}} \\
\hline & \\
\hline 3651 & 2 FT. RIVER DEPTH. \\
\hline 3652 & 2 FT. RIVER DEPTH. \\
\hline 3653 & 2 FT. RIVER DEPTH. \\
\hline 3654 & 1.5 FT. RIVER DEPTH. \\
\hline 3655 & 1.5 FT. RIVER DEPTH. \\
\hline 3656 & 1.5 FT. RIVER DEPTH. \\
\hline 3657 & 1.5 FT. RIVER DEPTH. \\
\hline 3658 & 1.5 FT. RIVER DEPTH. \\
\hline 3659 & 16 FT. RIVER DEPTH. \\
\hline 3660 & 16 FT. RIVER DEPTH. \\
\hline 3661 & 16 FT. RIVER DEPTH. \\
\hline 3662 & 16 FT. RIVER DEPTH. \\
\hline 3663 & 16 FT. RIVER DEPTH. \\
\hline 3664 & 23 FT. RIVER DEPTH. \\
\hline 3665 & 23 FT. RIVER DEPTH. \\
\hline 3666 & 23 FT. RIVER DEPTH. \\
\hline 3667 & 23 FT. RIVER DEPTH. \\
\hline 3668 & 23 FT. RIVER DEPTH. \\
\hline 3669 & 15 FT. RIVER DEPTH. \\
\hline 3670 & 15 FT. RIVER DEPTH. \\
\hline 3671 & 15 FT. RIVER DEPTH. \\
\hline 3672 & 15 FT. RIVER DEPTH. \\
\hline 3673 & 15 FT. RIVER DEPTH. \\
\hline 3674 & 11 FT. RIVER DEPTH. \\
\hline 3675 & 11 FT. RIVER DEPTH. \\
\hline 3676 & 11 FT. RIVER DEPTH. \\
\hline 3677 & 11 FT. RIVER DEPTH. \\
\hline 3678 & 11 FT. RIVER DEPTH. \\
\hline 3679 & 16 FT. RIVER DEPTH. \\
\hline 3680 & 16 FT. RIVER DEPTH. \\
\hline 3681 & 16 FT. RIVER DEPTH. \\
\hline 3682 & 16 FT. RIVER DEPTH. \\
\hline 3683 & 16 FT. RIVER DEPTH. \\
\hline 3684 & 26 FT. RIVER DEPTH. \\
\hline 3685 & 26 FT. RIVER DEPTH. \\
\hline 3686 & 26 FT. RIVER DEPTH. \\
\hline
\end{tabular}

368726 FT. RIVER DEPTH. 
$\mathrm{AC}$

\begin{tabular}{|c|c|}
\hline 3688 & 26 FT. RIVER DEPTH \\
\hline 3689 & 10 FT. RIVER DEPTH \\
\hline
\end{tabular}

\begin{tabular}{|l|l|}
\hline 3689 & 10 FT. RIVER DEPTH \\
\hline 3690 & 10 FT. RIVER DEPTH \\
\hline
\end{tabular}

3691 10 FT. RIVER DEPTH.

\begin{tabular}{l|l}
3692 & 10 FT. RIVER DEPTH. \\
\hline 369 & .
\end{tabular}

\begin{tabular}{|l|l}
3693 & 10 FT. RIVER DEPTH. \\
\hline 3694 & .
\end{tabular}

36944 FT. RIVER DEPTH.

\begin{tabular}{|l|l}
3695 & 4 FT. RIVER DEPTH.
\end{tabular}

\begin{tabular}{l|l}
3696 & 4 FT. RIVER DEPTH.
\end{tabular}

3697 4 FT. RIVER DEPTH.

3698 4 FT. RIVER DEPTH.

36999 FT. RIVER DEPTH.

3700 9 FT. RIVER DEPTH.

37019 FT. RIVER DEPTH.

3702 9 FT. RIVER DEPTH.

\begin{tabular}{l|l}
3703 & 9 FT. RIVER DEPTH.
\end{tabular}

370416 FT. RIVER DEPTH.

370516 FT. RIVER DEPTH.

\begin{tabular}{l|l}
3706 & 16 FT. RIVER DEPTH.
\end{tabular}

3707 16 FT. RIVER DEPTH.

3708 16 FT. RIVER DEPTH.

3709 20 FT. RIVER DEPTH.

\begin{tabular}{ll}
3710 & 20 FT. RIVER DEPTH. \\
\hline 3711 & 20 FT. RIVER DEPTH.
\end{tabular}

\begin{tabular}{|l|l}
3711 & 20 FT. RIVER DEPTH \\
\hline
\end{tabular}

371220 FT. RIVER DEPTH.

371320 FT. RIVER DEPTH.

\begin{tabular}{|l|l|}
\hline 3714 & 24 FT. RIVER DEPTH \\
\hline 3715 & 24 FT. RIVER DEPTH.
\end{tabular}

371524 FT. RIVER DEPTH.

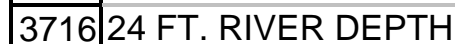

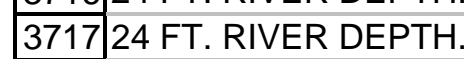

\begin{tabular}{|l|l}
\hline 3718 & 24 FT. RIVER DEPTH \\
\hline
\end{tabular}

37193 FT. RIVER DEPTH.

3720 3 FT. RIVER DEPTH.

\begin{tabular}{l|l}
3721 & 3 FT. RIVER DEPTH.
\end{tabular}

37223 FT. RIVER DEPTH.

$\begin{array}{ll}3723 & 3 \text { FT. RIVER DEPTH. }\end{array}$

$\begin{array}{ll}3724 & 33 \text { FT. RIVER DEPTH. }\end{array}$

\begin{tabular}{|l|l|}
3725 & 33 FT. RIVER DEPTH. \\
\hline
\end{tabular}

SAMP COMMENT 
AC

\begin{tabular}{|c|c|}
\hline 1 & SAMP_COMMENT \\
\hline 3726 & 33 FT. RIVER DEPTH. \\
\hline 3727 & 33 FT. RIVER DEPTH. \\
\hline 3728 & 33 FT. RIVER DEPTH. \\
\hline 3729. & 17 FT. RIVER DEPTH. \\
\hline 3730 & 17 FT. RIVER DEPTH. \\
\hline 3731 & 17 FT. RIVER DEPTH. \\
\hline 3732 & 17 FT. RIVER DEPTH. \\
\hline 3733 & 17 FT. RIVER DEPTH. \\
\hline 3734 & 22 FT. RIVER DEPTH. \\
\hline 3735 & 22 FT. RIVER DEPTH. \\
\hline 3736 & 22 FT. RIVER DEPTH. \\
\hline 3737 & 22 FT. RIVER DEPTH. \\
\hline 3738 & 22 FT. RIVER DEPTH. \\
\hline 3739 & 15 FT. RIVER DEPTH. \\
\hline 3740 & 15 FT. RIVER DEPTH. \\
\hline 3741 & 15 FT. RIVER DEPTH. \\
\hline 3742 & 15 FT. RIVER DEPTH. \\
\hline 3743 & 15 FT. RIVER DEPTH. \\
\hline 3744 & SAMPLE DEPTH 2 FT. \\
\hline 3745 & SAMPLE DEPTH 2 FT. \\
\hline 3746 & SAMPLE DEPTH 2 FT. \\
\hline 3747 & SAMPLE DEPTH 2 FT. \\
\hline 3748 & SAMPLE DEPTH 2 FT. \\
\hline 3749 & SAMPLE DEPTH 2 FT. \\
\hline 3750 & SAMPLE DEPTH 2 FT. \\
\hline 3751 & SAMPLE DEPTH 2 FT. \\
\hline 3752 & SAMPLE DEPTH 2 FT. \\
\hline 3753 & SAMPLE DEPTH 2 FT. \\
\hline 3754 & SAMPLE DEPTH 5.5 FT. \\
\hline 3755 & SAMPLE DEPTH 5.5 FT. \\
\hline 3756 & SAMPLE DEPTH 5.5 FT. \\
\hline 3757 & SAMPLE DEPTH 5.5 FT. \\
\hline 3758 & SAMPLE DEPTH 5.5 FT. \\
\hline 3759 & SAMPLE DEPTH 12 FT. \\
\hline 3760 & SAMPLE DEPTH 12 FT. \\
\hline 3761 & SAMPLE DEPTH 12 FT. \\
\hline 3762 & SAMPLE DEPTH 12 FT. \\
\hline 3763 & SAMPLE DEPTH 12 FT. \\
\hline
\end{tabular}

3763 SAMPLE DEPTH 12 FT. 
$A C$

\begin{tabular}{|c|c|}
\hline 3764 & SAMPLE DEPTH $11 \mathrm{FT}$ \\
\hline 3765 & SAMPLE DEPTH $11 \mathrm{FT}$ \\
\hline
\end{tabular}

\begin{tabular}{|l|l|}
\hline 3765 & SAMPLE DEPTH $11 \mathrm{FT}$ \\
\hline 3766 & SAMPLE DEPTH $11 \mathrm{FT}$ \\
\hline
\end{tabular}

\begin{tabular}{|l|l}
3767 & SAMPLE DEPTH $11 \mathrm{FT}$.
\end{tabular}

3768 SAMPLE DEPTH 11 FT.

3769 SAMPLE DEPTH 12 FT.

3770 SAMPLE DEPTH 12 FT.

3771 SAMPLE DEPTH $12 \mathrm{FT}$.

\begin{tabular}{|l|l|}
\hline 3772 & SAMPLE DEPTH $12 \mathrm{FT}$ \\
\hline 3773
\end{tabular}

3773 SAMPLE DEPTH 12 FT.

3774 SAMPLE DEPTH 13 FT.

3775 SAMPLE DEPTH 13 FT.

\begin{tabular}{|l|l}
3776 & SAMPLE DEPTH 13 FT. \\
\hline 3777 & SAMPLE DEPTH 13 FT.
\end{tabular}

3777 SAMPLE DEPTH $13 \mathrm{FT}$.

\begin{tabular}{|l|l|}
\hline 3778 & SAMPLE DEPTH $13 \mathrm{FT}$. \\
\hline
\end{tabular}

\begin{tabular}{l|l}
3779 & SAMPLE DEPTH 8 FT.
\end{tabular}

3780 SAMPLE DEPTH 8 FT.

3781 SAMPLE DEPTH 8 FT.

3782 SAMPLE DEPTH 8 FT.

\begin{tabular}{|l|l}
3783 & SAMPLE DEPTH 8 FT.
\end{tabular}

3784 SAMPLE DEPTH 6 FT.

3785 SAMPLE DEPTH 6 FT.

\begin{tabular}{|l|l|}
\hline 3786 & SAMPLE DEPTH 6 FT. \\
\hline 3787 & SAMPLE DEPTH 6 FT.
\end{tabular}

3787 SAMPLE DEPTH 6 FT.

3788 SAMPLE DEPTH 6 FT.

3789 SAMPLE DEPTH 4 FT.

3790 SAMPLE DEPTH 4 FT.

3791 SAMPLE DEPTH 4 FT.

3792 SAMPLE DEPTH $4 \mathrm{FT}$.

3793 SAMPLE DEPTH 4 FT.

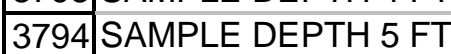

3795 SAMPLE DEPTH 5 FT.

3796 SAMPLE DEPTH 5 FT.

\begin{tabular}{l}
3797 \\
\hline $379 A M P L$ \\
\hline
\end{tabular}

3798 SAMPLE DEPTH 5 FT.

3799 SAMPLE DEPTH $10 \mathrm{FT}$

3800 SAMPLE DEPTH $10 \mathrm{FT}$.

\begin{tabular}{|l|l|}
\hline 3801 & SAMPLE DEPTH 10 FT.
\end{tabular}

SAMP COMMENT 
$A C$

\begin{tabular}{|c|c|}
\hline 1 & SAMP_COMMENT \\
\hline 3802 & SAMPLE DEPTH $10 \mathrm{FT}$. \\
\hline 3803 & SAMPLE DEPTH 10 FT. \\
\hline 3804 & SAMPLE DEPTH 12 FT. \\
\hline 3805 & SAMPLE DEPTH 12 FT. \\
\hline 3806 & SAMPLE DEPTH 12 FT. \\
\hline 3807 & SAMPLE DEPTH 12 FT. \\
\hline 3808 & SAMPLE DEPTH 12 FT. \\
\hline 3809 & SAMPLE DEPTH 6 FT. \\
\hline 3810 & SAMPLE DEPTH 6 FT. \\
\hline 3811 & SAMPLE DEPTH 6 FT. \\
\hline 3812 & SAMPLE DEPTH 6 FT. \\
\hline 3813 & SAMPLE DEPTH 6 FT. \\
\hline 3814 & SAMPLE DEPTH 2 FT. \\
\hline 3815 & SAMPLE DEPTH 2 FT. \\
\hline 3816 & SAMPLE DEPTH 2 FT. \\
\hline 3817 & SAMPLE DEPTH 2 FT. \\
\hline 3818 & SAMPLE DEPTH 2 FT. \\
\hline 3819 & SAMPLE DEPTH 10 FT. \\
\hline 3820 & SAMPLE DEPTH 10 FT. \\
\hline 3821 & SAMPLE DEPTH 10 FT. \\
\hline \begin{tabular}{|l|}
3822 \\
\end{tabular} & SAMPLE DEPTH 10 FT. \\
\hline 3823 & SAMPLE DEPTH 10 FT. \\
\hline 3824 & SAMPLE DEPTH 18 FT. \\
\hline 3825 & SAMPLE DEPTH 18 FT. \\
\hline 3826 & SAMPLE DEPTH $18 \mathrm{FT}$. \\
\hline 3827 & SAMPLE DEPTH 18 FT. \\
\hline 3828 & SAMPLE DEPTH 18 FT. \\
\hline 3829 & SAMPLE DEPTH 17 FT. \\
\hline 3830 & SAMPLE DEPTH 17 FT. \\
\hline 3831 & SAMPLE DEPTH 17 FT. \\
\hline 3832 & SAMPLE DEPTH 17 FT. \\
\hline 3833 & SAMPLE DEPTH 17 FT. \\
\hline 3834 & SAMPLE DEPTH 5 FT. \\
\hline 3835 & SAMPLE DEPTH 5 FT. \\
\hline 3836 & SAMPLE DEPTH 5 FT. \\
\hline 3837 & SAMPLE DEPTH 5 FT. \\
\hline 3838 & SAMPLE DEPTH 5 FT. \\
\hline 3839 & SAMPLE DEPTH 1 FT. \\
\hline
\end{tabular}

3839 SAMPLE DEPTH $1 \mathrm{FT}$. 
$\mathrm{AC}$

\begin{tabular}{|c|c|}
\hline 1 & $\begin{array}{l}\text { SAMP COMMENT } \\
\end{array}$ \\
\hline 3840 & SAMPLE DEPTH 1 FT. \\
\hline 3841 & SAMPLE DEPTH 1 FT. \\
\hline 3842 & SAMPLE DEPTH 1 FT. \\
\hline 3843 & SAMPLE DEPTH $1 \mathrm{FT}$. \\
\hline 3844 & SAMPLE DEPTH 2 FT. \\
\hline 3845 & SAMPLE DEPTH 2 FT. \\
\hline 3846 & SAMPLE DEPTH 2 FT. \\
\hline 3847 & SAMPLE DEPTH 2 FT. \\
\hline 3848 & SAMPLE DEPTH 2 FT. \\
\hline 3849 & SAMPLE DEPTH 5 FT. \\
\hline 3850 & SAMPLE DEPTH 5 FT. \\
\hline 3851 & SAMPLE DEPTH 5 FT. \\
\hline 3852 & SAMPLE DEPTH 5 FT. \\
\hline 3853 & SAMPLE DEPTH 5 FT. \\
\hline 3854 & SAMPLE DEPTH $4.5 \mathrm{FT}$. \\
\hline 3855 & SAMPLE DEPTH 4.5 FT. \\
\hline 3856 & SAMPLE DEPTH 4.5 FT. \\
\hline 3857 & SAMPLE DEPTH 4.5 FT. \\
\hline 3858 & SAMPLE DEPTH 4.5 FT. \\
\hline 3859 & SAMPLE DEPTH 1.5 FT. \\
\hline 3860 & SAMPLE DEPTH 1.5 FT. \\
\hline 3861 & SAMPLE DEPTH 1.5 FT. \\
\hline 3862 & SAMPLE DEPTH 1.5 FT. \\
\hline 3863 & SAMPLE DEPTH $1.5 \mathrm{FT}$. \\
\hline 3864 & SAMPLE DEPTH $2.5 \mathrm{FT}$. \\
\hline 3865 & SAMPLE DEPTH $2.5 \mathrm{FT}$. \\
\hline 3866 & SAMPLE DEPTH $2.5 \mathrm{FT}$. \\
\hline 3867 & SAMPLE DEPTH 2.5 FT. \\
\hline 3868 & SAMPLE DEPTH 2.5 FT. \\
\hline 3869 & SAMPLE DEPTH 1 FT. \\
\hline 3870 & SAMPLE DEPTH 1 FT. \\
\hline 3871 & SAMPLE DEPTH 1 FT. \\
\hline 3872 & SAMPLE DEPTH 1 FT. \\
\hline 3873 & SAMPLE DEPTH 1 FT. \\
\hline 3874 & SAMPLE DEPTH 5.5 FT. \\
\hline 3875 & SAMPLE DEPTH 5.5 FT. \\
\hline 3876 & SAMPLE DEPTH 5.5 FT. \\
\hline 3877 & SAMPLE DEPTH 5.5 FT. \\
\hline
\end{tabular}


AC

\begin{tabular}{|c|c|}
\hline \multirow{2}{*}{\begin{tabular}{c|c|c|}
1 \\
3878 \\
$n$
\end{tabular}} & \multirow{2}{*}{\begin{tabular}{|ll} 
& SAMP_COMMENT \\
\end{tabular}} \\
\hline & \\
\hline 3879 & SAMPLE DEPTH 9 FT. \\
\hline 3880 & SAMPLE DEPTH 9 FT. \\
\hline 3881 & SAMPLE DEPTH 9 FT. \\
\hline 3882 & SAMPLE DEPTH 9 FT. \\
\hline 3883 & SAMPLE DEPTH 9 FT. \\
\hline 3884 & SAMPLE DEPTH $10 \mathrm{FT}$. \\
\hline 3885 & SAMPLE DEPTH 10 FT. \\
\hline 3886 & SAMPLE DEPTH 10 FT. \\
\hline 3887 & SAMPLE DEPTH 10 FT. \\
\hline 3888 & SAMPLE DEPTH 10 FT. \\
\hline 3889 & SAMPLE DEPTH 1 FT. \\
\hline 3890 & SAMPLE DEPTH $1 \mathrm{FT}$. \\
\hline 3891 & SAMPLE DEPTH $1 \mathrm{FT}$. \\
\hline 3892 & SAMPLE DEPTH 1 FT. \\
\hline 3893 & SAMPLE DEPTH 1 FT. \\
\hline 3894 & SAMPLE DEPTH 1 FT. \\
\hline 3895 & SAMPLE DEPTH 1 FT. \\
\hline 3896 & SAMPLE DEPTH 1 FT. \\
\hline 3897 & SAMPLE DEPTH 1 FT. \\
\hline 3898 & SAMPLE DEPTH 1 FT. \\
\hline 3899 & SAMPLE DEPTH 8 FT. \\
\hline 3900 & SAMPLE DEPTH 8 FT. \\
\hline 3901 & SAMPLE DEPTH 8 FT. \\
\hline 3902 & SAMPLE DEPTH 8 FT. \\
\hline 3903 & SAMPLE DEPTH 8 FT. \\
\hline 3904 & SAMPLE DEPTH $11 \mathrm{FT}$. \\
\hline 3905 & SAMPLE DEPTH 11 FT. \\
\hline 3906 & SAMPLE DEPTH $11 \mathrm{FT}$. \\
\hline 3907 & SAMPLE DEPTH 11 FT. \\
\hline \begin{tabular}{|l|}
3908 \\
\end{tabular} & SAMPLE DEPTH 11 FT. \\
\hline 3909 & SAMPLE DEPTH 7 FT. \\
\hline 3910 & SAMPLE DEPTH 7 FT. \\
\hline \begin{tabular}{|l|}
3911 \\
\end{tabular} & SAMPLE DEPTH 7 FT. \\
\hline 3912 & SAMPLE DEPTH 7 FT. \\
\hline 3913 & SAMPLE DEPTH 7 FT. \\
\hline 3914 & SAMPLE DEPTH 2 FT. \\
\hline 3915 & SAMPLE DEPTH 2 FT. \\
\hline
\end{tabular}


$\mathrm{AC}$

\begin{tabular}{|c|c|}
\hline 1 & 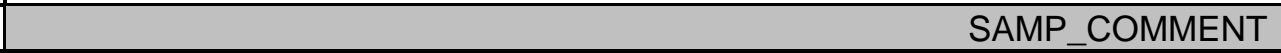 \\
\hline 3916 & SAMPLE DEPTH 2 FT. \\
\hline 3917 & SAMPLE DEPTH 2 FT. \\
\hline 3918 & SAMPLE DEPTH 2 FT. \\
\hline 3919 & SAMPLE DEPTH 2 FT. \\
\hline 3920 & SAMPLE DEPTH 2 FT. \\
\hline 3921 & SAMPLE DEPTH 2 FT. \\
\hline 3922 & SAMPLE DEPTH 2 FT. \\
\hline 3923 & SAMPLE DEPTH 2 FT. \\
\hline 3924 & SAMPLE DEPTH 5 FT. \\
\hline 3925 & SAMPLE DEPTH 5 FT. \\
\hline 3926 & SAMPLE DEPTH 5 FT. \\
\hline 3927 & SAMPLE DEPTH 5 FT. \\
\hline 3928 & SAMPLE DEPTH 5 FT. \\
\hline 3929 & SAMPLE DEPTH. \\
\hline 3930 & SAMPLE DEPTH. \\
\hline 3931 & SAMPLE DEPTH. \\
\hline 3932 & SAMPLE DEPTH. \\
\hline 3933 & SAMPLE DEPTH. \\
\hline 3934 & SAMPLE DEPTH $4.5 \mathrm{FT}$. \\
\hline 3935 & SAMPLE DEPTH 4.5 FT. \\
\hline 3936 & SAMPLE DEPTH 4.5 FT. \\
\hline 3937 & SAMPLE DEPTH 4.5 FT. \\
\hline 3938 & SAMPLE DEPTH 4.5 FT. \\
\hline 3939 & SAMPLE DEPTH 5 FT. \\
\hline 3940 & SAMPLE DEPTH 5 FT. \\
\hline 3941 & SAMPLE DEPTH 5 FT. \\
\hline 3942 & SAMPLE DEPTH 5 FT. \\
\hline 3943 & SAMPLE DEPTH 5 FT. \\
\hline 3944 & SAMPLE DEPTH $3.5 \mathrm{FT}$. \\
\hline 3945 & SAMPLE DEPTH 3.5 FT. \\
\hline 3946 & SAMPLE DEPTH 3.5 FT. \\
\hline 3947 & SAMPLE DEPTH 3.5 FT. \\
\hline 3948 & SAMPLE DEPTH 3.5 FT. \\
\hline 3949 & SAMPLE DEPTH 2 FT. \\
\hline 3950 & SAMPLE DEPTH 2 FT. \\
\hline 3951 & SAMPLE DEPTH 2 FT. \\
\hline 3952 & SAMPLE DEPTH 2 FT. \\
\hline 3953 & SAMPLE DEPTH 2 FT. \\
\hline
\end{tabular}


$\mathrm{AC}$

\begin{tabular}{|c|c|}
\hline 1 & $\begin{array}{l}\text { SAMP COMMENT } \\
\end{array}$ \\
\hline 3954 & SAMPLE DEPTH 1 FT. \\
\hline 3955 & SAMPLE DEPTH $1 \mathrm{FT}$. \\
\hline 3956 & SAMPLE DEPTH $1 \mathrm{FT}$. \\
\hline 3957 & SAMPLE DEPTH 1 FT. \\
\hline 3958 & SAMPLE DEPTH 1 FT. \\
\hline 3959 & SAMPLE DEPTH 2 FT. \\
\hline 3960 & SAMPLE DEPTH 2 FT. \\
\hline 3961 & SAMPLE DEPTH 2 FT. \\
\hline 3962 & SAMPLE DEPTH 2 FT. \\
\hline 3963 & SAMPLE DEPTH 2 FT. \\
\hline 3964 & SAMPLE DEPTH 1 FT. \\
\hline 3965 & SAMPLE DEPTH 1 FT. \\
\hline 3966 & SAMPLE DEPTH 1 FT. \\
\hline 3967 & SAMPLE DEPTH 1 FT. \\
\hline 3968 & SAMPLE DEPTH $1 \mathrm{FT}$. \\
\hline 3969 & SAMPLE DEPTH 1 FT. \\
\hline 3970 & SAMPLE DEPTH 1 FT. \\
\hline 3971 & SAMPLE DEPTH 1 FT. \\
\hline 3972 & SAMPLE DEPTH 1 FT. \\
\hline 3973 & SAMPLE DEPTH 1 FT. \\
\hline 3974 & SAMPLE DEPTH 4 FT. \\
\hline 3975 & SAMPLE DEPTH 4 FT. \\
\hline 3976 & SAMPLE DEPTH 4 FT. \\
\hline 3977 & SAMPLE DEPTH 4 FT. \\
\hline 3978 & SAMPLE DEPTH 4 FT. \\
\hline 3979 & SAMPLE DEPTH $10 \mathrm{FT}$. \\
\hline 3980 & SAMPLE DEPTH $10 \mathrm{FT}$. \\
\hline 3981 & SAMPLE DEPTH 10 FT. \\
\hline 3982 & SAMPLE DEPTH $10 \mathrm{FT}$. \\
\hline 3983 & SAMPLE DEPTH 10 FT. \\
\hline 3984 & SAMPLE DEPTH 15 FT. \\
\hline 3985 & SAMPLE DEPTH 15 FT. \\
\hline 3986 & SAMPLE DEPTH 15 FT. \\
\hline 3987 & SAMPLE DEPTH 15 FT. \\
\hline 3988 & SAMPLE DEPTH 15 FT. \\
\hline 3989 & SAMPLE DEPTH 15.5 FT. \\
\hline 3990 & SAMPLE DEPTH 15.5 FT. \\
\hline 3991 & SAMPLE DEPTH 15.5 FT. \\
\hline
\end{tabular}


$A C$

\begin{tabular}{|c|c|}
\hline 1 & \multirow{2}{*}{ SAMP_COMMENT } \\
\hline 3992 & \\
\hline 3993 & SAMPLE DEPTH 15.5 FT. \\
\hline 3994 & SAMPLE DEPTH 19 FT. \\
\hline 3995 & SAMPLE DEPTH 19 FT. \\
\hline 3996 & SAMPLE DEPTH 19 FT. \\
\hline 3997 & SAMPLE DEPTH 19 FT. \\
\hline 3998 & SAMPLE DEPTH 19 FT. \\
\hline 3999 & SAMPLE DEPTH 15 FT. \\
\hline 4000 & SAMPLE DEPTH 15 FT. \\
\hline 4001 & SAMPLE DEPTH 15 FT. \\
\hline 4002 & SAMPLE DEPTH 15 FT. \\
\hline 4003 & SAMPLE DEPTH 15 FT. \\
\hline 4004 & SAMPLE DEPTH 8 FT. \\
\hline 4005 & SAMPLE DEPTH 8 FT. \\
\hline 4006 & SAMPLE DEPTH 8 FT. \\
\hline 4007 & SAMPLE DEPTH 8 FT. \\
\hline \begin{tabular}{|l|}
4008 \\
\end{tabular} & SAMPLE DEPTH 8 FT. \\
\hline 4009 & SAMPLE DEPTH 4.5 FT. \\
\hline 4010 & SAMPLE DEPTH $4.5 \mathrm{FT}$. \\
\hline 4011 & SAMPLE DEPTH 4.5 FT. \\
\hline \begin{tabular}{|l|}
4012 \\
\end{tabular} & SAMPLE DEPTH 4.5 FT. \\
\hline 4013 & SAMPLE DEPTH 4.5 FT. \\
\hline 4014 & 12 FT. RIVER DEPTH. \\
\hline 4015 & 12 FT. RIVER DEPTH. \\
\hline 4016 & 12 FT. RIVER DEPTH. \\
\hline 4017 & 12 FT. RIVER DEPTH. \\
\hline 4018 & 12 FT. RIVER DEPTH. \\
\hline 4019 & 18 FT. RIVER DEPTH. \\
\hline 4020 & 18 FT. RIVER DEPTH. \\
\hline \begin{tabular}{|l|}
4021 \\
\end{tabular} & 18 FT. RIVER DEPTH. \\
\hline 4022 & 18 FT. RIVER DEPTH. \\
\hline 4023 & 18 FT. RIVER DEPTH. \\
\hline 4024. & 35 FT. RIVER DEPTH. \\
\hline 4025 & 35 FT. RIVER DEPTH. \\
\hline \begin{tabular}{|l|}
4026 \\
\end{tabular} & 35 FT. RIVER DEPTH. \\
\hline 4027 & 35 FT. RIVER DEPTH. \\
\hline 4028 & 35 FT. RIVER DEPTH. \\
\hline \begin{tabular}{|l|}
4029 \\
\end{tabular} & 15 FT. RIVER DEPTH. \\
\hline
\end{tabular}

\begin{tabular}{|l|l}
4029 & 35 FT. RIVER DEPTH. RIVER DEPTH. \\
\hline 4029 & FT.
\end{tabular} 
$A C$

\begin{tabular}{|c|c}
1 & \\
\hline 4030 & 15 FT. RIVER DEPTH.
\end{tabular}

\begin{tabular}{l|l}
4031 & 15 FT. RIVER DEPTH. \\
\hline
\end{tabular}

\begin{tabular}{|l|l}
4031 & 15 \\
\hline 4032 & FT. RIVER DEPTH.
\end{tabular}

403315 FT. RIVER DEPTH.

\begin{tabular}{|l|l}
4034 & 4 FT. RIVER DEPTH.
\end{tabular}

40354 FT. RIVER DEPTH.

4036 4 FT. RIVER DEPTH.

\begin{tabular}{|l|l}
4037 & 4 FT. RIVER DEPTH.
\end{tabular}

4038 4 FT. RIVER DEPTH.

40394 FT. RIVER DEPTH.

40404 FT. RIVER DEPTH.

4041 4 FT. RIVER DEPTH.

\begin{tabular}{|l|l}
4041 & 4 FT. RIVER DEPT. RIVER DEPTH. \\
\hline 4042 & 4 FT
\end{tabular}

40434 FT. RIVER DEPTH.

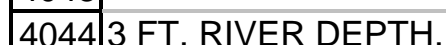

40453 FT. RIVER DEPTH.

40463 FT. RIVER DEPTH.

40473 FT. RIVER DEPTH.

40483 FT. RIVER DEPTH.

\begin{tabular}{l|l}
4049 & 15 FT. RIVER DEPTH. \\
\hline 4050 & .
\end{tabular}

405015 FT. RIVER DEPTH.

4051 15 FT. RIVER DEPTH.

\begin{tabular}{l|l}
4052 & 15 FT. RIVER DEPTH. \\
\hline
\end{tabular}

4053 15 FT. RIVER DEPTH.

405416 FT. RIVER DEPTH.

405516 FT. RIVER DEPTH.

\begin{tabular}{|l|l}
4056 & 16 FT. RIVER DEPTH. \\
\hline 4057 & .
\end{tabular}

405716 FT. RIVER DEPTH.

405816 FT. RIVER DEPTH.

405925 FT. RIVER DEPTH.

406025 FT. RIVER DEPTH.

406125 FT. RIVER DEPTH.

4062 25 FT. RIVER DEPTH.

\begin{tabular}{|l|l|}
\hline 4063 & 25 FT. RIVER DEPTH. \\
\hline
\end{tabular}

40644 FT. RIVER DEPTH.

40654 FT. RIVER DEPTH.

\begin{tabular}{|l|l}
4066 & 4 FT. RIVER DEPTH.
\end{tabular}

\begin{tabular}{|l|l|}
44067 & 4 FT. RIVER DEPTH.
\end{tabular}

SAMP COMMENT 
$A C$

\begin{tabular}{|c|c|}
\hline & \\
\hline 1 & SAMP_COMMENT \\
\hline 4068 & 4 FT. RIVER DEPTH. \\
\hline 4069 & 3 FT. RIVER DEPTH. \\
\hline 4070 & 3 FT. RIVER DEPTH. \\
\hline 4071 & 3 FT. RIVER DEPTH. \\
\hline 4072 & 3 FT. RIVER DEPTH. \\
\hline 4073 & 3 FT. RIVER DEPTH. \\
\hline 4074 & 16 FT. RIVER DEPTH. \\
\hline 4075 & 16 FT. RIVER DEPTH. \\
\hline 4076 & 16 FT. RIVER DEPTH. \\
\hline 4077 & 16 FT. RIVER DEPTH. \\
\hline 4078 & 16 FT. RIVER DEPTH. \\
\hline 4079 & 28 FT. RIVER DEPTH. \\
\hline 4080 & 28 FT. RIVER DEPTH. \\
\hline 4081 & 28 FT. RIVER DEPTH. \\
\hline 4082 & 28 FT. RIVER DEPTH. \\
\hline 4083 & 28 FT. RIVER DEPTH. \\
\hline 4084 & 14 FT. RIVER DEPTH. \\
\hline 4085 & 14 FT. RIVER DEPTH. \\
\hline 4086 & 14 FT. RIVER DEPTH. \\
\hline 4087 & 14 FT. RIVER DEPTH. \\
\hline 4088 & 14 FT. RIVER DEPTH. \\
\hline 4089 & \\
\hline 4090 & \\
\hline 4091 & \\
\hline 4092 & \\
\hline 4093 & \\
\hline 4094 & \\
\hline 4095 & \\
\hline 4096 & \\
\hline 4097 & \\
\hline 4098 & \\
\hline 4099 & \\
\hline 4100 & \\
\hline 4101 & \\
\hline 4102 & \\
\hline 4103 & \\
\hline 4104 & \\
\hline 4105 & \\
\hline
\end{tabular}




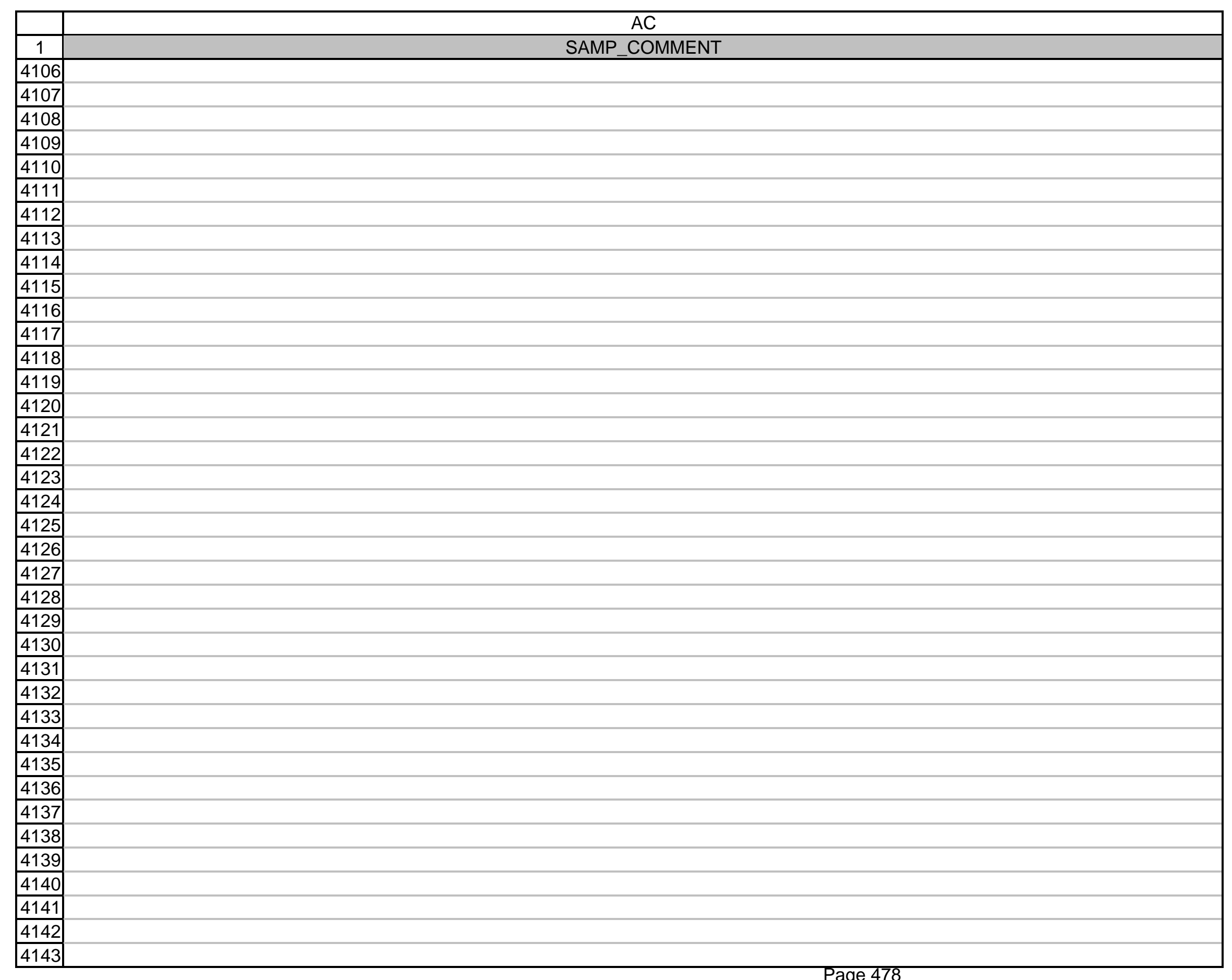




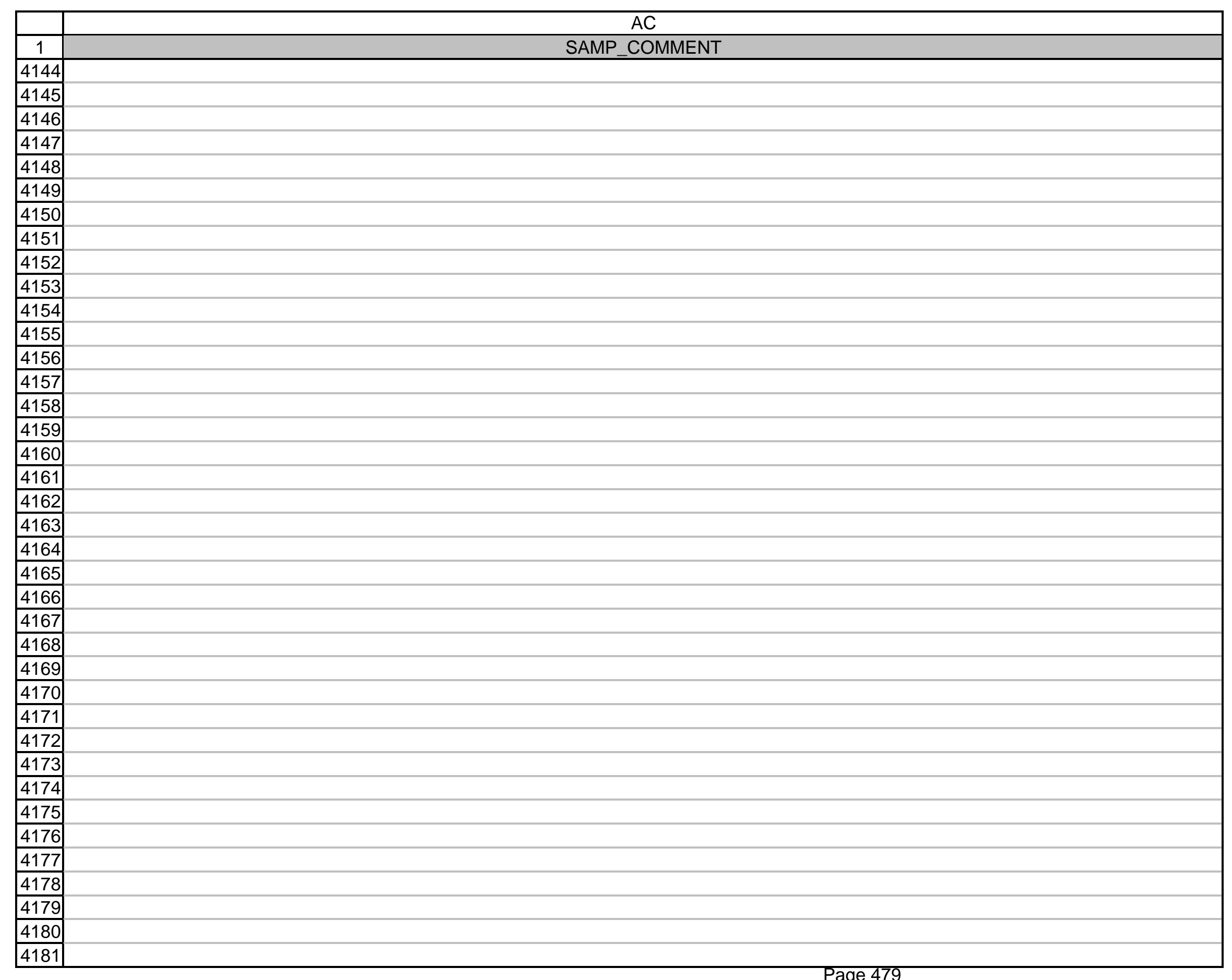




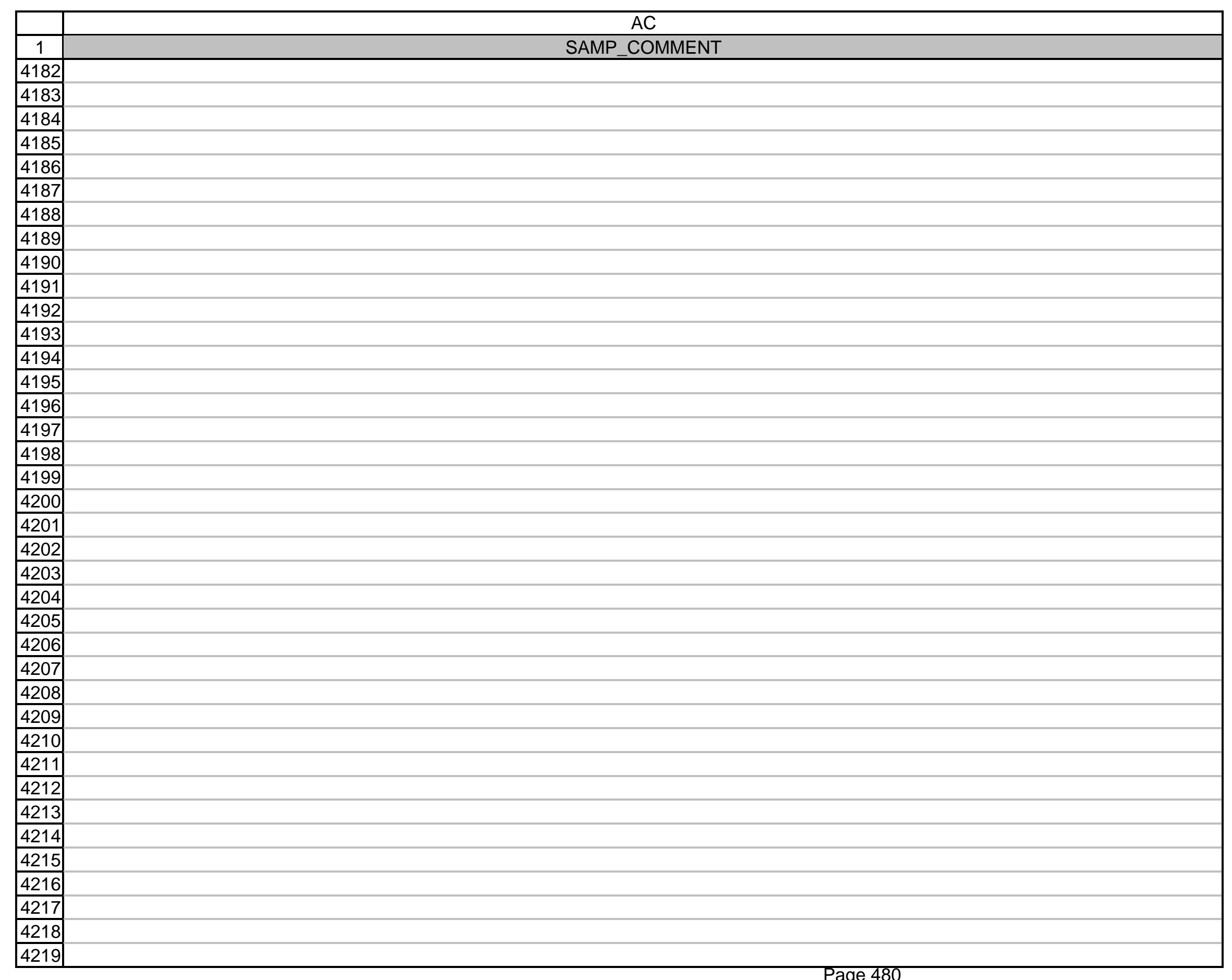




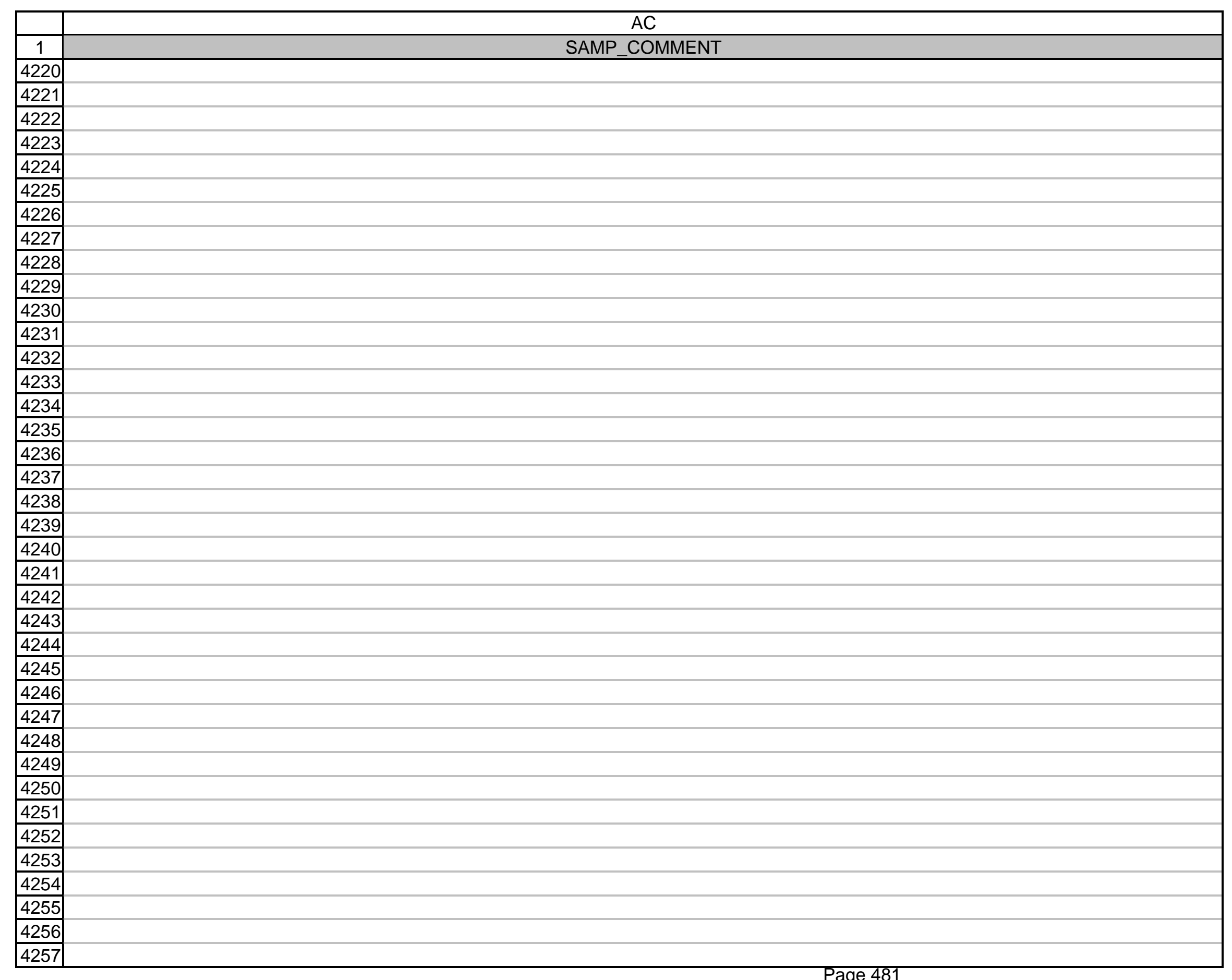




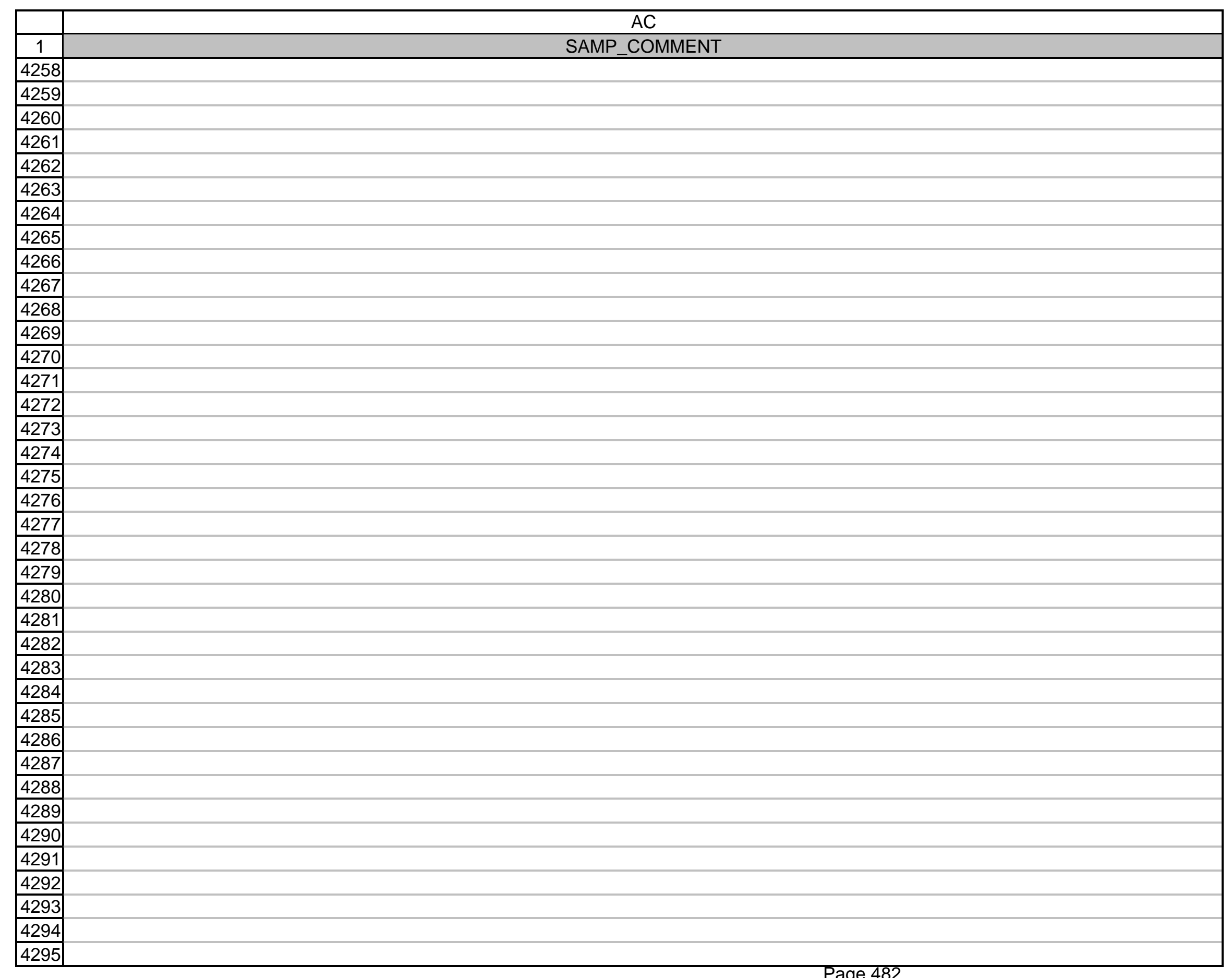




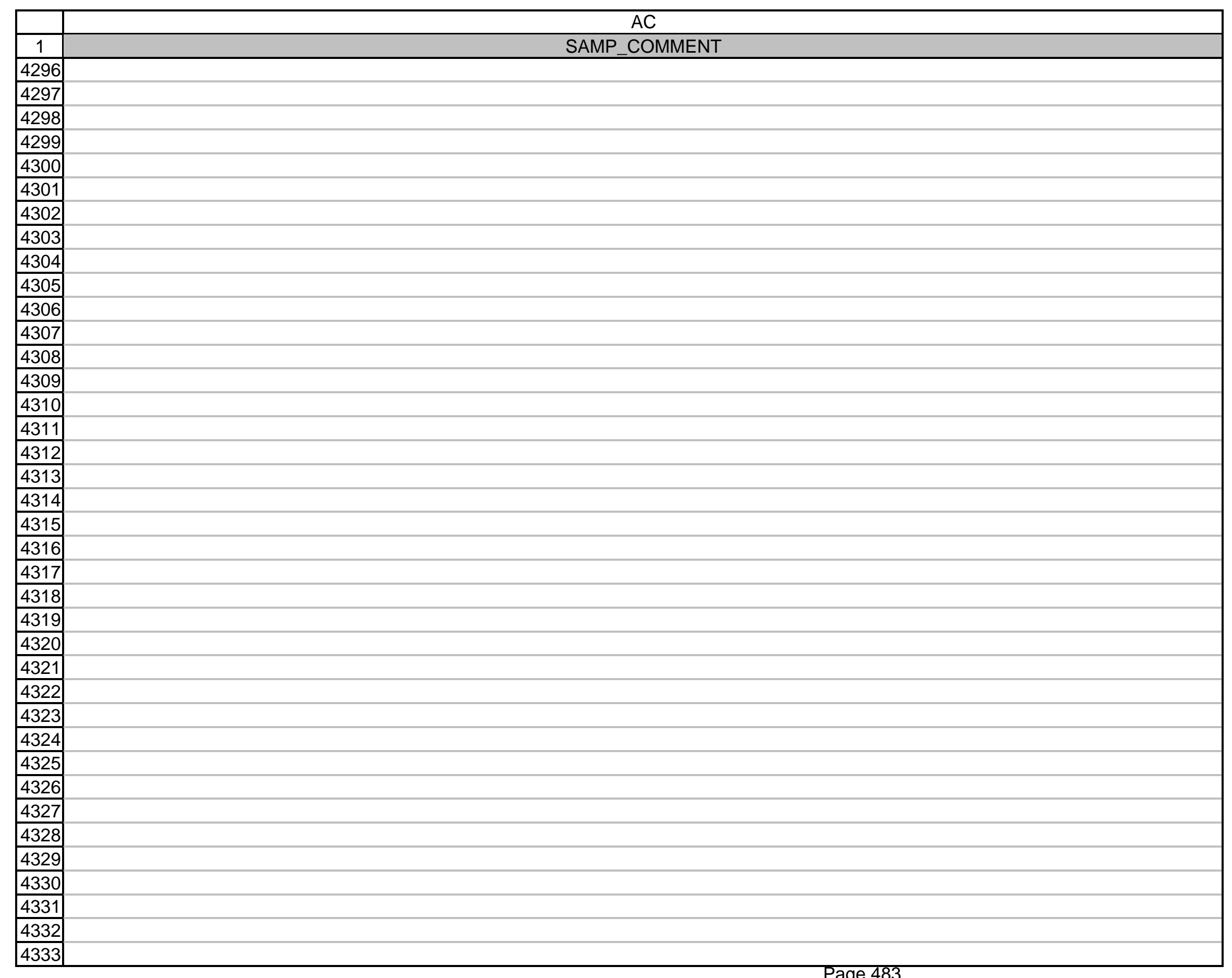




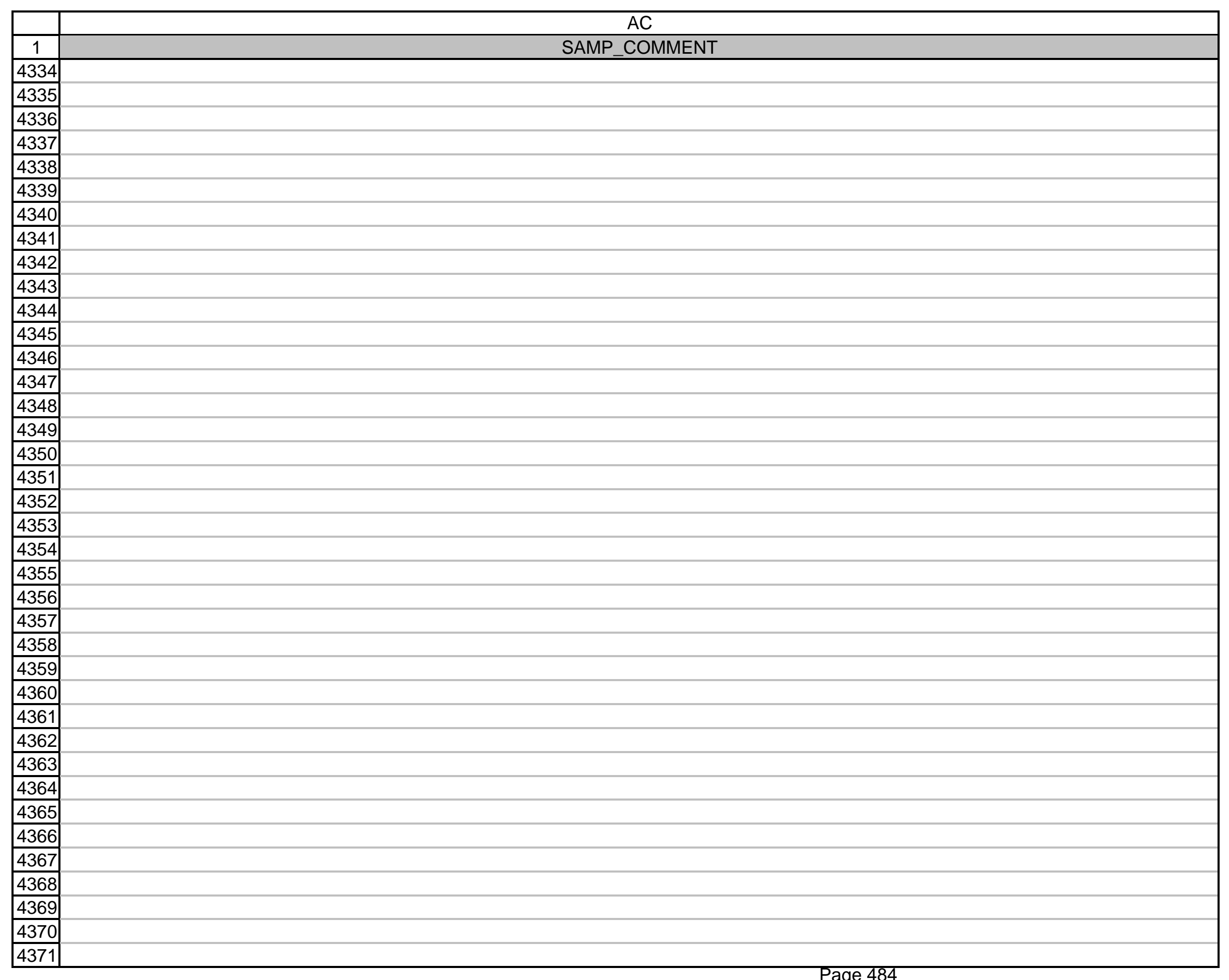




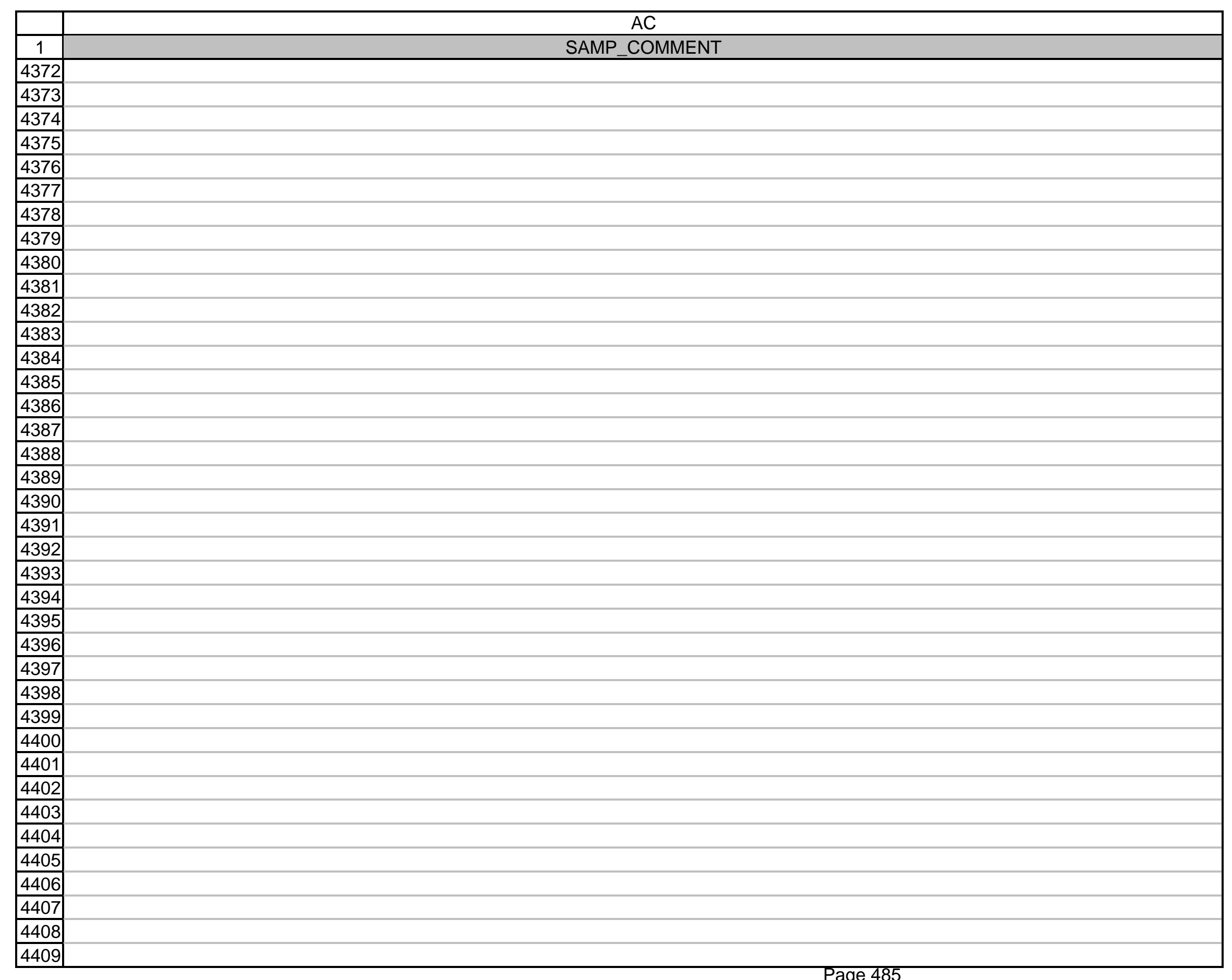




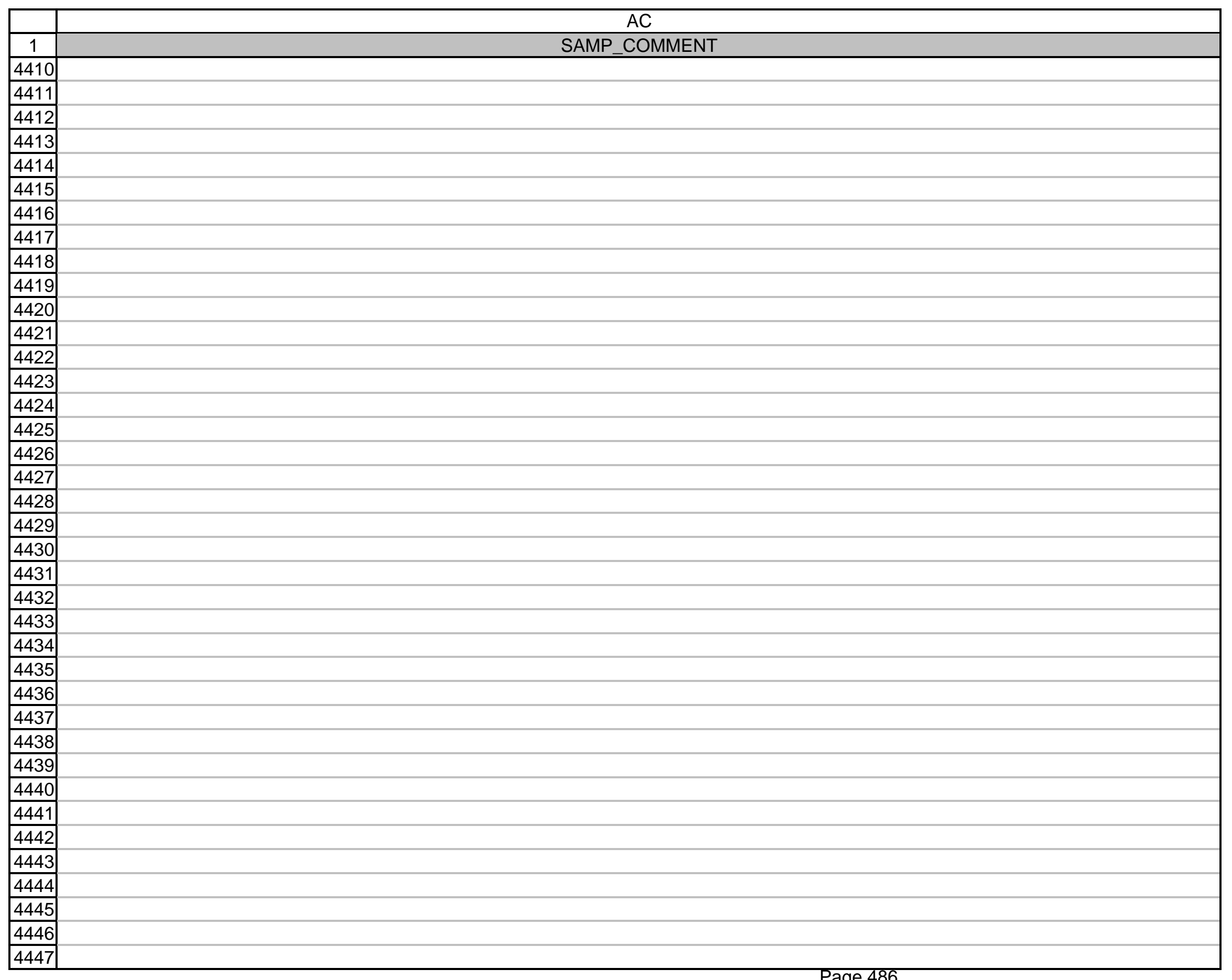




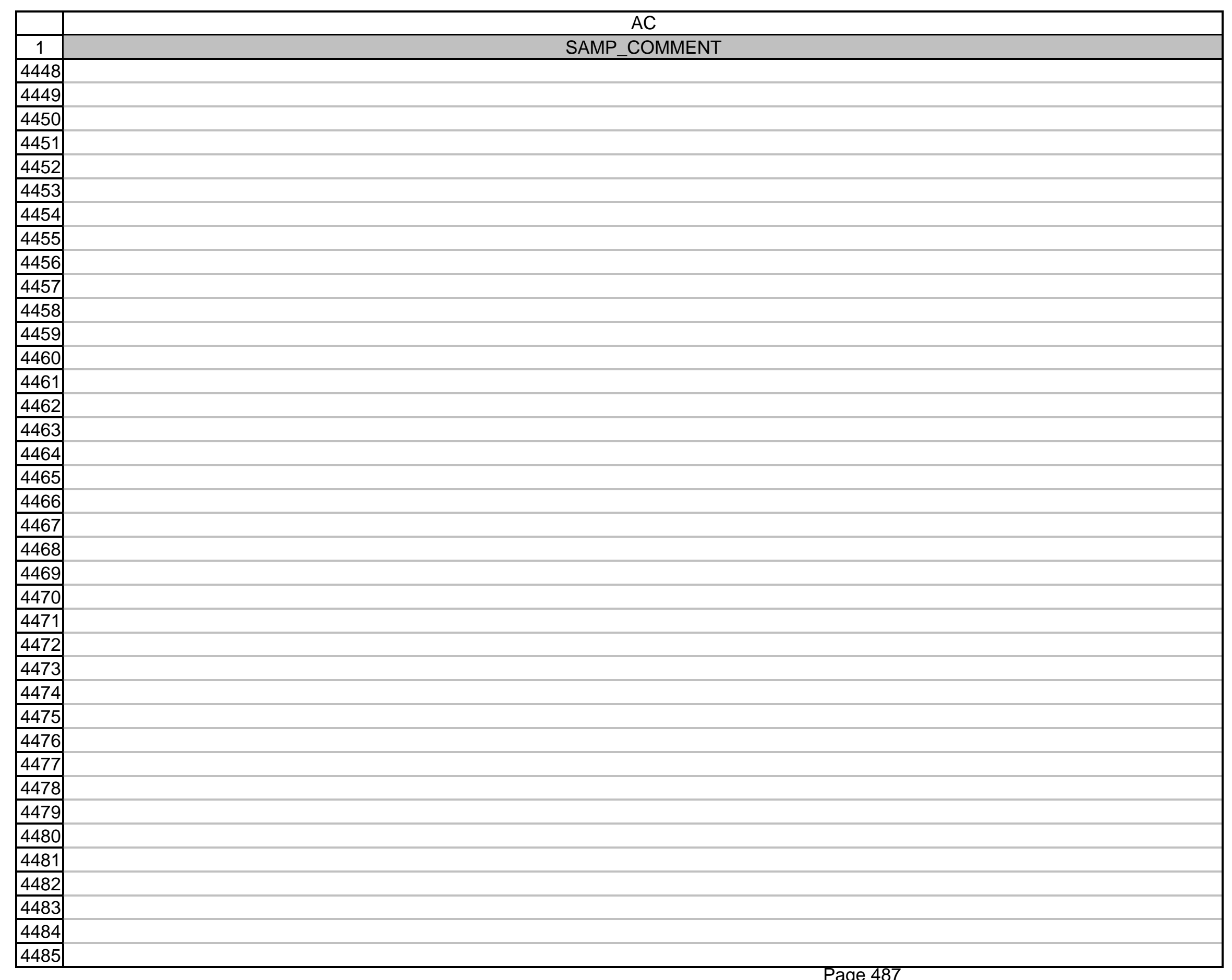




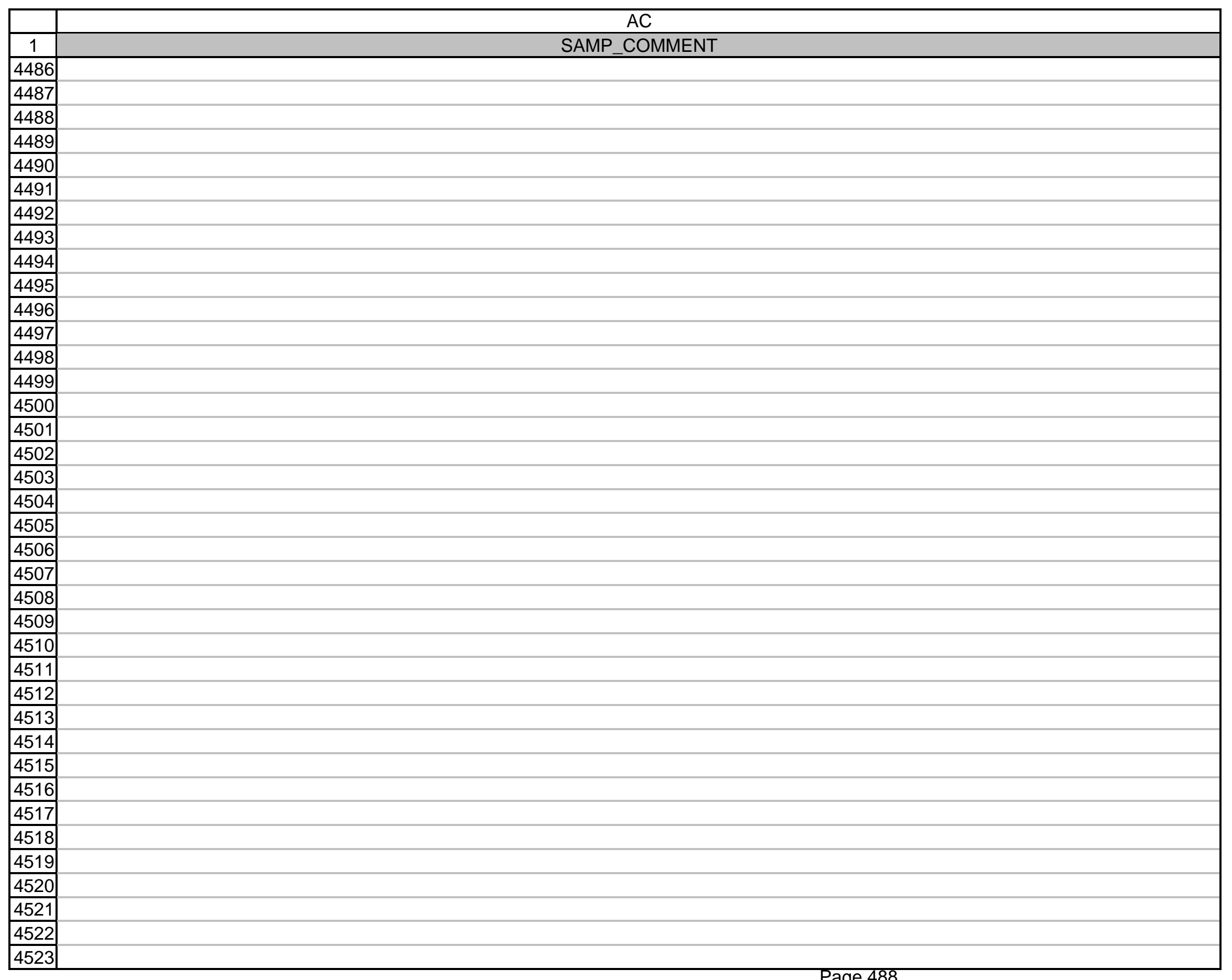




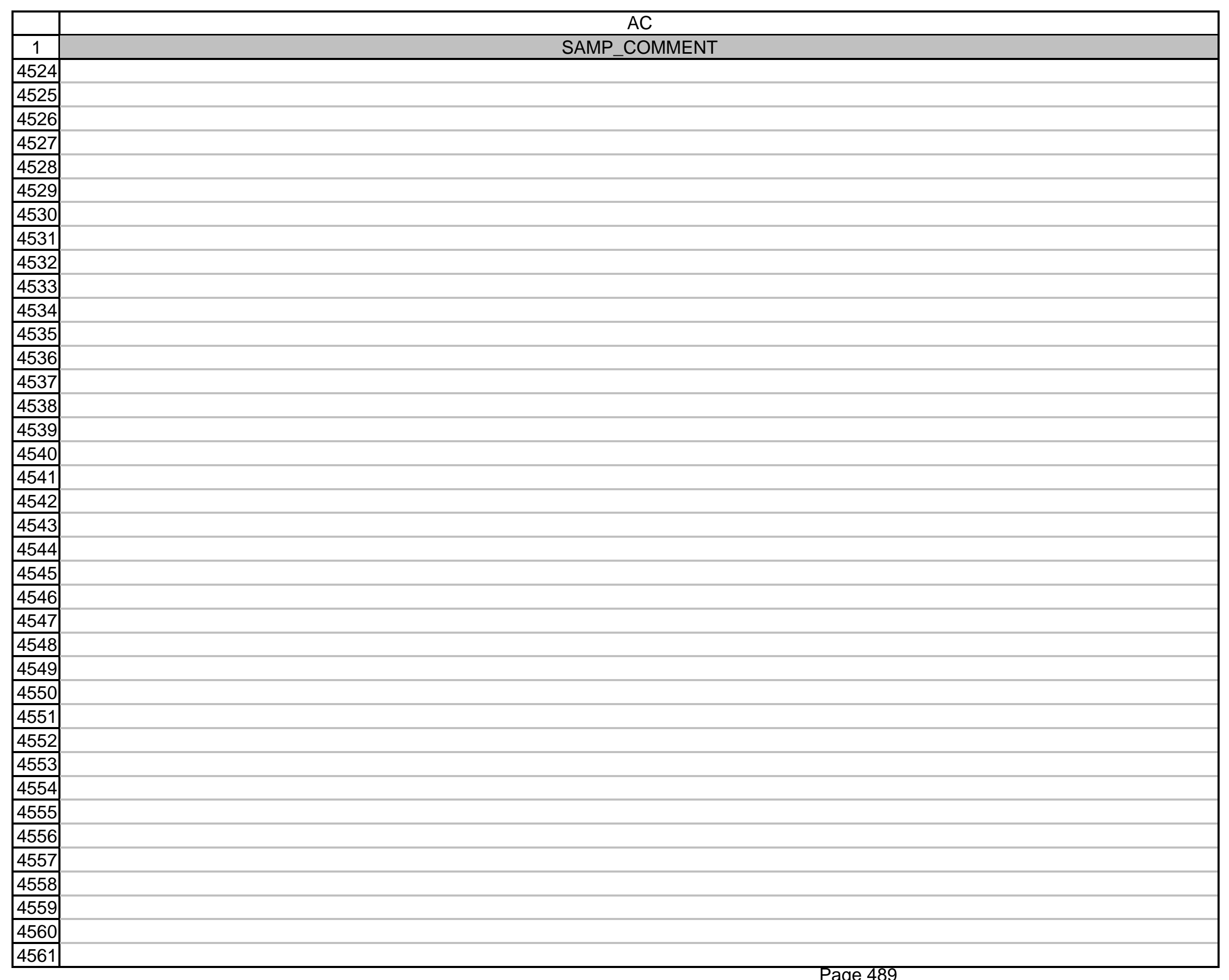




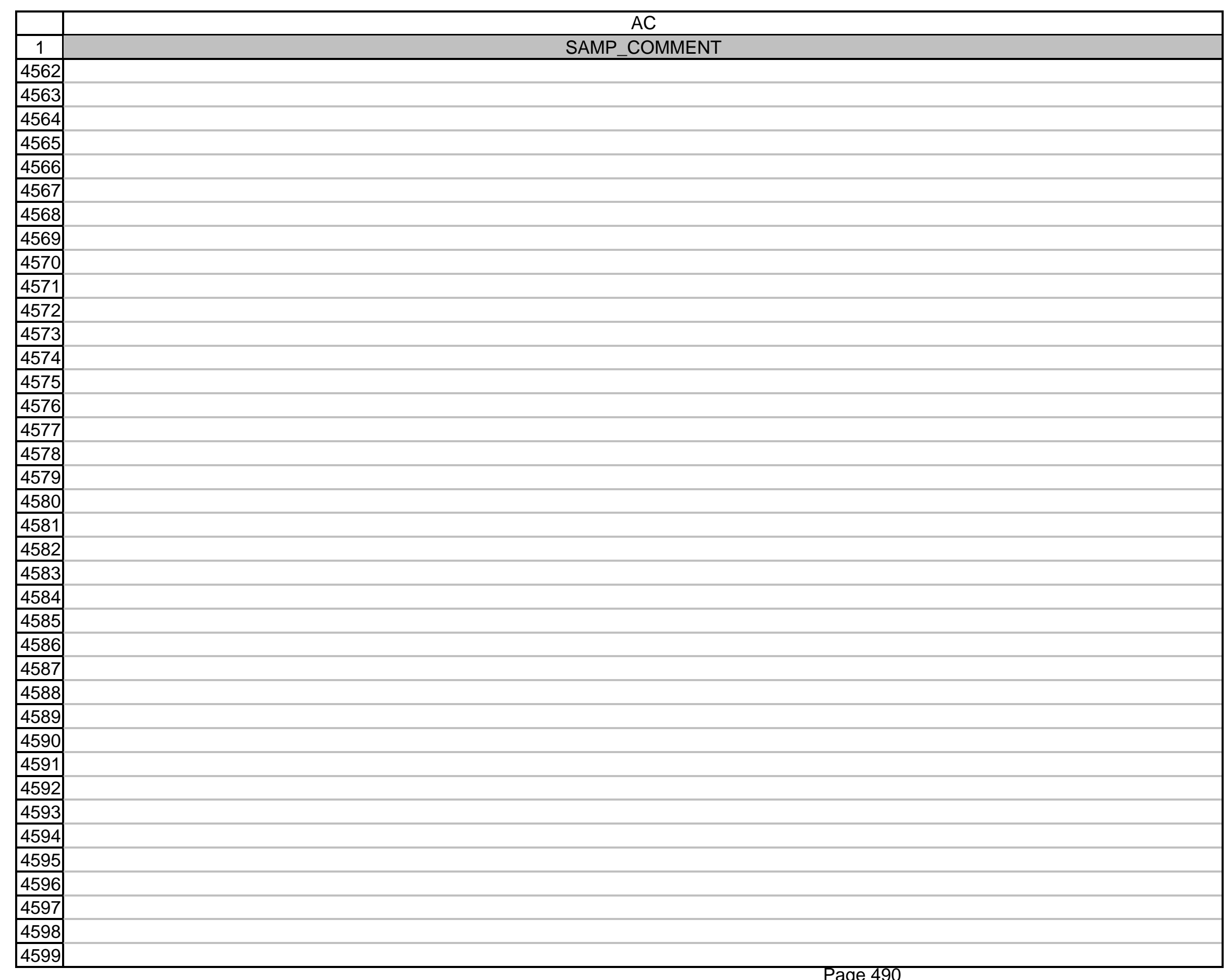




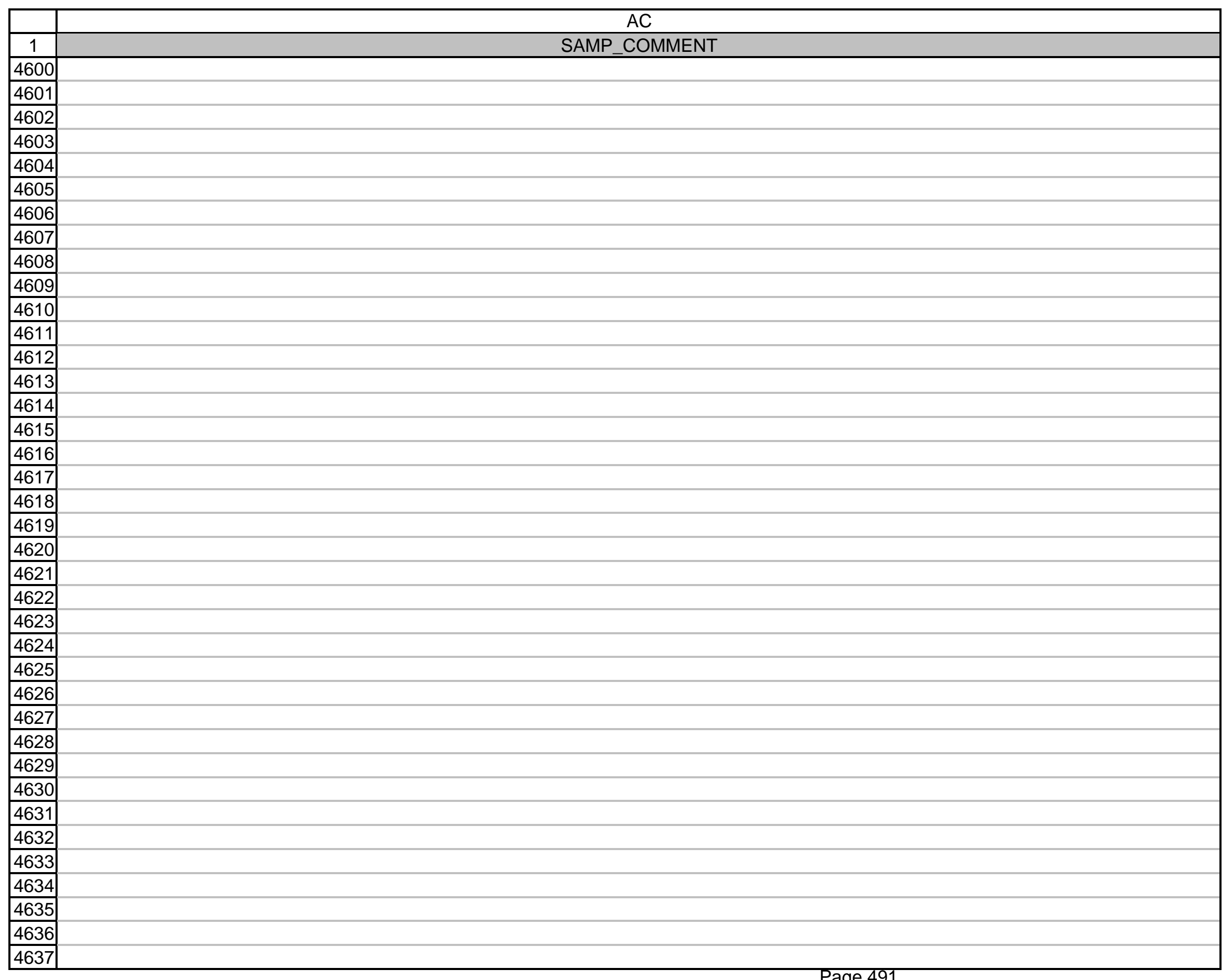




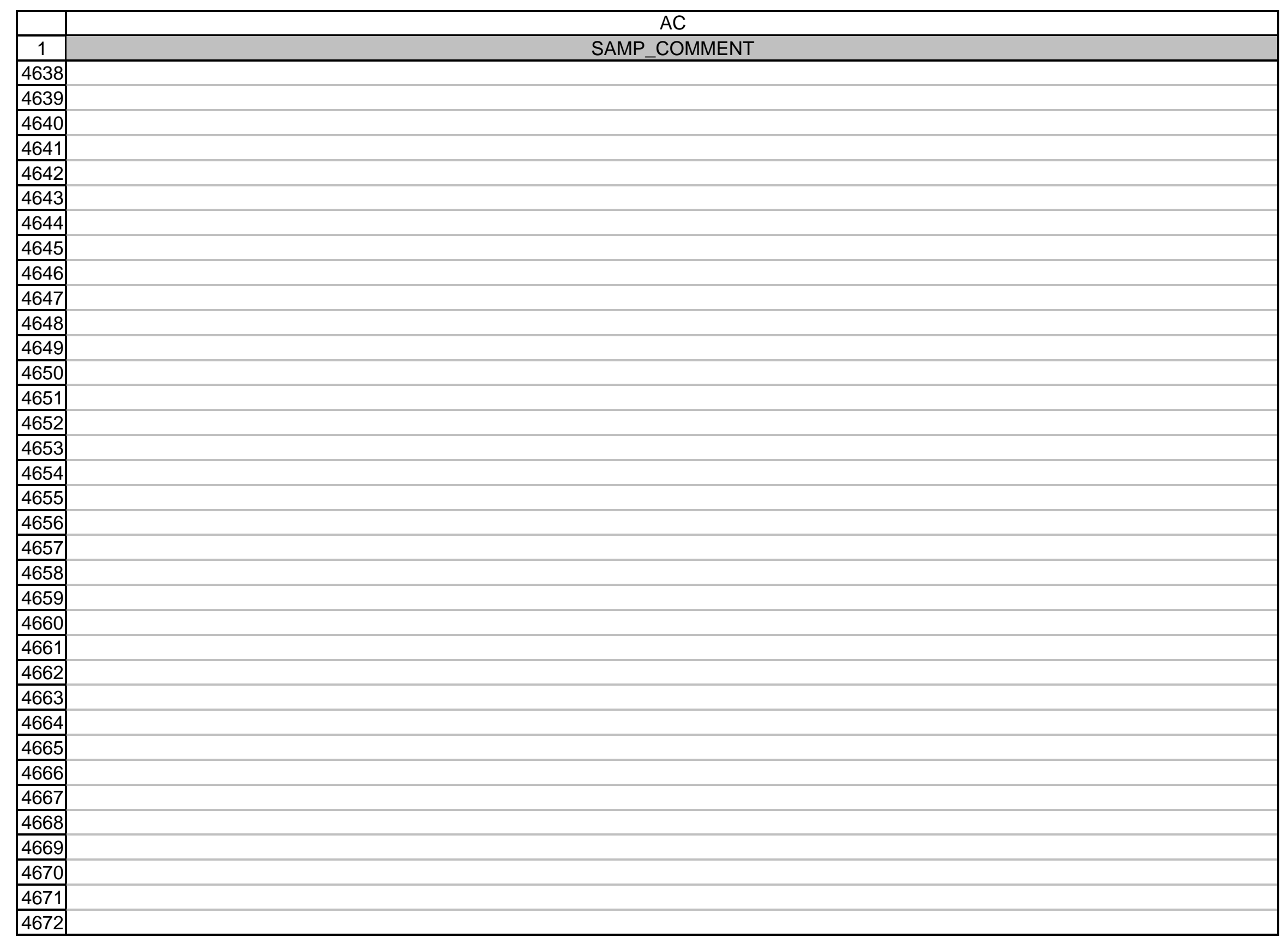

Page 492 


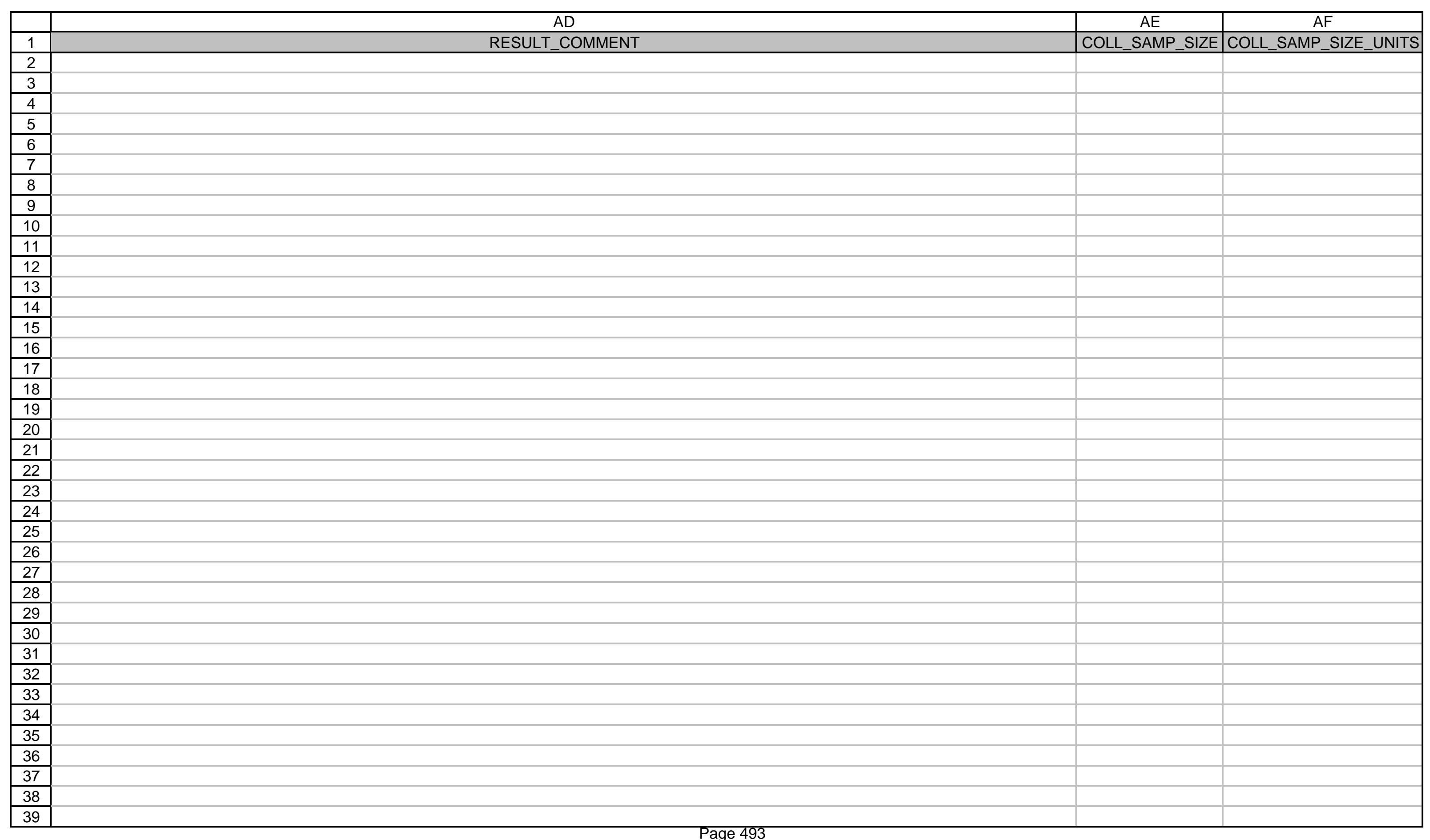




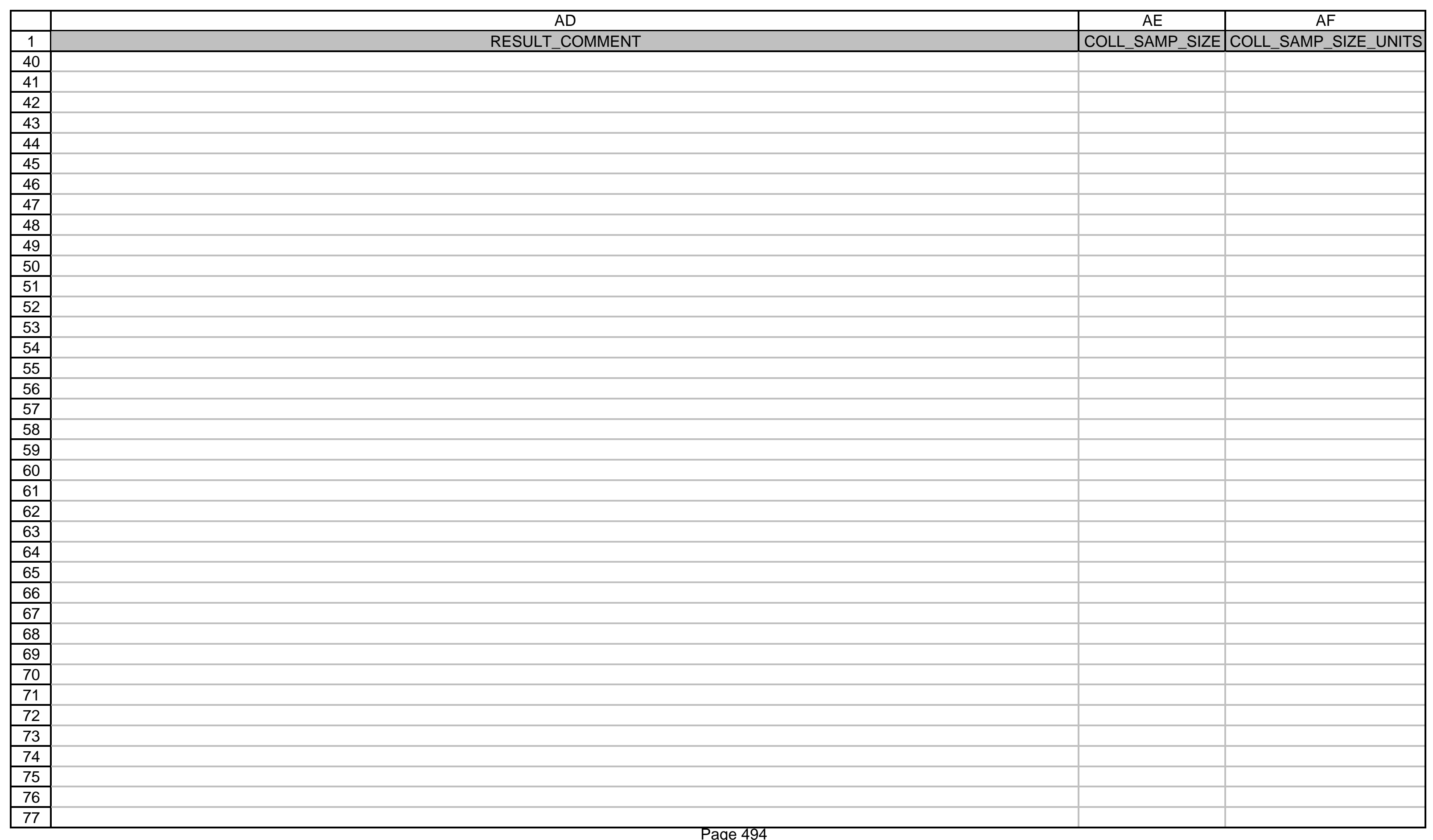




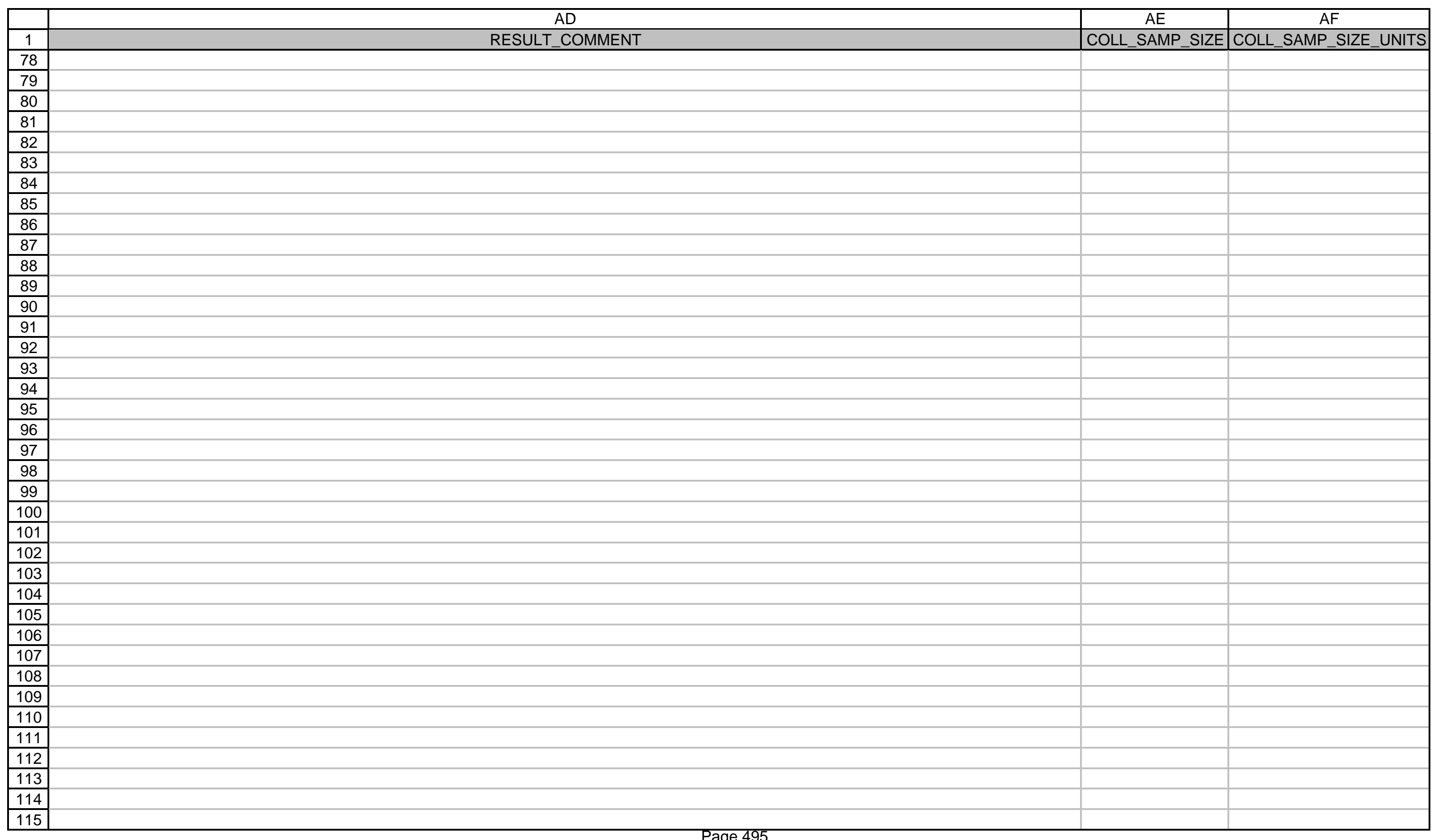




\begin{tabular}{|c|c|c|c|}
\hline & 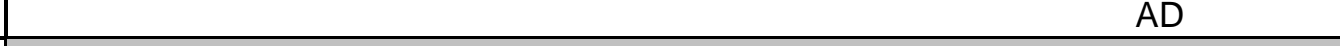 & $\mathrm{AE}$ & \\
\hline 1 & RESULT_COMMENT & COLL_SAMP_SIZE & COLL_SAMP_SIZE_UNITS \\
\hline \multicolumn{4}{|c|}{ 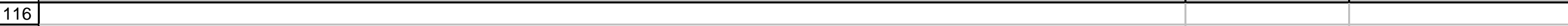 } \\
\hline 117 & See sample record for comment. & & \\
\hline 118 & See sample record for comment. & & \\
\hline 119 & See sample record for comment. & & \\
\hline 120 & See sample record for comment. & & \\
\hline 121 & See sample record for comment. & & \\
\hline \multicolumn{4}{|l|}{122} \\
\hline \multicolumn{4}{|l|}{123} \\
\hline \multicolumn{4}{|l|}{124} \\
\hline \multicolumn{4}{|l|}{125} \\
\hline \multicolumn{4}{|l|}{126} \\
\hline \multicolumn{4}{|l|}{127} \\
\hline \multicolumn{4}{|l|}{128} \\
\hline \multicolumn{4}{|l|}{129} \\
\hline \multicolumn{4}{|l|}{130} \\
\hline \multicolumn{4}{|l|}{131} \\
\hline \multicolumn{4}{|l|}{132} \\
\hline \multicolumn{4}{|l|}{133} \\
\hline \multicolumn{4}{|l|}{134} \\
\hline \multicolumn{4}{|l|}{135} \\
\hline \multicolumn{4}{|l|}{136} \\
\hline \multicolumn{4}{|l|}{137} \\
\hline \multicolumn{4}{|l|}{138} \\
\hline \multicolumn{4}{|l|}{139} \\
\hline \multicolumn{4}{|l|}{140} \\
\hline \multicolumn{4}{|l|}{141} \\
\hline \multicolumn{4}{|l|}{142} \\
\hline \multicolumn{4}{|l|}{143} \\
\hline \multicolumn{4}{|l|}{144} \\
\hline \multicolumn{4}{|l|}{145} \\
\hline \multicolumn{4}{|l|}{146} \\
\hline \multicolumn{4}{|l|}{147} \\
\hline 148 & & & \\
\hline 149 & & & \\
\hline 150 & & & \\
\hline 151 & & & \\
\hline 152 & & & \\
\hline 153 & $-T_{-2}$ & & \\
\hline
\end{tabular}




\begin{tabular}{|c|c|c|c|}
\hline & 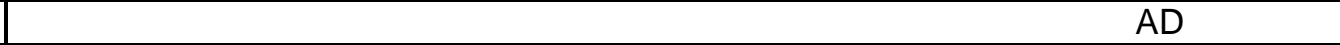 & $A E$ & $\mathrm{AF}$ \\
\hline 1 & $\begin{array}{r}\text { RESULT_COMMENT } \\
\end{array}$ & COLL_SAMP_SIZE & COLL_SAMP_SIZE_UNITS \\
\hline 154 & & & \\
\hline 155 & & & \\
\hline 156 & & & \\
\hline 157 & & & \\
\hline 158 & & & \\
\hline 159 & & & \\
\hline 160 & & & \\
\hline 161 & & & \\
\hline 162 & & & \\
\hline 163 & & & \\
\hline 164 & & & \\
\hline 165 & & & \\
\hline 166 & & & \\
\hline 167 & & & \\
\hline 168 & & & \\
\hline 169 & & & \\
\hline 170 & & & \\
\hline 171 & & & \\
\hline 172 & & & \\
\hline 173 & & & \\
\hline 174 & & & \\
\hline 175 & & & \\
\hline 176 & & & \\
\hline 177 & See sample record for comment. & & \\
\hline 178 & See sample record for comment. & & \\
\hline 179 & See sample record for comment. & & \\
\hline 180 & See sample record for comment. & & \\
\hline 181 & See sample record for comment. & & \\
\hline 182 & & & \\
\hline 183 & & & \\
\hline 184 & & & \\
\hline 185 & & & \\
\hline 186 & & & \\
\hline 187 & & & \\
\hline 188 & & & \\
\hline 189 & & & \\
\hline 190 & & & \\
\hline 191 & ( & & \\
\hline
\end{tabular}




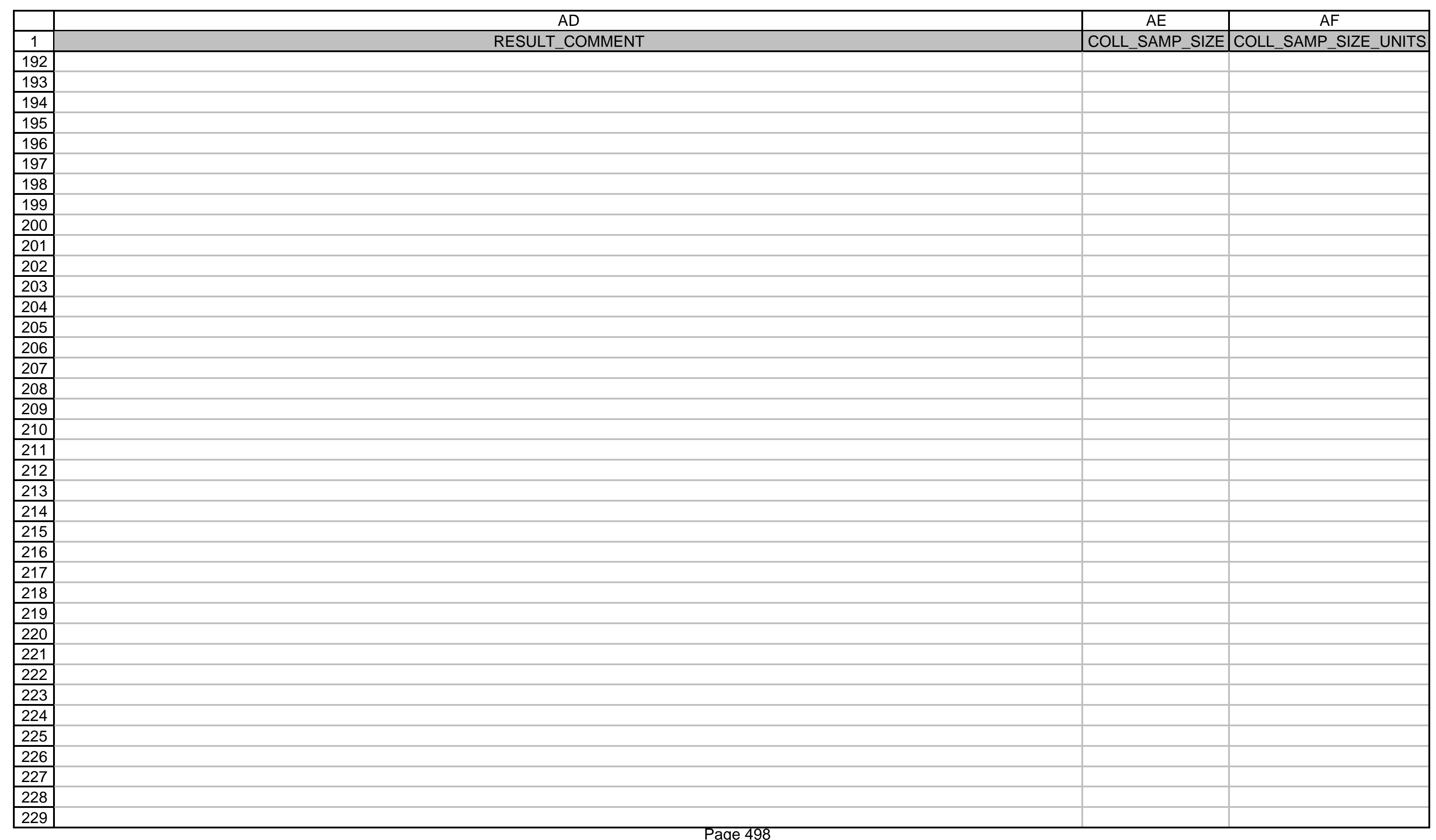




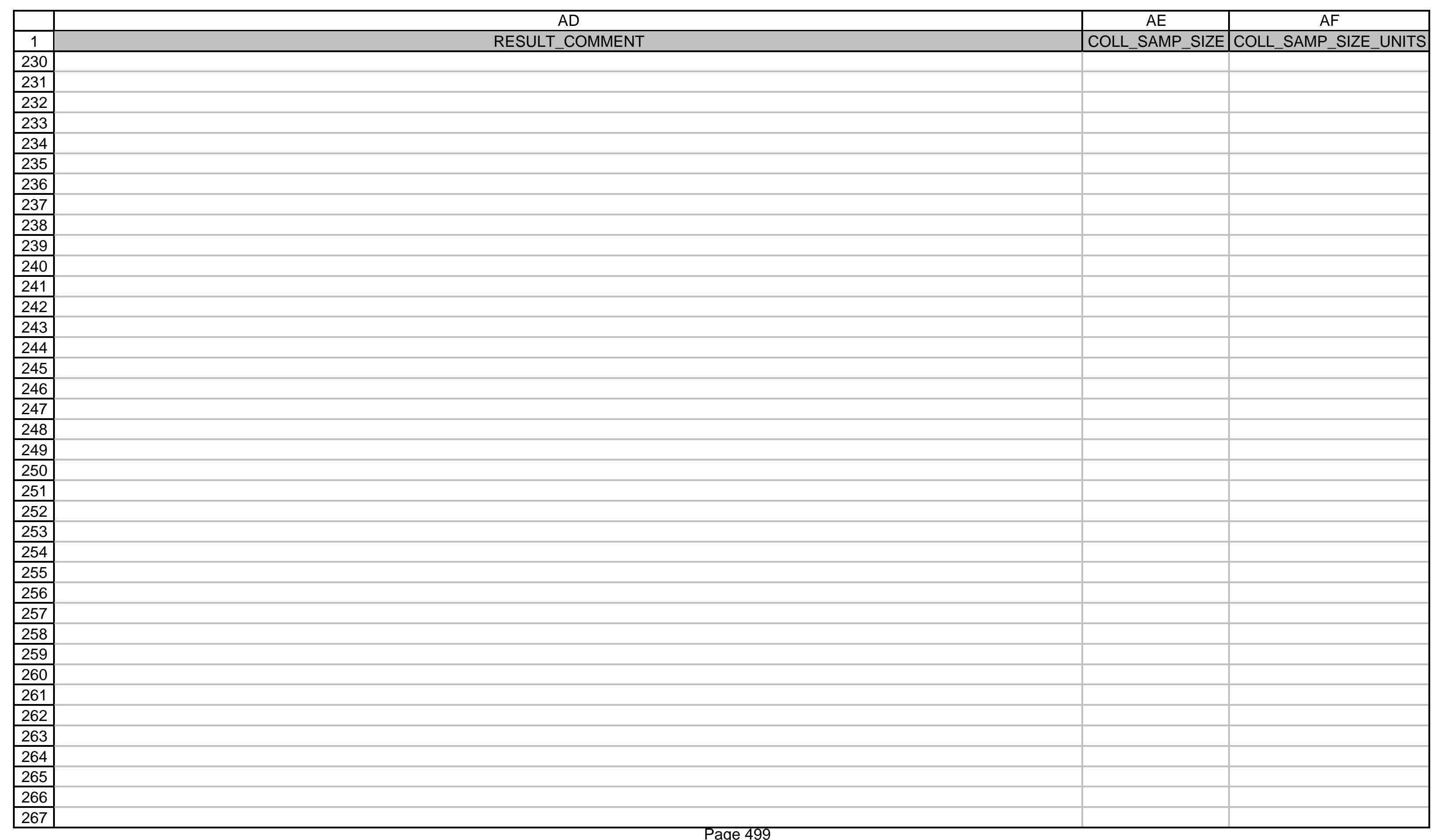




\begin{tabular}{|c|c|c|c|}
\hline & 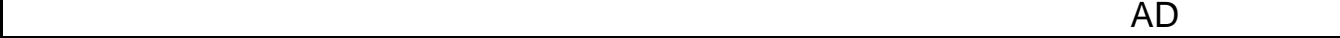 & & AF \\
\hline 1 & $\begin{array}{ll}\text { RESULT_COMMENT } \\
\end{array}$ & COLL_SAMP_SIZE & COLL_SAMP_SIZE_UNITS \\
\hline \multicolumn{4}{|c|}{ 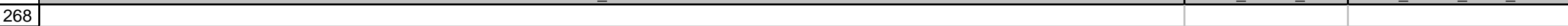 } \\
\hline \multicolumn{4}{|l|}{269} \\
\hline \multicolumn{4}{|l|}{270} \\
\hline \multicolumn{4}{|l|}{271} \\
\hline \multicolumn{4}{|l|}{272} \\
\hline \multicolumn{4}{|l|}{273} \\
\hline \multicolumn{4}{|l|}{274} \\
\hline \multicolumn{4}{|l|}{275} \\
\hline \multicolumn{4}{|l|}{276} \\
\hline \multicolumn{4}{|l|}{277} \\
\hline \multicolumn{4}{|l|}{278} \\
\hline \multicolumn{4}{|l|}{279} \\
\hline \multicolumn{4}{|l|}{280} \\
\hline 281 & See sample record for comment. & & \\
\hline 282 & See sample record for comment. & & \\
\hline 283 & See sample record for comment. & & \\
\hline 284 & See sample record for comment. & & \\
\hline 285 & See sample record for comment. & & \\
\hline \multicolumn{4}{|l|}{286} \\
\hline \multicolumn{4}{|l|}{287} \\
\hline \multicolumn{4}{|l|}{288} \\
\hline \multicolumn{4}{|l|}{289} \\
\hline \multicolumn{4}{|l|}{290} \\
\hline \multicolumn{4}{|l|}{291} \\
\hline \multicolumn{4}{|l|}{292} \\
\hline \multicolumn{4}{|l|}{293} \\
\hline \multicolumn{4}{|l|}{294} \\
\hline \multicolumn{4}{|l|}{295} \\
\hline \multicolumn{4}{|l|}{296} \\
\hline \multicolumn{4}{|l|}{297} \\
\hline \multicolumn{4}{|l|}{298} \\
\hline \multicolumn{4}{|l|}{299} \\
\hline 300 & & & \\
\hline 301 & & & \\
\hline 302 & & & \\
\hline 303 & & & \\
\hline 304 & & & \\
\hline 305 & & & \\
\hline
\end{tabular}




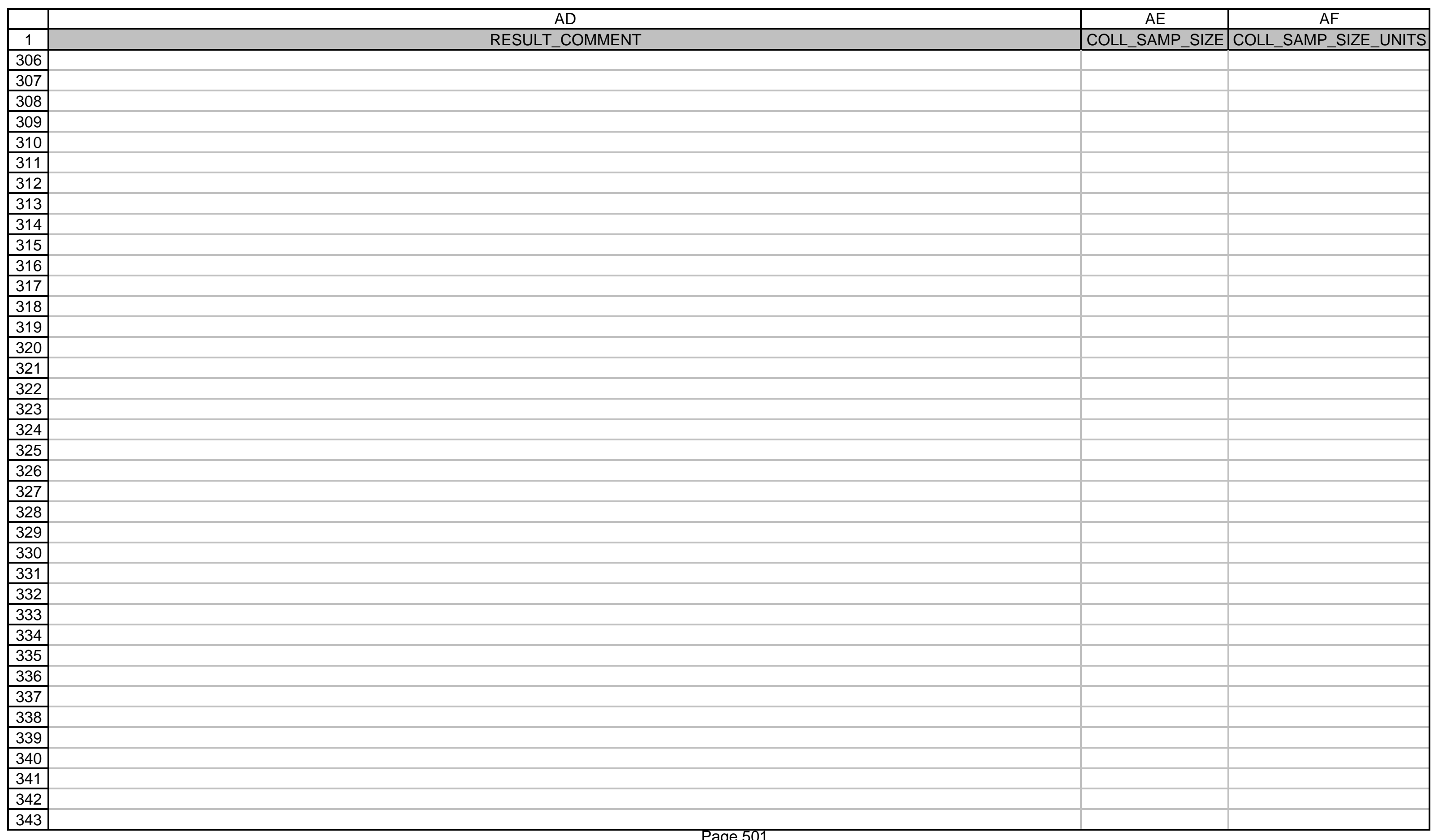




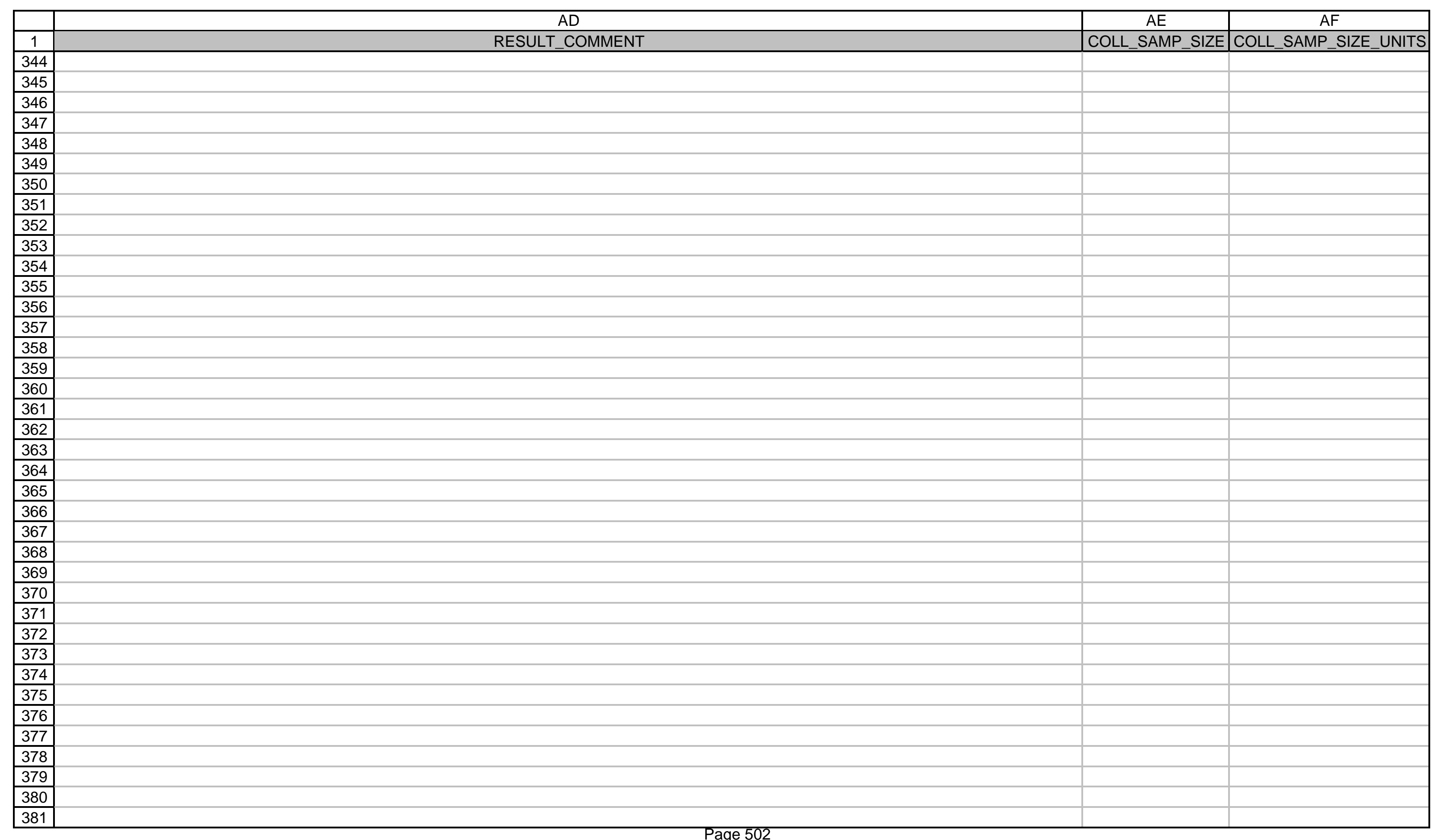




\begin{tabular}{|c|c|c|c|}
\hline & $A D$ & $\mathrm{AE}$ & AF \\
\hline 1 & RESULT_COMMENT & COLL_SAMP_SIZE & COLL_SAMP_SIZE_UNITS \\
\hline \multicolumn{4}{|c|}{ 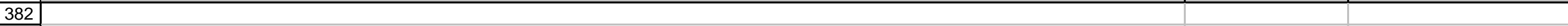 } \\
\hline \multicolumn{4}{|l|}{383} \\
\hline \multicolumn{4}{|l|}{384} \\
\hline \multicolumn{4}{|l|}{385} \\
\hline \multicolumn{4}{|l|}{386} \\
\hline \multicolumn{4}{|l|}{387} \\
\hline \multicolumn{4}{|l|}{388} \\
\hline \multicolumn{4}{|l|}{389} \\
\hline \multicolumn{4}{|l|}{390} \\
\hline \multicolumn{4}{|l|}{391} \\
\hline \multicolumn{4}{|l|}{392} \\
\hline \multicolumn{4}{|l|}{393} \\
\hline \multicolumn{4}{|l|}{394} \\
\hline \multicolumn{4}{|l|}{395} \\
\hline \multicolumn{4}{|l|}{396} \\
\hline \multicolumn{4}{|l|}{397} \\
\hline \multicolumn{4}{|l|}{398} \\
\hline \multicolumn{4}{|l|}{399} \\
\hline \multicolumn{4}{|l|}{400} \\
\hline \multicolumn{4}{|l|}{401} \\
\hline \multicolumn{4}{|l|}{402} \\
\hline \multicolumn{4}{|l|}{403} \\
\hline \multicolumn{4}{|l|}{404} \\
\hline \multicolumn{4}{|l|}{405} \\
\hline \multicolumn{4}{|l|}{406} \\
\hline \multicolumn{4}{|l|}{407} \\
\hline \multicolumn{4}{|l|}{408} \\
\hline 409 & & & \\
\hline 410 & & & \\
\hline 411 & See sample record for comment. & & \\
\hline 412 & See sample record for comment. & & \\
\hline 413 & See sample record for comment. & & \\
\hline 414 & See sample record for comment. & & \\
\hline 415 & See sample record for comment. & & \\
\hline 416 & & & \\
\hline 417 & & & \\
\hline 418 & & & \\
\hline 419 & 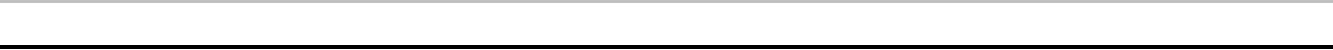 & & \\
\hline
\end{tabular}




\section{AD}

AE

AF

\begin{tabular}{|c|c|c|c|}
\hline & 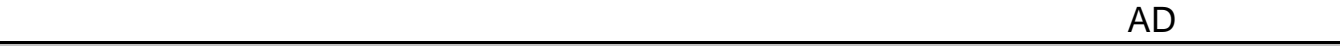 & $\mathrm{AE}$ & $\mathrm{AF}$ \\
\hline 1 & $\begin{array}{l}\text { RESULT_COMMENT } \\
\end{array}$ & COLL_SAMP_SIZE & COLL_SAMP_SIZE_UNITS \\
\hline \multicolumn{4}{|l|}{420} \\
\hline \multicolumn{4}{|l|}{421} \\
\hline \multicolumn{4}{|l|}{422} \\
\hline \multicolumn{4}{|l|}{423} \\
\hline \multicolumn{4}{|l|}{424} \\
\hline \multicolumn{4}{|l|}{425} \\
\hline \multicolumn{4}{|l|}{426} \\
\hline \multicolumn{4}{|l|}{427} \\
\hline \multicolumn{4}{|l|}{428} \\
\hline \multicolumn{4}{|l|}{429} \\
\hline \multicolumn{4}{|l|}{430} \\
\hline \multicolumn{4}{|l|}{431} \\
\hline \multicolumn{4}{|l|}{432} \\
\hline \multicolumn{4}{|l|}{433} \\
\hline \multicolumn{4}{|l|}{434} \\
\hline \multicolumn{4}{|l|}{435} \\
\hline \multicolumn{4}{|l|}{436} \\
\hline \multicolumn{4}{|l|}{437} \\
\hline \multicolumn{4}{|l|}{438} \\
\hline \multicolumn{4}{|l|}{439} \\
\hline \multicolumn{4}{|l|}{440} \\
\hline \multicolumn{4}{|l|}{441} \\
\hline \multicolumn{4}{|l|}{442} \\
\hline \multicolumn{4}{|l|}{443} \\
\hline \multicolumn{4}{|l|}{444} \\
\hline \multicolumn{4}{|l|}{445} \\
\hline \multicolumn{4}{|c|}{446 See sample record for comment. } \\
\hline 447 & See sample record for comment. & & \\
\hline 448 & See sample record for comment. & & \\
\hline 449 & See sample record for comment. & & \\
\hline 450 & See sample record for comment. & & \\
\hline 451 & & & \\
\hline 452 & & & \\
\hline 453 & & & \\
\hline 454 & & & \\
\hline 455 & & & \\
\hline 456 & & & \\
\hline 457 & & & \\
\hline
\end{tabular}




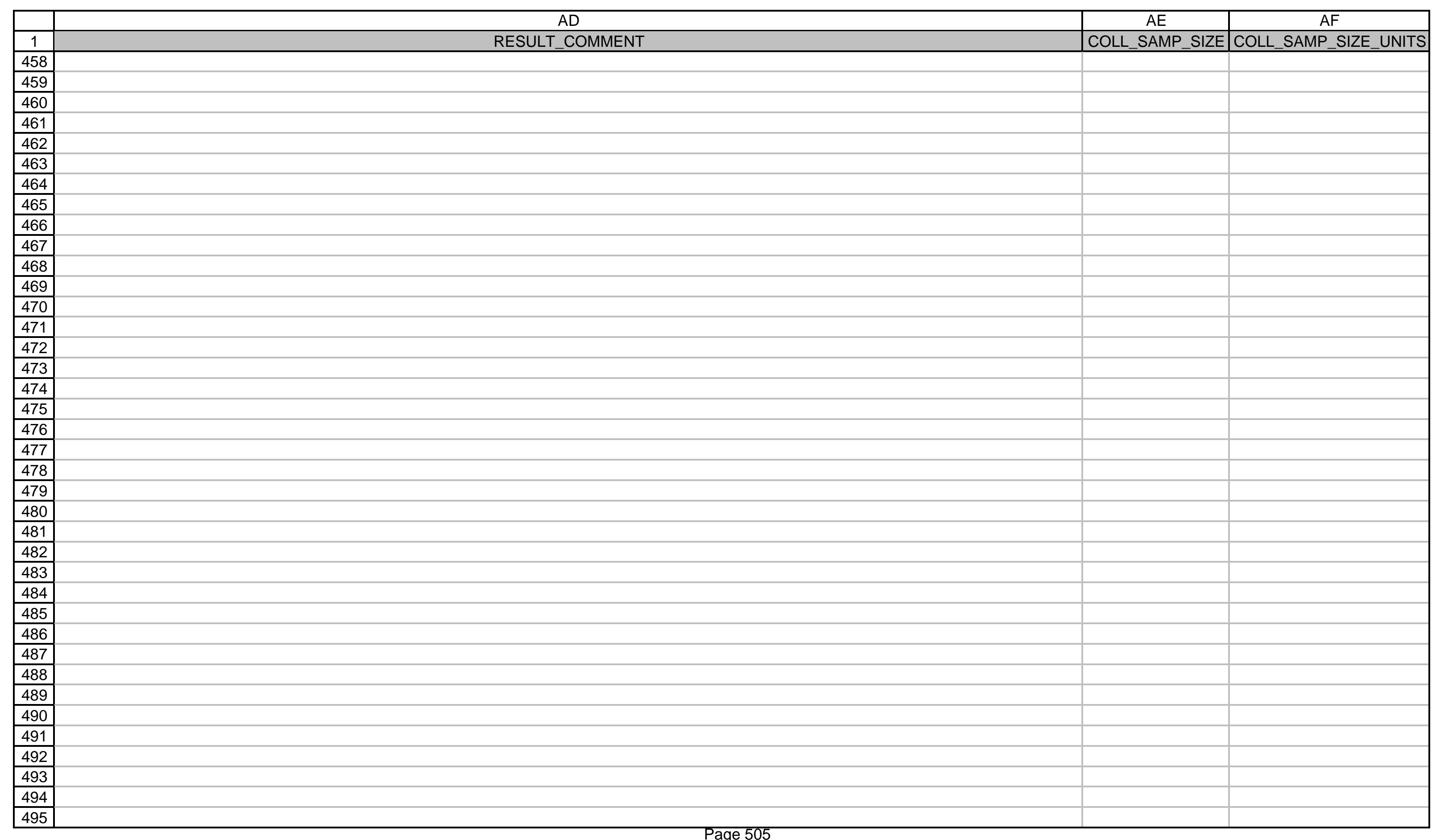




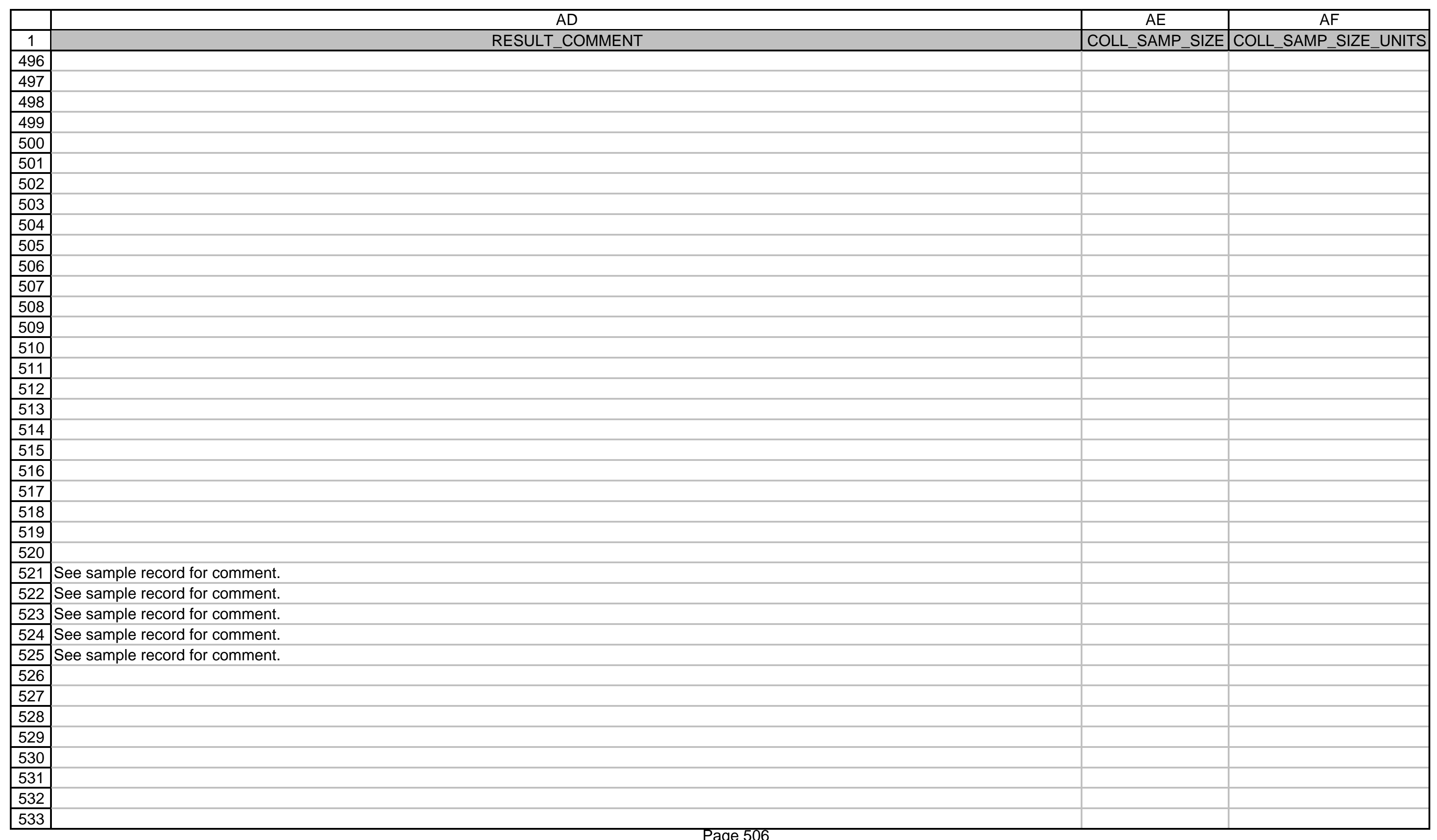




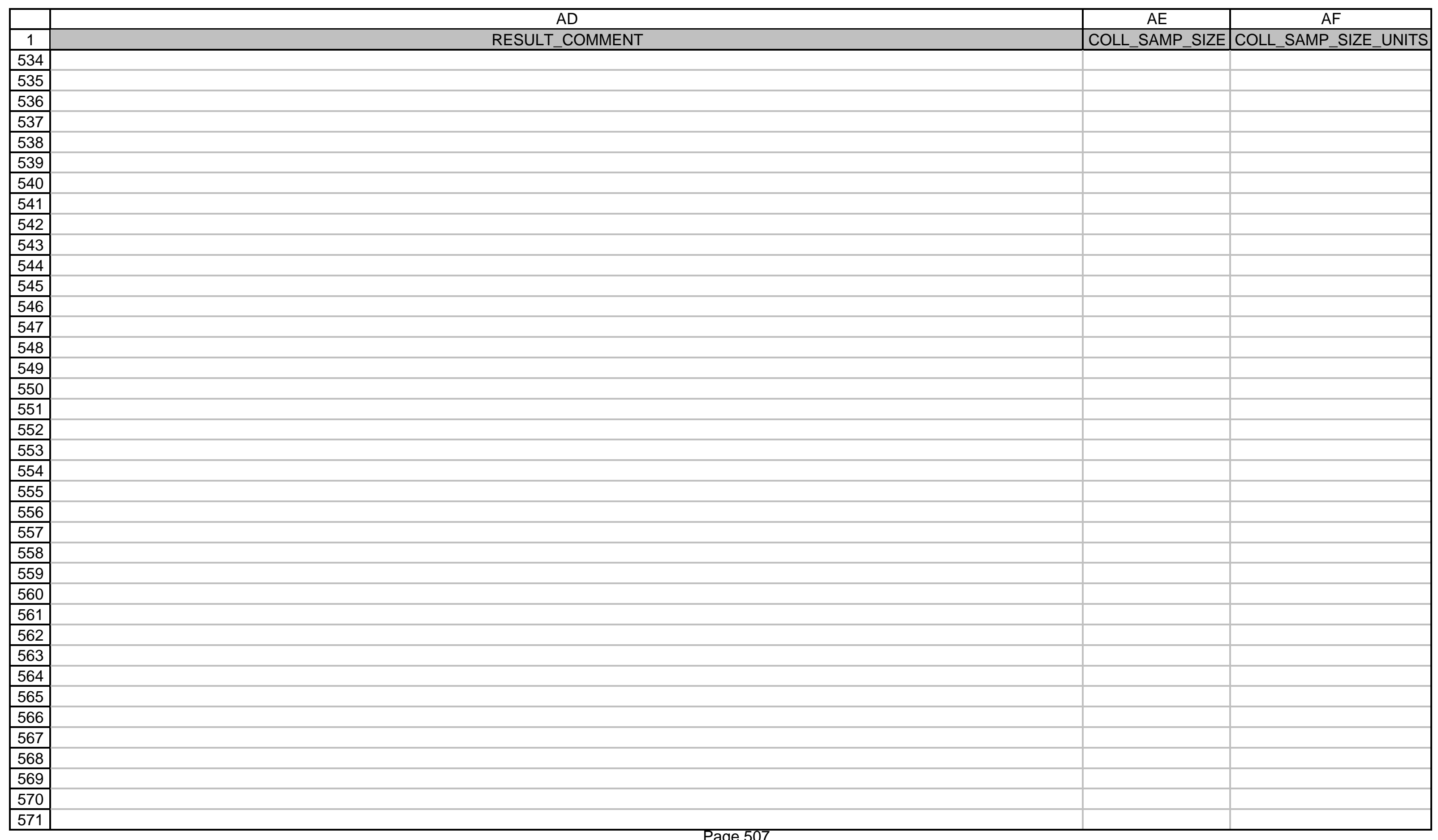




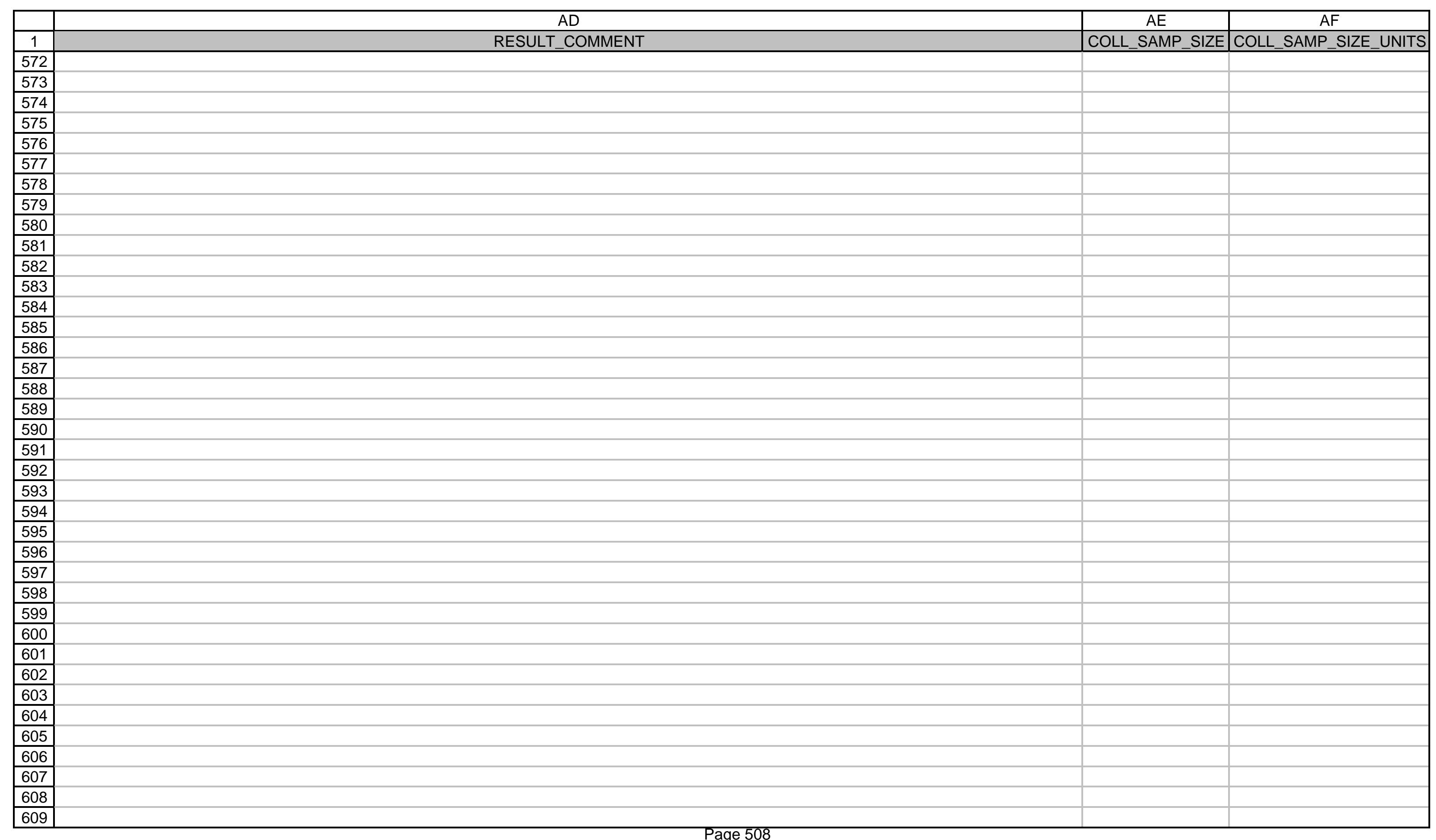




\begin{tabular}{|c|c|c|c|}
\hline & 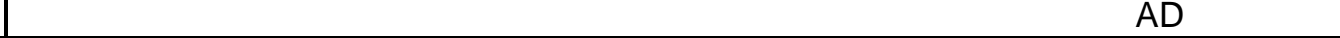 & $\mathrm{AE}$ & AF \\
\hline 1 & $\begin{array}{ll} & \text { RESULT_COMMENT } \\
\end{array}$ & COLL_SAMP_SIZE & COLL_SAMP_SIZE_UNITS \\
\hline 610 & & & \\
\hline 611 & & & \\
\hline 612 & & & \\
\hline 613 & & & \\
\hline 614 & & & \\
\hline 615 & & & \\
\hline 616 & & & \\
\hline 617 & & & \\
\hline 618 & & & \\
\hline 619 & & & \\
\hline 620 & & & \\
\hline 621 & & & \\
\hline 622 & & & \\
\hline 623 & & & \\
\hline 624 & & & \\
\hline 625 & & & \\
\hline 626 & & & \\
\hline 627 & & & \\
\hline 628 & & & \\
\hline 629 & & & \\
\hline 630 & & & \\
\hline 631 & & & \\
\hline 632 & & & \\
\hline 633 & & & \\
\hline 634 & & & \\
\hline 635 & & & \\
\hline 636 & & & \\
\hline 637 & & & \\
\hline 638 & & & \\
\hline 639 & & & \\
\hline 640 & & & \\
\hline 641 & & & \\
\hline 642 & See sample record for comment. & & \\
\hline 643 & See sample record for comment. & & \\
\hline 644 & See sample record for comment. & & \\
\hline 645 & See sample record for comment. & & \\
\hline 646 & See sample record for comment. & & \\
\hline 647 & See sample record for comment. & & \\
\hline
\end{tabular}


AD

AE

AF

\begin{tabular}{|c|c|c|c|}
\hline & 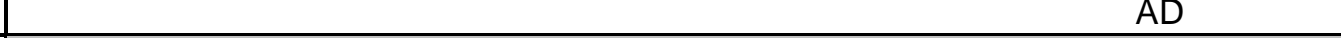 & & \\
\hline 1 & 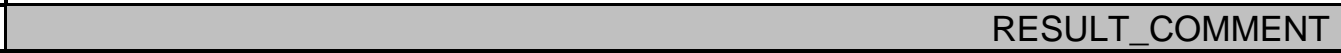 & COLL_SAMP_SIZE & COLL_SAMP_SIZE_UNITS \\
\hline 648 & See sample record for comment. & & \\
\hline 649 & See sample record for comment. & & \\
\hline 650 & See sample record for comment. & & \\
\hline 651 & See sample record for comment. & & \\
\hline 652 & See sample record for comment. & & \\
\hline 653 & See sample record for comment. & & \\
\hline 654 & & & \\
\hline 655 & & & \\
\hline 656 & & & \\
\hline 657 & & & \\
\hline 658 & & & \\
\hline 659 & & & \\
\hline 660 & & & \\
\hline 661 & & & \\
\hline 662 & & & \\
\hline 663 & & & \\
\hline 664 & & & \\
\hline 665 & & & \\
\hline 666 & & & \\
\hline 667 & & & \\
\hline 668 & & & \\
\hline 669 & & & \\
\hline 670 & & & \\
\hline 671 & & & \\
\hline 672 & & & \\
\hline 673 & & & \\
\hline 674 & & & \\
\hline 675 & & & \\
\hline 676 & & & \\
\hline 677 & & & \\
\hline 678 & & & \\
\hline 679 & & & \\
\hline 680 & & & \\
\hline 681 & & & \\
\hline 682 & & & \\
\hline 683 & & & \\
\hline 684 & & & \\
\hline 685 & 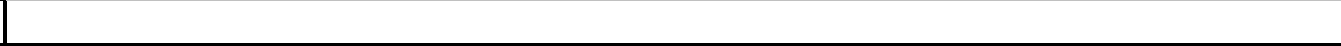 & & \\
\hline
\end{tabular}




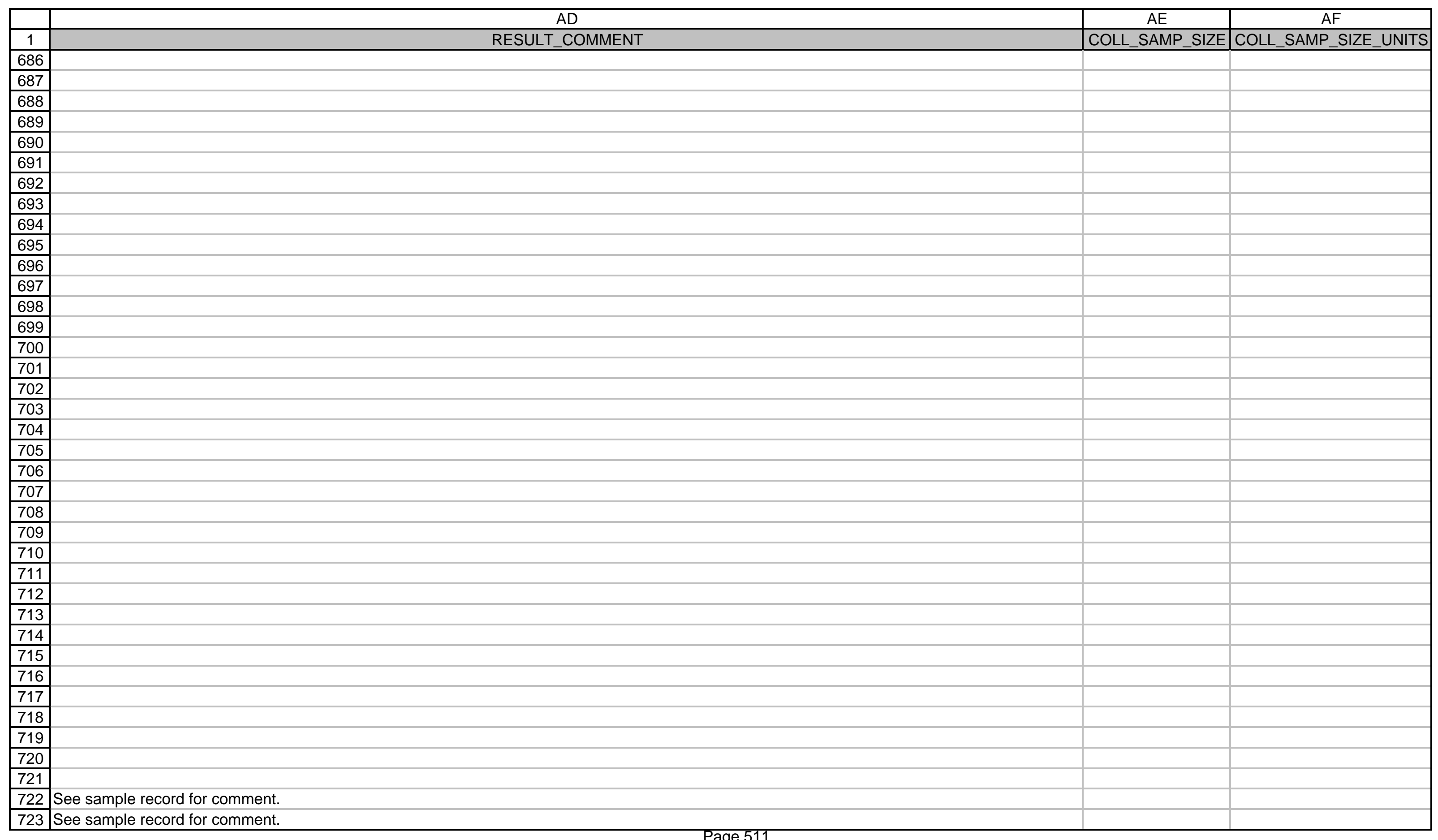




\begin{tabular}{|c|c|c|c|}
\hline & & & \\
\hline 1 & $\begin{array}{ll}\text { RESULT_COMMENT } \\
\end{array}$ & COLL_SAMP_SIZE & COLL_SAMP_SIZE_UNITS \\
\hline 724 & See sample record for comment. & & \\
\hline 725 & See sample record for comment. & & \\
\hline 726 & See sample record for comment. & & \\
\hline 727 & See sample record for comment. & & \\
\hline 728 & See sample record for comment. & & \\
\hline 729 & See sample record for comment. & & \\
\hline 730 & See sample record for comment. & & \\
\hline 731 & See sample record for comment. & & \\
\hline 732 & See sample record for comment. & & \\
\hline 733 & See sample record for comment. & & \\
\hline 734 & & & \\
\hline 735 & & & \\
\hline 736 & & & \\
\hline 737 & & & \\
\hline 738 & & & \\
\hline 739 & See sample record for comment. & & \\
\hline 740 & See sample record for comment. & & \\
\hline 741 & See sample record for comment. & & \\
\hline 742 & See sample record for comment. & & \\
\hline 743 & See sample record for comment. & & \\
\hline 744 & & & \\
\hline 745 & & & \\
\hline 746 & & & \\
\hline 747 & & & \\
\hline 748 & & & \\
\hline 749 & & & \\
\hline 750 & & & \\
\hline 751 & & & \\
\hline 752 & & & \\
\hline 753 & & & \\
\hline 754 & & & \\
\hline 755 & & & \\
\hline 756 & & & \\
\hline 757 & & & \\
\hline 758 & & & \\
\hline 759 & & & \\
\hline 760 & & & \\
\hline 761 & & & \\
\hline
\end{tabular}




\begin{tabular}{|c|c|c|c|}
\hline & 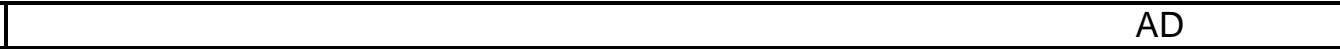 & $\mathrm{AE}$ & $\mathrm{AF}$ \\
\hline 1 & 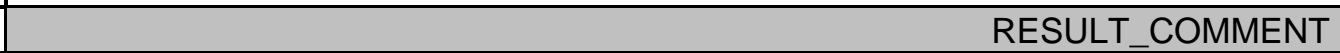 & COLL_SAMP_SIZE & COLL_SAMP_SIZE_UNITS \\
\hline 762 & & & \\
\hline 763 & & & \\
\hline 764 & & & \\
\hline 765 & & & \\
\hline 766 & & & \\
\hline 767 & & & \\
\hline 768 & & & \\
\hline 769 & & & \\
\hline 770 & & & \\
\hline 771 & & & \\
\hline 772 & & & \\
\hline 773 & & & \\
\hline 774 & See sample record for comment. & & \\
\hline 775 & See sample record for comment. & & \\
\hline 776 & See sample record for comment. & & \\
\hline 777 & See sample record for comment. & & \\
\hline 778 & See sample record for comment. & & \\
\hline 779 & See sample record for comment. & & \\
\hline 780 & See sample record for comment. & & \\
\hline 781 & See sample record for comment. & & \\
\hline 782 & See sample record for comment. & & \\
\hline 783 & See sample record for comment. & & \\
\hline 784 & See sample record for comment. & & \\
\hline 785 & See sample record for comment. & & \\
\hline 786 & & & \\
\hline 787 & & & \\
\hline 788 & & & \\
\hline 789 & & & \\
\hline 790 & & & \\
\hline 791 & & & \\
\hline 792 & See sample record for comment. & & \\
\hline 793 & See sample record for comment. & & \\
\hline 794 & See sample record for comment. & & \\
\hline 795 & See sample record for comment. & & \\
\hline 796 & See sample record for comment. & & \\
\hline 797 & See sample record for comment. & & \\
\hline 798 & See sample record for comment. & & \\
\hline 799 & See sample record for comment. & & \\
\hline
\end{tabular}


801 See sample record for comment.

802 See sample record for comment.

803 See sample record for comment.

\begin{tabular}{|l|l}
804 & See sample record for comment.
\end{tabular}

805 See sample record for comment.

806 See sample record for comment.

807 See sample record for comment.

\begin{tabular}{|l|l}
808 & See sample record for comment.
\end{tabular}

809 See sample record for comment.

810 See sample record for comment.

811 See sample record for comment.

\begin{tabular}{|l|l}
812 & See sample record for comment.
\end{tabular}

813 See sample record for comment.

814 See sample record for comment.

\begin{tabular}{|l|l}
815 & See sample record for comment.
\end{tabular}

\begin{tabular}{|l|l}
816 & See sample record for comment.
\end{tabular}

817 See sample record for comment.

818 See sample record for comment.

\begin{tabular}{|l|l|}
819 & See sample record for comment.
\end{tabular}

820 See sample record for comment.

821 See sample record for comment.

\begin{tabular}{|l|l}
822 & See sample record for comment. \\
\hline 823 & See samplecord for comment.
\end{tabular}

823 See sample record for comment.

824 See sample record for comment.

825 See sample record for comment.

\begin{tabular}{|l|l}
826 & See sample record for comment. \\
\hline 827 & See samplecord for comment.
\end{tabular}

827 See sample record for comment.

828 See sample record for comment.

\begin{tabular}{|l|l}
829 & See sample record for comment.
\end{tabular}

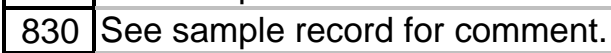

831 See sample record for comment.

832 See sample record for comment.

833 See sample record for comment.

834 See sample record for comment.

835 See sample record for comment.

\begin{tabular}{|l|l|}
836 & See sample record for comment. \\
\hline 837 & See samplecord for comment.
\end{tabular}

\begin{tabular}{|l|l|}
\hline 837 & See sample record for comment.
\end{tabular}

\begin{tabular}{|l|}
\hline \\
\hline \\
\hline \\
\hline \\
\hline \\
\hline \\
\hline \\
\hline \\
\hline
\end{tabular}




\begin{tabular}{|c|c|c|c|}
\hline 1 & \begin{tabular}{cc}
\multicolumn{2}{c}{ AD } \\
RESULT COMMENT
\end{tabular} & \begin{tabular}{|c|} 
AE \\
COII SAMP SI7F
\end{tabular} & $\frac{\text { AF }}{\text { COII SAMP SIZF UNITS }}$ \\
\hline $\begin{array}{ll}1 \\
838\end{array}$ & See sample record for comment. & CULL_SAIVIP_SILE & |CULL_SAIVIT_SILE_UNIIS \\
\hline 839 & See sample record for comment. & & \\
\hline 840 & See sample record for comment. & & \\
\hline 841 & See sample record for comment. & & \\
\hline 842 & See sample record for comment. & & \\
\hline 843 & See sample record for comment. & & \\
\hline 844 & See sample record for comment. & & \\
\hline 845 & See sample record for comment. & & \\
\hline 846 & & & \\
\hline 847 & & & \\
\hline 848 & & & \\
\hline 849 & & & \\
\hline 850 & & & \\
\hline 851 & See sample record for comment. & & \\
\hline 852 & See sample record for comment. & & \\
\hline 853 & See sample record for comment. & & \\
\hline 854 & See sample record for comment. & & \\
\hline 855 & See sample record for comment. & & \\
\hline 856 & See sample record for comment. & & \\
\hline 857 & See sample record for comment. & & \\
\hline 858 & See sample record for comment. & & \\
\hline 859 & See sample record for comment. & & \\
\hline 860 & See sample record for comment. & & \\
\hline 861 & & & \\
\hline 862 & & & \\
\hline 863 & & & \\
\hline 864 & & & \\
\hline 865 & & & \\
\hline 866 & & & \\
\hline 867 & & & \\
\hline 868 & & & \\
\hline 869 & & & \\
\hline 870 & & & \\
\hline 871 & & & \\
\hline 872 & & & \\
\hline 873 & & & \\
\hline 874 & & & \\
\hline 875 & & & \\
\hline
\end{tabular}




\begin{tabular}{|c|c|c|c|}
\hline & 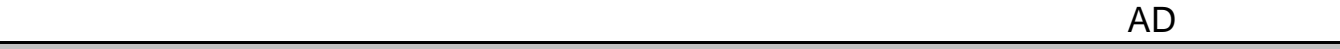 & $\mathrm{AE}$ & AF \\
\hline 1 & 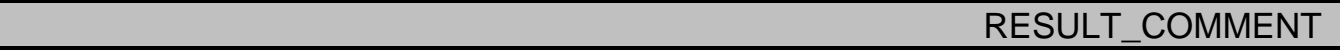 & COLL_SAMP_SIZE & COLL_SAMP_SIZE_UNITS \\
\hline 876 & & & \\
\hline 877 & & & \\
\hline 878 & & & \\
\hline 879 & & & \\
\hline 880 & & & \\
\hline 881 & & & \\
\hline 882 & & & \\
\hline 883 & & & \\
\hline 884 & & & \\
\hline 885 & & & \\
\hline 886 & & & \\
\hline 887 & & & \\
\hline 888 & & & \\
\hline 889 & & & \\
\hline 890 & & & \\
\hline 891 & & & \\
\hline 892 & & & \\
\hline 893 & & & \\
\hline 894 & & & \\
\hline 895 & & & \\
\hline 896 & See sample record for comment. & & \\
\hline 897 & See sample record for comment. & & \\
\hline 898 & See sample record for comment. & & \\
\hline 899 & See sample record for comment. & & \\
\hline 900 & See sample record for comment. & & \\
\hline 901 & See sample record for comment. & & \\
\hline 902 & See sample record for comment. & & \\
\hline 903 & See sample record for comment. & & \\
\hline 904 & See sample record for comment. & & \\
\hline 905 & See sample record for comment. & & \\
\hline 906 & See sample record for comment. & & \\
\hline 907 & See sample record for comment. & & \\
\hline 908 & See sample record for comment. & & \\
\hline 909 & See sample record for comment. & & \\
\hline 910 & See sample record for comment. & & \\
\hline 911 & See sample record for comment. & & \\
\hline 912 & See sample record for comment. & & \\
\hline 913 & See sample record for comment. & & \\
\hline
\end{tabular}


915 See sample record for comment.

916 See sample record for comment.

917 See sample record for comment.

918 See sample record for comment.

919 See sample record for comment.

920 See sample record for comment.

921 See sample record for comment.

\begin{tabular}{|l|l}
922 & See sample record for comment.
\end{tabular}

923 See sample record for comment.

924 See sample record for comment.

925 See sample record for comment.

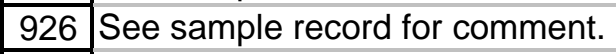

927 See sample record for comment.

928 See sample record for comment.

$\begin{array}{ll}929 & \text { See sample record for comment. }\end{array}$

930 See sample record for comment.

931 See sample record for comment.

932 See sample record for comment.

\begin{tabular}{|l|l}
933 & See sample record for comment. \\
\hline
\end{tabular}

934 See sample record for comment.

935 See sample record for comment.

\begin{tabular}{|l|l}
936 & See sample record for comment. \\
\hline 937 & See samplecord for comment.
\end{tabular}

937 See sample record for comment.

938 See sample record for comment.

\begin{tabular}{|l|l}
939 & See sample record for comment.
\end{tabular}

\begin{tabular}{|l|l}
940 & See sample record for comment. \\
\hline 941 & See samplecord for comment.
\end{tabular}

941 See sample record for comment.

942 See sample record for comment.

943 See sample record for comment.

944 See sample record for comment.

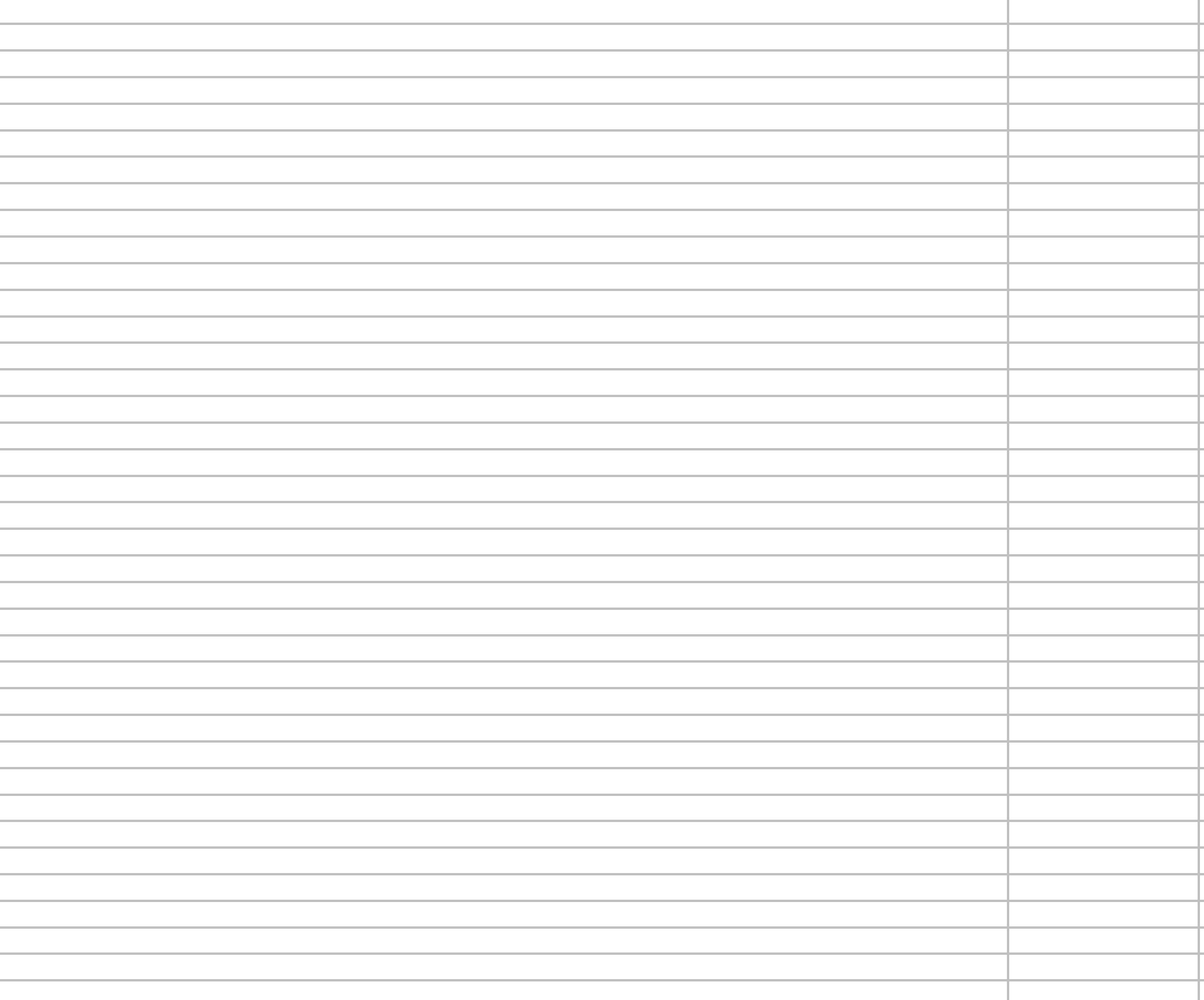

\begin{tabular}{|l|}
\hline \\
\hline \\
\hline \\
\hline \\
\hline \\
\hline \\
\hline \\
\hline \\
\hline \\
\hline \\
\hline
\end{tabular}




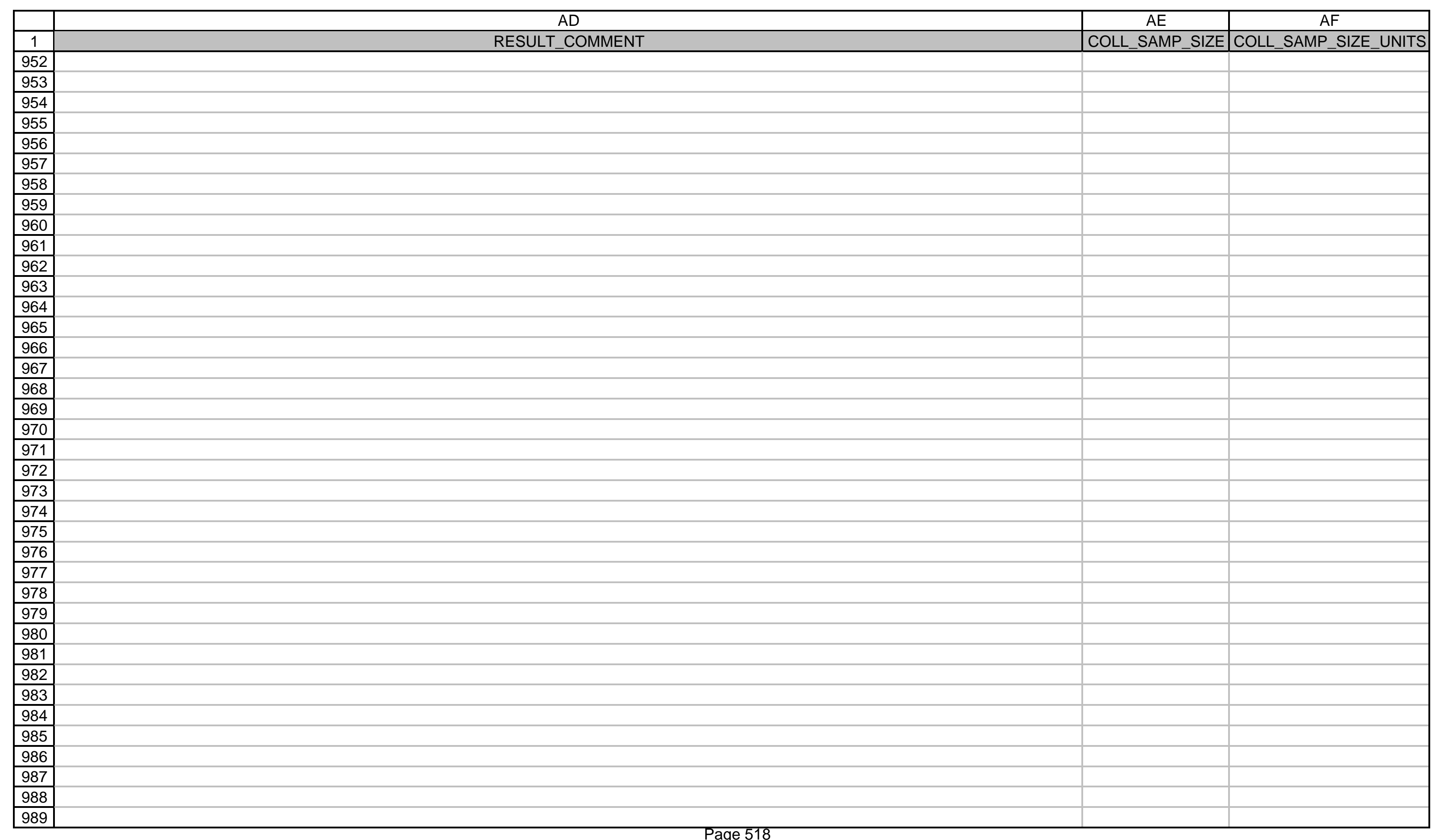




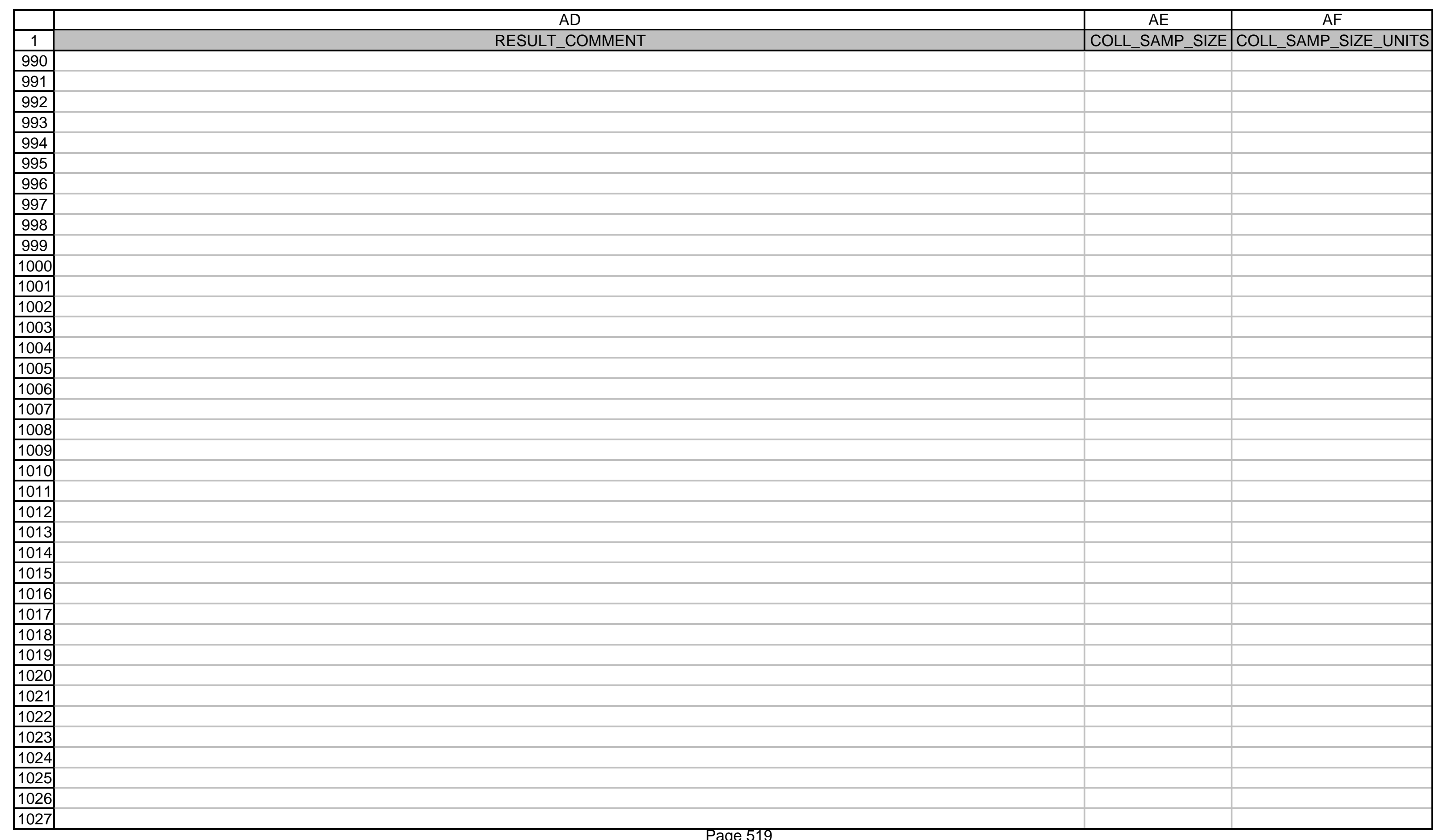




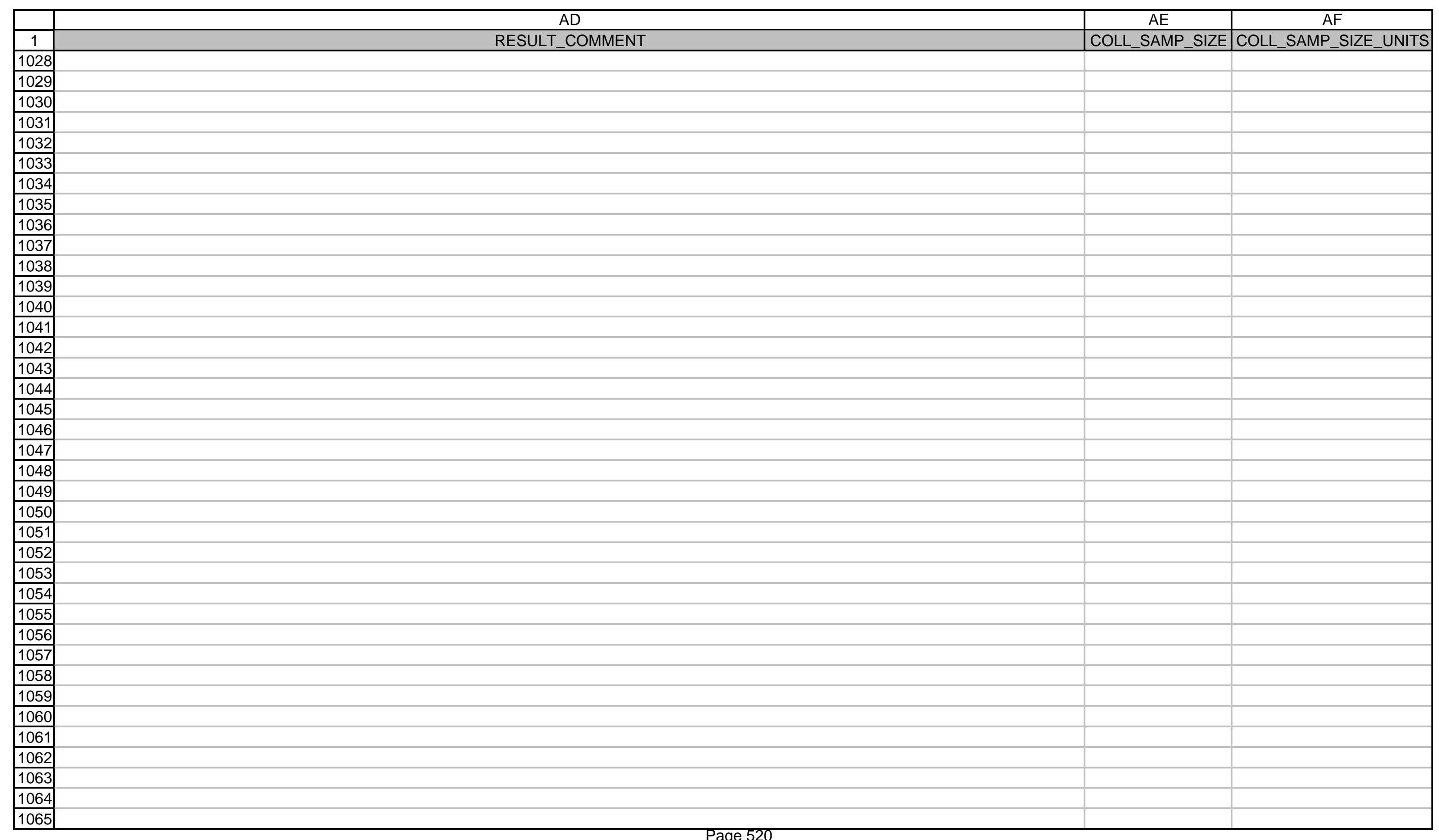




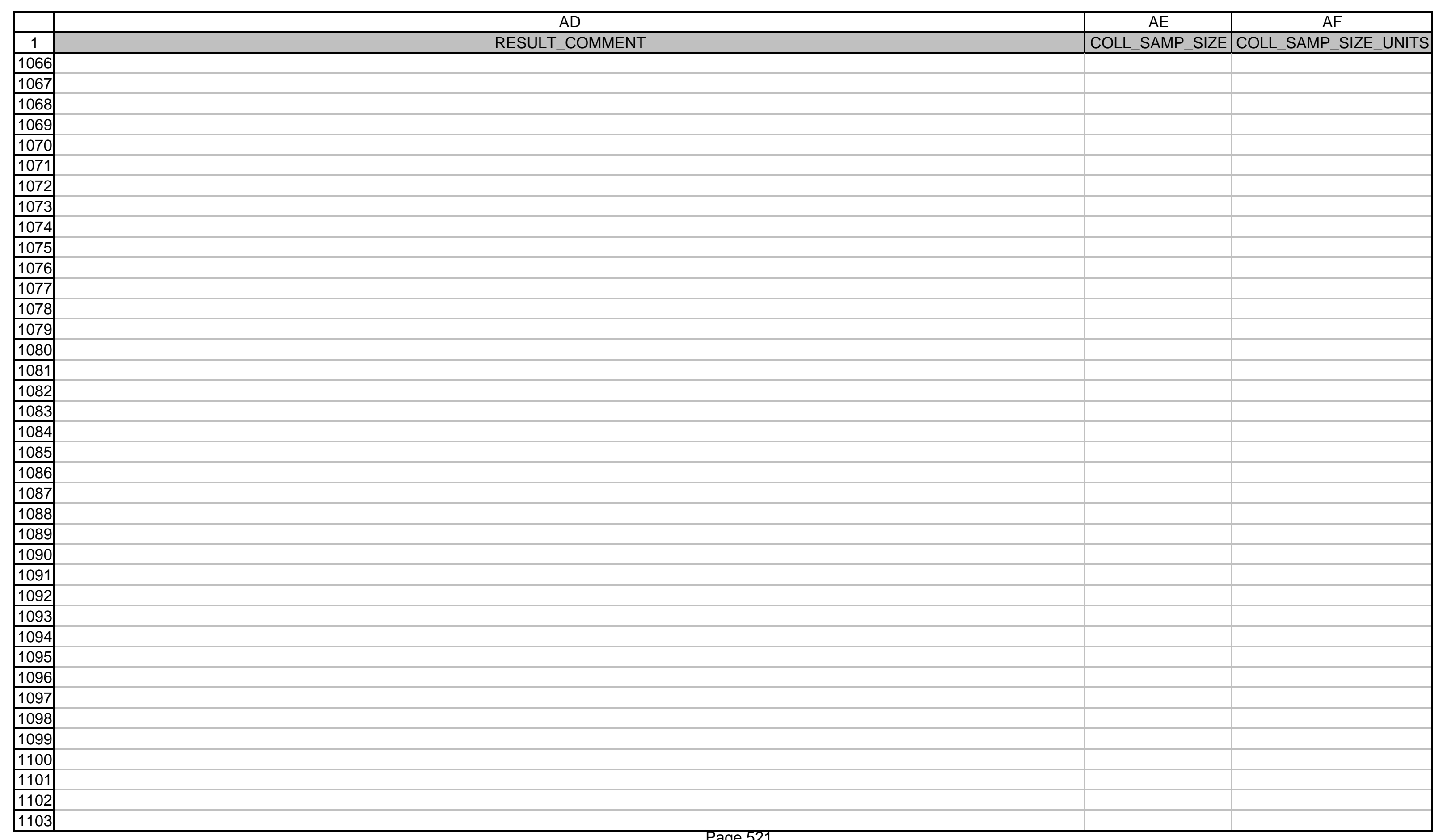




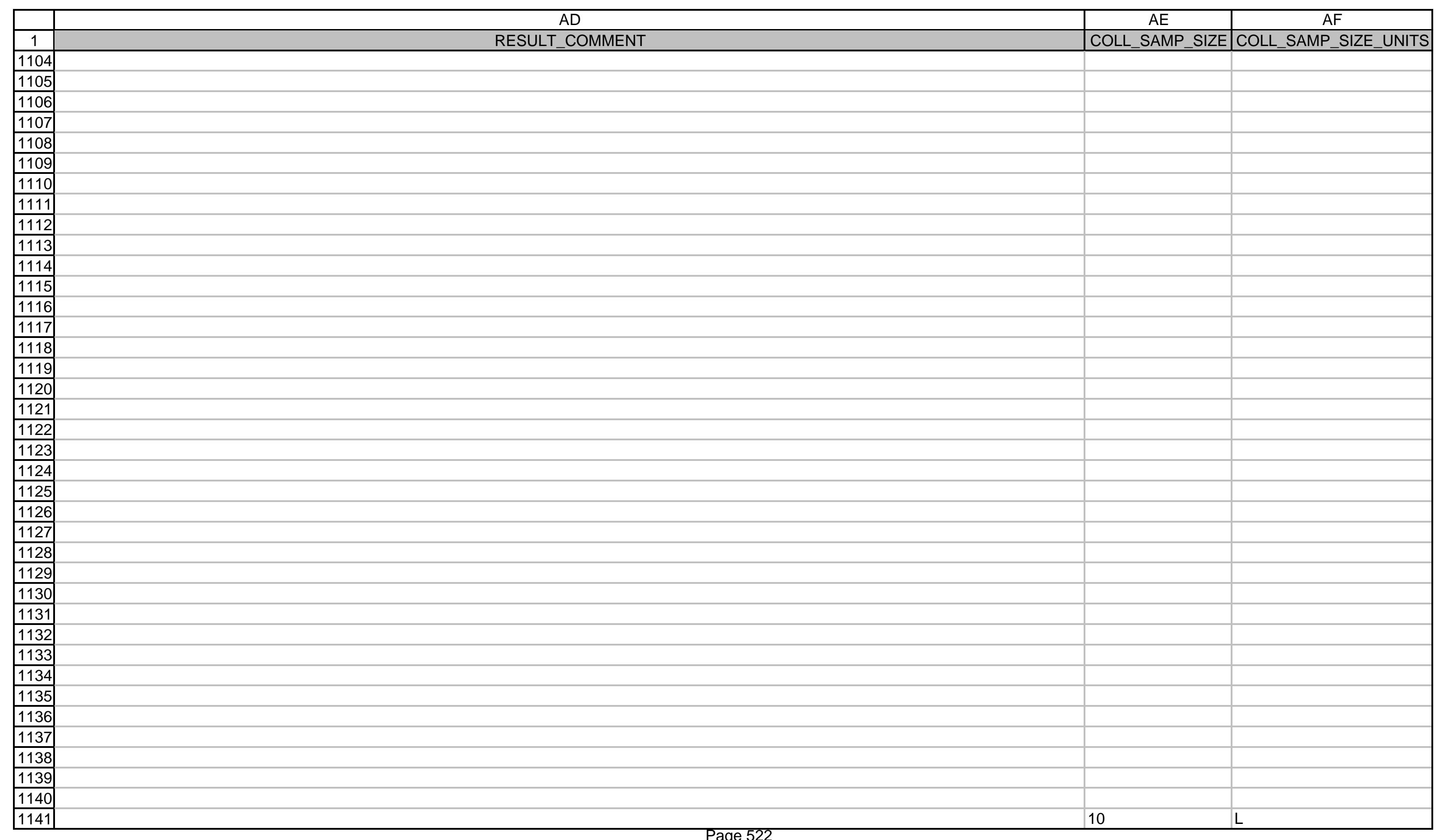




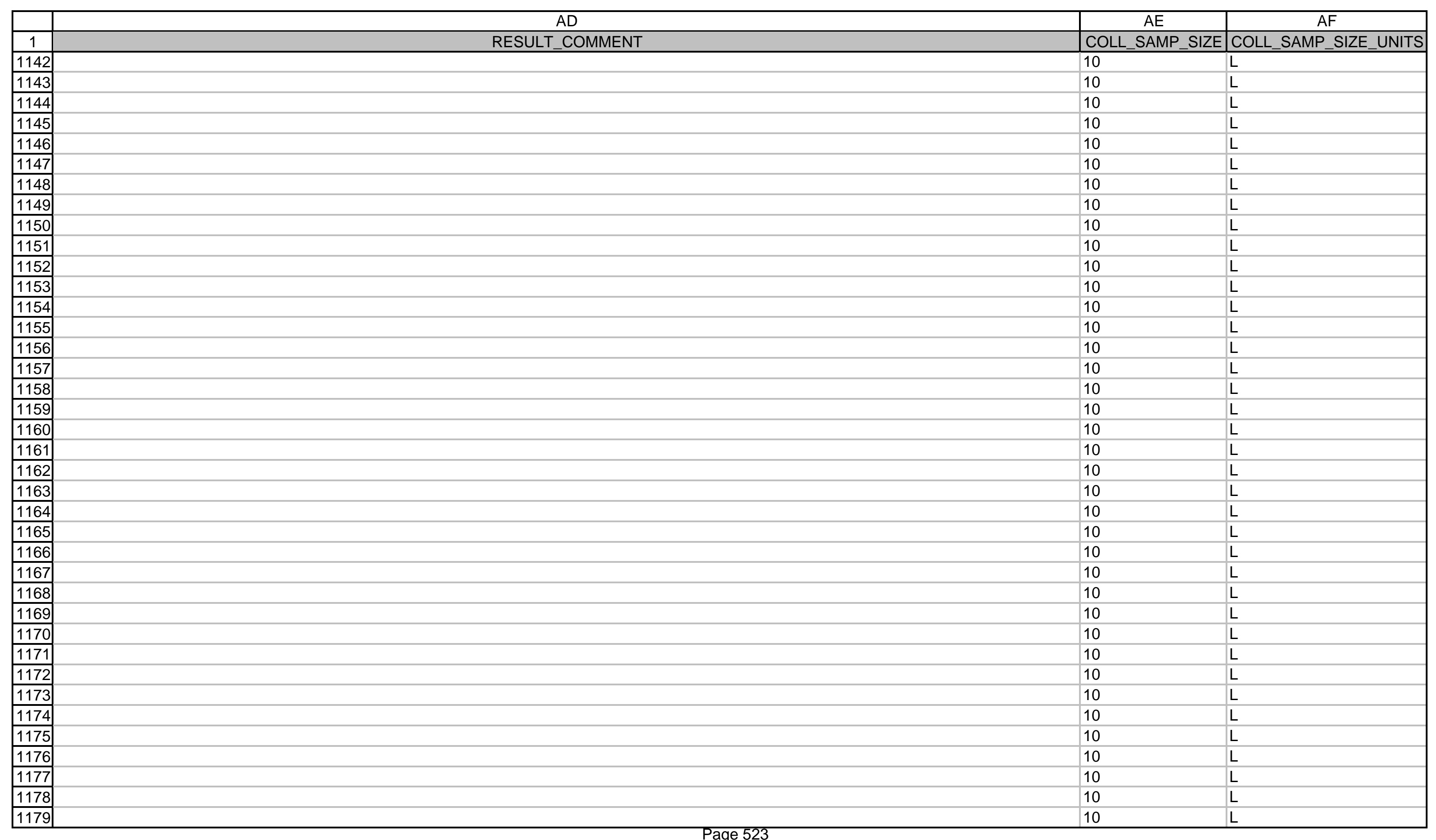




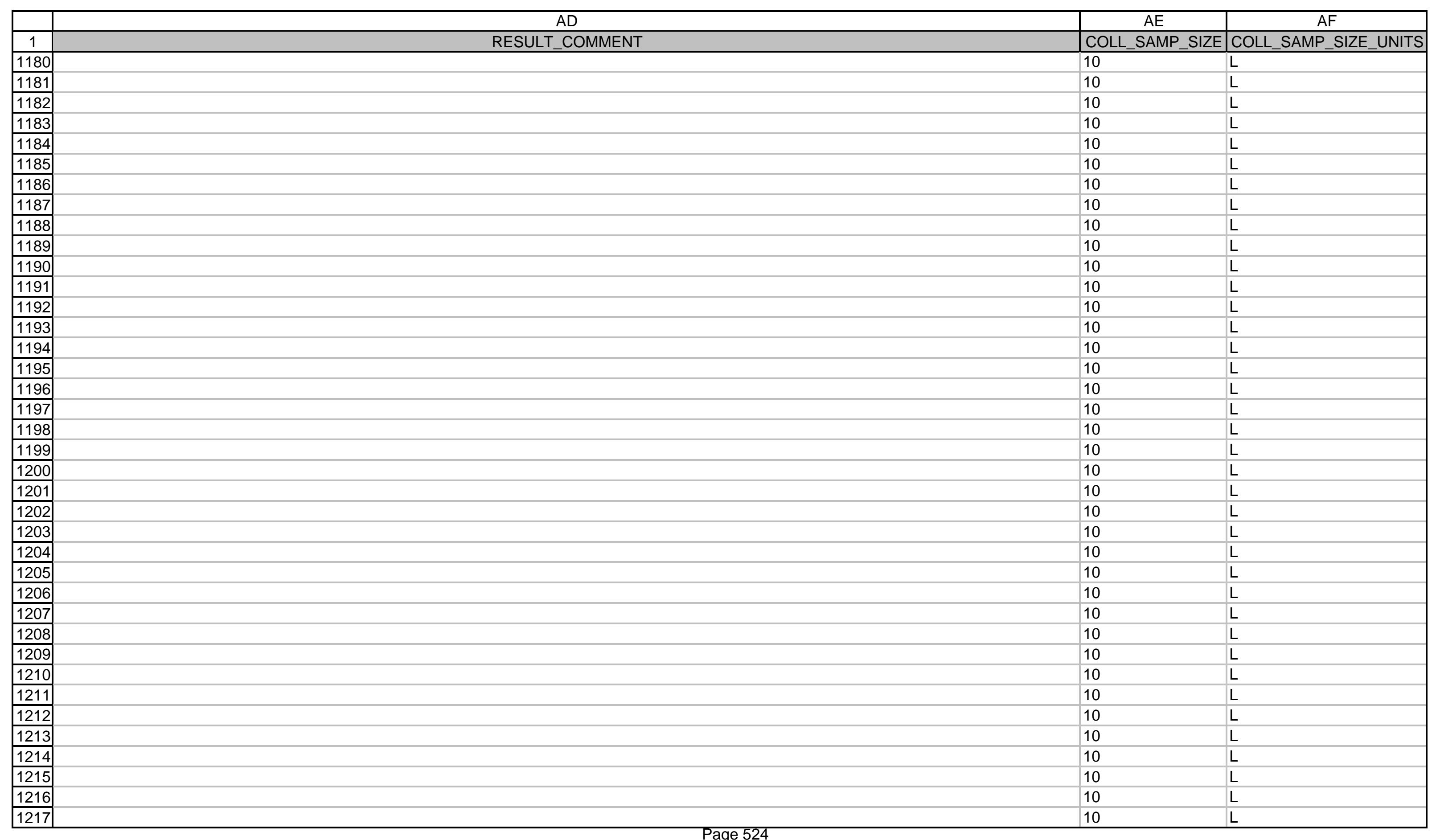




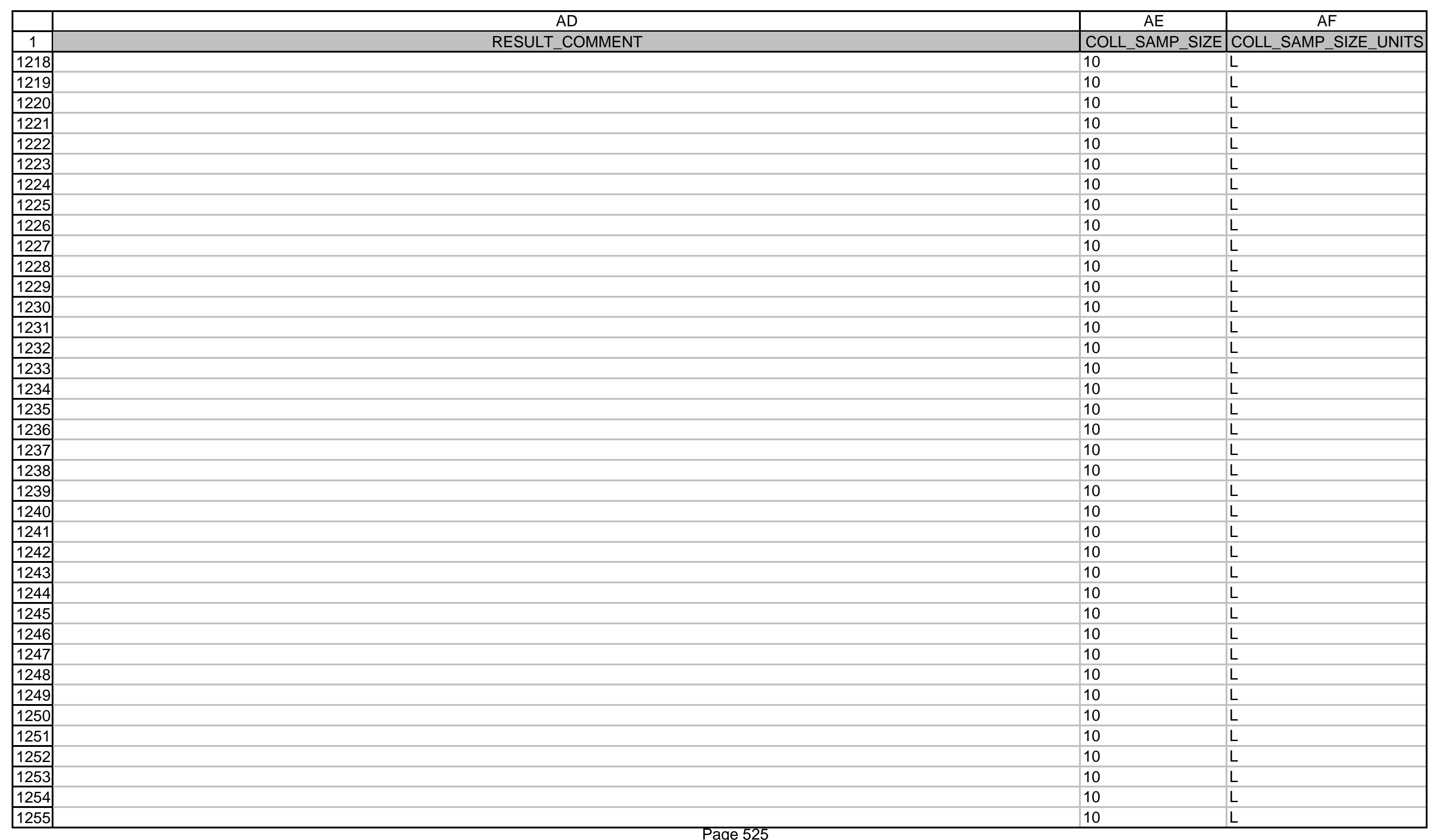




\begin{tabular}{|c|c|c|c|}
\hline & $A D$ & $\mathrm{AE}$ & $\mathrm{AF}$ \\
\hline 1 & RESULT_COMMENT & COLL_SAMP_SIZE & COLL_SAMP_SIZE_UNITS \\
\hline 1256 & & 10 & $\mathrm{~L}$ \\
\hline \begin{tabular}{|l|}
1257 \\
\end{tabular} & & 10 & $\mathrm{~L}$ \\
\hline \begin{tabular}{|l|}
1258 \\
\end{tabular} & & 10 & $\mathrm{~L}$ \\
\hline \begin{tabular}{|l|}
1259 \\
\end{tabular} & & 10 & $\mathrm{~L}$ \\
\hline 1260 & & 10 & $\mathrm{~L}$ \\
\hline \begin{tabular}{|l|}
1261 \\
\end{tabular} & & 10 & $\mathrm{~L}$ \\
\hline \begin{tabular}{|l|}
1262 \\
\end{tabular} & & 10 & $\bar{L}$ \\
\hline \begin{tabular}{|l|}
1263 \\
\end{tabular} & & 10 & $\mathrm{~L}$ \\
\hline \begin{tabular}{|l|}
1264 \\
\end{tabular} & & 10 & $\mathrm{~L}$ \\
\hline \begin{tabular}{|l|}
1265 \\
\end{tabular} & & 10 & $\mathrm{~L}$ \\
\hline \begin{tabular}{|l|}
1266 \\
\end{tabular} & & 10 & $\mathrm{~L}$ \\
\hline 1267 & & 10 & $\mathrm{~L}$ \\
\hline 1268 & & 10 & L \\
\hline 1269 & & 10 & $\mathrm{~L}$ \\
\hline \begin{tabular}{|l|}
1270 \\
\end{tabular} & & 10 & $\mathrm{~L}$ \\
\hline \multicolumn{4}{|l|}{\begin{tabular}{|l|}
1271 \\
\end{tabular}} \\
\hline \multicolumn{4}{|l|}{\begin{tabular}{|l|}
1272 \\
\end{tabular}} \\
\hline \begin{tabular}{|l|}
1273 \\
\end{tabular} & & 10 & $\mathrm{~L}$ \\
\hline \begin{tabular}{|l|}
1274 \\
\end{tabular} & & 10 & $\mathrm{~L}$ \\
\hline \begin{tabular}{|l|}
1275 \\
\end{tabular} & & 10 & $\bar{L}$ \\
\hline \begin{tabular}{|l|}
1276 \\
\end{tabular} & & 10 & $\mathrm{~L}$ \\
\hline \begin{tabular}{|l|}
1277 \\
\end{tabular} & & 10 & $\mathrm{~L}$ \\
\hline \multicolumn{4}{|l|}{1278} \\
\hline \multicolumn{4}{|l|}{\begin{tabular}{|l|}
1279 \\
\end{tabular}} \\
\hline 1280 & & 10 & $\mathrm{~L}$ \\
\hline 1281 & & 10 & L \\
\hline \begin{tabular}{|l|}
1282 \\
\end{tabular} & & 10 & $\mathrm{~L}$ \\
\hline \begin{tabular}{|l|}
1283 \\
\end{tabular} & & 10 & $\mathrm{~L}$ \\
\hline \begin{tabular}{|l|}
1284 \\
\end{tabular} & & 10 & $\mathrm{~L}$ \\
\hline \multicolumn{4}{|l|}{\begin{tabular}{|l|l|}
1285 \\
128
\end{tabular}} \\
\hline \multicolumn{4}{|l|}{\begin{tabular}{|l|l|}
1286 \\
\end{tabular}} \\
\hline \begin{tabular}{|l|}
1287 \\
\end{tabular} & & 10 & L \\
\hline \begin{tabular}{|l|}
1288 \\
\end{tabular} & & 10 & $\mathrm{~L}$ \\
\hline \begin{tabular}{|l|}
1289 \\
\end{tabular} & & 10 & $\mathrm{~L}$ \\
\hline \begin{tabular}{|l|}
1290 \\
\end{tabular} & & 10 & $\mathrm{~L}$ \\
\hline \begin{tabular}{|l|l|}
1291 \\
\end{tabular} & & 10 & $L$ \\
\hline \multicolumn{4}{|l|}{\begin{tabular}{|l|}
1292 \\
\end{tabular}} \\
\hline \begin{tabular}{|l|}
1293 \\
\end{tabular} & & & \\
\hline
\end{tabular}




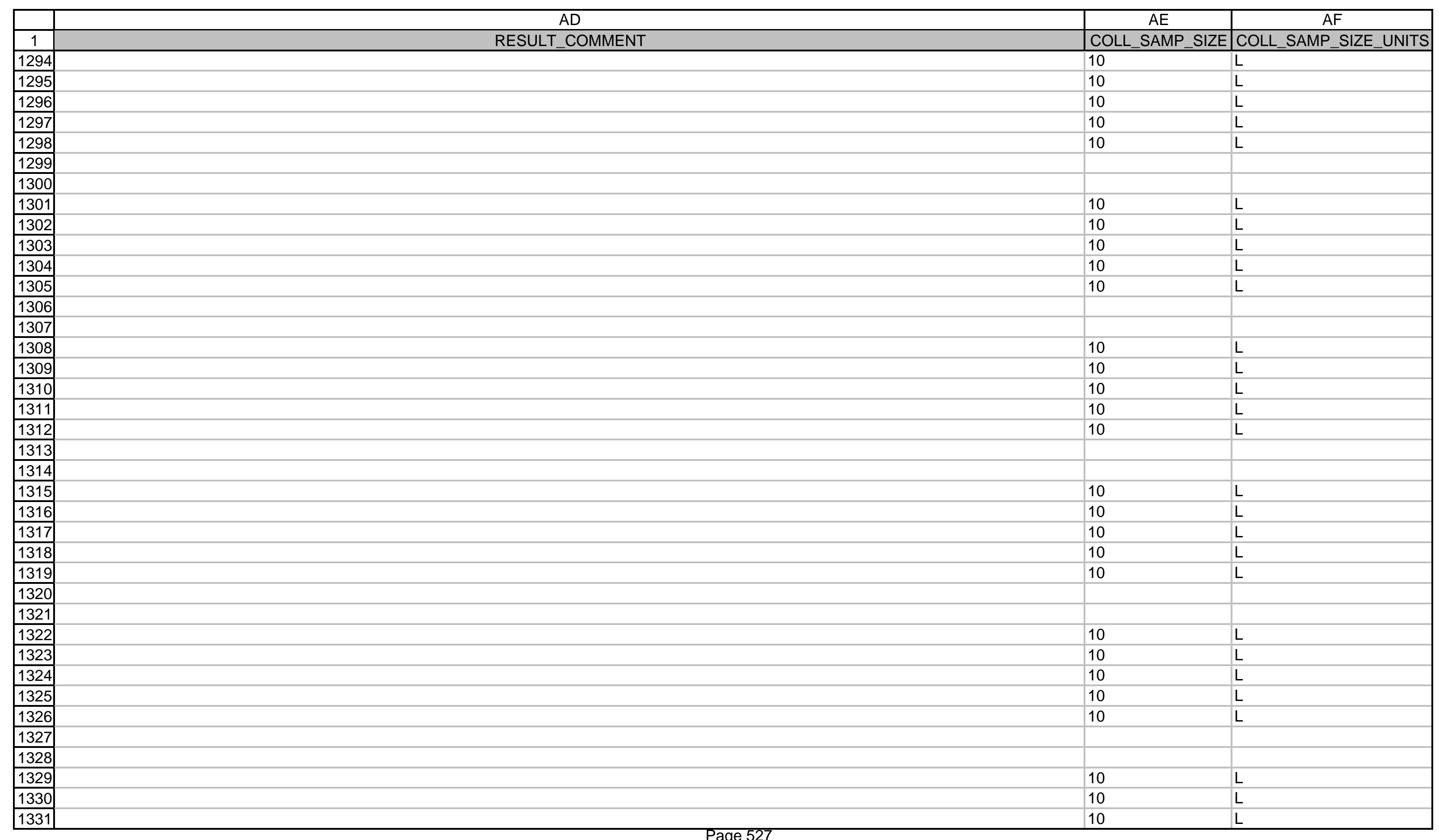




\begin{tabular}{|c|c|c|c|}
\hline & $\overline{A D}$ & $\mathrm{AE}$ & $\mathrm{AF}$ \\
\hline 1 & RESULT_COMMENT & COLL_SAMP_SIZE & COLL_SAMP_SIZE_UNITS \\
\hline 1332 & & 10 & $\mathrm{~L}$ \\
\hline 1333 & & 10 & $\mathrm{~L}$ \\
\hline \multicolumn{4}{|l|}{\begin{tabular}{|l|}
1334 \\
\end{tabular}} \\
\hline \multicolumn{4}{|l|}{\begin{tabular}{|l|}
1335 \\
\end{tabular}} \\
\hline 1336 & & 10 & $\mathrm{~L}$ \\
\hline 1337 & & 10 & $\mathrm{~L}$ \\
\hline \begin{tabular}{|l|}
1338 \\
\end{tabular} & & 10 & $\bar{L}$ \\
\hline 1339 & & 10 & $\mathrm{~L}$ \\
\hline 1340 & & 10 & $\mathrm{~L}$ \\
\hline \multicolumn{4}{|l|}{1341} \\
\hline \multicolumn{4}{|l|}{\begin{tabular}{|l|}
1342 \\
\end{tabular}} \\
\hline 1343 & & 10 & $\mathrm{~L}$ \\
\hline 1344 & & 10 & $\mathrm{~L}$ \\
\hline 1345 & & 10 & $\mathrm{~L}$ \\
\hline 1346 & & 10 & $\mathrm{~L}$ \\
\hline \begin{tabular}{|l|}
1347 \\
\end{tabular} & & 10 & $\mathrm{~L}$ \\
\hline \begin{tabular}{|l|}
1348 \\
\end{tabular} & & 10 & $\bar{L}$ \\
\hline 1349 & & 10 & $\mathrm{~L}$ \\
\hline \multicolumn{4}{|l|}{\begin{tabular}{|l|}
1350 \\
\end{tabular}} \\
\hline \multicolumn{4}{|l|}{1351} \\
\hline 1352 & & 10 & $\mathrm{~L}$ \\
\hline 1353 & & 10 & $\mathrm{~L}$ \\
\hline \begin{tabular}{|l|}
1354 \\
\end{tabular} & & 10 & $L$ \\
\hline 1355 & & 10 & $\mathrm{~L}$ \\
\hline 1356 & & 10 & $\mathrm{~L}$ \\
\hline 1357 & & 10 & $\mathrm{~L}$ \\
\hline 1358 & & 10 & $\mathrm{~L}$ \\
\hline \multicolumn{4}{|l|}{1359} \\
\hline \multicolumn{4}{|l|}{\begin{tabular}{|l|}
1360 \\
\end{tabular}} \\
\hline 1361 & & 10 & $\mathrm{~L}$ \\
\hline 1362 & & 10 & $\mathrm{~L}$ \\
\hline \begin{tabular}{|l|}
1363 \\
\end{tabular} & & 10 & $\mathrm{~L}$ \\
\hline \begin{tabular}{|l|}
1364 \\
\end{tabular} & & 10 & $\mathrm{~L}$ \\
\hline 1365 & & 10 & $\mathrm{~L}$ \\
\hline 1366 & & 10 & $\mathrm{~L}$ \\
\hline \begin{tabular}{|l|}
1367 \\
1
\end{tabular} & & 10 & $\mathrm{~L}$ \\
\hline \multicolumn{4}{|l|}{\begin{tabular}{|l|}
1368 \\
\end{tabular}} \\
\hline \begin{tabular}{|l|}
1369 \\
\end{tabular} & & & \\
\hline
\end{tabular}




\begin{tabular}{|c|c|c|c|}
\hline & +2 & $\mathrm{AE}$ & $\mathrm{AF}$ \\
\hline 1 & $\begin{array}{ll} & \text { RESULT_COMMENT }\end{array}$ & COLL_SAMP_SIZE & COLL_SAMP_SIZE_UNITS \\
\hline 1370 & & 10 & $\mathrm{~L}$ \\
\hline 1371 & & 10 & $\mathrm{~L}$ \\
\hline 1372 & & 10 & $\mathrm{~L}$ \\
\hline 1373 & & 10 & L \\
\hline 1374 & 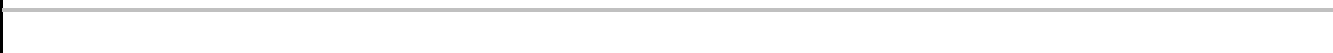 & 10 & $\mathrm{~L}$ \\
\hline 1375 & 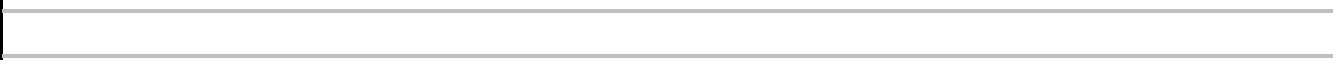 & 10 & $\mathrm{~L}$ \\
\hline 1376 & & 10 & $\mathrm{~L}$ \\
\hline \multicolumn{4}{|l|}{1377} \\
\hline \multicolumn{4}{|l|}{1378} \\
\hline 1379 & & 10 & $\mathrm{~L}$ \\
\hline 1380 & & 10 & L \\
\hline 1381 & & 10 & L \\
\hline 1382 & & 10 & L \\
\hline \begin{tabular}{|l}
1383 \\
\end{tabular} & & 10 & $\mathrm{~L}$ \\
\hline 1384 & & 10 & L \\
\hline 1385 & & 10 & L \\
\hline \multicolumn{4}{|l|}{\begin{tabular}{|l|}
1386 \\
\end{tabular}} \\
\hline \multicolumn{4}{|l|}{1387} \\
\hline 1389 & & 10 & L \\
\hline 1390 & & 10 & L \\
\hline 1391 & & 10 & L \\
\hline \begin{tabular}{|l|}
1392 \\
\end{tabular} & & 10 & L \\
\hline 1393 & & 10 & L \\
\hline 1394 & & 10 & L \\
\hline \multicolumn{4}{|l|}{\begin{tabular}{|l|}
1395 \\
\end{tabular}} \\
\hline \multicolumn{4}{|c|}{\begin{tabular}{|l|}
1396 \\
\end{tabular}} \\
\hline 1397 & & 10 & L \\
\hline \begin{tabular}{|l|}
1398 \\
\end{tabular} & & 10 & L \\
\hline \begin{tabular}{|l|}
1399 \\
\end{tabular} & & 10 & L \\
\hline 1400 & & 10 & L \\
\hline \begin{tabular}{|l|}
1401 \\
\end{tabular} & & 10 & $\mathrm{~L}$ \\
\hline \begin{tabular}{|l|l}
1402 \\
\end{tabular} & & 10 & L \\
\hline 1403 & & 10 & L \\
\hline \multicolumn{4}{|c|}{\begin{tabular}{|l|l|}
1404 \\
\end{tabular}} \\
\hline \multicolumn{4}{|c|}{\begin{tabular}{|l|}
1405 \\
\end{tabular}} \\
\hline 1406 & & 10 & L \\
\hline
\end{tabular}




\begin{tabular}{|c|c|c|c|}
\hline & $A D$ & $\mathrm{AE}$ & $\mathrm{AF}$ \\
\hline 1 & RESULT_COMMENT & COLL_SAMP_SIZE & EOLL_SAMP_SIZE_UNITS \\
\hline 1408 & & 10 & $\mathrm{~L}$ \\
\hline 1409 & & 10 & L \\
\hline 1410 & & 10 & L \\
\hline 1411 & & 10 & L \\
\hline 1412 & & 10 & L \\
\hline \multicolumn{4}{|l|}{1413} \\
\hline \multicolumn{4}{|l|}{1414} \\
\hline 1415 & & 10 & L \\
\hline 1416 & & 10 & L \\
\hline \begin{tabular}{|l|l|}
1417 \\
1417
\end{tabular} & & 10 & L \\
\hline 1418 & & 10 & $L$ \\
\hline 1419 & & 10 & L \\
\hline 1420 & & 10 & L \\
\hline 1421 & & 10 & L \\
\hline \multicolumn{4}{|l|}{1422} \\
\hline \multicolumn{4}{|l|}{1423} \\
\hline \begin{tabular}{|l|l|}
1424 \\
\end{tabular} & & 10 & $\mathrm{~L}$ \\
\hline \begin{tabular}{|l|l|}
1425 \\
142 \\
\end{tabular} & & 10 & L \\
\hline \begin{tabular}{|c|c|}
1426 \\
\end{tabular} & & 10 & L \\
\hline \begin{tabular}{|l|l|}
1427 \\
\end{tabular} & & 10 & L \\
\hline \begin{tabular}{|l|l|}
1428 \\
\end{tabular} & & 10 & L \\
\hline 1429 & & 10 & L \\
\hline 1430 & & 10 & $\mathrm{~L}$ \\
\hline \multicolumn{4}{|l|}{$\begin{array}{ll}\mid+730 \\
1431\end{array}$} \\
\hline \multicolumn{4}{|l|}{1432} \\
\hline 1433 & & 10 & $\mathrm{~L}$ \\
\hline 1434 & & 10 & L \\
\hline 1435 & & 10 & L \\
\hline \begin{tabular}{|l}
1436 \\
\end{tabular} & & 10 & L \\
\hline \begin{tabular}{|l|}
1437 \\
\end{tabular} & & 10 & $\mathrm{~L}$ \\
\hline \multicolumn{4}{|l|}{\begin{tabular}{|c|}
1438 \\
\end{tabular}} \\
\hline \multicolumn{4}{|l|}{\begin{tabular}{|c|}
1439 \\
\end{tabular}} \\
\hline \begin{tabular}{|l|}
1440 \\
\end{tabular} & & 10 & L \\
\hline \begin{tabular}{|l|}
1441 \\
\end{tabular} & & 10 & L \\
\hline \begin{tabular}{|l|}
1442 \\
\end{tabular} & & 10 & L \\
\hline \begin{tabular}{|l|}
1443 \\
\end{tabular} & & 10 & $L$ \\
\hline \begin{tabular}{|l|}
1444 \\
\end{tabular} & & 10 & $\mathrm{~L}$ \\
\hline \begin{tabular}{|l}
1445 \\
\end{tabular} & . & & \\
\hline
\end{tabular}




\begin{tabular}{|c|c|c|c|}
\hline & 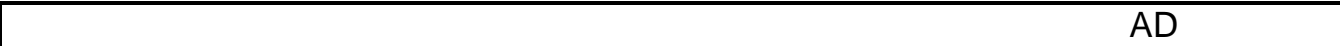 & $\mathrm{AE}$ & AF \\
\hline 1 & $\begin{array}{ll} & \text { RESULT_COMMENT } \\
\end{array}$ & COLL_SAMP_SIZE & COLL_SAMP_SIZE_UNITS \\
\hline \multicolumn{4}{|c|}{1446} \\
\hline 1447 & & 10 & L \\
\hline 1448 & & 10 & L \\
\hline 1449 & & 10 & L \\
\hline 1450 & 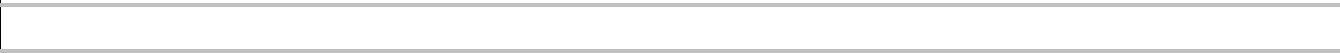 & 10 & L \\
\hline 1451 & & 10 & L \\
\hline \multicolumn{4}{|l|}{1452} \\
\hline \multicolumn{4}{|l|}{1453} \\
\hline 1454 & & 10 & $L$ \\
\hline 1455 & & 10 & $L$ \\
\hline 1456 & & 10 & L \\
\hline 1457 & & 10 & L \\
\hline 1458 & & 10 & L \\
\hline \multicolumn{4}{|l|}{1459} \\
\hline \multicolumn{4}{|l|}{1460} \\
\hline 1461 & & 10 & L \\
\hline 1462 & & 10 & L \\
\hline 1463 & & 10 & L \\
\hline 1464 & & 10 & $L$ \\
\hline 1465 & & 10 & L \\
\hline \multicolumn{4}{|l|}{1466} \\
\hline \multicolumn{4}{|l|}{1467} \\
\hline 1468 & & 10 & L \\
\hline 1469 & & 10 & L \\
\hline 1470 & & 10 & $\mathrm{~L}$ \\
\hline 1471 & & 10 & $\mathrm{~L}$ \\
\hline 1472 & & 10 & $L$ \\
\hline \multicolumn{4}{|l|}{1473} \\
\hline \multicolumn{4}{|l|}{1474} \\
\hline 1475 & & 10 & L \\
\hline 1476 & & 10 & $L$ \\
\hline 1477 & & 10 & L \\
\hline 1478 & & 10 & L \\
\hline 1479 & & 10 & $L$ \\
\hline \multicolumn{4}{|l|}{1480} \\
\hline \multicolumn{4}{|l|}{1481} \\
\hline 1482 & & 10 & L \\
\hline 1483 & 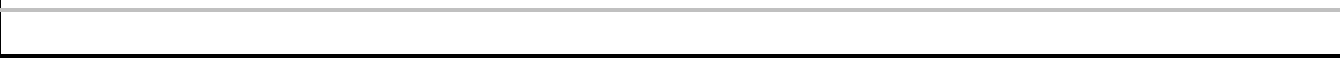 & 10 & $\mathrm{~L}$ \\
\hline
\end{tabular}




\begin{tabular}{|c|c|c|c|}
\hline & $\overline{A D}$ & $\mathrm{AE}$ & $\mathrm{AF}$ \\
\hline 1 & RESULT_COMMENT & COLL_SAMP_SIZE & COLL_SAMP_SIZE_UNITS \\
\hline 1484 & & 10 & $\mathrm{~L}$ \\
\hline 1485 & & 10 & $\mathrm{~L}$ \\
\hline 1486 & & 10 & $\mathrm{~L}$ \\
\hline \multicolumn{4}{|l|}{\begin{tabular}{|l|}
1487 \\
\end{tabular}} \\
\hline \multicolumn{4}{|l|}{\begin{tabular}{|l|}
1488 \\
\end{tabular}} \\
\hline 1489 & & 10 & $\mathrm{~L}$ \\
\hline 1490 & & 10 & L \\
\hline 1491 & & 10 & $\mathrm{~L}$ \\
\hline \begin{tabular}{|l|}
1492 \\
\end{tabular} & & 10 & $\mathrm{~L}$ \\
\hline \begin{tabular}{|l|}
1493 \\
\end{tabular} & & 10 & $\mathrm{~L}$ \\
\hline \multicolumn{4}{|l|}{1494} \\
\hline \multicolumn{4}{|l|}{1495} \\
\hline 1496 & & 10 & L \\
\hline 1497 & & 10 & $\mathrm{~L}$ \\
\hline 1498 & & 10 & $\mathrm{~L}$ \\
\hline \begin{tabular}{|l|}
1499 \\
\end{tabular} & & 10 & $\mathrm{~L}$ \\
\hline 1500 & & 10 & $\mathrm{~L}$ \\
\hline \multicolumn{4}{|l|}{1501} \\
\hline \multicolumn{4}{|l|}{1502} \\
\hline 1503 & & 10 & $\mathrm{~L}$ \\
\hline 1504 & & 10 & $\mathrm{~L}$ \\
\hline 1505 & & 10 & $\mathrm{~L}$ \\
\hline \begin{tabular}{|l|}
1506 \\
\end{tabular} & & 10 & $\mathrm{~L}$ \\
\hline 1507 & & 10 & $\mathrm{~L}$ \\
\hline \multicolumn{4}{|l|}{1508} \\
\hline \multicolumn{4}{|l|}{1509} \\
\hline 1510 & & 10 & $\mathrm{~L}$ \\
\hline 1511 & & 10 & $\mathrm{~L}$ \\
\hline \begin{tabular}{|l|}
1512 \\
\end{tabular} & & 10 & $\mathrm{~L}$ \\
\hline 1513 & & 10 & L \\
\hline \begin{tabular}{|l|}
1514 \\
\end{tabular} & & 10 & $\mathrm{~L}$ \\
\hline \multicolumn{4}{|l|}{1515} \\
\hline \multicolumn{4}{|l|}{1516} \\
\hline 1517 & & 10 & $\mathrm{~L}$ \\
\hline \begin{tabular}{|l|}
1518 \\
\end{tabular} & & 10 & $\mathrm{~L}$ \\
\hline 1519 & & 10 & $\mathrm{~L}$ \\
\hline 1520 & & 10 & $\mathrm{~L}$ \\
\hline 1521 & & 10 & $\mathrm{~L}$ \\
\hline
\end{tabular}




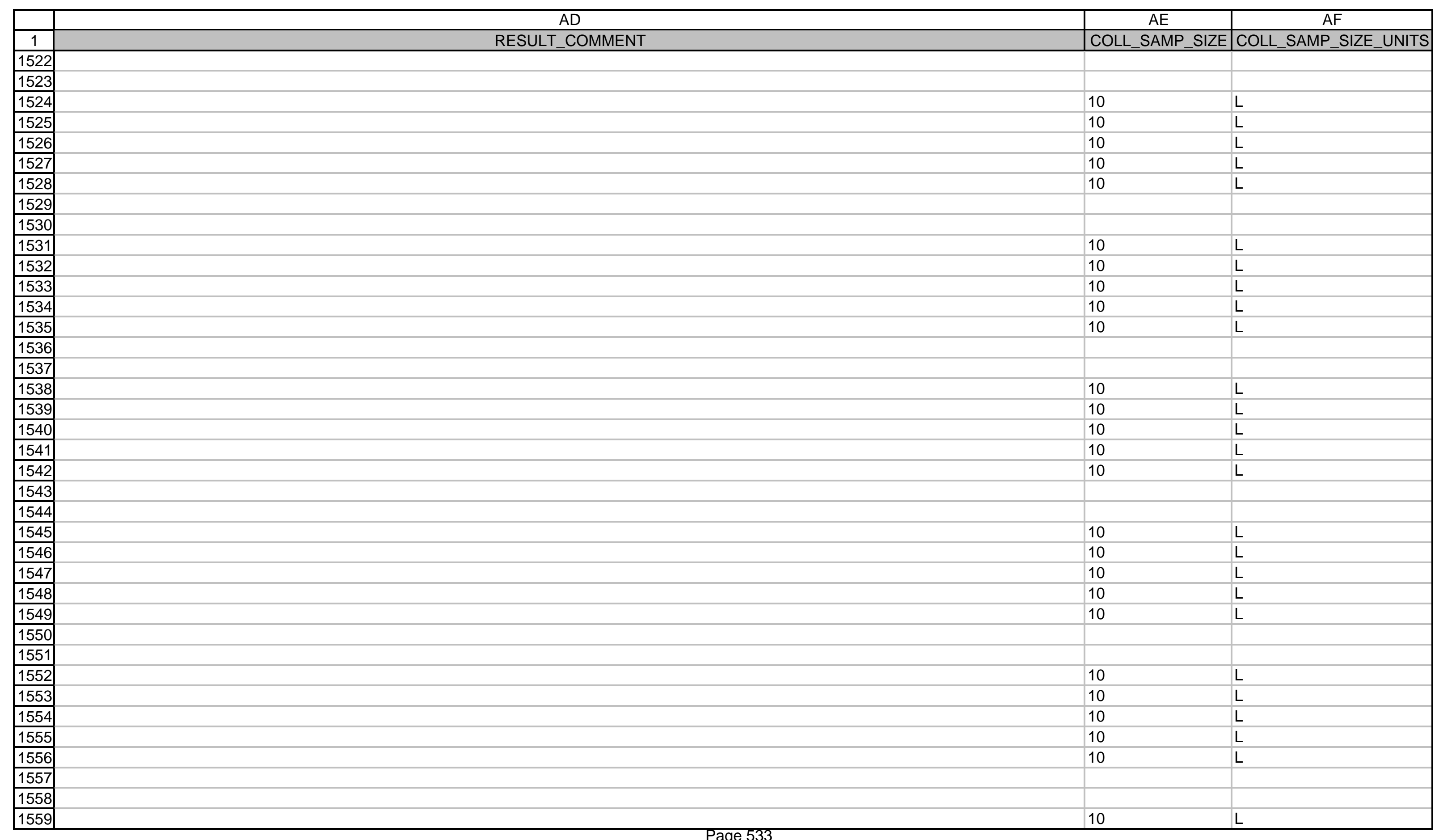




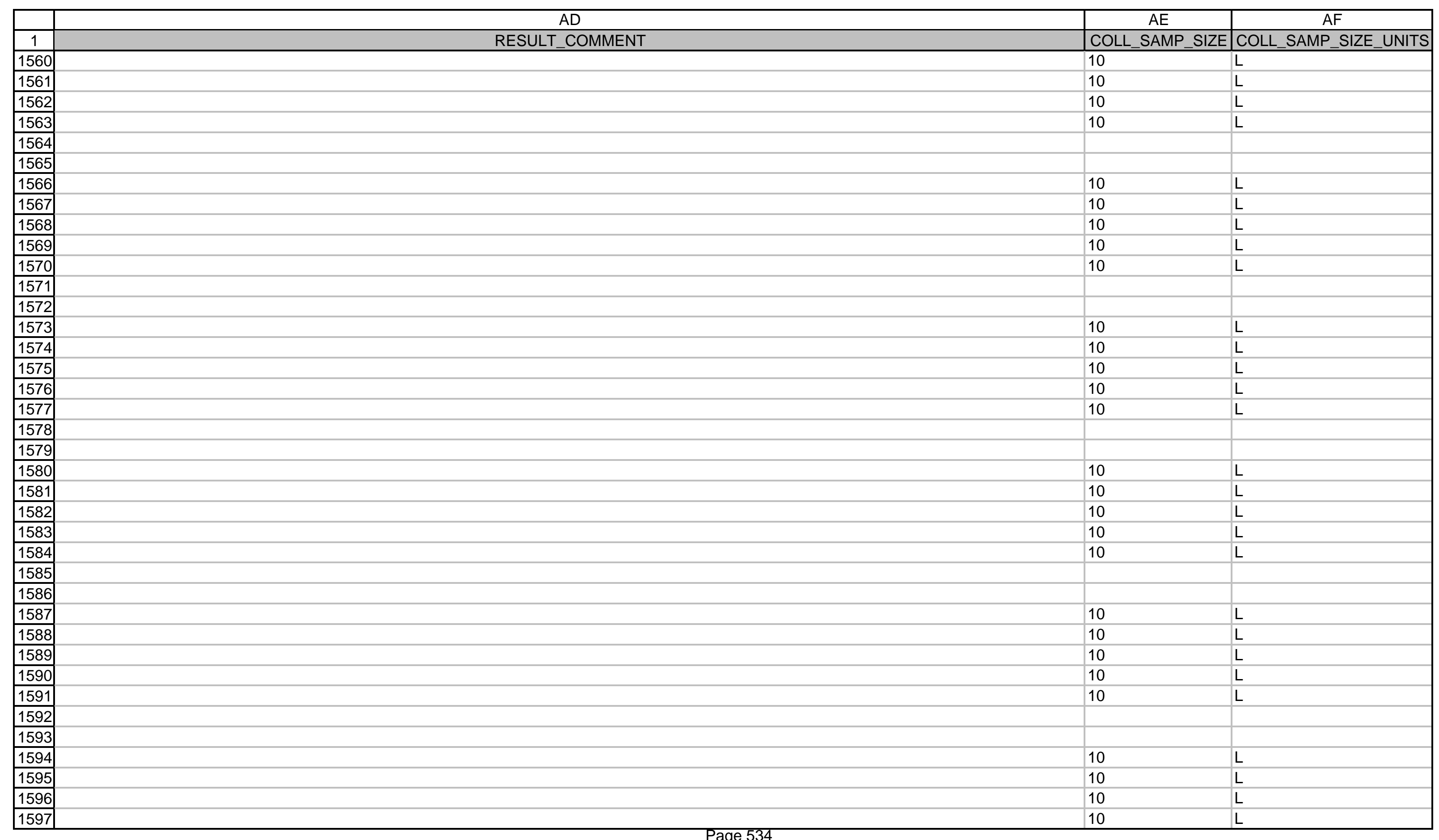




\begin{tabular}{|c|c|c|c|}
\hline & 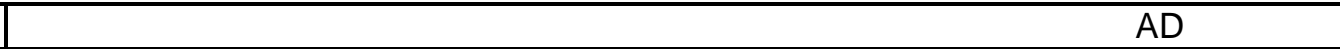 & $\mathrm{AE}$ & $\mathrm{AF}$ \\
\hline 1 & RESULT_COMMENT & COLL_SAMP_SIZE & COLL_SAMP_SIZE_UNITS \\
\hline 1598 & & 10 & $\mathrm{~L}$ \\
\hline \multicolumn{4}{|l|}{1599} \\
\hline \multicolumn{4}{|l|}{1600} \\
\hline 1601 & & 10 & L \\
\hline 1602 & & 10 & $\mathrm{~L}$ \\
\hline $\mid 1603$ & & 10 & $\mathrm{~L}$ \\
\hline 1604 & & 10 & L \\
\hline 1605 & 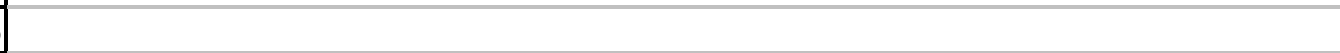 & 10 & L \\
\hline 1606 & 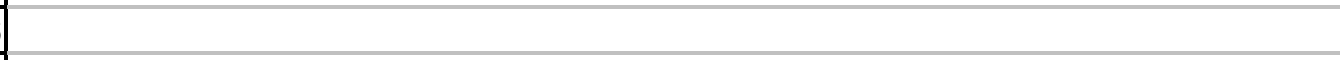 & 10 & L \\
\hline \multicolumn{4}{|l|}{1607} \\
\hline \multicolumn{4}{|l|}{1608} \\
\hline 1609 & & 10 & L \\
\hline 1610 & & 10 & $\mathrm{~L}$ \\
\hline 1611 & & 10 & $\mathrm{~L}$ \\
\hline 1612 & RESULT LOST AT LAB. & 10 & L \\
\hline 1613 & RESULT LOST AT LAB. & 10 & L \\
\hline 1614 & RESULT LOST AT LAB. & 10 & L \\
\hline \multicolumn{4}{|c|}{\begin{tabular}{|l|l|}
1615 \\
\end{tabular}} \\
\hline \multicolumn{4}{|l|}{1616} \\
\hline 1617 & & 10 & L \\
\hline \begin{tabular}{|l|}
1618 \\
\end{tabular} & & 10 & L \\
\hline 1619 & & 10 & L \\
\hline 1620 & & 10 & L \\
\hline 1621 & & 10 & L \\
\hline 1622 & & 10 & $L$ \\
\hline \multicolumn{4}{|l|}{1623} \\
\hline \multicolumn{4}{|l|}{1624} \\
\hline 1625 & & 10 & L \\
\hline 1626 & & 10 & L \\
\hline \begin{tabular}{|l|}
1627 \\
\end{tabular} & & 10 & L \\
\hline 1628 & & 10 & $\mathrm{~L}$ \\
\hline \begin{tabular}{|l|l|}
1629 \\
\end{tabular} & & 10 & $\mathrm{~L}$ \\
\hline 1630 & & 10 & L \\
\hline \multicolumn{4}{|l|}{1631} \\
\hline \multicolumn{4}{|l|}{1632} \\
\hline \begin{tabular}{|l|}
1633 \\
\end{tabular} & & 10 & $L$ \\
\hline \begin{tabular}{|l|}
1634 \\
\end{tabular} & & 10 & $\mathrm{~L}$ \\
\hline \begin{tabular}{|l|}
1635 \\
\end{tabular} & 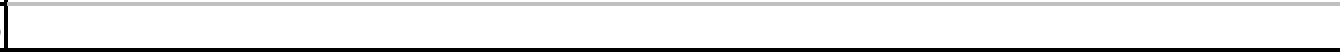 & 10 & $\mathrm{~L}$ \\
\hline
\end{tabular}




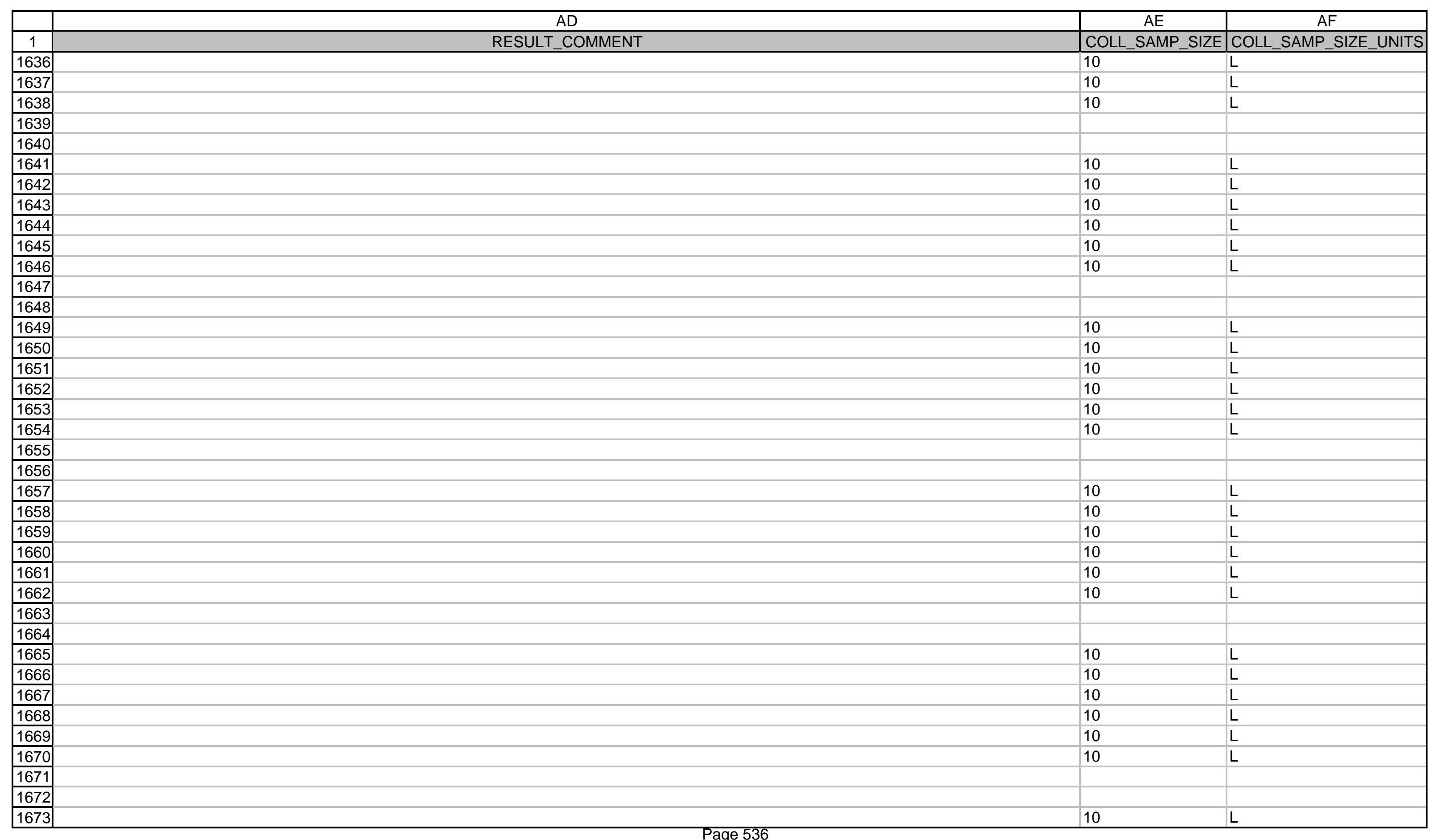




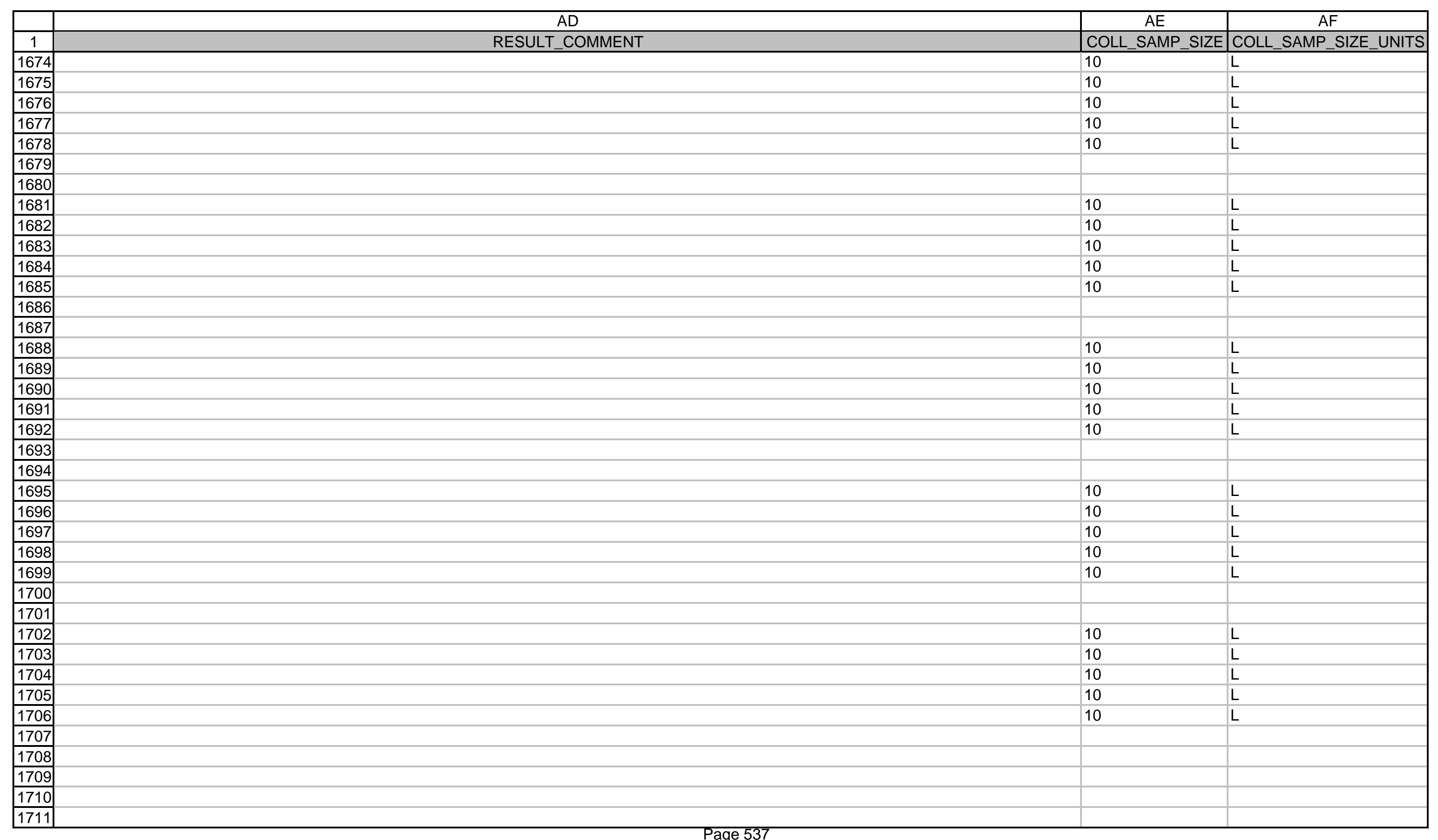


AD

AE

$\mathrm{AF}$

\begin{tabular}{|c|c|c|c|}
\hline & $A D$ & $\mathrm{AE}$ & AF \\
\hline 1 & RESULT_COMMENT & COLL_SAMP_SIZE & COLL_SAMP_SIZE_UNITS \\
\hline \multicolumn{4}{|c|}{ 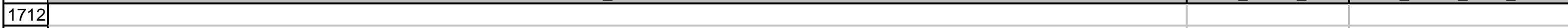 } \\
\hline \multicolumn{4}{|l|}{1713} \\
\hline \multicolumn{4}{|l|}{1714} \\
\hline \multicolumn{4}{|l|}{1715} \\
\hline \multicolumn{4}{|l|}{1716} \\
\hline \multicolumn{4}{|l|}{1717} \\
\hline \multicolumn{4}{|l|}{1718} \\
\hline \multicolumn{4}{|l|}{1719} \\
\hline \multicolumn{4}{|l|}{1720} \\
\hline \multicolumn{4}{|l|}{1721} \\
\hline \multicolumn{4}{|l|}{1722} \\
\hline \multicolumn{4}{|l|}{1723} \\
\hline \multicolumn{4}{|l|}{1724} \\
\hline \multicolumn{4}{|l|}{1725} \\
\hline \multicolumn{4}{|l|}{1726} \\
\hline 1727 & & 10 & L \\
\hline 1728 & & 10 & L \\
\hline 1729 & REFER TO 3/24/95 INCIDENT REPORT, RESULT LOST AT LAB. & 10 & $\mathrm{~L}$ \\
\hline 1730 & REFER TO 3/24/95 INCIDENT REPORT, RESULT LOST AT LAB. & 10 & L \\
\hline 1731 & REFER TO 3/24/95 INCIDENT REPORT, RESULT LOST AT LAB. & 10 & L \\
\hline 1732 & & 10 & L \\
\hline 1733 & & 10 & $\mathrm{~L}$ \\
\hline 1734 & U-ISOTOPIC FRACTION LOST AT LAB. RETRIEVED SAMPLES FROM DATACHEM AND SUBMITTED TO IT ON 4/3/95. & 10 & L \\
\hline 1735 & U-ISOTOPIC FRACTION LOST AT LAB. RETRIEVED SAMPLES FROM DATACHEM AND SUBMITTED TO IT ON 4/3/95. & 10 & L \\
\hline 1736 & U-ISOTOPIC FRACTION LOST AT LAB. RETRIEVED SAMPLES FROM DATACHEM AND SUBMITTED TO IT ON 4/3/95. & 10 & L \\
\hline 1737 & & 10 & $\mathrm{~L}$ \\
\hline 1738 & & 10 & L \\
\hline 1739 & U-ISOTOPIC FRACTION LOST AT LAB. RETRIEVED SAMPLES FROM DATACHEM AND SUBMITTED TO IT ON 4/3/95. & 10 & L \\
\hline 1740 & U-ISOTOPIC FRACTION LOST AT LAB. RETRIEVED SAMPLES FROM DATACHEM AND SUBMITTED TO IT ON 4/3/95. & 10 & L \\
\hline 1741 & U-ISOTOPIC FRACTION LOST AT LAB. RETRIEVED SAMPLES FROM DATACHEM AND SUBMITTED TO IT ON 4/3/95. & 10 & L \\
\hline 1742 & & 10 & L \\
\hline 1743 & & 10 & L \\
\hline 1744 & U-ISOTOPIC FRACTION LOST AT LAB. RETRIEVED SAMPLES FROM DATACHEM AND SUBMITTED TO IT ON 4/3/95. & 10 & L \\
\hline 1745 & U-ISOTOPIC FRACTION LOST AT LAB. RETRIEVED SAMPLES FROM DATACHEM AND SUBMITTED TO IT ON 4/3/95. & 10 & L \\
\hline 1746 & U-ISOTOPIC FRACTION LOST AT LAB. RETRIEVED SAMPLES FROM DATACHEM AND SUBMITTED TO IT ON 4/3/95. & 10 & L \\
\hline 1747 & & 10 & L \\
\hline 1748 & & 10 & L \\
\hline 1749 & U-ISOTOPIC FRACTION LOST AT LAB. RETRIEVED SAMPLES FROM DATACHEM AND SUBMITTED TO IT ON 4/3/95. & 10 & $\mathrm{~L}$ \\
\hline
\end{tabular}


1750 U-ISOTOPIC FRACTION LOST AT LAB. RETRIEVED SAMPLES FROM DATACHEM AND SUBMITTED TO IT ON 4/3/95.

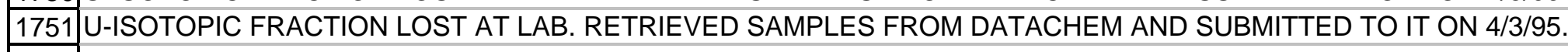

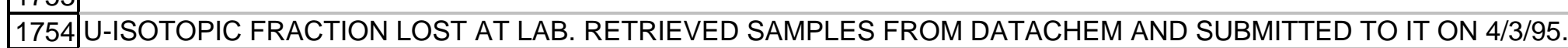

1755 U-ISOTOPIC FRACTION LOST AT LAB. RETRIEVED SAMPLES FROM DATACHEM AND SUBMITTED TO IT ON 4/3/95.

1756 U-ISOTOPIC FRACTION LOST AT LAB. RETRIEVED SAMPLES FROM DATACHEM AND SUBMITTED TO IT ON 4/3/95.

1757

1758

1759 U-ISOTOPIC FRACTION LOST AT LAB. RETRIEVED SAMPLES FROM DATACHEM AND SUBMITTED TO IT ON 4/3/95.

1760 U-ISOTOPIC FRACTION LOST AT LAB. RETRIEVED SAMPLES FROM DATACHEM AND SUBMITTED TO IT ON 4/3/95.

1761 U-ISOTOPIC FRACTION LOST AT LAB. RETRIEVED SAMPLES FROM DATACHEM AND SUBMITTED TO IT ON 4/3/95.

1762

1763

1764 U-ISOTOPIC FRACTION LOST AT LAB. RETRIEVED SAMPLES FROM DATACHEM AND SUBMITTED TO IT ON 4/3/95.

1765 U-ISOTOPIC FRACTION LOST AT LAB. RETRIEVED SAMPLES FROM DATACHEM AND SUBMITTED TO IT ON 4/3/95.

1766 U-ISOTOPIC FRACTION LOST AT LAB. RETRIEVED SAMPLES FROM DATACHEM AND SUBMITTED TO IT ON 4/3/95.

1767

1768

1769 U-ISOTOPIC FRACTION LOST AT LAB. RETRIEVED SAMPLES FROM DATACHEM AND SUBMITTED TO IT ON 4/3/95.

1770 U-ISOTOPIC FRACTION LOST AT LAB. RETRIEVED SAMPLES FROM DATACHEM AND SUBMITTED TO IT ON 4/3/95.

1771 U-ISOTOPIC FRACTION LOST AT LAB. RETRIEVED SAMPLES FROM DATACHEM AND SUBMITTED TO IT ON 4/3/95.

\section{2}

1773

1774 U-ISOTOPIC FRACTION LOST AT LAB. RETRIEVED SAMPLES FROM DATACHEM AND SUBMITTED TO IT ON 4/3/95.

1775 U-ISOTOPIC FRACTION LOST AT LAB. RETRIEVED SAMPLES FROM DATACHEM AND SUBMITTED TO IT ON 4/3/95.

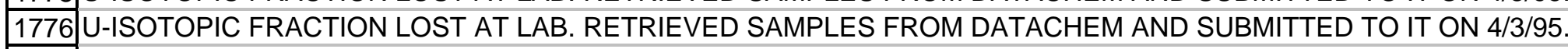

1777

1778

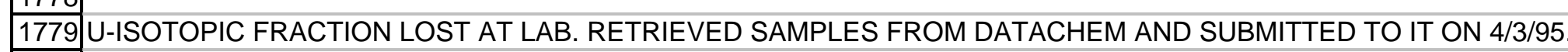

1780 U-ISOTOPIC FRACTION LOST AT LAB. RETRIEVED SAMPLES FROM DATACHEM AND SUBMITTED TO IT ON 4/3/95.

1781 U-ISOTOPIC FRACTION LOST AT LAB. RETRIEVED SAMPLES FROM DATACHEM AND SUBMITTED TO IT ON 4/3/95.

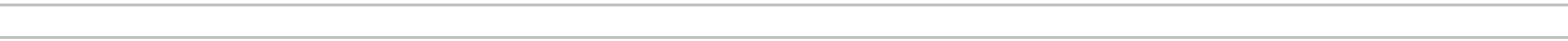
20
10

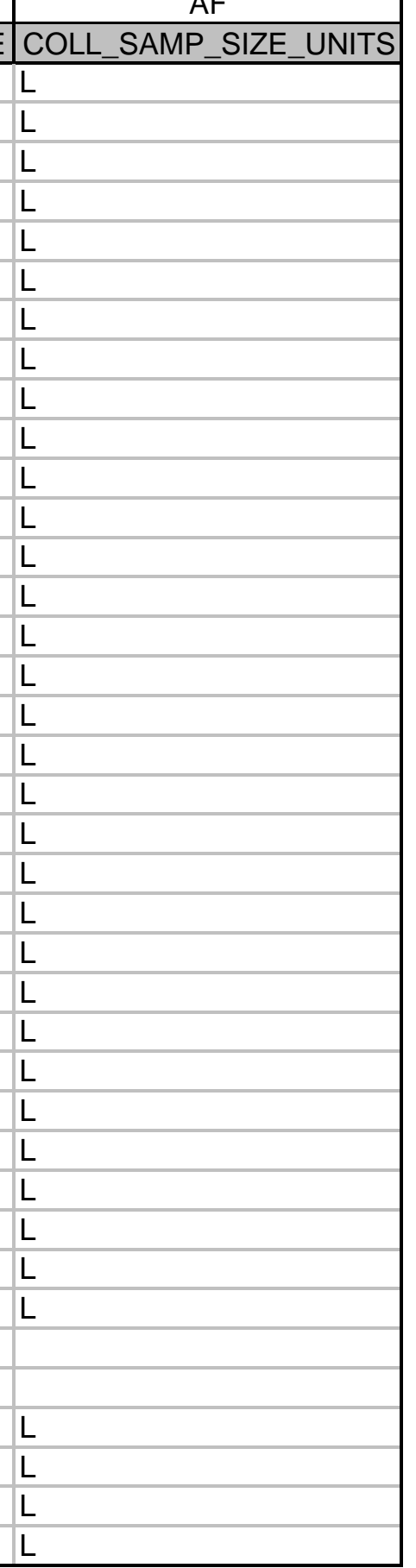




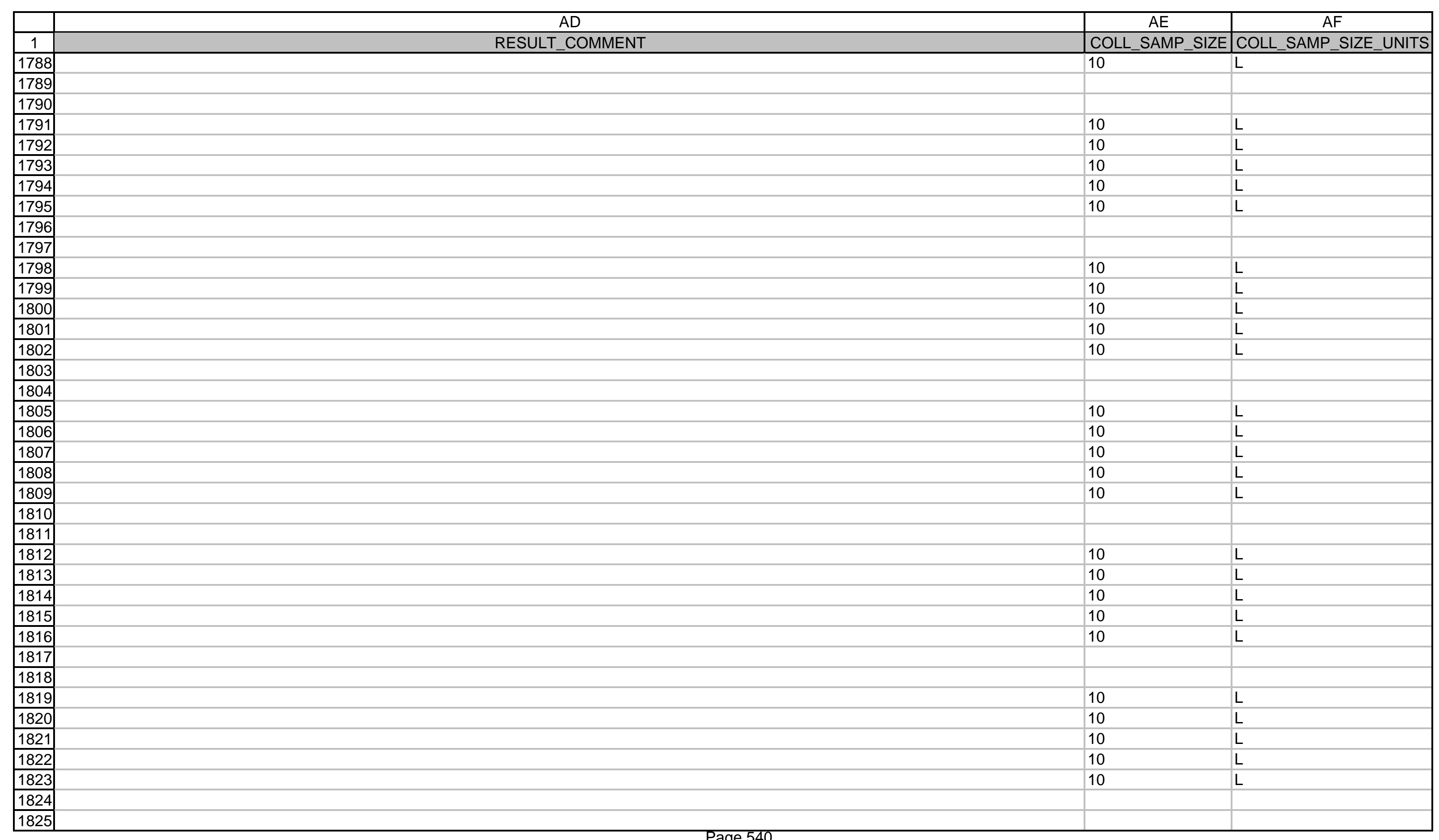




\begin{tabular}{|c|c|c|c|}
\hline & $\overline{A D}$ & $\mathrm{AE}$ & $\mathrm{AF}$ \\
\hline 1 & RESULT_COMMENT & COLL_SAMP_SIZE & COLL_SAMP_SIZE_UNITS \\
\hline 1826 & & 10 & $\mathrm{~L}$ \\
\hline 1827 & & 10 & $\mathrm{~L}$ \\
\hline 1828 & & 10 & $\mathrm{~L}$ \\
\hline 1829 & & 10 & $\bar{L}$ \\
\hline 1830 & & 10 & $\mathrm{~L}$ \\
\hline \multicolumn{4}{|l|}{1831} \\
\hline \multicolumn{4}{|l|}{1832} \\
\hline 1833 & & 10 & L \\
\hline 1834 & & 10 & $\mathrm{~L}$ \\
\hline 1835 & & 10 & $\bar{L}$ \\
\hline 1836 & & 10 & $\mathrm{~L}$ \\
\hline 1837 & & 10 & $\mathrm{~L}$ \\
\hline \multicolumn{4}{|l|}{1838} \\
\hline \multicolumn{4}{|l|}{1839} \\
\hline 1840 & & 10 & $\mathrm{~L}$ \\
\hline 1841 & & 10 & $\bar{L}$ \\
\hline 1842 & & 10 & $\bar{L}$ \\
\hline 1843 & & 10 & L \\
\hline 1844 & & 10 & $\mathrm{~L}$ \\
\hline \multicolumn{4}{|l|}{1845} \\
\hline \multicolumn{4}{|l|}{1846} \\
\hline 1847 & & 10 & $\mathrm{~L}$ \\
\hline 1848 & & 10 & L \\
\hline 1849 & & 10 & L \\
\hline 1850 & & 10 & L \\
\hline 1851 & & 10 & L \\
\hline \multicolumn{4}{|l|}{1852} \\
\hline \multicolumn{4}{|l|}{1853} \\
\hline 1854 & & 10 & $\mathrm{~L}$ \\
\hline 1855 & & 10 & $\bar{L}$ \\
\hline 1856 & & 10 & $\mathrm{~L}$ \\
\hline 1857 & & 10 & $\bar{L}$ \\
\hline 1858 & & 10 & L \\
\hline \multicolumn{4}{|l|}{1859} \\
\hline \multicolumn{4}{|l|}{1860} \\
\hline $\begin{array}{ll}1861 \\
1801\end{array}$ & & 10 & L \\
\hline 1862 & & 10 & $\mathrm{~L}$ \\
\hline 1863 & & 10 & $\mathrm{~L}$ \\
\hline
\end{tabular}




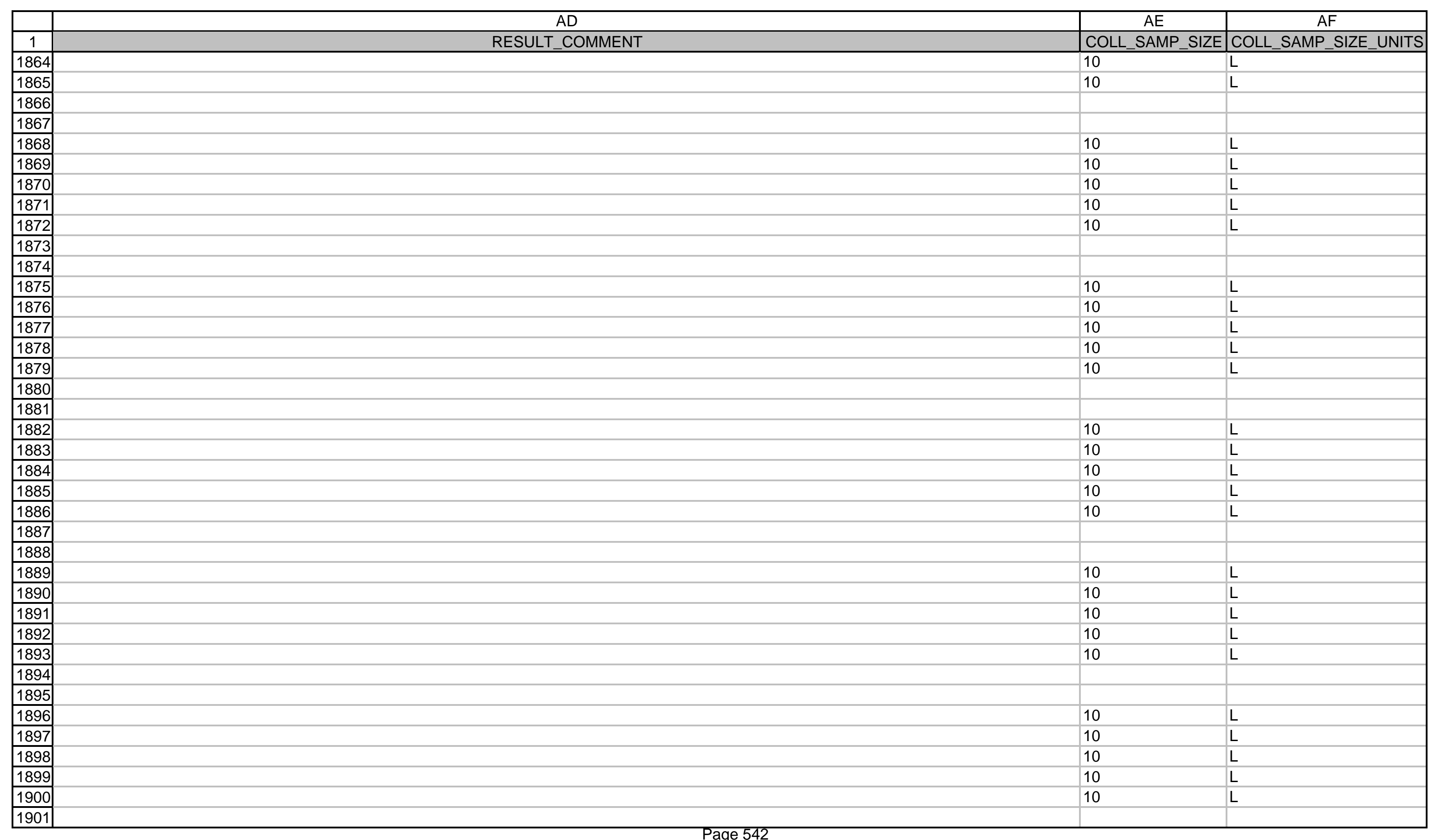




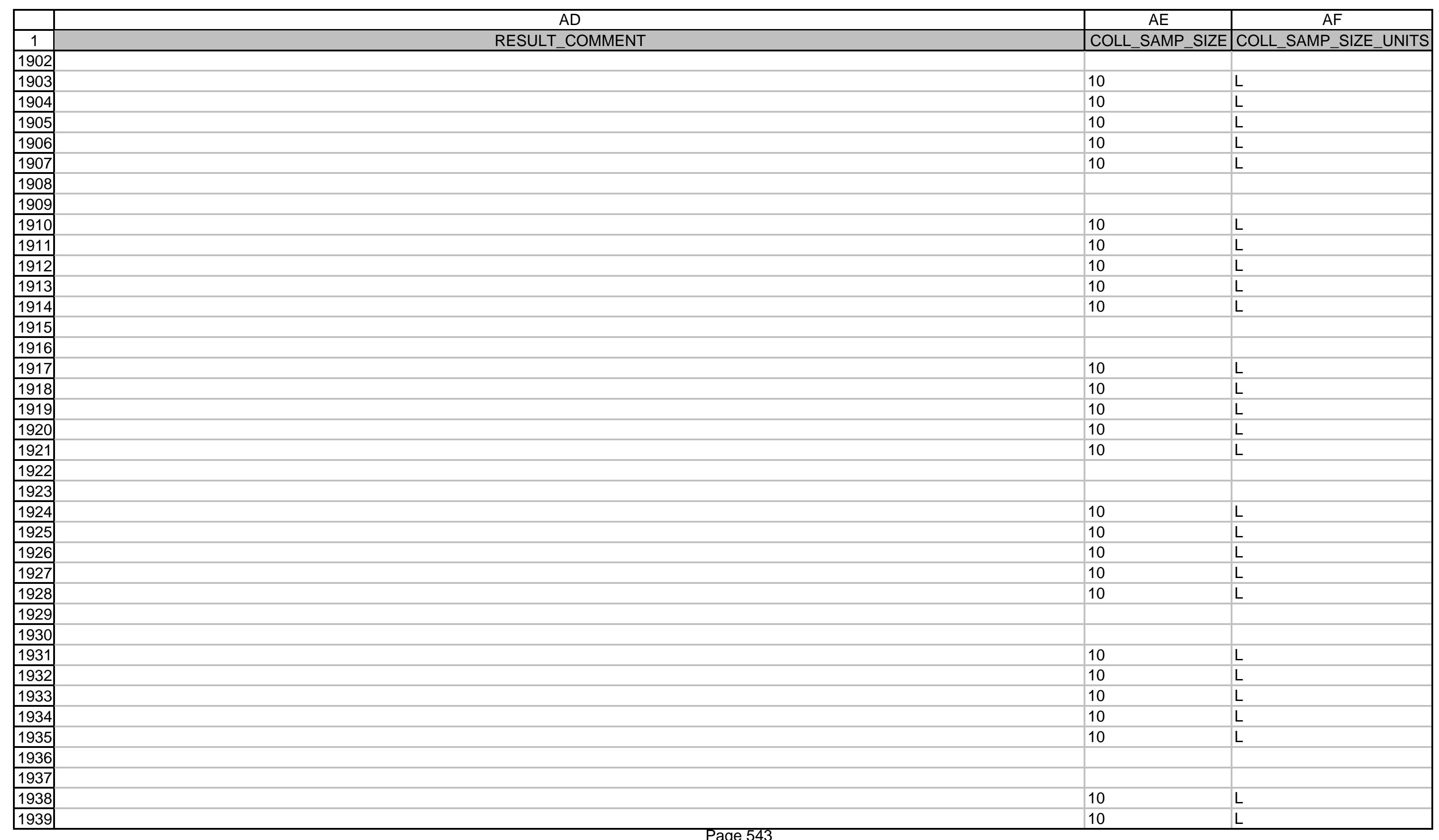




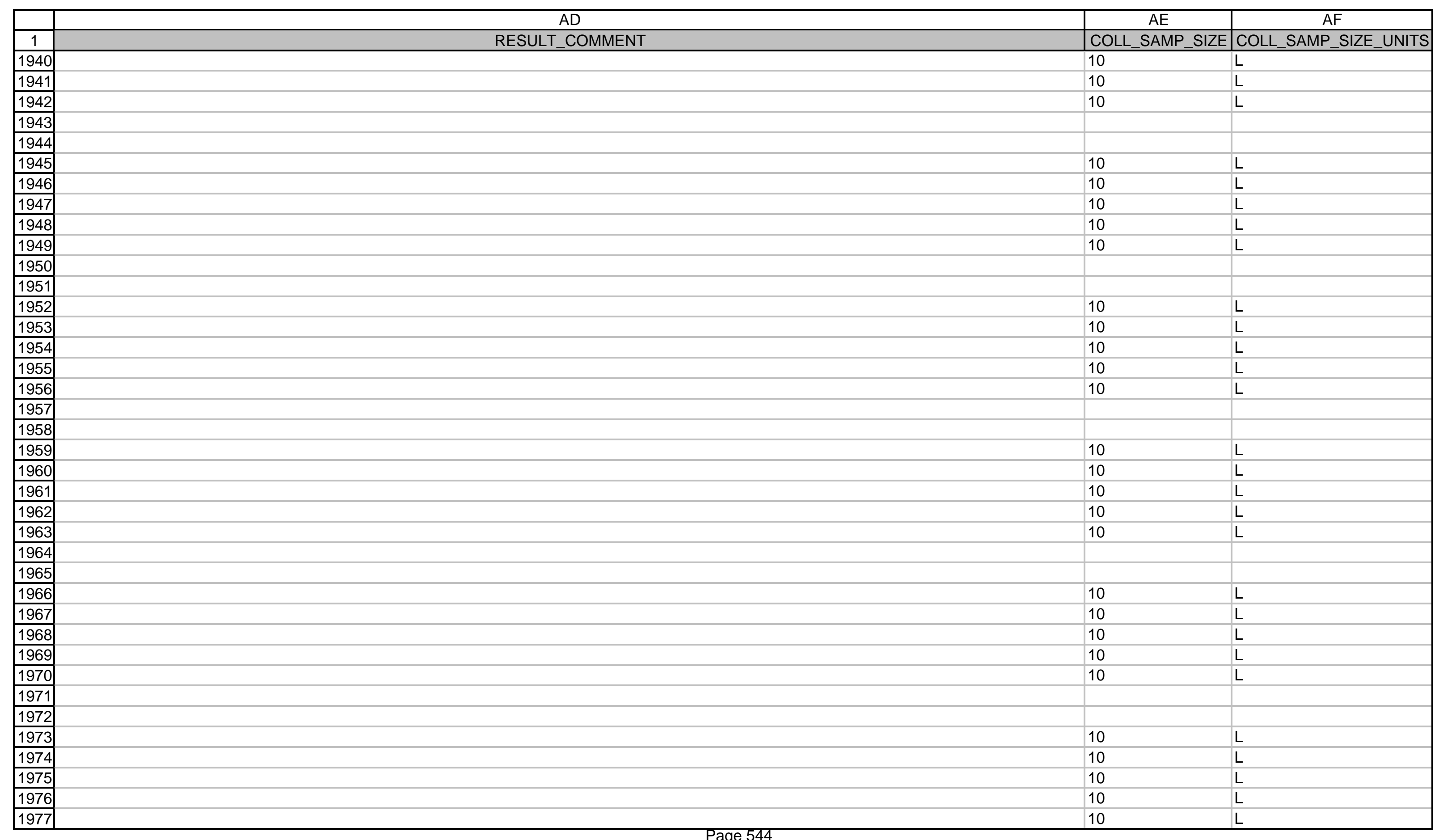




\begin{tabular}{|c|c|c|c|}
\hline & $A D$ & $\mathrm{AE}$ & $\mathrm{AF}$ \\
\hline 1 & RESULT_COMMENT & COLL_SAMP_SIZE & COLL_SAMP_SIZE_UNITS \\
\hline \multicolumn{4}{|c|}{ 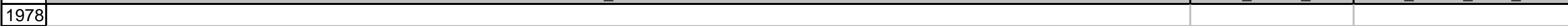 } \\
\hline \multicolumn{4}{|l|}{1979} \\
\hline 1980 & & 10 & L \\
\hline 1981 & & 10 & $\mathrm{~L}$ \\
\hline 1982 & & 10 & L \\
\hline 1983 & & 10 & L \\
\hline 1984 & & 10 & L \\
\hline 1985 & & 10 & L \\
\hline 1986 & & 10 & L \\
\hline \multicolumn{4}{|l|}{1987} \\
\hline \multicolumn{4}{|l|}{1988} \\
\hline 1989 & & 10 & L \\
\hline 1990 & & 10 & L \\
\hline \begin{tabular}{|l|}
1991 \\
\end{tabular} & & 10 & $\mathrm{~L}$ \\
\hline 1992 & & 10 & L \\
\hline 1993 & & 10 & L \\
\hline 1994 & & 10 & L \\
\hline 1995 & & 10 & L \\
\hline \multicolumn{4}{|l|}{1996} \\
\hline \multicolumn{4}{|c|}{1997} \\
\hline 1998 & & 10 & L \\
\hline 1999 & & 10 & L \\
\hline 2000 & & 10 & L \\
\hline 2001 & & 10 & L \\
\hline 2002 & & 10 & L \\
\hline 2003 & & 10 & L \\
\hline 2004 & & 10 & $\mathrm{~L}$ \\
\hline \multicolumn{4}{|l|}{\begin{tabular}{|l|}
2005 \\
\end{tabular}} \\
\hline \multicolumn{4}{|l|}{2006} \\
\hline 2007 & & 10 & L \\
\hline 2008 & & 10 & L \\
\hline 2009 & REFER TO 1/24/96 INCIDENT REPORT, RESULT NOT LOST AT LAB, MODIFIED RESULT REPORTED 2/21/96. & 10 & $\mathrm{~L}$ \\
\hline 2010 & & 10 & L \\
\hline 2011 & & 10 & $\mathrm{~L}$ \\
\hline 2012 & & 10 & $\mathrm{~L}$ \\
\hline 2013 & & 10 & L \\
\hline 2014 & & & \\
\hline 2015 & & & \\
\hline
\end{tabular}




\begin{tabular}{|c|c|c|c|}
\hline & $A D$ & $A E$ & $\mathrm{AF}$ \\
\hline 1 & RESULT_COMMENT & COLL_SAMP_SIZE & COLL_SAMP_SIZE_UNITS \\
\hline \begin{tabular}{|l|} 
\\
\end{tabular} & & 10 & L \\
\hline 2017 & RESULT MODIFIED, REFER TO FOLLOW-UP ANALYSIS ORDER W030. & 10 & L \\
\hline 2018 & & 10 & L \\
\hline 2019 & & 10 & L \\
\hline 2020 & & 10 & L \\
\hline 2021 & & 10 & L \\
\hline 2022 & . & 10 & L \\
\hline \multicolumn{4}{|l|}{\begin{tabular}{|l|}
2023 \\
\end{tabular}} \\
\hline \multicolumn{4}{|l|}{\begin{tabular}{|l|}
2024 \\
\end{tabular}} \\
\hline 2025 & & 10 & L \\
\hline 2026 & & 10 & L \\
\hline \begin{tabular}{|l|}
2027 \\
\end{tabular} & & 10 & L \\
\hline \begin{tabular}{|l|}
2028 \\
\end{tabular} & & 10 & L \\
\hline \begin{tabular}{|l|}
2029 \\
\end{tabular} & & 10 & L \\
\hline \begin{tabular}{|l|}
2030 \\
\end{tabular} & & 10 & L \\
\hline \begin{tabular}{|l|}
2031 \\
\end{tabular} & & 10 & L \\
\hline \multicolumn{4}{|l|}{\begin{tabular}{|l|}
2032 \\
\end{tabular}} \\
\hline \multicolumn{4}{|l|}{\begin{tabular}{|l|}
2033 \\
\end{tabular}} \\
\hline \begin{tabular}{|l|}
2034 \\
\end{tabular} & & 10 & L \\
\hline \begin{tabular}{|l|}
2035 \\
\end{tabular} & & 10 & L \\
\hline \begin{tabular}{|l|}
2036 \\
\end{tabular} & & 10 & L \\
\hline \begin{tabular}{|l|}
2037 \\
\end{tabular} & & 10 & L \\
\hline \begin{tabular}{|l|}
2038 \\
\end{tabular} & & 10 & L \\
\hline \begin{tabular}{|l|}
2039 \\
\end{tabular} & & 10 & L \\
\hline \begin{tabular}{|l|}
2040 \\
\end{tabular} & & 10 & L \\
\hline \multicolumn{4}{|l|}{\begin{tabular}{|l|}
2041 \\
\end{tabular}} \\
\hline \multicolumn{4}{|l|}{\begin{tabular}{|l|}
2042 \\
\end{tabular}} \\
\hline \begin{tabular}{|l|}
2043 \\
\end{tabular} & & 10 & L \\
\hline \begin{tabular}{|l|}
2044 \\
\end{tabular} & & 10 & L \\
\hline \begin{tabular}{|l|}
2045 \\
\end{tabular} & & 10 & L \\
\hline \begin{tabular}{|l|}
2046 \\
\end{tabular} & & 10 & L \\
\hline \begin{tabular}{|l|}
2047 \\
\end{tabular} & & 10 & $\mathrm{~L}$ \\
\hline \begin{tabular}{|l|}
2048 \\
\end{tabular} & & 10 & L \\
\hline \begin{tabular}{|l|}
2049 \\
\end{tabular} & & 10 & L \\
\hline \multicolumn{4}{|l|}{\begin{tabular}{|l|}
2050 \\
\end{tabular}} \\
\hline \multicolumn{4}{|l|}{\begin{tabular}{|l|}
2051 \\
\end{tabular}} \\
\hline \begin{tabular}{|l|}
2052 \\
\end{tabular} & & 10 & $\mathrm{~L}$ \\
\hline \begin{tabular}{|l|}
2053 \\
\end{tabular} & & 10 & $\mathrm{~L}$ \\
\hline
\end{tabular}




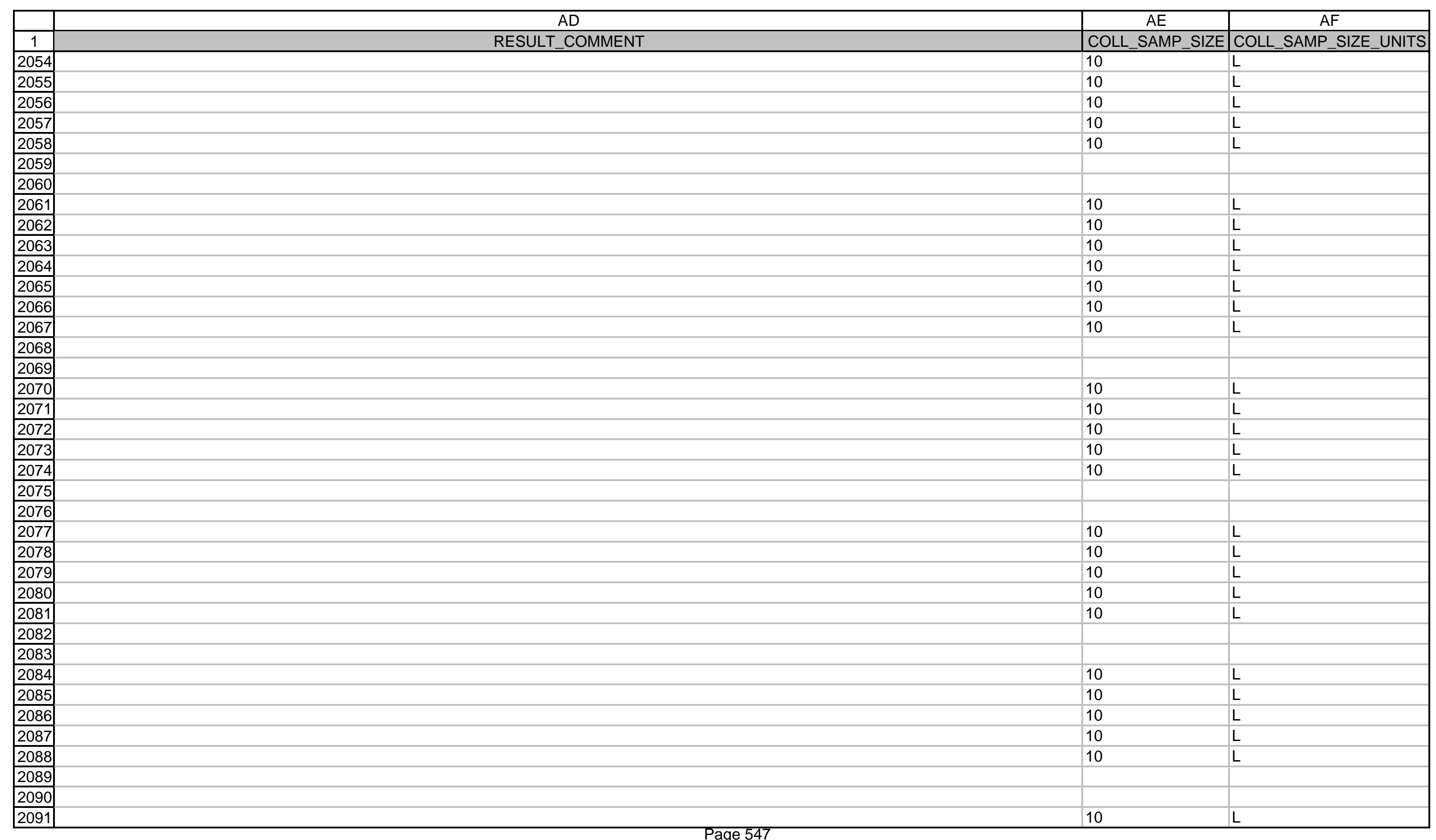




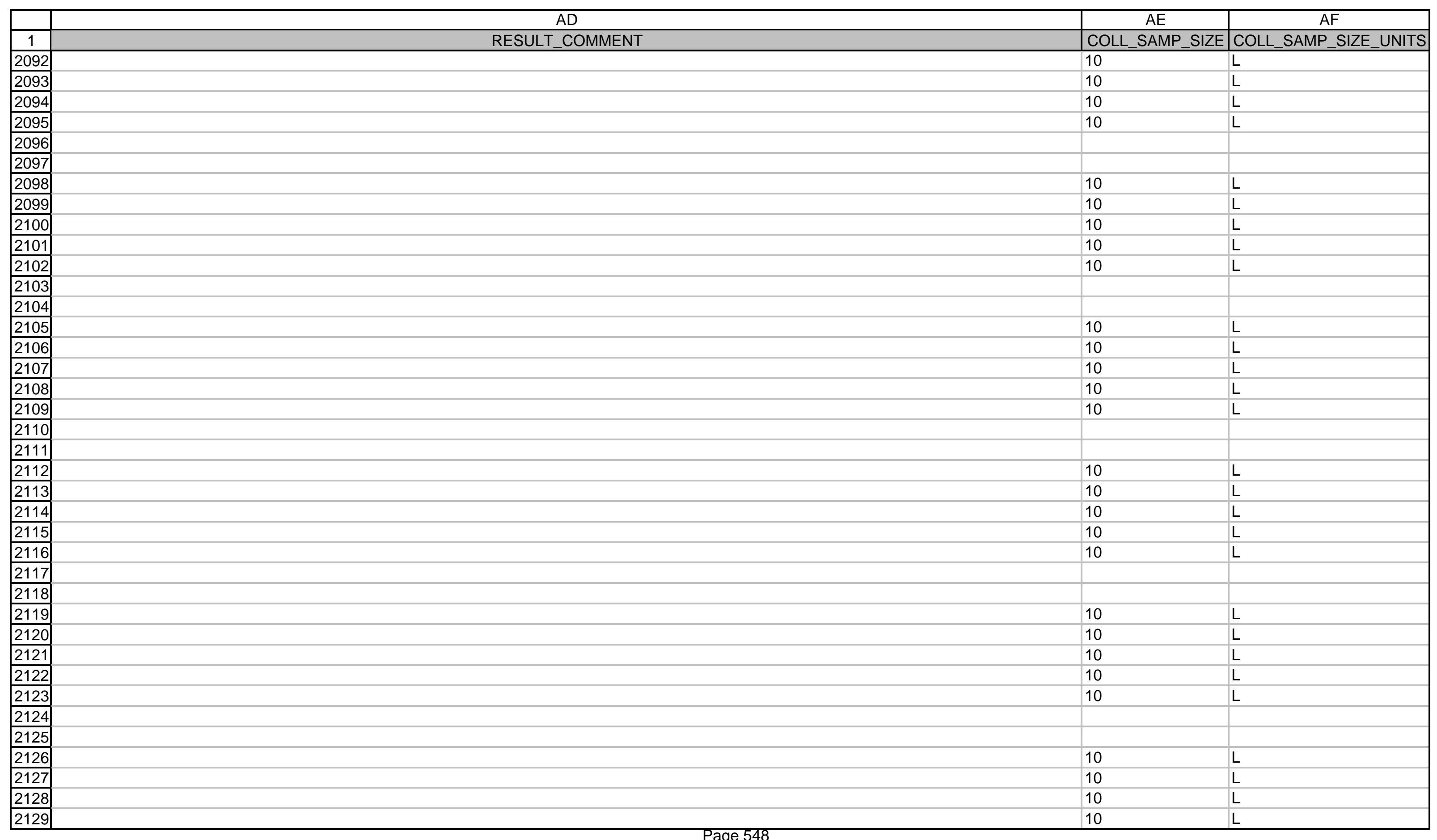




\begin{tabular}{|c|c|c|c|}
\hline & $A D$ & $\mathrm{AE}$ & $\mathrm{AF}$ \\
\hline 1 & RESULT_COMMENT & COLL_SAMP_SIZE & COLL_SAMP_SIZE_UNITS \\
\hline 2130 & & 10 & $\mathrm{~L}$ \\
\hline \multicolumn{4}{|l|}{2131} \\
\hline \multicolumn{4}{|l|}{2132} \\
\hline 2133 & & 10 & L \\
\hline 2134 & & 10 & $\mathrm{~L}$ \\
\hline 2135 & & 10 & $L$ \\
\hline 2136 & & 10 & L \\
\hline 2137 & & 10 & L \\
\hline \multicolumn{4}{|l|}{2138} \\
\hline \multicolumn{4}{|l|}{2139} \\
\hline 2140 & & 10 & L \\
\hline 2141 & & 10 & L \\
\hline 2142 & & 10 & L \\
\hline 2143 & & 10 & L \\
\hline 2144 & & 10 & L \\
\hline \multicolumn{4}{|l|}{2145} \\
\hline \multicolumn{4}{|l|}{2146} \\
\hline 2147 & & 10 & $\mathrm{~L}$ \\
\hline 2148 & & 10 & L \\
\hline 2149 & & 10 & L \\
\hline 2150 & & 10 & L \\
\hline 2151 & & 10 & L \\
\hline \multicolumn{4}{|l|}{2152} \\
\hline \multicolumn{4}{|l|}{2153} \\
\hline 2154 & & 10 & L \\
\hline 2155 & & 10 & L \\
\hline 2156 & & 10 & L \\
\hline 2157 & & 10 & L \\
\hline 2158 & & 10 & L \\
\hline \multicolumn{4}{|l|}{2159} \\
\hline \multicolumn{4}{|l|}{2160} \\
\hline 2161 & & 10 & L \\
\hline 2162 & & 10 & L \\
\hline 2163 & & 10 & L \\
\hline 2164 & & 10 & L \\
\hline 2165 & & 10 & L \\
\hline \multicolumn{4}{|l|}{2166} \\
\hline 2167 & & & \\
\hline
\end{tabular}




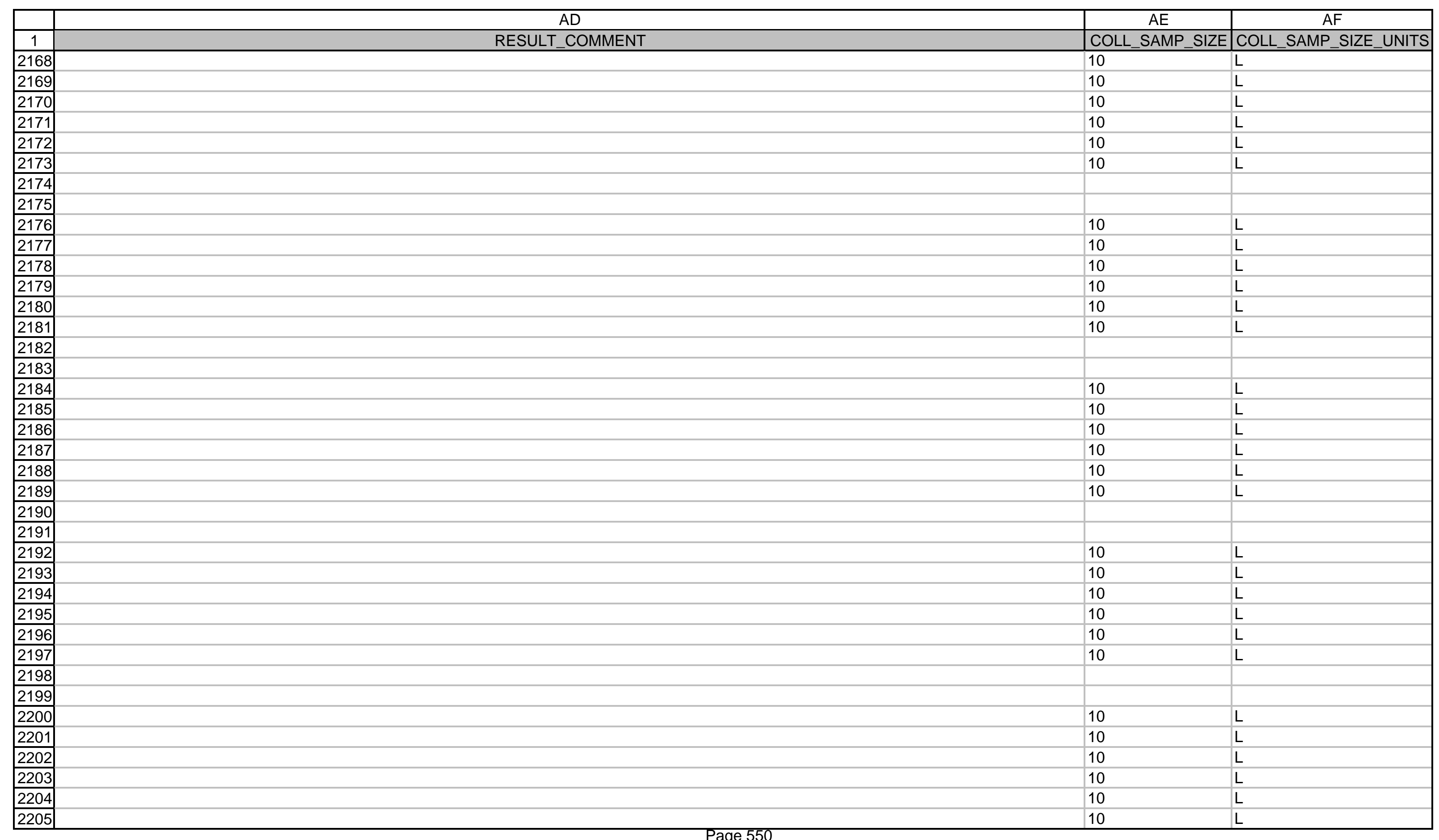




\begin{tabular}{|c|c|c|c|}
\hline & $A D$ & $\mathrm{AE}$ & AF \\
\hline 1 & RESULT_COMMENT & COLL_SAMP_SIZE & COLL_SAMP_SIZE_UNITS \\
\hline \multicolumn{4}{|l|}{2206} \\
\hline \multicolumn{4}{|l|}{2207} \\
\hline 2208 & & 10 & $\mathrm{~L}$ \\
\hline 2209 & & 10 & $\mathrm{~L}$ \\
\hline 2210 & & 10 & $\mathrm{~L}$ \\
\hline 2211 & & 10 & $\mathrm{~L}$ \\
\hline \begin{tabular}{|l|}
2212 \\
\end{tabular} & & 10 & $\bar{L}$ \\
\hline 2213 & & 10 & $\mathrm{~L}$ \\
\hline \multicolumn{4}{|l|}{2214} \\
\hline \multicolumn{4}{|l|}{2215} \\
\hline 2216 & & 10 & $\mathrm{~L}$ \\
\hline 2217 & & 10 & $\mathrm{~L}$ \\
\hline 2218 & & 10 & $\mathrm{~L}$ \\
\hline 2219 & & 10 & $\mathrm{~L}$ \\
\hline 2220 & & 10 & $\mathrm{~L}$ \\
\hline 2221 & & 10 & $\mathrm{~L}$ \\
\hline \multicolumn{4}{|l|}{\begin{tabular}{|l|}
2222 \\
222
\end{tabular}} \\
\hline \multicolumn{4}{|l|}{\begin{tabular}{|l|l|}
2223 \\
\end{tabular}} \\
\hline 2224 & & 10 & $\mathrm{~L}$ \\
\hline 2225 & & 10 & $\mathrm{~L}$ \\
\hline 2226 & & 10 & $\mathrm{~L}$ \\
\hline 2227 & & 10 & $\mathrm{~L}$ \\
\hline \begin{tabular}{|l|}
2228 \\
\end{tabular} & & 10 & $\bar{L}$ \\
\hline 2229 & & 10 & $\mathrm{~L}$ \\
\hline \multicolumn{4}{|l|}{2230} \\
\hline \multicolumn{4}{|l|}{2231} \\
\hline 2232 & & 10 & $\mathrm{~L}$ \\
\hline 2233 & & 10 & $\mathrm{~L}$ \\
\hline 2234 & & 10 & $\mathrm{~L}$ \\
\hline 2235 & & 10 & $\mathrm{~L}$ \\
\hline 2236 & & 10 & $\mathrm{~L}$ \\
\hline 2237 & & 10 & $\mathrm{~L}$ \\
\hline \multicolumn{4}{|l|}{2238} \\
\hline \multicolumn{4}{|l|}{2239} \\
\hline 2240 & & 10 & $\mathrm{~L}$ \\
\hline 2241 & & 10 & $\mathrm{~L}$ \\
\hline \begin{tabular}{|l|}
2242 \\
\end{tabular} & & 10 & $\mathrm{~L}$ \\
\hline 2243 & & 10 & $\mathrm{~L}$ \\
\hline
\end{tabular}




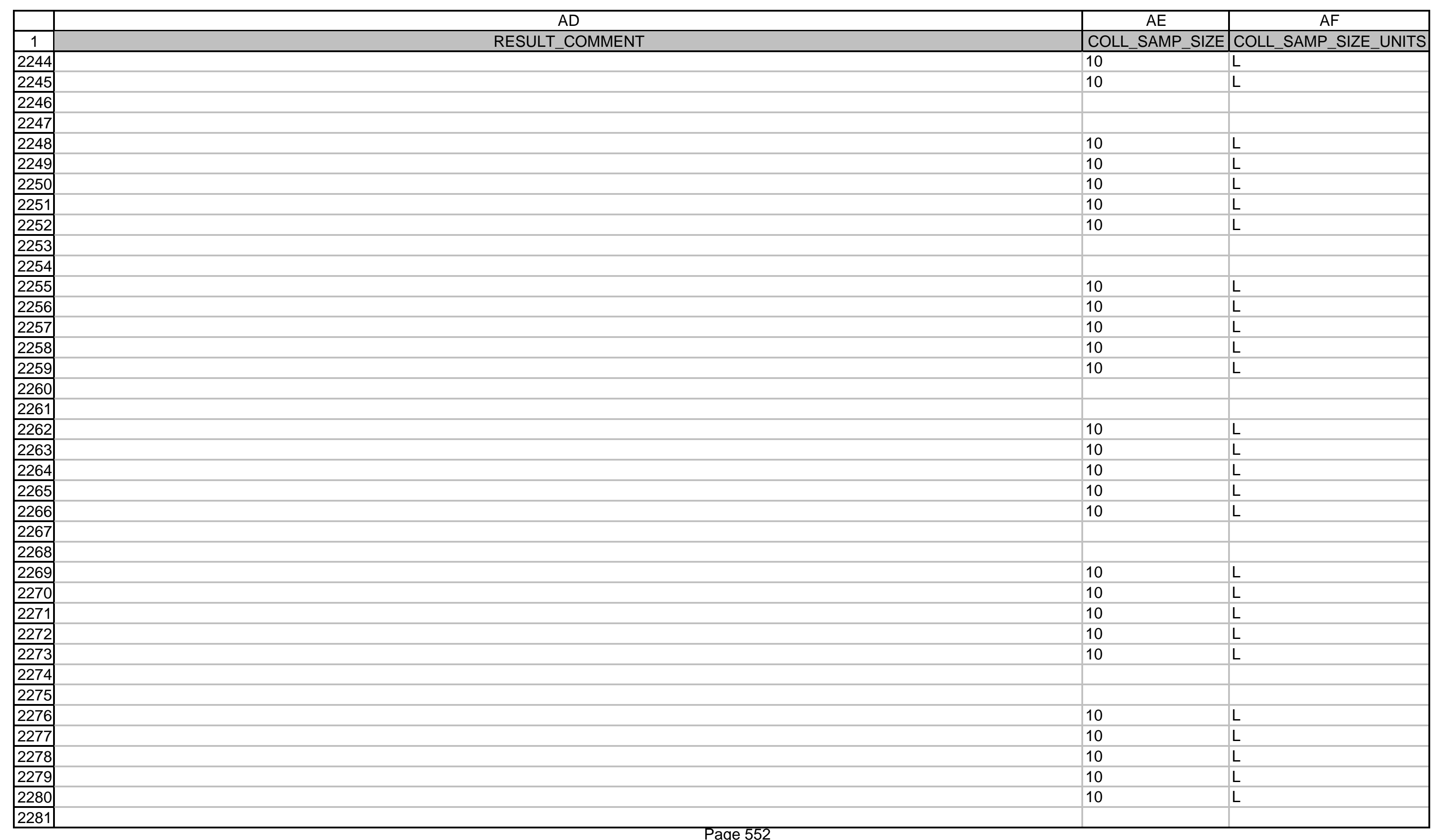




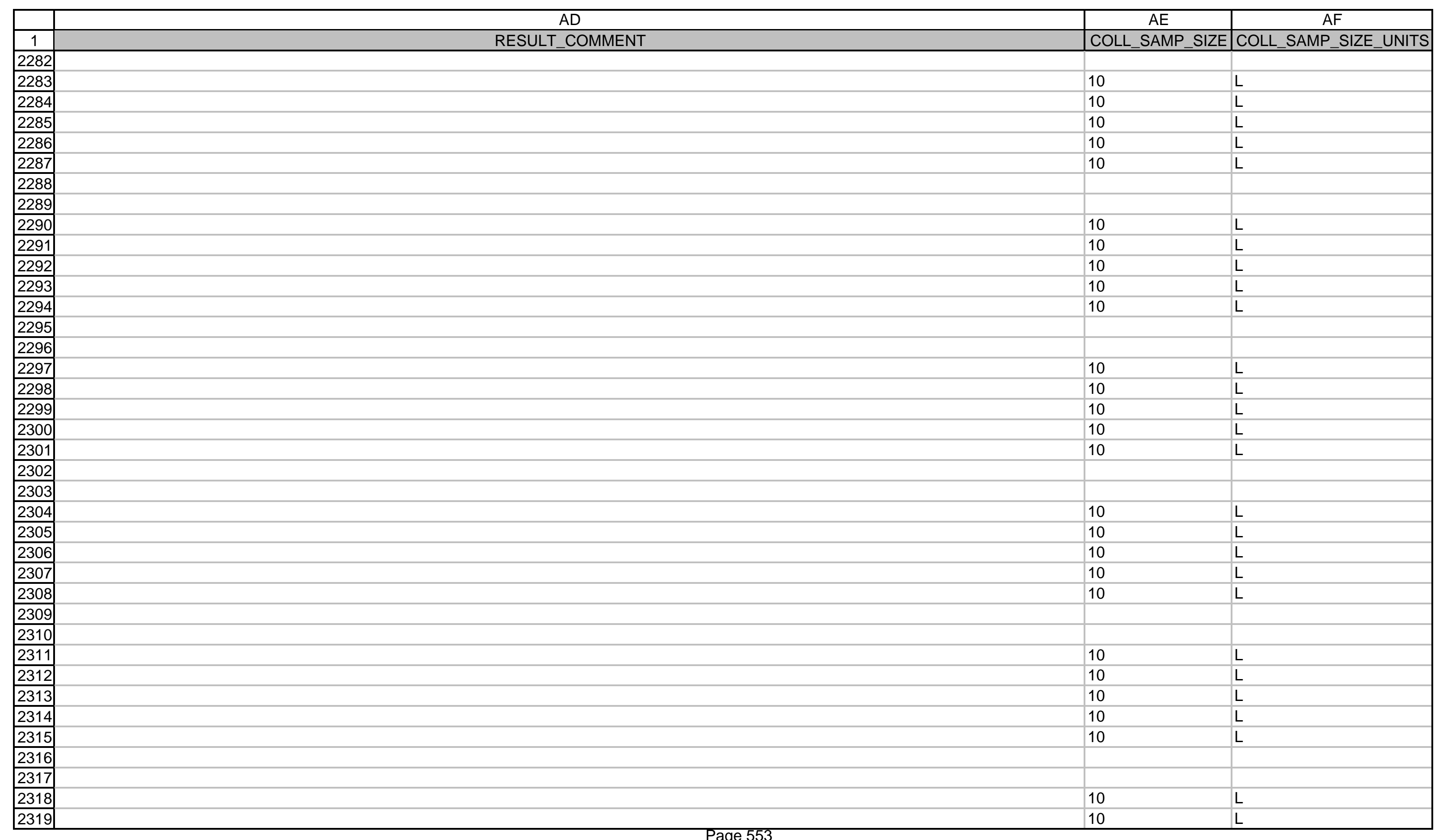




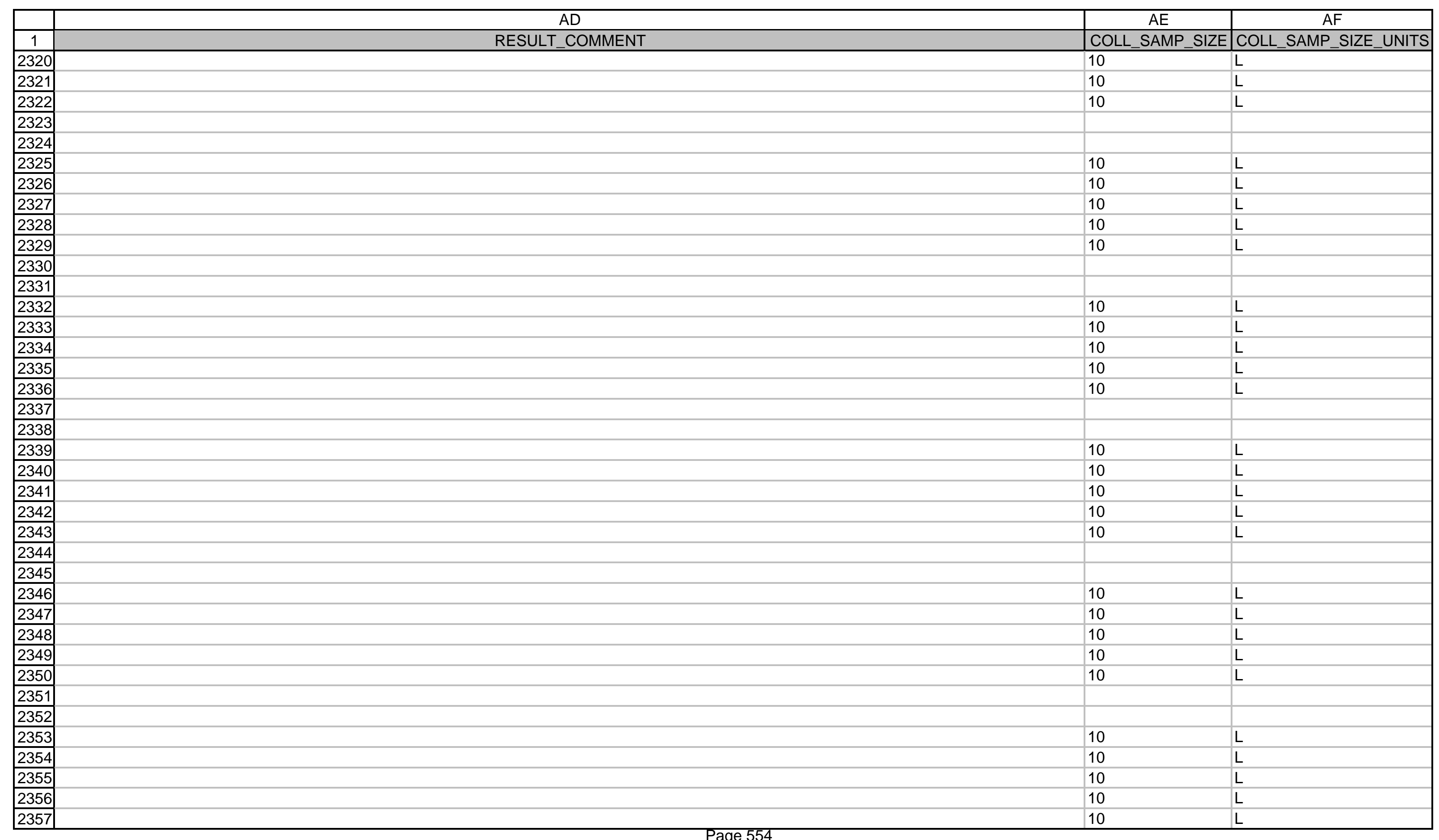




\begin{tabular}{|c|c|c|c|}
\hline & $A D$ & $\mathrm{AE}$ & $\mathrm{AF}$ \\
\hline 1 & RESULT_COMMENT & $\mid$ COLL_SAMP_SIZE & COLL_SAMP_SIZE_UNITS \\
\hline \multicolumn{4}{|c|}{ - } \\
\hline \multicolumn{4}{|l|}{2359} \\
\hline \begin{tabular}{|l|}
2360 \\
\end{tabular} & & 10 & L \\
\hline \begin{tabular}{|l|}
2361 \\
\end{tabular} & & 10 & L \\
\hline \begin{tabular}{|l|}
2362 \\
\end{tabular} & & 10 & $\mathrm{~L}$ \\
\hline \begin{tabular}{|l|}
2363 \\
\end{tabular} & & 10 & L \\
\hline \begin{tabular}{|l|}
2364 \\
\end{tabular} & & 10 & $\mathrm{~L}$ \\
\hline \multicolumn{4}{|l|}{2365} \\
\hline \multicolumn{4}{|l|}{2366} \\
\hline \begin{tabular}{|l|}
2367 \\
\end{tabular} & & 10 & L \\
\hline \begin{tabular}{|l|}
2368 \\
\end{tabular} & & 10 & $L$ \\
\hline 2369 & & 10 & L \\
\hline 2370 & & 10 & $\mathrm{~L}$ \\
\hline \begin{tabular}{|l|}
2371 \\
\end{tabular} & & 10 & L \\
\hline \multicolumn{4}{|l|}{2372} \\
\hline \multicolumn{4}{|l|}{2373} \\
\hline \begin{tabular}{|l|}
2374 \\
\end{tabular} & & 10 & L \\
\hline \begin{tabular}{|l|}
2375 \\
\end{tabular} & & 10 & L \\
\hline \begin{tabular}{|l|}
2376 \\
\end{tabular} & & 10 & L \\
\hline \begin{tabular}{|l|}
2377 \\
\end{tabular} & & 10 & L \\
\hline \begin{tabular}{|l|}
2378 \\
\end{tabular} & & 10 & L \\
\hline \multicolumn{4}{|l|}{2379} \\
\hline \multicolumn{4}{|l|}{2380} \\
\hline \begin{tabular}{|l|}
2381 \\
\end{tabular} & & 10 & L \\
\hline \begin{tabular}{|l|}
2382 \\
\end{tabular} & & 10 & L \\
\hline \begin{tabular}{|l|}
2383 \\
\end{tabular} & & 10 & L \\
\hline \begin{tabular}{|l|}
2384 \\
\end{tabular} & & 10 & L \\
\hline \begin{tabular}{|l|}
2385 \\
\end{tabular} & & 10 & L \\
\hline \multicolumn{4}{|l|}{2386} \\
\hline \multicolumn{4}{|l|}{2387} \\
\hline \begin{tabular}{|l|}
2388 \\
\end{tabular} & REFER TO 1/24/96 INCIDENT REPORT. SAMPLE HAD CHEMICAL YIELD LESS THAN 20 PERCENT. & 10 & L \\
\hline 2389 & & 10 & L \\
\hline \begin{tabular}{|c|c|}
2390 \\
\end{tabular} & & 10 & L \\
\hline 2391 & & 10 & L \\
\hline 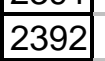 & & 10 & L \\
\hline \multicolumn{4}{|l|}{2393} \\
\hline \multicolumn{4}{|l|}{$\frac{2004}{2394}$} \\
\hline 2395 & 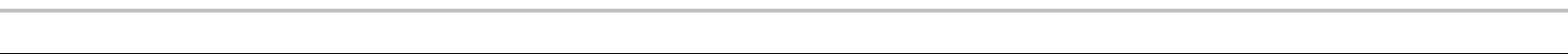 & 10 & L \\
\hline
\end{tabular}




\begin{tabular}{|c|c|c|c|}
\hline & $A D$ & $\mathrm{AE}$ & $\mathrm{AF}$ \\
\hline 1 & RESULT_COMMENT & COLL_SAMP_SIZE & COLL_SAMP_SIZE_UNITS \\
\hline 2396 & & 10 & $\mathrm{~L}$ \\
\hline 2397 & & 10 & L \\
\hline 2398 & & 10 & L \\
\hline 2399 & & 10 & L \\
\hline \multicolumn{4}{|l|}{2400} \\
\hline \multicolumn{4}{|l|}{2401} \\
\hline 2402 & REFER TO 1/24/96 INCIDENT REPORT. SAMPLE HAD CHEMICAL YIELD LESS THAN 20 PERCENT. & 10 & L \\
\hline 2403 & & 10 & L \\
\hline 2404 & & 10 & L \\
\hline 2405 & & 10 & L \\
\hline 2406 & & 10 & L \\
\hline \multicolumn{4}{|l|}{2407} \\
\hline \multicolumn{4}{|l|}{2408} \\
\hline 2409 & & 10 & L \\
\hline 2410 & & 10 & L \\
\hline 2411 & & 10 & L \\
\hline 2412 & & 10 & L \\
\hline 2413 & & 10 & L \\
\hline \multicolumn{4}{|l|}{2414} \\
\hline \multicolumn{4}{|l|}{2415} \\
\hline 2416 & REFER TO 1/24/96 INCIDENT REPORT, RESULT LOST AT LAB. & 10 & L \\
\hline 2417 & & 10 & L \\
\hline 2418 & & 10 & L \\
\hline 2419 & & 10 & L \\
\hline 2420 & & 10 & $\mathrm{~L}$ \\
\hline \multicolumn{4}{|l|}{\begin{tabular}{|l|}
2421 \\
\end{tabular}} \\
\hline \multicolumn{4}{|l|}{\begin{tabular}{|l|}
2422 \\
\end{tabular}} \\
\hline 2423 & & 10 & L \\
\hline 2424 & & 10 & L \\
\hline 2425 & & 10 & L \\
\hline 2426 & & 10 & L \\
\hline 2427 & & 10 & $\mathrm{~L}$ \\
\hline \multicolumn{4}{|l|}{\begin{tabular}{|l|}
2428 \\
\end{tabular}} \\
\hline \multicolumn{4}{|l|}{2429} \\
\hline 2430 & & 10 & L \\
\hline 2431 & & 10 & $\mathrm{~L}$ \\
\hline \begin{tabular}{|l|}
2432 \\
\end{tabular} & & 10 & L \\
\hline 2433 & 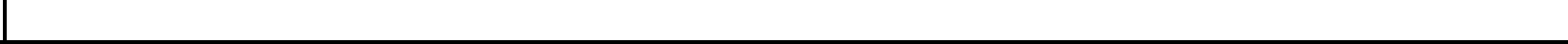 & 10 & $\mathrm{~L}$ \\
\hline
\end{tabular}




\begin{tabular}{|c|c|c|c|}
\hline & $A D$ & $\mathrm{AE}$ & AF \\
\hline 1 & RESULT_COMMENT & COLL_SAMP_SIZE & COLL_SAMP_SIZE_UNITS \\
\hline 2434 & & 10 & $L$ \\
\hline \multicolumn{4}{|l|}{2435} \\
\hline \multicolumn{4}{|l|}{2436} \\
\hline 2437 & & 10 & L \\
\hline 2438 & & 10 & $\mathrm{~L}$ \\
\hline 2439 & & 10 & L \\
\hline 2440 & & 10 & L \\
\hline 2441 & & 10 & $\mathrm{~L}$ \\
\hline \multicolumn{4}{|c|}{2442} \\
\hline \multicolumn{4}{|l|}{2443} \\
\hline 2444 & & 10 & L \\
\hline 2445 & & 10 & L \\
\hline 2446 & & 10 & $\mathrm{~L}$ \\
\hline 2447 & & 10 & $\mathrm{~L}$ \\
\hline 2448 & & 10 & L \\
\hline \multicolumn{4}{|c|}{\begin{tabular}{|l|}
2449 \\
\end{tabular}} \\
\hline \multicolumn{4}{|c|}{\begin{tabular}{|l|}
2450 \\
\end{tabular}} \\
\hline 2451 & & 10 & $\mathrm{~L}$ \\
\hline 2452 & & 10 & L \\
\hline 2453 & & 10 & L \\
\hline 2454 & & 10 & L \\
\hline 2455 & & 10 & $\mathrm{~L}$ \\
\hline \multicolumn{4}{|c|}{2456} \\
\hline \multicolumn{4}{|l|}{2457} \\
\hline 2458 & & 10 & L \\
\hline 2459 & & 10 & L \\
\hline 2460 & & 10 & L \\
\hline 2461 & & 10 & L \\
\hline 2462 & & 10 & L \\
\hline \multicolumn{4}{|c|}{\begin{tabular}{|l|}
2463 \\
\end{tabular}} \\
\hline \multicolumn{4}{|c|}{\begin{tabular}{|l|}
2464 \\
\end{tabular}} \\
\hline 2465 & REFER TO 1/24/96 INCIDENT REPORT. SAMPLE HAD CHEMICAL YIELD LESS THAN 20 PERCENT. & 10 & L \\
\hline 2466 & & 10 & $\mathrm{~L}$ \\
\hline 2467 & & 10 & $\mathrm{~L}$ \\
\hline 2468 & & 10 & L \\
\hline 2469 & & 10 & L \\
\hline \multicolumn{4}{|c|}{2470} \\
\hline 2471 & 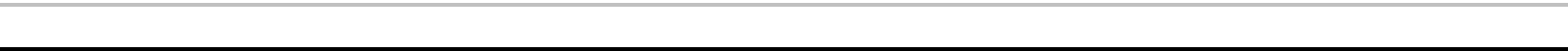 & & \\
\hline
\end{tabular}




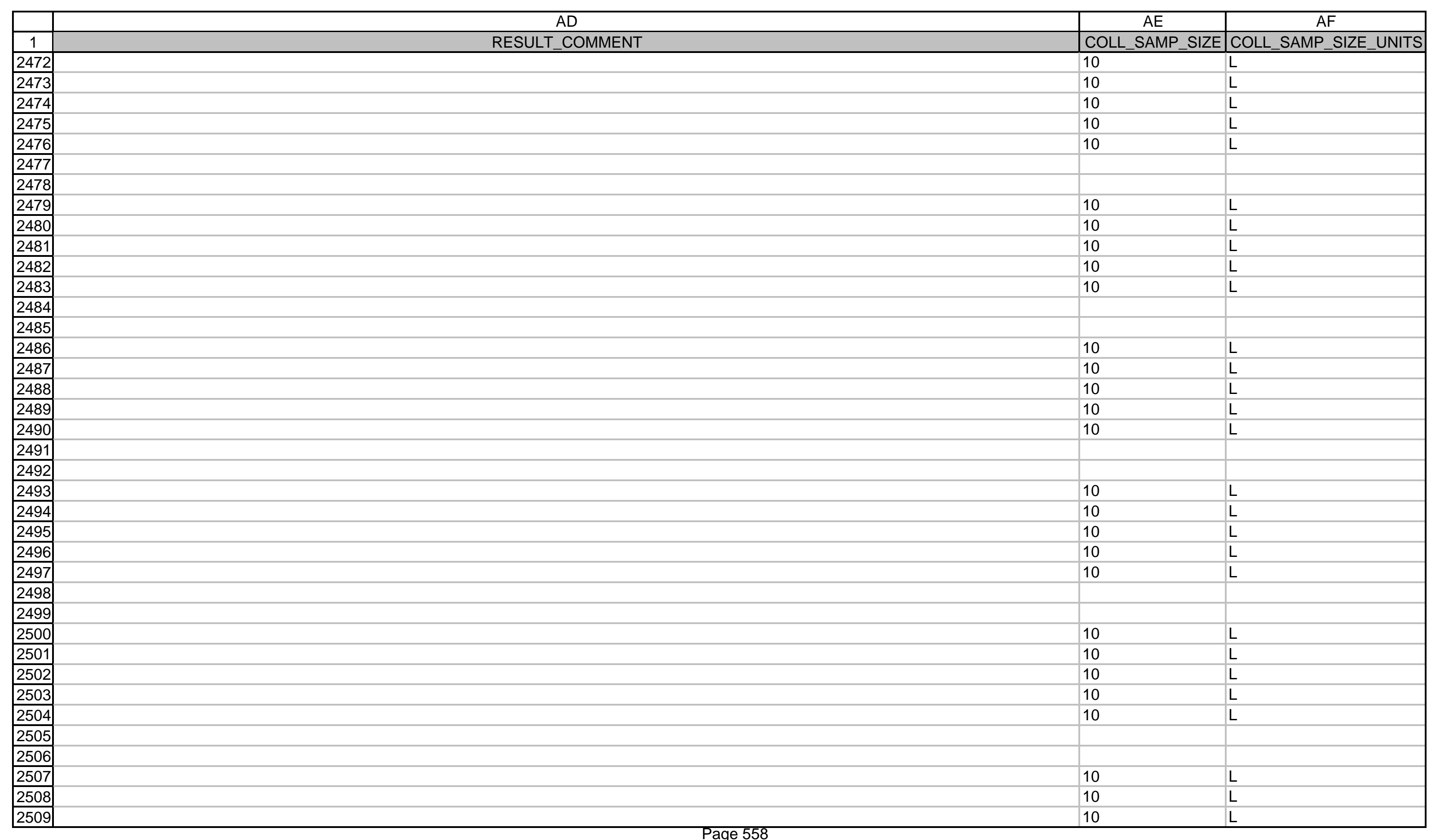




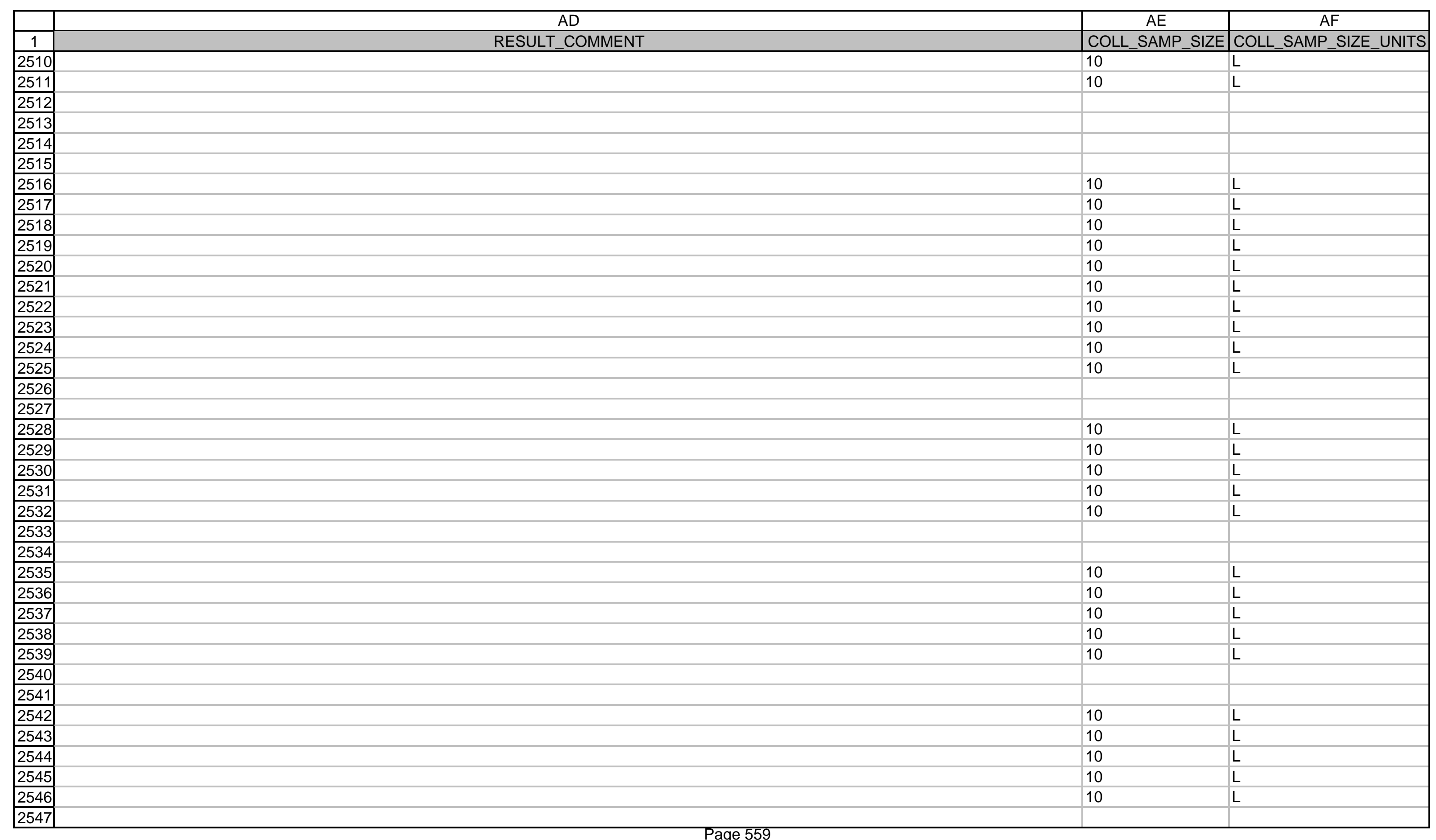




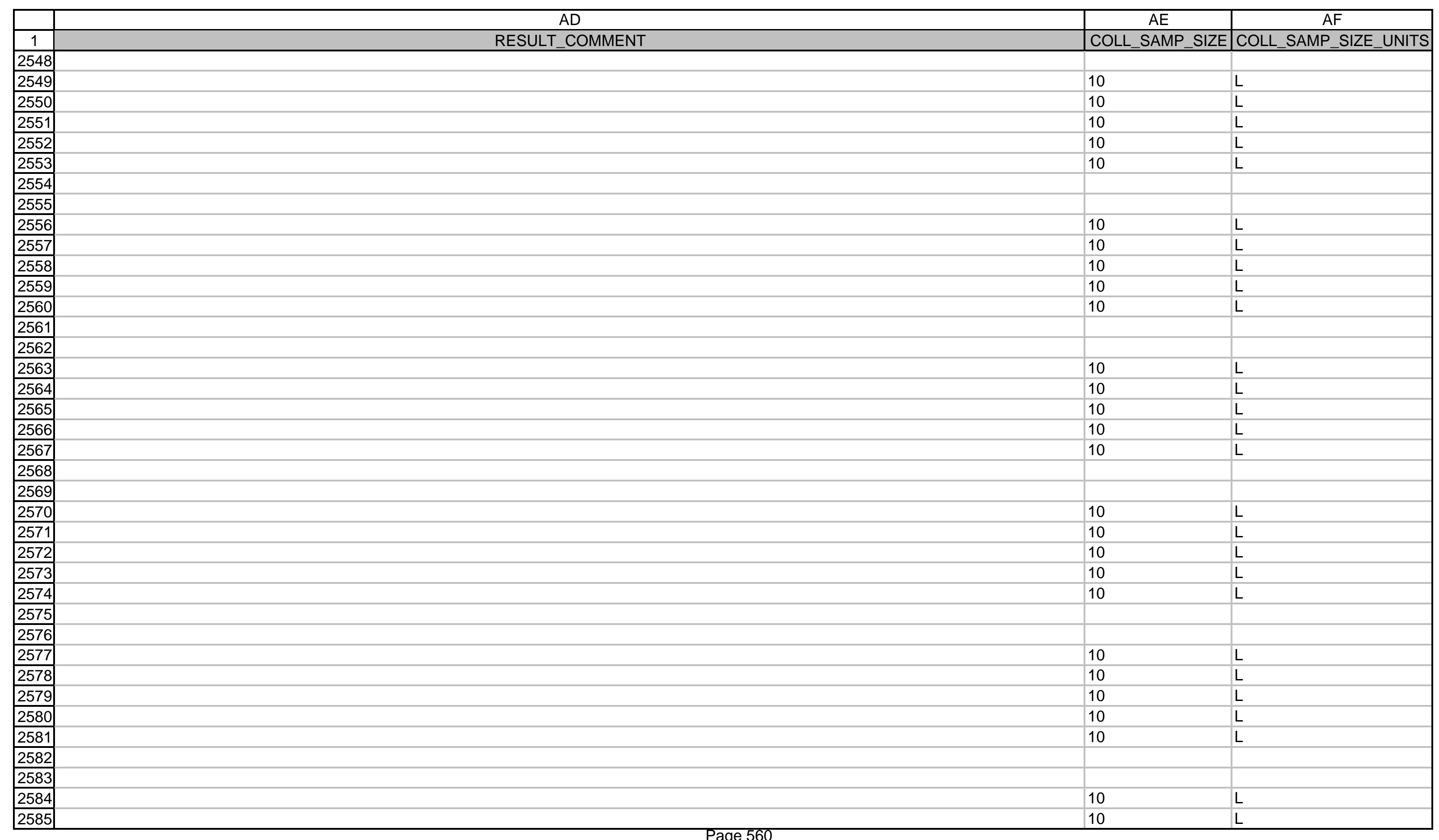




\begin{tabular}{|c|c|c|c|}
\hline & $A D$ & $\mathrm{AE}$ & AF \\
\hline 1 & RESULT_COMMENT & COLL_SAMP_SIZE & COLL_SAMP_SIZE_UNITS \\
\hline 2586 & & 10 & $\mathrm{~L}$ \\
\hline 2587 & & 10 & $\mathrm{~L}$ \\
\hline 2588 & & 10 & $\mathrm{~L}$ \\
\hline \multicolumn{4}{|l|}{\begin{tabular}{|l|}
2589 \\
\end{tabular}} \\
\hline \multicolumn{4}{|l|}{\begin{tabular}{|l|}
2590 \\
\end{tabular}} \\
\hline 2591 & & 10 & $\mathrm{~L}$ \\
\hline 2592 & & 10 & L \\
\hline 2593 & & 10 & $\mathrm{~L}$ \\
\hline 2594 & & 10 & $\mathrm{~L}$ \\
\hline 2595 & & 10 & $\bar{L}$ \\
\hline \multicolumn{4}{|l|}{\begin{tabular}{|l|}
2596 \\
\end{tabular}} \\
\hline \multicolumn{4}{|l|}{2597} \\
\hline 2598 & & 10 & $\mathrm{~L}$ \\
\hline 2599 & & 10 & $\mathrm{~L}$ \\
\hline 2600 & & 10 & $\mathrm{~L}$ \\
\hline 2601 & & 10 & $\mathrm{~L}$ \\
\hline 2602 & & 10 & $\mathrm{~L}$ \\
\hline \multicolumn{4}{|l|}{2603} \\
\hline \multicolumn{4}{|l|}{2604} \\
\hline 2605 & & 10 & $\mathrm{~L}$ \\
\hline 2606 & & 10 & $\mathrm{~L}$ \\
\hline 2607 & & 10 & $\mathrm{~L}$ \\
\hline \begin{tabular}{|l|}
2608 \\
\end{tabular} & & 10 & $\bar{L}$ \\
\hline 2609 & & 10 & $\mathrm{~L}$ \\
\hline \multicolumn{4}{|l|}{2610} \\
\hline \multicolumn{4}{|l|}{2611} \\
\hline 2612 & & 10 & $\mathrm{~L}$ \\
\hline 2613 & & 10 & $\mathrm{~L}$ \\
\hline 2614 & & 10 & $\mathrm{~L}$ \\
\hline 2615 & & 10 & L \\
\hline 2616 & & 10 & $\mathrm{~L}$ \\
\hline \multicolumn{4}{|l|}{2617} \\
\hline \multicolumn{4}{|l|}{2618} \\
\hline 2619 & & 10 & $\mathrm{~L}$ \\
\hline 2620 & & 10 & $\mathrm{~L}$ \\
\hline 2621 & & 10 & $\mathrm{~L}$ \\
\hline 2622 & & 10 & $\mathrm{~L}$ \\
\hline 2623 & & 10 & $\mathrm{~L}$ \\
\hline
\end{tabular}




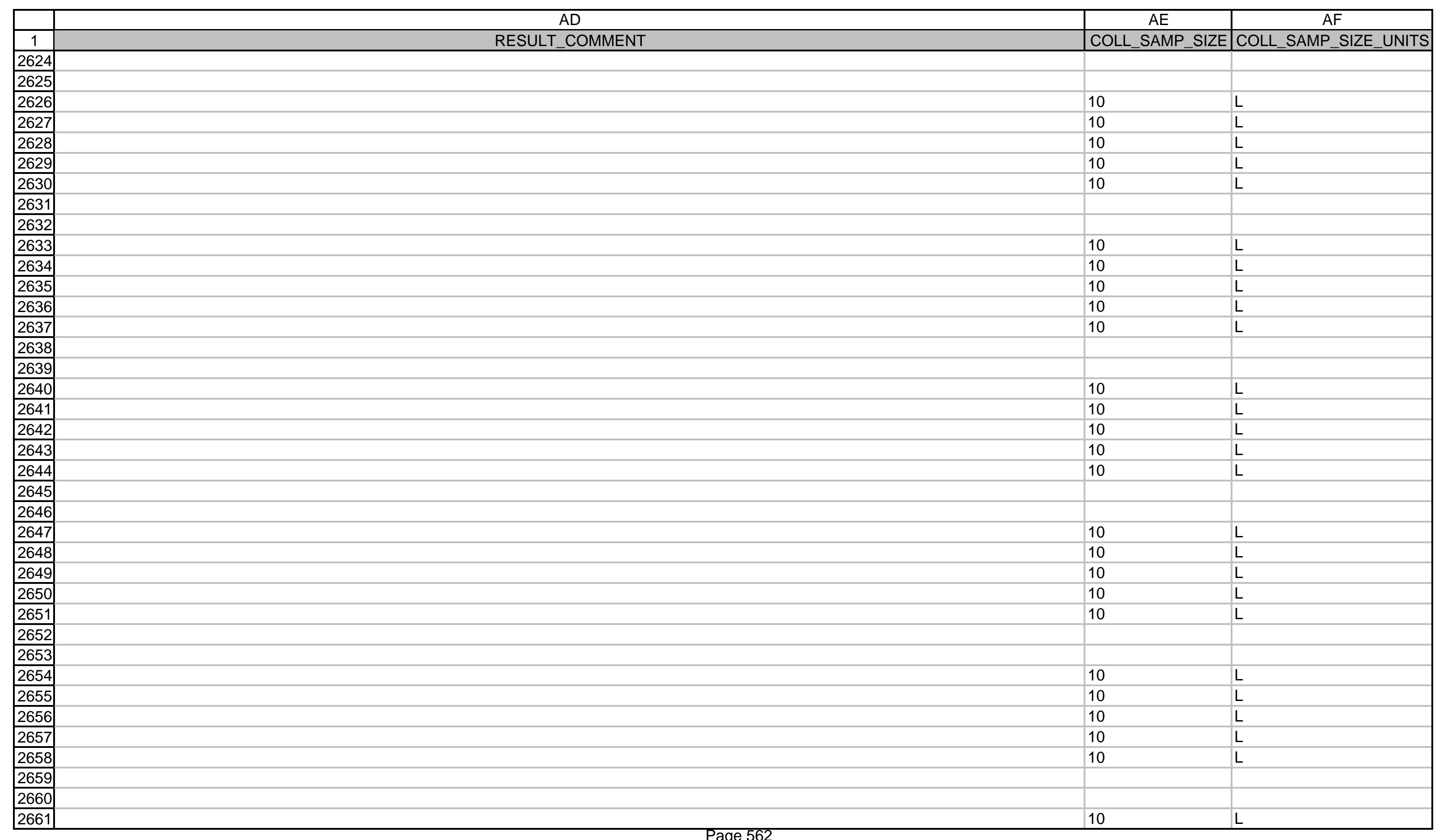




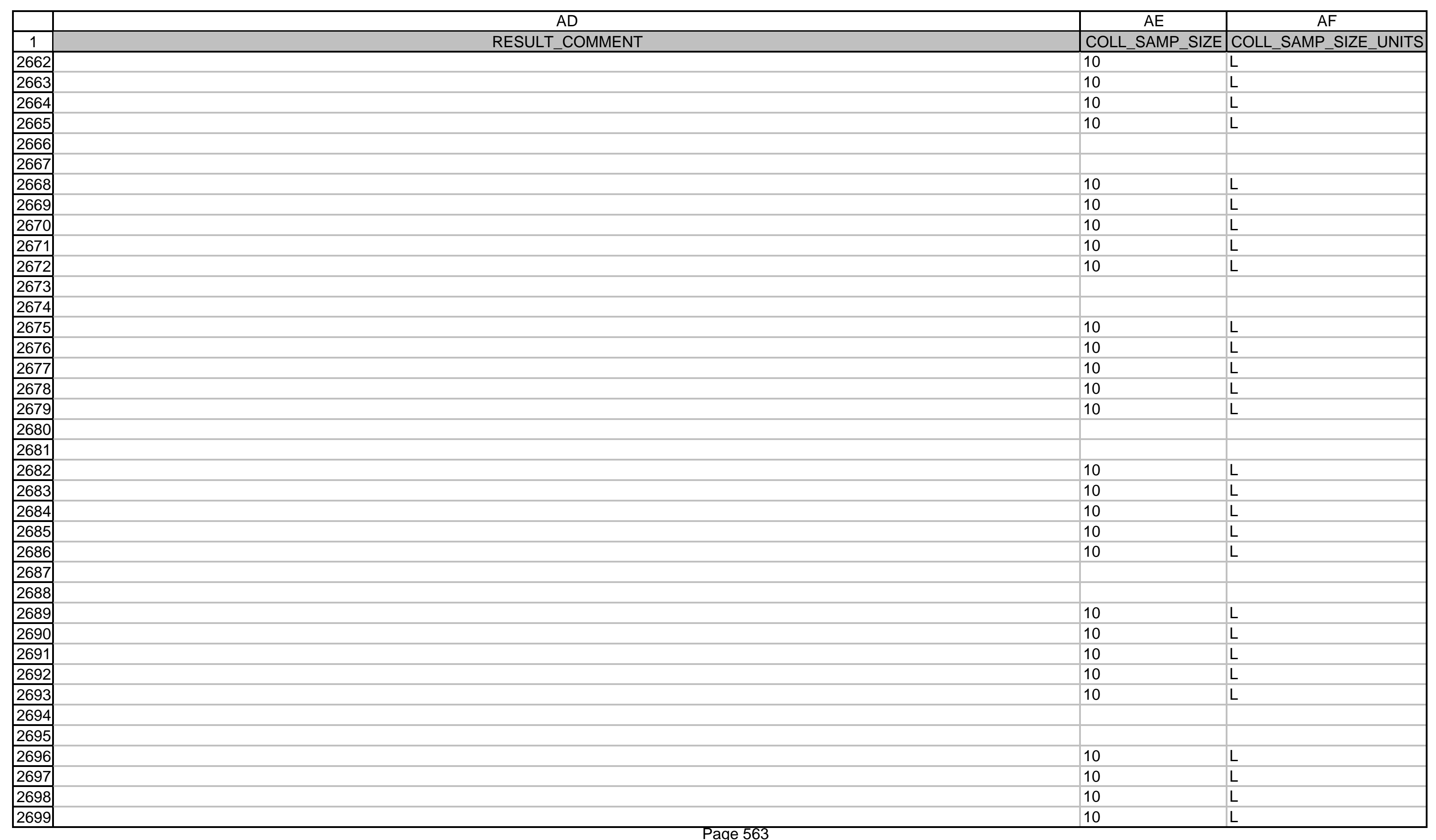




\begin{tabular}{|c|c|c|c|}
\hline & $A D$ & $\mathrm{AE}$ & $\mathrm{AF}$ \\
\hline 1 & RESULT_COMMENT & COLL_SAMP_SIZE & COLL_SAMP_SIZE_UNITS \\
\hline 2700 & & 10 & $\mathrm{~L}$ \\
\hline \multicolumn{4}{|l|}{2701} \\
\hline \multicolumn{4}{|l|}{2702} \\
\hline 2703 & & 10 & L \\
\hline 2704 & & 10 & $\mathrm{~L}$ \\
\hline 2705 & & 10 & L \\
\hline 2706 & & 10 & L \\
\hline 2707 & & 10 & L \\
\hline \multicolumn{4}{|l|}{2708} \\
\hline \multicolumn{4}{|l|}{2709} \\
\hline 2710 & & 10 & L \\
\hline 2711 & & 10 & L \\
\hline 2712 & & 10 & L \\
\hline 2713 & & 10 & L \\
\hline 2714 & & 10 & L \\
\hline \multicolumn{4}{|l|}{2715} \\
\hline \multicolumn{4}{|l|}{2716} \\
\hline 2717 & & 10 & L \\
\hline 2718 & & 10 & L \\
\hline 2719 & & 10 & $L$ \\
\hline 2720 & & 10 & L \\
\hline 2721 & & 10 & L \\
\hline \multicolumn{4}{|l|}{2722} \\
\hline \multicolumn{4}{|l|}{2723} \\
\hline 2724 & & 10 & L \\
\hline 2725 & & 10 & L \\
\hline 2726 & & 10 & L \\
\hline 2727 & & 10 & L \\
\hline 2728 & & 10 & L \\
\hline \multicolumn{4}{|l|}{2729} \\
\hline \multicolumn{4}{|l|}{2730} \\
\hline 2731 & & 10 & L \\
\hline 2732 & & 10 & L \\
\hline 2733 & & 10 & L \\
\hline 2734 & & 10 & L \\
\hline 2735 & & 10 & L \\
\hline \multicolumn{4}{|l|}{2736} \\
\hline 2737 & & & \\
\hline
\end{tabular}




\begin{tabular}{|c|c|c|c|}
\hline & 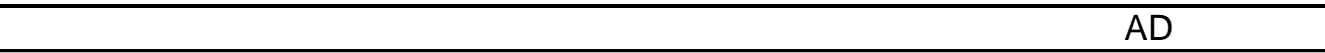 & $\mathrm{AE}$ & $\mathrm{AF}$ \\
\hline 1 & $\begin{array}{ll} & \text { RESULT_COMMENT } \\
\end{array}$ & COLL_SAMP_SIZE & EOLL_SAMP_SIZE_UNITS \\
\hline 2738 & & 10 & $\mathrm{~L}$ \\
\hline 2739 & 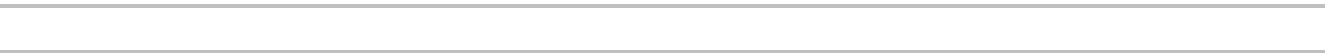 & 10 & $\mathrm{~L}$ \\
\hline 2740 & 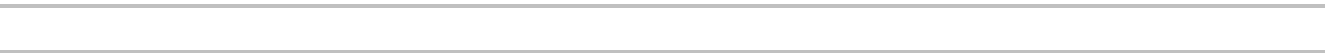 & 10 & $\mathrm{~L}$ \\
\hline 2741 & & 10 & $\mathrm{~L}$ \\
\hline 2742 & & 10 & $\mathrm{~L}$ \\
\hline \multicolumn{4}{|l|}{2743} \\
\hline \multicolumn{4}{|l|}{2744} \\
\hline 2745 & & 10 & L \\
\hline \begin{tabular}{|l|}
2746 \\
\end{tabular} & & 10 & $L$ \\
\hline 2747 & & 10 & $L$ \\
\hline \begin{tabular}{|l|}
2748 \\
\end{tabular} & & 10 & L \\
\hline \begin{tabular}{|l|}
2749 \\
\end{tabular} & & 10 & L \\
\hline \multicolumn{4}{|l|}{2750} \\
\hline \multicolumn{4}{|l|}{2751} \\
\hline \begin{tabular}{|l|}
2752 \\
\end{tabular} & . & 10 & L \\
\hline \begin{tabular}{|l|}
2753 \\
\end{tabular} & 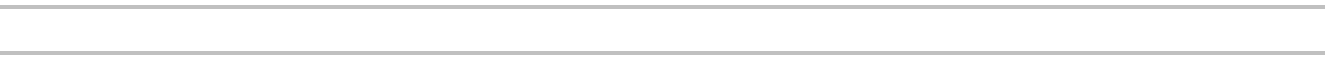 & 10 & L \\
\hline 2754 & & 10 & L \\
\hline \begin{tabular}{|l|}
2755 \\
\end{tabular} & & 10 & L \\
\hline \begin{tabular}{|l|}
2756 \\
\end{tabular} & & 10 & $L$ \\
\hline \multicolumn{4}{|l|}{2757} \\
\hline \multicolumn{4}{|l|}{\begin{tabular}{|l|}
2758 \\
\end{tabular}} \\
\hline \begin{tabular}{|l|}
2759 \\
\end{tabular} & & 10 & $L$ \\
\hline 2760 & & 10 & $L$ \\
\hline \begin{tabular}{|l|}
2761 \\
\end{tabular} & & 10 & L \\
\hline \begin{tabular}{|l|}
2762 \\
\end{tabular} & & 10 & $L$ \\
\hline \begin{tabular}{|l|}
2763 \\
\end{tabular} & & 10 & L \\
\hline \multicolumn{4}{|l|}{2764} \\
\hline \multicolumn{4}{|l|}{2765} \\
\hline \begin{tabular}{|l|}
2766 \\
\end{tabular} & & 10 & L \\
\hline \begin{tabular}{|l|}
2767 \\
\end{tabular} & & 10 & L \\
\hline \begin{tabular}{|l|}
2768 \\
\end{tabular} & & 10 & $L$ \\
\hline \begin{tabular}{|l|}
2769 \\
\end{tabular} & & 10 & $L$ \\
\hline 2770 & & 10 & L \\
\hline \multicolumn{4}{|l|}{2771} \\
\hline \multicolumn{4}{|l|}{\begin{tabular}{|l|}
2772 \\
\end{tabular}} \\
\hline \begin{tabular}{|l|}
2773 \\
\end{tabular} & & 10 & L \\
\hline \begin{tabular}{|l|}
2774 \\
\end{tabular} & & 10 & $L$ \\
\hline 2775 & 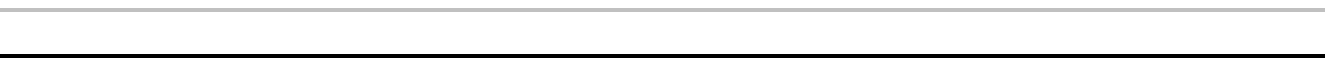 & 10 & $\mathrm{~L}$ \\
\hline
\end{tabular}




\begin{tabular}{|c|c|c|c|}
\hline & $A D$ & $\mathrm{AE}$ & $\mathrm{AF}$ \\
\hline 1 & RESULT_COMMENT & COLL_SAMP_SIZE & COLL_SAMP_SIZE_UNITS \\
\hline 2776 & & 10 & $\mathrm{~L}$ \\
\hline 2777 & & 10 & L \\
\hline \multicolumn{4}{|l|}{2778} \\
\hline \multicolumn{4}{|l|}{2779} \\
\hline 2780 & & 10 & $\mathrm{~L}$ \\
\hline 2781 & & 10 & L \\
\hline 2782 & & 10 & L \\
\hline 2783 & & 10 & L \\
\hline 2784 & & 10 & L \\
\hline \multicolumn{4}{|l|}{2785} \\
\hline \multicolumn{4}{|l|}{2786} \\
\hline 2787 & & 10 & L \\
\hline 2788 & & 10 & L \\
\hline 2789 & & 10 & L \\
\hline 2790 & & 10 & L \\
\hline 2791 & & 10 & L \\
\hline \multicolumn{4}{|l|}{2792} \\
\hline \multicolumn{4}{|l|}{2793} \\
\hline 2794 & & 10 & L \\
\hline 2795 & & 10 & $\mathrm{~L}$ \\
\hline 2796 & & 10 & L \\
\hline 2797 & & 10 & L \\
\hline 2798 & & 10 & L \\
\hline \multicolumn{4}{|l|}{2799} \\
\hline \multicolumn{4}{|l|}{2800} \\
\hline 2801 & & 10 & L \\
\hline 2802 & & 10 & $\mathrm{~L}$ \\
\hline 2803 & & 10 & L \\
\hline 2804 & & 10 & L \\
\hline 2805 & & 10 & L \\
\hline \multicolumn{4}{|l|}{2806} \\
\hline \multicolumn{4}{|l|}{2807} \\
\hline 2808 & & 10 & L \\
\hline 2809 & & 10 & L \\
\hline 2810 & & 10 & L \\
\hline 2811 & & 10 & L \\
\hline 2812 & & 10 & L \\
\hline 2813 & & & \\
\hline
\end{tabular}




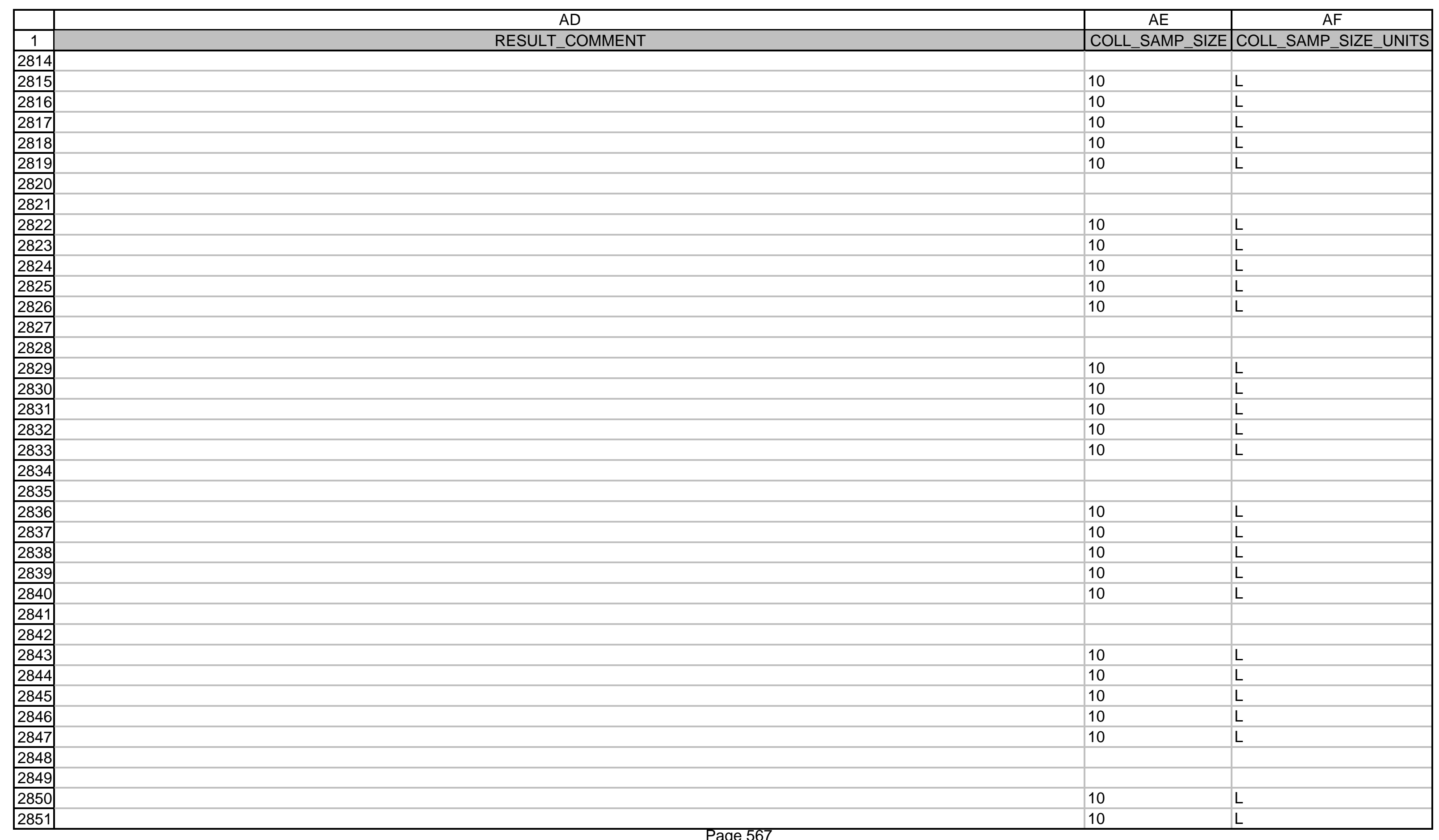




\begin{tabular}{|c|c|c|c|}
\hline & & & \\
\hline 1 & RESULT_COMMENT & COLL_SAMP_SIZE & COLL_SAMP_SIZE_UNITS \\
\hline 2852 & & 10 & L \\
\hline 2853 & & 10 & L \\
\hline 2854 & & 10 & L \\
\hline \multicolumn{4}{|c|}{2855} \\
\hline \multicolumn{4}{|l|}{2856} \\
\hline 2857 & REFER TO 1/23/97 AND 1/24/97 INCIDENT REPORTS. EXPIRED TRACER USED FOR SR-90 ANALYSIS AND RESULT LOST AT LAB. & 10 & L \\
\hline 2858 & REFER TO 1/23/97 INCIDENT REPORT. EXPIRED TRACER USED FOR SR-90 ANALYSIS. & 10 & L \\
\hline 2859 & & 10 & $\mathrm{~L}$ \\
\hline 2860 & & 10 & L \\
\hline 2861 & & 10 & L \\
\hline 2862 & & 10 & L \\
\hline 2863 & & 10 & L \\
\hline 2864 & & 10 & $\mathrm{~L}$ \\
\hline 2865 & & 10 & L \\
\hline 2866 & & 10 & L \\
\hline \multicolumn{4}{|l|}{2867} \\
\hline \multicolumn{4}{|l|}{2868} \\
\hline 2869 & REFER TO 1/23/97 INCIDENT REPORT. EXPIRED TRACER USED FOR SR-90 ANALYSIS. & 10 & L \\
\hline 2870 & & 10 & $\mathrm{~L}$ \\
\hline 2871 & & 10 & L \\
\hline 2872 & & 10 & $\mathrm{~L}$ \\
\hline 2873 & & 10 & L \\
\hline \multicolumn{4}{|l|}{2874} \\
\hline \multicolumn{4}{|l|}{2875} \\
\hline 2876 & REFER TO 1/23/97 INCIDENT REPORT. EXPIRED TRACER USED FOR SR-90 ANALYSIS. & 10 & L \\
\hline 2877 & & 10 & L \\
\hline 2878 & & 10 & L \\
\hline 2879 & & 10 & $\mathrm{~L}$ \\
\hline 2880 & & 10 & L \\
\hline \multicolumn{4}{|l|}{ 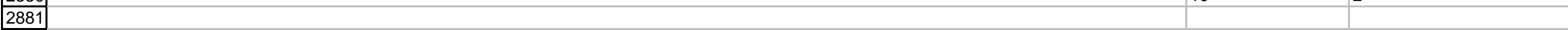 } \\
\hline \multicolumn{4}{|l|}{2882} \\
\hline 2883 & REFER TO 1/23/97 INCIDENT REPORT. EXPIRED TRACER USED FOR SR-90 ANALYSIS. & 10 & L \\
\hline 2884 & & 10 & L \\
\hline 2885 & & 10 & L \\
\hline 2886 & & 10 & L \\
\hline 2887 & & 10 & L \\
\hline 2888 & & & \\
\hline \begin{tabular}{|l|}
2889 \\
\end{tabular} & & & \\
\hline
\end{tabular}


2897 REFER TO 1/23/97 INCIDENT REPORT. EXPIRED TRACER USED FOR SR-90 ANALYSIS.

\begin{tabular}{|l|l|}
2898 & SAMPLE WAS NOT ANALYZED FOR ENRICHED TRITIUM. \\
\hline 2899 &
\end{tabular}

\begin{tabular}{|l|l|l}
2904 & REFER TO 1/23/97 INCIDENT REPORT. EXPIRED TRACER USED FOR SR-90 ANALYSIS.
\end{tabular}

\begin{tabular}{|l|l}
2911 & REFER TO 1/23/97 INCIDENT REPORT. EXPIRED TRACER USED FOR SR-90 ANALYSIS.
\end{tabular}

REFER TO 1/23/97 INCIDENT REPORT. EXPIRED TRACER USED FOR SR-90 ANALYSIS.

10

10

10

\begin{tabular}{|l|l|}
\hline & 10 \\
\hline & 10 \\
10 \\
\hline
\end{tabular}

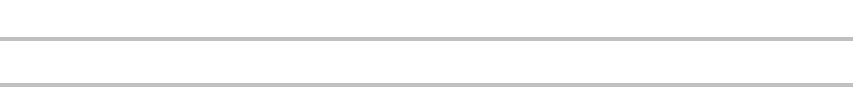$$
L
$$$$
\text { L }
$$$$
\text { L }
$$$$
\text { L }
$$$$
\text { L }
$$$$
\text { - }
$$$$
\text { L }
$$

\begin{tabular}{|l|l}
\hline & $L$ \\
\hline & $L$ \\
\hline & $L$ \\
\hline & $L$ \\
\hline & $L$ \\
\hline & $L$ \\
\hline
\end{tabular}

\begin{tabular}{l}
\hline \\
\hline \\
\hline \\
\hline \\
\hline \\
\hline \\
\hline$L$ \\
\hline$L$ \\
\hline$L$ \\
\hline$L$ \\
\hline \\
\hline \\
\hline \\
\hline \\
\hline
\end{tabular}




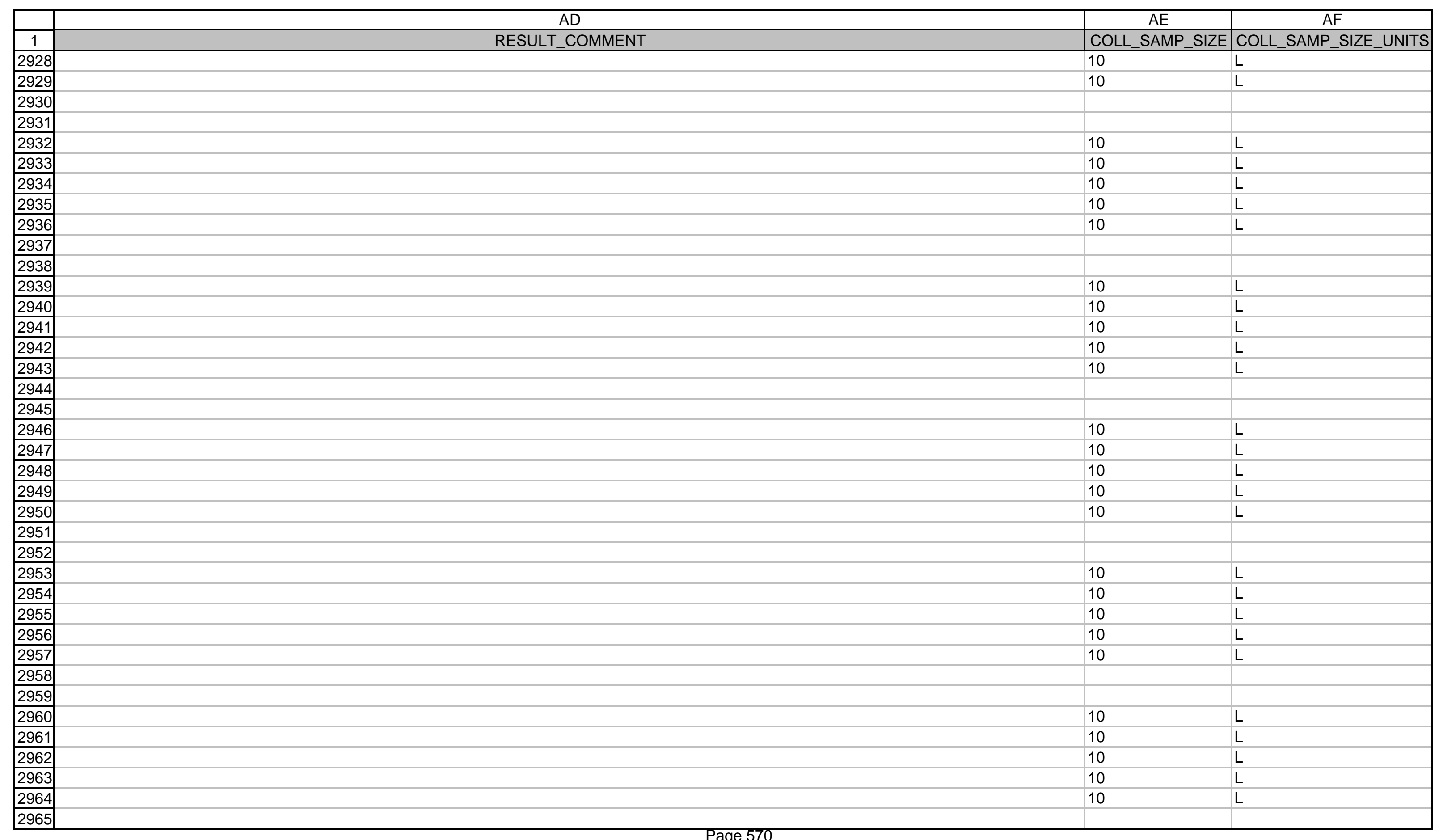




\begin{tabular}{|c|c|c|c|}
\hline & +2 & $\mathrm{AE}$ & $\mathrm{AF}$ \\
\hline 1 & $\begin{array}{lc} & \text { RESULT_COMMENT } \\
\end{array}$ & COLL_SAMP_SIZE & COLL_SAMP_SIZE_UNITS \\
\hline \multicolumn{4}{|c|}{ 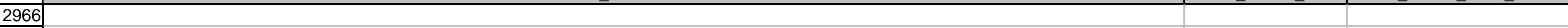 } \\
\hline 2967 & & 10 & $\mathrm{~L}$ \\
\hline 2968 & & 10 & $\mathrm{~L}$ \\
\hline 2969 & I & 10 & $\mathrm{~L}$ \\
\hline 2970 & 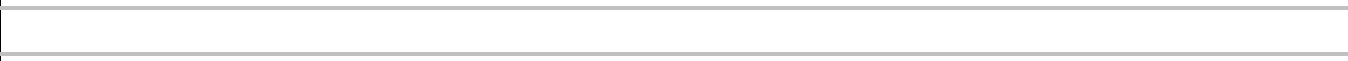 & 10 & $\mathrm{~L}$ \\
\hline 2971 & 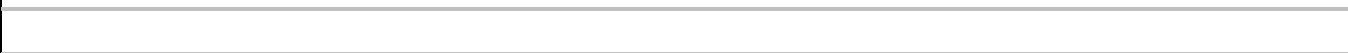 & 10 & $\mathrm{~L}$ \\
\hline \multicolumn{4}{|l|}{2972} \\
\hline \multicolumn{4}{|l|}{2973} \\
\hline 2974 & & 10 & L \\
\hline 2975 & & 10 & L \\
\hline 2976 & & 10 & L \\
\hline 2977 & & 10 & L \\
\hline 2978 & & 10 & L \\
\hline \multicolumn{4}{|l|}{2979} \\
\hline \multicolumn{4}{|l|}{2980} \\
\hline 2981 & & 10 & L \\
\hline 2982 & 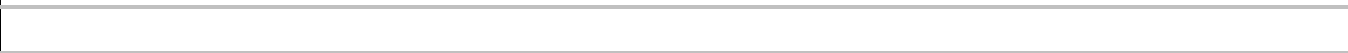 & 10 & L \\
\hline 2983 & & 10 & L \\
\hline 2984 & & 10 & L \\
\hline 2985 & & 10 & L \\
\hline \multicolumn{4}{|l|}{2986} \\
\hline \multicolumn{4}{|l|}{2987} \\
\hline 2988 & & 10 & $L$ \\
\hline 2989 & & 10 & L \\
\hline 2990 & & 10 & L \\
\hline 2991 & & 10 & L \\
\hline 2992 & & 10 & L \\
\hline \multicolumn{4}{|l|}{2993} \\
\hline \multicolumn{4}{|l|}{2994} \\
\hline 2995 & & 10 & L \\
\hline 2996 & & 10 & L \\
\hline 2997 & & 10 & L \\
\hline 2998 & & 10 & L \\
\hline 2999 & & 10 & L \\
\hline \multicolumn{4}{|l|}{3000} \\
\hline \multicolumn{4}{|l|}{3001} \\
\hline 3002 & & 10 & L \\
\hline 3003 & 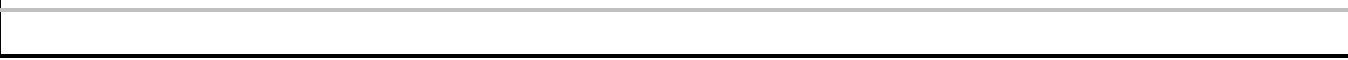 & 10 & $\mathrm{~L}$ \\
\hline
\end{tabular}




\begin{tabular}{|c|c|c|}
\hline & $A D$ & $\mathrm{AF}$ \\
\hline 1 & RESULT_COMMENT & COLL_SAMP_SIZE_UNITS \\
\hline 3004 & & $\mathrm{~L}$ \\
\hline 3005 & & L \\
\hline 3006 & & L \\
\hline \multicolumn{3}{|l|}{3007} \\
\hline \multicolumn{3}{|l|}{3008} \\
\hline 3009 & & L \\
\hline 3010 & & L \\
\hline 3011 & 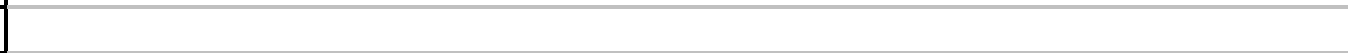 & L \\
\hline 3012 & 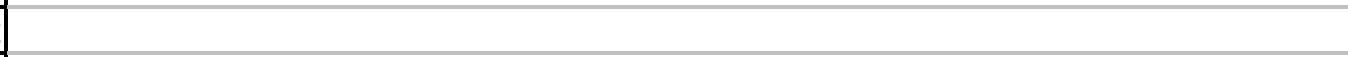 & L \\
\hline 3013 & & L \\
\hline \multicolumn{3}{|l|}{3014} \\
\hline \multicolumn{3}{|l|}{3015} \\
\hline 3016 & & $\mathrm{~L}$ \\
\hline 3017 & & $\mathrm{~L}$ \\
\hline 3018 & & L \\
\hline 3019 & & L \\
\hline 3020 & 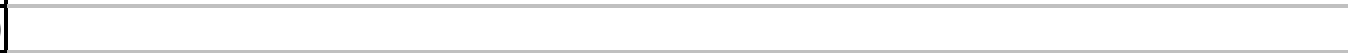 & L \\
\hline \multicolumn{3}{|l|}{3021} \\
\hline \multicolumn{3}{|l|}{3022} \\
\hline 3023 & & L \\
\hline 3024 & & L \\
\hline 3025 & & L \\
\hline 3026 & & L \\
\hline 3027 & & $\mathrm{~L}$ \\
\hline \multicolumn{3}{|l|}{\begin{tabular}{|l|}
3028 \\
\end{tabular}} \\
\hline \multicolumn{3}{|l|}{\begin{tabular}{|l|}
3029 \\
\end{tabular}} \\
\hline 3030 & & $\mathrm{~L}$ \\
\hline 3031 & & L \\
\hline 3032 & & L \\
\hline 3033 & & L \\
\hline 3034 & & $\mathrm{~L}$ \\
\hline \multicolumn{3}{|l|}{3035} \\
\hline \multicolumn{3}{|l|}{\begin{tabular}{|l|}
3036 \\
\end{tabular}} \\
\hline 3037 & & $\mathrm{~L}$ \\
\hline 3038 & & L \\
\hline 3039 & & L \\
\hline 3040 & & $L$ \\
\hline 3041 & $x_{0}^{-1}$ & $\mathrm{~L}$ \\
\hline
\end{tabular}




\begin{tabular}{|c|c|c|c|}
\hline & $A D$ & $A E$ & $\mathrm{AF}$ \\
\hline 1 & RESULT_COMMENT & COLL_SAMP_SIZE & COLL_SAMP_SIZE_UNITS \\
\hline \multicolumn{4}{|l|}{3042} \\
\hline \multicolumn{4}{|c|}{\begin{tabular}{|l|}
3043 \\
\end{tabular}} \\
\hline 3044 & & 10 & L \\
\hline 3045 & & 10 & $\mathrm{~L}$ \\
\hline 3046 & & 10 & L \\
\hline 3047 & & 10 & L \\
\hline 3048 & & 10 & L \\
\hline \multicolumn{4}{|l|}{3049} \\
\hline \multicolumn{4}{|l|}{3050} \\
\hline 3051 & & 10 & L \\
\hline 3052 & & 10 & L \\
\hline 3053 & & 10 & $\mathrm{~L}$ \\
\hline 3054 & & 10 & $\mathrm{~L}$ \\
\hline 3055 & & 10 & $\mathrm{~L}$ \\
\hline \multicolumn{4}{|l|}{3056} \\
\hline \multicolumn{4}{|l|}{3057} \\
\hline 3058 & & 10 & L \\
\hline 3059 & & 10 & L \\
\hline 3060 & & 10 & L \\
\hline 3061 & & 10 & L \\
\hline 3062 & & 10 & $\mathrm{~L}$ \\
\hline \multicolumn{4}{|l|}{3063} \\
\hline \multicolumn{4}{|l|}{3064} \\
\hline 3065 & & 10 & L \\
\hline 3066 & & 10 & L \\
\hline 3067 & & 10 & $\mathrm{~L}$ \\
\hline 3068 & & 10 & $\mathrm{~L}$ \\
\hline 3069 & & 10 & L \\
\hline \multicolumn{4}{|l|}{3070} \\
\hline \multicolumn{4}{|l|}{3071} \\
\hline 3072 & REANALYZED RESULTS REPORTED ON 5/23/97, DATABASE MODIFIED PER TASK MANAGER. & 20 & $\mathrm{~L}$ \\
\hline 3073 & & 20 & L \\
\hline 3074 & & 20 & $\mathrm{~L}$ \\
\hline 3075 & & 20 & $\mathrm{~L}$ \\
\hline 3076 & & 20 & $\mathrm{~L}$ \\
\hline \multicolumn{4}{|l|}{3077} \\
\hline \multicolumn{4}{|l|}{3078} \\
\hline 3079 & REANALYZED RESULTS REPORTED ON 5/23/97, DATABASE MODIFIED PER TASK MANAGER. & 20 & $\mathrm{~L}$ \\
\hline
\end{tabular}




\begin{tabular}{|c|c|c|c|}
\hline & $A D$ & $\mathrm{AE}$ & $\mathrm{AF}$ \\
\hline 1 & RESULT_COMMENT & COLL_SAMP_SIZE & COLL_SAMP_SIZE_UNITS \\
\hline 3080 & & $20=$ & L \\
\hline 3081 & & 20 & $\mathrm{~L}$ \\
\hline 3082 & & 20 & $\mathrm{~L}$ \\
\hline 3083 & & 20 & L \\
\hline \multicolumn{4}{|l|}{3084} \\
\hline \multicolumn{4}{|l|}{3085} \\
\hline 3086 & REANALYZED RESULTS REPORTED ON 5/23/97, DATABASE MODIFIED PER TASK MANAGER. & 20 & $\mathrm{~L}$ \\
\hline 3087 & & 20 & L \\
\hline 3088 & & 20 & $\mathrm{~L}$ \\
\hline 3089 & & 20 & L \\
\hline 3090 & & 20 & L \\
\hline \multicolumn{4}{|l|}{3091} \\
\hline \multicolumn{4}{|l|}{3092} \\
\hline 3093 & REANALYZED RESULTS REPORTED ON 5/23/97, DATABASE MODIFIED PER TASK MANAGER. & 20 & L \\
\hline 3094 & & 20 & $\mathrm{~L}$ \\
\hline 3095 & & 20 & L \\
\hline 3096 & & 20 & $\mathrm{~L}$ \\
\hline 3097 & & 20 & $\mathrm{~L}$ \\
\hline \multicolumn{4}{|l|}{3099} \\
\hline 3100 & REANALYZED RESULTS REPORTED ON 5/23/97, DATABASE MODIFIED PER TASK MANAGER. & 20 & $\mathrm{~L}$ \\
\hline 3101 & & 20 & $\mathrm{~L}$ \\
\hline 3102 & & 20 & L \\
\hline 3103 & & 20 & L \\
\hline 3104 & & 20 & $\mathrm{~L}$ \\
\hline 3105 & & 20 & $\mathrm{~L}$ \\
\hline 3106 & & 20 & $\mathrm{~L}$ \\
\hline 3107 & & 20 & $\mathrm{~L}$ \\
\hline 3108 & & 20 & $\mathrm{~L}$ \\
\hline 3109 & & 20 & $\mathrm{~L}$ \\
\hline \multicolumn{4}{|l|}{3110} \\
\hline \multicolumn{4}{|l|}{3111} \\
\hline 3112 & REANALYZED RESULTS REPORTED ON 5/23/97, DATABASE MODIFIED PER TASK MANAGER. & 20 & $\mathrm{~L}$ \\
\hline 3113 & & 20 & $\mathrm{~L}$ \\
\hline 3114 & & 20 & $\mathrm{~L}$ \\
\hline 3115 & & 20 & L \\
\hline 3116 & & 20 & L \\
\hline
\end{tabular}




\begin{tabular}{|c|c|c|c|}
\hline & $\mathrm{AD}$ & $\mathrm{AE}$ & $\mathrm{AF}$ \\
\hline 1 & RESULT_COMMENT & COLL_SAMP_SIZE & COLL_SAMP_SIZE_UNITS \\
\hline \multicolumn{4}{|l|}{3118} \\
\hline 3119 & REANALYZED RESULTS REPORTED ON 5/23/97, DATABASE MODIFIED PER TASK MANAGER. & 20 & $\mathrm{~L}$ \\
\hline 3120 & & 20 & $\mathrm{~L}$ \\
\hline 3121 & & 20 & L \\
\hline 3122 & & 20 & L \\
\hline 3123 & & 20 & L \\
\hline \multicolumn{4}{|l|}{\begin{tabular}{|l|l|}
3124 \\
\end{tabular}} \\
\hline \multicolumn{4}{|l|}{\begin{tabular}{|l|l|}
3125 \\
\end{tabular}} \\
\hline 3126 & REFER TO FOLLOW-UP REQUEST \#W037 REGARDING REANALYSIS. DATABASE MODIFIED PER TASK MANAGER. & 20 & $\mathrm{~L}$ \\
\hline 3127 & & 20 & L \\
\hline 3128 & & 20 & L \\
\hline 3129 & & 20 & $\mathrm{~L}$ \\
\hline 3130 & & 20 & $\mathrm{~L}$ \\
\hline \multicolumn{4}{|l|}{3131} \\
\hline \multicolumn{4}{|l|}{3132} \\
\hline 3133 & REANALYZED RESULTS REPORTED ON 5/23/97, DATABASE MODIFIED PER TASK MANAGER. & 20 & $\mathrm{~L}$ \\
\hline 3134 & & 20 & L \\
\hline 3135 & & 20 & $\mathrm{~L}$ \\
\hline 3136 & & 20 & $\mathrm{~L}$ \\
\hline 3137 & & 20 & L \\
\hline \multicolumn{4}{|l|}{3138} \\
\hline \multicolumn{4}{|l|}{3139} \\
\hline 3140 & REANALYZED RESULTS REPORTED ON 5/23/97, DATABASE MODIFIED PER TASK MANAGER. & 20 & $\mathrm{~L}$ \\
\hline 3141 & & 20 & $\mathrm{~L}$ \\
\hline 3142 & & 20 & $\mathrm{~L}$ \\
\hline 3143 & & 20 & L \\
\hline 3144 & & 20 & $\mathrm{~L}$ \\
\hline \multicolumn{4}{|l|}{3145} \\
\hline \multicolumn{4}{|c|}{3146} \\
\hline 3147 & REANALYZED RESULTS REPORTED ON 5/23/97, DATABASE MODIFIED PER TASK MANAGER. & 20 & $\mathrm{~L}$ \\
\hline 3148 & & 20 & $\mathrm{~L}$ \\
\hline 3149 & & 20 & L \\
\hline 3150 & & 20 & L \\
\hline 3151 & & 20 & $\mathrm{~L}$ \\
\hline \multicolumn{4}{|l|}{3152} \\
\hline \multicolumn{4}{|l|}{3153} \\
\hline 3154 & REANALYZED RESULTS REPORTED ON 5/23/97, DATABASE MODIFIED PER TASK MANAGER. & 20 & L \\
\hline 3155 & & 20 & $\mathrm{~L}$ \\
\hline
\end{tabular}




\begin{tabular}{|c|c|}
\hline $\mathrm{AE}$ & $\mathrm{AF}$ \\
\hline COLL_SAMP_SIZE & COLL_SAMP_SIZE_UNITS \\
\hline 20 & $\mathrm{~L}$ \\
\hline 20 & L \\
\hline 20 & $\mathrm{~L}$ \\
\hline & \\
\hline 20 & L \\
\hline 20 & L \\
\hline 20 & L \\
\hline 20 & L \\
\hline 20 & L \\
\hline 20 & L \\
\hline 20 & $\mathrm{~L}$ \\
\hline 20 & $\mathrm{~L}$ \\
\hline 20 & L \\
\hline 20 & L \\
\hline 20 & L \\
\hline 20 & $\mathrm{~L}$ \\
\hline 20 & L \\
\hline 20 & $\mathrm{~L}$ \\
\hline 20 & L \\
\hline 20 & L \\
\hline 20 & L \\
\hline 20 & L \\
\hline 20 & L \\
\hline 20 & L \\
\hline 20 & L \\
\hline 20 & L \\
\hline 20 & L \\
\hline 20 & L \\
\hline 20 & L \\
\hline 20 & L \\
\hline 20 & $L$ \\
\hline 20 & L \\
\hline 20 & L \\
\hline 20 & L \\
\hline 20 & $\mathrm{~L}$ \\
\hline
\end{tabular}




\begin{tabular}{|c|c|c|c|}
\hline & $A D$ & $\mathrm{AE}$ & $\mathrm{AF}$ \\
\hline 1 & RESULT_COMMENT & COLL_SAMP_SIZE & COLL_SAMP_SIZE_UNITS \\
\hline 3194 & & 20 & $\mathrm{~L}$ \\
\hline 3195 & & 20 & L \\
\hline 3196 & & 20 & L \\
\hline 3197 & & 20 & $\mathrm{~L}$ \\
\hline 3198 & & 18 & $\mathrm{~L}$ \\
\hline 3199 & 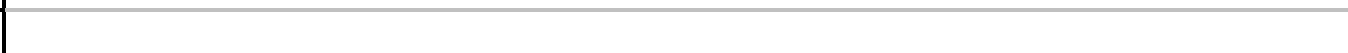 & 18 & L \\
\hline 3200 & 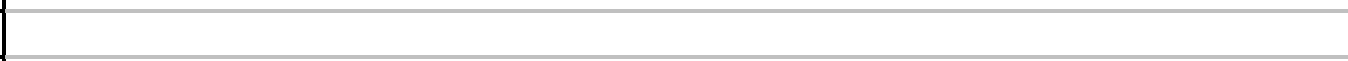 & 18 & $\mathrm{~L}$ \\
\hline 3201 & 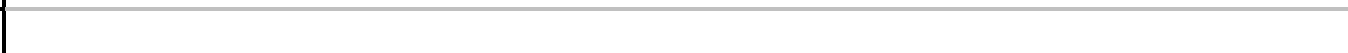 & 18 & L \\
\hline 3202 & 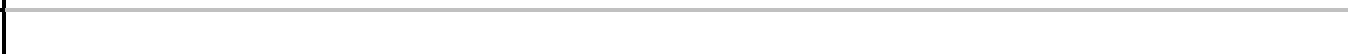 & 18 & L \\
\hline 3203 & 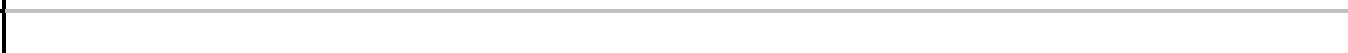 & 20 & $\mathrm{~L}$ \\
\hline 3204 & & 20 & L \\
\hline 3205 & & 20 & L \\
\hline 3206 & & 20 & L \\
\hline 3207 & & 20 & L \\
\hline 3208 & & 20 & L \\
\hline 3209 & & 20 & $\mathrm{~L}$ \\
\hline 3210 & & 20 & $\mathrm{~L}$ \\
\hline 3211 & & 20 & L \\
\hline 3212 & & 20 & L \\
\hline 3213 & & 20 & $\mathrm{~L}$ \\
\hline 3214 & & 20 & L \\
\hline 3215 & & 20 & L \\
\hline 3216 & & 20 & $\mathrm{~L}$ \\
\hline \begin{tabular}{|l|}
3217 \\
3
\end{tabular} & & 20 & $\mathrm{~L}$ \\
\hline 3218 & & 20 & $\mathrm{~L}$ \\
\hline 3219 & & 20 & L \\
\hline 3220 & & 20 & L \\
\hline 3221 & & 20 & L \\
\hline 3222 & & 20 & $\mathrm{~L}$ \\
\hline 3223 & & 20 & $\mathrm{~L}$ \\
\hline 3224 & & 20 & L \\
\hline 3225 & & 20 & $\mathrm{~L}$ \\
\hline 3226 & & 20 & $\mathrm{~L}$ \\
\hline 3227 & & 20 & L \\
\hline 3228 & & 20 & $\mathrm{~L}$ \\
\hline 3229 & & 20 & $\mathrm{~L}$ \\
\hline 3230 & & 20 & L \\
\hline 3231 & 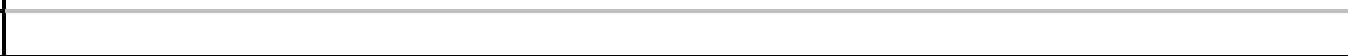 & 20 & L \\
\hline
\end{tabular}




\begin{tabular}{|c|c|c|c|}
\hline & $A D$ & $\mathrm{AE}$ & $\mathrm{AF}$ \\
\hline 1 & RESULT_COMMENT & COLL_SAMP_SIZE & COLL_SAMP_SIZE_UNITS \\
\hline 3232 & & 20 & L \\
\hline 3233 & & 20 & $\mathrm{~L}$ \\
\hline 3234 & & 20 & L \\
\hline 3235 & & 20 & $\mathrm{~L}$ \\
\hline 3236 & & 20 & $\mathrm{~L}$ \\
\hline 3237 & 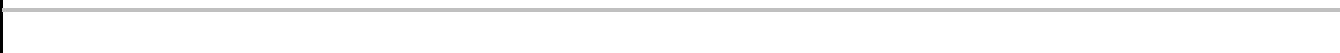 & 20 & $\mathrm{~L}$ \\
\hline 3238 & . & 20 & $\mathrm{~L}$ \\
\hline 3239 & 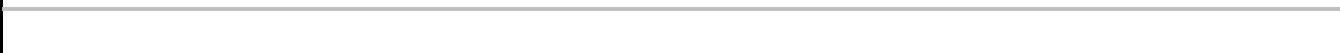 & 20 & $\mathrm{~L}$ \\
\hline 3240 & & 20 & $\mathrm{~L}$ \\
\hline 3241 & & 20 & $L$ \\
\hline 3242 & & 20 & $\mathrm{~L}$ \\
\hline 3243 & & 20 & $\mathrm{~L}$ \\
\hline 3244 & & 20 & L \\
\hline 3245 & & 20 & $\mathrm{~L}$ \\
\hline 3246 & & 20 & $\mathrm{~L}$ \\
\hline 3247 & & 20 & $\mathrm{~L}$ \\
\hline 3248 & & 20 & $\mathrm{~L}$ \\
\hline 3249 & & 20 & $\mathrm{~L}$ \\
\hline 3250 & & 20 & L \\
\hline \begin{tabular}{|l|l|}
3251 \\
\end{tabular} & & 20 & $\mathrm{~L}$ \\
\hline \begin{tabular}{|l|}
3252 \\
325
\end{tabular} & & 20 & $\mathrm{~L}$ \\
\hline 3253 & & 15 & $\mathrm{~L}$ \\
\hline 3254 & & 15 & $\mathrm{~L}$ \\
\hline \begin{tabular}{|l|}
3255 \\
\end{tabular} & & 15 & $\mathrm{~L}$ \\
\hline \begin{tabular}{|l|}
3256 \\
\end{tabular} & & 15 & L \\
\hline \begin{tabular}{|l|}
3257 \\
\end{tabular} & & 15 & L \\
\hline \begin{tabular}{|l|}
3258 \\
\end{tabular} & & 15 & $\mathrm{~L}$ \\
\hline \begin{tabular}{|l|}
3259 \\
\end{tabular} & & 15 & $\mathrm{~L}$ \\
\hline \begin{tabular}{|l|}
3260 \\
\end{tabular} & & 15 & $\mathrm{~L}$ \\
\hline \begin{tabular}{|l|}
3261 \\
\end{tabular} & & 15 & $\mathrm{~L}$ \\
\hline \begin{tabular}{|l|}
3262 \\
\end{tabular} & & 15 & $\mathrm{~L}$ \\
\hline \begin{tabular}{|l|}
3263 \\
\end{tabular} & & 15 & $\mathrm{~L}$ \\
\hline \begin{tabular}{|l|}
3264 \\
\end{tabular} & & 15 & $\mathrm{~L}$ \\
\hline \begin{tabular}{|l|}
3265 \\
\end{tabular} & & 15 & $\mathrm{~L}$ \\
\hline \begin{tabular}{|l|}
3266 \\
\end{tabular} & & 15 & $\mathrm{~L}$ \\
\hline \begin{tabular}{|l|}
3267 \\
\end{tabular} & & 15 & $\mathrm{~L}$ \\
\hline \begin{tabular}{|l|}
3268 \\
\end{tabular} & & 20 & $\mathrm{~L}$ \\
\hline \begin{tabular}{|l|}
3269 \\
\end{tabular} & 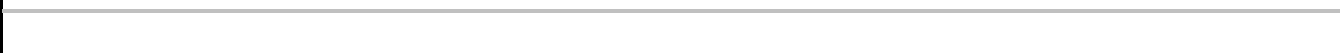 & 20 & $\mathrm{~L}$ \\
\hline
\end{tabular}




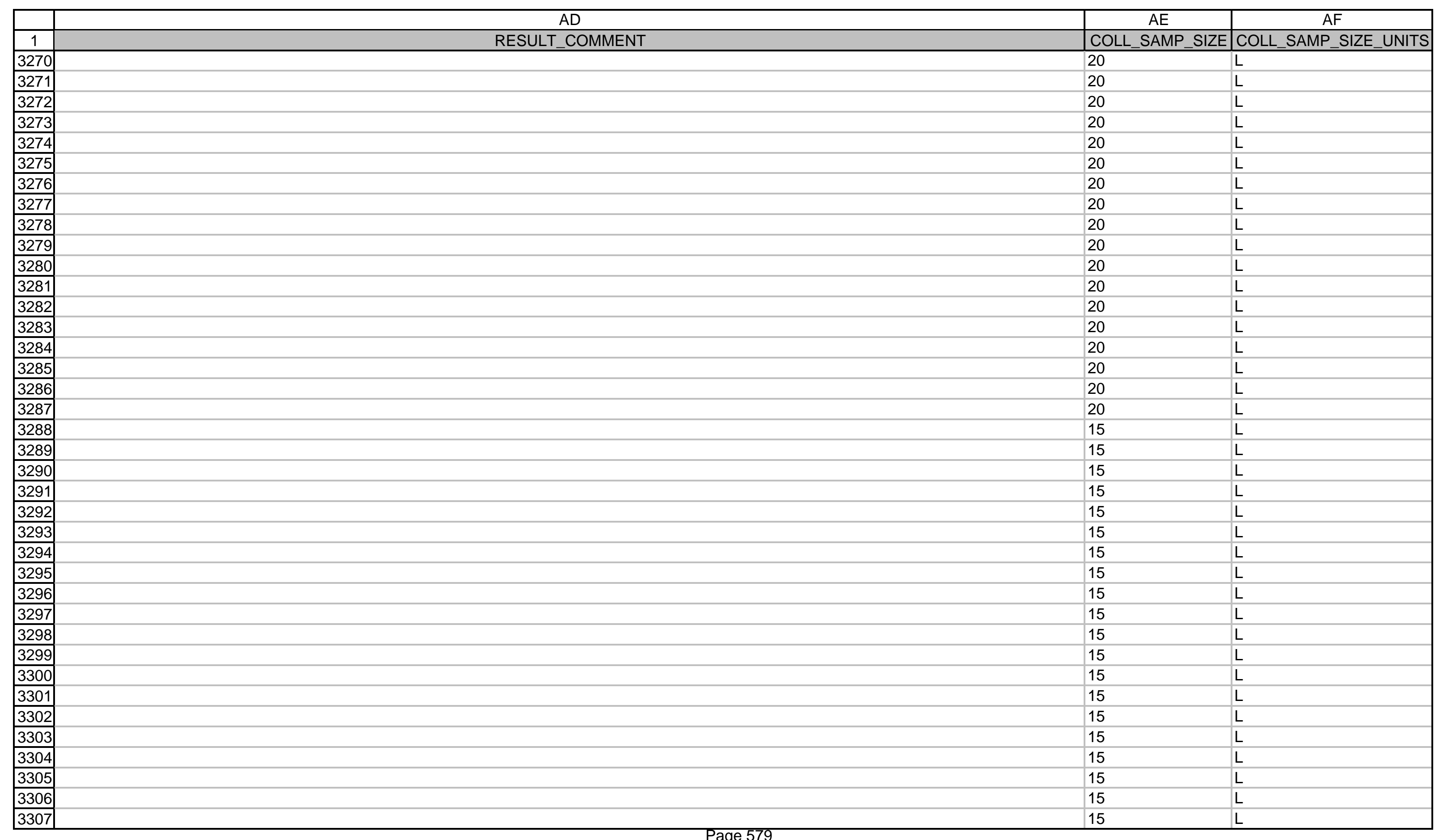




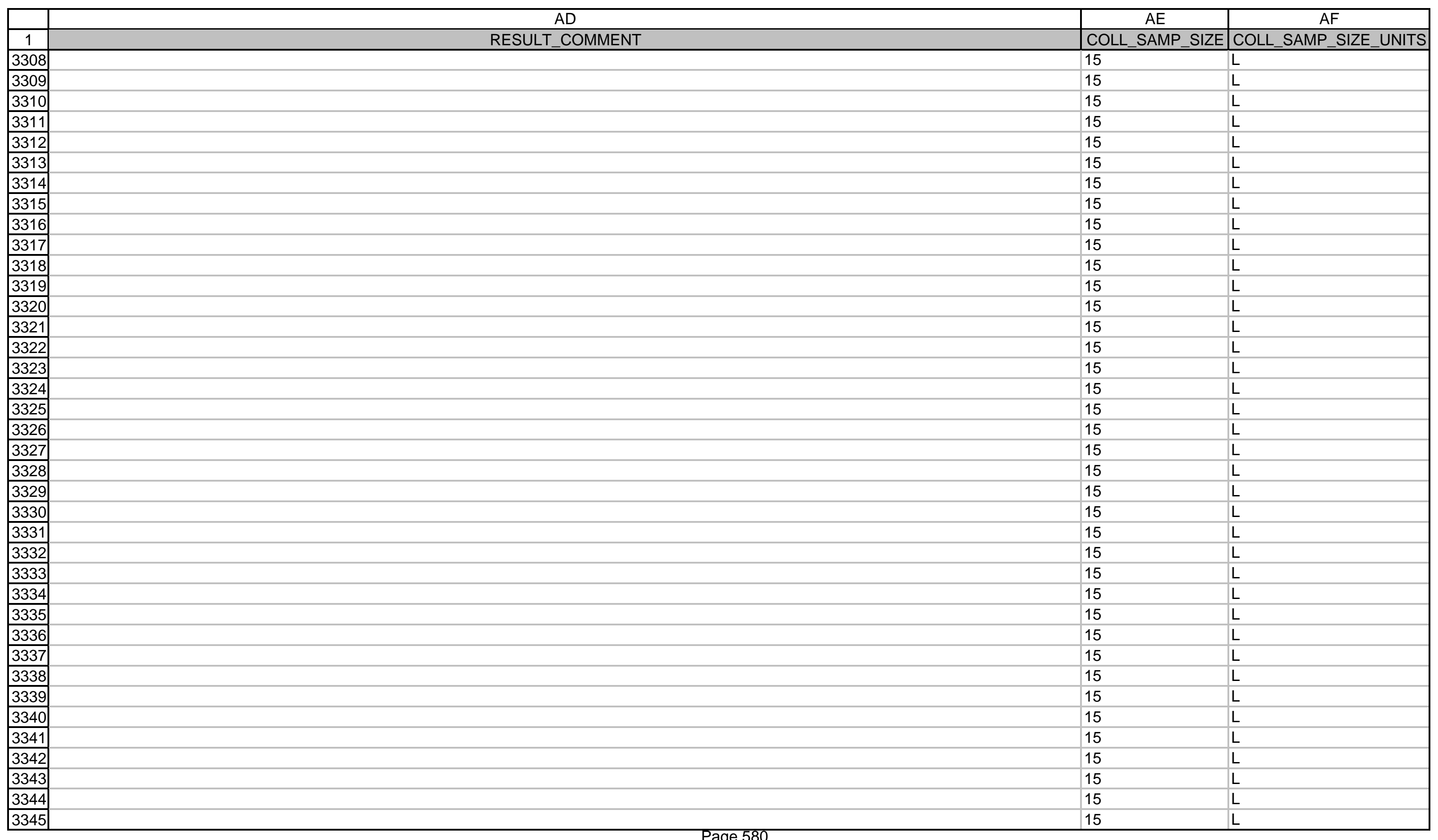




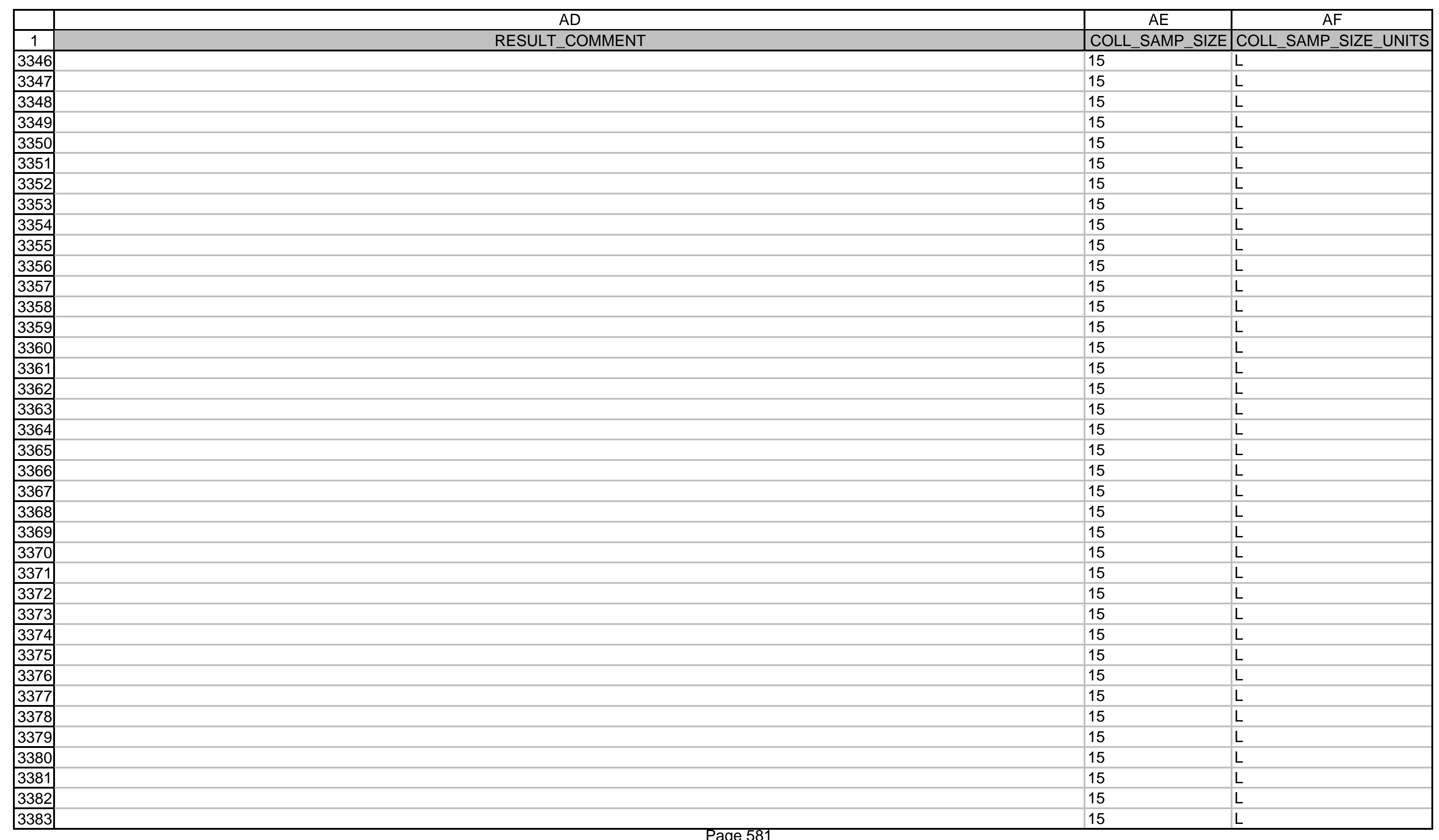




\begin{tabular}{|c|c|c|}
\hline & $A D$ & AF \\
\hline 1 & RESULT_COMMENT & COLL_SAMP_SIZE_UNITS \\
\hline 3384 & & $\mathrm{~L}$ \\
\hline 3385 & & L \\
\hline 3386 & & L \\
\hline 3387 & & L \\
\hline 3388 & & L \\
\hline 3389 & & $\mathrm{~L}$ \\
\hline 3390 & & L \\
\hline 3391 & 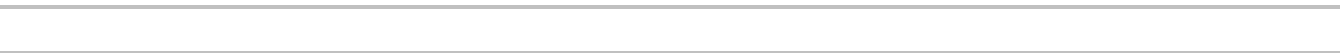 & $\mathrm{L}$ \\
\hline 3392 & 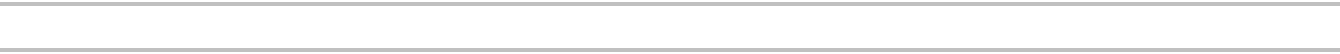 & $L$ \\
\hline 3393 & - & L \\
\hline 3394 & 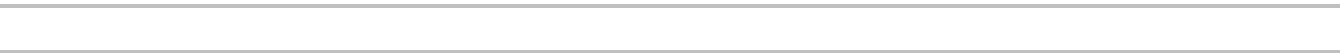 & $\mathrm{L}$ \\
\hline 3395 & & L \\
\hline 3396 & & L \\
\hline 3397 & & $\mathrm{~L}$ \\
\hline 3398 & & L \\
\hline 3399 & & L \\
\hline 3400 & & $\mathrm{~L}$ \\
\hline 3401 & & $\mathrm{~L}$ \\
\hline 3402 & & $\mathrm{~L}$ \\
\hline 3403 & & $\mathrm{~L}$ \\
\hline 3404 & & $\mathrm{~L}$ \\
\hline 3405 & & L \\
\hline 3406 & & $\mathrm{~L}$ \\
\hline 3407 & & $\mathrm{~L}$ \\
\hline 3408 & & L \\
\hline 3409 & & $\mathrm{~L}$ \\
\hline 3410 & & $\mathrm{~L}$ \\
\hline 3411 & & L \\
\hline 3412 & & L \\
\hline 3413 & & $\mathrm{~L}$ \\
\hline 3414 & & $\mathrm{~L}$ \\
\hline 3415 & & $\mathrm{~L}$ \\
\hline 3416 & & $\mathrm{~L}$ \\
\hline 3417 & & L \\
\hline 3418 & & L \\
\hline 3419 & & $\mathrm{~L}$ \\
\hline 3420 & & $L$ \\
\hline 3421 & 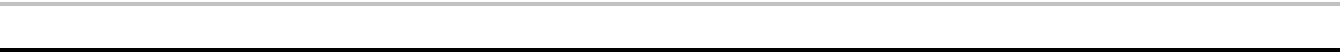 & $\mathrm{L}$ \\
\hline
\end{tabular}




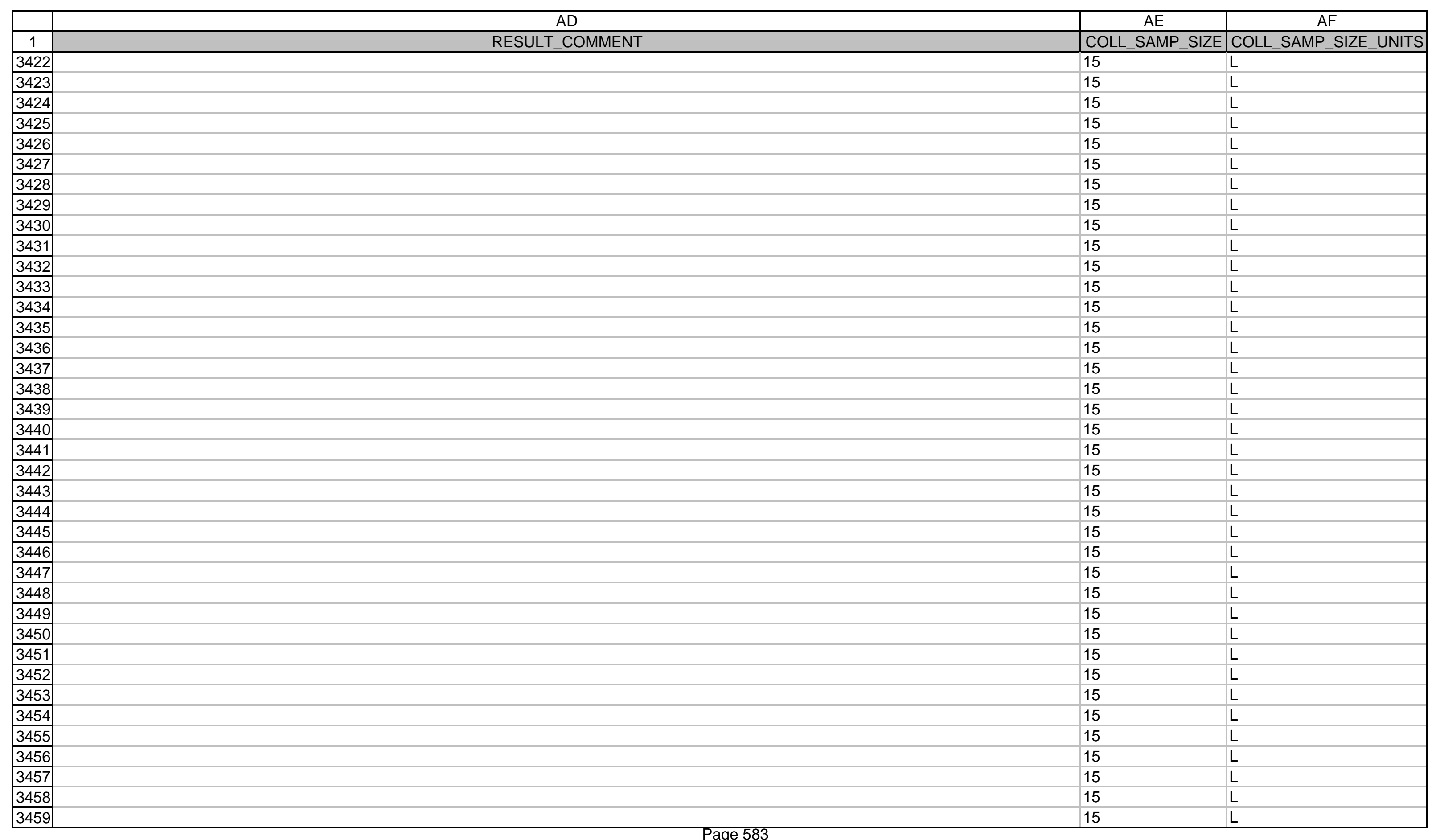




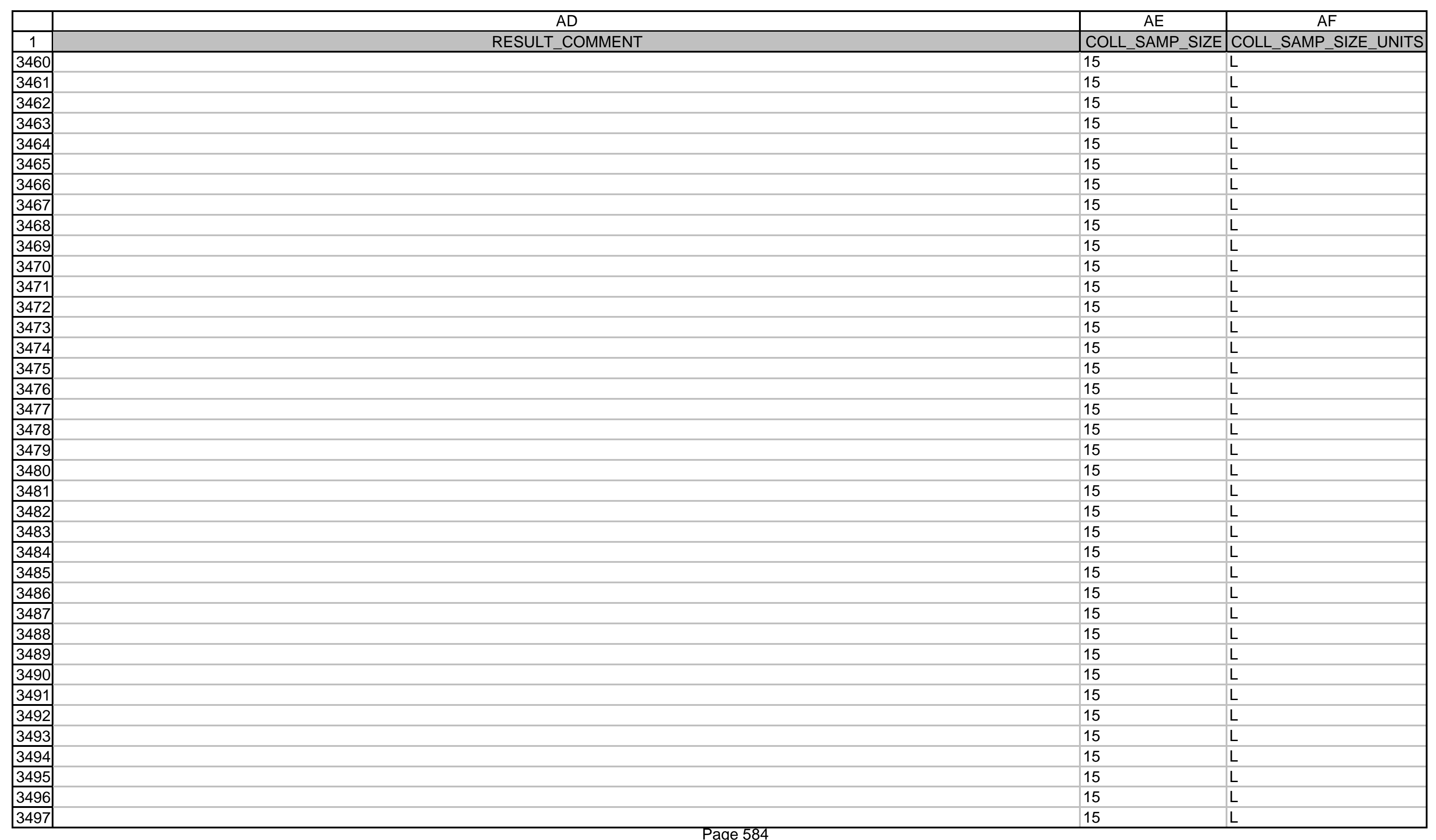




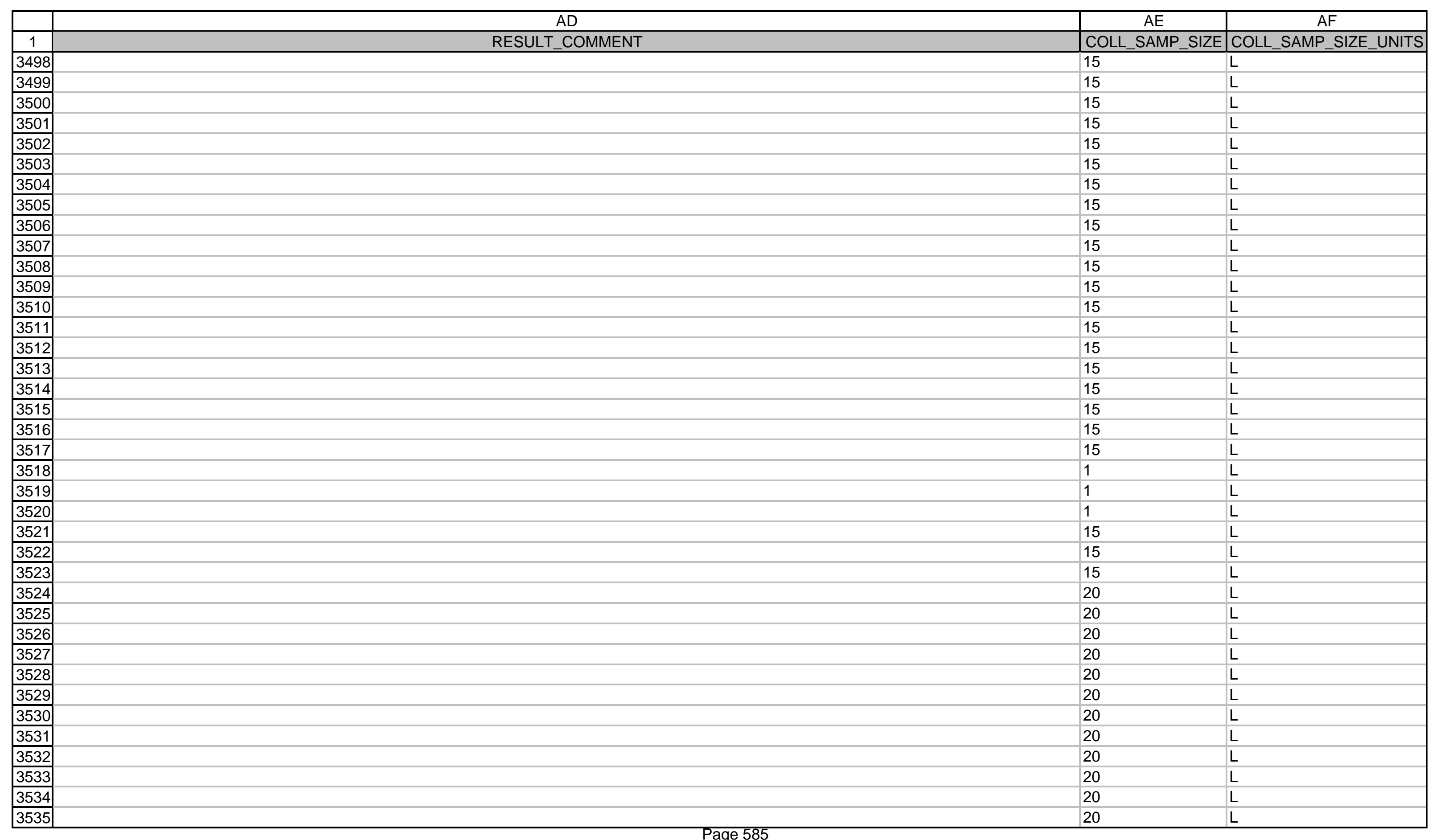




\begin{tabular}{|c|c|}
\hline $\mathrm{AE}$ & AF \\
\hline SAMP_SIZE & COLL_SAMP_SIZE_UNITS \\
\hline & $\mathrm{L}$ \\
\hline & L \\
\hline & L \\
\hline & L \\
\hline & L \\
\hline & L \\
\hline & L \\
\hline & $\mathrm{L}$ \\
\hline & $\mathrm{L}$ \\
\hline & L \\
\hline & L \\
\hline & $\mathrm{L}$ \\
\hline & $\mathrm{L}$ \\
\hline & L \\
\hline & L \\
\hline & L \\
\hline & L \\
\hline & L \\
\hline & L \\
\hline & L \\
\hline & $\mathrm{L}$ \\
\hline & L \\
\hline & $\mathrm{L}$ \\
\hline & L \\
\hline & $\mathrm{L}$ \\
\hline & L \\
\hline & L \\
\hline & L \\
\hline & L \\
\hline & L \\
\hline & L \\
\hline & $\mathrm{L}$ \\
\hline & L \\
\hline & L \\
\hline & L \\
\hline & L \\
\hline & L \\
\hline & $\mathrm{L}$ \\
\hline
\end{tabular}




\begin{tabular}{|c|c|}
\hline$A E$ & $\mathrm{AF}$ \\
\hline SAMP_SIZE & COLL_SAMP_SIZE_UNITS \\
\hline & L \\
\hline & L \\
\hline & $\mathrm{L}$ \\
\hline & $\mathrm{L}$ \\
\hline & L \\
\hline & L \\
\hline & L \\
\hline & L \\
\hline & L \\
\hline & L \\
\hline & L \\
\hline & L \\
\hline & L \\
\hline & L \\
\hline & $\mathrm{L}$ \\
\hline & L \\
\hline & L \\
\hline & L \\
\hline & L \\
\hline & L \\
\hline & L \\
\hline & L \\
\hline & L \\
\hline & L \\
\hline & L \\
\hline & L \\
\hline & $\mathrm{L}$ \\
\hline & $\mathrm{L}$ \\
\hline & L \\
\hline & L \\
\hline & L \\
\hline & L \\
\hline & L \\
\hline & L \\
\hline & L \\
\hline & L \\
\hline & L \\
\hline & $\mathrm{L}$ \\
\hline
\end{tabular}




\begin{tabular}{|c|c|c|c|}
\hline & $A D$ & $\mathrm{AE}$ & $\mathrm{AF}$ \\
\hline 1 & (2) & COLL_SAMP_SIZE & COLL_SAMP_SIZE_UNITS \\
\hline \begin{tabular}{|l|}
3612 \\
\end{tabular} & & 20 & L \\
\hline 3613 & & 20 & L \\
\hline 3614 & & 20 & L \\
\hline 3615 & & 20 & L \\
\hline 3616 & & 20 & $\mathrm{~L}$ \\
\hline \begin{tabular}{|l|}
3617 \\
\end{tabular} & & 20 & L \\
\hline 3618 & & 20 & L \\
\hline 3619 & 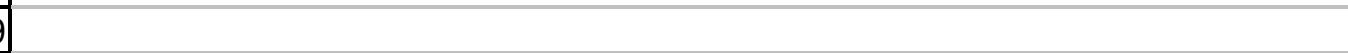 & 20 & L \\
\hline 3620 & 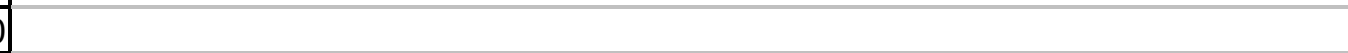 & 20 & L \\
\hline 3621 & 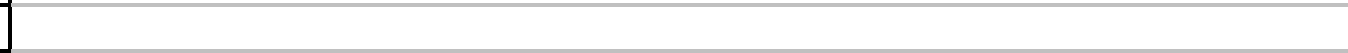 & 20 & L \\
\hline 3622 & t & 20 & L \\
\hline 3623 & & 20 & L \\
\hline 3624 & & 20 & L \\
\hline \begin{tabular}{|l|}
3625 \\
\end{tabular} & & 20 & L \\
\hline 3626 & & 20 & L \\
\hline 3627 & & 20 & L \\
\hline 3628 & & 20 & L \\
\hline 3629 & & 20 & L \\
\hline 3630 & & 20 & L \\
\hline 3631 & & 20 & L \\
\hline 3632 & & 20 & L \\
\hline \begin{tabular}{|l|}
3633 \\
\end{tabular} & & 20 & L \\
\hline 3634 & & 20 & L \\
\hline \begin{tabular}{|l|}
3635 \\
\end{tabular} & & 20 & L \\
\hline \begin{tabular}{|l|}
3636 \\
\end{tabular} & & 20 & L \\
\hline \begin{tabular}{|l|}
3637 \\
\end{tabular} & & 20 & L \\
\hline \begin{tabular}{|l|}
3638 \\
\end{tabular} & & 20 & L \\
\hline \begin{tabular}{|l|}
3639 \\
\end{tabular} & & 20 & L \\
\hline \begin{tabular}{|l|}
3640 \\
\end{tabular} & & 20 & L \\
\hline \begin{tabular}{|l|}
3641 \\
\end{tabular} & & 20 & L \\
\hline \begin{tabular}{|l|}
3642 \\
\end{tabular} & & 20 & L \\
\hline \begin{tabular}{|l|}
3643 \\
\end{tabular} & & 20 & L \\
\hline \begin{tabular}{|l|}
3644 \\
\end{tabular} & & 20 & L \\
\hline \begin{tabular}{|l|}
3645 \\
\end{tabular} & & 20 & L \\
\hline \begin{tabular}{|l|}
3646 \\
\end{tabular} & & 20 & L \\
\hline \begin{tabular}{|l|}
3647 \\
\end{tabular} & & 20 & L \\
\hline \begin{tabular}{|l|}
3648 \\
\end{tabular} & & 20 & L \\
\hline \begin{tabular}{|l|}
3649 \\
\end{tabular} & 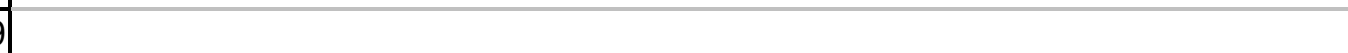 & 20 & L \\
\hline
\end{tabular}




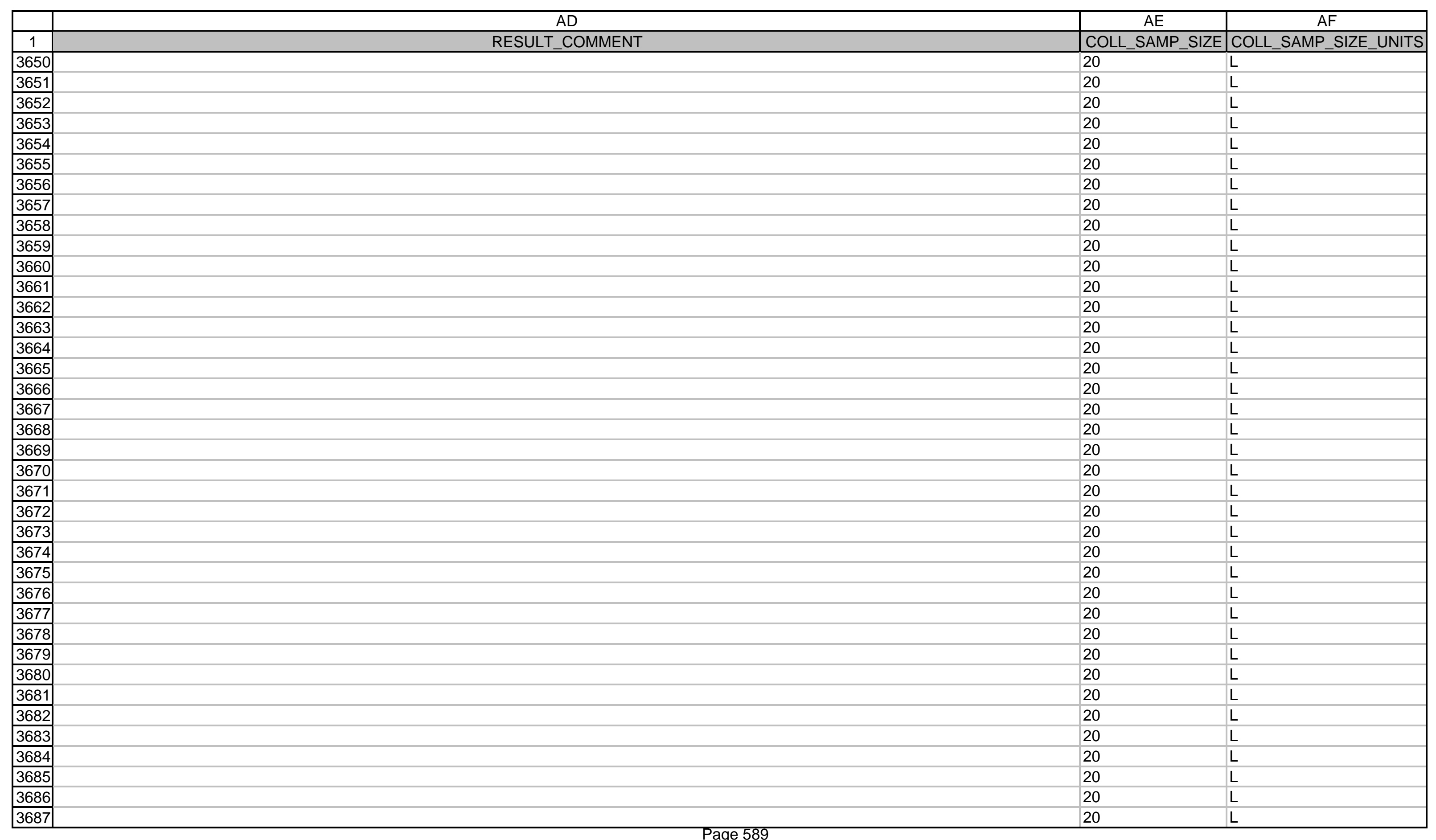




\begin{tabular}{|c|c|c|c|}
\hline & 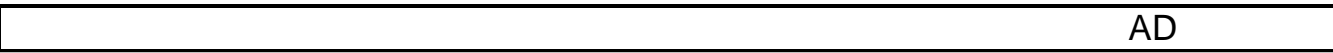 & $\mathrm{AE}$ & $\mathrm{AF}$ \\
\hline 1 & $\begin{array}{lc} & \text { RESULT_COMMENT } \\
\end{array}$ & COLL_SAMP_SIZE & EOLL_SAMP_SIZE_UNITS \\
\hline 3688 & & 20 & $\mathrm{~L}$ \\
\hline 3689 & 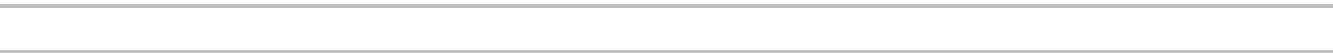 & 20 & $\mathrm{~L}$ \\
\hline 3690 & & 20 & $\mathrm{~L}$ \\
\hline 3691 & 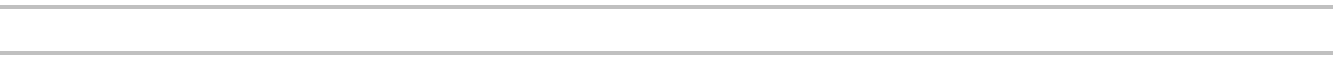 & 20 & L \\
\hline 3692 & & 20 & $\mathrm{~L}$ \\
\hline 3693 & & 20 & L \\
\hline 3694 & & 20 & L \\
\hline \begin{tabular}{|l|}
3695 \\
\end{tabular} & & 20 & L \\
\hline \begin{tabular}{|l|}
3696 \\
\end{tabular} & & 20 & $L$ \\
\hline \begin{tabular}{|l|}
3697 \\
\end{tabular} & & 20 & L \\
\hline \begin{tabular}{|l|}
3698 \\
\end{tabular} & & 20 & L \\
\hline \begin{tabular}{|l|}
3699 \\
\end{tabular} & & 20 & L \\
\hline \begin{tabular}{|l|}
3700 \\
\end{tabular} & & 20 & L \\
\hline \begin{tabular}{|l|}
3701 \\
\end{tabular} & & 20 & L \\
\hline \begin{tabular}{|l|}
3702 \\
\end{tabular} & & 20 & L \\
\hline \begin{tabular}{|l|}
3703 \\
\end{tabular} & & 20 & L \\
\hline \begin{tabular}{|l|}
3704 \\
\end{tabular} & & 20 & $L$ \\
\hline \begin{tabular}{|l|}
3705 \\
\end{tabular} & & 20 & L \\
\hline \begin{tabular}{|l|}
3706 \\
\end{tabular} & & 20 & L \\
\hline \begin{tabular}{|l|}
3707 \\
\end{tabular} & & 20 & L \\
\hline \begin{tabular}{|l|}
3708 \\
\end{tabular} & & 20 & L \\
\hline \begin{tabular}{|l|}
3709 \\
\end{tabular} & & 20 & L \\
\hline \begin{tabular}{|l|}
3710 \\
\end{tabular} & & 20 & L \\
\hline \begin{tabular}{|l|}
3711 \\
\end{tabular} & & 20 & L \\
\hline \begin{tabular}{|l|}
3712 \\
\end{tabular} & & 20 & L \\
\hline \begin{tabular}{|l|}
3713 \\
\end{tabular} & & 20 & L \\
\hline \begin{tabular}{|l|}
3714 \\
\end{tabular} & & 20 & L \\
\hline \begin{tabular}{|l|}
3715 \\
\end{tabular} & & 20 & L \\
\hline \begin{tabular}{|l|}
3716 \\
\end{tabular} & & 20 & L \\
\hline \begin{tabular}{|l|}
3717 \\
\end{tabular} & & 20 & L \\
\hline \begin{tabular}{|l|}
3718 \\
\end{tabular} & & 20 & L \\
\hline \begin{tabular}{|l|}
3719 \\
\end{tabular} & & 20 & $L$ \\
\hline \begin{tabular}{|l|}
3720 \\
\end{tabular} & & 20 & L \\
\hline \begin{tabular}{|l|}
3721 \\
\end{tabular} & & 20 & L \\
\hline \begin{tabular}{|l|}
3722 \\
\end{tabular} & & 20 & L \\
\hline \begin{tabular}{|l|}
3723 \\
\end{tabular} & & 20 & L \\
\hline \begin{tabular}{|l|}
3724 \\
\end{tabular} & & 20 & L \\
\hline \begin{tabular}{|l|}
3725 \\
\end{tabular} & L & 20 & $\mathrm{~L}$ \\
\hline
\end{tabular}




\begin{tabular}{|c|c|}
\hline $\mathrm{AE}$ & AF \\
\hline SAMP_SIZE & COLL_SAMP_SIZE_UNITS \\
\hline & $\mathrm{L}$ \\
\hline & L \\
\hline & L \\
\hline & L \\
\hline & L \\
\hline & L \\
\hline & L \\
\hline & $\mathrm{L}$ \\
\hline & $\mathrm{L}$ \\
\hline & L \\
\hline & L \\
\hline & $\mathrm{L}$ \\
\hline & L \\
\hline & L \\
\hline & L \\
\hline & L \\
\hline & L \\
\hline & L \\
\hline & L \\
\hline & L \\
\hline & $\mathrm{L}$ \\
\hline & L \\
\hline & $\mathrm{L}$ \\
\hline & L \\
\hline & $\mathrm{L}$ \\
\hline & L \\
\hline & L \\
\hline & L \\
\hline & L \\
\hline & L \\
\hline & L \\
\hline & L \\
\hline & L \\
\hline & L \\
\hline & L \\
\hline & L \\
\hline & L \\
\hline & $\mathrm{L}$ \\
\hline
\end{tabular}




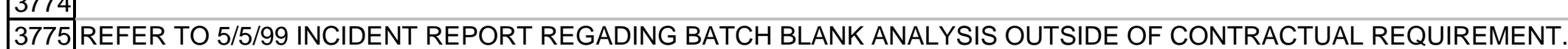

REFER TO 5/5/99 INCIDENT REPORT REGADING BATCH BLANK ANALYSIS OUTSIDE OF CONTRACTUAL REQUIREMENT.

20

REFER TO 5/5/99 INCIDENT REPORT REGADING BATCH BLANK ANALYSIS OUTSIDE OF CONTRACTUAL REQUIREMENT.

RESULT LOST. REFER TO 5/6/99 INCIDENT REPORT REGADING BATCH BLANK ANALYSIS OUTSIDE OF CONTRACTUAL REQUIREMENT. \begin{tabular}{|l|l|l|l|}
10 \\
\hline
\end{tabular}

ESULT LOST. REFER TO 5/6/99 INCIDENT REPORT REGADING BATCH BLANK ANALYSIS OUTSIDE OF CONTRACTUAL REQUIREMENT. 10

\begin{tabular}{|c|c|}
\hline$\overline{A E}$ & $\mathrm{AF}$ \\
\hline COLL_SAMP_SIZE & COLL_SAMP_SIZE_UNITS \\
\hline 20 & L \\
\hline 20 & L \\
\hline 20 & $\mathrm{~L}$ \\
\hline 20 & $\mathrm{~L}$ \\
\hline 20 & L \\
\hline 20 & L \\
\hline 20 & L \\
\hline 20 & $L$ \\
\hline 20 & L \\
\hline 20 & L \\
\hline 10.5 & $L$ \\
\hline 10.5 & L \\
\hline 10.5 & L \\
\hline 10.5 & L \\
\hline 10.5 & L \\
\hline 10.5 & L \\
\hline 10.5 & L \\
\hline 10.5 & L \\
\hline 10.5 & L \\
\hline 10.5 & L \\
\hline 10 & L \\
\hline 10 & L \\
\hline 10 & $L$ \\
\hline 10 & $L$ \\
\hline 10 & L \\
\hline 10 & $L$ \\
\hline 10 & $L$ \\
\hline 10 & $L$ \\
\hline 10 & $L$ \\
\hline 10 & L \\
\hline 20 & L \\
\hline 20 & $L$ \\
\hline 20 & L \\
\hline 20 & L \\
\hline 20 & L \\
\hline 20 & L \\
\hline 20 & L \\
\hline & $\mathrm{L}$ \\
\hline
\end{tabular}




\begin{tabular}{|c|c|c|c|}
\hline & $A D$ & $\mathrm{AE}$ & $\mathrm{AF}$ \\
\hline 1 & RESULT_COMMENT & COLL_SAMP_SIZE & COLL_SAMP_SIZE_UNITS \\
\hline 3802 & & 20 & $\mathrm{~L}$ \\
\hline 3803 & & 20 & L \\
\hline 3804 & & 20 & L \\
\hline 3805 & REFER TO 5/5/99 INCIDENT REPORT REGADING BATCH BLANK ANALYSIS OUTSIDE OF CONTRACTUAL REQUIREMENT. & 20 & $\mathrm{~L}$ \\
\hline 3806 & & 20 & L \\
\hline 3807 & & 20 & L \\
\hline 3808 & & 20 & L \\
\hline 3809 & & 20 & L \\
\hline 3810 & REFER TO 5/5/99 INCIDENT REPORT REGADING BATCH BLANK ANALYSIS OUTSIDE OF CONTRACTUAL REQUIREMENT. & 20 & $\mathrm{~L}$ \\
\hline 3811 & & 20 & $L$ \\
\hline 3812 & & 20 & L \\
\hline 3813 & & 20 & L \\
\hline 3814 & & 20 & L \\
\hline 3815 & & 20 & L \\
\hline \begin{tabular}{|l|}
3816 \\
\end{tabular} & & 20 & L \\
\hline \begin{tabular}{|l|}
3817 \\
\end{tabular} & & 20 & $\mathrm{~L}$ \\
\hline \begin{tabular}{|l|}
3818 \\
\end{tabular} & & 20 & $\mathrm{~L}$ \\
\hline \begin{tabular}{|l|}
3819 \\
\end{tabular} & & 20 & L \\
\hline \begin{tabular}{|l}
3820 \\
\end{tabular} & & 20 & L \\
\hline \begin{tabular}{|l|}
3821 \\
\end{tabular} & & 20 & $\mathrm{~L}$ \\
\hline \begin{tabular}{|l|}
3822 \\
\end{tabular} & & 20 & L \\
\hline \begin{tabular}{|l|}
3823 \\
\end{tabular} & & 20 & L \\
\hline \begin{tabular}{|l|}
3824 \\
\end{tabular} & & 20 & $\mathrm{~L}$ \\
\hline 3825 & & 20 & L \\
\hline 3826 & & 20 & $\mathrm{~L}$ \\
\hline 3827 & & 20 & L \\
\hline \begin{tabular}{|l|}
3828 \\
\end{tabular} & & 20 & L \\
\hline \begin{tabular}{|l|}
3829 \\
\end{tabular} & & 20 & L \\
\hline \begin{tabular}{|l}
3830 \\
\end{tabular} & & 20 & L \\
\hline \begin{tabular}{|l|}
3831 \\
\end{tabular} & & 20 & $\mathrm{~L}$ \\
\hline \begin{tabular}{|l|}
3832 \\
\end{tabular} & & 20 & L \\
\hline \begin{tabular}{|l|}
3833 \\
\end{tabular} & & 20 & $\mathrm{~L}$ \\
\hline 3834 & & 20 & $\mathrm{~L}$ \\
\hline 3835 & & 20 & L \\
\hline \begin{tabular}{|l}
3836 \\
\end{tabular} & & 20 & $\mathrm{~L}$ \\
\hline 3837 & & 20 & $\mathrm{~L}$ \\
\hline \begin{tabular}{|l|}
3838 \\
\end{tabular} & & 20 & L \\
\hline 3839 & & 20 & L \\
\hline
\end{tabular}


\begin{tabular}{l|l}
$\mathrm{AE}$ & $\mathrm{AF}$ \\
\hline
\end{tabular}

\section{0 \\ 3841 REFER TO 11/05/98 INCIDENT REPORT REGARDING ANALYSIS LOST AT LAB.}

RESULT_COMMENT

3842 REFER TO 11/05/98 INCIDENT REPORT REGARDING ANALYSIS LOST AT LAB.

3843 REFER TO 11/05/98 INCIDENT REPORT REGARDING ANALYSIS LOST AT LAB.

3844

3845

3846

3847

3848

3857 REFER TO 11/05/98 INCIDENT REPORT REGARDING ANALYSIS LOST AT LAB.

3858 REFER TO 11/05/98 INCIDENT REPORT REGARDING ANALYSIS LOST AT LAB. 


\begin{tabular}{|c|c|c|c|}
\hline & $A D$ & $\mathrm{AE}$ & AF \\
\hline 1 & RESULT_COMMENT & COLL_SAMP_SIZE & COLL_SAMP_SIZE_UNITS \\
\hline 3878 & & 20 & L \\
\hline 3879 & & 20 & $\mathrm{~L}$ \\
\hline 3880 & & 20 & L \\
\hline 3881 & & 20 & L \\
\hline 3882 & & 20 & $\mathrm{~L}$ \\
\hline 3883 & & 20 & $\mathrm{~L}$ \\
\hline 3884 & & 20 & L \\
\hline 3885 & & 20 & L \\
\hline 3886 & & 20 & $L$ \\
\hline 3887 & & 20 & L \\
\hline \begin{tabular}{|l|}
3888 \\
\end{tabular} & & 20 & L \\
\hline 3889 & & 20 & L \\
\hline 3890 & & 20 & L \\
\hline 3891 & & 20 & L \\
\hline 3892 & & 20 & L \\
\hline 3893 & & 20 & L \\
\hline 3894 & & 20 & L \\
\hline 3895 & & 20 & L \\
\hline 3896 & & 20 & L \\
\hline 3897 & & 20 & L \\
\hline 3898 & & 20 & L \\
\hline 3899 & & 20 & L \\
\hline 3900 & & 20 & L \\
\hline 3901 & & 20 & L \\
\hline 3902 & & 20 & L \\
\hline 3903 & & 20 & L \\
\hline 3904 & & 20 & L \\
\hline 3905 & & 20 & L \\
\hline 3906 & & 20 & L \\
\hline 3907 & & 20 & L \\
\hline 3908 & & 20 & L \\
\hline 3909 & & 20 & L \\
\hline 3910 & & 20 & L \\
\hline 3911 & & 20 & $\mathrm{~L}$ \\
\hline 3912 & & 20 & L \\
\hline 3913 & & 20 & L \\
\hline 3914 & & 20 & L \\
\hline 3915 & REFER TO 5/5/99 INCIDENT REPORT REGADING BATCH BLANK ANALYSIS OUTSIDE OF CONTRACTUAL REQUIREMENT. & 20 & L \\
\hline
\end{tabular}




\begin{tabular}{|c|c|c|c|}
\hline & $A D$ & $\mathrm{AE}$ & $\mathrm{AF}$ \\
\hline 1 & RESULT COMMENT & COLL SAMP SIZE & COLL SAMP SIZE UNITS \\
\hline 3916 & & 20 & L \\
\hline 3917 & & 20 & L \\
\hline 3918 & & 20 & $\mathrm{~L}$ \\
\hline 3919 & & 20 & $\mathrm{~L}$ \\
\hline 3920 & REFER TO 5/5/99 INCIDENT REPORT REGADING BATCH BLANK ANALYSIS OUTSIDE OF CONTRACTUAL REQUIREMENT. & 20 & $\mathrm{~L}$ \\
\hline 3921 & & 20 & $\mathrm{~L}$ \\
\hline 3922 & & 20 & $\mathrm{~L}$ \\
\hline 3923 & & 20 & $\mathrm{~L}$ \\
\hline 3924 & & 20 & $\mathrm{~L}$ \\
\hline 3925 & REFER TO 5/5/99 INCIDENT REPORT REGADING BATCH BLANK ANALYSIS OUTSIDE OF CONTRACTUAL REQUIREMENT. & 20 & L \\
\hline 3926 & & 20 & $\mathrm{~L}$ \\
\hline 3927 & & 20 & $\mathrm{~L}$ \\
\hline 3928 & & 20 & L \\
\hline 3929 & & 20 & L \\
\hline 3930 & REFER TO 5/5/99 INCIDENT REPORT REGADING BATCH BLANK ANALYSIS OUTSIDE OF CONTRACTUAL REQUIREMENT. & 20 & L \\
\hline 3931 & & 20 & $\mathrm{~L}$ \\
\hline 3932 & & 20 & L \\
\hline 3933 & & 20 & $\mathrm{~L}$ \\
\hline 3934 & & 20 & $\mathrm{~L}$ \\
\hline 3935 & REFER TO 5/5/99 INCIDENT REPORT REGADING BATCH BLANK ANALYSIS OUTSIDE OF CONTRACTUAL REQUIREMENT. & 20 & $\mathrm{~L}$ \\
\hline 3936 & & 20 & L \\
\hline 3937 & & 20 & L \\
\hline 3938 & & 20 & L \\
\hline 3939 & & 20 & L \\
\hline 3940 & REFER TO 5/5/99 INCIDENT REPORT REGADING BATCH BLANK ANALYSIS OUTSIDE OF CONTRACTUAL REQUIREMENT. & 20 & $\mathrm{~L}$ \\
\hline 3941 & & 20 & L \\
\hline 3942 & & 20 & L \\
\hline 3943 & & 20 & $\mathrm{~L}$ \\
\hline 3944 & & 20 & L \\
\hline 3945 & REFER TO 5/5/99 INCIDENT REPORT REGADING BATCH BLANK ANALYSIS OUTSIDE OF CONTRACTUAL REQUIREMENT. & 20 & L \\
\hline 3946 & & 20 & $\mathrm{~L}$ \\
\hline 3947 & & 20 & $\mathrm{~L}$ \\
\hline 3948 & & 20 & L \\
\hline 3949 & & 20 & L \\
\hline 3950 & REFER TO 5/5/99 INCIDENT REPORT REGADING BATCH BLANK ANALYSIS OUTSIDE OF CONTRACTUAL REQUIREMENT. & 20 & $\mathrm{~L}$ \\
\hline 3951 & & 20 & L \\
\hline 3952 & & 20 & L \\
\hline 3953 & & 20 & L \\
\hline
\end{tabular}




\begin{tabular}{|c|c|c|c|}
\hline & $A D$ & $\mathrm{AE}$ & $\mathrm{AF}$ \\
\hline 1 & RESULT_COMMENT & COLL_SAMP_SIZE & COLL_SAMP_SIZE_UNITS \\
\hline 3954 & & 20 & $\mathrm{~L}$ \\
\hline 3955 & REFER TO 5/5/99 INCIDENT REPORT REGADING BATCH BLANK ANALYSIS OUTSIDE OF CONTRACTUAL REQUIREMENT. & 20 & L \\
\hline 3956 & & 20 & L \\
\hline \begin{tabular}{|c|}
3957 \\
\end{tabular} & & 20 & $\mathrm{~L}$ \\
\hline \begin{tabular}{|c|}
3958 \\
\end{tabular} & & 20 & $\mathrm{~L}$ \\
\hline 3959 & & 20 & L \\
\hline 3960 & REFER TO 5/5/99 INCIDENT REPORT REGADING BATCH BLANK ANALYSIS OUTSIDE OF CONTRACTUAL REQUIREMENT. & 20 & $\mathrm{~L}$ \\
\hline 3961 & & 20 & L \\
\hline 3962 & & 20 & $\mathrm{~L}$ \\
\hline 3963 & & 20 & $L$ \\
\hline 3964 & & 20 & L \\
\hline 3965 & & 20 & L \\
\hline 3966 & & 20 & L \\
\hline \begin{tabular}{|l|}
3967 \\
\end{tabular} & & 20 & L \\
\hline \begin{tabular}{|l|}
3968 \\
\end{tabular} & & 20 & L \\
\hline \begin{tabular}{|l|}
3969 \\
\end{tabular} & & 20 & $\mathrm{~L}$ \\
\hline \begin{tabular}{|l|}
3970 \\
\end{tabular} & & 20 & $\mathrm{~L}$ \\
\hline \begin{tabular}{|l|}
3971 \\
\end{tabular} & & 20 & L \\
\hline \begin{tabular}{|l|}
3972 \\
\end{tabular} & & 20 & L \\
\hline \begin{tabular}{|l|}
3973 \\
\end{tabular} & & 20 & $\mathrm{~L}$ \\
\hline \begin{tabular}{|l|}
3974 \\
\end{tabular} & & 20 & L \\
\hline \begin{tabular}{|l|}
3975 \\
\end{tabular} & & 20 & L \\
\hline \begin{tabular}{|l|}
3976 \\
\end{tabular} & & 20 & $\mathrm{~L}$ \\
\hline \begin{tabular}{|l|}
3977 \\
\end{tabular} & & 20 & L \\
\hline 3978 & & 20 & $\mathrm{~L}$ \\
\hline \begin{tabular}{|l|}
3979 \\
\end{tabular} & & 20 & L \\
\hline \begin{tabular}{|l|}
3980 \\
\end{tabular} & & 20 & L \\
\hline \begin{tabular}{|l|}
3981 \\
\end{tabular} & & 20 & L \\
\hline \begin{tabular}{|l|}
3982 \\
\end{tabular} & & 20 & L \\
\hline \begin{tabular}{|l|}
3983 \\
\end{tabular} & & 20 & $\mathrm{~L}$ \\
\hline \begin{tabular}{|l|}
3984 \\
\end{tabular} & & 20 & L \\
\hline \begin{tabular}{|l|}
3985 \\
\end{tabular} & & 20 & $\mathrm{~L}$ \\
\hline \begin{tabular}{|l|}
3986 \\
\end{tabular} & & 20 & $\mathrm{~L}$ \\
\hline \begin{tabular}{|l|}
3987 \\
\end{tabular} & & 20 & L \\
\hline \begin{tabular}{|l|}
3988 \\
\end{tabular} & & 20 & $\mathrm{~L}$ \\
\hline \begin{tabular}{|l|}
3989 \\
\end{tabular} & & 20 & $\mathrm{~L}$ \\
\hline \begin{tabular}{|l|}
3990 \\
\end{tabular} & & 20 & L \\
\hline \begin{tabular}{|l|}
3991 \\
\end{tabular} & & 20 & L \\
\hline
\end{tabular}




\begin{tabular}{|c|c|c|c|}
\hline & $A D$ & $\mathrm{AE}$ & $\mathrm{AF}$ \\
\hline 1 & RESULT_COMMENT & COLL_SAMP_SIZE & COLL_SAMP_SIZE_UNITS \\
\hline 3992 & & 20 & $\mathrm{~L}$ \\
\hline \begin{tabular}{|l|}
3993 \\
\end{tabular} & & 20 & L \\
\hline \begin{tabular}{|l|}
3994 \\
\end{tabular} & & 20 & L \\
\hline \begin{tabular}{|l|}
3995 \\
\end{tabular} & & 20 & L \\
\hline \begin{tabular}{|l|}
3996 \\
\end{tabular} & & 20 & L \\
\hline \begin{tabular}{|c|}
3997 \\
\end{tabular} & & 20 & $\mathrm{~L}$ \\
\hline \begin{tabular}{|l|}
3998 \\
\end{tabular} & & 20 & L \\
\hline \begin{tabular}{|l|}
3999 \\
\end{tabular} & ) & 20 & L \\
\hline \begin{tabular}{|l|}
4000 \\
\end{tabular} & ) & 20 & L \\
\hline \begin{tabular}{|l|}
4001 \\
\end{tabular} & 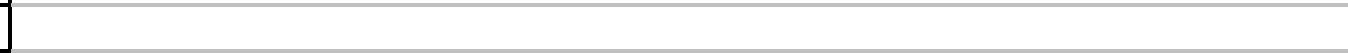 & 20 & L \\
\hline \begin{tabular}{|l|}
4002 \\
\end{tabular} & . & 20 & L \\
\hline \begin{tabular}{|l|}
4003 \\
\end{tabular} & & 20 & L \\
\hline \begin{tabular}{|l|}
4004 \\
\end{tabular} & & 20 & L \\
\hline 4005 & & 20 & $\mathrm{~L}$ \\
\hline \begin{tabular}{|l|}
4006 \\
\end{tabular} & & 20 & L \\
\hline \begin{tabular}{|l|}
4007 \\
\end{tabular} & & 20 & L \\
\hline \begin{tabular}{|l|}
4008 \\
\end{tabular} & & 20 & L \\
\hline \begin{tabular}{|l|}
4009 \\
\end{tabular} & & 20 & L \\
\hline \begin{tabular}{|l|}
4010 \\
\end{tabular} & & 20 & $\mathrm{~L}$ \\
\hline \begin{tabular}{|l|}
4011 \\
\end{tabular} & & 20 & L \\
\hline \begin{tabular}{|l|}
4012 \\
\end{tabular} & & 20 & L \\
\hline \begin{tabular}{|l|}
4013 \\
\end{tabular} & & 20 & L \\
\hline \begin{tabular}{|l|}
4014 \\
\end{tabular} & & 20 & L \\
\hline \begin{tabular}{|l|}
4015 \\
\end{tabular} & & 20 & L \\
\hline \begin{tabular}{|l|}
4016 \\
\end{tabular} & & 20 & $\mathrm{~L}$ \\
\hline \begin{tabular}{|l|}
4017 \\
\end{tabular} & & 20 & L \\
\hline \begin{tabular}{|l|}
4018 \\
\end{tabular} & & 20 & L \\
\hline \begin{tabular}{|l|}
4019 \\
\end{tabular} & & 20 & L \\
\hline \begin{tabular}{|l|}
4020 \\
\end{tabular} & & 20 & L \\
\hline \begin{tabular}{|l|}
4021 \\
\end{tabular} & & 20 & L \\
\hline \begin{tabular}{|l|}
4022 \\
\end{tabular} & & 20 & L \\
\hline \begin{tabular}{|l|}
4023 \\
\end{tabular} & & 20 & $\mathrm{~L}$ \\
\hline \begin{tabular}{|l|}
4024 \\
\end{tabular} & & 20 & L \\
\hline \begin{tabular}{|l|}
4025 \\
\end{tabular} & & 20 & L \\
\hline \begin{tabular}{|l|}
4026 \\
\end{tabular} & & 20 & L \\
\hline \begin{tabular}{|l|}
4027 \\
\end{tabular} & & 20 & L \\
\hline \begin{tabular}{|l|}
4028 \\
\end{tabular} & & 20 & L \\
\hline \begin{tabular}{|l|}
4029 \\
\end{tabular} & 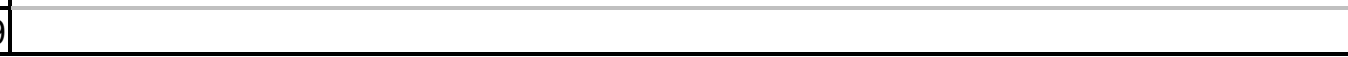 & 20 & $\mathrm{~L}$ \\
\hline
\end{tabular}




\begin{tabular}{|c|c|c|c|}
\hline & $A D$ & $A E$ & $\mathrm{AF}$ \\
\hline 1 & RESULT_COMMENT & COLL_SAMP_SIZE & COLL_SAMP_SIZE_UNITS \\
\hline 4030 & & 20 & L \\
\hline 4031 & & 20 & L \\
\hline 4032 & & 20 & L \\
\hline 4033 & & 20 & L \\
\hline 4034 & & 20 & $\mathrm{~L}$ \\
\hline \begin{tabular}{|l|}
4035 \\
\end{tabular} & & 20 & L \\
\hline 4036 & & 20 & L \\
\hline \begin{tabular}{|l|}
4037 \\
\end{tabular} & & 20 & L \\
\hline 4038 & & 20 & L \\
\hline 4039 & & 20 & L \\
\hline 4040 & & 20 & L \\
\hline 4041 & & 20 & L \\
\hline \begin{tabular}{|l|l|} 
\\
\end{tabular} & & 20 & L \\
\hline \begin{tabular}{|l|}
4043 \\
\end{tabular} & & 20 & L \\
\hline 4044 & & 20 & L \\
\hline \begin{tabular}{|l|}
4045 \\
\end{tabular} & RESULT REPORTED AS LOST, BUT WAS FOUND AND REPORTED ON 7/20/99. & 20 & L \\
\hline 4046 & & 20 & L \\
\hline 4047 & & 20 & L \\
\hline 4048 & & 20 & $\mathrm{~L}$ \\
\hline 4049 & & 20 & L \\
\hline 4050 & & 20 & L \\
\hline \begin{tabular}{|l|l|}
4051 \\
\end{tabular} & & 20 & L \\
\hline 4052 & & 20 & L \\
\hline 4053 & & 20 & L \\
\hline \begin{tabular}{|l|}
4054 \\
\end{tabular} & & 20 & L \\
\hline \begin{tabular}{|l|}
4055 \\
\end{tabular} & & 20 & L \\
\hline \begin{tabular}{|l|}
4056 \\
\end{tabular} & & 20 & L \\
\hline \begin{tabular}{|l|}
4057 \\
\end{tabular} & & 20 & L \\
\hline \begin{tabular}{|l|}
4058 \\
\end{tabular} & & 20 & L \\
\hline \begin{tabular}{|l|}
4059 \\
\end{tabular} & & 20 & L \\
\hline \begin{tabular}{|l|}
4060 \\
\end{tabular} & & 20 & L \\
\hline \begin{tabular}{|l|}
4061 \\
\end{tabular} & & 20 & $\mathrm{~L}$ \\
\hline \begin{tabular}{|l|}
4062 \\
\end{tabular} & & 20 & L \\
\hline \begin{tabular}{|l|}
4063 \\
\end{tabular} & & 20 & L \\
\hline \begin{tabular}{|l|}
4064 \\
\end{tabular} & & 20 & L \\
\hline \begin{tabular}{|l|}
4065 \\
\end{tabular} & & 20 & L \\
\hline \begin{tabular}{|l|}
4066 \\
\end{tabular} & & 20 & $L$ \\
\hline \begin{tabular}{|l|}
4067 \\
\end{tabular} & 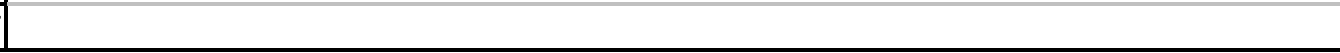 & 20 & $\mathrm{~L}$ \\
\hline
\end{tabular}




\begin{tabular}{|c|c|c|c|}
\hline & $A D$ & $\mathrm{AE}$ & $\mathrm{AF}$ \\
\hline 1 & RESULT_COMMENT & COLL_SAMP_SIZE & EOLL_SAMP_SIZE_UNITS \\
\hline 4068 & & 20 & $\mathrm{~L}$ \\
\hline 4069 & & 20 & L \\
\hline 4070 & & 20 & L \\
\hline \begin{tabular}{|l|}
4071 \\
\end{tabular} & & 20 & L \\
\hline \begin{tabular}{|l|}
4072 \\
\end{tabular} & & 20 & L \\
\hline \begin{tabular}{|l|l|}
4073 \\
\end{tabular} & & 20 & L \\
\hline \begin{tabular}{|l|l|}
4074 \\
\end{tabular} & 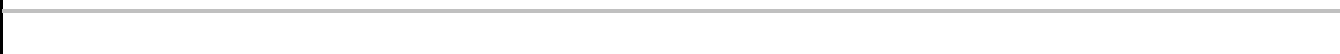 & 20 & L \\
\hline 4075 & 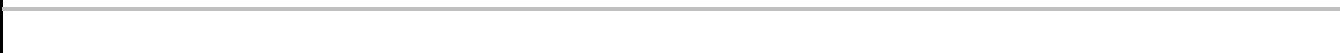 & 20 & L \\
\hline 4076 & & 20 & L \\
\hline \begin{tabular}{|l|l|}
4077 \\
407
\end{tabular} & & 20 & $\mathrm{~L}$ \\
\hline 4078 & & 20 & $L$ \\
\hline 4079 & & 20 & L \\
\hline \begin{tabular}{|l|}
4080 \\
\end{tabular} & & 20 & L \\
\hline 4081 & & 20 & L \\
\hline 4082 & & 20 & L \\
\hline \begin{tabular}{|l|l|}
4083 \\
\end{tabular} & & 20 & L \\
\hline \begin{tabular}{|l|l|}
4084 \\
\end{tabular} & & 20 & L \\
\hline $\begin{array}{l} \\
4085 \\
7085\end{array}$ & & 20 & $L$ \\
\hline \begin{tabular}{|l|l|}
4086 \\
\end{tabular} & & 20 & L \\
\hline 4087 & & 20 & $\mathrm{~L}$ \\
\hline \begin{tabular}{|l|l|}
4088 \\
\end{tabular} & & 20 & L \\
\hline 4089 & & 20 & L \\
\hline \begin{tabular}{|l|l|}
4090 \\
\end{tabular} & & 20 & $\mathrm{~L}$ \\
\hline \begin{tabular}{|l|}
4091 \\
\end{tabular} & & 20 & L \\
\hline \begin{tabular}{|l|}
4092 \\
\end{tabular} & & 20 & L \\
\hline \begin{tabular}{|l|}
4093 \\
\end{tabular} & & 20 & L \\
\hline \begin{tabular}{|l|}
4094 \\
\end{tabular} & & 20 & L \\
\hline \begin{tabular}{|l|}
4095 \\
\end{tabular} & & 20 & L \\
\hline \begin{tabular}{|l|}
4096 \\
\end{tabular} & & 20 & L \\
\hline \begin{tabular}{|l|}
4097 \\
\end{tabular} & & 20 & L \\
\hline \begin{tabular}{|l|}
4098 \\
\end{tabular} & & 20 & L \\
\hline \begin{tabular}{|l|}
4099 \\
\end{tabular} & & 20 & L \\
\hline \begin{tabular}{|l|}
4100 \\
\end{tabular} & & 20 & $\mathrm{~L}$ \\
\hline \begin{tabular}{|l|}
4101 \\
\end{tabular} & & 20 & L \\
\hline \begin{tabular}{|l|}
4102 \\
\end{tabular} & & 20 & L \\
\hline \begin{tabular}{|l|}
4103 \\
4103 \\
\end{tabular} & & 20 & $L$ \\
\hline \begin{tabular}{|l|}
4104 \\
\end{tabular} & & 20 & L \\
\hline \begin{tabular}{|l|}
4105 \\
\end{tabular} & 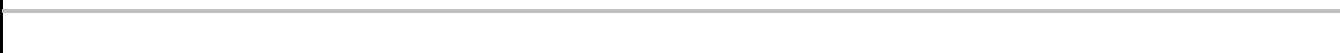 & 20 & L \\
\hline
\end{tabular}




\begin{tabular}{|c|c|c|c|}
\hline & $A D$ & $\mathrm{AE}$ & $\mathrm{AF}$ \\
\hline 1 & RESULT_COMMENT & COLL_SAMP_SIZE & COLL_SAMP_SIZE_UNITS \\
\hline 4106 & & 20 & $\mathrm{~L}$ \\
\hline 4107 & & 20 & L \\
\hline 4108 & & 20 & L \\
\hline 4109 & & 20 & L \\
\hline 4110 & & 20 & $\mathrm{~L}$ \\
\hline 4111 & IR-0008 Blank>CRDL & 20 & $\mathrm{~L}$ \\
\hline 4112 & IR-0008 Blank>CRDL & 20 & L \\
\hline 4113 & & 20 & $\mathrm{~L}$ \\
\hline 4114 & & 20 & L \\
\hline 4115 & & 20 & L \\
\hline 4116 & & 20 & L \\
\hline 4117 & & 20 & L \\
\hline 4118 & & 20 & L \\
\hline 4119 & & 20 & $\mathrm{~L}$ \\
\hline 4120 & IR-0008 Blank>CRDL & 20 & L \\
\hline 4121 & & 20 & L \\
\hline 4122 & & 20 & L \\
\hline 4123 & & 20 & L \\
\hline 4124 & & 20 & $\mathrm{~L}$ \\
\hline 4125 & IR-0008 Blank>CRDL & 20 & L \\
\hline 4126 & & 20 & L \\
\hline 4127 & & 20 & L \\
\hline 4128 & & 20 & L \\
\hline 4129 & & 20 & L \\
\hline 4130 & IR-0008 Blank>CRDL & 20 & $\mathrm{~L}$ \\
\hline 4131 & & 20 & L \\
\hline 4132 & & 20 & L \\
\hline 4133 & & 20 & L \\
\hline 4134 & & 20 & L \\
\hline 4135 & IR-0008 Blank>CRDL & 20 & L \\
\hline 4136 & & 20 & $\mathrm{~L}$ \\
\hline 4137 & & 20 & $\mathrm{~L}$ \\
\hline 4138 & & 20 & L \\
\hline 4139 & & 20 & $\mathrm{~L}$ \\
\hline 4140 & IR-0008 Blank>CRDL & 20 & L \\
\hline 4141 & & 20 & L \\
\hline 4142 & & 20 & $\mathrm{~L}$ \\
\hline 4143 & 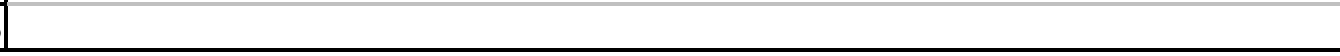 & 20 & $\mathrm{~L}$ \\
\hline
\end{tabular}




\begin{tabular}{|c|c|c|c|}
\hline & $A D$ & $\mathrm{AE}$ & $\mathrm{AF}$ \\
\hline 1 & RESULT_COMMENT & COLL_SAMP_SIZE & COLL_SAMP_SIZE_UNITS \\
\hline 4144 & & 20 & $L$ \\
\hline \begin{tabular}{|l|}
4145 \\
\end{tabular} & IR-0008 Blank>CRDL & 20 & L \\
\hline \begin{tabular}{|l|}
4146 \\
\end{tabular} & & 20 & L \\
\hline \begin{tabular}{|l|l|}
4147 \\
\end{tabular} & & 20 & L \\
\hline 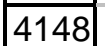 & & 20 & $\mathrm{~L}$ \\
\hline 4149 & & 20 & L \\
\hline \begin{tabular}{|l|}
4150 \\
\end{tabular} & IR-0008 Blank>CRDL & 20 & L \\
\hline \begin{tabular}{|l|}
4151 \\
\end{tabular} & & 20 & L \\
\hline 4152 & & 20 & L \\
\hline 4153 & & 20 & $\mathrm{~L}$ \\
\hline 4154 & & 20 & L \\
\hline \begin{tabular}{|l|l|}
4155 \\
\end{tabular} & IR-0008 Blank>CRDL & 20 & L \\
\hline 4156 & & 20 & L \\
\hline 4157 & & 20 & L \\
\hline 4158 & & 20 & L \\
\hline \begin{tabular}{|l|}
4159 \\
\end{tabular} & & 20 & L \\
\hline \begin{tabular}{|l|}
4160 \\
\end{tabular} & IR-0008 Blank>CRDL & 20 & L \\
\hline \begin{tabular}{|l|}
4161 \\
\end{tabular} & & 20 & $\mathrm{~L}$ \\
\hline 4162 & & 20 & $\mathrm{~L}$ \\
\hline 4163 & & 20 & L \\
\hline 4164 & & 20 & L \\
\hline \begin{tabular}{|l|l|}
4165 \\
\end{tabular} & & 20 & $\mathrm{~L}$ \\
\hline 4166 & IR-0019 Blank result > CRDL & 20 & L \\
\hline 4167 & IR-0019 Blank result > CRDL & 20 & L \\
\hline 4168 & & 20 & L \\
\hline 4169 & & 20 & L \\
\hline 4170 & & 20 & L \\
\hline \begin{tabular}{|l|}
4171 \\
\end{tabular} & & 20 & L \\
\hline \begin{tabular}{|l|}
4172 \\
\end{tabular} & & 20 & L \\
\hline \begin{tabular}{|l|l|}
4173 \\
\end{tabular} & & 20 & $\mathrm{~L}$ \\
\hline 4174 & & 20 & $\mathrm{~L}$ \\
\hline \begin{tabular}{|l|l|}
4175 \\
\end{tabular} & IR-0019 Blank result > CRDL & 20 & L \\
\hline 4176 & & 20 & L \\
\hline \begin{tabular}{|l|}
4177 \\
\end{tabular} & & 20 & L \\
\hline 4178 & & 20 & $\mathrm{~L}$ \\
\hline 4179 & & 20 & L \\
\hline 4180 & IR-0019 Blank result > CRDL & 20 & L \\
\hline 4181 & 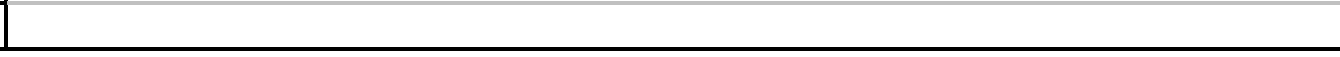 & 20 & $\mathrm{~L}$ \\
\hline
\end{tabular}




\begin{tabular}{|c|c|c|c|}
\hline & $A D$ & $\mathrm{AE}$ & AF \\
\hline 1 & RESULT_COMMENT & COLL_SAMP_SIZE & COLL_SAMP_SIZE_UNITS \\
\hline 4182 & & 20 & L \\
\hline 4183 & & 20 & L \\
\hline 4184 & & 20 & L \\
\hline $\begin{array}{ll}4185 \\
\end{array}$ & IR-0019 Blank result > CRDL & 20 & L \\
\hline 4186 & & 20 & L \\
\hline 4187 & & 20 & L \\
\hline 4188 & & 20 & L \\
\hline 4189 & & 20 & L \\
\hline | & IR-0019 Blank result > CRDL & 20 & L \\
\hline 4191 & & 20 & $\mathrm{~L}$ \\
\hline 4192 & & 20 & L \\
\hline 4193 & & 20 & L \\
\hline 4194 & & 20 & L \\
\hline |l & IR-0019 Blank result > CRDL & 20 & L \\
\hline 4196 & & 20 & L \\
\hline 4197 & & 20 & L \\
\hline 4198 & & 20 & L \\
\hline 4199 & & 20 & L \\
\hline $\begin{array}{ll}4200 & 1\end{array}$ & IR-0019 Blank result > CRDL & 20 & L \\
\hline 4201 & & 20 & L \\
\hline 4202 & & 20 & $\mathrm{~L}$ \\
\hline 4203 & & 20 & L \\
\hline 4204 & & 20 & $\mathrm{~L}$ \\
\hline 4205 & IR-0019 Blank result > CRDL & 20 & L \\
\hline 4206 & & 20 & L \\
\hline 4207 & & 20 & L \\
\hline 4208 & & 20 & L \\
\hline 4209 & & 20 & L \\
\hline 4210 ا & IR-0019 Blank result > CRDL & 20 & L \\
\hline 4211 & & 20 & $\mathrm{~L}$ \\
\hline 4212 & & 20 & L \\
\hline 4213 & & 20 & L \\
\hline 4214 & & 20 & $\mathrm{~L}$ \\
\hline 4215 & IR-0019 Blank result > CRDL & 20 & L \\
\hline 4216 & & 20 & $\mathrm{~L}$ \\
\hline 4217 & & 20 & L \\
\hline 4218 & & 20 & L \\
\hline 4219 & & 20 & $\mathrm{~L}$ \\
\hline
\end{tabular}




\begin{tabular}{|c|c|c|c|}
\hline & $A D$ & $\mathrm{AE}$ & $\mathrm{AF}$ \\
\hline 1 & RESULT_COMMENT & $\mid$ COLL_SAMP_SIZE & COLL_SAMP_SIZE_UNITS \\
\hline \begin{tabular}{|l|l|}
4220 \\
\end{tabular} & IR-0019 Blank result > CRDL & 20 & L \\
\hline \begin{tabular}{|l|}
4221 \\
\end{tabular} & & 20 & L \\
\hline \begin{tabular}{|l|l|}
4222 \\
\end{tabular} & & 20 & L \\
\hline \begin{tabular}{|l|l|}
4223 \\
\end{tabular} & & 20 & L \\
\hline \begin{tabular}{|l|}
4224 \\
\end{tabular} & & 20 & $\mathrm{~L}$ \\
\hline \begin{tabular}{|l|l|}
4225 \\
\end{tabular} & IR-0019 Blank result > CRDL & 20 & L \\
\hline \begin{tabular}{|l|l|}
4226 \\
\end{tabular} & & 20 & L \\
\hline \begin{tabular}{|l|}
4227 \\
\end{tabular} & & 20 & L \\
\hline \begin{tabular}{|l|l|}
4228 \\
\end{tabular} & & 20 & L \\
\hline \begin{tabular}{|l|}
4229 \\
\end{tabular} & & 20 & L \\
\hline \begin{tabular}{|l|l|}
4230 \\
\end{tabular} & IR-0019 Blank result > CRDL & 20 & $L$ \\
\hline \begin{tabular}{|l|}
4231 \\
\end{tabular} & & 20 & L \\
\hline 4232 & & 20 & $\mathrm{~L}$ \\
\hline \begin{tabular}{|l|}
4233 \\
\end{tabular} & & 20 & L \\
\hline \begin{tabular}{|l|}
4234 \\
\end{tabular} & & 20 & L \\
\hline $4 \mid$ & IR-0019 Blank result > CRDL & 20 & L \\
\hline \begin{tabular}{|l|l|}
4236 \\
\end{tabular} & & 20 & L \\
\hline \begin{tabular}{|l|}
4237 \\
\end{tabular} & & 20 & L \\
\hline \begin{tabular}{|l|}
4238 \\
\end{tabular} & & 20 & L \\
\hline \begin{tabular}{|l|l|}
4239 \\
\end{tabular} & & 20 & L \\
\hline \begin{tabular}{|l|l|}
4240 \\
\end{tabular} & & 20 & L \\
\hline \begin{tabular}{|l|}
4241 \\
\end{tabular} & & 20 & L \\
\hline \begin{tabular}{|l|l|}
4242 \\
\end{tabular} & & 20 & $\mathrm{~L}$ \\
\hline \begin{tabular}{|l|}
4243 \\
\end{tabular} & & 20 & L \\
\hline \begin{tabular}{|l|}
4244 \\
\end{tabular} & & 20 & L \\
\hline \begin{tabular}{|l|}
4245 \\
\end{tabular} & & 20 & L \\
\hline \begin{tabular}{|l|}
4246 \\
\end{tabular} & & 20 & L \\
\hline \begin{tabular}{|l|}
4247 \\
\end{tabular} & & 20 & L \\
\hline \begin{tabular}{|l|}
4248 \\
\end{tabular} & & 20 & L \\
\hline \begin{tabular}{|l|}
4249 \\
\end{tabular} & & 20 & L \\
\hline \begin{tabular}{|l|}
4250 \\
4250
\end{tabular} & & 20 & $L$ \\
\hline \begin{tabular}{|l|}
4251 \\
\end{tabular} & & 20 & L \\
\hline \begin{tabular}{|l|}
4252 \\
\end{tabular} & & 20 & $\mathrm{~L}$ \\
\hline \begin{tabular}{|l|}
4253 \\
\end{tabular} & & 20 & $\mathrm{~L}$ \\
\hline \begin{tabular}{|l|}
4254 \\
\end{tabular} & & 20 & L \\
\hline \begin{tabular}{|l|}
4255 \\
\end{tabular} & & 20 & $L$ \\
\hline \begin{tabular}{|l|}
4256 \\
\end{tabular} & & 20 & L \\
\hline \begin{tabular}{|l|}
4257 \\
\end{tabular} & & 20 & L \\
\hline
\end{tabular}




\begin{tabular}{|c|c|c|c|}
\hline & $A D$ & $\mathrm{AE}$ & $\mathrm{AF}$ \\
\hline 1 & RESULT_COMMENT & COLL_SAMP_SIZE & COLL_SAMP_SIZE_UNITS \\
\hline \begin{tabular}{|l|}
4258 \\
\end{tabular} & & 20 & $\bar{L}$ \\
\hline 4259 & & 20 & L \\
\hline 4260 & & 20 & L \\
\hline 4261 & & 20 & L \\
\hline 4262 & & 20 & L \\
\hline 4263 & & 20 & L \\
\hline \begin{tabular}{|l|}
4264 \\
\end{tabular} & & 20 & L \\
\hline \begin{tabular}{|l}
4265 \\
\end{tabular} & & 20 & L \\
\hline 4266 & & 20 & L \\
\hline \begin{tabular}{|l|l|}
4267 \\
\end{tabular} & & 20 & $\mathrm{~L}$ \\
\hline \begin{tabular}{|c|}
4268 \\
\end{tabular} & & 20 & L \\
\hline 4269 & & 20 & L \\
\hline \begin{tabular}{|l|l|}
4270 \\
\end{tabular} & REFER TO FOLLOW-UP ANALYSIS ORDER \#W051. & 20 & $\mathrm{~L}$ \\
\hline 4271 & & 20 & L \\
\hline 4272 & & 20 & L \\
\hline \begin{tabular}{|l|l}
4273 \\
\end{tabular} & & 20 & L \\
\hline 4274 & & 20 & L \\
\hline 4275 & REFER TO FOLLOW-UP ANALYSIS ORDER \#W051. & 20 & L \\
\hline \begin{tabular}{|l|}
4276 \\
\end{tabular} & & 20 & L \\
\hline \begin{tabular}{|l|}
4277 \\
\end{tabular} & & 20 & $\mathrm{~L}$ \\
\hline \begin{tabular}{|l|}
4278 \\
\end{tabular} & & 20 & $\mathrm{~L}$ \\
\hline \begin{tabular}{|l|}
4279 \\
\end{tabular} & & 20 & L \\
\hline \begin{tabular}{|l|}
4280 \\
\end{tabular} & & 20 & L \\
\hline \begin{tabular}{|l|}
4281 \\
\end{tabular} & & 20 & $\mathrm{~L}$ \\
\hline \begin{tabular}{|l|}
4282 \\
\end{tabular} & & 20 & L \\
\hline \begin{tabular}{|l|}
4283 \\
\end{tabular} & & 20 & $\mathrm{~L}$ \\
\hline \begin{tabular}{|l|}
4284 \\
\end{tabular} & & 20 & L \\
\hline \begin{tabular}{|l|}
4285 \\
\end{tabular} & & 20 & L \\
\hline 4286 & & 20 & L \\
\hline \begin{tabular}{|l|}
4287 \\
\end{tabular} & & 20 & L \\
\hline \begin{tabular}{|l|}
4288 \\
\end{tabular} & & 20 & L \\
\hline \begin{tabular}{|l|}
4289 \\
\end{tabular} & & 20 & $\mathrm{~L}$ \\
\hline \begin{tabular}{|l|}
4290 \\
\end{tabular} & & 20 & $\mathrm{~L}$ \\
\hline \begin{tabular}{|l|}
4291 \\
\end{tabular} & & 20 & $L$ \\
\hline \begin{tabular}{|l|}
4292 \\
\end{tabular} & & 20 & $\mathrm{~L}$ \\
\hline \begin{tabular}{|l|}
4293 \\
\end{tabular} & & 20 & $\mathrm{~L}$ \\
\hline \begin{tabular}{|l|}
4294 \\
\end{tabular} & & 20 & $\mathrm{~L}$ \\
\hline \begin{tabular}{|l}
4295 \\
\end{tabular} & & 20 & $\mathrm{~L}$ \\
\hline
\end{tabular}




\begin{tabular}{|c|c|c|c|}
\hline & $A D$ & $\mathrm{AE}$ & $\mathrm{AF}$ \\
\hline 1 & RESULT_COMMENT & COLL_SAMP_SIZE & EOLL_SAMP_SIZE_UNITS \\
\hline \begin{tabular}{|l|l|}
4296 \\
\end{tabular} & & 20 & $\mathrm{~L}$ \\
\hline \begin{tabular}{|l|}
4297 \\
\end{tabular} & & 20 & L \\
\hline \begin{tabular}{|l|}
4298 \\
\end{tabular} & & 20 & L \\
\hline \begin{tabular}{|l|}
4299 \\
\end{tabular} & & 20 & L \\
\hline \begin{tabular}{|l|}
4300 \\
\end{tabular} & & 20 & L \\
\hline \begin{tabular}{|l|}
4301 \\
\end{tabular} & & 20 & $\mathrm{~L}$ \\
\hline \begin{tabular}{|l|l|}
4302 \\
\end{tabular} & & 20 & L \\
\hline \begin{tabular}{|l|}
4303 \\
\end{tabular} & 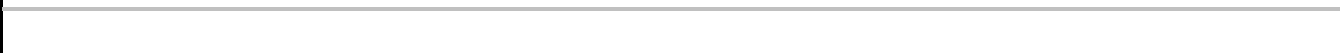 & 20 & L \\
\hline \begin{tabular}{|l|l|}
4304 \\
\end{tabular} & & 20 & L \\
\hline \begin{tabular}{|l|l|}
4305 \\
\end{tabular} & & 20 & L \\
\hline \begin{tabular}{|l|l|}
4306 \\
\end{tabular} & & 20 & $L$ \\
\hline \begin{tabular}{|l|}
4307 \\
\end{tabular} & & 20 & L \\
\hline \begin{tabular}{|l|}
4308 \\
\end{tabular} & & 20 & L \\
\hline \begin{tabular}{|l|}
4309 \\
\end{tabular} & & 20 & L \\
\hline \begin{tabular}{|l|l|}
4310 \\
\end{tabular} & & 20 & L \\
\hline \begin{tabular}{|l|}
4311 \\
\end{tabular} & & 20 & L \\
\hline \begin{tabular}{|l|l|}
4312 \\
\end{tabular} & & 20 & L \\
\hline \begin{tabular}{|l|l|}
4313 \\
\end{tabular} & & 20 & L \\
\hline \begin{tabular}{|l|}
4314 \\
\end{tabular} & 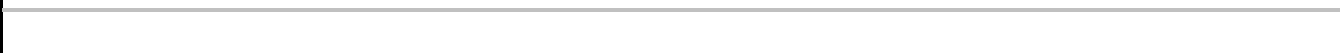 & 20 & L \\
\hline 4315 & & 20 & $\mathrm{~L}$ \\
\hline \begin{tabular}{|l|l|}
4316 \\
4316
\end{tabular} & & 20 & L \\
\hline \begin{tabular}{|l|}
4317 \\
\end{tabular} & & 20 & L \\
\hline \begin{tabular}{|l|l|}
4318 \\
4318
\end{tabular} & & 20 & L \\
\hline \begin{tabular}{|l|}
4319 \\
\end{tabular} & & 20 & L \\
\hline \begin{tabular}{|l|}
4320 \\
\end{tabular} & & 20 & L \\
\hline \begin{tabular}{|l|}
4321 \\
\end{tabular} & & 20 & L \\
\hline \begin{tabular}{|l|}
4322 \\
\end{tabular} & & 20 & L \\
\hline \begin{tabular}{|l|}
4323 \\
\end{tabular} & & 20 & L \\
\hline \begin{tabular}{|l|}
4324 \\
\end{tabular} & & 20 & L \\
\hline \begin{tabular}{|l|}
4325 \\
\end{tabular} & & 20 & L \\
\hline \begin{tabular}{|l|}
4326 \\
\end{tabular} & & 20 & L \\
\hline \begin{tabular}{|l|}
4327 \\
\end{tabular} & & 20 & L \\
\hline \begin{tabular}{|l|}
4328 \\
\end{tabular} & & 20 & $\mathrm{~L}$ \\
\hline \begin{tabular}{|l|}
4329 \\
\end{tabular} & & 20 & L \\
\hline \begin{tabular}{|l|}
4330 \\
\end{tabular} & & 20 & L \\
\hline \begin{tabular}{|l}
4331 \\
\end{tabular} & & 20 & $L$ \\
\hline \begin{tabular}{|l|}
4332 \\
\end{tabular} & & 20 & L \\
\hline \begin{tabular}{|l|}
4333 \\
\end{tabular} & 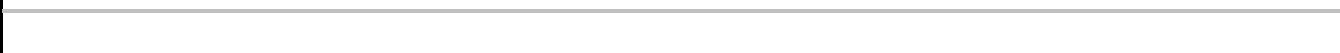 & 20 & L \\
\hline
\end{tabular}




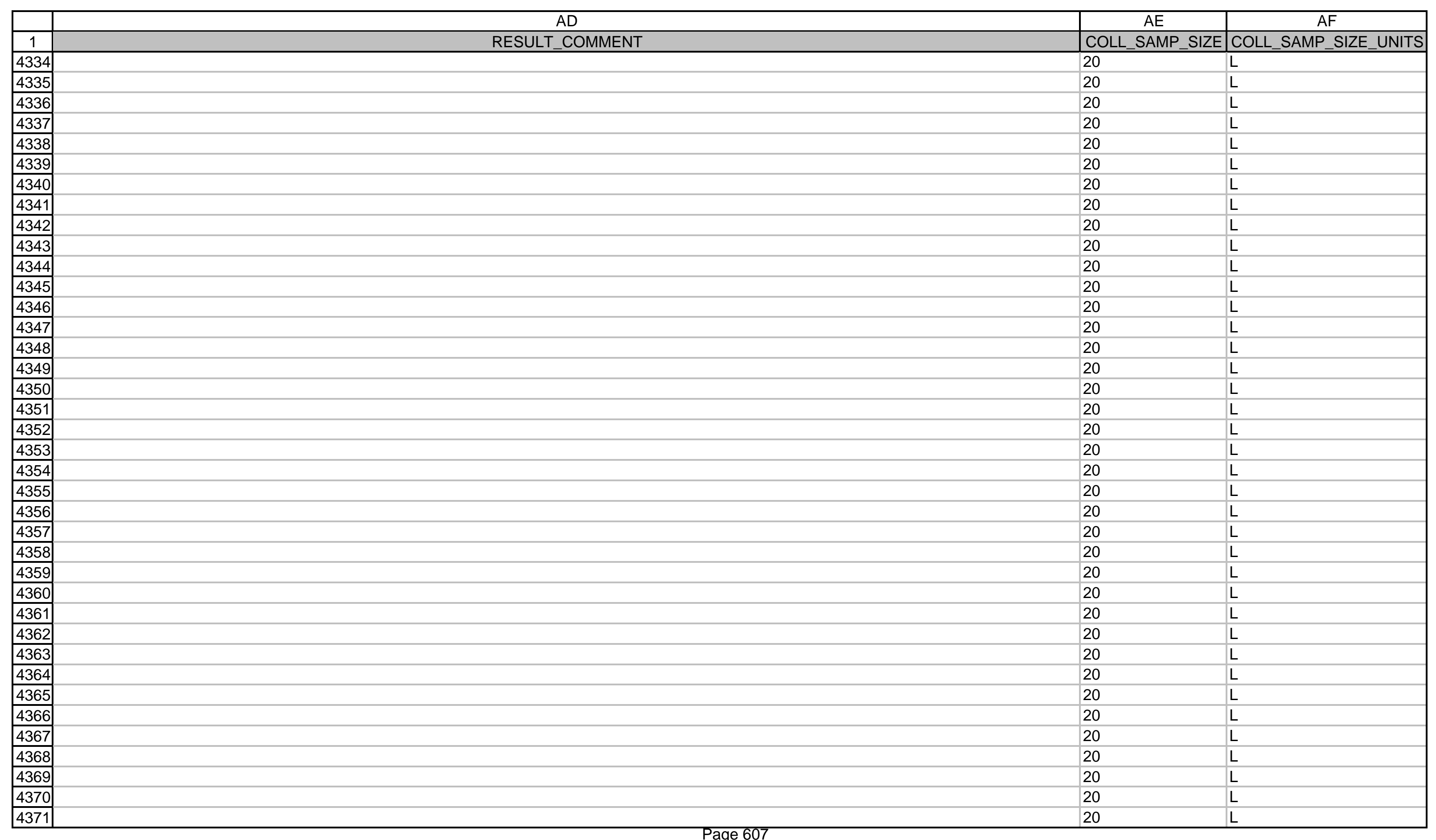




\begin{tabular}{|c|c|c|c|}
\hline & $A D$ & $\mathrm{AE}$ & $\mathrm{AF}$ \\
\hline 1 & RESULT_COMMENT & COLL_SAMP_SIZE & COLL_SAMP_SIZE_UNITS \\
\hline \begin{tabular}{|l|}
4372 \\
\end{tabular} & & 20 & L \\
\hline \begin{tabular}{|l|}
4373 \\
\end{tabular} & & 20 & $\mathrm{~L}$ \\
\hline \begin{tabular}{|l|}
4374 \\
\end{tabular} & & 20 & L \\
\hline \begin{tabular}{|l|l|}
4375 \\
\end{tabular} & & 20 & $\mathrm{~L}$ \\
\hline \begin{tabular}{|l|}
4376 \\
\end{tabular} & & 20 & $\mathrm{~L}$ \\
\hline \begin{tabular}{|l|}
4377 \\
\end{tabular} & & 20 & $\mathrm{~L}$ \\
\hline \begin{tabular}{|l|}
4378 \\
\end{tabular} & 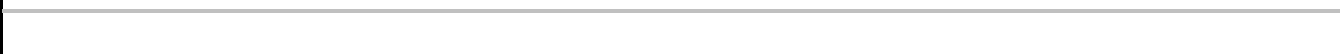 & 20 & $\mathrm{~L}$ \\
\hline \begin{tabular}{|l|}
4379 \\
\end{tabular} & 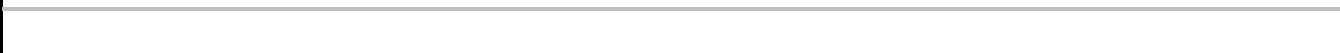 & 20 & $\mathrm{~L}$ \\
\hline \begin{tabular}{|l|l|}
4380 \\
\end{tabular} & & 20 & $\mathrm{~L}$ \\
\hline \begin{tabular}{|l|l|}
4381 \\
43
\end{tabular} & & 20 & $\mathrm{~L}$ \\
\hline \begin{tabular}{|l|}
4382 \\
\end{tabular} & & 20 & $\mathrm{~L}$ \\
\hline \begin{tabular}{|l|l|}
4383 \\
\end{tabular} & & 20 & $\mathrm{~L}$ \\
\hline \begin{tabular}{|l|}
4384 \\
\end{tabular} & & 20 & L \\
\hline \begin{tabular}{|l|l|}
4385 \\
\end{tabular} & & 20 & $\mathrm{~L}$ \\
\hline \begin{tabular}{|l|l|}
4386 \\
\end{tabular} & & 20 & $\mathrm{~L}$ \\
\hline \begin{tabular}{|l|l|}
4387 \\
\end{tabular} & & 20 & $\mathrm{~L}$ \\
\hline \begin{tabular}{|l|}
4388 \\
\end{tabular} & & 20 & $\mathrm{~L}$ \\
\hline \begin{tabular}{|l|}
43809 \\
4389
\end{tabular} & & 20 & $L$ \\
\hline \begin{tabular}{|l|l|}
4390 \\
\end{tabular} & - & 20 & L \\
\hline \begin{tabular}{|l|}
4391 \\
\end{tabular} & & 20 & $\mathrm{~L}$ \\
\hline \begin{tabular}{|l|l|}
4392 \\
\end{tabular} & & 20 & L \\
\hline \begin{tabular}{|l|l|}
4393 \\
\end{tabular} & & 20 & $\mathrm{~L}$ \\
\hline \begin{tabular}{|l|l|}
4394 \\
\end{tabular} & & 20 & $L$ \\
\hline \begin{tabular}{|l|}
4395 \\
\end{tabular} & & 20 & $\mathrm{~L}$ \\
\hline \begin{tabular}{|l|}
4396 \\
\end{tabular} & & 20 & L \\
\hline \begin{tabular}{|l|}
4397 \\
\end{tabular} & & 20 & L \\
\hline \begin{tabular}{|l|}
4398 \\
\end{tabular} & & 20 & $\mathrm{~L}$ \\
\hline \begin{tabular}{|l|}
4399 \\
\end{tabular} & & 20 & $\mathrm{~L}$ \\
\hline \begin{tabular}{|l|}
4400 \\
\end{tabular} & & 20 & $\mathrm{~L}$ \\
\hline \begin{tabular}{|l|}
4401 \\
\end{tabular} & & 20 & $\mathrm{~L}$ \\
\hline \begin{tabular}{|l|}
4402 \\
4402 \\
\end{tabular} & & 20 & $L$ \\
\hline \begin{tabular}{|l|}
4403 \\
\end{tabular} & & 20 & $\mathrm{~L}$ \\
\hline \begin{tabular}{|l|}
4404 \\
\end{tabular} & & 20 & $\mathrm{~L}$ \\
\hline \begin{tabular}{|l|}
4405 \\
\end{tabular} & & 20 & $\mathrm{~L}$ \\
\hline \begin{tabular}{|l|}
4406 \\
\end{tabular} & & 20 & $\mathrm{~L}$ \\
\hline \begin{tabular}{|l|}
4407 \\
\end{tabular} & & 20 & $\mathrm{~L}$ \\
\hline \begin{tabular}{|l|}
4408 \\
\end{tabular} & & 20 & $\mathrm{~L}$ \\
\hline \begin{tabular}{|l|}
4409 \\
\end{tabular} & 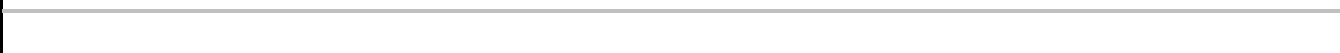 & 20 & $\mathrm{~L}$ \\
\hline
\end{tabular}




\begin{tabular}{|c|c|c|c|}
\hline & $A D$ & $\mathrm{AE}$ & $\mathrm{AF}$ \\
\hline 1 & RESULT_COMMENT & COLL_SAMP_SIZE & COLL_SAMP_SIZE_UNITS \\
\hline 4410 & & 20 & $\mathrm{~L}$ \\
\hline \begin{tabular}{|l|}
4411 \\
\end{tabular} & & 20 & L \\
\hline \begin{tabular}{|l|}
4412 \\
\end{tabular} & & 20 & L \\
\hline \begin{tabular}{|l|}
4413 \\
\end{tabular} & & 20 & L \\
\hline \begin{tabular}{|l|}
4414 \\
\end{tabular} & & 20 & L \\
\hline \begin{tabular}{|l|}
4415 \\
\end{tabular} & & 20 & L \\
\hline \begin{tabular}{|l|}
4416 \\
\end{tabular} & & 20 & L \\
\hline \begin{tabular}{|l|}
4417 \\
\end{tabular} & 7) & 20 & L \\
\hline \begin{tabular}{|l|}
4418 \\
\end{tabular} & 3 & 20 & L \\
\hline \begin{tabular}{|l|}
4419 \\
\end{tabular} & ) & 20 & L \\
\hline \begin{tabular}{|l|}
4420 \\
\end{tabular} & & 20 & L \\
\hline \begin{tabular}{|l|}
4421 \\
\end{tabular} & & 20 & $\mathrm{~L}$ \\
\hline \begin{tabular}{|l|}
4422 \\
\end{tabular} & & 20 & L \\
\hline 4423 & & 20 & $\mathrm{~L}$ \\
\hline 4424 & & 20 & L \\
\hline \begin{tabular}{|l|}
4425 \\
\end{tabular} & & 20 & L \\
\hline \begin{tabular}{|l|}
4426 \\
\end{tabular} & & 20 & L \\
\hline \begin{tabular}{|l|}
4427 \\
\end{tabular} & & 20 & L \\
\hline \begin{tabular}{|l|}
4428 \\
\end{tabular} & & 20 & L \\
\hline \begin{tabular}{|l|}
4429 \\
\end{tabular} & & 20 & L \\
\hline \begin{tabular}{|l|}
4430 \\
\end{tabular} & & 20 & L \\
\hline \begin{tabular}{|l|}
4431 \\
\end{tabular} & & 20 & L \\
\hline \begin{tabular}{|l|}
4432 \\
\end{tabular} & & 20 & L \\
\hline \begin{tabular}{|l|}
4433 \\
\end{tabular} & & 20 & L \\
\hline \begin{tabular}{|l|}
4434 \\
\end{tabular} & & 20 & $\mathrm{~L}$ \\
\hline \begin{tabular}{|l|}
4435 \\
\end{tabular} & & 20 & L \\
\hline \begin{tabular}{|l|}
4436 \\
\end{tabular} & & 20 & L \\
\hline \begin{tabular}{|l|}
4437 \\
\end{tabular} & & 20 & $\mathrm{~L}$ \\
\hline \begin{tabular}{|l|}
4438 \\
\end{tabular} & & 20 & L \\
\hline \begin{tabular}{|l|}
4439 \\
\end{tabular} & & 20 & L \\
\hline \begin{tabular}{|l|}
4440 \\
\end{tabular} & & 20 & L \\
\hline \begin{tabular}{|l|}
4441 \\
\end{tabular} & & 20 & $\mathrm{~L}$ \\
\hline \begin{tabular}{|l|}
4442 \\
\end{tabular} & & 20 & L \\
\hline \begin{tabular}{|l|}
4443 \\
\end{tabular} & & 20 & L \\
\hline \begin{tabular}{|l|}
4444 \\
\end{tabular} & & 20 & L \\
\hline \begin{tabular}{|l|}
4445 \\
\end{tabular} & & 20 & L \\
\hline \begin{tabular}{|l|}
4446 \\
\end{tabular} & & 20 & L \\
\hline \begin{tabular}{|l|}
4447 \\
\end{tabular} & 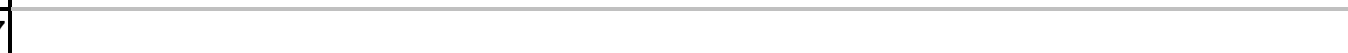 & 20 & $\mathrm{~L}$ \\
\hline
\end{tabular}




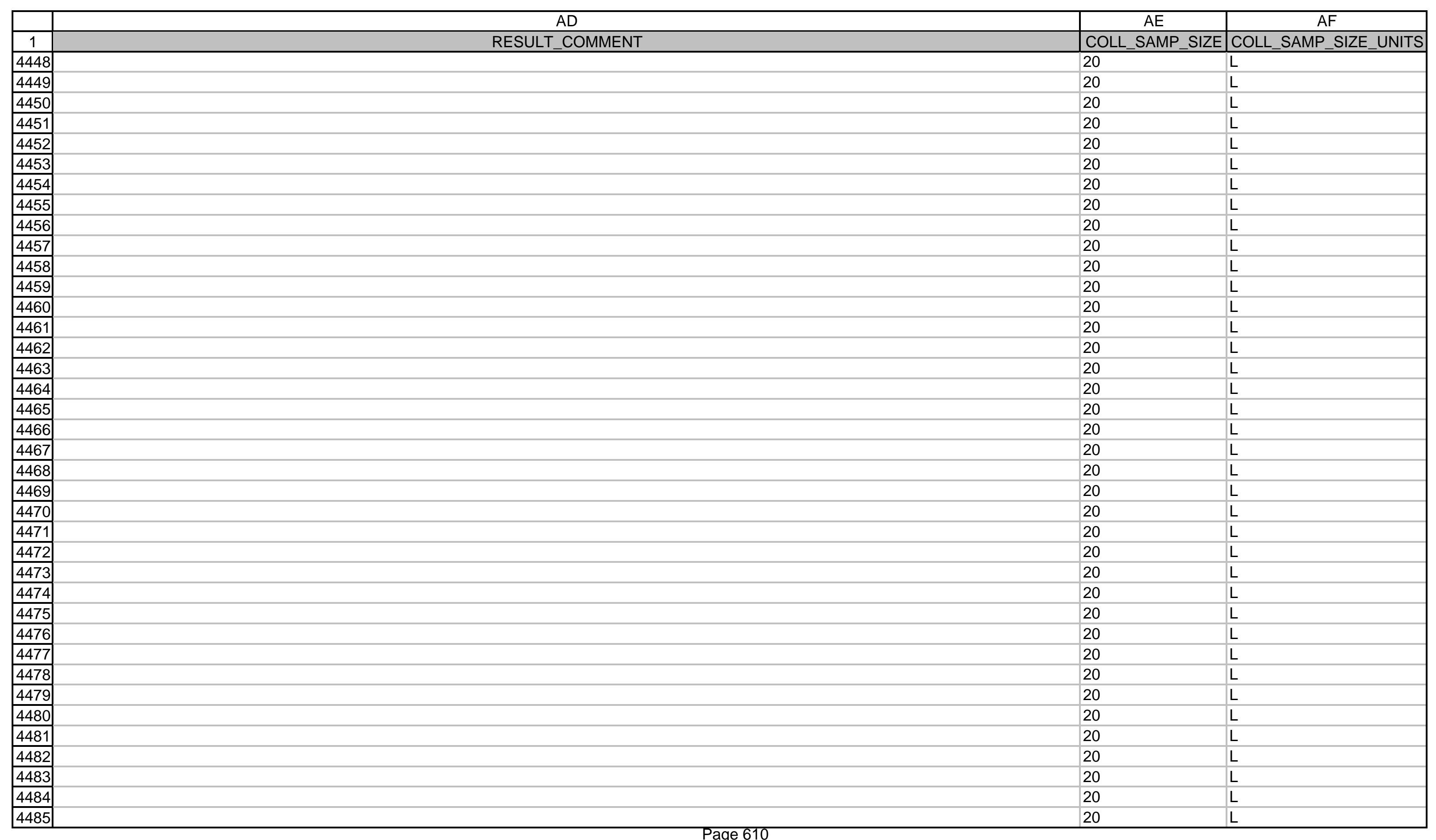




\begin{tabular}{|c|c|c|c|}
\hline & $A D$ & $\mathrm{AE}$ & $\mathrm{AF}$ \\
\hline 1 & RESULT_COMMENT & COLL_SAMP_SIZE & COLL_SAMP_SIZE_UNITS \\
\hline \begin{tabular}{|l|l|}
4486 \\
\end{tabular} & & 20 & L \\
\hline \begin{tabular}{|l|}
4487 \\
\end{tabular} & & 20 & $\mathrm{~L}$ \\
\hline \begin{tabular}{|l|}
4488 \\
\end{tabular} & & 20 & L \\
\hline \begin{tabular}{|l|l|}
4489 \\
\end{tabular} & & 20 & $\mathrm{~L}$ \\
\hline \begin{tabular}{|l|}
4490 \\
\end{tabular} & & 20 & $\mathrm{~L}$ \\
\hline \begin{tabular}{|l|}
4491 \\
\end{tabular} & 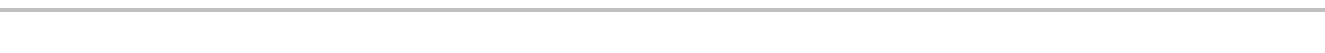 & 20 & $\mathrm{~L}$ \\
\hline \begin{tabular}{|l|l|}
4492 \\
\end{tabular} & - & 20 & $\mathrm{~L}$ \\
\hline \begin{tabular}{|l|}
4493 \\
\end{tabular} & & 20 & $\mathrm{~L}$ \\
\hline \begin{tabular}{|l|l|}
4494 \\
\end{tabular} & & & \\
\hline \begin{tabular}{|l|l|}
4495 \\
\end{tabular} & & & \\
\hline \begin{tabular}{|l|l|}
4496 \\
\end{tabular} & & & \\
\hline \begin{tabular}{|l|}
4497 \\
\end{tabular} & & & \\
\hline \begin{tabular}{|l|}
4498 \\
\end{tabular} & & & \\
\hline \begin{tabular}{|l|}
4499 \\
\end{tabular} & & & \\
\hline \begin{tabular}{|l|l|}
4500 \\
\end{tabular} & & & \\
\hline \begin{tabular}{|l|}
4501 \\
\end{tabular} & & & \\
\hline \begin{tabular}{|l|}
4502 \\
\end{tabular} & & & \\
\hline \begin{tabular}{|l|l|}
4503 \\
\end{tabular} & & & \\
\hline \begin{tabular}{|l|}
4504 \\
\end{tabular} & & 20 & L \\
\hline 4505 & & 20 & $\mathrm{~L}$ \\
\hline \begin{tabular}{|l|l|}
4506 \\
4
\end{tabular} & & 20 & L \\
\hline \begin{tabular}{|l|l|}
4507 \\
\end{tabular} & & 20 & $\mathrm{~L}$ \\
\hline \begin{tabular}{|l|}
4508 \\
450
\end{tabular} & & 20 & $L$ \\
\hline \begin{tabular}{|l|}
4509 \\
\end{tabular} & & 20 & $\mathrm{~L}$ \\
\hline \begin{tabular}{|l|}
4510 \\
\end{tabular} & & 20 & $\mathrm{~L}$ \\
\hline \begin{tabular}{|l|}
4511 \\
\end{tabular} & & 20 & $\mathrm{~L}$ \\
\hline \begin{tabular}{|l|}
4512 \\
\end{tabular} & & 20 & $\mathrm{~L}$ \\
\hline \begin{tabular}{|l|}
4513 \\
\end{tabular} & & 20 & $\mathrm{~L}$ \\
\hline \begin{tabular}{|l|}
4514 \\
\end{tabular} & & 20 & $\mathrm{~L}$ \\
\hline \begin{tabular}{|l|}
4515 \\
\end{tabular} & & 20 & $\mathrm{~L}$ \\
\hline \begin{tabular}{|l|}
4516 \\
\end{tabular} & & 20 & $\mathrm{~L}$ \\
\hline \begin{tabular}{|l|}
4517 \\
\end{tabular} & & 20 & $\mathrm{~L}$ \\
\hline \begin{tabular}{|l|}
4518 \\
\end{tabular} & & 20 & $\mathrm{~L}$ \\
\hline \begin{tabular}{|l|}
4519 \\
\end{tabular} & & 20 & $\mathrm{~L}$ \\
\hline \begin{tabular}{|l|}
4520 \\
\end{tabular} & & 20 & $\mathrm{~L}$ \\
\hline \begin{tabular}{|l|}
4521 \\
\end{tabular} & & 20 & $L$ \\
\hline \begin{tabular}{|l|}
4522 \\
\end{tabular} & & 20 & $\mathrm{~L}$ \\
\hline \begin{tabular}{|l|}
4523 \\
\end{tabular} & 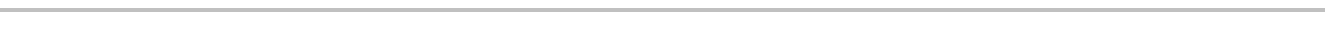 & 20 & $\mathrm{~L}$ \\
\hline
\end{tabular}




\begin{tabular}{|c|c|c|c|}
\hline & $A D$ & $\mathrm{AE}$ & $\mathrm{AF}$ \\
\hline 1 & RESULT_COMMENT & COLL_SAMP_SIZE & COLL_SAMP_SIZE_UNITS \\
\hline \multicolumn{4}{|c|}{ 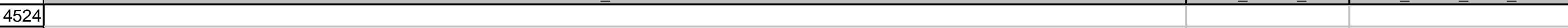 } \\
\hline \multicolumn{4}{|l|}{4525} \\
\hline \multicolumn{4}{|l|}{4526} \\
\hline \multicolumn{4}{|l|}{4527} \\
\hline \begin{tabular}{|l|}
4528 \\
\end{tabular} & & 20 & L \\
\hline \begin{tabular}{|l|}
4529 \\
\end{tabular} & & 20 & L \\
\hline \begin{tabular}{|l|}
4530 \\
\end{tabular} & 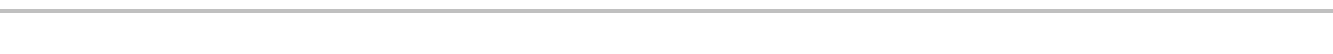 & 20 & L \\
\hline \begin{tabular}{|l|}
4531 \\
\end{tabular} & 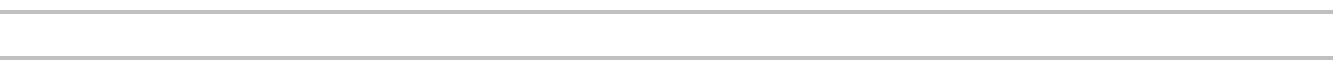 & 20 & L \\
\hline \begin{tabular}{|l|}
4532 \\
\end{tabular} & 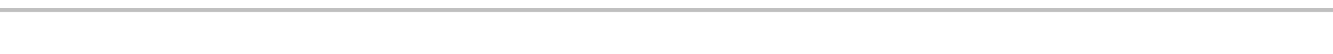 & 20 & L \\
\hline \begin{tabular}{|l|}
4533 \\
\end{tabular} & & 20 & L \\
\hline \begin{tabular}{|l|}
4534 \\
\end{tabular} & & 20 & $L$ \\
\hline \begin{tabular}{|l|}
4535 \\
\end{tabular} & & 20 & L \\
\hline \begin{tabular}{|l|}
4536 \\
\end{tabular} & & 20 & $L$ \\
\hline \begin{tabular}{|l|}
4537 \\
\end{tabular} & & 20 & L \\
\hline \begin{tabular}{|l|}
4538 \\
\end{tabular} & & 20 & L \\
\hline \begin{tabular}{|l|}
4539 \\
\end{tabular} & & 20 & L \\
\hline \begin{tabular}{|l|}
4540 \\
\end{tabular} & & 20 & L \\
\hline \begin{tabular}{|l|}
4541 \\
\end{tabular} & & 20 & L \\
\hline \begin{tabular}{|l|}
4542 \\
\end{tabular} & 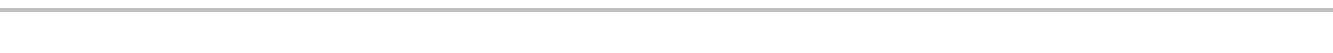 & 20 & L \\
\hline \begin{tabular}{|l|}
4543 \\
\end{tabular} & 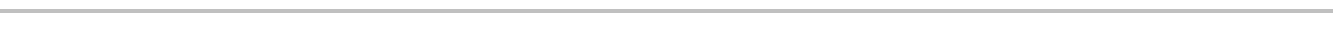 & 20 & L \\
\hline \begin{tabular}{|l|}
4544 \\
\end{tabular} & 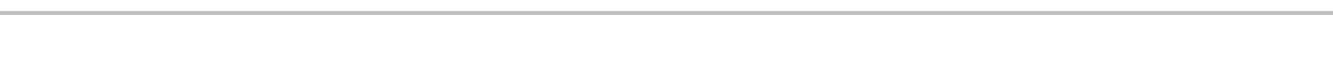 & 20 & L \\
\hline \begin{tabular}{|l|}
4545 \\
\end{tabular} & & 20 & L \\
\hline \begin{tabular}{|l|}
4546 \\
\end{tabular} & & 20 & $\mathrm{~L}$ \\
\hline \begin{tabular}{|l|}
4547 \\
\end{tabular} & & 20 & L \\
\hline \begin{tabular}{|l|}
4548 \\
\end{tabular} & & 20 & L \\
\hline \begin{tabular}{|l|}
4549 \\
\end{tabular} & & 20 & L \\
\hline \begin{tabular}{|l|}
4550 \\
\end{tabular} & & 20 & L \\
\hline \begin{tabular}{|l|}
4551 \\
\end{tabular} & & 20 & L \\
\hline \begin{tabular}{|l|}
4552 \\
\end{tabular} & & 20 & L \\
\hline \begin{tabular}{|l|}
4553 \\
\end{tabular} & & 20 & L \\
\hline \begin{tabular}{|l|}
4554 \\
\end{tabular} & & 20 & L \\
\hline \begin{tabular}{|l|}
4555 \\
\end{tabular} & & 20 & L \\
\hline \begin{tabular}{|l|}
4556 \\
\end{tabular} & & 20 & L \\
\hline \begin{tabular}{|l|}
4557 \\
\end{tabular} & & 20 & L \\
\hline \begin{tabular}{|l|}
4558 \\
\end{tabular} & & 20 & L \\
\hline \begin{tabular}{|l|}
4559 \\
\end{tabular} & & 20 & $L$ \\
\hline \begin{tabular}{|l|}
4560 \\
\end{tabular} & & 20 & L \\
\hline \begin{tabular}{|l|}
4561 \\
\end{tabular} & 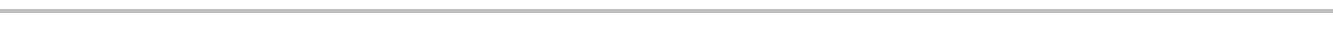 & 20 & L \\
\hline
\end{tabular}




\begin{tabular}{|c|c|c|c|}
\hline & 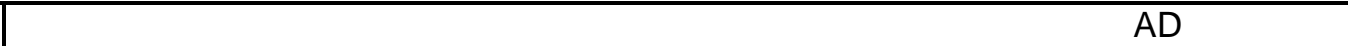 & $\mathrm{AE}$ & $\mathrm{AF}$ \\
\hline 1 & $\begin{array}{lc} & \text { RESULT_COMMENT } \\
\end{array}$ & COLL_SAMP_SIZE & COLL_SAMP_SIZE_UNITS \\
\hline 4562 & & 20 & $\mathrm{~L}$ \\
\hline 4563 & & 20 & L \\
\hline 4564 & & 20 & L \\
\hline 4565 & 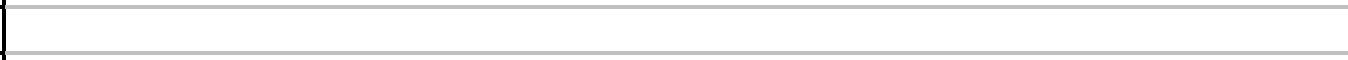 & 20 & L \\
\hline 4566 & 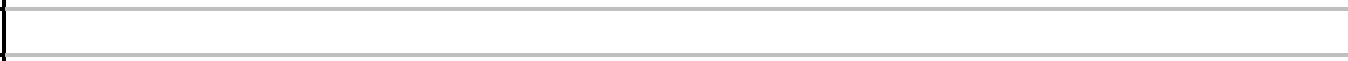 & 20 & L \\
\hline 4567 & & 20 & L \\
\hline 4568 & & 20 & L \\
\hline 4569 & & 20 & L \\
\hline 4570 & & 20 & L \\
\hline 4571 & & 20 & L \\
\hline 4572 & & 20 & L \\
\hline 4573 & & 20 & L \\
\hline 4574 & & 20 & $\mathrm{~L}$ \\
\hline 4575 & & 20 & L \\
\hline 4576 & & 20 & L \\
\hline 4577 & & 20 & $L$ \\
\hline 4578 & & 20 & L \\
\hline 4579 & & 20 & L \\
\hline 4580 & & 20 & $\mathrm{~L}$ \\
\hline 4581 & & 20 & L \\
\hline 4582 & & 20 & L \\
\hline 4583 & & 20 & L \\
\hline 4584 & & 20 & L \\
\hline 4585 & & 20 & L \\
\hline 4586 & & 20 & $\mathrm{~L}$ \\
\hline 4587 & & 20 & L \\
\hline \begin{tabular}{|l|l|}
4588 \\
\end{tabular} & & 20 & L \\
\hline 4589 & & 20 & $L$ \\
\hline 4590 & & 20 & L \\
\hline 4591 & & 20 & L \\
\hline 4592 & & 20 & $L$ \\
\hline 4593 & & 20 & $L$ \\
\hline 4594 & & 20 & L \\
\hline 4595 & & 20 & L \\
\hline \begin{tabular}{|l|}
4596 \\
\end{tabular} & & 20 & L \\
\hline 4597 & & 20 & L \\
\hline 4598 & & 20 & L \\
\hline 4599 & 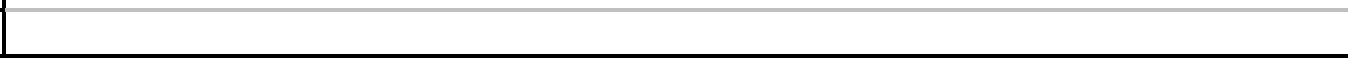 & 20 & $\mathrm{~L}$ \\
\hline
\end{tabular}




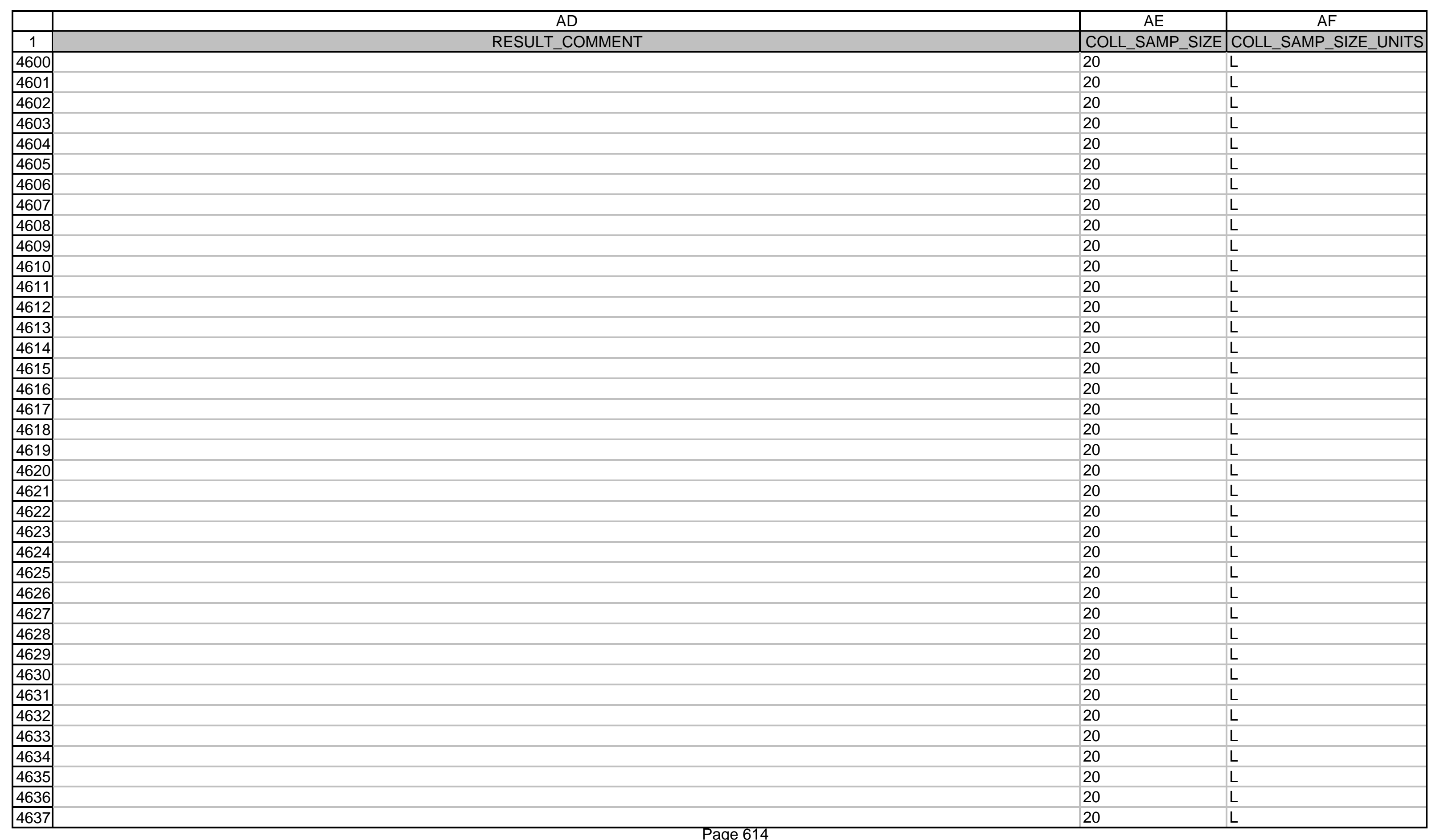




\begin{tabular}{|c|c|c|}
\hline & 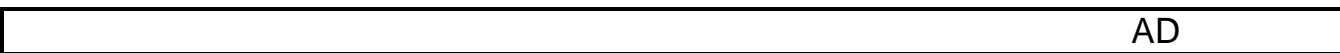 & $\mathrm{AF}$ \\
\hline 1 & RESULT_COMMENT & COLL_SAMP_SIZE_UNITS \\
\hline \begin{tabular}{|l|l|}
4638 \\
\end{tabular} & & $\mathrm{~L}$ \\
\hline 4639 & & L \\
\hline 4640 & & L \\
\hline 4641 & & L \\
\hline 4642 & & $\mathrm{~L}$ \\
\hline 4643 & & $\mathrm{~L}$ \\
\hline \begin{tabular}{|l|}
4644 \\
\end{tabular} & & L \\
\hline 4645 & & $\mathrm{~L}$ \\
\hline 4646 & - & $\mathrm{L}$ \\
\hline \begin{tabular}{|l|l|}
4647 \\
\end{tabular} & 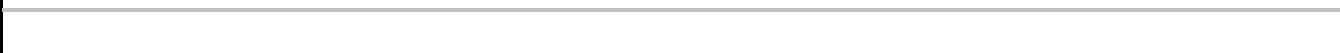 & $\mathrm{L}$ \\
\hline 4648 & 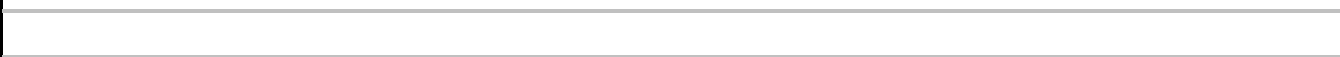 & L \\
\hline 4649 & & $\mathrm{~L}$ \\
\hline 4650 & & L \\
\hline 4651 & & $\mathrm{~L}$ \\
\hline 4652 & & L \\
\hline 4653 & & L \\
\hline 4654 & & $\mathrm{~L}$ \\
\hline 4655 & & $\mathrm{~L}$ \\
\hline 4656 & & $\mathrm{~L}$ \\
\hline 4657 & & $\mathrm{~L}$ \\
\hline 4658 & & $\mathrm{~L}$ \\
\hline 4659 & & $\mathrm{~L}$ \\
\hline 4660 & & $\mathrm{~L}$ \\
\hline 4661 & & $\mathrm{~L}$ \\
\hline 4662 & & $\bar{L}$ \\
\hline 4663 & & $\mathrm{~L}$ \\
\hline 4664 & & $\mathrm{~L}$ \\
\hline 4665 & & L \\
\hline 4666 & & L \\
\hline \begin{tabular}{|l|}
4667 \\
\end{tabular} & & L \\
\hline \begin{tabular}{|l|}
4668 \\
\end{tabular} & & $\mathrm{~L}$ \\
\hline \begin{tabular}{|l|}
4669 \\
\end{tabular} & & $\mathrm{~L}$ \\
\hline \begin{tabular}{|l|}
4670 \\
\end{tabular} & & $\mathrm{~L}$ \\
\hline \begin{tabular}{|l|}
4671 \\
\end{tabular} & & $\mathrm{~L}$ \\
\hline \begin{tabular}{|l|}
4672 \\
\end{tabular} & 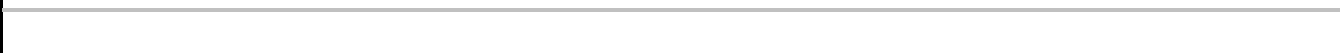 & $\mathrm{L}$ \\
\hline
\end{tabular}




\begin{tabular}{|c|l|l|l|}
\hline & AG & AH & \\
\hline 1 & DIST_CLASS & OWNER_ID & COLLECTION_PURPOSE \\
\hline 2 & OFFSITE & SESPMNT & $\mathrm{R}$ \\
\hline 3 & OFFSITE & SESPMNT & $\mathrm{R}$ \\
\hline 4 & OFFSITE & SESPMNT & $\mathrm{R}$ \\
\hline 5 & OFFSITE & SESPMNT & $\mathrm{R}$ \\
\hline 6 & OFFSITE & SESPMNT & $\mathrm{R}$ \\
\hline 7 & OFFSITE & SESPMNT & $\mathrm{R}$ \\
\hline 8 & OFFSITE & SESPMNT & $\mathrm{R}$ \\
\hline 9 & OFFSITE & SESPMNT & $\mathrm{R}$ \\
\hline 10 & OFFSITE & SESPMNT & $\mathrm{R}$ \\
\hline 11 & OFFSITE & SESPMNT & $\mathrm{R}$ \\
\hline 12 & OFFSITE & SESPMNT & $\mathrm{R}$ \\
\hline 13 & OFFSITE & SESPMNT & $\mathrm{R}$ \\
\hline 14 & OFFSITE & SESPMNT & $\mathrm{R}$ \\
\hline 15 & OFFSITE & SESPMNT & $\mathrm{R}$ \\
\hline 16 & OFFSITE & SESPMNT & $\mathrm{R}$ \\
\hline 17 & OFFSITE & SESPMNT & $\mathrm{R}$ \\
\hline 18 & OFFSITE & SESPMNT & $\mathrm{R}$ \\
\hline 19 & OFFSITE & SESPMNT & $\mathrm{R}$ \\
\hline 20 & OFFSITE & SESPMNT & $\mathrm{R}$ \\
\hline 21 & OFFSITE & SESPMNT & $\mathrm{R}$ \\
\hline 22 & OFFSITE & SESPMNT & $\mathrm{R}$ \\
\hline 23 & OFFSITE & SESPMNT & $\mathrm{R}$ \\
\hline 24 & OFFSITE & SESPMNT & $\mathrm{R}$ \\
\hline 25 & OFFSITE & SESPMNT & $\mathrm{R}$ \\
\hline 26 & OFFSITE & SESPMNT & $\mathrm{R}$ \\
\hline 27 & OFFSITE & SESPMNT & $\mathrm{R}$ \\
\hline 28 & OFFSITE & SESPMNT & $\mathrm{R}$ \\
\hline 29 & OFFSITE & SESPMNT & $\mathrm{R}$ \\
\hline 30 & OFFSITE & SESPMNT & $\mathrm{R}$ \\
\hline 31 & OFFSITE & SESPMNT & $\mathrm{R}$ \\
\hline 32 & OFFSITE & SESPMNT & $\mathrm{R}$ \\
\hline 33 & OFFSITE & SESPMNT & $\mathrm{R}$ \\
\hline 34 & OFFSITE & SESPMNT & $\mathrm{R}$ \\
\hline 35 & OFFSITE & SESPMNT & $\mathrm{R}$ \\
\hline 36 & OFFSITE & SESPMNT & $\mathrm{R}$ \\
\hline 37 & OFFSITE & SESPMNT & $\mathrm{R}$ \\
\hline 38 & OFFSITE & SESPMNT & $\mathrm{R}$ \\
\hline 39 & OFFSITE & SESPMNT & $\mathrm{R}$ \\
\hline & & & \\
\hline
\end{tabular}




\begin{tabular}{|c|l|l|l|}
\hline & AG & AH & \\
\hline 1 & DIST_CLASS & OWNER_ID & COLLECTION_PURPOSE \\
\hline 40 & OFFSITE & SESPMNT & $\mathrm{R}$ \\
\hline 41 & OFFSITE & SESPMNT & $\mathrm{R}$ \\
\hline 42 & OFFSITE & SESPMNT & $\mathrm{R}$ \\
\hline 43 & OFFSITE & SESPMNT & $\mathrm{R}$ \\
\hline 44 & OFFSITE & SESPMNT & $\mathrm{R}$ \\
\hline 45 & OFFSITE & SESPMNT & $\mathrm{R}$ \\
\hline 46 & OFFSITE & SESPMNT & $\mathrm{R}$ \\
\hline 47 & OFFSITE & SESPMNT & $\mathrm{R}$ \\
\hline 48 & OFFSITE & SESPMNT & $\mathrm{R}$ \\
\hline 49 & OFFSITE & SESPMNT & $\mathrm{R}$ \\
\hline 50 & OFFSITE & SESPMNT & $\mathrm{R}$ \\
\hline 51 & OFFSITE & SESPMNT & $\mathrm{R}$ \\
\hline 52 & OFFSITE & SESPMNT & $\mathrm{R}$ \\
\hline 53 & OFFSITE & SESPMNT & $\mathrm{R}$ \\
\hline 54 & OFFSITE & SESPMNT & $\mathrm{R}$ \\
\hline 55 & OFFSITE & SESPMNT & $\mathrm{R}$ \\
\hline 56 & OFFSITE & SESPMNT & $\mathrm{R}$ \\
\hline 57 & OFFSITE & SESPMNT & $\mathrm{R}$ \\
\hline 58 & OFFSITE & SESPMNT & $\mathrm{R}$ \\
\hline 59 & OFFSITE & SESPMNT & $\mathrm{R}$ \\
\hline 60 & OFFSITE & SESPMNT & $\mathrm{R}$ \\
\hline 61 & OFFSITE & SESPMNT & $\mathrm{R}$ \\
\hline 62 & OFFSITE & SESPMNT & $\mathrm{R}$ \\
\hline 63 & OFFSITE & SESPMNT & $\mathrm{R}$ \\
\hline 64 & OFFSITE & SESPMNT & $\mathrm{R}$ \\
\hline 65 & OFFSITE & SESPMNT & $\mathrm{R}$ \\
\hline 66 & OFFSITE & SESPMNT & $\mathrm{R}$ \\
\hline 67 & OFFSITE & SESPMNT & $\mathrm{R}$ \\
\hline 68 & OFFSITE & SESPMNT & $\mathrm{R}$ \\
\hline 69 & OFFSITE & SESPMNT & $\mathrm{R}$ \\
\hline 70 & OFFSITE & SESPMNT & $\mathrm{R}$ \\
\hline 71 & OFFSITE & SESPMNT & $\mathrm{R}$ \\
\hline 72 & OFFSITE & SESPMNT & $\mathrm{R}$ \\
\hline 73 & OFFSITE & SESPMNT & $\mathrm{R}$ \\
\hline 74 & OFFSITE & SESPMNT & $\mathrm{R}$ \\
\hline 75 & OFFSITE & SESPMNT & $\mathrm{R}$ \\
\hline 76 & OFFSITE & SESPMNT & $\mathrm{R}$ \\
\hline 77 & OFFSITE & SESPMNT & $\mathrm{R}$ \\
\hline & & & \\
\hline
\end{tabular}




\begin{tabular}{|r|l|l|l|}
\hline & AG & AH & \\
\hline 1 & DIST_CLASS & OWNER_ID & COLLECTION_PURPOSE \\
\hline 78 & OFFSITE & SESPMNT & $\mathrm{R}$ \\
\hline 79 & OFFSITE & SESPMNT & $\mathrm{R}$ \\
\hline 80 & OFFSITE & SESPMNT & $\mathrm{R}$ \\
\hline 81 & OFFSITE & SESPMNT & $\mathrm{R}$ \\
\hline 82 & OFFSITE & SESPMNT & $\mathrm{R}$ \\
\hline 83 & OFFSITE & SESPMNT & $\mathrm{R}$ \\
\hline 84 & OFFSITE & SESPMNT & $\mathrm{R}$ \\
\hline 85 & OFFSITE & SESPMNT & $\mathrm{R}$ \\
\hline 86 & OFFSITE & SESPMNT & $\mathrm{R}$ \\
\hline 87 & OFFSITE & SESPMNT & $\mathrm{R}$ \\
\hline 88 & OFFSITE & SESPMNT & $\mathrm{R}$ \\
\hline 89 & OFFSITE & SESPMNT & $\mathrm{R}$ \\
\hline 90 & OFFSITE & SESPMNT & $\mathrm{R}$ \\
\hline 91 & OFFSITE & SESPMNT & $\mathrm{R}$ \\
\hline 92 & OFFSITE & SESPMNT & $\mathrm{R}$ \\
\hline 93 & OFFSITE & SESPMNT & $\mathrm{R}$ \\
\hline 94 & OFFSITE & SESPMNT & $\mathrm{R}$ \\
\hline 95 & OFFSITE & SESPMNT & $\mathrm{R}$ \\
\hline 96 & OFFSITE & SESPMNT & $\mathrm{R}$ \\
\hline 97 & OFFSITE & SESPMNT & $\mathrm{R}$ \\
\hline 98 & OFFSITE & SESPMNT & $\mathrm{R}$ \\
\hline 99 & OFFSITE & SESPMNT & $\mathrm{R}$ \\
\hline 100 & OFFSITE & SESPMNT & $\mathrm{R}$ \\
\hline 101 & OFFSITE & SESPMNT & $\mathrm{R}$ \\
\hline 102 & OFFSITE & SESPMNT & $\mathrm{R}$ \\
\hline 103 & OFFSITE & SESPMNT & $\mathrm{R}$ \\
\hline 104 & OFFSITE & SESPMNT & $\mathrm{R}$ \\
\hline 105 & OFFSITE & SESPMNT & $\mathrm{R}$ \\
\hline 106 & OFFSITE & SESPMNT & $\mathrm{R}$ \\
\hline 107 & OFFSITE & SESPMNT & $\mathrm{R}$ \\
\hline 108 & OFFSITE & SESPMNT & $\mathrm{R}$ \\
\hline 109 & OFFSITE & SESPMNT & $\mathrm{R}$ \\
\hline 110 & OFFSITE & SESPMNT & $\mathrm{R}$ \\
\hline 111 & OFFSITE & SESPMNT & $\mathrm{R}$ \\
\hline 112 & OFFSITE & SESPMNT & $\mathrm{R}$ \\
\hline 113 & OFFSITE & SESPMNT & $\mathrm{R}$ \\
\hline 114 & OFFSITE & SESPMNT & $\mathrm{R}$ \\
\hline 115 & OFFSITE & SESPMNT & $\mathrm{R}$ \\
\hline & & & \\
\hline
\end{tabular}




\begin{tabular}{|c|l|l|l|}
\hline & AG & AH & \\
\hline 1 & DIST_CLASS & OWNER_ID & COLLECTION_PURPOSE \\
\hline 116 & OFFSITE & SESPMNT & $\mathrm{R}$ \\
\hline 117 & OFFSITE & SESPMNT & $\mathrm{R}$ \\
\hline 118 & OFFSITE & SESPMNT & $\mathrm{R}$ \\
\hline 119 & OFFSITE & SESPMNT & $\mathrm{R}$ \\
\hline 120 & OFFSITE & SESPMNT & $\mathrm{R}$ \\
\hline 121 & OFFSITE & SESPMNT & $\mathrm{R}$ \\
\hline 122 & OFFSITE & SESPMNT & $\mathrm{R}$ \\
\hline 123 & OFFSITE & SESPMNT & $\mathrm{R}$ \\
\hline 124 & OFFSITE & SESPMNT & $\mathrm{R}$ \\
\hline 125 & OFFSITE & SESPMNT & $\mathrm{R}$ \\
\hline 126 & OFFSITE & SESPMNT & $\mathrm{R}$ \\
\hline 127 & OFFSITE & SESPMNT & $\mathrm{R}$ \\
\hline 128 & OFFSITE & SESPMNT & $\mathrm{R}$ \\
\hline 129 & OFFSITE & SESPMNT & $\mathrm{R}$ \\
\hline 130 & OFFSITE & SESPMNT & $\mathrm{R}$ \\
\hline 131 & OFFSITE & SESPMNT & $\mathrm{R}$ \\
\hline 132 & OFFSITE & SESPMNT & $\mathrm{R}$ \\
\hline 133 & OFFSITE & SESPMNT & $\mathrm{R}$ \\
\hline 134 & OFFSITE & SESPMNT & $\mathrm{R}$ \\
\hline 135 & OFFSITE & SESPMNT & $\mathrm{R}$ \\
\hline 136 & OFFSITE & SESPMNT & $\mathrm{R}$ \\
\hline 137 & OFFSITE & SESPMNT & $\mathrm{R}$ \\
\hline 138 & OFFSITE & SESPMNT & $\mathrm{R}$ \\
\hline 139 & OFFSITE & SESPMNT & $\mathrm{R}$ \\
\hline 140 & OFFSITE & SESPMNT & $\mathrm{R}$ \\
\hline 141 & OFFSITE & SESPMNT & $\mathrm{R}$ \\
\hline 142 & OFFSITE & SESPMNT & $\mathrm{R}$ \\
\hline 143 & OFFSITE & SESPMNT & $\mathrm{R}$ \\
\hline 144 & OFFSITE & SESPMNT & $\mathrm{R}$ \\
\hline 145 & OFFSITE & SESPMNT & $\mathrm{R}$ \\
\hline 146 & OFFSITE & SESPMNT & $\mathrm{R}$ \\
\hline 147 & OFFSITE & SESPMNT & $\mathrm{R}$ \\
\hline 148 & OFFSITE & SESPMNT & $\mathrm{R}$ \\
\hline 149 & OFFSITE & SESPMNT & $\mathrm{R}$ \\
\hline 150 & OFFSITE & SESPMNT & $\mathrm{R}$ \\
\hline 151 & OFFSITE & SESPMNT & $\mathrm{R}$ \\
\hline 152 & OFFSITE & SESPMNT & $\mathrm{R}$ \\
\hline 153 & OFFSITE & SESPMNT & $\mathrm{R}$ \\
\hline & & & \\
\hline
\end{tabular}




\begin{tabular}{|c|l|l|l|}
\hline & AG & AH & \\
\hline 1 & DIST_CLASS & OWNER_ID & COLLECTION_PURPOSE \\
\hline 154 & OFFSITE & SESPMNT & $\mathrm{R}$ \\
\hline 155 & OFFSITE & SESPMNT & $\mathrm{R}$ \\
\hline 156 & OFFSITE & SESPMNT & $\mathrm{R}$ \\
\hline 157 & OFFSITE & SESPMNT & $\mathrm{R}$ \\
\hline 158 & OFFSITE & SESPMNT & $\mathrm{R}$ \\
\hline 159 & OFFSITE & SESPMNT & $\mathrm{R}$ \\
\hline 160 & OFFSITE & SESPMNT & $\mathrm{R}$ \\
\hline 161 & OFFSITE & SESPMNT & $\mathrm{R}$ \\
\hline 162 & OFFSITE & SESPMNT & $\mathrm{R}$ \\
\hline 163 & OFFSITE & SESPMNT & $\mathrm{R}$ \\
\hline 164 & OFFSITE & SESPMNT & $\mathrm{R}$ \\
\hline 165 & OFFSITE & SESPMNT & $\mathrm{R}$ \\
\hline 166 & OFFSITE & SESPMNT & $\mathrm{R}$ \\
\hline 167 & OFFSITE & SESPMNT & $\mathrm{R}$ \\
\hline 168 & OFFSITE & SESPMNT & $\mathrm{R}$ \\
\hline 169 & OFFSITE & SESPMNT & $\mathrm{R}$ \\
\hline 170 & OFFSITE & SESPMNT & $\mathrm{R}$ \\
\hline 171 & OFFSITE & SESPMNT & $\mathrm{R}$ \\
\hline 172 & OFFSITE & SESPMNT & $\mathrm{R}$ \\
\hline 173 & OFFSITE & SESPMNT & $\mathrm{R}$ \\
\hline 174 & OFFSITE & SESPMNT & $\mathrm{R}$ \\
\hline 175 & OFFSITE & SESPMNT & $\mathrm{R}$ \\
\hline 176 & OFFSITE & SESPMNT & $\mathrm{R}$ \\
\hline 177 & OFFSITE & SESPMNT & $\mathrm{R}$ \\
\hline 178 & OFFSITE & SESPMNT & $\mathrm{R}$ \\
\hline 179 & OFFSITE & SESPMNT & $\mathrm{R}$ \\
\hline 180 & OFFSITE & SESPMNT & $\mathrm{R}$ \\
\hline 181 & OFFSITE & SESPMNT & $\mathrm{R}$ \\
\hline 182 & OFFSITE & SESPMNT & $\mathrm{R}$ \\
\hline 183 & OFFSITE & SESPMNT & $\mathrm{R}$ \\
\hline 184 & OFFSITE & SESPMNT & $\mathrm{R}$ \\
\hline 185 & OFFSITE & SESPMNT & $\mathrm{R}$ \\
\hline 186 & OFFSITE & SESPMNT & $\mathrm{R}$ \\
\hline 187 & OFFSITE & SESPMNT & $\mathrm{R}$ \\
\hline 188 & OFFSITE & SESPMNT & $\mathrm{R}$ \\
\hline 189 & OFFSITE & SESPMNT & $\mathrm{R}$ \\
\hline 190 & OFFSITE & SESPMNT & $\mathrm{R}$ \\
\hline 191 & OFFSITE & SESPMNT & $\mathrm{R}$ \\
\hline & & & \\
\hline
\end{tabular}




\begin{tabular}{|c|l|l|l|}
\hline & AG & AH & \\
\hline 1 & DIST_CLASS & OWNER_ID & COLLECTION_PURPOSE \\
\hline 192 & OFFSITE & SESPMNT & $\mathrm{R}$ \\
\hline 193 & OFFSITE & SESPMNT & $\mathrm{R}$ \\
\hline 194 & OFFSITE & SESPMNT & $\mathrm{R}$ \\
\hline 195 & OFFSITE & SESPMNT & $\mathrm{R}$ \\
\hline 196 & OFFSITE & SESPMNT & $\mathrm{R}$ \\
\hline 197 & OFFSITE & SESPMNT & $\mathrm{R}$ \\
\hline 198 & OFFSITE & SESPMNT & $\mathrm{R}$ \\
\hline 199 & OFFSITE & SESPMNT & $\mathrm{R}$ \\
\hline 200 & OFFSITE & SESPMNT & $\mathrm{R}$ \\
\hline 201 & OFFSITE & SESPMNT & $\mathrm{R}$ \\
\hline 202 & OFFSITE & SESPMNT & $\mathrm{R}$ \\
\hline 203 & OFFSITE & SESPMNT & $\mathrm{R}$ \\
\hline 204 & OFFSITE & SESPMNT & $\mathrm{R}$ \\
\hline 205 & OFFSITE & SESPMNT & $\mathrm{R}$ \\
\hline 206 & OFFSITE & SESPMNT & $\mathrm{R}$ \\
\hline 207 & OFFSITE & SESPMNT & $\mathrm{R}$ \\
\hline 208 & OFFSITE & SESPMNT & $\mathrm{R}$ \\
\hline 209 & OFFSITE & SESPMNT & $\mathrm{R}$ \\
\hline 210 & OFFSITE & SESPMNT & $\mathrm{R}$ \\
\hline 211 & OFFSITE & SESPMNT & $\mathrm{R}$ \\
\hline 212 & OFFSITE & SESPMNT & $\mathrm{R}$ \\
\hline 213 & OFFSITE & SESPMNT & $\mathrm{R}$ \\
\hline 214 & OFFSITE & SESPMNT & $\mathrm{R}$ \\
\hline 215 & OFFSITE & SESPMNT & $\mathrm{R}$ \\
\hline 216 & OFFSITE & SESPMNT & $\mathrm{R}$ \\
\hline 217 & OFFSITE & SESPMNT & $\mathrm{R}$ \\
\hline 218 & OFFSITE & SESPMNT & $\mathrm{R}$ \\
\hline 219 & OFFSITE & SESPMNT & $\mathrm{R}$ \\
\hline 220 & OFFSITE & SESPMNT & $\mathrm{R}$ \\
\hline 221 & OFFSITE & SESPMNT & $\mathrm{R}$ \\
\hline 222 & OFFSITE & SESPMNT & $\mathrm{R}$ \\
\hline 223 & OFFSITE & SESPMNT & $\mathrm{R}$ \\
\hline 224 & OFFSITE & SESPMNT & $\mathrm{R}$ \\
\hline 225 & OFFSITE & SESPMNT & $\mathrm{R}$ \\
\hline 226 & OFFSITE & SESPMNT & $\mathrm{R}$ \\
\hline 227 & OFFSITE & SESPMNT & $\mathrm{R}$ \\
\hline 228 & OFFSITE & SESPMNT & $\mathrm{R}$ \\
\hline 229 & OFFSITE & SESPMNT & $\mathrm{R}$ \\
\hline & & & \\
\hline
\end{tabular}




\begin{tabular}{|c|l|l|l|}
\hline & AG & AH & \\
\hline 1 & DIST_CLASS & OWNER_ID & COLLECTION_PURPOSE \\
\hline 230 & OFFSITE & SESPMNT & $\mathrm{R}$ \\
\hline 231 & OFFSITE & SESPMNT & $\mathrm{R}$ \\
\hline 232 & OFFSITE & SESPMNT & $\mathrm{R}$ \\
\hline 233 & OFFSITE & SESPMNT & $\mathrm{R}$ \\
\hline 234 & OFFSITE & SESPMNT & $\mathrm{R}$ \\
\hline 235 & OFFSITE & SESPMNT & $\mathrm{R}$ \\
\hline 236 & OFFSITE & SESPMNT & $\mathrm{R}$ \\
\hline 237 & OFFSITE & SESPMNT & $\mathrm{R}$ \\
\hline 238 & OFFSITE & SESPMNT & $\mathrm{R}$ \\
\hline 239 & OFFSITE & SESPMNT & $\mathrm{R}$ \\
\hline 240 & OFFSITE & SESPMNT & $\mathrm{R}$ \\
\hline 241 & OFFSITE & SESPMNT & $\mathrm{R}$ \\
\hline 242 & OFFSITE & SESPMNT & $\mathrm{R}$ \\
\hline 243 & OFFSITE & SESPMNT & $\mathrm{R}$ \\
\hline 244 & OFFSITE & SESPMNT & $\mathrm{R}$ \\
\hline 245 & OFFSITE & SESPMNT & $\mathrm{R}$ \\
\hline 246 & OFFSITE & SESPMNT & $\mathrm{R}$ \\
\hline 247 & OFFSITE & SESPMNT & $\mathrm{R}$ \\
\hline 248 & OFFSITE & SESPMNT & $\mathrm{R}$ \\
\hline 249 & OFFSITE & SESPMNT & $\mathrm{R}$ \\
\hline 250 & OFFSITE & SESPMNT & $\mathrm{R}$ \\
\hline 251 & OFFSITE & SESPMNT & $\mathrm{R}$ \\
\hline 252 & OFFSITE & SESPMNT & $\mathrm{R}$ \\
\hline 253 & OFFSITE & SESPMNT & $\mathrm{R}$ \\
\hline 254 & OFFSITE & SESPMNT & $\mathrm{R}$ \\
\hline 255 & OFFSITE & SESPMNT & $\mathrm{R}$ \\
\hline 256 & OFFSITE & SESPMNT & $\mathrm{R}$ \\
\hline 257 & OFFSITE & SESPMNT & $\mathrm{R}$ \\
\hline 258 & OFFSITE & SESPMNT & $\mathrm{R}$ \\
\hline 259 & OFFSITE & SESPMNT & $\mathrm{R}$ \\
\hline 260 & OFFSITE & SESPMNT & $\mathrm{R}$ \\
\hline 261 & OFFSITE & SESPMNT & $\mathrm{R}$ \\
\hline 262 & OFFSITE & SESPMNT & $\mathrm{R}$ \\
\hline 263 & OFFSITE & SESPMNT & $\mathrm{R}$ \\
\hline 264 & OFFSITE & SESPMNT & $\mathrm{R}$ \\
\hline 265 & OFFSITE & SESPMNT & $\mathrm{R}$ \\
\hline 266 & OFFSITE & SESPMNT & $\mathrm{R}$ \\
\hline 267 & OFFSITE & SESPMNT & $\mathrm{R}$ \\
\hline & & & \\
\hline
\end{tabular}




\begin{tabular}{|c|l|l|l|}
\hline & AG & AH & \\
\hline 1 & DIST_CLASS & OWNER_ID & COLLECTION_PURPOSE \\
\hline 268 & OFFSITE & SESPMNT & $\mathrm{R}$ \\
\hline 269 & OFFSITE & SESPMNT & $\mathrm{R}$ \\
\hline 270 & OFFSITE & SESPMNT & $\mathrm{R}$ \\
\hline 271 & OFFSITE & SESPMNT & $\mathrm{R}$ \\
\hline 272 & OFFSITE & SESPMNT & $\mathrm{R}$ \\
\hline 273 & OFFSITE & SESPMNT & $\mathrm{R}$ \\
\hline 274 & OFFSITE & SESPMNT & $\mathrm{R}$ \\
\hline 275 & OFFSITE & SESPMNT & $\mathrm{R}$ \\
\hline 276 & OFFSITE & SESPMNT & $\mathrm{R}$ \\
\hline 277 & OFFSITE & SESPMNT & $\mathrm{R}$ \\
\hline 278 & OFFSITE & SESPMNT & $\mathrm{R}$ \\
\hline 279 & OFFSITE & SESPMNT & $\mathrm{R}$ \\
\hline 280 & OFFSITE & SESPMNT & $\mathrm{R}$ \\
\hline 281 & OFFSITE & SESPMNT & $\mathrm{R}$ \\
\hline 282 & OFFSITE & SESPMNT & $\mathrm{R}$ \\
\hline 283 & OFFSITE & SESPMNT & $\mathrm{R}$ \\
\hline 284 & OFFSITE & SESPMNT & $\mathrm{R}$ \\
\hline 285 & OFFSITE & SESPMNT & $\mathrm{R}$ \\
\hline 286 & OFFSITE & SESPMNT & $\mathrm{R}$ \\
\hline 287 & OFFSITE & SESPMNT & $\mathrm{R}$ \\
\hline 288 & OFFSITE & SESPMNT & $\mathrm{R}$ \\
\hline 289 & OFFSITE & SESPMNT & $\mathrm{R}$ \\
\hline 290 & OFFSITE & SESPMNT & $\mathrm{R}$ \\
\hline 291 & OFFSITE & SESPMNT & $\mathrm{R}$ \\
\hline 292 & OFFSITE & SESPMNT & $\mathrm{R}$ \\
\hline 293 & OFFSITE & SESPMNT & $\mathrm{R}$ \\
\hline 294 & OFFSITE & SESPMNT & $\mathrm{R}$ \\
\hline 295 & OFFSITE & SESPMNT & $\mathrm{R}$ \\
\hline 296 & OFFSITE & SESPMNT & $\mathrm{R}$ \\
\hline 297 & OFFSITE & SESPMNT & $\mathrm{R}$ \\
\hline 298 & OFFSITE & SESPMNT & $\mathrm{R}$ \\
\hline 299 & OFFSITE & SESPMNT & $\mathrm{R}$ \\
\hline 300 & OFFSITE & SESPMNT & $\mathrm{R}$ \\
\hline 301 & OFFSITE & SESPMNT & $\mathrm{R}$ \\
\hline 302 & OFFSITE & SESPMNT & $\mathrm{R}$ \\
\hline 303 & OFFSITE & SESPMNT & $\mathrm{R}$ \\
\hline 304 & OFFSITE & SESPMNT & $\mathrm{R}$ \\
\hline 305 & OFFSITE & SESPMNT & $\mathrm{R}$ \\
\hline & & & \\
\hline
\end{tabular}




\begin{tabular}{|c|l|l|l|}
\hline & AG & AH & \\
\hline 1 & DIST_CLASS & OWNER_ID & COLLECTION_PURPOSE \\
\hline 306 & OFFSITE & SESPMNT & $\mathrm{R}$ \\
\hline 307 & OFFSITE & SESPMNT & $\mathrm{R}$ \\
\hline 308 & OFFSITE & SESPMNT & $\mathrm{R}$ \\
\hline 309 & OFFSITE & SESPMNT & $\mathrm{R}$ \\
\hline 310 & OFFSITE & SESPMNT & $\mathrm{R}$ \\
\hline 311 & OFFSITE & SESPMNT & $\mathrm{R}$ \\
\hline 312 & OFFSITE & SESPMNT & $\mathrm{R}$ \\
\hline 313 & OFFSITE & SESPMNT & $\mathrm{R}$ \\
\hline 314 & OFFSITE & SESPMNT & $\mathrm{R}$ \\
\hline 315 & OFFSITE & SESPMNT & $\mathrm{R}$ \\
\hline 316 & OFFSITE & SESPMNT & $\mathrm{R}$ \\
\hline 317 & OFFSITE & SESPMNT & $\mathrm{R}$ \\
\hline 318 & OFFSITE & SESPMNT & $\mathrm{R}$ \\
\hline 319 & OFFSITE & SESPMNT & $\mathrm{R}$ \\
\hline 320 & OFFSITE & SESPMNT & $\mathrm{R}$ \\
\hline 321 & OFFSITE & SESPMNT & $\mathrm{R}$ \\
\hline 322 & OFFSITE & SESPMNT & $\mathrm{R}$ \\
\hline 323 & OFFSITE & SESPMNT & $\mathrm{R}$ \\
\hline 324 & OFFSITE & SESPMNT & $\mathrm{R}$ \\
\hline 325 & OFFSITE & SESPMNT & $\mathrm{R}$ \\
\hline 326 & OFFSITE & SESPMNT & $\mathrm{R}$ \\
\hline 327 & OFFSITE & SESPMNT & $\mathrm{R}$ \\
\hline 328 & OFFSITE & SESPMNT & $\mathrm{R}$ \\
\hline 329 & OFFSITE & SESPMNT & $\mathrm{R}$ \\
\hline 330 & OFFSITE & SESPMNT & $\mathrm{R}$ \\
\hline 331 & OFFSITE & SESPMNT & $\mathrm{R}$ \\
\hline 332 & OFFSITE & SESPMNT & $\mathrm{R}$ \\
\hline 333 & OFFSITE & SESPMNT & $\mathrm{R}$ \\
\hline 334 & OFFSITE & SESPMNT & $\mathrm{R}$ \\
\hline 335 & OFFSITE & SESPMNT & $\mathrm{R}$ \\
\hline 336 & OFFSITE & SESPMNT & $\mathrm{R}$ \\
\hline 337 & OFFSITE & SESPMNT & $\mathrm{R}$ \\
\hline 338 & OFFSITE & SESPMNT & $\mathrm{R}$ \\
\hline 339 & OFFSITE & SESPMNT & $\mathrm{R}$ \\
\hline 340 & OFFSITE & SESPMNT & $\mathrm{R}$ \\
\hline 341 & OFFSITE & SESPMNT & $\mathrm{R}$ \\
\hline 342 & OFFSITE & SESPMNT & $\mathrm{R}$ \\
\hline 343 & OFFSITE & SESPMNT & $\mathrm{R}$ \\
\hline & & & \\
\hline
\end{tabular}




\begin{tabular}{|c|l|l|l|}
\hline & AG & AH & \\
\hline 1 & DIST_CLASS & OWNER_ID & COLLECTION_PURPOSE \\
\hline 344 & OFFSITE & SESPMNT & $\mathrm{R}$ \\
\hline 345 & OFFSITE & SESPMNT & $\mathrm{R}$ \\
\hline 346 & OFFSITE & SESPMNT & $\mathrm{R}$ \\
\hline 347 & OFFSITE & SESPMNT & $\mathrm{R}$ \\
\hline 348 & OFFSITE & SESPMNT & $\mathrm{R}$ \\
\hline 349 & OFFSITE & SESPMNT & $\mathrm{R}$ \\
\hline 350 & OFFSITE & SESPMNT & $\mathrm{R}$ \\
\hline 351 & OFFSITE & SESPMNT & $\mathrm{R}$ \\
\hline 352 & OFFSITE & SESPMNT & $\mathrm{R}$ \\
\hline 353 & OFFSITE & SESPMNT & $\mathrm{R}$ \\
\hline 354 & OFFSITE & SESPMNT & $\mathrm{R}$ \\
\hline 355 & OFFSITE & SESPMNT & $\mathrm{R}$ \\
\hline 356 & OFFSITE & SESPMNT & $\mathrm{R}$ \\
\hline 357 & OFFSITE & SESPMNT & $\mathrm{R}$ \\
\hline 358 & OFFSITE & SESPMNT & $\mathrm{R}$ \\
\hline 359 & OFFSITE & SESPMNT & $\mathrm{R}$ \\
\hline 360 & OFFSITE & SESPMNT & $\mathrm{R}$ \\
\hline 361 & OFFSITE & SESPMNT & $\mathrm{R}$ \\
\hline 362 & OFFSITE & SESPMNT & $\mathrm{R}$ \\
\hline 363 & OFFSITE & SESPMNT & $\mathrm{R}$ \\
\hline 364 & OFFSITE & SESPMNT & $\mathrm{R}$ \\
\hline 365 & OFFSITE & SESPMNT & $\mathrm{R}$ \\
\hline 366 & OFFSITE & SESPMNT & $\mathrm{R}$ \\
\hline 367 & OFFSITE & SESPMNT & $\mathrm{R}$ \\
\hline 368 & OFFSITE & SESPMNT & $\mathrm{R}$ \\
\hline 369 & OFFSITE & SESPMNT & $\mathrm{R}$ \\
\hline 370 & OFFSITE & SESPMNT & $\mathrm{R}$ \\
\hline 371 & OFFSITE & SESPMNT & $\mathrm{R}$ \\
\hline 372 & OFFSITE & SESPMNT & $\mathrm{R}$ \\
\hline 373 & OFFSITE & SESPMNT & $\mathrm{R}$ \\
\hline 374 & OFFSITE & SESPMNT & $\mathrm{R}$ \\
\hline 375 & OFFSITE & SESPMNT & $\mathrm{R}$ \\
\hline 376 & OFFSITE & SESPMNT & $\mathrm{R}$ \\
\hline 377 & OFFSITE & SESPMNT & $\mathrm{R}$ \\
\hline 378 & OFFSITE & SESPMNT & $\mathrm{R}$ \\
\hline 379 & OFFSITE & SESPMNT & $\mathrm{R}$ \\
\hline 380 & OFFSITE & SESPMNT & $\mathrm{R}$ \\
\hline 381 & OFFSITE & SESPMNT & $\mathrm{R}$ \\
\hline & & & \\
\hline
\end{tabular}




\begin{tabular}{|c|l|l|l|}
\hline & AG & AH & \\
\hline 1 & DIST_CLASS & OWNER_ID & COLLECTION_PURPOSE \\
\hline 382 & OFFSITE & SESPMNT & $\mathrm{R}$ \\
\hline 383 & OFFSITE & SESPMNT & $\mathrm{R}$ \\
\hline 384 & OFFSITE & SESPMNT & $\mathrm{R}$ \\
\hline 385 & OFFSITE & SESPMNT & $\mathrm{R}$ \\
\hline 386 & OFFSITE & SESPMNT & $\mathrm{R}$ \\
\hline 387 & OFFSITE & SESPMNT & $\mathrm{R}$ \\
\hline 388 & OFFSITE & SESPMNT & $\mathrm{R}$ \\
\hline 389 & OFFSITE & SESPMNT & $\mathrm{R}$ \\
\hline 390 & OFFSITE & SESPMNT & $\mathrm{R}$ \\
\hline 391 & OFFSITE & SESPMNT & $\mathrm{R}$ \\
\hline 392 & OFFSITE & SESPMNT & $\mathrm{R}$ \\
\hline 393 & OFFSITE & SESPMNT & $\mathrm{R}$ \\
\hline 394 & OFFSITE & SESPMNT & $\mathrm{R}$ \\
\hline 395 & OFFSITE & SESPMNT & $\mathrm{R}$ \\
\hline 396 & OFFSITE & SESPMNT & $\mathrm{R}$ \\
\hline 397 & OFFSITE & SESPMNT & $\mathrm{R}$ \\
\hline 398 & OFFSITE & SESPMNT & $\mathrm{R}$ \\
\hline 399 & OFFSITE & SESPMNT & $\mathrm{R}$ \\
\hline 400 & OFFSITE & SESPMNT & $\mathrm{R}$ \\
\hline 401 & OFFSITE & SESPMNT & $\mathrm{R}$ \\
\hline 402 & OFFSITE & SESPMNT & $\mathrm{R}$ \\
\hline 403 & OFFSITE & SESPMNT & $\mathrm{R}$ \\
\hline 404 & OFFSITE & SESPMNT & $\mathrm{R}$ \\
\hline 405 & OFFSITE & SESPMNT & $\mathrm{R}$ \\
\hline 406 & OFFSITE & SESPMNT & $\mathrm{R}$ \\
\hline 407 & OFFSITE & SESPMNT & $\mathrm{R}$ \\
\hline 408 & OFFSITE & SESPMNT & $\mathrm{R}$ \\
\hline 409 & OFFSITE & SESPMNT & $\mathrm{R}$ \\
\hline 410 & OFFSITE & SESPMNT & $\mathrm{R}$ \\
\hline 411 & OFFSITE & SESPMNT & $\mathrm{R}$ \\
\hline 412 & OFFSITE & SESPMNT & $\mathrm{R}$ \\
\hline 413 & OFFSITE & SESPMNT & $\mathrm{R}$ \\
\hline 414 & OFFSITE & SESPMNT & $\mathrm{R}$ \\
\hline 415 & OFFSITE & SESPMNT & $\mathrm{R}$ \\
\hline 416 & OFFSITE & SESPMNT & $\mathrm{R}$ \\
\hline 417 & OFFSITE & SESPMNT & $\mathrm{R}$ \\
\hline 418 & OFFSITE & SESPMNT & $\mathrm{R}$ \\
\hline 419 & OFFSITE & SESPMNT & $\mathrm{R}$ \\
\hline & & & \\
\hline
\end{tabular}




\begin{tabular}{|c|l|l|l|}
\hline & AG & AH & \\
\hline 1 & DIST_CLASS & OWNER_ID & COLLECTION_PURPOSE \\
\hline 420 & OFFSITE & SESPMNT & $\mathrm{R}$ \\
\hline 421 & OFFSITE & SESPMNT & $\mathrm{R}$ \\
\hline 422 & OFFSITE & SESPMNT & $\mathrm{R}$ \\
\hline 423 & OFFSITE & SESPMNT & $\mathrm{R}$ \\
\hline 424 & OFFSITE & SESPMNT & $\mathrm{R}$ \\
\hline 425 & OFFSITE & SESPMNT & $\mathrm{R}$ \\
\hline 426 & OFFSITE & SESPMNT & $\mathrm{R}$ \\
\hline 427 & OFFSITE & SESPMNT & $\mathrm{R}$ \\
\hline 428 & OFFSITE & SESPMNT & $\mathrm{R}$ \\
\hline 429 & OFFSITE & SESPMNT & $\mathrm{R}$ \\
\hline 430 & OFFSITE & SESPMNT & $\mathrm{R}$ \\
\hline 431 & OFFSITE & SESPMNT & $\mathrm{R}$ \\
\hline 432 & OFFSITE & SESPMNT & $\mathrm{R}$ \\
\hline 433 & OFFSITE & SESPMNT & $\mathrm{R}$ \\
\hline 434 & OFFSITE & SESPMNT & $\mathrm{R}$ \\
\hline 435 & OFFSITE & SESPMNT & $\mathrm{R}$ \\
\hline 436 & OFFSITE & SESPMNT & $\mathrm{R}$ \\
\hline 437 & OFFSITE & SESPMNT & $\mathrm{R}$ \\
\hline 438 & OFFSITE & SESPMNT & $\mathrm{R}$ \\
\hline 439 & OFFSITE & SESPMNT & $\mathrm{R}$ \\
\hline 440 & OFFSITE & SESPMNT & $\mathrm{R}$ \\
\hline 441 & OFFSITE & SESPMNT & $\mathrm{R}$ \\
\hline 442 & OFFSITE & SESPMNT & $\mathrm{R}$ \\
\hline 443 & OFFSITE & SESPMNT & $\mathrm{R}$ \\
\hline 444 & OFFSITE & SESPMNT & $\mathrm{R}$ \\
\hline 445 & OFFSITE & SESPMNT & $\mathrm{R}$ \\
\hline 446 & OFFSITE & SESPMNT & $\mathrm{R}$ \\
\hline 447 & OFFSITE & SESPMNT & $\mathrm{R}$ \\
\hline 448 & OFFSITE & SESPMNT & $\mathrm{R}$ \\
\hline 449 & OFFSITE & SESPMNT & $\mathrm{R}$ \\
\hline 450 & OFFSITE & SESPMNT & $\mathrm{R}$ \\
\hline 451 & OFFSITE & SESPMNT & $\mathrm{R}$ \\
\hline 452 & OFFSITE & SESPMNT & $\mathrm{R}$ \\
\hline 453 & OFFSITE & SESPMNT & $\mathrm{R}$ \\
\hline 454 & OFFSITE & SESPMNT & $\mathrm{R}$ \\
\hline 455 & OFFSITE & SESPMNT & $\mathrm{R}$ \\
\hline 456 & OFFSITE & SESPMNT & $\mathrm{R}$ \\
\hline 457 & OFFSITE & SESPMNT & $\mathrm{R}$ \\
\hline & & & \\
\hline
\end{tabular}




\begin{tabular}{|c|l|l|l|}
\hline & AG & AH & \\
\hline 1 & DIST_CLASS & OWNER_ID & COLLECTION_PURPOSE \\
\hline 458 & OFFSITE & SESPMNT & $\mathrm{R}$ \\
\hline 459 & OFFSITE & SESPMNT & $\mathrm{R}$ \\
\hline 460 & OFFSITE & SESPMNT & $\mathrm{R}$ \\
\hline 461 & OFFSITE & SESPMNT & $\mathrm{R}$ \\
\hline 462 & OFFSITE & SESPMNT & $\mathrm{R}$ \\
\hline 463 & OFFSITE & SESPMNT & $\mathrm{R}$ \\
\hline 464 & OFFSITE & SESPMNT & $\mathrm{R}$ \\
\hline 465 & OFFSITE & SESPMNT & $\mathrm{R}$ \\
\hline 466 & OFFSITE & SESPMNT & $\mathrm{R}$ \\
\hline 467 & OFFSITE & SESPMNT & $\mathrm{R}$ \\
\hline 468 & OFFSITE & SESPMNT & $\mathrm{R}$ \\
\hline 469 & OFFSITE & SESPMNT & $\mathrm{R}$ \\
\hline 470 & OFFSITE & SESPMNT & $\mathrm{R}$ \\
\hline 471 & OFFSITE & SESPMNT & $\mathrm{R}$ \\
\hline 472 & OFFSITE & SESPMNT & $\mathrm{R}$ \\
\hline 473 & OFFSITE & SESPMNT & $\mathrm{R}$ \\
\hline 474 & OFFSITE & SESPMNT & $\mathrm{R}$ \\
\hline 475 & OFFSITE & SESPMNT & $\mathrm{R}$ \\
\hline 476 & OFFSITE & SESPMNT & $\mathrm{R}$ \\
\hline 477 & OFFSITE & SESPMNT & $\mathrm{R}$ \\
\hline 478 & OFFSITE & SESPMNT & $\mathrm{R}$ \\
\hline 479 & OFFSITE & SESPMNT & $\mathrm{R}$ \\
\hline 480 & OFFSITE & SESPMNT & $\mathrm{R}$ \\
\hline 481 & OFFSITE & SESPMNT & $\mathrm{R}$ \\
\hline 482 & OFFSITE & SESPMNT & $\mathrm{R}$ \\
\hline 483 & OFFSITE & SESPMNT & $\mathrm{R}$ \\
\hline 484 & OFFSITE & SESPMNT & $\mathrm{R}$ \\
\hline 485 & OFFSITE & SESPMNT & $\mathrm{R}$ \\
\hline 486 & OFFSITE & SESPMNT & $\mathrm{R}$ \\
\hline 487 & OFFSITE & SESPMNT & $\mathrm{R}$ \\
\hline 488 & OFFSITE & SESPMNT & $\mathrm{R}$ \\
\hline 489 & OFFSITE & SESPMNT & $\mathrm{R}$ \\
\hline 490 & OFFSITE & SESPMNT & $\mathrm{R}$ \\
\hline 491 & OFFSITE & SESPMNT & $\mathrm{R}$ \\
\hline 492 & OFFSITE & SESPMNT & $\mathrm{R}$ \\
\hline 493 & OFFSITE & SESPMNT & $\mathrm{R}$ \\
\hline 494 & OFFSITE & SESPMNT & $\mathrm{R}$ \\
\hline 495 & OFFSITE & SESPMNT & $\mathrm{R}$ \\
\hline & & & \\
\hline
\end{tabular}




\begin{tabular}{|c|l|l|l|}
\hline & AG & AH & \\
\hline 1 & DIST_CLASS & OWNER_ID & COLLECTION_PURPOSE \\
\hline 496 & OFFSITE & SESPMNT & $\mathrm{R}$ \\
\hline 497 & OFFSITE & SESPMNT & $\mathrm{R}$ \\
\hline 498 & OFFSITE & SESPMNT & $\mathrm{R}$ \\
\hline 499 & OFFSITE & SESPMNT & $\mathrm{R}$ \\
\hline 500 & OFFSITE & SESPMNT & $\mathrm{R}$ \\
\hline 501 & OFFSITE & SESPMNT & $\mathrm{R}$ \\
\hline 502 & OFFSITE & SESPMNT & $\mathrm{R}$ \\
\hline 503 & OFFSITE & SESPMNT & $\mathrm{R}$ \\
\hline 504 & OFFSITE & SESPMNT & $\mathrm{R}$ \\
\hline 505 & OFFSITE & SESPMNT & $\mathrm{R}$ \\
\hline 506 & OFFSITE & SESPSPEC & $\mathrm{S}$ \\
\hline 507 & OFFSITE & SESPSPEC & $\mathrm{S}$ \\
\hline 508 & OFFSITE & SESPSPEC & $\mathrm{S}$ \\
\hline 509 & OFFSITE & SESPSPEC & $\mathrm{S}$ \\
\hline 510 & OFFSITE & SESPSPEC & $\mathrm{S}$ \\
\hline 511 & OFFSITE & SESPSPEC & $\mathrm{S}$ \\
\hline 512 & OFFSITE & SESPSPEC & $\mathrm{S}$ \\
\hline 513 & OFFSITE & SESPSPEC & $\mathrm{S}$ \\
\hline 514 & OFFSITE & SESPSPEC & $\mathrm{S}$ \\
\hline 515 & OFFSITE & SESPSPEC & $\mathrm{S}$ \\
\hline 516 & OFFSITE & SESPMNT & $\mathrm{R}$ \\
\hline 517 & OFFSITE & SESPMNT & $\mathrm{R}$ \\
\hline 518 & OFFSITE & SESPMNT & $\mathrm{R}$ \\
\hline 519 & OFFSITE & SESPMNT & $\mathrm{R}$ \\
\hline 520 & OFFSITE & SESPMNT & $\mathrm{R}$ \\
\hline 521 & OFFSITE & SESPMNT & $\mathrm{R}$ \\
\hline 522 & OFFSITE & SESPMNT & $\mathrm{R}$ \\
\hline 523 & OFFSITE & SESPMNT & $\mathrm{R}$ \\
\hline 524 & OFFSITE & SESPMNT & $\mathrm{R}$ \\
\hline 525 & OFFSITE & SESPMNT & $\mathrm{R}$ \\
\hline 526 & OFFSITE & SESPMNT & $\mathrm{R}$ \\
\hline 527 & OFFSITE & SESPMNT & $\mathrm{R}$ \\
\hline 528 & OFFSITE & SESPMNT & $\mathrm{R}$ \\
\hline 529 & OFFSITE & SESPMNT & $\mathrm{R}$ \\
\hline 530 & OFFSITE & SESPMNT & $\mathrm{R}$ \\
\hline 531 & OFFSITE & SESPMNT & $\mathrm{R}$ \\
\hline 532 & OFFSITE & SESPMNT & $\mathrm{R}$ \\
\hline 533 & OFFSITE & SESPMNT & $\mathrm{R}$ \\
\hline & & & \\
\hline
\end{tabular}




\begin{tabular}{|c|l|l|l|}
\hline & AG & AH & \\
\hline 1 & DIST_CLASS & OWNER_ID & COLLECTION_PURPOSE \\
\hline 534 & OFFSITE & SESPMNT & $\mathrm{R}$ \\
\hline 535 & OFFSITE & SESPMNT & $\mathrm{R}$ \\
\hline 536 & OFFSITE & SESPMNT & $\mathrm{R}$ \\
\hline 537 & OFFSITE & SESPMNT & $\mathrm{R}$ \\
\hline 538 & OFFSITE & SESPMNT & $\mathrm{R}$ \\
\hline 539 & OFFSITE & SESPMNT & $\mathrm{R}$ \\
\hline 540 & OFFSITE & SESPMNT & $\mathrm{R}$ \\
\hline 541 & OFFSITE & SESPMNT & $\mathrm{R}$ \\
\hline 542 & OFFSITE & SESPMNT & $\mathrm{R}$ \\
\hline 543 & OFFSITE & SESPMNT & $\mathrm{R}$ \\
\hline 544 & OFFSITE & SESPMNT & $\mathrm{R}$ \\
\hline 545 & OFFSITE & SESPMNT & $\mathrm{R}$ \\
\hline 546 & OFFSITE & SESPMNT & $\mathrm{R}$ \\
\hline 547 & OFFSITE & SESPMNT & $\mathrm{R}$ \\
\hline 548 & OFFSITE & SESPMNT & $\mathrm{R}$ \\
\hline 549 & OFFSITE & SESPMNT & $\mathrm{R}$ \\
\hline 550 & OFFSITE & SESPMNT & $\mathrm{R}$ \\
\hline 551 & OFFSITE & SESPMNT & $\mathrm{R}$ \\
\hline 552 & OFFSITE & SESPMNT & $\mathrm{R}$ \\
\hline 553 & OFFSITE & SESPMNT & $\mathrm{R}$ \\
\hline 554 & OFFSITE & SESPMNT & $\mathrm{R}$ \\
\hline 555 & OFFSITE & SESPMNT & $\mathrm{R}$ \\
\hline 556 & OFFSITE & SESPMNT & $\mathrm{R}$ \\
\hline 557 & OFFSITE & SESPMNT & $\mathrm{R}$ \\
\hline 558 & OFFSITE & SESPMNT & $\mathrm{R}$ \\
\hline 559 & OFFSITE & SESPMNT & $\mathrm{R}$ \\
\hline 560 & OFFSITE & SESPMNT & $\mathrm{R}$ \\
\hline 561 & OFFSITE & SESPMNT & $\mathrm{R}$ \\
\hline 562 & OFFSITE & SESPMNT & $\mathrm{R}$ \\
\hline 563 & OFFSITE & SESPMNT & $\mathrm{R}$ \\
\hline 564 & OFFSITE & SESPMNT & $\mathrm{R}$ \\
\hline 565 & OFFSITE & SESPMNT & $\mathrm{R}$ \\
\hline 566 & OFFSITE & SESPMNT & $\mathrm{R}$ \\
\hline 567 & OFFSITE & SESPMNT & $\mathrm{R}$ \\
\hline 568 & OFFSITE & SESPMNT & $\mathrm{R}$ \\
\hline 569 & OFFSITE & SESPMNT & $\mathrm{R}$ \\
\hline 570 & OFFSITE & SESPMNT & $\mathrm{R}$ \\
\hline 571 & OFFSITE & SESPMNT & $\mathrm{R}$ \\
\hline & & & \\
\hline 5 & & \\
\hline 5
\end{tabular}




\begin{tabular}{|c|l|l|l|}
\hline & AG & AH & \\
\hline 1 & DIST_CLASS & OWNER_ID & COLLECTION_PURPOSE \\
\hline 572 & OFFSITE & SESPMNT & $\mathrm{R}$ \\
\hline 573 & OFFSITE & SESPMNT & $\mathrm{R}$ \\
\hline 574 & OFFSITE & SESPMNT & $\mathrm{R}$ \\
\hline 575 & OFFSITE & SESPMNT & $\mathrm{R}$ \\
\hline 576 & OFFSITE & SESPMNT & $\mathrm{R}$ \\
\hline 577 & OFFSITE & SESPMNT & $\mathrm{R}$ \\
\hline 578 & OFFSITE & SESPMNT & $\mathrm{R}$ \\
\hline 579 & OFFSITE & SESPMNT & $\mathrm{R}$ \\
\hline 580 & OFFSITE & SESPMNT & $\mathrm{R}$ \\
\hline 581 & OFFSITE & SESPMNT & $\mathrm{R}$ \\
\hline 582 & OFFSITE & SESPMNT & $\mathrm{R}$ \\
\hline 583 & OFFSITE & SESPMNT & $\mathrm{R}$ \\
\hline 584 & OFFSITE & SESPMNT & $\mathrm{R}$ \\
\hline 585 & OFFSITE & SESPMNT & $\mathrm{R}$ \\
\hline 586 & OFFSITE & SESPMNT & $\mathrm{R}$ \\
\hline 587 & OFFSITE & SESPMNT & $\mathrm{R}$ \\
\hline 588 & OFFSITE & SESPMNT & $\mathrm{R}$ \\
\hline 589 & OFFSITE & SESPMNT & $\mathrm{R}$ \\
\hline 590 & OFFSITE & SESPMNT & $\mathrm{R}$ \\
\hline 591 & OFFSITE & SESPMNT & $\mathrm{R}$ \\
\hline 592 & OFFSITE & SESPMNT & $\mathrm{R}$ \\
\hline 593 & OFFSITE & SESPMNT & $\mathrm{R}$ \\
\hline 594 & OFFSITE & SESPMNT & $\mathrm{R}$ \\
\hline 595 & OFFSITE & SESPMNT & $\mathrm{R}$ \\
\hline 596 & OFFSITE & SESPMNT & $\mathrm{R}$ \\
\hline 597 & OFFSITE & SESPMNT & $\mathrm{R}$ \\
\hline 598 & OFFSITE & SESPMNT & $\mathrm{R}$ \\
\hline 599 & OFFSITE & SESPMNT & $\mathrm{R}$ \\
\hline 600 & OFFSITE & SESPMNT & $\mathrm{R}$ \\
\hline 601 & OFFSITE & SESPMNT & $\mathrm{R}$ \\
\hline 602 & OFFSITE & SESPMNT & $\mathrm{R}$ \\
\hline 603 & OFFSITE & SESPMNT & $\mathrm{R}$ \\
\hline 604 & OFFSITE & SESPMNT & $\mathrm{R}$ \\
\hline 605 & OFFSITE & SESPMNT & $\mathrm{R}$ \\
\hline 606 & OFFSITE & SESPMNT & $\mathrm{R}$ \\
\hline 607 & OFFSITE & SESPMNT & $\mathrm{R}$ \\
\hline 608 & OFFSITE & SESPMNT & $\mathrm{R}$ \\
\hline 609 & OFFSITE & SESPMNT & $\mathrm{R}$ \\
\hline & & & \\
\hline
\end{tabular}




\begin{tabular}{|c|l|l|l|}
\hline & AG & AH & \\
\hline 1 & DIST_CLASS & OWNER_ID & COLLECTION_PURPOSE \\
\hline 610 & OFFSITE & SESPMNT & $\mathrm{R}$ \\
\hline 611 & OFFSITE & SESPMNT & $\mathrm{R}$ \\
\hline 612 & OFFSITE & SESPMNT & $\mathrm{R}$ \\
\hline 613 & OFFSITE & SESPMNT & $\mathrm{R}$ \\
\hline 614 & OFFSITE & SESPMNT & $\mathrm{R}$ \\
\hline 615 & OFFSITE & SESPMNT & $\mathrm{R}$ \\
\hline 616 & OFFSITE & SESPMNT & $\mathrm{R}$ \\
\hline 617 & OFFSITE & SESPMNT & $\mathrm{R}$ \\
\hline 618 & OFFSITE & SESPMNT & $\mathrm{R}$ \\
\hline 619 & OFFSITE & SESPMNT & $\mathrm{R}$ \\
\hline 620 & OFFSITE & SESPMNT & $\mathrm{R}$ \\
\hline 621 & OFFSITE & SESPMNT & $\mathrm{R}$ \\
\hline 622 & OFFSITE & SESPMNT & $\mathrm{R}$ \\
\hline 623 & OFFSITE & SESPMNT & $\mathrm{R}$ \\
\hline 624 & OFFSITE & SESPMNT & $\mathrm{R}$ \\
\hline 625 & OFFSITE & SESPMNT & $\mathrm{R}$ \\
\hline 626 & OFFSITE & SESPMNT & $\mathrm{R}$ \\
\hline 627 & OFFSITE & SESPMNT & $\mathrm{R}$ \\
\hline 628 & OFFSITE & SESPMNT & $\mathrm{R}$ \\
\hline 629 & OFFSITE & SESPMNT & $\mathrm{R}$ \\
\hline 630 & OFFSITE & SESPMNT & $\mathrm{R}$ \\
\hline 631 & OFFSITE & SESPMNT & $\mathrm{R}$ \\
\hline 632 & OFFSITE & SESPMNT & $\mathrm{R}$ \\
\hline 633 & OFFSITE & SESPMNT & $\mathrm{R}$ \\
\hline 634 & OFFSITE & SESPMNT & $\mathrm{R}$ \\
\hline 635 & OFFSITE & SESPMNT & $\mathrm{R}$ \\
\hline 636 & OFFSITE & SESPMNT & $\mathrm{R}$ \\
\hline 637 & OFFSITE & SESPMNT & $\mathrm{R}$ \\
\hline 638 & OFFSITE & SESPMNT & $\mathrm{R}$ \\
\hline 639 & OFFSITE & SESPMNT & $\mathrm{R}$ \\
\hline 640 & OFFSITE & SESPMNT & $\mathrm{R}$ \\
\hline 641 & OFFSITE & SESPMNT & $\mathrm{R}$ \\
\hline 642 & OFFSITE & SESPMNT & $\mathrm{R}$ \\
\hline 643 & OFFSITE & SESPMNT & $\mathrm{R}$ \\
\hline 644 & OFFSITE & SESPMNT & $\mathrm{R}$ \\
\hline 645 & OFFSITE & SESPMNT & $\mathrm{R}$ \\
\hline 646 & OFFSITE & SESPMNT & $\mathrm{R}$ \\
\hline 647 & OFFSITE & SESPMNT & $\mathrm{R}$ \\
\hline & & & \\
\hline
\end{tabular}




\begin{tabular}{|c|l|l|l|}
\hline & \multicolumn{1}{|c|}{ AG } & AH & \\
\hline 1 & DIST_CLASS & OWNER_ID & COLLECTION_PURPOSE \\
\hline 648 & OFFSITE & SESPMNT & $\mathrm{R}$ \\
\hline 649 & OFFSITE & SESPMNT & $\mathrm{R}$ \\
\hline 650 & OFFSITE & SESPMNT & $\mathrm{R}$ \\
\hline 651 & OFFSITE & SESPMNT & $\mathrm{R}$ \\
\hline 652 & OFFSITE & SESPMNT & $\mathrm{R}$ \\
\hline 653 & OFFSITE & SESPMNT & $\mathrm{R}$ \\
\hline 654 & OFFSITE & SESPMNT & $\mathrm{R}$ \\
\hline 655 & OFFSITE & SESPMNT & $\mathrm{R}$ \\
\hline 656 & OFFSITE & SESPMNT & $\mathrm{R}$ \\
\hline 657 & OFFSITE & SESPMNT & $\mathrm{R}$ \\
\hline 658 & OFFSITE & SESPMNT & $\mathrm{R}$ \\
\hline 659 & OFFSITE & SESPMNT & $\mathrm{R}$ \\
\hline 660 & ONSITE & SESPMNT & $\mathrm{R}$ \\
\hline 661 & ONSITE & SESPMNT & $\mathrm{R}$ \\
\hline 662 & ONSITE & SESPMNT & $\mathrm{R}$ \\
\hline 663 & ONSITE & SESPMNT & $\mathrm{R}$ \\
\hline 664 & ONSITE & SESPMNT & $\mathrm{R}$ \\
\hline 665 & ONSITE & SESPMNT & $\mathrm{R}$ \\
\hline 666 & ONSITE & SESPMNT & $\mathrm{R}$ \\
\hline 667 & ONSITE & SESPMNT & $\mathrm{R}$ \\
\hline 668 & ONSITE & SESPMNT & $\mathrm{R}$ \\
\hline 669 & ONSITE & SESPMNT & $\mathrm{R}$ \\
\hline 670 & ONSITE & SESPMNT & $\mathrm{R}$ \\
\hline 671 & ONSITE & SESPMNT & $\mathrm{R}$ \\
\hline 672 & ONSITE & SESPMNT & $\mathrm{R}$ \\
\hline 673 & ONSITE & SESPMNT & $\mathrm{R}$ \\
\hline 674 & ONSITE & SESPMNT & $\mathrm{R}$ \\
\hline 675 & ONSITE & SESPMNT & $\mathrm{R}$ \\
\hline 676 & ONSITE & SESPMNT & $\mathrm{R}$ \\
\hline 677 & ONSITE & SESPMNT & $\mathrm{R}$ \\
\hline 678 & ONSITE & SESPMNT & $\mathrm{R}$ \\
\hline 679 & ONSITE & SESPMNT & $\mathrm{R}$ \\
\hline 680 & ONSITE & SESPMNT & $\mathrm{R}$ \\
\hline 681 & ONSITE & SESPMNT & $\mathrm{R}$ \\
\hline 682 & ONSITE & SESPMNT & $\mathrm{R}$ \\
\hline 683 & ONSITE & SESPMNT & $\mathrm{R}$ \\
\hline 684 & ONSITE & SESPMNT & $\mathrm{R}$ \\
\hline 685 & ONSITE & SESPMNT & $\mathrm{R}$ \\
\hline & & & \\
\hline
\end{tabular}




\begin{tabular}{|c|l|l|l|}
\hline & \multicolumn{1}{|c|}{ AG } & \multicolumn{1}{|c|}{ AH } & \\
\hline 1 & DIST_CLASS & OWNER_ID & COLLECTION_PURPOSE \\
\hline 686 & ONSITE & SESPMNT & $\mathrm{R}$ \\
\hline 687 & ONSITE & SESPMNT & $\mathrm{R}$ \\
\hline 688 & ONSITE & SESPMNT & $\mathrm{R}$ \\
\hline 689 & ONSITE & SESPMNT & $\mathrm{R}$ \\
\hline 690 & ONSITE & SESPMNT & $\mathrm{R}$ \\
\hline 691 & ONSITE & SESPMNT & $\mathrm{R}$ \\
\hline 692 & ONSITE & SESPMNT & $\mathrm{R}$ \\
\hline 693 & ONSITE & SESPMNT & $\mathrm{R}$ \\
\hline 694 & ONSITE & SESPMNT & $\mathrm{R}$ \\
\hline 695 & ONSITE & SESPMNT & $\mathrm{R}$ \\
\hline 696 & ONSITE & SESPMNT & $\mathrm{R}$ \\
\hline 697 & ONSITE & SESPMNT & $\mathrm{R}$ \\
\hline 698 & ONSITE & SESPMNT & $\mathrm{R}$ \\
\hline 699 & ONSITE & SESPMNT & $\mathrm{R}$ \\
\hline 700 & ONSITE & SESPMNT & $\mathrm{R}$ \\
\hline 701 & ONSITE & SESPMNT & $\mathrm{R}$ \\
\hline 702 & ONSITE & SESPMNT & $\mathrm{R}$ \\
\hline 703 & ONSITE & SESPMNT & $\mathrm{R}$ \\
\hline 704 & ONSITE & SESPMNT & $\mathrm{R}$ \\
\hline 705 & ONSITE & SESPMNT & $\mathrm{R}$ \\
\hline 706 & ONSITE & SESPMNT & $\mathrm{R}$ \\
\hline 707 & ONSITE & SESPMNT & $\mathrm{R}$ \\
\hline 708 & ONSITE & SESPMNT & $\mathrm{R}$ \\
\hline 709 & ONSITE & SESPMNT & $\mathrm{R}$ \\
\hline 710 & & SESPSPEC & $\mathrm{S}$ \\
\hline 711 & & SESPSPEC & $\mathrm{S}$ \\
\hline 712 & & SESPSPEC & $\mathrm{S}$ \\
\hline 713 & & SESPSPEC & $\mathrm{S}$ \\
\hline 714 & & SESPSPEC & $\mathrm{S}$ \\
\hline 715 & & SESPSPEC & $\mathrm{S}$ \\
\hline 716 & & SESPSPEC & $\mathrm{S}$ \\
\hline 717 & ONSITE & SESPMNT & $\mathrm{R}$ \\
\hline 718 & ONSITE & SESPMNT & $\mathrm{R}$ \\
\hline 719 & ONSITE & SESPMNT & $\mathrm{R}$ \\
\hline 720 & ONSITE & SESPMNT & $\mathrm{R}$ \\
\hline 721 & ONSITE & SESPMNT & $\mathrm{R}$ \\
\hline 722 & ONSITE & SESPMNT & $\mathrm{R}$ \\
\hline 723 & ONSITE & SESPMNT & $\mathrm{R}$ \\
\hline & & & \\
\hline
\end{tabular}




\begin{tabular}{|c|c|c|c|}
\hline & AG & $\mathrm{AH}$ & $\mathrm{Al}$ \\
\hline 1 & DIST_CLASS & OWNER_ID & COLLECTION_PURPOSE \\
\hline 724 & ONSITE & SESPMNT & $\mathrm{R}$ \\
\hline 725 & ONSITE & SESPMNT & $\mathrm{R}$ \\
\hline 726 & ONSITE & SESPMNT & $\mathrm{R}$ \\
\hline 727 & & SESPSPEC & S \\
\hline 728 & & SESPSPEC & S \\
\hline 729 & & SESPSPEC & S \\
\hline 730 & & SESPSPEC & S \\
\hline 731 & & SESPSPEC & S \\
\hline 732 & & SESPSPEC & S \\
\hline 733 & & SESPSPEC & S \\
\hline 734 & ONSITE & SESPMNT & $\mathrm{R}$ \\
\hline 735 & ONSITE & SESPMNT & $\mathrm{R}$ \\
\hline 736 & ONSITE & SESPMNT & $\mathrm{R}$ \\
\hline 737 & ONSITE & SESPMNT & $\mathrm{R}$ \\
\hline 738 & ONSITE & SESPMNT & $\mathrm{R}$ \\
\hline 739 & ONSITE & SESPMNT & $\mathrm{R}$ \\
\hline 740 & ONSITE & SESPMNT & $\mathrm{R}$ \\
\hline 741 & ONSITE & SESPMNT & $\mathrm{R}$ \\
\hline 742 & ONSITE & SESPMNT & $\mathrm{R}$ \\
\hline 743 & ONSITE & SESPMNT & $\mathrm{R}$ \\
\hline 744 & ONSITE & SESPMNT & $\mathrm{R}$ \\
\hline 745 & ONSITE & SESPMNT & $\mathrm{R}$ \\
\hline 746 & ONSITE & SESPMNT & $\mathrm{R}$ \\
\hline 747 & ONSITE & SESPMNT & $\mathrm{R}$ \\
\hline 748 & ONSITE & SESPMNT & $\mathrm{R}$ \\
\hline 749 & ONSITE & SESPMNT & $\mathrm{R}$ \\
\hline 750 & ONSITE & SESPMNT & $\mathrm{R}$ \\
\hline 751 & ONSITE & SESPMNT & $\mathrm{R}$ \\
\hline 752 & ONSITE & SESPMNT & $\mathrm{R}$ \\
\hline 753 & ONSITE & SESPMNT & $\mathrm{R}$ \\
\hline 754 & ONSITE & SESPMNT & $\mathrm{R}$ \\
\hline 755 & ONSITE & SESPMNT & $\mathrm{R}$ \\
\hline 756 & ONSITE & SESPMNT & $\mathrm{R}$ \\
\hline 757 & ONSITE & SESPMNT & $\mathrm{R}$ \\
\hline 758 & ONSITE & SESPMNT & $\mathrm{R}$ \\
\hline 759 & ONSITE & SESPMNT & $\mathrm{R}$ \\
\hline 760 & ONSITE & SESPMNT & $\mathrm{R}$ \\
\hline 761 & ONSITE & SESPMNT & $\mathrm{R}$ \\
\hline
\end{tabular}




\begin{tabular}{|c|c|c|c|}
\hline & AG & $\mathrm{AH}$ & $\mathrm{Al}$ \\
\hline 1 & DIST_CLASS & OWNER_ID & COLLECTION_PURPOSE \\
\hline 762 & ONSITE & SESPMNT & $\mathrm{R}$ \\
\hline 763 & ONSITE & SESPMNT & $\mathrm{R}$ \\
\hline 764 & ONSITE & SESPMNT & $\mathrm{R}$ \\
\hline 765 & ONSITE & SESPMNT & $\mathrm{R}$ \\
\hline 766 & ONSITE & SESPMNT & $\mathrm{R}$ \\
\hline 767 & ONSITE & SESPMNT & $\mathrm{R}$ \\
\hline 768 & ONSITE & SESPMNT & $\mathrm{R}$ \\
\hline 769 & ONSITE & SESPMNT & $\mathrm{R}$ \\
\hline 770 & ONSITE & SESPMNT & $\mathrm{R}$ \\
\hline 771 & ONSITE & SESPMNT & $\mathrm{R}$ \\
\hline 772 & ONSITE & SESPMNT & $\mathrm{R}$ \\
\hline 773 & ONSITE & SESPMNT & $\mathrm{R}$ \\
\hline 774 & & SESPSPEC & S \\
\hline 775 & & SESPSPEC & S \\
\hline 776 & & SESPSPEC & $S$ \\
\hline 777 & & SESPSPEC & S \\
\hline 778 & & SESPSPEC & $S$ \\
\hline 779 & & SESPSPEC & S \\
\hline 780 & & SESPSPEC & S \\
\hline 781 & & SESPSPEC & S \\
\hline 782 & & SESPSPEC & S \\
\hline 783 & & SESPSPEC & S \\
\hline 784 & & SESPSPEC & S \\
\hline 785 & & SESPSPEC & S \\
\hline 786 & & SESPSPEC & S \\
\hline 787 & & SESPSPEC & S \\
\hline 788 & & SESPSPEC & S \\
\hline 789 & & SESPSPEC & S \\
\hline 790 & & SESPSPEC & $S$ \\
\hline 791 & & SESPSPEC & S \\
\hline 792 & & SESPSPEC & S \\
\hline 793 & & SESPSPEC & S \\
\hline 794 & & SESPSPEC & S \\
\hline 795 & & SESPSPEC & S \\
\hline 796 & & SESPSPEC & S \\
\hline 797 & & SESPSPEC & $S$ \\
\hline 798 & & SESPSPEC & S \\
\hline 799 & & SESPSPEC & $\mathrm{S}$ \\
\hline
\end{tabular}




\begin{tabular}{|c|c|c|c|}
\hline & AG & $\mathrm{AH}$ & $\mathrm{Al}$ \\
\hline 1 & DIST_CLASS & OWNER_ID & COLLECTION_PURPOSE \\
\hline 800 & & SESPSPEC & S \\
\hline 801 & & SESPSPEC & S \\
\hline 802 & & SESPSPEC & $\mathrm{S}$ \\
\hline 803 & & SESPSPEC & S \\
\hline 804 & & SESPSPEC & S \\
\hline 805 & & SESPSPEC & S \\
\hline 806 & & SESPSPEC & S \\
\hline 807 & & SESPSPEC & S \\
\hline 808 & & SESPSPEC & S \\
\hline 809 & & SESPSPEC & S \\
\hline 810 & & SESPSPEC & S \\
\hline 811 & & SESPSPEC & S \\
\hline 812 & & SESPSPEC & $S$ \\
\hline 813 & & SESPSPEC & S \\
\hline 814 & & SESPSPEC & $S$ \\
\hline 815 & & SESPSPEC & S \\
\hline 816 & & SESPSPEC & $S$ \\
\hline 817 & & SESPSPEC & S \\
\hline 818 & & SESPSPEC & S \\
\hline 819 & & SESPSPEC & S \\
\hline 820 & & SESPSPEC & S \\
\hline 821 & & SESPSPEC & S \\
\hline 822 & & SESPSPEC & S \\
\hline 823 & & SESPSPEC & S \\
\hline 824 & & SESPSPEC & S \\
\hline 825 & & SESPSPEC & $S$ \\
\hline 826 & & SESPSPEC & S \\
\hline 827 & & SESPSPEC & S \\
\hline 828 & & SESPSPEC & S \\
\hline 829 & & SESPSPEC & S \\
\hline 830 & & SESPSPEC & S \\
\hline 831 & & SESPSPEC & S \\
\hline 832 & & SESPSPEC & S \\
\hline 833 & & SESPSPEC & S \\
\hline 834 & & SESPSPEC & S \\
\hline 835 & & SESPSPEC & $S$ \\
\hline 836 & & SESPSPEC & S \\
\hline 837 & & SESPSPEC & $S$ \\
\hline
\end{tabular}




\begin{tabular}{|c|l|l|l|}
\hline & AG & AH & \\
\hline 1 & DIST_CLASS & OWNER_ID & COLLECTION_PURPOSE \\
\hline 838 & & SESPSPEC & $\mathrm{S}$ \\
\hline 839 & & SESPSPEC & $\mathrm{S}$ \\
\hline 840 & & SESPSPEC & $\mathrm{S}$ \\
\hline 841 & & SESPSPEC & $\mathrm{S}$ \\
\hline 842 & & SESPSPEC & $\mathrm{S}$ \\
\hline 843 & & SESPSPEC & $\mathrm{S}$ \\
\hline 844 & & SESPSPEC & $\mathrm{S}$ \\
\hline 845 & & SESPSPEC & $\mathrm{S}$ \\
\hline 846 & ONSITE & SESPMNT & $\mathrm{R}$ \\
\hline 847 & ONSITE & SESPMNT & $\mathrm{R}$ \\
\hline 848 & ONSITE & SESPMNT & $\mathrm{R}$ \\
\hline 849 & ONSITE & SESPMNT & $\mathrm{R}$ \\
\hline 850 & ONSITE & SESPMNT & $\mathrm{R}$ \\
\hline 851 & ONSITE & SESPMNT & $\mathrm{R}$ \\
\hline 852 & ONSITE & SESPMNT & $\mathrm{R}$ \\
\hline 853 & ONSITE & SESPMNT & $\mathrm{R}$ \\
\hline 854 & ONSITE & SESPMNT & $\mathrm{R}$ \\
\hline 855 & ONSITE & SESPMNT & $\mathrm{R}$ \\
\hline 856 & ONSITE & SESPMNT & $\mathrm{R}$ \\
\hline 857 & ONSITE & SESPMNT & $\mathrm{R}$ \\
\hline 858 & ONSITE & SESPMNT & $\mathrm{R}$ \\
\hline 859 & ONSITE & SESPMNT & $\mathrm{R}$ \\
\hline 860 & ONSITE & SESPMNT & $\mathrm{R}$ \\
\hline 861 & ONSITE & SESPMNT & $\mathrm{R}$ \\
\hline 862 & ONSITE & SESPMNT & $\mathrm{R}$ \\
\hline 863 & ONSITE & SESPMNT & $\mathrm{R}$ \\
\hline 864 & ONSITE & SESPMNT & $\mathrm{R}$ \\
\hline 865 & ONSITE & SESPMNT & $\mathrm{R}$ \\
\hline 866 & ONSITE & SESPMNT & $\mathrm{R}$ \\
\hline 867 & ONSITE & SESPMNT & $\mathrm{R}$ \\
\hline 868 & ONSITE & SESPMNT & $\mathrm{R}$ \\
\hline 869 & ONSITE & SESPMNT & $\mathrm{R}$ \\
\hline 870 & ONSITE & SESPMNT & $\mathrm{R}$ \\
\hline 871 & ONSITE & SESPMNT & $\mathrm{R}$ \\
\hline 872 & ONSITE & SESPMNT & $\mathrm{R}$ \\
\hline 873 & ONSITE & SESPMNT & $\mathrm{R}$ \\
\hline 874 & ONSITE & SESPMNT & $\mathrm{R}$ \\
\hline 875 & ONSITE & SESPMNT & $\mathrm{R}$ \\
\hline & & & \\
\hline
\end{tabular}




\begin{tabular}{|c|l|l|l|}
\hline & AG & AH & \\
\hline 1 & DIST_CLASS & OWNER_ID & COLLECTION_PURPOSE \\
\hline 876 & ONSITE & SESPMNT & $\mathrm{R}$ \\
\hline 877 & ONSITE & SESPMNT & $\mathrm{R}$ \\
\hline 878 & ONSITE & SESPMNT & $\mathrm{R}$ \\
\hline 879 & ONSITE & SESPMNT & $\mathrm{R}$ \\
\hline 880 & ONSITE & SESPMNT & $\mathrm{R}$ \\
\hline 881 & ONSITE & SESPMNT & $\mathrm{R}$ \\
\hline 882 & ONSITE & SESPMNT & $\mathrm{R}$ \\
\hline 883 & ONSITE & SESPMNT & $\mathrm{R}$ \\
\hline 884 & ONSITE & SESPMNT & $\mathrm{R}$ \\
\hline 885 & ONSITE & SESPMNT & $\mathrm{R}$ \\
\hline 886 & ONSITE & SESPMNT & $\mathrm{R}$ \\
\hline 887 & ONSITE & SESPMNT & $\mathrm{R}$ \\
\hline 888 & ONSITE & SESPMNT & $\mathrm{R}$ \\
\hline 889 & ONSITE & SESPMNT & $\mathrm{R}$ \\
\hline 890 & ONSITE & SESPMNT & $\mathrm{R}$ \\
\hline 891 & ONSITE & SESPMNT & $\mathrm{R}$ \\
\hline 892 & ONSITE & SESPMNT & $\mathrm{R}$ \\
\hline 893 & ONSITE & SESPMNT & $\mathrm{R}$ \\
\hline 894 & ONSITE & SESPMNT & $\mathrm{R}$ \\
\hline 895 & ONSITE & SESPMNT & $\mathrm{R}$ \\
\hline 896 & & SESPSPEC & $\mathrm{S}$ \\
\hline 897 & & SESPSPEC & $\mathrm{S}$ \\
\hline 898 & & SESPSPEC & $\mathrm{S}$ \\
\hline 899 & & SESPSPEC & $\mathrm{S}$ \\
\hline 900 & & SESPSPEC & $\mathrm{S}$ \\
\hline 901 & & SESPSPEC & $\mathrm{S}$ \\
\hline 902 & & SESPSPEC & $\mathrm{S}$ \\
\hline 903 & & SESPSPEC & $\mathrm{S}$ \\
\hline 904 & & SESPSPEC & $\mathrm{S}$ \\
\hline 905 & & SESPSPEC & $\mathrm{S}$ \\
\hline 906 & & SESPSPEC & $\mathrm{S}$ \\
\hline 907 & & SESPSPEC & $\mathrm{S}$ \\
\hline 908 & & SESPSPEC & $\mathrm{S}$ \\
\hline 909 & & SESPSPEC & $\mathrm{S}$ \\
\hline 910 & & SESPSPEC & $\mathrm{S}$ \\
\hline 911 & & SESPSPEC & $\mathrm{S}$ \\
\hline 912 & & SESPSPEC & $\mathrm{S}$ \\
\hline 913 & & SESPSPEC & $\mathrm{S}$ \\
\hline & & & \\
\hline & & & \\
\hline$n n n$
\end{tabular}




\begin{tabular}{|c|c|c|c|}
\hline & $A G$ & $\mathrm{AH}$ & $\mathrm{Al}$ \\
\hline 1 & DIST_CLASS & OWNER_ID & |COLLECTION_PURPOSE \\
\hline 914 & & SESPSPEC & S \\
\hline 915 & & SESPSPEC & S \\
\hline 916 & & SESPSPEC & S \\
\hline 917 & & SESPSPEC & S \\
\hline 918 & & SESPSPEC & S \\
\hline 919 & & SESPSPEC & $\mathrm{S}$ \\
\hline 920 & & SESPSPEC & S \\
\hline 921 & & SESPSPEC & S \\
\hline 922 & & SESPSPEC & S \\
\hline 923 & & SESPSPEC & S \\
\hline 924 & & SESPSPEC & S \\
\hline 925 & & SESPSPEC & S \\
\hline 926 & & SESPSPEC & S \\
\hline 927 & & SESPSPEC & S \\
\hline 928 & & SESPSPEC & S \\
\hline 929 & & SESPSPEC & S \\
\hline 930 & & SESPSPEC & S \\
\hline 931 & & SESPSPEC & S \\
\hline 932 & & SESPSPEC & $\mathrm{S}$ \\
\hline 933 & & SESPSPEC & S \\
\hline 934 & & SESPSPEC & S \\
\hline 935 & & SESPSPEC & S \\
\hline 936 & & SESPSPEC & S \\
\hline 937 & & SESPSPEC & S \\
\hline 938 & & SESPSPEC & S \\
\hline 939 & & SESPSPEC & S \\
\hline 940 & & SESPSPEC & S \\
\hline 941 & & SESPSPEC & S \\
\hline 942 & & SESPSPEC & S \\
\hline 943 & & SESPSPEC & S \\
\hline 944 & & SESPSPEC & S \\
\hline 945 & & SESPSPEC & S \\
\hline 946 & & SESPSPEC & S \\
\hline 947 & & SESPSPEC & S \\
\hline 948 & & SESPSPEC & S \\
\hline 949 & & SESPSPEC & S \\
\hline 950 & & SESPSPEC & S \\
\hline 951 & & SESPSPEC & S \\
\hline
\end{tabular}




\begin{tabular}{|c|l|l|l|}
\hline & AG & \multicolumn{1}{|c|}{ AH } & \\
\hline 1 & DIST_CLASS & OWNER_ID & COLLECTION_PURPOSE \\
\hline 952 & & SESPSPEC & S \\
\hline 953 & & SESPSPEC & S \\
\hline 954 & & SESPSPEC & S \\
\hline 955 & & SESPSPEC & S \\
\hline 956 & & SESPSPEC & S \\
\hline 957 & & SESPSPEC & S \\
\hline 958 & & SESPSPEC & S \\
\hline 959 & & SESPSPEC & S \\
\hline 960 & & SESPSPEC & S \\
\hline 961 & & SESPSPEC & S \\
\hline 962 & & SESPSPEC & S \\
\hline 963 & & SESPSPEC & S \\
\hline 964 & & SESPSPEC & S \\
\hline 965 & & SESPSPEC & S \\
\hline 966 & OFFSITE & SESPMNT & $\mathrm{R}$ \\
\hline 967 & OFFSITE & SESPMNT & $\mathrm{R}$ \\
\hline 968 & OFFSITE & SESPMNT & $\mathrm{R}$ \\
\hline 969 & OFFSITE & SESPMNT & $\mathrm{R}$ \\
\hline 970 & OFFSITE & SESPMNT & $\mathrm{R}$ \\
\hline 971 & OFFSITE & SESPMNT & $\mathrm{R}$ \\
\hline 972 & OFFSITE & SESPMNT & $\mathrm{R}$ \\
\hline 973 & OFFSITE & SESPMNT & $\mathrm{R}$ \\
\hline 974 & OFFSITE & SESPMNT & $\mathrm{R}$ \\
\hline 975 & OFFSITE & SESPMNT & $\mathrm{R}$ \\
\hline 976 & OFFSITE & SESPMNT & $\mathrm{R}$ \\
\hline 977 & OFFSITE & SESPMNT & $\mathrm{R}$ \\
\hline 978 & OFFSITE & SESPMNT & $\mathrm{R}$ \\
\hline 979 & OFFSITE & SESPMNT & $\mathrm{R}$ \\
\hline 980 & OFFSITE & SESPMNT & $\mathrm{R}$ \\
\hline 981 & OFFSITE & SESPMNT & $\mathrm{R}$ \\
\hline 982 & OFFSITE & SESPMNT & $\mathrm{R}$ \\
\hline 983 & OFFSITE & SESPMNT & $\mathrm{R}$ \\
\hline 984 & OFFSITE & SESPMNT & $\mathrm{R}$ \\
\hline 985 & OFFSITE & SESPMNT & $\mathrm{R}$ \\
\hline 986 & OFFSITE & SESPMNT & $\mathrm{R}$ \\
\hline 987 & OFFSITE & SESPMNT & $\mathrm{R}$ \\
\hline 988 & OFFSITE & SESPMNT & $\mathrm{R}$ \\
\hline 989 & OFFSITE & SESPMNT & $\mathrm{R}$ \\
\hline & & & \\
\hline
\end{tabular}




\begin{tabular}{|c|l|l|l|}
\hline & AG & \multicolumn{1}{|c|}{ AH } & \\
\hline 1 & DIST_CLASS & OWNER_ID & COLLECTION_PURPOSE \\
\hline 990 & OFFSITE & SESPMNT & $\mathrm{R}$ \\
\hline 991 & OFFSITE & SESPMNT & $\mathrm{R}$ \\
\hline 992 & OFFSITE & SESPMNT & $\mathrm{R}$ \\
\hline 993 & OFFSITE & SESPMNT & $\mathrm{R}$ \\
\hline 994 & OFFSITE & SESPMNT & $\mathrm{R}$ \\
\hline 995 & OFFSITE & SESPMNT & $\mathrm{R}$ \\
\hline 996 & OFFSITE & SESPMNT & $\mathrm{R}$ \\
\hline 997 & OFFSITE & SESPMNT & $\mathrm{R}$ \\
\hline 998 & OFFSITE & SESPMNT & $\mathrm{R}$ \\
\hline 999 & OFFSITE & SESPMNT & $\mathrm{R}$ \\
\hline 1000 & OFFSITE & SESPMNT & $\mathrm{R}$ \\
\hline 1001 & OFFSITE & SESPMNT & $\mathrm{R}$ \\
\hline 1002 & OFFSITE & SESPMNT & $\mathrm{R}$ \\
\hline 1003 & OFFSITE & SESPMNT & $\mathrm{R}$ \\
\hline 1004 & OFFSITE & SESPMNT & $\mathrm{R}$ \\
\hline 1005 & OFFSITE & SESPMNT & $\mathrm{R}$ \\
\hline 1006 & OFFSITE & SESPMNT & $\mathrm{R}$ \\
\hline 1007 & OFFSITE & SESPMNT & $\mathrm{R}$ \\
\hline 1008 & OFFSITE & SESPMNT & $\mathrm{R}$ \\
\hline 1009 & OFFSITE & SESPMNT & $\mathrm{R}$ \\
\hline 1010 & OFFSITE & SESPMNT & $\mathrm{R}$ \\
\hline 1011 & OFFSITE & SESPMNT & $\mathrm{R}$ \\
\hline 1012 & OFFSITE & SESPMNT & $\mathrm{R}$ \\
\hline 1013 & OFFSITE & SESPMNT & $\mathrm{R}$ \\
\hline 1014 & OFFSITE & SESPMNT & $\mathrm{R}$ \\
\hline 1015 & OFFSITE & SESPMNT & $\mathrm{R}$ \\
\hline 1016 & ONSITE & SESPMNT & $\mathrm{R}$ \\
\hline 1017 & ONSITE & SESPMNT & $\mathrm{R}$ \\
\hline 1018 & ONSITE & SESPMNT & $\mathrm{R}$ \\
\hline 1019 & ONSITE & SESPMNT & $\mathrm{R}$ \\
\hline 1020 & ONSITE & SESPMNT & $\mathrm{R}$ \\
\hline 1021 & ONSITE & SESPMNT & $\mathrm{R}$ \\
\hline 1022 & ONSITE & SESPMNT & $\mathrm{R}$ \\
\hline 1023 & ONSITE & SESPMNT & $\mathrm{R}$ \\
\hline 1024 & ONSITE & SESPMNT & $\mathrm{R}$ \\
\hline 1025 & ONSITE & SESPMNT & $\mathrm{R}$ \\
\hline 1026 & ONSITE & SESPMNT & $\mathrm{R}$ \\
\hline 1027 & ONSITE & SESPMNT & $\mathrm{R}$ \\
\hline & & & \\
\hline
\end{tabular}




\begin{tabular}{|c|l|l|l|}
\hline & \multicolumn{1}{|c|}{ AG } & AH & \\
\hline 1 & DIST_CLASS & OWNER_ID & COLLECTION_PURPOSE \\
\hline 1028 & ONSITE & SESPMNT & $\mathrm{R}$ \\
\hline 1029 & ONSITE & SESPMNT & $\mathrm{R}$ \\
\hline 1030 & ONSITE & SESPMNT & $\mathrm{R}$ \\
\hline 1031 & ONSITE & SESPMNT & $\mathrm{R}$ \\
\hline 1032 & ONSITE & SESPMNT & $\mathrm{R}$ \\
\hline 1033 & ONSITE & SESPMNT & $\mathrm{R}$ \\
\hline 1034 & ONSITE & SESPMNT & $\mathrm{R}$ \\
\hline 1035 & ONSITE & SESPMNT & $\mathrm{R}$ \\
\hline 1036 & ONSITE & SESPMNT & $\mathrm{R}$ \\
\hline 1037 & ONSITE & SESPMNT & $\mathrm{R}$ \\
\hline 1038 & ONSITE & SESPMNT & $\mathrm{R}$ \\
\hline 1039 & ONSITE & SESPMNT & $\mathrm{R}$ \\
\hline 1040 & ONSITE & SESPMNT & $\mathrm{R}$ \\
\hline 1041 & ONSITE & SESPMNT & $\mathrm{R}$ \\
\hline 1042 & ONSITE & SESPMNT & $\mathrm{R}$ \\
\hline 1043 & ONSITE & SESPMNT & $\mathrm{R}$ \\
\hline 1044 & ONSITE & SESPMNT & $\mathrm{R}$ \\
\hline 1045 & ONSITE & SESPMNT & $\mathrm{R}$ \\
\hline 1046 & ONSITE & SESPMNT & $\mathrm{R}$ \\
\hline 1047 & ONSITE & SESPMNT & $\mathrm{R}$ \\
\hline 1048 & ONSITE & SESPMNT & $\mathrm{R}$ \\
\hline 1049 & ONSITE & SESPMNT & $\mathrm{R}$ \\
\hline 1050 & ONSITE & SESPMNT & $\mathrm{R}$ \\
\hline 1051 & ONSITE & SESPMNT & $\mathrm{R}$ \\
\hline 1052 & ONSITE & SESPMNT & $\mathrm{R}$ \\
\hline 1053 & ONSITE & SESPMNT & $\mathrm{R}$ \\
\hline 1054 & ONSITE & SESPMNT & $\mathrm{R}$ \\
\hline 1055 & ONSITE & SESPMNT & $\mathrm{R}$ \\
\hline 1056 & ONSITE & SESPMNT & $\mathrm{R}$ \\
\hline 1057 & ONSITE & SESPMNT & $\mathrm{R}$ \\
\hline 1058 & ONSITE & SESPMNT & $\mathrm{R}$ \\
\hline 1059 & ONSITE & SESPMNT & $\mathrm{R}$ \\
\hline 1060 & ONSITE & SESPMNT & $\mathrm{R}$ \\
\hline 1061 & ONSITE & SESPMNT & $\mathrm{R}$ \\
\hline 1062 & ONSITE & SESPMNT & $\mathrm{R}$ \\
\hline 1063 & ONSITE & SESPMNT & $\mathrm{R}$ \\
\hline 1064 & ONSITE & SESPMNT & $\mathrm{R}$ \\
\hline 1065 & ONSITE & SESPMNT & $\mathrm{R}$ \\
\hline & & & \\
\hline
\end{tabular}




\begin{tabular}{|c|l|l|l|}
\hline & AG & \multicolumn{1}{|c|}{ AH } & \\
\hline 1 & DIST_CLASS & OWNER_ID & COLLECTION_PURPOSE \\
\hline 1066 & OFFSITE & SESPMNT & $\mathrm{R}$ \\
\hline 1067 & OFFSITE & SESPMNT & $\mathrm{R}$ \\
\hline 1068 & OFFSITE & SESPMNT & $\mathrm{R}$ \\
\hline 1069 & OFFSITE & SESPMNT & $\mathrm{R}$ \\
\hline 1070 & OFFSITE & SESPMNT & $\mathrm{R}$ \\
\hline 1071 & OFFSITE & SESPMNT & $\mathrm{R}$ \\
\hline 1072 & OFFSITE & SESPMNT & $\mathrm{R}$ \\
\hline 1073 & OFFSITE & SESPMNT & $\mathrm{R}$ \\
\hline 1074 & OFFSITE & SESPMNT & $\mathrm{R}$ \\
\hline 1075 & OFFSITE & SESPMNT & $\mathrm{R}$ \\
\hline 1076 & OFFSITE & SESPMNT & $\mathrm{R}$ \\
\hline 1077 & OFFSITE & SESPMNT & $\mathrm{R}$ \\
\hline 1078 & OFFSITE & SESPMNT & $\mathrm{R}$ \\
\hline 1079 & OFFSITE & SESPMNT & $\mathrm{R}$ \\
\hline 1080 & OFFSITE & SESPMNT & $\mathrm{R}$ \\
\hline 1081 & OFFSITE & SESPMNT & $\mathrm{R}$ \\
\hline 1082 & OFFSITE & SESPMNT & $\mathrm{R}$ \\
\hline 1083 & OFFSITE & SESPMNT & $\mathrm{R}$ \\
\hline 1084 & OFFSITE & SESPMNT & $\mathrm{R}$ \\
\hline 1085 & OFFSITE & SESPMNT & $\mathrm{R}$ \\
\hline 1086 & OFFSITE & SESPSPEC & $\mathrm{S}$ \\
\hline 1087 & OFFSITE & SESPSPEC & $\mathrm{S}$ \\
\hline 1088 & OFFSITE & SESPSPEC & $\mathrm{S}$ \\
\hline 1089 & OFFSITE & SESPSPEC & $\mathrm{S}$ \\
\hline 1090 & OFFSITE & SESPSPEC & $\mathrm{S}$ \\
\hline 1091 & OFFSITE & SESPMNT & $\mathrm{R}$ \\
\hline 1092 & OFFSITE & SESPMNT & $\mathrm{R}$ \\
\hline 1093 & OFFSITE & SESPMNT & $\mathrm{R}$ \\
\hline 1094 & OFFSITE & SESPMNT & $\mathrm{R}$ \\
\hline 1095 & OFFSITE & SESPMNT & $\mathrm{R}$ \\
\hline 1096 & OFFSITE & SESPMNT & $\mathrm{R}$ \\
\hline 1097 & OFFSITE & SESPMNT & $\mathrm{R}$ \\
\hline 1098 & OFFSITE & SESPMNT & $\mathrm{R}$ \\
\hline 1099 & OFFSITE & SESPMNT & $\mathrm{R}$ \\
\hline 1100 & OFFSITE & SESPMNT & $\mathrm{R}$ \\
\hline 1101 & OFFSITE & SESPMNT & $\mathrm{R}$ \\
\hline 1102 & OFFSITE & SESPMNT & $\mathrm{R}$ \\
\hline 1103 & OFFSITE & SESPMNT & $\mathrm{R}$ \\
\hline & & & \\
\hline
\end{tabular}




\begin{tabular}{|c|l|l|l|}
\hline & AG & \multicolumn{1}{|c|}{ AH } & \\
\hline 1 & DIST_CLASS & OWNER_ID & COLLECTION_PURPOSE \\
\hline 1104 & OFFSITE & SESPMNT & $\mathrm{R}$ \\
\hline 1105 & OFFSITE & SESPMNT & $\mathrm{R}$ \\
\hline 1106 & OFFSITE & SESPMNT & $\mathrm{R}$ \\
\hline 1107 & OFFSITE & SESPMNT & $\mathrm{R}$ \\
\hline 1108 & OFFSITE & SESPMNT & $\mathrm{R}$ \\
\hline 1109 & OFFSITE & SESPMNT & $\mathrm{R}$ \\
\hline 1110 & OFFSITE & SESPMNT & $\mathrm{R}$ \\
\hline 1111 & OFFSITE & SESPMNT & $\mathrm{R}$ \\
\hline 1112 & OFFSITE & SESPMNT & $\mathrm{R}$ \\
\hline 1113 & OFFSITE & SESPMNT & $\mathrm{R}$ \\
\hline 1114 & OFFSITE & SESPMNT & $\mathrm{R}$ \\
\hline 1115 & OFFSITE & SESPMNT & $\mathrm{R}$ \\
\hline 1116 & OFFSITE & SESPMNT & $\mathrm{R}$ \\
\hline 1117 & OFFSITE & SESPMNT & $\mathrm{R}$ \\
\hline 1118 & OFFSITE & SESPMNT & $\mathrm{R}$ \\
\hline 1119 & OFFSITE & SESPMNT & $\mathrm{R}$ \\
\hline 1120 & OFFSITE & SESPMNT & $\mathrm{R}$ \\
\hline 1121 & OFFSITE & SESPMNT & $\mathrm{R}$ \\
\hline 1122 & OFFSITE & SESPMNT & $\mathrm{R}$ \\
\hline 1123 & OFFSITE & SESPMNT & $\mathrm{R}$ \\
\hline 1124 & OFFSITE & SESPMNT & $\mathrm{R}$ \\
\hline 1125 & OFFSITE & SESPMNT & $\mathrm{R}$ \\
\hline 1126 & OFFSITE & SESPMNT & $\mathrm{R}$ \\
\hline 1127 & OFFSITE & SESPMNT & $\mathrm{R}$ \\
\hline 1128 & OFFSITE & SESPMNT & $\mathrm{R}$ \\
\hline 1129 & OFFSITE & SESPMNT & $\mathrm{R}$ \\
\hline 1130 & OFFSITE & SESPMNT & $\mathrm{R}$ \\
\hline 1131 & OFFSITE & SESPMNT & $\mathrm{R}$ \\
\hline 1132 & OFFSITE & SESPMNT & $\mathrm{R}$ \\
\hline 1133 & OFFSITE & SESPMNT & $\mathrm{R}$ \\
\hline 1134 & OFFSITE & SESPMNT & $\mathrm{R}$ \\
\hline 1135 & OFFSITE & SESPMNT & $\mathrm{R}$ \\
\hline 1136 & OFFSITE & SESPMNT & $\mathrm{R}$ \\
\hline 1137 & OFFSITE & SESPMNT & $\mathrm{R}$ \\
\hline 1138 & OFFSITE & SESPMNT & $\mathrm{R}$ \\
\hline 1139 & OFFSITE & SESPMNT & $\mathrm{R}$ \\
\hline 1140 & OFFSITE & SESPMNT & $\mathrm{R}$ \\
\hline 1141 & OFFSITE & SESPMNT & $\mathrm{R}$ \\
\hline & & & \\
\hline
\end{tabular}




\begin{tabular}{|c|l|l|l|}
\hline & AG & AH & \\
\hline 1 & DIST_CLASS & OWNER_ID & COLLECTION_PURPOSE \\
\hline 1142 & OFFSITE & SESPMNT & $\mathrm{R}$ \\
\hline 1143 & OFFSITE & SESPMNT & $\mathrm{R}$ \\
\hline 1144 & OFFSITE & SESPMNT & $\mathrm{R}$ \\
\hline 1145 & OFFSITE & SESPMNT & $\mathrm{R}$ \\
\hline 1146 & OFFSITE & SESPMNT & $\mathrm{R}$ \\
\hline 1147 & OFFSITE & SESPMNT & $\mathrm{R}$ \\
\hline 1148 & OFFSITE & SESPMNT & $\mathrm{R}$ \\
\hline 1149 & OFFSITE & SESPMNT & $\mathrm{R}$ \\
\hline 1150 & OFFSITE & SESPMNT & $\mathrm{R}$ \\
\hline 1151 & OFFSITE & SESPMNT & $\mathrm{R}$ \\
\hline 1152 & OFFSITE & SESPMNT & $\mathrm{R}$ \\
\hline 1153 & OFFSITE & SESPMNT & $\mathrm{R}$ \\
\hline 1154 & OFFSITE & SESPMNT & $\mathrm{R}$ \\
\hline 1155 & OFFSITE & SESPMNT & $\mathrm{R}$ \\
\hline 1156 & OFFSITE & SESPMNT & $\mathrm{R}$ \\
\hline 1157 & OFFSITE & SESPMNT & $\mathrm{R}$ \\
\hline 1158 & OFFSITE & SESPMNT & $\mathrm{R}$ \\
\hline 1159 & OFFSITE & SESPMNT & $\mathrm{R}$ \\
\hline 1160 & OFFSITE & SESPMNT & $\mathrm{R}$ \\
\hline 1161 & OFFSITE & SESPMNT & $\mathrm{R}$ \\
\hline 1162 & OFFSITE & SESPMNT & $\mathrm{R}$ \\
\hline 1163 & OFFSITE & SESPMNT & $\mathrm{R}$ \\
\hline 1164 & OFFSITE & SESPMNT & $\mathrm{R}$ \\
\hline 1165 & OFFSITE & SESPMNT & $\mathrm{R}$ \\
\hline 1166 & OFFSITE & SESPMNT & $\mathrm{R}$ \\
\hline 1167 & OFFSITE & SESPMNT & $\mathrm{R}$ \\
\hline 1168 & OFFSITE & SESPMNT & $\mathrm{R}$ \\
\hline 1169 & OFFSITE & SESPMNT & $\mathrm{R}$ \\
\hline 1170 & OFFSITE & SESPMNT & $\mathrm{R}$ \\
\hline 1171 & OFFSITE & SESPMNT & $\mathrm{R}$ \\
\hline 1172 & OFFSITE & SESPMNT & $\mathrm{R}$ \\
\hline 1173 & OFFSITE & SESPMNT & $\mathrm{R}$ \\
\hline 1174 & OFFSITE & SESPMNT & $\mathrm{R}$ \\
\hline 1175 & OFFSITE & SESPMNT & $\mathrm{R}$ \\
\hline 1176 & OFFSITE & SESPMNT & $\mathrm{R}$ \\
\hline 1177 & OFFSITE & SESPMNT & $\mathrm{R}$ \\
\hline 1178 & OFFSITE & SESPMNT & $\mathrm{R}$ \\
\hline 1179 & OFFSITE & SESPMNT & $\mathrm{R}$ \\
\hline & & & \\
\hline
\end{tabular}




\begin{tabular}{|c|l|l|l|}
\hline & AG & \multicolumn{1}{|c|}{ AH } & \\
\hline 1 & DIST_CLASS & OWNER_ID & COLLECTION_PURPOSE \\
\hline 1180 & OFFSITE & SESPMNT & $\mathrm{R}$ \\
\hline 1181 & OFFSITE & SESPMNT & $\mathrm{R}$ \\
\hline 1182 & OFFSITE & SESPMNT & $\mathrm{R}$ \\
\hline 1183 & OFFSITE & SESPMNT & $\mathrm{R}$ \\
\hline 1184 & OFFSITE & SESPMNT & $\mathrm{R}$ \\
\hline 1185 & OFFSITE & SESPMNT & $\mathrm{R}$ \\
\hline 1186 & OFFSITE & SESPMNT & $\mathrm{R}$ \\
\hline 1187 & OFFSITE & SESPMNT & $\mathrm{R}$ \\
\hline 1188 & OFFSITE & SESPMNT & $\mathrm{R}$ \\
\hline 1189 & OFFSITE & SESPMNT & $\mathrm{R}$ \\
\hline 1190 & OFFSITE & SESPMNT & $\mathrm{R}$ \\
\hline 1191 & OFFSITE & SESPMNT & $\mathrm{R}$ \\
\hline 1192 & OFFSITE & SESPMNT & $\mathrm{R}$ \\
\hline 1193 & OFFSITE & SESPMNT & $\mathrm{R}$ \\
\hline 1194 & OFFSITE & SESPMNT & $\mathrm{R}$ \\
\hline 1195 & OFFSITE & SESPMNT & $\mathrm{R}$ \\
\hline 1196 & OFFSITE & SESPMNT & $\mathrm{R}$ \\
\hline 1197 & OFFSITE & SESPMNT & $\mathrm{R}$ \\
\hline 1198 & OFFSITE & SESPMNT & $\mathrm{R}$ \\
\hline 1199 & OFFSITE & SESPMNT & $\mathrm{R}$ \\
\hline 1200 & OFFSITE & SESPMNT & $\mathrm{R}$ \\
\hline 1201 & OFFSITE & SESPMNT & $\mathrm{R}$ \\
\hline 1202 & OFFSITE & SESPMNT & $\mathrm{R}$ \\
\hline 1203 & OFFSITE & SESPMNT & $\mathrm{R}$ \\
\hline 1204 & OFFSITE & SESPMNT & $\mathrm{R}$ \\
\hline 1205 & OFFSITE & SESPMNT & $\mathrm{R}$ \\
\hline 1206 & OFFSITE & SESPMNT & $\mathrm{R}$ \\
\hline 1207 & OFFSITE & SESPMNT & $\mathrm{R}$ \\
\hline 1208 & OFFSITE & SESPMNT & $\mathrm{R}$ \\
\hline 1209 & OFFSITE & SESPMNT & $\mathrm{R}$ \\
\hline 1210 & OFFSITE & SESPMNT & $\mathrm{R}$ \\
\hline 1211 & OFFSITE & SESPMNT & $\mathrm{R}$ \\
\hline 1212 & OFFSITE & SESPMNT & $\mathrm{R}$ \\
\hline 1213 & OFFSITE & SESPMNT & $\mathrm{R}$ \\
\hline 1214 & OFFSITE & SESPMNT & $\mathrm{R}$ \\
\hline 1215 & OFFSITE & SESPMNT & $\mathrm{R}$ \\
\hline 1216 & OFFSITE & SESPMNT & $\mathrm{R}$ \\
\hline 1217 & OFFSITE & SESPMNT & $\mathrm{R}$ \\
\hline & & & \\
\hline
\end{tabular}




\begin{tabular}{|c|l|l|l|}
\hline & AG & \multicolumn{1}{|c|}{ AH } & \\
\hline 1 & DIST_CLASS & OWNER_ID & COLLECTION_PURPOSE \\
\hline 1218 & OFFSITE & SESPMNT & $\mathrm{R}$ \\
\hline 1219 & OFFSITE & SESPMNT & $\mathrm{R}$ \\
\hline 1220 & OFFSITE & SESPMNT & $\mathrm{R}$ \\
\hline 1221 & OFFSITE & SESPMNT & $\mathrm{R}$ \\
\hline 1222 & OFFSITE & SESPMNT & $\mathrm{R}$ \\
\hline 1223 & OFFSITE & SESPMNT & $\mathrm{R}$ \\
\hline 1224 & OFFSITE & SESPMNT & $\mathrm{R}$ \\
\hline 1225 & OFFSITE & SESPMNT & $\mathrm{R}$ \\
\hline 1226 & OFFSITE & SESPMNT & $\mathrm{R}$ \\
\hline 1227 & OFFSITE & SESPMNT & $\mathrm{R}$ \\
\hline 1228 & OFFSITE & SESPMNT & $\mathrm{R}$ \\
\hline 1229 & OFFSITE & SESPMNT & $\mathrm{R}$ \\
\hline 1230 & OFFSITE & SESPMNT & $\mathrm{R}$ \\
\hline 1231 & OFFSITE & SESPMNT & $\mathrm{R}$ \\
\hline 1232 & OFFSITE & SESPMNT & $\mathrm{R}$ \\
\hline 1233 & OFFSITE & SESPMNT & $\mathrm{R}$ \\
\hline 1234 & OFFSITE & SESPMNT & $\mathrm{R}$ \\
\hline 1235 & OFFSITE & SESPMNT & $\mathrm{R}$ \\
\hline 1236 & OFFSITE & SESPMNT & $\mathrm{R}$ \\
\hline 1237 & OFFSITE & SESPMNT & $\mathrm{R}$ \\
\hline 1238 & OFFSITE & SESPMNT & $\mathrm{R}$ \\
\hline 1239 & OFFSITE & SESPMNT & $\mathrm{R}$ \\
\hline 1240 & OFFSITE & SESPMNT & $\mathrm{R}$ \\
\hline 1241 & OFFSITE & SESPMNT & $\mathrm{R}$ \\
\hline 1242 & OFFSITE & SESPMNT & $\mathrm{R}$ \\
\hline 1243 & OFFSITE & SESPMNT & $\mathrm{R}$ \\
\hline 1244 & OFFSITE & SESPMNT & $\mathrm{R}$ \\
\hline 1245 & OFFSITE & SESPMNT & $\mathrm{R}$ \\
\hline 1246 & OFFSITE & SESPMNT & $\mathrm{R}$ \\
\hline 1247 & OFFSITE & SESPMNT & $\mathrm{R}$ \\
\hline 1248 & OFFSITE & SESPMNT & $\mathrm{R}$ \\
\hline 1249 & OFFSITE & SESPMNT & $\mathrm{R}$ \\
\hline 1250 & OFFSITE & SESPMNT & $\mathrm{R}$ \\
\hline 1251 & OFFSITE & SESPMNT & $\mathrm{R}$ \\
\hline 1252 & OFFSITE & SESPMNT & $\mathrm{R}$ \\
\hline 1253 & OFFSITE & SESPMNT & $\mathrm{R}$ \\
\hline 1254 & OFFSITE & SESPMNT & $\mathrm{R}$ \\
\hline 1255 & OFFSITE & SESPMNT & $\mathrm{R}$ \\
\hline & & & \\
\hline
\end{tabular}




\begin{tabular}{|c|l|l|l|}
\hline & AG & \multicolumn{1}{|c|}{ AH } & \\
\hline 1 & DIST_CLASS & OWNER_ID & COLLECTION_PURPOSE \\
\hline 1256 & OFFSITE & SESPMNT & $\mathrm{R}$ \\
\hline 1257 & OFFSITE & SESPMNT & $\mathrm{R}$ \\
\hline 1258 & OFFSITE & SESPMNT & $\mathrm{R}$ \\
\hline 1259 & OFFSITE & SESPMNT & $\mathrm{R}$ \\
\hline 1260 & OFFSITE & SESPMNT & $\mathrm{R}$ \\
\hline 1261 & OFFSITE & SESPMNT & $\mathrm{R}$ \\
\hline 1262 & OFFSITE & SESPMNT & $\mathrm{R}$ \\
\hline 1263 & OFFSITE & SESPMNT & $\mathrm{R}$ \\
\hline 1264 & OFFSITE & SESPMNT & $\mathrm{R}$ \\
\hline 1265 & OFFSITE & SESPMNT & $\mathrm{R}$ \\
\hline 1266 & OFFSITE & SESPMNT & $\mathrm{R}$ \\
\hline 1267 & OFFSITE & SESPMNT & $\mathrm{R}$ \\
\hline 1268 & OFFSITE & SESPMNT & $\mathrm{R}$ \\
\hline 1269 & OFFSITE & SESPMNT & $\mathrm{R}$ \\
\hline 1270 & OFFSITE & SESPMNT & $\mathrm{R}$ \\
\hline 1271 & ONSITE & SESPMNT & $\mathrm{R}$ \\
\hline 1272 & ONSITE & SESPMNT & $\mathrm{R}$ \\
\hline 1273 & ONSITE & SESPMNT & $\mathrm{R}$ \\
\hline 1274 & ONSITE & SESPMNT & $\mathrm{R}$ \\
\hline 1275 & ONSITE & SESPMNT & $\mathrm{R}$ \\
\hline 1276 & ONSITE & SESPMNT & $\mathrm{R}$ \\
\hline 1277 & ONSITE & SESPMNT & $\mathrm{R}$ \\
\hline 1278 & ONSITE & SESPMNT & $\mathrm{R}$ \\
\hline 1279 & ONSITE & SESPMNT & $\mathrm{R}$ \\
\hline 1280 & ONSITE & SESPMNT & $\mathrm{R}$ \\
\hline 1281 & ONSITE & SESPMNT & $\mathrm{R}$ \\
\hline 1282 & ONSITE & SESPMNT & $\mathrm{R}$ \\
\hline 1283 & ONSITE & SESPMNT & $\mathrm{R}$ \\
\hline 1284 & ONSITE & SESPMNT & $\mathrm{R}$ \\
\hline 1285 & ONSITE & SESPMNT & $\mathrm{R}$ \\
\hline 1286 & ONSITE & SESPMNT & $\mathrm{R}$ \\
\hline 1287 & ONSITE & SESPMNT & $\mathrm{R}$ \\
\hline 1288 & ONSITE & SESPMNT & $\mathrm{R}$ \\
\hline 1289 & ONSITE & SESPMNT & $\mathrm{R}$ \\
\hline 1290 & ONSITE & SESPMNT & $\mathrm{R}$ \\
\hline 1291 & ONSITE & SESPMNT & $\mathrm{R}$ \\
\hline 1292 & ONSITE & SESPMNT & $\mathrm{R}$ \\
\hline 1293 & ONSITE & SESPMNT & $\mathrm{R}$ \\
\hline & & & \\
\hline
\end{tabular}




\begin{tabular}{|c|l|l|l|}
\hline & \multicolumn{1}{|c|}{ AG } & \multicolumn{1}{|c|}{ AH } & \\
\hline 1 & DIST_CLASS & OWNER_ID & COLLECTION_PURPOSE \\
\hline 1294 & ONSITE & SESPMNT & $\mathrm{R}$ \\
\hline 1295 & ONSITE & SESPMNT & $\mathrm{R}$ \\
\hline 1296 & ONSITE & SESPMNT & $\mathrm{R}$ \\
\hline 1297 & ONSITE & SESPMNT & $\mathrm{R}$ \\
\hline 1298 & ONSITE & SESPMNT & $\mathrm{R}$ \\
\hline 1299 & ONSITE & SESPMNT & $\mathrm{R}$ \\
\hline 1300 & ONSITE & SESPMNT & $\mathrm{R}$ \\
\hline 1301 & ONSITE & SESPMNT & $\mathrm{R}$ \\
\hline 1302 & ONSITE & SESPMNT & $\mathrm{R}$ \\
\hline 1303 & ONSITE & SESPMNT & $\mathrm{R}$ \\
\hline 1304 & ONSITE & SESPMNT & $\mathrm{R}$ \\
\hline 1305 & ONSITE & SESPMNT & $\mathrm{R}$ \\
\hline 1306 & ONSITE & SESPMNT & $\mathrm{R}$ \\
\hline 1307 & ONSITE & SESPMNT & $\mathrm{R}$ \\
\hline 1308 & ONSITE & SESPMNT & $\mathrm{R}$ \\
\hline 1309 & ONSITE & SESPMNT & $\mathrm{R}$ \\
\hline 1310 & ONSITE & SESPMNT & $\mathrm{R}$ \\
\hline 1311 & ONSITE & SESPMNT & $\mathrm{R}$ \\
\hline 1312 & ONSITE & SESPMNT & $\mathrm{R}$ \\
\hline 1313 & ONSITE & SESPMNT & $\mathrm{R}$ \\
\hline 1314 & ONSITE & SESPMNT & $\mathrm{R}$ \\
\hline 1315 & ONSITE & SESPMNT & $\mathrm{R}$ \\
\hline 1316 & ONSITE & SESPMNT & $\mathrm{R}$ \\
\hline 1317 & ONSITE & SESPMNT & $\mathrm{R}$ \\
\hline 1318 & ONSITE & SESPMNT & $\mathrm{R}$ \\
\hline 1319 & ONSITE & SESPMNT & $\mathrm{R}$ \\
\hline 1320 & ONSITE & SESPMNT & $\mathrm{R}$ \\
\hline 1321 & ONSITE & SESPMNT & $\mathrm{R}$ \\
\hline 1322 & ONSITE & SESPMNT & $\mathrm{R}$ \\
\hline 1323 & ONSITE & SESPMNT & $\mathrm{R}$ \\
\hline 1324 & ONSITE & SESPMNT & $\mathrm{R}$ \\
\hline 1325 & ONSITE & SESPMNT & $\mathrm{R}$ \\
\hline 1326 & ONSITE & SESPMNT & $\mathrm{R}$ \\
\hline 1327 & ONSITE & SESPMNT & $\mathrm{R}$ \\
\hline 1328 & ONSITE & SESPMNT & $\mathrm{R}$ \\
\hline 1329 & ONSITE & SESPMNT & $\mathrm{R}$ \\
\hline 1330 & ONSITE & SESPMNT & $\mathrm{R}$ \\
\hline 1331 & ONSITE & SESPMNT & $\mathrm{R}$ \\
\hline & & & \\
\hline
\end{tabular}




\begin{tabular}{|c|l|l|l|}
\hline & AG & AH & \\
\hline 1 & DIST_CLASS & OWNER_ID & COLLECTION_PURPOSE \\
\hline 1332 & ONSITE & SESPMNT & $\mathrm{R}$ \\
\hline 1333 & ONSITE & SESPMNT & $\mathrm{R}$ \\
\hline 1334 & ONSITE & SESPMNT & $\mathrm{R}$ \\
\hline 1335 & ONSITE & SESPMNT & $\mathrm{R}$ \\
\hline 1336 & ONSITE & SESPMNT & $\mathrm{R}$ \\
\hline 1337 & ONSITE & SESPMNT & $\mathrm{R}$ \\
\hline 1338 & ONSITE & SESPMNT & $\mathrm{R}$ \\
\hline 1339 & ONSITE & SESPMNT & $\mathrm{R}$ \\
\hline 1340 & ONSITE & SESPMNT & $\mathrm{R}$ \\
\hline 1341 & ONSITE & SESPMNT & $\mathrm{R}$ \\
\hline 1342 & ONSITE & SESPMNT & $\mathrm{R}$ \\
\hline 1343 & ONSITE & SESPMNT & $\mathrm{R}$ \\
\hline 1344 & ONSITE & SESPMNT & $\mathrm{R}$ \\
\hline 1345 & ONSITE & SESPMNT & $\mathrm{R}$ \\
\hline 1346 & ONSITE & SESPMNT & $\mathrm{R}$ \\
\hline 1347 & ONSITE & SESPMNT & $\mathrm{R}$ \\
\hline 1348 & ONSITE & SESPMNT & $\mathrm{R}$ \\
\hline 1349 & ONSITE & SESPMNT & $\mathrm{R}$ \\
\hline 1350 & ONSITE & SESPMNT & $\mathrm{R}$ \\
\hline 1351 & ONSITE & SESPMNT & $\mathrm{R}$ \\
\hline 1352 & ONSITE & SESPMNT & $\mathrm{R}$ \\
\hline 1353 & ONSITE & SESPMNT & $\mathrm{R}$ \\
\hline 1354 & ONSITE & SESPMNT & $\mathrm{R}$ \\
\hline 1355 & ONSITE & SESPMNT & $\mathrm{R}$ \\
\hline 1356 & ONSITE & SESPMNT & $\mathrm{R}$ \\
\hline 1357 & ONSITE & SESPMNT & $\mathrm{R}$ \\
\hline 1358 & ONSITE & SESPMNT & $\mathrm{R}$ \\
\hline 1359 & ONSITE & SESPMNT & $\mathrm{R}$ \\
\hline 1360 & ONSITE & SESPMNT & $\mathrm{R}$ \\
\hline 1361 & ONSITE & SESPMNT & $\mathrm{R}$ \\
\hline 1362 & ONSITE & SESPMNT & $\mathrm{R}$ \\
\hline 1363 & ONSITE & SESPMNT & $\mathrm{R}$ \\
\hline 1364 & ONSITE & SESPMNT & $\mathrm{R}$ \\
\hline 1365 & ONSITE & SESPMNT & $\mathrm{R}$ \\
\hline 1366 & ONSITE & SESPMNT & $\mathrm{R}$ \\
\hline 1367 & ONSITE & SESPMNT & $\mathrm{R}$ \\
\hline 1368 & ONSITE & SESPMNT & $\mathrm{R}$ \\
\hline 1369 & ONSITE & SESPMNT & $\mathrm{R}$ \\
\hline & & & \\
\hline
\end{tabular}




\begin{tabular}{|c|l|l|l|}
\hline & \multicolumn{1}{|c|}{ AG } & \multicolumn{1}{|c|}{ AH } & \\
\hline 1 & DIST_CLASS & OWNER_ID & COLLECTION_PURPOSE \\
\hline 1370 & ONSITE & SESPMNT & $\mathrm{R}$ \\
\hline 1371 & ONSITE & SESPMNT & $\mathrm{R}$ \\
\hline 1372 & ONSITE & SESPMNT & $\mathrm{R}$ \\
\hline 1373 & ONSITE & SESPMNT & $\mathrm{R}$ \\
\hline 1374 & ONSITE & SESPMNT & $\mathrm{R}$ \\
\hline 1375 & ONSITE & SESPMNT & $\mathrm{R}$ \\
\hline 1376 & ONSITE & SESPMNT & $\mathrm{R}$ \\
\hline 1377 & ONSITE & SESPMNT & $\mathrm{R}$ \\
\hline 1378 & ONSITE & SESPMNT & $\mathrm{R}$ \\
\hline 1379 & ONSITE & SESPMNT & $\mathrm{R}$ \\
\hline 1380 & ONSITE & SESPMNT & $\mathrm{R}$ \\
\hline 1381 & ONSITE & SESPMNT & $\mathrm{R}$ \\
\hline 1382 & ONSITE & SESPMNT & $\mathrm{R}$ \\
\hline 1383 & ONSITE & SESPMNT & $\mathrm{R}$ \\
\hline 1384 & ONSITE & SESPMNT & $\mathrm{R}$ \\
\hline 1385 & ONSITE & SESPMNT & $\mathrm{R}$ \\
\hline 1386 & ONSITE & SESPMNT & $\mathrm{R}$ \\
\hline 1387 & ONSITE & SESPMNT & $\mathrm{R}$ \\
\hline 1388 & ONSITE & SESPMNT & $\mathrm{R}$ \\
\hline 1389 & ONSITE & SESPMNT & $\mathrm{R}$ \\
\hline 1390 & ONSITE & SESPMNT & $\mathrm{R}$ \\
\hline 1391 & ONSITE & SESPMNT & $\mathrm{R}$ \\
\hline 1392 & ONSITE & SESPMNT & $\mathrm{R}$ \\
\hline 1393 & ONSITE & SESPMNT & $\mathrm{R}$ \\
\hline 1394 & ONSITE & SESPMNT & $\mathrm{R}$ \\
\hline 1395 & ONSITE & SESPMNT & $\mathrm{R}$ \\
\hline 1396 & ONSITE & SESPMNT & $\mathrm{R}$ \\
\hline 1397 & ONSITE & SESPMNT & $\mathrm{R}$ \\
\hline 1398 & ONSITE & SESPMNT & $\mathrm{R}$ \\
\hline 1399 & ONSITE & SESPMNT & $\mathrm{R}$ \\
\hline 1400 & ONSITE & SESPMNT & $\mathrm{R}$ \\
\hline 1401 & ONSITE & SESPMNT & $\mathrm{R}$ \\
\hline 1402 & ONSITE & SESPMNT & $\mathrm{R}$ \\
\hline 1403 & ONSITE & SESPMNT & $\mathrm{R}$ \\
\hline 1404 & ONSITE & SESPMNT & $\mathrm{R}$ \\
\hline 1405 & ONSITE & SESPMNT & $\mathrm{R}$ \\
\hline 1406 & ONSITE & SESPMNT & $\mathrm{R}$ \\
\hline 1407 & ONSITE & SESPMNT & $\mathrm{R}$ \\
\hline & & & \\
\hline
\end{tabular}




\begin{tabular}{|c|l|l|l|}
\hline & AG & AH & \\
\hline 1 & DIST_CLASS & OWNER_ID & COLLECTION_PURPOSE \\
\hline 1408 & ONSITE & SESPMNT & $\mathrm{R}$ \\
\hline 1409 & ONSITE & SESPMNT & $\mathrm{R}$ \\
\hline 1410 & ONSITE & SESPMNT & $\mathrm{R}$ \\
\hline 1411 & ONSITE & SESPMNT & $\mathrm{R}$ \\
\hline 1412 & ONSITE & SESPMNT & $\mathrm{R}$ \\
\hline 1413 & ONSITE & SESPMNT & $\mathrm{R}$ \\
\hline 1414 & ONSITE & SESPMNT & $\mathrm{R}$ \\
\hline 1415 & ONSITE & SESPMNT & $\mathrm{R}$ \\
\hline 1416 & ONSITE & SESPMNT & $\mathrm{R}$ \\
\hline 1417 & ONSITE & SESPMNT & $\mathrm{R}$ \\
\hline 1418 & ONSITE & SESPMNT & $\mathrm{R}$ \\
\hline 1419 & ONSITE & SESPMNT & $\mathrm{R}$ \\
\hline 1420 & ONSITE & SESPMNT & $\mathrm{R}$ \\
\hline 1421 & ONSITE & SESPMNT & $\mathrm{R}$ \\
\hline 1422 & ONSITE & SESPMNT & $\mathrm{R}$ \\
\hline 1423 & ONSITE & SESPMNT & $\mathrm{R}$ \\
\hline 1424 & ONSITE & SESPMNT & $\mathrm{R}$ \\
\hline 1425 & ONSITE & SESPMNT & $\mathrm{R}$ \\
\hline 1426 & ONSITE & SESPMNT & $\mathrm{R}$ \\
\hline 1427 & ONSITE & SESPMNT & $\mathrm{R}$ \\
\hline 1428 & ONSITE & SESPMNT & $\mathrm{R}$ \\
\hline 1429 & ONSITE & SESPMNT & $\mathrm{R}$ \\
\hline 1430 & ONSITE & SESPMNT & $\mathrm{R}$ \\
\hline 1431 & OFFSITE & SESPMNT & $\mathrm{R}$ \\
\hline 1432 & OFFSITE & SESPMNT & $\mathrm{R}$ \\
\hline 1433 & OFFSITE & SESPMNT & $\mathrm{R}$ \\
\hline 1434 & OFFSITE & SESPMNT & $\mathrm{R}$ \\
\hline 1435 & OFFSITE & SESPMNT & $\mathrm{R}$ \\
\hline 1436 & OFFSITE & SESPMNT & $\mathrm{R}$ \\
\hline 1437 & OFFSITE & SESPMNT & $\mathrm{R}$ \\
\hline 1438 & OFFSITE & SESPMNT & $\mathrm{R}$ \\
\hline 1439 & OFFSITE & SESPMNT & $\mathrm{R}$ \\
\hline 1440 & OFFSITE & SESPMNT & $\mathrm{R}$ \\
\hline 1441 & OFFSITE & SESPMNT & $\mathrm{R}$ \\
\hline 1442 & OFFSITE & SESPMNT & $\mathrm{R}$ \\
\hline 1443 & OFFSITE & SESPMNT & $\mathrm{R}$ \\
\hline 1444 & OFFSITE & SESPMNT & $\mathrm{R}$ \\
\hline 1445 & OFFSITE & SESPMNT & $\mathrm{R}$ \\
\hline & & & \\
\hline
\end{tabular}




\begin{tabular}{|c|l|l|l|}
\hline & AG & \multicolumn{1}{|c|}{ AH } & \\
\hline 1 & DIST_CLASS & OWNER_ID & COLLECTION_PURPOSE \\
\hline 1446 & OFFSITE & SESPMNT & $\mathrm{R}$ \\
\hline 1447 & OFFSITE & SESPMNT & $\mathrm{R}$ \\
\hline 1448 & OFFSITE & SESPMNT & $\mathrm{R}$ \\
\hline 1449 & OFFSITE & SESPMNT & $\mathrm{R}$ \\
\hline 1450 & OFFSITE & SESPMNT & $\mathrm{R}$ \\
\hline 1451 & OFFSITE & SESPMNT & $\mathrm{R}$ \\
\hline 1452 & OFFSITE & SESPMNT & $\mathrm{R}$ \\
\hline 1453 & OFFSITE & SESPMNT & $\mathrm{R}$ \\
\hline 1454 & OFFSITE & SESPMNT & $\mathrm{R}$ \\
\hline 1455 & OFFSITE & SESPMNT & $\mathrm{R}$ \\
\hline 1456 & OFFSITE & SESPMNT & $\mathrm{R}$ \\
\hline 1457 & OFFSITE & SESPMNT & $\mathrm{R}$ \\
\hline 1458 & OFFSITE & SESPMNT & $\mathrm{R}$ \\
\hline 1459 & ONSITE & SESPMNT & $\mathrm{R}$ \\
\hline 1460 & ONSITE & SESPMNT & $\mathrm{R}$ \\
\hline 1461 & ONSITE & SESPMNT & $\mathrm{R}$ \\
\hline 1462 & ONSITE & SESPMNT & $\mathrm{R}$ \\
\hline 1463 & ONSITE & SESPMNT & $\mathrm{R}$ \\
\hline 1464 & ONSITE & SESPMNT & $\mathrm{R}$ \\
\hline 1465 & ONSITE & SESPMNT & $\mathrm{R}$ \\
\hline 1466 & ONSITE & SESPMNT & $\mathrm{R}$ \\
\hline 1467 & ONSITE & SESPMNT & $\mathrm{R}$ \\
\hline 1468 & ONSITE & SESPMNT & $\mathrm{R}$ \\
\hline 1469 & ONSITE & SESPMNT & $\mathrm{R}$ \\
\hline 1470 & ONSITE & SESPMNT & $\mathrm{R}$ \\
\hline 1471 & ONSITE & SESPMNT & $\mathrm{R}$ \\
\hline 1472 & ONSITE & SESPMNT & $\mathrm{R}$ \\
\hline 1473 & ONSITE & SESPMNT & $\mathrm{R}$ \\
\hline 1474 & ONSITE & SESPMNT & $\mathrm{R}$ \\
\hline 1475 & ONSITE & SESPMNT & $\mathrm{R}$ \\
\hline 1476 & ONSITE & SESPMNT & $\mathrm{R}$ \\
\hline 1477 & ONSITE & SESPMNT & $\mathrm{R}$ \\
\hline 1478 & ONSITE & SESPMNT & $\mathrm{R}$ \\
\hline 1479 & ONSITE & SESPMNT & $\mathrm{R}$ \\
\hline 1480 & ONSITE & SESPMNT & $\mathrm{R}$ \\
\hline 1481 & ONSITE & SESPMNT & $\mathrm{R}$ \\
\hline 1482 & ONSITE & SESPMNT & $\mathrm{R}$ \\
\hline 1483 & ONSITE & SESPMNT & $\mathrm{R}$ \\
\hline & & & \\
\hline
\end{tabular}




\begin{tabular}{|c|l|l|l|}
\hline & \multicolumn{1}{|c|}{ AG } & \multicolumn{1}{|c|}{ AH } & \\
\hline 1 & DIST_CLASS & OWNER_ID & COLLECTION_PURPOSE \\
\hline 1484 & ONSITE & SESPMNT & $\mathrm{R}$ \\
\hline 1485 & ONSITE & SESPMNT & $\mathrm{R}$ \\
\hline 1486 & ONSITE & SESPMNT & $\mathrm{R}$ \\
\hline 1487 & ONSITE & SESPMNT & $\mathrm{R}$ \\
\hline 1488 & ONSITE & SESPMNT & $\mathrm{R}$ \\
\hline 1489 & ONSITE & SESPMNT & $\mathrm{R}$ \\
\hline 1490 & ONSITE & SESPMNT & $\mathrm{R}$ \\
\hline 1491 & ONSITE & SESPMNT & $\mathrm{R}$ \\
\hline 1492 & ONSITE & SESPMNT & $\mathrm{R}$ \\
\hline 1493 & ONSITE & SESPMNT & $\mathrm{R}$ \\
\hline 1494 & ONSITE & SESPMNT & $\mathrm{R}$ \\
\hline 1495 & ONSITE & SESPMNT & $\mathrm{R}$ \\
\hline 1496 & ONSITE & SESPMNT & $\mathrm{R}$ \\
\hline 1497 & ONSITE & SESPMNT & $\mathrm{R}$ \\
\hline 1498 & ONSITE & SESPMNT & $\mathrm{R}$ \\
\hline 1499 & ONSITE & SESPMNT & $\mathrm{R}$ \\
\hline 1500 & ONSITE & SESPMNT & $\mathrm{R}$ \\
\hline 1501 & ONSITE & SESPMNT & $\mathrm{R}$ \\
\hline 1502 & ONSITE & SESPMNT & $\mathrm{R}$ \\
\hline 1503 & ONSITE & SESPMNT & $\mathrm{R}$ \\
\hline 1504 & ONSITE & SESPMNT & $\mathrm{R}$ \\
\hline 1505 & ONSITE & SESPMNT & $\mathrm{R}$ \\
\hline 1506 & ONSITE & SESPMNT & $\mathrm{R}$ \\
\hline 1507 & ONSITE & SESPMNT & $\mathrm{R}$ \\
\hline 1508 & ONSITE & SESPMNT & $\mathrm{R}$ \\
\hline 1509 & ONSITE & SESPMNT & $\mathrm{R}$ \\
\hline 1510 & ONSITE & SESPMNT & $\mathrm{R}$ \\
\hline 1511 & ONSITE & SESPMNT & $\mathrm{R}$ \\
\hline 1512 & ONSITE & SESPMNT & $\mathrm{R}$ \\
\hline 1513 & ONSITE & SESPMNT & $\mathrm{R}$ \\
\hline 1514 & ONSITE & SESPMNT & $\mathrm{R}$ \\
\hline 1515 & ONSITE & SESPMNT & $\mathrm{R}$ \\
\hline 1516 & ONSITE & SESPMNT & $\mathrm{R}$ \\
\hline 1517 & ONSITE & SESPMNT & $\mathrm{R}$ \\
\hline 1518 & ONSITE & SESPMNT & $\mathrm{R}$ \\
\hline 1519 & ONSITE & SESPMNT & $\mathrm{R}$ \\
\hline 1520 & ONSITE & SESPMNT & $\mathrm{R}$ \\
\hline 1521 & ONSITE & SESPMNT & $\mathrm{R}$ \\
\hline & & & \\
\hline
\end{tabular}




\begin{tabular}{|c|l|l|l|}
\hline & AG & \multicolumn{1}{|c|}{ AH } & \\
\hline 1 & DIST_CLASS & OWNER_ID & COLLECTION_PURPOSE \\
\hline 1522 & ONSITE & SESPMNT & $\mathrm{R}$ \\
\hline 1523 & ONSITE & SESPMNT & $\mathrm{R}$ \\
\hline 1524 & ONSITE & SESPMNT & $\mathrm{R}$ \\
\hline 1525 & ONSITE & SESPMNT & $\mathrm{R}$ \\
\hline 1526 & ONSITE & SESPMNT & $\mathrm{R}$ \\
\hline 1527 & ONSITE & SESPMNT & $\mathrm{R}$ \\
\hline 1528 & ONSITE & SESPMNT & $\mathrm{R}$ \\
\hline 1529 & OFFSITE & SESPMNT & $\mathrm{R}$ \\
\hline 1530 & OFFSITE & SESPMNT & $\mathrm{R}$ \\
\hline 1531 & OFFSITE & SESPMNT & $\mathrm{R}$ \\
\hline 1532 & OFFSITE & SESPMNT & $\mathrm{R}$ \\
\hline 1533 & OFFSITE & SESPMNT & $\mathrm{R}$ \\
\hline 1534 & OFFSITE & SESPMNT & $\mathrm{R}$ \\
\hline 1535 & OFFSITE & SESPMNT & $\mathrm{R}$ \\
\hline 1536 & OFFSITE & SESPMNT & $\mathrm{R}$ \\
\hline 1537 & OFFSITE & SESPMNT & $\mathrm{R}$ \\
\hline 1538 & OFFSITE & SESPMNT & $\mathrm{R}$ \\
\hline 1539 & OFFSITE & SESPMNT & $\mathrm{R}$ \\
\hline 1540 & OFFSITE & SESPMNT & $\mathrm{R}$ \\
\hline 1541 & OFFSITE & SESPMNT & $\mathrm{R}$ \\
\hline 1542 & OFFSITE & SESPMNT & $\mathrm{R}$ \\
\hline 1543 & OFFSITE & SESPMNT & $\mathrm{R}$ \\
\hline 1544 & OFFSITE & SESPMNT & $\mathrm{R}$ \\
\hline 1545 & OFFSITE & SESPMNT & $\mathrm{R}$ \\
\hline 1546 & OFFSITE & SESPMNT & $\mathrm{R}$ \\
\hline 1547 & OFFSITE & SESPMNT & $\mathrm{R}$ \\
\hline 1548 & OFFSITE & SESPMNT & $\mathrm{R}$ \\
\hline 1549 & OFFSITE & SESPMNT & $\mathrm{R}$ \\
\hline 1550 & OFFSITE & SESPMNT & $\mathrm{R}$ \\
\hline 1551 & OFFSITE & SESPMNT & $\mathrm{R}$ \\
\hline 1552 & OFFSITE & SESPMNT & $\mathrm{R}$ \\
\hline 1553 & OFFSITE & SESPMNT & $\mathrm{R}$ \\
\hline 1554 & OFFSITE & SESPMNT & $\mathrm{R}$ \\
\hline 1555 & OFFSITE & SESPMNT & $\mathrm{R}$ \\
\hline 1556 & OFFSITE & SESPMNT & $\mathrm{R}$ \\
\hline 1557 & OFFSITE & SESPMNT & $\mathrm{R}$ \\
\hline 1558 & OFFSITE & SESPMNT & $\mathrm{R}$ \\
\hline 1559 & OFFSITE & SESPMNT & $\mathrm{R}$ \\
\hline & & & \\
\hline
\end{tabular}




\begin{tabular}{|c|l|l|l|}
\hline & AG & \multicolumn{1}{|c|}{ AH } & \\
\hline 1 & DIST_CLASS & OWNER_ID & COLLECTION_PURPOSE \\
\hline 1560 & OFFSITE & SESPMNT & $\mathrm{R}$ \\
\hline 1561 & OFFSITE & SESPMNT & $\mathrm{R}$ \\
\hline 1562 & OFFSITE & SESPMNT & $\mathrm{R}$ \\
\hline 1563 & OFFSITE & SESPMNT & $\mathrm{R}$ \\
\hline 1564 & OFFSITE & SESPMNT & $\mathrm{R}$ \\
\hline 1565 & OFFSITE & SESPMNT & $\mathrm{R}$ \\
\hline 1566 & OFFSITE & SESPMNT & $\mathrm{R}$ \\
\hline 1567 & OFFSITE & SESPMNT & $\mathrm{R}$ \\
\hline 1568 & OFFSITE & SESPMNT & $\mathrm{R}$ \\
\hline 1569 & OFFSITE & SESPMNT & $\mathrm{R}$ \\
\hline 1570 & OFFSITE & SESPMNT & $\mathrm{R}$ \\
\hline 1571 & OFFSITE & SESPMNT & $\mathrm{R}$ \\
\hline 1572 & OFFSITE & SESPMNT & $\mathrm{R}$ \\
\hline 1573 & OFFSITE & SESPMNT & $\mathrm{R}$ \\
\hline 1574 & OFFSITE & SESPMNT & $\mathrm{R}$ \\
\hline 1575 & OFFSITE & SESPMNT & $\mathrm{R}$ \\
\hline 1576 & OFFSITE & SESPMNT & $\mathrm{R}$ \\
\hline 1577 & OFFSITE & SESPMNT & $\mathrm{R}$ \\
\hline 1578 & OFFSITE & SESPMNT & $\mathrm{R}$ \\
\hline 1579 & OFFSITE & SESPMNT & $\mathrm{R}$ \\
\hline 1580 & OFFSITE & SESPMNT & $\mathrm{R}$ \\
\hline 1581 & OFFSITE & SESPMNT & $\mathrm{R}$ \\
\hline 1582 & OFFSITE & SESPMNT & $\mathrm{R}$ \\
\hline 1583 & OFFSITE & SESPMNT & $\mathrm{R}$ \\
\hline 1584 & OFFSITE & SESPMNT & $\mathrm{R}$ \\
\hline 1585 & OFFSITE & SESPMNT & $\mathrm{R}$ \\
\hline 1586 & OFFSITE & SESPMNT & $\mathrm{R}$ \\
\hline 1587 & OFFSITE & SESPMNT & $\mathrm{R}$ \\
\hline 1588 & OFFSITE & SESPMNT & $\mathrm{R}$ \\
\hline 1589 & OFFSITE & SESPMNT & $\mathrm{R}$ \\
\hline 1590 & OFFSITE & SESPMNT & $\mathrm{R}$ \\
\hline 1591 & OFFSITE & SESPMNT & $\mathrm{R}$ \\
\hline 1592 & OFFSITE & SESPMNT & $\mathrm{R}$ \\
\hline 1593 & OFFSITE & SESPMNT & $\mathrm{R}$ \\
\hline 1594 & OFFSITE & SESPMNT & $\mathrm{R}$ \\
\hline 1595 & OFFSITE & SESPMNT & $\mathrm{R}$ \\
\hline 1596 & OFFSITE & SESPMNT & $\mathrm{R}$ \\
\hline 1597 & OFFSITE & SESPMNT & $\mathrm{R}$ \\
\hline & & & \\
\hline
\end{tabular}




\begin{tabular}{|c|l|l|l|}
\hline & AG & AH & \\
\hline 1 & DIST_CLASS & OWNER_ID & COLLECTION_PURPOSE \\
\hline 1598 & OFFSITE & SESPMNT & $\mathrm{R}$ \\
\hline 1599 & ONSITE & SESPMNT & $\mathrm{R}$ \\
\hline 1600 & ONSITE & SESPMNT & $\mathrm{R}$ \\
\hline 1601 & ONSITE & SESPMNT & $\mathrm{R}$ \\
\hline 1602 & ONSITE & SESPMNT & $\mathrm{R}$ \\
\hline 1603 & ONSITE & SESPMNT & $\mathrm{R}$ \\
\hline 1604 & ONSITE & SESPMNT & $\mathrm{R}$ \\
\hline 1605 & ONSITE & SESPMNT & $\mathrm{R}$ \\
\hline 1606 & ONSITE & SESPMNT & $\mathrm{R}$ \\
\hline 1607 & ONSITE & SESPMNT & $\mathrm{R}$ \\
\hline 1608 & ONSITE & SESPMNT & $\mathrm{R}$ \\
\hline 1609 & ONSITE & SESPMNT & $\mathrm{R}$ \\
\hline 1610 & ONSITE & SESPMNT & $\mathrm{R}$ \\
\hline 1611 & ONSITE & SESPMNT & $\mathrm{R}$ \\
\hline 1612 & ONSITE & SESPMNT & $\mathrm{R}$ \\
\hline 1613 & ONSITE & SESPMNT & $\mathrm{R}$ \\
\hline 1614 & ONSITE & SESPMNT & $\mathrm{R}$ \\
\hline 1615 & ONSITE & SESPMNT & $\mathrm{R}$ \\
\hline 1616 & ONSITE & SESPMNT & $\mathrm{R}$ \\
\hline 1617 & ONSITE & SESPMNT & $\mathrm{R}$ \\
\hline 1618 & ONSITE & SESPMNT & $\mathrm{R}$ \\
\hline 1619 & ONSITE & SESPMNT & $\mathrm{R}$ \\
\hline 1620 & ONSITE & SESPMNT & $\mathrm{R}$ \\
\hline 1621 & ONSITE & SESPMNT & $\mathrm{R}$ \\
\hline 1622 & ONSITE & SESPMNT & $\mathrm{R}$ \\
\hline 1623 & ONSITE & SESPMNT & $\mathrm{R}$ \\
\hline 1624 & ONSITE & SESPMNT & $\mathrm{R}$ \\
\hline 1625 & ONSITE & SESPMNT & $\mathrm{R}$ \\
\hline 1626 & ONSITE & SESPMNT & $\mathrm{R}$ \\
\hline 1627 & ONSITE & SESPMNT & $\mathrm{R}$ \\
\hline 1628 & ONSITE & SESPMNT & $\mathrm{R}$ \\
\hline 1629 & ONSITE & SESPMNT & $\mathrm{R}$ \\
\hline 1630 & ONSITE & SESPMNT & $\mathrm{R}$ \\
\hline 1631 & ONSITE & SESPMNT & $\mathrm{R}$ \\
\hline 1632 & ONSITE & SESPMNT & $\mathrm{R}$ \\
\hline 1633 & ONSITE & SESPMNT & $\mathrm{R}$ \\
\hline 1634 & ONSITE & SESPMNT & $\mathrm{R}$ \\
\hline 1635 & ONSITE & SESPMNT & $\mathrm{R}$ \\
\hline & & & \\
\hline
\end{tabular}




\begin{tabular}{|c|l|l|l|}
\hline & AG & AH & \\
\hline 1 & DIST_CLASS & OWNER_ID & COLLECTION_PURPOSE \\
\hline 1636 & ONSITE & SESPMNT & $\mathrm{R}$ \\
\hline 1637 & ONSITE & SESPMNT & $\mathrm{R}$ \\
\hline 1638 & ONSITE & SESPMNT & $\mathrm{R}$ \\
\hline 1639 & ONSITE & SESPMNT & $\mathrm{R}$ \\
\hline 1640 & ONSITE & SESPMNT & $\mathrm{R}$ \\
\hline 1641 & ONSITE & SESPMNT & $\mathrm{R}$ \\
\hline 1642 & ONSITE & SESPMNT & $\mathrm{R}$ \\
\hline 1643 & ONSITE & SESPMNT & $\mathrm{R}$ \\
\hline 1644 & ONSITE & SESPMNT & $\mathrm{R}$ \\
\hline 1645 & ONSITE & SESPMNT & $\mathrm{R}$ \\
\hline 1646 & ONSITE & SESPMNT & $\mathrm{R}$ \\
\hline 1647 & ONSITE & SESPMNT & $\mathrm{R}$ \\
\hline 1648 & ONSITE & SESPMNT & $\mathrm{R}$ \\
\hline 1649 & ONSITE & SESPMNT & $\mathrm{R}$ \\
\hline 1650 & ONSITE & SESPMNT & $\mathrm{R}$ \\
\hline 1651 & ONSITE & SESPMNT & $\mathrm{R}$ \\
\hline 1652 & ONSITE & SESPMNT & $\mathrm{R}$ \\
\hline 1653 & ONSITE & SESPMNT & $\mathrm{R}$ \\
\hline 1654 & ONSITE & SESPMNT & $\mathrm{R}$ \\
\hline 1655 & ONSITE & SESPMNT & $\mathrm{R}$ \\
\hline 1656 & ONSITE & SESPMNT & $\mathrm{R}$ \\
\hline 1657 & ONSITE & SESPMNT & $\mathrm{R}$ \\
\hline 1658 & ONSITE & SESPMNT & $\mathrm{R}$ \\
\hline 1659 & ONSITE & SESPMNT & $\mathrm{R}$ \\
\hline 1660 & ONSITE & SESPMNT & $\mathrm{R}$ \\
\hline 1661 & ONSITE & SESPMNT & $\mathrm{R}$ \\
\hline 1662 & ONSITE & SESPMNT & $\mathrm{R}$ \\
\hline 1663 & ONSITE & SESPMNT & $\mathrm{R}$ \\
\hline 1664 & ONSITE & SESPMNT & $\mathrm{R}$ \\
\hline 1665 & ONSITE & SESPMNT & $\mathrm{R}$ \\
\hline 1666 & ONSITE & SESPMNT & $\mathrm{R}$ \\
\hline 1667 & ONSITE & SESPMNT & $\mathrm{R}$ \\
\hline 1668 & ONSITE & SESPMNT & $\mathrm{R}$ \\
\hline 1669 & ONSITE & SESPMNT & $\mathrm{R}$ \\
\hline 1670 & ONSITE & SESPMNT & $\mathrm{R}$ \\
\hline 1671 & ONSITE & SESPMNT & $\mathrm{R}$ \\
\hline 1672 & ONSITE & SESPMNT & $\mathrm{R}$ \\
\hline 1673 & ONSITE & SESPMNT & $\mathrm{R}$ \\
\hline & & & \\
\hline
\end{tabular}




\begin{tabular}{|c|l|l|l|}
\hline & AG & \multicolumn{1}{|c|}{ AH } & \\
\hline 1 & DIST_CLASS & OWNER_ID & COLLECTION_PURPOSE \\
\hline 1674 & ONSITE & SESPMNT & $\mathrm{R}$ \\
\hline 1675 & ONSITE & SESPMNT & $\mathrm{R}$ \\
\hline 1676 & ONSITE & SESPMNT & $\mathrm{R}$ \\
\hline 1677 & ONSITE & SESPMNT & $\mathrm{R}$ \\
\hline 1678 & ONSITE & SESPMNT & $\mathrm{R}$ \\
\hline 1679 & OFFSITE & SESPMNT & $\mathrm{R}$ \\
\hline 1680 & OFFSITE & SESPMNT & $\mathrm{R}$ \\
\hline 1681 & OFFSITE & SESPMNT & $\mathrm{R}$ \\
\hline 1682 & OFFSITE & SESPMNT & $\mathrm{R}$ \\
\hline 1683 & OFFSITE & SESPMNT & $\mathrm{R}$ \\
\hline 1684 & OFFSITE & SESPMNT & $\mathrm{R}$ \\
\hline 1685 & OFFSITE & SESPMNT & $\mathrm{R}$ \\
\hline 1686 & OFFSITE & SESPMNT & $\mathrm{R}$ \\
\hline 1687 & OFFSITE & SESPMNT & $\mathrm{R}$ \\
\hline 1688 & OFFSITE & SESPMNT & $\mathrm{R}$ \\
\hline 1689 & OFFSITE & SESPMNT & $\mathrm{R}$ \\
\hline 1690 & OFFSITE & SESPMNT & $\mathrm{R}$ \\
\hline 1691 & OFFSITE & SESPMNT & $\mathrm{R}$ \\
\hline 1692 & OFFSITE & SESPMNT & $\mathrm{R}$ \\
\hline 1693 & OFFSITE & SESPMNT & $\mathrm{R}$ \\
\hline 1694 & OFFSITE & SESPMNT & $\mathrm{R}$ \\
\hline 1695 & OFFSITE & SESPMNT & $\mathrm{R}$ \\
\hline 1696 & OFFSITE & SESPMNT & $\mathrm{R}$ \\
\hline 1697 & OFFSITE & SESPMNT & $\mathrm{R}$ \\
\hline 1698 & OFFSITE & SESPMNT & $\mathrm{R}$ \\
\hline 1699 & OFFSITE & SESPMNT & $\mathrm{R}$ \\
\hline 1700 & OFFSITE & SESPMNT & $\mathrm{R}$ \\
\hline 1701 & OFFSITE & SESPMNT & $\mathrm{R}$ \\
\hline 1702 & OFFSITE & SESPMNT & $\mathrm{R}$ \\
\hline 1703 & OFFSITE & SESPMNT & $\mathrm{R}$ \\
\hline 1704 & OFFSITE & SESPMNT & $\mathrm{R}$ \\
\hline 1705 & OFFSITE & SESPMNT & $\mathrm{R}$ \\
\hline 1706 & OFFSITE & SESPMNT & $\mathrm{R}$ \\
\hline 1707 & OFFSITE & SESPMNT & $\mathrm{R}$ \\
\hline 1708 & OFFSITE & SESPMNT & $\mathrm{R}$ \\
\hline 1709 & OFFSITE & SESPMNT & $\mathrm{R}$ \\
\hline 1710 & OFFSITE & SESPMNT & $\mathrm{R}$ \\
\hline 1711 & OFFSITE & SESPMNT & $\mathrm{R}$ \\
\hline & & & \\
\hline
\end{tabular}




\begin{tabular}{|c|l|l|l|}
\hline & AG & \multicolumn{1}{|c|}{ AH } & \\
\hline 1 & DIST_CLASS & OWNER_ID & COLLECTION_PURPOSE \\
\hline 1712 & OFFSITE & SESPMNT & $\mathrm{R}$ \\
\hline 1713 & OFFSITE & SESPMNT & $\mathrm{R}$ \\
\hline 1714 & OFFSITE & SESPMNT & $\mathrm{R}$ \\
\hline 1715 & OFFSITE & SESPMNT & $\mathrm{R}$ \\
\hline 1716 & OFFSITE & SESPMNT & $\mathrm{R}$ \\
\hline 1717 & OFFSITE & SESPMNT & $\mathrm{R}$ \\
\hline 1718 & OFFSITE & SESPMNT & $\mathrm{R}$ \\
\hline 1719 & OFFSITE & SESPMNT & $\mathrm{R}$ \\
\hline 1720 & OFFSITE & SESPMNT & $\mathrm{R}$ \\
\hline 1721 & OFFSITE & SESPMNT & $\mathrm{R}$ \\
\hline 1722 & OFFSITE & SESPMNT & $\mathrm{R}$ \\
\hline 1723 & OFFSITE & SESPMNT & $\mathrm{R}$ \\
\hline 1724 & OFFSITE & SESPMNT & $\mathrm{R}$ \\
\hline 1725 & OFFSITE & SESPMNT & $\mathrm{R}$ \\
\hline 1726 & OFFSITE & SESPMNT & $\mathrm{R}$ \\
\hline 1727 & OFFSITE & SESPMNT & $\mathrm{R}$ \\
\hline 1728 & OFFSITE & SESPMNT & $\mathrm{R}$ \\
\hline 1729 & OFFSITE & SESPMNT & $\mathrm{R}$ \\
\hline 1730 & OFFSITE & SESPMNT & $\mathrm{R}$ \\
\hline 1731 & OFFSITE & SESPMNT & $\mathrm{R}$ \\
\hline 1732 & OFFSITE & SESPMNT & $\mathrm{R}$ \\
\hline 1733 & OFFSITE & SESPMNT & $\mathrm{R}$ \\
\hline 1734 & OFFSITE & SESPMNT & $\mathrm{R}$ \\
\hline 1735 & OFFSITE & SESPMNT & $\mathrm{R}$ \\
\hline 1736 & OFFSITE & SESPMNT & $\mathrm{R}$ \\
\hline 1737 & OFFSITE & SESPMNT & $\mathrm{R}$ \\
\hline 1738 & OFFSITE & SESPMNT & $\mathrm{R}$ \\
\hline 1739 & OFFSITE & SESPMNT & $\mathrm{R}$ \\
\hline 1740 & OFFSITE & SESPMNT & $\mathrm{R}$ \\
\hline 1741 & OFFSITE & SESPMNT & $\mathrm{R}$ \\
\hline 1742 & OFFSITE & SESPMNT & $\mathrm{R}$ \\
\hline 1743 & OFFSITE & SESPMNT & $\mathrm{R}$ \\
\hline 1744 & OFFSITE & SESPMNT & $\mathrm{R}$ \\
\hline 1745 & OFFSITE & SESPMNT & $\mathrm{R}$ \\
\hline 1746 & OFFSITE & SESPMNT & $\mathrm{R}$ \\
\hline 1747 & OFFSITE & SESPMNT & $\mathrm{R}$ \\
\hline 1748 & OFFSITE & SESPMNT & $\mathrm{R}$ \\
\hline 1749 & OFFSITE & SESPMNT & $\mathrm{R}$ \\
\hline & & & \\
\hline
\end{tabular}




\begin{tabular}{|c|l|l|l|}
\hline & AG & \multicolumn{1}{|c|}{ AH } & \multicolumn{1}{|c|}{ AI } \\
\hline 1 & DIST_CLASS & OWNER_ID & COLLECTION_PURPOSE \\
\hline 1750 & OFFSITE & SESPMNT & $\mathrm{R}$ \\
\hline 1751 & OFFSITE & SESPMNT & $\mathrm{R}$ \\
\hline 1752 & OFFSITE & SESPMNT & $\mathrm{R}$ \\
\hline 1753 & OFFSITE & SESPMNT & $\mathrm{R}$ \\
\hline 1754 & OFFSITE & SESPMNT & $\mathrm{R}$ \\
\hline 1755 & OFFSITE & SESPMNT & $\mathrm{R}$ \\
\hline 1756 & OFFSITE & SESPMNT & $\mathrm{R}$ \\
\hline 1757 & OFFSITE & SESPMNT & $\mathrm{R}$ \\
\hline 1758 & OFFSITE & SESPMNT & $\mathrm{R}$ \\
\hline 1759 & OFFSITE & SESPMNT & $\mathrm{R}$ \\
\hline 1760 & OFFSITE & SESPMNT & $\mathrm{R}$ \\
\hline 1761 & OFFSITE & SESPMNT & $\mathrm{R}$ \\
\hline 1762 & OFFSITE & SESPMNT & $\mathrm{R}$ \\
\hline 1763 & OFFSITE & SESPMNT & $\mathrm{R}$ \\
\hline 1764 & OFFSITE & SESPMNT & $\mathrm{R}$ \\
\hline 1765 & OFFSITE & SESPMNT & $\mathrm{R}$ \\
\hline 1766 & OFFSITE & SESPMNT & $\mathrm{R}$ \\
\hline 1767 & OFFSITE & SESPMNT & $\mathrm{R}$ \\
\hline 1768 & OFFSITE & SESPMNT & $\mathrm{R}$ \\
\hline 1769 & OFFSITE & SESPMNT & $\mathrm{R}$ \\
\hline 1770 & OFFSITE & SESPMNT & $\mathrm{R}$ \\
\hline 1771 & OFFSITE & SESPMNT & $\mathrm{R}$ \\
\hline 1772 & OFFSITE & SESPMNT & $\mathrm{R}$ \\
\hline 1773 & OFFSITE & SESPMNT & $\mathrm{R}$ \\
\hline 1774 & OFFSITE & SESPMNT & $\mathrm{R}$ \\
\hline 1775 & OFFSITE & SESPMNT & $\mathrm{R}$ \\
\hline 1776 & OFFSITE & SESPMNT & $\mathrm{R}$ \\
\hline 1777 & OFFSITE & SESPMNT & $\mathrm{R}$ \\
\hline 1778 & OFFSITE & SESPMNT & $\mathrm{R}$ \\
\hline 1779 & OFFSITE & SESPMNT & $\mathrm{R}$ \\
\hline 1780 & OFFSITE & SESPMNT & $\mathrm{R}$ \\
\hline 1781 & OFFSITE & SESPMNT & $\mathrm{R}$ \\
\hline 1782 & OFFSITE & SESPMNT & $\mathrm{R}$ \\
\hline 1783 & OFFSITE & SESPMNT & $\mathrm{R}$ \\
\hline 1784 & OFFSITE & SESPMNT & $\mathrm{R}$ \\
\hline 1785 & OFFSITE & SESPMNT & $\mathrm{R}$ \\
\hline 1786 & OFFSITE & SESPMNT & $\mathrm{R}$ \\
\hline 1787 & OFFSITE & SESPMNT & $\mathrm{R}$ \\
\hline & & & \\
\hline
\end{tabular}




\begin{tabular}{|c|l|l|l|}
\hline & AG & \multicolumn{1}{|c|}{ AH } & \\
\hline 1 & DIST_CLASS & OWNER_ID & COLLECTION_PURPOSE \\
\hline 1788 & OFFSITE & SESPMNT & $\mathrm{R}$ \\
\hline 1789 & OFFSITE & SESPMNT & $\mathrm{R}$ \\
\hline 1790 & OFFSITE & SESPMNT & $\mathrm{R}$ \\
\hline 1791 & OFFSITE & SESPMNT & $\mathrm{R}$ \\
\hline 1792 & OFFSITE & SESPMNT & $\mathrm{R}$ \\
\hline 1793 & OFFSITE & SESPMNT & $\mathrm{R}$ \\
\hline 1794 & OFFSITE & SESPMNT & $\mathrm{R}$ \\
\hline 1795 & OFFSITE & SESPMNT & $\mathrm{R}$ \\
\hline 1796 & OFFSITE & SESPMNT & $\mathrm{R}$ \\
\hline 1797 & OFFSITE & SESPMNT & $\mathrm{R}$ \\
\hline 1798 & OFFSITE & SESPMNT & $\mathrm{R}$ \\
\hline 1799 & OFFSITE & SESPMNT & $\mathrm{R}$ \\
\hline 1800 & OFFSITE & SESPMNT & $\mathrm{R}$ \\
\hline 1801 & OFFSITE & SESPMNT & $\mathrm{R}$ \\
\hline 1802 & OFFSITE & SESPMNT & $\mathrm{R}$ \\
\hline 1803 & OFFSITE & SESPMNT & $\mathrm{R}$ \\
\hline 1804 & OFFSITE & SESPMNT & $\mathrm{R}$ \\
\hline 1805 & OFFSITE & SESPMNT & $\mathrm{R}$ \\
\hline 1806 & OFFSITE & SESPMNT & $\mathrm{R}$ \\
\hline 1807 & OFFSITE & SESPMNT & $\mathrm{R}$ \\
\hline 1808 & OFFSITE & SESPMNT & $\mathrm{R}$ \\
\hline 1809 & OFFSITE & SESPMNT & $\mathrm{R}$ \\
\hline 1810 & OFFSITE & SESPMNT & $\mathrm{R}$ \\
\hline 1811 & OFFSITE & SESPMNT & $\mathrm{R}$ \\
\hline 1812 & OFFSITE & SESPMNT & $\mathrm{R}$ \\
\hline 1813 & OFFSITE & SESPMNT & $\mathrm{R}$ \\
\hline 1814 & OFFSITE & SESPMNT & $\mathrm{R}$ \\
\hline 1815 & OFFSITE & SESPMNT & $\mathrm{R}$ \\
\hline 1816 & OFFSITE & SESPMNT & $\mathrm{R}$ \\
\hline 1817 & OFFSITE & SESPMNT & $\mathrm{R}$ \\
\hline 1818 & OFFSITE & SESPMNT & $\mathrm{R}$ \\
\hline 1819 & OFFSITE & SESPMNT & $\mathrm{R}$ \\
\hline 1820 & OFFSITE & SESPMNT & $\mathrm{R}$ \\
\hline 1821 & OFFSITE & SESPMNT & $\mathrm{R}$ \\
\hline 1822 & OFFSITE & SESPMNT & $\mathrm{R}$ \\
\hline 1823 & OFFSITE & SESPMNT & $\mathrm{R}$ \\
\hline 1824 & OFFSITE & SESPMNT & $\mathrm{R}$ \\
\hline 1825 & OFFSITE & SESPMNT & $\mathrm{R}$ \\
\hline & & & \\
\hline
\end{tabular}




\begin{tabular}{|c|l|l|l|}
\hline & AG & \multicolumn{1}{|c|}{ AH } & \\
\hline 1 & DIST_CLASS & OWNER_ID & COLLECTION_PURPOSE \\
\hline 1826 & OFFSITE & SESPMNT & $\mathrm{R}$ \\
\hline 1827 & OFFSITE & SESPMNT & $\mathrm{R}$ \\
\hline 1828 & OFFSITE & SESPMNT & $\mathrm{R}$ \\
\hline 1829 & OFFSITE & SESPMNT & $\mathrm{R}$ \\
\hline 1830 & OFFSITE & SESPMNT & $\mathrm{R}$ \\
\hline 1831 & OFFSITE & SESPMNT & $\mathrm{R}$ \\
\hline 1832 & OFFSITE & SESPMNT & $\mathrm{R}$ \\
\hline 1833 & OFFSITE & SESPMNT & $\mathrm{R}$ \\
\hline 1834 & OFFSITE & SESPMNT & $\mathrm{R}$ \\
\hline 1835 & OFFSITE & SESPMNT & $\mathrm{R}$ \\
\hline 1836 & OFFSITE & SESPMNT & $\mathrm{R}$ \\
\hline 1837 & OFFSITE & SESPMNT & $\mathrm{R}$ \\
\hline 1838 & OFFSITE & SESPMNT & $\mathrm{R}$ \\
\hline 1839 & OFFSITE & SESPMNT & $\mathrm{R}$ \\
\hline 1840 & OFFSITE & SESPMNT & $\mathrm{R}$ \\
\hline 1841 & OFFSITE & SESPMNT & $\mathrm{R}$ \\
\hline 1842 & OFFSITE & SESPMNT & $\mathrm{R}$ \\
\hline 1843 & OFFSITE & SESPMNT & $\mathrm{R}$ \\
\hline 1844 & OFFSITE & SESPMNT & $\mathrm{R}$ \\
\hline 1845 & OFFSITE & SESPMNT & $\mathrm{R}$ \\
\hline 1846 & OFFSITE & SESPMNT & $\mathrm{R}$ \\
\hline 1847 & OFFSITE & SESPMNT & $\mathrm{R}$ \\
\hline 1848 & OFFSITE & SESPMNT & $\mathrm{R}$ \\
\hline 1849 & OFFSITE & SESPMNT & $\mathrm{R}$ \\
\hline 1850 & OFFSITE & SESPMNT & $\mathrm{R}$ \\
\hline 1851 & OFFSITE & SESPMNT & $\mathrm{R}$ \\
\hline 1852 & OFFSITE & SESPMNT & $\mathrm{R}$ \\
\hline 1853 & OFFSITE & SESPMNT & $\mathrm{R}$ \\
\hline 1854 & OFFSITE & SESPMNT & $\mathrm{R}$ \\
\hline 1855 & OFFSITE & SESPMNT & $\mathrm{R}$ \\
\hline 1856 & OFFSITE & SESPMNT & $\mathrm{R}$ \\
\hline 1857 & OFFSITE & SESPMNT & $\mathrm{R}$ \\
\hline 1858 & OFFSITE & SESPMNT & $\mathrm{R}$ \\
\hline 1859 & OFFSITE & SESPMNT & $\mathrm{R}$ \\
\hline 1860 & OFFSITE & SESPMNT & $\mathrm{R}$ \\
\hline 1861 & OFFSITE & SESPMNT & $\mathrm{R}$ \\
\hline 1862 & OFFSITE & SESPMNT & $\mathrm{R}$ \\
\hline 1863 & OFFSITE & SESPMNT & $\mathrm{R}$ \\
\hline & & & \\
\hline
\end{tabular}




\begin{tabular}{|c|l|l|l|}
\hline & AG & \multicolumn{1}{|c|}{ AH } & \\
\hline 1 & DIST_CLASS & OWNER_ID & COLLECTION_PURPOSE \\
\hline 1864 & OFFSITE & SESPMNT & $\mathrm{R}$ \\
\hline 1865 & OFFSITE & SESPMNT & $\mathrm{R}$ \\
\hline 1866 & OFFSITE & SESPMNT & $\mathrm{R}$ \\
\hline 1867 & OFFSITE & SESPMNT & $\mathrm{R}$ \\
\hline 1868 & OFFSITE & SESPMNT & $\mathrm{R}$ \\
\hline 1869 & OFFSITE & SESPMNT & $\mathrm{R}$ \\
\hline 1870 & OFFSITE & SESPMNT & $\mathrm{R}$ \\
\hline 1871 & OFFSITE & SESPMNT & $\mathrm{R}$ \\
\hline 1872 & OFFSITE & SESPMNT & $\mathrm{R}$ \\
\hline 1873 & OFFSITE & SESPMNT & $\mathrm{R}$ \\
\hline 1874 & OFFSITE & SESPMNT & $\mathrm{R}$ \\
\hline 1875 & OFFSITE & SESPMNT & $\mathrm{R}$ \\
\hline 1876 & OFFSITE & SESPMNT & $\mathrm{R}$ \\
\hline 1877 & OFFSITE & SESPMNT & $\mathrm{R}$ \\
\hline 1878 & OFFSITE & SESPMNT & $\mathrm{R}$ \\
\hline 1879 & OFFSITE & SESPMNT & $\mathrm{R}$ \\
\hline 1880 & OFFSITE & SESPMNT & $\mathrm{R}$ \\
\hline 1881 & OFFSITE & SESPMNT & $\mathrm{R}$ \\
\hline 1882 & OFFSITE & SESPMNT & $\mathrm{R}$ \\
\hline 1883 & OFFSITE & SESPMNT & $\mathrm{R}$ \\
\hline 1884 & OFFSITE & SESPMNT & $\mathrm{R}$ \\
\hline 1885 & OFFSITE & SESPMNT & $\mathrm{R}$ \\
\hline 1886 & OFFSITE & SESPMNT & $\mathrm{R}$ \\
\hline 1887 & OFFSITE & SESPMNT & $\mathrm{R}$ \\
\hline 1888 & OFFSITE & SESPMNT & $\mathrm{R}$ \\
\hline 1889 & OFFSITE & SESPMNT & $\mathrm{R}$ \\
\hline 1890 & OFFSITE & SESPMNT & $\mathrm{R}$ \\
\hline 1891 & OFFSITE & SESPMNT & $\mathrm{R}$ \\
\hline 1892 & OFFSITE & SESPMNT & $\mathrm{R}$ \\
\hline 1893 & OFFSITE & SESPMNT & $\mathrm{R}$ \\
\hline 1894 & OFFSITE & SESPMNT & $\mathrm{R}$ \\
\hline 1895 & OFFSITE & SESPMNT & $\mathrm{R}$ \\
\hline 1896 & OFFSITE & SESPMNT & $\mathrm{R}$ \\
\hline 1897 & OFFSITE & SESPMNT & $\mathrm{R}$ \\
\hline 1898 & OFFSITE & SESPMNT & $\mathrm{R}$ \\
\hline 1899 & OFFSITE & SESPMNT & $\mathrm{R}$ \\
\hline 1900 & OFFSITE & SESPMNT & $\mathrm{R}$ \\
\hline 1901 & OFFSITE & SESPMNT & $\mathrm{R}$ \\
\hline & & & \\
\hline
\end{tabular}




\begin{tabular}{|c|l|l|l|}
\hline & AG & \multicolumn{1}{|c|}{ AH } & \\
\hline 1 & DIST_CLASS & OWNER_ID & COLLECTION_PURPOSE \\
\hline 1902 & OFFSITE & SESPMNT & $\mathrm{R}$ \\
\hline 1903 & OFFSITE & SESPMNT & $\mathrm{R}$ \\
\hline 1904 & OFFSITE & SESPMNT & $\mathrm{R}$ \\
\hline 1905 & OFFSITE & SESPMNT & $\mathrm{R}$ \\
\hline 1906 & OFFSITE & SESPMNT & $\mathrm{R}$ \\
\hline 1907 & OFFSITE & SESPMNT & $\mathrm{R}$ \\
\hline 1908 & OFFSITE & SESPMNT & $\mathrm{R}$ \\
\hline 1909 & OFFSITE & SESPMNT & $\mathrm{R}$ \\
\hline 1910 & OFFSITE & SESPMNT & $\mathrm{R}$ \\
\hline 1911 & OFFSITE & SESPMNT & $\mathrm{R}$ \\
\hline 1912 & OFFSITE & SESPMNT & $\mathrm{R}$ \\
\hline 1913 & OFFSITE & SESPMNT & $\mathrm{R}$ \\
\hline 1914 & OFFSITE & SESPMNT & $\mathrm{R}$ \\
\hline 1915 & OFFSITE & SESPMNT & $\mathrm{R}$ \\
\hline 1916 & OFFSITE & SESPMNT & $\mathrm{R}$ \\
\hline 1917 & OFFSITE & SESPMNT & $\mathrm{R}$ \\
\hline 1918 & OFFSITE & SESPMNT & $\mathrm{R}$ \\
\hline 1919 & OFFSITE & SESPMNT & $\mathrm{R}$ \\
\hline 1920 & OFFSITE & SESPMNT & $\mathrm{R}$ \\
\hline 1921 & OFFSITE & SESPMNT & $\mathrm{R}$ \\
\hline 1922 & OFFSITE & SESPMNT & $\mathrm{R}$ \\
\hline 1923 & OFFSITE & SESPMNT & $\mathrm{R}$ \\
\hline 1924 & OFFSITE & SESPMNT & $\mathrm{R}$ \\
\hline 1925 & OFFSITE & SESPMNT & $\mathrm{R}$ \\
\hline 1926 & OFFSITE & SESPMNT & $\mathrm{R}$ \\
\hline 1927 & OFFSITE & SESPMNT & $\mathrm{R}$ \\
\hline 1928 & OFFSITE & SESPMNT & $\mathrm{R}$ \\
\hline 1929 & OFFSITE & SESPMNT & $\mathrm{R}$ \\
\hline 1930 & OFFSITE & SESPMNT & $\mathrm{R}$ \\
\hline 1931 & OFFSITE & SESPMNT & $\mathrm{R}$ \\
\hline 1932 & OFFSITE & SESPMNT & $\mathrm{R}$ \\
\hline 1933 & OFFSITE & SESPMNT & $\mathrm{R}$ \\
\hline 1934 & OFFSITE & SESPMNT & $\mathrm{R}$ \\
\hline 1935 & OFFSITE & SESPMNT & $\mathrm{R}$ \\
\hline 1936 & OFFSITE & SESPMNT & $\mathrm{R}$ \\
\hline 1937 & OFFSITE & SESPMNT & $\mathrm{R}$ \\
\hline 1938 & OFFSITE & SESPMNT & $\mathrm{R}$ \\
\hline 1939 & OFFSITE & SESPMNT & $\mathrm{R}$ \\
\hline & & & \\
\hline
\end{tabular}




\begin{tabular}{|c|l|l|l|}
\hline & AG & \multicolumn{1}{|c|}{ AH } & \\
\hline 1 & DIST_CLASS & OWNER_ID & COLLECTION_PURPOSE \\
\hline 1940 & OFFSITE & SESPMNT & $\mathrm{R}$ \\
\hline 1941 & OFFSITE & SESPMNT & $\mathrm{R}$ \\
\hline 1942 & OFFSITE & SESPMNT & $\mathrm{R}$ \\
\hline 1943 & OFFSITE & SESPMNT & $\mathrm{R}$ \\
\hline 1944 & OFFSITE & SESPMNT & $\mathrm{R}$ \\
\hline 1945 & OFFSITE & SESPMNT & $\mathrm{R}$ \\
\hline 1946 & OFFSITE & SESPMNT & $\mathrm{R}$ \\
\hline 1947 & OFFSITE & SESPMNT & $\mathrm{R}$ \\
\hline 1948 & OFFSITE & SESPMNT & $\mathrm{R}$ \\
\hline 1949 & OFFSITE & SESPMNT & $\mathrm{R}$ \\
\hline 1950 & OFFSITE & SESPMNT & $\mathrm{R}$ \\
\hline 1951 & OFFSITE & SESPMNT & $\mathrm{R}$ \\
\hline 1952 & OFFSITE & SESPMNT & $\mathrm{R}$ \\
\hline 1953 & OFFSITE & SESPMNT & $\mathrm{R}$ \\
\hline 1954 & OFFSITE & SESPMNT & $\mathrm{R}$ \\
\hline 1955 & OFFSITE & SESPMNT & $\mathrm{R}$ \\
\hline 1956 & OFFSITE & SESPMNT & $\mathrm{R}$ \\
\hline 1957 & OFFSITE & SESPMNT & $\mathrm{R}$ \\
\hline 1958 & OFFSITE & SESPMNT & $\mathrm{R}$ \\
\hline 1959 & OFFSITE & SESPMNT & $\mathrm{R}$ \\
\hline 1960 & OFFSITE & SESPMNT & $\mathrm{R}$ \\
\hline 1961 & OFFSITE & SESPMNT & $\mathrm{R}$ \\
\hline 1962 & OFFSITE & SESPMNT & $\mathrm{R}$ \\
\hline 1963 & OFFSITE & SESPMNT & $\mathrm{R}$ \\
\hline 1964 & OFFSITE & SESPMNT & $\mathrm{R}$ \\
\hline 1965 & OFFSITE & SESPMNT & $\mathrm{R}$ \\
\hline 1966 & OFFSITE & SESPMNT & $\mathrm{R}$ \\
\hline 1967 & OFFSITE & SESPMNT & $\mathrm{R}$ \\
\hline 1968 & OFFSITE & SESPMNT & $\mathrm{R}$ \\
\hline 1969 & OFFSITE & SESPMNT & $\mathrm{R}$ \\
\hline 1970 & OFFSITE & SESPMNT & $\mathrm{R}$ \\
\hline 1971 & OFFSITE & SESPMNT & $\mathrm{R}$ \\
\hline 1972 & OFFSITE & SESPMNT & $\mathrm{R}$ \\
\hline 1973 & OFFSITE & SESPMNT & $\mathrm{R}$ \\
\hline 1974 & OFFSITE & SESPMNT & $\mathrm{R}$ \\
\hline 1975 & OFFSITE & SESPMNT & $\mathrm{R}$ \\
\hline 1976 & OFFSITE & SESPMNT & $\mathrm{R}$ \\
\hline 1977 & OFFSITE & SESPMNT & $\mathrm{R}$ \\
\hline & & & \\
\hline
\end{tabular}




\begin{tabular}{|c|l|l|l|}
\hline & \multicolumn{1}{|c|}{ AG } & \multicolumn{1}{|c|}{ AH } & \\
\hline 1 & DIST_CLASS & OWNER_ID & COLLECTION_PURPOSE \\
\hline 1978 & ONSITE & SESPMNT & $\mathrm{R}$ \\
\hline 1979 & ONSITE & SESPMNT & $\mathrm{R}$ \\
\hline 1980 & ONSITE & SESPMNT & $\mathrm{R}$ \\
\hline 1981 & ONSITE & SESPMNT & $\mathrm{R}$ \\
\hline 1982 & ONSITE & SESPMNT & $\mathrm{R}$ \\
\hline 1983 & ONSITE & SESPMNT & $\mathrm{R}$ \\
\hline 1984 & ONSITE & SESPMNT & $\mathrm{R}$ \\
\hline 1985 & ONSITE & SESPMNT & $\mathrm{R}$ \\
\hline 1986 & ONSITE & SESPMNT & $\mathrm{R}$ \\
\hline 1987 & ONSITE & SESPMNT & $\mathrm{R}$ \\
\hline 1988 & ONSITE & SESPMNT & $\mathrm{R}$ \\
\hline 1989 & ONSITE & SESPMNT & $\mathrm{R}$ \\
\hline 1990 & ONSITE & SESPMNT & $\mathrm{R}$ \\
\hline 1991 & ONSITE & SESPMNT & $\mathrm{R}$ \\
\hline 1992 & ONSITE & SESPMNT & $\mathrm{R}$ \\
\hline 1993 & ONSITE & SESPMNT & $\mathrm{R}$ \\
\hline 1994 & ONSITE & SESPMNT & $\mathrm{R}$ \\
\hline 1995 & ONSITE & SESPMNT & $\mathrm{R}$ \\
\hline 1996 & ONSITE & SESPMNT & $\mathrm{R}$ \\
\hline 1997 & ONSITE & SESPMNT & $\mathrm{R}$ \\
\hline 1998 & ONSITE & SESPMNT & $\mathrm{R}$ \\
\hline 1999 & ONSITE & SESPMNT & $\mathrm{R}$ \\
\hline 2000 & ONSITE & SESPMNT & $\mathrm{R}$ \\
\hline 2001 & ONSITE & SESPMNT & $\mathrm{R}$ \\
\hline 2002 & ONSITE & SESPMNT & $\mathrm{R}$ \\
\hline 2003 & ONSITE & SESPMNT & $\mathrm{R}$ \\
\hline 2004 & ONSITE & SESPMNT & $\mathrm{R}$ \\
\hline 2005 & ONSITE & SESPMNT & $\mathrm{R}$ \\
\hline 2006 & ONSITE & SESPMNT & $\mathrm{R}$ \\
\hline 2007 & ONSITE & SESPMNT & $\mathrm{R}$ \\
\hline 2008 & ONSITE & SESPMNT & $\mathrm{R}$ \\
\hline 2009 & ONSITE & SESPMNT & $\mathrm{R}$ \\
\hline 2010 & ONSITE & SESPMNT & $\mathrm{R}$ \\
\hline 2011 & ONSITE & SESPMNT & $\mathrm{R}$ \\
\hline 2012 & ONSITE & SESPMNT & $\mathrm{R}$ \\
\hline 2013 & ONSITE & SESPMNT & $\mathrm{R}$ \\
\hline 2014 & ONSITE & SESPMNT & $\mathrm{R}$ \\
\hline 2015 & ONSITE & SESPMNT & $\mathrm{R}$ \\
\hline
\end{tabular}




\begin{tabular}{|c|l|l|l|}
\hline & AG & AH & \\
\hline 1 & DIST_CLASS & OWNER_ID & COLLECTION_PURPOSE \\
\hline 2016 & ONSITE & SESPMNT & $\mathrm{R}$ \\
\hline 2017 & ONSITE & SESPMNT & $\mathrm{R}$ \\
\hline 2018 & ONSITE & SESPMNT & $\mathrm{R}$ \\
\hline 2019 & ONSITE & SESPMNT & $\mathrm{R}$ \\
\hline 2020 & ONSITE & SESPMNT & $\mathrm{R}$ \\
\hline 2021 & ONSITE & SESPMNT & $\mathrm{R}$ \\
\hline 2022 & ONSITE & SESPMNT & $\mathrm{R}$ \\
\hline 2023 & ONSITE & SESPMNT & $\mathrm{R}$ \\
\hline 2024 & ONSITE & SESPMNT & $\mathrm{R}$ \\
\hline 2025 & ONSITE & SESPMNT & $\mathrm{R}$ \\
\hline 2026 & ONSITE & SESPMNT & $\mathrm{R}$ \\
\hline 2027 & ONSITE & SESPMNT & $\mathrm{R}$ \\
\hline 2028 & ONSITE & SESPMNT & $\mathrm{R}$ \\
\hline 2029 & ONSITE & SESPMNT & $\mathrm{R}$ \\
\hline 2030 & ONSITE & SESPMNT & $\mathrm{R}$ \\
\hline 2031 & ONSITE & SESPMNT & $\mathrm{R}$ \\
\hline 2032 & ONSITE & SESPMNT & $\mathrm{R}$ \\
\hline 2033 & ONSITE & SESPMNT & $\mathrm{R}$ \\
\hline 2034 & ONSITE & SESPMNT & $\mathrm{R}$ \\
\hline 2035 & ONSITE & SESPMNT & $\mathrm{R}$ \\
\hline 2036 & ONSITE & SESPMNT & $\mathrm{R}$ \\
\hline 2037 & ONSITE & SESPMNT & $\mathrm{R}$ \\
\hline 2038 & ONSITE & SESPMNT & $\mathrm{R}$ \\
\hline 2039 & ONSITE & SESPMNT & $\mathrm{R}$ \\
\hline 2040 & ONSITE & SESPMNT & $\mathrm{R}$ \\
\hline 2041 & ONSITE & SESPMNT & $\mathrm{R}$ \\
\hline 2042 & ONSITE & SESPMNT & $\mathrm{R}$ \\
\hline 2043 & ONSITE & SESPMNT & $\mathrm{R}$ \\
\hline 2044 & ONSITE & SESPMNT & $\mathrm{R}$ \\
\hline 2045 & ONSITE & SESPMNT & $\mathrm{R}$ \\
\hline 2046 & ONSITE & SESPMNT & $\mathrm{R}$ \\
\hline 2047 & ONSITE & SESPMNT & $\mathrm{R}$ \\
\hline 2048 & ONSITE & SESPMNT & $\mathrm{R}$ \\
\hline 2049 & ONSITE & SESPMNT & $\mathrm{R}$ \\
\hline 2050 & ONSITE & SESPMNT & $\mathrm{R}$ \\
\hline 2051 & ONSITE & SESPMNT & $\mathrm{R}$ \\
\hline 2052 & ONSITE & SESPMNT & $\mathrm{R}$ \\
\hline 2053 & ONSITE & SESPMNT & $\mathrm{R}$ \\
\hline & & & \\
\hline
\end{tabular}




\begin{tabular}{|c|l|l|l|}
\hline & AG & \multicolumn{1}{|c|}{ AH } & \\
\hline 1 & DIST_CLASS & OWNER_ID & COLLECTION_PURPOSE \\
\hline 2054 & ONSITE & SESPMNT & $\mathrm{R}$ \\
\hline 2055 & ONSITE & SESPMNT & $\mathrm{R}$ \\
\hline 2056 & ONSITE & SESPMNT & $\mathrm{R}$ \\
\hline 2057 & ONSITE & SESPMNT & $\mathrm{R}$ \\
\hline 2058 & ONSITE & SESPMNT & $\mathrm{R}$ \\
\hline 2059 & ONSITE & SESPMNT & $\mathrm{R}$ \\
\hline 2060 & ONSITE & SESPMNT & $\mathrm{R}$ \\
\hline 2061 & ONSITE & SESPMNT & $\mathrm{R}$ \\
\hline 2062 & ONSITE & SESPMNT & $\mathrm{R}$ \\
\hline 2063 & ONSITE & SESPMNT & $\mathrm{R}$ \\
\hline 2064 & ONSITE & SESPMNT & $\mathrm{R}$ \\
\hline 2065 & ONSITE & SESPMNT & $\mathrm{R}$ \\
\hline 2066 & ONSITE & SESPMNT & $\mathrm{R}$ \\
\hline 2067 & ONSITE & SESPMNT & $\mathrm{R}$ \\
\hline 2068 & OFFSITE & SESPMNT & $\mathrm{R}$ \\
\hline 2069 & OFFSITE & SESPMNT & $\mathrm{R}$ \\
\hline 2070 & OFFSITE & SESPMNT & $\mathrm{R}$ \\
\hline 2071 & OFFSITE & SESPMNT & $\mathrm{R}$ \\
\hline 2072 & OFFSITE & SESPMNT & $\mathrm{R}$ \\
\hline 2073 & OFFSITE & SESPMNT & $\mathrm{R}$ \\
\hline 2074 & OFFSITE & SESPMNT & $\mathrm{R}$ \\
\hline 2075 & OFFSITE & SESPMNT & $\mathrm{R}$ \\
\hline 2076 & OFFSITE & SESPMNT & $\mathrm{R}$ \\
\hline 2077 & OFFSITE & SESPMNT & $\mathrm{R}$ \\
\hline 2078 & OFFSITE & SESPMNT & $\mathrm{R}$ \\
\hline 2079 & OFFSITE & SESPMNT & $\mathrm{R}$ \\
\hline 2080 & OFFSITE & SESPMNT & $\mathrm{R}$ \\
\hline 2081 & OFFSITE & SESPMNT & $\mathrm{R}$ \\
\hline 2082 & OFFSITE & SESPMNT & $\mathrm{R}$ \\
\hline 2083 & OFFSITE & SESPMNT & $\mathrm{R}$ \\
\hline 2084 & OFFSITE & SESPMNT & $\mathrm{R}$ \\
\hline 2085 & OFFSITE & SESPMNT & $\mathrm{R}$ \\
\hline 2086 & OFFSITE & SESPMNT & $\mathrm{R}$ \\
\hline 2087 & OFFSITE & SESPMNT & $\mathrm{R}$ \\
\hline 2088 & OFFSITE & SESPMNT & $\mathrm{R}$ \\
\hline 2089 & OFFSITE & SESPMNT & $\mathrm{R}$ \\
\hline 2090 & OFFSITE & SESPMNT & $\mathrm{R}$ \\
\hline 2091 & OFFSITE & SESPMNT & $\mathrm{R}$ \\
\hline & & & \\
\hline
\end{tabular}




\begin{tabular}{|c|l|l|l|}
\hline & AG & AH & \\
\hline 1 & DIST_CLASS & OWNER_ID & COLLECTION_PURPOSE \\
\hline 2092 & OFFSITE & SESPMNT & $\mathrm{R}$ \\
\hline 2093 & OFFSITE & SESPMNT & $\mathrm{R}$ \\
\hline 2094 & OFFSITE & SESPMNT & $\mathrm{R}$ \\
\hline 2095 & OFFSITE & SESPMNT & $\mathrm{R}$ \\
\hline 2096 & ONSITE & SESPMNT & $\mathrm{R}$ \\
\hline 2097 & ONSITE & SESPMNT & $\mathrm{R}$ \\
\hline 2098 & ONSITE & SESPMNT & $\mathrm{R}$ \\
\hline 2099 & ONSITE & SESPMNT & $\mathrm{R}$ \\
\hline 2100 & ONSITE & SESPMNT & $\mathrm{R}$ \\
\hline 2101 & ONSITE & SESPMNT & $\mathrm{R}$ \\
\hline 2102 & ONSITE & SESPMNT & $\mathrm{R}$ \\
\hline 2103 & ONSITE & SESPMNT & $\mathrm{R}$ \\
\hline 2104 & ONSITE & SESPMNT & $\mathrm{R}$ \\
\hline 2105 & ONSITE & SESPMNT & $\mathrm{R}$ \\
\hline 2106 & ONSITE & SESPMNT & $\mathrm{R}$ \\
\hline 2107 & ONSITE & SESPMNT & $\mathrm{R}$ \\
\hline 2108 & ONSITE & SESPMNT & $\mathrm{R}$ \\
\hline 2109 & ONSITE & SESPMNT & $\mathrm{R}$ \\
\hline 2110 & ONSITE & SESPMNT & $\mathrm{R}$ \\
\hline 2111 & ONSITE & SESPMNT & $\mathrm{R}$ \\
\hline 2112 & ONSITE & SESPMNT & $\mathrm{R}$ \\
\hline 2113 & ONSITE & SESPMNT & $\mathrm{R}$ \\
\hline 2114 & ONSITE & SESPMNT & $\mathrm{R}$ \\
\hline 2115 & ONSITE & SESPMNT & $\mathrm{R}$ \\
\hline 2116 & ONSITE & SESPMNT & $\mathrm{R}$ \\
\hline 2117 & ONSITE & SESPMNT & $\mathrm{R}$ \\
\hline 2118 & ONSITE & SESPMNT & $\mathrm{R}$ \\
\hline 2119 & ONSITE & SESPMNT & $\mathrm{R}$ \\
\hline 2120 & ONSITE & SESPMNT & $\mathrm{R}$ \\
\hline 2121 & ONSITE & SESPMNT & $\mathrm{R}$ \\
\hline 2122 & ONSITE & SESPMNT & $\mathrm{R}$ \\
\hline 2123 & ONSITE & SESPMNT & $\mathrm{R}$ \\
\hline 2124 & ONSITE & SESPMNT & $\mathrm{R}$ \\
\hline 2125 & ONSITE & SESPMNT & $\mathrm{R}$ \\
\hline 2126 & ONSITE & SESPMNT & $\mathrm{R}$ \\
\hline 2127 & ONSITE & SESPMNT & $\mathrm{R}$ \\
\hline 2128 & ONSITE & SESPMNT & $\mathrm{R}$ \\
\hline 2129 & ONSITE & SESPMNT & $\mathrm{R}$ \\
\hline & & & \\
\hline
\end{tabular}




\begin{tabular}{|c|l|l|l|}
\hline & AG & AH & \\
\hline 1 & DIST_CLASS & OWNER_ID & COLLECTION_PURPOSE \\
\hline 2130 & ONSITE & SESPMNT & $\mathrm{R}$ \\
\hline 2131 & ONSITE & SESPMNT & $\mathrm{R}$ \\
\hline 2132 & ONSITE & SESPMNT & $\mathrm{R}$ \\
\hline 2133 & ONSITE & SESPMNT & $\mathrm{R}$ \\
\hline 2134 & ONSITE & SESPMNT & $\mathrm{R}$ \\
\hline 2135 & ONSITE & SESPMNT & $\mathrm{R}$ \\
\hline 2136 & ONSITE & SESPMNT & $\mathrm{R}$ \\
\hline 2137 & ONSITE & SESPMNT & $\mathrm{R}$ \\
\hline 2138 & ONSITE & SESPMNT & $\mathrm{R}$ \\
\hline 2139 & ONSITE & SESPMNT & $\mathrm{R}$ \\
\hline 2140 & ONSITE & SESPMNT & $\mathrm{R}$ \\
\hline 2141 & ONSITE & SESPMNT & $\mathrm{R}$ \\
\hline 2142 & ONSITE & SESPMNT & $\mathrm{R}$ \\
\hline 2143 & ONSITE & SESPMNT & $\mathrm{R}$ \\
\hline 2144 & ONSITE & SESPMNT & $\mathrm{R}$ \\
\hline 2145 & ONSITE & SESPMNT & $\mathrm{R}$ \\
\hline 2146 & ONSITE & SESPMNT & $\mathrm{R}$ \\
\hline 2147 & ONSITE & SESPMNT & $\mathrm{R}$ \\
\hline 2148 & ONSITE & SESPMNT & $\mathrm{R}$ \\
\hline 2149 & ONSITE & SESPMNT & $\mathrm{R}$ \\
\hline 2150 & ONSITE & SESPMNT & $\mathrm{R}$ \\
\hline 2151 & ONSITE & SESPMNT & $\mathrm{R}$ \\
\hline 2152 & ONSITE & SESPMNT & $\mathrm{R}$ \\
\hline 2153 & ONSITE & SESPMNT & $\mathrm{R}$ \\
\hline 2154 & ONSITE & SESPMNT & $\mathrm{R}$ \\
\hline 2155 & ONSITE & SESPMNT & $\mathrm{R}$ \\
\hline 2156 & ONSITE & SESPMNT & $\mathrm{R}$ \\
\hline 2157 & ONSITE & SESPMNT & $\mathrm{R}$ \\
\hline 2158 & ONSITE & SESPMNT & $\mathrm{R}$ \\
\hline 2159 & ONSITE & SESPMNT & $\mathrm{R}$ \\
\hline 2160 & ONSITE & SESPMNT & $\mathrm{R}$ \\
\hline 2161 & ONSITE & SESPMNT & $\mathrm{R}$ \\
\hline 2162 & ONSITE & SESPMNT & $\mathrm{R}$ \\
\hline 2163 & ONSITE & SESPMNT & $\mathrm{R}$ \\
\hline 2164 & ONSITE & SESPMNT & $\mathrm{R}$ \\
\hline 2165 & ONSITE & SESPMNT & $\mathrm{R}$ \\
\hline 2166 & ONSITE & SESPMNT & $\mathrm{R}$ \\
\hline 2167 & ONSITE & SESPMNT & $\mathrm{R}$ \\
\hline & & & \\
\hline
\end{tabular}




\begin{tabular}{|c|l|l|l|}
\hline & \multicolumn{1}{|c|}{ AG } & AH & \\
\hline 1 & DIST_CLASS & OWNER_ID & COLLECTION_PURPOSE \\
\hline 2168 & ONSITE & SESPMNT & $\mathrm{R}$ \\
\hline 2169 & ONSITE & SESPMNT & $\mathrm{R}$ \\
\hline 2170 & ONSITE & SESPMNT & $\mathrm{R}$ \\
\hline 2171 & ONSITE & SESPMNT & $\mathrm{R}$ \\
\hline 2172 & ONSITE & SESPMNT & $\mathrm{R}$ \\
\hline 2173 & ONSITE & SESPMNT & $\mathrm{R}$ \\
\hline 2174 & ONSITE & SESPMNT & $\mathrm{R}$ \\
\hline 2175 & ONSITE & SESPMNT & $\mathrm{R}$ \\
\hline 2176 & ONSITE & SESPMNT & $\mathrm{R}$ \\
\hline 2177 & ONSITE & SESPMNT & $\mathrm{R}$ \\
\hline 2178 & ONSITE & SESPMNT & $\mathrm{R}$ \\
\hline 2179 & ONSITE & SESPMNT & $\mathrm{R}$ \\
\hline 2180 & ONSITE & SESPMNT & $\mathrm{R}$ \\
\hline 2181 & ONSITE & SESPMNT & $\mathrm{R}$ \\
\hline 2182 & ONSITE & SESPMNT & $\mathrm{R}$ \\
\hline 2183 & ONSITE & SESPMNT & $\mathrm{R}$ \\
\hline 2184 & ONSITE & SESPMNT & $\mathrm{R}$ \\
\hline 2185 & ONSITE & SESPMNT & $\mathrm{R}$ \\
\hline 2186 & ONSITE & SESPMNT & $\mathrm{R}$ \\
\hline 2187 & ONSITE & SESPMNT & $\mathrm{R}$ \\
\hline 2188 & ONSITE & SESPMNT & $\mathrm{R}$ \\
\hline 2189 & ONSITE & SESPMNT & $\mathrm{R}$ \\
\hline 2190 & ONSITE & SESPMNT & $\mathrm{R}$ \\
\hline 2191 & ONSITE & SESPMNT & $\mathrm{R}$ \\
\hline 2192 & ONSITE & SESPMNT & $\mathrm{R}$ \\
\hline 2193 & ONSITE & SESPMNT & $\mathrm{R}$ \\
\hline 2194 & ONSITE & SESPMNT & $\mathrm{R}$ \\
\hline 2195 & ONSITE & SESPMNT & $\mathrm{R}$ \\
\hline 2196 & ONSITE & SESPMNT & $\mathrm{R}$ \\
\hline 2197 & ONSITE & SESPMNT & $\mathrm{R}$ \\
\hline 2198 & ONSITE & SESPMNT & $\mathrm{R}$ \\
\hline 2199 & ONSITE & SESPMNT & $\mathrm{R}$ \\
\hline 2200 & ONSITE & SESPMNT & $\mathrm{R}$ \\
\hline 2201 & ONSITE & SESPMNT & $\mathrm{R}$ \\
\hline 2202 & ONSITE & SESPMNT & $\mathrm{R}$ \\
\hline 2203 & ONSITE & SESPMNT & $\mathrm{R}$ \\
\hline 2204 & ONSITE & SESPMNT & $\mathrm{R}$ \\
\hline 2205 & ONSITE & SESPMNT & $\mathrm{R}$ \\
\hline & & & \\
\hline
\end{tabular}




\begin{tabular}{|c|l|l|l|}
\hline & AG & AH & \\
\hline 1 & DIST_CLASS & OWNER_ID & COLLECTION_PURPOSE \\
\hline 2206 & ONSITE & SESPMNT & $\mathrm{R}$ \\
\hline 2207 & ONSITE & SESPMNT & $\mathrm{R}$ \\
\hline 2208 & ONSITE & SESPMNT & $\mathrm{R}$ \\
\hline 2209 & ONSITE & SESPMNT & $\mathrm{R}$ \\
\hline 2210 & ONSITE & SESPMNT & $\mathrm{R}$ \\
\hline 2211 & ONSITE & SESPMNT & $\mathrm{R}$ \\
\hline 2212 & ONSITE & SESPMNT & $\mathrm{R}$ \\
\hline 2213 & ONSITE & SESPMNT & $\mathrm{R}$ \\
\hline 2214 & ONSITE & SESPMNT & $\mathrm{R}$ \\
\hline 2215 & ONSITE & SESPMNT & $\mathrm{R}$ \\
\hline 2216 & ONSITE & SESPMNT & $\mathrm{R}$ \\
\hline 2217 & ONSITE & SESPMNT & $\mathrm{R}$ \\
\hline 2218 & ONSITE & SESPMNT & $\mathrm{R}$ \\
\hline 2219 & ONSITE & SESPMNT & $\mathrm{R}$ \\
\hline 2220 & ONSITE & SESPMNT & $\mathrm{R}$ \\
\hline 2221 & ONSITE & SESPMNT & $\mathrm{R}$ \\
\hline 2222 & ONSITE & SESPMNT & $\mathrm{R}$ \\
\hline 2223 & ONSITE & SESPMNT & $\mathrm{R}$ \\
\hline 2224 & ONSITE & SESPMNT & $\mathrm{R}$ \\
\hline 2225 & ONSITE & SESPMNT & $\mathrm{R}$ \\
\hline 2226 & ONSITE & SESPMNT & $\mathrm{R}$ \\
\hline 2227 & ONSITE & SESPMNT & $\mathrm{R}$ \\
\hline 2228 & ONSITE & SESPMNT & $\mathrm{R}$ \\
\hline 2229 & ONSITE & SESPMNT & $\mathrm{R}$ \\
\hline 2230 & ONSITE & SESPMNT & $\mathrm{R}$ \\
\hline 2231 & ONSITE & SESPMNT & $\mathrm{R}$ \\
\hline 2232 & ONSITE & SESPMNT & $\mathrm{R}$ \\
\hline 2233 & ONSITE & SESPMNT & $\mathrm{R}$ \\
\hline 2234 & ONSITE & SESPMNT & $\mathrm{R}$ \\
\hline 2235 & ONSITE & SESPMNT & $\mathrm{R}$ \\
\hline 2236 & ONSITE & SESPMNT & $\mathrm{R}$ \\
\hline 2237 & ONSITE & SESPMNT & $\mathrm{R}$ \\
\hline 2238 & ONSITE & SESPMNT & $\mathrm{R}$ \\
\hline 2239 & ONSITE & SESPMNT & $\mathrm{R}$ \\
\hline 2240 & ONSITE & SESPMNT & $\mathrm{R}$ \\
\hline 2241 & ONSITE & SESPMNT & $\mathrm{R}$ \\
\hline 2242 & ONSITE & SESPMNT & $\mathrm{R}$ \\
\hline 2243 & ONSITE & SESPMNT & $\mathrm{R}$ \\
\hline & & & \\
\hline
\end{tabular}




\begin{tabular}{|c|l|l|l|}
\hline & AG & AH & \\
\hline 1 & DIST_CLASS & OWNER_ID & COLLECTION_PURPOSE \\
\hline 2244 & ONSITE & SESPMNT & $\mathrm{R}$ \\
\hline 2245 & ONSITE & SESPMNT & $\mathrm{R}$ \\
\hline 2246 & ONSITE & SESPMNT & $\mathrm{R}$ \\
\hline 2247 & ONSITE & SESPMNT & $\mathrm{R}$ \\
\hline 2248 & ONSITE & SESPMNT & $\mathrm{R}$ \\
\hline 2249 & ONSITE & SESPMNT & $\mathrm{R}$ \\
\hline 2250 & ONSITE & SESPMNT & $\mathrm{R}$ \\
\hline 2251 & ONSITE & SESPMNT & $\mathrm{R}$ \\
\hline 2252 & ONSITE & SESPMNT & $\mathrm{R}$ \\
\hline 2253 & ONSITE & SESPMNT & $\mathrm{R}$ \\
\hline 2254 & ONSITE & SESPMNT & $\mathrm{R}$ \\
\hline 2255 & ONSITE & SESPMNT & $\mathrm{R}$ \\
\hline 2256 & ONSITE & SESPMNT & $\mathrm{R}$ \\
\hline 2257 & ONSITE & SESPMNT & $\mathrm{R}$ \\
\hline 2258 & ONSITE & SESPMNT & $\mathrm{R}$ \\
\hline 2259 & ONSITE & SESPMNT & $\mathrm{R}$ \\
\hline 2260 & ONSITE & SESPMNT & $\mathrm{R}$ \\
\hline 2261 & ONSITE & SESPMNT & $\mathrm{R}$ \\
\hline 2262 & ONSITE & SESPMNT & $\mathrm{R}$ \\
\hline 2263 & ONSITE & SESPMNT & $\mathrm{R}$ \\
\hline 2264 & ONSITE & SESPMNT & $\mathrm{R}$ \\
\hline 2265 & ONSITE & SESPMNT & $\mathrm{R}$ \\
\hline 2266 & ONSITE & SESPMNT & $\mathrm{R}$ \\
\hline 2267 & ONSITE & SESPMNT & $\mathrm{R}$ \\
\hline 2268 & ONSITE & SESPMNT & $\mathrm{R}$ \\
\hline 2269 & ONSITE & SESPMNT & $\mathrm{R}$ \\
\hline 2270 & ONSITE & SESPMNT & $\mathrm{R}$ \\
\hline 2271 & ONSITE & SESPMNT & $\mathrm{R}$ \\
\hline 2272 & ONSITE & SESPMNT & $\mathrm{R}$ \\
\hline 2273 & ONSITE & SESPMNT & $\mathrm{R}$ \\
\hline 2274 & ONSITE & SESPMNT & $\mathrm{R}$ \\
\hline 2275 & ONSITE & SESPMNT & $\mathrm{R}$ \\
\hline 2276 & ONSITE & SESPMNT & $\mathrm{R}$ \\
\hline 2277 & ONSITE & SESPMNT & $\mathrm{R}$ \\
\hline 2278 & ONSITE & SESPMNT & $\mathrm{R}$ \\
\hline 2279 & ONSITE & SESPMNT & $\mathrm{R}$ \\
\hline 2280 & ONSITE & SESPMNT & $\mathrm{R}$ \\
\hline 2281 & ONSITE & SESPMNT & $\mathrm{R}$ \\
\hline & & & \\
\hline
\end{tabular}




\begin{tabular}{|c|l|l|l|}
\hline & AG & AH & \\
\hline 1 & DIST_CLASS & OWNER_ID & COLLECTION_PURPOSE \\
\hline 2282 & ONSITE & SESPMNT & $\mathrm{R}$ \\
\hline 2283 & ONSITE & SESPMNT & $\mathrm{R}$ \\
\hline 2284 & ONSITE & SESPMNT & $\mathrm{R}$ \\
\hline 2285 & ONSITE & SESPMNT & $\mathrm{R}$ \\
\hline 2286 & ONSITE & SESPMNT & $\mathrm{R}$ \\
\hline 2287 & ONSITE & SESPMNT & $\mathrm{R}$ \\
\hline 2288 & ONSITE & SESPMNT & $\mathrm{R}$ \\
\hline 2289 & ONSITE & SESPMNT & $\mathrm{R}$ \\
\hline 2290 & ONSITE & SESPMNT & $\mathrm{R}$ \\
\hline 2291 & ONSITE & SESPMNT & $\mathrm{R}$ \\
\hline 2292 & ONSITE & SESPMNT & $\mathrm{R}$ \\
\hline 2293 & ONSITE & SESPMNT & $\mathrm{R}$ \\
\hline 2294 & ONSITE & SESPMNT & $\mathrm{R}$ \\
\hline 2295 & ONSITE & SESPMNT & $\mathrm{R}$ \\
\hline 2296 & ONSITE & SESPMNT & $\mathrm{R}$ \\
\hline 2297 & ONSITE & SESPMNT & $\mathrm{R}$ \\
\hline 2298 & ONSITE & SESPMNT & $\mathrm{R}$ \\
\hline 2299 & ONSITE & SESPMNT & $\mathrm{R}$ \\
\hline 2300 & ONSITE & SESPMNT & $\mathrm{R}$ \\
\hline 2301 & ONSITE & SESPMNT & $\mathrm{R}$ \\
\hline 2302 & ONSITE & SESPMNT & $\mathrm{R}$ \\
\hline 2303 & ONSITE & SESPMNT & $\mathrm{R}$ \\
\hline 2304 & ONSITE & SESPMNT & $\mathrm{R}$ \\
\hline 2305 & ONSITE & SESPMNT & $\mathrm{R}$ \\
\hline 2306 & ONSITE & SESPMNT & $\mathrm{R}$ \\
\hline 2307 & ONSITE & SESPMNT & $\mathrm{R}$ \\
\hline 2308 & ONSITE & SESPMNT & $\mathrm{R}$ \\
\hline 2309 & ONSITE & SESPMNT & $\mathrm{R}$ \\
\hline 2310 & ONSITE & SESPMNT & $\mathrm{R}$ \\
\hline 2311 & ONSITE & SESPMNT & $\mathrm{R}$ \\
\hline 2312 & ONSITE & SESPMNT & $\mathrm{R}$ \\
\hline 2313 & ONSITE & SESPMNT & $\mathrm{R}$ \\
\hline 2314 & ONSITE & SESPMNT & $\mathrm{R}$ \\
\hline 2315 & ONSITE & SESPMNT & $\mathrm{R}$ \\
\hline 2316 & OFFSITE & SESPMNT & $\mathrm{R}$ \\
\hline 2317 & OFFSITE & SESPMNT & $\mathrm{R}$ \\
\hline 2318 & OFFSITE & SESPMNT & $\mathrm{R}$ \\
\hline 2319 & OFFSITE & SESPMNT & $\mathrm{R}$ \\
\hline & & & \\
\hline
\end{tabular}




\begin{tabular}{|c|l|l|l|}
\hline & AG & \multicolumn{1}{|c|}{ AH } & \\
\hline 1 & DIST_CLASS & OWNER_ID & COLLECTION_PURPOSE \\
\hline 2320 & OFFSITE & SESPMNT & $\mathrm{R}$ \\
\hline 2321 & OFFSITE & SESPMNT & $\mathrm{R}$ \\
\hline 2322 & OFFSITE & SESPMNT & $\mathrm{R}$ \\
\hline 2323 & OFFSITE & SESPMNT & $\mathrm{R}$ \\
\hline 2324 & OFFSITE & SESPMNT & $\mathrm{R}$ \\
\hline 2325 & OFFSITE & SESPMNT & $\mathrm{R}$ \\
\hline 2326 & OFFSITE & SESPMNT & $\mathrm{R}$ \\
\hline 2327 & OFFSITE & SESPMNT & $\mathrm{R}$ \\
\hline 2328 & OFFSITE & SESPMNT & $\mathrm{R}$ \\
\hline 2329 & OFFSITE & SESPMNT & $\mathrm{R}$ \\
\hline 2330 & OFFSITE & SESPMNT & $\mathrm{R}$ \\
\hline 2331 & OFFSITE & SESPMNT & $\mathrm{R}$ \\
\hline 2332 & OFFSITE & SESPMNT & $\mathrm{R}$ \\
\hline 2333 & OFFSITE & SESPMNT & $\mathrm{R}$ \\
\hline 2334 & OFFSITE & SESPMNT & $\mathrm{R}$ \\
\hline 2335 & OFFSITE & SESPMNT & $\mathrm{R}$ \\
\hline 2336 & OFFSITE & SESPMNT & $\mathrm{R}$ \\
\hline 2337 & OFFSITE & SESPMNT & $\mathrm{R}$ \\
\hline 2338 & OFFSITE & SESPMNT & $\mathrm{R}$ \\
\hline 2339 & OFFSITE & SESPMNT & $\mathrm{R}$ \\
\hline 2340 & OFFSITE & SESPMNT & $\mathrm{R}$ \\
\hline 2341 & OFFSITE & SESPMNT & $\mathrm{R}$ \\
\hline 2342 & OFFSITE & SESPMNT & $\mathrm{R}$ \\
\hline 2343 & OFFSITE & SESPMNT & $\mathrm{R}$ \\
\hline 2344 & OFFSITE & SESPMNT & $\mathrm{R}$ \\
\hline 2345 & OFFSITE & SESPMNT & $\mathrm{R}$ \\
\hline 2346 & OFFSITE & SESPMNT & $\mathrm{R}$ \\
\hline 2347 & OFFSITE & SESPMNT & $\mathrm{R}$ \\
\hline 2348 & OFFSITE & SESPMNT & $\mathrm{R}$ \\
\hline 2349 & OFFSITE & SESPMNT & $\mathrm{R}$ \\
\hline 2350 & OFFSITE & SESPMNT & $\mathrm{R}$ \\
\hline 2351 & OFFSITE & SESPMNT & $\mathrm{R}$ \\
\hline 2352 & OFFSITE & SESPMNT & $\mathrm{R}$ \\
\hline 2353 & OFFSITE & SESPMNT & $\mathrm{R}$ \\
\hline 2354 & OFFSITE & SESPMNT & $\mathrm{R}$ \\
\hline 2355 & OFFSITE & SESPMNT & $\mathrm{R}$ \\
\hline 2356 & OFFSITE & SESPMNT & $\mathrm{R}$ \\
\hline 2357 & OFFSITE & SESPMNT & $\mathrm{R}$ \\
\hline & & & \\
\hline
\end{tabular}




\begin{tabular}{|c|l|l|l|}
\hline & AG & \multicolumn{1}{|c|}{ AH } & \\
\hline 1 & DIST_CLASS & OWNER_ID & COLLECTION_PURPOSE \\
\hline 2358 & OFFSITE & SESPMNT & $\mathrm{R}$ \\
\hline 2359 & OFFSITE & SESPMNT & $\mathrm{R}$ \\
\hline 2360 & OFFSITE & SESPMNT & $\mathrm{R}$ \\
\hline 2361 & OFFSITE & SESPMNT & $\mathrm{R}$ \\
\hline 2362 & OFFSITE & SESPMNT & $\mathrm{R}$ \\
\hline 2363 & OFFSITE & SESPMNT & $\mathrm{R}$ \\
\hline 2364 & OFFSITE & SESPMNT & $\mathrm{R}$ \\
\hline 2365 & OFFSITE & SESPMNT & $\mathrm{R}$ \\
\hline 2366 & OFFSITE & SESPMNT & $\mathrm{R}$ \\
\hline 2367 & OFFSITE & SESPMNT & $\mathrm{R}$ \\
\hline 2368 & OFFSITE & SESPMNT & $\mathrm{R}$ \\
\hline 2369 & OFFSITE & SESPMNT & $\mathrm{R}$ \\
\hline 2370 & OFFSITE & SESPMNT & $\mathrm{R}$ \\
\hline 2371 & OFFSITE & SESPMNT & $\mathrm{R}$ \\
\hline 2372 & OFFSITE & SESPMNT & $\mathrm{R}$ \\
\hline 2373 & OFFSITE & SESPMNT & $\mathrm{R}$ \\
\hline 2374 & OFFSITE & SESPMNT & $\mathrm{R}$ \\
\hline 2375 & OFFSITE & SESPMNT & $\mathrm{R}$ \\
\hline 2376 & OFFSITE & SESPMNT & $\mathrm{R}$ \\
\hline 2377 & OFFSITE & SESPMNT & $\mathrm{R}$ \\
\hline 2378 & OFFSITE & SESPMNT & $\mathrm{R}$ \\
\hline 2379 & OFFSITE & SESPMNT & $\mathrm{R}$ \\
\hline 2380 & OFFSITE & SESPMNT & $\mathrm{R}$ \\
\hline 2381 & OFFSITE & SESPMNT & $\mathrm{R}$ \\
\hline 2382 & OFFSITE & SESPMNT & $\mathrm{R}$ \\
\hline 2383 & OFFSITE & SESPMNT & $\mathrm{R}$ \\
\hline 2384 & OFFSITE & SESPMNT & $\mathrm{R}$ \\
\hline 2385 & OFFSITE & SESPMNT & $\mathrm{R}$ \\
\hline 2386 & OFFSITE & SESPMNT & $\mathrm{R}$ \\
\hline 2387 & OFFSITE & SESPMNT & $\mathrm{R}$ \\
\hline 2388 & OFFSITE & SESPMNT & $\mathrm{R}$ \\
\hline 2389 & OFFSITE & SESPMNT & $\mathrm{R}$ \\
\hline 2390 & OFFSITE & SESPMNT & $\mathrm{R}$ \\
\hline 2391 & OFFSITE & SESPMNT & $\mathrm{R}$ \\
\hline 2392 & OFFSITE & SESPMNT & $\mathrm{R}$ \\
\hline 2393 & OFFSITE & SESPMNT & $\mathrm{R}$ \\
\hline 2394 & OFFSITE & SESPMNT & $\mathrm{R}$ \\
\hline 2395 & OFFSITE & SESPMNT & $\mathrm{R}$ \\
\hline & & & \\
\hline
\end{tabular}




\begin{tabular}{|c|l|l|l|}
\hline & AG & \multicolumn{1}{|c|}{ AH } & \\
\hline 1 & DIST_CLASS & OWNER_ID & COLLECTION_PURPOSE \\
\hline 2396 & OFFSITE & SESPMNT & $\mathrm{R}$ \\
\hline 2397 & OFFSITE & SESPMNT & $\mathrm{R}$ \\
\hline 2398 & OFFSITE & SESPMNT & $\mathrm{R}$ \\
\hline 2399 & OFFSITE & SESPMNT & $\mathrm{R}$ \\
\hline 2400 & OFFSITE & SESPMNT & $\mathrm{R}$ \\
\hline 2401 & OFFSITE & SESPMNT & $\mathrm{R}$ \\
\hline 2402 & OFFSITE & SESPMNT & $\mathrm{R}$ \\
\hline 2403 & OFFSITE & SESPMNT & $\mathrm{R}$ \\
\hline 2404 & OFFSITE & SESPMNT & $\mathrm{R}$ \\
\hline 2405 & OFFSITE & SESPMNT & $\mathrm{R}$ \\
\hline 2406 & OFFSITE & SESPMNT & $\mathrm{R}$ \\
\hline 2407 & OFFSITE & SESPMNT & $\mathrm{R}$ \\
\hline 2408 & OFFSITE & SESPMNT & $\mathrm{R}$ \\
\hline 2409 & OFFSITE & SESPMNT & $\mathrm{R}$ \\
\hline 2410 & OFFSITE & SESPMNT & $\mathrm{R}$ \\
\hline 2411 & OFFSITE & SESPMNT & $\mathrm{R}$ \\
\hline 2412 & OFFSITE & SESPMNT & $\mathrm{R}$ \\
\hline 2413 & OFFSITE & SESPMNT & $\mathrm{R}$ \\
\hline 2414 & OFFSITE & SESPMNT & $\mathrm{R}$ \\
\hline 2415 & OFFSITE & SESPMNT & $\mathrm{R}$ \\
\hline 2416 & OFFSITE & SESPMNT & $\mathrm{R}$ \\
\hline 2417 & OFFSITE & SESPMNT & $\mathrm{R}$ \\
\hline 2418 & OFFSITE & SESPMNT & $\mathrm{R}$ \\
\hline 2419 & OFFSITE & SESPMNT & $\mathrm{R}$ \\
\hline 2420 & OFFSITE & SESPMNT & $\mathrm{R}$ \\
\hline 2421 & OFFSITE & SESPMNT & $\mathrm{R}$ \\
\hline 2422 & OFFSITE & SESPMNT & $\mathrm{R}$ \\
\hline 2423 & OFFSITE & SESPMNT & $\mathrm{R}$ \\
\hline 2424 & OFFSITE & SESPMNT & $\mathrm{R}$ \\
\hline 2425 & OFFSITE & SESPMNT & $\mathrm{R}$ \\
\hline 2426 & OFFSITE & SESPMNT & $\mathrm{R}$ \\
\hline 2427 & OFFSITE & SESPMNT & $\mathrm{R}$ \\
\hline 2428 & OFFSITE & SESPMNT & $\mathrm{R}$ \\
\hline 2429 & OFFSITE & SESPMNT & $\mathrm{R}$ \\
\hline 2430 & OFFSITE & SESPMNT & $\mathrm{R}$ \\
\hline 2431 & OFFSITE & SESPMNT & $\mathrm{R}$ \\
\hline 2432 & OFFSITE & SESPMNT & $\mathrm{R}$ \\
\hline 2433 & OFFSITE & SESPMNT & $\mathrm{R}$ \\
\hline & & & \\
\hline
\end{tabular}




\begin{tabular}{|c|l|l|l|}
\hline & AG & \multicolumn{1}{|c|}{ AH } & \\
\hline 1 & DIST_CLASS & OWNER_ID & COLLECTION_PURPOSE \\
\hline 2434 & OFFSITE & SESPMNT & $\mathrm{R}$ \\
\hline 2435 & OFFSITE & SESPMNT & $\mathrm{R}$ \\
\hline 2436 & OFFSITE & SESPMNT & $\mathrm{R}$ \\
\hline 2437 & OFFSITE & SESPMNT & $\mathrm{R}$ \\
\hline 2438 & OFFSITE & SESPMNT & $\mathrm{R}$ \\
\hline 2439 & OFFSITE & SESPMNT & $\mathrm{R}$ \\
\hline 2440 & OFFSITE & SESPMNT & $\mathrm{R}$ \\
\hline 2441 & OFFSITE & SESPMNT & $\mathrm{R}$ \\
\hline 2442 & OFFSITE & SESPMNT & $\mathrm{R}$ \\
\hline 2443 & OFFSITE & SESPMNT & $\mathrm{R}$ \\
\hline 2444 & OFFSITE & SESPMNT & $\mathrm{R}$ \\
\hline 2445 & OFFSITE & SESPMNT & $\mathrm{R}$ \\
\hline 2446 & OFFSITE & SESPMNT & $\mathrm{R}$ \\
\hline 2447 & OFFSITE & SESPMNT & $\mathrm{R}$ \\
\hline 2448 & OFFSITE & SESPMNT & $\mathrm{R}$ \\
\hline 2449 & OFFSITE & SESPMNT & $\mathrm{R}$ \\
\hline 2450 & OFFSITE & SESPMNT & $\mathrm{R}$ \\
\hline 2451 & OFFSITE & SESPMNT & $\mathrm{R}$ \\
\hline 2452 & OFFSITE & SESPMNT & $\mathrm{R}$ \\
\hline 2453 & OFFSITE & SESPMNT & $\mathrm{R}$ \\
\hline 2454 & OFFSITE & SESPMNT & $\mathrm{R}$ \\
\hline 2455 & OFFSITE & SESPMNT & $\mathrm{R}$ \\
\hline 2456 & OFFSITE & SESPMNT & $\mathrm{R}$ \\
\hline 2457 & OFFSITE & SESPMNT & $\mathrm{R}$ \\
\hline 2458 & OFFSITE & SESPMNT & $\mathrm{R}$ \\
\hline 2459 & OFFSITE & SESPMNT & $\mathrm{R}$ \\
\hline 2460 & OFFSITE & SESPMNT & $\mathrm{R}$ \\
\hline 2461 & OFFSITE & SESPMNT & $\mathrm{R}$ \\
\hline 2462 & OFFSITE & SESPMNT & $\mathrm{R}$ \\
\hline 2463 & OFFSITE & SESPMNT & $\mathrm{R}$ \\
\hline 2464 & OFFSITE & SESPMNT & $\mathrm{R}$ \\
\hline 2465 & OFFSITE & SESPMNT & $\mathrm{R}$ \\
\hline 2466 & OFFSITE & SESPMNT & $\mathrm{R}$ \\
\hline 2467 & OFFSITE & SESPMNT & $\mathrm{R}$ \\
\hline 2468 & OFFSITE & SESPMNT & $\mathrm{R}$ \\
\hline 2469 & OFFSITE & SESPMNT & $\mathrm{R}$ \\
\hline 2470 & OFFSITE & SESPMNT & $\mathrm{R}$ \\
\hline 2471 & OFFSITE & SESPMNT & $\mathrm{R}$ \\
\hline & & & \\
\hline
\end{tabular}




\begin{tabular}{|c|l|l|l|}
\hline & AG & \multicolumn{1}{|c|}{ AH } & \\
\hline 1 & DIST_CLASS & OWNER_ID & COLLECTION_PURPOSE \\
\hline 2472 & OFFSITE & SESPMNT & $\mathrm{R}$ \\
\hline 2473 & OFFSITE & SESPMNT & $\mathrm{R}$ \\
\hline 2474 & OFFSITE & SESPMNT & $\mathrm{R}$ \\
\hline 2475 & OFFSITE & SESPMNT & $\mathrm{R}$ \\
\hline 2476 & OFFSITE & SESPMNT & $\mathrm{R}$ \\
\hline 2477 & OFFSITE & SESPMNT & $\mathrm{R}$ \\
\hline 2478 & OFFSITE & SESPMNT & $\mathrm{R}$ \\
\hline 2479 & OFFSITE & SESPMNT & $\mathrm{R}$ \\
\hline 2480 & OFFSITE & SESPMNT & $\mathrm{R}$ \\
\hline 2481 & OFFSITE & SESPMNT & $\mathrm{R}$ \\
\hline 2482 & OFFSITE & SESPMNT & $\mathrm{R}$ \\
\hline 2483 & OFFSITE & SESPMNT & $\mathrm{R}$ \\
\hline 2484 & OFFSITE & SESPMNT & $\mathrm{R}$ \\
\hline 2485 & OFFSITE & SESPMNT & $\mathrm{R}$ \\
\hline 2486 & OFFSITE & SESPMNT & $\mathrm{R}$ \\
\hline 2487 & OFFSITE & SESPMNT & $\mathrm{R}$ \\
\hline 2488 & OFFSITE & SESPMNT & $\mathrm{R}$ \\
\hline 2489 & OFFSITE & SESPMNT & $\mathrm{R}$ \\
\hline 2490 & OFFSITE & SESPMNT & $\mathrm{R}$ \\
\hline 2491 & OFFSITE & SESPMNT & $\mathrm{R}$ \\
\hline 2492 & OFFSITE & SESPMNT & $\mathrm{R}$ \\
\hline 2493 & OFFSITE & SESPMNT & $\mathrm{R}$ \\
\hline 2494 & OFFSITE & SESPMNT & $\mathrm{R}$ \\
\hline 2495 & OFFSITE & SESPMNT & $\mathrm{R}$ \\
\hline 2496 & OFFSITE & SESPMNT & $\mathrm{R}$ \\
\hline 2497 & OFFSITE & SESPMNT & $\mathrm{R}$ \\
\hline 2498 & OFFSITE & SESPMNT & $\mathrm{R}$ \\
\hline 2499 & OFFSITE & SESPMNT & $\mathrm{R}$ \\
\hline 2500 & OFFSITE & SESPMNT & $\mathrm{R}$ \\
\hline 2501 & OFFSITE & SESPMNT & $\mathrm{R}$ \\
\hline 2502 & OFFSITE & SESPMNT & $\mathrm{R}$ \\
\hline 2503 & OFFSITE & SESPMNT & $\mathrm{R}$ \\
\hline 2504 & OFFSITE & SESPMNT & $\mathrm{R}$ \\
\hline 2505 & OFFSITE & SESPMNT & $\mathrm{R}$ \\
\hline 2506 & OFFSITE & SESPMNT & $\mathrm{R}$ \\
\hline 2507 & OFFSITE & SESPMNT & $\mathrm{R}$ \\
\hline 2508 & OFFSITE & SESPMNT & $\mathrm{R}$ \\
\hline 2509 & OFFSITE & SESPMNT & $\mathrm{R}$ \\
\hline & & & \\
\hline
\end{tabular}




\begin{tabular}{|c|l|l|l|}
\hline & \multicolumn{1}{|c|}{ AG } & \multicolumn{1}{|c|}{ AH } & \\
\hline 1 & DIST_CLASS & OWNER_ID & COLLECTION_PURPOSE \\
\hline 2510 & OFFSITE & SESPMNT & $\mathrm{R}$ \\
\hline 2511 & OFFSITE & SESPMNT & $\mathrm{R}$ \\
\hline 2512 & OFFSITE & SESPMNT & $\mathrm{R}$ \\
\hline 2513 & OFFSITE & SESPSPEC & $\mathrm{S}$ \\
\hline 2514 & OFFSITE & SESPMNT & $\mathrm{R}$ \\
\hline 2515 & OFFSITE & SESPSPEC & $\mathrm{S}$ \\
\hline 2516 & OFFSITE & SESPMNT & $\mathrm{R}$ \\
\hline 2517 & OFFSITE & SESPSPEC & $\mathrm{S}$ \\
\hline 2518 & OFFSITE & SESPMNT & $\mathrm{R}$ \\
\hline 2519 & OFFSITE & SESPSPEC & $\mathrm{S}$ \\
\hline 2520 & OFFSITE & SESPMNT & $\mathrm{R}$ \\
\hline 2521 & OFFSITE & SESPMNT & $\mathrm{R}$ \\
\hline 2522 & OFFSITE & SESPMNT & $\mathrm{R}$ \\
\hline 2523 & OFFSITE & SESPSPEC & $\mathrm{S}$ \\
\hline 2524 & OFFSITE & SESPSPEC & $\mathrm{S}$ \\
\hline 2525 & OFFSITE & SESPSPEC & $\mathrm{S}$ \\
\hline 2526 & OFFSITE & SESPMNT & $\mathrm{R}$ \\
\hline 2527 & OFFSITE & SESPMNT & $\mathrm{R}$ \\
\hline 2528 & OFFSITE & SESPMNT & $\mathrm{R}$ \\
\hline 2529 & OFFSITE & SESPMNT & $\mathrm{R}$ \\
\hline 2530 & OFFSITE & SESPMNT & $\mathrm{R}$ \\
\hline 2531 & OFFSITE & SESPMNT & $\mathrm{R}$ \\
\hline 2532 & OFFSITE & SESPMNT & $\mathrm{R}$ \\
\hline 2533 & OFFSITE & SESPMNT & $\mathrm{R}$ \\
\hline 2534 & OFFSITE & SESPMNT & $\mathrm{R}$ \\
\hline 2535 & OFFSITE & SESPMNT & $\mathrm{R}$ \\
\hline 2536 & OFFSITE & SESPMNT & $\mathrm{R}$ \\
\hline 2537 & OFFSITE & SESPMNT & $\mathrm{R}$ \\
\hline 2538 & OFFSITE & SESPMNT & $\mathrm{R}$ \\
\hline 2539 & OFFSITE & SESPMNT & $\mathrm{R}$ \\
\hline 2540 & OFFSITE & SESPMNT & $\mathrm{R}$ \\
\hline 2541 & OFFSITE & SESPMNT & $\mathrm{R}$ \\
\hline 2542 & OFFSITE & SESPMNT & $\mathrm{R}$ \\
\hline 2543 & OFFSITE & SESPMNT & $\mathrm{R}$ \\
\hline 2544 & OFFSITE & SESPMNT & $\mathrm{R}$ \\
\hline 2545 & OFFSITE & SESPMNT & $\mathrm{R}$ \\
\hline 2546 & OFFSITE & SESPMNT & $\mathrm{R}$ \\
\hline 2547 & OFFSITE & SESPMNT & $\mathrm{R}$ \\
\hline & & & \\
\hline
\end{tabular}




\begin{tabular}{|c|l|l|l|}
\hline & AG & \multicolumn{1}{|c|}{ AH } & \\
\hline 1 & DIST_CLASS & OWNER_ID & COLLECTION_PURPOSE \\
\hline 2548 & OFFSITE & SESPMNT & $\mathrm{R}$ \\
\hline 2549 & OFFSITE & SESPMNT & $\mathrm{R}$ \\
\hline 2550 & OFFSITE & SESPMNT & $\mathrm{R}$ \\
\hline 2551 & OFFSITE & SESPMNT & $\mathrm{R}$ \\
\hline 2552 & OFFSITE & SESPMNT & $\mathrm{R}$ \\
\hline 2553 & OFFSITE & SESPMNT & $\mathrm{R}$ \\
\hline 2554 & OFFSITE & SESPMNT & $\mathrm{R}$ \\
\hline 2555 & OFFSITE & SESPMNT & $\mathrm{R}$ \\
\hline 2556 & OFFSITE & SESPMNT & $\mathrm{R}$ \\
\hline 2557 & OFFSITE & SESPMNT & $\mathrm{R}$ \\
\hline 2558 & OFFSITE & SESPMNT & $\mathrm{R}$ \\
\hline 2559 & OFFSITE & SESPMNT & $\mathrm{R}$ \\
\hline 2560 & OFFSITE & SESPMNT & $\mathrm{R}$ \\
\hline 2561 & OFFSITE & SESPMNT & $\mathrm{R}$ \\
\hline 2562 & OFFSITE & SESPMNT & $\mathrm{R}$ \\
\hline 2563 & OFFSITE & SESPMNT & $\mathrm{R}$ \\
\hline 2564 & OFFSITE & SESPMNT & $\mathrm{R}$ \\
\hline 2565 & OFFSITE & SESPMNT & $\mathrm{R}$ \\
\hline 2566 & OFFSITE & SESPMNT & $\mathrm{R}$ \\
\hline 2567 & OFFSITE & SESPMNT & $\mathrm{R}$ \\
\hline 2568 & OFFSITE & SESPMNT & $\mathrm{R}$ \\
\hline 2569 & OFFSITE & SESPMNT & $\mathrm{R}$ \\
\hline 2570 & OFFSITE & SESPMNT & $\mathrm{R}$ \\
\hline 2571 & OFFSITE & SESPMNT & $\mathrm{R}$ \\
\hline 2572 & OFFSITE & SESPMNT & $\mathrm{R}$ \\
\hline 2573 & OFFSITE & SESPMNT & $\mathrm{R}$ \\
\hline 2574 & OFFSITE & SESPMNT & $\mathrm{R}$ \\
\hline 2575 & OFFSITE & SESPMNT & $\mathrm{R}$ \\
\hline 2576 & OFFSITE & SESPMNT & $\mathrm{R}$ \\
\hline 2577 & OFFSITE & SESPMNT & $\mathrm{R}$ \\
\hline 2578 & OFFSITE & SESPMNT & $\mathrm{R}$ \\
\hline 2579 & OFFSITE & SESPMNT & $\mathrm{R}$ \\
\hline 2580 & OFFSITE & SESPMNT & $\mathrm{R}$ \\
\hline 2581 & OFFSITE & SESPMNT & $\mathrm{R}$ \\
\hline 2582 & OFFSITE & SESPMNT & $\mathrm{R}$ \\
\hline 2583 & OFFSITE & SESPMNT & $\mathrm{R}$ \\
\hline 2584 & OFFSITE & SESPMNT & $\mathrm{R}$ \\
\hline 2585 & OFFSITE & SESPMNT & $\mathrm{R}$ \\
\hline & & & \\
\hline
\end{tabular}




\begin{tabular}{|c|l|l|l|}
\hline & AG & \multicolumn{1}{|c|}{ AH } & \\
\hline 1 & DIST_CLASS & OWNER_ID & COLLECTION_PURPOSE \\
\hline 2586 & OFFSITE & SESPMNT & $\mathrm{R}$ \\
\hline 2587 & OFFSITE & SESPMNT & $\mathrm{R}$ \\
\hline 2588 & OFFSITE & SESPMNT & $\mathrm{R}$ \\
\hline 2589 & OFFSITE & SESPMNT & $\mathrm{R}$ \\
\hline 2590 & OFFSITE & SESPMNT & $\mathrm{R}$ \\
\hline 2591 & OFFSITE & SESPMNT & $\mathrm{R}$ \\
\hline 2592 & OFFSITE & SESPMNT & $\mathrm{R}$ \\
\hline 2593 & OFFSITE & SESPMNT & $\mathrm{R}$ \\
\hline 2594 & OFFSITE & SESPMNT & $\mathrm{R}$ \\
\hline 2595 & OFFSITE & SESPMNT & $\mathrm{R}$ \\
\hline 2596 & OFFSITE & SESPMNT & $\mathrm{R}$ \\
\hline 2597 & OFFSITE & SESPMNT & $\mathrm{R}$ \\
\hline 2598 & OFFSITE & SESPMNT & $\mathrm{R}$ \\
\hline 2599 & OFFSITE & SESPMNT & $\mathrm{R}$ \\
\hline 2600 & OFFSITE & SESPMNT & $\mathrm{R}$ \\
\hline 2601 & OFFSITE & SESPMNT & $\mathrm{R}$ \\
\hline 2602 & OFFSITE & SESPMNT & $\mathrm{R}$ \\
\hline 2603 & OFFSITE & SESPMNT & $\mathrm{R}$ \\
\hline 2604 & OFFSITE & SESPMNT & $\mathrm{R}$ \\
\hline 2605 & OFFSITE & SESPMNT & $\mathrm{R}$ \\
\hline 2606 & OFFSITE & SESPMNT & $\mathrm{R}$ \\
\hline 2607 & OFFSITE & SESPMNT & $\mathrm{R}$ \\
\hline 2608 & OFFSITE & SESPMNT & $\mathrm{R}$ \\
\hline 2609 & OFFSITE & SESPMNT & $\mathrm{R}$ \\
\hline 2610 & OFFSITE & SESPMNT & $\mathrm{R}$ \\
\hline 2611 & OFFSITE & SESPMNT & $\mathrm{R}$ \\
\hline 2612 & OFFSITE & SESPMNT & $\mathrm{R}$ \\
\hline 2613 & OFFSITE & SESPMNT & $\mathrm{R}$ \\
\hline 2614 & OFFSITE & SESPMNT & $\mathrm{R}$ \\
\hline 2615 & OFFSITE & SESPMNT & $\mathrm{R}$ \\
\hline 2616 & OFFSITE & SESPMNT & $\mathrm{R}$ \\
\hline 2617 & OFFSITE & SESPMNT & $\mathrm{R}$ \\
\hline 2618 & OFFSITE & SESPMNT & $\mathrm{R}$ \\
\hline 2619 & OFFSITE & SESPMNT & $\mathrm{R}$ \\
\hline 2620 & OFFSITE & SESPMNT & $\mathrm{R}$ \\
\hline 2621 & OFFSITE & SESPMNT & $\mathrm{R}$ \\
\hline 2622 & OFFSITE & SESPMNT & $\mathrm{R}$ \\
\hline 2623 & OFFSITE & SESPMNT & $\mathrm{R}$ \\
\hline & & & \\
\hline
\end{tabular}




\begin{tabular}{|c|l|l|l|}
\hline & AG & AH & \\
\hline 1 & DIST_CLASS & OWNER_ID & COLLECTION_PURPOSE \\
\hline 2624 & OFFSITE & SESPMNT & $\mathrm{R}$ \\
\hline 2625 & OFFSITE & SESPMNT & $\mathrm{R}$ \\
\hline $\mathbf{2 6 2 6}$ & OFFSITE & SESPMNT & $\mathrm{R}$ \\
\hline 2627 & OFFSITE & SESPMNT & $\mathrm{R}$ \\
\hline 2628 & OFFSITE & SESPMNT & $\mathrm{R}$ \\
\hline 2629 & OFFSITE & SESPMNT & $\mathrm{R}$ \\
\hline 2630 & OFFSITE & SESPMNT & $\mathrm{R}$ \\
\hline 2631 & OFFSITE & SESPMNT & $\mathrm{R}$ \\
\hline 2632 & OFFSITE & SESPMNT & $\mathrm{R}$ \\
\hline 2633 & OFFSITE & SESPMNT & $\mathrm{R}$ \\
\hline 2634 & OFFSITE & SESPMNT & $\mathrm{R}$ \\
\hline 2635 & OFFSITE & SESPMNT & $\mathrm{R}$ \\
\hline 2636 & OFFSITE & SESPMNT & $\mathrm{R}$ \\
\hline 2637 & OFFSITE & SESPMNT & $\mathrm{R}$ \\
\hline 2638 & OFFSITE & SESPMNT & $\mathrm{R}$ \\
\hline 2639 & OFFSITE & SESPMNT & $\mathrm{R}$ \\
\hline 2640 & OFFSITE & SESPMNT & $\mathrm{R}$ \\
\hline 2641 & OFFSITE & SESPMNT & $\mathrm{R}$ \\
\hline 2642 & OFFSITE & SESPMNT & $\mathrm{R}$ \\
\hline 2643 & OFFSITE & SESPMNT & $\mathrm{R}$ \\
\hline 2644 & OFFSITE & SESPMNT & $\mathrm{R}$ \\
\hline 2645 & OFFSITE & SESPMNT & $\mathrm{R}$ \\
\hline 2646 & OFFSITE & SESPMNT & $\mathrm{R}$ \\
\hline 2647 & OFFSITE & SESPMNT & $\mathrm{R}$ \\
\hline 2648 & OFFSITE & SESPMNT & $\mathrm{R}$ \\
\hline 2649 & OFFSITE & SESPMNT & $\mathrm{R}$ \\
\hline 2650 & OFFSITE & SESPMNT & $\mathrm{R}$ \\
\hline 2651 & OFFSITE & SESPMNT & $\mathrm{R}$ \\
\hline 2652 & OFFSITE & SESPMNT & $\mathrm{R}$ \\
\hline 2653 & OFFSITE & SESPMNT & $\mathrm{R}$ \\
\hline 2654 & OFFSITE & SESPMNT & $\mathrm{R}$ \\
\hline 2655 & OFFSITE & SESPMNT & $\mathrm{R}$ \\
\hline 2656 & OFFSITE & SESPMNT & $\mathrm{R}$ \\
\hline 2657 & OFFSITE & SESPMNT & $\mathrm{R}$ \\
\hline 2658 & OFFSITE & SESPMNT & $\mathrm{R}$ \\
\hline 2659 & OFFSITE & SESPMNT & $\mathrm{R}$ \\
\hline 2660 & OFFSITE & SESPMNT & $\mathrm{R}$ \\
\hline 2661 & OFFSITE & SESPMNT & $\mathrm{R}$ \\
\hline & & & \\
\hline
\end{tabular}




\begin{tabular}{|c|l|l|l|}
\hline & AG & \multicolumn{1}{|c|}{ AH } & \\
\hline 1 & DIST_CLASS & OWNER_ID & COLLECTION_PURPOSE \\
\hline 2662 & OFFSITE & SESPMNT & $\mathrm{R}$ \\
\hline 2663 & OFFSITE & SESPMNT & $\mathrm{R}$ \\
\hline 2664 & OFFSITE & SESPMNT & $\mathrm{R}$ \\
\hline 2665 & OFFSITE & SESPMNT & $\mathrm{R}$ \\
\hline 2666 & OFFSITE & SESPMNT & $\mathrm{R}$ \\
\hline 2667 & OFFSITE & SESPMNT & $\mathrm{R}$ \\
\hline 2668 & OFFSITE & SESPMNT & $\mathrm{R}$ \\
\hline 2669 & OFFSITE & SESPMNT & $\mathrm{R}$ \\
\hline 2670 & OFFSITE & SESPMNT & $\mathrm{R}$ \\
\hline 2671 & OFFSITE & SESPMNT & $\mathrm{R}$ \\
\hline 2672 & OFFSITE & SESPMNT & $\mathrm{R}$ \\
\hline 2673 & OFFSITE & SESPMNT & $\mathrm{R}$ \\
\hline 2674 & OFFSITE & SESPMNT & $\mathrm{R}$ \\
\hline 2675 & OFFSITE & SESPMNT & $\mathrm{R}$ \\
\hline 2676 & OFFSITE & SESPMNT & $\mathrm{R}$ \\
\hline 2677 & OFFSITE & SESPMNT & $\mathrm{R}$ \\
\hline 2678 & OFFSITE & SESPMNT & $\mathrm{R}$ \\
\hline 2679 & OFFSITE & SESPMNT & $\mathrm{R}$ \\
\hline 2680 & OFFSITE & SESPMNT & $\mathrm{R}$ \\
\hline 2681 & OFFSITE & SESPMNT & $\mathrm{R}$ \\
\hline 2682 & OFFSITE & SESPMNT & $\mathrm{R}$ \\
\hline 2683 & OFFSITE & SESPMNT & $\mathrm{R}$ \\
\hline 2684 & OFFSITE & SESPMNT & $\mathrm{R}$ \\
\hline 2685 & OFFSITE & SESPMNT & $\mathrm{R}$ \\
\hline 2686 & OFFSITE & SESPMNT & $\mathrm{R}$ \\
\hline 2687 & OFFSITE & SESPMNT & $\mathrm{R}$ \\
\hline 2688 & OFFSITE & SESPMNT & $\mathrm{R}$ \\
\hline 2689 & OFFSITE & SESPMNT & $\mathrm{R}$ \\
\hline 2690 & OFFSITE & SESPMNT & $\mathrm{R}$ \\
\hline 2691 & OFFSITE & SESPMNT & $\mathrm{R}$ \\
\hline 2692 & OFFSITE & SESPMNT & $\mathrm{R}$ \\
\hline 2693 & OFFSITE & SESPMNT & $\mathrm{R}$ \\
\hline 2694 & OFFSITE & SESPMNT & $\mathrm{R}$ \\
\hline 2695 & OFFSITE & SESPMNT & $\mathrm{R}$ \\
\hline 2696 & OFFSITE & SESPMNT & $\mathrm{R}$ \\
\hline 2697 & OFFSITE & SESPMNT & $\mathrm{R}$ \\
\hline 2698 & OFFSITE & SESPMNT & $\mathrm{R}$ \\
\hline 2699 & OFFSITE & SESPMNT & $\mathrm{R}$ \\
\hline & & & \\
\hline
\end{tabular}




\begin{tabular}{|c|l|l|l|}
\hline & AG & \multicolumn{1}{|c|}{ AH } & \\
\hline 1 & DIST_CLASS & OWNER_ID & COLLECTION_PURPOSE \\
\hline 2700 & OFFSITE & SESPMNT & $\mathrm{R}$ \\
\hline 2701 & OFFSITE & SESPMNT & $\mathrm{R}$ \\
\hline 2702 & OFFSITE & SESPMNT & $\mathrm{R}$ \\
\hline 2703 & OFFSITE & SESPMNT & $\mathrm{R}$ \\
\hline 2704 & OFFSITE & SESPMNT & $\mathrm{R}$ \\
\hline 2705 & OFFSITE & SESPMNT & $\mathrm{R}$ \\
\hline 2706 & OFFSITE & SESPMNT & $\mathrm{R}$ \\
\hline 2707 & OFFSITE & SESPMNT & $\mathrm{R}$ \\
\hline 2708 & OFFSITE & SESPMNT & $\mathrm{R}$ \\
\hline 2709 & OFFSITE & SESPMNT & $\mathrm{R}$ \\
\hline 2710 & OFFSITE & SESPMNT & $\mathrm{R}$ \\
\hline 2711 & OFFSITE & SESPMNT & $\mathrm{R}$ \\
\hline 2712 & OFFSITE & SESPMNT & $\mathrm{R}$ \\
\hline 2713 & OFFSITE & SESPMNT & $\mathrm{R}$ \\
\hline 2714 & OFFSITE & SESPMNT & $\mathrm{R}$ \\
\hline 2715 & ONSITE & SESPMNT & $\mathrm{R}$ \\
\hline 2716 & ONSITE & SESPMNT & $\mathrm{R}$ \\
\hline 2717 & ONSITE & SESPMNT & $\mathrm{R}$ \\
\hline 2718 & ONSITE & SESPMNT & $\mathrm{R}$ \\
\hline 2719 & ONSITE & SESPMNT & $\mathrm{R}$ \\
\hline 2720 & ONSITE & SESPMNT & $\mathrm{R}$ \\
\hline 2721 & ONSITE & SESPMNT & $\mathrm{R}$ \\
\hline 2722 & ONSITE & SESPMNT & $\mathrm{R}$ \\
\hline 2723 & ONSITE & SESPMNT & $\mathrm{R}$ \\
\hline 2724 & ONSITE & SESPMNT & $\mathrm{R}$ \\
\hline 2725 & ONSITE & SESPMNT & $\mathrm{R}$ \\
\hline 2726 & ONSITE & SESPMNT & $\mathrm{R}$ \\
\hline 2727 & ONSITE & SESPMNT & $\mathrm{R}$ \\
\hline 2728 & ONSITE & SESPMNT & $\mathrm{R}$ \\
\hline 2729 & ONSITE & SESPMNT & $\mathrm{R}$ \\
\hline 2730 & ONSITE & SESPMNT & $\mathrm{R}$ \\
\hline 2731 & ONSITE & SESPMNT & $\mathrm{R}$ \\
\hline 2732 & ONSITE & SESPMNT & $\mathrm{R}$ \\
\hline 2733 & ONSITE & SESPMNT & $\mathrm{R}$ \\
\hline 2734 & ONSITE & SESPMNT & $\mathrm{R}$ \\
\hline 2735 & ONSITE & SESPMNT & $\mathrm{R}$ \\
\hline 2736 & ONSITE & SESPMNT & $\mathrm{R}$ \\
\hline 2737 & ONSITE & SESPMNT & $\mathrm{R}$ \\
\hline & & & \\
\hline
\end{tabular}




\begin{tabular}{|c|l|l|l|}
\hline & \multicolumn{1}{|c|}{ AG } & \multicolumn{1}{|c|}{ AH } & \\
\hline 1 & DIST_CLASS & OWNER_ID & COLLECTION_PURPOSE \\
\hline 2738 & ONSITE & SESPMNT & $\mathrm{R}$ \\
\hline 2739 & ONSITE & SESPMNT & $\mathrm{R}$ \\
\hline 2740 & ONSITE & SESPMNT & $\mathrm{R}$ \\
\hline 2741 & ONSITE & SESPMNT & $\mathrm{R}$ \\
\hline 2742 & ONSITE & SESPMNT & $\mathrm{R}$ \\
\hline 2743 & ONSITE & SESPMNT & $\mathrm{R}$ \\
\hline 2744 & ONSITE & SESPMNT & $\mathrm{R}$ \\
\hline 2745 & ONSITE & SESPMNT & $\mathrm{R}$ \\
\hline 2746 & ONSITE & SESPMNT & $\mathrm{R}$ \\
\hline 2747 & ONSITE & SESPMNT & $\mathrm{R}$ \\
\hline 2748 & ONSITE & SESPMNT & $\mathrm{R}$ \\
\hline 2749 & ONSITE & SESPMNT & $\mathrm{R}$ \\
\hline 2750 & ONSITE & SESPMNT & $\mathrm{R}$ \\
\hline 2751 & ONSITE & SESPMNT & $\mathrm{R}$ \\
\hline 2752 & ONSITE & SESPMNT & $\mathrm{R}$ \\
\hline 2753 & ONSITE & SESPMNT & $\mathrm{R}$ \\
\hline 2754 & ONSITE & SESPMNT & $\mathrm{R}$ \\
\hline 2755 & ONSITE & SESPMNT & $\mathrm{R}$ \\
\hline 2756 & ONSITE & SESPMNT & $\mathrm{R}$ \\
\hline 2757 & ONSITE & SESPMNT & $\mathrm{R}$ \\
\hline 2758 & ONSITE & SESPMNT & $\mathrm{R}$ \\
\hline 2759 & ONSITE & SESPMNT & $\mathrm{R}$ \\
\hline 2760 & ONSITE & SESPMNT & $\mathrm{R}$ \\
\hline 2761 & ONSITE & SESPMNT & $\mathrm{R}$ \\
\hline 2762 & ONSITE & SESPMNT & $\mathrm{R}$ \\
\hline 2763 & ONSITE & SESPMNT & $\mathrm{R}$ \\
\hline 2764 & ONSITE & SESPMNT & $\mathrm{R}$ \\
\hline 2765 & ONSITE & SESPMNT & $\mathrm{R}$ \\
\hline 2766 & ONSITE & SESPMNT & $\mathrm{R}$ \\
\hline 2767 & ONSITE & SESPMNT & $\mathrm{R}$ \\
\hline 2768 & ONSITE & SESPMNT & $\mathrm{R}$ \\
\hline 2769 & ONSITE & SESPMNT & $\mathrm{R}$ \\
\hline 2770 & ONSITE & SESPMNT & $\mathrm{R}$ \\
\hline 2771 & ONSITE & SESPMNT & $\mathrm{R}$ \\
\hline 2772 & ONSITE & SESPMNT & $\mathrm{R}$ \\
\hline 2773 & ONSITE & SESPMNT & $\mathrm{R}$ \\
\hline 2774 & ONSITE & SESPMNT & $\mathrm{R}$ \\
\hline 2775 & ONSITE & SESPMNT & $\mathrm{R}$ \\
\hline & & & \\
\hline
\end{tabular}




\begin{tabular}{|c|l|l|l|}
\hline & AG & AH & \\
\hline 1 & DIST_CLASS & OWNER_ID & COLLECTION_PURPOSE \\
\hline 2776 & ONSITE & SESPMNT & $\mathrm{R}$ \\
\hline 2777 & ONSITE & SESPMNT & $\mathrm{R}$ \\
\hline 2778 & ONSITE & SESPMNT & $\mathrm{R}$ \\
\hline 2779 & ONSITE & SESPMNT & $\mathrm{R}$ \\
\hline 2780 & ONSITE & SESPMNT & $\mathrm{R}$ \\
\hline 2781 & ONSITE & SESPMNT & $\mathrm{R}$ \\
\hline 2782 & ONSITE & SESPMNT & $\mathrm{R}$ \\
\hline 2783 & ONSITE & SESPMNT & $\mathrm{R}$ \\
\hline 2784 & ONSITE & SESPMNT & $\mathrm{R}$ \\
\hline 2785 & OFFSITE & SESPMNT & $\mathrm{R}$ \\
\hline 2786 & OFFSITE & SESPMNT & $\mathrm{R}$ \\
\hline 2787 & OFFSITE & SESPMNT & $\mathrm{R}$ \\
\hline 2788 & OFFSITE & SESPMNT & $\mathrm{R}$ \\
\hline 2789 & OFFSITE & SESPMNT & $\mathrm{R}$ \\
\hline 2790 & OFFSITE & SESPMNT & $\mathrm{R}$ \\
\hline 2791 & OFFSITE & SESPMNT & $\mathrm{R}$ \\
\hline 2792 & OFFSITE & SESPMNT & $\mathrm{R}$ \\
\hline 2793 & OFFSITE & SESPMNT & $\mathrm{R}$ \\
\hline 2794 & OFFSITE & SESPMNT & $\mathrm{R}$ \\
\hline 2795 & OFFSITE & SESPMNT & $\mathrm{R}$ \\
\hline 2796 & OFFSITE & SESPMNT & $\mathrm{R}$ \\
\hline 2797 & OFFSITE & SESPMNT & $\mathrm{R}$ \\
\hline 2798 & OFFSITE & SESPMNT & $\mathrm{R}$ \\
\hline 2799 & OFFSITE & SESPMNT & $\mathrm{R}$ \\
\hline 2800 & OFFSITE & SESPMNT & $\mathrm{R}$ \\
\hline 2801 & OFFSITE & SESPMNT & $\mathrm{R}$ \\
\hline 2802 & OFFSITE & SESPMNT & $\mathrm{R}$ \\
\hline 2803 & OFFSITE & SESPMNT & $\mathrm{R}$ \\
\hline 2804 & OFFSITE & SESPMNT & $\mathrm{R}$ \\
\hline 2805 & OFFSITE & SESPMNT & $\mathrm{R}$ \\
\hline 2806 & OFFSITE & SESPMNT & $\mathrm{R}$ \\
\hline 2807 & OFFSITE & SESPMNT & $\mathrm{R}$ \\
\hline 2808 & OFFSITE & SESPMNT & $\mathrm{R}$ \\
\hline 2809 & OFFSITE & SESPMNT & $\mathrm{R}$ \\
\hline 2810 & OFFSITE & SESPMNT & $\mathrm{R}$ \\
\hline 2811 & OFFSITE & SESPMNT & $\mathrm{R}$ \\
\hline 2812 & OFFSITE & SESPMNT & $\mathrm{R}$ \\
\hline 2813 & OFFSITE & SESPMNT & $\mathrm{R}$ \\
\hline & & & \\
\hline
\end{tabular}




\begin{tabular}{|c|l|l|l|}
\hline & AG & \multicolumn{1}{|c|}{ AH } & \\
\hline 1 & DIST_CLASS & OWNER_ID & COLLECTION_PURPOSE \\
\hline 2814 & OFFSITE & SESPMNT & $\mathrm{R}$ \\
\hline 2815 & OFFSITE & SESPMNT & $\mathrm{R}$ \\
\hline 2816 & OFFSITE & SESPMNT & $\mathrm{R}$ \\
\hline 2817 & OFFSITE & SESPMNT & $\mathrm{R}$ \\
\hline 2818 & OFFSITE & SESPMNT & $\mathrm{R}$ \\
\hline 2819 & OFFSITE & SESPMNT & $\mathrm{R}$ \\
\hline 2820 & OFFSITE & SESPMNT & $\mathrm{R}$ \\
\hline 2821 & OFFSITE & SESPMNT & $\mathrm{R}$ \\
\hline 2822 & OFFSITE & SESPMNT & $\mathrm{R}$ \\
\hline 2823 & OFFSITE & SESPMNT & $\mathrm{R}$ \\
\hline 2824 & OFFSITE & SESPMNT & $\mathrm{R}$ \\
\hline 2825 & OFFSITE & SESPMNT & $\mathrm{R}$ \\
\hline 2826 & OFFSITE & SESPMNT & $\mathrm{R}$ \\
\hline 2827 & OFFSITE & SESPMNT & $\mathrm{R}$ \\
\hline 2828 & OFFSITE & SESPMNT & $\mathrm{R}$ \\
\hline 2829 & OFFSITE & SESPMNT & $\mathrm{R}$ \\
\hline 2830 & OFFSITE & SESPMNT & $\mathrm{R}$ \\
\hline 2831 & OFFSITE & SESPMNT & $\mathrm{R}$ \\
\hline 2832 & OFFSITE & SESPMNT & $\mathrm{R}$ \\
\hline 2833 & OFFSITE & SESPMNT & $\mathrm{R}$ \\
\hline 2834 & OFFSITE & SESPMNT & $\mathrm{R}$ \\
\hline 2835 & OFFSITE & SESPMNT & $\mathrm{R}$ \\
\hline 2836 & OFFSITE & SESPMNT & $\mathrm{R}$ \\
\hline 2837 & OFFSITE & SESPMNT & $\mathrm{R}$ \\
\hline 2838 & OFFSITE & SESPMNT & $\mathrm{R}$ \\
\hline 2839 & OFFSITE & SESPMNT & $\mathrm{R}$ \\
\hline 2840 & OFFSITE & SESPMNT & $\mathrm{R}$ \\
\hline 2841 & OFFSITE & SESPMNT & $\mathrm{R}$ \\
\hline 2842 & OFFSITE & SESPMNT & $\mathrm{R}$ \\
\hline 2843 & OFFSITE & SESPMNT & $\mathrm{R}$ \\
\hline 2844 & OFFSITE & SESPMNT & $\mathrm{R}$ \\
\hline 2845 & OFFSITE & SESPMNT & $\mathrm{R}$ \\
\hline 2846 & OFFSITE & SESPMNT & $\mathrm{R}$ \\
\hline 2847 & OFFSITE & SESPMNT & $\mathrm{R}$ \\
\hline 2848 & OFFSITE & SESPMNT & $\mathrm{R}$ \\
\hline 2849 & OFFSITE & SESPMNT & $\mathrm{R}$ \\
\hline 2850 & OFFSITE & SESPMNT & $\mathrm{R}$ \\
\hline 2851 & OFFSITE & SESPMNT & $\mathrm{R}$ \\
\hline & & & \\
\hline
\end{tabular}




\begin{tabular}{|c|l|l|l|}
\hline & AG & \multicolumn{1}{|c|}{ AH } & \multicolumn{1}{|c|}{ AI } \\
\hline 1 & DIST_CLASS & OWNER_ID & COLLECTION_PURPOSE \\
\hline 2852 & OFFSITE & SESPMNT & $\mathrm{R}$ \\
\hline 2853 & OFFSITE & SESPMNT & $\mathrm{R}$ \\
\hline $\mathbf{2 8 5 4}$ & OFFSITE & SESPMNT & $\mathrm{R}$ \\
\hline 2855 & ONSITE & SESPMNT & $\mathrm{R}$ \\
\hline 2856 & ONSITE & SESPMNT & $\mathrm{R}$ \\
\hline 2857 & ONSITE & SESPMNT & $\mathrm{R}$ \\
\hline 2858 & ONSITE & SESPSPEC & $\mathrm{S}$ \\
\hline 2859 & ONSITE & SESPMNT & $\mathrm{R}$ \\
\hline 2860 & ONSITE & SESPSPEC & $\mathrm{S}$ \\
\hline 2861 & ONSITE & SESPMNT & $\mathrm{R}$ \\
\hline 2862 & ONSITE & SESPMNT & $\mathrm{R}$ \\
\hline 2863 & ONSITE & SESPMNT & $\mathrm{R}$ \\
\hline 2864 & ONSITE & SESPSPEC & $\mathrm{S}$ \\
\hline 2865 & ONSITE & SESPSPEC & $\mathrm{S}$ \\
\hline 2866 & ONSITE & SESPSPEC & $\mathrm{S}$ \\
\hline 2867 & ONSITE & SESPMNT & $\mathrm{R}$ \\
\hline 2868 & ONSITE & SESPMNT & $\mathrm{R}$ \\
\hline 2869 & ONSITE & SESPMNT & $\mathrm{R}$ \\
\hline 2870 & ONSITE & SESPMNT & $\mathrm{R}$ \\
\hline 2871 & ONSITE & SESPMNT & $\mathrm{R}$ \\
\hline 2872 & ONSITE & SESPMNT & $\mathrm{R}$ \\
\hline 2873 & ONSITE & SESPMNT & $\mathrm{R}$ \\
\hline 2874 & ONSITE & SESPMNT & $\mathrm{R}$ \\
\hline 2875 & ONSITE & SESPMNT & $\mathrm{R}$ \\
\hline 2876 & ONSITE & SESPMNT & $\mathrm{R}$ \\
\hline 2877 & ONSITE & SESPMNT & $\mathrm{R}$ \\
\hline 2878 & ONSITE & SESPMNT & $\mathrm{R}$ \\
\hline 2879 & ONSITE & SESPMNT & $\mathrm{R}$ \\
\hline 2880 & ONSITE & SESPMNT & $\mathrm{R}$ \\
\hline 2881 & ONSITE & SESPMNT & $\mathrm{R}$ \\
\hline 2882 & ONSITE & SESPMNT & $\mathrm{R}$ \\
\hline 2883 & ONSITE & SESPMNT & $\mathrm{R}$ \\
\hline 2884 & ONSITE & SESPMNT & $\mathrm{R}$ \\
\hline 2885 & ONSITE & SESPMNT & $\mathrm{R}$ \\
\hline 2886 & ONSITE & SESPMNT & $\mathrm{R}$ \\
\hline 2887 & ONSITE & SESPMNT & $\mathrm{R}$ \\
\hline 2888 & ONSITE & SESPMNT & $\mathrm{R}$ \\
\hline 2889 & ONSITE & SESPMNT & $\mathrm{R}$ \\
\hline & & & \\
\hline
\end{tabular}




\begin{tabular}{|c|l|l|l|}
\hline & \multicolumn{1}{|c|}{ AG } & \multicolumn{1}{|c|}{ AH } & \\
\hline 1 & DIST_CLASS & OWNER_ID & COLLECTION_PURPOSE \\
\hline 2890 & ONSITE & SESPMNT & $\mathrm{R}$ \\
\hline 2891 & ONSITE & SESPMNT & $\mathrm{R}$ \\
\hline 2892 & ONSITE & SESPMNT & $\mathrm{R}$ \\
\hline 2893 & ONSITE & SESPMNT & $\mathrm{R}$ \\
\hline 2894 & ONSITE & SESPMNT & $\mathrm{R}$ \\
\hline 2895 & ONSITE & SESPMNT & $\mathrm{R}$ \\
\hline 2896 & ONSITE & SESPMNT & $\mathrm{R}$ \\
\hline 2897 & ONSITE & SESPMNT & $\mathrm{R}$ \\
\hline 2898 & ONSITE & SESPMNT & $\mathrm{R}$ \\
\hline 2899 & ONSITE & SESPMNT & $\mathrm{R}$ \\
\hline 2900 & ONSITE & SESPMNT & $\mathrm{R}$ \\
\hline 2901 & ONSITE & SESPMNT & $\mathrm{R}$ \\
\hline 2902 & ONSITE & SESPMNT & $\mathrm{R}$ \\
\hline 2903 & ONSITE & SESPMNT & $\mathrm{R}$ \\
\hline 2904 & ONSITE & SESPMNT & $\mathrm{R}$ \\
\hline 2905 & ONSITE & SESPMNT & $\mathrm{R}$ \\
\hline 2906 & ONSITE & SESPMNT & $\mathrm{R}$ \\
\hline 2907 & ONSITE & SESPMNT & $\mathrm{R}$ \\
\hline 2908 & ONSITE & SESPMNT & $\mathrm{R}$ \\
\hline 2909 & ONSITE & SESPMNT & $\mathrm{R}$ \\
\hline 2910 & ONSITE & SESPMNT & $\mathrm{R}$ \\
\hline 2911 & ONSITE & SESPMNT & $\mathrm{R}$ \\
\hline 2912 & ONSITE & SESPMNT & $\mathrm{R}$ \\
\hline 2913 & ONSITE & SESPMNT & $\mathrm{R}$ \\
\hline 2914 & ONSITE & SESPMNT & $\mathrm{R}$ \\
\hline 2915 & ONSITE & SESPMNT & $\mathrm{R}$ \\
\hline 2916 & ONSITE & SESPMNT & $\mathrm{R}$ \\
\hline 2917 & ONSITE & SESPMNT & $\mathrm{R}$ \\
\hline 2918 & ONSITE & SESPMNT & $\mathrm{R}$ \\
\hline 2919 & ONSITE & SESPMNT & $\mathrm{R}$ \\
\hline 2920 & ONSITE & SESPMNT & $\mathrm{R}$ \\
\hline 2921 & ONSITE & SESPMNT & $\mathrm{R}$ \\
\hline 2922 & ONSITE & SESPMNT & $\mathrm{R}$ \\
\hline 2923 & ONSITE & SESPMNT & $\mathrm{R}$ \\
\hline 2924 & ONSITE & SESPMNT & $\mathrm{R}$ \\
\hline 2925 & ONSITE & SESPMNT & $\mathrm{R}$ \\
\hline 2926 & ONSITE & SESPMNT & $\mathrm{R}$ \\
\hline 2927 & ONSITE & SESPMNT & $\mathrm{R}$ \\
\hline & & & \\
\hline
\end{tabular}




\begin{tabular}{|c|l|l|l|}
\hline & AG & AH & \\
\hline 1 & DIST_CLASS & OWNER_ID & COLLECTION_PURPOSE \\
\hline 2928 & ONSITE & SESPMNT & $\mathrm{R}$ \\
\hline 2929 & ONSITE & SESPMNT & $\mathrm{R}$ \\
\hline 2930 & ONSITE & SESPMNT & $\mathrm{R}$ \\
\hline 2931 & ONSITE & SESPMNT & $\mathrm{R}$ \\
\hline 2932 & ONSITE & SESPMNT & $\mathrm{R}$ \\
\hline 2933 & ONSITE & SESPMNT & $\mathrm{R}$ \\
\hline 2934 & ONSITE & SESPMNT & $\mathrm{R}$ \\
\hline 2935 & ONSITE & SESPMNT & $\mathrm{R}$ \\
\hline 2936 & ONSITE & SESPMNT & $\mathrm{R}$ \\
\hline 2937 & ONSITE & SESPMNT & $\mathrm{R}$ \\
\hline 2938 & ONSITE & SESPMNT & $\mathrm{R}$ \\
\hline 2939 & ONSITE & SESPMNT & $\mathrm{R}$ \\
\hline 2940 & ONSITE & SESPMNT & $\mathrm{R}$ \\
\hline 2941 & ONSITE & SESPMNT & $\mathrm{R}$ \\
\hline 2942 & ONSITE & SESPMNT & $\mathrm{R}$ \\
\hline 2943 & ONSITE & SESPMNT & $\mathrm{R}$ \\
\hline 2944 & ONSITE & SESPMNT & $\mathrm{R}$ \\
\hline 2945 & ONSITE & SESPMNT & $\mathrm{R}$ \\
\hline 2946 & ONSITE & SESPMNT & $\mathrm{R}$ \\
\hline 2947 & ONSITE & SESPMNT & $\mathrm{R}$ \\
\hline 2948 & ONSITE & SESPMNT & $\mathrm{R}$ \\
\hline 2949 & ONSITE & SESPMNT & $\mathrm{R}$ \\
\hline 2950 & ONSITE & SESPMNT & $\mathrm{R}$ \\
\hline 2951 & ONSITE & SESPMNT & $\mathrm{R}$ \\
\hline 2952 & ONSITE & SESPMNT & $\mathrm{R}$ \\
\hline 2953 & ONSITE & SESPMNT & $\mathrm{R}$ \\
\hline 2954 & ONSITE & SESPMNT & $\mathrm{R}$ \\
\hline 2955 & ONSITE & SESPMNT & $\mathrm{R}$ \\
\hline 2956 & ONSITE & SESPMNT & $\mathrm{R}$ \\
\hline 2957 & ONSITE & SESPMNT & $\mathrm{R}$ \\
\hline 2958 & ONSITE & SESPMNT & $\mathrm{R}$ \\
\hline 2959 & ONSITE & SESPMNT & $\mathrm{R}$ \\
\hline 2960 & ONSITE & SESPMNT & $\mathrm{R}$ \\
\hline 2961 & ONSITE & SESPMNT & $\mathrm{R}$ \\
\hline 2962 & ONSITE & SESPMNT & $\mathrm{R}$ \\
\hline 2963 & ONSITE & SESPMNT & $\mathrm{R}$ \\
\hline 2964 & ONSITE & SESPMNT & $\mathrm{R}$ \\
\hline 2965 & ONSITE & SESPMNT & $\mathrm{R}$ \\
\hline & & & \\
\hline
\end{tabular}




\begin{tabular}{|c|l|l|l|}
\hline & \multicolumn{1}{|c|}{ AG } & \multicolumn{1}{|c|}{ AH } & \\
\hline 1 & DIST_CLASS & OWNER_ID & COLLECTION_PURPOSE \\
\hline 2966 & ONSITE & SESPMNT & $\mathrm{R}$ \\
\hline 2967 & ONSITE & SESPMNT & $\mathrm{R}$ \\
\hline 2968 & ONSITE & SESPMNT & $\mathrm{R}$ \\
\hline 2969 & ONSITE & SESPMNT & $\mathrm{R}$ \\
\hline 2970 & ONSITE & SESPMNT & $\mathrm{R}$ \\
\hline 2971 & ONSITE & SESPMNT & $\mathrm{R}$ \\
\hline 2972 & ONSITE & SESPMNT & $\mathrm{R}$ \\
\hline 2973 & ONSITE & SESPMNT & $\mathrm{R}$ \\
\hline 2974 & ONSITE & SESPMNT & $\mathrm{R}$ \\
\hline 2975 & ONSITE & SESPMNT & $\mathrm{R}$ \\
\hline 2976 & ONSITE & SESPMNT & $\mathrm{R}$ \\
\hline 2977 & ONSITE & SESPMNT & $\mathrm{R}$ \\
\hline 2978 & ONSITE & SESPMNT & $\mathrm{R}$ \\
\hline 2979 & ONSITE & SESPMNT & $\mathrm{R}$ \\
\hline 2980 & ONSITE & SESPMNT & $\mathrm{R}$ \\
\hline 2981 & ONSITE & SESPMNT & $\mathrm{R}$ \\
\hline 2982 & ONSITE & SESPMNT & $\mathrm{R}$ \\
\hline 2983 & ONSITE & SESPMNT & $\mathrm{R}$ \\
\hline 2984 & ONSITE & SESPMNT & $\mathrm{R}$ \\
\hline 2985 & ONSITE & SESPMNT & $\mathrm{R}$ \\
\hline 2986 & ONSITE & SESPMNT & $\mathrm{R}$ \\
\hline 2987 & ONSITE & SESPMNT & $\mathrm{R}$ \\
\hline 2988 & ONSITE & SESPMNT & $\mathrm{R}$ \\
\hline 2989 & ONSITE & SESPMNT & $\mathrm{R}$ \\
\hline 2990 & ONSITE & SESPMNT & $\mathrm{R}$ \\
\hline 2991 & ONSITE & SESPMNT & $\mathrm{R}$ \\
\hline 2992 & ONSITE & SESPMNT & $\mathrm{R}$ \\
\hline 2993 & ONSITE & SESPMNT & $\mathrm{R}$ \\
\hline 2994 & ONSITE & SESPMNT & $\mathrm{R}$ \\
\hline 2995 & ONSITE & SESPMNT & $\mathrm{R}$ \\
\hline 2996 & ONSITE & SESPMNT & $\mathrm{R}$ \\
\hline 2997 & ONSITE & SESPMNT & $\mathrm{R}$ \\
\hline 2998 & ONSITE & SESPMNT & $\mathrm{R}$ \\
\hline 2999 & ONSITE & SESPMNT & $\mathrm{R}$ \\
\hline 3000 & ONSITE & SESPMNT & $\mathrm{R}$ \\
\hline 3001 & ONSITE & SESPMNT & $\mathrm{R}$ \\
\hline 3002 & ONSITE & SESPMNT & $\mathrm{R}$ \\
\hline 3003 & ONSITE & SESPMNT & $\mathrm{R}$ \\
\hline & & & \\
\hline
\end{tabular}




\begin{tabular}{|c|l|l|l|}
\hline & \multicolumn{1}{|c|}{ AG } & \multicolumn{1}{|c|}{ AH } & \\
\hline 1 & DIST_CLASS & OWNER_ID & COLLECTION_PURPOSE \\
\hline 3004 & ONSITE & SESPMNT & $\mathrm{R}$ \\
\hline 3005 & ONSITE & SESPMNT & $\mathrm{R}$ \\
\hline 3006 & ONSITE & SESPMNT & $\mathrm{R}$ \\
\hline 3007 & ONSITE & SESPMNT & $\mathrm{R}$ \\
\hline 3008 & ONSITE & SESPMNT & $\mathrm{R}$ \\
\hline 3009 & ONSITE & SESPMNT & $\mathrm{R}$ \\
\hline 3010 & ONSITE & SESPMNT & $\mathrm{R}$ \\
\hline 3011 & ONSITE & SESPMNT & $\mathrm{R}$ \\
\hline 3012 & ONSITE & SESPMNT & $\mathrm{R}$ \\
\hline 3013 & ONSITE & SESPMNT & $\mathrm{R}$ \\
\hline 3014 & ONSITE & SESPMNT & $\mathrm{R}$ \\
\hline 3015 & ONSITE & SESPMNT & $\mathrm{R}$ \\
\hline 3016 & ONSITE & SESPMNT & $\mathrm{R}$ \\
\hline 3017 & ONSITE & SESPMNT & $\mathrm{R}$ \\
\hline 3018 & ONSITE & SESPMNT & $\mathrm{R}$ \\
\hline 3019 & ONSITE & SESPMNT & $\mathrm{R}$ \\
\hline 3020 & ONSITE & SESPMNT & $\mathrm{R}$ \\
\hline 3021 & ONSITE & SESPMNT & $\mathrm{R}$ \\
\hline 3022 & ONSITE & SESPMNT & $\mathrm{R}$ \\
\hline 3023 & ONSITE & SESPMNT & $\mathrm{R}$ \\
\hline 3024 & ONSITE & SESPMNT & $\mathrm{R}$ \\
\hline 3025 & ONSITE & SESPMNT & $\mathrm{R}$ \\
\hline 3026 & ONSITE & SESPMNT & $\mathrm{R}$ \\
\hline 3027 & ONSITE & SESPMNT & $\mathrm{R}$ \\
\hline 3028 & ONSITE & SESPMNT & $\mathrm{R}$ \\
\hline 3029 & ONSITE & SESPMNT & $\mathrm{R}$ \\
\hline 3030 & ONSITE & SESPMNT & $\mathrm{R}$ \\
\hline 3031 & ONSITE & SESPMNT & $\mathrm{R}$ \\
\hline 3032 & ONSITE & SESPMNT & $\mathrm{R}$ \\
\hline 3033 & ONSITE & SESPMNT & $\mathrm{R}$ \\
\hline 3034 & ONSITE & SESPMNT & $\mathrm{R}$ \\
\hline 3035 & ONSITE & SESPMNT & $\mathrm{R}$ \\
\hline 3036 & ONSITE & SESPMNT & $\mathrm{R}$ \\
\hline 3037 & ONSITE & SESPMNT & $\mathrm{R}$ \\
\hline 3038 & ONSITE & SESPMNT & $\mathrm{R}$ \\
\hline 3039 & ONSITE & SESPMNT & $\mathrm{R}$ \\
\hline 3040 & ONSITE & SESPMNT & $\mathrm{R}$ \\
\hline 3041 & ONSITE & SESPMNT & $\mathrm{R}$ \\
\hline & & & \\
\hline
\end{tabular}




\begin{tabular}{|c|l|l|l|}
\hline & AG & AH & \\
\hline 1 & DIST_CLASS & OWNER_ID & COLLECTION_PURPOSE \\
\hline 3042 & ONSITE & SESPMNT & $\mathrm{R}$ \\
\hline 3043 & ONSITE & SESPMNT & $\mathrm{R}$ \\
\hline 3044 & ONSITE & SESPMNT & $\mathrm{R}$ \\
\hline 3045 & ONSITE & SESPMNT & $\mathrm{R}$ \\
\hline 3046 & ONSITE & SESPMNT & $\mathrm{R}$ \\
\hline 3047 & ONSITE & SESPMNT & $\mathrm{R}$ \\
\hline 3048 & ONSITE & SESPMNT & $\mathrm{R}$ \\
\hline 3049 & ONSITE & SESPMNT & $\mathrm{R}$ \\
\hline 3050 & ONSITE & SESPMNT & $\mathrm{R}$ \\
\hline 3051 & ONSITE & SESPMNT & $\mathrm{R}$ \\
\hline 3052 & ONSITE & SESPMNT & $\mathrm{R}$ \\
\hline 3053 & ONSITE & SESPMNT & $\mathrm{R}$ \\
\hline 3054 & ONSITE & SESPMNT & $\mathrm{R}$ \\
\hline 3055 & ONSITE & SESPMNT & $\mathrm{R}$ \\
\hline 3056 & ONSITE & SESPMNT & $\mathrm{R}$ \\
\hline 3057 & ONSITE & SESPMNT & $\mathrm{R}$ \\
\hline 3058 & ONSITE & SESPMNT & $\mathrm{R}$ \\
\hline 3059 & ONSITE & SESPMNT & $\mathrm{R}$ \\
\hline 3060 & ONSITE & SESPMNT & $\mathrm{R}$ \\
\hline 3061 & ONSITE & SESPMNT & $\mathrm{R}$ \\
\hline 3062 & ONSITE & SESPMNT & $\mathrm{R}$ \\
\hline 3063 & ONSITE & SESPMNT & $\mathrm{R}$ \\
\hline 3064 & ONSITE & SESPMNT & $\mathrm{R}$ \\
\hline 3065 & ONSITE & SESPMNT & $\mathrm{R}$ \\
\hline 3066 & ONSITE & SESPMNT & $\mathrm{R}$ \\
\hline 3067 & ONSITE & SESPMNT & $\mathrm{R}$ \\
\hline 3068 & ONSITE & SESPMNT & $\mathrm{R}$ \\
\hline 3069 & ONSITE & SESPMNT & $\mathrm{R}$ \\
\hline 3070 & OFFSITE & SESPMNT & $\mathrm{R}$ \\
\hline 3071 & OFFSITE & SESPMNT & $\mathrm{R}$ \\
\hline 3072 & OFFSITE & SESPMNT & $\mathrm{R}$ \\
\hline 3073 & OFFSITE & SESPMNT & $\mathrm{R}$ \\
\hline 3074 & OFFSITE & SESPMNT & $\mathrm{R}$ \\
\hline 3075 & OFFSITE & SESPMNT & $\mathrm{R}$ \\
\hline 3076 & OFFSITE & SESPMNT & $\mathrm{R}$ \\
\hline 3077 & OFFSITE & SESPMNT & $\mathrm{R}$ \\
\hline 3078 & OFFSITE & SESPMNT & $\mathrm{R}$ \\
\hline 3079 & OFFSITE & SESPMNT & $\mathrm{R}$ \\
\hline & & & \\
\hline
\end{tabular}




\begin{tabular}{|c|l|l|l|}
\hline & AG & \multicolumn{1}{|c|}{ AH } & \\
\hline 1 & DIST_CLASS & OWNER_ID & COLLECTION_PURPOSE \\
\hline 3080 & OFFSITE & SESPMNT & $\mathrm{R}$ \\
\hline 3081 & OFFSITE & SESPMNT & $\mathrm{R}$ \\
\hline 3082 & OFFSITE & SESPMNT & $\mathrm{R}$ \\
\hline 3083 & OFFSITE & SESPMNT & $\mathrm{R}$ \\
\hline 3084 & OFFSITE & SESPMNT & $\mathrm{R}$ \\
\hline 3085 & OFFSITE & SESPMNT & $\mathrm{R}$ \\
\hline 3086 & OFFSITE & SESPMNT & $\mathrm{R}$ \\
\hline 3087 & OFFSITE & SESPMNT & $\mathrm{R}$ \\
\hline 3088 & OFFSITE & SESPMNT & $\mathrm{R}$ \\
\hline 3089 & OFFSITE & SESPMNT & $\mathrm{R}$ \\
\hline 3090 & OFFSITE & SESPMNT & $\mathrm{R}$ \\
\hline 3091 & OFFSITE & SESPMNT & $\mathrm{R}$ \\
\hline 3092 & OFFSITE & SESPMNT & $\mathrm{R}$ \\
\hline 3093 & OFFSITE & SESPMNT & $\mathrm{R}$ \\
\hline 3094 & OFFSITE & SESPMNT & $\mathrm{R}$ \\
\hline 3095 & OFFSITE & SESPMNT & $\mathrm{R}$ \\
\hline 3096 & OFFSITE & SESPMNT & $\mathrm{R}$ \\
\hline 3097 & OFFSITE & SESPMNT & $\mathrm{R}$ \\
\hline 3098 & OFFSITE & SESPMNT & $\mathrm{R}$ \\
\hline 3099 & OFFSITE & SESPMNT & $\mathrm{R}$ \\
\hline 3100 & OFFSITE & SESPMNT & $\mathrm{R}$ \\
\hline 3101 & OFFSITE & SESPSPEC & $\mathrm{S}$ \\
\hline 3102 & OFFSITE & SESPMNT & $\mathrm{R}$ \\
\hline 3103 & OFFSITE & SESPSPEC & $\mathrm{S}$ \\
\hline 3104 & OFFSITE & SESPMNT & $\mathrm{R}$ \\
\hline 3105 & OFFSITE & SESPMNT & $\mathrm{R}$ \\
\hline 3106 & OFFSITE & SESPMNT & $\mathrm{R}$ \\
\hline 3107 & OFFSITE & SESPSPEC & $\mathrm{S}$ \\
\hline 3108 & OFFSITE & SESPSPEC & $\mathrm{S}$ \\
\hline 3109 & OFFSITE & SESPSPEC & $\mathrm{S}$ \\
\hline 3110 & OFFSITE & SESPMNT & $\mathrm{R}$ \\
\hline 3111 & OFFSITE & SESPMNT & $\mathrm{R}$ \\
\hline 3112 & OFFSITE & SESPMNT & $\mathrm{R}$ \\
\hline 3113 & OFFSITE & SESPMNT & $\mathrm{R}$ \\
\hline 3114 & OFFSITE & SESPMNT & $\mathrm{R}$ \\
\hline 3115 & OFFSITE & SESPMNT & $\mathrm{R}$ \\
\hline 3116 & OFFSITE & SESPMNT & $\mathrm{R}$ \\
\hline 3117 & OFFSITE & SESPMNT & $\mathrm{R}$ \\
\hline & & & \\
\hline
\end{tabular}




\begin{tabular}{|c|l|l|l|}
\hline & AG & \multicolumn{1}{|c|}{ AH } & \\
\hline 1 & DIST_CLASS & OWNER_ID & COLLECTION_PURPOSE \\
\hline 3118 & OFFSITE & SESPMNT & $\mathrm{R}$ \\
\hline 3119 & OFFSITE & SESPMNT & $\mathrm{R}$ \\
\hline 3120 & OFFSITE & SESPMNT & $\mathrm{R}$ \\
\hline 3121 & OFFSITE & SESPMNT & $\mathrm{R}$ \\
\hline 3122 & OFFSITE & SESPMNT & $\mathrm{R}$ \\
\hline 3123 & OFFSITE & SESPMNT & $\mathrm{R}$ \\
\hline 3124 & OFFSITE & SESPMNT & $\mathrm{R}$ \\
\hline 3125 & OFFSITE & SESPMNT & $\mathrm{R}$ \\
\hline 3126 & OFFSITE & SESPMNT & $\mathrm{R}$ \\
\hline 3127 & OFFSITE & SESPMNT & $\mathrm{R}$ \\
\hline 3128 & OFFSITE & SESPMNT & $\mathrm{R}$ \\
\hline 3129 & OFFSITE & SESPMNT & $\mathrm{R}$ \\
\hline 3130 & OFFSITE & SESPMNT & $\mathrm{R}$ \\
\hline 3131 & OFFSITE & SESPMNT & $\mathrm{R}$ \\
\hline 3132 & OFFSITE & SESPMNT & $\mathrm{R}$ \\
\hline 3133 & OFFSITE & SESPMNT & $\mathrm{R}$ \\
\hline 3134 & OFFSITE & SESPMNT & $\mathrm{R}$ \\
\hline 3135 & OFFSITE & SESPMNT & $\mathrm{R}$ \\
\hline 3136 & OFFSITE & SESPMNT & $\mathrm{R}$ \\
\hline 3137 & OFFSITE & SESPMNT & $\mathrm{R}$ \\
\hline 3138 & OFFSITE & SESPMNT & $\mathrm{R}$ \\
\hline 3139 & OFFSITE & SESPMNT & $\mathrm{R}$ \\
\hline 3140 & OFFSITE & SESPMNT & $\mathrm{R}$ \\
\hline 3141 & OFFSITE & SESPMNT & $\mathrm{R}$ \\
\hline 3142 & OFFSITE & SESPMNT & $\mathrm{R}$ \\
\hline 3143 & OFFSITE & SESPMNT & $\mathrm{R}$ \\
\hline 3144 & OFFSITE & SESPMNT & $\mathrm{R}$ \\
\hline 3145 & OFFSITE & SESPMNT & $\mathrm{R}$ \\
\hline 3146 & OFFSITE & SESPMNT & $\mathrm{R}$ \\
\hline 3147 & OFFSITE & SESPMNT & $\mathrm{R}$ \\
\hline 3148 & OFFSITE & SESPMNT & $\mathrm{R}$ \\
\hline 3149 & OFFSITE & SESPMNT & $\mathrm{R}$ \\
\hline 3150 & OFFSITE & SESPMNT & $\mathrm{R}$ \\
\hline 3151 & OFFSITE & SESPMNT & $\mathrm{R}$ \\
\hline 3152 & OFFSITE & SESPMNT & $\mathrm{R}$ \\
\hline 3153 & OFFSITE & SESPMNT & $\mathrm{R}$ \\
\hline 3154 & OFFSITE & SESPMNT & $\mathrm{R}$ \\
\hline 3155 & OFFSITE & SESPMNT & $\mathrm{R}$ \\
\hline & & & \\
\hline
\end{tabular}




\begin{tabular}{|c|l|l|l|}
\hline & AG & \multicolumn{1}{|c|}{ AH } & \\
\hline 1 & DIST_CLASS & OWNER_ID & COLLECTION_PURPOSE \\
\hline 3156 & OFFSITE & SESPMNT & $\mathrm{R}$ \\
\hline 3157 & OFFSITE & SESPMNT & $\mathrm{R}$ \\
\hline 3158 & OFFSITE & SESPMNT & $\mathrm{R}$ \\
\hline 3159 & OFFSITE & SESPMNT & $\mathrm{R}$ \\
\hline 3160 & OFFSITE & SESPMNT & $\mathrm{R}$ \\
\hline 3161 & OFFSITE & SESPMNT & $\mathrm{R}$ \\
\hline 3162 & OFFSITE & SESPMNT & $\mathrm{R}$ \\
\hline 3163 & OFFSITE & SESPMNT & $\mathrm{R}$ \\
\hline 3164 & OFFSITE & SESPMNT & $\mathrm{R}$ \\
\hline 3165 & OFFSITE & SESPMNT & $\mathrm{R}$ \\
\hline 3166 & OFFSITE & SESPMNT & $\mathrm{R}$ \\
\hline 3167 & OFFSITE & SESPMNT & $\mathrm{R}$ \\
\hline 3168 & OFFSITE & SESPMNT & $\mathrm{R}$ \\
\hline 3169 & OFFSITE & SESPMNT & $\mathrm{R}$ \\
\hline 3170 & OFFSITE & SESPMNT & $\mathrm{R}$ \\
\hline 3171 & OFFSITE & SESPMNT & $\mathrm{R}$ \\
\hline 3172 & OFFSITE & SESPMNT & $\mathrm{R}$ \\
\hline 3173 & OFFSITE & SESPMNT & $\mathrm{R}$ \\
\hline 3174 & OFFSITE & SESPMNT & $\mathrm{R}$ \\
\hline 3175 & OFFSITE & SESPMNT & $\mathrm{R}$ \\
\hline 3176 & OFFSITE & SESPMNT & $\mathrm{R}$ \\
\hline 3177 & OFFSITE & SESPMNT & $\mathrm{R}$ \\
\hline 3178 & OFFSITE & SESPMNT & $\mathrm{R}$ \\
\hline 3179 & OFFSITE & SESPMNT & $\mathrm{R}$ \\
\hline 3180 & OFFSITE & SESPMNT & $\mathrm{R}$ \\
\hline 3181 & OFFSITE & SESPMNT & $\mathrm{R}$ \\
\hline 3182 & OFFSITE & SESPMNT & $\mathrm{R}$ \\
\hline 3183 & OFFSITE & SESPMNT & $\mathrm{R}$ \\
\hline 3184 & OFFSITE & SESPMNT & $\mathrm{R}$ \\
\hline 3185 & OFFSITE & SESPMNT & $\mathrm{R}$ \\
\hline 3186 & OFFSITE & SESPMNT & $\mathrm{R}$ \\
\hline 3187 & OFFSITE & SESPMNT & $\mathrm{R}$ \\
\hline 3188 & OFFSITE & SESPMNT & $\mathrm{R}$ \\
\hline 3189 & OFFSITE & SESPMNT & $\mathrm{R}$ \\
\hline 3190 & OFFSITE & SESPMNT & $\mathrm{R}$ \\
\hline 3191 & OFFSITE & SESPMNT & $\mathrm{R}$ \\
\hline 3192 & OFFSITE & SESPMNT & $\mathrm{R}$ \\
\hline 3193 & OFFSITE & SESPMNT & $\mathrm{R}$ \\
\hline & & & \\
\hline
\end{tabular}




\begin{tabular}{|c|l|l|l|}
\hline & AG & \multicolumn{1}{|c|}{ AH } & \\
\hline 1 & DIST_CLASS & OWNER_ID & COLLECTION_PURPOSE \\
\hline 3194 & OFFSITE & SESPMNT & $\mathrm{R}$ \\
\hline 3195 & OFFSITE & SESPMNT & $\mathrm{R}$ \\
\hline 3196 & OFFSITE & SESPMNT & $\mathrm{R}$ \\
\hline 3197 & OFFSITE & SESPMNT & $\mathrm{R}$ \\
\hline 3198 & OFFSITE & SESPMNT & $\mathrm{R}$ \\
\hline 3199 & OFFSITE & SESPMNT & $\mathrm{R}$ \\
\hline 3200 & OFFSITE & SESPMNT & $\mathrm{R}$ \\
\hline 3201 & OFFSITE & SESPMNT & $\mathrm{R}$ \\
\hline 3202 & OFFSITE & SESPMNT & $\mathrm{R}$ \\
\hline 3203 & OFFSITE & SESPMNT & $\mathrm{R}$ \\
\hline 3204 & OFFSITE & SESPMNT & $\mathrm{R}$ \\
\hline 3205 & OFFSITE & SESPMNT & $\mathrm{R}$ \\
\hline 3206 & OFFSITE & SESPMNT & $\mathrm{R}$ \\
\hline 3207 & OFFSITE & SESPMNT & $\mathrm{R}$ \\
\hline 3208 & OFFSITE & SESPMNT & $\mathrm{R}$ \\
\hline 3209 & OFFSITE & SESPMNT & $\mathrm{R}$ \\
\hline 3210 & OFFSITE & SESPMNT & $\mathrm{R}$ \\
\hline 3211 & OFFSITE & SESPMNT & $\mathrm{R}$ \\
\hline 3212 & OFFSITE & SESPMNT & $\mathrm{R}$ \\
\hline 3213 & OFFSITE & SESPMNT & $\mathrm{R}$ \\
\hline 3214 & OFFSITE & SESPMNT & $\mathrm{R}$ \\
\hline 3215 & OFFSITE & SESPMNT & $\mathrm{R}$ \\
\hline 3216 & OFFSITE & SESPMNT & $\mathrm{R}$ \\
\hline 3217 & OFFSITE & SESPMNT & $\mathrm{R}$ \\
\hline 3218 & OFFSITE & SESPMNT & $\mathrm{R}$ \\
\hline 3219 & OFFSITE & SESPMNT & $\mathrm{R}$ \\
\hline 3220 & OFFSITE & SESPMNT & $\mathrm{R}$ \\
\hline 3221 & OFFSITE & SESPMNT & $\mathrm{R}$ \\
\hline 3222 & OFFSITE & SESPMNT & $\mathrm{R}$ \\
\hline 3223 & OFFSITE & SESPMNT & $\mathrm{R}$ \\
\hline 3224 & OFFSITE & SESPMNT & $\mathrm{R}$ \\
\hline 3225 & OFFSITE & SESPMNT & $\mathrm{R}$ \\
\hline 3226 & OFFSITE & SESPMNT & $\mathrm{R}$ \\
\hline 3227 & OFFSITE & SESPMNT & $\mathrm{R}$ \\
\hline 3228 & OFFSITE & SESPMNT & $\mathrm{R}$ \\
\hline 3229 & OFFSITE & SESPMNT & $\mathrm{R}$ \\
\hline 3230 & OFFSITE & SESPMNT & $\mathrm{R}$ \\
\hline 3231 & OFFSITE & SESPMNT & $\mathrm{R}$ \\
\hline & & & \\
\hline
\end{tabular}




\begin{tabular}{|c|l|l|l|}
\hline & \multicolumn{1}{|c|}{ AG } & \multicolumn{1}{|c|}{ AH } & \\
\hline 1 & DIST_CLASS & OWNER_ID & COLLECTION_PURPOSE \\
\hline 3232 & OFFSITE & SESPMNT & $\mathrm{R}$ \\
\hline 3233 & OFFSITE & SESPMNT & $\mathrm{R}$ \\
\hline 3234 & OFFSITE & SESPMNT & $\mathrm{R}$ \\
\hline 3235 & OFFSITE & SESPMNT & $\mathrm{R}$ \\
\hline 3236 & OFFSITE & SESPMNT & $\mathrm{R}$ \\
\hline 3237 & OFFSITE & SESPMNT & $\mathrm{R}$ \\
\hline 3238 & OFFSITE & SESPMNT & $\mathrm{R}$ \\
\hline 3239 & OFFSITE & SESPMNT & $\mathrm{R}$ \\
\hline 3240 & OFFSITE & SESPMNT & $\mathrm{R}$ \\
\hline 3241 & OFFSITE & SESPMNT & $\mathrm{R}$ \\
\hline 3242 & OFFSITE & SESPMNT & $\mathrm{R}$ \\
\hline 3243 & ONSITE & SESPMNT & $\mathrm{R}$ \\
\hline 3244 & ONSITE & SESPSPEC & $\mathrm{S}$ \\
\hline 3245 & ONSITE & SESPMNT & $\mathrm{R}$ \\
\hline 3246 & ONSITE & SESPSPEC & $\mathrm{S}$ \\
\hline 3247 & ONSITE & SESPMNT & $\mathrm{R}$ \\
\hline 3248 & ONSITE & SESPMNT & $\mathrm{R}$ \\
\hline 3249 & ONSITE & SESPMNT & $\mathrm{R}$ \\
\hline 3250 & ONSITE & SESPSPEC & $\mathrm{S}$ \\
\hline 3251 & ONSITE & SESPSPEC & $\mathrm{S}$ \\
\hline 3252 & ONSITE & SESPSPEC & $\mathrm{S}$ \\
\hline 3253 & ONSITE & SESPMNT & $\mathrm{R}$ \\
\hline 3254 & ONSITE & SESPMNT & $\mathrm{R}$ \\
\hline 3255 & ONSITE & SESPMNT & $\mathrm{R}$ \\
\hline 3256 & ONSITE & SESPMNT & $\mathrm{R}$ \\
\hline 3257 & ONSITE & SESPMNT & $\mathrm{R}$ \\
\hline 3258 & ONSITE & SESPMNT & $\mathrm{R}$ \\
\hline 3259 & ONSITE & SESPMNT & $\mathrm{R}$ \\
\hline 3260 & ONSITE & SESPMNT & $\mathrm{R}$ \\
\hline 3261 & ONSITE & SESPMNT & $\mathrm{R}$ \\
\hline 3262 & ONSITE & SESPMNT & $\mathrm{R}$ \\
\hline 3263 & ONSITE & SESPMNT & $\mathrm{R}$ \\
\hline 3264 & ONSITE & SESPMNT & $\mathrm{R}$ \\
\hline 3265 & ONSITE & SESPMNT & $\mathrm{R}$ \\
\hline 3266 & ONSITE & SESPMNT & $\mathrm{R}$ \\
\hline 3267 & ONSITE & SESPMNT & $\mathrm{R}$ \\
\hline 3268 & OFFSITE & SESPMNT & $\mathrm{R}$ \\
\hline 3269 & OFFSITE & SESPMNT & $\mathrm{R}$ \\
\hline & & & \\
\hline
\end{tabular}




\begin{tabular}{|c|l|l|l|}
\hline & AG & \multicolumn{1}{|c|}{ AH } & \\
\hline 1 & DIST_CLASS & OWNER_ID & COLLECTION_PURPOSE \\
\hline 3270 & OFFSITE & SESPMNT & $\mathrm{R}$ \\
\hline 3271 & OFFSITE & SESPMNT & $\mathrm{R}$ \\
\hline 3272 & OFFSITE & SESPMNT & $\mathrm{R}$ \\
\hline 3273 & OFFSITE & SESPMNT & $\mathrm{R}$ \\
\hline 3274 & OFFSITE & SESPMNT & $\mathrm{R}$ \\
\hline 3275 & OFFSITE & SESPMNT & $\mathrm{R}$ \\
\hline 3276 & OFFSITE & SESPMNT & $\mathrm{R}$ \\
\hline 3277 & OFFSITE & SESPMNT & $\mathrm{R}$ \\
\hline 3278 & OFFSITE & SESPMNT & $\mathrm{R}$ \\
\hline 3279 & OFFSITE & SESPMNT & $\mathrm{R}$ \\
\hline 3280 & OFFSITE & SESPMNT & $\mathrm{R}$ \\
\hline 3281 & OFFSITE & SESPMNT & $\mathrm{R}$ \\
\hline 3282 & OFFSITE & SESPMNT & $\mathrm{R}$ \\
\hline 3283 & OFFSITE & SESPMNT & $\mathrm{R}$ \\
\hline 3284 & OFFSITE & SESPMNT & $\mathrm{R}$ \\
\hline 3285 & OFFSITE & SESPMNT & $\mathrm{R}$ \\
\hline 3286 & OFFSITE & SESPMNT & $\mathrm{R}$ \\
\hline 3287 & OFFSITE & SESPMNT & $\mathrm{R}$ \\
\hline 3288 & ONSITE & SESPMNT & $\mathrm{R}$ \\
\hline 3289 & ONSITE & SESPMNT & $\mathrm{R}$ \\
\hline 3290 & ONSITE & SESPMNT & $\mathrm{R}$ \\
\hline 3291 & ONSITE & SESPMNT & $\mathrm{R}$ \\
\hline 3292 & ONSITE & SESPMNT & $\mathrm{R}$ \\
\hline 3293 & ONSITE & SESPMNT & $\mathrm{R}$ \\
\hline 3294 & ONSITE & SESPMNT & $\mathrm{R}$ \\
\hline 3295 & ONSITE & SESPMNT & $\mathrm{R}$ \\
\hline 3296 & ONSITE & SESPMNT & $\mathrm{R}$ \\
\hline 3297 & ONSITE & SESPMNT & $\mathrm{R}$ \\
\hline 3298 & ONSITE & SESPMNT & $\mathrm{R}$ \\
\hline 3299 & ONSITE & SESPMNT & $\mathrm{R}$ \\
\hline 3300 & ONSITE & SESPMNT & $\mathrm{R}$ \\
\hline 3301 & ONSITE & SESPMNT & $\mathrm{R}$ \\
\hline 3302 & ONSITE & SESPMNT & $\mathrm{R}$ \\
\hline 3303 & ONSITE & SESPMNT & $\mathrm{R}$ \\
\hline 3304 & ONSITE & SESPMNT & $\mathrm{R}$ \\
\hline 3305 & ONSITE & SESPMNT & $\mathrm{R}$ \\
\hline 3306 & ONSITE & SESPMNT & $\mathrm{R}$ \\
\hline 3307 & ONSITE & SESPMNT & $\mathrm{R}$ \\
\hline & & & \\
\hline
\end{tabular}




\begin{tabular}{|c|l|l|l|}
\hline & AG & \multicolumn{1}{|c|}{ AH } & \\
\hline 1 & DIST_CLASS & OWNER_ID & COLLECTION_PURPOSE \\
\hline 3308 & ONSITE & SESPMNT & $\mathrm{R}$ \\
\hline 3309 & ONSITE & SESPMNT & $\mathrm{R}$ \\
\hline 3310 & ONSITE & SESPMNT & $\mathrm{R}$ \\
\hline 3311 & ONSITE & SESPMNT & $\mathrm{R}$ \\
\hline 3312 & ONSITE & SESPMNT & $\mathrm{R}$ \\
\hline 3313 & ONSITE & SESPMNT & $\mathrm{R}$ \\
\hline 3314 & ONSITE & SESPMNT & $\mathrm{R}$ \\
\hline 3315 & ONSITE & SESPMNT & $\mathrm{R}$ \\
\hline 3316 & ONSITE & SESPMNT & $\mathrm{R}$ \\
\hline 3317 & ONSITE & SESPMNT & $\mathrm{R}$ \\
\hline 3318 & ONSITE & SESPMNT & $\mathrm{R}$ \\
\hline 3319 & ONSITE & SESPMNT & $\mathrm{R}$ \\
\hline 3320 & ONSITE & SESPMNT & $\mathrm{R}$ \\
\hline 3321 & ONSITE & SESPMNT & $\mathrm{R}$ \\
\hline 3322 & ONSITE & SESPMNT & $\mathrm{R}$ \\
\hline 3323 & ONSITE & SESPMNT & $\mathrm{R}$ \\
\hline 3324 & ONSITE & SESPMNT & $\mathrm{R}$ \\
\hline 3325 & ONSITE & SESPMNT & $\mathrm{R}$ \\
\hline 3326 & ONSITE & SESPMNT & $\mathrm{R}$ \\
\hline 3327 & ONSITE & SESPMNT & $\mathrm{R}$ \\
\hline 3328 & ONSITE & SESPMNT & $\mathrm{R}$ \\
\hline 3329 & ONSITE & SESPMNT & $\mathrm{R}$ \\
\hline 3330 & ONSITE & SESPMNT & $\mathrm{R}$ \\
\hline 3331 & ONSITE & SESPMNT & $\mathrm{R}$ \\
\hline 3332 & ONSITE & SESPMNT & $\mathrm{R}$ \\
\hline 3333 & ONSITE & SESPMNT & $\mathrm{R}$ \\
\hline 3334 & ONSITE & SESPMNT & $\mathrm{R}$ \\
\hline 3335 & ONSITE & SESPMNT & $\mathrm{R}$ \\
\hline 3336 & ONSITE & SESPMNT & $\mathrm{R}$ \\
\hline 3337 & ONSITE & SESPMNT & $\mathrm{R}$ \\
\hline 3338 & ONSITE & SESPMNT & $\mathrm{R}$ \\
\hline 3339 & ONSITE & SESPMNT & $\mathrm{R}$ \\
\hline 3340 & ONSITE & SESPMNT & $\mathrm{R}$ \\
\hline 3341 & ONSITE & SESPMNT & $\mathrm{R}$ \\
\hline 3342 & ONSITE & SESPMNT & $\mathrm{R}$ \\
\hline 3343 & ONSITE & SESPMNT & $\mathrm{R}$ \\
\hline 3344 & ONSITE & SESPMNT & $\mathrm{R}$ \\
\hline 3345 & ONSITE & SESPMNT & $\mathrm{R}$ \\
\hline & & & \\
\hline
\end{tabular}




\begin{tabular}{|c|l|l|l|}
\hline & AG & \multicolumn{1}{|c|}{ AH } & \\
\hline 1 & DIST_CLASS & OWNER_ID & COLLECTION_PURPOSE \\
\hline 3346 & ONSITE & SESPMNT & $\mathrm{R}$ \\
\hline 3347 & ONSITE & SESPMNT & $\mathrm{R}$ \\
\hline 3348 & ONSITE & SESPMNT & $\mathrm{R}$ \\
\hline 3349 & ONSITE & SESPMNT & $\mathrm{R}$ \\
\hline 3350 & ONSITE & SESPMNT & $\mathrm{R}$ \\
\hline 3351 & ONSITE & SESPMNT & $\mathrm{R}$ \\
\hline 3352 & ONSITE & SESPMNT & $\mathrm{R}$ \\
\hline 3353 & ONSITE & SESPMNT & $\mathrm{R}$ \\
\hline 3354 & ONSITE & SESPMNT & $\mathrm{R}$ \\
\hline 3355 & ONSITE & SESPMNT & $\mathrm{R}$ \\
\hline 3356 & ONSITE & SESPMNT & $\mathrm{R}$ \\
\hline 3357 & ONSITE & SESPMNT & $\mathrm{R}$ \\
\hline 3358 & ONSITE & SESPMNT & $\mathrm{R}$ \\
\hline 3359 & ONSITE & SESPMNT & $\mathrm{R}$ \\
\hline 3360 & ONSITE & SESPMNT & $\mathrm{R}$ \\
\hline 3361 & ONSITE & SESPMNT & $\mathrm{R}$ \\
\hline 3362 & ONSITE & SESPMNT & $\mathrm{R}$ \\
\hline 3363 & ONSITE & SESPMNT & $\mathrm{R}$ \\
\hline 3364 & ONSITE & SESPMNT & $\mathrm{R}$ \\
\hline 3365 & ONSITE & SESPMNT & $\mathrm{R}$ \\
\hline 3366 & ONSITE & SESPMNT & $\mathrm{R}$ \\
\hline 3367 & ONSITE & SESPMNT & $\mathrm{R}$ \\
\hline 3368 & ONSITE & SESPMNT & $\mathrm{R}$ \\
\hline 3369 & ONSITE & SESPMNT & $\mathrm{R}$ \\
\hline 3370 & ONSITE & SESPMNT & $\mathrm{R}$ \\
\hline 3371 & ONSITE & SESPMNT & $\mathrm{R}$ \\
\hline 3372 & ONSITE & SESPMNT & $\mathrm{R}$ \\
\hline 3373 & ONSITE & SESPMNT & $\mathrm{R}$ \\
\hline 3374 & ONSITE & SESPMNT & $\mathrm{R}$ \\
\hline 3375 & ONSITE & SESPMNT & $\mathrm{R}$ \\
\hline 3376 & ONSITE & SESPMNT & $\mathrm{R}$ \\
\hline 3377 & ONSITE & SESPMNT & $\mathrm{R}$ \\
\hline 3378 & ONSITE & SESPMNT & $\mathrm{R}$ \\
\hline 3379 & ONSITE & SESPMNT & $\mathrm{R}$ \\
\hline 3380 & ONSITE & SESPMNT & $\mathrm{R}$ \\
\hline 3381 & ONSITE & SESPMNT & $\mathrm{R}$ \\
\hline 3382 & ONSITE & SESPMNT & $\mathrm{R}$ \\
\hline 3383 & ONSITE & SESPMNT & $\mathrm{R}$ \\
\hline & & & \\
\hline
\end{tabular}




\begin{tabular}{|c|l|l|l|}
\hline & \multicolumn{1}{|c|}{ AG } & \multicolumn{1}{|c|}{ AH } & \\
\hline 1 & DIST_CLASS & OWNER_ID & COLLECTION_PURPOSE \\
\hline 3384 & ONSITE & SESPMNT & $\mathrm{R}$ \\
\hline 3385 & ONSITE & SESPMNT & $\mathrm{R}$ \\
\hline 3386 & ONSITE & SESPMNT & $\mathrm{R}$ \\
\hline 3387 & ONSITE & SESPMNT & $\mathrm{R}$ \\
\hline 3388 & ONSITE & SESPMNT & $\mathrm{R}$ \\
\hline 3389 & ONSITE & SESPMNT & $\mathrm{R}$ \\
\hline 3390 & ONSITE & SESPMNT & $\mathrm{R}$ \\
\hline 3391 & ONSITE & SESPMNT & $\mathrm{R}$ \\
\hline 3392 & ONSITE & SESPMNT & $\mathrm{R}$ \\
\hline 3393 & ONSITE & SESPMNT & $\mathrm{R}$ \\
\hline 3394 & ONSITE & SESPMNT & $\mathrm{R}$ \\
\hline 3395 & ONSITE & SESPMNT & $\mathrm{R}$ \\
\hline 3396 & ONSITE & SESPMNT & $\mathrm{R}$ \\
\hline 3397 & ONSITE & SESPMNT & $\mathrm{R}$ \\
\hline 3398 & ONSITE & SESPMNT & $\mathrm{R}$ \\
\hline 3399 & ONSITE & SESPMNT & $\mathrm{R}$ \\
\hline 3400 & ONSITE & SESPMNT & $\mathrm{R}$ \\
\hline 3401 & ONSITE & SESPMNT & $\mathrm{R}$ \\
\hline 3402 & ONSITE & SESPMNT & $\mathrm{R}$ \\
\hline 3403 & ONSITE & SESPMNT & $\mathrm{R}$ \\
\hline 3404 & ONSITE & SESPMNT & $\mathrm{R}$ \\
\hline 3405 & ONSITE & SESPMNT & $\mathrm{R}$ \\
\hline 3406 & ONSITE & SESPMNT & $\mathrm{R}$ \\
\hline 3407 & ONSITE & SESPMNT & $\mathrm{R}$ \\
\hline 3408 & ONSITE & SESPMNT & $\mathrm{R}$ \\
\hline 3409 & ONSITE & SESPMNT & $\mathrm{R}$ \\
\hline 3410 & ONSITE & SESPMNT & $\mathrm{R}$ \\
\hline 3411 & ONSITE & SESPMNT & $\mathrm{R}$ \\
\hline 3412 & ONSITE & SESPMNT & $\mathrm{R}$ \\
\hline 3413 & ONSITE & SESPMNT & $\mathrm{R}$ \\
\hline 3414 & ONSITE & SESPMNT & $\mathrm{R}$ \\
\hline 3415 & ONSITE & SESPMNT & $\mathrm{R}$ \\
\hline 3416 & ONSITE & SESPMNT & $\mathrm{R}$ \\
\hline 3417 & ONSITE & SESPMNT & $\mathrm{R}$ \\
\hline 3418 & ONSITE & SESPMNT & $\mathrm{R}$ \\
\hline 3419 & ONSITE & SESPMNT & $\mathrm{R}$ \\
\hline 3420 & ONSITE & SESPMNT & $\mathrm{R}$ \\
\hline 3421 & ONSITE & SESPMNT & $\mathrm{R}$ \\
\hline & & & \\
\hline
\end{tabular}




\begin{tabular}{|c|l|l|l|}
\hline & AG & \multicolumn{1}{|c|}{ AH } & \\
\hline 1 & DIST_CLASS & OWNER_ID & COLLECTION_PURPOSE \\
\hline 3422 & ONSITE & SESPMNT & $\mathrm{R}$ \\
\hline 3423 & ONSITE & SESPMNT & $\mathrm{R}$ \\
\hline 3424 & ONSITE & SESPMNT & $\mathrm{R}$ \\
\hline 3425 & ONSITE & SESPMNT & $\mathrm{R}$ \\
\hline 3426 & ONSITE & SESPMNT & $\mathrm{R}$ \\
\hline 3427 & ONSITE & SESPMNT & $\mathrm{R}$ \\
\hline 3428 & ONSITE & SESPMNT & $\mathrm{R}$ \\
\hline 3429 & ONSITE & SESPMNT & $\mathrm{R}$ \\
\hline 3430 & ONSITE & SESPMNT & $\mathrm{R}$ \\
\hline 3431 & ONSITE & SESPMNT & $\mathrm{R}$ \\
\hline 3432 & ONSITE & SESPMNT & $\mathrm{R}$ \\
\hline 3433 & ONSITE & SESPMNT & $\mathrm{R}$ \\
\hline 3434 & ONSITE & SESPMNT & $\mathrm{R}$ \\
\hline 3435 & ONSITE & SESPMNT & $\mathrm{R}$ \\
\hline 3436 & ONSITE & SESPMNT & $\mathrm{R}$ \\
\hline 3437 & ONSITE & SESPMNT & $\mathrm{R}$ \\
\hline 3438 & OFFSITE & SESPMNT & $\mathrm{R}$ \\
\hline 3439 & OFFSITE & SESPMNT & $\mathrm{R}$ \\
\hline 3440 & OFFSITE & SESPMNT & $\mathrm{R}$ \\
\hline 3441 & OFFSITE & SESPMNT & $\mathrm{R}$ \\
\hline 3442 & OFFSITE & SESPMNT & $\mathrm{R}$ \\
\hline 3443 & OFFSITE & SESPMNT & $\mathrm{R}$ \\
\hline 3444 & OFFSITE & SESPMNT & $\mathrm{R}$ \\
\hline 3445 & OFFSITE & SESPMNT & $\mathrm{R}$ \\
\hline 3446 & OFFSITE & SESPMNT & $\mathrm{R}$ \\
\hline 3447 & OFFSITE & SESPMNT & $\mathrm{R}$ \\
\hline 3448 & OFFSITE & SESPMNT & $\mathrm{R}$ \\
\hline 3449 & OFFSITE & SESPMNT & $\mathrm{R}$ \\
\hline 3450 & OFFSITE & SESPMNT & $\mathrm{R}$ \\
\hline 3451 & OFFSITE & SESPMNT & $\mathrm{R}$ \\
\hline 3452 & OFFSITE & SESPMNT & $\mathrm{R}$ \\
\hline 3453 & OFFSITE & SESPMNT & $\mathrm{R}$ \\
\hline 3454 & OFFSITE & SESPMNT & $\mathrm{R}$ \\
\hline 3455 & OFFSITE & SESPMNT & $\mathrm{R}$ \\
\hline 3456 & OFFSITE & SESPMNT & $\mathrm{R}$ \\
\hline 3457 & OFFSITE & SESPMNT & $\mathrm{R}$ \\
\hline 3458 & OFFSITE & SESPMNT & $\mathrm{R}$ \\
\hline 3459 & OFFSITE & SESPMNT & $\mathrm{R}$ \\
\hline & & & \\
\hline
\end{tabular}




\begin{tabular}{|c|l|l|l|}
\hline & \multicolumn{1}{|c|}{ AG } & \multicolumn{1}{|c|}{ AH } & \\
\hline 1 & DIST_CLASS & OWNER_ID & COLLECTION_PURPOSE \\
\hline 3460 & OFFSITE & SESPMNT & $\mathrm{R}$ \\
\hline 3461 & OFFSITE & SESPMNT & $\mathrm{R}$ \\
\hline 3462 & OFFSITE & SESPMNT & $\mathrm{R}$ \\
\hline 3463 & OFFSITE & SESPMNT & $\mathrm{R}$ \\
\hline 3464 & OFFSITE & SESPMNT & $\mathrm{R}$ \\
\hline 3465 & OFFSITE & SESPMNT & $\mathrm{R}$ \\
\hline 3466 & OFFSITE & SESPMNT & $\mathrm{R}$ \\
\hline 3467 & OFFSITE & SESPMNT & $\mathrm{R}$ \\
\hline 3468 & OFFSITE & SESPMNT & $\mathrm{R}$ \\
\hline 3469 & OFFSITE & SESPMNT & $\mathrm{R}$ \\
\hline 3470 & OFFSITE & SESPMNT & $\mathrm{R}$ \\
\hline 3471 & OFFSITE & SESPMNT & $\mathrm{R}$ \\
\hline 3472 & OFFSITE & SESPMNT & $\mathrm{R}$ \\
\hline 3473 & OFFSITE & SESPMNT & $\mathrm{R}$ \\
\hline 3474 & OFFSITE & SESPMNT & $\mathrm{R}$ \\
\hline 3475 & OFFSITE & SESPMNT & $\mathrm{R}$ \\
\hline 3476 & OFFSITE & SESPMNT & $\mathrm{R}$ \\
\hline 3477 & OFFSITE & SESPMNT & $\mathrm{R}$ \\
\hline 3478 & OFFSITE & SESPMNT & $\mathrm{R}$ \\
\hline 3479 & OFFSITE & SESPMNT & $\mathrm{R}$ \\
\hline 3480 & OFFSITE & SESPMNT & $\mathrm{R}$ \\
\hline 3481 & OFFSITE & SESPMNT & $\mathrm{R}$ \\
\hline 3482 & OFFSITE & SESPMNT & $\mathrm{R}$ \\
\hline 3483 & OFFSITE & SESPMNT & $\mathrm{R}$ \\
\hline 3484 & OFFSITE & SESPMNT & $\mathrm{R}$ \\
\hline 3485 & OFFSITE & SESPMNT & $\mathrm{R}$ \\
\hline 3486 & OFFSITE & SESPMNT & $\mathrm{R}$ \\
\hline 3487 & OFFSITE & SESPMNT & $\mathrm{R}$ \\
\hline 3488 & ONSITE & SESPSPEC & $\mathrm{S}$ \\
\hline 3489 & ONSITE & SESPSPEC & $\mathrm{S}$ \\
\hline 3490 & ONSITE & SESPSPEC & $\mathrm{S}$ \\
\hline 3491 & ONSITE & SESPSPEC & $\mathrm{S}$ \\
\hline 3492 & ONSITE & SESPSPEC & $\mathrm{S}$ \\
\hline 3493 & ONSITE & SESPSPEC & $\mathrm{S}$ \\
\hline 3494 & ONSITE & SESPSPEC & $\mathrm{S}$ \\
\hline 3495 & ONSITE & SESPSPEC & $\mathrm{S}$ \\
\hline 3496 & ONSITE & SESPSPEC & $\mathrm{S}$ \\
\hline 3497 & ONSITE & SESPSPEC & $\mathrm{S}$ \\
\hline
\end{tabular}




\begin{tabular}{|c|l|l|l|}
\hline & AG & AH & \multicolumn{1}{|c|}{ AI } \\
\hline 1 & DIST_CLASS & OWNER_ID & COLLECTION_PURPOSE \\
\hline 3498 & ONSITE & SESPSPEC & S \\
\hline 3499 & ONSITE & SESPSPEC & S \\
\hline 3500 & ONSITE & SESPSPEC & S \\
\hline 3501 & ONSITE & SESPSPEC & S \\
\hline 3502 & ONSITE & SESPSPEC & S \\
\hline 3503 & ONSITE & SESPSPEC & S \\
\hline 3504 & ONSITE & SESPSPEC & S \\
\hline 3505 & ONSITE & SESPSPEC & S \\
\hline 3506 & ONSITE & SESPSPEC & S \\
\hline 3507 & ONSITE & SESPSPEC & S \\
\hline 3508 & ONSITE & SESPSPEC & $\mathrm{S}$ \\
\hline 3509 & ONSITE & SESPSPEC & $\mathrm{S}$ \\
\hline 3510 & ONSITE & SESPSPEC & $\mathrm{S}$ \\
\hline 3511 & ONSITE & SESPSPEC & $\mathrm{S}$ \\
\hline 3512 & ONSITE & SESPSPEC & $\mathrm{S}$ \\
\hline 3513 & ONSITE & SESPSPEC & $\mathrm{S}$ \\
\hline 3514 & ONSITE & SESPSPEC & $\mathrm{S}$ \\
\hline 3515 & ONSITE & SESPSPEC & $\mathrm{S}$ \\
\hline 3516 & ONSITE & SESPSPEC & $\mathrm{S}$ \\
\hline 3517 & ONSITE & SESPSPEC & $\mathrm{S}$ \\
\hline 3518 & ONSITE & SESPSPEC & $\mathrm{S}$ \\
\hline 3519 & ONSITE & SESPSPEC & $\mathrm{S}$ \\
\hline 3520 & ONSITE & SESPSPEC & $\mathrm{S}$ \\
\hline 3521 & ONSITE & SESPSPEC & $\mathrm{S}$ \\
\hline 3522 & ONSITE & SESPSPEC & $\mathrm{S}$ \\
\hline 3523 & ONSITE & SESPSPEC & $\mathrm{S}$ \\
\hline 3524 & OFFSITE & SESPMNT & $\mathrm{R}$ \\
\hline 3525 & OFFSITE & SESPMNT & $\mathrm{R}$ \\
\hline 3526 & OFFSITE & SESPMNT & $\mathrm{R}$ \\
\hline 3527 & OFFSITE & SESPMNT & $\mathrm{R}$ \\
\hline 3528 & OFFSITE & SESPMNT & $\mathrm{R}$ \\
\hline 3529 & OFFSITE & SESPMNT & $\mathrm{R}$ \\
\hline 3530 & OFFSITE & SESPMNT & $\mathrm{R}$ \\
\hline 3531 & OFFSITE & SESPMNT & $\mathrm{R}$ \\
\hline 3532 & OFFSITE & SESPMNT & $\mathrm{R}$ \\
\hline 3533 & OFFSITE & SESPMNT & $\mathrm{R}$ \\
\hline 3534 & OFFSITE & SESPMNT & $\mathrm{R}$ \\
\hline 3535 & OFFSITE & SESPMNT & $\mathrm{R}$ \\
\hline & & & \\
\hline
\end{tabular}




\begin{tabular}{|c|l|l|l|}
\hline & AG & \multicolumn{1}{|c|}{ AH } & \\
\hline 1 & DIST_CLASS & OWNER_ID & COLLECTION_PURPOSE \\
\hline 3536 & OFFSITE & SESPMNT & $\mathrm{R}$ \\
\hline 3537 & OFFSITE & SESPMNT & $\mathrm{R}$ \\
\hline 3538 & OFFSITE & SESPMNT & $\mathrm{R}$ \\
\hline 3539 & OFFSITE & SESPMNT & $\mathrm{R}$ \\
\hline 3540 & OFFSITE & SESPMNT & $\mathrm{R}$ \\
\hline 3541 & OFFSITE & SESPMNT & $\mathrm{R}$ \\
\hline 3542 & OFFSITE & SESPMNT & $\mathrm{R}$ \\
\hline 3543 & OFFSITE & SESPMNT & $\mathrm{R}$ \\
\hline 3544 & OFFSITE & SESPMNT & $\mathrm{R}$ \\
\hline 3545 & OFFSITE & SESPMNT & $\mathrm{R}$ \\
\hline 3546 & OFFSITE & SESPMNT & $\mathrm{R}$ \\
\hline 3547 & OFFSITE & SESPMNT & $\mathrm{R}$ \\
\hline 3548 & OFFSITE & SESPMNT & $\mathrm{R}$ \\
\hline 3549 & OFFSITE & SESPMNT & $\mathrm{R}$ \\
\hline 3550 & OFFSITE & SESPMNT & $\mathrm{R}$ \\
\hline 3551 & OFFSITE & SESPMNT & $\mathrm{R}$ \\
\hline 3552 & OFFSITE & SESPMNT & $\mathrm{R}$ \\
\hline 3553 & OFFSITE & SESPMNT & $\mathrm{R}$ \\
\hline 3554 & OFFSITE & SESPMNT & $\mathrm{R}$ \\
\hline 3555 & OFFSITE & SESPMNT & $\mathrm{R}$ \\
\hline 3556 & OFFSITE & SESPMNT & $\mathrm{R}$ \\
\hline 3557 & OFFSITE & SESPMNT & $\mathrm{R}$ \\
\hline 3558 & OFFSITE & SESPMNT & $\mathrm{R}$ \\
\hline 3559 & OFFSITE & SESPMNT & $\mathrm{R}$ \\
\hline 3560 & OFFSITE & SESPMNT & $\mathrm{R}$ \\
\hline 3561 & OFFSITE & SESPMNT & $\mathrm{R}$ \\
\hline 3562 & OFFSITE & SESPMNT & $\mathrm{R}$ \\
\hline 3563 & OFFSITE & SESPMNT & $\mathrm{R}$ \\
\hline 3564 & OFFSITE & SESPMNT & $\mathrm{R}$ \\
\hline 3565 & OFFSITE & SESPMNT & $\mathrm{R}$ \\
\hline 3566 & OFFSITE & SESPMNT & $\mathrm{R}$ \\
\hline 3567 & OFFSITE & SESPMNT & $\mathrm{R}$ \\
\hline 3568 & OFFSITE & SESPMNT & $\mathrm{R}$ \\
\hline 3569 & OFFSITE & SESPMNT & $\mathrm{R}$ \\
\hline 3570 & OFFSITE & SESPMNT & $\mathrm{R}$ \\
\hline 3571 & OFFSITE & SESPMNT & $\mathrm{R}$ \\
\hline 3572 & OFFSITE & SESPMNT & $\mathrm{R}$ \\
\hline 3573 & OFFSITE & SESPMNT & $\mathrm{R}$ \\
\hline & & & \\
\hline
\end{tabular}




\begin{tabular}{|c|l|l|l|}
\hline & AG & \multicolumn{1}{|c|}{ AH } & \\
\hline 1 & DIST_CLASS & OWNER_ID & COLLECTION_PURPOSE \\
\hline 3574 & OFFSITE & SESPMNT & $\mathrm{R}$ \\
\hline 3575 & OFFSITE & SESPMNT & $\mathrm{R}$ \\
\hline 3576 & OFFSITE & SESPMNT & $\mathrm{R}$ \\
\hline 3577 & OFFSITE & SESPMNT & $\mathrm{R}$ \\
\hline 3578 & OFFSITE & SESPMNT & $\mathrm{R}$ \\
\hline 3579 & OFFSITE & SESPMNT & $\mathrm{R}$ \\
\hline 3580 & OFFSITE & SESPMNT & $\mathrm{R}$ \\
\hline 3581 & OFFSITE & SESPMNT & $\mathrm{R}$ \\
\hline 3582 & OFFSITE & SESPMNT & $\mathrm{R}$ \\
\hline 3583 & OFFSITE & SESPMNT & $\mathrm{R}$ \\
\hline 3584 & OFFSITE & SESPMNT & $\mathrm{R}$ \\
\hline 3585 & OFFSITE & SESPMNT & $\mathrm{R}$ \\
\hline 3586 & OFFSITE & SESPMNT & $\mathrm{R}$ \\
\hline 3587 & OFFSITE & SESPMNT & $\mathrm{R}$ \\
\hline 3588 & OFFSITE & SESPMNT & $\mathrm{R}$ \\
\hline 3589 & OFFSITE & SESPSPEC & $\mathrm{S}$ \\
\hline 3590 & OFFSITE & SESPMNT & $\mathrm{R}$ \\
\hline 3591 & OFFSITE & SESPSPEC & $\mathrm{S}$ \\
\hline 3592 & OFFSITE & SESPMNT & $\mathrm{R}$ \\
\hline 3593 & OFFSITE & SESPSPEC & $\mathrm{S}$ \\
\hline 3594 & OFFSITE & SESPSPEC & $\mathrm{S}$ \\
\hline 3595 & OFFSITE & SESPSPEC & $\mathrm{S}$ \\
\hline 3596 & OFFSITE & SESPMNT & $\mathrm{R}$ \\
\hline 3597 & OFFSITE & SESPMNT & $\mathrm{R}$ \\
\hline 3598 & OFFSITE & SESPMNT & $\mathrm{R}$ \\
\hline 3599 & OFFSITE & SESPMNT & $\mathrm{R}$ \\
\hline 3600 & OFFSITE & SESPMNT & $\mathrm{R}$ \\
\hline 3601 & OFFSITE & SESPMNT & $\mathrm{R}$ \\
\hline 3602 & OFFSITE & SESPMNT & $\mathrm{R}$ \\
\hline 3603 & OFFSITE & SESPMNT & $\mathrm{R}$ \\
\hline 3604 & OFFSITE & SESPMNT & $\mathrm{R}$ \\
\hline 3605 & OFFSITE & SESPMNT & $\mathrm{R}$ \\
\hline 3606 & OFFSITE & SESPMNT & $\mathrm{R}$ \\
\hline 3607 & OFFSITE & SESPMNT & $\mathrm{R}$ \\
\hline 3608 & OFFSITE & SESPMNT & $\mathrm{R}$ \\
\hline 3609 & OFFSITE & SESPMNT & $\mathrm{R}$ \\
\hline 3610 & OFFSITE & SESPMNT & $\mathrm{R}$ \\
\hline 3611 & OFFSITE & SESPMNT & $\mathrm{R}$ \\
\hline & & & \\
\hline
\end{tabular}




\begin{tabular}{|c|l|l|l|}
\hline & AG & \multicolumn{1}{|c|}{ AH } & \\
\hline 1 & DIST_CLASS & OWNER_ID & COLLECTION_PURPOSE \\
\hline 3612 & OFFSITE & SESPMNT & $\mathrm{R}$ \\
\hline 3613 & OFFSITE & SESPMNT & $\mathrm{R}$ \\
\hline 3614 & OFFSITE & SESPMNT & $\mathrm{R}$ \\
\hline 3615 & OFFSITE & SESPMNT & $\mathrm{R}$ \\
\hline 3616 & OFFSITE & SESPMNT & $\mathrm{R}$ \\
\hline 3617 & OFFSITE & SESPMNT & $\mathrm{R}$ \\
\hline 3618 & OFFSITE & SESPMNT & $\mathrm{R}$ \\
\hline 3619 & OFFSITE & SESPSPEC & $\mathrm{S}$ \\
\hline 3620 & OFFSITE & SESPMNT & $\mathrm{R}$ \\
\hline 3621 & OFFSITE & SESPSPEC & $\mathrm{S}$ \\
\hline 3622 & OFFSITE & SESPMNT & $\mathrm{R}$ \\
\hline 3623 & OFFSITE & SESPSPEC & $\mathrm{S}$ \\
\hline 3624 & OFFSITE & SESPSPEC & $\mathrm{S}$ \\
\hline 3625 & OFFSITE & SESPSPEC & $\mathrm{S}$ \\
\hline 3626 & OFFSITE & SESPMNT & $\mathrm{R}$ \\
\hline 3627 & OFFSITE & SESPMNT & $\mathrm{R}$ \\
\hline 3628 & OFFSITE & SESPMNT & $\mathrm{R}$ \\
\hline 3629 & OFFSITE & SESPMNT & $\mathrm{R}$ \\
\hline 3630 & OFFSITE & SESPMNT & $\mathrm{R}$ \\
\hline 3631 & OFFSITE & SESPMNT & $\mathrm{R}$ \\
\hline 3632 & OFFSITE & SESPMNT & $\mathrm{R}$ \\
\hline 3633 & OFFSITE & SESPMNT & $\mathrm{R}$ \\
\hline 3634 & OFFSITE & SESPMNT & $\mathrm{R}$ \\
\hline 3635 & OFFSITE & SESPMNT & $\mathrm{R}$ \\
\hline 3636 & OFFSITE & SESPMNT & $\mathrm{R}$ \\
\hline 3637 & OFFSITE & SESPMNT & $\mathrm{R}$ \\
\hline 3638 & OFFSITE & SESPMNT & $\mathrm{R}$ \\
\hline 3639 & OFFSITE & SESPMNT & $\mathrm{R}$ \\
\hline 3640 & OFFSITE & SESPMNT & $\mathrm{R}$ \\
\hline 3641 & OFFSITE & SESPMNT & $\mathrm{R}$ \\
\hline 3642 & OFFSITE & SESPMNT & $\mathrm{R}$ \\
\hline 3643 & OFFSITE & SESPMNT & $\mathrm{R}$ \\
\hline 3644 & OFFSITE & SESPMNT & $\mathrm{R}$ \\
\hline 3645 & OFFSITE & SESPMNT & $\mathrm{R}$ \\
\hline 3646 & OFFSITE & SESPMNT & $\mathrm{R}$ \\
\hline 3647 & OFFSITE & SESPMNT & $\mathrm{R}$ \\
\hline 3648 & OFFSITE & SESPMNT & $\mathrm{R}$ \\
\hline 3649 & OFFSITE & SESPMNT & $\mathrm{R}$ \\
\hline & & & \\
\hline
\end{tabular}




\begin{tabular}{|c|l|l|l|}
\hline & AG & \multicolumn{1}{|c|}{ AH } & \multicolumn{1}{|c|}{ AI } \\
\hline 1 & DIST_CLASS & OWNER_ID & COLLECTION_PURPOSE \\
\hline 3650 & OFFSITE & SESPMNT & $\mathrm{R}$ \\
\hline 3651 & OFFSITE & SESPMNT & $\mathrm{R}$ \\
\hline 3652 & OFFSITE & SESPMNT & $\mathrm{R}$ \\
\hline 3653 & OFFSITE & SESPMNT & $\mathrm{R}$ \\
\hline 3654 & OFFSITE & SESPMNT & $\mathrm{R}$ \\
\hline 3655 & OFFSITE & SESPMNT & $\mathrm{R}$ \\
\hline 3656 & OFFSITE & SESPMNT & $\mathrm{R}$ \\
\hline 3657 & OFFSITE & SESPMNT & $\mathrm{R}$ \\
\hline 3658 & OFFSITE & SESPMNT & $\mathrm{R}$ \\
\hline 3659 & OFFSITE & SESPMNT & $\mathrm{R}$ \\
\hline 3660 & OFFSITE & SESPMNT & $\mathrm{R}$ \\
\hline 3661 & OFFSITE & SESPMNT & $\mathrm{R}$ \\
\hline 3662 & OFFSITE & SESPMNT & $\mathrm{R}$ \\
\hline 3663 & OFFSITE & SESPMNT & $\mathrm{R}$ \\
\hline 3664 & OFFSITE & SESPMNT & $\mathrm{R}$ \\
\hline 3665 & OFFSITE & SESPMNT & $\mathrm{R}$ \\
\hline 3666 & OFFSITE & SESPMNT & $\mathrm{R}$ \\
\hline 3667 & OFFSITE & SESPMNT & $\mathrm{R}$ \\
\hline 3668 & OFFSITE & SESPMNT & $\mathrm{R}$ \\
\hline 3669 & OFFSITE & SESPMNT & $\mathrm{R}$ \\
\hline 3670 & OFFSITE & SESPMNT & $\mathrm{R}$ \\
\hline 3671 & OFFSITE & SESPMNT & $\mathrm{R}$ \\
\hline 3672 & OFFSITE & SESPMNT & $\mathrm{R}$ \\
\hline 3673 & OFFSITE & SESPMNT & $\mathrm{R}$ \\
\hline 3674 & OFFSITE & SESPMNT & $\mathrm{R}$ \\
\hline 3675 & OFFSITE & SESPMNT & $\mathrm{R}$ \\
\hline 3676 & OFFSITE & SESPMNT & $\mathrm{R}$ \\
\hline 3677 & OFFSITE & SESPMNT & $\mathrm{R}$ \\
\hline 3678 & OFFSITE & SESPMNT & $\mathrm{R}$ \\
\hline 3679 & OFFSITE & SESPMNT & $\mathrm{R}$ \\
\hline 3680 & OFFSITE & SESPMNT & $\mathrm{R}$ \\
\hline 3681 & OFFSITE & SESPMNT & $\mathrm{R}$ \\
\hline 3682 & OFFSITE & SESPMNT & $\mathrm{R}$ \\
\hline 3683 & OFFSITE & SESPMNT & $\mathrm{R}$ \\
\hline 3684 & OFFSITE & SESPMNT & $\mathrm{R}$ \\
\hline 3685 & OFFSITE & SESPMNT & $\mathrm{R}$ \\
\hline 3686 & OFFSITE & SESPMNT & $\mathrm{R}$ \\
\hline 3687 & OFFSITE & SESPMNT & $\mathrm{R}$ \\
\hline & & & \\
\hline
\end{tabular}




\begin{tabular}{|c|l|l|l|}
\hline & AG & \multicolumn{1}{|c|}{ AH } & \multicolumn{1}{|c|}{ AI } \\
\hline 1 & DIST_CLASS & OWNER_ID & COLLECTION_PURPOSE \\
\hline 3688 & OFFSITE & SESPMNT & $\mathrm{R}$ \\
\hline 3689 & OFFSITE & SESPMNT & $\mathrm{R}$ \\
\hline 3690 & OFFSITE & SESPMNT & $\mathrm{R}$ \\
\hline 3691 & OFFSITE & SESPMNT & $\mathrm{R}$ \\
\hline 3692 & OFFSITE & SESPMNT & $\mathrm{R}$ \\
\hline 3693 & OFFSITE & SESPMNT & $\mathrm{R}$ \\
\hline 3694 & OFFSITE & SESPMNT & $\mathrm{R}$ \\
\hline 3695 & OFFSITE & SESPMNT & $\mathrm{R}$ \\
\hline 3696 & OFFSITE & SESPMNT & $\mathrm{R}$ \\
\hline 3697 & OFFSITE & SESPMNT & $\mathrm{R}$ \\
\hline 3698 & OFFSITE & SESPMNT & $\mathrm{R}$ \\
\hline 3699 & OFFSITE & SESPMNT & $\mathrm{R}$ \\
\hline 3700 & OFFSITE & SESPMNT & $\mathrm{R}$ \\
\hline 3701 & OFFSITE & SESPMNT & $\mathrm{R}$ \\
\hline 3702 & OFFSITE & SESPMNT & $\mathrm{R}$ \\
\hline 3703 & OFFSITE & SESPMNT & $\mathrm{R}$ \\
\hline 3704 & OFFSITE & SESPMNT & $\mathrm{R}$ \\
\hline 3705 & OFFSITE & SESPMNT & $\mathrm{R}$ \\
\hline 3706 & OFFSITE & SESPMNT & $\mathrm{R}$ \\
\hline 3707 & OFFSITE & SESPMNT & $\mathrm{R}$ \\
\hline 3708 & OFFSITE & SESPMNT & $\mathrm{R}$ \\
\hline 3709 & OFFSITE & SESPMNT & $\mathrm{R}$ \\
\hline 3710 & OFFSITE & SESPMNT & $\mathrm{R}$ \\
\hline 3711 & OFFSITE & SESPMNT & $\mathrm{R}$ \\
\hline 3712 & OFFSITE & SESPMNT & $\mathrm{R}$ \\
\hline 3713 & OFFSITE & SESPMNT & $\mathrm{R}$ \\
\hline 3714 & OFFSITE & SESPMNT & $\mathrm{R}$ \\
\hline 3715 & OFFSITE & SESPMNT & $\mathrm{R}$ \\
\hline 3716 & OFFSITE & SESPMNT & $\mathrm{R}$ \\
\hline 3717 & OFFSITE & SESPMNT & $\mathrm{R}$ \\
\hline 3718 & OFFSITE & SESPMNT & $\mathrm{R}$ \\
\hline 3719 & OFFSITE & SESPMNT & $\mathrm{R}$ \\
\hline 3720 & OFFSITE & SESPMNT & $\mathrm{R}$ \\
\hline 3721 & OFFSITE & SESPMNT & $\mathrm{R}$ \\
\hline 3722 & OFFSITE & SESPMNT & $\mathrm{R}$ \\
\hline 3723 & OFFSITE & SESPMNT & $\mathrm{R}$ \\
\hline 3724 & OFFSITE & SESPMNT & $\mathrm{R}$ \\
\hline 3725 & OFFSITE & SESPMNT & $\mathrm{R}$ \\
\hline & & & \\
\hline
\end{tabular}




\begin{tabular}{|c|l|l|l|}
\hline & AG & AH & \\
\hline 1 & DIST_CLASS & OWNER_ID & COLLECTION_PURPOSE \\
\hline 3726 & OFFSITE & SESPMNT & $\mathrm{R}$ \\
\hline 3727 & OFFSITE & SESPMNT & $\mathrm{R}$ \\
\hline 3728 & OFFSITE & SESPMNT & $\mathrm{R}$ \\
\hline 3729 & OFFSITE & SESPMNT & $\mathrm{R}$ \\
\hline 3730 & OFFSITE & SESPMNT & $\mathrm{R}$ \\
\hline 3731 & OFFSITE & SESPMNT & $\mathrm{R}$ \\
\hline 3732 & OFFSITE & SESPMNT & $\mathrm{R}$ \\
\hline 3733 & OFFSITE & SESPMNT & $\mathrm{R}$ \\
\hline 3734 & OFFSITE & SESPMNT & $\mathrm{R}$ \\
\hline 3735 & OFFSITE & SESPMNT & $\mathrm{R}$ \\
\hline 3736 & OFFSITE & SESPMNT & $\mathrm{R}$ \\
\hline 3737 & OFFSITE & SESPMNT & $\mathrm{R}$ \\
\hline 3738 & OFFSITE & SESPMNT & $\mathrm{R}$ \\
\hline 3739 & OFFSITE & SESPMNT & $\mathrm{R}$ \\
\hline 3740 & OFFSITE & SESPMNT & $\mathrm{R}$ \\
\hline 3741 & OFFSITE & SESPMNT & $\mathrm{R}$ \\
\hline 3742 & OFFSITE & SESPMNT & $\mathrm{R}$ \\
\hline 3743 & OFFSITE & SESPMNT & $\mathrm{R}$ \\
\hline 3744 & ONSITE & SESPMNT & $\mathrm{R}$ \\
\hline 3745 & ONSITE & SESPMNT & $\mathrm{R}$ \\
\hline 3746 & ONSITE & SESPMNT & $\mathrm{R}$ \\
\hline 3747 & ONSITE & SESPMNT & $\mathrm{R}$ \\
\hline 3748 & ONSITE & SESPMNT & $\mathrm{R}$ \\
\hline 3749 & ONSITE & SESPMNT & $\mathrm{R}$ \\
\hline 3750 & ONSITE & SESPMNT & $\mathrm{R}$ \\
\hline 3751 & ONSITE & SESPMNT & $\mathrm{R}$ \\
\hline 3752 & ONSITE & SESPMNT & $\mathrm{R}$ \\
\hline 3753 & ONSITE & SESPMNT & $\mathrm{R}$ \\
\hline 3754 & ONSITE & SESPMNT & $\mathrm{R}$ \\
\hline 3755 & ONSITE & SESPMNT & $\mathrm{R}$ \\
\hline 3756 & ONSITE & SESPMNT & $\mathrm{R}$ \\
\hline 3757 & ONSITE & SESPMNT & $\mathrm{R}$ \\
\hline 3758 & ONSITE & SESPMNT & $\mathrm{R}$ \\
\hline 3759 & ONSITE & SESPMNT & $\mathrm{R}$ \\
\hline 3760 & ONSITE & SESPMNT & $\mathrm{R}$ \\
\hline 3761 & ONSITE & SESPMNT & $\mathrm{R}$ \\
\hline 3762 & ONSITE & SESPMNT & $\mathrm{R}$ \\
\hline 3763 & ONSITE & SESPMNT & $\mathrm{R}$ \\
\hline & & & \\
\hline
\end{tabular}




\begin{tabular}{|c|l|l|l|}
\hline & AG & AH & \\
\hline 1 & DIST_CLASS & OWNER_ID & COLLECTION_PURPOSE \\
\hline 3764 & ONSITE & SESPMNT & $\mathrm{R}$ \\
\hline 3765 & ONSITE & SESPMNT & $\mathrm{R}$ \\
\hline 3766 & ONSITE & SESPMNT & $\mathrm{R}$ \\
\hline 3767 & ONSITE & SESPMNT & $\mathrm{R}$ \\
\hline 3768 & ONSITE & SESPMNT & $\mathrm{R}$ \\
\hline 3769 & ONSITE & SESPMNT & $\mathrm{R}$ \\
\hline 3770 & ONSITE & SESPMNT & $\mathrm{R}$ \\
\hline 3771 & ONSITE & SESPMNT & $\mathrm{R}$ \\
\hline 3772 & ONSITE & SESPMNT & $\mathrm{R}$ \\
\hline 3773 & ONSITE & SESPMNT & $\mathrm{R}$ \\
\hline 3774 & ONSITE & SESPMNT & $\mathrm{R}$ \\
\hline 3775 & ONSITE & SESPMNT & $\mathrm{R}$ \\
\hline 3776 & ONSITE & SESPMNT & $\mathrm{R}$ \\
\hline 3777 & ONSITE & SESPMNT & $\mathrm{R}$ \\
\hline 3778 & ONSITE & SESPMNT & $\mathrm{R}$ \\
\hline 3779 & ONSITE & SESPMNT & $\mathrm{R}$ \\
\hline 3780 & ONSITE & SESPMNT & $\mathrm{R}$ \\
\hline 3781 & ONSITE & SESPMNT & $\mathrm{R}$ \\
\hline 3782 & ONSITE & SESPMNT & $\mathrm{R}$ \\
\hline 3783 & ONSITE & SESPMNT & $\mathrm{R}$ \\
\hline 3784 & ONSITE & SESPMNT & $\mathrm{R}$ \\
\hline 3785 & ONSITE & SESPMNT & $\mathrm{R}$ \\
\hline 3786 & ONSITE & SESPMNT & $\mathrm{R}$ \\
\hline 3787 & ONSITE & SESPMNT & $\mathrm{R}$ \\
\hline 3788 & ONSITE & SESPMNT & $\mathrm{R}$ \\
\hline 3789 & ONSITE & SESPMNT & $\mathrm{R}$ \\
\hline 3790 & ONSITE & SESPMNT & $\mathrm{R}$ \\
\hline 3791 & ONSITE & SESPMNT & $\mathrm{R}$ \\
\hline 3792 & ONSITE & SESPMNT & $\mathrm{R}$ \\
\hline 3793 & ONSITE & SESPMNT & $\mathrm{R}$ \\
\hline 3794 & OFFSITE & SESPMNT & $\mathrm{R}$ \\
\hline 3795 & OFFSITE & SESPMNT & $\mathrm{R}$ \\
\hline 3796 & OFFSITE & SESPMNT & $\mathrm{R}$ \\
\hline 3797 & OFFSITE & SESPMNT & $\mathrm{R}$ \\
\hline 3798 & OFFSITE & SESPMNT & $\mathrm{R}$ \\
\hline 3799 & OFFSITE & SESPMNT & $\mathrm{R}$ \\
\hline 3800 & OFFSITE & SESPMNT & $\mathrm{R}$ \\
\hline 3801 & OFFSITE & SESPMNT & $\mathrm{R}$ \\
\hline & & & \\
\hline
\end{tabular}




\begin{tabular}{|c|l|l|l|}
\hline & AG & \multicolumn{1}{|c|}{ AH } & \\
\hline 1 & DIST_CLASS & OWNER_ID & COLLECTION_PURPOSE \\
\hline 3802 & OFFSITE & SESPMNT & $\mathrm{R}$ \\
\hline 3803 & OFFSITE & SESPMNT & $\mathrm{R}$ \\
\hline 3804 & OFFSITE & SESPMNT & $\mathrm{R}$ \\
\hline 3805 & OFFSITE & SESPMNT & $\mathrm{R}$ \\
\hline 3806 & OFFSITE & SESPMNT & $\mathrm{R}$ \\
\hline 3807 & OFFSITE & SESPMNT & $\mathrm{R}$ \\
\hline 3808 & OFFSITE & SESPMNT & $\mathrm{R}$ \\
\hline 3809 & OFFSITE & SESPMNT & $\mathrm{R}$ \\
\hline 3810 & OFFSITE & SESPMNT & $\mathrm{R}$ \\
\hline 3811 & OFFSITE & SESPMNT & $\mathrm{R}$ \\
\hline 3812 & OFFSITE & SESPMNT & $\mathrm{R}$ \\
\hline 3813 & OFFSITE & SESPMNT & $\mathrm{R}$ \\
\hline 3814 & ONSITE & SESPMNT & $\mathrm{R}$ \\
\hline 3815 & ONSITE & SESPMNT & $\mathrm{R}$ \\
\hline 3816 & ONSITE & SESPMNT & $\mathrm{R}$ \\
\hline 3817 & ONSITE & SESPMNT & $\mathrm{R}$ \\
\hline 3818 & ONSITE & SESPMNT & $\mathrm{R}$ \\
\hline 3819 & ONSITE & SESPMNT & $\mathrm{R}$ \\
\hline 3820 & ONSITE & SESPMNT & $\mathrm{R}$ \\
\hline 3821 & ONSITE & SESPMNT & $\mathrm{R}$ \\
\hline 3822 & ONSITE & SESPMNT & $\mathrm{R}$ \\
\hline 3823 & ONSITE & SESPMNT & $\mathrm{R}$ \\
\hline 3824 & ONSITE & SESPMNT & $\mathrm{R}$ \\
\hline 3825 & ONSITE & SESPMNT & $\mathrm{R}$ \\
\hline 3826 & ONSITE & SESPMNT & $\mathrm{R}$ \\
\hline 3827 & ONSITE & SESPMNT & $\mathrm{R}$ \\
\hline 3828 & ONSITE & SESPMNT & $\mathrm{R}$ \\
\hline 3829 & ONSITE & SESPMNT & $\mathrm{R}$ \\
\hline 3830 & ONSITE & SESPMNT & $\mathrm{R}$ \\
\hline 3831 & ONSITE & SESPMNT & $\mathrm{R}$ \\
\hline 3832 & ONSITE & SESPMNT & $\mathrm{R}$ \\
\hline 3833 & ONSITE & SESPMNT & $\mathrm{R}$ \\
\hline 3834 & ONSITE & SESPMNT & $\mathrm{R}$ \\
\hline 3835 & ONSITE & SESPMNT & $\mathrm{R}$ \\
\hline 3836 & ONSITE & SESPMNT & $\mathrm{R}$ \\
\hline 3837 & ONSITE & SESPMNT & $\mathrm{R}$ \\
\hline 3838 & ONSITE & SESPMNT & $\mathrm{R}$ \\
\hline 3839 & ONSITE & SESPMNT & $\mathrm{R}$ \\
\hline & & & \\
\hline
\end{tabular}




\begin{tabular}{|c|l|l|l|}
\hline & AG & AH & \\
\hline 1 & DIST_CLASS & OWNER_ID & COLLECTION_PURPOSE \\
\hline 3840 & ONSITE & SESPMNT & $\mathrm{R}$ \\
\hline 3841 & ONSITE & SESPMNT & $\mathrm{R}$ \\
\hline 3842 & ONSITE & SESPMNT & $\mathrm{R}$ \\
\hline 3843 & ONSITE & SESPMNT & $\mathrm{R}$ \\
\hline 3844 & ONSITE & SESPMNT & $\mathrm{R}$ \\
\hline 3845 & ONSITE & SESPMNT & $\mathrm{R}$ \\
\hline 3846 & ONSITE & SESPMNT & $\mathrm{R}$ \\
\hline 3847 & ONSITE & SESPMNT & $\mathrm{R}$ \\
\hline 3848 & ONSITE & SESPMNT & $\mathrm{R}$ \\
\hline 3849 & ONSITE & SESPMNT & $\mathrm{R}$ \\
\hline 3850 & ONSITE & SESPMNT & $\mathrm{R}$ \\
\hline 3851 & ONSITE & SESPMNT & $\mathrm{R}$ \\
\hline 3852 & ONSITE & SESPMNT & $\mathrm{R}$ \\
\hline 3853 & ONSITE & SESPMNT & $\mathrm{R}$ \\
\hline 3854 & ONSITE & SESPMNT & $\mathrm{R}$ \\
\hline 3855 & ONSITE & SESPMNT & $\mathrm{R}$ \\
\hline 3856 & ONSITE & SESPMNT & $\mathrm{R}$ \\
\hline 3857 & ONSITE & SESPMNT & $\mathrm{R}$ \\
\hline 3858 & ONSITE & SESPMNT & $\mathrm{R}$ \\
\hline 3859 & ONSITE & SESPMNT & $\mathrm{R}$ \\
\hline 3860 & ONSITE & SESPMNT & $\mathrm{R}$ \\
\hline 3861 & ONSITE & SESPMNT & $\mathrm{R}$ \\
\hline 3862 & ONSITE & SESPMNT & $\mathrm{R}$ \\
\hline 3863 & ONSITE & SESPMNT & $\mathrm{R}$ \\
\hline 3864 & OFFSITE & SESPMNT & $\mathrm{R}$ \\
\hline 3865 & OFFSITE & SESPMNT & $\mathrm{R}$ \\
\hline 3866 & OFFSITE & SESPMNT & $\mathrm{R}$ \\
\hline 3867 & OFFSITE & SESPMNT & $\mathrm{R}$ \\
\hline 3868 & OFFSITE & SESPMNT & $\mathrm{R}$ \\
\hline 3869 & OFFSITE & SESPMNT & $\mathrm{R}$ \\
\hline 3870 & OFFSITE & SESPMNT & $\mathrm{R}$ \\
\hline 3871 & OFFSITE & SESPMNT & $\mathrm{R}$ \\
\hline 3872 & OFFSITE & SESPMNT & $\mathrm{R}$ \\
\hline 3873 & OFFSITE & SESPMNT & $\mathrm{R}$ \\
\hline 3874 & OFFSITE & SESPMNT & $\mathrm{R}$ \\
\hline 3875 & OFFSITE & SESPMNT & $\mathrm{R}$ \\
\hline 3876 & OFFSITE & SESPMNT & $\mathrm{R}$ \\
\hline 3877 & OFFSITE & SESPMNT & $\mathrm{R}$ \\
\hline & & & \\
\hline
\end{tabular}




\begin{tabular}{|c|l|l|l|}
\hline & AG & \multicolumn{1}{|c|}{ AH } & \\
\hline 1 & DIST_CLASS & OWNER_ID & COLLECTION_PURPOSE \\
\hline 3878 & OFFSITE & SESPMNT & $\mathrm{R}$ \\
\hline 3879 & OFFSITE & SESPMNT & $\mathrm{R}$ \\
\hline 3880 & OFFSITE & SESPMNT & $\mathrm{R}$ \\
\hline 3881 & OFFSITE & SESPMNT & $\mathrm{R}$ \\
\hline 3882 & OFFSITE & SESPMNT & $\mathrm{R}$ \\
\hline 3883 & OFFSITE & SESPMNT & $\mathrm{R}$ \\
\hline 3884 & OFFSITE & SESPMNT & $\mathrm{R}$ \\
\hline 3885 & OFFSITE & SESPMNT & $\mathrm{R}$ \\
\hline 3886 & OFFSITE & SESPMNT & $\mathrm{R}$ \\
\hline 3887 & OFFSITE & SESPMNT & $\mathrm{R}$ \\
\hline 3888 & OFFSITE & SESPMNT & $\mathrm{R}$ \\
\hline 3889 & OFFSITE & SESPMNT & $\mathrm{R}$ \\
\hline 3890 & OFFSITE & SESPMNT & $\mathrm{R}$ \\
\hline 3891 & OFFSITE & SESPMNT & $\mathrm{R}$ \\
\hline 3892 & OFFSITE & SESPMNT & $\mathrm{R}$ \\
\hline 3893 & OFFSITE & SESPMNT & $\mathrm{R}$ \\
\hline 3894 & OFFSITE & SESPMNT & $\mathrm{R}$ \\
\hline 3895 & OFFSITE & SESPMNT & $\mathrm{R}$ \\
\hline 3896 & OFFSITE & SESPMNT & $\mathrm{R}$ \\
\hline 3897 & OFFSITE & SESPMNT & $\mathrm{R}$ \\
\hline 3898 & OFFSITE & SESPMNT & $\mathrm{R}$ \\
\hline 3899 & OFFSITE & SESPMNT & $\mathrm{R}$ \\
\hline 3900 & OFFSITE & SESPMNT & $\mathrm{R}$ \\
\hline 3901 & OFFSITE & SESPMNT & $\mathrm{R}$ \\
\hline 3902 & OFFSITE & SESPMNT & $\mathrm{R}$ \\
\hline 3903 & OFFSITE & SESPMNT & $\mathrm{R}$ \\
\hline 3904 & OFFSITE & SESPMNT & $\mathrm{R}$ \\
\hline 3905 & OFFSITE & SESPMNT & $\mathrm{R}$ \\
\hline 3906 & OFFSITE & SESPMNT & $\mathrm{R}$ \\
\hline 3907 & OFFSITE & SESPMNT & $\mathrm{R}$ \\
\hline 3908 & OFFSITE & SESPMNT & $\mathrm{R}$ \\
\hline 3909 & OFFSITE & SESPMNT & $\mathrm{R}$ \\
\hline 3910 & OFFSITE & SESPMNT & $\mathrm{R}$ \\
\hline 3911 & OFFSITE & SESPMNT & $\mathrm{R}$ \\
\hline 3912 & OFFSITE & SESPMNT & $\mathrm{R}$ \\
\hline 3913 & OFFSITE & SESPMNT & $\mathrm{R}$ \\
\hline 3914 & ONSITE & SESPMNT & $\mathrm{R}$ \\
\hline 3915 & ONSITE & SESPMNT & $\mathrm{R}$ \\
\hline & & & \\
\hline
\end{tabular}




\begin{tabular}{|c|l|l|l|}
\hline & \multicolumn{1}{|c|}{ AG } & \multicolumn{1}{|c|}{ AH } & \\
\hline 1 & DIST_CLASS & OWNER_ID & COLLECTION_PURPOSE \\
\hline 3916 & ONSITE & SESPMNT & $\mathrm{R}$ \\
\hline 3917 & ONSITE & SESPMNT & $\mathrm{R}$ \\
\hline 3918 & ONSITE & SESPMNT & $\mathrm{R}$ \\
\hline 3919 & ONSITE & SESPMNT & $\mathrm{R}$ \\
\hline 3920 & ONSITE & SESPMNT & $\mathrm{R}$ \\
\hline 3921 & ONSITE & SESPMNT & $\mathrm{R}$ \\
\hline 3922 & ONSITE & SESPMNT & $\mathrm{R}$ \\
\hline 3923 & ONSITE & SESPMNT & $\mathrm{R}$ \\
\hline 3924 & ONSITE & SESPMNT & $\mathrm{R}$ \\
\hline 3925 & ONSITE & SESPMNT & $\mathrm{R}$ \\
\hline 3926 & ONSITE & SESPMNT & $\mathrm{R}$ \\
\hline 3927 & ONSITE & SESPMNT & $\mathrm{R}$ \\
\hline 3928 & ONSITE & SESPMNT & $\mathrm{R}$ \\
\hline 3929 & ONSITE & SESPMNT & $\mathrm{R}$ \\
\hline 3930 & ONSITE & SESPMNT & $\mathrm{R}$ \\
\hline 3931 & ONSITE & SESPMNT & $\mathrm{R}$ \\
\hline 3932 & ONSITE & SESPMNT & $\mathrm{R}$ \\
\hline 3933 & ONSITE & SESPMNT & $\mathrm{R}$ \\
\hline 3934 & ONSITE & SESPMNT & $\mathrm{R}$ \\
\hline 3935 & ONSITE & SESPMNT & $\mathrm{R}$ \\
\hline 3936 & ONSITE & SESPMNT & $\mathrm{R}$ \\
\hline 3937 & ONSITE & SESPMNT & $\mathrm{R}$ \\
\hline 3938 & ONSITE & SESPMNT & $\mathrm{R}$ \\
\hline 3939 & ONSITE & SESPMNT & $\mathrm{R}$ \\
\hline 3940 & ONSITE & SESPMNT & $\mathrm{R}$ \\
\hline 3941 & ONSITE & SESPMNT & $\mathrm{R}$ \\
\hline 3942 & ONSITE & SESPMNT & $\mathrm{R}$ \\
\hline 3943 & ONSITE & SESPMNT & $\mathrm{R}$ \\
\hline 3944 & ONSITE & SESPMNT & $\mathrm{R}$ \\
\hline 3945 & ONSITE & SESPMNT & $\mathrm{R}$ \\
\hline 3946 & ONSITE & SESPMNT & $\mathrm{R}$ \\
\hline 3947 & ONSITE & SESPMNT & $\mathrm{R}$ \\
\hline 3948 & ONSITE & SESPMNT & $\mathrm{R}$ \\
\hline 3949 & ONSITE & SESPMNT & $\mathrm{R}$ \\
\hline 3950 & ONSITE & SESPMNT & $\mathrm{R}$ \\
\hline 3951 & ONSITE & SESPMNT & $\mathrm{R}$ \\
\hline 3952 & ONSITE & SESPMNT & $\mathrm{R}$ \\
\hline 3953 & ONSITE & SESPMNT & $\mathrm{R}$ \\
\hline & & & \\
\hline
\end{tabular}




\begin{tabular}{|c|l|l|l|}
\hline & \multicolumn{1}{|c|}{ AG } & \multicolumn{1}{|c|}{ AH } & \\
\hline 1 & DIST_CLASS & OWNER_ID & COLLECTION_PURPOSE \\
\hline 3954 & ONSITE & SESPMNT & $\mathrm{R}$ \\
\hline 3955 & ONSITE & SESPMNT & $\mathrm{R}$ \\
\hline 3956 & ONSITE & SESPMNT & $\mathrm{R}$ \\
\hline 3957 & ONSITE & SESPMNT & $\mathrm{R}$ \\
\hline 3958 & ONSITE & SESPMNT & $\mathrm{R}$ \\
\hline 3959 & ONSITE & SESPMNT & $\mathrm{R}$ \\
\hline 3960 & ONSITE & SESPMNT & $\mathrm{R}$ \\
\hline 3961 & ONSITE & SESPMNT & $\mathrm{R}$ \\
\hline 3962 & ONSITE & SESPMNT & $\mathrm{R}$ \\
\hline 3963 & ONSITE & SESPMNT & $\mathrm{R}$ \\
\hline 3964 & ONSITE & SESPMNT & $\mathrm{R}$ \\
\hline 3965 & ONSITE & SESPMNT & $\mathrm{R}$ \\
\hline 3966 & ONSITE & SESPMNT & $\mathrm{R}$ \\
\hline 3967 & ONSITE & SESPMNT & $\mathrm{R}$ \\
\hline 3968 & ONSITE & SESPMNT & $\mathrm{R}$ \\
\hline 3969 & ONSITE & SESPMNT & $\mathrm{R}$ \\
\hline 3970 & ONSITE & SESPMNT & $\mathrm{R}$ \\
\hline 3971 & ONSITE & SESPMNT & $\mathrm{R}$ \\
\hline 3972 & ONSITE & SESPMNT & $\mathrm{R}$ \\
\hline 3973 & ONSITE & SESPMNT & $\mathrm{R}$ \\
\hline 3974 & ONSITE & SESPMNT & $\mathrm{R}$ \\
\hline 3975 & ONSITE & SESPMNT & $\mathrm{R}$ \\
\hline 3976 & ONSITE & SESPMNT & $\mathrm{R}$ \\
\hline 3977 & ONSITE & SESPMNT & $\mathrm{R}$ \\
\hline 3978 & ONSITE & SESPMNT & $\mathrm{R}$ \\
\hline 3979 & ONSITE & SESPMNT & $\mathrm{R}$ \\
\hline 3980 & ONSITE & SESPMNT & $\mathrm{R}$ \\
\hline 3981 & ONSITE & SESPMNT & $\mathrm{R}$ \\
\hline 3982 & ONSITE & SESPMNT & $\mathrm{R}$ \\
\hline 3983 & ONSITE & SESPMNT & $\mathrm{R}$ \\
\hline 3984 & ONSITE & SESPMNT & $\mathrm{R}$ \\
\hline 3985 & ONSITE & SESPMNT & $\mathrm{R}$ \\
\hline 3986 & ONSITE & SESPMNT & $\mathrm{R}$ \\
\hline 3987 & ONSITE & SESPMNT & $\mathrm{R}$ \\
\hline 3988 & ONSITE & SESPMNT & $\mathrm{R}$ \\
\hline 3989 & ONSITE & SESPMNT & $\mathrm{R}$ \\
\hline 3990 & ONSITE & SESPMNT & $\mathrm{R}$ \\
\hline 3991 & ONSITE & SESPMNT & $\mathrm{R}$ \\
\hline & & & \\
\hline
\end{tabular}




\begin{tabular}{|c|l|l|l|}
\hline & \multicolumn{1}{|c|}{ AG } & \multicolumn{1}{|c|}{ AH } & \\
\hline 1 & DIST_CLASS & OWNER_ID & COLLECTION_PURPOSE \\
\hline 3992 & ONSITE & SESPMNT & $\mathrm{R}$ \\
\hline 3993 & ONSITE & SESPMNT & $\mathrm{R}$ \\
\hline 3994 & ONSITE & SESPMNT & $\mathrm{R}$ \\
\hline 3995 & ONSITE & SESPMNT & $\mathrm{R}$ \\
\hline 3996 & ONSITE & SESPMNT & $\mathrm{R}$ \\
\hline 3997 & ONSITE & SESPMNT & $\mathrm{R}$ \\
\hline 3998 & ONSITE & SESPMNT & $\mathrm{R}$ \\
\hline 3999 & ONSITE & SESPMNT & $\mathrm{R}$ \\
\hline 4000 & ONSITE & SESPMNT & $\mathrm{R}$ \\
\hline 4001 & ONSITE & SESPMNT & $\mathrm{R}$ \\
\hline 4002 & ONSITE & SESPMNT & $\mathrm{R}$ \\
\hline 4003 & ONSITE & SESPMNT & $\mathrm{R}$ \\
\hline 4004 & ONSITE & SESPMNT & $\mathrm{R}$ \\
\hline 4005 & ONSITE & SESPMNT & $\mathrm{R}$ \\
\hline 4006 & ONSITE & SESPMNT & $\mathrm{R}$ \\
\hline 4007 & ONSITE & SESPMNT & $\mathrm{R}$ \\
\hline 4008 & ONSITE & SESPMNT & $\mathrm{R}$ \\
\hline 4009 & ONSITE & SESPMNT & $\mathrm{R}$ \\
\hline 4010 & ONSITE & SESPMNT & $\mathrm{R}$ \\
\hline 4011 & ONSITE & SESPMNT & $\mathrm{R}$ \\
\hline 4012 & ONSITE & SESPMNT & $\mathrm{R}$ \\
\hline 4013 & ONSITE & SESPMNT & $\mathrm{R}$ \\
\hline 4014 & OFFSITE & SESPMNT & $\mathrm{R}$ \\
\hline 4015 & OFFSITE & SESPMNT & $\mathrm{R}$ \\
\hline 4016 & OFFSITE & SESPMNT & $\mathrm{R}$ \\
\hline 4017 & OFFSITE & SESPMNT & $\mathrm{R}$ \\
\hline 4018 & OFFSITE & SESPMNT & $\mathrm{R}$ \\
\hline 4019 & OFFSITE & SESPMNT & $\mathrm{R}$ \\
\hline 4020 & OFFSITE & SESPMNT & $\mathrm{R}$ \\
\hline 4021 & OFFSITE & SESPMNT & $\mathrm{R}$ \\
\hline 4022 & OFFSITE & SESPMNT & $\mathrm{R}$ \\
\hline 4023 & OFFSITE & SESPMNT & $\mathrm{R}$ \\
\hline 4024 & OFFSITE & SESPMNT & $\mathrm{R}$ \\
\hline 4025 & OFFSITE & SESPMNT & $\mathrm{R}$ \\
\hline 4026 & OFFSITE & SESPMNT & $\mathrm{R}$ \\
\hline 4027 & OFFSITE & SESPMNT & $\mathrm{R}$ \\
\hline 4028 & OFFSITE & SESPMNT & $\mathrm{R}$ \\
\hline 4029 & OFFSITE & SESPMNT & $\mathrm{R}$ \\
\hline & & & \\
\hline
\end{tabular}




\begin{tabular}{|c|l|l|l|}
\hline & \multicolumn{1}{|c|}{ AG } & \multicolumn{1}{|c|}{ AH } & \\
\hline 1 & DIST_CLASS & OWNER_ID & COLLECTION_PURPOSE \\
\hline 4030 & OFFSITE & SESPMNT & $\mathrm{R}$ \\
\hline 4031 & OFFSITE & SESPMNT & $\mathrm{R}$ \\
\hline 4032 & OFFSITE & SESPMNT & $\mathrm{R}$ \\
\hline 4033 & OFFSITE & SESPMNT & $\mathrm{R}$ \\
\hline 4034 & OFFSITE & SESPSPEC & $\mathrm{S}$ \\
\hline 4035 & OFFSITE & SESPMNT & $\mathrm{R}$ \\
\hline 4036 & OFFSITE & SESPSPEC & $\mathrm{S}$ \\
\hline 4037 & OFFSITE & SESPMNT & $\mathrm{R}$ \\
\hline 4038 & OFFSITE & SESPSPEC & $\mathrm{S}$ \\
\hline 4039 & OFFSITE & SESPSPEC & $\mathrm{S}$ \\
\hline 4040 & OFFSITE & SESPSPEC & $\mathrm{S}$ \\
\hline 4041 & OFFSITE & SESPMNT & $\mathrm{R}$ \\
\hline 4042 & OFFSITE & SESPMNT & $\mathrm{R}$ \\
\hline 4043 & OFFSITE & SESPMNT & $\mathrm{R}$ \\
\hline 4044 & OFFSITE & SESPMNT & $\mathrm{R}$ \\
\hline 4045 & OFFSITE & SESPMNT & $\mathrm{R}$ \\
\hline 4046 & OFFSITE & SESPMNT & $\mathrm{R}$ \\
\hline 4047 & OFFSITE & SESPMNT & $\mathrm{R}$ \\
\hline 4048 & OFFSITE & SESPMNT & $\mathrm{R}$ \\
\hline 4049 & OFFSITE & SESPMNT & $\mathrm{R}$ \\
\hline 4050 & OFFSITE & SESPMNT & $\mathrm{R}$ \\
\hline 4051 & OFFSITE & SESPMNT & $\mathrm{R}$ \\
\hline 4052 & OFFSITE & SESPMNT & $\mathrm{R}$ \\
\hline 4053 & OFFSITE & SESPMNT & $\mathrm{R}$ \\
\hline 4054 & OFFSITE & SESPMNT & $\mathrm{R}$ \\
\hline 4055 & OFFSITE & SESPMNT & $\mathrm{R}$ \\
\hline 4056 & OFFSITE & SESPMNT & $\mathrm{R}$ \\
\hline 4057 & OFFSITE & SESPMNT & $\mathrm{R}$ \\
\hline 4058 & OFFSITE & SESPMNT & $\mathrm{R}$ \\
\hline 4059 & OFFSITE & SESPMNT & $\mathrm{R}$ \\
\hline 4060 & OFFSITE & SESPMNT & $\mathrm{R}$ \\
\hline 4061 & OFFSITE & SESPMNT & $\mathrm{R}$ \\
\hline 4062 & OFFSITE & SESPMNT & $\mathrm{R}$ \\
\hline 4063 & OFFSITE & SESPMNT & $\mathrm{R}$ \\
\hline 4064 & OFFSITE & SESPMNT & $\mathrm{R}$ \\
\hline 4065 & OFFSITE & SESPMNT & $\mathrm{R}$ \\
\hline 4066 & OFFSITE & SESPMNT & $\mathrm{R}$ \\
\hline 4067 & OFFSITE & SESPMNT & $\mathrm{R}$ \\
\hline & & & \\
\hline
\end{tabular}




\begin{tabular}{|c|l|l|l|}
\hline & AG & \multicolumn{1}{|c|}{ AH } & \\
\hline 1 & DIST_CLASS & OWNER_ID & COLLECTION_PURPOSE \\
\hline 4068 & OFFSITE & SESPMNT & $\mathrm{R}$ \\
\hline 4069 & OFFSITE & SESPMNT & $\mathrm{R}$ \\
\hline 4070 & OFFSITE & SESPMNT & $\mathrm{R}$ \\
\hline 4071 & OFFSITE & SESPMNT & $\mathrm{R}$ \\
\hline 4072 & OFFSITE & SESPMNT & $\mathrm{R}$ \\
\hline 4073 & OFFSITE & SESPMNT & $\mathrm{R}$ \\
\hline 4074 & OFFSITE & SESPMNT & $\mathrm{R}$ \\
\hline 4075 & OFFSITE & SESPMNT & $\mathrm{R}$ \\
\hline 4076 & OFFSITE & SESPMNT & $\mathrm{R}$ \\
\hline 4077 & OFFSITE & SESPMNT & $\mathrm{R}$ \\
\hline 4078 & OFFSITE & SESPMNT & $\mathrm{R}$ \\
\hline 4079 & OFFSITE & SESPMNT & $\mathrm{R}$ \\
\hline 4080 & OFFSITE & SESPMNT & $\mathrm{R}$ \\
\hline 4081 & OFFSITE & SESPMNT & $\mathrm{R}$ \\
\hline 4082 & OFFSITE & SESPMNT & $\mathrm{R}$ \\
\hline 4083 & OFFSITE & SESPMNT & $\mathrm{R}$ \\
\hline 4084 & OFFSITE & SESPMNT & $\mathrm{R}$ \\
\hline 4085 & OFFSITE & SESPMNT & $\mathrm{R}$ \\
\hline 4086 & OFFSITE & SESPMNT & $\mathrm{R}$ \\
\hline 4087 & OFFSITE & SESPMNT & $\mathrm{R}$ \\
\hline 4088 & OFFSITE & SESPMNT & $\mathrm{R}$ \\
\hline 4089 & OFFSITE & SESPMNT & $\mathrm{R}$ \\
\hline 4090 & OFFSITE & SESPMNT & $\mathrm{R}$ \\
\hline 4091 & OFFSITE & SESPMNT & $\mathrm{R}$ \\
\hline 4092 & OFFSITE & SESPMNT & $\mathrm{R}$ \\
\hline 4093 & OFFSITE & SESPMNT & $\mathrm{R}$ \\
\hline 4094 & OFFSITE & SESPMNT & $\mathrm{R}$ \\
\hline 4095 & OFFSITE & SESPMNT & $\mathrm{R}$ \\
\hline 4096 & OFFSITE & SESPMNT & $\mathrm{R}$ \\
\hline 4097 & OFFSITE & SESPMNT & $\mathrm{R}$ \\
\hline 4098 & OFFSITE & SESPMNT & $\mathrm{R}$ \\
\hline 4099 & OFFSITE & SESPMNT & $\mathrm{R}$ \\
\hline 4100 & OFFSITE & SESPMNT & $\mathrm{R}$ \\
\hline 4101 & OFFSITE & SESPMNT & $\mathrm{R}$ \\
\hline 4102 & OFFSITE & SESPMNT & $\mathrm{R}$ \\
\hline 4103 & OFFSITE & SESPMNT & $\mathrm{R}$ \\
\hline 4104 & OFFSITE & SESPMNT & $\mathrm{R}$ \\
\hline 4105 & OFFSITE & SESPMNT & $\mathrm{R}$ \\
\hline & & & \\
\hline
\end{tabular}




\begin{tabular}{|c|l|l|l|}
\hline & AG & \multicolumn{1}{|c|}{ AH } & \\
\hline 1 & DIST_CLASS & OWNER_ID & COLLECTION_PURPOSE \\
\hline 4106 & OFFSITE & SESPMNT & $\mathrm{R}$ \\
\hline 4107 & OFFSITE & SESPMNT & $\mathrm{R}$ \\
\hline 4108 & OFFSITE & SESPMNT & $\mathrm{R}$ \\
\hline 4109 & OFFSITE & SESPSPEC & $\mathrm{S}$ \\
\hline 4110 & OFFSITE & SESPMNT & $\mathrm{R}$ \\
\hline 4111 & OFFSITE & SESPSPEC & $\mathrm{S}$ \\
\hline 4112 & OFFSITE & SESPMNT & $\mathrm{R}$ \\
\hline 4113 & OFFSITE & SESPSPEC & $\mathrm{S}$ \\
\hline 4114 & OFFSITE & SESPSPEC & $\mathrm{S}$ \\
\hline 4115 & OFFSITE & SESPSPEC & $\mathrm{S}$ \\
\hline 4116 & OFFSITE & SESPMNT & $\mathrm{R}$ \\
\hline 4117 & OFFSITE & SESPMNT & $\mathrm{R}$ \\
\hline 4118 & OFFSITE & SESPMNT & $\mathrm{R}$ \\
\hline 4119 & OFFSITE & SESPMNT & $\mathrm{R}$ \\
\hline 4120 & OFFSITE & SESPMNT & $\mathrm{R}$ \\
\hline 4121 & OFFSITE & SESPMNT & $\mathrm{R}$ \\
\hline 4122 & OFFSITE & SESPMNT & $\mathrm{R}$ \\
\hline 4123 & OFFSITE & SESPMNT & $\mathrm{R}$ \\
\hline 4124 & OFFSITE & SESPMNT & $\mathrm{R}$ \\
\hline 4125 & OFFSITE & SESPMNT & $\mathrm{R}$ \\
\hline 4126 & OFFSITE & SESPMNT & $\mathrm{R}$ \\
\hline 4127 & OFFSITE & SESPMNT & $\mathrm{R}$ \\
\hline 4128 & OFFSITE & SESPMNT & $\mathrm{R}$ \\
\hline 4129 & OFFSITE & SESPMNT & $\mathrm{R}$ \\
\hline 4130 & OFFSITE & SESPMNT & $\mathrm{R}$ \\
\hline 4131 & OFFSITE & SESPMNT & $\mathrm{R}$ \\
\hline 4132 & OFFSITE & SESPMNT & $\mathrm{R}$ \\
\hline 4133 & OFFSITE & SESPMNT & $\mathrm{R}$ \\
\hline 4134 & OFFSITE & SESPMNT & $\mathrm{R}$ \\
\hline 4135 & OFFSITE & SESPMNT & $\mathrm{R}$ \\
\hline 4136 & OFFSITE & SESPMNT & $\mathrm{R}$ \\
\hline 4137 & OFFSITE & SESPMNT & $\mathrm{R}$ \\
\hline 4138 & OFFSITE & SESPMNT & $\mathrm{R}$ \\
\hline 4139 & OFFSITE & SESPMNT & $\mathrm{R}$ \\
\hline 4140 & OFFSITE & SESPMNT & $\mathrm{R}$ \\
\hline 4141 & OFFSITE & SESPMNT & $\mathrm{R}$ \\
\hline 4142 & OFFSITE & SESPMNT & $\mathrm{R}$ \\
\hline 4143 & OFFSITE & SESPMNT & $\mathrm{R}$ \\
\hline & & & \\
\hline
\end{tabular}




\begin{tabular}{|c|l|l|l|}
\hline & \multicolumn{1}{|c|}{ AG } & \multicolumn{1}{|c|}{ AH } & \\
\hline 1 & DIST_CLASS & OWNER_ID & COLLECTION_PURPOSE \\
\hline 4144 & OFFSITE & SESPMNT & $\mathrm{R}$ \\
\hline 4145 & OFFSITE & SESPMNT & $\mathrm{R}$ \\
\hline 4146 & OFFSITE & SESPMNT & $\mathrm{R}$ \\
\hline 4147 & OFFSITE & SESPMNT & $\mathrm{R}$ \\
\hline 4148 & OFFSITE & SESPMNT & $\mathrm{R}$ \\
\hline 4149 & OFFSITE & SESPMNT & $\mathrm{R}$ \\
\hline 4150 & OFFSITE & SESPMNT & $\mathrm{R}$ \\
\hline 4151 & OFFSITE & SESPMNT & $\mathrm{R}$ \\
\hline 4152 & OFFSITE & SESPMNT & $\mathrm{R}$ \\
\hline 4153 & OFFSITE & SESPMNT & $\mathrm{R}$ \\
\hline 4154 & OFFSITE & SESPMNT & $\mathrm{R}$ \\
\hline 4155 & OFFSITE & SESPMNT & $\mathrm{R}$ \\
\hline 4156 & OFFSITE & SESPMNT & $\mathrm{R}$ \\
\hline 4157 & OFFSITE & SESPMNT & $\mathrm{R}$ \\
\hline 4158 & OFFSITE & SESPMNT & $\mathrm{R}$ \\
\hline 4159 & OFFSITE & SESPMNT & $\mathrm{R}$ \\
\hline 4160 & OFFSITE & SESPMNT & $\mathrm{R}$ \\
\hline 4161 & OFFSITE & SESPMNT & $\mathrm{R}$ \\
\hline 4162 & OFFSITE & SESPMNT & $\mathrm{R}$ \\
\hline 4163 & OFFSITE & SESPMNT & $\mathrm{R}$ \\
\hline 4164 & ONSITE & SESPSPEC & $\mathrm{S}$ \\
\hline 4165 & ONSITE & SESPMNT & $\mathrm{R}$ \\
\hline 4166 & ONSITE & SESPSPEC & $\mathrm{S}$ \\
\hline 4167 & ONSITE & SESPMNT & $\mathrm{R}$ \\
\hline 4168 & ONSITE & SESPSPEC & $\mathrm{S}$ \\
\hline 4169 & ONSITE & SESPSPEC & $\mathrm{S}$ \\
\hline 4170 & ONSITE & SESPSPEC & $\mathrm{S}$ \\
\hline 4171 & ONSITE & SESPMNT & $\mathrm{R}$ \\
\hline 4172 & ONSITE & SESPMNT & $\mathrm{R}$ \\
\hline 4173 & ONSITE & SESPMNT & $\mathrm{R}$ \\
\hline 4174 & ONSITE & SESPMNT & $\mathrm{R}$ \\
\hline 4175 & ONSITE & SESPMNT & $\mathrm{R}$ \\
\hline 4176 & ONSITE & SESPMNT & $\mathrm{R}$ \\
\hline 4177 & ONSITE & SESPMNT & $\mathrm{R}$ \\
\hline 4178 & ONSITE & SESPMNT & $\mathrm{R}$ \\
\hline 4179 & ONSITE & SESPMNT & $\mathrm{R}$ \\
\hline 4180 & ONSITE & SESPMNT & $\mathrm{R}$ \\
\hline 4181 & ONSITE & SESPMNT & $\mathrm{R}$ \\
\hline
\end{tabular}




\begin{tabular}{|c|l|l|l|}
\hline & \multicolumn{1}{|c|}{ AG } & \multicolumn{1}{|c|}{ AH } & \\
\hline 1 & DIST_CLASS & OWNER_ID & COLLECTION_PURPOSE \\
\hline 4182 & ONSITE & SESPMNT & $\mathrm{R}$ \\
\hline 4183 & ONSITE & SESPMNT & $\mathrm{R}$ \\
\hline 4184 & ONSITE & SESPMNT & $\mathrm{R}$ \\
\hline 4185 & ONSITE & SESPMNT & $\mathrm{R}$ \\
\hline 4186 & ONSITE & SESPMNT & $\mathrm{R}$ \\
\hline 4187 & ONSITE & SESPMNT & $\mathrm{R}$ \\
\hline 4188 & ONSITE & SESPMNT & $\mathrm{R}$ \\
\hline 4189 & ONSITE & SESPMNT & $\mathrm{R}$ \\
\hline 4190 & ONSITE & SESPMNT & $\mathrm{R}$ \\
\hline 4191 & ONSITE & SESPMNT & $\mathrm{R}$ \\
\hline 4192 & ONSITE & SESPMNT & $\mathrm{R}$ \\
\hline 4193 & ONSITE & SESPMNT & $\mathrm{R}$ \\
\hline 4194 & ONSITE & SESPMNT & $\mathrm{R}$ \\
\hline 4195 & ONSITE & SESPMNT & $\mathrm{R}$ \\
\hline 4196 & ONSITE & SESPMNT & $\mathrm{R}$ \\
\hline 4197 & ONSITE & SESPMNT & $\mathrm{R}$ \\
\hline 4198 & ONSITE & SESPMNT & $\mathrm{R}$ \\
\hline 4199 & ONSITE & SESPMNT & $\mathrm{R}$ \\
\hline 4200 & ONSITE & SESPMNT & $\mathrm{R}$ \\
\hline 4201 & ONSITE & SESPMNT & $\mathrm{R}$ \\
\hline 4202 & ONSITE & SESPMNT & $\mathrm{R}$ \\
\hline 4203 & ONSITE & SESPMNT & $\mathrm{R}$ \\
\hline 4204 & ONSITE & SESPMNT & $\mathrm{R}$ \\
\hline 4205 & ONSITE & SESPMNT & $\mathrm{R}$ \\
\hline 4206 & ONSITE & SESPMNT & $\mathrm{R}$ \\
\hline 4207 & ONSITE & SESPMNT & $\mathrm{R}$ \\
\hline 4208 & ONSITE & SESPMNT & $\mathrm{R}$ \\
\hline 4209 & ONSITE & SESPMNT & $\mathrm{R}$ \\
\hline 4210 & ONSITE & SESPMNT & $\mathrm{R}$ \\
\hline 4211 & ONSITE & SESPMNT & $\mathrm{R}$ \\
\hline 4212 & ONSITE & SESPMNT & $\mathrm{R}$ \\
\hline 4213 & ONSITE & SESPMNT & $\mathrm{R}$ \\
\hline 4214 & ONSITE & SESPMNT & $\mathrm{R}$ \\
\hline 4215 & ONSITE & SESPMNT & $\mathrm{R}$ \\
\hline 4216 & ONSITE & SESPMNT & $\mathrm{R}$ \\
\hline 4217 & ONSITE & SESPMNT & $\mathrm{R}$ \\
\hline 4218 & ONSITE & SESPMNT & $\mathrm{R}$ \\
\hline 4219 & OFFSITE & SESPMNT & $\mathrm{R}$ \\
\hline & & & \\
\hline
\end{tabular}




\begin{tabular}{|c|l|l|l|}
\hline & AG & \multicolumn{1}{|c|}{ AH } & \\
\hline 1 & DIST_CLASS & OWNER_ID & COLLECTION_PURPOSE \\
\hline 4220 & OFFSITE & SESPMNT & $\mathrm{R}$ \\
\hline 4221 & OFFSITE & SESPMNT & $\mathrm{R}$ \\
\hline 4222 & OFFSITE & SESPMNT & $\mathrm{R}$ \\
\hline 4223 & OFFSITE & SESPMNT & $\mathrm{R}$ \\
\hline 4224 & OFFSITE & SESPMNT & $\mathrm{R}$ \\
\hline 4225 & OFFSITE & SESPMNT & $\mathrm{R}$ \\
\hline 4226 & OFFSITE & SESPMNT & $\mathrm{R}$ \\
\hline 4227 & OFFSITE & SESPMNT & $\mathrm{R}$ \\
\hline 4228 & OFFSITE & SESPMNT & $\mathrm{R}$ \\
\hline 4229 & OFFSITE & SESPMNT & $\mathrm{R}$ \\
\hline 4230 & OFFSITE & SESPMNT & $\mathrm{R}$ \\
\hline 4231 & OFFSITE & SESPMNT & $\mathrm{R}$ \\
\hline 4232 & OFFSITE & SESPMNT & $\mathrm{R}$ \\
\hline 4233 & OFFSITE & SESPMNT & $\mathrm{R}$ \\
\hline 4234 & OFFSITE & SESPMNT & $\mathrm{R}$ \\
\hline 4235 & OFFSITE & SESPMNT & $\mathrm{R}$ \\
\hline 4236 & OFFSITE & SESPMNT & $\mathrm{R}$ \\
\hline 4237 & OFFSITE & SESPMNT & $\mathrm{R}$ \\
\hline 4238 & OFFSITE & SESPMNT & $\mathrm{R}$ \\
\hline 4239 & ONSITE & SESPMNT & $\mathrm{R}$ \\
\hline 4240 & ONSITE & SESPMNT & $\mathrm{R}$ \\
\hline 4241 & ONSITE & SESPMNT & $\mathrm{R}$ \\
\hline 4242 & ONSITE & SESPMNT & $\mathrm{R}$ \\
\hline 4243 & ONSITE & SESPMNT & $\mathrm{R}$ \\
\hline 4244 & ONSITE & SESPMNT & $\mathrm{R}$ \\
\hline 4245 & ONSITE & SESPMNT & $\mathrm{R}$ \\
\hline 4246 & ONSITE & SESPMNT & $\mathrm{R}$ \\
\hline 4247 & ONSITE & SESPMNT & $\mathrm{R}$ \\
\hline 4248 & ONSITE & SESPMNT & $\mathrm{R}$ \\
\hline 4249 & ONSITE & SESPMNT & $\mathrm{R}$ \\
\hline 4250 & ONSITE & SESPMNT & $\mathrm{R}$ \\
\hline 4251 & ONSITE & SESPMNT & $\mathrm{R}$ \\
\hline 4252 & ONSITE & SESPMNT & $\mathrm{R}$ \\
\hline 4253 & ONSITE & SESPMNT & $\mathrm{R}$ \\
\hline 4254 & ONSITE & SESPMNT & $\mathrm{R}$ \\
\hline 4255 & ONSITE & SESPMNT & $\mathrm{R}$ \\
\hline 4256 & ONSITE & SESPMNT & $\mathrm{R}$ \\
\hline 4257 & ONSITE & SESPMNT & $\mathrm{R}$ \\
\hline & & & \\
\hline
\end{tabular}




\begin{tabular}{|c|l|l|l|}
\hline & \multicolumn{1}{|c|}{ AG } & \multicolumn{1}{|c|}{ AH } & \\
\hline 1 & DIST_CLASS & OWNER_ID & COLLECTION_PURPOSE \\
\hline 4258 & ONSITE & SESPMNT & $\mathrm{R}$ \\
\hline 4259 & ONSITE & SESPMNT & $\mathrm{R}$ \\
\hline 4260 & ONSITE & SESPMNT & $\mathrm{R}$ \\
\hline 4261 & ONSITE & SESPMNT & $\mathrm{R}$ \\
\hline 4262 & ONSITE & SESPMNT & $\mathrm{R}$ \\
\hline 4263 & ONSITE & SESPMNT & $\mathrm{R}$ \\
\hline 4264 & ONSITE & SESPMNT & $\mathrm{R}$ \\
\hline 4265 & ONSITE & SESPMNT & $\mathrm{R}$ \\
\hline 4266 & ONSITE & SESPMNT & $\mathrm{R}$ \\
\hline 4267 & ONSITE & SESPMNT & $\mathrm{R}$ \\
\hline 4268 & ONSITE & SESPMNT & $\mathrm{R}$ \\
\hline 4269 & ONSITE & SESPMNT & $\mathrm{R}$ \\
\hline 4270 & ONSITE & SESPMNT & $\mathrm{R}$ \\
\hline 4271 & ONSITE & SESPMNT & $\mathrm{R}$ \\
\hline 4272 & ONSITE & SESPMNT & $\mathrm{R}$ \\
\hline 4273 & ONSITE & SESPMNT & $\mathrm{R}$ \\
\hline 4274 & ONSITE & SESPMNT & $\mathrm{R}$ \\
\hline 4275 & ONSITE & SESPMNT & $\mathrm{R}$ \\
\hline 4276 & ONSITE & SESPMNT & $\mathrm{R}$ \\
\hline 4277 & ONSITE & SESPMNT & $\mathrm{R}$ \\
\hline 4278 & ONSITE & SESPMNT & $\mathrm{R}$ \\
\hline 4279 & ONSITE & SESPMNT & $\mathrm{R}$ \\
\hline 4280 & ONSITE & SESPMNT & $\mathrm{R}$ \\
\hline 4281 & ONSITE & SESPMNT & $\mathrm{R}$ \\
\hline 4282 & ONSITE & SESPMNT & $\mathrm{R}$ \\
\hline 4283 & ONSITE & SESPMNT & $\mathrm{R}$ \\
\hline 4284 & ONSITE & SESPMNT & $\mathrm{R}$ \\
\hline 4285 & ONSITE & SESPMNT & $\mathrm{R}$ \\
\hline 4286 & ONSITE & SESPMNT & $\mathrm{R}$ \\
\hline 4287 & ONSITE & SESPMNT & $\mathrm{R}$ \\
\hline 4288 & ONSITE & SESPMNT & $\mathrm{R}$ \\
\hline 4289 & OFFSITE & SESPMNT & $\mathrm{R}$ \\
\hline 4290 & OFFSITE & SESPMNT & $\mathrm{R}$ \\
\hline 4291 & OFFSITE & SESPMNT & $\mathrm{R}$ \\
\hline 4292 & OFFSITE & SESPMNT & $\mathrm{R}$ \\
\hline 4293 & OFFSITE & SESPMNT & $\mathrm{R}$ \\
\hline 4294 & OFFSITE & SESPMNT & $\mathrm{R}$ \\
\hline 4295 & OFFSITE & SESPMNT & $\mathrm{R}$ \\
\hline & & & \\
\hline
\end{tabular}




\begin{tabular}{|c|l|l|l|}
\hline & AG & \multicolumn{1}{|c|}{ AH } & \\
\hline 1 & DIST_CLASS & OWNER_ID & COLLECTION_PURPOSE \\
\hline 4296 & OFFSITE & SESPMNT & $\mathrm{R}$ \\
\hline 4297 & OFFSITE & SESPMNT & $\mathrm{R}$ \\
\hline 4298 & OFFSITE & SESPMNT & $\mathrm{R}$ \\
\hline 4299 & OFFSITE & SESPMNT & $\mathrm{R}$ \\
\hline 4300 & OFFSITE & SESPMNT & $\mathrm{R}$ \\
\hline 4301 & OFFSITE & SESPMNT & $\mathrm{R}$ \\
\hline 4302 & OFFSITE & SESPMNT & $\mathrm{R}$ \\
\hline 4303 & OFFSITE & SESPMNT & $\mathrm{R}$ \\
\hline 4304 & OFFSITE & SESPMNT & $\mathrm{R}$ \\
\hline 4305 & OFFSITE & SESPMNT & $\mathrm{R}$ \\
\hline 4306 & OFFSITE & SESPMNT & $\mathrm{R}$ \\
\hline 4307 & OFFSITE & SESPMNT & $\mathrm{R}$ \\
\hline 4308 & OFFSITE & SESPMNT & $\mathrm{R}$ \\
\hline 4309 & OFFSITE & SESPMNT & $\mathrm{R}$ \\
\hline 4310 & OFFSITE & SESPMNT & $\mathrm{R}$ \\
\hline 4311 & OFFSITE & SESPMNT & $\mathrm{R}$ \\
\hline 4312 & OFFSITE & SESPMNT & $\mathrm{R}$ \\
\hline 4313 & OFFSITE & SESPMNT & $\mathrm{R}$ \\
\hline 4314 & OFFSITE & SESPMNT & $\mathrm{R}$ \\
\hline 4315 & OFFSITE & SESPMNT & $\mathrm{R}$ \\
\hline 4316 & OFFSITE & SESPMNT & $\mathrm{R}$ \\
\hline 4317 & OFFSITE & SESPMNT & $\mathrm{R}$ \\
\hline 4318 & OFFSITE & SESPMNT & $\mathrm{R}$ \\
\hline 4319 & OFFSITE & SESPMNT & $\mathrm{R}$ \\
\hline 4320 & OFFSITE & SESPMNT & $\mathrm{R}$ \\
\hline 4321 & OFFSITE & SESPMNT & $\mathrm{R}$ \\
\hline 4322 & OFFSITE & SESPMNT & $\mathrm{R}$ \\
\hline 4323 & OFFSITE & SESPMNT & $\mathrm{R}$ \\
\hline 4324 & OFFSITE & SESPMNT & $\mathrm{R}$ \\
\hline 4325 & OFFSITE & SESPMNT & $\mathrm{R}$ \\
\hline 4326 & OFFSITE & SESPMNT & $\mathrm{R}$ \\
\hline 4327 & OFFSITE & SESPMNT & $\mathrm{R}$ \\
\hline 4328 & OFFSITE & SESPMNT & $\mathrm{R}$ \\
\hline 4329 & OFFSITE & SESPMNT & $\mathrm{R}$ \\
\hline 4330 & OFFSITE & SESPMNT & $\mathrm{R}$ \\
\hline 4331 & OFFSITE & SESPMNT & $\mathrm{R}$ \\
\hline 4332 & OFFSITE & SESPMNT & $\mathrm{R}$ \\
\hline 4333 & OFFSITE & SESPMNT & $\mathrm{R}$ \\
\hline & & & \\
\hline
\end{tabular}




\begin{tabular}{|c|l|l|l|}
\hline & \multicolumn{1}{|c|}{ AG } & \multicolumn{1}{|c|}{ AH } & \\
\hline 1 & DIST_CLASS & OWNER_ID & COLLECTION_PURPOSE \\
\hline 4334 & OFFSITE & SESPMNT & $\mathrm{R}$ \\
\hline 4335 & OFFSITE & SESPMNT & $\mathrm{R}$ \\
\hline 4336 & OFFSITE & SESPMNT & $\mathrm{R}$ \\
\hline 4337 & OFFSITE & SESPMNT & $\mathrm{R}$ \\
\hline 4338 & OFFSITE & SESPMNT & $\mathrm{R}$ \\
\hline 4339 & ONSITE & SESPMNT & $\mathrm{R}$ \\
\hline 4340 & ONSITE & SESPMNT & $\mathrm{R}$ \\
\hline 4341 & ONSITE & SESPMNT & $\mathrm{R}$ \\
\hline 4342 & ONSITE & SESPMNT & $\mathrm{R}$ \\
\hline 4343 & ONSITE & SESPMNT & $\mathrm{R}$ \\
\hline 4344 & ONSITE & SESPMNT & $\mathrm{R}$ \\
\hline 4345 & ONSITE & SESPMNT & $\mathrm{R}$ \\
\hline 4346 & ONSITE & SESPMNT & $\mathrm{R}$ \\
\hline 4347 & ONSITE & SESPMNT & $\mathrm{R}$ \\
\hline 4348 & ONSITE & SESPMNT & $\mathrm{R}$ \\
\hline 4349 & ONSITE & SESPMNT & $\mathrm{R}$ \\
\hline 4350 & ONSITE & SESPMNT & $\mathrm{R}$ \\
\hline 4351 & ONSITE & SESPMNT & $\mathrm{R}$ \\
\hline 4352 & ONSITE & SESPMNT & $\mathrm{R}$ \\
\hline 4353 & ONSITE & SESPMNT & $\mathrm{R}$ \\
\hline 4354 & ONSITE & SESPMNT & $\mathrm{R}$ \\
\hline 4355 & ONSITE & SESPMNT & $\mathrm{R}$ \\
\hline 4356 & ONSITE & SESPMNT & $\mathrm{R}$ \\
\hline 4357 & ONSITE & SESPMNT & $\mathrm{R}$ \\
\hline 4358 & ONSITE & SESPMNT & $\mathrm{R}$ \\
\hline 4359 & ONSITE & SESPMNT & $\mathrm{R}$ \\
\hline 4360 & ONSITE & SESPMNT & $\mathrm{R}$ \\
\hline 4361 & ONSITE & SESPMNT & $\mathrm{R}$ \\
\hline 4362 & ONSITE & SESPMNT & $\mathrm{R}$ \\
\hline 4363 & ONSITE & SESPMNT & $\mathrm{R}$ \\
\hline 4364 & ONSITE & SESPMNT & $\mathrm{R}$ \\
\hline 4365 & ONSITE & SESPMNT & $\mathrm{R}$ \\
\hline 4366 & ONSITE & SESPMNT & $\mathrm{R}$ \\
\hline 4367 & ONSITE & SESPMNT & $\mathrm{R}$ \\
\hline 4368 & ONSITE & SESPMNT & $\mathrm{R}$ \\
\hline 4369 & ONSITE & SESPMNT & $\mathrm{R}$ \\
\hline 4370 & ONSITE & SESPMNT & $\mathrm{R}$ \\
\hline 4371 & ONSITE & SESPMNT & $\mathrm{R}$ \\
\hline & & & \\
\hline
\end{tabular}




\begin{tabular}{|c|l|l|l|}
\hline & \multicolumn{1}{|c|}{ AG } & \multicolumn{1}{|c|}{ AH } & \\
\hline 1 & DIST_CLASS & OWNER_ID & COLLECTION_PURPOSE \\
\hline 4372 & ONSITE & SESPMNT & $\mathrm{R}$ \\
\hline 4373 & ONSITE & SESPMNT & $\mathrm{R}$ \\
\hline 4374 & ONSITE & SESPMNT & $\mathrm{R}$ \\
\hline 4375 & ONSITE & SESPMNT & $\mathrm{R}$ \\
\hline 4376 & ONSITE & SESPMNT & $\mathrm{R}$ \\
\hline 4377 & ONSITE & SESPMNT & $\mathrm{R}$ \\
\hline 4378 & ONSITE & SESPMNT & $\mathrm{R}$ \\
\hline 4379 & ONSITE & SESPMNT & $\mathrm{R}$ \\
\hline 4380 & ONSITE & SESPMNT & $\mathrm{R}$ \\
\hline 4381 & ONSITE & SESPMNT & $\mathrm{R}$ \\
\hline 4382 & ONSITE & SESPMNT & $\mathrm{R}$ \\
\hline 4383 & ONSITE & SESPMNT & $\mathrm{R}$ \\
\hline 4384 & ONSITE & SESPMNT & $\mathrm{R}$ \\
\hline 4385 & ONSITE & SESPMNT & $\mathrm{R}$ \\
\hline 4386 & ONSITE & SESPMNT & $\mathrm{R}$ \\
\hline 4387 & ONSITE & SESPMNT & $\mathrm{R}$ \\
\hline 4388 & ONSITE & SESPMNT & $\mathrm{R}$ \\
\hline 4389 & ONSITE & SESPMNT & $\mathrm{R}$ \\
\hline 4390 & ONSITE & SESPMNT & $\mathrm{R}$ \\
\hline 4391 & ONSITE & SESPMNT & $\mathrm{R}$ \\
\hline 4392 & ONSITE & SESPMNT & $\mathrm{R}$ \\
\hline 4393 & ONSITE & SESPMNT & $\mathrm{R}$ \\
\hline 4394 & ONSITE & SESPMNT & $\mathrm{R}$ \\
\hline 4395 & ONSITE & SESPMNT & $\mathrm{R}$ \\
\hline 4396 & ONSITE & SESPMNT & $\mathrm{R}$ \\
\hline 4397 & ONSITE & SESPMNT & $\mathrm{R}$ \\
\hline 4398 & ONSITE & SESPMNT & $\mathrm{R}$ \\
\hline 4399 & ONSITE & SESPMNT & $\mathrm{R}$ \\
\hline 4400 & ONSITE & SESPMNT & $\mathrm{R}$ \\
\hline 4401 & ONSITE & SESPMNT & $\mathrm{R}$ \\
\hline 4402 & ONSITE & SESPMNT & $\mathrm{R}$ \\
\hline 4403 & ONSITE & SESPMNT & $\mathrm{R}$ \\
\hline 4404 & ONSITE & SESPMNT & $\mathrm{R}$ \\
\hline 4405 & ONSITE & SESPMNT & $\mathrm{R}$ \\
\hline 4406 & ONSITE & SESPMNT & $\mathrm{R}$ \\
\hline 4407 & ONSITE & SESPMNT & $\mathrm{R}$ \\
\hline 4408 & ONSITE & SESPMNT & $\mathrm{R}$ \\
\hline 4409 & ONSITE & SESPMNT & $\mathrm{R}$ \\
\hline & & & \\
\hline
\end{tabular}




\begin{tabular}{|c|l|l|l|}
\hline & \multicolumn{1}{|c|}{ AG } & \multicolumn{1}{|c|}{ AH } & \\
\hline 1 & DIST_CLASS & OWNER_ID & COLLECTION_PURPOSE \\
\hline 4410 & ONSITE & SESPMNT & $\mathrm{R}$ \\
\hline 4411 & ONSITE & SESPMNT & $\mathrm{R}$ \\
\hline 4412 & ONSITE & SESPMNT & $\mathrm{R}$ \\
\hline 4413 & ONSITE & SESPMNT & $\mathrm{R}$ \\
\hline 4414 & ONSITE & SESPMNT & $\mathrm{R}$ \\
\hline 4415 & ONSITE & SESPMNT & $\mathrm{R}$ \\
\hline 4416 & ONSITE & SESPMNT & $\mathrm{R}$ \\
\hline 4417 & ONSITE & SESPMNT & $\mathrm{R}$ \\
\hline 4418 & ONSITE & SESPMNT & $\mathrm{R}$ \\
\hline 4419 & ONSITE & SESPMNT & $\mathrm{R}$ \\
\hline 4420 & ONSITE & SESPMNT & $\mathrm{R}$ \\
\hline 4421 & ONSITE & SESPMNT & $\mathrm{R}$ \\
\hline 4422 & ONSITE & SESPMNT & $\mathrm{R}$ \\
\hline 4423 & ONSITE & SESPMNT & $\mathrm{R}$ \\
\hline 4424 & ONSITE & SESPMNT & $\mathrm{R}$ \\
\hline 4425 & ONSITE & SESPMNT & $\mathrm{R}$ \\
\hline 4426 & ONSITE & SESPMNT & $\mathrm{R}$ \\
\hline 4427 & ONSITE & SESPMNT & $\mathrm{R}$ \\
\hline 4428 & ONSITE & SESPMNT & $\mathrm{R}$ \\
\hline 4429 & ONSITE & SESPMNT & $\mathrm{R}$ \\
\hline 4430 & ONSITE & SESPMNT & $\mathrm{R}$ \\
\hline 4431 & ONSITE & SESPMNT & $\mathrm{R}$ \\
\hline 4432 & ONSITE & SESPMNT & $\mathrm{R}$ \\
\hline 4433 & ONSITE & SESPMNT & $\mathrm{R}$ \\
\hline 4434 & ONSITE & SESPMNT & $\mathrm{R}$ \\
\hline 4435 & ONSITE & SESPMNT & $\mathrm{R}$ \\
\hline 4436 & ONSITE & SESPMNT & $\mathrm{R}$ \\
\hline 4437 & ONSITE & SESPMNT & $\mathrm{R}$ \\
\hline 4438 & ONSITE & SESPMNT & $\mathrm{R}$ \\
\hline 4439 & OFFSITE & SESPMNT & $\mathrm{R}$ \\
\hline 4440 & OFFSITE & SESPMNT & $\mathrm{R}$ \\
\hline 4441 & OFFSITE & SESPMNT & $\mathrm{R}$ \\
\hline 4442 & OFFSITE & SESPMNT & $\mathrm{R}$ \\
\hline 4443 & OFFSITE & SESPMNT & $\mathrm{R}$ \\
\hline 4444 & OFFSITE & SESPMNT & $\mathrm{R}$ \\
\hline 4445 & OFFSITE & SESPMNT & $\mathrm{R}$ \\
\hline 4446 & OFFSITE & SESPMNT & $\mathrm{R}$ \\
\hline 4447 & OFFSITE & SESPMNT & $\mathrm{R}$ \\
\hline & & & \\
\hline
\end{tabular}




\begin{tabular}{|c|l|l|l|}
\hline & \multicolumn{1}{|c|}{ AG } & \multicolumn{1}{|c|}{ AH } & \\
\hline 1 & DIST_CLASS & OWNER_ID & COLLECTION_PURPOSE \\
\hline 4448 & OFFSITE & SESPMNT & $\mathrm{R}$ \\
\hline 4449 & OFFSITE & SESPMNT & $\mathrm{R}$ \\
\hline 4450 & OFFSITE & SESPMNT & $\mathrm{R}$ \\
\hline 4451 & OFFSITE & SESPMNT & $\mathrm{R}$ \\
\hline 4452 & OFFSITE & SESPMNT & $\mathrm{R}$ \\
\hline 4453 & OFFSITE & SESPMNT & $\mathrm{R}$ \\
\hline 4454 & OFFSITE & SESPMNT & $\mathrm{R}$ \\
\hline 4455 & OFFSITE & SESPMNT & $\mathrm{R}$ \\
\hline 4456 & OFFSITE & SESPMNT & $\mathrm{R}$ \\
\hline 4457 & OFFSITE & SESPMNT & $\mathrm{R}$ \\
\hline 4458 & OFFSITE & SESPMNT & $\mathrm{R}$ \\
\hline 4459 & OFFSITE & SESPSPEC & $\mathrm{S}$ \\
\hline 4460 & OFFSITE & SESPMNT & $\mathrm{R}$ \\
\hline 4461 & OFFSITE & SESPSPEC & $\mathrm{S}$ \\
\hline 4462 & OFFSITE & SESPMNT & $\mathrm{R}$ \\
\hline 4463 & OFFSITE & SESPSPEC & $\mathrm{S}$ \\
\hline 4464 & OFFSITE & SESPSPEC & $\mathrm{S}$ \\
\hline 4465 & OFFSITE & SESPSPEC & $\mathrm{S}$ \\
\hline 4466 & OFFSITE & SESPMNT & $\mathrm{R}$ \\
\hline 4467 & OFFSITE & SESPMNT & $\mathrm{R}$ \\
\hline 4468 & OFFSITE & SESPMNT & $\mathrm{R}$ \\
\hline 4469 & OFFSITE & SESPMNT & $\mathrm{R}$ \\
\hline 4470 & OFFSITE & SESPMNT & $\mathrm{R}$ \\
\hline 4471 & OFFSITE & SESPMNT & $\mathrm{R}$ \\
\hline 4472 & OFFSITE & SESPMNT & $\mathrm{R}$ \\
\hline 4473 & OFFSITE & SESPMNT & $\mathrm{R}$ \\
\hline 4474 & OFFSITE & SESPMNT & $\mathrm{R}$ \\
\hline 4475 & OFFSITE & SESPMNT & $\mathrm{R}$ \\
\hline 4476 & OFFSITE & SESPMNT & $\mathrm{R}$ \\
\hline 4477 & OFFSITE & SESPMNT & $\mathrm{R}$ \\
\hline 4478 & OFFSITE & SESPMNT & $\mathrm{R}$ \\
\hline 4479 & OFFSITE & SESPMNT & $\mathrm{R}$ \\
\hline 4480 & OFFSITE & SESPMNT & $\mathrm{R}$ \\
\hline 4481 & OFFSITE & SESPMNT & $\mathrm{R}$ \\
\hline 4482 & OFFSITE & SESPMNT & $\mathrm{R}$ \\
\hline 4483 & OFFSITE & SESPMNT & $\mathrm{R}$ \\
\hline 4484 & OFFSITE & SESPMNT & $\mathrm{R}$ \\
\hline 4485 & OFFSITE & SESPMNT & $\mathrm{R}$ \\
\hline & & & \\
\hline
\end{tabular}




\begin{tabular}{|c|l|l|l|}
\hline & AG & \multicolumn{1}{|c|}{ AH } & \multicolumn{1}{c|}{ AI } \\
\hline 1 & DIST_CLASS & OWNER_ID & COLLECTION_PURPOSE \\
\hline 4486 & OFFSITE & SESPMNT & $\mathrm{R}$ \\
\hline 4487 & OFFSITE & SESPMNT & $\mathrm{R}$ \\
\hline 4488 & OFFSITE & SESPMNT & $\mathrm{R}$ \\
\hline 4489 & OFFSITE & SESPMNT & $\mathrm{R}$ \\
\hline 4490 & OFFSITE & SESPMNT & $\mathrm{R}$ \\
\hline 4491 & OFFSITE & SESPMNT & $\mathrm{R}$ \\
\hline 4492 & OFFSITE & SESPMNT & $\mathrm{R}$ \\
\hline 4493 & OFFSITE & SESPMNT & $\mathrm{R}$ \\
\hline 4494 & OFFSITE & SESPMNT & \\
\hline 4495 & OFFSITE & SESPMNT & \\
\hline 4496 & OFFSITE & SESPMNT & \\
\hline 4497 & OFFSITE & SESPMNT & \\
\hline 4498 & OFFSITE & SESPMNT & \\
\hline 4499 & OFFSITE & SESPMNT & \\
\hline 4500 & OFFSITE & SESPMNT & \\
\hline 4501 & OFFSITE & SESPMNT & \\
\hline 4502 & OFFSITE & SESPMNT & \\
\hline 4503 & OFFSITE & SESPMNT & \\
\hline 4504 & OFFSITE & SESPMNT & $\mathrm{R}$ \\
\hline 4505 & OFFSITE & SESPMNT & $\mathrm{R}$ \\
\hline 4506 & OFFSITE & SESPMNT & $\mathrm{R}$ \\
\hline 4507 & OFFSITE & SESPMNT & $\mathrm{R}$ \\
\hline 4508 & OFFSITE & SESPMNT & $\mathrm{R}$ \\
\hline 4509 & OFFSITE & SESPMNT & $\mathrm{R}$ \\
\hline 4510 & OFFSITE & SESPMNT & $\mathrm{R}$ \\
\hline 4511 & OFFSITE & SESPMNT & $\mathrm{R}$ \\
\hline 4512 & OFFSITE & SESPMNT & $\mathrm{R}$ \\
\hline 4513 & OFFSITE & SESPMNT & $\mathrm{R}$ \\
\hline 4514 & OFFSITE & SESPMNT & $\mathrm{R}$ \\
\hline 4515 & OFFSITE & SESPMNT & $\mathrm{R}$ \\
\hline 4516 & OFFSITE & SESPMNT & $\mathrm{R}$ \\
\hline 4517 & OFFSITE & SESPMNT & $\mathrm{R}$ \\
\hline 4518 & OFFSITE & SESPMNT & $\mathrm{R}$ \\
\hline 4519 & OFFSITE & SESPMNT & $\mathrm{R}$ \\
\hline 4520 & OFFSITE & SESPMNT & $\mathrm{R}$ \\
\hline 4521 & OFFSITE & SESPMNT & $\mathrm{R}$ \\
\hline 4522 & OFFSITE & SESPMNT & $\mathrm{R}$ \\
\hline 4523 & OFFSITE & SESPMNT & $\mathrm{R}$ \\
\hline & & & \\
\hline
\end{tabular}




\begin{tabular}{|c|l|l|l|}
\hline & AG & \multicolumn{1}{|c|}{ AH } & \\
\hline 1 & DIST_CLASS & OWNER_ID & COLLECTION_PURPOSE \\
\hline 4524 & OFFSITE & SESPMNT & \\
\hline 4525 & OFFSITE & SESPMNT & \\
\hline 4526 & OFFSITE & SESPMNT & \\
\hline 4527 & OFFSITE & SESPMNT & \\
\hline 4528 & OFFSITE & SESPMNT & $\mathrm{R}$ \\
\hline 4529 & OFFSITE & SESPMNT & $\mathrm{R}$ \\
\hline 4530 & OFFSITE & SESPMNT & $\mathrm{R}$ \\
\hline 4531 & OFFSITE & SESPMNT & $\mathrm{R}$ \\
\hline 4532 & OFFSITE & SESPMNT & $\mathrm{R}$ \\
\hline 4533 & OFFSITE & SESPMNT & $\mathrm{R}$ \\
\hline 4534 & OFFSITE & SESPMNT & $\mathrm{R}$ \\
\hline 4535 & OFFSITE & SESPMNT & $\mathrm{R}$ \\
\hline 4536 & OFFSITE & SESPMNT & $\mathrm{R}$ \\
\hline 4537 & OFFSITE & SESPMNT & $\mathrm{R}$ \\
\hline 4538 & OFFSITE & SESPMNT & $\mathrm{R}$ \\
\hline 4539 & OFFSITE & SESPMNT & $\mathrm{R}$ \\
\hline 4540 & OFFSITE & SESPMNT & $\mathrm{R}$ \\
\hline 4541 & OFFSITE & SESPMNT & $\mathrm{R}$ \\
\hline 4542 & OFFSITE & SESPMNT & $\mathrm{R}$ \\
\hline 4543 & OFFSITE & SESPMNT & $\mathrm{R}$ \\
\hline 4544 & OFFSITE & SESPMNT & $\mathrm{R}$ \\
\hline 4545 & OFFSITE & SESPMNT & $\mathrm{R}$ \\
\hline 4546 & OFFSITE & SESPMNT & $\mathrm{R}$ \\
\hline 4547 & OFFSITE & SESPMNT & $\mathrm{R}$ \\
\hline 4548 & OFFSITE & SESPMNT & $\mathrm{R}$ \\
\hline 4549 & OFFSITE & SESPMNT & $\mathrm{R}$ \\
\hline 4550 & OFFSITE & SESPMNT & $\mathrm{R}$ \\
\hline 4551 & OFFSITE & SESPMNT & $\mathrm{R}$ \\
\hline 4552 & OFFSITE & SESPMNT & $\mathrm{R}$ \\
\hline 4553 & OFFSITE & SESPMNT & $\mathrm{R}$ \\
\hline 4554 & OFFSITE & SESPMNT & $\mathrm{R}$ \\
\hline 4555 & OFFSITE & SESPMNT & $\mathrm{R}$ \\
\hline 4556 & OFFSITE & SESPMNT & $\mathrm{R}$ \\
\hline 4557 & OFFSITE & SESPMNT & $\mathrm{R}$ \\
\hline 4558 & OFFSITE & SESPMNT & $\mathrm{R}$ \\
\hline 4559 & OFFSITE & SESPMNT & $\mathrm{R}$ \\
\hline 4560 & OFFSITE & SESPMNT & $\mathrm{R}$ \\
\hline 4561 & OFFSITE & SESPMNT & $\mathrm{R}$ \\
\hline & & & \\
\hline
\end{tabular}




\begin{tabular}{|c|l|l|l|}
\hline & \multicolumn{1}{|c|}{ AG } & \multicolumn{1}{|c|}{ AH } & \\
\hline 1 & DIST_CLASS & OWNER_ID & COLLECTION_PURPOSE \\
\hline 4562 & OFFSITE & SESPMNT & $\mathrm{R}$ \\
\hline 4563 & OFFSITE & SESPMNT & $\mathrm{R}$ \\
\hline 4564 & OFFSITE & SESPMNT & $\mathrm{R}$ \\
\hline 4565 & OFFSITE & SESPMNT & $\mathrm{R}$ \\
\hline 4566 & OFFSITE & SESPMNT & $\mathrm{R}$ \\
\hline 4567 & OFFSITE & SESPMNT & $\mathrm{R}$ \\
\hline 4568 & OFFSITE & SESPSPEC & $\mathrm{S}$ \\
\hline 4569 & OFFSITE & SESPMNT & $\mathrm{R}$ \\
\hline 4570 & OFFSITE & SESPSPEC & $\mathrm{S}$ \\
\hline 4571 & OFFSITE & SESPMNT & $\mathrm{R}$ \\
\hline 4572 & OFFSITE & SESPSPEC & $\mathrm{S}$ \\
\hline 4573 & OFFSITE & SESPSPEC & $\mathrm{S}$ \\
\hline 4574 & OFFSITE & SESPSPEC & $\mathrm{S}$ \\
\hline 4575 & OFFSITE & SESPMNT & $\mathrm{R}$ \\
\hline 4576 & OFFSITE & SESPMNT & $\mathrm{R}$ \\
\hline 4577 & OFFSITE & SESPMNT & $\mathrm{R}$ \\
\hline 4578 & OFFSITE & SESPMNT & $\mathrm{R}$ \\
\hline 4579 & OFFSITE & SESPMNT & $\mathrm{R}$ \\
\hline 4580 & OFFSITE & SESPMNT & $\mathrm{R}$ \\
\hline 4581 & OFFSITE & SESPMNT & $\mathrm{R}$ \\
\hline 4582 & OFFSITE & SESPMNT & $\mathrm{R}$ \\
\hline 4583 & OFFSITE & SESPMNT & $\mathrm{R}$ \\
\hline 4584 & OFFSITE & SESPMNT & $\mathrm{R}$ \\
\hline 4585 & OFFSITE & SESPMNT & $\mathrm{R}$ \\
\hline 4586 & OFFSITE & SESPMNT & $\mathrm{R}$ \\
\hline 4587 & OFFSITE & SESPMNT & $\mathrm{R}$ \\
\hline 4588 & OFFSITE & SESPMNT & $\mathrm{R}$ \\
\hline 4589 & OFFSITE & SESPMNT & $\mathrm{R}$ \\
\hline 4590 & OFFSITE & SESPMNT & $\mathrm{R}$ \\
\hline 4591 & OFFSITE & SESPMNT & $\mathrm{R}$ \\
\hline 4592 & OFFSITE & SESPMNT & $\mathrm{R}$ \\
\hline 4593 & OFFSITE & SESPMNT & $\mathrm{R}$ \\
\hline 4594 & OFFSITE & SESPMNT & $\mathrm{R}$ \\
\hline 4595 & OFFSITE & SESPMNT & $\mathrm{R}$ \\
\hline 4596 & OFFSITE & SESPMNT & $\mathrm{R}$ \\
\hline 4597 & OFFSITE & SESPMNT & $\mathrm{R}$ \\
\hline 4598 & OFFSITE & SESPMNT & $\mathrm{R}$ \\
\hline 4599 & OFFSITE & SESPMNT & $\mathrm{R}$ \\
\hline & & & \\
\hline
\end{tabular}




\begin{tabular}{|c|l|l|l|}
\hline & AG & \multicolumn{1}{|c|}{ AH } & \\
\hline 1 & DIST_CLASS & OWNER_ID & COLLECTION_PURPOSE \\
\hline 4600 & OFFSITE & SESPMNT & $\mathrm{R}$ \\
\hline 4601 & OFFSITE & SESPMNT & $\mathrm{R}$ \\
\hline 4602 & OFFSITE & SESPMNT & $\mathrm{R}$ \\
\hline 4603 & OFFSITE & SESPMNT & $\mathrm{R}$ \\
\hline 4604 & OFFSITE & SESPMNT & $\mathrm{R}$ \\
\hline 4605 & OFFSITE & SESPMNT & $\mathrm{R}$ \\
\hline 4606 & OFFSITE & SESPMNT & $\mathrm{R}$ \\
\hline 4607 & OFFSITE & SESPMNT & $\mathrm{R}$ \\
\hline 4608 & OFFSITE & SESPMNT & $\mathrm{R}$ \\
\hline 4609 & OFFSITE & SESPMNT & $\mathrm{R}$ \\
\hline 4610 & OFFSITE & SESPMNT & $\mathrm{R}$ \\
\hline 4611 & OFFSITE & SESPMNT & $\mathrm{R}$ \\
\hline 4612 & OFFSITE & SESPMNT & $\mathrm{R}$ \\
\hline 4613 & OFFSITE & SESPMNT & $\mathrm{R}$ \\
\hline 4614 & OFFSITE & SESPMNT & $\mathrm{R}$ \\
\hline 4615 & OFFSITE & SESPMNT & $\mathrm{R}$ \\
\hline 4616 & OFFSITE & SESPMNT & $\mathrm{R}$ \\
\hline 4617 & OFFSITE & SESPMNT & $\mathrm{R}$ \\
\hline 4618 & OFFSITE & SESPMNT & $\mathrm{R}$ \\
\hline 4619 & OFFSITE & SESPMNT & $\mathrm{R}$ \\
\hline 4620 & OFFSITE & SESPMNT & $\mathrm{R}$ \\
\hline 4621 & OFFSITE & SESPMNT & $\mathrm{R}$ \\
\hline 4622 & OFFSITE & SESPMNT & $\mathrm{R}$ \\
\hline 4623 & OFFSITE & SESPMNT & $\mathrm{R}$ \\
\hline 4624 & OFFSITE & SESPMNT & $\mathrm{R}$ \\
\hline 4625 & OFFSITE & SESPMNT & $\mathrm{R}$ \\
\hline 4626 & OFFSITE & SESPMNT & $\mathrm{R}$ \\
\hline 4627 & OFFSITE & SESPMNT & $\mathrm{R}$ \\
\hline 4628 & OFFSITE & SESPMNT & $\mathrm{R}$ \\
\hline 4629 & OFFSITE & SESPMNT & $\mathrm{R}$ \\
\hline 4630 & OFFSITE & SESPMNT & $\mathrm{R}$ \\
\hline 4631 & OFFSITE & SESPMNT & $\mathrm{R}$ \\
\hline 4632 & OFFSITE & SESPMNT & $\mathrm{R}$ \\
\hline 4633 & OFFSITE & SESPMNT & $\mathrm{R}$ \\
\hline 4634 & OFFSITE & SESPMNT & $\mathrm{R}$ \\
\hline 4635 & OFFSITE & SESPMNT & $\mathrm{R}$ \\
\hline 4636 & OFFSITE & SESPMNT & $\mathrm{R}$ \\
\hline 4637 & OFFSITE & SESPMNT & $\mathrm{R}$ \\
\hline & & & \\
\hline
\end{tabular}




\begin{tabular}{|c|c|c|c|}
\hline & $\mathrm{AG}$ & $\overline{\mathrm{AH}}$ & $\mathrm{Al}$ \\
\hline 1 & DIST CLASS & OWNER ID & COLLECTION PURPOSE \\
\hline 4638 & OFFSITE & SESPMNT & $\mathrm{R}$ \\
\hline 4639 & OFFSITE & SESPMNT & $\mathrm{R}$ \\
\hline 4640 & OFFSITE & SESPMNT & $\mathrm{R}$ \\
\hline 4641 & OFFSITE & SESPMNT & $\mathrm{R}$ \\
\hline 4642 & OFFSITE & SESPMNT & $\mathrm{R}$ \\
\hline 4643 & OFFSITE & SESPMNT & $\mathrm{R}$ \\
\hline 4644 & OFFSITE & SESPMNT & $\mathrm{R}$ \\
\hline 4645 & OFFSITE & SESPMNT & $\mathrm{R}$ \\
\hline 4646 & OFFSITE & SESPMNT & $\mathrm{R}$ \\
\hline 4647 & OFFSITE & SESPMNT & $\mathrm{R}$ \\
\hline 4648 & OFFSITE & SESPMNT & $\mathrm{R}$ \\
\hline 4649 & OFFSITE & SESPMNT & $\mathrm{R}$ \\
\hline 4650 & OFFSITE & SESPMNT & $\mathrm{R}$ \\
\hline 4651 & OFFSITE & SESPMNT & $\mathrm{R}$ \\
\hline 4652 & OFFSITE & SESPMNT & $\mathrm{R}$ \\
\hline 4653 & OFFSITE & SESPMNT & $\mathrm{R}$ \\
\hline 4654 & OFFSITE & SESPMNT & $\mathrm{R}$ \\
\hline 4655 & OFFSITE & SESPMNT & $\mathrm{R}$ \\
\hline 4656 & OFFSITE & SESPMNT & $\mathrm{R}$ \\
\hline 4657 & OFFSITE & SESPMNT & $\mathrm{R}$ \\
\hline 4658 & OFFSITE & SESPMNT & $\mathrm{R}$ \\
\hline 4659 & OFFSITE & SESPMNT & $\mathrm{R}$ \\
\hline 4660 & OFFSITE & SESPMNT & $\mathrm{R}$ \\
\hline 4661 & OFFSITE & SESPMNT & $\mathrm{R}$ \\
\hline 4662 & OFFSITE & SESPMNT & $\mathrm{R}$ \\
\hline 4663 & OFFSITE & SESPMNT & $\mathrm{R}$ \\
\hline 4664 & OFFSITE & SESPMNT & $\mathrm{R}$ \\
\hline 4665 & OFFSITE & SESPMNT & $\mathrm{R}$ \\
\hline 4666 & OFFSITE & SESPMNT & $\mathrm{R}$ \\
\hline 4667 & OFFSITE & SESPMNT & $\mathrm{R}$ \\
\hline 4668 & OFFSITE & SESPMNT & $\mathrm{R}$ \\
\hline 4669 & OFFSITE & SESPMNT & $\mathrm{R}$ \\
\hline 4670 & OFFSITE & SESPMNT & $\mathrm{R}$ \\
\hline 4671 & OFFSITE & SESPMNT & $\mathrm{R}$ \\
\hline 4672 & OFFSITE & SESPMNT & $\mathrm{R}$ \\
\hline
\end{tabular}




\section{0 - 95 Sediment}




\begin{tabular}{|c|c|c|c|c|c|c|c|c|c|c|c|}
\hline & $A$ & $B$ & $C$ & $\mathrm{D}$ & $E$ & $\mathrm{~F}$ & $\mathrm{G}$ & $\mathrm{H}$ & $\mathrm{I}$ & $\mathrm{J}$ & $\mathrm{K}$ \\
\hline 1 & SAMPLE NUM ASSIGNED TO & SAMPLER & MEDIA & BIOTA MEDIA & TAG ID & SAMP NUM & SAMP DATE TIME & SAMP DATE TIME ON & CON ID & CON SHORT NAME & METHOD NAME \\
\hline 2 & PDMS & & SO & & & E119895 & 14-Dec-90 & & $15117-96-1$ & U-235 & UISO AEA \\
\hline 3 & PDMS & & SO & & & E119896 & 14-Dec-90 & & 15117-96-1 & U-235 & UISO_AEA \\
\hline 4 & PDMS & & SO & & & E119894 & 14-Dec-90 & & 15117-96-1 & U-235 & UISO_AEA \\
\hline 5 & PDMS & & SO & & & E119897 & 14-Dec-90 & & 15117-96-1 & U-235 & UISO_AEA \\
\hline 6 & PDMS & & SO & & & E118450 & 18-Dec-90 & & 15117-96-1 & U-235 & UISO_AEA \\
\hline 7 & PDMS & & SO & & & E118451 & 18-Dec-90 & & 15117-96-1 & U-235 & UISO_AEA \\
\hline 8 & PDMS & & SO & & & E118452 & 18-Dec-90 & & $15117-96-1$ & U-235 & UISO_AEA \\
\hline 9 & PDMS & & SO & & & E118449 & 18-Dec-90 & & 15117-96-1 & U-235 & UISO_AEA \\
\hline 11 & PDMS & & SO & & & E119892 & 18-Jan-91 & & $15117-96-1$ & U-235 & UISO_AEA \\
\hline 12 & PDMS & & SO & & & E119890 & 18-Jan-91 & & $15117-96-1$ & U-235 & UISO_AEA \\
\hline 13 & PDMS & & SO & & & E119893 & 18-Jan-91 & & 15117-96-1 & U-235 & UISO_AEA \\
\hline 14 & PDMS & & SO & & & E119899 & 25-Nov-91 & & $15117-96-1$ & U-235 & UISO_AEA \\
\hline 15 & PDMS & & SO & & & E119900 & 25-Nov-91 & & 15117-96-1 & U-235 & UISO_AEA \\
\hline 16 & PDMS & & SO & & & E119898 & 25-Nov-91 & & 15117-96-1 & U-235 & UISO_AEA \\
\hline 17 & PDMS & & SO & & & E119901 & 25-Nov-91 & & 15117-96-1 & U-235 & UISO_AEA \\
\hline 18 & PDMS & & SO & & & E118323 & 27-Nov-91 & & 15117-96-1 & U-235 & UISO_AEA \\
\hline 19 & PDMS & & SO & & & E118322 & 27-Nov-91 & & 15117-96-1 & U-235 & UISO_AEA \\
\hline 21 & PDMS & & SO & & & E118324 & 02-Dec-91 & & $15117-96-1$ & U-235 & UISO_AEA \\
\hline 22 & PDMS & & SO & & & E119903 & 13-Dec-91 & & $15117-96-1$ & U-235 & UISO AEA \\
\hline 23 & PDMS & & SO & & & E119904 & 13-Dec-91 & & $15117-96-1$ & U-235 & UISO_AEA \\
\hline 24 & PDMS & & SO & & & E119902 & 13-Dec-91 & & $15117-96-1$ & U-235 & UISO_AEA \\
\hline 25 & PDMS & & SO & & & E119905 & 13-Dec-91 & & $15117-96-1$ & U-235 & UISO_AEA \\
\hline 26 & PDMS & & SO & & & E121541 & 21-Sep-92 & & 15117-96-1 & U-235 & GAMM̄A_GS \\
\hline 27 & PDMS & & SO & & & E121543 & 21-Sep-92 & & $15117-96-1$ & U-235 & GAMMA_GS \\
\hline 28 & PDMS & & SO & & & E121542 & 21-Sep-92 & & 15117-96-1 & U-235 & GAMMA_GS \\
\hline 29 & PDMS & & SO & & & E121544 & 22-Sep-92 & & 15117-96-1 & U-235 & GAMMA_GS \\
\hline 30 & PDMS & & SO & & & E033017 & 29-Sep-92 & & 15117-96-1 & U-235 & GAMMA_GS \\
\hline 31 & PDMS & & SO & & & E033018 & 29-Sep-92 & & 15117-96-1 & U-235 & GAMMA_GS \\
\hline 32 & PDMS & & SO & & & E033016 & 29-Sep-92 & & 15117-96-1 & U-235 & GAMMA_GS \\
\hline 33 & PDMS & & SO & & & E033019 & 29-Sep-92 & & 15117-96-1 & U-235 & GAMMA_GS \\
\hline 34 & PDMS & & SO & & & E033013 & 01-Oct-92 & & $15117-96-1$ & $\mathrm{U}-235$ & GAMMA GS \\
\hline 35 & PDMS & & SO & & & E033014 & 01-Oct-92 & & $15117-96-1$ & U-235 & GAMMA_GS \\
\hline 36 & PDMS & & SO & & & E033012 & 01-Oct-92 & & 15117-96-1 & U-235 & GAMMA_GS \\
\hline 37 & PDMS & & SO & & & E033015 & 01-Oct-92 & & $15117-96-1$ & U-235 & GAMMA_GS \\
\hline 38 & PDMS & & SO & & & E124478 & 20-Aug-93 & & $15117-96-1$ & U-235 & GAMMA_GS \\
\hline
\end{tabular}




\begin{tabular}{|c|c|c|c|c|c|c|c|c|c|c|c|}
\hline & A & $\mathrm{B}$ & $\mathrm{C}$ & $\mathrm{D}$ & $E$ & $\mathrm{~F}$ & G & $\mathrm{H}$ & I & $\mathrm{J}$ & $\mathrm{K}$ \\
\hline 1 & SAMPLE_NUM_ASSIGNED_TO & SAMPLER & MEDIA & BIOTA_MEDIA & TAG_ID & SAMP_NUM & SAMP_DATE_TIME & SAMP_DATE_TIME_ON & CON_ID & CON_SHORT_NAME & METHOD_NAME \\
\hline 40 & PDMS & & SO & & & E124477 & 20-Aug-93 & & $15117-96-1$ & $\mathrm{U}-235$ & GAMMA_GS \\
\hline 41 & PDMS & & SO & & & E124479 & 20-Aug-93 & & 15117-96-1 & U-235 & GAMMA_GS \\
\hline 42 & PDMS & & SO & & & E124526 & 02-Sep-93 & & 15117-96-1 & U-235 & GAMMA_GS \\
\hline 43 & PDMS & & SO & & & E124524 & 02-Sep-93 & & 15117-96-1 & U-235 & GAMMA_GS \\
\hline 44 & PDMS & & SO & & & E124527 & 02-Sep-93 & & 15117-96-1 & U-235 & GAMMA_GS \\
\hline 45 & PDMS & & SO & & & E124525 & 02-Sep-93 & & 15117-96-1 & U-235 & GAMMA_GS \\
\hline 46 & PDMS & & SO & & & E124607 & 20-Sep-93 & & 15117-96-1 & U-235 & GAMMA_GS \\
\hline 47 & PDMS & & SO & & & E124545 & 20-Sep-93 & & 15117-96-1 & U-235 & GAMMA_GS \\
\hline 49 & PDMS & & SO & & & E124779 & 28-Sep-93 & & 15117-96-1 & U-235 & GAMMA_GS \\
\hline 50 & PDMS & & SO & & & E124778 & 28-Sep-93 & & 15117-96-1 & U-235 & GAMMA_GS \\
\hline 51 & PDMS & & SO & & & E124780 & 28-Sep-93 & & 15117-96-1 & U-235 & GAMMA_GS \\
\hline 52 & SESPSCH & & SO & & & B0BRS5 & 11-Apr-94 & & 15117-96-1 & U-235 & GAMMA_GS \\
\hline 53 & SESPSCH & & SO & & & B0BRS7 & 11-Apr-94 & & 15117-96-1 & U-235 & GAMMA_GS \\
\hline 54 & SESPSCH & & SO & & & B0BRS3 & 11-Apr-94 & & 15117-96-1 & U-235 & GAMMA_GS \\
\hline 55 & SESPSCH & & SO & & & B0BRS9 & 11-Apr-94 & & $15117-96-1$ & U-235 & GAMMA_GS \\
\hline 56 & SESPSCH & & SO & & & B0BRT8 & 14-Apr-94 & & 15117-96-1 & U-235 & GAMMA_GS \\
\hline 57 & SESPSCH & & SO & & & BOBRVO & 14-Apr-94 & & $15117-96-1$ & U-235 & GAMMA_GS \\
\hline 59 & SESPSCH & & SO & & & BOBRV2 & 14-Apr-94 & & 15117-96-1 & U-235 & GAMMA_GS \\
\hline 60 & SESPSCH & & SO & & & B0BRV6 & 15-Apr-94 & & 15117-96-1 & U-235 & GAMMA_GS \\
\hline 61 & SESPSCH & & SO & & & B0BRV8 & 15-Apr-94 & & $15117-96-1$ & U-235 & GAMMA_GS \\
\hline 62 & SESPSCH & & SO & & & BOBRW0 & 15-Apr-94 & & 15117-96-1 & U-235 & GAMMA_GS \\
\hline 63 & SESPSCH & & SO & & & B0BRV4 & 15-Apr-94 & & 15117-96-1 & U-235 & GAMMA_GS \\
\hline 64 & SESPSCH & & SO & & & B0CDM6 & 29-Aug-94 & & $15117-96-1$ & U-235 & GAMMA_GS \\
\hline 65 & SESPSCH & & SO & & & B0CDL3 & 22-Sep-94 & & 15117-96-1 & U-235 & GAMMA_GS \\
\hline 66 & SESPSCH & & SO & & & B0CDL5 & 22-Sep-94 & & 15117-96-1 & U-235 & GAMMA_GS \\
\hline 67 & SESPSCH & & SO & & & B0CDL1 & 22-Sep-94 & & 15117-96-1 & U-235 & GAMMA_GS \\
\hline 68 & SESPSCH & & SO & & & B0CDL7 & 22-Sep-94 & & $15117-96-1$ & U-235 & GAMMA_GS \\
\hline 69 & SESPSCH & & SO & & & B0CDK5 & 23-Sep-94 & & $15117-96-1$ & U-235 & GAMMA_GS \\
\hline 70 & SESPSCH & & SO & & & B0CDK7 & 23-Sep-94 & & 15117-96-1 & U-235 & GAMMA_GS \\
\hline 71 & SESPSCH & & SO & & & B0CDK3 & 23-Sep-94 & & 15117-96-1 & U-235 & GAMMA_GS \\
\hline 72 & SESPSCH & & SO & & & B0CDK9 & 23-Sep-94 & & 15117-96-1 & U-235 & GAMMA_GS \\
\hline 73 & SESPSCH & & SO & & & BOCDMO & 24-Sep-94 & & 15117-96-1 & U-235 & UISO_AEA \\
\hline 74 & SESPSCH & & SO & & & B0CDL9 & 24-Sep-94 & & $15117-96-1$ & U-235 & UISO_AEA \\
\hline 75 & SESPSCH & & SO & & & B0CDM1 & 25-Sep-94 & & 15117-96-1 & U-235 & UISO_AEA \\
\hline 76 & SESPSCH & & SO & & & B0CDM5 & 25-Sep-94 & & 15117-96-1 & U-235 & UISO_AEA \\
\hline
\end{tabular}




\begin{tabular}{|c|c|c|c|c|c|c|c|c|c|c|c|}
\hline & $\mathrm{A}$ & $\mathrm{B}$ & $\mathrm{C}$ & $\mathrm{D}$ & $E$ & $\mathrm{~F}$ & $\mathrm{G}$ & $\mathrm{H}$ & $\mathrm{I}$ & $\mathrm{J}$ & $\mathrm{K}$ \\
\hline 1 & SAMPLE_NUM_ASSIGNED_TO & SAMPLER & MEDIA & BIOTA_MEDIA & TAG_ID & SAMP_NUM & SAMP_DATE_TIME & SAMP_DATE_TIME_ON & CON_ID & CON_SHORT_NAME & METHOD_NAME \\
\hline 78 & SESPSCH & & SO & & & BOG8W0 & 21-Jul-95 & & $15117-96-1$ & U-235 & UISO_AEA \\
\hline 79 & SESPSCH & & SO & & & B0G8W1 & 21-Jul-95 & & 15117-96-1 & U-235 & GAMMA_GS \\
\hline 80 & SESPSCH & & SO & & & B0G8W2 & 21-Jul-95 & & 15117-96-1 & U-235 & GAMMA_GS \\
\hline 81 & SESPSCH & & SO & & & B0G8V9 & 21-Jul-95 & & 15117-96-1 & U-235 & GAMMA_GS \\
\hline 82 & SESPSCH & & SO & & & B0G8V3 & 24-Jul-95 & & 15117-96-1 & U-235 & GAMMA_GS \\
\hline 83 & SESPSCH & & SO & & & B0G8V5 & 24-Jul-95 & & 15117-96-1 & U-235 & GAMMA_GS \\
\hline 84 & SESPSCH & & SO & & & B0G8V1 & 24-Jul-95 & & 15117-96-1 & U-235 & GAMMA_GS \\
\hline 85 & SESPSCH & & SO & & & B0G8V7 & 24-Jul-95 & & 15117-96-1 & U-235 & GAMMA_GS \\
\hline 87 & SESPSCH & & SO & & & B0G8T7 & 28-Jul-95 & & 15117-96-1 & U-235 & GAMMA_GS \\
\hline 88 & SESPSCH & & SO & & & B0G8T3 & 28-Jul-95 & & 15117-96-1 & U-235 & GAMMA_GS \\
\hline 89 & SESPSCH & & SO & & & B0G8T9 & 28-Jul-95 & & 15117-96-1 & U-235 & GAMMA_GS \\
\hline 90 & SESPSCH & & SO & & & B0G8W6 & 28-Aug-95 & & $15117-96-1$ & U-235 & GAMMA_GS \\
\hline 91 & SESPSCH & & SO & & & B0GJY7 & 05-Sep-95 & & $15117-96-1$ & U-235 & GAMMA_GS \\
\hline 92 & SESPSCH & & SO & & & B0G8W5 & 05-Sep-95 & & $15117-96-1$ & U-235 & GAMMA_GS \\
\hline 93 & SESPSCH & & SO & & & B0G8W4 & 05-Sep-95 & & 15117-96-1 & U-235 & GAMMA_GS \\
\hline 94 & SESPSCH & & SO & & & B0GKL4 & 12-Sep-95 & & 15117-96-1 & U-235 & GAMMA_GS \\
\hline 95 & PDMS & & SO & & & E119895 & 14-Dec-90 & & PU-239/240 & PU-239/240 & PUISO_PLATE_AEA \\
\hline 97 & PDMS & & SO & & & E119894 & 14-Dec-90 & & PU-239/240 & PU-239/240 & PUISO_PLATE_AEA \\
\hline 98 & PDMS & & SO & & & E119897 & 14-Dec-90 & & PU-239/240 & PU-239/240 & PUISO_PLATE_AEA \\
\hline 99 & PDMS & & SO & & & E118450 & 18-Dec-90 & & PU-239/240 & PU-239/240 & PUISO_PLATE_AEA \\
\hline 100 & PDMS & & SO & & & E118451 & 18-Dec-90 & & PU-239/240 & PU-239/240 & PUISO_PLATE_AEA \\
\hline 101 & PDMS & & SO & & & E118452 & 18-Dec-90 & & PU-239/240 & PU-239/240 & PUISO_PLATE_AEA \\
\hline 102 & PDMS & & SO & & & E118449 & 18-Dec-90 & & PU-239/240 & PU-239/240 & PUISO_PLATE_AEA \\
\hline 103 & PDMS & & SO & & & E119891 & 18-Jan-91 & & PU-239/240 & PU-239/240 & PUISO_PLATE_AEA \\
\hline 104 & PDMS & & SO & & & E119892 & 18-Jan-91 & & PU-239/240 & PU-239/240 & PUISO_PLATE_AEA \\
\hline 105 & PDMS & & SO & & & E119890 & 18-Jan-91 & & PU-239/240 & PU-239/240 & PUISO_PLATE_AEA \\
\hline 106 & PDMS & & SO & & & E119893 & 18-Jan-91 & & PU-239/240 & PU-239/240 & PUISO_PLATE_AEA \\
\hline 107 & PDMS & & SO & & & E119899 & 25-Nov-91 & & PU-239/240 & PU-239/240 & PUISO_PLATE_AEA \\
\hline 108 & PDMS & & SO & & & E119900 & 25-Nov-91 & & PU-239/240 & PU-239/240 & PUISO_PLATE_AEA \\
\hline 109 & PDMS & & SO & & & E119898 & 25-Nov-91 & & PU-239/240 & PU-239/240 & PUISO_PLATE_AEA \\
\hline 110 & PDMS & & SO & & & E119901 & 25-Nov-91 & & PU-239/240 & PU-239/240 & PUISO_PLATE_AEA \\
\hline 111 & PDMS & & SO & & & E118323 & 27-Nov-91 & & PU-239/240 & PU-239/240 & PUISO_PLATE_AEA \\
\hline 112 & PDMS & & SO & & & E118322 & 27-Nov-91 & & PU-239/240 & PU-239/240 & PUISO_PLATE_AEA \\
\hline 113 & PDMS & & SO & & & E118321 & 02-Dec-91 & & PU-239/240 & PU-239/240 & PUISO_PLATE_AEA \\
\hline 114 & PDMS & & SO & & & E118324 & 02-Dec-91 & & PU-239/240 & PU-239/240 & PUISO_PLATE_AEA \\
\hline
\end{tabular}




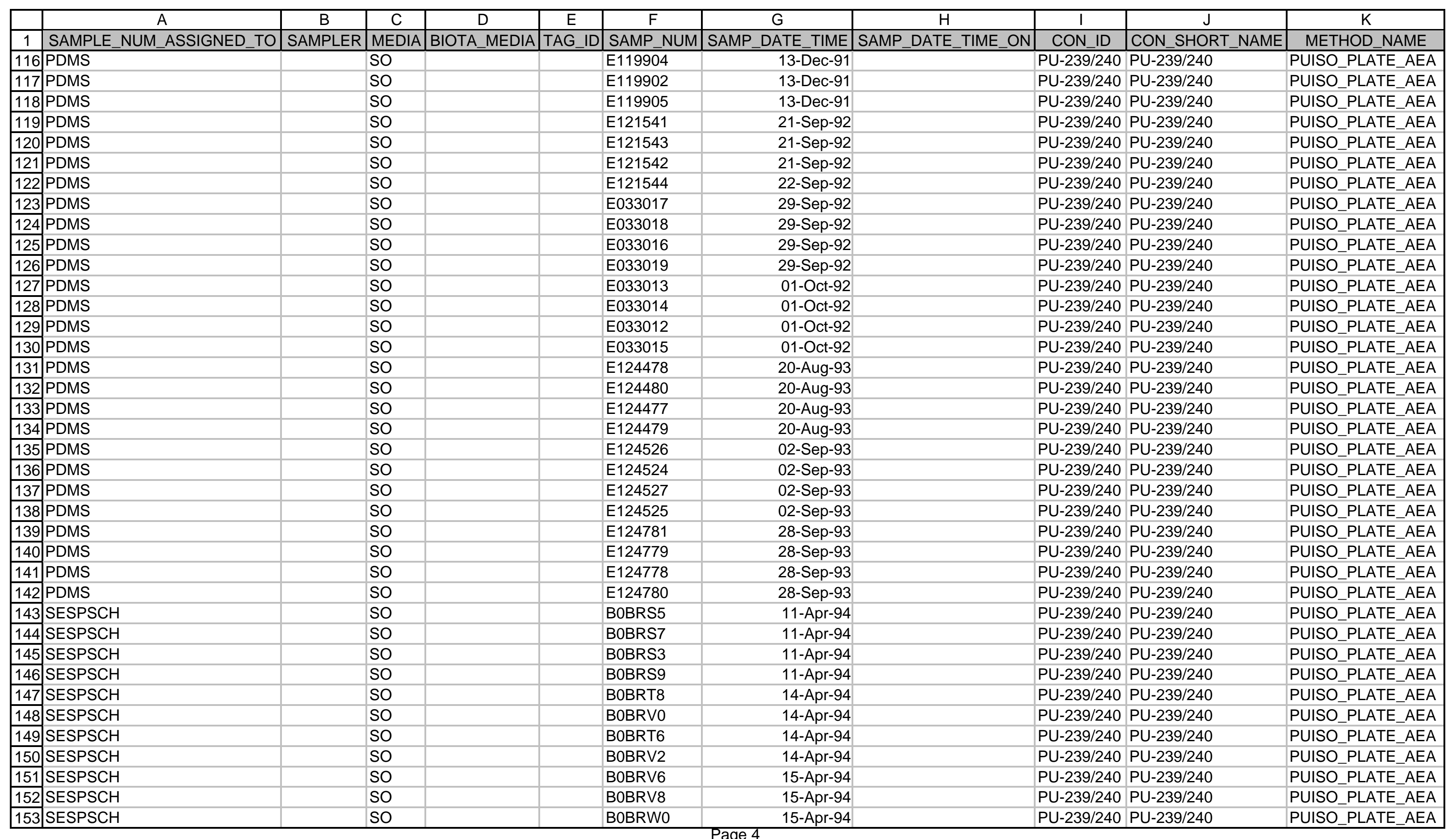




\begin{tabular}{|c|c|c|c|c|c|c|c|c|c|c|c|}
\hline & A & $\mathrm{B}$ & C & $\mathrm{D}$ & $E$ & $\mathrm{~F}$ & $G$ & $\mathrm{H}$ & $\mathrm{I}$ & $\mathrm{J}$ & $\mathrm{K}$ \\
\hline 1 & SAMPLE_NUM_ASSIGNED_TO & SAMPLER & MEDIA & BIOTA_MEDIA & TAG_ID & SAMP_NUM & SAMP_DATE_TIME & SAMP_DATE_TIME_ON & CON_ID & CON_SHORT_NAME & METHOD_NAME \\
\hline 154 & SESPSCH & & SO & & & B0BRV4 & 15-Apr-94 & & PU-239/240 & PU-239/240 & PUISO_PLATE_AEA \\
\hline 155 & SESPSCH & & SO & & & B0CDL3 & 22-Sep-94 & & PU-239/240 & PU-239/240 & PUISO_PLATE_AEA \\
\hline 156 & SESPSCH & & SO & & & B0CDL5 & 22-Sep-94 & & PU-239/240 & PU-239/240 & PUISO_PLATE_AEA \\
\hline 157 & SESPSCH & & SO & & & B0CDL1 & 22-Sep-94 & & PU-239/240 & PU-239/240 & PUISO_PLATE_AEA \\
\hline 158 & SESPSCH & & SO & & & B0CDL7 & 22-Sep-94 & & PU-239/240 & PU-239/240 & PUISO_PLATE_AEA \\
\hline 159 & SESPSCH & & SO & & & B0CDK5 & 23-Sep-94 & & PU-239/240 & PU-239/240 & PUISO_PLATE_AEA \\
\hline 160 & SESPSCH & & SO & & & B0CDK7 & 23-Sep-94 & & PU-239/240 & PU-239/240 & PUISO_PLATE_AEA \\
\hline 161 & SESPSCH & & SO & & & B0CDK3 & 23-Sep-94 & & PU-239/240 & PU-239/240 & PUISO_PLATE_AEA \\
\hline 162 & SESPSCH & & SO & & & B0CDK9 & 23-Sep-94 & & PU-239/240 & PU-239/240 & PUISO_PLATE_AEA \\
\hline 163 & SESPSCH & & SO & & & BOCDMO & 24-Sep-94 & & PU-239/240 & PU-239/240 & PUISO_PLATE_AEA \\
\hline 164 & SESPSCH & & SO & & & B0CDL9 & 24-Sep-94 & & PU-239/240 & PU-239/240 & PUISO_PLATE_AEA \\
\hline 165 & SESPSCH & & SO & & & B0CDM1 & 25-Sep-94 & & PU-239/240 & PU-239/240 & PUISO_PLATE_AEA \\
\hline 166 & SESPSCH & & SO & & & B0CDM5 & 25-Sep-94 & & PU-239/240 & PU-239/240 & PUISO_PLATE_AEA \\
\hline 167 & SESPSCH & & SO & & & B0CDM2 & 25-Sep-94 & & PU-239/240 & PU-239/240 & PUISO_PLATE_AEA \\
\hline 168 & SESPSCH & & SO & & & BOG8W0 & 21-Jul-95 & & PU-239/240 & PU-239/240 & PUISO_PLATE_AEA \\
\hline 169 & SESPSCH & & SO & & & B0G8W1 & 21-Jul-95 & & PU-239/240 & PU-239/240 & PUISO_PLATE_AEA \\
\hline 170 & SESPSCH & & SO & & & B0G8W2 & 21-Jul-95 & & PU-239/240 & PU-239/240 & PUISO_PLATE_AEA \\
\hline 171 & SESPSCH & & SO & & & B0G8V9 & 21-Jul-95 & & PU-239/240 & PU-239/240 & PUISO_PLATE_AEA \\
\hline 173 & SESPSCH & & SO & & & B0G8V5 & 24-Jul-95 & & PU-239/240 & PU-239/240 & PUISO_PLATE_AEA \\
\hline 174 & SESPSCH & & SO & & & B0G8V1 & 24-Jul-95 & & PU-239/240 & PU-239/240 & PUISO_PLATE_AEA \\
\hline 175 & SESPSCH & & SO & & & B0G8V7 & 24-Jul-95 & & PU-239/240 & PU-239/240 & PUISO_PLATE_AEA \\
\hline 176 & SESPSCH & & SO & & & B0G8T5 & 28-Jul-95 & & PU-239/240 & PU-239/240 & PUISO_PLATE_AEA \\
\hline 177 & SESPSCH & & SO & & & B0G8T7 & 28-Jul-95 & & PU-239/240 & PU-239/240 & PUISO_PLATE_AEA \\
\hline 178 & SESPSCH & & SO & & & B0G8T3 & 28-Jul-95 & & PU-239/240 & PU-239/240 & PUISO_PLATE_AEA \\
\hline 179 & SESPSCH & & SO & & & B0G8T9 & 28-Jul-95 & & PU-239/240 & PU-239/240 & PUISO_PLATE_AEA \\
\hline
\end{tabular}




\begin{tabular}{|c|c|c|c|c|c|c|c|c|c|}
\hline & $L$ & $M$ & $\mathrm{~N}$ & 0 & $\mathrm{P}$ & $\mathrm{Q}$ & $\mathrm{R}$ & $\mathrm{S}$ & $\mathrm{T}$ \\
\hline 1 & LAB_CODE & VALUE_RPTD & ANAL_UNITS_RPTD & |COUNTING_ERROR| & TOTAL_ANAL_ERROR & MIN_DETECTABLE_ACTIVITY & PCNT_MOISTURE & LAB_QUALIFIER & REVIEW_QUALIFIER \\
\hline 2 & ITASRL & 0.0333 & $\mathrm{pCi} / \mathrm{g}$ & 0.0187 & 0.0195 & & & & \\
\hline 3 & ITASRL & 0.0344 & $\mathrm{pCi} / \mathrm{g}$ & 0.0192 & 0.02 & & & & \\
\hline 4 & ITASRL & 0.04 & $\mathrm{pCi} / \mathrm{g}$ & 0.0234 & 0.0242 & & & & \\
\hline 5 & ITASRL & 0.0229 & $\mathrm{pCi} / \mathrm{g}$ & 0.0162 & 0.0169 & & & & \\
\hline 6 & ITASRL & 0.0587 & $\mathrm{pCi} / \mathrm{g}$ & 0.0256 & 0.0266 & & & & \\
\hline 7 & ITASRL & 0.0325 & $\mathrm{pCi} / \mathrm{g}$ & 0.0203 & 0.021 & & & & \\
\hline 8 & ITASRL & 0.101 & $\mathrm{pCi} / \mathrm{g}$ & 0.0374 & 0.0389 & & & & \\
\hline 9 & ITASRL & 0.0267 & $\mathrm{pCi} / \mathrm{g}$ & 0.0241 & 0.0246 & & & & \\
\hline 10 & ITASRL & 0.0342 & $\mathrm{pCi} / \mathrm{g}$ & 0.0203 & 0.021 & & & & \\
\hline 11 & ITASRL & 0.0263 & $\mathrm{pCi} / \mathrm{g}$ & 0.0182 & 0.0189 & & & & \\
\hline 12 & ITASRL & 0.0224 & $\mathrm{pCi} / \mathrm{g}$ & 0.0165 & 0.0172 & & & & \\
\hline 13 & ITASRL & 0.0269 & $\mathrm{pCi} / \mathrm{g}$ & 0.0196 & 0.0203 & & & & \\
\hline 14 & ITASRL & 0.0444 & $\mathrm{pCi} / \mathrm{g}$ & 0.0194 & 0.0203 & & & & \\
\hline 15 & ITASRL & 0.0281 & $\mathrm{pCi} / \mathrm{g}$ & 0.0152 & 0.016 & & & & \\
\hline 16 & ITASRL & 0.0454 & $\mathrm{pCi} / \mathrm{g}$ & 0.0194 & 0.0203 & & & & \\
\hline 17 & ITASRL & 0.0288 & $\mathrm{pCi} / \mathrm{g}$ & 0.0164 & 0.0172 & & & & \\
\hline 18 & ITASRL & 0.123 & $\mathrm{pCi} / \mathrm{g}$ & 0.031 & 0.0334 & & & & \\
\hline 19 & ITASRL & 0.191 & $\mathrm{pCi} / \mathrm{g}$ & 0.0403 & 0.0444 & & & & \\
\hline 20 & ITASRL & 0.0563 & $\mathrm{pCi} / \mathrm{g}$ & 0.0226 & 0.0236 & & & & \\
\hline 21 & ITASRL & 0.0794 & $\mathrm{pCi} / \mathrm{g}$ & 0.0249 & 0.0263 & & & & \\
\hline 22 & ITASRL & 0.0268 & $\mathrm{pCi} / \mathrm{g}$ & 0.016 & 0.0168 & & & & \\
\hline 23 & ITASRL & 0.0416 & $\mathrm{pCi} / \mathrm{g}$ & 0.0189 & 0.0198 & & & & \\
\hline 24 & ITASRL & 0.0599 & $\mathrm{pCi} / \mathrm{g}$ & 0.0234 & 0.0245 & & & & \\
\hline 25 & ITASRL & 0.0653 & $\mathrm{pCi} / \mathrm{g}$ & 0.0258 & 0.0269 & & & & \\
\hline 26 & ITASRL & -0.0193 & $\mathrm{pCi} / \mathrm{g}$ & 0.0721 & 0.0721 & & & U & \\
\hline 27 & ITASRL & 0.0853 & $\mathrm{pCi} / \mathrm{g}$ & 0.0679 & 0.0679 & & & & \\
\hline 28 & ITASRL & 0.0401 & $\mathrm{pCi} / \mathrm{g}$ & 0.0672 & 0.0672 & & & U & \\
\hline 29 & ITASRL & 0.0761 & $\mathrm{pCi} / \mathrm{g}$ & 0.0812 & 0.0812 & & & U & \\
\hline 30 & ITASRL & 0.108 & $\mathrm{pCi} / \mathrm{g}$ & 0.0488 & 0.0488 & & & & \\
\hline 31 & ITASRL & 0.00319 & $\mathrm{pCi} / \mathrm{g}$ & 0.08 & 0.08 & & & U & \\
\hline 32 & ITASRL & 0.0999 & $\mathrm{pCi} / \mathrm{g}$ & 0.0656 & 0.0656 & & & & \\
\hline 33 & ITASRL & 0.0494 & $\mathrm{pCi} / \mathrm{g}$ & 0.0772 & 0.0772 & & & U & \\
\hline 34 & ITASRL & 0.00094 & $\mathrm{pCi} / \mathrm{g}$ & 0.0668 & 0.0668 & & & U & \\
\hline 35 & ITASRL & 0.0971 & $\mathrm{pCi} / \mathrm{g}$ & 0.0601 & 0.0601 & & & & \\
\hline 36 & ITASRL & 0.0846 & $\mathrm{pCi} / \mathrm{g}$ & 0.0704 & 0.0704 & & & & \\
\hline 37 & ITASRL & 0.0266 & $\mathrm{pCi} / \mathrm{g}$ & 0.066 & 0.066 & & & U & \\
\hline 38 & ITASRL & 0.119 & $\mathrm{pCi} / \mathrm{g}$ & 0.0659 & 0.0669 & & & & \\
\hline 39 & ITASRL & 0.113 & $\mathrm{pCi} / \mathrm{g}$ & 0.0719 & 0.0728 & & & & \\
\hline
\end{tabular}




\begin{tabular}{|c|c|c|c|c|c|c|c|c|c|}
\hline & $\mathrm{L}$ & $\mathrm{M}$ & $\mathrm{N}$ & $\mathrm{O}$ & $\mathrm{P}$ & $\mathrm{Q}$ & $\mathrm{R}$ & $\mathrm{S}$ & $\mathrm{T}$ \\
\hline 1 & LAB_CODE & VALUE_RPTD & ANAL_UNITS_RPTD & COUNTING_ERROR & TOTAL_ANAL_ERROR & MIN_DETECTABLE_ACTIVITY & PCNT_MOISTURE & LAB_QUALIFIER & REVIEW_QUALIFIER \\
\hline 40 & ITASRL & -0.00679 & $\mathrm{pCi} / \mathrm{g}$ & 0.0669 & 0.0669 & & & $U$ & \\
\hline 41 & ITASRL & -0.0027 & $\mathrm{pCi} / \mathrm{g}$ & 0.0883 & 0.0883 & & & $U$ & \\
\hline 42 & ITASRL & 0.0352 & $\mathrm{pCi} / \mathrm{g}$ & 0.0926 & 0.0926 & & & $U$ & \\
\hline 43 & ITASRL & 0.0839 & $\mathrm{pCi} / \mathrm{g}$ & 0.0921 & 0.0924 & & & $U$ & \\
\hline 44 & ITASRL & 0.136 & $\mathrm{pCi} / \mathrm{g}$ & 0.0788 & 0.08 & & & & \\
\hline 45 & ITASRL & -0.0159 & $\mathrm{pCi} / \mathrm{g}$ & 0.0714 & 0.0715 & & & $U$ & \\
\hline 46 & ITASRL & 0.124 & $\mathrm{pCi} / \mathrm{g}$ & 0.0774 & 0.0784 & & & & \\
\hline 47 & ITASRL & -0.0024 & $\mathrm{pCi} / \mathrm{g}$ & 0.0708 & 0.0708 & & & $U$ & \\
\hline 48 & ITASRL & 0.0269 & $\mathrm{pCi} / \mathrm{g}$ & 0.0688 & 0.0689 & & & $U$ & \\
\hline 49 & ITASRL & 0.0526 & $\mathrm{pCi} / \mathrm{g}$ & 0.0932 & 0.0933 & & & $U$ & \\
\hline 50 & ITASRL & 0.0673 & $\mathrm{pCi} / \mathrm{g}$ & 0.0851 & 0.0854 & & & $U$ & \\
\hline 51 & ITASRL & 0.0873 & $\mathrm{pCi} / \mathrm{g}$ & 0.0917 & 0.0922 & & & $U$ & \\
\hline 52 & ITASRL & -0.0588 & $\mathrm{pCi} / \mathrm{g}$ & 0.0877 & 0.0879 & & & $U$ & \\
\hline 53 & ITASRL & 0.121 & $\mathrm{pCi} / \mathrm{g}$ & 0.0717 & 0.0727 & & & & \\
\hline 54 & ITASRL & 0.00647 & $\mathrm{pCi} / \mathrm{g}$ & 0.098 & 0.098 & & & $U$ & \\
\hline 55 & ITASRL & 0.0833 & $\mathrm{pCi} / \mathrm{g}$ & 0.0724 & 0.0729 & & & & \\
\hline 56 & ITASRL & 0.164 & $\mathrm{pCi} / \mathrm{g}$ & 0.0701 & 0.072 & & & & \\
\hline 57 & ITASRL & -0.0266 & $\mathrm{pCi} / \mathrm{g}$ & 0.0711 & 0.0712 & & & $U$ & \\
\hline 58 & ITASRL & 0.139 & $\mathrm{pCi} / \mathrm{g}$ & 0.0928 & 0.0938 & & & & \\
\hline 59 & ITASRL & 0.0549 & $\mathrm{pCi} / \mathrm{g}$ & 0.0879 & 0.0881 & & & U & \\
\hline 60 & ITASRL & 0.054 & $\mathrm{pCi} / \mathrm{g}$ & 0.0683 & 0.0685 & & & $U$ & \\
\hline 61 & ITASRL & 0.044 & $\mathrm{pCi} / \mathrm{g}$ & 0.066 & 0.0661 & & & $U$ & \\
\hline 62 & ITASRL & & & & & & & & \\
\hline 63 & ITASRL & 0.032 & $\mathrm{pCi} / \mathrm{g}$ & 0.0985 & 0.0985 & & & $U$ & \\
\hline 64 & ITASRL & 0.0188 & $\mathrm{pCi} / \mathrm{g}$ & 0.158 & 0.159 & & & $U$ & \\
\hline 65 & ITASRL & -0.000394 & $+\mathrm{pCi} / \mathrm{g}$ & 0.0956 & 0.0956 & & & U & \\
\hline 66 & ITASRL & -0.0382 & $\mathrm{pCi} / \mathrm{g}$ & 0.155 & 0.155 & & & $U$ & \\
\hline 67 & ITASRL & 0.115 & $\mathrm{pCi} / \mathrm{g}$ & 0.194 & 0.194 & & & $U$ & \\
\hline 68 & ITASRL & 0.137 & $\mathrm{pCi} / \mathrm{g}$ & 0.154 & 0.155 & & & $U$ & \\
\hline 69 & ITASRL & 0.062 & $\mathrm{pCi} / \mathrm{g}$ & 0.252 & 0.252 & & & U & \\
\hline 70 & ITASRL & 0.0206 & $\mathrm{pCi} / \mathrm{g}$ & 0.194 & 0.194 & & & $U$ & \\
\hline 71 & ITASRL & -0.046 & $\mathrm{pCi} / \mathrm{g}$ & 0.163 & 0.163 & & & U & \\
\hline 72 & ITASRL & 0.0706 & $\mathrm{pCi} / \mathrm{g}$ & 0.128 & 0.128 & & & $U$ & \\
\hline 73 & ITASRL & 0.00924 & $\mathrm{pCi} / \mathrm{g}$ & 0.00597 & 0.00615 & & & & \\
\hline 74 & ITASRL & 0.00619 & $\mathrm{pCi} / \mathrm{g}$ & 0.00661 & 0.00673 & & & $U$ & \\
\hline 75 & ITASRL & 0.0089 & $\mathrm{pCi} / \mathrm{g}$ & 0.00526 & 0.00543 & & & & \\
\hline 76 & ITASRL & 0.0232 & $\mathrm{pCi} / \mathrm{g}$ & 0.0062 & 0.00684 & & & & \\
\hline 77 & ITASRL & 0.00354 & $+\mathrm{pCi} / \mathrm{g}$ & 0.00244 & 0.0025 & & & & \\
\hline
\end{tabular}




\begin{tabular}{|c|c|c|c|c|c|c|c|c|c|}
\hline & $\mathrm{L}$ & $\mathrm{M}$ & $\bar{N}$ & $\mathrm{O}$ & $P$ & $\mathrm{Q}$ & $\bar{R}$ & $\mathrm{~S}$ & $T$ \\
\hline 1 & LAB_CODE & VALUE_RPTD & ANAL_UNITS_RPTD & COUNTING_ERROR & TOTAL_ANAL_ERROR & MIN_DETECTABLE_ACTIVITY & PCNT_MOISTURE & LAB_QUALIFIER & REVIEW_QUALIFIER \\
\hline 78 & ITASRL & 0.00191 & $\mathrm{pCi} / \mathrm{g}$ & 0.00155 & 0.0016 & & & & \\
\hline 79 & ITASRL & 0.235 & $\mathrm{pCi} / \mathrm{g}$ & 0.157 & 0.159 & & & & \\
\hline 80 & ITASRL & -0.112 & $\mathrm{pCi} / \mathrm{g}$ & 0.135 & 0.136 & & & $U$ & \\
\hline 81 & ITASRL & 0.155 & $\mathrm{pCi} / \mathrm{g}$ & 0.118 & 0.119 & & & & \\
\hline 82 & ITASRL & 0.053 & $\mathrm{pCi} / \mathrm{g}$ & 0.137 & 0.137 & & & $U$ & \\
\hline 83 & ITASRL & 0.197 & $\mathrm{pCi} / \mathrm{g}$ & 0.156 & 0.157 & & & & \\
\hline 84 & ITASRL & 0.317 & $\mathrm{pCi} / \mathrm{g}$ & 0.164 & 0.167 & & & & $\mathrm{~F}$ \\
\hline 85 & ITASRL & 0.0779 & $\mathrm{pCi} / \mathrm{g}$ & 0.183 & 0.183 & & & $U$ & \\
\hline 86 & ITASRL & 0.197 & $\mathrm{pCi} / \mathrm{g}$ & 0.127 & 0.129 & & & & \\
\hline 87 & ITASRL & 0.0958 & $\mathrm{pCi} / \mathrm{g}$ & 0.139 & 0.139 & & & $U$ & \\
\hline 88 & ITASRL & 0.201 & $\mathrm{pCi} / \mathrm{g}$ & 0.158 & 0.159 & & & & \\
\hline 89 & ITASRL & 0.168 & $\mathrm{pCi} / \mathrm{g}$ & 0.147 & 0.147 & & & & \\
\hline 90 & ITASRL & -0.0166 & $\mathrm{pCi} / \mathrm{g}$ & 0.136 & 0.136 & & & $U$ & \\
\hline 91 & ITASRL & 0.205 & $\mathrm{pCi} / \mathrm{g}$ & 0.134 & 0.135 & & & & \\
\hline 92 & ITASRL & 0.406 & $\mathrm{pCi} / \mathrm{g}$ & 0.16 & 0.165 & & & & $\mathrm{~F}$ \\
\hline 93 & ITASRL & 0.234 & $\mathrm{pCi} / \mathrm{g}$ & 0.139 & 0.141 & & & & \\
\hline 94 & ITASRL & 0.173 & $\mathrm{pCi} / \mathrm{g}$ & 0.132 & 0.134 & & & & \\
\hline 95 & ITASRL & 0.00433 & $\mathrm{pCi} / \mathrm{g}$ & 0.000669 & 0.000797 & & & & \\
\hline 96 & ITASRL & 0.00881 & $\mathrm{pCi} / \mathrm{g}$ & 0.000938 & 0.00128 & & & & \\
\hline 97 & ITASRL & 0.00791 & $\mathrm{pCi} / \mathrm{g}$ & 0.000927 & 0.00122 & & & & \\
\hline 98 & ITASRL & 0.0115 & $\mathrm{pCi} / \mathrm{g}$ & 0.00103 & 0.00153 & & & & \\
\hline 99 & ITASRL & 0.000907 & $\mathrm{pCi} / \mathrm{g}$ & 0.000315 & 0.000329 & & & & \\
\hline 100 & ITASRL & 0.000773 & $3 \mathrm{pCi} / \mathrm{g}$ & 0.000277 & 0.000289 & & & & \\
\hline 101 & ITASRL & 0.00115 & $\mathrm{pCi} / \mathrm{g}$ & 0.000353 & 0.000373 & & & & \\
\hline 102 & ITASRL & 0.00303 & $3 \mathrm{pCi} / \mathrm{g}$ & 0.000669 & 0.000743 & & & & \\
\hline 103 & ITASRL & 0.00701 & $\mathrm{pCi} / \mathrm{g}$ & 0.000846 & 0.0011 & & & & \\
\hline 104 & ITASRL & 0.0071 & $\mathrm{pCi} / \mathrm{g}$ & 0.000861 & 0.00112 & & & & \\
\hline 105 & ITASRL & 0.00725 & $\mathrm{pCi} / \mathrm{g}$ & 0.000961 & 0.00122 & & & & \\
\hline 106 & ITASRL & 0.0144 & $\mathrm{pCi} / \mathrm{g}$ & 0.00113 & 0.00181 & & & & \\
\hline 107 & ITASRL & 0.00589 & $\mathrm{pCi} / \mathrm{g}$ & 0.000749 & 0.000948 & & & & \\
\hline 108 & ITASRL & 0.0091 & $\mathrm{pCi} / \mathrm{g}$ & 0.00101 & 0.00136 & & & & \\
\hline 109 & ITASRL & 0.00648 & $\mathrm{pCi} / \mathrm{g}$ & 0.000792 & 0.00102 & & & & \\
\hline 110 & ITASRL & 0.00747 & $\mathrm{pCi} / \mathrm{g}$ & 0.000883 & 0.00116 & & & & \\
\hline 111 & ITASRL & 0.00323 & $\mathrm{pCi} / \mathrm{g}$ & 0.000635 & 0.000717 & & & & \\
\hline 112 & ITASRL & 0.000953 & $\mathrm{pCi} / \mathrm{g}$ & 0.000382 & 0.000397 & & & & \\
\hline 113 & ITASRL & 0.000444 & $\mathrm{pCi} / \mathrm{g}$ & 0.000282 & 0.000288 & & & & \\
\hline 114 & ITASRL & 0.00304 & $\mathrm{pCi} / \mathrm{g}$ & 0.000635 & 0.000711 & & & & \\
\hline 115 & ITASRL & 0.00667 & $\mathrm{pCi} / \mathrm{g}$ & 0.000851 & 0.00109 & & & & \\
\hline
\end{tabular}




\begin{tabular}{|c|c|c|c|c|c|c|c|c|c|}
\hline & $\mathrm{L}$ & $\mathrm{M}$ & $\mathrm{N}$ & $\mathrm{O}$ & $\mathrm{P}$ & $\mathrm{Q}$ & $\mathrm{R}$ & $\mathrm{S}$ & $\mathrm{T}$ \\
\hline 1 & LAB_CODE & VALUE_RPTD & ANAL_UNITS_RPTD & COUNTING_ERROR & TOTAL_ANAL_ERROR & MIN_DETECTABLE_ACTIVITY & PCNT_MOISTURE & LAB_QUALIFIER & REVIEW_QUALIFIER \\
\hline 116 & ITASRL & 0.0137 & $\mathrm{pCi} / \mathrm{g}$ & 0.00138 & 0.002 & & & & \\
\hline 117 & ITASRL & 0.00789 & $\mathrm{pCi} / \mathrm{g}$ & 0.000897 & 0.00119 & & & & \\
\hline 118 & ITASRL & 0.00784 & $\mathrm{pCi} / \mathrm{g}$ & 0.000866 & 0.00116 & & & & \\
\hline 119 & ITASRL & 0.00153 & $\mathrm{pCi} / \mathrm{g}$ & 0.000661 & 0.000689 & & & & \\
\hline 120 & ITASRL & 0.000633 & $\mathrm{pCi} / \mathrm{g}$ & 0.000351 & 0.00036 & & & & \\
\hline 121 & ITASRL & 0.00414 & $\mathrm{pCi} / \mathrm{g}$ & 0.000961 & 0.00107 & & & & \\
\hline 122 & ITASRL & 0.0023 & $\mathrm{pCi} / \mathrm{g}$ & 0.00113 & 0.00118 & & & & \\
\hline 123 & ITASRL & 0.00968 & $\mathrm{pCi} / \mathrm{g}$ & 0.00179 & 0.00218 & & & & \\
\hline 124 & ITASRL & 0.0115 & $\mathrm{pCi} / \mathrm{g}$ & 0.0023 & 0.00284 & & & & \\
\hline 125 & ITASRL & 0.0107 & $\mathrm{pCi} / \mathrm{g}$ & 0.0016 & 0.00204 & & & & \\
\hline 126 & ITASRL & 0.0099 & $\mathrm{pCi} / \mathrm{g}$ & 0.00148 & 0.00187 & & & & \\
\hline 127 & ITASRL & 0.00901 & $\mathrm{pCi} / \mathrm{g}$ & 0.00154 & 0.00189 & & & & \\
\hline \begin{tabular}{|l|}
128 \\
\end{tabular} & ITASRL & 0.0091 & $\mathrm{pCi} / \mathrm{g}$ & 0.00144 & 0.00179 & & & & \\
\hline 129 & ITASRL & 0.00823 & $\mathrm{pCi} / \mathrm{g}$ & 0.00138 & 0.00169 & & & & \\
\hline 130 & ITASRL & 0.0144 & $\mathrm{pCi} / \mathrm{g}$ & 0.00239 & 0.0031 & & & & \\
\hline 131 & ITASRL & 0.0084 & $\mathrm{pCi} / \mathrm{g}$ & 0.00121 & 0.00153 & & & & \\
\hline 132 & ITASRL & 0.0119 & $\mathrm{pCi} / \mathrm{g}$ & 0.00209 & 0.00263 & & & & \\
\hline 133 & ITASRL & 0.00135 & $\mathrm{pCi} / \mathrm{g}$ & 0.000458 & 0.000481 & & & & \\
\hline 134 & ITASRL & 0.00253 & $\mathrm{pCi} / \mathrm{g}$ & 0.000639 & 0.000698 & & & & \\
\hline 135 & ITASRL & 0.000701 & $\mathrm{pCi} / \mathrm{g}$ & 0.000425 & 0.000432 & & & & \\
\hline 136 & ITASRL & 0.00174 & $\mathrm{pCi} / \mathrm{g}$ & 0.000573 & 0.000598 & & & & \\
\hline 137 & ITASRL & 0.00199 & $\mathrm{pCi} / \mathrm{g}$ & 0.000685 & 0.000715 & & & & \\
\hline 138 & ITASRL & 0.00402 & $\mathrm{pCi} / \mathrm{g}$ & 0.00106 & 0.00114 & & & & \\
\hline 139 & ITASRL & 0.0125 & $\mathrm{pCi} / \mathrm{g}$ & 0.00217 & 0.00276 & & & & \\
\hline 140 & ITASRL & 0.0111 & $\mathrm{pCi} / \mathrm{g}$ & 0.00139 & 0.00185 & & & & \\
\hline 141 & ITASRL & 0.00854 & $\mathrm{pCi} / \mathrm{g}$ & 0.000935 & 0.00127 & & & & \\
\hline 142 & ITASRL & 0.0126 & $\mathrm{pCi} / \mathrm{g}$ & 0.00153 & 0.00209 & & & & \\
\hline 143 & ITASRL & 0.00782 & $\mathrm{pCi} / \mathrm{g}$ & 0.00312 & 0.00324 & & & & \\
\hline 144 & ITASRL & 0.0135 & $\mathrm{pCi} / \mathrm{g}$ & 0.00325 & 0.00403 & & & & \\
\hline 145 & ITASRL & 0.0124 & $\mathrm{pCi} / \mathrm{g}$ & 0.0012 & 0.00174 & & & & \\
\hline 146 & ITASRL & 0.00981 & $\mathrm{pCi} / \mathrm{g}$ & 0.00186 & 0.00228 & & & & \\
\hline 147 & ITASRL & 0.00948 & $\mathrm{pCi} / \mathrm{g}$ & 0.00108 & 0.00146 & & & & \\
\hline 148 & ITASRL & 0.00452 & $\mathrm{pCi} / \mathrm{g}$ & 0.000937 & 0.00107 & & & & \\
\hline 149 & ITASRL & 0.00543 & $\mathrm{pCi} / \mathrm{g}$ & 0.00102 & 0.00119 & & & & \\
\hline 150 & ITASRL & 0.00952 & $\mathrm{pCi} / \mathrm{g}$ & 0.00119 & 0.00157 & & & & \\
\hline 151 & ITASRL & 0.00147 & $\mathrm{pCi} / \mathrm{g}$ & 0.000462 & 0.00049 & & & & \\
\hline 152 & ITASRL & 0.00566 & $\mathrm{pCi} / \mathrm{g}$ & 0.000896 & 0.00108 & & & & \\
\hline 153 & ITASRL & & & & & & & & \\
\hline
\end{tabular}


M

$\mathrm{N}$

O

P Q

$\mathrm{R}$

$\mathrm{S}$

$\mathrm{T}$

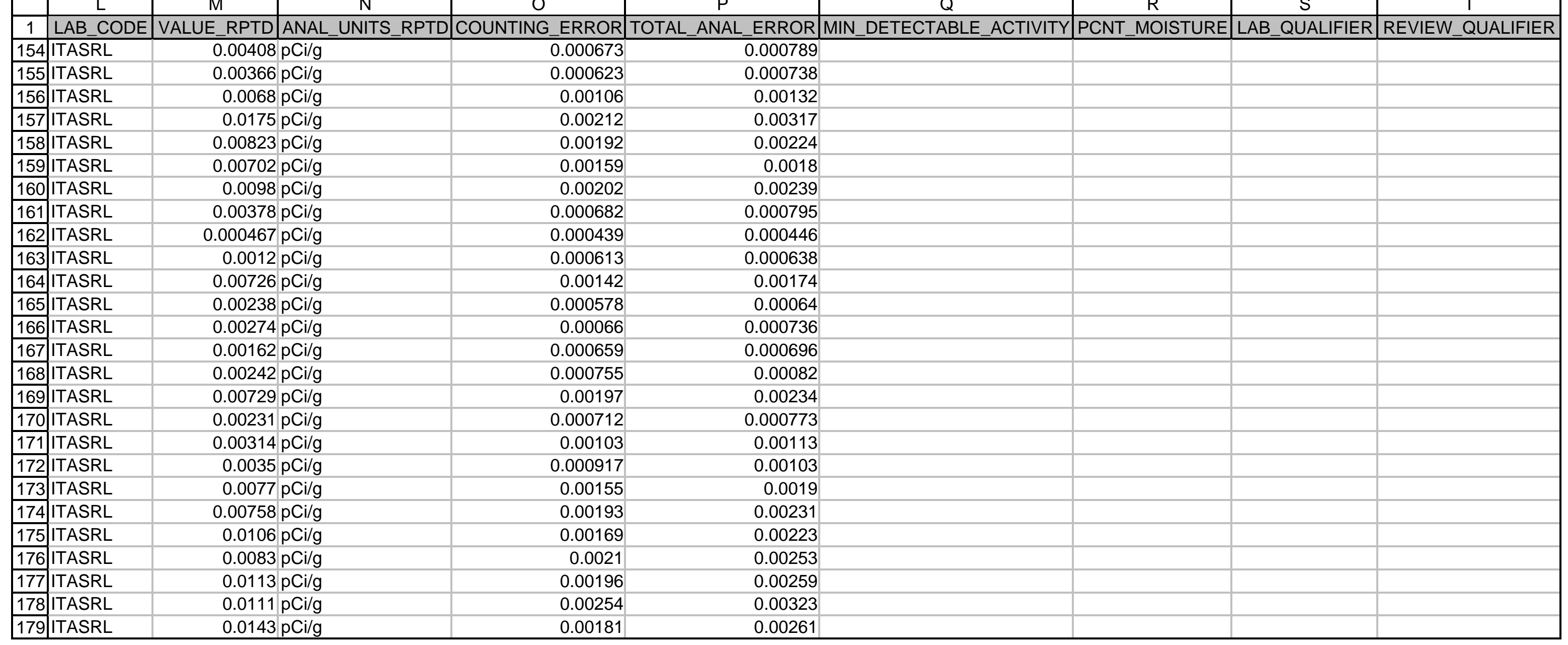




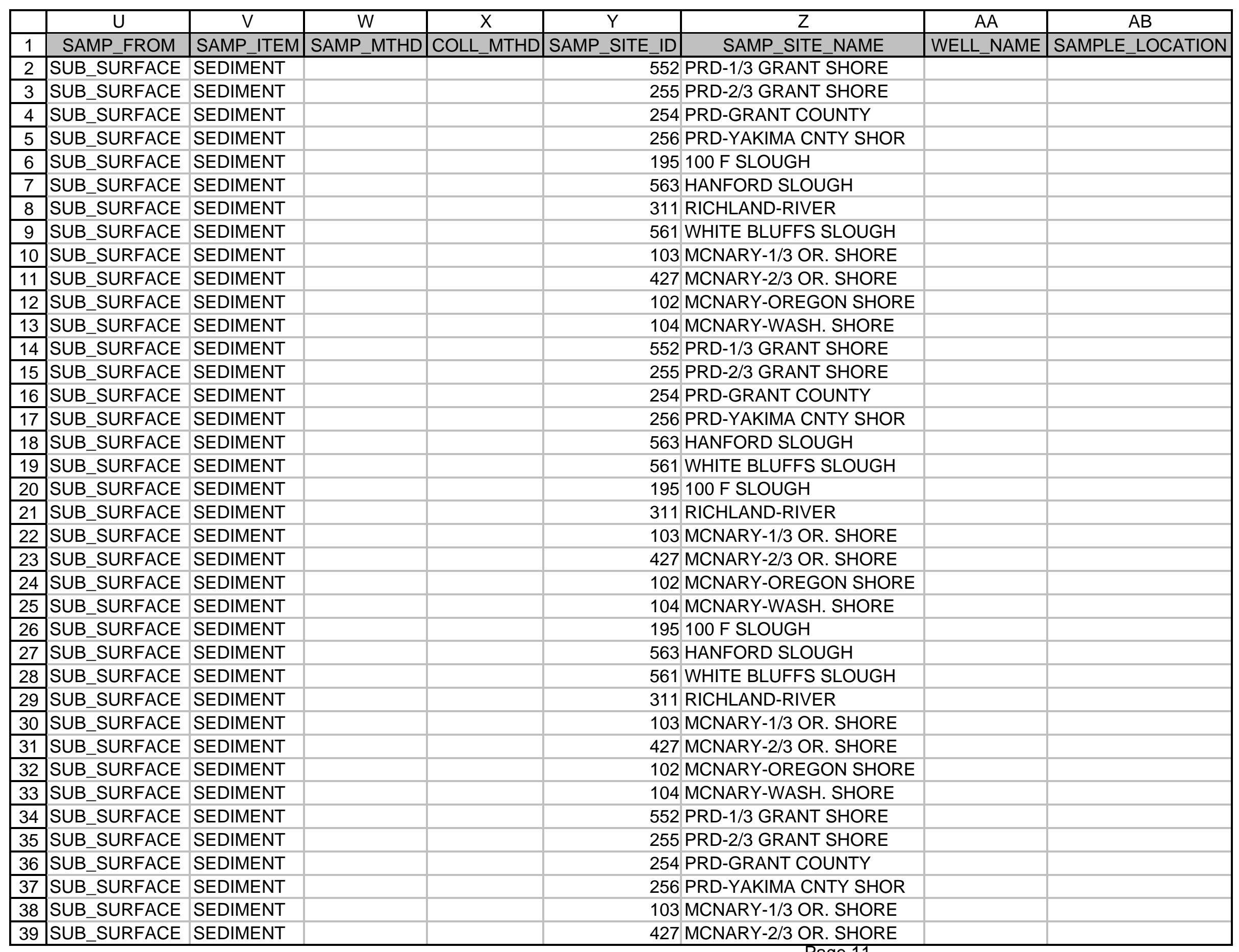




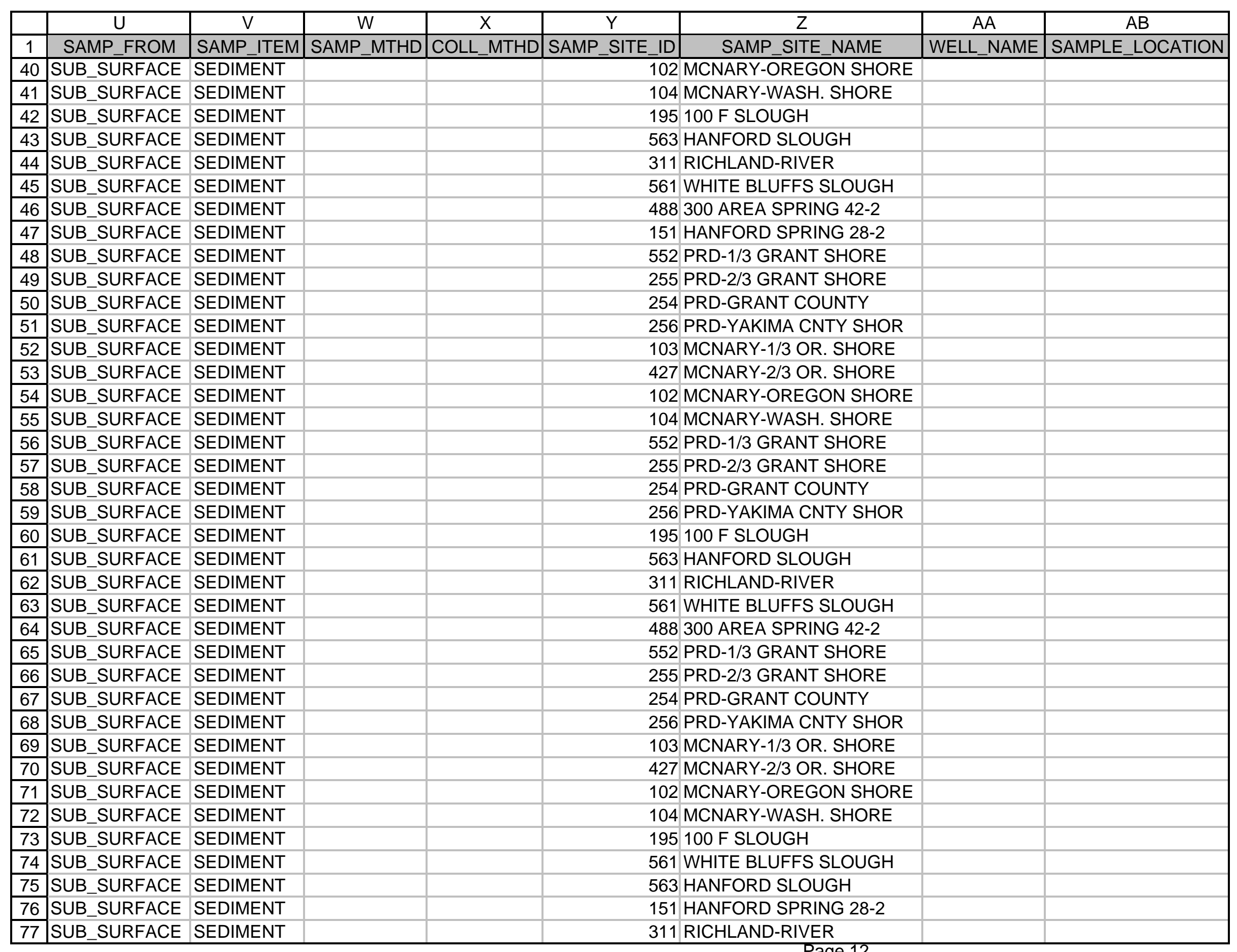

Page 12 


\begin{tabular}{|c|c|c|c|c|c|c|c|c|c|}
\hline & $U$ & $\mathrm{~V}$ & $\mathrm{~W}$ & $\mathrm{X}$ & & $\mathrm{Y}$ & Z & $\overline{A A}$ & $A B$ \\
\hline 1 & SAMP_FROM & SAMP_ITEM & SAMP_MTHD & COLL_MTHD & SAMP & SITE_ID & SAMP_SITE_NAME & WELL_NAME & SAMPLE_LOCATION \\
\hline 79 & SUB_SURFACE & SEDIMENT & & & & 563 & 3 HANFORD SLOUGH & & \\
\hline 81 & SUB_SURFACE & SEDIMENT & & & & 561 & WHITE BLUFFS SLOUGH & & \\
\hline 82 & SUB_SURFACE & SEDIMENT & & & & 552 & PRD-1/3 GRANT SHORE & & \\
\hline 83 & SUB SURFACE & SEDIMENT & & & & 255 & PRD-2/3 GRANT SHORE & & \\
\hline 84 & SUB_SURFACE & SEDIMENT & & & & 254 & PRD-GRANT COUNTY & & \\
\hline 87 & SUB_SURFACE & SEDIMENT & & & & 427 & MCNARY-2/3 OR. SHORE & & \\
\hline 88 & SUB_SURFACE & SEDIMENT & & & & 102 & MCNARY-OREGON SHORE & & \\
\hline 89 & SUB_SURFACE & SEDIMENT & & & & 104 & MCNARY-WASH. SHORE & & \\
\hline 90 & SUB_SURFACE & SEDIMENT & & & & 52 & 100-B SPRING & & \\
\hline 91 & SUB_SURFACE & SEDIMENT & & & & 533 & 3 100-K SPRING & & \\
\hline 92 & SUB_SURFACE & SEDIMENT & & & & 488 & 300 AREA SPRING 42-2 & & \\
\hline 97 & SUB_SURFACE & SEDIMENT & & & & 254 & PRD-GRANT COUNTY & & \\
\hline 98 & SUB_SURFACE & SEDIMENT & & & & 256 & PRD-YAKIMA CNTY SHOR & & \\
\hline 99 & SUB_SURFACE & SEDIMENT & & & & 195 & 100 F SLOUGH & & \\
\hline 100 & SUB_SURFACE & SEDIMENT & & & & 563 & 3 HANFORD SLOUGH & & \\
\hline 101 & SUB_SURFACE & SEDIMENT & & & & 311 & RICHLAND-RIVER & & \\
\hline 102 & SUB_SURFACE & SEDIMENT & & & & 561 & WHITE BLUFFS SLOUGH & & \\
\hline 103 & SUB_SURFACE & SEDIMENT & & & & 103 & 3 MCNARY-1/3 OR. SHORE & & \\
\hline 104 & SUB_SURFACE & SEDIMENT & & & & 427 & MCNARY-2/3 OR. SHORE & & \\
\hline 105 & SUB_SURFACE & SEDIMENT & & & & 102 & MCNARY-OREGON SHORE & & \\
\hline 106 & SUB_SURFACE & SEDIMENT & & & & 104 & MCNARY-WASH. SHORE & & \\
\hline 107 & SUB_SURFACE & SEDIMENT & & & & 552 & PRD-1/3 GRANT SHORE & & \\
\hline 108 & SUB_SURFACE & SEDIMENT & & & & 255 & PRD-2/3 GRANT SHORE & & \\
\hline
\end{tabular}




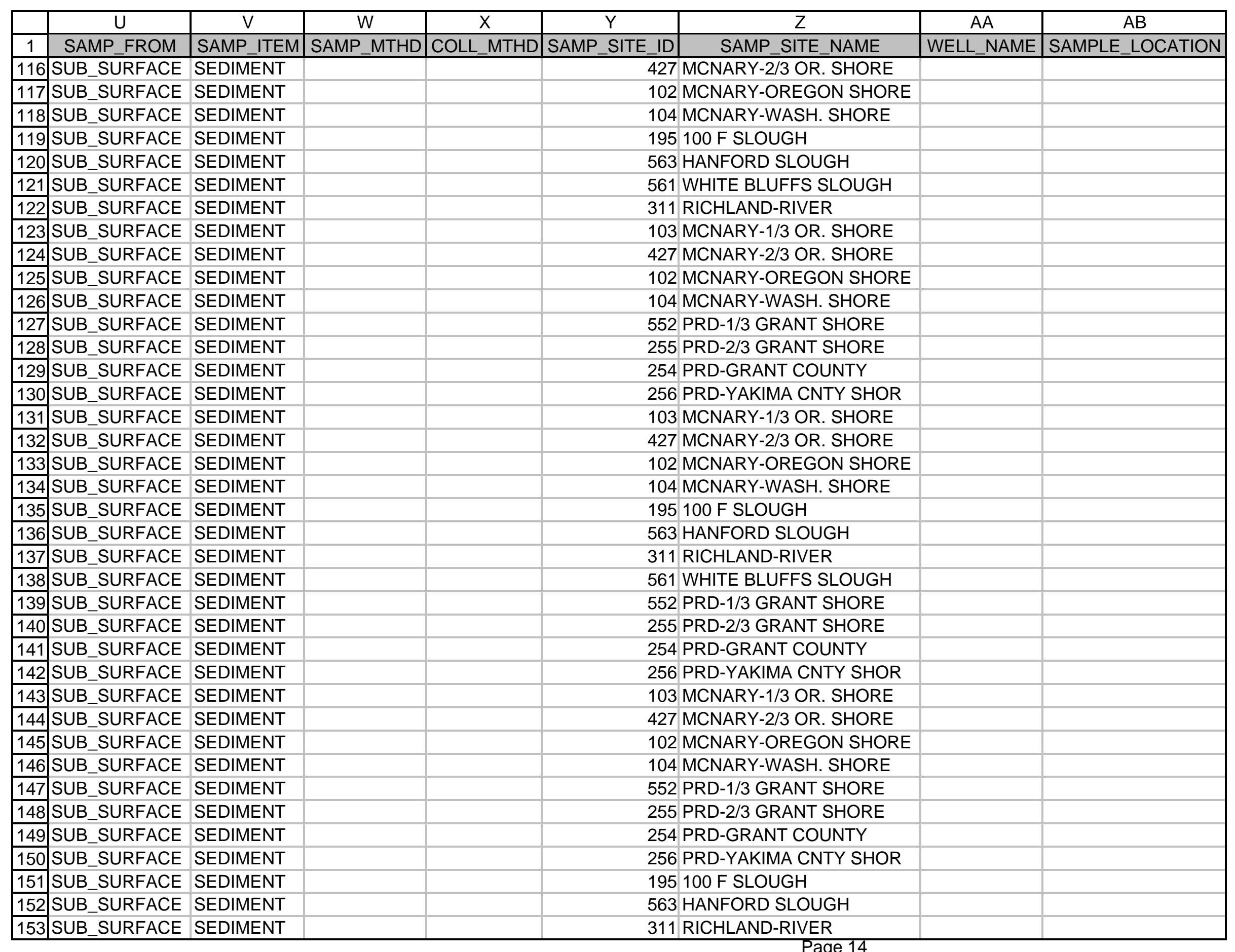




\begin{tabular}{|c|c|c|c|c|c|c|c|c|}
\hline & $\bar{U}$ & $\mathrm{~V}$ & $\mathrm{~W}$ & $\bar{X}$ & $\bar{Y}$ & $\bar{Z}$ & $\overline{A A}$ & $\overline{A B}$ \\
\hline 1 & SAMP_FROM & SAMP_ITEM & SAMP_MTHD & COLL_MTHD & SAMP_SITE_IL & SAMP_SITE_NAME & WELL_NAME & SAMPLE_LOCATION \\
\hline 154 & SUB_SURFACE & SEDIMENT & & & & 1 WHITE BLUFFS SLOUGH & & \\
\hline 155 & SUB SURFACE & SEDIMENT & & & 55 & 2 PRD-1/3 GRANT SHORE & & \\
\hline 156 & SUB SURFACE & SEDIMENT & & & 25 & 5 PRD-2/3 GRANT SHORE & & \\
\hline 157 & SUB_SURFACE & SEDIMENT & & & 25 & 4 PRD-GRANT COUNTY & & \\
\hline 158 & SUB_SURFACE & SEDIMENT & & & 25 & 6 PRD-YAKIMA CNTY SHOR & & \\
\hline 159 & SUB_SURFACE & SEDIMENT & & & 10 & 3 MCNARY-1/3 OR. SHORE & & \\
\hline 160 & SUB_SURFACE & SEDIMENT & & & 42 & 7 MCNARY-2/3 OR. SHORE & & \\
\hline 161 & SUB_SURFACE & SEDIMENT & & & 10 & 2 MCNARY-OREGON SHORE & & \\
\hline 162 & SUB_SURFACE & SEDIMENT & & & 10 & 4 MCNARY-WASH. SHORE & & \\
\hline 163 & SUB_SURFACE & SEDIMENT & & & 19 & 5100 F SLOUGH & & \\
\hline 164 & SUB_SURFACE & SEDIMENT & & & 56 & 1 WHITE BLUFFS SLOUGH & & \\
\hline 165 & SUB_SURFACE & SEDIMENT & & & 56 & 3 HANFORD SLOUGH & & \\
\hline 166 & SUB_SURFACE & SEDIMENT & & & 15 & 1 HANFORD SPRING 28-2 & & \\
\hline 167 & SUB_SURFACE & SEDIMENT & & & 31 & 1 RICHLAND-RIVER & & \\
\hline 168 & SUB_SURFACE & SEDIMENT & & & 19 & 5100 F SLOUGH & & \\
\hline 169 & SUB SURFACE & SEDIMENT & & & 56 & 3 HANFORD SLOUGH & & \\
\hline 170 & SUB_SURFACE & SEDIMENT & & & 31 & 1 RICHLAND-RIVER & & \\
\hline 171 & SUB_SURFACE & SEDIMENT & & & 56 & 1 WHITE BLUFFS SLOUGH & & \\
\hline 172 & SUB_SURFACE & SEDIMENT & & & 55 & 2 PRD-1/3 GRANT SHORE & & \\
\hline 173 & SUB_SURFACE & SEDIMENT & & & 25 & 5 PRD-2/3 GRANT SHORE & & \\
\hline 174 & SUB_SURFACE & SEDIMENT & & & 25 & 4 PRD-GRANT COUNTY & & \\
\hline 175 & SUB_SURFACE & SEDIMENT & & & 25 & 6 PRD-YAKIMA CNTY SHOR & & \\
\hline 176 & SUB_SURFACE & SEDIMENT & & & 10 & 3 MCNARY-1/3 OR. SHORE & & \\
\hline 177 & SUB_SURFACE & SEDIMENT & & & 42 & 7 MCNARY-2/3 OR. SHORE & & \\
\hline 178 & SUB_SURFACE & SEDIMENT & & & 10 & 2 MCNARY-OREGON SHORE & & \\
\hline 179 & SUB_SURFACE & SEDIMENT & & & 10 & 4 MCNARY-WASH. SHORE & & \\
\hline
\end{tabular}




\begin{tabular}{|c|c|c|c|c|c|}
\hline & $\mathrm{AC}$ & $A D$ & $\mathrm{AE}$ & $\mathrm{AF}$ & $A G$ \\
\hline 1 & SAMP COMMENT & RESULT COMMENT & COLL SAMP SIZE & COLL SAMP SIZE UNITS & DIST CLASS \\
\hline 2 & 1/3 FROM GRANT COUNTY SHORE & See sample record for comment. & & & OFFSITE \\
\hline 3 & 2/3 FROM GRANT COUNTY SHORE & See sample record for comment. & & & OFFSITE \\
\hline 4 & GRANT COUNTY SHORE & See sample record for comment. & & & OFFSITE \\
\hline 5 & YAKIMA COUNTY SHORE & See sample record for comment. & & & OFFSITE \\
\hline 6 & & & & & ONSITE \\
\hline 7 & & & & & RIVER_SHORELINE \\
\hline 8 & & & & & RIVER SHORELINE \\
\hline 9 & & & & & RIVER_SHORELINE \\
\hline 10 & 1/3 FROM OREGON SHORE & See sample record for comment. & & & OFFSITE \\
\hline 11 & $2 / 3$ FROM OREGON SHORE & See sample record for comment. & & & OFFSITE \\
\hline 12 & OREGON SHORE & See sample record for comment. & & & OFFSITE \\
\hline 13 & WASHINGTON SHORE & See sample record for comment. & & & OFFSITE \\
\hline 14 & 1/3 FROM GRANT COUNTY SHORE & See sample record for comment. & & & OFFSITE \\
\hline 15 & 2/3 FROM GRANT COUNT SHORE & See sample record for comment. & & & OFFSITE \\
\hline 16 & GRANT COUNTY SHORE & See sample record for comment. & & & OFFSITE \\
\hline 17 & YAKIMA COUNTY SHORE & See sample record for comment. & & & OFFSITE \\
\hline 18 & REFER TO INCIDENT REPORT 1/15/92 REGARDING U-ISO. DETECTION LIMIT. & See sample record for comment. & & & RIVER_SHORELINE \\
\hline 19 & REFER TO INCIDENT REPORT 1/15/92 REGARDING U-ISO. DETECTION LIMIT. & See sample record for comment. & & & RIVER_SHORELINE \\
\hline 20 & REFER TO INCIDENT REPORT 1/15/92 REGARDING U-ISO. DETECTION LIMIT. & See sample record for comment. & & & ONSITE \\
\hline 21 & REFER TO INCIDENT REPORT 1/15/92 REGARDING U-ISO. DETECTION LIMIT. & See sample record for comment. & & & RIVER_SHORELINE \\
\hline 22 & 1/3 FROM OREGON SHORE & See sample record for comment. & & & OFFSITE \\
\hline 23 & 2/3 FROM OREGON SHORE & See sample record for comment. & & & OFFSITE \\
\hline 24 & OREGON SHORE & See sample record for comment. & & & OFFSITE \\
\hline 25 & WASHINGTON SHORE & See sample record for comment. & & & OFFSITE \\
\hline 26 & & & & & ONSITE \\
\hline 27 & & & & & RIVER_SHORELINE \\
\hline 28 & & & & & RIVER_SHORELINE \\
\hline 29 & & & & & RIVER_SHORELINE \\
\hline 30 & & & & & OFFSITE \\
\hline 31 & & & & & OFFSITE \\
\hline 32 & & & & & OFFSITE \\
\hline 33 & & & & & OFFSITE \\
\hline 34 & & & & & OFFSITE \\
\hline 35 & & & & & OFFSITE \\
\hline 36 & & & & & OFFSITE \\
\hline 37 & & & & & OFFSITE \\
\hline 38 & & & & & OFFSITE \\
\hline 39 & & & & & OFFSITE \\
\hline
\end{tabular}




\begin{tabular}{|c|c|c|c|c|c|}
\hline & $\mathrm{AC}$ & $\mathrm{AD}$ & $\mathrm{AE}$ & $\mathrm{AF}$ & $A G$ \\
\hline 1 & SAMP_COMMENT & RESULT_COMMENT & COLL_SAMP_SIZE & COLL_SAMP_SIZE_UNITS & DIST_CLASS \\
\hline 40 & & & & & OFFSITE \\
\hline 41 & & & & & OFFSITE \\
\hline 42 & & & & & ONSITE \\
\hline 43 & & & & & RIVER_SHORELINE \\
\hline 44 & & & & & RIVER_SHORELINE \\
\hline 45 & & & & & RIVER_SHORELINE \\
\hline 46 & & & & & ONSITE \\
\hline 47 & REFER TO INCIDENT REPORT 9/22/93 REGARDING TEAR IN SAMPLING CONTAINER. & See sample record for comment. & & & ONSITE \\
\hline 48 & & & & & OFFSITE \\
\hline 49 & & & & & OFFSITE \\
\hline 50 & & & & & OFFSITE \\
\hline 51 & & & & & OFFSITE \\
\hline 52 & & & 1008 & $\mathrm{~g}$ & OFFSITE \\
\hline 53 & & & 1579 & $\mathrm{~g}$ & OFFSITE \\
\hline 54 & & & 2824 & $\mathrm{~g}$ & OFFSITE \\
\hline 55 & & & 897 & $\mathrm{~g}$ & OFFSITE \\
\hline 56 & & & 1767 & $\mathrm{~g}$ & OFFSITE \\
\hline 57 & & & 1220 & $\mathrm{~g}$ & OFFSITE \\
\hline 58 & & & 1273 & $\mathrm{~g}$ & OFFSITE \\
\hline 59 & & & 952 & $\mathrm{~g}$ & OFFSITE \\
\hline 60 & & & 2090 & $\mathrm{~g}$ & ONSITE \\
\hline 61 & & & 1521 & $\mathrm{~g}$ & RIVER_SHORELINE \\
\hline 62 & SAMPLE CONTAINER BROKE AT LAB, ALL ANALYSES LOST, REFER TO 5/4/94 IR. & & 1614 & $\mathrm{~g}$ & RIVER_SHORELINE \\
\hline 63 & & & 1711 & g & RIVER_SHORELINE \\
\hline 64 & & & 580 & $\mathrm{~g}$ & ONSITE \\
\hline 65 & & & 500 & $\mathrm{~g}$ & OFFSITE \\
\hline 66 & & & 500 & $\mathrm{~g}$ & OFFSITE \\
\hline 67 & & & 500 & $\mathrm{~g}$ & OFFSITE \\
\hline 68 & & & 500 & $\mathrm{~g}$ & OFFSITE \\
\hline 69 & & & 480 & $\mathrm{~g}$ & OFFSITE \\
\hline 70 & & & 551 & $\mathrm{~g}$ & OFFSITE \\
\hline 71 & & & 477 & $\mathrm{~g}$ & OFFSITE \\
\hline 72 & & & 548 & $\mathrm{~g}$ & OFFSITE \\
\hline 73 & & & 4904 & $\mathrm{~g}$ & ONSITE \\
\hline 74 & & & 4636 & $\mathrm{~g}$ & RIVER_SHORELINE \\
\hline 75 & & & 7000 & $\mathrm{~g}$ & RIVER_SHORELINE \\
\hline 76 & & & 7247 & $\mathrm{~g}$ & ONSITE \\
\hline 77 & & & 4738 & $\mathrm{~g}$ & RIVER_SHORELINE \\
\hline
\end{tabular}




\begin{tabular}{|c|c|c|c|c|c|}
\hline & $A C$ & $A D$ & $\mathrm{AE}$ & $\mathrm{AF}$ & $A G$ \\
\hline 1 & SAMP_COMMENT & RESULT_COMMENT & COLL_SAMP_SIZE & COLL_SAMP_SIZE_UNITS & DIST_CLASS \\
\hline 78 & & & 500 & $\mathrm{~g}$ & ONSITE \\
\hline 79 & & & 500 & g & RIVER_SHORELINE \\
\hline 80 & & & 500 & $\mathrm{~g}$ & RIVER_SHORELINE \\
\hline 81 & & & 500 & $\mathrm{~g}$ & RIVER_SHORELINE \\
\hline 82 & & & 500 & $\mathrm{~g}$ & OFFSITE \\
\hline 83 & & & 500 & $\mathrm{~g}$ & OFFSITE \\
\hline 84 & & & 500 & $\mathrm{~g}$ & OFFSITE \\
\hline 85 & & & 500 & $\mathrm{~g}$ & OFFSITE \\
\hline 86 & & & 500 & $\mathrm{~g}$ & OFFSITE \\
\hline 87 & & & 500 & $\mathrm{~g}$ & OFFSITE \\
\hline 88 & & & 500 & $\mathrm{~g}$ & OFFSITE \\
\hline 89 & & & 500 & $\mathrm{~g}$ & OFFSITE \\
\hline 90 & & & 500 & $\mathrm{~g}$ & ONSITE \\
\hline 91 & & & 500 & $\mathrm{~g}$ & ONSITE \\
\hline 92 & & & 500 & $\mathrm{~g}$ & ONSITE \\
\hline 93 & & & 500 & $\mathrm{~g}$ & ONSITE \\
\hline 94 & & & 500 & $\mathrm{~g}$ & ONSITE \\
\hline 95 & 1/3 FROM GRANT COUNTY SHORE & See sample record for comment. & & & OFFSITE \\
\hline 96 & 2/3 FROM GRANT COUNTY SHORE & See sample record for comment. & & & OFFSITE \\
\hline 97 & GRANT COUNTY SHORE & See sample record for comment. & & & OFFSITE \\
\hline 98 & YAKIMA COUNTY SHORE & See sample record for comment. & & & OFFSITE \\
\hline 99 & & & & & ONSITE \\
\hline 100 & & & & & RIVER_SHORELINE \\
\hline 101 & & & & & RIVER_SHORELINE \\
\hline 102 & & & & & RIVER_SHORELINE \\
\hline 103 & 1/3 FROM OREGON SHORE & See sample record for comment. & & & OFFSITE \\
\hline 104 & 2/3 FROM OREGON SHORE & See sample record for comment. & & & OFFSITE \\
\hline 105 & OREGON SHORE & See sample record for comment. & & & OFFSITE \\
\hline 106 & WASHINGTON SHORE & See sample record for comment. & & & OFFSITE \\
\hline 107 & 1/3 FROM GRANT COUNTY SHORE & See sample record for comment. & & & OFFSITE \\
\hline 108 & 2/3 FROM GRANT COUNT SHORE & See sample record for comment. & & & OFFSITE \\
\hline 109 & GRANT COUNTY SHORE & See sample record for comment. & & & OFFSITE \\
\hline 110 & YAKIMA COUNTY SHORE & See sample record for comment. & & & OFFSITE \\
\hline 111 & REFER TO INCIDENT REPORT 1/15/92 REGARDING U-ISO. DETECTION LIMIT. & See sample record for comment. & & & RIVER_SHORELINE \\
\hline 112 & REFER TO INCIDENT REPORT 1/15/92 REGARDING U-ISO. DETECTION LIMIT. & See sample record for comment. & & & RIVER_SHORELINE \\
\hline 113 & REFER TO INCIDENT REPORT 1/15/92 REGARDING U-ISO. DETECTION LIMIT. & See sample record for comment. & & & ONSITE \\
\hline 114 & REFER TO INCIDENT REPORT 1/15/92 REGARDING U-ISO. DETECTION LIMIT. & See sample record for comment. & & & RIVER_SHORELINE \\
\hline 115 & 1/3 FROM OREGON SHORE & See sample record for comment. & & & OFFSITE \\
\hline
\end{tabular}




\begin{tabular}{|c|c|c|c|c|c|}
\hline & $A C$ & $\mathrm{AD}$ & $\mathrm{AE}$ & $\mathrm{AF}$ & $A G$ \\
\hline 1 & SAMP_COMMENT & RESULT_COMMENT & COLL_SAMP_SIZE & COLL_SAMP_SIZE_UNITS & DIST_CLASS \\
\hline 116 & 2/3 FROM OREGON SHORE & See sample record for comment. & & & OFFSITE \\
\hline 117 & OREGON SHORE & See sample record for comment. & & & OFFSITE \\
\hline 118 & WASHINGTON SHORE & See sample record for comment. & & & OFFSITE \\
\hline 119 & & & & & ONSITE \\
\hline 120 & & & & & RIVER_SHORELINE \\
\hline 121 & & & & & RIVER_SHORELINE \\
\hline 122 & & & & & RIVER_SHORELINE \\
\hline 123 & & & & & OFFSITE \\
\hline 124 & & & & & OFFSITE \\
\hline 125 & & & & & OFFSITE \\
\hline 126 & & & & & OFFSITE \\
\hline 127 & & & & & OFFSITE \\
\hline 128 & & & & & OFFSITE \\
\hline 129 & & & & & OFFSITE \\
\hline 130 & & & & & OFFSITE \\
\hline 131 & & & & & OFFSITE \\
\hline 132 & & & & & OFFSITE \\
\hline 133 & & & & & OFFSITE \\
\hline 134 & & & & & OFFSITE \\
\hline 135 & & & & & ONSITE \\
\hline 136 & & & & & RIVER_SHORELINE \\
\hline 137 & & & & & RIVER_SHORELINE \\
\hline 138 & & & & & RIVER_SHORELINE \\
\hline 139 & & & & & OFFSITE \\
\hline 140 & & & & & OFFSITE \\
\hline 141 & & & & & OFFSITE \\
\hline 142 & & & & & OFFSITE \\
\hline 143 & & & 1008 & g & OFFSITE \\
\hline 144 & & & 1579 & $\mathrm{~g}$ & OFFSITE \\
\hline 145 & & & 2824 & $g$ & OFFSITE \\
\hline 146 & & & 897 & g & OFFSITE \\
\hline 147 & & & 1767 & $\mathrm{~g}$ & OFFSITE \\
\hline 148 & & & 1220 & $\mathrm{~g}$ & OFFSITE \\
\hline 149 & & & 1273 & $\mathrm{~g}$ & OFFSITE \\
\hline 150 & & & 952 & $\mathrm{~g}$ & OFFSITE \\
\hline 151 & & & 2090 & $\mathrm{~g}$ & ONSITE \\
\hline 152 & & & 1521 & g & RIVER_SHORELINE \\
\hline 153 & SAMPLE CONTAINER BROKE AT LAB, ALL ANALYSES LOST, REFER TO 5/4/94 IR. & & 1614 & $\mathrm{~g}$ & RIVER_SHORELINE \\
\hline
\end{tabular}




\begin{tabular}{|c|c|c|c|c|c|}
\hline & $A C$ & $A D$ & $\mathrm{AE}$ & $\mathrm{AF}$ & $A G$ \\
\hline \begin{tabular}{|l}
1 \\
\end{tabular} & SAMP_COMMENT & RESULT_COMMENT & COLL_SAMP_SIZE & COLL_SAMP_SIZE_UNITS & DIST_CLASS \\
\hline 154 & & & 1711 & $\mathrm{~g}$ & RIVER_SHORELINE \\
\hline 155 & & & 500 & $\mathrm{~g}$ & OFFSITE \\
\hline 156 & & & 500 & $\mathrm{~g}$ & OFFSITE \\
\hline 157 & & & 500 & $\mathrm{~g}$ & OFFSITE \\
\hline 158 & & & 500 & $g$ & OFFSITE \\
\hline 159 & & & 480 & $\mathrm{~g}$ & OFFSITE \\
\hline 160 & & & 551 & $\mathrm{~g}$ & OFFSITE \\
\hline 161 & & & 477 & $g$ & OFFSITE \\
\hline \begin{tabular}{|l|}
162 \\
\end{tabular} & & & 548 & $\mathrm{~g}$ & OFFSITE \\
\hline \begin{tabular}{|l|l|}
163 \\
\end{tabular} & & & 4904 & $\mathrm{~g}$ & ONSITE \\
\hline \begin{tabular}{|l|l|}
164 \\
\end{tabular} & & & 4636 & $\mathrm{~g}$ & RIVER_SHORELINE \\
\hline 165 & & & 7000 & $\mathrm{~g}$ & RIVER_SHORELINE \\
\hline \begin{tabular}{|l|l|}
166 \\
\end{tabular} & & & 7247 & $\mathrm{~g}$ & ONSITE \\
\hline \begin{tabular}{|l|}
167 \\
\end{tabular} & & & 4738 & $\mathrm{~g}$ & RIVER_SHORELINE \\
\hline 168 & & & 500 & $\mathrm{~g}$ & ONSITE \\
\hline \begin{tabular}{|l|}
169 \\
\end{tabular} & & & 500 & $\mathrm{~g}$ & RIVER_SHORELINE \\
\hline 170 & & & 500 & $\mathrm{~g}$ & RIVER SHORELINE \\
\hline \begin{tabular}{|l|}
171 \\
\end{tabular} & & & 500 & $\mathrm{~g}$ & RIVER_SHORELINE \\
\hline \begin{tabular}{|l|}
172 \\
\end{tabular} & & & 500 & $\mathrm{~g}$ & OFFSITE \\
\hline \begin{tabular}{|l|l|}
173 \\
\end{tabular} & & & 500 & $\mathrm{~g}$ & OFFSITE \\
\hline \begin{tabular}{|l|}
174 \\
\end{tabular} & & & 500 & $\mathrm{~g}$ & OFFSITE \\
\hline \begin{tabular}{|l|}
175 \\
\end{tabular} & & & 500 & $\mathrm{~g}$ & OFFSITE \\
\hline \begin{tabular}{|l|}
176 \\
\end{tabular} & & & 500 & $\mathrm{~g}$ & OFFSITE \\
\hline \begin{tabular}{|l|}
177 \\
\end{tabular} & & & 500 & $\mathrm{~g}$ & OFFSITE \\
\hline \begin{tabular}{|l|}
178 \\
\end{tabular} & & & 500 & $\mathrm{~g}$ & OFFSITE \\
\hline \begin{tabular}{|l|}
179 \\
\end{tabular} & & & 500 & $\mathrm{~g}$ & OFFSITE \\
\hline
\end{tabular}




\begin{tabular}{|r|l|l|}
\hline & \multicolumn{1}{|c|}{ AH } & \\
\hline 1 & OWNER_ID & COLLECTION_PURPOSE \\
\hline 2 & SESPMNT & $\mathrm{R}$ \\
\hline 3 & SESPMNT & $\mathrm{R}$ \\
\hline 4 & SESPMNT & $\mathrm{R}$ \\
\hline 5 & SESPMNT & $\mathrm{R}$ \\
\hline 6 & SESPMNT & $\mathrm{R}$ \\
\hline 7 & SESPMNT & $\mathrm{R}$ \\
\hline 8 & SESPMNT & $\mathrm{R}$ \\
\hline 9 & SESPMNT & $\mathrm{R}$ \\
\hline 10 & SESPMNT & $\mathrm{R}$ \\
\hline 11 & SESPMNT & $\mathrm{R}$ \\
\hline 12 & SESPMNT & $\mathrm{R}$ \\
\hline 13 & SESPMNT & $\mathrm{R}$ \\
\hline 14 & SESPMNT & $\mathrm{R}$ \\
\hline 15 & SESPMNT & $\mathrm{R}$ \\
\hline 16 & SESPMNT & $\mathrm{R}$ \\
\hline 17 & SESPMNT & $\mathrm{R}$ \\
\hline 18 & SESPMNT & $\mathrm{R}$ \\
\hline 19 & SESPMNT & $\mathrm{R}$ \\
\hline 20 & SESPMNT & $\mathrm{R}$ \\
\hline 21 & SESPMNT & $\mathrm{R}$ \\
\hline 22 & SESPMNT & $\mathrm{R}$ \\
\hline 23 & SESPMNT & $\mathrm{R}$ \\
\hline 24 & SESPMNT & $\mathrm{R}$ \\
\hline 25 & SESPMNT & $\mathrm{R}$ \\
\hline 26 & SESPMNT & $\mathrm{R}$ \\
\hline 27 & SESPMNT & $\mathrm{R}$ \\
\hline 28 & SESPMNT & $\mathrm{R}$ \\
\hline 29 & SESPMNT & $\mathrm{R}$ \\
\hline 30 & SESPMNT & $\mathrm{R}$ \\
\hline 31 & SESPMNT & $\mathrm{R}$ \\
\hline 32 & SESPMNT & $\mathrm{R}$ \\
\hline 33 & SESPMNT & $\mathrm{R}$ \\
\hline 34 & SESPMNT & $\mathrm{R}$ \\
\hline 35 & SESPMNT & $\mathrm{R}$ \\
\hline 36 & SESPMNT & $\mathrm{R}$ \\
\hline 37 & SESPMNT & $\mathrm{R}$ \\
\hline 38 & SESPMNT & $\mathrm{R}$ \\
\hline 39 & SESPMNT & $\mathrm{R}$ \\
\hline & & \\
\hline
\end{tabular}




\begin{tabular}{|c|l|l|}
\hline & \multicolumn{1}{|c|}{ AH } & \\
\hline 1 & OWNER_ID & COLLECTION_PURPOSE \\
\hline 40 & SESPMNT & $\mathrm{R}$ \\
\hline 41 & SESPMNT & $\mathrm{R}$ \\
\hline 42 & SESPMNT & $\mathrm{R}$ \\
\hline 43 & SESPMNT & $\mathrm{R}$ \\
\hline 44 & SESPMNT & $\mathrm{R}$ \\
\hline 45 & SESPMNT & $\mathrm{R}$ \\
\hline 46 & SESPMNT & $\mathrm{R}$ \\
\hline 47 & SESPMNT & $\mathrm{R}$ \\
\hline 48 & SESPMNT & $\mathrm{R}$ \\
\hline 49 & SESPMNT & $\mathrm{R}$ \\
\hline 50 & SESPMNT & $\mathrm{R}$ \\
\hline 51 & SESPMNT & $\mathrm{R}$ \\
\hline 52 & SESPSPEC & $\mathrm{S}$ \\
\hline 53 & SESPSPEC & $\mathrm{S}$ \\
\hline 54 & SESPSPEC & $\mathrm{S}$ \\
\hline 55 & SESPSPEC & $\mathrm{S}$ \\
\hline 56 & SESPSPEC & $\mathrm{S}$ \\
\hline 57 & SESPSPEC & $\mathrm{S}$ \\
\hline 58 & SESPSPEC & $\mathrm{S}$ \\
\hline 59 & SESPSPEC & $\mathrm{S}$ \\
\hline 60 & SESPSPEC & $\mathrm{S}$ \\
\hline 61 & SESPSPEC & $\mathrm{S}$ \\
\hline 62 & SESPSPEC & $\mathrm{S}$ \\
\hline 63 & SESPSPEC & $\mathrm{S}$ \\
\hline 64 & SESPMNT & $\mathrm{R}$ \\
\hline 65 & SESPMNT & $\mathrm{R}$ \\
\hline 66 & SESPMNT & $\mathrm{R}$ \\
\hline 67 & SESPMNT & $\mathrm{R}$ \\
\hline 68 & SESPMNT & $\mathrm{R}$ \\
\hline 69 & SESPMNT & $\mathrm{R}$ \\
\hline 70 & SESPMNT & $\mathrm{R}$ \\
\hline 71 & SESPMNT & $\mathrm{R}$ \\
\hline 72 & SESPMNT & $\mathrm{R}$ \\
\hline 73 & SESPMNT & $\mathrm{R}$ \\
\hline 74 & SESPMNT & $\mathrm{R}$ \\
\hline 75 & SESPMNT & $\mathrm{R}$ \\
\hline 76 & SESPMNT & $\mathrm{R}$ \\
\hline 77 & SESPMNT & $\mathrm{R}$ \\
\hline & & \\
\hline
\end{tabular}




\begin{tabular}{|c|l|l|}
\hline & \multicolumn{1}{|c|}{ AH } & \\
\hline 1 & OWNER_ID & COLLECTION_PURPOSE \\
\hline 78 & SESPMNT & $\mathrm{R}$ \\
\hline 79 & SESPMNT & $\mathrm{R}$ \\
\hline 80 & SESPMNT & $\mathrm{R}$ \\
\hline 81 & SESPMNT & $\mathrm{R}$ \\
\hline 82 & SESPMNT & $\mathrm{R}$ \\
\hline 83 & SESPMNT & $\mathrm{R}$ \\
\hline 84 & SESPMNT & $\mathrm{R}$ \\
\hline 85 & SESPMNT & $\mathrm{R}$ \\
\hline 86 & SESPMNT & $\mathrm{R}$ \\
\hline 87 & SESPMNT & $\mathrm{R}$ \\
\hline 88 & SESPMNT & $\mathrm{R}$ \\
\hline 89 & SESPMNT & $\mathrm{R}$ \\
\hline 90 & SESPMNT & $\mathrm{R}$ \\
\hline 91 & SESPMNT & $\mathrm{R}$ \\
\hline 92 & SESPMNT & $\mathrm{R}$ \\
\hline 93 & SESPMNT & $\mathrm{R}$ \\
\hline 94 & SESPMNT & $\mathrm{R}$ \\
\hline 95 & SESPMNT & $\mathrm{R}$ \\
\hline 96 & SESPMNT & $\mathrm{R}$ \\
\hline 97 & SESPMNT & $\mathrm{R}$ \\
\hline 98 & SESPMNT & $\mathrm{R}$ \\
\hline 99 & SESPMNT & $\mathrm{R}$ \\
\hline 100 & SESPMNT & $\mathrm{R}$ \\
\hline 101 & SESPMNT & $\mathrm{R}$ \\
\hline 102 & SESPMNT & $\mathrm{R}$ \\
\hline 103 & SESPMNT & $\mathrm{R}$ \\
\hline 104 & SESPMNT & $\mathrm{R}$ \\
\hline 105 & SESPMNT & $\mathrm{R}$ \\
\hline 106 & SESPMNT & $\mathrm{R}$ \\
\hline 107 & SESPMNT & $\mathrm{R}$ \\
\hline 108 & SESPMNT & $\mathrm{R}$ \\
\hline 109 & SESPMNT & $\mathrm{R}$ \\
\hline 110 & SESPMNT & $\mathrm{R}$ \\
\hline 111 & SESPMNT & $\mathrm{R}$ \\
\hline 112 & SESPMNT & $\mathrm{R}$ \\
\hline 113 & SESPMNT & $\mathrm{R}$ \\
\hline 114 & SESPMNT & $\mathrm{R}$ \\
\hline 115 & SESPMNT & $\mathrm{R}$ \\
\hline & & \\
\hline
\end{tabular}




\begin{tabular}{|c|l|l|}
\hline & \multicolumn{1}{|c|}{ AH } & \\
\hline 1 & OWNER_ID & COLLECTION_PURPOSE \\
\hline 116 & SESPMNT & $\mathrm{R}$ \\
\hline 117 & SESPMNT & $\mathrm{R}$ \\
\hline 118 & SESPMNT & $\mathrm{R}$ \\
\hline 119 & SESPMNT & $\mathrm{R}$ \\
\hline 120 & SESPMNT & $\mathrm{R}$ \\
\hline 121 & SESPMNT & $\mathrm{R}$ \\
\hline 122 & SESPMNT & $\mathrm{R}$ \\
\hline 123 & SESPMNT & $\mathrm{R}$ \\
\hline 124 & SESPMNT & $\mathrm{R}$ \\
\hline 125 & SESPMNT & $\mathrm{R}$ \\
\hline 126 & SESPMNT & $\mathrm{R}$ \\
\hline 127 & SESPMNT & $\mathrm{R}$ \\
\hline 128 & SESPMNT & $\mathrm{R}$ \\
\hline 129 & SESPMNT & $\mathrm{R}$ \\
\hline 130 & SESPMNT & $\mathrm{R}$ \\
\hline 131 & SESPMNT & $\mathrm{R}$ \\
\hline 132 & SESPMNT & $\mathrm{R}$ \\
\hline 133 & SESPMNT & $\mathrm{R}$ \\
\hline 134 & SESPMNT & $\mathrm{R}$ \\
\hline 135 & SESPMNT & $\mathrm{R}$ \\
\hline 136 & SESPMNT & $\mathrm{R}$ \\
\hline 137 & SESPMNT & $\mathrm{R}$ \\
\hline 138 & SESPMNT & $\mathrm{R}$ \\
\hline 139 & SESPMNT & $\mathrm{R}$ \\
\hline 140 & SESPMNT & $\mathrm{R}$ \\
\hline 141 & SESPMNT & $\mathrm{R}$ \\
\hline 142 & SESPMNT & $\mathrm{R}$ \\
\hline 143 & SESPSPEC & $\mathrm{S}$ \\
\hline 144 & SESPSPEC & $\mathrm{S}$ \\
\hline 145 & SESPSPEC & $\mathrm{S}$ \\
\hline 146 & SESPSPEC & $\mathrm{S}$ \\
\hline 147 & SESPSPEC & $\mathrm{S}$ \\
\hline 148 & SESPSPEC & $\mathrm{S}$ \\
\hline 149 & SESPSPEC & $\mathrm{S}$ \\
\hline 150 & SESPSPEC & $\mathrm{S}$ \\
\hline 151 & SESPSPEC & $\mathrm{S}$ \\
\hline 152 & SESPSPEC & $\mathrm{S}$ \\
\hline 153 & SESPSPEC & $\mathrm{S}$ \\
\hline & & \\
\hline
\end{tabular}




\begin{tabular}{|c|l|l|}
\hline & \multicolumn{1}{|c|}{ AH } & \multicolumn{1}{c|}{ Al } \\
\hline 1 & OWNER_ID & COLLECTION_PURPOSE \\
\hline 154 & SESPSPEC & $\mathrm{S}$ \\
\hline 155 & SESPMNT & $\mathrm{R}$ \\
\hline 156 & SESPMNT & $\mathrm{R}$ \\
\hline 157 & SESPMNT & $\mathrm{R}$ \\
\hline 158 & SESPMNT & $\mathrm{R}$ \\
\hline 159 & SESPMNT & $\mathrm{R}$ \\
\hline 160 & SESPMNT & $\mathrm{R}$ \\
\hline 161 & SESPMNT & $\mathrm{R}$ \\
\hline 162 & SESPMNT & $\mathrm{R}$ \\
\hline 163 & SESPMNT & $\mathrm{R}$ \\
\hline 164 & SESPMNT & $\mathrm{R}$ \\
\hline 165 & SESPMNT & $\mathrm{R}$ \\
\hline 166 & SESPMNT & $\mathrm{R}$ \\
\hline 167 & SESPMNT & $\mathrm{R}$ \\
\hline 168 & SESPMNT & $\mathrm{R}$ \\
\hline 169 & SESPMNT & $\mathrm{R}$ \\
\hline 170 & SESPMNT & $\mathrm{R}$ \\
\hline 171 & SESPMNT & $\mathrm{R}$ \\
\hline 172 & SESPMNT & $\mathrm{R}$ \\
\hline 173 & SESPMNT & $\mathrm{R}$ \\
\hline 174 & SESPMNT & $\mathrm{R}$ \\
\hline 175 & SESPMNT & $\mathrm{R}$ \\
\hline 176 & SESPMNT & $\mathrm{R}$ \\
\hline 177 & SESPMNT & $\mathrm{R}$ \\
\hline 178 & SESPMNT & $\mathrm{R}$ \\
\hline 179 & SESPMNT & $\mathrm{R}$ \\
\hline
\end{tabular}


$90-95$ SW 


\begin{tabular}{|c|c|c|c|c|c|c|c|c|c|c|c|}
\hline & A & $\mathrm{B}$ & $\mathrm{C}$ & $\mathrm{D}$ & $E$ & $\mathrm{~F}$ & G & $\mathrm{H}$ & $\mathrm{I}$ & $\mathrm{J}$ & $\mathrm{K}$ \\
\hline 1 & SAMPLE_NUM_ASSIGNED_TO & SAMPLER & MEDIA & BIOTA_MEDIA & TAG_ID & SAMP_NUM & SAMP_DATE_TIME & SAMP_DATE_TIME_ON & CON_ID & CON_SHORT_NAME & METHOD_NAME \\
\hline 2 & PDMS & & SW & & & E111249 & 03-Jan-90 & & $15117-96-1$ & U-235 & UISO_AEA \\
\hline 3 & PDMS & & SW & & & E111248 & 03-Jan-90 & & $15117-96-1$ & U-235 & UISO_AEA \\
\hline 4 & PDMS & & SW & & & E112521 & $30-J a n-90$ & & $15117-96-1$ & U-235 & UISO_AEA \\
\hline 5 & PDMS & & SW & & & E112520 & 30-Jan-90 & & $15117-96-1$ & U-235 & UISO_AEA \\
\hline 6 & PDMS & & SW & & & E112805 & 27-Feb-90 & & 15117-96-1 & U-235 & UISO_AEA \\
\hline 7 & PDMS & & SW & & & E112806 & 27-Feb-90 & & $15117-96-1$ & U-235 & UISO_AEA \\
\hline 8 & PDMS & & SW & & & E113120 & 03-Apr-90 & & $15117-96-1$ & U-235 & UISO_IE_PLATE_AEA \\
\hline 9 & PDMS & & SW & & & E113119 & 03-Apr-90 & & 15117-96-1 & U-235 & UISO_AEA \\
\hline 10 & PDMS & & SW & & & E113118 & 03-Apr-90 & & $15117-96-1$ & U-235 & UISO_AEA \\
\hline 11 & PDMS & & SW & & & E033077 & 26-Jun-90 & & $15117-96-1$ & U-235 & UISO IE PLATE AEA \\
\hline 12 & PDMS & & SW & & & E115795 & 03-Jul-90 & & $15117-96-1$ & $\mathrm{U}-235$ & UISOAAEA \\
\hline 13 & PDMS & & SW & & & E114227 & 03-Jul-90 & & $15117-96-1$ & U-235 & UISO_AEA \\
\hline 14 & PDMS & & SW & & & E115796 & 31-Jul-90 & & $15117-96-1$ & U-235 & UISO_AEA \\
\hline 15 & PDMS & & SW & & & E115269 & 31-Jul-90 & & 15117-96-1 & U-235 & UISO_AEA \\
\hline 16 & PDMS & & SW & & & E115797 & 28-Aug-90 & & $15117-96-1$ & U-235 & UISO_AEA \\
\hline 17 & PDMS & & SW & & & E115297 & 28-Aug-90 & & $15117-96-1$ & U-235 & UISO_AEA \\
\hline 18 & PDMS & & SW & & & E033075 & 18-Sep-90 & & $15117-96-1$ & $\mathrm{U}-235$ & UISO IE PLATE AEA \\
\hline 19 & PDMS & & SW & & & E115798 & 02-Oct-90 & & 15117-96-1 & U-235 & UISO_AEA \\
\hline 20 & PDMS & & SW & & & E115332 & $02-$ Oct-90 & & $15117-96-1$ & U-235 & UISO_AEA \\
\hline 21 & PDMS & & SW & & & E115799 & 29-Oct-90 & & $15117-96-1$ & $\mathrm{U}-235$ & UISO_AEA \\
\hline 22 & PDMS & & SW & & & E115359 & 29-Oct-90 & & $15117-96-1$ & U-235 & UISO_AEA \\
\hline 23 & PDMS & & SW & & & E115800 & 27-Nov-90 & & $15117-96-1$ & U-235 & UISO_AEA \\
\hline 24 & PDMS & & SW & & & E115389 & 28-Nov-90 & & $15117-96-1$ & U-235 & UISO_AEA \\
\hline 25 & PDMS & & SW & & & E115801 & 31-Dec-90 & & $15117-96-1$ & $\mathrm{U}-235$ & UISO AEA \\
\hline 26 & PDMS & & SW & & & E115422 & 31-Dec-90 & & $15117-96-1$ & U-235 & UISO_AEA \\
\hline 27 & PDMS & & SW & & & E033076 & 08-Jan-91 & & $15117-96-1$ & $\mathrm{U}-235$ & UISO_IE_PLATE_AEA \\
\hline 28 & PDMS & & SW & & & E117635 & 29-Jan-91 & & 15117-96-1 & U-235 & UISO_AEA \\
\hline 29 & PDMS & & SW & & & E117632 & 29-Jan-91 & & $15117-96-1$ & U-235 & UISO_AEA \\
\hline 30 & PDMS & & SW & & & E117636 & 26-Feb-91 & & $15117-96-1$ & U-235 & UISO_AEA \\
\hline 31 & PDMS & & SW & & & E117633 & 26-Feb-91 & & 15117-96-1 & U-235 & UISO_AEA \\
\hline 32 & PDMS & & SW & & & E117638 & 02-Apr-91 & & $15117-96-1$ & U-235 & UISO_IE_PLATE_AEA \\
\hline 33 & PDMS & & SW & & & E117637 & 02-Apr-91 & & $15117-96-1$ & U-235 & UISO_AEA \\
\hline 34 & PDMS & & SW & & & E117634 & 02-Apr-91 & & $15117-96-1$ & $\mathrm{U}-235$ & UISO AEA \\
\hline 35 & PDMS & & SW & & & E116362 & 30-Apr-91 & & 15117-96-1 & U-235 & UISO_AEA \\
\hline 36 & PDMS & & SW & & & E116363 & 30-Apr-91 & & $15117-96-1$ & U-235 & UISO_AEA \\
\hline 37 & PDMS & & SW & & & E117312 & 28-May-91 & & $15117-96-1$ & $\mathrm{U}-235$ & UISO_AEA \\
\hline 38 & PDMS & & SW & & & E117311 & 28-May-91 & & $15117-96-1$ & U-235 & UISO_AEA \\
\hline 39 & PDMS & & SW & & & E117313 & 25-Jun-91 & & $15117-96-1$ & U-235 & UISO_IE_PLATE_AEA \\
\hline
\end{tabular}




\begin{tabular}{|c|c|c|c|c|c|c|c|c|c|c|c|}
\hline & $A$ & $B$ & $C$ & $\mathrm{D}$ & $E$ & $\mathrm{~F}$ & $G$ & $\mathrm{H}$ & $\mathrm{I}$ & $\mathrm{J}$ & $\mathrm{K}$ \\
\hline 1 & SAMPLE NUM ASSIGNED TO & SAMPLER & MEDIA & BIOTA MEDIA & TAG ID & SAMP NUM & SAMP DATE TIME & SAMP DATE TIME ON & CON ID & CON SHORT NAME & METHOD NAME \\
\hline 40 & PDMS & & SW & & & E117440 & 02-Jul-91 & & 15117-96-1 & U-235 & UISO_AEA \\
\hline 41 & PDMS & & SW & & & E117441 & 02-Jul-91 & & $15117-96-1$ & U-235 & UISO_AEA \\
\hline 42 & PDMS & & SW & & & E117332 & 30-Jul-91 & & 15117-96-1 & U-235 & UISO_AEA \\
\hline 43 & PDMS & & SW & & & E117333 & 30-Jul-91 & & $15117-96-1$ & U-235 & UISO_AEA \\
\hline 44 & PDMS & & SW & & & E117334 & 07-Aug-91 & & 15117-96-1 & U-235 & UISO_AEA \\
\hline 45 & PDMS & & SW & & & E117335 & 07-Aug-91 & & 15117-96-1 & U-235 & UISO_AEA \\
\hline 46 & PDMS & & SW & & & E117336 & 07-Aug-91 & & $15117-96-1$ & U-235 & UISO_AEA \\
\hline 47 & PDMS & & SW & & & E117337 & 07-Aug-91 & & 15117-96-1 & U-235 & UISO_AEA \\
\hline 48 & PDMS & & SW & & & E117338 & 08-Aug-91 & & $15117-96-1$ & U-235 & UISO_AEA \\
\hline 49 & PDMS & & SW & & & E117339 & 08-Aug-91 & & 15117-96-1 & U-235 & UISO_AEA \\
\hline 50 & PDMS & & SW & & & E117340 & 08-Aug-91 & & 15117-96-1 & U-235 & UISO_AEA \\
\hline 51 & PDMS & & SW & & & E117341 & 08-Aug-91 & & $15117-96-1$ & U-235 & UISO_AEA \\
\hline 52 & PDMS & & SW & & & E117646 & 03-Sep-91 & & $15117-96-1$ & U-235 & UISO_AEA \\
\hline 53 & PDMS & & SW & & & E117647 & 03-Sep-91 & & 15117-96-1 & U-235 & UISO_AEA \\
\hline 54 & PDMS & & SW & & & E117991 & 17-Sep-91 & & $15117-96-1$ & U-235 & UISO_IE_PLATE_AEA \\
\hline 55 & PDMS & & SW & & & E118570 & 24-Sep-91 & & $15117-96-1$ & U-235 & UISO_AEA \\
\hline 56 & PDMS & & SW & & & E118571 & 24-Sep-91 & & $15117-96-1$ & U-235 & UISO_AEA \\
\hline 57 & PDMS & & SW & & & E118572 & 24-Sep-91 & & 15117-96-1 & U-235 & UISO_AEA \\
\hline 58 & PDMS & & SW & & & E118573 & 24-Sep-91 & & $15117-96-1$ & U-235 & UISO_AEA \\
\hline 59 & PDMS & & SW & & & E117946 & 25-Sep-91 & & $15117-96-1$ & $\mathrm{U}-235$ & UISO_AEA \\
\hline 60 & PDMS & & SW & & & E117947 & 25-Sep-91 & & $15117-96-1$ & U-235 & UISO_AEA \\
\hline 61 & PDMS & & SW & & & E117948 & 25-Sep-91 & & $15117-96-1$ & U-235 & UISO_AEA \\
\hline 62 & PDMS & & SW & & & E117949 & 25-Sep-91 & & $15117-96-1$ & U-235 & UISO_AEA \\
\hline 63 & PDMS & & SW & & & E118574 & 01-Oct-91 & & 15117-96-1 & U-235 & UISO_AEA \\
\hline 64 & PDMS & & SW & & & E118575 & 01-Oct-91 & & $15117-96-1$ & U-235 & UISO_AEA \\
\hline 65 & PDMS & & SW & & & E118587 & $17-$ Oct-91 & & $15117-96-1$ & $\mathrm{U}-235$ & UISO_AEA \\
\hline 66 & PDMS & & SW & & & E118588 & 17-Oct-91 & & 15117-96-1 & U-235 & UISO_AEA \\
\hline 67 & PDMS & & SW & & & E118589 & 17-Oct-91 & & $15117-96-1$ & U-235 & UISO_AEA \\
\hline 68 & PDMS & & SW & & & E118590 & $17-$ Oct-91 & & $15117-96-1$ & U-235 & UISO_AEA \\
\hline 69 & PDMS & & SW & & & E118579 & 17-Oct-91 & & 15117-96-1 & U-235 & UISO_AEA \\
\hline 70 & PDMS & & SW & & & E118580 & 17-Oct-91 & & 15117-96-1 & U-235 & UISO_AEA \\
\hline 71 & PDMS & & SW & & & E118581 & 17-Oct-91 & & 15117-96-1 & U-235 & UISO_AEA \\
\hline 72 & PDMS & & SW & & & E118582 & 17-Oct-91 & & $15117-96-1$ & U-235 & UISO AEA \\
\hline 73 & PDMS & & SW & & & E118802 & 29-Oct-91 & & 15117-96-1 & U-235 & UISO_AEA \\
\hline 74 & PDMS & & SW & & & E118801 & 29-Oct-91 & & 15117-96-1 & U-235 & UISO_AEA \\
\hline 75 & PDMS & & SW & & & E118278 & 25-Nov-91 & & $15117-96-1$ & U-235 & UISO_AEA \\
\hline 76 & PDMS & & SW & & & E118279 & 25-Nov-91 & & $15117-96-1$ & U-235 & UISO_AEA \\
\hline 77 & PDMS & & SW & & & E118280 & 25-Nov-91 & & 15117-96-1 & U-235 & UISO_AEA \\
\hline
\end{tabular}




\begin{tabular}{|c|c|c|c|c|c|c|c|c|c|c|c|}
\hline & A & $\mathrm{B}$ & C & $\mathrm{D}$ & $\mathrm{E}$ & $\mathrm{F}$ & $\mathrm{G}$ & $\mathrm{H}$ & $\mathrm{I}$ & $\mathrm{J}$ & $\mathrm{K}$ \\
\hline 1 & SAMPLE_NUM_ASSIGNED_TO & SAMPLER & MEDIA & BIOTA_MEDIA & TAG_ID & SAMP_NUM & SAMP_DATE_TIME & SAMP_DATE_TIME_ON & CON_ID & CON_SHORT_NAME & METHOD_NAME \\
\hline 78 & PDMS & & SW & & & E118281 & 25-Nov-91 & & 15117-96-1 & U-235 & UISO_AEA \\
\hline 79 & PDMS & & SW & & & E118274 & 27-Nov-91 & & $15117-96-1$ & U-235 & UISO_AEA \\
\hline 80 & PDMS & & SW & & & E118275 & 27-Nov-91 & & 15117-96-1 & $\mathrm{U}-235$ & UISO_AEA \\
\hline 81 & PDMS & & SW & & & E118276 & 27-Nov-91 & & 15117-96-1 & U-235 & UISO_AEA \\
\hline 82 & PDMS & & SW & & & E118277 & 27-Nov-91 & & $15117-96-1$ & U-235 & UISO_AEA \\
\hline 83 & PDMS & & SW & & & E118273 & 03-Dec-91 & & $15117-96-1$ & U-235 & UISO_AEA \\
\hline 84 & PDMS & & SW & & & E118272 & 03-Dec-91 & & $15117-96-1$ & U-235 & UISO_AEA \\
\hline 85 & PDMS & & SW & & & E118380 & 17-Dec-91 & & $15117-96-1$ & U-235 & UISO_AEA \\
\hline 86 & PDMS & & SW & & & E118381 & 17-Dec-91 & & 15117-96-1 & U-235 & UISO_AEA \\
\hline 87 & PDMS & & SW & & & E118382 & 17-Dec-91 & & 15117-96-1 & U-235 & UISO_AEA \\
\hline 88 & PDMS & & SW & & & E118383 & 17-Dec-91 & & $15117-96-1$ & U-235 & UISO_AEA \\
\hline 89 & PDMS & & SW & & & E118803 & 18-Dec-91 & & 15117-96-1 & U-235 & UISO_AEA \\
\hline 90 & PDMS & & SW & & & E118804 & 18-Dec-91 & & 15117-96-1 & U-235 & UISO_AEA \\
\hline 91 & PDMS & & SW & & & E118805 & 18-Dec-91 & & $15117-96-1$ & U-235 & UISO_AEA \\
\hline 92 & PDMS & & SW & & & E118806 & 18-Dec-91 & & $15117-96-1$ & U-235 & UISO_AEA \\
\hline 93 & PDMS & & SW & & & E118378 & 31-Dec-91 & & 15117-96-1 & U-235 & UISO_AEA \\
\hline 94 & PDMS & & SW & & & E118377 & 31-Dec-91 & & 15117-96-1 & U-235 & UISO_AEA \\
\hline 95 & PDMS & & SW & & & E118657 & 07-Jan-92 & & $15117-96-1$ & U-235 & UISO_IE_PLATE_AEA \\
\hline 96 & PDMS & & SW & & & E120046 & 28-Jan-92 & & 15117-96-1 & U-235 & UISO_AEA \\
\hline 97 & PDMS & & SW & & & E044687 & 28-Jan-92 & & 15117-96-1 & U-235 & UISO_AEA \\
\hline 98 & PDMS & & SW & & & E120226 & 03-Mar-92 & & 15117-96-1 & U-235 & UISO_AEA \\
\hline 99 & PDMS & & SW & & & E120225 & 03-Mar-92 & & 15117-96-1 & U-235 & UISO_AEA \\
\hline 100 & PDMS & & SW & & & E120423 & 25-Mar-92 & & $15117-96-1$ & U-235 & UISO_AEA \\
\hline 101 & PDMS & & SW & & & E120424 & 25-Mar-92 & & $15117-96-1$ & U-235 & UISO_AEA \\
\hline 102 & PDMS & & SW & & & E120425 & 25-Mar-92 & & 15117-96-1 & U-235 & UISO_AEA \\
\hline 103 & PDMS & & SW & & & E120426 & 25-Mar-92 & & 15117-96-1 & U-235 & UISO_AEA \\
\hline 104 & PDMS & & SW & & & E120344 & 30-Mar-92 & & 15117-96-1 & U-235 & UISO_AEA \\
\hline 105 & PDMS & & SW & & & E120353 & 30-Mar-92 & & $15117-96-1$ & U-235 & UISO_AEA \\
\hline 106 & PDMS & & SW & & & E120345 & 30-Mar-92 & & 15117-96-1 & U-235 & UISO_AEA \\
\hline 107 & PDMS & & SW & & & E120346 & 30-Mar-92 & & 15117-96-1 & U-235 & UISO_AEA \\
\hline 108 & PDMS & & SW & & & E120347 & 30-Mar-92 & & $15117-96-1$ & U-235 & UISO_AEA \\
\hline 109 & PDMS & & SW & & & E120348 & 30-Mar-92 & & $15117-96-1$ & U-235 & UISO_AEA \\
\hline 110 & PDMS & & SW & & & E120349 & 30-Mar-92 & & 15117-96-1 & U-235 & UISO_AEA \\
\hline 111 & PDMS & & SW & & & E120350 & 30-Mar-92 & & $15117-96-1$ & U-235 & UISO_AEA \\
\hline 112 & PDMS & & SW & & & E120351 & 30-Mar-92 & & $15117-96-1$ & U-235 & UISO_AEA \\
\hline 113 & PDMS & & SW & & & E120352 & 30-Mar-92 & & 15117-96-1 & U-235 & UISO_AEA \\
\hline 114 & PDMS & & SW & & & E120435 & 31-Mar-92 & & 15117-96-1 & U-235 & UISO_IE_PLATE_AEA \\
\hline 115 & PDMS & & SW & & & E120434 & 31-Mar-92 & & $15117-96-1$ & U-235 & UISO_AEA \\
\hline
\end{tabular}




\begin{tabular}{|c|c|c|c|c|c|c|c|c|c|c|c|}
\hline & $A$ & $B$ & $C$ & $\mathrm{D}$ & $E$ & $\mathrm{~F}$ & $G$ & $\mathrm{H}$ & $\mathrm{I}$ & $\mathrm{J}$ & $\mathrm{K}$ \\
\hline 1 & SAMPLE NUM ASSIGNED TO & SAMPLER & MEDIA & BIOTA MEDIA & TAG ID & SAMP NUM & SAMP DATE TIME & SAMP DATE TIME ON & CON ID & CON SHORT NAME & METHOD NAME \\
\hline 116 & PDMS & & SW & & & E120433 & 31-Mar-92 & & 15117-96-1 & U-235 & UISO_AEA \\
\hline 117 & PDMS & & SW & & & E120643 & 28-Apr-92 & & 15117-96-1 & U-235 & UISO_AEA \\
\hline 118 & PDMS & & SW & & & E120644 & 28-Apr-92 & & 15117-96-1 & U-235 & UISO_AEA \\
\hline 119 & PDMS & & SW & & & E120831 & 02-Jun-92 & & 15117-96-1 & U-235 & UISO_AEA \\
\hline 120 & PDMS & & SW & & & E120830 & 02-Jun-92 & & 15117-96-1 & U-235 & UISO_AEA \\
\hline \begin{tabular}{|l|}
121 \\
\end{tabular} & PDMS & & SW & & & E120954 & 11-Jun-92 & & 15117-96-1 & U-235 & UISO_AEA \\
\hline 122 & PDMS & & SW & & & E120955 & 11-Jun-92 & & $15117-96-1$ & U-235 & UISO AEA \\
\hline \begin{tabular}{|l|}
123 \\
\end{tabular} & PDMS & & SW & & & E120956 & 11-Jun-92 & & 15117-96-1 & U-235 & UISO_AEA \\
\hline 124 & PDMS & & SW & & & E120957 & 11-Jun-92 & & $15117-96-1$ & U-235 & UISO ${ }^{-}$AEA \\
\hline \begin{tabular}{|l|}
125 \\
\end{tabular} & PDMS & & SW & & & E120890 & 12-Jun-92 & & $15117-96-1$ & U-235 & UISO_AEA \\
\hline \begin{tabular}{|l|}
126 \\
\end{tabular} & PDMS & & SW & & & E120899 & 12-Jun-92 & & 15117-96-1 & U-235 & UISO_AEA \\
\hline 127 & PDMS & & SW & & & E120891 & 12-Jun-92 & & 15117-96-1 & U-235 & UISO_AEA \\
\hline 128 & PDMS & & SW & & & E120892 & 12-Jun-92 & & $15117-96-1$ & U-235 & UISO_AEA \\
\hline 129 & PDMS & & SW & & & E120893 & 12-Jun-92 & & 15117-96-1 & U-235 & UISO_AEA \\
\hline 130 & PDMS & & SW & & & E120894 & 12-Jun-92 & & 15117-96-1 & U-235 & UISO_AEA \\
\hline \begin{tabular}{|l|}
131 \\
\end{tabular} & PDMS & & SW & & & E120895 & 12-Jun-92 & & 15117-96-1 & U-235 & UISO_AEA \\
\hline 132 & PDMS & & SW & & & E120896 & 12-Jun-92 & & 15117-96-1 & U-235 & UISO_AEA \\
\hline 133 & PDMS & & SW & & & E120897 & 12-Jun-92 & & 15117-96-1 & U-235 & UISO_AEA \\
\hline \begin{tabular}{|l|}
134 \\
\end{tabular} & PDMS & & SW & & & E120898 & 12-Jun-92 & & $15117-96-1$ & U-235 & UISO_AEA \\
\hline \begin{tabular}{|l|}
135 \\
\end{tabular} & PDMS & & SW & & & E120947 & 23-Jun-92 & & $15117-96-1$ & U-235 & UISO_IE_PLATE_AEA \\
\hline 136 & PDMS & & SW & & & E121010 & 30-Jun-92 & & 15117-96-1 & U-235 & UISO_AEA \\
\hline 137 & PDMS & & SW & & & E083310 & 30-Jun-92 & & $15117-96-1$ & U-235 & UISO_AEA \\
\hline 138 & PDMS & & SW & & & E121225 & 28-Jul-92 & & 15117-96-1 & $U-235$ & UISO_AEA \\
\hline 139 & PDMS & & SW & & & E121224 & 28-Jul-92 & & 15117-96-1 & $U-235$ & UISO_AEA \\
\hline 140 & PDMS & & SW & & & E121431 & 01-Sep-92 & & 15117-96-1 & U-235 & UISO_AEA \\
\hline 141 & PDMS & & SW & & & E121430 & 01-Sep-92 & & 15117-96-1 & $U-235$ & UISO_AEA \\
\hline 142 & PDMS & & SW & & & E121587 & 29-Sep-92 & & 15117-96-1 & U-235 & UISO_AEA \\
\hline 143 & PDMS & & SW & & & E121586 & 29-Sep-92 & & 15117-96-1 & U-235 & UISO_AEA \\
\hline 144 & PDMS & & SW & & & E121756 & 30-Sep-92 & & 15117-96-1 & U-235 & UISO_AEA \\
\hline 145 & PDMS & & SW & & & E121757 & 30-Sep-92 & & 15117-96-1 & $U-235$ & UISO_AEA \\
\hline 146 & PDMS & & SW & & & E121758 & 30-Sep-92 & & 15117-96-1 & U-235 & UISO_AEA \\
\hline 147 & PDMS & & SW & & & E121759 & 30-Sep-92 & & $15117-96-1$ & $U-235$ & UISO_AEA \\
\hline 148 & PDMS & & SW & & & E121794 & 02-Oct-92 & & 15117-96-1 & U-235 & UISO_AEA \\
\hline 149 & PDMS & & SW & & & E121803 & 02-Oct-92 & & 15117-96-1 & U-235 & UISO_AEA \\
\hline \begin{tabular}{|l|}
150 \\
\end{tabular} & PDMS & & SW & & & E121795 & 02-Oct-92 & & $15117-96-1$ & U-235 & UISO_AEA \\
\hline \begin{tabular}{|l|}
151 \\
\end{tabular} & PDMS & & SW & & & E121796 & 02-Oct-92 & & 15117-96-1 & U-235 & UISO_AEA \\
\hline \begin{tabular}{|l|}
152 \\
\end{tabular} & PDMS & & SW & & & E121797 & 02-Oct-92 & & 15117-96-1 & U-235 & UISO_AEA \\
\hline \begin{tabular}{|l|}
153 \\
\end{tabular} & PDMS & & SW & & & E121798 & 02-Oct-92 & & $15117-96-1$ & U-235 & UISO_AEA \\
\hline
\end{tabular}




\begin{tabular}{|c|c|c|c|c|c|c|c|c|c|c|c|}
\hline & $A$ & $\mathrm{~B}$ & $C$ & $\mathrm{D}$ & $E$ & $\mathrm{~F}$ & $G$ & $\mathrm{H}$ & $\mathrm{I}$ & $\mathrm{J}$ & $\mathrm{K}$ \\
\hline 1 & SAMPLE NUM ASSIGNED TO & SAMPLER & MEDIA & BIOTA MEDIA & TAG ID & SAMP NUM & SAMP DATE TIME & SAMP DATE TIME ON & CON ID & CON SHORT NAME & METHOD NAME \\
\hline 154 & PDMS & & SW & & & E121799 & 02-Oct-92 & & 15117-96-1 & U-235 & UISO_AEA \\
\hline 155 & PDMS & & SW & & & E121800 & $02-$ Oct-92 & & 15117-96-1 & U-235 & UISO_AEA \\
\hline 156 & PDMS & & SW & & & E121801 & $02-O c t-92$ & & $15117-96-1$ & U-235 & UISO_AEA \\
\hline 157 & PDMS & & SW & & & E121802 & 02-Oct-92 & & 15117-96-1 & U-235 & UISO_AEA \\
\hline 158 & PDMS & & SW & & & E032849 & 13-Oct-92 & & 15117-96-1 & $U-235$ & UISO_IE_PLATE_AEA \\
\hline 159 & PDMS & & SW & & & E032942 & 03-Nov-92 & & 15117-96-1 & U-235 & UISO_AEA \\
\hline 160 & PDMS & & SW & & & E032941 & 03-Nov-92 & & $15117-96-1$ & U-235 & UISO AEA \\
\hline 161 & PDMS & & SW & & & E033356 & 01-Dec-92 & & 15117-96-1 & $U-235$ & UISO_AEA \\
\hline 162 & PDMS & & SW & & & E033355 & 01-Dec-92 & & $15117-96-1$ & U-235 & UISO ${ }^{-} \mathrm{AEA}$ \\
\hline 163 & PDMS & & SW & & & E119997 & 10-Dec-92 & & 15117-96-1 & U-235 & UISO_AEA \\
\hline 164 & PDMS & & SW & & & E120006 & 10-Dec-92 & & 15117-96-1 & $U-235$ & UISO_AEA \\
\hline 165 & PDMS & & SW & & & E119998 & 10-Dec-92 & & 15117-96-1 & U-235 & UISO_AEA \\
\hline 166 & PDMS & & SW & & & E119999 & 10-Dec-92 & & 15117-96-1 & $U-235$ & UISO_AEA \\
\hline 167 & PDMS & & SW & & & E120000 & 10-Dec-92 & & 15117-96-1 & U-235 & UISO_AEA \\
\hline 168 & PDMS & & SW & & & E120001 & 10-Dec-92 & & 15117-96-1 & U-235 & UISO_AEA \\
\hline 169 & PDMS & & SW & & & E120002 & 10-Dec-92 & & 15117-96-1 & U-235 & UISO_AEA \\
\hline 170 & PDMS & & SW & & & E120003 & 10-Dec-92 & & 15117-96-1 & $U-235$ & UISO_AEA \\
\hline 171 & PDMS & & SW & & & E120004 & 10-Dec-92 & & 15117-96-1 & $U-235$ & UISO_AEA \\
\hline 172 & PDMS & & SW & & & E120005 & 10-Dec-92 & & $15117-96-1$ & $U-235$ & UISO_AEA \\
\hline \begin{tabular}{|l|}
173 \\
\end{tabular} & PDMS & & SW & & & E120007 & 11-Dec-92 & & $15117-96-1$ & U-235 & UISO AEA \\
\hline 174 & PDMS & & SW & & & E120008 & 11-Dec-92 & & 15117-96-1 & U-235 & UISO_AEA \\
\hline 175 & PDMS & & SW & & & E120009 & 11-Dec-92 & & $15117-96-1$ & U-235 & UISO_AEA \\
\hline 176 & PDMS & & SW & & & E120010 & 11-Dec-92 & & $15117-96-1$ & $\mathrm{U}-235$ & UISO_AEA \\
\hline 177 & PDMS & & SW & & & E119962 & 30-Dec-92 & & 15117-96-1 & $U-235$ & UISO_AEA \\
\hline 178 & PDMS & & SW & & & E119963 & 30-Dec-92 & & 15117-96-1 & U-235 & UISO_AEA \\
\hline 179 & PDMS & & SW & & & E120011 & 05-Jan-93 & & 15117-96-1 & $U-235$ & UISO_IE_PLATE_AEA \\
\hline 180 & PDMS & & SW & & & E122051 & 02-Feb-93 & & 15117-96-1 & U-235 & UISO_AEA \\
\hline \begin{tabular}{|l|}
181 \\
\end{tabular} & PDMS & & SW & & & E122050 & 02-Feb-93 & & 15117-96-1 & U-235 & UISO_AEA \\
\hline 182 & PDMS & & SW & & & E123361 & 02-Mar-93 & & 15117-96-1 & U-235 & UISO_AEA \\
\hline 183 & PDMS & & SW & & & E123360 & 02-Mar-93 & & 15117-96-1 & $U-235$ & UISO_AEA \\
\hline 184 & PDMS & & SW & & & E123569 & 25-Mar-93 & & 15117-96-1 & U-235 & UISO_AEA \\
\hline 185 & PDMS & & SW & & & E123570 & 25-Mar-93 & & $15117-96-1$ & $U-235$ & UISO_AEA \\
\hline \begin{tabular}{|l|}
186 \\
\end{tabular} & PDMS & & SW & & & E123571 & 25-Mar-93 & & 15117-96-1 & U-235 & UISO_AEA \\
\hline \begin{tabular}{|l|}
187 \\
\end{tabular} & PDMS & & SW & & & E123572 & 25-Mar-93 & & 15117-96-1 & U-235 & UISO_AEA \\
\hline \begin{tabular}{|l|}
188 \\
\end{tabular} & PDMS & & SW & & & E123599 & 26-Mar-93 & & $15117-96-1$ & U-235 & UISO_AEA \\
\hline 189 & PDMS & & SW & & & E123598 & 26-Mar-93 & & 15117-96-1 & U-235 & UISO_AEA \\
\hline 190 & PDMS & & SW & & & E123588 & 26-Mar-93 & & 15117-96-1 & U-235 & UISO_AEA \\
\hline \begin{tabular}{|l|}
191 \\
\end{tabular} & PDMS & & SW & & & E123597 & 26-Mar-93 & & $15117-96-1$ & U-235 & UISO_AEA \\
\hline
\end{tabular}




\begin{tabular}{|c|c|c|c|c|c|c|c|c|c|c|c|}
\hline & $A$ & $\mathrm{~B}$ & $C$ & $\mathrm{D}$ & $E$ & $\mathrm{~F}$ & $G$ & $\mathrm{H}$ & $\mathrm{I}$ & $\mathrm{J}$ & $\mathrm{K}$ \\
\hline 1 & SAMPLE NUM ASSIGNED TO & SAMPLER & MEDIA & BIOTA MEDIA & TAG ID & SAMP NUM & SAMP DATE TIME & SAMP DATE TIME ON & CON ID & CON SHORT NAME & METHOD NAME \\
\hline 192 & PDMS & & SW & & & E123589 & 26-Mar-93 & & $15117-96-1$ & $\mathrm{U}-235$ & UISO_AEA \\
\hline 193 & PDMS & & SW & & & E123590 & 26-Mar-93 & & 15117-96-1 & U-235 & UISO_AEA \\
\hline 194 & PDMS & & SW & & & E123591 & 26-Mar-93 & & 15117-96-1 & U-235 & UISO_AEA \\
\hline 195 & PDMS & & SW & & & E123592 & 26-Mar-93 & & 15117-96-1 & U-235 & UISO_AEA \\
\hline 196 & PDMS & & SW & & & E123593 & 26-Mar-93 & & 15117-96-1 & U-235 & UISO_AEA \\
\hline 197 & PDMS & & SW & & & E123594 & 26-Mar-93 & & 15117-96-1 & U-235 & UISO_AEA \\
\hline 198 & PDMS & & SW & & & E123595 & 26-Mar-93 & & $15117-96-1$ & U-235 & UISO AEA \\
\hline 199 & PDMS & & SW & & & E123596 & 26-Mar-93 & & 15117-96-1 & $U-235$ & UISO_AEA \\
\hline 200 & PDMS & & SW & & & E123530 & 30-Mar-93 & & 15117-96-1 & U-235 & UISO_IE_PLATE_AEA \\
\hline 201 & PDMS & & SW & & & E123476 & 30-Mar-93 & & 15117-96-1 & U-235 & UISO_AEA \\
\hline 202 & PDMS & & SW & & & E123475 & 30-Mar-93 & & 15117-96-1 & $U-235$ & UISO_AEA \\
\hline 203 & PDMS & & SW & & & E123718 & 27-Apr-93 & & 15117-96-1 & U-235 & UISO_AEA \\
\hline 204 & PDMS & & SW & & & E123717 & 27-Apr-93 & & 15117-96-1 & U-235 & UISO_AEA \\
\hline 205 & PDMS & & SW & & & E123842 & 01-Jun-93 & & 15117-96-1 & U-235 & UISO_AEA \\
\hline 206 & PDMS & & SW & & & E123841 & 01-Jun-93 & & 15117-96-1 & U-235 & UISO_AEA \\
\hline 207 & PDMS & & SW & & & E124066 & 22-Jun-93 & & $15117-96-1$ & U-235 & UISO_IE_PLATE_AEA \\
\hline 208 & PDMS & & SW & & & E124069 & 29-Jun-93 & & 15117-96-1 & $U-235$ & UISO_AE- AEA \\
\hline 209 & PDMS & & SW & & & E124070 & 29-Jun-93 & & 15117-96-1 & $U-235$ & UISO_AEA \\
\hline 210 & PDMS & & SW & & & E124100 & 01-Jul-93 & & $15117-96-1$ & U-235 & UISO_AEA \\
\hline 211 & PDMS & & SW & & & E124101 & 01-Jul-93 & & 15117-96-1 & U-235 & UISO_AEA \\
\hline 212 & PDMS & & SW & & & E124102 & 01-Jul-93 & & 15117-96-1 & U-235 & UISO_AEA \\
\hline \begin{tabular}{|l|}
213 \\
\end{tabular} & PDMS & & SW & & & E124103 & 01-Jul-93 & & $15117-96-1$ & U-235 & UISO_AEA \\
\hline 214 & PDMS & & SW & & & E124158 & 02-Jul-93 & & $15117-96-1$ & U-235 & UISO_AEA \\
\hline 215 & PDMS & & SW & & & E124167 & 02-Jul-93 & & 15117-96-1 & U-235 & UISO_AEA \\
\hline \begin{tabular}{|l|}
216 \\
\end{tabular} & PDMS & & SW & & & E124159 & 02-Jul-93 & & 15117-96-1 & U-235 & UISO_AEA \\
\hline 217 & PDMS & & SW & & & E124160 & 02-Jul-93 & & 15117-96-1 & U-235 & UISO_AEA \\
\hline 218 & PDMS & & SW & & & E124161 & 02-Jul-93 & & 15117-96-1 & U-235 & UISO_AEA \\
\hline 219 & PDMS & & SW & & & E124162 & 02-Jul-93 & & $15117-96-1$ & U-235 & UISO_AEA \\
\hline 220 & PDMS & & SW & & & E124163 & 02-Jul-93 & & 15117-96-1 & U-235 & UISO_AEA \\
\hline 221 & PDMS & & SW & & & E124164 & 02-Jul-93 & & 15117-96-1 & U-235 & UISO_AEA \\
\hline 222 & PDMS & & SW & & & E124165 & 02-Jul-93 & & 15117-96-1 & U-235 & UISO_AEA \\
\hline \begin{tabular}{|l|}
223 \\
\end{tabular} & PDMS & & SW & & & E124166 & 02-Jul-93 & & $15117-96-1$ & U-235 & UISO_AEA \\
\hline 224 & PDMS & & SW & & & E124351 & 03-Aug-93 & & 15117-96-1 & U-235 & UISO_AEA \\
\hline 225 & PDMS & & SW & & & E124352 & 03-Aug-93 & & 15117-96-1 & U-235 & UISO_AEA \\
\hline \begin{tabular}{|l|}
226 \\
\end{tabular} & PDMS & & SW & & & E124529 & 31-Aug-93 & & $15117-96-1$ & U-235 & UISO_AEA \\
\hline 227 & PDMS & & SW & & & E124528 & 31-Aug-93 & & 15117-96-1 & U-235 & UISO_AEA \\
\hline 228 & PDMS & & SW & & & E124774 & 16-Sep-93 & & 15117-96-1 & U-235 & UISO_AEA \\
\hline \begin{tabular}{|l|}
229 \\
\end{tabular} & PDMS & & SW & & & E124775 & 16-Sep-93 & & $15117-96-1$ & U-235 & UISO_AEA \\
\hline
\end{tabular}




\begin{tabular}{|c|c|c|c|c|c|c|c|c|c|c|c|}
\hline & $A$ & $B$ & $C$ & $\mathrm{D}$ & $E$ & $\mathrm{~F}$ & $G$ & $\mathrm{H}$ & $\mathrm{I}$ & $\mathrm{J}$ & $\mathrm{K}$ \\
\hline 1 & SAMPLE NUM ASSIGNED TO & SAMPLER & MEDIA & BIOTA MEDIA & TAG ID & SAMP NUM & SAMP DATE TIME & SAMP DATE TIME ON & CON ID & CON SHORT NAME & METHOD NAME \\
\hline 230 & PDMS & & SW & & & E124776 & 16-Sep-93 & & 15117-96-1 & U-235 & UISO_AEA \\
\hline 231 & PDMS & & SW & & & E124777 & 16-Sep-93 & & 15117-96-1 & U-235 & UISO_AEA \\
\hline 232 & PDMS & & SW & & & E124683 & 17-Sep-93 & & 15117-96-1 & U-235 & UISO_AEA \\
\hline 233 & PDMS & & SW & & & E124684 & 17-Sep-93 & & 15117-96-1 & U-235 & UISO_AEA \\
\hline 234 & PDMS & & SW & & & E124685 & 17-Sep-93 & & 15117-96-1 & U-235 & UISO_AEA \\
\hline 235 & PDMS & & SW & & & E124686 & 17-Sep-93 & & 15117-96-1 & U-235 & UISO_AEA \\
\hline 236 & PDMS & & SW & & & E124687 & 17-Sep-93 & & $15117-96-1$ & U-235 & UISO AEA \\
\hline 237 & PDMS & & SW & & & E124688 & 17-Sep-93 & & 15117-96-1 & U-235 & UISO_AEA \\
\hline 238 & PDMS & & SW & & & E124689 & 17-Sep-93 & & 15117-96-1 & U-235 & UISO_AEA \\
\hline 239 & PDMS & & SW & & & E124690 & 17-Sep-93 & & 15117-96-1 & U-235 & UISO_AEA \\
\hline 240 & PDMS & & SW & & & E124691 & 17-Sep-93 & & 15117-96-1 & U-235 & UISO_AEA \\
\hline \begin{tabular}{|l|}
241 \\
\end{tabular} & PDMS & & SW & & & E124950 & 27-Sep-93 & & 15117-96-1 & U-235 & UISO_AEA \\
\hline 242 & PDMS & & SW & & & E124959 & 27-Sep-93 & & $15117-96-1$ & U-235 & UISO_AEA \\
\hline 243 & PDMS & & SW & & & E124951 & 27-Sep-93 & & 15117-96-1 & U-235 & UISO_AEA \\
\hline 244 & PDMS & & SW & & & E124952 & 27-Sep-93 & & 15117-96-1 & U-235 & UISO_AEA \\
\hline 245 & PDMS & & SW & & & E124953 & 27-Sep-93 & & 15117-96-1 & U-235 & UISO_AEA \\
\hline 246 & PDMS & & SW & & & E124954 & 27-Sep-93 & & 15117-96-1 & U-235 & UISO_AEA \\
\hline 247 & PDMS & & SW & & & E124955 & 27-Sep-93 & & 15117-96-1 & U-235 & UISO_AEA \\
\hline \begin{tabular}{|l|}
248 \\
\end{tabular} & PDMS & & SW & & & E124956 & 27-Sep-93 & & $15117-96-1$ & U-235 & UISO_AEA \\
\hline \begin{tabular}{|l|}
249 \\
\end{tabular} & PDMS & & SW & & & E124957 & 27-Sep-93 & & $15117-96-1$ & U-235 & UISO AEA \\
\hline 250 & PDMS & & SW & & & E124958 & 27-Sep-93 & & 15117-96-1 & U-235 & UISO_AEA \\
\hline \begin{tabular}{|l|}
251 \\
\end{tabular} & PDMS & & SW & & & E124940 & 27-Sep-93 & & $15117-96-1$ & U-235 & UISO_AEA \\
\hline 252 & PDMS & & SW & & & E124949 & 27-Sep-93 & & $15117-96-1$ & U-235 & UISO_AEA \\
\hline 253 & PDMS & & SW & & & E124941 & 27-Sep-93 & & 15117-96-1 & U-235 & UISO_AEA \\
\hline 254 & PDMS & & SW & & & E124942 & 27-Sep-93 & & 15117-96-1 & U-235 & UISO_AEA \\
\hline 255 & PDMS & & SW & & & E124943 & 27-Sep-93 & & 15117-96-1 & U-235 & UISO_AEA \\
\hline 256 & PDMS & & SW & & & E124944 & 27-Sep-93 & & 15117-96-1 & U-235 & UISO_AEA \\
\hline 257 & PDMS & & SW & & & E124945 & 27-Sep-93 & & 15117-96-1 & U-235 & UISO_AEA \\
\hline 258 & PDMS & & SW & & & E124946 & 27-Sep-93 & & 15117-96-1 & U-235 & UISO_AEA \\
\hline 259 & PDMS & & SW & & & E124947 & 27-Sep-93 & & 15117-96-1 & U-235 & UISO_AEA \\
\hline 260 & PDMS & & SW & & & E124948 & 27-Sep-93 & & 15117-96-1 & U-235 & UISO_AEA \\
\hline \begin{tabular}{|l|}
261 \\
\end{tabular} & PDMS & & SW & & & E124679 & 28-Sep-93 & & $15117-96-1$ & U-235 & UISO_AEA \\
\hline 262 & PDMS & & SW & & & E124678 & 28-Sep-93 & & 15117-96-1 & U-235 & UISO_AEA \\
\hline 263 & PDMS & & SW & & & E124874 & 30-Sep-93 & & 15117-96-1 & U-235 & UISO_AEA \\
\hline \begin{tabular}{|l|}
264 \\
\end{tabular} & PDMS & & SW & & & E124883 & 30-Sep-93 & & $15117-96-1$ & U-235 & UISO_AEA \\
\hline 265 & PDMS & & SW & & & E124875 & 30-Sep-93 & & 15117-96-1 & U-235 & UISO_AEA \\
\hline 266 & PDMS & & SW & & & E124876 & 30-Sep-93 & & 15117-96-1 & U-235 & UISO_AEA \\
\hline \begin{tabular}{|l|}
267 \\
\end{tabular} & PDMS & & SW & & & E124877 & 30-Sep-93 & & $15117-96-1$ & U-235 & UISO_AEA \\
\hline
\end{tabular}




\begin{tabular}{|c|c|c|c|c|c|c|c|c|c|c|c|}
\hline & $A$ & $\mathrm{~B}$ & $C$ & $\mathrm{D}$ & $E$ & $\mathrm{~F}$ & $G$ & $\mathrm{H}$ & $\mathrm{I}$ & $\mathrm{J}$ & $\mathrm{K}$ \\
\hline 1 & SAMPLE NUM ASSIGNED TO & SAMPLER & MEDIA & BIOTA MEDIA & TAG ID & SAMP NUM & SAMP DATE TIME & SAMP DATE TIME ON & CON ID & CON SHORT NAME & METHOD NAME \\
\hline 268 & PDMS & & SW & & & E124878 & 30-Sep-93 & & 15117-96-1 & U-235 & UISO_AEA \\
\hline 269 & PDMS & & SW & & & E124879 & 30-Sep-93 & & 15117-96-1 & U-235 & UISO_AEA \\
\hline 270 & PDMS & & SW & & & E124880 & 30-Sep-93 & & 15117-96-1 & U-235 & UISO_AEA \\
\hline 271 & PDMS & & SW & & & E124881 & 30-Sep-93 & & 15117-96-1 & $U-235$ & UISO_AEA \\
\hline 272 & PDMS & & SW & & & E124882 & 30-Sep-93 & & 15117-96-1 & $U-235$ & UISO_AEA \\
\hline 273 & PDMS & & SW & & & E124897 & 12-Oct-93 & & 15117-96-1 & U-235 & UISO_IE_PLATE_AEA \\
\hline 274 & PDMS & & SW & & & E124982 & 02-Nov-93 & & $15117-96-1$ & U-235 & UISO AËA \\
\hline 275 & PDMS & & SW & & & E124981 & 02-Nov-93 & & 15117-96-1 & $U-235$ & UISO_AEA \\
\hline 276 & PDMS & & SW & & & E125158 & 22-Nov-93 & & 15117-96-1 & U-235 & UISO_AEA \\
\hline 277 & PDMS & & SW & & & E125167 & 22-Nov-93 & & 15117-96-1 & U-235 & UISO_AEA \\
\hline 278 & PDMS & & SW & & & E125159 & 22-Nov-93 & & 15117-96-1 & $U-235$ & UISO_AEA \\
\hline 279 & PDMS & & SW & & & E125160 & 22-Nov-93 & & 15117-96-1 & U-235 & UISO_AEA \\
\hline 280 & PDMS & & SW & & & E125161 & 22-Nov-93 & & $15117-96-1$ & $U-235$ & UISO_AEA \\
\hline 281 & PDMS & & SW & & & E125162 & 22-Nov-93 & & 15117-96-1 & U-235 & UISO_AEA \\
\hline 282 & PDMS & & SW & & & E125163 & 22-Nov-93 & & 15117-96-1 & U-235 & UISO_AEA \\
\hline 283 & PDMS & & SW & & & E125164 & 22-Nov-93 & & $15117-96-1$ & U-235 & UISO_AEA \\
\hline 284 & PDMS & & SW & & & E125165 & 22-Nov-93 & & 15117-96-1 & $U-235$ & UISO_AEA \\
\hline 285 & PDMS & & SW & & & E125166 & 22-Nov-93 & & 15117-96-1 & $U-235$ & UISO_AEA \\
\hline 286 & PDMS & & SW & & & E125097 & 30-Nov-93 & & $15117-96-1$ & $U-235$ & UISO_AEA \\
\hline \begin{tabular}{|l|}
287 \\
287
\end{tabular} & PDMS & & SW & & & E125098 & 30-Nov-93 & & $15117-96-1$ & $\mathrm{U}-235$ & UISO_AEA \\
\hline 288 & PDMS & & SW & & & E125332 & 07-Dec-93 & & 15117-96-1 & U-235 & UISO_IE_PLATE_AEA \\
\hline 289 & PDMS & & SW & & & E125267 & 16-Dec-93 & & $15117-96-1$ & U-235 & UISO_AEA \\
\hline 290 & PDMS & & SW & & & E125268 & 16-Dec-93 & & 15117-96-1 & $U-235$ & UISO_AEA \\
\hline 291 & PDMS & & SW & & & E125269 & 16-Dec-93 & & 15117-96-1 & $U-235$ & UISO_AEA \\
\hline 292 & PDMS & & SW & & & E125270 & 16-Dec-93 & & 15117-96-1 & U-235 & UISO_AEA \\
\hline 293 & PDMS & & SW & & & E125331 & 17-Dec-93 & & 15117-96-1 & $U-235$ & UISO_AEA \\
\hline 294 & PDMS & & SW & & & E125315 & 17-Dec-93 & & 15117-96-1 & U-235 & UISO_AEA \\
\hline 295 & PDMS & & SW & & & E125324 & 17-Dec-93 & & 15117-96-1 & U-235 & UISO_AEA \\
\hline 296 & PDMS & & SW & & & E125316 & 17-Dec-93 & & 15117-96-1 & U-235 & UISO_AEA \\
\hline 297 & PDMS & & SW & & & E125317 & 17-Dec-93 & & 15117-96-1 & U-235 & UISO_AEA \\
\hline 298 & PDMS & & SW & & & E125318 & 17-Dec-93 & & 15117-96-1 & U-235 & UISO_AEA \\
\hline \begin{tabular}{|l|}
299 \\
\end{tabular} & PDMS & & SW & & & E125319 & 17-Dec-93 & & $15117-96-1$ & U-235 & UISO_AEA \\
\hline 300 & PDMS & & SW & & & E125320 & 17-Dec-93 & & 15117-96-1 & U-235 & UISO_AEA \\
\hline 301 & PDMS & & SW & & & E125321 & 17-Dec-93 & & 15117-96-1 & U-235 & UISO_AEA \\
\hline \begin{tabular}{|l|}
302 \\
\end{tabular} & PDMS & & SW & & & E125322 & 17-Dec-93 & & $15117-96-1$ & U-235 & UISO_AEA \\
\hline 303 & PDMS & & SW & & & E125323 & 17-Dec-93 & & 15117-96-1 & U-235 & UISO_AEA \\
\hline 304 & PDMS & & SW & & & E125266 & 28-Dec-93 & & 15117-96-1 & U-235 & UISO_AEA \\
\hline 305 & PDMS & & SW & & & E125265 & 28-Dec-93 & & $15117-96-1$ & U-235 & UISO_AEA \\
\hline
\end{tabular}




\begin{tabular}{|c|c|c|c|c|c|c|c|c|c|c|c|}
\hline & $A$ & $\mathrm{~B}$ & $C$ & $\mathrm{D}$ & $E$ & $\mathrm{~F}$ & $G$ & $\mathrm{H}$ & $\mathrm{I}$ & $\mathrm{J}$ & $\mathrm{K}$ \\
\hline 1 & SAMPLE NUM ASSIGNED TO & SAMPLER & MEDIA & BIOTA MEDIA & TAG ID & SAMP NUM & SAMP DATE TIME & SAMP DATE TIME ON & CON ID & CON SHORT NAME & METHOD NAME \\
\hline 306 & SESPSCH & & SW & & & B09QP3 & 25-Jan-94 & & 15117-96-1 & U-235 & UISO_AEA \\
\hline 307 & SESPSCH & & SW & & & B09QQ3 & 25-Jan-94 & & 15117-96-1 & U-235 & UISO_AEA \\
\hline 308 & SESPSCH & & SW & & & BOBCK3 & 22-Feb-94 & & 15117-96-1 & U-235 & UISO_AEA \\
\hline 309 & SESPSCH & & SW & & & B0BCL3 & 22-Feb-94 & & 15117-96-1 & U-235 & UISO_AEA \\
\hline 310 & SESPSCH & & SW & & & B0BG75 & 17-Mar-94 & & 15117-96-1 & $U-235$ & UISO_AEA \\
\hline 311 & SESPSCH & & SW & & & B0BG76 & 17-Mar-94 & & 15117-96-1 & U-235 & UISO_AEA \\
\hline 312 & SESPSCH & & SW & & & B09QT2 & 29-Mar-94 & & $15117-96-1$ & U-235 & UISO_IE_PLATE_AEA \\
\hline 313 & SESPSCH & & SW & & & B0BG45 & 29-Mar-94 & & 15117-96-1 & $U-235$ & UISO_AËA \\
\hline 314 & SESPSCH & & SW & & & B0BG57 & 29-Mar-94 & & 15117-96-1 & U-235 & UISO_AEA \\
\hline 315 & SESPSCH & & SW & & & BOBG79 & 31-Mar-94 & & 15117-96-1 & U-235 & UISO_AEA \\
\hline 316 & SESPSCH & & SW & & & B0BG88 & 31-Mar-94 & & 15117-96-1 & $U-235$ & UISO_AEA \\
\hline 317 & SESPSCH & & SW & & & B0BG80 & 31-Mar-94 & & 15117-96-1 & U-235 & UISO_AEA \\
\hline 318 & SESPSCH & & SW & & & B0BG81 & 31-Mar-94 & & $15117-96-1$ & $\mathrm{U}-235$ & UISO AEA \\
\hline 319 & SESPSCH & & SW & & & B0BG82 & 31-Mar-94 & & 15117-96-1 & U-235 & UISO_AEA \\
\hline 320 & SESPSCH & & SW & & & B0BG83 & 31-Mar-94 & & 15117-96-1 & U-235 & UISO_AEA \\
\hline 321 & SESPSCH & & SW & & & B0BG84 & 31-Mar-94 & & $15117-96-1$ & U-235 & UISO_AEA \\
\hline 322 & SESPSCH & & SW & & & B0BG85 & 31-Mar-94 & & 15117-96-1 & $U-235$ & UISO_AEA \\
\hline 323 & SESPSCH & & SW & & & B0BG86 & 31-Mar-94 & & 15117-96-1 & U-235 & UISO_AEA \\
\hline 324 & SESPSCH & & SW & & & B0BG87 & 31-Mar-94 & & $15117-96-1$ & $U-235$ & UISO_AEA \\
\hline 325 & SESPSCH & & SW & & & B0BP48 & 03-May-94 & & $15117-96-1$ & U-235 & UISO AEA \\
\hline 326 & SESPSCH & & SW & & & B0BP55 & 03-May-94 & & 15117-96-1 & U-235 & UISO_AEA \\
\hline 327 & SESPSCH & & SW & & & B0BVQ2 & 07-Jun-94 & & $15117-96-1$ & U-235 & UISO_AEA \\
\hline 328 & SESPSCH & & SW & & & BOBVQ9 & 07-Jun-94 & & $15117-96-1$ & $U-235$ & UISO_AEA \\
\hline 329 & SESPSCH & & SW & & & B0BP89 & 21-Jun-94 & & 15117-96-1 & U-235 & UISO_IE_PLATE_AEA \\
\hline 330 & SESPSCH & & SW & & & B0BQM7 & 30-Jun-94 & & 15117-96-1 & U-235 & UISO_AEA \\
\hline 331 & SESPSCH & & SW & & & B0BQM8 & 30-Jun-94 & & 15117-96-1 & $U-235$ & UISO_AEA \\
\hline 332 & SESPSCH & & SW & & & B0BQM9 & 30-Jun-94 & & 15117-96-1 & U-235 & UISO_AEA \\
\hline 333 & SESPSCH & & SW & & & BOBQNO & 30-Jun-94 & & 15117-96-1 & U-235 & UISO_AEA \\
\hline 334 & SESPSCH & & SW & & & B0BQN1 & 01-Jul-94 & & 15117-96-1 & U-235 & UISO_AEA \\
\hline 335 & SESPSCH & & SW & & & BOBQPO & 01-Jul-94 & & 15117-96-1 & $U-235$ & UISO_AEA \\
\hline 336 & SESPSCH & & SW & & & BOBQN2 & 01-Jul-94 & & 15117-96-1 & U-235 & UISO_AEA \\
\hline 337 & SESPSCH & & SW & & & BOBQN3 & 01-Jul-94 & & $15117-96-1$ & U-235 & UISO_AEA \\
\hline 338 & SESPSCH & & SW & & & BOBQN4 & 01-Jul-94 & & 15117-96-1 & U-235 & UISO_AEA \\
\hline 339 & SESPSCH & & SW & & & B0BQN5 & 01-Jul-94 & & 15117-96-1 & U-235 & UISO_AEA \\
\hline 340 & SESPSCH & & SW & & & BOBQN6 & 01-Jul-94 & & $15117-96-1$ & U-235 & UISO_AEA \\
\hline 341 & SESPSCH & & SW & & & BOBQN7 & 01-Jul-94 & & 15117-96-1 & U-235 & UISO_AEA \\
\hline 342 & SESPSCH & & SW & & & BOBQN8 & 01-Jul-94 & & 15117-96-1 & U-235 & UISO_AEA \\
\hline 343 & SESPSCH & & SW & & & BOBQN9 & 01-Jul-94 & & $15117-96-1$ & U-235 & UISO_AEA \\
\hline
\end{tabular}




\begin{tabular}{|c|c|c|c|c|c|c|c|c|c|c|c|}
\hline & A & $\mathrm{B}$ & $C$ & $\mathrm{D}$ & $E$ & $\mathrm{~F}$ & $G$ & $\mathrm{H}$ & $\mathrm{I}$ & $\mathrm{J}$ & $\mathrm{K}$ \\
\hline 1 & SAMPLE_NUM_ASSIGNED_TO & SAMPLER & MEDIA & BIOTA_MEDIA & TAG_ID & SAMP_NUM & SAMP_DATE_TIME & SAMP_DATE_TIME_ON & CON_ID & CON_SHORT_NAME & METHOD_NAME \\
\hline 344 & SESPSCH & & SW & & & B0C155 & 06-Jul-94 & & $15117-96-1$ & U-235 & UISO_AEA \\
\hline 345 & SESPSCH & & SW & & & B0C166 & 06-Jul-94 & & 15117-96-1 & U-235 & UISO_AEA \\
\hline 346 & SESPSCH & & SW & & & B0C442 & 01-Aug-94 & & 15117-96-1 & U-235 & UISO_AEA \\
\hline 347 & SESPSCH & & SW & & & B0C448 & 01-Aug-94 & & 15117-96-1 & U-235 & UISO_AEA \\
\hline 348 & SESPSCH & & SW & & & B0C588 & 25-Aug-94 & & 15117-96-1 & U-235 & UISO_AEA \\
\hline 349 & SESPSCH & & SW & & & B0C598 & 25-Aug-94 & & 15117-96-1 & U-235 & UISO_AEA \\
\hline 350 & SESPSCH & & SW & & & B0C592 & 25-Aug-94 & & 15117-96-1 & U-235 & UISO_AEA \\
\hline 351 & SESPSCH & & SW & & & B0C589 & 25-Aug-94 & & 15117-96-1 & U-235 & UISO_AEA \\
\hline 352 & SESPSCH & & SW & & & B0C593 & 25-Aug-94 & & 15117-96-1 & U-235 & UISO_AEA \\
\hline 353 & SESPSCH & & SW & & & B0C594 & 25-Aug-94 & & 15117-96-1 & U-235 & UISO_AEA \\
\hline 354 & SESPSCH & & SW & & & B0C595 & 25-Aug-94 & & 15117-96-1 & U-235 & UISO_AEA \\
\hline 355 & SESPSCH & & SW & & & B0C596 & 25-Aug-94 & & 15117-96-1 & U-235 & UISO_AEA \\
\hline 356 & SESPSCH & & SW & & & B0C597 & 25-Aug-94 & & $15117-96-1$ & U-235 & UISO_AEA \\
\hline 357 & SESPSCH & & SW & & & B0C599 & 25-Aug-94 & & $15117-96-1$ & U-235 & UISO_AEA \\
\hline 358 & SESPSCH & & SW & & & B0C584 & 25-Aug-94 & & $15117-96-1$ & U-235 & UISO_AEA \\
\hline 359 & SESPSCH & & SW & & & B0C5H2 & 25-Aug-94 & & 15117-96-1 & U-235 & UISO_AEA \\
\hline 360 & SESPSCH & & SW & & & B0C585 & 25-Aug-94 & & 15117-96-1 & U-235 & UISO_AEA \\
\hline 361 & SESPSCH & & SW & & & B0C5H5 & 25-Aug-94 & & 15117-96-1 & U-235 & UISO_AEA \\
\hline 362 & SESPSCH & & SW & & & B0C5H6 & 25-Aug-94 & & 15117-96-1 & U-235 & UISO_AEA \\
\hline 363 & SESPSCH & & SW & & & B0C587 & 25-Aug-94 & & 15117-96-1 & U-235 & UISO_AEA \\
\hline 364 & SESPSCH & & SW & & & B0C5H8 & 25-Aug-94 & & 15117-96-1 & U-235 & UISO_AEA \\
\hline 365 & SESPSCH & & SW & & & B0C5H7 & 25-Aug-94 & & 15117-96-1 & U-235 & UISO_AEA \\
\hline 366 & SESPSCH & & SW & & & B0C5H9 & 25-Aug-94 & & 15117-96-1 & U-235 & UISO_AEA \\
\hline 367 & SESPSCH & & SW & & & B0C5J0 & 25-Aug-94 & & 15117-96-1 & U-235 & UISO_AEA \\
\hline 368 & SESPSCH & & SW & & & B0C580 & 25-Aug-94 & & 15117-96-1 & U-235 & UISO_AEA \\
\hline 369 & SESPSCH & & SW & & & B0C581 & 25-Aug-94 & & $15117-96-1$ & U-235 & UISO_AEA \\
\hline 370 & SESPSCH & & SW & & & B0C582 & 25-Aug-94 & & $15117-96-1$ & U-235 & UISO_AEA \\
\hline 371 & SESPSCH & & SW & & & B0C583 & 25-Aug-94 & & $15117-96-1$ & U-235 & UISO_AEA \\
\hline 372 & SESPSCH & & SW & & & B0C5B7 & 26-Aug-94 & & 15117-96-1 & U-235 & UISO_AEA \\
\hline 373 & SESPSCH & & SW & & & B0C5B8 & 26-Aug-94 & & 15117-96-1 & U-235 & UISO_AEA \\
\hline 374 & SESPSCH & & SW & & & B0C5B9 & 26-Aug-94 & & 15117-96-1 & U-235 & UISO_AEA \\
\hline 375 & SESPSCH & & SW & & & B0C5C0 & 26-Aug-94 & & 15117-96-1 & U-235 & UISO_AEA \\
\hline 376 & SESPSCH & & SW & & & B0C5C1 & 26-Aug-94 & & 15117-96-1 & U-235 & UISO_AEA \\
\hline 377 & SESPSCH & & SW & & & B0C $5 \mathrm{C} 2$ & 26-Aug-94 & & 15117-96-1 & U-235 & UISO_AEA \\
\hline 378 & SESPSCH & & SW & & & B0C5C3 & 26-Aug-94 & & 15117-96-1 & U-235 & UISO_AEA \\
\hline 379 & SESPSCH & & SW & & & B0C5C4 & 26-Aug-94 & & 15117-96-1 & U-235 & UISO_AEA \\
\hline 380 & SESPSCH & & SW & & & B0C5C5 & 26-Aug-94 & & $15117-96-1$ & U-235 & UISO_AEA \\
\hline 381 & SESPSCH & & SW & & & B0C5C7 & 26-Aug-94 & & 15117-96-1 & U-235 & UISO_AEA \\
\hline
\end{tabular}




\begin{tabular}{|c|c|c|c|c|c|c|c|c|c|c|c|}
\hline & A & $\mathrm{B}$ & C & $\mathrm{D}$ & $\mathrm{E}$ & $\mathrm{F}$ & $\mathrm{G}$ & $\mathrm{H}$ & $\mathrm{I}$ & $\mathrm{J}$ & $\mathrm{K}$ \\
\hline 1 & SAMPLE_NUM_ASSIGNED_TO & SAMPLER & MEDIA & BIOTA_MEDIA & TAG_ID & SAMP_NUM & SAMP_DATE_TIME & SAMP_DATE_TIME_ON & CON_ID & CON_SHORT_NAME & METHOD_NAME \\
\hline 382 & SESPSCH & & SW & & & B0C5D6 & 26-Aug-94 & & $15117-96-1$ & U-235 & UISO_AEA \\
\hline 383 & SESPSCH & & SW & & & B0C5C8 & 26-Aug-94 & & 15117-96-1 & U-235 & UISO_AEA \\
\hline 384 & SESPSCH & & SW & & & B0C5C9 & 26-Aug-94 & & 15117-96-1 & $\mathrm{U}-235$ & UISO_AEA \\
\hline 385 & SESPSCH & & SW & & & B0C5D0 & 26-Aug-94 & & 15117-96-1 & U-235 & UISO_AEA \\
\hline 386 & SESPSCH & & SW & & & B0C5D1 & 26-Aug-94 & & 15117-96-1 & U-235 & UISO_AEA \\
\hline 387 & SESPSCH & & SW & & & B0C5D2 & 26-Aug-94 & & 15117-96-1 & U-235 & UISO_AEA \\
\hline 388 & SESPSCH & & SW & & & B0C5D3 & 26-Aug-94 & & 15117-96-1 & U-235 & UISO_AEA \\
\hline 389 & SESPSCH & & SW & & & B0C5D4 & 26-Aug-94 & & 15117-96-1 & U-235 & UISO_AEA \\
\hline 390 & SESPSCH & & SW & & & B0C5D5 & 26-Aug-94 & & 15117-96-1 & U-235 & UISO_AEA \\
\hline 391 & SESPSCH & & SW & & & B0C5B0 & 02-Sep-94 & & 15117-96-1 & U-235 & UISO_AEA \\
\hline 392 & SESPSCH & & SW & & & B0C5B5 & 02-Sep-94 & & 15117-96-1 & U-235 & UISO_AEA \\
\hline 393 & SESPSCH & & SW & & & B0C5B1 & 02-Sep-94 & & 15117-96-1 & U-235 & UISO_AEA \\
\hline 394 & SESPSCH & & SW & & & B0C5B6 & 02-Sep-94 & & 15117-96-1 & U-235 & UISO_AEA \\
\hline 395 & SESPSCH & & SW & & & B0C586 & 02-Sep-94 & & 15117-96-1 & U-235 & UISO_AEA \\
\hline 396 & SESPSCH & & SW & & & B0C590 & 02-Sep-94 & & 15117-96-1 & U-235 & UISO_AEA \\
\hline 397 & SESPSCH & & SW & & & B0C591 & 02-Sep-94 & & 15117-96-1 & U-235 & UISO_AEA \\
\hline 398 & SESPSCH & & SW & & & B0C5B2 & 02-Sep-94 & & 15117-96-1 & U-235 & UISO_AEA \\
\hline 399 & SESPSCH & & SW & & & B0C5B3 & 02-Sep-94 & & 15117-96-1 & U-235 & UISO_AEA \\
\hline 400 & SESPSCH & & SW & & & B0C5B4 & 02-Sep-94 & & 15117-96-1 & U-235 & UISO_AEA \\
\hline 401 & SESPSCH & & SW & & & B0CDF2 & 06-Sep-94 & & 15117-96-1 & U-235 & UISO_AEA \\
\hline 402 & SESPSCH & & SW & & & B0CDF9 & 06-Sep-94 & & 15117-96-1 & U-235 & UISO_AEA \\
\hline 403 & SESPSCH & & SW & & & B0CXG4 & 04-Oct-94 & & 15117-96-1 & U-235 & UISO_AEA \\
\hline 404 & SESPSCH & & SW & & & BOCXHO & 04-Oct-94 & & 15117-96-1 & U-235 & UISO_AEA \\
\hline 405 & SESPSCH & & SW & & & B0C477 & 11-Oct-94 & & 15117-96-1 & U-235 & UISO_IE_PLATE_AEA \\
\hline 406 & SESPSCH & & SW & & & B0D0V3 & 01-Nov-94 & & 15117-96-1 & U-235 & UISO_AEA \\
\hline 407 & SESPSCH & & SW & & & B0D0V9 & 01-Nov-94 & & 15117-96-1 & U-235 & UISO_AEA \\
\hline 408 & SESPSCH & & SW & & & B0D5W8 & 06-Dec-94 & & 15117-96-1 & U-235 & UISO_AEA \\
\hline 409 & SESPSCH & & SW & & & B0D5X5 & 06-Dec-94 & & 15117-96-1 & U-235 & UISO_AEA \\
\hline 410 & SESPSCH & & SW & & & B0D1Y0 & 15-Dec-94 & & 15117-96-1 & U-235 & UISO_AEA \\
\hline 411 & SESPSCH & & SW & & & B0D1Y1 & 15-Dec-94 & & 15117-96-1 & U-235 & UISO_AEA \\
\hline 412 & SESPSCH & & SW & & & B0D1Y2 & 15-Dec-94 & & 15117-96-1 & U-235 & UISO_AEA \\
\hline 413 & SESPSCH & & SW & & & B0D1Y3 & 15-Dec-94 & & 15117-96-1 & U-235 & UISO_AEA \\
\hline 414 & SESPSCH & & SW & & & B0DG81 & 21-Dec-94 & & 15117-96-1 & U-235 & UISO_AEA \\
\hline 415 & SESPSCH & & SW & & & B0D1Y4 & 21-Dec-94 & & 15117-96-1 & U-235 & UISO_AEA \\
\hline 416 & SESPSCH & & SW & & & B0D1Y5 & 21-Dec-94 & & 15117-96-1 & U-235 & UISO_AEA \\
\hline 417 & SESPSCH & & SW & & & B0D1Y6 & 21-Dec-94 & & 15117-96-1 & U-235 & UISO_AEA \\
\hline 418 & SESPSCH & & SW & & & B0D1Y7 & 21-Dec-94 & & 15117-96-1 & U-235 & UISO_AEA \\
\hline 419 & SESPSCH & & SW & & & B0D1Y8 & 21-Dec-94 & & 15117-96-1 & U-235 & UISO_AEA \\
\hline
\end{tabular}




\begin{tabular}{|c|c|c|c|c|c|c|c|c|c|c|c|}
\hline & $A$ & $\mathrm{~B}$ & $\mathrm{C}$ & $\mathrm{D}$ & $E$ & $\mathrm{~F}$ & $G$ & $\mathrm{H}$ & $\mathrm{I}$ & $\mathrm{J}$ & $\mathrm{K}$ \\
\hline 1 & SAMPLE_NUM_ASSIGNED_TO & SAMPLER & MEDIA & BIOTA_MEDIA & TAG_ID & SAMP_NUM & SAMP_DATE_TIME & SAMP_DATE_TIME_ON & CON_ID & CON_SHORT_NAME & METHOD_NAME \\
\hline 420 & SESPSCH & & SW & & & B0D1Y9 & 21-Dec-94 & & 15117-96-1 & U-235 & UISO_AEA \\
\hline 421 & SESPSCH & & SW & & & B0D1Z0 & 21-Dec-94 & & 15117-96-1 & U-235 & UISO_AEA \\
\hline 422 & SESPSCH & & SW & & & B0D1Z1 & 21-Dec-94 & & 15117-96-1 & U-235 & UISO_AEA \\
\hline 423 & SESPSCH & & SW & & & B0D1Z2 & 21-Dec-94 & & 15117-96-1 & U-235 & UISO_AEA \\
\hline 424 & SESPSCH & & SW & & & B0D1Z3 & 21-Dec-94 & & 15117-96-1 & $\mathrm{U}-235$ & UISO_AEA \\
\hline 425 & SESPSCH & & SW & & & B0D0Y8 & 03-Jan-95 & & 15117-96-1 & $\mathrm{U}-235$ & UISO_IE_PLATE_AEA \\
\hline 426 & SESPSCH & & SW & & & BODFN2 & 03-Jan-95 & & 15117-96-1 & U-235 & UISO_AEA \\
\hline 427 & SESPSCH & & SW & & & BODFN8 & 03-Jan-95 & & 15117-96-1 & $\mathrm{U}-235$ & UISO_AEA \\
\hline 428 & SESPSCH & & SW & & & B0DK64 & 07-Feb-95 & & 15117-96-1 & $\mathrm{U}-235$ & UISO_AEA \\
\hline 429 & SESPSCH & & SW & & & B0DK76 & 07-Feb-95 & & 15117-96-1 & U-235 & UISO_AEA \\
\hline 430 & SESPSCH & & SW & & & BODTPO & 07-Mar-95 & & 15117-96-1 & $U-235$ & UISO_AEA \\
\hline 431 & SESPSCH & & SW & & & B0DTP5 & 07-Mar-95 & & 15117-96-1 & U-235 & UISO_AEA \\
\hline 432 & SESPSCH & & SW & & & B0DX17 & 16-Mar-95 & & 15117-96-1 & U-235 & UISO_AEA \\
\hline 433 & SESPSCH & & SW & & & B0DX18 & 16-Mar-95 & & 15117-96-1 & U-235 & UISO_AEA \\
\hline 434 & SESPSCH & & SW & & & B0DX19 & 16-Mar-95 & & 15117-96-1 & U-235 & UISO_AEA \\
\hline 435 & SESPSCH & & SW & & & B0DX20 & 16-Mar-95 & & 15117-96-1 & U-235 & UISO_AEA \\
\hline 436 & SESPSCH & & SW & & & B0DX24 & 17-Mar-95 & & 15117-96-1 & U-235 & UISO_AEA \\
\hline 437 & SESPSCH & & SW & & & B0DX33 & 17-Mar-95 & & 15117-96-1 & U-235 & UISO_AEA \\
\hline 438 & SESPSCH & & SW & & & B0DX25 & 17-Mar-95 & & 15117-96-1 & U-235 & UISO_AEA \\
\hline 439 & SESPSCH & & SW & & & BoDX26 & 17-Mar-95 & & 15117-96-1 & U-235 & UISO_AEA \\
\hline 440 & SESPSCH & & SW & & & B0DX27 & 17-Mar-95 & & 15117-96-1 & U-235 & UISO_AEA \\
\hline 441 & SESPSCH & & SW & & & B0DX28 & 17-Mar-95 & & 15117-96-1 & U-235 & UISO_AEA \\
\hline 442 & SESPSCH & & SW & & & B0DX29 & 17-Mar-95 & & 15117-96-1 & U-235 & UISO_AEA \\
\hline 443 & SESPSCH & & SW & & & B0DX30 & 17-Mar-95 & & 15117-96-1 & U-235 & UISO_AEA \\
\hline 444 & SESPSCH & & SW & & & B0DX31 & 17-Mar-95 & & 15117-96-1 & U-235 & UISO_AEA \\
\hline 445 & SESPSCH & & SW & & & B0DX32 & 17-Mar-95 & & 15117-96-1 & U-235 & UISO_AEA \\
\hline 446 & SESPSCH & & SW & & & B0DKB6 & 27-Mar-95 & & 15117-96-1 & U-235 & UISO_IE_PLATE_AEA \\
\hline 447 & SESPSCH & & SW & & & BODZC4 & 04-Apr-95 & & 15117-96-1 & $\mathrm{U}-235$ & UISO_AEA \\
\hline 448 & SESPSCH & & SW & & & BODZC9 & 04-Apr-95 & & 15117-96-1 & U-235 & UISO_AEA \\
\hline 449 & SESPSCH & & SW & & & B0F8X5 & 02-May-95 & & 15117-96-1 & U-235 & UISO_AEA \\
\hline 450 & SESPSCH & & SW & & & B0F8Y0 & 02-May-95 & & 15117-96-1 & $\mathrm{U}-235$ & UISO_AEA \\
\hline 451 & SESPSCH & & SW & & & B0FGL7 & 06-Jun-95 & & 15117-96-1 & U-235 & UISO_AEA \\
\hline 452 & SESPSCH & & SW & & & B0FGM3 & 06-Jun-95 & & 15117-96-1 & U-235 & UISO_AEA \\
\hline 453 & SESPSCH & & SW & & & BOFL62 & 15-Jun-95 & & 15117-96-1 & $\mathrm{U}-235$ & UISO_AEA \\
\hline 454 & SESPSCH & & SW & & & BOFL63 & 15-Jun-95 & & 15117-96-1 & $\mathrm{U}-235$ & UISO_AEA \\
\hline 455 & SESPSCH & & SW & & & BOFL64 & 15-Jun-95 & & 15117-96-1 & $\mathrm{U}-235$ & UISO_AEA \\
\hline 456 & SESPSCH & & SW & & & B0FL65 & 15-Jun-95 & & 15117-96-1 & $\mathrm{U}-235$ & UISO_AEA \\
\hline 457 & SESPSCH & & SW & & & BOFL66 & 16-Jun-95 & & 15117-96-1 & U-235 & UISO_AEA \\
\hline
\end{tabular}




\begin{tabular}{|c|c|c|c|c|c|c|c|c|c|c|c|}
\hline & $A$ & $B$ & $\mathrm{C}$ & $\mathrm{D}$ & $E$ & $F$ & $G$ & $\mathrm{H}$ & $\mathrm{I}$ & $\mathrm{J}$ & $\mathrm{K}$ \\
\hline 1 & SAMPLE_NUM_ASSIGNED_TO & SAMPLER & MEDIA & BIOTA_MEDIA & TAG_ID & SAMP_NUM & SAMP_DATE_TIME & SAMP_DATE_TIME_ON & CON_ID & CON_SHORT_NAME & METHOD_NAME \\
\hline 458 & SESPSCH & & SW & & & B0FL75 & 16-Jun-95 & & 15117-96-1 & U-235 & UISO_AEA \\
\hline 459 & SESPSCH & & SW & & & B0FL67 & 16-Jun-95 & & 15117-96-1 & $\mathrm{U}-235$ & UISO_AEA \\
\hline 460 & SESPSCH & & SW & & & B0FL68 & 16-Jun-95 & & 15117-96-1 & U-235 & UISO_AEA \\
\hline 461 & SESPSCH & & SW & & & B0FL69 & 16-Jun-95 & & $15117-96-1$ & U-235 & UISO_AEA \\
\hline 462 & SESPSCH & & SW & & & BOFL70 & 16-Jun-95 & & 15117-96-1 & U-235 & UISO_AEA \\
\hline \begin{tabular}{|l|}
463 \\
\end{tabular} & SESPSCH & & SW & & & BOFL71 & 16-Jun-95 & & 15117-96-1 & U-235 & UISO_AEA \\
\hline 464 & SESPSCH & & SW & & & BOFL72 & 16-Jun-95 & & 15117-96-1 & $\mathrm{U}-235$ & UISO_AEA \\
\hline 465 & SESPSCH & & SW & & & BOFL73 & 16-Jun-95 & & 15117-96-1 & U-235 & UISO_AEA \\
\hline 466 & SESPSCH & & SW & & & BOFL74 & 16-Jun-95 & & 15117-96-1 & $\mathrm{U}-235$ & UISO_AEA \\
\hline 467 & SESPSCH & & SW & & & B0F909 & 20-Jun-95 & & 15117-96-1 & U-235 & UISO_IE_PLATE_AEA \\
\hline 468 & SESPSCH & & SW & & & B0FMP8 & 05-Jul-95 & & 15117-96-1 & U-235 & UISO_AEA \\
\hline 469 & SESPSCH & & SW & & & B0FMQ3 & 05-Jul-95 & & $15117-96-1$ & $\mathrm{U}-235$ & UISO_AEA \\
\hline 470 & SESPSCH & & SW & & & B0G503 & 01-Aug-95 & & $15117-96-1$ & U-235 & UISO_AEA \\
\hline 471 & SESPSCH & & SW & & & B0G508 & 01-Aug-95 & & 15117-96-1 & U-235 & UISO_AEA \\
\hline 472 & SESPSCH & & SW & & & B0GBK4 & 06-Sep-95 & & 15117-96-1 & U-235 & UISO_AEA \\
\hline 473 & SESPSCH & & SW & & & B0GBLO & 06-Sep-95 & & 15117-96-1 & $\mathrm{U}-235$ & UISO_AEA \\
\hline 474 & SESPSCH & & SW & & & B0G868 & 11-Sep-95 & & 15117-96-1 & U-235 & UISO_AEA \\
\hline 475 & SESPSCH & & SW & & & B0G8C4 & 11-Sep-95 & & 15117-96-1 & U-235 & UISO_AEA \\
\hline 476 & SESPSCH & & SW & & & B0G870 & 11-Sep-95 & & 15117-96-1 & U-235 & UISO_AEA \\
\hline 477 & SESPSCH & & SW & & & B0G8C5 & 11-Sep-95 & & 15117-96-1 & U-235 & UISO_AEA \\
\hline 478 & SESPSCH & & SW & & & B0G8C6 & 11-Sep-95 & & 15117-96-1 & $\mathrm{U}-235$ & UISO_AEA \\
\hline 479 & SESPSCH & & SW & & & B0G873 & 11-Sep-95 & & 15117-96-1 & $\mathrm{U}-235$ & UISO_AEA \\
\hline 480 & SESPSCH & & SW & & & B0G8C9 & 11-Sep-95 & & 15117-96-1 & U-235 & UISO_AEA \\
\hline \begin{tabular}{|l|}
481 \\
\end{tabular} & SESPSCH & & SW & & & B0G8C8 & 11-Sep-95 & & 15117-96-1 & U-235 & UISO_AEA \\
\hline 482 & SESPSCH & & SW & & & B0G8D0 & 11-Sep-95 & & 15117-96-1 & U-235 & UISO_AEA \\
\hline 483 & SESPSCH & & SW & & & B0G8D1 & 11-Sep-95 & & $15117-96-1$ & U-235 & UISO_AEA \\
\hline 484 & SESPSCH & & SW & & & B0GBM4 & 11-Sep-95 & & 15117-96-1 & U-235 & UISO_AEA \\
\hline 485 & SESPSCH & & SW & & & B0GBM5 & 11-Sep-95 & & 15117-96-1 & U-235 & UISO_AEA \\
\hline 486 & SESPSCH & & SW & & & B0GBM6 & 11-Sep-95 & & 15117-96-1 & U-235 & UISO_AEA \\
\hline 487 & SESPSCH & & SW & & & B0GBM7 & 11-Sep-95 & & 15117-96-1 & U-235 & UISO_AEA \\
\hline 48 & SESPSCH & & SW & & & B0G874 & 12-Sep-95 & & 15117-96-1 & U-235 & UISO_AEA \\
\hline 489 & SESPSCH & & SW & & & B0G884 & 12-Sep-95 & & 15117-96-1 & U-235 & UISO_AEA \\
\hline 490 & SESPSCH & & SW & & & B0G878 & 12-Sep-95 & & 15117-96-1 & U-235 & UISO_AEA \\
\hline 491 & SESPSCH & & SW & & & B0G875 & 12-Sep-95 & & 15117-96-1 & U-235 & UISO_AEA \\
\hline 492 & SESPSCH & & SW & & & B0G879 & 12-Sep-95 & & $15117-96-1$ & U-235 & UISO_AEA \\
\hline 493 & SESPSCH & & SW & & & B0G880 & 12-Sep-95 & & 15117-96-1 & U-235 & UISO_AEA \\
\hline 494 & SESPSCH & & SW & & & B0G881 & 12-Sep-95 & & 15117-96-1 & U-235 & UISO_AEA \\
\hline 495 & SESPSCH & & SW & & & B0G882 & 12-Sep-95 & & 15117-96-1 & U-235 & UISO_AEA \\
\hline
\end{tabular}




\begin{tabular}{|c|c|c|c|c|c|c|c|c|c|c|c|}
\hline & A & $\mathrm{B}$ & $\mathrm{C}$ & $\mathrm{D}$ & $E$ & $\mathrm{~F}$ & G & $\mathrm{H}$ & $\mathrm{I}$ & $\mathrm{J}$ & $\mathrm{K}$ \\
\hline 1 & SAMPLE_NUM_ASSIGNED_TO & SAMPLER & MEDIA & BIOTA_MEDIA & TAG_ID & SAMP_NUM & SAMP_DATE_TIME & SAMP_DATE_TIME_ON & CON_ID & CON_SHORT_NAME & METHOD_NAME \\
\hline 496 & SESPSCH & & SW & & & B0G883 & 12-Sep-95 & & 15117-96-1 & U-235 & UISO_AEA \\
\hline 497 & SESPSCH & & SW & & & B0G885 & 12-Sep-95 & & 15117-96-1 & U-235 & UISO_AEA \\
\hline 498 & SESPSCH & & SW & & & B0G886 & 12-Sep-95 & & 15117-96-1 & U-235 & UISO_AEA \\
\hline 499 & SESPSCH & & SW & & & B0G891 & 12-Sep-95 & & 15117-96-1 & U-235 & UISO_AEA \\
\hline 500 & SESPSCH & & SW & & & B0G887 & 12-Sep-95 & & 15117-96-1 & U-235 & UISO_AEA \\
\hline 501 & SESPSCH & & SW & & & B0G892 & 12-Sep-95 & & 15117-96-1 & U-235 & UISO_AEA \\
\hline 502 & SESPSCH & & SW & & & B0G872 & 12-Sep-95 & & 15117-96-1 & U-235 & UISO_AEA \\
\hline 503 & SESPSCH & & SW & & & B0G876 & 12-Sep-95 & & 15117-96-1 & U-235 & UISO_AEA \\
\hline 504 & SESPSCH & & SW & & & B0G877 & 12-Sep-95 & & 15117-96-1 & U-235 & UISO_AEA \\
\hline 505 & SESPSCH & & SW & & & B0G888 & 12-Sep-95 & & 15117-96-1 & U-235 & UISO_AEA \\
\hline 506 & SESPSCH & & SW & & & B0G889 & 12-Sep-95 & & 15117-96-1 & U-235 & UISO_AEA \\
\hline 507 & SESPSCH & & SW & & & B0G890 & 12-Sep-95 & & 15117-96-1 & U-235 & UISO_AEA \\
\hline 508 & SESPSCH & & SW & & & B0G893 & 18-Sep-95 & & 15117-96-1 & U-235 & UISO_AEA \\
\hline 509 & SESPSCH & & SW & & & B0G894 & 18-Sep-95 & & 15117-96-1 & U-235 & UISO_AEA \\
\hline 510 & SESPSCH & & SW & & & B0G895 & 18-Sep-95 & & 15117-96-1 & U-235 & UISO_AEA \\
\hline 511 & SESPSCH & & SW & & & B0G896 & 18-Sep-95 & & 15117-96-1 & U-235 & UISO_AEA \\
\hline 512 & SESPSCH & & SW & & & B0G897 & 18-Sep-95 & & 15117-96-1 & U-235 & UISO_AEA \\
\hline 513 & SESPSCH & & SW & & & B0G898 & 18-Sep-95 & & 15117-96-1 & U-235 & UISO_AEA \\
\hline 514 & SESPSCH & & SW & & & B0G899 & 18-Sep-95 & & 15117-96-1 & U-235 & UISO_AEA \\
\hline 515 & SESPSCH & & SW & & & B0G8B0 & 18-Sep-95 & & 15117-96-1 & U-235 & UISO_AEA \\
\hline 516 & SESPSCH & & SW & & & B0G8B1 & 18-Sep-95 & & 15117-96-1 & U-235 & UISO_AEA \\
\hline 517 & SESPSCH & & SW & & & B0GBM8 & 18-Sep-95 & & 15117-96-1 & U-235 & UISO_AEA \\
\hline 518 & SESPSCH & & SW & & & B0GBN7 & 18-Sep-95 & & 15117-96-1 & U-235 & UISO_AEA \\
\hline 519 & SESPSCH & & SW & & & B0GBM9 & 18-Sep-95 & & 15117-96-1 & U-235 & UISO_AEA \\
\hline 520 & SESPSCH & & SW & & & BOGBNO & 18-Sep-95 & & $15117-96-1$ & U-235 & UISO_AEA \\
\hline 521 & SESPSCH & & SW & & & B0GBN1 & 18-Sep-95 & & $15117-96-1$ & U-235 & UISO_AEA \\
\hline 522 & SESPSCH & & SW & & & B0GBN2 & 18-Sep-95 & & 15117-96-1 & U-235 & UISO_AEA \\
\hline 523 & SESPSCH & & SW & & & B0GBN3 & 18-Sep-95 & & 15117-96-1 & U-235 & UISO_AEA \\
\hline 524 & SESPSCH & & SW & & & B0GBN4 & 18-Sep-95 & & 15117-96-1 & U-235 & UISO_AEA \\
\hline 525 & SESPSCH & & SW & & & B0GBN5 & 18-Sep-95 & & 15117-96-1 & U-235 & UISO_AEA \\
\hline 526 & SESPSCH & & SW & & & B0GBN6 & 18-Sep-95 & & 15117-96-1 & $U-235$ & UISO_AEA \\
\hline 527 & SESPSCH & & SW & & & B0GNP9 & 26-Sep-95 & & 15117-96-1 & U-235 & UISO_AEA \\
\hline 528 & SESPSCH & & SW & & & B0GHY1 & 03-Oct-95 & & 15117-96-1 & U-235 & UISO_AEA \\
\hline 529 & SESPSCH & & SW & & & B0GHY6 & 03-Oct-95 & & 15117-96-1 & $\mathrm{U}-235$ & UISO_AEA \\
\hline 530 & SESPSCH & & SW & & & B0G537 & 10-Oct-95 & & 15117-96-1 & U-235 & UISO_IE_PLATE_AEA \\
\hline 531 & SESPSCH & & SW & & & B0GW44 & 30-Oct-95 & & 15117-96-1 & U-235 & UISO_AEA \\
\hline 532 & SESPSCH & & SW & & & B0GWM1 & 02-Nov-95 & & 15117-96-1 & U-235 & UISO_AEA \\
\hline 533 & SESPSCH & & SW & & & BOGWMO & 02-Nov-95 & & 15117-96-1 & U-235 & UISO_AEA \\
\hline
\end{tabular}




\begin{tabular}{|c|c|c|c|c|c|c|c|c|c|c|c|}
\hline & $A$ & $B$ & $C$ & $\mathrm{D}$ & $E$ & $\mathrm{~F}$ & $G$ & $\mathrm{H}$ & $I$ & $\mathrm{~J}$ & $\mathrm{~K}$ \\
\hline 1 & SAMPLE_NUM_ASSIGNED_TO & SAMPLER & MEDIA & BIOTA_MEDIA & TAG_ID & SAMP_NUM & SAMP_DATE_TIME & SAMP_DATE_TIME_ON & CON_ID & CON_SHORT_NAME & METHOD_NAME \\
\hline 534 & SESPSCH & & SW & & & B0GMG6 & 08-Nov-95 & & 15117-96-1 & U-235 & UISO_AEA \\
\hline 535 & SESPSCH & & SW & & & BOGMH2 & 08-Nov-95 & & 15117-96-1 & U-235 & UISO_AEA \\
\hline 536 & SESPSCH & & SW & & & B0GW08 & 05-Dec-95 & & 15117-96-1 & U-235 & UISO_AEA \\
\hline 537 & SESPSCH & & SW & & & B0GW13 & 05-Dec-95 & & 15117-96-1 & U-235 & UISO_AEA \\
\hline 538 & SESPSCH & & SW & & & B0GVY1 & 07-Dec-95 & & $15117-96-1$ & U-235 & UISO_AEA \\
\hline 539 & SESPSCH & & SW & & & BOGVZO & 07-Dec-95 & & 15117-96-1 & U-235 & UISO_AEA \\
\hline 540 & SESPSCH & & SW & & & B0GVY2 & 07-Dec-95 & & 15117-96-1 & U-235 & UISO_AEA \\
\hline 541 & SESPSCH & & SW & & & B0GVY3 & 07-Dec-95 & & 15117-96-1 & U-235 & UISO_AEA \\
\hline 542 & SESPSCH & & SW & & & B0GVY4 & 07-Dec-95 & & 15117-96-1 & U-235 & UISO_AEA \\
\hline 543 & SESPSCH & & SW & & & B0GVY5 & 07-Dec-95 & & 15117-96-1 & U-235 & UISO_AEA \\
\hline 544 & SESPSCH & & SW & & & BOGVY6 & 07-Dec-95 & & 15117-96-1 & U-235 & UISO_AEA \\
\hline 545 & SESPSCH & & SW & & & B0GVY7 & 07-Dec-95 & & 15117-96-1 & $U-235$ & UISO_AEA \\
\hline 546 & SESPSCH & & SW & & & BOGVY8 & 07-Dec-95 & & 15117-96-1 & U-235 & UISO_AEA \\
\hline 547 & SESPSCH & & SW & & & B0GVY9 & 07-Dec-95 & & 15117-96-1 & U-235 & UISO_AEA \\
\hline 548 & SESPSCH & & SW & & & B0GVX7 & 08-Dec-95 & & 15117-96-1 & U-235 & UISO_AEA \\
\hline 549 & SESPSCH & & SW & & & B0GVX8 & 08-Dec-95 & & 15117-96-1 & U-235 & UISO_AEA \\
\hline 550 & SESPSCH & & SW & & & B0GVX9 & 08-Dec-95 & & 15117-96-1 & U-235 & UISO_AEA \\
\hline 551 & SESPSCH & & SW & & & BOGVYO & 08-Dec-95 & & 15117-96-1 & U-235 & UISO_AEA \\
\hline 552 & JL JULYA/LE BISPING & & SW & & & B09J78 & 25-Aug-94 & & $56-23-5$ & CARBTET & 8240_VOA_GCMS \\
\hline 553 & JL JULYA/LE BISPING & & SW & & & B09J87 & 25-Aug-94 & & $56-23-5$ & CARBTET & 8240_VOA_GCMS \\
\hline 554 & JL JULYA/LE BISPING & & SW & & & B09J79 & 25-Aug-94 & & $56-23-5$ & CARBTET & 8240_VOA_GCMS \\
\hline 555 & JL JULYA/LE BISPING & & SW & & & B09J80 & 25-Aug-94 & & $56-23-5$ & CARBTET & 8240_VOA_GCMS \\
\hline 556 & JL JULYA/LE BISPING & & SW & & & B09J81 & 25-Aug-94 & & $56-23-5$ & CARBTET & 8240_VOA_GCMS \\
\hline 557 & JL JULYA/LE BISPING & & SW & & & B09J82 & 25-Aug-94 & & $56-23-5$ & CARBTET & 8240_VOA_GCMS \\
\hline 558 & JL JULYA/LE BISPING & & SW & & & B09J83 & 25-Aug-94 & & $56-23-5$ & CARBTET & 8240_VOA_GCMS \\
\hline 559 & JL JULYA/LE BISPING & & SW & & & B09J84 & 25-Aug-94 & & $56-23-5$ & CARBTET & 8240_VOA_GCMS \\
\hline 560 & JL JULYA/LE BISPING & & SW & & & B09J85 & 25-Aug-94 & & $56-23-5$ & CARBTET & 8240_VOA_GCMS \\
\hline 561 & JL JULYA/LE BISPING & & SW & & & B09J86 & 25-Aug-94 & & $56-23-5$ & CARBTET & 8240_VOA_GCMS \\
\hline 562 & JL JULYA/LE BISPING & & SW & & & B09J68 & 25-Aug-94 & & $56-23-5$ & CARBTET & 8240_VOA_GCMS \\
\hline 563 & JL JULYA/LE BISPING & & SW & & & B09J77 & 25-Aug-94 & & $56-23-5$ & CARBTET & 8240_VOA_GCMS \\
\hline 564 & JL JULYA/LE BISPING & & SW & & & B09J69 & 25-Aug-94 & & $56-23-5$ & CARBTET & 8240_VOA_GCMS \\
\hline 565 & JL JULYA/LE BISPING & & SW & & & B09J70 & 25-Aug-94 & & $56-23-5$ & CARBTET & 8240_VOA_GCMS \\
\hline 566 & JL JULYA/LE BISPING & & SW & & & B09J71 & 25-Aug-94 & & $56-23-5$ & CARBTET & 8240_VOA_GCMS \\
\hline 567 & JL JULYA/LE BISPING & & SW & & & B09J72 & 25-Aug-94 & & $56-23-5$ & CARBTET & 8240_VOA_GCMS \\
\hline 568 & JL JULYA/LE BISPING & & SW & & & B09J73 & 25-Aug-94 & & $56-23-5$ & CARBTET & 8240_VOA_GCMS \\
\hline 569 & JL JULYA/LE BISPING & & SW & & & B09J74 & 25-Aug-94 & & $56-23-5$ & CARBTET & 8240_VOA_GCMS \\
\hline 570 & JL JULYA/LE BISPING & & SW & & & B09J75 & 25-Aug-94 & & $56-23-5$ & CARBTET & 8240_VOA_GCMS \\
\hline 571 & JL JULYA/LE BISPING & & SW & & & B09J76 & 25-Aug-94 & & $56-23-5$ & CARBTET & 8240_VOA_GCMS \\
\hline
\end{tabular}




\begin{tabular}{|c|c|c|c|c|c|c|c|c|c|c|c|}
\hline & A & $\mathrm{B}$ & $\mathrm{C}$ & $\mathrm{D}$ & $E$ & $\mathrm{~F}$ & G & $\mathrm{H}$ & $\mathrm{I}$ & $\mathrm{J}$ & $\mathrm{K}$ \\
\hline 1 & SAMPLE_NUM_ASSIGNED_TO & SAMPLER & MEDIA & BIOTA_MEDIA & TAG_ID & SAMP_NUM & SAMP_DATE_TIME & SAMP_DATE_TIME_ON & CON_ID & CON_SHORT_NAME & METHOD_NAME \\
\hline 572 & JL JULYA/LE BISPING & & SW & & & B09J64 & 25-Aug-94 & & $56-23-5$ & CARBTET & 8240_VOA_GCMS \\
\hline 573 & JL JULYA/LE BISPING & & SW & & & B09J65 & 25-Aug-94 & & $56-23-5$ & CARBTET & 8240_VOA_GCMS \\
\hline 574 & JL JULYA/LE BISPING & & SW & & & B09J66 & 25-Aug-94 & & $56-23-5$ & CARBTET & 8240_VOA_GCMS \\
\hline 575 & JL JULYA/LE BISPING & & SW & & & B09J67 & 25-Aug-94 & & $56-23-5$ & CARBTET & 8240_VOA_GCMS \\
\hline 576 & JL JULYA/LE BISPING & & SW & & & B09J98 & 26-Aug-94 & & $56-23-5$ & CARBTET & 8240_VOA_GCMS \\
\hline 577 & JL JULYA/LE BISPING & & SW & & & B09J99 & 26-Aug-94 & & $56-23-5$ & CARBTET & 8240_VOA_GCMS \\
\hline 578 & JL JULYA/LE BISPING & & SW & & & B09JB0 & 26-Aug-94 & & $56-23-5$ & CARBTET & 8240_VOA_GCMS \\
\hline 579 & JL JULYA/LE BISPING & & SW & & & B09J60 & 26-Aug-94 & & $56-23-5$ & CARBTET & 8240_VOA_GCMS \\
\hline 580 & PS HENRY/LE BISPING & & SW & & & B0CSC3 & 26-Aug-94 & & $56-23-5$ & CARBTET & 8240_VOA_GCMS \\
\hline 581 & PS HENRY/LE BISPING & & SW & & & B0CSC4 & 26-Aug-94 & & $56-23-5$ & CARBTET & 8240_VOA_GCMS \\
\hline 582 & PS HENRY/LE BISPING & & SW & & & B0CSC5 & 26-Aug-94 & & $56-23-5$ & CARBTET & 8240_VOA_GCMS \\
\hline 583 & PS HENRY/LE BISPING & & SW & & & B0CSC6 & 26-Aug-94 & & $56-23-5$ & CARBTET & 8240_VOA_GCMS \\
\hline 584 & PS HENRY/LE BISPING & & SW & & & B0CSC7 & 26-Aug-94 & & $56-23-5$ & CARBTET & 8240_VOA_GCMS \\
\hline 585 & PS HENRY/LE BISPING & & SW & & & B0CSC9 & 26-Aug-94 & & $56-23-5$ & CARBTET & 8240_VOA_GCMS \\
\hline 586 & PS HENRY/LE BISPING & & SW & & & B0CSD8 & 26-Aug-94 & & $56-23-5$ & CARBTET & 8240_VOA_GCMS \\
\hline 587 & PS HENRY/LE BISPING & & SW & & & BOCSDO & 26-Aug-94 & & $56-23-5$ & CARBTET & 8240_VOA_GCMS \\
\hline 588 & PS HENRY/LE BISPING & & SW & & & B0CSD1 & 26-Aug-94 & & $56-23-5$ & CARBTET & 8240_VOA_GCMS \\
\hline 589 & PS HENRY/LE BISPING & & SW & & & B0CSD2 & 26-Aug-94 & & $56-23-5$ & CARBTET & 8240_VOA_GCMS \\
\hline 590 & PS HENRY/LE BISPING & & SW & & & B0CSD3 & 26-Aug-94 & & $56-23-5$ & CARBTET & 8240_VOA_GCMS \\
\hline 591 & PS HENRY/LE BISPING & & SW & & & B0CSD4 & 26-Aug-94 & & $56-23-5$ & CARBTET & 8240_VOA_GCMS \\
\hline 592 & PS HENRY/LE BISPING & & SW & & & B0CSD5 & 26-Aug-94 & & $56-23-5$ & CARBTET & 8240_VOA_GCMS \\
\hline 593 & PS HENRY/LE BISPING & & SW & & & B0CSD6 & 26-Aug-94 & & $56-23-5$ & CARBTET & 8240_VOA_GCMS \\
\hline 594 & PS HENRY/LE BISPING & & SW & & & B0CSD7 & 26-Aug-94 & & $56-23-5$ & CARBTET & 8240_VOA_GCMS \\
\hline 595 & JL JULYA/LE BISPING & & SW & & & B09J88 & 02-Sep-94 & & $56-23-5$ & CARBTET & 8240_VOA_GCMS \\
\hline 596 & JL JULYA/LE BISPING & & SW & & & B09J97 & 02-Sep-94 & & $56-23-5$ & CARBTET & 8240_VOA_GCMS \\
\hline 597 & JL JULYA/LE BISPING & & SW & & & B09J89 & 02-Sep-94 & & $56-23-5$ & CARBTET & 8240_VOA_GCMS \\
\hline 598 & JL JULYA/LE BISPING & & SW & & & B09J90 & 02-Sep-94 & & $56-23-5$ & CARBTET & 8240_VOA_GCMS \\
\hline 599 & JL JULYA/LE BISPING & & SW & & & B09J91 & 02-Sep-94 & & $56-23-5$ & CARBTET & 8240_VOA_GCMS \\
\hline 600 & JL JULYA/LE BISPING & & SW & & & B09J92 & 02-Sep-94 & & $56-23-5$ & CARBTET & 8240_VOA_GCMS \\
\hline 601 & JL JULYA/LE BISPING & & SW & & & B09J93 & 02-Sep-94 & & $56-23-5$ & CARBTET & 8240_VOA_GCMS \\
\hline 602 & JL JULYA/LE BISPING & & SW & & & B09J94 & 02-Sep-94 & & $56-23-5$ & CARBTET & 8240_VOA_GCMS \\
\hline 603 & JL JULYA/LE BISPING & & SW & & & B09J95 & 02-Sep-94 & & $56-23-5$ & CARBTET & 8240_VOA_GCMS \\
\hline 604 & JL JULYA/LE BISPING & & SW & & & B09J96 & 02-Sep-94 & & $56-23-5$ & CARBTET & 8240_VOA_GCMS \\
\hline 605 & PS HENRY/LE BISPING & & SW & & & B0CV80 & 15-Dec-94 & & $56-23-5$ & CARBTET & 8240_VOA_GCMS \\
\hline 606 & PS HENRY/LE BISPING & & SW & & & B0CV81 & 15-Dec-94 & & $56-23-5$ & CARBTET & 8240_VOA_GCMS \\
\hline 607 & PS HENRY/LE BISPING & & SW & & & B0CV82 & 15-Dec-94 & & $56-23-5$ & CARBTET & 8240_VOA_GCMS \\
\hline 608 & PS HENRY/LE BISPING & & SW & & & B0CV83 & 15-Dec-94 & & $56-23-5$ & CARBTET & 8240_VOA_GCMS \\
\hline 609 & PS HENRY/LE BISPING & & SW & & & B0CV84 & 21-Dec-94 & & $56-23-5$ & CARBTET & 8240_VOA_GCMS \\
\hline
\end{tabular}




\begin{tabular}{|l|l|}
\hline & $S W$ \\
\hline & $S W$ \\
\hline & $S W$ \\
\hline & $S W$ \\
\hline & $S W$ \\
\hline & $S W$ \\
\hline & $S W$ \\
\hline & $S W$ \\
\hline & $S W$ \\
\hline & $S W$ \\
\hline & $S W$ \\
\hline & $S W$ \\
\hline & $S W$ \\
\hline & $S W$ \\
\hline & $S W$ \\
\hline & $S W$ \\
\hline & $S W$ \\
\hline & $S W$ \\
\hline & $S W$ \\
\hline & $S W$ \\
\hline & $S W$ \\
\hline & $S W$ \\
\hline & $S W$ \\
\hline & $S W$ \\
\hline & $S W$
\end{tabular}

\begin{tabular}{|l|}
\hline SW \\
\hline SW \\
\hline SW \\
\hline SW \\
\hline SW \\
\hline SW \\
\hline SW \\
\hline SW \\
\hline SW \\
\hline SW \\
\hline SW \\
\hline SW \\
\hline SW \\
\hline SW \\
\hline SW \\
\hline SW \\
\hline SW \\
\hline SW \\
\hline SW \\
\hline SW \\
\hline SW \\
\hline SW \\
\hline SW \\
\hline SW \\
\hline SW \\
\hline SW \\
\hline SW \\
\hline SW \\
\hline SW \\
\hline SW \\
\hline SW \\
\hline SW \\
\hline SW \\
\hline SW \\
\hline SW \\
\hline \\
\hline
\end{tabular}

\begin{tabular}{|l|l|}
\hline & \\
\hline & \\
\hline & \\
\hline & \\
\hline & \\
\hline & \\
\hline & \\
\hline & \\
\hline & \\
\hline & \\
\hline & \\
\hline & \\
\hline & \\
\hline & \\
\hline & \\
\hline & \\
\hline & \\
\hline & \\
\hline & \\
\hline & \\
\hline & \\
\hline & \\
\hline & \\
\hline & \\
\hline
\end{tabular}

B0CV8

21-Dec-94

BOCV87

21-Dec-94

\begin{tabular}{l|l} 
B0CV89 21-Dec-94 & 21-Dec-94
\end{tabular}

BOCV90 21-Dec-94

BOCV91 21-Dec-94

BOCV92 21-Dec-94

B0CVY1 16-Mar-95

16-Mar-95

BOCVY3 16-Mar-95

BOCVY4 16-Mar-95

BOCVY5

BOCVZ4

17-Mar-95

BOCVY7 17-Mar-95

BOCVY8

17-Mar-95

BOCVZ0 17-Mar-95

BOCVZ1

BOCVZ2

17-Mar-95

B0CW59 15-Jun-95

BOCW60 15-Jun-95

B0CW61 15-Jun-95

B0CW62 15-Jun-95

B0CW63 16-Jun-95

B0CW72 16-Jun-95

B0CW64 16-Jun-95

B0CW65 16-Jun-95

B0CW66 16-Jun-95

B0CW67 16-Jun-95

B0CW68 16-Jun-95

B0CW69 16-Jun-95

B0CW70 16-Jun-95

B0CW71 16-Jun-95

Page 17

$\frac{\mathrm{I}}{\mathrm{CON}}$

CON

56-23-5

56-23-5

$56-23-5$

56-23-5

56-23-5

$56-23-5$

56-23-5

56-23-5

56-23-5

56-23-5

$56-23-5$

56-23-5

$56-23-5$

56-23-5

$56-23-5$

56-23-5

56-23-5

$56-23-5$

56-23-5

$56-23-5$

$56-23-5$

$56-23-5$

$56-23-5$

56-23-5

$56-23-5$

$56-23-5$

$56-23-5$

$56-23-5$

$56-23-5$

$56-23-5$

$56-23-5$

56-23-5

56-23-5

56-23-5

$56-23-5$

56-23-5

CON_SHORT NAME
CARBTET

CARBTET

CARBTET

CARBTET

CARBTET

CARBTET

CARBTET

CARBTET

CARBTET

CARBTET

CARBTET

CARBTET

CARBTET

CARBTET

CARBTET

CARBTET

CARBTET

CARBTET

CARBTET

CARBTET

CARBTET

CARBTET

CARBTET

CARBTET

CARBTET

CARBTET

CARBTET

CARBTET

CARBTET

CARBTET

CARBTET

CARBTET

CARBTET

CARBTET

CARBTET

CARBTET

CARBTET

CARBTET 


\begin{tabular}{|c|c|c|c|c|c|c|c|c|c|c|c|}
\hline & $A$ & $\mathrm{~B}$ & $\mathrm{C}$ & $\mathrm{D}$ & $E$ & $\mathrm{~F}$ & G & $\mathrm{H}$ & $\mathrm{I}$ & $\mathrm{J}$ & $\mathrm{K}$ \\
\hline 1 & SAMPLE_NUM_ASSIGNED_TO & SAMPLER & MEDIA & BIOTA_MEDIA & TAG_ID & SAMP_NUM & SAMP_DATE_TIME & SAMP_DATE_TIME_ON & CON_ID & CON_SHORT_NAME & METHOD_NAME \\
\hline 648 & PS HENRY/LE BISPING & & SW & & & BOG0T2 & 11-Sep-95 & & $56-23-5$ & CARBTET & 8260_VOA_GCMS \\
\hline 649 & PS HENRY/LE BISPING & & SW & & & B0G0S4 & 11-Sep-95 & & $56-23-5$ & CARBTET & 8260_VOA_GCMS \\
\hline 650 & PS HENRY/LE BISPING & & SW & & & B0G0S5 & 11-Sep-95 & & $56-23-5$ & CARBTET & 8260_VOA_GCMS \\
\hline 651 & PS HENRY/LE BISPING & & SW & & & B0G0S6 & 11-Sep-95 & & $56-23-5$ & CARBTET & 8260_VOA_GCMS \\
\hline 652 & PS HENRY/LE BISPING & & SW & & & B0G0S7 & 11-Sep-95 & & $56-23-5$ & CARBTET & 8260_VOA_GCMS \\
\hline 653 & PS HENRY/LE BISPING & & SW & & & BOG0S8 & 11-Sep-95 & & $56-23-5$ & CARBTET & 8260_VOA_GCMS \\
\hline 654 & PS HENRY/LE BISPING & & SW & & & B0G0S9 & 11-Sep-95 & & $56-23-5$ & CARBTET & 8260_VOA_GCMS \\
\hline 655 & PS HENRY/LE BISPING & & SW & & & BOGOT0 & 11-Sep-95 & & $56-23-5$ & CARBTET & 8260_VOA_GCMS \\
\hline 656 & PS HENRY/LE BISPING & & SW & & & B0G0T1 & 11-Sep-95 & & $56-23-5$ & CARBTET & 8260_VOA_GCMS \\
\hline 657 & PS HENRY/LE BISPING & & SW & & & B0G0Q9 & 11-Sep-95 & & $56-23-5$ & CARBTET & 8260_VOA_GCMS \\
\hline 658 & PS HENRY/LE BISPING & & SW & & & BOGORO & 11-Sep-95 & & $56-23-5$ & CARBTET & 8260_VOA_GCMS \\
\hline 659 & PS HENRY/LE BISPING & & SW & & & B0G0R1 & 11-Sep-95 & & $56-23-5$ & CARBTET & 8260_VOA_GCMS \\
\hline 660 & PS HENRY/LE BISPING & & SW & & & BOG0R2 & 11-Sep-95 & & $56-23-5$ & CARBTET & 8260_VOA_GCMS \\
\hline 661 & PS HENRY/LE BISPING & & SW & & & BOG0T3 & 12-Sep-95 & & $56-23-5$ & CARBTET & 8260_VOA_GCMS \\
\hline 662 & PS HENRY/LE BISPING & & SW & & & B0G0V2 & 12-Sep-95 & & $56-23-5$ & CARBTET & 8260_VOA_GCMS \\
\hline 663 & PS HENRY/LE BISPING & & SW & & & B0G0T4 & 12-Sep-95 & & $56-23-5$ & CARBTET & 8260_VOA_GCMS \\
\hline 664 & PS HENRY/LE BISPING & & SW & & & B0G0T5 & 12-Sep-95 & & $56-23-5$ & CARBTET & 8260_VOA_GCMS \\
\hline 665 & PS HENRY/LE BISPING & & SW & & & B0G0T6 & 12-Sep-95 & & $56-23-5$ & CARBTET & 8260_VOA_GCMS \\
\hline 666 & PS HENRY/LE BISPING & & SW & & & B0G0T7 & 12-Sep-95 & & $56-23-5$ & CARBTET & 8260_VOA_GCMS \\
\hline 667 & PS HENRY/LE BISPING & & SW & & & B0G0T8 & 12-Sep-95 & & $56-23-5$ & CARBTET & 8260_VOA_GCMS \\
\hline 668 & PS HENRY/LE BISPING & & SW & & & B0G0T9 & 12-Sep-95 & & $56-23-5$ & CARBTET & 8260_VOA_GCMS \\
\hline 669 & PS HENRY/LE BISPING & & SW & & & BOGOVO & 12-Sep-95 & & $56-23-5$ & CARBTET & 8260_VOA_GCMS \\
\hline 670 & PS HENRY/LE BISPING & & SW & & & B0G0V1 & 12-Sep-95 & & $56-23-5$ & CARBTET & 8260_VOA_GCMS \\
\hline 671 & PS HENRY/LE BISPING & & SW & & & BOG0V3 & 12-Sep-95 & & $56-23-5$ & CARBTET & 8260_VOA_GCMS \\
\hline 672 & PS HENRY/LE BISPING & & SW & & & BOGOW2 & 12-Sep-95 & & $56-23-5$ & CARBTET & 8260_VOA_GCMS \\
\hline 673 & PS HENRY/LE BISPING & & SW & & & BOG0V4 & 12-Sep-95 & & $56-23-5$ & CARBTET & 8260_VOA_GCMS \\
\hline 674 & PS HENRY/LE BISPING & & SW & & & B0G0V5 & 12-Sep-95 & & $56-23-5$ & CARBTET & 8260_VOA_GCMS \\
\hline 675 & PS HENRY/LE BISPING & & SW & & & B0G0V6 & 12-Sep-95 & & $56-23-5$ & CARBTET & 8260_VOA_GCMS \\
\hline 676 & PS HENRY/LE BISPING & & SW & & & B0G0V7 & 12-Sep-95 & & $56-23-5$ & CARBTET & 8260_VOA_GCMS \\
\hline 677 & PS HENRY/LE BISPING & & SW & & & B0G0V8 & 12-Sep-95 & & $56-23-5$ & CARBTET & 8260_VOA_GCMS \\
\hline 678 & PS HENRY/LE BISPING & & SW & & & B0G0V9 & 12-Sep-95 & & $56-23-5$ & CARBTET & 8260_VOA_GCMS \\
\hline 679 & PS HENRY/LE BISPING & & SW & & & BOGOWO & 12-Sep-95 & & $56-23-5$ & CARBTET & 8260_VOA_GCMS \\
\hline 680 & PS HENRY/LE BISPING & & SW & & & B0G0W1 & 12-Sep-95 & & $56-23-5$ & CARBTET & 8260_VOA_GCMS \\
\hline 681 & PS HENRY/LE BISPING & & SW & & & B0G0W3 & 18-Sep-95 & & $56-23-5$ & CARBTET & 8260_VOA_GCMS \\
\hline 682 & PS HENRY/LE BISPING & & SW & & & B0G0W4 & 18-Sep-95 & & $56-23-5$ & CARBTET & 8260_VOA_GCMS \\
\hline 683 & PS HENRY/LE BISPING & & SW & & & B0G0W5 & 18-Sep-95 & & $56-23-5$ & CARBTET & 8260_VOA_GCMS \\
\hline 684 & PS HENRY/LE BISPING & & SW & & & B0G0W6 & 18-Sep-95 & & $56-23-5$ & CARBTET & 8260_VOA_GCMS \\
\hline 685 & PS HENRY/LE BISPING & & SW & & & B0G0W7 & 18-Sep-95 & & $56-23-5$ & CARBTET & 8260_VOA_GCMS \\
\hline
\end{tabular}




\begin{tabular}{|c|c|c|c|c|c|c|c|c|c|c|c|}
\hline & $A$ & $\mathrm{~B}$ & $\mathrm{C}$ & $\mathrm{D}$ & $E$ & $\mathrm{~F}$ & G & $\mathrm{H}$ & $\mathrm{I}$ & $\mathrm{J}$ & $\mathrm{K}$ \\
\hline 1 & SAMPLE_NUM_ASSIGNED_TO & SAMPLER & MEDIA & BIOTA_MEDIA & TAG_ID & SAMP_NUM & SAMP_DATE_TIME & SAMP_DATE_TIME_ON & CON_ID & CON_SHORT_NAME & METHOD_NAME \\
\hline 686 & PS HENRY/LE BISPING & & SW & & & B0G0W8 & 18-Sep-95 & & $56-23-5$ & CARBTET & 8260_VOA_GCMS \\
\hline 687 & PS HENRY/LE BISPING & & SW & & & B0G0W9 & 18-Sep-95 & & $56-23-5$ & CARBTET & 8260_VOA_GCMS \\
\hline 688 & PS HENRY/LE BISPING & & SW & & & BOGOXO & 18-Sep-95 & & $56-23-5$ & CARBTET & 8260_VOA_GCMS \\
\hline 689 & PS HENRY/LE BISPING & & SW & & & B0G0X1 & 18-Sep-95 & & $56-23-5$ & CARBTET & 8260_VOA_GCMS \\
\hline 690 & PS HENRY/LE BISPING & & SW & & & B0G0R3 & 18-Sep-95 & & $56-23-5$ & CARBTET & 8260_VOA_GCMS \\
\hline 691 & PS HENRY/LE BISPING & & SW & & & BOG0S2 & 18-Sep-95 & & $56-23-5$ & CARBTET & 8260_VOA_GCMS \\
\hline 692 & PS HENRY/LE BISPING & & SW & & & B0G0R4 & 18-Sep-95 & & $56-23-5$ & CARBTET & 8260_VOA_GCMS \\
\hline 693 & PS HENRY/LE BISPING & & SW & & & B0G0R5 & 18-Sep-95 & & $56-23-5$ & CARBTET & 8260_VOA_GCMS \\
\hline 694 & PS HENRY/LE BISPING & & SW & & & B0G0R6 & 18-Sep-95 & & $56-23-5$ & CARBTET & 8260_VOA_GCMS \\
\hline 695 & PS HENRY/LE BISPING & & SW & & & B0G0R7 & 18-Sep-95 & & $56-23-5$ & CARBTET & 8260_VOA_GCMS \\
\hline 696 & PS HENRY/LE BISPING & & SW & & & B0G0R8 & 18-Sep-95 & & $56-23-5$ & CARBTET & 8260_VOA_GCMS \\
\hline 697 & PS HENRY/LE BISPING & & SW & & & BOG0R9 & 18-Sep-95 & & $56-23-5$ & CARBTET & 8260_VOA_GCMS \\
\hline 698 & PS HENRY/LE BISPING & & SW & & & BOGOSO & 18-Sep-95 & & $56-23-5$ & CARBTET & 8260_VOA_GCMS \\
\hline 699 & PS HENRY/LE BISPING & & SW & & & B0G0S1 & 18-Sep-95 & & $56-23-5$ & CARBTET & 8260_VOA_GCMS \\
\hline 700 & PS HENRY/LE BISPING & & SW & & & B0G132 & 07-Dec-95 & & $56-23-5$ & CARBTET & 8260_VOA_GCMS \\
\hline 701 & PS HENRY/LE BISPING & & SW & & & B0G145 & 07-Dec-95 & & $56-23-5$ & CARBTET & 8260_VOA_GCMS \\
\hline 702 & PS HENRY/LE BISPING & & SW & & & B0G133 & 07-Dec-95 & & $56-23-5$ & CARBTET & 8260_VOA_GCMS \\
\hline 703 & PS HENRY/LE BISPING & & SW & & & B0G134 & 07-Dec-95 & & $56-23-5$ & CARBTET & 8260_VOA_GCMS \\
\hline 704 & PS HENRY/LE BISPING & & SW & & & B0G135 & 07-Dec-95 & & $56-23-5$ & CARBTET & 8260_VOA_GCMS \\
\hline 705 & PS HENRY/LE BISPING & & SW & & & B0G140 & 07-Dec-95 & & $56-23-5$ & CARBTET & 8260_VOA_GCMS \\
\hline 706 & PS HENRY/LE BISPING & & SW & & & B0G141 & 07-Dec-95 & & $56-23-5$ & CARBTET & 8260_VOA_GCMS \\
\hline 707 & PS HENRY/LE BISPING & & SW & & & B0G142 & 07-Dec-95 & & $56-23-5$ & CARBTET & 8260_VOA_GCMS \\
\hline 708 & PS HENRY/LE BISPING & & SW & & & B0G143 & 07-Dec-95 & & $56-23-5$ & CARBTET & 8260_VOA_GCMS \\
\hline 709 & PS HENRY/LE BISPING & & SW & & & B0G144 & 07-Dec-95 & & $56-23-5$ & CARBTET & 8260_VOA_GCMS \\
\hline 710 & PS HENRY/LE BISPING & & SW & & & B0G136 & 08-Dec-95 & & $56-23-5$ & CARBTET & 8260_VOA_GCMS \\
\hline 711 & PS HENRY/LE BISPING & & SW & & & B0G137 & 08-Dec-95 & & $56-23-5$ & CARBTET & 8260_VOA_GCMS \\
\hline 712 & PS HENRY/LE BISPING & & SW & & & B0G138 & 08-Dec-95 & & $56-23-5$ & CARBTET & 8260_VOA_GCMS \\
\hline 713 & PS HENRY/LE BISPING & & SW & & & B0G139 & 08-Dec-95 & & $56-23-5$ & CARBTET & 8260_VOA_GCMS \\
\hline 714 & PDMS & & SW & & & E113160 & 03-Apr-90 & & PU-239/240 & PU-239/240 & PUISO_IE_AEA \\
\hline 715 & PDMS & & SW & & & E113163 & 03-Apr-90 & & PU-239/240 & PU-239/240 & PUISO_IE_AEA \\
\hline 716 & PDMS & & SW & & & E113161 & 03-Apr-90 & & PU-239/240 & PU-239/240 & PUISO_IE_AEA \\
\hline 717 & PDMS & & SW & & & E113164 & 03-Apr-90 & & PU-239/240 & PU-239/240 & PUISO_IE_AEA \\
\hline 718 & PDMS & & SW & & & E113162 & 03-Apr-90 & & PU-239/240 & PU-239/240 & PUISO_IE_AEA \\
\hline 719 & PDMS & & SW & & & E113165 & 03-Apr-90 & & PU-239/240 & PU-239/240 & PUISO_IE_AEA \\
\hline 720 & PDMS & & SW & & & E120495 & 02-Oct-90 & & PU-239/240 & PU-239/240 & PUISO_IE_AEA \\
\hline 721 & PDMS & & SW & & & E120636 & 02-Oct-90 & & PU-239/240 & PU-239/240 & PUISO_IE_AEA \\
\hline 722 & PDMS & & SW & & & E120497 & 02-Oct-90 & & PU-239/240 & PU-239/240 & PUISO_IE_AEA \\
\hline 723 & PDMS & & SW & & & E120638 & 02-Oct-90 & & PU-239/240 & PU-239/240 & PUISO_IE_AEA \\
\hline
\end{tabular}




\begin{tabular}{|c|c|c|c|c|c|c|c|c|c|c|c|}
\hline & A & $\mathrm{B}$ & $\mathrm{C}$ & $\mathrm{D}$ & $E$ & $\mathrm{~F}$ & G & $\mathrm{H}$ & $\mathrm{I}$ & $\mathrm{J}$ & $\mathrm{K}$ \\
\hline 1 & SAMPLE_NUM_ASSIGNED_TO & SAMPLER & MEDIA & BIOTA_MEDIA & TAG_ID & SAMP_NUM & SAMP_DATE_TIME & SAMP_DATE_TIME_ON & CON_ID & CON_SHORT_NAME & METHOD_NAME \\
\hline 724 & PDMS & & SW & & & E033251 & 02-Oct-90 & & PU-239/240 & PU-239/240 & PUISO_IE_AEA \\
\hline 725 & PDMS & & SW & & & E120499 & 02-Oct-90 & & PU-239/240 & PU-239/240 & PUISO_IE_AEA \\
\hline 726 & PDMS & & SW & & & E120496 & 27-Dec-90 & & PU-239/240 & PU-239/240 & PUISO_IE_AEA \\
\hline 727 & PDMS & & SW & & & E120637 & 27-Dec-90 & & PU-239/240 & PU-239/240 & PUISO_IE_AEA \\
\hline 728 & PDMS & & SW & & & E120498 & 27-Dec-90 & & PU-239/240 & PU-239/240 & PUISO_IE_AEA \\
\hline 729 & PDMS & & SW & & & E120639 & 27-Dec-90 & & PU-239/240 & PU-239/240 & PUISO_IE_AEA \\
\hline 730 & PDMS & & SW & & & E120500 & 27-Dec-90 & & PU-239/240 & PU-239/240 & PUISO_IE_AEA \\
\hline 731 & PDMS & & SW & & & E120640 & 27-Dec-90 & & PU-239/240 & PU-239/240 & PUISO_IE_AEA \\
\hline 732 & PDMS & & SW & & & E120224 & 02-Apr-91 & & PU-239/240 & PU-239/240 & PUISO_IE_AEA \\
\hline 733 & PDMS & & SW & & & E120441 & 02-Apr-91 & & PU-239/240 & PU-239/240 & PUISO_IE_AEA \\
\hline 734 & PDMS & & SW & & & E120222 & 02-Apr-91 & & PU-239/240 & PU-239/240 & PUISO_IE_AEA \\
\hline 735 & PDMS & & SW & & & E120439 & 02-Apr-91 & & PU-239/240 & PU-239/240 & PUISO_IE_AEA \\
\hline 736 & PDMS & & SW & & & E120223 & 02-Apr-91 & & PU-239/240 & PU-239/240 & PUISO_IE_AEA \\
\hline 737 & PDMS & & SW & & & E120440 & 02-Apr-91 & & PU-239/240 & PU-239/240 & PUISO_IE_AEA \\
\hline 738 & PDMS & & SW & & & E120177 & 25-Jun-91 & & PU-239/240 & PU-239/240 & PUISO_IE_AEA \\
\hline 739 & PDMS & & SW & & & E120445 & 25-Jun-91 & & PU-239/240 & PU-239/240 & PUISO_IE_AEA \\
\hline 740 & PDMS & & SW & & & E120175 & 25-Jun-91 & & PU-239/240 & PU-239/240 & PUISO_IE_AEA \\
\hline 741 & PDMS & & SW & & & E120443 & 25-Jun-91 & & PU-239/240 & PU-239/240 & PUISO_IE_AEA \\
\hline 742 & PDMS & & SW & & & E120176 & 25-Jun-91 & & PU-239/240 & PU-239/240 & PUISO_IE_AEA \\
\hline 743 & PDMS & & SW & & & E120444 & 25-Jun-91 & & PU-239/240 & PU-239/240 & PUISO_IE_AEA \\
\hline 744 & PDMS & & SW & & & E120232 & 01-Oct-91 & & PU-239/240 & PU-239/240 & PUISO_IE_AEA \\
\hline 745 & PDMS & & SW & & & E120230 & 01-Oct-91 & & PU-239/240 & PU-239/240 & PUISO_IE_AEA \\
\hline 746 & PDMS & & SW & & & E120231 & 01-Oct-91 & & PU-239/240 & PU-239/240 & PUISO_IE_AEA \\
\hline 747 & PDMS & & SW & & & E120338 & 01-Oct-91 & & PU-239/240 & PU-239/240 & PUISO_IE_AEA \\
\hline 748 & PDMS & & SW & & & E120339 & 04-Oct-91 & & PU-239/240 & PU-239/240 & PUISO_IE_AEA \\
\hline 749 & PDMS & & SW & & & E120337 & 04-Oct-91 & & PU-239/240 & PU-239/240 & PUISO_IE_AEA \\
\hline 750 & PDMS & & SW & & & E120319 & 07-Jan-92 & & PU-239/240 & PU-239/240 & PUISO_IE_AEA \\
\hline 751 & PDMS & & SW & & & E120509 & 07-Jan-92 & & PU-239/240 & PU-239/240 & PUISO_IE_AEA \\
\hline 752 & PDMS & & SW & & & E120317 & 07-Jan-92 & & PU-239/240 & PU-239/240 & PUISO_IE_AEA \\
\hline 753 & PDMS & & SW & & & E120507 & 07-Jan-92 & & PU-239/240 & PU-239/240 & PUISO_IE_AEA \\
\hline 754 & PDMS & & SW & & & E120318 & 07-Jan-92 & & PU-239/240 & PU-239/240 & PUISO_IE_AEA \\
\hline 755 & PDMS & & SW & & & E120508 & 07-Jan-92 & & PU-239/240 & PU-239/240 & PUISO_IE_AEA \\
\hline 756 & PDMS & & SW & & & E120340 & 31-Mar-92 & & PU-239/240 & PU-239/240 & PUISO_IE_AEA \\
\hline 757 & PDMS & & SW & & & E120420 & 31-Mar-92 & & PU-239/240 & PU-239/240 & PUISO_IE_AEA \\
\hline 758 & PDMS & & SW & & & E120341 & 31-Mar-92 & & PU-239/240 & PU-239/240 & PUISO_IE_AEA \\
\hline 759 & PDMS & & SW & & & E120421 & 31-Mar-92 & & PU-239/240 & PU-239/240 & PUISO_IE_AEA \\
\hline 760 & PDMS & & SW & & & E120342 & 31-Mar-92 & & PU-239/240 & PU-239/240 & PUISO_IE_AEA \\
\hline 761 & PDMS & & SW & & & E120422 & 31-Mar-92 & & PU-239/240 & PU-239/240 & PUISO_IE_AEA \\
\hline
\end{tabular}




\begin{tabular}{|c|c|c|c|c|c|c|c|c|c|c|c|}
\hline & $A$ & $B$ & $\mathrm{C}$ & $\mathrm{D}$ & $E$ & $\mathrm{~F}$ & $G$ & $\mathrm{H}$ & $\mathrm{I}$ & $\mathrm{J}$ & $\mathrm{K}$ \\
\hline 1 & SAMPLE_NUM_ASSIGNED_TO & SAMPLER & MEDIA & BIOTA_MEDIA & TAG_ID & SAMP_NUM & SAMP_DATE_TIME & SAMP_DATE_TIME_ON & CON_ID & CON_SHORT_NAME & METHOD_NAME \\
\hline 762 & PDMS & & SW & & & E121086 & 07-Jul-92 & & PU-239/240 & PU-239/240 & PUISO_IE_AEA \\
\hline 763 & PDMS & & SW & & & E121128 & 07-Jul-92 & & PU-239/240 & PU-239/240 & PUISO_IE_AEA \\
\hline 764 & PDMS & & SW & & & E121087 & 07-Jul-92 & & PU-239/240 & PU-239/240 & PUISO_IE_AEA \\
\hline 765 & PDMS & & SW & & & E121129 & 07-Jul-92 & & PU-239/240 & PU-239/240 & PUISO_IE_AEA \\
\hline 766 & PDMS & & SW & & & E121088 & 07-Jul-92 & & PU-239/240 & PU-239/240 & PUISO_IE_AEA \\
\hline \begin{tabular}{|l|}
767 \\
\end{tabular} & PDMS & & SW & & & E121130 & 07-Jul-92 & & PU-239/240 & PU-239/240 & PUISO_IE_AEA \\
\hline 768 & PDMS & & SW & & & E121571 & 29-Sep-92 & & PU-239/240 & PU-239/240 & PUISO_IE_AEA \\
\hline 769 & PDMS & & SW & & & E121574 & 29-Sep-92 & & PU-239/240 & PU-239/240 & PUISO_IE_AEA \\
\hline 770 & PDMS & & SW & & & E121570 & 29-Sep-92 & & PU-239/240 & PU-239/240 & PUISO_IE_AEA \\
\hline 771 & PDMS & & SW & & & E121573 & 29-Sep-92 & & PU-239/240 & PU-239/240 & PUISO_IE_AEA \\
\hline 772 & PDMS & & SW & & & E121572 & 29-Sep-92 & & PU-239/240 & PU-239/240 & PUISO_IE_AEA \\
\hline 773 & PDMS & & SW & & & E121575 & 29-Sep-92 & & PU-239/240 & PU-239/240 & PUISO_IE_AEA \\
\hline 774 & PDMS & & SW & & & E119985 & 05-Jan-93 & & PU-239/240 & PU-239/240 & PUISO_IE_AEA \\
\hline 775 & PDMS & & SW & & & E119986 & 05-Jan-93 & & PU-239/240 & PU-239/240 & PUISO_IE_AEA \\
\hline 776 & PDMS & & SW & & & E119984 & 05-Jan-93 & & PU-239/240 & PU-239/240 & PUISO_IE_AEA \\
\hline 777 & PDMS & & SW & & & E119987 & 05-Jan-93 & & PU-239/240 & PU-239/240 & PUISO_IE_AEA \\
\hline 778 & PDMS & & SW & & & E119983 & 05-Jan-93 & & PU-239/240 & PU-239/240 & PUISO_IE_AEA \\
\hline 779 & PDMS & & SW & & & E119988 & 05-Jan-93 & & PU-239/240 & PU-239/240 & PUISO_IE_AEA \\
\hline 780 & PDMS & & SW & & & E123497 & 30-Mar-93 & & PU-239/240 & PU-239/240 & PUISO_IE_AEA \\
\hline 781 & PDMS & & SW & & & E123477 & 30-Mar-93 & & PU-239/240 & PU-239/240 & PUISO_IE_AEA \\
\hline 782 & PDMS & & SW & & & E123496 & 30-Mar-93 & & PU-239/240 & PU-239/240 & PUISO_IE_AEA \\
\hline 783 & PDMS & & SW & & & E123478 & 30-Mar-93 & & PU-239/240 & PU-239/240 & PUISO_IE_AEA \\
\hline 784 & PDMS & & SW & & & E124094 & 07-Jul-93 & & PU-239/240 & PU-239/240 & PUISO_IE_AEA \\
\hline 785 & PDMS & & SW & & & E124098 & 07-Jul-93 & & PU-239/240 & PU-239/240 & PUISO_IE_AEA \\
\hline 786 & PDMS & & SW & & & E124095 & 07-Jul-93 & & PU-239/240 & PU-239/240 & PUISO_IE_AEA \\
\hline 787 & PDMS & & SW & & & E124099 & 07-Jul-93 & & PU-239/240 & PU-239/240 & PUISO_IE_AEA \\
\hline 788 & PDMS & & SW & & & E124699 & 28-Sep-93 & & PU-239/240 & PU-239/240 & PUISO_IE_AEA \\
\hline 789 & PDMS & & SW & & & E124681 & 28-Sep-93 & & PU-239/240 & PU-239/240 & PUISO_IE_AEA \\
\hline 790 & PDMS & & SW & & & E124682 & 28-Sep-93 & & PU-239/240 & PU-239/240 & PUISO_IE_AEA \\
\hline 791 & PDMS & & SW & & & E124700 & 28-Sep-93 & & PU-239/240 & PU-239/240 & PUISO_IE_AEA \\
\hline 792 & PDMS & & SW & & & E125271 & 21-Dec-93 & & PU-239/240 & PU-239/240 & PUISO_IE_AEA \\
\hline 793 & PDMS & & SW & & & E125282 & 21-Dec-93 & & PU-239/240 & PU-239/240 & PUISO_IE_AEA \\
\hline 794 & PDMS & & SW & & & E125272 & 21-Dec-93 & & PU-239/240 & PU-239/240 & PUISO_IE_AEA \\
\hline 795 & PDMS & & SW & & & E125285 & 21-Dec-93 & & PU-239/240 & PU-239/240 & PUISO_IE_AEA \\
\hline 796 & SESPSCH & & SW & & & B09QQ8 & 29-Mar-94 & & PU-239/240 & PU-239/240 & PUISO_IE_AEA \\
\hline 797 & SESPSCH & & SW & & & B09QR4 & 29-Mar-94 & & PU-239/240 & PU-239/240 & PUISO_IE_AEA \\
\hline 798 & SESPSCH & & SW & & & B09QS6 & 29-Mar-94 & & PU-239/240 & PU-239/240 & PUISO_IE_AEA \\
\hline 799 & SESPSCH & & SW & & & B09QS0 & 29-Mar-94 & & PU-239/240 & PU-239/240 & PUISO_IE_AEA \\
\hline
\end{tabular}




\begin{tabular}{|c|c|c|c|c|c|c|c|c|c|c|c|}
\hline & A & $\mathrm{B}$ & $\mathrm{C}$ & $\mathrm{D}$ & $\mathrm{E}$ & $\mathrm{F}$ & $G$ & $\mathrm{H}$ & $\mathrm{I}$ & $\mathrm{J}$ & $\mathrm{K}$ \\
\hline 1 & SAMPLE_NUM_ASSIGNED_TO & SAMPLER & MEDIA & BIOTA_MEDIA & TAG_ID & SAMP_NUM & SAMP_DATE_TIME & SAMP_DATE_TIME_ON & CON_ID & CON_SHORT_NAME & METHOD_NAME \\
\hline 800 & SESPSCH & & SW & & & B0BP61 & 01-Aug-94 & & PU-239/240 & PU-239/240 & PUISO_IE_AEA \\
\hline 801 & SESPSCH & & SW & & & B0BP68 & 01-Aug-94 & & PU-239/240 & PU-239/240 & PUISO_IE_AEA \\
\hline 802 & SESPSCH & & SW & & & B0BP75 & 01-Aug-94 & & PU-239/240 & PU-239/240 & PUISO_IE_AEA \\
\hline 803 & SESPSCH & & SW & & & B0BP82 & 01-Aug-94 & & PU-239/240 & PU-239/240 & PUISO_IE_AEA \\
\hline 804 & SESPSCH & & SW & & & B0C453 & 25-Oct-94 & & PU-239/240 & PU-239/240 & PUISO_IE_AEA \\
\hline 805 & SESPSCH & & SW & & & B0C459 & 25-Oct-94 & & PU-239/240 & PU-239/240 & PUISO_IE_AEA \\
\hline 806 & SESPSCH & & SW & & & B0C465 & 25-Oct-94 & & PU-239/240 & PU-239/240 & PUISO_IE_AEA \\
\hline 807 & SESPSCH & & SW & & & B0C471 & 25-Oct-94 & & PU-239/240 & PU-239/240 & PUISO_IE_AEA \\
\hline 808 & SESPSCH & & SW & & & BODOW4 & 03-Jan-95 & & PU-239/240 & PU-239/240 & PUISO_IE_AEA \\
\hline 809 & SESPSCH & & SW & & & BODOX0 & 03-Jan-95 & & PU-239/240 & PU-239/240 & PUISO_IE_AEA \\
\hline 810 & SESPSCH & & SW & & & B0D0Y2 & 03-Jan-95 & & PU-239/240 & PU-239/240 & PUISO_IE_AEA \\
\hline 811 & SESPSCH & & SW & & & B0D0X6 & 03-Jan-95 & & PU-239/240 & PU-239/240 & PUISO_IE_AEA \\
\hline 812 & SESPSCH & & SW & & & B0DK82 & 27-Mar-95 & & PU-239/240 & PU-239/240 & PUISO_IE_AEA \\
\hline 813 & SESPSCH & & SW & & & B0DK88 & 27-Mar-95 & & PU-239/240 & PU-239/240 & PUISO_IE_AEA \\
\hline 814 & SESPSCH & & SW & & & B0DK94 & 27-Mar-95 & & PU-239/240 & PU-239/240 & PUISO_IE_AEA \\
\hline 815 & SESPSCH & & SW & & & BODKBO & 27-Mar-95 & & PU-239/240 & PU-239/240 & PUISO_IE_AEA \\
\hline 816 & SESPSCH & & SW & & & B0F8Y5 & 05-Jul-95 & & PU-239/240 & PU-239/240 & PUISO_IE_AEA \\
\hline 817 & SESPSCH & & SW & & & B0F8Z1 & 05-Jul-95 & & PU-239/240 & PU-239/240 & PUISO_IE_AEA \\
\hline 818 & SESPSCH & & SW & & & B0G513 & 27-Sep-95 & & PU-239/240 & PU-239/240 & PUISO_IE_AEA \\
\hline 819 & SESPSCH & & SW & & & B0G519 & 27-Sep-95 & & PU-239/240 & PU-239/240 & PUISO_IE_AEA \\
\hline
\end{tabular}




\begin{tabular}{|c|c|c|c|c|c|c|c|c|c|}
\hline & $\mathrm{L}$ & $\mathrm{M}$ & $\mathrm{N}$ & $\mathrm{O}$ & $\mathrm{P}$ & $\mathrm{Q}$ & $\mathrm{R}$ & $\mathrm{S}$ & $\mathrm{T}$ \\
\hline 1 & LAB_CODE & VALUE_RPTD & ANAL_UNITS_RPTD & COUNTING_ERROR & TOTAL_ANAL_ERROR & MIN_DETECTABLE_ACTIVITY & PCNT_MOISTURE & LAB_QUALIFIER & REVIEW_QUALIFIER \\
\hline 2 & USTEST & 0.0385 & $\mathrm{pCi} / \mathrm{L}$ & 0.0174 & 0.0178 & & & & \\
\hline 3 & USTEST & 0.0172 & $\mathrm{pCi} / \mathrm{L}$ & 0.0117 & 0.0119 & & & & \\
\hline 4 & USTEST & -0.0157 & $\mathrm{pCi} / \mathrm{L}$ & 0.0159 & 0.016 & & & U & \\
\hline 5 & USTEST & 0.0168 & $\mathrm{pCi} / \mathrm{L}$ & 0.017 & 0.0171 & & & U & \\
\hline 6 & USTEST & 0.0181 & $\mathrm{pCi} / \mathrm{L}$ & 0.0129 & 0.0131 & & & & \\
\hline 7 & USTEST & 0.00664 & $\mathrm{pCi} / \mathrm{L}$ & 0.00972 & 0.00979 & & & $U$ & \\
\hline 8 & USTEST & -0.0124 & $\mathrm{pCi} / \mathrm{L}$ & 0.0157 & 0.0157 & & & $U$ & \\
\hline 9 & USTEST & 0.00782 & $\mathrm{pCi} / \mathrm{L}$ & 0.0136 & 0.0137 & & & $U$ & \\
\hline 10 & USTEST & 0.0113 & $\mathrm{pCi} / \mathrm{L}$ & 0.0103 & 0.0104 & & & & \\
\hline 11 & ITASRL & 0.0241 & $\mathrm{pCi} / \mathrm{L}$ & 0.0139 & 0.0141 & & & & \\
\hline 12 & ITASRL & 0.000952 & $\mathrm{pCi} / \mathrm{L}$ & 0.0045 & 0.00458 & & & $U$ & \\
\hline 13 & ITASRL & 0.0128 & $\mathrm{pCi} / \mathrm{L}$ & 0.0107 & 0.0108 & & & & \\
\hline 14 & ITASRL & 0.00277 & $\mathrm{pCi} / \mathrm{L}$ & 0.00576 & 0.00583 & & & U & \\
\hline 15 & ITASRL & 0.00652 & $\mathrm{pCi} / \mathrm{L}$ & 0.00782 & 0.0079 & & & U & \\
\hline 16 & ITASRL & 0.0112 & $\mathrm{pCi} / \mathrm{L}$ & 0.0102 & 0.0103 & & & & \\
\hline 17 & ITASRL & 0.00657 & $\mathrm{pCi} / \mathrm{L}$ & 0.00939 & 0.00946 & & & $U$ & \\
\hline 18 & ITASRL & 0.00535 & $\mathrm{pCi} / \mathrm{L}$ & 0.00809 & 0.00811 & & & U & \\
\hline 19 & ITASRL & 0.00696 & $\mathrm{pCi} / \mathrm{L}$ & 0.00826 & 0.00834 & & & U & \\
\hline 20 & ITASRL & 0.00895 & $\mathrm{pCi} / \mathrm{L}$ & 0.00917 & 0.00926 & & & $U$ & \\
\hline 21 & ITASRL & 0.00666 & $\mathrm{pCi} / \mathrm{L}$ & 0.00796 & 0.00804 & & & $U$ & \\
\hline 22 & ITASRL & 0.0104 & $\mathrm{pCi} / \mathrm{L}$ & 0.00956 & 0.00966 & & & & \\
\hline 23 & ITASRL & 0.000628 & $\mathrm{pCi} / \mathrm{L}$ & 0.00385 & 0.00395 & & & $U$ & \\
\hline 24 & ITASRL & 0.000871 & $\mathrm{pCi} / \mathrm{L}$ & 0.00694 & 0.007 & & & U & \\
\hline 25 & ITASRL & 0.00817 & $\mathrm{pCi} / \mathrm{L}$ & 0.00847 & 0.00856 & & & $U$ & \\
\hline 26 & ITASRL & 0.00465 & $\mathrm{pCi} / \mathrm{L}$ & 0.00859 & 0.00865 & & & $U$ & \\
\hline 27 & ITASRL & 0.00998 & $\mathrm{pCi} / \mathrm{L}$ & 0.00965 & 0.0097 & & & & \\
\hline 28 & ITASRL & 0.0183 & $\mathrm{pCi} / \mathrm{L}$ & 0.0154 & 0.0156 & & & & \\
\hline 29 & ITASRL & -0.00000966 & $\mathrm{pCi} / \mathrm{L}$ & 0.00793 & 0.00798 & & & $U$ & \\
\hline 30 & ITASRL & 0.00318 & $\mathrm{pCi} / \mathrm{L}$ & 0.00995 & 0.00999 & & & $U$ & \\
\hline 31 & ITASRL & 0.00218 & $\mathrm{pCi} / \mathrm{L}$ & 0.00823 & 0.00828 & & & U & \\
\hline 32 & ITASRL & -0.0013 & $\mathrm{pCi} / \mathrm{L}$ & 0 & 0.00771 & & & U & \\
\hline 33 & ITASRL & 0.00163 & $\mathrm{pCi} / \mathrm{L}$ & 0.00805 & 0.0081 & & & U & \\
\hline 34 & ITASRL & 0.012 & $\mathrm{pCi} / \mathrm{L}$ & 0.0134 & 0.0135 & & & $U$ & \\
\hline 35 & ITASRL & 0.0181 & $\mathrm{pCi} / \mathrm{L}$ & 0.0143 & 0.0145 & & & & \\
\hline 36 & ITASRL & 0.0127 & $\mathrm{pCi} / \mathrm{L}$ & 0.0161 & 0.0162 & & & U & \\
\hline 37 & ITASRL & 0.0112 & $\mathrm{pCi} / \mathrm{L}$ & 0.00981 & 0.00991 & & & & \\
\hline 38 & ITASRL & 0.0125 & $\mathrm{pCi} / \mathrm{L}$ & 0.0101 & 0.0103 & & & & \\
\hline 39 & ITASRL & 0.013 & $\mathrm{pCi} / \mathrm{L}$ & 0.0108 & 0.011 & & & & \\
\hline
\end{tabular}




\begin{tabular}{|c|c|c|c|c|c|c|c|c|c|}
\hline & $\mathrm{L}$ & $M$ & $\mathrm{~N}$ & $\mathrm{O}$ & $\mathrm{P}$ & $\mathrm{Q}$ & $\mathrm{R}$ & $\mathrm{S}$ & $\mathrm{T}$ \\
\hline 1 & LAB_CODE & VALUE_RPTD & ANAL_UNITS_RPTD & COUNTING_ERROR & TOTAL_ANAL_ERROR & MIN_DETECTABLE_ACTIVITY & PCNT_MOISTURE & LAB_QUALIFIER & REVIEW_QUALIFIER \\
\hline 40 & ITASRL & 0.0175 & $\mathrm{pCi} / \mathrm{L}$ & 0.0125 & 0.0127 & & & & \\
\hline 41 & ITASRL & 0.00389 & $\mathrm{pCi} / \mathrm{L}$ & 0.00709 & 0.00716 & & & U & \\
\hline 42 & ITASRL & 0.0214 & $\mathrm{pCi} / \mathrm{L}$ & 0.0249 & 0.0251 & & & U & \\
\hline 43 & ITASRL & 0.00954 & $\mathrm{pCi} / \mathrm{L}$ & 0.00885 & 0.00895 & & & & \\
\hline 44 & ITASRL & 0.00713 & $\mathrm{pCi} / \mathrm{L}$ & 0.0151 & 0.0152 & & & U & \\
\hline 45 & ITASRL & -0.000668 & $\mathrm{pCi} / \mathrm{L}$ & 0.00721 & 0.00726 & & & U & \\
\hline 46 & ITASRL & 0.00711 & $\mathrm{pCi} / \mathrm{L}$ & 0.0109 & 0.011 & & & U & \\
\hline 47 & ITASRL & 0.0154 & $\mathrm{pCi} / \mathrm{L}$ & 0.0167 & 0.0169 & & & U & \\
\hline 48 & ITASRL & 0.00745 & $\mathrm{pCi} / \mathrm{L}$ & 0.0101 & 0.0102 & & & $U$ & \\
\hline 49 & ITASRL & 0.00209 & $\mathrm{pCi} / \mathrm{L}$ & 0.00676 & 0.00682 & & & U & \\
\hline 50 & ITASRL & 0.00209 & $\mathrm{pCi} / \mathrm{L}$ & 0.00676 & 0.00682 & & & U & \\
\hline 51 & ITASRL & 0.00169 & $\mathrm{pCi} / \mathrm{L}$ & 0.00821 & 0.00826 & & & U & \\
\hline 52 & ITASRL & 0.00367 & $\mathrm{pCi} / \mathrm{L}$ & 0.00965 & 0.0097 & & & U & \\
\hline 53 & ITASRL & 0.00337 & $\mathrm{pCi} / \mathrm{L}$ & 0.0114 & 0.0115 & & & U & \\
\hline 54 & ITASRL & 0.014 & $\mathrm{pCi} / \mathrm{L}$ & 0.0116 & 0.0117 & & & & \\
\hline 55 & ITASRL & 0.00593 & $\mathrm{pCi} / \mathrm{L}$ & 0.00777 & 0.00785 & & & U & \\
\hline 56 & ITASRL & 0.00298 & $\mathrm{pCi} / \mathrm{L}$ & 0.00701 & 0.00708 & & & U & \\
\hline 57 & ITASRL & 0.0105 & $\mathrm{pCi} / \mathrm{L}$ & 0.0111 & 0.0112 & & & $U$ & \\
\hline 58 & ITASRL & 0.0145 & $\mathrm{pCi} / \mathrm{L}$ & 0.012 & 0.0122 & & & & \\
\hline 59 & ITASRL & 0.0126 & $\mathrm{pCi} / \mathrm{L}$ & 0.0139 & 0.014 & & & U & \\
\hline 60 & ITASRL & 0.0113 & $\mathrm{pCi} / \mathrm{L}$ & 0.0122 & 0.0123 & & & U & \\
\hline 61 & ITASRL & 0.00376 & $\mathrm{pCi} / \mathrm{L}$ & 0.00692 & 0.00698 & & & U & \\
\hline 62 & ITASRL & 0.0156 & $\mathrm{pCi} / \mathrm{L}$ & 0.0137 & 0.0138 & & & & \\
\hline 63 & ITASRL & 0.00749 & $\mathrm{pCi} / \mathrm{L}$ & 0.00786 & 0.00795 & & & U & \\
\hline 64 & ITASRL & 0.0122 & $\mathrm{pCi} / \mathrm{L}$ & 0.0102 & 0.0103 & & & & \\
\hline 65 & ITASRL & 0.00693 & $\mathrm{pCi} / \mathrm{L}$ & 0.00882 & 0.00889 & & & U & \\
\hline 66 & ITASRL & 0.00063 & $\mathrm{pCi} / \mathrm{L}$ & 0.00385 & 0.00395 & & & $U$ & \\
\hline 67 & ITASRL & 0.0245 & $\mathrm{pCi} / \mathrm{L}$ & 0.015 & 0.0152 & & & & \\
\hline 68 & ITASRL & 0.00708 & $\mathrm{pCi} / \mathrm{L}$ & 0.00793 & 0.00802 & & & U & \\
\hline 69 & ITASRL & 0.00329 & $\mathrm{pCi} / \mathrm{L}$ & 0.00649 & 0.00656 & & & U & \\
\hline 70 & ITASRL & 0.0135 & $\mathrm{pCi} / \mathrm{L}$ & 0.0108 & 0.011 & & & & \\
\hline 71 & ITASRL & 0.0268 & $\mathrm{pCi} / \mathrm{L}$ & 0.0167 & 0.0169 & & & & \\
\hline 72 & ITASRL & 0.675 & $\mathrm{pCi} / \mathrm{L}$ & 0.0777 & 0.101 & & & & \\
\hline 73 & ITASRL & 0.0105 & $\mathrm{pCi} / \mathrm{L}$ & 0.0105 & 0.0106 & & & U & \\
\hline 74 & ITASRL & 0.00758 & $\mathrm{pCi} / \mathrm{L}$ & 0.00888 & 0.00896 & & & $U$ & \\
\hline 75 & ITASRL & 0.0139 & $\mathrm{pCi} / \mathrm{L}$ & 0.0316 & 0.0316 & & & $U$ & \\
\hline 76 & ITASRL & 0.00222 & $\mathrm{pCi} / \mathrm{L}$ & 0.00576 & 0.00584 & & & U & \\
\hline 77 & ITASRL & 0.014 & $\mathrm{pCi} / \mathrm{L}$ & 0.0164 & 0.0165 & & & $\mathrm{U}$ & \\
\hline
\end{tabular}




\begin{tabular}{|c|c|c|c|c|c|c|c|c|c|}
\hline & $\mathrm{L}$ & $M$ & $\mathrm{~N}$ & $\mathrm{O}$ & $\mathrm{P}$ & $\mathrm{Q}$ & $\mathrm{R}$ & $\mathrm{S}$ & $\mathrm{T}$ \\
\hline 1 & LAB_CODE & VALUE_RPTD & ANAL_UNITS_RPTD & |COUNTING_ERROR| & TOTAL_ANAL_ERROR & MIN_DETECTABLE_ACTIVITY & PCNT_MOISTURE & LAB_QUALIFIER & REVIEW_QUALIFIER \\
\hline 78 & ITASRL & 0.00571 & $\mathrm{pCi} / \mathrm{L}$ & 0.0106 & 0.0106 & & & U & \\
\hline 79 & ITASRL & 0.0136 & $\mathrm{pCi} / \mathrm{L}$ & 0.0105 & 0.0106 & & & & \\
\hline 80 & ITASRL & 0.00202 & $\mathrm{pCi} / \mathrm{L}$ & 0.0119 & 0.012 & & & U & \\
\hline 81 & ITASRL & 0.00666 & $\mathrm{pCi} / \mathrm{L}$ & 0.00796 & 0.00804 & & & U & \\
\hline 82 & ITASRL & 0.0143 & $\mathrm{pCi} / \mathrm{L}$ & 0.0156 & 0.0157 & & & U & \\
\hline 83 & ITASRL & 0.00203 & $\mathrm{pCi} / \mathrm{L}$ & 0.00612 & 0.00618 & & & $\mathrm{U}$ & \\
\hline 84 & ITASRL & 0.0134 & $\mathrm{pCi} / \mathrm{L}$ & 0.0118 & 0.0119 & & & & \\
\hline 85 & ITASRL & 0.0479 & $\mathrm{pCi} / \mathrm{L}$ & 0.0231 & 0.0236 & & & & \\
\hline 86 & ITASRL & 0.00707 & $\mathrm{pCi} / \mathrm{L}$ & 0.00919 & 0.00926 & & & $U$ & \\
\hline 87 & ITASRL & 0.00914 & $\mathrm{pCi} / \mathrm{L}$ & 0.00925 & 0.00934 & & & U & \\
\hline 88 & ITASRL & 0.00652 & $\mathrm{pCi} / \mathrm{L}$ & 0.00886 & 0.00893 & & & U & \\
\hline 89 & ITASRL & 0.00159 & $\mathrm{pCi} / \mathrm{L}$ & 0.00733 & 0.00738 & & & $\mathrm{U}$ & \\
\hline 90 & ITASRL & 0.00407 & $\mathrm{pCi} / \mathrm{L}$ & 0.00734 & 0.00741 & & & U & \\
\hline 91 & ITASRL & 0.00183 & $\mathrm{pCi} / \mathrm{L}$ & 0.00575 & 0.00582 & & & U & \\
\hline 92 & ITASRL & 0.0255 & $\mathrm{pCi} / \mathrm{L}$ & 0.016 & 0.0163 & & & & \\
\hline 93 & ITASRL & 0.00806 & $\mathrm{pCi} / \mathrm{L}$ & 0.00936 & 0.00944 & & & U & \\
\hline 94 & ITASRL & 0.017 & $\mathrm{pCi} / \mathrm{L}$ & 0.0183 & 0.0184 & & & U & \\
\hline 95 & ITASRL & 0.034 & $\mathrm{pCi} / \mathrm{L}$ & 0.0189 & 0.0192 & & & & \\
\hline 96 & ITASRL & 0.0143 & $\mathrm{pCi} / \mathrm{L}$ & 0.0118 & 0.0119 & & & & \\
\hline 97 & ITASRL & 0.00794 & $\mathrm{pCi} / \mathrm{L}$ & 0.00912 & 0.0092 & & & U & \\
\hline 98 & ITASRL & 0.0218 & $\mathrm{pCi} / \mathrm{L}$ & 0.0136 & 0.0138 & & & & \\
\hline 99 & ITASRL & 0.0382 & $\mathrm{pCi} / \mathrm{L}$ & 0.0186 & 0.019 & & & & \\
\hline 100 & ITASRL & 0.00642 & $\mathrm{pCi} / \mathrm{L}$ & 0.00772 & 0.0078 & & & U & \\
\hline 101 & ITASRL & 0.00796 & $\mathrm{pCi} / \mathrm{L}$ & 0.00828 & 0.00837 & & & U & \\
\hline 102 & ITASRL & 0.0139 & $\mathrm{pCi} / \mathrm{L}$ & 0.012 & 0.0121 & & & & \\
\hline 103 & ITASRL & 0.0158 & $\mathrm{pCi} / \mathrm{L}$ & 0.0127 & 0.0129 & & & & \\
\hline 104 & ITASRL & 0.0138 & $\mathrm{pCi} / \mathrm{L}$ & 0.0159 & 0.016 & & & $U$ & \\
\hline 105 & ITASRL & 0.025 & $\mathrm{pCi} / \mathrm{L}$ & 0.015 & 0.0153 & & & & \\
\hline 106 & ITASRL & 0.0154 & $\mathrm{pCi} / \mathrm{L}$ & 0.0134 & 0.0135 & & & & \\
\hline 107 & ITASRL & 0.0152 & $\mathrm{pCi} / \mathrm{L}$ & 0.0132 & 0.0133 & & & & \\
\hline 108 & ITASRL & 0.0144 & $\mathrm{pCi} / \mathrm{L}$ & 0.0126 & 0.0127 & & & & \\
\hline 109 & ITASRL & 0.00986 & $\mathrm{pCi} / \mathrm{L}$ & 0.00989 & 0.00998 & & & U & \\
\hline 110 & ITASRL & 0.00999 & $\mathrm{pCi} / \mathrm{L}$ & 0.0109 & 0.011 & & & $U$ & \\
\hline 111 & ITASRL & 0.00897 & $\mathrm{pCi} / \mathrm{L}$ & 0.00993 & 0.01 & & & $U$ & \\
\hline 112 & ITASRL & 0.00616 & $\mathrm{pCi} / \mathrm{L}$ & 0.00918 & 0.00925 & & & $U$ & \\
\hline 113 & ITASRL & 0.0095 & $\mathrm{pCi} / \mathrm{L}$ & 0.0104 & 0.0105 & & & $U$ & \\
\hline 114 & ITASRL & 0.0104 & $\mathrm{pCi} / \mathrm{L}$ & 0.0102 & 0.0103 & & & & \\
\hline 115 & ITASRL & 0.00183 & $\mathrm{pCi} / \mathrm{L}$ & 0.00647 & 0.00653 & & & $\mathrm{U}$ & \\
\hline
\end{tabular}




\begin{tabular}{|c|c|c|c|c|c|c|c|c|c|}
\hline & $\mathrm{L}$ & $\mathrm{M}$ & $\mathrm{N}$ & $\mathrm{O}$ & $\mathrm{P}$ & $\mathrm{Q}$ & $\mathrm{R}$ & $\mathrm{S}$ & $\mathrm{T}$ \\
\hline 1 & LAB_CODE & VALUE_RPTD & ANAL_UNITS_RPTD & |COUNTING_ERROR| & TOTAL_ANAL_ERROR & MIN_DETECTABLE_ACTIVITY & PCNT_MOISTURE & LAB_QUALIFIER & REVIEW_QUALIFIER \\
\hline 116 & ITASRL & 0.00646 & $\mathrm{pCi} / \mathrm{L}$ & 0.00852 & 0.0086 & & & U & \\
\hline 117 & ITASRL & 0.0102 & $\mathrm{pCi} / \mathrm{L}$ & 0.0103 & 0.0104 & & & U & \\
\hline 118 & ITASRL & 0.0117 & $\mathrm{pCi} / \mathrm{L}$ & 0.0106 & 0.0107 & & & & \\
\hline 119 & ITASRL & 0.00673 & $\mathrm{pCi} / \mathrm{L}$ & 0.0094 & 0.00947 & & & U & \\
\hline 120 & ITASRL & 0.0104 & $\mathrm{pCi} / \mathrm{L}$ & 0.0102 & 0.0103 & & & & \\
\hline \begin{tabular}{|l|}
121 \\
\end{tabular} & ITASRL & 0.0127 & $\mathrm{pCi} / \mathrm{L}$ & 0.0105 & 0.0107 & & & & \\
\hline 122 & ITASRL & 0.0643 & $\mathrm{pCi} / \mathrm{L}$ & 0.0587 & 0.0592 & & & & \\
\hline 123 & ITASRL & 0.0151 & $\mathrm{pCi} / \mathrm{L}$ & 0.0122 & 0.0123 & & & & \\
\hline \begin{tabular}{|l|}
124 \\
\end{tabular} & ITASRL & 0.00627 & $\mathrm{pCi} / \mathrm{L}$ & 0.00874 & 0.00881 & & & $U$ & \\
\hline \begin{tabular}{|l|}
125 \\
\end{tabular} & ITASRL & 0.00962 & $\mathrm{pCi} / \mathrm{L}$ & 0.0103 & 0.0104 & & & U & \\
\hline 126 & ITASRL & 0.00461 & $\mathrm{pCi} / \mathrm{L}$ & 0.0104 & 0.0105 & & & $U$ & \\
\hline 127 & ITASRL & 0.0119 & $\mathrm{pCi} / \mathrm{L}$ & 0.0118 & 0.0119 & & & & \\
\hline \begin{tabular}{|l|}
128 \\
\end{tabular} & ITASRL & 0.00947 & $\mathrm{pCi} / \mathrm{L}$ & 0.0106 & 0.0107 & & & U & \\
\hline \begin{tabular}{|l|}
129 \\
\end{tabular} & ITASRL & 0.0116 & $\mathrm{pCi} / \mathrm{L}$ & 0.00975 & 0.00987 & & & & \\
\hline 130 & ITASRL & 0.0078 & $\mathrm{pCi} / \mathrm{L}$ & 0.00879 & 0.00887 & & & U & \\
\hline \begin{tabular}{|l|}
131 \\
\end{tabular} & ITASRL & 0.00466 & $\mathrm{pCi} / \mathrm{L}$ & 0.00774 & 0.0078 & & & U & \\
\hline 132 & ITASRL & 0.00772 & $\mathrm{pCi} / \mathrm{L}$ & 0.00902 & 0.0091 & & & U & \\
\hline 133 & ITASRL & 0.0099 & $\mathrm{pCi} / \mathrm{L}$ & 0.00993 & 0.01 & & & $U$ & \\
\hline 134 & ITASRL & 0.00884 & $\mathrm{pCi} / \mathrm{L}$ & 0.0122 & 0.0122 & & & U & \\
\hline 135 & ITASRL & 0.00654 & $\mathrm{pCi} / \mathrm{L}$ & 0.00773 & 0.00782 & & & U & \\
\hline 136 & ITASRL & 0.00763 & $\mathrm{pCi} / \mathrm{L}$ & 0.0098 & 0.00988 & & & $U$ & \\
\hline 137 & ITASRL & 0.00808 & $\mathrm{pCi} / \mathrm{L}$ & 0.0105 & 0.0105 & & & $U$ & \\
\hline 138 & ITASRL & -0.0013 & $\mathrm{pCi} / \mathrm{L}$ & 0.0085 & 0.00855 & & & U & \\
\hline 139 & ITASRL & 0.00287 & $\mathrm{pCi} / \mathrm{L}$ & 0.00834 & 0.00839 & & & U & \\
\hline 140 & ITASRL & 0.0147 & $\mathrm{pCi} / \mathrm{L}$ & 0.012 & 0.0122 & & & & \\
\hline 141 & ITASRL & 0.0111 & $\mathrm{pCi} / \mathrm{L}$ & 0.0101 & 0.0102 & & & & \\
\hline 142 & ITASRL & 0.00602 & $\mathrm{pCi} / \mathrm{L}$ & 0.0111 & 0.0111 & & & U & \\
\hline 143 & ITASRL & 0.0142 & $\mathrm{pCi} / \mathrm{L}$ & 0.0137 & 0.0138 & & & & \\
\hline \begin{tabular}{|l|}
144 \\
\end{tabular} & ITASRL & 0.00416 & $\mathrm{pCi} / \mathrm{L}$ & 0.00772 & 0.00779 & & & U & \\
\hline 145 & ITASRL & 0.001 & $\mathrm{pCi} / \mathrm{L}$ & 0.00897 & 0.00901 & & & U & \\
\hline 146 & ITASRL & 0.0218 & $\mathrm{pCi} / \mathrm{L}$ & 0.0139 & 0.0141 & & & & \\
\hline 147 & ITASRL & 0.00247 & $\mathrm{pCi} / \mathrm{L}$ & 0.00693 & 0.00699 & & & U & \\
\hline 148 & ITASRL & 0.00077 & $\mathrm{pCi} / \mathrm{L}$ & 0.00413 & 0.00422 & & & $U$ & \\
\hline 149 & ITASRL & 0.0167 & $\mathrm{pCi} / \mathrm{L}$ & 0.0127 & 0.0129 & & & & \\
\hline 150 & ITASRL & 0.00789 & $\mathrm{pCi} / \mathrm{L}$ & 0.00821 & 0.0083 & & & $U$ & \\
\hline \begin{tabular}{|l|}
151 \\
\end{tabular} & ITASRL & 0.00521 & $\mathrm{pCi} / \mathrm{L}$ & 0.0089 & 0.00896 & & & $U$ & \\
\hline 152 & ITASRL & -0.0013 & $\mathrm{pCi} / \mathrm{L}$ & 0.0066 & 0.00665 & & & $U$ & \\
\hline 153 & ITASRL & 0.00288 & $\mathrm{pCi} / \mathrm{L}$ & 0.00865 & 0.0087 & & & U & \\
\hline
\end{tabular}




\begin{tabular}{|c|c|c|c|c|c|c|c|c|c|}
\hline & $\mathrm{L}$ & $\mathrm{M}$ & $\mathrm{N}$ & $\mathrm{O}$ & $\mathrm{P}$ & $\mathrm{Q}$ & $\mathrm{R}$ & $\mathrm{S}$ & $\mathrm{T}$ \\
\hline 1 & LAB_CODE & VALUE_RPTD & ANAL_UNITS_RPTD & |COUNTING_ERROR| & TOTAL_ANAL_ERROR & MIN_DETECTABLE_ACTIVITY & PCNT_MOISTURE & LAB_QUALIFIER & REVIEW_QUALIFIER \\
\hline 154 & ITASRL & 0.0104 & $\mathrm{pCi} / \mathrm{L}$ & 0.0137 & 0.0138 & & & $U$ & \\
\hline 155 & ITASRL & 0.0156 & $\mathrm{pCi} / \mathrm{L}$ & 0.0119 & 0.0121 & & & & \\
\hline 156 & ITASRL & 0.00505 & $\mathrm{pCi} / \mathrm{L}$ & 0.00733 & 0.0074 & & & $U$ & \\
\hline 157 & ITASRL & 0.00116 & $\mathrm{pCi} / \mathrm{L}$ & 0.00624 & 0.0063 & & & $U$ & \\
\hline 158 & ITASRL & 0.0192 & $\mathrm{pCi} / \mathrm{L}$ & 0.0155 & 0.0156 & & & & \\
\hline 159 & ITASRL & 0.00425 & $\mathrm{pCi} / \mathrm{L}$ & 0.00758 & 0.00765 & & & $\mathrm{U}$ & \\
\hline 160 & ITASRL & 0.00181 & $\mathrm{pCi} / \mathrm{L}$ & 0.0057 & 0.00578 & & & $U$ & \\
\hline 161 & ITASRL & 0.0077 & $\mathrm{pCi} / \mathrm{L}$ & 0.0126 & 0.0126 & & & U & \\
\hline 162 & ITASRL & 0.000378 & $\mathrm{pCi} / \mathrm{L}$ & 0.00659 & 0.00665 & & & U & \\
\hline 163 & ITASRL & 0.00339 & $\mathrm{pCi} / \mathrm{L}$ & 0.00776 & 0.00782 & & & $U$ & \\
\hline 164 & ITASRL & 0.0284 & $\mathrm{pCi} / \mathrm{L}$ & 0.0176 & 0.0179 & & & & \\
\hline 165 & ITASRL & 0.0064 & $\mathrm{pCi} / \mathrm{L}$ & 0.00948 & 0.00954 & & & $U$ & \\
\hline \begin{tabular}{|l|}
166 \\
\end{tabular} & ITASRL & 0.00584 & $\mathrm{pCi} / \mathrm{L}$ & 0.00998 & 0.01 & & & $U$ & \\
\hline 167 & ITASRL & 0.00404 & $\mathrm{pCi} / \mathrm{L}$ & 0.00884 & 0.0089 & & & U & \\
\hline 168 & ITASRL & 0.0017 & $\mathrm{pCi} / \mathrm{L}$ & 0.0076 & 0.00765 & & & $U$ & \\
\hline 169 & ITASRL & 0.00402 & $\mathrm{pCi} / \mathrm{L}$ & 0.011 & 0.0111 & & & $U$ & \\
\hline 170 & ITASRL & 0.0108 & $\mathrm{pCi} / \mathrm{L}$ & 0.0108 & 0.0109 & & & $U$ & \\
\hline 171 & ITASRL & 0.00908 & $\mathrm{pCi} / \mathrm{L}$ & 0.0142 & 0.0142 & & & $\mathrm{U}$ & \\
\hline 172 & ITASRL & 0.00261 & $\mathrm{pCi} / \mathrm{L}$ & 0.0107 & 0.0107 & & & $U$ & \\
\hline 173 & ITASRL & 0.00367 & $\mathrm{pCi} / \mathrm{L}$ & 0.00892 & 0.00897 & & & $U$ & \\
\hline 174 & ITASRL & 0.00783 & $\mathrm{pCi} / \mathrm{L}$ & 0.01 & 0.0101 & & & U & \\
\hline 175 & ITASRL & 0.0141 & $\mathrm{pCi} / \mathrm{L}$ & 0.0123 & 0.0124 & & & & \\
\hline 176 & ITASRL & 0.00718 & $\mathrm{pCi} / \mathrm{L}$ & 0.0104 & 0.0105 & & & $U$ & \\
\hline 177 & ITASRL & 0.00687 & $\mathrm{pCi} / \mathrm{L}$ & 0.00925 & 0.00932 & & & $U$ & \\
\hline 178 & ITASRL & 0.00399 & $\mathrm{pCi} / \mathrm{L}$ & 0.00949 & 0.00955 & & & $U$ & \\
\hline 179 & ITASRL & 0.0182 & $\mathrm{pCi} / \mathrm{L}$ & 0.0156 & 0.0157 & & & & \\
\hline 180 & ITASRL & 0.00645 & $\mathrm{pCi} / \mathrm{L}$ & 0.0101 & 0.0102 & & & U & \\
\hline 181 & ITASRL & 0.00465 & $\mathrm{pCi} / \mathrm{L}$ & 0.00687 & 0.00694 & & & U & \\
\hline 182 & ITASRL & 0.012 & $\mathrm{pCi} / \mathrm{L}$ & 0.0127 & 0.0128 & & & $U$ & \\
\hline 183 & ITASRL & 0.022 & $\mathrm{pCi} / \mathrm{L}$ & 0.0154 & 0.0156 & & & & \\
\hline 184 & ITASRL & 0.00656 & $\mathrm{pCi} / \mathrm{L}$ & 0.0115 & 0.0116 & & & U & \\
\hline 185 & ITASRL & 0.0241 & $\mathrm{pCi} / \mathrm{L}$ & 0.0178 & 0.018 & & & & \\
\hline 186 & ITASRL & 0.00308 & $\mathrm{pCi} / \mathrm{L}$ & 0.00725 & 0.00731 & & & $U$ & \\
\hline 187 & ITASRL & 0.00449 & $\mathrm{pCi} / \mathrm{L}$ & 0.00875 & 0.00881 & & & U & \\
\hline \begin{tabular}{|l|}
188 \\
\end{tabular} & ITASRL & 0.00756 & $\mathrm{pCi} / \mathrm{L}$ & 0.0115 & 0.0116 & & & $U$ & \\
\hline 189 & ITASRL & 0.0105 & $\mathrm{pCi} / \mathrm{L}$ & 0.0103 & 0.0104 & & & & \\
\hline 190 & ITASRL & 0.0149 & $\mathrm{pCi} / \mathrm{L}$ & 0.0128 & 0.0129 & & & & \\
\hline 191 & ITASRL & 0.0261 & $\mathrm{pCi} / \mathrm{L}$ & 0.0453 & 0.0455 & & & $\mathrm{U}$ & \\
\hline
\end{tabular}




\begin{tabular}{|c|c|c|c|c|c|c|c|c|c|}
\hline & $\mathrm{L}$ & $\mathrm{M}$ & $\mathrm{N}$ & $\mathrm{O}$ & $\mathrm{P}$ & $\mathrm{Q}$ & $\mathrm{R}$ & $\mathrm{S}$ & $\mathrm{T}$ \\
\hline 1 & LAB_CODE & VALUE_RPTD & ANAL_UNITS_RPTD & COUNTING_ERROR & TOTAL_ANAL_ERROR & MIN_DETECTABLE_ACTIVITY & PCNT_MOISTURE & LAB_QUALIFIER & REVIEW_QUALIFIER \\
\hline 192 & ITASRL & 0.018 & $\mathrm{pCi} / \mathrm{L}$ & 0.0136 & 0.0138 & & & & \\
\hline 193 & ITASRL & 0.0343 & $\mathrm{pCi} / \mathrm{L}$ & 0.0184 & 0.0187 & & & & \\
\hline 194 & ITASRL & 0.00522 & $\mathrm{pCi} / \mathrm{L}$ & 0.00847 & 0.00853 & & & U & \\
\hline 195 & ITASRL & 0.00688 & $\mathrm{pCi} / \mathrm{L}$ & 0.00898 & 0.00905 & & & U & \\
\hline 196 & ITASRL & 0.00252 & $\mathrm{pCi} / \mathrm{L}$ & 0.00791 & 0.00797 & & & $U$ & \\
\hline 197 & ITASRL & 0.00958 & $\mathrm{pCi} / \mathrm{L}$ & 0.0105 & 0.0106 & & & $U$ & \\
\hline 198 & ITASRL & 0.0127 & $\mathrm{pCi} / \mathrm{L}$ & 0.012 & 0.0121 & & & & \\
\hline 199 & ITASRL & 0.0141 & $\mathrm{pCi} / \mathrm{L}$ & 0.0136 & 0.0137 & & & & \\
\hline 200 & ITASRL & 0.0221 & $\mathrm{pCi} / \mathrm{L}$ & 0.0185 & 0.0186 & & & & \\
\hline 201 & ITASRL & 0.0186 & $\mathrm{pCi} / \mathrm{L}$ & 0.0131 & 0.0133 & & & & \\
\hline 202 & ITASRL & 0.0159 & $\mathrm{pCi} / \mathrm{L}$ & 0.0127 & 0.0128 & & & & \\
\hline 203 & ITASRL & 0.0197 & $\mathrm{pCi} / \mathrm{L}$ & 0.0138 & 0.0139 & & & & \\
\hline 204 & ITASRL & 0.00867 & $\mathrm{pCi} / \mathrm{L}$ & 0.0102 & 0.0103 & & & U & \\
\hline 205 & ITASRL & 0.019 & $\mathrm{pCi} / \mathrm{L}$ & 0.0133 & 0.0135 & & & & \\
\hline 206 & ITASRL & 0.00937 & $\mathrm{pCi} / \mathrm{L}$ & 0.00946 & 0.00955 & & & $U$ & \\
\hline 207 & ITASRL & 0.00878 & $\mathrm{pCi} / \mathrm{L}$ & 0.0118 & 0.0119 & & & U & \\
\hline 208 & ITASRL & 0.00622 & $\mathrm{pCi} / \mathrm{L}$ & 0.0124 & 0.0125 & & & U & \\
\hline 209 & ITASRL & 0.000243 & $\mathrm{pCi} / \mathrm{L}$ & 0.0125 & 0.0125 & & & $U$ & \\
\hline 210 & ITASRL & 0.0175 & $\mathrm{pCi} / \mathrm{L}$ & 0.0148 & 0.015 & & & & \\
\hline 211 & ITASRL & 0.0257 & $\mathrm{pCi} / \mathrm{L}$ & 0.019 & 0.0192 & & & & \\
\hline 212 & ITASRL & 0.000912 & $\mathrm{pCi} / \mathrm{L}$ & 0.00869 & 0.00874 & & & $U$ & \\
\hline 213 & ITASRL & 0.00353 & $\mathrm{pCi} / \mathrm{L}$ & 0.01 & 0.0101 & & & $U$ & \\
\hline 214 & ITASRL & 0.00156 & $\mathrm{pCi} / \mathrm{L}$ & 0.0134 & 0.0134 & & & $U$ & \\
\hline 215 & ITASRL & 0.0131 & $\mathrm{pCi} / \mathrm{L}$ & 0.0147 & 0.0148 & & & $U$ & \\
\hline 216 & ITASRL & 0.00924 & $\mathrm{pCi} / \mathrm{L}$ & 0.0116 & 0.0116 & & & $U$ & \\
\hline 217 & ITASRL & -0.000449 & $\mathrm{pCi} / \mathrm{L}$ & 0.00688 & 0.00694 & & & U & \\
\hline 218 & ITASRL & 0.00729 & $\mathrm{pCi} / \mathrm{L}$ & 0.0106 & 0.0107 & & & U & \\
\hline 219 & ITASRL & 0.0296 & $\mathrm{pCi} / \mathrm{L}$ & 0.033 & 0.0332 & & & $U$ & \\
\hline 220 & ITASRL & -0.00183 & $\mathrm{pCi} / \mathrm{L}$ & 0.00654 & 0.0066 & & & $U$ & \\
\hline 221 & ITASRL & 0.0189 & $\mathrm{pCi} / \mathrm{L}$ & 0.0149 & 0.015 & & & & \\
\hline 222 & ITASRL & 0.0103 & $\mathrm{pCi} / \mathrm{L}$ & 0.0202 & 0.0202 & & & $U$ & \\
\hline 223 & ITASRL & 0.00491 & $\mathrm{pCi} / \mathrm{L}$ & 0.00877 & 0.00884 & & & U & \\
\hline 224 & ITASRL & 0.0236 & $\mathrm{pCi} / \mathrm{L}$ & 0.0149 & 0.0151 & & & & \\
\hline 225 & ITASRL & -0.000505 & $\mathrm{pCi} / \mathrm{L}$ & 0.00643 & 0.00648 & & & $U$ & \\
\hline 226 & ITASRL & -0.000265 & $\mathrm{pCi} / \mathrm{L}$ & 0.00837 & 0.00842 & & & $U$ & \\
\hline 227 & ITASRL & 0.011 & $\mathrm{pCi} / \mathrm{L}$ & 0.0144 & 0.0145 & & & $\mathrm{U}$ & \\
\hline 228 & ITASRL & 0.00768 & $\mathrm{pCi} / \mathrm{L}$ & 0.00986 & 0.00993 & & & $U$ & \\
\hline 229 & ITASRL & & & & & & & U & \\
\hline
\end{tabular}




\begin{tabular}{|c|c|c|c|c|c|c|c|c|c|}
\hline & $\mathrm{L}$ & $\mathrm{M}$ & $\mathrm{N}$ & $\mathrm{O}$ & $\mathrm{P}$ & $\mathrm{Q}$ & $\mathrm{R}$ & $\mathrm{S}$ & $\mathrm{T}$ \\
\hline 1 & LAB_CODE & VALUE_RPTD & ANAL_UNITS_RPTD & COUNTING_ERROR & TOTAL_ANAL_ERROR & MIN_DETECTABLE_ACTIVITY & PCNT_MOISTURE & LAB_QUALIFIER & REVIEW_QUALIFIER \\
\hline 230 & ITASRL & 0.00825 & $\mathrm{pCi} / \mathrm{L}$ & 0.0108 & 0.0109 & & & U & \\
\hline 231 & ITASRL & 0.00337 & $\mathrm{pCi} / \mathrm{L}$ & 0.0102 & 0.0103 & & & U & \\
\hline 232 & ITASRL & 0.0107 & $\mathrm{pCi} / \mathrm{L}$ & 0.0114 & 0.0114 & & & U & \\
\hline 233 & ITASRL & 0.00669 & $\mathrm{pCi} / \mathrm{L}$ & 0.0117 & 0.0118 & & & U & \\
\hline 234 & ITASRL & 0.0129 & $\mathrm{pCi} / \mathrm{L}$ & 0.0151 & 0.0152 & & & $U$ & \\
\hline 235 & ITASRL & 0.0136 & $\mathrm{pCi} / \mathrm{L}$ & 0.0119 & 0.012 & & & & \\
\hline 236 & ITASRL & 0.0121 & $\mathrm{pCi} / \mathrm{L}$ & 0.0118 & 0.0119 & & & & \\
\hline \begin{tabular}{|l|}
237 \\
\end{tabular} & ITASRL & 0.0216 & $\mathrm{pCi} / \mathrm{L}$ & 0.0151 & 0.0153 & & & & \\
\hline 238 & ITASRL & 0.00647 & $\mathrm{pCi} / \mathrm{L}$ & 0.0101 & 0.0102 & & & $U$ & \\
\hline 239 & ITASRL & 0.013 & $\mathrm{pCi} / \mathrm{L}$ & 0.0135 & 0.0136 & & & U & \\
\hline 240 & ITASRL & 0.00769 & $\mathrm{pCi} / \mathrm{L}$ & 0.0117 & 0.0118 & & & $U$ & \\
\hline 241 & ITASRL & 0.00787 & $\mathrm{pCi} / \mathrm{L}$ & 0.0113 & 0.0114 & & & U & \\
\hline \begin{tabular}{|l|}
242 \\
\end{tabular} & ITASRL & 0.00974 & $\mathrm{pCi} / \mathrm{L}$ & 0.0129 & 0.013 & & & U & \\
\hline 243 & ITASRL & 0.0059 & $\mathrm{pCi} / \mathrm{L}$ & 0.00935 & 0.00942 & & & U & \\
\hline 244 & ITASRL & 0.00391 & $\mathrm{pCi} / \mathrm{L}$ & 0.00935 & 0.0094 & & & U & \\
\hline 245 & ITASRL & 0.00332 & $\mathrm{pCi} / \mathrm{L}$ & 0.00848 & 0.00853 & & & U & \\
\hline 246 & ITASRL & 0.0148 & $\mathrm{pCi} / \mathrm{L}$ & 0.0144 & 0.0145 & & & & \\
\hline 247 & ITASRL & 0.00509 & $\mathrm{pCi} / \mathrm{L}$ & 0.012 & 0.012 & & & U & \\
\hline 248 & ITASRL & 0.01 & $\mathrm{pCi} / \mathrm{L}$ & 0.0113 & 0.0114 & & & U & \\
\hline 249 & ITASRL & 0.0133 & $\mathrm{pCi} / \mathrm{L}$ & 0.0125 & 0.0126 & & & & \\
\hline 250 & ITASRL & 0.00494 & $\mathrm{pCi} / \mathrm{L}$ & 0.0112 & 0.0113 & & & $U$ & \\
\hline 251 & ITASRL & 0.00409 & $\mathrm{pCi} / \mathrm{L}$ & 0.00891 & 0.00897 & & & $U$ & \\
\hline 252 & ITASRL & 0.01 & $\mathrm{pCi} / \mathrm{L}$ & 0.0235 & 0.0235 & & & U & \\
\hline 253 & ITASRL & 0.00873 & $\mathrm{pCi} / \mathrm{L}$ & 0.0106 & 0.0107 & & & U & \\
\hline 254 & ITASRL & 0.0154 & $\mathrm{pCi} / \mathrm{L}$ & 0.0132 & 0.0133 & & & & \\
\hline 255 & ITASRL & 0.00871 & $\mathrm{pCi} / \mathrm{L}$ & 0.0112 & 0.0113 & & & U & \\
\hline 256 & ITASRL & 0.0179 & $\mathrm{pCi} / \mathrm{L}$ & 0.0154 & 0.0156 & & & & \\
\hline 257 & ITASRL & 0.0141 & $\mathrm{pCi} / \mathrm{L}$ & 0.0123 & 0.0125 & & & & \\
\hline 258 & ITASRL & 0.00812 & $\mathrm{pCi} / \mathrm{L}$ & 0.00998 & 0.0101 & & & U & \\
\hline 259 & ITASRL & 0.00795 & $\mathrm{pCi} / \mathrm{L}$ & 0.0129 & 0.013 & & & U & \\
\hline 260 & ITASRL & 0.00925 & $\mathrm{pCi} / \mathrm{L}$ & 0.0118 & 0.0119 & & & $U$ & \\
\hline 261 & ITASRL & 0.0263 & $\mathrm{pCi} / \mathrm{L}$ & 0.0173 & 0.0175 & & & & \\
\hline 262 & ITASRL & 0.0153 & $\mathrm{pCi} / \mathrm{L}$ & 0.0136 & 0.0137 & & & & \\
\hline 263 & ITASRL & 0.0021 & $\mathrm{pCi} / \mathrm{L}$ & 0.00862 & 0.00867 & & & $U$ & \\
\hline 264 & ITASRL & 0.00531 & $\mathrm{pCi} / \mathrm{L}$ & 0.00999 & 0.01 & & & $U$ & \\
\hline 265 & ITASRL & 0.00635 & $\mathrm{pCi} / \mathrm{L}$ & 0.0116 & 0.0116 & & & $U$ & \\
\hline 266 & ITASRL & 0.000243 & $\mathrm{pCi} / \mathrm{L}$ & 0.00553 & 0.00559 & & & $U$ & \\
\hline 267 & ITASRL & -0.0022 & $\mathrm{pCi} / \mathrm{L}$ & 0.00777 & 0.00782 & & & U & \\
\hline
\end{tabular}

Page 29 


\begin{tabular}{|c|c|c|c|c|c|c|c|c|c|}
\hline & $\mathrm{L}$ & $\mathrm{M}$ & $\mathrm{N}$ & $\mathrm{O}$ & $P$ & $\mathrm{Q}$ & $\mathrm{R}$ & $\mathrm{S}$ & $\mathrm{T}$ \\
\hline 1 & LAB_CODE & VALUE_RPTD & ANAL_UNITS_RPTD & COUNTING_ERROR| & TOTAL_ANAL_ERROR & MIN_DETECTABLE_ACTIVITY & PCNT_MOISTURE & LAB_QUALIFIER & REVIEW_QUALIFIER \\
\hline 268 & ITASRL & -0.00085 & $\mathrm{pCi} / \mathrm{L}$ & 0.00904 & 0.00908 & & & $U$ & \\
\hline 269 & ITASRL & 0.0237 & $\mathrm{pCi} / \mathrm{L}$ & 0.0165 & 0.0167 & & & & \\
\hline 270 & ITASRL & 0.00554 & $\mathrm{pCi} / \mathrm{L}$ & 0.0123 & 0.0123 & & & U & \\
\hline 271 & ITASRL & 0.00843 & $\mathrm{pCi} / \mathrm{L}$ & 0.00961 & 0.00969 & & & $U$ & \\
\hline 272 & ITASRL & 0.00946 & $\mathrm{pCi} / \mathrm{L}$ & 0.0104 & 0.0105 & & & $U$ & \\
\hline 273 & ITASRL & 0.0168 & $\mathrm{pCi} / \mathrm{L}$ & 0.0145 & 0.0147 & & & & \\
\hline 274 & ITASRL & 0.00837 & $\mathrm{pCi} / \mathrm{L}$ & 0.0135 & 0.0136 & & & $U$ & \\
\hline 275 & ITASRL & 0.0144 & $\mathrm{pCi} / \mathrm{L}$ & 0.0155 & 0.0156 & & & $U$ & \\
\hline 276 & ITASRL & 0.00737 & $\mathrm{pCi} / \mathrm{L}$ & 0.0131 & 0.0132 & & & $U$ & \\
\hline 277 & ITASRL & 0.0159 & $\mathrm{pCi} / \mathrm{L}$ & 0.0153 & 0.0154 & & & & \\
\hline 278 & ITASRL & 0.00391 & $p C i / L$ & 0.00957 & 0.00962 & & & $U$ & \\
\hline 279 & ITASRL & 0.0104 & $\mathrm{pCi} / \mathrm{L}$ & 0.0137 & 0.0138 & & & $U$ & \\
\hline 280 & ITASRL & -0.021 & $\mathrm{pCi} / \mathrm{L}$ & 0.0237 & 0.0238 & & & $U$ & \\
\hline 281 & ITASRL & 0.0177 & $\mathrm{pCi} / \mathrm{L}$ & 0.0367 & 0.0368 & & & $U$ & \\
\hline 282 & ITASRL & 0.0145 & $\mathrm{pCi} / \mathrm{L}$ & 0.0185 & 0.0186 & & & U & \\
\hline 283 & ITASRL & 0.00884 & $\mathrm{pCi} / \mathrm{L}$ & 0.0115 & 0.0116 & & & $U$ & \\
\hline 284 & ITASRL & 0.0314 & $\mathrm{pCi} / \mathrm{L}$ & 0.023 & 0.0233 & & & & \\
\hline 285 & ITASRL & 0.00861 & $\mathrm{pCi} / \mathrm{L}$ & 0.0159 & 0.016 & & & $U$ & \\
\hline 286 & ITASRL & 0.0181 & $\mathrm{pCi} / \mathrm{L}$ & 0.0172 & 0.0174 & & & & \\
\hline 287 & ITASRL & 0.00738 & $\mathrm{pCi} / \mathrm{L}$ & 0.0156 & 0.0156 & & & $U$ & \\
\hline 288 & ITASRL & 0.0219 & $\mathrm{pCi} / \mathrm{L}$ & 0.0155 & 0.0157 & & & & \\
\hline 289 & ITASRL & 0.0177 & $\mathrm{pCi} / \mathrm{L}$ & 0.015 & 0.0151 & & & & \\
\hline 290 & ITASRL & 0.00917 & $\mathrm{pCi} / \mathrm{L}$ & 0.0105 & 0.0105 & & & $U$ & \\
\hline 291 & ITASRL & 0.0229 & $\mathrm{pCi} / \mathrm{L}$ & 0.0145 & 0.0147 & & & & \\
\hline 292 & ITASRL & 0.0153 & $\mathrm{pCi} / \mathrm{L}$ & 0.0134 & 0.0135 & & & & \\
\hline 293 & ITASRL & 0.00536 & $\mathrm{pCi} / \mathrm{L}$ & 0.00755 & 0.00762 & & & $U$ & \\
\hline 294 & ITASRL & 0.00788 & $\mathrm{pCi} / \mathrm{L}$ & 0.012 & 0.012 & & & $U$ & \\
\hline 295 & ITASRL & 0.017 & $\mathrm{pCi} / \mathrm{L}$ & 0.0122 & 0.0124 & & & & \\
\hline 296 & ITASRL & 0.019 & $\mathrm{pCi} / \mathrm{L}$ & 0.0152 & 0.0154 & & & & \\
\hline 297 & ITASRL & 0.0129 & $\mathrm{pCi} / \mathrm{L}$ & 0.0108 & 0.0109 & & & & \\
\hline 298 & ITASRL & 0.00322 & $\mathrm{pCi} / \mathrm{L}$ & 0.00811 & 0.00817 & & & $U$ & \\
\hline 299 & ITASRL & 0.0177 & $\mathrm{pCi} / \mathrm{L}$ & 0.0127 & 0.0128 & & & & \\
\hline 300 & ITASRL & 0.00582 & $\mathrm{pCi} / \mathrm{L}$ & 0.00806 & 0.00813 & & & $U$ & \\
\hline 301 & ITASRL & 0.00545 & $\mathrm{pCi} / \mathrm{L}$ & 0.0115 & 0.0116 & & & $U$ & \\
\hline 302 & ITASRL & 0.0135 & $\mathrm{pCi} / \mathrm{L}$ & 0.0146 & 0.0147 & & & $U$ & \\
\hline 303 & ITASRL & 0.0119 & $\mathrm{pCi} / \mathrm{L}$ & 0.0122 & 0.0123 & & & $U$ & \\
\hline 304 & ITASRL & 0.0102 & $\mathrm{pCi} / \mathrm{L}$ & 0.0102 & 0.0103 & & & $U$ & \\
\hline 305 & ITASRL & 0.0219 & $\mathrm{pCi} / \mathrm{L}$ & 0.0146 & 0.0148 & & & & \\
\hline
\end{tabular}




\begin{tabular}{|c|c|c|c|c|c|c|c|c|c|}
\hline & $\mathrm{L}$ & $\mathrm{M}$ & $\mathrm{N}$ & $\mathrm{O}$ & $\mathrm{P}$ & $\mathrm{Q}$ & $\mathrm{R}$ & $S$ & $\mathrm{~T}$ \\
\hline 1 & LAB_CODE & VALUE_RPTD & ANAL_UNITS_RPTD & COUNTING_ERROR & TOTAL_ANAL_ERROR & MIN_DETECTABLE_ACTIVITY & |PCNT_MOISTURE & LAB_QUALIFIER & REVIEW_QUALIFIER \\
\hline 306 & ITASRL & 0.0183 & $3 \mathrm{pCi} / \mathrm{L}$ & 0.0189 & 0.0191 & & & $U$ & \\
\hline 307 & ITASRL & 0.00771 & $\mathrm{pCi} / \mathrm{L}$ & 0.0111 & 0.0112 & & & U & \\
\hline 308 & ITASRL & 0.0097 & $\mathrm{pCi} / \mathrm{L}$ & 0.0123 & 0.0124 & & & $U$ & \\
\hline 309 & ITASRL & 0.0143 & $3 \mathrm{pCi} / \mathrm{L}$ & 0.0127 & 0.0128 & & & & \\
\hline 310 & ITASRL & 0.0183 & $\mathrm{pCi} / \mathrm{L}$ & 0.0139 & 0.014 & & & & \\
\hline 311 & ITASRL & 0.0165 & $\mathrm{pCi} / \mathrm{L}$ & 0.0155 & 0.0156 & & & & \\
\hline 312 & ITASRL & 0.00496 & $\mathrm{pCi} / \mathrm{L}$ & 0.00855 & 0.00861 & & & $U$ & \\
\hline 313 & ITASRL & 0.0108 & $\mathrm{pCi} / \mathrm{L}$ & 0.0117 & 0.0118 & & & $U$ & \\
\hline 314 & ITASRL & 0.00456 & $\mathrm{pCi} / \mathrm{L}$ & 0.011 & 0.011 & & & U & \\
\hline 315 & ITASRL & 0.1 & $\mathrm{pCi} / \mathrm{L}$ & 0.0273 & 0.029 & & & & $\mathrm{~F}$ \\
\hline 316 & ITASRL & 0.0917 & $\mathrm{pCi} / \mathrm{L}$ & 0.027 & 0.0284 & & & & $\mathrm{~F}$ \\
\hline 317 & ITASRL & 0.0103 & $\mathrm{pCi} / \mathrm{L}$ & 0.00937 & 0.00947 & & & & \\
\hline 318 & ITASRL & 0.0498 & $\mathrm{pCi} / \mathrm{L}$ & 0.0196 & 0.0202 & & & & $F$ \\
\hline 319 & ITASRL & 0.0175 & $\mathrm{pCi} / \mathrm{L}$ & 0.0123 & 0.0124 & & & & \\
\hline 320 & ITASRL & 0.0215 & $\mathrm{pCi} / \mathrm{L}$ & 0.0136 & 0.0138 & & & & \\
\hline 321 & ITASRL & 0.0179 & $\mathrm{pCi} / \mathrm{L}$ & 0.0133 & 0.0134 & & & & \\
\hline 322 & ITASRL & 0.0729 & $\mathrm{pCi} / \mathrm{L}$ & 0.0234 & 0.0244 & & & & $\mathrm{~F}$ \\
\hline 323 & ITASRL & 0.00652 & $\mathrm{pCi} / \mathrm{L}$ & 0.00772 & 0.0078 & & & U & \\
\hline 324 & ITASRL & 0.0399 & $\mathrm{pCi} / \mathrm{L}$ & 0.0171 & 0.0176 & & & & \\
\hline 325 & ITASRL & -0.00319 & $\mathrm{pCi} / \mathrm{L}$ & 0.00808 & 0.00813 & & & $U$ & \\
\hline 326 & ITASRL & 0.00349 & $\mathrm{pCi} / \mathrm{L}$ & 0.00859 & 0.00865 & & & U & \\
\hline 327 & ITASRL & 0.00347 & $\mathrm{pCi} / \mathrm{L}$ & 0.0079 & 0.00796 & & & U & \\
\hline 328 & ITASRL & 0.0101 & $\mathrm{pCi} / \mathrm{L}$ & 0.0117 & 0.0117 & & & $U$ & \\
\hline 329 & ITASRL & 0.0178 & $\mathrm{pCi} / \mathrm{L}$ & 0.0131 & 0.0132 & & & & \\
\hline 330 & ITASRL & 0.00919 & $\mathrm{pCi} / \mathrm{L}$ & 0.012 & 0.0121 & & & $U$ & \\
\hline 331 & ITASRL & 0.00463 & $3 \mathrm{pCi} / \mathrm{L}$ & 0.00972 & 0.00978 & & & $U$ & \\
\hline 332 & ITASRL & 0.00674 & $\mathrm{pCi} / \mathrm{L}$ & 0.00928 & 0.00935 & & & U & \\
\hline 333 & ITASRL & 0.0101 & $\mathrm{pCi} / \mathrm{L}$ & 0.0132 & 0.0133 & & & U & \\
\hline 334 & ITASRL & 0.00804 & $\mathrm{pCi} / \mathrm{L}$ & 0.0107 & 0.0108 & & & $U$ & \\
\hline 335 & ITASRL & 0.00736 & $\mathrm{pCi} / \mathrm{L}$ & 0.0093 & 0.00938 & & & $U$ & \\
\hline 336 & ITASRL & 0.0163 & $\mathrm{pCi} / \mathrm{L}$ & 0.0118 & 0.0119 & & & & \\
\hline 337 & ITASRL & 0.014 & $\mathrm{pCi} / \mathrm{L}$ & 0.0116 & 0.0117 & & & & \\
\hline 338 & ITASRL & 0.00551 & $\mathrm{pCi} / \mathrm{L}$ & 0.00786 & 0.00794 & & & U & \\
\hline 339 & ITASRL & 0.00777 & $\mathrm{pCi} / \mathrm{L}$ & 0.0105 & 0.0106 & & & U & \\
\hline 340 & ITASRL & 0.0141 & $\mathrm{pCi} / \mathrm{L}$ & 0.0153 & 0.0154 & & & $U$ & \\
\hline 341 & ITASRL & 0.0105 & $\mathrm{pCi} / \mathrm{L}$ & 0.0101 & 0.0102 & & & & \\
\hline 342 & ITASRL & 0.0093 & $\mathrm{pCi} / \mathrm{L}$ & 0.00948 & 0.00957 & & & U & \\
\hline 343 & ITASRL & 0.0144 & $\mathrm{pCi} / \mathrm{L}$ & 0.0129 & 0.013 & & & & \\
\hline
\end{tabular}




\begin{tabular}{|c|c|c|c|c|c|c|c|c|c|}
\hline & $\mathrm{L}$ & $\mathrm{M}$ & $\mathrm{N}$ & $\mathrm{O}$ & $\mathrm{P}$ & $\mathrm{Q}$ & $\mathrm{R}$ & $\mathrm{S}$ & $\mathrm{T}$ \\
\hline 1 & LAB_CODE & VALUE_RPTD & ANAL_UNITS_RPTD & COUNTING_ERROR & TOTAL_ANAL_ERROR & MIN_DETECTABLE_ACTIVITY & PCNT_MOISTURE & LAB_QUALIFIER & REVIEW_QUALIFIER \\
\hline 344 & ITASRL & 0.0079 & $\mathrm{pCi} / \mathrm{L}$ & 0.0256 & 0.0256 & & & U & \\
\hline 345 & ITASRL & 0.0104 & $\mathrm{pCi} / \mathrm{L}$ & 0.0113 & 0.0114 & & & $U$ & \\
\hline 346 & ITASRL & 0.00647 & $\mathrm{pCi} / \mathrm{L}$ & 0.00889 & 0.00896 & & & U & \\
\hline 347 & ITASRL & 0.00835 & $\mathrm{pCi} / \mathrm{L}$ & 0.00914 & 0.00922 & & & U & \\
\hline 348 & ITASRL & 0.00473 & $\mathrm{pCi} / \mathrm{L}$ & 0.0202 & 0.0203 & & & $U$ & \\
\hline 349 & ITASRL & 0.0111 & $\mathrm{pCi} / \mathrm{L}$ & 0.0111 & 0.0112 & & & $U$ & \\
\hline 350 & ITASRL & 0.0153 & $\mathrm{pCi} / \mathrm{L}$ & 0.0139 & 0.014 & & & & \\
\hline 351 & ITASRL & -0.00562 & $\mathrm{pCi} / \mathrm{L}$ & 0.00612 & 0.0062 & & & $U$ & \\
\hline 352 & ITASRL & 0.015 & $\mathrm{pCi} / \mathrm{L}$ & 0.0132 & 0.0133 & & & & \\
\hline 353 & ITASRL & 0.00946 & $\mathrm{pCi} / \mathrm{L}$ & 0.0115 & 0.0116 & & & $U$ & \\
\hline 354 & ITASRL & -0.000441 & $\mathrm{pCi} / \mathrm{L}$ & 0.00695 & 0.007 & & & $U$ & \\
\hline 355 & ITASRL & 0.0031 & $\mathrm{pCi} / \mathrm{L}$ & 0.00728 & 0.00734 & & & $U$ & \\
\hline 356 & ITASRL & 0.00703 & $\mathrm{pCi} / \mathrm{L}$ & 0.00832 & 0.0084 & & & U & \\
\hline 357 & ITASRL & 0.00209 & $\mathrm{pCi} / \mathrm{L}$ & 0.00556 & 0.00564 & & & U & \\
\hline 358 & ITASRL & 0.011 & $\mathrm{pCi} / \mathrm{L}$ & 0.0151 & 0.0151 & & & U & \\
\hline 359 & ITASRL & 0.00482 & $\mathrm{pCi} / \mathrm{L}$ & 0.0084 & 0.00846 & & & $U$ & \\
\hline 360 & ITASRL & 0.000943 & $\mathrm{pCi} / \mathrm{L}$ & 0.00873 & 0.00877 & & & U & \\
\hline 361 & ITASRL & 0.0117 & $\mathrm{pCi} / \mathrm{L}$ & 0.0104 & 0.0105 & & & & \\
\hline 362 & ITASRL & 0.0108 & $\mathrm{pCi} / \mathrm{L}$ & 0.0119 & 0.012 & & & $U$ & \\
\hline 363 & ITASRL & 0.01 & $\mathrm{pCi} / \mathrm{L}$ & 0.0139 & 0.014 & & & $U$ & \\
\hline 364 & ITASRL & 0.0127 & $\mathrm{pCi} / \mathrm{L}$ & 0.0117 & 0.0118 & & & & \\
\hline 365 & ITASRL & 0.00893 & $\mathrm{pCi} / \mathrm{L}$ & 0.0169 & 0.017 & & & $U$ & \\
\hline 366 & ITASRL & 0.00515 & $\mathrm{pCi} / \mathrm{L}$ & 0.00744 & 0.00751 & & & $U$ & \\
\hline 367 & ITASRL & -0.000172 & $\mathrm{pCi} / \mathrm{L}$ & 0.00477 & 0.00485 & & & $U$ & \\
\hline 368 & ITASRL & 0.00567 & $\mathrm{pCi} / \mathrm{L}$ & 0.00748 & 0.00756 & & & $U$ & \\
\hline 369 & ITASRL & 0.0217 & $\mathrm{pCi} / \mathrm{L}$ & 0.023 & 0.0232 & & & U & \\
\hline 370 & ITASRL & 0.0109 & $\mathrm{pCi} / \mathrm{L}$ & 0.0274 & 0.0274 & & & U & \\
\hline 371 & ITASRL & 0.00718 & $\mathrm{pCi} / \mathrm{L}$ & 0.00837 & 0.00845 & & & $U$ & \\
\hline 372 & ITASRL & 0.0695 & $\mathrm{pCi} / \mathrm{L}$ & 0.0976 & 0.098 & & & $U$ & $\mathrm{~F}$ \\
\hline 373 & ITASRL & 0.00282 & $\mathrm{pCi} / \mathrm{L}$ & 0.0335 & 0.0335 & & & U & \\
\hline 374 & ITASRL & 0.117 & $\mathrm{pCi} / \mathrm{L}$ & 0.129 & 0.132 & & & $U$ & $\mathrm{~F}$ \\
\hline 375 & ITASRL & 0.0103 & $\mathrm{pCi} / \mathrm{L}$ & 0.0213 & 0.0214 & & & U & \\
\hline 376 & ITASRL & -0.00775 & $\mathrm{pCi} / \mathrm{L}$ & 0.0129 & 0.013 & & & $U$ & \\
\hline 377 & ITASRL & 0.00907 & $\mathrm{pCi} / \mathrm{L}$ & 0.0283 & 0.0283 & & & $U$ & \\
\hline 378 & ITASRL & 0.000346 & $\mathrm{pCi} / \mathrm{L}$ & 0.0189 & 0.0189 & & & $U$ & \\
\hline 379 & ITASRL & 0.00514 & $\mathrm{pCi} / \mathrm{L}$ & 0.0177 & 0.0177 & & & $\mathrm{U}$ & \\
\hline 380 & ITASRL & 0.0255 & $\mathrm{pCi} / \mathrm{L}$ & 0.052 & 0.0522 & & & $U$ & \\
\hline 381 & ITASRL & 0.00514 & $\mathrm{pCi} / \mathrm{L}$ & 0.0561 & 0.0561 & & & U & \\
\hline
\end{tabular}




\begin{tabular}{|c|c|c|c|c|c|c|c|c|c|}
\hline & $\mathrm{L}$ & $\mathrm{M}$ & $\mathrm{N}$ & $\mathrm{O}$ & $\mathrm{P}$ & $\mathrm{Q}$ & $\mathrm{R}$ & $\mathrm{S}$ & $\mathrm{T}$ \\
\hline 1 & LAB_CODE & VALUE_RPTD & ANAL_UNITS_RPTD & |COUNTING_ERROR| & TOTAL_ANAL_ERROR & MIN_DETECTABLE_ACTIVITY & PCNT_MOISTURE & LAB_QUALIFIER & REVIEW_QUALIFIER \\
\hline 382 & ITASRL & -0.00293 & $\mathrm{pCi} / \mathrm{L}$ & 0.0141 & 0.0141 & & & U & \\
\hline 383 & ITASRL & 0.00537 & $\mathrm{pCi} / \mathrm{L}$ & 0.0239 & 0.024 & & & $U$ & \\
\hline 384 & ITASRL & -0.00599 & $\mathrm{pCi} / \mathrm{L}$ & 0.0126 & 0.0126 & & & U & \\
\hline 385 & ITASRL & -0.00245 & $\mathrm{pCi} / \mathrm{L}$ & 0.0562 & 0.0562 & & & U & \\
\hline 386 & ITASRL & 0.0103 & $\mathrm{pCi} / \mathrm{L}$ & 0.0255 & 0.0256 & & & $U$ & \\
\hline 387 & ITASRL & 0.00925 & $\mathrm{pCi} / \mathrm{L}$ & 0.0231 & 0.0232 & & & U & \\
\hline 388 & ITASRL & -0.00618 & $\mathrm{pCi} / \mathrm{L}$ & 0.0131 & 0.0132 & & & $u$ & \\
\hline 389 & ITASRL & 0.00828 & $\mathrm{pCi} / \mathrm{L}$ & 0.0159 & 0.0159 & & & U & \\
\hline 390 & ITASRL & 0.0132 & $\mathrm{pCi} / \mathrm{L}$ & 0.0199 & 0.0199 & & & $U$ & \\
\hline 391 & ITASRL & 0.0148 & $\mathrm{pCi} / \mathrm{L}$ & 0.0171 & 0.0172 & & & $U$ & \\
\hline 392 & ITASRL & & & & & & & & \\
\hline 393 & ITASRL & 0.000486 & $\mathrm{pCi} / \mathrm{L}$ & 0.0118 & 0.0119 & & & $U$ & \\
\hline 394 & ITASRL & 0.0249 & $\mathrm{pCi} / \mathrm{L}$ & 0.0342 & 0.0344 & & & U & \\
\hline 395 & ITASRL & 0.01 & $\mathrm{pCi} / \mathrm{L}$ & 0.0147 & 0.0148 & & & U & \\
\hline 396 & ITASRL & 0.00689 & $\mathrm{pCi} / \mathrm{L}$ & 0.0116 & 0.0116 & & & $U$ & \\
\hline 397 & ITASRL & 0.00493 & $\mathrm{pCi} / \mathrm{L}$ & 0.0102 & 0.0103 & & & $U$ & \\
\hline 398 & ITASRL & 0.0153 & $\mathrm{pCi} / \mathrm{L}$ & 0.015 & 0.0151 & & & & \\
\hline 399 & ITASRL & 0.0056 & $\mathrm{pCi} / \mathrm{L}$ & 0.00869 & 0.00876 & & & $U$ & \\
\hline 400 & ITASRL & 0.00784 & $\mathrm{pCi} / \mathrm{L}$ & 0.00866 & 0.00874 & & & $U$ & \\
\hline 401 & ITASRL & 0.0316 & $\mathrm{pCi} / \mathrm{L}$ & 0.039 & 0.0393 & & & $U$ & \\
\hline 402 & ITASRL & 0.0248 & $\mathrm{pCi} / \mathrm{L}$ & 0.0314 & 0.0317 & & & $U$ & \\
\hline 403 & ITASRL & 0.00471 & $\mathrm{pCi} / \mathrm{L}$ & 0.00995 & 0.0101 & & & $U$ & \\
\hline 404 & ITASRL & 0.00432 & $\mathrm{pCi} / \mathrm{L}$ & 0.00724 & 0.0074 & & & $U$ & \\
\hline 405 & ITASRL & 0.0209 & $\mathrm{pCi} / \mathrm{L}$ & 0.0152 & 0.0154 & & & & \\
\hline 406 & ITASRL & 0.00916 & $\mathrm{pCi} / \mathrm{L}$ & 0.0108 & 0.011 & & & $U$ & \\
\hline 407 & ITASRL & 0.0386 & $\mathrm{pCi} / \mathrm{L}$ & 0.024 & 0.0245 & & & & \\
\hline 408 & ITASRL & 0.00792 & $\mathrm{pCi} / \mathrm{L}$ & 0.0154 & 0.0155 & & & U & \\
\hline 409 & ITASRL & 0.00978 & $\mathrm{pCi} / \mathrm{L}$ & 0.0135 & 0.0136 & & & U & \\
\hline 410 & ITASRL & 0.0156 & $\mathrm{pCi} / \mathrm{L}$ & 0.0143 & 0.0144 & & & & \\
\hline 411 & ITASRL & 0.0504 & $\mathrm{pCi} / \mathrm{L}$ & 0.022 & 0.0227 & & & & $\mathrm{~F}$ \\
\hline 412 & ITASRL & 0.00299 & $\mathrm{pCi} / \mathrm{L}$ & 0.00826 & 0.00838 & & & $U$ & \\
\hline 413 & ITASRL & 0.00725 & $\mathrm{pCi} / \mathrm{L}$ & 0.0116 & 0.0117 & & & U & \\
\hline 414 & ITASRL & & & & & & & & \\
\hline 415 & ITASRL & 0.00619 & $\mathrm{pCi} / \mathrm{L}$ & 0.045 & 0.0454 & & & $U$ & \\
\hline 416 & ITASRL & 0.00581 & $\mathrm{pCi} / \mathrm{L}$ & 0.0212 & 0.0215 & & & $U$ & \\
\hline 417 & ITASRL & -0.00271 & $\mathrm{pCi} / \mathrm{L}$ & 0.0177 & 0.018 & & & U & \\
\hline 418 & ITASRL & 0.0131 & $\mathrm{pCi} / \mathrm{L}$ & 0.0216 & 0.0219 & & & $U$ & \\
\hline 419 & ITASRL & 0.00244 & $\mathrm{pCi} / \mathrm{L}$ & 0.0218 & 0.0221 & & & U & \\
\hline
\end{tabular}




\begin{tabular}{|c|c|c|c|c|c|c|c|c|c|}
\hline & $\mathrm{L}$ & $\mathrm{M}$ & $\mathrm{N}$ & $\mathrm{O}$ & $\mathrm{P}$ & $\mathrm{Q}$ & $\mathrm{R}$ & $\mathrm{S}$ & $\mathrm{T}$ \\
\hline 1 & LAB_CODE & VALUE_RPTD & ANAL_UNITS_RPTD & |COUNTING_ERROR| & TOTAL_ANAL_ERROR & MIN_DETECTABLE_ACTIVITY & PCNT_MOISTURE & LAB_QUALIFIER & REVIEW_QUALIFIER \\
\hline 420 & ITASRL & -0.0226 & $\mathrm{pCi} / \mathrm{L}$ & 0.0183 & 0.0186 & & & U & \\
\hline 421 & ITASRL & 0.0193 & $\mathrm{pCi} / \mathrm{L}$ & 0.0218 & 0.0222 & & & $U$ & \\
\hline 422 & ITASRL & 0.00491 & $\mathrm{pCi} / \mathrm{L}$ & 0.0169 & 0.0173 & & & $U$ & \\
\hline 423 & ITASRL & 0.00341 & $\mathrm{pCi} / \mathrm{L}$ & 0.0147 & 0.0151 & & & U & \\
\hline 424 & ITASRL & -0.000955 & $\mathrm{pCi} / \mathrm{L}$ & 0.0251 & 0.0259 & & & $U$ & \\
\hline 425 & ITASRL & 0.0233 & $\mathrm{pCi} / \mathrm{L}$ & 0.0177 & 0.018 & & & & \\
\hline 426 & ITASRL & 0.00000557 & $\mathrm{pCi} / \mathrm{L}$ & 0.00000839 & 0.00000853 & & & $U$ & \\
\hline \begin{tabular}{|l|}
427 \\
\end{tabular} & ITASRL & 0.00000826 & $\mathrm{pCi} / \mathrm{L}$ & 0.00000913 & 0.00000929 & & & $U$ & \\
\hline 428 & ITASRL & 0.00914 & $\mathrm{pCi} / \mathrm{L}$ & 0.0119 & 0.0121 & & & $U$ & \\
\hline 429 & ITASRL & 0.00676 & $\mathrm{pCi} / \mathrm{L}$ & 0.0108 & 0.0109 & & & $U$ & \\
\hline 430 & ITASRL & -0.00204 & $\mathrm{pCi} / \mathrm{L}$ & 0.00716 & 0.00728 & & & $U$ & \\
\hline 431 & ITASRL & 0.0244 & $\mathrm{pCi} / \mathrm{L}$ & 0.0193 & 0.0196 & & & & \\
\hline 432 & ITASRL & 0.00688 & $\mathrm{pCi} / \mathrm{L}$ & 0.0128 & 0.0129 & & & U & \\
\hline 433 & ITASRL & 0.0018 & $\mathrm{pCi} / \mathrm{L}$ & 0.0103 & 0.0104 & & & U & \\
\hline 434 & ITASRL & 0.00644 & $\mathrm{pCi} / \mathrm{L}$ & 0.00993 & 0.0101 & & & U & \\
\hline 435 & ITASRL & 0.00714 & $\mathrm{pCi} / \mathrm{L}$ & 0.0118 & 0.0119 & & & $U$ & \\
\hline 436 & ITASRL & 0.00627 & $\mathrm{pCi} / \mathrm{L}$ & 0.00787 & 0.00803 & & & U & \\
\hline 437 & ITASRL & 0.00887 & $\mathrm{pCi} / \mathrm{L}$ & 0.0111 & 0.0113 & & & $U$ & \\
\hline 438 & ITASRL & 0.0172 & $\mathrm{pCi} / \mathrm{L}$ & 0.0125 & 0.0127 & & & & \\
\hline 439 & ITASRL & 0.00626 & $\mathrm{pCi} / \mathrm{L}$ & 0.00821 & 0.00837 & & & $U$ & \\
\hline 440 & ITASRL & 0.00514 & $\mathrm{pCi} / \mathrm{L}$ & 0.00764 & 0.00779 & & & U & \\
\hline 441 & ITASRL & 0.00382 & $\mathrm{pCi} / \mathrm{L}$ & 0.00778 & 0.00792 & & & $U$ & \\
\hline 442 & ITASRL & 0.01 & $\mathrm{pCi} / \mathrm{L}$ & 0.0106 & 0.0107 & & & $U$ & \\
\hline 443 & ITASRL & 0.0197 & $\mathrm{pCi} / \mathrm{L}$ & 0.0126 & 0.0128 & & & & \\
\hline 444 & ITASRL & 0.00316 & $\mathrm{pCi} / \mathrm{L}$ & 0.00831 & 0.00844 & & & $U$ & \\
\hline 445 & ITASRL & 0.0126 & $\mathrm{pCi} / \mathrm{L}$ & 0.0138 & 0.0139 & & & $U$ & \\
\hline 446 & ITASRL & 0.0287 & $\mathrm{pCi} / \mathrm{L}$ & 0.0275 & 0.0278 & & & & \\
\hline 447 & ITASRL & 0.0293 & $\mathrm{pCi} / \mathrm{L}$ & 0.0156 & 0.016 & & & & \\
\hline 448 & ITASRL & 0.0481 & $\mathrm{pCi} / \mathrm{L}$ & 0.0213 & 0.022 & & & & $\mathrm{~F}$ \\
\hline 449 & ITASRL & 0.00459 & $\mathrm{pCi} / \mathrm{L}$ & 0.00851 & 0.00865 & & & U & \\
\hline 450 & ITASRL & 0.00292 & $\mathrm{pCi} / \mathrm{L}$ & 0.00675 & 0.0069 & & & $U$ & \\
\hline 451 & ITASRL & 0.00506 & $\mathrm{pCi} / \mathrm{L}$ & 0.00983 & 0.00996 & & & U & \\
\hline 452 & ITASRL & -0.000244 & $\mathrm{pCi} / \mathrm{L}$ & 0.00679 & 0.00692 & & & $U$ & \\
\hline 453 & ITASRL & 0.0139 & $\mathrm{pCi} / \mathrm{L}$ & 0.0112 & 0.0114 & & & & \\
\hline 454 & ITASRL & 0.00966 & $\mathrm{pCi} / \mathrm{L}$ & 0.0102 & 0.0104 & & & $U$ & \\
\hline 455 & ITASRL & 0.0086 & $\mathrm{pCi} / \mathrm{L}$ & 0.0106 & 0.0107 & & & U & \\
\hline 456 & ITASRL & -0.00139 & $\mathrm{pCi} / \mathrm{L}$ & 0.00604 & 0.00618 & & & U & \\
\hline 457 & ITASRL & 0.00899 & $\mathrm{pCi} / \mathrm{L}$ & 0.0109 & 0.0111 & & & $\mathrm{U}$ & \\
\hline
\end{tabular}




\begin{tabular}{|c|c|c|c|c|c|c|c|c|c|}
\hline & $\mathrm{L}$ & $M$ & $\mathrm{~N}$ & $\mathrm{O}$ & $P$ & $\mathrm{Q}$ & $\mathrm{R}$ & $\mathrm{S}$ & $T$ \\
\hline 1 & LAB_CODE & VALUE_RPTD & ANAL_UNITS_RPTD & COUNTING_ERROR & TOTAL_ANAL_ERROR & MIN_DETECTABLE_ACTIVITY & PCNT_MOISTURE & LAB_QUALIFIER & REVIEW_QUALIFIER \\
\hline 458 & ITASRL & 0.0112 & $\mathrm{pCi} / \mathrm{L}$ & 0.0113 & 0.0115 & & & $U$ & \\
\hline 459 & ITASRL & 0.00441 & $p C i / L$ & 0.0102 & 0.0104 & & & $U$ & \\
\hline 460 & ITASRL & 0.00543 & $\mathrm{pCi} / \mathrm{L}$ & 0.0109 & 0.011 & & & $U$ & \\
\hline 461 & ITASRL & 0.00446 & $\mathrm{pCi} / \mathrm{L}$ & 0.00909 & 0.00921 & & & $U$ & \\
\hline 462 & ITASRL & 0.00974 & $p C i / L$ & 0.011 & 0.0112 & & & $U$ & \\
\hline 463 & ITASRL & -0.00517 & $\mathrm{pCi} / \mathrm{L}$ & 0.00604 & 0.00619 & & & $U$ & \\
\hline 464 & ITASRL & 0.000254 & $\mathrm{pCi} / \mathrm{L}$ & 0.00883 & 0.00894 & & & $U$ & \\
\hline 465 & ITASRL & 0.0082 & $\mathrm{pCi} / \mathrm{L}$ & 0.00908 & 0.00925 & & & $U$ & \\
\hline 466 & ITASRL & 0.00236 & $\mathrm{pCi} / \mathrm{L}$ & 0.00798 & 0.0081 & & & $U$ & \\
\hline 467 & ITASRL & 0.00777 & $\mathrm{pCi} / \mathrm{L}$ & 0.0109 & 0.0111 & & & $U$ & \\
\hline 468 & ITASRL & 0.00361 & $\mathrm{pCi} / \mathrm{L}$ & 0.0117 & 0.0118 & & & $U$ & \\
\hline 469 & ITASRL & 0.00477 & $\mathrm{pCi} / \mathrm{L}$ & 0.0116 & 0.0117 & & & $U$ & \\
\hline 470 & ITASRL & 0.0109 & $\mathrm{pCi} / \mathrm{L}$ & 0.0116 & 0.0118 & & & $U$ & \\
\hline 471 & ITASRL & 0.0107 & $\mathrm{pCi} / \mathrm{L}$ & 0.0117 & 0.0118 & & & $U$ & \\
\hline 472 & ITASRL & -0.000358 & $\mathrm{pCi} / \mathrm{L}$ & 0.00698 & 0.00711 & & & $U$ & \\
\hline 473 & ITASRL & 0.00408 & $\mathrm{pCi} / \mathrm{L}$ & 0.00912 & 0.00924 & & & $U$ & \\
\hline 474 & ITASRL & 0.00634 & $\mathrm{pCi} / \mathrm{L}$ & 0.00672 & 0.00683 & & & $U$ & \\
\hline 475 & ITASRL & 0.00421 & $\mathrm{pCi} / \mathrm{L}$ & 0.0107 & 0.0108 & & & $U$ & \\
\hline 476 & ITASRL & 0.0122 & $\mathrm{pCi} / \mathrm{L}$ & 0.0114 & 0.0116 & & & & \\
\hline 477 & ITASRL & -0.000403 & $\mathrm{pCi} / \mathrm{L}$ & 0.00631 & 0.00645 & & & $U$ & \\
\hline 478 & ITASRL & 0.0111 & $\mathrm{pCi} / \mathrm{L}$ & 0.0111 & 0.0112 & & & U & \\
\hline 479 & ITASRL & 0.000395 & $\mathrm{pCi} / \mathrm{L}$ & 0.0131 & 0.0131 & & & $U$ & \\
\hline 480 & ITASRL & 0.0137 & $\mathrm{pCi} / \mathrm{L}$ & 0.0131 & 0.0133 & & & & \\
\hline 481 & ITASRL & 0.0101 & $\mathrm{pCi} / \mathrm{L}$ & 0.0129 & 0.013 & & & $U$ & \\
\hline 482 & ITASRL & 0.00307 & $\mathrm{pCi} / \mathrm{L}$ & 0.00818 & 0.00831 & & & $U$ & \\
\hline 483 & ITASRL & 0.00868 & $\mathrm{pCi} / \mathrm{L}$ & 0.0147 & 0.0148 & & & $U$ & \\
\hline 484 & ITASRL & 0.00101 & $\mathrm{pCi} / \mathrm{L}$ & 0.00813 & 0.00824 & & & $U$ & \\
\hline 485 & ITASRL & 0.0101 & $\mathrm{pCi} / \mathrm{L}$ & 0.0122 & 0.0124 & & & $U$ & \\
\hline 486 & ITASRL & 0.00659 & $\mathrm{pCi} / \mathrm{L}$ & 0.0106 & 0.0108 & & & $U$ & \\
\hline 487 & ITASRL & -0.00277 & $\mathrm{pCi} / \mathrm{L}$ & 0.00598 & 0.00611 & & & $U$ & \\
\hline 488 & ITASRL & 0.011 & $\mathrm{pCi} / \mathrm{L}$ & 0.0133 & 0.0135 & & & $U$ & \\
\hline 489 & ITASRL & 0.00619 & $\mathrm{pCi} / \mathrm{L}$ & 0.01 & 0.0101 & & & $U$ & \\
\hline 490 & ITASRL & 0.00767 & $\mathrm{pCi} / \mathrm{L}$ & 0.0112 & 0.0114 & & & $U$ & \\
\hline 491 & ITASRL & 0.00934 & $\mathrm{pCi} / \mathrm{L}$ & 0.013 & 0.0131 & & & $U$ & \\
\hline 492 & ITASRL & -0.00292 & $\mathrm{pCi} / \mathrm{L}$ & 0.00776 & 0.00787 & & & $U$ & \\
\hline 493 & ITASRL & 0.00558 & $\mathrm{pCi} / \mathrm{L}$ & 0.0111 & 0.0112 & & & $U$ & \\
\hline 494 & ITASRL & 0.00498 & $\mathrm{pCi} / \mathrm{L}$ & 0.0112 & 0.0113 & & & $U$ & \\
\hline 495 & ITASRL & 0.0119 & $\mathrm{pCi} / \mathrm{L}$ & 0.0117 & 0.0118 & & & & \\
\hline
\end{tabular}




\begin{tabular}{|c|c|c|c|c|c|c|c|c|c|}
\hline & $L$ & $\mathrm{M}$ & $\mathrm{N}$ & $\mathrm{O}$ & $P$ & $\mathrm{Q}$ & $\mathrm{R}$ & $\mathrm{S}$ & $\mathrm{T}$ \\
\hline 1 & LAB_CODE & VALUE_RPTD & ANAL_UNITS_RPTD & |COUNTING_ERROR| & TOTAL_ANAL_ERROR & MIN_DETECTABLE_ACTIVITY & PCNT_MOISTURE & LAB_QUALIFIER & REVIEW_QUALIFIER \\
\hline 496 & ITASRL & 0.00563 & $\mathrm{pCi} / \mathrm{L}$ & 0.00939 & 0.00953 & & & $U$ & \\
\hline 497 & ITASRL & 0.00468 & $\mathrm{pCi} / \mathrm{L}$ & 0.0106 & 0.0107 & & & $U$ & \\
\hline 498 & ITASRL & 0.00912 & $\mathrm{pCi} / \mathrm{L}$ & 0.00983 & 0.00999 & & & U & \\
\hline 499 & ITASRL & 0.0158 & $\mathrm{pCi} / \mathrm{L}$ & 0.0141 & 0.0143 & & & & \\
\hline 500 & ITASRL & 0.00413 & $\mathrm{pCi} / \mathrm{L}$ & 0.00916 & 0.00929 & & & $U$ & \\
\hline 501 & ITASRL & 0.00359 & $\mathrm{pCi} / \mathrm{L}$ & 0.0105 & 0.0106 & & & $U$ & \\
\hline 502 & ITASRL & 0.00444 & $\mathrm{pCi} / \mathrm{L}$ & 0.00834 & 0.00848 & & & $U$ & \\
\hline 503 & ITASRL & 0.00371 & $\mathrm{pCi} / \mathrm{L}$ & 0.0128 & 0.0129 & & & $U$ & \\
\hline 504 & ITASRL & 0.0184 & $\mathrm{pCi} / \mathrm{L}$ & 0.0151 & 0.0153 & & & & \\
\hline 505 & ITASRL & 0.00876 & $\mathrm{pCi} / \mathrm{L}$ & 0.011 & 0.0112 & & & $U$ & \\
\hline 506 & ITASRL & 0.00371 & $\mathrm{pCi} / \mathrm{L}$ & 0.00926 & 0.00937 & & & $\mathrm{U}$ & \\
\hline 507 & ITASRL & 0.0131 & $\mathrm{pCi} / \mathrm{L}$ & 0.0131 & 0.0133 & & & $U$ & \\
\hline 508 & ITASRL & 0.0161 & $\mathrm{pCi} / \mathrm{L}$ & 0.0133 & 0.0135 & & & & \\
\hline 509 & ITASRL & 0.00484 & $\mathrm{pCi} / \mathrm{L}$ & 0.015 & 0.015 & & & $U$ & \\
\hline 510 & ITASRL & 0.00775 & $\mathrm{pCi} / \mathrm{L}$ & 0.0109 & 0.0111 & & & $U$ & \\
\hline 511 & ITASRL & 0.000782 & $\mathrm{pCi} / \mathrm{L}$ & 0.00767 & 0.00779 & & & $U$ & \\
\hline 512 & ITASRL & 0.00783 & $\mathrm{pCi} / \mathrm{L}$ & 0.014 & 0.0141 & & & $U$ & \\
\hline 513 & ITASRL & 0.00597 & $\mathrm{pCi} / \mathrm{L}$ & 0.0116 & 0.0117 & & & $U$ & \\
\hline 514 & ITASRL & 0.000862 & $\mathrm{pCi} / \mathrm{L}$ & 0.0105 & 0.0106 & & & $U$ & \\
\hline 515 & ITASRL & 0.0132 & $\mathrm{pCi} / \mathrm{L}$ & 0.0129 & 0.0131 & & & & \\
\hline 516 & ITASRL & 0.00274 & $\mathrm{pCi} / \mathrm{L}$ & 0.0102 & 0.0103 & & & U & \\
\hline 517 & ITASRL & 0.00664 & $\mathrm{pCi} / \mathrm{L}$ & 0.00956 & 0.0097 & & & $U$ & \\
\hline 518 & ITASRL & 0.0163 & $\mathrm{pCi} / \mathrm{L}$ & 0.0144 & 0.0146 & & & & \\
\hline 519 & ITASRL & 0.00211 & $\mathrm{pCi} / \mathrm{L}$ & 0.00833 & 0.00845 & & & $U$ & \\
\hline 520 & ITASRL & 0.00689 & $\mathrm{pCi} / \mathrm{L}$ & 0.0121 & 0.0122 & & & $U$ & \\
\hline 521 & ITASRL & 0.0113 & $\mathrm{pCi} / \mathrm{L}$ & 0.0185 & 0.0187 & & & $U$ & \\
\hline 522 & ITASRL & 0.00953 & $\mathrm{pCi} / \mathrm{L}$ & 0.0139 & 0.014 & & & U & \\
\hline 523 & ITASRL & 0.00416 & $\mathrm{pCi} / \mathrm{L}$ & 0.0118 & 0.0119 & & & U & \\
\hline 524 & ITASRL & -0.00245 & $\mathrm{pCi} / \mathrm{L}$ & 0.00541 & 0.00557 & & & $U$ & \\
\hline 525 & ITASRL & 0.00533 & $\mathrm{pCi} / \mathrm{L}$ & 0.0148 & 0.0149 & & & $U$ & \\
\hline 526 & ITASRL & 0.011 & $\mathrm{pCi} / \mathrm{L}$ & 0.0153 & 0.0154 & & & $U$ & \\
\hline 527 & ITASRL & 0.0604 & $\mathrm{pCi} / \mathrm{L}$ & 0.0227 & 0.0237 & & & & \\
\hline 528 & ITASRL & 0.00113 & $\mathrm{pCi} / \mathrm{L}$ & 0.00839 & 0.0085 & & & $U$ & \\
\hline 529 & ITASRL & -0.000739 & $\mathrm{pCi} / \mathrm{L}$ & 0.0112 & 0.0113 & & & $U$ & \\
\hline 530 & ITASRL & 0.00975 & $\mathrm{pCi} / \mathrm{L}$ & 0.014 & 0.0142 & & & $U$ & \\
\hline 531 & ITASRL & 0.00952 & $\mathrm{pCi} / \mathrm{L}$ & 0.0117 & 0.0119 & & & $U$ & \\
\hline 532 & ITASRL & 0.0589 & $\mathrm{pCi} / \mathrm{L}$ & 0.0224 & 0.0234 & & & & \\
\hline 533 & ITASRL & 0.0299 & $\mathrm{pCi} / \mathrm{L}$ & 0.0159 & 0.0163 & & & & \\
\hline
\end{tabular}




\begin{tabular}{|c|c|c|c|c|c|c|c|c|c|}
\hline & $\mathrm{L}$ & $\mathrm{M}$ & $\mathrm{N}$ & $\mathrm{O}$ & $\mathrm{P}$ & $\mathrm{Q}$ & $\mathrm{R}$ & $\mathrm{S}$ & $\mathrm{T}$ \\
\hline 1 & LAB_CODE & VALUE_RPTD & ANAL_UNITS_RPTD & COUNTING_ERROR & TOTAL_ANAL_ERROR & MIN_DETECTABLE_ACTIVITY & PCNT_MOISTURE & LAB_QUALIFIER & REVIEW_QUALIFIER \\
\hline 534 & ITASRL & -0.00182 & $\mathrm{pCi} / \mathrm{L}$ & 0.00394 & 0.00415 & & & U & \\
\hline 535 & ITASRL & 0.00382 & $\mathrm{pCi} / \mathrm{L}$ & 0.00763 & 0.00777 & & & $U$ & \\
\hline 536 & ITASRL & 0.00839 & $\mathrm{pCi} / \mathrm{L}$ & 0.0112 & 0.0113 & & & U & \\
\hline 537 & ITASRL & 0.0102 & $\mathrm{pCi} / \mathrm{L}$ & 0.0106 & 0.0108 & & & U & \\
\hline 538 & ITASRL & 0.0084 & $\mathrm{pCi} / \mathrm{L}$ & 0.0116 & 0.0118 & & & $U$ & \\
\hline 539 & ITASRL & 0.00316 & $\mathrm{pCi} / \mathrm{L}$ & 0.0117 & 0.0118 & & & U & \\
\hline 540 & ITASRL & 0.00885 & $\mathrm{pCi} / \mathrm{L}$ & 0.012 & 0.0122 & & & $U$ & \\
\hline 541 & ITASRL & 0.00653 & $\mathrm{pCi} / \mathrm{L}$ & 0.0112 & 0.0114 & & & U & \\
\hline 542 & ITASRL & -0.00034 & $\mathrm{pCi} / \mathrm{L}$ & 0.00551 & 0.00581 & & & $U$ & \\
\hline 543 & ITASRL & 0.00462 & $\mathrm{pCi} / \mathrm{L}$ & 0.00875 & 0.00897 & & & $U$ & \\
\hline 544 & ITASRL & 0.01 & $\mathrm{pCi} / \mathrm{L}$ & 0.0118 & 0.0121 & & & $U$ & \\
\hline 545 & ITASRL & 0.00579 & $\mathrm{pCi} / \mathrm{L}$ & 0.00902 & 0.00924 & & & $U$ & \\
\hline 546 & ITASRL & 0.0114 & $\mathrm{pCi} / \mathrm{L}$ & 0.012 & 0.0122 & & & U & \\
\hline 547 & ITASRL & 0.0168 & $\mathrm{pCi} / \mathrm{L}$ & 0.0151 & 0.0154 & & & & \\
\hline 548 & ITASRL & 0.00114 & $\mathrm{pCi} / \mathrm{L}$ & 0.00742 & 0.00765 & & & U & \\
\hline 549 & ITASRL & 0.0209 & $\mathrm{pCi} / \mathrm{L}$ & 0.0159 & 0.0162 & & & & \\
\hline 550 & ITASRL & 0.00806 & $\mathrm{pCi} / \mathrm{L}$ & 0.0101 & 0.0103 & & & U & \\
\hline 551 & ITASRL & 0.0024 & $\mathrm{pCi} / \mathrm{L}$ & 0.00868 & 0.00889 & & & U & \\
\hline 552 & DATACH & & & & & & & $U$ & \\
\hline 553 & DATACH & & & & & & & $U$ & \\
\hline 554 & DATACH & & & & & & & $U$ & \\
\hline 555 & DATACH & & & & & & & $U$ & \\
\hline 556 & DATACH & & & & & & & $U$ & \\
\hline 557 & DATACH & & & & & & & U & \\
\hline 558 & DATACH & & & & & & & $U$ & \\
\hline 559 & DATACH & & & & & & & $U$ & \\
\hline 560 & DATACH & & & & & & & U & \\
\hline 561 & DATACH & & & & & & & $U$ & \\
\hline 562 & DATACH & & & & & & & $U$ & \\
\hline 563 & DATACH & & & & & & & U & \\
\hline 564 & DATACH & & & & & & & U & \\
\hline 565 & DATACH & & & & & & & U & \\
\hline 566 & DATACH & & & & & & & U & \\
\hline 567 & DATACH & & & & & & & $U$ & \\
\hline 568 & DATACH & & & & & & & $U$ & \\
\hline 569 & DATACH & & & & & & & U & \\
\hline 570 & DATACH & & & & & & & U & \\
\hline 571 & DATACH & & & & & & & U & \\
\hline
\end{tabular}




\begin{tabular}{|c|c|c|c|c|c|c|c|c|c|}
\hline & $\mathrm{L}$ & $\mathrm{M}$ & $\mathrm{N}$ & $\mathrm{O}$ & $\mathrm{P}$ & $\mathrm{Q}$ & $\mathrm{R}$ & $\mathrm{S}$ & $\mathrm{T}$ \\
\hline 1 & LAB_CODE & VALUE_RPTD & |ANAL_UNITS_RPTD & COUNTING_ERROR & TOTAL_ANAL_ERROR & MIN_DETECTABLE_ACTIVITY & PCNT_MOISTURE & LAB_QUALIFIER & REVIEW_QUALIFIER \\
\hline 572 & DATACH & & & & & & & $U$ & \\
\hline 573 & DATACH & & & & & & & U & \\
\hline 574 & DATACH & & & & & & & 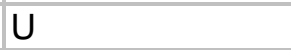 & \\
\hline 575 & DATACH & & & & & & & $U$ & \\
\hline 576 & DATACH & & & & & & & $U$ & \\
\hline 577 & DATACH & & & & & & & $U$ & \\
\hline 578 & DATACH & & & & & & & $U$ & \\
\hline 579 & DATACH & & & & & & & $\mathrm{U}$ & \\
\hline 580 & DATACH & & & & & & & U & \\
\hline 581 & DATACH & & & & & & & $U$ & \\
\hline 582 & DATACH & & & & & & & $U$ & \\
\hline 583 & DATACH & & & & & & & U & \\
\hline 584 & DATACH & & & & & & & $U$ & \\
\hline 585 & DATACH & & & & & & & U & \\
\hline 586 & DATACH & & & & & & & $U$ & \\
\hline 587 & DATACH & & & & & & & $U$ & \\
\hline 588 & DATACH & & & & & & & $U$ & \\
\hline 589 & DATACH & & & & & & & U & \\
\hline 590 & DATACH & & & & & & & $U$ & \\
\hline 591 & DATACH & & & & & & & $U$ & \\
\hline 592 & DATACH & & & & & & & U & \\
\hline 593 & DATACH & & & & & & & U & \\
\hline 594 & DATACH & & & & & & & $U$ & \\
\hline 595 & DATACH & & & & & & & U & \\
\hline 596 & DATACH & & & & & & & $U$ & \\
\hline 597 & DATACH & & & & & & & $U$ & \\
\hline 598 & DATACH & & & & & & & $U$ & \\
\hline 599 & DATACH & & & & & & & U & \\
\hline 600 & DATACH & & & & & & & $U$ & \\
\hline 601 & DATACH & & & & & & & U & \\
\hline 602 & DATACH & & & & & & & U & \\
\hline 603 & DATACH & & & & & & & $U$ & \\
\hline 604 & DATACH & & & & & & & $U$ & \\
\hline 605 & DATACH & & & & & & & $U$ & \\
\hline 606 & DATACH & & & & & & & $U$ & \\
\hline 607 & DATACH & & & & & & & $U$ & \\
\hline 608 & DATACH & & & & & & & U & \\
\hline 609 & DATACH & & & & 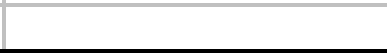 & & & U & \\
\hline
\end{tabular}


M N O P Q $\mathrm{R}$ $\mathrm{S}$ $\mathrm{T}$

\begin{tabular}{|c|c|c|c|c|c|c|c|c|c|}
\hline 1 & LAB CODE & VALUE RPTD & ANAL UNITS RPTD & COUNTING ERROR & TOTAL ANAL ERROR & MIN DETECTABLE ACTIVITY & PCNT MOISTURE & LAB QUALIFIER & REVIEW QUALIFIER \\
\hline 610 & DATACH & & & & & & & $U$ & \\
\hline 611 & DATACH & & & & & & & U & \\
\hline 612 & DATACH & & & & & & & $U$ & \\
\hline 613 & DATACH & & & & & & & U & \\
\hline 614 & DATACH & & & & & & & $U$ & \\
\hline 615 & DATACH & & & & & & & $U$ & \\
\hline 616 & DATACH & & & & & & & $U$ & \\
\hline 617 & DATACH & & & & & & & $U$ & \\
\hline 618 & DATACH & & & & & & & $\mathrm{U}$ & \\
\hline 619 & DATACH & & & & & & & $U$ & \\
\hline 620 & DATACH & & & & & & & $U$ & \\
\hline 621 & DATACH & & & & & & & 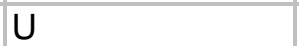 & \\
\hline 622 & DATACH & & & & & & & $U$ & \\
\hline 623 & DATACH & & & & & & & U & \\
\hline 624 & DATACH & & & & & & & U & \\
\hline 625 & DATACH & & & & & & & $U$ & \\
\hline 626 & DATACH & & & & & & & $U$ & \\
\hline 627 & DATACH & & & & & & & $U$ & \\
\hline 628 & DATACH & & & & & & & $U$ & \\
\hline 629 & DATACH & & & & & & & $U$ & \\
\hline 630 & DATACH & & & & & & & $U$ & \\
\hline 631 & DATACH & & & & & & & $U$ & \\
\hline 632 & DATACH & & & & & & & $U$ & \\
\hline 633 & DATACH & & & & & & & $U$ & \\
\hline 634 & DATACH & & & & & & & 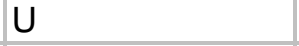 & \\
\hline 635 & DATACH & & & & & & & U & \\
\hline 636 & DATACH & & & & & & & $U$ & \\
\hline 637 & DATACH & & & & & & & $U$ & \\
\hline 638 & DATACH & & & & & & & $U$ & \\
\hline 639 & DATACH & & & & & & & $U$ & \\
\hline 640 & DATACH & & & & & & & $U$ & \\
\hline 641 & DATACH & & & & & & & U & \\
\hline 642 & DATACH & & & & & & & $U$ & \\
\hline 643 & DATACH & & & & & & & $U$ & \\
\hline 644 & DATACH & & & & & & & $U$ & \\
\hline 645 & DATACH & & & & & & & $U$ & \\
\hline 646 & DATACH & & & & & & & $U$ & \\
\hline 647 & DATACH & & & & & & & $\mathrm{U}$ & \\
\hline
\end{tabular}


M N O P Q $\mathrm{R}$ $\mathrm{S}$ $T$

\begin{tabular}{|c|c|c|c|c|c|c|c|c|c|}
\hline & & & & & & & & & \\
\hline 1 & LAB_CODE & VALUE_RPTD & ANAL_UNITS_RPTD & COUNTING_ERROR & TOTAL_ANAL_ERROR & MIN_DETECTABLE_ACTIVITY & PCNT_MOISTURE & LAB_QUALIFIER & REVIEW_QUALIFIER \\
\hline 648 & DATACH & & & & & & & $U$ & \\
\hline 649 & DATACH & & & & & & & $\mathrm{U}$ & \\
\hline 650 & DATACH & & & & & & & $\mathrm{U}$ & \\
\hline 651 & DATACH & & & & & & & U & \\
\hline 652 & DATACH & & & & & & & U & \\
\hline 653 & DATACH & & & & & & & $\mathrm{U}$ & \\
\hline 654 & DATACH & & & & & & & U & \\
\hline 655 & DATACH & & & & & & & $\mathrm{U}$ & \\
\hline 656 & DATACH & & & & & & & U & \\
\hline 657 & DATACH & & & & & & & $\mathrm{U}$ & \\
\hline 658 & DATACH & & & & & & & U & \\
\hline 659 & DATACH & & & & & & & U & \\
\hline 660 & DATACH & & & & & & & U & \\
\hline 661 & DATACH & & & & & & & $\mathrm{U}$ & \\
\hline 662 & DATACH & & & & & & & $\mathrm{U}$ & \\
\hline 663 & DATACH & & & & & & & $\mathrm{U}$ & \\
\hline 664 & DATACH & & & & & & & $\mathrm{U}$ & \\
\hline 665 & DATACH & & & & & & & U & \\
\hline 666 & DATACH & & & & & & & U & \\
\hline 667 & DATACH & & & & & & & $\mathrm{U}$ & \\
\hline 668 & DATACH & & & & & & & U & \\
\hline 669 & DATACH & & & & & & & U & \\
\hline 670 & DATACH & & & & & & & U & \\
\hline 671 & DATACH & & & & & & & U & \\
\hline 672 & DATACH & & & & & & & U & \\
\hline 673 & DATACH & & & & & & & U & \\
\hline 674 & DATACH & & & & & & & U & \\
\hline 675 & DATACH & & & & & & & U & \\
\hline 676 & DATACH & & & & & & & U & \\
\hline 677 & DATACH & & & & & & & U & \\
\hline 678 & DATACH & & & & & & & U & \\
\hline 679 & DATACH & & & & & & & U & \\
\hline 680 & DATACH & & & & & & & U & \\
\hline 681 & DATACH & & & & & & & U & \\
\hline 682 & DATACH & & & & & & & $\mathrm{U}$ & \\
\hline 683 & DATACH & & & & & & & U & \\
\hline 684 & DATACH & & & & & & & U & \\
\hline 685 & DATACH & & & & & & & U & \\
\hline
\end{tabular}


M

$\mathrm{N}$

O

P

Q

$\mathrm{R}$

$\mathrm{S}$

$\mathrm{T}$

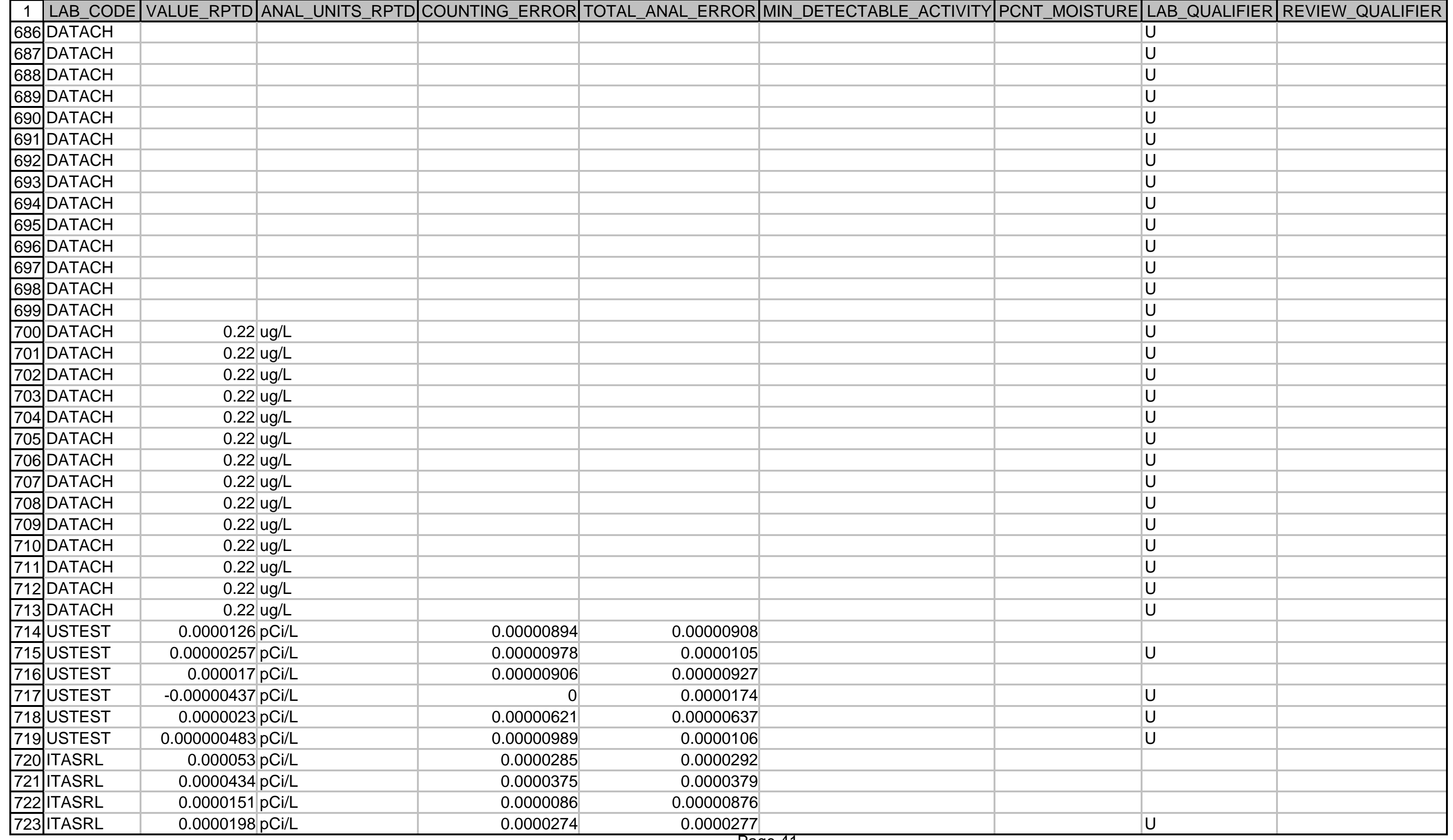

Page 41 


\begin{tabular}{|c|c|c|c|c|c|c|c|c|c|}
\hline & $\mathrm{L}$ & $\mathrm{M}$ & $\mathrm{N}$ & $\mathrm{O}$ & $\mathrm{P}$ & $\mathrm{Q}$ & $\mathrm{R}$ & $\mathrm{S}$ & $\mathrm{T}$ \\
\hline 1 & LAB_CODE & VALUE_RPTD & ANAL_UNITS_RPTD & |COUNTING_ERROR| & TOTAL_ANAL_ERROR & MIN_DETECTABLE_ACTIVITY & PCNT_MOISTURE & LAB_QUALIFIER & REVIEW_QUALIFIER \\
\hline 724 & ITASRL & 0.0000415 & $\mathrm{pCi} / \mathrm{L}$ & 0.0000374 & 0.0000379 & & & & \\
\hline 725 & ITASRL & 0.0000262 & $\mathrm{pCi} / \mathrm{L}$ & 0.0000112 & 0.0000116 & & & & \\
\hline 726 & ITASRL & 0.0000351 & $\mathrm{pCi} / \mathrm{L}$ & 0.0000174 & 0.0000178 & & & & \\
\hline 727 & ITASRL & 0.0000738 & $\mathrm{pCi} / \mathrm{L}$ & 0.0000472 & 0.0000479 & & & & \\
\hline 728 & ITASRL & 0.0000267 & $\mathrm{pCi} / \mathrm{L}$ & 0.0000179 & 0.0000182 & & & & \\
\hline 729 & ITASRL & 0.0000527 & $\mathrm{pCi} / \mathrm{L}$ & 0.0000433 & 0.0000438 & & & & \\
\hline 730 & ITASRL & 0.0000698 & $\mathrm{pCi} / \mathrm{L}$ & 0.0000275 & 0.0000285 & & & & \\
\hline \begin{tabular}{|l|}
731 \\
\end{tabular} & ITASRL & 0.00215 & $\mathrm{pCi} / \mathrm{L}$ & 0.000302 & 0.000376 & & & & \\
\hline 732 & ITASRL & 0.0000159 & $\mathrm{pCi} / \mathrm{L}$ & 0.000011 & 0.0000112 & & & & \\
\hline 733 & ITASRL & 0.0000233 & $\mathrm{pCi} / \mathrm{L}$ & 0.0000403 & 0.0000406 & & & $U$ & \\
\hline 734 & ITASRL & 0.0000113 & $\mathrm{pCi} / \mathrm{L}$ & 0.00000837 & 0.00000849 & & & & \\
\hline 735 & ITASRL & 0.0000559 & $\mathrm{pCi} / \mathrm{L}$ & 0.0000367 & 0.0000373 & & & & \\
\hline 736 & ITASRL & 0.00000873 & $\mathrm{pCi} / \mathrm{L}$ & 0.00000725 & 0.00000736 & & & & \\
\hline 737 & ITASRL & 0.000017 & $\mathrm{pCi} / \mathrm{L}$ & 0.0000279 & 0.0000283 & & & U & \\
\hline 738 & ITASRL & 0.00000589 & $\mathrm{pCi} / \mathrm{L}$ & 0.00000948 & 0.00000955 & & & U & \\
\hline 739 & ITASRL & 0.0000299 & $\mathrm{pCi} / \mathrm{L}$ & 0.0000331 & 0.0000336 & & & $U$ & \\
\hline 740 & ITASRL & 0.0000353 & $\mathrm{pCi} / \mathrm{L}$ & 0.0000159 & 0.0000163 & & & & \\
\hline 741 & ITASRL & -0.00000422 & $\mathrm{pCi} / \mathrm{L}$ & 0.0000247 & 0.0000333 & & & U & \\
\hline 742 & ITASRL & 0.00000606 & $\mathrm{pCi} / \mathrm{L}$ & 0.0000137 & 0.0000138 & & & $U$ & \\
\hline 743 & ITASRL & 0.0000291 & $\mathrm{pCi} / \mathrm{L}$ & 0.0000321 & 0.0000325 & & & $U$ & \\
\hline 744 & ITASRL & 0.0000301 & $\mathrm{pCi} / \mathrm{L}$ & 0.0000146 & 0.0000149 & & & & \\
\hline 745 & ITASRL & 0.0000229 & $\mathrm{pCi} / \mathrm{L}$ & 0.0000124 & 0.0000127 & & & & \\
\hline 746 & ITASRL & 0.000032 & $\mathrm{pCi} / \mathrm{L}$ & 0.000017 & 0.0000174 & & & & \\
\hline 747 & ITASRL & 0.0000049 & $\mathrm{pCi} / \mathrm{L}$ & 0.0000493 & 0.0000494 & & & U & \\
\hline 748 & ITASRL & 0.000107 & $\mathrm{pCi} / \mathrm{L}$ & 0.0000568 & 0.000058 & & & & \\
\hline 749 & ITASRL & 0.000044 & $\mathrm{pCi} / \mathrm{L}$ & 0.0000365 & 0.000037 & & & & \\
\hline 750 & ITASRL & 0.0000246 & $\mathrm{pCi} / \mathrm{L}$ & 0.0000142 & 0.0000145 & & & & \\
\hline 751 & ITASRL & 0.0000112 & $\mathrm{pCi} / \mathrm{L}$ & 0.0000658 & 0.000066 & & & U & \\
\hline 752 & ITASRL & 0.0000371 & $\mathrm{pCi} / \mathrm{L}$ & 0.0000219 & 0.0000223 & & & & \\
\hline 753 & ITASRL & 0.0000271 & $\mathrm{pCi} / \mathrm{L}$ & 0.0000474 & 0.0000477 & & & $U$ & \\
\hline 754 & ITASRL & 0.0000249 & $\mathrm{pCi} / \mathrm{L}$ & 0.0000151 & 0.0000153 & & & & \\
\hline 755 & ITASRL & 0.000617 & $\mathrm{pCi} / \mathrm{L}$ & 0.000192 & 0.000204 & & & & \\
\hline 756 & ITASRL & 0.00000872 & $\mathrm{pCi} / \mathrm{L}$ & 0.00000887 & 0.00000897 & & & U & \\
\hline 757 & ITASRL & 0.00000569 & $\mathrm{pCi} / \mathrm{L}$ & 0.000021 & 0.0000214 & & & U & \\
\hline 758 & ITASRL & 0.000025 & $\mathrm{pCi} / \mathrm{L}$ & 0.0000122 & 0.0000125 & & & & \\
\hline 759 & ITASRL & 0.0000412 & $\mathrm{pCi} / \mathrm{L}$ & 0.0000434 & 0.0000439 & & & $U$ & \\
\hline 760 & ITASRL & 0.000007 & $\mathrm{pCi} / \mathrm{L}$ & 0.0000071 & 0.0000072 & & & $\mathrm{U}$ & \\
\hline 761 & ITASRL & 0.000115 & $\mathrm{pCi} / \mathrm{L}$ & 0.000113 & 0.000114 & & & & \\
\hline
\end{tabular}




\begin{tabular}{|c|c|c|c|c|c|c|c|c|c|}
\hline & $\mathrm{L}$ & $\mathrm{M}$ & $\mathrm{N}$ & $\mathrm{O}$ & $\mathrm{P}$ & $\mathrm{Q}$ & $\mathrm{R}$ & $\mathrm{S}$ & $T$ \\
\hline 1 & LAB_CODE & VALUE_RPTD & ANAL_UNITS_RPTD & |COUNTING_ERROR| & TOTAL_ANAL_ERROR & MIN_DETECTABLE_ACTIVITY & PCNT_MOISTURE & LAB_QUALIFIER & REVIEW_QUALIFIER \\
\hline 762 & ITASRL & 0.00002 & $\mathrm{pCi} / \mathrm{L}$ & 0.0000132 & 0.0000134 & & & & \\
\hline 763 & ITASRL & 0.0000514 & $\mathrm{pCi} / \mathrm{L}$ & 0.0000446 & 0.0000451 & & & & \\
\hline 764 & ITASRL & 0.0000439 & $\mathrm{pCi} / \mathrm{L}$ & 0.0000242 & 0.0000247 & & & & \\
\hline 765 & ITASRL & 0.0000649 & $\mathrm{pCi} / \mathrm{L}$ & 0.000052 & 0.0000526 & & & & \\
\hline 766 & ITASRL & 0.00000841 & $\mathrm{pCi} / \mathrm{L}$ & 0.00000849 & 0.00000857 & & & $U$ & \\
\hline 767 & ITASRL & 0.00000993 & $\mathrm{pCi} / \mathrm{L}$ & 0.0000241 & 0.0000243 & & & U & \\
\hline 768 & ITASRL & 0.00001 & $\mathrm{pCi} / \mathrm{L}$ & 0.0000502 & 0.0000503 & & & $U$ & \\
\hline 769 & ITASRL & 0.000018 & $\mathrm{pCi} / \mathrm{L}$ & 0.0000114 & 0.0000116 & & & & \\
\hline 770 & ITASRL & 0.0000555 & $\mathrm{pCi} / \mathrm{L}$ & 0.000099 & 0.0000994 & & & $U$ & \\
\hline 771 & ITASRL & 0.00000786 & $\mathrm{pCi} / \mathrm{L}$ & 0.0000104 & 0.0000105 & & & $U$ & \\
\hline 772 & ITASRL & -0.0000041 & $\mathrm{pCi} / \mathrm{L}$ & 0 & 0.0000819 & & & $U$ & \\
\hline 773 & ITASRL & 0.0000138 & $\mathrm{pCi} / \mathrm{L}$ & 0.0000124 & 0.0000125 & & & & \\
\hline 774 & ITASRL & 0.0000205 & $\mathrm{pCi} / \mathrm{L}$ & 0.0000113 & 0.0000116 & & & & \\
\hline 775 & ITASRL & 0.000577 & $\mathrm{pCi} / \mathrm{L}$ & 0.000255 & 0.000266 & & & & \\
\hline 776 & ITASRL & 0.0000378 & $\mathrm{pCi} / \mathrm{L}$ & 0.0000192 & 0.0000196 & & & & \\
\hline 777 & ITASRL & 0.000627 & $\mathrm{pCi} / \mathrm{L}$ & 0.000194 & 0.000207 & & & & \\
\hline 778 & ITASRL & 0.0000172 & $\mathrm{pCi} / \mathrm{L}$ & 0.0000164 & 0.0000165 & & & & \\
\hline 779 & ITASRL & 0.000197 & $\mathrm{pCi} / \mathrm{L}$ & 0.000122 & 0.000125 & & & & \\
\hline 780 & ITASRL & 0.000201 & $\mathrm{pCi} / \mathrm{L}$ & 0.0000908 & 0.0000932 & & & & \\
\hline 781 & ITASRL & 0.0000969 & $\mathrm{pCi} / \mathrm{L}$ & 0.0000382 & 0.0000395 & & & & \\
\hline 782 & ITASRL & 0.0000782 & $\mathrm{pCi} / \mathrm{L}$ & 0.0000694 & 0.0000702 & & & & \\
\hline 783 & ITASRL & 0.00000325 & $\mathrm{pCi} / \mathrm{L}$ & 0.0000145 & 0.0000148 & & & $U$ & \\
\hline 784 & ITASRL & 0.0000414 & $\mathrm{pCi} / \mathrm{L}$ & 0.0000464 & 0.0000468 & & & $U$ & \\
\hline 785 & ITASRL & 0.0000227 & $\mathrm{pCi} / \mathrm{L}$ & 0.0000111 & 0.0000114 & & & & \\
\hline 786 & ITASRL & 0.0000226 & $\mathrm{pCi} / \mathrm{L}$ & 0.0000544 & 0.0000547 & & & $U$ & \\
\hline 787 & ITASRL & 0.0000204 & $\mathrm{pCi} / \mathrm{L}$ & 0.0000101 & 0.0000103 & & & & \\
\hline 788 & ITASRL & 0.0000133 & $\mathrm{pCi} / \mathrm{L}$ & 0.0000355 & 0.0000358 & & & U & \\
\hline 789 & ITASRL & 0.00000889 & $\mathrm{pCi} / \mathrm{L}$ & 0.00000923 & 0.00000941 & & & $U$ & \\
\hline 790 & ITASRL & 0.0000136 & $\mathrm{pCi} / \mathrm{L}$ & 0.00000839 & 0.00000857 & & & & \\
\hline 791 & ITASRL & 0.0000227 & $\mathrm{pCi} / \mathrm{L}$ & 0.0000482 & 0.0000484 & & & U & \\
\hline 792 & ITASRL & 0.00000188 & $\mathrm{pCi} / \mathrm{L}$ & 0.00000558 & 0.00000567 & & & $U$ & \\
\hline 793 & ITASRL & 0.0000507 & $\mathrm{pCi} / \mathrm{L}$ & 0.0000777 & 0.0000781 & & & U & \\
\hline 794 & ITASRL & 0.00000822 & $\mathrm{pCi} / \mathrm{L}$ & 0.00000867 & 0.00000878 & & & U & \\
\hline 795 & ITASRL & 0.0000315 & $\mathrm{pCi} / \mathrm{L}$ & 0.000051 & 0.0000513 & & & $U$ & \\
\hline 796 & ITASRL & 0.000000029 & $\mathrm{pCi} / \mathrm{L}$ & 1.68E-08 & 1.71E-08 & & & & $\mathrm{F}$ \\
\hline 797 & ITASRL & 0.0000539 & $\mathrm{pCi} / \mathrm{L}$ & 0.0000683 & 0.0000688 & & & $U$ & \\
\hline 798 & ITASRL & 0.000000024 & $\mathrm{pCi} / \mathrm{L}$ & $1.45 E-08$ & 1.48E-08 & & & & $\mathrm{F}$ \\
\hline 799 & ITASRL & 0.0000332 & $\mathrm{pCi} / \mathrm{L}$ & 0.0000948 & 0.000095 & & & U & \\
\hline
\end{tabular}




\begin{tabular}{|c|c|c|c|c|c|c|c|c|c|}
\hline & $\mathrm{L}$ & $\mathrm{M}$ & $\mathrm{N}$ & $\mathrm{O}$ & $\mathrm{P}$ & $\mathrm{Q}$ & $\mathrm{R}$ & $\mathrm{S}$ & $\mathrm{T}$ \\
\hline 1 & LAB_CODE & VALUE_RPTD & ANAL_UNITS_RPTD & COUNTING_ERROR & TOTAL_ANAL_ERROR & MIN_DETECTABLE_ACTIVITY & PCNT_MOISTURE & LAB_QUALIFIER & REVIEW_QUALIFIER \\
\hline 800 & ITASRL & -0.00000892 & $\mathrm{pCi} / \mathrm{L}$ & 0.0000175 & 0.0000176 & & & $U$ & \\
\hline 801 & ITASRL & -0.000166 & $\mathrm{pCi} / \mathrm{L}$ & 0.000192 & 0.000195 & & & $U$ & \\
\hline 802 & ITASRL & 0.0000226 & $\mathrm{pCi} / \mathrm{L}$ & 0.0000826 & 0.0000827 & & & $U$ & \\
\hline 803 & ITASRL & 0.0000323 & $3 \mathrm{pCi} / \mathrm{L}$ & 0.0000162 & 0.0000167 & & & & \\
\hline 804 & ITASRL & 0.0000131 & $\mathrm{pCi} / \mathrm{L}$ & 0.0000117 & 0.0000118 & & & & \\
\hline 805 & ITASRL & 0.0000609 & $\mathrm{pCi} / \mathrm{L}$ & 0.0000527 & 0.0000532 & & & & $F$ \\
\hline 806 & ITASRL & 0.000109 & $\mathrm{pCi} / \mathrm{L}$ & 0.0000631 & 0.0000643 & & & & $\mathrm{~F}$ \\
\hline 807 & ITASRL & 0.0000177 & $\mathrm{pCi} / \mathrm{L}$ & 0.00000883 & 0.00000901 & & & & \\
\hline 808 & ITASRL & 0.0000136 & $\mathrm{pCi} / \mathrm{L}$ & 0.0000112 & 0.0000113 & & & & \\
\hline 809 & ITASRL & 0.0000513 & $3 \mathrm{pCi} / \mathrm{L}$ & 0.0000543 & 0.0000547 & & & $U$ & \\
\hline 810 & ITASRL & 0.0000146 & $\mathrm{pCi} / \mathrm{L}$ & 0.0000132 & 0.0000133 & & & & \\
\hline 811 & ITASRL & 0.0000695 & $\mathrm{pCi} / \mathrm{L}$ & 0.0000802 & 0.0000809 & & & $U$ & \\
\hline 812 & ITASRL & 0.000031 & $\mathrm{pCi} / \mathrm{L}$ & 0.0000161 & 0.0000165 & & & & \\
\hline 813 & ITASRL & & $\mathrm{pCi} / \mathrm{L}$ & 0.0000441 & 0.0000468 & & & U & \\
\hline 814 & ITASRL & 0.000024 & $4 \mathrm{pCi} / \mathrm{L}$ & 0.000048 & 0.0000482 & & & $U$ & \\
\hline 815 & ITASRL & 0.0000564 & $4 \mathrm{pCi} / \mathrm{L}$ & 0.0000247 & 0.0000256 & & & & $F$ \\
\hline 816 & ITASRL & 0.0000549 & $\mathrm{pCi} / \mathrm{L}$ & 0.000029 & 0.0000298 & & & & $\mathrm{~F}$ \\
\hline 817 & ITASRL & 0.0000309 & $\mathrm{pCi} / \mathrm{L}$ & 0.0000437 & 0.0000439 & & & $U$ & \\
\hline 818 & ITASRL & 0.0000104 & $4 \mathrm{pCi} / \mathrm{L}$ & 0.0000055 & 0.00000564 & & & & \\
\hline 819 & ITASRL & 0.00000519 & $\mathrm{pCi} / \mathrm{L}$ & 0.0000186 & 0.0000188 & & & U & \\
\hline
\end{tabular}




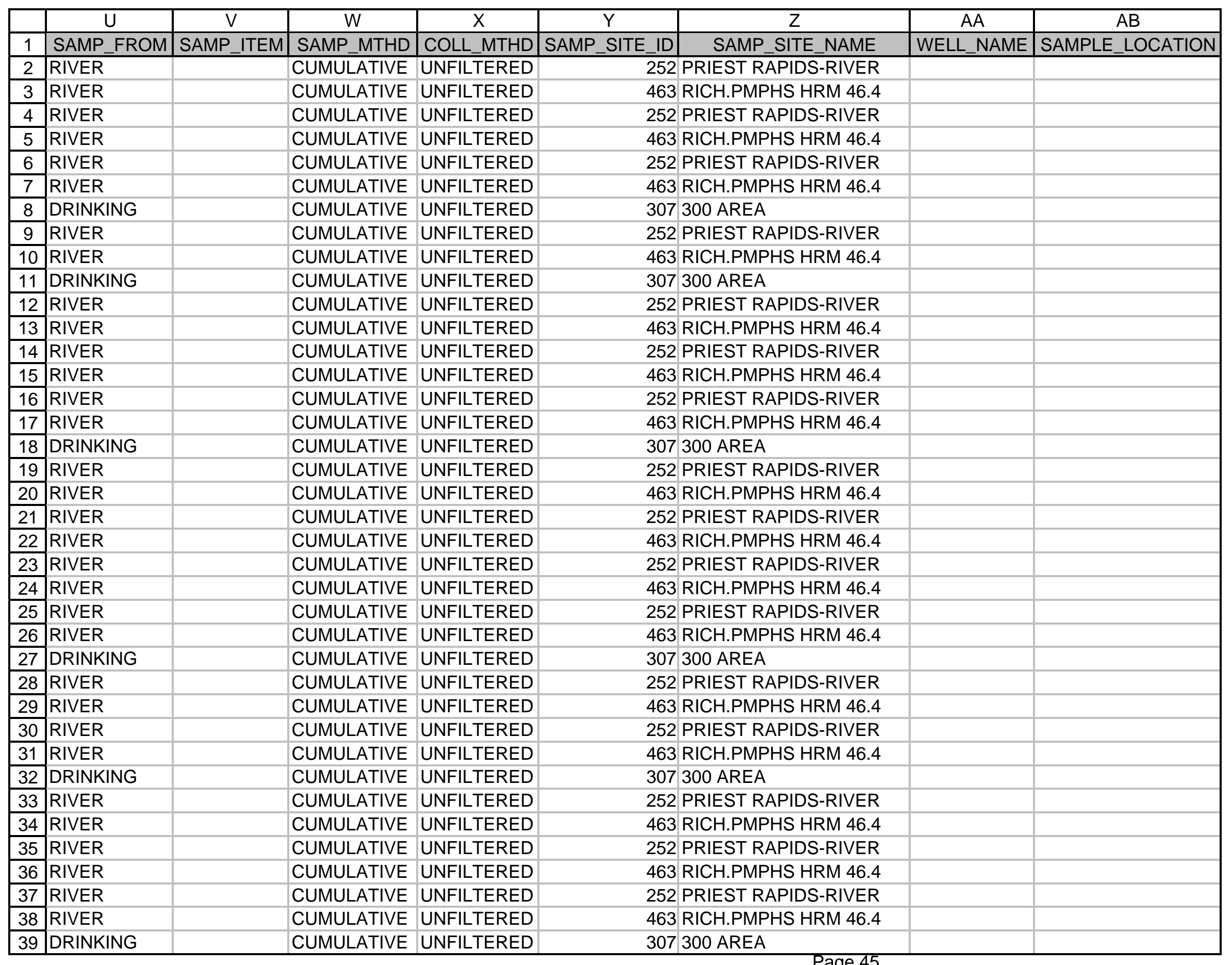

Page 45 


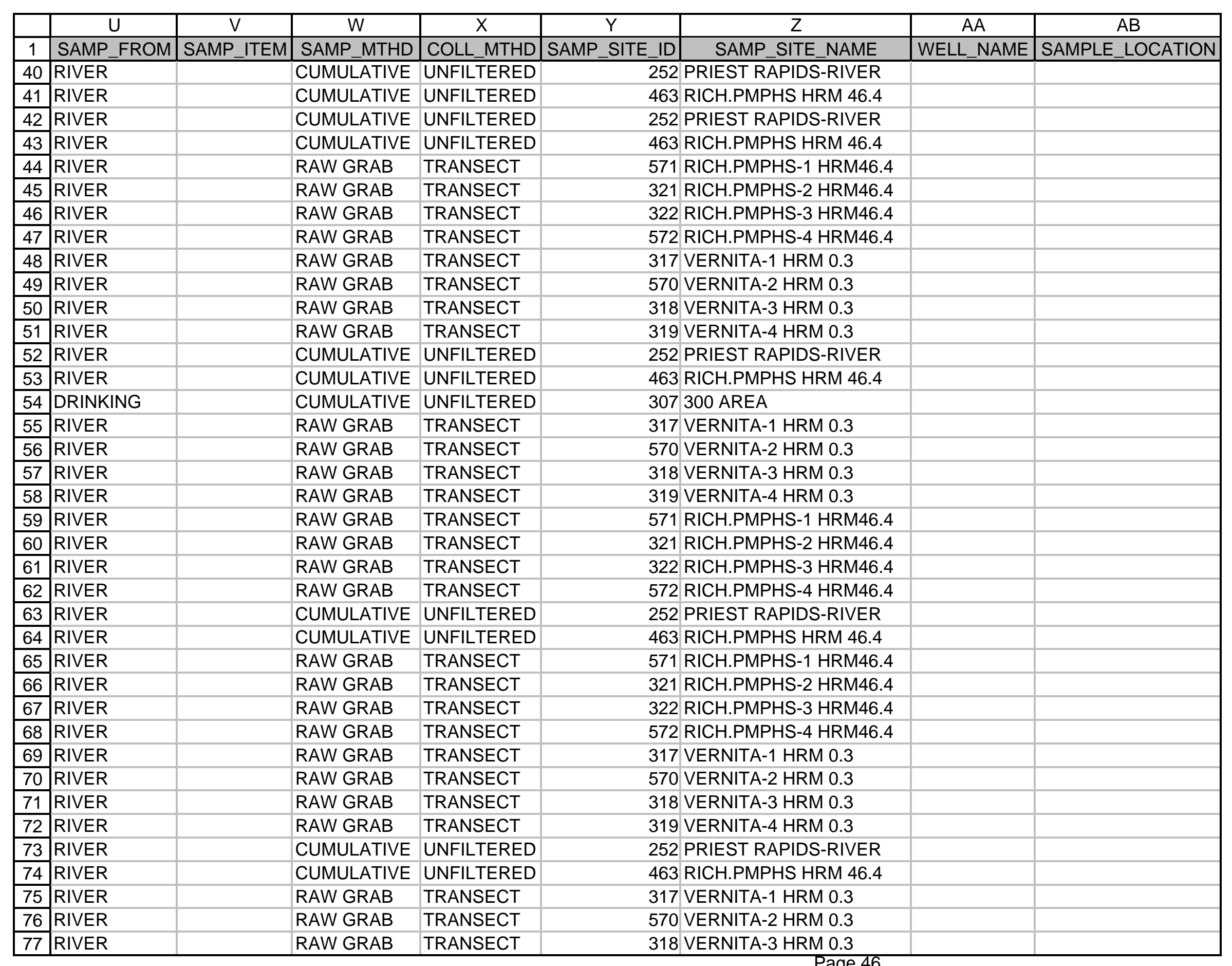




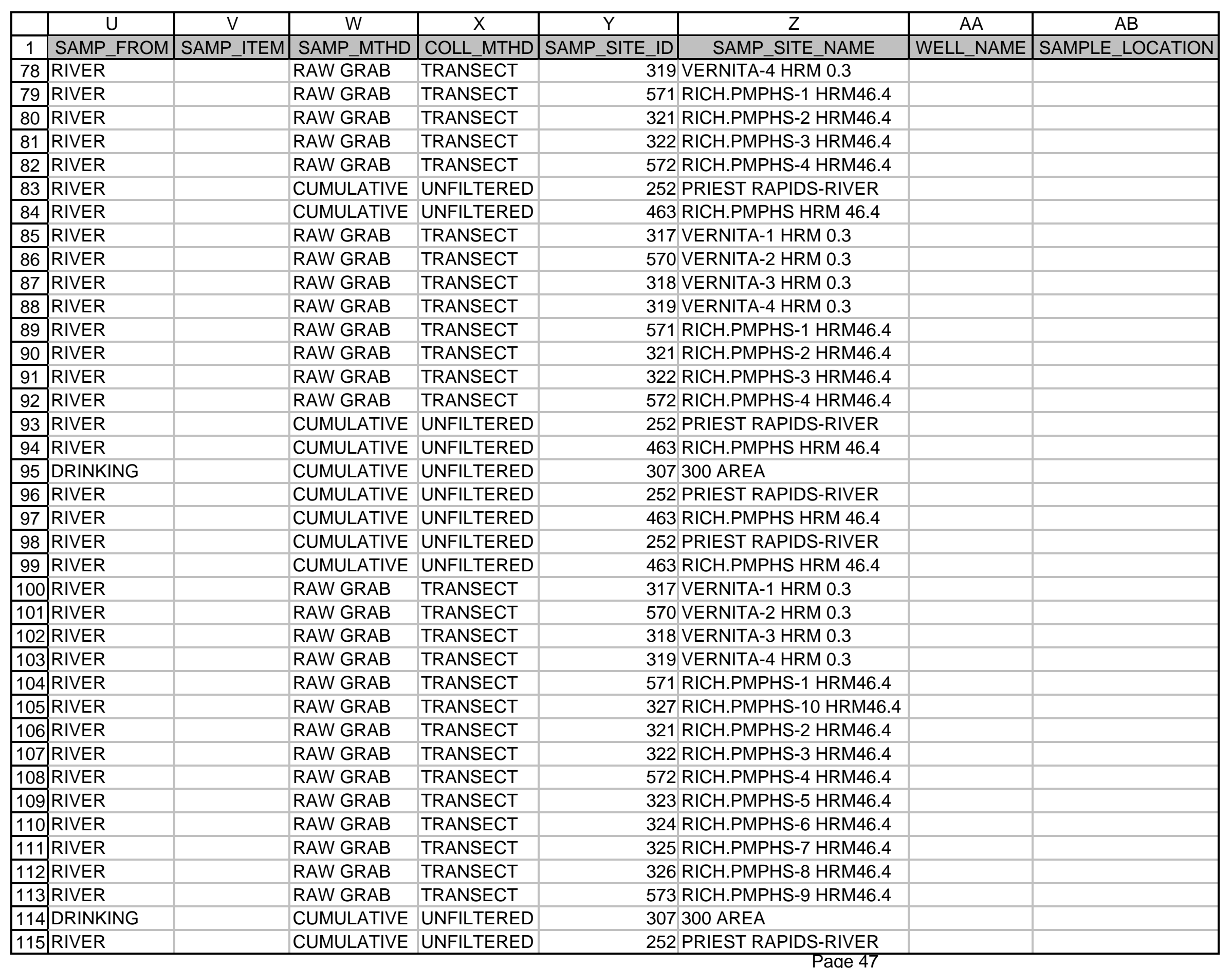




\begin{tabular}{|c|c|c|c|c|c|c|c|c|}
\hline & $\bar{U}$ & $\mathrm{~V}$ & $\mathrm{~W}$ & $\mathrm{X}$ & $\bar{Y}$ & $\mathrm{Z}$ & AA & $A B$ \\
\hline 1 & SAMP_FROM & SAMP_ITEM & SAMP_MTHD & COLL_MTHD & SAMP_SITE_ID & SAMP_SITE_NAME & WELL_NAME & SAMPLE_LOCATION \\
\hline 116 & RIVER & & CUMULATIVE & UNFILTERED & 463 & RICH.PMPHS HRM 46.4 & & \\
\hline 117 & RIVER & & CUMULATIVE & UNFILTERED & 252 & PRIEST RAPIDS-RIVER & & \\
\hline 118 & RIVER & & CUMULATIVE & UNFILTERED & 463 & RICH.PMPHS HRM 46.4 & & \\
\hline 119 & RIVER & & CUMULATIVE & UNFILTERED & 252 & PRIEST RAPIDS-RIVER & & \\
\hline 120 & RIVER & & CUMULATIVE & UNFILTERED & 463 & RICH.PMPHS HRM 46.4 & & \\
\hline 121 & RIVER & & RAW GRAB & TRANSECT & 317 & VERNITA-1 HRM 0.3 & & \\
\hline 122 & RIVER & & RAW GRAB & TRANSECT & 570 & VERNITA-2 HRM 0.3 & & \\
\hline 123 & RIVER & & RAW GRAB & TRANSECT & 318 & VERNITA-3 HRM 0.3 & & \\
\hline 124 & RIVER & & RAW GRAB & TRANSECT & 319 & VERNITA-4 HRM 0.3 & & \\
\hline 125 & RIVER & & RAW GRAB & TRANSECT & 571 & RICH.PMPHS-1 HRM46.4 & & \\
\hline 126 & RIVER & & RAW GRAB & TRANSECT & 327 & RICH.PMPHS-10 HRM46.4 & & \\
\hline 127 & RIVER & & RAW GRAB & TRANSECT & 321 & RICH.PMPHS-2 HRM46.4 & & \\
\hline 128 & RIVER & & RAW GRAB & TRANSECT & 322 & RICH.PMPHS-3 HRM46.4 & & \\
\hline 129 & RIVER & & RAW GRAB & TRANSECT & 572 & RICH.PMPHS-4 HRM46.4 & & \\
\hline 130 & RIVER & & RAW GRAB & TRANSECT & 323 & RICH.PMPHS-5 HRM46.4 & & \\
\hline 131 & RIVER & & RAW GRAB & TRANSECT & 324 & RICH.PMPHS-6 HRM46.4 & & \\
\hline 132 & RIVER & & RAW GRAB & TRANSECT & 325 & RICH.PMPHS-7 HRM46.4 & & \\
\hline 133 & RIVER & & RAW GRAB & TRANSECT & 326 & RICH.PMPHS-8 HRM46.4 & & \\
\hline 134 & RIVER & & RAW GRAB & TRANSECT & 573 & RICH.PMPHS-9 HRM46.4 & & \\
\hline 135 & DRINKING & & CUMULATIVE & UNFILTERED & 307 & 300 AREA & & \\
\hline 136 & RIVER & & CUMULATIVE & UNFILTERED & 252 & PRIEST RAPIDS-RIVER & & \\
\hline 137 & RIVER & & CUMULATIVE & UNFILTERED & 463 & RICH.PMPHS HRM 46.4 & & \\
\hline 138 & RIVER & & CUMULATIVE & UNFILTERED & 252 & PRIEST RAPIDS-RIVER & & \\
\hline 139 & RIVER & & CUMULATIVE & UNFILTERED & 463 & RICH.PMPHS HRM 46.4 & & \\
\hline 140 & RIVER & & CUMULATIVE & UNFILTERED & 252 & PRIEST RAPIDS-RIVER & & \\
\hline 141 & RIVER & & CUMULATIVE & UNFILTERED & 463 & RICH.PMPHS HRM 46.4 & & \\
\hline 142 & RIVER & & CUMULATIVE & UNFILTERED & 252 & PRIEST RAPIDS-RIVER & & \\
\hline 143 & RIVER & & CUMULATIVE & UNFILTERED & 463 & RICH.PMPHS HRM 46.4 & & \\
\hline 144 & RIVER & & RAW GRAB & TRANSECT & 317 & VERNITA-1 HRM 0.3 & & \\
\hline 145 & RIVER & & RAW GRAB & TRANSECT & 570 & VERNITA-2 HRM 0.3 & & \\
\hline 146 & RIVER & & RAW GRAB & TRANSECT & 318 & VERNITA-3 HRM 0.3 & & \\
\hline 147 & RIVER & & RAW GRAB & TRANSECT & 319 & VERNITA-4 HRM 0.3 & & \\
\hline 148 & RIVER & & RAW GRAB & TRANSECT & 571 & RICH.PMPHS-1 HRM46.4 & & \\
\hline 149 & RIVER & & RAW GRAB & TRANSECT & 327 & RICH.PMPHS-10 HRM46.4 & & \\
\hline 150 & RIVER & & RAW GRAB & TRANSECT & 321 & RICH.PMPHS-2 HRM46.4 & & \\
\hline 151 & RIVER & & RAW GRAB & TRANSECT & 322 & RICH.PMPHS-3 HRM46.4 & & \\
\hline 152 & RIVER & & RAW GRAB & TRANSECT & 572 & RICH.PMPHS-4 HRM46.4 & & \\
\hline 153 & RIVER & & RAW GRAB & TRANSECT & 323 & RICH.PMPHS-5 HRM46.4 & & \\
\hline
\end{tabular}




\begin{tabular}{|c|c|c|c|c|c|c|c|c|}
\hline & $\bar{U}$ & $\mathrm{~V}$ & $\mathrm{~W}$ & $\bar{X}$ & $\bar{Y}$ & $\bar{Z}$ & AA & $A B$ \\
\hline 1 & SAMP_FROM & SAMP_ITEM & SAMP_MTHD & COLL_MTHD & SAMP_SITE_ID & SAMP_SITE_NAME & WELL_NAME & SAMPLE_LOCATION \\
\hline 155 & RIVER & & RAW GRAB & TRANSECT & 325 & RICH.PMPHS-7 HRM46.4 & & \\
\hline 157 & RIVER & & RAW GRAB & TRANSECT & 573 & RICH.PMPHS-9 HRM46.4 & & \\
\hline 158 & DRINKING & & CUMULATIVE & UNFILTERED & 307 & 300 AREA & & \\
\hline 159 & RIVER & & CUMULATIVE & UNFILTERED & 252 & PRIEST RAPIDS-RIVER & & \\
\hline 160 & RIVER & & CUMULATIVE & UNFILTERED & 463 & RICH.PMPHS HRM 46.4 & & \\
\hline 162 & RIVER & & CUMULATIVE & UNFILTERED & 463 & RICH.PMPHS HRM 46.4 & & \\
\hline 163 & RIVER & & RAW GRAB & TRANSECT & 571 & RICH.PMPHS-1 HRM46.4 & & \\
\hline 164 & RIVER & & RAW GRAB & TRANSECT & 327 & RICH.PMPHS-10 HRM46.4 & & \\
\hline 165 & RIVER & & RAW GRAB & TRANSECT & 321 & RICH.PMPHS-2 HRM46.4 & & \\
\hline 166 & RIVER & & RAW GRAB & TRANSECT & 322 & RICH.PMPHS-3 HRM46.4 & & \\
\hline 167 & RIVER & & RAW GRAB & TRANSECT & 572 & RICH.PMPHS-4 HRM46.4 & & \\
\hline 168 & RIVER & & RAW GRAB & TRANSECT & 323 & RICH.PMPHS-5 HRM46.4 & & \\
\hline 173 & RIVER & & RAW GRAB & TRANSECT & 317 & VERNITA-1 HRM 0.3 & & \\
\hline 174 & RIVER & & RAW GRAB & TRANSECT & 570 & VERNITA-2 HRM 0.3 & & \\
\hline 175 & RIVER & & RAW GRAB & TRANSECT & 318 & VERNITA-3 HRM 0.3 & & \\
\hline 176 & RIVER & & RAW GRAB & TRANSECT & 319 & VERNITA-4 HRM 0.3 & & \\
\hline 177 & RIVER & & CUMULATIVE & UNFILTERED & 252 & PRIEST RAPIDS-RIVER & & \\
\hline 178 & RIVER & & CUMULATIVE & UNFILTERED & 463 & RICH.PMPHS HRM 46.4 & & \\
\hline 179 & DRINKING & & CUMULATIVE & UNFILTERED & 307 & 300 AREA & & \\
\hline 180 & RIVER & & CUMULATIVE & UNFILTERED & 252 & PRIEST RAPIDS-RIVER & & \\
\hline 181 & RIVER & & CUMULATIVE & UNFILTERED & 463 & RICH.PMPHS HRM 46.4 & & \\
\hline 182 & RIVER & & CUMULATIVE & UNFILTERED & 252 & PRIEST RAPIDS-RIVER & & \\
\hline 183 & RIVER & & CUMULATIVE & UNFILTERED & 463 & RICH.PMPHS HRM 46.4 & & \\
\hline 184 & RIVER & & RAW GRAB & TRANSECT & 317 & VERNITA-1 HRM 0.3 & & \\
\hline
\end{tabular}




\begin{tabular}{|c|c|c|c|c|c|c|c|c|}
\hline & $\bar{U}$ & $\mathrm{~V}$ & $\mathrm{~W}$ & $\mathrm{X}$ & $\bar{Y}$ & $\bar{Z}$ & $\overline{A A}$ & $A B$ \\
\hline 1 & SAMP_FROM & SAMP_ITEM & SAMP_MTHD & COLL_MTHD & SAMP_SITE_ID & SAMP_SITE_NAME & WELL_NAME & SAMPLE_LOCATION \\
\hline 193 & RIVER & & RAW GRAB & TRANSECT & 322 & RICH.PMPHS-3 HRM46.4 & & \\
\hline 195 & RIVER & & RAW GRAB & TRANSECT & 323 & RICH.PMPHS-5 HRM46.4 & & \\
\hline 196 & RIVER & & RAW GRAB & TRANSECT & 324 & RICH.PMPHS-6 HRM46.4 & & \\
\hline 197 & RIVER & & RAW GRAB & TRANSECT & 325 & RICH.PMPHS-7 HRM46.4 & & \\
\hline 198 & RIVER & & RAW GRAB & TRANSECT & 326 & RICH.PMPHS-8 HRM46.4 & & \\
\hline 200 & DRINKING & & CUMULATIVE & UNFILTERED & 307 & 300 AREA & & \\
\hline 201 & RIVER & & CUMULATIVE & UNFILTERED & 252 & PRIEST RAPIDS-RIVER & & \\
\hline 202 & RIVER & & CUMULATIVE & UNFILTERED & 463 & RICH.PMPHS HRM 46.4 & & \\
\hline 203 & RIVER & & CUMULATIVE & UNFILTERED & 252 & PRIEST RAPIDS-RIVER & & \\
\hline 204 & RIVER & & CUMULATIVE & UNFILTERED & 463 & RICH.PMPHS HRM 46.4 & & \\
\hline 205 & RIVER & & CUMULATIVE & UNFILTERED & 252 & PRIEST RAPIDS-RIVER & & \\
\hline 206 & RIVER & & CUMULATIVE & UNFILTERED & 463 & RICH.PMPHS HRM 46.4 & & \\
\hline 211 & RIVER & & RAW GRAB & TRANSECT & 570 & VERNITA-2 HRM 0.3 & & \\
\hline 212 & RIVER & & RAW GRAB & TRANSECT & 318 & VERNITA-3 HRM 0.3 & & \\
\hline 213 & RIVER & & RAW GRAB & TRANSECT & 319 & VERNITA-4 HRM 0.3 & & \\
\hline 214 & RIVER & & RAW GRAB & TRANSECT & 571 & RICH.PMPHS-1 HRM46.4 & & \\
\hline 215 & RIVER & & RAW GRAB & TRANSECT & 327 & RICH.PMPHS-10 HRM46.4 & & \\
\hline 216 & RIVER & & RAW GRAB & TRANSECT & 321 & RICH.PMPHS-2 HRM46.4 & & \\
\hline 217 & RIVER & & RAW GRAB & TRANSECT & 322 & RICH.PMPHS-3 HRM46.4 & & \\
\hline 218 & RIVER & & RAW GRAB & TRANSECT & 572 & RICH.PMPHS-4 HRM46.4 & & \\
\hline 219 & RIVER & & RAW GRAB & TRANSECT & 323 & RICH.PMPHS-5 HRM46.4 & & \\
\hline 220 & RIVER & & RAW GRAB & TRANSECT & 324 & RICH.PMPHS-6 HRM46.4 & & \\
\hline 221 & RIVER & & RAW GRAB & TRANSECT & 325 & RICH.PMPHS-7 HRM46.4 & & \\
\hline 222 & RIVER & & RAW GRAB & TRANSECT & 326 & RICH.PMPHS-8 HRM46.4 & & \\
\hline
\end{tabular}




\begin{tabular}{|c|c|c|c|c|c|c|c|c|c|}
\hline & $\bar{U}$ & $\mathrm{~V}$ & $\mathrm{~W}$ & $\bar{X}$ & & $\bar{Y}$ & $\bar{Z}$ & $\overline{A A}$ & $A B$ \\
\hline 1 & SAMP_FROM & SAMP_ITEM & SAMP_MTHD & COLL_MTHD & SAMP & SITE_ID & SAMP_SITE_NAME & WELL_NAME & SAMPLE_LOCATION \\
\hline 230 & RIVER & & RAW GRAB & TRANSECT & & 318 & VERNITA-3 HRM 0.3 & & \\
\hline 231 & RIVER & & RAW GRAB & TRANSECT & & 319 & VERNITA-4 HRM 0.3 & & \\
\hline 232 & RIVER & & RAW GRAB & TRANSECT & & 348 & 300 AREA -1 HRM 43.1 & & \\
\hline 233 & RIVER & & RAW GRAB & TRANSECT & & 470 & 300 AREA -2 HRM 43.1 & & \\
\hline 234 & RIVER & & RAW GRAB & TRANSECT & & 580 & 300 AREA -3 HRM 43.1 & & \\
\hline 235 & RIVER & & RAW GRAB & TRANSECT & & 349 & 300 AREA -4 HRM 43.1 & & \\
\hline 236 & RIVER & & RAW GRAB & TRANSECT & & 607 & 300 AREA -5 HRM 43.1 & & \\
\hline 237 & RIVER & & RAW GRAB & TRANSECT & & 350 & 300 AREA -6 HRM 43.1 & & \\
\hline 238 & RIVER & & RAW GRAB & TRANSECT & & 351 & 300 AREA -7 HRM 43.1 & & \\
\hline 239 & RIVER & & RAW GRAB & TRANSECT & & 581 & 300 AREA -8 HRM 43.1 & & \\
\hline 240 & RIVER & & RAW GRAB & TRANSECT & & 471 & 300 AREA -9 HRM 43.1 & & \\
\hline 241 & RIVER & & RAW GRAB & TRANSECT & & 328 & 3100 N -1 HRM 9.5 & & \\
\hline 242 & RIVER & & RAW GRAB & TRANSECT & & 335 & 100 N -10 HRM 9.5 & & \\
\hline 243 & RIVER & & RAW GRAB & TRANSECT & & 329 & 100 N -2 HRM 9.5 & & \\
\hline 244 & RIVER & & RAW GRAB & TRANSECT & & 330 & 100 N -3 HRM 9.5 & & \\
\hline 245 & RIVER & & RAW GRAB & TRANSECT & & 574 & 100 N -4 HRM 9.5 & & \\
\hline 246 & RIVER & & RAW GRAB & TRANSECT & & 331 & 100 N -5 HRM 9.5 & & \\
\hline 247 & RIVER & & RAW GRAB & TRANSECT & & 332 & 100 N -6 HRM 9.5 & & \\
\hline 248 & RIVER & & RAW GRAB & TRANSECT & & 333 & 100 N - 7 HRM 9.5 & & \\
\hline 249 & RIVER & & RAW GRAB & TRANSECT & & 575 & 100 N -8 HRM 9.5 & & \\
\hline 250 & RIVER & & RAW GRAB & TRANSECT & & 334 & 100 N -9 HRM 9.5 & & \\
\hline 251 & RIVER & & RAW GRAB & TRANSECT & & 578 & HANFRD TS-1 HRM 28.7 & & \\
\hline 252 & RIVER & & RAW GRAB & TRANSECT & & 347 & HANFRD TS-10 HRM 28.7 & & \\
\hline 253 & RIVER & & RAW GRAB & TRANSECT & & 342 & HANFRD TS-2 HRM 28.7 & & \\
\hline 254 & RIVER & & RAW GRAB & TRANSECT & & 468 & 3 HANFRD TS-3 HRM 28.7 & & \\
\hline 255 & RIVER & & RAW GRAB & TRANSECT & & 343 & HANFRD TS-4 HRM 28.7 & & \\
\hline 256 & RIVER & & RAW GRAB & TRANSECT & & 344 & HANFRD TS-5 HRM 28.7 & & \\
\hline 257 & RIVER & & RAW GRAB & TRANSECT & & 345 & HANFRD TS-6 HRM 28.7 & & \\
\hline 258 & RIVER & & RAW GRAB & TRANSECT & & 469 & HANFRD TS-7 HRM 28.7 & & \\
\hline 259 & RIVER & & RAW GRAB & TRANSECT & & 579 & HANFRD TS-8 HRM 28.7 & & \\
\hline 260 & RIVER & & RAW GRAB & TRANSECT & & 346 & HANFRD TS-9 HRM 28.7 & & \\
\hline 261 & RIVER & & CUMULATIVE & UNFILTERED & & 252 & PRIEST RAPIDS-RIVER & & \\
\hline 262 & RIVER & & CUMULATIVE & UNFILTERED & & 463 & RICH.PMPHS HRM 46.4 & & \\
\hline 263 & RIVER & & RAW GRAB & TRANSECT & & 571 & RICH.PMPHS-1 HRM46.4 & & \\
\hline 264 & RIVER & & RAW GRAB & TRANSECT & & 327 & RICH.PMPHS-10 HRM46.4 & & \\
\hline 265 & RIVER & & RAW GRAB & TRANSECT & & 321 & RICH.PMPHS-2 HRM46.4 & & \\
\hline 266 & RIVER & & RAW GRAB & TRANSECT & & 322 & RICH.PMPHS-3 HRM46.4 & & \\
\hline 267 & RIVER & & RAW GRAB & TRANSECT & & 572 & RICH.PMPHS-4 HRM46.4 & & \\
\hline
\end{tabular}




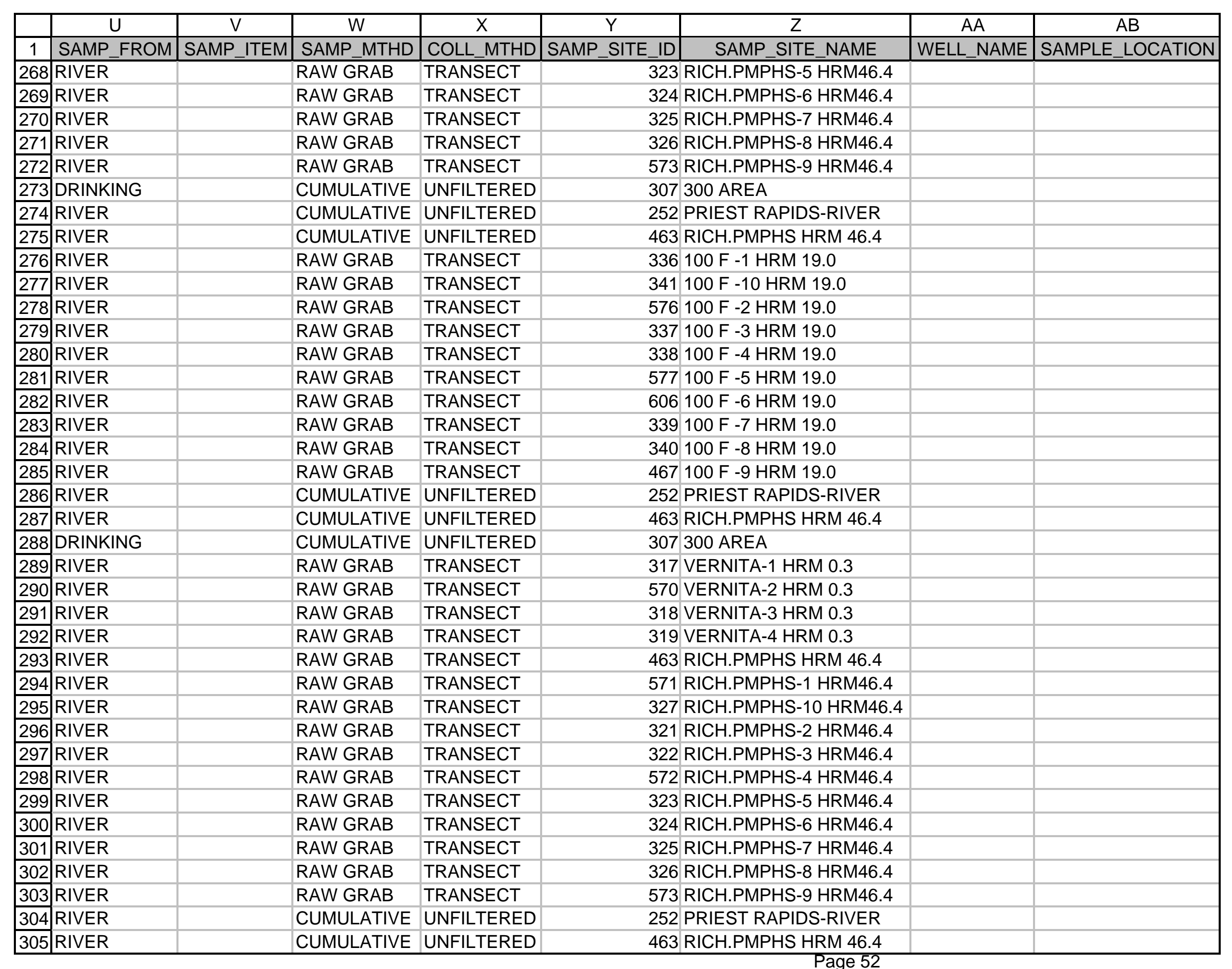




\begin{tabular}{|c|c|c|c|c|c|c|c|c|c|}
\hline & $\bar{U}$ & $\mathrm{~V}$ & $\mathrm{~W}$ & $\bar{X}$ & & $\bar{Y}$ & $\bar{Z}$ & $\overline{A A}$ & $A B$ \\
\hline 1 & SAMP_FROM & SAMP_ITEM & SAMP_MTHD & COLL_MTHD & SAMP & SITE_ID & SAMP_SITE_NAME & WELL_NAME & SAMPLE_LOCATION \\
\hline 306 & RIVER & & CUMULATIVE & UNFILTERED & & 252 & PRIEST RAPIDS-RIVER & & \\
\hline 307 & RIVER & & CUMULATIVE & UNFILTERED & & 463 & RICH.PMPHS HRM 46.4 & & \\
\hline 308 & RIVER & & CUMULATIVE & UNFILTERED & & 252 & PRIEST RAPIDS-RIVER & & \\
\hline 309 & RIVER & & CUMULATIVE & UNFILTERED & & 463 & RICH.PMPHS HRM 46.4 & & \\
\hline 310 & RIVER & & RAW GRAB & TRANSECT & & 317 & VERNITA-1 HRM 0.3 & & \\
\hline 311 & RIVER & & RAW GRAB & TRANSECT & & 570 & VERNITA-2 HRM 0.3 & & \\
\hline 312 & DRINKING & & CUMULATIVE & UNFILTERED & & 307 & 300 AREA & & \\
\hline 313 & RIVER & & CUMULATIVE & UNFILTERED & & 252 & PRIEST RAPIDS-RIVER & & \\
\hline 314 & RIVER & & CUMULATIVE & UNFILTERED & & 463 & RICH.PMPHS HRM 46.4 & & \\
\hline 315 & RIVER & & RAW GRAB & TRANSECT & & 571 & RICH.PMPHS-1 HRM46.4 & & \\
\hline 316 & RIVER & & RAW GRAB & TRANSECT & & 327 & RICH.PMPHS-10 HRM46.4 & & \\
\hline 317 & RIVER & & RAW GRAB & TRANSECT & & 321 & RICH.PMPHS-2 HRM46.4 & & \\
\hline 318 & RIVER & & RAW GRAB & TRANSECT & & 322 & RICH.PMPHS-3 HRM46.4 & & \\
\hline 319 & RIVER & & RAW GRAB & TRANSECT & & 572 & RICH.PMPHS-4 HRM46.4 & & \\
\hline 320 & RIVER & & RAW GRAB & TRANSECT & & 323 & RICH.PMPHS-5 HRM46.4 & & \\
\hline 321 & RIVER & & RAW GRAB & TRANSECT & & 324 & RICH.PMPHS-6 HRM46.4 & & \\
\hline 322 & RIVER & & RAW GRAB & TRANSECT & & 325 & RICH.PMPHS-7 HRM46.4 & & \\
\hline 323 & RIVER & & RAW GRAB & TRANSECT & & 326 & RICH.PMPHS-8 HRM46.4 & & \\
\hline 324 & RIVER & & RAW GRAB & TRANSECT & & 573 & RICH.PMPHS-9 HRM46.4 & & \\
\hline 325 & RIVER & & CUMULATIVE & UNFILTERED & & 252 & PRIEST RAPIDS-RIVER & & \\
\hline 326 & RIVER & & CUMULATIVE & UNFILTERED & & 463 & RICH.PMPHS HRM 46.4 & & \\
\hline 327 & RIVER & & CUMULATIVE & UNFILTERED & & 252 & PRIEST RAPIDS-RIVER & & \\
\hline 328 & RIVER & & CUMULATIVE & UNFILTERED & & 463 & RICH.PMPHS HRM 46.4 & & \\
\hline 329 & DRINKING & & CUMULATIVE & UNFILTERED & & 307 & 300 AREA & & \\
\hline 330 & RIVER & & RAW GRAB & TRANSECT & & 317 & VERNITA-1 HRM 0.3 & & \\
\hline 331 & RIVER & & RAW GRAB & TRANSECT & & 570 & VERNITA-2 HRM 0.3 & & \\
\hline 332 & RIVER & & RAW GRAB & TRANSECT & & 318 & VERNITA-3 HRM 0.3 & & \\
\hline 333 & RIVER & & RAW GRAB & TRANSECT & & 319 & VERNITA-4 HRM 0.3 & & \\
\hline 334 & RIVER & & RAW GRAB & TRANSECT & & 571 & RICH.PMPHS-1 HRM46.4 & & \\
\hline 335 & RIVER & & RAW GRAB & TRANSECT & & 327 & RICH.PMPHS-10 HRM46.4 & & \\
\hline 336 & RIVER & & RAW GRAB & TRANSECT & & 321 & RICH.PMPHS-2 HRM46.4 & & \\
\hline 337 & RIVER & & RAW GRAB & TRANSECT & & 322 & RICH.PMPHS-3 HRM46.4 & & \\
\hline 338 & RIVER & & RAW GRAB & TRANSECT & & 572 & RICH.PMPHS-4 HRM46.4 & & \\
\hline 339 & RIVER & & RAW GRAB & TRANSECT & & 323 & RICH.PMPHS-5 HRM46.4 & & \\
\hline 340 & RIVER & & RAW GRAB & TRANSECT & & 324 & RICH.PMPHS-6 HRM46.4 & & \\
\hline 341 & RIVER & & RAW GRAB & TRANSECT & & 325 & RICH.PMPHS-7 HRM46.4 & & \\
\hline 342 & RIVER & & RAW GRAB & TRANSECT & & 326 & RICH.PMPHS-8 HRM46.4 & & \\
\hline 343 & RIVER & & RAW GRAB & TRANSECT & & 573 & RICH.PMPHS-9 HRM46.4 & & \\
\hline
\end{tabular}




\begin{tabular}{|c|c|c|c|c|c|c|c|c|}
\hline & $\bar{U}$ & $\mathrm{~V}$ & $\mathrm{~W}$ & $\bar{x}$ & $\bar{Y}$ & $\bar{Z}$ & $\overline{\mathrm{AA}}$ & $A B$ \\
\hline 1 & SAMP_FROM & SAMP_ITEM & SAMP_MTHD & COLL_MTHD & SAMP_SITE_ID & SAMP_SITE_NAME & WELL_NAME & SAMPLE_LOCATION \\
\hline 344 & RIVER & & CUMULATIVE & UNFILTERED & $\overline{252}$ & PRIEST RAPIDS-RIVER & & \\
\hline 345 & RIVER & & CUMULATIVE & UNFILTERED & 463 & RICH.PMPHS HRM 46.4 & & \\
\hline 346 & RIVER & & CUMULATIVE & UNFILTERED & 252 & PRIEST RAPIDS-RIVER & & \\
\hline 347 & RIVER & & CUMULATIVE & UNFILTERED & 463 & RICH.PMPHS HRM 46.4 & & \\
\hline 348 & RIVER & & RAW GRAB & TRANSECT & 336 & 100 F -1 HRM 19.0 & & \\
\hline 349 & RIVER & & RAW GRAB & TRANSECT & 341 & 100 F -10 HRM 19.0 & & \\
\hline 350 & RIVER & & RAW GRAB & TRANSECT & 576 & 100 F -2 HRM 19.0 & & \\
\hline 351 & RIVER & & RAW GRAB & TRANSECT & 337 & 100 F -3 HRM 19.0 & & \\
\hline 352 & RIVER & & RAW GRAB & TRANSECT & 338 & 100 F -4 HRM 19.0 & & \\
\hline 353 & RIVER & & RAW GRAB & TRANSECT & 577 & 100 F -5 HRM 19.0 & & \\
\hline 354 & RIVER & & RAW GRAB & TRANSECT & 606 & 100 F -6 HRM 19.0 & & \\
\hline 355 & RIVER & & RAW GRAB & TRANSECT & 339 & 100 F -7 HRM 19.0 & & \\
\hline 356 & RIVER & & RAW GRAB & TRANSECT & 340 & 100 F -8 HRM 19.0 & & \\
\hline 357 & RIVER & & RAW GRAB & TRANSECT & 467 & 100 F -9 HRM 19.0 & & \\
\hline 358 & RIVER & & RAW GRAB & TRANSECT & 328 & 100 N -1 HRM 9.5 & & \\
\hline 359 & RIVER & & RAW GRAB & TRANSECT & 335 & 100 N -10 HRM 9.5 & & \\
\hline 360 & RIVER & & RAW GRAB & TRANSECT & 329 & 100 N -2 HRM 9.5 & & \\
\hline 361 & RIVER & & RAW GRAB & TRANSECT & 330 & 100 N -3 HRM 9.5 & & \\
\hline 362 & RIVER & & RAW GRAB & TRANSECT & 574 & 100 N -4 HRM 9.5 & & \\
\hline 363 & RIVER & & RAW GRAB & TRANSECT & 331 & 100 N -5 HRM 9.5 & & \\
\hline 364 & RIVER & & RAW GRAB & TRANSECT & 332 & 100 N -6 HRM 9.5 & & \\
\hline 365 & RIVER & & RAW GRAB & TRANSECT & 333 & 100 N - 7 HRM 9.5 & & \\
\hline 366 & RIVER & & RAW GRAB & TRANSECT & 575 & 100 N -8 HRM 9.5 & & \\
\hline 367 & RIVER & & RAW GRAB & TRANSECT & 334 & 100 N - 9 HRM 9.5 & & \\
\hline 368 & RIVER & & RAW GRAB & TRANSECT & 317 & VERNITA-1 HRM 0.3 & & \\
\hline 369 & RIVER & & RAW GRAB & TRANSECT & 570 & VERNITA-2 HRM 0.3 & & \\
\hline 370 & RIVER & & RAW GRAB & TRANSECT & 318 & VERNITA-3 HRM 0.3 & & \\
\hline 371 & RIVER & & RAW GRAB & TRANSECT & 319 & VERNITA-4 HRM 0.3 & & \\
\hline 372 & RIVER & & RAW GRAB & TRANSECT & 348 & 300 AREA -1 HRM 43.1 & & \\
\hline 373 & RIVER & & RAW GRAB & TRANSECT & 470 & 300 AREA -2 HRM 43.1 & & \\
\hline 374 & RIVER & & RAW GRAB & TRANSECT & 580 & 300 AREA -3 HRM 43.1 & & \\
\hline 375 & RIVER & & RAW GRAB & TRANSECT & 349 & 300 AREA -4 HRM 43.1 & & \\
\hline 376 & RIVER & & RAW GRAB & TRANSECT & 607 & 300 AREA -5 HRM 43.1 & & \\
\hline 377 & RIVER & & RAW GRAB & TRANSECT & 350 & 300 AREA - 6 HRM 43.1 & & \\
\hline 378 & RIVER & & RAW GRAB & TRANSECT & 351 & 300 AREA -7 HRM 43.1 & & \\
\hline 379 & RIVER & & RAW GRAB & TRANSECT & 581 & 300 AREA -8 HRM 43.1 & & \\
\hline 380 & RIVER & & RAW GRAB & TRANSECT & 471 & 300 AREA -9 HRM 43.1 & & \\
\hline 381 & RIVER & & RAW GRAB & TRANSECT & 571 & RICH.PMPHS-1 HRM46.4 & & \\
\hline
\end{tabular}




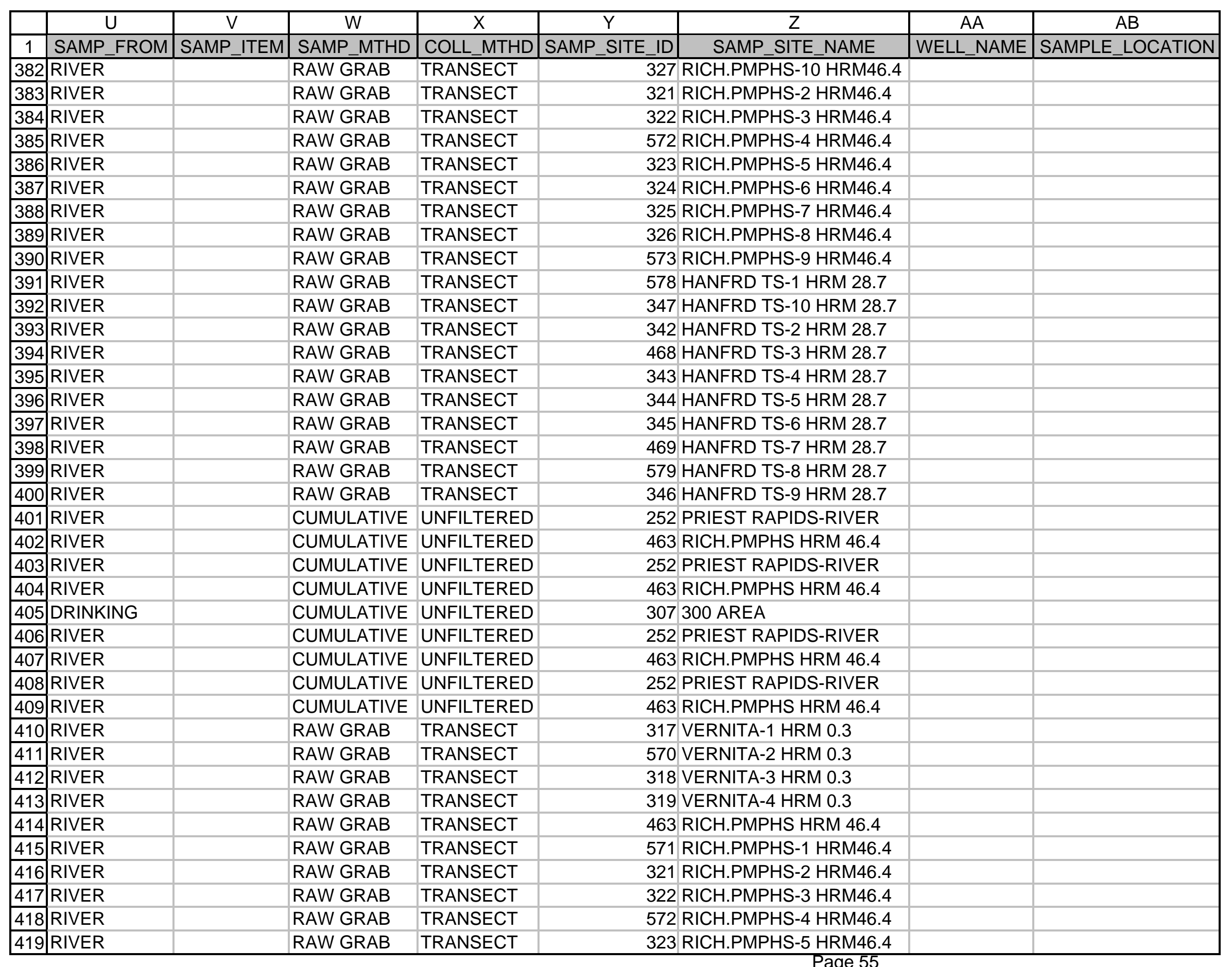




\begin{tabular}{|c|c|c|c|c|c|c|c|c|}
\hline & $\bar{U}$ & $\mathrm{~V}$ & $\mathrm{~W}$ & $\bar{X}$ & $\bar{Y}$ & $\bar{Z}$ & AA & $A B$ \\
\hline 1 & SAMP_FROM & SAMP_ITEM & SAMP_MTHD & COLL_MTHD & SAMP_SITE_ID & SAMP_SITE_NAME & WELL_NAME & SAMPLE_LOCATION \\
\hline 421 & RIVER & & RAW GRAB & TRANSECT & 325 & RICH.PMPHS-7 HRM46.4 & & \\
\hline 423 & RIVER & & RAW GRAB & TRANSECT & 573 & RICH.PMPHS-9 HRM46.4 & & \\
\hline 424 & RIVER & & RAW GRAB & TRANSECT & 327 & RICH.PMPHS-10 HRM46.4 & & \\
\hline 425 & DRINKING & & CUMULATIVE & UNFILTERED & 307 & 300 AREA & & \\
\hline 426 & RIVER & & CUMULATIVE & UNFILTERED & 252 & PRIEST RAPIDS-RIVER & & \\
\hline 429 & RIVER & & CUMULATIVE & UNFILTERED & 463 & RICH.PMPHS HRM 46.4 & & \\
\hline 430 & RIVER & & CUMULATIVE & UNFILTERED & 252 & PRIEST RAPIDS-RIVER & & \\
\hline 431 & RIVER & & CUMULATIVE & UNFILTERED & 463 & RICH.PMPHS HRM 46.4 & & \\
\hline 432 & RIVER & & RAW GRAB & TRANSECT & 317 & VERNITA-1 HRM 0.3 & & \\
\hline 433 & RIVER & & RAW GRAB & TRANSECT & 570 & VERNITA-2 HRM 0.3 & & \\
\hline 434 & RIVER & & RAW GRAB & TRANSECT & 318 & VERNITA-3 HRM 0.3 & & \\
\hline 439 & RIVER & & RAW GRAB & TRANSECT & 322 & RICH.PMPHS-3 HRM46.4 & & \\
\hline 440 & RIVER & & RAW GRAB & TRANSECT & 572 & RICH.PMPHS-4 HRM46.4 & & \\
\hline 441 & RIVER & & RAW GRAB & TRANSECT & 323 & RICH.PMPHS-5 HRM46.4 & & \\
\hline 442 & RIVER & & RAW GRAB & TRANSECT & 324 & RICH.PMPHS-6 HRM46.4 & & \\
\hline 443 & RIVER & & RAW GRAB & TRANSECT & 325 & RICH.PMPHS-7 HRM46.4 & & \\
\hline 444 & RIVER & & RAW GRAB & TRANSECT & 326 & RICH.PMPHS-8 HRM46.4 & & \\
\hline 445 & RIVER & & RAW GRAB & TRANSECT & 573 & RICH.PMPHS-9 HRM46.4 & & \\
\hline 446 & DRINKING & & CUMULATIVE & UNFILTERED & 307 & 300 AREA & & \\
\hline 447 & RIVER & & CUMULATIVE & UNFILTERED & 252 & PRIEST RAPIDS-RIVER & & \\
\hline 448 & RIVER & & CUMULATIVE & UNFILTERED & 463 & RICH.PMPHS HRM 46.4 & & \\
\hline 449 & RIVER & & CUMULATIVE & UNFILTERED & 252 & PRIEST RAPIDS-RIVER & & \\
\hline 450 & RIVER & & CUMULATIVE & UNFILTERED & 463 & RICH.PMPHS HRM 46.4 & & \\
\hline
\end{tabular}




\begin{tabular}{|c|c|c|c|c|c|c|c|c|c|}
\hline & $\bar{U}$ & $\mathrm{~V}$ & $\mathrm{~W}$ & $\bar{X}$ & & $\bar{Y}$ & $\bar{Z}$ & AA & $A B$ \\
\hline 1 & SAMP_FROM & SAMP_ITEM & SAMP_MTHD & COLL_MTHD & SAMP & SITE_ID & SAMP_SITE_NAME & WELL_NAME & SAMPLE_LOCATION \\
\hline 459 & RIVER & & RAW GRAB & TRANSECT & & 321 & RICH.PMPHS-2 HRM46.4 & & \\
\hline 461 & RIVER & & RAW GRAB & TRANSECT & & 572 & RICH.PMPHS-4 HRM46.4 & & \\
\hline 462 & RIVER & & RAW GRAB & TRANSECT & & 323 & RICH.PMPHS-5 HRM46.4 & & \\
\hline 463 & RIVER & & RAW GRAB & TRANSECT & & 324 & RICH.PMPHS-6 HRM46.4 & & \\
\hline 464 & RIVER & & RAW GRAB & TRANSECT & & 325 & RICH.PMPHS-7 HRM46.4 & & \\
\hline 467 & DRINKING & & CUMULATIVE & UNFILTERED & & 307 & 300 AREA & & \\
\hline 468 & RIVER & & CUMULATIVE & UNFILTERED & & 252 & PRIEST RAPIDS-RIVER & & \\
\hline 469 & RIVER & & CUMULATIVE & UNFILTERED & & 463 & RICH.PMPHS HRM 46.4 & & \\
\hline 470 & RIVER & & CUMULATIVE & UNFILTERED & & 252 & PRIEST RAPIDS-RIVER & & \\
\hline 471 & RIVER & & CUMULATIVE & UNFILTERED & & 463 & RICH.PMPHS HRM 46.4 & & \\
\hline 472 & RIVER & & CUMULATIVE & UNFILTERED & & 252 & PRIEST RAPIDS-RIVER & & \\
\hline 477 & RIVER & & RAW GRAB & TRANSECT & & 330 & 100 N -3 HRM 9.5 & & \\
\hline 478 & RIVER & & RAW GRAB & TRANSECT & & 574 & 100 N -4 HRM 9.5 & & \\
\hline 479 & RIVER & & RAW GRAB & TRANSECT & & 331 & 100 N -5 HRM 9.5 & & \\
\hline 480 & RIVER & & RAW GRAB & TRANSECT & & 332 & 100 N -6 HRM 9.5 & & \\
\hline 481 & RIVER & & RAW GRAB & TRANSECT & & 333 & 100 N -7 HRM 9.5 & & \\
\hline 482 & RIVER & & RAW GRAB & TRANSECT & & 575 & 100 N -8 HRM 9.5 & & \\
\hline 483 & RIVER & & RAW GRAB & TRANSECT & & 334 & 100 N -9 HRM 9.5 & & \\
\hline 484 & RIVER & & RAW GRAB & TRANSECT & & 317 & VERNITA-1 HRM 0.3 & & \\
\hline 485 & RIVER & & RAW GRAB & TRANSECT & & 570 & VERNITA-2 HRM 0.3 & & \\
\hline 486 & RIVER & & RAW GRAB & TRANSECT & & 318 & VERNITA-3 HRM 0.3 & & \\
\hline 487 & RIVER & & RAW GRAB & TRANSECT & & 319 & VERNITA-4 HRM 0.3 & & \\
\hline 488 & RIVER & & RAW GRAB & TRANSECT & & 336 & 100 F -1 HRM 19.0 & & \\
\hline
\end{tabular}

Page 57 


\begin{tabular}{|c|c|c|c|c|c|c|c|c|c|}
\hline & $\bar{U}$ & $\mathrm{~V}$ & $\mathrm{~W}$ & $\bar{x}$ & & $\bar{Y}$ & $\mathrm{Z}$ & AA & $A B$ \\
\hline 1 & SAMP_FROM & SAMP_ITEM & SAMP_MTHD & COLL_MTHD & SAMP & SITE_ID & SAMP_SITE_NAME & WELL_NAME & SAMPLE_LOCATION \\
\hline 496 & RIVER & & RAW GRAB & TRANSECT & & 340 & 100 F -8 HRM 19.0 & & \\
\hline 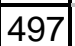 & RIVER & & RAW GRAB & TRANSECT & & 467 & 100 F -9 HRM 19.0 & & \\
\hline 498 & RIVER & & RAW GRAB & TRANSECT & & 578 & HANFRD TS-1 HRM 28.7 & & \\
\hline 499 & RIVER & & RAW GRAB & TRANSECT & & 347 & HANFRD TS-10 HRM 28.7 & & \\
\hline 500 & RIVER & & RAW GRAB & TRANSECT & & 342 & HANFRD TS-2 HRM 28.7 & & \\
\hline 501 & RIVER & & RAW GRAB & TRANSECT & & 468 & 3 HANFRD TS-3 HRM 28.7 & & \\
\hline 502 & RIVER & & RAW GRAB & TRANSECT & & 343 & HANFRD TS-4 HRM 28.7 & & \\
\hline 503 & RIVER & & RAW GRAB & TRANSECT & & 344 & HANFRD TS-5 HRM 28.7 & & \\
\hline 504 & RIVER & & RAW GRAB & TRANSECT & & 345 & HANFRD TS-6 HRM 28.7 & & \\
\hline 505 & RIVER & & RAW GRAB & TRANSECT & & 469 & HANFRD TS-7 HRM 28.7 & & \\
\hline 506 & RIVER & & RAW GRAB & TRANSECT & & 579 & HANFRD TS-8 HRM 28.7 & & \\
\hline 507 & RIVER & & RAW GRAB & TRANSECT & & 346 & HANFRD TS-9 HRM 28.7 & & \\
\hline 508 & RIVER & & RAW GRAB & TRANSECT & & 348 & 300 AREA -1 HRM 43.1 & & \\
\hline 509 & RIVER & & RAW GRAB & TRANSECT & & 470 & 300 AREA -2 HRM 43.1 & & \\
\hline 510 & RIVER & & RAW GRAB & TRANSECT & & 580 & 300 AREA -3 HRM 43.1 & & \\
\hline 511 & RIVER & & RAW GRAB & TRANSECT & & 349 & 300 AREA -4 HRM 43.1 & & \\
\hline 512 & RIVER & & RAW GRAB & TRANSECT & & 607 & 300 AREA -5 HRM 43.1 & & \\
\hline 513 & RIVER & & RAW GRAB & TRANSECT & & 350 & 300 AREA -6 HRM 43.1 & & \\
\hline 514 & RIVER & & RAW GRAB & TRANSECT & & 351 & 300 AREA -7 HRM 43.1 & & \\
\hline 515 & RIVER & & RAW GRAB & TRANSECT & & 581 & 300 AREA -8 HRM 43.1 & & \\
\hline 516 & RIVER & & RAW GRAB & TRANSECT & & 471 & 300 AREA -9 HRM 43.1 & & \\
\hline 517 & RIVER & & RAW GRAB & TRANSECT & & 571 & RICH.PMPHS-1 HRM46.4 & & \\
\hline 518 & RIVER & & RAW GRAB & TRANSECT & & 327 & RICH.PMPHS-10 HRM46.4 & & \\
\hline 519 & RIVER & & RAW GRAB & TRANSECT & & 321 & RICH.PMPHS-2 HRM46.4 & & \\
\hline 520 & RIVER & & RAW GRAB & TRANSECT & & 322 & RICH.PMPHS-3 HRM46.4 & & \\
\hline 521 & RIVER & & RAW GRAB & TRANSECT & & 572 & RICH.PMPHS-4 HRM46.4 & & \\
\hline 522 & RIVER & & RAW GRAB & TRANSECT & & 323 & RICH.PMPHS-5 HRM46.4 & & \\
\hline 523 & RIVER & & RAW GRAB & TRANSECT & & 324 & RICH.PMPHS-6 HRM46.4 & & \\
\hline 524 & RIVER & & RAW GRAB & TRANSECT & & 325 & RICH.PMPHS-7 HRM46.4 & & \\
\hline 525 & RIVER & & RAW GRAB & TRANSECT & & 326 & RICH.PMPHS-8 HRM46.4 & & \\
\hline 526 & RIVER & & RAW GRAB & TRANSECT & & 573 & RICH.PMPHS-9 HRM46.4 & & \\
\hline 527 & RIVER & & RAW GRAB & UNFILTERED & & 149 & HANFORD TOWNSITE & & \\
\hline 528 & RIVER & & CUMULATIVE & UNFILTERED & & 252 & PRIEST RAPIDS-RIVER & & \\
\hline 529 & RIVER & & CUMULATIVE & UNFILTERED & & 463 & RICH.PMPHS HRM 46.4 & & \\
\hline 530 & DRINKING & & CUMULATIVE & UNFILTERED & & 307 & 300 AREA & & \\
\hline 531 & RIVER & & RAW GRAB & UNFILTERED & & 282 & $100 \mathrm{H}$ AREA & & \\
\hline 532 & RIVER & & RAW GRAB & UNFILTERED & & 282 & $100 \mathrm{H}$ AREA & & \\
\hline 533 & RIVER & & RAW GRAB & UNFILTERED & & 282 & $100 \mathrm{H}$ AREA & & \\
\hline
\end{tabular}




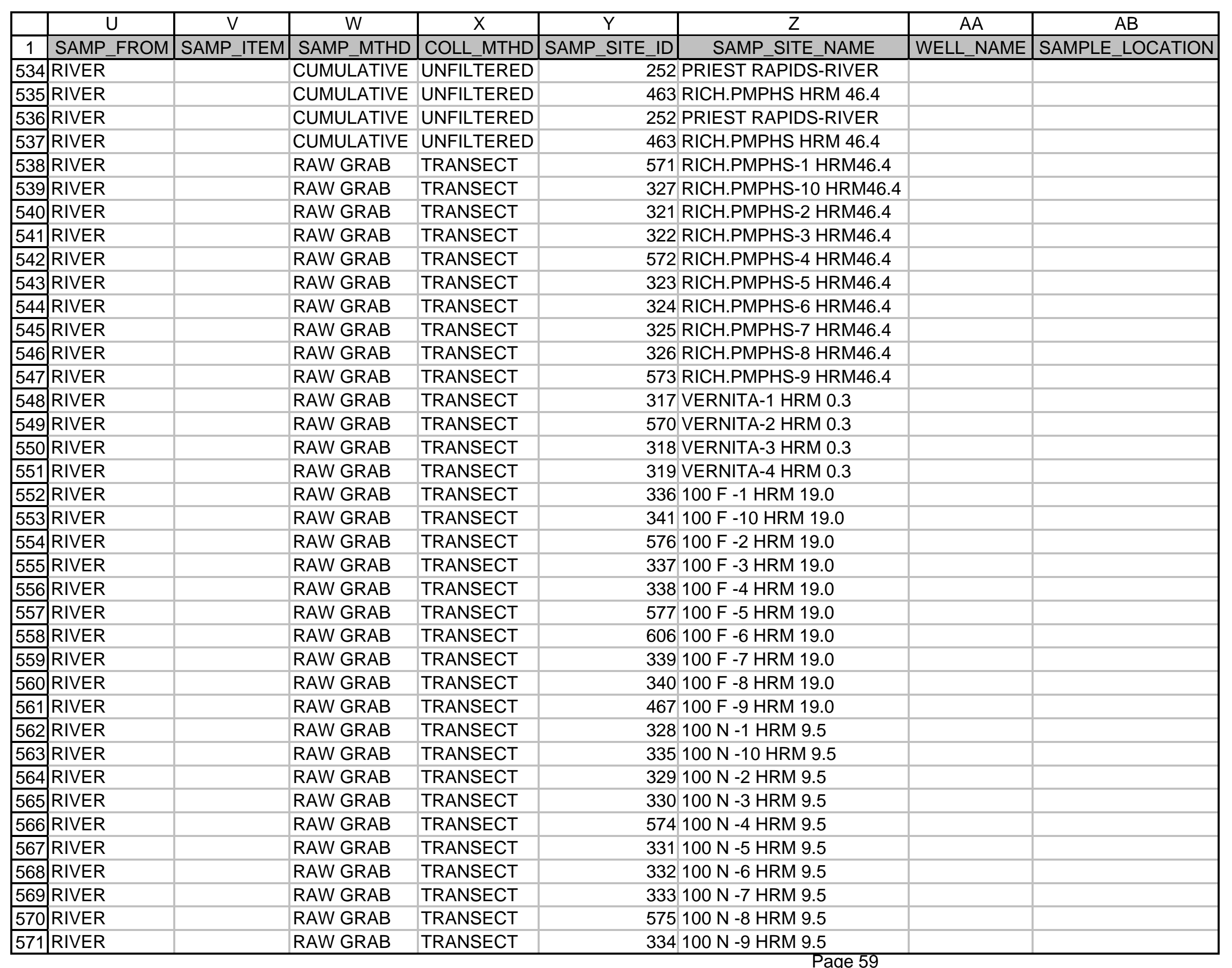




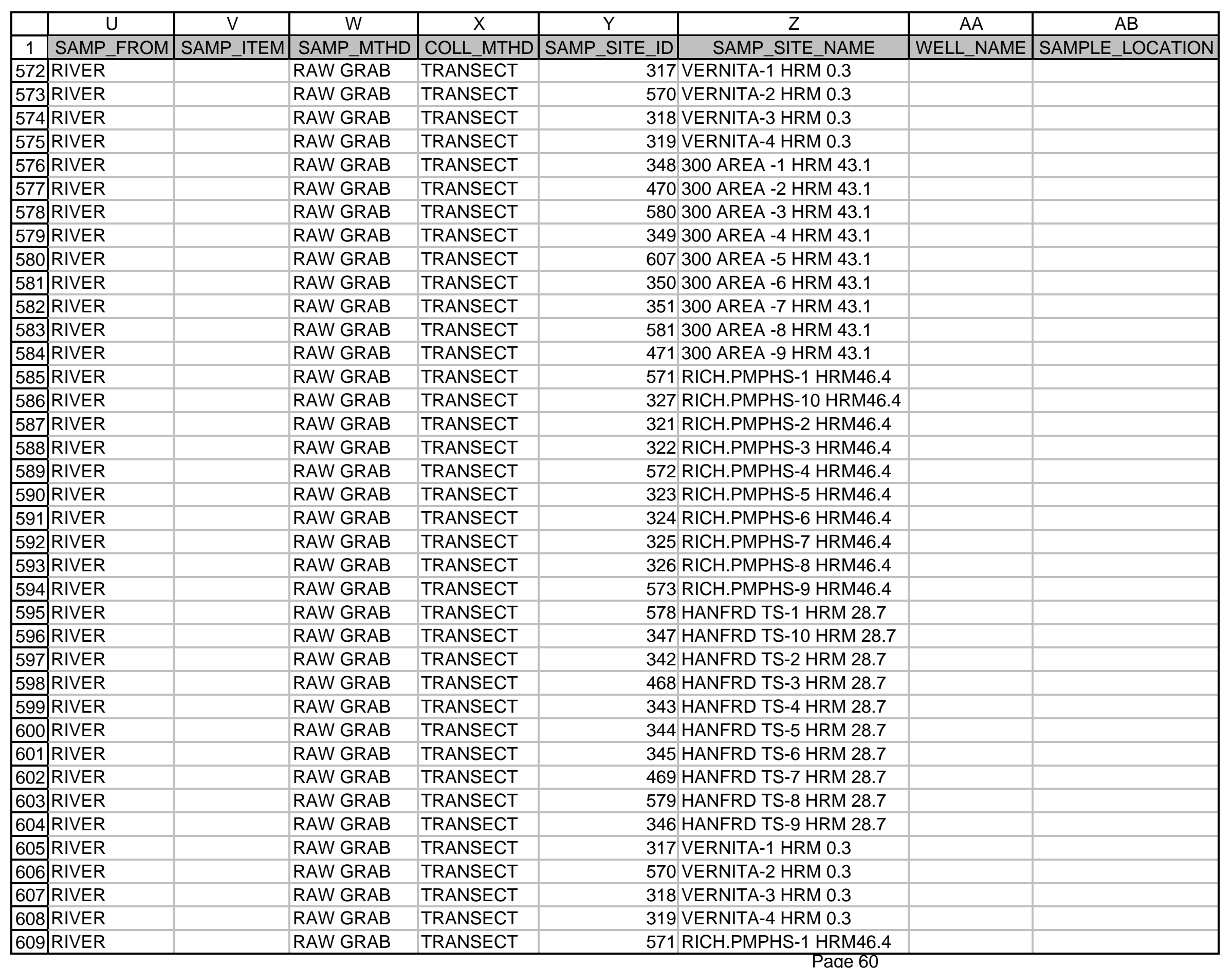




\begin{tabular}{|c|c|c|c|c|c|c|c|c|}
\hline & $\bar{U}$ & $\mathrm{~V}$ & $\mathrm{~W}$ & $\bar{X}$ & $\bar{Y}$ & $\mathrm{Z}$ & AA & $A B$ \\
\hline 1 & SAMP_FROM & SAMP_ITEM & SAMP_MTHD & COLL_MTHD & SAMP_SITE_ID & SAMP_SITE_NAME & WELL_NAME & SAMPLE_LOCATION \\
\hline 611 & RIVER & & RAW GRAB & TRANSECT & 321 & RICH.PMPHS-2 HRM46.4 & & \\
\hline 613 & RIVER & & RAW GRAB & TRANSECT & 572 & RICH.PMPHS-4 HRM46.4 & & \\
\hline 614 & RIVER & & RAW GRAB & TRANSECT & 323 & RICH.PMPHS-5 HRM46.4 & & \\
\hline 615 & RIVER & & RAW GRAB & TRANSECT & 324 & RICH.PMPHS-6 HRM46.4 & & \\
\hline 616 & RIVER & & RAW GRAB & TRANSECT & 325 & RICH.PMPHS-7 HRM46.4 & & \\
\hline 618 & RIVER & & RAW GRAB & TRANSECT & 573 & RICH.PMPHS-9 HRM46.4 & & \\
\hline 619 & RIVER & & RAW GRAB & TRANSECT & 317 & VERNITA-1 HRM 0.3 & & \\
\hline 620 & RIVER & & RAW GRAB & TRANSECT & 570 & VERNITA-2 HRM 0.3 & & \\
\hline 621 & RIVER & & RAW GRAB & TRANSECT & 318 & VERNITA-3 HRM 0.3 & & \\
\hline 622 & RIVER & & RAW GRAB & TRANSECT & 319 & VERNITA-4 HRM 0.3 & & \\
\hline 623 & RIVER & & RAW GRAB & TRANSECT & 571 & RICH.PMPHS-1 HRM46.4 & & \\
\hline 624 & RIVER & & RAW GRAB & TRANSECT & 327 & RICH.PMPHS-10 HRM46.4 & & \\
\hline 629 & RIVER & & RAW GRAB & TRANSECT & 324 & RICH.PMPHS-6 HRM46.4 & & \\
\hline 630 & RIVER & & RAW GRAB & TRANSECT & 325 & RICH.PMPHS-7 HRM46.4 & & \\
\hline 631 & RIVER & & RAW GRAB & TRANSECT & 326 & RICH.PMPHS-8 HRM46.4 & & \\
\hline 632 & RIVER & & RAW GRAB & TRANSECT & 573 & RICH.PMPHS-9 HRM46.4 & & \\
\hline 633 & RIVER & & RAW GRAB & TRANSECT & 317 & VERNITA-1 HRM 0.3 & & \\
\hline 634 & RIVER & & RAW GRAB & TRANSECT & 570 & VERNITA-2 HRM 0.3 & & \\
\hline 635 & RIVER & & RAW GRAB & TRANSECT & 318 & VERNITA-3 HRM 0.3 & & \\
\hline 636 & RIVER & & RAW GRAB & TRANSECT & 319 & VERNITA-4 HRM 0.3 & & \\
\hline 637 & RIVER & & RAW GRAB & TRANSECT & 571 & RICH.PMPHS-1 HRM46.4 & & \\
\hline 638 & RIVER & & RAW GRAB & TRANSECT & 327 & RICH.PMPHS-10 HRM46.4 & & \\
\hline 639 & RIVER & & RAW GRAB & TRANSECT & 321 & RICH.PMPHS-2 HRM46.4 & & \\
\hline 640 & RIVER & & RAW GRAB & TRANSECT & 322 & RICH.PMPHS-3 HRM46.4 & & \\
\hline
\end{tabular}




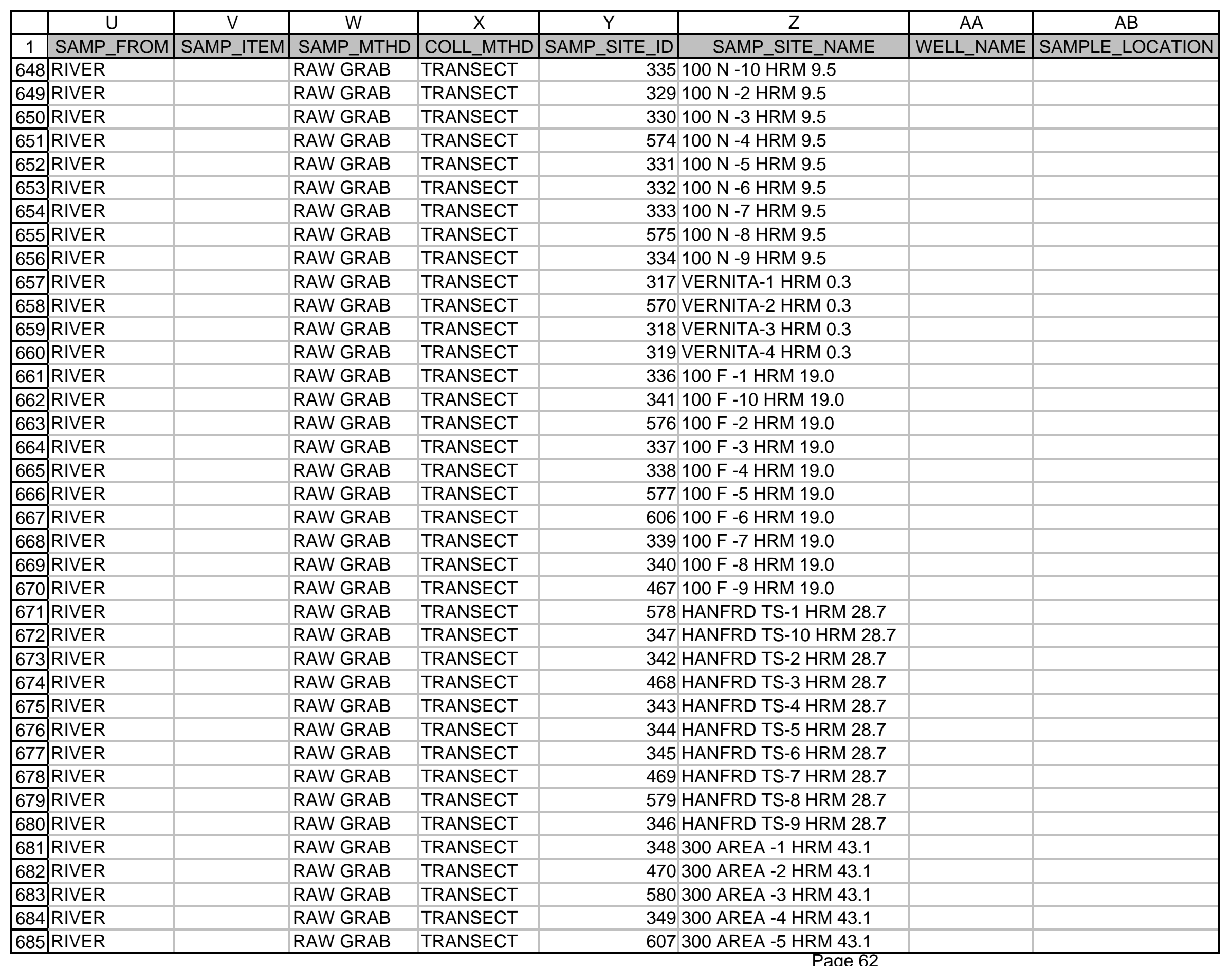

Page 62 


\begin{tabular}{|c|c|c|c|c|c|c|c|c|}
\hline & $\bar{U}$ & $\mathrm{~V}$ & $\mathrm{~W}$ & $\bar{x}$ & $\bar{Y}$ & $\bar{Z}$ & AA & $A B$ \\
\hline 1 & SAMP_FROM & SAMP_ITEM & SAMP_MTHD & COLL_MTHD & SAMP_SITE_ID & SAMP_SITE_NAME & WELL_NAME & SAMPLE_LOCATION \\
\hline 686 & RIVER & & RAW GRAB & TRANSECT & 350 & 300 AREA -6 HRM 43.1 & & \\
\hline 687 & RIVER & & RAW GRAB & TRANSECT & 351 & 300 AREA -7 HRM 43.1 & & \\
\hline 688 & RIVER & & RAW GRAB & TRANSECT & 581 & 300 AREA -8 HRM 43.1 & & \\
\hline 689 & RIVER & & RAW GRAB & TRANSECT & 471 & 300 AREA -9 HRM 43.1 & & \\
\hline 690 & RIVER & & RAW GRAB & TRANSECT & 571 & RICH.PMPHS-1 HRM46.4 & & \\
\hline 691 & RIVER & & RAW GRAB & TRANSECT & 327 & RICH.PMPHS-10 HRM46.4 & & \\
\hline 692 & RIVER & & RAW GRAB & TRANSECT & 321 & RICH.PMPHS-2 HRM46.4 & & \\
\hline 693 & RIVER & & RAW GRAB & TRANSECT & 322 & RICH.PMPHS-3 HRM46.4 & & \\
\hline 694 & RIVER & & RAW GRAB & TRANSECT & 572 & RICH.PMPHS-4 HRM46.4 & & \\
\hline 695 & RIVER & & RAW GRAB & TRANSECT & 323 & RICH.PMPHS-5 HRM46.4 & & \\
\hline 696 & RIVER & & RAW GRAB & TRANSECT & 324 & RICH.PMPHS-6 HRM46.4 & & \\
\hline 697 & RIVER & & RAW GRAB & TRANSECT & 325 & RICH.PMPHS-7 HRM46.4 & & \\
\hline 698 & RIVER & & RAW GRAB & TRANSECT & 326 & RICH.PMPHS-8 HRM46.4 & & \\
\hline 699 & RIVER & & RAW GRAB & TRANSECT & 573 & RICH.PMPHS-9 HRM46.4 & & \\
\hline 700 & RIVER & & RAW GRAB & TRANSECT & 571 & RICH.PMPHS-1 HRM46.4 & & \\
\hline 701 & RIVER & & RAW GRAB & TRANSECT & 327 & RICH.PMPHS-10 HRM46.4 & & \\
\hline 702 & RIVER & & RAW GRAB & TRANSECT & 321 & RICH.PMPHS-2 HRM46.4 & & \\
\hline 703 & RIVER & & RAW GRAB & TRANSECT & 322 & RICH.PMPHS-3 HRM46.4 & & \\
\hline 704 & RIVER & & RAW GRAB & TRANSECT & 572 & RICH.PMPHS-4 HRM46.4 & & \\
\hline 705 & RIVER & & RAW GRAB & TRANSECT & 323 & RICH.PMPHS-5 HRM46.4 & & \\
\hline 706 & RIVER & & RAW GRAB & TRANSECT & 324 & RICH.PMPHS-6 HRM46.4 & & \\
\hline 707 & RIVER & & RAW GRAB & TRANSECT & 325 & RICH.PMPHS-7 HRM46.4 & & \\
\hline 708 & RIVER & & RAW GRAB & TRANSECT & 326 & RICH.PMPHS-8 HRM46.4 & & \\
\hline 709 & RIVER & & RAW GRAB & TRANSECT & 573 & RICH.PMPHS-9 HRM46.4 & & \\
\hline 710 & RIVER & & RAW GRAB & TRANSECT & 317 & VERNITA-1 HRM 0.3 & & \\
\hline 711 & RIVER & & RAW GRAB & TRANSECT & 570 & VERNITA-2 HRM 0.3 & & \\
\hline 712 & RIVER & & RAW GRAB & TRANSECT & 318 & VERNITA-3 HRM 0.3 & & \\
\hline 713 & RIVER & & RAW GRAB & TRANSECT & 319 & VERNITA-4 HRM 0.3 & & \\
\hline 714 & RIVER & & CONTINUOUS & FILTER & 307 & 300 AREA & & \\
\hline 715 & RIVER & & CONTINUOUS & RESIN & 307 & 300 AREA & & \\
\hline 716 & RIVER & & CONTINUOUS & FILTER & 252 & PRIEST RAPIDS-RIVER & & \\
\hline 717 & RIVER & & CONTINUOUS & RESIN & 252 & PRIEST RAPIDS-RIVER & & \\
\hline 718 & RIVER & & CONTINUOUS & FILTER & 463 & RICH.PMPHS HRM 46.4 & & \\
\hline 719 & RIVER & & CONTINUOUS & RESIN & 463 & RICH.PMPHS HRM 46.4 & & \\
\hline 720 & RIVER & & CONTINUOUS & FILTER & 307 & 300 AREA & & \\
\hline 721 & RIVER & & CONTINUOUS & RESIN & 307 & 300 AREA & & \\
\hline 722 & RIVER & & CONTINUOUS & FILTER & 252 & PRIEST RAPIDS-RIVER & & \\
\hline 723 & RIVER & & CONTINUOUS & RESIN & 252 & PRIEST RAPIDS-RIVER & & \\
\hline
\end{tabular}




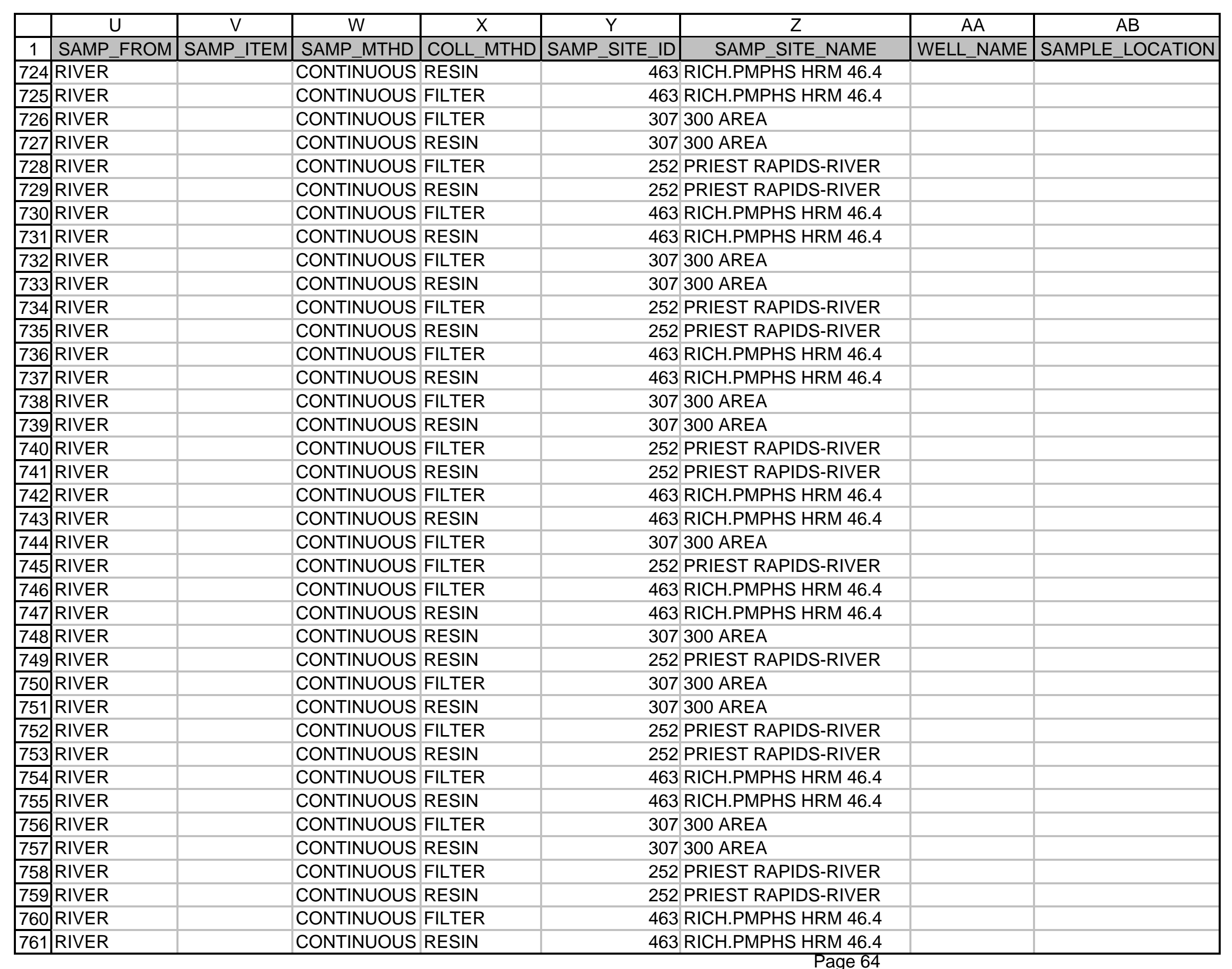

Page 64 


\begin{tabular}{|c|c|c|c|c|c|c|c|c|}
\hline & $\mathrm{U}$ & $\mathrm{V}$ & $\mathrm{W}$ & $\mathrm{X}$ & $\bar{Y}$ & $Z$ & AA & $A B$ \\
\hline 1 & SAMP_FROM & SAMP_ITEM & SAMP_MTHD & COLL_MTHD & SAMP_SITE_ID & SAMP_SITE_NAME & WELL_NAME & SAMPLE_LOCATION \\
\hline 763 & RIVER & & CONTINUOUS & RESIN & 307 & 300 AREA & & \\
\hline 765 & RIVER & & CONTINUOUS & RESIN & 252 & PRIEST RAPIDS-RIVER & & \\
\hline 766 & RIVER & & CONTINUOUS & FILTER & 463 & RICH.PMPHS HRM 46.4 & & \\
\hline 767 & RIVER & & CONTINUOUS & RESIN & 463 & RICH.PMPHS HRM 46.4 & & \\
\hline 768 & RIVER & & CONTINUOUS & RESIN & 307 & 300 AREA & & \\
\hline 771 & RIVER & & CONTINUOUS & FILTER & 252 & PRIEST RAPIDS-RIVER & & \\
\hline 772 & RIVER & & CONTINUOUS & RESIN & 463 & RICH.PMPHS HRM 46.4 & & \\
\hline 773 & RIVER & & CONTINUOUS & FILTER & 463 & RICH.PMPHS HRM 46.4 & & \\
\hline 774 & RIVER & & CONTINUOUS & FILTER & 307 & 300 AREA & & \\
\hline 775 & RIVER & & CONTINUOUS & RESIN & 307 & 300 AREA & & \\
\hline 776 & RIVER & & CONTINUOUS & FILTER & 252 & PRIEST RAPIDS-RIVER & & \\
\hline 781 & RIVER & & CONTINUOUS & FILTER & 252 & PRIEST RAPIDS-RIVER & & \\
\hline 782 & RIVER & & CONTINUOUS & RESIN & 463 & RICH.PMPHS HRM 46.4 & & \\
\hline 783 & RIVER & & CONTINUOUS & FILTER & 463 & RICH.PMPHS HRM 46.4 & & \\
\hline 784 & RIVER & & CONTINUOUS & RESIN & 252 & PRIEST RAPIDS-RIVER & & \\
\hline 785 & RIVER & & CONTINUOUS & FILTER & 252 & PRIEST RAPIDS-RIVER & & \\
\hline 786 & RIVER & & CONTINUOUS & RESIN & 463 & RICH.PMPHS HRM 46.4 & & \\
\hline 787 & RIVER & & CONTINUOUS & FILTER & 463 & RICH.PMPHS HRM 46.4 & & \\
\hline 788 & RIVER & & CONTINUOUS & RESIN & 252 & PRIEST RAPIDS-RIVER & & \\
\hline 789 & RIVER & & CONTINUOUS & FILTER & 252 & PRIEST RAPIDS-RIVER & & \\
\hline 790 & RIVER & & CONTINUOUS & FILTER & 463 & RICH.PMPHS HRM 46.4 & & \\
\hline 791 & RIVER & & CONTINUOUS & RESIN & 463 & RICH.PMPHS HRM 46.4 & & \\
\hline 792 & RIVER & & CONTINUOUS & FILTER & 252 & PRIEST RAPIDS-RIVER & & \\
\hline
\end{tabular}

Page 65 


\begin{tabular}{|c|c|c|c|c|c|c|c|c|}
\hline & $\bar{U}$ & $\mathrm{~V}$ & $\mathrm{~W}$ & $\bar{x}$ & $\bar{Y}$ & $\bar{Z}$ & $\overline{A A}$ & $A B$ \\
\hline 1 & SAMP_FROM & SAMP_ITEM & SAMP_MTHD & COLL_MTHD & SAMP_SITE_ID & SAMP_SITE_NAME & WELL_NAME & SAMPLE_LOCATION \\
\hline 800 & RIVER & & CONTINUOUS & FILTER & 252 & PRIEST RAPIDS-RIVER & & \\
\hline 801 & RIVER & & CONTINUOUS & RESIN & 252 & PRIEST RAPIDS-RIVER & & \\
\hline 802 & RIVER & & CONTINUOUS & RESIN & 463 & RICH.PMPHS HRM 46.4 & & \\
\hline 803 & RIVER & & CONTINUOUS & FILTER & 463 & RICH.PMPHS HRM 46.4 & & \\
\hline 804 & RIVER & & CONTINUOUS & FILTER & 252 & PRIEST RAPIDS-RIVER & & \\
\hline 805 & RIVER & & CONTINUOUS & RESIN & 252 & PRIEST RAPIDS-RIVER & & \\
\hline 806 & RIVER & & CONTINUOUS & RESIN & 463 & RICH.PMPHS HRM 46.4 & & \\
\hline 807 & RIVER & & CONTINUOUS & FILTER & 463 & RICH.PMPHS HRM 46.4 & & \\
\hline 808 & RIVER & & CONTINUOUS & FILTER & 252 & PRIEST RAPIDS-RIVER & & \\
\hline 809 & RIVER & & CONTINUOUS & RESIN & 252 & PRIEST RAPIDS-RIVER & & \\
\hline 810 & RIVER & & CONTINUOUS & FILTER & 463 & RICH.PMPHS HRM 46.4 & & \\
\hline 811 & RIVER & & CONTINUOUS & RESIN & 463 & RICH.PMPHS HRM 46.4 & & \\
\hline 812 & RIVER & & CONTINUOUS & FILTER & 252 & PRIEST RAPIDS-RIVER & & \\
\hline 813 & RIVER & & CONTINUOUS & RESIN & 252 & PRIEST RAPIDS-RIVER & & \\
\hline 814 & RIVER & & CONTINUOUS & RESIN & 463 & RICH.PMPHS HRM 46.4 & & \\
\hline 815 & RIVER & & CONTINUOUS & FILTER & 463 & RICH.PMPHS HRM 46.4 & & \\
\hline 816 & RIVER & & CONTINUOUS & FILTER & 252 & PRIEST RAPIDS-RIVER & & \\
\hline 817 & RIVER & & CONTINUOUS & RESIN & 252 & PRIEST RAPIDS-RIVER & & \\
\hline 818 & RIVER & & CONTINUOUS & FILTER & 252 & PRIEST RAPIDS-RIVER & & \\
\hline 819 & RIVER & & CONTINUOUS & RESIN & 252 & PRIEST RAPIDS-RIVER & & \\
\hline
\end{tabular}




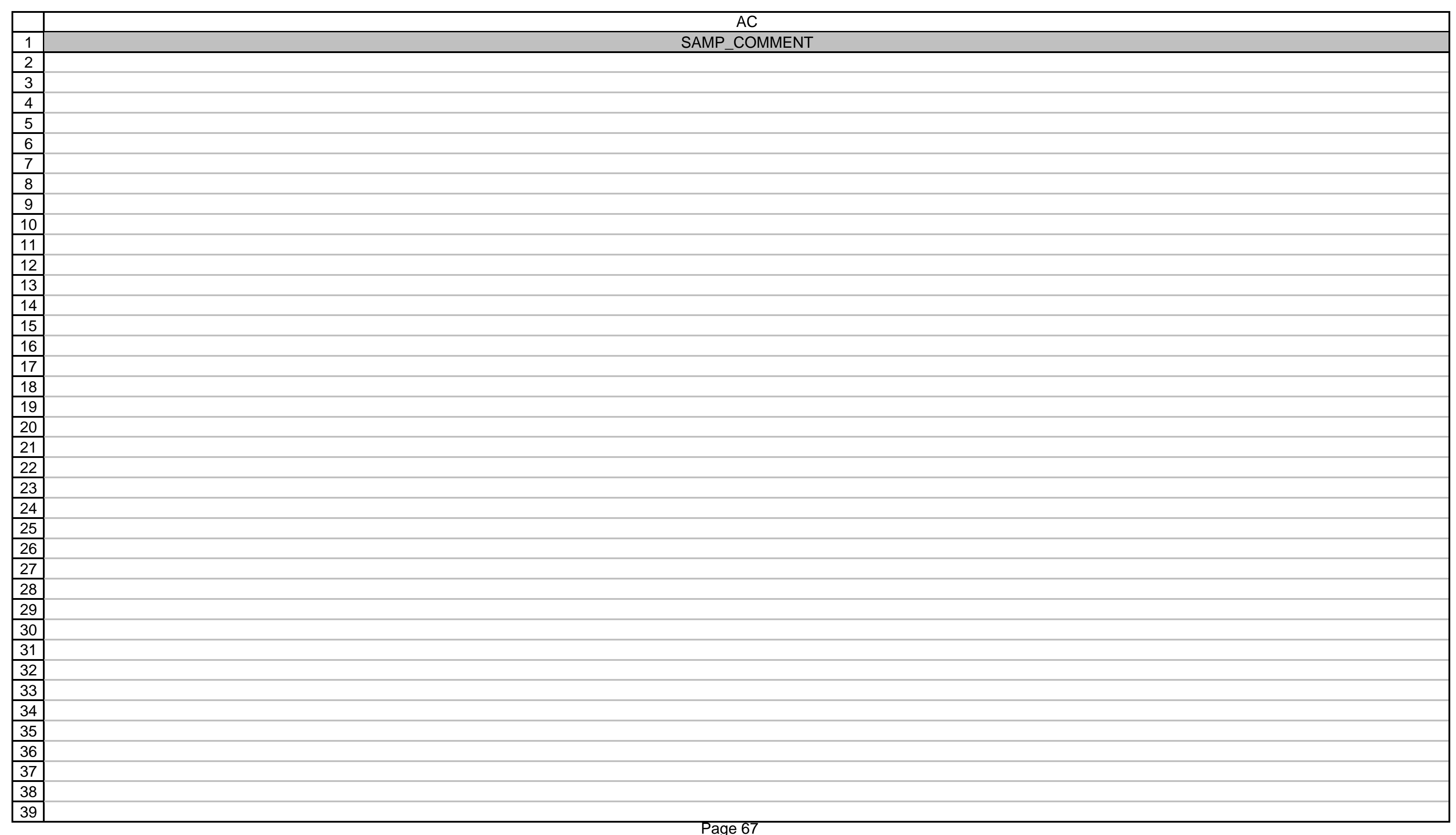




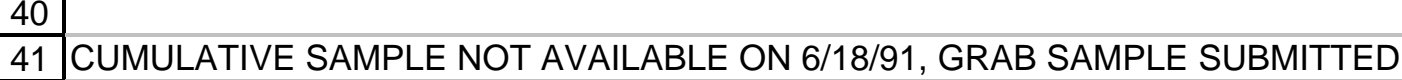

53 CUMULATIVE SAMPLE NOT AVAILABLE FOR 8/13/91, GRAB SAMPLE SUBMITTED. 


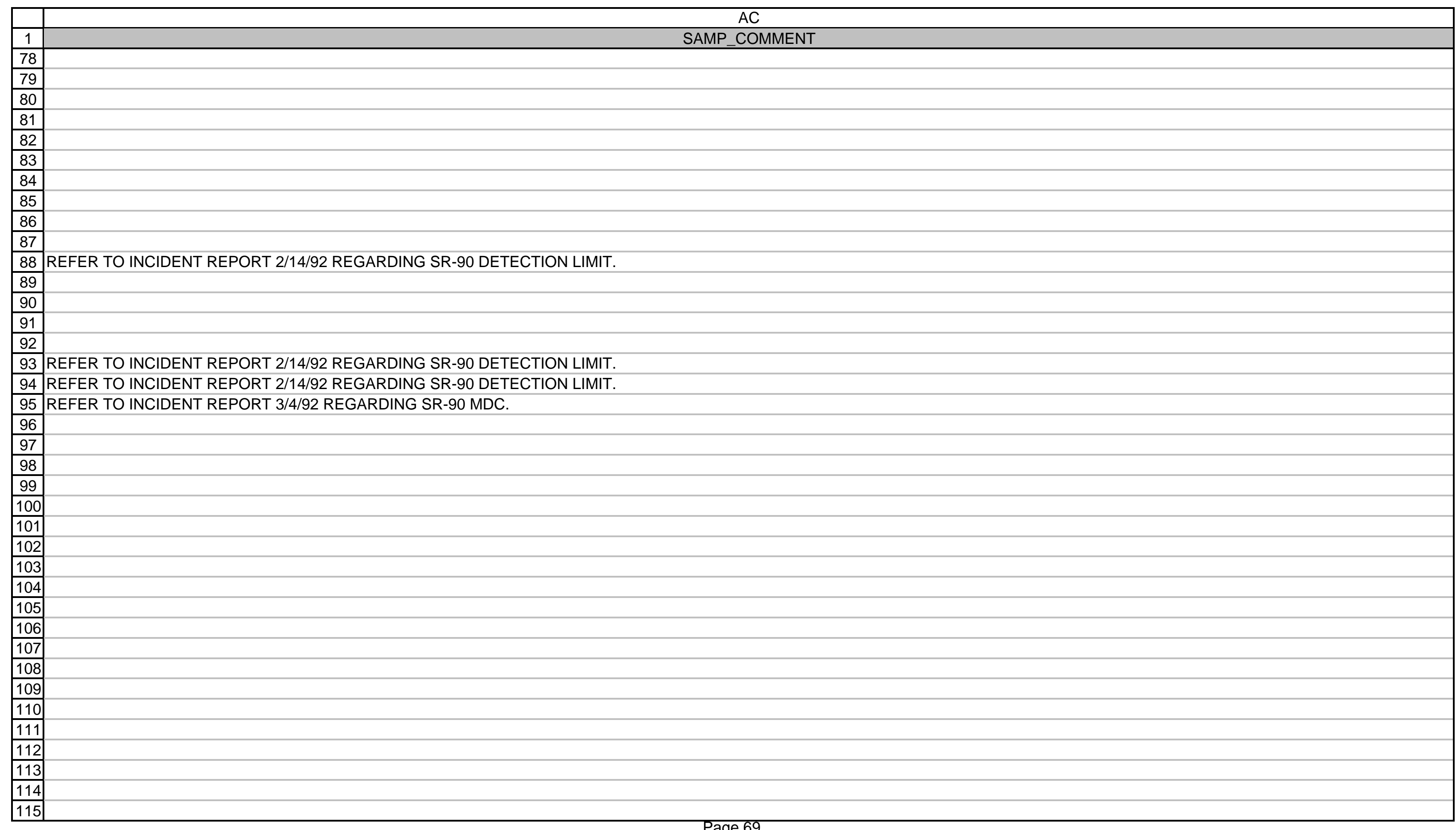




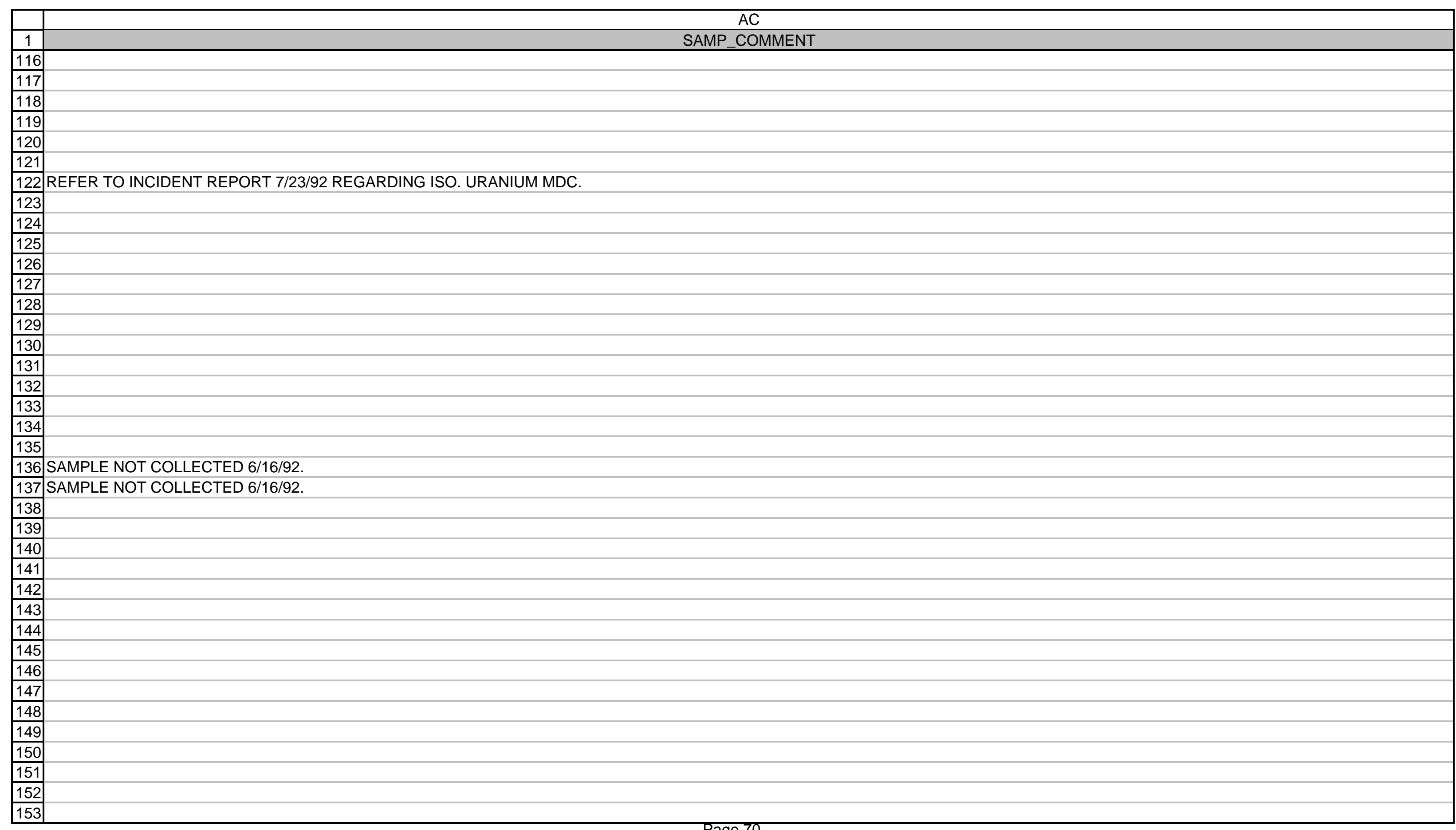




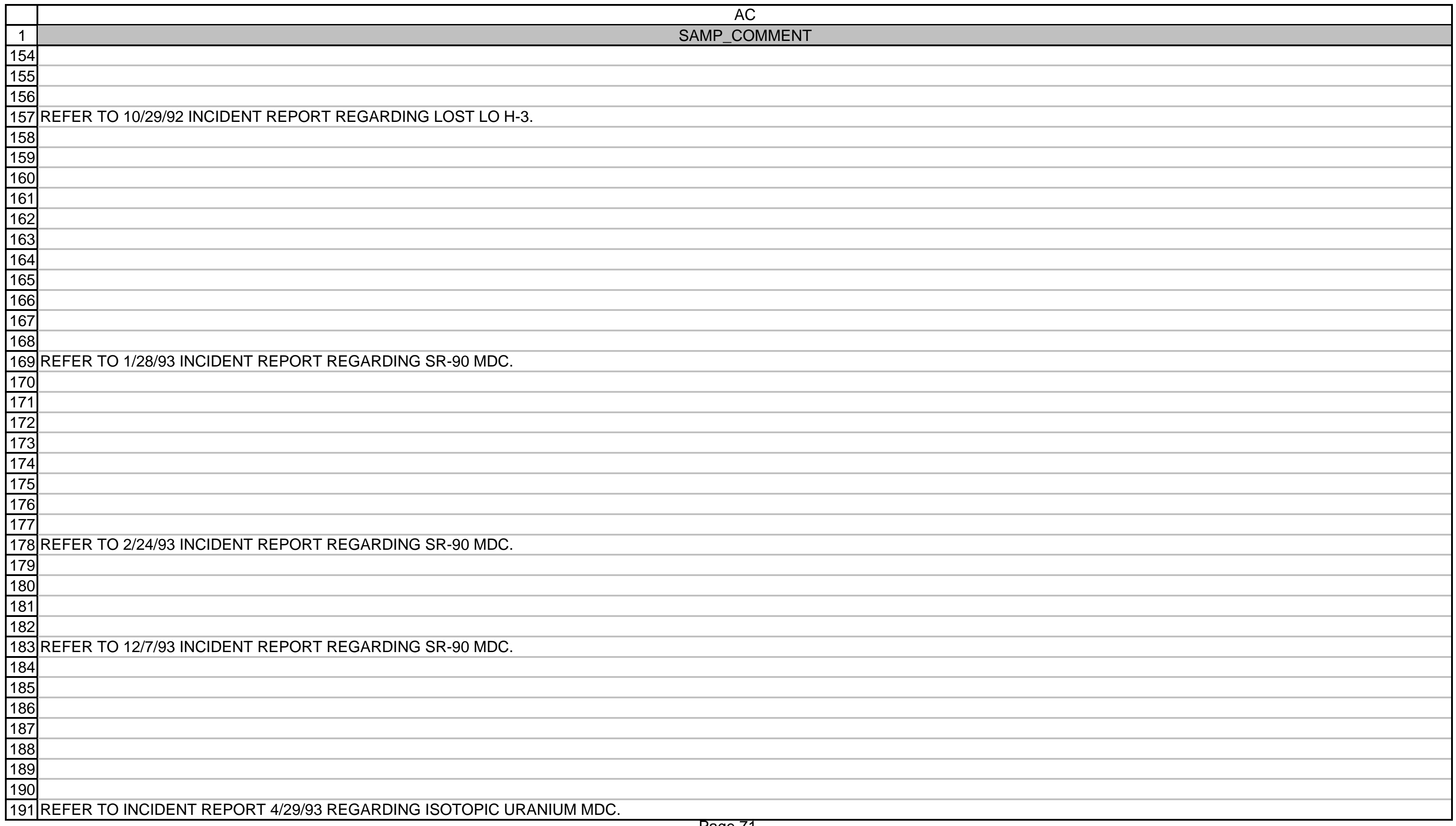




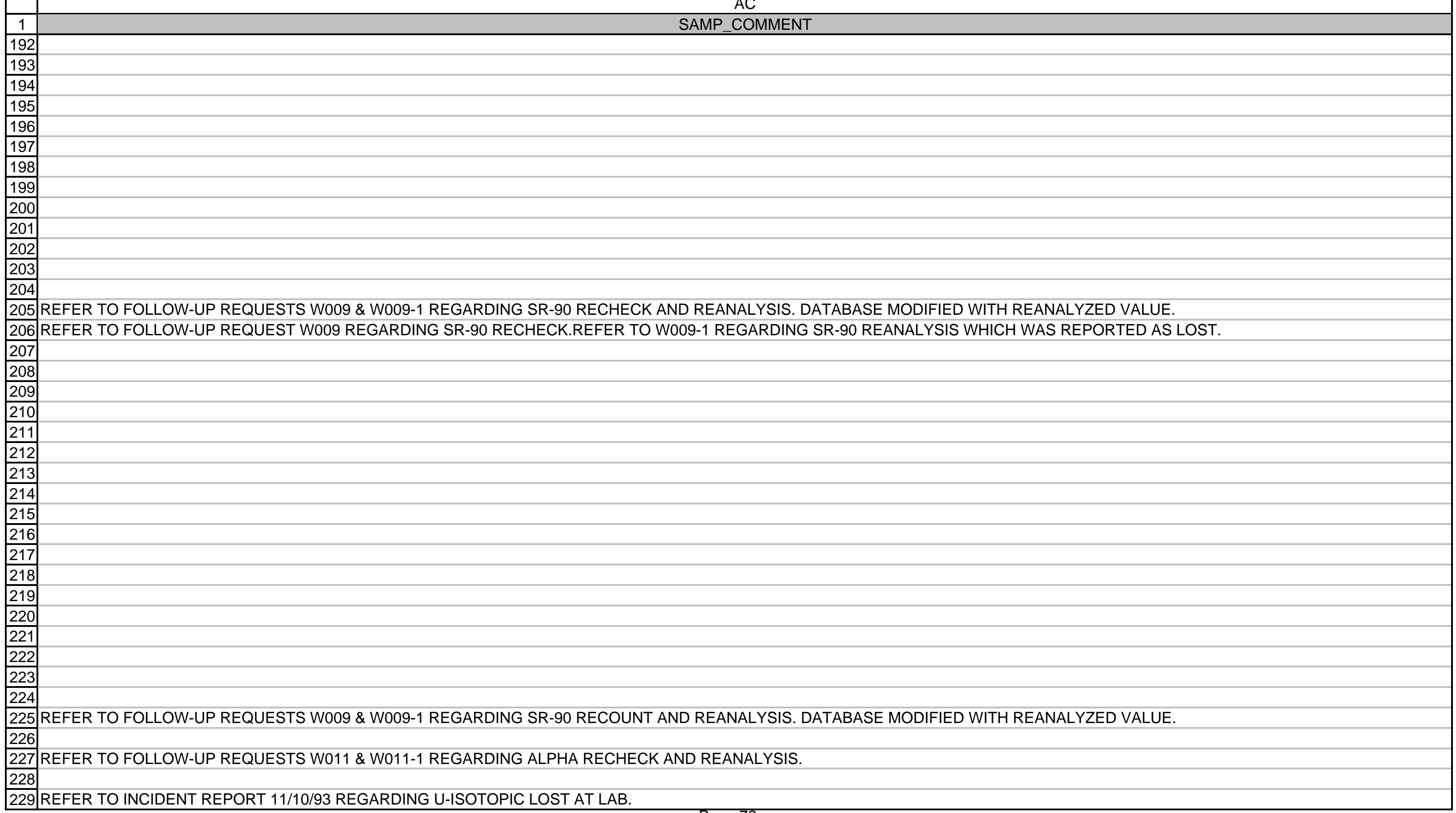




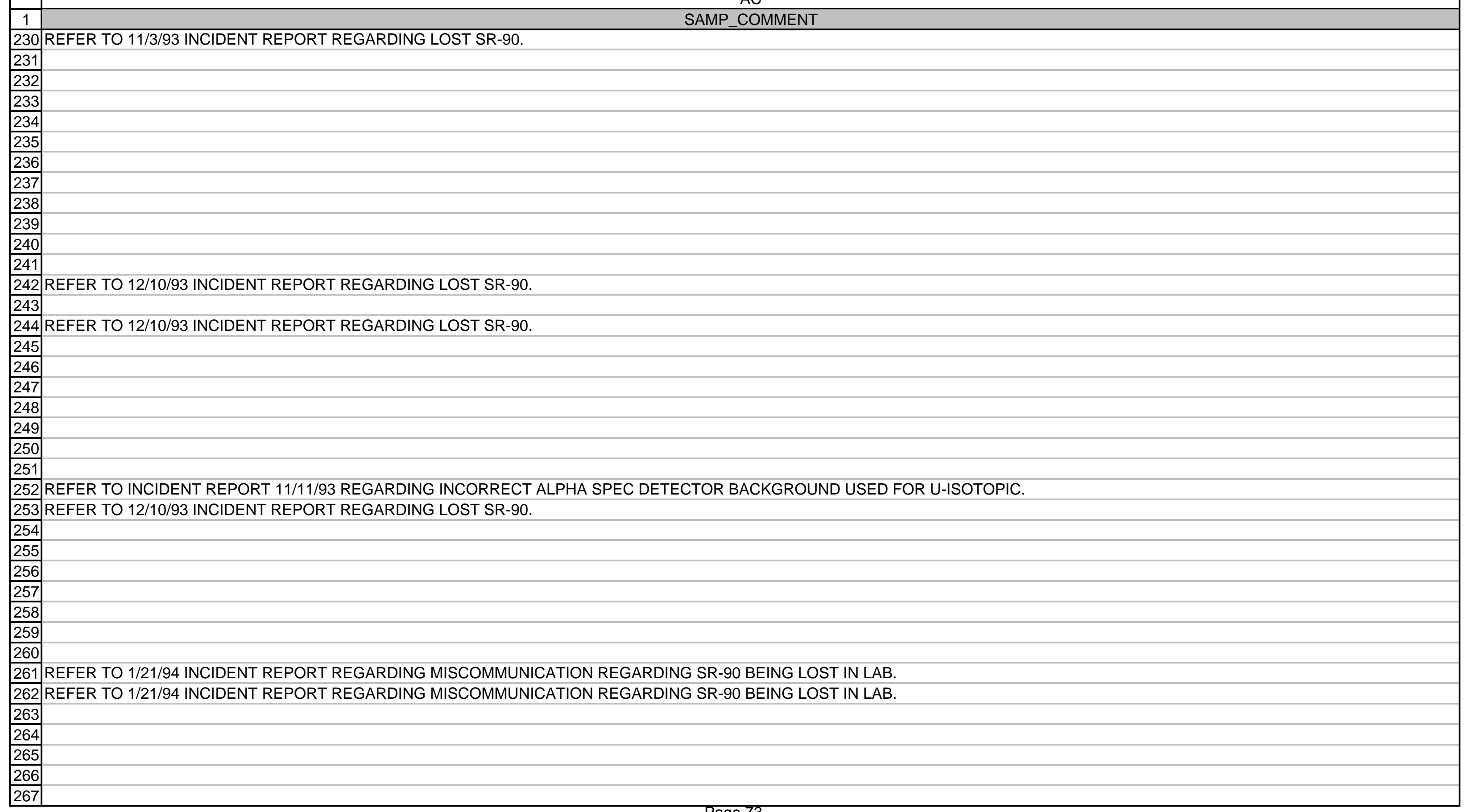


271

\begin{tabular}{|l|}
\hline 272 \\
\hline 273 \\
\hline
\end{tabular}

274 REFER TO 1/25/94 INCIDENT REPORT REGARDING SR-90 ANALYSIS BEING CONTAMINATED IN LAB.

275 CONTAMINATED IN LAB

276

277

278

\begin{tabular}{l}
279 \\
280 \\
\hline
\end{tabular}

280

282

283

285

286

287

288

289

290

291

292.

293

294

(95)

296

297.

299

300

301

302

303

304 


\section{AC}

\begin{tabular}{|l|}
\hline 310 \\
\hline 311 \\
\hline
\end{tabular}

311.

\begin{tabular}{|l|}
312 \\
\hline 313 \\
\hline 314
\end{tabular}

\begin{tabular}{|l|}
313 \\
\hline 314 \\
\hline 315
\end{tabular}

316

\begin{tabular}{|l|}
\hline 317 \\
\hline 318 \\
\hline
\end{tabular}

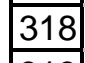

\begin{tabular}{|l|}
319 \\
\hline 320 \\
\hline 322 \\
\hline
\end{tabular}

\begin{tabular}{|l|}
\hline 321 \\
\hline 322 \\
\hline
\end{tabular}

322

323

\begin{tabular}{|l|}
\hline 324 \\
\hline 325 \\
\hline 326 \\
\hline
\end{tabular}

\begin{tabular}{l}
326 \\
327 \\
\hline
\end{tabular}

328

330

\begin{tabular}{|l|}
\hline 331 \\
\hline 332 \\
\hline 333 \\
\hline
\end{tabular}

332

\begin{tabular}{|l|}
\hline 333 \\
\hline 335 \\
\hline
\end{tabular}

\begin{tabular}{l}
334 \\
\hline 335 \\
\hline
\end{tabular}

336.

337

338

339

340

341

\begin{tabular}{|l|}
\hline 342 \\
\hline 343 \\
\hline
\end{tabular} 


\section{AC}

\begin{tabular}{|l|}
\hline 348 \\
\hline 349 \\
\hline 350 \\
\hline
\end{tabular}

351

\begin{tabular}{|l|}
\hline 352 \\
\hline 352 \\
\hline
\end{tabular}

358

372

373

375

\begin{tabular}{|l|}
\hline 376 \\
\hline 377 \\
\hline 378 \\
\hline
\end{tabular} 


\section{AC}

406

\begin{tabular}{l}
407 \\
408 \\
\hline 409
\end{tabular}

408

410

411

412

413 


\section{AC}

430

\begin{tabular}{|l|}
\hline 431 \\
\hline 432 \\
\hline
\end{tabular}

432

433

435

437

\begin{tabular}{l}
438 \\
439 \\
\hline 440
\end{tabular}

439

441

442

443

\begin{tabular}{l}
443 \\
\hline 445 \\
\hline
\end{tabular}

445

446

447

448

\begin{tabular}{l}
449 \\
450 \\
\hline
\end{tabular}

450

\begin{tabular}{|l|}
\hline 451 \\
\hline 452 \\
\hline 453 \\
\hline
\end{tabular}

\begin{tabular}{|l|}
\hline 452 \\
\hline 453 \\
\hline 454 \\
\hline
\end{tabular}

\begin{tabular}{l}
454 \\
\hline 455 \\
\hline
\end{tabular}

456

457 
461

\begin{tabular}{l}
462 \\
463 \\
\hline
\end{tabular}

463

464

65

\begin{tabular}{|l|}
\hline 466 \\
\hline 467 \\
\hline
\end{tabular}

469

472

473

475

\begin{tabular}{l}
476 \\
477 \\
\hline 478 \\
\hline
\end{tabular}

$\frac{479}{479}$

480

\begin{tabular}{|l|}
481 \\
\hline 482 \\
\hline 483 \\
\hline
\end{tabular}

483

484

485

486

487

488

489

490

491

492

493

\begin{tabular}{|l|}
\hline 494 \\
\hline 495 \\
\hline
\end{tabular}

495 


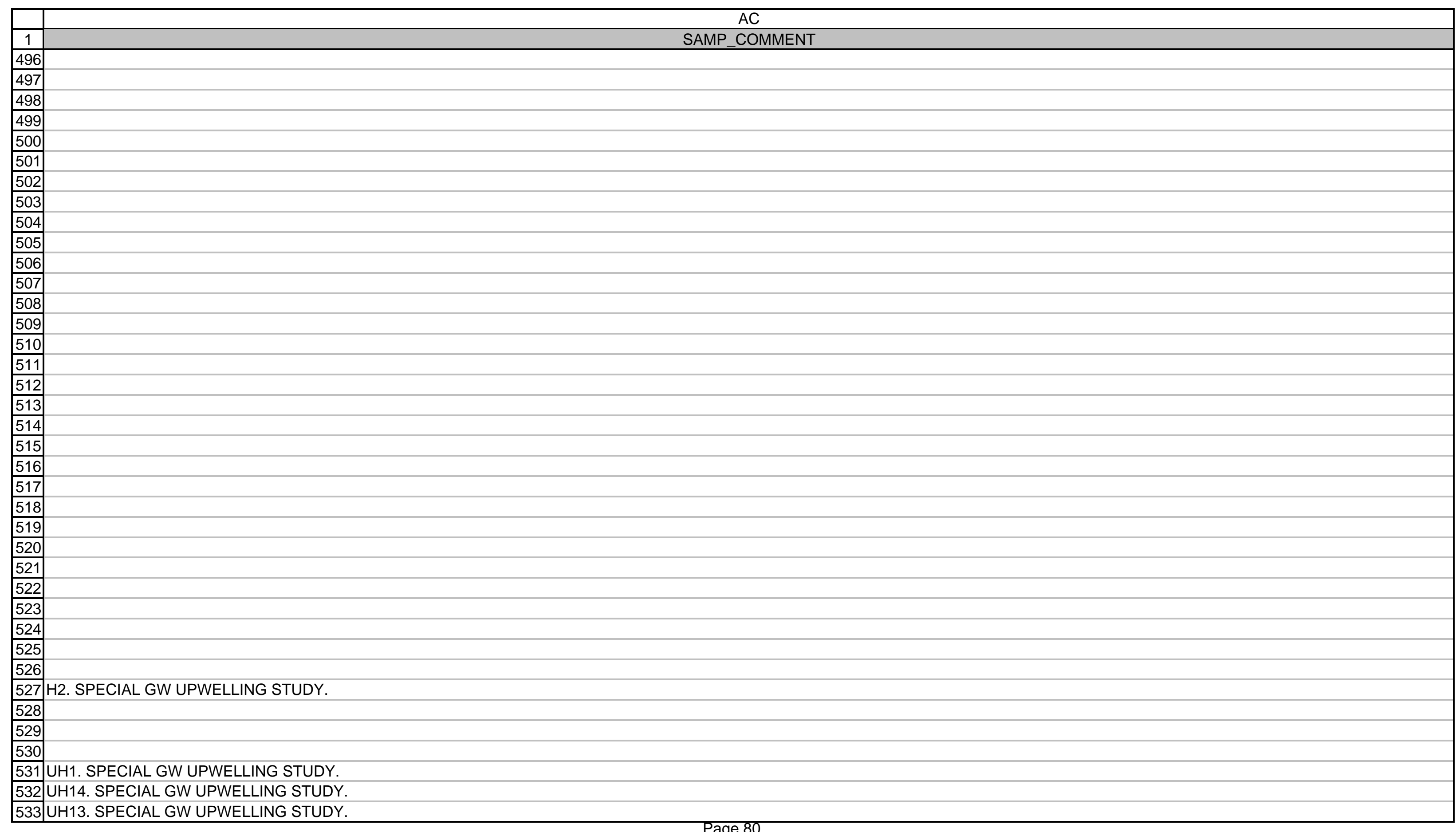




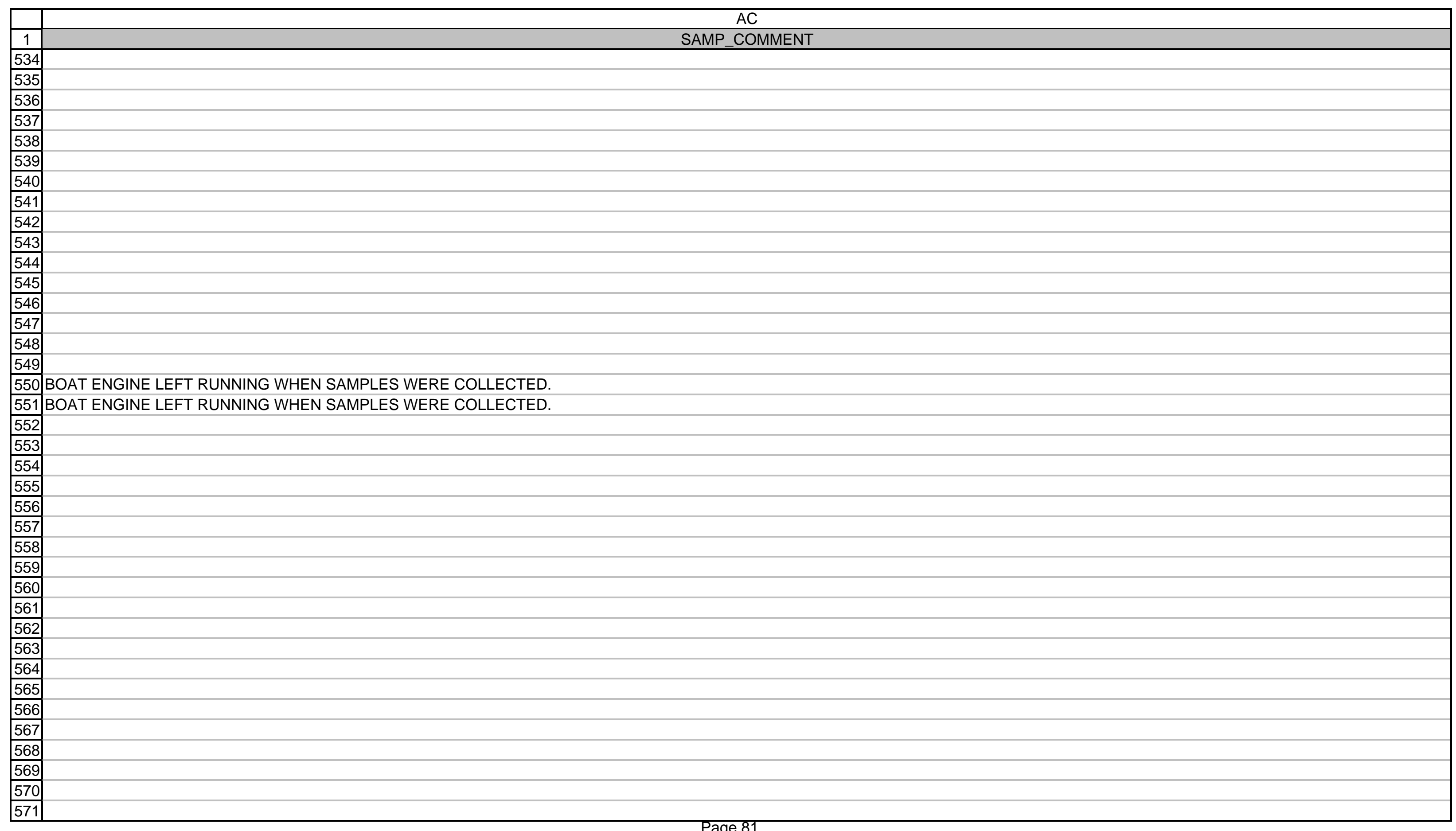


\begin{tabular}{|l|}
602 \\
\hline 603 \\
\hline 604 \\
\hline
\end{tabular}

604

605

606

607

\begin{tabular}{|l|}
\hline 608 \\
\hline 609 \\
\hline
\end{tabular} 


\section{AC}

631

632

638

641

\begin{tabular}{|l|}
\hline 642 \\
\hline 643 \\
\hline
\end{tabular}

643

644

645

646

647 
661

662

672

\begin{tabular}{l}
673 \\
674 \\
\hline 675
\end{tabular}

674

675

676

679

680

681

682

683

\begin{tabular}{|l|}
\hline 684 \\
\hline 685 \\
\hline
\end{tabular} 


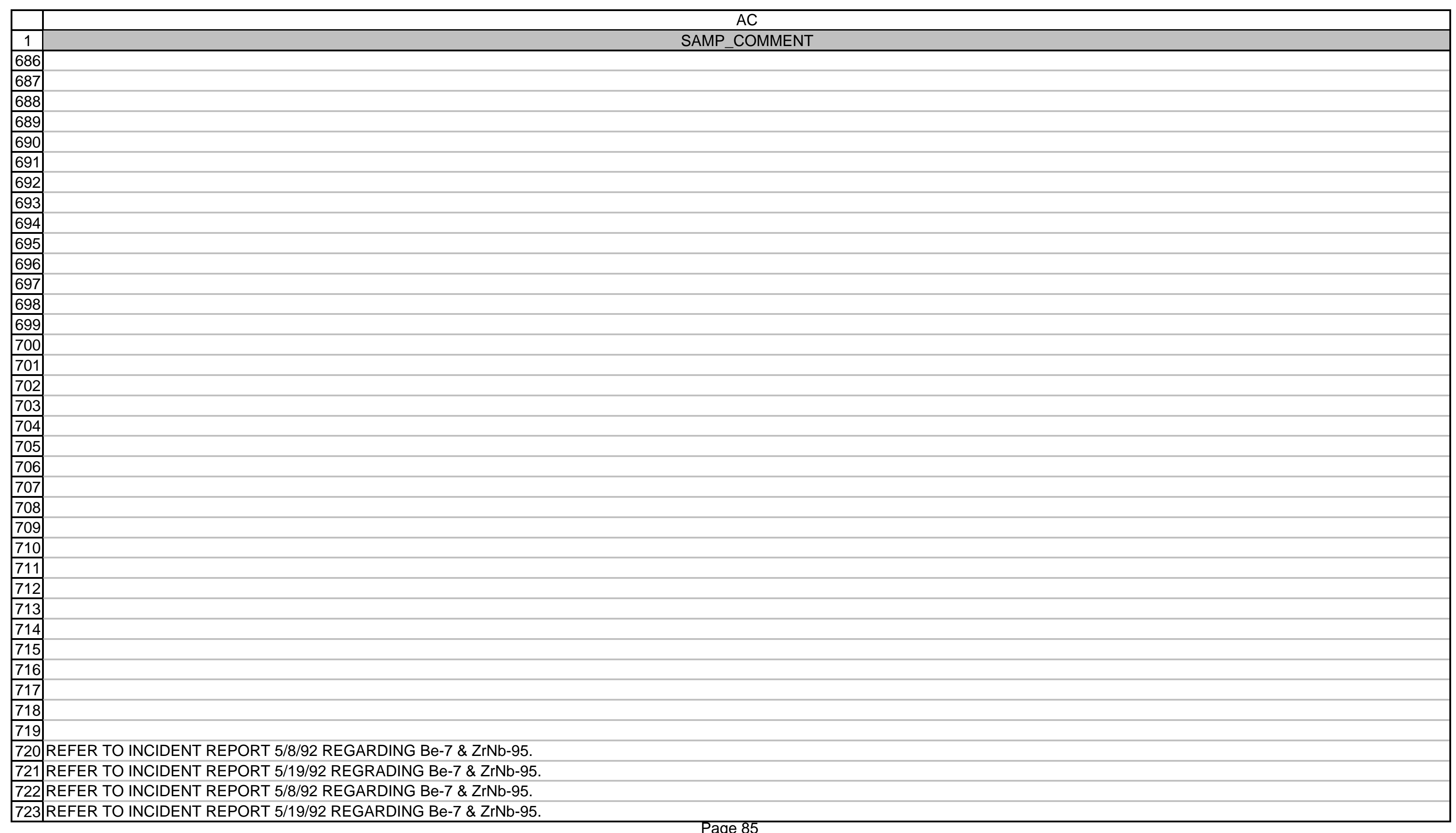


724 REFER TO INCIDENT REPORT 5/8/92 REGARDING Be-7 \& ZrNb-95.

726 REFER TO INCIDENT REPORT 5/8/92 REGARDING Be-7 \& ZrNb-95.

727 REFER TO INCIDENT REPORT'S 5/19/92 REGARDING Be-7 \& ZrNb-95.

728 REFER TO INCIDENT REPORT 5/8/92 REGARDING Be-7 \& ZrNb-95.

729 REFER TO INCIDENT REPORT 5/19/92 REGARDING Be-7 \& ZrNb-95.

730 REFER TO INCIDENT REPORT 5/8/92 REGARDING Be-7 \& ZrNb-95.

731

\begin{tabular}{|l|}
\hline 732 \\
\hline 733 \\
\hline 734 \\
\hline
\end{tabular}

733

\begin{tabular}{|l|}
734 \\
\hline 735 \\
\hline 736 \\
\hline
\end{tabular}

\begin{tabular}{l}
735 \\
\hline 736 \\
\hline
\end{tabular}

737

\begin{tabular}{|l|}
\hline 738 \\
\hline 739 \\
\hline 72
\end{tabular}

\begin{tabular}{|l|}
\hline 739 \\
\hline 740 \\
\hline 741 \\
\hline
\end{tabular}

753 REFER TO INCIDENT REPORT 5/19/92 REGARDING GAMMA MDC

755 REFER TO INCIDENT REPORT 5/19/92 REGARDING GAMMA MDC

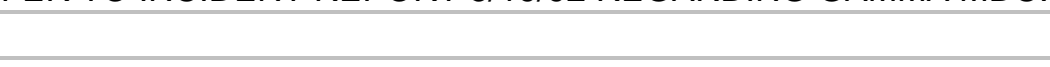




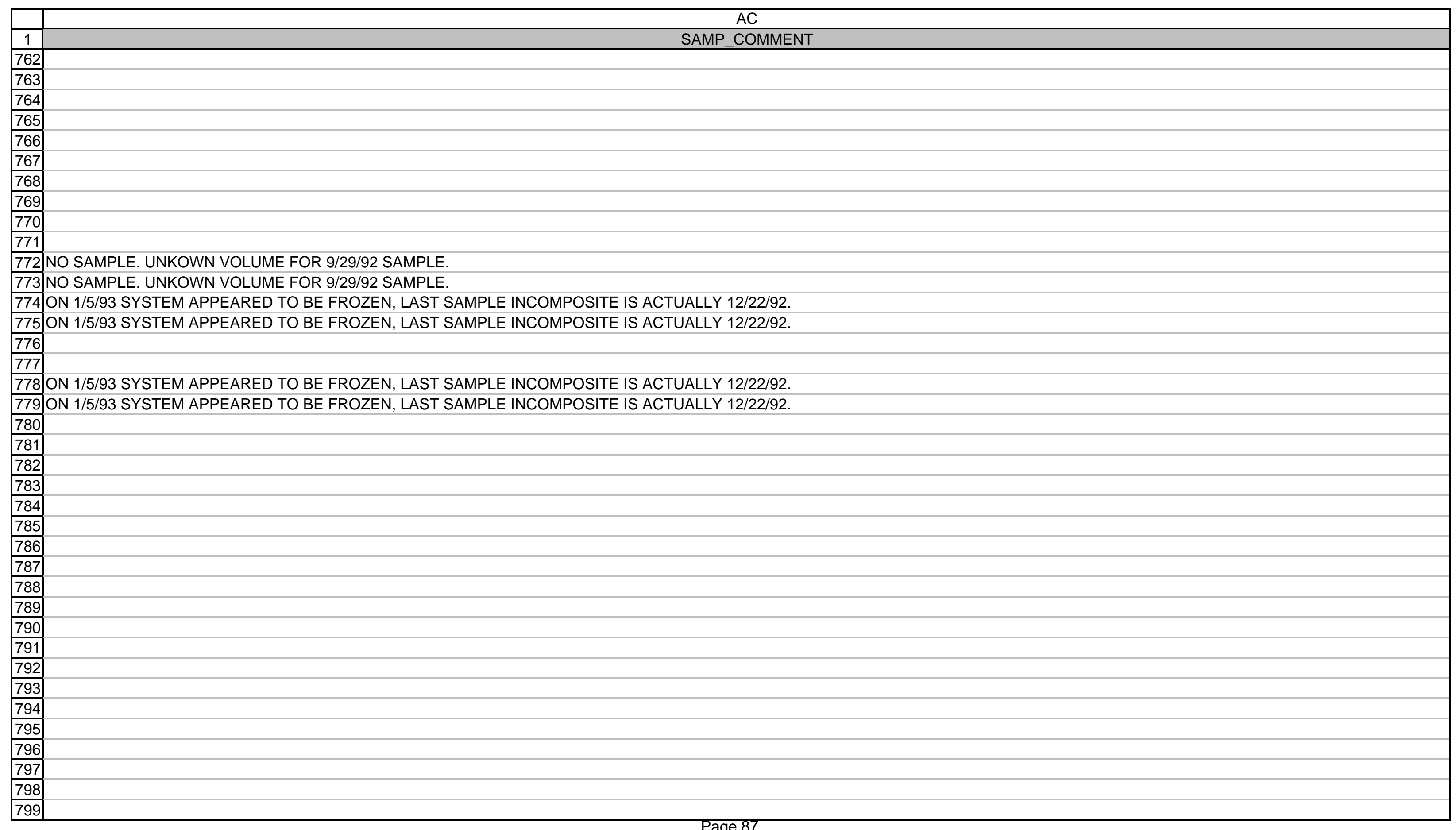


\begin{tabular}{|l|}
\hline 806 \\
\hline 807 \\
\hline 808 \\
\hline
\end{tabular}

808

809

\begin{tabular}{|l|}
\hline 811 \\
\hline 812 \\
\hline 813 \\
\hline
\end{tabular}

\begin{tabular}{|l|}
\hline 812 \\
\hline 813 \\
\hline
\end{tabular}

813

\begin{tabular}{|l|}
814 \\
\hline 815 \\
\hline 816 \\
\hline
\end{tabular}

816

817

\begin{tabular}{|l|}
\hline 818 \\
\hline 819 \\
\hline
\end{tabular}

Page 88 


\begin{tabular}{|c|c|c|c|c|}
\hline & $A D$ & $\mathrm{AE}$ & $\mathrm{AF}$ & $A G$ \\
\hline 1 & RESULT_COMMENT & COLL_SAMP_SIZE & COLL_SAMP_SIZE_UNITS & DIST_CLASS \\
\hline 2 & & & & OFFSITE \\
\hline 3 & & & & OFFSITE \\
\hline 4 & & & & OFFSITE \\
\hline 5 & & & & OFFSITE \\
\hline 6 & & & & OFFSITE \\
\hline 7 & & & & OFFSITE \\
\hline 8 & & & & ONSITE \\
\hline 9 & & & & OFFSITE \\
\hline 10 & & & & OFFSITE \\
\hline 11 & & & & ONSITE \\
\hline 12 & & & & OFFSITE \\
\hline 13 & & & & OFFSITE \\
\hline 14 & & & & OFFSITE \\
\hline 15 & & & & OFFSITE \\
\hline 16 & & & & OFFSITE \\
\hline 17 & & & & OFFSITE \\
\hline 18 & & & & ONSITE \\
\hline 19 & & & & OFFSITE \\
\hline 20 & & & & OFFSITE \\
\hline 21 & & & & OFFSITE \\
\hline 22 & & & & OFFSITE \\
\hline 23 & & & & OFFSITE \\
\hline 24 & & & & OFFSITE \\
\hline 25 & & & & OFFSITE \\
\hline 26 & & & & OFFSITE \\
\hline 27 & & & & ONSITE \\
\hline 28 & & & & OFFSITE \\
\hline 29 & & & & OFFSITE \\
\hline 30 & & & & OFFSITE \\
\hline 31 & & & & OFFSITE \\
\hline 32 & & & & ONSITE \\
\hline 33 & & & & OFFSITE \\
\hline 34 & & & & OFFSITE \\
\hline 35 & & & & OFFSITE \\
\hline 36 & & & & OFFSITE \\
\hline 37 & & & & OFFSITE \\
\hline 38 & & & & OFFSITE \\
\hline 39 & 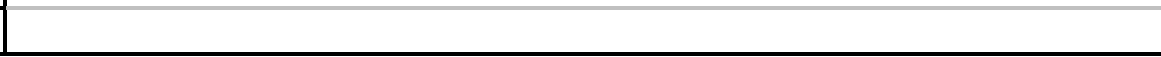 & & & ONSITE \\
\hline
\end{tabular}




\begin{tabular}{|c|c|c|c|c|}
\hline & $A D$ & $\mathrm{AE}$ & $\mathrm{AF}$ & $\overline{A G}$ \\
\hline 1 & RESULT_COMMENT & COLL_SAMP_SIZE & COLL_SAMP_SIZE_UNITS & DIST_CLASS \\
\hline 40 & & & & OFFSITE \\
\hline 41 & See sample record for comment. & & & OFFSITE \\
\hline 42 & & & & OFFSITE \\
\hline 43 & & & & OFFSITE \\
\hline 44 & & & & OFFSITE \\
\hline 45 & & & & OFFSITE \\
\hline 46 & & & & OFFSITE \\
\hline 47 & & & & OFFSITE \\
\hline 48 & & & & OFFSITE \\
\hline 49 & & & & OFFSITE \\
\hline 50 & & & & OFFSITE \\
\hline 51 & & & & OFFSITE \\
\hline 52 & & & & OFFSITE \\
\hline 53 & See sample record for comment. & & & OFFSITE \\
\hline 54 & & & & ONSITE \\
\hline 55 & & & & OFFSITE \\
\hline 56 & & & & OFFSITE \\
\hline 57 & & & & OFFSITE \\
\hline 58 & & & & OFFSITE \\
\hline 59 & & & & OFFSITE \\
\hline 60 & & & & OFFSITE \\
\hline 61 & & & & OFFSITE \\
\hline 62 & & & & OFFSITE \\
\hline 63 & & & & OFFSITE \\
\hline 64 & & & & OFFSITE \\
\hline 65 & & & & OFFSITE \\
\hline 66 & & & & OFFSITE \\
\hline 67 & & & & OFFSITE \\
\hline 68 & & & & OFFSITE \\
\hline 69 & & & & OFFSITE \\
\hline 70 & & & & OFFSITE \\
\hline 71 & & & & OFFSITE \\
\hline 72 & See sample record for comment. & & & OFFSITE \\
\hline 73 & & & & OFFSITE \\
\hline 74 & & & & OFFSITE \\
\hline 75 & & & & OFFSITE \\
\hline 76 & & & & OFFSITE \\
\hline 77 & & & & OFFSITE \\
\hline
\end{tabular}




\begin{tabular}{|c|c|c|c|c|}
\hline & $A D$ & $\mathrm{AE}$ & $\mathrm{AF}$ & AG \\
\hline 1 & RESULT_COMMENT & COLL_SAMP_SIZE & COLL_SAMP_SIZE_UNITS & DIST_CLASS \\
\hline 78 & & & & OFFSITE \\
\hline 79 & & & & OFFSITE \\
\hline 80 & & & & OFFSITE \\
\hline 81 & & & & OFFSITE \\
\hline 82 & & & & OFFSITE \\
\hline 83 & & & & OFFSITE \\
\hline 84 & & & & OFFSITE \\
\hline 85 & & & & OFFSITE \\
\hline 86 & & & & OFFSITE \\
\hline 87 & & & & OFFSITE \\
\hline 88 & See sample record for comment. & & & OFFSITE \\
\hline 89 & & & & OFFSITE \\
\hline 90 & & & & OFFSITE \\
\hline 91 & & & & OFFSITE \\
\hline 92 & & & & OFFSITE \\
\hline 93 & See sample record for comment. & & & OFFSITE \\
\hline 94 & See sample record for comment. & & & OFFSITE \\
\hline 95 & See sample record for comment. & & & ONSITE \\
\hline 96 & & & & OFFSITE \\
\hline 97 & & & & OFFSITE \\
\hline 98 & & & & OFFSITE \\
\hline 99 & & & & OFFSITE \\
\hline 100 & & & & OFFSITE \\
\hline 101 & & & & OFFSITE \\
\hline 102 & & & & OFFSITE \\
\hline 103 & & & & OFFSITE \\
\hline 104 & & & & OFFSITE \\
\hline 105 & & & & OFFSITE \\
\hline 106 & & & & OFFSITE \\
\hline 107 & & & & OFFSITE \\
\hline 108 & & & & OFFSITE \\
\hline 109 & & & & OFFSITE \\
\hline 110 & & & & OFFSITE \\
\hline 111 & & & & OFFSITE \\
\hline 112 & & & & OFFSITE \\
\hline 113 & & & & OFFSITE \\
\hline 114 & & & & ONSITE \\
\hline 115 & 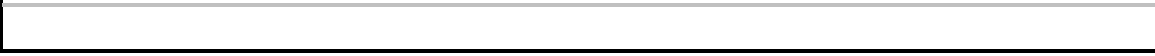 & & & OFFSITE \\
\hline
\end{tabular}




\begin{tabular}{|c|c|c|c|c|}
\hline & $A D$ & $\mathrm{AE}$ & $\mathrm{AF}$ & $\overline{A G}$ \\
\hline 1 & RESULT_COMMENT & COLL_SAMP_SIZE & COLL_SAMP_SIZE_UNITS & DIST_CLASS \\
\hline 116 & & & & OFFSITE \\
\hline 117 & & & & OFFSITE \\
\hline 118 & & & & OFFSITE \\
\hline 119 & & & & OFFSITE \\
\hline \begin{tabular}{|c|}
120 \\
120
\end{tabular} & & & & OFFSITE \\
\hline 121 & & & & OFFSITE \\
\hline \begin{tabular}{|c|}
122 \\
\end{tabular} & See sample record for comment. & & & OFFSITE \\
\hline \begin{tabular}{|l|l|}
123 \\
124
\end{tabular} & & & & OFFSITE \\
\hline 124 & & & & OFFSITE \\
\hline 125 & & & & OFFSITE \\
\hline 126 & & & & OFFSITE \\
\hline 127 & & & & OFFSITE \\
\hline 128 & & & & OFFSITE \\
\hline \begin{tabular}{|l|l|}
129 \\
129
\end{tabular} & & & & OFFSITE \\
\hline 130 & & & & OFFSITE \\
\hline \begin{tabular}{|c|}
131 \\
\end{tabular} & & & & OFFSITE \\
\hline \begin{tabular}{|c|}
132 \\
\end{tabular} & & & & OFFSITE \\
\hline 133 & & & & OFFSITE \\
\hline 134 & & & & OFFSITE \\
\hline \begin{tabular}{|c|c|}
135 \\
\end{tabular} & & & & ONSITE \\
\hline \begin{tabular}{|l|l|}
136 \\
\end{tabular} & See sample record for comment. & & & OFFSITE \\
\hline 137 & See sample record for comment. & & & OFFSITE \\
\hline \begin{tabular}{|l|l|}
138 \\
\end{tabular} & & & & OFFSITE \\
\hline \begin{tabular}{|l|l|}
139 \\
139
\end{tabular} & & & & OFFSITE \\
\hline 140 & & & & OFFSITE \\
\hline \begin{tabular}{|l|l|}
141 \\
\end{tabular} & & & & OFFSITE \\
\hline \begin{tabular}{|l|l|}
142 \\
\end{tabular} & & & & OFFSITE \\
\hline 143 & & & & OFFSITE \\
\hline 144 & & & & OFFSITE \\
\hline \begin{tabular}{|l|}
145 \\
\end{tabular} & & & & OFFSITE \\
\hline \begin{tabular}{|l|}
146 \\
\end{tabular} & & & & OFFSITE \\
\hline \begin{tabular}{|l|}
147 \\
\end{tabular} & & & & OFFSITE \\
\hline \begin{tabular}{|l|}
148 \\
\end{tabular} & & & & OFFSITE \\
\hline \begin{tabular}{|l|}
149 \\
\end{tabular} & & & & OFFSITE \\
\hline \begin{tabular}{|l|}
150 \\
\end{tabular} & & & & OFFSITE \\
\hline \begin{tabular}{|l|}
151 \\
\end{tabular} & & & & OFFSITE \\
\hline 152 & & & & OFFSITE \\
\hline 153 & & & & OFFSITE \\
\hline
\end{tabular}




\begin{tabular}{|c|c|c|c|c|}
\hline & $\mathrm{AD}$ & $\mathrm{AE}$ & $\mathrm{AF}$ & $\overline{A G}$ \\
\hline 1 & RESULT_COMMENT & COLL_SAMP_SIZE & COLL_SAMP_SIZE_UNITS & DIST_CLASS \\
\hline 154 & & & & OFFSITE \\
\hline 155 & & & & OFFSITE \\
\hline 156 & & & & OFFSITE \\
\hline 157 & See sample record for comment. & & & OFFSITE \\
\hline 158 & & & & ONSITE \\
\hline 159 & & & & OFFSITE \\
\hline 160 & & & & OFFSITE \\
\hline 161 & & & & OFFSITE \\
\hline 162 & & & & OFFSITE \\
\hline 163 & & & & OFFSITE \\
\hline 164 & & & & OFFSITE \\
\hline 165 & & & & OFFSITE \\
\hline 166 & & & & OFFSITE \\
\hline 167 & & & & OFFSITE \\
\hline 168 & & & & OFFSITE \\
\hline 169 & See sample record for comment. & & & OFFSITE \\
\hline 170 & & & & OFFSITE \\
\hline 171 & & & & OFFSITE \\
\hline 172 & & & & OFFSITE \\
\hline 173 & & & & OFFSITE \\
\hline 174 & & & & OFFSITE \\
\hline 175 & & & & OFFSITE \\
\hline 176 & & & & OFFSITE \\
\hline 177 & & & & OFFSITE \\
\hline 178 & See sample record for comment. & & & OFFSITE \\
\hline 179 & & & & ONSITE \\
\hline 180 & & & & OFFSITE \\
\hline 181 & & & & OFFSITE \\
\hline 182 & & & & OFFSITE \\
\hline 183 & See sample record for comment. & & & OFFSITE \\
\hline 184 & & & & OFFSITE \\
\hline 185 & & & & OFFSITE \\
\hline 186 & & & & OFFSITE \\
\hline 187 & & & & OFFSITE \\
\hline 188 & & & & OFFSITE \\
\hline 189 & & & & OFFSITE \\
\hline 190 & & & & OFFSITE \\
\hline 191 & See sample record for comment. & & & OFFSITE \\
\hline
\end{tabular}




\begin{tabular}{|c|c|c|c|c|}
\hline & $\mathrm{AD}$ & $\mathrm{AE}$ & $\mathrm{AF}$ & $A G$ \\
\hline 1 & RESULT_COMMENT & COLL_SAMP_SIZE & COLL_SAMP_SIZE_UNITS & DIST_CLASS \\
\hline 192 & & & & OFFSITE \\
\hline 193 & & & & OFFSITE \\
\hline 194 & & & & OFFSITE \\
\hline 195 & & & & OFFSITE \\
\hline 196 & & & & OFFSITE \\
\hline 197 & & & & OFFSITE \\
\hline 198 & & & & OFFSITE \\
\hline 199 & & & & OFFSITE \\
\hline 200 & & & & ONSITE \\
\hline 201 & & & & OFFSITE \\
\hline 202 & & & & OFFSITE \\
\hline 203 & & & & OFFSITE \\
\hline 204 & & & & OFFSITE \\
\hline 205 & See sample record for comment. & & & OFFSITE \\
\hline 206 & See sample record for comment. & & & OFFSITE \\
\hline 207 & & & & ONSITE \\
\hline 208 & & & & OFFSITE \\
\hline 209 & & & & OFFSITE \\
\hline 210 & & & & OFFSITE \\
\hline 211 & & & & OFFSITE \\
\hline 212 & & & & OFFSITE \\
\hline 213 & & & & OFFSITE \\
\hline 214 & & & & OFFSITE \\
\hline 215 & & & & OFFSITE \\
\hline 216 & & & & OFFSITE \\
\hline 217 & & & & OFFSITE \\
\hline 218 & & & & OFFSITE \\
\hline 219 & & & & OFFSITE \\
\hline 220 & & & & OFFSITE \\
\hline 221 & & & & OFFSITE \\
\hline 222 & & & & OFFSITE \\
\hline 223 & & & & OFFSITE \\
\hline 224 & & & & OFFSITE \\
\hline 225 & See sample record for comment. & & & OFFSITE \\
\hline 226 & & & & OFFSITE \\
\hline 227 & See sample record for comment. & & & OFFSITE \\
\hline 228 & & & & OFFSITE \\
\hline 229 & See sample record for comment. & & & OFFSITE \\
\hline
\end{tabular}




\begin{tabular}{|c|c|c|c|c|}
\hline & $A D$ & $\mathrm{AE}$ & $\mathrm{AF}$ & AG \\
\hline 1 & RESULT_COMMENT & COLL_SAMP_SIZE & COLL_SAMP_SIZE_UNITS & DIST_CLASS \\
\hline 230 & See sample record for comment. & & & OFFSITE \\
\hline 231 & & & & OFFSITE \\
\hline 232 & & & & ONSITE \\
\hline 233 & & & & ONSITE \\
\hline 234 & & & & ONSITE \\
\hline 235 & & & & ONSITE \\
\hline 236 & & & & ONSITE \\
\hline 237 & & & & ONSITE \\
\hline 238 & & & & ONSITE \\
\hline 239 & & & & ONSITE \\
\hline 240 & & & & ONSITE \\
\hline 241 & & & & ONSITE \\
\hline 242 & See sample record for comment. & & & ONSITE \\
\hline 243 & & & & ONSITE \\
\hline 244 & See sample record for comment. & & & ONSITE \\
\hline 245 & & & & ONSITE \\
\hline 246 & & & & ONSITE \\
\hline 247 & & & & ONSITE \\
\hline 248 & & & & ONSITE \\
\hline 249 & & & & ONSITE \\
\hline 250 & & & & ONSITE \\
\hline 251 & & & & ONSITE \\
\hline 252 & See sample record for comment. & & & ONSITE \\
\hline 253 & See sample record for comment. & & & ONSITE \\
\hline 254 & & & & ONSITE \\
\hline 255 & & & & ONSITE \\
\hline 256 & & & & ONSITE \\
\hline 257 & & & & ONSITE \\
\hline 258 & & & & ONSITE \\
\hline 259 & & & & ONSITE \\
\hline 260 & & & & ONSITE \\
\hline 261 & See sample record for comment. & & & OFFSITE \\
\hline 262 & See sample record for comment. & & & OFFSITE \\
\hline 263 & & & & OFFSITE \\
\hline 264 & & & & OFFSITE \\
\hline 265 & & & & OFFSITE \\
\hline 266 & & & & OFFSITE \\
\hline 267 & 1 & & & OFFSITE \\
\hline
\end{tabular}




\begin{tabular}{|c|c|c|c|c|}
\hline & $A D$ & $\mathrm{AE}$ & $\mathrm{AF}$ & $A G$ \\
\hline \begin{tabular}{|l|} 
\\
\end{tabular} & RESULT_COMMENT & COLL_SAMP_SIZE & COLL_SAMP_SIZE_UNITS & DIST_CLASS \\
\hline 268 & & & & OFFSITE \\
\hline 269 & & & & OFFSITE \\
\hline 270 & & & & OFFSITE \\
\hline 271 & & & & OFFSITE \\
\hline 272 & & & & OFFSITE \\
\hline 273 & & & & ONSITE \\
\hline 274 & See sample record for comment. & & & OFFSITE \\
\hline 275 & See sample record for comment. & & & OFFSITE \\
\hline 276 & & & & ONSITE \\
\hline 277 & & & & ONSITE \\
\hline 278 & & & & ONSITE \\
\hline 279 & & & & ONSITE \\
\hline 280 & & & & ONSITE \\
\hline 281 & & & & ONSITE \\
\hline 282 & & & & ONSITE \\
\hline 283 & & & & ONSITE \\
\hline 284 & & & & ONSITE \\
\hline 285 & & & & ONSITE \\
\hline 286 & & & & OFFSITE \\
\hline 287 & & & & OFFSITE \\
\hline 288 & & & & ONSITE \\
\hline 289 & & & & OFFSITE \\
\hline 290 & & & & OFFSITE \\
\hline 291 & & & & OFFSITE \\
\hline 292 & & & & OFFSITE \\
\hline 293 & & & & OFFSITE \\
\hline 294 & & & & OFFSITE \\
\hline 295 & & & & OFFSITE \\
\hline 296 & & & & OFFSITE \\
\hline 297 & & & & OFFSITE \\
\hline 298 & & & & OFFSITE \\
\hline 299 & & & & OFFSITE \\
\hline 300 & & & & OFFSITE \\
\hline 301 & & & & OFFSITE \\
\hline 302 & & & & OFFSITE \\
\hline 303 & & & & OFFSITE \\
\hline 304 & & & & OFFSITE \\
\hline 305 & & & & OFFSITE \\
\hline
\end{tabular}




\begin{tabular}{|c|c|c|c|c|}
\hline & $A D$ & $\mathrm{AE}$ & $\mathrm{AF}$ & AG \\
\hline 1 & RESULT_COMMENT & COLL_SAMP_SIZE & COLL_SAMP_SIZE_UNITS & DIST_CLASS \\
\hline 306 & & 10 & L & OFFSITE \\
\hline \begin{tabular}{|l|}
307 \\
\end{tabular} & & 10 & $\mathrm{~L}$ & OFFSITE \\
\hline \begin{tabular}{|l|}
308 \\
\end{tabular} & & 10 & L & OFFSITE \\
\hline \begin{tabular}{|l|}
309 \\
\end{tabular} & & 10 & L & OFFSITE \\
\hline 310 & & 10 & L & OFFSITE \\
\hline \begin{tabular}{|l|}
311 \\
\end{tabular} & & 10 & $\mathrm{~L}$ & OFFSITE \\
\hline \begin{tabular}{|l|}
312 \\
\end{tabular} & & & & ONSITE \\
\hline \begin{tabular}{|l|}
313 \\
\end{tabular} & 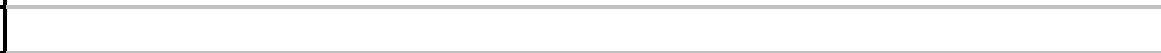 & 10 & L & OFFSITE \\
\hline \begin{tabular}{|l|}
314 \\
\end{tabular} & & 10 & L & OFFSITE \\
\hline \begin{tabular}{|l|}
315 \\
\end{tabular} & & 10 & L & OFFSITE \\
\hline \begin{tabular}{|l|}
316 \\
\end{tabular} & & 10 & L & OFFSITE \\
\hline \begin{tabular}{|l|}
317 \\
\end{tabular} & & 10 & L & OFFSITE \\
\hline \begin{tabular}{|l|}
318 \\
\end{tabular} & & 10 & L & OFFSITE \\
\hline 319 & & 10 & $\mathrm{~L}$ & OFFSITE \\
\hline 320 & & 10 & L & OFFSITE \\
\hline \begin{tabular}{|l|}
321 \\
\end{tabular} & & 10 & L & OFFSITE \\
\hline \begin{tabular}{|l|}
322 \\
\end{tabular} & & 10 & L & OFFSITE \\
\hline \begin{tabular}{|l|}
323 \\
\end{tabular} & & 10 & L & OFFSITE \\
\hline \begin{tabular}{|l|}
324 \\
\end{tabular} & & 10 & L & OFFSITE \\
\hline \begin{tabular}{|l|}
325 \\
\end{tabular} & & 10 & L & OFFSITE \\
\hline \begin{tabular}{|l|}
326 \\
\end{tabular} & & 10 & L & OFFSITE \\
\hline \begin{tabular}{|l|}
327 \\
\end{tabular} & & 10 & L & OFFSITE \\
\hline \begin{tabular}{|l|}
328 \\
\end{tabular} & & 10 & L & OFFSITE \\
\hline \begin{tabular}{|l|}
329 \\
\end{tabular} & & & & ONSITE \\
\hline \begin{tabular}{|l|}
330 \\
\end{tabular} & & 10 & L & OFFSITE \\
\hline 331 & & 10 & L & OFFSITE \\
\hline 332 & & 10 & $\mathrm{~L}$ & OFFSITE \\
\hline 333 & & 10 & L & OFFSITE \\
\hline 334 & & 10 & L & OFFSITE \\
\hline \begin{tabular}{|l|}
335 \\
\end{tabular} & & 10 & L & OFFSITE \\
\hline \begin{tabular}{|l|}
336 \\
\end{tabular} & & 10 & L & OFFSITE \\
\hline \begin{tabular}{|l|}
337 \\
\end{tabular} & & 10 & L & OFFSITE \\
\hline \begin{tabular}{|l|}
338 \\
\end{tabular} & & 10 & L & OFFSITE \\
\hline \begin{tabular}{|l|}
339 \\
\end{tabular} & & 10 & L & OFFSITE \\
\hline 340 & & 10 & L & OFFSITE \\
\hline \begin{tabular}{|l|}
341 \\
\end{tabular} & & 10 & L & OFFSITE \\
\hline \begin{tabular}{|l|}
342 \\
\end{tabular} & & 10 & L & OFFSITE \\
\hline \begin{tabular}{|l|}
343 \\
\end{tabular} & 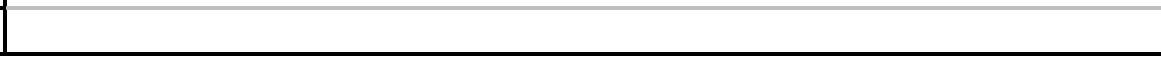 & 10 & L & OFFSITE \\
\hline
\end{tabular}




\begin{tabular}{|c|c|c|c|c|}
\hline & $A D$ & $\mathrm{AE}$ & $\mathrm{AF}$ & AG \\
\hline 1 & $\begin{array}{lc}\text { RESULT_COMMENT } \\
\end{array}$ & COLL_SAMP_SIZE & COLL_SAMP_SIZE_UNITS & DIST_CLASS \\
\hline 344 & & 10 & L & OFFSITE \\
\hline 345 & & 10 & $\mathrm{~L}$ & OFFSITE \\
\hline 346 & & 10 & $\mathrm{~L}$ & OFFSITE \\
\hline \begin{tabular}{|l|l|l}
347 \\
\end{tabular} & & 10 & L & OFFSITE \\
\hline 348 & 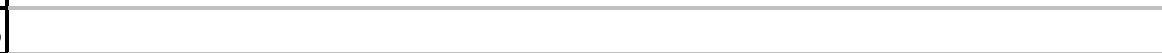 & 10 & L & ONSITE \\
\hline 349 & & 10 & L & ONSITE \\
\hline 350 & & 10 & L & ONSITE \\
\hline \begin{tabular}{|l|}
351 \\
\end{tabular} & & 10 & L & ONSITE \\
\hline \begin{tabular}{|l|}
352 \\
\end{tabular} & & 10 & L & ONSITE \\
\hline \begin{tabular}{|l|}
353 \\
\end{tabular} & & 10 & L & ONSITE \\
\hline \begin{tabular}{|l|}
354 \\
\end{tabular} & & 10 & L & ONSITE \\
\hline 355 & & 10 & L & ONSITE \\
\hline \begin{tabular}{|l|}
356 \\
\end{tabular} & & 10 & $\mathrm{~L}$ & ONSITE \\
\hline \begin{tabular}{|l|}
357 \\
\end{tabular} & & 10 & $\mathrm{~L}$ & ONSITE \\
\hline \begin{tabular}{|l|}
358 \\
\end{tabular} & & 10 & $\mathrm{~L}$ & ONSITE \\
\hline \begin{tabular}{|l|}
359 \\
\end{tabular} & & 10 & $\mathrm{~L}$ & ONSITE \\
\hline 360 & & 10 & $\mathrm{~L}$ & ONSITE \\
\hline \begin{tabular}{|l|}
361 \\
\end{tabular} & & 10 & L & ONSITE \\
\hline \begin{tabular}{|l|}
362 \\
\end{tabular} & & 10 & L & ONSITE \\
\hline 363 & & 10 & L & ONSITE \\
\hline 364 & & 10 & $\mathrm{~L}$ & ONSITE \\
\hline 365 & & 10 & L & ONSITE \\
\hline \begin{tabular}{|l|}
366 \\
\end{tabular} & & 10 & L & ONSITE \\
\hline \begin{tabular}{|l|}
367 \\
\end{tabular} & & 10 & $\mathrm{~L}$ & ONSITE \\
\hline 368 & & 10 & L & OFFSITE \\
\hline 369 & & 10 & L & OFFSITE \\
\hline \begin{tabular}{|l|}
370 \\
\end{tabular} & & 10 & $\mathrm{~L}$ & OFFSITE \\
\hline \begin{tabular}{|l|}
371 \\
\end{tabular} & & 10 & L & OFFSITE \\
\hline \begin{tabular}{|l|}
372 \\
\end{tabular} & & 10 & L & ONSITE \\
\hline \begin{tabular}{|l|}
373 \\
\end{tabular} & & 10 & L & ONSITE \\
\hline 374 & & 10 & L & ONSITE \\
\hline 375 & & 10 & L & ONSITE \\
\hline \begin{tabular}{|l|}
376 \\
\end{tabular} & & 10 & L & ONSITE \\
\hline \begin{tabular}{|l|}
377 \\
\end{tabular} & & 10 & L & ONSITE \\
\hline 378 & & 10 & L & ONSITE \\
\hline 379 & & 10 & L & ONSITE \\
\hline 380 & & 10 & L & ONSITE \\
\hline \begin{tabular}{|l|}
381 \\
\end{tabular} & 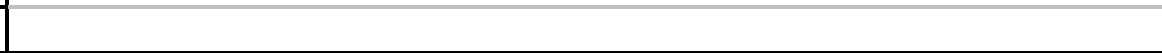 & 10 & L & OFFSITE \\
\hline
\end{tabular}




\begin{tabular}{|c|c|c|c|c|}
\hline & $A D$ & $\mathrm{AE}$ & $\mathrm{AF}$ & $A G$ \\
\hline \begin{tabular}{|l|}
1 \\
\end{tabular} & RESULT_COMMENT & COLL_SAMP_SIZE & COLL_SAMP_SIZE_UNITS & DIST_CLASS \\
\hline 382 & & 10 & $\mathrm{~L}$ & OFFSITE \\
\hline 383 & & 10 & $\mathrm{~L}$ & OFFSITE \\
\hline 384 & & 10 & L & OFFSITE \\
\hline 385 & & 10 & $\mathrm{~L}$ & OFFSITE \\
\hline 386 & & 10 & $\mathrm{~L}$ & OFFSITE \\
\hline 387 & & 10 & L & OFFSITE \\
\hline 388 & & 10 & $\mathrm{~L}$ & OFFSITE \\
\hline 389 & & 10 & $\mathrm{~L}$ & OFFSITE \\
\hline 390 & & 10 & L & OFFSITE \\
\hline 391 & & 10 & $\mathrm{~L}$ & ONSITE \\
\hline 392 & RESULT LOST AT LAB. & 10 & $\mathrm{~L}$ & ONSITE \\
\hline 393 & & 10 & L & ONSITE \\
\hline 394 & & 10 & $L$ & ONSITE \\
\hline 395 & & 10 & L & ONSITE \\
\hline 396 & & 10 & L & ONSITE \\
\hline 397 & & 10 & L & ONSITE \\
\hline 398 & & 10 & $\mathrm{~L}$ & ONSITE \\
\hline 399 & & 10 & $\mathrm{~L}$ & ONSITE \\
\hline 400 & & 10 & L & ONSITE \\
\hline \begin{tabular}{|l|}
401 \\
\end{tabular} & & 10 & L & OFFSITE \\
\hline 402 & & 10 & $\mathrm{~L}$ & OFFSITE \\
\hline 403 & & 10 & $\mathrm{~L}$ & OFFSITE \\
\hline 404 & & 10 & L & OFFSITE \\
\hline 405 & & & & ONSITE \\
\hline 406 & & 10 & $\mathrm{~L}$ & OFFSITE \\
\hline 407 & REFER TO FOLLOW-UP REQUEST \#W021. REANALYSIS ENTERED IN DATABASE. & 10 & $\mathrm{~L}$ & OFFSITE \\
\hline 408 & & 10 & L & OFFSITE \\
\hline 409 & & 10 & $\mathrm{~L}$ & OFFSITE \\
\hline 410 & & 10 & L & OFFSITE \\
\hline 411 & & 10 & $\mathrm{~L}$ & OFFSITE \\
\hline 412 & & 10 & $\mathrm{~L}$ & OFFSITE \\
\hline 413 & & 10 & $\mathrm{~L}$ & OFFSITE \\
\hline \begin{tabular}{|l|l|l|l|}
414 \\
\end{tabular} & REFER TO 3/24/95 INCIDENT REPORT, RESULT LOST AT LAB. & 10 & L & OFFSITE \\
\hline 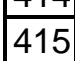 & U-ISOTOPIC FRACTION LOST AT LAB. RETRIEVED SAMPLES FROM DATACHEM AND SUBMITTED TO IT ON 4/3/95. & 10 & $\mathrm{~L}$ & OFFSITE \\
\hline 416 & U-ISOTOPIC FRACTION LOST AT LAB. RETRIEVED SAMPLES FROM DATACHEM AND SUBMITTED TO IT ON 4/3/95. & 10 & L & OFFSITE \\
\hline 417 & U-ISOTOPIC FRACTION LOST AT LAB. RETRIEVED SAMPLES FROM DATACHEM AND SUBMITTED TO IT ON 4/3/95. & 10 & L & OFFSITE \\
\hline 418 & U-ISOTOPIC FRACTION LOST AT LAB. RETRIEVED SAMPLES FROM DATACHEM AND SUBMITTED TO IT ON 4/3/95. & 10 & $\mathrm{~L}$ & OFFSITE \\
\hline 419 & U-ISOTOPIC FRACTION LOST AT LAB. RETRIEVED SAMPLES FROM DATACHEM AND SUBMITTED TO IT ON 4/3/95. & 10 & L & OFFSITE \\
\hline
\end{tabular}




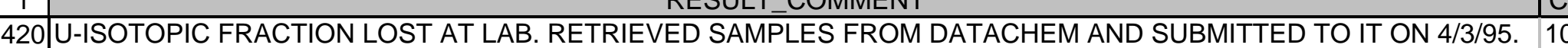
\begin{tabular}{|l|l|l|l}
\hline 421 & U-ISOTOPIC FRACTION LOST AT LAB. RETRIEVED SAMPLES FROM DATACHEM AND SUBMITTED TO IT ON 4/3/95. 10 \\
\hline 422
\end{tabular}

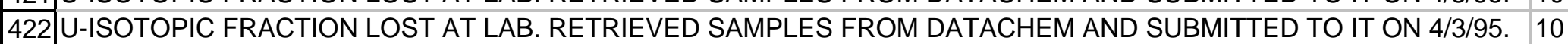
423 U-ISOTOPIC FRACTION LOST AT LAB. RETRIEVED SAMPLES FROM DATACHEM AND SUBMITTED TO IT ON 4/3/95. 10

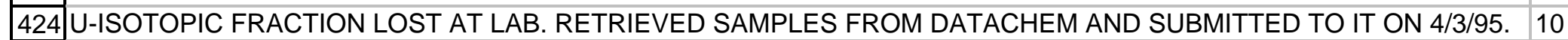
425 MODIFIED RESULT ENTERED IN DATABASE PER FOLLOW-UP ORDER W034. 


\begin{tabular}{|c|c|c|c|c|}
\hline & $\mathrm{AD}$ & $\mathrm{AE}$ & $\mathrm{AF}$ & AG \\
\hline 1 & $\begin{array}{lc}\text { RESULT_COMMENT } \\
\end{array}$ & COLL_SAMP_SIZE & COLL_SAMP_SIZE_UNITS & DIST_CLASS \\
\hline 458 & & 10 & L & OFFSITE \\
\hline 459 & & 10 & $\mathrm{~L}$ & OFFSITE \\
\hline 460 & & 10 & $\mathrm{~L}$ & OFFSITE \\
\hline 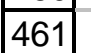 & & 10 & L & OFFSITE \\
\hline \begin{tabular}{|l|l|l|l}
462 \\
\end{tabular} & 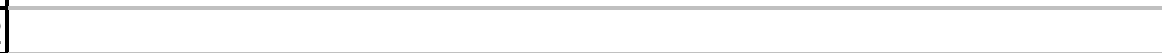 & 10 & L & OFFSITE \\
\hline \begin{tabular}{|l|}
463 \\
\end{tabular} & & 10 & $\mathrm{~L}$ & OFFSITE \\
\hline \begin{tabular}{|l|}
464 \\
\end{tabular} & & 10 & L & OFFSITE \\
\hline 465 & & 10 & L & OFFSITE \\
\hline \begin{tabular}{|l|}
466 \\
\end{tabular} & & 10 & $\bar{L}$ & OFFSITE \\
\hline \begin{tabular}{|l|}
467 \\
\end{tabular} & & & & ONSITE \\
\hline \begin{tabular}{|l|}
468 \\
\end{tabular} & & 10 & $\mathrm{~L}$ & OFFSITE \\
\hline \begin{tabular}{|l|}
469 \\
\end{tabular} & & 10 & $\mathrm{~L}$ & OFFSITE \\
\hline 470 & & 10 & $\mathrm{~L}$ & OFFSITE \\
\hline \begin{tabular}{|l|}
471 \\
\end{tabular} & & 10 & $\mathrm{~L}$ & OFFSITE \\
\hline \begin{tabular}{|l|}
472 \\
\end{tabular} & & 10 & $\mathrm{~L}$ & OFFSITE \\
\hline \begin{tabular}{|l|}
473 \\
\end{tabular} & & 10 & $\mathrm{~L}$ & OFFSITE \\
\hline \begin{tabular}{|l|}
474 \\
\end{tabular} & & 10 & $\mathrm{~L}$ & ONSITE \\
\hline \begin{tabular}{|l|}
475 \\
\end{tabular} & & 10 & L & ONSITE \\
\hline \begin{tabular}{|l|}
476 \\
\end{tabular} & & 10 & $\mathrm{~L}$ & ONSITE \\
\hline 477 & & 10 & L & ONSITE \\
\hline \begin{tabular}{|l|}
478 \\
\end{tabular} & & 10 & L & ONSITE \\
\hline 479 & & 10 & L & ONSITE \\
\hline 480 & & 10 & L & ONSITE \\
\hline \begin{tabular}{|l|}
481 \\
\end{tabular} & & 10 & $\mathrm{~L}$ & ONSITE \\
\hline \begin{tabular}{|l|}
482 \\
\end{tabular} & & 10 & L & ONSITE \\
\hline \begin{tabular}{|l|}
483 \\
\end{tabular} & & 10 & L & ONSITE \\
\hline \begin{tabular}{|l|}
484 \\
\end{tabular} & & 10 & $\mathrm{~L}$ & OFFSITE \\
\hline \begin{tabular}{|l|}
485 \\
\end{tabular} & & 10 & $\mathrm{~L}$ & OFFSITE \\
\hline \begin{tabular}{|l|}
486 \\
\end{tabular} & & 10 & L & OFFSITE \\
\hline \begin{tabular}{|l|}
487 \\
\end{tabular} & & 10 & $\mathrm{~L}$ & OFFSITE \\
\hline 488 & & 10 & L & ONSITE \\
\hline 489 & & 10 & $\mathrm{~L}$ & ONSITE \\
\hline 490 & & 10 & $\mathrm{~L}$ & ONSITE \\
\hline \begin{tabular}{|l|}
491 \\
\end{tabular} & & 10 & L & ONSITE \\
\hline \begin{tabular}{|l|}
492 \\
\end{tabular} & & 10 & L & ONSITE \\
\hline 493 & & 10 & L & ONSITE \\
\hline 494 & & 10 & L & ONSITE \\
\hline \begin{tabular}{|l|}
495 \\
\end{tabular} & 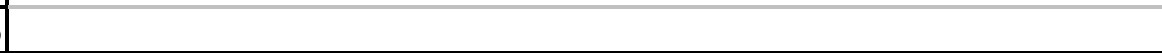 & 10 & L & ONSITE \\
\hline
\end{tabular}




\begin{tabular}{|c|c|c|c|c|}
\hline & $A D$ & $\mathrm{AE}$ & $\mathrm{AF}$ & AG \\
\hline \begin{tabular}{|l|l|} 
\\
\end{tabular} & RESULT_COMMENT & COLL_SAMP_SIZE & COLL_SAMP_SIZE_UNITS & DIST_CLASS \\
\hline 496 & & 10 & $\mathrm{~L}$ & ONSITE \\
\hline 497 & & 10 & L & ONSITE \\
\hline 498 & & 10 & L & ONSITE \\
\hline 499 & & 10 & L & ONSITE \\
\hline 500 & & 10 & $\mathrm{~L}$ & ONSITE \\
\hline 501 & & 10 & L & ONSITE \\
\hline 502 & & 10 & L & ONSITE \\
\hline 503 & & 10 & $\mathrm{~L}$ & ONSITE \\
\hline 504 & & 10 & $\mathrm{~L}$ & ONSITE \\
\hline 505 & & 10 & L & ONSITE \\
\hline 506 & & 10 & $\mathrm{~L}$ & ONSITE \\
\hline 507 & & 10 & L & ONSITE \\
\hline 508 & & 10 & $\mathrm{~L}$ & ONSITE \\
\hline 509 & & 10 & $\mathrm{~L}$ & ONSITE \\
\hline 510 & & 10 & L & ONSITE \\
\hline 511 & & 10 & L & ONSITE \\
\hline 512 & & 10 & $\mathrm{~L}$ & ONSITE \\
\hline 513 & & 10 & $\mathrm{~L}$ & ONSITE \\
\hline 514 & & 10 & L & ONSITE \\
\hline 515 & & 10 & $\mathrm{~L}$ & ONSITE \\
\hline 516 & & 10 & L & ONSITE \\
\hline 517 & & 10 & $\mathrm{~L}$ & OFFSITE \\
\hline 518 & & 10 & $\mathrm{~L}$ & OFFSITE \\
\hline 519 & & 10 & $\mathrm{~L}$ & OFFSITE \\
\hline 520 & & 10 & $\mathrm{~L}$ & OFFSITE \\
\hline 521 & & 10 & $\mathrm{~L}$ & OFFSITE \\
\hline 522 & & 10 & L & OFFSITE \\
\hline 523 & & 10 & L & OFFSITE \\
\hline 524 & & 10 & L & OFFSITE \\
\hline 525 & & 10 & $\mathrm{~L}$ & OFFSITE \\
\hline 526 & & 10 & $\mathrm{~L}$ & OFFSITE \\
\hline 527 & & 10 & $\mathrm{~L}$ & ONSITE \\
\hline 528 & & 10 & L & OFFSITE \\
\hline 529 & & 10 & $\mathrm{~L}$ & OFFSITE \\
\hline 530 & ORIGINAL RESULT NOT BLANK SUBTRACTED, MODIFIED RESULT PER 1/9/96 REPORT. & & & ONSITE \\
\hline 531 & & 10 & $\mathrm{~L}$ & RIVER_SHORELINE \\
\hline 532 & & 10 & $\mathrm{~L}$ & RIVER_SHORELINE \\
\hline 533 & 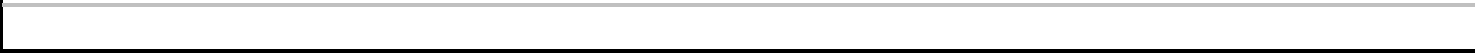 & 10 & L & RIVER_SHORELINE \\
\hline
\end{tabular}




\begin{tabular}{|c|c|c|c|c|}
\hline & $A D$ & $\mathrm{AE}$ & $\mathrm{AF}$ & AG \\
\hline 1 & RESULT_COMMENT & COLL_SAMP_SIZE & COLL_SAMP_SIZE_UNITS & DIST_CLASS \\
\hline 534 & & 10 & $\mathrm{~L}$ & OFFSITE \\
\hline 535 & & 10 & L & OFFSITE \\
\hline 536 & & 10 & L & OFFSITE \\
\hline \begin{tabular}{|l|}
537 \\
\end{tabular} & & 10 & L & OFFSITE \\
\hline 538 & & 10 & $\mathrm{~L}$ & OFFSITE \\
\hline \begin{tabular}{|l|}
539 \\
\end{tabular} & & 10 & $\mathrm{~L}$ & OFFSITE \\
\hline 540 & & 10 & L & OFFSITE \\
\hline \begin{tabular}{|l|}
541 \\
\end{tabular} & & 10 & $\mathrm{~L}$ & OFFSITE \\
\hline \begin{tabular}{|l|}
542 \\
\end{tabular} & & 10 & L & OFFSITE \\
\hline \begin{tabular}{|l|}
543 \\
\end{tabular} & & 10 & L & OFFSITE \\
\hline 544 & & 10 & $\mathrm{~L}$ & OFFSITE \\
\hline 545 & & 10 & $\mathrm{~L}$ & OFFSITE \\
\hline \begin{tabular}{|l|}
546 \\
\end{tabular} & & 10 & L & OFFSITE \\
\hline 547 & & 10 & $\mathrm{~L}$ & OFFSITE \\
\hline 548 & & 10 & L & OFFSITE \\
\hline \begin{tabular}{|l|}
549 \\
\end{tabular} & & 10 & L & OFFSITE \\
\hline 550 & & 10 & $\mathrm{~L}$ & OFFSITE \\
\hline \begin{tabular}{|l|}
551 \\
\end{tabular} & & 10 & $\mathrm{~L}$ & OFFSITE \\
\hline \begin{tabular}{|l|}
552 \\
\end{tabular} & & & & ONSITE \\
\hline 553 & & & & ONSITE \\
\hline \begin{tabular}{|l|}
554 \\
\end{tabular} & & & & ONSITE \\
\hline \begin{tabular}{|l|}
555 \\
\end{tabular} & & & & ONSITE \\
\hline \begin{tabular}{|l|}
556 \\
\end{tabular} & & & & ONSITE \\
\hline \begin{tabular}{|l|}
557 \\
\end{tabular} & & & & ONSITE \\
\hline \begin{tabular}{|l|}
558 \\
\end{tabular} & & & & ONSITE \\
\hline 559 & & & & ONSITE \\
\hline 560 & & & & ONSITE \\
\hline \begin{tabular}{|l|}
561 \\
\end{tabular} & & & & ONSITE \\
\hline \begin{tabular}{|l|}
562 \\
\end{tabular} & & & & ONSITE \\
\hline 563 & & & & ONSITE \\
\hline \begin{tabular}{|l|}
564 \\
\end{tabular} & & & & ONSITE \\
\hline 565 & & & & ONSITE \\
\hline \begin{tabular}{|l|}
566 \\
\end{tabular} & & & & ONSITE \\
\hline \begin{tabular}{|l|}
567 \\
\end{tabular} & & & & ONSITE \\
\hline \begin{tabular}{|l|}
568 \\
\end{tabular} & & & & ONSITE \\
\hline 569 & & & & ONSITE \\
\hline \begin{tabular}{|l|}
570 \\
\end{tabular} & & & & ONSITE \\
\hline \begin{tabular}{|l|}
571 \\
\end{tabular} & 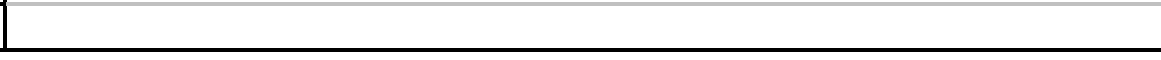 & & & ONSITE \\
\hline
\end{tabular}




\begin{tabular}{|c|c|c|c|c|}
\hline & $A D$ & $\mathrm{AE}$ & $\mathrm{AF}$ & $A G$ \\
\hline 1 & RESULT_COMMENT & COLL_SAMP_SIZE & COLL_SAMP_SIZE_UNITS & DIST_CLASS \\
\hline \begin{tabular}{|l|l}
572 \\
\end{tabular} & & & & OFFSITE \\
\hline \begin{tabular}{|l|}
573 \\
\end{tabular} & & & & OFFSITE \\
\hline \begin{tabular}{|l|}
574 \\
\end{tabular} & & & & OFFSITE \\
\hline \begin{tabular}{|l|}
575 \\
\end{tabular} & & & & OFFSITE \\
\hline \begin{tabular}{|l|}
576 \\
\end{tabular} & & & & ONSITE \\
\hline \begin{tabular}{|l|}
577 \\
\end{tabular} & & & & ONSITE \\
\hline \begin{tabular}{|l|}
578 \\
\end{tabular} & & & & ONSITE \\
\hline \begin{tabular}{|l|}
579 \\
\end{tabular} & & & & ONSITE \\
\hline 580 & & & & ONSITE \\
\hline \begin{tabular}{|l|}
581 \\
\end{tabular} & & & & ONSITE \\
\hline \begin{tabular}{|l|}
582 \\
\end{tabular} & & & & ONSITE \\
\hline 58 & & & & ONSITE \\
\hline 584 & & & & ONSITE \\
\hline 585 & & & & OFFSITE \\
\hline \begin{tabular}{|l|}
586 \\
\end{tabular} & & & & OFFSITE \\
\hline \begin{tabular}{|l|}
587 \\
\end{tabular} & & & & OFFSITE \\
\hline \begin{tabular}{|l|}
588 \\
\end{tabular} & & & & OFFSITE \\
\hline 589 & & & & OFFSITE \\
\hline 590 & & & & OFFSITE \\
\hline \begin{tabular}{|l|}
591 \\
\end{tabular} & & & & OFFSITE \\
\hline \begin{tabular}{|l|}
592 \\
\end{tabular} & & & & OFFSITE \\
\hline \begin{tabular}{|l|}
593 \\
\end{tabular} & & & & OFFSITE \\
\hline \begin{tabular}{|l|l|}
594 \\
\end{tabular} & & & & OFFSITE \\
\hline \begin{tabular}{|l|l|}
595 \\
595
\end{tabular} & & & & ONSITE \\
\hline \begin{tabular}{|l|l|}
596 \\
\end{tabular} & & & & ONSITE \\
\hline \begin{tabular}{|l|}
597 \\
\end{tabular} & & & & ONSITE \\
\hline \begin{tabular}{|l|}
598 \\
\end{tabular} & & & & ONSITE \\
\hline \begin{tabular}{|l|}
599 \\
\end{tabular} & & & & ONSITE \\
\hline \begin{tabular}{|l|l|}
600 \\
\end{tabular} & & & & ONSITE \\
\hline \begin{tabular}{|l|}
601 \\
\end{tabular} & & & & ONSITE \\
\hline \begin{tabular}{|l|}
602 \\
\end{tabular} & & & & ONSITE \\
\hline \begin{tabular}{|l|l|}
603 \\
\end{tabular} & & & & ONSITE \\
\hline \begin{tabular}{|l|l|}
604 \\
\end{tabular} & & & & ONSITE \\
\hline \begin{tabular}{|l|}
605 \\
\end{tabular} & & & & OFFSITE \\
\hline \begin{tabular}{|l|l|}
606 \\
\end{tabular} & & & & OFFSITE \\
\hline \begin{tabular}{|l|}
607 \\
\end{tabular} & & & & OFFSITE \\
\hline \begin{tabular}{|l|}
608 \\
\end{tabular} & & & & OFFSITE \\
\hline 609 & 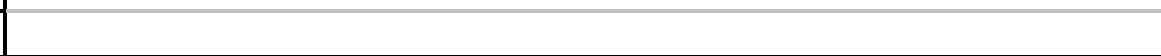 & & & OFFSITE \\
\hline
\end{tabular}




\begin{tabular}{|c|c|c|c|c|}
\hline & $A D$ & $\mathrm{AE}$ & $\mathrm{AF}$ & AG \\
\hline 1 & $\begin{array}{lc}\text { RESULT_COMMENT } \\
\end{array}$ & COLL_SAMP_SIZE & COLL_SAMP_SIZE_UNITS & DIST_CLASS \\
\hline 610 & & & & OFFSITE \\
\hline 611 & & & & OFFSITE \\
\hline 612 & & & & OFFSITE \\
\hline \begin{tabular}{|c|c|}
613 \\
\end{tabular} & & & & OFFSITE \\
\hline 614 & 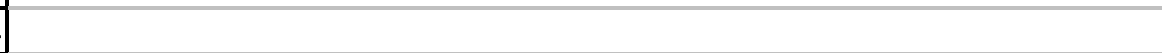 & & & OFFSITE \\
\hline 615 & & & & OFFSITE \\
\hline 616 & & & & OFFSITE \\
\hline 617 & & & & OFFSITE \\
\hline 618 & & & & OFFSITE \\
\hline 619 & & & & OFFSITE \\
\hline 620 & & & & OFFSITE \\
\hline 621 & & & & OFFSITE \\
\hline 622 & & & & OFFSITE \\
\hline 623 & & & & OFFSITE \\
\hline 624 & & & & OFFSITE \\
\hline \begin{tabular}{|l|}
625 \\
\end{tabular} & & & & OFFSITE \\
\hline 626 & & & & OFFSITE \\
\hline \begin{tabular}{|l|}
627 \\
\end{tabular} & & & & OFFSITE \\
\hline 628 & & & & OFFSITE \\
\hline 629 & & & & OFFSITE \\
\hline 630 & & & & OFFSITE \\
\hline 631 & & & & OFFSITE \\
\hline 632 & & & & OFFSITE \\
\hline 633 & & & & OFFSITE \\
\hline 634 & & & & OFFSITE \\
\hline 635 & & & & OFFSITE \\
\hline 636 & & & & OFFSITE \\
\hline \begin{tabular}{|l|}
637 \\
\end{tabular} & & & & OFFSITE \\
\hline 638 & & & & OFFSITE \\
\hline 639 & & & & OFFSITE \\
\hline 640 & & & & OFFSITE \\
\hline 641 & & & & OFFSITE \\
\hline 642 & & & & OFFSITE \\
\hline 643 & & & & OFFSITE \\
\hline 644 & & & & OFFSITE \\
\hline 645 & & & & OFFSITE \\
\hline 646 & & & & OFFSITE \\
\hline \begin{tabular}{|l|}
647 \\
\end{tabular} & 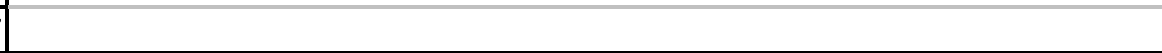 & & & ONSITE \\
\hline
\end{tabular}




\begin{tabular}{|c|c|c|c|c|}
\hline & $\mathrm{AD}$ & $\mathrm{AE}$ & $\mathrm{AF}$ & $A G$ \\
\hline 1 & 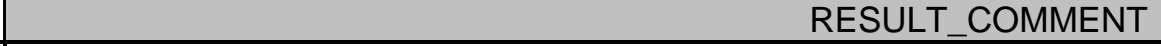 & COLL_SAMP_SIZE & COLL_SAMP_SIZE_UNITS & DIST_CLASS \\
\hline 648 & & & & ONSITE \\
\hline 649 & & & & ONSITE \\
\hline 650 & & & & ONSITE \\
\hline 651 & & & & ONSITE \\
\hline 652 & & & & ONSITE \\
\hline 653 & & & & ONSITE \\
\hline 654 & & & & ONSITE \\
\hline 655 & & & & ONSITE \\
\hline 656 & 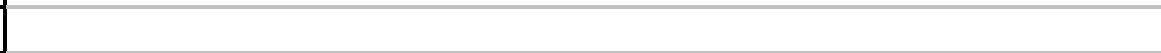 & & & ONSITE \\
\hline 657 & & & & OFFSITE \\
\hline 658 & & & & OFFSITE \\
\hline 659 & & & & OFFSITE \\
\hline 660 & & & & OFFSITE \\
\hline 661 & & & & ONSITE \\
\hline 662 & & & & ONSITE \\
\hline 663 & & & & ONSITE \\
\hline 664 & & & & ONSITE \\
\hline 665 & & & & ONSITE \\
\hline 666 & & & & ONSITE \\
\hline 667 & & & & ONSITE \\
\hline 668 & & & & ONSITE \\
\hline 669 & & & & ONSITE \\
\hline 670 & & & & ONSITE \\
\hline 671 & & & & ONSITE \\
\hline 672 & & & & ONSITE \\
\hline 673 & & & & ONSITE \\
\hline 674 & & & & ONSITE \\
\hline 675 & & & & ONSITE \\
\hline 676 & & & & ONSITE \\
\hline 677 & & & & ONSITE \\
\hline 678 & & & & ONSITE \\
\hline 679 & & & & ONSITE \\
\hline 680 & & & & ONSITE \\
\hline 681 & & & & ONSITE \\
\hline 682 & & & & ONSITE \\
\hline 683 & & & & ONSITE \\
\hline 684 & & & & ONSITE \\
\hline 685 & 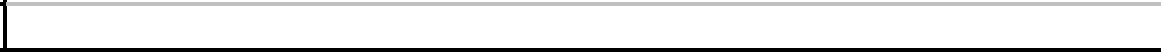 & & & ONSITE \\
\hline
\end{tabular}




\begin{tabular}{|c|c|c|c|c|}
\hline & $A D$ & $\mathrm{AE}$ & $\mathrm{AF}$ & $A G$ \\
\hline 1 & RESULT_COMMENT & COLL_SAMP_SIZE & COLL_SAMP_SIZE_UNITS & DIST_CLASS \\
\hline 686 & & & & ONSITE \\
\hline \begin{tabular}{|l|}
687 \\
\end{tabular} & & & & ONSITE \\
\hline 68 & & & & ONSITE \\
\hline 689 & & & & ONSITE \\
\hline 690 & & & & OFFSITE \\
\hline \begin{tabular}{|l|}
691 \\
\end{tabular} & & & & OFFSITE \\
\hline 692 & & & & OFFSITE \\
\hline 693 & & & & OFFSITE \\
\hline 694 & & & & OFFSITE \\
\hline \begin{tabular}{|l|}
695 \\
\end{tabular} & & & & OFFSITE \\
\hline 696 & & & & OFFSITE \\
\hline \begin{tabular}{|l|}
697 \\
\end{tabular} & & & & OFFSITE \\
\hline 698 & & & & OFFSITE \\
\hline 699 & & & & OFFSITE \\
\hline 700 & & & & OFFSITE \\
\hline 701 & & & & OFFSITE \\
\hline 702 & & & & OFFSITE \\
\hline 703 & & & & OFFSITE \\
\hline 704 & & & & OFFSITE \\
\hline 705 & & & & OFFSITE \\
\hline 706 & & & & OFFSITE \\
\hline 707 & & & & OFFSITE \\
\hline 708 & & & & OFFSITE \\
\hline 709 & & & & OFFSITE \\
\hline \begin{tabular}{|l|}
710 \\
\end{tabular} & & & & OFFSITE \\
\hline 711 & & & & OFFSITE \\
\hline 712 & & & & OFFSITE \\
\hline 713 & & & & OFFSITE \\
\hline 714 & & & & ONSITE \\
\hline 715 & & & & ONSITE \\
\hline 716 & & & & OFFSITE \\
\hline 717 & & & & OFFSITE \\
\hline 718 & & & & OFFSITE \\
\hline 719 & & & & OFFSITE \\
\hline 720 & See sample record for comment. & & & ONSITE \\
\hline 721 & See sample record for comment. & & & ONSITE \\
\hline 722 & See sample record for comment. & & & OFFSITE \\
\hline 723 & See sample record for comment. & & & OFFSITE \\
\hline
\end{tabular}




\begin{tabular}{|c|c|c|c|c|}
\hline & $A D$ & $\mathrm{AE}$ & $\mathrm{AF}$ & $A G$ \\
\hline 1 & RESULT_COMMENT & COLL_SAMP_SIZE & COLL_SAMP_SIZE_UNITS & DIST_CLASS \\
\hline 724 & & & & OFFSITE \\
\hline 725 & See sample record for comment. & & & OFFSITE \\
\hline 726 & See sample record for comment. & & & ONSITE \\
\hline 727 & See sample record for comment. & & & ONSITE \\
\hline 728 & See sample record for comment. & & & OFFSITE \\
\hline 729 & See sample record for comment. & & & OFFSITE \\
\hline 730 & See sample record for comment. & & & OFFSITE \\
\hline 731 & & & & OFFSITE \\
\hline 732 & & & & ONSITE \\
\hline 733 & & & & ONSITE \\
\hline 734 & & & & OFFSITE \\
\hline 735 & & & & OFFSITE \\
\hline 736 & & & & OFFSITE \\
\hline 737 & & & & OFFSITE \\
\hline 738 & & & & ONSITE \\
\hline 739 & & & & ONSITE \\
\hline 740 & & & & OFFSITE \\
\hline 741 & & & & OFFSITE \\
\hline 742 & & & & OFFSITE \\
\hline 743 & & & & OFFSITE \\
\hline 744 & & & & ONSITE \\
\hline 745 & & & & OFFSITE \\
\hline 746 & & & & OFFSITE \\
\hline 747 & & & & OFFSITE \\
\hline 748 & & & & ONSITE \\
\hline 749 & & & & OFFSITE \\
\hline 750 & & & & ONSITE \\
\hline 751 & & & & ONSITE \\
\hline 752 & & & & OFFSITE \\
\hline 753 & See sample record for comment. & & & OFFSITE \\
\hline 754 & 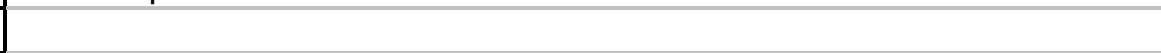 & & & OFFSITE \\
\hline 755 & See sample record for comment. & & & OFFSITE \\
\hline 756 & & & & ONSITE \\
\hline \begin{tabular}{|l|}
757 \\
\end{tabular} & & & & ONSITE \\
\hline 758 & & & & OFFSITE \\
\hline 759 & & & & OFFSITE \\
\hline 760 & & & & OFFSITE \\
\hline \begin{tabular}{|l|}
761 \\
\end{tabular} & 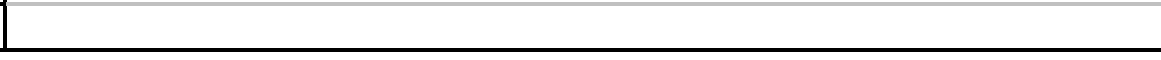 & & & OFFSITE \\
\hline
\end{tabular}




\begin{tabular}{|c|c|c|c|c|}
\hline & $A D$ & $\mathrm{AE}$ & $\mathrm{AF}$ & AG \\
\hline 1 & RESULT_COMMENT & COLL_SAMP_SIZE & COLL_SAMP_SIZE_UNITS & DIST_CLASS \\
\hline 762 & & & & ONSITE \\
\hline 763 & & & & ONSITE \\
\hline 764 & & & & OFFSITE \\
\hline 765 & & & & OFFSITE \\
\hline 766 & & & & OFFSITE \\
\hline 767 & & & & OFFSITE \\
\hline 768 & & & & ONSITE \\
\hline 769 & & & & ONSITE \\
\hline 770 & & & & OFFSITE \\
\hline 771 & & & & OFFSITE \\
\hline 772 & See sample record for comment. & & & OFFSITE \\
\hline 773 & See sample record for comment. & & & OFFSITE \\
\hline 774 & See sample record for comment. & & & ONSITE \\
\hline 775 & See sample record for comment. & & & ONSITE \\
\hline 776 & & & & OFFSITE \\
\hline 777 & & & & OFFSITE \\
\hline 778 & See sample record for comment. & & & OFFSITE \\
\hline 779 & See sample record for comment. & & & OFFSITE \\
\hline 780 & & & & OFFSITE \\
\hline 781 & & & & OFFSITE \\
\hline 782 & & & & OFFSITE \\
\hline 783 & & & & OFFSITE \\
\hline 784 & & & & OFFSITE \\
\hline 785 & & & & OFFSITE \\
\hline 786 & & & & OFFSITE \\
\hline 787 & & & & OFFSITE \\
\hline 788 & & & & OFFSITE \\
\hline \begin{tabular}{|l|}
789 \\
\end{tabular} & & & & OFFSITE \\
\hline 790 & & & & OFFSITE \\
\hline \begin{tabular}{|l|}
791 \\
\end{tabular} & & & & OFFSITE \\
\hline 792 & & & & OFFSITE \\
\hline 793 & & & & OFFSITE \\
\hline 794 & & & & OFFSITE \\
\hline 795 & & & & OFFSITE \\
\hline 796 & & & & OFFSITE \\
\hline 797 & & & & OFFSITE \\
\hline 798 & & & & OFFSITE \\
\hline 799 & & & & OFFSITE \\
\hline
\end{tabular}


AD

AE

AF

AG

\begin{tabular}{|c|c|c|c|c|}
\hline & AD & $\mathrm{AE}$ & AF & $A G$ \\
\hline 1 & RESULT_COMMENT & COLL_SAMP_SIZE & COLL_SAMP_SIZE_UNITS & DIST_CLASS \\
\hline \begin{tabular}{|l|}
800 \\
\end{tabular} & & & & OFFSITE \\
\hline 801 & & & & OFFSITE \\
\hline 802 & & & & OFFSITE \\
\hline 803 & & & & OFFSITE \\
\hline \begin{tabular}{|l|}
804 \\
\end{tabular} & & & & OFFSITE \\
\hline 805 & & & & OFFSITE \\
\hline 806 & & & & OFFSITE \\
\hline 807 & & & & OFFSITE \\
\hline 808 & & & & OFFSITE \\
\hline 809 & & & & OFFSITE \\
\hline 810 & & & & OFFSITE \\
\hline 811 & & & & OFFSITE \\
\hline 812 & & & & OFFSITE \\
\hline 813 & & & & OFFSITE \\
\hline 814 & & & & OFFSITE \\
\hline 815 & & & & OFFSITE \\
\hline \begin{tabular}{|l|}
816 \\
\end{tabular} & & & & OFFSITE \\
\hline \begin{tabular}{|l|}
817 \\
\end{tabular} & & & & OFFSITE \\
\hline 818 & & & & OFFSITE \\
\hline 819 & ORIGINAL RESULT NOT BLANK SUBTRACTED, MODIFIED RESULT PER 1/9/96 REPORT. & & & OFFSITE \\
\hline
\end{tabular}




\begin{tabular}{|r|l|l|}
\hline & \multicolumn{1}{|c|}{ AH } & \\
\hline 1 & OWNER_ID & COLLECTION_PURPOSE \\
\hline 2 & SESPMNT & $\mathrm{R}$ \\
\hline 3 & SESPMNT & $\mathrm{R}$ \\
\hline 4 & SESPMNT & $\mathrm{R}$ \\
\hline 5 & SESPMNT & $\mathrm{R}$ \\
\hline 6 & SESPMNT & $\mathrm{R}$ \\
\hline 7 & SESPMNT & $\mathrm{R}$ \\
\hline 8 & SESPMNT & $\mathrm{R}$ \\
\hline 9 & SESPMNT & $\mathrm{R}$ \\
\hline 10 & SESPMNT & $\mathrm{R}$ \\
\hline 11 & SESPMNT & $\mathrm{R}$ \\
\hline 12 & SESPMNT & $\mathrm{R}$ \\
\hline 13 & SESPMNT & $\mathrm{R}$ \\
\hline 14 & SESPMNT & $\mathrm{R}$ \\
\hline 15 & SESPMNT & $\mathrm{R}$ \\
\hline 16 & SESPMNT & $\mathrm{R}$ \\
\hline 17 & SESPMNT & $\mathrm{R}$ \\
\hline 18 & SESPMNT & $\mathrm{R}$ \\
\hline 19 & SESPMNT & $\mathrm{R}$ \\
\hline 20 & SESPMNT & $\mathrm{R}$ \\
\hline 21 & SESPMNT & $\mathrm{R}$ \\
\hline 22 & SESPMNT & $\mathrm{R}$ \\
\hline 23 & SESPMNT & $\mathrm{R}$ \\
\hline 24 & SESPMNT & $\mathrm{R}$ \\
\hline 25 & SESPMNT & $\mathrm{R}$ \\
\hline 26 & SESPMNT & $\mathrm{R}$ \\
\hline 27 & SESPMNT & $\mathrm{R}$ \\
\hline 28 & SESPMNT & $\mathrm{R}$ \\
\hline 29 & SESPMNT & $\mathrm{R}$ \\
\hline 30 & SESPMNT & $\mathrm{R}$ \\
\hline 31 & SESPMNT & $\mathrm{R}$ \\
\hline 32 & SESPMNT & $\mathrm{R}$ \\
\hline 33 & SESPMNT & $\mathrm{R}$ \\
\hline 34 & SESPMNT & $\mathrm{R}$ \\
\hline 35 & SESPMNT & $\mathrm{R}$ \\
\hline 36 & SESPMNT & $\mathrm{R}$ \\
\hline 37 & SESPMNT & $\mathrm{R}$ \\
\hline 38 & SESPMNT & $\mathrm{R}$ \\
\hline 39 & SESPMNT & $\mathrm{R}$ \\
\hline & & \\
\hline
\end{tabular}




\begin{tabular}{|c|l|l|}
\hline & \multicolumn{1}{|c|}{ AH } & \\
\hline 1 & OWNER_ID & COLLECTION_PURPOSE \\
\hline 40 & SESPMNT & $\mathrm{R}$ \\
\hline 41 & SESPMNT & $\mathrm{R}$ \\
\hline 42 & SESPMNT & $\mathrm{R}$ \\
\hline 43 & SESPMNT & $\mathrm{R}$ \\
\hline 44 & SESPMNT & $\mathrm{R}$ \\
\hline 45 & SESPMNT & $\mathrm{R}$ \\
\hline 46 & SESPMNT & $\mathrm{R}$ \\
\hline 47 & SESPMNT & $\mathrm{R}$ \\
\hline 48 & SESPMNT & $\mathrm{R}$ \\
\hline 49 & SESPMNT & $\mathrm{R}$ \\
\hline 50 & SESPMNT & $\mathrm{R}$ \\
\hline 51 & SESPMNT & $\mathrm{R}$ \\
\hline 52 & SESPMNT & $\mathrm{R}$ \\
\hline 53 & SESPMNT & $\mathrm{R}$ \\
\hline 54 & SESPMNT & $\mathrm{R}$ \\
\hline 55 & SESPMNT & $\mathrm{R}$ \\
\hline 56 & SESPMNT & $\mathrm{R}$ \\
\hline 57 & SESPMNT & $\mathrm{R}$ \\
\hline 58 & SESPMNT & $\mathrm{R}$ \\
\hline 59 & SESPMNT & $\mathrm{R}$ \\
\hline 60 & SESPMNT & $\mathrm{R}$ \\
\hline 61 & SESPMNT & $\mathrm{R}$ \\
\hline 62 & SESPMNT & $\mathrm{R}$ \\
\hline 63 & SESPMNT & $\mathrm{R}$ \\
\hline 64 & SESPMNT & $\mathrm{R}$ \\
\hline 65 & SESPMNT & $\mathrm{R}$ \\
\hline 66 & SESPMNT & $\mathrm{R}$ \\
\hline 67 & SESPMNT & $\mathrm{R}$ \\
\hline 68 & SESPMNT & $\mathrm{R}$ \\
\hline 69 & SESPMNT & $\mathrm{R}$ \\
\hline 70 & SESPMNT & $\mathrm{R}$ \\
\hline 71 & SESPMNT & $\mathrm{R}$ \\
\hline 72 & SESPMNT & $\mathrm{R}$ \\
\hline 73 & SESPMNT & $\mathrm{R}$ \\
\hline 74 & SESPMNT & $\mathrm{R}$ \\
\hline 75 & SESPMNT & $\mathrm{R}$ \\
\hline 76 & SESPMNT & $\mathrm{R}$ \\
\hline 77 & SESPMNT & $\mathrm{R}$ \\
\hline & & \\
\hline
\end{tabular}




\begin{tabular}{|c|l|l|}
\hline & \multicolumn{1}{|c|}{ AH } & \\
\hline 1 & OWNER_ID & COLLECTION_PURPOSE \\
\hline 78 & SESPMNT & $\mathrm{R}$ \\
\hline 79 & SESPMNT & $\mathrm{R}$ \\
\hline 80 & SESPMNT & $\mathrm{R}$ \\
\hline 81 & SESPMNT & $\mathrm{R}$ \\
\hline 82 & SESPMNT & $\mathrm{R}$ \\
\hline 83 & SESPMNT & $\mathrm{R}$ \\
\hline 84 & SESPMNT & $\mathrm{R}$ \\
\hline 85 & SESPMNT & $\mathrm{R}$ \\
\hline 86 & SESPMNT & $\mathrm{R}$ \\
\hline 87 & SESPMNT & $\mathrm{R}$ \\
\hline 88 & SESPMNT & $\mathrm{R}$ \\
\hline 89 & SESPMNT & $\mathrm{R}$ \\
\hline 90 & SESPMNT & $\mathrm{R}$ \\
\hline 91 & SESPMNT & $\mathrm{R}$ \\
\hline 92 & SESPMNT & $\mathrm{R}$ \\
\hline 93 & SESPMNT & $\mathrm{R}$ \\
\hline 94 & SESPMNT & $\mathrm{R}$ \\
\hline 95 & SESPMNT & $\mathrm{R}$ \\
\hline 96 & SESPMNT & $\mathrm{R}$ \\
\hline 97 & SESPMNT & $\mathrm{R}$ \\
\hline 98 & SESPMNT & $\mathrm{R}$ \\
\hline 99 & SESPMNT & $\mathrm{R}$ \\
\hline 100 & SESPMNT & $\mathrm{R}$ \\
\hline 101 & SESPMNT & $\mathrm{R}$ \\
\hline 102 & SESPMNT & $\mathrm{R}$ \\
\hline 103 & SESPMNT & $\mathrm{R}$ \\
\hline 104 & SESPMNT & $\mathrm{R}$ \\
\hline 105 & SESPMNT & $\mathrm{R}$ \\
\hline 106 & SESPMNT & $\mathrm{R}$ \\
\hline 107 & SESPMNT & $\mathrm{R}$ \\
\hline 108 & SESPMNT & $\mathrm{R}$ \\
\hline 109 & SESPMNT & $\mathrm{R}$ \\
\hline 110 & SESPMNT & $\mathrm{R}$ \\
\hline 111 & SESPMNT & $\mathrm{R}$ \\
\hline 112 & SESPMNT & $\mathrm{R}$ \\
\hline 113 & SESPMNT & $\mathrm{R}$ \\
\hline 114 & SESPMNT & $\mathrm{R}$ \\
\hline 115 & SESPMNT & $\mathrm{R}$ \\
\hline & & \\
\hline
\end{tabular}




\begin{tabular}{|c|l|l|}
\hline & \multicolumn{1}{|c|}{ AH } & \\
\hline 1 & OWNER_ID & COLLECTION_PURPOSE \\
\hline 116 & SESPMNT & $\mathrm{R}$ \\
\hline 117 & SESPMNT & $\mathrm{R}$ \\
\hline 118 & SESPMNT & $\mathrm{R}$ \\
\hline 119 & SESPMNT & $\mathrm{R}$ \\
\hline 120 & SESPMNT & $\mathrm{R}$ \\
\hline 121 & SESPMNT & $\mathrm{R}$ \\
\hline 122 & SESPMNT & $\mathrm{R}$ \\
\hline 123 & SESPMNT & $\mathrm{R}$ \\
\hline 124 & SESPMNT & $\mathrm{R}$ \\
\hline 125 & SESPMNT & $\mathrm{R}$ \\
\hline 126 & SESPMNT & $\mathrm{R}$ \\
\hline 127 & SESPMNT & $\mathrm{R}$ \\
\hline 128 & SESPMNT & $\mathrm{R}$ \\
\hline 129 & SESPMNT & $\mathrm{R}$ \\
\hline 130 & SESPMNT & $\mathrm{R}$ \\
\hline 131 & SESPMNT & $\mathrm{R}$ \\
\hline 132 & SESPMNT & $\mathrm{R}$ \\
\hline 133 & SESPMNT & $\mathrm{R}$ \\
\hline 134 & SESPMNT & $\mathrm{R}$ \\
\hline 135 & SESPMNT & $\mathrm{R}$ \\
\hline 136 & SESPMNT & $\mathrm{R}$ \\
\hline 137 & SESPMNT & $\mathrm{R}$ \\
\hline 138 & SESPMNT & $\mathrm{R}$ \\
\hline 139 & SESPMNT & $\mathrm{R}$ \\
\hline 140 & SESPMNT & $\mathrm{R}$ \\
\hline 141 & SESPMNT & $\mathrm{R}$ \\
\hline 142 & SESPMNT & $\mathrm{R}$ \\
\hline 143 & SESPMNT & $\mathrm{R}$ \\
\hline 144 & SESPMNT & $\mathrm{R}$ \\
\hline 145 & SESPMNT & $\mathrm{R}$ \\
\hline 146 & SESPMNT & $\mathrm{R}$ \\
\hline 147 & SESPMNT & $\mathrm{R}$ \\
\hline 148 & SESPMNT & $\mathrm{R}$ \\
\hline 149 & SESPMNT & $\mathrm{R}$ \\
\hline 150 & SESPMNT & $\mathrm{R}$ \\
\hline 151 & SESPMNT & $\mathrm{R}$ \\
\hline 152 & SESPMNT & $\mathrm{R}$ \\
\hline 153 & SESPMNT & $\mathrm{R}$ \\
\hline & & \\
\hline
\end{tabular}




\begin{tabular}{|c|l|l|}
\hline & AH & \\
\hline 1 & OWNER_ID & COLLECTION_PURPOSE \\
\hline 154 & SESPMNT & $\mathrm{R}$ \\
\hline 155 & SESPMNT & $\mathrm{R}$ \\
\hline 156 & SESPMNT & $\mathrm{R}$ \\
\hline 157 & SESPMNT & $\mathrm{R}$ \\
\hline 158 & SESPMNT & $\mathrm{R}$ \\
\hline 159 & SESPMNT & $\mathrm{R}$ \\
\hline 160 & SESPMNT & $\mathrm{R}$ \\
\hline 161 & SESPMNT & $\mathrm{R}$ \\
\hline 162 & SESPMNT & $\mathrm{R}$ \\
\hline 163 & SESPMNT & $\mathrm{R}$ \\
\hline 164 & SESPMNT & $\mathrm{R}$ \\
\hline 165 & SESPMNT & $\mathrm{R}$ \\
\hline 166 & SESPMNT & $\mathrm{R}$ \\
\hline 167 & SESPMNT & $\mathrm{R}$ \\
\hline 168 & SESPMNT & $\mathrm{R}$ \\
\hline 169 & SESPMNT & $\mathrm{R}$ \\
\hline 170 & SESPMNT & $\mathrm{R}$ \\
\hline 171 & SESPMNT & $\mathrm{R}$ \\
\hline 172 & SESPMNT & $\mathrm{R}$ \\
\hline 173 & SESPMNT & $\mathrm{R}$ \\
\hline 174 & SESPMNT & $\mathrm{R}$ \\
\hline 175 & SESPMNT & $\mathrm{R}$ \\
\hline 176 & SESPMNT & $\mathrm{R}$ \\
\hline 177 & SESPMNT & $\mathrm{R}$ \\
\hline 178 & SESPMNT & $\mathrm{R}$ \\
\hline 179 & SESPMNT & $\mathrm{R}$ \\
\hline 180 & SESPMNT & $\mathrm{R}$ \\
\hline 181 & SESPMNT & $\mathrm{R}$ \\
\hline 182 & SESPMNT & $\mathrm{R}$ \\
\hline 183 & SESPMNT & $\mathrm{R}$ \\
\hline 184 & SESPMNT & $\mathrm{R}$ \\
\hline 185 & SESPMNT & $\mathrm{R}$ \\
\hline 186 & SESPMNT & $\mathrm{R}$ \\
\hline 187 & SESPMNT & $\mathrm{R}$ \\
\hline 188 & SESPSPEC & $\mathrm{S}$ \\
\hline 189 & SESPSPEC & $\mathrm{S}$ \\
\hline 190 & SESPMNT & $\mathrm{R}$ \\
\hline 191 & SESPMNT & $\mathrm{R}$ \\
\hline
\end{tabular}




\begin{tabular}{|c|l|l|}
\hline & \multicolumn{1}{|c|}{ AH } & \\
\hline 1 & OWNER_ID & COLLECTION_PURPOSE \\
\hline 192 & SESPMNT & $\mathrm{R}$ \\
\hline 193 & SESPMNT & $\mathrm{R}$ \\
\hline 194 & SESPMNT & $\mathrm{R}$ \\
\hline 195 & SESPMNT & $\mathrm{R}$ \\
\hline 196 & SESPMNT & $\mathrm{R}$ \\
\hline 197 & SESPMNT & $\mathrm{R}$ \\
\hline 198 & SESPMNT & $\mathrm{R}$ \\
\hline 199 & SESPMNT & $\mathrm{R}$ \\
\hline 200 & SESPMNT & $\mathrm{R}$ \\
\hline 201 & SESPMNT & $\mathrm{R}$ \\
\hline 202 & SESPMNT & $\mathrm{R}$ \\
\hline 203 & SESPMNT & $\mathrm{R}$ \\
\hline 204 & SESPMNT & $\mathrm{R}$ \\
\hline 205 & SESPMNT & $\mathrm{R}$ \\
\hline 206 & SESPMNT & $\mathrm{R}$ \\
\hline 207 & SESPMNT & $\mathrm{R}$ \\
\hline 208 & SESPMNT & $\mathrm{R}$ \\
\hline 209 & SESPMNT & $\mathrm{R}$ \\
\hline 210 & SESPMNT & $\mathrm{R}$ \\
\hline 211 & SESPMNT & $\mathrm{R}$ \\
\hline 212 & SESPMNT & $\mathrm{R}$ \\
\hline 213 & SESPMNT & $\mathrm{R}$ \\
\hline 214 & SESPMNT & $\mathrm{R}$ \\
\hline 215 & SESPMNT & $\mathrm{R}$ \\
\hline 216 & SESPMNT & $\mathrm{R}$ \\
\hline 217 & SESPMNT & $\mathrm{R}$ \\
\hline 218 & SESPMNT & $\mathrm{R}$ \\
\hline 219 & SESPMNT & $\mathrm{R}$ \\
\hline 220 & SESPMNT & $\mathrm{R}$ \\
\hline 221 & SESPMNT & $\mathrm{R}$ \\
\hline 222 & SESPMNT & $\mathrm{R}$ \\
\hline 223 & SESPMNT & $\mathrm{R}$ \\
\hline 224 & SESPMNT & $\mathrm{R}$ \\
\hline 225 & SESPMNT & $\mathrm{R}$ \\
\hline 226 & SESPMNT & $\mathrm{R}$ \\
\hline 227 & SESPMNT & $\mathrm{R}$ \\
\hline 228 & SESPMNT & $\mathrm{R}$ \\
\hline 229 & SESPMNT & $\mathrm{R}$ \\
\hline & & \\
\hline
\end{tabular}




\begin{tabular}{|c|l|l|}
\hline & \multicolumn{1}{|c|}{ AH } & \\
\hline 1 & OWNER_ID & COLLECTION_PURPOSE \\
\hline 230 & SESPMNT & $\mathrm{R}$ \\
\hline 231 & SESPMNT & $\mathrm{R}$ \\
\hline 232 & SESPMNT & $\mathrm{R}$ \\
\hline 233 & SESPMNT & $\mathrm{R}$ \\
\hline 234 & SESPMNT & $\mathrm{R}$ \\
\hline 235 & SESPMNT & $\mathrm{R}$ \\
\hline 236 & SESPMNT & $\mathrm{R}$ \\
\hline 237 & SESPMNT & $\mathrm{R}$ \\
\hline 238 & SESPMNT & $\mathrm{R}$ \\
\hline 239 & SESPMNT & $\mathrm{R}$ \\
\hline 240 & SESPMNT & $\mathrm{R}$ \\
\hline 241 & SESPMNT & $\mathrm{R}$ \\
\hline 242 & SESPMNT & $\mathrm{R}$ \\
\hline 243 & SESPMNT & $\mathrm{R}$ \\
\hline 244 & SESPMNT & $\mathrm{R}$ \\
\hline 245 & SESPMNT & $\mathrm{R}$ \\
\hline 246 & SESPMNT & $\mathrm{R}$ \\
\hline 247 & SESPMNT & $\mathrm{R}$ \\
\hline 248 & SESPMNT & $\mathrm{R}$ \\
\hline 249 & SESPMNT & $\mathrm{R}$ \\
\hline 250 & SESPMNT & $\mathrm{R}$ \\
\hline 251 & SESPMNT & $\mathrm{R}$ \\
\hline 252 & SESPMNT & $\mathrm{R}$ \\
\hline 253 & SESPMNT & $\mathrm{R}$ \\
\hline 254 & SESPMNT & $\mathrm{R}$ \\
\hline 255 & SESPMNT & $\mathrm{R}$ \\
\hline 256 & SESPMNT & $\mathrm{R}$ \\
\hline 257 & SESPMNT & $\mathrm{R}$ \\
\hline 258 & SESPMNT & $\mathrm{R}$ \\
\hline 259 & SESPMNT & $\mathrm{R}$ \\
\hline 260 & SESPMNT & $\mathrm{R}$ \\
\hline 261 & SESPMNT & $\mathrm{R}$ \\
\hline 262 & SESPMNT & $\mathrm{R}$ \\
\hline 263 & SESPMNT & $\mathrm{R}$ \\
\hline 264 & SESPMNT & $\mathrm{R}$ \\
\hline 265 & SESPMNT & $\mathrm{R}$ \\
\hline 266 & SESPMNT & $\mathrm{R}$ \\
\hline 267 & SESPMNT & $\mathrm{R}$ \\
\hline & & \\
\hline
\end{tabular}




\begin{tabular}{|c|l|l|}
\hline & \multicolumn{1}{|c|}{ AH } & \\
\hline 1 & OWNER_ID & COLLECTION_PURPOSE \\
\hline 268 & SESPMNT & $\mathrm{R}$ \\
\hline 269 & SESPMNT & $\mathrm{R}$ \\
\hline 270 & SESPMNT & $\mathrm{R}$ \\
\hline 271 & SESPMNT & $\mathrm{R}$ \\
\hline 272 & SESPMNT & $\mathrm{R}$ \\
\hline 273 & SESPMNT & $\mathrm{R}$ \\
\hline 274 & SESPMNT & $\mathrm{R}$ \\
\hline 275 & SESPMNT & $\mathrm{R}$ \\
\hline 276 & SESPMNT & $\mathrm{R}$ \\
\hline 277 & SESPMNT & $\mathrm{R}$ \\
\hline 278 & SESPMNT & $\mathrm{R}$ \\
\hline 279 & SESPMNT & $\mathrm{R}$ \\
\hline 280 & SESPMNT & $\mathrm{R}$ \\
\hline 281 & SESPMNT & $\mathrm{R}$ \\
\hline 282 & SESPMNT & $\mathrm{R}$ \\
\hline 283 & SESPMNT & $\mathrm{R}$ \\
\hline 284 & SESPMNT & $\mathrm{R}$ \\
\hline 285 & SESPMNT & $\mathrm{R}$ \\
\hline 286 & SESPMNT & $\mathrm{R}$ \\
\hline 287 & SESPMNT & $\mathrm{R}$ \\
\hline 288 & SESPMNT & $\mathrm{R}$ \\
\hline 289 & SESPMNT & $\mathrm{R}$ \\
\hline 290 & SESPMNT & $\mathrm{R}$ \\
\hline 291 & SESPMNT & $\mathrm{R}$ \\
\hline 292 & SESPMNT & $\mathrm{R}$ \\
\hline 293 & SESPSPEC & $\mathrm{S}$ \\
\hline 294 & SESPMNT & $\mathrm{R}$ \\
\hline 295 & SESPMNT & $\mathrm{R}$ \\
\hline 296 & SESPMNT & $\mathrm{R}$ \\
\hline 297 & SESPMNT & $\mathrm{R}$ \\
\hline 298 & SESPMNT & $\mathrm{R}$ \\
\hline 299 & SESPMNT & $\mathrm{R}$ \\
\hline 300 & SESPMNT & $\mathrm{R}$ \\
\hline 301 & SESPMNT & $\mathrm{R}$ \\
\hline 302 & SESPMNT & $\mathrm{R}$ \\
\hline 303 & SESPMNT & $\mathrm{R}$ \\
\hline 304 & SESPMNT & $\mathrm{R}$ \\
\hline 305 & SESPMNT & $\mathrm{R}$ \\
\hline & & \\
\hline
\end{tabular}




\begin{tabular}{|c|l|l|}
\hline & \multicolumn{1}{|c|}{ AH } & \\
\hline 1 & OWNER_ID & COLLECTION_PURPOSE \\
\hline 306 & SESPMNT & $\mathrm{R}$ \\
\hline 307 & SESPMNT & $\mathrm{R}$ \\
\hline 308 & SESPMNT & $\mathrm{R}$ \\
\hline 309 & SESPMNT & $\mathrm{R}$ \\
\hline 310 & SESPMNT & $\mathrm{R}$ \\
\hline 311 & SESPMNT & $\mathrm{R}$ \\
\hline 312 & SESPMNT & $\mathrm{R}$ \\
\hline 313 & SESPMNT & $\mathrm{R}$ \\
\hline 314 & SESPMNT & $\mathrm{R}$ \\
\hline 315 & SESPMNT & $\mathrm{R}$ \\
\hline 316 & SESPMNT & $\mathrm{R}$ \\
\hline 317 & SESPMNT & $\mathrm{R}$ \\
\hline 318 & SESPMNT & $\mathrm{R}$ \\
\hline 319 & SESPMNT & $\mathrm{R}$ \\
\hline 320 & SESPMNT & $\mathrm{R}$ \\
\hline 321 & SESPMNT & $\mathrm{R}$ \\
\hline 322 & SESPMNT & $\mathrm{R}$ \\
\hline 323 & SESPMNT & $\mathrm{R}$ \\
\hline 324 & SESPMNT & $\mathrm{R}$ \\
\hline 325 & SESPMNT & $\mathrm{R}$ \\
\hline 326 & SESPMNT & $\mathrm{R}$ \\
\hline 327 & SESPMNT & $\mathrm{R}$ \\
\hline 328 & SESPMNT & $\mathrm{R}$ \\
\hline 329 & SESPMNT & $\mathrm{R}$ \\
\hline 330 & SESPMNT & $\mathrm{R}$ \\
\hline 331 & SESPMNT & $\mathrm{R}$ \\
\hline 332 & SESPMNT & $\mathrm{R}$ \\
\hline 333 & SESPMNT & $\mathrm{R}$ \\
\hline 334 & SESPMNT & $\mathrm{R}$ \\
\hline 335 & SESPMNT & $\mathrm{R}$ \\
\hline 336 & SESPMNT & $\mathrm{R}$ \\
\hline 337 & SESPMNT & $\mathrm{R}$ \\
\hline 338 & SESPMNT & $\mathrm{R}$ \\
\hline 339 & SESPMNT & $\mathrm{R}$ \\
\hline 340 & SESPMNT & $\mathrm{R}$ \\
\hline 341 & SESPMNT & $\mathrm{R}$ \\
\hline 342 & SESPMNT & $\mathrm{R}$ \\
\hline 343 & SESPMNT & $\mathrm{R}$ \\
\hline & & \\
\hline
\end{tabular}




\begin{tabular}{|c|l|l|}
\hline & AH & \\
\hline 1 & OWNER_ID & COLLECTION_PURPOSE \\
\hline 344 & SESPMNT & $\mathrm{R}$ \\
\hline 345 & SESPMNT & $\mathrm{R}$ \\
\hline 346 & SESPMNT & $\mathrm{R}$ \\
\hline 347 & SESPMNT & $\mathrm{R}$ \\
\hline 348 & SESPMNT & $\mathrm{R}$ \\
\hline 349 & SESPMNT & $\mathrm{R}$ \\
\hline 350 & SESPMNT & $\mathrm{R}$ \\
\hline 351 & SESPMNT & $\mathrm{R}$ \\
\hline 352 & SESPMNT & $\mathrm{R}$ \\
\hline 353 & SESPMNT & $\mathrm{R}$ \\
\hline 354 & SESPMNT & $\mathrm{R}$ \\
\hline 355 & SESPMNT & $\mathrm{R}$ \\
\hline 356 & SESPMNT & $\mathrm{R}$ \\
\hline 357 & SESPMNT & $\mathrm{R}$ \\
\hline 358 & SESPMNT & $\mathrm{R}$ \\
\hline 359 & SESPMNT & $\mathrm{R}$ \\
\hline 360 & SESPMNT & $\mathrm{R}$ \\
\hline 361 & SESPMNT & $\mathrm{R}$ \\
\hline 362 & SESPMNT & $\mathrm{R}$ \\
\hline 363 & SESPMNT & $\mathrm{R}$ \\
\hline 364 & SESPMNT & $\mathrm{R}$ \\
\hline 365 & SESPMNT & $\mathrm{R}$ \\
\hline 366 & SESPMNT & $\mathrm{R}$ \\
\hline 367 & SESPMNT & $\mathrm{R}$ \\
\hline 368 & SESPMNT & $\mathrm{R}$ \\
\hline 369 & SESPMNT & $\mathrm{R}$ \\
\hline 370 & SESPMNT & $\mathrm{R}$ \\
\hline 371 & SESPMNT & $\mathrm{R}$ \\
\hline 372 & SESPMNT & $\mathrm{R}$ \\
\hline 373 & SESPMNT & $\mathrm{R}$ \\
\hline 374 & SESPMNT & $\mathrm{R}$ \\
\hline 375 & SESPMNT & $\mathrm{R}$ \\
\hline 376 & SESPMNT & $\mathrm{R}$ \\
\hline 377 & SESPMNT & $\mathrm{R}$ \\
\hline 378 & SESPMNT & $\mathrm{R}$ \\
\hline 379 & SESPMNT & $\mathrm{R}$ \\
\hline 380 & SESPMNT & $\mathrm{R}$ \\
\hline 381 & SESPMNT & $\mathrm{R}$ \\
\hline
\end{tabular}




\begin{tabular}{|c|l|l|}
\hline & AH & \\
\hline 1 & OWNER_ID & COLLECTION_PURPOSE \\
\hline 382 & SESPMNT & $\mathrm{R}$ \\
\hline 383 & SESPMNT & $\mathrm{R}$ \\
\hline 384 & SESPMNT & $\mathrm{R}$ \\
\hline 385 & SESPMNT & $\mathrm{R}$ \\
\hline 386 & SESPMNT & $\mathrm{R}$ \\
\hline 387 & SESPMNT & $\mathrm{R}$ \\
\hline 388 & SESPMNT & $\mathrm{R}$ \\
\hline 389 & SESPMNT & $\mathrm{R}$ \\
\hline 390 & SESPMNT & $\mathrm{R}$ \\
\hline 391 & SESPMNT & $\mathrm{R}$ \\
\hline 392 & SESPMNT & $\mathrm{R}$ \\
\hline 393 & SESPMNT & $\mathrm{R}$ \\
\hline 394 & SESPMNT & $\mathrm{R}$ \\
\hline 395 & SESPMNT & $\mathrm{R}$ \\
\hline 396 & SESPMNT & $\mathrm{R}$ \\
\hline 397 & SESPMNT & $\mathrm{R}$ \\
\hline 398 & SESPMNT & $\mathrm{R}$ \\
\hline 399 & SESPMNT & $\mathrm{R}$ \\
\hline 400 & SESPMNT & $\mathrm{R}$ \\
\hline 401 & SESPMNT & $\mathrm{R}$ \\
\hline 402 & SESPMNT & $\mathrm{R}$ \\
\hline 403 & SESPMNT & $\mathrm{R}$ \\
\hline 404 & SESPMNT & $\mathrm{R}$ \\
\hline 405 & SESPMNT & $\mathrm{R}$ \\
\hline 406 & SESPMNT & $\mathrm{R}$ \\
\hline 407 & SESPMNT & $\mathrm{R}$ \\
\hline 408 & SESPMNT & $\mathrm{R}$ \\
\hline 409 & SESPMNT & $\mathrm{R}$ \\
\hline 410 & SESPMNT & $\mathrm{R}$ \\
\hline 411 & SESPMNT & $\mathrm{R}$ \\
\hline 412 & SESPMNT & $\mathrm{R}$ \\
\hline 413 & SESPMNT & $\mathrm{R}$ \\
\hline 414 & SESPMNT & $\mathrm{R}$ \\
\hline 415 & SESPMNT & $\mathrm{R}$ \\
\hline 416 & SESPMNT & $\mathrm{R}$ \\
\hline 417 & SESPMNT & $\mathrm{R}$ \\
\hline 418 & SESPMNT & $\mathrm{R}$ \\
\hline 419 & SESPMNT & $\mathrm{R}$ \\
\hline & & \\
\hline
\end{tabular}




\begin{tabular}{|c|l|l|}
\hline & \multicolumn{1}{|c|}{ AH } & \\
\hline 1 & OWNER_ID & COLLECTION_PURPOSE \\
\hline 420 & SESPMNT & $\mathrm{R}$ \\
\hline 421 & SESPMNT & $\mathrm{R}$ \\
\hline 422 & SESPMNT & $\mathrm{R}$ \\
\hline 423 & SESPMNT & $\mathrm{R}$ \\
\hline 424 & SESPMNT & $\mathrm{R}$ \\
\hline 425 & SESPMNT & $\mathrm{R}$ \\
\hline 426 & SESPMNT & $\mathrm{R}$ \\
\hline 427 & SESPMNT & $\mathrm{R}$ \\
\hline 428 & SESPMNT & $\mathrm{R}$ \\
\hline 429 & SESPMNT & $\mathrm{R}$ \\
\hline 430 & SESPMNT & $\mathrm{R}$ \\
\hline 431 & SESPMNT & $\mathrm{R}$ \\
\hline 432 & SESPMNT & $\mathrm{R}$ \\
\hline 433 & SESPMNT & $\mathrm{R}$ \\
\hline 434 & SESPMNT & $\mathrm{R}$ \\
\hline 435 & SESPMNT & $\mathrm{R}$ \\
\hline 436 & SESPMNT & $\mathrm{R}$ \\
\hline 437 & SESPMNT & $\mathrm{R}$ \\
\hline 438 & SESPMNT & $\mathrm{R}$ \\
\hline 439 & SESPMNT & $\mathrm{R}$ \\
\hline 440 & SESPMNT & $\mathrm{R}$ \\
\hline 441 & SESPMNT & $\mathrm{R}$ \\
\hline 442 & SESPMNT & $\mathrm{R}$ \\
\hline 443 & SESPMNT & $\mathrm{R}$ \\
\hline 444 & SESPMNT & $\mathrm{R}$ \\
\hline 445 & SESPMNT & $\mathrm{R}$ \\
\hline 446 & SESPMNT & $\mathrm{R}$ \\
\hline 447 & SESPMNT & $\mathrm{R}$ \\
\hline 448 & SESPMNT & $\mathrm{R}$ \\
\hline 449 & SESPMNT & $\mathrm{R}$ \\
\hline 450 & SESPMNT & $\mathrm{R}$ \\
\hline 451 & SESPMNT & $\mathrm{R}$ \\
\hline 452 & SESPMNT & $\mathrm{R}$ \\
\hline 453 & SESPMNT & $\mathrm{R}$ \\
\hline 454 & SESPMNT & $\mathrm{R}$ \\
\hline 455 & SESPMNT & $\mathrm{R}$ \\
\hline 456 & SESPMNT & $\mathrm{R}$ \\
\hline 457 & SESPMNT & $\mathrm{R}$ \\
\hline & & \\
\hline
\end{tabular}




\begin{tabular}{|c|l|l|}
\hline & \multicolumn{1}{|c|}{ AH } & \\
\hline 1 & OWNER_ID & COLLECTION_PURPOSE \\
\hline 458 & SESPMNT & $\mathrm{R}$ \\
\hline 459 & SESPMNT & $\mathrm{R}$ \\
\hline 460 & SESPMNT & $\mathrm{R}$ \\
\hline 461 & SESPMNT & $\mathrm{R}$ \\
\hline 462 & SESPMNT & $\mathrm{R}$ \\
\hline 463 & SESPMNT & $\mathrm{R}$ \\
\hline 464 & SESPMNT & $\mathrm{R}$ \\
\hline 465 & SESPMNT & $\mathrm{R}$ \\
\hline 466 & SESPMNT & $\mathrm{R}$ \\
\hline 467 & SESPMNT & $\mathrm{R}$ \\
\hline 468 & SESPMNT & $\mathrm{R}$ \\
\hline 469 & SESPMNT & $\mathrm{R}$ \\
\hline 470 & SESPMNT & $\mathrm{R}$ \\
\hline 471 & SESPMNT & $\mathrm{R}$ \\
\hline 472 & SESPMNT & $\mathrm{R}$ \\
\hline 473 & SESPMNT & $\mathrm{R}$ \\
\hline 474 & SESPMNT & $\mathrm{R}$ \\
\hline 475 & SESPMNT & $\mathrm{R}$ \\
\hline 476 & SESPMNT & $\mathrm{R}$ \\
\hline 477 & SESPMNT & $\mathrm{R}$ \\
\hline 478 & SESPMNT & $\mathrm{R}$ \\
\hline 479 & SESPMNT & $\mathrm{R}$ \\
\hline 480 & SESPMNT & $\mathrm{R}$ \\
\hline 481 & SESPMNT & $\mathrm{R}$ \\
\hline 482 & SESPMNT & $\mathrm{R}$ \\
\hline 483 & SESPMNT & $\mathrm{R}$ \\
\hline 484 & SESPMNT & $\mathrm{R}$ \\
\hline 485 & SESPMNT & $\mathrm{R}$ \\
\hline 486 & SESPMNT & $\mathrm{R}$ \\
\hline 487 & SESPMNT & $\mathrm{R}$ \\
\hline 488 & SESPMNT & $\mathrm{R}$ \\
\hline 489 & SESPMNT & $\mathrm{R}$ \\
\hline 490 & SESPMNT & $\mathrm{R}$ \\
\hline 491 & SESPMNT & $\mathrm{R}$ \\
\hline 492 & SESPMNT & $\mathrm{R}$ \\
\hline 493 & SESPMNT & $\mathrm{R}$ \\
\hline 494 & SESPMNT & $\mathrm{R}$ \\
\hline 495 & SESPMNT & $\mathrm{R}$ \\
\hline & & \\
\hline
\end{tabular}




\begin{tabular}{|c|l|l|}
\hline & \multicolumn{1}{|c|}{ AH } & \\
\hline 1 & OWNER_ID & COLLECTION_PURPOSE \\
\hline 496 & SESPMNT & $\mathrm{R}$ \\
\hline 497 & SESPMNT & $\mathrm{R}$ \\
\hline 498 & SESPMNT & $\mathrm{R}$ \\
\hline 499 & SESPMNT & $\mathrm{R}$ \\
\hline 500 & SESPMNT & $\mathrm{R}$ \\
\hline 501 & SESPMNT & $\mathrm{R}$ \\
\hline 502 & SESPMNT & $\mathrm{R}$ \\
\hline 503 & SESPMNT & $\mathrm{R}$ \\
\hline 504 & SESPMNT & $\mathrm{R}$ \\
\hline 505 & SESPMNT & $\mathrm{R}$ \\
\hline 506 & SESPMNT & $\mathrm{R}$ \\
\hline 507 & SESPMNT & $\mathrm{R}$ \\
\hline 508 & SESPMNT & $\mathrm{R}$ \\
\hline 509 & SESPMNT & $\mathrm{R}$ \\
\hline 510 & SESPMNT & $\mathrm{R}$ \\
\hline 511 & SESPMNT & $\mathrm{R}$ \\
\hline 512 & SESPMNT & $\mathrm{R}$ \\
\hline 513 & SESPMNT & $\mathrm{R}$ \\
\hline 514 & SESPMNT & $\mathrm{R}$ \\
\hline 515 & SESPMNT & $\mathrm{R}$ \\
\hline 516 & SESPMNT & $\mathrm{R}$ \\
\hline 517 & SESPMNT & $\mathrm{R}$ \\
\hline 518 & SESPMNT & $\mathrm{R}$ \\
\hline 519 & SESPMNT & $\mathrm{R}$ \\
\hline 520 & SESPMNT & $\mathrm{R}$ \\
\hline 521 & SESPMNT & $\mathrm{R}$ \\
\hline 522 & SESPMNT & $\mathrm{R}$ \\
\hline 523 & SESPMNT & $\mathrm{R}$ \\
\hline 524 & SESPMNT & $\mathrm{R}$ \\
\hline 525 & SESPMNT & $\mathrm{R}$ \\
\hline 526 & SESPMNT & $\mathrm{R}$ \\
\hline 527 & SESPSPEC & $\mathrm{S}$ \\
\hline 528 & SESPMNT & $\mathrm{R}$ \\
\hline 529 & SESPMNT & $\mathrm{R}$ \\
\hline 530 & SESPMNT & $\mathrm{R}$ \\
\hline 531 & SESPSPEC & $\mathrm{S}$ \\
\hline 532 & SESPSPEC & $\mathrm{S}$ \\
\hline 533 & SESPSPEC & $\mathrm{S}$ \\
\hline & & \\
\hline
\end{tabular}




\begin{tabular}{|c|l|l|}
\hline & AH & \\
\hline 1 & OWNER_ID & COLLECTION_PURPOSE \\
\hline 534 & SESPMNT & $\mathrm{R}$ \\
\hline 535 & SESPMNT & $\mathrm{R}$ \\
\hline 536 & SESPMNT & $\mathrm{R}$ \\
\hline 537 & SESPMNT & $\mathrm{R}$ \\
\hline 538 & SESPMNT & $\mathrm{R}$ \\
\hline 539 & SESPMNT & $\mathrm{R}$ \\
\hline 540 & SESPMNT & $\mathrm{R}$ \\
\hline 541 & SESPMNT & $\mathrm{R}$ \\
\hline 542 & SESPMNT & $\mathrm{R}$ \\
\hline 543 & SESPMNT & $\mathrm{R}$ \\
\hline 544 & SESPMNT & $\mathrm{R}$ \\
\hline 545 & SESPMNT & $\mathrm{R}$ \\
\hline 546 & SESPMNT & $\mathrm{R}$ \\
\hline 547 & SESPMNT & $\mathrm{R}$ \\
\hline 548 & SESPMNT & $\mathrm{R}$ \\
\hline 549 & SESPMNT & $\mathrm{R}$ \\
\hline 550 & SESPMNT & $\mathrm{R}$ \\
\hline 551 & SESPMNT & $\mathrm{R}$ \\
\hline 552 & SESPMNT & $\mathrm{R}$ \\
\hline 553 & SESPMNT & $\mathrm{R}$ \\
\hline 554 & SESPMNT & $\mathrm{R}$ \\
\hline 555 & SESPMNT & $\mathrm{R}$ \\
\hline 556 & SESPMNT & $\mathrm{R}$ \\
\hline 557 & SESPMNT & $\mathrm{R}$ \\
\hline 558 & SESPMNT & $\mathrm{R}$ \\
\hline 559 & SESPMNT & $\mathrm{R}$ \\
\hline 560 & SESPMNT & $\mathrm{R}$ \\
\hline 561 & SESPMNT & $\mathrm{R}$ \\
\hline 562 & SESPMNT & $\mathrm{R}$ \\
\hline 563 & SESPMNT & $\mathrm{R}$ \\
\hline 564 & SESPMNT & $\mathrm{R}$ \\
\hline 565 & SESPMNT & $\mathrm{R}$ \\
\hline 566 & SESPMNT & $\mathrm{R}$ \\
\hline 567 & SESPMNT & $\mathrm{R}$ \\
\hline 568 & SESPMNT & $\mathrm{R}$ \\
\hline 569 & SESPMNT & $\mathrm{R}$ \\
\hline 570 & SESPMNT & $\mathrm{R}$ \\
\hline 571 & SESPMNT & $\mathrm{R}$ \\
\hline
\end{tabular}




\begin{tabular}{|c|l|l|}
\hline & AH & \\
\hline 1 & OWNER_ID & COLLECTION_PURPOSE \\
\hline 572 & SESPMNT & $\mathrm{R}$ \\
\hline 573 & SESPMNT & $\mathrm{R}$ \\
\hline 574 & SESPMNT & $\mathrm{R}$ \\
\hline 575 & SESPMNT & $\mathrm{R}$ \\
\hline 576 & SESPMNT & $\mathrm{R}$ \\
\hline 577 & SESPMNT & $\mathrm{R}$ \\
\hline 578 & SESPMNT & $\mathrm{R}$ \\
\hline 579 & SESPMNT & $\mathrm{R}$ \\
\hline 580 & SESPMNT & $\mathrm{R}$ \\
\hline 581 & SESPMNT & $\mathrm{R}$ \\
\hline 582 & SESPMNT & $\mathrm{R}$ \\
\hline 583 & SESPMNT & $\mathrm{R}$ \\
\hline 584 & SESPMNT & $\mathrm{R}$ \\
\hline 585 & SESPMNT & $\mathrm{R}$ \\
\hline 586 & SESPMNT & $\mathrm{R}$ \\
\hline 587 & SESPMNT & $\mathrm{R}$ \\
\hline 588 & SESPMNT & $\mathrm{R}$ \\
\hline 589 & SESPMNT & $\mathrm{R}$ \\
\hline 590 & SESPMNT & $\mathrm{R}$ \\
\hline 591 & SESPMNT & $\mathrm{R}$ \\
\hline 592 & SESPMNT & $\mathrm{R}$ \\
\hline 593 & SESPMNT & $\mathrm{R}$ \\
\hline 594 & SESPMNT & $\mathrm{R}$ \\
\hline 595 & SESPMNT & $\mathrm{R}$ \\
\hline 596 & SESPMNT & $\mathrm{R}$ \\
\hline 597 & SESPMNT & $\mathrm{R}$ \\
\hline 598 & SESPMNT & $\mathrm{R}$ \\
\hline 599 & SESPMNT & $\mathrm{R}$ \\
\hline 600 & SESPMNT & $\mathrm{R}$ \\
\hline 601 & SESPMNT & $\mathrm{R}$ \\
\hline 602 & SESPMNT & $\mathrm{R}$ \\
\hline 603 & SESPMNT & $\mathrm{R}$ \\
\hline 604 & SESPMNT & $\mathrm{R}$ \\
\hline 605 & SESPMNT & $\mathrm{R}$ \\
\hline 606 & SESPMNT & $\mathrm{R}$ \\
\hline 607 & SESPMNT & $\mathrm{R}$ \\
\hline 608 & SESPMNT & $\mathrm{R}$ \\
\hline 609 & SESPMNT & $\mathrm{R}$ \\
\hline & & \\
\hline
\end{tabular}




\begin{tabular}{|c|l|l|}
\hline & \multicolumn{1}{|c|}{ AH } & \\
\hline 1 & OWNER_ID & COLLECTION_PURPOSE \\
\hline 610 & SESPMNT & $\mathrm{R}$ \\
\hline 611 & SESPMNT & $\mathrm{R}$ \\
\hline 612 & SESPMNT & $\mathrm{R}$ \\
\hline 613 & SESPMNT & $\mathrm{R}$ \\
\hline 614 & SESPMNT & $\mathrm{R}$ \\
\hline 615 & SESPMNT & $\mathrm{R}$ \\
\hline 616 & SESPMNT & $\mathrm{R}$ \\
\hline 617 & SESPMNT & $\mathrm{R}$ \\
\hline 618 & SESPMNT & $\mathrm{R}$ \\
\hline 619 & SESPMNT & $\mathrm{R}$ \\
\hline 620 & SESPMNT & $\mathrm{R}$ \\
\hline 621 & SESPMNT & $\mathrm{R}$ \\
\hline 622 & SESPMNT & $\mathrm{R}$ \\
\hline 623 & SESPMNT & $\mathrm{R}$ \\
\hline 624 & SESPMNT & $\mathrm{R}$ \\
\hline 625 & SESPMNT & $\mathrm{R}$ \\
\hline 626 & SESPMNT & $\mathrm{R}$ \\
\hline 627 & SESPMNT & $\mathrm{R}$ \\
\hline 628 & SESPMNT & $\mathrm{R}$ \\
\hline 629 & SESPMNT & $\mathrm{R}$ \\
\hline 630 & SESPMNT & $\mathrm{R}$ \\
\hline 631 & SESPMNT & $\mathrm{R}$ \\
\hline 632 & SESPMNT & $\mathrm{R}$ \\
\hline 633 & SESPMNT & $\mathrm{R}$ \\
\hline 634 & SESPMNT & $\mathrm{R}$ \\
\hline 635 & SESPMNT & $\mathrm{R}$ \\
\hline 636 & SESPMNT & $\mathrm{R}$ \\
\hline 637 & SESPMNT & $\mathrm{R}$ \\
\hline 638 & SESPMNT & $\mathrm{R}$ \\
\hline 639 & SESPMNT & $\mathrm{R}$ \\
\hline 640 & SESPMNT & $\mathrm{R}$ \\
\hline 641 & SESPMNT & $\mathrm{R}$ \\
\hline 642 & SESPMNT & $\mathrm{R}$ \\
\hline 643 & SESPMNT & $\mathrm{R}$ \\
\hline 644 & SESPMNT & $\mathrm{R}$ \\
\hline 645 & SESPMNT & $\mathrm{R}$ \\
\hline 646 & SESPMNT & $\mathrm{R}$ \\
\hline 647 & SESPMNT & $\mathrm{R}$ \\
\hline & & \\
\hline
\end{tabular}




\begin{tabular}{|c|l|l|}
\hline & AH & \\
\hline 1 & OWNER_ID & COLLECTION_PURPOSE \\
\hline 648 & SESPMNT & $\mathrm{R}$ \\
\hline 649 & SESPMNT & $\mathrm{R}$ \\
\hline 650 & SESPMNT & $\mathrm{R}$ \\
\hline 651 & SESPMNT & $\mathrm{R}$ \\
\hline 652 & SESPMNT & $\mathrm{R}$ \\
\hline 653 & SESPMNT & $\mathrm{R}$ \\
\hline 654 & SESPMNT & $\mathrm{R}$ \\
\hline 655 & SESPMNT & $\mathrm{R}$ \\
\hline 656 & SESPMNT & $\mathrm{R}$ \\
\hline 657 & SESPMNT & $\mathrm{R}$ \\
\hline 658 & SESPMNT & $\mathrm{R}$ \\
\hline 659 & SESPMNT & $\mathrm{R}$ \\
\hline 660 & SESPMNT & $\mathrm{R}$ \\
\hline 661 & SESPMNT & $\mathrm{R}$ \\
\hline 662 & SESPMNT & $\mathrm{R}$ \\
\hline 663 & SESPMNT & $\mathrm{R}$ \\
\hline 664 & SESPMNT & $\mathrm{R}$ \\
\hline 665 & SESPMNT & $\mathrm{R}$ \\
\hline 666 & SESPMNT & $\mathrm{R}$ \\
\hline 667 & SESPMNT & $\mathrm{R}$ \\
\hline 668 & SESPMNT & $\mathrm{R}$ \\
\hline 669 & SESPMNT & $\mathrm{R}$ \\
\hline 670 & SESPMNT & $\mathrm{R}$ \\
\hline 671 & SESPMNT & $\mathrm{R}$ \\
\hline 672 & SESPMNT & $\mathrm{R}$ \\
\hline 673 & SESPMNT & $\mathrm{R}$ \\
\hline 674 & SESPMNT & $\mathrm{R}$ \\
\hline 675 & SESPMNT & $\mathrm{R}$ \\
\hline 676 & SESPMNT & $\mathrm{R}$ \\
\hline 677 & SESPMNT & $\mathrm{R}$ \\
\hline 678 & SESPMNT & $\mathrm{R}$ \\
\hline 679 & SESPMNT & $\mathrm{R}$ \\
\hline 680 & SESPMNT & $\mathrm{R}$ \\
\hline 681 & SESPMNT & $\mathrm{R}$ \\
\hline 682 & SESPMNT & $\mathrm{R}$ \\
\hline 683 & SESPMNT & $\mathrm{R}$ \\
\hline 684 & SESPMNT & $\mathrm{R}$ \\
\hline 685 & SESPMNT & $\mathrm{R}$ \\
\hline & & \\
\hline
\end{tabular}




\begin{tabular}{|c|l|l|}
\hline & AH & \\
\hline 1 & OWNER_ID & COLLECTION_PURPOSE \\
\hline 686 & SESPMNT & $\mathrm{R}$ \\
\hline 687 & SESPMNT & $\mathrm{R}$ \\
\hline 688 & SESPMNT & $\mathrm{R}$ \\
\hline 689 & SESPMNT & $\mathrm{R}$ \\
\hline 690 & SESPMNT & $\mathrm{R}$ \\
\hline 691 & SESPMNT & $\mathrm{R}$ \\
\hline 692 & SESPMNT & $\mathrm{R}$ \\
\hline 693 & SESPMNT & $\mathrm{R}$ \\
\hline 694 & SESPMNT & $\mathrm{R}$ \\
\hline 695 & SESPMNT & $\mathrm{R}$ \\
\hline 696 & SESPMNT & $\mathrm{R}$ \\
\hline 697 & SESPMNT & $\mathrm{R}$ \\
\hline 698 & SESPMNT & $\mathrm{R}$ \\
\hline 699 & SESPMNT & $\mathrm{R}$ \\
\hline 700 & SESPMNT & $\mathrm{R}$ \\
\hline 701 & SESPMNT & $\mathrm{R}$ \\
\hline 702 & SESPMNT & $\mathrm{R}$ \\
\hline 703 & SESPMNT & $\mathrm{R}$ \\
\hline 704 & SESPMNT & $\mathrm{R}$ \\
\hline 705 & SESPMNT & $\mathrm{R}$ \\
\hline 706 & SESPMNT & $\mathrm{R}$ \\
\hline 707 & SESPMNT & $\mathrm{R}$ \\
\hline 708 & SESPMNT & $\mathrm{R}$ \\
\hline 709 & SESPMNT & $\mathrm{R}$ \\
\hline 710 & SESPMNT & $\mathrm{R}$ \\
\hline 711 & SESPMNT & $\mathrm{R}$ \\
\hline 712 & SESPMNT & $\mathrm{R}$ \\
\hline 713 & SESPMNT & $\mathrm{R}$ \\
\hline 714 & SESPMNT & $\mathrm{R}$ \\
\hline 715 & SESPMNT & $\mathrm{R}$ \\
\hline 716 & SESPMNT & $\mathrm{R}$ \\
\hline 717 & SESPMNT & $\mathrm{R}$ \\
\hline 718 & SESPMNT & $\mathrm{R}$ \\
\hline 719 & SESPMNT & $\mathrm{R}$ \\
\hline 720 & SESPMNT & $\mathrm{R}$ \\
\hline 721 & SESPMNT & $\mathrm{R}$ \\
\hline 722 & SESPMNT & $\mathrm{R}$ \\
\hline 723 & SESPMNT & $\mathrm{R}$ \\
\hline & & \\
\hline
\end{tabular}




\begin{tabular}{|c|l|l|}
\hline & AH & \\
\hline 1 & OWNER_ID & COLLECTION_PURPOSE \\
\hline 724 & SESPMNT & $\mathrm{R}$ \\
\hline 725 & SESPMNT & $\mathrm{R}$ \\
\hline 726 & SESPMNT & $\mathrm{R}$ \\
\hline 727 & SESPMNT & $\mathrm{R}$ \\
\hline 728 & SESPMNT & $\mathrm{R}$ \\
\hline 729 & SESPMNT & $\mathrm{R}$ \\
\hline 730 & SESPMNT & $\mathrm{R}$ \\
\hline 731 & SESPMNT & $\mathrm{R}$ \\
\hline 732 & SESPMNT & $\mathrm{R}$ \\
\hline 733 & SESPMNT & $\mathrm{R}$ \\
\hline 734 & SESPMNT & $\mathrm{R}$ \\
\hline 735 & SESPMNT & $\mathrm{R}$ \\
\hline 736 & SESPMNT & $\mathrm{R}$ \\
\hline 737 & SESPMNT & $\mathrm{R}$ \\
\hline 738 & SESPMNT & $\mathrm{R}$ \\
\hline 739 & SESPMNT & $\mathrm{R}$ \\
\hline 740 & SESPMNT & $\mathrm{R}$ \\
\hline 741 & SESPMNT & $\mathrm{R}$ \\
\hline 742 & SESPMNT & $\mathrm{R}$ \\
\hline 743 & SESPMNT & $\mathrm{R}$ \\
\hline 744 & SESPMNT & $\mathrm{R}$ \\
\hline 745 & SESPMNT & $\mathrm{R}$ \\
\hline 746 & SESPMNT & $\mathrm{R}$ \\
\hline 747 & SESPMNT & $\mathrm{R}$ \\
\hline 748 & SESPMNT & $\mathrm{R}$ \\
\hline 749 & SESPMNT & $\mathrm{R}$ \\
\hline 750 & SESPMNT & $\mathrm{R}$ \\
\hline 751 & SESPMNT & $\mathrm{R}$ \\
\hline 752 & SESPMNT & $\mathrm{R}$ \\
\hline 753 & SESPMNT & $\mathrm{R}$ \\
\hline 754 & SESPMNT & $\mathrm{R}$ \\
\hline 755 & SESPMNT & $\mathrm{R}$ \\
\hline 756 & SESPMNT & $\mathrm{R}$ \\
\hline 757 & SESPMNT & $\mathrm{R}$ \\
\hline 758 & SESPMNT & $\mathrm{R}$ \\
\hline 759 & SESPMNT & $\mathrm{R}$ \\
\hline 760 & SESPMNT & $\mathrm{R}$ \\
\hline 761 & SESPMNT & $\mathrm{R}$ \\
\hline & & \\
\hline
\end{tabular}




\begin{tabular}{|c|l|l|}
\hline & AH & \\
\hline 1 & OWNER_ID & COLLECTION_PURPOSE \\
\hline 762 & SESPMNT & $\mathrm{R}$ \\
\hline 763 & SESPMNT & $\mathrm{R}$ \\
\hline 764 & SESPMNT & $\mathrm{R}$ \\
\hline 765 & SESPMNT & $\mathrm{R}$ \\
\hline 766 & SESPMNT & $\mathrm{R}$ \\
\hline 767 & SESPMNT & $\mathrm{R}$ \\
\hline 768 & SESPMNT & $\mathrm{R}$ \\
\hline 769 & SESPMNT & $\mathrm{R}$ \\
\hline 770 & SESPMNT & $\mathrm{R}$ \\
\hline 771 & SESPMNT & $\mathrm{R}$ \\
\hline 772 & SESPMNT & $\mathrm{R}$ \\
\hline 773 & SESPMNT & $\mathrm{R}$ \\
\hline 774 & SESPMNT & $\mathrm{R}$ \\
\hline 775 & SESPMNT & $\mathrm{R}$ \\
\hline 776 & SESPMNT & $\mathrm{R}$ \\
\hline 777 & SESPMNT & $\mathrm{R}$ \\
\hline 778 & SESPMNT & $\mathrm{R}$ \\
\hline 779 & SESPMNT & $\mathrm{R}$ \\
\hline 780 & SESPMNT & $\mathrm{R}$ \\
\hline 781 & SESPMNT & $\mathrm{R}$ \\
\hline 782 & SESPMNT & $\mathrm{R}$ \\
\hline 783 & SESPMNT & $\mathrm{R}$ \\
\hline 784 & SESPMNT & $\mathrm{R}$ \\
\hline 785 & SESPMNT & $\mathrm{R}$ \\
\hline 786 & SESPMNT & $\mathrm{R}$ \\
\hline 787 & SESPMNT & $\mathrm{R}$ \\
\hline 788 & SESPMNT & $\mathrm{R}$ \\
\hline 789 & SESPMNT & $\mathrm{R}$ \\
\hline 790 & SESPMNT & $\mathrm{R}$ \\
\hline 791 & SESPMNT & $\mathrm{R}$ \\
\hline 792 & SESPMNT & $\mathrm{R}$ \\
\hline 793 & SESPMNT & $\mathrm{R}$ \\
\hline 794 & SESPMNT & $\mathrm{R}$ \\
\hline 795 & SESPMNT & $\mathrm{R}$ \\
\hline 796 & SESPMNT & $\mathrm{R}$ \\
\hline 797 & SESPMNT & $\mathrm{R}$ \\
\hline 798 & SESPMNT & $\mathrm{R}$ \\
\hline 799 & SESPMNT & $\mathrm{R}$ \\
\hline & & \\
\hline
\end{tabular}




\begin{tabular}{|c|l|l|}
\hline 1 & OWNER_ID & COLLECTION_PURPOSE \\
\hline 800 & SESPMNT & $\mathrm{R}$ \\
\hline 801 & SESPMNT & $\mathrm{R}$ \\
\hline 802 & SESPMNT & $\mathrm{R}$ \\
\hline 803 & SESPMNT & $\mathrm{R}$ \\
\hline 804 & SESPMNT & $\mathrm{R}$ \\
\hline 805 & SESPMNT & $\mathrm{R}$ \\
\hline 806 & SESPMNT & $\mathrm{R}$ \\
\hline 807 & SESPMNT & $\mathrm{R}$ \\
\hline 808 & SESPMNT & $\mathrm{R}$ \\
\hline 809 & SESPMNT & $\mathrm{R}$ \\
\hline 810 & SESPMNT & $\mathrm{R}$ \\
\hline 811 & SESPMNT & $\mathrm{R}$ \\
\hline 812 & SESPMNT & $\mathrm{R}$ \\
\hline 813 & SESPMNT & $\mathrm{R}$ \\
\hline 814 & SESPMNT & $\mathrm{R}$ \\
\hline 815 & SESPMNT & $\mathrm{R}$ \\
\hline 816 & SESPMNT & $\mathrm{R}$ \\
\hline 817 & SESPMNT & $\mathrm{R}$ \\
\hline 818 & SESPMNT & $\mathrm{R}$ \\
\hline 819 & SESPMNT & $\mathrm{R}$ \\
\hline
\end{tabular}


90_pres C-14 


\begin{tabular}{|c|c|c|c|c|c|c|c|c|c|c|c|c|c|c|c|c|c|}
\hline & $\mathrm{A}$ & $\mathrm{B}$ & $\mathrm{C}$ & $\mathrm{D}$ & $E$ & $\mathrm{~F}$ & $G$ & $\mathrm{H}$ & $\mathrm{I}$ & $\mathrm{J}$ & $\mathrm{K}$ & $\mathrm{L}$ & $\mathrm{M}$ & $\mathrm{N}$ & $\mathrm{O}$ & $P$ & $\mathrm{Q}$ \\
\hline 1 & MEDIA & SAMP_NUMh & IP_DATE_T & P_DATE_TIMB & HORT & METHOD_NAME & LAB_CODE & ALUE_RPT| & L_UNITS_F & RWTING_EN & NAL_ERROI & 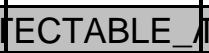 & T_MOIS & QUALI & N_QUA & $A P \_F$ & AMP_MTH \\
\hline 3021 & SW & B018Q4 & $16-$ Oct-91 & & C-14 & SAS COUNT LSC & TELDYN & & $\mathrm{pCi} / \mathrm{L}$ & & & 20 & & $U$ & & RF & \\
\hline 3022 & SW & B0M6×5 & 05-Nov-97 & & C-14 & C14_CHEM_LSC & QTESRL & 2.34 & $\mathrm{pCi} / \mathrm{L}$ & 3.24 & 4.98 & 7.5 & & $U$ & & EP & \\
\hline 3023 & SW & B0M6X7 & 05-Nov-97 & & C-14 & C14_CHEM_LSC & QTESRL & 2.34 & $\mathrm{pCi} / \mathrm{L}$ & 3.24 & 4.98 & 7.5 & & U & & EP & \\
\hline 3024 & SW & B11BT1 & 23-Jan-01 & & C-14 & C14_COX_LSC & TMANC & -93 & $\mathrm{pCi} / \mathrm{L}$ & 310 & 310 & 520 & & $U$ & & & \\
\hline 3025 & SW & B16WL1 & 30-Apr-03 & & C-14 & C14_CHEM_LSC & STLRL & -0.895 & $\mathrm{pCi} / \mathrm{L}$ & 3.2 & 3.5 & 7.78 & & U & & ER & IVE \\
\hline 3026 & SW & B16WL2 & 30-Apr-03 & & C-14 & C14_CHEM_LSC & STLRL & -0.308 & $\mathrm{pCi} / \mathrm{L}$ & 3.2 & 3.5 & 7.78 & & $U$ & & ER & IVE \\
\hline 3027 & SW & B16WL3 & 07-May-03 & & C-14 & C14_CHEM_LSC & STLRL & 3.34 & $\mathrm{pCi} / \mathrm{L}$ & 3.3 & 3.7 & 7.77 & & $U$ & & ER & IVE \\
\hline 3028 & SW & B16WL4 & 07-May-03 & & C-14 & C14_CHEM_LSC & STLRL & 1.4 & $\mathrm{pCi} / \mathrm{L}$ & 3.3 & 3.6 & 7.77 & & $U$ & & ER & IVE \\
\hline 3029 & SW & B170P7 & 13-May-03 & & C-14 & C14_CHEM_LSC & STLRL & 3.45 & $\mathrm{pCi} / \mathrm{L}$ & 3.3 & 3.7 & 7.77 & & $U$ & & ER & IVE \\
\hline 3030 & SW & B170P8 & 13-May-03 & & C-14 & C14_CHEM_LSC & STLRL & 3.41 & $\mathrm{pCi} / \mathrm{L}$ & 3.3 & 3.7 & 7.78 & & U & & ER & IVE \\
\hline 3031 & SW & B170X7 & 21-May-03 & & C-14 & C14_CHEM_LSC & STLRL & 8.02 & $\mathrm{pCi} / \mathrm{L}$ & 6.8 & 7.5 & 15.9 & & $U$ & & ER & IVE \\
\hline 3032 & SW & B170X8 & 21-May-03 & & C-14 & C14_CHEM_LSC & STLRL & 10 & $\mathrm{pCi} / \mathrm{L}$ & 3.6 & 4 & 7.95 & & & & ER & IVE \\
\hline 3033 & SW & B17140 & 28-May-03 & & C-14 & C14_CHEM_LSC & STLRL & 8.77 & $\mathrm{pCi} / \mathrm{L}$ & 3.6 & 3.9 & 7.95 & & & & ER & IVE \\
\hline 3034 & SW & B17141 & 28-May-03 & & C-14 & C14_CHEM_LSC & STLRL & 9.22 & $\mathrm{pCi} / \mathrm{L}$ & 3.6 & 4 & 7.95 & & & & ER & IVE \\
\hline 3035 & SW & B171K6 & 05-Jun-03 & & C-14 & C14_CHEM_LSC & STLRL & 1.52 & $\mathrm{pCi} / \mathrm{L}$ & 6.8 & 7.5 & 16.3 & & $U$ & & ER & IVE \\
\hline 3036 & SW & B171K7 & 05-Jun-03 & & C-14 & C14_CHEM_LSC & STLRL & -1.93 & $\mathrm{pCi} / \mathrm{L}$ & 3.3 & 3.6 & 8.14 & & $U$ & & ER & IVE \\
\hline 3037 & SW & B17214 & 12-Jun-03 & & C-14 & C14_CHEM_LSC & STLRL & 2.7 & $\mathrm{pCi} / \mathrm{L}$ & 6.6 & 7.2 & 15.7 & & U & & ER & IVE \\
\hline 3038 & SW & B17215 & 12-Jun-03 & & C-14 & C14_CHEM_LSC & STLRL & -1.75 & $\mathrm{pCi} / \mathrm{L}$ & 3.2 & 3.5 & 7.87 & & $U$ & & ER & IVE \\
\hline 3039 & SW & B172F1 & 18-Jun-03 & & C-14 & C14_CHEM_LSC & STLRL & -0.506 & $\mathrm{pCi} / \mathrm{L}$ & 3.2 & 3.5 & 7.87 & & $U$ & & ER & IVE \\
\hline 3040 & SW & B172F2 & 18-Jun-03 & & C-14 & C14_CHEM_LSC & STLRL & 1.16 & $\mathrm{pCi} / \mathrm{L}$ & 3.3 & 3.6 & 7.86 & & U & & ER & IVE \\
\hline 3041 & SW & B172K0 & 25-Jun-03 & & C-14 & C14_CHEM_LSC & STLRL & -2.75 & $\mathrm{pCi} / \mathrm{L}$ & 3.2 & 3.5 & 8.05 & & U & & ER & IVE \\
\hline 3042 & SW & B172K1 & 25-Jun-03 & & C-14 & C14_CHEM_LSC & STLRL & -2.57 & $\mathrm{pCi} / \mathrm{L}$ & 3.2 & 3.6 & 8.05 & & $U$ & & ER & IVE \\
\hline 3043 & SW & B17997 & 24-Jul-03 & & C-14 & C14_CHEM_LSC & STLRL & -3.5 & $\mathrm{pCi} / \mathrm{L}$ & 3.1 & 3.6 & 7.8 & & $U$ & & NKI & GRAB \\
\hline 3044 & SW & B17JR2 & 20-Oct-03 & & C-14 & C14_CHEM_LSC & STLRL & 608 & $\mathrm{pCi} / \mathrm{L}$ & 13 & 26 & 7.82 & & & & EP & \\
\hline 3045 & SW & B17LF3 & 10-Nov-03 & & C-14 & C14_CHEM_LSC & STLRL & 97 & $\mathrm{pCi} / \mathrm{L}$ & 5.8 & 7.2 & 7.79 & & & & EP & \\
\hline
\end{tabular}

Page 1 


\begin{tabular}{|c|c|c|c|c|c|c|}
\hline & $R$ & $\mathrm{~S}$ & $T$ & $\bar{U}$ & $\bar{V}$ & $\mathrm{~W}$ \\
\hline 1 & SAMP_SITE_NAME & SAMP_COMMENT & LT_COM & OWNER_ID & ON & WELL_NAME \\
\hline 3022 & & & & PNLGW & & \\
\hline 3024 & & 2390 & & HEISPROD & $\mathrm{T}$ & \\
\hline 3025 & PRIEST RAPIDS-RIVER & & & SESPSPEC & $S$ & \\
\hline 3026 & RICH.PMPHS HRM 46.4 & & & SESPSPEC & $\mathrm{S}$ & \\
\hline 3029 & PRIEST RAPIDS-RIVER & & & SESPSPEC & $\mathrm{S}$ & \\
\hline 3030 & RICH.PMPHS HRM 46.4 & & & SESPSPEC & $S$ & \\
\hline 3031 & PRIEST RAPIDS-RIVER & & & SESPSPEC & $S$ & \\
\hline 3032 & RICH.PMPHS HRM 46.4 & & & SESPSPEC & $S$ & \\
\hline 3033 & PRIEST RAPIDS-RIVER & & & SESPSPEC & $\mathrm{S}$ & \\
\hline 3034 & RICH.PMPHS HRM 46.4 & & & SESPSPEC & $S$ & \\
\hline 3040 & RICH.PMPHS HRM 46.4 & & & SESPSPEC & $\mathrm{S}$ & \\
\hline 3041 & PRIEST RAPIDS-RIVER & & & SESPSPEC & $S$ & \\
\hline 3042 & RICH.PMPHS HRM 46.4 & & & SESPSPEC & $\mathrm{S}$ & \\
\hline 3043 & LIGO & & & SESPSPEC & $S$ & \\
\hline 3044 & 100-K SPRING 63-1 & & & PNLGW & $\mathrm{R}$ & \\
\hline 3045 & 100-K SPRING 68-1 & & & PNLGW & $\mathrm{R}$ & \\
\hline
\end{tabular}




\section{6 - 99 Sediment_FNL}




\begin{tabular}{|c|c|c|c|c|c|c|c|c|c|c|}
\hline & A & $B$ & $\mathrm{C}$ & $\mathrm{D}$ & $E$ & $\mathrm{~F}$ & G & $\mathrm{H}$ & $\mathrm{I}$ & $\mathrm{J}$ \\
\hline 1 & SAMPLE_NUM_ASSIGNED_TO & SAMPLER & MEDIA & BIOTA_MEDIA & TAG_ID & SAMP_NUM & SAMP_DATE_TIME & SAMP_DATE_TIME_ON & CON_ID & CON_SHORT_NAME \\
\hline 2 & TRAPP, KF & BOWERS & SO & & & B0JK24 & 04-Nov-96 & & $7440-47-3$ & CR \\
\hline 3 & TRAPP, KF & BOWERS & SO & & & B0JKY9 & 31-Oct-96 & & $7440-47-3$ & CR \\
\hline 4 & TRAPP, KF & BOWERS & SO & & & B0JKY6 & 29-Oct-96 & & $7440-47-3$ & $\mathrm{CR}$ \\
\hline 5 & TRAPP, KF & BOWERS & SO & & & BOJJV9 & 29-Oct-96 & & $7440-47-3$ & $\mathrm{CR}$ \\
\hline 6 & TRAPP, KF & BOWERS & SO & & & B0JKZ3 & 01-Nov-96 & & $7440-47-3$ & CR \\
\hline 7 & TRAPP, KF & BOWERS & SO & & & BOJK30 & 31-Oct-96 & & $7440-47-3$ & CR \\
\hline 8 & TRAPP, KF & BOWERS & SO & & & B0JK28 & $31-$ Oct-96 & & $7440-47-3$ & $\mathrm{CR}$ \\
\hline 9 & TRAPP, KF & BOWERS & SO & & & B0JK26 & 04-Nov-96 & & $7440-47-3$ & CR \\
\hline 10 & TRAPP, KF & BOWERS & SO & & & B0JK22 & 30-Oct-96 & & $7440-47-3$ & $\mathrm{CR}$ \\
\hline 11 & TRAPP, KF & BOWERS & SO & & & BOJJYO & 28-Oct-96 & & $7440-47-3$ & $\mathrm{CR}$ \\
\hline 12 & TRAPP, KF & BOWERS & SO & & & B0JJX8 & 28-Oct-96 & & $7440-47-3$ & CR \\
\hline 13 & TRAPP, KF & BOWERS & SO & & & B0JJW1 & 06-Nov-96 & & $7440-47-3$ & $\mathrm{CR}$ \\
\hline 14 & SESP SCHEDULING & & SO & & & B0R0F6 & 20-Oct-98 & & $10045-97-3$ & CS-137 \\
\hline 15 & SESP SCHEDULING & & SO & & & B0R0F6 & 20-Oct-98 & & $10098-97-2$ & SR-90 \\
\hline 16 & SESP SCHEDULING & & SO & & & B0R0F6 & 20-Oct-98 & & 13966-29-5 & U-234 \\
\hline 17 & SESP SCHEDULING & & SO & & & B0ROF6 & 20-Oct-98 & & $13966-29-5$ & U-234 \\
\hline 18 & SESP SCHEDULING & & SO & & & B0ROF6 & 20-Oct-98 & & $15117-96-1$ & U-235 \\
\hline 19 & SESP SCHEDULING & & SO & & & BOROF6 & $20-$ Oct-98 & & $15117-96-1$ & U-235 \\
\hline 20 & SESP SCHEDULING & & SO & & & B0R0F6 & 20-Oct-98 & & U-238 & $U-238$ \\
\hline 21 & SESP SCHEDULING & & SO & & & B0ROF6 & 20-Oct-98 & & U-238 & $\mathrm{U}-238$ \\
\hline 22 & SESP SCHEDULING & & SO & & & BOROF7 & 24-Sep-98 & & $10045-97-3$ & CS-137 \\
\hline 23 & SESP SCHEDULING & & SO & & & BOROF7 & 24-Sep-98 & & $10098-97-2$ & SR-90 \\
\hline 24 & SESP SCHEDULING & & SO & & & BOROF7 & 24-Sep-98 & & 13966-29-5 & $U-234$ \\
\hline 25 & SESP SCHEDULING & & SO & & & BOROF7 & 24-Sep-98 & & 15117-96-1 & U-235 \\
\hline 26 & SESP SCHEDULING & & SO & & & BOROF7 & 24-Sep-98 & & U-238 & U-238 \\
\hline 27 & PS HENRY & & SO & & & B0G1G2 & 11-Nov-96 & & $7440-47-3$ & CR \\
\hline 28 & PS HENRY & & SO & & & B0G1G1 & 21-Nov-96 & & $7440-47-3$ & $\mathrm{CR}$ \\
\hline 29 & PS HENRY & & SO & & & B0G1C4 & 11-Nov-96 & & $7440-47-3$ & $\mathrm{CR}$ \\
\hline 30 & PS HENRY & & SO & & & B0G1B1 & 04-Nov-96 & & $7440-47-3$ & $\mathrm{CR}$ \\
\hline 31 & PS HENRY & & SO & & & B0G195 & 20-Aug-96 & & $7440-47-3$ & CR \\
\hline 32 & PS HENRY & & SO & & & B0G194 & 20-Aug-96 & & $7440-47-3$ & CR \\
\hline 33 & PS HENRY & & SO & & & B0G193 & 15-Aug-96 & & $7440-47-3$ & $\mathrm{CR}$ \\
\hline 34 & PS HENRY & & SO & & & B0G192 & 15-Aug-96 & & $7440-47-3$ & CR \\
\hline 35 & PS HENRY & & SO & & & B0G191 & 15-Aug-96 & & $7440-47-3$ & $\mathrm{CR}$ \\
\hline 36 & PS HENRY & & SO & & & B0G190 & 15-Aug-96 & & $7440-47-3$ & $\mathrm{CR}$ \\
\hline 37 & PS HENRY & & SO & & & B0G189 & 16-Aug-96 & & $7440-47-3$ & CR \\
\hline 38 & PS HENRY & & SO & & & B0G188 & 16-Aug-96 & & $7440-47-3$ & CR \\
\hline 39 & PS HENRY & & SO & & & B0G187 & 16-Aug-96 & & $7440-47-3$ & CR \\
\hline
\end{tabular}




\begin{tabular}{|c|c|c|c|c|c|c|c|c|c|c|}
\hline & $A$ & $B$ & $\mathrm{C}$ & $\mathrm{D}$ & $E$ & $\mathrm{~F}$ & $G$ & $\mathrm{H}$ & $\mathrm{I}$ & $\mathrm{J}$ \\
\hline 1 & SAMPLE NUM ASSIGNED TO & SAMPLER & MEDIA & BIOTA MEDIA & TAG ID & SAMP NUM & SAMP DATE TIME & SAMP DATE TIME ON & CON ID & CON SHORT NAME \\
\hline 40 & PS HENRY & & SO & & & B0G186 & 16-Aug-96 & & $7440-47-3$ & $\mathrm{CR}$ \\
\hline 41 & PS HENRY & & SO & & & B0G185 & 20-Aug-96 & & $7440-47-3$ & CR \\
\hline 42 & PS HENRY & & SO & & & B0G184 & 20-Aug-96 & & $7440-47-3$ & CR \\
\hline 43 & SESPSCH & & SO & & & B0J5F2 & 16-Aug-96 & & $10045-97-3$ & CS-137 \\
\hline 44 & SESPSCH & & SO & & & B0J5F2 & 16-Aug-96 & & 10098-97-2 & SR-90 \\
\hline 45 & SESPSCH & & SO & & & B0J5F2 & 16-Aug-96 & & 15117-96-1 & U-235 \\
\hline 46 & SESPSCH & & SO & & & B0J5F2 & 16-Aug-96 & & PU-239/240 & PU-239/240 \\
\hline 47 & SESPSCH & & SO & & & B0J5F2 & 16-Aug-96 & & U-238 & U-238 \\
\hline 48 & SESPSCH & & SO & & & B0J5F0 & 16-Aug-96 & & $10045-97-3$ & CS-137 \\
\hline 49 & SESPSCH & & SO & & & B0J5F0 & 16-Aug-96 & & $10098-97-2$ & SR-90 \\
\hline 50 & SESPSCH & & SO & & & B0J5F0 & 16-Aug-96 & & 15117-96-1 & U-235 \\
\hline 51 & SESPSCH & & SO & & & B0J5F0 & 16-Aug-96 & & PU-239/240 & PU-239/240 \\
\hline 52 & SESPSCH & & SO & & & B0J5F0 & 16-Aug-96 & & U-238 & U-238 \\
\hline 53 & SESPSCH & & SO & & & B0J5D8 & 16-Aug-96 & & $10045-97-3$ & CS-137 \\
\hline 54 & SESPSCH & & SO & & & B0J5D8 & 16-Aug-96 & & $10098-97-2$ & SR-90 \\
\hline 55 & SESPSCH & & SO & & & B0J5D8 & 16-Aug-96 & & 15117-96-1 & U-235 \\
\hline 56 & SESPSCH & & SO & & & B0J5D8 & 16-Aug-96 & & PU-239/240 & PU-239/240 \\
\hline 57 & SESPSCH & & SO & & & B0J5D8 & 16-Aug-96 & & U-238 & U-238 \\
\hline 58 & SESPSCH & & SO & & & B0J5D6 & 16-Aug-96 & & $10045-97-3$ & CS-137 \\
\hline 59 & SESPSCH & & SO & & & B0J5D6 & 16-Aug-96 & & $10098-97-2$ & SR-90 \\
\hline 60 & SESPSCH & & SO & & & B0J5D6 & 16-Aug-96 & & 15117-96-1 & U-235 \\
\hline 61 & SESPSCH & & SO & & & B0J5D6 & 16-Aug-96 & & PU-239/240 & PU-239/240 \\
\hline 62 & SESPSCH & & SO & & & B0J5D6 & 16-Aug-96 & & U-238 & U-238 \\
\hline 63 & SESPSCH & & SO & & & B0J5G8 & 20-Aug-96 & & $10045-97-3$ & CS-137 \\
\hline 64 & SESPSCH & & SO & & & B0J5G8 & 20-Aug-96 & & 10098-97-2 & SR-90 \\
\hline 65 & SESPSCH & & SO & & & B0J5G8 & 20-Aug-96 & & $15117-96-1$ & U-235 \\
\hline 66 & SESPSCH & & SO & & & B0J5G8 & 20-Aug-96 & & PU-239/240 & PU-239/240 \\
\hline 67 & SESPSCH & & SO & & & B0J5G8 & 20-Aug-96 & & U-238 & U-238 \\
\hline 68 & SESPSCH & & SO & & & B0J5G6 & 20-Aug-96 & & $10045-97-3$ & CS-137 \\
\hline 69 & SESPSCH & & SO & & & B0J5G6 & 20-Aug-96 & & 10098-97-2 & SR-90 \\
\hline 70 & SESPSCH & & SO & & & B0J5G6 & 20-Aug-96 & & 15117-96-1 & U-235 \\
\hline 71 & SESPSCH & & SO & & & B0J5G6 & 20-Aug-96 & & PU-239/240 & PU-239/240 \\
\hline 72 & SESPSCH & & SO & & & B0J5G6 & 20-Aug-96 & & U-238 & $\mathrm{U}-238$ \\
\hline 73 & SESPSCH & & SO & & & B0J5G4 & 20-Aug-96 & & $10045-97-3$ & CS-137 \\
\hline 74 & SESPSCH & & SO & & & B0J5G4 & 20-Aug-96 & & $10098-97-2$ & SR-90 \\
\hline 75 & SESPSCH & & SO & & & B0J5G4 & 20-Aug-96 & & $15117-96-1$ & U-235 \\
\hline 76 & SESPSCH & & SO & & & B0J5G4 & 20-Aug-96 & & PU-239/240 & PU-239/240 \\
\hline 77 & SESPSCH & & SO & & & B0J5G4 & 20-Aug-96 & & U-238 & U-238 \\
\hline
\end{tabular}




\begin{tabular}{|c|c|c|c|c|c|c|c|c|c|c|}
\hline & A & $\mathrm{B}$ & $\mathrm{C}$ & $\mathrm{D}$ & $\mathrm{E}$ & $\mathrm{F}$ & $\mathrm{G}$ & $\mathrm{H}$ & $\mathrm{I}$ & $\mathrm{J}$ \\
\hline 1 & SAMPLE_NUM_ASSIGNED_TO & SAMPLER & MEDIA & BIOTA_MEDIA & TAG_ID & SAMP_NUM & SAMP_DATE_TIME & SAMP_DATE_TIME_ON & CON_ID & CON_SHORT_NAME \\
\hline 78 & SESPSCH & & SO & & & B0J5G2 & 20-Aug-96 & & $10045-97-3$ & CS-137 \\
\hline 79 & SESPSCH & & SO & & & B0J5G2 & 20-Aug-96 & & $10098-97-2$ & SR-90 \\
\hline 80 & SESPSCH & & SO & & & B0J5G2 & 20-Aug-96 & & 15117-96-1 & U-235 \\
\hline 81 & SESPSCH & & SO & & & B0J5G2 & 20-Aug-96 & & PU-239/240 & PU-239/240 \\
\hline 82 & SESPSCH & & SO & & & B0J5G2 & 20-Aug-96 & & $\mathrm{U}-238$ & $\mathrm{U}-238$ \\
\hline 83 & SESPSCH & & SO & & & B0J5H8 & 11-Nov-96 & & $10045-97-3$ & CS-137 \\
\hline 84 & SESPSCH & & SO & & & B0J5H8 & 11-Nov-96 & & $10098-97-2$ & SR-90 \\
\hline 85 & SESPSCH & & SO & & & B0J5H8 & 11-Nov-96 & & $15117-96-1$ & U-235 \\
\hline 86 & SESPSCH & & SO & & & B0J5H8 & 11-Nov-96 & & U-238 & U-238 \\
\hline \begin{tabular}{|l}
87 \\
\end{tabular} & SESPSCH & & SO & & & B0J5H6 & 04-Nov-96 & & $10045-97-3$ & CS-137 \\
\hline 88 & SESPSCH & & SO & & & B0J5H6 & 04-Nov-96 & & $10098-97-2$ & SR-90 \\
\hline 89 & SESPSCH & & SO & & & B0J5H6 & 04-Nov-96 & & $15117-96-1$ & U-235 \\
\hline 90 & SESPSCH & & SO & & & B0J5H6 & 04-Nov-96 & & U-238 & U-238 \\
\hline \begin{tabular}{|l|}
91 \\
\end{tabular} & SESPSCH & & SO & & & B0J5H2 & 21-Nov-96 & & $10045-97-3$ & CS-137 \\
\hline 92 & SESPSCH & & SO & & & B0J5H2 & 21-Nov-96 & & $10098-97-2$ & SR-90 \\
\hline 93 & SESPSCH & & SO & & & B0J5H2 & 21-Nov-96 & & 15117-96-1 & U-235 \\
\hline \begin{tabular}{|l|}
94 \\
\end{tabular} & SESPSCH & & SO & & & B0J5H2 & 21-Nov-96 & & U-238 & U-238 \\
\hline 95 & SESPSCH & & SO & & & B0J5H1 & 11-Nov-96 & & $10045-97-3$ & CS-137 \\
\hline 96 & SESPSCH & & SO & & & B0J5H1 & 11-Nov-96 & & $10098-97-2$ & SR-90 \\
\hline 97 & SESPSCH & & SO & & & B0J5H1 & 11-Nov-96 & & $15117-96-1$ & U-235 \\
\hline \begin{tabular}{|l}
98 \\
\end{tabular} & SESPSCH & & SO & & & B0J5H1 & 11-Nov-96 & & U-238 & $\mathrm{U}-238$ \\
\hline 99 & SESPSCH & & SO & & & B0J5G0 & 15-Aug-96 & & $10045-97-3$ & CS-137 \\
\hline 100 & SESPSCH & & SO & & & B0J5G0 & 15-Aug-96 & & $10098-97-2$ & SR-90 \\
\hline 101 & SESPSCH & & SO & & & B0J5G0 & 15-Aug-96 & & $15117-96-1$ & U-235 \\
\hline 102 & SESPSCH & & SO & & & B0J5G0 & 15-Aug-96 & & PU-239/240 & PU-239/240 \\
\hline 103 & SESPSCH & & SO & & & B0J5G0 & 15-Aug-96 & & U-238 & U-238 \\
\hline 104 & SESPSCH & & SO & & & B0J5F8 & 15-Aug-96 & & $10045-97-3$ & CS-137 \\
\hline 105 & SESPSCH & & SO & & & B0J5F8 & 15-Aug-96 & & $10098-97-2$ & SR-90 \\
\hline 106 & SESPSCH & & SO & & & B0J5F8 & 15-Aug-96 & & 15117-96-1 & U-235 \\
\hline 107 & SESPSCH & & SO & & & B0J5F8 & 15-Aug-96 & & PU-239/240 & PU-239/240 \\
\hline 108 & SESPSCH & & SO & & & B0J5F8 & 15-Aug-96 & & U-238 & $\mathrm{U}-238$ \\
\hline 109 & SESPSCH & & SO & & & B0J5F6 & 15-Aug-96 & & $10045-97-3$ & CS-137 \\
\hline 110 & SESPSCH & & SO & & & B0J5F6 & 15-Aug-96 & & $10098-97-2$ & SR-90 \\
\hline 111 & SESPSCH & & SO & & & B0J5F6 & 15-Aug-96 & & $15117-96-1$ & U-235 \\
\hline 112 & SESPSCH & & SO & & & B0J5F6 & 15-Aug-96 & & PU-239/240 & PU-239/240 \\
\hline 113 & SESPSCH & & SO & & & B0J5F6 & 15-Aug-96 & & U-238 & $\mathrm{U}-238$ \\
\hline 114 & SESPSCH & & SO & & & B0J5F4 & 15-Aug-96 & & $10045-97-3$ & CS-137 \\
\hline 115 & SESPSCH & & SO & & & B0J5F4 & 15-Aug-96 & & $10098-97-2$ & SR-90 \\
\hline
\end{tabular}




\begin{tabular}{|c|c|c|c|c|c|c|c|c|c|c|}
\hline & A & $\mathrm{B}$ & $\mathrm{C}$ & $\mathrm{D}$ & $E$ & $\mathrm{~F}$ & G & $\mathrm{H}$ & $\mathrm{I}$ & $\mathrm{J}$ \\
\hline 1 & SAMPLE_NUM_ASSIGNED_TO & SAMPLER & MEDIA & BIOTA_MEDIA & TAG_ID & SAMP_NUM & SAMP_DATE_TIME & SAMP_DATE_TIME_ON & CON_ID & CON_SHORT_NAME \\
\hline 116 & SESPSCH & & SO & & & B0J5F4 & 15-Aug-96 & & $15117-96-1$ & U-235 \\
\hline 117 & SESPSCH & & so & & & B0J5F4 & 15-Aug-96 & & PU-239/240 & PU-239/240 \\
\hline 118 & SESPSCH & & SO & & & B0J5F4 & 15-Aug-96 & & U-238 & U-238 \\
\hline 119 & SESPSCH & & So & & & BOLV71 & 15-Aug-97 & & $10045-97-3$ & CS-137 \\
\hline 120 & SESPSCH & & SO & & & BOLV71 & 15-Aug-97 & & $10098-97-2$ & SR-90 \\
\hline 121 & SESPSCH & & so & & & BOLV71 & 15-Aug-97 & & $15117-96-1$ & U-235 \\
\hline 122 & SESPSCH & & SO & & & BOLV71 & 15-Aug-97 & & PU-239/240 & PU-239/240 \\
\hline 123 & SESPSCH & & so & & & BOLV71 & 15-Aug-97 & & U-238 & U-238 \\
\hline 124 & SESPSCH & & SO & & & BOLV73 & 15-Aug-97 & & $10045-97-3$ & CS-137 \\
\hline 125 & SESPSCH & & SO & & & BOLV73 & 15-Aug-97 & & $10098-97-2$ & SR-90 \\
\hline 126 & SESPSCH & & SO & & & BOLV73 & 15-Aug-97 & & $15117-96-1$ & U-235 \\
\hline 127 & SESPSCH & & SO & & & BOLV73 & 15-Aug-97 & & PU-239/240 & PU-239/240 \\
\hline 128 & SESPSCH & & so & & & B0LV73 & 15-Aug-97 & & U-238 & U-238 \\
\hline 129 & SESPSCH & & SO & & & B0LV87 & 18-Aug-97 & & $10045-97-3$ & CS-137 \\
\hline 130 & SESPSCH & & SO & & & BOLV87 & 18-Aug-97 & & $10098-97-2$ & SR-90 \\
\hline 131 & SESPSCH & & SO & & & BOLV87 & 18-Aug-97 & & 15117-96-1 & U-235 \\
\hline 132 & SESPSCH & & SO & & & BOLV87 & 18-Aug-97 & & PU-239/240 & PU-239/240 \\
\hline 133 & SESPSCH & & SO & & & BOLV87 & 18-Aug-97 & & U-238 & U-238 \\
\hline 134 & SESPSCH & & SO & & & BOLV88 & 18-Aug-97 & & $10045-97-3$ & CS-137 \\
\hline 135 & SESPSCH & & SO & & & BOLV88 & 18-Aug-97 & & $10098-97-2$ & SR-90 \\
\hline 136 & SESPSCH & & SO & & & BOLV88 & 18-Aug-97 & & 15117-96-1 & U-235 \\
\hline 137 & SESPSCH & & SO & & & BOLV88 & 18-Aug-97 & & PU-239/240 & PU-239/240 \\
\hline 138 & SESPSCH & & SO & & & BOLV88 & 18-Aug-97 & & U-238 & U-238 \\
\hline 139 & SESPSCH & & SO & & & BOLV89 & 18-Aug-97 & & $10045-97-3$ & CS-137 \\
\hline 140 & SESPSCH & & SO & & & BOLV89 & 18-Aug-97 & & $10098-97-2$ & SR-90 \\
\hline 141 & SESPSCH & & SO & & & BOLV89 & 18-Aug-97 & & $15117-96-1$ & U-235 \\
\hline 142 & SESPSCH & & SO & & & BOLV89 & 18-Aug-97 & & PU-239/240 & PU-239/240 \\
\hline 143 & SESPSCH & & SO & & & BOLV89 & 18-Aug-97 & & U-238 & U-238 \\
\hline 144 & SESPSCH & & SO & & & BOLV90 & 15-Aug-97 & & $10045-97-3$ & CS-137 \\
\hline 145 & SESPSCH & & SO & & & BOLV90 & 15-Aug-97 & & $10098-97-2$ & SR-90 \\
\hline 146 & SESPSCH & & SO & & & BOLV90 & 15-Aug-97 & & 15117-96-1 & U-235 \\
\hline 147 & SESPSCH & & SO & & & BOLV90 & 15-Aug-97 & & PU-239/240 & PU-239/240 \\
\hline 148 & SESPSCH & & SO & & & BOLV90 & 15-Aug-97 & & U-238 & $\mathrm{U}-238$ \\
\hline 149 & SESPSCH & & SO & & & SOLV75 & 15-Aug-97 & & $10045-97-3$ & CS-137 \\
\hline 150 & SESPSCH & & SO & & & SOLV75 & 15-Aug-97 & & $10098-97-2$ & SR-90 \\
\hline 151 & SESPSCH & & SO & & & SOLV75 & 15-Aug-97 & & $15117-96-1$ & U-235 \\
\hline 152 & SESPSCH & & SO & & & SOLV75 & 15-Aug-97 & & PU-239/240 & PU-239/240 \\
\hline 153 & SESPSCH & & SO & & & SOLV75 & 15-Aug-97 & & U-238 & U-238 \\
\hline
\end{tabular}




\begin{tabular}{|c|c|c|c|c|c|c|c|c|c|c|}
\hline & A & $\mathrm{B}$ & $\mathrm{C}$ & $\mathrm{D}$ & $E$ & $\mathrm{~F}$ & G & $\mathrm{H}$ & $\mathrm{I}$ & $\mathrm{J}$ \\
\hline $1 \mid$ & SAMPLE_NUM_ASSIGNED_TO & SAMPLER & MEDIA & BIOTA_MEDIA & TAG_ID & SAMP_NUM & SAMP_DATE_TIME & SAMP_DATE_TIME_ON & CON_ID & CON_SHORT_NAME \\
\hline 154 & SESPSCH & & SO & & & SOLV77 & 15-Aug-97 & & $10045-97-3$ & CS-137 \\
\hline 155 & SESPSCH & & so & & & SOLV77 & 15-Aug-97 & & $10098-97-2$ & SR-90 \\
\hline 156 & SESPSCH & & SO & & & SOLV77 & 15-Aug-97 & & 15117-96-1 & U-235 \\
\hline 157 & SESPSCH & & So & & & SOLV77 & 15-Aug-97 & & PU-239/240 & PU-239/240 \\
\hline 158 & SESPSCH & & SO & & & SOLV77 & 15-Aug-97 & & U-238 & U-238 \\
\hline 159 & SESPSCH & & so & & & SOLV79 & 18-Aug-97 & & $10045-97-3$ & CS-137 \\
\hline 160 & SESPSCH & & SO & & & SOLV79 & 18-Aug-97 & & $10098-97-2$ & SR-90 \\
\hline 161 & SESPSCH & & so & & & SOLV79 & 18-Aug-97 & & $15117-96-1$ & U-235 \\
\hline 162 & SESPSCH & & SO & & & SOLV79 & 18-Aug-97 & & PU-239/240 & PU-239/240 \\
\hline 163 & SESPSCH & & SO & & & SOLV79 & 18-Aug-97 & & U-238 & U-238 \\
\hline 164 & SESPSCH & & SO & & & SOLV83 & 18-Aug-97 & & $10045-97-3$ & CS-137 \\
\hline 165 & SESPSCH & & so & & & SOLV83 & 18-Aug-97 & & $10098-97-2$ & SR-90 \\
\hline 166 & SESPSCH & & so & & & SOLV83 & 18-Aug-97 & & $15117-96-1$ & U-235 \\
\hline 167 & SESPSCH & & SO & & & SOLV83 & 18-Aug-97 & & PU-239/240 & PU-239/240 \\
\hline 168 & SESPSCH & & so & & & SOLV83 & 18-Aug-97 & & U-238 & U-238 \\
\hline 169 & SESPSCH & & SO & & & SOLV85 & 18-Aug-97 & & $10045-97-3$ & CS-137 \\
\hline 170 & SESPSCH & & SO & & & SOLV85 & 18-Aug-97 & & $10098-97-2$ & SR-90 \\
\hline 171 & SESPSCH & & SO & & & SOLV85 & 18-Aug-97 & & $15117-96-1$ & U-235 \\
\hline 172 & SESPSCH & & SO & & & SOLV85 & 18-Aug-97 & & PU-239/240 & PU-239/240 \\
\hline 173 & SESPSCH & & SO & & & SOLV85 & 18-Aug-97 & & U-238 & U-238 \\
\hline 174 & SESPSCH & & SO & & & SOLWD6 & 08-Sep-97 & & $10045-97-3$ & CS-137 \\
\hline 175 & SESPSCH & & SO & & & SOLWD6 & 08-Sep-97 & & $10098-97-2$ & SR-90 \\
\hline 176 & SESPSCH & & SO & & & SOLWD7 & 09-Sep-97 & & $10045-97-3$ & CS-137 \\
\hline 177. & SESPSCH & & SO & & & SOLWD7 & 09-Sep-97 & & $10098-97-2$ & SR-90 \\
\hline 178 & SESPSCH & & SO & & & SOLWD8 & 09-Sep-97 & & $10045-97-3$ & CS-137 \\
\hline 179 & SESPSCH & & SO & & & SOLWD8 & 09-Sep-97 & & $10098-97-2$ & SR-90 \\
\hline 180 & SESP Scheduling & & so & & & B0M7T5 & 03-Nov-97 & & $10045-97-3$ & CS-137 \\
\hline 181 & SESP Scheduling & & SO & & & B0M7T5 & 03-Nov-97 & & $10098-97-2$ & SR-90 \\
\hline 182 & SESP Scheduling & & SO & & & B0M7T5 & 03-Nov-97 & & $15117-96-1$ & U-235 \\
\hline 183 & SESP Scheduling & & SO & & & B0M7T5 & 03-Nov-97 & & U-238 & $\mathrm{U}-238$ \\
\hline 184 & SESP Scheduling & & so & & & B0M7T7 & 23-Oct-97 & & $10045-97-3$ & CS-137 \\
\hline 185 & SESP Scheduling & & SO & & & B0M7T7 & 23-Oct-97 & & $10098-97-2$ & SR-90 \\
\hline 186 & SESP Scheduling & & SO & & & B0M7T7 & 23-Oct-97 & & $15117-96-1$ & U-235 \\
\hline 187 & SESP Scheduling & & SO & & & B0M7T7 & 23-Oct-97 & & U-238 & $\mathrm{U}-238$ \\
\hline 188 & SESP Scheduling & & SO & & & B0M7T8 & 23-Oct-97 & & $10045-97-3$ & CS-137 \\
\hline 189 & SESP Scheduling & & SO & & & B0M7T8 & 23-Oct-97 & & $10098-97-2$ & SR-90 \\
\hline 190 & SESP Scheduling & & SO & & & B0M7T8 & 23-Oct-97 & & $15117-96-1$ & U-235 \\
\hline 191 & SESP Scheduling & & SO & & & B0M7T8 & 23-Oct-97 & & U-238 & $\mathrm{U}-238$ \\
\hline
\end{tabular}




\begin{tabular}{|c|c|c|c|c|c|c|c|c|c|c|}
\hline & $A$ & $\mathrm{~B}$ & $C$ & $\mathrm{D}$ & $E$ & $\mathrm{~F}$ & $\mathrm{G}$ & $\mathrm{H}$ & $\mathrm{I}$ & $\mathrm{J}$ \\
\hline 1 & SAMPLE_NUM_ASSIGNED_TO & SAMPLER & MEDIA & BIOTA_MEDIA & TAG_ID & SAMP_NUM & SAMP_DATE_TIME & SAMP_DATE_TIME_ON & CON_ID & CON_SHORT_NAME \\
\hline 192 & SESP Scheduling & & SO & & & B0M7V2 & 27-Oct-97 & & $10045-97-3$ & CS-137 \\
\hline 193 & SESP Scheduling & & so & & & B0M7V2 & 27-Oct-97 & & $10098-97-2$ & SR-90 \\
\hline 194 & SESP Scheduling & & SO & & & B0M7V2 & 27-Oct-97 & & 15117-96-1 & U-235 \\
\hline 195 & SESP Scheduling & & SO & & & B0M7V2 & 27-Oct-97 & & U-238 & U-238 \\
\hline 196 & SESP Scheduling & & SO & & & B0M7V3 & 27-Oct-97 & & $10045-97-3$ & CS-137 \\
\hline 197 & SESP Scheduling & & so & & & B0M7V3 & 27-Oct-97 & & $10098-97-2$ & SR-90 \\
\hline 198 & SESP Scheduling & & SO & & & B0M7V3 & 27-Oct-97 & & $15117-96-1$ & U-235 \\
\hline 199 & SESP Scheduling & & so & & & B0M7V3 & 27-Oct-97 & & $\mathrm{U}-238$ & $\mathrm{U}-238$ \\
\hline 200 & SESP SCHEDULING & & SO & & & B0PK81 & 07-Aug-98 & & $10045-97-3$ & CS-137 \\
\hline 201 & SESP SCHEDULING & & SO & & & B0PK81 & 07-Aug-98 & & $10098-97-2$ & SR-90 \\
\hline 202 & SESP SCHEDULING & & SO & & & B0PK81 & 07-Aug-98 & & $13966-29-5$ & $\mathrm{U}-234$ \\
\hline 203 & SESP SCHEDULING & & so & & & B0PK81 & 07-Aug-98 & & $15117-96-1$ & $\mathrm{U}-235$ \\
\hline 204 & SESP SCHEDULING & & So & & & B0PK81 & 07-Aug-98 & & PU-239/240 & PU-239/240 \\
\hline 205 & SESP SCHEDULING & & So & & & B0PK81 & 07-Aug-98 & & U-238 & $\mathrm{U}-238$ \\
\hline 206 & SESP SCHEDULING & & so & & & B0PKB3 & 07-Aug-98 & & $10045-97-3$ & CS-137 \\
\hline 207 & SESP SCHEDULING & & SO & & & B0PKB3 & 07-Aug-98 & & $10098-97-2$ & SR-90 \\
\hline 208 & SESP SCHEDULING & & SO & & & B0PKB3 & 07-Aug-98 & & $13966-29-5$ & U-234 \\
\hline 209 & SESP SCHEDULING & & SO & & & B0PKB3 & 07-Aug-98 & & $15117-96-1$ & U-235 \\
\hline 210 & SESP SCHEDULING & & SO & & & B0PKB3 & 07-Aug-98 & & PU-239/240 & PU-239/240 \\
\hline 211 & SESP SCHEDULING & & SO & & & B0PKB3 & 07-Aug-98 & & U-238 & $\mathrm{U}-238$ \\
\hline 212 & SESP SCHEDULING & & SO & & & B0PKB4 & 07-Aug-98 & & $10045-97-3$ & CS-137 \\
\hline 213 & SESP SCHEDULING & & SO & & & B0PKB4 & 07-Aug-98 & & $10098-97-2$ & SR-90 \\
\hline 214 & SESP SCHEDULING & & SO & & & B0PKB4 & 07-Aug-98 & & $13966-29-5$ & U-234 \\
\hline 215 & SESP SCHEDULING & & SO & & & B0PKB4 & 07-Aug-98 & & $15117-96-1$ & U-235 \\
\hline 216 & SESP SCHEDULING & & SO & & & B0PKB4 & 07-Aug-98 & & PU-239/240 & PU-239/240 \\
\hline 217. & SESP SCHEDULING & & SO & & & BOPKB4 & 07-Aug-98 & & U-238 & U-238 \\
\hline 218 & SESP SCHEDULING & & SO & & & B0PM83 & 17-Aug-98 & & $10045-97-3$ & CS-137 \\
\hline 219 & SESP SCHEDULING & & SO & & & B0PM83 & 17-Aug-98 & & $10098-97-2$ & SR-90 \\
\hline 220 & SESP SCHEDULING & & SO & & & B0PM83 & 17-Aug-98 & & $13966-29-5$ & U-234 \\
\hline 221 & SESP SCHEDULING & & SO & & & B0PM83 & 17-Aug-98 & & $15117-96-1$ & U-235 \\
\hline 222 & SESP SCHEDULING & & so & & & B0PM83 & 17-Aug-98 & & PU-239/240 & PU-239/240 \\
\hline 223 & SESP SCHEDULING & & SO & & & B0PM83 & 17-Aug-98 & & U-238 & $\mathrm{U}-238$ \\
\hline 224 & SESP SCHEDULING & & SO & & & B0PM85 & 11-Aug-98 & & $10045-97-3$ & CS-137 \\
\hline 225 & SESP SCHEDULING & & SO & & & B0PM85 & 11-Aug-98 & & $10098-97-2$ & SR-90 \\
\hline 226 & SESP SCHEDULING & & SO & & & B0PM85 & 11-Aug-98 & & $13966-29-5$ & $\mathrm{U}-234$ \\
\hline 227 & SESP SCHEDULING & & SO & & & B0PM85 & 11-Aug-98 & & $15117-96-1$ & U-235 \\
\hline 228 & SESP SCHEDULING & & SO & & & B0PM85 & 11-Aug-98 & & PU-239/240 & PU-239/240 \\
\hline 229 & SESP SCHEDULING & & So & & & B0PM85 & 11-Aug-98 & & U-238 & $\mathrm{U}-238$ \\
\hline
\end{tabular}




\begin{tabular}{|c|c|c|c|c|c|c|c|c|c|c|}
\hline & A & $\mathrm{B}$ & $\mathrm{C}$ & $\mathrm{D}$ & $E$ & $\mathrm{~F}$ & G & $\mathrm{H}$ & $\mathrm{I}$ & $\mathrm{J}$ \\
\hline 1 & SAMPLE_NUM_ASSIGNED_TO & SAMPLER & MEDIA & BIOTA_MEDIA & TAG_ID & SAMP_NUM & SAMP_DATE_TIME & SAMP_DATE_TIME_ON & CON_ID & CON_SHORT_NAME \\
\hline 230 & SESP SCHEDULING & & SO & & & B0PM87 & 11-Aug-98 & & $10045-97-3$ & CS-137 \\
\hline 231 & SESP SCHEDULING & & so & & & B0PM87 & 11-Aug-98 & & $10098-97-2$ & SR-90 \\
\hline 232 & SESP SCHEDULING & & SO & & & B0PM87 & 11-Aug-98 & & $13966-29-5$ & U-234 \\
\hline 233 & SESP SCHEDULING & & SO & & & B0PM87 & 11-Aug-98 & & $15117-96-1$ & U-235 \\
\hline 234 & SESP SCHEDULING & & SO & & & B0PM87 & 11-Aug-98 & & PU-239/240 & PU-239/240 \\
\hline 235 & SESP SCHEDULING & & so & & & B0PM87 & 11-Aug-98 & & $\mathrm{U}-238$ & $\mathrm{U}-238$ \\
\hline 236 & SESP SCHEDULING & & SO & & & B0PM89 & 11-Aug-98 & & $10045-97-3$ & CS-137 \\
\hline 237 & SESP SCHEDULING & & SO & & & B0PM89 & 11-Aug-98 & & $10098-97-2$ & SR-90 \\
\hline 238 & SESP SCHEDULING & & SO & & & B0PM89 & 11-Aug-98 & & $13966-29-5$ & $\mathrm{U}-234$ \\
\hline 239 & SESP SCHEDULING & & SO & & & B0PM89 & 11-Aug-98 & & $15117-96-1$ & U-235 \\
\hline 240 & SESP SCHEDULING & & SO & & & B0PM89 & 11-Aug-98 & & PU-239/240 & PU-239/240 \\
\hline 241 & SESP SCHEDULING & & so & & & B0PM89 & 11-Aug-98 & & U-238 & $\mathrm{U}-238$ \\
\hline 242 & SESP SCHEDULING & & so & & & B0PM91 & 11-Aug-98 & & $10045-97-3$ & CS-137 \\
\hline 243 & SESP SCHEDULING & & So & & & B0PM91 & 11-Aug-98 & & $10098-97-2$ & SR-90 \\
\hline 244 & SESP SCHEDULING & & so & & & B0PM91 & 11-Aug-98 & & $13966-29-5$ & $\mathrm{U}-234$ \\
\hline 245 & SESP SCHEDULING & & SO & & & B0PM91 & 11-Aug-98 & & $15117-96-1$ & U-235 \\
\hline 246 & SESP SCHEDULING & & SO & & & B0PM91 & 11-Aug-98 & & PU-239/240 & PU-239/240 \\
\hline 247 & SESP SCHEDULING & & SO & & & B0PM91 & 11-Aug-98 & & $\mathrm{U}-238$ & $\mathrm{U}-238$ \\
\hline 248 & SESP SCHEDULING & & SO & & & B0PM93 & 10-Aug-98 & & $10045-97-3$ & CS-137 \\
\hline 249 & SESP SCHEDULING & & SO & & & B0РM93 & 10-Aug-98 & & $10098-97-2$ & SR-90 \\
\hline 250 & SESP SCHEDULING & & SO & & & B0РM93 & 10-Aug-98 & & $13966-29-5$ & $\mathrm{U}-234$ \\
\hline 251 & SESP SCHEDULING & & SO & & & B0PM93 & 10-Aug-98 & & $15117-96-1$ & $\mathrm{U}-235$ \\
\hline 252 & SESP SCHEDULING & & SO & & & B0PM93 & 10-Aug-98 & & PU-239/240 & PU-239/240 \\
\hline 253 & SESP SCHEDULING & & SO & & & B0РM93 & 10-Aug-98 & & U-238 & $\mathrm{U}-238$ \\
\hline 254 & SESP SCHEDULING & & SO & & & B0PM95 & 10-Aug-98 & & $10045-97-3$ & CS-137 \\
\hline 255 & SESP SCHEDULING & & SO & & & B0PM95 & 10-Aug-98 & & $10098-97-2$ & SR-90 \\
\hline 256 & SESP SCHEDULING & & SO & & & B0PM95 & 10-Aug-98 & & $13966-29-5$ & $\mathrm{U}-234$ \\
\hline 257 & SESP SCHEDULING & & SO & & & B0PM95 & 10-Aug-98 & & $15117-96-1$ & $\mathrm{U}-235$ \\
\hline 258 & SESP SCHEDULING & & SO & & & B0PM95 & 10-Aug-98 & & PU-239/240 & PU-239/240 \\
\hline 259 & SESP SCHEDULING & & SO & & & B0PM95 & 10-Aug-98 & & $\mathrm{U}-238$ & $\mathrm{U}-238$ \\
\hline 260 & SESP SCHEDULING & & SO & & & B0PM97 & 10-Aug-98 & & $10045-97-3$ & CS-137 \\
\hline 261 & SESP SCHEDULING & & SO & & & B0PM97 & 10-Aug-98 & & $10098-97-2$ & SR-90 \\
\hline 262 & SESP SCHEDULING & & SO & & & B0PM97 & 10-Aug-98 & & $13966-29-5$ & $\mathrm{U}-234$ \\
\hline 263 & SESP SCHEDULING & & SO & & & B0PM97 & 10-Aug-98 & & $15117-96-1$ & $\mathrm{U}-235$ \\
\hline 264 & SESP SCHEDULING & & SO & & & B0PM97 & 10-Aug-98 & & PU-239/240 & PU-239/240 \\
\hline 265 & SESP SCHEDULING & & SO & & & B0PM97 & 10-Aug-98 & & U-238 & $\mathrm{U}-238$ \\
\hline 266 & SESP SCHEDULING & & so & & & B0PM99 & 10-Aug-98 & & $10045-97-3$ & CS-137 \\
\hline 267 & SESP SCHEDULING & & So & & & B0PM99 & 10-Aug-98 & & $10098-97-2$ & SR-90 \\
\hline
\end{tabular}




\begin{tabular}{|c|c|c|c|c|c|c|c|c|c|c|}
\hline & A & $\mathrm{B}$ & $\mathrm{C}$ & $\mathrm{D}$ & $E$ & $\mathrm{~F}$ & $G$ & $\mathrm{H}$ & $\mathrm{I}$ & $\mathrm{J}$ \\
\hline 1 & SAMPLE_NUM_ASSIGNED_TO & SAMPLER & MEDIA & BIOTA_MEDIA & TAG_ID & SAMP_NUM & SAMP_DATE_TIME| & SAMP_DATE_TIME_ON & CON_ID & CON_SHORT_NAME \\
\hline 268 & SESP SCHEDULING & & SO & & & B0PM99 & 10-Aug-98 & & 13966-29-5 & U-234 \\
\hline 269 & SESP SCHEDULING & & SO & & & B0PM99 & 10-Aug-98 & & 15117-96-1 & $\mathrm{U}-235$ \\
\hline 270 & SESP SCHEDULING & & SO & & & B0PM99 & 10-Aug-98 & & PU-239/240 & PU-239/240 \\
\hline 271 & SESP SCHEDULING & & SO & & & B0PM99 & 10-Aug-98 & & U-238 & U-238 \\
\hline 272 & SESP SCHEDULING & & SO & & & B0PMB2 & 17-Aug-98 & & $10045-97-3$ & CS-137 \\
\hline 273 & SESP SCHEDULING & & SO & & & BOPMB2 & 17-Aug-98 & & $10098-97-2$ & SR-90 \\
\hline 274 & SESP SCHEDULING & & SO & & & B0PMB2 & 17-Aug-98 & & 13966-29-5 & $\mathrm{U}-234$ \\
\hline 275 & SESP SCHEDULING & & SO & & & BOPMB2 & 17-Aug-98 & & 15117-96-1 & U-235 \\
\hline 276 & SESP SCHEDULING & & SO & & & B0PMB2 & 17-Aug-98 & & PU-239/240 & PU-239/240 \\
\hline 277 & SESP SCHEDULING & & SO & & & B0PMB2 & 17-Aug-98 & & U-238 & $\mathrm{U}-238$ \\
\hline 278 & SESP SCHEDULING & & SO & & & B0PMB6 & 10-Aug-98 & & $10045-97-3$ & CS-137 \\
\hline 279 & SESP SCHEDULING & & $\mathrm{SO}$ & & & B0PMB6 & 10-Aug-98 & & $10098-97-2$ & SR-90 \\
\hline 280 & SESP SCHEDULING & & SO & & & B0PMB6 & 10-Aug-98 & & 13966-29-5 & U-234 \\
\hline 281 & SESP SCHEDULING & & SO & & & B0PMB6 & 10-Aug-98 & & 15117-96-1 & U-235 \\
\hline 282 & SESP SCHEDULING & & SO & & & B0PMB6 & 10-Aug-98 & & PU-239/240 & PU-239/240 \\
\hline 283 & SESP SCHEDULING & & SO & & & B0PMB6 & 10-Aug-98 & & U-238 & $\mathrm{U}-238$ \\
\hline 284 & SESP SCHEDULING & & SO & & & BOPMB7 & 10-Aug-98 & & $10045-97-3$ & CS-137 \\
\hline 285 & SESP SCHEDULING & & SO & & & BOPMB7 & 10-Aug-98 & & $10098-97-2$ & SR-90 \\
\hline 286 & SESP SCHEDULING & & SO & & & B0PMB7 & 10-Aug-98 & & 13966-29-5 & U-234 \\
\hline 287 & SESP SCHEDULING & & SO & & & BOPMB7 & 10-Aug-98 & & 15117-96-1 & U-235 \\
\hline 288 & SESP SCHEDULING & & SO & & & B0PMB7 & 10-Aug-98 & & PU-239/240 & PU-239/240 \\
\hline 289 & SESP SCHEDULING & & $\mathrm{SO}$ & & & B0PMB7 & 10-Aug-98 & & U-238 & $\mathrm{U}-238$ \\
\hline 290 & SESP SCHEDULING & & SO & & & B0PMH5 & 11-Aug-98 & & $10045-97-3$ & CS-137 \\
\hline 291 & SESP SCHEDULING & & SO & & & B0PMH5 & 11-Aug-98 & & $10098-97-2$ & SR-90 \\
\hline 292 & SESP SCHEDULING & & SO & & & B0PMH5 & 11-Aug-98 & & 13966-29-5 & U-234 \\
\hline 293 & SESP SCHEDULING & & SO & & & B0PMH5 & 11-Aug-98 & & 15117-96-1 & U-235 \\
\hline 294 & SESP SCHEDULING & & SO & & & B0PMH5 & 11-Aug-98 & & PU-239/240 & PU-239/240 \\
\hline 295 & SESP SCHEDULING & & SO & & & B0PMH5 & 11-Aug-98 & & U-238 & $\mathrm{U}-238$ \\
\hline 296 & SESP SCHEDULING & & SO & & & B0PMH6 & 11-Aug-98 & & $10045-97-3$ & CS-137 \\
\hline 297 & SESP SCHEDULING & & SO & & & B0PMH6 & 11-Aug-98 & & $10098-97-2$ & SR-90 \\
\hline 298 & SESP SCHEDULING & & SO & & & B0PMH6 & 11-Aug-98 & & 13966-29-5 & $\mathrm{U}-234$ \\
\hline 299 & SESP SCHEDULING & & SO & & & B0PMH6 & 11-Aug-98 & & 15117-96-1 & U-235 \\
\hline 300 & SESP SCHEDULING & & SO & & & B0PMH6 & 11-Aug-98 & & PU-239/240 & PU-239/240 \\
\hline 301 & SESP SCHEDULING & & $\mathrm{SO}$ & & & B0PMH6 & 11-Aug-98 & & U-238 & $\mathrm{U}-238$ \\
\hline 302 & SESP SCHEDULING & & SO & & & B0PM84 & 17-Aug-98 & & $10045-97-3$ & CS-137 \\
\hline 303 & SESP SCHEDULING & & SO & & & B0PM84 & 17-Aug-98 & & $10098-97-2$ & SR-90 \\
\hline 304 & SESP SCHEDULING & & SO & & & B0PM84 & 17-Aug-98 & & $13966-29-5$ & U-234 \\
\hline 305 & SESP SCHEDULING & & SO & & & B0PM84 & 17-Aug-98 & & $15117-96-1$ & U-235 \\
\hline
\end{tabular}




\begin{tabular}{|c|c|c|c|c|c|c|c|c|c|c|}
\hline & A & $\mathrm{B}$ & $\mathrm{C}$ & $\mathrm{D}$ & $E$ & $\mathrm{~F}$ & $\mathrm{G}$ & $\mathrm{H}$ & $\mathrm{I}$ & $\mathrm{J}$ \\
\hline 1 & SAMPLE_NUM_ASSIGNED_TO & SAMPLER & MEDIA & BIOTA_MEDIA & TAG_ID & SAMP_NUM & SAMP_DATE_TIME & SAMP_DATE_TIME_ON & CON_ID & CON_SHORT_NAME \\
\hline 306 & SESP SCHEDULING & & SO & & & B0PM84 & 17-Aug-98 & & PU-239/240 & PU-239/240 \\
\hline 307 & SESP SCHEDULING & & so & & & B0PM84 & 17-Aug-98 & & U-238 & $\mathrm{U}-238$ \\
\hline 308 & SESP SCHEDULING & & SO & & & B0PMB1 & 17-Aug-98 & & $10045-97-3$ & CS-137 \\
\hline 309 & SESP SCHEDULING & & SO & & & B0PMB1 & 17-Aug-98 & & $10098-97-2$ & SR-90 \\
\hline 310 & SESP SCHEDULING & & SO & & & B0PMB1 & 17-Aug-98 & & $13966-29-5$ & U-234 \\
\hline 311 & SESP SCHEDULING & & SO & & & B0PMB1 & 17-Aug-98 & & 15117-96-1 & U-235 \\
\hline 312 & SESP SCHEDULING & & SO & & & B0PMB1 & 17-Aug-98 & & PU-239/240 & PU-239/240 \\
\hline 313 & SESP SCHEDULING & & SO & & & B0PMB1 & 17-Aug-98 & & U-238 & $\mathrm{U}-238$ \\
\hline 314 & SESP SCHEDULING & & SO & & & B0W3L5 & 03-Aug-99 & & $10045-97-3$ & CS-137 \\
\hline 315 & SESP SCHEDULING & & SO & & & B0W3L5 & 03-Aug-99 & & $10098-97-2$ & SR-90 \\
\hline 316 & SESP SCHEDULING & & SO & & & B0W3L5 & 03-Aug-99 & & $13966-29-5$ & $\mathrm{U}-234$ \\
\hline 317 & SESP SCHEDULING & & SO & & & BOW3L5 & 03-Aug-99 & & 15117-96-1 & U-235 \\
\hline 318 & SESP SCHEDULING & & so & & & BOW3L5 & 03-Aug-99 & & PU-239/240 & PU-239/240 \\
\hline 319 & SESP SCHEDULING & & SO & & & BOW3L5 & 03-Aug-99 & & U-238 & U-238 \\
\hline 320 & SESP SCHEDULING & & so & & & B0W3L7 & 03-Aug-99 & & $10045-97-3$ & CS-137 \\
\hline 321 & SESP SCHEDULING & & SO & & & B0W3L7 & 03-Aug-99 & & $10098-97-2$ & SR-90 \\
\hline 322 & SESP SCHEDULING & & SO & & & B0W3L7 & 03-Aug-99 & & $13966-29-5$ & U-234 \\
\hline 323 & SESP SCHEDULING & & SO & & & B0W3L7 & 03-Aug-99 & & $15117-96-1$ & U-235 \\
\hline 324 & SESP SCHEDULING & & SO & & & B0W3L7 & 03-Aug-99 & & PU-239/240 & PU-239/240 \\
\hline 325 & SESP SCHEDULING & & SO & & & B0W3L7 & 03-Aug-99 & & U-238 & $\mathrm{U}-238$ \\
\hline 326 & SESP SCHEDULING & & SO & & & B0W3L9 & 09-Aug-99 & & $10045-97-3$ & CS-137 \\
\hline 327 & SESP SCHEDULING & & SO & & & B0W3L9 & 09-Aug-99 & & $10098-97-2$ & SR-90 \\
\hline 328 & SESP SCHEDULING & & SO & & & B0W3L9 & 09-Aug-99 & & $13966-29-5$ & U-234 \\
\hline 329 & SESP SCHEDULING & & SO & & & BOW3L9 & 09-Aug-99 & & $15117-96-1$ & $\mathrm{U}-235$ \\
\hline 330 & SESP SCHEDULING & & SO & & & B0W3L9 & 09-Aug-99 & & PU-239/240 & PU-239/240 \\
\hline 331 & SESP SCHEDULING & & SO & & & BOW3L9 & 09-Aug-99 & & U-238 & U-238 \\
\hline 332 & SESP SCHEDULING & & so & & & BOW3M0 & 09-Aug-99 & & $10045-97-3$ & CS-137 \\
\hline 333 & SESP SCHEDULING & & SO & & & BOW3M0 & 09-Aug-99 & & $10098-97-2$ & SR-90 \\
\hline 334 & SESP SCHEDULING & & SO & & & BOW3M0 & 09-Aug-99 & & $13966-29-5$ & U-234 \\
\hline 335 & SESP SCHEDULING & & SO & & & BOW3M0 & 09-Aug-99 & & $15117-96-1$ & U-235 \\
\hline 336 & SESP SCHEDULING & & so & & & BOW3M0 & 09-Aug-99 & & PU-239/240 & PU-239/240 \\
\hline 337 & SESP SCHEDULING & & SO & & & BOW3M0 & 09-Aug-99 & & U-238 & $\mathrm{U}-238$ \\
\hline 338 & SESP SCHEDULING & & SO & & & BOW3C4 & 16-Aug-99 & & $10045-97-3$ & CS-137 \\
\hline 339 & SESP SCHEDULING & & SO & & & BOW3C4 & 16-Aug-99 & & $10098-97-2$ & SR-90 \\
\hline 340 & SESP SCHEDULING & & SO & & & B0W3C4 & 16-Aug-99 & & $13966-29-5$ & $\mathrm{U}-234$ \\
\hline 341 & SESP SCHEDULING & & SO & & & B0W3C4 & 16-Aug-99 & & $15117-96-1$ & U-235 \\
\hline 342 & SESP SCHEDULING & & SO & & & BOW3C4 & 16-Aug-99 & & PU-239/240 & PU-239/240 \\
\hline 343 & SESP SCHEDULING & & So & & & BOW3C4 & 16-Aug-99 & & U-238 & U-238 \\
\hline
\end{tabular}




\begin{tabular}{|c|c|c|c|c|c|c|c|c|c|c|}
\hline & A & $\mathrm{B}$ & $\mathrm{C}$ & $\mathrm{D}$ & $\mathrm{E}$ & $\mathrm{F}$ & $\mathrm{G}$ & $\mathrm{H}$ & $\mathrm{I}$ & $\mathrm{J}$ \\
\hline 1 & SAMPLE_NUM_ASSIGNED_TO & SAMPLER & MEDIA & BIOTA_MEDIA & TAG_ID & SAMP_NUM & SAMP_DATE_TIME & SAMP_DATE_TIME_ON & CON_ID & CON_SHORT_NAME \\
\hline 344 & SESP SCHEDULING & & SO & & & B0W3D1 & 16-Aug-99 & & $10045-97-3$ & CS-137 \\
\hline 345 & SESP SCHEDULING & & SO & & & B0W3D1 & 16-Aug-99 & & $10098-97-2$ & SR-90 \\
\hline 346 & SESP SCHEDULING & & SO & & & B0W3D1 & 16-Aug-99 & & $13966-29-5$ & $\mathrm{U}-234$ \\
\hline 347 & SESP SCHEDULING & & SO & & & B0W3D1 & 16-Aug-99 & & 15117-96-1 & U-235 \\
\hline 348 & SESP SCHEDULING & & SO & & & B0W3D1 & 16-Aug-99 & & PU-239/240 & PU-239/240 \\
\hline 349 & SESP SCHEDULING & & SO & & & B0W3D1 & 16-Aug-99 & & U-238 & $\mathrm{U}-238$ \\
\hline 350 & SESP SCHEDULING & & SO & & & B0W3D2 & 16-Aug-99 & & $10045-97-3$ & CS-137 \\
\hline 351 & SESP SCHEDULING & & SO & & & B0W3D2 & 16-Aug-99 & & $10098-97-2$ & SR-90 \\
\hline 352 & SESP SCHEDULING & & SO & & & B0W3D2 & 16-Aug-99 & & $13966-29-5$ & $\mathrm{U}-234$ \\
\hline 353 & SESP SCHEDULING & & SO & & & B0W3D2 & 16-Aug-99 & & $15117-96-1$ & U-235 \\
\hline 354 & SESP SCHEDULING & & SO & & & B0W3D2 & 16-Aug-99 & & PU-239/240 & PU-239/240 \\
\hline 355 & SESP SCHEDULING & & SO & & & B0W3D2 & 16-Aug-99 & & U-238 & $\mathrm{U}-238$ \\
\hline 356 & SESP SCHEDULING & & SO & & & BOW3H7 & 09-Aug-99 & & $10045-97-3$ & CS-137 \\
\hline 357 & SESP SCHEDULING & & SO & & & BOW3H7 & 09-Aug-99 & & $10098-97-2$ & SR-90 \\
\hline 358 & SESP SCHEDULING & & SO & & & BOW3H7 & 09-Aug-99 & & $13966-29-5$ & $\mathrm{U}-234$ \\
\hline 359 & SESP SCHEDULING & & SO & & & BOW3H7 & 09-Aug-99 & & $15117-96-1$ & U-235 \\
\hline 360 & SESP SCHEDULING & & SO & & & BOW3H7 & 09-Aug-99 & & PU-239/240 & PU-239/240 \\
\hline 361 & SESP SCHEDULING & & SO & & & BOW3H7 & 09-Aug-99 & & U-238 & $\mathrm{U}-238$ \\
\hline 362 & SESP SCHEDULING & & SO & & & BOW3H8 & 03-Sep-99 & & $10045-97-3$ & CS-137 \\
\hline 363 & SESP SCHEDULING & & SO & & & BOW3H8 & 03-Sep-99 & & $10098-97-2$ & SR-90 \\
\hline 364 & SESP SCHEDULING & & SO & & & BOW3H8 & 03-Sep-99 & & $13966-29-5$ & $\mathrm{U}-234$ \\
\hline 365 & SESP SCHEDULING & & SO & & & BOW3H8 & 03-Sep-99 & & $15117-96-1$ & $\mathrm{U}-235$ \\
\hline 366 & SESP SCHEDULING & & SO & & & BOW3H8 & 03-Sep-99 & & PU-239/240 & PU-239/240 \\
\hline 367 & SESP SCHEDULING & & SO & & & BOW3H8 & 03-Sep-99 & & U-238 & $\mathrm{U}-238$ \\
\hline 368 & SESP SCHEDULING & & SO & & & B0W3K3 & 02-Aug-99 & & $10045-97-3$ & CS-137 \\
\hline 369 & SESP SCHEDULING & & SO & & & B0W3K3 & 02-Aug-99 & & $10098-97-2$ & SR-90 \\
\hline 370 & SESP SCHEDULING & & SO & & & B0W3K3 & 02-Aug-99 & & $13966-29-5$ & $\mathrm{U}-234$ \\
\hline 371 & SESP SCHEDULING & & SO & & & B0W3K3 & 02-Aug-99 & & $15117-96-1$ & $\mathrm{U}-235$ \\
\hline 372 & SESP SCHEDULING & & SO & & & B0W3K3 & 02-Aug-99 & & PU-239/240 & PU-239/240 \\
\hline 373 & SESP SCHEDULING & & SO & & & B0W3K3 & 02-Aug-99 & & $\mathrm{U}-238$ & $\mathrm{U}-238$ \\
\hline 374 & SESP SCHEDULING & & SO & & & B0W3K5 & 02-Aug-99 & & $10045-97-3$ & CS-137 \\
\hline 375 & SESP SCHEDULING & & SO & & & B0W3K5 & 02-Aug-99 & & $10098-97-2$ & SR-90 \\
\hline 376 & SESP SCHEDULING & & SO & & & B0W3K5 & 02-Aug-99 & & $13966-29-5$ & $\mathrm{U}-234$ \\
\hline 377 & SESP SCHEDULING & & SO & & & B0W3K5 & 02-Aug-99 & & $15117-96-1$ & $\mathrm{U}-235$ \\
\hline 378 & SESP SCHEDULING & & SO & & & B0W3K5 & 02-Aug-99 & & PU-239/240 & PU-239/240 \\
\hline 379 & SESP SCHEDULING & & SO & & & BOW3K5 & 02-Aug-99 & & $\mathrm{U}-238$ & $\mathrm{U}-238$ \\
\hline 380 & SESP SCHEDULING & & SO & & & B0W3K7 & 02-Aug-99 & & $10045-97-3$ & CS-137 \\
\hline 381 & SESP SCHEDULING & & SO & & & B0W3K7 & 02-Aug-99 & & $10098-97-2$ & SR-90 \\
\hline
\end{tabular}




\begin{tabular}{|c|c|c|c|c|c|c|c|c|c|c|}
\hline & A & $\mathrm{B}$ & C & $\mathrm{D}$ & $\mathrm{E}$ & $\mathrm{F}$ & G & $\mathrm{H}$ & $\mathrm{I}$ & $\mathrm{J}$ \\
\hline 1 & SAMPLE_NUM_ASSIGNED_TO & SAMPLER & MEDIA & BIOTA_MEDIA & TAG_ID & SAMP_NUM & SAMP_DATE_TIME & SAMP_DATE_TIME_ON & CON_ID & CON_SHORT_NAME \\
\hline 382 & SESP SCHEDULING & & SO & & & B0W3K7 & 02-Aug-99 & & $13966-29-5$ & $\mathrm{U}-234$ \\
\hline 383 & SESP SCHEDULING & & SO & & & BOW3K7 & 02-Aug-99 & & 15117-96-1 & U-235 \\
\hline 384 & SESP SCHEDULING & & SO & & & BOW3K7 & 02-Aug-99 & & PU-239/240 & PU-239/240 \\
\hline 385 & SESP SCHEDULING & & SO & & & BOW3K7 & 02-Aug-99 & & U-238 & $\mathrm{U}-238$ \\
\hline 386 & SESP SCHEDULING & & SO & & & BOW3K9 & 02-Aug-99 & & $10045-97-3$ & CS-137 \\
\hline 387 & SESP SCHEDULING & & SO & & & BOW3K9 & 02-Aug-99 & & $10098-97-2$ & SR-90 \\
\hline 388 & SESP SCHEDULING & & SO & & & BOW3K9 & 02-Aug-99 & & 13966-29-5 & $\mathrm{U}-234$ \\
\hline 389 & SESP SCHEDULING & & SO & & & B0W3K9 & 02-Aug-99 & & 15117-96-1 & $\mathrm{U}-235$ \\
\hline 390 & SESP SCHEDULING & & SO & & & BOW3K9 & 02-Aug-99 & & PU-239/240 & PU-239/240 \\
\hline 391 & SESP SCHEDULING & & SO & & & BOW3K9 & 02-Aug-99 & & $\mathrm{U}-238$ & $\mathrm{U}-238$ \\
\hline 392 & SESP SCHEDULING & & SO & & & BOW3L1 & 03-Aug-99 & & $10045-97-3$ & CS-137 \\
\hline 393 & SESP SCHEDULING & & $\mathrm{SO}$ & & & B0W3L1 & 03-Aug-99 & & $10098-97-2$ & SR-90 \\
\hline 394 & SESP SCHEDULING & & SO & & & BOW3L1 & 03-Aug-99 & & 13966-29-5 & U-234 \\
\hline 395 & SESP SCHEDULING & & SO & & & B0W3L1 & 03-Aug-99 & & 15117-96-1 & $\mathrm{U}-235$ \\
\hline 396 & SESP SCHEDULING & & SO & & & B0W3L1 & 03-Aug-99 & & PU-239/240 & PU-239/240 \\
\hline 397 & SESP SCHEDULING & & SO & & & B0W3L1 & 03-Aug-99 & & U-238 & $\mathrm{U}-238$ \\
\hline 398 & SESP SCHEDULING & & SO & & & B0W3L3 & 03-Aug-99 & & $10045-97-3$ & CS-137 \\
\hline 399 & SESP SCHEDULING & & SO & & & B0W3L3 & 03-Aug-99 & & $10098-97-2$ & SR-90 \\
\hline 400 & SESP SCHEDULING & & SO & & & B0W3L3 & 03-Aug-99 & & 13966-29-5 & U-234 \\
\hline 401 & SESP SCHEDULING & & SO & & & B0W3L3 & 03-Aug-99 & & 15117-96-1 & U-235 \\
\hline 402 & SESP SCHEDULING & & SO & & & B0W3L3 & 03-Aug-99 & & PU-239/240 & PU-239/240 \\
\hline 403 & SESP SCHEDULING & & $\mathrm{SO}$ & & & B0W3L3 & 03-Aug-99 & & U-238 & $\mathrm{U}-238$ \\
\hline 404 & SESP SCHEDULING & & SO & & & B0W3N6 & 03-Aug-99 & & $10045-97-3$ & CS-137 \\
\hline 405 & SESP SCHEDULING & & SO & & & B0W3N6 & 03-Aug-99 & & $10098-97-2$ & SR-90 \\
\hline 406 & SESP SCHEDULING & & SO & & & B0W3N6 & 03-Aug-99 & & 13966-29-5 & U-234 \\
\hline 407 & SESP SCHEDULING & & SO & & & BOW3N6 & 03-Aug-99 & & 15117-96-1 & U-235 \\
\hline 408 & SESP SCHEDULING & & SO & & & B0W3N6 & 03-Aug-99 & & PU-239/240 & PU-239/240 \\
\hline 409 & SESP SCHEDULING & & SO & & & B0W3N6 & 03-Aug-99 & & U-238 & $\mathrm{U}-238$ \\
\hline 410 & SESP SCHEDULING & & SO & & & B0W3N7 & 03-Aug-99 & & $10045-97-3$ & CS-137 \\
\hline 411 & SESP SCHEDULING & & SO & & & B0W3N7 & 03-Aug-99 & & $10098-97-2$ & SR-90 \\
\hline 412 & SESP SCHEDULING & & SO & & & BOW3N7 & 03-Aug-99 & & 13966-29-5 & $\mathrm{U}-234$ \\
\hline 413 & SESP SCHEDULING & & SO & & & BOW3N7 & 03-Aug-99 & & 15117-96-1 & U-235 \\
\hline 414 & SESP SCHEDULING & & SO & & & BOW3N7 & 03-Aug-99 & & PU-239/240 & PU-239/240 \\
\hline 415 & SESP SCHEDULING & & $\mathrm{SO}$ & & & BOW3N7 & 03-Aug-99 & & U-238 & $\mathrm{U}-238$ \\
\hline 416 & SESP SCHEDULING & & SO & & & B0W3P2 & 02-Aug-99 & & $10045-97-3$ & CS-137 \\
\hline 417 & SESP SCHEDULING & & SO & & & B0W3P2 & 02-Aug-99 & & $10098-97-2$ & SR-90 \\
\hline 418 & SESP SCHEDULING & & SO & & & B0W3P2 & 02-Aug-99 & & 13966-29-5 & $\mathrm{U}-234$ \\
\hline 419 & SESP SCHEDULING & & SO & & & B0W3P2 & 02-Aug-99 & & 15117-96-1 & U-235 \\
\hline
\end{tabular}




\begin{tabular}{|c|c|c|c|c|c|c|c|c|c|c|}
\hline & A & $\mathrm{B}$ & $\mathrm{C}$ & $\mathrm{D}$ & $E$ & $\mathrm{~F}$ & G & $\mathrm{H}$ & $\mathrm{I}$ & $\mathrm{J}$ \\
\hline 1 & SAMPLE_NUM_ASSIGNED_TO & SAMPLER & MEDIA & BIOTA_MEDIA & TAG_ID & SAMP_NUM & SAMP_DATE_TIME & SAMP_DATE_TIME_ON & CON_ID & CON_SHORT_NAME \\
\hline 420 & SESP SCHEDULING & & SO & & & BOW3P2 & 02-Aug-99 & & PU-239/240 & PU-239/240 \\
\hline 421 & SESP SCHEDULING & & so & & & B0W3P2 & 02-Aug-99 & & U-238 & $\mathrm{U}-238$ \\
\hline 422 & SESP SCHEDULING & & SO & & & B0W3P3 & 02-Aug-99 & & $10045-97-3$ & CS-137 \\
\hline 423 & SESP SCHEDULING & & SO & & & B0W3P3 & 02-Aug-99 & & $10098-97-2$ & SR-90 \\
\hline 424 & SESP SCHEDULING & & SO & & & B0W3P3 & 02-Aug-99 & & $13966-29-5$ & U-234 \\
\hline 425 & SESP SCHEDULING & & so & & & BOW3P3 & 02-Aug-99 & & $15117-96-1$ & U-235 \\
\hline 426 & SESP SCHEDULING & & SO & & & B0W3P3 & 02-Aug-99 & & PU-239/240 & PU-239/240 \\
\hline 427 & SESP SCHEDULING & & SO & & & B0W3P3 & 02-Aug-99 & & U-238 & $\mathrm{U}-238$ \\
\hline 428 & SESP SCHEDULING & & SO & & & B0WDL8 & 01-Nov-99 & & $10045-97-3$ & CS-137 \\
\hline 429 & SESP SCHEDULING & & SO & & & BOWDL8 & 01-Nov-99 & & $10098-97-2$ & SR-90 \\
\hline 430 & SESP SCHEDULING & & SO & & & BOWDL8 & 01-Nov-99 & & $13966-29-5$ & $\mathrm{U}-234$ \\
\hline 431 & SESP SCHEDULING & & so & & & B0WDL8 & 01-Nov-99 & & $15117-96-1$ & $\mathrm{U}-235$ \\
\hline 432 & SESP SCHEDULING & & so & & & BOWDL8 & 01-Nov-99 & & U-238 & U-238 \\
\hline 433 & SESP SCHEDULING & & SO & & & BOWDP4 & 25-Oct-99 & & $10045-97-3$ & CS-137 \\
\hline 434 & SESP SCHEDULING & & so & & & BOWDP4 & 25-Oct-99 & & $10098-97-2$ & SR-90 \\
\hline 435 & SESP SCHEDULING & & SO & & & BOWDP4 & 25-Oct-99 & & $13966-29-5$ & U-234 \\
\hline 436 & SESP SCHEDULING & & SO & & & BOWDP4 & 25-Oct-99 & & $15117-96-1$ & U-235 \\
\hline 437 & SESP SCHEDULING & & SO & & & BOWDP4 & 25-Oct-99 & & $\mathrm{U}-238$ & $\mathrm{U}-238$ \\
\hline 438 & SESP SCHEDULING & & SO & & & B0WDP8 & 01-Nov-99 & & $10045-97-3$ & CS-137 \\
\hline 439 & SESP SCHEDULING & & SO & & & B0WDP8 & 01-Nov-99 & & $10098-97-2$ & SR-90 \\
\hline 440 & SESP SCHEDULING & & SO & & & B0WDP8 & 01-Nov-99 & & $13966-29-5$ & $\mathrm{U}-234$ \\
\hline 441 & SESP SCHEDULING & & SO & & & B0WDP8 & 01-Nov-99 & & $15117-96-1$ & $\mathrm{U}-235$ \\
\hline 442 & SESP SCHEDULING & & SO & & & B0WDP8 & 01-Nov-99 & & U-238 & $\mathrm{U}-238$ \\
\hline 443 & SESP SCHEDULING & & SO & & & BOWDRO & 01-Nov-99 & & $10045-97-3$ & CS-137 \\
\hline 444 & SESP SCHEDULING & & SO & & & BOWDR0 & 01-Nov-99 & & $10098-97-2$ & SR-90 \\
\hline 445 & SESP SCHEDULING & & SO & & & BOWDRO & 01-Nov-99 & & $13966-29-5$ & U-234 \\
\hline 446 & SESP SCHEDULING & & so & & & BOWDRO & 01-Nov-99 & & 15117-96-1 & U-235 \\
\hline 447 & SESP SCHEDULING & & SO & & & BOWDR0 & 01-Nov-99 & & U-238 & $\mathrm{U}-238$ \\
\hline 448 & SESP SCHEDULING & & SO & & & BOWDR2 & 01-Nov-99 & & $10045-97-3$ & CS-137 \\
\hline 449 & SESP SCHEDULING & & SO & & & BOWDR2 & 01-Nov-99 & & $10098-97-2$ & SR-90 \\
\hline 450 & SESP SCHEDULING & & so & & & BOWDR2 & 01-Nov-99 & & $13966-29-5$ & $\mathrm{U}-234$ \\
\hline 451 & SESP SCHEDULING & & SO & & & BOWDR2 & 01-Nov-99 & & $15117-96-1$ & U-235 \\
\hline 452 & SESP SCHEDULING & & SO & & & BOWDR2 & 01-Nov-99 & & U-238 & $\mathrm{U}-238$ \\
\hline 453 & SESP SCHEDULING & & SO & & & B0WDR8 & 01-Nov-99 & & $10045-97-3$ & CS-137 \\
\hline 454 & SESP SCHEDULING & & SO & & & B0WDR8 & 01-Nov-99 & & $10098-97-2$ & SR-90 \\
\hline 455 & SESP SCHEDULING & & SO & & & B0WDR8 & 01-Nov-99 & & $13966-29-5$ & U-234 \\
\hline 456 & SESP SCHEDULING & & SO & & & BOWDR8 & 01-Nov-99 & & $15117-96-1$ & U-235 \\
\hline 457 & SESP SCHEDULING & & SO & & & B0WDR8 & 01-Nov-99 & & U-238 & $U-238$ \\
\hline
\end{tabular}




\begin{tabular}{|c|c|c|c|c|c|c|c|c|c|}
\hline & $\mathrm{K}$ & $\mathrm{L}$ & $\mathrm{M}$ & $\mathrm{N}$ & $\mathrm{O}$ & $\mathrm{P}$ & $\mathrm{Q}$ & $\mathrm{R}$ & $\mathrm{S}$ \\
\hline 1 & METHOD_NAME & LAB_CODE & VALUE_RPTD & ANAL_UNITS_RPTD & |COUNTING_ERROR & TOTAL_ANAL_ERROR & MIN_DETECTABLE_ACTIVITY & PCNT_MOISTURE & LAB_QUALIFIER \\
\hline 2 & 6010_METALS_ICP & QTESSL & & $u g / g$ & & & & & \\
\hline 3 & 6010_METALS_ICP & QTESSL & 24.2 & $u g / g$ & & & & & \\
\hline 4 & 6010_METALS_ICP & QTESSL & 58.4 & $\mathrm{mg} / \mathrm{kg}$ & & & & & \\
\hline 5 & 6010_METALS_ICP & QTESSL & 13.1 & $\mathrm{mg} / \mathrm{kg}$ & & & & & \\
\hline 6 & 6010_METALS_ICP & QTESSL & 40.5 & $u g / g$ & & & & & \\
\hline 7 & 6010_METALS_ICP & QTESSL & 25.7 & $\mathrm{ug} / \mathrm{g}$ & & & & & \\
\hline 8 & 6010_METALS_ICP & QTESSL & 15 & $u g / g$ & & & & & \\
\hline 9 & 6010_METALS_ICP & QTESSL & 84.2 & $u g / g$ & & & & & \\
\hline 11 & 6010_METALS_ICP & QTESSL & 42.5 & $\mathrm{mg} / \mathrm{kg}$ & & & & & \\
\hline 12 & 6010_METALS_ICP & QTESSL & 94.8 & $\mathrm{mg} / \mathrm{kg}$ & & & & & \\
\hline 13 & 6010_METALS_ICP & QTESSL & 26.5 & $\mathrm{mg} / \mathrm{kg}$ & & & & & \\
\hline 14 & GAMMA_GS & QTESRL & 0.145 & $\mathrm{pCi} / \mathrm{g}$ & 0.019 & 0.019 & 0.0105 & & \\
\hline 15 & SRISO_SEP_PRECIP_GPC & QTESRL & 0.00412 & $\mathrm{pCi} / \mathrm{g}$ & 0.0069 & 0.0071 & 0.013 & & U \\
\hline 16 & GAMMA_GS & QTESRL & 0.694 & $\mathrm{pCi} / \mathrm{g}$ & 0.078 & 0.078 & 0.0393 & & \\
\hline 17 & UISO_IE_PLATE_AEA & QTESRL & 0.492 & $\mathrm{pCi} / \mathrm{g}$ & 0.042 & 0.066 & 0.0082 & & $\mathrm{~J}$ \\
\hline 18 & GAMMA_GS & QTESRL & 0.0509 & $\mathrm{pCi} / \mathrm{g}$ & 0.047 & 0.047 & 0.066 & & $U$ \\
\hline 19 & UISO_IE_PLATE_AEA & QTESRL & 0.0216 & $\mathrm{pCi} / \mathrm{g}$ & 0.0092 & 0.0095 & 0.00607 & & $\mathrm{~J}$ \\
\hline 21 & UISO_IE_PLATE_AEA & QTESRL & 0.429 & $\mathrm{pCi} / \mathrm{g}$ & 0.039 & 0.059 & 0.00607 & & $\mathrm{~J}$ \\
\hline 22 & GAMMA_GS & QTESRL & 0.1 & $\mathrm{pCi} / \mathrm{g}$ & 0.023 & 0.023 & 0.0234 & & \\
\hline 23 & SRISO_SEP_PRECIP_GPC & QTESRL & 0.00412 & $\mathrm{pCi} / \mathrm{g}$ & 0.0081 & 0.0083 & 0.0152 & & U \\
\hline 24 & UISO_IE_PLATE_AEA & QTESRL & 0.262 & $\mathrm{pCi} / \mathrm{g}$ & 0.046 & 0.056 & 0.0199 & & $\mathrm{~J}$ \\
\hline 25 & UISO_IE_PLATE_AEA & QTESRL & 0.0291 & $\mathrm{pCi} / \mathrm{g}$ & 0.016 & 0.016 & 0.013 & & $\mathrm{~J}$ \\
\hline 26 & UISO_IE_PLATE_AEA & QTESRL & 0.257 & $\mathrm{pCi} / \mathrm{g}$ & 0.045 & 0.055 & 0.0148 & & $\mathrm{~J}$ \\
\hline 27 & 6010_METALS_ICP & DATACH & 12000 & ug/kg & & 6120 & & & \\
\hline 28 & 6010_METALS_ICP & DATACH & 10000 & $\mathrm{ug} / \mathrm{kg}$ & & 5100 & & & \\
\hline 29 & 6010_METALS_ICP & DATACH & 23000 & $\mathrm{ug} / \mathrm{kg}$ & & 11700 & & & \\
\hline 30 & 6010_METALS_ICP & DATACH & 23000 & $\mathrm{ug} / \mathrm{kg}$ & & 11700 & & & \\
\hline 31 & 6010_METALS_ICP & DATACH & 13000 & $\mathrm{ug} / \mathrm{kg}$ & & 6630 & & & \\
\hline 32 & 6010_METALS_ICP & DATACH & 12000 & $\mathrm{ug} / \mathrm{kg}$ & & 6120 & & & \\
\hline 33 & 6010_METALS_ICP & DATACH & 17000 & $\mathrm{ug} / \mathrm{kg}$ & & 8670 & & & B \\
\hline 34 & 6010_METALS_ICP & DATACH & 4800 & $\mathrm{ug} / \mathrm{kg}$ & & 2450 & & & B \\
\hline 35 & 6010_METALS_ICP & DATACH & 16000 & $\mathrm{ug} / \mathrm{kg}$ & & 8160 & & & B \\
\hline 36 & 6010_METALS_ICP & DATACH & 9900 & $\mathrm{ug} / \mathrm{kg}$ & & 5050 & & & B \\
\hline 37 & 6010_METALS_ICP & DATACH & 11000 & $\mathrm{ug} / \mathrm{kg}$ & & 5610 & & & \\
\hline 38 & 6010_METALS_ICP & DATACH & 13000 & $\mathrm{ug} / \mathrm{kg}$ & & 6630 & & & \\
\hline
\end{tabular}




\begin{tabular}{|c|c|c|c|c|c|c|c|c|c|}
\hline & $\mathrm{K}$ & $\mathrm{L}$ & $\mathrm{M}$ & $\mathrm{N}$ & $\mathrm{O}$ & $P$ & $\mathrm{Q}$ & $\mathrm{R}$ & $S$ \\
\hline 1 & METHOD_NAME & LAB_CODE & VALUE_RPTD & ANAL_UNITS_RPTD & COUNTING_ERROR| & TOTAL_ANAL_ERROR|I & MIN_DETECTABLE_ACTIVITY & PCNT_MOISTURE & LAB_QUALIFIER \\
\hline 40 & 6010_METALS_ICP & DATACH & 12000 & ug/kg & & 6120 & & & \\
\hline 41 & 6010_METALS_ICP & DATACH & 14000 & ug/kg & & 7140 & & & \\
\hline 42 & 6010_METALS_ICP & DATACH & 10000 & $\mathrm{ug} / \mathrm{kg}$ & & 5100 & & & \\
\hline 43 & GAMMA_GS & ITASRL & 0.536 & $\mathrm{pCi} / \mathrm{g}$ & 0.0283 & 0.0606 & & & \\
\hline 44 & SRTOT_SEP_PRECIP_GPC & ITASRL & 0.0477 & $\mathrm{pCi} / \mathrm{g}$ & 0.00486 & 0.0113 & & & \\
\hline 45 & GAMMA_GS & ITASRL & 0.045 & $\mathrm{pCi} / \mathrm{g}$ & 0.134 & 0.134 & & & $U$ \\
\hline 46 & PUISO_PLATE_AEA & ITASRL & 0.00775 & $\mathrm{pCi} / \mathrm{g}$ & 0.00215 & 0.00257 & & & \\
\hline 47 & GAMMA_GS & ITASRL & 1.35 & $\mathrm{pCi} / \mathrm{g}$ & 0.977 & 0.986 & & & \\
\hline 49 & SRTOT_SEP_PRECIP_GPC & ITASRL & 0.0285 & $\mathrm{pCi} / \mathrm{g}$ & 0.00469 & 0.00801 & & & \\
\hline 50 & GAMMA_GS & ITASRL & -0.0193 & $\mathrm{pCi} / \mathrm{g}$ & 0.138 & 0.138 & & & $U$ \\
\hline 51 & PUISO_PLATE_AEA & ITASRL & 0.00832 & $\mathrm{pCi} / \mathrm{g}$ & 0.00186 & 0.00228 & & & \\
\hline 52 & GAMMA_GS & ITASRL & 1.89 & $\mathrm{pCi} / \mathrm{g}$ & 0.763 & 0.786 & & & \\
\hline 53 & GAMMA_GS & ITASRL & 0.47 & $\mathrm{pCi} / \mathrm{g}$ & 0.0326 & 0.0572 & & & \\
\hline 54 & SRTOT_SEP_PRECIP_GPC & ITASRL & 0.0224 & $\mathrm{pCi} / \mathrm{g}$ & 0.00457 & 0.00677 & & & \\
\hline 55 & GAMMA_GS & ITASRL & -0.000883 & $\mathrm{pCi} / \mathrm{g}$ & 0.186 & 0.186 & & & U \\
\hline 56 & PUISO_PLATE_AEA & ITASRL & 0.0097 & $\mathrm{pCi} / \mathrm{g}$ & 0.00196 & 0.00248 & & & \\
\hline 57 & GAMMA_GS & ITASRL & 0.968 & $\mathrm{pCi} / \mathrm{g}$ & 0.743 & 0.749 & & & \\
\hline 59 & SRTOT_SEP_PRECIP_GPC & ITASRL & 0.0241 & $\mathrm{pCi} / \mathrm{g}$ & 0.00452 & 0.00649 & & & \\
\hline 60 & GAMMA_GS & ITASRL & 0.122 & $\mathrm{pCi} / \mathrm{g}$ & 0.154 & 0.155 & & & U \\
\hline 61 & PUISO_PLATE_AEA & ITASRL & 0.00668 & $\mathrm{pCi} / \mathrm{g}$ & 0.00155 & 0.00184 & & & \\
\hline 62 & GAMMA_GS & ITASRL & 2.32 & $\mathrm{pCi} / \mathrm{g}$ & 0.777 & 0.811 & & & \\
\hline 63 & GAMMA_GS & ITASRL & 0.243 & $\mathrm{pCi} / \mathrm{g}$ & 0.0221 & 0.0329 & & & \\
\hline 64 & SRTOT_SEP_PRECIP_GPC & ITASRL & 0.00504 & $\mathrm{pCi} / \mathrm{g}$ & 0.00323 & 0.00347 & & & \\
\hline 65 & GAMMA_GS & ITASRL & 0.0683 & $\mathrm{pCi} / \mathrm{g}$ & 0.133 & 0.133 & & & $\mathrm{U}$ \\
\hline 66 & PUISO_PLATE_AEA & ITASRL & 0.00204 & $\mathrm{pCi} / \mathrm{g}$ & 0.000636 & 0.000684 & & & \\
\hline 67 & GAMMA_GS & ITASRL & 2.09 & $\mathrm{pCi} / \mathrm{g}$ & 0.496 & 0.538 & & & \\
\hline 68 & GAMMA_GS & ITASRL & 0.593 & $\mathrm{pCi} / \mathrm{g}$ & 0.0326 & 0.0677 & & & \\
\hline 69 & SRTOT_SEP_PRECIP_GPC & ITASRL & 0.0163 & $\mathrm{pCi} / \mathrm{g}$ & 0.00838 & 0.009 & & & \\
\hline 70 & GAMMA_GS & ITASRL & 0.16 & $\mathrm{pCi} / \mathrm{g}$ & 0.145 & 0.146 & & & \\
\hline 71 & PUISO_PLATE_AEA & ITASRL & 0.00758 & $\mathrm{pCi} / \mathrm{g}$ & 0.00106 & 0.00138 & & & \\
\hline 72 & GAMMA_GS & ITASRL & 1.69 & $\mathrm{pCi} / \mathrm{g}$ & 0.639 & 0.661 & & & \\
\hline 73 & GAMMA_GS & ITASRL & 0.472 & $\mathrm{pCi} / \mathrm{g}$ & 0.0249 & 0.0533 & & & \\
\hline 74 & SRTOT_SEP_PRECIP_GPC & ITASRL & 0.00615 & $\mathrm{pCi} / \mathrm{g}$ & 0.00451 & 0.00474 & & & \\
\hline 75 & GAMMA_GS & ITASRL & & $\mathrm{pCi} / \mathrm{g}$ & 0.113 & 0.113 & & & $U$ \\
\hline 76 & PUISO_PLATE_AEA & ITASRL & 0.00239 & $\mathrm{pCi} / \mathrm{g}$ & 0.000657 & 0.000719 & & & \\
\hline
\end{tabular}




\begin{tabular}{|c|c|c|c|c|c|c|c|c|c|}
\hline & $\mathrm{K}$ & $\mathrm{L}$ & $\mathrm{M}$ & $\mathrm{N}$ & $\mathrm{O}$ & $P$ & $\mathrm{Q}$ & $\mathrm{R}$ & $S$ \\
\hline 1 & METHOD_NAME & LAB_CODE & VALUE_RPTD & ANAL_UNITS_RPTD & COUNTING_ERROR & \begin{tabular}{|l||} 
TOTAL_ANAL_ERROR \\
\end{tabular} & MIN_DETECTABLE_ACTIVITY & PCNT_MOISTURE & LAB_QUALIFIER \\
\hline $78 \mathrm{C}$ & GAMMA_GS & ITASRL & 0.46 & $\mathrm{pCi} / \mathrm{g}$ & 0.027 & 0.0533 & & & \\
\hline 79 & SRTOT_SEP_PRECIP_GPC & ITASRL & 0.0103 & $\mathrm{pCi} / \mathrm{g}$ & 0.00526 & 0.00572 & & & \\
\hline 80 & GAMMA_GS & ITASRL & 0.137 & $\mathrm{pCi} / \mathrm{g}$ & 0.138 & 0.139 & & & U \\
\hline 81 & PUISO_PLATE_AEA & ITASRL & 0.00492 & $\mathrm{pCi} / \mathrm{g}$ & 0.000799 & 0.00097 & & & \\
\hline 82 & GAMMA_GS & ITASRL & 1.87 & $\mathrm{pCi} / \mathrm{g}$ & 0.481 & 0.516 & & & \\
\hline 83 & GAMMA_GS & ITASRL & 0.324 & $\mathrm{pCi} / \mathrm{g}$ & 0.024 & 0.0403 & & & \\
\hline 84 & SRTOT_SEP_PRECIP_GPC & ITASRL & 0.00965 & $\mathrm{pCi} / \mathrm{g}$ & 0.00976 & 0.00996 & & & $U$ \\
\hline 85 & GAMMĀGS & ITASRL & 0.165 & $\mathrm{pCi} / \mathrm{g}$ & 0.0738 & 0.0756 & & & $U$ \\
\hline 87 & GAMMA_GS & ITASRL & 0.0237 & $\mathrm{pCi} / \mathrm{g}$ & 0.0129 & 0.0132 & & & \\
\hline 88 & SRTOT_SEP_PRECIP_GPC & ITASRL & 0.00267 & $\mathrm{pCi} / \mathrm{g}$ & 0.00314 & 0.00326 & & & U \\
\hline 89 & GAMMA_GS & ITASRL & 0.101 & $\mathrm{pCi} / \mathrm{g}$ & 0.0791 & 0.0797 & & & U \\
\hline 90 & GAMMA_GS & ITASRL & 1.25 & $\mathrm{pCi} / \mathrm{g}$ & 0.358 & 0.379 & & & \\
\hline 91 & GAMMA_GS & ITASRL & 0.15 & $\mathrm{pCi} / \mathrm{g}$ & 0.0207 & 0.0256 & & & \\
\hline $92 \leq$ & SRTOT_SEP_PRECIP_GPC & ITASRL & 0.00734 & $\mathrm{pCi} / \mathrm{g}$ & 0.0105 & 0.0108 & & & $\mathrm{U}$ \\
\hline 93 & GAMMA_GS & ITASRL & 0.0727 & $\mathrm{pCi} / \mathrm{g}$ & 0.0705 & 0.0709 & & & U \\
\hline 94 & GAMMA_GS & ITASRL & 1.22 & $\mathrm{pCi} / \mathrm{g}$ & 0.46 & 0.476 & & & \\
\hline 95 & GAMMA_GS & ITASRL & 0.169 & $\mathrm{pCi} / \mathrm{g}$ & 0.0164 & 0.0235 & & & \\
\hline 97 & GAMMA_GS & ITASRL & 0.0254 & $\mathrm{pCi} / \mathrm{g}$ & 0.0766 & 0.0766 & & & $U$ \\
\hline 98 & GAMMA_GS & ITASRL & 1.32 & $\mathrm{pCi} / \mathrm{g}$ & 0.335 & 0.36 & & & \\
\hline 99 & GAMMA_GS & ITASRL & 0.674 & $\mathrm{pCi} / \mathrm{g}$ & 0.038 & 0.0773 & & & \\
\hline 100 & SRTOT_SEP_PRECIP_GPC & ITASRL & 0.0143 & $\mathrm{pCi} / \mathrm{g}$ & 0.00379 & 0.00469 & & & \\
\hline 101 & GAMMA_GS & ITASRL & 0.168 & $\mathrm{pCi} / \mathrm{g}$ & 0.154 & 0.155 & & & \\
\hline $102 \mathrm{~F}$ & PUISO_PLATE_AEA & ITASRL & 0.0139 & $\mathrm{pCi} / \mathrm{g}$ & 0.00272 & 0.00364 & & & \\
\hline 103 & GAMMA_GS & ITASRL & 0.767 & $\mathrm{pCi} / \mathrm{g}$ & 0.843 & 0.846 & & & $\mathrm{U}$ \\
\hline 104 & GAMMA_GS & ITASRL & 0.411 & $\mathrm{pCi} / \mathrm{g}$ & 0.0301 & 0.0509 & & & \\
\hline 105 & SRTOT_SEP_PRECIP_GPC & ITASRL & 0.0115 & $\mathrm{pCi} / \mathrm{g}$ & 0.00348 & 0.0042 & & & \\
\hline 106 & GAMMA_GS & ITASRL & -0.0248 & $\mathrm{pCi} / \mathrm{g}$ & 0.137 & 0.137 & & & $U$ \\
\hline $107 \mathrm{~F}$ & PUISO_PLATE_AEA & ITASRL & 0.00779 & $\mathrm{pCi} / \mathrm{g}$ & 0.00187 & 0.00225 & & & \\
\hline 108 & GAMMA_GS & ITASRL & 1.54 & $\mathrm{pCi} / \mathrm{g}$ & 0.543 & 0.564 & & & \\
\hline 109 & GAMMA_GS & ITASRL & 0.17 & $\mathrm{pCi} / \mathrm{g}$ & 0.0164 & 0.0237 & & & \\
\hline 110 & SRTOT_SEP_PRECIP_GPC & ITASRL & 0.00533 & $\mathrm{pCi} / \mathrm{g}$ & 0.00248 & 0.00276 & & & \\
\hline 111 & GAMMA_GS & ITASRL & 0.0985 & $\mathrm{pCi} / \mathrm{g}$ & 0.114 & 0.114 & & & U \\
\hline $112 \mathrm{~F}$ & PUISO_PLATE_AEA & ITASRL & 0.00312 & $\mathrm{pCi} / \mathrm{g}$ & 0.000833 & 0.000926 & & & \\
\hline 113 & GAMMA_GS & ITASRL & 0.981 & $\mathrm{pCi} / \mathrm{g}$ & 0.389 & 0.402 & & & \\
\hline 114 & GAMMA_GS & ITASRL & 0.296 & $\mathrm{pCi} / \mathrm{g}$ & 0.0318 & 0.0434 & & & \\
\hline
\end{tabular}




\begin{tabular}{|c|c|c|c|c|c|c|c|c|c|}
\hline & $\mathrm{K}$ & $\mathrm{L}$ & $\mathrm{M}$ & $\mathrm{N}$ & $\mathrm{O}$ & $P$ & $\mathrm{Q}$ & $\mathrm{R}$ & $\mathrm{S}$ \\
\hline 1 & METHOD_NAME & LAB_CODE & VALUE_RPTD & ANAL_UNITS_RPTD & COUNTING_ERROR & TOTAL_ANAL_ERROR & MIN_DETECTABLE_ACTIVITY & PCNT_MOISTURE & LAB_QUALIFIER \\
\hline 116 & GAMMA_GS & ITASRL & -0.0231 & $\mathrm{pCi} / \mathrm{g}$ & 0.143 & 0.143 & & & $\mathrm{U}$ \\
\hline 117 & PUISO_PLATE_AEA & ITASRL & 0.00695 & $\mathrm{pCi} / \mathrm{g}$ & 0.00139 & 0.00169 & & & \\
\hline 118 & GAMMA_GS & ITASRL & 0.985 & $\mathrm{pCi} / \mathrm{g}$ & 0.522 & 0.531 & & & \\
\hline 119 & GAMMA_GS & ITASRL & 0.241 & $\mathrm{pCi} / \mathrm{g}$ & 0.0299 & 0.0384 & & & \\
\hline 120 & SRTOT_SEP_PRECIP_GPC & ITASRL & 0.00687 & $\mathrm{pCi} / \mathrm{g}$ & 0.00311 & 0.00344 & & & \\
\hline 121 & GAMMA_GS & ITASRL & 0.015 & $\mathrm{pCi} / \mathrm{g}$ & 0.0996 & 0.0996 & & & $U$ \\
\hline 122 & PUISO_PLATE_AEA & ITASRL & 0.00655 & $\mathrm{pCi} / \mathrm{g}$ & 0.00123 & 0.00149 & & & \\
\hline 123 & GAMMA_GS & ITASRL & 1.67 & $\mathrm{pCi} / \mathrm{g}$ & 0.331 & 0.371 & & & U \\
\hline 125 & SRTOT_SEP_PRECIP_GPC & ITASRL & 0.0238 & $\mathrm{pCi} / \mathrm{g}$ & 0.00522 & 0.00681 & & & \\
\hline 126 & GAMMA_GS & ITASRL & 0.206 & $\mathrm{pCi} / \mathrm{g}$ & 0.101 & 0.103 & & & U \\
\hline 127 & PUISO_PLATE_AEA & ITASRL & 0.007 & $\mathrm{pCi} / \mathrm{g}$ & 0.00118 & 0.00147 & & & \\
\hline 128 & GAMMA_GS & ITASRL & 1.94 & $\mathrm{pCi} / \mathrm{g}$ & 0.447 & 0.487 & & & \\
\hline 129 & GAMMA_GS & ITASRL & 0.533 & $\mathrm{pCi} / \mathrm{g}$ & 0.0262 & 0.0594 & & & \\
\hline 130 & SRTOT_SEP_PRECIP_GPC & ITASRL & 0.00497 & $\mathrm{pCi} / \mathrm{g}$ & 0.00323 & 0.00346 & & & \\
\hline 131 & GAMMA_GS & ITASRL & -0.00339 & $\mathrm{pCi} / \mathrm{g}$ & 0.0734 & 0.0734 & & & $U$ \\
\hline 132 & PUISO_PLATE_AEA & ITASRL & 0.00393 & $\mathrm{pCi} / \mathrm{g}$ & 0.000761 & 0.000887 & & & \\
\hline 133 & GAMMA_GS & ITASRL & 0.999 & $\mathrm{pCi} / \mathrm{g}$ & 0.351 & 0.365 & & & \\
\hline 135 & SRTOT_SEP_PRECIP_GPC & ITASRL & 0.00317 & $\mathrm{pCi} / \mathrm{g}$ & 0.0027 & 0.00287 & & & \\
\hline 136 & GAMMA_GS & ITASRL & 0.064 & $\mathrm{pCi} / \mathrm{g}$ & 0.0682 & 0.0685 & & & $U$ \\
\hline 137 & PUISO_PLATE_AEA & ITASRL & 0.00203 & $\mathrm{pCi} / \mathrm{g}$ & 0.000422 & 0.000473 & & & \\
\hline 138 & GAMMA_GS & ITASRL & 0.994 & $\mathrm{pCi} / \mathrm{g}$ & 0.318 & 0.333 & & & \\
\hline 139 & GAMMA_GS & ITASRL & 0.254 & $\mathrm{pCi} / \mathrm{g}$ & 0.0255 & 0.036 & & & \\
\hline 140 & SRTOT_SEP_PRECIP_GPC & ITASRL & 0.0105 & $\mathrm{pCi} / \mathrm{g}$ & 0.00467 & 0.00515 & & & \\
\hline 141 & GAMMA_GS & ITASRL & 0.04 & $\mathrm{pCi} / \mathrm{g}$ & 0.0769 & 0.077 & & & U \\
\hline 142 & PUISO_PLATE_AEA & ITASRL & 0.00368 & $\mathrm{pCi} / \mathrm{g}$ & 0.000715 & 0.000828 & & & \\
\hline 143 & GAMMA_GS & ITASRL & 1.39 & $\mathrm{pCi} / \mathrm{g}$ & 0.405 & 0.429 & & & \\
\hline 144 & GAMMA_GS & ITASRL & 0.221 & $\mathrm{pCi} / \mathrm{g}$ & 0.023 & 0.0319 & & & \\
\hline 145 & SRTOT_SEP_PRECIP_GPC & ITASRL & 0.00429 & $\mathrm{pCi} / \mathrm{g}$ & 0.00271 & 0.00292 & & & \\
\hline 146 & GAMMA_GS & ITASRL & 0.0534 & $\mathrm{pCi} / \mathrm{g}$ & 0.0743 & 0.0745 & & & $U$ \\
\hline 147 & PUISO_PLATE_AEA & ITASRL & 0.00337 & $\mathrm{pCi} / \mathrm{g}$ & 0.00063 & 0.000732 & & & \\
\hline 148 & GAMMA_GS & ITASRL & 0.831 & $\mathrm{pCi} / \mathrm{g}$ & 0.264 & 0.277 & & & U \\
\hline 149 & GAMMA_GS & ITASRL & 0.343 & $\mathrm{pCi} / \mathrm{g}$ & 0.0292 & 0.045 & & & \\
\hline 150 & SRTOT_SEP_PRECIP_GPC & ITASRL & 0.0241 & $\mathrm{pCi} / \mathrm{g}$ & 0.00568 & 0.00738 & & & \\
\hline 151 & GAMMA_GS & ITASRL & 0.112 & $\mathrm{pCi} / \mathrm{g}$ & 0.0806 & 0.0814 & & & $\mathrm{U}$ \\
\hline 152 & PUISO_PLATE_AEA & ITASRL & 0.00773 & $\mathrm{pCi} / \mathrm{g}$ & 0.0011 & 0.00143 & & & \\
\hline
\end{tabular}




\begin{tabular}{|c|c|c|c|c|c|c|c|c|c|}
\hline & $\mathrm{K}$ & $\mathrm{L}$ & $\mathrm{M}$ & $\mathrm{N}$ & $\mathrm{O}$ & $\mathrm{P}$ & $\mathrm{Q}$ & $\mathrm{R}$ & $\mathrm{S}$ \\
\hline 1 & METHOD_NAME & LAB_CODE & VALUE_RPTD & ANAL_UNITS_RPTD & |COUNTING_ERROR & TOTAL_ANAL_ERROR & MIN_DETECTABLE_ACTIVITY & PCNT_MOISTURE & LAB_QUALIFIER \\
\hline 154 & GAMMA_GS & ITASRL & 0.343 & $\mathrm{pCi} / \mathrm{g}$ & 0.0244 & 0.042 & & & \\
\hline 155 & SRTOT_SEP_PRECIP_GPC & ITASRL & 0.0111 & $\mathrm{pCi} / \mathrm{g}$ & 0.00445 & 0.00496 & & & \\
\hline 156 & GAMMA_GS & ITASRL & 0.02 & $\mathrm{pCi} / \mathrm{g}$ & 0.0694 & 0.0694 & & & U \\
\hline 157 & PUISO_PLATE_AEA & ITASRL & 0.00562 & $\mathrm{pCi} / \mathrm{g}$ & 0.000987 & 0.0012 & & & \\
\hline 158 & GAMMA_GS & ITASRL & 1.03 & $\mathrm{pCi} / \mathrm{g}$ & 0.352 & 0.367 & & & \\
\hline 159 & GAMMA_GS & ITASRL & 0.215 & $\mathrm{pCi} / \mathrm{g}$ & 0.0241 & 0.0323 & & & \\
\hline 160 & SRTOT_SEP_PRECIP_GPC & ITASRL & 0.00808 & $\mathrm{pCi} / \mathrm{g}$ & 0.00349 & 0.00392 & & & \\
\hline 161 & GAMMA_GS & ITASRL & 0.136 & $\mathrm{pCi} / \mathrm{g}$ & 0.0851 & 0.0862 & & & $U$ \\
\hline 163 & GAMMA_GS & ITASRL & 1.44 & $\mathrm{pCi} / \mathrm{g}$ & 0.475 & 0.496 & & & \\
\hline 164 & GAMMA_GS & ITASRL & 0.345 & $\mathrm{pCi} / \mathrm{g}$ & 0.0254 & 0.0428 & & & \\
\hline 165 & SRTOT_SEP_PRECIP_GPC & ITASRL & 0.00951 & $\mathrm{pCi} / \mathrm{g}$ & 0.00431 & 0.00479 & & & \\
\hline 166 & GAMMA_GS & ITASRL & 0.0997 & $\mathrm{pCi} / \mathrm{g}$ & 0.0903 & 0.0908 & & & $\mathrm{U}$ \\
\hline 167 & PUISO_PLATE_AEA & ITASRL & 0.0173 & $\mathrm{pCi} / \mathrm{g}$ & 0.00198 & 0.00299 & & & \\
\hline 168 & GAMMA_GS & ITASRL & 1.15 & $\mathrm{pCi} / \mathrm{g}$ & 0.459 & 0.473 & & & \\
\hline 169 & GAMMA_GS & ITASRL & 0.522 & $\mathrm{pCi} / \mathrm{g}$ & 0.0303 & 0.0604 & & & \\
\hline 170 & SRTOT_SEP_PRECIP_GPC & ITASRL & 0.0149 & $\mathrm{pCi} / \mathrm{g}$ & 0.00656 & 0.00722 & & & \\
\hline 171 & GAMMA_GS & ITASRL & 0.079 & $\mathrm{pCi} / \mathrm{g}$ & 0.096 & 0.0963 & & & U \\
\hline 173 & GAMMA_GS & ITASRL & 1.04 & $\mathrm{pCi} / \mathrm{g}$ & 0.446 & 0.458 & & & \\
\hline 174 & GAMMA_GS & ITASRL & 0.369 & $\mathrm{pCi} / \mathrm{g}$ & 0.0296 & 0.0473 & & & \\
\hline 175 & SRTOT_SEP_PRECIP_GPC & ITASRL & 0.0135 & $\mathrm{pCi} / \mathrm{g}$ & 0.00421 & 0.00546 & & & \\
\hline 176 & GAMMĀGS & ITASRL & 0.0911 & $\mathrm{pCi} / \mathrm{g}$ & 0.0195 & 0.0215 & & & \\
\hline 177 & SRTOT_SEP_PRECIP_GPC & ITASRL & 46.7 & $\mathrm{pCi} / \mathrm{g}$ & 0.433 & 8.53 & & & \\
\hline 178 & GAMMA_GS & ITASRL & 0.246 & $\mathrm{pCi} / \mathrm{g}$ & 0.0273 & 0.0368 & & & \\
\hline 179 & SRTOT_SEP_PRECIP_GPC & ITASRL & 0.0847 & $\mathrm{pCi} / \mathrm{g}$ & 0.00677 & 0.0197 & & & \\
\hline 180 & GAMMA_GS & ITASRL & 0.194 & $\mathrm{pCi} / \mathrm{g}$ & 0.0419 & 0.0462 & & & \\
\hline 181 & SRTOT_SEP_PRECIP_GPC & ITASRL & 0.00846 & $\mathrm{pCi} / \mathrm{g}$ & 0.00434 & 0.00476 & & & \\
\hline 182 & GAMMA_GS & ITASRL & 0.135 & $\mathrm{pCi} / \mathrm{g}$ & 0.0639 & 0.0653 & & & $U$ \\
\hline 183 & GAMMA_GS & ITASRL & 0.819 & $\mathrm{pCi} / \mathrm{g}$ & 0.231 & 0.245 & & & U \\
\hline 184 & GAMMA_GS & ITASRL & 0.115 & $\mathrm{pCi} / \mathrm{g}$ & 0.0387 & 0.0404 & & & \\
\hline 185 & SRTOT_SEP_PRECIP_GPC & ITASRL & 0.00869 & $\mathrm{pCi} / \mathrm{g}$ & 0.00653 & 0.00686 & & & \\
\hline 186 & GAMMA_GS & ITASRL & 0.0831 & $\mathrm{pCi} / \mathrm{g}$ & 0.112 & 0.112 & & & U \\
\hline 187 & GAMMA_GS & ITASRL & 0.972 & $\mathrm{pCi} / \mathrm{g}$ & 0.418 & 0.43 & & & $U$ \\
\hline 188 & GAMMA_GS & ITASRL & 0.079 & $\mathrm{pCi} / \mathrm{g}$ & 0.0307 & 0.0317 & & & \\
\hline 189 & SRTOT_SEP_PRECIP_GPC & ITASRL & 0.00137 & $\mathrm{pCi} / \mathrm{g}$ & 0.00316 & 0.00323 & & & U \\
\hline 190 & GAMMA_GS & ITASRL & 0.199 & $\mathrm{pCi} / \mathrm{g}$ & 0.0997 & 0.102 & & & $\mathrm{U}$ \\
\hline
\end{tabular}




\begin{tabular}{|c|c|c|c|c|c|c|c|c|c|}
\hline & $\mathrm{K}$ & $\mathrm{L}$ & $\mathrm{M}$ & $\mathrm{N}$ & $\mathrm{O}$ & $P$ & $\mathrm{Q}$ & $\mathrm{R}$ & $\mathrm{S}$ \\
\hline 1 & METHOD_NAME & LAB_CODE & VALUE_RPTD & ANAL_UNITS_RPTD & COUNTING_ERROR & TOTAL_ANAL_ERROR & MIN_DETECTABLE_ACTIVITY & PCNT_MOISTURE & LAB_QUALIFIER \\
\hline 192 & GAMMA_GS & ITASRL & 0.251 & $\mathrm{pCi} / \mathrm{g}$ & 0.0526 & 0.0583 & & & \\
\hline 193 & SRTOT_SEP_PRECIP_GPC & ITASRL & 0.00566 & $\mathrm{pCi} / \mathrm{g}$ & 0.0037 & 0.00392 & & & \\
\hline 194 & GAMMA_GS & ITASRL & 0.017 & $\mathrm{pCi} / \mathrm{g}$ & 0.114 & 0.114 & & & $U$ \\
\hline 195 & GAMMA_GS & ITASRL & 1.6 & $\mathrm{pCi} / \mathrm{g}$ & 0.534 & 0.557 & & & \\
\hline 196 & GAMMA_GS & ITASRL & 0.077 & $\mathrm{pCi} / \mathrm{g}$ & 0.0417 & 0.0424 & & & \\
\hline 197 & SRTOT_SEP_PRECIP_GPC & ITASRL & 0.00547 & $\mathrm{pCi} / \mathrm{g}$ & 0.00544 & 0.00559 & & & $\mathrm{U}$ \\
\hline 198 & GAMMA_GS & ITASRL & 0.194 & $\mathrm{pCi} / \mathrm{g}$ & 0.112 & 0.114 & & & 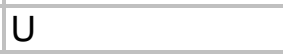 \\
\hline 199 & GAMMA_GS & ITASRL & 1.98 & $\mathrm{pCi} / \mathrm{g}$ & 0.541 & 0.576 & & & \\
\hline 201 & SRISO_SEP_PRECIP_GPC & ITASRL & 0.0192 & $\mathrm{pCi} / \mathrm{g}$ & 0.00868 & 0.00946 & & & \\
\hline 202 & UISO_IE_PLATE_AEA & ITASRL & 0.917 & $\mathrm{pCi} / \mathrm{g}$ & 0.056 & 0.108 & & & \\
\hline 203 & UISO_IE_PLATE_AEA & ITASRL & 0.0271 & $\mathrm{pCi} / \mathrm{g}$ & 0.0102 & 0.0106 & & & \\
\hline 204 & PUISO_PLATE_AEA & ITASRL & 0.00867 & $\mathrm{pCi} / \mathrm{g}$ & 0.0015 & 0.0019 & & & \\
\hline 205 & UISO_IE_PLATE_AEA & ITASRL & 0.665 & $\mathrm{pCi} / \mathrm{g}$ & 0.0476 & 0.082 & & & \\
\hline 206 & GAMMA_GS & ITASRL & 0.0679 & $\mathrm{pCi} / \mathrm{g}$ & 0.0167 & 0.0181 & & & \\
\hline 207 & SRISO_SEP_PRECIP_GPC & ITASRL & 0.00608 & $\mathrm{pCi} / \mathrm{g}$ & 0.0034 & 0.00367 & & & \\
\hline 208 & UISO_IE_PLATE_AEA & ITASRL & 0.467 & $\mathrm{pCi} / \mathrm{g}$ & 0.0441 & 0.0659 & & & \\
\hline 209 & UISO_IE_PLATE_AEA & ITASRL & 0.0142 & $\mathrm{pCi} / \mathrm{g}$ & 0.00814 & 0.00833 & & & \\
\hline 211 & UISO_IE_PLATE_AEA & ITASRL & 0.381 & $\mathrm{pCi} / \mathrm{g}$ & 0.0395 & 0.056 & & & \\
\hline 212 & GAMMA_GS & ITASRL & 0.226 & $\mathrm{pCi} / \mathrm{g}$ & 0.0271 & 0.0353 & & & \\
\hline 213 & SRISO_SEP_PRECIP_GPC & ITASRL & 0.018 & $\mathrm{pCi} / \mathrm{g}$ & 0.00487 & 0.00598 & & & \\
\hline 214 & UISO_IE_PLATTE_AEA & ITASRL & 1.1 & $\mathrm{pCi} / \mathrm{g}$ & 0.0632 & 0.127 & & & \\
\hline 215 & UISO_IE_PLATE_AEA & ITASRL & 0.0182 & $\mathrm{pCi} / \mathrm{g}$ & 0.00846 & 0.00871 & & & \\
\hline 216 & PUISO_PLATE_AEA & ITASRL & 0.00853 & $\mathrm{pCi} / \mathrm{g}$ & 0.00108 & 0.00137 & & & \\
\hline 217 & UISO_IE_PLATE_AEA & ITASRL & 0.732 & $\mathrm{pCi} / \mathrm{g}$ & 0.0515 & 0.0899 & & & \\
\hline 218 & GAMMA_GS & ITASRL & 0.126 & $\mathrm{pCi} / \mathrm{g}$ & 0.0166 & 0.0209 & & & \\
\hline 219 & SRISO_SEP_PRECIP_GPC & ITASRL & 0.00365 & $\mathrm{pCi} / \mathrm{g}$ & 0.00341 & 0.00355 & & & \\
\hline 220 & UISO_IE_PLATE_AEA & ITASRL & 0.277 & $\mathrm{pCi} / \mathrm{g}$ & 0.0341 & 0.045 & & & \\
\hline 221 & UISO_IE_PLATE_AEA & ITASRL & 0.00901 & $\mathrm{pCi} / \mathrm{g}$ & 0.0067 & 0.00682 & & & \\
\hline 222 & PUISO_PLATE_AEA & ITASRL & 0.00135 & $\mathrm{pCi} / \mathrm{g}$ & 0.000362 & 0.00039 & & & \\
\hline 223 & UISO_IE_PLATE_AEA & ITASRL & 0.273 & $\mathrm{pCi} / \mathrm{g}$ & 0.0334 & 0.0439 & & & \\
\hline 224 & GAMMA_GS & ITASRL & 0.244 & $\mathrm{pCi} / \mathrm{g}$ & 0.031 & 0.0394 & & & \\
\hline 225 & SRISO_SEP_PRECIP_GPC & ITASRL & 0.0206 & $\mathrm{pCi} / \mathrm{g}$ & 0.00614 & 0.0073 & & & \\
\hline 226 & UISO_IE_PLATE_AEA & ITASRL & 0.641 & $\mathrm{pCi} / \mathrm{g}$ & 0.0464 & 0.0794 & & & \\
\hline 227 & UISO_IE_PLATE_AEA & ITASRL & 0.0303 & $\mathrm{pCi} / \mathrm{g}$ & 0.0104 & 0.0108 & & & \\
\hline 228 & PUISO_PLATE_AEA & ITASRL & 0.00623 & $\mathrm{pCi} / \mathrm{g}$ & 0.00105 & 0.00129 & & & \\
\hline
\end{tabular}




\begin{tabular}{|c|c|c|c|c|c|c|c|c|c|}
\hline & $\mathrm{K}$ & $\mathrm{L}$ & $\mathrm{M}$ & $\mathrm{N}$ & $\mathrm{O}$ & $P$ & $\mathrm{Q}$ & $\mathrm{R}$ & $\mathrm{S}$ \\
\hline 1 & METHOD_NAME & LAB_CODE & VALUE_RPTD & ANAL_UNITS_RPTD & COUNTING_ERROR & TOTAL_ANAL_ERROR & MIN_DETECTABLE_ACTIVITY & PCNT_MOISTURE & LAB_QUALIFIER \\
\hline 230 & GAMMA_GS & ITASRL & 0.376 & $\mathrm{pCi} / \mathrm{g}$ & 0.0367 & 0.0526 & & & \\
\hline 231 & SRISO_SEP_PRECIP_GPC & ITASRL & 0.0197 & $\mathrm{pCi} / \mathrm{g}$ & 0.00626 & 0.00733 & & & \\
\hline 232 & UISO_IE_PLATE_AEA & ITASRL & 0.861 & $\mathrm{pCi} / \mathrm{g}$ & 0.0582 & 0.106 & & & \\
\hline 233 & UISO_IE_PLATE_AEA & ITASRL & 0.0271 & $\mathrm{pCi} / \mathrm{g}$ & 0.0107 & 0.0111 & & & \\
\hline 234 & PUISO_PLATE_AEA & ITASRL & 0.00762 & $\mathrm{pCi} / \mathrm{g}$ & 0.00104 & 0.00136 & & & \\
\hline 235 & UISO_IE_PLATE_AEA & ITASRL & 0.673 & $\mathrm{pCi} / \mathrm{g}$ & 0.0512 & 0.0862 & & & \\
\hline 236 & GAMMA_GS & ITASRL & 0.433 & $\mathrm{pCi} / \mathrm{g}$ & 0.0359 & 0.0562 & & & \\
\hline 237 & SRISO_SEP_PRECIP_GPC & ITASRL & 0.0199 & $\mathrm{pCi} / \mathrm{g}$ & 0.00545 & 0.00659 & & & \\
\hline 239 & UISO_IE_PLATE_AEA & ITASRL & 0.0292 & $\mathrm{pCi} / \mathrm{g}$ & 0.0112 & 0.0117 & & & \\
\hline 240 & PUISO_PLATE_AEA & ITASRL & 0.00921 & $\mathrm{pCi} / \mathrm{g}$ & 0.00099 & 0.0014 & & & \\
\hline 241 & UISO_IE_PLATE_AEA & ITASRL & 0.621 & $\mathrm{pCi} / \mathrm{g}$ & 0.0497 & 0.081 & & & \\
\hline 242 & GAMMA_GS & ITASRL & 0.811 & $\mathrm{pCi} / \mathrm{g}$ & 0.0381 & 0.0897 & & & \\
\hline 243 & SRISO_SEP_PRECIP_GPC & ITASRL & 0.0317 & $\mathrm{pCi} / \mathrm{g}$ & 0.00613 & 0.00841 & & & \\
\hline 244 & UISO_IE_PLATE_AEA & ITASRL & 0.485 & $\mathrm{pCi} / \mathrm{g}$ & 0.0421 & 0.0649 & & & \\
\hline 245 & UISO_IE_PLATE_AEA & ITASRL & 0.0128 & $\mathrm{pCi} / \mathrm{g}$ & 0.00718 & 0.00735 & & & \\
\hline 246 & PUISO_PLATE_AEA & ITASRL & 0.0127 & $\mathrm{pCi} / \mathrm{g}$ & 0.00128 & 0.00192 & & & \\
\hline 247 & UISO_IE_PLATE_AEA & ITASRL & 0.408 & $\mathrm{pCi} / \mathrm{g}$ & 0.0385 & 0.0565 & & & \\
\hline 249 & SRISO_SEP_PRECIP_GPC & ITASRL & 0.0167 & $\mathrm{pCi} / \mathrm{g}$ & 0.00437 & 0.00547 & & & \\
\hline 250 & UISO_IE_PLATE_AEA & ITASRL & 0.503 & $\mathrm{pCi} / \mathrm{g}$ & 0.0444 & 0.0683 & & & \\
\hline 251 & UISO_IE_PLATE_AEA & ITASRL & 0.0152 & $\mathrm{pCi} / \mathrm{g}$ & 0.00852 & 0.00871 & & & \\
\hline 252 & PUISO_PLATE_AEA & ITASRL & 0.0064 & $\mathrm{pCi} / \mathrm{g}$ & 0.00116 & 0.00142 & & & \\
\hline 253 & UISO_IE_PLATE_AEA & ITASRL & 0.419 & $\mathrm{pCi} / \mathrm{g}$ & 0.0406 & 0.0591 & & & \\
\hline 254 & GAMMA_GS & ITASRL & 0.304 & $\mathrm{pCi} / \mathrm{g}$ & 0.0288 & 0.0419 & & & \\
\hline 255 & SRISO_SEP_PRECIP_GPC & ITASRL & 0.00752 & $\mathrm{pCi} / \mathrm{g}$ & 0.00456 & 0.00485 & & & \\
\hline 256 & UISO_IE_PLATE_AEA & ITASRL & 0.312 & $\mathrm{pCi} / \mathrm{g}$ & 0.034 & 0.0468 & & & \\
\hline 257 & UISO_IE_PLATE_AEA & ITASRL & 0.00706 & $\mathrm{pCi} / \mathrm{g}$ & 0.00574 & 0.00583 & & & \\
\hline 258 & PUISO_PLATE_AEA & ITASRL & 0.0132 & $\mathrm{pCi} / \mathrm{g}$ & 0.00237 & 0.00318 & & & \\
\hline 259 & UISO_IE_PLATE_AEA & ITASRL & 0.274 & $\mathrm{pCi} / \mathrm{g}$ & 0.0317 & 0.0423 & & & \\
\hline 260 & GAMMA_GS & ITASRL & 0.237 & $\mathrm{pCi} / \mathrm{g}$ & 0.0232 & 0.0331 & & & \\
\hline 261 & SRISO_SEP_PRECIP_GPC & ITASRL & 0.00672 & $\mathrm{pCi} / \mathrm{g}$ & 0.00406 & 0.00434 & & & \\
\hline 262 & UISO_IE_PLATE_AEA & ITASRL & 0.339 & $\mathrm{pCi} / \mathrm{g}$ & 0.0356 & 0.0498 & & & \\
\hline 263 & UISO_IE_PLATE_AEA & ITASRL & 0.0108 & $\mathrm{pCi} / \mathrm{g}$ & 0.00686 & 0.007 & & & \\
\hline 264 & PUISO_PLATE_AEA & ITASRL & 0.00508 & $\mathrm{pCi} / \mathrm{g}$ & 0.000815 & 0.000996 & & & \\
\hline 265 & UISO_IE_PLATE_AEA & ITASRL & 0.292 & $\mathrm{pCi} / \mathrm{g}$ & 0.0329 & 0.0444 & & & \\
\hline 266 & GAMMA_GS & ITASRL & 0.34 & $\mathrm{pCi} / \mathrm{g}$ & 0.0339 & 0.048 & & & \\
\hline
\end{tabular}




\begin{tabular}{|c|c|c|c|c|c|c|c|c|c|}
\hline & $\mathrm{K}$ & $\mathrm{L}$ & $\mathrm{M}$ & $\mathrm{N}$ & $\mathrm{O}$ & $P$ & $\mathrm{Q}$ & $\mathrm{R}$ & $\mathrm{S}$ \\
\hline 1 & METHOD_NAME & LAB_CODE & VALUE_RPTD & ANAL_UNITS_RPTD & COUNTING_ERROR & TOTAL_ANAL_ERROR & MIN_DETECTABLE_ACTIVITY & PCNT_MOISTURE & LAB_QUALIFIER \\
\hline 268 & UISO_IE_PLATE_AEA & ITASRL & 0.51 & $\mathrm{pCi} / \mathrm{g}$ & 0.0449 & 0.0695 & & & \\
\hline 269 & UISO_IE_PLATE_AEA & ITASRL & 0.0278 & $\mathrm{pCi} / \mathrm{g}$ & 0.0111 & 0.0115 & & & \\
\hline 270 & PUISO_PLATE_AEA & ITASRL & 0.00745 & $\mathrm{pCi} / \mathrm{g}$ & 0.000958 & 0.00127 & & & \\
\hline 271 & UISO_IE_PLATE_AEA & ITASRL & 0.469 & $\mathrm{pCi} / \mathrm{g}$ & 0.0429 & 0.0647 & & & \\
\hline 272 & GAMMA_GS & ITASRL & 0.357 & $\mathrm{pCi} / \mathrm{g}$ & 0.0212 & 0.0415 & & & \\
\hline 273 & SRISO_SEP_PRECIP_GPC & ITASRL & 0.0052 & $\mathrm{pCi} / \mathrm{g}$ & 0.00346 & 0.0037 & & & \\
\hline 274 & UISO_IE_PLATE_AEA & ITASRL & 0.108 & $\mathrm{pCi} / \mathrm{g}$ & 0.0207 & 0.0239 & & & \\
\hline 275 & UISO_IE_PLATE_AEA & ITASRL & 0.00222 & $\mathrm{pCi} / \mathrm{g}$ & 0.00517 & 0.00522 & & & U \\
\hline 277 & UISO_IE_PLATE_AEA & ITASRL & 0.101 & $\mathrm{pCi} / \mathrm{g}$ & 0.0198 & 0.0225 & & & \\
\hline 278 & GAMMA_GS & ITASRL & 0.521 & $\mathrm{pCi} / \mathrm{g}$ & 0.0389 & 0.065 & & & \\
\hline 279 & SRISO_SEP_PRECIP_GPC & ITASRL & 0.0191 & $\mathrm{pCi} / \mathrm{g}$ & 0.00635 & 0.0074 & & & \\
\hline 280 & ]UISO_IE_PLATE_AEA & ITASRL & 0.814 & $\mathrm{pCi} / \mathrm{g}$ & 0.0539 & 0.0987 & & & \\
\hline 281 & UISO_IE_PLATE_AEA & ITASRL & 0.0171 & $\mathrm{pCi} / \mathrm{g}$ & 0.00831 & 0.00854 & & & \\
\hline 282 & PUISO_PLATE_AEA & ITASRL & 0.0114 & $\mathrm{pCi} / \mathrm{g}$ & 0.00113 & 0.00168 & & & \\
\hline 283 & UISO_IE_PLATE_AEA & ITASRL & 0.704 & $\mathrm{pCi} / \mathrm{g}$ & 0.0501 & 0.0871 & & & \\
\hline 284 & GAMMA_GS & ITASRL & 0.33 & $\mathrm{pCi} / \mathrm{g}$ & 0.033 & 0.0467 & & & \\
\hline 285 & SRISO_SEP_PRECIP_GPC & ITASRL & 0.0171 & $\mathrm{pCi} / \mathrm{g}$ & 0.00519 & 0.00623 & & & \\
\hline 287 & UISO_IE_PLATE_AEA & ITASRL & 0.0216 & $\mathrm{pCi} / \mathrm{g}$ & 0.00929 & 0.0096 & & & \\
\hline 288 & PUISO_PLATE_AEA & ITASRL & 0.00857 & $\mathrm{pCi} / \mathrm{g}$ & 0.0011 & 0.00148 & & & \\
\hline 289 & UISO_IE_PLATE_AEA & ITASRL & 0.565 & $\mathrm{pCi} / \mathrm{g}$ & 0.046 & 0.0737 & & & \\
\hline 290 & GAMMA_GS & ITASRL & 0.271 & $\mathrm{pCi} / \mathrm{g}$ & 0.0326 & 0.0424 & & & \\
\hline 291 & SRISO_SEP_PRECIP_GPC & ITASRL & 0.0154 & $\mathrm{pCi} / \mathrm{g}$ & 0.00507 & 0.00595 & & & \\
\hline 292 & UISO_IE_PLATE_AEA & ITASRL & 0.817 & $\mathrm{pCi} / \mathrm{g}$ & 0.0542 & 0.0991 & & & \\
\hline 293 & UISO_IE_PLATE_AEA & ITASRL & 0.0274 & $\mathrm{pCi} / \mathrm{g}$ & 0.0106 & 0.011 & & & \\
\hline 294 & PUISO_PLATE_AEA & ITASRL & 0.0069 & $\mathrm{pCi} / \mathrm{g}$ & 0.000943 & 0.00122 & & & \\
\hline 295 & UISO_IE_PLATE_AEA & ITASRL & 0.639 & $\mathrm{pCi} / \mathrm{g}$ & 0.0479 & 0.0805 & & & \\
\hline 296 & GAMMA_GS & ITASRL & 0.35 & $\mathrm{pCi} / \mathrm{g}$ & 0.0281 & 0.0449 & & & \\
\hline 297 & SRISO_SEP_PRECIP_GPC & ITASRL & 0.0394 & $\mathrm{pCi} / \mathrm{g}$ & 0.00688 & 0.01 & & & \\
\hline 298 & UISO_IE_PLATE_AEA & ITASRL & 0.704 & $\mathrm{pCi} / \mathrm{g}$ & 0.0528 & 0.0894 & & & \\
\hline 299 & UISO_IE_PLATE_AEA & ITASRL & 0.03 & $\mathrm{pCi} / \mathrm{g}$ & 0.0115 & 0.0119 & & & \\
\hline 300 & PUISO_PLATE_AEA & ITASRL & 0.00947 & $\mathrm{pCi} / \mathrm{g}$ & 0.00102 & 0.00144 & & & \\
\hline 301 & UISO_IE_PLATE_AEA & ITASRL & 0.62 & $\mathrm{pCi} / \mathrm{g}$ & 0.0495 & 0.0803 & & & \\
\hline 302 & GAMMA-GS - & ITASRL & 0.0858 & $\mathrm{pCi} / \mathrm{g}$ & 0.0155 & 0.0177 & & & \\
\hline 303 & SRISO_SEP_PRECIP_GPC & ITASRL & 0.0041 & $\mathrm{pCi} / \mathrm{g}$ & 0.00401 & 0.00415 & & & U \\
\hline 304 & UISO_IE_PLATE_AEA & ITASRL & 0.235 & $\mathrm{pCi} / \mathrm{g}$ & 0.0306 & 0.0394 & & & \\
\hline
\end{tabular}




\begin{tabular}{|c|c|c|c|c|c|c|c|c|c|}
\hline & $\mathrm{K}$ & $\mathrm{L}$ & $\mathrm{M}$ & $\mathrm{N}$ & $\mathrm{O}$ & $P$ & $\mathrm{Q}$ & $\mathrm{R}$ & $\mathrm{S}$ \\
\hline 1 & METHOD_NAME & LAB_CODE & VALUE_RPTD & ANAL_UNITS_RPTD & COUNTING_ERROR & TOTAL_ANAL_ERROR & MIN_DETECTABLE_ACTIVITY & PCNT_MOISTURE & LAB_QUALIFIER \\
\hline 306 & PUISO_PLATE_AEA & ITASRL & 0.0014 & $\mathrm{pCi} / \mathrm{g}$ & 0.000578 & 0.000607 & & & \\
\hline 307 & UISO_IE_PLATE_AEA & ITASRL & 0.237 & $\mathrm{pCi} / \mathrm{g}$ & 0.0302 & 0.0389 & & & \\
\hline 308 & GAMMA_GS & ITASRL & 0.599 & $\mathrm{pCi} / \mathrm{g}$ & 0.031 & 0.0674 & & & \\
\hline 309 & SRISO_SEP_PRECIP_GPC & ITASRL & 0.00822 & $\mathrm{pCi} / \mathrm{g}$ & 0.00462 & 0.00493 & & & \\
\hline 310 & UISO_IE_PLATE_AEA & ITASRL & 0.301 & $\mathrm{pCi} / \mathrm{g}$ & 0.0341 & 0.0463 & & & \\
\hline 311 & UISO_IE_PLATE_AEA & ITASRL & 0.00874 & $\mathrm{pCi} / \mathrm{g}$ & 0.00623 & 0.00634 & & & \\
\hline 312 & PUISO_PLATE_AEA & ITASRL & 0.00499 & $\mathrm{pCi} / \mathrm{g}$ & 0.000974 & 0.00115 & & & \\
\hline 313 & UISO_IE_PLATE_AEA & ITASRL & 0.26 & $\mathrm{pCi} / \mathrm{g}$ & 0.0315 & 0.0414 & & & \\
\hline 315 & SRISO_SEP_PRECIP_GPC & QTESRL & 0.00995 & $\mathrm{pCi} / \mathrm{g}$ & 0.0032 & 0.0044 & 0.005 & 55.5 & \\
\hline 316 & UISO_IE_PLATE_AEA & QTESRL & 0.37 & $\mathrm{pCi} / \mathrm{g}$ & 0.037 & 0.068 & 0.011 & 55.5 & \\
\hline 317 & UISO_IE_PLATE_AEA & QTESRL & 0.0146 & $\mathrm{pCi} / \mathrm{g}$ & 0.0078 & 0.0089 & 0.0101 & 55.5 & \\
\hline 318 & PUISO_IE_PLATE_AEA & QTESRL & 0.00871 & $\mathrm{pCi} / \mathrm{g}$ & 0.001 & 0.0017 & 0.000226 & 55.5 & \\
\hline 319 & UISO_IE_PLATE_AEA & QTESRL & 0.327 & $\mathrm{pCi} / \mathrm{g}$ & 0.036 & 0.062 & 0.0222 & 55.5 & \\
\hline 320 & GAMMA_GS & QTESRL & 0.597 & $\mathrm{pCi} / \mathrm{g}$ & 0.11 & 0.11 & 0.0695 & 51.3 & \\
\hline 321 & SRISO_SEP_PRECIP_GPC & QTESRL & 0.0149 & $\mathrm{pCi} / \mathrm{g}$ & 0.0041 & 0.0058 & 0.00597 & 51.3 & \\
\hline 322 & UISO_IE_PLATE_AEA & QTESRL & 0.487 & $\mathrm{pCi} / \mathrm{g}$ & 0.046 & 0.088 & 0.00853 & 51.3 & \\
\hline 323 & UISO_IE_PLATE_AEA & QTESRL & 0.0135 & $\mathrm{pCi} / \mathrm{g}$ & 0.0077 & 0.0089 & 0.00853 & 51.3 & \\
\hline 325 & UISO_IE_PLATE_AEA & QTESRL & 0.436 & $\mathrm{pCi} / \mathrm{g}$ & 0.044 & 0.08 & 0.0108 & 51.3 & \\
\hline 326 & GAMMA_GS & QTESRL & 0.324 & $\mathrm{pCi} / \mathrm{g}$ & 0.045 & 0.045 & 0.0228 & 72.3 & \\
\hline 327 & SRISO_SEP_PRECIP_GPC & QTESRL & 0.00233 & $\mathrm{pCi} / \mathrm{g}$ & 0.002 & 0.0033 & 0.00563 & 72.3 & \\
\hline 328 & UISO_IE_PLATE_AEA & QTESRL & 0.152 & $\mathrm{pCi} / \mathrm{g}$ & 0.021 & 0.033 & 0.00998 & 72.3 & \\
\hline 329 & UISO_IE_PLATE_AEA & QTESRL & 0.00646 & $\mathrm{pCi} / \mathrm{g}$ & 0.0049 & 0.0062 & 0.00892 & 72.3 & $U$ \\
\hline 330 & PUISO_IE_PLATE_AEA & QTESRL & 0.00239 & $\mathrm{pCi} / \mathrm{g}$ & 0.0005 & 0.00063 & 0.0000712 & 72.3 & \\
\hline 331 & UISO_IE_PLATE_AEA & QTESRL & 0.152 & $\mathrm{pCi} / \mathrm{g}$ & 0.023 & 0.033 & 0.0196 & 72.3 & \\
\hline 332 & GAMMA_GS & QTESRL & 0.225 & $\mathrm{pCi} / \mathrm{g}$ & 0.034 & 0.034 & 0.0252 & 62.3 & \\
\hline 333 & SRISO_SEP_PRECIP_GPC & QTESRL & 0.00172 & $\mathrm{pCi} / \mathrm{g}$ & 0.0036 & 0.0061 & 0.0117 & 62.3 & $U$ \\
\hline 334 & UISO_IE_PLATE_AEA & QTESRL & 0.161 & $\mathrm{pCi} / \mathrm{g}$ & 0.022 & 0.034 & 0.0114 & 62.3 & \\
\hline 335 & UISO_IE_PLATE_AEA & QTESRL & 0.00576 & $\mathrm{pCi} / \mathrm{g}$ & 0.0049 & 0.0063 & 0.01 & 62.3 & $U$ \\
\hline 336 & PUISO_IE_PLATE_AEA & QTESRL & 0.00182 & $\mathrm{pCi} / \mathrm{g}$ & 0.00065 & 0.00072 & 0.000157 & 62.3 & \\
\hline 337 & UISO_IE_PLATE_AEA & QTESRL & 0.151 & $\mathrm{pCi} / \mathrm{g}$ & 0.024 & 0.034 & 0.0251 & 62.3 & \\
\hline 338 & GAMMA_GS & QTESRL & 0.27 & $\mathrm{pCi} / \mathrm{g}$ & 0.044 & 0.044 & 0.0315 & 51.6 & \\
\hline 339 & SRISO_SEP_PRECIP_GPC & QTESRL & 0.0236 & $\mathrm{pCi} / \mathrm{g}$ & 0.0055 & 0.008 & 0.00716 & 51.6 & \\
\hline 340 & UISO_IE_PLATE_AEA & QTESRL & 0.842 & $\mathrm{pCi} / \mathrm{g}$ & 0.058 & 0.14 & 0.0107 & 51.6 & \\
\hline 341 & UISO_IE_PLATE_AEA & QTESRL & 0.0313 & $\mathrm{pCi} / \mathrm{g}$ & 0.012 & 0.013 & 0.0107 & 51.6 & \\
\hline 342 & PUISO_IE_PLATE_AEA & QTESRL & 0.00742 & $\mathrm{pCi} / \mathrm{g}$ & 0.00076 & 0.0014 & 0.0000527 & 51.6 & \\
\hline
\end{tabular}




\begin{tabular}{|c|c|c|c|c|c|c|c|c|c|}
\hline & $\mathrm{K}$ & $\mathrm{L}$ & $\mathrm{M}$ & $\mathrm{N}$ & $\mathrm{O}$ & $P$ & $\mathrm{Q}$ & $\mathrm{R}$ & $\mathrm{S}$ \\
\hline 1 & METHOD_NAME & LAB_CODE & VALUE_RPTD & ANAL_UNITS_RPTD & COUNTING_ERROR & TOTAL_ANAL_ERROR & MIN_DETECTABLE_ACTIVITY & PCNT_MOISTURE & LAB_QUALIFIER \\
\hline 344 & GAMMA_GS & QTESRL & 0.0307 & $\mathrm{pCi} / \mathrm{g}$ & 0.015 & 0.015 & 0.0202 & 68.9 & \\
\hline 345 & SRISO_SEP_PRECIP_GPC & QTESRL & 0.00843 & $\mathrm{pCi} / \mathrm{g}$ & 0.0033 & 0.0045 & 0.00604 & 68.9 & \\
\hline 346 & UISO_IE_PLATE_AEA & QTESRL & 0.303 & $\mathrm{pCi} / \mathrm{g}$ & 0.036 & 0.059 & 0.00988 & 68.9 & \\
\hline 347 & UISO_IE_PLATE_AEA & QTESRL & 0.0146 & $\mathrm{pCi} / \mathrm{g}$ & 0.0084 & 0.0096 & 0.0112 & 68.9 & \\
\hline 348 & PUISO_IE_PLATE_AEA & QTESRL & 0.000766 & $\mathrm{pCi} / \mathrm{g}$ & 0.00044 & 0.00047 & 0.000447 & 68.9 & \\
\hline 349 & UISO_IE_PLATE_AEA & QTESRL & 0.233 & $\mathrm{pCi} / \mathrm{g}$ & 0.033 & 0.049 & 0.0219 & 68.9 & \\
\hline 350 & GAMMA_GS & QTESRL & 0.281 & $\mathrm{pCi} / \mathrm{g}$ & 0.039 & 0.039 & 0.0251 & 51.5 & \\
\hline 351 & SRISO_SEP_PRECIP_GPC & QTESRL & 0.0202 & $\mathrm{pCi} / \mathrm{g}$ & 0.0049 & 0.0071 & 0.00688 & 51.5 & \\
\hline 353 & UISO_IE_PLATE_AEA & QTESRL & 0.0555 & $\mathrm{pCi} / \mathrm{g}$ & 0.016 & 0.019 & 0.0126 & 51.5 & \\
\hline 354 & PUISO_IE_PLATE_AEA & QTESRL & 0.00949 & $\mathrm{pCi} / \mathrm{g}$ & 0.00091 & 0.0017 & 0.0000593 & 51.5 & \\
\hline 355 & UISO_IE_PLATE_AEA & QTESRL & 0.636 & $\mathrm{pCi} / \mathrm{g}$ & 0.054 & 0.11 & 0.00985 & 51.5 & \\
\hline 356 & GAMMA_GS & QTESRL & 0.161 & $\mathrm{pCi} / \mathrm{g}$ & 0.033 & 0.033 & 0.0303 & 57.2 & \\
\hline 357 & SRISO_SEP_PRECIP_GPC & QTESRL & 0.0043 & $\mathrm{pCi} / \mathrm{g}$ & 0.004 & 0.0056 & 0.00984 & 57.2 & $\mathrm{U}$ \\
\hline 358 & UISO_IE_PLATE_AEA & QTESRL & 0.213 & $\mathrm{pCi} / \mathrm{g}$ & 0.026 & 0.042 & 0.00864 & 57.2 & \\
\hline 359 & UISO_IE_PLATE_AEA & QTESRL & 0.0087 & $\mathrm{pCi} / \mathrm{g}$ & 0.0055 & 0.0067 & 0.00822 & 57.2 & \\
\hline 360 & PUISO_IE_PLATE_AEA & QTESRL & 0.00298 & $\mathrm{pCi} / \mathrm{g}$ & 0.00054 & 0.00071 & 0.0000668 & 57.2 & \\
\hline 361 & UISO_IE_PLATE_AEA & QTESRL & 0.199 & $\mathrm{pCi} / \mathrm{g}$ & 0.025 & 0.04 & 0.00611 & 57.2 & \\
\hline 363 & SRISO_SEP_PRECIP_GPC & QTESRL & 0.00634 & $\mathrm{pCi} / \mathrm{g}$ & 0.0029 & 0.0041 & 0.00589 & 71.1 & \\
\hline 364 & UISO_IE_PLATE_AEA & QTESRL & 0.178 & $\mathrm{pCi} / \mathrm{g}$ & 0.029 & 0.041 & 0.0123 & 71.1 & \\
\hline 365 & UISO_IE_PLATE_AEA & QTESRL & 0.00493 & $\mathrm{pCi} / \mathrm{g}$ & 0.0044 & 0.0059 & 0.0066 & 71.1 & $U$ \\
\hline 366 & PUISO_IE_PLATE_AEA & QTESRL & 0.00213 & $\mathrm{pCi} / \mathrm{g}$ & 0.00045 & 0.00056 & 0.0000663 & 71.1 & \\
\hline 367 & UISO_IE_PLATE_AEA & QTESRL & 0.192 & $\mathrm{pCi} / \mathrm{g}$ & 0.03 & 0.042 & 0.00911 & 71.1 & \\
\hline 368 & GAMMA_GS & QTESRL & 0.29 & $\mathrm{pCi} / \mathrm{g}$ & 0.042 & 0.042 & 0.0322 & 44.5 & \\
\hline 369 & SRISO_SEP_PRECIP_GPC & QTESRL & 0.0186 & $\mathrm{pCi} / \mathrm{g}$ & 0.0064 & 0.0082 & 0.00972 & 44.5 & \\
\hline 370 & UISO_IE_PLATE_AEA & QTESRL & 0.725 & $\mathrm{pCi} / \mathrm{g}$ & 0.074 & 0.14 & 0.0188 & 44.5 & \\
\hline 371 & UISO_IE_PLATE_AEA & QTESRL & 0.0248 & $\mathrm{pCi} / \mathrm{g}$ & 0.015 & 0.016 & 0.0176 & 44.5 & \\
\hline 372 & PUISO_IE_PLATE_AEA & QTESRL & 0.00736 & $\mathrm{pCi} / \mathrm{g}$ & 0.00093 & 0.0015 & 0.000168 & 44.5 & \\
\hline 373 & UISO_IE_PLATE_AEA & QTESRL & 0.534 & $\mathrm{pCi} / \mathrm{g}$ & 0.064 & 0.11 & 0.0148 & 44.5 & \\
\hline 374 & GAMMA_GS & QTESRL & 0.431 & $\mathrm{pCi} / \mathrm{g}$ & 0.058 & 0.058 & 0.0304 & 42.8 & \\
\hline 375 & SRISO_SEP_PRECIP_GPC & QTESRL & 0.0241 & $\mathrm{pCi} / \mathrm{g}$ & 0.0065 & 0.009 & 0.00955 & 42.8 & \\
\hline 376 & UISO_IE_PLATE_AEA & QTESRL & 0.711 & $\mathrm{pCi} / \mathrm{g}$ & 0.05 & 0.12 & 0.00892 & 42.8 & \\
\hline 377 & UISO_IE_PLATE_AEA & QTESRL & 0.0222 & $\mathrm{pCi} / \mathrm{g}$ & 0.0094 & 0.011 & 0.0104 & 42.8 & \\
\hline 378 & PUISO_IE_PLATE_AEA & QTESRL & 0.00839 & $\mathrm{pCi} / \mathrm{g}$ & 0.0012 & 0.0018 & 0.000125 & 42.8 & \\
\hline 379 & UISO_IE_PLATE_AEA & QTESRL & 0.59 & $\mathrm{pCi} / \mathrm{g}$ & 0.046 & 0.1 & 0.0154 & 42.8 & \\
\hline 380 & GAMMA_GS & QTESRL & 0.288 & $\mathrm{pCi} / \mathrm{g}$ & 0.039 & 0.039 & 0.0227 & 47.9 & \\
\hline
\end{tabular}




\begin{tabular}{|c|c|c|c|c|c|c|c|c|c|}
\hline & $\mathrm{K}$ & $\mathrm{L}$ & $\mathrm{M}$ & $\mathrm{N}$ & $\mathrm{O}$ & $\mathrm{P}$ & $\mathrm{Q}$ & $\mathrm{R}$ & $\mathrm{S}$ \\
\hline 1 & METHOD_NAME & LAB_CODE & VALUE_RPTD & ANAL_UNITS_RPTD & |COUNTING_ERROR & TOTAL_ANAL_ERROR & MIN_DETECTABLE_ACTIVITY & PCNT_MOISTURE & LAB_QUALIFIER \\
\hline 382 & UISO_IE_PLATE_AEA & QTESRL & 0.702 & $\mathrm{pCi} / \mathrm{g}$ & 0.059 & 0.12 & 0.0159 & 47.9 & \\
\hline 383 & UISO_IE_PLATE_AEA & QTESRL & 0.0262 & $\mathrm{pCi} / \mathrm{g}$ & 0.012 & 0.013 & 0.0122 & 47.9 & \\
\hline 384 & PUISO_IE_PLATE_AEA & QTESRL & 0.00704 & $\mathrm{pCi} / \mathrm{g}$ & 0.0012 & 0.0016 & 0.000135 & 47.9 & \\
\hline 385 & UISO_IE_PLATE_AEA & QTESRL & 0.517 & $\mathrm{pCi} / \mathrm{g}$ & 0.051 & 0.095 & 0.0159 & 47.9 & \\
\hline 386 & GAMMA_GS & QTESRL & 0.532 & $\mathrm{pCi} / \mathrm{g}$ & 0.061 & 0.061 & 0.0223 & 64.1 & \\
\hline 387 & SRISO_SEP_PRECIP_GPC & QTESRL & 0.0176 & $\mathrm{pCi} / \mathrm{g}$ & 0.0057 & 0.0075 & 0.00869 & 64.1 & \\
\hline 388 & UISO_IE_PLATE_AEA & QTESRL & 0.439 & $\mathrm{pCi} / \mathrm{g}$ & 0.044 & 0.081 & 0.011 & 64.1 & \\
\hline 389 & UISO_IE_PLATE_AEA & QTESRL & 0.0213 & $\mathrm{pCi} / \mathrm{g}$ & 0.0097 & 0.011 & 0.00862 & 64.1 & \\
\hline 391 & UISO_IE_PLATE_AEA & QTESRL & 0.369 & $\mathrm{pCi} / \mathrm{g}$ & 0.04 & 0.07 & 0.00862 & 64.1 & \\
\hline 392 & GAMMA_GS & QTESRL & 0.234 & $\mathrm{pCi} / \mathrm{g}$ & 0.074 & 0.074 & 0.0629 & 41.1 & \\
\hline 393 & SRISO_SEP_PRECIP_GPC & QTESRL & 0.0134 & $\mathrm{pCi} / \mathrm{g}$ & 0.0036 & 0.0051 & 0.00509 & 41.1 & \\
\hline 394 & UISO_IE_PLATE_AEA & QTESRL & 0.429 & $\mathrm{pCi} / \mathrm{g}$ & 0.045 & 0.081 & 0.0119 & 41.1 & \\
\hline 395 & UISO_IE_PLATE_AEA & QTESRL & 0.0104 & $\mathrm{pCi} / \mathrm{g}$ & 0.0075 & 0.0088 & 0.0112 & 41.1 & $U$ \\
\hline 396 & PUISO_IE_PLATE_AEA & QTESRL & 0.00702 & $\mathrm{pCi} / \mathrm{g}$ & 0.0009 & 0.0014 & 0.0000792 & 41.1 & \\
\hline 397 & UISO_IE_PLATE_AEA & QTESRL & 0.349 & $\mathrm{pCi} / \mathrm{g}$ & 0.041 & 0.068 & 0.0104 & 41.1 & \\
\hline 398 & GAMMA_GS & QTESRL & 0.157 & $\mathrm{pCi} / \mathrm{g}$ & 0.048 & 0.048 & 0.0402 & 73.1 & \\
\hline 399 & SRISO_SEP_PRECIP_GPC & QTESRL & 0.00515 & $\mathrm{pCi} / \mathrm{g}$ & 0.0024 & 0.0033 & 0.00469 & 73.1 & \\
\hline 401 & UISO_IE_PLATE_AEA & QTESRL & 0.00815 & $\mathrm{pCi} / \mathrm{g}$ & 0.0056 & 0.0069 & 0.00777 & 73.1 & \\
\hline 402 & PUISO_IE_PLATE_AEA & QTESRL & 0.00498 & $\mathrm{pCi} / \mathrm{g}$ & 0.00061 & 0.00097 & 0.000051 & 73.1 & \\
\hline 403 & UISO_IE_PLATE_AEA & QTESRL & 0.151 & $\mathrm{pCi} / \mathrm{g}$ & 0.024 & 0.034 & 0.00924 & 73.1 & \\
\hline 404 & GAMMA_GS & QTESRL & 0.388 & $\mathrm{pCi} / \mathrm{g}$ & 0.098 & 0.098 & 0.0744 & 38.9 & \\
\hline 405 & SRISO_SEP_PRECIP_GPC & QTESRL & 0.019 & $\mathrm{pCi} / \mathrm{g}$ & 0.0055 & 0.0075 & 0.00781 & 38.9 & \\
\hline 406 & UISO_IE_PLATE_AEA & QTESRL & 0.741 & $\mathrm{pCi} / \mathrm{g}$ & 0.053 & 0.12 & 0.0118 & 38.9 & \\
\hline 407 & UISO_IE_PLATE_AEA & QTESRL & 0.0175 & $\mathrm{pCi} / \mathrm{g}$ & 0.009 & 0.01 & 0.0122 & 38.9 & \\
\hline 408 & PUISO_IE_PLATE_AEA & QTESRL & 0.0105 & $\mathrm{pCi} / \mathrm{g}$ & 0.0015 & 0.0023 & 0.000313 & 38.9 & \\
\hline 409 & UISO_IE_PLATE_AEA & QTESRL & 0.658 & $\mathrm{pCi} / \mathrm{g}$ & 0.052 & 0.11 & 0.0301 & 38.9 & \\
\hline 410 & GAMMA_GS & QTESRL & 0.405 & $\mathrm{pCi} / \mathrm{g}$ & 0.093 & 0.093 & 0.0711 & 34.4 & \\
\hline 411 & SRISO_SEP_PRECIP_GPC & QTESRL & 0.0157 & $\mathrm{pCi} / \mathrm{g}$ & 0.0062 & 0.0077 & 0.0104 & 34.4 & \\
\hline 412 & UISO_IE_PLATE_AEA & QTESRL & 0.829 & $\mathrm{pCi} / \mathrm{g}$ & 0.057 & 0.14 & 0.00977 & 34.4 & \\
\hline 413 & UISO_IE_PLATE_AEA & QTESRL & 0.0372 & $\mathrm{pCi} / \mathrm{g}$ & 0.013 & 0.014 & 0.0114 & 34.4 & \\
\hline 414 & PUISO_IE_PLATE_AEA & QTESRL & 0.0102 & $\mathrm{pCi} / \mathrm{g}$ & 0.0011 & 0.002 & 0.000183 & 34.4 & \\
\hline 415 & UISO_IE_PLATE_AEA & QTESRL & 0.73 & $\mathrm{pCi} / \mathrm{g}$ & 0.054 & 0.12 & 0.0156 & 34.4 & \\
\hline 416 & GAMMA_GS & QTESRL & 0.301 & $\mathrm{pCi} / \mathrm{g}$ & 0.047 & 0.047 & 0.035 & 46 & \\
\hline 417 & SRISO_SEP_PRECIP_GPC & QTESRL & 0.0229 & $\mathrm{pCi} / \mathrm{g}$ & 0.005 & 0.0076 & 0.00637 & 46 & \\
\hline 418 & UISO_IE_PLATE_AEA & QTESRL & 0.813 & $\mathrm{pCi} / \mathrm{g}$ & 0.062 & 0.14 & 0.0147 & 46 & \\
\hline
\end{tabular}




\begin{tabular}{|c|c|c|c|c|c|c|c|c|c|}
\hline & $\mathrm{K}$ & $\mathrm{L}$ & $\bar{M}$ & $\mathrm{~N}$ & $\mathrm{O}$ & $P$ & $\mathrm{Q}$ & $\bar{R}$ & $\mathrm{~S}$ \\
\hline 1 & METHOD_NAME & LAB_CODE & VALUE_RPTD & ANAL_UNITS_RPTD & COUNTING_ERROR & TOTAL_ANAL_ERROR & MIN_DETECTABLE_ACTIVITY & PCNT_MOISTURE & LAB_QUALIFIER \\
\hline 420 & PUISO_IE_PLATE_AEA & QTESRL & 0.00721 & $\mathrm{pCi} / \mathrm{g}$ & 0.0011 & 0.0016 & 0.000106 & 46 & \\
\hline 421 & UISO_IE_PLATE_AEA & QTESRL & 0.6 & $\mathrm{pCi} / \mathrm{g}$ & 0.053 & 0.11 & 0.00921 & 46 & \\
\hline 422 & GAMMA_GS & QTESRL & 0.507 & $\mathrm{pCi} / \mathrm{g}$ & 0.064 & 0.064 & 0.0297 & 52.9 & \\
\hline 423 & SRISO_SEP_PRECIP_GPC & QTESRL & 0.0226 & $\mathrm{pCi} / \mathrm{g}$ & 0.0059 & 0.0082 & 0.00805 & 52.9 & \\
\hline 424 & UISO_IE_PLATE_AEA & QTESRL & 0.573 & $\mathrm{pCi} / \mathrm{g}$ & 0.05 & 0.1 & 0.00857 & 52.9 & \\
\hline 425 & UISO_IE_PLATE_AEA & QTESRL & 0.0176 & $\mathrm{pCi} / \mathrm{g}$ & 0.0085 & 0.0098 & 0.0062 & 52.9 & \\
\hline 426 & PUISO_IE_PLATE_AEA & QTESRL & 0.0094 & $\mathrm{pCi} / \mathrm{g}$ & 0.0016 & 0.0022 & 0.000179 & 52.9 & \\
\hline 427 & UISO_IE_PLATE_AEA & QTESRL & 0.482 & $\mathrm{pCi} / \mathrm{g}$ & 0.046 & 0.087 & 0.00754 & 52.9 & \\
\hline 429 & SRISO_SEP_PRECIP_GPC & QTESRL & 0.0114 & $\mathrm{pCi} / \mathrm{g}$ & 0.011 & 0.023 & 0.0396 & 74.4 & $U$ \\
\hline 430 & UISO_IE_PLATE_AEA & QTESRL & 2.56 & $\mathrm{pCi} / \mathrm{g}$ & 0.099 & 0.4 & 0.01 & 74.4 & \\
\hline 431 & UISO_IE_PLATE_AEA & QTESRL & 0.0949 & $\mathrm{pCi} / \mathrm{g}$ & 0.019 & 0.024 & 0.00655 & 74.4 & \\
\hline 432 & UISO_IE_PLATE_AEA & QTESRL & 2.24 & $\mathrm{pCi} / \mathrm{g}$ & 0.092 & 0.35 & 0.00946 & 74.4 & \\
\hline 433 & GAMMA_GS & QTESRL & 0.143 & $\mathrm{pCi} / \mathrm{g}$ & 0.026 & 0.026 & 0.0204 & 59.9 & \\
\hline 434 & SRISO_SEP_PRECIP_GPC & QTESRL & -0.00485 & $\mathrm{pCi} / \mathrm{g}$ & 0.016 & 0.022 & 0.0427 & 59.9 & $U$ \\
\hline 435 & UISO_IE_PLATE_AEA & QTESRL & 0.486 & $\mathrm{pCi} / \mathrm{g}$ & 0.043 & 0.087 & 0.00845 & 59.9 & \\
\hline 436 & UISO_IE_PLATE_AEA & QTESRL & 0.0151 & $\mathrm{pCi} / \mathrm{g}$ & 0.0079 & 0.0091 & 0.00913 & 59.9 & \\
\hline 437 & UISO_IE_PLATE_AEA & QTESRL & 0.402 & $\mathrm{pCi} / \mathrm{g}$ & 0.04 & 0.073 & 0.00768 & 59.9 & \\
\hline 439 & SRISO_SEP_PRECIP_GPC & QTESRL & 0.00709 & $\mathrm{pCi} / \mathrm{g}$ & 0.0082 & 0.022 & 0.0396 & 79.5 & $U$ \\
\hline 440 & UISO_IE_PLATE_AEA & QTESRL & 0.52 & $\mathrm{pCi} / \mathrm{g}$ & 0.044 & 0.091 & 0.0107 & 79.5 & \\
\hline 441 & UISO_IE_PLATE_AEA & QTESRL & 0.0241 & $\mathrm{pCi} / \mathrm{g}$ & 0.0094 & 0.011 & 0.00723 & 79.5 & \\
\hline 442 & UISO_IE_PLATE_AEA & QTESRL & 0.441 & $\mathrm{pCi} / \mathrm{g}$ & 0.041 & 0.079 & 0.0123 & 79.5 & \\
\hline 443 & GAMMA_GS & QTESRL & 0.201 & $\mathrm{pCi} / \mathrm{g}$ & 0.03 & 0.03 & 0.022 & 68.9 & \\
\hline 444 & SRISO_SEP_PRECIP_GPC & QTESRL & -0.0088 & $\mathrm{pCi} / \mathrm{g}$ & 0.02 & 0.02 & 0.0395 & 68.9 & $\mathrm{U}$ \\
\hline 445 & UISO_IE_PLATE_AEA & QTESRL & 0.753 & $\mathrm{pCi} / \mathrm{g}$ & 0.055 & 0.13 & 0.0127 & 68.9 & \\
\hline 446 & UISO_IE_PLATE_AEA & QTESRL & 0.0171 & $\mathrm{pCi} / \mathrm{g}$ & 0.0085 & 0.0097 & 0.00876 & 68.9 & \\
\hline 447 & UISO_IE_PLATE_AEA & QTESRL & 0.595 & $\mathrm{pCi} / \mathrm{g}$ & 0.049 & 0.1 & 0.00947 & 68.9 & \\
\hline 448 & GAMMA_GS & QTESRL & 0.211 & $\mathrm{pCi} / \mathrm{g}$ & 0.029 & 0.029 & 0.0145 & 79.5 & \\
\hline 449 & SRISO_SEP_PRECIP_GPC & QTESRL & 0.000507 & $\mathrm{pCi} / \mathrm{g}$ & 0.00076 & 0.02 & 0.0366 & 79.5 & $U$ \\
\hline 450 & UISO_IE_PLATE_AEA & QTESRL & 3.89 & $\mathrm{pCi} / \mathrm{g}$ & 0.12 & 0.6 & 0.0102 & 79.5 & \\
\hline 451 & UISO_IE_PLATE_AEA & QTESRL & 0.177 & $\mathrm{pCi} / \mathrm{g}$ & 0.025 & 0.037 & 0.0102 & 79.5 & \\
\hline 452 & UISO_IE_PLATE_AEA & QTESRL & 3.71 & $\mathrm{pCi} / \mathrm{g}$ & 0.11 & 0.57 & 0.019 & 79.5 & \\
\hline 453 & GAMMA_GS & QTESRL & 0.205 & $\mathrm{pCi} / \mathrm{g}$ & 0.035 & 0.035 & 0.0196 & 66.3 & \\
\hline 454 & SRISO_SEP_PRECIP_GPC & QTESRL & -0.00826 & $\mathrm{pCi} / \mathrm{g}$ & 0.019 & 0.019 & 0.0379 & 66.3 & $U$ \\
\hline 455 & UISO_IE_PLATE_AEA & QTESRL & 0.284 & $\mathrm{pCi} / \mathrm{g}$ & 0.033 & 0.055 & 0.0056 & 66.3 & \\
\hline 456 & UISO_IE_PLATE_AEA & QTESRL & 0.0176 & $\mathrm{pCi} / \mathrm{g}$ & 0.0084 & 0.0097 & 0.00851 & 66.3 & \\
\hline
\end{tabular}




\begin{tabular}{|c|c|c|c|c|c|c|c|c|}
\hline & $\mathrm{T}$ & $\mathrm{U}$ & V & W & $\mathrm{X}$ & $\mathrm{Y}$ & Z & $A A$ \\
\hline 1 & REVIEW_QUALIFIER & SAMP_FROM & SAMP_ITEM & SAMP_MTHD & COLL_MTHD & SAMP_SITE_ID & SAMP_SITE_NAME & WELL_NAME \\
\hline 2 & & SUB_SURFACE & SEDIMENT & & & 2228 & 3 SEEP 110-1 & \\
\hline 3 & & SUB_SURFACE & SEDIMENT & & & 5307 & 7 SEEP 150-3 & \\
\hline 4 & & SUB_SURFACE & SEDIMENT & & & 5304 & 4 SEEP $082-2$ & \\
\hline 5 & & SUB_SURFACE & SEDIMENT & & & 5303 & 3 SEEP 057-3 & \\
\hline 6 & & SUB_SURFACE & SEDIMENT & & & 5325 & 5 SEEP 153-1 & \\
\hline 7 & & SUB_SURFACE & SEDIMENT & & & 5306 & SEEP 145-2 & \\
\hline 8 & & SUB_SURFACE & SEDIMENT & & & 2232 & SEEP 144-1 & \\
\hline 9 & & SUB_SURFACE & SEDIMENT & & & 2229 & SEEP 110-2 & \\
\hline 10 & & SUB_SURFACE & SEDIMENT & & & 4721 & SD-098-1 & \\
\hline 11 & & SUB_SURFACE & SEDIMENT & & & 2218 & 3 SEEP 039-2 & \\
\hline 12 & & SUB_SURFACE & SEDIMENT & & & 2216 & SEEP 037-1 & \\
\hline 13 & & SUB_SURFACE & SEDIMENT & & & 5326 & SEEP 102-1 & \\
\hline 14 & & SUB_SURFACE & SEDIMENT & & & 196 & 100-F SPRING & \\
\hline 15 & $\mathrm{~F}$ & SUB_SURFACE & SEDIMENT & & & 196 & 100-F SPRING & \\
\hline 16 & & SUB_SURFACE & SEDIMENT & & & 196 & 100-F SPRING & \\
\hline 17 & & SUB_SURFACE & SEDIMENT & & & 196 & 100-F SPRING & \\
\hline 18 & & SUB_SURFACE & SEDIMENT & & & 196 & 100-F SPRING & \\
\hline 19 & & SUB_SURFACE & SEDIMENT & & & 196 & 100-F SPRING & \\
\hline 20 & & SUB_SURFACE & SEDIMENT & & & 196 & 100-F SPRING & \\
\hline 21 & & SUB_SURFACE & SEDIMENT & & & 196 & 100-F SPRING & \\
\hline 22 & & SUB_SURFACE & SEDIMENT & & & 52 & 100-B SPRING & \\
\hline 23 & $F$ & SUB_SURFACE & SEDIMENT & & & 52 & 100-B SPRING & \\
\hline 24 & & SUB_SURFACE & SEDIMENT & & & 52 & 100-B SPRING & \\
\hline 25 & & SUB_SURFACE & SEDIMENT & & & 52 & 100-B SPRING & \\
\hline 26 & & SUB_SURFACE & SEDIMENT & & & 52 & 100-B SPRING & \\
\hline 27 & & SUB_SURFACE & SEDIMENT & & & 151 & LANFORD SPRING 28-2 & \\
\hline 28 & & SUB_SURFACE & SEDIMENT & & & 488 & 300 AREA SPRING 42-2 & \\
\hline 29 & & SUB_SURFACE & SEDIMENT & & & 196 & 100-F SPRING & \\
\hline 30 & & SUB_SURFACE & SEDIMENT & & & 52 & 100-B SPRING & \\
\hline 31 & & SUB_SURFACE & SEDIMENT & & & 561 & WHITE BLUFFS SLOUGH & \\
\hline 32 & & SUB_SURFACE & SEDIMENT & & & 311 & RICHLAND-RIVER & \\
\hline 33 & & SUB_SURFACE & SEDIMENT & & & 256 & PRD-YAKIMA CNTY SHOR & \\
\hline 34 & & SUB_SURFACE & SEDIMENT & & & 254 & PRD-GRANT COUNTY & \\
\hline 35 & & SUB_SURFACE & SEDIMENT & & & 255 & 5 PRD-2/3 GRANT SHORE & \\
\hline 36 & & SUB_SURFACE & SEDIMENT & & & 552 & PRD-1/3 GRANT SHORE & \\
\hline 37 & & SUB_SURFACE & SEDIMENT & & & 104 & MCNARY-WASH. SHORE & \\
\hline 38 & & SUB_SURFACE & SEDIMENT & & & 102 & MCNARY-OREGON SHORE & \\
\hline 39 & & SUB_SURFACE & SEDIMENT & & & 427 & MCNARY-2/3 OR. SHORE & \\
\hline
\end{tabular}




\begin{tabular}{|c|c|c|c|c|c|c|c|c|}
\hline & $\mathrm{T}$ & $U$ & $\mathrm{~V}$ & W & $\mathrm{X}$ & $\mathrm{Y}$ & Z & $A A$ \\
\hline 1 & REVIEW_QUALIFIER & SAMP_FROM & SAMP_ITEM & SAMP_MTHD & COLL_MTHD & SAMP_SITE_ID & SAMP_SITE_NAME & WELL_NAME \\
\hline 40 & & SUB_SURFACE & SEDIMENT & & & 103 & 3 MCNARY-1/3 OR. SHORE & \\
\hline 41 & & SUB_SURFACE & SEDIMENT & & & 563 & 3 HANFORD SLOUGH & \\
\hline 42 & & SUB_SURFACE & SEDIMENT & & & 195 & 100 F SLOUGH & \\
\hline 43 & & SUB_SURFACE & SEDIMENT & & & 104 & 4 MCNARY-WASH. SHORE & \\
\hline 44 & & SUB_SURFACE & SEDIMENT & & & 104 & 4 MCNARY-WASH. SHORE & \\
\hline 45 & & SUB_SURFACE & SEDIMENT & & & 104 & MCNARY-WASH. SHORE & \\
\hline 46 & & SUB_SURFACE & SEDIMENT & & & 104 & 4 MCNARY-WASH. SHORE & \\
\hline 47 & & SUB_SURFACE & SEDIMENT & & & 104 & MCNARY-WASH. SHORE & \\
\hline 48 & & SUB_SURFACE & SEDIMENT & & & 427 & MCNARY-2/3 OR. SHORE & \\
\hline 49 & & SUB_SURFACE & SEDIMENT & & & 427 & MCNARY-2/3 OR. SHORE & \\
\hline 50 & & SUB_SURFACE & SEDIMENT & & & 427 & MCNARY-2/3 OR. SHORE & \\
\hline 51 & & SUB_SURFACE & SEDIMENT & & & 427 & MCNARY-2/3 OR. SHORE & \\
\hline 52 & $F$ & SUB_SURFACE & SEDIMENT & & & 427 & MCNARY-2/3 OR. SHORE & \\
\hline 53 & & SUB_SURFACE & SEDIMENT & & & 103 & 3 MCNARY-1/3 OR. SHORE & \\
\hline 54 & & SUB_SURFACE & SEDIMENT & & & 103 & 3 MCNARY-1/3 OR. SHORE & \\
\hline 55 & & SUB_SURFACE & SEDIMENT & & & 103 & 3 MCNARY-1/3 OR. SHORE & \\
\hline 56 & & SUB_SURFACE & SEDIMENT & & & 103 & 3 MCNARY-1/3 OR. SHORE & \\
\hline 57 & & SUB_SURFACE & SEDIMENT & & & 103 & 3 MCNARY-1/3 OR. SHORE & \\
\hline 58 & & SUB_SURFACE & SEDIMENT & & & 102 & MCNARY-OREGON SHORE & \\
\hline 59 & & SUB_SURFACE & SEDIMENT & & & 102 & - MCNARY-OREGON SHORE & \\
\hline 60 & & SUB_SURFACE & SEDIMENT & & & 102 & MCNARY-OREGON SHORE & \\
\hline 61 & & SUB_SURFACE & SEDIMENT & & & 102 & MCNARY-OREGON SHORE & \\
\hline 62 & $F$ & SUB_SURFACE & SEDIMENT & & & 102 & MCNARY-OREGON SHORE & \\
\hline 63 & & SUB_SURFACE & SEDIMENT & & & 311 & RICHLAND-RIVER & \\
\hline 64 & & SUB_SURFACE & SEDIMENT & & & 311 & RICHLAND-RIVER & \\
\hline 65 & & SUB_SURFACE & SEDIMENT & & & 311 & RICHLAND-RIVER & \\
\hline 66 & & SUB_SURFACE & SEDIMENT & & & 311 & RICHLAND-RIVER & \\
\hline 67 & $F$ & SUB_SURFACE & SEDIMENT & & & 311 & RICHLAND-RIVER & \\
\hline 68 & & SUB_SURFACE & SEDIMENT & & & 563 & 3 HANFORD SLOUGH & \\
\hline 69 & & SUB_SURFACE & SEDIMENT & & & 563 & 3 HANFORD SLOUGH & \\
\hline 70 & & SUB_SURFACE & SEDIMENT & & & 563 & 3 HANFORD SLOUGH & \\
\hline 71 & & SUB_SURFACE & SEDIMENT & & & 563 & 3 HANFORD SLOUGH & \\
\hline 72 & $F$ & SUB_SURFACE & SEDIMENT & & & 563 & 3 HANFORD SLOUGH & \\
\hline 73 & & SUB_SURFACE & SEDIMENT & & & 195 & 100 F SLOUGH & \\
\hline 74 & & SUB_SURFACE & SEDIMENT & & & 195 & 100 F SLOUGH & \\
\hline 75 & & SUB_SURFACE & SEDIMENT & & & 195 & 100 F SLOUGH & \\
\hline 76 & & SUB_SURFACE & SEDIMENT & & & 195 & 100 F SLOUGH & \\
\hline 77 & & SUB_SURFACE & SEDIMENT & & & 195 & 100 F SLOUGH & \\
\hline
\end{tabular}




\begin{tabular}{|c|c|c|c|c|c|c|c|c|}
\hline & $\mathrm{T}$ & $U$ & $\mathrm{~V}$ & $\mathrm{~W}$ & $\mathrm{x}$ & $\mathrm{Y}$ & Z & $\mathrm{AA}$ \\
\hline \begin{tabular}{l|l}
1 \\
\end{tabular} & REVIEW_QUALIFIER & SAMP_FROM & SAMP_ITEM & SAMP_MTHD & COLL_MTHD & SAMP_SITE_ID & SAMP_SITE_NAME & WELL_NAME \\
\hline 78 & & SUB_SURFACE & SEDIMENT & & & 561 & WHITE BLUFFS SLOUGH & \\
\hline 79 & & SUB_SURFACE & SEDIMENT & & & 561 & I WHITE BLUFFS SLOUGH & \\
\hline 80 & & SUB_SURFACE & SEDIMENT & & & 561 & WHITE BLUFFS SLOUGH & \\
\hline 81 & & SUB_SURFACE & SEDIMENT & & & 561 & WHITE BLUFFS SLOUGH & \\
\hline 82 & $F$ & SUB_SURFACE & SEDIMENT & & & 561 & WHITE BLUFFS SLOUGH & \\
\hline 83 & & SUB_SURFACE & SEDIMENT & & & 196 & 100-F SPRING & \\
\hline 84 & $F$ & SUB_SURFACE & SEDIMENT & & & 196 & 100-F SPRING & \\
\hline 85 & & SUB_SURFACE & SEDIMENT & & & 196 & 100-F SPRING & \\
\hline 86 & & SUB_SURFACE & SEDIMENT & & & 196 & 100-F SPRING & \\
\hline 87 & & SUB_SURFACE & SEDIMENT & & & 52 & 100-B SPRING & \\
\hline 88 & & SUB_SURFACE & SEDIMENT & & & 52 & 100-B SPRING & \\
\hline 89 & & SUB_SURFACE & SEDIMENT & & & 52 & 100-B SPRING & \\
\hline 90 & & SUB_SURFACE & SEDIMENT & & & 52 & 100-B SPRING & \\
\hline 91 & & SUB_SURFACE & SEDIMENT & & & 488 & 300 AREA SPRING 42-2 & \\
\hline 92 & $F$ & SUB_SURFACE & SEDIMENT & & & 488 & 300 AREA SPRING 42-2 & \\
\hline 93 & & SUB_SURFACE & SEDIMENT & & & 488 & 300 AREA SPRING 42-2 & \\
\hline 94 & & SUB_SURFACE & SEDIMENT & & & 488 & 300 AREA SPRING 42-2 & \\
\hline 95 & & SUB_SURFACE & SEDIMENT & & & 151 & HANFORD SPRING 28-2 & \\
\hline 96 & & SUB_SURFACE & SEDIMENT & & & 151 & HANFORD SPRING 28-2 & \\
\hline 97 & & SUB_SURFACE & SEDIMENT & & & 151 & HANFORD SPRING 28-2 & \\
\hline 98 & & SUB_SURFACE & SEDIMENT & & & 151 & HANFORD SPRING 28-2 & \\
\hline 99 & & SUB_SURFACE & SEDIMENT & & & 256 & PRD-YAKIMA CNTY SHOR & \\
\hline 100 & & SUB_SURFACE & SEDIMENT & & & 256 & PRD-YAKIMA CNTY SHOR & \\
\hline 101 & & SUB_SURFACE & SEDIMENT & & & 256 & PRD-YAKIMA CNTY SHOR & \\
\hline 102 & & SUB_SURFACE & SEDIMENT & & & 256 & PRD-YAKIMA CNTY SHOR & \\
\hline 103 & & SUB_SURFACE & SEDIMENT & & & 256 & PRD-YAKIMA CNTY SHOR & \\
\hline 104 & & SUB_SURFACE & SEDIMENT & & & 255 & 5 PRD-2/3 GRANT SHORE & \\
\hline 105 & & SUB_SURFACE & SEDIMENT & & & 255 & PRD-2/3 GRANT SHORE & \\
\hline 106 & & SUB_SURFACE & SEDIMENT & & & 255 & PRD-2/3 GRANT SHORE & \\
\hline 107 & & SUB_SURFACE & SEDIMENT & & & 255 & PRD-2/3 GRANT SHORE & \\
\hline 108 & $F$ & SUB_SURFACE & SEDIMENT & & & 255 & PRD-2/3 GRANT SHORE & \\
\hline 109 & & SUB_SURFACE & SEDIMENT & & & 552 & PRD-1/3 GRANT SHORE & \\
\hline 110 & & SUB_SURFACE & SEDIMENT & & & 552 & PRD-1/3 GRANT SHORE & \\
\hline 111 & & SUB_SURFACE & SEDIMENT & & & 552 & PRD-1/3 GRANT SHORE & \\
\hline 112 & & SUB_SURFACE & SEDIMENT & & & 552 & PRD-1/3 GRANT SHORE & \\
\hline 113 & & SUB_SURFACE & SEDIMENT & & & 552 & PRD-1/3 GRANT SHORE & \\
\hline 114 & & SUB_SURFACE & SEDIMENT & & & 254 & PRD-GRANT COUNTY & \\
\hline 115 & & SUB_SURFACE & SEDIMENT & & & 254 & 4 PRD-GRANT COUNTY & \\
\hline
\end{tabular}




\begin{tabular}{|c|c|c|c|c|c|c|c|c|}
\hline & $\mathrm{T}$ & $U$ & $\mathrm{~V}$ & $\mathrm{~W}$ & $\mathrm{X}$ & $\mathrm{Y}$ & $Z$ & $\mathrm{AA}$ \\
\hline 1 & REVIEW_QUALIFIER & SAMP_FROM & SAMP_ITEM & SAMP_MTHD & COLL_MTHD & SAMP_SITE_ID & SAMP_SITE_NAME & WELL_NAME \\
\hline 116 & & SUB_SURFACE & SEDIMENT & & & 254 & 4 PRD-GRANT COUNTY & \\
\hline 117 & & SUB_SURFACE & SEDIMENT & & & 254 & PRD-GRANT COUNTY & \\
\hline 118 & & SUB_SURFACE & SEDIMENT & & & 254 & 4 PRD-GRANT COUNTY & \\
\hline 119 & & SUB_SURFACE & SEDIMENT & & & 102 & - MCNARY-OREGON SHORE & \\
\hline 120 & & SUB_SURFACE & SEDIMENT & & & 102 & 2 MCNARY-OREGON SHORE & \\
\hline 121 & & SUB_SURFACE & SEDIMENT & & & 102 & MCNARY-OREGON SHORE & \\
\hline 122 & & SUB_SURFACE & SEDIMENT & & & 102 & I MCNARY-OREGON SHORE & \\
\hline 123 & $F$ & SUB_SURFACE & SEDIMENT & & & 102 & MCNARY-OREGON SHORE & \\
\hline 124 & & SUB_SURFACE & SEDIMENT & & & 103 & 3 MCNARY-1/3 OR. SHORE & \\
\hline 125 & & SUB_SURFACE & SEDIMENT & & & 103 & 3 MCNARY-1/3 OR. SHORE & \\
\hline 126 & & SUB_SURFACE & SEDIMENT & & & 103 & 3 MCNARY-1/3 OR. SHORE & \\
\hline 127 & & SUB_SURFACE & SEDIMENT & & & 103 & 3 MCNARY-1/3 OR. SHORE & \\
\hline 128 & $\mathrm{~F}$ & SUB_SURFACE & SEDIMENT & & & 103 & 3 MCNARY-1/3 OR. SHORE & \\
\hline 129 & & SUB_SURFACE & SEDIMENT & & & 561 & WHITE BLUFFS SLOUGH & \\
\hline 130 & $F$ & SUB_SURFACE & SEDIMENT & & & 561 & WHITE BLUFFS SLOUGH & \\
\hline 131 & & SUB_SURFACE & SEDIMENT & & & 561 & WHITE BLUFFS SLOUGH & \\
\hline 132 & & SUB_SURFACE & SEDIMENT & & & 561 & WHITE BLUFFS SLOUGH & \\
\hline 133 & & SUB_SURFACE & SEDIMENT & & & 561 & WHITE BLUFFS SLOUGH & \\
\hline 134 & & SUB_SURFACE & SEDIMENT & & & 195 & 100 F SLOUGH & \\
\hline 135 & $F$ & SUB_SURFACE & SEDIMENT & & & 195 & 100 F SLOUGH & \\
\hline 136 & & SUB_SURFACE & SEDIMENT & & & 195 & 100 F SLOUGH & \\
\hline 137 & & SUB_SURFACE & SEDIMENT & & & 195 & 100 F SLOUGH & \\
\hline 138 & & SUB_SURFACE & SEDIMENT & & & 195 & 100 F SLOUGH & \\
\hline 139 & & SUB_SURFACE & SEDIMENT & & & 563 & 3 HANFORD SLOUGH & \\
\hline 140 & & SUB_SURFACE & SEDIMENT & & & 563 & 3 HANFORD SLOUGH & \\
\hline 141 & & SUB_SURFACE & SEDIMENT & & & 563 & 3 HANFORD SLOUGH & \\
\hline 142 & & SUB_SURFACE & SEDIMENT & & & 563 & 3 HANFORD SLOUGH & \\
\hline 143 & & SUB_SURFACE & SEDIMENT & & & 563 & 3 HANFORD SLOUGH & \\
\hline 144 & & SUB_SURFACE & SEDIMENT & & & 311 & RICHLAND-RIVER & \\
\hline 145 & $F$ & SUB_SURFACE & SEDIMENT & & & 311 & RICHLAND-RIVER & \\
\hline 146 & & SUB_SURFACE & SEDIMENT & & & 311 & RICHLAND-RIVER & \\
\hline 147 & & SUB_SURFACE & SEDIMENT & & & 311 & RICHLAND-RIVER & \\
\hline 148 & & SUB_SURFACE & SEDIMENT & & & 311 & RICHLAND-RIVER & \\
\hline 149 & & SUB_SURFACE & SEDIMENT & & & 427 & MCNARY-2/3 OR. SHORE & \\
\hline 150 & & SUB_SURFACE & SEDIMENT & & & 427 & MCNARY-2/3 OR. SHORE & \\
\hline \begin{tabular}{|l|}
151 \\
\end{tabular} & & SUB_SURFACE & SEDIMENT & & & 427 & MCNARY-2/3 OR. SHORE & \\
\hline 152 & & SUB_SURFACE & SEDIMENT & & & 427 & MCNARY-2/3 OR. SHORE & \\
\hline \begin{tabular}{|l|l|}
153 \\
\end{tabular} & $F$ & SUB_SURFACE & SEDIMENT & & & 427 & MCNARY-2/3 OR. SHORE & \\
\hline
\end{tabular}




\begin{tabular}{|c|c|c|c|c|c|c|c|c|}
\hline & $\mathrm{T}$ & $\mathrm{U}$ & $\mathrm{V}$ & $\mathrm{W}$ & $\mathrm{X}$ & $\mathrm{Y}$ & Z & $\mathrm{AA}$ \\
\hline 1 & REVIEW_QUALIFIER & SAMP_FROM & SAMP_ITEM & SAMP_MTHD & COLL_MTHD & SAMP_SITE_ID & SAMP_SITE_NAME & WELL_NAME \\
\hline 154 & & SUB_SURFACE & SEDIMENT & & & 104 & 4 MCNARY-WASH. SHORE & \\
\hline 155 & & SUB_SURFACE & SEDIMENT & & & 104 & 4 MCNARY-WASH. SHORE & \\
\hline 156 & & SUB_SURFACE & SEDIMENT & & & 104 & MCNARY-WASH. SHORE & \\
\hline 157 & & SUB_SURFACE & SEDIMENT & & & 104 & 4 MCNARY-WASH. SHORE & \\
\hline 158 & & SUB_SURFACE & SEDIMENT & & & 104 & MCNARY-WASH. SHORE & \\
\hline 159 & & SUB_SURFACE & SEDIMENT & & & 254 & PRD-GRANT COUNTY & \\
\hline 160 & & SUB_SURFACE & SEDIMENT & & & 254 & PRD-GRANT COUNTY & \\
\hline 161 & & SUB_SURFACE & SEDIMENT & & & 254 & 4 PRD-GRANT COUNTY & \\
\hline 162 & & SUB_SURFACE & SEDIMENT & & & 254 & 4 PRD-GRANT COUNTY & \\
\hline 163 & & SUB_SURFACE & SEDIMENT & & & 254 & 4 PRD-GRANT COUNTY & \\
\hline 164 & & SUB_SURFACE & SEDIMENT & & & 255 & PRD-2/3 GRANT SHORE & \\
\hline 165 & & SUB_SURFACE & SEDIMENT & & & 255 & PRD-2/3 GRANT SHORE & \\
\hline 166 & & SUB_SURFACE & SEDIMENT & & & 255 & 5 PRD-2/3 GRANT SHORE & \\
\hline 167 & & SUB_SURFACE & SEDIMENT & & & 255 & 5 PRD-2/3 GRANT SHORE & \\
\hline 168 & & SUB_SURFACE & SEDIMENT & & & 255 & PRD-2/3 GRANT SHORE & \\
\hline 169 & & SUB_SURFACE & SEDIMENT & & & 256 & PRD-YAKIMA CNTY SHOR & \\
\hline 170 & & SUB_SURFACE & SEDIMENT & & & 256 & PRD-YAKIMA CNTY SHOR & \\
\hline 171 & & SUB_SURFACE & SEDIMENT & & & 256 & PRD-YAKIMA CNTY SHOR & \\
\hline \begin{tabular}{|l|l|}
172 \\
\end{tabular} & & SUB_SURFACE & SEDIMENT & & & 256 & PRD-YAKIMA CNTY SHOR & \\
\hline 173 & & SUB_SURFACE & SEDIMENT & & & 256 & PRD-YAKIMA CNTY SHOR & \\
\hline \begin{tabular}{|l|l|}
174 \\
\end{tabular} & & SUB_SURFACE & SEDIMENT & & & 5523 & 100 N SPRING-1 & \\
\hline 175 & & SUB_SURFACE & SEDIMENT & & & 5523 & 100 N SPRING-1 & \\
\hline 176 & & SUB_SURFACE & SEDIMENT & & & 5525 & 100 N SPRING-3 & \\
\hline 177 & & SUB_SURFACE & SEDIMENT & & & 5525 & 100 N SPRING-3 & \\
\hline 178 & & SUB_SURFACE & SEDIMENT & & & 5539 & 100 N SPRING-5 & \\
\hline 179 & & SUB_SURFACE & SEDIMENT & & & 5539 & 100 N SPRING-5 & \\
\hline 180 & & SUB_SURFACE & SEDIMENT & & & 533 & 3 100-K SPRING & \\
\hline 181 & & SUB_SURFACE & SEDIMENT & & & 533 & 100-K SPRING & \\
\hline 182 & & SUB_SURFACE & SEDIMENT & & & 533 & 100-K SPRING & \\
\hline 183 & & SUB_SURFACE & SEDIMENT & & & 533 & 100-K SPRING & \\
\hline 184 & & SUB_SURFACE & SEDIMENT & & & 196 & 100-F SPRING & \\
\hline 185 & & SUB_SURFACE & SEDIMENT & & & 196 & 100-F SPRING & \\
\hline 186 & & SUB_SURFACE & SEDIMENT & & & 196 & 100-F SPRING & \\
\hline \begin{tabular}{|l|}
187 \\
\end{tabular} & & SUB_SURFACE & SEDIMENT & & & 196 & 100-F SPRING & \\
\hline 188 & $F$ & SUB_SURFACE & SEDIMENT & & & 52 & 100-B SPRING & \\
\hline 189 & & SUB_SURFACE & SEDIMENT & & & 52 & 100-B SPRING & \\
\hline 190 & & SUB_SURFACE & SEDIMENT & & & 52 & 100-B SPRING & \\
\hline \begin{tabular}{|l|}
191 \\
\end{tabular} & & SUB_SURFACE & SEDIMENT & & & 52 & 100-B SPRING & \\
\hline
\end{tabular}




\begin{tabular}{|c|c|c|c|c|c|c|c|c|}
\hline & $\mathrm{T}$ & $\bar{U}$ & $\mathrm{~V}$ & $\mathrm{~W}$ & $\mathrm{X}$ & $\mathrm{Y}$ & Z & $\mathrm{AA}$ \\
\hline 1 & REVIEW_QUALIFIER & SAMP_FROM & SAMP_ITEM & SAMP_MTHD & COLL_MTHD & SAMP_SITE_ID & SAMP_SITE_NAME & WELL_NAME \\
\hline 192 & & SUB_SURFACE & SEDIMENT & & & 151 & HANFORD SPRING 28-2 & \\
\hline 193 & & SUB_SURFACE & SEDIMENT & & & 151 & HANFORD SPRING 28-2 & \\
\hline 194 & & SUB_SURFACE & SEDIMENT & & & 151 & HANFORD SPRING 28-2 & \\
\hline 195 & $F$ & SUB_SURFACE & SEDIMENT & & & 151 & HANFORD SPRING 28-2 & \\
\hline 196 & $F$ & SUB_SURFACE & SEDIMENT & & & 488 & 300 AREA SPRING 42-2 & \\
\hline 197 & & SUB_SURFACE & SEDIMENT & & & 488 & 300 AREA SPRING 42-2 & \\
\hline 198 & & SUB_SURFACE & SEDIMENT & & & 488 & 300 AREA SPRING 42-2 & \\
\hline 199 & $\mathrm{~F}$ & SUB_SURFACE & SEDIMENT & & & 488 & 300 AREA SPRING 42-2 & \\
\hline 200 & & SUB_SURFACE & SEDIMENT & & & 7736 & ICE HARBOR-WALLA WALLA SHORE & \\
\hline 201 & & SUB_SURFACE & SEDIMENT & & & 7736 & ICE HARBOR-WALLA WALLA SHORE & \\
\hline 202 & & SUB_SURFACE & SEDIMENT & & & 7736 & ICE HARBOR-WALLA WALLA SHORE & \\
\hline 203 & & SUB_SURFACE & SEDIMENT & & & 7736 & ICE HARBOR-WALLA WALLA SHORE & \\
\hline 204 & & SUB_SURFACE & SEDIMENT & & & 7736 & ICE HARBOR-WALLA WALLA SHORE & \\
\hline 205 & & SUB_SURFACE & SEDIMENT & & & 7736 & ICE HARBOR-WALLA WALLA SHORE & \\
\hline 206 & $F$ & SUB_SURFACE & SEDIMENT & & & 7734 & I ICE HARBOR-FRANKLIN SHORE & \\
\hline 207 & & SUB_SURFACE & SEDIMENT & & & 7734 & ICE HARBOR-FRANKLIN SHORE & \\
\hline 208 & & SUB_SURFACE & SEDIMENT & & & 7734 & ICE HARBOR-FRANKLIN SHORE & \\
\hline 209 & & SUB_SURFACE & SEDIMENT & & & 7734 & ICE HARBOR-FRANKLIN SHORE & \\
\hline 210 & & SUB_SURFACE & SEDIMENT & & & 7734 & ICE HARBOR-FRANKLIN SHORE & \\
\hline 211 & & SUB_SURFACE & SEDIMENT & & & 7734 & ICE HARBOR-FRANKLIN SHORE & \\
\hline 212 & & SUB_SURFACE & SEDIMENT & & & 7735 & ICE HARBOR-MID RIVER & \\
\hline 213 & & SUB_SURFACE & SEDIMENT & & & 7735 & ICE HARBOR-MID RIVER & \\
\hline 214 & & SUB_SURFACE & SEDIMENT & & & 7735 & ICE HARBOR-MID RIVER & \\
\hline 215 & & SUB_SURFACE & SEDIMENT & & & 7735 & ICE HARBOR-MID RIVER & \\
\hline 216 & & SUB_SURFACE & SEDIMENT & & & 7735 & ICE HARBOR-MID RIVER & \\
\hline 217 & & SUB_SURFACE & SEDIMENT & & & 7735 & ICE HARBOR-MID RIVER & \\
\hline 218 & & SUB_SURFACE & SEDIMENT & & & 563 & HANFORD SLOUGH & \\
\hline 219 & $\mathrm{~F}$ & SUB_SURFACE & SEDIMENT & & & 563 & HANFORD SLOUGH & \\
\hline 220 & & SUB_SURFACE & SEDIMENT & & & 563 & HANFORD SLOUGH & \\
\hline 221 & & SUB_SURFACE & SEDIMENT & & & 563 & HANFORD SLOUGH & \\
\hline 222 & & SUB_SURFACE & SEDIMENT & & & 563 & HANFORD SLOUGH & \\
\hline 223 & & SUB_SURFACE & SEDIMENT & & & 563 & HANFORD SLOUGH & \\
\hline 224 & & SUB_SURFACE & SEDIMENT & & & 102 & MCNARY-OREGON SHORE & \\
\hline 225 & & SUB_SURFACE & SEDIMENT & & & 102 & MCNARY-OREGON SHORE & \\
\hline 226 & & SUB_SURFACE & SEDIMENT & & & 102 & MCNARY-OREGON SHORE & \\
\hline 227 & & SUB_SURFACE & SEDIMENT & & & 102 & MCNARY-OREGON SHORE & \\
\hline 228 & & SUB_SURFACE & SEDIMENT & & & 102 & MCNARY-OREGON SHORE & \\
\hline 229 & & SUB_SURFACE & SEDIMENT & & & 102 & MCNARY-OREGON SHORE & \\
\hline
\end{tabular}




\begin{tabular}{|c|c|c|c|c|c|c|c|c|}
\hline & $\mathrm{T}$ & $\mathrm{U}$ & $\mathrm{V}$ & W & $\mathrm{X}$ & $\mathrm{Y}$ & Z & $A A$ \\
\hline 1 & REVIEW_QUALIFIER & SAMP_FROM & SAMP_ITEM & SAMP_MTHD & COLL_MTHD & SAMP_SITE_ID & SAMP_SITE_NAME & WELL_NAME \\
\hline 230 & & SUB_SURFACE & SEDIMENT & & & 103 & 3 MCNARY-1/3 OR. SHORE & \\
\hline 231 & & SUB_SURFACE & SEDIMENT & & & 103 & 3 MCNARY-1/3 OR. SHORE & \\
\hline 232 & & SUB_SURFACE & SEDIMENT & & & 103 & 3 MCNARY-1/3 OR. SHORE & \\
\hline 233 & & SUB_SURFACE & SEDIMENT & & & 103 & 3 MCNARY-1/3 OR. SHORE & \\
\hline 234 & & SUB_SURFACE & SEDIMENT & & & 103 & 3 MCNARY-1/3 OR. SHORE & \\
\hline 235 & & SUB_SURFACE & SEDIMENT & & & 103 & 3 MCNARY-1/3 OR. SHORE & \\
\hline 236 & & SUB_SURFACE & SEDIMENT & & & 427 & MCNARY-2/3 OR. SHORE & \\
\hline 237 & & SUB_SURFACE & SEDIMENT & & & 427 & MCNARY-2/3 OR. SHORE & \\
\hline 238 & & SUB_SURFACE & SEDIMENT & & & 427 & MCNARY-2/3 OR. SHORE & \\
\hline 239 & & SUB_SURFACE & SEDIMENT & & & 427 & MCNARY-2/3 OR. SHORE & \\
\hline 240 & & SUB_SURFACE & SEDIMENT & & & 427 & MCNARY-2/3 OR. SHORE & \\
\hline 241 & & SUB_SURFACE & SEDIMENT & & & 427 & MCNARY-2/3 OR. SHORE & \\
\hline 242 & & SUB_SURFACE & SEDIMENT & & & 104 & 4 MCNARY-WASH. SHORE & \\
\hline 243 & & SUB_SURFACE & SEDIMENT & & & 104 & 4 MCNARY-WASH. SHORE & \\
\hline 244 & & SUB_SURFACE & SEDIMENT & & & 104 & MCNARY-WASH. SHORE & \\
\hline 245 & & SUB_SURFACE & SEDIMENT & & & 104 & 4 MCNARY-WASH. SHORE & \\
\hline 246 & & SUB_SURFACE & SEDIMENT & & & 104 & MCNARY-WASH. SHORE & \\
\hline 247 & & SUB_SURFACE & SEDIMENT & & & 104 & MCNARY-WASH. SHORE & \\
\hline 248 & & SUB_SURFACE & SEDIMENT & & & 254 & PRD-GRANT COUNTY & \\
\hline 249 & & SUB_SURFACE & SEDIMENT & & & 254 & PRD-GRANT COUNTY & \\
\hline 250 & & SUB_SURFACE & SEDIMENT & & & 254 & PRD-GRANT COUNTY & \\
\hline 251 & & SUB_SURFACE & SEDIMENT & & & 254 & PRD-GRANT COUNTY & \\
\hline 252 & & SUB_SURFACE & SEDIMENT & & & 254 & PRD-GRANT COUNTY & \\
\hline 253 & & SUB_SURFACE & SEDIMENT & & & 254 & 4 PRD-GRANT COUNTY & \\
\hline 254 & & SUB_SURFACE & SEDIMENT & & & 552 & PRD-1/3 GRANT SHORE & \\
\hline 255 & & SUB_SURFACE & SEDIMENT & & & 552 & PRD-1/3 GRANT SHORE & \\
\hline 256 & & SUB_SURFACE & SEDIMENT & & & 552 & PRD-1/3 GRANT SHORE & \\
\hline 257 & & SUB_SURFACE & SEDIMENT & & & 552 & PRD-1/3 GRANT SHORE & \\
\hline 258 & & SUB_SURFACE & SEDIMENT & & & 552 & PRD-1/3 GRANT SHORE & \\
\hline 259 & & SUB_SURFACE & SEDIMENT & & & 552 & PRD-1/3 GRANT SHORE & \\
\hline 260 & & SUB_SURFACE & SEDIMENT & & & 255 & PRD-2/3 GRANT SHORE & \\
\hline 261 & & SUB_SURFACE & SEDIMENT & & & 255 & PRD-2/3 GRANT SHORE & \\
\hline 262 & & SUB_SURFACE & SEDIMENT & & & 255 & 5 PRD-2/3 GRANT SHORE & \\
\hline 263 & & SUB_SURFACE & SEDIMENT & & & 255 & PRD-2/3 GRANT SHORE & \\
\hline 264 & & SUB_SURFACE & SEDIMENT & & & 255 & PRD-2/3 GRANT SHORE & \\
\hline 265 & & SUB_SURFACE & SEDIMENT & & & 255 & PRD-2/3 GRANT SHORE & \\
\hline 266 & & SUB_SURFACE & SEDIMENT & & & 256 & PRD-YAKIMA CNTY SHOR & \\
\hline 267 & & SUB_SURFACE & SEDIMENT & & & 256 & PRD-YAKIMA CNTY SHOR & \\
\hline
\end{tabular}




\begin{tabular}{|c|c|c|c|c|c|c|c|c|}
\hline & $\mathrm{T}$ & $\mathrm{U}$ & $\mathrm{V}$ & W & $\mathrm{X}$ & $\mathrm{Y}$ & Z & $A A$ \\
\hline 1 & REVIEW_QUALIFIER & SAMP_FROM & SAMP_ITEM & SAMP_MTHD & COLL_MTHD & SAMP_SITE_ID & SAMP_SITE_NAME & WELL_NAME \\
\hline 268 & & SUB_SURFACE & SEDIMENT & & & 256 & 6 PRD-YAKIMA CNTY SHOR & \\
\hline 269 & & SUB_SURFACE & SEDIMENT & & & 256 & 6 PRD-YAKIMA CNTY SHOR & \\
\hline 270 & & SUB_SURFACE & SEDIMENT & & & 256 & 6 PRD-YAKIMA CNTY SHOR & \\
\hline 271 & & SUB_SURFACE & SEDIMENT & & & 256 & 6 PRD-YAKIMA CNTY SHOR & \\
\hline 272 & & SUB_SURFACE & SEDIMENT & & & 195 & 5100 F SLOUGH & \\
\hline 273 & & SUB_SURFACE & SEDIMENT & & & 195 & 5100 F SLOUGH & \\
\hline 274 & & SUB_SURFACE & SEDIMENT & & & 195 & 5100 F SLOUGH & \\
\hline 275 & & SUB_SURFACE & SEDIMENT & & & 195 & 5100 F SLOUGH & \\
\hline 276 & & SUB_SURFACE & SEDIMENT & & & 195 & 5100 F SLOUGH & \\
\hline 277 & & SUB_SURFACE & SEDIMENT & & & 195 & 5100 F SLOUGH & \\
\hline 278 & & SUB_SURFACE & SEDIMENT & & & 7737 & 7 PRD-YAKIMA SIDE NEAR DAM & \\
\hline 279 & & SUB_SURFACE & SEDIMENT & & & 7737 & 7 PRD-YAKIMA SIDE NEAR DAM & \\
\hline 280 & & SUB_SURFACE & SEDIMENT & & & 7737 & 7 PRD-YAKIMA SIDE NEAR DAM & \\
\hline 281 & & SUB_SURFACE & SEDIMENT & & & 7737 & 7 PRD-YAKIMA SIDE NEAR DAM & \\
\hline 282 & & SUB_SURFACE & SEDIMENT & & & 7737 & 7 PRD-YAKIMA SIDE NEAR DAM & \\
\hline 283 & & SUB_SURFACE & SEDIMENT & & & 7737 & 7 PRD-YAKIMA SIDE NEAR DAM & \\
\hline 284 & & SUB_SURFACE & SEDIMENT & & & 7738 & 8 PRD-GRANT SIDE NEAR DAM & \\
\hline 285 & & SUB_SURFACE & SEDIMENT & & & 7738 & 8 PRD-GRANT SIDE NEAR DAM & \\
\hline 286 & & SUB_SURFACE & SEDIMENT & & & 7738 & 8 PRD-GRANT SIDE NEAR DAM & \\
\hline 287 & & SUB_SURFACE & SEDIMENT & & & 7738 & 8 PRD-GRANT SIDE NEAR DAM & \\
\hline 288 & & SUB_SURFACE & SEDIMENT & & & 7738 & 8 PRD-GRANT SIDE NEAR DAM & \\
\hline 289 & & SUB_SURFACE & SEDIMENT & & & 7738 & 8 PRD-GRANT SIDE NEAR DAM & \\
\hline 290 & & SUB_SURFACE & SEDIMENT & & & 7739 & 9 MCNARY-OR.SIDE NEAR DAM & \\
\hline 291 & & SUB_SURFACE & SEDIMENT & & & 7739 & 9 MCNARY-OR.SIDE NEAR DAM & \\
\hline 292 & & SUB_SURFACE & SEDIMENT & & & 7739 & 9 MCNARY-OR.SIDE NEAR DAM & \\
\hline 293 & & SUB_SURFACE & SEDIMENT & & & 7739 & 9 MCNARY-OR.SIDE NEAR DAM & \\
\hline 294 & & SUB_SURFACE & SEDIMENT & & & 7739 & 9 MCNARY-OR.SIDE NEAR DAM & \\
\hline 295 & & SUB_SURFACE & SEDIMENT & & & 7739 & 9 MCNARY-OR.SIDE NEAR DAM & \\
\hline 296 & & SUB_SURFACE & SEDIMENT & & & 7740 & O MCNARY-WASH.SIDE NEAR DAM & \\
\hline 297 & & SUB_SURFACE & SEDIMENT & & & 7740 & MCNARY-WASH.SIDE NEAR DAM & \\
\hline 298 & & SUB_SURFACE & SEDIMENT & & & 7740 & O MCNARY-WASH.SIDE NEAR DAM & \\
\hline 299 & & SUB_SURFACE & SEDIMENT & & & 7740 & O MCNARY-WASH.SIDE NEAR DAM & \\
\hline 300 & & SUB_SURFACE & SEDIMENT & & & 7740 & O MCNARY-WASH.SIDE NEAR DAM & \\
\hline 301 & & SUB_SURFACE & SEDIMENT & & & 7740 & O MCNARY-WASH.SIDE NEAR DAM & \\
\hline 302 & $\mathrm{~F}$ & SUB_SURFACE & SEDIMENT & & & 311 & 1 RICHLAND-RIVER & \\
\hline 303 & $\mathrm{~F}$ & SUB_SURFACE & SEDIMENT & & & 311 & 1 RICHLAND-RIVER & \\
\hline 304 & & SUB_SURFACE & SEDIMENT & & & 311 & 1 RICHLAND-RIVER & \\
\hline 305 & & SUB_SURFACE & SEDIMENT & & & 311 & 1 RICHLAND-RIVER & \\
\hline
\end{tabular}




\begin{tabular}{|c|c|c|c|c|c|c|c|c|}
\hline & $T$ & $U$ & $\mathrm{~V}$ & $\mathrm{~W}$ & $\mathrm{X}$ & $\bar{Y}$ & $Z$ & $\overline{A A}$ \\
\hline 1 & REVIEW_QUALIFIER & SAMP_FROM & SAMP_ITEM & SAMP_MTHD & COLL_MTHD & SAMP_SITE_ID & SAMP_SITE_NAME & WELL_NAME \\
\hline 306 & & SUB_SURFACE & SEDIMENT & & & 311 & LICHLAND-RIVER & \\
\hline 307 & & SUB_SURFACE & SEDIMENT & & & 311 & RICHLAND-RIVER & \\
\hline 308 & & SUB_SURFACE & SEDIMENT & & & 561 & WHITE BLUFFS SLOUGH & \\
\hline 309 & & SUB_SURFACE & SEDIMENT & & & 561 & WHITE BLUFFS SLOUGH & \\
\hline 310 & & SUB_SURFACE & SEDIMENT & & & 561 & WHITE BLUFFS SLOUGH & \\
\hline 311 & & SUB SURFACE & SEDIMENT & & & 561 & WHITE BLUFFS SLOUGH & \\
\hline 312 & & SUB_SURFACE & SEDIMENT & & & 561 & WHITE BLUFFS SLOUGH & \\
\hline 313 & & SUB_SURFACE & SEDIMENT & & & 561 & WHITE BLUFFS SLOUGH & \\
\hline 314 & & SUB_SURFACE & SEDIMENT & & & 255 & PRD-2/3 GRANT SHORE & \\
\hline 315 & & SUB_SURFACE & SEDIMENT & & & 255 & 5 PRD-2/3 GRANT SHORE & \\
\hline 316 & & SUB_SURFACE & SEDIMENT & & & 255 & PRD-2/3 GRANT SHORE & \\
\hline 317 & & SUB_SURFACE & SEDIMENT & & & 255 & 5 PRD-2/3 GRANT SHORE & \\
\hline 318 & & SUB_SURFACE & SEDIMENT & & & 255 & 5 PRD-2/3 GRANT SHORE & \\
\hline 319 & & SUB_SURFACE & SEDIMENT & & & 255 & 5 PRD-2/3 GRANT SHORE & \\
\hline 320 & & SUB_SURFACE & SEDIMENT & & & 256 & PRD-YAKIMA CNTY SHOR & \\
\hline 321 & & SUB_SURFACE & SEDIMENT & & & 256 & PRD-YAKIMA CNTY SHOR & \\
\hline 322 & & SUB_SURFACE & SEDIMENT & & & 256 & PRD-YAKIMA CNTY SHOR & \\
\hline 323 & & SUB_SURFACE & SEDIMENT & & & 256 & PRD-YAKIMA CNTY SHOR & \\
\hline 324 & & SUB_SURFACE & SEDIMENT & & & 256 & PRD-YAKIMA CNTY SHOR & \\
\hline 325 & & SUB_SURFACE & SEDIMENT & & & 256 & PRD-YAKIMA CNTY SHOR & \\
\hline 326 & & SUB_SURFACE & SEDIMENT & & & 561 & WHITE BLUFFS SLOUGH & \\
\hline 327 & $\mathrm{~F}$ & SUB_SURFACE & SEDIMENT & & & 561 & WHITE BLUFFS SLOUGH & \\
\hline 328 & & SUB_SURFACE & SEDIMENT & & & 561 & WHITE BLUFFS SLOUGH & \\
\hline 329 & & SUB_SURFACE & SEDIMENT & & & 561 & WHITE BLUFFS SLOUGH & \\
\hline 330 & & SUB_SURFACE & SEDIMENT & & & 561 & I WHITE BLUFFS SLOUGH & \\
\hline 331 & & SUB_SURFACE & SEDIMENT & & & 561 & I WHITE BLUFFS SLOUGH & \\
\hline 332 & & SUB_SURFACE & SEDIMENT & & & 195 & 100 F SLOUGH & \\
\hline 333 & & SUB_SURFACE & SEDIMENT & & & 195 & 100 F SLOUGH & \\
\hline 334 & & SUB_SURFACE & SEDIMENT & & & 195 & 100 F SLOUGH & \\
\hline 335 & & SUB_SURFACE & SEDIMENT & & & 195 & 100 F SLOUGH & \\
\hline 336 & & SUB_SURFACE & SEDIMENT & & & 195 & 100 F SLOUGH & \\
\hline 337 & & SUB_SURFACE & SEDIMENT & & & 195 & 100 F SLOUGH & \\
\hline 338 & & SUB_SURFACE & SEDIMENT & & & 7736 & ICE HARBOR-WALLA WALLA SHORE & \\
\hline 339 & & SUB_SURFACE & SEDIMENT & & & 7736 & ICE HARBOR-WALLA WALLA SHORE & \\
\hline 340 & & SUB_SURFACE & SEDIMENT & & & 7736 & ICE HARBOR-WALLA WALLA SHORE & \\
\hline 341 & & SUB_SURFACE & SEDIMENT & & & 7736 & ICE HARBOR-WALLA WALLA SHORE & \\
\hline 342 & & SUB_SURFACE & SEDIMENT & & & 7736 & ICE HARBOR-WALLA WALLA SHORE & \\
\hline 343 & & SUB_SURFACE & SEDIMENT & & & 7736 & ICE HARBOR-WALLA WALLA SHORE & \\
\hline
\end{tabular}




\begin{tabular}{|c|c|c|c|c|c|c|c|c|}
\hline & $\mathrm{T}$ & $U$ & $\mathrm{~V}$ & $\mathrm{~W}$ & $\mathrm{x}$ & $\mathrm{Y}$ & $Z$ & $\mathrm{AA}$ \\
\hline 1 & REVIEW_QUALIFIER & SAMP_FROM & SAMP_ITEM & SAMP_MTHD & COLL_MTHD & SAMP_SITE_ID & SAMP_SITE_NAME & WELL_NAME \\
\hline 344 & $\mathrm{~F}$ & SUB_SURFACE & SEDIMENT & & & 7734 & 4 ICE HARBOR-FRANKLIN SHORE & \\
\hline 345 & & SUB_SURFACE & SEDIMENT & & & 7734 & 4 ICE HARBOR-FRANKLIN SHORE & \\
\hline 346 & & SUB_SURFACE & SEDIMENT & & & 7734 & 4 ICE HARBOR-FRANKLIN SHORE & \\
\hline 347 & & SUB_SURFACE & SEDIMENT & & & 7734 & 4 ICE HARBOR-FRANKLIN SHORE & \\
\hline 348 & $F$ & SUB_SURFACE & SEDIMENT & & & 7734 & 4 ICE HARBOR-FRANKLIN SHORE & \\
\hline 349 & & SUB_SURFACE & SEDIMENT & & & 7734 & 4 ICE HARBOR-FRANKLIN SHORE & \\
\hline 350 & & SUB_SURFACE & SEDIMENT & & & 7735 & 5 ICE HARBOR-MID RIVER & \\
\hline 351 & & SUB_SURFACE & SEDIMENT & & & 7735 & 5 ICE HARBOR-MID RIVER & \\
\hline 352 & & SUB_SURFACE & SEDIMENT & & & 7735 & 5 ICE HARBOR-MID RIVER & \\
\hline 353 & & SUB_SURFACE & SEDIMENT & & & 7735 & 5 ICE HARBOR-MID RIVER & \\
\hline 354 & & SUB_SURFACE & SEDIMENT & & & 7735 & 5 ICE HARBOR-MID RIVER & \\
\hline 355 & & SUB_SURFACE & SEDIMENT & & & 7735 & 5 ICE HARBOR-MID RIVER & \\
\hline 356 & & SUB_SURFACE & SEDIMENT & & & 563 & 3 HANFORD SLOUGH & \\
\hline 357 & $F$ & SUB_SURFACE & SEDIMENT & & & 563 & 3 HANFORD SLOUGH & \\
\hline 358 & & SUB_SURFACE & SEDIMENT & & & & 3 HANFORD SLOUGH & \\
\hline 359 & & SUB_SURFACE & SEDIMENT & & & 563 & 3 HANFORD SLOUGH & \\
\hline 360 & & SUB_SURFACE & SEDIMENT & & & 563 & 3 HANFORD SLOUGH & \\
\hline 361 & & SUB_SURFACE & SEDIMENT & & & 563 & 3 HANFORD SLOUGH & \\
\hline 362 & & SUB_SURFACE & SEDIMENT & & & 311 & 1 RICHLAND-RIVER & \\
\hline 363 & & SUB_SURFACE & SEDIMENT & & & 311 & 1 RICHLAND-RIVER & \\
\hline 364 & & SUB_SURFACE & SEDIMENT & & & 311 & 1 RICHLAND-RIVER & \\
\hline 365 & & SUB_SURFACE & SEDIMENT & & & 311 & 1 RICHLAND-RIVER & \\
\hline 366 & & SUB_SURFACE & SEDIMENT & & & 311 & 1 RICHLAND-RIVER & \\
\hline 367 & & SUB_SURFACE & SEDIMENT & & & 311 & 1 RICHLAND-RIVER & \\
\hline 368 & & SUB_SURFACE & SEDIMENT & & & 102 & 2 MCNARY-OREGON SHORE & \\
\hline 369 & & SUB_SURFACE & SEDIMENT & & & 102 & 2 MCNARY-OREGON SHORE & \\
\hline 370 & & SUB_SURFACE & SEDIMENT & & & 102 & 2 MCNARY-OREGON SHORE & \\
\hline 371 & & SUB_SURFACE & SEDIMENT & & & 102 & 2 MCNARY-OREGON SHORE & \\
\hline 372 & & SUB_SURFACE & SEDIMENT & & & 102 & 2 MCNARY-OREGON SHORE & \\
\hline 373 & & SUB_SURFACE & SEDIMENT & & & 102 & 2 MCNARY-OREGON SHORE & \\
\hline 374 & & SUB_SURFACE & SEDIMENT & & & 103 & 3 MCNARY-1/3 OR. SHORE & \\
\hline 375 & & SUB_SURFACE & SEDIMENT & & & 103 & 3 MCNARY-1/3 OR. SHORE & \\
\hline 376 & & SUB_SURFACE & SEDIMENT & & & 103 & 3 MCNARY-1/3 OR. SHORE & \\
\hline 377 & & SUB_SURFACE & SEDIMENT & & & 103 & 3 MCNARY-1/3 OR. SHORE & \\
\hline 378 & & SUB_SURFACE & SEDIMENT & & & 103 & 3 MCNARY-1/3 OR. SHORE & \\
\hline 379 & & SUB_SURFACE & SEDIMENT & & & 103 & 3 MCNARY-1/3 OR. SHORE & \\
\hline 380 & & SUB_SURFACE & SEDIMENT & & & 427 & 7 MCNARY-2/3 OR. SHORE & \\
\hline 381 & & SUB_SURFACE & SEDIMENT & & & 427 & 7 MCNARY-2/3 OR. SHORE & \\
\hline
\end{tabular}




\begin{tabular}{|c|c|c|c|c|c|c|c|c|}
\hline & $T$ & $U$ & $\mathrm{~V}$ & $\mathrm{~W}$ & $\mathrm{X}$ & $\bar{Y}$ & $Z$ & $\overline{A A}$ \\
\hline 1 & REVIEW_QUALIFIER & SAMP_FROM & SAMP_ITEM & SAMP_MTHD & COLL_MTHD & SAMP_SITE_ID & SAMP_SITE_NAME & WELL_NAME \\
\hline 382 & & SUB_SURFACE & SEDIMENT & & & 427 & MCNARY-2/3 OR. SHORE & \\
\hline 383 & & SUB_SURFACE & SEDIMENT & & & 427 & MCNARY-2/3 OR. SHORE & \\
\hline 384 & & SUB_SURFACE & SEDIMENT & & & 427 & MCNARY-2/3 OR. SHORE & \\
\hline 385 & & SUB_SURFACE & SEDIMENT & & & 427 & MCNARY-2/3 OR. SHORE & \\
\hline 386 & & SUB_SURFACE & SEDIMENT & & & 104 & MCNARY-WASH. SHORE & \\
\hline 387 & & SUB_SURFACE & SEDIMENT & & & 104 & 4 MCNARY-WASH. SHORE & \\
\hline 388 & & SUB_SURFACE & SEDIMENT & & & 104 & 4 MCNARY-WASH. SHORE & \\
\hline 389 & & SUB_SURFACE & SEDIMENT & & & 104 & MCNARY-WASH. SHORE & \\
\hline 390 & & SUB_SURFACE & SEDIMENT & & & 104 & MCNARY-WASH. SHORE & \\
\hline 391 & & SUB_SURFACE & SEDIMENT & & & 104 & 4 MCNARY-WASH. SHORE & \\
\hline 392 & & SUB_SURFACE & SEDIMENT & & & 254 & PRD-GRANT COUNTY & \\
\hline 393 & & SUB_SURFACE & SEDIMENT & & & 254 & 4 PRD-GRANT COUNTY & \\
\hline 394 & & SUB_SURFACE & SEDIMENT & & & 254 & A PRD-GRANT COUNTY & \\
\hline 395 & & SUB_SURFACE & SEDIMENT & & & 254 & 4 PRD-GRANT COUNTY & \\
\hline 396 & & SUB_SURFACE & SEDIMENT & & & 254 & PRD-GRANT COUNTY & \\
\hline 397 & & SUB_SURFACE & SEDIMENT & & & 254 & 4 PRD-GRANT COUNTY & \\
\hline 398 & & SUB_SURFACE & SEDIMENT & & & 552 & PRD-1/3 GRANT SHORE & \\
\hline 399 & & SUB_SURFACE & SEDIMENT & & & 552 & PRD-1/3 GRANT SHORE & \\
\hline 400 & & SUB_SURFACE & SEDIMENT & & & 552 & PRD-1/3 GRANT SHORE & \\
\hline 401 & & SUB_SURFACE & SEDIMENT & & & 552 & PRD-1/3 GRANT SHORE & \\
\hline 402 & & SUB_SURFACE & SEDIMENT & & & 552 & PRD-1/3 GRANT SHORE & \\
\hline 403 & & SUB_SURFACE & SEDIMENT & & & 552 & PRD-1/3 GRANT SHORE & \\
\hline 404 & & SUB_SURFACE & SEDIMENT & & & 7737 & PRD-YAKIMA SIDE NEAR DAM & \\
\hline 405 & & SUB_SURFACE & SEDIMENT & & & 7737 & PRD-YAKIMA SIDE NEAR DAM & \\
\hline 406 & & SUB_SURFACE & SEDIMENT & & & 7737 & PRD-YAKIMA SIDE NEAR DAM & \\
\hline 407 & & SUB_SURFACE & SEDIMENT & & & 7737 & 7 PRD-YAKIMA SIDE NEAR DAM & \\
\hline 408 & & SUB_SURFACE & SEDIMENT & & & 7737 & PRD-YAKIMA SIDE NEAR DAM & \\
\hline 409 & & SUB_SURFACE & SEDIMENT & & & 7737 & PRD-YAKIMA SIDE NEAR DAM & \\
\hline 410 & & SUB_SURFACE & SEDIMENT & & & 7738 & 3 PRD-GRANT SIDE NEAR DAM & \\
\hline 411 & & SUB_SURFACE & SEDIMENT & & & 7738 & 3 PRD-GRANT SIDE NEAR DAM & \\
\hline 412 & & SUB_SURFACE & SEDIMENT & & & 7738 & 3 PRD-GRANT SIDE NEAR DAM & \\
\hline 413 & & SUB_SURFACE & SEDIMENT & & & 7738 & 3 PRD-GRANT SIDE NEAR DAM & \\
\hline 414 & & SUB_SURFACE & SEDIMENT & & & 7738 & 3 PRD-GRANT SIDE NEAR DAM & \\
\hline 415 & & SUB_SURFACE & SEDIMENT & & & 7738 & 3 PRD-GRANT SIDE NEAR DAM & \\
\hline 416 & & SUB_SURFACE & SEDIMENT & & & 7739 & MCNARY-OR.SIDE NEAR DAM & \\
\hline 417 & & SUB_SURFACE & SEDIMENT & & & 7739 & MCNARY-OR.SIDE NEAR DAM & \\
\hline 418 & & SUB_SURFACE & SEDIMENT & & & 7739 & MCNARY-OR.SIDE NEAR DAM & \\
\hline 419 & & SUB_SURFACE & SEDIMENT & & & 7739 & MCNARY-OR.SIDE NEAR DAM & \\
\hline
\end{tabular}




\begin{tabular}{|c|c|c|c|c|c|c|c|c|}
\hline & $T$ & $U$ & $\mathrm{~V}$ & $\mathrm{~W}$ & $\mathrm{X}$ & $\bar{Y}$ & Z & $\overline{A A}$ \\
\hline 1 & REVIEW_QUALIFIER & SAMP_FROM & SAMP_ITEM & SAMP_MTHD & COLL_MTHD & SAMP_SITE_ID & SAMP_SITE_NAME & WELL_NAME \\
\hline 420 & & SUB_SURFACE & SEDIMENT & & & 7739 & MCNARY-OR.SIDE NEAR DAM & \\
\hline 421 & & SUB_SURFACE & SEDIMENT & & & 7739 & MCNARY-OR.SIDE NEAR DAM & \\
\hline 422 & & SUB_SURFACE & SEDIMENT & & & 7740 & MCNARY-WASH.SIDE NEAR DAM & \\
\hline \begin{tabular}{|l|l|}
423 \\
\end{tabular} & & SUB_SURFACE & SEDIMENT & & & 7740 & MCNARY-WASH.SIDE NEAR DAM & \\
\hline \begin{tabular}{|l|l|}
424 \\
\end{tabular} & & SUB_SURFACE & SEDIMENT & & & 7740 & MCNARY-WASH.SIDE NEAR DAM & \\
\hline 425 & & SUB SURFACE & SEDIMENT & & & 7740 & MCNARY-WASH.SIDE NEAR DAM & \\
\hline 426 & & SUB_SURFACE & SEDIMENT & & & 7740 & MCNARY-WASH.SIDE NEAR DAM & \\
\hline \begin{tabular}{|l|l|}
427 \\
\end{tabular} & & SUB_SURFACE & SEDIMENT & & & 7740 & MCNARY-WASH.SIDE NEAR DAM & \\
\hline 428 & $\mathrm{~F}$ & SUB_SURFACE & SEDIMENT & & & 488 & 300 AREA SPRING 42-2 & \\
\hline \begin{tabular}{|l|l|}
429 \\
\end{tabular} & & SUB_SURFACE & SEDIMENT & & & 488 & 300 AREA SPRING 42-2 & \\
\hline 430 & & SUB_SURFACE & SEDIMENT & & & 488 & 3300 AREA SPRING 42-2 & \\
\hline 431 & & SUB_SURFACE & SEDIMENT & & & 488 & 300 AREA SPRING 42-2 & \\
\hline 432 & & SUB_SURFACE & SEDIMENT & & & 488 & 300 AREA SPRING 42-2 & \\
\hline 433 & & SUB_SURFACE & SEDIMENT & & & 8140 & 100-B SPRING 38-3 & \\
\hline 434 & & SUB_SURFACE & SEDIMENT & & & 8140 & 100-B SPRING 38-3 & \\
\hline \begin{tabular}{|l|}
435 \\
\end{tabular} & & SUB_SURFACE & SEDIMENT & & & 8140 & 100-B SPRING 38-3 & \\
\hline \begin{tabular}{|l|}
436 \\
\end{tabular} & & SUB_SURFACE & SEDIMENT & & & 8140 & 100-B SPRING 38-3 & \\
\hline \begin{tabular}{|l|l|}
437 \\
\end{tabular} & & SUB_SURFACE & SEDIMENT & & & 8140 & 100-B SPRING 38-3 & \\
\hline 438 & & SUB_SURFACE & SEDIMENT & & & 8142 & HANFORD SPR UR 28-2 & \\
\hline \begin{tabular}{|l|}
439 \\
\end{tabular} & & SUB_SURFACE & SEDIMENT & & & 8142 & - HANFORD SPR UR 28-2 & \\
\hline 440 & & SUB_SURFACE & SEDIMENT & & & 8142 & HANFORD SPR UR 28-2 & \\
\hline 441 & & SUB_SURFACE & SEDIMENT & & & 8142 & HANFORD SPR UR 28-2 & \\
\hline \begin{tabular}{|l|}
442 \\
\end{tabular} & & SUB_SURFACE & SEDIMENT & & & 8142 & - HANFORD SPR UR 28-2 & \\
\hline 443 & & SUB_SURFACE & SEDIMENT & & & 8143 & 3 HANFORD SPR DR 28-2 & \\
\hline 444 & & SUB_SURFACE & SEDIMENT & & & 8143 & 3 HANFORD SPR DR 28-2 & \\
\hline 445 & & SUB_SURFACE & SEDIMENT & & & 8143 & 3 HANFORD SPR DR 28-2 & \\
\hline 446 & & SUB_SURFACE & SEDIMENT & & & 8143 & 3 HANFORD SPR DR 28-2 & \\
\hline 447 & & SUB_SURFACE & SEDIMENT & & & 8143 & 3 HANFORD SPR DR 28-2 & \\
\hline 44 & & SUB_SURFACE & SEDIMENT & & & 8144 & 400 AREA SPR DR 42-2 & \\
\hline \begin{tabular}{|l|}
449 \\
\end{tabular} & & SUB_SURFACE & SEDIMENT & & & 8144 & 300 AREA SPR DR 42-2 & \\
\hline 450 & & SUB_SURFACE & SEDIMENT & & & 8144 & 400 AREA SPR DR 42-2 & \\
\hline \begin{tabular}{|l|}
451 \\
\end{tabular} & & SUB_SURFACE & SEDIMENT & & & 8144 & 400 AREA SPR DR 42-2 & \\
\hline 452 & & SUB_SURFACE & SEDIMENT & & & 8144 & 300 AREA SPR DR 42-2 & \\
\hline 453 & & SUB_SURFACE & SEDIMENT & & & 8147 & 100-F SPRING 207-1 & \\
\hline 454 & & SUB_SURFACE & SEDIMENT & & & 8147 & 100-F SPRING 207-1 & \\
\hline 455 & & SUB_SURFACE & SEDIMENT & & & 8147 & 100-F SPRING 207-1 & \\
\hline 456 & & SUB_SURFACE & SEDIMENT & & & 8147 & 100-F SPRING 207-1 & \\
\hline \begin{tabular}{|l|}
457 \\
\end{tabular} & & SUB_SURFACE & SEDIMENT & & & 8147 & 100-F SPRING 207-1 & \\
\hline
\end{tabular}




\begin{tabular}{|c|c|c|}
\hline & 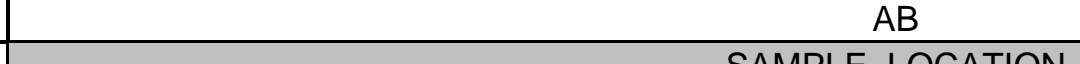 & $\frac{\mathrm{AC}}{\mathrm{CAMD} C \mathrm{CMMCNT}}$ \\
\hline 1 & $\begin{array}{r}\text { SAMPLE_LOCATION } \\
\end{array}$ & SAMP_COMMENT \\
\hline 2 & $110-1$ & ANALYSIS BY QUANTERRA. \\
\hline 3 & $150-3$ & ANALYSIS BY QUANTERRA. \\
\hline 4 & $082-2$ & ANALYSIS BY QUANTERRA. \\
\hline 5 & $057-3$ & ANALYSIS BY QUANTERRA. \\
\hline 6 & $153-1$ & ANALYSIS BY QUANTERRA. \\
\hline 7 & $145-1$ & ANALYSIS BY QUANTERRA. \\
\hline 8 & $144-1$ & ANALYSIS BY QUANTERRA. \\
\hline 9 & $110-2$ & ANALYSIS BY QUANTERRA. \\
\hline 10 & $098-1$ & ANALYSIS BY QUANTERRA. \\
\hline 11 & $039-2$ & ANALYSIS BY QUANTERRA. \\
\hline 12 & 037-1 & ANALYSIS BY QUANTERRA. \\
\hline 13 & $102-1$ & ANALYSIS BY QUANTERRA. \\
\hline 14 & & \\
\hline 15 & & \\
\hline 16 & & \\
\hline 17 & & \\
\hline 18 & & \\
\hline 19 & & \\
\hline 20 & & \\
\hline 21 & & \\
\hline 22 & & \\
\hline 23 & & \\
\hline 24 & & \\
\hline 25 & & \\
\hline 26 & & \\
\hline 27 & & \\
\hline 28 & & \\
\hline 29 & & \\
\hline 30 & & \\
\hline 31 & & \\
\hline 32 & & \\
\hline 33 & & \\
\hline 34 & & \\
\hline 35 & & \\
\hline 36 & & \\
\hline 37 & & \\
\hline 38 & & \\
\hline 39 & & \\
\hline
\end{tabular}


$A B$

$A C$

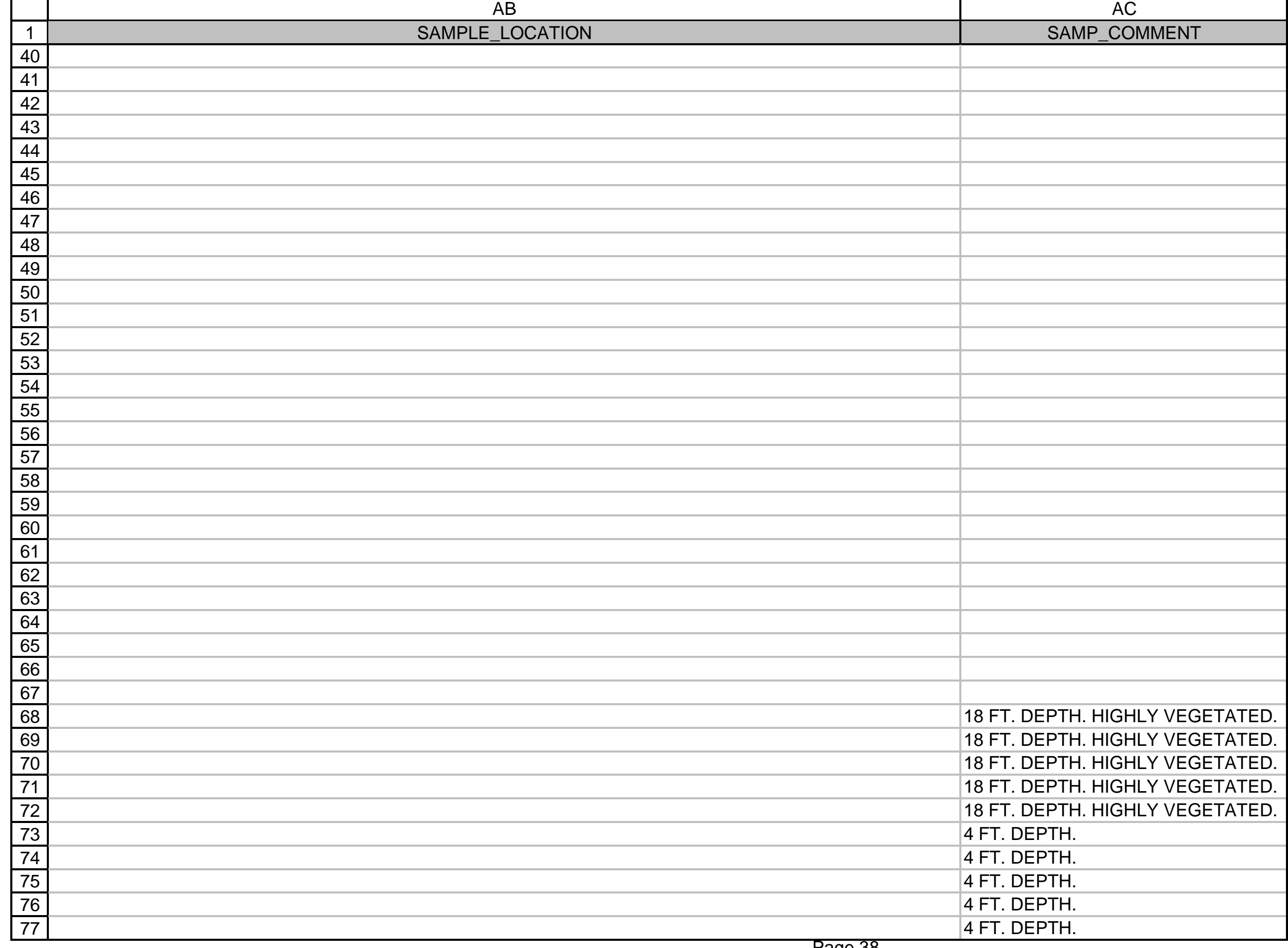

Page 38

4 FT. DEPTH. 


\begin{tabular}{|c|c|c|}
\hline & 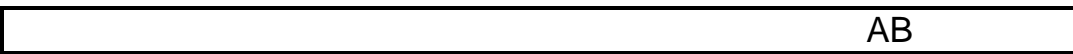 & $A C$ \\
\hline 1 & $x_{1}^{2}$ & SAMP_COMMENT \\
\hline 78 & & 10 FT. DEPTH. \\
\hline 79 & & 10 FT. DEPTH. \\
\hline 80 & & 10 FT. DEPTH. \\
\hline 81 & & 10 FT. DEPTH. \\
\hline 82 & & 10 FT. DEPTH. \\
\hline 83 & & \\
\hline 84 & & \\
\hline 85 & & \\
\hline 86 & & \\
\hline 87 & & \\
\hline 88 & & \\
\hline 89 & & \\
\hline 90 & & \\
\hline 91 & & \\
\hline 92 & & \\
\hline 93 & & \\
\hline 94 & & \\
\hline 95 & & \\
\hline 96 & & \\
\hline 97 & & \\
\hline 98 & & \\
\hline 99 & & 24 FT. DEPTH. \\
\hline 100 & & 24 FT. DEPTH. \\
\hline 101 & & 24 FT. DEPTH. \\
\hline 102 & & 24 FT. DEPTH. \\
\hline 103 & & 24 FT. DEPTH. \\
\hline 104 & & 69 FT. DEPTH. \\
\hline 105 & & 69 FT. DEPTH. \\
\hline 106 & & 69 FT. DEPTH. \\
\hline 107 & & 69 FT. DEPTH. \\
\hline 108 & & 69 FT. DEPTH. \\
\hline 109 & & 12 FT. DEPTH. \\
\hline 110 & & 12 FT. DEPTH. \\
\hline 111 & & 12 FT. DEPTH. \\
\hline 112 & & 12 FT. DEPTH. \\
\hline 113 & & 12 FT. DEPTH. \\
\hline 114 & & 25 FT. DEPTH. \\
\hline 115 & 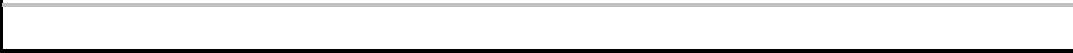 & 25 FT. DEPTH. \\
\hline
\end{tabular}




\begin{tabular}{|c|c|c|}
\hline & $A B$ & $A C$ \\
\hline 1 & SAMPLE_LOCATION & SAMP_COMMENT \\
\hline 116 & & 25 FT. DEPTH. \\
\hline 117 & & 25 FT. DEPTH. \\
\hline 118 & & 25 FT. DEPTH. \\
\hline 119 & & 50 FT. DEPTH. \\
\hline 120 & & 50 FT. DEPTH. \\
\hline 121 & & 50 FT. DEPTH. \\
\hline 122 & & 50 FT. DEPTH. \\
\hline 123 & & 50 FT. DEPTH. \\
\hline 124 & & 75 FT. DEPTH. \\
\hline 125 & & 75 FT. DEPTH. \\
\hline 126 & & 75 FT. DEPTH. \\
\hline 127 & & 75 FT. DEPTH. \\
\hline 128 & & 75 FT. DEPTH. \\
\hline 129 & 3 FT. DEPTH, COLLECTED OFFSHORE FROM TLD LOCATION. & \\
\hline 130 & 3 FT. DEPTH, COLLECTED OFFSHORE FROM TLD LOCATION. & \\
\hline 131 & 3 FT. DEPTH, COLLECTED OFFSHORE FROM TLD LOCATION. & \\
\hline 132 & 3 FT. DEPTH, COLLECTED OFFSHORE FROM TLD LOCATION. & \\
\hline 133 & 3 FT. DEPTH, COLLECTED OFFSHORE FROM TLD LOCATION. & \\
\hline 134 & & 8 FT. DEPTH. \\
\hline 135 & & 8 FT. DEPTH. \\
\hline 136 & & 8 FT. DEPTH. \\
\hline 137 & & 8 FT. DEPTH. \\
\hline 138 & & 8 FT. DEPTH. \\
\hline 139 & & 19 FT. DEPTH. \\
\hline 140 & & 19 FT. DEPTH. \\
\hline 141 & & 19 FT. DEPTH. \\
\hline 142 & & 19 FT. DEPTH. \\
\hline 143 & & 19 FT. DEPTH. \\
\hline 144 & 3 FT. DEPTH. WALKED IN FROM SHORE TO COLLECT SAMPLE. & \\
\hline 145 & 3 FT. DEPTH. WALKED IN FROM SHORE TO COLLECT SAMPLE. & \\
\hline 146 & 3 FT. DEPTH. WALKED IN FROM SHORE TO COLLECT SAMPLE. & \\
\hline 147 & 3 FT. DEPTH. WALKED IN FROM SHORE TO COLLECT SAMPLE. & \\
\hline 148 & 3 FT. DEPTH. WALKED IN FROM SHORE TO COLLECT SAMPLE. & \\
\hline 149 & & 70 FT. DEPTH. \\
\hline 150 & & 70 FT. DEPTH. \\
\hline 151 & & 70 FT. DEPTH. \\
\hline 152 & & 70 FT. DEPTH. \\
\hline 153 & & 70 FT. DEPTH. \\
\hline
\end{tabular}




\begin{tabular}{|c|c|c|}
\hline & $A B$ & $A C$ \\
\hline 1 & 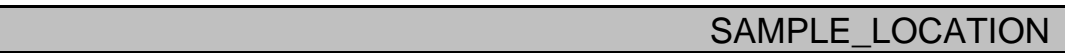 & SAMP_COMMENT \\
\hline 154 & & 12 FT. DEPTH. \\
\hline 155 & & 12 FT. DEPTH. \\
\hline 156 & & 12 FT. DEPTH. \\
\hline 157 & & 12 FT. DEPTH. \\
\hline 158 & & 12 FT. DEPTH. \\
\hline 159 & & 20 FT. DEPTH. \\
\hline 160 & & 20 FT. DEPTH. \\
\hline 161 & & 20 FT. DEPTH. \\
\hline 162 & & 20 FT. DEPTH. \\
\hline 163 & & 20 FT. DEPTH. \\
\hline 164 & & 75 FT. DEPTH. \\
\hline 165 & & 75 FT. DEPTH. \\
\hline 166 & & 75 FT. DEPTH. \\
\hline 167 & & 75 FT. DEPTH. \\
\hline 168 & & 75 FT. DEPTH. \\
\hline 169 & & 23 FT. DEPTH. \\
\hline 170 & & 23 FT. DEPTH. \\
\hline 171 & & 23 FT. DEPTH. \\
\hline 172 & & 23 FT. DEPTH. \\
\hline 173 & & 23 FT. DEPTH. \\
\hline \multicolumn{3}{|l|}{174} \\
\hline \multicolumn{3}{|l|}{175} \\
\hline \multicolumn{3}{|l|}{176} \\
\hline \multicolumn{3}{|l|}{177} \\
\hline \multicolumn{3}{|l|}{178} \\
\hline \multicolumn{3}{|l|}{179} \\
\hline \multicolumn{3}{|l|}{180} \\
\hline \multicolumn{3}{|l|}{181} \\
\hline \multicolumn{3}{|l|}{\begin{tabular}{|c|} 
\\
182
\end{tabular}} \\
\hline \multicolumn{3}{|l|}{\begin{tabular}{|l|}
183 \\
\end{tabular}} \\
\hline \multicolumn{3}{|l|}{184} \\
\hline \multicolumn{3}{|l|}{185} \\
\hline \multicolumn{3}{|l|}{186} \\
\hline \multicolumn{3}{|l|}{187} \\
\hline \multicolumn{3}{|l|}{188} \\
\hline \multicolumn{3}{|l|}{189} \\
\hline \multicolumn{3}{|l|}{190} \\
\hline 191 & . & \\
\hline
\end{tabular}


$A B$

AC

\begin{tabular}{|c|c|c|}
\hline & $\mathrm{AB}$ & $A C$ \\
\hline 1 & SAMPLE_LOCATION & SAMP_COMMENT \\
\hline \multicolumn{3}{|l|}{192} \\
\hline \multicolumn{3}{|l|}{193} \\
\hline \multicolumn{3}{|l|}{194} \\
\hline \multicolumn{3}{|l|}{195} \\
\hline \multicolumn{3}{|l|}{196} \\
\hline \multicolumn{3}{|l|}{197} \\
\hline \multicolumn{3}{|l|}{198} \\
\hline \multicolumn{3}{|l|}{199} \\
\hline 200 & & 68 FT. RIVER DEPTH. \\
\hline 201 & & 68 FT. RIVER DEPTH. \\
\hline 202 & & 68 FT. RIVER DEPTH. \\
\hline 203 & & 68 FT. RIVER DEPTH. \\
\hline 204 & & 68 FT. RIVER DEPTH. \\
\hline 205 & & 68 FT. RIVER DEPTH. \\
\hline 206 & & DRIFTING 35-90 FT. RIVER DEPTH. \\
\hline 207 & & DRIFTING 35-90 FT. RIVER DEPTH. \\
\hline 208 & & DRIFTING 35-90 FT. RIVER DEPTH. \\
\hline 209 & & DRIFTING 35-90 FT. RIVER DEPTH. \\
\hline 210 & & DRIFTING 35-90 FT. RIVER DEPTH. \\
\hline 211 & & DRIFTING 35-90 FT. RIVER DEPTH. \\
\hline 212 & & 85 FT. RIVER DEPTH. \\
\hline 213 & & 85 FT. RIVER DEPTH. \\
\hline 214 & & 85 FT. RIVER DEPTH. \\
\hline 215 & & 85 FT. RIVER DEPTH. \\
\hline 216 & & 85 FT. RIVER DEPTH. \\
\hline 217 & & 85 FT. RIVER DEPTH. \\
\hline 218 & & 3 FT. RIVER DEPTH. \\
\hline 219 & & 3 FT. RIVER DEPTH. \\
\hline 220 & & 3 FT. RIVER DEPTH. \\
\hline 221 & & 3 FT. RIVER DEPTH. \\
\hline 222 & & 3 FT. RIVER DEPTH. \\
\hline 223 & & 3 FT. RIVER DEPTH. \\
\hline 224 & & 75 FT. RIVER DEPTH. \\
\hline 225 & & 75 FT. RIVER DEPTH. \\
\hline 226 & & 75 FT. RIVER DEPTH. \\
\hline 227 & & 75 FT. RIVER DEPTH. \\
\hline 228 & & 75 FT. RIVER DEPTH. \\
\hline 229 & & 75 FT. RIVER DEPTH. \\
\hline
\end{tabular}

Page 42 
$\mathrm{AB}$

\begin{tabular}{|c|c|c|}
\hline & $A B$ & $\mathrm{AC}$ \\
\hline 1 & SAMPLE_LOCATION & SAMP_COMMENT \\
\hline 230 & & 75 FT. RIVER DEPTH. \\
\hline 231 & & 75 FT. RIVER DEPTH. \\
\hline 232 & & 75 FT. RIVER DEPTH. \\
\hline 233 & & 75 FT. RIVER DEPTH. \\
\hline 234 & & 75 FT. RIVER DEPTH. \\
\hline 235 & & 75 FT. RIVER DEPTH. \\
\hline 236 & & 60 FT. RIVER DEPTH. \\
\hline 237 & & 60 FT. RIVER DEPTH. \\
\hline 238 & & 60 FT. RIVER DEPTH. \\
\hline 239 & & 60 FT. RIVER DEPTH. \\
\hline 240 & & 60 FT. RIVER DEPTH. \\
\hline 241 & & 60 FT. RIVER DEPTH. \\
\hline 242 & & 20 FT. RIVER DEPTH. \\
\hline 243 & & 20 FT. RIVER DEPTH. \\
\hline 244 & & 20 FT. RIVER DEPTH. \\
\hline 245 & & 20 FT. RIVER DEPTH. \\
\hline 246 & & 20 FT. RIVER DEPTH. \\
\hline 247 & & 20 FT. RIVER DEPTH. \\
\hline 248 & & 25 FT. RIVER DEPTH. \\
\hline 249 & & 25 FT. RIVER DEPTH. \\
\hline 250 & & 25 FT. RIVER DEPTH. \\
\hline 251 & & 25 FT. RIVER DEPTH. \\
\hline 252 & & 25 FT. RIVER DEPTH. \\
\hline 253 & & 25 FT. RIVER DEPTH. \\
\hline 254 & & 25 FT. RIVER DEPTH. \\
\hline 255 & & 25 FT. RIVER DEPTH. \\
\hline 256 & & 25 FT. RIVER DEPTH. \\
\hline 257 & & 25 FT. RIVER DEPTH. \\
\hline 258 & & 25 FT. RIVER DEPTH. \\
\hline 259 & & 25 FT. RIVER DEPTH. \\
\hline 260 & & 50 FT. RIVER DEPTH. \\
\hline 261 & & 50 FT. RIVER DEPTH. \\
\hline 262 & & 50 FT. RIVER DEPTH. \\
\hline 263 & & 50 FT. RIVER DEPTH. \\
\hline 264 & & 50 FT. RIVER DEPTH. \\
\hline 265 & & 50 FT. RIVER DEPTH. \\
\hline 266 & & 15 FT. RIVER DEPTH. \\
\hline 267 & & 15 FT. RIVER DEPTH. \\
\hline
\end{tabular}

Page 43 


\begin{tabular}{|c|c|c|}
\hline & $A B$ & $\mathrm{AC}$ \\
\hline 1 & SAMPLE_LOCATION & SAMP_COMMENT \\
\hline 268 & & 15 FT. RIVER DEPTH. \\
\hline 269 & & 15 FT. RIVER DEPTH. \\
\hline 270 & & 15 FT. RIVER DEPTH. \\
\hline 271 & & 15 FT. RIVER DEPTH. \\
\hline 272 & & 2 FT. RIVER DEPTH. \\
\hline 273 & & 2 FT. RIVER DEPTH. \\
\hline 274 & & 2 FT. RIVER DEPTH. \\
\hline 275 & & 2 FT. RIVER DEPTH. \\
\hline 276 & & 2 FT. RIVER DEPTH. \\
\hline 277 & & 2 FT. RIVER DEPTH. \\
\hline 278 & & 75 FT. RIVER DEPTH. \\
\hline 279 & & 75 FT. RIVER DEPTH. \\
\hline 280 & & 75 FT. RIVER DEPTH. \\
\hline 281 & & 75 FT. RIVER DEPTH. \\
\hline 282 & & 75 FT. RIVER DEPTH. \\
\hline 283 & & 75 FT. RIVER DEPTH. \\
\hline 284 & & 45 FT. RIVER DEPTH. \\
\hline 285 & & 45 FT. RIVER DEPTH. \\
\hline 286 & & 45 FT. RIVER DEPTH. \\
\hline 287 & & 45 FT. RIVER DEPTH. \\
\hline 288 & & 45 FT. RIVER DEPTH. \\
\hline 289 & & 45 FT. RIVER DEPTH. \\
\hline 290 & & 80 FT. RIVER DEPTH. \\
\hline 291 & & 80 FT. RIVER DEPTH. \\
\hline 292 & & 80 FT. RIVER DEPTH. \\
\hline 293 & & 80 FT. RIVER DEPTH. \\
\hline 294 & & 80 FT. RIVER DEPTH. \\
\hline 295 & & 80 FT. RIVER DEPTH. \\
\hline 296 & & 80 FT. RIVER DEPTH. \\
\hline 297 & & 80 FT. RIVER DEPTH. \\
\hline 298 & & 80 FT. RIVER DEPTH. \\
\hline 299 & & 80 FT. RIVER DEPTH. \\
\hline 300 & & 80 FT. RIVER DEPTH. \\
\hline 301 & & 80 FT. RIVER DEPTH. \\
\hline 302 & SAMPLE COLLECTED 1-2 FT. WALK-IN FROM SHORE. & \\
\hline 303 & SAMPLE COLLECTED 1-2 FT. WALK-IN FROM SHORE. & \\
\hline 304 & SAMPLE COLLECTED 1-2 FT. WALK-IN FROM SHORE. & \\
\hline 305 & SAMPLE COLLECTED 1-2 FT. WALK-IN FROM SHORE. & \\
\hline
\end{tabular}




\begin{tabular}{|c|c|c|}
\hline & $A B$ & $A C$ \\
\hline 1 & SAMPLE_LOCATION & SAMP_COMMENT \\
\hline 306 & SAMPLE COLLECTED 1-2 FT. WALK-IN FROM SHORE. & \\
\hline 307 & SAMPLE COLLECTED 1-2 FT. WALK-IN FROM SHORE. & \\
\hline 308 & & 6 FT. RIVER DEPTH. \\
\hline 309 & & 6 FT. RIVER DEPTH. \\
\hline 310 & & 6 FT. RIVER DEPTH. \\
\hline 311 & & 6 FT. RIVER DEPTH. \\
\hline 312 & & 6 FT. RIVER DEPTH. \\
\hline 313 & & 6 FT. RIVER DEPTH. \\
\hline 314 & & \\
\hline 315 & & \\
\hline 316 & & \\
\hline 317 & & \\
\hline 318 & & \\
\hline 319 & & \\
\hline 320 & & \\
\hline 321 & & \\
\hline 322 & & \\
\hline 323 & & \\
\hline 324 & & \\
\hline 325 & & \\
\hline 326 & SAMPLED BETWEEN EAGLE BUOY AND TIP OF PENINSULA. & \\
\hline 327 & SAMPLED BETWEEN EAGLE BUOY AND TIP OF PENINSULA. & \\
\hline 328 & SAMPLED BETWEEN EAGLE BUOY AND TIP OF PENINSULA. & \\
\hline 329 & SAMPLED BETWEEN EAGLE BUOY AND TIP OF PENINSULA. & \\
\hline 330 & SAMPLED BETWEEN EAGLE BUOY AND TIP OF PENINSULA. & \\
\hline 331 & SAMPLED BETWEEN EAGLE BUOY AND TIP OF PENINSULA. & \\
\hline 332 & & \\
\hline 333 & & \\
\hline 334 & & \\
\hline 335 & & \\
\hline 336 & & \\
\hline 337 & & \\
\hline 338 & & \\
\hline 339 & & \\
\hline 340 & & \\
\hline 341 & & \\
\hline 342 & & \\
\hline 343 & 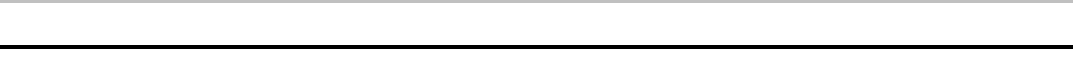 & \\
\hline
\end{tabular}




\begin{tabular}{|c|c|c|}
\hline & $\mathrm{AB}$ & $\mathrm{AC}$ \\
\hline 1 & SAMPLE_LOCATION & SAMP_COMMENT \\
\hline \multicolumn{3}{|l|}{344} \\
\hline \multicolumn{3}{|c|}{345} \\
\hline \multicolumn{3}{|c|}{346} \\
\hline \multicolumn{3}{|l|}{347} \\
\hline \multicolumn{3}{|l|}{348} \\
\hline \multicolumn{3}{|l|}{349} \\
\hline \multicolumn{3}{|l|}{350} \\
\hline \multicolumn{3}{|l|}{351} \\
\hline \multicolumn{3}{|l|}{352} \\
\hline \multicolumn{3}{|l|}{353} \\
\hline \multicolumn{3}{|l|}{354} \\
\hline \multicolumn{3}{|l|}{355} \\
\hline \multicolumn{3}{|c|}{356 SAMPLED 100 METERS UPRIVER OF POWERLINES, IN SLOUGH 40 METERS FROM HANFORD SHORE. } \\
\hline \multirow{2}{*}{\begin{tabular}{|l|}
357 \\
358 \\
\end{tabular}} & SAMPLED 100 METERS UPRIVER OF POWERLINES, IN SLOUGH 40 METERS FROM HANFORD SHORE. & \\
\hline & SAMPLED 100 METERS UPRIVER OF POWERLINES, IN SLOUGH 40 METERS FROM HANFORD SHORE. & \\
\hline \multirow{2}{*}{\begin{tabular}{|l|}
359 \\
360 \\
\end{tabular}} & SAMPLED 100 METERS UPRIVER OF POWERLINES, IN SLOUGH 40 METERS FROM HANFORD SHORE. & \\
\hline & SAMPLED 100 METERS UPRIVER OF POWERLINES, IN SLOUGH 40 METERS FROM HANFORD SHORE. & \\
\hline \multirow{2}{*}{\begin{tabular}{|l|}
361 \\
362
\end{tabular}} & SAMPLED 100 METERS UPRIVER OF POWERLINES, IN SLOUGH 40 METERS FROM HANFORD SHORE. & \\
\hline & \multicolumn{2}{|c|}{362} \\
\hline \multicolumn{3}{|l|}{363} \\
\hline \multicolumn{3}{|l|}{364} \\
\hline \multicolumn{3}{|l|}{365} \\
\hline \multicolumn{3}{|l|}{366} \\
\hline \multicolumn{3}{|l|}{367} \\
\hline \multicolumn{3}{|l|}{368} \\
\hline \multicolumn{3}{|l|}{369} \\
\hline \multicolumn{3}{|l|}{370} \\
\hline \multicolumn{3}{|l|}{371} \\
\hline \multicolumn{3}{|l|}{372} \\
\hline \multicolumn{3}{|l|}{373} \\
\hline 374 & & \\
\hline 375 & & \\
\hline \begin{tabular}{|l|}
376 \\
\end{tabular} & & \\
\hline 377 & & \\
\hline 378 & & \\
\hline 379 & & \\
\hline 380 & & \\
\hline 381 & & \\
\hline
\end{tabular}




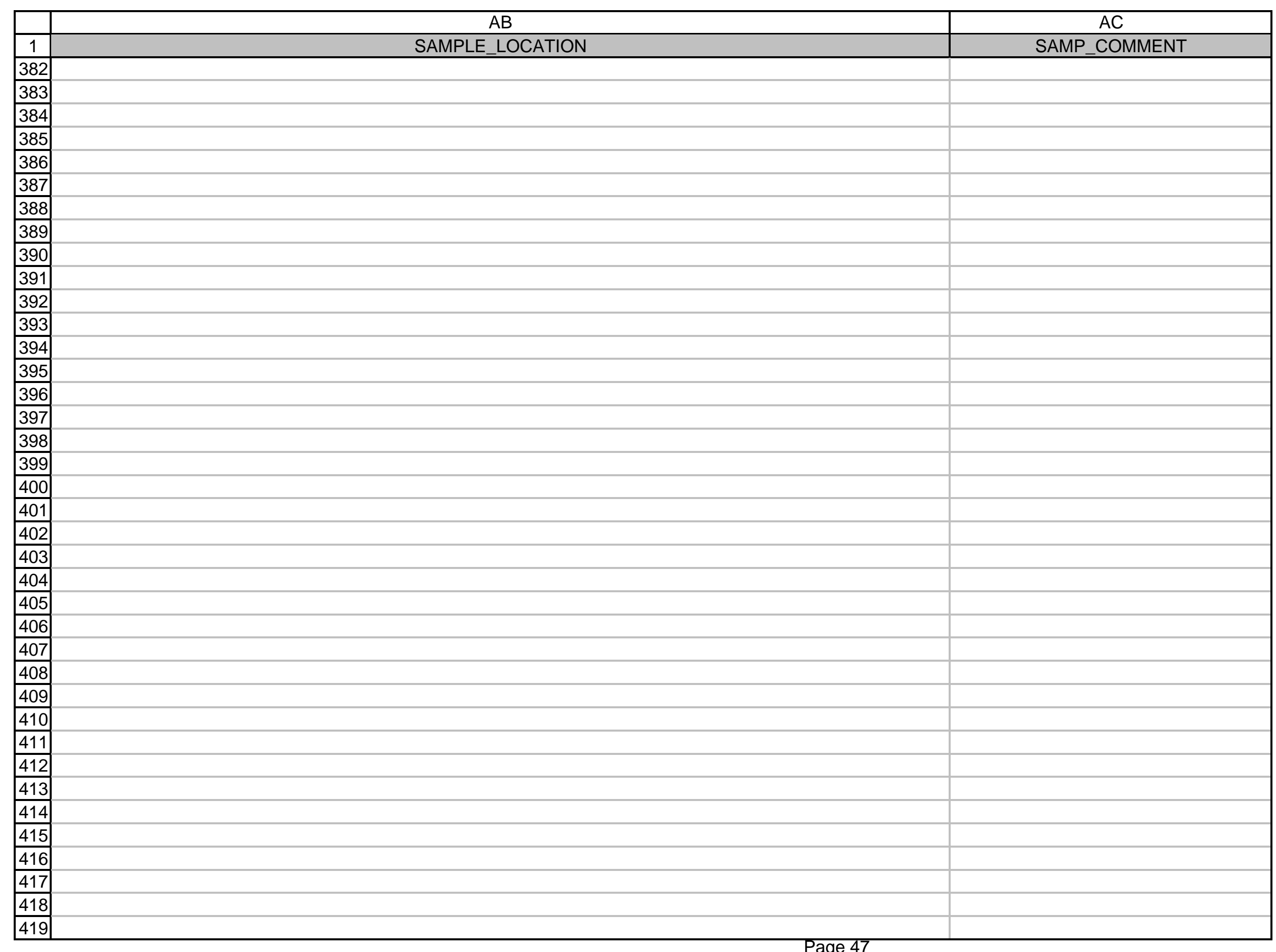




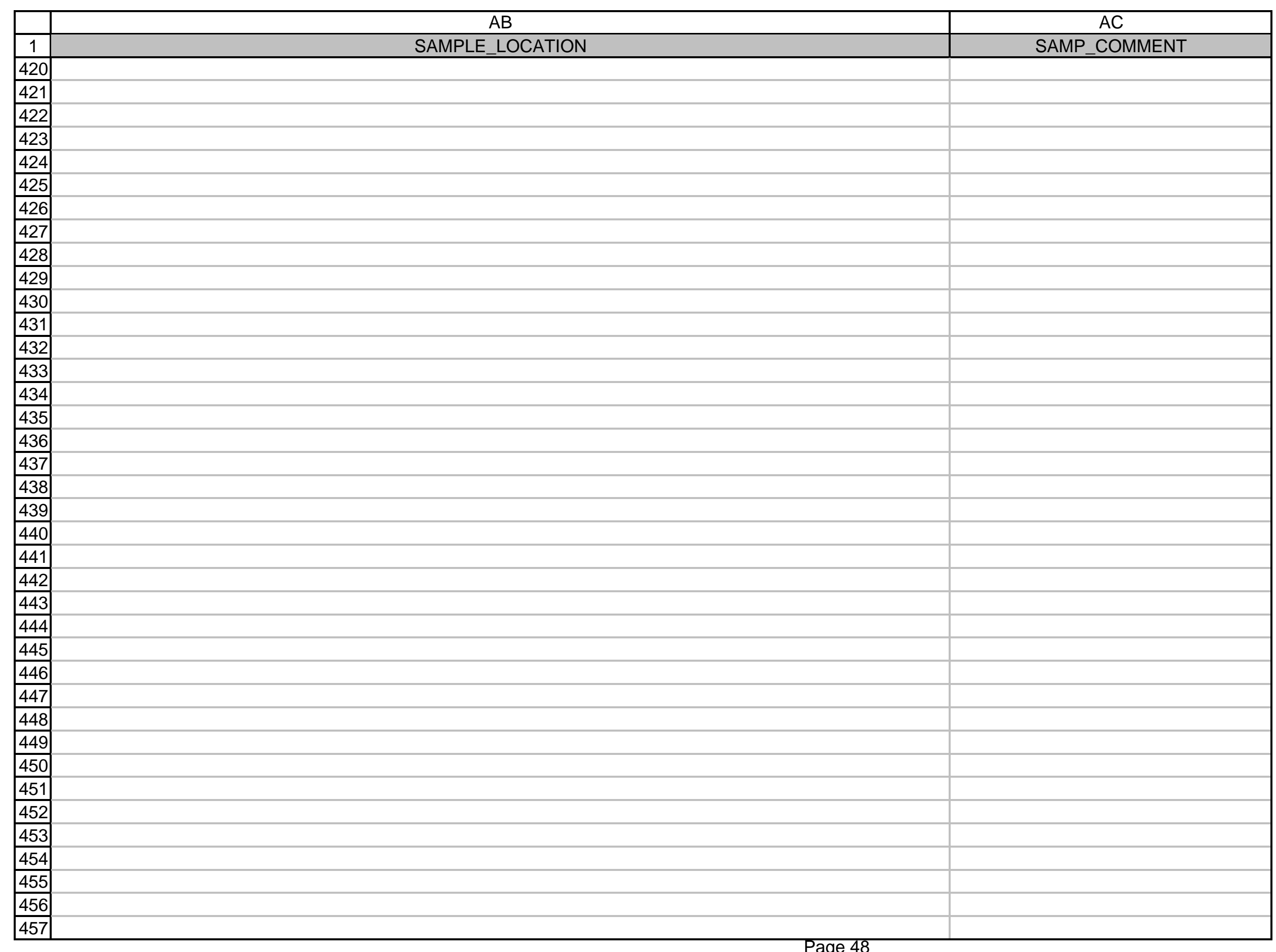




\begin{tabular}{|c|c|c|c|}
\hline & 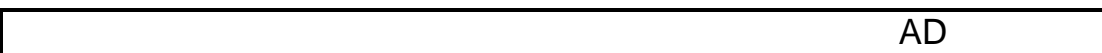 & $\mathrm{AF}$ & $A G$ \\
\hline 1 & RESULT_COMMENT & COLL_SAMP_SIZE_UNITS & DIST_CLASS \\
\hline 2 & & & \\
\hline 3 & & & \\
\hline 4 & & & \\
\hline 5 & & & \\
\hline 6 & & & \\
\hline 7 & & & \\
\hline 8 & & & \\
\hline 9 & & & \\
\hline 10 & & & \\
\hline 11 & & & \\
\hline 12 & & & \\
\hline 13 & & & \\
\hline 14 & & g & ONSITE \\
\hline 15 & & $\mathrm{~g}$ & ONSITE \\
\hline 16 & & g & ONSITE \\
\hline 17 & & g & ONSITE \\
\hline 18 & & g & ONSITE \\
\hline 19 & & $\mathrm{~g}$ & ONSITE \\
\hline 20 & & $\mathrm{~g}$ & ONSITE \\
\hline 21 & & 9 & ONSITE \\
\hline 22 & & g & ONSITE \\
\hline 23 & & g & ONSITE \\
\hline 24 & & g & ONSITE \\
\hline 25 & & g & ONSITE \\
\hline 26 & & $g$ & ONSITE \\
\hline 27 & & & ONSITE \\
\hline 28 & & & ONSITE \\
\hline 29 & & & ONSITE \\
\hline 30 & & & ONSITE \\
\hline 31 & & & RIVER_SHORELINE \\
\hline 32 & & & RIVER_SHORELINE \\
\hline 33 & & & OFFSITE \\
\hline 34 & & & OFFSITE \\
\hline 35 & & & OFFSITE \\
\hline 36 & & & OFFSITE \\
\hline 37 & & & OFFSITE \\
\hline 38 & & & OFFSITE \\
\hline 39 & $x_{1}^{2}$ & & OFFSITE \\
\hline
\end{tabular}




\begin{tabular}{|c|c|c|c|c|}
\hline & $A D$ & $\mathrm{AE}$ & $\mathrm{AF}$ & $A G$ \\
\hline 1 & RESULT_COMMENT & COLL_SAMP_SIZE & COLL_SAMP_SIZE_UNITS & DIST_CLASS \\
\hline 40 & & & & OFFSITE \\
\hline 41 & & & & RIVER_SHORELINE \\
\hline 42 & & & & ONSITE \\
\hline 43 & & 1900 & g & OFFSITE \\
\hline 44 & & 1900 & g & OFFSITE \\
\hline 45 & & 1900 & g & OFFSITE \\
\hline 46 & REFER TO 10/08/96 INCIDENT REPORT. SAMPLE HAD CHEMICAL YIELD LESS THAN 20 PERCENT. & 1900 & g & OFFSITE \\
\hline 47 & & 1900 & g & OFFSITE \\
\hline 48 & & 2001 & g & OFFSITE \\
\hline 49 & & 2001 & g & OFFSITE \\
\hline 50 & & 2001 & g & OFFSITE \\
\hline 51 & REFER TO 10/08/96 INCIDENT REPORT. SAMPLE HAD CHEMICAL YIELD LESS THAN 20 PERCENT. & 2001 & g & OFFSITE \\
\hline 52 & & 2001 & g & OFFSITE \\
\hline 53 & & 2800 & g & OFFSITE \\
\hline 54 & & 2800 & g & OFFSITE \\
\hline 55 & & 2800 & g & OFFSITE \\
\hline 56 & REFER TO 10/08/96 INCIDENT REPORT. SAMPLE HAD CHEMICAL YIELD LESS THAN 20 PERCENT. & 2800 & g & OFFSITE \\
\hline 57 & & 2800 & g & OFFSITE \\
\hline 58 & & 3000 & g & OFFSITE \\
\hline 59 & & 3000 & g & OFFSITE \\
\hline 60 & & 3000 & g & OFFSITE \\
\hline 61 & REFER TO 10/08/96 INCIDENT REPORT. SAMPLE HAD CHEMICAL YIELD LESS THAN 20 PERCENT. & 3000 & g & OFFSITE \\
\hline 62 & & 3000 & g & OFFSITE \\
\hline 63 & & 4602 & g & RIVER_SHORELINE \\
\hline 64 & & 4602 & $\mathrm{~g}$ & RIVER_SHORELINE \\
\hline 65 & & 4602 & g & RIVER_SHORELINE \\
\hline 66 & & 4602 & $\mathrm{~g}$ & RIVER_SHORELINE \\
\hline 67 & & 4602 & $\mathrm{~g}$ & RIVER_SHORELINE \\
\hline 68 & & 4923 & $\mathrm{~g}$ & RIVER_SHORELINE \\
\hline 69 & & 4923 & $\mathrm{~g}$ & RIVER_SHORELINE \\
\hline 70 & & 4923 & $\mathrm{~g}$ & RIVER_SHORELINE \\
\hline 71 & & 4923 & $\mathrm{~g}$ & RIVER_SHORELINE \\
\hline 72 & & 4923 & $\mathrm{~g}$ & RIVER_SHORELINE \\
\hline 73 & & 5077 & $\mathrm{~g}$ & ONSITE \\
\hline 74 & & 5077 & $\mathrm{~g}$ & ONSITE \\
\hline 75 & & 5077 & $\mathrm{~g}$ & ONSITE \\
\hline 76 & & 5077 & $\mathrm{~g}$ & ONSITE \\
\hline 77 & & 5077 & $\mathrm{~g}$ & ONSITE \\
\hline
\end{tabular}




\begin{tabular}{|c|c|c|c|c|}
\hline & $\mathrm{AD}$ & $\mathrm{AE}$ & $\mathrm{AF}$ & $A G$ \\
\hline 1 & RESULT_COMMENT & COLL_SAMP_SIZE & COLL_SAMP_SIZE_UNITS & DIST_CLASS \\
\hline 78 & & 6412 & $\mathrm{~g}$ & RIVER_SHORELINE \\
\hline 79 & & 6412 & g & RIVER_SHORELINE \\
\hline 80 & & 6412 & $\mathrm{~g}$ & RIVER_SHORELINE \\
\hline 81 & & 6412 & $\mathrm{~g}$ & RIVER_SHORELINE \\
\hline 82 & & 6412 & $\mathrm{~g}$ & RIVER_SHORELINE \\
\hline 83 & & 500 & $\mathrm{~g}$ & ONSITE \\
\hline 84 & & 500 & $\mathrm{~g}$ & ONSITE \\
\hline 85 & & 500 & $\mathrm{~g}$ & ONSITE \\
\hline 86 & & 500 & $\mathrm{~g}$ & ONSITE \\
\hline 87 & & 3390 & $\mathrm{~g}$ & ONSITE \\
\hline 88 & & 3390 & $\mathrm{~g}$ & ONSITE \\
\hline 89 & & 3390 & $\mathrm{~g}$ & ONSITE \\
\hline 90 & & 3390 & $\mathrm{~g}$ & ONSITE \\
\hline 91 & & 4700 & $\mathrm{~g}$ & ONSITE \\
\hline 92 & & 4700 & $\mathrm{~g}$ & ONSITE \\
\hline 93 & & 4700 & $\mathrm{~g}$ & ONSITE \\
\hline 94 & & 4700 & $\mathrm{~g}$ & ONSITE \\
\hline 95 & & 500 & $\mathrm{~g}$ & ONSITE \\
\hline 96 & & 500 & $\mathrm{~g}$ & ONSITE \\
\hline 97 & & 500 & $\mathrm{~g}$ & ONSITE \\
\hline 98 & & 500 & $\mathrm{~g}$ & ONSITE \\
\hline 99 & & 6900 & $\mathrm{~g}$ & OFFSITE \\
\hline 100 & & 6900 & $\mathrm{~g}$ & OFFSITE \\
\hline 101 & & 6900 & $\mathrm{~g}$ & OFFSITE \\
\hline 102 & REFER TO 10/08/96 INCIDENT REPORT. SAMPLE HAD CHEMICAL YIELD LESS THAN 20 PERCENT. & 6900 & g & OFFSITE \\
\hline 103 & & 6900 & $\mathrm{~g}$ & OFFSITE \\
\hline 104 & & 6400 & $\mathrm{~g}$ & OFFSITE \\
\hline 105 & & 6400 & g & OFFSITE \\
\hline 106 & & 6400 & g & OFFSITE \\
\hline 107 & REFER TO 10/08/96 INCIDENT REPORT. SAMPLE HAD CHEMICAL YIELD LESS THAN 20 PERCENT. & 6400 & $\mathrm{~g}$ & OFFSITE \\
\hline 108 & & 6400 & g & OFFSITE \\
\hline 109 & & 7500 & g & OFFSITE \\
\hline 110 & & 7500 & $\mathrm{~g}$ & OFFSITE \\
\hline 111 & & 7500 & g & OFFSITE \\
\hline 112 & & 7500 & $\mathrm{~g}$ & OFFSITE \\
\hline 113 & & 7500 & $\mathrm{~g}$ & OFFSITE \\
\hline 114 & & 8650 & $\mathrm{~g}$ & OFFSITE \\
\hline 115 & & 8650 & $\mathrm{~g}$ & OFFSITE \\
\hline
\end{tabular}




\begin{tabular}{|c|c|c|c|c|}
\hline & $\mathrm{AD}$ & $\mathrm{AE}$ & $\mathrm{AF}$ & $\mathrm{AG}$ \\
\hline 1 & RESULT_COMMENT & COLL_SAMP_SIZE & COLL_SAMP_SIZE_UNITS & DIST_CLASS \\
\hline 116 & & 8650 & $\mathrm{~g}$ & OFFSITE \\
\hline 117 & REFER TO 10/08/96 INCIDENT REPORT. SAMPLE HAD CHEMICAL YIELD LESS THAN 20 PERCENT. & 8650 & g & OFFSITE \\
\hline 118 & & 8650 & $\mathrm{~g}$ & OFFSITE \\
\hline 119 & & 500 & $\mathrm{~g}$ & OFFSITE \\
\hline 120 & & 500 & $\mathrm{~g}$ & OFFSITE \\
\hline 121 & & 500 & $\mathrm{~g}$ & OFFSITE \\
\hline 122 & & 500 & $\mathrm{~g}$ & OFFSITE \\
\hline 123 & & 500 & $\mathrm{~g}$ & OFFSITE \\
\hline 124 & & 500 & $\mathrm{~g}$ & OFFSITE \\
\hline 125 & & 500 & $\mathrm{~g}$ & OFFSITE \\
\hline 126 & & 500 & $\mathrm{~g}$ & OFFSITE \\
\hline 127 & & 500 & $\mathrm{~g}$ & OFFSITE \\
\hline 128 & & 500 & $\mathrm{~g}$ & OFFSITE \\
\hline 129 & & 500 & $\mathrm{~g}$ & RIVER_SHORELINE \\
\hline 130 & & 500 & $\mathrm{~g}$ & RIVER_SHORELINE \\
\hline 131 & & 500 & $\mathrm{~g}$ & RIVER_SHORELINE \\
\hline 132 & & 500 & $\mathrm{~g}$ & RIVER_SHORELINE \\
\hline 133 & & 500 & $\mathrm{~g}$ & RIVER_SHORELINE \\
\hline 134 & & 500 & $\mathrm{~g}$ & ONSITE \\
\hline 135 & & 500 & $\mathrm{~g}$ & ONSITE \\
\hline 136 & & 500 & $\mathrm{~g}$ & ONSITE \\
\hline 137 & & 500 & $\mathrm{~g}$ & ONSITE \\
\hline 138 & & 500 & $\mathrm{~g}$ & ONSITE \\
\hline 139 & & 500 & $\mathrm{~g}$ & RIVER_SHORELINE \\
\hline 140 & & 500 & $\mathrm{~g}$ & RIVER_SHORELINE \\
\hline 141 & & 500 & $\mathrm{~g}$ & RIVER_SHORELINE \\
\hline 142 & & 500 & $\mathrm{~g}$ & RIVER_SHORELINE \\
\hline 143 & & 500 & $\mathrm{~g}$ & RIVER_SHORELINE \\
\hline 144 & & 500 & $\mathrm{~g}$ & RIVER_SHORELINE \\
\hline 145 & & 500 & $\mathrm{~g}$ & RIVER_SHORELINE \\
\hline 146 & & 500 & $\mathrm{~g}$ & RIVER_SHORELINE \\
\hline 147 & & 500 & $\mathrm{~g}$ & RIVER_SHORELINE \\
\hline 148 & & 500 & $\mathrm{~g}$ & RIVER_SHORELINE \\
\hline 149 & & 500 & $\mathrm{~g}$ & OFFSITE \\
\hline 150 & & 500 & $\mathrm{~g}$ & OFFSITE \\
\hline 151 & & 500 & $\mathrm{~g}$ & OFFSITE \\
\hline 152 & & 500 & $\mathrm{~g}$ & OFFSITE \\
\hline 153 & & 500 & $\mathrm{~g}$ & OFFSITE \\
\hline
\end{tabular}




\begin{tabular}{|c|c|c|c|c|}
\hline & $A D$ & $\mathrm{AE}$ & $\mathrm{AF}$ & $A G$ \\
\hline 1 & RESULT_COMMENT & COLL_SAMP_SIZE & COLL_SAMP_SIZE_UNITS & DIST_CLASS \\
\hline 154 & & 500 & $\mathrm{~g}$ & OFFSITE \\
\hline 155 & & 500 & g & OFFSITE \\
\hline \begin{tabular}{|l|}
156 \\
\end{tabular} & & 500 & $\mathrm{~g}$ & OFFSITE \\
\hline \begin{tabular}{|l|}
157 \\
\end{tabular} & & 500 & $\mathrm{~g}$ & OFFSITE \\
\hline \begin{tabular}{|l|}
158 \\
\end{tabular} & & 500 & $\mathrm{~g}$ & OFFSITE \\
\hline \begin{tabular}{|l|}
159 \\
\end{tabular} & & 500 & $\mathrm{~g}$ & OFFSITE \\
\hline 160 & & 500 & $\mathrm{~g}$ & OFFSITE \\
\hline \begin{tabular}{|l|}
161 \\
\end{tabular} & & 500 & $\mathrm{~g}$ & OFFSITE \\
\hline \begin{tabular}{|l|}
162 \\
\end{tabular} & & 500 & $\mathrm{~g}$ & OFFSITE \\
\hline \begin{tabular}{|l|}
163 \\
\end{tabular} & & 500 & $\mathrm{~g}$ & OFFSITE \\
\hline \begin{tabular}{|l|}
164 \\
\end{tabular} & & 500 & $\mathrm{~g}$ & OFFSITE \\
\hline \begin{tabular}{|l|}
165 \\
\end{tabular} & & 500 & $\mathrm{~g}$ & OFFSITE \\
\hline \begin{tabular}{|l|}
166 \\
\end{tabular} & & 500 & $\mathrm{~g}$ & OFFSITE \\
\hline 167 & & 500 & $\mathrm{~g}$ & OFFSITE \\
\hline 168 & & 500 & $\mathrm{~g}$ & OFFSITE \\
\hline \begin{tabular}{|l|}
169 \\
\end{tabular} & & 500 & $\mathrm{~g}$ & OFFSITE \\
\hline 170 & & 500 & $\mathrm{~g}$ & OFFSITE \\
\hline \begin{tabular}{|l|}
171 \\
\end{tabular} & & 500 & $\mathrm{~g}$ & OFFSITE \\
\hline \begin{tabular}{|l|}
172 \\
\end{tabular} & & 500 & $\mathrm{~g}$ & OFFSITE \\
\hline \begin{tabular}{|l|}
173 \\
\end{tabular} & & 500 & $\mathrm{~g}$ & OFFSITE \\
\hline \begin{tabular}{|l|}
174 \\
\end{tabular} & & 1000 & $\mathrm{~g}$ & ONSITE \\
\hline \begin{tabular}{|l|}
175 \\
\end{tabular} & & 1000 & $\mathrm{~g}$ & ONSITE \\
\hline \begin{tabular}{|l|}
176 \\
\end{tabular} & & 1000 & $\mathrm{~g}$ & ONSITE \\
\hline \begin{tabular}{|l|}
177 \\
\end{tabular} & & 1000 & $\mathrm{~g}$ & ONSITE \\
\hline \begin{tabular}{|l|}
178 \\
\end{tabular} & & 1000 & $\mathrm{~g}$ & ONSITE \\
\hline \begin{tabular}{|l|}
179 \\
\end{tabular} & & 1000 & $\mathrm{~g}$ & ONSITE \\
\hline 180 & & 790 & $\mathrm{~g}$ & ONSITE \\
\hline 181 & & 790 & $\mathrm{~g}$ & ONSITE \\
\hline \begin{tabular}{|l|}
182 \\
\end{tabular} & & 790 & $\mathrm{~g}$ & ONSITE \\
\hline \begin{tabular}{|l|}
183 \\
\end{tabular} & & 790 & $\mathrm{~g}$ & ONSITE \\
\hline \begin{tabular}{|l|}
184 \\
\end{tabular} & & 3381 & $\mathrm{~g}$ & ONSITE \\
\hline \begin{tabular}{|l|}
185 \\
\end{tabular} & & 3381 & $\mathrm{~g}$ & ONSITE \\
\hline \begin{tabular}{|l|}
186 \\
\end{tabular} & & 3381 & $\mathrm{~g}$ & ONSITE \\
\hline \begin{tabular}{|l|}
187 \\
\end{tabular} & & 3381 & $\mathrm{~g}$ & ONSITE \\
\hline \begin{tabular}{|l|}
188 \\
\end{tabular} & & 2907 & $\mathrm{~g}$ & ONSITE \\
\hline 189 & & 2907 & $\mathrm{~g}$ & ONSITE \\
\hline \begin{tabular}{|l|}
190 \\
\end{tabular} & & 2907 & $\mathrm{~g}$ & ONSITE \\
\hline \begin{tabular}{|l|}
191 \\
\end{tabular} & 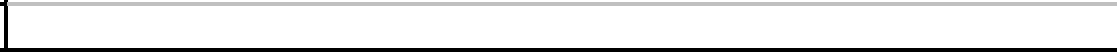 & 2907 & $\mathrm{~g}$ & ONSITE \\
\hline
\end{tabular}




\begin{tabular}{|c|c|c|c|c|}
\hline & $A D$ & $\mathrm{AE}$ & $\mathrm{AF}$ & $A G$ \\
\hline 1 & RESULT_COMMENT & COLL_SAMP_SIZE & COLL_SAMP_SIZE_UNITS & DIST_CLASS \\
\hline 192 & & 1735 & $\mathrm{~g}$ & ONSITE \\
\hline 193 & & 1735 & g & ONSITE \\
\hline 194 & & 1735 & $\mathrm{~g}$ & ONSITE \\
\hline 195 & & 1735 & $\mathrm{~g}$ & ONSITE \\
\hline 196 & & 2526 & $\mathrm{~g}$ & ONSITE \\
\hline \begin{tabular}{|l|}
197 \\
\end{tabular} & & 2526 & $\mathrm{~g}$ & ONSITE \\
\hline 198 & & 2526 & $\mathrm{~g}$ & ONSITE \\
\hline 199 & & 2526 & $\mathrm{~g}$ & ONSITE \\
\hline 200 & & 1200 & $\mathrm{~g}$ & OFFSITE \\
\hline 201 & & 1200 & $\mathrm{~g}$ & OFFSITE \\
\hline 202 & & 1200 & $\mathrm{~g}$ & OFFSITE \\
\hline 203 & & 1200 & $\mathrm{~g}$ & OFFSITE \\
\hline 204 & & 1200 & $\mathrm{~g}$ & OFFSITE \\
\hline 205 & & 1200 & $\mathrm{~g}$ & OFFSITE \\
\hline 206 & & 1200 & g & OFFSITE \\
\hline 207 & & 1200 & $\mathrm{~g}$ & OFFSITE \\
\hline 208 & & 1200 & $\mathrm{~g}$ & OFFSITE \\
\hline 209 & & 1200 & $\mathrm{~g}$ & OFFSITE \\
\hline 210 & & 1200 & $\mathrm{~g}$ & OFFSITE \\
\hline 211 & & 1200 & $\mathrm{~g}$ & OFFSITE \\
\hline 212 & & 1200 & $\mathrm{~g}$ & OFFSITE \\
\hline 213 & & 1200 & $\mathrm{~g}$ & OFFSITE \\
\hline 214 & & 1200 & $\mathrm{~g}$ & OFFSITE \\
\hline 215 & & 1200 & $\mathrm{~g}$ & OFFSITE \\
\hline 216 & & 1200 & $\mathrm{~g}$ & OFFSITE \\
\hline 217 & & 1200 & $\mathrm{~g}$ & OFFSITE \\
\hline 218 & & 1200 & $\mathrm{~g}$ & RIVER_SHORELINE \\
\hline 219 & & 1200 & $\mathrm{~g}$ & RIVER_SHORELINE \\
\hline 220 & & 1200 & $\mathrm{~g}$ & RIVER_SHORELINE \\
\hline 221 & & 1200 & $\mathrm{~g}$ & RIVER_SHORELINE \\
\hline 222 & & 1200 & $\mathrm{~g}$ & RIVER_SHORELINE \\
\hline \begin{tabular}{|l|}
223 \\
\end{tabular} & & 1200 & $\mathrm{~g}$ & RIVER_SHORELINE \\
\hline 224 & & 1200 & $\mathrm{~g}$ & OFFSITE \\
\hline 225 & & 1200 & $\mathrm{~g}$ & OFFSITE \\
\hline 226 & & 1200 & $\mathrm{~g}$ & OFFSITE \\
\hline 227 & & 1200 & $\mathrm{~g}$ & OFFSITE \\
\hline 228 & & 1200 & $\mathrm{~g}$ & OFFSITE \\
\hline 229 & t & 1200 & $\mathrm{~g}$ & OFFSITE \\
\hline
\end{tabular}




\begin{tabular}{|c|c|c|c|c|}
\hline & $A D$ & $\mathrm{AE}$ & $\mathrm{AF}$ & $A G$ \\
\hline 1 & RESULT_COMMENT & COLL_SAMP_SIZE & COLL_SAMP_SIZE_UNITS & DIST_CLASS \\
\hline 230 & & 1200 & g & OFFSITE \\
\hline 231 & & 1200 & g & OFFSITE \\
\hline \begin{tabular}{|l|}
232 \\
\end{tabular} & & 1200 & $\mathrm{~g}$ & OFFSITE \\
\hline 233 & & 1200 & $\mathrm{~g}$ & OFFSITE \\
\hline 234 & & 1200 & $\mathrm{~g}$ & OFFSITE \\
\hline \begin{tabular}{|l|}
235 \\
\end{tabular} & & 1200 & $\mathrm{~g}$ & OFFSITE \\
\hline 236 & & 1200 & $\mathrm{~g}$ & OFFSITE \\
\hline \begin{tabular}{|l|}
237 \\
\end{tabular} & 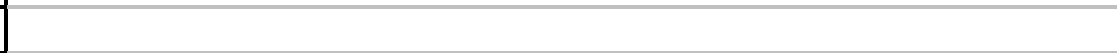 & 1200 & $\mathrm{~g}$ & OFFSITE \\
\hline \begin{tabular}{|l|}
238 \\
\end{tabular} & & 1200 & $\mathrm{~g}$ & OFFSITE \\
\hline 239 & & 1200 & $\mathrm{~g}$ & OFFSITE \\
\hline 240 & & 1200 & $\mathrm{~g}$ & OFFSITE \\
\hline \begin{tabular}{|l|}
241 \\
\end{tabular} & & 1200 & $\mathrm{~g}$ & OFFSITE \\
\hline \begin{tabular}{|l|}
242 \\
\end{tabular} & & 1200 & $\mathrm{~g}$ & OFFSITE \\
\hline 243 & & 1200 & $\mathrm{~g}$ & OFFSITE \\
\hline 244 & & 1200 & $\mathrm{~g}$ & OFFSITE \\
\hline 245 & & 1200 & $\mathrm{~g}$ & OFFSITE \\
\hline 246 & & 1200 & $\mathrm{~g}$ & OFFSITE \\
\hline 247 & & 1200 & $\mathrm{~g}$ & OFFSITE \\
\hline \begin{tabular}{|l|}
248 \\
\end{tabular} & & 1200 & $\mathrm{~g}$ & OFFSITE \\
\hline 249 & & 1200 & $\mathrm{~g}$ & OFFSITE \\
\hline 250 & & 1200 & $\mathrm{~g}$ & OFFSITE \\
\hline \begin{tabular}{|l|}
251 \\
\end{tabular} & & 1200 & $\mathrm{~g}$ & OFFSITE \\
\hline 252 & & 1200 & $\mathrm{~g}$ & OFFSITE \\
\hline \begin{tabular}{|l|}
253 \\
\end{tabular} & & 1200 & $\mathrm{~g}$ & OFFSITE \\
\hline \begin{tabular}{|l|}
254 \\
\end{tabular} & & 1200 & $\mathrm{~g}$ & OFFSITE \\
\hline 255 & & 1200 & $\mathrm{~g}$ & OFFSITE \\
\hline \begin{tabular}{|l|}
256 \\
\end{tabular} & & 1200 & $\mathrm{~g}$ & OFFSITE \\
\hline 257 & & 1200 & $\mathrm{~g}$ & OFFSITE \\
\hline \begin{tabular}{|l|}
258 \\
\end{tabular} & & 1200 & $\mathrm{~g}$ & OFFSITE \\
\hline 259 & & 1200 & $\mathrm{~g}$ & OFFSITE \\
\hline 260 & & 1200 & $\mathrm{~g}$ & OFFSITE \\
\hline \begin{tabular}{|l|}
261 \\
\end{tabular} & & 1200 & $\mathrm{~g}$ & OFFSITE \\
\hline \begin{tabular}{|l|}
262 \\
\end{tabular} & & 1200 & $\mathrm{~g}$ & OFFSITE \\
\hline \begin{tabular}{|l|}
263 \\
\end{tabular} & & 1200 & $\mathrm{~g}$ & OFFSITE \\
\hline \begin{tabular}{|l|}
264 \\
\end{tabular} & & 1200 & $\mathrm{~g}$ & OFFSITE \\
\hline 265 & & 1200 & $\mathrm{~g}$ & OFFSITE \\
\hline \begin{tabular}{|l|}
266 \\
\end{tabular} & & 1200 & $\mathrm{~g}$ & OFFSITE \\
\hline \begin{tabular}{|l|}
267 \\
\end{tabular} & 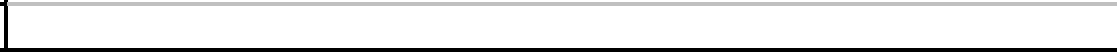 & 1200 & $\mathrm{~g}$ & OFFSITE \\
\hline
\end{tabular}




\begin{tabular}{|c|c|c|c|c|}
\hline & $A D$ & $\mathrm{AE}$ & $\mathrm{AF}$ & $A G$ \\
\hline 1 & RESULT_COMMENT & COLL_SAMP_SIZE & COLL_SAMP_SIZE_UNITS & DIST_CLASS \\
\hline 268 & & 1200 & g & OFFSITE \\
\hline 269 & & 1200 & g & OFFSITE \\
\hline 270 & & 1200 & $\mathrm{~g}$ & OFFSITE \\
\hline 271 & & 1200 & $\mathrm{~g}$ & OFFSITE \\
\hline \begin{tabular}{|l|}
272 \\
\end{tabular} & & 1200 & $\mathrm{~g}$ & ONSITE \\
\hline \begin{tabular}{|l|}
273 \\
\end{tabular} & & 1200 & $\mathrm{~g}$ & ONSITE \\
\hline 274 & & 1200 & $\mathrm{~g}$ & ONSITE \\
\hline \begin{tabular}{|l|}
275 \\
\end{tabular} & 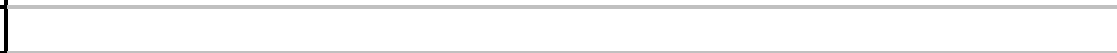 & 1200 & $\mathrm{~g}$ & ONSITE \\
\hline \begin{tabular}{|l|}
276 \\
\end{tabular} & & 1200 & $\mathrm{~g}$ & ONSITE \\
\hline \begin{tabular}{|l|}
277 \\
\end{tabular} & & 1200 & $\mathrm{~g}$ & ONSITE \\
\hline \begin{tabular}{|l|}
278 \\
\end{tabular} & & 1200 & $\mathrm{~g}$ & OFFSITE \\
\hline 279 & & 1200 & $\mathrm{~g}$ & OFFSITE \\
\hline 280 & & 1200 & $\mathrm{~g}$ & OFFSITE \\
\hline 281 & & 1200 & $\mathrm{~g}$ & OFFSITE \\
\hline 282 & & 1200 & $\mathrm{~g}$ & OFFSITE \\
\hline 283 & & 1200 & $\mathrm{~g}$ & OFFSITE \\
\hline 284 & & 1200 & $\mathrm{~g}$ & OFFSITE \\
\hline 285 & & 1200 & $\mathrm{~g}$ & OFFSITE \\
\hline \begin{tabular}{|l|}
286 \\
\end{tabular} & & 1200 & $\mathrm{~g}$ & OFFSITE \\
\hline 287 & & 1200 & $\mathrm{~g}$ & OFFSITE \\
\hline \begin{tabular}{|l|}
288 \\
\end{tabular} & & 1200 & $\mathrm{~g}$ & OFFSITE \\
\hline \begin{tabular}{|l|}
289 \\
\end{tabular} & & 1200 & $\mathrm{~g}$ & OFFSITE \\
\hline 290 & & 1200 & $\mathrm{~g}$ & OFFSITE \\
\hline \begin{tabular}{|l|}
291 \\
\end{tabular} & & 1200 & $\mathrm{~g}$ & OFFSITE \\
\hline \begin{tabular}{|l|}
292 \\
\end{tabular} & & 1200 & $\mathrm{~g}$ & OFFSITE \\
\hline 293 & & 1200 & $\mathrm{~g}$ & OFFSITE \\
\hline 294 & & 1200 & $\mathrm{~g}$ & OFFSITE \\
\hline 295 & & 1200 & $\mathrm{~g}$ & OFFSITE \\
\hline 296 & & 1200 & $\mathrm{~g}$ & OFFSITE \\
\hline \begin{tabular}{|l|}
297 \\
\end{tabular} & & 1200 & $\mathrm{~g}$ & OFFSITE \\
\hline \begin{tabular}{|l|}
298 \\
\end{tabular} & & 1200 & $\mathrm{~g}$ & OFFSITE \\
\hline \begin{tabular}{|l|}
299 \\
\end{tabular} & & 1200 & $\mathrm{~g}$ & OFFSITE \\
\hline 300 & & 1200 & $\mathrm{~g}$ & OFFSITE \\
\hline \begin{tabular}{|l|}
301 \\
\end{tabular} & & 1200 & $\mathrm{~g}$ & OFFSITE \\
\hline \begin{tabular}{|l|}
302 \\
\end{tabular} & & 1200 & $\mathrm{~g}$ & RIVER_SHORELINE \\
\hline \begin{tabular}{|l|}
303 \\
\end{tabular} & & 1200 & $\mathrm{~g}$ & RIVER_SHORELINE \\
\hline 304 & & 1200 & $\mathrm{~g}$ & RIVER_SHORELINE \\
\hline 305 & 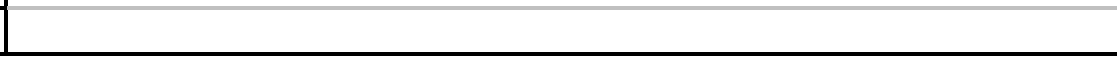 & 1200 & $\mathrm{~g}$ & RIVER SHORELINE \\
\hline
\end{tabular}




\begin{tabular}{|c|c|c|c|c|}
\hline & $A D$ & $\mathrm{AE}$ & $\mathrm{AF}$ & $A G$ \\
\hline 1 & RESULT_COMMENT & COLL_SAMP_SIZE & COLL_SAMP_SIZE_UNITS & DIST_CLASS \\
\hline 306 & & 1200 & g & RIVER_SHORELINE \\
\hline 307 & & 1200 & $\mathrm{~g}$ & RIVER_SHORELINE \\
\hline 308 & & 1200 & g & RIVER_SHORELINE \\
\hline 309 & & 1200 & g & RIVER_SHORELINE \\
\hline 310 & & 1200 & $\mathrm{~g}$ & RIVER_SHORELINE \\
\hline 311 & & 1200 & g & RIVER_SHORELINE \\
\hline 312 & & 1200 & g & RIVER_SHORELINE \\
\hline 313 & & 1200 & g & RIVER_SHORELINE \\
\hline 314 & & 1200 & g & OFFSITE \\
\hline 315 & & 1200 & g & OFFSITE \\
\hline 316 & & 1200 & $\mathrm{~g}$ & OFFSITE \\
\hline 317 & & 1200 & g & OFFSITE \\
\hline 318 & & 1200 & g & OFFSITE \\
\hline 319 & & 1200 & $\mathrm{~g}$ & OFFSITE \\
\hline 320 & & 1200 & g & OFFSITE \\
\hline 321 & & 1200 & g & OFFSITE \\
\hline 322 & & 1200 & g & OFFSITE \\
\hline 323 & & 1200 & $\mathrm{~g}$ & OFFSITE \\
\hline 324 & & 1200 & g & OFFSITE \\
\hline 325 & & 1200 & g & OFFSITE \\
\hline 326 & & 1200 & g & RIVER SHORELINE \\
\hline 327 & IR-0003 MDA not met & 1200 & g & RIVER_SHORELINE \\
\hline 328 & REFER TO 10/4/1999 INCIDENT REPORT, DUPLICATES ASSOCIATED TO THIS SAMPLE WAS OUT OF LIMITS. & 1200 & g & RIVER_SHORELINE \\
\hline 329 & REFER TO 10/4/1999 INCIDENT REPORT, DUPLICATES ASSOCIATED TO THIS SAMPLE WAS OUT OF LIMITS. & 1200 & g & RIVER_SHORELINE \\
\hline 330 & & 1200 & g & RIVER_SHORELINE \\
\hline 331 & REFER TO 10/4/1999 INCIDENT REPORT, DUPLICATES ASSOCIATED TO THIS SAMPLE WAS OUT OF LIMITS. & 1200 & g & RIVER_SHORELINE \\
\hline 332 & & 1200 & g & ONSITE \\
\hline 333 & IR-0003 MDA not met & 1200 & g & ONSITE \\
\hline 334 & REFER TO 10/4/1999 INCIDENT REPORT, DUPLICATES ASSOCIATED TO THIS SAMPLE WAS OUT OF LIMITS. & 1200 & g & ONSITE \\
\hline 335 & REFER TO 10/4/1999 INCIDENT REPORT, DUPLICATES ASSOCIATED TO THIS SAMPLE WAS OUT OF LIMITS. & 1200 & g & ONSITE \\
\hline 336 & & 1200 & g & ONSITE \\
\hline 337 & REFER TO 10/4/1999 INCIDENT REPORT, DUPLICATES ASSOCIATED TO THIS SAMPLE WAS OUT OF LIMITS. & 1200 & g & ONSITE \\
\hline 338 & & 1200 & g & OFFSITE \\
\hline 339 & IR-0013 MDA > CRDL & 1200 & g & OFFSITE \\
\hline 340 & & 1200 & g & OFFSITE \\
\hline 341 & & 1200 & g & OFFSITE \\
\hline 342 & & 1200 & $\mathrm{~g}$ & OFFSITE \\
\hline 343 & & 1200 & g & OFFSITE \\
\hline
\end{tabular}




\begin{tabular}{|c|c|c|c|c|}
\hline & $A D$ & $\mathrm{AE}$ & $\mathrm{AF}$ & $A G$ \\
\hline 1 & RESULT_COMMENT & COLL_SAMP_SIZE & COLL_SAMP_SIZE_UNITS & DIST_CLASS \\
\hline 344 & & 1200 & $\mathrm{~g}$ & OFFSITE \\
\hline 345 & IR-0013 MDA > CRDL & 1200 & g & OFFSITE \\
\hline 346 & & 1200 & $\mathrm{~g}$ & OFFSITE \\
\hline 347 & & 1200 & $\mathrm{~g}$ & OFFSITE \\
\hline 348 & & 1200 & $\mathrm{~g}$ & OFFSITE \\
\hline 349 & & 1200 & $\mathrm{~g}$ & OFFSITE \\
\hline 350 & & 1200 & $\mathrm{~g}$ & OFFSITE \\
\hline \begin{tabular}{|l|}
351 \\
\end{tabular} & IR-0013 MDA > CRDL & 1200 & $\mathrm{~g}$ & OFFSITE \\
\hline 352 & & 1200 & $\mathrm{~g}$ & OFFSITE \\
\hline 353 & & 1200 & $\mathrm{~g}$ & OFFSITE \\
\hline 354 & & 1200 & $\mathrm{~g}$ & OFFSITE \\
\hline 355 & & 1200 & $\mathrm{~g}$ & OFFSITE \\
\hline 356 & & 1200 & $\mathrm{~g}$ & RIVER_SHORELINE \\
\hline 357 & IR-0003 MDA not met & 1200 & $\mathrm{~g}$ & RIVER_SHORELINE \\
\hline 358 & REFER TO 10/4/1999 INCIDENT REPORT, DUPLICATES ASSOCIATED TO THIS SAMPLE WAS OUT OF LIMITS. & 1200 & $\mathrm{~g}$ & RIVER_SHORELINE \\
\hline 359 & REFER TO 10/4/1999 INCIDENT REPORT, DUPLICATES ASSOCIATED TO THIS SAMPLE WAS OUT OF LIMITS. & 1200 & $\mathrm{~g}$ & RIVER_SHORELINE \\
\hline 360 & & 1200 & $\mathrm{~g}$ & RIVER_SHORELINE \\
\hline 361 & REFER TO 10/4/1999 INCIDENT REPORT, DUPLICATES ASSOCIATED TO THIS SAMPLE WAS OUT OF LIMITS. & 1200 & g & RIVER_SHORELINE \\
\hline 362 & & 3953 & $\mathrm{~g}$ & RIVER_SHORELINE \\
\hline 363 & IR-0013 MDA > CRDL & 3953 & $\mathrm{~g}$ & RIVER_SHORELINE \\
\hline 364 & & 3953 & $\mathrm{~g}$ & RIVER_SHORELINE \\
\hline 365 & & 3953 & $\mathrm{~g}$ & RIVER_SHORELINE \\
\hline 366 & & 3953 & $\mathrm{~g}$ & RIVER_SHORELINE \\
\hline 367 & & 3953 & $\mathrm{~g}$ & RIVER_SHORELINE \\
\hline 368 & & 1200 & $\mathrm{~g}$ & OFFSITE \\
\hline 369 & & 1200 & $\mathrm{~g}$ & OFFSITE \\
\hline 370 & & 1200 & $\mathrm{~g}$ & OFFSITE \\
\hline 371 & & 1200 & g & OFFSITE \\
\hline 372 & & 1200 & $\mathrm{~g}$ & OFFSITE \\
\hline 373 & & 1200 & $\mathrm{~g}$ & OFFSITE \\
\hline 374 & & 1200 & $\mathrm{~g}$ & OFFSITE \\
\hline 375 & & 1200 & $\mathrm{~g}$ & OFFSITE \\
\hline 376 & & 1200 & $\mathrm{~g}$ & OFFSITE \\
\hline 377 & & 1200 & $\mathrm{~g}$ & OFFSITE \\
\hline 378 & & 1200 & $\mathrm{~g}$ & OFFSITE \\
\hline 379 & & 1200 & $\mathrm{~g}$ & OFFSITE \\
\hline 380 & & 1200 & $\mathrm{~g}$ & OFFSITE \\
\hline 381 & & 1200 & $\mathrm{~g}$ & OFFSITE \\
\hline
\end{tabular}




\begin{tabular}{|c|c|c|c|c|}
\hline & $\overline{A D}$ & $\mathrm{AE}$ & $\mathrm{AF}$ & $A G$ \\
\hline 1 & RESULT_COMMENT & COLL_SAMP_SIZE & COLL_SAMP_SIZE_UNITS & DIST_CLASS \\
\hline 382 & & 1200 & g & OFFSITE \\
\hline 383 & & 1200 & $\mathrm{~g}$ & OFFSITE \\
\hline 384 & & 1200 & $\mathrm{~g}$ & OFFSITE \\
\hline 385 & & 1200 & $g$ & OFFSITE \\
\hline 386 & & 1200 & $\mathrm{~g}$ & OFFSITE \\
\hline 387 & & 1200 & g & OFFSITE \\
\hline 388 & & 1200 & g & OFFSITE \\
\hline 389 & & 1200 & g & OFFSITE \\
\hline 390 & & 1200 & g & OFFSITE \\
\hline \begin{tabular}{|l|}
391 \\
\end{tabular} & & 1200 & g & OFFSITE \\
\hline 392 & & 1200 & $\mathrm{~g}$ & OFFSITE \\
\hline 393 & & 1200 & g & OFFSITE \\
\hline 394 & & 1200 & $g$ & OFFSITE \\
\hline 395 & & 1200 & g & OFFSITE \\
\hline 396 & & 1200 & g & OFFSITE \\
\hline 397 & & 1200 & g & OFFSITE \\
\hline 398 & & 1200 & g & OFFSITE \\
\hline 399 & & 1200 & $\mathrm{~g}$ & OFFSITE \\
\hline 400 & & 1200 & $\mathrm{~g}$ & OFFSITE \\
\hline \begin{tabular}{|l|l|}
401 \\
\end{tabular} & & 1200 & 9 & OFFSITE \\
\hline 402 & & 1200 & g & OFFSITE \\
\hline 403 & & 1200 & g & OFFSITE \\
\hline 404 & & 1200 & g & OFFSITE \\
\hline 405 & & 1200 & g & OFFSITE \\
\hline 406 & REFER TO 9/10/99 INCIDENT REPORT, PORTION OF SAMPLE SPILLED POSSIBLY INTO BLANK SAMPLE. & 1200 & g & OFFSITE \\
\hline 407 & REFER TO 9/10/99 INCIDENT REPORT, PORTION OF SAMPLE SPILLED POSSIBLY INTO BLANK SAMPLE. & 1200 & g & OFFSITE \\
\hline 408 & REFER TO 9/10/99 INCIDENT REPORT, PORTION OF SAMPLE SPILLED POSSIBLY INTO BLANK SAMPLE. & 1200 & g & OFFSITE \\
\hline 409 & REFER TO 9/10/99 INCIDENT REPORT, PORTION OF SAMPLE SPILLED POSSIBLY INTO BLANK SAMPLE. & 1200 & g & OFFSITE \\
\hline 410 & & 1200 & g & OFFSITE \\
\hline 411 & & 1200 & g & OFFSITE \\
\hline \begin{tabular}{|l|}
412 \\
\end{tabular} & & 1200 & $\mathrm{~g}$ & OFFSITE \\
\hline \begin{tabular}{|l|}
413 \\
\end{tabular} & & 1200 & g & OFFSITE \\
\hline \begin{tabular}{|l|}
414 \\
\end{tabular} & & 1200 & $\mathrm{~g}$ & OFFSITE \\
\hline 415 & & 1200 & $\mathrm{~g}$ & OFFSITE \\
\hline \begin{tabular}{|l|}
416 \\
\end{tabular} & & 1200 & g & OFFSITE \\
\hline \begin{tabular}{|l|}
417 \\
\end{tabular} & & 1200 & $\mathrm{~g}$ & OFFSITE \\
\hline \begin{tabular}{|l|}
418 \\
\end{tabular} & & 1200 & $\mathrm{~g}$ & OFFSITE \\
\hline \begin{tabular}{|l|}
419 \\
\end{tabular} & & 1200 & g & OFFSITE \\
\hline
\end{tabular}




\begin{tabular}{|c|c|c|c|c|}
\hline & $A D$ & $\overline{A E}$ & $\mathrm{AF}$ & $A G$ \\
\hline 1 & RESULT_COMMENT & COLL_SAMP_SIZE & COLL_SAMP_SIZE_UNITS & DIST_CLASS \\
\hline 420 & & 1200 & g & OFFSITE \\
\hline 421 & & 1200 & g & OFFSITE \\
\hline 422 & & 1200 & g & OFFSITE \\
\hline 423 & & 1200 & g & OFFSITE \\
\hline 424 & & 1200 & $\mathrm{~g}$ & OFFSITE \\
\hline 425 & & 1200 & g & OFFSITE \\
\hline 426 & & 1200 & g & OFFSITE \\
\hline 427 & & 1200 & g & OFFSITE \\
\hline 428 & & 1200 & g & ONSITE \\
\hline 429 & & 1200 & g & ONSITE \\
\hline 430 & IR-0029 Matrix Blank > CRDL & 1200 & g & ONSITE \\
\hline 431 & IR-0029 Matrix Blank > CRDL & 1200 & g & ONSITE \\
\hline 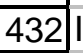 & IR-0029 Matrix Blank > CRDL & 1200 & g & ONSITE \\
\hline 433 & & 1200 & g & ONSITE \\
\hline 434 & & 1200 & g & ONSITE \\
\hline 435 & & 1200 & g & ONSITE \\
\hline 436 & & 1200 & g & ONSITE \\
\hline 437 & & 1200 & g & ONSITE \\
\hline 438 & & 1200 & g & ONSITE \\
\hline 439 & & 1200 & g & ONSITE \\
\hline 440 & IR-0029 Martix Blank > CRDL & 1200 & g & ONSITE \\
\hline 441 & IR-0029 Martix Blank > CRDL & 1200 & g & ONSITE \\
\hline 442 & IR-0029 Martix Blank > CRDL & 1200 & g & ONSITE \\
\hline 443 & & 1200 & g & ONSITE \\
\hline 444 & & 1200 & g & ONSITE \\
\hline 445 & IR-0029 Matrix Blank > CRDL & 1200 & g & ONSITE \\
\hline $\begin{array}{ll}446 \\
\end{array}$ & IR-0029 Matrix Blank > CRDL & 1200 & g & ONSITE \\
\hline 447 & IR-0029 Matrix Blank > CRDL & 1200 & g & ONSITE \\
\hline 448 & & 1200 & g & ONSITE \\
\hline 449 & & 1200 & g & ONSITE \\
\hline 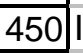 & IR-0029 Matrix Blank > CRDL & 1200 & g & ONSITE \\
\hline 451 & IR-0029 Matrix Blank > CRDL & 1200 & g & ONSITE \\
\hline 452 I & IR-0029 Matrix Blank > CRDL & 1200 & g & ONSITE \\
\hline 453 & & 1200 & g & ONSITE \\
\hline 454 & & 1200 & g & ONSITE \\
\hline 455 & IR-0029 Matrix Blank > CRDL & 1200 & g & ONSITE \\
\hline $\begin{array}{ll}456 \\
4\end{array}$ & IR-0029 Matrix Blank > CRDL & 1200 & g & ONSITE \\
\hline 457| & IR-0029 Matrix Blank > CRDL & 1200 & g & ONSITE \\
\hline
\end{tabular}




\begin{tabular}{|r|l|l|}
\hline & \multicolumn{1}{|c|}{ AH } & \\
\hline 1 & OWNER_ID & COLLECTION_PURPOSE \\
\hline 2 & HEISPROD & \\
\hline 3 & HEISPROD & \\
\hline 4 & HEISPROD & \\
\hline 5 & HEISPROD & \\
\hline 6 & HEISPROD & \\
\hline 7 & HEISPROD & \\
\hline 8 & HEISPROD & \\
\hline 9 & HEISPROD & \\
\hline 10 & HEISPROD & \\
\hline 11 & HEISPROD & \\
\hline 12 & HEISPROD & \\
\hline 13 & HEISPROD & \\
\hline 14 & SESPMNT & $\mathrm{R}$ \\
\hline 15 & SESPMNT & $\mathrm{R}$ \\
\hline 16 & SESPMNT & $\mathrm{R}$ \\
\hline 17 & SESPMNT & $\mathrm{R}$ \\
\hline 18 & SESPMNT & $\mathrm{R}$ \\
\hline 19 & SESPMNT & $\mathrm{R}$ \\
\hline 20 & SESPMNT & $\mathrm{R}$ \\
\hline 21 & SESPMNT & $\mathrm{R}$ \\
\hline 22 & SESPMNT & $\mathrm{R}$ \\
\hline 23 & SESPMNT & $\mathrm{R}$ \\
\hline 24 & SESPMNT & $\mathrm{R}$ \\
\hline 25 & SESPMNT & $\mathrm{R}$ \\
\hline 26 & SESPMNT & $\mathrm{R}$ \\
\hline 27 & SESPMNT & $\mathrm{R}$ \\
\hline 28 & SESPMNT & $\mathrm{R}$ \\
\hline 29 & SESPMNT & $\mathrm{R}$ \\
\hline 30 & SESPMNT & $\mathrm{R}$ \\
\hline 31 & SESPMNT & $\mathrm{R}$ \\
\hline 32 & SESPMNT & $\mathrm{R}$ \\
\hline 33 & SESPMNT & $\mathrm{R}$ \\
\hline 34 & SESPMNT & $\mathrm{R}$ \\
\hline 35 & SESPMNT & $\mathrm{R}$ \\
\hline 36 & SESPMNT & $\mathrm{R}$ \\
\hline 37 & SESPMNT & $\mathrm{R}$ \\
\hline 38 & SESPMNT & $\mathrm{R}$ \\
\hline 39 & SESPMNT & $\mathrm{R}$ \\
\hline & & \\
\hline
\end{tabular}




\begin{tabular}{|c|l|l|}
\hline & \multicolumn{1}{|c|}{ AH } & \\
\hline 1 & OWNER_ID & COLLECTION_PURPOSE \\
\hline 40 & SESPMNT & $\mathrm{R}$ \\
\hline 41 & SESPMNT & $\mathrm{R}$ \\
\hline 42 & SESPMNT & $\mathrm{R}$ \\
\hline 43 & SESPMNT & $\mathrm{R}$ \\
\hline 44 & SESPMNT & $\mathrm{R}$ \\
\hline 45 & SESPMNT & $\mathrm{R}$ \\
\hline 46 & SESPMNT & $\mathrm{R}$ \\
\hline 47 & SESPMNT & $\mathrm{R}$ \\
\hline 48 & SESPMNT & $\mathrm{R}$ \\
\hline 49 & SESPMNT & $\mathrm{R}$ \\
\hline 50 & SESPMNT & $\mathrm{R}$ \\
\hline 51 & SESPMNT & $\mathrm{R}$ \\
\hline 52 & SESPMNT & $\mathrm{R}$ \\
\hline 53 & SESPMNT & $\mathrm{R}$ \\
\hline 54 & SESPMNT & $\mathrm{R}$ \\
\hline 55 & SESPMNT & $\mathrm{R}$ \\
\hline 56 & SESPMNT & $\mathrm{R}$ \\
\hline 57 & SESPMNT & $\mathrm{R}$ \\
\hline 58 & SESPMNT & $\mathrm{R}$ \\
\hline 59 & SESPMNT & $\mathrm{R}$ \\
\hline 60 & SESPMNT & $\mathrm{R}$ \\
\hline 61 & SESPMNT & $\mathrm{R}$ \\
\hline 62 & SESPMNT & $\mathrm{R}$ \\
\hline 63 & SESPMNT & $\mathrm{R}$ \\
\hline 64 & SESPMNT & $\mathrm{R}$ \\
\hline 65 & SESPMNT & $\mathrm{R}$ \\
\hline 66 & SESPMNT & $\mathrm{R}$ \\
\hline 67 & SESPMNT & $\mathrm{R}$ \\
\hline 68 & SESPMNT & $\mathrm{R}$ \\
\hline 69 & SESPMNT & $\mathrm{R}$ \\
\hline 70 & SESPMNT & $\mathrm{R}$ \\
\hline 71 & SESPMNT & $\mathrm{R}$ \\
\hline 72 & SESPMNT & $\mathrm{R}$ \\
\hline 73 & SESPMNT & $\mathrm{R}$ \\
\hline 74 & SESPMNT & $\mathrm{R}$ \\
\hline 75 & SESPMNT & $\mathrm{R}$ \\
\hline 76 & SESPMNT & $\mathrm{R}$ \\
\hline 77 & SESPMNT & $\mathrm{R}$ \\
\hline & & \\
\hline
\end{tabular}




\begin{tabular}{|c|l|l|}
\hline & \multicolumn{1}{|c|}{ AH } & \\
\hline 1 & OWNER_ID & COLLECTION_PURPOSE \\
\hline 78 & SESPMNT & $\mathrm{R}$ \\
\hline 79 & SESPMNT & $\mathrm{R}$ \\
\hline 80 & SESPMNT & $\mathrm{R}$ \\
\hline 81 & SESPMNT & $\mathrm{R}$ \\
\hline 82 & SESPMNT & $\mathrm{R}$ \\
\hline 83 & SESPMNT & $\mathrm{R}$ \\
\hline 84 & SESPMNT & $\mathrm{R}$ \\
\hline 85 & SESPMNT & $\mathrm{R}$ \\
\hline 86 & SESPMNT & $\mathrm{R}$ \\
\hline 87 & SESPMNT & $\mathrm{R}$ \\
\hline 88 & SESPMNT & $\mathrm{R}$ \\
\hline 89 & SESPMNT & $\mathrm{R}$ \\
\hline 90 & SESPMNT & $\mathrm{R}$ \\
\hline 91 & SESPMNT & $\mathrm{R}$ \\
\hline 92 & SESPMNT & $\mathrm{R}$ \\
\hline 93 & SESPMNT & $\mathrm{R}$ \\
\hline 94 & SESPMNT & $\mathrm{R}$ \\
\hline 95 & SESPMNT & $\mathrm{R}$ \\
\hline 96 & SESPMNT & $\mathrm{R}$ \\
\hline 97 & SESPMNT & $\mathrm{R}$ \\
\hline 98 & SESPMNT & $\mathrm{R}$ \\
\hline 99 & SESPMNT & $\mathrm{R}$ \\
\hline 100 & SESPMNT & $\mathrm{R}$ \\
\hline 101 & SESPMNT & $\mathrm{R}$ \\
\hline 102 & SESPMNT & $\mathrm{R}$ \\
\hline 103 & SESPMNT & $\mathrm{R}$ \\
\hline 104 & SESPMNT & $\mathrm{R}$ \\
\hline 105 & SESPMNT & $\mathrm{R}$ \\
\hline 106 & SESPMNT & $\mathrm{R}$ \\
\hline 107 & SESPMNT & $\mathrm{R}$ \\
\hline 108 & SESPMNT & $\mathrm{R}$ \\
\hline 109 & SESPMNT & $\mathrm{R}$ \\
\hline 110 & SESPMNT & $\mathrm{R}$ \\
\hline 111 & SESPMNT & $\mathrm{R}$ \\
\hline 112 & SESPMNT & $\mathrm{R}$ \\
\hline 113 & SESPMNT & $\mathrm{R}$ \\
\hline 114 & SESPMNT & $\mathrm{R}$ \\
\hline 115 & SESPMNT & $\mathrm{R}$ \\
\hline & & \\
\hline
\end{tabular}




\begin{tabular}{|c|l|l|}
\hline & \multicolumn{1}{|c|}{ AH } & \\
\hline 1 & OWNER_ID & COLLECTION_PURPOSE \\
\hline 116 & SESPMNT & $\mathrm{R}$ \\
\hline 117 & SESPMNT & $\mathrm{R}$ \\
\hline 118 & SESPMNT & $\mathrm{R}$ \\
\hline 119 & SESPMNT & $\mathrm{R}$ \\
\hline 120 & SESPMNT & $\mathrm{R}$ \\
\hline 121 & SESPMNT & $\mathrm{R}$ \\
\hline 122 & SESPMNT & $\mathrm{R}$ \\
\hline 123 & SESPMNT & $\mathrm{R}$ \\
\hline 124 & SESPMNT & $\mathrm{R}$ \\
\hline 125 & SESPMNT & $\mathrm{R}$ \\
\hline 126 & SESPMNT & $\mathrm{R}$ \\
\hline 127 & SESPMNT & $\mathrm{R}$ \\
\hline 128 & SESPMNT & $\mathrm{R}$ \\
\hline 129 & SESPMNT & $\mathrm{R}$ \\
\hline 130 & SESPMNT & $\mathrm{R}$ \\
\hline 131 & SESPMNT & $\mathrm{R}$ \\
\hline 132 & SESPMNT & $\mathrm{R}$ \\
\hline 133 & SESPMNT & $\mathrm{R}$ \\
\hline 134 & SESPMNT & $\mathrm{R}$ \\
\hline 135 & SESPMNT & $\mathrm{R}$ \\
\hline 136 & SESPMNT & $\mathrm{R}$ \\
\hline 137 & SESPMNT & $\mathrm{R}$ \\
\hline 138 & SESPMNT & $\mathrm{R}$ \\
\hline 139 & SESPMNT & $\mathrm{R}$ \\
\hline 140 & SESPMNT & $\mathrm{R}$ \\
\hline 141 & SESPMNT & $\mathrm{R}$ \\
\hline 142 & SESPMNT & $\mathrm{R}$ \\
\hline 143 & SESPMNT & $\mathrm{R}$ \\
\hline 144 & SESPMNT & $\mathrm{R}$ \\
\hline 145 & SESPMNT & $\mathrm{R}$ \\
\hline 146 & SESPMNT & $\mathrm{R}$ \\
\hline 147 & SESPMNT & $\mathrm{R}$ \\
\hline 148 & SESPMNT & $\mathrm{R}$ \\
\hline 149 & SESPMNT & $\mathrm{R}$ \\
\hline 150 & SESPMNT & $\mathrm{R}$ \\
\hline 151 & SESPMNT & $\mathrm{R}$ \\
\hline 152 & SESPMNT & $\mathrm{R}$ \\
\hline 153 & SESPMNT & $\mathrm{R}$ \\
\hline & & \\
\hline
\end{tabular}




\begin{tabular}{|c|l|l|}
\hline & \multicolumn{1}{|c|}{ AH } & \\
\hline 1 & OWNER_ID & COLLECTION_PURPOSE \\
\hline 154 & SESPMNT & $\mathrm{R}$ \\
\hline 155 & SESPMNT & $\mathrm{R}$ \\
\hline 156 & SESPMNT & $\mathrm{R}$ \\
\hline 157 & SESPMNT & $\mathrm{R}$ \\
\hline 158 & SESPMNT & $\mathrm{R}$ \\
\hline 159 & SESPMNT & $\mathrm{R}$ \\
\hline 160 & SESPMNT & $\mathrm{R}$ \\
\hline 161 & SESPMNT & $\mathrm{R}$ \\
\hline 162 & SESPMNT & $\mathrm{R}$ \\
\hline 163 & SESPMNT & $\mathrm{R}$ \\
\hline 164 & SESPMNT & $\mathrm{R}$ \\
\hline 165 & SESPMNT & $\mathrm{R}$ \\
\hline 166 & SESPMNT & $\mathrm{R}$ \\
\hline 167 & SESPMNT & $\mathrm{R}$ \\
\hline 168 & SESPMNT & $\mathrm{R}$ \\
\hline 169 & SESPMNT & $\mathrm{R}$ \\
\hline 170 & SESPMNT & $\mathrm{R}$ \\
\hline 171 & SESPMNT & $\mathrm{R}$ \\
\hline 172 & SESPMNT & $\mathrm{R}$ \\
\hline 173 & SESPMNT & $\mathrm{R}$ \\
\hline 174 & SESPSPEC & $\mathrm{S}$ \\
\hline 175 & SESPSPEC & $\mathrm{S}$ \\
\hline 176 & SESPSPEC & $\mathrm{S}$ \\
\hline 177 & SESPSPEC & $\mathrm{S}$ \\
\hline 178 & SESPSPEC & $\mathrm{S}$ \\
\hline 179 & SESPSPEC & $\mathrm{S}$ \\
\hline 180 & SESPMNT & $\mathrm{R}$ \\
\hline 181 & SESPMNT & $\mathrm{R}$ \\
\hline 182 & SESPMNT & $\mathrm{R}$ \\
\hline 183 & SESPMNT & $\mathrm{R}$ \\
\hline 184 & SESPMNT & $\mathrm{R}$ \\
\hline 185 & SESPMNT & $\mathrm{R}$ \\
\hline 186 & SESPMNT & $\mathrm{R}$ \\
\hline 187 & SESPMNT & $\mathrm{R}$ \\
\hline 188 & SESPMNT & $\mathrm{R}$ \\
\hline 189 & SESPMNT & $\mathrm{R}$ \\
\hline 190 & SESPMNT & $\mathrm{R}$ \\
\hline 191 & SESPMNT & $\mathrm{R}$ \\
\hline & & \\
\hline
\end{tabular}




\begin{tabular}{|c|l|l|}
\hline & \multicolumn{1}{|c|}{ AH } & \\
\hline 1 & OWNER_ID & COLLECTION_PURPOSE \\
\hline 192 & SESPMNT & $\mathrm{R}$ \\
\hline 193 & SESPMNT & $\mathrm{R}$ \\
\hline 194 & SESPMNT & $\mathrm{R}$ \\
\hline 195 & SESPMNT & $\mathrm{R}$ \\
\hline 196 & SESPMNT & $\mathrm{R}$ \\
\hline 197 & SESPMNT & $\mathrm{R}$ \\
\hline 198 & SESPMNT & $\mathrm{R}$ \\
\hline 199 & SESPMNT & $\mathrm{R}$ \\
\hline 200 & SESPSPEC & $\mathrm{S}$ \\
\hline 201 & SESPSPEC & $\mathrm{S}$ \\
\hline 202 & SESPSPEC & $\mathrm{S}$ \\
\hline 203 & SESPSPEC & $\mathrm{S}$ \\
\hline 204 & SESPSPEC & $\mathrm{S}$ \\
\hline 205 & SESPSPEC & $\mathrm{S}$ \\
\hline 206 & SESPSPEC & $\mathrm{S}$ \\
\hline 207 & SESPSPEC & $\mathrm{S}$ \\
\hline 208 & SESPSPEC & $\mathrm{S}$ \\
\hline 209 & SESPSPEC & $\mathrm{S}$ \\
\hline 210 & SESPSPEC & $\mathrm{S}$ \\
\hline 211 & SESPSPEC & $\mathrm{S}$ \\
\hline 212 & SESPSPEC & $\mathrm{S}$ \\
\hline 213 & SESPSPEC & $\mathrm{S}$ \\
\hline 214 & SESPSPEC & $\mathrm{S}$ \\
\hline 215 & SESPSPEC & $\mathrm{S}$ \\
\hline 216 & SESPSPEC & $\mathrm{S}$ \\
\hline 217 & SESPSPEC & $\mathrm{S}$ \\
\hline 218 & SESPMNT & $\mathrm{R}$ \\
\hline 219 & SESPMNT & $\mathrm{R}$ \\
\hline 220 & SESPMNT & $\mathrm{R}$ \\
\hline 221 & SESPMNT & $\mathrm{R}$ \\
\hline 222 & SESPMNT & $\mathrm{R}$ \\
\hline 223 & SESPMNT & $\mathrm{R}$ \\
\hline 224 & SESPMNT & $\mathrm{R}$ \\
\hline 225 & SESPMNT & $\mathrm{R}$ \\
\hline 226 & SESPMNT & $\mathrm{R}$ \\
\hline 227 & SESPMNT & $\mathrm{R}$ \\
\hline 228 & SESPMNT & $\mathrm{R}$ \\
\hline 229 & SESPMNT & $\mathrm{R}$ \\
\hline
\end{tabular}




\begin{tabular}{|c|l|l|}
\hline & \multicolumn{1}{|c|}{ AH } & \\
\hline 1 & OWNER_ID & COLLECTION_PURPOSE \\
\hline 230 & SESPMNT & $\mathrm{R}$ \\
\hline 231 & SESPMNT & $\mathrm{R}$ \\
\hline 232 & SESPMNT & $\mathrm{R}$ \\
\hline 233 & SESPMNT & $\mathrm{R}$ \\
\hline 234 & SESPMNT & $\mathrm{R}$ \\
\hline 235 & SESPMNT & $\mathrm{R}$ \\
\hline 236 & SESPMNT & $\mathrm{R}$ \\
\hline 237 & SESPMNT & $\mathrm{R}$ \\
\hline 238 & SESPMNT & $\mathrm{R}$ \\
\hline 239 & SESPMNT & $\mathrm{R}$ \\
\hline 240 & SESPMNT & $\mathrm{R}$ \\
\hline 241 & SESPMNT & $\mathrm{R}$ \\
\hline 242 & SESPMNT & $\mathrm{R}$ \\
\hline 243 & SESPMNT & $\mathrm{R}$ \\
\hline 244 & SESPMNT & $\mathrm{R}$ \\
\hline 245 & SESPMNT & $\mathrm{R}$ \\
\hline 246 & SESPMNT & $\mathrm{R}$ \\
\hline 247 & SESPMNT & $\mathrm{R}$ \\
\hline 248 & SESPMNT & $\mathrm{R}$ \\
\hline 249 & SESPMNT & $\mathrm{R}$ \\
\hline 250 & SESPMNT & $\mathrm{R}$ \\
\hline 251 & SESPMNT & $\mathrm{R}$ \\
\hline 252 & SESPMNT & $\mathrm{R}$ \\
\hline 253 & SESPMNT & $\mathrm{R}$ \\
\hline 254 & SESPMNT & $\mathrm{R}$ \\
\hline 255 & SESPMNT & $\mathrm{R}$ \\
\hline 256 & SESPMNT & $\mathrm{R}$ \\
\hline 257 & SESPMNT & $\mathrm{R}$ \\
\hline 258 & SESPMNT & $\mathrm{R}$ \\
\hline 259 & SESPMNT & $\mathrm{R}$ \\
\hline 260 & SESPMNT & $\mathrm{R}$ \\
\hline 261 & SESPMNT & $\mathrm{R}$ \\
\hline 262 & SESPMNT & $\mathrm{R}$ \\
\hline 263 & SESPMNT & $\mathrm{R}$ \\
\hline 264 & SESPMNT & $\mathrm{R}$ \\
\hline 265 & SESPMNT & $\mathrm{R}$ \\
\hline 266 & SESPMNT & $\mathrm{R}$ \\
\hline 267 & SESPMNT & $\mathrm{R}$ \\
\hline & & \\
\hline
\end{tabular}




\begin{tabular}{|c|l|l|}
\hline & \multicolumn{1}{|c|}{ AH } & \\
\hline 1 & OWNER_ID & COLLECTION_PURPOSE \\
\hline 268 & SESPMNT & $\mathrm{R}$ \\
\hline 269 & SESPMNT & $\mathrm{R}$ \\
\hline 270 & SESPMNT & $\mathrm{R}$ \\
\hline 271 & SESPMNT & $\mathrm{R}$ \\
\hline 272 & SESPMNT & $\mathrm{R}$ \\
\hline 273 & SESPMNT & $\mathrm{R}$ \\
\hline 274 & SESPMNT & $\mathrm{R}$ \\
\hline 275 & SESPMNT & $\mathrm{R}$ \\
\hline 276 & SESPMNT & $\mathrm{R}$ \\
\hline 277 & SESPMNT & $\mathrm{R}$ \\
\hline 278 & SESPSPEC & $\mathrm{S}$ \\
\hline 279 & SESPSPEC & $\mathrm{S}$ \\
\hline 280 & SESPSPEC & $\mathrm{S}$ \\
\hline 281 & SESPSPEC & $\mathrm{S}$ \\
\hline 282 & SESPSPEC & $\mathrm{S}$ \\
\hline 283 & SESPSPEC & $\mathrm{S}$ \\
\hline 284 & SESPSPEC & $\mathrm{S}$ \\
\hline 285 & SESPSPEC & $\mathrm{S}$ \\
\hline 286 & SESPSPEC & $\mathrm{S}$ \\
\hline 287 & SESPSPEC & $\mathrm{S}$ \\
\hline 288 & SESPSPEC & $\mathrm{S}$ \\
\hline 289 & SESPSPEC & $\mathrm{S}$ \\
\hline 290 & SESPSPEC & $\mathrm{S}$ \\
\hline 291 & SESPSPEC & $\mathrm{S}$ \\
\hline 292 & SESPSPEC & $\mathrm{S}$ \\
\hline 293 & SESPSPEC & $\mathrm{S}$ \\
\hline 294 & SESPSPEC & $\mathrm{S}$ \\
\hline 295 & SESPSPEC & $\mathrm{S}$ \\
\hline 296 & SESPSPEC & $\mathrm{S}$ \\
\hline 297 & SESPSPEC & $\mathrm{S}$ \\
\hline 298 & SESPSPEC & $\mathrm{S}$ \\
\hline 299 & SESPSPEC & $\mathrm{S}$ \\
\hline 300 & SESPSPEC & $\mathrm{S}$ \\
\hline 301 & SESPSPEC & $\mathrm{S}$ \\
\hline 302 & SESPMNT & $\mathrm{R}$ \\
\hline 303 & SESPMNT & $\mathrm{R}$ \\
\hline 304 & SESPMNT & $\mathrm{R}$ \\
\hline 305 & SESPMNT & $\mathrm{R}$ \\
\hline & & \\
\hline
\end{tabular}




\begin{tabular}{|c|l|l|}
\hline & \multicolumn{1}{|c|}{ AH } & \\
\hline 1 & OWNER_ID & COLLECTION_PURPOSE \\
\hline 306 & SESPMNT & $\mathrm{R}$ \\
\hline 307 & SESPMNT & $\mathrm{R}$ \\
\hline 308 & SESPMNT & $\mathrm{R}$ \\
\hline 309 & SESPMNT & $\mathrm{R}$ \\
\hline 310 & SESPMNT & $\mathrm{R}$ \\
\hline 311 & SESPMNT & $\mathrm{R}$ \\
\hline 312 & SESPMNT & $\mathrm{R}$ \\
\hline 313 & SESPMNT & $\mathrm{R}$ \\
\hline 314 & SESPMNT & $\mathrm{R}$ \\
\hline 315 & SESPMNT & $\mathrm{R}$ \\
\hline 316 & SESPMNT & $\mathrm{R}$ \\
\hline 317 & SESPMNT & $\mathrm{R}$ \\
\hline 318 & SESPMNT & $\mathrm{R}$ \\
\hline 319 & SESPMNT & $\mathrm{R}$ \\
\hline 320 & SESPMNT & $\mathrm{R}$ \\
\hline 321 & SESPMNT & $\mathrm{R}$ \\
\hline 322 & SESPMNT & $\mathrm{R}$ \\
\hline 323 & SESPMNT & $\mathrm{R}$ \\
\hline 324 & SESPMNT & $\mathrm{R}$ \\
\hline 325 & SESPMNT & $\mathrm{R}$ \\
\hline 326 & SESPMNT & $\mathrm{R}$ \\
\hline 327 & SESPMNT & $\mathrm{R}$ \\
\hline 328 & SESPMNT & $\mathrm{R}$ \\
\hline 329 & SESPMNT & $\mathrm{R}$ \\
\hline 330 & SESPMNT & $\mathrm{R}$ \\
\hline 331 & SESPMNT & $\mathrm{R}$ \\
\hline 332 & SESPMNT & $\mathrm{R}$ \\
\hline 333 & SESPMNT & $\mathrm{R}$ \\
\hline 334 & SESPMNT & $\mathrm{R}$ \\
\hline 335 & SESPMNT & $\mathrm{R}$ \\
\hline 336 & SESPMNT & $\mathrm{R}$ \\
\hline 337 & SESPMNT & $\mathrm{R}$ \\
\hline 338 & SESPSPEC & $\mathrm{S}$ \\
\hline 339 & SESPSPEC & $\mathrm{S}$ \\
\hline 340 & SESPSPEC & $\mathrm{S}$ \\
\hline 341 & SESPSPEC & $\mathrm{S}$ \\
\hline 342 & SESPSPEC & $\mathrm{S}$ \\
\hline 343 & SESPSPEC & $\mathrm{S}$ \\
\hline & & \\
\hline
\end{tabular}




\begin{tabular}{|c|l|l|}
\hline & AH & \\
\hline 1 & OWNER_ID & COLLECTION_PURPOSE \\
\hline 344 & SESPSPEC & $\mathrm{S}$ \\
\hline 345 & SESPSPEC & $\mathrm{S}$ \\
\hline 346 & SESPSPEC & $\mathrm{S}$ \\
\hline 347 & SESPSPEC & $\mathrm{S}$ \\
\hline 348 & SESPSPEC & $\mathrm{S}$ \\
\hline 349 & SESPSPEC & $\mathrm{S}$ \\
\hline 350 & SESPSPEC & $\mathrm{S}$ \\
\hline 351 & SESPSPEC & $\mathrm{S}$ \\
\hline 352 & SESPSPEC & $\mathrm{S}$ \\
\hline 353 & SESPSPEC & $\mathrm{S}$ \\
\hline 354 & SESPSPEC & $\mathrm{S}$ \\
\hline 355 & SESPSPEC & $\mathrm{S}$ \\
\hline 356 & SESPMNT & $\mathrm{R}$ \\
\hline 357 & SESPMNT & $\mathrm{R}$ \\
\hline 358 & SESPMNT & $\mathrm{R}$ \\
\hline 359 & SESPMNT & $\mathrm{R}$ \\
\hline 360 & SESPMNT & $\mathrm{R}$ \\
\hline 361 & SESPMNT & $\mathrm{R}$ \\
\hline 362 & SESPMNT & $\mathrm{R}$ \\
\hline 363 & SESPMNT & $\mathrm{R}$ \\
\hline 364 & SESPMNT & $\mathrm{R}$ \\
\hline 365 & SESPMNT & $\mathrm{R}$ \\
\hline 366 & SESPMNT & $\mathrm{R}$ \\
\hline 367 & SESPMNT & $\mathrm{R}$ \\
\hline 368 & SESPMNT & $\mathrm{R}$ \\
\hline 369 & SESPMNT & $\mathrm{R}$ \\
\hline 370 & SESPMNT & $\mathrm{R}$ \\
\hline 371 & SESPMNT & $\mathrm{R}$ \\
\hline 372 & SESPMNT & $\mathrm{R}$ \\
\hline 373 & SESPMNT & $\mathrm{R}$ \\
\hline 374 & SESPMNT & $\mathrm{R}$ \\
\hline 375 & SESPMNT & $\mathrm{R}$ \\
\hline 376 & SESPMNT & $\mathrm{R}$ \\
\hline 377 & SESPMNT & $\mathrm{R}$ \\
\hline 378 & SESPMNT & $\mathrm{R}$ \\
\hline 379 & SESPMNT & $\mathrm{R}$ \\
\hline 380 & SESPMNT & $\mathrm{R}$ \\
\hline 381 & SESPMNT & $\mathrm{R}$ \\
\hline
\end{tabular}




\begin{tabular}{|c|l|l|}
\hline & \multicolumn{1}{|c|}{ AH } & \\
\hline 1 & OWNER_ID & COLLECTION_PURPOSE \\
\hline 382 & SESPMNT & $\mathrm{R}$ \\
\hline 383 & SESPMNT & $\mathrm{R}$ \\
\hline 384 & SESPMNT & $\mathrm{R}$ \\
\hline 385 & SESPMNT & $\mathrm{R}$ \\
\hline 386 & SESPMNT & $\mathrm{R}$ \\
\hline 387 & SESPMNT & $\mathrm{R}$ \\
\hline 388 & SESPMNT & $\mathrm{R}$ \\
\hline 389 & SESPMNT & $\mathrm{R}$ \\
\hline 390 & SESPMNT & $\mathrm{R}$ \\
\hline 391 & SESPMNT & $\mathrm{R}$ \\
\hline 392 & SESPMNT & $\mathrm{R}$ \\
\hline 393 & SESPMNT & $\mathrm{R}$ \\
\hline 394 & SESPMNT & $\mathrm{R}$ \\
\hline 395 & SESPMNT & $\mathrm{R}$ \\
\hline 396 & SESPMNT & $\mathrm{R}$ \\
\hline 397 & SESPMNT & $\mathrm{R}$ \\
\hline 398 & SESPMNT & $\mathrm{R}$ \\
\hline 399 & SESPMNT & $\mathrm{R}$ \\
\hline 400 & SESPMNT & $\mathrm{R}$ \\
\hline 401 & SESPMNT & $\mathrm{R}$ \\
\hline 402 & SESPMNT & $\mathrm{R}$ \\
\hline 403 & SESPMNT & $\mathrm{R}$ \\
\hline 404 & SESPSPEC & $\mathrm{S}$ \\
\hline 405 & SESPSPEC & $\mathrm{S}$ \\
\hline 406 & SESPSPEC & $\mathrm{S}$ \\
\hline 407 & SESPSPEC & $\mathrm{S}$ \\
\hline 408 & SESPSPEC & $\mathrm{S}$ \\
\hline 409 & SESPSPEC & $\mathrm{S}$ \\
\hline 410 & SESPSPEC & $\mathrm{S}$ \\
\hline 411 & SESPSPEC & $\mathrm{S}$ \\
\hline 412 & SESPSPEC & $\mathrm{S}$ \\
\hline 413 & SESPSPEC & $\mathrm{S}$ \\
\hline 414 & SESPSPEC & $\mathrm{S}$ \\
\hline 415 & SESPSPEC & $\mathrm{S}$ \\
\hline 416 & SESPSPEC & $\mathrm{S}$ \\
\hline 417 & SESPSPEC & $\mathrm{S}$ \\
\hline 418 & SESPSPEC & $\mathrm{S}$ \\
\hline 419 & SESPSPEC & $\mathrm{S}$ \\
\hline & & \\
\hline
\end{tabular}




\begin{tabular}{|c|l|l|}
\hline & \multicolumn{1}{|c|}{ AH } & \\
\hline 1 & OWNER_ID & COLLECTION_PURPOSE \\
\hline 420 & SESPSPEC & $\mathrm{S}$ \\
\hline 421 & SESPSPEC & $\mathrm{S}$ \\
\hline 422 & SESPSPEC & $\mathrm{S}$ \\
\hline 423 & SESPSPEC & $\mathrm{S}$ \\
\hline 424 & SESPSPEC & $\mathrm{S}$ \\
\hline 425 & SESPSPEC & $\mathrm{S}$ \\
\hline 426 & SESPSPEC & $\mathrm{S}$ \\
\hline 427 & SESPSPEC & $\mathrm{S}$ \\
\hline 428 & SESPMNT & $\mathrm{R}$ \\
\hline 429 & SESPMNT & $\mathrm{R}$ \\
\hline 430 & SESPMNT & $\mathrm{R}$ \\
\hline 431 & SESPMNT & $\mathrm{R}$ \\
\hline 432 & SESPMNT & $\mathrm{R}$ \\
\hline 433 & SESPMNT & $\mathrm{R}$ \\
\hline 434 & SESPMNT & $\mathrm{R}$ \\
\hline 435 & SESPMNT & $\mathrm{R}$ \\
\hline 436 & SESPMNT & $\mathrm{R}$ \\
\hline 437 & SESPMNT & $\mathrm{R}$ \\
\hline 438 & SESPMNT & $\mathrm{R}$ \\
\hline 439 & SESPMNT & $\mathrm{R}$ \\
\hline 440 & SESPMNT & $\mathrm{R}$ \\
\hline 441 & SESPMNT & $\mathrm{R}$ \\
\hline 442 & SESPMNT & $\mathrm{R}$ \\
\hline 443 & SESPMNT & $\mathrm{R}$ \\
\hline 444 & SESPMNT & $\mathrm{R}$ \\
\hline 445 & SESPMNT & $\mathrm{R}$ \\
\hline 446 & SESPMNT & $\mathrm{R}$ \\
\hline 447 & SESPMNT & $\mathrm{R}$ \\
\hline 448 & SESPMNT & $\mathrm{R}$ \\
\hline 449 & SESPMNT & $\mathrm{R}$ \\
\hline 450 & SESPMNT & $\mathrm{R}$ \\
\hline 451 & SESPMNT & $\mathrm{R}$ \\
\hline 452 & SESPMNT & $\mathrm{R}$ \\
\hline 453 & SESPMNT & $\mathrm{R}$ \\
\hline 454 & SESPMNT & $\mathrm{R}$ \\
\hline 455 & SESPMNT & $\mathrm{R}$ \\
\hline 456 & SESPMNT & $\mathrm{R}$ \\
\hline 457 & SESPMNT & $\mathrm{R}$ \\
\hline & & \\
\hline
\end{tabular}


96 - 99 SW_FNL 


\begin{tabular}{|c|c|c|c|c|c|c|c|c|c|c|c|}
\hline & A & $\mathrm{B}$ & $\mathrm{C}$ & $\mathrm{D}$ & $E$ & $\mathrm{~F}$ & G & $\mathrm{H}$ & $\mathrm{I}$ & $\mathrm{J}$ & $\mathrm{K}$ \\
\hline \multicolumn{12}{|l|}{1} \\
\hline \multirow{2}{*}{\multicolumn{12}{|c|}{ CON SHORT NAME }} \\
\hline & & & & & & & & & & & \\
\hline 4 & SAMPLE_LOCATION & Data & CS-137 & $\mathrm{I}-129$ & PU-239/240 & SR-90 & TC-99 & TRITIUM & $\mathrm{U}-234$ & $\mathrm{U}-235$ & $\mathrm{U}-238$ \\
\hline 5 & \multirow[t]{4}{*}{$100 \mathrm{~F}$} & Count of VALUE_RPTD & 1 & & & 41 & & 45 & 40 & 40 & 40 \\
\hline 6 & & Max of VALUE_RPTD2 & -0.635 & & & 1.12 & & 96 & 0.315 & 0.0287 & 0.279 \\
\hline 7 & & Average of VALUE_RPTD3 & -0.635 & & & 0.106863415 & & 34.26222222 & 0.2257 & 0.005860448 & 0.176 \\
\hline 8 & & StdDev of VALUE_RPTD4 & \#DIV/O! & & & 0.16317812 & & 18.50513797 & 0.034299735 & 0.006927011 & 0.028641014 \\
\hline 9 & \multirow[t]{4}{*}{$100 \mathrm{H}$} & Count of VALUE_RPTD & 1 & & & 1 & & 2 & & & \\
\hline 10 & & Max of VALUE_RPTD2 & 0.0223 & & & 2.63 & & 26 & & & \\
\hline 11 & & Average of VALUE_RPTD3 & 0.0223 & & & 2.63 & & 21.25 & & & \\
\hline 12 & & StdDev of VALUE_RPTD4 & \#DIV/O! & & & \#DIV/O! & & 6.717514421 & & & \\
\hline 13 & \multirow[t]{4}{*}{$100 \mathrm{~N}$} & Count of VALUE_RPTD & 9 & & & 38 & & 47 & 37 & 37 & 37 \\
\hline 14 & & Max of VALUE_RPTD2 & 4.9 & & & 109 & & 207 & 0.303 & 0.0327 & 0.275 \\
\hline 15 & & Average of VALUE_RPTD3 & 1.570444444 & & & 3.086967568 & & 32.13636364 & 0.219783784 & 0.006458011 & 0.173783784 \\
\hline 16 & & StdDev of VALUE_RPTD4 & 2.030188545 & & & 17.89685675 & & 65.8762197 & 0.036041747 & 0.007146095 & 0.030407542 \\
\hline 17 & \multirow[t]{4}{*}{$300 A$} & Count of VALUE_RPTD & 13 & & & 49 & 13 & 51 & 49 & 49 & 49 \\
\hline 18 & & Max of VALUE_RPTD2 & 2.3 & & & 0.417 & 0.663 & 2660 & 1.33 & 0.0561 & 1.29 \\
\hline 19 & & Average of VALUE_RPTD3 & -0.125110769 & & & 0.086655102 & 0.164123846 & 176.3019608 & 0.355510638 & 0.012376911 & 0.304910638 \\
\hline 20 & & StdDev of VALUE RPTD4 & 1.535062131 & & & 0.071198008 & 0.211814328 & 402.3799709 & 0.250053722 & 0.015058311 & 0.237165841 \\
\hline 21 & \multirow[t]{4}{*}{ Hanford TS } & Count of VALUE_RPTD & & & & 40 & & 43 & 40 & 40 & 40 \\
\hline 22 & & Max of VALUE_RPTD2 & & & & 0.125 & & 9000 & 0.355 & 0.0167 & 0.248 \\
\hline 23 & & Average of VALUE_RPTD3 & & & & 0.0839525 & & 449.0325581 & 0.224 & 0.0048257 & 0.169375 \\
\hline 24 & & StdDev of VALUE_RPTD4 & & & & 0.018818635 & & 1534.142344 & 0.044001748 & 0.005232209 & 0.028845021 \\
\hline 25 & \multirow[t]{4}{*}{ PRD } & Count of VALUE_RPTD & 87 & 17 & 34 & 49 & 49 & 47 & 49 & 49 & 49 \\
\hline 26 & & Max of VALUE_RPTD2 & 3.48 & 0.0000223 & 0.000282 & 0.127 & 1.61 & 205 & 0.418 & 0.0251 & 0.38 \\
\hline 27 & & Average of VALUEE_RPTD3 & 0.162070277 & 9.90588E-06 & 3.78007E-05 & 0.079157143 & 0.044575306 & 36.63212766 & 0.251510204 & 0.008261886 & 0.202816327 \\
\hline 28 & & StdDev of VALUE_RPTD4 & 0.709562989 & $6.5918 \mathrm{E}-06$ & 5.32209E-05 & 0.019119885 & 0.271518799 & 26.5461082 & 0.052154068 & 0.00675089 & 0.04995109 \\
\hline 29 & \multirow[t]{4}{*}{ Richland PH } & Count of VALUE_RPTD & 85 & 16 & 32 & 196 & 49 & 194 & 196 & 196 & 196 \\
\hline 30 & & Max of VALUE_RPTD2 & 3.66 & 0.0001626 & 0.000166 & 0.305 & 0.534 & 244 & 0.501 & 0.0237 & 0.364 \\
\hline 31 & & Average of VALUE_RPTD3 & 0.216405115 & 0.000104744 & 4.44913E-05 & 0.082485265 & 0.048579388 & 55.52474227 & 0.255556122 & 0.007466125 & 0.206637755 \\
\hline 32 & & StdDev of VALUE_RPTD4 & 0.780636131 & 4.53145E-05 & 4.43298E-05 & 0.028306131 & 0.159060283 & 29.25420666 & 0.055203232 & 0.005950824 & 0.041973818 \\
\hline 33 & \multirow[t]{4}{*}{ Verntia $\mathrm{Br}$} & Count of VALUE_RPTD & 1 & & & 57 & & 58 & 56 & 56 & 56 \\
\hline 34 & & Max of VALUE_RPTD2 & 0.561 & & & 0.147 & & 85.4 & 0.33 & 0.0277 & 0.265 \\
\hline 35 & & Average of VALUE_RPTD3 & 0.561 & & & 0.080363158 & & 32.94310345 & 0.238767857 & 0.008100429 & 0.186375 \\
\hline 36 & & StdDev of VALUE_RPTD4 & \#DIV/O! & & & 0.018936554 & & 19.60001891 & 0.035587021 & 0.006114653 & 0.030634243 \\
\hline
\end{tabular}


99-03 SD SAC 


\begin{tabular}{|c|c|c|c|c|c|c|c|c|c|c|}
\hline & $A$ & $\mathrm{~B}$ & $\mathrm{C}$ & $\mathrm{D}$ & $E$ & $\mathrm{~F}$ & $G$ & $\mathrm{H}$ & $\mathrm{I}$ & $\mathrm{J}$ \\
\hline 1 & SAMPLE NUM ASSIGNED TO & \begin{tabular}{|l} 
SAMPLER \\
\end{tabular} & MEDIA & BIOTA MEDIA & TAG ID & SAMP NUM & SAMP DATE TIME & SAMP DATE TIME ON & CON ID & CON SHORT NAME \\
\hline 2 & SESP SCHEDULING & & SO & & & B0YRB6 & 12-Jun-00 & & $10045-97-3$ & CS-137 \\
\hline 3 & SESP SCHEDULING & & SO & & & B0YWC5 & 07-Aug-00 & & $10045-97-3$ & CS-137 \\
\hline 4 & SESP SCHEDULING & & SO & & & BOYWC7 & 07-Aug-00 & & $10045-97-3$ & CS-137 \\
\hline 5 & SESP SCHEDULING & & SO & & & B0YW87 & $08-A u g-00$ & & $10045-97-3$ & CS-137 \\
\hline 6 & SESP SCHEDULING & & SO & & & B0YWD3 & 08-Aug-00 & & $10045-97-3$ & CS-137 \\
\hline 7 & SESP SCHEDULING & & SO & & & B0YWD5 & $08-A u g-00$ & & $10045-97-3$ & CS-137 \\
\hline 8 & SESP SCHEDULING & & SO & & & BOYY70 & 15-Aug-00 & & $10045-97-3$ & CS-137 \\
\hline 9 & SESP SCHEDULING & & SO & & & BOYY71 & 15-Aug-00 & & $10045-97-3$ & CS-137 \\
\hline 10 & SESP SCHEDULING & & SO & & & BOYY72 & 15-Aug-00 & & $10045-97-3$ & CS-137 \\
\hline 11 & SESP SCHEDULING & & SO & & & B10908 & 27-Sep-00 & & $10045-97-3$ & CS-137 \\
\hline 12 & SESP SCHEDULING & & SO & & & B10917 & 27-Sep-00 & & $10045-97-3$ & CS-137 \\
\hline 13 & SESP SCHEDULING & & SO & & & B10919 & 27-Sep-00 & & $10045-97-3$ & CS-137 \\
\hline 14 & SESP SCHEDULING & & SO & & & B10922 & 27-Sep-00 & & $10045-97-3$ & CS-137 \\
\hline 15 & SESP SCHEDULING & & SO & & & B10915 & 25-Oct-00 & & $10045-97-3$ & CS-137 \\
\hline 16 & SESP SCHEDULING & & SO & & & B10929 & 07-Nov-00 & & $10045-97-3$ & CS-137 \\
\hline 17 & SESP SCHEDULING & & SO & & & B12CK8 & 19-Jul-01 & & $10045-97-3$ & CS-137 \\
\hline 18 & SESP SCHEDULING & & SO & & & B12CL5 & 19-Jul-01 & & $10045-97-3$ & CS-137 \\
\hline 19 & SESP SCHEDULING & & SO & & & B12CL6 & 19-Jul-01 & & $10045-97-3$ & CS-137 \\
\hline 20 & SESP SCHEDULING & & SO & & & B12CM2 & 19-Jul-01 & & $10045-97-3$ & CS-137 \\
\hline 21 & SESP SCHEDULING & & SO & & & B12CM4 & 19-Jul-01 & & $10045-97-3$ & CS-137 \\
\hline 22 & SESP SCHEDULING & & SO & & & B12CL1 & 20-Jul-01 & & $10045-97-3$ & CS-137 \\
\hline 23 & SESP SCHEDULING & & SO & & & B12CL2 & 20-Jul-01 & & $10045-97-3$ & CS-137 \\
\hline 24 & SESP SCHEDULING & & SO & & & B12CL3 & 20-Jul-01 & & $10045-97-3$ & CS-137 \\
\hline 25 & SESP SCHEDULING & & SO & & & B12CL4 & 20-Jul-01 & & $10045-97-3$ & CS-137 \\
\hline 26 & SESP SCHEDULING & & SO & & & $\mathrm{B} 12 \mathrm{CH} 3$ & 23-Jul-01 & & $10045-97-3$ & CS-137 \\
\hline 27 & SESP SCHEDULING & & SO & & & B12CJ0 & 23-Jul-01 & & $10045-97-3$ & CS-137 \\
\hline 28 & SESP SCHEDULING & & SO & & & B12CJ1 & 23-Jul-01 & & $10045-97-3$ & CS-137 \\
\hline 29 & SESP SCHEDULING & & SO & & & B12CK9 & 23-Jul-01 & & $10045-97-3$ & CS-137 \\
\hline 30 & SESP SCHEDULING & & SO & & & $\mathrm{B} 12 \mathrm{CNO}$ & 23-Jul-01 & & $10045-97-3$ & CS-137 \\
\hline 31 & SESP SCHEDULING & & SO & & & B12CN2 & 23-Jul-01 & & $10045-97-3$ & CS-137 \\
\hline 32 & SESP SCHEDULING & & SO & & & B12RL9 & 27-Aug-01 & & $10045-97-3$ & CS-137 \\
\hline 33 & SESP SCHEDULING & & SO & & & B134L5 & $08-O c t-01$ & & $10045-97-3$ & CS-137 \\
\hline 34 & SESP SCHEDULING & & SO & & & $\mathrm{B} 12 \times 20$ & 22-Oct-01 & & $10045-97-3$ & CS-137 \\
\hline 35 & SESP SCHEDULING & & SO & & & B12X35 & 22-Oct-01 & & 10045-97-3 & CS-137 \\
\hline 36 & SESP SCHEDULING & & SO & & & B141M8 & 14-Feb-02 & & $10045-97-3$ & CS-137 \\
\hline 37 & SESP SCHEDULING & & SO & & & B14CV4 & 08-Apr-02 & & $10045-97-3$ & CS-137 \\
\hline 38 & SESP SCHEDULING & & SO & & & B14YJ7 & 08-Jul-02 & & $10045-97-3$ & CS-137 \\
\hline 39 & SESP SCHEDULING & & SO & & & B14YM5 & 19-Jul-02 & & $10045-97-3$ & CS-137 \\
\hline
\end{tabular}




\begin{tabular}{|c|c|c|c|c|c|c|c|c|c|c|}
\hline & A & $\mathrm{B}$ & $\mathrm{C}$ & $\mathrm{D}$ & $E$ & $\mathrm{~F}$ & $G$ & $\mathrm{H}$ & $\mathrm{I}$ & $\mathrm{J}$ \\
\hline 1 & SAMPLE_NUM_ASSIGNED_TO & SAMPLER & MEDIA & BIOTA_MEDIA & TAG_ID & SAMP_NUM & SAMP_DATE_TIME & SAMP_DATE_TIME_ON & CON_ID & CON_SHORT_NAME \\
\hline 40 & SESP SCHEDULING & & SO & & & B14YP1 & 19-Jul-02 & & $10045-97-3$ & CS-137 \\
\hline 41 & SESP SCHEDULING & & $\mathrm{SO}$ & & & B14YP3 & 19-Jul-02 & & $10045-97-3$ & CS-137 \\
\hline 42 & SESP SCHEDULING & & SO & & & B14YM4 & 23-Jul-02 & & $10045-97-3$ & CS-137 \\
\hline 43 & SESP SCHEDULING & & SO & & & B14YM6 & 23-Jul-02 & & $10045-97-3$ & CS-137 \\
\hline 44 & SESP SCHEDULING & & SO & & & B14YM7 & 23-Jul-02 & & $10045-97-3$ & CS-137 \\
\hline 45 & SESP SCHEDULING & & SO & & & B14YN3 & 23-Jul-02 & & $10045-97-3$ & CS-137 \\
\hline 46 & SESP SCHEDULING & & SO & & & B14YN5 & 23-Jul-02 & & $10045-97-3$ & CS-137 \\
\hline 47 & SESP SCHEDULING & & SO & & & B15C40 & 16-Sep-02 & & $10045-97-3$ & CS-137 \\
\hline 48 & SESP SCHEDULING & & SO & & & B15C49 & 16-Sep-02 & & $10045-97-3$ & CS-137 \\
\hline 49 & SESP SCHEDULING & & SO & & & B15C07 & 07-Oct-02 & & $10045-97-3$ & CS-137 \\
\hline 50 & SESP SCHEDULING & & SO & & & B15C44 & 07-Oct-02 & & $10045-97-3$ & CS-137 \\
\hline 51 & SESP SCHEDULING & & SO & & & B15C47 & 07-Oct-02 & & $10045-97-3$ & CS-137 \\
\hline 52 & SESP SCHEDULING & & SO & & & B15LL9 & 09-Oct-02 & & $10045-97-3$ & CS-137 \\
\hline 53 & SESP SCHEDULING & & SO & & & B15C52 & 29-Oct-02 & & $10045-97-3$ & CS-137 \\
\hline 54 & SESP SCHEDULING & & SO & & & B0YWC5 & 07-Aug-00 & & PU-239/240 & PU-239/240 \\
\hline 55 & SESP SCHEDULING & & SO & & & BOYWC7 & 07-Aug-00 & & PU-239/240 & PU-239/240 \\
\hline 56 & SESP SCHEDULING & & SO & & & B0YW87 & 08-Aug-00 & & PU-239/240 & PU-239/240 \\
\hline 57 & SESP SCHEDULING & & SO & & & B0YWD3 & 08-Aug-00 & & PU-239/240 & PU-239/240 \\
\hline 58 & SESP SCHEDULING & & SO & & & B0YWD5 & 08-Aug-00 & & PU-239/240 & PU-239/240 \\
\hline 59 & SESP SCHEDULING & & SO & & & BOYY70 & 15-Aug-00 & & PU-239/240 & PU-239/240 \\
\hline 60 & SESP SCHEDULING & & SO & & & B0YY71 & 15-Aug-00 & & PU-239/240 & PU-239/240 \\
\hline 61 & SESP SCHEDULING & & SO & & & BOYY72 & 15-Aug-00 & & PU-239/240 & PU-239/240 \\
\hline 62 & SESP SCHEDULING & & SO & & & B12CK8 & 19-Jul-01 & & PU-239/240 & PU-239/240 \\
\hline 63 & SESP SCHEDULING & & SO & & & B12CL5 & 19-Jul-01 & & PU-239/240 & PU-239/240 \\
\hline 64 & SESP SCHEDULING & & SO & & & B12CL6 & 19-Jul-01 & & PU-239/240 & PU-239/240 \\
\hline 65 & SESP SCHEDULING & & SO & & & B12CM2 & 19-Jul-01 & & PU-239/240 & PU-239/240 \\
\hline 66 & SESP SCHEDULING & & SO & & & B12CM4 & 19-Jul-01 & & PU-239/240 & PU-239/240 \\
\hline 67 & SESP SCHEDULING & & SO & & & B12CL1 & 20-Jul-01 & & PU-239/240 & PU-239/240 \\
\hline 68 & SESP SCHEDULING & & SO & & & B12CL2 & 20-Jul-01 & & PU-239/240 & PU-239/240 \\
\hline 69 & SESP SCHEDULING & & SO & & & B12CL3 & 20-Jul-01 & & PU-239/240 & PU-239/240 \\
\hline 70 & SESP SCHEDULING & & SO & & & B12CL4 & 20-Jul-01 & & PU-239/240 & PU-239/240 \\
\hline 71 & SESP SCHEDULING & & SO & & & $\mathrm{B} 12 \mathrm{CH} 3$ & 23-Jul-01 & & PU-239/240 & PU-239/240 \\
\hline 72 & SESP SCHEDULING & & SO & & & B12CJ0 & 23-Jul-01 & & PU-239/240 & PU-239/240 \\
\hline 73 & SESP SCHEDULING & & SO & & & B12CJ1 & 23-Jul-01 & & PU-239/240 & PU-239/240 \\
\hline 74 & SESP SCHEDULING & & SO & & & B12CK9 & 23-Jul-01 & & PU-239/240 & PU-239/240 \\
\hline 75 & SESP SCHEDULING & & SO & & & $\mathrm{B} 12 \mathrm{CNO}$ & 23-Jul-01 & & PU-239/240 & PU-239/240 \\
\hline 76 & SESP SCHEDULING & & SO & & & B12CN2 & 23-Jul-01 & & PU-239/240 & PU-239/240 \\
\hline 77 & SESP SCHEDULING & & SO & & & B14YM5 & 19-Jul-02 & & PU-239/240 & PU-239/240 \\
\hline
\end{tabular}




\begin{tabular}{|c|c|c|c|c|c|c|c|c|c|c|}
\hline & $\mathrm{A}$ & $\mathrm{B}$ & $\mathrm{C}$ & $\mathrm{D}$ & $E$ & $\mathrm{~F}$ & $G$ & $\mathrm{H}$ & $\mathrm{I}$ & $\mathrm{J}$ \\
\hline 1 & SAMPLE_NUM_ASSIGNED_TO & SAMPLER & MEDIA & BIOTA_MEDIA & TAG_ID & SAMP_NUM & SAMP_DATE_TIME & SAMP_DATE_TIME_ON & CON_ID & |CON_SHORT_NAME \\
\hline 78 & SESP SCHEDULING & & SO & & & B14YP1 & 19-Jul-02 & & PU-239/240 & PU-239/240 \\
\hline 79 & SESP SCHEDULING & & SO & & & B14YP3 & 19-Jul-02 & & PU-239/240 & PU-239/240 \\
\hline 80 & SESP SCHEDULING & & SO & & & B14YM4 & 23-Jul-02 & & PU-239/240 & PU-239/240 \\
\hline 81 & SESP SCHEDULING & & SO & & & B14YM6 & 23-Jul-02 & & PU-239/240 & PU-239/240 \\
\hline 82 & SESP SCHEDULING & & SO & & & B14YM7 & 23-Jul-02 & & PU-239/240 & PU-239/240 \\
\hline 83 & SESP SCHEDULING & & SO & & & B14YN3 & 23-Jul-02 & & PU-239/240 & PU-239/240 \\
\hline 84 & SESP SCHEDULING & & SO & & & B14YN5 & 23-Jul-02 & & PU-239/240 & PU-239/240 \\
\hline 85 & SESP SCHEDULING & & SO & & & B0YRB6 & 12-Jun-00 & & $10098-97-2$ & SR-90 \\
\hline 86 & SESP SCHEDULING & & SO & & & B0YWC5 & 07-Aug-00 & & $10098-97-2$ & SR-90 \\
\hline 87 & SESP SCHEDULING & & SO & & & BOYWC7 & 07-Aug-00 & & $10098-97-2$ & SR-90 \\
\hline 88 & SESP SCHEDULING & & SO & & & B0YW87 & 08-Aug-00 & & $10098-97-2$ & SR-90 \\
\hline 89 & SESP SCHEDULING & & SO & & & B0YWD3 & 08-Aug-00 & & $10098-97-2$ & SR-90 \\
\hline 90 & SESP SCHEDULING & & SO & & & B0YWD5 & 08-Aug-00 & & $10098-97-2$ & SR-90 \\
\hline 91 & SESP SCHEDULING & & SO & & & BOYY70 & 15-Aug-00 & & $10098-97-2$ & SR-90 \\
\hline 92 & SESP SCHEDULING & & SO & & & B0YY71 & 15-Aug-00 & & $10098-97-2$ & SR-90 \\
\hline 93 & SESP SCHEDULING & & SO & & & B0YY72 & 15-Aug-00 & & $10098-97-2$ & SR-90 \\
\hline 94 & SESP SCHEDULING & & SO & & & B10908 & 27-Sep-00 & & $10098-97-2$ & SR-90 \\
\hline 95 & SESP SCHEDULING & & SO & & & B10917 & 27-Sep-00 & & $10098-97-2$ & SR-90 \\
\hline 96 & SESP SCHEDULING & & SO & & & B10919 & 27-Sep-00 & & $10098-97-2$ & SR-90 \\
\hline 97 & SESP SCHEDULING & & SO & & & B10922 & 27-Sep-00 & & $10098-97-2$ & SR-90 \\
\hline 98 & SESP SCHEDULING & & SO & & & B10915 & 25-Oct-00 & & $10098-97-2$ & SR-90 \\
\hline 99 & SESP SCHEDULING & & SO & & & B10929 & 07-Nov-00 & & $10098-97-2$ & SR-90 \\
\hline 100 & SESP SCHEDULING & & SO & & & B12CK8 & 19-Jul-01 & & $10098-97-2$ & SR-90 \\
\hline 101 & SESP SCHEDULING & & SO & & & B12CL5 & 19-Jul-01 & & $10098-97-2$ & SR-90 \\
\hline 102 & SESP SCHEDULING & & SO & & & B12CL6 & 19-Jul-01 & & $10098-97-2$ & SR-90 \\
\hline 103 & SESP SCHEDULING & & SO & & & B12CM2 & 19-Jul-01 & & $10098-97-2$ & SR-90 \\
\hline 104 & SESP SCHEDULING & & SO & & & B12CM4 & 19-Jul-01 & & $10098-97-2$ & SR-90 \\
\hline 105 & SESP SCHEDULING & & SO & & & B12CL1 & 20-Jul-01 & & $10098-97-2$ & SR-90 \\
\hline 106 & SESP SCHEDULING & & SO & & & B12CL2 & 20-Jul-01 & & $10098-97-2$ & SR-90 \\
\hline 107 & SESP SCHEDULING & & SO & & & B12CL3 & 20-Jul-01 & & $10098-97-2$ & SR-90 \\
\hline 108 & SESP SCHEDULING & & SO & & & B12CL4 & 20-Jul-01 & & $10098-97-2$ & SR-90 \\
\hline 109 & SESP SCHEDULING & & SO & & & $\mathrm{B} 12 \mathrm{CH} 3$ & 23-Jul-01 & & $10098-97-2$ & SR-90 \\
\hline 110 & SESP SCHEDULING & & SO & & & $\mathrm{B} 12 \mathrm{CJO}$ & 23-Jul-01 & & $10098-97-2$ & SR-90 \\
\hline 111 & SESP SCHEDULING & & SO & & & B12CJ1 & 23-Jul-01 & & $10098-97-2$ & SR-90 \\
\hline 112 & SESP SCHEDULING & & SO & & & B12CK9 & 23-Jul-01 & & $10098-97-2$ & SR-90 \\
\hline 113 & SESP SCHEDULING & & SO & & & B12CN0 & 23-Jul-01 & & $10098-97-2$ & SR-90 \\
\hline 114 & SESP SCHEDULING & & SO & & & B12CN2 & 23-Jul-01 & & $10098-97-2$ & SR-90 \\
\hline 115 & SESP SCHEDULING & & $\mathrm{SO}$ & & & B12RL9 & 27-Aug-01 & & $10098-97-2$ & SR-90 \\
\hline
\end{tabular}




\begin{tabular}{|c|c|c|c|c|c|c|c|c|c|c|}
\hline & A & $\mathrm{B}$ & $\mathrm{C}$ & $\mathrm{D}$ & $E$ & $\mathrm{~F}$ & $\mathrm{G}$ & $\mathrm{H}$ & $\mathrm{I}$ & $\mathrm{J}$ \\
\hline 1 & SAMPLE_NUM_ASSIGNED_TO & SAMPLER & MEDIA & BIOTA_MEDIA & TAG_ID & SAMP_NUM & SAMP_DATE_TIME & SAMP_DATE_TIME_ON & CON_ID & CON_SHORT_NAME \\
\hline 116 & SESP SCHEDULING & & $\mathrm{SO}$ & & & B134L5 & $08-$-Oct-01 & & $10098-97-2$ & SR-90 \\
\hline 117 & SESP SCHEDULING & & SO & & & $\mathrm{B} 12 \times 20$ & 22-Oct-01 & & $10098-97-2$ & SR-90 \\
\hline 118 & SESP SCHEDULING & & SO & & & B12X35 & 22-Oct-01 & & $10098-97-2$ & SR-90 \\
\hline 119 & SESP SCHEDULING & & SO & & & B141M8 & 14-Feb-02 & & $10098-97-2$ & SR-90 \\
\hline 120 & SESP SCHEDULING & & SO & & & B14CV4 & 08-Apr-02 & & $10098-97-2$ & SR-90 \\
\hline 121 & SESP SCHEDULING & & SO & & & B14YJ7 & 08-Jul-02 & & $10098-97-2$ & SR-90 \\
\hline 122 & SESP SCHEDULING & & SO & & & B14YM5 & 19-Jul-02 & & $10098-97-2$ & SR-90 \\
\hline 123 & SESP SCHEDULING & & SO & & & B14YP1 & 19-Jul-02 & & $10098-97-2$ & SR-90 \\
\hline 124 & SESP SCHEDULING & & SO & & & B14YP3 & 19-Jul-02 & & $10098-97-2$ & SR-90 \\
\hline 125 & SESP SCHEDULING & & SO & & & B14YM4 & 23-Jul-02 & & $10098-97-2$ & SR-90 \\
\hline 126 & SESP SCHEDULING & & SO & & & B14YM6 & 23-Jul-02 & & $10098-97-2$ & SR-90 \\
\hline 127 & SESP SCHEDULING & & SO & & & B14YM7 & 23-Jul-02 & & $10098-97-2$ & SR-90 \\
\hline 128 & SESP SCHEDULING & & SO & & & B14YN3 & 23-Jul-02 & & $10098-97-2$ & SR-90 \\
\hline 129 & SESP SCHEDULING & & SO & & & B14YN5 & 23-Jul-02 & & $10098-97-2$ & SR-90 \\
\hline 130 & SESP SCHEDULING & & SO & & & B15C40 & 16-Sep-02 & & $10098-97-2$ & SR-90 \\
\hline 131 & SESP SCHEDULING & & SO & & & B15C49 & 16-Sep-02 & & $10098-97-2$ & SR-90 \\
\hline 132 & SESP SCHEDULING & & SO & & & B15C07 & 07-Oct-02 & & $10098-97-2$ & SR-90 \\
\hline 133 & SESP SCHEDULING & & SO & & & B15C44 & 07-Oct-02 & & $10098-97-2$ & SR-90 \\
\hline 134 & SESP SCHEDULING & & SO & & & B15C47 & 07-Oct-02 & & $10098-97-2$ & SR-90 \\
\hline 135 & SESP SCHEDULING & & SO & & & B15LL9 & 09-Oct-02 & & $10098-97-2$ & SR-90 \\
\hline 136 & SESP SCHEDULING & & SO & & & B15C52 & 29-Oct-02 & & $10098-97-2$ & SR-90 \\
\hline 137 & SESP SCHEDULING & & SO & & & B134L5 & 08-Oct-01 & & 14133-76-7 & TC-99 \\
\hline 138 & SESP SCHEDULING & & SO & & & B141M8 & 14-Feb-02 & & $14133-76-7$ & TC-99 \\
\hline 139 & SESP SCHEDULING & & SO & & & B14CV4 & 08-Apr-02 & & $14133-76-7$ & TC-99 \\
\hline 140 & SESP SCHEDULING & & SO & & & B14YJ7 & 08-Jul-02 & & $14133-76-7$ & TC-99 \\
\hline 141 & SESP SCHEDULING & & SO & & & B15LL9 & 09-Oct-02 & & 14133-76-7 & TC-99 \\
\hline 142 & SESP SCHEDULING & & SO & & & B0YRB6 & 12-Jun-00 & & $13966-29-5$ & U-234 \\
\hline 143 & SESP SCHEDULING & & SO & & & B0YWC5 & 07-Aug-00 & & $13966-29-5$ & U-234 \\
\hline 144 & SESP SCHEDULING & & SO & & & BOYWC7 & 07-Aug-00 & & $13966-29-5$ & $\mathrm{U}-234$ \\
\hline 145 & SESP SCHEDULING & & SO & & & B0YW87 & 08-Aug-00 & & $13966-29-5$ & U-234 \\
\hline 146 & SESP SCHEDULING & & SO & & & B0YWD3 & 08-Aug-00 & & $13966-29-5$ & $\mathrm{U}-234$ \\
\hline 147 & SESP SCHEDULING & & SO & & & B0YWD5 & 08-Aug-00 & & $13966-29-5$ & $\mathrm{U}-234$ \\
\hline 148 & SESP SCHEDULING & & SO & & & BOYY70 & 15-Aug-00 & & $13966-29-5$ & U-234 \\
\hline 149 & SESP SCHEDULING & & SO & & & B0YY71 & 15-Aug-00 & & $13966-29-5$ & U-234 \\
\hline 150 & SESP SCHEDULING & & SO & & & BOYY72 & 15-Aug-00 & & $13966-29-5$ & U-234 \\
\hline 151 & SESP SCHEDULING & & SO & & & B10908 & 27-Sep-00 & & $13966-29-5$ & U-234 \\
\hline 152 & SESP SCHEDULING & & SO & & & B10917 & 27-Sep-00 & & $13966-29-5$ & U-234 \\
\hline 153 & SESP SCHEDULING & & $\mathrm{SO}$ & & & B10919 & 27-Sep-00 & & 13966-29-5 & $\mathrm{U}-234$ \\
\hline
\end{tabular}




\begin{tabular}{|c|c|c|c|c|c|c|c|c|c|c|}
\hline & A & $\mathrm{B}$ & $\mathrm{C}$ & $\mathrm{D}$ & $E$ & $\mathrm{~F}$ & $\mathrm{G}$ & $\mathrm{H}$ & $\mathrm{I}$ & $\mathrm{J}$ \\
\hline 1 & SAMPLE_NUM_ASSIGNED_TO & SAMPLER & MEDIA & BIOTA_MEDIA & TAG_ID & SAMP_NUM & SAMP_DATE_TIME & SAMP_DATE_TIME_ON & CON_ID & CON_SHORT_NAME \\
\hline 154 & SESP SCHEDULING & & $\mathrm{SO}$ & & & B10922 & 27-Sep-00 & & $13966-29-5$ & U-234 \\
\hline 155 & SESP SCHEDULING & & SO & & & B10915 & 25-Oct-00 & & 13966-29-5 & U-234 \\
\hline 156 & SESP SCHEDULING & & SO & & & B10929 & 07-Nov-00 & & 13966-29-5 & $\mathrm{U}-234$ \\
\hline 157 & SESP SCHEDULING & & SO & & & B12CK8 & 19-Jul-01 & & 13966-29-5 & $\mathrm{U}-234$ \\
\hline 158 & SESP SCHEDULING & & SO & & & B12CL5 & 19-Jul-01 & & $13966-29-5$ & U-234 \\
\hline 159 & SESP SCHEDULING & & SO & & & B12CL6 & 19-Jul-01 & & $13966-29-5$ & U-234 \\
\hline 160 & SESP SCHEDULING & & SO & & & B12CM2 & 19-Jul-01 & & $13966-29-5$ & U-234 \\
\hline 161 & SESP SCHEDULING & & SO & & & B12CM4 & 19-Jul-01 & & $13966-29-5$ & U-234 \\
\hline 162 & SESP SCHEDULING & & SO & & & B12CL1 & 20-Jul-01 & & 13966-29-5 & U-234 \\
\hline 163 & SESP SCHEDULING & & SO & & & B12CL2 & 20-Jul-01 & & $13966-29-5$ & U-234 \\
\hline 164 & SESP SCHEDULING & & SO & & & B12CL3 & 20-Jul-01 & & $13966-29-5$ & U-234 \\
\hline 165 & SESP SCHEDULING & & SO & & & B12CL4 & 20-Jul-01 & & $13966-29-5$ & U-234 \\
\hline 166 & SESP SCHEDULING & & SO & & & $\mathrm{B} 12 \mathrm{CH} 3$ & 23-Jul-01 & & $13966-29-5$ & U-234 \\
\hline 167 & SESP SCHEDULING & & SO & & & B12CJ0 & 23-Jul-01 & & $13966-29-5$ & U-234 \\
\hline 168 & SESP SCHEDULING & & SO & & & B12CJ1 & 23-Jul-01 & & 13966-29-5 & U-234 \\
\hline 169 & SESP SCHEDULING & & SO & & & B12CK9 & 23-Jul-01 & & 13966-29-5 & $\mathrm{U}-234$ \\
\hline 170 & SESP SCHEDULING & & SO & & & $\mathrm{B} 12 \mathrm{CNO}$ & 23-Jul-01 & & $13966-29-5$ & $\mathrm{U}-234$ \\
\hline 171 & SESP SCHEDULING & & SO & & & B12CN2 & 23-Jul-01 & & $13966-29-5$ & U-234 \\
\hline 172 & SESP SCHEDULING & & SO & & & B12RL9 & 27-Aug-01 & & 13966-29-5 & $\mathrm{U}-234$ \\
\hline 173 & SESP SCHEDULING & & SO & & & B12RY9 & 27-Aug-01 & & $13966-29-5$ & U-234 \\
\hline 174 & SESP SCHEDULING & & SO & & & B12T01 & 27-Aug-01 & & $13966-29-5$ & U-234 \\
\hline 175 & SESP SCHEDULING & & SO & & & B12T04 & 27-Aug-01 & & 13966-29-5 & U-234 \\
\hline 176 & SESP SCHEDULING & & SO & & & B12T05 & 27-Aug-01 & & $13966-29-5$ & U-234 \\
\hline 177 & SESP SCHEDULING & & SO & & & B12T06 & 27-Aug-01 & & $13966-29-5$ & U-234 \\
\hline 178 & SESP SCHEDULING & & SO & & & B12T07 & 27-Aug-01 & & 13966-29-5 & U-234 \\
\hline 179 & SESP SCHEDULING & & SO & & & B12RY5 & 28-Aug-01 & & $13966-29-5$ & $\mathrm{U}-234$ \\
\hline 180 & SESP SCHEDULING & & SO & & & B12T03 & 28-Aug-01 & & $13966-29-5$ & U-234 \\
\hline 181 & SESP SCHEDULING & & SO & & & B134L5 & 08-Oct-01 & & $13966-29-5$ & U-234 \\
\hline 182 & SESP SCHEDULING & & SO & & & B12X20 & 22-Oct-01 & & $13966-29-5$ & U-234 \\
\hline 183 & SESP SCHEDULING & & SO & & & B12X35 & 22-Oct-01 & & $13966-29-5$ & U-234 \\
\hline 184 & SESP SCHEDULING & & SO & & & B141M8 & 14-Feb-02 & & 13966-29-5 & $\mathrm{U}-234$ \\
\hline 185 & SESP SCHEDULING & & SO & & & B14CV4 & 08-Apr-02 & & $13966-29-5$ & $\mathrm{U}-234$ \\
\hline 186 & SESP SCHEDULING & & SO & & & B14YJ7 & 08-Jul-02 & & $13966-29-5$ & U-234 \\
\hline 187 & SESP SCHEDULING & & SO & & & B14YM5 & 19-Jul-02 & & $13966-29-5$ & U-234 \\
\hline 188 & SESP SCHEDULING & & SO & & & B14YP1 & 19-Jul-02 & & $13966-29-5$ & U-234 \\
\hline 189 & SESP SCHEDULING & & SO & & & B14YP3 & 19-Jul-02 & & $13966-29-5$ & U-234 \\
\hline 190 & SESP SCHEDULING & & SO & & & B14YM4 & 23-Jul-02 & & $13966-29-5$ & U-234 \\
\hline \begin{tabular}{|l|}
191 \\
\end{tabular} & SESP SCHEDULING & & $\mathrm{SO}$ & & & B14YM6 & 23-Jul-02 & & 13966-29-5 & $\mathrm{U}-234$ \\
\hline
\end{tabular}




\begin{tabular}{|c|c|c|c|c|c|c|c|c|c|c|}
\hline & A & $\mathrm{B}$ & $\mathrm{C}$ & $\mathrm{D}$ & $E$ & $\mathrm{~F}$ & G & $\mathrm{H}$ & $\mathrm{I}$ & $\mathrm{J}$ \\
\hline 1 & SAMPLE_NUM_ASSIGNED_TO & SAMPLER & MEDIA & BIOTA_MEDIA & TAG_ID & SAMP_NUM & SAMP_DATE_TIME & SAMP_DATE_TIME_ON & CON_ID & |CON_SHORT_NAME \\
\hline 192 & SESP SCHEDULING & & SO & & & B14YM7 & 23-Jul-02 & & $13966-29-5$ & U-234 \\
\hline 193 & SESP SCHEDULING & & SO & & & B14YN3 & 23-Jul-02 & & $13966-29-5$ & $\mathrm{U}-234$ \\
\hline 194 & SESP SCHEDULING & & SO & & & B14YN5 & 23-Jul-02 & & $13966-29-5$ & U-234 \\
\hline 195 & SESP SCHEDULING & & SO & & & B15C40 & 16-Sep-02 & & $13966-29-5$ & U-234 \\
\hline 196 & SESP SCHEDULING & & SO & & & B15C49 & 16-Sep-02 & & $13966-29-5$ & $\mathrm{U}-234$ \\
\hline 197 & SESP SCHEDULING & & SO & & & B15C07 & 07-Oct-02 & & $13966-29-5$ & $\mathrm{U}-234$ \\
\hline 198 & SESP SCHEDULING & & SO & & & B15C44 & 07-Oct-02 & & $13966-29-5$ & $\mathrm{U}-234$ \\
\hline 199 & SESP SCHEDULING & & SO & & & B15C47 & 07-Oct-02 & & $13966-29-5$ & U-234 \\
\hline 200 & SESP SCHEDULING & & SO & & & B15LL9 & 09-Oct-02 & & $13966-29-5$ & $\mathrm{U}-234$ \\
\hline 201 & SESP SCHEDULING & & SO & & & B15C52 & 29-Oct-02 & & $13966-29-5$ & $\mathrm{U}-234$ \\
\hline 202 & SESP SCHEDULING & & SO & & & B0YRB6 & 12-Jun-00 & & 15117-96-1 & U-235 \\
\hline 203 & SESP SCHEDULING & & SO & & & B0YWC5 & 07-Aug-00 & & 15117-96-1 & $\mathrm{U}-235$ \\
\hline 204 & SESP SCHEDULING & & SO & & & BOYWC7 & 07-Aug-00 & & $15117-96-1$ & U-235 \\
\hline 205 & SESP SCHEDULING & & SO & & & B0YW87 & $08-A u g-00$ & & 15117-96-1 & U-235 \\
\hline 206 & SESP SCHEDULING & & SO & & & B0YWD3 & 08-Aug-00 & & 15117-96-1 & $\mathrm{U}-235$ \\
\hline 207 & SESP SCHEDULING & & SO & & & B0YWD5 & 08-Aug-00 & & 15117-96-1 & U-235 \\
\hline 208 & SESP SCHEDULING & & SO & & & BOYY70 & 15-Aug-00 & & 15117-96-1 & U-235 \\
\hline 209 & SESP SCHEDULING & & SO & & & B0YY71 & 15-Aug-00 & & $15117-96-1$ & U-235 \\
\hline 210 & SESP SCHEDULING & & SO & & & BOYY72 & 15-Aug-00 & & 15117-96-1 & U-235 \\
\hline 211 & SESP SCHEDULING & & SO & & & B10908 & 27-Sep-00 & & 15117-96-1 & U-235 \\
\hline 212 & SESP SCHEDULING & & SO & & & B10917 & 27-Sep-00 & & 15117-96-1 & $\mathrm{U}-235$ \\
\hline 213 & SESP SCHEDULING & & SO & & & B10919 & 27-Sep-00 & & 15117-96-1 & $\mathrm{U}-235$ \\
\hline 214 & SESP SCHEDULING & & SO & & & B10922 & 27-Sep-00 & & 15117-96-1 & U-235 \\
\hline 215 & SESP SCHEDULING & & SO & & & B10915 & 25-Oct-00 & & 15117-96-1 & U-235 \\
\hline 216 & SESP SCHEDULING & & SO & & & B10929 & 07-Nov-00 & & 15117-96-1 & U-235 \\
\hline 217 & SESP SCHEDULING & & SO & & & B12CK8 & 19-Jul-01 & & 15117-96-1 & U-235 \\
\hline 218 & SESP SCHEDULING & & SO & & & B12CL5 & 19-Jul-01 & & 15117-96-1 & U-235 \\
\hline 219 & SESP SCHEDULING & & SO & & & B12CL6 & 19-Jul-01 & & 15117-96-1 & $\mathrm{U}-235$ \\
\hline 220 & SESP SCHEDULING & & SO & & & B12CM2 & 19-Jul-01 & & 15117-96-1 & U-235 \\
\hline 221 & SESP SCHEDULING & & SO & & & B12CM4 & 19-Jul-01 & & 15117-96-1 & U-235 \\
\hline 222 & SESP SCHEDULING & & SO & & & B12CL1 & 20-Jul-01 & & $15117-96-1$ & U-235 \\
\hline 223 & SESP SCHEDULING & & SO & & & B12CL2 & 20-Jul-01 & & 15117-96-1 & U-235 \\
\hline 224 & SESP SCHEDULING & & SO & & & B12CL3 & 20-Jul-01 & & 15117-96-1 & U-235 \\
\hline 225 & SESP SCHEDULING & & SO & & & B12CL4 & 20-Jul-01 & & 15117-96-1 & $\mathrm{U}-235$ \\
\hline 226 & SESP SCHEDULING & & SO & & & $\mathrm{B} 12 \mathrm{CH} 3$ & 23-Jul-01 & & 15117-96-1 & U-235 \\
\hline 227 & SESP SCHEDULING & & SO & & & $\mathrm{B} 12 \mathrm{CJO}$ & 23-Jul-01 & & 15117-96-1 & U-235 \\
\hline 228 & SESP SCHEDULING & & SO & & & B12CJ1 & 23-Jul-01 & & 15117-96-1 & $\mathrm{U}-235$ \\
\hline 229 & SESP SCHEDULING & & SO & & & B12CK9 & 23-Jul-01 & & $15117-96-1$ & U-235 \\
\hline
\end{tabular}




\begin{tabular}{|c|c|c|c|c|c|c|c|c|c|c|}
\hline & A & $\mathrm{B}$ & $\mathrm{C}$ & $\mathrm{D}$ & $E$ & $\mathrm{~F}$ & G & $\mathrm{H}$ & $\mathrm{I}$ & $\mathrm{J}$ \\
\hline 1 & SAMPLE_NUM_ASSIGNED_TO & SAMPLER & MEDIA & BIOTA_MEDIA & TAG_ID & SAMP_NUM & SAMP_DATE_TIME & SAMP_DATE_TIME_ON & CON_ID & |CON_SHORT_NAME \\
\hline 230 & SESP SCHEDULING & & SO & & & B12CNO & 23-Jul-01 & & 15117-96-1 & U-235 \\
\hline 231 & SESP SCHEDULING & & SO & & & B12CN2 & 23-Jul-01 & & $15117-96-1$ & $\mathrm{U}-235$ \\
\hline 232 & SESP SCHEDULING & & SO & & & B12RL9 & 27-Aug-01 & & 15117-96-1 & U-235 \\
\hline 233 & SESP SCHEDULING & & SO & & & B12RY9 & 27-Aug-01 & & 15117-96-1 & U-235 \\
\hline 234 & SESP SCHEDULING & & SO & & & B12T01 & 27-Aug-01 & & 15117-96-1 & U-235 \\
\hline 235 & SESP SCHEDULING & & SO & & & B12T04 & 27-Aug-01 & & 15117-96-1 & U-235 \\
\hline 236 & SESP SCHEDULING & & SO & & & B12T05 & 27-Aug-01 & & 15117-96-1 & U-235 \\
\hline 237 & SESP SCHEDULING & & SO & & & B12T06 & 27-Aug-01 & & 15117-96-1 & U-235 \\
\hline 238 & SESP SCHEDULING & & SO & & & B12T07 & 27-Aug-01 & & 15117-96-1 & $\mathrm{U}-235$ \\
\hline 239 & SESP SCHEDULING & & SO & & & B12RY5 & 28-Aug-01 & & 15117-96-1 & $\mathrm{U}-235$ \\
\hline 240 & SESP SCHEDULING & & SO & & & B12T03 & 28-Aug-01 & & 15117-96-1 & U-235 \\
\hline 241 & SESP SCHEDULING & & SO & & & B134L5 & 08-Oct-01 & & 15117-96-1 & $\mathrm{U}-235$ \\
\hline 242 & SESP SCHEDULING & & SO & & & B12X20 & 22-Oct-01 & & $15117-96-1$ & U-235 \\
\hline 243 & SESP SCHEDULING & & SO & & & B12X35 & 22-Oct-01 & & 15117-96-1 & U-235 \\
\hline 244 & SESP SCHEDULING & & SO & & & B141M8 & 14-Feb-02 & & 15117-96-1 & U-235 \\
\hline 245 & SESP SCHEDULING & & SO & & & B14CV4 & 08-Apr-02 & & 15117-96-1 & U-235 \\
\hline 246 & SESP SCHEDULING & & SO & & & B14YJ7 & 08-Jul-02 & & 15117-96-1 & U-235 \\
\hline 247 & SESP SCHEDULING & & SO & & & B14YM5 & 19-Jul-02 & & $15117-96-1$ & U-235 \\
\hline 248 & SESP SCHEDULING & & SO & & & B14YP1 & 19-Jul-02 & & 15117-96-1 & U-235 \\
\hline 249 & SESP SCHEDULING & & SO & & & B14YP3 & 19-Jul-02 & & 15117-96-1 & U-235 \\
\hline 250 & SESP SCHEDULING & & SO & & & B14YM4 & 23-Jul-02 & & 15117-96-1 & $\mathrm{U}-235$ \\
\hline 251 & SESP SCHEDULING & & SO & & & B14YM6 & 23-Jul-02 & & 15117-96-1 & $\mathrm{U}-235$ \\
\hline 252 & SESP SCHEDULING & & SO & & & B14YM7 & 23-Jul-02 & & 15117-96-1 & U-235 \\
\hline 253 & SESP SCHEDULING & & SO & & & B14YN3 & 23-Jul-02 & & 15117-96-1 & $\mathrm{U}-235$ \\
\hline 254 & SESP SCHEDULING & & SO & & & B14YN5 & 23-Jul-02 & & 15117-96-1 & U-235 \\
\hline 255 & SESP SCHEDULING & & SO & & & B15C40 & 16-Sep-02 & & $15117-96-1$ & U-235 \\
\hline 256 & SESP SCHEDULING & & SO & & & B15C49 & 16-Sep-02 & & 15117-96-1 & U-235 \\
\hline 257 & SESP SCHEDULING & & SO & & & B15C07 & 07-Oct-02 & & 15117-96-1 & $\mathrm{U}-235$ \\
\hline 258 & SESP SCHEDULING & & SO & & & B15C44 & 07-Oct-02 & & 15117-96-1 & U-235 \\
\hline 259 & SESP SCHEDULING & & SO & & & B15C47 & 07-Oct-02 & & 15117-96-1 & U-235 \\
\hline 260 & SESP SCHEDULING & & SO & & & B15LL9 & 09-Oct-02 & & $15117-96-1$ & U-235 \\
\hline 261 & SESP SCHEDULING & & SO & & & B15C52 & 29-Oct-02 & & 15117-96-1 & U-235 \\
\hline 262 & SESP SCHEDULING & & SO & & & B0YRB6 & 12-Jun-00 & & U-238 & U-238 \\
\hline 263 & SESP SCHEDULING & & SO & & & B0YWC5 & 07-Aug-00 & & $\mathrm{U}-238$ & $\mathrm{U}-238$ \\
\hline 264 & SESP SCHEDULING & & SO & & & BOYWC7 & 07-Aug-00 & & $\mathrm{U}-238$ & $\mathrm{U}-238$ \\
\hline 265 & SESP SCHEDULING & & SO & & & B0YW87 & 08-Aug-00 & & $\mathrm{U}-238$ & $\mathrm{U}-238$ \\
\hline 266 & SESP SCHEDULING & & SO & & & B0YWD3 & 08-Aug-00 & & $\mathrm{U}-238$ & $\mathrm{U}-238$ \\
\hline 267 & SESP SCHEDULING & & SO & & & B0YWD5 & 08-Aug-00 & & U-238 & U-238 \\
\hline
\end{tabular}




\begin{tabular}{|c|c|c|c|c|c|c|c|c|c|c|}
\hline & A & $\mathrm{B}$ & $\mathrm{C}$ & $\mathrm{D}$ & $E$ & $\mathrm{~F}$ & G & $\mathrm{H}$ & $\mathrm{I}$ & $\mathrm{J}$ \\
\hline 1 & SAMPLE_NUM_ASSIGNED_TO & SAMPLER & MEDIA & BIOTA_MEDIA & TAG_ID & SAMP_NUM & SAMP_DATE_TIME & SAMP_DATE_TIME_ON & CON_ID & |CON_SHORT_NAME \\
\hline 268 & SESP SCHEDULING & & SO & & & BOYY70 & 15-Aug-00 & & U-238 & U-238 \\
\hline 269 & SESP SCHEDULING & & SO & & & B0YY71 & 15-Aug-00 & & $\mathrm{U}-238$ & $\mathrm{U}-238$ \\
\hline 270 & SESP SCHEDULING & & SO & & & BOYY72 & 15-Aug-00 & & U-238 & U-238 \\
\hline 271 & SESP SCHEDULING & & SO & & & B10908 & 27-Sep-00 & & U-238 & U-238 \\
\hline 272 & SESP SCHEDULING & & SO & & & B10917 & 27-Sep-00 & & $\mathrm{U}-238$ & $\mathrm{U}-238$ \\
\hline 273 & SESP SCHEDULING & & SO & & & B10919 & 27-Sep-00 & & $\mathrm{U}-238$ & $\mathrm{U}-238$ \\
\hline 274 & SESP SCHEDULING & & SO & & & B10922 & 27-Sep-00 & & $\mathrm{U}-238$ & $\mathrm{U}-238$ \\
\hline 275 & SESP SCHEDULING & & SO & & & B10915 & 25-Oct-00 & & $\mathrm{U}-238$ & U-238 \\
\hline 276 & SESP SCHEDULING & & SO & & & B10929 & 07-Nov-00 & & $\mathrm{U}-238$ & $\mathrm{U}-238$ \\
\hline 277 & SESP SCHEDULING & & SO & & & B12CK8 & 19-Jul-01 & & $\mathrm{U}-238$ & $\mathrm{U}-238$ \\
\hline 278 & SESP SCHEDULING & & SO & & & B12CL5 & 19-Jul-01 & & $\mathrm{U}-238$ & $\mathrm{U}-238$ \\
\hline 279 & SESP SCHEDULING & & SO & & & B12CL6 & 19-Jul-01 & & $\mathrm{U}-238$ & $\mathrm{U}-238$ \\
\hline 280 & SESP SCHEDULING & & SO & & & B12CM2 & 19-Jul-01 & & $\mathrm{U}-238$ & U-238 \\
\hline 281 & SESP SCHEDULING & & SO & & & B12CM4 & 19-Jul-01 & & U-238 & U-238 \\
\hline 282 & SESP SCHEDULING & & SO & & & B12CL1 & 20-Jul-01 & & $\mathrm{U}-238$ & $\mathrm{U}-238$ \\
\hline 283 & SESP SCHEDULING & & SO & & & B12CL2 & 20-Jul-01 & & $\mathrm{U}-238$ & $\mathrm{U}-238$ \\
\hline 284 & SESP SCHEDULING & & SO & & & B12CL3 & 20-Jul-01 & & $\mathrm{U}-238$ & U-238 \\
\hline 285 & SESP SCHEDULING & & SO & & & B12CL4 & 20-Jul-01 & & $\mathrm{U}-238$ & U-238 \\
\hline 286 & SESP SCHEDULING & & SO & & & $\mathrm{B} 12 \mathrm{CH} 3$ & 23-Jul-01 & & $\mathrm{U}-238$ & $\mathrm{U}-238$ \\
\hline 287 & SESP SCHEDULING & & SO & & & $\mathrm{B} 12 \mathrm{CJO}$ & 23-Jul-01 & & $\mathrm{U}-238$ & $\mathrm{U}-238$ \\
\hline 288 & SESP SCHEDULING & & SO & & & B12CJ1 & 23-Jul-01 & & U-238 & U-238 \\
\hline 289 & SESP SCHEDULING & & SO & & & B12CK9 & 23-Jul-01 & & $\mathrm{U}-238$ & $\mathrm{U}-238$ \\
\hline 290 & SESP SCHEDULING & & SO & & & B12CN0 & 23-Jul-01 & & $\mathrm{U}-238$ & $\mathrm{U}-238$ \\
\hline 291 & SESP SCHEDULING & & SO & & & B12CN2 & 23-Jul-01 & & $\mathrm{U}-238$ & $\mathrm{U}-238$ \\
\hline 292 & SESP SCHEDULING & & SO & & & B12RL9 & 27-Aug-01 & & $\mathrm{U}-238$ & $\mathrm{U}-238$ \\
\hline 293 & SESP SCHEDULING & & SO & & & B12RY9 & 27-Aug-01 & & U-238 & U-238 \\
\hline 294 & SESP SCHEDULING & & SO & & & B12T01 & 27-Aug-01 & & U-238 & U-238 \\
\hline 295 & SESP SCHEDULING & & SO & & & B12T04 & 27-Aug-01 & & $\mathrm{U}-238$ & $\mathrm{U}-238$ \\
\hline 296 & SESP SCHEDULING & & SO & & & B12T05 & 27-Aug-01 & & $\mathrm{U}-238$ & $\mathrm{U}-238$ \\
\hline 297 & SESP SCHEDULING & & SO & & & B12T06 & 27-Aug-01 & & $\mathrm{U}-238$ & U-238 \\
\hline 298 & SESP SCHEDULING & & SO & & & B12T07 & 27-Aug-01 & & $\mathrm{U}-238$ & $\mathrm{U}-238$ \\
\hline 299 & SESP SCHEDULING & & SO & & & B12RY5 & 28-Aug-01 & & $\mathrm{U}-238$ & U-238 \\
\hline 300 & SESP SCHEDULING & & SO & & & B12T03 & 28-Aug-01 & & $\mathrm{U}-238$ & U-238 \\
\hline 301 & SESP SCHEDULING & & SO & & & B134L5 & 08-Oct-01 & & U-238 & U-238 \\
\hline 302 & SESP SCHEDULING & & SO & & & B12X20 & 22-Oct-01 & & $\mathrm{U}-238$ & $\mathrm{U}-238$ \\
\hline 303 & SESP SCHEDULING & & SO & & & B12X35 & 22-Oct-01 & & $\mathrm{U}-238$ & $\mathrm{U}-238$ \\
\hline 304 & SESP SCHEDULING & & SO & & & B141M8 & 14-Feb-02 & & $\mathrm{U}-238$ & $\mathrm{U}-238$ \\
\hline 305 & SESP SCHEDULING & & SO & & & B14CV4 & 08-Apr-02 & & U-238 & U-238 \\
\hline
\end{tabular}




\begin{tabular}{|c|c|c|c|c|c|c|c|c|c|c|}
\hline & A & $B$ & $\mathrm{C}$ & $\mathrm{D}$ & $\mathrm{E}$ & $\mathrm{F}$ & $G$ & $\mathrm{H}$ & $\mathrm{I}$ & $\mathrm{J}$ \\
\hline 1 & SAMPLE_NUM_ASSIGNED_TO & SAMPLER & MEDIA & BIOTA_MEDIA & TAG_ID & SAMP_NUM & SAMP_DATE_TIME & SAMP_DATE_TIME_ON & CON_ID & CON_SHORT_NAME \\
\hline 306 & SESP SCHEDULING & & so & & & B14YJ7 & 08-Jul-02 & & $\mathrm{U}-238$ & $\mathrm{U}-238$ \\
\hline 307 & SESP SCHEDULING & & SO & & & B14YM5 & 19-Jul-02 & & U-238 & U-238 \\
\hline 308 & SESP SCHEDULING & & SO & & & B14YP1 & 19-Jul-02 & & U-238 & U-238 \\
\hline 309 & SESP SCHEDULING & & SO & & & B14YP3 & 19-Jul-02 & & $\mathrm{U}-238$ & $\mathrm{U}-238$ \\
\hline 310 & SESP SCHEDULING & & SO & & & B14YM4 & 23-Jul-02 & & $\mathrm{U}-238$ & U-238 \\
\hline 311 & SESP SCHEDULING & & SO & & & B14YM6 & 23-Jul-02 & & U-238 & U-238 \\
\hline 312 & SESP SCHEDULING & & SO & & & B14YM7 & 23-Jul-02 & & $\mathrm{U}-238$ & $\mathrm{U}-238$ \\
\hline 313 & SESP SCHEDULING & & SO & & & B14YN3 & 23-Jul-02 & & $\mathrm{U}-238$ & $U-238$ \\
\hline 314 & SESP SCHEDULING & & SO & & & B14YN5 & 23-Jul-02 & & $\mathrm{U}-238$ & U-238 \\
\hline 315 & SESP SCHEDULING & & SO & & & B15C40 & 16-Sep-02 & & $\mathrm{U}-238$ & $\mathrm{U}-238$ \\
\hline 316 & SESP SCHEDULING & & SO & & & B15C49 & 16-Sep-02 & & $\mathrm{U}-238$ & $\mathrm{U}-238$ \\
\hline 317 & SESP SCHEDULING & & SO & & & B15C07 & $07-$ Oct-02 & & U-238 & U-238 \\
\hline 318 & SESP SCHEDULING & & so & & & B15C44 & 07-Oct-02 & & U-238 & U-238 \\
\hline 319 & SESP SCHEDULING & & SO & & & B15C47 & $07-$ Oct-02 & & $\mathrm{U}-238$ & U-238 \\
\hline 320 & SESP SCHEDULING & & SO & & & B15LL9 & 09-Oct-02 & & U-238 & U-238 \\
\hline 321 & SESP SCHEDULING & & SO & & & B15C52 & 29-Oct-02 & & $\mathrm{U}-238$ & U-238 \\
\hline
\end{tabular}




\begin{tabular}{|c|c|c|c|c|c|c|c|c|c|}
\hline & $\mathrm{K}$ & $\mathrm{L}$ & $\mathrm{M}$ & $\mathrm{N}$ & $\mathrm{O}$ & $\mathrm{P}$ & $\mathrm{Q}$ & $\mathrm{R}$ & $\mathrm{S}$ \\
\hline 1 & METHOD_NAME & LAB_CODE & VALUE_RPTD & ANAL_UNITS_RPTD & |COUNTING_ERROR| & TOTAL_ANAL_ERROR & MIN_DETECTABLE_ACTIVITY & PCNT_MOISTURE & LAB_QUALIFIER \\
\hline 2 & GAMMA_GS & STLRL & 1.05 & $\mathrm{pCi} / \mathrm{g}$ & 0.13 & 0.13 & 0.0387 & 59.6 & \\
\hline 3 & GAMMA_GS & STLRL & 0.442 & $\mathrm{pCi} / \mathrm{g}$ & 0.056 & 0.056 & 0.0261 & 41 & \\
\hline 4 & GAMMA_GS & STLRL & 0.331 & $\mathrm{pCi} / \mathrm{g}$ & 0.052 & 0.052 & 0.0251 & 39.7 & \\
\hline 5 & GAMMA_GS & STLRL & 0.23 & $\mathrm{pCi} / \mathrm{g}$ & 0.051 & 0.051 & 0.0449 & 67.8 & \\
\hline 6 & GAMMA_GS & STLRL & 0.276 & $\mathrm{pCi} / \mathrm{g}$ & 0.066 & 0.066 & 0.0617 & 42.5 & \\
\hline 7 & GAMMA_GS & STLRL & 0.449 & $\mathrm{pCi} / \mathrm{g}$ & 0.079 & 0.079 & 0.0496 & 50.9 & \\
\hline 8 & GAMMA_GS & STLRL & 0.0108 & $\mathrm{pCi} / \mathrm{g}$ & 0.012 & 0.012 & 0.0208 & 64.5 & U \\
\hline 9 & GAMMA_GS & STLRL & 0.534 & $\mathrm{pCi} / \mathrm{g}$ & 0.061 & 0.061 & 0.0222 & 56.6 & \\
\hline 11 & GAMMA_GS & STLRL & 0.0556 & $\mathrm{pCi} / \mathrm{g}$ & 0.021 & 0.021 & 0.0235 & 75.2 & \\
\hline 12 & GAMMA_GS & STLRL & 0.223 & $\mathrm{pCi} / \mathrm{g}$ & 0.031 & 0.031 & 0.0171 & 78.7 & \\
\hline 13 & GAMMA_GS & STLRL & 0.166 & $\mathrm{pCi} / \mathrm{g}$ & 0.031 & 0.031 & 0.0226 & 65.9 & \\
\hline 14 & GAMMA_GS & STLRL & 0.269 & $\mathrm{pCi} / \mathrm{g}$ & 0.035 & 0.035 & 0.0199 & 71.5 & \\
\hline 15 & GAMMA_GS & STLRL & 0.0629 & $\mathrm{pCi} / \mathrm{g}$ & 0.019 & 0.019 & 0.0191 & 74.6 & \\
\hline 16 & GAMMA_GS & STLRL & 0.092 & $\mathrm{pCi} / \mathrm{g}$ & 0.045 & 0.045 & 0.055 & 59.5 & \\
\hline 17 & GAMMA_GS & STLRL & 0.027 & $\mathrm{pCi} / \mathrm{g}$ & 0.026 & 0.026 & 0.0473 & 74.2 & U \\
\hline 18 & GAMMA_GS & STLRL & 0.578 & $\mathrm{pCi} / \mathrm{g}$ & 0.098 & 0.098 & 0.0518 & 39.9 & \\
\hline 19 & GAMMA_GS & STLRL & 0.159 & $\mathrm{pCi} / \mathrm{g}$ & 0.045 & 0.045 & 0.0385 & 72.9 & \\
\hline 21 & GAMMA_GS & STLRL & 0.502 & $\mathrm{pCi} / \mathrm{g}$ & 0.09 & 0.09 & 0.0422 & 50.8 & \\
\hline 22 & GAMMA_GS & STLRL & 0.221 & $\mathrm{pCi} / \mathrm{g}$ & 0.049 & 0.049 & 0.0499 & 47.9 & \\
\hline 23 & GAMMA_GS & STLRL & 0.246 & $\mathrm{pCi} / \mathrm{g}$ & 0.068 & 0.068 & 0.054 & 43.1 & \\
\hline 24 & GAMMA_GS & STLRL & 0.389 & $\mathrm{pCi} / \mathrm{g}$ & 0.077 & 0.077 & 0.052 & 49.7 & \\
\hline 25 & GAMMA_GS & STLRL & 0.528 & $\mathrm{pCi} / \mathrm{g}$ & 0.099 & 0.099 & 0.0572 & 44.6 & \\
\hline 26 & GAMMA_GS & STLRL & 0.355 & $\mathrm{pCi} / \mathrm{g}$ & 0.078 & 0.078 & 0.0567 & 44.3 & \\
\hline 27 & GAMMA_GS & STLRL & 0.196 & $\mathrm{pCi} / \mathrm{g}$ & 0.05 & 0.05 & 0.0482 & 45.9 & \\
\hline 28 & GAMMA_GS & STLRL & 0.129 & $\mathrm{pCi} / \mathrm{g}$ & 0.044 & 0.044 & 0.0489 & 36.3 & \\
\hline 29 & GAMMA_GS & STLRL & 0.241 & $\mathrm{pCi} / \mathrm{g}$ & 0.049 & 0.049 & 0.0402 & 64.8 & \\
\hline 30 & GAMMA_GS & STLRL & 1.1 & $\mathrm{pCi} / \mathrm{g}$ & 0.15 & 0.15 & 0.0549 & 43.2 & \\
\hline 31 & GAMMA_GS & STLRL & 0.442 & $\mathrm{pCi} / \mathrm{g}$ & 0.085 & 0.085 & 0.0492 & 44.2 & \\
\hline 32 & GAMMA_GS & STLRL & 0.0379 & $\mathrm{pCi} / \mathrm{g}$ & 0.013 & 0.013 & 0.0148 & 75.1 & \\
\hline 33 & GAMMA_GS & STLRL & 1.88 & $\mathrm{pCi} / \mathrm{g}$ & 0.23 & 0.23 & 0.02 & 39.4 & \\
\hline 34 & GAMMA_GS & STLRL & 0.0747 & $\mathrm{pCi} / \mathrm{g}$ & 0.019 & 0.019 & 0.0197 & 75.2 & \\
\hline 35 & GAMMA_GS & STLRL & 0.145 & $\mathrm{pCi} / \mathrm{g}$ & 0.025 & 0.025 & 0.0241 & 69.6 & \\
\hline 36 & GAMMA_GS & STLRL & 0.276 & $\mathrm{pCi} / \mathrm{g}$ & 0.038 & 0.038 & 0.0173 & 16.7 & \\
\hline 37 & GAMMA_GS & STLRL & 0.696 & $\mathrm{pCi} / \mathrm{g}$ & 0.087 & 0.087 & 0.0209 & 36.1 & \\
\hline 38 & GAMMA_GS & STLRL & 1.21 & $\mathrm{pCi} / \mathrm{g}$ & 0.15 & 0.15 & 0.0199 & 63.3 & \\
\hline
\end{tabular}




\begin{tabular}{|c|c|c|c|c|c|c|c|c|c|}
\hline & $\mathrm{K}$ & $\mathrm{L}$ & $\mathrm{M}$ & $\mathrm{N}$ & $\mathrm{O}$ & $\mathrm{P}$ & $\mathrm{Q}$ & $\mathrm{R}$ & $\mathrm{S}$ \\
\hline 1 & METHOD_NAME & LAB_CODE & VALUE_RPTD & ANAL_UNITS_RPTD & COUNTING_ERROR & TOTAL_ANAL_ERROR & MIN_DETECTABLE_ACTIVITY & PCNT_MOISTURE & LAB_QUALIFIER \\
\hline 40 & GAMMA_GS & STLRL & 0.223 & $\mathrm{pCi} / \mathrm{g}$ & 0.041 & 0.041 & 0.0281 & 43.7 & \\
\hline 41 & GAMMA_GS & STLRL & 0.254 & $\mathrm{pCi} / \mathrm{g}$ & 0.039 & 0.039 & 0.0246 & 47.1 & \\
\hline 42 & GAMMA_GS & STLRL & 0.00714 & $\mathrm{pCi} / \mathrm{g}$ & 0.013 & 0.013 & 0.023 & 74 & U \\
\hline 43 & GAMMA_GS & STLRL & 0.645 & $\mathrm{pCi} / \mathrm{g}$ & 0.089 & 0.089 & 0.0339 & 31 & \\
\hline 44 & GAMMA_GS & STLRL & 0.299 & $\mathrm{pCi} / \mathrm{g}$ & 0.041 & 0.041 & 0.0168 & 78.9 & \\
\hline 45 & GAMMA_GS & STLRL & 0.649 & $\mathrm{pCi} / \mathrm{g}$ & 0.086 & 0.086 & 0.0266 & 47.6 & \\
\hline 46 & GAMMA_GS & STLRL & 0.416 & $\mathrm{pCi} / \mathrm{g}$ & 0.06 & 0.06 & 0.0266 & 34.8 & \\
\hline 47 & GAMMA_GS & STLRL & 0.0591 & $\mathrm{pCi} / \mathrm{g}$ & 0.016 & 0.016 & 0.018 & 73.6 & \\
\hline 49 & GAMMA_GS & STLRL & 0.0566 & $\mathrm{pCi} / \mathrm{g}$ & 0.019 & 0.019 & 0.0224 & 77 & \\
\hline 50 & GAMMA_GS & STLRL & 0.0987 & $\mathrm{pCi} / \mathrm{g}$ & 0.024 & 0.024 & 0.0203 & 70 & \\
\hline 51 & GAMMA_GS & STLRL & 0.25 & $\mathrm{pCi} / \mathrm{g}$ & 0.038 & 0.038 & 0.0179 & 60.5 & \\
\hline 52 & GAMMA_GS & STLRL & 1.82 & $\mathrm{pCi} / \mathrm{g}$ & 0.22 & 0.22 & 0.022 & 48 & \\
\hline 53 & GAMMA_GS & STLRL & 0.0713 & $\mathrm{pCi} / \mathrm{g}$ & 0.019 & 0.019 & 0.0188 & 59.1 & \\
\hline 54 & PUISO_IE_PLATE_AEA & STLRL & 0.00956 & $\mathrm{pCi} / \mathrm{g}$ & 0.0011 & 0.0017 & 0.000253 & 41 & \\
\hline 55 & PUISO_IE_PLATE_AEA & STLRL & 0.00784 & $\mathrm{pCi} / \mathrm{g}$ & 0.00084 & 0.0014 & 0.000155 & 39.7 & \\
\hline 56 & PUISO_IE_PLATE_AEA & STLRL & 0.00112 & $\mathrm{pCi} / \mathrm{g}$ & 0.00045 & 0.0005 & 0.000306 & 67.8 & \\
\hline 57 & PUISO_IE_PLATE_AEA & STLRL & 0.00713 & $\mathrm{pCi} / \mathrm{g}$ & 0.0013 & 0.0017 & 0.000327 & 42.5 & \\
\hline 59 & PUISO_IE_PLATE_AEA & STLRL & 0.000641 & $\mathrm{pCi} / \mathrm{g}$ & 0.00021 & 0.00023 & 0.0000463 & 64.5 & \\
\hline 60 & PUISO_IE_PLATE_AEA & STLRL & 0.00578 & $\mathrm{pCi} / \mathrm{g}$ & 0.00075 & 0.0011 & 0.000067 & 56.6 & \\
\hline 61 & PUISO_IE_PLATE_AEA & STLRL & 0.0023 & $\mathrm{pCi} / \mathrm{g}$ & 0.00043 & 0.00054 & 0.000112 & 72.5 & \\
\hline 62 & PUISO_IE_PLATE_AEA & STLRL & 0.000398 & $\mathrm{pCi} / \mathrm{g}$ & 0.00023 & 0.00023 & 0.000164 & 74.2 & \\
\hline 63 & PUISO_IE_PLATE_AEA & STLRL & 0.0044 & $\mathrm{pCi} / \mathrm{g}$ & 0.0008 & 0.001 & 0.000247 & 39.9 & \\
\hline 64 & PUISO_IE_PLATE_AEA & STLRL & 0.00197 & $\mathrm{pCi} / \mathrm{g}$ & 0.00045 & 0.00054 & 0.0000702 & 72.9 & \\
\hline 65 & PUISO_IE_PLATE_AEA & STLRL & 0.00959 & $\mathrm{pCi} / \mathrm{g}$ & 0.00088 & 0.0016 & 0.000115 & 37.8 & \\
\hline 66 & PUISO_IE_PLATE_AEA & STLRL & 0.00962 & $\mathrm{pCi} / \mathrm{g}$ & 0.00076 & 0.0015 & 0.0000838 & 50.8 & \\
\hline 67 & PUISO_IE_PLATE_AEA & STLRL & 0.00509 & $\mathrm{pCi} / \mathrm{g}$ & 0.00062 & 0.00094 & 0.0000502 & 47.9 & \\
\hline 68 & PUISO_IE_PLATE_AEA & STLRL & 0.00795 & $\mathrm{pCi} / \mathrm{g}$ & 0.00076 & 0.0013 & 0.000125 & 43.1 & \\
\hline 69 & PUISO_IE_PLATE_AEA & STLRL & 0.00787 & $\mathrm{pCi} / \mathrm{g}$ & 0.00078 & 0.0014 & 0.000109 & 49.7 & \\
\hline 70 & PUISO_IE_PLATE_AEA & STLRL & 0.0104 & $\mathrm{pCi} / \mathrm{g}$ & 0.00087 & 0.0017 & 0.000102 & 44.6 & \\
\hline 71 & PUISO_IE_PLATE_AEA & STLRL & 0.00996 & $\mathrm{pCi} / \mathrm{g}$ & 0.00089 & 0.0017 & 0.000155 & 44.3 & \\
\hline 72 & PUISO_IE_PLATE_AEA & STLRL & 0.00573 & $\mathrm{pCi} / \mathrm{g}$ & 0.00071 & 0.0011 & 0.0000589 & 45.9 & \\
\hline 73 & PUISO_IE_PLATE_AEA & STLRL & 0.0048 & $\mathrm{pCi} / \mathrm{g}$ & 0.00078 & 0.001 & 0.0000855 & 36.3 & \\
\hline 74 & PUISO_IE_PLATE_AEA & STLRL & 0.00163 & $\mathrm{pCi} / \mathrm{g}$ & 0.00043 & 0.00049 & 0.00016 & 64.8 & \\
\hline 75 & PUISO_IE_PLATE_AEA & STLRL & 0.0316 & $\mathrm{pCi} / \mathrm{g}$ & 0.0018 & 0.0048 & 0.000208 & 43.2 & \\
\hline 76 & PUISO_IE_PLATE_AEA & STLRL & 0.0091 & $\mathrm{pCi} / \mathrm{g}$ & 0.0011 & 0.0017 & 0.0000858 & 44.2 & \\
\hline
\end{tabular}




\begin{tabular}{|c|c|c|c|c|c|c|c|c|c|}
\hline & $\mathrm{K}$ & $\mathrm{L}$ & $\mathrm{M}$ & $\mathrm{N}$ & $\mathrm{O}$ & $P$ & $\mathrm{Q}$ & $\mathrm{R}$ & $\mathrm{S}$ \\
\hline 1 & METHOD_NAME & LAB_CODE & VALUE_RPTD & ANAL_UNITS_RPTD & COUNTING_ERROR & TOTAL_ANAL_ERROR & MIN_DETECTABLE_ACTIVITY & PCNT_MOISTURE & LAB_QUALIFIER \\
\hline 78 & PUISO_IE_PLATE_AEA & STLRL & 0.0073 & $\mathrm{pCi} / \mathrm{g}$ & 0.0013 & 0.0017 & 0.000312 & 43.7 & \\
\hline 79 & PUISO_IE_PLATE_AEA & STLRL & 0.00892 & $\mathrm{pCi} / \mathrm{g}$ & 0.0012 & 0.0017 & 0.000101 & 47.1 & \\
\hline 80 & PUISO_IE_PLATE_AEA & STLRL & 0.0045 & $\mathrm{pCi} / \mathrm{g}$ & 0.00067 & 0.00093 & 0.0000681 & 74 & \\
\hline 81 & PUISO_IE_PLATE_AEA & STLRL & 0.00769 & $\mathrm{pCi} / \mathrm{g}$ & 0.0013 & 0.0017 & 0.000139 & 31 & \\
\hline 82 & PUISO_IE_PLATE_AEA & STLRL & 0.00136 & $\mathrm{pCi} / \mathrm{g}$ & 0.00034 & 0.00039 & 0.0000567 & 78.9 & \\
\hline 83 & PUISO_IE_PLATE_AEA & STLRL & 0.0146 & $\mathrm{pCi} / \mathrm{g}$ & 0.0013 & 0.0024 & 0.0000738 & 47.6 & \\
\hline 84 & PUISO_IE_PLATE_AEA & STLRL & 0.01 & $\mathrm{pCi} / \mathrm{g}$ & 0.0012 & 0.0019 & 0.0000991 & 34.8 & \\
\hline 85 & SRISO_SEP_PRECIP_GPC & STLRL & 0.376 & $\mathrm{pCi} / \mathrm{g}$ & 0.059 & 0.11 & 0.0606 & 59.6 & \\
\hline 87 & SRISO_SEP_PRECIP_GPC & STLRL & 0.0281 & $\mathrm{pCi} / \mathrm{g}$ & 0.017 & 0.028 & 0.0445 & 39.7 & \\
\hline 88 & SRISO_SEP_PRECIP_GPC & STLRL & 0.000654 & $\mathrm{pCi} / \mathrm{g}$ & 0.001 & 0.02 & 0.0362 & 67.8 & \\
\hline 89 & SRISO_SEP_PRECIP_GPC & STLRL & 0.0292 & $\mathrm{pCi} / \mathrm{g}$ & 0.02 & 0.03 & 0.047 & 42.5 & \\
\hline 90 & SRISO_SEP_PRECIP_GPC & STLRL & 0.0119 & $\mathrm{pCi} / \mathrm{g}$ & 0.014 & 0.028 & 0.0489 & 50.9 & \\
\hline 91 & SRISO_SEP_PRECIP_GPC & STLRL & 0.000154 & $\mathrm{pCi} / \mathrm{g}$ & 0.024 & 0.024 & 0.0448 & 64.5 & \\
\hline 92 & SRISO_SEP_PRECIP_GPC & STLRL & -0.00409 & $\mathrm{pCi} / \mathrm{g}$ & 0.022 & 0.023 & 0.0443 & 56.6 & \\
\hline 93 & SRISO_SEP_PRECIP_GPC & STLRL & -0.00948 & $\mathrm{pCi} / \mathrm{g}$ & 0.02 & 0.02 & 0.0395 & 72.5 & \\
\hline 94 & SRISO_SEP_PRECIP_GPC & STLRL & -0.0122 & $\mathrm{pCi} / \mathrm{g}$ & 0.026 & 0.026 & 0.0525 & 75.2 & \\
\hline 95 & SRISO_SEP_PRECIP_GPC & STLRL & 0.00208 & $\mathrm{pCi} / \mathrm{g}$ & 0.025 & 0.025 & 0.0455 & 78.7 & \\
\hline 97 & SRISO_SEP_PRECIP_GPC & STLRL & 0.00748 & $\mathrm{pCi} / \mathrm{g}$ & 0.025 & 0.026 & 0.0469 & 71.5 & \\
\hline 98 & SRISO_SEP_PRECIP_GPC & STLRL & 0.00205 & $\mathrm{pCi} / \mathrm{g}$ & 0.022 & 0.024 & 0.0439 & 74.6 & $\mathrm{U}$ \\
\hline 99 & SRISO_SEP_PRECIP_GPC & STLRL & 0.013 & $\mathrm{pCi} / \mathrm{g}$ & 0.032 & 0.032 & 0.0592 & 59.5 & $\mathrm{U}$ \\
\hline 100 & SRISO_SEP_PRECIP_GPC & STLRL & 0.00207 & $\mathrm{pCi} / \mathrm{g}$ & 0.02 & 0.02 & 0.0358 & 74.2 & $U$ \\
\hline 101 & SRISO_SEP_PRECIP_GPC & STLRL & -0.0135 & $\mathrm{pCi} / \mathrm{g}$ & 0.015 & 0.017 & 0.0342 & 39.9 & $U$ \\
\hline 102 & SRISO_SEP_PRECIP_GPC & STLRL & -0.0103 & $\mathrm{pCi} / \mathrm{g}$ & 0.018 & 0.018 & 0.0338 & 72.9 & \\
\hline 103 & SRISO_SEP_PRECIP_GPC & STLRL & 0.0217 & $\mathrm{pCi} / \mathrm{g}$ & 0.021 & 0.023 & 0.0359 & 37.8 & $U$ \\
\hline 104 & SRISO_SEP_PRECIP_GPC & STLRL & -0.00432 & $\mathrm{pCi} / \mathrm{g}$ & 0.017 & 0.019 & 0.0362 & 50.8 & $\mathrm{U}$ \\
\hline 105 & SRISO_SEP_PRECIP_GPC & STLRL & -0.00999 & $\mathrm{pCi} / \mathrm{g}$ & 0.016 & 0.019 & 0.0382 & 47.9 & $\mathrm{U}$ \\
\hline 106 & SRISO_SEP_PRECIP_GPC & STLRL & 0.00637 & $\mathrm{pCi} / \mathrm{g}$ & 0.022 & 0.023 & 0.0401 & 43.1 & $U$ \\
\hline 107 & SRISO_SEP_PRECIP_GPC & STLRL & 0.00252 & $\mathrm{pCi} / \mathrm{g}$ & 0.02 & 0.021 & 0.0376 & 49.7 & $U$ \\
\hline 108 & SRISO_SEP_PRECIP_GPC & STLRL & 0.00824 & $\mathrm{pCi} / \mathrm{g}$ & 0.02 & 0.021 & 0.0365 & 44.6 & $\mathrm{U}$ \\
\hline 109 & SRISO_SEP_PRECIP_GPC & STLRL & 0.0088 & $\mathrm{pCi} / \mathrm{g}$ & 0.018 & 0.02 & 0.0348 & 44.3 & $U$ \\
\hline 110 & SRISO_SEP_PRECIP_GPC & STLRL & 0.00286 & $\mathrm{pCi} / \mathrm{g}$ & 0.019 & 0.021 & 0.0374 & 45.9 & $U$ \\
\hline 111 & SRISO_SEP_PRECIP_GPC & STLRL & -0.00338 & $\mathrm{pCi} / \mathrm{g}$ & 0.02 & 0.02 & 0.038 & 36.3 & $\mathrm{U}$ \\
\hline 112 & SRISO_SEP_PRECIP_GPC & STLRL & -0.00733 & $\mathrm{pCi} / \mathrm{g}$ & 0.023 & 0.023 & 0.0432 & 64.8 & $U$ \\
\hline 113 & SRISO_SEP_PRECIP_GPC & STLRL & 0.0434 & $\mathrm{pCi} / \mathrm{g}$ & 0.025 & 0.028 & 0.04 & 43.2 & \\
\hline 114 & SRISO_SEP_PRECIP_GPC & STLRL & 0.0182 & $\mathrm{pCi} / \mathrm{g}$ & 0.02 & 0.023 & 0.0367 & 44.2 & $\mathrm{U}$ \\
\hline
\end{tabular}


$0.752 \mathrm{pCi} / \mathrm{g}$

$0.00297 \mathrm{pCi} / \mathrm{g}$

$0.0018 \mathrm{pCi} / \mathrm{g}$

$0.112 \mathrm{pCi} / \mathrm{g}$

$0.25 \mathrm{pCi} / \mathrm{g}$

$0.518 \mathrm{pCi} / \mathrm{g}$

$-0.0246 \mathrm{pCi} / \mathrm{g}$

$0.027 \mathrm{pCi} / \mathrm{g}$

$0.0232 \mathrm{pCi} / \mathrm{g}$

$0.00587 \mathrm{pCi} / \mathrm{g}$

$0.00284 \mathrm{pCi} / \mathrm{g}$

$0.00541 \mathrm{pCi} / \mathrm{g}$

$-0.00226 \mathrm{pCi} / \mathrm{g}$

$-0.00755 \mathrm{pCi} / \mathrm{g}$

$-0.00361 \mathrm{pCi} / \mathrm{g}$

$0.0147 \mathrm{pCi} / \mathrm{g}$

$0.00649 \mathrm{pCi} / \mathrm{g}$

$0.0216 \mathrm{pCi} / \mathrm{g}$

$-0.00709 \mathrm{pCi} / \mathrm{g}$

$0.698 \mathrm{pCi} / \mathrm{g}$

$-0.00802 \mathrm{pCi} / \mathrm{g}$

$-0.0488 \mathrm{pCi} / \mathrm{g}$

$-0.114 \mathrm{pCi} / \mathrm{g}$

$0.122 \mathrm{pCi} / \mathrm{g}$

$-0.117 \mathrm{pCi} / \mathrm{g}$

$0.215 \mathrm{pCi} / \mathrm{g}$

$1.18 \mathrm{pCi} / \mathrm{g}$

$0.731 \mathrm{pCi} / \mathrm{g}$

$0.635 \mathrm{pCi} / \mathrm{g}$

$0.248 \mathrm{pCi} / \mathrm{g}$

$0.836 \mathrm{pCi} / \mathrm{g}$

$0.737 \mathrm{pCi} / \mathrm{g}$

$0.369 \mathrm{pCi} / \mathrm{g}$

$0.687 \mathrm{pCi} / \mathrm{g}$

$0.309 \mathrm{pCi} / \mathrm{g}$

$1.97 \mathrm{pCi} / \mathrm{g}$

$0.412 \mathrm{pCi} / \mathrm{g}$

$0.633 \mathrm{pCi} / \mathrm{g}$

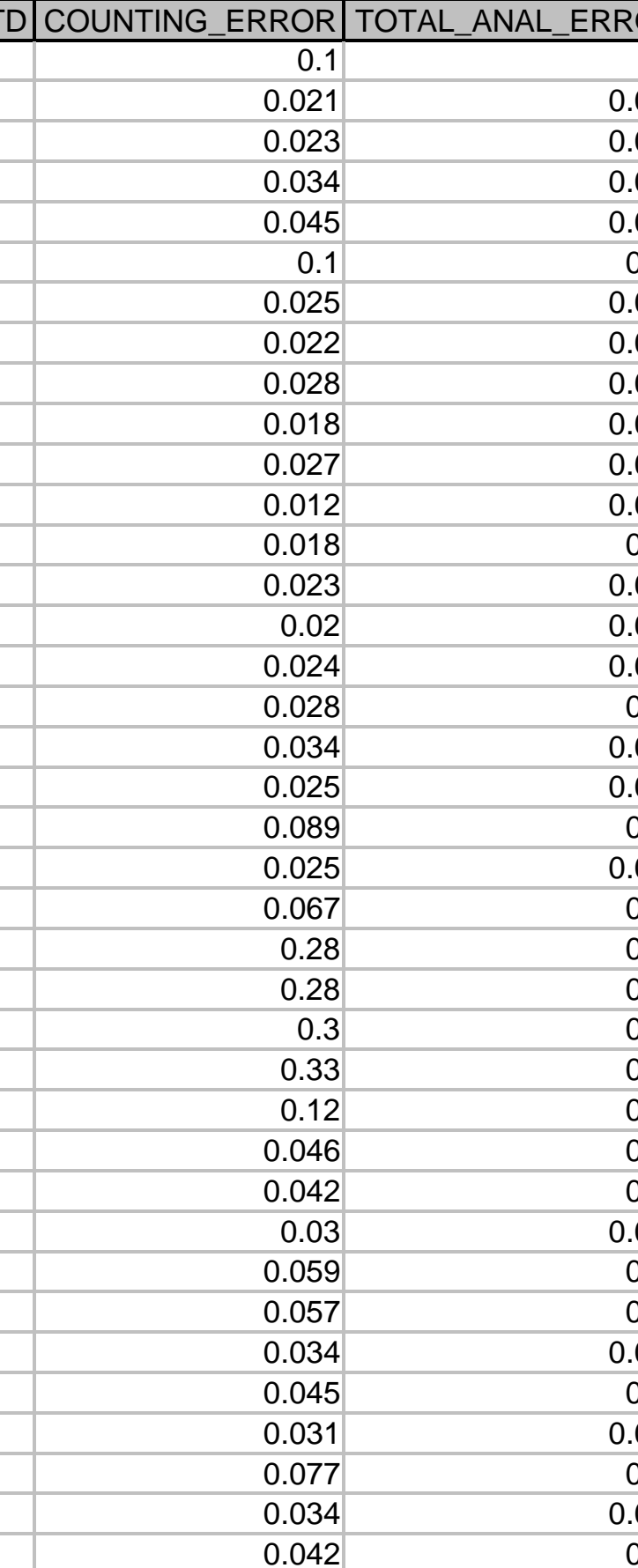

0.042

\begin{tabular}{|c|c|c|}
\hline 0.2 & 0.0936 & 39.4 \\
\hline 0.025 & 0.042 & $75.2 \mathrm{U}$ \\
\hline 0.025 & 0.0429 & $69.6 U$ \\
\hline 0.042 & 0.0539 & 16.7 \\
\hline 0.074 & 0.0471 & 36.1 \\
\hline 0.15 & 0.124 & 63.3 \\
\hline 0.025 & 0.0498 & $77.1 \mathrm{U}$ \\
\hline 0.023 & 0.0371 & $43.7 \mathrm{U}$ \\
\hline 0.034 & 0.0604 & $47.1 \mathrm{U}$ \\
\hline 0.019 & 0.0347 & $74 \mathrm{U}$ \\
\hline 0.029 & 0.0554 & $31 \mathrm{U}$ \\
\hline 0.013 & 0.0219 & $78.9 \mathrm{U}$ \\
\hline 0.02 & 0.0366 & $47.6 \mathrm{U}$ \\
\hline 0.026 & 0.0498 & $34.8 \mathrm{U}$ \\
\hline 0.021 & 0.0408 & $73.6 \mathrm{U}$ \\
\hline 0.024 & 0.0409 & $72.2 \mathrm{U}$ \\
\hline 0.03 & 0.0561 & $77 \mathrm{U}$ \\
\hline 0.035 & 0.0636 & $70 \mathrm{U}$ \\
\hline 0.032 & 0.0617 & $60.5 \mathrm{U}$ \\
\hline 0.17 & 0.0911 & 48 \\
\hline 0.027 & 0.0534 & $59.1 \mathrm{U}$ \\
\hline 0.14 & 0.161 & $39.4 \mathrm{U}$ \\
\hline 0.45 & 0.687 & $16.7 U$ \\
\hline 0.44 & 0.654 & $36.1 \mathrm{U}$ \\
\hline 0.48 & 0.735 & $63.3 \mathrm{U}$ \\
\hline 0.56 & 0.797 & $48 U$ \\
\hline 0.25 & 0.0422 & 59.6 \\
\hline 0.13 & 0.00201 & 41 \\
\hline 0.12 & 0.00398 & 39.7 \\
\hline 0.053 & 0.0106 & 67.8 \\
\hline 0.16 & 0.0161 & 42.5 \\
\hline 0.14 & 0.0121 & 50.9 \\
\hline 0.072 & 0.00442 & 64.5 \\
\hline 0.13 & 0.00425 & 56.6 \\
\hline 0.062 & 0.00454 & 72.5 \\
\hline 0.35 & 0.00205 & 75.2 \\
\hline 0.079 & 0.00398 & 78.7 \\
\hline 0.12 & 0.00542 & 65.9 \\
\hline
\end{tabular}

0.00542

65.9 


\begin{tabular}{|c|c|c|c|c|c|c|c|c|c|}
\hline & $\mathrm{K}$ & $L$ & $\mathrm{M}$ & $\mathrm{N}$ & $\mathrm{O}$ & $\mathrm{P}$ & $\mathrm{Q}$ & $\mathrm{R}$ & $\mathrm{S}$ \\
\hline 1 & METHOD_NAME & LAB_CODE & VALUE_RPTD & ANAL_UNITS_RPTD & COUNTING_ERROR & TOTAL_ANAL_ERROR & MIN_DETECTABLE_ACTIVITY & PCNT_MOISTURE & LAB_QUALIFIER \\
\hline 154 & UISO_IE_PLATE_AEA & STLRL & 3.01 & $\mathrm{pCi} / \mathrm{g}$ & 0.094 & 0.52 & 0.00501 & 71.5 & \\
\hline 155 & UISO_IE_PLATE_AEA & STLRL & 0.236 & $\mathrm{pCi} / \mathrm{g}$ & 0.025 & 0.049 & 0.00472 & 74.6 & \\
\hline 156 & UISO_IE_PLATE_AEA & STLRL & 0.359 & $\mathrm{pCi} / \mathrm{g}$ & 0.034 & 0.071 & 0.00619 & 59.5 & \\
\hline 157 & UISO_IE_PLATE_AEA & STLRL & 0.125 & $\mathrm{pCi} / \mathrm{g}$ & 0.02 & 0.03 & 0.00409 & 74.2 & \\
\hline 158 & UISO_IE_PLATE_AEA & STLRL & 0.474 & $\mathrm{pCi} / \mathrm{g}$ & 0.037 & 0.093 & 0.00554 & 39.9 & \\
\hline 159 & UISO_IE_PLATE_AEA & STLRL & 0.133 & $\mathrm{pCi} / \mathrm{g}$ & 0.021 & 0.032 & 0.00439 & 72.9 & \\
\hline 160 & UISO_IE_PLATE_AEA & STLRL & 0.818 & $\mathrm{pCi} / \mathrm{g}$ & 0.051 & 0.15 & 0.0044 & 37.8 & \\
\hline 161 & UISO_IE_PLATE_AEA & STLRL & 0.459 & $\mathrm{pCi} / \mathrm{g}$ & 0.039 & 0.091 & 0.00218 & 50.8 & \\
\hline 163 & UISO_IE_PLATE_AEA & STLRL & 0.808 & $\mathrm{pCi} / \mathrm{g}$ & 0.05 & 0.15 & 0.00206 & 43.1 & \\
\hline 164 & UISO_IE_PLATE_AEA & STLRL & 0.803 & $\mathrm{pCi} / \mathrm{g}$ & 0.05 & 0.15 & 0.00213 & 49.7 & \\
\hline 165 & UISO_IE_PLATE_AEA & STLRL & 0.862 & $\mathrm{pCi} / \mathrm{g}$ & 0.052 & 0.16 & 0.0021 & 44.6 & \\
\hline 166 & UISO_IE_PLATE_AEA & STLRL & 1.09 & $\mathrm{pCi} / \mathrm{g}$ & 0.058 & 0.2 & 0.00533 & 44.3 & \\
\hline 167 & UISO_IE_PLATE_AEA & STLRL & 0.878 & $\mathrm{pCi} / \mathrm{g}$ & 0.067 & 0.17 & 0.0153 & 45.9 & \\
\hline 168 & UISO_IE_PLATE_AEA & STLRL & 0.796 & $\mathrm{pCi} / \mathrm{g}$ & 0.05 & 0.15 & 0.00612 & 36.3 & \\
\hline 169 & UISO_IE_PLATE_AEA & STLRL & 0.165 & $\mathrm{pCi} / \mathrm{g}$ & 0.032 & 0.044 & 0.0148 & 64.8 & \\
\hline 170 & UISO_IE_PLATE_AEA & STLRL & 0.731 & $\mathrm{pCi} / \mathrm{g}$ & 0.061 & 0.15 & 0.0131 & 43.2 & \\
\hline 171 & UISO_IE_PLATE_AEA & STLRL & 0.873 & $\mathrm{pCi} / \mathrm{g}$ & 0.06 & 0.17 & 0.0101 & 44.2 & \\
\hline 173 & UISO_IE_PLATE_AEA & STLRL & 1.85 & $\mathrm{pCi} / \mathrm{g}$ & 0.076 & 0.34 & 0.00433 & 78.3 & \\
\hline 174 & UISO_IE_PLATE_AEA & STLRL & 0.328 & $\mathrm{pCi} / \mathrm{g}$ & 0.033 & 0.068 & 0.00564 & 75.6 & \\
\hline 175 & RADISOTOPES_ICPMS & PNL329 & 0.896003955 & $\mathrm{pCi} / \mathrm{g}$ & & & & & \\
\hline 176 & RADISOTOPES_ICPMS & PNL329 & 1.470445515 & $\mathrm{pCi} / \mathrm{g}$ & & & & & \\
\hline 177 & RADISOTOPES_ICPMS & PNL329 & 1.472943087 & $\mathrm{pCi} / \mathrm{g}$ & & & & & \\
\hline 178 & RADISOTOPES_ICPMS & PNL329 & 0.070556409 & $\mathrm{pCi} / \mathrm{g}$ & & & & & \\
\hline 179 & UISO_IE_PLATE_AEA & STLRL & 0.23 & $\mathrm{pCi} / \mathrm{g}$ & 0.027 & 0.05 & 0.00215 & 74.3 & \\
\hline 180 & RADISOTOPES_ICPMS & PNL329 & 0.064312479 & $\mathrm{pCi} / \mathrm{g}$ & & & & & \\
\hline 181 & UISO_IE_PLATE_AEA & STLRL & 2.46 & $\mathrm{pCi} / \mathrm{g}$ & 0.091 & 0.45 & 0.0047 & 39.4 & \\
\hline 182 & UISO_IE_PLATE_AEA & STLRL & 0.476 & $\mathrm{pCi} / \mathrm{g}$ & 0.045 & 0.097 & 0.00283 & 75.2 & \\
\hline 183 & UISO_IE_PLATE_AEA & STLRL & 0.702 & $\mathrm{pCi} / \mathrm{g}$ & 0.052 & 0.14 & 0.00656 & 69.6 & \\
\hline 184 & UISO_IE_PLATE_AEA & STLRL & 0.289 & $\mathrm{pCi} / \mathrm{g}$ & 0.029 & 0.06 & 0.00619 & 16.7 & \\
\hline 185 & UISO_IE_PLATE_AEA & STLRL & 0.625 & $\mathrm{pCi} / \mathrm{g}$ & 0.051 & 0.12 & 0.00284 & 36.1 & \\
\hline 186 & UISO_IE_PLATE_AEA & STLRL & 4.83 & $\mathrm{pCi} / \mathrm{g}$ & 0.13 & 0.87 & 0.00828 & 63.3 & \\
\hline 187 & UISO_IE_PLATE_AEA & STLRL & 0.117 & $\mathrm{pCi} / \mathrm{g}$ & 0.02 & 0.03 & 0.00215 & 77.1 & \\
\hline 188 & UISO_IE_PLATE_AEA & STLRL & 0.791 & $\mathrm{pCi} / \mathrm{g}$ & 0.052 & 0.15 & 0.0062 & 43.7 & \\
\hline 189 & UISO_IE_PLATE_AEA & STLRL & 0.853 & $\mathrm{pCi} / \mathrm{g}$ & 0.055 & 0.16 & 0.00235 & 47.1 & \\
\hline 190 & UISO_IE_PLATE_AEA & STLRL & 0.53 & $\mathrm{pCi} / \mathrm{g}$ & 0.04 & 0.1 & 0.00206 & 74 & \\
\hline
\end{tabular}




\begin{tabular}{|c|c|c|c|c|c|c|c|c|c|}
\hline & $\mathrm{K}$ & $\mathrm{L}$ & $\mathrm{M}$ & $\mathrm{N}$ & $\mathrm{O}$ & $\mathrm{P}$ & $\mathrm{Q}$ & $\mathrm{R}$ & $\mathrm{S}$ \\
\hline 1 & METHOD_NAME & LAB_CODE & VALUE_RPTD & ANAL_UNITS_RPTD & COUNTING_ERROR & TOTAL_ANAL_ERROR & MIN_DETECTABLE_ACTIVITY & PCNT_MOISTURE & LAB_QUALIFIER \\
\hline 192 & UISO_IE_PLATE_AEA & STLRL & 0.173 & $\mathrm{pCi} / \mathrm{g}$ & 0.021 & 0.037 & 0.00362 & 78.9 & \\
\hline 193 & UISO_IE_PLATE_AEA & STLRL & 0.526 & $\mathrm{pCi} / \mathrm{g}$ & 0.039 & 0.1 & 0.00194 & 47.6 & \\
\hline 194 & UISO_IE_PLATE_AEA & STLRL & 0.721 & $\mathrm{pCi} / \mathrm{g}$ & 0.046 & 0.14 & 0.00198 & 34.8 & \\
\hline 195 & UISO_IE_PLATE_AEA & STLRL & 0.192 & $\mathrm{pCi} / \mathrm{g}$ & 0.023 & 0.042 & 0.00181 & 73.6 & \\
\hline 196 & UISO_IE_PLATE_AEA & STLRL & 0.298 & $\mathrm{pCi} / \mathrm{g}$ & 0.035 & 0.065 & 0.00937 & 72.2 & \\
\hline 197 & UISO_IE_PLATE_AEA & STLRL & 0.872 & $\mathrm{pCi} / \mathrm{g}$ & 0.051 & 0.16 & 0.00198 & 77 & \\
\hline 198 & UISO_IE_PLATE_AEA & STLRL & 0.571 & $\mathrm{pCi} / \mathrm{g}$ & 0.04 & 0.11 & 0.00655 & 70 & \\
\hline 199 & UISO_IE_PLATE_AEA & STLRL & 11.3 & $\mathrm{pCi} / \mathrm{g}$ & 0.18 & 2 & 0.0019 & 60.5 & \\
\hline 201 & UISO_IE_PLATE_AEA & STLRL & 0.521 & $\mathrm{pCi} / \mathrm{g}$ & 0.04 & 0.1 & 0.00545 & 59.1 & \\
\hline 202 & UISO_IE_PLATE_AEA & STLRL & 0.0632 & $\mathrm{pCi} / \mathrm{g}$ & 0.027 & 0.031 & 0.02 & 59.6 & \\
\hline 203 & UISO_IE_PLATE_AEA & STLRL & 0.0258 & $\mathrm{pCi} / \mathrm{g}$ & 0.0085 & 0.01 & 0.00201 & 41 & \\
\hline 204 & UISO_IE_PLATE_AEA & STLRL & 0.0202 & $\mathrm{pCi} / \mathrm{g}$ & 0.0072 & 0.0087 & 0.00191 & 39.7 & \\
\hline 205 & UISO_IE_PLATE_AEA & STLRL & 0.00956 & $\mathrm{pCi} / \mathrm{g}$ & 0.0078 & 0.0093 & 0.0151 & 67.8 & \\
\hline 206 & UISO_IE_PLATE_AEA & STLRL & 0.022 & $\mathrm{pCi} / \mathrm{g}$ & 0.011 & 0.013 & 0.0158 & 42.5 & \\
\hline 207 & UISO_IE_PLATE_AEA & STLRL & 0.0177 & $\mathrm{pCi} / \mathrm{g}$ & 0.0092 & 0.011 & 0.0109 & 50.9 & \\
\hline 208 & UISO_IE_PLATE_AEA & STLRL & 0.0125 & $\mathrm{pCi} / \mathrm{g}$ & 0.0059 & 0.007 & 0.00212 & 64.5 & \\
\hline 209 & UISO_IE_PLATE_AEA & STLRL & 0.0267 & $\mathrm{pCi} / \mathrm{g}$ & 0.0088 & 0.01 & 0.00425 & 56.6 & \\
\hline 211 & UISO_IE_PLATE_AEA & STLRL & 0.0677 & $\mathrm{pCi} / \mathrm{g}$ & 0.014 & 0.019 & 0.00427 & 75.2 & \\
\hline 212 & UISO_IE_PLATE_AEA & STLRL & 0.0132 & $\mathrm{pCi} / \mathrm{g}$ & 0.0057 & 0.0069 & 0.00191 & 78.7 & \\
\hline 213 & UISO_IE_PLATE_AEA & STLRL & 0.0185 & $\mathrm{pCi} / \mathrm{g}$ & 0.0069 & 0.0082 & 0.00188 & 65.9 & \\
\hline 214 & UISO_IE_PLATE_AEA & STLRL & 0.129 & $\mathrm{pCi} / \mathrm{g}$ & 0.019 & 0.03 & 0.00198 & 71.5 & \\
\hline 215 & UISO_IE_PLATE_AEA & STLRL & 0.00528 & $\mathrm{pCi} / \mathrm{g}$ & 0.0033 & 0.0045 & 0.00186 & 74.6 & \\
\hline 216 & UISO_IE_PLATE_AEA & STLRL & 0.016 & $\mathrm{pCi} / \mathrm{g}$ & 0.0077 & 0.0082 & 0.00545 & 59.5 & \\
\hline 217 & UISO_IE_PLATE_AEA & STLRL & 0.00459 & $\mathrm{pCi} / \mathrm{g}$ & 0.0044 & 0.0045 & 0.00409 & 74.2 & \\
\hline 218 & UISO_IE_PLATE_AEA & STLRL & 0.0133 & $\mathrm{pCi} / \mathrm{g}$ & 0.0065 & 0.007 & 0.00192 & 39.9 & \\
\hline 219 & UISO_IE_PLATE_AEA & STLRL & 0.00226 & $\mathrm{pCi} / \mathrm{g}$ & 0.0035 & 0.0036 & 0.0021 & 72.9 & $U$ \\
\hline 220 & UISO_IE_PLATE_AEA & STLRL & 0.0367 & $\mathrm{pCi} / \mathrm{g}$ & 0.011 & 0.013 & 0.00535 & 37.8 & \\
\hline 221 & UISO_IE_PLATE_AEA & STLRL & 0.0182 & $\mathrm{pCi} / \mathrm{g}$ & 0.0081 & 0.0088 & 0.00454 & 50.8 & \\
\hline 222 & UISO_IE_PLATE_AEA & STLRL & 0.0213 & $\mathrm{pCi} / \mathrm{g}$ & 0.0084 & 0.0093 & 0.00424 & 47.9 & \\
\hline 223 & UISO_IE_PLATE_AEA & STLRL & 0.0212 & $\mathrm{pCi} / \mathrm{g}$ & 0.0083 & 0.0093 & 0.00206 & 43.1 & \\
\hline 224 & UISO_IE_PLATE_AEA & STLRL & 0.0196 & $\mathrm{pCi} / \mathrm{g}$ & 0.0082 & 0.009 & 0.00213 & 49.7 & \\
\hline 225 & UISO_IE_PLATE_AEA & STLRL & 0.0324 & $\mathrm{pCi} / \mathrm{g}$ & 0.01 & 0.012 & 0.0021 & 44.6 & \\
\hline 226 & UISO_IE_PLATE_AEA & STLRL & 0.0151 & $\mathrm{pCi} / \mathrm{g}$ & 0.0073 & 0.0079 & 0.00438 & 44.3 & \\
\hline 227 & UISO_IE_PLATE_AEA & STLRL & 0.0217 & $\mathrm{pCi} / \mathrm{g}$ & 0.012 & 0.012 & 0.0109 & 45.9 & \\
\hline 228 & UISO_IE_PLATE_AEA & STLRL & 0.0172 & $\mathrm{pCi} / \mathrm{g}$ & 0.0081 & 0.0088 & 0.00728 & 36.3 & \\
\hline
\end{tabular}




\begin{tabular}{|c|c|c|c|c|c|c|c|c|c|}
\hline & $\mathrm{K}$ & $L$ & $\mathrm{M}$ & $\mathrm{N}$ & $\mathrm{O}$ & $\mathrm{P}$ & $\mathrm{Q}$ & $\mathrm{R}$ & $\mathrm{S}$ \\
\hline 1 & METHOD_NAME & LAB_CODE & VALUE_RPTD & ANAL_UNITS_RPTD & COUNTING_ERROR & TOTAL_ANAL_ERROR & MIN_DETECTABLE_ACTIVITY & PCNT_MOISTURE & LAB_QUALIFIER \\
\hline 230 & UISO_IE_PLATE_AEA & STLRL & 0.0197 & $\mathrm{pCi} / \mathrm{g}$ & 0.01 & 0.011 & 0.0034 & 43.2 & \\
\hline 231 & UISO_IE_PLATE_AEA & STLRL & 0.0278 & $3 \mathrm{pCi} / \mathrm{g}$ & 0.011 & 0.012 & 0.00796 & 44.2 & \\
\hline 232 & UISO_IE_PLATE_AEA & STLRL & 0.102 & $\mathrm{pCi} / \mathrm{g}$ & 0.018 & 0.026 & 0.00212 & 75.1 & \\
\hline 233 & UISO_IE_PLATE_AEA & STLRL & 0.0757 & $\mathrm{pCi} / \mathrm{g}$ & 0.015 & 0.021 & 0.00208 & 78.3 & \\
\hline 234 & UISO_IE_PLATE_AEA & STLRL & 0.00987 & $\mathrm{pCi} / \mathrm{g}$ & 0.0061 & 0.0065 & 0.00222 & 75.6 & \\
\hline 235 & RADISOTOPES_ICPMS & PNL329 & 0.040367683 & $\mathrm{pCi} / \mathrm{g}$ & & & & & \\
\hline 236 & RADISOTOPES_ICPMS & PNL329 & 0.066543535 & $\mathrm{pCi} / \mathrm{g}$ & & & & & \\
\hline 237 & RADISOTOPES_ICPMS & PNL329 & 0.065762909 & $\mathrm{pCi} / \mathrm{g}$ & & & & & \\
\hline 239 & UISO_IE_PLATE_AEA & STLRL & 0.0055 & $\mathrm{pCi} / \mathrm{g}$ & 0.0048 & 0.0049 & 0.00215 & 74.3 & \\
\hline 240 & RADISOTOPES_ICPMS & PNL329 & 0.002681376 & $\mathrm{pCi} / \mathrm{g}$ & & & & & \\
\hline 241 & UISO_IE_PLATE_AEA & STLRL & 0.0949 & $\mathrm{pCi} / \mathrm{g}$ & 0.018 & 0.025 & 0.00225 & 39.4 & \\
\hline 242 & UISO_IE_PLATE_AEA & STLRL & 0.0139 & $\mathrm{pCi} / \mathrm{g}$ & 0.0085 & 0.0089 & 0.00816 & 75.2 & \\
\hline 243 & UISO_IE_PLATE_AEA & STLRL & 0.0597 & $\mathrm{pCi} / \mathrm{g}$ & 0.015 & 0.019 & 0.00656 & 69.6 & \\
\hline 244 & UISO_IE_PLATE_AEA & STLRL & 0.0099 & $\mathrm{pCi} / \mathrm{g}$ & 0.0058 & 0.0061 & 0.00195 & 16.7 & \\
\hline 245 & UISO_IE_PLATE_AEA & STLRL & 0.0162 & $\mathrm{pCi} / \mathrm{g}$ & 0.0086 & 0.0092 & 0.00284 & 36.1 & \\
\hline 246 & UISO_IE_PLATE_AEA & STLRL & 0.184 & $\mathrm{pCi} / \mathrm{g}$ & 0.026 & 0.042 & 0.00696 & 63.3 & \\
\hline 247 & UISO_IE_PLATE_AEA & STLRL & 0.00473 & $\mathrm{pCi} / \mathrm{g}$ & 0.0045 & 0.0046 & 0.00215 & 77.1 & \\
\hline 249 & UISO_IE_PLATE_AEA & STLRL & 0.0167 & $\mathrm{pCi} / \mathrm{g}$ & 0.0082 & 0.0088 & 0.00594 & 47.1 & \\
\hline 250 & UISO_IE_PLATE_AEA & STLRL & 0.0167 & $\mathrm{pCi} / \mathrm{g}$ & 0.0071 & 0.0077 & 0.00206 & 74 & \\
\hline 251 & UISO_IE_PLATE_AEA & STLRL & 0.0529 & $\mathrm{pCi} / \mathrm{g}$ & 0.013 & 0.016 & 0.00221 & 31 & \\
\hline 252 & UISO_IE_PLATE_AEA & STLRL & 0.0113 & $\mathrm{pCi} / \mathrm{g}$ & 0.0055 & 0.0058 & 0.00362 & 78.9 & \\
\hline 253 & UISO_IE_PLATE_AEA & STLRL & 0.0214 & $\mathrm{pCi} / \mathrm{g}$ & 0.0078 & 0.0087 & 0.00194 & 47.6 & \\
\hline 254 & UISO_IE_PLATE_AEA & STLRL & 0.0238 & $\mathrm{pCi} / \mathrm{g}$ & 0.0084 & 0.0094 & 0.00413 & 34.8 & \\
\hline 255 & UISO_IE_PLATE_AEA & STLRL & 0.00977 & $\mathrm{pCi} / \mathrm{g}$ & 0.0055 & 0.0059 & 0.00181 & 73.6 & \\
\hline 256 & UISO_IE_PLATE_AEA & STLRL & 0.00852 & $\mathrm{pCi} / \mathrm{g}$ & 0.0064 & 0.0066 & 0.00274 & 72.2 & \\
\hline 257 & UISO_IE_PLATE_AEA & STLRL & 0.0297 & $\mathrm{pCi} / \mathrm{g}$ & 0.0096 & 0.011 & 0.00198 & 77 & \\
\hline 258 & UISO_IE_PLATE_AEA & STLRL & 0.0146 & $\mathrm{pCi} / \mathrm{g}$ & 0.0068 & 0.0073 & 0.00191 & 70 & \\
\hline 259 & UISO_IE_PLATE_AEA & STLRL & 0.381 & $\mathrm{pCi} / \mathrm{g}$ & 0.033 & 0.075 & 0.0019 & 60.5 & \\
\hline 260 & UISO_IE_PLATE_AEA & STLRL & 0.0751 & $\mathrm{pCi} / \mathrm{g}$ & 0.015 & 0.02 & 0.00194 & 48 & \\
\hline 261 & UISO_IE_PLATE_AEA & STLRL & 0.0236 & $\mathrm{pCi} / \mathrm{g}$ & 0.0086 & 0.0097 & 0.00201 & 59.1 & \\
\hline 262 & UISO_IE_PLATE_AEA & STLRL & 1.2 & $\mathrm{pCi} / \mathrm{g}$ & 0.12 & 0.25 & 0.0337 & 59.6 & \\
\hline 263 & UISO_IE_PLATE_AEA & STLRL & 0.651 & $\mathrm{pCi} / \mathrm{g}$ & 0.044 & 0.12 & 0.00201 & 41 & \\
\hline 264 & UISO_IE_PLATE_AEA & STLRL & 0.56 & $\mathrm{pCi} / \mathrm{g}$ & 0.04 & 0.1 & 0.00191 & 39.7 & \\
\hline 265 & UISO_IE_PLATE_AEA & STLRL & 0.245 & $\mathrm{pCi} / \mathrm{g}$ & 0.03 & 0.053 & 0.0157 & 67.8 & \\
\hline 266 & UISO_IE_PLATE_AEA & STLRL & 0.672 & $\mathrm{pCi} / \mathrm{g}$ & 0.053 & 0.13 & 0.0187 & 42.5 & \\
\hline
\end{tabular}




\begin{tabular}{|c|c|c|c|c|c|c|c|c|c|}
\hline & $\mathrm{K}$ & $\mathrm{L}$ & $\mathrm{M}$ & $\mathrm{N}$ & $\mathrm{O}$ & $\mathrm{P}$ & $\mathrm{Q}$ & $\mathrm{R}$ & $\mathrm{S}$ \\
\hline 1 & METHOD_NAME & LAB_CODE & VALUE_RPTD & ANAL_UNITS_RPTD & COUNTING_ERROR & TOTAL_ANAL_ERROR & MIN_DETECTABLE_ACTIVITY & PCNT_MOISTURE & LAB_QUALIFIER \\
\hline 268 & UISO_IE_PLATE_AEA & STLRL & 0.341 & $\mathrm{pCi} / \mathrm{g}$ & 0.032 & 0.067 & 0.00537 & 64.5 & \\
\hline 269 & UISO_IE_PLATE_AEA & STLRL & 0.587 & $\mathrm{pCi} / \mathrm{g}$ & 0.042 & 0.11 & 0.00425 & 56.6 & \\
\hline 270 & UISO_IE_PLATE_AEA & STLRL & 0.289 & $\mathrm{pCi} / \mathrm{g}$ & 0.03 & 0.058 & 0.00454 & 72.5 & \\
\hline 271 & UISO_IE_PLATE_AEA & STLRL & 1.86 & $\mathrm{pCi} / \mathrm{g}$ & 0.075 & 0.33 & 0.0052 & 75.2 & \\
\hline 272 & UISO_IE_PLATE_AEA & STLRL & 0.331 & $\mathrm{pCi} / \mathrm{g}$ & 0.03 & 0.065 & 0.00191 & 78.7 & \\
\hline 273 & UISO IE_PLATE AEA & STLRL & 0.473 & $3 \mathrm{pCi} / \mathrm{g}$ & 0.036 & 0.089 & 0.00392 & 65.9 & \\
\hline 274 & UISO_IE_PLATE_AEA & STLRL & 2.62 & $\mathrm{pCi} / \mathrm{g}$ & 0.087 & 0.45 & 0.00198 & 71.5 & \\
\hline 275 & UISO_IE_PLATE_AEA & STLRL & 0.209 & $\mathrm{pCi} / \mathrm{g}$ & 0.024 & 0.044 & 0.00186 & 74.6 & \\
\hline 277 & UISO_IE_PLATE_AEA & STLRL & 0.133 & $\mathrm{pCi} / \mathrm{g}$ & 0.02 & 0.031 & 0.00409 & 74.2 & \\
\hline 278 & UISO_IE_PLATE_AEA & STLRL & 0.378 & $\mathrm{pCi} / \mathrm{g}$ & 0.033 & 0.075 & 0.00488 & 39.9 & \\
\hline 279 & UISO_IE_PLATE_AEA & STLRL & 0.122 & $\mathrm{pCi} / \mathrm{g}$ & 0.02 & 0.03 & 0.0021 & 72.9 & \\
\hline 280 & UISO_IE_PLATE_AEA & STLRL & 0.6 & $\mathrm{pCi} / \mathrm{g}$ & 0.043 & 0.12 & 0.0044 & 37.8 & \\
\hline 281 & UISO_IE_PLATE_AEA & STLRL & 0.46 & $\mathrm{pCi} / \mathrm{g}$ & 0.039 & 0.091 & 0.00218 & 50.8 & \\
\hline 282 & UISO_IE_PLATE_AEA & STLRL & 0.394 & $\mathrm{pCi} / \mathrm{g}$ & 0.035 & 0.078 & 0.00203 & 47.9 & \\
\hline 283 & UISO_IE_PLATE_AEA & STLRL & 0.65 & $\mathrm{pCi} / \mathrm{g}$ & 0.045 & 0.12 & 0.00206 & 43.1 & \\
\hline 284 & UISO_IE_PLATE_AEA & STLRL & 0.635 & $\mathrm{pCi} / \mathrm{g}$ & 0.045 & 0.12 & 0.00444 & 49.7 & \\
\hline 285 & UISO_IE_PLATE_AEA & STLRL & 0.612 & $\mathrm{pCi} / \mathrm{g}$ & 0.044 & 0.12 & 0.00437 & 44.6 & \\
\hline 287 & UISO_IE_PLATE_AEA & STLRL & 0.649 & $\mathrm{pCi} / \mathrm{g}$ & 0.058 & 0.13 & 0.0109 & 45.9 & \\
\hline 288 & UISO_IE_PLATE_AEA & STLRL & 0.524 & $\mathrm{pCi} / \mathrm{g}$ & 0.041 & 0.1 & 0.00778 & 36.3 & \\
\hline 289 & UISO_IE_PLATE_AEA & STLRL & 0.117 & $\mathrm{pCi} / \mathrm{g}$ & 0.026 & 0.034 & 0.0121 & 64.8 & \\
\hline 290 & UISO_IE_PLATE_AEA & STLRL & 0.591 & $\mathrm{pCi} / \mathrm{g}$ & 0.055 & 0.12 & 0.00861 & 43.2 & \\
\hline 291 & UISO_IE_PLATE_AEA & STLRL & 0.652 & $\mathrm{pCi} / \mathrm{g}$ & 0.052 & 0.13 & 0.00876 & 44.2 & \\
\hline 292 & UISO_IE_PLATE_AEA & STLRL & 2.45 & $\mathrm{pCi} / \mathrm{g}$ & 0.088 & 0.44 & 0.00442 & 75.1 & \\
\hline 293 & UISO_IE_PLATE_AEA & STLRL & 1.79 & $\mathrm{pCi} / \mathrm{g}$ & 0.074 & 0.33 & 0.00208 & 78.3 & \\
\hline 294 & UISO_IE_PLATE_AEA & STLRL & 0.346 & $\mathrm{pCi} / \mathrm{g}$ & 0.034 & 0.071 & 0.00564 & 75.6 & \\
\hline 295 & RADISOTOPES_ICPMS & PNL329 & 0.784637652 & $\mathrm{pCi} / \mathrm{g}$ & & & & & \\
\hline 296 & RADISOTOPES_ICPMS & PNL329 & 1.270972224 & $\mathrm{pCi} / \mathrm{g}$ & & & & & \\
\hline 297 & RADISOTOPES_ICPMS & PNL329 & 1.271307396 & $\mathrm{pCi} / \mathrm{g}$ & & & & & \\
\hline 298 & RADISOTOPES_ICPMS & PNL329 & 0.050610972 & $\mathrm{pCi} / \mathrm{g}$ & & & & & \\
\hline 299 & UISO_IE_PLATE_AEA & STLRL & 0.186 & $\mathrm{pCi} / \mathrm{g}$ & 0.025 & 0.042 & 0.00448 & 74.3 & \\
\hline 300 & RADISOTOPES_ICPMS & PNL329 & 0.062677164 & $\mathrm{pCi} / \mathrm{g}$ & & & & & \\
\hline 301 & UISO_IE_PLATE_AEA & STLRL & 2.4 & $\mathrm{pCi} / \mathrm{g}$ & 0.09 & 0.44 & 0.0047 & 39.4 & \\
\hline 302 & UISO_IE_PLATE_AEA & STLRL & 0.413 & $\mathrm{pCi} / \mathrm{g}$ & 0.042 & 0.085 & 0.00591 & 75.2 & \\
\hline 303 & UISO_IE_PLATE_AEA & STLRL & 0.654 & $\mathrm{pCi} / \mathrm{g}$ & 0.05 & 0.13 & 0.00887 & 69.6 & \\
\hline 304 & UISO_IE_PLATE_AEA & STLRL & 0.291 & $\mathrm{pCi} / \mathrm{g}$ & 0.029 & 0.06 & 0.00407 & 16.7 & \\
\hline
\end{tabular}




\begin{tabular}{|c|c|c|c|c|c|c|c|c|c|}
\hline & $\mathrm{K}$ & $\mathrm{L}$ & $M$ & $\mathrm{~N}$ & $\mathrm{O}$ & $\mathrm{P}$ & $\mathrm{Q}$ & $\mathrm{R}$ & $S$ \\
\hline 1 & METHOD_NAME & LAB_CODE & VALUE_RPTD & ANAL_UNITS_RPTD & COUNTING_ERROR & TOTAL_ANAL_ERROR & MIN_DETECTABLE_ACTIVITY & PCNT_MOISTURE & LAB_QUALIFIER \\
\hline 306 & UISO_IE_PLATE_AEA & STLRL & 4.3 & $\mathrm{pCi} / \mathrm{g}$ & 0.12 & 0.78 & 0.00696 & 63.3 & \\
\hline 307 & UISO_IE_PLATE_AEA & STLRL & 0.131 & $\mathrm{pCi} / \mathrm{g}$ & 0.021 & 0.032 & 0.00215 & 77.1 & \\
\hline 308 & UISO IE PLATE AEA & STLRL & 0.696 & $\mathrm{pCi} / \mathrm{g}$ & 0.049 & 0.13 & 0.00228 & 43.7 & \\
\hline 309 & UISO_IE_PLATE_AEA & STLRL & 0.697 & $\mathrm{pCi} / \mathrm{g}$ & 0.049 & 0.13 & 0.00489 & 47.1 & \\
\hline 310 & UISO_IE_PLATE_AEA & STLRL & 0.47 & $\mathrm{pCi} / \mathrm{g}$ & 0.038 & 0.092 & 0.00206 & 74 & \\
\hline 311 & UISO_IE_PLATE_AEA & STLRL & 1.32 & $\mathrm{pCi} / \mathrm{g}$ & 0.066 & 0.24 & 0.00756 & 31 & \\
\hline 312 & UISO_IE_PLATE_AEA & STLRL & 0.167 & $\mathrm{pCi} / \mathrm{g}$ & 0.021 & 0.036 & 0.0055 & 78.9 & \\
\hline 313 & UISO_IE_PLATE_AEA & STLRL & 0.457 & $\mathrm{pCi} / \mathrm{g}$ & 0.036 & 0.089 & 0.00194 & 47.6 & \\
\hline 314 & UISO_IE_PLATE_AEA & STLRL & 0.623 & $\mathrm{pCi} / \mathrm{g}$ & 0.043 & 0.12 & 0.0057 & 34.8 & \\
\hline 315 & UISO_IE_PLATE_AEA & STLRL & 0.197 & $\mathrm{pCi} / \mathrm{g}$ & 0.023 & 0.042 & 0.00181 & 73.6 & \\
\hline 316 & UISO_IE_PLATE_AEA & STLRL & 0.276 & $\mathrm{pCi} / \mathrm{g}$ & 0.034 & 0.06 & 0.00743 & 72.2 & \\
\hline 317 & UISO_IE_PLATE_AEA & STLRL & 0.832 & $\mathrm{pCi} / \mathrm{g}$ & 0.049 & 0.16 & 0.00198 & 77 & \\
\hline 318 & UISO_IE_PLATE_AEA & STLRL & 0.454 & $\mathrm{pCi} / \mathrm{g}$ & 0.036 & 0.089 & 0.00519 & 70 & \\
\hline 319 & UISO_IE_PLATE_AEA & STLRL & 9.97 & $\mathrm{pCi} / \mathrm{g}$ & 0.17 & 1.8 & 0.00515 & 60.5 & \\
\hline 320 & UISO_IE_PLATE_AEA & STLRL & 2.08 & $\mathrm{pCi} / \mathrm{g}$ & 0.077 & 0.38 & 0.00528 & 48 & \\
\hline 321 & UISO_IE_PLATE_AEA & STLRL & 0.42 & $\mathrm{pCi} / \mathrm{g}$ & 0.035 & 0.083 & 0.00201 & 59.1 & \\
\hline
\end{tabular}




\begin{tabular}{|c|c|c|c|c|c|c|c|c|c|}
\hline & $\mathrm{T}$ & $\mathrm{U}$ & $\mathrm{V}$ & $\mathrm{W}$ & $\mathrm{X}$ & $\bar{Y}$ & $Z$ & $\overline{A A}$ & $A B$ \\
\hline 1 & REVIEW_QUALIFIER & SAMP_FROM & SAMP_ITEM & SAMP_MTHD & COLL_MTHD & SAMP_SITE_ID & SAMP_SITE_NAME & WELL_NAME & SAMPLE_LOCATION \\
\hline 2 & & SUB_SURFACE & SEDIMENT & & & 147 & 7 WEST LAKE & & \\
\hline 3 & & SUB_SURFACE & SEDIMENT & & & 7737 & 7 PRD-YAKIMA SIDE NEAR DAM & & \\
\hline 4 & & SUB_SURFACE & SEDIMENT & & & 7738 & 3 PRD-GRANT SIDE NEAR DAM & & \\
\hline 5 & & SUB_SURFACE & SEDIMENT & & & 311 & RICHLAND-RIVER & & \\
\hline 6 & & SUB_SURFACE & SEDIMENT & & & 7739 & MCNARY-OR.SIDE NEAR DAM & & \\
\hline 7 & & SUB_SURFACE & SEDIMENT & & & 7740 & MCNARY-WASH.SIDE NEAR DAM & & \\
\hline 8 & & SUB_SURFACE & SEDIMENT & & & 563 & 3 HANFORD SLOUGH & & \\
\hline 9 & & SUB_SURFACE & SEDIMENT & & & 561 & WHITE BLUFFS SLOUGH & & \\
\hline 10 & & SUB_SURFACE & SEDIMENT & & & 195 & 100 F SLOUGH & & \\
\hline 11 & $F$ & SUB_SURFACE & SEDIMENT & & & 488 & 300 AREA SPRING 42-2 & & \\
\hline 12 & & SUB_SURFACE & SEDIMENT & & & 8142 & HANFORD SPR UR 28-2 & & \\
\hline 13 & & SUB_SURFACE & SEDIMENT & & & 8143 & 3 HANFORD SPR DR 28-2 & & \\
\hline 14 & & SUB_SURFACE & SEDIMENT & & & 8144 & 300 AREA SPR DR 42-2 & & \\
\hline 15 & $F$ & SUB_SURFACE & SEDIMENT & & & 8140 & 100-B SPRING 38-3 & & \\
\hline 16 & $F$ & SUB_SURFACE & SEDIMENT & & & 8147 & 100-F SPRING 207-1 & & \\
\hline 17 & $\mathrm{~F}$ & SUB_SURFACE & SEDIMENT & & & 563 & 3 HANFORD SLOUGH & & \\
\hline 18 & & SUB_SURFACE & SEDIMENT & & & 561 & WHITE BLUFFS SLOUGH & & \\
\hline 19 & & SUB_SURFACE & SEDIMENT & & & 195 & 100 F SLOUGH & & \\
\hline 20 & & SUB_SURFACE & SEDIMENT & & & 7737 & 7 PRD-YAKIMA SIDE NEAR DAM & & \\
\hline 21 & & SUB_SURFACE & SEDIMENT & & & 7738 & 3 PRD-GRANT SIDE NEAR DAM & & \\
\hline 22 & & SUB_SURFACE & SEDIMENT & & & 102 & MCNARY-OREGON SHORE & & \\
\hline 23 & & SUB_SURFACE & SEDIMENT & & & 103 & 3 MCNARY-1/3 OR. SHORE & & \\
\hline 24 & & SUB_SURFACE & SEDIMENT & & & 427 & MCNARY-2/3 OR. SHORE & & \\
\hline 25 & & SUB_SURFACE & SEDIMENT & & & 104 & MCNARY-WASH. SHORE & & \\
\hline 26 & & SUB_SURFACE & SEDIMENT & & & 7736 & ICE HARBOR-WALLA WALLA SHORE & & \\
\hline 27 & & SUB_SURFACE & SEDIMENT & & & 7734 & 4 ICE HARBOR-FRANKLIN SHORE & & \\
\hline 28 & & SUB_SURFACE & SEDIMENT & & & 7735 & 5 ICE HARBOR-MID RIVER & & \\
\hline 29 & & SUB_SURFACE & SEDIMENT & & & 311 & RICHLAND-RIVER & & \\
\hline 30 & $F$ & SUB_SURFACE & SEDIMENT & & & 7739 & MCNARY-OR.SIDE NEAR DAM & & \\
\hline 31 & & SUB_SURFACE & SEDIMENT & & & 7740 & MCNARY-WASH.SIDE NEAR DAM & & \\
\hline 32 & $F$ & SUB_SURFACE & SEDIMENT & & & 488 & 300 AREA SPRING 42-2 & & \\
\hline 33 & & SUB_SURFACE & SEDIMENT & & & 147 & WEST LAKE & & \\
\hline 34 & $F$ & SUB_SURFACE & SEDIMENT & & & 8140 & 100-B SPRING 38-3 & & \\
\hline 35 & & SUB_SURFACE & SEDIMENT & & & 8147 & 100-F SPRING 207-1 & & \\
\hline 36 & & SUB_SURFACE & SEDIMENT & & & 147 & 7 WEST LAKE & & \\
\hline 37 & & SUB_SURFACE & SEDIMENT & & & 147 & 7 WEST LAKE & & \\
\hline 38 & $F$ & SUB_SURFACE & SEDIMENT & & & 147 & 7 WEST LAKE & & \\
\hline 39 & & SUB_SURFACE & SEDIMENT & & & 311 & RICHLAND-RIVER & & \\
\hline
\end{tabular}




\begin{tabular}{|c|c|c|c|c|c|c|c|c|c|}
\hline & $T$ & $U$ & $\mathrm{~V}$ & $\mathrm{~W}$ & $\mathrm{X}$ & $\bar{Y}$ & $Z$ & $\overline{A A}$ & $A B$ \\
\hline 1 & REVIEW_QUALIFIER & SAMP_FROM & SAMP_ITEM & SAMP_MTHD & COLL_MTHD & SAMP_SITE_ID & SAMP_SITE_NAME & WELL_NAME & SAMPLE_LOCATION \\
\hline 40 & & SUB_SURFACE & SEDIMENT & & & 7739 & MCNARY-OR.SIDE NEAR DAM & & \\
\hline 41 & & SUB_SURFACE & SEDIMENT & & & 7740 & MCNARY-WASH.SIDE NEAR DAM & & \\
\hline 42 & & SUB_SURFACE & SEDIMENT & & & 563 & HANFORD SLOUGH & & \\
\hline 43 & & SUB_SURFACE & SEDIMENT & & & 561 & WHITE BLUFFS SLOUGH & & \\
\hline 44 & & SUB_SURFACE & SEDIMENT & & & 195 & 100 F SLOUGH & & \\
\hline 45 & & SUB_SURFACE & SEDIMENT & & & 7737 & PRD-YAKIMA SIDE NEAR DAM & & \\
\hline 46 & & SUB_SURFACE & SEDIMENT & & & 7738 & PRD-GRANT SIDE NEAR DAM & & \\
\hline 47 & $F$ & SUB_SURFACE & SEDIMENT & & & 9106 & 100-B SPRING 37-1 & & \\
\hline 48 & $F$ & SUB_SURFACE & SEDIMENT & & & 13792 & 100-K SPRING 63-2 & & \\
\hline 49 & $F$ & SUB_SURFACE & SEDIMENT & & & 488 & 300 AREA SPRING 42-2 & & \\
\hline 50 & $F$ & SUB_SURFACE & SEDIMENT & & & 8143 & HANFORD SPR DR 28-2 & & \\
\hline 51 & & SUB_SURFACE & SEDIMENT & & & 8144 & 300 AREA SPR DR 42-2 & & \\
\hline 52 & $\mathrm{~F}$ & SUB_SURFACE & SEDIMENT & & & 147 & WEST LAKE & & \\
\hline 53 & $\mathrm{~F}$ & SUB_SURFACE & SEDIMENT & & & 8147 & 100-F SPRING 207-1 & & \\
\hline 54 & & SUB_SURFACE & SEDIMENT & & & 7737 & PRD-YAKIMA SIDE NEAR DAM & & \\
\hline 55 & & SUB_SURFACE & SEDIMENT & & & 7738 & PRD-GRANT SIDE NEAR DAM & & \\
\hline 56 & & SUB_SURFACE & SEDIMENT & & & 311 & RICHLAND-RIVER & & \\
\hline 57 & & SUB_SURFACE & SEDIMENT & & & 7739 & MCNARY-OR.SIDE NEAR DAM & & \\
\hline 58 & & SUB_SURFACE & SEDIMENT & & & 7740 & MCNARY-WASH.SIDE NEAR DAM & & \\
\hline 59 & $\mathrm{~F}$ & SUB_SURFACE & SEDIMENT & & & 563 & 3 HANFORD SLOUGH & & \\
\hline 60 & & SUB_SURFACE & SEDIMENT & & & 561 & WHITE BLUFFS SLOUGH & & \\
\hline 61 & & SUB_SURFACE & SEDIMENT & & & 195 & 100 F SLOUGH & & \\
\hline 62 & $F$ & SUB_SURFACE & SEDIMENT & & & 563 & HANFORD SLOUGH & & \\
\hline 63 & & SUB_SURFACE & SEDIMENT & & & 561 & WHITE BLUFFS SLOUGH & & \\
\hline 64 & & SUB_SURFACE & SEDIMENT & & & 195 & 100 F SLOUGH & & \\
\hline 65 & & SUB_SURFACE & SEDIMENT & & & 7737 & PRD-YAKIMA SIDE NEAR DAM & & \\
\hline 66 & & SUB_SURFACE & SEDIMENT & & & 7738 & PRD-GRANT SIDE NEAR DAM & & \\
\hline 67 & & SUB_SURFACE & SEDIMENT & & & 102 & MCNARY-OREGON SHORE & & \\
\hline 68 & & SUB_SURFACE & SEDIMENT & & & 103 & 3 MCNARY-1/3 OR. SHORE & & \\
\hline 69 & & SUB_SURFACE & SEDIMENT & & & 427 & MCNARY-2/3 OR. SHORE & & \\
\hline 70 & & SUB_SURFACE & SEDIMENT & & & 104 & MCNARY-WASH. SHORE & & \\
\hline 71 & & SUB_SURFACE & SEDIMENT & & & 7736 & ICE HARBOR-WALLA WALLA SHORE & & \\
\hline 72 & & SUB_SURFACE & SEDIMENT & & & 7734 & ICE HARBOR-FRANKLIN SHORE & & \\
\hline 73 & & SUB_SURFACE & SEDIMENT & & & 7735 & ICE HARBOR-MID RIVER & & \\
\hline 74 & & SUB_SURFACE & SEDIMENT & & & 311 & RICHLAND-RIVER & & \\
\hline 75 & $\mathrm{~F}$ & SUB_SURFACE & SEDIMENT & & & 7739 & MCNARY-OR.SIDE NEAR DAM & & \\
\hline 76 & & SUB_SURFACE & SEDIMENT & & & 7740 & MCNARY-WASH.SIDE NEAR DAM & & \\
\hline 77 & & SUB_SURFACE & SEDIMENT & & & 311 & RICHLAND-RIVER & & \\
\hline
\end{tabular}




\begin{tabular}{|c|c|c|c|c|c|c|c|c|c|}
\hline & $\mathrm{T}$ & $\mathrm{U}$ & $\mathrm{V}$ & $\mathrm{W}$ & $\mathrm{X}$ & $\bar{Y}$ & $Z$ & $\overline{A A}$ & $A B$ \\
\hline 1 & REVIEW_QUALIFIER & SAMP_FROM & SAMP_ITEM & SAMP_MTHD & COLL_MTHD & SAMP_SITE_ID & SAMP_SITE_NAME & WELL_NAME & SAMPLE_LOCATION \\
\hline 78 & & SUB_SURFACE & SEDIMENT & & & 7739 & 9 MCNARY-OR.SIDE NEAR DAM & & \\
\hline 79 & & SUB_SURFACE & SEDIMENT & & & 7740 & MCNARY-WASH.SIDE NEAR DAM & & \\
\hline 80 & & SUB_SURFACE & SEDIMENT & & & 563 & 3 HANFORD SLOUGH & & \\
\hline 81 & & SUB_SURFACE & SEDIMENT & & & 561 & WHITE BLUFFS SLOUGH & & \\
\hline 82 & & SUB_SURFACE & SEDIMENT & & & 195 & 100 F SLOUGH & & \\
\hline 83 & & SUB_SURFACE & SEDIMENT & & & 7737 & 7 PRD-YAKIMA SIDE NEAR DAM & & \\
\hline 84 & & SUB_SURFACE & SEDIMENT & & & 7738 & 3 PRD-GRANT SIDE NEAR DAM & & \\
\hline 85 & & SUB_SURFACE & SEDIMENT & & & 147 & 7 WEST LAKE & & \\
\hline 86 & & SUB_SURFACE & SEDIMENT & & & 7737 & 7 PRD-YAKIMA SIDE NEAR DAM & & \\
\hline 87 & & SUB_SURFACE & SEDIMENT & & & 7738 & 3 PRD-GRANT SIDE NEAR DAM & & \\
\hline 88 & & SUB_SURFACE & SEDIMENT & & & 311 & RICHLAND-RIVER & & \\
\hline 89 & & SUB_SURFACE & SEDIMENT & & & 7739 & MCNARY-OR.SIDE NEAR DAM & & \\
\hline 90 & & SUB_SURFACE & SEDIMENT & & & 7740 & MCNARY-WASH.SIDE NEAR DAM & & \\
\hline 91 & & SUB_SURFACE & SEDIMENT & & & 563 & 3 HANFORD SLOUGH & & \\
\hline 92 & & SUB_SURFACE & SEDIMENT & & & 561 & WHITE BLUFFS SLOUGH & & \\
\hline 93 & & SUB_SURFACE & SEDIMENT & & & 195 & 100 F SLOUGH & & \\
\hline 94 & & SUB_SURFACE & SEDIMENT & & & 488 & 300 AREA SPRING 42-2 & & \\
\hline 95 & & SUB_SURFACE & SEDIMENT & & & 8142 & - HANFORD SPR UR 28-2 & & \\
\hline 96 & & SUB_SURFACE & SEDIMENT & & & 8143 & 3 HANFORD SPR DR 28-2 & & \\
\hline 97 & & SUB_SURFACE & SEDIMENT & & & 8144 & 300 AREA SPR DR 42-2 & & \\
\hline 98 & & SUB_SURFACE & SEDIMENT & & & 8140 & 100-B SPRING 38-3 & & \\
\hline 99 & & SUB_SURFACE & SEDIMENT & & & 8147 & 100-F SPRING 207-1 & & \\
\hline 100 & & SUB_SURFACE & SEDIMENT & & & 563 & 3 HANFORD SLOUGH & & \\
\hline 101 & & SUB_SURFACE & SEDIMENT & & & 561 & WHITE BLUFFS SLOUGH & & \\
\hline 102 & & SUB_SURFACE & SEDIMENT & & & 195 & 100 F SLOUGH & & \\
\hline 103 & & SUB_SURFACE & SEDIMENT & & & 7737 & 7 PRD-YAKIMA SIDE NEAR DAM & & \\
\hline 104 & & SUB_SURFACE & SEDIMENT & & & 7738 & 3 PRD-GRANT SIDE NEAR DAM & & \\
\hline 105 & & SUB_SURFACE & SEDIMENT & & & 102 & MCNARY-OREGON SHORE & & \\
\hline 106 & & SUB_SURFACE & SEDIMENT & & & 103 & 3 MCNARY-1/3 OR. SHORE & & \\
\hline 107 & & SUB_SURFACE & SEDIMENT & & & 427 & MCNARY-2/3 OR. SHORE & & \\
\hline 108 & & SUB_SURFACE & SEDIMENT & & & 104 & MCNARY-WASH. SHORE & & \\
\hline 109 & & SUB_SURFACE & SEDIMENT & & & 7736 & ICE HARBOR-WALLA WALLA SHORE & & \\
\hline 110 & & SUB_SURFACE & SEDIMENT & & & 7734 & 4 ICE HARBOR-FRANKLIN SHORE & & \\
\hline 111 & & SUB_SURFACE & SEDIMENT & & & 7735 & 5 ICE HARBOR-MID RIVER & & \\
\hline 112 & & SUB_SURFACE & SEDIMENT & & & 311 & RICHLAND-RIVER & & \\
\hline 113 & & SUB_SURFACE & SEDIMENT & & & 7739 & MCNARY-OR.SIDE NEAR DAM & & \\
\hline 114 & & SUB_SURFACE & SEDIMENT & & & 7740 & MCNARY-WASH.SIDE NEAR DAM & & \\
\hline 115 & & SUB_SURFACE & SEDIMENT & & & 488 & 300 AREA SPRING 42-2 & & \\
\hline
\end{tabular}




\begin{tabular}{|c|c|c|c|c|c|c|c|c|c|}
\hline & $T$ & $\mathrm{U}$ & $\mathrm{V}$ & $\mathrm{W}$ & $\mathrm{X}$ & $\mathrm{Y}$ & Z & $\overline{A A}$ & $A B$ \\
\hline 1 & REVIEW_QUALIFIER & SAMP_FROM & SAMP_ITEM & SAMP_MTHD & COLL_MTHD & SAMP_SITE_ID & SAMP_SITE_NAME & WELL_NAME & SAMPLE_LOCATION \\
\hline 116 & & SUB_SURFACE & SEDIMENT & & & 147 & WEST LAKE & & \\
\hline 117 & & SUB_SURFACE & SEDIMENT & & & 8140 & 100-B SPRING 38-3 & & \\
\hline 118 & & SUB_SURFACE & SEDIMENT & & & 8147 & 100-F SPRING 207-1 & & \\
\hline 119 & & SUB_SURFACE & SEDIMENT & & & 147 & WEST LAKE & & \\
\hline 120 & & SUB_SURFACE & SEDIMENT & & & 147 & WEST LAKE & & \\
\hline 121 & $F$ & SUB_SURFACE & SEDIMENT & & & 147 & WEST LAKE & & \\
\hline 122 & & SUB_SURFACE & SEDIMENT & & & 311 & RICHLAND-RIVER & & \\
\hline 123 & & SUB_SURFACE & SEDIMENT & & & 7739 & MCNARY-OR.SIDE NEAR DAM & & \\
\hline 124 & & SUB_SURFACE & SEDIMENT & & & 7740 & MCNARY-WASH.SIDE NEAR DAM & & \\
\hline 125 & & SUB_SURFACE & SEDIMENT & & & 563 & HANFORD SLOUGH & & \\
\hline \begin{tabular}{|l|l|}
126 \\
\end{tabular} & & SUB_SURFACE & SEDIMENT & & & 561 & WHITE BLUFFS SLOUGH & & \\
\hline 127 & & SUB_SURFACE & SEDIMENT & & & 195 & 100 F SLOUGH & & \\
\hline 128 & & SUB_SURFACE & SEDIMENT & & & 7737 & PRD-YAKIMA SIDE NEAR DAM & & \\
\hline 129 & & SUB_SURFACE & SEDIMENT & & & 7738 & PRD-GRANT SIDE NEAR DAM & & \\
\hline 130 & & SUB_SURFACE & SEDIMENT & & & 9106 & 100-B SPRING 37-1 & & \\
\hline 131 & & SUB_SURFACE & SEDIMENT & & & 13792 & 100-K SPRING 63-2 & & \\
\hline 132 & & SUB_SURFACE & SEDIMENT & & & 488 & 300 AREA SPRING 42-2 & & \\
\hline 133 & & SUB_SURFACE & SEDIMENT & & & 8143 & HANFORD SPR DR 28-2 & & \\
\hline 134 & & SUB_SURFACE & SEDIMENT & & & 8144 & 300 AREA SPR DR 42-2 & & \\
\hline 135 & $F$ & SUB_SURFACE & SEDIMENT & & & 147 & WEST LAKE & & \\
\hline 136 & & SUB_SURFACE & SEDIMENT & & & 8147 & 100-F SPRING 207-1 & & \\
\hline 137 & & SUB_SURFACE & SEDIMENT & & & 147 & WEST LAKE & & \\
\hline 138 & & SUB_SURFACE & SEDIMENT & & & 147 & WEST LAKE & & \\
\hline 139 & & SUB_SURFACE & SEDIMENT & & & 147 & WEST LAKE & & \\
\hline 140 & & SUB_SURFACE & SEDIMENT & & & 147 & WEST LAKE & & \\
\hline 141 & & SUB_SURFACE & SEDIMENT & & & 147 & WEST LAKE & & \\
\hline 142 & & SUB_SURFACE & SEDIMENT & & & 147 & WEST LAKE & & \\
\hline 143 & & SUB_SURFACE & SEDIMENT & & & 7737 & PRD-YAKIMA SIDE NEAR DAM & & \\
\hline 144 & & SUB_SURFACE & SEDIMENT & & & 7738 & PRD-GRANT SIDE NEAR DAM & & \\
\hline 145 & & SUB_SURFACE & SEDIMENT & & & 311 & RICHLAND-RIVER & & \\
\hline 146 & & SUB_SURFACE & SEDIMENT & & & 7739 & MCNARY-OR.SIDE NEAR DAM & & \\
\hline 147 & & SUB_SURFACE & SEDIMENT & & & 7740 & MCNARY-WASH.SIDE NEAR DAM & & \\
\hline 148 & & SUB_SURFACE & SEDIMENT & & & 563 & HANFORD SLOUGH & & \\
\hline 149 & & SUB_SURFACE & SEDIMENT & & & 561 & WHITE BLUFFS SLOUGH & & \\
\hline 150 & & SUB_SURFACE & SEDIMENT & & & 195 & 100 F SLOUGH & & \\
\hline 151 & & SUB_SURFACE & SEDIMENT & & & 488 & 300 AREA SPRING 42-2 & & \\
\hline 152 & & SUB_SURFACE & SEDIMENT & & & 8142 & HANFORD SPR UR 28-2 & & \\
\hline \begin{tabular}{|l|l|}
153 \\
\end{tabular} & & SUB_SURFACE & SEDIMENT & & & 8143 & HANFORD SPR DR 28-2 & & \\
\hline
\end{tabular}




\begin{tabular}{|c|c|c|c|c|c|c|c|c|c|}
\hline & $\mathrm{T}$ & $\mathrm{U}$ & $\mathrm{V}$ & W & $\mathrm{X}$ & $\mathrm{Y}$ & Z & AA & $A B$ \\
\hline 1 & REVIEW_QUALIFIER & SAMP_FROM & SAMP_ITEM & SAMP_MTHD & COLL_MTHD & SAMP_SITE_ID & SAMP_SITE_NAME & WELL_NAME & SAMPLE_LOCATION \\
\hline 154 & & SUB_SURFACE & SEDIMENT & & & 8144 & 300 AREA SPR DR 42-2 & & \\
\hline 155 & & SUB_SURFACE & SEDIMENT & & & 8140 & 100-B SPRING 38-3 & & \\
\hline 156 & & SUB_SURFACE & SEDIMENT & & & 8147 & 100-F SPRING 207-1 & & \\
\hline 157 & & SUB_SURFACE & SEDIMENT & & & 563 & HANFORD SLOUGH & & \\
\hline 158 & & SUB_SURFACE & SEDIMENT & & & 561 & WHITE BLUFFS SLOUGH & & \\
\hline 159 & & SUB_SURFACE & SEDIMENT & & & 195 & 100 F SLOUGH & & \\
\hline 160 & & SUB_SURFACE & SEDIMENT & & & 7737 & PRD-YAKIMA SIDE NEAR DAM & & \\
\hline 161 & & SUB_SURFACE & SEDIMENT & & & 7738 & PRD-GRANT SIDE NEAR DAM & & \\
\hline 162 & & SUB_SURFACE & SEDIMENT & & & 102 & MCNARY-OREGON SHORE & & \\
\hline 163 & & SUB_SURFACE & SEDIMENT & & & 103 & MCNARY-1/3 OR. SHORE & & \\
\hline 164 & & SUB_SURFACE & SEDIMENT & & & 427 & MCNARY-2/3 OR. SHORE & & \\
\hline 165 & & SUB_SURFACE & SEDIMENT & & & 104 & MCNARY-WASH. SHORE & & \\
\hline 166 & & SUB_SURFACE & SEDIMENT & & & 7736 & ICE HARBOR-WALLA WALLA SHORE & & \\
\hline 167 & & SUB_SURFACE & SEDIMENT & & & 7734 & ICE HARBOR-FRANKLIN SHORE & & \\
\hline 168 & & SUB_SURFACE & SEDIMENT & & & 7735 & ICE HARBOR-MID RIVER & & \\
\hline 169 & & SUB_SURFACE & SEDIMENT & & & 311 & RICHLAND-RIVER & & \\
\hline 170 & & SUB_SURFACE & SEDIMENT & & & 7739 & MCNARY-OR.SIDE NEAR DAM & & \\
\hline 171 & & SUB_SURFACE & SEDIMENT & & & 7740 & MCNARY-WASH.SIDE NEAR DAM & & \\
\hline 172 & & SUB_SURFACE & SEDIMENT & & & 488 & 300 AREA SPRING 42-2 & & \\
\hline 173 & & SUB_SURFACE & SEDIMENT & & & 9225 & 300 SPR 11 & & \\
\hline 174 & & SUB_SURFACE & SEDIMENT & & & 9232 & 300 SPR 14 & & \\
\hline 175 & & SUB_SURFACE & SEDIMENT & & & 488 & 300 AREA SPRING 42-2 & & \\
\hline 176 & & SUB_SURFACE & SEDIMENT & & & 8144 & 300 AREA SPR DR 42-2 & & \\
\hline 177 & & SUB_SURFACE & SEDIMENT & & & 9225 & 300 SPR 11 & & \\
\hline 178 & & SUB_SURFACE & SEDIMENT & & & 9232 & 300 SPR 14 & & \\
\hline 179 & & SUB_SURFACE & SEDIMENT & & & 9236 & VERNITA BRIDGE -1 & & \\
\hline 180 & & SUB_SURFACE & SEDIMENT & & & 9236 & VERNITA BRIDGE -1 & & \\
\hline 181 & & SUB_SURFACE & SEDIMENT & & & 147 & WEST LAKE & & \\
\hline 182 & & SUB_SURFACE & SEDIMENT & & & 8140 & 100-B SPRING 38-3 & & \\
\hline 183 & & SUB_SURFACE & SEDIMENT & & & 8147 & 100-F SPRING 207-1 & & \\
\hline 184 & & SUB_SURFACE & SEDIMENT & & & 147 & WEST LAKE & & \\
\hline 185 & & SUB_SURFACE & SEDIMENT & & & 147 & WEST LAKE & & \\
\hline 186 & $F$ & SUB_SURFACE & SEDIMENT & & & 147 & WEST LAKE & & \\
\hline 187 & & SUB_SURFACE & SEDIMENT & & & 311 & RICHLAND-RIVER & & \\
\hline 188 & & SUB_SURFACE & SEDIMENT & & & 7739 & MCNARY-OR.SIDE NEAR DAM & & \\
\hline 189 & & SUB_SURFACE & SEDIMENT & & & 7740 & MCNARY-WASH.SIDE NEAR DAM & & \\
\hline 190 & & SUB_SURFACE & SEDIMENT & & & 563 & HANFORD SLOUGH & & \\
\hline 191 & & SUB_SURFACE & SEDIMENT & & & 561 & WHITE BLUFFS SLOUGH & & \\
\hline
\end{tabular}




\begin{tabular}{|c|c|c|c|c|c|c|c|c|c|}
\hline & $T$ & $\mathrm{U}$ & $\mathrm{V}$ & W & $\mathrm{X}$ & $\bar{Y}$ & Z & AA & $A B$ \\
\hline 1 & REVIEW_QUALIFIER & SAMP_FROM & SAMP_ITEM & SAMP_MTHD & COLL_MTHD & SAMP_SITE_ID & SAMP_SITE_NAME & WELL_NAME & SAMPLE_LOCATION \\
\hline 192 & & SUB_SURFACE & SEDIMENT & & & 195 & 100 F SLOUGH & & \\
\hline 193 & & SUB_SURFACE & SEDIMENT & & & 7737 & PRD-YAKIMA SIDE NEAR DAM & & \\
\hline 194 & & SUB_SURFACE & SEDIMENT & & & 7738 & PRD-GRANT SIDE NEAR DAM & & \\
\hline 195 & & SUB_SURFACE & SEDIMENT & & & 9106 & 100-B SPRING 37-1 & & \\
\hline 196 & & SUB_SURFACE & SEDIMENT & & & 13792 & 100-K SPRING 63-2 & & \\
\hline 197 & & SUB_SURFACE & SEDIMENT & & & 488 & 300 AREA SPRING 42-2 & & \\
\hline 198 & & SUB_SURFACE & SEDIMENT & & & 8143 & HANFORD SPR DR 28-2 & & \\
\hline 199 & & SUB_SURFACE & SEDIMENT & & & 8144 & 300 AREA SPR DR 42-2 & & \\
\hline 200 & $F$ & SUB_SURFACE & SEDIMENT & & & 147 & WEST LAKE & & \\
\hline 201 & & SUB_SURFACE & SEDIMENT & & & 8147 & 100-F SPRING 207-1 & & \\
\hline 202 & & SUB_SURFACE & SEDIMENT & & & 147 & WEST LAKE & & \\
\hline 203 & & SUB_SURFACE & SEDIMENT & & & 7737 & PRD-YAKIMA SIDE NEAR DAM & & \\
\hline 204 & & SUB_SURFACE & SEDIMENT & & & 7738 & PRD-GRANT SIDE NEAR DAM & & \\
\hline 205 & & SUB_SURFACE & SEDIMENT & & & 311 & RICHLAND-RIVER & & \\
\hline 206 & & SUB_SURFACE & SEDIMENT & & & 7739 & MCNARY-OR.SIDE NEAR DAM & & \\
\hline 207 & & SUB_SURFACE & SEDIMENT & & & 7740 & MCNARY-WASH.SIDE NEAR DAM & & \\
\hline 208 & & SUB_SURFACE & SEDIMENT & & & 563 & HANFORD SLOUGH & & \\
\hline 209 & & SUB_SURFACE & SEDIMENT & & & 561 & WHITE BLUFFS SLOUGH & & \\
\hline 210 & & SUB_SURFACE & SEDIMENT & & & 195 & 100 F SLOUGH & & \\
\hline 211 & & SUB_SURFACE & SEDIMENT & & & 488 & 300 AREA SPRING 42-2 & & \\
\hline 212 & & SUB_SURFACE & SEDIMENT & & & 8142 & HANFORD SPR UR 28-2 & & \\
\hline 213 & & SUB_SURFACE & SEDIMENT & & & 8143 & HANFORD SPR DR 28-2 & & \\
\hline 214 & & SUB_SURFACE & SEDIMENT & & & 8144 & 300 AREA SPR DR 42-2 & & \\
\hline 215 & & SUB_SURFACE & SEDIMENT & & & 8140 & 100-B SPRING 38-3 & & \\
\hline 216 & & SUB_SURFACE & SEDIMENT & & & 8147 & 100-F SPRING 207-1 & & \\
\hline 217 & & SUB_SURFACE & SEDIMENT & & & 563 & HANFORD SLOUGH & & \\
\hline 218 & & SUB_SURFACE & SEDIMENT & & & 561 & WHITE BLUFFS SLOUGH & & \\
\hline 219 & & SUB_SURFACE & SEDIMENT & & & 195 & 100 F SLOUGH & & \\
\hline 220 & & SUB_SURFACE & SEDIMENT & & & 7737 & PRD-YAKIMA SIDE NEAR DAM & & \\
\hline 221 & & SUB_SURFACE & SEDIMENT & & & 7738 & 3 PRD-GRANT SIDE NEAR DAM & & \\
\hline 222 & & SUB_SURFACE & SEDIMENT & & & 102 & MCNARY-OREGON SHORE & & \\
\hline 223 & & SUB_SURFACE & SEDIMENT & & & 103 & 3 MCNARY-1/3 OR. SHORE & & \\
\hline 224 & & SUB_SURFACE & SEDIMENT & & & 427 & MCNARY-2/3 OR. SHORE & & \\
\hline 225 & & SUB_SURFACE & SEDIMENT & & & 104 & MCNARY-WASH. SHORE & & \\
\hline 226 & & SUB_SURFACE & SEDIMENT & & & 7736 & ICE HARBOR-WALLA WALLA SHORE & & \\
\hline 227 & & SUB_SURFACE & SEDIMENT & & & 7734 & ICE HARBOR-FRANKLIN SHORE & & \\
\hline 228 & & SUB_SURFACE & SEDIMENT & & & 7735 & 5 ICE HARBOR-MID RIVER & & \\
\hline 229 & & SUB_SURFACE & SEDIMENT & & & 311 & RICHLAND-RIVER & & \\
\hline
\end{tabular}




\begin{tabular}{|c|c|c|c|c|c|c|c|c|c|}
\hline & $\mathrm{T}$ & $U$ & $\mathrm{~V}$ & $\mathrm{~W}$ & $\mathrm{X}$ & $\bar{Y}$ & $Z$ & $\overline{A A}$ & $A B$ \\
\hline 1 & REVIEW_QUALIFIER & SAMP_FROM & SAMP_ITEM & SAMP_MTHD & COLL_MTHD & SAMP_SITE_ID & SAMP_SITE_NAME & WELL_NAME & SAMPLE_LOCATION \\
\hline 230 & & SUB_SURFACE & SEDIMENT & & & 7739 & 9 MCNARY-OR.SIDE NEAR DAM & & \\
\hline 231 & & SUB_SURFACE & SEDIMENT & & & 7740 & MCNARY-WASH.SIDE NEAR DAM & & \\
\hline 232 & & SUB_SURFACE & SEDIMENT & & & 488 & 300 AREA SPRING 42-2 & & \\
\hline 233 & & SUB_SURFACE & SEDIMENT & & & 9225 & 300 SPR 11 & & \\
\hline 234 & & SUB_SURFACE & SEDIMENT & & & 9232 & 2300 SPR 14 & & \\
\hline 235 & & SUB_SURFACE & SEDIMENT & & & 488 & 300 AREA SPRING 42-2 & & \\
\hline 236 & & SUB_SURFACE & SEDIMENT & & & 8144 & 300 AREA SPR DR 42-2 & & \\
\hline 23 & & SUB_SURFACE & SEDIMENT & & & 9225 & 500 SPR 11 & & \\
\hline 238 & & SUB_SURFACE & SEDIMENT & & & 9232 & 300 SPR 14 & & \\
\hline 239 & & SUB_SURFACE & SEDIMENT & & & 9236 & VERNITA BRIDGE -1 & & \\
\hline 240 & & SUB_SURFACE & SEDIMENT & & & 9236 & VERNITA BRIDGE -1 & & \\
\hline 241 & & SUB_SURFACE & SEDIMENT & & & 147 & 7 WEST LAKE & & \\
\hline 242 & & SUB_SURFACE & SEDIMENT & & & 8140 & 100-B SPRING 38-3 & & \\
\hline 243 & & SUB_SURFACE & SEDIMENT & & & 8147 & 100-F SPRING 207-1 & & \\
\hline 244 & & SUB_SURFACE & SEDIMENT & & & 147 & 7 WEST LAKE & & \\
\hline 245 & & SUB_SURFACE & SEDIMENT & & & 147 & 7 WEST LAKE & & \\
\hline 246 & $\mathrm{~F}$ & SUB_SURFACE & SEDIMENT & & & 147 & 7 WEST LAKE & & \\
\hline 247 & & SUB_SURFACE & SEDIMENT & & & 311 & RICHLAND-RIVER & & \\
\hline 248 & & SUB_SURFACE & SEDIMENT & & & 7739 & MCNARY-OR.SIDE NEAR DAM & & \\
\hline 249 & & SUB_SURFACE & SEDIMENT & & & 7740 & MCNARY-WASH.SIDE NEAR DAM & & \\
\hline 250 & & SUB_SURFACE & SEDIMENT & & & 563 & 3 HANFORD SLOUGH & & \\
\hline 251 & & SUB_SURFACE & SEDIMENT & & & 561 & WHITE BLUFFS SLOUGH & & \\
\hline 252 & & SUB_SURFACE & SEDIMENT & & & 195 & 100 F SLOUGH & & \\
\hline 253 & & SUB_SURFACE & SEDIMENT & & & 7737 & 7 PRD-YAKIMA SIDE NEAR DAM & & \\
\hline 254 & & SUB_SURFACE & SEDIMENT & & & 7738 & 3 PRD-GRANT SIDE NEAR DAM & & \\
\hline 255 & & SUB_SURFACE & SEDIMENT & & & 9106 & 100-B SPRING 37-1 & & \\
\hline 256 & & SUB_SURFACE & SEDIMENT & & & 13792 & 100-K SPRING 63-2 & & \\
\hline 257 & & SUB_SURFACE & SEDIMENT & & & 488 & 300 AREA SPRING 42-2 & & \\
\hline 258 & & SUB_SURFACE & SEDIMENT & & & 8143 & 3 HANFORD SPR DR 28-2 & & \\
\hline 259 & & SUB_SURFACE & SEDIMENT & & & 8144 & 400 AREA SPR DR 42-2 & & \\
\hline 260 & & SUB_SURFACE & SEDIMENT & & & 147 & 7 WEST LAKE & & \\
\hline 261 & & SUB_SURFACE & SEDIMENT & & & 8147 & 100-F SPRING 207-1 & & \\
\hline 262 & & SUB_SURFACE & SEDIMENT & & & 147 & 7 WEST LAKE & & \\
\hline 263 & & SUB_SURFACE & SEDIMENT & & & 7737 & 7 PRD-YAKIMA SIDE NEAR DAM & & \\
\hline 264 & & SUB_SURFACE & SEDIMENT & & & 7738 & 3 PRD-GRANT SIDE NEAR DAM & & \\
\hline 265 & & SUB_SURFACE & SEDIMENT & & & 311 & RICHLAND-RIVER & & \\
\hline 266 & & SUB_SURFACE & SEDIMENT & & & 7739 & 9 MCNARY-OR.SIDE NEAR DAM & & \\
\hline \begin{tabular}{|l|l|}
267 & -1 \\
\end{tabular} & & SUB_SURFACE & SEDIMENT & & & 7740 & MCNARY-WASH.SIDE NEAR DAM & & \\
\hline
\end{tabular}




\begin{tabular}{|c|c|c|c|c|c|c|c|c|c|}
\hline & $\mathrm{T}$ & $\mathrm{U}$ & $\mathrm{V}$ & W & $\mathrm{X}$ & $\mathrm{Y}$ & Z & $\mathrm{AA}$ & $A B$ \\
\hline 1 & REVIEW_QUALIFIER & SAMP_FROM & SAMP_ITEM & SAMP_MTHD & COLL_MTHD & SAMP_SITE_ID & SAMP_SITE_NAME & WELL_NAME & SAMPLE_LOCATION \\
\hline 268 & & SUB_SURFACE & SEDIMENT & & & 563 & 3 HANFORD SLOUGH & & \\
\hline 269 & & SUB_SURFACE & SEDIMENT & & & 561 & WHITE BLUFFS SLOUGH & & \\
\hline 270 & & SUB_SURFACE & SEDIMENT & & & 195 & 100 F SLOUGH & & \\
\hline 271 & & SUB_SURFACE & SEDIMENT & & & 488 & 300 AREA SPRING 42-2 & & \\
\hline 272 & & SUB_SURFACE & SEDIMENT & & & 8142 & HANFORD SPR UR 28-2 & & \\
\hline 273 & & SUB_SURFACE & SEDIMENT & & & 8143 & 3 HANFORD SPR DR 28-2 & & \\
\hline 274 & & SUB_SURFACE & SEDIMENT & & & 8144 & 300 AREA SPR DR 42-2 & & \\
\hline 275 & & SUB_SURFACE & SEDIMENT & & & 8140 & 100-B SPRING 38-3 & & \\
\hline 276 & & SUB_SURFACE & SEDIMENT & & & 8147 & 100-F SPRING 207-1 & & \\
\hline 277 & & SUB_SURFACE & SEDIMENT & & & 563 & 3 HANFORD SLOUGH & & \\
\hline 278 & & SUB_SURFACE & SEDIMENT & & & 561 & I WHITE BLUFFS SLOUGH & & \\
\hline 279 & & SUB_SURFACE & SEDIMENT & & & 195 & 100 F SLOUGH & & \\
\hline 280 & & SUB_SURFACE & SEDIMENT & & & 7737 & 7 PRD-YAKIMA SIDE NEAR DAM & & \\
\hline 281 & & SUB_SURFACE & SEDIMENT & & & 7738 & 3 PRD-GRANT SIDE NEAR DAM & & \\
\hline 282 & & SUB_SURFACE & SEDIMENT & & & 102 & MCNARY-OREGON SHORE & & \\
\hline 283 & & SUB_SURFACE & SEDIMENT & & & 103 & 3 MCNARY-1/3 OR. SHORE & & \\
\hline 284 & & SUB_SURFACE & SEDIMENT & & & 427 & MCNARY-2/3 OR. SHORE & & \\
\hline 285 & & SUB_SURFACE & SEDIMENT & & & 104 & MCNARY-WASH. SHORE & & \\
\hline 286 & & SUB_SURFACE & SEDIMENT & & & 7736 & ICE HARBOR-WALLA WALLA SHORE & & \\
\hline 287 & & SUB_SURFACE & SEDIMENT & & & 7734 & 4 ICE HARBOR-FRANKLIN SHORE & & \\
\hline 288 & & SUB_SURFACE & SEDIMENT & & & 7735 & 5 ICE HARBOR-MID RIVER & & \\
\hline 289 & & SUB_SURFACE & SEDIMENT & & & 311 & RICHLAND-RIVER & & \\
\hline 290 & & SUB_SURFACE & SEDIMENT & & & 7739 & MCNARY-OR.SIDE NEAR DAM & & \\
\hline 291 & & SUB_SURFACE & SEDIMENT & & & 7740 & MCNARY-WASH.SIDE NEAR DAM & & \\
\hline 292 & & SUB_SURFACE & SEDIMENT & & & 488 & 300 AREA SPRING 42-2 & & \\
\hline 293 & & SUB_SURFACE & SEDIMENT & & & 9225 & 500 SPR 11 & & \\
\hline 294 & & SUB_SURFACE & SEDIMENT & & & 9232 & 300 SPR 14 & & \\
\hline 295 & & SUB_SURFACE & SEDIMENT & & & 488 & 300 AREA SPRING 42-2 & & \\
\hline 296 & & SUB_SURFACE & SEDIMENT & & & 8144 & 300 AREA SPR DR 42-2 & & \\
\hline 297 & & SUB_SURFACE & SEDIMENT & & & 9225 & 300 SPR 11 & & \\
\hline 298 & & SUB_SURFACE & SEDIMENT & & & 9232 & 2300 SPR 14 & & \\
\hline 299 & & SUB_SURFACE & SEDIMENT & & & 9236 & VERNITA BRIDGE -1 & & \\
\hline 300 & & SUB_SURFACE & SEDIMENT & & & 9236 & VERNITA BRIDGE -1 & & \\
\hline 301 & & SUB_SURFACE & SEDIMENT & & & 147 & 7 WEST LAKE & & \\
\hline 302 & & SUB_SURFACE & SEDIMENT & & & 8140 & 100-B SPRING 38-3 & & \\
\hline 303 & & SUB_SURFACE & SEDIMENT & & & 8147 & 100-F SPRING 207-1 & & \\
\hline 304 & & SUB_SURFACE & SEDIMENT & & & 147 & 7 WEST LAKE & & \\
\hline 305 & & SUB_SURFACE & SEDIMENT & & & 147 & 7 WEST LAKE & & \\
\hline
\end{tabular}




\begin{tabular}{|c|c|c|c|c|c|c|c|c|c|}
\hline & $T$ & $\bar{U}$ & $\mathrm{~V}$ & $\mathrm{~W}$ & $x$ & $\mathrm{Y}$ & $Z$ & $\overline{\mathrm{AA}}$ & $A B$ \\
\hline 1 & REVIEW_QUALIFIER & SAMP_FROM & SAMP_ITEM & SAMP_MTHD & COLL_MTHD & SAMP_SITE_ID & SAMP_SITE_NAME & WELL_NAME & SAMPLE_LOCATION \\
\hline 306 & $\bar{F}$ & SUB_SURFACE & SEDIMENT & & & 147 & WEST LAKE & & \\
\hline 307 & & SUB_SURFACE & SEDIMENT & & & 311 & RICHLAND-RIVER & & \\
\hline 308 & & SUB_SURFACE & SEDIMENT & & & 7739 & MCNARY-OR.SIDE NEAR DAM & & \\
\hline 309 & & SUB_SURFACE & SEDIMENT & & & 7740 & MCNARY-WASH.SIDE NEAR DAM & & \\
\hline 310 & & SUB_SURFACE & SEDIMENT & & & 563 & HANFORD SLOUGH & & \\
\hline 311 & & SUB_SURFACE & SEDIMENT & & & 561 & WHITE BLUFFS SLOUGH & & \\
\hline 312 & & SUB_SURFACE & SEDIMENT & & & 195 & 100 F SLOUGH & & \\
\hline 313 & & SUB_SURFACE & SEDIMENT & & & 7737 & PRD-YAKIMA SIDE NEAR DAM & & \\
\hline 314 & & SUB_SURFACE & SEDIMENT & & & 7738 & PRD-GRANT SIDE NEAR DAM & & \\
\hline 315 & & SUB_SURFACE & SEDIMENT & & & 9106 & 100-B SPRING 37-1 & & \\
\hline 316 & & SUB_SURFACE & SEDIMENT & & & 13792 & 100-K SPRING 63-2 & & \\
\hline 317 & & SUB_SURFACE & SEDIMENT & & & 488 & 300 AREA SPRING 42-2 & & \\
\hline 318 & & SUB_SURFACE & SEDIMENT & & & 8143 & HANFORD SPR DR 28-2 & & \\
\hline 319 & & SUB_SURFACE & SEDIMENT & & & 8144 & 300 AREA SPR DR 42-2 & & \\
\hline $320 \mathrm{~F}$ & $\mathrm{~F}$ & SUB_SURFACE & SEDIMENT & & & 147 & WEST LAKE & & \\
\hline 321 & & SUB_SURFACE & SEDIMENT & & & 8147 & 100-F SPRING 207-1 & & \\
\hline
\end{tabular}




\begin{tabular}{|c|c|c|c|c|c|c|}
\hline & $A C$ & $A D$ & $\mathrm{AE}$ & $\mathrm{AF}$ & $A G$ & $\mathrm{AH}$ \\
\hline 1 & SAMP_COMMENT & RESULT_COMMENT & COLL_SAMP_SIZE & COLL_SAMP_SIZE_UNITS & DIST_CLASS & OWNER_ID \\
\hline 2 & & \#IR-0060 MDA > CRDL & 1859 & g & ONSITE & SESPSPEC \\
\hline 3 & & \#IR-0063 MDA > CRDL & 3750 & g & OFFSITE & SESPMNT \\
\hline 4 & & & 4072 & g & OFFSITE & SESPMNT \\
\hline 5 & & \#IR-0064 MDA > CRDL & 1942 & g & RIVER_SHORELINE & SESPMNT \\
\hline 6 & & \#IR-0064 MDA > CRDL & 2947 & g & OFFSITE & SESPMNT \\
\hline 7 & & \#IR-0064 MDA > CRDL & 2711 & g & OFFSITE & SESPMNT \\
\hline 8 & MILFOIL PRESENT. & & 2163 & g & RIVER_SHORELINE & SESPMNT \\
\hline 9 & & & 8504 & g & RIVER_SHORELINE & SESPMNT \\
\hline 11 & & & 2857 & g & ONSITE & SESPMNT \\
\hline 12 & & & 2622 & g & ONSITE & SESPMNT \\
\hline 13 & & & 2075 & 9 & ONSITE & SESPMNT \\
\hline 14 & & & 1774 & g & ONSITE & SESPMNT \\
\hline 15 & & & 1100 & g & ONSITE & SESPMNT \\
\hline 16 & & \#IR-0083 Achieved MDAs > CRDL & 1555 & 9 & ONSITE & SESPMNT \\
\hline 17 & & & 1200 & g & RIVER_SHORELINE & SESPMNT \\
\hline 18 & & & 1200 & g & RIVER_SHORELINE & SESPMNT \\
\hline 19 & & & 1200 & g & ONSITE & SESPMNT \\
\hline 21 & & & 1200 & g & OFFSITE & SESPMNT \\
\hline 22 & & & 1200 & g & OFFSITE & SESPSPEC \\
\hline 23 & & & 1200 & $\mathrm{~g}$ & OFFSITE & SESPSPEC \\
\hline 24 & & & 1200 & g & OFFSITE & SESPSPEC \\
\hline 25 & & IR-0146; MDA > CRDL & 1200 & g & OFFSITE & SESPSPEC \\
\hline 26 & & IR-0146; MDA > CRDL & 1200 & g & OFFSITE & SESPSPEC \\
\hline 27 & & & 1200 & g & OFFSITE & SESPSPEC \\
\hline 28 & & & 1200 & g & OFFSITE & SESPSPEC \\
\hline 29 & & & 1200 & g & RIVER_SHORELINE & SESPMNT \\
\hline 30 & & & 1200 & g & OFFSITE & SESPMNT \\
\hline 31 & & & 1200 & g & OFFSITE & SESPMNT \\
\hline 32 & & & 3000 & g & ONSITE & SESPMNT \\
\hline 33 & & & 2465 & g & ONSITE & SESPMNT \\
\hline 34 & & & 1100 & g & ONSITE & SESPMNT \\
\hline 35 & & & 1100 & g & ONSITE & SESPMNT \\
\hline 36 & & & 8673 & g & ONSITE & SESPMNT \\
\hline 37 & & & 2526 & g & ONSITE & SESPMNT \\
\hline 38 & & IR-0212: Variance on seive size. & 2370 & g & ONSITE & SESPMNT \\
\hline
\end{tabular}




\begin{tabular}{|c|c|c|c|c|c|c|}
\hline & $\mathrm{AC}$ & $A D$ & $\mathrm{AE}$ & $\mathrm{AF}$ & AG & $\mathrm{AH}$ \\
\hline 1 & SAMP_COMMENT & RESULT_COMMENT & COLL_SAMP_SIZE & COLL_SAMP_SIZE_UNITS & DIST_CLASS & OWNER_ID \\
\hline 40 & & & 2000 & g & OFFSITE & SESPMNT \\
\hline 41 & & & 2000 & g & OFFSITE & SESPMNT \\
\hline 42 & & IR-0216: MDA>CRDL due to low sample density. & 1900 & g & RIVER_SHORELINE & SESPMNT \\
\hline 43 & & IR-0216: MDA>CRDL due to low sample density. & 1900 & g & RIVER_SHORELINE & SESPMNT \\
\hline 44 & SAMPLE WEIGHT UNKNOWN. & IR-0216: MDA>CRDL due to low sample density. & 1836 & g & ONSITE & SESPMNT \\
\hline 45 & & IR-0216: MDA>CRDL due to low sample density. & 1900 & g & OFFSITE & SESPMNT \\
\hline 46 & & IR-0216: MDA>CRDL due to low sample density. & 1900 & g & OFFSITE & SESPMNT \\
\hline 47 & & & 1100 & g & ONSITE & SESPMNT \\
\hline 48 & SAMPLE COLLECTED AT SPRING B. & & 1800 & g & ONSITE & SESPMNT \\
\hline 49 & & & 1536 & g & ONSITE & SESPMNT \\
\hline 50 & & & 1536 & g & ONSITE & SESPMNT \\
\hline 51 & & & 1536 & g & ONSITE & SESPMNT \\
\hline 52 & & & 2590 & g & ONSITE & SESPMNT \\
\hline 53 & & & 1536 & g & ONSITE & SESPMNT \\
\hline 54 & & & 3750 & g & OFFSITE & SESPMNT \\
\hline 55 & & & 4072 & g & OFFSITE & SESPMNT \\
\hline 56 & & & 1942 & g & RIVER_SHORELINE & SESPMNT \\
\hline 57 & & & 2947 & g & OFFSITE & SESPMNT \\
\hline 59 & MILFOIL PRESENT. & & 2163 & g & RIVER_SHORELINE & SESPMNT \\
\hline 60 & & & 8504 & g & RIVER_SHORELINE & SESPMNT \\
\hline 61 & & & 2447 & g & ONSITE & SESPMNT \\
\hline 62 & & & 1200 & g & RIVER_SHORELINE & SESPMNT \\
\hline 63 & & & 1200 & g & RIVER_SHORELINE & SESPMNT \\
\hline 64 & & & 1200 & g & ONSITE & SESPMNT \\
\hline 65 & & & 1200 & g & OFFSITE & SESPMNT \\
\hline 66 & & & 1200 & g & OFFSITE & SESPMNT \\
\hline 67 & & & 1200 & g & OFFSITE & SESPSPEC \\
\hline 68 & & & 1200 & g & OFFSITE & SESPSPEC \\
\hline 69 & & & 1200 & g & OFFSITE & SESPSPEC \\
\hline 70 & & & 1200 & g & OFFSITE & SESPSPEC \\
\hline 71 & & & 1200 & g & OFFSITE & SESPSPEC \\
\hline 72 & & & 1200 & g & OFFSITE & SESPSPEC \\
\hline 73 & & & 1200 & g & OFFSITE & SESPSPEC \\
\hline 74 & & & 1200 & g & RIVER_SHORELINE & SESPMNT \\
\hline 75 & & & 1200 & g & OFFSITE & SESPMNT \\
\hline 76 & & & 1200 & g & OFFSITE & SESPMNT \\
\hline
\end{tabular}




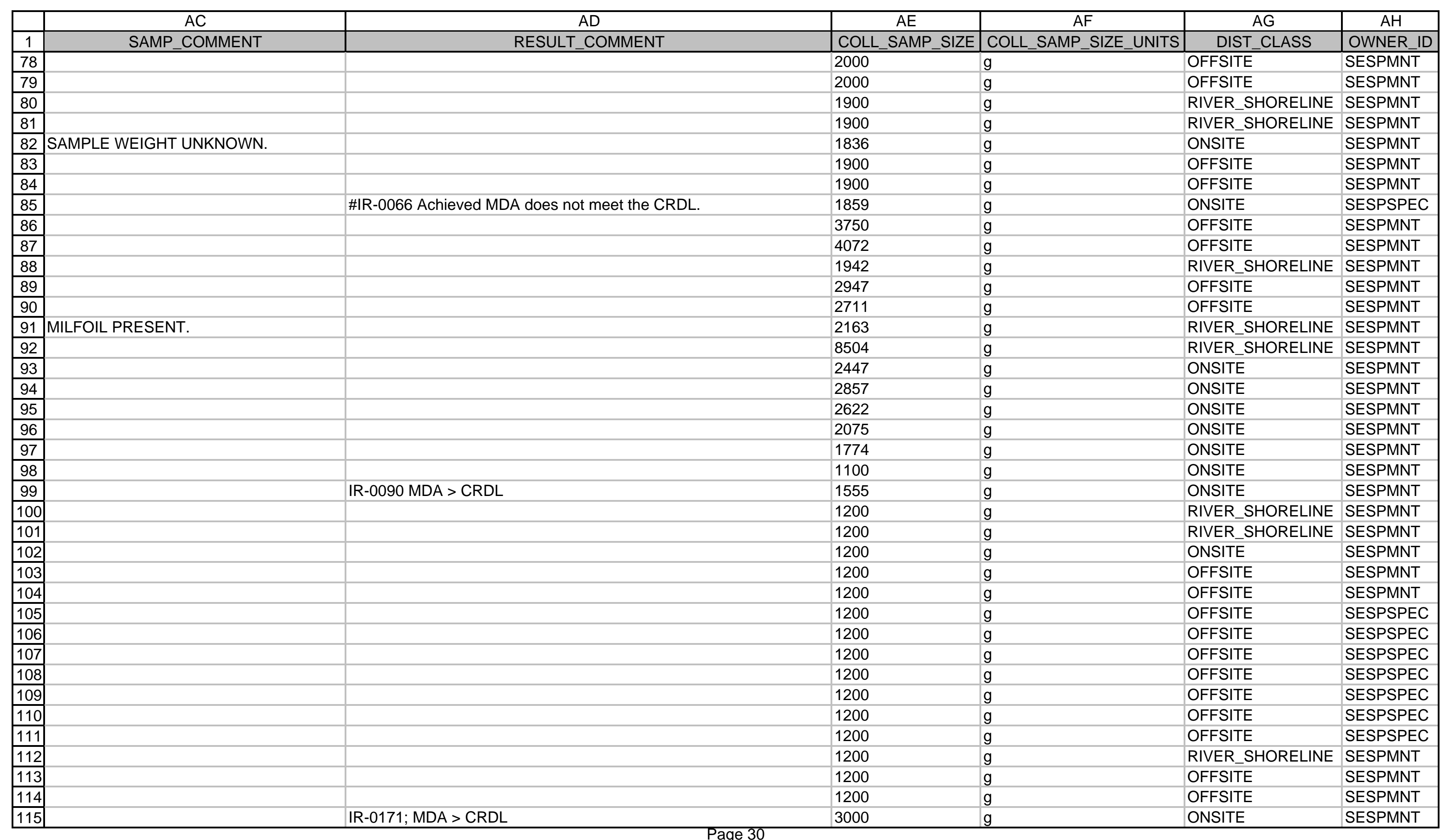




\begin{tabular}{|c|c|c|c|c|c|c|}
\hline & $\mathrm{AC}$ & $A D$ & $\mathrm{AE}$ & $\mathrm{AF}$ & $A G$ & $\overline{\mathrm{AH}}$ \\
\hline 1 & SAMP_COMMENT & RESULT_COMMENT & COLL_SAMP_SIZE & COLL_SAMP_SIZE_UNITS & DIST_CLASS & OWNER_ID \\
\hline 116 & & IR-0184; MDA > CRDL & 2465 & g & ONSITE & SESPMNT \\
\hline 117 & & & 1100 & $\mathrm{~g}$ & ONSITE & SESPMNT \\
\hline 118 & & & 1100 & $\mathrm{~g}$ & ONSITE & SESPMNT \\
\hline 119 & & & 8673 & $\mathrm{~g}$ & ONSITE & SESPMNT \\
\hline \begin{tabular}{|c|}
+20 \\
120 \\
\end{tabular} & & & 2526 & g & ONSITE & SESPMNT \\
\hline \begin{tabular}{|l|}
120 \\
121 \\
\end{tabular} & & IR-0217: Spike $>400 \%$ due to $\mathrm{Sr}-90$ contamination in Sr-85 tracer. & 2370 & g & ONSITE & SESPMNT \\
\hline 122 & COLLECTED BY HAND FROM SHORE. & & 2000 & g & RIVER_SHORELINE & SESPMNT \\
\hline \begin{tabular}{|c|c|}
123 \\
123 \\
\end{tabular} & & & 2000 & g & OFFSITE & SESPMNT \\
\hline 125 & & & 1900 & g & RIVER_SHORELINE & SESPMNT \\
\hline 126 & & IR-0224: MDA>CRDL. & 1900 & $\mathrm{~g}$ & RIVER_SHORELINE & SESPMNT \\
\hline 127 & SAMPLE WEIGHT UNKNOWN. & & 1836 & $\mathrm{~g}$ & ONSITE & SESPMNT \\
\hline 128 & & & 1900 & g & OFFSITE & SESPMNT \\
\hline 129 & & & 1900 & $\mathrm{~g}$ & OFFSITE & SESPMNT \\
\hline 130 & & & 1100 & $\mathrm{~g}$ & ONSITE & SESPMNT \\
\hline \begin{tabular}{|c|}
131 \\
\end{tabular} & SAMPLE COLLECTED AT SPRING B. & & 1800 & $\mathrm{~g}$ & ONSITE & SESPMNT \\
\hline \begin{tabular}{|c|c|}
132 \\
132
\end{tabular} & & IR-0246: MDA>CRDL. & 1536 & g & ONSITE & SESPMNT \\
\hline \begin{tabular}{|l|l|}
+42 \\
133 \\
\end{tabular} & & IR-0246: MDA>CRDL. & 1536 & g & ONSITE & SESPMNT \\
\hline 135 & & & 2590 & $g$ & ONSITE & SESPMNT \\
\hline \begin{tabular}{|l|l|}
+4 \\
136 \\
\end{tabular} & & & 1536 & g & ONSITE & SESPMNT \\
\hline 137 & & & 2465 & $\mathrm{~g}$ & ONSITE & SESPMNT \\
\hline 138 & & & 8673 & g & ONSITE & SESPMNT \\
\hline 139 & & & 2526 & $\mathrm{~g}$ & ONSITE & SESPMNT \\
\hline 140 & & & 2370 & $\mathrm{~g}$ & ONSITE & SESPMNT \\
\hline 141 & & IR-0248: Matrix blank>CRDL. & 2590 & g & ONSITE & SESPMNT \\
\hline 142 & & \#IR-0058 MDA > CRDL & 1859 & $\mathrm{~g}$ & ONSITE & SESPSPEC \\
\hline 143 & & & 3750 & $\mathrm{~g}$ & OFFSITE & SESPMNT \\
\hline 144 & & & 4072 & $\mathrm{~g}$ & OFFSITE & SESPMNT \\
\hline \begin{tabular}{|l|l|}
145 \\
145
\end{tabular} & & & 1942 & 9 & RIVER_SHORELINE & SESPMNT \\
\hline \begin{tabular}{|l|l|}
146 \\
146
\end{tabular} & & & 2947 & g & OFFSITE & SESPMNT \\
\hline \begin{tabular}{|l|l|}
147 \\
\end{tabular} & & & 2711 & g & OFFSITE & SESPMNT \\
\hline \begin{tabular}{|l|}
148 \\
\end{tabular} & MILFOIL PRESENT. & & 2163 & g & RIVER SHORELINE & SESPMNT \\
\hline \begin{tabular}{|c|}
149 \\
\end{tabular} & & & 8504 & g & RIVER SHORELINE & SESPMNT \\
\hline \begin{tabular}{|l|}
+150 \\
150
\end{tabular} & & & 2447 & g & ONSITE & SESPMNT \\
\hline \begin{tabular}{|l|l|}
151 \\
\end{tabular} & & & 2857 & g & ONSITE & SESPMNT \\
\hline 152 & & & 2622 & $\mathrm{~g}$ & ONSITE & SESPMNT \\
\hline
\end{tabular}




\begin{tabular}{|c|c|c|c|c|c|c|}
\hline & $\mathrm{AC}$ & $A D$ & $\mathrm{AE}$ & $\mathrm{AF}$ & $\mathrm{AG}$ & $\mathrm{AH}$ \\
\hline 1 & SAMP_COMMENT & RESULT_COMMENT & COLL_SAMP_SIZE & COLL_SAMP_SIZE_UNITS & DIST_CLASS & OWNER_ID \\
\hline 154 & & & 1774 & g & ONSITE & SESPMNT \\
\hline 155 & & & 1100 & $\mathrm{~g}$ & ONSITE & SESPMNT \\
\hline 156 & & & 1555 & $\mathrm{~g}$ & ONSITE & SESPMNT \\
\hline \begin{tabular}{|l|l|}
157 \\
150
\end{tabular} & & & 1200 & g & RIVER_SHORELINE & SESPMNT \\
\hline 158 & & & 1200 & $\mathrm{~g}$ & RIVER_SHORELINE & SESPMNT \\
\hline 159 & & & 1200 & $\mathrm{~g}$ & ONSITE & SESPMNT \\
\hline \begin{tabular}{|c|}
160 \\
160
\end{tabular} & & & 1200 & $\mathrm{~g}$ & OFFSITE & SESPMNT \\
\hline 161 & & & 1200 & $\mathrm{~g}$ & OFFSITE & SESPMNT \\
\hline 162 & & & 1200 & $\mathrm{~g}$ & OFFSITE & SESPSPEC \\
\hline 163 & & & 1200 & $\mathrm{~g}$ & OFFSITE & SESPSPEC \\
\hline 164 & & & 1200 & $\mathrm{~g}$ & OFFSITE & SESPSPEC \\
\hline 165 & & & 1200 & g & OFFSITE & SESPSPEC \\
\hline 166 & & & 1200 & $\mathrm{~g}$ & OFFSITE & SESPSPEC \\
\hline 167 & & & 1200 & $\mathrm{~g}$ & OFFSITE & SESPSPEC \\
\hline 168 & & & 1200 & $\mathrm{~g}$ & OFFSITE & SESPSPEC \\
\hline \begin{tabular}{|c|}
169 \\
\end{tabular} & & & 1200 & $\mathrm{~g}$ & RIVER_SHORELINE & SESPMNT \\
\hline \begin{tabular}{|l|}
170 \\
170
\end{tabular} & & & 1200 & g & OFFSITE & SESPMNT \\
\hline \begin{tabular}{|l|}
171 \\
\end{tabular} & & & 1200 & $g$ & OFFSITE & SESPMNT \\
\hline 173 & & & 3000 & $\mathrm{~g}$ & ONSITE & SESPSPEC \\
\hline \begin{tabular}{|l|}
174 \\
\end{tabular} & & & 3000 & $g$ & ONSITE & SESPSPEC \\
\hline \begin{tabular}{|l|}
175 \\
\end{tabular} & & & 960 & g & ONSITE & SESPSPEC \\
\hline 176 & & & 225 & $\mathrm{~g}$ & ONSITE & SESPSPEC \\
\hline 177 & & & 702 & $\mathrm{~g}$ & ONSITE & SESPSPEC \\
\hline 178 & & & 409 & g & ONSITE & SESPSPEC \\
\hline 179 & & & 2877 & $\mathrm{~g}$ & ONSITE & SESPSPEC \\
\hline 180 & & & 1414 & $\mathrm{~g}$ & ONSITE & SESPSPEC \\
\hline \begin{tabular}{|l|}
181 \\
\end{tabular} & & & 2465 & $\mathrm{~g}$ & ONSITE & SESPMNT \\
\hline \begin{tabular}{|l|}
182 \\
182 \\
\end{tabular} & & & 1100 & g & ONSITE & SESPMNT \\
\hline 183 & & & 1100 & $\mathrm{~g}$ & ONSITE & SESPMNT \\
\hline 184 & & & 8673 & $\mathrm{~g}$ & ONSITE & SESPMNT \\
\hline 185 & & & 2526 & $g$ & ONSITE & SESPMNT \\
\hline 186 & & & 2370 & $\mathrm{~g}$ & ONSITE & SESPMNT \\
\hline 187 & COLLECTED BY HAND FROM SHORE. & & 2000 & g & RIVER_SHORELINE & SESPMNT \\
\hline 188 & & & 2000 & $\mathrm{~g}$ & OFFSITE & SESPMNT \\
\hline 189 & & & 2000 & $\mathrm{~g}$ & OFFSITE & SESPMNT \\
\hline 190 & & & 1900 & g & RIVER SHORELINE & SESPMNT \\
\hline
\end{tabular}




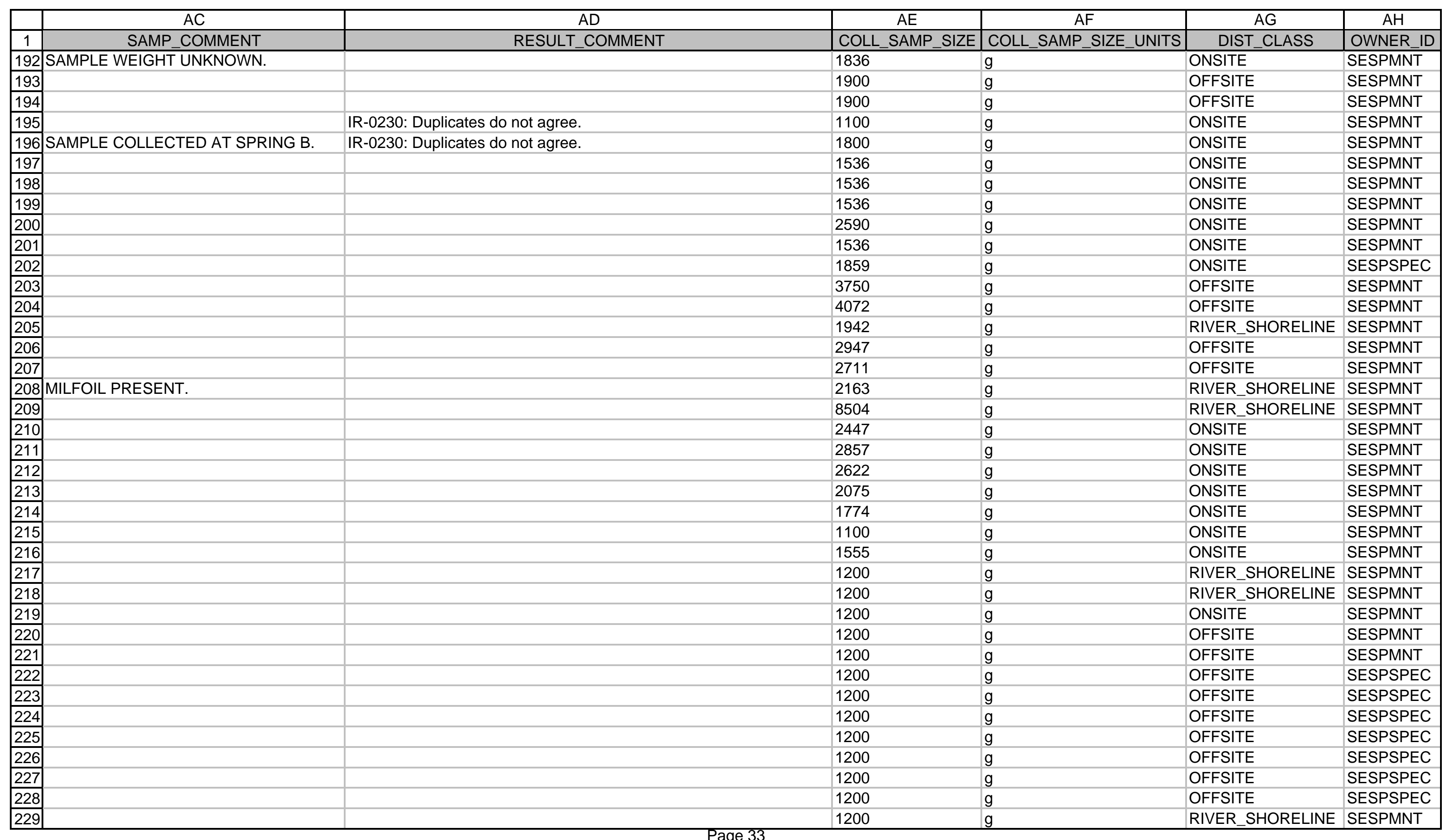




\begin{tabular}{|c|c|c|c|c|c|c|}
\hline & $A C$ & $A D$ & $\mathrm{AE}$ & $\mathrm{AF}$ & $A G$ & $\overline{\mathrm{AH}}$ \\
\hline 1 & SAMP_COMMENT & RESULT_COMMENT & COLL_SAMP_SIZE & COLL_SAMP_SIZE_UNITS & DIST_CLASS & OWNER_ID \\
\hline 230 & & & 1200 & g & OFFSITE & SESPMNT \\
\hline 231 & & & 1200 & $\mathrm{~g}$ & OFFSITE & SESPMNT \\
\hline 232 & & & 3000 & $\mathrm{~g}$ & ONSITE & SESPMNT \\
\hline \begin{tabular}{|l|}
233 \\
\end{tabular} & & & 3000 & $\mathrm{~g}$ & ONSITE & SESPSPEC \\
\hline 234 & & & 3000 & $\mathrm{~g}$ & ONSITE & SESPSPEC \\
\hline 235 & & & 960 & $\mathrm{~g}$ & ONSITE & SESPSPEC \\
\hline 236 & & & 225 & $g$ & ONSITE & SESPSPEC \\
\hline 237 & & & 702 & $\mathrm{~g}$ & ONSITE & SESPSPEC \\
\hline \begin{tabular}{|l|l|}
239 \\
230 \\
\end{tabular} & & & 2877 & g & ONSITE & SESPSPEC \\
\hline 240 & & & 1414 & $\mathrm{~g}$ & ONSITE & SESPSPEC \\
\hline 241 & & & 2465 & $\mathrm{~g}$ & ONSITE & SESPMNT \\
\hline 242 & & & 1100 & g & ONSITE & SESPMNT \\
\hline 243 & & & 1100 & $\mathrm{~g}$ & ONSITE & SESPMNT \\
\hline 244 & & & 8673 & $\mathrm{~g}$ & ONSITE & SESPMNT \\
\hline 245 & & & 2526 & $\mathrm{~g}$ & ONSITE & SESPMNT \\
\hline \begin{tabular}{|l|l|}
246 \\
\end{tabular} & & & 2370 & g & ONSITE & SESPMNT \\
\hline \begin{tabular}{|l|l|}
247 \\
247 \\
\end{tabular} & COLLECTED BY HAND FROM SHORE. & & 2000 & g & RIVER_SHORELINE & SESPMNT \\
\hline 249 & & & 2000 & $g$ & OFFSITE & SESPMNT \\
\hline \begin{tabular}{|l|l|}
250 \\
\end{tabular} & & & 1900 & g & RIVER_SHORELINE & SESPMNT \\
\hline 251 & & & 1900 & $\mathrm{~g}$ & RIVER_SHORELINE & SESPMNT \\
\hline 252 & SAMPLE WEIGHT UNKNOWN. & & 1836 & g & ONSITE & SESPMNT \\
\hline 253 & & & 1900 & $\mathrm{~g}$ & OFFSITE & SESPMNT \\
\hline 254 & & & 1900 & $\mathrm{~g}$ & OFFSITE & SESPMNT \\
\hline 255 & & IR-0230: Duplicates do not agree. & 1100 & g & ONSITE & SESPMNT \\
\hline 256 & SAMPLE COLLECTED AT SPRING B. & IR-0230: Duplicates do not agree. & 1800 & g & ONSITE & SESPMNT \\
\hline 257 & & & 1536 & $\mathrm{~g}$ & ONSITE & SESPMNT \\
\hline 258 & & & 1536 & $\mathrm{~g}$ & ONSITE & SESPMNT \\
\hline \begin{tabular}{|l|l|}
259 \\
259 \\
\end{tabular} & & & 1536 & g & ONSITE & SESPMNT \\
\hline \begin{tabular}{|l|l|}
260 \\
260 \\
\end{tabular} & & & 2590 & g & ONSITE & SESPMNT \\
\hline \begin{tabular}{|l|l|}
261 \\
261
\end{tabular} & & & 1536 & g & ONSITE & SESPMNT \\
\hline \begin{tabular}{|l|l|}
262 \\
262 \\
\end{tabular} & & \#IR-0058 MDA > CRDL & 1859 & g & ONSITE & SESPSPEC \\
\hline \begin{tabular}{|l|l|}
263 \\
263 \\
\end{tabular} & & & 3750 & g & OFFSITE & SESPMNT \\
\hline \begin{tabular}{|l|l|}
264 \\
264 \\
\end{tabular} & & & 4072 & g & OFFSITE & SESPMNT \\
\hline 265 & & & 1942 & g & RIVER_SHORELINE & SESPMNT \\
\hline 266 & & & 2947 & $\mathrm{~g}$ & OFFSITE & SESPMNT \\
\hline
\end{tabular}




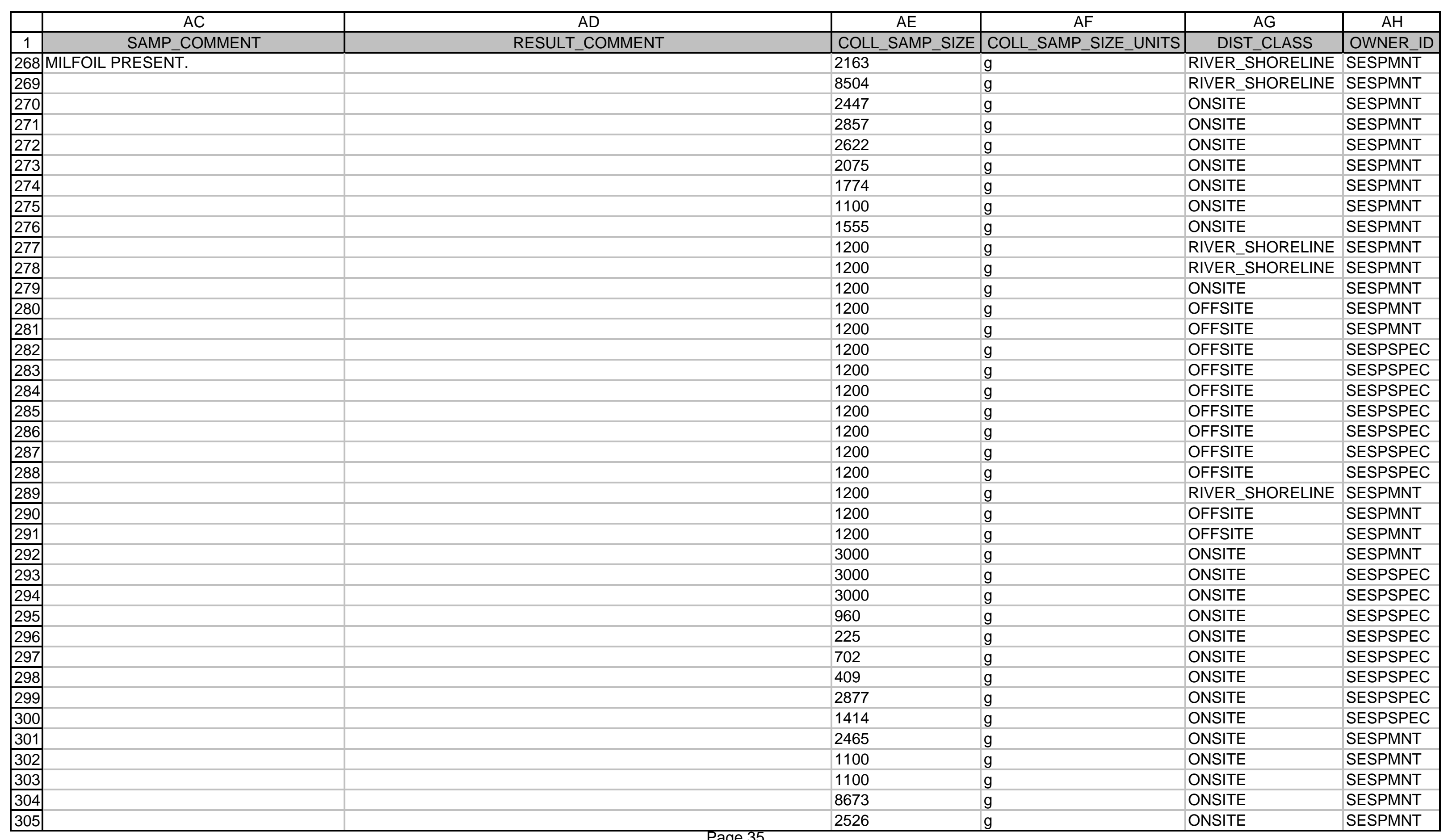




\begin{tabular}{|c|c|c|c|c|c|c|}
\hline & $A C$ & AD & $\mathrm{AE}$ & $\mathrm{AF}$ & $\overline{A G}$ & $\mathrm{AH}$ \\
\hline 1 & SAMP_COMMENT & RESULT_COMMENT & COLL_SAMP_SIZE & COLL_SAMP_SIZE_UNITS & DIST_CLASS & OWNER_ID \\
\hline 306 & & & 2370 & g & ONSITE & SESPMNT \\
\hline 307 & COLLECTED BY HAND FROM SHORE. & & 2000 & $\mathrm{~g}$ & RIVER_SHORELINE & SESPMNT \\
\hline 308 & & & 2000 & g & OFFSITE & SESPMNT \\
\hline 309 & & & 2000 & g & OFFSITE & SESPMNT \\
\hline 310 & & & 1900 & g & RIVER_SHORELINE & SESPMNT \\
\hline 311 & & & 1900 & $\mathrm{~g}$ & RIVER_SHORELINE & SESPMNT \\
\hline 312 & SAMPLE WEIGHT UNKNOWN. & & 1836 & g & ONSITE & SESPMNT \\
\hline 313 & & & 1900 & $\mathrm{~g}$ & OFFSITE & SESPMNT \\
\hline 315 & & IR-0230: Duplicates do not agree. & 1100 & g & ONSITE & SESPMNT \\
\hline 316 & SAMPLE COLLECTED AT SPRING B. & IR-0230: Duplicates do not agree. & 1800 & g & ONSITE & SESPMNT \\
\hline 317 & & & 1536 & $\mathrm{~g}$ & ONSITE & SESPMNT \\
\hline 318 & & & 1536 & g & ONSITE & SESPMNT \\
\hline 319 & & & 1536 & g & ONSITE & SESPMNT \\
\hline 320 & & & 2590 & g & ONSITE & SESPMNT \\
\hline 321 & & IR-0255: Matrix Blank U-238>CRDL. & 1536 & g & ONSITE & SESPMNT \\
\hline
\end{tabular}




\begin{tabular}{|c|c|c|c|c|c|c|c|c|c|}
\hline & & AJ & AK & AL & AM & & $\mathrm{AO}$ & AP & \\
\hline 1 & COLLECTION_PURPOSE & X_Y_COORD_FINAL.SAMP_NUM & COORDINATE SYSTEM & DATUM & LATITUDE ${ }^{\circ} \mathrm{N}$ & LATITUDE MIN_MIN & LATITUDE MIN_SEC & $\mid$ LONGITUDE $^{\circ} \mathrm{W}$ & LONGITUDE MIN_MIN \\
\hline 2 & $\mathrm{~S}$ & & & & & & & & \\
\hline 3 & $\mathrm{R}$ & & & & & & & & \\
\hline 4 & $\mathrm{R}$ & & & & & & & & \\
\hline 5 & $\mathrm{R}$ & & & & & & & & \\
\hline 6 & $\mathrm{R}$ & & & & & & & & \\
\hline 7 & $\mathrm{R}$ & & & & & & & & \\
\hline 8 & $\mathrm{R}$ & & & & & & & & \\
\hline 9 & $\mathrm{R}$ & & & & & & & & \\
\hline 10 & $\mathrm{R}$ & & & & & & & & \\
\hline 11 & $\mathrm{R}$ & B10908 & STATE PLANE & & & & & & \\
\hline 12 & $\mathrm{R}$ & B10917 & STATE PLANE & & & & & & \\
\hline 13 & $\mathrm{R}$ & B10919 & STATE PLANE & & & & & & \\
\hline 14 & $\mathrm{R}$ & B10922 & STATE PLANE & & & & & & \\
\hline 15 & $\mathrm{R}$ & & & & & & & & \\
\hline 16 & $\mathrm{R}$ & & & & & & & & \\
\hline 17 & $\mathrm{R}$ & B12CK8 & LAT/LONG & & 46 & & 35.24 .685 & 119 & \\
\hline 18 & $\mathrm{R}$ & B12CL5 & LAT/LONG & & 46 & & 40.40 .882 & 119 & \\
\hline 19 & $\mathrm{R}$ & & & & & & & & \\
\hline 20 & $\mathrm{R}$ & B12CM2 & LAT/LONG & & 46 & & 38.49 .822 & 119 & \\
\hline 21 & $\mathrm{R}$ & B12CM4 & LAT/LONG & & 46 & & 39.13.366 & 119 & \\
\hline 22 & S & B12CL1 & LAT/LONG & & 45 & & 55.52 .061 & 119 & \\
\hline 23 & S & B12CL2 & LAT/LONG & & 45 & & 56.62 .863 & 119 & \\
\hline 24 & S & B12CL3 & LAT/LONG & & 45 & & 56.33 .1352 & 119 & \\
\hline 25 & S & B12CL4 & LAT/LONG & & 45 & & 56.45 .2625 & 119 & \\
\hline 26 & S & $\mathrm{B} 12 \mathrm{CH} 3$ & LAT/LONG & & 46 & & 14.56.3853 & 118 & \\
\hline 27 & S & B12CJ0 & LAT/LONG & & 46 & & 15.27 .8170 & 118 & \\
\hline 28 & S & B12CJ1 & LAT/LONG & & 46 & & 15.12 .1645 & 118 & \\
\hline 29 & $\mathrm{R}$ & B12CK9 & LAT/LONG & & 46 & & 18.49.3916 & 119 & \\
\hline 30 & $\mathrm{R}$ & B12CNO & LAT/LONG & & 45 & & 55.55 .7549 & 119 & \\
\hline 31 & $\mathrm{R}$ & B12CN2 & LAT/LONG & & 45 & & 56.29.1919 & 119 & \\
\hline 32 & $\mathrm{R}$ & & & & & & & & \\
\hline 33 & $\mathrm{R}$ & & & & & & & & \\
\hline 34 & $\mathrm{R}$ & & & & & & & & \\
\hline 35 & $\mathrm{R}$ & & & & & & & & \\
\hline 36 & $\mathrm{R}$ & & & & & & & & \\
\hline 37 & $\mathrm{R}$ & & & & & & & & \\
\hline 38 & $\mathrm{R}$ & & & & & & & & \\
\hline 39 & $\mathrm{R}$ & B14YM5 & LAT/LONG & & 46 & & 18.49 .93 & 119 & \\
\hline
\end{tabular}




\begin{tabular}{|c|c|c|c|c|c|c|c|c|c|}
\hline & $\mathrm{Al}$ & AJ & AK & $\mathrm{AL}$ & AM & AN & $\mathrm{AO}$ & AP & $\mathrm{AQ}$ \\
\hline 1 & COLLECTION_PURPOSE & X_Y_COORD_FINAL.SAMP_NUM & COORDINATE SYSTEM & DATUM & LATITUDE ${ }^{\circ} \mathrm{N}$ & LATITUDE MIN_MIN & LATITUDE MIN_SEC & LONGITUDE ${ }^{\circ} \mathrm{W}$ & LONGITUDE MIN_MIN \\
\hline 40 & $\mathrm{R}$ & B14YP1 & LAT/LONG & & 45 & & 56.00 .751 & 119 & \\
\hline 41 & $R$ & B14YP3 & LAT/LONG & & 45 & & 56.28 .053 & 119 & \\
\hline 42 & $\mathrm{R}$ & B14YM4 & LAT/LONG & & 46 & & 35.24 .020 & 119 & \\
\hline 43 & $\mathrm{R}$ & B14YM6 & LAT/LONG & & 46 & & 40.44 .579 & 119 & \\
\hline 44 & $\mathrm{R}$ & B14YM7 & LAT/LONG & & 46 & & 38.10.776 & 119 & \\
\hline 45 & $\mathrm{R}$ & B14YN3 & LAT/LONG & & 46 & & 39.49 .096 & 119 & \\
\hline 46 & $\mathrm{R}$ & B14YN5 & LAT/LONG & & 46 & & 39.11.37211 & 119 & \\
\hline 47 & $R$ & & & & & & & & \\
\hline 48 & $R$ & & & & & & & & \\
\hline 49 & $\mathrm{R}$ & & & & & & & & \\
\hline 50 & $R$ & B15C44 & LAT/LONG & & 46 & & 33.50 .499 & 119 & \\
\hline 51 & $\mathrm{R}$ & & & & & & & & \\
\hline 52 & $R$ & & & & & & & & \\
\hline 53 & $R$ & B15C52 & LAT/LONG & & 46 & & 38.14 .440 & 119 & \\
\hline 54 & $R$ & & & & & & & & \\
\hline 55 & $R$ & & & & & & & & \\
\hline 56 & $\mathrm{R}$ & & & & & & & & \\
\hline 57 & $\mathrm{R}$ & & & & & & & & \\
\hline 58 & $R$ & & & & & & & & \\
\hline 59 & $R$ & & & & & & & & \\
\hline 60 & $R$ & & & & & & & & \\
\hline 61 & $R$ & & & & & & & & \\
\hline 62 & $R$ & B12CK8 & LAT/LONG & & 46 & & 35.24 .685 & 119 & \\
\hline 63 & $R$ & B12CL5 & LAT/LONG & & 46 & & 40.40 .882 & 119 & \\
\hline 64 & $R$ & & & & & & & & \\
\hline 65 & $R$ & B12CM2 & LAT/LONG & & 46 & & 38.49 .822 & 119 & \\
\hline 66 & $R$ & B12CM4 & LAT/LONG & & 46 & & 39.13.366 & 119 & \\
\hline 67 & S & B12CL1 & LAT/LONG & & 45 & & 55.52 .061 & 119 & \\
\hline 68 & s & B12CL2 & LAT/LONG & & 45 & & 56.62.863 & 119 & \\
\hline 69 & s & B12CL3 & LAT/LONG & & 45 & & 56.33 .1352 & 119 & \\
\hline 70 & S & B12CL4 & LAT/LONG & & 45 & & 56.45 .2625 & 119 & \\
\hline 71 & S & $\mathrm{B} 12 \mathrm{CH} 3$ & LAT/LONG & & 46 & & 14.56 .3853 & 118 & \\
\hline 72 & S & B12CJ0 & LAT/LONG & & 46 & & 15.27 .8170 & 118 & \\
\hline 73 & S & B12CJ1 & LAT/LONG & & 46 & & 15.12 .1645 & 118 & \\
\hline 74 & $R$ & B12CK9 & LAT/LONG & & 46 & & 18.49 .3916 & 119 & \\
\hline 75 & $R$ & B12CN0 & LAT/LONG & & 45 & & 55.55 .7549 & 119 & \\
\hline 76 & $\mathrm{R}$ & B12CN2 & LAT/LONG & & 45 & & 56.29 .1919 & 119 & \\
\hline 77 & $R$ & B14YM5 & LAT/LONG & & 46 & & 18.49.93 & 119 & \\
\hline
\end{tabular}




\begin{tabular}{|c|c|c|c|c|c|c|c|c|c|}
\hline & $\mathrm{Al}$ & AJ & AK & $\overline{A L}$ & AM & AN & $\mathrm{AO}$ & $\mathrm{AP}$ & $\mathrm{AQ}$ \\
\hline 1 & COLLECTION_PURPOSE & X_Y_COORD_FINAL.SAMP_NUM & | COORDINATE SYSTEM & DATUM & LATITUDE ${ }^{\circ} \mathrm{N}$ & LATITUDE MIN_MIN & LATITUDE MIN_SEC & LONGITUDE ${ }^{\circ} \mathrm{W}$ & LONGITUDE MIN_MIN \\
\hline 78 & $\mathrm{R}$ & B14YP1 & LAT/LONG & & 45 & & 56.00 .751 & 119 & \\
\hline 79 & $R$ & B14YP3 & LAT/LONG & & 45 & & 56.28 .053 & 119 & \\
\hline 80 & $R$ & B14YM4 & LAT/LONG & & 46 & & 35.24 .020 & 119 & \\
\hline 81 & $R$ & B14YM6 & LAT/LONG & & 46 & & 40.44 .579 & 119 & \\
\hline 82 & $R$ & B14YM7 & LAT/LONG & & 46 & & 38.10 .776 & 119 & \\
\hline 83 & $R$ & B14YN3 & LAT/LONG & & 46 & & 39.49 .096 & 119 & \\
\hline 84 & $R$ & B14YN5 & LAT/LONG & & 46 & & 39.11.37211 & 119 & \\
\hline 85 & s & & & & & & & & \\
\hline 86 & $R$ & & & & & & & & \\
\hline 87 & $R$ & & & & & & & & \\
\hline 88 & $R$ & & & & & & & & \\
\hline 89 & $R$ & & & & & & & & \\
\hline 90 & $R$ & & & & & & & & \\
\hline 91 & $R$ & & & & & & & & \\
\hline 92 & $R$ & & & & & & & & \\
\hline 93 & $R$ & & & & & & & & \\
\hline 94 & $R$ & B10908 & STATE PLANE & & & & & & \\
\hline \begin{tabular}{|l|}
95 \\
95
\end{tabular} & $R$ & B10917 & STATE PLANE & & & & & & \\
\hline 96 & $R$ & B10919 & STATE PLANE & & & & & & \\
\hline 97 & $R$ & B10922 & STATE PLANE & & & & & & \\
\hline 98 & $R$ & & & & & & & & \\
\hline 99 & $R$ & & & & & & & & \\
\hline 100 & $R$ & B12CK8 & LAT/LONG & & 46 & & 35.24 .685 & 119 & \\
\hline 101 & $R$ & B12CL5 & LAT/LONG & & 46 & & 40.40 .882 & 119 & \\
\hline 102 & $R$ & & & & & & & & \\
\hline 103 & $R$ & B12CM2 & LAT/LONG & & 46 & & 38.49 .822 & 119 & \\
\hline 104 & $R$ & B12CM4 & LAT/LONG & & 46 & & 39.13 .366 & 119 & \\
\hline 105 & 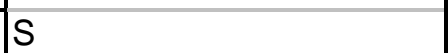 & B12CL1 & LAT/LONG & & 45 & & 55.52 .061 & 119 & \\
\hline 106 & s & B12CL2 & LAT/LONG & & 45 & & 56.62 .863 & 119 & \\
\hline 107 & s & B12CL3 & LAT/LONG & & 45 & & 56.33 .1352 & 119 & \\
\hline \begin{tabular}{|l|}
108 \\
108
\end{tabular} & S & B12CL4 & LAT/LONG & & 45 & & 56.45 .2625 & 119 & \\
\hline 109 & s & $\mathrm{B} 12 \mathrm{CH} 3$ & LAT/LONG & & 46 & & 14.56 .3853 & 118 & \\
\hline 110 & s & B12CJ0 & LAT/LONG & & 46 & & 15.27 .8170 & 118 & \\
\hline 111 & s & B12CJ1 & LAT/LONG & & 46 & & 15.12 .1645 & 118 & \\
\hline 112 & $R$ & B12CK9 & LAT/LONG & & 46 & & 18.49 .3916 & 119 & \\
\hline 113 & $R$ & B12CNO & LAT/LONG & & 45 & & 55.55 .7549 & 119 & \\
\hline 114 & $R$ & B12CN2 & LAT/LONG & & 45 & & 56.29 .1919 & 119 & \\
\hline 115 & $R$ & & & & & & & & \\
\hline
\end{tabular}




\begin{tabular}{|c|c|c|c|c|c|c|c|c|c|}
\hline & & AJ & AK & $\mathrm{AL}$ & AM & AN & $\mathrm{AO}$ & AP & \\
\hline 1 & COLLECTION_PURPOSE & X_Y_COORD_FINAL.SAMP_NUM & COORDINATE SYSTEM & DATUM & LATITUDE ${ }^{\circ} \mathrm{N}$ & LATITUDE MIN_MIN & LATITUDE MIN_SEC & LONGITUDE ${ }^{\circ} \mathrm{W}$ & LONGITUDE MIN_MIN \\
\hline \multicolumn{10}{|c|}{$116 \mathrm{R}$} \\
\hline \multicolumn{10}{|c|}{\begin{tabular}{l|l}
117 & $R$ \\
\end{tabular}} \\
\hline \multicolumn{10}{|c|}{$118 \mathrm{R}$} \\
\hline \multicolumn{10}{|c|}{\begin{tabular}{l|l}
119 & $R$ \\
\end{tabular}} \\
\hline \multicolumn{10}{|c|}{\begin{tabular}{l|l}
120 & $R$ \\
\end{tabular}} \\
\hline \multicolumn{10}{|c|}{$121 \mid R$} \\
\hline 122 & $\mathrm{R}$ & B14YM5 & LAT/LONG & & 46 & & 18.49 .93 & 119 & \\
\hline 123 & $\mathrm{R}$ & B14YP1 & LAT/LONG & & 45 & & 56.00 .751 & 119 & \\
\hline 124 & $\mathrm{R}$ & B14YP3 & LAT/LONG & & 45 & & 56.28 .053 & 119 & \\
\hline 125 & $\mathrm{R}$ & B14YM4 & LAT/LONG & & 46 & & 35.24 .020 & 119 & \\
\hline 126 & $\mathrm{R}$ & B14YM6 & LAT/LONG & & 46 & & 40.44 .579 & 119 & \\
\hline 127 & $\mathrm{R}$ & B14YM7 & LAT/LONG & & 46 & & 38.10 .776 & 119 & \\
\hline 128 & $\mathrm{R}$ & B14YN3 & LAT/LONG & & 46 & & 39.49 .096 & 119 & \\
\hline 129 & $\mathrm{R}$ & B14YN5 & LAT/LONG & & 46 & & 39.11 .37211 & 119 & \\
\hline \multicolumn{10}{|c|}{$130 \mathrm{R}$} \\
\hline \multicolumn{10}{|c|}{$131 \mathrm{R}$} \\
\hline \multicolumn{10}{|c|}{$132 \mathrm{R}$} \\
\hline 133 & $\mathrm{R}$ & B15C44 & LAT/LONG & & 46 & & 33.50 .499 & 119 & \\
\hline \multicolumn{10}{|c|}{$134 \mid R$} \\
\hline \multicolumn{10}{|c|}{$135 \mathrm{R}$} \\
\hline 136 & $\mathrm{R}$ & B15C52 & LAT/LONG & & 46 & & 38.14 .440 & 119 & \\
\hline \multicolumn{10}{|c|}{$137 \mathrm{R}$} \\
\hline \multicolumn{10}{|c|}{\begin{tabular}{l|l|l|l}
138 & $R$
\end{tabular}} \\
\hline \multicolumn{10}{|c|}{\begin{tabular}{l|l}
139 & $R$ \\
\end{tabular}} \\
\hline \multicolumn{10}{|c|}{\begin{tabular}{l|l}
140 & $R$ \\
\end{tabular}} \\
\hline \multicolumn{10}{|c|}{\begin{tabular}{l|l}
141 & $R$ \\
\end{tabular}} \\
\hline \multicolumn{10}{|c|}{\begin{tabular}{l|l}
142 & $S$ \\
\end{tabular}} \\
\hline \multicolumn{10}{|c|}{\begin{tabular}{l|l}
143 & $R$ \\
\end{tabular}} \\
\hline \multicolumn{10}{|c|}{$144 \mathrm{R}$} \\
\hline \multicolumn{10}{|c|}{\begin{tabular}{l|l}
145 & $R$ \\
\end{tabular}} \\
\hline \multicolumn{10}{|c|}{\begin{tabular}{l|l}
146 & $R$ \\
\end{tabular}} \\
\hline \multicolumn{10}{|c|}{\begin{tabular}{l|l}
147 & $R$ \\
\end{tabular}} \\
\hline \multicolumn{10}{|c|}{$\begin{array}{lll}148 & R \\
\end{array}$} \\
\hline \multicolumn{10}{|c|}{$149 \mathrm{R}$} \\
\hline 150 & $\mathrm{R}$ & & & & & & & & \\
\hline 151 & $\mathrm{R}$ & B10908 & STATE PLANE & & & & & & \\
\hline 152 & $\mathrm{R}$ & B10917 & STATE PLANE & & & & & & \\
\hline 153 & $\mathrm{R}$ & B10919 & STATE PLANE & & & & & & \\
\hline
\end{tabular}




\begin{tabular}{|c|c|c|c|c|c|c|c|c|c|}
\hline & $\mathrm{Al}$ & AJ & AK & $\overline{A L}$ & AM & AN & $\mathrm{AO}$ & AP & $\mathrm{AQ}$ \\
\hline 1 & COLLECTION_PURPOSE & X_Y_COORD_FINAL.SAMP_NUM & \begin{tabular}{|l|} 
COORDINATE SYSTEM \\
\end{tabular} & DATUM & LATITUDE ${ }^{\circ} \mathrm{N}$ & LATITUDE MIN_MIN & LATITUDE MIN_SEC & LONGITUDE ${ }^{\circ} \mathrm{W}$ & LONGITUDE MIN_MIN \\
\hline 154 & $\mathrm{R}$ & B10922 & STATE PLANE & & & & & & \\
\hline \multicolumn{10}{|c|}{\begin{tabular}{l|l}
155 & $R$ \\
\end{tabular}} \\
\hline \multicolumn{10}{|c|}{\begin{tabular}{l|l}
156 & $R$
\end{tabular}} \\
\hline \begin{tabular}{|c|}
157 \\
\end{tabular} & $\mathrm{R}$ & B12CK8 & LAT/LONG & & 46 & & 35.24 .685 & 119 & \\
\hline 158 & $\mathrm{R}$ & B12CL5 & LAT/LONG & & 46 & & 40.40 .882 & 119 & \\
\hline \multicolumn{10}{|c|}{\begin{tabular}{|l|l|}
159 & $R$ \\
\end{tabular}} \\
\hline 160 & $\mathrm{R}$ & B12CM2 & LAT/LONG & & 46 & & 38.49 .822 & 119 & \\
\hline 161 & $\mathrm{R}$ & B12CM4 & LAT/LONG & & 46 & & 39.13.366 & 119 & \\
\hline 162 & S & B12CL1 & LAT/LONG & & 45 & & 55.52 .061 & 119 & \\
\hline \begin{tabular}{|c|}
163 \\
\end{tabular} & S & B12CL2 & LAT/LONG & & 45 & & 56.62 .863 & 119 & \\
\hline 164 & $S$ & B12CL3 & LAT/LONG & & 45 & & 56.33 .1352 & 119 & \\
\hline 165 & $\mathrm{~S}$ & B12CL4 & LAT/LONG & & 45 & & 56.45 .2625 & 119 & \\
\hline 166 & $\mathrm{~S}$ & $\mathrm{~B} 12 \mathrm{CH} 3$ & LAT/LONG & & 46 & & 14.56 .3853 & 118 & \\
\hline \begin{tabular}{|l|}
167 \\
\end{tabular} & $S$ & B12CJ0 & LAT/LONG & & 46 & & 15.27 .8170 & 118 & \\
\hline \begin{tabular}{|l|}
168 \\
\end{tabular} & $S$ & B12CJ1 & LAT/LONG & & 46 & & 15.12 .1645 & 118 & \\
\hline \begin{tabular}{|l|}
169 \\
\end{tabular} & $\mathrm{R}$ & B12CK9 & LAT/LONG & & 46 & & 18.49.3916 & 119 & \\
\hline \begin{tabular}{|l|}
170 \\
\end{tabular} & $\mathrm{R}$ & B12CNO & LAT/LONG & & 45 & & 55.55 .7549 & 119 & \\
\hline \begin{tabular}{|l|}
171 \\
\end{tabular} & $R$ & B12CN2 & LAT/LONG & & 45 & & 56.29 .1919 & 119 & \\
\hline \multicolumn{10}{|c|}{\begin{tabular}{l|l}
172 & $R$ \\
\end{tabular}} \\
\hline \multicolumn{10}{|c|}{$173 \mathrm{~s}$} \\
\hline \multicolumn{10}{|c|}{$174 \mathrm{~s}$} \\
\hline \multicolumn{10}{|c|}{$175 \mathrm{~s}$} \\
\hline \multicolumn{10}{|c|}{\begin{tabular}{l|l}
176 & $\mathrm{~S}$
\end{tabular}} \\
\hline \multicolumn{10}{|c|}{\begin{tabular}{|l|l|}
177 & $S$ \\
\end{tabular}} \\
\hline \multicolumn{10}{|c|}{$178 \mathrm{~S}$} \\
\hline \multicolumn{10}{|c|}{\begin{tabular}{|l|l|}
179 & $S$ \\
\end{tabular}} \\
\hline \multicolumn{10}{|c|}{\begin{tabular}{|l|l|}
180 & $\mathrm{~S}$ \\
\end{tabular}} \\
\hline \multicolumn{10}{|c|}{\begin{tabular}{|l|l}
181 & $R$ \\
\end{tabular}} \\
\hline \multicolumn{10}{|c|}{\begin{tabular}{|l|l|}
182 & $R$ \\
\end{tabular}} \\
\hline \multicolumn{10}{|c|}{\begin{tabular}{|l|l|}
183 & $R$ \\
\end{tabular}} \\
\hline \multicolumn{10}{|c|}{\begin{tabular}{|l|l|}
184 & $R$ \\
184
\end{tabular}} \\
\hline \multicolumn{10}{|c|}{\begin{tabular}{|l|l}
185 & $R$ \\
\end{tabular}} \\
\hline \multicolumn{10}{|c|}{\begin{tabular}{|l|l|}
186 & $\mathrm{R}$ \\
\end{tabular}} \\
\hline \begin{tabular}{|l|}
187 \\
\end{tabular} & $\mathrm{R}$ & B14YM5 & LAT/LONG & & 46 & & 18.49 .93 & 119 & \\
\hline \begin{tabular}{|l|}
188 \\
\end{tabular} & $\mathrm{R}$ & B14YP1 & LAT/LONG & & 45 & & 56.00 .751 & 119 & \\
\hline \begin{tabular}{|l|}
189 \\
189
\end{tabular} & $\mathrm{R}$ & B14YP3 & LAT/LONG & & 45 & & 56.28 .053 & 119 & \\
\hline 190 & $\mathrm{R}$ & B14YM4 & LAT/LONG & & 46 & & 35.24 .020 & 119 & \\
\hline \begin{tabular}{|l|}
191 \\
\end{tabular} & $\mathrm{R}$ & B14YM6 & LAT/LONG & & 46 & & 40.44 .579 & 119 & \\
\hline
\end{tabular}




\begin{tabular}{|c|c|c|c|c|c|c|c|c|c|}
\hline & $\mathrm{Al}$ & AJ & AK & $\overline{A L}$ & AM & AN & $\mathrm{AO}$ & AP & $\mathrm{AQ}$ \\
\hline 1 & COLLECTION_PURPOSE & X_Y_COORD_FINAL.SAMP_NUM & \begin{tabular}{|l|} 
COORDINATE SYSTEM \\
\end{tabular} & DATUM & LATITUDE ${ }^{\circ} \mathrm{N}$ & LATITUDE MIN_MIN & LATITUDE MIN_SEC & LONGITUDE ${ }^{\circ} \mathrm{W}$ & LONGITUDE MIN_MIN \\
\hline \begin{tabular}{|l|}
192 \\
\end{tabular} & $\mathrm{R}$ & B14YM7 & LAT/LONG & & 46 & & 38.10 .776 & 119 & \\
\hline 193 & $\mathrm{R}$ & B14YN3 & LAT/LONG & & 46 & & 39.49 .096 & 119 & \\
\hline 194 & $\mathrm{R}$ & B14YN5 & LAT/LONG & & 46 & & 39.11.37211 & 119 & \\
\hline \multicolumn{10}{|c|}{$195 \mathrm{R}$} \\
\hline \multicolumn{10}{|c|}{\begin{tabular}{|l|l|l|}
196 & $R$
\end{tabular}} \\
\hline \multicolumn{10}{|c|}{\begin{tabular}{|l|l|l|}
197 & $R$ \\
\end{tabular}} \\
\hline \begin{tabular}{|c|}
198 \\
\end{tabular} & $\mathrm{R}$ & B15C44 & LAT/LONG & & 46 & & 33.50 .499 & 119 & \\
\hline \multicolumn{10}{|c|}{\begin{tabular}{l|l}
199 & $R$
\end{tabular}} \\
\hline \multicolumn{10}{|c|}{$200 R$} \\
\hline \begin{tabular}{|l|l|}
201 \\
\end{tabular} & $\mathrm{R}$ & B15C52 & LAT/LONG & & 46 & & 38.14 .440 & 119 & \\
\hline \multicolumn{10}{|c|}{$202 \mathrm{~S}$} \\
\hline \multicolumn{10}{|c|}{\begin{tabular}{|l|l|}
203 & $R$ \\
\end{tabular}} \\
\hline \multicolumn{10}{|c|}{\begin{tabular}{|l|l|l|}
204 & $R$ \\
\end{tabular}} \\
\hline \multicolumn{10}{|c|}{$205 R$} \\
\hline \multicolumn{10}{|c|}{\begin{tabular}{|l|l|}
206 & $R$ \\
\end{tabular}} \\
\hline \multicolumn{10}{|c|}{\begin{tabular}{l|l}
207 & $R$ \\
207
\end{tabular}} \\
\hline \multicolumn{10}{|c|}{\begin{tabular}{l|l}
$\mathrm{R}$ \\
208 & $\mathrm{R}$
\end{tabular}} \\
\hline \multicolumn{10}{|c|}{\begin{tabular}{|l|l|}
209 & $R$ \\
\end{tabular}} \\
\hline \multicolumn{10}{|c|}{$\begin{array}{ll}\mathrm{R} \\
210\end{array}$} \\
\hline 211 & $\mathrm{R}$ & B10908 & STATE PLANE & & & & & & \\
\hline 212 & $\mathrm{R}$ & B10917 & STATE PLANE & & & & & & \\
\hline 213 & $\mathrm{R}$ & B10919 & STATE PLANE & & & & & & \\
\hline \begin{tabular}{|l|}
214 \\
214 \\
\end{tabular} & $\mathrm{R}$ & B10922 & STATE PLANE & & & & & & \\
\hline \multicolumn{10}{|c|}{\begin{tabular}{l|l} 
\\
215 & $R$
\end{tabular}} \\
\hline \multicolumn{10}{|c|}{\begin{tabular}{|l|l|}
216 & $R$ \\
\end{tabular}} \\
\hline \begin{tabular}{|l|}
217 \\
\end{tabular} & $\mathrm{R}$ & B12CK8 & LAT/LONG & & 46 & & 35.24 .685 & 119 & \\
\hline 218 & $\mathrm{R}$ & B12CL5 & LAT/LONG & & 46 & & 40.40 .882 & 119 & \\
\hline \multicolumn{10}{|c|}{\begin{tabular}{l|l}
219 & $R$ \\
\end{tabular}} \\
\hline 22 & $\mathrm{R}$ & B12CM2 & LAT/LONG & & 46 & & 38.49 .822 & 119 & \\
\hline \begin{tabular}{|l|}
221 \\
\end{tabular} & $\mathrm{R}$ & B12CM4 & LAT/LONG & & 46 & & 39.13 .366 & 119 & \\
\hline 222 & S & B12CL1 & LAT/LONG & & 45 & & 55.52 .061 & 119 & \\
\hline \begin{tabular}{|l|}
223 \\
\end{tabular} & $\mathrm{~S}$ & B12CL2 & LAT/LONG & & 45 & & 56.62 .863 & 119 & \\
\hline \begin{tabular}{|l|}
224 \\
224
\end{tabular} & S & B12CL3 & LAT/LONG & & 45 & & 56.33 .1352 & 119 & \\
\hline 225 & S & B12CL4 & LAT/LONG & & 45 & & 56.45 .2625 & 119 & \\
\hline \begin{tabular}{|l|}
226 \\
\end{tabular} & S & $\mathrm{B} 12 \mathrm{CH} 3$ & LAT/LONG & & 46 & & 14.56 .3853 & 118 & \\
\hline \begin{tabular}{|l|}
227 \\
\end{tabular} & S & B12CJ0 & LAT/LONG & & 46 & & 15.27 .8170 & 118 & \\
\hline 228 & $S$ & B12CJ1 & LAT/LONG & & 46 & & 15.12 .1645 & 118 & \\
\hline 229 & $\mathrm{R}$ & B12CK9 & LAT/LONG & & 46 & & 18.49 .3916 & 119 & \\
\hline
\end{tabular}




\begin{tabular}{|c|c|c|c|c|c|c|c|c|c|}
\hline & $\mathrm{Al}$ & $\mathrm{AJ}$ & $\mathrm{AK}$ & $\mathrm{AL}$ & AM & AN & $\mathrm{AO}$ & $\mathrm{AP}$ & $\mathrm{AQ}$ \\
\hline 1 & COLLECTION_PURPOSE & X_Y_COORD_FINAL.SAMP_NUM & COORDINATE SYSTEM & DATUM & LATITUDE ${ }^{\circ} \mathrm{N}$ & LATITUDE MIN_MIN & LATITUDE MIN_SEC & LONGITUDE ${ }^{\circ} \mathrm{W}$ & LONGITUDE MIN_MIN \\
\hline \begin{tabular}{|l|l|}
230 & $F$ \\
\end{tabular} & $\mathrm{R}$ & $\mathrm{B} 12 \mathrm{CNO}$ & LAT/LONG & & 45 & & 55.55 .7549 & 119 & \\
\hline $231 \mathrm{~F}$ & $\mathrm{R}$ & B12CN2 & LAT/LONG & & 45 & & 56.29 .1919 & 119 & \\
\hline \begin{tabular}{|l|l}
232 & $F$ \\
\end{tabular} & $\mathrm{R}$ & & & & & & & & \\
\hline $233 \leq$ & S & & & & & & & & \\
\hline $234 \mathrm{~s}$ & $\mathrm{~S}$ & & & & & & & & \\
\hline 235 & S & & & & & & & & \\
\hline 236 & $S$ & & & & & & & & \\
\hline 237 s & S & & & & & & & & \\
\hline $238 \mathrm{~s}$ & S & & & & & & & & \\
\hline $239 \leq$ & S & & & & & & & & \\
\hline $240 \leqslant$ & $\mathrm{~S}$ & & & & & & & & \\
\hline $241 \mathrm{~F}$ & $\mathrm{R}$ & & & & & & & & \\
\hline $242 \mathrm{~F}$ & $\mathrm{R}$ & & & & & & & & \\
\hline $243 \mathrm{~F}$ & $\mathrm{R}$ & & & & & & & & \\
\hline $244 \mathrm{~F}$ & $\mathrm{R}$ & & & & & & & & \\
\hline 245 & $\mathrm{R}$ & & & & & & & & \\
\hline $246 \mathrm{~F}$ & $\mathrm{R}$ & & & & & & & & \\
\hline $247 \mathrm{~F}$ & $\mathrm{R}$ & B14YM5 & LAT/LONG & & 46 & & 18.49 .93 & 119 & \\
\hline $248 \mathrm{~F}$ & $\mathrm{R}$ & B14YP1 & LAT/LONG & & 45 & & 56.00 .751 & 119 & \\
\hline $249 \mathrm{~F}$ & $\mathrm{R}$ & B14YP3 & LAT/LONG & & 45 & & 56.28.053 & 119 & \\
\hline $250 \mathrm{~F}$ & $\mathrm{R}$ & B14YM4 & LAT/LONG & & 46 & & 35.24 .020 & 119 & \\
\hline $251 \mathrm{~F}$ & $\mathrm{R}$ & B14YM6 & LAT/LONG & & 46 & & 40.44 .579 & 119 & \\
\hline \begin{tabular}{|l|l}
$252 \mathrm{~F}$ \\
\end{tabular} & $\mathrm{R}$ & B14YM7 & LAT/LONG & & 46 & & 38.10.776 & 119 & \\
\hline $253 \mathrm{~F}$ & $\mathrm{R}$ & B14YN3 & LAT/LONG & & 46 & & 39.49 .096 & 119 & \\
\hline $254 \mathrm{~F}$ & $\mathrm{R}$ & B14YN5 & LAT/LONG & & 46 & & 39.11.37211 & 119 & \\
\hline $255 \mathrm{~F}$ & $\mathrm{R}$ & & & & & & & & \\
\hline \begin{tabular}{|l|l}
256 & $F$ \\
\end{tabular} & $\mathrm{R}$ & & & & & & & & \\
\hline $257 \mathrm{~F}$ & $\mathrm{R}$ & & & & & & & & \\
\hline $258 \mathrm{~F}$ & $\mathrm{R}$ & B15C44 & LAT/LONG & & 46 & & 33.50 .499 & 119 & \\
\hline $259 \mathrm{~F}$ & $\mathrm{R}$ & & & & & & & & \\
\hline $260 \mathrm{~F}$ & $\mathrm{R}$ & & & & & & & & \\
\hline $261 \mathrm{~F}$ & $\mathrm{R}$ & B15C52 & LAT/LONG & & 46 & & 38.14 .440 & 119 & \\
\hline $262 \leq$ & S & & & & & & & & \\
\hline $263 \mathrm{~F}$ & $\mathrm{R}$ & & & & & & & & \\
\hline $264 \mathrm{~F}$ & $\mathrm{R}$ & & & & & & & & \\
\hline \begin{tabular}{|l|l|}
265 \\
\end{tabular} & $\mathrm{R}$ & & & & & & & & \\
\hline \begin{tabular}{|l|l}
266 & $F$ \\
\end{tabular} & $\mathrm{R}$ & & & & & & & & \\
\hline \begin{tabular}{|l|l|}
267 & $F$ \\
\end{tabular} & $\mathrm{R}$ & & & & & & & & \\
\hline
\end{tabular}




\begin{tabular}{|c|c|c|c|c|c|c|c|c|c|}
\hline & $\mathrm{Al}$ & AJ & AK & $\overline{A L}$ & AM & AN & $\mathrm{AO}$ & $\mathrm{AP}$ & $\mathrm{AQ}$ \\
\hline 1 & COLLECTION_PURPOSE & X_Y_COORD_FINAL.SAMP_NUM & COORDINATE SYSTEM & DATUM & LATITUDE ${ }^{\circ} \mathrm{N}$ & LATITUDE MIN_MIN & LATITUDE MIN_SEC & LONGITUDE ${ }^{\circ} \mathrm{W}$ & LONGITUDE MIN_MIN \\
\hline \multicolumn{10}{|c|}{\begin{tabular}{|l|l|l|}
268 & $R$ & \\
\end{tabular}} \\
\hline \multicolumn{10}{|c|}{\begin{tabular}{|l|l|}
269 & $R$ \\
\end{tabular}} \\
\hline \multicolumn{10}{|c|}{$270 \mathrm{R}$} \\
\hline 271 & $\mathrm{R}$ & B10908 & STATE PLANE & & & & & & \\
\hline 272 & $\mathrm{R}$ & B10917 & STATE PLANE & & & & & & \\
\hline 273 & $\mathrm{R}$ & B10919 & STATE PLANE & & & & & & \\
\hline 274 & $\mathrm{R}$ & B10922 & STATE PLANE & & & & & & \\
\hline \multicolumn{10}{|c|}{\begin{tabular}{|l|l|}
275 & $R$ \\
\end{tabular}} \\
\hline \multicolumn{10}{|c|}{\begin{tabular}{|l|l|}
276 & $R$ \\
\end{tabular}} \\
\hline 277 & $\mathrm{R}$ & B12CK8 & LAT/LONG & & 46 & & 35.24 .685 & 119 & \\
\hline 278 & $\mathrm{R}$ & B12CL5 & LAT/LONG & & 46 & & 40.40 .882 & 119 & \\
\hline \multicolumn{10}{|c|}{\begin{tabular}{|l|l|}
279 & $R$ \\
\end{tabular}} \\
\hline 280 & $\mathrm{R}$ & B12CM2 & LAT/LONG & & 46 & & 38.49 .822 & 119 & \\
\hline 281 & $\mathrm{R}$ & B12CM4 & LAT/LONG & & 46 & & 39.13.366 & 119 & \\
\hline 282 & $S$ & B12CL1 & LAT/LONG & & 45 & & 55.52 .061 & 119 & \\
\hline 283 & S & $\mathrm{B} 12 \mathrm{CL} 2$ & LAT/LONG & & 45 & & 56.62 .863 & 119 & \\
\hline 284 & $\mathrm{~S}$ & $\mathrm{~B} 12 \mathrm{CL} 3$ & LAT/LONG & & 45 & & 56.33 .1352 & 119 & \\
\hline 285 & $S$ & B12CL4 & LAT/LONG & & 45 & & 56.45 .2625 & 119 & \\
\hline 286 & S & B12CH3 & LAT/LONG & & 46 & & 14.56 .3853 & 118 & \\
\hline 287 & $S$ & B12CJ0 & LAT/LONG & & 46 & & 15.27 .8170 & 118 & \\
\hline 288 & $S$ & B12CJ1 & LAT/LONG & & 46 & & 15.12 .1645 & 118 & \\
\hline 289 & $\mathrm{R}$ & B12CK9 & LAT/LONG & & 46 & & 18.49 .3916 & 119 & \\
\hline 290 & $\mathrm{R}$ & B12CNO & LAT/LONG & & 45 & & 55.55 .7549 & 119 & \\
\hline 291 & $\mathrm{R}$ & B12CN2 & LAT/LONG & & 45 & & 56.29.1919 & 119 & \\
\hline \multicolumn{10}{|c|}{\begin{tabular}{l|l}
292 & $R$ \\
\end{tabular}} \\
\hline \multicolumn{10}{|c|}{$293 \mathrm{~S}$} \\
\hline \multicolumn{10}{|c|}{$294 \mathrm{~S}$} \\
\hline \multicolumn{10}{|c|}{$295 \mathrm{~S}$} \\
\hline \multicolumn{10}{|c|}{\begin{tabular}{l|l} 
\\
296
\end{tabular}} \\
\hline \multicolumn{10}{|c|}{\begin{tabular}{|l|l|l|}
297 & $S$
\end{tabular}} \\
\hline \multicolumn{10}{|c|}{\begin{tabular}{l|l} 
& \\
298 & $S$
\end{tabular}} \\
\hline \multicolumn{10}{|c|}{$299 \mathrm{~S}$} \\
\hline \multicolumn{10}{|c|}{$300 \mathrm{~s}$} \\
\hline \multicolumn{10}{|c|}{\begin{tabular}{l|l}
301 & $R$
\end{tabular}} \\
\hline \multicolumn{10}{|c|}{\begin{tabular}{l|l}
302 & $R$
\end{tabular}} \\
\hline \multicolumn{10}{|c|}{\begin{tabular}{|l|l|}
303 & $R$ \\
\end{tabular}} \\
\hline \multicolumn{10}{|c|}{\begin{tabular}{|l|l|}
304 & $R$ \\
\end{tabular}} \\
\hline $305 \mathrm{~F}$ & $\mathrm{R}$ & & & & & & & & \\
\hline
\end{tabular}




\begin{tabular}{|c|c|c|c|c|c|c|c|c|c|}
\hline & $\mathrm{Al}$ & $\mathrm{AJ}$ & AK & $\mathrm{AL}$ & AM & AN & $\mathrm{AO}$ & $\mathrm{AP}$ & $\mathrm{AQ}$ \\
\hline 1 & COLLECTION_PURPOSE & X_Y_COORD_FINAL.SAMP_NUM & COORDINATE SYSTEM & DATUM & LATITUDE ${ }^{\circ} \mathrm{N}$ & LATITUDE MIN_MIN & LATITUDE MIN_SEC & LONGITUDE ${ }^{\circ} \mathrm{W}$ & LONGITUDE MIN_MIN \\
\hline \multicolumn{10}{|c|}{ 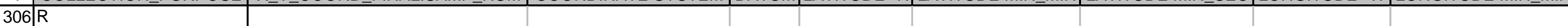 } \\
\hline 307 & $\mathrm{R}$ & B14YM5 & LAT/LONG & & 46 & & 18.49 .93 & 119 & \\
\hline 308 & $\mathrm{R}$ & B14YP1 & LAT/LONG & & 45 & & 56.00 .751 & 119 & \\
\hline 309 & $\mathrm{R}$ & B14YP3 & LAT/LONG & & 45 & & 56.28 .053 & 119 & \\
\hline 310 & $\mathrm{R}$ & B14YM4 & LAT/LONG & & 46 & & 35.24 .020 & 119 & \\
\hline 311 & $\mathrm{R}$ & B14YM6 & LAT/LONG & & 46 & & 40.44 .579 & 119 & \\
\hline 312 & $\mathrm{R}$ & B14YM7 & LAT/LONG & & 46 & & 38.10 .776 & 119 & \\
\hline 313 & $\mathrm{R}$ & B14YN3 & LAT/LONG & & 46 & & 39.49 .096 & 119 & \\
\hline 314 & $\mathrm{R}$ & B14YN5 & LAT/LONG & & 46 & & 39.11 .37211 & 119 & \\
\hline \multicolumn{10}{|c|}{$\begin{array}{ll}315 & R \\
\end{array}$} \\
\hline \multicolumn{10}{|c|}{\begin{tabular}{l|l}
316 & $R$
\end{tabular}} \\
\hline \multicolumn{10}{|c|}{\begin{tabular}{l|l}
317 & $R$
\end{tabular}} \\
\hline \begin{tabular}{|l|}
318 \\
318
\end{tabular} & $\mathrm{R}$ & B15C44 & LAT/LONG & & 46 & & 33.50 .499 & 119 & \\
\hline \multicolumn{10}{|c|}{\begin{tabular}{l|l} 
\\
319 & $R$
\end{tabular}} \\
\hline \multicolumn{10}{|c|}{$\begin{array}{lll} & \\
320 & R\end{array}$} \\
\hline \begin{tabular}{|l|}
321 \\
321 \\
\end{tabular} & $\mathrm{R}$ & B15C52 & LAT/LONG & & 46 & & 38.14 .440 & 119 & \\
\hline
\end{tabular}




\begin{tabular}{|c|c|c|c|c|c|}
\hline & $\overline{A R}$ & $\overline{A S}$ & $\overline{\mathrm{AT}}$ & $\overline{A U}$ & $\overline{A V}$ \\
\hline 1 & LONGITUDE MIN_SEC & X_COORD (EW) & Y_COORD (NW) & 3 D HAE/ALTITUDE & INSTRUMENT \\
\hline 2 & & & & & \\
\hline 3 & & & & & \\
\hline 4 & & & & & \\
\hline 5 & & & & & \\
\hline 6 & & & & & \\
\hline 7 & & & & & \\
\hline 8 & & & & & \\
\hline 9 & & & & & \\
\hline 10 & & & & & \\
\hline 11 & & 594392 & 116700 & 82 & \\
\hline 12 & & 588808 & 137808 & 94 & \\
\hline 13 & & 589438 & 137451 & & \\
\hline 14 & & 594489 & 116208 & 82.5 & \\
\hline 15 & & & & & \\
\hline 16 & & & & & \\
\hline 17 & 23.3 .084 & & & & \\
\hline 18 & 27.42 .478 & & & & \\
\hline 19 & & & & & \\
\hline 20 & 55.19 .604 & & & & \\
\hline 21 & 54.16 .200 & & & & \\
\hline 22 & 16.18 .731 & & & & \\
\hline 23 & 16.22 .43894 & & & & \\
\hline 24 & 16.24 .38 & & & & \\
\hline 25 & 16.34 .21523 & & & & \\
\hline 26 & 51.41 .558 & & & & \\
\hline 27 & 51.53 .707 & & & & \\
\hline 28 & 51.47 .9098 & & & & \\
\hline 29 & 15.37 .05820 & & & & \\
\hline 30 & 17.22 .57042 & & & & \\
\hline 31 & 17.32 .8546 & & & & \\
\hline 32 & & & & & \\
\hline 33 & & & & & \\
\hline 34 & & & & & \\
\hline 35 & & & & & \\
\hline 36 & & & & & \\
\hline 37 & & & & & \\
\hline 38 & & & & & \\
\hline 39 & 15.38 .075 & & & & \\
\hline
\end{tabular}




\begin{tabular}{|c|c|c|c|c|c|}
\hline & AR & AS & AT & $A U$ & AV \\
\hline 1 & LONGITUDE MIN_SEC & X_COORD (EW) & Y_COORD (NW) & 3 D HAE/ALTITUDE & INSTRUMENT \\
\hline 40 & 17.14.874 & & & & \\
\hline 41 & 17.04 .227 & & & & \\
\hline 42 & 23.03 .780 & & & & \\
\hline 43 & 27.42 .909 & & & & \\
\hline 44 & 25.07 .836 & & & & \\
\hline 45 & 55.14 .481 & & & & \\
\hline 46 & 54.12 .8496 & & & & \\
\hline 47 & & & & & \\
\hline 48 & & & & & \\
\hline 49 & & & & & \\
\hline 50 & 20.00 .067 & & & & \\
\hline 51 & & & & & \\
\hline 52 & & & & & \\
\hline 53 & 25.12 .798 & & & & \\
\hline 54 & & & & & \\
\hline 55 & & & & & \\
\hline 56 & & & & & \\
\hline 57 & & & & & \\
\hline 58 & & & & & \\
\hline 59 & & & & & \\
\hline 60 & & & & & \\
\hline 61 & & & & & \\
\hline 62 & 23.3.084 & & & & \\
\hline 63 & 27.42 .478 & & & & \\
\hline 64 & & & & & \\
\hline 65 & 55.19 .604 & & & & \\
\hline 66 & 54.16 .200 & & & & \\
\hline 67 & 16.18 .731 & & & & \\
\hline 68 & 16.22 .43894 & & & & \\
\hline 69 & 16.24 .38 & & & & \\
\hline 70 & 16.34 .21523 & & & & \\
\hline 71 & 51.41 .558 & & & & \\
\hline 72 & 51.53 .707 & & & & \\
\hline 73 & 51.47 .9098 & & & & \\
\hline 74 & 15.37 .05820 & & & & \\
\hline 75 & 17.22 .57042 & & & & \\
\hline 76 & 17.32 .8546 & & & & \\
\hline 77 & 15.38 .075 & & & & \\
\hline
\end{tabular}




\begin{tabular}{|c|c|c|c|c|c|}
\hline & AR & AS & AT & $A U$ & AV \\
\hline 1 & LONGITUDE MIN_SEC & X_COORD (EW) & Y_COORD (NW) & 3 D HAE/ALTITUDE & INSTRUMENT \\
\hline 78 & 17.14.874 & & & & \\
\hline 79 & 17.04 .227 & & & & \\
\hline 80 & 23.03 .780 & & & & \\
\hline 81 & 27.42 .909 & & & & \\
\hline 82 & 25.07 .836 & & & & \\
\hline 83 & 55.14 .481 & & & & \\
\hline 84 & 54.12 .8496 & & & & \\
\hline 85 & & & & & \\
\hline 86 & & & & & \\
\hline 87 & & & & & \\
\hline 88 & & & & & \\
\hline 89 & & & & & \\
\hline 90 & & & & & \\
\hline 91 & & & & & \\
\hline 92 & & & & & \\
\hline 93 & & & & & \\
\hline 94 & & 594392 & 116700 & 82 & \\
\hline 95 & & 588808 & 137808 & 94 & \\
\hline 96 & & 589438 & 137451 & & \\
\hline 97 & & 594489 & 116208 & 82.5 & \\
\hline 98 & & & & & \\
\hline 99 & & & & & \\
\hline 100 & 23.3.084 & & & & \\
\hline 101 & 27.42 .478 & & & & \\
\hline 102 & & & & & \\
\hline 103 & 55.19 .604 & & & & \\
\hline 104 & 54.16 .200 & & & & \\
\hline 105 & 16.18 .731 & & & & \\
\hline 106 & 16.22 .43894 & & & & \\
\hline 107 & 16.24 .38 & & & & \\
\hline 108 & 16.34 .21523 & & & & \\
\hline 109 & 51.41 .558 & & & & \\
\hline 110 & 51.53 .707 & & & & \\
\hline 111 & 51.47 .9098 & & & & \\
\hline 112 & 15.37 .05820 & & & & \\
\hline 113 & 17.22 .57042 & & & & \\
\hline 114 & 17.32 .8546 & & & & \\
\hline 115 & & & & & \\
\hline
\end{tabular}




\begin{tabular}{|c|c|c|c|c|c|}
\hline & AR & AS & AT & $A U$ & AV \\
\hline 1 & LONGITUDE MIN_SEC & X_COORD (EW) & Y_COORD (NW) & 3 D HAE/ALTITUDE & INSTRUMENT \\
\hline 116 & & & & & \\
\hline 117 & & & & & \\
\hline 118 & & & & & \\
\hline 119 & & & & & \\
\hline 120 & & & & & \\
\hline 121 & & & & & \\
\hline 122 & 15.38 .075 & & & & \\
\hline 123 & 17.14 .874 & & & & \\
\hline 124 & 17.04 .227 & & & & \\
\hline 125 & 23.03.780 & & & & \\
\hline 126 & 27.42 .909 & & & & \\
\hline 127 & 25.07 .836 & & & & \\
\hline 128 & 55.14 .481 & & & & \\
\hline 129 & 54.12 .8496 & & & & \\
\hline 130 & & & & & \\
\hline 131 & & & & & \\
\hline 132 & & & & & \\
\hline 133 & 20.00 .067 & & & & \\
\hline 134 & & & & & \\
\hline 135 & & & & & \\
\hline 136 & 25.12 .798 & & & & \\
\hline 137 & & & & & \\
\hline 138 & & & & & \\
\hline 139 & & & & & \\
\hline 140 & & & & & \\
\hline 141 & & & & & \\
\hline 142 & & & & & \\
\hline 143 & & & & & \\
\hline 144 & & & & & \\
\hline 145 & & & & & \\
\hline 146 & & & & & \\
\hline 147 & & & & & \\
\hline 148 & & & & & \\
\hline 149 & & & & & \\
\hline 150 & & & & & \\
\hline 151 & & 594392 & 116700 & 82 & \\
\hline 152 & & 588808 & 137808 & 94 & \\
\hline 153 & & 589438 & 137451 & & \\
\hline
\end{tabular}




\begin{tabular}{|c|c|c|c|c|c|}
\hline & AR & AS & AT & $\mathrm{AU}$ & AV \\
\hline 1 & LONGITUDE MIN_SEC & X_COORD (EW) & Y_COORD (NW) & 3 D HAE/ALTITUDE & INSTRUMENT \\
\hline 154 & & 594489 & 116208 & 82.5 & \\
\hline 155 & & & & & \\
\hline 156 & & & & & \\
\hline 157 & 23.3.084 & & & & \\
\hline 158 & 27.42 .478 & & & & \\
\hline 159 & & & & & \\
\hline 160 & 55.19 .604 & & & & \\
\hline 161 & 54.16 .200 & & & & \\
\hline 162 & 16.18 .731 & & & & \\
\hline 163 & 16.22 .43894 & & & & \\
\hline 164 & 16.24 .38 & & & & \\
\hline 165 & 16.34 .21523 & & & & \\
\hline 166 & 51.41 .558 & & & & \\
\hline 167. & 51.53 .707 & & & & \\
\hline 168 & 51.47 .9098 & & & & \\
\hline 169 & 15.37 .05820 & & & & \\
\hline 170 & 17.22 .57042 & & & & \\
\hline 171 & 17.32 .8546 & & & & \\
\hline 172 & & & & & \\
\hline 173 & & & & & \\
\hline 174 & & & & & \\
\hline 175 & & & & & \\
\hline 176 & & & & & \\
\hline 177 & & & & & \\
\hline 178 & & & & & \\
\hline 179 & & & & & \\
\hline 180 & & & & & \\
\hline 181 & & & & & \\
\hline 182 & & & & & \\
\hline 183 & & & & & \\
\hline 184 & & & & & \\
\hline 185 & & & & & \\
\hline 186 & & & & & \\
\hline 187 & 15.38 .075 & & & & \\
\hline 188 & 17.14 .874 & & & & \\
\hline 189 & 17.04 .227 & & & & \\
\hline 190 & 23.03 .780 & & & & \\
\hline 191 & 27.42 .909 & & & & \\
\hline
\end{tabular}




\begin{tabular}{|c|c|c|c|c|c|}
\hline & AR & AS & AT & $A U$ & AV \\
\hline 1 & LONGITUDE MIN_SEC & X_COORD (EW) & $\mathrm{Y}_{\text {_COORD }(\mathrm{NW})}$ & 3 D HAE/ALTITUDE & INSTRUMENT \\
\hline 192 & 25.07.836 & & & & \\
\hline 193 & 55.14 .481 & & & & \\
\hline 194 & 54.12 .8496 & & & & \\
\hline 195 & & & & & \\
\hline 196 & & & & & \\
\hline 197 & & & & & \\
\hline 198 & 20.00 .067 & & & & \\
\hline 199 & & & & & \\
\hline 200 & & & & & \\
\hline 201 & 25.12 .798 & & & & \\
\hline 202 & & & & & \\
\hline 203 & & & & & \\
\hline 204 & & & & & \\
\hline 205 & & & & & \\
\hline 206 & & & & & \\
\hline 207 & & & & & \\
\hline 208 & & & & & \\
\hline 209 & & & & & \\
\hline 210 & & & & & \\
\hline 211 & & 594392 & 116700 & 82 & \\
\hline 212 & & 588808 & 137808 & 94 & \\
\hline 213 & & 589438 & 137451 & & \\
\hline 214 & & 594489 & 116208 & 82.5 & \\
\hline 215 & & & & & \\
\hline 216 & & & & & \\
\hline 217 & 23.3.084 & & & & \\
\hline 218 & 27.42 .478 & & & & \\
\hline 219 & & & & & \\
\hline 220 & 55.19 .604 & & & & \\
\hline 221 & 54.16 .200 & & & & \\
\hline 222 & 16.18 .731 & & & & \\
\hline 223 & 16.22 .43894 & & & & \\
\hline 224 & 16.24 .38 & & & & \\
\hline 225 & 16.34 .21523 & & & & \\
\hline 226 & 51.41 .558 & & & & \\
\hline 227 & 51.53 .707 & & & & \\
\hline 228 & 51.47 .9098 & & & & \\
\hline 229 & 15.37 .05820 & & & & \\
\hline
\end{tabular}




\begin{tabular}{|c|c|c|c|c|c|}
\hline & AR & AS & AT & $A U$ & AV \\
\hline 1 & LONGITUDE MIN_SEC & X_COORD (EW) & Y_COORD (NW) & 3 D HAE/ALTITUDE & INSTRUMENT \\
\hline 230 & 17.22 .57042 & & & & \\
\hline 231 & 17.32 .8546 & & & & \\
\hline 232 & & & & & \\
\hline 233 & & & & & \\
\hline 234 & & & & & \\
\hline 235 & & & & & \\
\hline 236 & & & & & \\
\hline 237 & & & & & \\
\hline 238 & & & & & \\
\hline 239 & & & & & \\
\hline 240 & & & & & \\
\hline 241 & & & & & \\
\hline 242 & & & & & \\
\hline 243 & & & & & \\
\hline 244 & & & & & \\
\hline 245 & & & & & \\
\hline 246 & & & & & \\
\hline 247 & 15.38 .075 & & & & \\
\hline 248 & 17.14 .874 & & & & \\
\hline 249 & 17.04.227 & & & & \\
\hline 250 & 23.03 .780 & & & & \\
\hline 251 & 27.42 .909 & & & & \\
\hline 252 & 25.07 .836 & & & & \\
\hline 253 & 55.14 .481 & & & & \\
\hline 254 & 54.12 .8496 & & & & \\
\hline 255 & & & & & \\
\hline 256 & & & & & \\
\hline 257 & & & & & \\
\hline 258 & 20.00 .067 & & & & \\
\hline 259 & & & & & \\
\hline 260 & & & & & \\
\hline 261 & 25.12 .798 & & & & \\
\hline 262 & & & & & \\
\hline 263 & & & & & \\
\hline 264 & & & & & \\
\hline 265 & & & & & \\
\hline 266 & & & & & \\
\hline 267 & & & & & \\
\hline
\end{tabular}




\begin{tabular}{|c|c|c|c|c|c|}
\hline & AR & AS & AT & $\mathrm{AU}$ & $\mathrm{AV}$ \\
\hline 1 & LONGITUDE MIN_SEC & X_COORD $(E W)$ & Y_COORD (NW) & 3 D HAE/ALTITUDE & INSTRUMENT \\
\hline \multirow{2}{*}{\multicolumn{6}{|c|}{268}} \\
\hline & & & & & \\
\hline \multicolumn{6}{|l|}{270} \\
\hline 271 & & 594392 & 116700 & 82 & \\
\hline 272 & & 588808 & 137808 & 94 & \\
\hline 273 & & 589438 & 137451 & & \\
\hline 274 & & 594489 & 116208 & 82.5 & \\
\hline \multicolumn{6}{|l|}{275} \\
\hline \multicolumn{6}{|l|}{276} \\
\hline \multicolumn{6}{|c|}{\begin{tabular}{l|l|}
277 & 23.3 .084 \\
\end{tabular}} \\
\hline \multirow{2}{*}{\multicolumn{6}{|c|}{\begin{tabular}{l|l}
278 & 27.42 .478 \\
270 &
\end{tabular}}} \\
\hline & & & & & \\
\hline \multicolumn{6}{|c|}{\begin{tabular}{|l|l}
280 & 55.19 .604
\end{tabular}} \\
\hline \multicolumn{6}{|c|}{28154.16 .200} \\
\hline \multirow{2}{*}{\multicolumn{6}{|c|}{\begin{tabular}{r|r|}
282 & 16.18 .731 \\
202 & 1.22 .12891
\end{tabular}}} \\
\hline & & & & & \\
\hline \multicolumn{6}{|c|}{\begin{tabular}{|l|l|l|}
284 & 16.24 .38 \\
\end{tabular}} \\
\hline \multicolumn{6}{|c|}{\begin{tabular}{|l|l|}
285 & 16.34 .21523 \\
\end{tabular}} \\
\hline \multirow{2}{*}{\multicolumn{6}{|c|}{\begin{tabular}{l|l|}
286 & 51.41 .558 \\
887 & 51.53707
\end{tabular}}} \\
\hline 287 & & & & & \\
\hline \multicolumn{6}{|c|}{\begin{tabular}{|l|l|l|}
51.47 .9098 \\
288
\end{tabular}} \\
\hline \multicolumn{6}{|c|}{28915.37 .05820} \\
\hline \multirow{2}{*}{\multicolumn{6}{|c|}{\begin{tabular}{l|l}
290 & 17.22 .57042 \\
291 & 17328546
\end{tabular}}} \\
\hline \multirow{2}{*}{\multicolumn{6}{|c|}{\begin{tabular}{l|l}
291 & 17.32 .8546 \\
292 &
\end{tabular}}} \\
\hline & & & & & \\
\hline \multicolumn{6}{|l|}{293} \\
\hline \multirow{2}{*}{\multicolumn{6}{|c|}{$\frac{294}{295}$}} \\
\hline & & & & & \\
\hline \multicolumn{6}{|l|}{296} \\
\hline \multicolumn{6}{|l|}{297} \\
\hline \multicolumn{6}{|l|}{298} \\
\hline \multirow{2}{*}{\multicolumn{6}{|c|}{$\frac{299}{300}$}} \\
\hline & & & & & \\
\hline \multicolumn{6}{|l|}{301} \\
\hline \multicolumn{6}{|l|}{302} \\
\hline \multicolumn{6}{|l|}{$\frac{303}{301}$} \\
\hline 304 & & & & & \\
\hline 305 & & & & & \\
\hline
\end{tabular}


AR

AS

$\mathrm{AU}$

AV

\begin{tabular}{|c|c|c|c|c|c|}
\hline & AR & AS & AT & $\mathrm{AU}$ & AV \\
\hline \multirow{2}{*}{$\begin{array}{c}1 \\
306 \\
\end{array}$} & LONGITUDE MIN_SEC & X_COORD (EW) & Y_COORD (NW) & 3 D HAE/ALTITUDE & INSTRUMENT \\
\hline & & & & & \\
\hline \begin{tabular}{|l|l}
306 & \\
307 & 15,38075 \\
\end{tabular} & 15.38 .075 & & & & \\
\hline 308 & 17.14.874 & & & & \\
\hline \multirow{2}{*}{\begin{tabular}{|l|}
309 \\
310 \\
\end{tabular}} & 17.04 .227 & & & & \\
\hline & 23.03 .780 & & & & \\
\hline 311 & 27.42 .909 & & & & \\
\hline \multirow{2}{*}{$\frac{312}{313}$} & 25.07 .836 & & & & \\
\hline & 55.14 .481 & & & & \\
\hline 314 & 54.12 .8496 & & & & \\
\hline \multicolumn{6}{|l|}{315} \\
\hline \multicolumn{6}{|l|}{316} \\
\hline \multicolumn{6}{|l|}{317} \\
\hline \multicolumn{6}{|c|}{318 20.00.067 } \\
\hline \multicolumn{6}{|l|}{319} \\
\hline \multicolumn{6}{|l|}{320} \\
\hline 321 & 25.12 .798 & & & & \\
\hline
\end{tabular}




\section{9- 03 SW SAC}




\begin{tabular}{|c|c|c|c|c|c|c|c|c|c|c|}
\hline & $A$ & $B$ & $\mathrm{C}$ & $\mathrm{D}$ & $E$ & $\mathrm{~F}$ & $G$ & $\mathrm{H}$ & $\mathrm{I}$ & $\mathrm{J}$ \\
\hline 1 & SAMPLE_NUM_ASSIGNED_TO & SAMPLER & MEDIA & BIOTA_MEDIA & TAG_ID & SAMP_NUM & SAMP_DATE_TIME & SAMP_DATE_TIME_ON & CON_ID & CON_SHORT_NAME \\
\hline 2 & PSHENRY & RADFORD, E & SW & & & B106N8 & 14-Sep-00 & & $56-23-5$ & CARBTET \\
\hline 3 & PSHENRY & RADFORD, E & SW & & & B106N9 & 14-Sep-00 & & $56-23-5$ & CARBTET \\
\hline 4 & PSHENRY & RADFORD, E & SW & & & B106P0 & 14-Sep-00 & & $56-23-5$ & CARBTET \\
\hline 5 & PSHENRY & RADFORD, E & SW & & & B106P1 & 14-Sep-00 & & $56-23-5$ & CARBTET \\
\hline 6 & PSHENRY & RADFORD, E & SW & & & B106M8 & 19-Sep-00 & & $56-23-5$ & CARBTET \\
\hline 7 & PSHENRY & RADFORD, E & SW & & & B106M9 & 19-Sep-00 & & $56-23-5$ & CARBTET \\
\hline 8 & PSHENRY & RADFORD, E & SW & & & B106N0 & 19-Sep-00 & & $56-23-5$ & CARBTET \\
\hline 9 & PSHENRY & RADFORD, E & SW & & & B106N1 & 19-Sep-00 & & $56-23-5$ & CARBTET \\
\hline 10 & PSHENRY & RADFORD, E & SW & & & B106N2 & 19-Sep-00 & & $56-23-5$ & CARBTET \\
\hline 11 & PSHENRY & RADFORD, E & SW & & & B106N3 & 19-Sep-00 & & $56-23-5$ & CARBTET \\
\hline 12 & PSHENRY & RADFORD, E & SW & & & B106N4 & 19-Sep-00 & & $56-23-5$ & CARBTET \\
\hline 13 & PSHENRY & RADFORD, E & SW & & & B106N5 & 19-Sep-00 & & $56-23-5$ & CARBTET \\
\hline 14 & PSHENRY & RADFORD, E & SW & & & B106N6 & 19-Sep-00 & & $56-23-5$ & CARBTET \\
\hline 15 & PSHENRY & RADFORD, E & SW & & & B106N7 & 19-Sep-00 & & $56-23-5$ & CARBTET \\
\hline 16 & PSHENRY & RADFORD, E & SW & & & B108Y5 & 27-Sep-00 & & $56-23-5$ & CARBTET \\
\hline 17 & PSHENRY & RADFORD, E & SW & & & B108Y4 & 27-Sep-00 & & $56-23-5$ & CARBTET \\
\hline 18 & PSHENRY & RADFORD, E & SW & & & B10JP2 & 17-Oct-00 & & $56-23-5$ & CARBTET \\
\hline 19 & PSHENRY & RADFORD, E & SW & & & B10JP0 & 25-Oct-00 & & $56-23-5$ & CARBTET \\
\hline 20 & PSHENRY & RADFORD, E & SW & & & B10JP3 & $25-$ Oct-00 & & $56-23-5$ & CARBTET \\
\hline 21 & PSHENRY & RADFORD, E & SW & & & B10JP6 & 01-Nov-00 & & $56-23-5$ & CARBTET \\
\hline 22 & PSHENRY & RADFORD, E & SW & & & B10FC2 & 07-Nov-00 & & $56-23-5$ & CARBTET \\
\hline 23 & PSHENRY & HAWKES, M & SW & & & B12RL2 & 27-Aug-01 & & $56-23-5$ & CARBTET \\
\hline 24 & PSHENRY & HAWKES, M & SW & & & B12RL1 & 27-Aug-01 & & $56-23-5$ & CARBTET \\
\hline 25 & PSHENRY & HAWKES, M & SW & & & B12RL5 & 27-Aug-01 & & $56-23-5$ & CARBTET \\
\hline 26 & PSHENRY & HAWKES, M & SW & & & B12RL3 & 27-Aug-01 & & $56-23-5$ & CARBTET \\
\hline 27 & PSHENRY & HAWKES, M & SW & & & B12VM6 & 07-Sep-01 & & $56-23-5$ & CARBTET \\
\hline 28 & PSHENRY & HAWKES, M & SW & & & B12VM5 & 07-Sep-01 & & $56-23-5$ & CARBTET \\
\hline 29 & PSHENRY & HAWKES, M & SW & & & B12VM4 & 07-Sep-01 & & $56-23-5$ & CARBTET \\
\hline 30 & PSHENRY & HAWKES, M & SW & & & B12VM3 & 07-Sep-01 & & $56-23-5$ & CARBTET \\
\hline 31 & PSHENRY & HAWKES, M & SW & & & B12VL9 & 13-Sep-01 & & $56-23-5$ & CARBTET \\
\hline 32 & PSHENRY & HAWKES, M & SW & & & B12VM0 & 13-Sep-01 & & $56-23-5$ & CARBTET \\
\hline 33 & PSHENRY & HAWKES, M & SW & & & B12VM1 & 13-Sep-01 & & $56-23-5$ & CARBTET \\
\hline 34 & PSHENRY & HAWKES, M & SW & & & B12VM2 & 13-Sep-01 & & $56-23-5$ & CARBTET \\
\hline 35 & PSHENRY & HAWKES, M & SW & & & B12VL7 & 13-Sep-01 & & $56-23-5$ & CARBTET \\
\hline 36 & PSHENRY & HAWKES, M & SW & & & B12VL6 & 13-Sep-01 & & $56-23-5$ & CARBTET \\
\hline 37 & PSHENRY & HAWKES, M & SW & & & B12VL5 & 13-Sep-01 & & $56-23-5$ & CARBTET \\
\hline 38 & PSHENRY & HAWKES, M & SW & & & B12VL4 & 13-Sep-01 & & $56-23-5$ & CARBTET \\
\hline 39 & PSHENRY & HAWKES, M & SW & & & B12VL3 & 13-Sep-01 & & $56-23-5$ & CARBTET \\
\hline
\end{tabular}




\begin{tabular}{|c|c|c|c|c|c|c|c|c|c|c|}
\hline & $\mathrm{A}$ & $B$ & $\mathrm{C}$ & $\mathrm{D}$ & $E$ & $\mathrm{~F}$ & $G$ & $\mathrm{H}$ & $\mathrm{I}$ & $\mathrm{J}$ \\
\hline 1 & SAMPLE_NUM_ASSIGNED_TO & SAMPLER & MEDIA & BIOTA_MEDIA & TAG_ID & SAMP_NUM & SAMP_DATE_TIME & SAMP_DATE_TIME_ON & CON_ID & CON_SHORT_NAME \\
\hline 40 & PSHENRY & HAWKES, M & SW & & & B12VL8 & 15-Sep-01 & & $56-23-5$ & CARBTET \\
\hline 41 & PSHENRY & HAWKES, M & SW & & & B135P3 & 22-Oct-01 & & $56-23-5$ & CARBTET \\
\hline 42 & PSHENRY & HAWKES, M & SW & & & B135P5 & 25-Oct-01 & & $56-23-5$ & CARBTET \\
\hline 43 & PSHENRY & HAWKES, M & SW & & & B135P6 & 25-Oct-01 & & $56-23-5$ & CARBTET \\
\hline 44 & PSHENRY & RADFORD, E & SW & & & B15994 & 05-Sep-02 & & $56-23-5$ & CARBTET \\
\hline 45 & PSHENRY & RADFORD, E & SW & & & B15993 & 05-Sep-02 & & $56-23-5$ & CARBTET \\
\hline 46 & PSHENRY & RADFORD, E & SW & & & B15992 & 05-Sep-02 & & $56-23-5$ & CARBTET \\
\hline 47 & PSHENRY & RADFORD, E & SW & & & B15991 & 05-Sep-02 & & $56-23-5$ & CARBTET \\
\hline 48 & PSHENRY & RADFORD, E & SW & & & B15987 & 10-Sep-02 & & $56-23-5$ & CARBTET \\
\hline 49 & PSHENRY & RADFORD, E & SW & & & B15988 & 10-Sep-02 & & $56-23-5$ & CARBTET \\
\hline 50 & PSHENRY & RADFORD, E & SW & & & B15989 & 10-Sep-02 & & $56-23-5$ & CARBTET \\
\hline 51 & PSHENRY & RADFORD, E & SW & & & B15990 & 10-Sep-02 & & $56-23-5$ & CARBTET \\
\hline 52 & PSHENRY & RADFORD, E & SW & & & B15986 & 10-Sep-02 & & $56-23-5$ & CARBTET \\
\hline 53 & PSHENRY & RADFORD, E & SW & & & B15985 & 10-Sep-02 & & $56-23-5$ & CARBTET \\
\hline 54 & PSHENRY & RADFORD, E & SW & & & B15984 & 10-Sep-02 & & $56-23-5$ & CARBTET \\
\hline 55 & PSHENRY & RADFORD, E & SW & & & B15983 & 10-Sep-02 & & $56-23-5$ & CARBTET \\
\hline 56 & PSHENRY & RADFORD, E & SW & & & B15982 & 10-Sep-02 & & $56-23-5$ & CARBTET \\
\hline 57 & PSHENRY & RADFORD, E & SW & & & B15981 & 10-Sep-02 & & $56-23-5$ & CARBTET \\
\hline 58 & PSHENRY & RADFORD, E & SW & & & B15CC3 & 16-Sep-02 & & $56-23-5$ & CARBTET \\
\hline 59 & PSHENRY & RADFORD, E & SW & & & B15CD0 & 16-Sep-02 & & $56-23-5$ & CARBTET \\
\hline 60 & PSHENRY & RADFORD, E & SW & & & B15CD5 & 07-Oct-02 & & $56-23-5$ & CARBTET \\
\hline 61 & PSHENRY & RADFORD, E & SW & & & B15CD1 & 21-Oct-02 & & $56-23-5$ & CARBTET \\
\hline 62 & PSHENRY & RADFORD, E & SW & & & B10JP8 & 17-Oct-00 & & $7440-47-3$ & CR \\
\hline 63 & PSHENRY & RADFORD, E & SW & & & B10JP9 & 17-Oct-00 & & $7440-47-3$ & CR \\
\hline 64 & PSHENRY & RADFORD, E & SW & & & B10JR9 & 17-Oct-00 & & $7440-47-3$ & CR \\
\hline 65 & PSHENRY & RADFORD, E & SW & & & B10JT0 & 17-Oct-00 & & 7440-47-3 & CR \\
\hline 66 & PSHENRY & RADFORD, E & SW & & & B10JP7 & 17-Oct-00 & & 7440-47-3 & CR \\
\hline 67 & PSHENRY & RADFORD, E & SW & & & B10JR8 & 17-Oct-00 & & 7440-47-3 & CR \\
\hline 68 & PSHENRY & RADFORD, E & SW & & & B10FB1 & 25-Oct-00 & & $7440-47-3$ & CR \\
\hline 69 & PSHENRY & RADFORD, E & SW & & & B10JR3 & 01-Nov-00 & & $7440-47-3$ & CR \\
\hline 70 & PSHENRY & RADFORD, E & SW & & & B10JT4 & 01-Nov-00 & & $7440-47-3$ & CR \\
\hline 71 & PSHENRY & RADFORD, E & SW & & & B10FC3 & 07-Nov-00 & & 7440-47-3 & CR \\
\hline 72 & DEAYRES & DEROOS, GB & SW & & & B14DR7 & 09-May-02 & & 7440-47-3 & CR \\
\hline 73 & DEAYRES & DEROOS, GB & SW & & & B14DR8 & 09-May-02 & & 7440-47-3 & CR \\
\hline 74 & DEAYRES & DEROOS, GB & SW & & & B154X9 & 14-Aug-02 & & $7440-47-3$ & CR \\
\hline 75 & DEAYRES & DEROOS, GB & SW & & & B154Y0 & 14-Aug-02 & & 7440-47-3 & CR \\
\hline 76 & SESP SCHEDULING & & SW & & & B0X8R8 & 04-Jan-00 & & $10045-97-3$ & CS-137 \\
\hline 77 & SESP SCHEDULING & & SW & & & B0X8R9 & 04-Jan-00 & & $10045-97-3$ & CS-137 \\
\hline
\end{tabular}




\begin{tabular}{|c|c|c|c|c|c|c|c|c|c|c|}
\hline & $\mathrm{A}$ & $B$ & $\mathrm{C}$ & $\mathrm{D}$ & $E$ & $\mathrm{~F}$ & $G$ & $\mathrm{H}$ & $\mathrm{I}$ & $\mathrm{J}$ \\
\hline 1 & SAMPLE_NUM_ASSIGNED_TO & SAMPLER & MEDIA & BIOTA_MEDIA & TAG_ID & SAMP_NUM & SAMP_DATE_TIME & SAMP_DATE_TIME_ON & CON_ID & CON_SHORT_NAME \\
\hline 78 & SESP SCHEDULING & & SW & & & B0X7M6 & 26-Jan-00 & 29-Dec-99 & $10045-97-3$ & CS-137 \\
\hline 79 & SESP SCHEDULING & & SW & & & B0X7N3 & 26-Jan-00 & & $10045-97-3$ & CS-137 \\
\hline 80 & SESP SCHEDULING & & SW & & & B0X7L2 & 26-Jan-00 & & $10045-97-3$ & CS-137 \\
\hline 81 & SESP SCHEDULING & & SW & & & B0X7L9 & 26-Jan-00 & 29-Dec-99 & $10045-97-3$ & CS-137 \\
\hline 82 & SESP SCHEDULING & & SW & & & B0X7M7 & 08-Mar-00 & 26-Jan-00 & $10045-97-3$ & CS-137 \\
\hline 83 & SESP SCHEDULING & & SW & & & B0X7N4 & 08-Mar-00 & 26-Jan-00 & $10045-97-3$ & CS-137 \\
\hline 84 & SESP SCHEDULING & & SW & & & B0X7L3 & 08-Mar-00 & 26-Jan-00 & $10045-97-3$ & CS-137 \\
\hline 85 & SESP SCHEDULING & & SW & & & B0X7M0 & 08-Mar-00 & 26-Jan-00 & $10045-97-3$ & CS-137 \\
\hline 86 & SESP SCHEDULING & & SW & & & BOXWWO & 04-Apr-00 & & $10045-97-3$ & CS-137 \\
\hline 87 & SESP SCHEDULING & & SW & & & B0XWW1 & 04-Apr-00 & & $10045-97-3$ & CS-137 \\
\hline 88 & SESP SCHEDULING & & SW & & & B0XWW2 & 04-Apr-00 & & $10045-97-3$ & CS-137 \\
\hline 89 & SESP SCHEDULING & & SW & & & B0X7M8 & 05-Apr-00 & 08-Mar-00 & $10045-97-3$ & CS-137 \\
\hline 90 & SESP SCHEDULING & & SW & & & B0X7N5 & 05-Apr-00 & 08-Mar-00 & $10045-97-3$ & CS-137 \\
\hline 91 & SESP SCHEDULING & & SW & & & B0X7L4 & 05-Apr-00 & 08-Mar-00 & $10045-97-3$ & CS-137 \\
\hline 92 & SESP SCHEDULING & & SW & & & B0X7M1 & 05-Apr-00 & 08-Mar-00 & $10045-97-3$ & CS-137 \\
\hline 93 & SESP SCHEDULING & & SW & & & B0XVP7 & 03-May-00 & 05-Apr-00 & $10045-97-3$ & CS-137 \\
\hline 94 & SESP SCHEDULING & & SW & & & B0XVR3 & 03-May-00 & 05-Apr-00 & $10045-97-3$ & CS-137 \\
\hline 95 & SESP SCHEDULING & & SW & & & BOXVR9 & 03-May-00 & 05-Apr-00 & $10045-97-3$ & CS-137 \\
\hline 96 & SESP SCHEDULING & & SW & & & B0XVT5 & 03-May-00 & 05-Apr-00 & $10045-97-3$ & CS-137 \\
\hline 97 & SESP SCHEDULING & & SW & & & B0Y2Y6 & 11-May-00 & & $10045-97-3$ & CS-137 \\
\hline 98 & SESP SCHEDULING & & SW & & & B0XVP8 & 31-May-00 & 03-May-00 & $10045-97-3$ & CS-137 \\
\hline 99 & SESP SCHEDULING & & SW & & & B0XVR4 & 31-May-00 & 03-May-00 & $10045-97-3$ & CS-137 \\
\hline 100 & SESP SCHEDULING & & SW & & & BOXVTO & 31-May-00 & 03-May-00 & $10045-97-3$ & CS-137 \\
\hline 101 & SESP SCHEDULING & & SW & & & B0XVT6 & 31-May-00 & 03-May-00 & $10045-97-3$ & CS-137 \\
\hline 102 & SESP SCHEDULING & & SW & & & B0YB90 & 08-Jun-00 & & $10045-97-3$ & CS-137 \\
\hline 103 & SESP SCHEDULING & & SW & & & BOYRB8 & 12-Jun-00 & & $10045-97-3$ & CS-137 \\
\hline 104 & SESP SCHEDULING & & SW & & & BOYRCO & 12-Jun-00 & & $10045-97-3$ & CS-137 \\
\hline 105 & SESP SCHEDULING & & SW & & & B0XVP9 & 29-Jun-00 & 31-May-00 & $10045-97-3$ & CS-137 \\
\hline 106 & SESP SCHEDULING & & SW & & & B0XVR5 & 29-Jun-00 & 31-May-00 & $10045-97-3$ & CS-137 \\
\hline 107 & SESP SCHEDULING & & SW & & & B0XVT1 & 29-Jun-00 & 31-May-00 & $10045-97-3$ & CS-137 \\
\hline 108 & SESP SCHEDULING & & SW & & & BOXVT7 & 29-Jun-00 & 31-May-00 & $10045-97-3$ & CS-137 \\
\hline 109 & SESP SCHEDULING & & SW & & & B0YM20 & 05-Jul-00 & & $10045-97-3$ & CS-137 \\
\hline 110 & SESP SCHEDULING & & SW & & & B0YM21 & 05-Jul-00 & & $10045-97-3$ & CS-137 \\
\hline 111 & SESP SCHEDULING & & SW & & & B0YM22 & 07-Jul-00 & & $10045-97-3$ & CS-137 \\
\hline 112 & SESP SCHEDULING & & SW & & & BOYKW9 & 10-Aug-00 & 29-Jun-00 & $10045-97-3$ & CS-137 \\
\hline 113 & SESP SCHEDULING & & SW & & & BOYKX6 & 10-Aug-00 & 29-Jun-00 & $10045-97-3$ & CS-137 \\
\hline 114 & SESP SCHEDULING & & SW & & & BOYKY3 & 10-Aug-00 & 29-Jun-00 & $10045-97-3$ & CS-137 \\
\hline 115 & SESP SCHEDULING & & SW & & & BOYLO0 & 10-Aug-00 & 29-Jun-00 & $10045-97-3$ & CS-137 \\
\hline
\end{tabular}




\begin{tabular}{|c|c|c|c|c|c|c|c|c|c|c|c|}
\hline & $\mathrm{A}$ & $B$ & $\mathrm{C}$ & $\mathrm{D}$ & $\mathrm{E}$ & $\mathrm{F}$ & $G$ & $\mathrm{H}$ & & I & $\mathrm{J}$ \\
\hline 1 & SAMPLE_NUM_ASSIGNED_TO & SAMPLER & MEDIA & BIOTA_MEDIA & TAG_ID & SAMP_NUM & SAMP_DATE_TIME & SAMP_DATE & TIME_ON & CON_ID & CON_SHORT_NAME \\
\hline 116 & SESP SCHEDULING & & SW & & & BOYKXO & 07-Sep-00 & \multicolumn{3}{|c|}{ 10-Aug-00 10045-97-3 } & CS-137 \\
\hline 117 & SESP SCHEDULING & & SW & & & B0YKX7 & 07-Sep-00 & \multicolumn{3}{|c|}{ 10-Aug-00 10045-97-3 } & CS-137 \\
\hline 118 & SESP SCHEDULING & & SW & & & BOYKY4 & 07-Sep-00 & \multicolumn{3}{|c|}{ 10-Aug-00 10045-97-3 } & CS-137 \\
\hline 119 & SESP SCHEDULING & & SW & & & BOYL01 & 07-Sep-00 & \multicolumn{3}{|c|}{ 10-Aug-00 10045-97-3 } & CS-137 \\
\hline 120 & SESP SCHEDULING & & SW & & & B10912 & 27-Sep-00 & \multicolumn{3}{|c|}{$10045-97-3$} & CS-137 \\
\hline 121 & SESP SCHEDULING & & SW & & & B10913 & 27-Sep-00 & \multicolumn{3}{|c|}{$10045-97-3$} & CS-137 \\
\hline 122 & SESP SCHEDULING & & SW & & & B10945 & 27-Sep-00 & \multicolumn{3}{|c|}{$10045-97-3$} & CS-137 \\
\hline 123 & SESP SCHEDULING & & SW & & & B10946 & 27-Sep-00 & \multicolumn{3}{|c|}{\begin{tabular}{|l|l}
$10045-97-3$ \\
\end{tabular}} & CS-137 \\
\hline 124 & SESP SCHEDULING & & SW & & & B10949 & 27-Sep-00 & \multicolumn{3}{|c|}{\begin{tabular}{|l|l}
$10045-97-3$ \\
\end{tabular}} & CS-137 \\
\hline 125 & SESP SCHEDULING & & SW & & & B109J0 & 27-Sep-00 & \multicolumn{3}{|c|}{\begin{tabular}{|l|l}
$10045-97-3$ \\
\end{tabular}} & CS-137 \\
\hline 126 & SESP SCHEDULING & & SW & & & B0YKX1 & 05-Oct-00 & \multicolumn{3}{|c|}{ 07-Sep-00 10045-97-3 } & CS-137 \\
\hline 127 & SESP SCHEDULING & & SW & & & BOYKX8 & 05-Oct-00 & \multicolumn{3}{|c|}{ 07-Sep-00 10045-97-3 } & CS-137 \\
\hline 128 & SESP SCHEDULING & & SW & & & B0YKY5 & 05-Oct-00 & \multicolumn{3}{|c|}{ 07-Sep-00 10045-97-3 } & CS-137 \\
\hline 129 & SESP SCHEDULING & & SW & & & BOYL02 & 05-Oct-00 & \multicolumn{3}{|c|}{ 07-Sep-00 10045-97-3 } & CS-137 \\
\hline 130 & SESP SCHEDULING & & SW & & & B10F33 & 09-Oct-00 & \multicolumn{3}{|c|}{ 10045-97-3 } & CS-137 \\
\hline 131 & SESP SCHEDULING & & SW & & & B10F34 & 09-Oct-00 & \multicolumn{3}{|c|}{\begin{tabular}{|l|l|}
$10045-97-3$ \\
\end{tabular}} & CS-137 \\
\hline 132 & SESP SCHEDULING & & SW & & & B10910 & 17-Oct-00 & \multicolumn{3}{|c|}{\begin{tabular}{|l|l}
$10045-97-3$ \\
\end{tabular}} & CS-137 \\
\hline 133 & SESP SCHEDULING & & SW & & & B10934 & 17-Oct-00 & \multicolumn{3}{|c|}{\begin{tabular}{|l|l|}
$10045-97-3$ \\
\end{tabular}} & CS-137 \\
\hline 134 & SESP SCHEDULING & & SW & & & B10939 & $17-$ Oct-00 & \multicolumn{3}{|c|}{\begin{tabular}{|l|l|}
$10045-97-3$ \\
\end{tabular}} & CS-137 \\
\hline 135 & SESP SCHEDULING & & SW & & & B10940 & 17-Oct-00 & \multicolumn{3}{|c|}{\begin{tabular}{|l|l}
$10045-97-3$ \\
\end{tabular}} & CS-137 \\
\hline 136 & SESP SCHEDULING & & SW & & & B10932 & 25-Oct-00 & \multicolumn{3}{|c|}{\begin{tabular}{|l|l}
$10045-97-3$ \\
\end{tabular}} & CS-137 \\
\hline 137 & SESP SCHEDULING & & SW & & & B10936 & $25-$ Oct-00 & \multicolumn{3}{|c|}{\begin{tabular}{|l|l}
$10045-97-3$
\end{tabular}} & CS-137 \\
\hline 138 & SESP SCHEDULING & & SW & & & B10941 & 01-Nov-00 & \multicolumn{3}{|c|}{\begin{tabular}{|l|l}
$10045-97-3$ \\
\end{tabular}} & CS-137 \\
\hline 139 & SESP SCHEDULING & & SW & & & B10977 & 01-Nov-00 & & (n) & $10045-97-3$ & CS-137 \\
\hline 140 & SESP SCHEDULING & & SW & & & B10C26 & 02-Nov-00 & & 05-Oct-00 & $10045-97-3$ & CS-137 \\
\hline 141 & SESP SCHEDULING & & SW & & & B10C32 & 02-Nov-00 & & 05-Oct-00 & $10045-97-3$ & CS-137 \\
\hline 142 & SESP SCHEDULING & & SW & & & B10C44 & 02-Nov-00 & & 05-Oct-00 & $10045-97-3$ & CS-137 \\
\hline 143 & SESP SCHEDULING & & SW & & & B10C38 & 02-Nov-00 & & 05-Oct-00 & $10045-97-3$ & CS-137 \\
\hline 144 & SESP SCHEDULING & & SW & & & B10942 & 07-Nov-00 & & & $10045-97-3$ & CS-137 \\
\hline 145 & SESP SCHEDULING & & SW & & & B10C27 & 29-Nov-00 & & 02-Nov-00 & $10045-97-3$ & CS-137 \\
\hline 146 & SESP SCHEDULING & & SW & & & B10C33 & 29-Nov-00 & & 02-Nov-00 & $10045-97-3$ & CS-137 \\
\hline 147 & SESP SCHEDULING & & SW & & & B10C39 & 29-Nov-00 & & 02-Nov-00 & $10045-97-3$ & CS-137 \\
\hline 148 & SESP SCHEDULING & & SW & & & B10C45 & 29-Nov-00 & & 02-Nov-00 & $10045-97-3$ & CS-137 \\
\hline 149 & SESP SCHEDULING & & SW & & & B10C28 & 28-Dec-00 & & 29-Nov-00 & $10045-97-3$ & CS-137 \\
\hline 150 & SESP SCHEDULING & & SW & & & B10C34 & 28-Dec-00 & & 29-Nov-00 & $10045-97-3$ & CS-137 \\
\hline 151 & SESP SCHEDULING & & SW & & & $\mathrm{B} 10 \mathrm{C} 40$ & 28-Dec-00 & & 29-Nov-00 & $10045-97-3$ & CS-137 \\
\hline 152 & SESP SCHEDULING & & SW & & & B10C46 & 28-Dec-00 & & 29-Nov-00 & $10045-97-3$ & CS-137 \\
\hline 153 & SESP SCHEDULING & & SW & & & B114T7 & 07-Feb-01 & & 28-Dec-00 & $10045-97-3$ & CS-137 \\
\hline
\end{tabular}




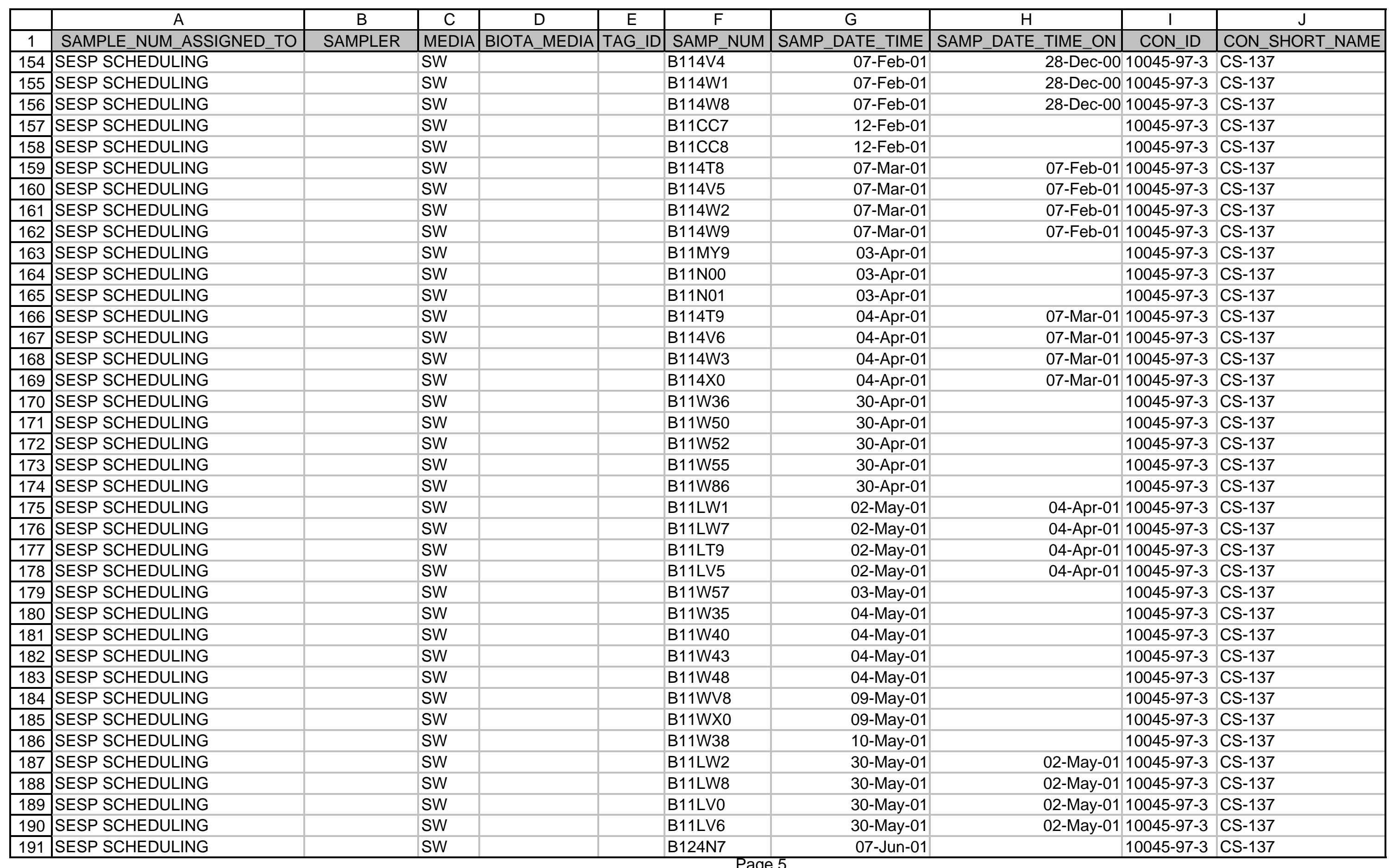




\begin{tabular}{|c|c|c|c|c|c|c|c|c|c|c|}
\hline & $A$ & $\mathrm{~B}$ & $\mathrm{C}$ & $\mathrm{D}$ & $E$ & $\mathrm{~F}$ & $G$ & $\mathrm{H}$ & $\mathrm{I}$ & $\mathrm{J}$ \\
\hline 1 & SAMPLE_NUM_ASSIGNED_TO & SAMPLER & MEDIA & BIOTA_MEDIA & TAG_ID & SAMP_NUM & SAMP_DATE_TIME & SAMP_DATE_TIME_ON & CON_ID & CON_SHORT_NAME \\
\hline 192 & SESP SCHEDULING & & SW & & & B124P7 & 07-Jun-01 & & $10045-97-3$ & CS-137 \\
\hline 193 & SESP SCHEDULING & & SW & & & B11LW3 & 28-Jun-01 & 30-May-01 & $10045-97-3$ & CS-137 \\
\hline 194 & SESP SCHEDULING & & SW & & & B11LW9 & 28-Jun-01 & 30-May-01 & $10045-97-3$ & CS-137 \\
\hline 195 & SESP SCHEDULING & & SW & & & B11LV1 & 28-Jun-01 & 30-May-01 & $10045-97-3$ & CS-137 \\
\hline 196 & SESP SCHEDULING & & SW & & & B11LV7 & 28-Jun-01 & 30-May-01 & $10045-97-3$ & CS-137 \\
\hline 197 & SESP SCHEDULING & & SW & & & B12992 & 09-Jul-01 & & $10045-97-3$ & CS-137 \\
\hline 198 & SESP SCHEDULING & & SW & & & B12993 & 09-Jul-01 & & $10045-97-3$ & CS-137 \\
\hline 199 & SESP SCHEDULING & & SW & & & B12994 & 09-Jul-01 & & $10045-97-3$ & CS-137 \\
\hline 200 & SESP SCHEDULING & & SW & & & B129B4 & 09-Jul-01 & & $10045-97-3$ & CS-137 \\
\hline 201 & SESP SCHEDULING & & SW & & & B12863 & 09-Aug-01 & 28-Jun-01 & $10045-97-3$ & CS-137 \\
\hline 202 & SESP SCHEDULING & & SW & & & B12870 & 09-Aug-01 & 28-Jun-01 & $10045-97-3$ & CS-137 \\
\hline 203 & SESP SCHEDULING & & SW & & & B12849 & 09-Aug-01 & 28-Jun-01 & $10045-97-3$ & CS-137 \\
\hline 204 & SESP SCHEDULING & & SW & & & B12856 & 09-Aug-01 & 28-Jun-01 & $10045-97-3$ & CS-137 \\
\hline 205 & SESP SCHEDULING & & SW & & & B12RM1 & 27-Aug-01 & & $10045-97-3$ & CS-137 \\
\hline 206 & SESP SCHEDULING & & SW & & & B12RM6 & 27-Aug-01 & & $10045-97-3$ & CS-137 \\
\hline 207 & SESP SCHEDULING & & SW & & & B12RP9 & 27-Aug-01 & & $10045-97-3$ & CS-137 \\
\hline 208 & SESP SCHEDULING & & SW & & & B12RR1 & 27-Aug-01 & & $10045-97-3$ & CS-137 \\
\hline 209 & SESP SCHEDULING & & SW & & & B12RR3 & 27-Aug-01 & & $10045-97-3$ & CS-137 \\
\hline 210 & SESP SCHEDULING & & SW & & & B12RR5 & 27-Aug-01 & & $10045-97-3$ & CS-137 \\
\hline 211 & SESP SCHEDULING & & SW & & & B12RR7 & 27-Aug-01 & & $10045-97-3$ & CS-137 \\
\hline 212 & SESP SCHEDULING & & SW & & & B12RR9 & 27-Aug-01 & & $10045-97-3$ & CS-137 \\
\hline 213 & SESP SCHEDULING & & SW & & & B12RT1 & 27-Aug-01 & & $10045-97-3$ & CS-137 \\
\hline 214 & SESP SCHEDULING & & SW & & & B12RT3 & 27-Aug-01 & & $10045-97-3$ & CS-137 \\
\hline 215 & SESP SCHEDULING & & SW & & & B12RT5 & 27-Aug-01 & & $10045-97-3$ & CS-137 \\
\hline 216 & SESP SCHEDULING & & SW & & & B12RT7 & 27-Aug-01 & & $10045-97-3$ & CS-137 \\
\hline 217 & SESP SCHEDULING & & SW & & & B12RT9 & 27-Aug-01 & & $10045-97-3$ & CS-137 \\
\hline 218 & SESP SCHEDULING & & SW & & & B12RV1 & 27-Aug-01 & & $10045-97-3$ & CS-137 \\
\hline 219 & SESP SCHEDULING & & SW & & & B12RV3 & 27-Aug-01 & & $10045-97-3$ & CS-137 \\
\hline 220 & SESP SCHEDULING & & SW & & & B12RV5 & 27-Aug-01 & & $10045-97-3$ & CS-137 \\
\hline 221 & SESP SCHEDULING & & SW & & & B12RV7 & 27-Aug-01 & & $10045-97-3$ & CS-137 \\
\hline 222 & SESP SCHEDULING & & SW & & & B12RV9 & 27-Aug-01 & & $10045-97-3$ & CS-137 \\
\hline 223 & SESP SCHEDULING & & SW & & & B12RW1 & 27-Aug-01 & & $10045-97-3$ & CS-137 \\
\hline 224 & SESP SCHEDULING & & SW & & & B12RW5 & 27-Aug-01 & & $10045-97-3$ & CS-137 \\
\hline 225 & SESP SCHEDULING & & SW & & & B12RW7 & 27-Aug-01 & & $10045-97-3$ & CS-137 \\
\hline 226 & SESP SCHEDULING & & SW & & & B12RW9 & 27-Aug-01 & & $10045-97-3$ & CS-137 \\
\hline 227 & SESP SCHEDULING & & SW & & & B12RX1 & 27-Aug-01 & & $10045-97-3$ & CS-137 \\
\hline 228 & SESP SCHEDULING & & SW & & & B12RX3 & 27-Aug-01 & & $10045-97-3$ & CS-137 \\
\hline 229 & SESP SCHEDULING & & SW & & & B12RX5 & 27-Aug-01 & & $10045-97-3$ & CS-137 \\
\hline
\end{tabular}




\begin{tabular}{|c|c|c|c|c|c|c|c|c|c|c|}
\hline & $\mathrm{A}$ & $\mathrm{B}$ & $\mathrm{C}$ & $\mathrm{D}$ & $E$ & $\mathrm{~F}$ & $G$ & $\mathrm{H}$ & $I$ & $\mathrm{~J}$ \\
\hline 1 & SAMPLE_NUM_ASSIGNED_TO & SAMPLER & MEDIA & BIOTA_MEDIA & TAG_ID & SAMP_NUM & SAMP_DATE_TIME & SAMP_DATE_TIME_ON & CON_ID & CON_SHORT_NAME \\
\hline 230 & SESP SCHEDULING & & SW & & & B12RX9 & 27-Aug-01 & & $10045-97-3$ & CS-137 \\
\hline 231 & SESP SCHEDULING & & SW & & & B12RY1 & 27-Aug-01 & & $10045-97-3$ & CS-137 \\
\hline 232 & SESP SCHEDULING & & SW & & & B12RY3 & 27-Aug-01 & & $10045-97-3$ & CS-137 \\
\hline 233 & SESP SCHEDULING & & SW & & & B12T11 & 27-Aug-01 & & $10045-97-3$ & CS-137 \\
\hline 234 & SESP SCHEDULING & & SW & & & B12T13 & 27-Aug-01 & & $10045-97-3$ & CS-137 \\
\hline 235 & SESP SCHEDULING & & SW & & & B12T15 & 27-Aug-01 & & $10045-97-3$ & CS-137 \\
\hline 236 & SESP SCHEDULING & & SW & & & B12T17 & 27-Aug-01 & & $10045-97-3$ & CS-137 \\
\hline 237 & SESP SCHEDULING & & SW & & & B12T19 & 27-Aug-01 & & $10045-97-3$ & CS-137 \\
\hline 238 & SESP SCHEDULING & & SW & & & B12T21 & 27-Aug-01 & & $10045-97-3$ & CS-137 \\
\hline 239 & SESP SCHEDULING & & SW & & & B12T23 & 27-Aug-01 & & $10045-97-3$ & CS-137 \\
\hline 240 & SESP SCHEDULING & & SW & & & B12T25 & 27-Aug-01 & & $10045-97-3$ & CS-137 \\
\hline 241 & SESP SCHEDULING & & SW & & & B12864 & 05-Sep-01 & 09-Aug-01 & $10045-97-3$ & CS-137 \\
\hline 242 & SESP SCHEDULING & & SW & & & B12871 & 05-Sep-01 & 09-Aug-01 & $10045-97-3$ & CS-137 \\
\hline 243 & SESP SCHEDULING & & SW & & & B12850 & 05-Sep-01 & 09-Aug-01 & $10045-97-3$ & CS-137 \\
\hline 244 & SESP SCHEDULING & & SW & & & B12857 & 05-Sep-01 & 09-Aug-01 & $10045-97-3$ & CS-137 \\
\hline 245 & SESP SCHEDULING & & SW & & & B12XK2 & 17-Sep-01 & & $10045-97-3$ & CS-137 \\
\hline 246 & SESP SCHEDULING & & SW & & & B12XK8 & 17-Sep-01 & & $10045-97-3$ & CS-137 \\
\hline 247 & SESP SCHEDULING & & SW & & & B12XK9 & 17-Sep-01 & & $10045-97-3$ & CS-137 \\
\hline 248 & SESP SCHEDULING & & SW & & & B12XL0 & 17-Sep-01 & & $10045-97-3$ & CS-137 \\
\hline 249 & SESP SCHEDULING & & SW & & & B12XL1 & 17-Sep-01 & & $10045-97-3$ & CS-137 \\
\hline 250 & SESP SCHEDULING & & SW & & & B12XL2 & 17-Sep-01 & & $10045-97-3$ & CS-137 \\
\hline 251 & SESP SCHEDULING & & SW & & & B12XK1 & 18-Sep-01 & & $10045-97-3$ & CS-137 \\
\hline 252 & SESP SCHEDULING & & SW & & & B12XK3 & 18-Sep-01 & & $10045-97-3$ & CS-137 \\
\hline 253 & SESP SCHEDULING & & SW & & & B12XK4 & 18-Sep-01 & & $10045-97-3$ & CS-137 \\
\hline 254 & SESP SCHEDULING & & SW & & & B12XK5 & 18-Sep-01 & & $10045-97-3$ & CS-137 \\
\hline 255 & SESP SCHEDULING & & SW & & & B12XK6 & 18-Sep-01 & & $10045-97-3$ & CS-137 \\
\hline 256 & SESP SCHEDULING & & SW & & & B12XK7 & 18-Sep-01 & & $10045-97-3$ & CS-137 \\
\hline 257 & SESP SCHEDULING & & SW & & & B12851 & 02-Oct-01 & 05-Sep-01 & $10045-97-3$ & CS-137 \\
\hline 258 & SESP SCHEDULING & & SW & & & B12858 & 02-Oct-01 & 05-Sep-01 & $10045-97-3$ & CS-137 \\
\hline 259 & SESP SCHEDULING & & SW & & & B12865 & 02-Oct-01 & 05-Sep-01 & $10045-97-3$ & CS-137 \\
\hline 260 & SESP SCHEDULING & & SW & & & B12872 & 02-Oct-01 & 05-Sep-01 & $10045-97-3$ & CS-137 \\
\hline 261 & SESP SCHEDULING & & SW & & & B134L0 & 08-Oct-01 & & $10045-97-3$ & CS-137 \\
\hline 262 & SESP SCHEDULING & & SW & & & B12X38 & 22-Oct-01 & & $10045-97-3$ & CS-137 \\
\hline 263 & SESP SCHEDULING & & SW & & & $\mathrm{B} 12 \times 50$ & 22-Oct-01 & & $10045-97-3$ & CS-137 \\
\hline 264 & SESP SCHEDULING & & SW & & & B12WY1 & 25-Oct-01 & & $10045-97-3$ & CS-137 \\
\hline 265 & SESP SCHEDULING & & SW & & & B12X41 & 25-Oct-01 & & $10045-97-3$ & CS-137 \\
\hline 266 & SESP SCHEDULING & & SW & & & $\mathrm{B} 12 \mathrm{X} 42$ & 25-Oct-01 & & $10045-97-3$ & CS-137 \\
\hline 267 & SESP SCHEDULING & & SW & & & B12X48 & 01-Nov-01 & & $10045-97-3$ & CS-137 \\
\hline
\end{tabular}




\begin{tabular}{|c|c|c|c|c|c|c|c|c|c|c|}
\hline & $\mathrm{A}$ & $\mathrm{B}$ & $\mathrm{C}$ & $\mathrm{D}$ & $E$ & $\mathrm{~F}$ & $G$ & $\mathrm{H}$ & $I$ & $\mathrm{~J}$ \\
\hline 1 & SAMPLE_NUM_ASSIGNED_TO & SAMPLER & MEDIA & BIOTA_MEDIA & TAG_ID & SAMP_NUM & SAMP_DATE_TIME & SAMP_DATE_TIME_ON & CON_ID & CON_SHORT_NAME \\
\hline 268 & SESP SCHEDULING & & SW & & & B12X85 & 01-Nov-01 & & $10045-97-3$ & CS-137 \\
\hline 269 & SESP SCHEDULING & & SW & & & B13122 & 01-Nov-01 & 02-Oct-01 & $10045-97-3$ & CS-137 \\
\hline 270 & SESP SCHEDULING & & SW & & & B13128 & 01-Nov-01 & 02-Oct-01 & $10045-97-3$ & CS-137 \\
\hline 271 & SESP SCHEDULING & & SW & & & B13110 & 01-Nov-01 & 02-Oct-01 & $10045-97-3$ & CS-137 \\
\hline 272 & SESP SCHEDULING & & SW & & & B13116 & 01-Nov-01 & 02-Oct-01 & $10045-97-3$ & CS-137 \\
\hline 273 & SESP SCHEDULING & & SW & & & B13J17 & 14-Nov-01 & & $10045-97-3$ & CS-137 \\
\hline 274 & SESP SCHEDULING & & SW & & & B13111 & 27-Nov-01 & 01-Nov-01 & $10045-97-3$ & CS-137 \\
\hline 275 & SESP SCHEDULING & & SW & & & B13117 & 27-Nov-01 & 01-Nov-01 & $10045-97-3$ & CS-137 \\
\hline 276 & SESP SCHEDULING & & SW & & & B13123 & 27-Nov-01 & 01-Nov-01 & $10045-97-3$ & CS-137 \\
\hline 277 & SESP SCHEDULING & & SW & & & B13129 & 27-Nov-01 & 01-Nov-01 & $10045-97-3$ & CS-137 \\
\hline 278 & SESP SCHEDULING & & SW & & & B13112 & 27-Dec-01 & 27-Nov-01 & $10045-97-3$ & CS-137 \\
\hline 279 & SESP SCHEDULING & & SW & & & B13118 & 27-Dec-01 & 27-Nov-01 & $10045-97-3$ & CS-137 \\
\hline 280 & SESP SCHEDULING & & SW & & & B13124 & 27-Dec-01 & 27-Nov-01 & $10045-97-3$ & CS-137 \\
\hline 281 & SESP SCHEDULING & & SW & & & B13130 & 27-Dec-01 & 27-Nov-01 & $10045-97-3$ & CS-137 \\
\hline 282 & SESP SCHEDULING & & SW & & & B13VL1 & 06-Feb-02 & 27-Dec-01 & $10045-97-3$ & CS-137 \\
\hline 283 & SESP SCHEDULING & & SW & & & B13VL8 & 06-Feb-02 & 27-Dec-01 & $10045-97-3$ & CS-137 \\
\hline 284 & SESP SCHEDULING & & SW & & & B13VJ7 & 06-Feb-02 & 27-Dec-01 & $10045-97-3$ & CS-137 \\
\hline 285 & SESP SCHEDULING & & SW & & & B13VK4 & 06-Feb-02 & 27-Dec-01 & $10045-97-3$ & CS-137 \\
\hline 286 & SESP SCHEDULING & & SW & & & B141L4 & 14-Feb-02 & & $10045-97-3$ & CS-137 \\
\hline 287 & SESP SCHEDULING & & SW & & & B13VJ8 & 07-Mar-02 & 06-Feb-02 & $10045-97-3$ & CS-137 \\
\hline 288 & SESP SCHEDULING & & SW & & & B13VK5 & 07-Mar-02 & 06-Feb-02 & $10045-97-3$ & CS-137 \\
\hline 289 & SESP SCHEDULING & & SW & & & B13VL2 & 07-Mar-02 & 06-Feb-02 & $10045-97-3$ & CS-137 \\
\hline 290 & SESP SCHEDULING & & SW & & & B13VL9 & 07-Mar-02 & 06-Feb-02 & $10045-97-3$ & CS-137 \\
\hline 291 & SESP SCHEDULING & & SW & & & B13VJ9 & 03-Apr-02 & 07-Mar-02 & $10045-97-3$ & CS-137 \\
\hline 292 & SESP SCHEDULING & & SW & & & B13VK6 & 03-Apr-02 & 07-Mar-02 & $10045-97-3$ & CS-137 \\
\hline 293 & SESP SCHEDULING & & SW & & & B13VL3 & 03-Apr-02 & 07-Mar-02 & $10045-97-3$ & CS-137 \\
\hline 294 & SESP SCHEDULING & & SW & & & B13VM0 & 03-Apr-02 & 07-Mar-02 & $10045-97-3$ & CS-137 \\
\hline 295 & SESP SCHEDULING & & SW & & & B14CT2 & 08-Apr-02 & & $10045-97-3$ & CS-137 \\
\hline 296 & SESP SCHEDULING & & SW & & & B14CV3 & 08-Apr-02 & & $10045-97-3$ & CS-137 \\
\hline 297 & SESP SCHEDULING & & SW & & & B14B50 & 01-May-02 & 03-Apr-02 & $10045-97-3$ & CS-137 \\
\hline 298 & SESP SCHEDULING & & SW & & & B14B56 & 01-May-02 & 03-Apr-02 & $10045-97-3$ & CS-137 \\
\hline 299 & SESP SCHEDULING & & SW & & & B14B62 & 01-May-02 & 03-Apr-02 & $10045-97-3$ & CS-137 \\
\hline 300 & SESP SCHEDULING & & SW & & & B14B68 & 01-May-02 & 03-Apr-02 & $10045-97-3$ & CS-137 \\
\hline 301 & SESP SCHEDULING & & SW & & & B14LJ0 & 23-May-02 & & $10045-97-3$ & CS-137 \\
\hline 302 & SESP SCHEDULING & & SW & & & B14LJ2 & 23-May-02 & & $10045-97-3$ & CS-137 \\
\hline 303 & SESP SCHEDULING & & SW & & & B14B51 & 29-May-02 & 01-May-02 & $10045-97-3$ & CS-137 \\
\hline 304 & SESP SCHEDULING & & SW & & & B14B57 & 29-May-02 & 01-May-02 & $10045-97-3$ & CS-137 \\
\hline 305 & SESP SCHEDULING & & SW & & & B14B63 & 29-May-02 & 01-May-02 & $10045-97-3$ & CS-137 \\
\hline
\end{tabular}




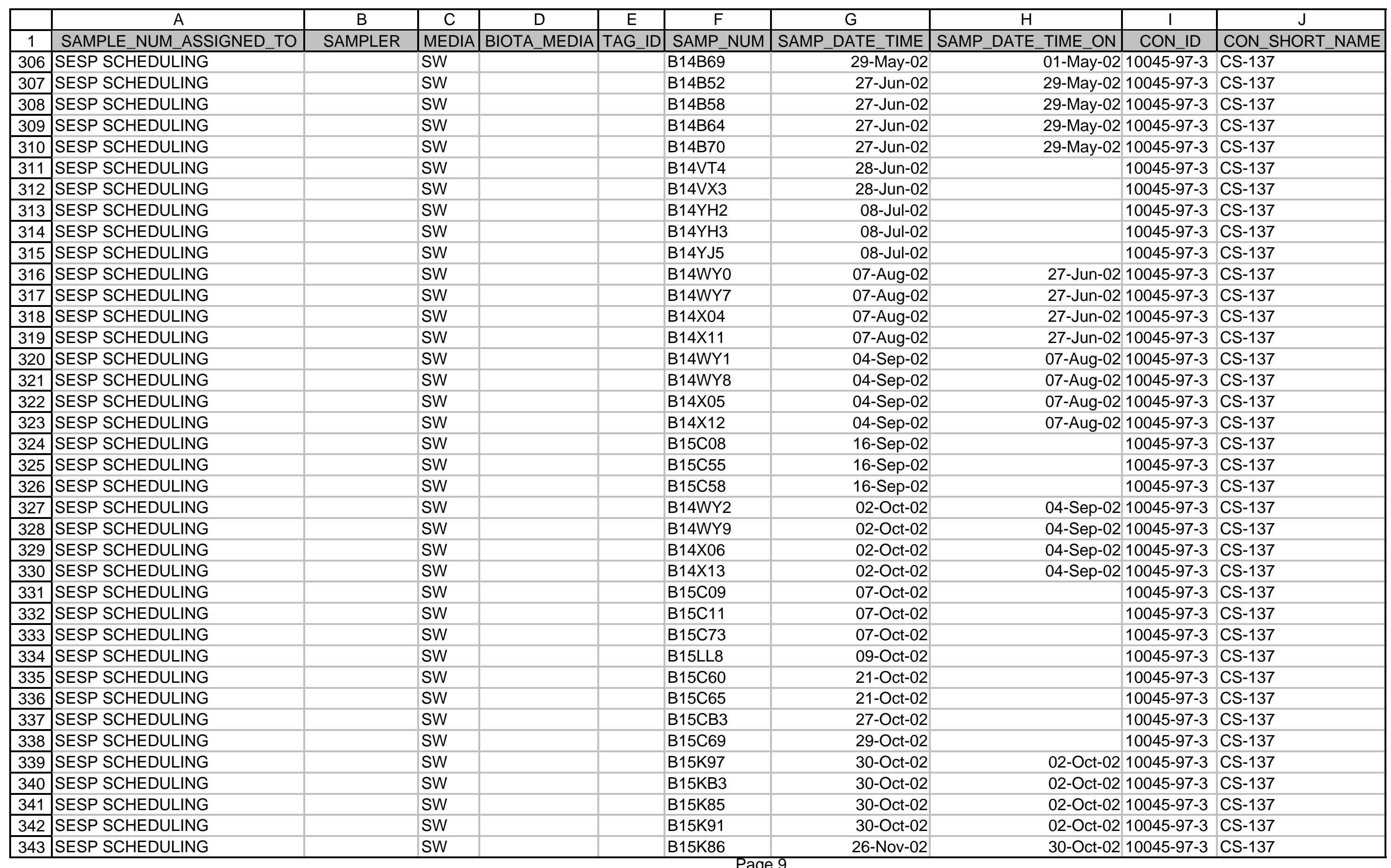




\begin{tabular}{|c|c|c|c|c|c|c|c|c|c|c|}
\hline & $\mathrm{A}$ & $\mathrm{B}$ & $\mathrm{C}$ & $\mathrm{D}$ & $E$ & $\mathrm{~F}$ & $G$ & $\mathrm{H}$ & $I$ & $\mathrm{~J}$ \\
\hline 1 & SAMPLE_NUM_ASSIGNED_TO & SAMPLER & MEDIA & BIOTA_MEDIA & TAG_ID & SAMP_NUM & SAMP_DATE_TIME & SAMP_DATE_TIME_ON & CON_ID & CON_SHORT_NAME \\
\hline 344 & SESP SCHEDULING & & SW & & & B15K92 & 26-Nov-02 & 30-Oct-02 & $10045-97-3$ & CS-137 \\
\hline 345 & SESP SCHEDULING & & SW & & & B15K98 & 26-Nov-02 & 30-Oct-02 & 10045-97-3 & CS-137 \\
\hline 346 & SESP SCHEDULING & & SW & & & B15KB4 & 26-Nov-02 & $30-O c t-02$ & $10045-97-3$ & CS-137 \\
\hline 347 & SESP SCHEDULING & & SW & & & B0X790 & 05-Apr-00 & & $15046-84-1$ & I-129 \\
\hline 348 & SESP SCHEDULING & & SW & & & B0X775 & 05-Apr-00 & & $15046-84-1$ & I-129 \\
\hline 349 & SESP SCHEDULING & & SW & & & B0XVD3 & 29-Jun-00 & & 15046-84-1 & I-129 \\
\hline 350 & SESP SCHEDULING & & SW & & & B0XVF7 & 29-Jun-00 & & $15046-84-1$ & I-129 \\
\hline 351 & SESP SCHEDULING & & SW & & & B10906 & 27-Sep-00 & & $15046-84-1$ & I-129 \\
\hline 352 & SESP SCHEDULING & & SW & & & B10907 & 27-Sep-00 & & $15046-84-1$ & I-129 \\
\hline 353 & SESP SCHEDULING & & SW & & & B10951 & 27-Sep-00 & & $15046-84-1$ & I-129 \\
\hline 354 & SESP SCHEDULING & & SW & & & B10952 & 27-Sep-00 & & $15046-84-1$ & $\mid-129$ \\
\hline 355 & SESP SCHEDULING & & SW & & & B10976 & 27-Sep-00 & & $15046-84-1$ & I-129 \\
\hline 356 & SESP SCHEDULING & & SW & & & BOYKJ9 & 05-Oct-00 & & 15046-84-1 & I-129 \\
\hline 357 & SESP SCHEDULING & & SW & & & BOYKL4 & 05-Oct-00 & & 15046-84-1 & I-129 \\
\hline 358 & SESP SCHEDULING & & SW & & & B10BR0 & 04-Jan-01 & & 15046-84-1 & I-129 \\
\hline 359 & SESP SCHEDULING & & SW & & & B10BT4 & 04-Jan-01 & & $15046-84-1$ & I-129 \\
\hline 360 & SESP SCHEDULING & & SW & & & B114F5 & 04-Apr-01 & & $15046-84-1$ & I-129 \\
\hline 361 & SESP SCHEDULING & & SW & & & $\mathrm{B} 114 \mathrm{H} 9$ & 04-Apr-01 & & $15046-84-1$ & I-129 \\
\hline 362 & SESP SCHEDULING & & SW & & & B11W33 & 30-Apr-01 & & 15046-84-1 & I-129 \\
\hline 363 & SESP SCHEDULING & & SW & & & B11W61 & 30-Apr-01 & & $15046-84-1$ & I-129 \\
\hline 364 & SESP SCHEDULING & & SW & & & B11W85 & 03-May-01 & & $15046-84-1$ & I-129 \\
\hline 365 & SESP SCHEDULING & & SW & & & B11W34 & 10-May-01 & & 15046-84-1 & $\mid-129$ \\
\hline 366 & SESP SCHEDULING & & SW & & & B11LJ7 & 28-Jun-01 & & $15046-84-1$ & I-129 \\
\hline 367 & SESP SCHEDULING & & SW & & & B11LH3 & 28-Jun-01 & & $15046-84-1$ & I-129 \\
\hline 368 & SESP SCHEDULING & & SW & & & B12RL8 & 27-Aug-01 & & $15046-84-1$ & I-129 \\
\hline 369 & SESP SCHEDULING & & SW & & & B12RN1 & 27-Aug-01 & & $15046-84-1$ & I-129 \\
\hline 370 & SESP SCHEDULING & & SW & & & B127T9 & 02-Oct-01 & & 15046-84-1 & I-129 \\
\hline 371 & SESP SCHEDULING & & SW & & & B127W4 & 02-Oct-01 & & 15046-84-1 & I-129 \\
\hline 372 & SESP SCHEDULING & & SW & & & B130P4 & 03-Jan-02 & & $15046-84-1$ & I-129 \\
\hline 373 & SESP SCHEDULING & & SW & & & B130M9 & 03-Jan-02 & & $15046-84-1$ & I-129 \\
\hline 374 & SESP SCHEDULING & & SW & & & B0X7M5 & 05-Apr-00 & 29-Dec-99 & PU-239/240 & PU-239/240 \\
\hline 375 & SESP SCHEDULING & & SW & & & B0X7N2 & 05-Apr-00 & 29-Dec-99 & PU-239/240 & PU-239/240 \\
\hline 376 & SESP SCHEDULING & & SW & & & B0X7L1 & 05-Apr-00 & 29-Dec-99 & PU-239/240 & PU-239/240 \\
\hline 377 & SESP SCHEDULING & & SW & & & B0X7L8 & 05-Apr-00 & 29-Dec-99 & PU-239/240 & PU-239/240 \\
\hline 378 & SESP SCHEDULING & & SW & & & B0XVP6 & 29-Jun-00 & 05-Apr-00 & PU-239/240 & PU-239/240 \\
\hline 379 & SESP SCHEDULING & & SW & & & B0XVR2 & 29-Jun-00 & 05-Apr-00 & PU-239/240 & PU-239/240 \\
\hline 380 & SESP SCHEDULING & & SW & & & B0XVR8 & 29-Jun-00 & 05-Apr-00 & PU-239/240 & PU-239/240 \\
\hline 381 & SESP SCHEDULING & & SW & & & B0XVT4 & 29-Jun-00 & 05-Apr-00 & PU-239/240 & PU-239/240 \\
\hline
\end{tabular}




\begin{tabular}{|c|c|c|c|c|c|c|c|c|c|c|}
\hline & $\mathrm{A}$ & $\mathrm{B}$ & $\mathrm{C}$ & $\mathrm{D}$ & $E$ & $\mathrm{~F}$ & $G$ & $\mathrm{H}$ & $\mathrm{I}$ & $\mathrm{J}$ \\
\hline 1 & SAMPLE_NUM_ASSIGNED_TO & SAMPLER & MEDIA & BIOTA_MEDIA & TAG_ID & SAMP_NUM & SAMP_DATE_TIME & SAMP_DATE_TIME_ON & CON_ID & CON_SHORT_NAME \\
\hline 382 & SESP SCHEDULING & & SW & & & BOYKW8 & 05-Oct-00 & 29-Jun-00 & PU-239/240 & PU-239/240 \\
\hline 383 & SESP SCHEDULING & & SW & & & B0YKX5 & 05-Oct-00 & 29-Jun-00 & PU-239/240 & PU-239/240 \\
\hline 384 & SESP SCHEDULING & & SW & & & BOYKY2 & 05-Oct-00 & 29-Jun-00 & PU-239/240 & PU-239/240 \\
\hline 385 & SESP SCHEDULING & & SW & & & BOYKY9 & 05-Oct-00 & 29-Jun-00 & PU-239/240 & PU-239/240 \\
\hline 386 & SESP SCHEDULING & & SW & & & B10C25 & 28-Dec-00 & $05-O c t-00$ & PU-239/240 & PU-239/240 \\
\hline 387 & SESP SCHEDULING & & SW & & & B10C31 & 28-Dec-00 & 05-Oct-00 & PU-239/240 & PU-239/240 \\
\hline 388 & SESP SCHEDULING & & SW & & & B10C37 & 28-Dec-00 & 05-Oct-00 & PU-239/240 & PU-239/240 \\
\hline 389 & SESP SCHEDULING & & SW & & & B10C43 & 28-Dec-00 & 05-Oct-00 & PU-239/240 & PU-239/240 \\
\hline 390 & SESP SCHEDULING & & SW & & & B114T6 & 04-Apr-01 & 28-Dec-00 & PU-239/240 & PU-239/240 \\
\hline 391 & SESP SCHEDULING & & SW & & & B114V3 & 04-Apr-01 & & PU-239/240 & PU-239/240 \\
\hline 392 & SESP SCHEDULING & & SW & & & B114W0 & 04-Apr-01 & & PU-239/240 & PU-239/240 \\
\hline 393 & SESP SCHEDULING & & SW & & & B114W7 & 04-Apr-01 & 28-Dec-00 & PU-239/240 & PU-239/240 \\
\hline 394 & SESP SCHEDULING & & SW & & & B11LW0 & 28-Jun-01 & 04-Apr-01 & PU-239/240 & PU-239/240 \\
\hline 395 & SESP SCHEDULING & & SW & & & B11LW6 & 28-Jun-01 & 04-Apr-01 & PU-239/240 & PU-239/240 \\
\hline 396 & SESP SCHEDULING & & SW & & & B11LT8 & 28-Jun-01 & 04-Apr-01 & PU-239/240 & PU-239/240 \\
\hline 397 & SESP SCHEDULING & & SW & & & B11LV4 & 28-Jun-01 & 04-Apr-01 & PU-239/240 & PU-239/240 \\
\hline 398 & SESP SCHEDULING & & SW & & & B12848 & 02-Oct-01 & 28-Jun-01 & PU-239/240 & PU-239/240 \\
\hline 399 & SESP SCHEDULING & & SW & & & B12855 & 02-Oct-01 & 28-Jun-01 & PU-239/240 & PU-239/240 \\
\hline 400 & SESP SCHEDULING & & SW & & & B12862 & 02-Oct-01 & 28-Jun-01 & PU-239/240 & PU-239/240 \\
\hline 401 & SESP SCHEDULING & & SW & & & B12869 & 02-Oct-01 & 28-Jun-01 & PU-239/240 & PU-239/240 \\
\hline 402 & SESP SCHEDULING & & SW & & & B13109 & 27-Dec-01 & 02-Oct-01 & PU-239/240 & PU-239/240 \\
\hline 403 & SESP SCHEDULING & & SW & & & B13115 & 27-Dec-01 & 02-Oct-01 & PU-239/240 & PU-239/240 \\
\hline 404 & SESP SCHEDULING & & SW & & & B13121 & 27-Dec-01 & 02-Oct-01 & PU-239/240 & PU-239/240 \\
\hline 405 & SESP SCHEDULING & & SW & & & B13127 & 27-Dec-01 & 02-Oct-01 & PU-239/240 & PU-239/240 \\
\hline 406 & SESP SCHEDULING & & SW & & & B13VJ6 & 03-Apr-02 & 27-Dec-01 & PU-239/240 & PU-239/240 \\
\hline 407 & SESP SCHEDULING & & SW & & & B13VK3 & 03-Apr-02 & 27-Dec-01 & PU-239/240 & PU-239/240 \\
\hline 408 & SESP SCHEDULING & & SW & & & B13VL0 & 03-Apr-02 & 27-Dec-01 & PU-239/240 & PU-239/240 \\
\hline 409 & SESP SCHEDULING & & SW & & & B13VL7 & 03-Apr-02 & 27-Dec-01 & PU-239/240 & PU-239/240 \\
\hline 410 & SESP SCHEDULING & & SW & & & B14B49 & 27-Jun-02 & 03-Apr-02 & PU-239/240 & PU-239/240 \\
\hline 411 & SESP SCHEDULING & & SW & & & B14B55 & 27-Jun-02 & 03-Apr-02 & PU-239/240 & PU-239/240 \\
\hline 412 & SESP SCHEDULING & & SW & & & B14B61 & 27-Jun-02 & 03-Apr-02 & PU-239/240 & PU-239/240 \\
\hline 413 & SESP SCHEDULING & & SW & & & B14B67 & 27-Jun-02 & 03-Apr-02 & PU-239/240 & PU-239/240 \\
\hline 414 & SESP SCHEDULING & & SW & & & B14WX9 & 02-Oct-02 & 27-Jun-02 & PU-239/240 & PU-239/240 \\
\hline 415 & SESP SCHEDULING & & SW & & & B14WY6 & 02-Oct-02 & 27-Jun-02 & PU-239/240 & PU-239/240 \\
\hline 416 & SESP SCHEDULING & & SW & & & B14X03 & 02-Oct-02 & 27-Jun-02 & PU-239/240 & PU-239/240 \\
\hline 417 & SESP SCHEDULING & & SW & & & B14X10 & 02-Oct-02 & 27-Jun-02 & PU-239/240 & PU-239/240 \\
\hline 418 & SESP SCHEDULING & & SW & & & B0X7K4 & 02-Feb-00 & 29-Dec-99 & $10098-97-2$ & SR-90 \\
\hline 419 & SESP SCHEDULING & & SW & & & B0X7J0 & 02-Feb-00 & 29-Dec-99 & $10098-97-2$ & SR-90 \\
\hline
\end{tabular}




\begin{tabular}{|c|c|c|c|c|c|c|c|c|c|c|}
\hline & $A$ & $B$ & $\mathrm{C}$ & $\mathrm{D}$ & $E$ & $\mathrm{~F}$ & $G$ & $\mathrm{H}$ & $\mathrm{I}$ & $\mathrm{J}$ \\
\hline 1 & SAMPLE_NUM_ASSIGNED_TO & SAMPLER & MEDIA & BIOTA_MEDIA & TAG_ID & SAMP_NUM & SAMP_DATE_TIME & SAMP_DATE_TIME_ON & CON_ID & CON_SHORT_NAME \\
\hline 420 & SESP SCHEDULING & & SW & & & B0XKV5 & 22-Feb-00 & & $10098-97-2$ & SR-90 \\
\hline 421 & SESP SCHEDULING & & SW & & & B0XKV6 & 22-Feb-00 & & $10098-97-2$ & SR-90 \\
\hline 422 & SESP SCHEDULING & & SW & & & B0XKV7 & 22-Feb-00 & & $10098-97-2$ & SR-90 \\
\hline 423 & SESP SCHEDULING & & SW & & & B0XKW7 & 22-Feb-00 & & $10098-97-2$ & SR-90 \\
\hline 424 & SESP SCHEDULING & & SW & & & BOXH28 & 08-Mar-00 & 02-Feb-00 & $10098-97-2$ & SR-90 \\
\hline 425 & SESP SCHEDULING & & SW & & & BOXH22 & 08-Mar-00 & 02-Feb-00 & $10098-97-2$ & SR-90 \\
\hline 426 & SESP SCHEDULING & & SW & & & BOXRK2 & 27-Mar-00 & & $10098-97-2$ & SR-90 \\
\hline 427 & SESP SCHEDULING & & SW & & & B0XRK3 & 27-Mar-00 & & $10098-97-2$ & SR-90 \\
\hline 428 & SESP SCHEDULING & & SW & & & BOXRK4 & 27-Mar-00 & & $10098-97-2$ & SR-90 \\
\hline 429 & SESP SCHEDULING & & SW & & & B0XRK5 & 27-Mar-00 & & $10098-97-2$ & SR-90 \\
\hline 430 & SESP SCHEDULING & & SW & & & BOXRKO & 28-Mar-00 & & $10098-97-2$ & SR-90 \\
\hline 431 & SESP SCHEDULING & & SW & & & B0XRK6 & 28-Mar-00 & & $10098-97-2$ & SR-90 \\
\hline 432 & SESP SCHEDULING & & SW & & & B0XRK7 & 28-Mar-00 & & $10098-97-2$ & SR-90 \\
\hline 433 & SESP SCHEDULING & & SW & & & BOXRK8 & 28-Mar-00 & & $10098-97-2$ & SR-90 \\
\hline 434 & SESP SCHEDULING & & SW & & & BOXRK9 & 28-Mar-00 & & $10098-97-2$ & SR-90 \\
\hline 435 & SESP SCHEDULING & & SW & & & BOXRLO & 28-Mar-00 & & $10098-97-2$ & SR-90 \\
\hline 436 & SESP SCHEDULING & & SW & & & B0XRL1 & 28-Mar-00 & & $10098-97-2$ & SR-90 \\
\hline 437 & SESP SCHEDULING & & SW & & & BOXRN8 & 28-Mar-00 & & $10098-97-2$ & SR-90 \\
\hline 438 & SESP SCHEDULING & & SW & & & BOXRN9 & 28-Mar-00 & & $10098-97-2$ & SR-90 \\
\hline 439 & SESP SCHEDULING & & SW & & & BOXRPO & 28-Mar-00 & & $10098-97-2$ & SR-90 \\
\hline 440 & SESP SCHEDULING & & SW & & & B0XRP1 & 28-Mar-00 & & $10098-97-2$ & SR-90 \\
\hline 441 & SESP SCHEDULING & & SW & & & B0XP21 & 05-Apr-00 & 08-Mar-00 & $10098-97-2$ & SR-90 \\
\hline 442 & SESP SCHEDULING & & SW & & & B0XP16 & 05-Apr-00 & 08-Mar-00 & $10098-97-2$ & SR-90 \\
\hline 443 & SESP SCHEDULING & & SW & & & B0Y265 & 02-May-00 & & $10098-97-2$ & SR-90 \\
\hline 444 & SESP SCHEDULING & & SW & & & B0Y266 & 02-May-00 & & $10098-97-2$ & SR-90 \\
\hline 445 & SESP SCHEDULING & & SW & & & B0Y267 & 02-May-00 & & $10098-97-2$ & SR-90 \\
\hline 446 & SESP SCHEDULING & & SW & & & B0Y269 & 02-May-00 & & $10098-97-2$ & SR-90 \\
\hline 447 & SESP SCHEDULING & & SW & & & B0XVN6 & 03-May-00 & 29-Mar-00 & $10098-97-2$ & SR-90 \\
\hline 448 & SESP SCHEDULING & & SW & & & B0XVP1 & 03-May-00 & 29-Mar-00 & $10098-97-2$ & SR-90 \\
\hline 449 & SESP SCHEDULING & & SW & & & B0Y2Y6 & 11-May-00 & & $10098-97-2$ & SR-90 \\
\hline 450 & SESP SCHEDULING & & SW & & & B0Y231 & 31-May-00 & 03-May-00 & $10098-97-2$ & SR-90 \\
\hline 451 & SESP SCHEDULING & & SW & & & B0Y237 & 31-May-00 & 03-May-00 & $10098-97-2$ & SR-90 \\
\hline 452 & SESP SCHEDULING & & SW & & & B0YB90 & 08-Jun-00 & & $10098-97-2$ & SR-90 \\
\hline 453 & SESP SCHEDULING & & SW & & & B0YCD7 & 19-Jun-00 & & $10098-97-2$ & SR-90 \\
\hline 454 & SESP SCHEDULING & & SW & & & B0YCD8 & 19-Jun-00 & & $10098-97-2$ & SR-90 \\
\hline 455 & SESP SCHEDULING & & SW & & & B0YCD9 & 19-Jun-00 & & $10098-97-2$ & SR-90 \\
\hline 456 & SESP SCHEDULING & & SW & & & BOYCFO & 19-Jun-00 & & $10098-97-2$ & SR-90 \\
\hline 457 & SESP SCHEDULING & & SW & & & B0YCF1 & 20-Jun-00 & & $10098-97-2$ & SR-90 \\
\hline
\end{tabular}




\begin{tabular}{|c|c|c|c|c|c|c|c|c|c|c|}
\hline & $\mathrm{A}$ & $\mathrm{B}$ & $\mathrm{C}$ & $\mathrm{D}$ & $E$ & $\mathrm{~F}$ & $G$ & $\mathrm{H}$ & $I$ & $\mathrm{~J}$ \\
\hline 1 & SAMPLE_NUM_ASSIGNED_TO & SAMPLER & MEDIA & BIOTA_MEDIA & TAG_ID & SAMP_NUM & SAMP_DATE_TIME & SAMP_DATE_TIME_ON & CON_ID & CON_SHORT_NAME \\
\hline 458 & SESP SCHEDULING & & SW & & & BOYCF2 & 20-Jun-00 & & $10098-97-2$ & SR-90 \\
\hline 459 & SESP SCHEDULING & & SW & & & BOYCF3 & 20-Jun-00 & & $10098-97-2$ & SR-90 \\
\hline 460 & SESP SCHEDULING & & SW & & & BOYCF4 & 20-Jun-00 & & $10098-97-2$ & SR-90 \\
\hline 461 & SESP SCHEDULING & & SW & & & B0YCF5 & 20-Jun-00 & & $10098-97-2$ & SR-90 \\
\hline 462 & SESP SCHEDULING & & SW & & & BOYCF6 & 20-Jun-00 & & $10098-97-2$ & SR-90 \\
\hline 463 & SESP SCHEDULING & & SW & & & BOYCK3 & 20-Jun-00 & & $10098-97-2$ & SR-90 \\
\hline 464 & SESP SCHEDULING & & SW & & & B0YCK4 & 20-Jun-00 & & $10098-97-2$ & SR-90 \\
\hline 465 & SESP SCHEDULING & & SW & & & B0YCK5 & 20-Jun-00 & & $10098-97-2$ & SR-90 \\
\hline 466 & SESP SCHEDULING & & SW & & & BOYCK6 & 20-Jun-00 & & $10098-97-2$ & SR-90 \\
\hline 467 & SESP SCHEDULING & & SW & & & B0YB64 & 29-Jun-00 & 31-May-00 & $10098-97-2$ & SR-90 \\
\hline 468 & SESP SCHEDULING & & SW & & & BOYB76 & 29-Jun-00 & 31-May-00 & $10098-97-2$ & SR-90 \\
\hline 469 & SESP SCHEDULING & & SW & & & B0YM22 & 07-Jul-00 & & $10098-97-2$ & SR-90 \\
\hline 470 & SESP SCHEDULING & & SW & & & B0YP01 & 25-Jul-00 & & $10098-97-2$ & SR-90 \\
\hline 471 & SESP SCHEDULING & & SW & & & B0YP02 & 25-Jul-00 & & $10098-97-2$ & SR-90 \\
\hline 472 & SESP SCHEDULING & & SW & & & BOYP03 & 25-Jul-00 & & $10098-97-2$ & SR-90 \\
\hline 473 & SESP SCHEDULING & & SW & & & B0YP05 & 25-Jul-00 & & $10098-97-2$ & SR-90 \\
\hline 474 & SESP SCHEDULING & & SW & & & BOYKV4 & 10-Aug-00 & 29-Jun-00 & $10098-97-2$ & SR-90 \\
\hline 475 & SESP SCHEDULING & & SW & & & B0YKW1 & 10-Aug-00 & 29-Jun-00 & $10098-97-2$ & SR-90 \\
\hline 476 & SESP SCHEDULING & & SW & & & B0YW65 & 07-Sep-00 & & $10098-97-2$ & SR-90 \\
\hline 477 & SESP SCHEDULING & & SW & & & BOYW70 & 07-Sep-00 & & $10098-97-2$ & SR-90 \\
\hline 478 & SESP SCHEDULING & & SW & & & B10672 & 14-Sep-00 & & $10098-97-2$ & SR-90 \\
\hline 479 & SESP SCHEDULING & & SW & & & B10673 & 14-Sep-00 & & $10098-97-2$ & SR-90 \\
\hline 480 & SESP SCHEDULING & & SW & & & B10674 & 14-Sep-00 & & $10098-97-2$ & SR-90 \\
\hline 481 & SESP SCHEDULING & & SW & & & B10675 & 14-Sep-00 & & $10098-97-2$ & SR-90 \\
\hline 482 & SESP SCHEDULING & & SW & & & B10676 & 14-Sep-00 & & $10098-97-2$ & SR-90 \\
\hline 483 & SESP SCHEDULING & & SW & & & B10677 & 14-Sep-00 & & $10098-97-2$ & SR-90 \\
\hline 484 & SESP SCHEDULING & & SW & & & B10679 & 14-Sep-00 & & $10098-97-2$ & SR-90 \\
\hline 485 & SESP SCHEDULING & & SW & & & B10681 & 14-Sep-00 & & $10098-97-2$ & SR-90 \\
\hline 486 & SESP SCHEDULING & & SW & & & B10683 & 14-Sep-00 & & $10098-97-2$ & SR-90 \\
\hline 487 & SESP SCHEDULING & & SW & & & B10684 & 14-Sep-00 & & $10098-97-2$ & SR-90 \\
\hline 488 & SESP SCHEDULING & & SW & & & B10686 & 14-Sep-00 & & $10098-97-2$ & SR-90 \\
\hline 489 & SESP SCHEDULING & & SW & & & B10698 & 14-Sep-00 & & $10098-97-2$ & SR-90 \\
\hline 490 & SESP SCHEDULING & & SW & & & B106B1 & 14-Sep-00 & & $10098-97-2$ & SR-90 \\
\hline 491 & SESP SCHEDULING & & SW & & & B106B4 & 14-Sep-00 & & $10098-97-2$ & SR-90 \\
\hline 492 & SESP SCHEDULING & & SW & & & B106B7 & 14-Sep-00 & & $10098-97-2$ & SR-90 \\
\hline 493 & SESP SCHEDULING & & SW & & & B106W1 & 18-Sep-00 & & $10098-97-2$ & SR-90 \\
\hline 494 & SESP SCHEDULING & & SW & & & B106W2 & 18-Sep-00 & & $10098-97-2$ & SR-90 \\
\hline 495 & SESP SCHEDULING & & SW & & & B106W3 & 18-Sep-00 & & 10098-97-2 & SR-90 \\
\hline
\end{tabular}




\begin{tabular}{|c|c|c|c|c|c|c|c|c|c|c|}
\hline & $\mathrm{A}$ & $B$ & $\mathrm{C}$ & $\mathrm{D}$ & $E$ & $\mathrm{~F}$ & $G$ & $\mathrm{H}$ & $\mathrm{I}$ & $\mathrm{J}$ \\
\hline 1 & SAMPLE_NUM_ASSIGNED_TO & SAMPLER & MEDIA & BIOTA_MEDIA & TAG_ID & SAMP_NUM & SAMP_DATE_TIME & SAMP_DATE_TIME_ON & CON_ID & CON_SHORT_NAME \\
\hline 496 & SESP SCHEDULING & & SW & & & B106W4 & 18-Sep-00 & & $10098-97-2$ & SR-90 \\
\hline 497 & SESP SCHEDULING & & SW & & & B106W5 & 18-Sep-00 & & $10098-97-2$ & SR-90 \\
\hline 498 & SESP SCHEDULING & & SW & & & B106W6 & 18-Sep-00 & & $10098-97-2$ & SR-90 \\
\hline 499 & SESP SCHEDULING & & SW & & & B106W7 & 18-Sep-00 & & $10098-97-2$ & SR-90 \\
\hline 500 & SESP SCHEDULING & & SW & & & B106W8 & 18-Sep-00 & & $10098-97-2$ & SR-90 \\
\hline 501 & SESP SCHEDULING & & SW & & & B106W9 & 18-Sep-00 & & $10098-97-2$ & SR-90 \\
\hline 502 & SESP SCHEDULING & & SW & & & B106X0 & 18-Sep-00 & & $10098-97-2$ & SR-90 \\
\hline 503 & SESP SCHEDULING & & SW & & & B106X1 & 18-Sep-00 & & $10098-97-2$ & SR-90 \\
\hline 504 & SESP SCHEDULING & & SW & & & B106X2 & 18-Sep-00 & & $10098-97-2$ & SR-90 \\
\hline 505 & SESP SCHEDULING & & SW & & & B10728 & 18-Sep-00 & & $10098-97-2$ & SR-90 \\
\hline 506 & SESP SCHEDULING & & SW & & & B10729 & 18-Sep-00 & & $10098-97-2$ & SR-90 \\
\hline 507 & SESP SCHEDULING & & SW & & & B10730 & 18-Sep-00 & & $10098-97-2$ & SR-90 \\
\hline 508 & SESP SCHEDULING & & SW & & & B10785 & 18-Sep-00 & & $10098-97-2$ & SR-90 \\
\hline 509 & SESP SCHEDULING & & SW & & & B10788 & 18-Sep-00 & & $10098-97-2$ & SR-90 \\
\hline 510 & SESP SCHEDULING & & SW & & & B10791 & 18-Sep-00 & & $10098-97-2$ & SR-90 \\
\hline 511 & SESP SCHEDULING & & SW & & & B10794 & 18-Sep-00 & & $10098-97-2$ & SR-90 \\
\hline 512 & SESP SCHEDULING & & SW & & & B106X3 & 19-Sep-00 & & $10098-97-2$ & SR-90 \\
\hline 513 & SESP SCHEDULING & & SW & & & B106X5 & 19-Sep-00 & & $10098-97-2$ & SR-90 \\
\hline 514 & SESP SCHEDULING & & SW & & & B106X7 & 19-Sep-00 & & $10098-97-2$ & SR-90 \\
\hline 515 & SESP SCHEDULING & & SW & & & B106X9 & 19-Sep-00 & & $10098-97-2$ & SR-90 \\
\hline 516 & SESP SCHEDULING & & SW & & & B106Y1 & 19-Sep-00 & & $10098-97-2$ & SR-90 \\
\hline 517 & SESP SCHEDULING & & SW & & & B106Y3 & 19-Sep-00 & & $10098-97-2$ & SR-90 \\
\hline 518 & SESP SCHEDULING & & SW & & & B106Y4 & 19-Sep-00 & & $10098-97-2$ & SR-90 \\
\hline 519 & SESP SCHEDULING & & SW & & & B106Y5 & 19-Sep-00 & & $10098-97-2$ & SR-90 \\
\hline 520 & SESP SCHEDULING & & SW & & & B106Y6 & 19-Sep-00 & & $10098-97-2$ & SR-90 \\
\hline 521 & SESP SCHEDULING & & SW & & & B106Y7 & 19-Sep-00 & & $10098-97-2$ & SR-90 \\
\hline 522 & SESP SCHEDULING & & SW & & & B106Y8 & 19-Sep-00 & & $10098-97-2$ & SR-90 \\
\hline 523 & SESP SCHEDULING & & SW & & & B106Y9 & 19-Sep-00 & & $10098-97-2$ & SR-90 \\
\hline 524 & SESP SCHEDULING & & SW & & & B10761 & 19-Sep-00 & & $10098-97-2$ & SR-90 \\
\hline 525 & SESP SCHEDULING & & SW & & & B10762 & 19-Sep-00 & & $10098-97-2$ & SR-90 \\
\hline 526 & SESP SCHEDULING & & SW & & & B10763 & 19-Sep-00 & & $10098-97-2$ & SR-90 \\
\hline 527 & SESP SCHEDULING & & SW & & & B10764 & 19-Sep-00 & & $10098-97-2$ & SR-90 \\
\hline 528 & SESP SCHEDULING & & SW & & & B10773 & 19-Sep-00 & & $10098-97-2$ & SR-90 \\
\hline 529 & SESP SCHEDULING & & SW & & & B10776 & 19-Sep-00 & & $10098-97-2$ & SR-90 \\
\hline 530 & SESP SCHEDULING & & SW & & & B10779 & 19-Sep-00 & & $10098-97-2$ & SR-90 \\
\hline 531 & SESP SCHEDULING & & SW & & & B10782 & 19-Sep-00 & & $10098-97-2$ & SR-90 \\
\hline 532 & SESP SCHEDULING & & SW & & & B10913 & 27-Sep-00 & & $10098-97-2$ & SR-90 \\
\hline 533 & SESP SCHEDULING & & SW & & & B10949 & 27-Sep-00 & & 10098-97-2 & SR-90 \\
\hline
\end{tabular}




\begin{tabular}{|c|c|c|c|c|c|c|c|c|c|c|}
\hline & $A$ & $\mathrm{~B}$ & $\mathrm{C}$ & $\mathrm{D}$ & $E$ & $\mathrm{~F}$ & $G$ & $\mathrm{H}$ & $\mathrm{I}$ & $\mathrm{J}$ \\
\hline 1 & SAMPLE_NUM_ASSIGNED_TO & SAMPLER & MEDIA & BIOTA_MEDIA & TAG_ID & SAMP_NUM & SAMP_DATE_TIME & SAMP_DATE_TIME_ON & CON_ID & CON_SHORT_NAME \\
\hline 534 & SESP SCHEDULING & & SW & & & B109J0 & 27-Sep-00 & & $10098-97-2$ & SR-90 \\
\hline 535 & SESP SCHEDULING & & SW & & & B103W8 & 05-Oct-00 & 07-Sep-00 & $10098-97-2$ & SR-90 \\
\hline 536 & SESP SCHEDULING & & SW & & & B103X3 & 05-Oct-00 & 07-Sep-00 & $10098-97-2$ & SR-90 \\
\hline 537 & SESP SCHEDULING & & SW & & & B10910 & 17-Oct-00 & & $10098-97-2$ & SR-90 \\
\hline 538 & SESP SCHEDULING & & SW & & & B10934 & 17-Oct-00 & & $10098-97-2$ & SR-90 \\
\hline 539 & SESP SCHEDULING & & SW & & & B10939 & $17-$ Oct-00 & & $10098-97-2$ & SR-90 \\
\hline 540 & SESP SCHEDULING & & SW & & & B10940 & 17-Oct-00 & & $10098-97-2$ & SR-90 \\
\hline 541 & SESP SCHEDULING & & SW & & & B10KN4 & 18-Oct-00 & & 10098-97-2 & SR-90 \\
\hline 542 & SESP SCHEDULING & & SW & & & B10KN5 & 18-Oct-00 & & $10098-97-2$ & SR-90 \\
\hline 543 & SESP SCHEDULING & & SW & & & B10KN6 & 18-Oct-00 & & $10098-97-2$ & SR-90 \\
\hline 544 & SESP SCHEDULING & & SW & & & B10KN8 & 18-Oct-00 & & $10098-97-2$ & SR-90 \\
\hline 545 & SESP SCHEDULING & & SW & & & B10932 & 25-Oct-00 & & $10098-97-2$ & SR-90 \\
\hline 546 & SESP SCHEDULING & & SW & & & B10936 & 25-Oct-00 & & $10098-97-2$ & SR-90 \\
\hline 547 & PSHENRY & RADFORD, E & SW & & & B10FB2 & $25-O c t-00$ & & $10098-97-2$ & SR-90 \\
\hline 548 & SESP SCHEDULING & & SW & & & B10941 & 01-Nov-00 & & $10098-97-2$ & SR-90 \\
\hline 549 & SESP SCHEDULING & & SW & & & B10977 & 01-Nov-00 & & $10098-97-2$ & SR-90 \\
\hline 550 & SESP SCHEDULING & & SW & & & B10C13 & 02-Nov-00 & 05-Oct-00 & $10098-97-2$ & SR-90 \\
\hline 551 & SESP SCHEDULING & & SW & & & B10C19 & 02-Nov-00 & 05-Oct-00 & 10098-97-2 & SR-90 \\
\hline 552 & SESP SCHEDULING & & SW & & & B10942 & 07-Nov-00 & & $10098-97-2$ & SR-90 \\
\hline 553 & PSHENRY & RADFORD, E & SW & & & B10FC4 & 07-Nov-00 & & $10098-97-2$ & SR-90 \\
\hline 554 & SESP SCHEDULING & & SW & & & B10MP5 & 29-Nov-00 & 02-Nov-00 & 10098-97-2 & SR-90 \\
\hline 555 & SESP SCHEDULING & & SW & & & B10MR1 & 29-Nov-00 & 02-Nov-00 & $10098-97-2$ & SR-90 \\
\hline 556 & SESP SCHEDULING & & SW & & & B11125 & 04-Dec-00 & & $10098-97-2$ & SR-90 \\
\hline 557 & SESP SCHEDULING & & SW & & & B11126 & 04-Dec-00 & & $10098-97-2$ & SR-90 \\
\hline 558 & SESP SCHEDULING & & SW & & & B11127 & 04-Dec-00 & & $10098-97-2$ & SR-90 \\
\hline 559 & SESP SCHEDULING & & SW & & & B11128 & 04-Dec-00 & & $10098-97-2$ & SR-90 \\
\hline 560 & SESP SCHEDULING & & SW & & & B11124 & 05-Dec-00 & & $10098-97-2$ & SR-90 \\
\hline 561 & SESP SCHEDULING & & SW & & & B11129 & 05-Dec-00 & & $10098-97-2$ & SR-90 \\
\hline 562 & SESP SCHEDULING & & SW & & & B11130 & 05-Dec-00 & & $10098-97-2$ & SR-90 \\
\hline 563 & SESP SCHEDULING & & SW & & & B11131 & 05-Dec-00 & & 10098-97-2 & SR-90 \\
\hline 564 & SESP SCHEDULING & & SW & & & B11132 & 05-Dec-00 & & $10098-97-2$ & SR-90 \\
\hline 565 & SESP SCHEDULING & & SW & & & B11133 & 05-Dec-00 & & $10098-97-2$ & SR-90 \\
\hline 566 & SESP SCHEDULING & & SW & & & B11134 & 05-Dec-00 & & 10098-97-2 & SR-90 \\
\hline 567 & SESP SCHEDULING & & SW & & & B11145 & 05-Dec-00 & & $10098-97-2$ & SR-90 \\
\hline 568 & SESP SCHEDULING & & SW & & & B11146 & 05-Dec-00 & & $10098-97-2$ & SR-90 \\
\hline 569 & SESP SCHEDULING & & SW & & & B11147 & 05-Dec-00 & & 10098-97-2 & SR-90 \\
\hline 570 & SESP SCHEDULING & & SW & & & B11148 & 05-Dec-00 & & $10098-97-2$ & SR-90 \\
\hline 571 & SESP SCHEDULING & & SW & & & B110Y4 & 04-Jan-01 & 29-Nov-00 & $10098-97-2$ & SR-90 \\
\hline
\end{tabular}




\begin{tabular}{|c|c|c|c|c|c|c|c|c|c|c|}
\hline & $\mathrm{A}$ & $\mathrm{B}$ & $\mathrm{C}$ & $\mathrm{D}$ & $E$ & $\mathrm{~F}$ & G & $\mathrm{H}$ & $I$ & $\mathrm{~J}$ \\
\hline 1 & SAMPLE_NUM_ASSIGNED_TO & SAMPLER & MEDIA & BIOTA_MEDIA & TAG_ID & SAMP_NUM & SAMP_DATE_TIME & SAMP_DATE_TIME_ON & CON_ID & CON_SHORT_NAME \\
\hline 572 & SESP SCHEDULING & & SW & & & B11100 & 04-Jan-01 & 29-Nov-00 & $10098-97-2$ & SR-90 \\
\hline 573 & SESP SCHEDULING & & SW & & & B114P8 & 31-Jan-01 & 04-Jan-01 & $10098-97-2$ & SR-90 \\
\hline 574 & SESP SCHEDULING & & SW & & & B114T0 & 31-Jan-01 & 04-Jan-01 & $10098-97-2$ & SR-90 \\
\hline 575 & SESP SCHEDULING & & SW & & & B11CX6 & 15-Feb-01 & & $10098-97-2$ & SR-90 \\
\hline 576 & SESP SCHEDULING & & SW & & & B11CX7 & 15-Feb-01 & & $10098-97-2$ & SR-90 \\
\hline 577 & SESP SCHEDULING & & SW & & & B11CX8 & 15-Feb-01 & & $10098-97-2$ & SR-90 \\
\hline 578 & SESP SCHEDULING & & SW & & & B11CY8 & 15-Feb-01 & & $10098-97-2$ & SR-90 \\
\hline 579 & SESP SCHEDULING & & SW & & & $\mathrm{B} 11 \mathrm{H} 38$ & 26-Feb-01 & & $10098-97-2$ & SR-90 \\
\hline 580 & SESP SCHEDULING & & SW & & & B11H43 & 26-Feb-01 & & $10098-97-2$ & SR-90 \\
\hline 581 & SESP SCHEDULING & & SW & & & $\mathrm{B} 11 \mathrm{H} 44$ & 26-Feb-01 & & $10098-97-2$ & SR-90 \\
\hline 582 & SESP SCHEDULING & & SW & & & $\mathrm{B} 11 \mathrm{H} 45$ & 26-Feb-01 & & $10098-97-2$ & SR-90 \\
\hline 583 & SESP SCHEDULING & & SW & & & $\mathrm{B} 11 \mathrm{H} 46$ & 26-Feb-01 & & $10098-97-2$ & SR-90 \\
\hline 584 & SESP SCHEDULING & & SW & & & B11H47 & 26-Feb-01 & & $10098-97-2$ & SR-90 \\
\hline 585 & SESP SCHEDULING & & SW & & & B11H48 & 26-Feb-01 & & $10098-97-2$ & SR-90 \\
\hline 586 & SESP SCHEDULING & & SW & & & B11H59 & 26-Feb-01 & & $10098-97-2$ & SR-90 \\
\hline 587 & SESP SCHEDULING & & SW & & & B11H60 & 26-Feb-01 & & $10098-97-2$ & SR-90 \\
\hline 588 & SESP SCHEDULING & & SW & & & B11H61 & 26-Feb-01 & & $10098-97-2$ & SR-90 \\
\hline 589 & SESP SCHEDULING & & SW & & & $\mathrm{B} 11 \mathrm{H} 62$ & 26-Feb-01 & & $10098-97-2$ & SR-90 \\
\hline 590 & SESP SCHEDULING & & SW & & & $\mathrm{B} 11 \mathrm{H} 39$ & 27-Feb-01 & & $10098-97-2$ & SR-90 \\
\hline 591 & SESP SCHEDULING & & SW & & & $\mathrm{B} 11 \mathrm{H} 40$ & 27-Feb-01 & & $10098-97-2$ & SR-90 \\
\hline 592 & SESP SCHEDULING & & SW & & & $\mathrm{B} 11 \mathrm{H} 41$ & 27-Feb-01 & & $10098-97-2$ & SR-90 \\
\hline 593 & SESP SCHEDULING & & SW & & & $\mathrm{B} 11 \mathrm{H} 42$ & 27-Feb-01 & & $10098-97-2$ & SR-90 \\
\hline 594 & SESP SCHEDULING & & SW & & & B11C04 & 01-Mar-01 & 31-Jan-01 & $10098-97-2$ & SR-90 \\
\hline 595 & SESP SCHEDULING & & SW & & & B11C10 & 01-Mar-01 & 31-Jan-01 & $10098-97-2$ & SR-90 \\
\hline 596 & SESP SCHEDULING & & SW & & & B11J40 & 04-Apr-01 & 01-Mar-01 & $10098-97-2$ & SR-90 \\
\hline 597 & SESP SCHEDULING & & SW & & & B11J46 & 04-Apr-01 & 01-Mar-01 & $10098-97-2$ & SR-90 \\
\hline 598 & SESP SCHEDULING & & SW & & & B11RY4 & 17-Apr-01 & & $10098-97-2$ & SR-90 \\
\hline 599 & SESP SCHEDULING & & SW & & & B11RY5 & 17-Apr-01 & & $10098-97-2$ & SR-90 \\
\hline 600 & SESP SCHEDULING & & SW & & & B11RY6 & 17-Apr-01 & & $10098-97-2$ & SR-90 \\
\hline 601 & SESP SCHEDULING & & SW & & & B11T05 & 17-Apr-01 & & $10098-97-2$ & SR-90 \\
\hline 602 & SESP SCHEDULING & & SW & & & B11W50 & 30-Apr-01 & & $10098-97-2$ & SR-90 \\
\hline 603 & SESP SCHEDULING & & SW & & & B11W52 & 30-Apr-01 & & $10098-97-2$ & SR-90 \\
\hline 604 & SESP SCHEDULING & & SW & & & B11W86 & 30-Apr-01 & & $10098-97-2$ & SR-90 \\
\hline 605 & SESP SCHEDULING & & SW & & & B11LT2 & 02-May-01 & 04-Apr-01 & $10098-97-2$ & SR-90 \\
\hline 606 & SESP SCHEDULING & & SW & & & B11LR6 & 02-May-01 & 04-Apr-01 & $10098-97-2$ & SR-90 \\
\hline 607 & SESP SCHEDULING & & SW & & & B11W57 & 03-May-01 & & $10098-97-2$ & SR-90 \\
\hline 608 & SESP SCHEDULING & & SW & & & B11W35 & 04-May-01 & & $10098-97-2$ & SR-90 \\
\hline 609 & SESP SCHEDULING & & SW & & & B11W40 & 04-May-01 & & 10098-97-2 & SR-90 \\
\hline
\end{tabular}




\begin{tabular}{|c|c|c|c|c|c|c|c|c|c|c|}
\hline & $\mathrm{A}$ & $B$ & $\mathrm{C}$ & $\mathrm{D}$ & $E$ & $\mathrm{~F}$ & $G$ & $\mathrm{H}$ & $\mathrm{I}$ & $\mathrm{J}$ \\
\hline 1 & SAMPLE_NUM_ASSIGNED_TO & SAMPLER & MEDIA & BIOTA_MEDIA & TAG_ID & SAMP_NUM & SAMP_DATE_TIME & SAMP_DATE_TIME_ON & CON_ID & CON_SHORT_NAME \\
\hline 610 & SESP SCHEDULING & & SW & & & B11W43 & 04-May-01 & & $10098-97-2$ & SR-90 \\
\hline 611 & SESP SCHEDULING & & SW & & & B11W48 & 04-May-01 & & $10098-97-2$ & SR-90 \\
\hline 612 & SESP SCHEDULING & & SW & & & B11WV8 & 09-May-01 & & $10098-97-2$ & SR-90 \\
\hline 613 & SESP SCHEDULING & & SW & & & B11WX0 & 09-May-01 & & $10098-97-2$ & SR-90 \\
\hline 614 & SESP SCHEDULING & & SW & & & B11W38 & 10-May-01 & & $10098-97-2$ & SR-90 \\
\hline 615 & SESP SCHEDULING & & SW & & & B11WT4 & 30-May-01 & 02-May-01 & $10098-97-2$ & SR-90 \\
\hline 616 & SESP SCHEDULING & & SW & & & B11WR8 & 30-May-01 & 02-May-01 & $10098-97-2$ & SR-90 \\
\hline 617 & SESP SCHEDULING & & SW & & & B124N7 & 07-Jun-01 & & $10098-97-2$ & SR-90 \\
\hline 618 & SESP SCHEDULING & & SW & & & B124P7 & 07-Jun-01 & & $10098-97-2$ & SR-90 \\
\hline 619 & SESP SCHEDULING & & SW & & & B12505 & 12-Jun-01 & & $10098-97-2$ & SR-90 \\
\hline 620 & SESP SCHEDULING & & SW & & & B12506 & 12-Jun-01 & & $10098-97-2$ & SR-90 \\
\hline 621 & SESP SCHEDULING & & SW & & & B12507 & 12-Jun-01 & & $10098-97-2$ & SR-90 \\
\hline 622 & SESP SCHEDULING & & SW & & & B12508 & 12-Jun-01 & & $10098-97-2$ & SR-90 \\
\hline 623 & SESP SCHEDULING & & SW & & & B12509 & 12-Jun-01 & & $10098-97-2$ & SR-90 \\
\hline 624 & SESP SCHEDULING & & SW & & & B12510 & 12-Jun-01 & & $10098-97-2$ & SR-90 \\
\hline 625 & SESP SCHEDULING & & SW & & & B12531 & 12-Jun-01 & & $10098-97-2$ & SR-90 \\
\hline 626 & SESP SCHEDULING & & SW & & & B12532 & 12-Jun-01 & & $10098-97-2$ & SR-90 \\
\hline 627 & SESP SCHEDULING & & SW & & & B12533 & 12-Jun-01 & & $10098-97-2$ & SR-90 \\
\hline 628 & SESP SCHEDULING & & SW & & & B12534 & 12-Jun-01 & & $10098-97-2$ & SR-90 \\
\hline 629 & SESP SCHEDULING & & SW & & & B12501 & 14-Jun-01 & & $10098-97-2$ & SR-90 \\
\hline 630 & SESP SCHEDULING & & SW & & & B12502 & 14-Jun-01 & & $10098-97-2$ & SR-90 \\
\hline 631 & SESP SCHEDULING & & SW & & & B12503 & 14-Jun-01 & & $10098-97-2$ & SR-90 \\
\hline 632 & SESP SCHEDULING & & SW & & & B12504 & 14-Jun-01 & & 10098-97-2 & SR-90 \\
\hline 633 & SESP SCHEDULING & & SW & & & B124M3 & 28-Jun-01 & 23-May-01 & $10098-97-2$ & SR-90 \\
\hline 634 & SESP SCHEDULING & & SW & & & B124L7 & 28-Jun-01 & 23-May-01 & $10098-97-2$ & SR-90 \\
\hline 635 & SESP SCHEDULING & & SW & & & B12994 & 09-Jul-01 & & $10098-97-2$ & SR-90 \\
\hline 636 & SESP SCHEDULING & & SW & & & B129B4 & 09-Jul-01 & & $10098-97-2$ & SR-90 \\
\hline 637 & SESP SCHEDULING & & SW & & & B129N8 & 09-Jul-01 & & $10098-97-2$ & SR-90 \\
\hline 638 & SESP SCHEDULING & & SW & & & B129N9 & 09-Jul-01 & & $10098-97-2$ & SR-90 \\
\hline 639 & SESP SCHEDULING & & SW & & & B129P0 & 09-Jul-01 & & $10098-97-2$ & SR-90 \\
\hline 640 & SESP SCHEDULING & & SW & & & B129P9 & 09-Jul-01 & & $10098-97-2$ & SR-90 \\
\hline 641 & SESP SCHEDULING & & SW & & & B12841 & 09-Aug-01 & 28-Jun-01 & $10098-97-2$ & SR-90 \\
\hline 642 & SESP SCHEDULING & & SW & & & B12834 & 09-Aug-01 & 28-Jun-01 & $10098-97-2$ & SR-90 \\
\hline 643 & SESP SCHEDULING & & SW & & & B12RM1 & 27-Aug-01 & & $10098-97-2$ & SR-90 \\
\hline 644 & SESP SCHEDULING & & SW & & & B12RM6 & 27-Aug-01 & & $10098-97-2$ & SR-90 \\
\hline 645 & SESP SCHEDULING & & SW & & & B12JT2 & 05-Sep-01 & & $10098-97-2$ & SR-90 \\
\hline 646 & SESP SCHEDULING & & SW & & & B12JR7 & 05-Sep-01 & 09-Aug-01 & $10098-97-2$ & SR-90 \\
\hline 647 & SESP SCHEDULING & & SW & & & B12T85 & 07-Sep-01 & & $10098-97-2$ & SR-90 \\
\hline
\end{tabular}




\begin{tabular}{|c|c|c|c|c|c|c|c|c|c|c|}
\hline & $\mathrm{A}$ & $B$ & $\mathrm{C}$ & $\mathrm{D}$ & $E$ & $\mathrm{~F}$ & $G$ & $\mathrm{H}$ & $\mathrm{I}$ & $\mathrm{J}$ \\
\hline 1 & SAMPLE_NUM_ASSIGNED_TO & SAMPLER & MEDIA & BIOTA_MEDIA & TAG_ID & SAMP_NUM & SAMP_DATE_TIME & SAMP_DATE_TIME_ON & CON_ID & CON_SHORT_NAME \\
\hline 648 & SESP SCHEDULING & & SW & & & B12T87 & 07-Sep-01 & & $10098-97-2$ & SR-90 \\
\hline 649 & SESP SCHEDULING & & SW & & & B12T88 & 07-Sep-01 & & $10098-97-2$ & SR-90 \\
\hline 650 & SESP SCHEDULING & & SW & & & B12T89 & 07-Sep-01 & & $10098-97-2$ & SR-90 \\
\hline 651 & SESP SCHEDULING & & SW & & & B12T90 & 07-Sep-01 & & $10098-97-2$ & SR-90 \\
\hline 652 & SESP SCHEDULING & & SW & & & B12T91 & 07-Sep-01 & & $10098-97-2$ & SR-90 \\
\hline 653 & SESP SCHEDULING & & SW & & & B12T92 & 07-Sep-01 & & $10098-97-2$ & SR-90 \\
\hline 654 & SESP SCHEDULING & & SW & & & B12T93 & 07-Sep-01 & & $10098-97-2$ & SR-90 \\
\hline 655 & SESP SCHEDULING & & SW & & & B12TD4 & 07-Sep-01 & & $10098-97-2$ & SR-90 \\
\hline 656 & SESP SCHEDULING & & SW & & & B12TD5 & 07-Sep-01 & & $10098-97-2$ & SR-90 \\
\hline 657 & SESP SCHEDULING & & SW & & & B12TD6 & 07-Sep-01 & & $10098-97-2$ & SR-90 \\
\hline 658 & SESP SCHEDULING & & SW & & & B12TN2 & 07-Sep-01 & & $10098-97-2$ & SR-90 \\
\hline 659 & SESP SCHEDULING & & SW & & & B12TN5 & 07-Sep-01 & & $10098-97-2$ & SR-90 \\
\hline 660 & SESP SCHEDULING & & SW & & & B12TN8 & 07-Sep-01 & & $10098-97-2$ & SR-90 \\
\hline 661 & SESP SCHEDULING & & SW & & & B12TP1 & 07-Sep-01 & & $10098-97-2$ & SR-90 \\
\hline 662 & SESP SCHEDULING & & SW & & & B12T94 & 10-Sep-01 & & $10098-97-2$ & SR-90 \\
\hline 663 & SESP SCHEDULING & & SW & & & B12T95 & 10-Sep-01 & & $10098-97-2$ & SR-90 \\
\hline 664 & SESP SCHEDULING & & SW & & & B12T96 & 10-Sep-01 & & $10098-97-2$ & SR-90 \\
\hline 665 & SESP SCHEDULING & & SW & & & B12T97 & 10-Sep-01 & & $10098-97-2$ & SR-90 \\
\hline 666 & SESP SCHEDULING & & SW & & & B12T98 & 10-Sep-01 & & $10098-97-2$ & SR-90 \\
\hline 667 & SESP SCHEDULING & & SW & & & B12T99 & 10-Sep-01 & & $10098-97-2$ & SR-90 \\
\hline 668 & SESP SCHEDULING & & SW & & & B12TB0 & 10-Sep-01 & & $10098-97-2$ & SR-90 \\
\hline 669 & SESP SCHEDULING & & SW & & & B12TB1 & 10-Sep-01 & & $10098-97-2$ & SR-90 \\
\hline 670 & SESP SCHEDULING & & SW & & & B12TB2 & 10-Sep-01 & & $10098-97-2$ & SR-90 \\
\hline 671 & SESP SCHEDULING & & SW & & & B12TB3 & 10-Sep-01 & & $10098-97-2$ & SR-90 \\
\hline 672 & SESP SCHEDULING & & SW & & & B12TB4 & 10-Sep-01 & & $10098-97-2$ & SR-90 \\
\hline 673 & SESP SCHEDULING & & SW & & & B12TB5 & 10-Sep-01 & & $10098-97-2$ & SR-90 \\
\hline 674 & SESP SCHEDULING & & SW & & & B12TF7 & 10-Sep-01 & & $10098-97-2$ & SR-90 \\
\hline 675 & SESP SCHEDULING & & SW & & & B12TF8 & 10-Sep-01 & & $10098-97-2$ & SR-90 \\
\hline 676 & SESP SCHEDULING & & SW & & & B12TF9 & 10-Sep-01 & & $10098-97-2$ & SR-90 \\
\hline 677 & SESP SCHEDULING & & SW & & & B12TT0 & 10-Sep-01 & & $10098-97-2$ & SR-90 \\
\hline 678 & SESP SCHEDULING & & SW & & & B12TT3 & 10-Sep-01 & & $10098-97-2$ & SR-90 \\
\hline 679 & SESP SCHEDULING & & SW & & & B12TT9 & 10-Sep-01 & & $10098-97-2$ & SR-90 \\
\hline 680 & SESP SCHEDULING & & SW & & & B12TB6 & 13-Sep-01 & & $10098-97-2$ & SR-90 \\
\hline 681 & SESP SCHEDULING & & SW & & & B12TB8 & 13-Sep-01 & & $10098-97-2$ & SR-90 \\
\hline 682 & SESP SCHEDULING & & SW & & & B12TC0 & 13-Sep-01 & & $10098-97-2$ & SR-90 \\
\hline 683 & SESP SCHEDULING & & SW & & & B12TC2 & 13-Sep-01 & & $10098-97-2$ & SR-90 \\
\hline 684 & SESP SCHEDULING & & SW & & & B12TC4 & 13-Sep-01 & & $10098-97-2$ & SR-90 \\
\hline 685 & SESP SCHEDULING & & SW & & & B12TC6 & 13-Sep-01 & & 10098-97-2 & SR-90 \\
\hline
\end{tabular}




\begin{tabular}{|c|c|c|c|c|c|c|c|c|c|c|}
\hline & $\mathrm{A}$ & $\mathrm{B}$ & $\mathrm{C}$ & $\mathrm{D}$ & $E$ & $\mathrm{~F}$ & $G$ & $\mathrm{H}$ & $I$ & $\mathrm{~J}$ \\
\hline 1 & SAMPLE_NUM_ASSIGNED_TO & SAMPLER & MEDIA & BIOTA_MEDIA & TAG_ID & SAMP_NUM & SAMP_DATE_TIME & SAMP_DATE_TIME_ON & CON_ID & CON_SHORT_NAME \\
\hline 686 & SESP SCHEDULING & & SW & & & B12TC8 & 13-Sep-01 & & $10098-97-2$ & SR-90 \\
\hline 687 & SESP SCHEDULING & & SW & & & B12TC9 & 13-Sep-01 & & $10098-97-2$ & SR-90 \\
\hline 688 & SESP SCHEDULING & & SW & & & B12TD0 & 13-Sep-01 & & $10098-97-2$ & SR-90 \\
\hline 689 & SESP SCHEDULING & & SW & & & B12TD1 & 13-Sep-01 & & $10098-97-2$ & SR-90 \\
\hline 690 & SESP SCHEDULING & & SW & & & B12TD2 & 13-Sep-01 & & $10098-97-2$ & SR-90 \\
\hline 691 & SESP SCHEDULING & & SW & & & B12TD3 & 13-Sep-01 & & $10098-97-2$ & SR-90 \\
\hline 692 & SESP SCHEDULING & & SW & & & B12TM0 & 13-Sep-01 & & $10098-97-2$ & SR-90 \\
\hline 693 & SESP SCHEDULING & & SW & & & B12TM1 & 13-Sep-01 & & $10098-97-2$ & SR-90 \\
\hline 694 & SESP SCHEDULING & & SW & & & B12TM2 & 13-Sep-01 & & $10098-97-2$ & SR-90 \\
\hline 695 & SESP SCHEDULING & & SW & & & B12TM3 & 13-Sep-01 & & $10098-97-2$ & SR-90 \\
\hline 696 & SESP SCHEDULING & & SW & & & B12TP4 & 13-Sep-01 & & $10098-97-2$ & SR-90 \\
\hline 697 & SESP SCHEDULING & & SW & & & B12TP8 & 13-Sep-01 & & 10098-97-2 & SR-90 \\
\hline 698 & SESP SCHEDULING & & SW & & & B12TR2 & 13-Sep-01 & & $10098-97-2$ & SR-90 \\
\hline 699 & SESP SCHEDULING & & SW & & & B12TR6 & 13-Sep-01 & & $10098-97-2$ & SR-90 \\
\hline 700 & SESP SCHEDULING & & SW & & & B12T28 & 02-Oct-01 & 05-Sep-01 & $10098-97-2$ & SR-90 \\
\hline 701 & SESP SCHEDULING & & SW & & & B12T33 & 02-Oct-01 & 05-Sep-01 & $10098-97-2$ & SR-90 \\
\hline 702 & SESP SCHEDULING & & SW & & & B134L1 & 08-Oct-01 & & $10098-97-2$ & SR-90 \\
\hline 703 & SESP SCHEDULING & & SW & & & B134L2 & 08-Oct-01 & & $10098-97-2$ & SR-90 \\
\hline 704 & SESP SCHEDULING & & SW & & & B134L3 & 08-Oct-01 & & 10098-97-2 & SR-90 \\
\hline 705 & SESP SCHEDULING & & SW & & & B134L4 & 08-Oct-01 & & $10098-97-2$ & SR-90 \\
\hline 706 & SESP SCHEDULING & & SW & & & B12X38 & 22-Oct-01 & & 10098-97-2 & SR-90 \\
\hline 707 & SESP SCHEDULING & & SW & & & B12X50 & 22-Oct-01 & & $10098-97-2$ & SR-90 \\
\hline 708 & SESP SCHEDULING & & SW & & & B12WY1 & 25-Oct-01 & & $10098-97-2$ & SR-90 \\
\hline 709 & SESP SCHEDULING & & SW & & & B12X41 & 25-Oct-01 & & $10098-97-2$ & SR-90 \\
\hline 710 & SESP SCHEDULING & & SW & & & B12X42 & 25-Oct-01 & & $10098-97-2$ & SR-90 \\
\hline 711 & SESP SCHEDULING & & SW & & & $\mathrm{B} 12 \mathrm{X} 48$ & 01-Nov-01 & & $10098-97-2$ & SR-90 \\
\hline 712 & SESP SCHEDULING & & SW & & & B12X85 & 01-Nov-01 & & $10098-97-2$ & SR-90 \\
\hline 713 & SESP SCHEDULING & & SW & & & B13103 & 01-Nov-01 & 02-Oct-01 & 10098-97-2 & SR-90 \\
\hline 714 & SESP SCHEDULING & & SW & & & B130Y7 & 01-Nov-01 & 02-Oct-01 & $10098-97-2$ & SR-90 \\
\hline 715 & SESP SCHEDULING & & SW & & & B13J17 & 14-Nov-01 & & $10098-97-2$ & SR-90 \\
\hline 716 & SESP SCHEDULING & & SW & & & B13CC6 & 27-Nov-01 & 01-Nov-01 & 10098-97-2 & SR-90 \\
\hline 717 & SESP SCHEDULING & & SW & & & B13CD2 & 27-Nov-01 & 01-Nov-01 & $10098-97-2$ & SR-90 \\
\hline 718 & SESP SCHEDULING & & SW & & & B13LF3 & 03-Dec-01 & & $10098-97-2$ & SR-90 \\
\hline 719 & SESP SCHEDULING & & SW & & & B13LF4 & 03-Dec-01 & & $10098-97-2$ & SR-90 \\
\hline 720 & SESP SCHEDULING & & SW & & & B13LF5 & 03-Dec-01 & & $10098-97-2$ & SR-90 \\
\hline 721 & SESP SCHEDULING & & SW & & & B13LF6 & 03-Dec-01 & & $10098-97-2$ & SR-90 \\
\hline 722 & SESP SCHEDULING & & SW & & & B13LF2 & 04-Dec-01 & & $10098-97-2$ & SR-90 \\
\hline 723 & SESP SCHEDULING & & SW & & & B13LF7 & 04-Dec-01 & & $10098-97-2$ & SR-90 \\
\hline
\end{tabular}




\begin{tabular}{|c|c|c|c|c|c|c|c|c|c|c|}
\hline & $\mathrm{A}$ & $\mathrm{B}$ & $\mathrm{C}$ & $\mathrm{D}$ & $E$ & $\mathrm{~F}$ & $G$ & $\mathrm{H}$ & $I$ & $\mathrm{~J}$ \\
\hline 1 & SAMPLE_NUM_ASSIGNED_TO & SAMPLER & MEDIA & BIOTA_MEDIA & TAG_ID & SAMP_NUM & SAMP_DATE_TIME & SAMP_DATE_TIME_ON & CON_ID & CON_SHORT_NAME \\
\hline 724 & SESP SCHEDULING & & SW & & & B13LF8 & 04-Dec-01 & & $10098-97-2$ & SR-90 \\
\hline 725 & SESP SCHEDULING & & SW & & & B13LF9 & 04-Dec-01 & & $10098-97-2$ & SR-90 \\
\hline 726 & SESP SCHEDULING & & SW & & & B13LH0 & 04-Dec-01 & & $10098-97-2$ & SR-90 \\
\hline 727 & SESP SCHEDULING & & SW & & & B13LH1 & 04-Dec-01 & & $10098-97-2$ & SR-90 \\
\hline 728 & SESP SCHEDULING & & SW & & & B13LH2 & 04-Dec-01 & & $10098-97-2$ & SR-90 \\
\hline 729 & SESP SCHEDULING & & SW & & & B13LJ3 & 04-Dec-01 & & 10098-97-2 & SR-90 \\
\hline 730 & SESP SCHEDULING & & SW & & & B13LJ4 & 04-Dec-01 & & $10098-97-2$ & SR-90 \\
\hline 731 & SESP SCHEDULING & & SW & & & B13LJ5 & 04-Dec-01 & & $10098-97-2$ & SR-90 \\
\hline 732 & SESP SCHEDULING & & SW & & & B13LJ6 & 04-Dec-01 & & $10098-97-2$ & SR-90 \\
\hline 733 & SESP SCHEDULING & & SW & & & B13LB3 & 03-Jan-02 & 27-Nov-01 & $10098-97-2$ & SR-90 \\
\hline 734 & SESP SCHEDULING & & SW & & & B13L96 & 03-Jan-02 & 27-Nov-01 & $10098-97-2$ & SR-90 \\
\hline 735 & SESP SCHEDULING & & SW & & & B13VJ0 & 06-Feb-02 & & 10098-97-2 & SR-90 \\
\hline 736 & SESP SCHEDULING & & SW & & & B13VF8 & 06-Feb-02 & & $10098-97-2$ & SR-90 \\
\hline 737 & SESP SCHEDULING & & SW & & & B14266 & 14-Feb-02 & & $10098-97-2$ & SR-90 \\
\hline 738 & SESP SCHEDULING & & SW & & & B14271 & 14-Feb-02 & & $10098-97-2$ & SR-90 \\
\hline 739 & SESP SCHEDULING & & SW & & & B14272 & 14-Feb-02 & & $10098-97-2$ & SR-90 \\
\hline 740 & SESP SCHEDULING & & SW & & & B14273 & 14-Feb-02 & & $10098-97-2$ & SR-90 \\
\hline 741 & SESP SCHEDULING & & SW & & & B140P7 & 07-Mar-02 & 06-Feb-02 & $10098-97-2$ & SR-90 \\
\hline 742 & SESP SCHEDULING & & SW & & & B140R2 & 07-Mar-02 & 06-Feb-02 & 10098-97-2 & SR-90 \\
\hline 743 & SESP SCHEDULING & & SW & & & B147T3 & 25-Mar-02 & & $10098-97-2$ & SR-90 \\
\hline 744 & SESP SCHEDULING & & SW & & & B147T4 & 25-Mar-02 & & 10098-97-2 & SR-90 \\
\hline 745 & SESP SCHEDULING & & SW & & & B147T5 & 25-Mar-02 & & $10098-97-2$ & SR-90 \\
\hline 746 & SESP SCHEDULING & & SW & & & B147T6 & 25-Mar-02 & & $10098-97-2$ & SR-90 \\
\hline 747 & SESP SCHEDULING & & SW & & & B147T2 & 26-Mar-02 & & $10098-97-2$ & SR-90 \\
\hline 748 & SESP SCHEDULING & & SW & & & B147T7 & 26-Mar-02 & & $10098-97-2$ & SR-90 \\
\hline 749 & SESP SCHEDULING & & SW & & & B147T8 & 26-Mar-02 & & $10098-97-2$ & SR-90 \\
\hline 750 & SESP SCHEDULING & & SW & & & B147T9 & 26-Mar-02 & & $10098-97-2$ & SR-90 \\
\hline 751 & SESP SCHEDULING & & SW & & & B147V0 & 26-Mar-02 & & 10098-97-2 & SR-90 \\
\hline 752 & SESP SCHEDULING & & SW & & & B147V1 & 26-Mar-02 & & $10098-97-2$ & SR-90 \\
\hline 753 & SESP SCHEDULING & & SW & & & B147V2 & 26-Mar-02 & & $10098-97-2$ & SR-90 \\
\hline 754 & SESP SCHEDULING & & SW & & & B147W3 & 26-Mar-02 & & 10098-97-2 & SR-90 \\
\hline 755 & SESP SCHEDULING & & SW & & & B147W4 & 26-Mar-02 & & $10098-97-2$ & SR-90 \\
\hline 756 & SESP SCHEDULING & & SW & & & B147W5 & 26-Mar-02 & & $10098-97-2$ & SR-90 \\
\hline 757 & SESP SCHEDULING & & SW & & & B147W6 & 26-Mar-02 & & $10098-97-2$ & SR-90 \\
\hline 758 & SESP SCHEDULING & & SW & & & B14408 & 03-Apr-02 & 07-Mar-02 & $10098-97-2$ & SR-90 \\
\hline 759 & SESP SCHEDULING & & SW & & & B14413 & 03-Apr-02 & 07-Mar-02 & $10098-97-2$ & SR-90 \\
\hline 760 & SESP SCHEDULING & & SW & & & B14CV5 & 08-Apr-02 & & $10098-97-2$ & SR-90 \\
\hline 761 & SESP SCHEDULING & & SW & & & B14CV7 & 08-Apr-02 & & $10098-97-2$ & SR-90 \\
\hline
\end{tabular}




\begin{tabular}{|c|c|c|c|c|c|c|c|c|c|c|}
\hline & $\mathrm{A}$ & $B$ & $\mathrm{C}$ & $\mathrm{D}$ & $E$ & $\mathrm{~F}$ & $G$ & $\mathrm{H}$ & $\mathrm{I}$ & $\mathrm{J}$ \\
\hline 1 & SAMPLE_NUM_ASSIGNED_TO & SAMPLER & MEDIA & BIOTA_MEDIA & TAG_ID & SAMP_NUM & SAMP_DATE_TIME & SAMP_DATE_TIME_ON & CON_ID & CON_SHORT_NAME \\
\hline 762 & SESP SCHEDULING & & SW & & & B14CV2 & 09-Apr-02 & & $10098-97-2$ & SR-90 \\
\hline 763 & SESP SCHEDULING & & SW & & & B14CV6 & 09-Apr-02 & & $10098-97-2$ & SR-90 \\
\hline 764 & SESP SCHEDULING & & SW & & & B14B37 & 01-May-02 & 03-Apr-02 & $10098-97-2$ & SR-90 \\
\hline 765 & SESP SCHEDULING & & SW & & & B14B43 & 01-May-02 & 03-Apr-02 & $10098-97-2$ & SR-90 \\
\hline 766 & SESP SCHEDULING & & SW & & & B14LJ0 & 23-May-02 & & $10098-97-2$ & SR-90 \\
\hline 767 & SESP SCHEDULING & & SW & & & B14LJ2 & 23-May-02 & & $10098-97-2$ & SR-90 \\
\hline 768 & SESP SCHEDULING & & SW & & & B14JF2 & 29-May-02 & 01-May-02 & $10098-97-2$ & SR-90 \\
\hline 769 & SESP SCHEDULING & & SW & & & B14JF8 & 29-May-02 & 01-May-02 & $10098-97-2$ & SR-90 \\
\hline 770 & SESP SCHEDULING & & SW & & & B14RV4 & 10-Jun-02 & & $10098-97-2$ & SR-90 \\
\hline 771 & SESP SCHEDULING & & SW & & & B14RV5 & 10-Jun-02 & & $10098-97-2$ & SR-90 \\
\hline 772 & SESP SCHEDULING & & SW & & & B14RV6 & 10-Jun-02 & & $10098-97-2$ & SR-90 \\
\hline 773 & SESP SCHEDULING & & SW & & & B14RV7 & 10-Jun-02 & & $10098-97-2$ & SR-90 \\
\hline 774 & SESP SCHEDULING & & SW & & & B14RV8 & 11-Jun-02 & & $10098-97-2$ & SR-90 \\
\hline 775 & SESP SCHEDULING & & SW & & & B14RV9 & 11-Jun-02 & & $10098-97-2$ & SR-90 \\
\hline 776 & SESP SCHEDULING & & SW & & & B14RW0 & 11-Jun-02 & & $10098-97-2$ & SR-90 \\
\hline 777 & SESP SCHEDULING & & SW & & & B14RW1 & 11-Jun-02 & & $10098-97-2$ & SR-90 \\
\hline 778 & SESP SCHEDULING & & SW & & & B14RW2 & 11-Jun-02 & & $10098-97-2$ & SR-90 \\
\hline 779 & SESP SCHEDULING & & SW & & & B14RW3 & 11-Jun-02 & & $10098-97-2$ & SR-90 \\
\hline 780 & SESP SCHEDULING & & SW & & & B14RX4 & 11-Jun-02 & & $10098-97-2$ & SR-90 \\
\hline 781 & SESP SCHEDULING & & SW & & & B14RX5 & 11-Jun-02 & & $10098-97-2$ & SR-90 \\
\hline 782 & SESP SCHEDULING & & SW & & & B14RX6 & 11-Jun-02 & & $10098-97-2$ & SR-90 \\
\hline 783 & SESP SCHEDULING & & SW & & & $\mathrm{B} 14 \mathrm{R} \times 7$ & 11-Jun-02 & & $10098-97-2$ & SR-90 \\
\hline 784 & SESP SCHEDULING & & SW & & & B14RL3 & 27-Jun-02 & 29-May-02 & $10098-97-2$ & SR-90 \\
\hline 785 & SESP SCHEDULING & & SW & & & B14RM5 & 27-Jun-02 & 29-May-02 & $10098-97-2$ & SR-90 \\
\hline 786 & SESP SCHEDULING & & SW & & & B14VT4 & 28-Jun-02 & & $10098-97-2$ & SR-90 \\
\hline 787 & SESP SCHEDULING & & SW & & & B14VX3 & 28-Jun-02 & & $10098-97-2$ & SR-90 \\
\hline 788 & SESP SCHEDULING & & SW & & & B14YH3 & 08-Jul-02 & & $10098-97-2$ & SR-90 \\
\hline 789 & SESP SCHEDULING & & SW & & & B14YJ2 & 08-Jul-02 & & $10098-97-2$ & SR-90 \\
\hline 790 & SESP SCHEDULING & & SW & & & B14YJ5 & 08-Jul-02 & & $10098-97-2$ & SR-90 \\
\hline 791 & SESP SCHEDULING & & SW & & & B14YJ8 & 08-Jul-02 & & $10098-97-2$ & SR-90 \\
\hline 792 & SESP SCHEDULING & & SW & & & B14YJ9 & 08-Jul-02 & & $10098-97-2$ & SR-90 \\
\hline 793 & SESP SCHEDULING & & SW & & & B14YKO & 08-Jul-02 & & $10098-97-2$ & SR-90 \\
\hline 794 & SESP SCHEDULING & & SW & & & B14WW5 & 07-Aug-02 & 27-Jun-02 & $10098-97-2$ & SR-90 \\
\hline 795 & SESP SCHEDULING & & SW & & & $\mathrm{B} 14 \mathrm{WX} 2$ & 07-Aug-02 & 27-Jun-02 & $10098-97-2$ & SR-90 \\
\hline 796 & SESP SCHEDULING & & SW & & & B153T9 & 04-Sep-02 & 07-Aug-02 & $10098-97-2$ & SR-90 \\
\hline 797 & SESP SCHEDULING & & SW & & & B153V4 & 04-Sep-02 & 07-Aug-02 & $10098-97-2$ & SR-90 \\
\hline 798 & SESP SCHEDULING & & SW & & & $\mathrm{B} 158 \mathrm{H} 3$ & 05-Sep-02 & & $10098-97-2$ & SR-90 \\
\hline 799 & SESP SCHEDULING & & SW & & & $\mathrm{B} 158 \mathrm{H} 4$ & 05-Sep-02 & & 10098-97-2 & SR-90 \\
\hline
\end{tabular}




\begin{tabular}{|c|c|c|c|c|c|c|c|c|c|c|}
\hline & $\mathrm{A}$ & $\mathrm{B}$ & $\mathrm{C}$ & $\mathrm{D}$ & $E$ & $\mathrm{~F}$ & $G$ & $\mathrm{H}$ & $I$ & $\mathrm{~J}$ \\
\hline 1 & SAMPLE_NUM_ASSIGNED_TO & SAMPLER & MEDIA & BIOTA_MEDIA & TAG_ID & SAMP_NUM & SAMP_DATE_TIME & SAMP_DATE_TIME_ON & CON_ID & CON_SHORT_NAME \\
\hline 800 & SESP SCHEDULING & & SW & & & B158H5 & 05-Sep-02 & & $10098-97-2$ & SR-90 \\
\hline 801 & SESP SCHEDULING & & SW & & & B158H6 & 05-Sep-02 & & $10098-97-2$ & SR-90 \\
\hline 802 & SESP SCHEDULING & & SW & & & $\mathrm{B} 158 \mathrm{H} 7$ & 05-Sep-02 & & $10098-97-2$ & SR-90 \\
\hline 803 & SESP SCHEDULING & & SW & & & $\mathrm{B} 158 \mathrm{H} 8$ & 05-Sep-02 & & $10098-97-2$ & SR-90 \\
\hline 804 & SESP SCHEDULING & & SW & & & $\mathrm{B} 158 \mathrm{H} 9$ & 05-Sep-02 & & $10098-97-2$ & SR-90 \\
\hline 805 & SESP SCHEDULING & & SW & & & B158J0 & 05-Sep-02 & & $10098-97-2$ & SR-90 \\
\hline 806 & SESP SCHEDULING & & SW & & & B158M7 & 05-Sep-02 & & $10098-97-2$ & SR-90 \\
\hline 807 & SESP SCHEDULING & & SW & & & B158M8 & 05-Sep-02 & & $10098-97-2$ & SR-90 \\
\hline 808 & SESP SCHEDULING & & SW & & & B158M9 & 05-Sep-02 & & $10098-97-2$ & SR-90 \\
\hline 809 & SESP SCHEDULING & & SW & & & B158W8 & 05-Sep-02 & & $10098-97-2$ & SR-90 \\
\hline 810 & SESP SCHEDULING & & SW & & & B158X1 & 05-Sep-02 & & $10098-97-2$ & SR-90 \\
\hline 811 & SESP SCHEDULING & & SW & & & B158X4 & 05-Sep-02 & & $10098-97-2$ & SR-90 \\
\hline 812 & SESP SCHEDULING & & SW & & & B158X7 & 05-Sep-02 & & $10098-97-2$ & SR-90 \\
\hline 813 & SESP SCHEDULING & & SW & & & B158J1 & 09-Sep-02 & & $10098-97-2$ & SR-90 \\
\hline 814 & SESP SCHEDULING & & SW & & & B158J3 & 09-Sep-02 & & $10098-97-2$ & SR-90 \\
\hline 815 & SESP SCHEDULING & & SW & & & B158J5 & 09-Sep-02 & & $10098-97-2$ & SR-90 \\
\hline 816 & SESP SCHEDULING & & SW & & & B158J7 & 09-Sep-02 & & $10098-97-2$ & SR-90 \\
\hline 817 & SESP SCHEDULING & & SW & & & B158J9 & 09-Sep-02 & & $10098-97-2$ & SR-90 \\
\hline 818 & SESP SCHEDULING & & SW & & & B158K1 & 09-Sep-02 & & $10098-97-2$ & SR-90 \\
\hline 819 & SESP SCHEDULING & & SW & & & B158K3 & 09-Sep-02 & & $10098-97-2$ & SR-90 \\
\hline 820 & SESP SCHEDULING & & SW & & & B158K5 & 09-Sep-02 & & $10098-97-2$ & SR-90 \\
\hline 821 & SESP SCHEDULING & & SW & & & B158K7 & 09-Sep-02 & & $10098-97-2$ & SR-90 \\
\hline 822 & SESP SCHEDULING & & SW & & & B158K9 & 09-Sep-02 & & $10098-97-2$ & SR-90 \\
\hline 823 & SESP SCHEDULING & & SW & & & B158L1 & 09-Sep-02 & & $10098-97-2$ & SR-90 \\
\hline 824 & SESP SCHEDULING & & SW & & & B158L3 & 09-Sep-02 & & $10098-97-2$ & SR-90 \\
\hline 825 & SESP SCHEDULING & & SW & & & B158NO & 09-Sep-02 & & $10098-97-2$ & SR-90 \\
\hline 826 & SESP SCHEDULING & & SW & & & B158N2 & 09-Sep-02 & & $10098-97-2$ & SR-90 \\
\hline 827 & SESP SCHEDULING & & SW & & & B158N4 & 09-Sep-02 & & $10098-97-2$ & SR-90 \\
\hline 828 & SESP SCHEDULING & & SW & & & B15902 & 09-Sep-02 & & $10098-97-2$ & SR-90 \\
\hline 829 & SESP SCHEDULING & & SW & & & B15906 & 09-Sep-02 & & $10098-97-2$ & SR-90 \\
\hline 830 & SESP SCHEDULING & & SW & & & B15910 & 09-Sep-02 & & $10098-97-2$ & SR-90 \\
\hline 831 & SESP SCHEDULING & & SW & & & B15914 & 09-Sep-02 & & $10098-97-2$ & SR-90 \\
\hline 832 & SESP SCHEDULING & & SW & & & B158L5 & 10-Sep-02 & & $10098-97-2$ & SR-90 \\
\hline 833 & SESP SCHEDULING & & SW & & & B158L6 & 10-Sep-02 & & $10098-97-2$ & SR-90 \\
\hline 834 & SESP SCHEDULING & & SW & & & B158L7 & 10-Sep-02 & & $10098-97-2$ & SR-90 \\
\hline 835 & SESP SCHEDULING & & SW & & & B158L8 & 10-Sep-02 & & $10098-97-2$ & SR-90 \\
\hline 836 & SESP SCHEDULING & & SW & & & B158L9 & 10-Sep-02 & & $10098-97-2$ & SR-90 \\
\hline 837 & SESP SCHEDULING & & SW & & & B158M0 & 10-Sep-02 & & 10098-97-2 & SR-90 \\
\hline
\end{tabular}




\begin{tabular}{|c|c|c|c|c|c|c|c|c|c|c|}
\hline & $\mathrm{A}$ & $\mathrm{B}$ & $\mathrm{C}$ & $\mathrm{D}$ & $E$ & $\mathrm{~F}$ & $G$ & $\mathrm{H}$ & $I$ & $\mathrm{~J}$ \\
\hline 1 & SAMPLE_NUM_ASSIGNED_TO & SAMPLER & MEDIA & BIOTA_MEDIA & TAG_ID & SAMP_NUM & SAMP_DATE_TIME & SAMP_DATE_TIME_ON & CON_ID & CON_SHORT_NAME \\
\hline 838 & SESP SCHEDULING & & SW & & & B158M1 & 10-Sep-02 & & $10098-97-2$ & SR-90 \\
\hline 839 & SESP SCHEDULING & & SW & & & B158M2 & 10-Sep-02 & & $10098-97-2$ & SR-90 \\
\hline 840 & SESP SCHEDULING & & SW & & & B158M3 & 10-Sep-02 & & $10098-97-2$ & SR-90 \\
\hline 841 & SESP SCHEDULING & & SW & & & B158M4 & 10-Sep-02 & & $10098-97-2$ & SR-90 \\
\hline 842 & SESP SCHEDULING & & SW & & & B158M5 & 10-Sep-02 & & $10098-97-2$ & SR-90 \\
\hline 843 & SESP SCHEDULING & & SW & & & B158M6 & 10-Sep-02 & & $10098-97-2$ & SR-90 \\
\hline 844 & SESP SCHEDULING & & SW & & & B158V6 & 10-Sep-02 & & $10098-97-2$ & SR-90 \\
\hline 845 & SESP SCHEDULING & & SW & & & B158V7 & 10-Sep-02 & & $10098-97-2$ & SR-90 \\
\hline 846 & SESP SCHEDULING & & SW & & & B158V8 & 10-Sep-02 & & $10098-97-2$ & SR-90 \\
\hline 847 & SESP SCHEDULING & & SW & & & B158V9 & 10-Sep-02 & & $10098-97-2$ & SR-90 \\
\hline 848 & SESP SCHEDULING & & SW & & & B158Y0 & 10-Sep-02 & & $10098-97-2$ & SR-90 \\
\hline 849 & SESP SCHEDULING & & SW & & & B158Y3 & 10-Sep-02 & & $10098-97-2$ & SR-90 \\
\hline 850 & SESP SCHEDULING & & SW & & & B158Y6 & 10-Sep-02 & & $10098-97-2$ & SR-90 \\
\hline 851 & SESP SCHEDULING & & SW & & & B158Y9 & 10-Sep-02 & & $10098-97-2$ & SR-90 \\
\hline 852 & SESP SCHEDULING & & SW & & & B15C08 & 16-Sep-02 & & $10098-97-2$ & SR-90 \\
\hline 853 & SESP SCHEDULING & & SW & & & B15C55 & 16-Sep-02 & & $10098-97-2$ & SR-90 \\
\hline 854 & SESP SCHEDULING & & SW & & & B15C58 & 16-Sep-02 & & $10098-97-2$ & SR-90 \\
\hline 855 & SESP SCHEDULING & & SW & & & B15948 & 02-Oct-02 & 04-Sep-02 & $10098-97-2$ & SR-90 \\
\hline 856 & SESP SCHEDULING & & SW & & & B15954 & 02-Oct-02 & 04-Sep-02 & $10098-97-2$ & SR-90 \\
\hline 857 & SESP SCHEDULING & & SW & & & B15C11 & 07-Oct-02 & & $10098-97-2$ & SR-90 \\
\hline 858 & SESP SCHEDULING & & SW & & & B15C60 & 21-Oct-02 & & $10098-97-2$ & SR-90 \\
\hline 859 & SESP SCHEDULING & & SW & & & B15CB3 & 27-Oct-02 & & $10098-97-2$ & SR-90 \\
\hline 860 & SESP SCHEDULING & & SW & & & B15C69 & 29-Oct-02 & & $10098-97-2$ & SR-90 \\
\hline 861 & SESP SCHEDULING & & SW & & & B15K74 & 30-Oct-02 & 02-Oct-02 & $10098-97-2$ & SR-90 \\
\hline 862 & SESP SCHEDULING & & SW & & & B15K79 & 30-Oct-02 & 02-Oct-02 & $10098-97-2$ & SR-90 \\
\hline 863 & SESP SCHEDULING & & SW & & & B15VN2 & 26-Nov-02 & 30-Oct-02 & $10098-97-2$ & SR-90 \\
\hline 864 & SESP SCHEDULING & & SW & & & B15VN8 & 26-Nov-02 & 30-Oct-02 & $10098-97-2$ & SR-90 \\
\hline 865 & SESP SCHEDULING & & SW & & & B16342 & 09-Dec-02 & & $10098-97-2$ & SR-90 \\
\hline 866 & SESP SCHEDULING & & SW & & & B16343 & 09-Dec-02 & & $10098-97-2$ & SR-90 \\
\hline 867 & SESP SCHEDULING & & SW & & & B16344 & 09-Dec-02 & & $10098-97-2$ & SR-90 \\
\hline 868 & SESP SCHEDULING & & SW & & & B16345 & 09-Dec-02 & & $10098-97-2$ & SR-90 \\
\hline 869 & SESP SCHEDULING & & SW & & & B16341 & 10-Dec-02 & & $10098-97-2$ & SR-90 \\
\hline 870 & SESP SCHEDULING & & SW & & & B16346 & 10-Dec-02 & & $10098-97-2$ & SR-90 \\
\hline 871 & SESP SCHEDULING & & SW & & & B16347 & 10-Dec-02 & & $10098-97-2$ & SR-90 \\
\hline 872 & SESP SCHEDULING & & SW & & & B16348 & 10-Dec-02 & & $10098-97-2$ & SR-90 \\
\hline 873 & SESP SCHEDULING & & SW & & & B16349 & 10-Dec-02 & & $10098-97-2$ & SR-90 \\
\hline 874 & SESP SCHEDULING & & SW & & & B16350 & 10-Dec-02 & & $10098-97-2$ & SR-90 \\
\hline 875 & SESP SCHEDULING & & SW & & & B16351 & 10-Dec-02 & & 10098-97-2 & SR-90 \\
\hline
\end{tabular}




\begin{tabular}{|c|c|c|c|c|c|c|c|c|c|c|}
\hline & $A$ & $B$ & $\mathrm{C}$ & $\mathrm{D}$ & $E$ & $\mathrm{~F}$ & $G$ & $\mathrm{H}$ & $\mathrm{I}$ & $\mathrm{J}$ \\
\hline 1 & SAMPLE NUM ASSIGNED TO & SAMPLER & MEDIA & BIOTA MEDIA & TAG ID & SAMP NUM & SAMP DATE TIME & SAMP DATE TIME ON & CON ID & CON SHORT NAME \\
\hline 876 & SESP SCHEDULING & & SW & & & B16362 & 10-Dec-02 & & $10098-97-2$ & SR-90 \\
\hline 877 & SESP SCHEDULING & & SW & & & B16363 & 10-Dec-02 & & $10098-97-2$ & SR-90 \\
\hline 878 & SESP SCHEDULING & & SW & & & B16364 & 10-Dec-02 & & $10098-97-2$ & SR-90 \\
\hline 879 & SESP SCHEDULING & & SW & & & B16365 & 10-Dec-02 & & $10098-97-2$ & SR-90 \\
\hline 880 & SESP SCHEDULING & & SW & & & B0X8R8 & 04-Jan-00 & & 14133-76-7 & TC-99 \\
\hline 881 & SESP SCHEDULING & & SW & & & B0X7K4 & 02-Feb-00 & 29-Dec-99 & $14133-76-7$ & TC-99 \\
\hline 882 & SESP SCHEDULING & & SW & & & B0X7J0 & 02-Feb-00 & 29-Dec-99 & $14133-76-7$ & TC-99 \\
\hline 883 & SESP SCHEDULING & & SW & & & BOXH28 & 08-Mar-00 & 02-Feb-00 & 14133-76-7 & TC-99 \\
\hline 884 & SESP SCHEDULING & & SW & & & BOXH22 & 08-Mar-00 & 02-Feb-00 & 14133-76-7 & TC-99 \\
\hline 885 & SESP SCHEDULING & & SW & & & BOXWWO & 04-Apr-00 & & $14133-76-7$ & TC-99 \\
\hline 886 & SESP SCHEDULING & & SW & & & B0XP21 & 05-Apr-00 & 08-Mar-00 & 14133-76-7 & TC-99 \\
\hline 887 & SESP SCHEDULING & & SW & & & B0XP16 & 05-Apr-00 & 08-Mar-00 & $14133-76-7$ & TC-99 \\
\hline 888 & SESP SCHEDULING & & SW & & & BOXVN6 & 03-May-00 & 29-Mar-00 & $14133-76-7$ & TC-99 \\
\hline 889 & SESP SCHEDULING & & SW & & & B0XVP1 & 03-May-00 & 29-Mar-00 & 14133-76-7 & TC-99 \\
\hline 890 & SESP SCHEDULING & & SW & & & B0Y231 & 31-May-00 & 03-May-00 & 14133-76-7 & TC-99 \\
\hline 891 & SESP SCHEDULING & & SW & & & B0Y237 & 31-May-00 & 03-May-00 & 14133-76-7 & TC-99 \\
\hline 892 & SESP SCHEDULING & & SW & & & B0YB64 & 29-Jun-00 & 31-May-00 & 14133-76-7 & TC-99 \\
\hline 893 & SESP SCHEDULING & & SW & & & B0YB76 & 29-Jun-00 & 31-May-00 & 14133-76-7 & TC-99 \\
\hline 894 & SESP SCHEDULING & & SW & & & B0YM20 & 05-Jul-00 & & $14133-76-7$ & TC-99 \\
\hline 895 & SESP SCHEDULING & & SW & & & BOYKV4 & 10-Aug-00 & 29-Jun-00 & $14133-76-7$ & TC-99 \\
\hline 896 & SESP SCHEDULING & & SW & & & BOYKW1 & 10-Aug-00 & 29-Jun-00 & 14133-76-7 & TC-99 \\
\hline 897 & SESP SCHEDULING & & SW & & & BoYW65 & 07-Sep-00 & & $14133-76-7$ & TC-99 \\
\hline 898 & SESP SCHEDULING & & SW & & & BOYW70 & 07-Sep-00 & & $14133-76-7$ & TC-99 \\
\hline 899 & SESP SCHEDULING & & SW & & & B10912 & 27-Sep-00 & & 14133-76-7 & TC-99 \\
\hline 900 & SESP SCHEDULING & & SW & & & B10913 & 27-Sep-00 & & $14133-76-7$ & TC-99 \\
\hline 901 & SESP SCHEDULING & & SW & & & B10945 & 27-Sep-00 & & $14133-76-7$ & TC-99 \\
\hline 902 & SESP SCHEDULING & & SW & & & B10946 & 27-Sep-00 & & 14133-76-7 & TC-99 \\
\hline 903 & SESP SCHEDULING & & SW & & & B10949 & 27-Sep-00 & & $14133-76-7$ & TC-99 \\
\hline 904 & SESP SCHEDULING & & SW & & & B103W8 & 05-Oct-00 & 07-Sep-00 & $14133-76-7$ & TC-99 \\
\hline 905 & SESP SCHEDULING & & SW & & & B103X3 & 05-Oct-00 & 07-Sep-00 & 14133-76-7 & TC-99 \\
\hline 906 & SESP SCHEDULING & & SW & & & B10F33 & 09-Oct-00 & & $14133-76-7$ & TC-99 \\
\hline 907 & SESP SCHEDULING & & SW & & & B10932 & $25-$ Oct-00 & & $14133-76-7$ & TC-99 \\
\hline 908 & SESP SCHEDULING & & SW & & & B10936 & 25-Oct-00 & & 14133-76-7 & TC-99 \\
\hline 909 & SESP SCHEDULING & & SW & & & B10941 & 01-Nov-00 & & 14133-76-7 & TC-99 \\
\hline 910 & SESP SCHEDULING & & SW & & & B10977 & 01-Nov-00 & & $14133-76-7$ & TC-99 \\
\hline 911 & SESP SCHEDULING & & SW & & & B10C13 & 02-Nov-00 & 05-Oct-00 & $14133-76-7$ & TC-99 \\
\hline 912 & SESP SCHEDULING & & SW & & & B10C19 & 02-Nov-00 & 05-Oct-00 & $14133-76-7$ & TC-99 \\
\hline 913 & SESP SCHEDULING & & SW & & & B10MP5 & 29-Nov-00 & 02-Nov-00 & $14133-76-7$ & TC-99 \\
\hline
\end{tabular}




\begin{tabular}{|c|c|c|c|c|c|c|c|c|c|c|}
\hline & $\mathrm{A}$ & $\mathrm{B}$ & $\mathrm{C}$ & $\mathrm{D}$ & $E$ & $\mathrm{~F}$ & $G$ & $\mathrm{H}$ & $I$ & $\mathrm{~J}$ \\
\hline 1 & SAMPLE_NUM_ASSIGNED_TO & SAMPLER & MEDIA & BIOTA_MEDIA & TAG_ID & SAMP_NUM & SAMP_DATE_TIME & SAMP_DATE_TIME_ON & CON_ID & CON_SHORT_NAME \\
\hline 914 & SESP SCHEDULING & & SW & & & B10MR1 & 29-Nov-00 & 02-Nov-00 & 14133-76-7 & TC-99 \\
\hline 915 & SESP SCHEDULING & & SW & & & B110Y4 & 04-Jan-01 & 29-Nov-00 & 14133-76-7 & TC-99 \\
\hline 916 & SESP SCHEDULING & & SW & & & B11100 & 04-Jan-01 & 29-Nov-00 & 14133-76-7 & TC-99 \\
\hline 917 & SESP SCHEDULING & & SW & & & B114P8 & 31-Jan-01 & 04-Jan-01 & $14133-76-7$ & TC-99 \\
\hline 918 & SESP SCHEDULING & & SW & & & B114T0 & 31-Jan-01 & 04-Jan-01 & 14133-76-7 & TC-99 \\
\hline 919 & SESP SCHEDULING & & SW & & & B11CC7 & 12-Feb-01 & & 14133-76-7 & TC-99 \\
\hline 920 & SESP SCHEDULING & & SW & & & B11C04 & 01-Mar-01 & 31-Jan-01 & $14133-76-7$ & TC-99 \\
\hline 921 & SESP SCHEDULING & & SW & & & B11C10 & 01-Mar-01 & 31-Jan-01 & $14133-76-7$ & TC-99 \\
\hline 922 & SESP SCHEDULING & & SW & & & B11MY9 & 03-Apr-01 & & 14133-76-7 & TC-99 \\
\hline 923 & SESP SCHEDULING & & SW & & & B11J40 & 04-Apr-01 & 01-Mar-01 & $14133-76-7$ & TC-99 \\
\hline 924 & SESP SCHEDULING & & SW & & & B11J46 & 04-Apr-01 & 01-Mar-01 & $14133-76-7$ & TC-99 \\
\hline 925 & SESP SCHEDULING & & SW & & & B11W36 & 30-Apr-01 & & $14133-76-7$ & TC-99 \\
\hline 926 & SESP SCHEDULING & & SW & & & B11W50 & 30-Apr-01 & & 14133-76-7 & TC-99 \\
\hline 927 & SESP SCHEDULING & & SW & & & B11W55 & 30-Apr-01 & & 14133-76-7 & TC-99 \\
\hline 928 & SESP SCHEDULING & & SW & & & B11W86 & 30-Apr-01 & & 14133-76-7 & TC-99 \\
\hline 929 & SESP SCHEDULING & & SW & & & B11LT2 & 02-May-01 & 04-Apr-01 & $14133-76-7$ & TC-99 \\
\hline 930 & SESP SCHEDULING & & SW & & & B11LR6 & 02-May-01 & 04-Apr-01 & $14133-76-7$ & TC-99 \\
\hline 931 & SESP SCHEDULING & & SW & & & B11W40 & 04-May-01 & & $14133-76-7$ & TC-99 \\
\hline 932 & SESP SCHEDULING & & SW & & & B11W43 & 04-May-01 & & 14133-76-7 & TC-99 \\
\hline 933 & SESP SCHEDULING & & SW & & & B11WT4 & 30-May-01 & 02-May-01 & $14133-76-7$ & TC-99 \\
\hline 934 & SESP SCHEDULING & & SW & & & B11WR8 & 30-May-01 & 02-May-01 & 14133-76-7 & TC-99 \\
\hline 935 & SESP SCHEDULING & & SW & & & B124M3 & 28-Jun-01 & 23-May-01 & $14133-76-7$ & TC-99 \\
\hline 936 & SESP SCHEDULING & & SW & & & B124L7 & 28-Jun-01 & 23-May-01 & $14133-76-7$ & TC-99 \\
\hline 937 & SESP SCHEDULING & & SW & & & B12992 & 09-Jul-01 & & $14133-76-7$ & TC-99 \\
\hline 938 & SESP SCHEDULING & & SW & & & B12841 & 09-Aug-01 & 28-Jun-01 & $14133-76-7$ & TC-99 \\
\hline 939 & SESP SCHEDULING & & SW & & & B12834 & 09-Aug-01 & 28-Jun-01 & $14133-76-7$ & TC-99 \\
\hline 940 & SESP SCHEDULING & & SW & & & B12JT2 & 05-Sep-01 & & 14133-76-7 & TC-99 \\
\hline 941 & SESP SCHEDULING & & SW & & & B12JR7 & 05-Sep-01 & 09-Aug-01 & 14133-76-7 & TC-99 \\
\hline 942 & SESP SCHEDULING & & SW & & & B12XK2 & 17-Sep-01 & & $14133-76-7$ & TC-99 \\
\hline 943 & SESP SCHEDULING & & SW & & & B12XK8 & 17-Sep-01 & & $14133-76-7$ & TC-99 \\
\hline 944 & SESP SCHEDULING & & SW & & & B12XK9 & 17-Sep-01 & & $14133-76-7$ & TC-99 \\
\hline 945 & SESP SCHEDULING & & SW & & & B12XLO & 17-Sep-01 & & $14133-76-7$ & TC-99 \\
\hline 946 & SESP SCHEDULING & & SW & & & B12XL1 & 17-Sep-01 & & $14133-76-7$ & TC-99 \\
\hline 947 & SESP SCHEDULING & & SW & & & B12XL2 & 17-Sep-01 & & $14133-76-7$ & TC-99 \\
\hline 948 & SESP SCHEDULING & & SW & & & B12XK1 & 18-Sep-01 & & $14133-76-7$ & TC-99 \\
\hline 949 & SESP SCHEDULING & & SW & & & B12XK3 & 18-Sep-01 & & 14133-76-7 & TC-99 \\
\hline 950 & SESP SCHEDULING & & SW & & & B12XK4 & 18-Sep-01 & & $14133-76-7$ & TC-99 \\
\hline 951 & SESP SCHEDULING & & SW & & & B12XK5 & 18-Sep-01 & & 14133-76-7 & TC-99 \\
\hline
\end{tabular}




\begin{tabular}{|c|c|c|c|c|c|c|c|c|c|c|}
\hline & $\mathrm{A}$ & $B$ & $\mathrm{C}$ & $\mathrm{D}$ & $E$ & $\mathrm{~F}$ & $G$ & $\mathrm{H}$ & $\mathrm{I}$ & $\mathrm{J}$ \\
\hline 1 & SAMPLE_NUM_ASSIGNED_TO & SAMPLER & MEDIA & BIOTA_MEDIA & TAG_ID & SAMP_NUM & SAMP_DATE_TIME & SAMP_DATE_TIME_ON & CON_ID & CON_SHORT_NAME \\
\hline 952 & SESP SCHEDULING & & SW & & & B12XK6 & 18-Sep-01 & & 14133-76-7 & TC-99 \\
\hline 953 & SESP SCHEDULING & & SW & & & B12XK7 & 18-Sep-01 & & 14133-76-7 & TC-99 \\
\hline 954 & SESP SCHEDULING & & SW & & & B12T28 & 02-Oct-01 & 05-Sep-01 & 14133-76-7 & TC-99 \\
\hline 955 & SESP SCHEDULING & & SW & & & B12T33 & 02-Oct-01 & 05-Sep-01 & $14133-76-7$ & TC-99 \\
\hline 956 & SESP SCHEDULING & & SW & & & B12X38 & 22-Oct-01 & & 14133-76-7 & TC-99 \\
\hline 957 & SESP SCHEDULING & & SW & & & B12X41 & 25-Oct-01 & & $14133-76-7$ & TC-99 \\
\hline 958 & SESP SCHEDULING & & SW & & & $\mathrm{B} 12 \mathrm{X} 42$ & 25-Oct-01 & & $14133-76-7$ & TC-99 \\
\hline 959 & SESP SCHEDULING & & SW & & & B12X48 & 01-Nov-01 & & $14133-76-7$ & TC-99 \\
\hline 960 & SESP SCHEDULING & & SW & & & B12X85 & 01-Nov-01 & & $14133-76-7$ & TC-99 \\
\hline 961 & SESP SCHEDULING & & SW & & & B13103 & 01-Nov-01 & 02-Oct-01 & $14133-76-7$ & TC-99 \\
\hline 962 & SESP SCHEDULING & & SW & & & B130Y7 & 01-Nov-01 & 02-Oct-01 & 14133-76-7 & TC-99 \\
\hline 963 & SESP SCHEDULING & & SW & & & B13CC6 & 27-Nov-01 & 01-Nov-01 & 14133-76-7 & TC-99 \\
\hline 964 & SESP SCHEDULING & & SW & & & B13CD2 & 27-Nov-01 & 01-Nov-01 & 14133-76-7 & TC-99 \\
\hline 965 & SESP SCHEDULING & & SW & & & B13LB3 & 03-Jan-02 & 27-Nov-01 & 14133-76-7 & TC-99 \\
\hline 966 & SESP SCHEDULING & & SW & & & B13L96 & 03-Jan-02 & 27-Nov-01 & $14133-76-7$ & TC-99 \\
\hline 967 & SESP SCHEDULING & & SW & & & B13VJ0 & 06-Feb-02 & & $14133-76-7$ & TC-99 \\
\hline 968 & SESP SCHEDULING & & SW & & & B13VF8 & 06-Feb-02 & & $14133-76-7$ & TC-99 \\
\hline 969 & SESP SCHEDULING & & SW & & & B140P7 & 07-Mar-02 & 06-Feb-02 & 14133-76-7 & TC-99 \\
\hline 970 & SESP SCHEDULING & & SW & & & B140R2 & 07-Mar-02 & 06-Feb-02 & $14133-76-7$ & TC-99 \\
\hline 971 & SESP SCHEDULING & & SW & & & B14408 & 03-Apr-02 & 07-Mar-02 & $14133-76-7$ & TC-99 \\
\hline 972 & SESP SCHEDULING & & SW & & & B14413 & 03-Apr-02 & 07-Mar-02 & $14133-76-7$ & TC-99 \\
\hline 973 & SESP SCHEDULING & & SW & & & B14B37 & 01-May-02 & 03-Apr-02 & $14133-76-7$ & TC-99 \\
\hline 974 & SESP SCHEDULING & & SW & & & B14B43 & 01-May-02 & 03-Apr-02 & 14133-76-7 & TC-99 \\
\hline 975 & SESP SCHEDULING & & SW & & & B14JF2 & 29-May-02 & 01-May-02 & $14133-76-7$ & TC-99 \\
\hline 976 & SESP SCHEDULING & & SW & & & B14JF8 & 29-May-02 & 01-May-02 & $14133-76-7$ & TC-99 \\
\hline 977 & SESP SCHEDULING & & SW & & & B14RL3 & 27-Jun-02 & 29-May-02 & 14133-76-7 & TC-99 \\
\hline 978 & SESP SCHEDULING & & SW & & & B14RM5 & 27-Jun-02 & 29-May-02 & 14133-76-7 & TC-99 \\
\hline 979 & SESP SCHEDULING & & SW & & & B14WW5 & 07-Aug-02 & 27-Jun-02 & $14133-76-7$ & TC-99 \\
\hline 980 & SESP SCHEDULING & & SW & & & B14WX2 & 07-Aug-02 & 27-Jun-02 & $14133-76-7$ & TC-99 \\
\hline 981 & SESP SCHEDULING & & SW & & & В153T9 & 04-Sep-02 & 07-Aug-02 & $14133-76-7$ & TC-99 \\
\hline 982 & SESP SCHEDULING & & SW & & & B153V4 & 04-Sep-02 & 07-Aug-02 & $14133-76-7$ & TC-99 \\
\hline 983 & SESP SCHEDULING & & SW & & & B15C55 & 16-Sep-02 & & 14133-76-7 & TC-99 \\
\hline 984 & SESP SCHEDULING & & SW & & & B15C58 & 16-Sep-02 & & $14133-76-7$ & TC-99 \\
\hline 985 & SESP SCHEDULING & & SW & & & B15948 & 02-Oct-02 & 04-Sep-02 & $14133-76-7$ & TC-99 \\
\hline 986 & SESP SCHEDULING & & SW & & & B15954 & 02-Oct-02 & 04-Sep-02 & $14133-76-7$ & TC-99 \\
\hline 987 & SESP SCHEDULING & & SW & & & B15C09 & 07-Oct-02 & & 14133-76-7 & TC-99 \\
\hline 988 & SESP SCHEDULING & & SW & & & B15C73 & 07-Oct-02 & & $14133-76-7$ & TC-99 \\
\hline 989 & SESP SCHEDULING & & SW & & & $\mathrm{B} 15 \mathrm{C} 60$ & 21-Oct-02 & & $14133-76-7$ & TC-99 \\
\hline
\end{tabular}




\begin{tabular}{|c|c|c|c|c|c|c|c|c|c|c|}
\hline & A & $B$ & $\mathrm{C}$ & $\mathrm{D}$ & $E$ & $\mathrm{~F}$ & $G$ & $\mathrm{H}$ & $I$ & $\mathrm{~J}$ \\
\hline 1 & SAMPLE_NUM_ASSIGNED_TO & SAMPLER & MEDIA & BIOTA_MEDIA & TAG_ID & SAMP_NUM & SAMP_DATE_TIME & SAMP_DATE_TIME_ON & CON_ID & CON_SHORT_NAME \\
\hline 990 & SESP SCHEDULING & & SW & & & B15CB3 & 27-Oct-02 & & 14133-76-7 & TC-99 \\
\hline 991 & SESP SCHEDULING & & SW & & & B15K74 & 30-Oct-02 & 02-Oct-02 & 14133-76-7 & TC-99 \\
\hline 992 & SESP SCHEDULING & & SW & & & B15K79 & 30-Oct-02 & 02-Oct-02 & 14133-76-7 & TC-99 \\
\hline 993 & SESP SCHEDULING & & SW & & & B15VN2 & 26-Nov-02 & $30-$ Oct-02 & 14133-76-7 & TC-99 \\
\hline 994 & SESP SCHEDULING & & SW & & & B15VN8 & 26-Nov-02 & 30-Oct-02 & 14133-76-7 & TC-99 \\
\hline 995 & SESP SCHEDULING & & SW & & & B0X8R8 & 04-Jan-00 & & $10028-17-8$ & TRITIUM \\
\hline 996 & SESP SCHEDULING & & SW & & & B0X8R9 & 04-Jan-00 & & $10028-17-8$ & TRITIUM \\
\hline 997 & SESP SCHEDULING & & SW & & & B0X7K4 & 02-Feb-00 & 29-Dec-99 & $10028-17-8$ & TRITIUM \\
\hline 998 & SESP SCHEDULING & & SW & & & B0X7J0 & 02-Feb-00 & 29-Dec-99 & $10028-17-8$ & TRITIUM \\
\hline 999 & SESP SCHEDULING & & SW & & & BOXJP2 & 04-Feb-00 & & $10028-17-8$ & TRITIUM \\
\hline 1000 & SESP SCHEDULING & & SW & & & BOXJP4 & 04-Feb-00 & & $10028-17-8$ & TRITIUM \\
\hline 1001 & SESP SCHEDULING & & SW & & & B0XJP6 & 04-Feb-00 & & $10028-17-8$ & TRITIUM \\
\hline 1002 & SESP SCHEDULING & & SW & & & B0XKV5 & 22-Feb-00 & & $10028-17-8$ & TRITIUM \\
\hline 1003 & SESP SCHEDULING & & SW & & & B0XKV6 & 22-Feb-00 & & $10028-17-8$ & TRITIUM \\
\hline 1004 & SESP SCHEDULING & & SW & & & B0XKV7 & 22-Feb-00 & & $10028-17-8$ & TRITIUM \\
\hline 1005 & SESP SCHEDULING & & SW & & & B0XKW7 & 22-Feb-00 & & $10028-17-8$ & TRITIUM \\
\hline 1006 & SESP SCHEDULING & & SW & & & B0XH28 & 08-Mar-00 & 02-Feb-00 & $10028-17-8$ & TRITIUM \\
\hline 1007 & SESP SCHEDULING & & SW & & & B0XH22 & 08-Mar-00 & 02-Feb-00 & $10028-17-8$ & TRITIUM \\
\hline 1008 & SESP SCHEDULING & & SW & & & BOXRK2 & 27-Mar-00 & & $10028-17-8$ & TRITIUM \\
\hline 1009 & SESP SCHEDULING & & SW & & & B0XRK3 & 27-Mar-00 & & $10028-17-8$ & TRITIUM \\
\hline 1010 & SESP SCHEDULING & & SW & & & B0XRK4 & 27-Mar-00 & & $10028-17-8$ & TRITIUM \\
\hline 1011 & SESP SCHEDULING & & SW & & & B0XRK5 & 27-Mar-00 & & $10028-17-8$ & TRITIUM \\
\hline 1012 & SESP SCHEDULING & & SW & & & BOXRKO & 28-Mar-00 & & $10028-17-8$ & TRITIUM \\
\hline 1013 & SESP SCHEDULING & & SW & & & B0XRK6 & 28-Mar-00 & & $10028-17-8$ & TRITIUM \\
\hline 1014 & SESP SCHEDULING & & SW & & & B0XRK7 & 28-Mar-00 & & $10028-17-8$ & TRITIUM \\
\hline 1015 & SESP SCHEDULING & & SW & & & B0XRK8 & 28-Mar-00 & & $10028-17-8$ & TRITIUM \\
\hline 1016 & SESP SCHEDULING & & SW & & & B0XRK9 & 28-Mar-00 & & $10028-17-8$ & TRITIUM \\
\hline 1017 & SESP SCHEDULING & & SW & & & BOXRLO & 28-Mar-00 & & $10028-17-8$ & TRITIUM \\
\hline 1018 & SESP SCHEDULING & & SW & & & B0XRL1 & 28-Mar-00 & & $10028-17-8$ & TRITIUM \\
\hline 1019 & SESP SCHEDULING & & SW & & & B0XRN8 & 28-Mar-00 & & $10028-17-8$ & TRITIUM \\
\hline 1020 & SESP SCHEDULING & & SW & & & BOXRN9 & 28-Mar-00 & & $10028-17-8$ & TRITIUM \\
\hline 1021 & SESP SCHEDULING & & SW & & & BOXRPO & 28-Mar-00 & & $10028-17-8$ & TRITIUM \\
\hline 1022 & SESP SCHEDULING & & SW & & & B0XRP1 & 28-Mar-00 & & $10028-17-8$ & TRITIUM \\
\hline 1023 & SESP SCHEDULING & & SW & & & BOXWWO & 04-Apr-00 & & $10028-17-8$ & TRITIUM \\
\hline 1024 & SESP SCHEDULING & & SW & & & B0XWW1 & 04-Apr-00 & & $10028-17-8$ & TRITIUM \\
\hline 1025 & SESP SCHEDULING & & SW & & & BoxWW2 & 04-Apr-00 & & $10028-17-8$ & TRITIUM \\
\hline 1026 & SESP SCHEDULING & & SW & & & B0XP21 & 05-Apr-00 & 08-Mar-00 & $10028-17-8$ & TRITIUM \\
\hline 1027 & SESP SCHEDULING & & SW & & & B0XP16 & 05-Apr-00 & 08-Mar-00 & $10028-17-8$ & TRITIUM \\
\hline
\end{tabular}




\begin{tabular}{|c|c|c|c|c|c|c|c|c|c|c|}
\hline & A & $\mathrm{B}$ & C & $\mathrm{D}$ & $E$ & $\mathrm{~F}$ & G & $\mathrm{H}$ & $\mathrm{I}$ & $\mathrm{J}$ \\
\hline 1 & SAMPLE_NUM_ASSIGNED_TO & SAMPLER & MEDIA & BIOTA_MEDIA & TAG_ID & SAMP_NUM & SAMP_DATE_TIME & SAMP_DATE_TIME_ON & CON_ID & CON_SHORT_NAME \\
\hline 1028 & SESP SCHEDULING & & SW & & & B0Y265 & 02-May-00 & & $10028-17-8$ & TRITIUM \\
\hline 1029 & SESP SCHEDULING & & SW & & & B0Y266 & 02-May-00 & & $10028-17-8$ & TRITIUM \\
\hline 1030 & SESP SCHEDULING & & SW & & & B0Y267 & 02-May-00 & & $10028-17-8$ & TRITIUM \\
\hline 1031 & SESP SCHEDULING & & SW & & & B0Y269 & 02-May-00 & & $10028-17-8$ & TRITIUM \\
\hline 1032 & SESP SCHEDULING & & SW & & & B0XVN6 & 03-May-00 & 29-Mar-00 & $10028-17-8$ & TRITIUM \\
\hline 1033 & SESP SCHEDULING & & SW & & & B0XVP1 & 03-May-00 & 29-Mar-00 & $10028-17-8$ & TRITIUM \\
\hline 1034 & SESP SCHEDULING & & SW & & & B0Y2Y6 & 11-May-00 & & $10028-17-8$ & TRITIUM \\
\hline 1035 & SESP SCHEDULING & & SW & & & B0Y231 & 31-May-00 & 03-May-00 & $10028-17-8$ & TRITIUM \\
\hline 1036 & SESP SCHEDULING & & SW & & & B0Y237 & 31-May-00 & 03-May-00 & $10028-17-8$ & TRITIUM \\
\hline 1037 & SESP SCHEDULING & & SW & & & BOYB90 & 08-Jun-00 & & $10028-17-8$ & TRITIUM \\
\hline 1038 & SESP SCHEDULING & & SW & & & BOYCD7 & 19-Jun-00 & & $10028-17-8$ & TRITIUM \\
\hline 1039 & SESP SCHEDULING & & SW & & & B0YCD8 & 19-Jun-00 & & $10028-17-8$ & TRITIUM \\
\hline 1040 & SESP SCHEDULING & & SW & & & B0YCD9 & 19-Jun-00 & & $10028-17-8$ & TRITIUM \\
\hline 1041 & SESP SCHEDULING & & SW & & & BOYCFO & 19-Jun-00 & & $10028-17-8$ & TRITIUM \\
\hline 1042 & SESP SCHEDULING & & SW & & & BOYCF1 & 20-Jun-00 & & $10028-17-8$ & TRITIUM \\
\hline 1043 & SESP SCHEDULING & & SW & & & BOYCF2 & 20-Jun-00 & & $10028-17-8$ & TRITIUM \\
\hline 1044 & SESP SCHEDULING & & SW & & & BOYCF3 & 20-Jun-00 & & $10028-17-8$ & TRITIUM \\
\hline 1045 & SESP SCHEDULING & & SW & & & BOYCF4 & 20-Jun-00 & & $10028-17-8$ & TRITIUM \\
\hline 1046 & SESP SCHEDULING & & SW & & & B0YCF5 & 20-Jun-00 & & $10028-17-8$ & TRITIUM \\
\hline 1047 & SESP SCHEDULING & & SW & & & BOYCF6 & 20-Jun-00 & & $10028-17-8$ & TRITIUM \\
\hline 1048 & SESP SCHEDULING & & SW & & & BOYCK3 & 20-Jun-00 & & $10028-17-8$ & TRITIUM \\
\hline 1049 & SESP SCHEDULING & & SW & & & BOYCK4 & 20-Jun-00 & & $10028-17-8$ & TRITIUM \\
\hline 1050 & SESP SCHEDULING & & SW & & & B0YCK5 & 20-Jun-00 & & $10028-17-8$ & TRITIUM \\
\hline 1051 & SESP SCHEDULING & & SW & & & B0YCK6 & 20-Jun-00 & & $10028-17-8$ & TRITIUM \\
\hline 1052 & SESP SCHEDULING & & SW & & & B0YB64 & 29-Jun-00 & 31-May-00 & $10028-17-8$ & TRITIUM \\
\hline 1053 & SESP SCHEDULING & & SW & & & B0YB76 & 29-Jun-00 & 31-May-00 & $10028-17-8$ & TRITIUM \\
\hline 1054 & SESP SCHEDULING & & SW & & & B0YM20 & 05-Jul-00 & & $10028-17-8$ & TRITIUM \\
\hline 1055 & SESP SCHEDULING & & SW & & & B0YM21 & 05-Jul-00 & & $10028-17-8$ & TRITIUM \\
\hline 1056 & SESP SCHEDULING & & SW & & & BOYVM2 & 06-Jul-00 & & $10028-17-8$ & TRITIUM \\
\hline 1057 & SESP SCHEDULING & & SW & & & BOYM22 & 07-Jul-00 & & $10028-17-8$ & TRITIUM \\
\hline 1058 & SESP SCHEDULING & & SW & & & BOYP01 & 25-Jul-00 & & $10028-17-8$ & TRITIUM \\
\hline 1059 & SESP SCHEDULING & & SW & & & B0YP02 & 25-Jul-00 & & $10028-17-8$ & TRITIUM \\
\hline 1060 & SESP SCHEDULING & & SW & & & B0YP03 & 25-Jul-00 & & $10028-17-8$ & TRITIUM \\
\hline 1061 & SESP SCHEDULING & & SW & & & B0YP05 & 25-Jul-00 & & $10028-17-8$ & TRITIUM \\
\hline 1062 & SESP SCHEDULING & & SW & & & BOYKV4 & 10-Aug-00 & 29-Jun-00 & 10028-17-8 & TRITIUM \\
\hline 1063 & SESP SCHEDULING & & SW & & & B0YKW1 & 10-Aug-00 & 29-Jun-00 & $10028-17-8$ & TRITIUM \\
\hline 1064 & SESP SCHEDULING & & SW & & & B0YW65 & 07-Sep-00 & & $10028-17-8$ & TRITIUM \\
\hline 1065 & SESP SCHEDULING & & SW & & & B0YW70 & 07-Sep-00 & & $10028-17-8$ & TRITIUM \\
\hline
\end{tabular}




\begin{tabular}{|c|c|c|c|c|c|c|c|c|c|c|}
\hline & A & $\mathrm{B}$ & C & $\mathrm{D}$ & $E$ & $\mathrm{~F}$ & G & $\mathrm{H}$ & $\mathrm{I}$ & $\mathrm{J}$ \\
\hline 1 & SAMPLE_NUM_ASSIGNED_TO & SAMPLER & MEDIA & BIOTA_MEDIA & TAG_ID & SAMP_NUM & SAMP_DATE_TIME & SAMP_DATE_TIME_ON & CON_ID & CON_SHORT_NAME \\
\hline 1066 & SESP SCHEDULING & & SW & & & B10672 & 14-Sep-00 & & $10028-17-8$ & TRITIUM \\
\hline 1067 & SESP SCHEDULING & & SW & & & B10673 & 14-Sep-00 & & $10028-17-8$ & TRITIUM \\
\hline 1068 & SESP SCHEDULING & & SW & & & B10674 & 14-Sep-00 & & $10028-17-8$ & TRITIUM \\
\hline 1069 & SESP SCHEDULING & & SW & & & B10675 & 14-Sep-00 & & $10028-17-8$ & TRITIUM \\
\hline 1070 & SESP SCHEDULING & & SW & & & B10676 & 14-Sep-00 & & $10028-17-8$ & TRITIUM \\
\hline 1071 & SESP SCHEDULING & & SW & & & B10677 & 14-Sep-00 & & $10028-17-8$ & TRITIUM \\
\hline 1072 & SESP SCHEDULING & & SW & & & B10679 & 14-Sep-00 & & $10028-17-8$ & TRITIUM \\
\hline 1073 & SESP SCHEDULING & & SW & & & B10681 & 14-Sep-00 & & $10028-17-8$ & TRITIUM \\
\hline 1074 & SESP SCHEDULING & & SW & & & B10683 & 14-Sep-00 & & $10028-17-8$ & TRITIUM \\
\hline 1075 & SESP SCHEDULING & & SW & & & B10684 & 14-Sep-00 & & $10028-17-8$ & TRITIUM \\
\hline 1076 & SESP SCHEDULING & & SW & & & B10686 & 14-Sep-00 & & $10028-17-8$ & TRITIUM \\
\hline 1077 & SESP SCHEDULING & & SW & & & B10698 & 14-Sep-00 & & $10028-17-8$ & TRITIUM \\
\hline 1078 & SESP SCHEDULING & & SW & & & B106B1 & 14-Sep-00 & & $10028-17-8$ & TRITIUM \\
\hline 1079 & SESP SCHEDULING & & SW & & & B106B4 & 14-Sep-00 & & $10028-17-8$ & TRITIUM \\
\hline 1080 & SESP SCHEDULING & & SW & & & B106B7 & 14-Sep-00 & & $10028-17-8$ & TRITIUM \\
\hline 1081 & SESP SCHEDULING & & SW & & & B109B4 & 14-Sep-00 & & $10028-17-8$ & TRITIUM \\
\hline 1082 & SESP SCHEDULING & & SW & & & B109B5 & 14-Sep-00 & & $10028-17-8$ & TRITIUM \\
\hline 1083 & SESP SCHEDULING & & SW & & & B109B6 & 14-Sep-00 & & $10028-17-8$ & TRITIUM \\
\hline 1084 & SESP SCHEDULING & & SW & & & B109B7 & 14-Sep-00 & & $10028-17-8$ & TRITIUM \\
\hline 1085 & SESP SCHEDULING & & SW & & & B109B8 & 14-Sep-00 & & $10028-17-8$ & TRITIUM \\
\hline 1086 & SESP SCHEDULING & & SW & & & B109B9 & 14-Sep-00 & & $10028-17-8$ & TRITIUM \\
\hline 1087 & SESP SCHEDULING & & SW & & & B106W1 & 18-Sep-00 & & $10028-17-8$ & TRITIUM \\
\hline 1088 & SESP SCHEDULING & & SW & & & B106W2 & 18-Sep-00 & & $10028-17-8$ & TRITIUM \\
\hline 1089 & SESP SCHEDULING & & SW & & & B106W3 & 18-Sep-00 & & $10028-17-8$ & TRITIUM \\
\hline 1090 & SESP SCHEDULING & & SW & & & B106W4 & 18-Sep-00 & & $10028-17-8$ & TRITIUM \\
\hline 1091 & SESP SCHEDULING & & SW & & & B106W5 & 18-Sep-00 & & $10028-17-8$ & TRITIUM \\
\hline 1092 & SESP SCHEDULING & & SW & & & B106W6 & 18-Sep-00 & & $10028-17-8$ & TRITIUM \\
\hline 1093 & SESP SCHEDULING & & SW & & & B106W7 & 18-Sep-00 & & $10028-17-8$ & TRITIUM \\
\hline 1094 & SESP SCHEDULING & & SW & & & B106W8 & 18-Sep-00 & & $10028-17-8$ & TRITIUM \\
\hline 1095 & SESP SCHEDULING & & SW & & & B106W9 & 18-Sep-00 & & $10028-17-8$ & TRITIUM \\
\hline 1096 & SESP SCHEDULING & & SW & & & B106X0 & 18-Sep-00 & & $10028-17-8$ & TRITIUM \\
\hline 1097 & SESP SCHEDULING & & SW & & & B106X1 & 18-Sep-00 & & $10028-17-8$ & TRITIUM \\
\hline 1098 & SESP SCHEDULING & & SW & & & B106X2 & 18-Sep-00 & & $10028-17-8$ & TRITIUM \\
\hline 1099 & SESP SCHEDULING & & SW & & & B10728 & 18-Sep-00 & & $10028-17-8$ & TRITIUM \\
\hline 1100 & SESP SCHEDULING & & SW & & & B10729 & 18-Sep-00 & & $10028-17-8$ & TRITIUM \\
\hline 1101 & SESP SCHEDULING & & SW & & & B10730 & 18-Sep-00 & & $10028-17-8$ & TRITIUM \\
\hline 1102 & SESP SCHEDULING & & SW & & & B10785 & 18-Sep-00 & & $10028-17-8$ & TRITIUM \\
\hline 1103 & SESP SCHEDULING & & SW & & & B10788 & 18-Sep-00 & & $10028-17-8$ & TRITIUM \\
\hline
\end{tabular}




\begin{tabular}{|c|c|c|c|c|c|c|c|c|c|c|}
\hline & A & $\mathrm{B}$ & C & $\mathrm{D}$ & $\mathrm{E}$ & $\mathrm{F}$ & G & $\mathrm{H}$ & $\mathrm{I}$ & $\mathrm{J}$ \\
\hline 1 & SAMPLE_NUM_ASSIGNED_TO & SAMPLER & MEDIA & BIOTA_MEDIA & TAG_ID & SAMP_NUM & SAMP_DATE_TIME & SAMP_DATE_TIME_ON & CON_ID & CON_SHORT_NAME \\
\hline 1104 & SESP SCHEDULING & & SW & & & B10791 & 18-Sep-00 & & $10028-17-8$ & TRITIUM \\
\hline 1105 & SESP SCHEDULING & & SW & & & B10794 & 18-Sep-00 & & $10028-17-8$ & TRITIUM \\
\hline 1106 & SESP SCHEDULING & & SW & & & B109C0 & 18-Sep-00 & & $10028-17-8$ & TRITIUM \\
\hline 1107 & SESP SCHEDULING & & SW & & & B109C1 & 18-Sep-00 & & $10028-17-8$ & TRITIUM \\
\hline 1108 & SESP SCHEDULING & & SW & & & B109C2 & 18-Sep-00 & & $10028-17-8$ & TRITIUM \\
\hline 1109 & SESP SCHEDULING & & SW & & & B109C3 & 18-Sep-00 & & $10028-17-8$ & TRITIUM \\
\hline 1110 & SESP SCHEDULING & & SW & & & B109C4 & 18-Sep-00 & & $10028-17-8$ & TRITIUM \\
\hline 1111 & SESP SCHEDULING & & SW & & & B109C5 & 18-Sep-00 & & $10028-17-8$ & TRITIUM \\
\hline 1112 & SESP SCHEDULING & & SW & & & B109C6 & 18-Sep-00 & & $10028-17-8$ & TRITIUM \\
\hline 1113 & SESP SCHEDULING & & SW & & & B106X3 & 19-Sep-00 & & $10028-17-8$ & TRITIUM \\
\hline 1114 & SESP SCHEDULING & & SW & & & B106X5 & 19-Sep-00 & & $10028-17-8$ & TRITIUM \\
\hline 1115 & SESP SCHEDULING & & SW & & & B106X7 & 19-Sep-00 & & $10028-17-8$ & TRITIUM \\
\hline 1116 & SESP SCHEDULING & & SW & & & B106X9 & 19-Sep-00 & & $10028-17-8$ & TRITIUM \\
\hline 1117 & SESP SCHEDULING & & SW & & & B106Y1 & 19-Sep-00 & & $10028-17-8$ & TRITIUM \\
\hline 1118 & SESP SCHEDULING & & SW & & & B106Y3 & 19-Sep-00 & & $10028-17-8$ & TRITIUM \\
\hline 1119 & SESP SCHEDULING & & SW & & & B106Y4 & 19-Sep-00 & & $10028-17-8$ & TRITIUM \\
\hline 1120 & SESP SCHEDULING & & SW & & & B106Y5 & 19-Sep-00 & & $10028-17-8$ & TRITIUM \\
\hline 1121 & SESP SCHEDULING & & SW & & & B106Y6 & 19-Sep-00 & & $10028-17-8$ & TRITIUM \\
\hline 1122 & SESP SCHEDULING & & SW & & & B106Y7 & 19-Sep-00 & & $10028-17-8$ & TRITIUM \\
\hline 1123 & SESP SCHEDULING & & SW & & & B106Y8 & 19-Sep-00 & & $10028-17-8$ & TRITIUM \\
\hline 1124 & SESP SCHEDULING & & SW & & & B106Y9 & 19-Sep-00 & & $10028-17-8$ & TRITIUM \\
\hline 1125 & SESP SCHEDULING & & SW & & & B10761 & 19-Sep-00 & & $10028-17-8$ & TRITIUM \\
\hline 1126 & SESP SCHEDULING & & SW & & & B10762 & 19-Sep-00 & & $10028-17-8$ & TRITIUM \\
\hline 1127 & SESP SCHEDULING & & SW & & & B10763 & 19-Sep-00 & & $10028-17-8$ & TRITIUM \\
\hline 1128 & SESP SCHEDULING & & SW & & & B10764 & 19-Sep-00 & & $10028-17-8$ & TRITIUM \\
\hline 1129 & SESP SCHEDULING & & SW & & & B10773 & 19-Sep-00 & & $10028-17-8$ & TRITIUM \\
\hline 1130 & SESP SCHEDULING & & SW & & & B10776 & 19-Sep-00 & & $10028-17-8$ & TRITIUM \\
\hline 1131 & SESP SCHEDULING & & SW & & & B10779 & 19-Sep-00 & & $10028-17-8$ & TRITIUM \\
\hline 1132 & SESP SCHEDULING & & SW & & & B10782 & 19-Sep-00 & & $10028-17-8$ & TRITIUM \\
\hline 1133 & SESP SCHEDULING & & SW & & & B109C7 & 19-Sep-00 & & $10028-17-8$ & TRITIUM \\
\hline 1134 & SESP SCHEDULING & & SW & & & B109C8 & 19-Sep-00 & & $10028-17-8$ & TRITIUM \\
\hline 1135 & SESP SCHEDULING & & SW & & & B109C9 & 19-Sep-00 & & $10028-17-8$ & TRITIUM \\
\hline 1136 & SESP SCHEDULING & & SW & & & B109D0 & 19-Sep-00 & & $10028-17-8$ & TRITIUM \\
\hline 1137 & SESP SCHEDULING & & SW & & & B109D1 & 19-Sep-00 & & $10028-17-8$ & TRITIUM \\
\hline 1138 & SESP SCHEDULING & & SW & & & B109D2 & 19-Sep-00 & & $10028-17-8$ & TRITIUM \\
\hline 1139 & SESP SCHEDULING & & SW & & & B109D3 & 19-Sep-00 & & $10028-17-8$ & TRITIUM \\
\hline 1140 & SESP SCHEDULING & & SW & & & B109D4 & 19-Sep-00 & & $10028-17-8$ & TRITIUM \\
\hline 1141 & SESP SCHEDULING & & SW & & & B109D5 & 19-Sep-00 & & $10028-17-8$ & TRITIUM \\
\hline
\end{tabular}




\begin{tabular}{|c|c|c|c|c|c|c|c|c|c|c|}
\hline & A & $\mathrm{B}$ & C & $\mathrm{D}$ & $\mathrm{E}$ & $\mathrm{F}$ & $\mathrm{G}$ & $\mathrm{H}$ & $\mathrm{I}$ & $\mathrm{J}$ \\
\hline 1 & SAMPLE_NUM_ASSIGNED_TO & SAMPLER & MEDIA & BIOTA_MEDIA & TAG_ID & SAMP_NUM & SAMP_DATE_TIME & SAMP_DATE_TIME_ON & CON_ID & CON_SHORT_NAME \\
\hline 1142 & SESP SCHEDULING & & SW & & & B109D6 & 19-Sep-00 & & $10028-17-8$ & TRITIUM \\
\hline 1143 & SESP SCHEDULING & & SW & & & B109D7 & 19-Sep-00 & & $10028-17-8$ & TRITIUM \\
\hline 1144 & SESP SCHEDULING & & SW & & & B10912 & 27-Sep-00 & & $10028-17-8$ & TRITIUM \\
\hline 1145 & SESP SCHEDULING & & SW & & & B10913 & 27-Sep-00 & & $10028-17-8$ & TRITIUM \\
\hline 1146 & SESP SCHEDULING & & SW & & & B10945 & 27-Sep-00 & & $10028-17-8$ & TRITIUM \\
\hline 1147 & SESP SCHEDULING & & SW & & & B10946 & 27-Sep-00 & & $10028-17-8$ & TRITIUM \\
\hline 1148 & SESP SCHEDULING & & SW & & & B10949 & 27-Sep-00 & & $10028-17-8$ & TRITIUM \\
\hline 1149 & SESP SCHEDULING & & SW & & & B103W8 & $05-$ Oct-00 & 07-Sep-00 & $10028-17-8$ & TRITIUM \\
\hline 1150 & SESP SCHEDULING & & SW & & & B103X3 & 05-Oct-00 & 07-Sep-00 & $10028-17-8$ & TRITIUM \\
\hline 1151 & SESP SCHEDULING & & SW & & & B10F33 & 09-Oct-00 & & $10028-17-8$ & TRITIUM \\
\hline 1152 & SESP SCHEDULING & & SW & & & B10F34 & 09-Oct-00 & & $10028-17-8$ & TRITIUM \\
\hline 1153 & SESP SCHEDULING & & SW & & & B10910 & 17-Oct-00 & & $10028-17-8$ & TRITIUM \\
\hline 1154 & SESP SCHEDULING & & SW & & & B10934 & 17-Oct-00 & & $10028-17-8$ & TRITIUM \\
\hline 1155 & SESP SCHEDULING & & SW & & & B10939 & 17-Oct-00 & & $10028-17-8$ & TRITIUM \\
\hline 1156 & SESP SCHEDULING & & SW & & & B10940 & 17-Oct-00 & & $10028-17-8$ & TRITIUM \\
\hline 1157 & PSHENRY & RADFORD, E & SW & & & B10JP8 & 17-Oct-00 & & $10028-17-8$ & TRITIUM \\
\hline 1158 & PSHENRY & RADFORD, E & SW & & & B10JP9 & $17-$ Oct-00 & & $10028-17-8$ & TRITIUM \\
\hline 1159 & PSHENRY & RADFORD, E & SW & & & B10JP7 & 17-Oct-00 & & $10028-17-8$ & TRITIUM \\
\hline 1160 & SESP SCHEDULING & & SW & & & B10KN4 & 18 -Oct-00 & & $10028-17-8$ & TRITIUM \\
\hline 1161 & SESP SCHEDULING & & SW & & & B10KN5 & 18-Oct-00 & & $10028-17-8$ & TRITIUM \\
\hline 1162 & SESP SCHEDULING & & SW & & & B10KN6 & $18-$ Oct-00 & & $10028-17-8$ & TRITIUM \\
\hline 1163 & SESP SCHEDULING & & SW & & & B10KN8 & $18-$ Oct-00 & & $10028-17-8$ & TRITIUM \\
\hline 1164 & SESP SCHEDULING & & SW & & & B10932 & 25-Oct-00 & & $10028-17-8$ & TRITIUM \\
\hline 1165 & SESP SCHEDULING & & SW & & & B10936 & 25-Oct-00 & & $10028-17-8$ & TRITIUM \\
\hline 1166 & PSHENRY & RADFORD, E & SW & & & B10FB2 & 25-Oct-00 & & $10028-17-8$ & TRITIUM \\
\hline 1167 & SESP SCHEDULING & & SW & & & B10941 & 01-Nov-00 & & $10028-17-8$ & TRITIUM \\
\hline 1168 & SESP SCHEDULING & & SW & & & B10977 & 01-Nov-00 & & $10028-17-8$ & TRITIUM \\
\hline 1169 & PSHENRY & RADFORD, E & SW & & & B10JR3 & 01-Nov-00 & & $10028-17-8$ & TRITIUM \\
\hline 1170 & SESP SCHEDULING & & SW & & & B10C13 & 02-Nov-00 & $05-O c t-00$ & $10028-17-8$ & TRITIUM \\
\hline 1171 & SESP SCHEDULING & & SW & & & B10C19 & 02-Nov-00 & $05-O c t-00$ & $10028-17-8$ & TRITIUM \\
\hline 1172 & SESP SCHEDULING & & SW & & & B10942 & 07-Nov-00 & & $10028-17-8$ & TRITIUM \\
\hline 1173 & PSHENRY & RADFORD, E & SW & & & B10FC4 & 07-Nov-00 & & $10028-17-8$ & TRITIUM \\
\hline 1174 & SESP SCHEDULING & & SW & & & B10MP5 & 29-Nov-00 & 02-Nov-00 & $10028-17-8$ & TRITIUM \\
\hline 1175 & SESP SCHEDULING & & SW & & & B10MR1 & 29-Nov-00 & 02-Nov-00 & $10028-17-8$ & TRITIUM \\
\hline 1176 & SESP SCHEDULING & & SW & & & B11125 & 04-Dec-00 & & $10028-17-8$ & TRITIUM \\
\hline 1177 & SESP SCHEDULING & & SW & & & B11126 & 04-Dec-00 & & $10028-17-8$ & TRITIUM \\
\hline 1178 & SESP SCHEDULING & & SW & & & B11127 & 04-Dec-00 & & $10028-17-8$ & TRITIUM \\
\hline 1179 & SESP SCHEDULING & & SW & & & B11128 & 04-Dec-00 & & $10028-17-8$ & TRITIUM \\
\hline
\end{tabular}




\begin{tabular}{|c|c|c|c|c|c|c|c|c|c|c|}
\hline & A & $\mathrm{B}$ & C & $\mathrm{D}$ & $E$ & $\mathrm{~F}$ & $G$ & $\mathrm{H}$ & $\mathrm{I}$ & $\mathrm{J}$ \\
\hline 1 & SAMPLE_NUM_ASSIGNED_TO & SAMPLER & MEDIA & BIOTA_MEDIA & TAG_ID & SAMP_NUM & SAMP_DATE_TIME & SAMP_DATE_TIME_ON & CON_ID & CON_SHORT_NAME \\
\hline 1180 & SESP SCHEDULING & & SW & & & B11124 & 05-Dec-00 & & $10028-17-8$ & TRITIUM \\
\hline 1181 & SESP SCHEDULING & & SW & & & B11129 & 05-Dec-00 & & $10028-17-8$ & TRITIUM \\
\hline 1182 & SESP SCHEDULING & & SW & & & B11130 & 05-Dec-00 & & $10028-17-8$ & TRITIUM \\
\hline 1183 & SESP SCHEDULING & & SW & & & B11131 & 05-Dec-00 & & $10028-17-8$ & TRITIUM \\
\hline 1184 & SESP SCHEDULING & & SW & & & B11132 & 05-Dec-00 & & 10028-17-8 & TRITIUM \\
\hline 1185 & SESP SCHEDULING & & SW & & & B11133 & 05-Dec-00 & & $10028-17-8$ & TRITIUM \\
\hline 1186 & SESP SCHEDULING & & SW & & & B11134 & 05-Dec-00 & & $10028-17-8$ & TRITIUM \\
\hline 1187 & SESP SCHEDULING & & SW & & & B11145 & 05-Dec-00 & & 10028-17-8 & TRITIUM \\
\hline 1188 & SESP SCHEDULING & & SW & & & B11146 & 05-Dec-00 & & $10028-17-8$ & TRITIUM \\
\hline 1189 & SESP SCHEDULING & & SW & & & B11147 & 05-Dec-00 & & $10028-17-8$ & TRITIUM \\
\hline 1190 & SESP SCHEDULING & & SW & & & B11148 & 05-Dec-00 & & $10028-17-8$ & TRITIUM \\
\hline 1191 & SESP SCHEDULING & & SW & & & B110Y4 & 04-Jan-01 & 29-Nov-00 & $10028-17-8$ & TRITIUM \\
\hline 1192 & SESP SCHEDULING & & SW & & & B11100 & 04-Jan-01 & 29-Nov-00 & $10028-17-8$ & TRITIUM \\
\hline 1193 & SESP SCHEDULING & & SW & & & B114P8 & 31-Jan-01 & 04-Jan-01 & $10028-17-8$ & TRITIUM \\
\hline 1194 & SESP SCHEDULING & & SW & & & B114T0 & 31-Jan-01 & 04-Jan-01 & $10028-17-8$ & TRITIUM \\
\hline 1195 & SESP SCHEDULING & & SW & & & B11CC7 & 12-Feb-01 & & $10028-17-8$ & TRITIUM \\
\hline 1196 & SESP SCHEDULING & & SW & & & B11CC8 & 12-Feb-01 & & $10028-17-8$ & TRITIUM \\
\hline 1197 & SESP SCHEDULING & & SW & & & B11CX6 & 15-Feb-01 & & $10028-17-8$ & TRITIUM \\
\hline 1198 & SESP SCHEDULING & & SW & & & B11CX7 & 15-Feb-01 & & $10028-17-8$ & TRITIUM \\
\hline 1199 & SESP SCHEDULING & & SW & & & B11CX8 & 15-Feb-01 & & $10028-17-8$ & TRITIUM \\
\hline 1200 & SESP SCHEDULING & & SW & & & B11CY8 & 15-Feb-01 & & $10028-17-8$ & TRITIUM \\
\hline 1201 & SESP SCHEDULING & & SW & & & B11H38 & 26-Feb-01 & & $10028-17-8$ & TRITIUM \\
\hline 1202 & SESP SCHEDULING & & SW & & & $\mathrm{B} 11 \mathrm{H} 43$ & 26-Feb-01 & & $10028-17-8$ & TRITIUM \\
\hline 1203 & SESP SCHEDULING & & SW & & & B11H44 & 26-Feb-01 & & $10028-17-8$ & TRITIUM \\
\hline 1204 & SESP SCHEDULING & & SW & & & B11H45 & 26-Feb-01 & & $10028-17-8$ & TRITIUM \\
\hline 1205 & SESP SCHEDULING & & SW & & & B11H46 & 26-Feb-01 & & $10028-17-8$ & TRITIUM \\
\hline 1206 & SESP SCHEDULING & & SW & & & B11H47 & 26-Feb-01 & & $10028-17-8$ & TRITIUM \\
\hline 1207 & SESP SCHEDULING & & SW & & & B11H48 & 26-Feb-01 & & $10028-17-8$ & TRITIUM \\
\hline 1208 & SESP SCHEDULING & & SW & & & B11H59 & 26-Feb-01 & & $10028-17-8$ & TRITIUM \\
\hline 1209 & SESP SCHEDULING & & SW & & & $\mathrm{B} 11 \mathrm{H} 60$ & 26-Feb-01 & & $10028-17-8$ & TRITIUM \\
\hline 1210 & SESP SCHEDULING & & SW & & & B11H61 & 26-Feb-01 & & $10028-17-8$ & TRITIUM \\
\hline 1211 & SESP SCHEDULING & & SW & & & B11H62 & 26-Feb-01 & & $10028-17-8$ & TRITIUM \\
\hline 1212 & SESP SCHEDULING & & SW & & & B11H39 & 27-Feb-01 & & $10028-17-8$ & TRITIUM \\
\hline 1213 & SESP SCHEDULING & & SW & & & $\mathrm{B} 11 \mathrm{H} 40$ & 27-Feb-01 & & $10028-17-8$ & TRITIUM \\
\hline 1214 & SESP SCHEDULING & & SW & & & B11H41 & 27-Feb-01 & & $10028-17-8$ & TRITIUM \\
\hline 1215 & SESP SCHEDULING & & SW & & & B11H42 & 27-Feb-01 & & $10028-17-8$ & TRITIUM \\
\hline 1216 & SESP SCHEDULING & & SW & & & B11C04 & 01-Mar-01 & 31-Jan-01 & $10028-17-8$ & TRITIUM \\
\hline 1217 & SESP SCHEDULING & & SW & & & B11C10 & 01-Mar-01 & 31-Jan-01 & $10028-17-8$ & TRITIUM \\
\hline
\end{tabular}




\begin{tabular}{|c|c|c|c|c|c|c|c|c|c|c|}
\hline & A & $\mathrm{B}$ & $\mathrm{C}$ & $\mathrm{D}$ & $\mathrm{E}$ & $\mathrm{F}$ & $\mathrm{G}$ & $\mathrm{H}$ & $\mathrm{I}$ & $\mathrm{J}$ \\
\hline 1 & SAMPLE_NUM_ASSIGNED_TO & SAMPLER & MEDIA & BIOTA_MEDIA & TAG_ID & SAMP_NUM & SAMP_DATE_TIME & SAMP_DATE_TIME_ON & CON_ID & CON_SHORT_NAME \\
\hline 1218 & SESP SCHEDULING & & SW & & & B11MY9 & 03-Apr-01 & & $10028-17-8$ & TRITIUM \\
\hline 1219 & SESP SCHEDULING & & SW & & & B11N00 & 03-Apr-01 & & $10028-17-8$ & TRITIUM \\
\hline 1220 & SESP SCHEDULING & & SW & & & B11N01 & 03-Apr-01 & & $10028-17-8$ & TRITIUM \\
\hline 1221 & SESP SCHEDULING & & SW & & & B11J40 & 04-Apr-01 & 01-Mar-01 & $10028-17-8$ & TRITIUM \\
\hline 1222 & SESP SCHEDULING & & SW & & & B11J46 & 04-Apr-01 & 01-Mar-01 & $10028-17-8$ & TRITIUM \\
\hline 1223 & SESP SCHEDULING & & SW & & & B11RY4 & 17-Apr-01 & & $10028-17-8$ & TRITIUM \\
\hline 1224 & SESP SCHEDULING & & SW & & & B11RY5 & 17-Apr-01 & & $10028-17-8$ & TRITIUM \\
\hline 1225 & SESP SCHEDULING & & SW & & & B11RY6 & 17-Apr-01 & & $10028-17-8$ & TRITIUM \\
\hline 1226 & SESP SCHEDULING & & SW & & & B11T05 & 17-Apr-01 & & $10028-17-8$ & TRITIUM \\
\hline 1227 & SESP SCHEDULING & & SW & & & B11W36 & 30-Apr-01 & & $10028-17-8$ & TRITIUM \\
\hline 1228 & SESP SCHEDULING & & SW & & & B11W50 & 30-Apr-01 & & $10028-17-8$ & TRITIUM \\
\hline 1229 & SESP SCHEDULING & & SW & & & B11W52 & 30-Apr-01 & & $10028-17-8$ & TRITIUM \\
\hline 1230 & SESP SCHEDULING & & SW & & & B11W55 & 30-Apr-01 & & $10028-17-8$ & TRITIUM \\
\hline 1231 & SESP SCHEDULING & & SW & & & B11W86 & 30-Apr-01 & & $10028-17-8$ & TRITIUM \\
\hline 1232 & SESP SCHEDULING & & SW & & & B11LT2 & 02-May-01 & 04-Apr-01 & $10028-17-8$ & TRITIUM \\
\hline 1233 & SESP SCHEDULING & & SW & & & B11LR6 & 02-May-01 & 04-Apr-01 & $10028-17-8$ & TRITIUM \\
\hline 1234 & SESP SCHEDULING & & SW & & & B11W57 & 03-May-01 & & $10028-17-8$ & TRITIUM \\
\hline 1235 & SESP SCHEDULING & & SW & & & B11W35 & 04-May-01 & & $10028-17-8$ & TRITIUM \\
\hline 1236 & SESP SCHEDULING & & SW & & & B11W40 & 04-May-01 & & $10028-17-8$ & TRITIUM \\
\hline 1237 & SESP SCHEDULING & & SW & & & B11W43 & 04-May-01 & & $10028-17-8$ & TRITIUM \\
\hline 1238 & SESP SCHEDULING & & SW & & & B11W48 & 04-May-01 & & $10028-17-8$ & TRITIUM \\
\hline 1239 & SESP SCHEDULING & & SW & & & B11WV8 & 09-May-01 & & $10028-17-8$ & TRITIUM \\
\hline 1240 & SESP SCHEDULING & & SW & & & B11WX0 & 09-May-01 & & $10028-17-8$ & TRITIUM \\
\hline 1241 & SESP SCHEDULING & & SW & & & B11W38 & 10-May-01 & & $10028-17-8$ & TRITIUM \\
\hline 1242 & SESP SCHEDULING & & SW & & & B121R8 & 17-May-01 & & $10028-17-8$ & TRITIUM \\
\hline 1243 & SESP SCHEDULING & & SW & & & B121R9 & 17-May-01 & & $10028-17-8$ & TRITIUM \\
\hline 1244 & SESP SCHEDULING & & SW & & & B121T0 & 17-May-01 & & $10028-17-8$ & TRITIUM \\
\hline 1245 & SESP SCHEDULING & & SW & & & B121T1 & 17-May-01 & & $10028-17-8$ & TRITIUM \\
\hline 1246 & SESP SCHEDULING & & SW & & & B121V4 & 17-May-01 & & $10028-17-8$ & TRITIUM \\
\hline 1247 & SESP SCHEDULING & & SW & & & B11WT4 & 30-May-01 & 02-May-01 & $10028-17-8$ & TRITIUM \\
\hline 1248 & SESP SCHEDULING & & SW & & & B11WR8 & 30-May-01 & 02-May-01 & $10028-17-8$ & TRITIUM \\
\hline 1249 & SESP SCHEDULING & & SW & & & B124N7 & 07-Jun-01 & & $10028-17-8$ & TRITIUM \\
\hline 1250 & SESP SCHEDULING & & SW & & & B124N7 & 07-Jun-01 & & $10028-17-8$ & TRITIUM \\
\hline 1251 & SESP SCHEDULING & & SW & & & B124P7 & 07-Jun-01 & & $10028-17-8$ & TRITIUM \\
\hline 1252 & SESP SCHEDULING & & SW & & & B124P7 & 07-Jun-01 & & $10028-17-8$ & TRITIUM \\
\hline 1253 & SESP SCHEDULING & & SW & & & B12505 & 12-Jun-01 & & $10028-17-8$ & TRITIUM \\
\hline 1254 & SESP SCHEDULING & & SW & & & B12506 & 12-Jun-01 & & $10028-17-8$ & TRITIUM \\
\hline 1255 & SESP SCHEDULING & & SW & & & B12507 & 12-Jun-01 & & $10028-17-8$ & TRITIUM \\
\hline
\end{tabular}




\begin{tabular}{|c|c|c|c|c|c|c|c|c|c|c|}
\hline & A & $\mathrm{B}$ & C & $\mathrm{D}$ & $\mathrm{E}$ & $\mathrm{F}$ & G & $\mathrm{H}$ & $\mathrm{I}$ & $\mathrm{J}$ \\
\hline 1 & SAMPLE_NUM_ASSIGNED_TO & SAMPLER & MEDIA & BIOTA_MEDIA & TAG_ID & SAMP_NUM & SAMP_DATE_TIME & SAMP_DATE_TIME_ON & CON_ID & CON_SHORT_NAME \\
\hline 1256 & SESP SCHEDULING & & SW & & & B12508 & 12-Jun-01 & & $10028-17-8$ & TRITIUM \\
\hline 1257 & SESP SCHEDULING & & SW & & & B12509 & 12-Jun-01 & & $10028-17-8$ & TRITIUM \\
\hline 1258 & SESP SCHEDULING & & SW & & & B12510 & 12-Jun-01 & & $10028-17-8$ & TRITIUM \\
\hline 1259 & SESP SCHEDULING & & SW & & & B12531 & 12-Jun-01 & & $10028-17-8$ & TRITIUM \\
\hline 1260 & SESP SCHEDULING & & SW & & & B12532 & 12-Jun-01 & & $10028-17-8$ & TRITIUM \\
\hline 1261 & SESP SCHEDULING & & SW & & & B12533 & 12-Jun-01 & & $10028-17-8$ & TRITIUM \\
\hline 1262 & SESP SCHEDULING & & SW & & & B12534 & 12-Jun-01 & & $10028-17-8$ & TRITIUM \\
\hline 1263 & SESP SCHEDULING & & SW & & & B12501 & 14-Jun-01 & & $10028-17-8$ & TRITIUM \\
\hline 1264 & SESP SCHEDULING & & SW & & & B12502 & 14-Jun-01 & & $10028-17-8$ & TRITIUM \\
\hline 1265 & SESP SCHEDULING & & SW & & & B12503 & 14-Jun-01 & & $10028-17-8$ & TRITIUM \\
\hline 1266 & SESP SCHEDULING & & SW & & & B12504 & 14-Jun-01 & & $10028-17-8$ & TRITIUM \\
\hline 1267 & SESP SCHEDULING & & SW & & & B124M3 & 28-Jun-01 & 23-May-01 & $10028-17-8$ & TRITIUM \\
\hline 1268 & SESP SCHEDULING & & SW & & & B124L7 & 28-Jun-01 & 23-May-01 & $10028-17-8$ & TRITIUM \\
\hline 1269 & SESP SCHEDULING & & SW & & & B12993 & 09-Jul-01 & & $10028-17-8$ & TRITIUM \\
\hline 1270 & SESP SCHEDULING & & SW & & & B12994 & 09-Jul-01 & & $10028-17-8$ & TRITIUM \\
\hline 1271 & SESP SCHEDULING & & SW & & & B129B4 & 09-Jul-01 & & $10028-17-8$ & TRITIUM \\
\hline 1272 & SESP SCHEDULING & & SW & & & B129N8 & 09-Jul-01 & & $10028-17-8$ & TRITIUM \\
\hline 1273 & SESP SCHEDULING & & SW & & & B129N9 & 09-Jul-01 & & $10028-17-8$ & TRITIUM \\
\hline 1274 & SESP SCHEDULING & & SW & & & B129P0 & 09-Jul-01 & & $10028-17-8$ & TRITIUM \\
\hline 1275 & SESP SCHEDULING & & SW & & & B129P9 & 09-Jul-01 & & $10028-17-8$ & TRITIUM \\
\hline 1276 & SESP SCHEDULING & & SW & & & B12841 & 09-Aug-01 & 28-Jun-01 & $10028-17-8$ & TRITIUM \\
\hline 1277 & SESP SCHEDULING & & SW & & & B12834 & 09-Aug-01 & 28-Jun-01 & $10028-17-8$ & TRITIUM \\
\hline 1278 & SESP SCHEDULING & & SW & & & B12MM7 & 14-Aug-01 & & $10028-17-8$ & TRITIUM \\
\hline 1279 & SESP SCHEDULING & & SW & & & B12RM1 & 27-Aug-01 & & $10028-17-8$ & TRITIUM \\
\hline 1280 & SESP SCHEDULING & & SW & & & B12RM6 & 27-Aug-01 & & $10028-17-8$ & TRITIUM \\
\hline 1281 & SESP SCHEDULING & & SW & & & B12JT2 & 05-Sep-01 & & $10028-17-8$ & TRITIUM \\
\hline 1282 & SESP SCHEDULING & & SW & & & B12JR7 & 05-Sep-01 & 09-Aug-01 & $10028-17-8$ & TRITIUM \\
\hline 1283 & SESP SCHEDULING & & SW & & & B12T85 & 07-Sep-01 & & $10028-17-8$ & TRITIUM \\
\hline 1284 & SESP SCHEDULING & & SW & & & B12T87 & 07-Sep-01 & & $10028-17-8$ & TRITIUM \\
\hline 1285 & SESP SCHEDULING & & SW & & & B12T88 & 07-Sep-01 & & $10028-17-8$ & TRITIUM \\
\hline 1286 & SESP SCHEDULING & & SW & & & B12T89 & 07-Sep-01 & & $10028-17-8$ & TRITIUM \\
\hline 1287 & SESP SCHEDULING & & SW & & & B12T90 & 07-Sep-01 & & $10028-17-8$ & TRITIUM \\
\hline 1288 & SESP SCHEDULING & & SW & & & B12T91 & 07-Sep-01 & & $10028-17-8$ & TRITIUM \\
\hline 1289 & SESP SCHEDULING & & SW & & & B12T92 & 07-Sep-01 & & $10028-17-8$ & TRITIUM \\
\hline 1290 & SESP SCHEDULING & & SW & & & B12T93 & 07-Sep-01 & & $10028-17-8$ & TRITIUM \\
\hline 1291 & SESP SCHEDULING & & SW & & & B12TD4 & 07-Sep-01 & & $10028-17-8$ & TRITIUM \\
\hline 1292 & SESP SCHEDULING & & SW & & & B12TD5 & 07-Sep-01 & & $10028-17-8$ & TRITIUM \\
\hline 1293 & SESP SCHEDULING & & SW & & & B12TD6 & 07-Sep-01 & & $10028-17-8$ & TRITIUM \\
\hline
\end{tabular}




\begin{tabular}{|c|c|c|c|c|c|c|c|c|c|c|}
\hline & A & $\mathrm{B}$ & C & $\mathrm{D}$ & $E$ & $\mathrm{~F}$ & G & $\mathrm{H}$ & $\mathrm{I}$ & $\mathrm{J}$ \\
\hline 1 & SAMPLE_NUM_ASSIGNED_TO & SAMPLER & MEDIA & BIOTA_MEDIA & TAG_ID & SAMP_NUM & SAMP_DATE_TIME & SAMP_DATE_TIME_ON & CON_ID & CON_SHORT_NAME \\
\hline 1294 & SESP SCHEDULING & & SW & & & B12TN2 & 07-Sep-01 & & $10028-17-8$ & TRITIUM \\
\hline 1295 & SESP SCHEDULING & & SW & & & B12TN5 & 07-Sep-01 & & $10028-17-8$ & TRITIUM \\
\hline 1296 & SESP SCHEDULING & & SW & & & B12TN8 & 07-Sep-01 & & $10028-17-8$ & TRITIUM \\
\hline 1297 & SESP SCHEDULING & & SW & & & B12TP1 & 07-Sep-01 & & $10028-17-8$ & TRITIUM \\
\hline 1298 & SESP SCHEDULING & & SW & & & B12T94 & 10-Sep-01 & & $10028-17-8$ & TRITIUM \\
\hline 1299 & SESP SCHEDULING & & SW & & & B12T95 & 10-Sep-01 & & $10028-17-8$ & TRITIUM \\
\hline 1300 & SESP SCHEDULING & & SW & & & B12T96 & 10-Sep-01 & & $10028-17-8$ & TRITIUM \\
\hline 1301 & SESP SCHEDULING & & SW & & & B12T97 & 10-Sep-01 & & $10028-17-8$ & TRITIUM \\
\hline 1302 & SESP SCHEDULING & & SW & & & B12T98 & 10-Sep-01 & & $10028-17-8$ & TRITIUM \\
\hline 1303 & SESP SCHEDULING & & SW & & & B12T99 & 10-Sep-01 & & $10028-17-8$ & TRITIUM \\
\hline 1304 & SESP SCHEDULING & & SW & & & B12TB0 & 10-Sep-01 & & $10028-17-8$ & TRITIUM \\
\hline 1305 & SESP SCHEDULING & & SW & & & B12TB1 & 10-Sep-01 & & $10028-17-8$ & TRITIUM \\
\hline 1306 & SESP SCHEDULING & & SW & & & B12TB2 & 10-Sep-01 & & $10028-17-8$ & TRITIUM \\
\hline 1307 & SESP SCHEDULING & & SW & & & B12TB3 & 10-Sep-01 & & $10028-17-8$ & TRITIUM \\
\hline 1308 & SESP SCHEDULING & & SW & & & B12TB4 & 10-Sep-01 & & $10028-17-8$ & TRITIUM \\
\hline 1309 & SESP SCHEDULING & & SW & & & B12TB5 & 10-Sep-01 & & $10028-17-8$ & TRITIUM \\
\hline 1310 & SESP SCHEDULING & & SW & & & B12TF7 & 10-Sep-01 & & $10028-17-8$ & TRITIUM \\
\hline 1311 & SESP SCHEDULING & & SW & & & B12TF8 & 10-Sep-01 & & $10028-17-8$ & TRITIUM \\
\hline 1312 & SESP SCHEDULING & & SW & & & B12TF9 & 10-Sep-01 & & $10028-17-8$ & TRITIUM \\
\hline 1313 & SESP SCHEDULING & & SW & & & B12TT0 & 10-Sep-01 & & $10028-17-8$ & TRITIUM \\
\hline 1314 & SESP SCHEDULING & & SW & & & B12TT3 & 10-Sep-01 & & $10028-17-8$ & TRITIUM \\
\hline 1315 & SESP SCHEDULING & & SW & & & B12TT9 & 10-Sep-01 & & $10028-17-8$ & TRITIUM \\
\hline 1316 & SESP SCHEDULING & & SW & & & B12TB6 & 13-Sep-01 & & $10028-17-8$ & TRITIUM \\
\hline 1317 & SESP SCHEDULING & & SW & & & B12TB8 & 13-Sep-01 & & $10028-17-8$ & TRITIUM \\
\hline 1318 & SESP SCHEDULING & & SW & & & B12TC0 & 13-Sep-01 & & $10028-17-8$ & TRITIUM \\
\hline 1319 & SESP SCHEDULING & & SW & & & B12TC2 & 13-Sep-01 & & $10028-17-8$ & TRITIUM \\
\hline 1320 & SESP SCHEDULING & & SW & & & B12TC4 & 13-Sep-01 & & $10028-17-8$ & TRITIUM \\
\hline 1321 & SESP SCHEDULING & & SW & & & B12TC6 & 13-Sep-01 & & $10028-17-8$ & TRITIUM \\
\hline 1322 & SESP SCHEDULING & & SW & & & B12TC8 & 13-Sep-01 & & $10028-17-8$ & TRITIUM \\
\hline 1323 & SESP SCHEDULING & & SW & & & B12TC9 & 13-Sep-01 & & $10028-17-8$ & TRITIUM \\
\hline 1324 & SESP SCHEDULING & & SW & & & B12TD0 & 13-Sep-01 & & $10028-17-8$ & TRITIUM \\
\hline 1325 & SESP SCHEDULING & & SW & & & B12TD1 & 13-Sep-01 & & $10028-17-8$ & TRITIUM \\
\hline 1326 & SESP SCHEDULING & & SW & & & B12TD2 & 13-Sep-01 & & $10028-17-8$ & TRITIUM \\
\hline 1327 & SESP SCHEDULING & & SW & & & B12TD3 & 13-Sep-01 & & $10028-17-8$ & TRITIUM \\
\hline 1328 & SESP SCHEDULING & & SW & & & B12TM0 & 13-Sep-01 & & $10028-17-8$ & TRITIUM \\
\hline 1329 & SESP SCHEDULING & & SW & & & B12TM1 & 13-Sep-01 & & $10028-17-8$ & TRITIUM \\
\hline 1330 & SESP SCHEDULING & & SW & & & B12TM2 & 13-Sep-01 & & $10028-17-8$ & TRITIUM \\
\hline 1331 & SESP SCHEDULING & & SW & & & B12TM3 & 13-Sep-01 & & $10028-17-8$ & TRITIUM \\
\hline
\end{tabular}




\begin{tabular}{|c|c|c|c|c|c|c|c|c|c|c|}
\hline & A & $\mathrm{B}$ & C & $\mathrm{D}$ & $\mathrm{E}$ & $\mathrm{F}$ & $\mathrm{G}$ & $\mathrm{H}$ & $\mathrm{I}$ & $\mathrm{J}$ \\
\hline 1 & SAMPLE_NUM_ASSIGNED_TO & SAMPLER & MEDIA & BIOTA_MEDIA & TAG_ID & SAMP_NUM & SAMP_DATE_TIME & SAMP_DATE_TIME_ON & CON_ID & CON_SHORT_NAME \\
\hline 1332 & SESP SCHEDULING & & SW & & & B12TP4 & 13-Sep-01 & & $10028-17-8$ & TRITIUM \\
\hline 1333 & SESP SCHEDULING & & SW & & & B12TP8 & 13-Sep-01 & & $10028-17-8$ & TRITIUM \\
\hline 1334 & SESP SCHEDULING & & SW & & & B12TR2 & 13-Sep-01 & & $10028-17-8$ & TRITIUM \\
\hline 1335 & SESP SCHEDULING & & SW & & & B12TR6 & 13-Sep-01 & & $10028-17-8$ & TRITIUM \\
\hline 1336 & SESP SCHEDULING & & SW & & & B12XK2 & 17-Sep-01 & & $10028-17-8$ & TRITIUM \\
\hline 1337 & SESP SCHEDULING & & SW & & & B12XK8 & 17-Sep-01 & & $10028-17-8$ & TRITIUM \\
\hline 1338 & SESP SCHEDULING & & SW & & & B12XK9 & 17-Sep-01 & & $10028-17-8$ & TRITIUM \\
\hline 1339 & SESP SCHEDULING & & SW & & & B12XL0 & 17-Sep-01 & & $10028-17-8$ & TRITIUM \\
\hline 1340 & SESP SCHEDULING & & SW & & & B12XL1 & 17-Sep-01 & & $10028-17-8$ & TRITIUM \\
\hline 1341 & SESP SCHEDULING & & SW & & & B12XL2 & 17-Sep-01 & & $10028-17-8$ & TRITIUM \\
\hline 1342 & SESP SCHEDULING & & SW & & & B12XK1 & 18-Sep-01 & & $10028-17-8$ & TRITIUM \\
\hline 1343 & SESP SCHEDULING & & SW & & & B12XK3 & 18-Sep-01 & & $10028-17-8$ & TRITIUM \\
\hline 1344 & SESP SCHEDULING & & SW & & & B12XK4 & 18-Sep-01 & & $10028-17-8$ & TRITIUM \\
\hline 1345 & SESP SCHEDULING & & SW & & & B12XK5 & 18-Sep-01 & & $10028-17-8$ & TRITIUM \\
\hline 1346 & SESP SCHEDULING & & SW & & & B12XK6 & 18-Sep-01 & & $10028-17-8$ & TRITIUM \\
\hline 1347 & SESP SCHEDULING & & SW & & & B12XK7 & 18-Sep-01 & & $10028-17-8$ & TRITIUM \\
\hline 1348 & SESP SCHEDULING & & SW & & & B12T28 & 02-Oct-01 & 05-Sep-01 & 10028-17-8 & TRITIUM \\
\hline 1349 & SESP SCHEDULING & & SW & & & B12T33 & 02-Oct-01 & 05-Sep-01 & $10028-17-8$ & TRITIUM \\
\hline 1350 & SESP SCHEDULING & & SW & & & B134K9 & 08-Oct-01 & & $10028-17-8$ & TRITIUM \\
\hline 1351 & SESP SCHEDULING & & SW & & & B134L0 & 08-Oct-01 & & $10028-17-8$ & TRITIUM \\
\hline 1352 & SESP SCHEDULING & & SW & & & B134L1 & 08-Oct-01 & & $10028-17-8$ & TRITIUM \\
\hline 1353 & SESP SCHEDULING & & SW & & & B134L2 & 08-Oct-01 & & $10028-17-8$ & TRITIUM \\
\hline 1354 & SESP SCHEDULING & & SW & & & B134L3 & 08-Oct-01 & & $10028-17-8$ & TRITIUM \\
\hline 1355 & SESP SCHEDULING & & SW & & & B134L4 & 08-Oct-01 & & $10028-17-8$ & TRITIUM \\
\hline 1356 & SESP SCHEDULING & & SW & & & B12X38 & 22-Oct-01 & & $10028-17-8$ & TRITIUM \\
\hline 1357 & SESP SCHEDULING & & SW & & & B12X50 & 22-Oct-01 & & $10028-17-8$ & TRITIUM \\
\hline 1358 & SESP SCHEDULING & & SW & & & B12WY1 & 25-Oct-01 & & $10028-17-8$ & TRITIUM \\
\hline 1359 & SESP SCHEDULING & & SW & & & B12X41 & 25-Oct-01 & & $10028-17-8$ & TRITIUM \\
\hline 1360 & SESP SCHEDULING & & SW & & & B12X42 & 25-Oct-01 & & $10028-17-8$ & TRITIUM \\
\hline 1361 & SESP SCHEDULING & & SW & & & B12X48 & 01-Nov-01 & & $10028-17-8$ & TRITIUM \\
\hline 1362 & SESP SCHEDULING & & SW & & & B12X85 & 01-Nov-01 & & $10028-17-8$ & TRITIUM \\
\hline 1363 & SESP SCHEDULING & & SW & & & B13103 & 01-Nov-01 & 02-Oct-01 & 10028-17-8 & TRITIUM \\
\hline 1364 & SESP SCHEDULING & & SW & & & B130Y7 & 01-Nov-01 & 02-Oct-01 & $10028-17-8$ & TRITIUM \\
\hline 1365 & SESP SCHEDULING & & SW & & & B13J17 & 14-Nov-01 & & $10028-17-8$ & TRITIUM \\
\hline 1366 & SESP SCHEDULING & & SW & & & B13CC6 & 27-Nov-01 & 01-Nov-01 & 10028-17-8 & TRITIUM \\
\hline 1367 & SESP SCHEDULING & & SW & & & B13CD2 & 27-Nov-01 & 01-Nov-01 & 10028-17-8 & TRITIUM \\
\hline 1368 & SESP SCHEDULING & & SW & & & B13LF3 & 03-Dec-01 & & $10028-17-8$ & TRITIUM \\
\hline 1369 & SESP SCHEDULING & & SW & & & B13LF4 & 03-Dec-01 & & $10028-17-8$ & TRITIUM \\
\hline
\end{tabular}




\begin{tabular}{|c|c|c|c|c|c|c|c|c|c|c|}
\hline & A & $\mathrm{B}$ & C & $\mathrm{D}$ & $E$ & $\mathrm{~F}$ & G & $\mathrm{H}$ & $\mathrm{I}$ & $\mathrm{J}$ \\
\hline 1 & SAMPLE_NUM_ASSIGNED_TO & SAMPLER & MEDIA & BIOTA_MEDIA & TAG_ID & SAMP_NUM & SAMP_DATE_TIME & SAMP_DATE_TIME_ON & CON_ID & CON_SHORT_NAME \\
\hline 1370 & SESP SCHEDULING & & SW & & & B13LF5 & 03-Dec-01 & & $10028-17-8$ & TRITIUM \\
\hline 1371 & SESP SCHEDULING & & SW & & & B13LF6 & 03-Dec-01 & & $10028-17-8$ & TRITIUM \\
\hline 1372 & SESP SCHEDULING & & SW & & & B13LF2 & 04-Dec-01 & & $10028-17-8$ & TRITIUM \\
\hline 1373 & SESP SCHEDULING & & SW & & & B13LF7 & 04-Dec-01 & & $10028-17-8$ & TRITIUM \\
\hline 1374 & SESP SCHEDULING & & SW & & & B13LF8 & 04-Dec-01 & & $10028-17-8$ & TRITIUM \\
\hline 1375 & SESP SCHEDULING & & SW & & & B13LF9 & 04-Dec-01 & & $10028-17-8$ & TRITIUM \\
\hline 1376 & SESP SCHEDULING & & SW & & & B13LHO & 04-Dec-01 & & $10028-17-8$ & TRITIUM \\
\hline 1377 & SESP SCHEDULING & & SW & & & B13LH1 & 04-Dec-01 & & $10028-17-8$ & TRITIUM \\
\hline 1378 & SESP SCHEDULING & & SW & & & B13LH2 & 04-Dec-01 & & $10028-17-8$ & TRITIUM \\
\hline 1379 & SESP SCHEDULING & & SW & & & B13LJ3 & 04-Dec-01 & & $10028-17-8$ & TRITIUM \\
\hline 1380 & SESP SCHEDULING & & SW & & & B13LJ4 & 04-Dec-01 & & $10028-17-8$ & TRITIUM \\
\hline 1381 & SESP SCHEDULING & & SW & & & B13LJ5 & 04-Dec-01 & & $10028-17-8$ & TRITIUM \\
\hline 1382 & SESP SCHEDULING & & SW & & & B13LJ6 & 04-Dec-01 & & $10028-17-8$ & TRITIUM \\
\hline 1383 & SESP SCHEDULING & & SW & & & B13LB3 & 03-Jan-02 & 27-Nov-01 & $10028-17-8$ & TRITIUM \\
\hline 1384 & SESP SCHEDULING & & SW & & & B13L96 & 03-Jan-02 & 27-Nov-01 & $10028-17-8$ & TRITIUM \\
\hline 1385 & SESP SCHEDULING & & SW & & & B13VJ0 & 06-Feb-02 & & $10028-17-8$ & TRITIUM \\
\hline 1386 & SESP SCHEDULING & & SW & & & B13VF8 & 06-Feb-02 & & $10028-17-8$ & TRITIUM \\
\hline 1387 & SESP SCHEDULING & & SW & & & B141L3 & 14-Feb-02 & & $10028-17-8$ & TRITIUM \\
\hline 1388 & SESP SCHEDULING & & SW & & & B141L4 & 14-Feb-02 & & $10028-17-8$ & TRITIUM \\
\hline 1389 & SESP SCHEDULING & & SW & & & B14266 & 14-Feb-02 & & $10028-17-8$ & TRITIUM \\
\hline 1390 & SESP SCHEDULING & & SW & & & B14271 & 14-Feb-02 & & $10028-17-8$ & TRITIUM \\
\hline 1391 & SESP SCHEDULING & & SW & & & B14272 & 14-Feb-02 & & $10028-17-8$ & TRITIUM \\
\hline 1392 & SESP SCHEDULING & & SW & & & B14273 & 14-Feb-02 & & $10028-17-8$ & TRITIUM \\
\hline 1393 & SESP SCHEDULING & & SW & & & B140P7 & 07-Mar-02 & 06-Feb-02 & $10028-17-8$ & TRITIUM \\
\hline 1394 & SESP SCHEDULING & & SW & & & B140R2 & 07-Mar-02 & 06-Feb-02 & $10028-17-8$ & TRITIUM \\
\hline 1395 & SESP SCHEDULING & & SW & & & B147T3 & 25-Mar-02 & & $10028-17-8$ & TRITIUM \\
\hline 1396 & SESP SCHEDULING & & SW & & & B147T4 & 25-Mar-02 & & $10028-17-8$ & TRITIUM \\
\hline 1397 & SESP SCHEDULING & & SW & & & B147T5 & 25-Mar-02 & & $10028-17-8$ & TRITIUM \\
\hline 1398 & SESP SCHEDULING & & SW & & & B147T6 & 25-Mar-02 & & $10028-17-8$ & TRITIUM \\
\hline 1399 & SESP SCHEDULING & & SW & & & B147T2 & 26-Mar-02 & & $10028-17-8$ & TRITIUM \\
\hline 1400 & SESP SCHEDULING & & SW & & & B147T7 & 26-Mar-02 & & $10028-17-8$ & TRITIUM \\
\hline 1401 & SESP SCHEDULING & & SW & & & B147T8 & 26-Mar-02 & & $10028-17-8$ & TRITIUM \\
\hline 1402 & SESP SCHEDULING & & SW & & & B147T9 & 26-Mar-02 & & $10028-17-8$ & TRITIUM \\
\hline 1403 & SESP SCHEDULING & & SW & & & B147V0 & 26-Mar-02 & & $10028-17-8$ & TRITIUM \\
\hline 1404 & SESP SCHEDULING & & SW & & & B147V1 & 26-Mar-02 & & $10028-17-8$ & TRITIUM \\
\hline 1405 & SESP SCHEDULING & & SW & & & B147V2 & 26-Mar-02 & & $10028-17-8$ & TRITIUM \\
\hline 1406 & SESP SCHEDULING & & SW & & & B147W3 & 26-Mar-02 & & $10028-17-8$ & TRITIUM \\
\hline 1407 & SESP SCHEDULING & & SW & & & B147W4 & 26-Mar-02 & & $10028-17-8$ & TRITIUM \\
\hline
\end{tabular}




\begin{tabular}{|c|c|c|c|c|c|c|c|c|c|c|}
\hline & A & $\mathrm{B}$ & C & $\mathrm{D}$ & $\mathrm{E}$ & $\mathrm{F}$ & $\mathrm{G}$ & $\mathrm{H}$ & $\mathrm{I}$ & $\mathrm{J}$ \\
\hline 1 & SAMPLE_NUM_ASSIGNED_TO & SAMPLER & MEDIA & BIOTA_MEDIA & TAG_ID & SAMP_NUM & SAMP_DATE_TIME & SAMP_DATE_TIME_ON & CON_ID & CON_SHORT_NAME \\
\hline 1408 & SESP SCHEDULING & & SW & & & B147W5 & 26-Mar-02 & & $10028-17-8$ & TRITIUM \\
\hline 1409 & SESP SCHEDULING & & SW & & & B147W6 & 26-Mar-02 & & $10028-17-8$ & TRITIUM \\
\hline 1410 & SESP SCHEDULING & & SW & & & B14408 & 03-Apr-02 & 07-Mar-02 & 2 10028-17-8 & TRITIUM \\
\hline 1411 & SESP SCHEDULING & & SW & & & B14413 & 03-Apr-02 & 07-Mar-02 & 2 10028-17-8 & TRITIUM \\
\hline 1412 & SESP SCHEDULING & & SW & & & B14CT1 & 08-Apr-02 & & $10028-17-8$ & TRITIUM \\
\hline 1413 & SESP SCHEDULING & & SW & & & B14CT2 & 08-Apr-02 & & $10028-17-8$ & TRITIUM \\
\hline 1414 & SESP SCHEDULING & & SW & & & B14CV3 & 08-Apr-02 & & $10028-17-8$ & TRITIUM \\
\hline 1415 & SESP SCHEDULING & & SW & & & B14CV5 & 08-Apr-02 & & $10028-17-8$ & TRITIUM \\
\hline 1416 & SESP SCHEDULING & & SW & & & B14CV7 & 08-Apr-02 & & $10028-17-8$ & TRITIUM \\
\hline 1417 & SESP SCHEDULING & & SW & & & B14CV2 & 09-Apr-02 & & $10028-17-8$ & TRITIUM \\
\hline 1418 & SESP SCHEDULING & & SW & & & B14CV6 & 09-Apr-02 & & $10028-17-8$ & TRITIUM \\
\hline 1419 & SESP SCHEDULING & & SW & & & B14B37 & 01-May-02 & 03-Apr-02 & 10028-17-8 & TRITIUM \\
\hline 1420 & SESP SCHEDULING & & SW & & & B14B43 & 01-May-02 & 03-Apr-02 & 2 10028-17-8 & TRITIUM \\
\hline 1421 & DEAYRES & DEROOS, GB & SW & & & B14DR7 & 09-May-02 & & $10028-17-8$ & TRITIUM \\
\hline 1422 & DEAYRES & DEROOS, GB & SW & & & B14DR8 & 09-May-02 & & $10028-17-8$ & TRITIUM \\
\hline 1423 & SESP SCHEDULING & & SW & & & B14LJ0 & 23-May-02 & & $10028-17-8$ & TRITIUM \\
\hline 1424 & SESP SCHEDULING & & SW & & & B14LJ2 & 23-May-02 & & $10028-17-8$ & TRITIUM \\
\hline 1425 & SESP SCHEDULING & & SW & & & B14JF2 & 29-May-02 & 01-May-02 & 10028-17-8 & TRITIUM \\
\hline 1426 & SESP SCHEDULING & & SW & & & B14JF8 & 29-May-02 & 01-May-02 & 2 10028-17-8 & TRITIUM \\
\hline 1427 & SESP SCHEDULING & & SW & & & B14RV4 & 10-Jun-02 & & $10028-17-8$ & TRITIUM \\
\hline 1428 & SESP SCHEDULING & & SW & & & B14RV5 & 10-Jun-02 & & $10028-17-8$ & TRITIUM \\
\hline 1429 & SESP SCHEDULING & & SW & & & B14RV6 & 10-Jun-02 & & $10028-17-8$ & TRITIUM \\
\hline 1430 & SESP SCHEDULING & & SW & & & B14RV7 & 10-Jun-02 & & $10028-17-8$ & TRITIUM \\
\hline 1431 & SESP SCHEDULING & & SW & & & B14RV8 & 11-Jun-02 & & $10028-17-8$ & TRITIUM \\
\hline 1432 & SESP SCHEDULING & & SW & & & B14RV9 & 11-Jun-02 & & $10028-17-8$ & TRITIUM \\
\hline 1433 & SESP SCHEDULING & & SW & & & B14RW0 & 11-Jun-02 & & $10028-17-8$ & TRITIUM \\
\hline 1434 & SESP SCHEDULING & & SW & & & B14RW1 & 11-Jun-02 & & $10028-17-8$ & TRITIUM \\
\hline 1435 & SESP SCHEDULING & & SW & & & B14RW2 & 11-Jun-02 & & $10028-17-8$ & TRITIUM \\
\hline 1436 & SESP SCHEDULING & & SW & & & B14RW3 & 11-Jun-02 & & $10028-17-8$ & TRITIUM \\
\hline 1437 & SESP SCHEDULING & & SW & & & B14RX4 & 11-Jun-02 & & $10028-17-8$ & TRITIUM \\
\hline 1438 & SESP SCHEDULING & & SW & & & B14RX5 & 11-Jun-02 & & $10028-17-8$ & TRITIUM \\
\hline 1439 & SESP SCHEDULING & & SW & & & B14RX6 & 11-Jun-02 & & $10028-17-8$ & TRITIUM \\
\hline 1440 & SESP SCHEDULING & & SW & & & B14RX7 & 11-Jun-02 & & $10028-17-8$ & TRITIUM \\
\hline 1441 & SESP SCHEDULING & & SW & & & B14RL3 & 27-Jun-02 & 29-May-02 & $210028-17-8$ & TRITIUM \\
\hline 1442 & SESP SCHEDULING & & SW & & & B14RM5 & 27-Jun-02 & 29-May-02 & 2 10028-17-8 & TRITIUM \\
\hline 1443 & SESP SCHEDULING & & SW & & & B14VT4 & 28-Jun-02 & & $10028-17-8$ & TRITIUM \\
\hline 1444 & SESP SCHEDULING & & SW & & & B14VX3 & 28-Jun-02 & & $10028-17-8$ & TRITIUM \\
\hline 1445 & SESP SCHEDULING & & SW & & & B14YH1 & 08-Jul-02 & & $10028-17-8$ & TRITIUM \\
\hline
\end{tabular}




\begin{tabular}{|c|c|c|c|c|c|c|c|c|c|c|}
\hline & A & $\mathrm{B}$ & C & $\mathrm{D}$ & $\mathrm{E}$ & $\mathrm{F}$ & $\mathrm{G}$ & $\mathrm{H}$ & $\mathrm{I}$ & $\mathrm{J}$ \\
\hline 1 & SAMPLE_NUM_ASSIGNED_TO & SAMPLER & MEDIA & BIOTA_MEDIA & TAG_ID & SAMP_NUM & SAMP_DATE_TIME & SAMP_DATE_TIME_ON & CON_ID & CON_SHORT_NAME \\
\hline 1446 & SESP SCHEDULING & & SW & & & B14YH2 & 08-Jul-02 & & $10028-17-8$ & TRITIUM \\
\hline 1447 & SESP SCHEDULING & & SW & & & B14YH3 & 08-Jul-02 & & $10028-17-8$ & TRITIUM \\
\hline 1448 & SESP SCHEDULING & & SW & & & B14YJ2 & 08-Jul-02 & & $10028-17-8$ & TRITIUM \\
\hline 1449 & SESP SCHEDULING & & SW & & & B14YJ5 & 08-Jul-02 & & $10028-17-8$ & TRITIUM \\
\hline 1450 & SESP SCHEDULING & & SW & & & B14YJ8 & 08-Jul-02 & & $10028-17-8$ & TRITIUM \\
\hline 1451 & SESP SCHEDULING & & SW & & & B14YJ9 & 08-Jul-02 & & $10028-17-8$ & TRITIUM \\
\hline 1452 & SESP SCHEDULING & & SW & & & B14YK0 & 08-Jul-02 & & $10028-17-8$ & TRITIUM \\
\hline 1453 & SESP SCHEDULING & & SW & & & B14WW5 & 07-Aug-02 & 27-Jun-02 & 2 10028-17-8 & TRITIUM \\
\hline 1454 & SESP SCHEDULING & & SW & & & B14WX2 & 07-Aug-02 & 27-Jun-02 & 2 10028-17-8 & TRITIUM \\
\hline 1455 & DEAYRES & DEROOS, GB & SW & & & B154X9 & 14-Aug-02 & & $10028-17-8$ & TRITIUM \\
\hline 1456 & DEAYRES & DEROOS, GB & SW & & & B154Y0 & 14-Aug-02 & & $10028-17-8$ & TRITIUM \\
\hline 1457 & SESP SCHEDULING & & SW & & & B153T9 & 04-Sep-02 & 07-Aug-02 & $210028-17-8$ & TRITIUM \\
\hline 1458 & SESP SCHEDULING & & SW & & & B153V4 & 04-Sep-02 & 07-Aug-02 & 2 10028-17-8 & TRITIUM \\
\hline 1459 & SESP SCHEDULING & & SW & & & B158H3 & 05-Sep-02 & & $10028-17-8$ & TRITIUM \\
\hline 1460 & SESP SCHEDULING & & SW & & & B158H4 & 05-Sep-02 & & $10028-17-8$ & TRITIUM \\
\hline 1461 & SESP SCHEDULING & & SW & & & B158H5 & 05-Sep-02 & & $10028-17-8$ & TRITIUM \\
\hline 1462 & SESP SCHEDULING & & SW & & & B158H6 & 05-Sep-02 & & $10028-17-8$ & TRITIUM \\
\hline 1463 & SESP SCHEDULING & & SW & & & B158H7 & 05-Sep-02 & & $10028-17-8$ & TRITIUM \\
\hline 1464 & SESP SCHEDULING & & SW & & & B158H8 & 05-Sep-02 & & $10028-17-8$ & TRITIUM \\
\hline 1465 & SESP SCHEDULING & & SW & & & B158H9 & 05-Sep-02 & & $10028-17-8$ & TRITIUM \\
\hline 1466 & SESP SCHEDULING & & SW & & & B158J0 & 05-Sep-02 & & $10028-17-8$ & TRITIUM \\
\hline 1467 & SESP SCHEDULING & & SW & & & B158M7 & 05-Sep-02 & & $10028-17-8$ & TRITIUM \\
\hline 1468 & SESP SCHEDULING & & SW & & & B158M8 & 05-Sep-02 & & $10028-17-8$ & TRITIUM \\
\hline 1469 & SESP SCHEDULING & & SW & & & B158M9 & 05-Sep-02 & & $10028-17-8$ & TRITIUM \\
\hline 1470 & SESP SCHEDULING & & SW & & & B158W8 & 05-Sep-02 & & $10028-17-8$ & TRITIUM \\
\hline 1471 & SESP SCHEDULING & & SW & & & B158X1 & 05-Sep-02 & & $10028-17-8$ & TRITIUM \\
\hline 1472 & SESP SCHEDULING & & SW & & & B158X4 & 05-Sep-02 & & $10028-17-8$ & TRITIUM \\
\hline 1473 & SESP SCHEDULING & & SW & & & B158X7 & 05-Sep-02 & & $10028-17-8$ & TRITIUM \\
\hline 1474 & SESP SCHEDULING & & SW & & & B158J1 & 09-Sep-02 & & $10028-17-8$ & TRITIUM \\
\hline 1475 & SESP SCHEDULING & & SW & & & B158J3 & 09-Sep-02 & & $10028-17-8$ & TRITIUM \\
\hline 1476 & SESP SCHEDULING & & SW & & & B158J5 & 09-Sep-02 & & $10028-17-8$ & TRITIUM \\
\hline 1477 & SESP SCHEDULING & & SW & & & B158J7 & 09-Sep-02 & & $10028-17-8$ & TRITIUM \\
\hline 1478 & SESP SCHEDULING & & SW & & & B158J9 & 09-Sep-02 & & $10028-17-8$ & TRITIUM \\
\hline 1479 & SESP SCHEDULING & & SW & & & B158K1 & 09-Sep-02 & & $10028-17-8$ & TRITIUM \\
\hline 1480 & SESP SCHEDULING & & SW & & & B158K3 & 09-Sep-02 & & $10028-17-8$ & TRITIUM \\
\hline 1481 & SESP SCHEDULING & & SW & & & B158K5 & 09-Sep-02 & & $10028-17-8$ & TRITIUM \\
\hline 1482 & SESP SCHEDULING & & SW & & & B158K7 & 09-Sep-02 & & $10028-17-8$ & TRITIUM \\
\hline 1483 & SESP SCHEDULING & & SW & & & B158K9 & 09-Sep-02 & & $10028-17-8$ & TRITIUM \\
\hline
\end{tabular}




\begin{tabular}{|c|c|c|c|c|c|c|c|c|c|c|}
\hline & $\mathrm{A}$ & $\mathrm{B}$ & $\mathrm{C}$ & $\mathrm{D}$ & $\mathrm{E}$ & $\mathrm{F}$ & $\mathrm{G}$ & $\mathrm{H}$ & $\mathrm{I}$ & $\mathrm{J}$ \\
\hline 1 & SAMPLE_NUM_ASSIGNED_TO & SAMPLER & MEDIA & BIOTA_MEDIA & TAG_ID & SAMP_NUM & SAMP_DATE_TIME & SAMP_DATE_TIME_ON & CON_ID & CON_SHORT_NAME \\
\hline 1484 & SESP SCHEDULING & & SW & & & B158L1 & 09-Sep-02 & & $10028-17-8$ & TRITIUM \\
\hline 1485 & SESP SCHEDULING & & SW & & & B158L3 & 09-Sep-02 & & $10028-17-8$ & TRITIUM \\
\hline 1486 & SESP SCHEDULING & & SW & & & B158N0 & 09-Sep-02 & & $10028-17-8$ & TRITIUM \\
\hline 1487 & SESP SCHEDULING & & SW & & & B158N2 & 09-Sep-02 & & $10028-17-8$ & TRITIUM \\
\hline 1488 & SESP SCHEDULING & & SW & & & B158N4 & 09-Sep-02 & & $10028-17-8$ & TRITIUM \\
\hline 1489 & SESP SCHEDULING & & SW & & & B15902 & 09-Sep-02 & & $10028-17-8$ & TRITIUM \\
\hline 1490 & SESP SCHEDULING & & SW & & & B15906 & 09-Sep-02 & & $10028-17-8$ & TRITIUM \\
\hline 1491 & SESP SCHEDULING & & SW & & & B15910 & 09-Sep-02 & & $10028-17-8$ & TRITIUM \\
\hline 1492 & SESP SCHEDULING & & SW & & & B15914 & 09-Sep-02 & & $10028-17-8$ & TRITIUM \\
\hline 1493 & SESP SCHEDULING & & SW & & & B158L5 & 10-Sep-02 & & $10028-17-8$ & TRITIUM \\
\hline 1494 & SESP SCHEDULING & & SW & & & B158L6 & 10-Sep-02 & & $10028-17-8$ & TRITIUM \\
\hline 1495 & SESP SCHEDULING & & SW & & & B158L7 & 10-Sep-02 & & $10028-17-8$ & TRITIUM \\
\hline 1496 & SESP SCHEDULING & & SW & & & B158L8 & 10-Sep-02 & & $10028-17-8$ & TRITIUM \\
\hline 1497 & SESP SCHEDULING & & SW & & & B158L9 & 10-Sep-02 & & $10028-17-8$ & TRITIUM \\
\hline 1498 & SESP SCHEDULING & & SW & & & B158M0 & 10-Sep-02 & & $10028-17-8$ & TRITIUM \\
\hline 1499 & SESP SCHEDULING & & SW & & & B158M1 & 10-Sep-02 & & $10028-17-8$ & TRITIUM \\
\hline 1500 & SESP SCHEDULING & & SW & & & B158M2 & 10-Sep-02 & & $10028-17-8$ & TRITIUM \\
\hline 1501 & SESP SCHEDULING & & SW & & & B158M3 & 10-Sep-02 & & $10028-17-8$ & TRITIUM \\
\hline 1502 & SESP SCHEDULING & & SW & & & B158M4 & 10-Sep-02 & & $10028-17-8$ & TRITIUM \\
\hline 1503 & SESP SCHEDULING & & SW & & & B158M5 & 10-Sep-02 & & $10028-17-8$ & TRITIUM \\
\hline 1504 & SESP SCHEDULING & & SW & & & B158M6 & 10-Sep-02 & & $10028-17-8$ & TRITIUM \\
\hline 1505 & SESP SCHEDULING & & SW & & & B158V6 & 10-Sep-02 & & $10028-17-8$ & TRITIUM \\
\hline 1506 & SESP SCHEDULING & & SW & & & B158V7 & 10-Sep-02 & & $10028-17-8$ & TRITIUM \\
\hline 1507 & SESP SCHEDULING & & SW & & & B158V8 & 10-Sep-02 & & $10028-17-8$ & TRITIUM \\
\hline 1508 & SESP SCHEDULING & & SW & & & B158V9 & 10-Sep-02 & & $10028-17-8$ & TRITIUM \\
\hline 1509 & SESP SCHEDULING & & SW & & & B158Y0 & 10-Sep-02 & & $10028-17-8$ & TRITIUM \\
\hline 1510 & SESP SCHEDULING & & SW & & & B158Y3 & 10-Sep-02 & & $10028-17-8$ & TRITIUM \\
\hline 1511 & SESP SCHEDULING & & SW & & & B158Y6 & 10-Sep-02 & & $10028-17-8$ & TRITIUM \\
\hline 1512 & SESP SCHEDULING & & SW & & & B158Y9 & 10-Sep-02 & & $10028-17-8$ & TRITIUM \\
\hline 1513 & SESP SCHEDULING & & SW & & & B15C08 & 16-Sep-02 & & $10028-17-8$ & TRITIUM \\
\hline 1514 & SESP SCHEDULING & & SW & & & B15C55 & 16-Sep-02 & & $10028-17-8$ & TRITIUM \\
\hline 1515 & SESP SCHEDULING & & SW & & & B15C58 & 16-Sep-02 & & $10028-17-8$ & TRITIUM \\
\hline 1516 & SESP SCHEDULING & & SW & & & B15948 & 02-Oct-02 & 04-Sep-02 & $10028-17-8$ & TRITIUM \\
\hline 1517 & SESP SCHEDULING & & SW & & & B15954 & 02-Oct-02 & 04-Sep-02 & $10028-17-8$ & TRITIUM \\
\hline 1518 & SESP SCHEDULING & & SW & & & B15C09 & 07-Oct-02 & & $10028-17-8$ & TRITIUM \\
\hline 1519 & SESP SCHEDULING & & SW & & & B15C11 & 07-Oct-02 & & $10028-17-8$ & TRITIUM \\
\hline 1520 & SESP SCHEDULING & & SW & & & B15C73 & 07-Oct-02 & & $10028-17-8$ & TRITIUM \\
\hline 1521 & SESP SCHEDULING & & SW & & & B15LK4 & 09-Oct-02 & & $10028-17-8$ & TRITIUM \\
\hline
\end{tabular}




\begin{tabular}{|c|c|c|c|c|c|c|c|c|c|c|}
\hline & A & $\mathrm{B}$ & $\mathrm{C}$ & $\mathrm{D}$ & $E$ & $\mathrm{~F}$ & G & $\mathrm{H}$ & $\mathrm{I}$ & $\mathrm{J}$ \\
\hline 1 & SAMPLE_NUM_ASSIGNED_TO & SAMPLER & MEDIA & BIOTA_MEDIA & TAG_ID & SAMP_NUM & SAMP_DATE_TIME & SAMP_DATE_TIME_ON & CON_ID & CON_SHORT_NAME \\
\hline 1522 & SESP SCHEDULING & & SW & & & B15LL8 & 09-Oct-02 & & $10028-17-8$ & TRITIUM \\
\hline 1523 & SESP SCHEDULING & & SW & & & B15LL7 & 09-Oct-02 & & $10028-17-8$ & TRITIUM \\
\hline 1524 & SESP SCHEDULING & & SW & & & B15C60 & 21-Oct-02 & & $10028-17-8$ & TRITIUM \\
\hline 1525 & SESP SCHEDULING & & SW & & & B15C65 & 21-Oct-02 & & $10028-17-8$ & TRITIUM \\
\hline 1526 & SESP SCHEDULING & & SW & & & B15CB3 & 27-Oct-02 & & $10028-17-8$ & TRITIUM \\
\hline 1527 & SESP SCHEDULING & & SW & & & B15C69 & 29-Oct-02 & & $10028-17-8$ & TRITIUM \\
\hline \begin{tabular}{|l|l|}
1528 \\
1528
\end{tabular} & SESP SCHEDULING & & SW & & & B15K74 & $30-$ Oct-02 & 02-Oct-02 & $10028-17-8$ & TRITIUM \\
\hline 1529 & SESP SCHEDULING & & SW & & & B15K79 & 30-Oct-02 & $02-$ Oct-02 & $10028-17-8$ & TRITIUM \\
\hline 1530 & SESP SCHEDULING & & SW & & & B15VN2 & 26-Nov-02 & $30-$ Oct-02 & $10028-17-8$ & TRITIUM \\
\hline \begin{tabular}{|l}
1531 \\
\end{tabular} & SESP SCHEDULING & & SW & & & B15VN8 & 26-Nov-02 & $30-$ Oct-02 & $10028-17-8$ & TRITIUM \\
\hline 1532 & SESP SCHEDULING & & SW & & & B0X8R8 & 04-Jan-00 & & $13966-29-5$ & U-234 \\
\hline 1533 & SESP SCHEDULING & & SW & & & B0X7K4 & 02-Feb-00 & 29-Dec-99 & $13966-29-5$ & U-234 \\
\hline 1534 & SESP SCHEDULING & & SW & & & B0X7J0 & 02-Feb-00 & 29-Dec-99 & $13966-29-5$ & U-234 \\
\hline 1535 & SESP SCHEDULING & & SW & & & BOXH28 & 08-Mar-00 & 02-Feb-00 & $13966-29-5$ & U-234 \\
\hline 1536 & SESP SCHEDULING & & SW & & & BOXH22 & 08-Mar-00 & 02-Feb-00 & $13966-29-5$ & U-234 \\
\hline \begin{tabular}{|l|}
1537 \\
\end{tabular} & SESP SCHEDULING & & SW & & & BOXRK2 & 27-Mar-00 & & $13966-29-5$ & $\mathrm{U}-234$ \\
\hline \begin{tabular}{|l|}
1538 \\
\end{tabular} & SESP SCHEDULING & & SW & & & BOXRK3 & 27-Mar-00 & & $13966-29-5$ & $\mathrm{U}-234$ \\
\hline 1539 & SESP SCHEDULING & & SW & & & BOXRK4 & 27-Mar-00 & & $13966-29-5$ & U-234 \\
\hline 1540 & SESP SCHEDULING & & SW & & & B0XRK5 & 27-Mar-00 & & $13966-29-5$ & U-234 \\
\hline 1541 & SESP SCHEDULING & & SW & & & BOXRKO & 28-Mar-00 & & $13966-29-5$ & $\mathrm{U}-234$ \\
\hline 1542 & SESP SCHEDULING & & SW & & & B0XRK6 & 28-Mar-00 & & $13966-29-5$ & U-234 \\
\hline 1543 & SESP SCHEDULING & & SW & & & B0XRK7 & 28-Mar-00 & & $13966-29-5$ & U-234 \\
\hline 1544 & SESP SCHEDULING & & SW & & & BOXRK8 & 28-Mar-00 & & $13966-29-5$ & U-234 \\
\hline 1545 & SESP SCHEDULING & & SW & & & BOXRK9 & 28-Mar-00 & & $13966-29-5$ & U-234 \\
\hline 1546 & SESP SCHEDULING & & SW & & & BOXRLO & 28-Mar-00 & & $13966-29-5$ & U-234 \\
\hline 1547 & SESP SCHEDULING & & SW & & & B0XRL1 & 28-Mar-00 & & $13966-29-5$ & U-234 \\
\hline 1548 & SESP SCHEDULING & & SW & & & BOXRN8 & 28-Mar-00 & & $13966-29-5$ & U-234 \\
\hline 1549 & SESP SCHEDULING & & SW & & & BOXRN9 & 28-Mar-00 & & $13966-29-5$ & U-234 \\
\hline 1550 & SESP SCHEDULING & & SW & & & BOXRPO & 28-Mar-00 & & $13966-29-5$ & U-234 \\
\hline \begin{tabular}{|l|}
1551 \\
150
\end{tabular} & SESP SCHEDULING & & SW & & & B0XRP1 & 28-Mar-00 & & $13966-29-5$ & U-234 \\
\hline 1552 & SESP SCHEDULING & & SW & & & BOXWWO & 04-Apr-00 & & $13966-29-5$ & U-234 \\
\hline \begin{tabular}{|l|}
1553 \\
\end{tabular} & SESP SCHEDULING & & SW & & & B0XP21 & 05-Apr-00 & 08-Mar-00 & $13966-29-5$ & $\mathrm{U}-234$ \\
\hline 1554 & SESP SCHEDULING & & SW & & & B0XP16 & 05-Apr-00 & 08-Mar-00 & $13966-29-5$ & U-234 \\
\hline 1555 & SESP SCHEDULING & & SW & & & B0XVN6 & 03-May-00 & 29-Mar-00 & $13966-29-5$ & U-234 \\
\hline 1556 & SESP SCHEDULING & & SW & & & BOXVP1 & 03-May-00 & 29-Mar-00 & $13966-29-5$ & U-234 \\
\hline 1557 & SESP SCHEDULING & & SW & & & BOY2Y6 & 11-May-00 & & $13966-29-5$ & $\mathrm{U}-234$ \\
\hline 1558 & SESP SCHEDULING & & SW & & & B0Y231 & 31-May-00 & 03-May-00 & $13966-29-5$ & U-234 \\
\hline 1559 & SESP SCHEDULING & & SW & & & B0Y237 & 31-May-00 & 03-May-00 & $13966-29-5$ & U-234 \\
\hline
\end{tabular}




\begin{tabular}{|c|c|c|c|c|c|c|c|c|c|c|}
\hline & A & $\mathrm{B}$ & C & $\mathrm{D}$ & $E$ & $\mathrm{~F}$ & G & $\mathrm{H}$ & $\mathrm{I}$ & $\mathrm{J}$ \\
\hline 1 & SAMPLE_NUM_ASSIGNED_TO & SAMPLER & MEDIA & BIOTA_MEDIA & TAG_ID & SAMP_NUM & SAMP_DATE_TIME & SAMP_DATE_TIME_ON & CON_ID & CON_SHORT_NAME \\
\hline 1560 & SESP SCHEDULING & & SW & & & B0YB90 & 08-Jun-00 & & $13966-29-5$ & $\mathrm{U}-234$ \\
\hline 1561 & SESP SCHEDULING & & SW & & & BOYRB8 & 12-Jun-00 & & $13966-29-5$ & U-234 \\
\hline 1562 & SESP SCHEDULING & & SW & & & BOYRCO & 12-Jun-00 & & $13966-29-5$ & U-234 \\
\hline 1563 & SESP SCHEDULING & & SW & & & BOYRC3 & 12-Jun-00 & & 13966-29-5 & $\mathrm{U}-234$ \\
\hline 1564 & SESP SCHEDULING & & SW & & & B0YCD7 & 19-Jun-00 & & $13966-29-5$ & $\mathrm{U}-234$ \\
\hline 1565 & SESP SCHEDULING & & SW & & & B0YCD8 & 19-Jun-00 & & $13966-29-5$ & $\mathrm{U}-234$ \\
\hline 1566 & SESP SCHEDULING & & SW & & & BOYCD9 & 19-Jun-00 & & 13966-29-5 & $\mathrm{U}-234$ \\
\hline 1567 & SESP SCHEDULING & & SW & & & BOYCFO & 19-Jun-00 & & $13966-29-5$ & $\mathrm{U}-234$ \\
\hline 1568 & SESP SCHEDULING & & SW & & & B0YCF1 & 20-Jun-00 & & 13966-29-5 & U-234 \\
\hline 1569 & SESP SCHEDULING & & SW & & & BOYCF2 & 20-Jun-00 & & $13966-29-5$ & $\mathrm{U}-234$ \\
\hline 1570 & SESP SCHEDULING & & SW & & & BOYCF3 & 20-Jun-00 & & $13966-29-5$ & U-234 \\
\hline 1571 & SESP SCHEDULING & & SW & & & BOYCF4 & 20-Jun-00 & & $13966-29-5$ & $\mathrm{U}-234$ \\
\hline 1572 & SESP SCHEDULING & & SW & & & B0YCF5 & 20-Jun-00 & & 13966-29-5 & U-234 \\
\hline 1573 & SESP SCHEDULING & & SW & & & B0YCF6 & 20-Jun-00 & & $13966-29-5$ & $\mathrm{U}-234$ \\
\hline 1574 & SESP SCHEDULING & & SW & & & BOYCK3 & 20-Jun-00 & & $13966-29-5$ & $\mathrm{U}-234$ \\
\hline 1575 & SESP SCHEDULING & & SW & & & BOYCK4 & 20-Jun-00 & & $13966-29-5$ & U-234 \\
\hline 1576 & SESP SCHEDULING & & SW & & & B0YCK5 & 20-Jun-00 & & 13966-29-5 & U-234 \\
\hline 1577 & SESP SCHEDULING & & SW & & & BOYCK6 & 20-Jun-00 & & $13966-29-5$ & U-234 \\
\hline 1578 & SESP SCHEDULING & & SW & & & B0YB64 & 29-Jun-00 & 31-May-00 & $13966-29-5$ & U-234 \\
\hline 1579 & SESP SCHEDULING & & SW & & & BOYB76 & 29-Jun-00 & 31-May-00 & $13966-29-5$ & $\mathrm{U}-234$ \\
\hline 1580 & SESP SCHEDULING & & SW & & & B0YM20 & 05-Jul-00 & & $13966-29-5$ & $\mathrm{U}-234$ \\
\hline 1581 & SESP SCHEDULING & & SW & & & B0YM22 & 07-Jul-00 & & $13966-29-5$ & $\mathrm{U}-234$ \\
\hline 1582 & SESP SCHEDULING & & SW & & & BOYKV4 & 10-Aug-00 & 29-Jun-00 & $13966-29-5$ & U-234 \\
\hline 1583 & SESP SCHEDULING & & SW & & & BOYKW1 & 10-Aug-00 & 29-Jun-00 & $13966-29-5$ & $\mathrm{U}-234$ \\
\hline 1584 & SESP SCHEDULING & & SW & & & B0YW65 & 07-Sep-00 & & $13966-29-5$ & U-234 \\
\hline 1585 & SESP SCHEDULING & & SW & & & BOYW70 & 07-Sep-00 & & 13966-29-5 & U-234 \\
\hline 1586 & SESP SCHEDULING & & SW & & & B10672 & 14-Sep-00 & & $13966-29-5$ & $\mathrm{U}-234$ \\
\hline 1587 & SESP SCHEDULING & & SW & & & B10673 & 14-Sep-00 & & $13966-29-5$ & $\mathrm{U}-234$ \\
\hline 1588 & SESP SCHEDULING & & SW & & & B10674 & 14-Sep-00 & & $13966-29-5$ & U-234 \\
\hline 1589 & SESP SCHEDULING & & SW & & & B10675 & 14-Sep-00 & & $13966-29-5$ & $\mathrm{U}-234$ \\
\hline 1590 & SESP SCHEDULING & & SW & & & B10676 & 14-Sep-00 & & $13966-29-5$ & U-234 \\
\hline 1591 & SESP SCHEDULING & & SW & & & B10677 & 14-Sep-00 & & $13966-29-5$ & $\mathrm{U}-234$ \\
\hline 1592 & SESP SCHEDULING & & SW & & & B10679 & 14-Sep-00 & & $13966-29-5$ & $\mathrm{U}-234$ \\
\hline 1593 & SESP SCHEDULING & & SW & & & B10681 & 14-Sep-00 & & $13966-29-5$ & $\mathrm{U}-234$ \\
\hline 1594 & SESP SCHEDULING & & SW & & & B10683 & 14-Sep-00 & & 13966-29-5 & U-234 \\
\hline 1595 & SESP SCHEDULING & & SW & & & B10684 & 14-Sep-00 & & $13966-29-5$ & $\mathrm{U}-234$ \\
\hline 1596 & SESP SCHEDULING & & SW & & & B10686 & 14-Sep-00 & & $13966-29-5$ & $\mathrm{U}-234$ \\
\hline 1597 & SESP SCHEDULING & & SW & & & B10698 & 14-Sep-00 & & 13966-29-5 & $\mathrm{U}-234$ \\
\hline
\end{tabular}




\begin{tabular}{|c|c|c|c|c|c|c|c|c|c|c|}
\hline & A & $\mathrm{B}$ & C & $\mathrm{D}$ & $E$ & $\mathrm{~F}$ & G & $\mathrm{H}$ & $\mathrm{I}$ & $\mathrm{J}$ \\
\hline 1 & SAMPLE_NUM_ASSIGNED_TO & SAMPLER & MEDIA & BIOTA_MEDIA & TAG_ID & SAMP_NUM & SAMP_DATE_TIME & SAMP_DATE_TIME_ON & CON_ID & CON_SHORT_NAME \\
\hline 1598 & SESP SCHEDULING & & SW & & & B106B1 & 14-Sep-00 & & $13966-29-5$ & $\mathrm{U}-234$ \\
\hline 1599 & SESP SCHEDULING & & SW & & & B106B4 & 14-Sep-00 & & $13966-29-5$ & U-234 \\
\hline 1600 & SESP SCHEDULING & & SW & & & B106B7 & 14-Sep-00 & & $13966-29-5$ & U-234 \\
\hline 1601 & SESP SCHEDULING & & SW & & & B106W1 & 18-Sep-00 & & 13966-29-5 & $\mathrm{U}-234$ \\
\hline 1602 & SESP SCHEDULING & & SW & & & B106W2 & 18-Sep-00 & & $13966-29-5$ & $\mathrm{U}-234$ \\
\hline 1603 & SESP SCHEDULING & & SW & & & B106W3 & 18-Sep-00 & & $13966-29-5$ & $\mathrm{U}-234$ \\
\hline 1604 & SESP SCHEDULING & & SW & & & B106W4 & 18-Sep-00 & & 13966-29-5 & $\mathrm{U}-234$ \\
\hline 1605 & SESP SCHEDULING & & SW & & & B106W5 & 18-Sep-00 & & 13966-29-5 & $\mathrm{U}-234$ \\
\hline 1606 & SESP SCHEDULING & & SW & & & B106W6 & 18-Sep-00 & & 13966-29-5 & U-234 \\
\hline 1607 & SESP SCHEDULING & & SW & & & B106W7 & 18-Sep-00 & & $13966-29-5$ & $\mathrm{U}-234$ \\
\hline 1608 & SESP SCHEDULING & & SW & & & B106W8 & 18-Sep-00 & & $13966-29-5$ & U-234 \\
\hline 1609 & SESP SCHEDULING & & SW & & & B106W9 & 18-Sep-00 & & $13966-29-5$ & $\mathrm{U}-234$ \\
\hline 1610 & SESP SCHEDULING & & SW & & & B106X0 & 18-Sep-00 & & 13966-29-5 & U-234 \\
\hline 1611 & SESP SCHEDULING & & SW & & & B106X1 & 18-Sep-00 & & $13966-29-5$ & $\mathrm{U}-234$ \\
\hline 1612 & SESP SCHEDULING & & SW & & & B106X2 & 18-Sep-00 & & $13966-29-5$ & $\mathrm{U}-234$ \\
\hline 1613 & SESP SCHEDULING & & SW & & & B10728 & 18-Sep-00 & & $13966-29-5$ & U-234 \\
\hline 1614 & SESP SCHEDULING & & SW & & & B10729 & 18-Sep-00 & & 13966-29-5 & U-234 \\
\hline 1615 & SESP SCHEDULING & & SW & & & B10730 & 18-Sep-00 & & $13966-29-5$ & U-234 \\
\hline 1616 & SESP SCHEDULING & & SW & & & B10785 & 18-Sep-00 & & $13966-29-5$ & U-234 \\
\hline 1617 & SESP SCHEDULING & & SW & & & B10788 & 18-Sep-00 & & 13966-29-5 & $\mathrm{U}-234$ \\
\hline 1618 & SESP SCHEDULING & & SW & & & B10791 & 18-Sep-00 & & $13966-29-5$ & $\mathrm{U}-234$ \\
\hline 1619 & SESP SCHEDULING & & SW & & & B10794 & 18-Sep-00 & & $13966-29-5$ & $\mathrm{U}-234$ \\
\hline 1620 & SESP SCHEDULING & & SW & & & B106X3 & 19-Sep-00 & & 13966-29-5 & U-234 \\
\hline 1621 & SESP SCHEDULING & & SW & & & B106X5 & 19-Sep-00 & & $13966-29-5$ & $\mathrm{U}-234$ \\
\hline 1622 & SESP SCHEDULING & & SW & & & B106X7 & 19-Sep-00 & & $13966-29-5$ & U-234 \\
\hline 1623 & SESP SCHEDULING & & SW & & & B106X9 & 19-Sep-00 & & 13966-29-5 & U-234 \\
\hline 1624 & SESP SCHEDULING & & SW & & & B106Y1 & 19-Sep-00 & & $13966-29-5$ & U-234 \\
\hline 1625 & SESP SCHEDULING & & SW & & & B106Y3 & 19-Sep-00 & & $13966-29-5$ & $\mathrm{U}-234$ \\
\hline 1626 & SESP SCHEDULING & & SW & & & B106Y4 & 19-Sep-00 & & $13966-29-5$ & U-234 \\
\hline 1627 & SESP SCHEDULING & & SW & & & B106Y5 & 19-Sep-00 & & $13966-29-5$ & $\mathrm{U}-234$ \\
\hline 1628 & SESP SCHEDULING & & SW & & & B106Y6 & 19-Sep-00 & & $13966-29-5$ & U-234 \\
\hline 1629 & SESP SCHEDULING & & SW & & & B106Y7 & 19-Sep-00 & & $13966-29-5$ & $\mathrm{U}-234$ \\
\hline 1630 & SESP SCHEDULING & & SW & & & B106Y8 & 19-Sep-00 & & $13966-29-5$ & $\mathrm{U}-234$ \\
\hline 1631 & SESP SCHEDULING & & SW & & & B106Y9 & 19-Sep-00 & & $13966-29-5$ & $\mathrm{U}-234$ \\
\hline 1632 & SESP SCHEDULING & & SW & & & B10761 & 19-Sep-00 & & 13966-29-5 & U-234 \\
\hline 1633 & SESP SCHEDULING & & SW & & & B10762 & 19-Sep-00 & & $13966-29-5$ & $\mathrm{U}-234$ \\
\hline 1634 & SESP SCHEDULING & & SW & & & B10763 & 19-Sep-00 & & $13966-29-5$ & $\mathrm{U}-234$ \\
\hline 1635 & SESP SCHEDULING & & SW & & & B10764 & 19-Sep-00 & & 13966-29-5 & $\mathrm{U}-234$ \\
\hline
\end{tabular}




\begin{tabular}{|c|c|c|c|c|c|c|c|c|c|c|}
\hline & A & $\mathrm{B}$ & C & $\mathrm{D}$ & $E$ & $\mathrm{~F}$ & G & $\mathrm{H}$ & $\mathrm{I}$ & $\mathrm{J}$ \\
\hline 1 & SAMPLE_NUM_ASSIGNED_TO & SAMPLER & MEDIA & BIOTA_MEDIA & TAG_ID & SAMP_NUM & SAMP_DATE_TIME & SAMP_DATE_TIME_ON & CON_ID & CON_SHORT_NAME \\
\hline 1636 & SESP SCHEDULING & & SW & & & B10773 & 19-Sep-00 & & $13966-29-5$ & $\mathrm{U}-234$ \\
\hline 1637 & SESP SCHEDULING & & SW & & & B10776 & 19-Sep-00 & & $13966-29-5$ & U-234 \\
\hline 1638 & SESP SCHEDULING & & SW & & & B10779 & 19-Sep-00 & & 13966-29-5 & U-234 \\
\hline 1639 & SESP SCHEDULING & & SW & & & B10782 & 19-Sep-00 & & 13966-29-5 & U-234 \\
\hline 1640 & SESP SCHEDULING & & SW & & & B10912 & 27-Sep-00 & & $13966-29-5$ & U-234 \\
\hline 1641 & SESP SCHEDULING & & SW & & & B10913 & 27-Sep-00 & & $13966-29-5$ & U-234 \\
\hline 1642 & SESP SCHEDULING & & SW & & & B10945 & 27-Sep-00 & & $13966-29-5$ & U-234 \\
\hline 1643 & SESP SCHEDULING & & SW & & & B10946 & 27-Sep-00 & & $13966-29-5$ & U-234 \\
\hline 1644 & SESP SCHEDULING & & SW & & & B10949 & 27-Sep-00 & & 13966-29-5 & U-234 \\
\hline 1645 & SESP SCHEDULING & & SW & & & B109J0 & 27-Sep-00 & & $13966-29-5$ & U-234 \\
\hline 1646 & SESP SCHEDULING & & SW & & & B103W8 & 05-Oct-00 & 07-Sep-00 & $13966-29-5$ & U-234 \\
\hline 1647 & SESP SCHEDULING & & SW & & & B103X3 & $05-O c t-00$ & 07-Sep-00 & $13966-29-5$ & U-234 \\
\hline 1648 & SESP SCHEDULING & & SW & & & B10F33 & 09-Oct-00 & & $13966-29-5$ & U-234 \\
\hline 1649 & SESP SCHEDULING & & SW & & & B10941 & 01-Nov-00 & & $13966-29-5$ & U-234 \\
\hline 1650 & SESP SCHEDULING & & SW & & & B10977 & 01-Nov-00 & & $13966-29-5$ & U-234 \\
\hline 1651 & SESP SCHEDULING & & SW & & & B10C13 & 02-Nov-00 & 05-Oct-00 & $13966-29-5$ & U-234 \\
\hline 1652 & SESP SCHEDULING & & SW & & & B10C19 & 02-Nov-00 & $05-$ Oct-00 & $13966-29-5$ & U-234 \\
\hline 1653 & SESP SCHEDULING & & SW & & & B10942 & 07-Nov-00 & & $13966-29-5$ & U-234 \\
\hline 1654 & SESP SCHEDULING & & SW & & & B10MP5 & 29-Nov-00 & 02-Nov-00 & $13966-29-5$ & U-234 \\
\hline 1655 & SESP SCHEDULING & & SW & & & B10MR1 & 29-Nov-00 & 02-Nov-00 & $13966-29-5$ & U-234 \\
\hline 1656 & SESP SCHEDULING & & SW & & & B11125 & 04-Dec-00 & & $13966-29-5$ & U-234 \\
\hline 1657 & SESP SCHEDULING & & SW & & & B11126 & 04-Dec-00 & & $13966-29-5$ & U-234 \\
\hline 1658 & SESP SCHEDULING & & SW & & & B11127 & 04-Dec-00 & & $13966-29-5$ & U-234 \\
\hline 1659 & SESP SCHEDULING & & SW & & & B11128 & 04-Dec-00 & & $13966-29-5$ & U-234 \\
\hline 1660 & SESP SCHEDULING & & SW & & & B11124 & 05-Dec-00 & & $13966-29-5$ & U-234 \\
\hline 1661 & SESP SCHEDULING & & SW & & & B11129 & 05-Dec-00 & & 13966-29-5 & U-234 \\
\hline 1662 & SESP SCHEDULING & & SW & & & B11130 & 05-Dec-00 & & $13966-29-5$ & U-234 \\
\hline 1663 & SESP SCHEDULING & & SW & & & B11131 & 05-Dec-00 & & $13966-29-5$ & U-234 \\
\hline 1664 & SESP SCHEDULING & & SW & & & B11132 & 05-Dec-00 & & $13966-29-5$ & U-234 \\
\hline 1665 & SESP SCHEDULING & & SW & & & B11133 & 05-Dec-00 & & $13966-29-5$ & U-234 \\
\hline 1666 & SESP SCHEDULING & & SW & & & B11134 & 05-Dec-00 & & $13966-29-5$ & U-234 \\
\hline 1667 & SESP SCHEDULING & & SW & & & B11145 & 05-Dec-00 & & $13966-29-5$ & U-234 \\
\hline 1668 & SESP SCHEDULING & & SW & & & B11146 & 05-Dec-00 & & $13966-29-5$ & U-234 \\
\hline 1669 & SESP SCHEDULING & & SW & & & B11147 & 05-Dec-00 & & $13966-29-5$ & U-234 \\
\hline 1670 & SESP SCHEDULING & & SW & & & B11148 & 05-Dec-00 & & 13966-29-5 & U-234 \\
\hline 1671 & SESP SCHEDULING & & SW & & & B110Y4 & 04-Jan-01 & 29-Nov-00 & $13966-29-5$ & U-234 \\
\hline 1672 & SESP SCHEDULING & & SW & & & B11100 & 04-Jan-01 & 29-Nov-00 & $13966-29-5$ & U-234 \\
\hline 1673 & SESP SCHEDULING & & SW & & & B114P8 & 31-Jan-01 & 04-Jan-01 & 13966-29-5 & U-234 \\
\hline
\end{tabular}




\begin{tabular}{|c|c|c|c|c|c|c|c|c|c|c|}
\hline & $A$ & $\mathrm{~B}$ & $\mathrm{C}$ & $\mathrm{D}$ & $E$ & $\mathrm{~F}$ & $G$ & $\mathrm{H}$ & $\mathrm{I}$ & $\mathrm{J}$ \\
\hline 1 & \begin{tabular}{|l|l} 
SAMPE_NUM_ASSIGNED_TO \\
\end{tabular} & SAMPLER & MEDIA & BIOTA_MEDIA & TAG_ID & SAMP_NUM & SAMP_DATE_TIME & SAMP_DATE_TIME_ON & CON_ID & CON_SHORT_NAME \\
\hline 1674 & SESP SCHEDULING & & SW & & & B114T0 & 31-Jan-01 & 04-Jan-01 & $13966-29-5$ & $\mathrm{U}-234$ \\
\hline 1675 & SESP SCHEDULING & & SW & & & B11CC7 & 12-Feb-01 & & $13966-29-5$ & U-234 \\
\hline 1676 & SESP SCHEDULING & & SW & & & B11H38 & 26-Feb-01 & & $13966-29-5$ & U-234 \\
\hline 1677 & SESP SCHEDULING & & SW & & & $\mathrm{B} 11 \mathrm{H} 43$ & 26-Feb-01 & & $13966-29-5$ & U-234 \\
\hline 1678 & SESP SCHEDULING & & SW & & & $\mathrm{B} 11 \mathrm{H} 44$ & 26-Feb-01 & & $13966-29-5$ & U-234 \\
\hline 1679 & SESP SCHEDULING & & SW & & & $\mathrm{B} 11 \mathrm{H} 45$ & 26-Feb-01 & & $13966-29-5$ & U-234 \\
\hline 1680 & SESP SCHEDULING & & SW & & & B11H46 & 26-Feb-01 & & $13966-29-5$ & $\mathrm{U}-234$ \\
\hline 1681 & SESP SCHEDULING & & SW & & & $\mathrm{B} 11 \mathrm{H} 47$ & 26-Feb-01 & & $13966-29-5$ & U-234 \\
\hline 1682 & SESP SCHEDULING & & SW & & & $\mathrm{B} 11 \mathrm{H} 48$ & 26-Feb-01 & & $13966-29-5$ & U-234 \\
\hline 1683 & SESP SCHEDULING & & SW & & & B11H59 & 26-Feb-01 & & $13966-29-5$ & $\mathrm{U}-234$ \\
\hline 1684 & SESP SCHEDULING & & SW & & & $\mathrm{B} 11 \mathrm{H} 60$ & 26-Feb-01 & & $13966-29-5$ & U-234 \\
\hline 1685 & SESP SCHEDULING & & SW & & & B11H61 & 26-Feb-01 & & $13966-29-5$ & U-234 \\
\hline 1686 & SESP SCHEDULING & & SW & & & B11H62 & 26-Feb-01 & & $13966-29-5$ & U-234 \\
\hline 1687 & SESP SCHEDULING & & SW & & & B11H39 & 27-Feb-01 & & $13966-29-5$ & U-234 \\
\hline 1688 & SESP SCHEDULING & & SW & & & $\mathrm{B} 11 \mathrm{H} 40$ & 27-Feb-01 & & $13966-29-5$ & U-234 \\
\hline \begin{tabular}{|l|}
1689 \\
\end{tabular} & SESP SCHEDULING & & SW & & & $\mathrm{B} 11 \mathrm{H} 41$ & 27-Feb-01 & & $13966-29-5$ & $\mathrm{U}-234$ \\
\hline \begin{tabular}{|l|}
1690 \\
\end{tabular} & SESP SCHEDULING & & SW & & & $\mathrm{B} 11 \mathrm{H} 42$ & 27-Feb-01 & & $13966-29-5$ & $\mathrm{U}-234$ \\
\hline 1691 & SESP SCHEDULING & & SW & & & B11C04 & 01-Mar-01 & 31-Jan-01 & $13966-29-5$ & U-234 \\
\hline 1692 & SESP SCHEDULING & & SW & & & B11C10 & 01-Mar-01 & 31-Jan-01 & $13966-29-5$ & U-234 \\
\hline 1693 & SESP SCHEDULING & & SW & & & B11MY9 & 03-Apr-01 & & $13966-29-5$ & $\mathrm{U}-234$ \\
\hline 1694 & SESP SCHEDULING & & SW & & & B11J40 & 04-Apr-01 & 01-Mar-01 & $13966-29-5$ & U-234 \\
\hline 1695 & SESP SCHEDULING & & SW & & & B11J46 & 04-Apr-01 & 01-Mar-01 & $13966-29-5$ & U-234 \\
\hline 1696 & SESP SCHEDULING & & SW & & & B11W36 & 30-Apr-01 & & $13966-29-5$ & U-234 \\
\hline 1697 & SESP SCHEDULING & & SW & & & B11W50 & 30-Apr-01 & & $13966-29-5$ & U-234 \\
\hline 1698 & SESP SCHEDULING & & SW & & & B11W52 & 30-Apr-01 & & $13966-29-5$ & U-234 \\
\hline 1699 & SESP SCHEDULING & & SW & & & B11W55 & 30-Apr-01 & & $13966-29-5$ & $\mathrm{U}-234$ \\
\hline 1700 & SESP SCHEDULING & & SW & & & B11W86 & 30-Apr-01 & & $13966-29-5$ & U-234 \\
\hline 1701 & SESP SCHEDULING & & SW & & & B11LT2 & 02-May-01 & 04-Apr-01 & $13966-29-5$ & U-234 \\
\hline 1702 & SESP SCHEDULING & & SW & & & B11LR6 & 02-May-01 & 04-Apr-01 & $13966-29-5$ & U-234 \\
\hline 1703 & SESP SCHEDULING & & SW & & & B11W57 & 03-May-01 & & $13966-29-5$ & U-234 \\
\hline 1704 & SESP SCHEDULING & & SW & & & B11WV8 & 09-May-01 & & $13966-29-5$ & U-234 \\
\hline \begin{tabular}{|l|}
1705 \\
\end{tabular} & SESP SCHEDULING & & SW & & & B11WX0 & 09-May-01 & & $13966-29-5$ & $\mathrm{U}-234$ \\
\hline 1706 & SESP SCHEDULING & & SW & & & B11W38 & 10-May-01 & & $13966-29-5$ & U-234 \\
\hline 1707 & SESP SCHEDULING & & SW & & & B121V4 & 17-May-01 & & $13966-29-5$ & U-234 \\
\hline 1708 & SESP SCHEDULING & & SW & & & B11WT4 & 30-May-01 & 02-May-01 & $13966-29-5$ & U-234 \\
\hline 1709 & SESP SCHEDULING & & SW & & & B11WR8 & 30-May-01 & 02-May-01 & $13966-29-5$ & U-234 \\
\hline 1710 & SESP SCHEDULING & & SW & & & B124N7 & 07-Jun-01 & & $13966-29-5$ & U-234 \\
\hline 1711 & SESP SCHEDULING & & SW & & & B124P7 & 07-Jun-01 & & $13966-29-5$ & U-234 \\
\hline
\end{tabular}




\begin{tabular}{|c|c|c|c|c|c|c|c|c|c|c|}
\hline & $\mathrm{A}$ & $\mathrm{B}$ & $\mathrm{C}$ & $\mathrm{D}$ & $\mathrm{E}$ & $\mathrm{F}$ & $\mathrm{G}$ & $\mathrm{H}$ & $\mathrm{I}$ & $\mathrm{J}$ \\
\hline 1 & SAMPLE_NUM_ASSIGNED_TO & SAMPLER & MEDIA & BIOTA_MEDIA & TAG_ID & SAMP_NUM & SAMP_DATE_TIME & SAMP_DATE_TIME_ON & CON_ID & CON_SHORT_NAME \\
\hline 1712 & SESP SCHEDULING & & SW & & & B12505 & 12-Jun-01 & & $13966-29-5$ & $\mathrm{U}-234$ \\
\hline 1713 & SESP SCHEDULING & & SW & & & B12506 & 12-Jun-01 & & $13966-29-5$ & $\mathrm{U}-234$ \\
\hline 1714 & SESP SCHEDULING & & SW & & & B12507 & 12-Jun-01 & & $13966-29-5$ & $\mathrm{U}-234$ \\
\hline 1715 & SESP SCHEDULING & & SW & & & B12508 & 12-Jun-01 & & $13966-29-5$ & $\mathrm{U}-234$ \\
\hline 1716 & SESP SCHEDULING & & SW & & & B12509 & 12-Jun-01 & & 13966-29-5 & $U-234$ \\
\hline 1717 & SESP SCHEDULING & & SW & & & B12510 & 12-Jun-01 & & $13966-29-5$ & U-234 \\
\hline 1718 & SESP SCHEDULING & & SW & & & B12531 & 12-Jun-01 & & $13966-29-5$ & $\mathrm{U}-234$ \\
\hline 1719 & SESP SCHEDULING & & SW & & & B12532 & 12-Jun-01 & & $13966-29-5$ & $\mathrm{U}-234$ \\
\hline 1720 & SESP SCHEDULING & & SW & & & B12533 & 12-Jun-01 & & $13966-29-5$ & U-234 \\
\hline 1721 & SESP SCHEDULING & & SW & & & B12534 & 12-Jun-01 & & $13966-29-5$ & $\mathrm{U}-234$ \\
\hline 1722 & SESP SCHEDULING & & SW & & & B12501 & 14-Jun-01 & & $13966-29-5$ & $\mathrm{U}-234$ \\
\hline 1723 & SESP SCHEDULING & & SW & & & B12502 & 14-Jun-01 & & $13966-29-5$ & U-234 \\
\hline 1724 & SESP SCHEDULING & & SW & & & B12503 & 14-Jun-01 & & 13966-29-5 & $\mathrm{U}-234$ \\
\hline 1725 & SESP SCHEDULING & & SW & & & B12504 & 14-Jun-01 & & $13966-29-5$ & $\mathrm{U}-234$ \\
\hline 1726 & SESP SCHEDULING & & SW & & & B124M3 & 28-Jun-01 & 23-May-01 & $13966-29-5$ & $\mathrm{U}-234$ \\
\hline 1727 & SESP SCHEDULING & & SW & & & B124L7 & 28-Jun-01 & 23-May-01 & $13966-29-5$ & U-234 \\
\hline 1728 & SESP SCHEDULING & & SW & & & B12992 & 09-Jul-01 & & $13966-29-5$ & U-234 \\
\hline 1729 & SESP SCHEDULING & & SW & & & B12994 & 09-Jul-01 & & $13966-29-5$ & U-234 \\
\hline 1730 & SESP SCHEDULING & & SW & & & B129B4 & 09-Jul-01 & & $13966-29-5$ & U-234 \\
\hline 1731 & SESP SCHEDULING & & SW & & & B12841 & 09-Aug-01 & 28-Jun-01 & $13966-29-5$ & $\mathrm{U}-234$ \\
\hline 1732 & SESP SCHEDULING & & SW & & & B12834 & 09-Aug-01 & 28-Jun-01 & $13966-29-5$ & U-234 \\
\hline 1733 & SESP SCHEDULING & & SW & & & B12RM1 & 27-Aug-01 & & $13966-29-5$ & $\mathrm{U}-234$ \\
\hline 1734 & SESP SCHEDULING & & SW & & & B12RM6 & 27-Aug-01 & & $13966-29-5$ & U-234 \\
\hline 1735 & SESP SCHEDULING & & SW & & & B12RN4 & 27-Aug-01 & & $13966-29-5$ & $\mathrm{U}-234$ \\
\hline 1736 & SESP SCHEDULING & & SW & & & B12RN5 & 27-Aug-01 & & $13966-29-5$ & U-234 \\
\hline 1737 & SESP SCHEDULING & & SW & & & B12RN6 & 27-Aug-01 & & 13966-29-5 & $\mathrm{U}-234$ \\
\hline 1738 & SESP SCHEDULING & & SW & & & B12RN7 & 27-Aug-01 & & $13966-29-5$ & U-234 \\
\hline 1739 & SESP SCHEDULING & & SW & & & B12RP9 & 27-Aug-01 & & $13966-29-5$ & U-234 \\
\hline 1740 & SESP SCHEDULING & & SW & & & B12RR1 & 27-Aug-01 & & $13966-29-5$ & $\mathrm{U}-234$ \\
\hline 1741 & SESP SCHEDULING & & SW & & & B12RR3 & 27-Aug-01 & & $13966-29-5$ & U-234 \\
\hline 1742 & SESP SCHEDULING & & SW & & & B12RR5 & 27-Aug-01 & & $13966-29-5$ & U-234 \\
\hline 1743 & SESP SCHEDULING & & SW & & & B12RR7 & 27-Aug-01 & & $13966-29-5$ & U-234 \\
\hline 1744 & SESP SCHEDULING & & SW & & & B12RR9 & 27-Aug-01 & & $13966-29-5$ & $\mathrm{U}-234$ \\
\hline 1745 & SESP SCHEDULING & & SW & & & B12RT1 & 27-Aug-01 & & $13966-29-5$ & $\mathrm{U}-234$ \\
\hline 1746 & SESP SCHEDULING & & SW & & & B12RT3 & 27-Aug-01 & & $13966-29-5$ & $\mathrm{U}-234$ \\
\hline 1747 & SESP SCHEDULING & & SW & & & B12RT5 & 27-Aug-01 & & $13966-29-5$ & $\mathrm{U}-234$ \\
\hline 1748 & SESP SCHEDULING & & SW & & & B12RT7 & 27-Aug-01 & & $13966-29-5$ & $\mathrm{U}-234$ \\
\hline 1749 & SESP SCHEDULING & & SW & & & B12RT9 & 27-Aug-01 & & 13966-29-5 & $\mathrm{U}-234$ \\
\hline
\end{tabular}




\begin{tabular}{|c|c|c|c|c|c|c|c|c|c|c|}
\hline & A & $\mathrm{B}$ & C & $\mathrm{D}$ & $\mathrm{E}$ & $\mathrm{F}$ & G & $\mathrm{H}$ & $\mathrm{I}$ & $\mathrm{J}$ \\
\hline 1 & SAMPLE_NUM_ASSIGNED_TO & SAMPLER & MEDIA & BIOTA_MEDIA & TAG_ID & SAMP_NUM & SAMP_DATE_TIME & SAMP_DATE_TIME_ON & CON_ID & CON_SHORT_NAME \\
\hline 1750 & SESP SCHEDULING & & SW & & & B12RV1 & 27-Aug-01 & & $13966-29-5$ & $\mathrm{U}-234$ \\
\hline 1751 & SESP SCHEDULING & & SW & & & B12RV3 & 27-Aug-01 & & $13966-29-5$ & $\mathrm{U}-234$ \\
\hline 1752 & SESP SCHEDULING & & SW & & & B12RV5 & 27-Aug-01 & & $13966-29-5$ & U-234 \\
\hline 1753 & SESP SCHEDULING & & SW & & & B12RV7 & 27-Aug-01 & & $13966-29-5$ & $\mathrm{U}-234$ \\
\hline 1754 & SESP SCHEDULING & & SW & & & B12RV9 & 27-Aug-01 & & $13966-29-5$ & $U-234$ \\
\hline 1755 & SESP SCHEDULING & & SW & & & B12RW1 & 27-Aug-01 & & $13966-29-5$ & U-234 \\
\hline 1756 & SESP SCHEDULING & & SW & & & B12RW5 & 27-Aug-01 & & $13966-29-5$ & U-234 \\
\hline 1757 & SESP SCHEDULING & & SW & & & B12RW7 & 27-Aug-01 & & $13966-29-5$ & U-234 \\
\hline 1758 & SESP SCHEDULING & & SW & & & B12RW9 & 27-Aug-01 & & $13966-29-5$ & U-234 \\
\hline 1759 & SESP SCHEDULING & & SW & & & B12RX1 & 27-Aug-01 & & $13966-29-5$ & U-234 \\
\hline 1760 & SESP SCHEDULING & & SW & & & B12RX3 & 27-Aug-01 & & $13966-29-5$ & U-234 \\
\hline 1761 & SESP SCHEDULING & & SW & & & B12RX5 & 27-Aug-01 & & $13966-29-5$ & U-234 \\
\hline 1762 & SESP SCHEDULING & & SW & & & B12RX9 & 27-Aug-01 & & $13966-29-5$ & U-234 \\
\hline 1763 & SESP SCHEDULING & & SW & & & B12RY1 & 27-Aug-01 & & $13966-29-5$ & U-234 \\
\hline 1764 & SESP SCHEDULING & & SW & & & B12RY3 & 27-Aug-01 & & $13966-29-5$ & U-234 \\
\hline 1765 & SESP SCHEDULING & & SW & & & B12T11 & 27-Aug-01 & & $13966-29-5$ & U-234 \\
\hline 1766 & SESP SCHEDULING & & SW & & & B12T13 & 27-Aug-01 & & $13966-29-5$ & U-234 \\
\hline 1767 & SESP SCHEDULING & & SW & & & B12T15 & 27-Aug-01 & & $13966-29-5$ & U-234 \\
\hline 1768 & SESP SCHEDULING & & SW & & & B12T17 & 27-Aug-01 & & $13966-29-5$ & U-234 \\
\hline 1769 & SESP SCHEDULING & & SW & & & B12T19 & 27-Aug-01 & & $13966-29-5$ & $U-234$ \\
\hline 1770 & SESP SCHEDULING & & SW & & & B12T21 & 27-Aug-01 & & $13966-29-5$ & U-234 \\
\hline 1771 & SESP SCHEDULING & & SW & & & B12T23 & 27-Aug-01 & & $13966-29-5$ & U-234 \\
\hline 1772 & SESP SCHEDULING & & SW & & & B12T25 & 27-Aug-01 & & $13966-29-5$ & U-234 \\
\hline 1773 & SESP SCHEDULING & & SW & & & B12JT2 & 05-Sep-01 & & $13966-29-5$ & U-234 \\
\hline 1774 & SESP SCHEDULING & & SW & & & B12JR7 & 05-Sep-01 & 09-Aug-01 & $13966-29-5$ & U-234 \\
\hline 1775 & SESP SCHEDULING & & SW & & & B12T85 & 07-Sep-01 & & $13966-29-5$ & U-234 \\
\hline 1776 & SESP SCHEDULING & & SW & & & B12T87 & 07-Sep-01 & & $13966-29-5$ & U-234 \\
\hline 1777 & SESP SCHEDULING & & SW & & & B12T88 & 07-Sep-01 & & $13966-29-5$ & U-234 \\
\hline 1778 & SESP SCHEDULING & & SW & & & B12T89 & 07-Sep-01 & & $13966-29-5$ & U-234 \\
\hline 1779 & SESP SCHEDULING & & SW & & & B12T90 & 07-Sep-01 & & $13966-29-5$ & U-234 \\
\hline 1780 & SESP SCHEDULING & & SW & & & B12T91 & 07-Sep-01 & & $13966-29-5$ & U-234 \\
\hline 1781 & SESP SCHEDULING & & SW & & & B12T92 & 07-Sep-01 & & $13966-29-5$ & U-234 \\
\hline 1782 & SESP SCHEDULING & & SW & & & B12T93 & 07-Sep-01 & & $13966-29-5$ & U-234 \\
\hline 1783 & SESP SCHEDULING & & SW & & & B12TD4 & 07-Sep-01 & & $13966-29-5$ & U-234 \\
\hline 1784 & SESP SCHEDULING & & SW & & & B12TD5 & 07-Sep-01 & & $13966-29-5$ & U-234 \\
\hline 1785 & SESP SCHEDULING & & SW & & & B12TD6 & 07-Sep-01 & & $13966-29-5$ & U-234 \\
\hline 1786 & SESP SCHEDULING & & SW & & & B12TN2 & 07-Sep-01 & & $13966-29-5$ & U-234 \\
\hline 1787 & SESP SCHEDULING & & SW & & & B12TN5 & 07-Sep-01 & & $13966-29-5$ & U-234 \\
\hline
\end{tabular}




\begin{tabular}{|c|c|c|c|c|c|c|c|c|c|c|}
\hline & A & $\mathrm{B}$ & $\mathrm{C}$ & $\mathrm{D}$ & $\mathrm{E}$ & $\mathrm{F}$ & G & $\mathrm{H}$ & $\mathrm{I}$ & $\mathrm{J}$ \\
\hline 1 & SAMPLE_NUM_ASSIGNED_TO & SAMPLER & MEDIA & BIOTA_MEDIA & TAG_ID & SAMP_NUM & SAMP_DATE_TIME & SAMP_DATE_TIME_ON & CON_ID & CON_SHORT_NAME \\
\hline 1788 & SESP SCHEDULING & & SW & & & B12TN8 & 07-Sep-01 & & $13966-29-5$ & U-234 \\
\hline 1789 & SESP SCHEDULING & & SW & & & B12TP1 & 07-Sep-01 & & $13966-29-5$ & U-234 \\
\hline 1790 & SESP SCHEDULING & & SW & & & B12T94 & 10-Sep-01 & & 13966-29-5 & U-234 \\
\hline 1791 & SESP SCHEDULING & & SW & & & B12T95 & 10-Sep-01 & & $13966-29-5$ & U-234 \\
\hline 1792 & SESP SCHEDULING & & SW & & & B12T96 & 10-Sep-01 & & $13966-29-5$ & U-234 \\
\hline 1793 & SESP SCHEDULING & & SW & & & B12T97 & 10-Sep-01 & & $13966-29-5$ & U-234 \\
\hline 1794 & SESP SCHEDULING & & SW & & & B12T98 & 10-Sep-01 & & $13966-29-5$ & U-234 \\
\hline 1795 & SESP SCHEDULING & & SW & & & B12T99 & 10-Sep-01 & & $13966-29-5$ & U-234 \\
\hline 1796 & SESP SCHEDULING & & SW & & & B12TB0 & 10-Sep-01 & & $13966-29-5$ & U-234 \\
\hline 1797 & SESP SCHEDULING & & SW & & & B12TB1 & 10-Sep-01 & & $13966-29-5$ & U-234 \\
\hline 1798 & SESP SCHEDULING & & SW & & & B12TB2 & 10-Sep-01 & & $13966-29-5$ & U-234 \\
\hline 1799 & SESP SCHEDULING & & SW & & & B12TB3 & 10-Sep-01 & & $13966-29-5$ & U-234 \\
\hline 1800 & SESP SCHEDULING & & SW & & & B12TB4 & 10-Sep-01 & & $13966-29-5$ & U-234 \\
\hline 1801 & SESP SCHEDULING & & SW & & & B12TB5 & 10-Sep-01 & & $13966-29-5$ & U-234 \\
\hline 1802 & SESP SCHEDULING & & SW & & & B12TF7 & 10-Sep-01 & & $13966-29-5$ & U-234 \\
\hline 1803 & SESP SCHEDULING & & SW & & & B12TF8 & 10-Sep-01 & & $13966-29-5$ & U-234 \\
\hline 1804 & SESP SCHEDULING & & SW & & & B12TF9 & 10-Sep-01 & & $13966-29-5$ & U-234 \\
\hline 1805 & SESP SCHEDULING & & SW & & & B12TT0 & 10-Sep-01 & & $13966-29-5$ & U-234 \\
\hline 1806 & SESP SCHEDULING & & SW & & & B12TT3 & 10-Sep-01 & & $13966-29-5$ & U-234 \\
\hline 1807 & SESP SCHEDULING & & SW & & & B12TT9 & 10-Sep-01 & & $13966-29-5$ & U-234 \\
\hline 1808 & SESP SCHEDULING & & SW & & & B12TB6 & 13-Sep-01 & & $13966-29-5$ & U-234 \\
\hline 1809 & SESP SCHEDULING & & SW & & & B12TB8 & 13-Sep-01 & & $13966-29-5$ & U-234 \\
\hline 1810 & SESP SCHEDULING & & SW & & & B12TC0 & 13-Sep-01 & & $13966-29-5$ & U-234 \\
\hline 1811 & SESP SCHEDULING & & SW & & & B12TC2 & 13-Sep-01 & & $13966-29-5$ & U-234 \\
\hline 1812 & SESP SCHEDULING & & SW & & & B12TC4 & 13-Sep-01 & & $13966-29-5$ & U-234 \\
\hline 1813 & SESP SCHEDULING & & SW & & & B12TC6 & 13-Sep-01 & & $13966-29-5$ & U-234 \\
\hline 1814 & SESP SCHEDULING & & SW & & & B12TC8 & 13-Sep-01 & & $13966-29-5$ & U-234 \\
\hline 1815 & SESP SCHEDULING & & SW & & & B12TC9 & 13-Sep-01 & & $13966-29-5$ & U-234 \\
\hline 1816 & SESP SCHEDULING & & SW & & & B12TD0 & 13-Sep-01 & & $13966-29-5$ & U-234 \\
\hline 1817 & SESP SCHEDULING & & SW & & & B12TD1 & 13-Sep-01 & & $13966-29-5$ & U-234 \\
\hline 1818 & SESP SCHEDULING & & SW & & & B12TD2 & 13-Sep-01 & & $13966-29-5$ & U-234 \\
\hline 1819 & SESP SCHEDULING & & SW & & & B12TD3 & 13-Sep-01 & & $13966-29-5$ & U-234 \\
\hline 1820 & SESP SCHEDULING & & SW & & & B12TM0 & 13-Sep-01 & & $13966-29-5$ & U-234 \\
\hline 1821 & SESP SCHEDULING & & SW & & & B12TM1 & 13-Sep-01 & & $13966-29-5$ & U-234 \\
\hline 1822 & SESP SCHEDULING & & SW & & & B12TM2 & 13-Sep-01 & & $13966-29-5$ & U-234 \\
\hline 1823 & SESP SCHEDULING & & SW & & & B12TM3 & 13-Sep-01 & & $13966-29-5$ & U-234 \\
\hline 1824 & SESP SCHEDULING & & SW & & & B12TP4 & 13-Sep-01 & & $13966-29-5$ & U-234 \\
\hline 1825 & SESP SCHEDULING & & SW & & & B12TP8 & 13-Sep-01 & & 13966-29-5 & $\mathrm{U}-234$ \\
\hline
\end{tabular}




\begin{tabular}{|c|c|c|c|c|c|c|c|c|c|c|}
\hline & A & $\mathrm{B}$ & $\mathrm{C}$ & $\mathrm{D}$ & $\mathrm{E}$ & $\mathrm{F}$ & G & $\mathrm{H}$ & $\mathrm{I}$ & $\mathrm{J}$ \\
\hline 1 & SAMPLE_NUM_ASSIGNED_TO & SAMPLER & MEDIA & BIOTA_MEDIA & TAG_ID & SAMP_NUM & SAMP_DATE_TIME & SAMP_DATE_TIME_ON & CON_ID & CON_SHORT_NAME \\
\hline 1826 & SESP SCHEDULING & & SW & & & B12TR2 & 13-Sep-01 & & $13966-29-5$ & U-234 \\
\hline 1827 & SESP SCHEDULING & & SW & & & B12TR6 & 13-Sep-01 & & $13966-29-5$ & U-234 \\
\hline 1828 & SESP SCHEDULING & & SW & & & B12XK2 & 17-Sep-01 & & 13966-29-5 & U-234 \\
\hline 1829 & SESP SCHEDULING & & SW & & & B12XK8 & 17-Sep-01 & & $13966-29-5$ & U-234 \\
\hline 1830 & SESP SCHEDULING & & SW & & & B12XK9 & 17-Sep-01 & & $13966-29-5$ & U-234 \\
\hline 1831 & SESP SCHEDULING & & SW & & & B12XL0 & 17-Sep-01 & & $13966-29-5$ & U-234 \\
\hline 1832 & SESP SCHEDULING & & SW & & & B12XL1 & 17-Sep-01 & & $13966-29-5$ & U-234 \\
\hline 1833 & SESP SCHEDULING & & SW & & & B12XL2 & 17-Sep-01 & & $13966-29-5$ & U-234 \\
\hline 1834 & SESP SCHEDULING & & SW & & & B12XK1 & 18-Sep-01 & & $13966-29-5$ & U-234 \\
\hline 1835 & SESP SCHEDULING & & SW & & & B12XK3 & 18-Sep-01 & & $13966-29-5$ & U-234 \\
\hline 1836 & SESP SCHEDULING & & SW & & & B12XK4 & 18-Sep-01 & & $13966-29-5$ & U-234 \\
\hline 1837 & SESP SCHEDULING & & SW & & & B12XK5 & 18-Sep-01 & & $13966-29-5$ & U-234 \\
\hline 1838 & SESP SCHEDULING & & SW & & & B12XK6 & 18-Sep-01 & & $13966-29-5$ & U-234 \\
\hline 1839 & SESP SCHEDULING & & SW & & & B12XK7 & 18-Sep-01 & & $13966-29-5$ & U-234 \\
\hline 1840 & SESP SCHEDULING & & SW & & & B12T28 & 02-Oct-01 & 05-Sep-01 & $13966-29-5$ & U-234 \\
\hline 1841 & SESP SCHEDULING & & SW & & & B12T33 & 02-Oct-01 & 05-Sep-01 & $13966-29-5$ & U-234 \\
\hline 1842 & SESP SCHEDULING & & SW & & & B12X50 & 22-Oct-01 & & $13966-29-5$ & U-234 \\
\hline 1843 & SESP SCHEDULING & & SW & & & B12X48 & 01-Nov-01 & & $13966-29-5$ & U-234 \\
\hline 1844 & SESP SCHEDULING & & SW & & & B12X85 & 01-Nov-01 & & $13966-29-5$ & U-234 \\
\hline 1845 & SESP SCHEDULING & & SW & & & B13103 & 01-Nov-01 & 02-Oct-01 & $13966-29-5$ & U-234 \\
\hline 1846 & SESP SCHEDULING & & SW & & & B130Y7 & 01-Nov-01 & 02-Oct-01 & $13966-29-5$ & U-234 \\
\hline 1847 & SESP SCHEDULING & & SW & & & B13J17 & 14-Nov-01 & & $13966-29-5$ & U-234 \\
\hline 1848 & SESP SCHEDULING & & SW & & & B13CC6 & 27-Nov-01 & 01-Nov-01 & $13966-29-5$ & U-234 \\
\hline 1849 & SESP SCHEDULING & & SW & & & B13CD2 & 27-Nov-01 & 01-Nov-01 & $13966-29-5$ & U-234 \\
\hline 1850 & SESP SCHEDULING & & SW & & & B13LF3 & 03-Dec-01 & & $13966-29-5$ & U-234 \\
\hline 1851 & SESP SCHEDULING & & SW & & & B13LF4 & 03-Dec-01 & & $13966-29-5$ & U-234 \\
\hline 1852 & SESP SCHEDULING & & SW & & & B13LF5 & 03-Dec-01 & & $13966-29-5$ & U-234 \\
\hline 1853 & SESP SCHEDULING & & SW & & & B13LF6 & 03-Dec-01 & & $13966-29-5$ & U-234 \\
\hline 1854 & SESP SCHEDULING & & SW & & & B13LF2 & 04-Dec-01 & & $13966-29-5$ & U-234 \\
\hline 1855 & SESP SCHEDULING & & SW & & & B13LF7 & 04-Dec-01 & & $13966-29-5$ & U-234 \\
\hline 1856 & SESP SCHEDULING & & SW & & & B13LF8 & 04-Dec-01 & & $13966-29-5$ & U-234 \\
\hline 1857 & SESP SCHEDULING & & SW & & & B13LF9 & 04-Dec-01 & & $13966-29-5$ & U-234 \\
\hline 1858 & SESP SCHEDULING & & SW & & & B13LH0 & 04-Dec-01 & & $13966-29-5$ & U-234 \\
\hline 1859 & SESP SCHEDULING & & SW & & & B13LH1 & 04-Dec-01 & & $13966-29-5$ & U-234 \\
\hline 1860 & SESP SCHEDULING & & SW & & & B13LH2 & 04-Dec-01 & & $13966-29-5$ & U-234 \\
\hline 1861 & SESP SCHEDULING & & SW & & & B13LJ3 & 04-Dec-01 & & $13966-29-5$ & U-234 \\
\hline 1862 & SESP SCHEDULING & & SW & & & B13LJ4 & 04-Dec-01 & & $13966-29-5$ & U-234 \\
\hline 1863 & SESP SCHEDULING & & SW & & & B13LJ5 & 04-Dec-01 & & 13966-29-5 & U-234 \\
\hline
\end{tabular}




\begin{tabular}{|c|c|c|c|c|c|c|c|c|c|c|}
\hline & A & $\mathrm{B}$ & C & $\mathrm{D}$ & $E$ & $\mathrm{~F}$ & G & $\mathrm{H}$ & $\mathrm{I}$ & $\mathrm{J}$ \\
\hline 1 & SAMPLE_NUM_ASSIGNED_TO & SAMPLER & MEDIA & BIOTA_MEDIA & TAG_ID & SAMP_NUM & SAMP_DATE_TIME & SAMP_DATE_TIME_ON & CON_ID & CON_SHORT_NAME \\
\hline 1864 & SESP SCHEDULING & & SW & & & B13LJ6 & 04-Dec-01 & & $13966-29-5$ & $\mathrm{U}-234$ \\
\hline 1865 & SESP SCHEDULING & & SW & & & B13LB3 & 03-Jan-02 & 27-Nov-01 & $13966-29-5$ & U-234 \\
\hline 1866 & SESP SCHEDULING & & SW & & & B13L96 & 03-Jan-02 & 27-Nov-01 & $13966-29-5$ & $\mathrm{U}-234$ \\
\hline 1867 & SESP SCHEDULING & & SW & & & B13VJ0 & 06-Feb-02 & & 13966-29-5 & $\mathrm{U}-234$ \\
\hline 1868 & SESP SCHEDULING & & SW & & & B13VF8 & 06-Feb-02 & & $13966-29-5$ & $\mathrm{U}-234$ \\
\hline 1869 & SESP SCHEDULING & & SW & & & B140P7 & 07-Mar-02 & 06-Feb-02 & $13966-29-5$ & $\mathrm{U}-234$ \\
\hline 1870 & SESP SCHEDULING & & SW & & & B140R2 & 07-Mar-02 & 06-Feb-02 & $13966-29-5$ & U-234 \\
\hline 1871 & SESP SCHEDULING & & SW & & & B147T3 & 25-Mar-02 & & $13966-29-5$ & U-234 \\
\hline 1872 & SESP SCHEDULING & & SW & & & B147T4 & 25-Mar-02 & & 13966-29-5 & U-234 \\
\hline 1873 & SESP SCHEDULING & & SW & & & B147T5 & 25-Mar-02 & & $13966-29-5$ & $\mathrm{U}-234$ \\
\hline 1874 & SESP SCHEDULING & & SW & & & B147T6 & 25-Mar-02 & & $13966-29-5$ & U-234 \\
\hline 1875 & SESP SCHEDULING & & SW & & & B147T2 & 26-Mar-02 & & $13966-29-5$ & $\mathrm{U}-234$ \\
\hline 1876 & SESP SCHEDULING & & SW & & & B147T7 & 26-Mar-02 & & 13966-29-5 & U-234 \\
\hline 1877 & SESP SCHEDULING & & SW & & & B147T8 & 26-Mar-02 & & $13966-29-5$ & $\mathrm{U}-234$ \\
\hline 1878 & SESP SCHEDULING & & SW & & & B147T9 & 26-Mar-02 & & $13966-29-5$ & $\mathrm{U}-234$ \\
\hline 1879 & SESP SCHEDULING & & SW & & & B147V0 & 26-Mar-02 & & $13966-29-5$ & U-234 \\
\hline 1880 & SESP SCHEDULING & & SW & & & B147V1 & 26-Mar-02 & & 13966-29-5 & U-234 \\
\hline 1881 & SESP SCHEDULING & & SW & & & B147V2 & 26-Mar-02 & & $13966-29-5$ & $\mathrm{U}-234$ \\
\hline 1882 & SESP SCHEDULING & & SW & & & B147W3 & 26-Mar-02 & & $13966-29-5$ & U-234 \\
\hline 1883 & SESP SCHEDULING & & SW & & & B147W4 & 26-Mar-02 & & $13966-29-5$ & $\mathrm{U}-234$ \\
\hline 1884 & SESP SCHEDULING & & SW & & & B147W5 & 26-Mar-02 & & $13966-29-5$ & $\mathrm{U}-234$ \\
\hline 1885 & SESP SCHEDULING & & SW & & & B147W6 & 26-Mar-02 & & $13966-29-5$ & $\mathrm{U}-234$ \\
\hline 1886 & SESP SCHEDULING & & SW & & & B14408 & 03-Apr-02 & 07-Mar-02 & $13966-29-5$ & U-234 \\
\hline 1887 & SESP SCHEDULING & & SW & & & B14413 & 03-Apr-02 & 07-Mar-02 & $13966-29-5$ & $\mathrm{U}-234$ \\
\hline 1888 & SESP SCHEDULING & & SW & & & B14B37 & 01-May-02 & 03-Apr-02 & $13966-29-5$ & U-234 \\
\hline 1889 & SESP SCHEDULING & & SW & & & B14B43 & 01-May-02 & 03-Apr-02 & $13966-29-5$ & U-234 \\
\hline 1890 & SESP SCHEDULING & & SW & & & B14LJ0 & 23-May-02 & & $13966-29-5$ & $\mathrm{U}-234$ \\
\hline 1891 & SESP SCHEDULING & & SW & & & B14LJ2 & 23-May-02 & & $13966-29-5$ & $\mathrm{U}-234$ \\
\hline 1892 & SESP SCHEDULING & & SW & & & B14JF2 & 29-May-02 & 01-May-02 & $13966-29-5$ & U-234 \\
\hline 1893 & SESP SCHEDULING & & SW & & & B14JF8 & 29-May-02 & 01-May-02 & $13966-29-5$ & $\mathrm{U}-234$ \\
\hline 1894 & SESP SCHEDULING & & SW & & & B14RV4 & 10-Jun-02 & & $13966-29-5$ & U-234 \\
\hline 1895 & SESP SCHEDULING & & SW & & & B14RV5 & 10-Jun-02 & & $13966-29-5$ & $\mathrm{U}-234$ \\
\hline 1896 & SESP SCHEDULING & & SW & & & B14RV6 & 10-Jun-02 & & $13966-29-5$ & $\mathrm{U}-234$ \\
\hline 1897 & SESP SCHEDULING & & SW & & & B14RV7 & 10-Jun-02 & & $13966-29-5$ & $\mathrm{U}-234$ \\
\hline 1898 & SESP SCHEDULING & & SW & & & B14RV8 & 11-Jun-02 & & 13966-29-5 & U-234 \\
\hline 1899 & SESP SCHEDULING & & SW & & & B14RV9 & 11-Jun-02 & & $13966-29-5$ & $\mathrm{U}-234$ \\
\hline 1900 & SESP SCHEDULING & & SW & & & B14RW0 & 11-Jun-02 & & $13966-29-5$ & $\mathrm{U}-234$ \\
\hline 1901 & SESP SCHEDULING & & SW & & & B14RW1 & 11-Jun-02 & & 13966-29-5 & $\mathrm{U}-234$ \\
\hline
\end{tabular}




\begin{tabular}{|c|c|c|c|c|c|c|c|c|c|c|}
\hline & A & $\mathrm{B}$ & C & $\mathrm{D}$ & $E$ & $\mathrm{~F}$ & G & $\mathrm{H}$ & $\mathrm{I}$ & $\mathrm{J}$ \\
\hline 1 & SAMPLE_NUM_ASSIGNED_TO & SAMPLER & MEDIA & BIOTA_MEDIA & TAG_ID & SAMP_NUM & SAMP_DATE_TIME & SAMP_DATE_TIME_ON & CON_ID & CON_SHORT_NAME \\
\hline 1902 & SESP SCHEDULING & & SW & & & B14RW2 & 11-Jun-02 & & $13966-29-5$ & $\mathrm{U}-234$ \\
\hline 1903 & SESP SCHEDULING & & SW & & & B14RW3 & 11-Jun-02 & & $13966-29-5$ & U-234 \\
\hline 1904 & SESP SCHEDULING & & SW & & & B14RX4 & 11-Jun-02 & & $13966-29-5$ & U-234 \\
\hline 1905 & SESP SCHEDULING & & SW & & & B14RX5 & 11-Jun-02 & & 13966-29-5 & $\mathrm{U}-234$ \\
\hline 1906 & SESP SCHEDULING & & SW & & & B14RX6 & 11-Jun-02 & & $13966-29-5$ & $\mathrm{U}-234$ \\
\hline 1907 & SESP SCHEDULING & & SW & & & B14RX7 & 11-Jun-02 & & $13966-29-5$ & $\mathrm{U}-234$ \\
\hline 1908 & SESP SCHEDULING & & SW & & & B14RL3 & 27-Jun-02 & 29-May-02 & $13966-29-5$ & $\mathrm{U}-234$ \\
\hline 1909 & SESP SCHEDULING & & SW & & & B14RM5 & 27-Jun-02 & 29-May-02 & $13966-29-5$ & $\mathrm{U}-234$ \\
\hline 1910 & SESP SCHEDULING & & SW & & & B14VT4 & 28-Jun-02 & & 13966-29-5 & U-234 \\
\hline 1911 & SESP SCHEDULING & & SW & & & B14VX3 & 28-Jun-02 & & $13966-29-5$ & $\mathrm{U}-234$ \\
\hline 1912 & SESP SCHEDULING & & SW & & & B14YH3 & 08-Jul-02 & & $13966-29-5$ & U-234 \\
\hline 1913 & SESP SCHEDULING & & SW & & & B14YJ5 & 08-Jul-02 & & $13966-29-5$ & $\mathrm{U}-234$ \\
\hline 1914 & SESP SCHEDULING & & SW & & & B14WW5 & 07-Aug-02 & 27-Jun-02 & $13966-29-5$ & U-234 \\
\hline 1915 & SESP SCHEDULING & & SW & & & B14WX2 & 07-Aug-02 & 27-Jun-02 & $13966-29-5$ & $\mathrm{U}-234$ \\
\hline 1916 & SESP SCHEDULING & & SW & & & B153T9 & 04-Sep-02 & 07-Aug-02 & $13966-29-5$ & $\mathrm{U}-234$ \\
\hline 1917 & SESP SCHEDULING & & SW & & & B153V4 & 04-Sep-02 & 07-Aug-02 & $13966-29-5$ & U-234 \\
\hline 1918 & SESP SCHEDULING & & SW & & & B158H3 & 05-Sep-02 & & 13966-29-5 & U-234 \\
\hline 1919 & SESP SCHEDULING & & SW & & & B158H4 & 05-Sep-02 & & $13966-29-5$ & U-234 \\
\hline 1920 & SESP SCHEDULING & & SW & & & B158H5 & 05-Sep-02 & & $13966-29-5$ & U-234 \\
\hline 1921 & SESP SCHEDULING & & SW & & & B158H6 & 05-Sep-02 & & $13966-29-5$ & $\mathrm{U}-234$ \\
\hline 1922 & SESP SCHEDULING & & SW & & & $\mathrm{B} 158 \mathrm{H} 7$ & 05-Sep-02 & & $13966-29-5$ & $\mathrm{U}-234$ \\
\hline 1923 & SESP SCHEDULING & & SW & & & $\mathrm{B} 158 \mathrm{H} 8$ & 05-Sep-02 & & $13966-29-5$ & $\mathrm{U}-234$ \\
\hline 1924 & SESP SCHEDULING & & SW & & & B158H9 & 05-Sep-02 & & 13966-29-5 & U-234 \\
\hline 1925 & SESP SCHEDULING & & SW & & & B158J0 & 05-Sep-02 & & $13966-29-5$ & $\mathrm{U}-234$ \\
\hline 1926 & SESP SCHEDULING & & SW & & & B158M7 & 05-Sep-02 & & $13966-29-5$ & U-234 \\
\hline 1927 & SESP SCHEDULING & & SW & & & B158M8 & 05-Sep-02 & & 13966-29-5 & U-234 \\
\hline 1928 & SESP SCHEDULING & & SW & & & B158M9 & 05-Sep-02 & & $13966-29-5$ & $\mathrm{U}-234$ \\
\hline 1929 & SESP SCHEDULING & & SW & & & B158W8 & 05-Sep-02 & & $13966-29-5$ & $\mathrm{U}-234$ \\
\hline 1930 & SESP SCHEDULING & & SW & & & B158X1 & 05-Sep-02 & & $13966-29-5$ & U-234 \\
\hline 1931 & SESP SCHEDULING & & SW & & & B158X4 & 05-Sep-02 & & $13966-29-5$ & U-234 \\
\hline 1932 & SESP SCHEDULING & & SW & & & B158X7 & 05-Sep-02 & & $13966-29-5$ & U-234 \\
\hline 1933 & SESP SCHEDULING & & SW & & & B158J1 & 09-Sep-02 & & $13966-29-5$ & $\mathrm{U}-234$ \\
\hline 1934 & SESP SCHEDULING & & SW & & & B158J3 & 09-Sep-02 & & $13966-29-5$ & $\mathrm{U}-234$ \\
\hline 1935 & SESP SCHEDULING & & SW & & & B158J5 & 09-Sep-02 & & $13966-29-5$ & $\mathrm{U}-234$ \\
\hline 1936 & SESP SCHEDULING & & SW & & & B158J7 & 09-Sep-02 & & 13966-29-5 & U-234 \\
\hline 1937 & SESP SCHEDULING & & SW & & & B158J9 & 09-Sep-02 & & $13966-29-5$ & $\mathrm{U}-234$ \\
\hline 1938 & SESP SCHEDULING & & SW & & & B158K1 & 09-Sep-02 & & $13966-29-5$ & $\mathrm{U}-234$ \\
\hline 1939 & SESP SCHEDULING & & SW & & & B158K3 & 09-Sep-02 & & 13966-29-5 & $\mathrm{U}-234$ \\
\hline
\end{tabular}




\begin{tabular}{|c|c|c|c|c|c|c|c|c|c|c|}
\hline & A & $\mathrm{B}$ & C & $\mathrm{D}$ & $E$ & $\mathrm{~F}$ & G & $\mathrm{H}$ & $\mathrm{I}$ & $\mathrm{J}$ \\
\hline 1 & SAMPLE_NUM_ASSIGNED_TO & SAMPLER & MEDIA & BIOTA_MEDIA & TAG_ID & SAMP_NUM & SAMP_DATE_TIME & SAMP_DATE_TIME_ON & CON_ID & CON_SHORT_NAME \\
\hline 1940 & SESP SCHEDULING & & SW & & & B158K5 & 09-Sep-02 & & $13966-29-5$ & $\mathrm{U}-234$ \\
\hline 1941 & SESP SCHEDULING & & SW & & & B158K7 & 09-Sep-02 & & $13966-29-5$ & U-234 \\
\hline 1942 & SESP SCHEDULING & & SW & & & B158K9 & 09-Sep-02 & & $13966-29-5$ & U-234 \\
\hline 1943 & SESP SCHEDULING & & SW & & & B158L1 & 09-Sep-02 & & 13966-29-5 & $\mathrm{U}-234$ \\
\hline 1944 & SESP SCHEDULING & & SW & & & B158L3 & 09-Sep-02 & & $13966-29-5$ & $\mathrm{U}-234$ \\
\hline 1945 & SESP SCHEDULING & & SW & & & B158NO & 09-Sep-02 & & $13966-29-5$ & $\mathrm{U}-234$ \\
\hline 1946 & SESP SCHEDULING & & SW & & & B158N2 & 09-Sep-02 & & 13966-29-5 & $\mathrm{U}-234$ \\
\hline 1947 & SESP SCHEDULING & & SW & & & B158N4 & 09-Sep-02 & & $13966-29-5$ & U-234 \\
\hline 1948 & SESP SCHEDULING & & SW & & & B15902 & 09-Sep-02 & & $13966-29-5$ & U-234 \\
\hline 1949 & SESP SCHEDULING & & SW & & & B15906 & 09-Sep-02 & & $13966-29-5$ & $\mathrm{U}-234$ \\
\hline 1950 & SESP SCHEDULING & & SW & & & B15910 & 09-Sep-02 & & $13966-29-5$ & U-234 \\
\hline 1951 & SESP SCHEDULING & & SW & & & B15914 & 09-Sep-02 & & $13966-29-5$ & $\mathrm{U}-234$ \\
\hline 1952 & SESP SCHEDULING & & SW & & & B158L5 & 10-Sep-02 & & $13966-29-5$ & U-234 \\
\hline 1953 & SESP SCHEDULING & & SW & & & B158L6 & 10-Sep-02 & & $13966-29-5$ & $\mathrm{U}-234$ \\
\hline 1954 & SESP SCHEDULING & & SW & & & B158L7 & 10-Sep-02 & & $13966-29-5$ & $\mathrm{U}-234$ \\
\hline 1955 & SESP SCHEDULING & & SW & & & B158L8 & 10-Sep-02 & & $13966-29-5$ & U-234 \\
\hline 1956 & SESP SCHEDULING & & SW & & & B158L9 & 10-Sep-02 & & $13966-29-5$ & U-234 \\
\hline 1957 & SESP SCHEDULING & & SW & & & B158M0 & 10-Sep-02 & & $13966-29-5$ & U-234 \\
\hline 1958 & SESP SCHEDULING & & SW & & & B158M1 & 10-Sep-02 & & $13966-29-5$ & U-234 \\
\hline 1959 & SESP SCHEDULING & & SW & & & B158M2 & 10-Sep-02 & & $13966-29-5$ & $\mathrm{U}-234$ \\
\hline 1960 & SESP SCHEDULING & & SW & & & B158M3 & 10-Sep-02 & & $13966-29-5$ & $\mathrm{U}-234$ \\
\hline 1961 & SESP SCHEDULING & & SW & & & B158M4 & 10-Sep-02 & & $13966-29-5$ & $\mathrm{U}-234$ \\
\hline 1962 & SESP SCHEDULING & & SW & & & B158M5 & 10-Sep-02 & & $13966-29-5$ & U-234 \\
\hline 1963 & SESP SCHEDULING & & SW & & & B158M6 & 10-Sep-02 & & $13966-29-5$ & $\mathrm{U}-234$ \\
\hline 1964 & SESP SCHEDULING & & SW & & & B158V6 & 10-Sep-02 & & $13966-29-5$ & U-234 \\
\hline 1965 & SESP SCHEDULING & & SW & & & B158V7 & 10-Sep-02 & & $13966-29-5$ & U-234 \\
\hline 1966 & SESP SCHEDULING & & SW & & & B158V8 & 10-Sep-02 & & $13966-29-5$ & $\mathrm{U}-234$ \\
\hline 1967 & SESP SCHEDULING & & SW & & & B158V9 & 10-Sep-02 & & $13966-29-5$ & $\mathrm{U}-234$ \\
\hline 1968 & SESP SCHEDULING & & SW & & & B158Y0 & 10-Sep-02 & & $13966-29-5$ & U-234 \\
\hline 1969 & SESP SCHEDULING & & SW & & & B158Y3 & 10-Sep-02 & & $13966-29-5$ & U-234 \\
\hline 1970 & SESP SCHEDULING & & SW & & & B158Y6 & 10-Sep-02 & & $13966-29-5$ & U-234 \\
\hline 1971 & SESP SCHEDULING & & SW & & & B158Y9 & 10-Sep-02 & & $13966-29-5$ & $\mathrm{U}-234$ \\
\hline 1972 & SESP SCHEDULING & & SW & & & B15948 & 02-Oct-02 & 04-Sep-02 & $13966-29-5$ & $\mathrm{U}-234$ \\
\hline 1973 & SESP SCHEDULING & & SW & & & B15954 & 02-Oct-02 & 04-Sep-02 & $13966-29-5$ & $\mathrm{U}-234$ \\
\hline 1974 & SESP SCHEDULING & & SW & & & B15C09 & 07-Oct-02 & & $13966-29-5$ & U-234 \\
\hline 1975 & SESP SCHEDULING & & SW & & & B15C11 & 07-Oct-02 & & $13966-29-5$ & $\mathrm{U}-234$ \\
\hline 1976 & SESP SCHEDULING & & SW & & & B15C73 & 07-Oct-02 & & $13966-29-5$ & $\mathrm{U}-234$ \\
\hline 1977 & SESP SCHEDULING & & SW & & & B15CB3 & 27-Oct-02 & & 13966-29-5 & U-234 \\
\hline
\end{tabular}




\begin{tabular}{|c|c|c|c|c|c|c|c|c|c|c|}
\hline & $A$ & $\mathrm{~B}$ & $\mathrm{C}$ & $\mathrm{D}$ & $E$ & $\mathrm{~F}$ & $G$ & $\mathrm{H}$ & $\mathrm{I}$ & $\mathrm{J}$ \\
\hline 1 & \begin{tabular}{|l|l} 
SAMPE_NUM_ASSIGNED_TO \\
\end{tabular} & SAMPLER & MEDIA & BIOTA_MEDIA & TAG_ID & SAMP_NUM & SAMP_DATE_TIME & SAMP_DATE_TIME_ON & CON_ID & CON_SHORT_NAME \\
\hline 1978 & SESP SCHEDULING & & SW & & & B15C69 & $29-$ Oct-02 & & $13966-29-5$ & $\mathrm{U}-234$ \\
\hline 1979 & SESP SCHEDULING & & SW & & & B15K74 & 30-Oct-02 & 02-Oct-02 & $13966-29-5$ & U-234 \\
\hline 1980 & SESP SCHEDULING & & SW & & & B15K79 & 30-Oct-02 & 02-Oct-02 & $13966-29-5$ & U-234 \\
\hline 1981 & SESP SCHEDULING & & SW & & & B15VN2 & 26-Nov-02 & $30-$ - ct-02 & $13966-29-5$ & U-234 \\
\hline 1982 & SESP SCHEDULING & & SW & & & B15VN8 & 26-Nov-02 & $30-O c t-02$ & $13966-29-5$ & U-234 \\
\hline 1983 & SESP SCHEDULING & & SW & & & B16342 & 09-Dec-02 & & $13966-29-5$ & U-234 \\
\hline 1984 & SESP SCHEDULING & & SW & & & B16343 & 09-Dec-02 & & $13966-29-5$ & U-234 \\
\hline 1985 & SESP SCHEDULING & & SW & & & B16344 & 09-Dec-02 & & $13966-29-5$ & U-234 \\
\hline 1986 & SESP SCHEDULING & & SW & & & B16345 & 09-Dec-02 & & $13966-29-5$ & U-234 \\
\hline \begin{tabular}{|l|}
1987 \\
\end{tabular} & SESP SCHEDULING & & SW & & & B16341 & 10-Dec-02 & & $13966-29-5$ & $\mathrm{U}-234$ \\
\hline 1988 & SESP SCHEDULING & & SW & & & B16346 & 10-Dec-02 & & $13966-29-5$ & $\mathrm{U}-234$ \\
\hline 1989 & SESP SCHEDULING & & SW & & & B16347 & 10-Dec-02 & & $13966-29-5$ & U-234 \\
\hline 1990 & SESP SCHEDULING & & SW & & & B16348 & 10-Dec-02 & & $13966-29-5$ & U-234 \\
\hline 1991 & SESP SCHEDULING & & SW & & & B16349 & 10-Dec-02 & & $13966-29-5$ & U-234 \\
\hline 1992 & SESP SCHEDULING & & SW & & & B16350 & 10-Dec-02 & & $13966-29-5$ & U-234 \\
\hline \begin{tabular}{|l}
1993 \\
\end{tabular} & SESP SCHEDULING & & SW & & & B16351 & 10-Dec-02 & & $13966-29-5$ & $\mathrm{U}-234$ \\
\hline \begin{tabular}{|l|}
1994 \\
\end{tabular} & SESP SCHEDULING & & SW & & & B16362 & 10-Dec-02 & & $13966-29-5$ & $\mathrm{U}-234$ \\
\hline 1995 & SESP SCHEDULING & & SW & & & B16363 & 10-Dec-02 & & $13966-29-5$ & U-234 \\
\hline 1996 & SESP SCHEDULING & & SW & & & B16364 & 10-Dec-02 & & $13966-29-5$ & U-234 \\
\hline 1997 & SESP SCHEDULING & & SW & & & B16365 & 10-Dec-02 & & $13966-29-5$ & $\mathrm{U}-234$ \\
\hline 1998 & SESP SCHEDULING & & SW & & & B0X8R8 & 04-Jan-00 & & 15117-96-1 & U-235 \\
\hline 1999 & SESP SCHEDULING & & SW & & & B0X7K4 & 02-Feb-00 & 29-Dec-99 & $15117-96-1$ & U-235 \\
\hline 2000 & SESP SCHEDULING & & SW & & & B0X7J0 & $02-F e b-00$ & 29-Dec-99 & $15117-96-1$ & U-235 \\
\hline 2001 & SESP SCHEDULING & & SW & & & BOXH28 & 08-Mar-00 & 02-Feb-00 & $15117-96-1$ & U-235 \\
\hline 2002 & SESP SCHEDULING & & SW & & & B0XH22 & 08-Mar-00 & 02-Feb-00 & $15117-96-1$ & U-235 \\
\hline 2003 & SESP SCHEDULING & & SW & & & BOXRK2 & 27-Mar-00 & & 15117-96-1 & U-235 \\
\hline 2004 & SESP SCHEDULING & & SW & & & BOXRK3 & 27-Mar-00 & & 15117-96-1 & U-235 \\
\hline 2005 & SESP SCHEDULING & & SW & & & B0XRK4 & 27-Mar-00 & & $15117-96-1$ & U-235 \\
\hline 2006 & SESP SCHEDULING & & SW & & & B0XRK5 & 27-Mar-00 & & $15117-96-1$ & U-235 \\
\hline 2007 & SESP SCHEDULING & & SW & & & BOXRKO & 28-Mar-00 & & 15117-96-1 & U-235 \\
\hline 2008 & SESP SCHEDULING & & SW & & & B0XRK6 & 28-Mar-00 & & $15117-96-1$ & U-235 \\
\hline \begin{tabular}{|l|}
2009 \\
\end{tabular} & SESP SCHEDULING & & SW & & & B0XRK7 & 28-Mar-00 & & $15117-96-1$ & U-235 \\
\hline 2010 & SESP SCHEDULING & & SW & & & BOXRK8 & 28-Mar-00 & & 15117-96-1 & U-235 \\
\hline 2011 & SESP SCHEDULING & & SW & & & BOXRK9 & 28-Mar-00 & & $15117-96-1$ & U-235 \\
\hline 2012 & SESP SCHEDULING & & SW & & & BOXRLO & 28-Mar-00 & & $15117-96-1$ & U-235 \\
\hline 2013 & SESP SCHEDULING & & SW & & & B0XRL1 & 28-Mar-00 & & $15117-96-1$ & U-235 \\
\hline 2014 & SESP SCHEDULING & & SW & & & BOXRN8 & 28-Mar-00 & & $15117-96-1$ & U-235 \\
\hline 2015 & SESP SCHEDULING & & SW & & & BOXRN9 & 28-Mar-00 & & $15117-96-1$ & U-235 \\
\hline
\end{tabular}




\begin{tabular}{|c|c|c|c|c|c|c|c|c|c|c|}
\hline & A & $\mathrm{B}$ & $\mathrm{C}$ & $\mathrm{D}$ & $E$ & $\mathrm{~F}$ & G & $\mathrm{H}$ & $\mathrm{I}$ & $\mathrm{J}$ \\
\hline 1 & SAMPLE_NUM_ASSIGNED_TO & SAMPLER & MEDIA & BIOTA_MEDIA & TAG_ID & SAMP_NUM & SAMP_DATE_TIME & SAMP_DATE_TIME_ON & CON_ID & CON_SHORT_NAME \\
\hline 2016 & SESP SCHEDULING & & SW & & & BOXRP0 & 28-Mar-00 & & $15117-96-1$ & U-235 \\
\hline 2017 & SESP SCHEDULING & & SW & & & B0XRP1 & 28-Mar-00 & & 15117-96-1 & U-235 \\
\hline 2018 & SESP SCHEDULING & & SW & & & BOXWWO & 04-Apr-00 & & $15117-96-1$ & U-235 \\
\hline 2019 & SESP SCHEDULING & & SW & & & B0XP21 & 05-Apr-00 & 08-Mar-00 & $15117-96-1$ & U-235 \\
\hline 2020 & SESP SCHEDULING & & SW & & & B0XP16 & 05-Apr-00 & 08-Mar-00 & $15117-96-1$ & U-235 \\
\hline 2021 & SESP SCHEDULING & & SW & & & B0XVN6 & 03-May-00 & 29-Mar-00 & $15117-96-1$ & U-235 \\
\hline 2022 & SESP SCHEDULING & & SW & & & BOXVP1 & 03-May-00 & 29-Mar-00 & $15117-96-1$ & $\mathrm{U}-235$ \\
\hline 2023 & SESP SCHEDULING & & SW & & & BOY2Y6 & 11-May-00 & & 15117-96-1 & U-235 \\
\hline 2024 & SESP SCHEDULING & & SW & & & B0Y231 & 31-May-00 & 03-May-00 & $15117-96-1$ & U-235 \\
\hline \begin{tabular}{|l|}
2025 \\
\end{tabular} & SESP SCHEDULING & & SW & & & B0Y237 & 31-May-00 & 03-May-00 & $15117-96-1$ & U-235 \\
\hline 2026 & SESP SCHEDULING & & SW & & & B0YB90 & 08-Jun-00 & & $15117-96-1$ & U-235 \\
\hline 2027 & SESP SCHEDULING & & SW & & & BOYRB8 & 12-Jun-00 & & $15117-96-1$ & U-235 \\
\hline 2028 & SESP SCHEDULING & & SW & & & BOYRCO & 12-Jun-00 & & $15117-96-1$ & U-235 \\
\hline 2029 & SESP SCHEDULING & & SW & & & BOYRC3 & 12-Jun-00 & & 15117-96-1 & U-235 \\
\hline 2030 & SESP SCHEDULING & & SW & & & BOYCD7 & 19-Jun-00 & & $15117-96-1$ & U-235 \\
\hline 2031 & SESP SCHEDULING & & SW & & & B0YCD8 & 19-Jun-00 & & $15117-96-1$ & U-235 \\
\hline \begin{tabular}{|l|}
2032 \\
\end{tabular} & SESP SCHEDULING & & SW & & & B0YCD9 & 19-Jun-00 & & $15117-96-1$ & U-235 \\
\hline 2033 & SESP SCHEDULING & & SW & & & BOYCFO & 19-Jun-00 & & $15117-96-1$ & U-235 \\
\hline 2034 & SESP SCHEDULING & & SW & & & BOYCF1 & 20-Jun-00 & & $15117-96-1$ & U-235 \\
\hline 2035 & SESP SCHEDULING & & SW & & & BOYCF2 & 20-Jun-00 & & 15117-96-1 & U-235 \\
\hline 2036 & SESP SCHEDULING & & SW & & & BOYCF3 & 20-Jun-00 & & $15117-96-1$ & U-235 \\
\hline 2037 & SESP SCHEDULING & & SW & & & BOYCF4 & 20-Jun-00 & & $15117-96-1$ & U-235 \\
\hline 2038 & SESP SCHEDULING & & SW & & & BOYCF5 & 20-Jun-00 & & $15117-96-1$ & U-235 \\
\hline 2039 & SESP SCHEDULING & & SW & & & B0YCF6 & 20-Jun-00 & & 15117-96-1 & U-235 \\
\hline 2040 & SESP SCHEDULING & & SW & & & BOYCK3 & 20-Jun-00 & & $15117-96-1$ & U-235 \\
\hline 2041 & SESP SCHEDULING & & SW & & & BOYCK4 & 20-Jun-00 & & 15117-96-1 & U-235 \\
\hline 2042 & SESP SCHEDULING & & SW & & & B0YCK5 & 20-Jun-00 & & $15117-96-1$ & U-235 \\
\hline 2043 & SESP SCHEDULING & & SW & & & B0YCK6 & 20-Jun-00 & & $15117-96-1$ & U-235 \\
\hline 2044 & SESP SCHEDULING & & SW & & & B0YB64 & 29-Jun-00 & 31-May-00 & $15117-96-1$ & U-235 \\
\hline \begin{tabular}{|l|}
2045 \\
\end{tabular} & SESP SCHEDULING & & SW & & & B0YB76 & 29-Jun-00 & 31-May-00 & $15117-96-1$ & U-235 \\
\hline 2046 & SESP SCHEDULING & & SW & & & B0YM20 & 05-Jul-00 & & $15117-96-1$ & U-235 \\
\hline \begin{tabular}{|l|}
2047 \\
\end{tabular} & SESP SCHEDULING & & SW & & & B0YM22 & 07-Jul-00 & & $15117-96-1$ & U-235 \\
\hline 2048 & SESP SCHEDULING & & SW & & & B0YKV4 & 10-Aug-00 & 29-Jun-00 & $15117-96-1$ & U-235 \\
\hline 2049 & SESP SCHEDULING & & SW & & & B0YKW1 & 10-Aug-00 & 29-Jun-00 & $15117-96-1$ & U-235 \\
\hline 2050 & SESP SCHEDULING & & SW & & & B0YW65 & 07-Sep-00 & & 15117-96-1 & U-235 \\
\hline 2051 & SESP SCHEDULING & & SW & & & B0YW70 & 07-Sep-00 & & $15117-96-1$ & $\mathrm{U}-235$ \\
\hline 2052 & SESP SCHEDULING & & SW & & & B10672 & 14-Sep-00 & & $15117-96-1$ & U-235 \\
\hline 2053 & SESP SCHEDULING & & SW & & & B10673 & 14-Sep-00 & & 15117-96-1 & $\mathrm{U}-235$ \\
\hline
\end{tabular}




\begin{tabular}{|c|c|c|c|c|c|c|c|c|c|c|}
\hline & A & $\mathrm{B}$ & C & $\mathrm{D}$ & $\mathrm{E}$ & $\mathrm{F}$ & $\mathrm{G}$ & $\mathrm{H}$ & $\mathrm{I}$ & $\mathrm{J}$ \\
\hline 1 & SAMPLE_NUM_ASSIGNED_TO & SAMPLER & MEDIA & BIOTA_MEDIA & TAG_ID & SAMP_NUM & SAMP_DATE_TIME & SAMP_DATE_TIME_ON & CON_ID & CON_SHORT_NAME \\
\hline 2054 & SESP SCHEDULING & & SW & & & B10674 & 14-Sep-00 & & $15117-96-1$ & U-235 \\
\hline 2055 & SESP SCHEDULING & & SW & & & B10675 & 14-Sep-00 & & $15117-96-1$ & U-235 \\
\hline 2056 & SESP SCHEDULING & & SW & & & B10676 & 14-Sep-00 & & $15117-96-1$ & U-235 \\
\hline 2057 & SESP SCHEDULING & & SW & & & B10677 & 14-Sep-00 & & $15117-96-1$ & U-235 \\
\hline 2058 & SESP SCHEDULING & & SW & & & B10679 & 14-Sep-00 & & $15117-96-1$ & U-235 \\
\hline 2059 & SESP SCHEDULING & & SW & & & B10681 & 14-Sep-00 & & 15117-96-1 & U-235 \\
\hline 2060 & SESP SCHEDULING & & SW & & & B10683 & 14-Sep-00 & & $15117-96-1$ & U-235 \\
\hline 2061 & SESP SCHEDULING & & SW & & & B10684 & 14-Sep-00 & & 15117-96-1 & U-235 \\
\hline 2062 & SESP SCHEDULING & & SW & & & B10686 & 14-Sep-00 & & $15117-96-1$ & U-235 \\
\hline 2063 & SESP SCHEDULING & & SW & & & B10698 & 14-Sep-00 & & $15117-96-1$ & U-235 \\
\hline 2064 & SESP SCHEDULING & & SW & & & B106B1 & 14-Sep-00 & & $15117-96-1$ & U-235 \\
\hline 2065 & SESP SCHEDULING & & SW & & & B106B4 & 14-Sep-00 & & $15117-96-1$ & U-235 \\
\hline 2066 & SESP SCHEDULING & & SW & & & B106B7 & 14-Sep-00 & & 15117-96-1 & U-235 \\
\hline 2067 & SESP SCHEDULING & & SW & & & B106W1 & 18-Sep-00 & & $15117-96-1$ & U-235 \\
\hline 2068 & SESP SCHEDULING & & SW & & & B106W2 & 18-Sep-00 & & $15117-96-1$ & U-235 \\
\hline 2069 & SESP SCHEDULING & & SW & & & B106W3 & 18-Sep-00 & & $15117-96-1$ & U-235 \\
\hline 2070 & SESP SCHEDULING & & SW & & & B106W4 & 18-Sep-00 & & $15117-96-1$ & U-235 \\
\hline 2071 & SESP SCHEDULING & & SW & & & B106W5 & 18-Sep-00 & & $15117-96-1$ & U-235 \\
\hline 2072 & SESP SCHEDULING & & SW & & & B106W6 & 18-Sep-00 & & $15117-96-1$ & U-235 \\
\hline 2073 & SESP SCHEDULING & & SW & & & B106W7 & 18-Sep-00 & & $15117-96-1$ & U-235 \\
\hline 2074 & SESP SCHEDULING & & SW & & & B106W8 & 18-Sep-00 & & $15117-96-1$ & U-235 \\
\hline 2075 & SESP SCHEDULING & & SW & & & B106W9 & 18-Sep-00 & & $15117-96-1$ & U-235 \\
\hline 2076 & SESP SCHEDULING & & SW & & & B106X0 & 18-Sep-00 & & 15117-96-1 & U-235 \\
\hline 2077 & SESP SCHEDULING & & SW & & & B106X1 & 18-Sep-00 & & $15117-96-1$ & U-235 \\
\hline 2078 & SESP SCHEDULING & & SW & & & B106X2 & 18-Sep-00 & & $15117-96-1$ & U-235 \\
\hline 2079 & SESP SCHEDULING & & SW & & & B10728 & 18-Sep-00 & & $15117-96-1$ & U-235 \\
\hline 2080 & SESP SCHEDULING & & SW & & & B10729 & 18-Sep-00 & & 15117-96-1 & U-235 \\
\hline 2081 & SESP SCHEDULING & & SW & & & B10730 & 18-Sep-00 & & $15117-96-1$ & U-235 \\
\hline 2082 & SESP SCHEDULING & & SW & & & B10785 & 18-Sep-00 & & 15117-96-1 & U-235 \\
\hline 2083 & SESP SCHEDULING & & SW & & & B10788 & 18-Sep-00 & & 15117-96-1 & U-235 \\
\hline 2084 & SESP SCHEDULING & & SW & & & B10791 & 18-Sep-00 & & $15117-96-1$ & U-235 \\
\hline 2085 & SESP SCHEDULING & & SW & & & B10794 & 18-Sep-00 & & $15117-96-1$ & U-235 \\
\hline 2086 & SESP SCHEDULING & & SW & & & B106X3 & 19-Sep-00 & & $15117-96-1$ & U-235 \\
\hline 2087 & SESP SCHEDULING & & SW & & & B106X5 & 19-Sep-00 & & $15117-96-1$ & U-235 \\
\hline 2088 & SESP SCHEDULING & & SW & & & B106X7 & 19-Sep-00 & & $15117-96-1$ & U-235 \\
\hline 2089 & SESP SCHEDULING & & SW & & & B106X9 & 19-Sep-00 & & $15117-96-1$ & U-235 \\
\hline 2090 & SESP SCHEDULING & & SW & & & B106Y1 & 19-Sep-00 & & $15117-96-1$ & U-235 \\
\hline 2091 & SESP SCHEDULING & & SW & & & B106Y3 & 19-Sep-00 & & 15117-96-1 & U-235 \\
\hline
\end{tabular}




\begin{tabular}{|c|c|c|c|c|c|c|c|c|c|c|}
\hline & A & $\mathrm{B}$ & C & $\mathrm{D}$ & $E$ & $\mathrm{~F}$ & G & $\mathrm{H}$ & $\mathrm{I}$ & $\mathrm{J}$ \\
\hline 1 & SAMPLE_NUM_ASSIGNED_TO & SAMPLER & MEDIA & BIOTA_MEDIA & TAG_ID & SAMP_NUM & SAMP_DATE_TIME & SAMP_DATE_TIME_ON & CON_ID & CON_SHORT_NAME \\
\hline 2092 & SESP SCHEDULING & & SW & & & B106Y4 & 19-Sep-00 & & $15117-96-1$ & $\mathrm{U}-235$ \\
\hline 2093 & SESP SCHEDULING & & SW & & & B106Y5 & 19-Sep-00 & & 15117-96-1 & U-235 \\
\hline 2094 & SESP SCHEDULING & & SW & & & B106Y6 & 19-Sep-00 & & 15117-96-1 & U-235 \\
\hline 2095 & SESP SCHEDULING & & SW & & & B106Y7 & 19-Sep-00 & & 15117-96-1 & U-235 \\
\hline 2096 & SESP SCHEDULING & & SW & & & B106Y8 & 19-Sep-00 & & 15117-96-1 & $\mathrm{U}-235$ \\
\hline 2097 & SESP SCHEDULING & & SW & & & B106Y9 & 19-Sep-00 & & 15117-96-1 & $\mathrm{U}-235$ \\
\hline 2098 & SESP SCHEDULING & & SW & & & B10761 & 19-Sep-00 & & 15117-96-1 & U-235 \\
\hline 2099 & SESP SCHEDULING & & SW & & & B10762 & 19-Sep-00 & & 15117-96-1 & U-235 \\
\hline 2100 & SESP SCHEDULING & & SW & & & B10763 & 19-Sep-00 & & 15117-96-1 & U-235 \\
\hline 2101 & SESP SCHEDULING & & SW & & & B10764 & 19-Sep-00 & & $15117-96-1$ & U-235 \\
\hline 2102 & SESP SCHEDULING & & SW & & & B10773 & 19-Sep-00 & & $15117-96-1$ & U-235 \\
\hline 2103 & SESP SCHEDULING & & SW & & & B10776 & 19-Sep-00 & & 15117-96-1 & U-235 \\
\hline 2104 & SESP SCHEDULING & & SW & & & B10779 & 19-Sep-00 & & 15117-96-1 & U-235 \\
\hline 2105 & SESP SCHEDULING & & SW & & & B10782 & 19-Sep-00 & & $15117-96-1$ & U-235 \\
\hline 2106 & SESP SCHEDULING & & SW & & & B10912 & 27-Sep-00 & & 15117-96-1 & U-235 \\
\hline 2107 & SESP SCHEDULING & & SW & & & B10913 & 27-Sep-00 & & 15117-96-1 & U-235 \\
\hline 2108 & SESP SCHEDULING & & SW & & & B10945 & 27-Sep-00 & & 15117-96-1 & U-235 \\
\hline 2109 & SESP SCHEDULING & & SW & & & B10946 & 27-Sep-00 & & 15117-96-1 & U-235 \\
\hline 2110 & SESP SCHEDULING & & SW & & & B10949 & 27-Sep-00 & & 15117-96-1 & U-235 \\
\hline 2111 & SESP SCHEDULING & & SW & & & B109J0 & 27-Sep-00 & & $15117-96-1$ & $\mathrm{U}-235$ \\
\hline 2112 & SESP SCHEDULING & & SW & & & B103W8 & $05-$ Oct-00 & 07-Sep-00 & $15117-96-1$ & $\mathrm{U}-235$ \\
\hline 2113 & SESP SCHEDULING & & SW & & & B103X3 & $05-O c t-00$ & 07-Sep-00 & $15117-96-1$ & U-235 \\
\hline 2114 & SESP SCHEDULING & & SW & & & B10F33 & $09-O c t-00$ & & 15117-96-1 & U-235 \\
\hline 2115 & SESP SCHEDULING & & SW & & & B10941 & 01-Nov-00 & & 15117-96-1 & U-235 \\
\hline 2116 & SESP SCHEDULING & & SW & & & B10977 & 01-Nov-00 & & 15117-96-1 & U-235 \\
\hline 2117 & SESP SCHEDULING & & SW & & & B10C13 & 02-Nov-00 & $05-$ Oct-00 & $15117-96-1$ & U-235 \\
\hline 2118 & SESP SCHEDULING & & SW & & & B10C19 & 02-Nov-00 & $05-O c t-00$ & $15117-96-1$ & U-235 \\
\hline 2119 & SESP SCHEDULING & & SW & & & B10942 & 07-Nov-00 & & 15117-96-1 & U-235 \\
\hline 2120 & SESP SCHEDULING & & SW & & & B10MP5 & 29-Nov-00 & 02-Nov-00 & $15117-96-1$ & U-235 \\
\hline 2121 & SESP SCHEDULING & & SW & & & B10MR1 & 29-Nov-00 & 02-Nov-00 & $15117-96-1$ & U-235 \\
\hline 2122 & SESP SCHEDULING & & SW & & & B11125 & 04-Dec-00 & & 15117-96-1 & U-235 \\
\hline 2123 & SESP SCHEDULING & & SW & & & B11126 & 04-Dec-00 & & $15117-96-1$ & U-235 \\
\hline 2124 & SESP SCHEDULING & & SW & & & B11127 & 04-Dec-00 & & 15117-96-1 & $\mathrm{U}-235$ \\
\hline 2125 & SESP SCHEDULING & & SW & & & B11128 & 04-Dec-00 & & 15117-96-1 & $\mathrm{U}-235$ \\
\hline 2126 & SESP SCHEDULING & & SW & & & B11124 & 05-Dec-00 & & $15117-96-1$ & U-235 \\
\hline 2127 & SESP SCHEDULING & & SW & & & B11129 & 05-Dec-00 & & 15117-96-1 & $\mathrm{U}-235$ \\
\hline 2128 & SESP SCHEDULING & & SW & & & B11130 & 05-Dec-00 & & 15117-96-1 & $\mathrm{U}-235$ \\
\hline 2129 & SESP SCHEDULING & & SW & & & B11131 & 05-Dec-00 & & 15117-96-1 & U-235 \\
\hline
\end{tabular}




\begin{tabular}{|c|c|c|c|c|c|c|c|c|c|c|}
\hline & A & $\mathrm{B}$ & $\mathrm{C}$ & $\mathrm{D}$ & $E$ & $\mathrm{~F}$ & G & $\mathrm{H}$ & $\mathrm{I}$ & $\mathrm{J}$ \\
\hline 1 & SAMPLE_NUM_ASSIGNED_TO & SAMPLER & MEDIA & BIOTA_MEDIA & TAG_ID & SAMP_NUM & SAMP_DATE_TIME & SAMP_DATE_TIME_ON & CON_ID & CON_SHORT_NAME \\
\hline 2130 & SESP SCHEDULING & & SW & & & B11132 & 05-Dec-00 & & $15117-96-1$ & $\mathrm{U}-235$ \\
\hline 2131 & SESP SCHEDULING & & SW & & & B11133 & 05-Dec-00 & & $15117-96-1$ & U-235 \\
\hline 2132 & SESP SCHEDULING & & SW & & & B11134 & 05-Dec-00 & & $15117-96-1$ & U-235 \\
\hline 2133 & SESP SCHEDULING & & SW & & & B11145 & 05-Dec-00 & & 15117-96-1 & U-235 \\
\hline 2134 & SESP SCHEDULING & & SW & & & B11146 & 05-Dec-00 & & 15117-96-1 & U-235 \\
\hline 2135 & SESP SCHEDULING & & SW & & & B11147 & 05-Dec-00 & & $15117-96-1$ & U-235 \\
\hline 2136 & SESP SCHEDULING & & SW & & & B11148 & 05-Dec-00 & & 15117-96-1 & $\mathrm{U}-235$ \\
\hline 2137 & SESP SCHEDULING & & SW & & & B110Y4 & 04-Jan-01 & 29-Nov-00 & $15117-96-1$ & U-235 \\
\hline 2138 & SESP SCHEDULING & & SW & & & B11100 & 04-Jan-01 & 29-Nov-00 & $15117-96-1$ & U-235 \\
\hline 2139 & SESP SCHEDULING & & SW & & & B114P8 & 31-Jan-01 & 04-Jan-01 & 15117-96-1 & U-235 \\
\hline 2140 & SESP SCHEDULING & & SW & & & B114T0 & 31-Jan-01 & 04-Jan-01 & 15117-96-1 & U-235 \\
\hline 2141 & SESP SCHEDULING & & SW & & & B11CC7 & 12-Feb-01 & & $15117-96-1$ & U-235 \\
\hline 2142 & SESP SCHEDULING & & SW & & & B11H38 & 26-Feb-01 & & 15117-96-1 & U-235 \\
\hline 2143 & SESP SCHEDULING & & SW & & & $\mathrm{B} 11 \mathrm{H} 43$ & 26-Feb-01 & & 15117-96-1 & U-235 \\
\hline 2144 & SESP SCHEDULING & & SW & & & $\mathrm{B} 11 \mathrm{H} 44$ & 26-Feb-01 & & $15117-96-1$ & U-235 \\
\hline 2145 & SESP SCHEDULING & & SW & & & $\mathrm{B} 11 \mathrm{H} 45$ & 26-Feb-01 & & $15117-96-1$ & U-235 \\
\hline 2146 & SESP SCHEDULING & & SW & & & B11H46 & 26-Feb-01 & & 15117-96-1 & U-235 \\
\hline 2147 & SESP SCHEDULING & & SW & & & $\mathrm{B} 11 \mathrm{H} 47$ & 26-Feb-01 & & $15117-96-1$ & U-235 \\
\hline 2148 & SESP SCHEDULING & & SW & & & $\mathrm{B} 11 \mathrm{H} 48$ & 26-Feb-01 & & 15117-96-1 & U-235 \\
\hline 2149 & SESP SCHEDULING & & SW & & & B11H59 & 26-Feb-01 & & 15117-96-1 & U-235 \\
\hline 2150 & SESP SCHEDULING & & SW & & & $\mathrm{B} 11 \mathrm{H} 60$ & 26-Feb-01 & & $15117-96-1$ & $\mathrm{U}-235$ \\
\hline 2151 & SESP SCHEDULING & & SW & & & B11H61 & 26-Feb-01 & & 15117-96-1 & U-235 \\
\hline 2152 & SESP SCHEDULING & & SW & & & B11H62 & 26-Feb-01 & & 15117-96-1 & U-235 \\
\hline 2153 & SESP SCHEDULING & & SW & & & B11H39 & 27-Feb-01 & & 15117-96-1 & U-235 \\
\hline 2154 & SESP SCHEDULING & & SW & & & $\mathrm{B} 11 \mathrm{H} 40$ & 27-Feb-01 & & 15117-96-1 & U-235 \\
\hline 2155 & SESP SCHEDULING & & SW & & & $\mathrm{B} 11 \mathrm{H} 41$ & 27-Feb-01 & & 15117-96-1 & U-235 \\
\hline 2156 & SESP SCHEDULING & & SW & & & $\mathrm{B} 11 \mathrm{H} 42$ & 27-Feb-01 & & $15117-96-1$ & U-235 \\
\hline 2157 & SESP SCHEDULING & & SW & & & B11C04 & 01-Mar-01 & 31-Jan-01 & $15117-96-1$ & U-235 \\
\hline 2158 & SESP SCHEDULING & & SW & & & B11C10 & 01-Mar-01 & 31-Jan-01 & 15117-96-1 & U-235 \\
\hline 2159 & SESP SCHEDULING & & SW & & & B11MY9 & 03-Apr-01 & & 15117-96-1 & U-235 \\
\hline 2160 & SESP SCHEDULING & & SW & & & B11J40 & 04-Apr-01 & 01-Mar-01 & $15117-96-1$ & $\mathrm{U}-235$ \\
\hline 2161 & SESP SCHEDULING & & SW & & & B11J46 & 04-Apr-01 & 01-Mar-01 & 15117-96-1 & U-235 \\
\hline 2162 & SESP SCHEDULING & & SW & & & B11W36 & 30-Apr-01 & & 15117-96-1 & U-235 \\
\hline 2163 & SESP SCHEDULING & & SW & & & B11W50 & 30-Apr-01 & & 15117-96-1 & U-235 \\
\hline 2164 & SESP SCHEDULING & & SW & & & B11W52 & 30-Apr-01 & & 15117-96-1 & U-235 \\
\hline 2165 & SESP SCHEDULING & & SW & & & B11W55 & 30-Apr-01 & & $15117-96-1$ & U-235 \\
\hline 2166 & SESP SCHEDULING & & SW & & & B11W86 & 30-Apr-01 & & $15117-96-1$ & U-235 \\
\hline 2167 & SESP SCHEDULING & & SW & & & B11LT2 & 02-May-01 & 04-Apr-01 & 15117-96-1 & U-235 \\
\hline
\end{tabular}




\begin{tabular}{|c|c|c|c|c|c|c|c|c|c|c|}
\hline & A & $\mathrm{B}$ & $\mathrm{C}$ & $\mathrm{D}$ & $E$ & $\mathrm{~F}$ & G & $\mathrm{H}$ & $\mathrm{I}$ & $\mathrm{J}$ \\
\hline 1 & SAMPLE_NUM_ASSIGNED_TO & SAMPLER & MEDIA & BIOTA_MEDIA & TAG_ID & SAMP_NUM & SAMP_DATE_TIME & SAMP_DATE_TIME_ON & CON_ID & CON_SHORT_NAME \\
\hline 2168 & SESP SCHEDULING & & SW & & & B11LR6 & 02-May-01 & 04-Apr-01 & $15117-96-1$ & U-235 \\
\hline 2169 & SESP SCHEDULING & & SW & & & B11W57 & 03-May-01 & & 15117-96-1 & U-235 \\
\hline 2170 & SESP SCHEDULING & & SW & & & B11WV8 & 09-May-01 & & 15117-96-1 & U-235 \\
\hline 2171 & SESP SCHEDULING & & SW & & & B11WX0 & 09-May-01 & & 15117-96-1 & U-235 \\
\hline 2172 & SESP SCHEDULING & & SW & & & B11W38 & 10-May-01 & & 15117-96-1 & U-235 \\
\hline 2173 & SESP SCHEDULING & & SW & & & B121V4 & 17-May-01 & & 15117-96-1 & U-235 \\
\hline 2174 & SESP SCHEDULING & & SW & & & B11WT4 & 30-May-01 & 02-May-01 & 15117-96-1 & U-235 \\
\hline 2175 & SESP SCHEDULING & & SW & & & B11WR8 & 30-May-01 & 02-May-01 & 15117-96-1 & U-235 \\
\hline 2176 & SESP SCHEDULING & & SW & & & B124N7 & 07-Jun-01 & & 15117-96-1 & U-235 \\
\hline 2177 & SESP SCHEDULING & & SW & & & B124P7 & 07-Jun-01 & & 15117-96-1 & U-235 \\
\hline 2178 & SESP SCHEDULING & & SW & & & B12505 & 12-Jun-01 & & 15117-96-1 & U-235 \\
\hline 2179 & SESP SCHEDULING & & SW & & & B12506 & 12-Jun-01 & & 15117-96-1 & U-235 \\
\hline 2180 & SESP SCHEDULING & & SW & & & B12507 & 12-Jun-01 & & 15117-96-1 & U-235 \\
\hline 2181 & SESP SCHEDULING & & SW & & & B12508 & 12-Jun-01 & & 15117-96-1 & U-235 \\
\hline 2182 & SESP SCHEDULING & & SW & & & B12509 & 12-Jun-01 & & 15117-96-1 & U-235 \\
\hline 2183 & SESP SCHEDULING & & SW & & & B12510 & 12-Jun-01 & & $15117-96-1$ & U-235 \\
\hline 2184 & SESP SCHEDULING & & SW & & & B12531 & 12-Jun-01 & & 15117-96-1 & U-235 \\
\hline 2185 & SESP SCHEDULING & & SW & & & B12532 & 12-Jun-01 & & 15117-96-1 & U-235 \\
\hline 2186 & SESP SCHEDULING & & SW & & & B12533 & 12-Jun-01 & & 15117-96-1 & U-235 \\
\hline 2187 & SESP SCHEDULING & & SW & & & B12534 & 12-Jun-01 & & $15117-96-1$ & U-235 \\
\hline 2188 & SESP SCHEDULING & & SW & & & B12501 & 14-Jun-01 & & 15117-96-1 & U-235 \\
\hline 2189 & SESP SCHEDULING & & SW & & & B12502 & 14-Jun-01 & & 15117-96-1 & U-235 \\
\hline 2190 & SESP SCHEDULING & & SW & & & B12503 & 14-Jun-01 & & 15117-96-1 & U-235 \\
\hline 2191 & SESP SCHEDULING & & SW & & & B12504 & 14-Jun-01 & & $15117-96-1$ & U-235 \\
\hline 2192 & SESP SCHEDULING & & SW & & & B124M3 & 28-Jun-01 & 23-May-01 & 15117-96-1 & U-235 \\
\hline 2193 & SESP SCHEDULING & & SW & & & B124L7 & 28-Jun-01 & 23-May-01 & 15117-96-1 & U-235 \\
\hline 2194 & SESP SCHEDULING & & SW & & & B12992 & 09-Jul-01 & & 15117-96-1 & U-235 \\
\hline 2195 & SESP SCHEDULING & & SW & & & B12994 & 09-Jul-01 & & 15117-96-1 & U-235 \\
\hline 2196 & SESP SCHEDULING & & SW & & & B129B4 & 09-Jul-01 & & $15117-96-1$ & U-235 \\
\hline 2197 & SESP SCHEDULING & & SW & & & B12841 & 09-Aug-01 & 28-Jun-01 & 15117-96-1 & U-235 \\
\hline 2198 & SESP SCHEDULING & & SW & & & B12834 & 09-Aug-01 & 28-Jun-01 & 15117-96-1 & U-235 \\
\hline 2199 & SESP SCHEDULING & & SW & & & B12RM1 & 27-Aug-01 & & 15117-96-1 & U-235 \\
\hline 2200 & SESP SCHEDULING & & SW & & & B12RM6 & 27-Aug-01 & & $15117-96-1$ & U-235 \\
\hline 2201 & SESP SCHEDULING & & SW & & & B12RN4 & 27-Aug-01 & & 15117-96-1 & U-235 \\
\hline 2202 & SESP SCHEDULING & & SW & & & B12RN5 & 27-Aug-01 & & 15117-96-1 & U-235 \\
\hline 2203 & SESP SCHEDULING & & SW & & & B12RN6 & 27-Aug-01 & & 15117-96-1 & U-235 \\
\hline 2204 & SESP SCHEDULING & & SW & & & B12RN7 & 27-Aug-01 & & 15117-96-1 & U-235 \\
\hline 2205 & SESP SCHEDULING & & SW & & & B12RP9 & 27-Aug-01 & & 15117-96-1 & U-235 \\
\hline
\end{tabular}




\begin{tabular}{|c|c|c|c|c|c|c|c|c|c|c|}
\hline & A & $\mathrm{B}$ & $\mathrm{C}$ & $\mathrm{D}$ & $\mathrm{E}$ & $\mathrm{F}$ & G & $\mathrm{H}$ & $\mathrm{I}$ & $\mathrm{J}$ \\
\hline 1 & SAMPLE_NUM_ASSIGNED_TO & SAMPLER & MEDIA & BIOTA_MEDIA & TAG_ID & SAMP_NUM & SAMP_DATE_TIME & SAMP_DATE_TIME_ON & CON_ID & CON_SHORT_NAME \\
\hline 2206 & SESP SCHEDULING & & SW & & & B12RR1 & 27-Aug-01 & & $15117-96-1$ & U-235 \\
\hline 2207 & SESP SCHEDULING & & SW & & & B12RR3 & 27-Aug-01 & & $15117-96-1$ & U-235 \\
\hline 2208 & SESP SCHEDULING & & SW & & & B12RR5 & 27-Aug-01 & & $15117-96-1$ & U-235 \\
\hline 2209 & SESP SCHEDULING & & SW & & & B12RR7 & 27-Aug-01 & & $15117-96-1$ & U-235 \\
\hline 2210 & SESP SCHEDULING & & SW & & & B12RR9 & 27-Aug-01 & & $15117-96-1$ & U-235 \\
\hline 2211 & SESP SCHEDULING & & SW & & & B12RT1 & 27-Aug-01 & & 15117-96-1 & U-235 \\
\hline 2212 & SESP SCHEDULING & & SW & & & B12RT3 & 27-Aug-01 & & $15117-96-1$ & U-235 \\
\hline 2213 & SESP SCHEDULING & & SW & & & B12RT5 & 27-Aug-01 & & 15117-96-1 & U-235 \\
\hline 2214 & SESP SCHEDULING & & SW & & & B12RT7 & 27-Aug-01 & & $15117-96-1$ & U-235 \\
\hline 2215 & SESP SCHEDULING & & SW & & & B12RT9 & 27-Aug-01 & & $15117-96-1$ & U-235 \\
\hline 2216 & SESP SCHEDULING & & SW & & & B12RV1 & 27-Aug-01 & & $15117-96-1$ & U-235 \\
\hline 2217 & SESP SCHEDULING & & SW & & & B12RV3 & 27-Aug-01 & & $15117-96-1$ & U-235 \\
\hline 2218 & SESP SCHEDULING & & SW & & & B12RV5 & 27-Aug-01 & & 15117-96-1 & U-235 \\
\hline 2219 & SESP SCHEDULING & & SW & & & B12RV7 & 27-Aug-01 & & $15117-96-1$ & U-235 \\
\hline 2220 & SESP SCHEDULING & & SW & & & B12RV9 & 27-Aug-01 & & $15117-96-1$ & U-235 \\
\hline 2221 & SESP SCHEDULING & & SW & & & B12RW1 & 27-Aug-01 & & $15117-96-1$ & U-235 \\
\hline 2222 & SESP SCHEDULING & & SW & & & B12RW5 & 27-Aug-01 & & $15117-96-1$ & U-235 \\
\hline 2223 & SESP SCHEDULING & & SW & & & B12RW7 & 27-Aug-01 & & 15117-96-1 & U-235 \\
\hline 2224 & SESP SCHEDULING & & SW & & & B12RW9 & 27-Aug-01 & & $15117-96-1$ & U-235 \\
\hline 2225 & SESP SCHEDULING & & SW & & & B12RX1 & 27-Aug-01 & & $15117-96-1$ & U-235 \\
\hline 2226 & SESP SCHEDULING & & SW & & & B12RX3 & 27-Aug-01 & & $15117-96-1$ & U-235 \\
\hline 2227 & SESP SCHEDULING & & SW & & & B12RX5 & 27-Aug-01 & & $15117-96-1$ & U-235 \\
\hline 2228 & SESP SCHEDULING & & SW & & & B12RX9 & 27-Aug-01 & & $15117-96-1$ & U-235 \\
\hline 2229 & SESP SCHEDULING & & SW & & & B12RY1 & 27-Aug-01 & & $15117-96-1$ & U-235 \\
\hline 2230 & SESP SCHEDULING & & SW & & & B12RY3 & 27-Aug-01 & & $15117-96-1$ & U-235 \\
\hline 2231 & SESP SCHEDULING & & SW & & & B12T11 & 27-Aug-01 & & $15117-96-1$ & U-235 \\
\hline 2232 & SESP SCHEDULING & & SW & & & B12T13 & 27-Aug-01 & & 15117-96-1 & U-235 \\
\hline 2233 & SESP SCHEDULING & & SW & & & B12T15 & 27-Aug-01 & & $15117-96-1$ & U-235 \\
\hline 2234 & SESP SCHEDULING & & SW & & & B12T17 & 27-Aug-01 & & $15117-96-1$ & U-235 \\
\hline 2235 & SESP SCHEDULING & & SW & & & B12T19 & 27-Aug-01 & & 15117-96-1 & U-235 \\
\hline 2236 & SESP SCHEDULING & & SW & & & B12T21 & 27-Aug-01 & & 15117-96-1 & U-235 \\
\hline 2237 & SESP SCHEDULING & & SW & & & B12T23 & 27-Aug-01 & & $15117-96-1$ & U-235 \\
\hline 2238 & SESP SCHEDULING & & SW & & & B12T25 & 27-Aug-01 & & $15117-96-1$ & U-235 \\
\hline 2239 & SESP SCHEDULING & & SW & & & B12JT2 & 05-Sep-01 & & $15117-96-1$ & U-235 \\
\hline 2240 & SESP SCHEDULING & & SW & & & B12JR7 & 05-Sep-01 & 09-Aug-01 & $15117-96-1$ & U-235 \\
\hline 2241 & SESP SCHEDULING & & SW & & & B12T85 & 07-Sep-01 & & $15117-96-1$ & U-235 \\
\hline 2242 & SESP SCHEDULING & & SW & & & B12T87 & 07-Sep-01 & & $15117-96-1$ & U-235 \\
\hline 2243 & SESP SCHEDULING & & SW & & & B12T88 & 07-Sep-01 & & 15117-96-1 & U-235 \\
\hline
\end{tabular}




\begin{tabular}{|c|c|c|c|c|c|c|c|c|c|c|}
\hline & $A$ & $\mathrm{~B}$ & $\mathrm{C}$ & $\mathrm{D}$ & $E$ & $\mathrm{~F}$ & G & $\mathrm{H}$ & $\mathrm{I}$ & $\mathrm{J}$ \\
\hline 1 & SAMPLE_NUM_ASSIGNED_TO & SAMPLER & MEDIA & BIOTA_MEDIA & TAG_ID & SAMP_NUM & SAMP_DATE_TIME & SAMP_DATE_TIME_ON & CON_ID & CON_SHORT_NAME \\
\hline 2244 & SESP SCHEDULING & & SW & & & B12T89 & 07-Sep-01 & & $15117-96-1$ & $\mathrm{U}-235$ \\
\hline 2245 & SESP SCHEDULING & & SW & & & B12T90 & 07-Sep-01 & & 15117-96-1 & U-235 \\
\hline 2246 & SESP SCHEDULING & & SW & & & B12T91 & 07-Sep-01 & & 15117-96-1 & U-235 \\
\hline 2247 & SESP SCHEDULING & & SW & & & B12T92 & 07-Sep-01 & & 15117-96-1 & U-235 \\
\hline 2248 & SESP SCHEDULING & & SW & & & B12T93 & 07-Sep-01 & & 15117-96-1 & $\mathrm{U}-235$ \\
\hline 2249 & SESP SCHEDULING & & SW & & & B12TD4 & 07-Sep-01 & & 15117-96-1 & $\mathrm{U}-235$ \\
\hline 2250 & SESP SCHEDULING & & SW & & & B12TD5 & 07-Sep-01 & & 15117-96-1 & U-235 \\
\hline 2251 & SESP SCHEDULING & & SW & & & B12TD6 & 07-Sep-01 & & 15117-96-1 & U-235 \\
\hline 2252 & SESP SCHEDULING & & SW & & & B12TN2 & 07-Sep-01 & & 15117-96-1 & U-235 \\
\hline 2253 & SESP SCHEDULING & & SW & & & B12TN5 & 07-Sep-01 & & 15117-96-1 & U-235 \\
\hline 2254 & SESP SCHEDULING & & SW & & & B12TN8 & 07-Sep-01 & & $15117-96-1$ & U-235 \\
\hline 2255 & SESP SCHEDULING & & SW & & & B12TP1 & 07-Sep-01 & & 15117-96-1 & U-235 \\
\hline 2256 & SESP SCHEDULING & & SW & & & B12T94 & 10-Sep-01 & & 15117-96-1 & U-235 \\
\hline 2257 & SESP SCHEDULING & & SW & & & B12T95 & 10-Sep-01 & & $15117-96-1$ & U-235 \\
\hline 2258 & SESP SCHEDULING & & SW & & & B12T96 & 10-Sep-01 & & 15117-96-1 & U-235 \\
\hline 2259 & SESP SCHEDULING & & SW & & & B12T97 & 10-Sep-01 & & 15117-96-1 & U-235 \\
\hline 2260 & SESP SCHEDULING & & SW & & & B12T98 & 10-Sep-01 & & 15117-96-1 & U-235 \\
\hline 2261 & SESP SCHEDULING & & SW & & & B12T99 & 10-Sep-01 & & 15117-96-1 & U-235 \\
\hline 2262 & SESP SCHEDULING & & SW & & & B12TB0 & 10-Sep-01 & & 15117-96-1 & U-235 \\
\hline 2263 & SESP SCHEDULING & & SW & & & B12TB1 & 10-Sep-01 & & 15117-96-1 & U-235 \\
\hline 2264 & SESP SCHEDULING & & SW & & & B12TB2 & 10-Sep-01 & & 15117-96-1 & U-235 \\
\hline 2265 & SESP SCHEDULING & & SW & & & B12TB3 & 10-Sep-01 & & 15117-96-1 & U-235 \\
\hline 2266 & SESP SCHEDULING & & SW & & & B12TB4 & 10-Sep-01 & & $15117-96-1$ & U-235 \\
\hline 2267 & SESP SCHEDULING & & SW & & & B12TB5 & 10-Sep-01 & & 15117-96-1 & U-235 \\
\hline 2268 & SESP SCHEDULING & & SW & & & B12TF7 & 10-Sep-01 & & 15117-96-1 & U-235 \\
\hline 2269 & SESP SCHEDULING & & SW & & & B12TF8 & 10-Sep-01 & & 15117-96-1 & U-235 \\
\hline 2270 & SESP SCHEDULING & & SW & & & B12TF9 & 10-Sep-01 & & 15117-96-1 & $\mathrm{U}-235$ \\
\hline 2271 & SESP SCHEDULING & & SW & & & B12TT0 & 10-Sep-01 & & 15117-96-1 & U-235 \\
\hline 2272 & SESP SCHEDULING & & SW & & & B12TT3 & 10-Sep-01 & & 15117-96-1 & U-235 \\
\hline 2273 & SESP SCHEDULING & & SW & & & B12TT9 & 10-Sep-01 & & 15117-96-1 & $\mathrm{U}-235$ \\
\hline 2274 & SESP SCHEDULING & & SW & & & B12TB6 & 13-Sep-01 & & 15117-96-1 & U-235 \\
\hline 2275 & SESP SCHEDULING & & SW & & & B12TB8 & 13-Sep-01 & & $15117-96-1$ & U-235 \\
\hline 2276 & SESP SCHEDULING & & SW & & & B12TC0 & 13-Sep-01 & & 15117-96-1 & $\mathrm{U}-235$ \\
\hline 2277 & SESP SCHEDULING & & SW & & & B12TC2 & 13-Sep-01 & & 15117-96-1 & $\mathrm{U}-235$ \\
\hline 2278 & SESP SCHEDULING & & SW & & & B12TC4 & 13-Sep-01 & & $15117-96-1$ & U-235 \\
\hline 2279 & SESP SCHEDULING & & SW & & & B12TC6 & 13-Sep-01 & & 15117-96-1 & $\mathrm{U}-235$ \\
\hline 2280 & SESP SCHEDULING & & SW & & & B12TC8 & 13-Sep-01 & & 15117-96-1 & $\mathrm{U}-235$ \\
\hline 2281 & SESP SCHEDULING & & SW & & & B12TC9 & 13-Sep-01 & & 15117-96-1 & U-235 \\
\hline
\end{tabular}




\begin{tabular}{|c|c|c|c|c|c|c|c|c|c|c|}
\hline & A & $\mathrm{B}$ & $\mathrm{C}$ & $\mathrm{D}$ & $\mathrm{E}$ & $\mathrm{F}$ & G & $\mathrm{H}$ & $\mathrm{I}$ & $\mathrm{J}$ \\
\hline 1 & SAMPLE_NUM_ASSIGNED_TO & SAMPLER & MEDIA & BIOTA_MEDIA & TAG_ID & SAMP_NUM & SAMP_DATE_TIME & SAMP_DATE_TIME_ON & CON_ID & CON_SHORT_NAME \\
\hline 2282 & SESP SCHEDULING & & SW & & & B12TD0 & 13-Sep-01 & & $15117-96-1$ & U-235 \\
\hline 2283 & SESP SCHEDULING & & SW & & & B12TD1 & 13-Sep-01 & & $15117-96-1$ & U-235 \\
\hline 2284 & SESP SCHEDULING & & SW & & & B12TD2 & 13-Sep-01 & & $15117-96-1$ & U-235 \\
\hline 2285 & SESP SCHEDULING & & SW & & & B12TD3 & 13-Sep-01 & & $15117-96-1$ & U-235 \\
\hline 2286 & SESP SCHEDULING & & SW & & & B12TM0 & 13-Sep-01 & & $15117-96-1$ & U-235 \\
\hline 2287 & SESP SCHEDULING & & SW & & & B12TM1 & 13-Sep-01 & & 15117-96-1 & U-235 \\
\hline 2288 & SESP SCHEDULING & & SW & & & B12TM2 & 13-Sep-01 & & $15117-96-1$ & U-235 \\
\hline 2289 & SESP SCHEDULING & & SW & & & B12TM3 & 13-Sep-01 & & 15117-96-1 & U-235 \\
\hline 2290 & SESP SCHEDULING & & SW & & & B12TP4 & 13-Sep-01 & & $15117-96-1$ & U-235 \\
\hline 2291 & SESP SCHEDULING & & SW & & & B12TP8 & 13-Sep-01 & & $15117-96-1$ & U-235 \\
\hline 2292 & SESP SCHEDULING & & SW & & & B12TR2 & 13-Sep-01 & & $15117-96-1$ & U-235 \\
\hline 2293 & SESP SCHEDULING & & SW & & & B12TR6 & 13-Sep-01 & & $15117-96-1$ & U-235 \\
\hline 2294 & SESP SCHEDULING & & SW & & & B12XK2 & 17-Sep-01 & & 15117-96-1 & U-235 \\
\hline 2295 & SESP SCHEDULING & & SW & & & B12XK8 & 17-Sep-01 & & $15117-96-1$ & U-235 \\
\hline 2296 & SESP SCHEDULING & & SW & & & B12XK9 & 17-Sep-01 & & $15117-96-1$ & U-235 \\
\hline 2297 & SESP SCHEDULING & & SW & & & B12XL0 & 17-Sep-01 & & $15117-96-1$ & U-235 \\
\hline 2298 & SESP SCHEDULING & & SW & & & B12XL1 & 17-Sep-01 & & $15117-96-1$ & U-235 \\
\hline 2299 & SESP SCHEDULING & & SW & & & B12XL2 & 17-Sep-01 & & 15117-96-1 & U-235 \\
\hline 2300 & SESP SCHEDULING & & SW & & & B12XK1 & 18-Sep-01 & & $15117-96-1$ & U-235 \\
\hline 2301 & SESP SCHEDULING & & SW & & & B12XK3 & 18-Sep-01 & & $15117-96-1$ & U-235 \\
\hline 2302 & SESP SCHEDULING & & SW & & & B12XK4 & 18-Sep-01 & & $15117-96-1$ & U-235 \\
\hline 2303 & SESP SCHEDULING & & SW & & & B12XK5 & 18-Sep-01 & & $15117-96-1$ & U-235 \\
\hline 2304 & SESP SCHEDULING & & SW & & & B12XK6 & 18-Sep-01 & & $15117-96-1$ & U-235 \\
\hline 2305 & SESP SCHEDULING & & SW & & & B12XK7 & 18-Sep-01 & & $15117-96-1$ & U-235 \\
\hline 2306 & SESP SCHEDULING & & SW & & & B12T28 & 02-Oct-01 & 05-Sep-01 & 15117-96-1 & U-235 \\
\hline 2307 & SESP SCHEDULING & & SW & & & B12T33 & 02-Oct-01 & 05-Sep-01 & $15117-96-1$ & U-235 \\
\hline 2308 & SESP SCHEDULING & & SW & & & B12X50 & 22-Oct-01 & & 15117-96-1 & U-235 \\
\hline 2309 & SESP SCHEDULING & & SW & & & B12X48 & 01-Nov-01 & & $15117-96-1$ & U-235 \\
\hline 2310 & SESP SCHEDULING & & SW & & & B12X85 & 01-Nov-01 & & $15117-96-1$ & U-235 \\
\hline 2311 & SESP SCHEDULING & & SW & & & B13103 & 01-Nov-01 & 02-Oct-01 & $15117-96-1$ & U-235 \\
\hline 2312 & SESP SCHEDULING & & SW & & & B130Y7 & 01-Nov-01 & 02-Oct-01 & $15117-96-1$ & U-235 \\
\hline 2313 & SESP SCHEDULING & & SW & & & B13J17 & 14-Nov-01 & & $15117-96-1$ & U-235 \\
\hline 2314 & SESP SCHEDULING & & SW & & & B13CC6 & 27-Nov-01 & 01-Nov-01 & $15117-96-1$ & U-235 \\
\hline 2315 & SESP SCHEDULING & & SW & & & B13CD2 & 27-Nov-01 & 01-Nov-01 & $15117-96-1$ & U-235 \\
\hline 2316 & SESP SCHEDULING & & SW & & & B13LF3 & 03-Dec-01 & & $15117-96-1$ & U-235 \\
\hline 2317 & SESP SCHEDULING & & SW & & & B13LF4 & 03-Dec-01 & & $15117-96-1$ & U-235 \\
\hline 2318 & SESP SCHEDULING & & SW & & & B13LF5 & 03-Dec-01 & & $15117-96-1$ & U-235 \\
\hline 2319 & SESP SCHEDULING & & SW & & & B13LF6 & 03-Dec-01 & & 15117-96-1 & U-235 \\
\hline
\end{tabular}




\begin{tabular}{|c|c|c|c|c|c|c|c|c|c|c|}
\hline & $A$ & $\mathrm{~B}$ & $\mathrm{C}$ & $\mathrm{D}$ & $E$ & $\mathrm{~F}$ & $G$ & $\mathrm{H}$ & $\mathrm{I}$ & $\mathrm{J}$ \\
\hline 1 & \begin{tabular}{|l|l} 
SAMPE_NUM_ASSIGNED_TO \\
\end{tabular} & SAMPLER & MEDIA & BIOTA_MEDIA & TAG_ID & SAMP_NUM & SAMP_DATE_TIME & SAMP_DATE_TIME_ON & CON_ID & CON_SHORT_NAME \\
\hline 2320 & SESP SCHEDULING & & SW & & & B13LF2 & 04-Dec-01 & & $15117-96-1$ & U-235 \\
\hline 2321 & SESP SCHEDULING & & SW & & & B13LF7 & 04-Dec-01 & & 15117-96-1 & U-235 \\
\hline 2322 & SESP SCHEDULING & & SW & & & B13LF8 & 04-Dec-01 & & 15117-96-1 & U-235 \\
\hline 2323 & SESP SCHEDULING & & SW & & & B13LF9 & 04-Dec-01 & & $15117-96-1$ & U-235 \\
\hline 2324 & SESP SCHEDULING & & SW & & & B13LH0 & 04-Dec-01 & & $15117-96-1$ & U-235 \\
\hline 2325 & SESP SCHEDULING & & SW & & & B13LH1 & 04-Dec-01 & & $15117-96-1$ & U-235 \\
\hline 2326 & SESP SCHEDULING & & SW & & & B13LH2 & 04-Dec-01 & & $15117-96-1$ & U-235 \\
\hline 2327 & SESP SCHEDULING & & SW & & & B13LJ3 & 04-Dec-01 & & 15117-96-1 & U-235 \\
\hline 2328 & SESP SCHEDULING & & SW & & & B13LJ4 & 04-Dec-01 & & $15117-96-1$ & U-235 \\
\hline 2329 & SESP SCHEDULING & & SW & & & B13LJ5 & 04-Dec-01 & & $15117-96-1$ & U-235 \\
\hline 2330 & SESP SCHEDULING & & SW & & & B13LJ6 & 04-Dec-01 & & 15117-96-1 & U-235 \\
\hline 2331 & SESP SCHEDULING & & SW & & & B13LB3 & 03-Jan-02 & 27-Nov-01 & $15117-96-1$ & U-235 \\
\hline 2332 & SESP SCHEDULING & & SW & & & B13L96 & 03-Jan-02 & 27-Nov-01 & $15117-96-1$ & U-235 \\
\hline 2333 & SESP SCHEDULING & & SW & & & B13VJ0 & 06-Feb-02 & & $15117-96-1$ & U-235 \\
\hline 2334 & SESP SCHEDULING & & SW & & & B13VF8 & 06-Feb-02 & & $15117-96-1$ & U-235 \\
\hline \begin{tabular}{|l|}
2335 \\
\end{tabular} & SESP SCHEDULING & & SW & & & B140P7 & 07-Mar-02 & 06-Feb-02 & $15117-96-1$ & U-235 \\
\hline \begin{tabular}{|l|}
2336 \\
\end{tabular} & SESP SCHEDULING & & SW & & & B140R2 & 07-Mar-02 & 06-Feb-02 & $15117-96-1$ & U-235 \\
\hline 2337 & SESP SCHEDULING & & SW & & & B147T3 & 25-Mar-02 & & $15117-96-1$ & U-235 \\
\hline 2338 & SESP SCHEDULING & & SW & & & B147T4 & 25-Mar-02 & & $15117-96-1$ & U-235 \\
\hline 2339 & SESP SCHEDULING & & SW & & & B147T5 & 25-Mar-02 & & $15117-96-1$ & U-235 \\
\hline 2340 & SESP SCHEDULING & & SW & & & B147T6 & 25-Mar-02 & & 15117-96-1 & U-235 \\
\hline 2341 & SESP SCHEDULING & & SW & & & B147T2 & 26-Mar-02 & & $15117-96-1$ & U-235 \\
\hline 2342 & SESP SCHEDULING & & SW & & & B147T7 & 26-Mar-02 & & $15117-96-1$ & U-235 \\
\hline 2343 & SESP SCHEDULING & & SW & & & B147T8 & 26-Mar-02 & & 15117-96-1 & U-235 \\
\hline 2344 & SESP SCHEDULING & & SW & & & B147T9 & 26-Mar-02 & & 15117-96-1 & U-235 \\
\hline 2345 & SESP SCHEDULING & & SW & & & B147V0 & 26-Mar-02 & & 15117-96-1 & U-235 \\
\hline 2346 & SESP SCHEDULING & & SW & & & B147V1 & 26-Mar-02 & & 15117-96-1 & U-235 \\
\hline 2347 & SESP SCHEDULING & & SW & & & B147V2 & 26-Mar-02 & & $15117-96-1$ & U-235 \\
\hline 2348 & SESP SCHEDULING & & SW & & & B147W3 & 26-Mar-02 & & $15117-96-1$ & U-235 \\
\hline 2349 & SESP SCHEDULING & & SW & & & B147W4 & 26-Mar-02 & & 15117-96-1 & U-235 \\
\hline 2350 & SESP SCHEDULING & & SW & & & B147W5 & 26-Mar-02 & & $15117-96-1$ & U-235 \\
\hline \begin{tabular}{|l|}
2351 \\
\end{tabular} & SESP SCHEDULING & & SW & & & B147W6 & 26-Mar-02 & & $15117-96-1$ & U-235 \\
\hline 2352 & SESP SCHEDULING & & SW & & & B14408 & 03-Apr-02 & 07-Mar-02 & $15117-96-1$ & U-235 \\
\hline 2353 & SESP SCHEDULING & & SW & & & B14413 & 03-Apr-02 & 07-Mar-02 & $15117-96-1$ & U-235 \\
\hline 2354 & SESP SCHEDULING & & SW & & & B14B37 & 01-May-02 & 03-Apr-02 & $15117-96-1$ & U-235 \\
\hline 2355 & SESP SCHEDULING & & SW & & & B14B43 & 01-May-02 & 03-Apr-02 & $15117-96-1$ & U-235 \\
\hline 2356 & SESP SCHEDULING & & SW & & & B14LJ0 & 23-May-02 & & $15117-96-1$ & U-235 \\
\hline 2357 & SESP SCHEDULING & & SW & & & B14LJ2 & 23-May-02 & & 15117-96-1 & U-235 \\
\hline
\end{tabular}




\begin{tabular}{|c|c|c|c|c|c|c|c|c|c|c|}
\hline & A & $\mathrm{B}$ & C & $\mathrm{D}$ & $E$ & $\mathrm{~F}$ & G & $\mathrm{H}$ & $\mathrm{I}$ & $\mathrm{J}$ \\
\hline 1 & SAMPLE_NUM_ASSIGNED_TO & SAMPLER & MEDIA & BIOTA_MEDIA & TAG_ID & SAMP_NUM & SAMP_DATE_TIME & SAMP_DATE_TIME_ON & CON_ID & CON_SHORT_NAME \\
\hline 2358 & SESP SCHEDULING & & SW & & & B14JF2 & 29-May-02 & 01-May-02 & $15117-96-1$ & $\mathrm{U}-235$ \\
\hline 2359 & SESP SCHEDULING & & SW & & & B14JF8 & 29-May-02 & 01-May-02 & $15117-96-1$ & U-235 \\
\hline 2360 & SESP SCHEDULING & & SW & & & B14RV4 & 10-Jun-02 & & 15117-96-1 & U-235 \\
\hline 2361 & SESP SCHEDULING & & SW & & & B14RV5 & 10-Jun-02 & & 15117-96-1 & U-235 \\
\hline 2362 & SESP SCHEDULING & & SW & & & B14RV6 & 10-Jun-02 & & 15117-96-1 & $\mathrm{U}-235$ \\
\hline 2363 & SESP SCHEDULING & & SW & & & B14RV7 & 10-Jun-02 & & 15117-96-1 & $\mathrm{U}-235$ \\
\hline 2364 & SESP SCHEDULING & & SW & & & B14RV8 & 11-Jun-02 & & 15117-96-1 & U-235 \\
\hline 2365 & SESP SCHEDULING & & SW & & & B14RV9 & 11-Jun-02 & & 15117-96-1 & U-235 \\
\hline 2366 & SESP SCHEDULING & & SW & & & B14RW0 & 11-Jun-02 & & 15117-96-1 & U-235 \\
\hline 2367 & SESP SCHEDULING & & SW & & & B14RW1 & 11-Jun-02 & & 15117-96-1 & U-235 \\
\hline 2368 & SESP SCHEDULING & & SW & & & B14RW2 & 11-Jun-02 & & $15117-96-1$ & U-235 \\
\hline 2369 & SESP SCHEDULING & & SW & & & B14RW3 & 11-Jun-02 & & 15117-96-1 & U-235 \\
\hline 2370 & SESP SCHEDULING & & SW & & & B14RX4 & 11-Jun-02 & & 15117-96-1 & U-235 \\
\hline 2371 & SESP SCHEDULING & & SW & & & B14RX5 & 11-Jun-02 & & 15117-96-1 & U-235 \\
\hline 2372 & SESP SCHEDULING & & SW & & & B14RX6 & 11-Jun-02 & & 15117-96-1 & U-235 \\
\hline 2373 & SESP SCHEDULING & & SW & & & B14RX7 & 11-Jun-02 & & 15117-96-1 & U-235 \\
\hline 2374 & SESP SCHEDULING & & SW & & & B14RL3 & 27-Jun-02 & 29-May-02 & 15117-96-1 & U-235 \\
\hline 2375 & SESP SCHEDULING & & SW & & & B14RM5 & 27-Jun-02 & 29-May-02 & $15117-96-1$ & U-235 \\
\hline 2376 & SESP SCHEDULING & & SW & & & B14VT4 & 28-Jun-02 & & 15117-96-1 & U-235 \\
\hline 2377 & SESP SCHEDULING & & SW & & & B14VX3 & 28-Jun-02 & & 15117-96-1 & U-235 \\
\hline 2378 & SESP SCHEDULING & & SW & & & B14YH3 & 08-Jul-02 & & 15117-96-1 & $\mathrm{U}-235$ \\
\hline 2379 & SESP SCHEDULING & & SW & & & B14YJ5 & 08-Jul-02 & & 15117-96-1 & $\mathrm{U}-235$ \\
\hline 2380 & SESP SCHEDULING & & SW & & & B14WW5 & 07-Aug-02 & 27-Jun-02 & 15117-96-1 & U-235 \\
\hline 2381 & SESP SCHEDULING & & SW & & & B14WX2 & 07-Aug-02 & 27-Jun-02 & 15117-96-1 & U-235 \\
\hline 2382 & SESP SCHEDULING & & SW & & & B153T9 & 04-Sep-02 & 07-Aug-02 & 15117-96-1 & U-235 \\
\hline 2383 & SESP SCHEDULING & & SW & & & B153V4 & 04-Sep-02 & 07-Aug-02 & 15117-96-1 & U-235 \\
\hline 2384 & SESP SCHEDULING & & SW & & & $\mathrm{B} 158 \mathrm{H} 3$ & 05-Sep-02 & & 15117-96-1 & U-235 \\
\hline 2385 & SESP SCHEDULING & & SW & & & $\mathrm{B} 158 \mathrm{H} 4$ & 05-Sep-02 & & 15117-96-1 & U-235 \\
\hline 2386 & SESP SCHEDULING & & SW & & & B158H5 & 05-Sep-02 & & 15117-96-1 & U-235 \\
\hline 2387 & SESP SCHEDULING & & SW & & & B158H6 & 05-Sep-02 & & $15117-96-1$ & $\mathrm{U}-235$ \\
\hline 2388 & SESP SCHEDULING & & SW & & & B158H7 & 05-Sep-02 & & 15117-96-1 & U-235 \\
\hline 2389 & SESP SCHEDULING & & SW & & & $\mathrm{B} 158 \mathrm{H} 8$ & 05-Sep-02 & & $15117-96-1$ & U-235 \\
\hline 2390 & SESP SCHEDULING & & SW & & & $\mathrm{B} 158 \mathrm{H} 9$ & 05-Sep-02 & & 15117-96-1 & $\mathrm{U}-235$ \\
\hline 2391 & SESP SCHEDULING & & SW & & & B158J0 & 05-Sep-02 & & 15117-96-1 & $\mathrm{U}-235$ \\
\hline 2392 & SESP SCHEDULING & & SW & & & B158M7 & 05-Sep-02 & & $15117-96-1$ & U-235 \\
\hline 2393 & SESP SCHEDULING & & SW & & & B158M8 & 05-Sep-02 & & $15117-96-1$ & $\mathrm{U}-235$ \\
\hline 2394 & SESP SCHEDULING & & SW & & & B158M9 & 05-Sep-02 & & 15117-96-1 & $\mathrm{U}-235$ \\
\hline 2395 & SESP SCHEDULING & & SW & & & B158W8 & 05-Sep-02 & & 15117-96-1 & U-235 \\
\hline
\end{tabular}




\begin{tabular}{|c|c|c|c|c|c|c|c|c|c|c|}
\hline & A & $\mathrm{B}$ & C & $\mathrm{D}$ & $\mathrm{E}$ & $\mathrm{F}$ & $\mathrm{G}$ & $\mathrm{H}$ & $\mathrm{I}$ & $\mathrm{J}$ \\
\hline 1 & SAMPLE_NUM_ASSIGNED_TO & SAMPLER & MEDIA & BIOTA_MEDIA & TAG_ID & SAMP_NUM & SAMP_DATE_TIME & SAMP_DATE_TIME_ON & CON_ID & CON_SHORT_NAME \\
\hline 2396 & SESP SCHEDULING & & SW & & & B158X1 & 05-Sep-02 & & $15117-96-1$ & U-235 \\
\hline 2397 & SESP SCHEDULING & & SW & & & B158X4 & 05-Sep-02 & & $15117-96-1$ & U-235 \\
\hline 2398 & SESP SCHEDULING & & SW & & & B158X7 & 05-Sep-02 & & $15117-96-1$ & U-235 \\
\hline 2399 & SESP SCHEDULING & & SW & & & B158J1 & 09-Sep-02 & & $15117-96-1$ & U-235 \\
\hline 2400 & SESP SCHEDULING & & SW & & & B158J3 & 09-Sep-02 & & $15117-96-1$ & U-235 \\
\hline 2401 & SESP SCHEDULING & & SW & & & B158J5 & 09-Sep-02 & & 15117-96-1 & U-235 \\
\hline 2402 & SESP SCHEDULING & & SW & & & B158J7 & 09-Sep-02 & & $15117-96-1$ & U-235 \\
\hline 2403 & SESP SCHEDULING & & SW & & & B158J9 & 09-Sep-02 & & 15117-96-1 & U-235 \\
\hline 2404 & SESP SCHEDULING & & SW & & & B158K1 & 09-Sep-02 & & $15117-96-1$ & U-235 \\
\hline 2405 & SESP SCHEDULING & & SW & & & B158K3 & 09-Sep-02 & & $15117-96-1$ & U-235 \\
\hline 2406 & SESP SCHEDULING & & SW & & & B158K5 & 09-Sep-02 & & $15117-96-1$ & U-235 \\
\hline 2407 & SESP SCHEDULING & & SW & & & B158K7 & 09-Sep-02 & & $15117-96-1$ & U-235 \\
\hline 2408 & SESP SCHEDULING & & SW & & & B158K9 & 09-Sep-02 & & 15117-96-1 & U-235 \\
\hline 2409 & SESP SCHEDULING & & SW & & & B158L1 & 09-Sep-02 & & $15117-96-1$ & U-235 \\
\hline 2410 & SESP SCHEDULING & & SW & & & B158L3 & 09-Sep-02 & & $15117-96-1$ & U-235 \\
\hline 2411 & SESP SCHEDULING & & SW & & & B158N0 & 09-Sep-02 & & $15117-96-1$ & U-235 \\
\hline 2412 & SESP SCHEDULING & & SW & & & B158N2 & 09-Sep-02 & & $15117-96-1$ & U-235 \\
\hline 2413 & SESP SCHEDULING & & SW & & & B158N4 & 09-Sep-02 & & $15117-96-1$ & U-235 \\
\hline 2414 & SESP SCHEDULING & & SW & & & B15902 & 09-Sep-02 & & $15117-96-1$ & U-235 \\
\hline 2415 & SESP SCHEDULING & & SW & & & B15906 & 09-Sep-02 & & $15117-96-1$ & U-235 \\
\hline 2416 & SESP SCHEDULING & & SW & & & B15910 & 09-Sep-02 & & $15117-96-1$ & U-235 \\
\hline 2417 & SESP SCHEDULING & & SW & & & B15914 & 09-Sep-02 & & $15117-96-1$ & U-235 \\
\hline 2418 & SESP SCHEDULING & & SW & & & B158L5 & 10-Sep-02 & & $15117-96-1$ & U-235 \\
\hline 2419 & SESP SCHEDULING & & SW & & & B158L6 & 10-Sep-02 & & $15117-96-1$ & U-235 \\
\hline 2420 & SESP SCHEDULING & & SW & & & B158L7 & 10-Sep-02 & & $15117-96-1$ & U-235 \\
\hline 2421 & SESP SCHEDULING & & SW & & & B158L8 & 10-Sep-02 & & $15117-96-1$ & U-235 \\
\hline 2422 & SESP SCHEDULING & & SW & & & B158L9 & 10-Sep-02 & & 15117-96-1 & U-235 \\
\hline 2423 & SESP SCHEDULING & & SW & & & B158M0 & 10-Sep-02 & & $15117-96-1$ & U-235 \\
\hline 2424 & SESP SCHEDULING & & SW & & & B158M1 & 10-Sep-02 & & $15117-96-1$ & U-235 \\
\hline 2425 & SESP SCHEDULING & & SW & & & B158M2 & 10-Sep-02 & & 15117-96-1 & U-235 \\
\hline 2426 & SESP SCHEDULING & & SW & & & B158M3 & 10-Sep-02 & & $15117-96-1$ & U-235 \\
\hline 2427 & SESP SCHEDULING & & SW & & & B158M4 & 10-Sep-02 & & $15117-96-1$ & U-235 \\
\hline 2428 & SESP SCHEDULING & & SW & & & B158M5 & 10-Sep-02 & & $15117-96-1$ & U-235 \\
\hline 2429 & SESP SCHEDULING & & SW & & & B158M6 & 10-Sep-02 & & $15117-96-1$ & U-235 \\
\hline 2430 & SESP SCHEDULING & & SW & & & B158V6 & 10-Sep-02 & & $15117-96-1$ & U-235 \\
\hline 2431 & SESP SCHEDULING & & SW & & & B158V7 & 10-Sep-02 & & $15117-96-1$ & U-235 \\
\hline 2432 & SESP SCHEDULING & & SW & & & B158V8 & 10-Sep-02 & & $15117-96-1$ & U-235 \\
\hline 2433 & SESP SCHEDULING & & SW & & & B158V9 & 10-Sep-02 & & 15117-96-1 & U-235 \\
\hline
\end{tabular}




\begin{tabular}{|c|c|c|c|c|c|c|c|c|c|c|}
\hline & $A$ & $\mathrm{~B}$ & $\mathrm{C}$ & $\mathrm{D}$ & $E$ & $\mathrm{~F}$ & G & $\mathrm{H}$ & $\mathrm{I}$ & $\mathrm{J}$ \\
\hline 1 & SAMPLE_NUM_ASSIGNED_TO & SAMPLER & MEDIA & BIOTA_MEDIA & TAG_ID & SAMP_NUM & SAMP_DATE_TIME & SAMP_DATE_TIME_ON & CON_ID & CON_SHORT_NAME \\
\hline 2434 & SESP SCHEDULING & & SW & & & B158Y0 & 10-Sep-02 & & $15117-96-1$ & $\mathrm{U}-235$ \\
\hline 2435 & SESP SCHEDULING & & SW & & & B158Y3 & 10-Sep-02 & & $15117-96-1$ & U-235 \\
\hline 2436 & SESP SCHEDULING & & SW & & & B158Y6 & 10-Sep-02 & & $15117-96-1$ & U-235 \\
\hline 2437 & SESP SCHEDULING & & SW & & & B158Y9 & 10-Sep-02 & & $15117-96-1$ & U-235 \\
\hline 2438 & SESP SCHEDULING & & SW & & & B15948 & 02-Oct-02 & 04-Sep-02 & 15117-96-1 & U-235 \\
\hline 2439 & SESP SCHEDULING & & SW & & & B15954 & 02-Oct-02 & 04-Sep-02 & 15117-96-1 & U-235 \\
\hline 2440 & SESP SCHEDULING & & SW & & & B15C09 & 07-Oct-02 & & 15117-96-1 & U-235 \\
\hline 2441 & SESP SCHEDULING & & SW & & & B15C11 & 07-Oct-02 & & 15117-96-1 & U-235 \\
\hline 2442 & SESP SCHEDULING & & SW & & & B15C73 & 07-Oct-02 & & $15117-96-1$ & U-235 \\
\hline 2443 & SESP SCHEDULING & & SW & & & B15CB3 & 27-Oct-02 & & $15117-96-1$ & U-235 \\
\hline 2444 & SESP SCHEDULING & & SW & & & B15C69 & 29-Oct-02 & & $15117-96-1$ & U-235 \\
\hline 2445 & SESP SCHEDULING & & SW & & & B15K74 & 30-Oct-02 & 02-Oct-02 & $15117-96-1$ & U-235 \\
\hline 2446 & SESP SCHEDULING & & SW & & & B15K79 & 30-Oct-02 & 02-Oct-02 & 15117-96-1 & U-235 \\
\hline 2447 & SESP SCHEDULING & & SW & & & B15VN2 & 26-Nov-02 & 30-Oct-02 & 15117-96-1 & U-235 \\
\hline 2448 & SESP SCHEDULING & & SW & & & B15VN8 & 26-Nov-02 & 30-Oct-02 & $15117-96-1$ & U-235 \\
\hline 2449 & SESP SCHEDULING & & SW & & & B16342 & 09-Dec-02 & & $15117-96-1$ & U-235 \\
\hline 2450 & SESP SCHEDULING & & SW & & & B16343 & 09-Dec-02 & & 15117-96-1 & U-235 \\
\hline 2451 & SESP SCHEDULING & & SW & & & B16344 & 09-Dec-02 & & $15117-96-1$ & U-235 \\
\hline 2452 & SESP SCHEDULING & & SW & & & B16345 & 09-Dec-02 & & $15117-96-1$ & U-235 \\
\hline 2453 & SESP SCHEDULING & & SW & & & B16341 & 10-Dec-02 & & $15117-96-1$ & U-235 \\
\hline 2454 & SESP SCHEDULING & & SW & & & B16346 & 10-Dec-02 & & $15117-96-1$ & U-235 \\
\hline 2455 & SESP SCHEDULING & & SW & & & B16347 & 10-Dec-02 & & $15117-96-1$ & U-235 \\
\hline 2456 & SESP SCHEDULING & & SW & & & B16348 & 10-Dec-02 & & $15117-96-1$ & U-235 \\
\hline 2457 & SESP SCHEDULING & & SW & & & B16349 & 10-Dec-02 & & $15117-96-1$ & U-235 \\
\hline 2458 & SESP SCHEDULING & & SW & & & B16350 & 10-Dec-02 & & $15117-96-1$ & U-235 \\
\hline 2459 & SESP SCHEDULING & & SW & & & B16351 & 10-Dec-02 & & 15117-96-1 & U-235 \\
\hline 2460 & SESP SCHEDULING & & SW & & & B16362 & 10-Dec-02 & & 15117-96-1 & U-235 \\
\hline 2461 & SESP SCHEDULING & & SW & & & B16363 & 10-Dec-02 & & $15117-96-1$ & U-235 \\
\hline 2462 & SESP SCHEDULING & & SW & & & B16364 & 10-Dec-02 & & 15117-96-1 & U-235 \\
\hline 2463 & SESP SCHEDULING & & SW & & & B16365 & 10-Dec-02 & & $15117-96-1$ & U-235 \\
\hline 2464 & SESP SCHEDULING & & SW & & & B0X8R8 & 04-Jan-00 & & $\mathrm{U}-238$ & U-238 \\
\hline 2465 & SESP SCHEDULING & & SW & & & B0X7K4 & 02-Feb-00 & 29-Dec-99 & $U-238$ & U-238 \\
\hline 2466 & SESP SCHEDULING & & SW & & & B0X7J0 & 02-Feb-00 & 29-Dec-99 & U-238 & U-238 \\
\hline 2467 & SESP SCHEDULING & & SW & & & B0XH28 & 08-Mar-00 & 02-Feb-00 & U-238 & U-238 \\
\hline 2468 & SESP SCHEDULING & & SW & & & B0XH22 & 08-Mar-00 & 02-Feb-00 & U-238 & U-238 \\
\hline 2469 & SESP SCHEDULING & & SW & & & BOXRK2 & 27-Mar-00 & & U-238 & U-238 \\
\hline 2470 & SESP SCHEDULING & & SW & & & B0XRK3 & 27-Mar-00 & & U-238 & U-238 \\
\hline 2471 & SESP SCHEDULING & & SW & & & BOXRK4 & 27-Mar-00 & & $\mathrm{U}-238$ & U-238 \\
\hline
\end{tabular}




\begin{tabular}{|c|c|c|c|c|c|c|c|c|c|c|}
\hline & $A$ & $\mathrm{~B}$ & $\mathrm{C}$ & $\mathrm{D}$ & $E$ & $\mathrm{~F}$ & $G$ & $\mathrm{H}$ & $\mathrm{I}$ & $\mathrm{J}$ \\
\hline 1 & \begin{tabular}{|l|l} 
SAMPE_NUM_ASSIGNED_TO \\
\end{tabular} & SAMPLER & MEDIA & BIOTA_MEDIA & TAG_ID & SAMP_NUM & SAMP_DATE_TIME & SAMP_DATE_TIME_ON & CON_ID & CON_SHORT_NAME \\
\hline 2472 & SESP SCHEDULING & & SW & & & B0XRK5 & 27-Mar-00 & & U-238 & $\mathrm{U}-238$ \\
\hline 2473 & SESP SCHEDULING & & SW & & & BOXRKO & 28-Mar-00 & & U-238 & U-238 \\
\hline 2474 & SESP SCHEDULING & & SW & & & B0XRK6 & 28-Mar-00 & & U-238 & U-238 \\
\hline 2475 & SESP SCHEDULING & & SW & & & B0XRK7 & 28-Mar-00 & & U-238 & U-238 \\
\hline 2476 & SESP SCHEDULING & & SW & & & BOXRK8 & 28-Mar-00 & & U-238 & U-238 \\
\hline 2477 & SESP SCHEDULING & & SW & & & BOXRK9 & 28-Mar-00 & & U-238 & U-238 \\
\hline \begin{tabular}{|l|}
2478 \\
\end{tabular} & SESP SCHEDULING & & SW & & & BOXRLO & 28-Mar-00 & & U-238 & $\mathrm{U}-238$ \\
\hline 2479 & SESP SCHEDULING & & SW & & & B0XRL1 & 28-Mar-00 & & U-238 & $\mathrm{U}-238$ \\
\hline 2480 & SESP SCHEDULING & & SW & & & BOXRN8 & 28-Mar-00 & & U-238 & $\mathrm{U}-238$ \\
\hline 2481 & SESP SCHEDULING & & SW & & & BOXRN9 & 28-Mar-00 & & U-238 & $\mathrm{U}-238$ \\
\hline 2482 & SESP SCHEDULING & & SW & & & BOXRPO & 28-Mar-00 & & U-238 & U-238 \\
\hline 2483 & SESP SCHEDULING & & SW & & & B0XRP1 & 28-Mar-00 & & U-238 & U-238 \\
\hline 2484 & SESP SCHEDULING & & SW & & & BOXWWO & 04-Apr-00 & & $\mathrm{U}-238$ & U-238 \\
\hline 2485 & SESP SCHEDULING & & SW & & & B0XP21 & 05-Apr-00 & 08-Mar-00 & U-238 & $\mathrm{U}-238$ \\
\hline 2486 & SESP SCHEDULING & & SW & & & B0XP16 & 05-Apr-00 & 08-Mar-00 & U-238 & U-238 \\
\hline \begin{tabular}{|l|}
2487 \\
\end{tabular} & SESP SCHEDULING & & SW & & & B0XVN6 & 03-May-00 & 29-Mar-00 & U-238 & $\mathrm{U}-238$ \\
\hline \begin{tabular}{|l|}
2488 \\
\end{tabular} & SESP SCHEDULING & & SW & & & B0XVP1 & 03-May-00 & 29-Mar-00 & U-238 & $\mathrm{U}-238$ \\
\hline 2489 & SESP SCHEDULING & & SW & & & BOY2Y6 & 11-May-00 & & U-238 & $\mathrm{U}-238$ \\
\hline 2490 & SESP SCHEDULING & & SW & & & B0Y231 & 31-May-00 & 03-May-00 & $U-238$ & U-238 \\
\hline 2491 & SESP SCHEDULING & & SW & & & B0Y237 & 31-May-00 & 03-May-00 & U-238 & $\mathrm{U}-238$ \\
\hline 2492 & SESP SCHEDULING & & SW & & & BOYB90 & 08-Jun-00 & & U-238 & U-238 \\
\hline 2493 & SESP SCHEDULING & & SW & & & B0YRB8 & 12-Jun-00 & & U-238 & $\mathrm{U}-238$ \\
\hline 2494 & SESP SCHEDULING & & SW & & & BOYRCO & 12-Jun-00 & & $\mathrm{U}-238$ & $\mathrm{U}-238$ \\
\hline 2495 & SESP SCHEDULING & & SW & & & BOYRC3 & 12-Jun-00 & & U-238 & U-238 \\
\hline 2496 & SESP SCHEDULING & & SW & & & BOYCD7 & 19-Jun-00 & & U-238 & U-238 \\
\hline 2497 & SESP SCHEDULING & & SW & & & B0YCD8 & 19-Jun-00 & & $\mathrm{U}-238$ & $\mathrm{U}-238$ \\
\hline 2498 & SESP SCHEDULING & & SW & & & B0YCD9 & 19-Jun-00 & & U-238 & U-238 \\
\hline 2499 & SESP SCHEDULING & & SW & & & BOYCFO & 19-Jun-00 & & U-238 & U-238 \\
\hline 2500 & SESP SCHEDULING & & SW & & & BOYCF1 & 20-Jun-00 & & U-238 & U-238 \\
\hline 2501 & SESP SCHEDULING & & SW & & & BOYCF2 & 20-Jun-00 & & U-238 & $U-238$ \\
\hline 2502 & SESP SCHEDULING & & SW & & & BOYCF3 & 20-Jun-00 & & U-238 & U-238 \\
\hline \begin{tabular}{|l|}
2503 \\
\end{tabular} & SESP SCHEDULING & & SW & & & BOYCF4 & 20-Jun-00 & & $\mathrm{U}-238$ & $\mathrm{U}-238$ \\
\hline 2504 & SESP SCHEDULING & & SW & & & B0YCF5 & 20-Jun-00 & & U-238 & $U-238$ \\
\hline 2505 & SESP SCHEDULING & & SW & & & BOYCF6 & 20-Jun-00 & & U-238 & U-238 \\
\hline 2506 & SESP SCHEDULING & & SW & & & BOYCK3 & 20-Jun-00 & & U-238 & $\mathrm{U}-238$ \\
\hline 2507 & SESP SCHEDULING & & SW & & & BOYCK4 & 20-Jun-00 & & $\mathrm{U}-238$ & $U-238$ \\
\hline 2508 & SESP SCHEDULING & & SW & & & BOYCK5 & 20-Jun-00 & & U-238 & U-238 \\
\hline 2509 & SESP SCHEDULING & & SW & & & BOYCK6 & 20-Jun-00 & & U-238 & $\mathrm{U}-238$ \\
\hline
\end{tabular}




\begin{tabular}{|c|c|c|c|c|c|c|c|c|c|c|}
\hline & A & $\mathrm{B}$ & C & $\mathrm{D}$ & $E$ & $\mathrm{~F}$ & G & $\mathrm{H}$ & $\mathrm{I}$ & $\mathrm{J}$ \\
\hline 1 & SAMPLE_NUM_ASSIGNED_TO & SAMPLER & MEDIA & BIOTA_MEDIA & TAG_ID & SAMP_NUM & SAMP_DATE_TIME & SAMP_DATE_TIME_ON & CON_ID & CON_SHORT_NAME \\
\hline 2510 & SESP SCHEDULING & & SW & & & B0YB64 & 29-Jun-00 & 31-May-00 & U-238 & $\mathrm{U}-238$ \\
\hline 2511 & SESP SCHEDULING & & SW & & & B0YB76 & 29-Jun-00 & 31-May-00 & $\mathrm{U}-238$ & U-238 \\
\hline 2512 & SESP SCHEDULING & & SW & & & B0YM20 & 05-Jul-00 & & $\mathrm{U}-238$ & $\mathrm{U}-238$ \\
\hline 2513 & SESP SCHEDULING & & SW & & & B0YM22 & 07-Jul-00 & & $\mathrm{U}-238$ & $\mathrm{U}-238$ \\
\hline 2514 & SESP SCHEDULING & & SW & & & B0YKV4 & 10-Aug-00 & 29-Jun-00 & $U-238$ & $\mathrm{U}-238$ \\
\hline 2515 & SESP SCHEDULING & & SW & & & B0YKW1 & 10-Aug-00 & 29-Jun-00 & $\mathrm{U}-238$ & $\mathrm{U}-238$ \\
\hline 2516 & SESP SCHEDULING & & SW & & & B0YW65 & 07-Sep-00 & & $\mathrm{U}-238$ & $\mathrm{U}-238$ \\
\hline 2517 & SESP SCHEDULING & & SW & & & BOYW70 & 07-Sep-00 & & $U-238$ & $\mathrm{U}-238$ \\
\hline 2518 & SESP SCHEDULING & & SW & & & B10672 & 14-Sep-00 & & $\mathrm{U}-238$ & $\mathrm{U}-238$ \\
\hline 2519 & SESP SCHEDULING & & SW & & & B10673 & 14-Sep-00 & & $\mathrm{U}-238$ & $\mathrm{U}-238$ \\
\hline 2520 & SESP SCHEDULING & & SW & & & B10674 & 14-Sep-00 & & U-238 & U-238 \\
\hline 2521 & SESP SCHEDULING & & SW & & & B10675 & 14-Sep-00 & & $\mathrm{U}-238$ & $\mathrm{U}-238$ \\
\hline 2522 & SESP SCHEDULING & & SW & & & B10676 & 14-Sep-00 & & $\mathrm{U}-238$ & $\mathrm{U}-238$ \\
\hline 2523 & SESP SCHEDULING & & SW & & & B10677 & 14-Sep-00 & & $U-238$ & $\mathrm{U}-238$ \\
\hline 2524 & SESP SCHEDULING & & SW & & & B10679 & 14-Sep-00 & & $\mathrm{U}-238$ & $\mathrm{U}-238$ \\
\hline 2525 & SESP SCHEDULING & & SW & & & B10681 & 14-Sep-00 & & $\mathrm{U}-238$ & $\mathrm{U}-238$ \\
\hline 2526 & SESP SCHEDULING & & SW & & & B10683 & 14-Sep-00 & & $\mathrm{U}-238$ & $\mathrm{U}-238$ \\
\hline 2527 & SESP SCHEDULING & & SW & & & B10684 & 14-Sep-00 & & $\mathrm{U}-238$ & $\mathrm{U}-238$ \\
\hline 2528 & SESP SCHEDULING & & SW & & & B10686 & 14-Sep-00 & & $U-238$ & $\mathrm{U}-238$ \\
\hline 2529 & SESP SCHEDULING & & SW & & & B10698 & 14-Sep-00 & & $\mathrm{U}-238$ & $\mathrm{U}-238$ \\
\hline 2530 & SESP SCHEDULING & & SW & & & B106B1 & 14-Sep-00 & & $\mathrm{U}-238$ & $\mathrm{U}-238$ \\
\hline 2531 & SESP SCHEDULING & & SW & & & B106B4 & 14-Sep-00 & & $\mathrm{U}-238$ & $\mathrm{U}-238$ \\
\hline 2532 & SESP SCHEDULING & & SW & & & B106B7 & 14-Sep-00 & & U-238 & $\mathrm{U}-238$ \\
\hline 2533 & SESP SCHEDULING & & SW & & & B106W1 & 18-Sep-00 & & $\mathrm{U}-238$ & $\mathrm{U}-238$ \\
\hline 2534 & SESP SCHEDULING & & SW & & & B106W2 & 18-Sep-00 & & $\mathrm{U}-238$ & $\mathrm{U}-238$ \\
\hline 2535 & SESP SCHEDULING & & SW & & & B106W3 & 18-Sep-00 & & $\mathrm{U}-238$ & $\mathrm{U}-238$ \\
\hline 2536 & SESP SCHEDULING & & SW & & & B106W4 & 18-Sep-00 & & $U-238$ & $\mathrm{U}-238$ \\
\hline 2537 & SESP SCHEDULING & & SW & & & B106W5 & 18-Sep-00 & & $\mathrm{U}-238$ & $\mathrm{U}-238$ \\
\hline 2538 & SESP SCHEDULING & & SW & & & B106W6 & 18-Sep-00 & & $\mathrm{U}-238$ & $\mathrm{U}-238$ \\
\hline 2539 & SESP SCHEDULING & & SW & & & B106W7 & 18-Sep-00 & & $\mathrm{U}-238$ & $\mathrm{U}-238$ \\
\hline 2540 & SESP SCHEDULING & & SW & & & B106W8 & 18-Sep-00 & & $\mathrm{U}-238$ & $\mathrm{U}-238$ \\
\hline 2541 & SESP SCHEDULING & & SW & & & B106W9 & 18-Sep-00 & & $\mathrm{U}-238$ & $\mathrm{U}-238$ \\
\hline 2542 & SESP SCHEDULING & & SW & & & B106X0 & 18-Sep-00 & & $\mathrm{U}-238$ & $\mathrm{U}-238$ \\
\hline 2543 & SESP SCHEDULING & & SW & & & B106X1 & 18-Sep-00 & & $\mathrm{U}-238$ & $\mathrm{U}-238$ \\
\hline 2544 & SESP SCHEDULING & & SW & & & B106X2 & 18-Sep-00 & & U-238 & U-238 \\
\hline 2545 & SESP SCHEDULING & & SW & & & B10728 & 18-Sep-00 & & $\mathrm{U}-238$ & $\mathrm{U}-238$ \\
\hline 2546 & SESP SCHEDULING & & SW & & & B10729 & 18-Sep-00 & & $\mathrm{U}-238$ & $\mathrm{U}-238$ \\
\hline 2547 & SESP SCHEDULING & & SW & & & B10730 & 18-Sep-00 & & U-238 & U-238 \\
\hline
\end{tabular}




\begin{tabular}{|c|c|c|c|c|c|c|c|c|c|c|}
\hline & A & $\mathrm{B}$ & C & $\mathrm{D}$ & $E$ & $\mathrm{~F}$ & G & $\mathrm{H}$ & $\mathrm{I}$ & $\mathrm{J}$ \\
\hline 1 & SAMPLE_NUM_ASSIGNED_TO & SAMPLER & MEDIA & BIOTA_MEDIA & TAG_ID & SAMP_NUM & SAMP_DATE_TIME & SAMP_DATE_TIME_ON & CON_ID & CON_SHORT_NAME \\
\hline 2548 & SESP SCHEDULING & & SW & & & B10785 & 18-Sep-00 & & U-238 & $\mathrm{U}-238$ \\
\hline 2549 & SESP SCHEDULING & & SW & & & B10788 & 18-Sep-00 & & U-238 & U-238 \\
\hline 2550 & SESP SCHEDULING & & SW & & & B10791 & 18-Sep-00 & & $\mathrm{U}-238$ & $\mathrm{U}-238$ \\
\hline 2551 & SESP SCHEDULING & & SW & & & B10794 & 18-Sep-00 & & $\mathrm{U}-238$ & $\mathrm{U}-238$ \\
\hline 2552 & SESP SCHEDULING & & SW & & & B106X3 & 19-Sep-00 & & $\mathrm{U}-238$ & $\mathrm{U}-238$ \\
\hline 2553 & SESP SCHEDULING & & SW & & & B106X5 & 19-Sep-00 & & $\mathrm{U}-238$ & $\mathrm{U}-238$ \\
\hline 2554 & SESP SCHEDULING & & SW & & & B106X7 & 19-Sep-00 & & $\mathrm{U}-238$ & $\mathrm{U}-238$ \\
\hline 2555 & SESP SCHEDULING & & SW & & & B106X9 & 19-Sep-00 & & $U-238$ & $\mathrm{U}-238$ \\
\hline 2556 & SESP SCHEDULING & & SW & & & B106Y1 & 19-Sep-00 & & $\mathrm{U}-238$ & $\mathrm{U}-238$ \\
\hline 2557 & SESP SCHEDULING & & SW & & & B106Y3 & 19-Sep-00 & & $\mathrm{U}-238$ & $\mathrm{U}-238$ \\
\hline 2558 & SESP SCHEDULING & & SW & & & B106Y4 & 19-Sep-00 & & U-238 & U-238 \\
\hline 2559 & SESP SCHEDULING & & SW & & & B106Y5 & 19-Sep-00 & & $\mathrm{U}-238$ & $\mathrm{U}-238$ \\
\hline 2560 & SESP SCHEDULING & & SW & & & B106Y6 & 19-Sep-00 & & $\mathrm{U}-238$ & $\mathrm{U}-238$ \\
\hline 2561 & SESP SCHEDULING & & SW & & & B106Y7 & 19-Sep-00 & & $U-238$ & $\mathrm{U}-238$ \\
\hline 2562 & SESP SCHEDULING & & SW & & & B106Y8 & 19-Sep-00 & & $\mathrm{U}-238$ & $\mathrm{U}-238$ \\
\hline 2563 & SESP SCHEDULING & & SW & & & B106Y9 & 19-Sep-00 & & $\mathrm{U}-238$ & $\mathrm{U}-238$ \\
\hline 2564 & SESP SCHEDULING & & SW & & & B10761 & 19-Sep-00 & & $\mathrm{U}-238$ & $\mathrm{U}-238$ \\
\hline 2565 & SESP SCHEDULING & & SW & & & B10762 & 19-Sep-00 & & $\mathrm{U}-238$ & $\mathrm{U}-238$ \\
\hline 2566 & SESP SCHEDULING & & SW & & & B10763 & 19-Sep-00 & & $U-238$ & $\mathrm{U}-238$ \\
\hline 2567 & SESP SCHEDULING & & SW & & & B10764 & 19-Sep-00 & & $\mathrm{U}-238$ & $\mathrm{U}-238$ \\
\hline 2568 & SESP SCHEDULING & & SW & & & B10773 & 19-Sep-00 & & $\mathrm{U}-238$ & $\mathrm{U}-238$ \\
\hline 2569 & SESP SCHEDULING & & SW & & & B10776 & 19-Sep-00 & & $\mathrm{U}-238$ & $\mathrm{U}-238$ \\
\hline 2570 & SESP SCHEDULING & & SW & & & B10779 & 19-Sep-00 & & U-238 & U-238 \\
\hline 2571 & SESP SCHEDULING & & SW & & & B10782 & 19-Sep-00 & & $\mathrm{U}-238$ & $\mathrm{U}-238$ \\
\hline 2572 & SESP SCHEDULING & & SW & & & B10912 & 27-Sep-00 & & $\mathrm{U}-238$ & $\mathrm{U}-238$ \\
\hline 2573 & SESP SCHEDULING & & SW & & & B10913 & 27-Sep-00 & & $\mathrm{U}-238$ & $\mathrm{U}-238$ \\
\hline 2574 & SESP SCHEDULING & & SW & & & B10945 & 27-Sep-00 & & $\mathrm{U}-238$ & $\mathrm{U}-238$ \\
\hline 2575 & SESP SCHEDULING & & SW & & & B10946 & 27-Sep-00 & & $\mathrm{U}-238$ & $\mathrm{U}-238$ \\
\hline 2576 & SESP SCHEDULING & & SW & & & B10949 & 27-Sep-00 & & $\mathrm{U}-238$ & $\mathrm{U}-238$ \\
\hline 2577 & SESP SCHEDULING & & SW & & & B109J0 & 27-Sep-00 & & $\mathrm{U}-238$ & $\mathrm{U}-238$ \\
\hline 2578 & SESP SCHEDULING & & SW & & & B103W8 & $05-O c t-00$ & 07-Sep-00 & $\mathrm{U}-238$ & $\mathrm{U}-238$ \\
\hline 2579 & SESP SCHEDULING & & SW & & & B103X3 & $05-O c t-00$ & 07-Sep-00 & $\mathrm{U}-238$ & $\mathrm{U}-238$ \\
\hline 2580 & SESP SCHEDULING & & SW & & & B10F33 & 09-Oct-00 & & $\mathrm{U}-238$ & $\mathrm{U}-238$ \\
\hline 2581 & SESP SCHEDULING & & SW & & & B10941 & 01-Nov-00 & & $\mathrm{U}-238$ & $\mathrm{U}-238$ \\
\hline 2582 & SESP SCHEDULING & & SW & & & B10977 & 01-Nov-00 & & U-238 & U-238 \\
\hline 2583 & SESP SCHEDULING & & SW & & & B10C13 & 02-Nov-00 & $05-$ Oct-00 & $\mathrm{U}-238$ & $\mathrm{U}-238$ \\
\hline 2584 & SESP SCHEDULING & & SW & & & B10C19 & 02-Nov-00 & $05-O c t-00$ & $\mathrm{U}-238$ & $\mathrm{U}-238$ \\
\hline 2585 & SESP SCHEDULING & & SW & & & B10942 & 07-Nov-00 & & U-238 & U-238 \\
\hline
\end{tabular}




\begin{tabular}{|c|c|c|c|c|c|c|c|c|c|c|}
\hline & A & $\mathrm{B}$ & $\mathrm{C}$ & $\mathrm{D}$ & $\mathrm{E}$ & $\mathrm{F}$ & G & $\mathrm{H}$ & $\mathrm{I}$ & $\mathrm{J}$ \\
\hline 1 & SAMPLE_NUM_ASSIGNED_TO & SAMPLER & MEDIA & BIOTA_MEDIA & TAG_ID & SAMP_NUM & SAMP_DATE_TIME & SAMP_DATE_TIME_ON & CON_ID & CON_SHORT_NAME \\
\hline 2586 & SESP SCHEDULING & & SW & & & B10MP5 & 29-Nov-00 & 02-Nov-00 & $\mathrm{U}-238$ & $\mathrm{U}-238$ \\
\hline 2587 & SESP SCHEDULING & & SW & & & B10MR1 & 29-Nov-00 & 02-Nov-00 & $\mathrm{U}-238$ & $\mathrm{U}-238$ \\
\hline 2588 & SESP SCHEDULING & & SW & & & B11125 & 04-Dec-00 & & $\mathrm{U}-238$ & $\mathrm{U}-238$ \\
\hline 2589 & SESP SCHEDULING & & SW & & & B11126 & 04-Dec-00 & & $\mathrm{U}-238$ & $\mathrm{U}-238$ \\
\hline 2590 & SESP SCHEDULING & & SW & & & B11127 & 04-Dec-00 & & $\mathrm{U}-238$ & $\mathrm{U}-238$ \\
\hline 2591 & SESP SCHEDULING & & SW & & & B11128 & 04-Dec-00 & & $\mathrm{U}-238$ & U-238 \\
\hline 2592 & SESP SCHEDULING & & SW & & & B11124 & 05-Dec-00 & & $\mathrm{U}-238$ & $\mathrm{U}-238$ \\
\hline 2593 & SESP SCHEDULING & & SW & & & B11129 & 05-Dec-00 & & $U-238$ & $\mathrm{U}-238$ \\
\hline 2594 & SESP SCHEDULING & & SW & & & B11130 & 05-Dec-00 & & $\mathrm{U}-238$ & $\mathrm{U}-238$ \\
\hline 2595 & SESP SCHEDULING & & SW & & & B11131 & 05-Dec-00 & & $\mathrm{U}-238$ & $\mathrm{U}-238$ \\
\hline 2596 & SESP SCHEDULING & & SW & & & B11132 & 05-Dec-00 & & $\mathrm{U}-238$ & $\mathrm{U}-238$ \\
\hline 2597 & SESP SCHEDULING & & SW & & & B11133 & 05-Dec-00 & & $\mathrm{U}-238$ & $\mathrm{U}-238$ \\
\hline 2598 & SESP SCHEDULING & & SW & & & B11134 & 05-Dec-00 & & $\mathrm{U}-238$ & $\mathrm{U}-238$ \\
\hline 2599 & SESP SCHEDULING & & SW & & & B11145 & 05-Dec-00 & & $\mathrm{U}-238$ & $\mathrm{U}-238$ \\
\hline 2600 & SESP SCHEDULING & & SW & & & B11146 & 05-Dec-00 & & $\mathrm{U}-238$ & $\mathrm{U}-238$ \\
\hline 2601 & SESP SCHEDULING & & SW & & & B11147 & 05-Dec-00 & & $\mathrm{U}-238$ & $\mathrm{U}-238$ \\
\hline 2602 & SESP SCHEDULING & & SW & & & B11148 & 05-Dec-00 & & $\mathrm{U}-238$ & $\mathrm{U}-238$ \\
\hline 2603 & SESP SCHEDULING & & SW & & & B110Y4 & 04-Jan-01 & 29-Nov-00 & $\mathrm{U}-238$ & $\mathrm{U}-238$ \\
\hline 2604 & SESP SCHEDULING & & SW & & & B11100 & 04-Jan-01 & 29-Nov-00 & $\mathrm{U}-238$ & $\mathrm{U}-238$ \\
\hline 2605 & SESP SCHEDULING & & SW & & & B114P8 & 31-Jan-01 & 04-Jan-01 & $\mathrm{U}-238$ & $\mathrm{U}-238$ \\
\hline 2606 & SESP SCHEDULING & & SW & & & B114T0 & 31-Jan-01 & 04-Jan-01 & $\mathrm{U}-238$ & $\mathrm{U}-238$ \\
\hline 2607 & SESP SCHEDULING & & SW & & & B11CC7 & 12-Feb-01 & & $\mathrm{U}-238$ & $\mathrm{U}-238$ \\
\hline 2608 & SESP SCHEDULING & & SW & & & $\mathrm{B} 11 \mathrm{H} 38$ & 26-Feb-01 & & $\mathrm{U}-238$ & $\mathrm{U}-238$ \\
\hline 2609 & SESP SCHEDULING & & SW & & & $\mathrm{B} 11 \mathrm{H} 43$ & 26-Feb-01 & & $\mathrm{U}-238$ & $\mathrm{U}-238$ \\
\hline 2610 & SESP SCHEDULING & & SW & & & $\mathrm{B} 11 \mathrm{H} 44$ & 26-Feb-01 & & $\mathrm{U}-238$ & $\mathrm{U}-238$ \\
\hline 2611 & SESP SCHEDULING & & SW & & & $\mathrm{B} 11 \mathrm{H} 45$ & 26-Feb-01 & & $\mathrm{U}-238$ & $\mathrm{U}-238$ \\
\hline 2612 & SESP SCHEDULING & & SW & & & $\mathrm{B} 11 \mathrm{H} 46$ & 26-Feb-01 & & $\mathrm{U}-238$ & $\mathrm{U}-238$ \\
\hline 2613 & SESP SCHEDULING & & SW & & & $\mathrm{B} 11 \mathrm{H} 47$ & 26-Feb-01 & & $\mathrm{U}-238$ & $\mathrm{U}-238$ \\
\hline 2614 & SESP SCHEDULING & & SW & & & $\mathrm{B} 11 \mathrm{H} 48$ & 26-Feb-01 & & $\mathrm{U}-238$ & $\mathrm{U}-238$ \\
\hline 2615 & SESP SCHEDULING & & SW & & & B11H59 & 26-Feb-01 & & $\mathrm{U}-238$ & $\mathrm{U}-238$ \\
\hline 2616 & SESP SCHEDULING & & SW & & & $\mathrm{B} 11 \mathrm{H} 60$ & 26-Feb-01 & & $\mathrm{U}-238$ & $\mathrm{U}-238$ \\
\hline 2617 & SESP SCHEDULING & & SW & & & B11H61 & 26-Feb-01 & & $\mathrm{U}-238$ & $\mathrm{U}-238$ \\
\hline 2618 & SESP SCHEDULING & & SW & & & B11H62 & 26-Feb-01 & & $\mathrm{U}-238$ & $\mathrm{U}-238$ \\
\hline 2619 & SESP SCHEDULING & & SW & & & B11H39 & 27-Feb-01 & & $\mathrm{U}-238$ & $\mathrm{U}-238$ \\
\hline 2620 & SESP SCHEDULING & & SW & & & $\mathrm{B} 11 \mathrm{H} 40$ & 27-Feb-01 & & $\mathrm{U}-238$ & $\mathrm{U}-238$ \\
\hline 2621 & SESP SCHEDULING & & SW & & & $\mathrm{B} 11 \mathrm{H} 41$ & 27-Feb-01 & & $\mathrm{U}-238$ & $\mathrm{U}-238$ \\
\hline 2622 & SESP SCHEDULING & & SW & & & $\mathrm{B} 11 \mathrm{H} 42$ & 27-Feb-01 & & $\mathrm{U}-238$ & $\mathrm{U}-238$ \\
\hline 2623 & SESP SCHEDULING & & SW & & & B11C04 & 01-Mar-01 & 31-Jan-01 & $\mathrm{U}-238$ & $\mathrm{U}-238$ \\
\hline
\end{tabular}




\begin{tabular}{|c|c|c|c|c|c|c|c|c|c|c|}
\hline & A & $\mathrm{B}$ & C & $\mathrm{D}$ & $E$ & $\mathrm{~F}$ & G & $\mathrm{H}$ & $\mathrm{I}$ & $\mathrm{J}$ \\
\hline 1 & SAMPLE_NUM_ASSIGNED_TO & SAMPLER & MEDIA & BIOTA_MEDIA & TAG_ID & SAMP_NUM & SAMP_DATE_TIME & SAMP_DATE_TIME_ON & CON_ID & CON_SHORT_NAME \\
\hline 2624 & SESP SCHEDULING & & SW & & & B11C10 & 01-Mar-01 & 31-Jan-01 & $U-238$ & $\mathrm{U}-238$ \\
\hline 2625 & SESP SCHEDULING & & SW & & & B11MY9 & 03-Apr-01 & & U-238 & U-238 \\
\hline 2626 & SESP SCHEDULING & & SW & & & B11J40 & 04-Apr-01 & 01-Mar-01 & $U-238$ & $\mathrm{U}-238$ \\
\hline 2627 & SESP SCHEDULING & & SW & & & B11J46 & 04-Apr-01 & 01-Mar-01 & $U-238$ & $\mathrm{U}-238$ \\
\hline 2628 & SESP SCHEDULING & & SW & & & B11W36 & 30-Apr-01 & & $\mathrm{U}-238$ & $\mathrm{U}-238$ \\
\hline 2629 & SESP SCHEDULING & & SW & & & B11W50 & 30-Apr-01 & & $\mathrm{U}-238$ & $\mathrm{U}-238$ \\
\hline 2630 & SESP SCHEDULING & & SW & & & B11W52 & 30-Apr-01 & & $\mathrm{U}-238$ & $\mathrm{U}-238$ \\
\hline 2631 & SESP SCHEDULING & & SW & & & B11W55 & 30-Apr-01 & & $U-238$ & $\mathrm{U}-238$ \\
\hline 2632 & SESP SCHEDULING & & SW & & & B11W86 & 30-Apr-01 & & $\mathrm{U}-238$ & $\mathrm{U}-238$ \\
\hline 2633 & SESP SCHEDULING & & SW & & & B11LT2 & 02-May-01 & 04-Apr-01 & $U-238$ & $\mathrm{U}-238$ \\
\hline 2634 & SESP SCHEDULING & & SW & & & B11LR6 & 02-May-01 & 04-Apr-01 & U-238 & U-238 \\
\hline 2635 & SESP SCHEDULING & & SW & & & B11W57 & 03-May-01 & & $\mathrm{U}-238$ & $\mathrm{U}-238$ \\
\hline 2636 & SESP SCHEDULING & & SW & & & B11WV8 & 09-May-01 & & $\mathrm{U}-238$ & $\mathrm{U}-238$ \\
\hline 2637 & SESP SCHEDULING & & SW & & & B11WX0 & 09-May-01 & & $U-238$ & $\mathrm{U}-238$ \\
\hline 2638 & SESP SCHEDULING & & SW & & & B11W38 & 10-May-01 & & $\mathrm{U}-238$ & $\mathrm{U}-238$ \\
\hline 2639 & SESP SCHEDULING & & SW & & & B121V4 & 17-May-01 & & $\mathrm{U}-238$ & $\mathrm{U}-238$ \\
\hline 2640 & SESP SCHEDULING & & SW & & & B11WT4 & 30-May-01 & 02-May-01 & $U-238$ & $\mathrm{U}-238$ \\
\hline 2641 & SESP SCHEDULING & & SW & & & B11WR8 & 30-May-01 & 02-May-01 & $\mathrm{U}-238$ & $\mathrm{U}-238$ \\
\hline 2642 & SESP SCHEDULING & & SW & & & B124N7 & 07-Jun-01 & & $U-238$ & $\mathrm{U}-238$ \\
\hline 2643 & SESP SCHEDULING & & SW & & & B124P7 & 07-Jun-01 & & $\mathrm{U}-238$ & $\mathrm{U}-238$ \\
\hline 2644 & SESP SCHEDULING & & SW & & & B12505 & 12-Jun-01 & & $\mathrm{U}-238$ & $\mathrm{U}-238$ \\
\hline 2645 & SESP SCHEDULING & & SW & & & B12506 & 12-Jun-01 & & $\mathrm{U}-238$ & $\mathrm{U}-238$ \\
\hline 2646 & SESP SCHEDULING & & SW & & & B12507 & 12-Jun-01 & & U-238 & $\mathrm{U}-238$ \\
\hline 2647 & SESP SCHEDULING & & SW & & & B12508 & 12-Jun-01 & & $\mathrm{U}-238$ & $\mathrm{U}-238$ \\
\hline 2648 & SESP SCHEDULING & & SW & & & B12509 & 12-Jun-01 & & $\mathrm{U}-238$ & $\mathrm{U}-238$ \\
\hline 2649 & SESP SCHEDULING & & SW & & & B12510 & 12-Jun-01 & & $\mathrm{U}-238$ & $\mathrm{U}-238$ \\
\hline 2650 & SESP SCHEDULING & & SW & & & B12531 & 12-Jun-01 & & $\mathrm{U}-238$ & $\mathrm{U}-238$ \\
\hline 2651 & SESP SCHEDULING & & SW & & & B12532 & 12-Jun-01 & & $\mathrm{U}-238$ & $\mathrm{U}-238$ \\
\hline 2652 & SESP SCHEDULING & & SW & & & B12533 & 12-Jun-01 & & $\mathrm{U}-238$ & $\mathrm{U}-238$ \\
\hline 2653 & SESP SCHEDULING & & SW & & & B12534 & 12-Jun-01 & & $\mathrm{U}-238$ & $\mathrm{U}-238$ \\
\hline 2654 & SESP SCHEDULING & & SW & & & B12501 & 14-Jun-01 & & $\mathrm{U}-238$ & $\mathrm{U}-238$ \\
\hline 2655 & SESP SCHEDULING & & SW & & & B12502 & 14-Jun-01 & & $\mathrm{U}-238$ & $\mathrm{U}-238$ \\
\hline 2656 & SESP SCHEDULING & & SW & & & B12503 & 14-Jun-01 & & $\mathrm{U}-238$ & $\mathrm{U}-238$ \\
\hline 2657 & SESP SCHEDULING & & SW & & & B12504 & 14-Jun-01 & & $\mathrm{U}-238$ & $\mathrm{U}-238$ \\
\hline 2658 & SESP SCHEDULING & & SW & & & B124M3 & 28-Jun-01 & 23-May-01 & $U-238$ & U-238 \\
\hline 2659 & SESP SCHEDULING & & SW & & & B124L7 & 28-Jun-01 & 23-May-01 & $\mathrm{U}-238$ & $\mathrm{U}-238$ \\
\hline 2660 & SESP SCHEDULING & & SW & & & B12992 & 09-Jul-01 & & $\mathrm{U}-238$ & $\mathrm{U}-238$ \\
\hline 2661 & SESP SCHEDULING & & SW & & & B12994 & 09-Jul-01 & & $\mathrm{U}-238$ & $\mathrm{U}-238$ \\
\hline
\end{tabular}




\begin{tabular}{|c|c|c|c|c|c|c|c|c|c|c|}
\hline & A & $\mathrm{B}$ & C & $\mathrm{D}$ & $E$ & $\mathrm{~F}$ & G & $\mathrm{H}$ & $\mathrm{I}$ & $\mathrm{J}$ \\
\hline 1 & SAMPLE_NUM_ASSIGNED_TO & SAMPLER & MEDIA & BIOTA_MEDIA & TAG_ID & SAMP_NUM & SAMP_DATE_TIME & SAMP_DATE_TIME_ON & CON_ID & CON_SHORT_NAME \\
\hline 2662 & SESP SCHEDULING & & SW & & & B129B4 & 09-Jul-01 & & U-238 & $\mathrm{U}-238$ \\
\hline 2663 & SESP SCHEDULING & & SW & & & B12841 & 09-Aug-01 & 28-Jun-01 & $\mathrm{U}-238$ & U-238 \\
\hline 2664 & SESP SCHEDULING & & SW & & & B12834 & 09-Aug-01 & 28-Jun-01 & $U-238$ & $\mathrm{U}-238$ \\
\hline 2665 & SESP SCHEDULING & & SW & & & B12RM1 & 27-Aug-01 & & $\mathrm{U}-238$ & $\mathrm{U}-238$ \\
\hline 2666 & SESP SCHEDULING & & SW & & & B12RM6 & 27-Aug-01 & & $\mathrm{U}-238$ & $\mathrm{U}-238$ \\
\hline 2667 & SESP SCHEDULING & & SW & & & B12RN4 & 27-Aug-01 & & $\mathrm{U}-238$ & $\mathrm{U}-238$ \\
\hline 2668 & SESP SCHEDULING & & SW & & & B12RN5 & 27-Aug-01 & & $\mathrm{U}-238$ & $\mathrm{U}-238$ \\
\hline 2669 & SESP SCHEDULING & & SW & & & B12RN6 & 27-Aug-01 & & $U-238$ & $\mathrm{U}-238$ \\
\hline 2670 & SESP SCHEDULING & & SW & & & B12RN7 & 27-Aug-01 & & $\mathrm{U}-238$ & $\mathrm{U}-238$ \\
\hline 2671 & SESP SCHEDULING & & SW & & & B12RP9 & 27-Aug-01 & & U-238 & U-238 \\
\hline 2672 & SESP SCHEDULING & & SW & & & B12RR1 & 27-Aug-01 & & U-238 & U-238 \\
\hline 2673 & SESP SCHEDULING & & SW & & & B12RR3 & 27-Aug-01 & & $\mathrm{U}-238$ & $\mathrm{U}-238$ \\
\hline 2674 & SESP SCHEDULING & & SW & & & B12RR5 & 27-Aug-01 & & $\mathrm{U}-238$ & $\mathrm{U}-238$ \\
\hline 2675 & SESP SCHEDULING & & SW & & & B12RR7 & 27-Aug-01 & & $U-238$ & $\mathrm{U}-238$ \\
\hline 2676 & SESP SCHEDULING & & SW & & & B12RR9 & 27-Aug-01 & & $\mathrm{U}-238$ & $\mathrm{U}-238$ \\
\hline 2677 & SESP SCHEDULING & & SW & & & B12RT1 & 27-Aug-01 & & $\mathrm{U}-238$ & $\mathrm{U}-238$ \\
\hline 2678 & SESP SCHEDULING & & SW & & & B12RT3 & 27-Aug-01 & & $\mathrm{U}-238$ & $\mathrm{U}-238$ \\
\hline 2679 & SESP SCHEDULING & & SW & & & B12RT5 & 27-Aug-01 & & $\mathrm{U}-238$ & $\mathrm{U}-238$ \\
\hline 2680 & SESP SCHEDULING & & SW & & & B12RT7 & 27-Aug-01 & & $U-238$ & $\mathrm{U}-238$ \\
\hline 2681 & SESP SCHEDULING & & SW & & & B12RT9 & 27-Aug-01 & & $\mathrm{U}-238$ & $\mathrm{U}-238$ \\
\hline 2682 & SESP SCHEDULING & & SW & & & B12RV1 & 27-Aug-01 & & $\mathrm{U}-238$ & $\mathrm{U}-238$ \\
\hline 2683 & SESP SCHEDULING & & SW & & & B12RV3 & 27-Aug-01 & & $\mathrm{U}-238$ & $\mathrm{U}-238$ \\
\hline 2684 & SESP SCHEDULING & & SW & & & B12RV5 & 27-Aug-01 & & U-238 & $\mathrm{U}-238$ \\
\hline 2685 & SESP SCHEDULING & & SW & & & B12RV7 & 27-Aug-01 & & $\mathrm{U}-238$ & $\mathrm{U}-238$ \\
\hline 2686 & SESP SCHEDULING & & SW & & & B12RV9 & 27-Aug-01 & & $\mathrm{U}-238$ & $\mathrm{U}-238$ \\
\hline 2687 & SESP SCHEDULING & & SW & & & B12RW1 & 27-Aug-01 & & $\mathrm{U}-238$ & $\mathrm{U}-238$ \\
\hline 2688 & SESP SCHEDULING & & SW & & & B12RW5 & 27-Aug-01 & & $\mathrm{U}-238$ & $\mathrm{U}-238$ \\
\hline 2689 & SESP SCHEDULING & & SW & & & B12RW7 & 27-Aug-01 & & $\mathrm{U}-238$ & $\mathrm{U}-238$ \\
\hline 2690 & SESP SCHEDULING & & SW & & & B12RW9 & 27-Aug-01 & & $\mathrm{U}-238$ & $\mathrm{U}-238$ \\
\hline 2691 & SESP SCHEDULING & & SW & & & B12RX1 & 27-Aug-01 & & $\mathrm{U}-238$ & $\mathrm{U}-238$ \\
\hline 2692 & SESP SCHEDULING & & SW & & & B12RX3 & 27-Aug-01 & & $\mathrm{U}-238$ & $\mathrm{U}-238$ \\
\hline 2693 & SESP SCHEDULING & & SW & & & B12RX5 & 27-Aug-01 & & $\mathrm{U}-238$ & $\mathrm{U}-238$ \\
\hline 2694 & SESP SCHEDULING & & SW & & & B12RX9 & 27-Aug-01 & & $\mathrm{U}-238$ & $\mathrm{U}-238$ \\
\hline 2695 & SESP SCHEDULING & & SW & & & B12RY1 & 27-Aug-01 & & $\mathrm{U}-238$ & $\mathrm{U}-238$ \\
\hline 2696 & SESP SCHEDULING & & SW & & & B12RY3 & 27-Aug-01 & & U-238 & U-238 \\
\hline 2697 & SESP SCHEDULING & & SW & & & B12T11 & 27-Aug-01 & & $\mathrm{U}-238$ & $\mathrm{U}-238$ \\
\hline 2698 & SESP SCHEDULING & & SW & & & B12T13 & 27-Aug-01 & & $\mathrm{U}-238$ & $\mathrm{U}-238$ \\
\hline 2699 & SESP SCHEDULING & & SW & & & B12T15 & 27-Aug-01 & & $\mathrm{U}-238$ & $\mathrm{U}-238$ \\
\hline
\end{tabular}




\begin{tabular}{|c|c|c|c|c|c|c|c|c|c|c|}
\hline & $A$ & $\mathrm{~B}$ & $\mathrm{C}$ & $\mathrm{D}$ & $E$ & $\mathrm{~F}$ & G & $\mathrm{H}$ & $\mathrm{I}$ & $\mathrm{J}$ \\
\hline 1 & SAMPLE_NUM_ASSIGNED_TO & SAMPLER & MEDIA & BIOTA_MEDIA & TAG_ID & SAMP_NUM & SAMP_DATE_TIME & SAMP_DATE_TIME_ON & CON_ID & CON_SHORT_NAME \\
\hline 2700 & SESP SCHEDULING & & SW & & & B12T17 & 27-Aug-01 & & U-238 & $\mathrm{U}-238$ \\
\hline 2701 & SESP SCHEDULING & & SW & & & B12T19 & 27-Aug-01 & & $\mathrm{U}-238$ & U-238 \\
\hline 2702 & SESP SCHEDULING & & SW & & & B12T21 & 27-Aug-01 & & $\mathrm{U}-238$ & $\mathrm{U}-238$ \\
\hline 2703 & SESP SCHEDULING & & SW & & & B12T23 & 27-Aug-01 & & $\mathrm{U}-238$ & $\mathrm{U}-238$ \\
\hline 2704 & SESP SCHEDULING & & SW & & & B12T25 & 27-Aug-01 & & $\mathrm{U}-238$ & $\mathrm{U}-238$ \\
\hline 2705 & SESP SCHEDULING & & SW & & & B12JT2 & 05-Sep-01 & & $\mathrm{U}-238$ & $\mathrm{U}-238$ \\
\hline 2706 & SESP SCHEDULING & & SW & & & B12JR7 & 05-Sep-01 & 09-Aug-01 & $U-238$ & $\mathrm{U}-238$ \\
\hline 2707 & SESP SCHEDULING & & SW & & & B12T85 & 07-Sep-01 & & U-238 & $\mathrm{U}-238$ \\
\hline 2708 & SESP SCHEDULING & & SW & & & B12T87 & 07-Sep-01 & & U-238 & U-238 \\
\hline 2709 & SESP SCHEDULING & & SW & & & B12T88 & 07-Sep-01 & & U-238 & U-238 \\
\hline 2710 & SESP SCHEDULING & & SW & & & B12T89 & 07-Sep-01 & & U-238 & U-238 \\
\hline 2711 & SESP SCHEDULING & & SW & & & B12T90 & 07-Sep-01 & & $\mathrm{U}-238$ & $\mathrm{U}-238$ \\
\hline 2712 & SESP SCHEDULING & & SW & & & B12T91 & 07-Sep-01 & & $\mathrm{U}-238$ & $\mathrm{U}-238$ \\
\hline 2713 & SESP SCHEDULING & & SW & & & B12T92 & 07-Sep-01 & & $\mathrm{U}-238$ & $\mathrm{U}-238$ \\
\hline 2714 & SESP SCHEDULING & & SW & & & B12T93 & 07-Sep-01 & & U-238 & $\mathrm{U}-238$ \\
\hline 2715 & SESP SCHEDULING & & SW & & & B12TD4 & 07-Sep-01 & & $\mathrm{U}-238$ & $\mathrm{U}-238$ \\
\hline 2716 & SESP SCHEDULING & & SW & & & B12TD5 & 07-Sep-01 & & $\mathrm{U}-238$ & $\mathrm{U}-238$ \\
\hline 2717 & SESP SCHEDULING & & SW & & & B12TD6 & 07-Sep-01 & & U-238 & $\mathrm{U}-238$ \\
\hline 2718 & SESP SCHEDULING & & SW & & & B12TN2 & 07-Sep-01 & & $\mathrm{U}-238$ & $\mathrm{U}-238$ \\
\hline 2719 & SESP SCHEDULING & & SW & & & B12TN5 & 07-Sep-01 & & $\mathrm{U}-238$ & $\mathrm{U}-238$ \\
\hline 2720 & SESP SCHEDULING & & SW & & & B12TN8 & 07-Sep-01 & & U-238 & $\mathrm{U}-238$ \\
\hline 2721 & SESP SCHEDULING & & SW & & & B12TP1 & 07-Sep-01 & & U-238 & $\mathrm{U}-238$ \\
\hline 2722 & SESP SCHEDULING & & SW & & & B12T94 & 10-Sep-01 & & U-238 & U-238 \\
\hline 2723 & SESP SCHEDULING & & SW & & & B12T95 & 10-Sep-01 & & U-238 & $\mathrm{U}-238$ \\
\hline 2724 & SESP SCHEDULING & & SW & & & B12T96 & 10-Sep-01 & & $\mathrm{U}-238$ & $\mathrm{U}-238$ \\
\hline 2725 & SESP SCHEDULING & & SW & & & B12T97 & 10-Sep-01 & & $\mathrm{U}-238$ & $\mathrm{U}-238$ \\
\hline 2726 & SESP SCHEDULING & & SW & & & B12T98 & 10-Sep-01 & & U-238 & $\mathrm{U}-238$ \\
\hline 2727 & SESP SCHEDULING & & SW & & & B12T99 & 10-Sep-01 & & U-238 & $\mathrm{U}-238$ \\
\hline 2728 & SESP SCHEDULING & & SW & & & B12TB0 & 10-Sep-01 & & $\mathrm{U}-238$ & $\mathrm{U}-238$ \\
\hline 2729 & SESP SCHEDULING & & SW & & & B12TB1 & 10-Sep-01 & & U-238 & $\mathrm{U}-238$ \\
\hline 2730 & SESP SCHEDULING & & SW & & & B12TB2 & 10-Sep-01 & & $\mathrm{U}-238$ & $\mathrm{U}-238$ \\
\hline 2731 & SESP SCHEDULING & & SW & & & B12TB3 & 10-Sep-01 & & $\mathrm{U}-238$ & $\mathrm{U}-238$ \\
\hline 2732 & SESP SCHEDULING & & SW & & & B12TB4 & 10-Sep-01 & & U-238 & $\mathrm{U}-238$ \\
\hline 2733 & SESP SCHEDULING & & SW & & & B12TB5 & 10-Sep-01 & & $\mathrm{U}-238$ & $\mathrm{U}-238$ \\
\hline 2734 & SESP SCHEDULING & & SW & & & B12TF7 & 10-Sep-01 & & U-238 & U-238 \\
\hline 2735 & SESP SCHEDULING & & SW & & & B12TF8 & 10-Sep-01 & & U-238 & $\mathrm{U}-238$ \\
\hline 2736 & SESP SCHEDULING & & SW & & & B12TF9 & 10-Sep-01 & & U-238 & $\mathrm{U}-238$ \\
\hline 2737 & SESP SCHEDULING & & SW & & & B12TT0 & 10-Sep-01 & & $\mathrm{U}-238$ & $\mathrm{U}-238$ \\
\hline
\end{tabular}




\begin{tabular}{|c|c|c|c|c|c|c|c|c|c|c|}
\hline & $A$ & $\mathrm{~B}$ & $\mathrm{C}$ & $\mathrm{D}$ & $E$ & $\mathrm{~F}$ & $G$ & $\mathrm{H}$ & $\mathrm{I}$ & $\mathrm{J}$ \\
\hline 1 & \begin{tabular}{|l|l} 
SAMPE_NUM_ASSIGNED_TO \\
\end{tabular} & SAMPLER & MEDIA & BIOTA_MEDIA & TAG_ID & SAMP_NUM & SAMP_DATE_TIME & SAMP_DATE_TIME_ON & CON_ID & CON_SHORT_NAME \\
\hline 2738 & SESP SCHEDULING & & SW & & & B12TT3 & 10-Sep-01 & & U-238 & $\mathrm{U}-238$ \\
\hline 2739 & SESP SCHEDULING & & SW & & & B12TT9 & 10-Sep-01 & & U-238 & U-238 \\
\hline 2740 & SESP SCHEDULING & & SW & & & B12TB6 & 13-Sep-01 & & U-238 & U-238 \\
\hline 2741 & SESP SCHEDULING & & SW & & & B12TB8 & 13-Sep-01 & & U-238 & U-238 \\
\hline 2742 & SESP SCHEDULING & & SW & & & B12TC0 & 13-Sep-01 & & U-238 & U-238 \\
\hline 2743 & SESP SCHEDULING & & SW & & & B12TC2 & 13-Sep-01 & & U-238 & U-238 \\
\hline 2744 & SESP SCHEDULING & & SW & & & B12TC4 & 13-Sep-01 & & $\mathrm{U}-238$ & U-238 \\
\hline 2745 & SESP SCHEDULING & & SW & & & B12TC6 & 13-Sep-01 & & U-238 & $\mathrm{U}-238$ \\
\hline 2746 & SESP SCHEDULING & & SW & & & B12TC8 & 13-Sep-01 & & U-238 & $\mathrm{U}-238$ \\
\hline 2747 & SESP SCHEDULING & & SW & & & B12TC9 & 13-Sep-01 & & U-238 & $\mathrm{U}-238$ \\
\hline 2748 & SESP SCHEDULING & & SW & & & B12TD0 & 13-Sep-01 & & U-238 & U-238 \\
\hline 2749 & SESP SCHEDULING & & SW & & & B12TD1 & 13-Sep-01 & & U-238 & U-238 \\
\hline 2750 & SESP SCHEDULING & & SW & & & B12TD2 & 13-Sep-01 & & U-238 & $\mathrm{U}-238$ \\
\hline 2751 & SESP SCHEDULING & & SW & & & B12TD3 & 13-Sep-01 & & U-238 & $\mathrm{U}-238$ \\
\hline 2752 & SESP SCHEDULING & & SW & & & B12TM0 & 13-Sep-01 & & U-238 & U-238 \\
\hline 2753 & SESP SCHEDULING & & SW & & & B12TM1 & 13-Sep-01 & & U-238 & $\mathrm{U}-238$ \\
\hline \begin{tabular}{|l|}
2754 \\
\end{tabular} & SESP SCHEDULING & & SW & & & B12TM2 & 13-Sep-01 & & $\mathrm{U}-238$ & $\mathrm{U}-238$ \\
\hline 2755 & SESP SCHEDULING & & SW & & & B12TM3 & 13-Sep-01 & & U-238 & $\mathrm{U}-238$ \\
\hline 2756 & SESP SCHEDULING & & SW & & & B12TP4 & 13-Sep-01 & & U-238 & U-238 \\
\hline 2757 & SESP SCHEDULING & & SW & & & B12TP8 & 13-Sep-01 & & $\mathrm{U}-238$ & $\mathrm{U}-238$ \\
\hline 2758 & SESP SCHEDULING & & SW & & & B12TR2 & 13-Sep-01 & & U-238 & U-238 \\
\hline 2759 & SESP SCHEDULING & & SW & & & B12TR6 & 13-Sep-01 & & U-238 & $\mathrm{U}-238$ \\
\hline 2760 & SESP SCHEDULING & & SW & & & B12XK2 & 17-Sep-01 & & $\mathrm{U}-238$ & $\mathrm{U}-238$ \\
\hline 2761 & SESP SCHEDULING & & SW & & & B12XK8 & 17-Sep-01 & & U-238 & U-238 \\
\hline 2762 & SESP SCHEDULING & & SW & & & B12XK9 & 17-Sep-01 & & U-238 & U-238 \\
\hline 2763 & SESP SCHEDULING & & SW & & & B12XL0 & 17-Sep-01 & & $\mathrm{U}-238$ & $\mathrm{U}-238$ \\
\hline 2764 & SESP SCHEDULING & & SW & & & B12XL1 & 17-Sep-01 & & U-238 & U-238 \\
\hline 2765 & SESP SCHEDULING & & SW & & & B12XL2 & 17-Sep-01 & & U-238 & U-238 \\
\hline 2766 & SESP SCHEDULING & & SW & & & B12XK1 & 18-Sep-01 & & U-238 & U-238 \\
\hline 2767 & SESP SCHEDULING & & SW & & & B12XK3 & 18-Sep-01 & & U-238 & $U-238$ \\
\hline 2768 & SESP SCHEDULING & & SW & & & B12XK4 & 18-Sep-01 & & U-238 & U-238 \\
\hline \begin{tabular}{|l|}
2769 \\
\end{tabular} & SESP SCHEDULING & & SW & & & B12XK5 & 18-Sep-01 & & $\mathrm{U}-238$ & $\mathrm{U}-238$ \\
\hline 2770 & SESP SCHEDULING & & SW & & & B12XK6 & 18-Sep-01 & & U-238 & $U-238$ \\
\hline 2771 & SESP SCHEDULING & & SW & & & B12XK7 & 18-Sep-01 & & U-238 & U-238 \\
\hline 2772 & SESP SCHEDULING & & SW & & & B12T28 & $02-O c t-01$ & 05-Sep-01 & $U-238$ & U-238 \\
\hline 2773 & SESP SCHEDULING & & SW & & & B12T33 & $02-O c t-01$ & 05-Sep-01 & $\mathrm{U}-238$ & $U-238$ \\
\hline 2774 & SESP SCHEDULING & & SW & & & B12X50 & 22-Oct-01 & & U-238 & U-238 \\
\hline 2775 & SESP SCHEDULING & & SW & & & B12X48 & 01-Nov-01 & & U-238 & U-238 \\
\hline
\end{tabular}




\begin{tabular}{|c|c|c|c|c|c|c|c|c|c|c|}
\hline & $A$ & $\mathrm{~B}$ & $\mathrm{C}$ & $\mathrm{D}$ & $E$ & $\mathrm{~F}$ & G & $\mathrm{H}$ & $\mathrm{I}$ & $\mathrm{J}$ \\
\hline 1 & SAMPLE_NUM_ASSIGNED_TO & SAMPLER & MEDIA & BIOTA_MEDIA & TAG_ID & SAMP_NUM & SAMP_DATE_TIME & SAMP_DATE_TIME_ON & CON_ID & CON_SHORT_NAME \\
\hline 2776 & SESP SCHEDULING & & SW & & & B12X85 & 01-Nov-01 & & U-238 & $\mathrm{U}-238$ \\
\hline 2777 & SESP SCHEDULING & & SW & & & B13103 & 01-Nov-01 & $02-$-Oct-01 & $\mathrm{U}-238$ & U-238 \\
\hline 2778 & SESP SCHEDULING & & SW & & & B130Y7 & 01-Nov-01 & 02-Oct-01 & $U-238$ & $\mathrm{U}-238$ \\
\hline 2779 & SESP SCHEDULING & & SW & & & B13J17 & 14-Nov-01 & & $\mathrm{U}-238$ & U-238 \\
\hline 2780 & SESP SCHEDULING & & SW & & & B13CC6 & 27-Nov-01 & 01-Nov-01 & $\mathrm{U}-238$ & U-238 \\
\hline 2781 & SESP SCHEDULING & & SW & & & B13CD2 & 27-Nov-01 & 01-Nov-01 & $\mathrm{U}-238$ & U-238 \\
\hline 2782 & SESP SCHEDULING & & SW & & & B13LF3 & 03-Dec-01 & & $\mathrm{U}-238$ & $\mathrm{U}-238$ \\
\hline 2783 & SESP SCHEDULING & & SW & & & B13LF4 & 03-Dec-01 & & $\mathrm{U}-238$ & $\mathrm{U}-238$ \\
\hline 2784 & SESP SCHEDULING & & SW & & & B13LF5 & 03-Dec-01 & & U-238 & U-238 \\
\hline 2785 & SESP SCHEDULING & & SW & & & B13LF6 & 03-Dec-01 & & $\mathrm{U}-238$ & U-238 \\
\hline 2786 & SESP SCHEDULING & & SW & & & B13LF2 & 04-Dec-01 & & U-238 & U-238 \\
\hline 2787 & SESP SCHEDULING & & SW & & & B13LF7 & 04-Dec-01 & & U-238 & U-238 \\
\hline 2788 & SESP SCHEDULING & & SW & & & B13LF8 & 04-Dec-01 & & $\mathrm{U}-238$ & U-238 \\
\hline 2789 & SESP SCHEDULING & & SW & & & B13LF9 & 04-Dec-01 & & $\mathrm{U}-238$ & U-238 \\
\hline 2790 & SESP SCHEDULING & & SW & & & B13LH0 & 04-Dec-01 & & U-238 & U-238 \\
\hline 2791 & SESP SCHEDULING & & SW & & & B13LH1 & 04-Dec-01 & & $\mathrm{U}-238$ & U-238 \\
\hline 2792 & SESP SCHEDULING & & SW & & & B13LH2 & 04-Dec-01 & & $\mathrm{U}-238$ & U-238 \\
\hline 2793 & SESP SCHEDULING & & SW & & & B13LJ3 & 04-Dec-01 & & U-238 & U-238 \\
\hline 2794 & SESP SCHEDULING & & SW & & & B13LJ4 & 04-Dec-01 & & $\mathrm{U}-238$ & U-238 \\
\hline 2795 & SESP SCHEDULING & & SW & & & B13LJ5 & 04-Dec-01 & & $\mathrm{U}-238$ & $\mathrm{U}-238$ \\
\hline 2796 & SESP SCHEDULING & & SW & & & B13LJ6 & 04-Dec-01 & & U-238 & U-238 \\
\hline 2797 & SESP SCHEDULING & & SW & & & B13LB3 & 03-Jan-02 & 27-Nov-01 & $\mathrm{U}-238$ & U-238 \\
\hline 2798 & SESP SCHEDULING & & SW & & & B13L96 & 03-Jan-02 & 27-Nov-01 & U-238 & U-238 \\
\hline 2799 & SESP SCHEDULING & & SW & & & B13VJ0 & 06-Feb-02 & & U-238 & U-238 \\
\hline 2800 & SESP SCHEDULING & & SW & & & B13VF8 & 06-Feb-02 & & $\mathrm{U}-238$ & U-238 \\
\hline 2801 & SESP SCHEDULING & & SW & & & B140P7 & 07-Mar-02 & 06-Feb-02 & U-238 & $\mathrm{U}-238$ \\
\hline 2802 & SESP SCHEDULING & & SW & & & B140R2 & 07-Mar-02 & 06-Feb-02 & U-238 & U-238 \\
\hline 2803 & SESP SCHEDULING & & SW & & & B147T3 & 25-Mar-02 & & U-238 & U-238 \\
\hline 2804 & SESP SCHEDULING & & SW & & & B147T4 & 25-Mar-02 & & $\mathrm{U}-238$ & U-238 \\
\hline 2805 & SESP SCHEDULING & & SW & & & B147T5 & 25-Mar-02 & & U-238 & U-238 \\
\hline 2806 & SESP SCHEDULING & & SW & & & B147T6 & 25-Mar-02 & & $\mathrm{U}-238$ & U-238 \\
\hline 2807 & SESP SCHEDULING & & SW & & & B147T2 & 26-Mar-02 & & $\mathrm{U}-238$ & U-238 \\
\hline 2808 & SESP SCHEDULING & & SW & & & B147T7 & 26-Mar-02 & & U-238 & U-238 \\
\hline 2809 & SESP SCHEDULING & & SW & & & B147T8 & 26-Mar-02 & & U-238 & U-238 \\
\hline 2810 & SESP SCHEDULING & & SW & & & B147T9 & 26-Mar-02 & & U-238 & U-238 \\
\hline 2811 & SESP SCHEDULING & & SW & & & B147V0 & 26-Mar-02 & & U-238 & U-238 \\
\hline 2812 & SESP SCHEDULING & & SW & & & B147V1 & 26-Mar-02 & & U-238 & U-238 \\
\hline 2813 & SESP SCHEDULING & & SW & & & B147V2 & 26-Mar-02 & & $\mathrm{U}-238$ & U-238 \\
\hline
\end{tabular}




\begin{tabular}{|c|c|c|c|c|c|c|c|c|c|c|}
\hline & A & $\mathrm{B}$ & $\mathrm{C}$ & $\mathrm{D}$ & $\mathrm{E}$ & $\mathrm{F}$ & G & $\mathrm{H}$ & $\mathrm{I}$ & $\mathrm{J}$ \\
\hline 1 & SAMPLE_NUM_ASSIGNED_TO & SAMPLER & MEDIA & BIOTA_MEDIA & TAG_ID & SAMP_NUM & SAMP_DATE_TIME & SAMP_DATE_TIME_ON & CON_ID & CON_SHORT_NAME \\
\hline 2814 & SESP SCHEDULING & & SW & & & B147W3 & 26-Mar-02 & & $\mathrm{U}-238$ & U-238 \\
\hline 2815 & SESP SCHEDULING & & SW & & & B147W4 & 26-Mar-02 & & $\mathrm{U}-238$ & U-238 \\
\hline 2816 & SESP SCHEDULING & & SW & & & B147W5 & 26-Mar-02 & & $\mathrm{U}-238$ & U-238 \\
\hline 2817 & SESP SCHEDULING & & SW & & & B147W6 & 26-Mar-02 & & U-238 & U-238 \\
\hline 2818 & SESP SCHEDULING & & SW & & & B14408 & 03-Apr-02 & 07-Mar-02 & U-238 & U-238 \\
\hline 2819 & SESP SCHEDULING & & SW & & & B14413 & 03-Apr-02 & 07-Mar-02 & U-238 & U-238 \\
\hline 2820 & SESP SCHEDULING & & SW & & & B14B37 & 01-May-02 & 03-Apr-02 & U-238 & U-238 \\
\hline 2821 & SESP SCHEDULING & & SW & & & B14B43 & 01-May-02 & 03-Apr-02 & U-238 & U-238 \\
\hline 2822 & SESP SCHEDULING & & SW & & & B14LJ0 & 23-May-02 & & U-238 & U-238 \\
\hline 2823 & SESP SCHEDULING & & SW & & & B14LJ2 & 23-May-02 & & $\mathrm{U}-238$ & U-238 \\
\hline 2824 & SESP SCHEDULING & & SW & & & B14JF2 & 29-May-02 & 01-May-02 & U-238 & U-238 \\
\hline 2825 & SESP SCHEDULING & & SW & & & B14JF8 & 29-May-02 & 01-May-02 & U-238 & U-238 \\
\hline 2826 & SESP SCHEDULING & & SW & & & B14RV4 & 10-Jun-02 & & $\mathrm{U}-238$ & U-238 \\
\hline 2827 & SESP SCHEDULING & & SW & & & B14RV5 & 10-Jun-02 & & U-238 & U-238 \\
\hline 2828 & SESP SCHEDULING & & SW & & & B14RV6 & 10-Jun-02 & & U-238 & U-238 \\
\hline 2829 & SESP SCHEDULING & & SW & & & B14RV7 & 10-Jun-02 & & U-238 & U-238 \\
\hline 2830 & SESP SCHEDULING & & SW & & & B14RV8 & 11-Jun-02 & & U-238 & U-238 \\
\hline 2831 & SESP SCHEDULING & & SW & & & B14RV9 & 11-Jun-02 & & $\mathrm{U}-238$ & U-238 \\
\hline 2832 & SESP SCHEDULING & & SW & & & B14RW0 & 11-Jun-02 & & U-238 & U-238 \\
\hline 2833 & SESP SCHEDULING & & SW & & & B14RW1 & 11-Jun-02 & & U-238 & U-238 \\
\hline 2834 & SESP SCHEDULING & & SW & & & B14RW2 & 11-Jun-02 & & $\mathrm{U}-238$ & U-238 \\
\hline 2835 & SESP SCHEDULING & & SW & & & B14RW3 & 11-Jun-02 & & $\mathrm{U}-238$ & U-238 \\
\hline 2836 & SESP SCHEDULING & & SW & & & B14RX4 & 11-Jun-02 & & U-238 & U-238 \\
\hline 2837 & SESP SCHEDULING & & SW & & & B14RX5 & 11-Jun-02 & & U-238 & U-238 \\
\hline 2838 & SESP SCHEDULING & & SW & & & B14RX6 & 11-Jun-02 & & $\mathrm{U}-238$ & U-238 \\
\hline 2839 & SESP SCHEDULING & & SW & & & B14RX7 & 11-Jun-02 & & U-238 & U-238 \\
\hline 2840 & SESP SCHEDULING & & SW & & & B14RL3 & 27-Jun-02 & 29-May-02 & U-238 & U-238 \\
\hline 2841 & SESP SCHEDULING & & SW & & & B14RM5 & 27-Jun-02 & 29-May-02 & U-238 & U-238 \\
\hline 2842 & SESP SCHEDULING & & SW & & & B14VT4 & 28-Jun-02 & & $\mathrm{U}-238$ & U-238 \\
\hline 2843 & SESP SCHEDULING & & SW & & & B14VX3 & 28-Jun-02 & & U-238 & U-238 \\
\hline 2844 & SESP SCHEDULING & & SW & & & B14YH3 & 08-Jul-02 & & $\mathrm{U}-238$ & U-238 \\
\hline 2845 & SESP SCHEDULING & & SW & & & B14YJ5 & 08-Jul-02 & & $\mathrm{U}-238$ & U-238 \\
\hline 2846 & SESP SCHEDULING & & SW & & & B14WW5 & 07-Aug-02 & 27-Jun-02 & U-238 & U-238 \\
\hline 2847 & SESP SCHEDULING & & SW & & & B14WX2 & 07-Aug-02 & 27-Jun-02 & U-238 & U-238 \\
\hline 2848 & SESP SCHEDULING & & SW & & & B153T9 & 04-Sep-02 & 07-Aug-02 & U-238 & U-238 \\
\hline 2849 & SESP SCHEDULING & & SW & & & B153V4 & 04-Sep-02 & 07-Aug-02 & U-238 & U-238 \\
\hline 2850 & SESP SCHEDULING & & SW & & & B158H3 & 05-Sep-02 & & U-238 & U-238 \\
\hline 2851 & SESP SCHEDULING & & SW & & & $\mathrm{B} 158 \mathrm{H} 4$ & 05-Sep-02 & & $\mathrm{U}-238$ & U-238 \\
\hline
\end{tabular}




\begin{tabular}{|c|c|c|c|c|c|c|c|c|c|c|}
\hline & A & $\mathrm{B}$ & $\mathrm{C}$ & $\mathrm{D}$ & $E$ & $\mathrm{~F}$ & G & $\mathrm{H}$ & $\mathrm{I}$ & $\mathrm{J}$ \\
\hline 1 & SAMPLE_NUM_ASSIGNED_TO & SAMPLER & MEDIA & BIOTA_MEDIA & TAG_ID & SAMP_NUM & SAMP_DATE_TIME & SAMP_DATE_TIME_ON & CON_ID & CON_SHORT_NAME \\
\hline 2852 & SESP SCHEDULING & & SW & & & B158H5 & 05-Sep-02 & & U-238 & U-238 \\
\hline 2853 & SESP SCHEDULING & & SW & & & B158H6 & 05-Sep-02 & & U-238 & U-238 \\
\hline 2854 & SESP SCHEDULING & & SW & & & $\mathrm{B} 158 \mathrm{H} 7$ & 05-Sep-02 & & U-238 & $\mathrm{U}-238$ \\
\hline 2855 & SESP SCHEDULING & & SW & & & $\mathrm{B} 158 \mathrm{H} 8$ & 05-Sep-02 & & $\mathrm{U}-238$ & $\mathrm{U}-238$ \\
\hline 2856 & SESP SCHEDULING & & SW & & & B158H9 & 05-Sep-02 & & U-238 & U-238 \\
\hline 2857 & SESP SCHEDULING & & SW & & & B158J0 & 05-Sep-02 & & U-238 & U-238 \\
\hline 2858 & SESP SCHEDULING & & SW & & & B158M7 & 05-Sep-02 & & $\mathrm{U}-238$ & $\mathrm{U}-238$ \\
\hline 2859 & SESP SCHEDULING & & SW & & & B158M8 & 05-Sep-02 & & $U-238$ & $\mathrm{U}-238$ \\
\hline 2860 & SESP SCHEDULING & & SW & & & B158M9 & 05-Sep-02 & & U-238 & $\mathrm{U}-238$ \\
\hline 2861 & SESP SCHEDULING & & SW & & & B158W8 & 05-Sep-02 & & U-238 & $\mathrm{U}-238$ \\
\hline 2862 & SESP SCHEDULING & & SW & & & B158X1 & 05-Sep-02 & & U-238 & $\mathrm{U}-238$ \\
\hline 2863 & SESP SCHEDULING & & SW & & & B158X4 & 05-Sep-02 & & U-238 & U-238 \\
\hline 2864 & SESP SCHEDULING & & SW & & & B158X7 & 05-Sep-02 & & U-238 & U-238 \\
\hline 2865 & SESP SCHEDULING & & SW & & & B158J1 & 09-Sep-02 & & U-238 & $\mathrm{U}-238$ \\
\hline 2866 & SESP SCHEDULING & & SW & & & B158J3 & 09-Sep-02 & & U-238 & U-238 \\
\hline \begin{tabular}{|l|}
2867 \\
\end{tabular} & SESP SCHEDULING & & SW & & & B158J5 & 09-Sep-02 & & U-238 & $\mathrm{U}-238$ \\
\hline \begin{tabular}{|l|}
2868 \\
\end{tabular} & SESP SCHEDULING & & SW & & & B158J7 & 09-Sep-02 & & $\mathrm{U}-238$ & $\mathrm{U}-238$ \\
\hline 2869 & SESP SCHEDULING & & SW & & & B158J9 & 09-Sep-02 & & U-238 & $\mathrm{U}-238$ \\
\hline 2870 & SESP SCHEDULING & & SW & & & B158K1 & 09-Sep-02 & & U-238 & U-238 \\
\hline 2871 & SESP SCHEDULING & & SW & & & B158K3 & 09-Sep-02 & & $\mathrm{U}-238$ & $\mathrm{U}-238$ \\
\hline 2872 & SESP SCHEDULING & & SW & & & B158K5 & 09-Sep-02 & & U-238 & U-238 \\
\hline 2873 & SESP SCHEDULING & & SW & & & B158K7 & 09-Sep-02 & & U-238 & $\mathrm{U}-238$ \\
\hline 2874 & SESP SCHEDULING & & SW & & & B158K9 & 09-Sep-02 & & $\mathrm{U}-238$ & U-238 \\
\hline 2875 & SESP SCHEDULING & & SW & & & B158L1 & 09-Sep-02 & & U-238 & $U-238$ \\
\hline 2876 & SESP SCHEDULING & & SW & & & B158L3 & 09-Sep-02 & & U-238 & U-238 \\
\hline 2877 & SESP SCHEDULING & & SW & & & B158NO & 09-Sep-02 & & $\mathrm{U}-238$ & $\mathrm{U}-238$ \\
\hline 2878 & SESP SCHEDULING & & SW & & & B158N2 & 09-Sep-02 & & U-238 & U-238 \\
\hline 2879 & SESP SCHEDULING & & SW & & & B158N4 & 09-Sep-02 & & U-238 & U-238 \\
\hline 2880 & SESP SCHEDULING & & SW & & & B15902 & 09-Sep-02 & & U-238 & U-238 \\
\hline 2881 & SESP SCHEDULING & & SW & & & B15906 & 09-Sep-02 & & U-238 & $U-238$ \\
\hline 2882 & SESP SCHEDULING & & SW & & & B15910 & 09-Sep-02 & & U-238 & U-238 \\
\hline \begin{tabular}{|l|}
2883 \\
\end{tabular} & SESP SCHEDULING & & SW & & & B15914 & 09-Sep-02 & & $\mathrm{U}-238$ & $\mathrm{U}-238$ \\
\hline 2884 & SESP SCHEDULING & & SW & & & B158L5 & 10-Sep-02 & & U-238 & $U-238$ \\
\hline 2885 & SESP SCHEDULING & & SW & & & B158L6 & 10-Sep-02 & & U-238 & U-238 \\
\hline 2886 & SESP SCHEDULING & & SW & & & B158L7 & 10-Sep-02 & & U-238 & $\mathrm{U}-238$ \\
\hline \begin{tabular}{|l|}
2887 \\
\end{tabular} & SESP SCHEDULING & & SW & & & B158L8 & 10-Sep-02 & & $\mathrm{U}-238$ & $\mathrm{U}-238$ \\
\hline 2888 & SESP SCHEDULING & & SW & & & B158L9 & 10-Sep-02 & & U-238 & U-238 \\
\hline 2889 & SESP SCHEDULING & & SW & & & B158M0 & 10-Sep-02 & & $\mathrm{U}-238$ & $\mathrm{U}-238$ \\
\hline
\end{tabular}




\begin{tabular}{|c|c|c|c|c|c|c|c|c|c|c|}
\hline & A & $\mathrm{B}$ & $\mathrm{C}$ & $\mathrm{D}$ & $E$ & $\mathrm{~F}$ & G & $\mathrm{H}$ & $\mathrm{I}$ & $\mathrm{J}$ \\
\hline 1 & SAMPLE_NUM_ASSIGNED_TO & SAMPLER & MEDIA & BIOTA_MEDIA & TAG_ID & SAMP_NUM & SAMP_DATE_TIME & SAMP_DATE_TIME_ON & CON_ID & CON_SHORT_NAME \\
\hline 2890 & SESP SCHEDULING & & SW & & & B158M1 & 10-Sep-02 & & U-238 & $\mathrm{U}-238$ \\
\hline 2891 & SESP SCHEDULING & & SW & & & B158M2 & 10-Sep-02 & & U-238 & U-238 \\
\hline 2892 & SESP SCHEDULING & & SW & & & B158M3 & 10-Sep-02 & & U-238 & U-238 \\
\hline 2893 & SESP SCHEDULING & & SW & & & B158M4 & 10-Sep-02 & & $\mathrm{U}-238$ & $\mathrm{U}-238$ \\
\hline 2894 & SESP SCHEDULING & & SW & & & B158M5 & 10-Sep-02 & & $U-238$ & $\mathrm{U}-238$ \\
\hline 2895 & SESP SCHEDULING & & SW & & & B158M6 & 10-Sep-02 & & $\mathrm{U}-238$ & $\mathrm{U}-238$ \\
\hline 2896 & SESP SCHEDULING & & SW & & & B158V6 & 10-Sep-02 & & $\mathrm{U}-238$ & $\mathrm{U}-238$ \\
\hline 2897 & SESP SCHEDULING & & SW & & & B158V7 & 10-Sep-02 & & $U-238$ & $\mathrm{U}-238$ \\
\hline 2898 & SESP SCHEDULING & & SW & & & B158V8 & 10-Sep-02 & & U-238 & U-238 \\
\hline 2899 & SESP SCHEDULING & & SW & & & B158V9 & 10-Sep-02 & & U-238 & U-238 \\
\hline 2900 & SESP SCHEDULING & & SW & & & B158Y0 & 10-Sep-02 & & U-238 & U-238 \\
\hline 2901 & SESP SCHEDULING & & SW & & & B158Y3 & 10-Sep-02 & & $\mathrm{U}-238$ & $\mathrm{U}-238$ \\
\hline 2902 & SESP SCHEDULING & & SW & & & B158Y6 & 10-Sep-02 & & U-238 & $\mathrm{U}-238$ \\
\hline 2903 & SESP SCHEDULING & & SW & & & B158Y9 & 10-Sep-02 & & $U-238$ & $\mathrm{U}-238$ \\
\hline 2904 & SESP SCHEDULING & & SW & & & B15948 & 02-Oct-02 & 04-Sep-02 & $U-238$ & U-238 \\
\hline \begin{tabular}{|l|}
2905 \\
\end{tabular} & SESP SCHEDULING & & SW & & & B15954 & $02-\mathrm{Oct}-02$ & 04-Sep-02 & $\mathrm{U}-238$ & $\mathrm{U}-238$ \\
\hline 2906 & SESP SCHEDULING & & SW & & & B15C09 & 07-Oct-02 & & $U-238$ & $\mathrm{U}-238$ \\
\hline 2907 & SESP SCHEDULING & & SW & & & B15C11 & 07-Oct-02 & & $\mathrm{U}-238$ & $\mathrm{U}-238$ \\
\hline 2908 & SESP SCHEDULING & & SW & & & B15C73 & 07-Oct-02 & & U-238 & $\mathrm{U}-238$ \\
\hline 2909 & SESP SCHEDULING & & SW & & & B15CB3 & 27-Oct-02 & & $U-238$ & $\mathrm{U}-238$ \\
\hline 2910 & SESP SCHEDULING & & SW & & & B15C69 & 29-Oct-02 & & $U-238$ & U-238 \\
\hline 2911 & SESP SCHEDULING & & SW & & & B15K74 & 30-Oct-02 & 02-Oct-02 & $\mathrm{U}-238$ & $\mathrm{U}-238$ \\
\hline 2912 & SESP SCHEDULING & & SW & & & B15K79 & 30-Oct-02 & 02-Oct-02 & $U-238$ & $\mathrm{U}-238$ \\
\hline 2913 & SESP SCHEDULING & & SW & & & B15VN2 & 26-Nov-02 & $30-$-Oct-02 & U-238 & U-238 \\
\hline 2914 & SESP SCHEDULING & & SW & & & B15VN8 & 26-Nov-02 & 30-Oct-02 & $\mathrm{U}-238$ & $\mathrm{U}-238$ \\
\hline 2915 & SESP SCHEDULING & & SW & & & B16342 & 09-Dec-02 & & $U-238$ & $\mathrm{U}-238$ \\
\hline 2916 & SESP SCHEDULING & & SW & & & B16343 & 09-Dec-02 & & U-238 & U-238 \\
\hline 2917 & SESP SCHEDULING & & SW & & & B16344 & 09-Dec-02 & & $U-238$ & U-238 \\
\hline 2918 & SESP SCHEDULING & & SW & & & B16345 & 09-Dec-02 & & $U-238$ & $\mathrm{U}-238$ \\
\hline 2919 & SESP SCHEDULING & & SW & & & B16341 & 10-Dec-02 & & $U-238$ & $U-238$ \\
\hline 2920 & SESP SCHEDULING & & SW & & & B16346 & 10-Dec-02 & & $\mathrm{U}-238$ & U-238 \\
\hline 2921 & SESP SCHEDULING & & SW & & & B16347 & 10-Dec-02 & & $U-238$ & $\mathrm{U}-238$ \\
\hline 2922 & SESP SCHEDULING & & SW & & & B16348 & 10-Dec-02 & & $U-238$ & $U-238$ \\
\hline 2923 & SESP SCHEDULING & & SW & & & B16349 & 10-Dec-02 & & $U-238$ & $\mathrm{U}-238$ \\
\hline 2924 & SESP SCHEDULING & & SW & & & B16350 & 10-Dec-02 & & U-238 & U-238 \\
\hline 2925 & SESP SCHEDULING & & SW & & & B16351 & 10-Dec-02 & & U-238 & U-238 \\
\hline 2926 & SESP SCHEDULING & & SW & & & B16362 & 10-Dec-02 & & $\mathrm{U}-238$ & $\mathrm{U}-238$ \\
\hline 2927 & SESP SCHEDULING & & SW & & & B16363 & 10-Dec-02 & & $\mathrm{U}-238$ & $\mathrm{U}-238$ \\
\hline
\end{tabular}




\begin{tabular}{|c|c|c|c|c|c|c|c|c|c|c|}
\hline & A & $\mathrm{B}$ & $\bar{C}$ & $\mathrm{D}$ & $\mathrm{E}$ & $\mathrm{F}$ & $\bar{G}$ & $\mathrm{H}$ & $\mathrm{T}$ & $\mathrm{J}$ \\
\hline 2928 & SESP SCHEDULING & & SW & & & B16364 & 10-Dec-02 & & $\mathrm{U}-238$ & $\mathrm{U}-238$ \\
\hline
\end{tabular}




\begin{tabular}{|c|c|c|c|c|c|c|c|c|c|}
\hline & $\mathrm{K}$ & $\mathrm{L}$ & $\mathrm{M}$ & $\mathrm{N}$ & $\mathrm{O}$ & $P$ & $\mathrm{Q}$ & $\mathrm{R}$ & $\mathrm{S}$ \\
\hline 1 & METHOD_NAME & LAB_CODE & VALUE_RPTD & ANAL_UNITS_RPTD & $\mid$ COUNTING_ERROR & TOTAL_ANAL_ERROR & MIN_DETECTABLE_ACTIVITY & PCNT_MOISTURE & LAB_QUALIFIER \\
\hline 2 & 8260_VOA_GCMS & $\overline{S T L S L}$ & 0.23 & $u g / L$ & & & & & $U$ \\
\hline 3 & 8260 VOA GCMS & STLSL & 0.23 & ug/L & & & & & $\mathrm{U}$ \\
\hline 4 & 8260_VOA_GCMS & STLSL & 0.23 & $\mathrm{ug} / \mathrm{L}$ & & & & & $U$ \\
\hline 5 & 8260_VOA_GCMS & STLSL & 0.23 & $\mathrm{ug} / \mathrm{L}$ & & & & & $U$ \\
\hline 6 & 8260_VOA_GCMS & STLSL & 0.23 & $\mathrm{ug} / \mathrm{L}$ & & & & & $U$ \\
\hline 7 & 8260_VOA_GCMS & STLSL & 0.23 & ug/L & & & & & $U$ \\
\hline 8 & 8260_VOA_GCMS & STLSL & 0.23 & $\mathrm{ug} / \mathrm{L}$ & & & & & $U$ \\
\hline 9 & 8260_VOA_GCMS & STLSL & 0.23 & $\mathrm{ug} / \mathrm{L}$ & & & & & $U$ \\
\hline 10 & 8260_VOA_GCMS & STLSL & 0.23 & $\mathrm{ug} / \mathrm{L}$ & & & & & $U$ \\
\hline 11 & 8260_VOA_GCMS & STLSL & 0.23 & $\mathrm{ug} / \mathrm{L}$ & & & & & $U$ \\
\hline 12 & 8260_VOA_GCMS & STLSL & 0.23 & $\mathrm{ug} / \mathrm{L}$ & & & & & $U$ \\
\hline 13 & 8260_VOA_GCMS & STLSL & 0.23 & ug/L & & & & & $U$ \\
\hline 14 & 8260_VOA_GCMS & STLSL & 0.23 & $\mathrm{ug} / \mathrm{L}$ & & & & & U \\
\hline 15 & 8260_VOA_GCMS & STLSL & 0.23 & $\mathrm{ug} / \mathrm{L}$ & & & & & $U$ \\
\hline 16 & 8260_VOA_GCMS & STLSL & 0.23 & ug/L & & & & & $U$ \\
\hline 17 & 8260_VOA_GCMS & STLSL & 0.23 & $\mathrm{ug} / \mathrm{L}$ & & & & & $U$ \\
\hline 18 & 8260_VOA_GCMS & STLSL & 0.23 & $\mathrm{ug} / \mathrm{L}$ & & & & & $U$ \\
\hline 19 & 8260_VOA_GCMS & STLSL & 0.23 & ug/L & & & & & $U$ \\
\hline 20 & 8260_VOA_GCMS & STLSL & 0.23 & $\mathrm{ug} / \mathrm{L}$ & & & & & $U$ \\
\hline 21 & 8260_VOA_GCMS & STLSL & 0.23 & $\mathrm{ug} / \mathrm{L}$ & & & & & $U$ \\
\hline 22 & 8260_VOA_GCMS & STLSL & 0.23 & $\mathrm{ug} / \mathrm{L}$ & & & & & $U$ \\
\hline 23 & 8260_VOA_GCMS & STLSL & 0.33 & $\mathrm{ug} / \mathrm{L}$ & & & & & U \\
\hline 24 & 8260_VOA_GCMS & STLSL & 0.33 & $\mathrm{ug} / \mathrm{L}$ & & & & & $U$ \\
\hline 25 & 8260_VOA_GCMS & STLSL & 0.33 & $\mathrm{ug} / \mathrm{L}$ & & & & & $U$ \\
\hline 26 & 8260_VOA_GCMS & STLSL & 0.33 & $\mathrm{ug} / \mathrm{L}$ & & & & & $U$ \\
\hline 27 & 8260_VOA_GCMS & STLSL & 0.33 & $\mathrm{ug} / \mathrm{L}$ & & & & & $U$ \\
\hline 28 & 8260_VOA_GCMS & STLSL & 0.33 & ug/L & & & & & $U$ \\
\hline 29 & 8260_VOA_GCMS & STLSL & 0.33 & ug/L & & & & & $U$ \\
\hline 30 & 8260_VOA_GCMS & STLSL & 0.33 & $\mathrm{ug} / \mathrm{L}$ & & & & & U \\
\hline 31 & 8260_VOA_GCMS & STLSL & 0.33 & $\mathrm{ug} / \mathrm{L}$ & & & & & $U$ \\
\hline 32 & 8260_VOA_GCMS & STLSL & 0.33 & $\mathrm{ug} / \mathrm{L}$ & & & & & $U$ \\
\hline 33 & 8260_VOA_GCMS & STLSL & 0.33 & $\mathrm{ug} / \mathrm{L}$ & & & & & $U$ \\
\hline 34 & 8260_VOA_GCMS & STLSL & 0.33 & $\mathrm{ug} / \mathrm{L}$ & & & & & $U$ \\
\hline 35 & 8260_VOA_GCMS & STLSL & 0.33 & ug/L & & & & & $U$ \\
\hline 36 & 8260_VOA_GCMS & STLSL & 0.33 & $\mathrm{ug} / \mathrm{L}$ & & & & & $U$ \\
\hline 37 & 8260_VOA_GCMS & STLSL & 0.33 & $\mathrm{ug} / \mathrm{L}$ & & & & & $U$ \\
\hline 38 & 8260_VOA_GCMS & STLSL & 0.33 & ug/L & & & & & $U$ \\
\hline 39 & 8260_VOA_GCMS & STLSL & 0.33 & $\mathrm{ug} / \mathrm{L}$ & & & & & U \\
\hline
\end{tabular}




\begin{tabular}{|c|c|c|c|c|c|c|c|c|c|}
\hline & $\mathrm{K}$ & $\mathrm{L}$ & $M$ & $\mathrm{~N}$ & $\mathrm{O}$ & $\mathrm{P}$ & $\mathrm{Q}$ & $\mathrm{R}$ & $\mathrm{S}$ \\
\hline 1 & METHOD_NAME & LAB_CODE & VALUE_RPTD & ANAL_UNITS_RPTD & COUNTING_ERROR & TOTAL_ANAL_ERROR & MIN_DETECTABLE_ACTIVITY & PCNT_MOISTURE & LAB_QUALIFIER \\
\hline 40 & 8260_VOA_GCMS & STLSL & 0.33 & $3 \mathrm{ug} / \mathrm{L}$ & & & & & $U$ \\
\hline 41 & 8260_VOA_GCMS & STLSL & 0.33 & ug/L & & & & & $\mathrm{U}$ \\
\hline 42 & 8260_VOA_GCMS & STLSL & 0.33 & $3 \mathrm{ug} / \mathrm{L}$ & & & & & $U$ \\
\hline 43 & 8260_VOA_GCMS & STLSL & 0.33 & $3 \mathrm{ug} / \mathrm{L}$ & & & & & $U$ \\
\hline 44 & 8260_VOA_GCMS & STLSL & 0.33 & ug/L & & & & & $U$ \\
\hline 45 & 8260_VOA_GCMS & STLSL & 0.33 & ug/L & & & & & U \\
\hline 46 & 8260_VOA_GCMS & STLSL & 0.33 & ug/L & & & & & $U$ \\
\hline 47 & 8260_VOA_GCMS & STLSL & 0.33 & $3 \mathrm{ug} / \mathrm{L}$ & & & & & U \\
\hline 48 & 8260_VOA_GCMS & STLSL & 0.33 & ug/L & & & & & $U$ \\
\hline 49 & 8260_VOA_GCMS & STLSL & 0.33 & $3 \mathrm{ug} / \mathrm{L}$ & & & & & $U$ \\
\hline 50 & 8260_VOA_GCMS & STLSL & 0.33 & ug/L & & & & & U \\
\hline 51 & 8260_VOA_GCMS & STLSL & 0.33 & ug/L & & & & & $U$ \\
\hline 52 & 8260_VOA_GCMS & STLSL & 0.33 & ug/L & & & & & $U$ \\
\hline 53 & 8260_VOA_GCMS & STLSL & 0.33 & ug/L & & & & & $U$ \\
\hline 54 & 8260_VOA_GCMS & STLSL & 0.33 & ug/L & & & & & U \\
\hline 55 & 8260_VOA_GCMS & STLSL & 0.33 & ug/L & & & & & $U$ \\
\hline 56 & 8260_VOA_GCMS & STLSL & 0.33 & $3 \mathrm{ug} / \mathrm{L}$ & & & & & U \\
\hline 57 & 8260_VOA_GCMS & STLSL & 0.33 & ug/L & & & & & U \\
\hline 58 & 8260_VOA_GCMS & STLSL & 0.33 & $3 \mathrm{ug} / \mathrm{L}$ & & & & & $U$ \\
\hline 59 & 8260_VOA_GCMS & STLSL & 0.33 & $3 \mathrm{ug} / \mathrm{L}$ & & & & & U \\
\hline 60 & 8260_VOA_GCMS & STLSL & 0.33 & $3 \mathrm{ug} / \mathrm{L}$ & & & & & U \\
\hline 61 & 8260_VOA_GCMS & STLSL & 4.1 & $\mathrm{ug} / \mathrm{L}$ & & & & & \\
\hline 62 & 6010_METALS_ICP & STLSL & 87.6 & $\mathrm{ug} / \mathrm{L}$ & & & & & \\
\hline 63 & 6010_METALS_ICP & STLSL & 87.2 & $\mathrm{ug} / \mathrm{L}$ & & & & & \\
\hline 64 & 6010_METALS_ICP & STLSL & 88.5 & $\mathrm{ug} / \mathrm{L}$ & & & & & \\
\hline 65 & 6010_METALS_ICP & STLSL & 88.9 & $\mathrm{ug} / \mathrm{L}$ & & & & & \\
\hline 66 & 6010_METALS_ICP & STLSL & 162 & $\mathrm{ug} / \mathrm{L}$ & & & & & \\
\hline 67 & 6010_METALS_ICP & STLSL & 160 & $\mathrm{ug} / \mathrm{L}$ & & & & & \\
\hline 68 & 6010_METALS_ICP & STLSL & 12.8 & $3 \mathrm{ug} / \mathrm{L}$ & & & & & \\
\hline 69 & 6010_METALS_ICP & STLSL & 13.8 & $3 \mathrm{ug} / \mathrm{L}$ & & & & & \\
\hline 70 & 6010_METALS_ICP & STLSL & 12.8 & ug/L & & & & & \\
\hline 71 & 6010_METALS_ICP & STLSL & 14 & ug/L & & & & & \\
\hline 72 & 6010_METALS_ICP & RLNP & & $\mathrm{ug} / \mathrm{L}$ & & & & & B \\
\hline 73 & 6010_METALS_ICP & RLNP & & $\mathrm{ug} / \mathrm{L}$ & & & & & B \\
\hline 74 & 6010_METALS_ICP & RLNP & & $\mathrm{ug} / \mathrm{L}$ & & & & & \\
\hline 75 & 6010_METALS_ICP & RLNP & & $\mathrm{ug} / \mathrm{L}$ & & & & & \\
\hline 76 & GAMMA_GS & QTESRL & 2.56 & $\mathrm{pCi} / \mathrm{L}$ & 6.3 & 6.3 & 12.2 & & U \\
\hline 77 & GAMMA_GS & QTESRL & -0.625 & $5 \mathrm{pCi} / \mathrm{L}$ & 1.3 & 1.3 & 2.12 & & $U$ \\
\hline
\end{tabular}




\begin{tabular}{|c|c|c|c|c|c|c|c|c|c|}
\hline & $\mathrm{K}$ & $\mathrm{L}$ & $\mathrm{M}$ & $\mathrm{N}$ & $\mathrm{O}$ & $\mathrm{P}$ & $\mathrm{Q}$ & $\mathrm{R}$ & $\mathrm{S}$ \\
\hline 1 & METHOD_NAME & LAB_CODE & VALUE_RPTD & ANAL_UNITS_RPTD & |COUNTING_ERROR & TOTAL_ANAL_ERROR & MIN_DETECTABLE_ACTIVITY & PCNT_MOISTURE & LAB_QUALIFIER \\
\hline 78 & GAMMA_GS & QTESRL & 0.00103 & $\mathrm{pCi} / \mathrm{L}$ & 0.0023 & 0.0023 & 0.00396 & & $\mathrm{U}$ \\
\hline 79 & GAMMA_GS & QTESRL & 0.000235 & $\mathrm{pCi} / \mathrm{L}$ & 0.00061 & 0.00061 & 0.00107 & & $\mathrm{U}$ \\
\hline 80 & GAMMA_GS & QTESRL & -0.000185 & $\mathrm{pCi} / \mathrm{L}$ & 0.00075 & 0.00075 & 0.00126 & & $\mathrm{U}$ \\
\hline 81 & GAMMA_GS & QTESRL & -0.000222 & $\mathrm{pCi} / \mathrm{L}$ & 0.0022 & 0.0022 & 0.00369 & & U \\
\hline 82 & GAMMA_GS & QTESRL & 0.0019 & $\mathrm{pCi} / \mathrm{L}$ & 0.002 & 0.002 & 0.00349 & & $\mathrm{U}$ \\
\hline 83 & GAMMA_GS & QTESRL & 0.000733 & $\mathrm{pCi} / \mathrm{L}$ & 0.00066 & 0.00066 & 0.00117 & & $\mathrm{U}$ \\
\hline 84 & GAMMA_GS & QTESRL & 0.000892 & $\mathrm{pCi} / \mathrm{L}$ & 0.00056 & 0.00056 & 0.000988 & & U \\
\hline 85 & GAMMA_GS & QTESRL & 0.00144 & $\mathrm{pCi} / \mathrm{L}$ & 0.0021 & 0.0021 & 0.00378 & & $U$ \\
\hline 86 & GAMMA_GS & QTESRL & 5.75 & $\mathrm{pCi} / \mathrm{L}$ & 6.9 & 6.9 & 13.9 & & $\mathrm{U}$ \\
\hline 87 & GAMMA_GS & QTESRL & 0.224 & $\mathrm{pCi} / \mathrm{L}$ & 0.69 & 0.69 & 1.44 & & $\mathrm{U}$ \\
\hline 88 & GAMMA_GS & QTESRL & -0.354 & $\mathrm{pCi} / \mathrm{L}$ & 1.2 & 1.2 & 2.04 & & $\mathrm{U}$ \\
\hline 89 & GAMMA_GS & QTESRL & 0.000839 & $\mathrm{pCi} / \mathrm{L}$ & 0.0023 & 0.0023 & 0.00396 & & $\mathrm{U}$ \\
\hline 90 & GAMMA_GS & QTESRL & 0.000507 & $\mathrm{pCi} / \mathrm{L}$ & 0.00069 & 0.00069 & 0.0012 & & $\mathrm{U}$ \\
\hline 91 & GAMMA_GS & QTESRL & 0.000448 & $\mathrm{pCi} / \mathrm{L}$ & 0.00066 & 0.00066 & 0.00116 & & $\mathrm{U}$ \\
\hline 92 & GAMMA_GS & QTESRL & 0.00161 & $\mathrm{pCi} / \mathrm{L}$ & 0.0021 & 0.0021 & 0.00374 & & $\mathrm{U}$ \\
\hline 93 & GAMMA_GS & QTESRL & 0.000716 & $\mathrm{pCi} / \mathrm{L}$ & 0.00065 & 0.00065 & 0.00117 & & $\mathrm{U}$ \\
\hline 94 & GAMMA_GS & STLRL & -0.000645 & $\mathrm{pCi} / \mathrm{L}$ & 0.0022 & 0.0022 & 0.00375 & & $U$ \\
\hline 95 & GAMMA_GS & STLRL & 0.000818 & $\mathrm{pCi} / \mathrm{L}$ & 0.0025 & 0.0025 & 0.00426 & & U \\
\hline 96 & GAMMA_GS & QTESRL & 0.000368 & $\mathrm{pCi} / \mathrm{L}$ & 0.00073 & 0.00073 & 0.00127 & & U \\
\hline 97 & GAMMA_GS & STLRL & 0.621 & $\mathrm{pCi} / \mathrm{L}$ & 0.95 & 0.95 & 1.99 & & U \\
\hline 98 & GAMMA_GS & STLRL & 0.00052 & $\mathrm{pCi} / \mathrm{L}$ & 0.0007 & 0.0007 & 0.00123 & & U \\
\hline 99 & GAMMA_GS & STLRL & 0.00134 & $\mathrm{pCi} / \mathrm{L}$ & 0.0022 & 0.0022 & 0.00383 & & U \\
\hline 100 & GAMMA_GS & STLRL & 0.00148 & $\mathrm{pCi} / \mathrm{L}$ & 0.0023 & 0.0023 & 0.00399 & & U \\
\hline 101 & GAMMA_GS & STLRL & 0.00109 & $\mathrm{pCi} / \mathrm{L}$ & 0.00072 & 0.00072 & 0.00129 & & U \\
\hline 102 & GAMMA_GS & STLRL & -0.501 & $\mathrm{pCi} / \mathrm{L}$ & 1.1 & 1.1 & 1.95 & & U \\
\hline 103 & GAMMA_GS & STLRL & -1.05 & $\mathrm{pCi} / \mathrm{L}$ & 4.1 & 4.1 & 7.45 & & $U$ \\
\hline 104 & GAMMA_GS & STLRL & 14.4 & $\mathrm{pCi} / \mathrm{L}$ & 13 & 13 & 26.9 & & $\mathrm{U}$ \\
\hline 105 & GAMMA_GS & STLRL & 0.000592 & $\mathrm{pCi} / \mathrm{L}$ & 0.0007 & 0.0007 & 0.00123 & & $\mathrm{U}$ \\
\hline 106 & GAMMA_GS & STLRL & 0.00108 & $\mathrm{pCi} / \mathrm{L}$ & 0.002 & 0.002 & 0.0035 & & U \\
\hline 107 & GAMMA_GS & STLRL & -0.0011 & $\mathrm{pCi} / \mathrm{L}$ & 0.0023 & 0.0023 & 0.00387 & & $U$ \\
\hline 108 & GAMMA_GS & STLRL & 0.000591 & $\mathrm{pCi} / \mathrm{L}$ & 0.00069 & 0.00069 & 0.00122 & & U \\
\hline 109 & GAMMA_GS & STLRL & 6.72 & $\mathrm{pCi} / \mathrm{L}$ & 12 & 12 & 24.4 & & U \\
\hline 110 & GAMMA_GS & STLRL & 0.0365 & $\mathrm{pCi} / \mathrm{L}$ & 0.99 & 0.99 & 1.88 & & U \\
\hline 111 & GAMMA_GS & STLRL & 0.206 & $\mathrm{pCi} / \mathrm{L}$ & 1.1 & 1.1 & 2.13 & & $\mathrm{U}$ \\
\hline 112 & GAMMA_GS & STLRL & -0.00000859 & $\mathrm{pCi} / \mathrm{L}$ & 0.00072 & 0.00072 & 0.00122 & & U \\
\hline 113 & GAMMA_GS & STLRL & 0.00305 & $\mathrm{pCi} / \mathrm{L}$ & 0.0019 & 0.0019 & 0.00347 & & U \\
\hline 114 & GAMMA_GS & STLRL & 0.000942 & $\mathrm{pCi} / \mathrm{L}$ & 0.0018 & 0.0018 & 0.00317 & & U \\
\hline 115 & GAMMA_GS & STLRL & 0.00121 & $\mathrm{pCi} / \mathrm{L}$ & 0.00074 & 0.00074 & 0.00132 & & U \\
\hline
\end{tabular}




\begin{tabular}{|c|c|c|c|c|c|c|c|c|c|}
\hline & $\mathrm{K}$ & $\mathrm{L}$ & $\mathrm{M}$ & $\mathrm{N}$ & $\mathrm{O}$ & $\mathrm{P}$ & $\mathrm{Q}$ & $\mathrm{R}$ & $\mathrm{S}$ \\
\hline 1 & METHOD_NAME & LAB_CODE & VALUE_RPTD & ANAL_UNITS_RPTD & |COUNTING_ERROR & TOTAL_ANAL_ERROR & MIN_DETECTABLE_ACTIVITY & PCNT_MOISTURE & LAB_QUALIFIER \\
\hline 116 & GAMMA_GS & STLRL & 0.000812 & $\mathrm{pCi} / \mathrm{L}$ & 0.001 & 0.001 & 0.00182 & & $\mathrm{U}$ \\
\hline 117 & GAMMA_GS & STLRL & -0.000173 & $\mathrm{pCi} / \mathrm{L}$ & 0.002 & 0.002 & 0.00346 & & $\mathrm{U}$ \\
\hline 118 & GAMMA_GS & STLRL & 0.00061 & $\mathrm{pCi} / \mathrm{L}$ & 0.002 & 0.002 & 0.00353 & & $\mathrm{U}$ \\
\hline 119 & GAMMA_GS & STLRL & 0.000177 & $\mathrm{pCi} / \mathrm{L}$ & 0.0011 & 0.0011 & 0.00188 & & U \\
\hline 120 & GAMMA_GS & STLRL & -0.845 & $\mathrm{pCi} / \mathrm{L}$ & 0.87 & 0.87 & 1.32 & & $U$ \\
\hline 121 & GAMMA_GS & STLRL & -0.226 & $\mathrm{pCi} / \mathrm{L}$ & 1.2 & 1.2 & 2.19 & & $U$ \\
\hline 122 & GAMMA_GS & STLRL & 0.324 & $\mathrm{pCi} / \mathrm{L}$ & 0.83 & 0.83 & 1.65 & & $\mathrm{U}$ \\
\hline 123 & GAMMA_GS & STLRL & 0.697 & $\mathrm{pCi} / \mathrm{L}$ & 1 & 1 & 2.08 & & $U$ \\
\hline 124 & GAMMA_GS & STLRL & 0.768 & $\mathrm{pCi} / \mathrm{L}$ & 1.2 & 1.2 & 2.37 & & $U$ \\
\hline 125 & GAMMA_GS & STLRL & 0.907 & $\mathrm{pCi} / \mathrm{L}$ & 1.1 & 1.1 & 2.31 & & $\mathrm{U}$ \\
\hline 126 & GAMMA_GS & STLRL & 0.000487 & $\mathrm{pCi} / \mathrm{L}$ & 0.00068 & 0.00068 & 0.00117 & & U \\
\hline 127 & GAMMA_GS & STLRL & 0.00111 & $\mathrm{pCi} / \mathrm{L}$ & 0.0017 & 0.0017 & 0.00303 & & $\mathrm{U}$ \\
\hline 128 & GAMMA_GS & STLRL & 0.000212 & $\mathrm{pCi} / \mathrm{L}$ & 0.0016 & 0.0016 & 0.00282 & & U \\
\hline 129 & GAMMA_GS & STLRL & -0.0000388 & $\mathrm{pCi} / \mathrm{L}$ & 0.00064 & 0.00064 & 0.00109 & & U \\
\hline 130 & GAMMA_GS & STLRL & -3.66 & $\mathrm{pCi} / \mathrm{L}$ & 14 & 14 & 24 & & $\mathrm{U}$ \\
\hline 131 & GAMMA_GS & STLRL & -1.1 & $\mathrm{pCi} / \mathrm{L}$ & 1.5 & 1.5 & 2.44 & & $\mathrm{U}$ \\
\hline 132 & GAMMA_GS & STLRL & 1.07 & $\mathrm{pCi} / \mathrm{L}$ & 0.97 & 0.97 & 2.11 & & $U$ \\
\hline 133 & GAMMA_GS & STLRL & -0.0718 & $\mathrm{pCi} / \mathrm{L}$ & 1.1 & 1.1 & 2.05 & & $U$ \\
\hline 134 & GAMMA_GS & STLRL & -0.85 & $\mathrm{pCi} / \mathrm{L}$ & 1.1 & 1.1 & 1.78 & & $\mathrm{U}$ \\
\hline 135 & GAMMA_GS & STLRL & 0.054 & $\mathrm{pCi} / \mathrm{L}$ & 0.89 & 0.89 & 1.71 & & $U$ \\
\hline 136 & GAMMA_GS & STLRL & -0.715 & $\mathrm{pCi} / \mathrm{L}$ & 1.1 & 1.1 & 1.77 & & $U$ \\
\hline 137 & GAMMA_GS & STLRL & -0.423 & $\mathrm{pCi} / \mathrm{L}$ & 1.2 & 1.2 & 2.15 & & U \\
\hline 138 & GAMMA_GS & STLRL & 0.295 & $\mathrm{pCi} / \mathrm{L}$ & 1.1 & 1.1 & 2.15 & & U \\
\hline 139 & GAMMA_GS & STLRL & -0.449 & $\mathrm{pCi} / \mathrm{L}$ & 1.1 & 1.1 & 1.95 & & U \\
\hline 140 & GAMMA_GS & STLRL & -0.00013 & $\mathrm{pCi} / \mathrm{L}$ & 0.00069 & 0.00069 & 0.00115 & & U \\
\hline 141 & GAMMA_GS & STLRL & 0.00136 & $\mathrm{pCi} / \mathrm{L}$ & 0.0017 & 0.0017 & 0.00294 & & U \\
\hline 142 & GAMMA_GS & STLRL & 0.000726 & $\mathrm{pCi} / \mathrm{L}$ & 0.0006 & 0.0006 & 0.00108 & & U \\
\hline 143 & GAMMA_GS & STLRL & 0.00229 & $\mathrm{pCi} / \mathrm{L}$ & 0.0014 & 0.0014 & 0.00256 & & $\mathrm{U}$ \\
\hline 144 & GAMMA_GS & STLRL & -1.06 & $\mathrm{pCi} / \mathrm{L}$ & 1.1 & 1.1 & 1.82 & & $\mathrm{U}$ \\
\hline 145 & GAMMA_GS & STLRL & -0.0000731 & $\mathrm{pCi} / \mathrm{L}$ & 0.00074 & 0.00074 & 0.00124 & & $U$ \\
\hline 146 & GAMMA_GS & STLRL & 0.000892 & $\mathrm{pCi} / \mathrm{L}$ & 0.0018 & 0.0018 & 0.00315 & & $U$ \\
\hline 147 & GAMMA_GS & STLRL & 0.000449 & $\mathrm{pCi} / \mathrm{L}$ & 0.0014 & 0.0014 & 0.00243 & & U \\
\hline 148 & GAMMA_GS & STLRL & 0.00031 & $\mathrm{pCi} / \mathrm{L}$ & 0.00069 & 0.00069 & 0.00119 & & U \\
\hline 149 & GAMMA_GS & STLRL & 0.00117 & $\mathrm{pCi} / \mathrm{L}$ & 0.00072 & 0.00072 & 0.00127 & & $U$ \\
\hline 150 & GAMMA_GS & STLRL & 0.00227 & $\mathrm{pCi} / \mathrm{L}$ & 0.0016 & 0.0016 & 0.0029 & & U \\
\hline 151 & GAMMA_GS & STLRL & 0.00154 & $\mathrm{pCi} / \mathrm{L}$ & 0.0015 & 0.0015 & 0.00273 & & U \\
\hline 152 & GAMMA_GS & STLRL & -0.0000458 & $\mathrm{pCi} / \mathrm{L}$ & 0.00067 & 0.00067 & 0.00113 & & $U$ \\
\hline 153 & GAMMA_GS & STLRL & 0.00086 & $\mathrm{pCi} / \mathrm{L}$ & 0.00067 & 0.00067 & 0.0012 & & U \\
\hline
\end{tabular}




\begin{tabular}{|c|c|c|c|c|c|c|c|c|c|}
\hline & $\mathrm{K}$ & $\mathrm{L}$ & $\mathrm{M}$ & $\mathrm{N}$ & $\mathrm{O}$ & $\mathrm{P}$ & $\mathrm{Q}$ & $\mathrm{R}$ & $\mathrm{S}$ \\
\hline 1 & METHOD_NAME & LAB_CODE & VALUE_RPTD & ANAL_UNITS_RPTD & COUNTING_ERROR & TOTAL_ANAL_ERROR & MIN_DETECTABLE_ACTIVITY & PCNT_MOISTURE & LAB_QUALIFIER \\
\hline 154 & GAMMA_GS & STLRL & 0.000697 & $\mathrm{pCi} / \mathrm{L}$ & 0.0015 & 0.0015 & 0.00257 & & $U$ \\
\hline 155 & GAMMA_GS & STLRL & 0.000681 & $\mathrm{pCi} / \mathrm{L}$ & 0.0015 & 0.0015 & 0.00257 & & $U$ \\
\hline 156 & GAMMA_GS & STLRL & 0.000532 & $\mathrm{pCi} / \mathrm{L}$ & 0.00075 & 0.00075 & 0.00131 & & $\mathrm{U}$ \\
\hline 157 & GAMMA_GS & STLRL & 26.5 & $\mathrm{pCi} / \mathrm{L}$ & 29 & 29 & 56.4 & & U \\
\hline 158 & GAMMA_GS & STLRL & 0.853 & $\mathrm{pCi} / \mathrm{L}$ & 2 & 2 & 3.95 & & $U$ \\
\hline 159 & GAMMA_GS & STLRL & 0.000542 & $\mathrm{pCi} / \mathrm{L}$ & 0.00074 & 0.00074 & 0.00128 & & $U$ \\
\hline 160 & GAMMA_GS & STLRL & 0.00108 & $\mathrm{pCi} / \mathrm{L}$ & 0.0017 & 0.0017 & 0.00291 & & $\mathrm{U}$ \\
\hline 161 & GAMMA_GS & STLRL & 0.000653 & $\mathrm{pCi} / \mathrm{L}$ & 0.0018 & 0.0018 & 0.00314 & & $U$ \\
\hline 162 & GAMMA_GS & STLRL & 0.000992 & $\mathrm{pCi} / \mathrm{L}$ & 0.00072 & 0.00072 & 0.0013 & & $U$ \\
\hline 163 & GAMMA_GS & STLRL & 4.06 & $\mathrm{pCi} / \mathrm{L}$ & 20 & 20 & 38.6 & & $\mathrm{U}$ \\
\hline 164 & GAMMA_GS & STLRL & -0.791 & $\mathrm{pCi} / \mathrm{L}$ & 2 & 2 & 3.43 & & $U$ \\
\hline 165 & GAMMA_GS & STLRL & -0.363 & $\mathrm{pCi} / \mathrm{L}$ & 2.3 & 2.3 & 4.19 & & $U$ \\
\hline 166 & GAMMA_GS & STLRL & 0.000461 & $\mathrm{pCi} / \mathrm{L}$ & 0.00067 & 0.00067 & 0.00118 & & U \\
\hline 167 & GAMMA_GS & STLRL & -0.000333 & $\mathrm{pCi} / \mathrm{L}$ & 0.0016 & 0.0016 & 0.00266 & & $U$ \\
\hline 168 & GAMMA_GS & STLRL & -0.000999 & $\mathrm{pCi} / \mathrm{L}$ & 0.0015 & 0.0015 & 0.00255 & & U \\
\hline 169 & GAMMA_GS & STLRL & -0.000155 & $\mathrm{pCi} / \mathrm{L}$ & 0.00062 & 0.00062 & 0.00105 & & $U$ \\
\hline 170 & GAMMA_GS & STLRL & 0.933 & $\mathrm{pCi} / \mathrm{L}$ & 2.9 & 2.9 & 5.63 & & $U$ \\
\hline 171 & GAMMA_GS & STLRL & 1.29 & $\mathrm{pCi} / \mathrm{L}$ & 2.3 & 2.3 & 4.58 & & $U$ \\
\hline 172 & GAMMA_GS & STLRL & 0.434 & $\mathrm{pCi} / \mathrm{L}$ & 2.1 & 2.1 & 4.08 & & U \\
\hline 173 & GAMMA_GS & STLRL & -0.999 & $\mathrm{pCi} / \mathrm{L}$ & 2.8 & 2.8 & 4.95 & & $\mathrm{U}$ \\
\hline 174 & GAMMA_GS & STLRL & -1 & $\mathrm{pCi} / \mathrm{L}$ & 2.4 & 2.4 & 4.19 & & U \\
\hline 175 & GAMMA_GS & STLRL & 0.00167 & $\mathrm{pCi} / \mathrm{L}$ & 0.0017 & 0.0017 & 0.00296 & & U \\
\hline 176 & GAMMA_GS & STLRL & 0.000338 & $\mathrm{pCi} / \mathrm{L}$ & 0.00073 & 0.00073 & 0.00126 & & U \\
\hline 177 & GAMMA_GS & STLRL & 0.000497 & $\mathrm{pCi} / \mathrm{L}$ & 0.00071 & 0.00071 & 0.00123 & & $U$ \\
\hline 178 & GAMMA_GS & STLRL & 0.0011 & $\mathrm{pCi} / \mathrm{L}$ & 0.0017 & 0.0017 & 0.00305 & & $U$ \\
\hline 179 & GAMMA_GS & STLRL & 0.0416 & $\mathrm{pCi} / \mathrm{L}$ & 2.7 & 2.7 & 4.86 & & $U$ \\
\hline 180 & GAMMA_GS & STLRL & -0.409 & $\mathrm{pCi} / \mathrm{L}$ & 2.7 & 2.7 & 4.75 & & $U$ \\
\hline 181 & GAMMA_GS & STLRL & -0.919 & $\mathrm{pCi} / \mathrm{L}$ & 2.5 & 2.5 & 4.33 & & U \\
\hline 182 & GAMMA_GS & STLRL & -0.731 & $p C i / L$ & 2.5 & 2.5 & 4.42 & & U \\
\hline 183 & GAMMA_GS & STLRL & 0.908 & $\mathrm{pCi} / \mathrm{L}$ & 2.4 & 2.4 & 4.64 & & $U$ \\
\hline 184 & GAMMA_GS & STLRL & -1.33 & $\mathrm{pCi} / \mathrm{L}$ & 2.6 & 2.6 & 4.41 & & $U$ \\
\hline 185 & GAMMA_GS & STLRL & 0.482 & $\mathrm{pCi} / \mathrm{L}$ & 2.1 & 2.1 & 4.08 & & $\mathrm{U}$ \\
\hline 186 & GAMMA_GS & STLRL & -1.93 & $\mathrm{pCi} / \mathrm{L}$ & 2.4 & 2.4 & 3.84 & & $\mathrm{U}$ \\
\hline 187 & GAMMA_GS & STLRL & 0.000374 & $\mathrm{pCi} / \mathrm{L}$ & 0.0017 & 0.0017 & 0.00286 & & $U$ \\
\hline 188 & GAMMA_GS & STLRL & 0.00128 & $\mathrm{pCi} / \mathrm{L}$ & 0.00068 & 0.00068 & 0.00122 & & U \\
\hline 189 & GAMMA_GS & STLRL & 0.000491 & $\mathrm{pCi} / \mathrm{L}$ & 0.00069 & 0.00069 & 0.00121 & & U \\
\hline 190 & GAMMA_GS & STLRL & -0.000011 & $\mathrm{pCi} / \mathrm{L}$ & 0.0016 & 0.0016 & 0.00272 & & $U$ \\
\hline 191 & GAMMA_GS & STLRL & 0.0157 & $\mathrm{pCi} / \mathrm{L}$ & 1.7 & 1.7 & 3.27 & & U \\
\hline
\end{tabular}




\begin{tabular}{|c|c|c|c|c|c|c|c|c|c|}
\hline & $\mathrm{K}$ & $\mathrm{L}$ & $\mathrm{M}$ & $\mathrm{N}$ & $\mathrm{O}$ & $\mathrm{P}$ & $\mathrm{Q}$ & $\mathrm{R}$ & $\mathrm{S}$ \\
\hline 1 & METHOD_NAME & LAB_CODE & VALUE_RPTD & ANAL_UNITS_RPTD & COUNTING_ERROR & TOTAL_ANAL_ERROR & MIN_DETECTABLE_ACTIVITY & PCNT_MOISTURE & LAB_QUALIFIER \\
\hline 192 & GAMMA_GS & STLRL & -0.0446 & $\mathrm{pCi} / \mathrm{L}$ & 2.2 & 2.2 & 3.98 & & $U$ \\
\hline 193 & GAMMA_GS & STLRL & 0.000741 & $\mathrm{pCi} / \mathrm{L}$ & 0.0016 & 0.0016 & 0.00275 & & $\mathrm{U}$ \\
\hline 194 & GAMMA_GS & STLRL & 0.00035 & $\mathrm{pCi} / \mathrm{L}$ & 0.00065 & 0.00065 & 0.00111 & & $\mathrm{U}$ \\
\hline 195 & GAMMA_GS & STLRL & 0.00185 & $\mathrm{pCi} / \mathrm{L}$ & 0.00066 & 0.00066 & 0.0012 & & $U$ \\
\hline 196 & GAMMA_GS & STLRL & 0.00211 & $\mathrm{pCi} / \mathrm{L}$ & 0.0027 & 0.0027 & 0.00468 & & $U$ \\
\hline 197 & GAMMA_GS & STLRL & 3.99 & $\mathrm{pCi} / \mathrm{L}$ & 23 & 23 & 44.2 & & $U$ \\
\hline 198 & GAMMA_GS & STLRL & -0.89 & $\mathrm{pCi} / \mathrm{L}$ & 2.4 & 2.4 & 4.09 & & $U$ \\
\hline 199 & GAMMA_GS & STLRL & -1.1 & $\mathrm{pCi} / \mathrm{L}$ & 2.6 & 2.6 & 4.5 & & $U$ \\
\hline 200 & GAMMA_GS & STLRL & -1 & $\mathrm{pCi} / \mathrm{L}$ & 2.7 & 2.7 & 4.64 & & $U$ \\
\hline 201 & GAMMA_GS & STLRL & 0.000698 & $\mathrm{pCi} / \mathrm{L}$ & 0.0017 & 0.0017 & 0.00303 & & $\mathrm{U}$ \\
\hline 202 & GAMMA_GS & STLRL & 0.000589 & $\mathrm{pCi} / \mathrm{L}$ & 0.0011 & 0.0011 & 0.00184 & & U \\
\hline 203 & GAMMA_GS & STLRL & 0.000962 & $\mathrm{pCi} / \mathrm{L}$ & 0.00068 & 0.00068 & 0.0012 & & $U$ \\
\hline 204 & GAMMA_GS & STLRL & 0.00016 & $\mathrm{pCi} / \mathrm{L}$ & 0.0018 & 0.0018 & 0.00311 & & U \\
\hline 205 & GAMMA_GS & STLRL & 0.269 & $\mathrm{pCi} / \mathrm{L}$ & 2.4 & 2.4 & 4.59 & & $U$ \\
\hline 206 & GAMMA_GS & STLRL & -0.678 & $\mathrm{pCi} / \mathrm{L}$ & 2.9 & 2.9 & 5.08 & & $\mathrm{U}$ \\
\hline 207 & GAMMA_GS & STLRL & 0.819 & $\mathrm{pCi} / \mathrm{L}$ & 2.1 & 2.1 & 4.21 & & U \\
\hline 208 & GAMMA_GS & STLRL & 0.629 & $\mathrm{pCi} / \mathrm{L}$ & 2 & 2 & 3.92 & & $U$ \\
\hline 209 & GAMMA_GS & STLRL & 2.7 & $\mathrm{pCi} / \mathrm{L}$ & 2.3 & 2.3 & 4.81 & & $U$ \\
\hline 210 & GAMMA_GS & STLRL & 0.704 & $\mathrm{pCi} / \mathrm{L}$ & 2.2 & 2.2 & 4.33 & & U \\
\hline 211 & GAMMA_GS & STLRL & 0.921 & $\mathrm{pCi} / \mathrm{L}$ & 2.3 & 2.3 & 4.37 & & $\mathrm{U}$ \\
\hline 212 & GAMMA_GS & STLRL & -0.358 & $\mathrm{pCi} / \mathrm{L}$ & 2 & 2 & 3.67 & & U \\
\hline 213 & GAMMA_GS & STLRL & 1.59 & $\mathrm{pCi} / \mathrm{L}$ & 2.1 & 2.1 & 4.41 & & U \\
\hline 214 & GAMMA_GS & STLRL & 2.86 & $\mathrm{pCi} / \mathrm{L}$ & 2.4 & 2.4 & 5.03 & & $\mathrm{U}$ \\
\hline 215 & GAMMA_GS & STLRL & 1.88 & $\mathrm{pCi} / \mathrm{L}$ & 2 & 2 & 4.27 & & $U$ \\
\hline 216 & GAMMA_GS & STLRL & -0.243 & $\mathrm{pCi} / \mathrm{L}$ & 2 & 2 & 3.75 & & $U$ \\
\hline 217 & GAMMA_GS & STLRL & -0.971 & $\mathrm{pCi} / \mathrm{L}$ & 1.8 & 1.8 & 2.96 & & $U$ \\
\hline 218 & GAMMA_GS & STLRL & 0.936 & $\mathrm{pCi} / \mathrm{L}$ & 2.3 & 2.3 & 4.52 & & $U$ \\
\hline 219 & GAMMA_GS & STLRL & 0.132 & $\mathrm{pCi} / \mathrm{L}$ & 2.1 & 2.1 & 4 & & U \\
\hline 220 & GAMMA_GS & STLRL & -0.244 & $\mathrm{pCi} / \mathrm{L}$ & 2 & 2 & 3.7 & & U \\
\hline 221 & GAMMA_GS & STLRL & 2.23 & $\mathrm{pCi} / \mathrm{L}$ & 2 & 2 & 4.24 & & $U$ \\
\hline 222 & GAMMA_GS & STLRL & 0.575 & $\mathrm{pCi} / \mathrm{L}$ & 2.5 & 2.5 & 4.74 & & $U$ \\
\hline 223 & GAMMA_GS & STLRL & 0.482 & $\mathrm{pCi} / \mathrm{L}$ & 2 & 2 & 3.9 & & $\mathrm{U}$ \\
\hline 224 & GAMMA_GS & STLRL & -0.337 & $\mathrm{pCi} / \mathrm{L}$ & 2.1 & 2.1 & 3.88 & & $U$ \\
\hline 225 & GAMMA_GS & STLRL & 0.0345 & $\mathrm{pCi} / \mathrm{L}$ & 2.1 & 2.1 & 3.79 & & $U$ \\
\hline 226 & GAMMA_GS & STLRL & 0.0126 & $\mathrm{pCi} / \mathrm{L}$ & 2.5 & 2.5 & 4.52 & & $\mathrm{U}$ \\
\hline 227 & GAMMA_GS & STLRL & 1.6 & $\mathrm{pCi} / \mathrm{L}$ & 2.3 & 2.3 & 4.65 & & U \\
\hline 228 & GAMMA_GS & STLRL & -0.215 & $\mathrm{pCi} / \mathrm{L}$ & 1.7 & 1.7 & 3.15 & & $U$ \\
\hline 229 & GAMMA_GS & STLRL & -1.85 & $\mathrm{pCi} / \mathrm{L}$ & 2 & 2 & 3.08 & & $\mathrm{U}$ \\
\hline
\end{tabular}




\begin{tabular}{|c|c|c|c|c|c|c|c|c|c|}
\hline & $\mathrm{K}$ & $\mathrm{L}$ & $\mathrm{M}$ & $\mathrm{N}$ & $\mathrm{O}$ & $\mathrm{P}$ & $\mathrm{Q}$ & $\mathrm{R}$ & $\mathrm{S}$ \\
\hline 1 & METHOD_NAME & LAB_CODE & VALUE_RPTD & ANAL_UNITS_RPTD & COUNTING_ERROR & TOTAL_ANAL_ERROR & MIN_DETECTABLE_ACTIVITY & PCNT_MOISTURE & LAB_QUALIFIER \\
\hline 230 & GAMMA_GS & STLRL & 0.301 & $\mathrm{pCi} / \mathrm{L}$ & 2.4 & 2.4 & 4.48 & & $U$ \\
\hline 231 & GAMMA_GS & STLRL & -0.301 & $\mathrm{pCi} / \mathrm{L}$ & 2.1 & 2.1 & 3.76 & & $\mathrm{U}$ \\
\hline 232 & GAMMA_GS & STLRL & 2.19 & $\mathrm{pCi} / \mathrm{L}$ & 2.2 & 2.2 & 4.6 & & $U$ \\
\hline 233 & GAMMA_GS & STLRL & -2.07 & $\mathrm{pCi} / \mathrm{L}$ & 1.8 & 1.8 & 2.51 & & $U$ \\
\hline 234 & GAMMA_GS & STLRL & 1.44 & $\mathrm{pCi} / \mathrm{L}$ & 1.9 & 1.9 & 3.98 & & $\mathrm{U}$ \\
\hline 235 & GAMMA_GS & STLRL & 1.04 & $\mathrm{pCi} / \mathrm{L}$ & 2.3 & 2.3 & 4.47 & & $U$ \\
\hline 236 & GAMMA_GS & STLRL & -1.13 & $\mathrm{pCi} / \mathrm{L}$ & 1.7 & 1.7 & 2.91 & & $\mathrm{U}$ \\
\hline 237 & GAMMA_GS & STLRL & -2.19 & $\mathrm{pCi} / \mathrm{L}$ & 2.3 & 2.3 & 3.66 & & $\mathrm{U}$ \\
\hline 238 & GAMMA_GS & STLRL & -2.89 & $\mathrm{pCi} / \mathrm{L}$ & 2.1 & 2.1 & 2.92 & & U \\
\hline 239 & GAMMA_GS & STLRL & 1.21 & $\mathrm{pCi} / \mathrm{L}$ & 2.4 & 2.4 & 4.62 & & $\mathrm{U}$ \\
\hline 240 & GAMMA_GS & STLRL & 0.934 & $\mathrm{pCi} / \mathrm{L}$ & 2.1 & 2.1 & 4.15 & & $\mathrm{U}$ \\
\hline 241 & GAMMA_GS & STLRL & 0.0014 & $\mathrm{pCi} / \mathrm{L}$ & 0.0015 & 0.0015 & 0.00269 & & U \\
\hline 242 & GAMMA_GS & STLRL & 0.000694 & $\mathrm{pCi} / \mathrm{L}$ & 0.00069 & 0.00069 & 0.00122 & & $\mathrm{U}$ \\
\hline 243 & GAMMA_GS & STLRL & 0.0032 & $\mathrm{pCi} / \mathrm{L}$ & 0.0013 & 0.0013 & 0.0014 & & \\
\hline 244 & GAMMA_GS & STLRL & 0.000171 & $\mathrm{pCi} / \mathrm{L}$ & 0.0018 & 0.0018 & 0.00303 & & $U$ \\
\hline 245 & GAMMA_GS & STLRL & -1.49 & $\mathrm{pCi} / \mathrm{L}$ & 2.4 & 2.4 & 4.01 & & $U$ \\
\hline 246 & GAMMA_GS & STLRL & -0.728 & $\mathrm{pCi} / \mathrm{L}$ & 2.8 & 2.8 & 4.86 & & $U$ \\
\hline 247 & GAMMA_GS & STLRL & -1.24 & $\mathrm{pCi} / \mathrm{L}$ & 2.9 & 2.9 & 4.86 & & $\mathrm{U}$ \\
\hline 248 & GAMMA_GS & STLRL & 0.937 & $\mathrm{pCi} / \mathrm{L}$ & 2.4 & 2.4 & 4.71 & & $U$ \\
\hline 249 & GAMMA_GS & STLRL & 0.246 & $\mathrm{pCi} / \mathrm{L}$ & 2 & 2 & 3.8 & & U \\
\hline 250 & GAMMA_GS & STLRL & 1.05 & $\mathrm{pCi} / \mathrm{L}$ & 2.3 & 2.3 & 4.45 & & U \\
\hline 251 & GAMMA_GS & STLRL & 0.0169 & $\mathrm{pCi} / \mathrm{L}$ & 2.1 & 2.1 & 4 & & U \\
\hline 252 & GAMMA_GS & STLRL & 2.56 & $\mathrm{pCi} / \mathrm{L}$ & 2.6 & 2.6 & 5.26 & & U \\
\hline 253 & GAMMA_GS & STLRL & 0.178 & $\mathrm{pCi} / \mathrm{L}$ & 1.9 & 1.9 & 3.66 & & U \\
\hline 254 & GAMMA_GS & STLRL & -0.533 & $\mathrm{pCi} / \mathrm{L}$ & 1.9 & 1.9 & 3.42 & & $\mathrm{U}$ \\
\hline 255 & GAMMA_GS & STLRL & 0.613 & $\mathrm{pCi} / \mathrm{L}$ & 2.4 & 2.4 & 4.5 & & $\mathrm{U}$ \\
\hline 256 & GAMMA_GS & STLRL & 0.865 & $\mathrm{pCi} / \mathrm{L}$ & 2.4 & 2.4 & 4.62 & & $U$ \\
\hline 257 & GAMMA_GS & STLRL & 0.000296 & $\mathrm{pCi} / \mathrm{L}$ & 0.0007 & 0.0007 & 0.00122 & & U \\
\hline 258 & GAMMA_GS & STLRL & 0.00342 & $\mathrm{pCi} / \mathrm{L}$ & 0.0021 & 0.0021 & 0.00383 & & $U$ \\
\hline 259 & GAMMA_GS & STLRL & 0.00223 & $\mathrm{pCi} / \mathrm{L}$ & 0.0019 & 0.0019 & 0.0034 & & U \\
\hline 260 & GAMMA_GS & STLRL & 0.000302 & $\mathrm{pCi} / \mathrm{L}$ & 0.00071 & 0.00071 & 0.00121 & & U \\
\hline 261 & GAMMA_GS & STLRL & 1.07 & $\mathrm{pCi} / \mathrm{L}$ & 2.3 & 2.3 & 4.46 & & U \\
\hline 262 & GAMMA_GS & STLRL & 2.03 & $\mathrm{pCi} / \mathrm{L}$ & 2.5 & 2.5 & 5 & & U \\
\hline 263 & GAMMA_GS & STLRL & 1.49 & $\mathrm{pCi} / \mathrm{L}$ & 2.5 & 2.5 & 4.85 & & U \\
\hline 264 & GAMMA_GS & STLRL & 1.3 & $\mathrm{pCi} / \mathrm{L}$ & 2.2 & 2.2 & 4.39 & & U \\
\hline 265 & GAMMA_GS & STLRL & 0.0802 & $\mathrm{pCi} / \mathrm{L}$ & 1.7 & 1.7 & 3.35 & & U \\
\hline 266 & GAMMA_GS & STLRL & 1.21 & $\mathrm{pCi} / \mathrm{L}$ & 2.3 & 2.3 & 4.54 & & U \\
\hline 267 & GAMMA_GS & STLRL & -0.75 & $\mathrm{pCi} / \mathrm{L}$ & 2 & 2 & 3.41 & & $\mathrm{U}$ \\
\hline
\end{tabular}

Page 85 


\begin{tabular}{|c|c|c|c|c|c|c|c|c|c|}
\hline & $\mathrm{K}$ & $\mathrm{L}$ & $\mathrm{M}$ & $\mathrm{N}$ & $\mathrm{O}$ & $\mathrm{P}$ & $\mathrm{Q}$ & $\mathrm{R}$ & $\mathrm{S}$ \\
\hline 1 & METHOD_NAME & LAB_CODE & VALUE_RPTD & ANAL_UNITS_RPTD & |COUNTING_ERROR & TOTAL_ANAL_ERROR & MIN_DETECTABLE_ACTIVITY & PCNT_MOISTURE & LAB_QUALIFIER \\
\hline 268 & GAMMA_GS & STLRL & -0.531 & $\mathrm{pCi} / \mathrm{L}$ & 2.2 & 2.2 & 3.99 & & $\mathrm{U}$ \\
\hline 269 & GAMMA_GS & STLRL & 0.00223 & $\mathrm{pCi} / \mathrm{L}$ & 0.0017 & 0.0017 & 0.00296 & & $\mathrm{U}$ \\
\hline 270 & GAMMA_GS & STLRL & 0.000609 & $\mathrm{pCi} / \mathrm{L}$ & 0.00063 & 0.00063 & 0.0011 & & $\mathrm{U}$ \\
\hline 271 & GAMMA_GS & STLRL & 0.000365 & $\mathrm{pCi} / \mathrm{L}$ & 0.00061 & 0.00061 & 0.00105 & & U \\
\hline 272 & GAMMA_GS & STLRL & 0.0000228 & $\mathrm{pCi} / \mathrm{L}$ & 0.0018 & 0.0018 & 0.00299 & & $U$ \\
\hline 273 & GAMMA_GS & STLRL & -1.22 & $\mathrm{pCi} / \mathrm{L}$ & 2.2 & 2.2 & 3.7 & & $\mathrm{U}$ \\
\hline 274 & GAMMA_GS & STLRL & 0.000663 & $\mathrm{pCi} / \mathrm{L}$ & 0.00068 & 0.00068 & 0.0012 & & U \\
\hline 275 & GAMMA_GS & STLRL & 0.00117 & $\mathrm{pCi} / \mathrm{L}$ & 0.0018 & 0.0018 & 0.00305 & & $U$ \\
\hline 276 & GAMMA_GS & STLRL & 0.000227 & $\mathrm{pCi} / \mathrm{L}$ & 0.0016 & 0.0016 & 0.00268 & & $U$ \\
\hline 277 & GAMMA_GS & STLRL & 0.000177 & $\mathrm{pCi} / \mathrm{L}$ & 0.00078 & 0.00078 & 0.00133 & & U \\
\hline 278 & GAMMA_GS & STLRL & 0.000166 & $\mathrm{pCi} / \mathrm{L}$ & 0.00064 & 0.00064 & 0.00108 & & U \\
\hline 279 & GAMMA_GS & STLRL & -0.000535 & $\mathrm{pCi} / \mathrm{L}$ & 0.0025 & 0.0025 & 0.00428 & & $\mathrm{U}$ \\
\hline 280 & GAMMA_GS & STLRL & 0.000715 & $\mathrm{pCi} / \mathrm{L}$ & 0.0029 & 0.0029 & 0.00493 & & $\mathrm{U}$ \\
\hline 281 & GAMMA_GS & STLRL & 0.00042 & $\mathrm{pCi} / \mathrm{L}$ & 0.00059 & 0.00059 & 0.00103 & & $\mathrm{U}$ \\
\hline 282 & GAMMA_GS & STLRL & 0.00156 & $\mathrm{pCi} / \mathrm{L}$ & 0.0018 & 0.0018 & 0.00319 & & $\mathrm{U}$ \\
\hline 283 & GAMMA_GS & STLRL & -0.000325 & $\mathrm{pCi} / \mathrm{L}$ & 0.00069 & 0.00069 & 0.00113 & & $\mathrm{U}$ \\
\hline 284 & GAMMA_GS & STLRL & 0.000964 & $\mathrm{pCi} / \mathrm{L}$ & 0.00071 & 0.00071 & 0.00126 & & $U$ \\
\hline 285 & GAMMA_GS & STLRL & 0.00116 & $\mathrm{pCi} / \mathrm{L}$ & 0.0019 & 0.0019 & 0.0033 & & $U$ \\
\hline 286 & GAMMA_GS & STLRL & 0.0178 & $\mathrm{pCi} / \mathrm{L}$ & 2.4 & 2.4 & 4.43 & & $\mathrm{U}$ \\
\hline 287 & GAMMA_GS & STLRL & -0.000304 & $\mathrm{pCi} / \mathrm{L}$ & 0.00068 & 0.00068 & 0.00114 & & $U$ \\
\hline 288 & GAMMA_GS & STLRL & 0.00247 & $\mathrm{pCi} / \mathrm{L}$ & 0.0025 & 0.0025 & 0.0044 & & $U$ \\
\hline 289 & GAMMA_GS & STLRL & -0.000306 & $\mathrm{pCi} / \mathrm{L}$ & 0.0018 & 0.0018 & 0.00302 & & U \\
\hline 290 & GAMMA_GS & STLRL & 0.000314 & $\mathrm{pCi} / \mathrm{L}$ & 0.00066 & 0.00066 & 0.00113 & & U \\
\hline 291 & GAMMA_GS & STLRL & -0.000243 & $\mathrm{pCi} / \mathrm{L}$ & 0.00066 & 0.00066 & 0.00109 & & $U$ \\
\hline 292 & GAMMA_GS & STLRL & 0.00196 & $\mathrm{pCi} / \mathrm{L}$ & 0.0018 & 0.0018 & 0.00315 & & U \\
\hline 293 & GAMMA_GS & STLRL & 0.00176 & $\mathrm{pCi} / \mathrm{L}$ & 0.0018 & 0.0018 & 0.00314 & & U \\
\hline 294 & GAMMA_GS & STLRL & 0.00042 & $\mathrm{pCi} / \mathrm{L}$ & 0.0007 & 0.0007 & 0.0012 & & $\mathrm{U}$ \\
\hline 295 & GAMMA_GS & STLRL & 0.491 & $\mathrm{pCi} / \mathrm{L}$ & 2.4 & 2.4 & 4.53 & & $\mathrm{U}$ \\
\hline 296 & GAMMA_GS & STLRL & -0.417 & $\mathrm{pCi} / \mathrm{L}$ & 2.5 & 2.5 & 4.35 & & $\mathrm{U}$ \\
\hline 297 & GAMMA_GS & STLRL & 0.000819 & $\mathrm{pCi} / \mathrm{L}$ & 0.00072 & 0.00072 & 0.00126 & & $U$ \\
\hline 298 & GAMMA_GS & STLRL & 0.00051 & $\mathrm{pCi} / \mathrm{L}$ & 0.0018 & 0.0018 & 0.00302 & & $U$ \\
\hline 299 & GAMMA_GS & STLRL & 0.000787 & $\mathrm{pCi} / \mathrm{L}$ & 0.0018 & 0.0018 & 0.00316 & & U \\
\hline 300 & GAMMA_GS & STLRL & -0.000108 & $\mathrm{pCi} / \mathrm{L}$ & 0.00057 & 0.00057 & 0.000975 & & U \\
\hline 301 & GAMMA_GS & STLRL & 0.517 & $\mathrm{pCi} / \mathrm{L}$ & 2.2 & 2.2 & 4.24 & & $U$ \\
\hline 302 & GAMMA_GS & STLRL & 0.125 & $\mathrm{pCi} / \mathrm{L}$ & 2.4 & 2.4 & 4.37 & & U \\
\hline 303 & GAMMA_GS & STLRL & 0.000262 & $\mathrm{pCi} / \mathrm{L}$ & 0.00073 & 0.00073 & 0.00125 & & U \\
\hline 304 & GAMMA_GS & STLRL & 0.0014 & $\mathrm{pCi} / \mathrm{L}$ & 0.0017 & 0.0017 & 0.003 & & $U$ \\
\hline 305 & GAMMA_GS & STLRL & -0.00147 & $\mathrm{pCi} / \mathrm{L}$ & 0.0018 & 0.0018 & 0.00291 & & U \\
\hline
\end{tabular}




\begin{tabular}{|c|c|c|c|c|c|c|c|c|c|}
\hline & $\mathrm{K}$ & $\mathrm{L}$ & $\mathrm{M}$ & $\mathrm{N}$ & $\mathrm{O}$ & $\mathrm{P}$ & $\mathrm{Q}$ & $\mathrm{R}$ & $\mathrm{S}$ \\
\hline 1 & METHOD_NAME & LAB_CODE & VALUE_RPTD & ANAL_UNITS_RPTD & |COUNTING_ERROR & TOTAL_ANAL_ERROR & MIN_DETECTABLE_ACTIVITY & PCNT_MOISTURE & LAB_QUALIFIER \\
\hline 306 & GAMMA_GS & STLRL & 0.000518 & $\mathrm{pCi} / \mathrm{L}$ & 0.00059 & 0.00059 & 0.00104 & & $\mathrm{U}$ \\
\hline 307 & GAMMA_GS & STLRL & 0.000622 & $\mathrm{pCi} / \mathrm{L}$ & 0.00069 & 0.00069 & 0.00119 & & $\mathrm{U}$ \\
\hline 308 & GAMMA_GS & STLRL & 0.000805 & $\mathrm{pCi} / \mathrm{L}$ & 0.0014 & 0.0014 & 0.00244 & & $\mathrm{U}$ \\
\hline 309 & GAMMA_GS & STLRL & 0.000772 & $\mathrm{pCi} / \mathrm{L}$ & 0.0014 & 0.0014 & 0.00244 & & U \\
\hline 310 & GAMMA_GS & STLRL & 0.000989 & $\mathrm{pCi} / \mathrm{L}$ & 0.00072 & 0.00072 & 0.00128 & & $\mathrm{U}$ \\
\hline 311 & GAMMA_GS & STLRL & 2.1 & $\mathrm{pCi} / \mathrm{L}$ & 2.2 & 2.2 & 4.57 & & $\mathrm{U}$ \\
\hline 312 & GAMMA_GS & STLRL & 0.278 & $\mathrm{pCi} / \mathrm{L}$ & 2.1 & 2.1 & 4.07 & & $\mathrm{U}$ \\
\hline 313 & GAMMA_GS & STLRL & 0.943 & $\mathrm{pCi} / \mathrm{L}$ & 2.3 & 2.3 & 4.5 & & $\mathrm{U}$ \\
\hline 314 & GAMMA_GS & STLRL & -0.669 & $\mathrm{pCi} / \mathrm{L}$ & 2.3 & 2.3 & 4.07 & & $\mathrm{U}$ \\
\hline 315 & GAMMA_GS & STLRL & 1.17 & $\mathrm{pCi} / \mathrm{L}$ & 2.5 & 2.5 & 4.77 & & $U$ \\
\hline 316 & GAMMA_GS & STLRL & 0.000543 & $\mathrm{pCi} / \mathrm{L}$ & 0.00064 & 0.00064 & 0.00113 & & $\mathrm{U}$ \\
\hline 317 & GAMMA_GS & STLRL & 0.00165 & $\mathrm{pCi} / \mathrm{L}$ & 0.0015 & 0.0015 & 0.00266 & & $\mathrm{U}$ \\
\hline 318 & GAMMA_GS & STLRL & -0.000871 & $\mathrm{pCi} / \mathrm{L}$ & 0.0019 & 0.0019 & 0.0032 & & $\mathrm{U}$ \\
\hline 319 & GAMMA_GS & STLRL & 0.00115 & $\mathrm{pCi} / \mathrm{L}$ & 0.00075 & 0.00075 & 0.00134 & & $\mathrm{U}$ \\
\hline 320 & GAMMA_GS & STLRL & 0.000451 & $\mathrm{pCi} / \mathrm{L}$ & 0.00067 & 0.00067 & 0.00117 & & $\mathrm{U}$ \\
\hline 321 & GAMMA_GS & STLRL & 0.00181 & $\mathrm{pCi} / \mathrm{L}$ & 0.0014 & 0.0014 & 0.00249 & & $\mathrm{U}$ \\
\hline 322 & GAMMA_GS & STLRL & 0.0012 & $\mathrm{pCi} / \mathrm{L}$ & 0.0014 & 0.0014 & 0.00253 & & $U$ \\
\hline 323 & GAMMA_GS & STLRL & 0.00095 & $\mathrm{pCi} / \mathrm{L}$ & 0.00068 & 0.00068 & 0.0012 & & $\mathrm{U}$ \\
\hline 324 & GAMMA_GS & STLRL & -2.56 & $\mathrm{pCi} / \mathrm{L}$ & 3 & 3 & 4.82 & & $\mathrm{U}$ \\
\hline 325 & GAMMA_GS & STLRL & 1.02 & $\mathrm{pCi} / \mathrm{L}$ & 2.3 & 2.3 & 4.52 & & U \\
\hline 326 & GAMMA_GS & STLRL & 1.54 & $\mathrm{pCi} / \mathrm{L}$ & 2.5 & 2.5 & 4.96 & & U \\
\hline 327 & GAMMA_GS & STLRL & 0.000156 & $\mathrm{pCi} / \mathrm{L}$ & 0.00065 & 0.00065 & 0.0011 & & U \\
\hline 328 & GAMMA_GS & STLRL & 0.00225 & $\mathrm{pCi} / \mathrm{L}$ & 0.0018 & 0.0018 & 0.0031 & & U \\
\hline 329 & GAMMA_GS & STLRL & 0.000856 & $\mathrm{pCi} / \mathrm{L}$ & 0.0016 & 0.0016 & 0.00289 & & $\mathrm{U}$ \\
\hline 330 & GAMMA_GS & STLRL & 0.000437 & $\mathrm{pCi} / \mathrm{L}$ & 0.00072 & 0.00072 & 0.00126 & & U \\
\hline 331 & GAMMA_GS & STLRL & 1.73 & $\mathrm{pCi} / \mathrm{L}$ & 2.7 & 2.7 & 5.22 & & $\mathrm{U}$ \\
\hline 332 & GAMMA_GS & STLRL & -0.556 & $\mathrm{pCi} / \mathrm{L}$ & 2.5 & 2.5 & 4.45 & & $\mathrm{U}$ \\
\hline 333 & GAMMA_GS & STLRL & 0.542 & $\mathrm{pCi} / \mathrm{L}$ & 2.4 & 2.4 & 4.61 & & $\mathrm{U}$ \\
\hline 334 & GAMMA_GS & STLRL & 1.82 & $\mathrm{pCi} / \mathrm{L}$ & 2.6 & 2.6 & 5.14 & & $\mathrm{U}$ \\
\hline 335 & GAMMA_GS & STLRL & 1.2 & $\mathrm{pCi} / \mathrm{L}$ & 2.7 & 2.7 & 5.14 & & U \\
\hline 336 & GAMMA_GS & STLRL & 0.131 & $\mathrm{pCi} / \mathrm{L}$ & 2.3 & 2.3 & 4.4 & & U \\
\hline 337 & GAMMA_GS & STLRL & 1.49 & $\mathrm{pCi} / \mathrm{L}$ & 2.7 & 2.7 & 5.3 & & U \\
\hline 338 & GAMMA_GS & STLRL & -1.9 & $\mathrm{pCi} / \mathrm{L}$ & 2.4 & 2.4 & 3.98 & & U \\
\hline 339 & GAMMA_GS & STLRL & 0.00116 & $\mathrm{pCi} / \mathrm{L}$ & 0.0015 & 0.0015 & 0.00269 & & U \\
\hline 340 & GAMMA_GS & STLRL & -0.0000359 & $\mathrm{pCi} / \mathrm{L}$ & 0.00067 & 0.00067 & 0.00115 & & U \\
\hline 341 & GAMMA_GS & STLRL & 0.000102 & $\mathrm{pCi} / \mathrm{L}$ & 0.00068 & 0.00068 & 0.00116 & & U \\
\hline 342 & GAMMA_GS & STLRL & 0.00169 & $\mathrm{pCi} / \mathrm{L}$ & 0.0019 & 0.0019 & 0.0034 & & U \\
\hline 343 & GAMMA_GS & STLRL & 0.0000488 & $\mathrm{pCi} / \mathrm{L}$ & 0.00068 & 0.00068 & 0.00117 & & U \\
\hline
\end{tabular}




\begin{tabular}{|c|c|c|c|c|c|c|c|c|c|}
\hline & $\mathrm{K}$ & $\mathrm{L}$ & $\mathrm{M}$ & $\mathrm{N}$ & $\mathrm{O}$ & $\mathrm{P}$ & $\mathrm{Q}$ & $\mathrm{R}$ & $\mathrm{S}$ \\
\hline 1 & METHOD_NAME & LAB_CODE & VALUE_RPTD & ANAL_UNITS_RPTD & |COUNTING_ERROR & TOTAL_ANAL_ERROR & MIN_DETECTABLE_ACTIVITY & PCNT_MOISTURE & LAB_QUALIFIER \\
\hline 344 & GAMMA_GS & STLRL & 0.000451 & $\mathrm{pCi} / \mathrm{L}$ & 0.002 & 0.002 & 0.00335 & & $\mathrm{U}$ \\
\hline 345 & GAMMA_GS & STLRL & 0.000534 & $\mathrm{pCi} / \mathrm{L}$ & 0.0012 & 0.0012 & 0.00216 & & $\mathrm{U}$ \\
\hline 346 & GAMMA_GS & STLRL & 0.000251 & $\mathrm{pCi} / \mathrm{L}$ & 0.00066 & 0.00066 & 0.00116 & & $\mathrm{U}$ \\
\hline 347 & I_SEP_TEMS & 320 & 0.0001191 & $\mathrm{pCi} / \mathrm{L}$ & & 5.7168E-06 & & & \\
\hline 348 & I_SEP_TEMS & 320 & 0.0000033 & $\mathrm{pCi} / \mathrm{L}$ & & 3.564E-07 & & & \\
\hline 349 & I_SEP_TEMS & 320 & 0.0000139 & $\mathrm{pCi} / \mathrm{L}$ & & 1.2232E-06 & & & \\
\hline 350 & I_SEP_TEMS & 320 & 0.0000576 & $\mathrm{pCi} / \mathrm{L}$ & & 3.9168E-06 & & & \\
\hline 351 & I_SEP_TEMS & 320 & 0.1545513 & $\mathrm{pCi} / \mathrm{L}$ & & 0.016382438 & & & \\
\hline 352 & I_SEP_TEMS & 320 & 0.0057375 & $\mathrm{pCi} / \mathrm{L}$ & & 0.00052785 & & & \\
\hline 353 & I_SEP_TEMS & 320 & 0.2697071 & $\mathrm{pCi} / \mathrm{L}$ & & 0.028588953 & & & \\
\hline 354 & I_SEP_TEMS & 320 & 0.0511546 & $\mathrm{pCi} / \mathrm{L}$ & & 0.004706223 & & & \\
\hline 355 & I_SEP_TEMS & 320 & 0.004972 & $\mathrm{pCi} / \mathrm{L}$ & & 0.000586696 & & & \\
\hline 356 & I_SEP_TEMS & 320 & 0.0000047 & $\mathrm{pCi} / \mathrm{L}$ & & 7.426E-07 & & & \\
\hline 357 & I_SEP_TEMS & 320 & 0.0001005 & $\mathrm{pCi} / \mathrm{L}$ & & 0.000007437 & & & \\
\hline 358 & I_SEP_TEMS & 320 & 0.0000108 & $\mathrm{pCi} / \mathrm{L}$ & & 1.0584E-06 & & & \\
\hline 359 & I_SEP_TEMS & 320 & 0.0000807 & $\mathrm{pCi} / \mathrm{L}$ & & 5.0034E-06 & & & \\
\hline 360 & I_SEP_TEMS & 320 & 0.0000034 & $\mathrm{pCi} / \mathrm{L}$ & & 5.236E-07 & & & \\
\hline 361 & I_SEP_TEMS & 320 & 0.0001882 & $\mathrm{pCi} / \mathrm{L}$ & & 2.14548E-05 & & & \\
\hline 362 & I_SEP_TEMS & 320 & 0.2173509 & $\mathrm{pCi} / \mathrm{L}$ & & 0.019996283 & & & \\
\hline 363 & I_SEP_TEMS & 320 & 0.2472086 & $\mathrm{pCi} / \mathrm{L}$ & & 0.022248774 & & & \\
\hline 364 & I_SEP_TEMS & 320 & 0.0034235 & $\mathrm{pCi} / \mathrm{L}$ & & 0.000335503 & & & \\
\hline 365 & I_SEP_TEMS & 320 & 0.0067254 & $\mathrm{pCi} / \mathrm{L}$ & & 0.000659089 & & & \\
\hline 366 & I_SEP_TEMS & 320 & 0.0001051 & $\mathrm{pCi} / \mathrm{L}$ & & 1.17712E-05 & & & \\
\hline 367 & I_SEP_TEMS & 320 & 0.0000035 & $\mathrm{pCi} / \mathrm{L}$ & & 0.000000455 & & & \\
\hline 368 & I_SEP_TEMS & 320 & 0.0033792 & $\mathrm{pCi} / \mathrm{L}$ & & 0.000398746 & & & \\
\hline 369 & I_SEP_TEMS & 320 & 0.0041188 & $\mathrm{pCi} / \mathrm{L}$ & & 0.000469543 & & & \\
\hline 370 & I_SEP_TEMS & 320 & 0.0000024 & $\mathrm{pCi} / \mathrm{L}$ & & 3.792E-07 & & & \\
\hline 371 & ]I_SEP_TEMS & 320 & 0.000098 & $\mathrm{pCi} / \mathrm{L}$ & & 0.0000098 & & & \\
\hline 372 & I_SEP_TEMS & 320 & 0.0000708 & $\mathrm{pCi} / \mathrm{L}$ & & 7.6464E-06 & & & \\
\hline 373 & I_SEP_TEMS & 320 & 0.000019 & $\mathrm{pCi} / \mathrm{L}$ & & 0.00000247 & & & \\
\hline 374 & PUISO_IE_PLATE_AEA & STLRL & 0.0000479 & $\mathrm{pCi} / \mathrm{L}$ & 0.000055 & 0.000056 & 0.0000433 & & $U$ \\
\hline 375 & PUISO_IE_PLATE_AEA & STLRL & 0.00000802 & $\mathrm{pCi} / \mathrm{L}$ & 0.0000069 & 0.0000078 & 0.0000102 & & U \\
\hline 376 & PUISO_IE_PLATE_AEA & STLRL & 0.0000131 & $\mathrm{pCi} / \mathrm{L}$ & 0.0000066 & 0.0000074 & 0.00000238 & & $\mathrm{~J}$ \\
\hline 377 & PUISO_IE_PLATE_AEA & STLRL & 0 & $\mathrm{pCi} / \mathrm{L}$ & 0.000062 & 0.000062 & 0.0000689 & & $U$ \\
\hline 378 & PUISO_IE_PLATE_AEA & STLRL & 0.0000255 & $\mathrm{pCi} / \mathrm{L}$ & 0.000011 & 0.000012 & 0.00000693 & & $\mathrm{~J}$ \\
\hline 379 & PUISO_IE_PLATE_AEA & STLRL & 0.0000475 & $\mathrm{pCi} / \mathrm{L}$ & 0.000079 & 0.000079 & 0.000148 & & U \\
\hline 380 & PUISO_IE_PLATE_AEA & STLRL & 0.0000632 & $\mathrm{pCi} / \mathrm{L}$ & 0.000089 & 0.00009 & 0.0000857 & & $U$ \\
\hline 381 & PUISO_IE_PLATE_AEA & STLRL & 0.0000157 & $\mathrm{pCi} / \mathrm{L}$ & 0.0000087 & 0.0000097 & 0.00000696 & & $\mathrm{~J}$ \\
\hline
\end{tabular}




\begin{tabular}{|c|c|c|c|c|c|c|c|c|c|}
\hline & $\mathrm{K}$ & $L$ & $\mathrm{M}$ & $\mathrm{N}$ & $\mathrm{O}$ & $\mathrm{P}$ & $\mathrm{Q}$ & $\mathrm{R}$ & $\mathrm{S}$ \\
\hline 1 & METHOD_NAME & LAB_CODE & VALUE_RPTD & ANAL_UNITS_RPTD & COUNTING_ERROR & TOTAL_ANAL_ERROR & MIN_DETECTABLE_ACTIVITY & PCNT_MOISTURE & LAB_QUALIFIER \\
\hline 382 & PUISO_IE_PLATE_AEA & STLRL & 0.000025 & $\mathrm{pCi} / \mathrm{L}$ & 0.000016 & 0.000017 & 0.0000159 & & $\mathrm{~J}$ \\
\hline 383 & PUISO_IE_PLATE_AEA & STLRL & 0.0000339 & $\mathrm{pCi} / \mathrm{L}$ & 0.00003 & 0.000031 & 0.0000184 & & $\mathrm{~J}$ \\
\hline 384 & PUISO_IE_PLATE_AEA & STLRL & & $\mathrm{pCi} / \mathrm{L}$ & 0.000019 & 0.000019 & 0.0000209 & & $U$ \\
\hline 385 & PUISO_IE_PLATE_AEA & STLRL & 0.0000279 & $\mathrm{pCi} / \mathrm{L}$ & 0.000012 & 0.000013 & 0.00000339 & & $\mathrm{~J}$ \\
\hline 386 & PUISO_IE_PLATE_AEA & STLRL & 0.0000143 & $\mathrm{pCi} / \mathrm{L}$ & 0.000011 & 0.000011 & 0.000014 & & $\mathrm{~J}$ \\
\hline 387 & PUISO_IE_PLATE_AEA & STLRL & 0.0000108 & $\mathrm{pCi} / \mathrm{L}$ & 0.000028 & 0.000028 & 0.000062 & & $U$ \\
\hline 388 & PUISO_IE_PLATE_AEA & STLRL & 0.0000189 & $\mathrm{pCi} / \mathrm{L}$ & 0.000035 & 0.000035 & 0.0000666 & & $\mathrm{U}$ \\
\hline 389 & PUISO_IE_PLATE_AEA & STLRL & 0.00000262 & $\mathrm{pCi} / \mathrm{L}$ & 0.0000067 & 0.0000067 & 0.0000128 & & $U$ \\
\hline 390 & PUISO_IE_PLATE_AEA & STLRL & 0.000025 & $\mathrm{pCi} / \mathrm{L}$ & 0.000019 & 0.00002 & 0.0000217 & & \\
\hline 391 & PUISO_IE_PLATE_AEA & STLRL & 0.00000166 & $\mathrm{pCi} / \mathrm{L}$ & 0.000019 & 0.000019 & 0.0000569 & & $U$ \\
\hline 392 & PUISO_IE_PLATE_AEA & STLRL & 0.00002 & $\mathrm{pCi} / \mathrm{L}$ & 0.000026 & 0.000026 & 0.000043 & & $U$ \\
\hline 393 & PUISO_IE_PLATE_AEA & STLRL & 0.0000332 & $\mathrm{pCi} / \mathrm{L}$ & 0.000021 & 0.000022 & 0.0000209 & & \\
\hline 394 & PUISO_IE_PLATE_AEA & STLRL & 0.0000256 & $\mathrm{pCi} / \mathrm{L}$ & 0.000029 & 0.000029 & 0.0000403 & & $U$ \\
\hline 395 & PUISO_IE_PLATE_AEA & STLRL & 0.0000146 & $\mathrm{pCi} / \mathrm{L}$ & 0.000013 & 0.000013 & 0.0000133 & & \\
\hline 396 & PUISO_IE_PLATE_AEA & STLRL & 0.000049 & $\mathrm{pCi} / \mathrm{L}$ & 0.000031 & 0.000033 & 0.0000266 & & \\
\hline 397 & PUISO_IE_PLATE_AEA & STLRL & 0.0000349 & $\mathrm{pCi} / \mathrm{L}$ & 0.000031 & 0.000032 & 0.0000189 & & \\
\hline 398 & PUISO_IE_PLATE_AEA & STLRL & 0.000179 & $\mathrm{pCi} / \mathrm{L}$ & 0.000062 & 0.000069 & 0.0000143 & & \\
\hline 399 & PUISO_IE_PLATE_AEA & STLRL & 0.0000548 & $\mathrm{pCi} / \mathrm{L}$ & 0.000071 & 0.000072 & 0.000118 & & $U$ \\
\hline 400 & PUISO_IE_PLATE_AEA & STLRL & 0.00015 & $\mathrm{pCi} / \mathrm{L}$ & 0.000067 & 0.00007 & 0.0000203 & & \\
\hline 401 & PUISO_IE_PLATE_AEA & STLRL & 0.0000734 & $\mathrm{pCi} / \mathrm{L}$ & 0.000041 & 0.000043 & 0.0000309 & & \\
\hline 402 & PUISO_IE_PLATE_AEA & STLRL & 0.000019 & $\mathrm{pCi} / \mathrm{L}$ & 0.000016 & 0.000016 & 0.0000199 & & $U$ \\
\hline 403 & PUISO_IE_PLATE_AEA & STLRL & 0.0000498 & $\mathrm{pCi} / \mathrm{L}$ & 0.00005 & 0.00005 & 0.0000337 & & $U$ \\
\hline 404 & PUISO_IE_PLATE_AEA & STLRL & 0.0000121 & $\mathrm{pCi} / \mathrm{L}$ & 0.000044 & 0.000044 & 0.000114 & & $U$ \\
\hline 405 & PUISO_IE_PLATE_AEA & STLRL & 0.00001 & $\mathrm{pCi} / \mathrm{L}$ & 0.000011 & 0.000011 & 0.00000744 & & $U$ \\
\hline 406 & PUISO_IE_PLATE_AEA & STLRL & 0.0000138 & $\mathrm{pCi} / \mathrm{L}$ & 0.0000096 & 0.00001 & 0.00000983 & & \\
\hline 407 & PUISO_IE_PLATE_AEA & STLRL & 0.000028 & $\mathrm{pCi} / \mathrm{L}$ & 0.00004 & 0.00004 & 0.000038 & & $U$ \\
\hline 408 & PUISO_IE_PLATE_AEA & STLRL & & $\mathrm{pCi} / \mathrm{L}$ & 0.000051 & 0.000051 & 0.0000567 & & $U$ \\
\hline 409 & PUISO_IE_PLATE_AEA & STLRL & 0.00000689 & $\mathrm{pCi} / \mathrm{L}$ & 0.0000055 & 0.0000057 & 0.00000262 & & \\
\hline 410 & PUISO_IE_PLATE_AEA & STLRL & 0.00004 & $\mathrm{pCi} / \mathrm{L}$ & 0.000021 & 0.000022 & 0.0000074 & & \\
\hline 411 & PUISO_IE_PLATE_AEA & STLRL & & $\mathrm{pCi} / \mathrm{L}$ & 0.000033 & 0.000033 & 0.0000363 & & $U$ \\
\hline 412 & PUISO_IE_PLATE_AEA & STLRL & 0.00000951 & $\mathrm{pCi} / \mathrm{L}$ & 0.000019 & 0.000019 & 0.0000258 & & $U$ \\
\hline 413 & PUISO_IE_PLATE_AEA & STLRL & 0.0000226 & $\mathrm{pCi} / \mathrm{L}$ & 0.000016 & 0.000016 & 0.0000183 & & \\
\hline 414 & PUISO_IE_PLATE_AEA & STLRL & 0.0000169 & $\mathrm{pCi} / \mathrm{L}$ & 0.000011 & 0.000011 & 0.0000101 & & \\
\hline 415 & PUISO_IE_PLATE_AEA & STLRL & 0.0000387 & $\mathrm{pCi} / \mathrm{L}$ & 0.000058 & 0.000058 & 0.0000949 & & $U$ \\
\hline 416 & PUISO_IE_PLATE_AEA & STLRL & -0.0000244 & $\mathrm{pCi} / \mathrm{L}$ & 0.000035 & 0.000035 & 0.000113 & & $U$ \\
\hline 417 & PUISO_IE_PLATE_AEA & STLRL & 0.00000766 & $\mathrm{pCi} / \mathrm{L}$ & 0.000012 & 0.000012 & 0.0000198 & & $\mathrm{U}$ \\
\hline 418 & SRISO_SËP_PRECIP_GPC & QTESRL & 0.0532 & $\mathrm{pCi} / \mathrm{L}$ & 0.02 & 0.029 & 0.039 & & $\mathrm{~J}$ \\
\hline 419 & SRISO_SEP_PRECIP_GPC & QTESRL & 0.0592 & $\mathrm{pCi} / \mathrm{L}$ & 0.02 & 0.031 & 0.0408 & & $\mathrm{~J}$ \\
\hline
\end{tabular}

Page 89 


\begin{tabular}{|c|c|c|c|c|c|c|c|c|c|}
\hline & $\mathrm{K}$ & $\mathrm{L}$ & $\mathrm{M}$ & $\mathrm{N}$ & $\mathrm{O}$ & $\mathrm{P}$ & $\mathrm{Q}$ & $\mathrm{R}$ & $\mathrm{S}$ \\
\hline 1 & METHOD_NAME & LAB_CODE & VALUE_RPTD & ANAL_UNITS_RPTD & COUNTING_ERROR & TOTAL_ANAL_ERROR & MIN_DETECTABLE_ACTIVITY & PCNT_MOISTURE & LAB_QUALIFIER \\
\hline 420 & 905.0_SR_GPC & QTESRL & 0.0922 & $\mathrm{pCi} / \mathrm{L}$ & 0.023 & 0.035 & 0.0314 & & \\
\hline 421 & 905.0_SR_GPC & QTESRL & 0.0895 & $\mathrm{pCi} / \mathrm{L}$ & 0.023 & 0.035 & 0.0347 & & \\
\hline 422 & 905.0_SR_GPC & QTESRL & 0.0023 & $\mathrm{pCi} / \mathrm{L}$ & 0.0035 & 0.023 & 0.0435 & & $\mathrm{U}$ \\
\hline 423 & 905.0_SR_GPC & QTESRL & 0.0671 & $\mathrm{pCi} / \mathrm{L}$ & 0.029 & 0.043 & 0.0595 & & \\
\hline 424 & SRISO_SĒP_PRECIP_GPC & QTESRL & 0.1 & $\mathrm{pCi} / \mathrm{L}$ & 0.025 & 0.037 & 0.0342 & & \\
\hline 425 & SRISO_SEP_PRECIP_GPC & QTESRL & 0.0709 & $\mathrm{pCi} / \mathrm{L}$ & 0.023 & 0.033 & 0.038 & & \\
\hline 426 & SRISO_SEP_PRECIP_GPC & QTESRL & 0.0783 & $\mathrm{pCi} / \mathrm{L}$ & 0.024 & 0.034 & 0.0374 & & \\
\hline 427 & SRISO_SEP_PRECIP_GPC & QTESRL & 0.0709 & $\mathrm{pCi} / \mathrm{L}$ & 0.024 & 0.033 & 0.0388 & & \\
\hline 428 & SRISO_SEP_PRECIP_GPC & QTESRL & 0.0696 & $\mathrm{pCi} / \mathrm{L}$ & 0.022 & 0.032 & 0.0364 & & \\
\hline 429 & SRISO_SEP_PRECIP_GPC & QTESRL & 0.0852 & $\mathrm{pCi} / \mathrm{L}$ & 0.023 & 0.034 & 0.0349 & & \\
\hline 430 & SRISO_SEP_PRECIP_GPC & QTESRL & 0.0942 & $\mathrm{pCi} / \mathrm{L}$ & 0.029 & 0.041 & 0.0468 & & \\
\hline 431 & SRISO_SEP_PRECIP_GPC & QTESRL & 0.0943 & $\mathrm{pCi} / \mathrm{L}$ & 0.033 & 0.044 & 0.0515 & & \\
\hline 432 & SRISO_SEP_PRECIP_GPC & QTESRL & 0.0664 & $\mathrm{pCi} / \mathrm{L}$ & 0.025 & 0.036 & 0.0462 & & \\
\hline 433 & ]SRISO_SEP_PRECIP_GPC & QTESRL & 0.0792 & $\mathrm{pCi} / \mathrm{L}$ & 0.026 & 0.037 & 0.043 & & \\
\hline 434 & SRISO_SEP_PRECIP_GPC & QTESRL & 0.0699 & $\mathrm{pCi} / \mathrm{L}$ & 0.025 & 0.034 & 0.0405 & & \\
\hline 435 & SRISO_SEP_PRECIP_GPC & QTESRL & 0.0699 & $\mathrm{pCi} / \mathrm{L}$ & 0.026 & 0.035 & 0.044 & & \\
\hline 436 & SRISO_SEP_PRECIP_GPC & QTESRL & 0.0637 & $\mathrm{pCi} / \mathrm{L}$ & 0.023 & 0.032 & 0.0399 & & \\
\hline 437 & SRISO_SEP_PRECIP_GPC & QTESRL & 0.0952 & $\mathrm{pCi} / \mathrm{L}$ & 0.031 & 0.043 & 0.0494 & & \\
\hline 438 & SRISO_SEP_PRECIP_GPC & QTESRL & 0.0817 & $\mathrm{pCi} / \mathrm{L}$ & 0.028 & 0.038 & 0.0448 & & \\
\hline 439 & SRISO_SEP_PRECIP_GPC & QTESRL & 0.0805 & $\mathrm{pCi} / \mathrm{L}$ & 0.028 & 0.038 & 0.0458 & & \\
\hline 440 & SRISO_SEP_PRECIP_GPC & QTESRL & 0.0873 & $\mathrm{pCi} / \mathrm{L}$ & 0.028 & 0.038 & 0.0425 & & \\
\hline 441 & SRISO_SEP_PRECIP_GPC & QTESRL & 0.0733 & $\mathrm{pCi} / \mathrm{L}$ & 0.021 & 0.031 & 0.0326 & & \\
\hline 442 & SRISO_SEP_PRECIP_GPC & QTESRL & 0.0631 & $\mathrm{pCi} / \mathrm{L}$ & 0.02 & 0.029 & 0.0331 & & \\
\hline 443 & 905.0_SR_GPC & STLRL & 0.069 & $\mathrm{pCi} / \mathrm{L}$ & 0.02 & 0.03 & 0.0312 & & \\
\hline 444 & 905.0_SR_GPC & STLRL & 0.0723 & $\mathrm{pCi} / \mathrm{L}$ & 0.021 & 0.031 & 0.0334 & & \\
\hline 445 & 905.0_SR_GPC & STLRL & -0.00638 & $\mathrm{pCi} / \mathrm{L}$ & 0.019 & 0.019 & 0.0377 & & $\mathrm{U}$ \\
\hline 446 & 905.0_SR_GPC & STLRL & 0.0694 & $\mathrm{pCi} / \mathrm{L}$ & 0.019 & 0.029 & 0.029 & & \\
\hline 447 & SRISO_SEP_PRECIP_GPC & STLRL & 0.0679 & $\mathrm{pCi} / \mathrm{L}$ & 0.02 & 0.03 & 0.0324 & & \\
\hline 448 & SRISO_SEP_PRECIP_GPC & STLRL & 0.0599 & $\mathrm{pCi} / \mathrm{L}$ & 0.018 & 0.027 & 0.0282 & & $\mathrm{~J}$ \\
\hline 449 & SRISO_SEP_PRECIP_GPC & STLRL & 0.0692 & $\mathrm{pCi} / \mathrm{L}$ & 0.023 & 0.033 & 0.0375 & & \\
\hline 450 & SRISO_SEP_PRECIP_GPC & STLRL & 0.0654 & $\mathrm{pCi} / \mathrm{L}$ & 0.02 & 0.029 & 0.0324 & & \\
\hline 451 & SRISO_SEP_PRECIP_GPC & STLRL & 0.0403 & $\mathrm{pCi} / \mathrm{L}$ & 0.016 & 0.025 & 0.0336 & & $\mathrm{~J}$ \\
\hline 452 & SRISO_SEP_PRECIP_GPC & STLRL & 0.0405 & $\mathrm{pCi} / \mathrm{L}$ & 0.02 & 0.03 & 0.0428 & & U \\
\hline 453 & SRISO_SEP_PRECIP_GPC & STLRL & 0.0771 & $\mathrm{pCi} / \mathrm{L}$ & 0.019 & 0.03 & 0.0279 & & \\
\hline 454 & SRISO_SEP_PRECIP_GPC & STLRL & 0.052 & $\mathrm{pCi} / \mathrm{L}$ & 0.017 & 0.025 & 0.0284 & & $\mathrm{~J}$ \\
\hline 455 & SRISO_SEP_PRECIP_GPC & STLRL & 0.0487 & $\mathrm{pCi} / \mathrm{L}$ & 0.017 & 0.025 & 0.0306 & & $\mathrm{~J}$ \\
\hline 456 & SRISO_SEP_PRECIP_GPC & STLRL & 0.0473 & $\mathrm{pCi} / \mathrm{L}$ & 0.017 & 0.025 & 0.0296 & & $\mathrm{~J}$ \\
\hline 457 & SRISO_SEP_PRECIP_GPC & STLRL & 0.0644 & $\mathrm{pCi} / \mathrm{L}$ & 0.018 & 0.028 & 0.0299 & & \\
\hline
\end{tabular}




\begin{tabular}{|c|c|c|c|c|c|c|c|c|c|}
\hline & $\mathrm{K}$ & $\mathrm{L}$ & $\mathrm{M}$ & $\mathrm{N}$ & $\mathrm{O}$ & $\mathrm{P}$ & $\mathrm{Q}$ & $\mathrm{R}$ & $\mathrm{S}$ \\
\hline 1 & METHOD_NAME & LAB_CODE & VALUE_RPTD & ANAL_UNITS_RPTD & COUNTING_ERROR & TOTAL_ANAL_ERROR & MIN_DETECTABLE_ACTIVITY & PCNT_MOISTURE & LAB_QUALIFIER \\
\hline 458 & SRISO_SEP_PRECIP_GPC & STLRL & 0.049 & $\mathrm{pCi} / \mathrm{L}$ & 0.016 & 0.024 & 0.0267 & & $\mathrm{~J}$ \\
\hline 459 & SRISO_SEP_PRECIP_GPC & STLRL & 0.0606 & $\mathrm{pCi} / \mathrm{L}$ & 0.018 & 0.027 & 0.0277 & & \\
\hline 460 & SRISO_SEP_PRECIP_GPC & STLRL & 0.0465 & $\mathrm{pCi} / \mathrm{L}$ & 0.016 & 0.024 & 0.0289 & & $\mathrm{~J}$ \\
\hline 461 & SRISO_SEP_PRECIP_GPC & STLRL & 0.0566 & $\mathrm{pCi} / \mathrm{L}$ & 0.019 & 0.027 & 0.0314 & & $\mathrm{~J}$ \\
\hline 462 & SRISO_SEP_PRECIP_GPC & STLRL & 0.0572 & $\mathrm{pCi} / \mathrm{L}$ & 0.019 & 0.027 & 0.0317 & & $\mathrm{~J}$ \\
\hline 463 & SRISO_SEP_PRECIP_GPC & STLRL & 0.0485 & $\mathrm{pCi} / \mathrm{L}$ & 0.017 & 0.025 & 0.0309 & & $\mathrm{~J}$ \\
\hline 464 & SRISO_SEP_PRECIP_GPC & STLRL & 0.0417 & pCi/L & 0.016 & 0.024 & 0.0319 & & $\mathrm{~J}$ \\
\hline 465 & SRISO_SEP_PRECIP_GPC & STLRL & 0.0635 & pCi/L & 0.019 & 0.028 & 0.0298 & & \\
\hline 466 & SRISO_SEP_PRECIP_GPC & STLRL & 0.0523 & $\mathrm{pCi} / \mathrm{L}$ & 0.018 & 0.026 & 0.0308 & & $\mathrm{~J}$ \\
\hline 467 & SRISO_SEP_PRECIP_GPC & STLRL & 0.0579 & $\mathrm{pCi} / \mathrm{L}$ & 0.02 & 0.029 & 0.0347 & & $\mathrm{~J}$ \\
\hline 468 & SRISO_SEP_PRECIP_GPC & STLRL & 0.0518 & pCi/L & 0.018 & 0.026 & 0.0314 & & $\mathrm{~J}$ \\
\hline 469 & SRISO_SEP_PRECIP_GPC & STLRL & 0.0728 & $\mathrm{pCi} / \mathrm{L}$ & 0.018 & 0.028 & 0.0263 & & \\
\hline 470 & $905.0 \_\overline{S R}$ SPPC & STLRL & 0.0436 & $\mathrm{pCi} / \mathrm{L}$ & 0.018 & 0.026 & 0.0356 & & $\mathrm{~J}$ \\
\hline 471 & 905.0_SR_GPC & STLRL & 0.0491 & pCi/L & 0.019 & 0.027 & 0.0357 & & $\mathrm{~J}$ \\
\hline 472 & 905.0_SR_GPC & STLRL & -0.00944 & $\mathrm{pCi} / \mathrm{L}$ & 0.026 & 0.026 & 0.053 & & $U$ \\
\hline 473 & 905.0_SR_GPC & STLRL & 0.0609 & $\mathrm{pCi} / \mathrm{L}$ & 0.021 & 0.03 & 0.0353 & & \\
\hline 474 & SRISO_SËP_PRECIP_GPC & STLRL & 0.0726 & $\mathrm{pCi} / \mathrm{L}$ & 0.021 & 0.031 & 0.033 & & \\
\hline 475 & SRISO_SEP_PRECIP_GPC & STLRL & 0.0598 & $\mathrm{pCi} / \mathrm{L}$ & 0.017 & 0.026 & 0.0269 & & $\mathrm{~J}$ \\
\hline 476 & SRISO_SEP_PRECIP_GPC & STLRL & 0.0814 & pCi/L & 0.022 & 0.032 & 0.0314 & & \\
\hline 477 & SRISO_SEP_PRECIP_GPC & STLRL & 0.0724 & pCi/L & 0.021 & 0.031 & 0.0332 & & \\
\hline 478 & SRISO_SEP_PRECIP_GPC & STLRL & 0.106 & $\mathrm{pCi} / \mathrm{L}$ & 0.026 & 0.04 & 0.0386 & & \\
\hline 479 & SRISO_SEP_PRECIP_GPC & STLRL & 0.0787 & $\mathrm{pCi} / \mathrm{L}$ & 0.03 & 0.041 & 0.0535 & & \\
\hline 480 & SRISO_SEP_PRECIP_GPC & STLRL & 0.0562 & pCi/L & 0.021 & 0.03 & 0.0379 & & $\mathrm{~J}$ \\
\hline 481 & SRISO_SEP_PRECIP_GPC & STLRL & 0.0703 & $\mathrm{pCi} / \mathrm{L}$ & 0.022 & 0.032 & 0.0374 & & \\
\hline 482 & SRISO_SEP_PRECIP_GPC & STLRL & 0.0721 & $\mathrm{pCi} / \mathrm{L}$ & 0.022 & 0.032 & 0.0372 & & \\
\hline 483 & SRISO_SEP_PRECIP_GPC & STLRL & 0.0677 & $\mathrm{pCi} / \mathrm{L}$ & 0.021 & 0.031 & 0.0346 & & \\
\hline 484 & SRISO_SEP_PRECIP_GPC & STLRL & 0.0579 & $\mathrm{pCi} / \mathrm{L}$ & 0.021 & 0.031 & 0.0399 & & $\mathrm{~J}$ \\
\hline 485 & SRISO_SEP_PRECIP_GPC & STLRL & 0.0673 & $\mathrm{pCi} / \mathrm{L}$ & 0.022 & 0.032 & 0.0379 & & \\
\hline 486 & SRISO_SEP_PRECIP_GPC & STLRL & 0.0746 & $\mathrm{pCi} / \mathrm{L}$ & 0.026 & 0.036 & 0.0436 & & \\
\hline 487 & SRISO_SEP_PRECIP_GPC & STLRL & 0.166 & pCi/L & 0.029 & 0.051 & 0.0336 & & \\
\hline 488 & SRISO_SEP_PRECIP_GPC & STLRL & 0.0687 & $\mathrm{pCi} / \mathrm{L}$ & 0.024 & 0.033 & 0.0405 & & \\
\hline 489 & SRISO_SEP_PRECIP_GPC & STLRL & 0.081 & pCi/L & 0.024 & 0.035 & 0.0379 & & \\
\hline 490 & SRISO_SEP_PRECIP_GPC & STLRL & 0.269 & $\mathrm{pCi} / \mathrm{L}$ & 0.04 & 0.076 & 0.0419 & & \\
\hline 491 & SRISO_SEP_PRECIP_GPC & STLRL & 0.107 & $\mathrm{pCi} / \mathrm{L}$ & 0.027 & 0.041 & 0.0395 & & \\
\hline 492 & SRISO_SEP_PRECIP_GPC & STLRL & 0.0775 & $\mathrm{pCi} / \mathrm{L}$ & 0.024 & 0.035 & 0.0403 & & \\
\hline 493 & SRISO_SEP_PRECIP_GPC & STLRL & 0.071 & pCi/L & 0.036 & 0.042 & 0.059 & & \\
\hline 494 & SRISO_SEP_PRECIP_GPC & STLRL & 0.0764 & $\mathrm{pCi} / \mathrm{L}$ & 0.031 & 0.037 & 0.0455 & & \\
\hline 495 & SRISO_SEP_PRECIP_GPC & STLRL & 0.0645 & $\mathrm{pCi} / \mathrm{L}$ & 0.023 & 0.029 & 0.0318 & & \\
\hline
\end{tabular}




\begin{tabular}{|c|c|c|c|c|c|c|c|c|}
\hline 1 & METHOD_NAME & LAB_CODE & \begin{tabular}{l|l|} 
VALUE_RPTD & ANAL_UNITS_RPTD \\
\end{tabular} & COUNTING_ERROR & TOTAL_ANAL_ERROR & MIN_DETECTABLE_ACTIVITY & PCNT_MOISTURE & LAB_QUALIFIER \\
\hline 496 & SRISO_SEP_PRECIP_GPC & STLRL & $0.0632 \mathrm{pCi} / \mathrm{L}$ & 0.033 & 0.038 & 0.0543 & & \\
\hline 497 & SRISO_SEP_PRECIP_GPC & STLRL & $0.0694 \mathrm{pCi} / \mathrm{L}$ & 0.027 & 0.033 & 0.04 & & \\
\hline 498 & SRISO_SEP_PRECIP_GPC & STLRL & $0.0616 \mathrm{pCi} / \mathrm{L}$ & 0.029 & 0.034 & 0.0452 & & \\
\hline 499 & SRISO_SEP_PRECIP_GPC & STLRL & $0.0717 \mathrm{pCi} / \mathrm{L}$ & 0.026 & 0.032 & 0.0352 & & \\
\hline 500 & SRISO_SEP_PRECIP_GPC & STLRL & $0.0812 \mathrm{pCi} / \mathrm{L}$ & 0.032 & 0.039 & 0.0482 & & \\
\hline 501 & SRISO_SEP_PRECIP_GPC & STLRL & $0.0458 \mathrm{pCi} / \mathrm{L}$ & 0.02 & 0.024 & 0.0301 & & $\mathrm{~J}$ \\
\hline 502 & SRISO_SEP_PRECIP_GPC & STLRL & $0.0573 \mathrm{pCi} / \mathrm{L}$ & 0.023 & 0.028 & 0.0334 & & $\mathrm{~J}$ \\
\hline 503 & SRISO_SEP_PRECIP_GPC & STLRL & $0.0625 \mathrm{pCi} / \mathrm{L}$ & 0.024 & 0.03 & 0.0353 & & \\
\hline 504 & SRISO_SEP_PRECIP_GPC & STLRL & $0.0517 \mathrm{pCi} / \mathrm{L}$ & 0.021 & 0.026 & 0.03 & & $\mathrm{~J}$ \\
\hline 505 & SRISO_SEP_PRECIP_GPC & STLRL & $0.071 \mathrm{pCi} / \mathrm{L}$ & 0.023 & 0.03 & 0.0311 & & \\
\hline 506 & SRISO_SEP_PRECIP_GPC & STLRL & $0.058 \mathrm{pCi} / \mathrm{L}$ & 0.023 & 0.028 & 0.0328 & & $\mathrm{~J}$ \\
\hline 507 & SRISO_SEP_PRECIP_GPC & STLRL & $0.0546 \mathrm{pCi} / \mathrm{L}$ & 0.023 & 0.028 & 0.0345 & & $\mathrm{~J}$ \\
\hline 508 & SRISO_SEP_PRECIP_GPC & STLRL & $0.0458 \mathrm{pCi} / \mathrm{L}$ & 0.022 & 0.026 & 0.0349 & & $\mathrm{~J}$ \\
\hline 509 & SRISO_SEP_PRECIP_GPC & STLRL & $0.0795 \mathrm{pCi} / \mathrm{L}$ & 0.029 & 0.036 & 0.04 & & \\
\hline 510 & ]SRISO_SEP_PRECIP_GPC & STLRL & $0.0501 \mathrm{pCi} / \mathrm{L}$ & 0.027 & 0.032 & 0.0447 & & $\mathrm{~J}$ \\
\hline 511 & SRISO_SEP_PRECIP_GPC & STLRL & $0.0739 \mathrm{pCi} / \mathrm{L}$ & 0.031 & 0.038 & 0.0476 & & \\
\hline 512 & SRISO_SEP_PRECIP_GPC & STLRL & $0.0552 \mathrm{pCi} / \mathrm{L}$ & 0.028 & 0.032 & 0.0429 & & $\mathrm{~J}$ \\
\hline 513 & SRISO_SEP_PRECIP_GPC & STLRL & $0.0735 \mathrm{pCi} / \mathrm{L}$ & 0.026 & 0.033 & 0.0361 & & \\
\hline 514 & SRISO_SEP_PRECIP_GPC & STLRL & $0.0592 \mathrm{pCi} / \mathrm{L}$ & 0.024 & 0.029 & 0.0336 & & $\mathrm{~J}$ \\
\hline 515 & SRISO_SEP_PRECIP_GPC & STLRL & $0.0681 \mathrm{pCi} / \mathrm{L}$ & 0.026 & 0.032 & 0.0376 & & \\
\hline 516 & SRISO_SEP_PRECIP_GPC & STLRL & $0.05 \mathrm{pCi} / \mathrm{L}$ & 0.028 & 0.032 & 0.0454 & & $\mathrm{~J}$ \\
\hline 517 & SRISO_SEP_PRECIP_GPC & STLRL & $0.0661 \mathrm{pCi} / \mathrm{L}$ & 0.024 & 0.03 & 0.0342 & & \\
\hline 518 & SRISO_SEP_PRECIP_GPC & STLRL & $0.0539 \mathrm{pCi} / \mathrm{L}$ & 0.026 & 0.031 & 0.0401 & & $\mathrm{~J}$ \\
\hline 519 & SRISO_SEP_PRECIP_GPC & STLRL & $0.0519 \mathrm{pCi} / \mathrm{L}$ & 0.024 & 0.029 & 0.0378 & & $\mathrm{~J}$ \\
\hline 520 & SRISO_SEP_PRECIP_GPC & STLRL & $0.0714 \mathrm{pCi} / \mathrm{L}$ & 0.025 & 0.032 & 0.0338 & & \\
\hline 521 & SRISO_SEP_PRECIP_GPC & STLRL & $0.0647 \mathrm{pCi} / \mathrm{L}$ & 0.025 & 0.031 & 0.0372 & & \\
\hline 522 & SRISO_SEP_PRECIP_GPC & STLRL & $0.0461 \mathrm{pCi} / \mathrm{L}$ & 0.022 & 0.027 & 0.0351 & & $\mathrm{~J}$ \\
\hline 523 & SRISO_SEP_PRECIP_GPC & STLRL & $0.0775 \mathrm{pCi} / \mathrm{L}$ & 0.026 & 0.033 & 0.036 & & \\
\hline 524 & SRISO_SEP_PRECIP_GPC & STLRL & $0.0765 \mathrm{pCi} / \mathrm{L}$ & 0.024 & 0.031 & 0.0305 & & \\
\hline 525 & SRISO_SEP_PRECIP_GPC & STLRL & $0.0835 \mathrm{pCi} / \mathrm{L}$ & 0.027 & 0.034 & 0.0356 & & \\
\hline 526 & SRISO_SEP_PRECIP_GPC & STLRL & $0.07 \mathrm{pCi} / \mathrm{L}$ & 0.025 & 0.031 & 0.0347 & & \\
\hline 527 & SRISO_SEP_PRECIP_GPC & STLRL & $0.068 \mathrm{pCi} / \mathrm{L}$ & 0.024 & 0.031 & 0.0351 & & \\
\hline 528 & SRISO_SEP_PRECIP_GPC & STLRL & $0.0652 \mathrm{pCi} / \mathrm{L}$ & 0.024 & 0.03 & 0.0337 & & \\
\hline 529 & SRISO_SEP_PRECIP_GPC & STLRL & $0.0477 \mathrm{pCi} / \mathrm{L}$ & 0.022 & 0.026 & 0.0337 & & $\mathrm{~J}$ \\
\hline 530 & SRISO_SEP_PRECIP_GPC & STLRL & $0.055 \mathrm{pCi} / \mathrm{L}$ & 0.024 & 0.029 & 0.038 & & $\mathrm{~J}$ \\
\hline 531 & SRISO_SEP_PRECIP_GPC & STLRL & $0.0716 \mathrm{pCi} / \mathrm{L}$ & 0.027 & 0.033 & 0.0372 & & \\
\hline 532 & SRISO_SEP_PRECIP_GPC & STLRL & $0.0923 \mathrm{pCi} / \mathrm{L}$ & 0.099 & 0.11 & 0.198 & & $\mathrm{U}$ \\
\hline 533 & SRISO_SEP_PRECIP_GPC & STLRL & $0.184 \mathrm{pCi} / \mathrm{L}$ & 0.047 & 0.071 & 0.0703 & & \\
\hline
\end{tabular}


M

N

O $\mathrm{P}$ Q $\mathrm{S}$

\begin{tabular}{|c|c|c|c|c|c|c|c|c|c|}
\hline & $\mathrm{K}$ & $\mathrm{L}$ & $\mathrm{M}$ & $\mathrm{N}$ & $\mathrm{O}$ & $\mathrm{P}$ & $\mathrm{Q}$ & $\mathrm{R}$ & $\mathrm{S}$ \\
\hline 1 & METHOD_NAME & LAB_CODE & VALUE_RPTD & ANAL_UNITS_RPTD & $\mid$ COUNTING_ERROR & TOTAL_ANAL_ERROR & MIN_DETECTABLE_ACTIVITY & PCNT_MOISTURE & LAB_QUALIFIER \\
\hline 534 & SRISO_SEP_PRECIP_GPC & STLRL & 0.081 & $\mathrm{pCi} / \mathrm{L}$ & 0.027 & 0.035 & 0.0369 & & \\
\hline 535 & SRISO SEP PRECIP GPC & STLRL & 0.082 & $\mathrm{pCi} / \mathrm{L}$ & 0.026 & 0.034 & 0.0361 & & \\
\hline 536 & SRISO_SEP_PRECIP_GPC & STLRL & 0.0672 & $\mathrm{pCi} / \mathrm{L}$ & 0.025 & 0.031 & 0.0369 & & \\
\hline 537 & SRISO_SEP_PRECIP_GPC & STLRL & -0.00261 & $\mathrm{pCi} / \mathrm{L}$ & 0.037 & 0.037 & 0.0682 & & U \\
\hline 538 & SRISO_SEP_PRECIP_GPC & STLRL & 0.0189 & $\mathrm{pCi} / \mathrm{L}$ & 0.035 & 0.038 & 0.0673 & & $U$ \\
\hline 539 & SRISO_SEP_PRECIP_GPC & STLRL & 1.36 & $\mathrm{pCi} / \mathrm{L}$ & 0.082 & 0.36 & 0.0579 & & \\
\hline 540 & SRISO_SEP_PRECIP_GPC & STLRL & 0.09 & $\mathrm{pCi} / \mathrm{L}$ & 0.04 & 0.05 & 0.0681 & & \\
\hline 541 & 905.0_SR_GPC & STLRL & 0.0761 & $\mathrm{pCi} / \mathrm{L}$ & 0.033 & 0.041 & 0.0546 & & \\
\hline 542 & 905.0_SR_GPC & STLRL & 0.089 & $\mathrm{pCi} / \mathrm{L}$ & 0.03 & 0.04 & 0.0477 & & \\
\hline 543 & 905.0_SR_GPC & STLRL & 0.0365 & $\mathrm{pCi} / \mathrm{L}$ & 0.036 & 0.038 & 0.0605 & & $U$ \\
\hline 544 & 905.0_SR_GPC & STLRL & 0.0729 & $\mathrm{pCi} / \mathrm{L}$ & 0.029 & 0.037 & 0.0459 & & \\
\hline 545 & SRISO_SEP_PRECIP_GPC & STLRL & 0.0318 & $\mathrm{pCi} / \mathrm{L}$ & 0.062 & 0.062 & 0.109 & & $U$ \\
\hline 546 & SRISO_SEP_PRECIP_GPC & STLRL & 2.12 & $\mathrm{pCi} / \mathrm{L}$ & 0.18 & 0.52 & 0.115 & & \\
\hline 547 & SRISO_SEP_PRECIP_GPC & STLRL & -0.0801 & $\mathrm{pCi} / \mathrm{L}$ & 0.17 & 0.17 & 0.434 & & $U$ \\
\hline 548 & SRISO_SEP_PRECIP_GPC & STLRL & 0.00301 & $\mathrm{pCi} / \mathrm{L}$ & 0.033 & 0.037 & 0.0635 & & $U$ \\
\hline 549 & SRISO_SEP_PRECIP_GPC & STLRL & 5.56 & $\mathrm{pCi} / \mathrm{L}$ & 0.18 & 1.3 & 0.0493 & & \\
\hline 550 & SRISO_SEP_PRECIP_GPC & STLRL & 0.0818 & $\mathrm{pCi} / \mathrm{L}$ & 0.028 & 0.036 & 0.0422 & & \\
\hline 551 & SRISO_SEP_PRECIP_GPC & STLRL & 0.0712 & $\mathrm{pCi} / \mathrm{L}$ & 0.026 & 0.033 & 0.0406 & & \\
\hline 552 & SRISO_SEP_PRECIP_GPC & STLRL & 0.0133 & $\mathrm{pCi} / \mathrm{L}$ & 0.024 & 0.027 & 0.0444 & & $\mathrm{U}$ \\
\hline 553 & SRISO_SEP_PRECIP_GPC & STLRL & 0.0585 & $\mathrm{pCi} / \mathrm{L}$ & 0.15 & 0.15 & 0.286 & & $U$ \\
\hline 554 & SRISO_SEP_PRECIP_GPC & STLRL & 0.0759 & $\mathrm{pCi} / \mathrm{L}$ & 0.023 & 0.033 & 0.0363 & & \\
\hline 555 & SRISO_SEP_PRECIP_GPC & STLRL & 0.0717 & $\mathrm{pCi} / \mathrm{L}$ & 0.022 & 0.031 & 0.0342 & & \\
\hline 556 & SRISO_SEP_PRECIP_GPC & STLRL & 0.0716 & $\mathrm{pCi} / \mathrm{L}$ & 0.027 & 0.034 & 0.0439 & & \\
\hline 557 & SRISO_SEP_PRECIP_GPC & STLRL & 0.0699 & $\mathrm{pCi} / \mathrm{L}$ & 0.023 & 0.032 & 0.0353 & & \\
\hline 558 & SRISO_SEP_PRECIP_GPC & STLRL & 0.0644 & $\mathrm{pCi} / \mathrm{L}$ & 0.024 & 0.031 & 0.0388 & & \\
\hline 559 & SRISO_SEP_PRECIP_GPC & STLRL & 0.064 & $\mathrm{pCi} / \mathrm{L}$ & 0.027 & 0.034 & 0.0422 & & \\
\hline 560 & SRISO_SEP_PRECIP_GPC & STLRL & 0.0557 & $\mathrm{pCi} / \mathrm{L}$ & 0.028 & 0.033 & 0.0428 & & $\mathrm{~J}$ \\
\hline 561 & SRISO_SEP_PRECIP_GPC & STLRL & 0.0589 & $\mathrm{pCi} / \mathrm{L}$ & 0.024 & 0.03 & 0.0362 & & $\mathrm{~J}$ \\
\hline 562 & SRISO_SEP_PRECIP_GPC & STLRL & 0.0708 & $\mathrm{pCi} / \mathrm{L}$ & 0.026 & 0.032 & 0.0377 & & \\
\hline 563 & SRISO_SEP_PRECIP_GPC & STLRL & 0.074 & $\mathrm{pCi} / \mathrm{L}$ & 0.026 & 0.033 & 0.0373 & & \\
\hline 564 & SRISO_SEP_PRECIP_GPC & STLRL & 0.0569 & $\mathrm{pCi} / \mathrm{L}$ & 0.024 & 0.029 & 0.0365 & & $\mathrm{~J}$ \\
\hline 565 & SRISO_SEP_PRECIP_GPC & STLRL & 0.133 & $\mathrm{pCi} / \mathrm{L}$ & 0.029 & 0.045 & 0.0343 & & \\
\hline 566 & SRISO_SEP_PRECIP_GPC & STLRL & 0.0456 & $\mathrm{pCi} / \mathrm{L}$ & 0.024 & 0.029 & 0.0389 & & $\mathrm{~J}$ \\
\hline 567 & ]SRISO_SEP_PRECIP_GPC & STLRL & 0.0638 & $\mathrm{pCi} / \mathrm{L}$ & 0.028 & 0.033 & 0.042 & & \\
\hline 568 & SRISO_SEP_PRECIP_GPC & STLRL & 0.0677 & $\mathrm{pCi} / \mathrm{L}$ & 0.03 & 0.035 & 0.0443 & & \\
\hline 569 & SRISO_SEP_PRECIP_GPC & STLRL & 0.0569 & $\mathrm{pCi} / \mathrm{L}$ & 0.028 & 0.032 & 0.0433 & & $\mathrm{~J}$ \\
\hline 570 & SRISO_SEP_PRECIP_GPC & STLRL & 0.0502 & $\mathrm{pCi} / \mathrm{L}$ & 0.026 & 0.03 & 0.0404 & & $\mathrm{~J}$ \\
\hline 571 & SRISO_SEP_PRECIP_GPC & STLRL & 0.0799 & $\mathrm{pCi} / \mathrm{L}$ & 0.028 & 0.035 & 0.039 & & \\
\hline
\end{tabular}

Page 93 


\begin{tabular}{|c|c|c|c|c|c|c|c|c|}
\hline 1 & METHOD_NAME & LAB_CODE & \begin{tabular}{l|l} 
VALUE_RPTD & ANAL_UNITS_RPTD
\end{tabular} & COUNTING_ERROR & TOTAL_ANAL_ERROR & MIN_DETECTABLE_ACTIVITY & PCNT_MOISTURE & LAB_QUALIFIER \\
\hline 572 & SRISO_SEP_PRECIP_GPC & STLRL & $0.0634 \mathrm{pCi} / \mathrm{L}$ & 0.027 & 0.032 & 0.0406 & & \\
\hline 573 & SRISO_SEP_PRECIP_GPC & STLRL & $0.062 \mathrm{pCi} / \mathrm{L}$ & 0.026 & 0.033 & 0.0433 & & \\
\hline 574 & SRISO_SEP_PRECIP_GPC & STLRL & $0.0628 \mathrm{pCi} / \mathrm{L}$ & 0.024 & 0.031 & 0.0391 & & \\
\hline 575 & 905.0_SR_GPC & STLRL & $0.083 \mathrm{pCi} / \mathrm{L}$ & 0.028 & 0.035 & 0.0383 & & \\
\hline 576 & 905.0_SR_GPC & STLRL & $0.0719 \mathrm{pCi} / \mathrm{L}$ & 0.027 & 0.033 & 0.0379 & & \\
\hline 577 & 905.0_SR_GPC & STLRL & $-0.0163 \mathrm{pCi} / \mathrm{L}$ & 0.0037 & 0.037 & 0.0741 & & $U$ \\
\hline 578 & 905.0_SR_GPC & STLRL & $0.0825 \mathrm{pCi} / \mathrm{L}$ & 0.03 & 0.037 & 0.042 & & \\
\hline 579 & SRISŌSĒP_PRECIP_GPC & STLRL & $0.067 \mathrm{pCi} / \mathrm{L}$ & 0.028 & 0.034 & 0.0417 & & \\
\hline 580 & SRISO_SEP_PRECIP_GPC & STLRL & $0.0758 \mathrm{pCi} / \mathrm{L}$ & 0.032 & 0.038 & 0.045 & & \\
\hline 581 & SRISO_SEP_PRECIP_GPC & STLRL & $0.0621 \mathrm{pCi} / \mathrm{L}$ & 0.025 & 0.031 & 0.0364 & & \\
\hline 582 & SRISO_SEP_PRECIP_GPC & STLRL & $0.0669 \mathrm{pCi} / \mathrm{L}$ & 0.028 & 0.034 & 0.0417 & & \\
\hline 583 & SRISO_SEP_PRECIP_GPC & STLRL & $0.0813 \mathrm{pCi} / \mathrm{L}$ & 0.029 & 0.036 & 0.0411 & & \\
\hline 584 & SRISO_SEP_PRECIP_GPC & STLRL & $0.067 \mathrm{pCi} / \mathrm{L}$ & 0.028 & 0.034 & 0.0435 & & \\
\hline 585 & SRISO_SEP_PRECIP_GPC & STLRL & $0.084 \mathrm{pCi} / \mathrm{L}$ & 0.027 & 0.035 & 0.0373 & & \\
\hline 586 & SRISO_SEP_PRECIP_GPC & STLRL & $0.0773 \mathrm{pCi} / \mathrm{L}$ & 0.028 & 0.035 & 0.0392 & & \\
\hline 587 & SRISO_SEP_PRECIP_GPC & STLRL & $0.115 \mathrm{pCi} / \mathrm{L}$ & 0.033 & 0.044 & 0.0411 & & \\
\hline 588 & SRISO_SEP_PRECIP_GPC & STLRL & $0.0744 \mathrm{pCi} / \mathrm{L}$ & 0.026 & 0.033 & 0.0357 & & \\
\hline 589 & SRISO_SEP_PRECIP_GPC & STLRL & $0.0603 \mathrm{pCi} / \mathrm{L}$ & 0.025 & 0.031 & 0.0384 & & \\
\hline 590 & SRISO_SEP_PRECIP_GPC & STLRL & $0.0813 \mathrm{pCi} / \mathrm{L}$ & 0.028 & 0.035 & 0.0383 & & \\
\hline 591 & SRISO_SEP_PRECIP_GPC & STLRL & $0.0768 \mathrm{pCi} / \mathrm{L}$ & 0.028 & 0.035 & 0.0407 & & \\
\hline 592 & SRISO SEP PRECIP GPC & STLRL & $0.0945 \mathrm{pCi} / \mathrm{L}$ & 0.032 & 0.04 & 0.0433 & & \\
\hline 593 & SRISO_SEP_PRECIP_GPC & STLRL & $0.0615 \mathrm{pCi} / \mathrm{L}$ & 0.028 & 0.033 & 0.0428 & & \\
\hline 594 & SRISO_SEP_PRECIP_GPC & STLRL & $0.0541 \mathrm{pCi} / \mathrm{L}$ & 0.028 & 0.033 & 0.0466 & & $\mathrm{~J}$ \\
\hline 595 & SRISO_SEP_PRECIP_GPC & STLRL & $0.0734 \mathrm{pCi} / \mathrm{L}$ & 0.026 & 0.035 & 0.0415 & & \\
\hline 596 & SRISO_SEP_PRECIP_GPC & STLRL & $0.0554 \mathrm{pCi} / \mathrm{L}$ & 0.027 & 0.032 & 0.0466 & & \\
\hline 597 & SRISO_SEP_PRECIP_GPC & STLRL & $0.0598 \mathrm{pCi} / \mathrm{L}$ & 0.026 & 0.032 & 0.0426 & & \\
\hline 598 & 905.0_SR_GPC & STLRL & $0.0784 \mathrm{pCi} / \mathrm{L}$ & 0.026 & 0.034 & 0.038 & & \\
\hline 599 & 905.0_SR_GPC & STLRL & $0.108 \mathrm{pCi} / \mathrm{L}$ & 0.031 & 0.041 & 0.0394 & & \\
\hline 600 & 905.0_SR_GPC & STLRL & $-0.0114 \mathrm{pCi} / \mathrm{L}$ & 0.0032 & 0.024 & 0.0494 & & $U$ \\
\hline 601 & 905.0_SR_GPC & STLRL & $0.0752 \mathrm{pCi} / \mathrm{L}$ & 0.026 & 0.033 & 0.0362 & & \\
\hline 602 & SRISO_SĒP_PRECIP_GPC & STLRL & $0.0248 \mathrm{pCi} / \mathrm{L}$ & 0.081 & 0.086 & 0.159 & & $U$ \\
\hline 603 & SRISO_SEP_PRECIP_GPC & STLRL & $-0.0261 \mathrm{pCi} / \mathrm{L}$ & 0.039 & 0.069 & 0.138 & & $U$ \\
\hline 604 & SRISO_SEP_PRECIP_GPC & STLRL & $14.2 \mathrm{pCi} / \mathrm{L}$ & 0.31 & 3.2 & 0.0549 & & \\
\hline 605 & SRISO_SEP_PRECIP_GPC & STLRL & $0.0795 \mathrm{pCi} / \mathrm{L}$ & 0.027 & 0.034 & 0.0384 & & \\
\hline 606 & SRISO_SEP_PRECIP_GPC & STLRL & $0.0921 \mathrm{pCi} / \mathrm{L}$ & 0.026 & 0.035 & 0.0322 & & \\
\hline 607 & SRISO_SEP_PRECIP_GPC & STLRL & $0.192 \mathrm{pCi} / \mathrm{L}$ & 0.064 & 0.081 & 0.0892 & & \\
\hline 608 & SRISO_SEP_PRECIP_GPC & STLRL & $0.0129 \mathrm{pCi} / \mathrm{L}$ & 0.043 & 0.043 & 0.0792 & & $\mathrm{U}$ \\
\hline 609 & SRISO_SEP_PRECIP_GPC & STLRL & $0.0255 \mathrm{pCi} / \mathrm{L}$ & 0.049 & 0.055 & 0.0997 & & $U$ \\
\hline
\end{tabular}




\begin{tabular}{|c|c|c|c|c|c|c|c|c|}
\hline 1 & METHOD_NAME & LAB_CODE & \begin{tabular}{l|l|} 
VALUE_RPTD & ANAL_UNITS_RPTD
\end{tabular} & COUNTING_ERROR & TOTAL_ANAL_ERROR & MIN_DETECTABLE_ACTIVITY & PCNT_MOISTURE & LAB_QUALIFIER \\
\hline 610 & SRISO_SEP_PRECIP_GPC & STLRL & $0.0275 \mathrm{pCi} / \mathrm{L}$ & 0.058 & 0.059 & 0.107 & & $\mathrm{U}$ \\
\hline 611 & SRISO_SEP_PRECIP_GPC & STLRL & $0.549 \mathrm{pCi} / \mathrm{L}$ & 0.1 & 0.17 & 0.115 & & \\
\hline 612 & SRISO_SEP_PRECIP_GPC & STLRL & $0.0825 \mathrm{pCi} / \mathrm{L}$ & 0.034 & 0.04 & 0.0507 & & \\
\hline 613 & SRISO_SEP_PRECIP_GPC & STLRL & $0.091 \mathrm{pCi} / \mathrm{L}$ & 0.038 & 0.044 & 0.0577 & & \\
\hline 615 & SRISO_SEP_PRECIP_GPC & STLRL & $0.0717 \mathrm{pCi} / \mathrm{L}$ & 0.03 & 0.035 & 0.048 & & \\
\hline 616 & SRISO_SEP_PRECIP_GPC & STLRL & $0.065 \mathrm{pCi} / \mathrm{L}$ & 0.03 & 0.035 & 0.0511 & & \\
\hline 617 & SRISO_SEP_PRECIP_GPC & STLRL & $0.0559 \mathrm{pCi} / \mathrm{L}$ & 0.024 & 0.028 & 0.0346 & & \\
\hline 618 & SRISO_SEP_PRECIP_GPC & STLRL & $0.075 \mathrm{pCi} / \mathrm{L}$ & 0.025 & 0.033 & 0.0352 & & \\
\hline 620 & SRISO_SEP_PRECIP_GPC & STLRL & $0.0721 \mathrm{pCi} / \mathrm{L}$ & 0.028 & 0.034 & 0.0396 & & \\
\hline 621 & SRISO_SEP_PRECIP_GPC & STLRL & $0.0643 \mathrm{pCi} / \mathrm{L}$ & 0.027 & 0.033 & 0.0413 & & \\
\hline 622 & SRISO_SEP_PRECIP_GPC & STLRL & $0.0766 \mathrm{pCi} / \mathrm{L}$ & 0.031 & 0.037 & 0.0463 & & \\
\hline 623 & SRISO_SEP_PRECIP_GPC & STLRL & $0.0696 \mathrm{pCi} / \mathrm{L}$ & 0.027 & 0.033 & 0.0388 & & \\
\hline 624 & SRISO_SEP_PRECIP_GPC & STLRL & $0.0662 \mathrm{pCi} / \mathrm{L}$ & 0.044 & 0.048 & 0.0707 & & $\mathrm{U}$ \\
\hline 625 & SRISO_SEP_PRECIP_GPC & STLRL & $0.0411 \mathrm{pCi} / \mathrm{L}$ & 0.025 & 0.028 & 0.0405 & & \\
\hline 626 & SRISO_SEP_PRECIP_GPC & STLRL & $0.0766 \mathrm{pCi} / \mathrm{L}$ & 0.027 & 0.035 & 0.0399 & & \\
\hline 627 & SRISO_SEP_PRECIP_GPC & STLRL & $0.0541 \mathrm{pCi} / \mathrm{L}$ & 0.023 & 0.028 & 0.0339 & & \\
\hline 628 & SRISO_SEP_PRECIP_GPC & STLRL & $0.0605 \mathrm{pCi} / \mathrm{L}$ & 0.025 & 0.03 & 0.0366 & & \\
\hline 630 & SRISO_SEP_PRECIP_GPC & STLRL & $0.0688 \mathrm{pCi} / \mathrm{L}$ & 0.026 & 0.033 & 0.0365 & & \\
\hline 631 & SRISO_SEP_PRECIP_GPC & STLRL & $0.0804 \mathrm{pCi} / \mathrm{L}$ & 0.027 & 0.034 & 0.0355 & & \\
\hline 632 & SRISO_SEP_PRECIP_GPC & STLRL & $0.0592 \mathrm{pCi} / \mathrm{L}$ & 0.024 & 0.029 & 0.0348 & & \\
\hline 633 & SRISO_SEP_PRECIP_GPC & STLRL & $0.0715 \mathrm{pCi} / \mathrm{L}$ & 0.028 & 0.033 & 0.0429 & & \\
\hline 634 & SRISO_SEP_PRECIP_GPC & STLRL & $0.0915 \mathrm{pCi} / \mathrm{L}$ & 0.028 & 0.035 & 0.0418 & & \\
\hline 635 & SRISO_SEP_PRECIP_GPC & STLRL & $0.0658 \mathrm{pCi} / \mathrm{L}$ & 0.027 & 0.033 & 0.0401 & & \\
\hline 636 & SRISO_SEP_PRECIP_GPC & STLRL & $0.0709 \mathrm{pCi} / \mathrm{L}$ & 0.028 & 0.034 & 0.0422 & & \\
\hline 637 & 905.0_SR_GPC & STLRL & $0.077 \mathrm{pCi} / \mathrm{L}$ & 0.027 & 0.034 & 0.037 & & \\
\hline 638 & 905.0_SR_GPC & STLRL & $0.11 \mathrm{pCi} / \mathrm{L}$ & 0.031 & 0.041 & 0.0404 & & \\
\hline 639 & 905.0_SR_GPC & STLRL & $0.000251 \mathrm{pCi} / \mathrm{L}$ & 0.024 & 0.024 & 0.0456 & & $U$ \\
\hline 640 & 905.0_SR_GPC & STLRL & $0.0878 \mathrm{pCi} / \mathrm{L}$ & 0.029 & 0.037 & 0.0397 & & \\
\hline 641 & SRISO_SEP_PRECIP_GPC & STLRL & $0.0692 \mathrm{pCi} / \mathrm{L}$ & 0.025 & 0.031 & 0.0339 & & \\
\hline 642 & SRISO_SEP_PRECIP_GPC & STLRL & $0.0688 \mathrm{pCi} / \mathrm{L}$ & 0.027 & 0.033 & 0.0395 & & \\
\hline 643 & SRISO_SEP_PRECIP_GPC & STLRL & $0.143 \mathrm{pCi} / \mathrm{L}$ & 0.048 & 0.06 & 0.0657 & & \\
\hline 644 & SRISO_SEP_PRECIP_GPC & STLRL & $0.202 \mathrm{pCi} / \mathrm{L}$ & 0.039 & 0.062 & 0.0427 & & \\
\hline 645 & SRISO_SEP_PRECIP_GPC & STLRL & $0.0625 \mathrm{pCi} / \mathrm{L}$ & 0.027 & 0.032 & 0.0456 & & \\
\hline 646 & SRISO_SEP_PRECIP_GPC & STLRL & $0.0827 \mathrm{pCi} / \mathrm{L}$ & 0.03 & 0.036 & 0.048 & & \\
\hline 647 & SRISO_SEP_PRECIP_GPC & STLRL & $0.131 \mathrm{pCi} / \mathrm{L}$ & 0.032 & 0.046 & 0.0409 & & \\
\hline
\end{tabular}




\begin{tabular}{|c|c|c|c|c|c|c|c|c|}
\hline 1 & METHOD_NAME & LAB_CODE & \begin{tabular}{|l|l|} 
VALUE_RPTD & ANAL_UNITS_RPTD \\
\end{tabular} & COUNTING_ERROR & TOTAL_ANAL_ERROR & MIN_DETECTABLE_ACTIVITY & PCNT_MOISTURE & LAB_QUALIFIER \\
\hline 648 & SRISO_SEP_PRECIP_GPC & STLRL & $0.0549 \mathrm{pCi} / \mathrm{L}$ & 0.026 & 0.03 & 0.0369 & & \\
\hline 649 & SRISO_SEP_PRECIP_GPC & STLRL & $0.0875 \mathrm{pCi} / \mathrm{L}$ & 0.03 & 0.037 & 0.0413 & & \\
\hline 650 & SRISO_SEP_PRECIP_GPC & STLRL & $0.0609 \mathrm{pCi} / \mathrm{L}$ & 0.026 & 0.031 & 0.0384 & & \\
\hline 651 & SRISO_SEP_PRECIP_GPC & STLRL & $0.0663 \mathrm{pCi} / \mathrm{L}$ & 0.026 & 0.032 & 0.0372 & & \\
\hline 653 & SRISO_SEP_PRECIP_GPC & STLRL & $0.0618 \mathrm{pCi} / \mathrm{L}$ & 0.027 & 0.032 & 0.0414 & & \\
\hline 654 & SRISO_SEP_PRECIP_GPC & STLRL & $0.0678 \mathrm{pCi} / \mathrm{L}$ & 0.027 & 0.033 & 0.0401 & & \\
\hline 655 & SRISO_SEP_PRECIP_GPC & STLRL & $0.0461 \mathrm{pCi} / \mathrm{L}$ & 0.025 & 0.029 & 0.0404 & & \\
\hline 656 & SRISO_SEP_PRECIP_GPC & STLRL & $0.0758 \mathrm{pCi} / \mathrm{L}$ & 0.027 & 0.033 & 0.0368 & & \\
\hline 658 & SRISO_SEP_PRECIP_GPC & STLRL & $0.0655 \mathrm{pCi} / \mathrm{L}$ & 0.027 & 0.032 & 0.0385 & & \\
\hline 659 & SRISO_SEP_PRECIP_GPC & STLRL & $0.285 \mathrm{pCi} / \mathrm{L}$ & 0.041 & 0.079 & 0.0387 & & \\
\hline 660 & SRISO_SEP_PRECIP_GPC & STLRL & $0.21 \mathrm{pCi} / \mathrm{L}$ & 0.04 & 0.064 & 0.0428 & & \\
\hline 661 & SRISO_SEP_PRECIP_GPC & STLRL & $0.0876 \mathrm{pCi} / \mathrm{L}$ & 0.028 & 0.036 & 0.0376 & & \\
\hline 662 & SRISO_SEP_PRECIP_GPC & STLRL & $0.0645 \mathrm{pCi} / \mathrm{L}$ & 0.025 & 0.031 & 0.0353 & & \\
\hline 663 & SRISO_SEP_PRECIP_GPC & STLRL & $0.0631 \mathrm{pCi} / \mathrm{L}$ & 0.025 & 0.031 & 0.0364 & & \\
\hline 664 & SRISO_SEP_PRECIP_GPC & STLRL & $0.0692 \mathrm{pCi} / \mathrm{L}$ & 0.026 & 0.032 & 0.0361 & & \\
\hline 665 & SRISO_SEP_PRECIP_GPC & STLRL & $0.0646 \mathrm{pCi} / \mathrm{L}$ & 0.027 & 0.032 & 0.0387 & & \\
\hline 666 & SRISO_SEP_PRECIP_GPC & STLRL & $0.0642 \mathrm{pCi} / \mathrm{L}$ & 0.024 & 0.03 & 0.0334 & & \\
\hline 668 & SRISO_SEP_PRECIP_GPC & STLRL & $0.0764 \mathrm{pCi} / \mathrm{L}$ & 0.026 & 0.033 & 0.0366 & & \\
\hline 669 & SRISO_SEP_PRECIP_GPC & STLRL & $0.0405 \mathrm{pCi} / \mathrm{L}$ & 0.024 & 0.028 & 0.0398 & & \\
\hline 670 & SRISO_SEP_PRECIP_GPC & STLRL & $0.0599 \mathrm{pCi} / \mathrm{L}$ & 0.024 & 0.029 & 0.0337 & & \\
\hline 671 & SRISO_SEP_PRECIP_GPC & STLRL & $0.0551 \mathrm{pCi} / \mathrm{L}$ & 0.025 & 0.029 & 0.0371 & & \\
\hline 672 & SRISO_SEP_PRECIP_GPC & STLRL & $0.0621 \mathrm{pCi} / \mathrm{L}$ & 0.024 & 0.03 & 0.0361 & & \\
\hline 673 & SRISO_SEP_PRECIP_GPC & STLRL & $0.0564 \mathrm{pCi} / \mathrm{L}$ & 0.024 & 0.029 & 0.0364 & & \\
\hline 674 & SRISO_SEP_PRECIP_GPC & STLRL & $0.0613 \mathrm{pCi} / \mathrm{L}$ & 0.025 & 0.03 & 0.0352 & & \\
\hline 675 & SRISO_SEP_PRECIP_GPC & STLRL & $0.0687 \mathrm{pCi} / \mathrm{L}$ & 0.028 & 0.033 & 0.0409 & & \\
\hline 676 & SRISO_SEP_PRECIP_GPC & STLRL & $0.0705 \mathrm{pCi} / \mathrm{L}$ & 0.026 & 0.033 & 0.0388 & & \\
\hline 677 & SRISO SEP PRECIP GPC & STLRL & $0.0769 \mathrm{pCi} / \mathrm{L}$ & 0.026 & 0.033 & 0.0367 & & \\
\hline 678 & SRISO_SEP_PRECIP_GPC & STLRL & $0.0554 \mathrm{pCi} / \mathrm{L}$ & 0.026 & 0.031 & 0.0407 & & \\
\hline 679 & SRISO_SEP_PRECIP_GPC & STLRL & $0.0716 \mathrm{pCi} / \mathrm{L}$ & 0.024 & 0.032 & 0.0346 & & \\
\hline 680 & SRISO_SEP_PRECIP_GPC & STLRL & $0.0744 \mathrm{pCi} / \mathrm{L}$ & 0.033 & 0.039 & 0.0485 & & \\
\hline 681 & SRISO_SEP_PRECIP_GPC & STLRL & $0.0709 \mathrm{pCi} / \mathrm{L}$ & 0.028 & 0.034 & 0.0407 & & \\
\hline 682 & SRISO_SEP_PRECIP_GPC & STLRL & $0.0566 \mathrm{pCi} / \mathrm{L}$ & 0.028 & 0.032 & 0.0427 & & \\
\hline 683 & SRISO_SEP_PRECIP_GPC & STLRL & $0.0634 \mathrm{pCi} / \mathrm{L}$ & 0.027 & 0.032 & 0.0386 & & \\
\hline 684 & SRISO_SEP_PRECIP_GPC & STLRL & $0.0536 \mathrm{pCi} / \mathrm{L}$ & 0.027 & 0.031 & 0.0403 & & \\
\hline 685 & SRISO_SEP_PRECIP_GPC & STLRL & $0.0628 \mathrm{pCi} / \mathrm{L}$ & 0.03 & 0.035 & 0.0455 & & \\
\hline
\end{tabular}




\begin{tabular}{|c|c|c|c|c|c|c|c|c|c|}
\hline & $\mathrm{K}$ & $\mathrm{L}$ & $\mathrm{M}$ & $\mathrm{N}$ & $\mathrm{O}$ & $\mathrm{P}$ & $\mathrm{Q}$ & $\mathrm{R}$ & $\mathrm{S}$ \\
\hline 1 & METHOD_NAME & LAB_CODE & VALUE_RPTD & ANAL_UNITS_RPTD & |COUNTING_ERROR & TOTAL_ANAL_ERROR & MIN_DETECTABLE_ACTIVITY & PCNT_MOISTURE & LAB_QUALIFIER \\
\hline 686 & SRISO_SEP_PRECIP_GPC & STLRL & 0.0533 & $\mathrm{pCi} / \mathrm{L}$ & 0.029 & 0.033 & 0.0448 & & \\
\hline 687 & SRISO_SEP_PRECIP_GPC & STLRL & 0.0743 & $\mathrm{pCi} / \mathrm{L}$ & 0.031 & 0.037 & 0.0454 & & \\
\hline 688 & SRISO_SEP_PRECIP_GPC & STLRL & 0.0586 & $\mathrm{pCi} / \mathrm{L}$ & 0.026 & 0.031 & 0.0389 & & \\
\hline 689 & SRISO_SEP_PRECIP_GPC & STLRL & 0.0748 & $\mathrm{pCi} / \mathrm{L}$ & 0.027 & 0.034 & 0.038 & & \\
\hline 690 & SRISO_SEP_PRECIP_GPC & STLRL & 0.0541 & $\mathrm{pCi} / \mathrm{L}$ & 0.027 & 0.031 & 0.043 & & \\
\hline 691 & SRISO_SEP_PRECIP_GPC & STLRL & 0.0577 & $\mathrm{pCi} / \mathrm{L}$ & 0.029 & 0.033 & 0.0446 & & \\
\hline 692 & SRISO_SEP_PRECIP_GPC & STLRL & 0.0699 & $\mathrm{pCi} / \mathrm{L}$ & 0.025 & 0.031 & 0.0347 & & \\
\hline 693 & SRISO_SEP_PRECIP_GPC & STLRL & 0.0657 & $\mathrm{pCi} / \mathrm{L}$ & 0.026 & 0.031 & 0.0374 & & \\
\hline 694 & SRISO_SEP_PRECIP_GPC & STLRL & 0.0616 & $\mathrm{pCi} / \mathrm{L}$ & 0.027 & 0.032 & 0.0406 & & \\
\hline 695 & SRISO_SEP_PRECIP_GPC & STLRL & 0.0685 & $\mathrm{pCi} / \mathrm{L}$ & 0.027 & 0.033 & 0.0406 & & \\
\hline 696 & SRISO_SEP_PRECIP_GPC & STLRL & 0.0818 & $\mathrm{pCi} / \mathrm{L}$ & 0.03 & 0.037 & 0.0423 & & \\
\hline 697 & SRISO_SEP_PRECIP_GPC & STLRL & 0.0759 & $\mathrm{pCi} / \mathrm{L}$ & 0.029 & 0.036 & 0.0423 & & \\
\hline 698 & SRISO_SEP_PRECIP_GPC & STLRL & 0.0594 & $\mathrm{pCi} / \mathrm{L}$ & 0.027 & 0.032 & 0.0411 & & \\
\hline 699 & SRISO_SEP_PRECIP_GPC & STLRL & 0.0683 & $\mathrm{pCi} / \mathrm{L}$ & 0.027 & 0.033 & 0.0398 & & \\
\hline 700 & SRISO_SEP_PRECIP_GPC & STLRL & 0.0688 & $\mathrm{pCi} / \mathrm{L}$ & 0.03 & 0.036 & 0.0472 & & \\
\hline 701 & SRISO_SEP_PRECIP_GPC & STLRL & 0.0573 & $\mathrm{pCi} / \mathrm{L}$ & 0.058 & 0.062 & 0.108 & & $\mathrm{U}$ \\
\hline 702 & 905.0_SR_GPC & STLRL & 0.0543 & $\mathrm{pCi} / \mathrm{L}$ & 0.097 & 0.097 & 0.18 & & $U$ \\
\hline 703 & 905.0_SR_GPC & STLRL & 0.0866 & $\mathrm{pCi} / \mathrm{L}$ & 0.039 & 0.046 & 0.0617 & & \\
\hline 704 & 905.0_SR_GPC & STLRL & -0.00696 & $\mathrm{pCi} / \mathrm{L}$ & 0.032 & 0.053 & 0.106 & & $\mathrm{U}$ \\
\hline 705 & 905.0_SR_GPC & STLRL & 0.0811 & $\mathrm{pCi} / \mathrm{L}$ & 0.041 & 0.048 & 0.0682 & & \\
\hline 706 & SRISO_SEP_PRECIP_GPC & STLRL & -0.0132 & $\mathrm{pCi} / \mathrm{L}$ & 0.047 & 0.047 & 0.0867 & & $\mathrm{U}$ \\
\hline 707 & SRISO_SEP_PRECIP_GPC & STLRL & -0.0205 & $\mathrm{pCi} / \mathrm{L}$ & 0.031 & 0.031 & 0.0582 & & U \\
\hline 708 & SRISO_SEP_PRECIP_GPC & STLRL & 0.0393 & $\mathrm{pCi} / \mathrm{L}$ & 0.044 & 0.044 & 0.0748 & & U \\
\hline 709 & SRISO_SEP_PRECIP_GPC & STLRL & 0.0435 & $\mathrm{pCi} / \mathrm{L}$ & 0.041 & 0.044 & 0.0718 & & $\mathrm{U}$ \\
\hline 710 & SRISO_SEP_PRECIP_GPC & STLRL & 1.96 & $\mathrm{pCi} / \mathrm{L}$ & 0.14 & 0.47 & 0.0808 & & \\
\hline 711 & SRISO_SEP_PRECIP_GPC & STLRL & 0.062 & $\mathrm{pCi} / \mathrm{L}$ & 0.06 & 0.063 & 0.11 & & U \\
\hline 712 & SRISO_SEP_PRECIP_GPC & STLRL & 3.77 & $\mathrm{pCi} / \mathrm{L}$ & 0.15 & 0.8 & 0.0733 & & \\
\hline 713 & SRISO_SEP_PRECIP_GPC & STLRL & 0.0659 & $\mathrm{pCi} / \mathrm{L}$ & 0.032 & 0.036 & 0.0494 & & \\
\hline 714 & SRISO_SEP_PRECIP_GPC & STLRL & 0.0777 & $\mathrm{pCi} / \mathrm{L}$ & 0.031 & 0.037 & 0.0447 & & \\
\hline 715 & SRISO_SEP_PRECIP_GPC & STLRL & 0.0264 & $\mathrm{pCi} / \mathrm{L}$ & 0.061 & 0.061 & 0.108 & & U \\
\hline 716 & SRISO_SEP_PRECIP_GPC & STLRL & 0.0694 & $\mathrm{pCi} / \mathrm{L}$ & 0.03 & 0.037 & 0.0447 & & \\
\hline 717 & SRISO_SEP_PRECIP_GPC & STLRL & 0.0156 & $\mathrm{pCi} / \mathrm{L}$ & 0.051 & 0.074 & 0.126 & & U \\
\hline 718 & SRISO_SEP_PRECIP_GPC & STLRL & 0.107 & $\mathrm{pCi} / \mathrm{L}$ & 0.14 & 0.15 & 0.236 & & U \\
\hline 719 & SRISO_SEP_PRECIP_GPC & STLRL & 0.0661 & $\mathrm{pCi} / \mathrm{L}$ & 0.028 & 0.034 & 0.0427 & & \\
\hline 720 & SRISO_SEP_PRECIP_GPC & STLRL & 0.0643 & $\mathrm{pCi} / \mathrm{L}$ & 0.026 & 0.031 & 0.0363 & & \\
\hline 721 & SRISO_SEP_PRECIP_GPC & STLRL & 0.0533 & $\mathrm{pCi} / \mathrm{L}$ & 0.026 & 0.03 & 0.0391 & & \\
\hline 722 & SRISO_SEP_PRECIP_GPC & STLRL & 0.0751 & $\mathrm{pCi} / \mathrm{L}$ & 0.029 & 0.036 & 0.0435 & & \\
\hline 723 & SRISO_SEP_PRECIP_GPC & STLRL & 0.0704 & $\mathrm{pCi} / \mathrm{L}$ & 0.035 & 0.04 & 0.0545 & & \\
\hline
\end{tabular}




\begin{tabular}{|c|c|c|c|c|c|c|c|c|c|}
\hline & $\mathrm{K}$ & $\mathrm{L}$ & $\mathrm{M}$ & $\mathrm{N}$ & $\mathrm{O}$ & $\mathrm{P}$ & $\mathrm{Q}$ & $\mathrm{R}$ & $\mathrm{S}$ \\
\hline 1 & METHOD_NAME & LAB_CODE & VALUE_RPTD & ANAL_UNITS_RPTD & |COUNTING_ERROR & TOTAL_ANAL_ERROR & MIN_DETECTABLE_ACTIVITY & PCNT_MOISTURE & LAB_QUALIFIER \\
\hline 724 & SRISO_SEP_PRECIP_GPC & STLRL & 0.0665 & $\mathrm{pCi} / \mathrm{L}$ & 0.03 & 0.035 & 0.047 & & \\
\hline 725 & SRISO_SEP_PRECIP_GPC & STLRL & 0.0558 & $\mathrm{pCi} / \mathrm{L}$ & 0.025 & 0.03 & 0.0381 & & \\
\hline 726 & SRISO_SEP_PRECIP_GPC & STLRL & 0.0759 & $\mathrm{pCi} / \mathrm{L}$ & 0.028 & 0.034 & 0.0382 & & \\
\hline 727 & SRISO_SEP_PRECIP_GPC & STLRL & 0.0625 & $\mathrm{pCi} / \mathrm{L}$ & 0.025 & 0.03 & 0.0341 & & \\
\hline 728 & SRISO_SEP_PRECIP_GPC & STLRL & 0.0681 & $\mathrm{pCi} / \mathrm{L}$ & 0.031 & 0.036 & 0.0467 & & \\
\hline 729 & SRISO_SEP_PRECIP_GPC & STLRL & 0.0742 & $\mathrm{pCi} / \mathrm{L}$ & 0.03 & 0.036 & 0.0435 & & \\
\hline 730 & SRISO_SEP_PRECIP_GPC & STLRL & 0.0888 & $\mathrm{pCi} / \mathrm{L}$ & 0.031 & 0.039 & 0.0419 & & \\
\hline 731 & SRISO_SEP_PRECIP_GPC & STLRL & 0.0872 & $\mathrm{pCi} / \mathrm{L}$ & 0.031 & 0.039 & 0.0445 & & \\
\hline 732 & SRISO_SEP_PRECIP_GPC & STLRL & 0.0824 & $\mathrm{pCi} / \mathrm{L}$ & 0.028 & 0.035 & 0.0363 & & \\
\hline 733 & SRISO_SEP_PRECIP_GPC & STLRL & 0.0938 & $\mathrm{pCi} / \mathrm{L}$ & 0.032 & 0.039 & 0.0505 & & \\
\hline 734 & SRISO_SEP_PRECIP_GPC & STLRL & 0.0911 & $\mathrm{pCi} / \mathrm{L}$ & 0.03 & 0.037 & 0.0464 & & \\
\hline 735 & SRISO_SEP_PRECIP_GPC & STLRL & 0.0642 & $\mathrm{pCi} / \mathrm{L}$ & 0.027 & 0.032 & 0.0407 & & \\
\hline 736 & SRISO_SEP_PRECIP_GPC & STLRL & 0.0816 & $\mathrm{pCi} / \mathrm{L}$ & 0.027 & 0.035 & 0.0387 & & \\
\hline 737 & 905.0_SR_GPC & STLRL & 0.0928 & $\mathrm{pCi} / \mathrm{L}$ & 0.028 & 0.036 & 0.0435 & & \\
\hline 738 & 905.0_SR_GPC & STLRL & 0.0894 & $\mathrm{pCi} / \mathrm{L}$ & 0.032 & 0.038 & 0.0496 & & \\
\hline 739 & 905.0_SR_GPC & STLRL & 0.0904 & $\mathrm{pCi} / \mathrm{L}$ & 0.031 & 0.037 & 0.048 & & \\
\hline 740 & 905.0_SR_GPC & STLRL & 0.00022 & $\mathrm{pCi} / \mathrm{L}$ & 0.028 & 0.031 & 0.0584 & & $U$ \\
\hline 741 & SRISO_SĒP_PRECIP_GPC & STLRL & 0.0745 & $\mathrm{pCi} / \mathrm{L}$ & 0.029 & 0.035 & 0.0435 & & \\
\hline 742 & SRISO_SEP_PRECIP_GPC & STLRL & 0.0802 & $\mathrm{pCi} / \mathrm{L}$ & 0.025 & 0.033 & 0.0346 & & \\
\hline 743 & SRISO_SEP_PRECIP_GPC & STLRL & 0.0613 & $\mathrm{pCi} / \mathrm{L}$ & 0.025 & 0.031 & 0.037 & & \\
\hline 744 & SRISO_SEP_PRECIP_GPC & STLRL & 0.0854 & $\mathrm{pCi} / \mathrm{L}$ & 0.029 & 0.036 & 0.0395 & & \\
\hline 745 & SRISO_SEP_PRECIP_GPC & STLRL & 0.0625 & $\mathrm{pCi} / \mathrm{L}$ & 0.027 & 0.032 & 0.0409 & & \\
\hline 746 & SRISO_SEP_PRECIP_GPC & STLRL & 0.0638 & $\mathrm{pCi} / \mathrm{L}$ & 0.028 & 0.033 & 0.0419 & & \\
\hline 747 & SRISO_SEP_PRECIP_GPC & STLRL & 0.0573 & $\mathrm{pCi} / \mathrm{L}$ & 0.029 & 0.033 & 0.0461 & & \\
\hline 748 & SRISO_SEP_PRECIP_GPC & STLRL & 0.0525 & $\mathrm{pCi} / \mathrm{L}$ & 0.026 & 0.03 & 0.0412 & & \\
\hline 749 & SRISO_SEP_PRECIP_GPC & STLRL & 0.0634 & $\mathrm{pCi} / \mathrm{L}$ & 0.026 & 0.032 & 0.0393 & & \\
\hline 750 & SRISO_SEP_PRECIP_GPC & STLRL & 0.0646 & $\mathrm{pCi} / \mathrm{L}$ & 0.026 & 0.032 & 0.0389 & & \\
\hline 751 & SRISO_SEP_PRECIP_GPC & STLRL & 0.0644 & $\mathrm{pCi} / \mathrm{L}$ & 0.026 & 0.033 & 0.0404 & & \\
\hline 752 & SRISO_SEP_PRECIP_GPC & STLRL & 0.0535 & $\mathrm{pCi} / \mathrm{L}$ & 0.025 & 0.03 & 0.0398 & & \\
\hline 753 & SRISO_SEP_PRECIP_GPC & STLRL & 0.0371 & $\mathrm{pCi} / \mathrm{L}$ & 0.024 & 0.027 & 0.0407 & & $U$ \\
\hline 754 & SRISO_SEP_PRECIP_GPC & STLRL & 0.0598 & $\mathrm{pCi} / \mathrm{L}$ & 0.028 & 0.032 & 0.0426 & & \\
\hline 755 & SRISO_SEP_PRECIP_GPC & STLRL & 0.0446 & $\mathrm{pCi} / \mathrm{L}$ & 0.026 & 0.029 & 0.041 & & \\
\hline 756 & SRISO_SEP_PRECIP_GPC & STLRL & 0.0825 & $\mathrm{pCi} / \mathrm{L}$ & 0.03 & 0.037 & 0.042 & & \\
\hline 757 & SRISO_SEP_PRECIP_GPC & STLRL & 0.0635 & $\mathrm{pCi} / \mathrm{L}$ & 0.026 & 0.031 & 0.0371 & & \\
\hline 758 & SRISO_SEP_PRECIP_GPC & STLRL & 0.0417 & $\mathrm{pCi} / \mathrm{L}$ & 0.023 & 0.027 & 0.0379 & & \\
\hline 759 & SRISO_SEP_PRECIP_GPC & STLRL & 0.0747 & $\mathrm{pCi} / \mathrm{L}$ & 0.027 & 0.032 & 0.0366 & & \\
\hline 760 & 905.0_SR_GPC & STLRL & 0.0648 & $\mathrm{pCi} / \mathrm{L}$ & 0.024 & 0.029 & 0.0332 & & \\
\hline 761 & 905.0_SR_GPC & STLRL & -0.00235 & $\mathrm{pCi} / \mathrm{L}$ & 0.026 & 0.028 & 0.0538 & & $\mathrm{U}$ \\
\hline
\end{tabular}




\begin{tabular}{|c|c|c|c|c|c|c|c|c|c|}
\hline & $\mathrm{K}$ & $\mathrm{L}$ & $\mathrm{M}$ & $\mathrm{N}$ & $\mathrm{O}$ & $\mathrm{P}$ & $\mathrm{Q}$ & $\mathrm{R}$ & $\mathrm{S}$ \\
\hline 1 & METHOD_NAME & LAB_CODE & VALUE_RPTD & ANAL_UNITS_RPTD & COUNTING_ERROR & TOTAL_ANAL_ERROR & MIN_DETECTABLE_ACTIVITY & PCNT_MOISTURE & LAB_QUALIFIER \\
\hline 762 & 905.0_SR_GPC & STLRL & 0.0592 & $\mathrm{pCi} / \mathrm{L}$ & 0.025 & 0.029 & 0.0366 & & \\
\hline 763 & 905.0_SR_GPC & STLRL & 0.0677 & $\mathrm{pCi} / \mathrm{L}$ & 0.024 & 0.03 & 0.0343 & & \\
\hline 764 & SRISO_SEP_PRECIP_GPC & STLRL & 0.0634 & $\mathrm{pCi} / \mathrm{L}$ & 0.026 & 0.03 & 0.0373 & & \\
\hline 765 & SRISO_SEP_PRECIP_GPC & STLRL & 0.0569 & $\mathrm{pCi} / \mathrm{L}$ & 0.026 & 0.03 & 0.0391 & & \\
\hline 766 & SRISO_SEP_PRECIP_GPC & STLRL & 0.0667 & $\mathrm{pCi} / \mathrm{L}$ & 0.022 & 0.027 & 0.0284 & & \\
\hline 767 & SRISO_SEP_PRECIP_GPC & STLRL & 0.0599 & $\mathrm{pCi} / \mathrm{L}$ & 0.021 & 0.026 & 0.0293 & & \\
\hline 768 & SRISO_SEP_PRECIP_GPC & STLRL & 0.0599 & $\mathrm{pCi} / \mathrm{L}$ & 0.022 & 0.026 & 0.0302 & & \\
\hline 769 & SRISO_SEP_PRECIP_GPC & STLRL & 0.061 & $\mathrm{pCi} / \mathrm{L}$ & 0.021 & 0.026 & 0.0299 & & \\
\hline 770 & SRISO_SEP_PRECIP_GPC & STLRL & 0.0497 & $\mathrm{pCi} / \mathrm{L}$ & 0.026 & 0.029 & 0.0401 & & \\
\hline 771 & SRISO_SEP_PRECIP_GPC & STLRL & 0.0533 & $\mathrm{pCi} / \mathrm{L}$ & 0.025 & 0.029 & 0.0382 & & \\
\hline 772 & SRISO_SEP_PRECIP_GPC & STLRL & 0.0516 & $\mathrm{pCi} / \mathrm{L}$ & 0.026 & 0.029 & 0.0403 & & \\
\hline 773 & SRISO_SEP_PRECIP_GPC & STLRL & 0.043 & $\mathrm{pCi} / \mathrm{L}$ & 0.032 & 0.035 & 0.0592 & & $U$ \\
\hline 774 & SRISO_SEP_PRECIP_GPC & STLRL & 0.0636 & $\mathrm{pCi} / \mathrm{L}$ & 0.037 & 0.04 & 0.063 & & \\
\hline 775 & ]SRISO_SEP_PRECIP_GPC & STLRL & 0.0599 & $\mathrm{pCi} / \mathrm{L}$ & 0.033 & 0.037 & 0.0571 & & \\
\hline 776 & SRISO_SEP_PRECIP_GPC & STLRL & 0.0644 & $\mathrm{pCi} / \mathrm{L}$ & 0.036 & 0.04 & 0.0618 & & \\
\hline 777 & SRISO_SEP_PRECIP_GPC & STLRL & 0.0549 & $\mathrm{pCi} / \mathrm{L}$ & 0.034 & 0.038 & 0.06 & & $U$ \\
\hline 778 & SRISO_SEP_PRECIP_GPC & STLRL & 0.0551 & $\mathrm{pCi} / \mathrm{L}$ & 0.032 & 0.035 & 0.0498 & & \\
\hline 779 & SRISO_SEP_PRECIP_GPC & STLRL & 0.0654 & $\mathrm{pCi} / \mathrm{L}$ & 0.029 & 0.034 & 0.045 & & \\
\hline 780 & SRISO_SEP_PRECIP_GPC & STLRL & 0.073 & $\mathrm{pCi} / \mathrm{L}$ & 0.029 & 0.034 & 0.0424 & & \\
\hline 781 & SRISO_SEP_PRECIP_GPC & STLRL & 0.0503 & $\mathrm{pCi} / \mathrm{L}$ & 0.027 & 0.03 & 0.0419 & & \\
\hline 782 & SRISO_SEP_PRECIP_GPC & STLRL & 0.0906 & $\mathrm{pCi} / \mathrm{L}$ & 0.032 & 0.039 & 0.0458 & & \\
\hline 783 & SRISO_SEP_PRECIP_GPC & STLRL & 0.073 & $\mathrm{pCi} / \mathrm{L}$ & 0.033 & 0.038 & 0.0493 & & \\
\hline 784 & SRISO_SEP_PRECIP_GPC & STLRL & 0.07 & $\mathrm{pCi} / \mathrm{L}$ & 0.026 & 0.031 & 0.0364 & & \\
\hline 785 & SRISO_SEP_PRECIP_GPC & STLRL & 0.0496 & $\mathrm{pCi} / \mathrm{L}$ & 0.025 & 0.028 & 0.0393 & & \\
\hline 786 & SRISO_SEP_PRECIP_GPC & STLRL & 0.0585 & $\mathrm{pCi} / \mathrm{L}$ & 0.024 & 0.029 & 0.0366 & & \\
\hline 787 & SRISO_SEP_PRECIP_GPC & STLRL & 0.0553 & $\mathrm{pCi} / \mathrm{L}$ & 0.025 & 0.028 & 0.0355 & & \\
\hline 788 & SRISO_SEP_PRECIP_GPC & STLRL & 0.0768 & $\mathrm{pCi} / \mathrm{L}$ & 0.03 & 0.034 & 0.0491 & & \\
\hline 789 & 905.0_SR_GPC & STLRL & 0.0989 & $\mathrm{pCi} / \mathrm{L}$ & 0.033 & 0.039 & 0.053 & & \\
\hline 790 & SRISO_SEP_PRECIP_GPC & STLRL & 0.0595 & $\mathrm{pCi} / \mathrm{L}$ & 0.029 & 0.032 & 0.0493 & & \\
\hline 791 & 905.0_SR_GPC & STLRL & 0.0653 & $\mathrm{pCi} / \mathrm{L}$ & 0.028 & 0.033 & 0.0499 & & \\
\hline 792 & 905.0_SR_GPC & STLRL & 0.0666 & $\mathrm{pCi} / \mathrm{L}$ & 0.026 & 0.029 & 0.038 & & \\
\hline 793 & 905.0_SR_GPC & STLRL & 0.021 & $\mathrm{pCi} / \mathrm{L}$ & 0.027 & 0.027 & 0.05 & & $U$ \\
\hline 794 & SRISO_SĒP_PRECIP_GPC & STLRL & 0.062 & $\mathrm{pCi} / \mathrm{L}$ & 0.023 & 0.028 & 0.0324 & & \\
\hline 795 & SRISO_SEP_PRECIP_GPC & STLRL & 0.0428 & $\mathrm{pCi} / \mathrm{L}$ & 0.021 & 0.024 & 0.0323 & & \\
\hline 796 & SRISO_SEP_PRECIP_GPC & STLRL & 0.0474 & $\mathrm{pCi} / \mathrm{L}$ & 0.018 & 0.022 & 0.026 & & \\
\hline 797 & SRISO_SEP_PRECIP_GPC & STLRL & 0.0361 & $\mathrm{pCi} / \mathrm{L}$ & 0.017 & 0.02 & 0.0266 & & \\
\hline 798 & SRISO_SEP_PRECIP_GPC & STLRL & 0.0739 & $\mathrm{pCi} / \mathrm{L}$ & 0.028 & 0.034 & 0.0401 & & \\
\hline 799 & SRISO_SEP_PRECIP_GPC & STLRL & 0.16 & $\mathrm{pCi} / \mathrm{L}$ & 0.037 & 0.051 & 0.0464 & & \\
\hline
\end{tabular}




\begin{tabular}{|c|c|c|c|c|c|c|c|c|c|}
\hline & $\mathrm{K}$ & $\mathrm{L}$ & $\mathrm{M}$ & $\mathrm{N}$ & $\mathrm{O}$ & $\mathrm{P}$ & $\mathrm{Q}$ & $\mathrm{R}$ & $\mathrm{S}$ \\
\hline 1 & METHOD_NAME & LAB_CODE & VALUE_RPTD & ANAL_UNITS_RPTD & COUNTING_ERROR & TOTAL_ANAL_ERROR & MIN_DETECTABLE_ACTIVITY & PCNT_MOISTURE & LAB_QUALIFIER \\
\hline 800 & SRISO_SEP_PRECIP_GPC & STLRL & 0.0605 & $\mathrm{pCi} / \mathrm{L}$ & 0.028 & 0.033 & 0.0494 & & \\
\hline 801 & SRISO_SEP_PRECIP_GPC & STLRL & 0.0707 & $\mathrm{pCi} / \mathrm{L}$ & 0.035 & 0.04 & 0.0602 & & \\
\hline 802 & SRISO_SEP_PRECIP_GPC & STLRL & 0.092 & $\mathrm{pCi} / \mathrm{L}$ & 0.073 & 0.076 & 0.128 & & $\mathrm{U}$ \\
\hline 803 & SRISO_SEP_PRECIP_GPC & STLRL & 0.0948 & $\mathrm{pCi} / \mathrm{L}$ & 0.028 & 0.036 & 0.0368 & & \\
\hline 804 & SRISO_SEP_PRECIP_GPC & STLRL & 0.0783 & $\mathrm{pCi} / \mathrm{L}$ & 0.029 & 0.035 & 0.0425 & & \\
\hline 805 & SRISO_SEP_PRECIP_GPC & STLRL & 0.0545 & $\mathrm{pCi} / \mathrm{L}$ & 0.023 & 0.028 & 0.0359 & & \\
\hline 806 & SRISO_SEP_PRECIP_GPC & STLRL & 0.0493 & $\mathrm{pCi} / \mathrm{L}$ & 0.033 & 0.035 & 0.0543 & & $\mathrm{U}$ \\
\hline 807 & SRISO_SEP_PRECIP_GPC & STLRL & 0.0665 & $\mathrm{pCi} / \mathrm{L}$ & 0.03 & 0.034 & 0.0453 & & \\
\hline 808 & SRISO_SEP_PRECIP_GPC & STLRL & 0.0504 & $\mathrm{pCi} / \mathrm{L}$ & 0.025 & 0.029 & 0.0395 & & \\
\hline 809 & SRISO_SEP_PRECIP_GPC & STLRL & 0.0449 & $\mathrm{pCi} / \mathrm{L}$ & 0.024 & 0.028 & 0.0403 & & \\
\hline 810 & SRISO_SEP_PRECIP_GPC & STLRL & 0.218 & $\mathrm{pCi} / \mathrm{L}$ & 0.046 & 0.066 & 0.053 & & \\
\hline 811 & SRISO_SEP_PRECIP_GPC & STLRL & 0.179 & $\mathrm{pCi} / \mathrm{L}$ & 0.048 & 0.063 & 0.0659 & & \\
\hline 812 & SRISO_SEP_PRECIP_GPC & STLRL & 0.0571 & $\mathrm{pCi} / \mathrm{L}$ & 0.038 & 0.042 & 0.0648 & & $U$ \\
\hline 813 & ]SRISO_SEP_PRECIP_GPC & STLRL & 0.0442 & $\mathrm{pCi} / \mathrm{L}$ & 0.025 & 0.028 & 0.0423 & & \\
\hline 814 & SRISO_SEP_PRECIP_GPC & STLRL & 0.0474 & $\mathrm{pCi} / \mathrm{L}$ & 0.023 & 0.027 & 0.0403 & & \\
\hline 815 & SRISO_SEP_PRECIP_GPC & STLRL & 0.0633 & $\mathrm{pCi} / \mathrm{L}$ & 0.033 & 0.037 & 0.0544 & & \\
\hline 816 & SRISO_SEP_PRECIP_GPC & STLRL & 0.0501 & $\mathrm{pCi} / \mathrm{L}$ & 0.025 & 0.028 & 0.0401 & & \\
\hline 817 & SRISO_SEP_PRECIP_GPC & STLRL & 0.0448 & $\mathrm{pCi} / \mathrm{L}$ & 0.023 & 0.027 & 0.0395 & & \\
\hline 818 & SRISO_SEP_PRECIP_GPC & STLRL & 0.0401 & $\mathrm{pCi} / \mathrm{L}$ & 0.018 & 0.021 & 0.0266 & & \\
\hline 819 & SRISO_SEP_PRECIP_GPC & STLRL & 0.0587 & $\mathrm{pCi} / \mathrm{L}$ & 0.021 & 0.026 & 0.0297 & & \\
\hline 820 & SRISO_SEP_PRECIP_GPC & STLRL & 0.0895 & $\mathrm{pCi} / \mathrm{L}$ & 0.036 & 0.041 & 0.0564 & & \\
\hline 821 & SRISO_SEP_PRECIP_GPC & STLRL & 0.057 & $\mathrm{pCi} / \mathrm{L}$ & 0.034 & 0.037 & 0.0565 & & \\
\hline 822 & SRISO_SEP_PRECIP_GPC & STLRL & 0.0871 & $\mathrm{pCi} / \mathrm{L}$ & 0.032 & 0.037 & 0.0428 & & \\
\hline 823 & SRISO_SEP_PRECIP_GPC & STLRL & 0.0591 & $\mathrm{pCi} / \mathrm{L}$ & 0.032 & 0.034 & 0.0509 & & \\
\hline 824 & SRISO_SEP_PRECIP_GPC & STLRL & 0.0757 & $\mathrm{pCi} / \mathrm{L}$ & 0.034 & 0.038 & 0.053 & & \\
\hline 825 & SRISO_SEP_PRECIP_GPC & STLRL & 0.0617 & $\mathrm{pCi} / \mathrm{L}$ & 0.028 & 0.032 & 0.0444 & & \\
\hline 826 & SRISO_SEP_PRECIP_GPC & STLRL & 0.0821 & $\mathrm{pCi} / \mathrm{L}$ & 0.033 & 0.037 & 0.047 & & \\
\hline 827 & SRISO_SEP_PRECIP_GPC & STLRL & 0.0701 & $\mathrm{pCi} / \mathrm{L}$ & 0.029 & 0.033 & 0.0436 & & \\
\hline 828 & SRISO_SEP_PRECIP_GPC & STLRL & 0.0563 & $\mathrm{pCi} / \mathrm{L}$ & 0.026 & 0.029 & 0.0398 & & \\
\hline 829 & SRISO_SEP_PRECIP_GPC & STLRL & 0.0388 & $\mathrm{pCi} / \mathrm{L}$ & 0.02 & 0.023 & 0.0321 & & \\
\hline 830 & SRISO_SEP_PRECIP_GPC & STLRL & 0.0543 & $\mathrm{pCi} / \mathrm{L}$ & 0.021 & 0.025 & 0.0303 & & \\
\hline 831 & SRISO_SEP_PRECIP_GPC & STLRL & 0.0197 & $\mathrm{pCi} / \mathrm{L}$ & 0.015 & 0.017 & 0.0251 & & $\mathrm{U}$ \\
\hline 832 & SRISO_SEP_PRECIP_GPC & STLRL & 0.066 & $\mathrm{pCi} / \mathrm{L}$ & 0.034 & 0.038 & 0.0553 & & \\
\hline 833 & SRISO_SEP_PRECIP_GPC & STLRL & 0.0637 & $\mathrm{pCi} / \mathrm{L}$ & 0.032 & 0.036 & 0.0508 & & \\
\hline 834 & SRISO_SEP_PRECIP_GPC & STLRL & 0.0662 & $\mathrm{pCi} / \mathrm{L}$ & 0.031 & 0.035 & 0.0513 & & \\
\hline 835 & SRISO_SEP_PRECIP_GPC & STLRL & 0.0422 & $\mathrm{pCi} / \mathrm{L}$ & 0.03 & 0.03 & 0.045 & & $\mathrm{U}$ \\
\hline 836 & SRISO_SEP_PRECIP_GPC & STLRL & 0.063 & $\mathrm{pCi} / \mathrm{L}$ & 0.03 & 0.033 & 0.0463 & & \\
\hline 837 & SRISO_SEP_PRECIP_GPC & STLRL & 0.0759 & $\mathrm{pCi} / \mathrm{L}$ & 0.031 & 0.035 & 0.0446 & & \\
\hline
\end{tabular}




\begin{tabular}{|c|c|c|c|c|c|c|c|c|c|}
\hline & $\mathrm{K}$ & $\mathrm{L}$ & $\mathrm{M}$ & $\mathrm{N}$ & $\mathrm{O}$ & $\mathrm{P}$ & $\mathrm{Q}$ & $\mathrm{R}$ & $\mathrm{S}$ \\
\hline 1 & METHOD_NAME & LAB_CODE & VALUE_RPTD & ANAL_UNITS_RPTD & COUNTING_ERROR & TOTAL_ANAL_ERROR & MIN_DETECTABLE_ACTIVITY & PCNT_MOISTURE & LAB_QUALIFIER \\
\hline 838 & SRISO_SEP_PRECIP_GPC & STLRL & 0.0796 & $\mathrm{pCi} / \mathrm{L}$ & 0.03 & 0.036 & 0.0438 & & \\
\hline 839 & SRISO_SEP_PRECIP_GPC & STLRL & 0.0966 & $\mathrm{pCi} / \mathrm{L}$ & 0.031 & 0.039 & 0.0427 & & \\
\hline 840 & SRISO_SEP_PRECIP_GPC & STLRL & 0.0685 & $\mathrm{pCi} / \mathrm{L}$ & 0.039 & 0.042 & 0.0602 & & \\
\hline 841 & SRISO_SEP_PRECIP_GPC & STLRL & 0.094 & $\mathrm{pCi} / \mathrm{L}$ & 0.033 & 0.04 & 0.0454 & & \\
\hline 842 & SRISO_SEP_PRECIP_GPC & STLRL & 0.0732 & $\mathrm{pCi} / \mathrm{L}$ & 0.029 & 0.034 & 0.0425 & & \\
\hline 843 & SRISO_SEP_PRECIP_GPC & STLRL & 0.0719 & $\mathrm{pCi} / \mathrm{L}$ & 0.028 & 0.034 & 0.0431 & & \\
\hline 844 & SRISO_SEP_PRECIP_GPC & STLRL & 0.0732 & $\mathrm{pCi} / \mathrm{L}$ & 0.029 & 0.034 & 0.0429 & & \\
\hline 845 & SRISO_SEP_PRECIP_GPC & STLRL & 0.0905 & $\mathrm{pCi} / \mathrm{L}$ & 0.028 & 0.035 & 0.0379 & & \\
\hline 846 & SRISO_SEP_PRECIP_GPC & STLRL & 0.0664 & $\mathrm{pCi} / \mathrm{L}$ & 0.031 & 0.036 & 0.0505 & & \\
\hline 847 & SRISO_SEP_PRECIP_GPC & STLRL & 0.0797 & $\mathrm{pCi} / \mathrm{L}$ & 0.03 & 0.036 & 0.0435 & & \\
\hline 848 & SRISO_SEP_PRECIP_GPC & STLRL & 0.0787 & $\mathrm{pCi} / \mathrm{L}$ & 0.031 & 0.037 & 0.051 & & \\
\hline 849 & SRISO_SEP_PRECIP_GPC & STLRL & 0.0753 & $\mathrm{pCi} / \mathrm{L}$ & 0.032 & 0.037 & 0.0538 & & \\
\hline 850 & SRISO_SEP_PRECIP_GPC & STLRL & 0.074 & $\mathrm{pCi} / \mathrm{L}$ & 0.031 & 0.036 & 0.0501 & & \\
\hline 851 & ]SRISO_SEP_PRECIP_GPC & STLRL & 0.0937 & $\mathrm{pCi} / \mathrm{L}$ & 0.033 & 0.04 & 0.0525 & & \\
\hline 852 & SRISO_SEP_PRECIP_GPC & STLRL & 0.00424 & $\mathrm{pCi} / \mathrm{L}$ & 0.0032 & 0.0034 & 0.00539 & & $\mathrm{U}$ \\
\hline 853 & SRISO_SEP_PRECIP_GPC & STLRL & -0.00188 & $\mathrm{pCi} / \mathrm{L}$ & 0.024 & 0.024 & 0.0453 & & $\mathrm{U}$ \\
\hline 854 & SRISO_SEP_PRECIP_GPC & STLRL & 0.0288 & $\mathrm{pCi} / \mathrm{L}$ & 0.025 & 0.027 & 0.0418 & & $\mathrm{U}$ \\
\hline 855 & SRISO_SEP_PRECIP_GPC & STLRL & 0.0736 & $\mathrm{pCi} / \mathrm{L}$ & 0.03 & 0.034 & 0.0455 & & \\
\hline 856 & SRISO_SEP_PRECIP_GPC & STLRL & 0.0532 & $\mathrm{pCi} / \mathrm{L}$ & 0.031 & 0.034 & 0.0522 & & \\
\hline 857 & SRISO_SEP_PRECIP_GPC & STLRL & & & & & & & \\
\hline 858 & SRISO_SEP_PRECIP_GPC & STLRL & 3.25 & $\mathrm{pCi} / \mathrm{L}$ & 0.23 & 0.72 & 0.128 & & \\
\hline 859 & SRISO_SEP_PRECIP_GPC & STLRL & 3.3 & $\mathrm{pCi} / \mathrm{L}$ & 0.13 & 0.71 & 0.0682 & & \\
\hline 860 & SRISO_SEP_PRECIP_GPC & STLRL & 0.0126 & $\mathrm{pCi} / \mathrm{L}$ & 0.028 & 0.032 & 0.0581 & & $\mathrm{U}$ \\
\hline 861 & SRISO_SEP_PRECIP_GPC & STLRL & 0.0586 & $\mathrm{pCi} / \mathrm{L}$ & 0.026 & 0.03 & 0.0389 & & \\
\hline 862 & SRISO_SEP_PRECIP_GPC & STLRL & 0.0609 & $\mathrm{pCi} / \mathrm{L}$ & 0.025 & 0.03 & 0.037 & & \\
\hline 863 & SRISO_SEP_PRECIP_GPC & STLRL & 0.068 & $\mathrm{pCi} / \mathrm{L}$ & 0.03 & 0.034 & 0.0457 & & \\
\hline 864 & SRISO_SEP_PRECIP_GPC & STLRL & 0.0702 & $\mathrm{pCi} / \mathrm{L}$ & 0.033 & 0.037 & 0.0523 & & \\
\hline 865 & SRISO_SEP_PRECIP_GPC & STLRL & 0.0845 & $\mathrm{pCi} / \mathrm{L}$ & 0.028 & 0.035 & 0.0452 & & \\
\hline 866 & SRISO_SEP_PRECIP_GPC & STLRL & 0.0812 & $\mathrm{pCi} / \mathrm{L}$ & 0.033 & 0.039 & 0.0534 & & \\
\hline 867 & SRISO_SEP_PRECIP_GPC & STLRL & 0.0641 & $\mathrm{pCi} / \mathrm{L}$ & 0.034 & 0.039 & 0.0592 & & \\
\hline 868 & SRISO_SEP_PRECIP_GPC & STLRL & 0.049 & $\mathrm{pCi} / \mathrm{L}$ & 0.033 & 0.037 & 0.0598 & & $U$ \\
\hline 869 & SRISO_SEP_PRECIP_GPC & STLRL & 0.0727 & $\mathrm{pCi} / \mathrm{L}$ & 0.028 & 0.034 & 0.0414 & & \\
\hline 870 & SRISO_SEP_PRECIP_GPC & STLRL & 0.0652 & $\mathrm{pCi} / \mathrm{L}$ & 0.028 & 0.034 & 0.0443 & & \\
\hline 871 & SRISO_SEP_PRECIP_GPC & STLRL & 0.07 & $\mathrm{pCi} / \mathrm{L}$ & 0.028 & 0.034 & 0.0428 & & \\
\hline 872 & SRISO_SEP_PRECIP_GPC & STLRL & 0.077 & $\mathrm{pCi} / \mathrm{L}$ & 0.028 & 0.034 & 0.0406 & & \\
\hline 873 & SRISO_SEP_PRECIP_GPC & STLRL & 0.0684 & $\mathrm{pCi} / \mathrm{L}$ & 0.029 & 0.034 & 0.0448 & & \\
\hline 874 & SRISO_SEP_PRECIP_GPC & STLRL & 0.0754 & $\mathrm{pCi} / \mathrm{L}$ & 0.029 & 0.035 & 0.0442 & & \\
\hline 875 & SRISO_SEP_PRECIP_GPC & STLRL & 0.0668 & $\mathrm{pCi} / \mathrm{L}$ & 0.028 & 0.033 & 0.0433 & & \\
\hline
\end{tabular}




\begin{tabular}{|c|c|c|c|c|c|c|c|c|}
\hline 1 & METHOD_NAME & LAB_CODE & \begin{tabular}{|l|l|} 
VALUE_RPTD & ANAL_UNITS_RPTD
\end{tabular} & $\mid$ COUNTING_ERROR & TOTAL_ANAL_ERROR & MIN_DETECTABLE_ACTIVITY & PCNT_MOISTURE & LAB_QUALIFIER \\
\hline 876 & SRISO_SEP_PRECIP_GPC & STLRL & $0.0958 \mathrm{pCi} / \mathrm{L}$ & 0.037 & 0.043 & 0.0606 & & \\
\hline 877 & SRISO_SEP_PRECIP_GPC & STLRL & $0.083 \mathrm{pCi} / \mathrm{L}$ & 0.03 & 0.036 & 0.0426 & & \\
\hline 878 & SRISO_SEP_PRECIP_GPC & STLRL & $0.0597 \mathrm{pCi} / \mathrm{L}$ & 0.035 & 0.039 & 0.0589 & & \\
\hline 879 & SRISO_SEP_PRECIP_GPC & STLRL & $0.068 \mathrm{pCi} / \mathrm{L}$ & 0.032 & 0.036 & 0.0491 & & \\
\hline 880 & TC99_SEP_LSC & QTESRL & $395 \mathrm{pCi} / \mathrm{L}$ & 6.6 & 33 & 5.65 & & \\
\hline 881 & TC99_SEP_LSC & QTESRL & $-0.0608 \mathrm{pCi} / \mathrm{L}$ & 0.0018 & 0.27 & 0.229 & & $U$ \\
\hline 882 & TC99_SEP_LSC & QTESRL & $-0.0481 \mathrm{pCi} / \mathrm{L}$ & 0.0014 & 0.27 & 0.232 & & $U$ \\
\hline 883 & TC99_SEP_LSC & QTESRL & $0.0206 \mathrm{pCi} / \mathrm{L}$ & 0.0006 & 0.29 & 0.235 & & $U$ \\
\hline 884 & TC99_SEP_LSC & QTESRL & $-0.0368 \mathrm{pCi} / \mathrm{L}$ & 0.0011 & 0.28 & 0.233 & & $U$ \\
\hline 885 & TC99_SEP_LSC & STLRL & $78.3 \mathrm{pCi} / \mathrm{L}$ & 1.2 & 7 & 1.85 & & \\
\hline 886 & TC99_SEP_LSC & QTESRL & $0.146 \mathrm{pCi} / \mathrm{L}$ & 0.0043 & 0.28 & 0.225 & & $\mathrm{U}$ \\
\hline 887 & TC99_SEP_LSC & QTESRL & $0.144 \mathrm{pCi} / \mathrm{L}$ & 0.0042 & 0.28 & 0.226 & & $U$ \\
\hline 888 & TC99_SEP_LSC & STLRL & $-0.0778 \mathrm{pCi} / \mathrm{L}$ & 0.0024 & 0.26 & 0.227 & & U \\
\hline 889 & TC99_SEP_LSC & STLRL & -0.0466 pCi/L & 0.0014 & 0.26 & 0.224 & & $\mathrm{U}$ \\
\hline 890 & TC99_SEP_LSC & STLRL & $-0.0297 \mathrm{pCi} / \mathrm{L}$ & 0.00088 & 0.27 & 0.227 & & U \\
\hline 891 & TC99_SEP_LSC & STLRL & $-0.1 \mathrm{pCi} / \mathrm{L}$ & 0.003 & 0.27 & 0.23 & & U \\
\hline 892 & TC99_SEP_LSC & STLRL & $0.158 \mathrm{pCi} / \mathrm{L}$ & 0.0046 & 0.28 & 0.226 & & $\mathrm{U}$ \\
\hline 893 & TC99_SEP_LSC & STLRL & $0.027 \mathrm{pCi} / \mathrm{L}$ & 0.0008 & 0.27 & 0.225 & & $U$ \\
\hline 894 & TC99_SEP_LSC & STLRL & & & & & & $U$ \\
\hline 895 & TC99_SEP_LSC & STLRL & $-0.269 \mathrm{pCi} / \mathrm{L}$ & 0.0086 & 0.24 & 0.228 & & $U$ \\
\hline 896 & TC99_SEP_LSC & STLRL & $-0.0827 \mathrm{pCi} / \mathrm{L}$ & 0.0026 & 0.25 & 0.227 & & $U$ \\
\hline 897 & TC99_SEP_LSC & STLRL & $-0.117 \mathrm{pCi} / \mathrm{L}$ & 0.0036 & 0.25 & 0.233 & & U \\
\hline 898 & TC99_SEP_LSC & STLRL & $-0.0637 \mathrm{pCi} / \mathrm{L}$ & 0.0019 & 0.26 & 0.23 & & $\mathrm{U}$ \\
\hline 899 & TC99_SEP_LSC & STLRL & $71.5 \mathrm{pCi} / \mathrm{L}$ & 0.83 & 5.6 & 0.915 & & \\
\hline 900 & TC99_SEP_LSC & STLRL & $15.6 \mathrm{pCi} / \mathrm{L}$ & 0.31 & 2 & 0.911 & & \\
\hline 901 & TC99_SEP_LSC & STLRL & $54.1 \mathrm{pCi} / \mathrm{L}$ & 0.7 & 4.5 & 0.912 & & \\
\hline 902 & TC99_SEP_LSC & STLRL & $79.7 \mathrm{pCi} / \mathrm{L}$ & 0.88 & 6.1 & 0.917 & & \\
\hline 903 & TC99_SEP_LSC & STLRL & $12.4 \mathrm{pCi} / \mathrm{L}$ & 0.26 & 1.8 & 0.912 & & $\mathrm{~J}$ \\
\hline 904 & TC99_SEP_LSC & STLRL & $0.0709 \mathrm{pCi} / \mathrm{L}$ & 0.0021 & 0.26 & 0.227 & & U \\
\hline 905 & TC99_SEP_LSC & STLRL & $0.00405 \mathrm{pCi} / \mathrm{L}$ & 0.00012 & 0.26 & 0.227 & & U \\
\hline 906 & TC99_SEP_LSC & STLRL & $792 \mathrm{pCi} / \mathrm{L}$ & 15 & 69 & 18 & & \\
\hline 907 & TC99_SEP_LSC & STLRL & $1.99 \mathrm{pCi} / \mathrm{L}$ & 0.12 & 0.36 & 0.221 & & $\mathrm{~J}$ \\
\hline 908 & TC99_SEP_LSC & STLRL & $0.272 \mathrm{pCi} / \mathrm{L}$ & 0.097 & 0.26 & 0.222 & & $\mathrm{~J}$ \\
\hline 909 & TC99_SEP_LSC & STLRL & $0.0663 \mathrm{pCi} / \mathrm{L}$ & 0.094 & 0.25 & 0.222 & & U \\
\hline 910 & TC99_SEP_LSC & STLRL & $0.3 \mathrm{pCi} / \mathrm{L}$ & 0.098 & 0.26 & 0.223 & & $\mathrm{~J}$ \\
\hline 911 & TC99_SEP_LSC & STLRL & $0.118 \mathrm{pCi} / \mathrm{L}$ & 0.095 & 0.25 & 0.223 & & $\mathrm{U}$ \\
\hline 912 & TC99_SEP_LSC & STLRL & $0.299 \mathrm{pCi} / \mathrm{L}$ & 0.097 & 0.26 & 0.222 & & $\mathrm{~J}$ \\
\hline 913 & TC99_SEP_LSC & STLRL & $-0.0237 \mathrm{pCi} / \mathrm{L}$ & 0.094 & 0.25 & 0.225 & & U \\
\hline
\end{tabular}




\begin{tabular}{|c|c|c|c|c|c|c|c|c|c|}
\hline & $\mathrm{K}$ & $\mathrm{L}$ & $\mathrm{M}$ & $\mathrm{N}$ & $\mathrm{O}$ & $\mathrm{P}$ & $\mathrm{Q}$ & $\mathrm{R}$ & $\mathrm{S}$ \\
\hline 1 & METHOD_NAME & LAB_CODE & VALUE_RPTD & ANAL_UNITS_RPTD & COUNTING_ERROR & TOTAL_ANAL_ERROR & MIN_DETECTABLE_ACTIVITY & PCNT_MOISTURE & LAB_QUALIFIER \\
\hline 914 & TC99_SEP_LSC & STLRL & -0.0482 & $\mathrm{pCi} / \mathrm{L}$ & 0.095 & 0.26 & 0.229 & & $U$ \\
\hline 915 & TC99_SEP_LSC & STLRL & -0.115 & $\mathrm{pCi} / \mathrm{L}$ & 0.095 & 0.26 & 0.23 & & $U$ \\
\hline 916 & TC99_SEP_LSC & STLRL & -0.0535 & $\mathrm{pCi} / \mathrm{L}$ & 0.095 & 0.26 & 0.228 & & $U$ \\
\hline 917 & TC99_SEP_LSC & STLRL & 0.258 & $\mathrm{pCi} / \mathrm{L}$ & 0.097 & 0.27 & 0.222 & & $U$ \\
\hline 918 & TC99_SEP_LSC & STLRL & 0.0943 & $\mathrm{pCi} / \mathrm{L}$ & 0.095 & 0.26 & 0.222 & & $\mathrm{U}$ \\
\hline 919 & TC99_SEP_LSC & STLRL & 27.1 & $\mathrm{pCi} / \mathrm{L}$ & 8.1 & 23 & 18.4 & & \\
\hline 920 & TC99_SEP_LSC & STLRL & 0.115 & $\mathrm{pCi} / \mathrm{L}$ & 0.098 & 0.27 & 0.229 & & $\mathrm{U}$ \\
\hline 921 & TC99_SEP_LSC & STLRL & 0.00808 & $\mathrm{pCi} / \mathrm{L}$ & 0.096 & 0.26 & 0.229 & & U \\
\hline 922 & TC99_SEP_LSC & STLRL & 26.7 & $\mathrm{pCi} / \mathrm{L}$ & 8 & 22 & 18.1 & & \\
\hline 923 & TC99_SEP_LSC & STLRL & -0.103 & $\mathrm{pCi} / \mathrm{L}$ & 0.093 & 0.25 & 0.224 & & $U$ \\
\hline 924 & TC99_SEP_LSC & STLRL & -0.0207 & $\mathrm{pCi} / \mathrm{L}$ & 0.094 & 0.25 & 0.227 & & U \\
\hline 925 & TC99_SEP_LSC & STLRL & 112 & $\mathrm{pCi} / \mathrm{L}$ & 0.58 & 7.5 & 0.23 & & \\
\hline 926 & TC99_SEP_LSC & STLRL & -0.29 & $\mathrm{pCi} / \mathrm{L}$ & 0.092 & 0.23 & 0.229 & & $\mathrm{U}$ \\
\hline 927 & TC99_SEP_LSC & STLRL & 81.8 & $\mathrm{pCi} / \mathrm{L}$ & 0.49 & 5.6 & 0.228 & & \\
\hline 928 & TC99_SEP_LSC & STLRL & 0.341 & $\mathrm{pCi} / \mathrm{L}$ & 0.1 & 0.27 & 0.228 & & \\
\hline 929 & TC99_SEP_LSC & STLRL & -0.058 & $\mathrm{pCi} / \mathrm{L}$ & 0.096 & 0.25 & 0.231 & & $U$ \\
\hline 930 & TC99_SEP_LSC & STLRL & -0.154 & $\mathrm{pCi} / \mathrm{L}$ & 0.095 & 0.24 & 0.231 & & $\mathrm{U}$ \\
\hline 931 & TC99_SEP_LSC & STLRL & 2.15 & $\mathrm{pCi} / \mathrm{L}$ & 0.12 & 0.38 & 0.228 & & \\
\hline 932 & TC99_SEP_LSC & STLRL & 0.236 & $\mathrm{pCi} / \mathrm{L}$ & 0.2 & 0.51 & 0.458 & & $U$ \\
\hline 933 & TC99_SEP_LSC & STLRL & 0.08 & $\mathrm{pCi} / \mathrm{L}$ & 0.096 & 0.25 & 0.227 & & U \\
\hline 934 & TC99_SEP_LSC & STLRL & 0.0147 & $\mathrm{pCi} / \mathrm{L}$ & 0.095 & 0.25 & 0.228 & & U \\
\hline 935 & TC99_SEP_LSC & STLRL & -0.0881 & $\mathrm{pCi} / \mathrm{L}$ & 0.091 & 0.24 & 0.221 & & U \\
\hline 936 & TC99_SEP_LSC & STLRL & -0.152 & $\mathrm{pCi} / \mathrm{L}$ & 0.09 & 0.24 & 0.22 & & U \\
\hline 937 & TC99_SEP_LSC & STLRL & 353 & $\mathrm{pCi} / \mathrm{L}$ & 12 & 41 & 18.1 & & \\
\hline 938 & TC99_SEP_LSC & STLRL & 0.0283 & $\mathrm{pCi} / \mathrm{L}$ & 0.088 & 0.19 & 0.208 & & $\mathrm{U}$ \\
\hline 939 & TC99_SEP_LSC & STLRL & 0.033 & $\mathrm{pCi} / \mathrm{L}$ & 0.088 & 0.19 & 0.208 & & $\mathrm{U}$ \\
\hline 940 & TC99_SEP_LSC & STLRL & 0.00644 & $\mathrm{pCi} / \mathrm{L}$ & 0.088 & 0.19 & 0.208 & & $U$ \\
\hline 941 & TC99_SEP_LSC & STLRL & -0.059 & $\mathrm{pCi} / \mathrm{L}$ & 0.09 & 0.19 & 0.218 & & U \\
\hline 942 & TC99_SEP_LSC & STLRL & 10.1 & $\mathrm{pCi} / \mathrm{L}$ & 0.34 & 0.95 & 0.512 & & \\
\hline 943 & TC99_SEP_LSC & STLRL & 9.43 & $\mathrm{pCi} / \mathrm{L}$ & 0.34 & 0.93 & 0.541 & & \\
\hline 944 & TC99_SEP_LSC & STLRL & 20.2 & $\mathrm{pCi} / \mathrm{L}$ & 0.43 & 1.5 & 0.518 & & \\
\hline 945 & TC99_SEP_LSC & STLRL & 12 & $\mathrm{pCi} / \mathrm{L}$ & 0.35 & 1 & 0.502 & & \\
\hline 946 & TC99_SEP_LSC & STLRL & 11.4 & $\mathrm{pCi} / \mathrm{L}$ & 0.72 & 1.7 & 1.33 & & \\
\hline 947 & TC99_SEP_LSC & STLRL & 10.7 & $\mathrm{pCi} / \mathrm{L}$ & 0.34 & 0.96 & 0.503 & & \\
\hline 948 & TC99_SEP_LSC & STLRL & 10.6 & $\mathrm{pCi} / \mathrm{L}$ & 0.33 & 0.96 & 0.496 & & \\
\hline 949 & TC99_SEP_LSC & STLRL & & & & & & & $\mathrm{U}$ \\
\hline 950 & TC99_SEP_LSC & STLRL & 9.93 & $\mathrm{pCi} / \mathrm{L}$ & 0.38 & 1 & 0.606 & & \\
\hline 951 & TC99_SEP_LSC & STLRL & 25.9 & $\mathrm{pCi} / \mathrm{L}$ & 0.75 & 2.2 & 1.08 & & \\
\hline
\end{tabular}




\begin{tabular}{|c|c|c|c|c|c|c|c|c|c|}
\hline & $\mathrm{K}$ & $\mathrm{L}$ & $\mathrm{M}$ & $\mathrm{N}$ & $\mathrm{O}$ & $\mathrm{P}$ & $\mathrm{Q}$ & $\mathrm{R}$ & $\mathrm{S}$ \\
\hline 1 & METHOD_NAME & LAB_CODE & VALUE_RPTD & ANAL_UNITS_RPTD & |COUNTING_ERROR & TOTAL_ANAL_ERROR & MIN_DETECTABLE_ACTIVITY & PCNT_MOISTURE & LAB_QUALIFIER \\
\hline 952 & TC99_SEP_LSC & STLRL & 9.07 & $\mathrm{pCi} / \mathrm{L}$ & 0.33 & 0.9 & 0.524 & & \\
\hline 953 & TC99_SEP_LSC & STLRL & & & & & & & U \\
\hline 954 & TC99_SEP_LSC & STLRL & 0.128 & $\mathrm{pCi} / \mathrm{L}$ & 0.09 & 0.19 & 0.211 & & U \\
\hline 955 & TC99_SEP_LSC & STLRL & 0.117 & $\mathrm{pCi} / \mathrm{L}$ & 0.09 & 0.19 & 0.209 & & U \\
\hline 956 & TC99_SEP_LSC & STLRL & 5.88 & $\mathrm{pCi} / \mathrm{L}$ & 0.16 & 0.5 & 0.23 & & \\
\hline 957 & TC99_SEP_LSC & STLRL & 2.31 & $\mathrm{pCi} / \mathrm{L}$ & 0.11 & 0.28 & 0.199 & & \\
\hline 958 & TC99_SEP_LSC & STLRL & 0.0347 & $\mathrm{pCi} / \mathrm{L}$ & 0.086 & 0.17 & 0.206 & & U \\
\hline 959 & TC99_SEP_LSC & STLRL & -0.0455 & $\mathrm{pCi} / \mathrm{L}$ & 0.084 & 0.17 & 0.202 & & U \\
\hline 960 & TC99_SEP_LSC & STLRL & 4.5 & $\mathrm{pCi} / \mathrm{L}$ & 0.14 & 0.41 & 0.203 & & \\
\hline 961 & TC99_SEP_LSC & STLRL & 0.0481 & $\mathrm{pCi} / \mathrm{L}$ & 0.086 & 0.17 & 0.202 & & U \\
\hline 962 & TC99_SEP_LSC & STLRL & -0.0962 & $\mathrm{pCi} / \mathrm{L}$ & 0.084 & 0.17 & 0.203 & & $\mathrm{U}$ \\
\hline 963 & TC99_SEP_LSC & STLRL & -0.0129 & $\mathrm{pCi} / \mathrm{L}$ & 0.085 & 0.17 & 0.203 & & U \\
\hline 964 & TC99_SEP_LSC & STLRL & 0.111 & $\mathrm{pCi} / \mathrm{L}$ & 0.097 & 0.2 & 0.226 & & U \\
\hline 965 & TC99_SEP_LSC & STLRL & -0.0348 & $\mathrm{pCi} / \mathrm{L}$ & 0.084 & 0.17 & 0.202 & & $U$ \\
\hline 966 & TC99_SEP_LSC & STLRL & 0.0157 & $\mathrm{pCi} / \mathrm{L}$ & 0.085 & 0.18 & 0.201 & & $\mathrm{U}$ \\
\hline 967 & TC99_SEP_LSC & STLRL & 0.0179 & $\mathrm{pCi} / \mathrm{L}$ & 0.23 & 0.48 & 0.543 & & U \\
\hline 968 & TC99_SEP_LSC & STLRL & -0.00175 & $\mathrm{pCi} / \mathrm{L}$ & 0.23 & 0.48 & 0.544 & & $\mathrm{U}$ \\
\hline 969 & TC99_SEP_LSC & STLRL & -0.254 & $\mathrm{pCi} / \mathrm{L}$ & 0.22 & 0.46 & 0.546 & & U \\
\hline 970 & TC99_SEP_LSC & STLRL & -0.32 & $\mathrm{pCi} / \mathrm{L}$ & 0.22 & 0.46 & 0.547 & & U \\
\hline 971 & TC99_SEP_LSC & STLRL & 0.299 & $\mathrm{pCi} / \mathrm{L}$ & 0.23 & 0.47 & 0.533 & & $\mathrm{U}$ \\
\hline 972 & TC99_SEP_LSC & STLRL & 0.172 & $\mathrm{pCi} / \mathrm{L}$ & 0.23 & 0.47 & 0.534 & & $\mathrm{U}$ \\
\hline 973 & TC99_SEP_LSC & STLRL & -0.0956 & $\mathrm{pCi} / \mathrm{L}$ & 0.22 & 0.45 & 0.528 & & $\mathrm{U}$ \\
\hline 974 & TC99_SEP_LSC & STLRL & -0.109 & $\mathrm{pCi} / \mathrm{L}$ & 0.22 & 0.45 & 0.514 & & $U$ \\
\hline 975 & TC99_SEP_LSC & STLRL & 0.127 & $\mathrm{pCi} / \mathrm{L}$ & 0.23 & 0.47 & 0.533 & & U \\
\hline 976 & TC99_SEP_LSC & STLRL & -0.0906 & $\mathrm{pCi} / \mathrm{L}$ & 0.22 & 0.46 & 0.535 & & $\mathrm{U}$ \\
\hline 977 & TC99_SEP_LSC & STLRL & 0.0015 & $\mathrm{pCi} / \mathrm{L}$ & 0.22 & 0.46 & 0.533 & & U \\
\hline 978 & TC99_SEP_LSC & STLRL & -0.0391 & $\mathrm{pCi} / \mathrm{L}$ & 0.22 & 0.45 & 0.529 & & U \\
\hline 979 & TC99_SEP_LSC & STLRL & 0.106 & $\mathrm{pCi} / \mathrm{L}$ & 0.23 & 0.47 & 0.543 & & U \\
\hline 980 & TC99_SEP_LSC & STLRL & -0.0266 & $\mathrm{pCi} / \mathrm{L}$ & 0.23 & 0.46 & 0.545 & & U \\
\hline 981 & TC99_SEP_LSC & STLRL & -0.317 & $\mathrm{pCi} / \mathrm{L}$ & 0.25 & 0.54 & 0.579 & & U \\
\hline 982 & TC99_SEP_LSC & STLRL & -0.387 & $\mathrm{pCi} / \mathrm{L}$ & 0.25 & 0.54 & 0.583 & & $U$ \\
\hline 983 & TC99_SEP_LSC & STLRL & 4.46 & $\mathrm{pCi} / \mathrm{L}$ & 0.31 & 0.77 & 0.604 & & \\
\hline 984 & TC99_SEP_LSC & STLRL & 1.12 & $\mathrm{pCi} / \mathrm{L}$ & 0.27 & 0.6 & 0.599 & & \\
\hline 985 & TC99_SEP_LSC & STLRL & 0.529 & $\mathrm{pCi} / \mathrm{L}$ & 0.25 & 0.55 & 0.593 & & U \\
\hline 986 & TC99_SEP_LSC & STLRL & 0.435 & $\mathrm{pCi} / \mathrm{L}$ & 0.25 & 0.55 & 0.595 & & $U$ \\
\hline 987 & TC99_SEP_LSC & STLRL & 74.8 & $\mathrm{pCi} / \mathrm{L}$ & 0.79 & 4.7 & 0.596 & & \\
\hline 988 & TC99_SEP_LSC & STLRL & 66.8 & $\mathrm{pCi} / \mathrm{L}$ & 0.75 & 4.3 & 0.592 & & \\
\hline 989 & TC99_SEP_LSC & STLRL & -0.266 & $\mathrm{pCi} / \mathrm{L}$ & 0.25 & 0.54 & 0.583 & & $\mathrm{U}$ \\
\hline
\end{tabular}




\begin{tabular}{|c|c|c|c|c|c|c|c|c|c|}
\hline & $\mathrm{K}$ & $\mathrm{L}$ & $\mathrm{M}$ & $\mathrm{N}$ & $\mathrm{O}$ & $\mathrm{P}$ & $\mathrm{Q}$ & $\mathrm{R}$ & $\mathrm{S}$ \\
\hline 1 & METHOD_NAME & LAB_CODE & VALUE_RPTD & ANAL_UNITS_RPTD & COUNTING_ERROR & TOTAL_ANAL_ERROR & MIN_DETECTABLE_ACTIVITY & PCNT_MOISTURE & LAB_QUALIFIER \\
\hline 990 & TC99_SEP_LSC & STLRL & 7.95 & $\mathrm{pCi} / \mathrm{L}$ & 0.35 & 0.97 & 0.587 & & \\
\hline 991 & TC99_SEP_LSC & STLRL & -0.148 & $\mathrm{pCi} / \mathrm{L}$ & 0.25 & 0.55 & 0.589 & & $\mathrm{U}$ \\
\hline 992 & TC99_SEP_LSC & STLRL & -0.14 & $\mathrm{pCi} / \mathrm{L}$ & 0.25 & 0.54 & 0.586 & & 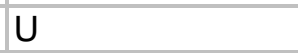 \\
\hline 993 & TC99_SEP_LSC & STLRL & 0.447 & $\mathrm{pCi} / \mathrm{L}$ & 0.25 & 0.53 & 0.569 & & $U$ \\
\hline 994 & TC99_SEP_LSC & STLRL & 0.456 & $\mathrm{pCi} / \mathrm{L}$ & 0.25 & 0.53 & 0.572 & & $U$ \\
\hline 995 & TRITIUM_DIST_LSC & QTESRL & 358 & $\mathrm{pCi} / \mathrm{L}$ & 34 & 140 & 257 & & \\
\hline 996 & TRITIUM_DIST_LSC & QTESRL & 5340 & $\mathrm{pCi} / \mathrm{L}$ & 160 & 330 & 143 & & \\
\hline 997 & TRITIUM_ELECT_LSC & QTESRL & 61.5 & $\mathrm{pCi} / \mathrm{L}$ & 1.5 & 7.5 & 4.44 & & \\
\hline 998 & TRITIUM_ELECT_LSC & QTESRL & 35.2 & $\mathrm{pCi} / \mathrm{L}$ & 0.97 & 5.5 & 4.38 & & \\
\hline 999 & TRITIUM_DIST_LSC & QTESRL & 113 & $\mathrm{pCi} / \mathrm{L}$ & 8.9 & 110 & 166 & & $U$ \\
\hline 1000 & TRITIUM_DIST_LSC & QTESRL & 132 & $\mathrm{pCi} / \mathrm{L}$ & 10 & 110 & 166 & & $\mathrm{U}$ \\
\hline 1001 & TRITIUM_DIST_LSC & QTESRL & 180 & $\mathrm{pCi} / \mathrm{L}$ & 14 & 110 & 165 & & \\
\hline 1002 & TRITIUM_ELECT_LSC & QTESRL & 159 & $\mathrm{pCi} / \mathrm{L}$ & 2.8 & 15 & 4.27 & & \\
\hline 1003 & 906.0_H3_LSC & QTESRL & 45.1 & $\mathrm{pCi} / \mathrm{L}$ & 3.6 & 110 & 173 & & $U$ \\
\hline 1004 & 906.0_H3_LSC & QTESRL & 4130 & $\mathrm{pCi} / \mathrm{L}$ & 140 & 300 & 174 & & \\
\hline 1005 & TRITIŪM_ELECT_LSC & QTESRL & 48.5 & $\mathrm{pCi} / \mathrm{L}$ & 1.3 & 6.4 & 4.31 & & \\
\hline 1006 & TRITIUM_ELECT_LSC & QTESRL & 98.1 & $\mathrm{pCi} / \mathrm{L}$ & 2 & 11 & 4.93 & & \\
\hline 1007 & TRITIUM_ELECT_LSC & QTESRL & 29.2 & $\mathrm{pCi} / \mathrm{L}$ & 0.79 & 5.5 & 4.85 & & \\
\hline 1008 & TRITIUM_ELECT_LSC & QTESRL & 29.8 & $\mathrm{pCi} / \mathrm{L}$ & 0.86 & 5.2 & 4.57 & & \\
\hline 1009 & TRITIUM_ELECT_LSC & QTESRL & 28.6 & $\mathrm{pCi} / \mathrm{L}$ & 0.83 & 5 & 4.32 & & \\
\hline 1010 & TRITIUM_ELECT_LSC & QTESRL & 29.8 & $\mathrm{pCi} / \mathrm{L}$ & 0.86 & 5 & 4.33 & & \\
\hline 1011 & TRITIUM_ELECT_LSC & QTESRL & 26.6 & $\mathrm{pCi} / \mathrm{L}$ & 0.78 & 4.8 & 4.35 & & \\
\hline 1012 & TRITIUM_ELECT_LSC & QTESRL & 84.8 & $\mathrm{pCi} / \mathrm{L}$ & 1.9 & 9.4 & 4.43 & & \\
\hline 1013 & TRITIUM_ELECT_LSC & QTESRL & 86.8 & $\mathrm{pCi} / \mathrm{L}$ & 1.9 & 9.6 & 4.51 & & \\
\hline 1014 & TRITIUM_ELECT_LSC & QTESRL & 50.5 & $\mathrm{pCi} / \mathrm{L}$ & 1.3 & 6.7 & 4.55 & & \\
\hline 1015 & TRITIUM_ELECT_LSC & QTESRL & 41.5 & $\mathrm{pCi} / \mathrm{L}$ & 1.1 & 6 & 4.47 & & \\
\hline 1016 & TRITIUM_ELECT_LSC & QTESRL & 41.8 & $\mathrm{pCi} / \mathrm{L}$ & 1.1 & 6 & 4.47 & & \\
\hline 1017 & TRITIUM_ELECT_LSC & QTESRL & 42.5 & $\mathrm{pCi} / \mathrm{L}$ & 1.1 & 6.1 & 4.52 & & \\
\hline 1018 & TRITIUM_ELECT_LSC & QTESRL & 43.4 & $\mathrm{pCi} / \mathrm{L}$ & 1.2 & 6.2 & 4.5 & & \\
\hline 1019 & TRITIUM_ELECT_LSC & QTESRL & 68.9 & $\mathrm{pCi} / \mathrm{L}$ & 1.6 & 8.2 & 4.54 & & \\
\hline 1020 & TRITIUM_ELECT_LSC & QTESRL & 69.9 & $\mathrm{pCi} / \mathrm{L}$ & 1.7 & 8.2 & 4.5 & & \\
\hline 1021 & TRITIUM_ELECT_LSC & QTESRL & 82 & $\mathrm{pCi} / \mathrm{L}$ & 1.9 & 9.2 & 4.58 & & \\
\hline 1022 & TRITIUM_ELECT_LSC & QTESRL & 70.7 & $\mathrm{pCi} / \mathrm{L}$ & 1.7 & 8.3 & 4.56 & & \\
\hline 1023 & TRITIUM_DIST_LSC & QTESRL & 146 & $\mathrm{pCi} / \mathrm{L}$ & 10 & 120 & 175 & & $U$ \\
\hline 1024 & TRITIUM_DIST_LSC & QTESRL & 3620 & $\mathrm{pCi} / \mathrm{L}$ & 120 & 270 & 174 & & \\
\hline 1025 & TRITIUM_DIST_LSC & QTESRL & 3490 & $\mathrm{pCi} / \mathrm{L}$ & 120 & 270 & 174 & & \\
\hline 1026 & TRITIUM_ELECTT_LSC & QTESRL & 96.7 & $\mathrm{pCi} / \mathrm{L}$ & 2.1 & 10 & 4.58 & & \\
\hline 1027 & TRITIUM_ELECT_LSC & QTESRL & 46.8 & $\mathrm{pCi} / \mathrm{L}$ & 1.2 & 6.5 & 4.59 & & \\
\hline
\end{tabular}




\begin{tabular}{|c|c|c|c|c|c|c|c|c|c|}
\hline & $\mathrm{K}$ & $\mathrm{L}$ & $\mathrm{M}$ & $\mathrm{N}$ & $\mathrm{O}$ & $\mathrm{P}$ & $\mathrm{Q}$ & $\mathrm{R}$ & $\mathrm{S}$ \\
\hline 1 & METHOD_NAME & LAB_CODE & VALUE_RPTD & ANAL_UNITS_RPTD & COUNTING_ERROR & TOTAL_ANAL_ERROR & MIN_DETECTABLE_ACTIVITY & PCNT_MOISTURE & LAB_QUALIFIER \\
\hline 1028 & TRITIUM_ELECT_LSC & STLRL & 56 & $\mathrm{pCi} / \mathrm{L}$ & 1.4 & 7 & 4.26 & & \\
\hline 1029 & 906.0_H3_LSC & STLRL & 22.3 & $\mathrm{pCi} / \mathrm{L}$ & 1.7 & 110 & 173 & & $U$ \\
\hline 1030 & 906.0_H3_LSC & STLRL & 3880 & $\mathrm{pCi} / \mathrm{L}$ & 130 & 280 & 173 & & \\
\hline 1031 & TRITIŪM_ELECT_LSC & STLRL & 40.1 & $\mathrm{pCi} / \mathrm{L}$ & 1.1 & 5.8 & 4.34 & & \\
\hline 1032 & TRITIUM_ELECT_LSC & STLRL & 41.6 & $\mathrm{pCi} / \mathrm{L}$ & 1.1 & 5.9 & 4.23 & & \\
\hline 1033 & TRITIUM_ELECT_LSC & STLRL & 52.6 & $\mathrm{pCi} / \mathrm{L}$ & 1.3 & 6.7 & 4.23 & & \\
\hline 1034 & TRITIUM_ELECT_LSC & STLRL & 85.5 & $\mathrm{pCi} / \mathrm{L}$ & 1.9 & 9.4 & 4.5 & & \\
\hline 1035 & TRITIUM_ELECT_LSC & STLRL & 27.8 & $\mathrm{pCi} / \mathrm{L}$ & 0.8 & 5 & 4.46 & & \\
\hline 1036 & TRITIUM_ELECT_LSC & STLRL & 49.9 & $\mathrm{pCi} / \mathrm{L}$ & 1.3 & 6.6 & 4.44 & & \\
\hline 1037 & TRITIUM_ELECT_LSC & STLRL & 26.8 & $\mathrm{pCi} / \mathrm{L}$ & 0.78 & 5 & 4.49 & & \\
\hline 1038 & TRITIUM_ELECT_LSC & STLRL & 40.6 & $\mathrm{pCi} / \mathrm{L}$ & 1.1 & 5.8 & 4.34 & & \\
\hline 1039 & TRITIUM_ELECT_LSC & STLRL & 33.4 & $\mathrm{pCi} / \mathrm{L}$ & 0.94 & 5.3 & 4.38 & & \\
\hline 1040 & TRITIUM_ELECT_LSC & STLRL & 38.5 & $\mathrm{pCi} / \mathrm{L}$ & 1 & 5.7 & 4.33 & & \\
\hline 1041 & TRITIUM_ELECT_LSC & STLRL & 44.3 & $\mathrm{pCi} / \mathrm{L}$ & 1.2 & 6.1 & 4.37 & & \\
\hline 1042 & TRITIUM_ELECT_LSC & STLRL & 68.9 & $\mathrm{pCi} / \mathrm{L}$ & 1.6 & 8 & 4.33 & & \\
\hline 1043 & TRITIUM_ELECT_LSC & STLRL & 120 & $\mathrm{pCi} / \mathrm{L}$ & 2.4 & 12 & 4.35 & & \\
\hline 1044 & TRITIUM_ELECT_LSC & STLRL & 130 & $\mathrm{pCi} / \mathrm{L}$ & 2.5 & 13 & 4.36 & & \\
\hline 1045 & TRITIUM_ELECT_LSC & STLRL & 119 & $\mathrm{pCi} / \mathrm{L}$ & 2.3 & 12 & 4.31 & & \\
\hline 1046 & TRITIUM_ELECT_LSC & STLRL & 131 & $\mathrm{pCi} / \mathrm{L}$ & 2.5 & 13 & 4.35 & & \\
\hline 1047 & TRITIUM_ELECT_LSC & STLRL & 147 & $\mathrm{pCi} / \mathrm{L}$ & 2.7 & 14 & 4.37 & & \\
\hline 1048 & TRITIUM_ELECT_LSC & STLRL & 42.3 & $\mathrm{pCi} / \mathrm{L}$ & 1.1 & 6 & 4.4 & & \\
\hline 1049 & TRITIUM_ELECT_LSC & STLRL & 36.3 & $\mathrm{pCi} / \mathrm{L}$ & 1 & 5.5 & 4.35 & & \\
\hline 1050 & TRITIUM_ELECT_LSC & STLRL & 33.3 & $\mathrm{pCi} / \mathrm{L}$ & 0.94 & 5.3 & 4.36 & & \\
\hline 1051 & TRITIUM_ELECT_LSC & STLRL & 34.9 & $\mathrm{pCi} / \mathrm{L}$ & 0.98 & 5.4 & 4.43 & & \\
\hline 1052 & TRITIUM_ELECT_LSC & STLRL & 53.3 & $\mathrm{pCi} / \mathrm{L}$ & 1.3 & 6.9 & 4.4 & & \\
\hline 1053 & TRITIUM_ELECT_LSC & STLRL & 80.6 & $\mathrm{pCi} / \mathrm{L}$ & 1.8 & 9 & 4.39 & & \\
\hline 1054 & TRITIUM_DIST_LSC & STLRL & 98.4 & $\mathrm{pCi} / \mathrm{L}$ & 7.8 & 97 & 149 & & $\mathrm{U}$ \\
\hline 1055 & TRITIUM_DIST_LSC & STLRL & 3160 & $\mathrm{pCi} / \mathrm{L}$ & 110 & 240 & 147 & & \\
\hline 1056 & TRITIUM_ELECT_LSC & STLRL & & & & & & & \\
\hline 1057 & TRITIUM_ELECT_LSC & STLRL & 34.9 & $\mathrm{pCi} / \mathrm{L}$ & 0.94 & 5.7 & 4.65 & & \\
\hline 1058 & TRITIUM_ELECT_LSC & STLRL & 128 & $\mathrm{pCi} / \mathrm{L}$ & 2.4 & 13 & 4.65 & & \\
\hline 1059 & 906.0_H3_LSC & STLRL & 99.6 & $\mathrm{pCi} / \mathrm{L}$ & 7.1 & 110 & 163 & & $U$ \\
\hline 1060 & 906.0_H3_LSC & STLRL & 3770 & $\mathrm{pCi} / \mathrm{L}$ & 120 & 270 & 163 & & \\
\hline 1061 & TRITIUM_ELECT_LSC & STLRL & 42.9 & $\mathrm{pCi} / \mathrm{L}$ & 1.1 & 6.3 & 4.71 & & \\
\hline 1062 & TRITIUM_ELECT_LSC & STLRL & 39.7 & $\mathrm{pCi} / \mathrm{L}$ & 1 & 6 & 4.68 & & \\
\hline 1063 & TRITIUM_ELECT_LSC & STLRL & 80.2 & $\mathrm{pCi} / \mathrm{L}$ & 1.8 & 9.1 & 4.62 & & \\
\hline 1064 & TRITIUM_ELECT_LSC & STLRL & 30.3 & $\mathrm{pCi} / \mathrm{L}$ & 3.3 & 6.1 & 6.13 & & \\
\hline 1065 & TRITIUM_ELECT_LSC & STLRL & 97 & $\mathrm{pCi} / \mathrm{L}$ & 4.4 & 11 & 6.07 & & \\
\hline
\end{tabular}




\begin{tabular}{|c|c|c|c|c|c|c|c|c|c|}
\hline & $\mathrm{K}$ & $\mathrm{L}$ & $\mathrm{M}$ & $\mathrm{N}$ & $\mathrm{O}$ & $\mathrm{P}$ & $\mathrm{Q}$ & $\mathrm{R}$ & $\mathrm{S}$ \\
\hline 1 & METHOD_NAME & LAB_CODE & VALUE_RPTD & ANAL_UNITS_RPTD & COUNTING_ERROR & TOTAL_ANAL_ERROR & MIN_DETECTABLE_ACTIVITY & PCNT_MOISTURE & LAB_QUALIFIER \\
\hline 1066 & TRITIUM_ELECT_LSC & STLRL & & $\mathrm{pCi} / \mathrm{L}$ & 1.7 & 8.6 & 4.42 & & \\
\hline 1067 & TRITIUM_ELECT_LSC & STLRL & 36.3 & $\mathrm{pCi} / \mathrm{L}$ & 1 & 5.6 & 4.38 & & \\
\hline 1068 & TRITIUM_ELECT_LSC & STLRL & 46.1 & $\mathrm{pCi} / \mathrm{L}$ & 1.2 & 6.3 & 4.33 & & \\
\hline 1069 & TRITIUM_ELECT_LSC & STLRL & 33.8 & $\mathrm{pCi} / \mathrm{L}$ & 0.95 & 5.4 & 4.43 & & \\
\hline 1070 & TRITIUM_ELECT_LSC & STLRL & 38.9 & $\mathrm{pCi} / \mathrm{L}$ & 1.1 & 5.7 & 4.35 & & \\
\hline 1071 & TRITIUM_ELECT_LSC & STLRL & 81.4 & $\mathrm{pCi} / \mathrm{L}$ & 1.8 & 9.1 & 4.51 & & \\
\hline 1072 & TRITIUM_ELECT_LSC & STLRL & 25.7 & $\mathrm{pCi} / \mathrm{L}$ & 0.76 & 4.8 & 4.46 & & \\
\hline 1073 & TRITIUM_ELECT_LSC & STLRL & 26.5 & $\mathrm{pCi} / \mathrm{L}$ & 0.78 & 4.8 & 4.32 & & \\
\hline 1074 & TRITIUM_ELECT_LSC & STLRL & 28.8 & $\mathrm{pCi} / \mathrm{L}$ & 0.83 & 5 & 4.36 & & \\
\hline 1075 & TRITIUM_ELECT_LSC & STLRL & 26.1 & $\mathrm{pCi} / \mathrm{L}$ & 0.77 & 4.9 & 4.45 & & \\
\hline 1076 & TRITIUM_ELECT_LSC & STLRL & 31.2 & $\mathrm{pCi} / \mathrm{L}$ & 0.89 & 5.2 & 4.39 & & \\
\hline 1077 & TRITIUM_ELECT_LSC & STLRL & 32.7 & $\mathrm{pCi} / \mathrm{L}$ & 0.93 & 5.3 & 4.39 & & \\
\hline 1078 & TRITIUM_ELECT_LSC & STLRL & 35.6 & $\mathrm{pCi} / \mathrm{L}$ & 0.99 & 5.5 & 4.43 & & \\
\hline 1079 & TRITIUM_ELECT_LSC & STLRL & 33 & $\mathrm{pCi} / \mathrm{L}$ & 0.93 & 5.3 & 4.42 & & \\
\hline 1080 & TRITIUM_ELECT_LSC & STLRL & 31.3 & $\mathrm{pCi} / \mathrm{L}$ & 0.9 & 5.2 & 4.45 & & \\
\hline 1081 & TRITIUM_ELECT_LSC & STLRL & 152 & $\mathrm{pCi} / \mathrm{L}$ & 5.2 & 16 & 6.26 & & \\
\hline 1082 & TRITIUM_ELECT_LSC & STLRL & 61.1 & $\mathrm{pCi} / \mathrm{L}$ & 3.9 & 8.4 & 6.26 & & \\
\hline 1083 & TRITIUM_ELECT_LSC & STLRL & 53.1 & $\mathrm{pCi} / \mathrm{L}$ & 3.7 & 7.8 & 6.13 & & \\
\hline 1084 & TRITIUM_ELECT_LSC & STLRL & 62.7 & $\mathrm{pCi} / \mathrm{L}$ & 3.9 & 8.5 & 6.17 & & \\
\hline 1085 & TRITIUM_ELECT_LSC & STLRL & 48.7 & $\mathrm{pCi} / \mathrm{L}$ & 3.7 & 7.5 & 6.28 & & \\
\hline 1086 & TRITIUM_ELECT_LSC & STLRL & 60.5 & $\mathrm{pCi} / \mathrm{L}$ & 3.8 & 8.3 & 6.19 & & \\
\hline 1087 & TRITIUM_ELECT_LSC & STLRL & 31.6 & $\mathrm{pCi} / \mathrm{L}$ & 2.3 & 5.2 & 4.35 & & \\
\hline 1088 & TRITIUM_ELECT_LSC & STLRL & 26.8 & $\mathrm{pCi} / \mathrm{L}$ & 2.2 & 4.8 & 4.3 & & \\
\hline 1089 & TRITIUM_ELECT_LSC & STLRL & 39.2 & $\mathrm{pCi} / \mathrm{L}$ & 3.4 & 6.7 & 6.09 & & \\
\hline 1090 & TRITIUM_ELECT_LSC & STLRL & 29.5 & $\mathrm{pCi} / \mathrm{L}$ & 2.3 & 5 & 4.33 & & \\
\hline 1091 & TRITIUM_ELECT_LSC & STLRL & 29 & $\mathrm{pCi} / \mathrm{L}$ & 2.3 & 5 & 4.3 & & \\
\hline 1092 & TRITIUM_ELECT_LSC & STLRL & 26.7 & $\mathrm{pCi} / \mathrm{L}$ & 2.3 & 4.8 & 4.36 & & \\
\hline 1093 & TRITIUM_ELECT_LSC & STLRL & 23.9 & $\mathrm{pCi} / \mathrm{L}$ & 2.2 & 4.7 & 4.34 & & \\
\hline 1094 & TRITIUM_ELECT_LSC & STLRL & 382 & $\mathrm{pCi} / \mathrm{L}$ & 5.4 & 35 & 4.35 & & \\
\hline 1095 & TRITIUM_ELECT_LSC & STLRL & 84.6 & $\mathrm{pCi} / \mathrm{L}$ & 4.2 & 10 & 6.13 & & \\
\hline 1096 & TRITIUM_ELECT_LSC & STLRL & 34.6 & $\mathrm{pCi} / \mathrm{L}$ & 3.3 & 6.4 & 6.05 & & \\
\hline 1097 & TRITIUM_ELECT_LSC & STLRL & 27 & $\mathrm{pCi} / \mathrm{L}$ & 3.2 & 5.8 & 6.08 & & \\
\hline 1098 & TRITIUM_ELECT_LSC & STLRL & 40.1 & $\mathrm{pCi} / \mathrm{L}$ & 3.5 & 6.8 & 6.13 & & \\
\hline 1099 & TRITIUM_ELECT_LSC & STLRL & 31 & $\mathrm{pCi} / \mathrm{L}$ & 3.3 & 6.2 & 6.13 & & \\
\hline 1100 & TRITIUM_ELECT_LSC & STLRL & 33.1 & $\mathrm{pCi} / \mathrm{L}$ & 3.3 & 6.3 & 6.07 & & \\
\hline 1101 & TRITIUM_ELECT_LSC & STLRL & 114 & $\mathrm{pCi} / \mathrm{L}$ & 4.7 & 13 & 6.07 & & \\
\hline 1102 & TRITIUM_ELECT_LSC & STLRL & 1920 & $\mathrm{pCi} / \mathrm{L}$ & 16 & 170 & 6.18 & & \\
\hline 1103 & TRITIUM_ELECT_LSC & STLRL & 29.9 & $\mathrm{pCi} / \mathrm{L}$ & 2.3 & 5.1 & 4.32 & & \\
\hline
\end{tabular}




\begin{tabular}{|c|c|c|c|c|c|c|c|c|c|}
\hline & $\mathrm{K}$ & $\mathrm{L}$ & $\bar{M}$ & $\mathrm{~N}$ & $\mathrm{O}$ & $P$ & $\mathrm{Q}$ & $\mathrm{R}$ & $\mathrm{S}$ \\
\hline 1 & METHOD_NAME & LAB_CODE & VALUE_RPTD & ANAL_UNITS_RPTD & COUNTING_ERROR & TOTAL_ANAL_ERROR & MIN_DETECTABLE_ACTIVITY & PCNT_MOISTURE & LAB_QUALIFIER \\
\hline 1104 & TRITIUM_ELECT_LSC & STLRL & 31.1 & $\mathrm{pCi} / \mathrm{L}$ & 2.3 & 5.2 & 4.36 & & \\
\hline 1105 & TRITIUM_ELECT_LSC & STLRL & 33.8 & $\mathrm{pCi} / \mathrm{L}$ & 2.4 & 5.4 & 4.33 & & \\
\hline 1106 & TRITIUM_ELECT_LSC & STLRL & 32.8 & $\mathrm{pCi} / \mathrm{L}$ & 3.3 & 6.3 & 6.2 & & \\
\hline 1107 & TRITIUM_ELECT_LSC & STLRL & 34 & $\mathrm{pCi} / \mathrm{L}$ & 3.4 & 6.4 & 6.21 & & \\
\hline 1108 & TRITIUM_ELECT_LSC & STLRL & 500 & $\mathrm{pCi} / \mathrm{L}$ & 8.6 & 45 & 6.13 & & \\
\hline 1109 & TRITIUM_ELECT_LSC & STLRL & 73.8 & $\mathrm{pCi} / \mathrm{L}$ & 4.1 & 9.4 & 6.17 & & \\
\hline 1110 & TRITIUM_ELECT_LSC & STLRL & 24.4 & $\mathrm{pCi} / \mathrm{L}$ & 3.2 & 5.8 & 6.32 & & \\
\hline 1111 & TRITIUM_ELECT_LSC & STLRL & 4100 & $\mathrm{pCi} / \mathrm{L}$ & 24 & 360 & 6.42 & & \\
\hline 1112 & TRITIUM_ELECT_LSC & STLRL & 157 & $\mathrm{pCi} / \mathrm{L}$ & 5.3 & 16 & 6.37 & & \\
\hline 1113 & |TRITIUM_ELECT_LSC & STLRL & 40.9 & $\mathrm{pCi} / \mathrm{L}$ & 3.5 & 6.8 & 6.1 & & \\
\hline 1114 & TRITIUM_ELECT_LSC & STLRL & 35.5 & $\mathrm{pCi} / \mathrm{L}$ & 3.4 & 6.4 & 6.08 & & \\
\hline 1115 & TRITIUM_ELECT_LSC & STLRL & 28.8 & $\mathrm{pCi} / \mathrm{L}$ & 3.2 & 6 & 6.04 & & \\
\hline 1116 & TRITIUM_ELECT_LSC & STLRL & 57.9 & $\mathrm{pCi} / \mathrm{L}$ & 3.8 & 8.1 & 6.12 & & \\
\hline 1117 & TRITIUM_ELECT_LSC & STLRL & 31.2 & $\mathrm{pCi} / \mathrm{L}$ & 3.3 & 6.1 & 6.07 & & \\
\hline 1118 & TRITIUM ELECT LSC & STLRL & 30.6 & $\mathrm{pCi} / \mathrm{L}$ & 3.3 & 6.1 & 6.13 & & \\
\hline 1119 & TRITIUM_ELECT_LSC & STLRL & 40.9 & $\mathrm{pCi} / \mathrm{L}$ & 3.5 & 6.9 & 6.22 & & \\
\hline 1120 & TRITIUM_ELECT_LSC & STLRL & 44.7 & $\mathrm{pCi} / \mathrm{L}$ & 3.5 & 7.1 & 6.14 & & \\
\hline 1121 & TRITIUM_ELECT_LSC & STLRL & 29.4 & $\mathrm{pCi} / \mathrm{L}$ & 3.3 & 6.1 & 6.17 & & \\
\hline 1122 & TRITIUM_ELECT_LSC & STLRL & 32.2 & $\mathrm{pCi} / \mathrm{L}$ & 3.3 & 6.3 & 6.21 & & \\
\hline 1123 & TRITIUM_ELECT_LSC & STLRL & 30 & $\mathrm{pCi} / \mathrm{L}$ & 3.3 & 6.1 & 6.25 & & \\
\hline 1124 & TRITIUM_ELECT_LSC & STLRL & 32.8 & $\mathrm{pCi} / \mathrm{L}$ & 3.3 & 6.3 & 6.18 & & \\
\hline 1125 & TRITIUM_ELECT_LSC & STLRL & 43.8 & $\mathrm{pCi} / \mathrm{L}$ & 3.5 & 7.1 & 6.17 & & \\
\hline 1126 & TRITIUM_ELECT_LSC & STLRL & 61 & $\mathrm{pCi} / \mathrm{L}$ & 3.9 & 8.4 & 6.24 & & \\
\hline 1127 & TRITIUM_ELECT_LSC & STLRL & 41.6 & $\mathrm{pCi} / \mathrm{L}$ & 3.5 & 6.9 & 6.25 & & \\
\hline 1128 & TRITIUM_ELECT_LSC & STLRL & 37.8 & $\mathrm{pCi} / \mathrm{L}$ & 3.5 & 6.7 & 6.32 & & \\
\hline 1129 & TRITIUM_ELECT_LSC & STLRL & 115 & $\mathrm{pCi} / \mathrm{L}$ & 4.7 & 13 & 6.2 & & \\
\hline 1130 & TRITIUM_ELECT_LSC & STLRL & 581 & $\mathrm{pCi} / \mathrm{L}$ & 9.2 & 52 & 6.13 & & \\
\hline 1131 & TRITIUM_ELECT_LSC & STLRL & 105 & $\mathrm{pCi} / \mathrm{L}$ & 4.6 & 12 & 6.24 & & \\
\hline 1132 & TRITIUM_ELECT_LSC & STLRL & 43.6 & $\mathrm{pCi} / \mathrm{L}$ & 3.5 & 7 & 6.09 & & \\
\hline 1133 & TRITIUM_ELECT_LSC & STLRL & 46.9 & $\mathrm{pCi} / \mathrm{L}$ & 3.7 & 7.4 & 6.41 & & \\
\hline 1134 & TRITIUM_ELECT_LSC & STLRL & 53.4 & $\mathrm{pCi} / \mathrm{L}$ & 3.8 & 7.9 & 6.32 & & \\
\hline 1135 & TRITIUM_ELECT_LSC & STLRL & 202 & $\mathrm{pCi} / \mathrm{L}$ & 5.8 & 20 & 6.24 & & \\
\hline 1136 & TRITIUM_ELECT_LSC & STLRL & 551 & $\mathrm{pCi} / \mathrm{L}$ & 9.1 & 50 & 6.35 & & \\
\hline 1137 & TRITIUM_ELECT_LSC & STLRL & 106 & $\mathrm{pCi} / \mathrm{L}$ & 4.6 & 12 & 6.28 & & \\
\hline 1138 & TRITIUM_ELECT_LSC & STLRL & 37.4 & $\mathrm{pCi} / \mathrm{L}$ & 3.4 & 6.7 & 6.26 & & \\
\hline 1139 & TRITIUM_ELECT_LSC & STLRL & 39.9 & $\mathrm{pCi} / \mathrm{L}$ & 3.5 & 6.9 & 6.27 & & \\
\hline 1140 & TRITIUM_ELECT_LSC & STLRL & 31.4 & $\mathrm{pCi} / \mathrm{L}$ & 3.3 & 6.3 & 6.32 & & \\
\hline 1141 & TRITIUM_ELECT_LSC & STLRL & 36.4 & $\mathrm{pCi} / \mathrm{L}$ & 3.4 & 6.6 & 6.29 & & \\
\hline
\end{tabular}




\begin{tabular}{|c|c|c|c|c|c|c|c|c|c|}
\hline & $\mathrm{K}$ & $\mathrm{L}$ & $\mathrm{M}$ & $\mathrm{N}$ & $\mathrm{O}$ & $P$ & $\mathrm{Q}$ & $\mathrm{R}$ & $\mathrm{S}$ \\
\hline 1 & METHOD_NAME & LAB_CODE & VALUE_RPTD & ANAL_UNITS_RPTD & COUNTING_ERROR & TOTAL_ANAL_ERROR & MIN_DETECTABLE_ACTIVITY & PCNT_MOISTURE & LAB_QUALIFIER \\
\hline 1142 & TRITIUM_ELECT_LSC & STLRL & 52.2 & $\mathrm{pCi} / \mathrm{L}$ & 3.7 & 7.8 & 6.33 & & \\
\hline 1143 & TRITIUM_ELECT_LSC & STLRL & 38.7 & $\mathrm{pCi} / \mathrm{L}$ & 3.5 & 6.8 & 6.25 & & \\
\hline 1144 & TRITIUM_DIST_LSC & STLRL & 61100 & $\mathrm{pCi} / \mathrm{L}$ & 560 & 2400 & 175 & & \\
\hline 1145 & TRITIUM_DIST_LSC & STLRL & 9940 & $\mathrm{pCi} / \mathrm{L}$ & 210 & 510 & 173 & & \\
\hline 1146 & TRITIUM_DIST_LSC & STLRL & 53900 & $\mathrm{pCi} / \mathrm{L}$ & 520 & 2100 & 175 & & \\
\hline 1147 & TRITIUM_DIST_LSC & STLRL & 79100 & $\mathrm{pCi} / \mathrm{L}$ & 630 & 3100 & 175 & & \\
\hline 1148 & TRITIUM_DIST_LSC & STLRL & 9130 & $\mathrm{pCi} / \mathrm{L}$ & 210 & 480 & 175 & & \\
\hline 1149 & TRITIUM_ELECT_LSC & STLRL & 28.8 & $\mathrm{pCi} / \mathrm{L}$ & 3.3 & 6.1 & 6.28 & & \\
\hline 1150 & TRITIUM_ELECT_LSC & STLRL & 75.6 & $\mathrm{pCi} / \mathrm{L}$ & 4.1 & 9.6 & 6.26 & & \\
\hline 1151 & TRITIUM_DIST_LSC & STLRL & 88.8 & $\mathrm{pCi} / \mathrm{L}$ & 7.2 & 96 & 149 & & $U$ \\
\hline 1152 & TRITIUM_DIST_LSC & STLRL & 3360 & $\mathrm{pCi} / \mathrm{L}$ & 120 & 250 & 146 & & \\
\hline 1153 & TRITIUM_DIST_LSC & STLRL & 17700 & $\mathrm{pCi} / \mathrm{L}$ & 300 & 800 & 175 & & \\
\hline 1154 & TRITIUM_DIST_LSC & STLRL & 5370 & $\mathrm{pCi} / \mathrm{L}$ & 150 & 340 & 175 & & \\
\hline 1155 & TRITIUM_DIST_LSC & STLRL & 1010 & $\mathrm{pCi} / \mathrm{L}$ & 52 & 160 & 175 & & \\
\hline 1156 & TRITIUM_DIST_LSC & STLRL & 21.3 & $\mathrm{pCi} / \mathrm{L}$ & 1.5 & 110 & 173 & & U \\
\hline 1157 & TRITIUM_ELECT_LSC & STLRL & 1310 & $\mathrm{pCi} / \mathrm{L}$ & 111 & 319 & 362 & & \\
\hline 1158 & TRITIUM_ELECT_LSC & STLRL & 1150 & $\mathrm{pCi} / \mathrm{L}$ & 100 & 307 & 360 & & \\
\hline 1159 & TRITIUM_ELECT_LSC & STLRL & 271 & $\mathrm{pCi} / \mathrm{L}$ & 30.1 & 246 & 366 & & U \\
\hline 1160 & TRITIUM_ELECT_LSC & STLRL & 154 & $\mathrm{pCi} / \mathrm{L}$ & 5.2 & 16 & 6.15 & & \\
\hline 1161 & TRITIUM_ELECT_LSC & STLRL & 71.2 & $\mathrm{pCi} / \mathrm{L}$ & 4 & 9.2 & 6.19 & & \\
\hline 1162 & TRITIUM_ELECT_LSC & STLRL & 3630 & $\mathrm{pCi} / \mathrm{L}$ & 22 & 310 & 6.31 & & \\
\hline 1163 & TRITIUM_ELECT_LSC & STLRL & 55.6 & $\mathrm{pCi} / \mathrm{L}$ & 3.8 & 8 & 6.21 & & \\
\hline 1164 & TRITIUM_DIST_LSC & STLRL & 7610 & $\mathrm{pCi} / \mathrm{L}$ & 190 & 420 & 167 & & \\
\hline 1165 & TRITIUM_DIST_LSC & STLRL & 125 & $\mathrm{pCi} / \mathrm{L}$ & 8.9 & 110 & 167 & & $U$ \\
\hline 1166 & 906.0_H3_LSC & STLRL & 7250 & $\mathrm{pCi} / \mathrm{L}$ & 340 & 640 & 333 & & \\
\hline 1167 & TRITIUM_DIST_LSC & STLRL & 386 & $\mathrm{pCi} / \mathrm{L}$ & 25 & 130 & 172 & & \\
\hline 1168 & TRITIUM_DIST_LSC & STLRL & 81 & $\mathrm{pCi} / \mathrm{L}$ & 6 & 120 & 180 & & $U$ \\
\hline 1169 & TRITIUM_ELECT_LSC & STLRL & 429 & $\mathrm{pCi} / \mathrm{L}$ & 162 & 243 & 341 & & \\
\hline 1170 & TRITIUM_ELECT_LSC & STLRL & 23.8 & $\mathrm{pCi} / \mathrm{L}$ & 3.2 & 5.8 & 6.29 & & \\
\hline 1171 & TRITIUM_ELECT_LSC & STLRL & 88.9 & $\mathrm{pCi} / \mathrm{L}$ & 4.4 & 11 & 6.3 & & \\
\hline 1172 & TRITIUM_DIST_LSC & STLRL & 873 & $\mathrm{pCi} / \mathrm{L}$ & 49 & 160 & 180 & & \\
\hline 1173 & 906.0_H3_LSC & STLRL & 768 & $\mathrm{pCi} / \mathrm{L}$ & 80 & 260 & 321 & & \\
\hline 1174 & TRITIUM_ELECT_LSC & STLRL & 27.3 & $\mathrm{pCi} / \mathrm{L}$ & 3.2 & 6 & 6.23 & & \\
\hline 1175 & TRITIUM_ELECT_LSC & STLRL & 62.7 & $\mathrm{pCi} / \mathrm{L}$ & 3.9 & 8.6 & 6.24 & & \\
\hline 1176 & TRITIUM_ELECT_LSC & STLRL & 27.1 & $\mathrm{pCi} / \mathrm{L}$ & 3.2 & 6 & 6.29 & & \\
\hline 1177 & TRITIUM_ELECT_LSC & STLRL & 38.3 & $\mathrm{pCi} / \mathrm{L}$ & 3.5 & 6.7 & 6.28 & & \\
\hline 1178 & TRITIUM_ELECT_LSC & STLRL & 42.3 & $\mathrm{pCi} / \mathrm{L}$ & 3.5 & 7 & 6.28 & & \\
\hline 1179 & TRITIUM_ELECT_LSC & STLRL & 29.7 & $\mathrm{pCi} / \mathrm{L}$ & 3.3 & 6.2 & 6.39 & & \\
\hline
\end{tabular}




\begin{tabular}{|c|c|c|c|c|c|c|c|c|c|}
\hline & $\mathrm{K}$ & $\mathrm{L}$ & $\mathrm{M}$ & $\mathrm{N}$ & $\mathrm{O}$ & $P$ & $\mathrm{Q}$ & $\mathrm{R}$ & $\mathrm{S}$ \\
\hline 1 & METHOD_NAME & LAB_CODE & VALUE_RPTD & ANAL_UNITS_RPTD & COUNTING_ERROR & TOTAL_ANAL_ERROR & MIN_DETECTABLE_ACTIVITY & PCNT_MOISTURE & LAB_QUALIFIER \\
\hline 1180 & TRITIUM_ELECT_LSC & STLRL & 48.1 & $\mathrm{pCi} / \mathrm{L}$ & 3.7 & 7.5 & 6.3 & & \\
\hline 1181 & TRITIUM_ELECT_LSC & STLRL & 40.8 & $\mathrm{pCi} / \mathrm{L}$ & 3.5 & 7 & 6.31 & & \\
\hline 1182 & TRITIUM_ELECT_LSC & STLRL & 38 & $\mathrm{pCi} / \mathrm{L}$ & 3.5 & 6.8 & 6.29 & & \\
\hline 1183 & TRITIUM_ELECT_LSC & STLRL & 22.9 & $\mathrm{pCi} / \mathrm{L}$ & 3.2 & 5.8 & 6.36 & & \\
\hline 1184 & TRITIUM_ELECT_LSC & STLRL & 26.3 & $\mathrm{pCi} / \mathrm{L}$ & 3.2 & 6 & 6.32 & & \\
\hline 1185 & TRITIUM_ELECT_LSC & STLRL & 36.3 & $\mathrm{pCi} / \mathrm{L}$ & 3.5 & 6.7 & 6.36 & & \\
\hline 1186 & TRITIUM_ELECT_LSC & STLRL & 43.8 & $\mathrm{pCi} / \mathrm{L}$ & 3.6 & 7.2 & 6.31 & & \\
\hline 1187 & TRITIUM_ELECT_LSC & STLRL & 38.8 & $\mathrm{pCi} / \mathrm{L}$ & 3.5 & 6.8 & 6.28 & & \\
\hline 1188 & TRITIUM_ELECT_LSC & STLRL & 39.1 & $\mathrm{pCi} / \mathrm{L}$ & 3.5 & 6.9 & 6.32 & & \\
\hline 1189 & TRITIUM_ELECT_LSC & STLRL & 35.8 & $\mathrm{pCi} / \mathrm{L}$ & 3.4 & 6.6 & 6.32 & & \\
\hline 1190 & TRITIUM_ELECT_LSC & STLRL & 33.2 & $\mathrm{pCi} / \mathrm{L}$ & 3.4 & 6.5 & 6.37 & & \\
\hline 1191 & TRITIUM_ELECT_LSC & STLRL & 41.4 & $\mathrm{pCi} / \mathrm{L}$ & 3.5 & 7 & 6.34 & & \\
\hline 1192 & TRITIUM_ELECT_LSC & STLRL & 81.6 & $\mathrm{pCi} / \mathrm{L}$ & 4.2 & 10 & 6.31 & & \\
\hline 1193 & TRITIUM_ELECT_LSC & STLRL & 42.7 & $\mathrm{pCi} / \mathrm{L}$ & 3.6 & 7.1 & 6.32 & & \\
\hline 1194 & TRITIUM_ELECT_LSC & STLRL & 76.3 & $\mathrm{pCi} / \mathrm{L}$ & 4.1 & 9.7 & 6.34 & & \\
\hline 1195 & TRITIUM_DIST_LSC & STLRL & 105 & $\mathrm{pCi} / \mathrm{L}$ & 80 & 120 & 185 & & U \\
\hline 1196 & TRITIUM_DIST_LSC & STLRL & 3340 & $\mathrm{pCi} / \mathrm{L}$ & 160 & 270 & 185 & & \\
\hline 1197 & TRITIUM_ELECT_LSC & STLRL & 145 & $\mathrm{pCi} / \mathrm{L}$ & 5.1 & 15 & 6.2 & & \\
\hline 1198 & 906.0_H3_LSC & STLRL & -10.5 & $\mathrm{pCi} / \mathrm{L}$ & 75 & 120 & 185 & & U \\
\hline 1199 & 906.0_H3_LSC & STLRL & 3510 & $\mathrm{pCi} / \mathrm{L}$ & 160 & 270 & 186 & & \\
\hline 1200 & TRITIUM_ELECT_LSC & STLRL & 49.7 & $\mathrm{pCi} / \mathrm{L}$ & 3.6 & 7.5 & 6.16 & & \\
\hline 1201 & TRITIUM_ELECT_LSC & STLRL & 62.8 & $\mathrm{pCi} / \mathrm{L}$ & 3.2 & 8.8 & 5.13 & & \\
\hline 1202 & TRITIUM_ELECT_LSC & STLRL & 64.5 & $\mathrm{pCi} / \mathrm{L}$ & 2.8 & 7.8 & 4.51 & & \\
\hline 1203 & TRITIUM_ELECT_LSC & STLRL & 53.3 & $\mathrm{pCi} / \mathrm{L}$ & 2.6 & 6.9 & 4.42 & & \\
\hline 1204 & TRITIUM_ELECT_LSC & STLRL & 40.5 & $\mathrm{pCi} / \mathrm{L}$ & 2.5 & 5.9 & 4.46 & & \\
\hline 1205 & TRITIUM_ELECT_LSC & STLRL & 40.3 & $\mathrm{pCi} / \mathrm{L}$ & 2.5 & 5.9 & 4.5 & & \\
\hline 1206 & TRITIUM_ELECT_LSC & STLRL & 38.1 & $\mathrm{pCi} / \mathrm{L}$ & 2.5 & 5.8 & 4.48 & & \\
\hline 1207 & TRITIUM_ELECT_LSC & STLRL & 33.4 & $\mathrm{pCi} / \mathrm{L}$ & 2.4 & 5.4 & 4.49 & & \\
\hline 1208 & TRITIUM_ELECT_LSC & STLRL & 62 & $\mathrm{pCi} / \mathrm{L}$ & 3.9 & 8.5 & 6.32 & & \\
\hline 1209 & TRITIUM_ELECT_LSC & STLRL & 49 & $\mathrm{pCi} / \mathrm{L}$ & 3.7 & 7.6 & 6.31 & & \\
\hline 1210 & TRITIUM_ELECT_LSC & STLRL & 49 & $\mathrm{pCi} / \mathrm{L}$ & 3.7 & 7.6 & 6.31 & & \\
\hline 1211 & TRITIUM_ELECT_LSC & STLRL & 47.4 & $\mathrm{pCi} / \mathrm{L}$ & 2.6 & 6.5 & 4.53 & & \\
\hline 1212 & TRITIUM_ELECT_LSC & STLRL & 34 & $\mathrm{pCi} / \mathrm{L}$ & 3.4 & 6.4 & 6.23 & & \\
\hline 1213 & TRITIUM_ELECT_LSC & STLRL & 28.5 & $\mathrm{pCi} / \mathrm{L}$ & 3.3 & 6.1 & 6.26 & & \\
\hline 1214 & TRITIUM_ELECT_LSC & STLRL & 27 & $\mathrm{pCi} / \mathrm{L}$ & 3.3 & 6 & 6.35 & & \\
\hline 1215 & TRITIUM_ELECT_LSC & STLRL & 29.2 & $\mathrm{pCi} / \mathrm{L}$ & 3.3 & 6.1 & 6.27 & & \\
\hline 1216 & TRITIUM_ELECT_LSC & STLRL & 33.2 & $\mathrm{pCi} / \mathrm{L}$ & 3.3 & 6.3 & 6.23 & & \\
\hline 1217 & TRITIUM_ELECT_LSC & STLRL & 75.7 & $\mathrm{pCi} / \mathrm{L}$ & 4.1 & 9.6 & 6.25 & & \\
\hline
\end{tabular}




\begin{tabular}{|c|c|c|c|c|c|c|c|c|c|}
\hline & $\mathrm{K}$ & $\mathrm{L}$ & $\bar{M}$ & $\mathrm{~N}$ & $\mathrm{O}$ & $P$ & $\mathrm{Q}$ & $\mathrm{R}$ & $\mathrm{S}$ \\
\hline 1 & METHOD_NAME & LAB_CODE & VALUE_RPTD & ANAL_UNITS_RPTD & $\mid$ COUNTING_ERROR & TOTAL_ANAL_ERROR & MIN_DETECTABLE_ACTIVITY & PCNT_MOISTURE & LAB_QUALIFIER \\
\hline 1218 & TRITIUM_ELECT_LSC & STLRL & 114 & $\mathrm{pCi} / \mathrm{L}$ & 4.7 & 13 & 6.39 & & \\
\hline 1219 & TRITIUM_DIST_LSC & STLRL & 3860 & $\mathrm{pCi} / \mathrm{L}$ & 160 & 280 & 180 & & \\
\hline 1220 & TRITIUM_DIST_LSC & STLRL & 3500 & $\mathrm{pCi} / \mathrm{L}$ & 150 & 260 & 165 & & \\
\hline 1221 & TRITIUM ËEECT LSC & STLRL & 32 & $\mathrm{pCi} / \mathrm{L}$ & 3.4 & 6.4 & 6.32 & & \\
\hline 1222 & TRITIUM_ELECT_LSC & STLRL & 90.7 & $\mathrm{pCi} / \mathrm{L}$ & 4.4 & 11 & 6.36 & & \\
\hline 1223 & TRITIUM_ELECT_LSC & STLRL & & & & & & & $u$ \\
\hline 1224 & 906.0_H3_LSC & STLRL & 46.1 & $\mathrm{pCi} / \mathrm{L}$ & 73 & 110 & 173 & & $U$ \\
\hline 1225 & 906.0_H3_LSC & STLRL & 3810 & $\mathrm{pCi} / \mathrm{L}$ & 160 & 280 & 174 & & \\
\hline 1226 & TRITIŪM_ELECT_LSC & STLRL & 55.7 & $\mathrm{pCi} / \mathrm{L}$ & 3.8 & 8.1 & 6.34 & & \\
\hline 1227 & TRITIUM_DIST_LSC & STLRL & 102000 & $\mathrm{pCi} / \mathrm{L}$ & 760 & 3900 & 174 & & \\
\hline 1228 & TRITIUM_ELECT_LSC & STLRL & 5460 & $\mathrm{pCi} / \mathrm{L}$ & 27 & 470 & 6.26 & & \\
\hline 1229 & TRITIUM_ELECT_LSC & STLRL & 1380 & $\mathrm{pCi} / \mathrm{L}$ & 14 & 120 & 6.47 & & \\
\hline 1230 & TRITIUM_DIST_LSC & STLRL & 107000 & $\mathrm{pCi} / \mathrm{L}$ & 770 & 4100 & 174 & & \\
\hline 1231 & TRITIUM_ELECT_LSC & STLRL & 836 & $\mathrm{pCi} / \mathrm{L}$ & 11 & 74 & 6.3 & & \\
\hline 1232 & TRITIUM_ELECT_LSC & STLRL & 119 & $\mathrm{pCi} / \mathrm{L}$ & 4.8 & 13 & 6.25 & & \\
\hline 1233 & TRITIUM_ELECT_LSC & STLRL & 65.9 & $\mathrm{pCi} / \mathrm{L}$ & 4 & 8.8 & 6.25 & & \\
\hline 1234 & TRITIUM ELECT ${ }^{-}$LSC $^{-1}$ & STLRL & 6400 & $\mathrm{pCi} / \mathrm{L}$ & 30 & 550 & 6.38 & & \\
\hline 1235 & TRITIUM_DIST_LSC & STLRL & 17300 & $\mathrm{pCi} / \mathrm{L}$ & 330 & 800 & 176 & & \\
\hline \begin{tabular}{|l|}
1236 \\
1230
\end{tabular} & TRITIUM_DIST_LSC & STLRL & 6120 & $\mathrm{pCi} / \mathrm{L}$ & 200 & 380 & 171 & & \\
\hline 1237 & TRITIUM_ELECT_LSC & STLRL & 6140 & $\mathrm{pCi} / \mathrm{L}$ & 29 & 530 & 6.29 & & \\
\hline 1238 & TRITIUM_ELECT_LSC & STLRL & 5170 & $\mathrm{pCi} / \mathrm{L}$ & 27 & 450 & 6.34 & & \\
\hline 1239 & TRITIUM_ELECT_LSC & STLRL & 130 & $\mathrm{pCi} / \mathrm{L}$ & 4.9 & 14 & 6.24 & & \\
\hline 1240 & TRITIUM_ELECT_LSC & STLRL & 33.9 & $\mathrm{pCi} / \mathrm{L}$ & 3.4 & 6.5 & 6.3 & & \\
\hline 1241 & TRITIUM_DIST_LSC & STLRL & 11700 & $\mathrm{pCi} / \mathrm{L}$ & 260 & 580 & 175 & & \\
\hline 1242 & TRITIUM_ELECT_LSC & STLRL & 126 & $\mathrm{pCi} / \mathrm{L}$ & 5.1 & 14 & 6.79 & & \\
\hline 1243 & TRITIUM_ELECT_LSC & STLRL & 125 & $\mathrm{pCi} / \mathrm{L}$ & 4.9 & 14 & 6.42 & & \\
\hline 1244 & TRITIUM_ELECT_LSC & STLRL & 60.1 & $\mathrm{pCi} / \mathrm{L}$ & 3.9 & 8.4 & 6.28 & & \\
\hline 1245 & TRITIUM_ELECT_LSC & STLRL & 49.1 & $\mathrm{pCi} / \mathrm{L}$ & 3.7 & 7.6 & 6.32 & & \\
\hline 1246 & TRITIUM_ELECT_LSC & STLRL & 232 & $\mathrm{pCi} / \mathrm{L}$ & 6.5 & 23 & 6.88 & & \\
\hline 1247 & TRITIUM_ELECT_LSC & STLRL & 130 & $\mathrm{pCi} / \mathrm{L}$ & 4.9 & 14 & 6.2 & & \\
\hline 1248 & TRITIUM_ELECT_LSC & STLRL & 43.2 & $\mathrm{pCi} / \mathrm{L}$ & 3.6 & 7.1 & 6.33 & & \\
\hline 1249 & TRITIUM_DIST_LSC & STLRL & 11.9 & $\mathrm{pCi} / \mathrm{L}$ & 75 & 110 & 180 & & U \\
\hline 1250 & TRITIUM_ELECT_LSC & STLRL & 123 & $\mathrm{pCi} / \mathrm{L}$ & 5.1 & 14 & 6.86 & & \\
\hline 1251 & TRITIUM_DIST_LSC & STLRL & -86.9 & $\mathrm{pCi} / \mathrm{L}$ & 71 & 110 & 182 & & $U$ \\
\hline 1252 & TRITIUM_ELECT_LSC & STLRL & 44.1 & $\mathrm{pCi} / \mathrm{L}$ & 3.6 & 7.3 & 6.49 & & \\
\hline 1253 & TRITIUM_ELECT_LSC & STLRL & 88.5 & $\mathrm{pCi} / \mathrm{L}$ & 4.5 & 11 & 6.57 & & \\
\hline 1254 & TRITIUM_ELECT_LSC & STLRL & 69.1 & $\mathrm{pCi} / \mathrm{L}$ & 4.5 & 9.6 & 7.21 & & \\
\hline 1255 & TRITIUM_ELECT_LSC & STLRL & 67.6 & $\mathrm{pCi} / \mathrm{L}$ & 4 & 8.9 & 6.15 & & \\
\hline
\end{tabular}




\begin{tabular}{|c|c|c|c|c|c|c|c|c|c|}
\hline & $\mathrm{K}$ & $\mathrm{L}$ & $\mathrm{M}$ & $\mathrm{N}$ & $\mathrm{O}$ & $\mathrm{P}$ & $\mathrm{Q}$ & $\mathrm{R}$ & $\mathrm{S}$ \\
\hline 1 & METHOD_NAME & LAB_CODE & VALUE_RPTD & ANAL_UNITS_RPTD & COUNTING_ERROR & TOTAL_ANAL_ERROR & MIN_DETECTABLE_ACTIVITY & PCNT_MOISTURE & LAB_QUALIFIER \\
\hline 1256 & TRITIUM_ELECT_LSC & STLRL & 74.8 & $\mathrm{pCi} / \mathrm{L}$ & 4.1 & 9.5 & 6.19 & & \\
\hline 1257 & TRITIUM_ELECT_LSC & STLRL & 36.7 & $\mathrm{pCi} / \mathrm{L}$ & 3.4 & 6.6 & 6.14 & & \\
\hline 1258 & TRITIUM_ELECT_LSC & STLRL & 42.9 & $\mathrm{pCi} / \mathrm{L}$ & 3.5 & 7 & 6.16 & & \\
\hline 1259 & TRITIUM_ELECT_LSC & STLRL & 28 & $\mathrm{pCi} / \mathrm{L}$ & 3.5 & 6.4 & 6.87 & & \\
\hline 1260 & TRITIUM_ELECT_LSC & STLRL & 42.9 & $\mathrm{pCi} / \mathrm{L}$ & 3.5 & 7 & 6.19 & & \\
\hline 1261 & TRITIUM_ELECT_LSC & STLRL & 44.8 & $\mathrm{pCi} / \mathrm{L}$ & 3.6 & 7.2 & 6.27 & & \\
\hline 1262 & TRITIUM_ELECT_LSC & STLRL & 44.2 & $\mathrm{pCi} / \mathrm{L}$ & 3.6 & 7.1 & 6.22 & & \\
\hline 1263 & TRITIUM_ELECT_LSC & STLRL & 70.2 & $\mathrm{pCi} / \mathrm{L}$ & 4.1 & 9.3 & 6.57 & & \\
\hline 1264 & TRITIUM_ELECT_LSC & STLRL & 79.8 & $\mathrm{pCi} / \mathrm{L}$ & 4.3 & 10 & 6.56 & & \\
\hline 1265 & TRITIUM_ELECT_LSC & STLRL & 66.9 & $\mathrm{pCi} / \mathrm{L}$ & 4.1 & 9.1 & 6.66 & & \\
\hline 1266 & TRITIUM_ELECT_LSC & STLRL & 67.2 & $\mathrm{pCi} / \mathrm{L}$ & 4.1 & 9.1 & 6.57 & & \\
\hline 1267 & TRITIUM_ELECT_LSC & STLRL & 36.4 & $\mathrm{pCi} / \mathrm{L}$ & 3.4 & 6.6 & 6.28 & & \\
\hline 1268 & TRITIUM_ELECT_LSC & STLRL & 21.7 & $\mathrm{pCi} / \mathrm{L}$ & 3.1 & 5.6 & 6.25 & & \\
\hline 1269 & TRITIUM_ELECT_LSC & STLRL & 3330 & $\mathrm{pCi} / \mathrm{L}$ & 21 & 290 & 6.29 & & \\
\hline 1270 & TRITIUM_ELECT_LSC & STLRL & 132 & $\mathrm{pCi} / \mathrm{L}$ & 5 & 14 & 6.48 & & \\
\hline 1271 & TRITIUM_ELECT_LSC & STLRL & 120 & $\mathrm{pCi} / \mathrm{L}$ & 4.8 & 13 & 6.36 & & \\
\hline 1272 & TRITIUM_ELECT_LSC & STLRL & 224 & $\mathrm{pCi} / \mathrm{L}$ & 6.1 & 22 & 6.43 & & \\
\hline 1273 & 906.0_H3_LSC & STLRL & 99.6 & $\mathrm{pCi} / \mathrm{L}$ & 73 & 110 & 167 & & 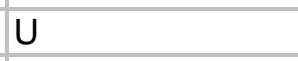 \\
\hline 1274 & 906.0_H3_LSC & STLRL & 3500 & $\mathrm{pCi} / \mathrm{L}$ & 160 & 270 & 166 & & \\
\hline 1275 & TRITIUM_ELECT_LSC & STLRL & 149 & $\mathrm{pCi} / \mathrm{L}$ & 5.2 & 16 & 6.41 & & \\
\hline 1276 & TRITIUM_ELECT_LSC & STLRL & 81 & $\mathrm{pCi} / \mathrm{L}$ & 4.1 & 10 & 6.09 & & \\
\hline 1277 & TRITIUM_ELECT_LSC & STLRL & 30.1 & $\mathrm{pCi} / \mathrm{L}$ & 3.3 & 6.2 & 6.29 & & \\
\hline 1278 & TRITIUM_ELECT_LSC & STLRL & 55.3 & $\mathrm{pCi} / \mathrm{L}$ & 3.8 & 8.1 & 6.38 & & \\
\hline 1279 & TRITIUM_DIST_LSC & STLRL & 6300 & $\mathrm{pCi} / \mathrm{L}$ & 200 & 380 & 169 & & \\
\hline 1280 & TRITIUM_DIST_LSC & STLRL & 8380 & $\mathrm{pCi} / \mathrm{L}$ & 230 & 460 & 169 & & \\
\hline 1281 & TRITIUM_ELECT_LSC & STLRL & 84.3 & $\mathrm{pCi} / \mathrm{L}$ & 4.3 & 10 & 6.32 & & \\
\hline 1282 & TRITIUM_ELECT_LSC & STLRL & 32.6 & $\mathrm{pCi} / \mathrm{L}$ & 3.4 & 6.4 & 6.41 & & \\
\hline 1283 & TRITIUM_ELECT_LSC & STLRL & 107 & $\mathrm{pCi} / \mathrm{L}$ & 4.7 & 12 & 6.47 & & \\
\hline 1284 & TRITIUM_ELECT_LSC & STLRL & 27.9 & $\mathrm{pCi} / \mathrm{L}$ & 3.4 & 6.2 & 6.59 & & \\
\hline 1285 & TRITIUM_ELECT_LSC & STLRL & 22.3 & $\mathrm{pCi} / \mathrm{L}$ & 3.2 & 5.8 & 6.56 & & \\
\hline 1286 & TRITIUM_ELECT_LSC & STLRL & 23.5 & $\mathrm{pCi} / \mathrm{L}$ & 3.3 & 6 & 6.73 & & \\
\hline 1287 & TRITIUM_ELECT_LSC & STLRL & 17.5 & $\mathrm{pCi} / \mathrm{L}$ & 3.1 & 5.5 & 6.52 & & \\
\hline 1288 & TRITIUM_ELECT_LSC & STLRL & 111 & $\mathrm{pCi} / \mathrm{L}$ & 4.8 & 13 & 6.67 & & \\
\hline 1289 & TRITIUM_ELECT_LSC & STLRL & 38 & $\mathrm{pCi} / \mathrm{L}$ & 3.5 & 6.9 & 6.47 & & \\
\hline 1290 & TRITIUM_ELECT_LSC & STLRL & 22.9 & $\mathrm{pCi} / \mathrm{L}$ & 3.2 & 5.9 & 6.47 & & \\
\hline 1291 & TRITIUM_ELECT_LSC & STLRL & 29 & $\mathrm{pCi} / \mathrm{L}$ & 3.4 & 6.3 & 6.52 & & \\
\hline 1292 & TRITIUM_ELECT_LSC & STLRL & 32.6 & $\mathrm{pCi} / \mathrm{L}$ & 3.4 & 6.5 & 6.5 & & \\
\hline 1293 & TRITIUM_ELECT_LSC & STLRL & 28.1 & $\mathrm{pCi} / \mathrm{L}$ & 3.4 & 6.2 & 6.53 & & \\
\hline
\end{tabular}




\begin{tabular}{|c|c|c|c|c|c|c|c|c|c|}
\hline & $\mathrm{K}$ & $\mathrm{L}$ & $\bar{M}$ & $\mathrm{~N}$ & $\mathrm{O}$ & $P$ & $\mathrm{Q}$ & $\mathrm{R}$ & $\mathrm{S}$ \\
\hline 1 & METHOD_NAME & LAB_CODE & VALUE_RPTD & ANAL_UNITS_RPTD & $\mid$ COUNTING_ERROR & TOTAL_ANAL_ERROR & MIN_DETECTABLE_ACTIVITY & PCNT_MOISTURE & LAB_QUALIFIER \\
\hline 1294 & TRITIUM_ELECT_LSC & STLRL & 131 & $\mathrm{pCi} / \mathrm{L}$ & 5 & 14 & 6.46 & & \\
\hline 1295 & TRITIUM_ELECT_LSC & STLRL & 63.1 & $\mathrm{pCi} / \mathrm{L}$ & 4.4 & 9.3 & 7.42 & & \\
\hline 1296 & TRITIUM ELECT LSC & STLRL & 62.1 & $\mathrm{pCi} / \mathrm{L}$ & 4 & 8.7 & 6.47 & & \\
\hline 1297 & TRITIUM E- ELECT ${ }^{-}$LSC $^{-1}$ & STLRL & 118 & $\mathrm{pCi} / \mathrm{L}$ & 5.2 & 14 & 7.22 & & \\
\hline 1298 & TRITIUM_ELECT_LSC & STLRL & 33.7 & $\mathrm{pCi} / \mathrm{L}$ & 3.5 & 6.6 & 6.57 & & \\
\hline 1299 & TRITIUM_ELECT_LSC & STLRL & 35.3 & $\mathrm{pCi} / \mathrm{L}$ & 3.6 & 6.9 & 6.79 & & \\
\hline 1300 & TRITIUM_ELECT_LSC & STLRL & 36.7 & $\mathrm{pCi} / \mathrm{L}$ & 3.5 & 6.7 & 6.38 & & \\
\hline 1301 & TRITIUM_ELECT_LSC & STLRL & 35.9 & $\mathrm{pCi} / \mathrm{L}$ & 3.5 & 6.8 & 6.58 & & \\
\hline 1302 & TRITIUM_ELECT_LSC & STLRL & 33.6 & $\mathrm{pCi} / \mathrm{L}$ & 3.5 & 6.5 & 6.46 & & \\
\hline 1303 & TRITIUM_ELECT_LSC & STLRL & 33.5 & $\mathrm{pCi} / \mathrm{L}$ & 3.5 & 6.6 & 6.49 & & \\
\hline 1304 & TRITIUM_ELECT_LSC & STLRL & 34 & $\mathrm{pCi} / \mathrm{L}$ & 3.7 & 6.9 & 6.89 & & \\
\hline 1305 & TRITIUM_ELECT_LSC & STLRL & 821 & $\mathrm{pCi} / \mathrm{L}$ & 11 & 73 & 6.42 & & \\
\hline 1306 & TRITIUM_ELECT_LSC & STLRL & 266 & $\mathrm{pCi} / \mathrm{L}$ & 6.8 & 26 & 6.81 & & \\
\hline 1307 & TRITIUM_ELECT_LSC & STLRL & 35.7 & $\mathrm{pCi} / \mathrm{L}$ & 3.5 & 6.7 & 6.32 & & \\
\hline 1308 & TRITIUM_ELECT_LSC & STLRL & 37.1 & $\mathrm{pCi} / \mathrm{L}$ & 3.5 & 6.8 & 6.36 & & \\
\hline 1309 & TRITIUM_ELECT_LSC & STLRL & 46.2 & $\mathrm{pCi} / \mathrm{L}$ & 3.7 & 7.4 & 6.36 & & \\
\hline \begin{tabular}{|l|}
1310 \\
\end{tabular} & TRITIUM_ELECT_LSC & STLRL & 39.9 & $\mathrm{pCi} / \mathrm{L}$ & 3.6 & 7 & 6.37 & & \\
\hline 1311 & TRITIUM_ELECT_LSC & STLRL & 41.4 & $\mathrm{pCi} / \mathrm{L}$ & 3.6 & 7.1 & 6.4 & & \\
\hline 1312 & TRITIUM_ELECT_LSC & STLRL & 1380 & $\mathrm{pCi} / \mathrm{L}$ & 14 & 120 & 6.37 & & \\
\hline 1313 & TRITIUM_ELECT_LSC & STLRL & 5140 & $\mathrm{pCi} / \mathrm{L}$ & 27 & 440 & 6.4 & & \\
\hline 1314 & TRITIUM_ELECT_LSC & STLRL & 34.7 & $\mathrm{pCi} / \mathrm{L}$ & 3.5 & 6.6 & 6.41 & & \\
\hline 1315 & TRITIUM_ELECT_LSC & STLRL & 36.8 & $\mathrm{pCi} / \mathrm{L}$ & 4.5 & 8.3 & 8.72 & & \\
\hline 1316 & TRITIUM_ELECT_LSC & STLRL & 43.3 & $\mathrm{pCi} / \mathrm{L}$ & 3.6 & 7.2 & 6.43 & & \\
\hline 1317 & TRITIUM_ELECT_LSC & STLRL & 48.7 & $\mathrm{pCi} / \mathrm{L}$ & 4.1 & 8.2 & 7.33 & & \\
\hline 1318 & TRITIUM_ELECT_LSC & STLRL & 29.7 & $\mathrm{pCi} / \mathrm{L}$ & 3.3 & 6.2 & 6.33 & & \\
\hline 1319 & TRITIUM_ELECT_LSC & STLRL & 33.9 & $\mathrm{pCi} / \mathrm{L}$ & 3.4 & 6.5 & 6.23 & & \\
\hline 1320 & TRITIUM_ELECT_LSC & STLRL & 30.4 & $\mathrm{pCi} / \mathrm{L}$ & 3.3 & 6.3 & 6.39 & & \\
\hline 1321 & TRITIUM_ELECT_LSC & STLRL & 32.5 & $\mathrm{pCi} / \mathrm{L}$ & 3.6 & 6.7 & 6.88 & & \\
\hline 1322 & TRITIUM_ELECT_LSC & STLRL & 87.8 & $\mathrm{pCi} / \mathrm{L}$ & 4.4 & 11 & 6.43 & & \\
\hline 1323 & TRITIUM_ELECT_LSC & STLRL & 41.4 & $\mathrm{pCi} / \mathrm{L}$ & 3.6 & 7.1 & 6.5 & & \\
\hline 1324 & TRITIUM_ELECT_LSC & STLRL & 34.4 & $\mathrm{pCi} / \mathrm{L}$ & 3.4 & 6.6 & 6.39 & & \\
\hline \begin{tabular}{|l|l|}
1325 \\
\end{tabular} & TRITIUM_ELECT_LSC & STLRL & 31.2 & $\mathrm{pCi} / \mathrm{L}$ & 3.4 & 6.3 & 6.37 & & \\
\hline 1326 & TRITIUM_ELECT_LSC & STLRL & 27.1 & $\mathrm{pCi} / \mathrm{L}$ & 3.3 & 6 & 6.33 & & \\
\hline 1327 & TRITIUM_ELECT_LSC & STLRL & 23.4 & $\mathrm{pCi} / \mathrm{L}$ & 3.2 & 5.8 & 6.42 & & \\
\hline 1328 & TRITIUM_ELECT_LSC & STLRL & 37.1 & $\mathrm{pCi} / \mathrm{L}$ & 3.5 & 6.7 & 6.4 & & \\
\hline 1329 & TRITIUM_ELECT_LSC & STLRL & 40.6 & $\mathrm{pCi} / \mathrm{L}$ & 3.7 & 7.2 & 6.67 & & \\
\hline 1330 & TRITIUM_ELECT_LSC & STLRL & 43.3 & $\mathrm{pCi} / \mathrm{L}$ & 3.6 & 7.2 & 6.45 & & \\
\hline 1331 & TRITIUM_ELECT_LSC & STLRL & 46.2 & $\mathrm{pCi} / \mathrm{L}$ & 3.7 & 7.4 & 6.47 & & \\
\hline
\end{tabular}




\begin{tabular}{|c|c|c|c|c|c|c|c|c|c|}
\hline & $\mathrm{K}$ & $\mathrm{L}$ & $\mathrm{M}$ & $\mathrm{N}$ & $\mathrm{O}$ & $\mathrm{P}$ & $\mathrm{Q}$ & $\mathrm{R}$ & $\mathrm{S}$ \\
\hline 1 & METHOD_NAME & LAB_CODE & VALUE_RPTD & ANAL_UNITS_RPTD & COUNTING_ERROR & TOTAL_ANAL_ERROR & MIN_DETECTABLE_ACTIVITY & PCNT_MOISTURE & LAB_QUALIFIER \\
\hline 1332 & TRITIUM_ELECT_LSC & STLRL & 135 & $\mathrm{pCi} / \mathrm{L}$ & 5 & 14 & 6.4 & & \\
\hline 1333 & TRITIUM_ELECT_LSC & STLRL & 547 & $\mathrm{pCi} / \mathrm{L}$ & 9.1 & 50 & 6.45 & & \\
\hline 1334 & TRITIUM_ELECT_LSC & STLRL & 103 & $\mathrm{pCi} / \mathrm{L}$ & 4.8 & 12 & 6.76 & & \\
\hline 1335 & TRITIUM_ELECT_LSC & STLRL & 48.7 & $\mathrm{pCi} / \mathrm{L}$ & 3.8 & 7.7 & 6.54 & & \\
\hline 1336 & TRITIUM_DIST_LSC & STLRL & 6940 & $\mathrm{pCi} / \mathrm{L}$ & 240 & 440 & 213 & & \\
\hline 1337 & TRITIUM_DIST_LSC & STLRL & 6060 & $\mathrm{pCi} / \mathrm{L}$ & 220 & 400 & 211 & & \\
\hline 1338 & TRITIUM_DIST_LSC & STLRL & 7590 & $\mathrm{pCi} / \mathrm{L}$ & 250 & 460 & 210 & & \\
\hline 1339 & TRITIUM_DIST_LSC & STLRL & 7800 & $\mathrm{pCi} / \mathrm{L}$ & 250 & 470 & 210 & & \\
\hline 1340 & TRITIUM_DIST_LSC & STLRL & 7620 & $\mathrm{pCi} / \mathrm{L}$ & 250 & 460 & 209 & & \\
\hline 1341 & TRITIUM_DIST_LSC & STLRL & 7160 & $\mathrm{pCi} / \mathrm{L}$ & 240 & 450 & 212 & & \\
\hline 1342 & TRITIUM_DIST_LSC & STLRL & 7410 & $\mathrm{pCi} / \mathrm{L}$ & 240 & 460 & 213 & & \\
\hline 1343 & TRITIUM_DIST_LSC & STLRL & 7560 & $\mathrm{pCi} / \mathrm{L}$ & 250 & 460 & 212 & & \\
\hline 1344 & TRITIUM_DIST_LSC & STLRL & 8020 & $\mathrm{pCi} / \mathrm{L}$ & 250 & 480 & 208 & & \\
\hline 1345 & TRITIUM_DIST_LSC & STLRL & 8390 & $\mathrm{pCi} / \mathrm{L}$ & 260 & 490 & 209 & & \\
\hline 1346 & TRITIUM_DIST_LSC & STLRL & 8420 & $\mathrm{pCi} / \mathrm{L}$ & 260 & 490 & 209 & & \\
\hline 1347 & TRITIUM_DIST_LSC & STLRL & 8660 & $\mathrm{pCi} / \mathrm{L}$ & 260 & 500 & 212 & & \\
\hline 1348 & TRITIUM_ELECT_LSC & STLRL & 30.7 & $\mathrm{pCi} / \mathrm{L}$ & 3.4 & 6.3 & 6.5 & & \\
\hline 1349 & TRITIUM_ELECT_LSC & STLRL & 79 & $\mathrm{pCi} / \mathrm{L}$ & 4.2 & 9.9 & 6.4 & & \\
\hline 1350 & TRITIUM_DIST_LSC & STLRL & 85.8 & $\mathrm{pCi} / \mathrm{L}$ & 81 & 120 & 190 & & $U$ \\
\hline 1351 & TRITIUM_ELECT_LSC & STLRL & 3140 & $\mathrm{pCi} / \mathrm{L}$ & 21 & 270 & 6.37 & & \\
\hline 1352 & 906.0_H3_LSC & STLRL & 177 & $\mathrm{pCi} / \mathrm{L}$ & 95 & 140 & 216 & & U \\
\hline 1353 & 906.0_H3_LSC & STLRL & -90.7 & $\mathrm{pCi} / \mathrm{L}$ & 83 & 130 & 211 & & $U$ \\
\hline 1354 & 906.0_H3_LSC & STLRL & 3010 & $\mathrm{pCi} / \mathrm{L}$ & 190 & 310 & 238 & & \\
\hline 1355 & 906.0_H3_LSC & STLRL & -77.1 & $\mathrm{pCi} / \mathrm{L}$ & 84 & 130 & 212 & & $\mathrm{U}$ \\
\hline 1356 & TRITIÜM_DIST_LSC & STLRL & 6380 & $\mathrm{pCi} / \mathrm{L}$ & 200 & 380 & 175 & & \\
\hline 1357 & TRITIUM_ELECT_LSC & STLRL & 1370 & $\mathrm{pCi} / \mathrm{L}$ & 14 & 120 & 6.61 & & \\
\hline 1358 & TRITIUM_DIST_LSC & STLRL & 6550 & $\mathrm{pCi} / \mathrm{L}$ & 230 & 430 & 215 & & \\
\hline 1359 & TRITIUM_ELECT_LSC & STLRL & 1200 & $\mathrm{pCi} / \mathrm{L}$ & 13 & 110 & 6.58 & & \\
\hline 1360 & TRITIUM_ELECT_LSC & STLRL & 55.7 & $\mathrm{pCi} / \mathrm{L}$ & 3.8 & 8.1 & 6.32 & & \\
\hline 1361 & TRITIUM_ELECT_LSC & STLRL & 1190 & $\mathrm{pCi} / \mathrm{L}$ & 13 & 100 & 6.31 & & \\
\hline 1362 & TRITIUM_ELECT_LSC & STLRL & 362 & $\mathrm{pCi} / \mathrm{L}$ & 8 & 34 & 7 & & \\
\hline 1363 & TRITIUM_ELECT_LSC & STLRL & 80.1 & $\mathrm{pCi} / \mathrm{L}$ & 4.3 & 10 & 6.36 & & \\
\hline 1364 & TRITIUM_ELECT_LSC & STLRL & 43.2 & $\mathrm{pCi} / \mathrm{L}$ & 3.6 & 7.2 & 6.31 & & \\
\hline 1365 & TRITIUM_ELECT_LSC & STLRL & 35.4 & $\mathrm{pCi} / \mathrm{L}$ & 3.7 & 6.9 & 6.86 & & \\
\hline 1366 & TRITIUM_ELECT_LSC & STLRL & 29.3 & $\mathrm{pCi} / \mathrm{L}$ & 3.3 & 6.2 & 6.38 & & \\
\hline 1367 & TRITIUM_ELECT_LSC & STLRL & 62.7 & $\mathrm{pCi} / \mathrm{L}$ & 3.9 & 8.6 & 6.25 & & \\
\hline 1368 & TRITIUM_ELECT_LSC & STLRL & 31.2 & $\mathrm{pCi} / \mathrm{L}$ & 3.4 & 6.4 & 6.45 & & \\
\hline 1369 & TRITIUM_ELECT_LSC & STLRL & 29.2 & $\mathrm{pCi} / \mathrm{L}$ & 3.3 & 6.2 & 6.4 & & \\
\hline
\end{tabular}




\begin{tabular}{|c|c|c|c|c|c|c|c|c|c|}
\hline & $\mathrm{K}$ & $\mathrm{L}$ & $\mathrm{M}$ & $\mathrm{N}$ & $\mathrm{O}$ & $P$ & $\mathrm{Q}$ & $\mathrm{R}$ & $\mathrm{S}$ \\
\hline 1 & METHOD_NAME & LAB_CODE & VALUE_RPTD & ANAL_UNITS_RPTD & COUNTING_ERROR & TOTAL_ANAL_ERROR & MIN_DETECTABLE_ACTIVITY & PCNT_MOISTURE & LAB_QUALIFIER \\
\hline 1370 & TRITIUM_ELECT_LSC & STLRL & 33.5 & $\mathrm{pCi} / \mathrm{L}$ & 3.4 & 6.5 & 6.41 & & \\
\hline 1371 & TRITIUM_ELECT_LSC & STLRL & 30.1 & $\mathrm{pCi} / \mathrm{L}$ & 3.3 & 6.3 & 6.34 & & \\
\hline 1372 & TRITIUM_ELECT_LSC & STLRL & 98.8 & $\mathrm{pCi} / \mathrm{L}$ & 4.5 & 11 & 6.27 & & \\
\hline 1373 & TRITIUM_ELECT_LSC & STLRL & 90.1 & $\mathrm{pCi} / \mathrm{L}$ & 4.3 & 11 & 6.26 & & \\
\hline 1374 & TRITIUM_ELECT_LSC & STLRL & 59.7 & $\mathrm{pCi} / \mathrm{L}$ & 3.9 & 8.4 & 6.36 & & \\
\hline 1375 & TRITIUM_ELECT_LSC & STLRL & 32.2 & $\mathrm{pCi} / \mathrm{L}$ & 3.4 & 6.4 & 6.29 & & \\
\hline 1376 & TRITIUM_ELECT_LSC & STLRL & 30.8 & $\mathrm{pCi} / \mathrm{L}$ & 3.4 & 6.4 & 6.5 & & \\
\hline 1377 & TRITIUM_ELECT_LSC & STLRL & 27.6 & $\mathrm{pCi} / \mathrm{L}$ & 3.3 & 6 & 6.27 & & \\
\hline 1378 & TRITIUM_ELECT_LSC & STLRL & 29.1 & $\mathrm{pCi} / \mathrm{L}$ & 3.5 & 6.4 & 6.71 & & \\
\hline 1379 & TRITIUM_ELECT_LSC & STLRL & 80.4 & $\mathrm{pCi} / \mathrm{L}$ & 4.2 & 10 & 6.3 & & \\
\hline 1380 & TRITIUM_ELECT_LSC & STLRL & 94.7 & $\mathrm{pCi} / \mathrm{L}$ & 4.4 & 11 & 6.27 & & \\
\hline 1381 & TRITIUM_ELECT_LSC & STLRL & 91.8 & $\mathrm{pCi} / \mathrm{L}$ & 4.4 & 11 & 6.31 & & \\
\hline 1382 & TRITIUM_ELECT_LSC & STLRL & 91.2 & $\mathrm{pCi} / \mathrm{L}$ & 4.4 & 11 & 6.24 & & \\
\hline 1383 & TRITIUM_ELECT_LSC & STLRL & 61.6 & $\mathrm{pCi} / \mathrm{L}$ & 3.9 & 8.6 & 6.42 & & \\
\hline 1384 & TRITIUM_ELECT_LSC & STLRL & 36.1 & $\mathrm{pCi} / \mathrm{L}$ & 3.5 & 6.7 & 6.43 & & \\
\hline 1385 & TRITIUM_ELECT_LSC & STLRL & 63.1 & $\mathrm{pCi} / \mathrm{L}$ & 4.3 & 9.2 & 7.22 & & \\
\hline 1386 & TRITIUM_ELECT_LSC & STLRL & 34.4 & $\mathrm{pCi} / \mathrm{L}$ & 3.5 & 6.7 & 6.58 & & \\
\hline 1387 & TRITIUM_ELECT_LSC & STLRL & 119 & $\mathrm{pCi} / \mathrm{L}$ & 4.8 & 13 & 6.26 & & \\
\hline 1388 & TRITIUM_DIST_LSC & STLRL & 3460 & $\mathrm{pCi} / \mathrm{L}$ & 170 & 280 & 193 & & \\
\hline 1389 & 906.0 H3 LSC & STLRL & -34.2 & $\mathrm{pCi} / \mathrm{L}$ & 74 & 110 & 185 & & $\mathrm{U}$ \\
\hline 1390 & 906.0_H3_LSC & STLRL & 67.4 & $\mathrm{pCi} / \mathrm{L}$ & 78 & 120 & 185 & & $U$ \\
\hline 1391 & 906.0_H3_LSC & STLRL & 234 & $\mathrm{pCi} / \mathrm{L}$ & 85 & 130 & 185 & & \\
\hline 1392 & $906.0 \mathrm{H} 3$ LSC & STLRL & 3250 & $\mathrm{pCi} / \mathrm{L}$ & 160 & 270 & 186 & & \\
\hline 1393 & TRITIŪM_ELECT_LSC & STLRL & 50.6 & $\mathrm{pCi} / \mathrm{L}$ & 3.9 & 8 & 6.75 & & \\
\hline 1394 & TRITIUM_ELECT_LSC & STLRL & 75.3 & $\mathrm{pCi} / \mathrm{L}$ & 4.3 & 9.8 & 6.62 & & \\
\hline 1395 & TRITIUM_ELECT_LSC & STLRL & 49.7 & $\mathrm{pCi} / \mathrm{L}$ & 3.7 & 7.7 & 6.43 & & \\
\hline 1396 & TRITIUM_ELECT_LSC & STLRL & 43.7 & $\mathrm{pCi} / \mathrm{L}$ & 3.6 & 7.2 & 6.32 & & \\
\hline 1397 & TRITIUM_ELECT_LSC & STLRL & 44 & $\mathrm{pCi} / \mathrm{L}$ & 3.6 & 7.2 & 6.36 & & \\
\hline 1398 & TRITIUM_ELECT_LSC & STLRL & 54.3 & $\mathrm{pCi} / \mathrm{L}$ & 3.8 & 8 & 6.35 & & \\
\hline 1399 & TRITIUM ELECT LSC & STLRL & 187 & $\mathrm{pCi} / \mathrm{L}$ & 5.6 & 19 & 6.26 & & \\
\hline 1400 & TRITIUM_ELECT_LSC & STLRL & 184 & $\mathrm{pCi} / \mathrm{L}$ & 5.6 & 19 & 6.37 & & \\
\hline 1401 & TRITIUM_ELECT_LSC & STLRL & 140 & $\mathrm{pCi} / \mathrm{L}$ & 5 & 15 & 6.3 & & \\
\hline 1402 & TRITIUM ELECT_LSC & STLRL & 80.1 & $\mathrm{pCi} / \mathrm{L}$ & 4.2 & 10 & 6.32 & & \\
\hline 1403 & TRITIUM_ELECT_LSC & STLRL & 66.1 & $\mathrm{pCi} / \mathrm{L}$ & 4 & 8.9 & 6.32 & & \\
\hline 1404 & TRITIUM_ELECT_LSC & STLRL & 57.2 & $\mathrm{pCi} / \mathrm{L}$ & 3.9 & 8.3 & 6.43 & & \\
\hline 1405 & TRITIUM_ELECT_LSC & STLRL & 46.5 & $\mathrm{pCi} / \mathrm{L}$ & 3.6 & 7.4 & 6.4 & & \\
\hline 1406 & TRITIUM_ELECT_LSC & STLRL & 166 & $\mathrm{pCi} / \mathrm{L}$ & 5.4 & 17 & 6.35 & & \\
\hline 1407 & TRITIUM_ELECT_LSC & STLRL & 187 & $\mathrm{pCi} / \mathrm{L}$ & 5.8 & 19 & 6.65 & & \\
\hline
\end{tabular}




\begin{tabular}{|c|c|c|c|c|c|c|c|c|c|}
\hline & $\mathrm{K}$ & $\mathrm{L}$ & $\mathrm{M}$ & $\mathrm{N}$ & $\mathrm{O}$ & $\mathrm{P}$ & $\mathrm{Q}$ & $\mathrm{R}$ & $\mathrm{S}$ \\
\hline 1 & METHOD_NAME & LAB_CODE & VALUE_RPTD & ANAL_UNITS_RPTD & COUNTING_ERROR & TOTAL_ANAL_ERROR & MIN_DETECTABLE_ACTIVITY & PCNT_MOISTURE & LAB_QUALIFIER \\
\hline 1408 & TRITIUM_ELECT_LSC & STLRL & 191 & $\mathrm{pCi} / \mathrm{L}$ & 5.9 & 19 & 6.76 & & \\
\hline 1409 & TRITIUM_ELECT_LSC & STLRL & 188 & $\mathrm{pCi} / \mathrm{L}$ & 5.6 & 19 & 6.33 & & \\
\hline 1410 & TRITIUM_ELECT_LSC & STLRL & 35.7 & $\mathrm{pCi} / \mathrm{L}$ & 3.6 & 6.8 & 6.67 & & \\
\hline 1411 & TRITIUM_ELECT_LSC & STLRL & 113 & $\mathrm{pCi} / \mathrm{L}$ & 4.9 & 13 & 6.61 & & \\
\hline 1412 & TRITIUM_ELECT_LSC & STLRL & 120 & $\mathrm{pCi} / \mathrm{L}$ & 5 & 13 & 6.77 & & \\
\hline 1413 & TRITIUM_DIST_LSC & STLRL & 3120 & $\mathrm{pCi} / \mathrm{L}$ & 140 & 170 & 162 & & \\
\hline 1414 & TRITIUM_DIST_LSC & STLRL & 3080 & $\mathrm{pCi} / \mathrm{L}$ & 140 & 170 & 166 & & \\
\hline 1415 & 906.0_H3_LSC & STLRL & 136 & $\mathrm{pCi} / \mathrm{L}$ & 71 & 77 & 161 & & $U$ \\
\hline 1416 & 906.0_H3_LSC & STLRL & 3330 & $\mathrm{pCi} / \mathrm{L}$ & 150 & 170 & 165 & & \\
\hline 1417 & 906.0_H3_LSC & STLRL & 45.3 & $\mathrm{pCi} / \mathrm{L}$ & 68 & 75 & 163 & & $U$ \\
\hline 1418 & 906.0_H3_LSC & STLRL & 224 & $\mathrm{pCi} / \mathrm{L}$ & 74 & 80 & 162 & & \\
\hline 1419 & TRITIUM_ELECT_LSC & STLRL & 53.5 & $\mathrm{pCi} / \mathrm{L}$ & 3.8 & 8 & 6.48 & & \\
\hline 1420 & TRITIUM_ELECT_LSC & STLRL & 63.6 & $\mathrm{pCi} / \mathrm{L}$ & 4 & 8.7 & 6.5 & & \\
\hline 1421 & 906.0_H3_LSC & TMANC & 4940 & $\mathrm{pCi} / \mathrm{L}$ & 250 & 550 & 200 & & \\
\hline 1422 & 906.0_H3_LSC & TMANC & 3850 & $\mathrm{pCi} / \mathrm{L}$ & 230 & 450 & 200 & & \\
\hline 1423 & TRITIŪM_ELECT_LSC & STLRL & 47.5 & $\mathrm{pCi} / \mathrm{L}$ & 3.7 & 7.5 & 6.47 & & \\
\hline 1424 & TRITIUM_ELECT_LSC & STLRL & 42.7 & $\mathrm{pCi} / \mathrm{L}$ & 3.6 & 7.2 & 6.47 & & \\
\hline 1425 & TRITIUM_ELECT_LSC & STLRL & 52.9 & $\mathrm{pCi} / \mathrm{L}$ & 3.8 & 7.9 & 6.48 & & \\
\hline 1426 & TRITIUM_ELECT_LSC & STLRL & 62.6 & $\mathrm{pCi} / \mathrm{L}$ & 3.9 & 8.7 & 6.47 & & \\
\hline 1427 & TRITIUM_ELECT_LSC & STLRL & 37.7 & $\mathrm{pCi} / \mathrm{L}$ & 2.8 & 4.6 & 5.1 & & \\
\hline 1428 & TRITIUM_ELECT_LSC & STLRL & 42.7 & $\mathrm{pCi} / \mathrm{L}$ & 2.9 & 4.9 & 5.06 & & \\
\hline 1429 & TRITIUM_ELECT_LSC & STLRL & 27.6 & $\mathrm{pCi} / \mathrm{L}$ & 2.7 & 3.9 & 5.15 & & \\
\hline 1430 & TRITIUM_ELECT_LSC & STLRL & 31.4 & $\mathrm{pCi} / \mathrm{L}$ & 2.9 & 4.3 & 5.44 & & \\
\hline 1431 & TRITIUM_ELECT_LSC & STLRL & 27.3 & $\mathrm{pCi} / \mathrm{L}$ & 2.7 & 3.8 & 5.06 & & \\
\hline 1432 & TRITIUM_ELECT_LSC & STLRL & 21.6 & $\mathrm{pCi} / \mathrm{L}$ & 2.6 & 3.5 & 5.06 & & \\
\hline 1433 & TRITIUM_ELECT_LSC & STLRL & 23.4 & $\mathrm{pCi} / \mathrm{L}$ & 2.6 & 3.6 & 5.01 & & \\
\hline 1434 & TRITIUM_ELECT_LSC & STLRL & 25.2 & $\mathrm{pCi} / \mathrm{L}$ & 2.6 & 3.7 & 4.99 & & \\
\hline 1435 & TRITIUM_ELECT_LSC & STLRL & 24.1 & $\mathrm{pCi} / \mathrm{L}$ & 2.6 & 3.6 & 5.02 & & \\
\hline 1436 & TRITIUM_ELECT_LSC & STLRL & 21.6 & $\mathrm{pCi} / \mathrm{L}$ & 2.5 & 3.5 & 4.98 & & \\
\hline 1437 & TRITIUM_ELECT_LSC & STLRL & 23.4 & $\mathrm{pCi} / \mathrm{L}$ & 2.6 & 3.6 & 5.1 & & \\
\hline 1438 & TRITIUM_ELECT_LSC & STLRL & 24.5 & $\mathrm{pCi} / \mathrm{L}$ & 2.6 & 3.7 & 5.07 & & \\
\hline 1439 & TRITIUM_ELECT_LSC & STLRL & 20.8 & $\mathrm{pCi} / \mathrm{L}$ & 2.5 & 3.4 & 5 & & \\
\hline 1440 & TRITIUM_ELECT_LSC & STLRL & 23.2 & $\mathrm{pCi} / \mathrm{L}$ & 2.6 & 3.6 & 5.02 & & \\
\hline 1441 & TRITIUM_ELECT_LSC & STLRL & 23.1 & $\mathrm{pCi} / \mathrm{L}$ & 2.6 & 3.6 & 5.06 & & \\
\hline 1442 & TRITIUM_ELECT_LSC & STLRL & 23.6 & $\mathrm{pCi} / \mathrm{L}$ & 2.6 & 3.6 & 5.1 & & \\
\hline 1443 & TRITIUM_ELECT_LSC & STLRL & 22.5 & $\mathrm{pCi} / \mathrm{L}$ & 2.6 & 3.6 & 5.16 & & \\
\hline 1444 & TRITIUM_ELECT_LSC & STLRL & 20.4 & $\mathrm{pCi} / \mathrm{L}$ & 2.5 & 3.4 & 5.04 & & \\
\hline 1445 & TRITIUM_ELECT_LSC & STLRL & 61.5 & $\mathrm{pCi} / \mathrm{L}$ & 3.2 & 6.3 & 5.12 & & \\
\hline
\end{tabular}




\begin{tabular}{|c|c|c|c|c|c|c|c|c|c|}
\hline & $\mathrm{K}$ & $\mathrm{L}$ & $\bar{M}$ & $\mathrm{~N}$ & $\mathrm{O}$ & $P$ & $\mathrm{Q}$ & $\mathrm{R}$ & $\mathrm{S}$ \\
\hline 1 & METHOD_NAME & LAB_CODE & VALUE_RPTD & ANAL_UNITS_RPTD & $\mid$ COUNTING_ERROR & TOTAL_ANAL_ERROR & MIN_DETECTABLE_ACTIVITY & PCNT_MOISTURE & LAB_QUALIFIER \\
\hline 1446 & TRITIUM_DIST_LSC & STLRL & 2440 & $\mathrm{pCi} / \mathrm{L}$ & 130 & 160 & 167 & & \\
\hline 1447 & TRITIUM ELECT_LSC & STLRL & 23.9 & $\mathrm{pCi} / \mathrm{L}$ & 2.6 & 3.6 & 5.15 & & \\
\hline 1448 & 906.0_H3_LSC & STLRL & -28 & $\mathrm{pCi} / \mathrm{L}$ & 60 & 92 & 146 & & $U$ \\
\hline 1449 & TRITIŪM ELECT LSC & STLRL & 28.9 & $\mathrm{pCi} / \mathrm{L}$ & 2.7 & 4 & 5.07 & & \\
\hline 1450 & 906.0_H3_LSC & STLRL & 31.8 & $\mathrm{pCi} / \mathrm{L}$ & 62 & 95 & 147 & & $U$ \\
\hline 1451 & 906.0_H3_LSC & STLRL & 6.07 & $\mathrm{pCi} / \mathrm{L}$ & 61 & 93 & 146 & & $U$ \\
\hline 1452 & 906.0_H3_LSC & STLRL & 3060 & $\mathrm{pCi} / \mathrm{L}$ & 130 & 230 & 147 & & \\
\hline 1453 & TRITIŪM_ELECT_LSC & STLRL & 14.1 & $\mathrm{pCi} / \mathrm{L}$ & 2.4 & 3.1 & 5.14 & & \\
\hline 1454 & TRITIUM_ELECT_LSC & STLRL & 25 & $\mathrm{pCi} / \mathrm{L}$ & 2.7 & 3.7 & 5.17 & & \\
\hline 1455 & 906.0_H3_LSC & TMANC & 10900 & $\mathrm{pCi} / \mathrm{L}$ & 310 & 1100 & 220 & & \\
\hline 1456 & 906.0_H3_LSC & TMANC & 10800 & $\mathrm{pCi} / \mathrm{L}$ & 310 & 1100 & 220 & & \\
\hline 1457 & TRITIUM_ELECT_LSC & STLRL & 20.3 & $\mathrm{pCi} / \mathrm{L}$ & 2.6 & 3.5 & 5.13 & & \\
\hline 1458 & TRITIUM_ELECT_LSC & STLRL & 59.2 & $\mathrm{pCi} / \mathrm{L}$ & 3.2 & 6.1 & 5.22 & & \\
\hline 1459 & TRITIUM_ELECT_LSC & STLRL & 37.6 & $\mathrm{pCi} / \mathrm{L}$ & 2.8 & 4.5 & 5.06 & & \\
\hline 1460 & TRITIUM_ELECT_LSC & STLRL & 19.8 & $\mathrm{pCi} / \mathrm{L}$ & 2.5 & 3.4 & 5.09 & & \\
\hline 1461 & TRITIUM_ELECT_LSC & STLRL & 46.1 & $\mathrm{pCi} / \mathrm{L}$ & 3 & 5.1 & 4.99 & & \\
\hline \begin{tabular}{|l|}
1462 \\
\end{tabular} & TRITIUM_ELECT_LSC & STLRL & 21.9 & $\mathrm{pCi} / \mathrm{L}$ & 2.5 & 3.5 & 4.99 & & \\
\hline 1463 & TRITIUM_ELECT_LSC & STLRL & 32.8 & $\mathrm{pCi} / \mathrm{L}$ & 2.7 & 4.2 & 5.03 & & \\
\hline 1464 & TRITIUM_ELECT_LSC & STLRL & 31.9 & $\mathrm{pCi} / \mathrm{L}$ & 2.7 & 4.1 & 5.05 & & \\
\hline 1465 & TRITIUM_ELECT_LSC & STLRL & 27.2 & $\mathrm{pCi} / \mathrm{L}$ & 2.7 & 3.8 & 5.08 & & \\
\hline 1466 & TRITIUM_ELECT_LSC & STLRL & 18.4 & $\mathrm{pCi} / \mathrm{L}$ & 2.5 & 3.3 & 5.06 & & \\
\hline 1467 & TRITIUM_ELECT_LSC & STLRL & 19.9 & $\mathrm{pCi} / \mathrm{L}$ & 2.6 & 3.5 & 5.23 & & \\
\hline 1468 & TRITIUM_ELECT_LSC & STLRL & 20.5 & $\mathrm{pCi} / \mathrm{L}$ & 2.5 & 3.4 & 4.99 & & \\
\hline 1469 & TRITIUM_ELECT_LSC & STLRL & 18.7 & $\mathrm{pCi} / \mathrm{L}$ & 2.6 & 3.4 & 5.25 & & \\
\hline 1470 & TRITIUM_ELECT_LSC & STLRL & 28.1 & $\mathrm{pCi} / \mathrm{L}$ & 2.7 & 3.9 & 5.02 & & \\
\hline 1471 & TRITIUM_ELECT_LSC & STLRL & 23.5 & $\mathrm{pCi} / \mathrm{L}$ & 2.8 & 3.8 & 5.52 & & \\
\hline 1472 & TRITIUM_ELECT_LSC & STLRL & 25.5 & $\mathrm{pCi} / \mathrm{L}$ & 2.8 & 3.9 & 5.38 & & \\
\hline 1473 & TRITIUM_ELECT_LSC & STLRL & 24.7 & $\mathrm{pCi} / \mathrm{L}$ & 2.6 & 3.7 & 5.11 & & \\
\hline 1474 & TRITIUM_ELECT_LSC & STLRL & 23.4 & $\mathrm{pCi} / \mathrm{L}$ & 2.6 & 3.6 & 5.06 & & \\
\hline 1475 & TRITIUM_ELECT_LSC & STLRL & 20.9 & $\mathrm{pCi} / \mathrm{L}$ & 2.6 & 3.5 & 5.16 & & \\
\hline 1476 & TRITIUM_ELECT_LSC & STLRL & 21 & $\mathrm{pCi} / \mathrm{L}$ & 2.6 & 3.5 & 5.19 & & \\
\hline \begin{tabular}{|l|}
1477 \\
\end{tabular} & TRITIUM_ELECT_LSC & STLRL & 21.3 & $\mathrm{pCi} / \mathrm{L}$ & 2.6 & 3.5 & 5.08 & & \\
\hline 1478 & TRITIUM_ELECT_LSC & STLRL & 20.8 & $\mathrm{pCi} / \mathrm{L}$ & 2.6 & 3.5 & 5.12 & & \\
\hline 1479 & TRITIUM_ELECT_LSC & STLRL & 21.1 & $\mathrm{pCi} / \mathrm{L}$ & 2.6 & 3.5 & 5.12 & & \\
\hline 1480 & TRITIUM_ELECT_LSC & STLRL & 29.5 & $\mathrm{pCi} / \mathrm{L}$ & 3 & 4.3 & 5.82 & & \\
\hline 1481 & TRITIUM_DIST_LSC & STLRL & 3120 & $\mathrm{pCi} / \mathrm{L}$ & 140 & 160 & 162 & & \\
\hline 1482 & TRITIUM_ELECT_LSC & STLRL & 441 & $\mathrm{pCi} / \mathrm{L}$ & 6.9 & 37 & 5.2 & & \\
\hline 1483 & TRITIUM_ELECT_LSC & STLRL & 19.6 & $\mathrm{pCi} / \mathrm{L}$ & 3 & 3.8 & 6.08 & & \\
\hline
\end{tabular}




\begin{tabular}{|c|c|c|c|c|c|c|c|c|c|}
\hline & $\mathrm{K}$ & $\mathrm{L}$ & $\bar{M}$ & $\mathrm{~N}$ & $\mathrm{O}$ & $P$ & $\mathrm{Q}$ & $\mathrm{R}$ & $\mathrm{S}$ \\
\hline 1 & METHOD_NAME & LAB_CODE & VALUE_RPTD & ANAL_UNITS_RPTD & $\mid$ COUNTING_ERROR & TOTAL_ANAL_ERROR & MIN_DETECTABLE_ACTIVITY & PCNT_MOISTURE & LAB_QUALIFIER \\
\hline 1484 & TRITIUM_ELECT_LSC & STLRL & 22.5 & $\mathrm{pCi} / \mathrm{L}$ & 2.7 & 3.7 & 5.31 & & \\
\hline 1485 & TRITIUM ELECT_LSC & STLRL & 27 & $\mathrm{pCi} / \mathrm{L}$ & 3.1 & 4.3 & 6.11 & & \\
\hline 1486 & TRITIUM_ELECT_LSC & STLRL & 22.5 & $\mathrm{pCi} / \mathrm{L}$ & 3.4 & 4.4 & 6.92 & & \\
\hline \begin{tabular}{|l|}
1487 \\
\end{tabular} & TRITIUM_ELECT_LSC & STLRL & 25.1 & $\mathrm{pCi} / \mathrm{L}$ & 2.9 & 4 & 5.64 & & \\
\hline 1488 & TRITIUM_ELECT_LSC & STLRL & 1760 & $\mathrm{pCi} / \mathrm{L}$ & 13 & 140 & 5.23 & & \\
\hline 1489 & TRITIUM_DIST_LSC & STLRL & 15500 & $\mathrm{pCi} / \mathrm{L}$ & 280 & 490 & 162 & & \\
\hline 1490 & TRITIUM_ELECT_LSC & STLRL & 26.8 & $\mathrm{pCi} / \mathrm{L}$ & 2.7 & 3.8 & 5.09 & & \\
\hline 1491 & TRITIUM_ELECT_LSC & STLRL & 21.4 & $\mathrm{pCi} / \mathrm{L}$ & 2.6 & 3.5 & 5.07 & & \\
\hline 1492 & TRITIUM_ELECT_LSC & STLRL & 23.5 & $\mathrm{pCi} / \mathrm{L}$ & 2.6 & 3.6 & 5.16 & & \\
\hline 1493 & TRITIUM_ELECT_LSC & STLRL & 43.2 & $\mathrm{pCi} / \mathrm{L}$ & 3 & 4.9 & 5.09 & & \\
\hline 1494 & TRITIUM_ELECT_LSC & STLRL & 47.5 & $\mathrm{pCi} / \mathrm{L}$ & 3 & 5.2 & 5.02 & & \\
\hline 1495 & TRITIUM_ELECT_LSC & STLRL & 32 & $\mathrm{pCi} / \mathrm{L}$ & 2.8 & 4.2 & 5.1 & & \\
\hline 1496 & TRITIUM_ELECT_LSC & STLRL & 37.5 & $\mathrm{pCi} / \mathrm{L}$ & 2.9 & 4.5 & 5.12 & & \\
\hline 1497 & TRITIUM_ELECT_LSC & STLRL & 39.1 & $\mathrm{pCi} / \mathrm{L}$ & 2.9 & 4.6 & 4.99 & & \\
\hline 1498 & TRITIUM_ELECT_LSC & STLRL & 41.7 & $\mathrm{pCi} / \mathrm{L}$ & 2.9 & 4.8 & 5.01 & & \\
\hline 1499 & TRITIUM_ELECT_LSC & STLRL & 43.4 & $\mathrm{pCi} / \mathrm{L}$ & 3 & 5 & 5.16 & & \\
\hline 1500 & TRITIUM_ELECT_LSC & STLRL & 29.2 & $\mathrm{pCi} / \mathrm{L}$ & 3.1 & 4.4 & 6.04 & & \\
\hline 1501 & TRITIUM_ELECT_LSC & STLRL & 36.6 & $\mathrm{pCi} / \mathrm{L}$ & 2.8 & 4.5 & 5.09 & & \\
\hline 1502 & TRITIUM_ELECT_LSC & STLRL & 18.7 & $\mathrm{pCi} / \mathrm{L}$ & 2.5 & 3.4 & 5.17 & & \\
\hline 1503 & TRITIUM_ELECT_LSC & STLRL & 31.6 & $\mathrm{pCi} / \mathrm{L}$ & 2.9 & 4.3 & 5.41 & & \\
\hline 1504 & TRITIUM_ELECT_LSC & STLRL & 25.4 & $\mathrm{pCi} / \mathrm{L}$ & 2.7 & 3.9 & 5.35 & & \\
\hline 1505 & TRITIUM_ELECT_LSC & STLRL & 45.5 & $\mathrm{pCi} / \mathrm{L}$ & 3.4 & 5.5 & 6.12 & & \\
\hline 1506 & TRITIUM_ELECT_LSC & STLRL & 43.3 & $\mathrm{pCi} / \mathrm{L}$ & 3 & 5 & 5.12 & & \\
\hline 1507 & TRITIUM_ELECT_LSC & STLRL & 43.6 & $\mathrm{pCi} / \mathrm{L}$ & 3.2 & 5.2 & 5.6 & & \\
\hline 1508 & TRITIUM_ELECT_LSC & STLRL & 33.5 & $\mathrm{pCi} / \mathrm{L}$ & 3 & 4.4 & 5.51 & & \\
\hline 1509 & TRITIUM_ELECT_LSC & STLRL & 65 & $\mathrm{pCi} / \mathrm{L}$ & 3.3 & 6.5 & 4.98 & & \\
\hline 1510 & TRITIUM_ELECT_LSC & STLRL & 70.1 & $\mathrm{pCi} / \mathrm{L}$ & 3.3 & 6.9 & 4.99 & & \\
\hline 1511 & TRITIUM_ELECT_LSC & STLRL & 44.5 & $\mathrm{pCi} / \mathrm{L}$ & 2.9 & 5 & 4.99 & & \\
\hline 1512 & TRITIUM_ELECT_LSC & STLRL & 34.2 & $\mathrm{pCi} / \mathrm{L}$ & 2.8 & 4.3 & 4.98 & & \\
\hline 1513 & TRITIUM_DIST_LSC & STLRL & 7140 & $\mathrm{pCi} / \mathrm{L}$ & 220 & 320 & 181 & & \\
\hline 1514 & TRITIUM_DIST_LSC & STLRL & 4330 & $\mathrm{pCi} / \mathrm{L}$ & 180 & 230 & 181 & & \\
\hline \begin{tabular}{|l|l|}
1515 \\
\end{tabular} & TRITIUM_DIST_LSC & STLRL & 1440 & $\mathrm{pCi} / \mathrm{L}$ & 120 & 130 & 181 & & \\
\hline 1516 & TRITIUM_ELECT_LSC & STLRL & 36.7 & $\mathrm{pCi} / \mathrm{L}$ & 2.8 & 4.5 & 5.05 & & \\
\hline 1517 & TRITIUM_ELECT_LSC & STLRL & 86.9 & $\mathrm{pCi} / \mathrm{L}$ & 3.6 & 8.2 & 5.03 & & \\
\hline 1518 & TRITIUM_DIST_LSC & STLRL & 58400 & $\mathrm{pCi} / \mathrm{L}$ & 610 & 1900 & 185 & & \\
\hline 1519 & TRITIUM_DIST_LSC & STLRL & 6910 & $\mathrm{pCi} / \mathrm{L}$ & 220 & 320 & 185 & & \\
\hline 1520 & TRITIUM_DIST_LSC & STLRL & 53900 & $\mathrm{pCi} / \mathrm{L}$ & 580 & 1800 & 185 & & \\
\hline 1521 & 906.0_H3_LSC & STLRL & 3000 & $\mathrm{pCi} / \mathrm{L}$ & 150 & 190 & 182 & & \\
\hline
\end{tabular}




\begin{tabular}{|c|c|c|c|c|c|c|c|c|c|}
\hline & $\mathrm{K}$ & $\mathrm{L}$ & $\bar{M}$ & $\mathrm{~N}$ & $\mathrm{O}$ & $P$ & $\mathrm{Q}$ & $\mathrm{R}$ & $\mathrm{S}$ \\
\hline 1 & METHOD_NAME & LAB_CODE & VALUE_RPTD & ANAL_UNITS_RPTD & $\mid$ COUNTING_ERROR & TOTAL_ANAL_ERROR & MIN_DETECTABLE_ACTIVITY & PCNT_MOISTURE & LAB_QUALIFIER \\
\hline 1522 & TRITIUM_ELECT_LSC & STLRL & 2340 & $\mathrm{pCi} / \mathrm{L}$ & 16 & 190 & 5.72 & & \\
\hline 1523 & TRITIUM_ELECT_LSC & STLRL & 129 & $\mathrm{pCi} / \mathrm{L}$ & 4.9 & 12 & 6.76 & & \\
\hline 1524 & TRITIUM_ELECT_LSC & STLRL & 46.8 & $\mathrm{pCi} / \mathrm{L}$ & 3 & 5.2 & 4.98 & & \\
\hline 1525 & TRITIUM E- ELECT ${ }^{-}$LSC $^{-1}$ & STLRL & 2050 & $\mathrm{pCi} / \mathrm{L}$ & 14 & 170 & 5.06 & & \\
\hline 1526 & TRITIUM_ELECT_LSC & STLRL & 279 & $\mathrm{pCi} / \mathrm{L}$ & 5.5 & 23 & 5 & & \\
\hline 1527 & TRITIUM_ELECT_LSC & STLRL & 1100 & $\mathrm{pCi} / \mathrm{L}$ & 10 & 90 & 4.99 & & \\
\hline 1528 & TRITIUM_ELECT_LSC & STLRL & 42 & $\mathrm{pCi} / \mathrm{L}$ & 2.9 & 4.8 & 4.97 & & \\
\hline 1529 & TRITIUM_ELECT_LSC & STLRL & 62.5 & $\mathrm{pCi} / \mathrm{L}$ & 3.8 & 6.8 & 6.27 & & \\
\hline 1530 & TRITIUM_ELECT_LSC & STLRL & 29.3 & $\mathrm{pCi} / \mathrm{L}$ & 2.7 & 3.9 & 5.02 & & \\
\hline \begin{tabular}{|l|}
1531 \\
\end{tabular} & TRITIUM_ELECT_LSC & STLRL & 43.6 & $\mathrm{pCi} / \mathrm{L}$ & 2.9 & 4.9 & 4.97 & & \\
\hline 1532 & UISO_IE_PLATE_AEA & QTESRL & 810 & $\mathrm{pCi} / \mathrm{L}$ & 11 & 130 & 0.463 & & \\
\hline 1533 & UISO_IE_PLATE_AEA & QTESRL & 0.242 & $\mathrm{pCi} / \mathrm{L}$ & 0.036 & 0.053 & 0.0107 & & \\
\hline 1534 & UISO_IE_PLATE_AEA & QTESRL & 0.244 & $\mathrm{pCi} / \mathrm{L}$ & 0.043 & 0.059 & 0.0151 & & \\
\hline 1535 & UISO_IE_PLATE_AEA & QTESRL & 0.327 & $\mathrm{pCi} / \mathrm{L}$ & 0.056 & 0.078 & 0.0168 & & \\
\hline 1536 & UISO_IE_PLATE_AEA & QTESRL & 0.211 & $\mathrm{pCi} / \mathrm{L}$ & 0.039 & 0.053 & 0.0205 & & \\
\hline \begin{tabular}{|l|}
1537 \\
\end{tabular} & UISO_IE_PLATE_AEA & QTESRL & 0.224 & $\mathrm{pCi} / \mathrm{L}$ & 0.044 & 0.058 & 0.0281 & & \\
\hline \begin{tabular}{|l|}
1538 \\
\end{tabular} & UISO IE PLATE AEA & QTESRL & 0.223 & $\mathrm{pCi} / \mathrm{L}$ & 0.04 & 0.055 & 0.0159 & & \\
\hline 1539 & UISO_IE_PLATE_AEA & QTESRL & 0.257 & $\mathrm{pCi} / \mathrm{L}$ & 0.043 & 0.06 & 0.0187 & & \\
\hline 1540 & UISO_IE_PLATE_AEA & QTESRL & 0.278 & $\mathrm{pCi} / \mathrm{L}$ & 0.046 & 0.064 & 0.0216 & & \\
\hline 1541 & UISO_IE_PLATE_AEA & QTESRL & 0.255 & $\mathrm{pCi} / \mathrm{L}$ & 0.041 & 0.058 & 0.0172 & & \\
\hline 1542 & UISO_IE_PLATE_AEA & QTESRL & 0.301 & $\mathrm{pCi} / \mathrm{L}$ & 0.045 & 0.066 & 0.0169 & & \\
\hline 1543 & UISO_IE_PLATE_AEA & QTESRL & 0.251 & $\mathrm{pCi} / \mathrm{L}$ & 0.042 & 0.059 & 0.0154 & & \\
\hline 1544 & UISO_IE_PLATE_AEA & QTESRL & 0.28 & $\mathrm{pCi} / \mathrm{L}$ & 0.044 & 0.064 & 0.0166 & & \\
\hline 1545 & UISO_IE_PLATE_AEA & QTESRL & 0.292 & $\mathrm{pCi} / \mathrm{L}$ & 0.047 & 0.067 & 0.0249 & & \\
\hline 1546 & UISO_IE_PLATE_AEA & QTESRL & 0.248 & $\mathrm{pCi} / \mathrm{L}$ & 0.042 & 0.059 & 0.021 & & \\
\hline 1547 & UISO_IE_PLATE_AEA & QTESRL & 0.534 & $\mathrm{pCi} / \mathrm{L}$ & 0.06 & 0.1 & 0.0144 & & \\
\hline 1548 & UISO_IE_PLATE_AEA & QTESRL & 0.228 & $\mathrm{pCi} / \mathrm{L}$ & 0.04 & 0.055 & 0.0167 & & \\
\hline 1549 & UISO_IE_PLATE_AEA & QTESRL & 0.227 & $\mathrm{pCi} / \mathrm{L}$ & 0.039 & 0.054 & 0.00976 & & \\
\hline 1550 & UISO_IE_PLATE_AEA & QTESRL & 0.245 & $\mathrm{pCi} / \mathrm{L}$ & 0.04 & 0.056 & 0.0128 & & \\
\hline 1551 & UISO_IE_PLATE_AEA & QTESRL & 0.271 & $\mathrm{pCi} / \mathrm{L}$ & 0.037 & 0.057 & 0.0103 & & \\
\hline 1552 & UISO_IE_PLATE_AEA & QTESRL & 539 & $\mathrm{pCi} / \mathrm{L}$ & 10 & 85 & 0.527 & & \\
\hline \begin{tabular}{|l|l|}
1553 \\
\end{tabular} & UISO IE PLATE AEA & QTESRL & 0.292 & $\mathrm{pCi} / \mathrm{L}$ & 0.044 & 0.065 & 0.0159 & & \\
\hline 1554 & UISO_IE_PLATE_AEA & QTESRL & 0.236 & $\mathrm{pCi} / \mathrm{L}$ & 0.039 & 0.055 & 0.0163 & & \\
\hline 1555 & UISO_IE_PLATE_AEA & STLRL & 0.258 & $\mathrm{pCi} / \mathrm{L}$ & 0.043 & 0.06 & 0.019 & & \\
\hline 1556 & UISO_IE_PLATE_AEA & STLRL & 0.205 & $\mathrm{pCi} / \mathrm{L}$ & 0.037 & 0.051 & 0.015 & & \\
\hline 1557 & UISO_IE_PLATE_AEA & STLRL & 0.295 & $\mathrm{pCi} / \mathrm{L}$ & 0.047 & 0.067 & 0.015 & & \\
\hline 1558 & UISO_IE_PLATE_AEA & STLRL & 0.23 & $\mathrm{pCi} / \mathrm{L}$ & 0.038 & 0.057 & 0.00929 & & \\
\hline 1559 & UISO_IE_PLATE_AEA & STLRL & 0.253 & $\mathrm{pCi} / \mathrm{L}$ & 0.04 & 0.061 & 0.0127 & & \\
\hline
\end{tabular}




\begin{tabular}{|c|c|c|c|c|c|c|c|c|c|}
\hline & $\mathrm{K}$ & $\mathrm{L}$ & $M$ & $\mathrm{~N}$ & $\mathrm{O}$ & $P$ & $\mathrm{Q}$ & $\mathrm{R}$ & $\mathrm{S}$ \\
\hline 1 & METHOD_NAME & LAB_CODE & VALUE_RPTD & ANAL_UNITS_RPTD & COUNTING_ERROR & \begin{tabular}{|l|} 
TOTAL_ANAL_ERROR \\
\end{tabular} & MIN_DETECTABLE_ACTIVITY & PCNT_MOISTURE & LAB_QUALIFIER \\
\hline 1560 & UISO_IE_PLATE_AEA & STLRL & 0.265 & $\mathrm{pCi} / \mathrm{L}$ & 0.041 & 0.063 & 0.0173 & & \\
\hline 1561 & UISO_IE_PLATE_AEA & STLRL & 29.1 & $\mathrm{pCi} / \mathrm{L}$ & 2 & 5.5 & 0.42 & & \\
\hline 1562 & UISO_IE_PLATE_AEA & STLRL & 1210 & $\mathrm{pCi} / \mathrm{L}$ & 14 & 210 & 0.501 & & \\
\hline 1563 & UISO_IE_PLATE_AEA & STLRL & 1360 & $\mathrm{pCi} / \mathrm{L}$ & 14 & 240 & 0.495 & & \\
\hline 1564 & UISO_IE_PLATE_AEA & STLRL & 0.224 & $\mathrm{pCi} / \mathrm{L}$ & 0.039 & 0.057 & 0.0197 & & \\
\hline 1565 & UISO_IE_PLATE_AEA & STLRL & 0.189 & $\mathrm{pCi} / \mathrm{L}$ & 0.035 & 0.05 & 0.0145 & & \\
\hline 1566 & UISO_IE_PLATE_AEA & STLRL & 0.219 & $\mathrm{pCi} / \mathrm{L}$ & 0.038 & 0.056 & 0.013 & & \\
\hline 1567 & UISO_IE_PLATE_AEA & STLRL & 0.154 & $\mathrm{pCi} / \mathrm{L}$ & 0.042 & 0.053 & 0.0304 & & \\
\hline 1568 & UISO_IE_PLATE_AEA & STLRL & 0.221 & $\mathrm{pCi} / \mathrm{L}$ & 0.04 & 0.057 & 0.0126 & & \\
\hline 1569 & UISO_IE_PLATE_AEA & STLRL & 0.221 & $\mathrm{pCi} / \mathrm{L}$ & 0.038 & 0.056 & 0.0131 & & \\
\hline 1570 & UISO_IE_PLATE_AEA & STLRL & 0.235 & $\mathrm{pCi} / \mathrm{L}$ & 0.036 & 0.056 & 0.0124 & & \\
\hline 1571 & UISO_IE_PLATE_AEA & STLRL & 0.203 & $\mathrm{pCi} / \mathrm{L}$ & 0.04 & 0.056 & 0.0176 & & \\
\hline 1572 & UISO_IE_PLATE_AEA & STLRL & 0.211 & $\mathrm{pCi} / \mathrm{L}$ & 0.04 & 0.056 & 0.0134 & & \\
\hline 1573 & UISO_IE_PLATE_AEA & STLRL & 0.255 & $\mathrm{pCi} / \mathrm{L}$ & 0.047 & 0.067 & 0.0215 & & \\
\hline 1574 & UISO_IE_PLATE_AEA & STLRL & 0.192 & $\mathrm{pCi} / \mathrm{L}$ & 0.039 & 0.053 & 0.0157 & & \\
\hline 1575 & UISO_IE_PLATE_AEA & STLRL & 0.183 & $\mathrm{pCi} / \mathrm{L}$ & 0.033 & 0.048 & 0.00894 & & \\
\hline 1576 & UISO_IE_PLATE_AEA & STLRL & 0.227 & $\mathrm{pCi} / \mathrm{L}$ & 0.04 & 0.058 & 0.0154 & & \\
\hline 1577 & UISO_IE_PLATE_AEA & STLRL & 0.194 & $\mathrm{pCi} / \mathrm{L}$ & 0.041 & 0.055 & 0.0203 & & \\
\hline 1578 & UISO_IE_PLATE_AEA & STLRL & 0.233 & $\mathrm{pCi} / \mathrm{L}$ & 0.044 & 0.062 & 0.0204 & & \\
\hline 1579 & UISO_IE_PLATE_AEA & STLRL & 0.207 & $\mathrm{pCi} / \mathrm{L}$ & 0.041 & 0.057 & 0.0118 & & \\
\hline 1580 & UISO_IE_PLATE_AEA & STLRL & 1990 & $\mathrm{pCi} / \mathrm{L}$ & 17 & 350 & 0.446 & & \\
\hline 1581 & UISO_IE_PLATE_AEA & STLRL & 0.216 & $\mathrm{pCi} / \mathrm{L}$ & 0.037 & 0.055 & 0.017 & & \\
\hline 1582 & UISO_IE_PLATE_AEA & STLRL & 0.235 & $\mathrm{pCi} / \mathrm{L}$ & 0.051 & 0.068 & 0.0345 & & \\
\hline 1583 & UISO_IE_PLATE_AEA & STLRL & 0.218 & $\mathrm{pCi} / \mathrm{L}$ & 0.045 & 0.061 & 0.022 & & \\
\hline 1584 & UISO_IE_PLATE_AEA & STLRL & 0.201 & $\mathrm{pCi} / \mathrm{L}$ & 0.04 & 0.055 & 0.0158 & & \\
\hline 1585 & UISO_IE_PLATE_AEA & STLRL & 0.284 & $\mathrm{pCi} / \mathrm{L}$ & 0.047 & 0.07 & 0.0112 & & \\
\hline 1586 & UISO_IE_PLATE_AEA & STLRL & 0.183 & $\mathrm{pCi} / \mathrm{L}$ & 0.029 & 0.044 & 0.00317 & & \\
\hline 1587 & UISO_IE_PLATE_AEA & STLRL & 0.175 & $\mathrm{pCi} / \mathrm{L}$ & 0.028 & 0.043 & 0.00317 & & \\
\hline 1588 & UISO_IE_PLATE_AEA & STLRL & 0.194 & $\mathrm{pCi} / \mathrm{L}$ & 0.03 & 0.047 & 0.00321 & & \\
\hline 1589 & UISO_IE_PLATE_AEA & STLRL & 0.205 & $\mathrm{pCi} / \mathrm{L}$ & 0.033 & 0.05 & 0.00765 & & \\
\hline 1590 & UISO_IE_PLATE_AEA & STLRL & 0.226 & $\mathrm{pCi} / \mathrm{L}$ & 0.036 & 0.055 & 0.00845 & & \\
\hline 1591 & UISO_IE_PLATE_AEA & STLRL & 0.198 & $\mathrm{pCi} / \mathrm{L}$ & 0.037 & 0.053 & 0.0156 & & \\
\hline 1592 & UISO_IE_PLATE_AEA & STLRL & 0.241 & $\mathrm{pCi} / \mathrm{L}$ & 0.045 & 0.064 & 0.0186 & & \\
\hline 1593 & UISO_IE_PLATE_AEA & STLRL & 0.205 & $\mathrm{pCi} / \mathrm{L}$ & 0.037 & 0.054 & 0.00481 & & \\
\hline 1594 & UISO_IE_PLATE_AEA & STLRL & 0.234 & $\mathrm{pCi} / \mathrm{L}$ & 0.045 & 0.063 & 0.0186 & & \\
\hline 1595 & UISO_IE_PLATE_AEA & STLRL & 0.162 & $\mathrm{pCi} / \mathrm{L}$ & 0.028 & 0.041 & 0.0106 & & \\
\hline 1596 & UISO_IE_PLATE_AEA & STLRL & 0.179 & $\mathrm{pCi} / \mathrm{L}$ & 0.043 & 0.056 & 0.0336 & & \\
\hline 1597 & UISO_IE_PLATE_AEA & STLRL & 0.2 & $\mathrm{pCi} / \mathrm{L}$ & 0.035 & 0.052 & 0.00444 & & \\
\hline
\end{tabular}




\begin{tabular}{|c|c|c|c|c|c|c|c|c|c|}
\hline & $\mathrm{K}$ & $\mathrm{L}$ & $\mathrm{M}$ & $\mathrm{N}$ & $\mathrm{O}$ & $\mathrm{P}$ & $\mathrm{Q}$ & $\mathrm{R}$ & $\mathrm{S}$ \\
\hline 1 & METHOD_NAME & LAB_CODE & VALUE_RPTD & ANAL_UNITS_RPTD & COUNTING_ERROR & TOTAL_ANAL_ERROR & MIN_DETECTABLE_ACTIVITY & PCNT_MOISTURE & LAB_QUALIFIER \\
\hline 1598 & UISO_IE_PLATE_AEA & STLRL & 0.171 & $\mathrm{pCi} / \mathrm{L}$ & 0.032 & 0.046 & 0.011 & & \\
\hline 1599 & UISO_IE_PLATE_AEA & STLRL & 0.196 & $\mathrm{pCi} / \mathrm{L}$ & 0.03 & 0.047 & 0.00672 & & \\
\hline 1600 & UISO_IE_PLATE_AEA & STLRL & 0.171 & $\mathrm{pCi} / \mathrm{L}$ & 0.03 & 0.045 & 0.00386 & & \\
\hline 1601 & UISO_IE_PLATE_AEA & STLRL & 0.176 & $\mathrm{pCi} / \mathrm{L}$ & 0.033 & 0.047 & 0.00444 & & \\
\hline 1602 & UISO_IE_PLATE_AEA & STLRL & 0.191 & $\mathrm{pCi} / \mathrm{L}$ & 0.043 & 0.057 & 0.0224 & & \\
\hline 1603 & UISO_IE_PLATE_AEA & STLRL & 0.208 & $\mathrm{pCi} / \mathrm{L}$ & 0.035 & 0.053 & 0.0108 & & \\
\hline 1604 & UISO_IE_PLATE_AEA & STLRL & 0.218 & $\mathrm{pCi} / \mathrm{L}$ & 0.044 & 0.061 & 0.018 & & \\
\hline 1605 & UISO_IE_PLATE_AEA & STLRL & 0.145 & $\mathrm{pCi} / \mathrm{L}$ & 0.036 & 0.047 & 0.0165 & & \\
\hline 1606 & UISO_IE_PLATE_AEA & STLRL & 0.218 & $\mathrm{pCi} / \mathrm{L}$ & 0.043 & 0.06 & 0.0235 & & \\
\hline 1607 & UISO_IE_PLATE_AEA & STLRL & 0.265 & $\mathrm{pCi} / \mathrm{L}$ & 0.042 & 0.064 & 0.0191 & & \\
\hline 1608 & UISO_IE_PLATE_AEA & STLRL & 0.191 & $\mathrm{pCi} / \mathrm{L}$ & 0.039 & 0.053 & 0.014 & & \\
\hline 1609 & UISO_IE_PLATE_AEA & STLRL & 0.189 & $\mathrm{pCi} / \mathrm{L}$ & 0.035 & 0.05 & 0.013 & & \\
\hline 1610 & UISO_IE_PLATE_AEA & STLRL & 0.192 & $\mathrm{pCi} / \mathrm{L}$ & 0.031 & 0.047 & 0.0128 & & \\
\hline 1611 & UISO_IE_PLATE_AEA & STLRL & 0.2 & $\mathrm{pCi} / \mathrm{L}$ & 0.032 & 0.049 & 0.00355 & & \\
\hline 1612 & UISO_IE_PLATE_AEA & STLRL & 0.19 & $\mathrm{pCi} / \mathrm{L}$ & 0.035 & 0.05 & 0.0144 & & \\
\hline 1613 & UISO_IE_PLATE_AEA & STLRL & 0.208 & $\mathrm{pCi} / \mathrm{L}$ & 0.038 & 0.055 & 0.0103 & & \\
\hline 1614 & UISO_IE_PLATE_AEA & STLRL & 0.242 & $\mathrm{pCi} / \mathrm{L}$ & 0.041 & 0.06 & 0.016 & & \\
\hline 1615 & UISO_IE_PLATE_AEA & STLRL & 0.177 & $\mathrm{pCi} / \mathrm{L}$ & 0.034 & 0.048 & 0.0119 & & \\
\hline 1616 & UISO_IE_PLATE_AEA & STLRL & 0.259 & $\mathrm{pCi} / \mathrm{L}$ & 0.042 & 0.064 & 0.015 & & \\
\hline 1617 & UISO_IE_PLATE_AEA & STLRL & 0.191 & $\mathrm{pCi} / \mathrm{L}$ & 0.034 & 0.049 & 0.00425 & & \\
\hline 1618 & UISO_IE_PLATE_AEA & STLRL & 0.233 & $\mathrm{pCi} / \mathrm{L}$ & 0.045 & 0.063 & 0.0173 & & \\
\hline 1619 & UISO_IE_PLATE_AEA & STLRL & 0.206 & $\mathrm{pCi} / \mathrm{L}$ & 0.042 & 0.058 & 0.0202 & & \\
\hline 1620 & UISO_IE_PLATE_AEA & STLRL & 0.174 & $\mathrm{pCi} / \mathrm{L}$ & 0.028 & 0.043 & 0.00823 & & \\
\hline 1621 & UISO_IE_PLATE_AEA & STLRL & 0.212 & $\mathrm{pCi} / \mathrm{L}$ & 0.033 & 0.051 & 0.00364 & & \\
\hline 1622 & UISO_IE_PLATE_AEA & STLRL & 0.196 & $\mathrm{pCi} / \mathrm{L}$ & 0.031 & 0.048 & 0.00352 & & \\
\hline 1623 & UISO_IE_PLATE_AEA & STLRL & 0.197 & $\mathrm{pCi} / \mathrm{L}$ & 0.039 & 0.055 & 0.0209 & & \\
\hline 1624 & UISO_IE_PLATE_AEA & STLRL & 0.21 & $\mathrm{pCi} / \mathrm{L}$ & 0.039 & 0.056 & 0.0171 & & \\
\hline 1625 & UISO_IE_PLATE_AEA & STLRL & 0.296 & $\mathrm{pCi} / \mathrm{L}$ & 0.044 & 0.07 & 0.0117 & & \\
\hline 1626 & UISO_IE_PLATE_AEA & STLRL & 0.181 & $\mathrm{pCi} / \mathrm{L}$ & 0.031 & 0.046 & 0.00383 & & \\
\hline 1627 & UISO_IE_PLATE_AEA & STLRL & 0.17 & $\mathrm{pCi} / \mathrm{L}$ & 0.029 & 0.043 & 0.00347 & & \\
\hline 1628 & UISO_IE_PLATE_AEA & STLRL & 0.185 & $\mathrm{pCi} / \mathrm{L}$ & 0.029 & 0.045 & 0.00328 & & \\
\hline 1629 & UISO_IE_PLATE_AEA & STLRL & 0.166 & $\mathrm{pCi} / \mathrm{L}$ & 0.039 & 0.051 & 0.0258 & & \\
\hline 1630 & UISO_IE_PLATE_AEA & STLRL & 0.243 & $\mathrm{pCi} / \mathrm{L}$ & 0.042 & 0.061 & 0.017 & & \\
\hline 1631 & UISO_IE_PLATE_AEA & STLRL & 0.327 & $\mathrm{pCi} / \mathrm{L}$ & 0.048 & 0.076 & 0.0123 & & \\
\hline 1632 & UISO_IE_PLATE_AEA & STLRL & 0.18 & $\mathrm{pCi} / \mathrm{L}$ & 0.031 & 0.046 & 0.014 & & \\
\hline 1633 & UISO_IE_PLATE_AEA & STLRL & 0.188 & $\mathrm{pCi} / \mathrm{L}$ & 0.035 & 0.05 & 0.0213 & & \\
\hline 1634 & UISO_IE_PLATE_AEA & STLRL & 0.184 & $\mathrm{pCi} / \mathrm{L}$ & 0.036 & 0.051 & 0.0191 & & \\
\hline 1635 & UISO_IE_PLATE_AEA & STLRL & 0.187 & $\mathrm{pCi} / \mathrm{L}$ & 0.037 & 0.051 & 0.0148 & & \\
\hline
\end{tabular}




\begin{tabular}{|c|c|c|c|c|c|c|c|c|c|}
\hline & $\mathrm{K}$ & $\mathrm{L}$ & $\mathrm{M}$ & $\mathrm{N}$ & $\mathrm{O}$ & $\mathrm{P}$ & $\mathrm{Q}$ & $\mathrm{R}$ & $\mathrm{S}$ \\
\hline 1 & METHOD_NAME & LAB_CODE & VALUE_RPTD & ANAL_UNITS_RPTD & COUNTING_ERROR & TOTAL_ANAL_ERROR & MIN_DETECTABLE_ACTIVITY & PCNT_MOISTURE & LAB_QUALIFIER \\
\hline 1636 & UISO_IE_PLATE_AEA & STLRL & 0.196 & $\mathrm{pCi} / \mathrm{L}$ & 0.037 & 0.052 & 0.0138 & & \\
\hline 1637 & UISO_IE_PLATE_AEA & STLRL & 0.335 & $\mathrm{pCi} / \mathrm{L}$ & 0.039 & 0.072 & 0.00924 & & \\
\hline 1638 & UISO_IE_PLATE_AEA & STLRL & 0.204 & $\mathrm{pCi} / \mathrm{L}$ & 0.032 & 0.05 & 0.00749 & & \\
\hline 1639 & UISO_IE_PLATE_AEA & STLRL & 0.264 & $\mathrm{pCi} / \mathrm{L}$ & 0.039 & 0.062 & 0.00405 & & \\
\hline 1640 & UISO_IE_PLATE_AEA & STLRL & 1.25 & $\mathrm{pCi} / \mathrm{L}$ & 0.084 & 0.23 & 0.00806 & & \\
\hline 1641 & UISO_IE_PLATE_AEA & STLRL & 25.8 & $\mathrm{pCi} / \mathrm{L}$ & 0.38 & 4.5 & 0.00384 & & \\
\hline 1642 & UISO_IE_PLATE_AEA & STLRL & 1.36 & $\mathrm{pCi} / \mathrm{L}$ & 0.085 & 0.25 & 0.00363 & & \\
\hline 1643 & UISO_IE_PLATE_AEA & STLRL & 1.9 & $\mathrm{pCi} / \mathrm{L}$ & 0.1 & 0.35 & 0.00357 & & \\
\hline 1644 & UISO_IE_PLATE_AEA & STLRL & 68.9 & $\mathrm{pCi} / \mathrm{L}$ & 6.6 & 14 & 1.09 & & \\
\hline 1645 & UISO_IE_PLATE_AEA & STLRL & 0.627 & $\mathrm{pCi} / \mathrm{L}$ & 0.062 & 0.13 & 0.00415 & & \\
\hline 1646 & UISO_IE_PLATE_AEA & STLRL & 0.225 & $\mathrm{pCi} / \mathrm{L}$ & 0.049 & 0.066 & 0.00756 & & \\
\hline 1647 & UISO_IE_PLATE_AEA & STLRL & 0.256 & $\mathrm{pCi} / \mathrm{L}$ & 0.036 & 0.059 & 0.00355 & & \\
\hline 1648 & UISO_IE_PLATE_AEA & STLRL & 2650 & $\mathrm{pCi} / \mathrm{L}$ & 19 & 460 & 0.091 & & \\
\hline 1649 & UISO_IE_PLATE_AEA & STLRL & 0.332 & $\mathrm{pCi} / \mathrm{L}$ & 0.045 & 0.075 & 0.00899 & & \\
\hline 1650 & UISO_IE_PLATE_AEA & STLRL & 0.176 & $\mathrm{pCi} / \mathrm{L}$ & 0.03 & 0.045 & 0.0104 & & \\
\hline 1651 & UISO_IE_PLATE_AEA & STLRL & 0.214 & $\mathrm{pCi} / \mathrm{L}$ & 0.035 & 0.053 & 0.00996 & & \\
\hline 1652 & UISO_IE_PLATE_AEA & STLRL & 0.215 & $\mathrm{pCi} / \mathrm{L}$ & 0.033 & 0.051 & 0.01 & & \\
\hline 1653 & UISO_IE_PLATE_AEA & STLRL & 1.33 & $\mathrm{pCi} / \mathrm{L}$ & 0.084 & 0.25 & 0.00355 & & \\
\hline 1654 & UISO_IE_PLATE_AEA & STLRL & 0.191 & $\mathrm{pCi} / \mathrm{L}$ & 0.038 & 0.051 & 0.0121 & & \\
\hline 1655 & UISO_IE_PLATE_AEA & STLRL & 0.212 & $\mathrm{pCi} / \mathrm{L}$ & 0.046 & 0.06 & 0.00652 & & \\
\hline 1656 & UISO_IE_PLATE_AEA & STLRL & 0.2 & $\mathrm{pCi} / \mathrm{L}$ & 0.032 & 0.048 & 0.00846 & & \\
\hline 1657 & UISO_IE_PLATE_AEA & STLRL & 0.181 & $\mathrm{pCi} / \mathrm{L}$ & 0.031 & 0.045 & 0.00341 & & \\
\hline 1658 & UISO_IE_PLATE_AEA & STLRL & 0.215 & $\mathrm{pCi} / \mathrm{L}$ & 0.041 & 0.057 & 0.0221 & & \\
\hline 1659 & UISO_IE_PLATE_AEA & STLRL & 0.193 & $\mathrm{pCi} / \mathrm{L}$ & 0.037 & 0.051 & 0.022 & & \\
\hline 1660 & UISO_IE_PLATE_AEA & STLRL & 0.198 & $\mathrm{pCi} / \mathrm{L}$ & 0.033 & 0.049 & 0.00923 & & \\
\hline 1661 & UISO_IE_PLATE_AEA & STLRL & 0.242 & $\mathrm{pCi} / \mathrm{L}$ & 0.039 & 0.058 & 0.00401 & & \\
\hline 1662 & UISO_IE_PLATE_AEA & STLRL & 0.217 & $\mathrm{pCi} / \mathrm{L}$ & 0.043 & 0.058 & 0.0246 & & \\
\hline 1663 & UISO_IE_PLATE_AEA & STLRL & 0.247 & $\mathrm{pCi} / \mathrm{L}$ & 0.044 & 0.062 & 0.0209 & & \\
\hline 1664 & UISO_IE_PLATE_AEA & STLRL & 0.199 & $\mathrm{pCi} / \mathrm{L}$ & 0.039 & 0.053 & 0.0138 & & \\
\hline 1665 & UISO_IE_PLATE_AEA & STLRL & 0.246 & $\mathrm{pCi} / \mathrm{L}$ & 0.044 & 0.062 & 0.017 & & \\
\hline 1666 & UISO_IE_PLATE_AEA & STLRL & 0.393 & $\mathrm{pCi} / \mathrm{L}$ & 0.056 & 0.089 & 0.0197 & & \\
\hline 1667 & UISO_IE_PLATE_AEA & STLRL & 0.226 & $\mathrm{pCi} / \mathrm{L}$ & 0.044 & 0.06 & 0.0218 & & \\
\hline 1668 & UISO_IE_PLATE_AEA & STLRL & 0.196 & $\mathrm{pCi} / \mathrm{L}$ & 0.033 & 0.048 & 0.0089 & & \\
\hline 1669 & UISO_IE_PLATE_AEA & STLRL & 0.219 & $\mathrm{pCi} / \mathrm{L}$ & 0.035 & 0.053 & 0.00913 & & \\
\hline 1670 & UISO_IE_PLATE_AEA & STLRL & 0.205 & $\mathrm{pCi} / \mathrm{L}$ & 0.048 & 0.061 & 0.0268 & & \\
\hline 1671 & UISO_IE_PLATE_AEA & STLRL & 0.187 & $\mathrm{pCi} / \mathrm{L}$ & 0.034 & 0.047 & 0.00806 & & \\
\hline 1672 & UISO_IE_PLATE_AEA & STLRL & 0.274 & $\mathrm{pCi} / \mathrm{L}$ & 0.036 & 0.061 & 0.00646 & & \\
\hline 1673 & UISO_IE_PLATE_AEA & STLRL & 0.209 & $\mathrm{pCi} / \mathrm{L}$ & 0.032 & 0.049 & 0.0078 & & \\
\hline
\end{tabular}




\begin{tabular}{|c|c|c|c|c|c|c|c|c|c|}
\hline & $\mathrm{K}$ & $\mathrm{L}$ & $\bar{M}$ & $\mathrm{~N}$ & $\mathrm{O}$ & $P$ & $\mathrm{Q}$ & $\mathrm{R}$ & $\mathrm{S}$ \\
\hline 1 & METHOD_NAME & LAB_CODE & VALUE_RPTD & ANAL_UNITS_RPTD & COUNTING_ERROR & TOTAL_ANAL_ERROR & MIN_DETECTABLE_ACTIVITY & PCNT_MOISTURE & LAB_QUALIFIER \\
\hline 1674 & UISO_IE_PLATE_AEA & STLRL & 0.26 & $\mathrm{pCi} / \mathrm{L}$ & 0.065 & 0.081 & 0.0297 & & \\
\hline 1675 & UISO_IE_PLATE_AEA & STLRL & 345 & $\mathrm{pCi} / \mathrm{L}$ & 5.9 & 60 & 0.141 & & \\
\hline 1676 & UISO_IE_PLATE_AEA & STLRL & 0.29 & $\mathrm{pCi} / \mathrm{L}$ & 0.039 & 0.065 & 0.00707 & & \\
\hline 1677 & UISO_IE_PLATE_AEA & STLRL & 0.254 & $\mathrm{pCi} / \mathrm{L}$ & 0.039 & 0.06 & 0.0097 & & \\
\hline 1678 & UISO_IE_PLATE_AEA & STLRL & 0.224 & $\mathrm{pCi} / \mathrm{L}$ & 0.054 & 0.068 & 0.0237 & & \\
\hline 1679 & UISO_IE_PLATE_AEA & STLRL & 0.265 & $\mathrm{pCi} / \mathrm{L}$ & 0.039 & 0.061 & 0.00792 & & \\
\hline 1680 & UISO_IE_PLATE_AEA & STLRL & 0.27 & $\mathrm{pCi} / \mathrm{L}$ & 0.038 & 0.062 & 0.00359 & & \\
\hline 1681 & UISO_IE_PLATE_AEA & STLRL & 0.22 & $\mathrm{pCi} / \mathrm{L}$ & 0.034 & 0.052 & 0.00332 & & \\
\hline 1682 & UISO_IE_PLATE_AEA & STLRL & 0.401 & $\mathrm{pCi} / \mathrm{L}$ & 0.065 & 0.097 & 0.025 & & \\
\hline 1683 & UISO_IE_PLATE_AEA & STLRL & 0.251 & $\mathrm{pCi} / \mathrm{L}$ & 0.046 & 0.065 & 0.0187 & & \\
\hline 1684 & UISO_IE_PLATE_AEA & STLRL & 0.312 & $\mathrm{pCi} / \mathrm{L}$ & 0.049 & 0.074 & 0.0143 & & \\
\hline 1685 & UISO_IE_PLATE_AEA & STLRL & 0.266 & $\mathrm{pCi} / \mathrm{L}$ & 0.051 & 0.07 & 0.0163 & & \\
\hline 1686 & UISO_IE_PLATE_AEA & STLRL & 0.264 & $\mathrm{pCi} / \mathrm{L}$ & 0.044 & 0.065 & 0.0183 & & \\
\hline 1687 & UISO_IE_PLATE_AEA & STLRL & 0.246 & $\mathrm{pCi} / \mathrm{L}$ & 0.073 & 0.086 & 0.0532 & & \\
\hline 1688 & UISO_IE_PLATE_AEA & STLRL & 0.21 & $\mathrm{pCi} / \mathrm{L}$ & 0.039 & 0.054 & 0.00461 & & \\
\hline 1689 & UISO_IE_PLATE_AEA & STLRL & 0.269 & $\mathrm{pCi} / \mathrm{L}$ & 0.038 & 0.061 & 0.0073 & & \\
\hline 1690 & UISO_IE_PLATE_AEA & STLRL & 0.237 & $\mathrm{pCi} / \mathrm{L}$ & 0.039 & 0.058 & 0.0089 & & \\
\hline 1691 & UISO_IE_PLATE_AEA & STLRL & 0.255 & $\mathrm{pCi} / \mathrm{L}$ & 0.035 & 0.057 & 0.00318 & & \\
\hline 1692 & UISO_IE_PLATE_AEA & STLRL & 0.315 & $\mathrm{pCi} / \mathrm{L}$ & 0.045 & 0.072 & 0.0155 & & \\
\hline 1693 & UISO_IE_PLATE_AEA & STLRL & 461 & $\mathrm{pCi} / \mathrm{L}$ & 7.2 & 81 & 0.503 & & \\
\hline 1694 & UISO_IE_PLATE_AEA & STLRL & 0.246 & $\mathrm{pCi} / \mathrm{L}$ & 0.038 & 0.058 & 0.00386 & & \\
\hline 1695 & UISO_IE_PLATE_AEA & STLRL & 0.301 & $\mathrm{pCi} / \mathrm{L}$ & 0.05 & 0.073 & 0.0182 & & \\
\hline 1696 & UISO_IE_PLATE_AEA & STLRL & 2.25 & $\mathrm{pCi} / \mathrm{L}$ & 0.13 & 0.42 & 0.0217 & & \\
\hline 1697 & UISO_IE_PLATE_AEA & STLRL & 1.32 & $\mathrm{pCi} / \mathrm{L}$ & 0.084 & 0.25 & 0.0115 & & \\
\hline 1698 & UISO_IE_PLATE_AEA & STLRL & 2.38 & $\mathrm{pCi} / \mathrm{L}$ & 0.11 & 0.43 & 0.00663 & & \\
\hline 1699 & UISO_IE_PLATE_AEA & STLRL & 2.29 & $\mathrm{pCi} / \mathrm{L}$ & 0.12 & 0.42 & 0.0162 & & \\
\hline 1700 & UISO_IE_PLATE_AEA & STLRL & 0.507 & $\mathrm{pCi} / \mathrm{L}$ & 0.049 & 0.1 & 0.00792 & & \\
\hline 1701 & UISO_IE_PLATE_AEA & STLRL & 0.315 & $\mathrm{pCi} / \mathrm{L}$ & 0.039 & 0.068 & 0.00326 & & \\
\hline 1702 & UISO_IE_PLATE_AEA & STLRL & 0.213 & $\mathrm{pCi} / \mathrm{L}$ & 0.033 & 0.05 & 0.00834 & & \\
\hline 1703 & UISO_IE_PLATE_AEA & STLRL & 37.9 & $\mathrm{pCi} / \mathrm{L}$ & 0.43 & 6.6 & 0.0083 & & \\
\hline 1704 & UISO_IE_PLATE_AEA & STLRL & 0.452 & $\mathrm{pCi} / \mathrm{L}$ & 0.048 & 0.093 & 0.0115 & & \\
\hline 1705 & UISO_IE_PLATE_AEA & STLRL & 0.306 & $\mathrm{pCi} / \mathrm{L}$ & 0.039 & 0.067 & 0.0032 & & \\
\hline 1706 & UISO_IE_PLATE_AEA & STLRL & 26.5 & $\mathrm{pCi} / \mathrm{L}$ & 0.79 & 5 & 0.0237 & & \\
\hline 1707 & UISO_IE_PLATE_AEA & STLRL & 0.844 & $\mathrm{pCi} / \mathrm{L}$ & 0.092 & 0.18 & 0.0168 & & \\
\hline 1708 & UISO_IE_PLATE_AEA & STLRL & 0.302 & $\mathrm{pCi} / \mathrm{L}$ & 0.039 & 0.065 & 0.00842 & & \\
\hline 1709 & UISO_IE_PLATE_AEA & STLRL & 0.259 & $\mathrm{pCi} / \mathrm{L}$ & 0.036 & 0.058 & 0.00347 & & \\
\hline 1710 & UISO_IE_PLATE_AEA & STLRL & 0.188 & $\mathrm{pCi} / \mathrm{L}$ & 0.035 & 0.048 & 0.0126 & & \\
\hline 1711 & UISO_IE_PLATE_AEA & STLRL & 0.274 & $\mathrm{pCi} / \mathrm{L}$ & 0.038 & 0.061 & 0.0102 & & \\
\hline
\end{tabular}




\begin{tabular}{|c|c|c|c|c|c|c|c|c|c|}
\hline & $\mathrm{K}$ & $\mathrm{L}$ & $\mathrm{M}$ & $\mathrm{N}$ & $\mathrm{O}$ & $P$ & $\mathrm{Q}$ & $\mathrm{R}$ & $\mathrm{S}$ \\
\hline 1 & METHOD_NAME & LAB_CODE & VALUE_RPTD & ANAL_UNITS_RPTD & COUNTING_ERROR & TOTAL_ANAL_ERROR & MIN_DETECTABLE_ACTIVITY & PCNT_MOISTURE & LAB_QUALIFIER \\
\hline 1712 & UISO_IE_PLATE_AEA & STLRL & 0.221 & $\mathrm{pCi} / \mathrm{L}$ & 0.035 & 0.052 & 0.00923 & & \\
\hline 1713 & UISO_IE_PLATE_AEA & STLRL & 0.217 & $7 \mathrm{pCi} / \mathrm{L}$ & 0.032 & 0.049 & 0.00663 & & \\
\hline 1714 & UISO_IE_PLATE_AEA & STLRL & 0.232 & $2 \mathrm{pCi} / \mathrm{L}$ & 0.035 & 0.053 & 0.00901 & & \\
\hline 1715 & UISO_IE_PLATE_AEA & STLRL & 0.252 & $\mathrm{pCi} / \mathrm{L}$ & 0.036 & 0.057 & 0.00735 & & \\
\hline 1716 & UISO_IE_PLATE_AEA & STLRL & 0.258 & $3 \mathrm{pCi} / \mathrm{L}$ & 0.037 & 0.058 & 0.00896 & & \\
\hline 1717 & UISO_IE_PLATE_AEA & STLRL & 0.377 & $7 \mathrm{pCi} / \mathrm{L}$ & 0.045 & 0.079 & 0.00755 & & \\
\hline 1718 & UISO_IE_PLATE_AEA & STLRL & 0.272 & $2 \mathrm{pCi} / \mathrm{L}$ & 0.039 & 0.061 & 0.0141 & & \\
\hline 1719 & UISO_IE_PLATE_AEA & STLRL & 0.252 & $2 \mathrm{pCi} / \mathrm{L}$ & 0.043 & 0.062 & 0.0182 & & \\
\hline 1720 & UISO_IE_PLATE_AEA & STLRL & 0.281 & $\mathrm{pCi} / \mathrm{L}$ & 0.044 & 0.066 & 0.00993 & & \\
\hline 1721 & UISO_IE_PLATE_AEA & STLRL & 0.271 & $\mathrm{pCi} / \mathrm{L}$ & 0.048 & 0.067 & 0.0212 & & \\
\hline 1722 & UISO_IE_PLATE_AEA & STLRL & 0.369 & $9 \mathrm{pCi} / \mathrm{L}$ & 0.077 & 0.1 & 0.0392 & & \\
\hline 1723 & UISO_IE_PLATE_AEA & STLRL & 0.253 & $3 \mathrm{pCi} / \mathrm{L}$ & 0.065 & 0.081 & 0.0223 & & \\
\hline 1724 & UISO_IE_PLATE_AEA & STLRL & 0.235 & $\mathrm{pCi} / \mathrm{L}$ & 0.044 & 0.062 & 0.0203 & & \\
\hline 1725 & UISO_IE_PLATE_AEA & STLRL & 0.251 & $\mathrm{pCi} / \mathrm{L}$ & 0.046 & 0.066 & 0.0114 & & \\
\hline 1726 & UISO_IE_PLATE_AEA & STLRL & 0.283 & $3 \mathrm{pCi} / \mathrm{L}$ & 0.046 & 0.07 & 0.0156 & & \\
\hline 1727 & UISO_IE_PLATE_AEA & STLRL & 0.205 & $\mathrm{pCi} / \mathrm{L}$ & 0.054 & 0.067 & 0.00938 & & \\
\hline 1728 & UISO IE_PLATE_AEA & STLRL & 2460 & $\mathrm{pCi} / \mathrm{L}$ & 57 & 450 & 4.44 & & \\
\hline 1729 & UISO_IE_PLATE_AEA & STLRL & 0.336 & $\mathrm{pCi} / \mathrm{L}$ & 0.071 & 0.095 & 0.0359 & & \\
\hline 1730 & UISO_IE_PLATE_AEA & STLRL & 0.31 & $1 \mathrm{pCi} / \mathrm{L}$ & 0.065 & 0.087 & 0.0387 & & \\
\hline 1731 & UISO_IE_PLATE_AEA & STLRL & 0.281 & $\mathrm{pCi} / \mathrm{L}$ & 0.045 & 0.069 & 0.0173 & & \\
\hline 1732 & UISO_IE_PLATE_AEA & STLRL & 0.238 & $3 \mathrm{pCi} / \mathrm{L}$ & 0.046 & 0.064 & 0.0203 & & \\
\hline 1733 & UISO_IE_PLATE_AEA & STLRL & 14.6 & $\mathrm{pCi} / \mathrm{L}$ & 0.3 & 2.7 & 0.00869 & & \\
\hline 1734 & UISO IE_PLATE_AEA & STLRL & 53.3 & $3 \mathrm{pCi} / \mathrm{L}$ & 0.53 & 9.6 & 0.0111 & & \\
\hline 1735 & RADISOTOTOPES_ICPMS & PNL329 & 0.10614681 & $1 \mathrm{pCi} / \mathrm{L}$ & & & & & \\
\hline 1736 & RADISOTOPES_ICPMS & PNL329 & 10.3024845 & $\mathrm{pCi} / \mathrm{L}$ & & & & & \\
\hline 1737 & RADISOTOPES ICPMS & PNL329 & 38.5250481 & $1 \mathrm{pCi} / \mathrm{L}$ & & & & & \\
\hline 1738 & RADISOTOPES ICPMS & PNL329 & 0.17483004 & $4 \mathrm{pCi} / \mathrm{L}$ & & & & & \\
\hline 1739 & UISO_IE_PLATE_AEA & STLRL & 0.218 & $3 \mathrm{pCi} / \mathrm{L}$ & 0.035 & 0.054 & 0.00935 & & \\
\hline 1740 & UISO_IE_PLATE_AEA & STLRL & 0.214 & $4 \mathrm{pCi} / \mathrm{L}$ & 0.036 & 0.054 & 0.0099 & & \\
\hline 1741 & UISO IE PLATE AEA & STLRL & 0.368 & $\mathrm{pCi} / \mathrm{L}$ & 0.049 & 0.084 & 0.00891 & & \\
\hline 1742 & UISO_IE_PLATE_AEA & STLRL & 5.14 & $4 \mathrm{pCi} / \mathrm{L}$ & 0.17 & 0.94 & 0.00979 & & \\
\hline 1743 & UISO IE_PLATE_AEA & STLRL & 1.77 & $7 \mathrm{pCi} / \mathrm{L}$ & 0.1 & 0.34 & 0.0125 & & \\
\hline 1744 & UISO IE PLATE AEA & STLRL & 0.56 & $\mathrm{pCi} / \mathrm{L}$ & 0.054 & 0.12 & 0.00348 & & \\
\hline 1745 & UISO_IE_PLATE_AEA & STLRL & 1.43 & $3 \mathrm{pCi} / \mathrm{L}$ & 0.089 & 0.27 & 0.00779 & & \\
\hline 1746 & UISO_IE_PLATE_AEA & STLRL & 0.606 & $\mathrm{pCi} / \mathrm{L}$ & 0.057 & 0.12 & 0.00907 & & \\
\hline 1747 & UISO_IE_PLATE_AEA & STLRL & 0.418 & $\mathrm{pCi} / \mathrm{L}$ & 0.049 & 0.091 & 0.00946 & & \\
\hline 1748 & UISO_IE_PLATE_AEA & STLRL & 0.479 & $\mathrm{pCi} / \mathrm{L}$ & 0.055 & 0.1 & 0.0107 & & \\
\hline 1749 & UISO_IE_PLATE_AEA & STLRL & 30.5 & $5 \mathrm{pCi} / \mathrm{L}$ & 0.42 & 5.5 & 0.00828 & & \\
\hline
\end{tabular}




\begin{tabular}{|c|c|c|c|c|c|c|c|c|c|}
\hline & $\mathrm{K}$ & $\mathrm{L}$ & $\bar{M}$ & $\mathrm{~N}$ & $\mathrm{O}$ & $P$ & $\mathrm{Q}$ & $\mathrm{R}$ & $\mathrm{S}$ \\
\hline 1 & METHOD_NAME & LAB_CODE & VALUE_RPTD & ANAL_UNITS_RPTD & COUNTING_ERROR & TOTAL_ANAL_ERROR & MIN_DETECTABLE_ACTIVITY & PCNT_MOISTURE & LAB_QUALIFIER \\
\hline 1750 & UISO_IE_PLATE_AEA & STLRL & 1.31 & $\mathrm{pCi} / \mathrm{L}$ & 0.11 & 0.26 & 0.0212 & & \\
\hline 1751 & UISO_IE_PLATE_AEA & STLRL & 0.356 & $\mathrm{pCi} / \mathrm{L}$ & 0.052 & 0.084 & 0.0178 & & \\
\hline 1752 & UISO_IE_PLATE_AEA & STLRL & 4.7 & $\mathrm{pCi} / \mathrm{L}$ & 0.16 & 0.86 & 0.0169 & & \\
\hline 1753 & UISO_IE_PLATE_AEA & STLRL & 5.27 & $\mathrm{pCi} / \mathrm{L}$ & 0.18 & 0.97 & 0.0102 & & \\
\hline 1754 & UISO_IE_PLATE_AEA & STLRL & 1.67 & $\mathrm{pCi} / \mathrm{L}$ & 0.095 & 0.32 & 0.0148 & & \\
\hline 1755 & UISO_IE_PLATE_AEA & STLRL & 0.538 & $\mathrm{pCi} / \mathrm{L}$ & 0.055 & 0.11 & 0.00778 & & \\
\hline 1756 & UISO_IE_PLATE_AEA & STLRL & 5.05 & $\mathrm{pCi} / \mathrm{L}$ & 0.16 & 0.92 & 0.0101 & & \\
\hline 1757 & UISO_IE_PLATE_AEA & STLRL & 0.703 & $\mathrm{pCi} / \mathrm{L}$ & 0.061 & 0.14 & 0.00909 & & \\
\hline 1758 & UISO_IE_PLATE_AEA & STLRL & 1.39 & $\mathrm{pCi} / \mathrm{L}$ & 0.086 & 0.27 & 0.00896 & & \\
\hline 1759 & UISO_IE_PLATE_AEA & STLRL & 2.59 & $\mathrm{pCi} / \mathrm{L}$ & 0.12 & 0.49 & 0.0102 & & \\
\hline 1760 & UISO_IE_PLATE_AEA & STLRL & 0.652 & $\mathrm{pCi} / \mathrm{L}$ & 0.061 & 0.13 & 0.00802 & & \\
\hline 1761 & UISO_IE_PLATE_AEA & STLRL & 0.493 & $\mathrm{pCi} / \mathrm{L}$ & 0.064 & 0.11 & 0.023 & & \\
\hline 1762 & UISO_IE_PLATE_AEA & STLRL & 0.459 & $\mathrm{pCi} / \mathrm{L}$ & 0.058 & 0.1 & 0.0207 & & \\
\hline 1763 & UISO_IE_PLATE_AEA & STLRL & 0.431 & $\mathrm{pCi} / \mathrm{L}$ & 0.05 & 0.094 & 0.0148 & & \\
\hline 1764 & UISO_IE_PLATE_AEA & STLRL & 0.542 & $\mathrm{pCi} / \mathrm{L}$ & 0.057 & 0.11 & 0.00825 & & \\
\hline 1765 & UISO_IE_PLATE_AEA & STLRL & 0.225 & $\mathrm{pCi} / \mathrm{L}$ & 0.042 & 0.06 & 0.0174 & & \\
\hline 1766 & UISO_IE_PLATE_AEA & STLRL & 0.418 & $\mathrm{pCi} / \mathrm{L}$ & 0.05 & 0.092 & 0.00836 & & \\
\hline 1767 & UISO_IE_PLATE_AEA & STLRL & 0.267 & $\mathrm{pCi} / \mathrm{L}$ & 0.041 & 0.064 & 0.00866 & & \\
\hline 1768 & UISO_IE_PLATE_AEA & STLRL & 0.263 & $\mathrm{pCi} / \mathrm{L}$ & 0.046 & 0.067 & 0.0214 & & \\
\hline 1769 & UISO_IE_PLATE_AEA & STLRL & 0.315 & $\mathrm{pCi} / \mathrm{L}$ & 0.042 & 0.071 & 0.00756 & & \\
\hline 1770 & UISO_IE_PLATE_AEA & STLRL & 0.719 & $\mathrm{pCi} / \mathrm{L}$ & 0.064 & 0.15 & 0.00803 & & \\
\hline 1771 & UISO_IE_PLATE_AEA & STLRL & 0.384 & $\mathrm{pCi} / \mathrm{L}$ & 0.054 & 0.089 & 0.0183 & & \\
\hline 1772 & UISO_IE_PLATE_AEA & STLRL & 0.371 & $\mathrm{pCi} / \mathrm{L}$ & 0.047 & 0.083 & 0.00801 & & \\
\hline 1773 & UISO_IE_PLATE_AEA & STLRL & 0.218 & $\mathrm{pCi} / \mathrm{L}$ & 0.041 & 0.058 & 0.017 & & \\
\hline 1774 & UISO_IE_PLATE_AEA & STLRL & 0.217 & $\mathrm{pCi} / \mathrm{L}$ & 0.037 & 0.054 & 0.00831 & & \\
\hline 1775 & UISO_IE_PLATE_AEA & STLRL & 0.226 & $\mathrm{pCi} / \mathrm{L}$ & 0.04 & 0.058 & 0.0115 & & \\
\hline 1776 & UISO_IE_PLATE_AEA & STLRL & 0.193 & $\mathrm{pCi} / \mathrm{L}$ & 0.037 & 0.052 & 0.00957 & & \\
\hline 1777 & UISO_IE_PLATE_AEA & STLRL & 0.291 & $\mathrm{pCi} / \mathrm{L}$ & 0.046 & 0.071 & 0.00979 & & \\
\hline 1778 & UISO_IE_PLATE_AEA & STLRL & 0.238 & $\mathrm{pCi} / \mathrm{L}$ & 0.049 & 0.066 & 0.0164 & & \\
\hline 1779 & UISO_IE_PLATE_AEA & STLRL & 0.238 & $\mathrm{pCi} / \mathrm{L}$ & 0.041 & 0.061 & 0.0118 & & \\
\hline 1780 & UISO_IE_PLATE_AEA & STLRL & 0.242 & $\mathrm{pCi} / \mathrm{L}$ & 0.04 & 0.06 & 0.00421 & & \\
\hline 1781 & UISO_IE_PLATE_AEA & STLRL & 0.217 & $\mathrm{pCi} / \mathrm{L}$ & 0.036 & 0.054 & 0.0112 & & \\
\hline 1782 & UISO_IE_PLATE_AEA & STLRL & 0.23 & $\mathrm{pCi} / \mathrm{L}$ & 0.045 & 0.062 & 0.00563 & & \\
\hline 1783 & UISO_IE_PLATE_AEA & STLRL & 0.277 & $\mathrm{pCi} / \mathrm{L}$ & 0.042 & 0.066 & 0.00876 & & \\
\hline 1784 & UISO_IE_PLATE_AEA & STLRL & 0.224 & $\mathrm{pCi} / \mathrm{L}$ & 0.042 & 0.059 & 0.00514 & & \\
\hline 1785 & UISO_IE_PLATE_AEA & STLRL & 0.263 & $\mathrm{pCi} / \mathrm{L}$ & 0.055 & 0.074 & 0.0155 & & \\
\hline 1786 & UISO_IE_PLATE_AEA & STLRL & 0.172 & $\mathrm{pCi} / \mathrm{L}$ & 0.04 & 0.051 & 0.0146 & & \\
\hline 1787 & UISO_IE_PLATE_AEA & STLRL & 0.209 & $\mathrm{pCi} / \mathrm{L}$ & 0.039 & 0.056 & 0.0137 & & \\
\hline
\end{tabular}




\begin{tabular}{|c|c|c|c|c|c|c|c|c|c|}
\hline & $\mathrm{K}$ & $\mathrm{L}$ & $\bar{M}$ & $\mathrm{~N}$ & $\mathrm{O}$ & $P$ & $\mathrm{Q}$ & $\mathrm{R}$ & $\mathrm{S}$ \\
\hline 1 & METHOD_NAME & LAB_CODE & VALUE_RPTD & ANAL_UNITS_RPTD & COUNTING_ERROR & TOTAL_ANAL_ERROR & MIN_DETECTABLE_ACTIVITY & PCNT_MOISTURE & LAB_QUALIFIER \\
\hline 1788 & UISO_IE_PLATE_AEA & STLRL & 0.247 & $\mathrm{pCi} / \mathrm{L}$ & 0.044 & 0.063 & 0.00503 & & \\
\hline 1789 & UISO_IE_PLATE_AEA & STLRL & 0.197 & $\mathrm{pCi} / \mathrm{L}$ & 0.038 & 0.053 & 0.0118 & & \\
\hline 1790 & UISO_IE_PLATE_AEA & STLRL & 0.214 & $\mathrm{pCi} / \mathrm{L}$ & 0.036 & 0.054 & 0.00983 & & \\
\hline 1791 & UISO_IE_PLATE_AEA & STLRL & 0.231 & $\mathrm{pCi} / \mathrm{L}$ & 0.038 & 0.057 & 0.00404 & & \\
\hline 1792 & UISO_IE_PLATE_AEA & STLRL & 0.212 & $\mathrm{pCi} / \mathrm{L}$ & 0.043 & 0.059 & 0.012 & & \\
\hline 1793 & UISO_IE_PLATE_AEA & STLRL & 0.238 & $\mathrm{pCi} / \mathrm{L}$ & 0.039 & 0.059 & 0.0086 & & \\
\hline 1794 & UISO_IE_PLATE_AEA & STLRL & 0.203 & $\mathrm{pCi} / \mathrm{L}$ & 0.035 & 0.051 & 0.00801 & & \\
\hline 1795 & UISO_IE_PLATE_AEA & STLRL & 0.231 & $\mathrm{pCi} / \mathrm{L}$ & 0.045 & 0.063 & 0.0145 & & \\
\hline 1796 & UISO_IE_PLATE_AEA & STLRL & 0.224 & $\mathrm{pCi} / \mathrm{L}$ & 0.036 & 0.055 & 0.00931 & & \\
\hline 1797 & UISO_IE_PLATE_AEA & STLRL & 0.279 & $\mathrm{pCi} / \mathrm{L}$ & 0.053 & 0.074 & 0.0164 & & \\
\hline 1798 & UISO_IE_PLATE_AEA & STLRL & 0.224 & $\mathrm{pCi} / \mathrm{L}$ & 0.037 & 0.056 & 0.0116 & & \\
\hline 1799 & UISO_IE_PLATE_AEA & STLRL & 0.238 & $\mathrm{pCi} / \mathrm{L}$ & 0.04 & 0.059 & 0.00892 & & \\
\hline 1800 & UISO_IE_PLATE_AEA & STLRL & 0.269 & $\mathrm{pCi} / \mathrm{L}$ & 0.039 & 0.063 & 0.00367 & & \\
\hline 1801 & UISO_IE_PLATE_AEA & STLRL & 0.212 & $\mathrm{pCi} / \mathrm{L}$ & 0.036 & 0.053 & 0.00387 & & \\
\hline 1802 & UISO_IE_PLATE_AEA & STLRL & 0.245 & $\mathrm{pCi} / \mathrm{L}$ & 0.038 & 0.059 & 0.00782 & & \\
\hline 1803 & UISO_IE_PLATE_AEA & STLRL & 0.23 & $\mathrm{pCi} / \mathrm{L}$ & 0.038 & 0.058 & 0.0105 & & \\
\hline 1804 & UISO_IE_PLATE_AEA & STLRL & 0.261 & $\mathrm{pCi} / \mathrm{L}$ & 0.044 & 0.065 & 0.0123 & & \\
\hline 1805 & UISO_IE_PLATE_AEA & STLRL & 0.344 & $\mathrm{pCi} / \mathrm{L}$ & 0.044 & 0.077 & 0.0107 & & \\
\hline 1806 & UISO_IE_PLATE_AEA & STLRL & 0.246 & $\mathrm{pCi} / \mathrm{L}$ & 0.04 & 0.06 & 0.00415 & & \\
\hline 1807 & UISO_IE_PLATE_AEA & STLRL & 0.223 & $\mathrm{pCi} / \mathrm{L}$ & 0.036 & 0.055 & 0.00386 & & \\
\hline 1808 & UISO_IE_PLATE_AEA & STLRL & 0.215 & $\mathrm{pCi} / \mathrm{L}$ & 0.034 & 0.052 & 0.00351 & & \\
\hline 1809 & UISO_IE_PLATE_AEA & STLRL & 0.227 & $\mathrm{pCi} / \mathrm{L}$ & 0.036 & 0.055 & 0.00932 & & \\
\hline 1810 & UISO_IE_PLATE_AEA & STLRL & 0.24 & $\mathrm{pCi} / \mathrm{L}$ & 0.037 & 0.058 & 0.0114 & & \\
\hline 1811 & UISO_IE_PLATE_AEA & STLRL & 0.269 & $\mathrm{pCi} / \mathrm{L}$ & 0.04 & 0.064 & 0.00393 & & \\
\hline 1812 & UISO_IE_PLATE_AEA & STLRL & 0.234 & $\mathrm{pCi} / \mathrm{L}$ & 0.038 & 0.058 & 0.00822 & & \\
\hline 1813 & UISO_IE_PLATE_AEA & STLRL & 0.972 & $\mathrm{pCi} / \mathrm{L}$ & 0.077 & 0.19 & 0.00844 & & \\
\hline 1814 & UISO_IE_PLATE_AEA & STLRL & 0.215 & $\mathrm{pCi} / \mathrm{L}$ & 0.035 & 0.053 & 0.00938 & & \\
\hline 1815 & UISO_IE_PLATE_AEA & STLRL & 0.193 & $\mathrm{pCi} / \mathrm{L}$ & 0.033 & 0.049 & 0.00914 & & \\
\hline 1816 & UISO_IE_PLATE_AEA & STLRL & 0.222 & $\mathrm{pCi} / \mathrm{L}$ & 0.035 & 0.054 & 0.0035 & & \\
\hline 1817 & UISO_IE_PLATE_AEA & STLRL & 0.231 & $\mathrm{pCi} / \mathrm{L}$ & 0.036 & 0.056 & 0.00926 & & \\
\hline 1818 & UISO_IE_PLATE_AEA & STLRL & 0.215 & $\mathrm{pCi} / \mathrm{L}$ & 0.035 & 0.053 & 0.0104 & & \\
\hline 1819 & UISO_IE_PLATE_AEA & STLRL & 0.542 & $\mathrm{pCi} / \mathrm{L}$ & 0.056 & 0.11 & 0.00386 & & \\
\hline 1820 & UISO_IE_PLATE_AEA & STLRL & 0.261 & $\mathrm{pCi} / \mathrm{L}$ & 0.04 & 0.063 & 0.01 & & \\
\hline 1821 & UISO_IE_PLATE_AEA & STLRL & 0.214 & $\mathrm{pCi} / \mathrm{L}$ & 0.038 & 0.055 & 0.0111 & & \\
\hline 1822 & UISO_IE_PLATE_AEA & STLRL & 0.23 & $\mathrm{pCi} / \mathrm{L}$ & 0.037 & 0.057 & 0.0082 & & \\
\hline 1823 & UISO_IE_PLATE_AEA & STLRL & 0.25 & $\mathrm{pCi} / \mathrm{L}$ & 0.041 & 0.062 & 0.0113 & & \\
\hline 1824 & UISO_IE_PLATE_AEA & STLRL & 0.249 & $\mathrm{pCi} / \mathrm{L}$ & 0.046 & 0.065 & 0.0158 & & \\
\hline 1825 & UISO_IE_PLATE_AEA & STLRL & 0.351 & $\mathrm{pCi} / \mathrm{L}$ & 0.045 & 0.079 & 0.0146 & & \\
\hline
\end{tabular}




\begin{tabular}{|c|c|c|c|c|c|c|c|c|c|}
\hline & $\mathrm{K}$ & $\mathrm{L}$ & $M$ & $\mathrm{~N}$ & $\mathrm{O}$ & $\mathrm{P}$ & $\mathrm{Q}$ & $\mathrm{R}$ & $\mathrm{S}$ \\
\hline 1 & METHOD_NAME & LAB_CODE & VALUE_RPTD & ANAL_UNITS_RPTD & COUNTING_ERROR & TOTAL_ANAL_ERROR & MIN_DETECTABLE_ACTIVITY & PCNT_MOISTURE & LAB_QUALIFIER \\
\hline 1826 & UISO IE PLATE AEA & STLRL & 0.262 & $\mathrm{pCi} / \mathrm{L}$ & 0.043 & 0.065 & 0.00943 & & \\
\hline 1827 & UISO_IE_PLATE_AEA & STLRL & 0.249 & $\mathrm{pCi} / \mathrm{L}$ & 0.038 & 0.06 & 0.00376 & & \\
\hline 1828 & UISO_IE_PLATE_AEA & STLRL & 36.3 & $\mathrm{pCi} / \mathrm{L}$ & 0.44 & 6.6 & 0.0126 & & \\
\hline 1829 & UISO_IE_PLATE_AEA & STLRL & 35.7 & $\mathrm{pCi} / \mathrm{L}$ & 0.52 & 6.5 & 0.0107 & & \\
\hline 1830 & UISO_IE_PLATE_AEA & STLRL & 53.7 & $\mathrm{pCi} / \mathrm{L}$ & 0.6 & 9.7 & 0.013 & & \\
\hline 1831 & UISO_IE_PLATE_AEA & STLRL & 56.6 & $\mathrm{pCi} / \mathrm{L}$ & 0.6 & 10 & 0.0137 & & \\
\hline 1832 & UISO_IE_PLATE_AEA & STLRL & 56.3 & $\mathrm{pCi} / \mathrm{L}$ & 0.62 & 10 & 0.0116 & & \\
\hline 1833 & UISO_IE_PLATE_AEA & STLRL & 70.5 & $\mathrm{pCi} / \mathrm{L}$ & 0.66 & 13 & 0.0131 & & \\
\hline 1834 & UISO_IE_PLATE_AEA & STLRL & 17.1 & $\mathrm{pCi} / \mathrm{L}$ & 0.31 & 3.1 & 0.00794 & & \\
\hline 1835 & UISO_IE_PLATE_AEA & STLRL & 62.8 & $\mathrm{pCi} / \mathrm{L}$ & 0.62 & 11 & 0.0121 & & \\
\hline 1836 & UISO_IE_PLATE_AEA & STLRL & 32.3 & $\mathrm{pCi} / \mathrm{L}$ & 0.46 & 5.9 & 0.0125 & & \\
\hline 1837 & UISO_IE_PLATE_AEA & STLRL & 33.7 & $\mathrm{pCi} / \mathrm{L}$ & 0.57 & 6.2 & 0.0188 & & \\
\hline 1838 & UISO_IE_PLATE_AEA & STLRL & 32.2 & $\mathrm{pCi} / \mathrm{L}$ & 0.52 & 5.9 & 0.0196 & & \\
\hline 1839 & UISO_IE_PLATE_AEA & STLRL & 37.4 & $\mathrm{pCi} / \mathrm{L}$ & 0.5 & 6.8 & 0.0219 & & \\
\hline 1840 & UISO_IE_PLATE_AEA & STLRL & 0.232 & $\mathrm{pCi} / \mathrm{L}$ & 0.047 & 0.064 & 0.0158 & & \\
\hline 1841 & UISO_IE_PLATE_AEA & STLRL & 0.31 & $\mathrm{pCi} / \mathrm{L}$ & 0.052 & 0.078 & 0.0118 & & \\
\hline 1842 & UISO_IE_PLATE_AEA & STLRL & 2.74 & $\mathrm{pCi} / \mathrm{L}$ & 0.16 & 0.52 & 0.0252 & & \\
\hline 1843 & UISO_IE_PLATE_AEA & STLRL & 0.482 & $\mathrm{pCi} / \mathrm{L}$ & 0.05 & 0.1 & 0.00348 & & \\
\hline 1844 & UISO_IE_PLATE_AEA & STLRL & 0.994 & $\mathrm{pCi} / \mathrm{L}$ & 0.073 & 0.19 & 0.00745 & & \\
\hline 1845 & UISO_IE_PLATE_AEA & STLRL & 0.262 & $\mathrm{pCi} / \mathrm{L}$ & 0.038 & 0.062 & 0.00901 & & \\
\hline 1846 & UISO_IE_PLATE_AEA & STLRL & 0.251 & $\mathrm{pCi} / \mathrm{L}$ & 0.037 & 0.06 & 0.00362 & & \\
\hline 1847 & UISO_IE_PLATE_AEA & STLRL & 0.246 & $\mathrm{pCi} / \mathrm{L}$ & 0.037 & 0.058 & 0.00799 & & \\
\hline 1848 & UISO_IE_PLATE_AEA & STLRL & 0.253 & $\mathrm{pCi} / \mathrm{L}$ & 0.044 & 0.063 & 0.0172 & & \\
\hline 1849 & UISO_IE_PLATE_AEA & STLRL & 0.256 & $\mathrm{pCi} / \mathrm{L}$ & 0.039 & 0.06 & 0.0144 & & \\
\hline 1850 & UISO_IE_PLATE_AEA & STLRL & 0.212 & $\mathrm{pCi} / \mathrm{L}$ & 0.035 & 0.053 & 0.00377 & & \\
\hline 1851 & UISO_IE_PLATE_AEA & STLRL & 0.237 & $\mathrm{pCi} / \mathrm{L}$ & 0.039 & 0.059 & 0.0104 & & \\
\hline 1852 & UISO_IE_PLATE_AEA & STLRL & 0.224 & $\mathrm{pCi} / \mathrm{L}$ & 0.035 & 0.055 & 0.00362 & & \\
\hline 1853 & UISO_IE_PLATE_AEA & STLRL & 0.243 & $\mathrm{pCi} / \mathrm{L}$ & 0.037 & 0.059 & 0.00377 & & \\
\hline 1854 & UISO_IE_PLATE_AEA & STLRL & 0.325 & $\mathrm{pCi} / \mathrm{L}$ & 0.044 & 0.075 & 0.00399 & & \\
\hline 1855 & UISO_IE_PLATE_AEA & STLRL & 0.295 & $\mathrm{pCi} / \mathrm{L}$ & 0.041 & 0.068 & 0.00368 & & \\
\hline 1856 & UISO_IE_PLATE_AEA & STLRL & 0.224 & $\mathrm{pCi} / \mathrm{L}$ & 0.043 & 0.06 & 0.0179 & & \\
\hline 1857 & UISO_IE_PLATE_AEA & STLRL & 0.237 & $\mathrm{pCi} / \mathrm{L}$ & 0.038 & 0.058 & 0.016 & & \\
\hline 1858 & UISO_IE_PLATE_AEA & STLRL & 0.235 & $\mathrm{pCi} / \mathrm{L}$ & 0.038 & 0.058 & 0.0125 & & \\
\hline 1859 & UISO_IE_PLATE_AEA & STLRL & 0.288 & $\mathrm{pCi} / \mathrm{L}$ & 0.042 & 0.068 & 0.00401 & & \\
\hline 1860 & UISO_IE_PLATE_AEA & STLRL & 0.471 & $\mathrm{pCi} / \mathrm{L}$ & 0.058 & 0.1 & 0.0047 & & \\
\hline 1861 & UISO_IE_PLATE_AEA & STLRL & 0.283 & $\mathrm{pCi} / \mathrm{L}$ & 0.042 & 0.067 & 0.00413 & & \\
\hline 1862 & UISO_IE_PLATE_AEA & STLRL & 0.277 & $\mathrm{pCi} / \mathrm{L}$ & 0.041 & 0.066 & 0.00828 & & \\
\hline 1863 & UISO_IE_PLATE_AEA & STLRL & 0.285 & $\mathrm{pCi} / \mathrm{L}$ & 0.041 & 0.067 & 0.00385 & & \\
\hline
\end{tabular}




\begin{tabular}{|c|c|c|c|c|c|c|c|c|c|}
\hline & $\mathrm{K}$ & $\mathrm{L}$ & $\mathrm{M}$ & $\mathrm{N}$ & $\mathrm{O}$ & $P$ & $\mathrm{Q}$ & $\mathrm{R}$ & $\mathrm{S}$ \\
\hline 1 & METHOD_NAME & LAB_CODE & VALUE_RPTD & ANAL_UNITS_RPTD & COUNTING_ERROR & TOTAL_ANAL_ERROR & MIN_DETECTABLE_ACTIVITY & PCNT_MOISTURE & LAB_QUALIFIER \\
\hline 1864 & UISO_IE_PLATE_AEA & STLRL & 0.267 & $\mathrm{pCi} / \mathrm{L}$ & 0.041 & 0.064 & 0.00861 & & \\
\hline 1865 & UISO_IE_PLATE_AEA & STLRL & 0.279 & $\mathrm{pCi} / \mathrm{L}$ & 0.041 & 0.066 & 0.00394 & & \\
\hline 1866 & UISO_IE_PLATE_AEA & STLRL & 0.244 & $\mathrm{pCi} / \mathrm{L}$ & 0.04 & 0.061 & 0.0091 & & \\
\hline 1867 & UISO_IE_PLATE_AEA & STLRL & 0.236 & $\mathrm{pCi} / \mathrm{L}$ & 0.036 & 0.056 & 0.0101 & & \\
\hline 1868 & UISO_IE_PLATE_AEA & STLRL & 0.202 & $\mathrm{pCi} / \mathrm{L}$ & 0.038 & 0.053 & 0.00938 & & \\
\hline 1869 & UISO_IE_PLATE_AEA & STLRL & 0.227 & $\mathrm{pCi} / \mathrm{L}$ & 0.04 & 0.058 & 0.00935 & & \\
\hline 1870 & UISO_IE_PLATE_AEA & STLRL & 0.269 & $\mathrm{pCi} / \mathrm{L}$ & 0.044 & 0.067 & 0.00974 & & \\
\hline 1871 & UISO_IE_PLATE_AEA & STLRL & 0.267 & $\mathrm{pCi} / \mathrm{L}$ & 0.04 & 0.064 & 0.00396 & & \\
\hline 1872 & UISO_IE_PLATE_AEA & STLRL & 0.271 & $\mathrm{pCi} / \mathrm{L}$ & 0.038 & 0.063 & 0.00894 & & \\
\hline 1873 & UISO_IE_PLATE_AEA & STLRL & 0.258 & $\mathrm{pCi} / \mathrm{L}$ & 0.037 & 0.06 & 0.00873 & & \\
\hline 1874 & UISO_IE_PLATE_AEA & STLRL & 0.304 & $\mathrm{pCi} / \mathrm{L}$ & 0.04 & 0.069 & 0.00348 & & \\
\hline 1875 & UISO_IE_PLATE_AEA & STLRL & 0.478 & $\mathrm{pCi} / \mathrm{L}$ & 0.053 & 0.1 & 0.00824 & & \\
\hline 1876 & UISO_IE_PLATE_AEA & STLRL & 0.429 & $\mathrm{pCi} / \mathrm{L}$ & 0.05 & 0.093 & 0.00967 & & \\
\hline 1877 & UISO_IE_PLATE_AEA & STLRL & 0.346 & $\mathrm{pCi} / \mathrm{L}$ & 0.044 & 0.077 & 0.00911 & & \\
\hline 1878 & UISO_IE_PLATE_AEA & STLRL & 0.281 & $\mathrm{pCi} / \mathrm{L}$ & 0.051 & 0.073 & 0.0238 & & \\
\hline 1879 & UISO_IE_PLATE_AEA & STLRL & 0.265 & $\mathrm{pCi} / \mathrm{L}$ & 0.041 & 0.064 & 0.0156 & & \\
\hline 1880 & UISO_IE_PLATE_AEA & STLRL & 0.283 & $\mathrm{pCi} / \mathrm{L}$ & 0.041 & 0.067 & 0.00397 & & \\
\hline 1881 & UISO_IE_PLATE_AEA & STLRL & 0.794 & $\mathrm{pCi} / \mathrm{L}$ & 0.066 & 0.16 & 0.0107 & & \\
\hline 1882 & UISO_IE_PLATE_AEA & STLRL & 0.424 & $\mathrm{pCi} / \mathrm{L}$ & 0.051 & 0.093 & 0.0103 & & \\
\hline 1883 & UISO_IE_PLATE_AEA & STLRL & 0.461 & $\mathrm{pCi} / \mathrm{L}$ & 0.056 & 0.1 & 0.00933 & & \\
\hline 1884 & UISO_IE_PLATE_AEA & STLRL & 0.449 & $\mathrm{pCi} / \mathrm{L}$ & 0.059 & 0.1 & 0.0205 & & \\
\hline 1885 & UISO_IE_PLATE_AEA & STLRL & 0.526 & $\mathrm{pCi} / \mathrm{L}$ & 0.055 & 0.11 & 0.00955 & & \\
\hline 1886 & UISO_IE_PLATE_AEA & STLRL & 0.221 & $\mathrm{pCi} / \mathrm{L}$ & 0.037 & 0.055 & 0.00992 & & \\
\hline 1887 & UISO_IE_PLATE_AEA & STLRL & 0.298 & $\mathrm{pCi} / \mathrm{L}$ & 0.044 & 0.07 & 0.0106 & & \\
\hline 1888 & UISO_IE_PLATE_AEA & STLRL & 0.232 & $\mathrm{pCi} / \mathrm{L}$ & 0.041 & 0.06 & 0.0119 & & \\
\hline 1889 & UISO_IE_PLATE_AEA & STLRL & 0.267 & $\mathrm{pCi} / \mathrm{L}$ & 0.055 & 0.075 & 0.0187 & & \\
\hline 1890 & UISO_IE_PLATE_AEA & STLRL & 0.256 & $\mathrm{pCi} / \mathrm{L}$ & 0.037 & 0.06 & 0.0112 & & \\
\hline 1891 & UISO_IE_PLATE_AEA & STLRL & 0.234 & $\mathrm{pCi} / \mathrm{L}$ & 0.035 & 0.056 & 0.00979 & & \\
\hline 1892 & UISO_IE_PLATE_AEA & STLRL & 0.241 & $\mathrm{pCi} / \mathrm{L}$ & 0.038 & 0.059 & 0.0146 & & \\
\hline 1893 & UISO_IE_PLATE_AEA & STLRL & 0.225 & $\mathrm{pCi} / \mathrm{L}$ & 0.035 & 0.054 & 0.0108 & & \\
\hline 1894 & UISO_IE_PLATE_AEA & STLRL & 0.247 & $\mathrm{pCi} / \mathrm{L}$ & 0.042 & 0.062 & 0.0177 & & \\
\hline 1895 & UISO_IE_PLATE_AEA & STLRL & 0.191 & $\mathrm{pCi} / \mathrm{L}$ & 0.033 & 0.048 & 0.00905 & & \\
\hline 1896 & UISO_IE_PLATE_AEA & STLRL & 0.257 & $\mathrm{pCi} / \mathrm{L}$ & 0.046 & 0.066 & 0.0202 & & \\
\hline 1897 & UISO_IE_PLATE_AEA & STLRL & 0.239 & $\mathrm{pCi} / \mathrm{L}$ & 0.038 & 0.059 & 0.01 & & \\
\hline 1898 & UISO_IE_PLATE_AEA & STLRL & 0.223 & $\mathrm{pCi} / \mathrm{L}$ & 0.035 & 0.054 & 0.0118 & & \\
\hline 1899 & UISO_IE_PLATE_AEA & STLRL & 0.203 & $\mathrm{pCi} / \mathrm{L}$ & 0.034 & 0.051 & 0.00368 & & \\
\hline 1900 & UISO_IE_PLATE_AEA & STLRL & 0.209 & $\mathrm{pCi} / \mathrm{L}$ & 0.034 & 0.052 & 0.00367 & & \\
\hline 1901 & UISO_IE_PLATE_AEA & STLRL & 0.195 & $\mathrm{pCi} / \mathrm{L}$ & 0.041 & 0.055 & 0.0213 & & \\
\hline
\end{tabular}




\begin{tabular}{|c|c|c|c|c|c|c|c|c|c|}
\hline & $\mathrm{K}$ & $\mathrm{L}$ & $\mathrm{M}$ & $\mathrm{N}$ & $\mathrm{O}$ & $P$ & $\mathrm{Q}$ & $\mathrm{R}$ & $\mathrm{S}$ \\
\hline 1 & METHOD_NAME & LAB_CODE & VALUE_RPTD & ANAL_UNITS_RPTD & COUNTING_ERROR & TOTAL_ANAL_ERROR & MIN_DETECTABLE_ACTIVITY & PCNT_MOISTURE & LAB_QUALIFIER \\
\hline 1902 & UISO_IE_PLATE_AEA & STLRL & 0.209 & $\mathrm{pCi} / \mathrm{L}$ & 0.035 & 0.052 & 0.00936 & & \\
\hline 1903 & UISO_IE_PLATE_AEA & STLRL & 0.232 & $\mathrm{pCi} / \mathrm{L}$ & 0.036 & 0.056 & 0.01 & & \\
\hline 1904 & UISO_IE_PLATE_AEA & STLRL & 0.239 & $\mathrm{pCi} / \mathrm{L}$ & 0.036 & 0.057 & 0.00354 & & \\
\hline 1905 & UISO_IE_PLATE_AEA & STLRL & 0.214 & $\mathrm{pCi} / \mathrm{L}$ & 0.035 & 0.053 & 0.0037 & & \\
\hline 1906 & UISO_IE_PLATE_AEA & STLRL & 0.223 & $\mathrm{pCi} / \mathrm{L}$ & 0.036 & 0.055 & 0.0124 & & \\
\hline 1907 & UISO_IE_PLATE_AEA & STLRL & 0.221 & $\mathrm{pCi} / \mathrm{L}$ & 0.036 & 0.055 & 0.0131 & & \\
\hline 1908 & UISO_IE_PLATE_AEA & STLRL & 0.187 & $\mathrm{pCi} / \mathrm{L}$ & 0.034 & 0.048 & 0.00388 & & \\
\hline 1909 & UISO_IE_PLATE_AEA & STLRL & 0.24 & $\mathrm{pCi} / \mathrm{L}$ & 0.042 & 0.061 & 0.00482 & & \\
\hline 1910 & UISO_IE_PLATE_AEA & STLRL & 0.209 & $\mathrm{pCi} / \mathrm{L}$ & 0.036 & 0.053 & 0.00397 & & \\
\hline 1911 & UISO_IE_PLATE_AEA & STLRL & 0.271 & $\mathrm{pCi} / \mathrm{L}$ & 0.05 & 0.071 & 0.00604 & & \\
\hline 1912 & UISO_IE_PLATE_AEA & STLRL & 0.259 & $\mathrm{pCi} / \mathrm{L}$ & 0.045 & 0.066 & 0.0139 & & \\
\hline 1913 & UISO_IE_PLATE_AEA & STLRL & 0.156 & $\mathrm{pCi} / \mathrm{L}$ & 0.033 & 0.044 & 0.0165 & & \\
\hline 1914 & UISO_IE_PLATE_AEA & STLRL & 0.186 & $\mathrm{pCi} / \mathrm{L}$ & 0.033 & 0.048 & 0.00991 & & \\
\hline 1915 & UISO_IE_PLATE_AEA & STLRL & 0.236 & $\mathrm{pCi} / \mathrm{L}$ & 0.037 & 0.057 & 0.00377 & & \\
\hline 1916 & UISO_IE_PLATE_AEA & STLRL & 0.224 & $\mathrm{pCi} / \mathrm{L}$ & 0.036 & 0.055 & 0.00379 & & \\
\hline 1917 & UISO_IE_PLATE_AEA & STLRL & 0.319 & $\mathrm{pCi} / \mathrm{L}$ & 0.044 & 0.073 & 0.0106 & & \\
\hline 1918 & UISO_IE_PLATE_AEA & STLRL & 0.194 & $\mathrm{pCi} / \mathrm{L}$ & 0.034 & 0.049 & 0.00788 & & \\
\hline 1919 & UISO_IE_PLATE_AEA & STLRL & 0.208 & $\mathrm{pCi} / \mathrm{L}$ & 0.04 & 0.056 & 0.019 & & \\
\hline 1920 & UISO_IE_PLATE_AEA & STLRL & 0.204 & $\mathrm{pCi} / \mathrm{L}$ & 0.033 & 0.05 & 0.00868 & & \\
\hline 1921 & UISO_IE_PLATE_AEA & STLRL & 0.21 & $\mathrm{pCi} / \mathrm{L}$ & 0.036 & 0.053 & 0.0186 & & \\
\hline 1922 & UISO_IE_PLATE_AEA & STLRL & 0.186 & $\mathrm{pCi} / \mathrm{L}$ & 0.032 & 0.047 & 0.0101 & & \\
\hline 1923 & UISO_IE_PLATE_AEA & STLRL & 0.206 & $\mathrm{pCi} / \mathrm{L}$ & 0.04 & 0.055 & 0.0164 & & \\
\hline 1924 & UISO_IE_PLATE_AEA & STLRL & 0.216 & $\mathrm{pCi} / \mathrm{L}$ & 0.034 & 0.053 & 0.00355 & & \\
\hline 1925 & UISO_IE_PLATE_AEA & STLRL & 0.2 & $\mathrm{pCi} / \mathrm{L}$ & 0.032 & 0.049 & 0.0033 & & \\
\hline 1926 & UISO_IE_PLATE_AEA & STLRL & 0.245 & $\mathrm{pCi} / \mathrm{L}$ & 0.036 & 0.058 & 0.0117 & & \\
\hline 1927 & UISO_IE_PLATE_AEA & STLRL & 0.216 & $\mathrm{pCi} / \mathrm{L}$ & 0.034 & 0.052 & 0.00345 & & \\
\hline 1928 & UISO_IE_PLATE_AEA & STLRL & 0.204 & $\mathrm{pCi} / \mathrm{L}$ & 0.035 & 0.052 & 0.00386 & & \\
\hline 1929 & UISO_IE_PLATE_AEA & STLRL & 0.19 & $\mathrm{pCi} / \mathrm{L}$ & 0.031 & 0.047 & 0.00884 & & \\
\hline 1930 & UISO_IE_PLATE_AEA & STLRL & 0.2 & $\mathrm{pCi} / \mathrm{L}$ & 0.033 & 0.05 & 0.00363 & & \\
\hline 1931 & UISO_IE_PLATE_AEA & STLRL & 0.202 & $\mathrm{pCi} / \mathrm{L}$ & 0.033 & 0.05 & 0.00343 & & \\
\hline 1932 & UISO_IE_PLATE_AEA & STLRL & 0.222 & $\mathrm{pCi} / \mathrm{L}$ & 0.035 & 0.054 & 0.00883 & & \\
\hline 1933 & UISO_IE_PLATE_AEA & STLRL & 0.181 & $\mathrm{pCi} / \mathrm{L}$ & 0.033 & 0.048 & 0.0132 & & \\
\hline 1934 & UISO_IE_PLATE_AEA & STLRL & 0.211 & $\mathrm{pCi} / \mathrm{L}$ & 0.041 & 0.057 & 0.00523 & & \\
\hline 1935 & UISO_IE_PLATE_AEA & STLRL & 0.209 & $\mathrm{pCi} / \mathrm{L}$ & 0.034 & 0.052 & 0.00361 & & \\
\hline 1936 & UISO_IE_PLATE_AEA & STLRL & 0.207 & $\mathrm{pCi} / \mathrm{L}$ & 0.037 & 0.054 & 0.0116 & & \\
\hline 1937 & UISO_IE_PLATE_AEA & STLRL & 0.165 & $\mathrm{pCi} / \mathrm{L}$ & 0.031 & 0.043 & 0.00363 & & \\
\hline 1938 & UISO_IE_PLATE_AEA & STLRL & 0.204 & $\mathrm{pCi} / \mathrm{L}$ & 0.039 & 0.055 & 0.0149 & & \\
\hline 1939 & UISO_IE_PLATE_AEA & STLRL & 0.226 & $\mathrm{pCi} / \mathrm{L}$ & 0.041 & 0.059 & 0.0405 & & \\
\hline
\end{tabular}




\begin{tabular}{|c|c|c|c|c|c|c|c|c|c|}
\hline & $\mathrm{K}$ & $\mathrm{L}$ & $\mathrm{M}$ & $\mathrm{N}$ & $\mathrm{O}$ & $P$ & $\mathrm{Q}$ & $\mathrm{R}$ & $\mathrm{S}$ \\
\hline 1 & METHOD_NAME & LAB_CODE & VALUE_RPTD & ANAL_UNITS_RPTD & COUNTING_ERROR & TOTAL_ANAL_ERROR & MIN_DETECTABLE_ACTIVITY & PCNT_MOISTURE & LAB_QUALIFIER \\
\hline 1940 & UISO_IE_PLATE_AEA & STLRL & 0.262 & $\mathrm{pCi} / \mathrm{L}$ & 0.04 & 0.063 & 0.00404 & & \\
\hline 1941 & UISO_IE_PLATE_AEA & STLRL & 0.2 & $\mathrm{pCi} / \mathrm{L}$ & 0.034 & 0.05 & 0.00371 & & \\
\hline 1942 & UISO_IE_PLATE_AEA & STLRL & 0.214 & $\mathrm{pCi} / \mathrm{L}$ & 0.034 & 0.052 & 0.012 & & \\
\hline 1943 & UISO_IE_PLATE_AEA & STLRL & 0.246 & $\mathrm{pCi} / \mathrm{L}$ & 0.036 & 0.058 & 0.00334 & & \\
\hline 1944 & UISO_IE_PLATE_AEA & STLRL & 0.185 & $\mathrm{pCi} / \mathrm{L}$ & 0.034 & 0.048 & 0.00394 & & \\
\hline 1945 & UISO_IE_PLATE_AEA & STLRL & 0.212 & $\mathrm{pCi} / \mathrm{L}$ & 0.034 & 0.052 & 0.00362 & & \\
\hline 1946 & UISO_IE_PLATE_AEA & STLRL & 0.21 & $\mathrm{pCi} / \mathrm{L}$ & 0.033 & 0.051 & 0.00328 & & \\
\hline 1947 & UISO_IE_PLATE_AEA & STLRL & 0.23 & $\mathrm{pCi} / \mathrm{L}$ & 0.036 & 0.056 & 0.00774 & & \\
\hline 1948 & UISO_IE_PLATE_AEA & STLRL & 0.641 & $\mathrm{pCi} / \mathrm{L}$ & 0.063 & 0.13 & 0.0132 & & \\
\hline 1949 & UISO_IE_PLATE_AEA & STLRL & 0.226 & $\mathrm{pCi} / \mathrm{L}$ & 0.039 & 0.058 & 0.0127 & & \\
\hline 1950 & UISO_IE_PLATE_AEA & STLRL & 0.182 & $\mathrm{pCi} / \mathrm{L}$ & 0.034 & 0.048 & 0.0127 & & \\
\hline 1951 & UISO_IE_PLATE_AEA & STLRL & 0.204 & $\mathrm{pCi} / \mathrm{L}$ & 0.036 & 0.052 & 0.0102 & & \\
\hline 1952 & UISO_IE_PLATE_AEA & STLRL & 0.2 & $\mathrm{pCi} / \mathrm{L}$ & 0.034 & 0.05 & 0.0105 & & \\
\hline 1953 & UISO_IE_PLATE_AEA & STLRL & 0.223 & $\mathrm{pCi} / \mathrm{L}$ & 0.035 & 0.054 & 0.0109 & & \\
\hline 1954 & UISO_IE_PLATE_AEA & STLRL & 0.21 & $\mathrm{pCi} / \mathrm{L}$ & 0.034 & 0.052 & 0.00917 & & \\
\hline 1955 & UISO_IE_PLATE_AEA & STLRL & 0.217 & $\mathrm{pCi} / \mathrm{L}$ & 0.038 & 0.055 & 0.0181 & & \\
\hline 1956 & UISO_IE_PLATE_AEA & STLRL & 0.293 & $\mathrm{pCi} / \mathrm{L}$ & 0.049 & 0.073 & 0.00542 & & \\
\hline 1957 & UISO_IE_PLATE_AEA & STLRL & 0.439 & $\mathrm{pCi} / \mathrm{L}$ & 0.059 & 0.1 & 0.018 & & \\
\hline 1958 & UISO_IE_PLATE_AEA & STLRL & 0.202 & $\mathrm{pCi} / \mathrm{L}$ & 0.034 & 0.051 & 0.0125 & & \\
\hline 1959 & UISO_IE_PLATE_AEA & STLRL & 0.177 & $\mathrm{pCi} / \mathrm{L}$ & 0.03 & 0.045 & 0.00904 & & \\
\hline 1960 & UISO_IE_PLATE_AEA & STLRL & 0.213 & $\mathrm{pCi} / \mathrm{L}$ & 0.034 & 0.053 & 0.00361 & & \\
\hline 1961 & UISO_IE_PLATE_AEA & STLRL & 0.21 & $\mathrm{pCi} / \mathrm{L}$ & 0.033 & 0.051 & 0.00332 & & \\
\hline 1962 & UISO_IE_PLATE_AEA & STLRL & 0.215 & $\mathrm{pCi} / \mathrm{L}$ & 0.036 & 0.053 & 0.0119 & & \\
\hline 1963 & UISO_IE_PLATE_AEA & STLRL & 0.359 & $\mathrm{pCi} / \mathrm{L}$ & 0.053 & 0.085 & 0.0604 & & \\
\hline 1964 & UISO_IE_PLATE_AEA & STLRL & 0.188 & $\mathrm{pCi} / \mathrm{L}$ & 0.038 & 0.052 & 0.018 & & \\
\hline 1965 & UISO_IE_PLATE_AEA & STLRL & 0.223 & $\mathrm{pCi} / \mathrm{L}$ & 0.035 & 0.054 & 0.00912 & & \\
\hline 1966 & UISO_IE_PLATE_AEA & STLRL & 0.185 & $\mathrm{pCi} / \mathrm{L}$ & 0.031 & 0.047 & 0.0108 & & \\
\hline 1967 & UISO_IE_PLATE_AEA & STLRL & 0.233 & $\mathrm{pCi} / \mathrm{L}$ & 0.037 & 0.057 & 0.0108 & & \\
\hline 1968 & UISO_IE_PLATE_AEA & STLRL & 0.214 & $\mathrm{pCi} / \mathrm{L}$ & 0.04 & 0.057 & 0.0162 & & \\
\hline 1969 & UISO_IE_PLATE_AEA & STLRL & 0.198 & $\mathrm{pCi} / \mathrm{L}$ & 0.035 & 0.051 & 0.00823 & & \\
\hline 1970 & UISO_IE_PLATE_AEA & STLRL & 0.237 & $\mathrm{pCi} / \mathrm{L}$ & 0.036 & 0.057 & 0.00358 & & \\
\hline 1971 & UISO_IE_PLATE_AEA & STLRL & 0.226 & $\mathrm{pCi} / \mathrm{L}$ & 0.053 & 0.068 & 0.00808 & & \\
\hline 1972 & UISO_IE_PLATE_AEA & STLRL & 0.199 & $\mathrm{pCi} / \mathrm{L}$ & 0.034 & 0.049 & 0.0147 & & \\
\hline 1973 & UISO_IE_PLATE_AEA & STLRL & 0.253 & $\mathrm{pCi} / \mathrm{L}$ & 0.037 & 0.059 & 0.0101 & & \\
\hline 1974 & UISO_IE_PLATE_AEA & STLRL & 1.4 & $\mathrm{pCi} / \mathrm{L}$ & 0.09 & 0.27 & 0.0133 & & \\
\hline 1975 & UISO_IE_PLATE_AEA & STLRL & 18.5 & $\mathrm{pCi} / \mathrm{L}$ & 0.31 & 3.4 & 0.00358 & & \\
\hline 1976 & UISO_IE_PLATE_AEA & STLRL & 1.46 & $\mathrm{pCi} / \mathrm{L}$ & 0.087 & 0.28 & 0.00943 & & \\
\hline 1977 & UISO_IE_PLATE_AEA & STLRL & 1.05 & $\mathrm{pCi} / \mathrm{L}$ & 0.073 & 0.2 & 0.0117 & & \\
\hline
\end{tabular}




\begin{tabular}{|c|c|c|c|c|c|c|c|c|c|}
\hline & $\mathrm{K}$ & $\mathrm{L}$ & $\bar{M}$ & $\mathrm{~N}$ & $\mathrm{O}$ & $P$ & $\mathrm{Q}$ & $\mathrm{R}$ & $\mathrm{S}$ \\
\hline 1 & METHOD_NAME & LAB_CODE & VALUE_RPTD & ANAL_UNITS_RPTD & COUNTING_ERROR & \begin{tabular}{|l|} 
TOTAL_ANAL_ERROR \\
\end{tabular} & MIN_DETECTABLE_ACTIVITY & PCNT_MOISTURE & LAB_QUALIFIER \\
\hline 1978 & UISO_IE_PLATE_AEA & STLRL & 2.22 & $\mathrm{pCi} / \mathrm{L}$ & 0.11 & 0.42 & 0.00345 & & \\
\hline 1979 & UISO IE_PLATE_AEA & STLRL & 0.193 & $\mathrm{pCi} / \mathrm{L}$ & 0.04 & 0.054 & 0.0181 & & \\
\hline 1980 & UISO_IE_PLATE_AEA & STLRL & 0.207 & $\mathrm{pCi} / \mathrm{L}$ & 0.036 & 0.053 & 0.0111 & & \\
\hline 1981 & UISO_IE_PLATE_AEA & STLRL & 0.207 & $\mathrm{pCi} / \mathrm{L}$ & 0.034 & 0.052 & 0.00373 & & \\
\hline 1982 & UISO_IE_PLATE_AEA & STLRL & 0.222 & $\mathrm{pCi} / \mathrm{L}$ & 0.034 & 0.054 & 0.00346 & & \\
\hline 1983 & UISO_IE_PLATE_AEA & STLRL & 0.215 & $\mathrm{pCi} / \mathrm{L}$ & 0.038 & 0.055 & 0.0183 & & \\
\hline 1984 & UISO_IE_PLATE_AEA & STLRL & 0.183 & $\mathrm{pCi} / \mathrm{L}$ & 0.044 & 0.056 & 0.0218 & & \\
\hline 1985 & UISO_IE_PLATE_AEA & STLRL & 0.218 & pCi/L & 0.059 & 0.072 & 0.0641 & & \\
\hline 1986 & UISO_IE_PLATE_AEA & STLRL & 0.229 & $\mathrm{pCi} / \mathrm{L}$ & 0.048 & 0.065 & 0.0309 & & \\
\hline 1987 & |UISO_IE_PLATE_AEA & STLRL & 0.181 & $\mathrm{pCi} / \mathrm{L}$ & 0.041 & 0.053 & 0.0157 & & \\
\hline 1988 & |UISO_IE_PLATE_AEA & STLRL & 0.217 & $\mathrm{pCi} / \mathrm{L}$ & 0.04 & 0.057 & 0.0125 & & \\
\hline 1989 & UISO_IE_PLATE_AEA & STLRL & 0.242 & $\mathrm{pCi} / \mathrm{L}$ & 0.046 & 0.064 & 0.015 & & \\
\hline 1990 & UISO_IE_PLATE_AEA & STLRL & 0.213 & $\mathrm{pCi} / \mathrm{L}$ & 0.044 & 0.06 & 0.00603 & & \\
\hline 1991 & UISO_IE_PLATE_AEA & STLRL & 0.229 & $\mathrm{pCi} / \mathrm{L}$ & 0.044 & 0.061 & 0.0179 & & \\
\hline 1992 & UISO_IE_PLATE_AEA & STLRL & 0.22 & $\mathrm{pCi} / \mathrm{L}$ & 0.043 & 0.06 & 0.0055 & & \\
\hline 1993 & UISO_IE_PLATE_AEA & STLRL & 0.353 & $\mathrm{pCi} / \mathrm{L}$ & 0.058 & 0.088 & 0.0132 & & \\
\hline 1994 & UISO_IE_PLATE_AEA & STLRL & 0.219 & $\mathrm{pCi} / \mathrm{L}$ & 0.036 & 0.054 & 0.0129 & & \\
\hline 1995 & UISO_IE_PLATE_AEA & STLRL & 0.203 & $\mathrm{pCi} / \mathrm{L}$ & 0.037 & 0.053 & 0.0167 & & \\
\hline 1996 & UISO_IE_PLATE_AEA & STLRL & 0.252 & $\mathrm{pCi} / \mathrm{L}$ & 0.039 & 0.061 & 0.015 & & \\
\hline 1997 & UISO_IE_PLATE_AEA & STLRL & 0.218 & pCi/L & 0.044 & 0.06 & 0.0163 & & \\
\hline 1998 & UISO_IE_PLATE_AEA & QTESRL & 29.8 & $\mathrm{pCi} / \mathrm{L}$ & 2 & 5 & 0.239 & & \\
\hline 1999 & |UISO_IE_PLATE_AEA & QTESRL & 0.00296 & $\mathrm{pCi} / \mathrm{L}$ & 0.0029 & 0.0065 & 0.00774 & & $U$ \\
\hline 2000 & UISO_IE_PLATE_AEA & QTESRL & 0.00558 & $\mathrm{pCi} / \mathrm{L}$ & 0.0055 & 0.0091 & 0.011 & & U \\
\hline 2001 & UISO_IE_PLATE_AEA & QTESRL & 0.00254 & $\mathrm{pCi} / \mathrm{L}$ & 0.0046 & 0.011 & 0.0211 & & $U$ \\
\hline 2002 & UISO_IE_PLATE_AEA & QTESRL & 0.00297 & $\mathrm{pCi} / \mathrm{L}$ & 0.0041 & 0.009 & 0.0159 & & $\mathrm{U}$ \\
\hline 2003 & UISO_IE_PLATE_AEA & QTESRL & 0.0148 & $\mathrm{pCi} / \mathrm{L}$ & 0.012 & 0.015 & 0.0209 & & U \\
\hline 2004 & UISO_IE_PLATE_AEA & QTESRL & 0.00962 & $\mathrm{pCi} / \mathrm{L}$ & 0.0073 & 0.01 & 0.00502 & & $U$ \\
\hline 2005 & UISO_IE_PLATE_AEA & QTESRL & 0.012 & $\mathrm{pCi} / \mathrm{L}$ & 0.0085 & 0.011 & 0.0101 & & $\mathrm{~J}$ \\
\hline 2006 & UISO_IE_PLATE_AEA & QTESRL & 0.0281 & pCi/L & 0.014 & 0.017 & 0.0146 & & $\mathrm{~J}$ \\
\hline 2007 & UISO_IE_PLATE_AEA & QTESRL & 0.00421 & pCi/L & 0.0042 & 0.0077 & 0.00927 & & $\mathrm{U}$ \\
\hline 2008 & UISO_IE_PLATE_AEA & QTESRL & 0.0124 & $\mathrm{pCi} / \mathrm{L}$ & 0.0087 & 0.011 & 0.0117 & & $\mathrm{~J}$ \\
\hline 2009 & UISO_IE_PLATE_AEA & QTESRL & 0.00454 & pCi/L & 0.0053 & 0.0095 & 0.0154 & & U \\
\hline 2010 & |UISO_IE_PLATE_AEA & QTESRL & 0.00954 & $\mathrm{pCi} / \mathrm{L}$ & 0.0076 & 0.011 & 0.0123 & & $\mathrm{U}$ \\
\hline 2011 & |UISO_IE_PLATE_AEA & QTESRL & 0.012 & $\mathrm{pCi} / \mathrm{L}$ & 0.01 & 0.013 & 0.0196 & & $\mathrm{U}$ \\
\hline 2012 & UISO_IE_PLATE_AEA & QTESRL & 0.0192 & pCi/L & 0.012 & 0.014 & 0.0156 & & $\mathrm{~J}$ \\
\hline 2013 & UISO_IE_PLATE_AEA & QTESRL & 0.0201 & pCi/L & 0.011 & 0.013 & 0.00454 & & $\mathrm{~J}$ \\
\hline 2014 & UISO_IE_PLATE_AEA & QTESRL & 0.0035 & $\mathrm{pCi} / \mathrm{L}$ & 0.0043 & 0.0087 & 0.014 & & $\mathrm{U}$ \\
\hline 2015 & UISO_IE_PLATE_AEA & QTESRL & 0.00322 & $\mathrm{pCi} / \mathrm{L}$ & 0.004 & 0.0083 & 0.0135 & & U \\
\hline
\end{tabular}




\begin{tabular}{|c|c|c|c|c|c|c|c|c|c|}
\hline & $\mathrm{K}$ & $\mathrm{L}$ & $\mathrm{M}$ & $\mathrm{N}$ & $\mathrm{O}$ & $\mathrm{P}$ & $\mathrm{Q}$ & $\mathrm{R}$ & $\mathrm{S}$ \\
\hline 1 & METHOD_NAME & LAB_CODE & VALUE_RPTD & ANAL_UNITS_RPTD & COUNTING_ERROR & TOTAL_ANAL_ERROR & MIN_DETECTABLE_ACTIVITY & PCNT_MOISTURE & LAB_QUALIFIER \\
\hline 2016 & UISO_IE_PLATE_AEA & QTESRL & 0.00486 & $\mathrm{pCi} / \mathrm{L}$ & 0.0054 & 0.0094 & 0.0152 & & $U$ \\
\hline 2017 & UISO_IE_PLATE_AEA & QTESRL & 0.0114 & $\mathrm{pCi} / \mathrm{L}$ & 0.0072 & 0.0097 & 0.00906 & & $\mathrm{~J}$ \\
\hline 2018 & UISO_IE_PLATE_AEA & QTESRL & 19.7 & $\mathrm{pCi} / \mathrm{L}$ & 1.9 & 3.7 & 0.328 & & \\
\hline 2019 & UISO_IE_PLATE_AEA & QTESRL & 0.00317 & $\mathrm{pCi} / \mathrm{L}$ & 0.0039 & 0.0083 & 0.0134 & & $U$ \\
\hline 2020 & UISO_IE_PLATE_AEA & QTESRL & 0.00192 & $\mathrm{pCi} / \mathrm{L}$ & 0.0025 & 0.0071 & 0.0113 & & U \\
\hline 2021 & UISO_IE_PLATE_AEA & STLRL & 0.00425 & $\mathrm{pCi} / \mathrm{L}$ & 0.0047 & 0.0086 & 0.0124 & & U \\
\hline 2022 & UISO_IE_PLATE_AEA & STLRL & 0.0135 & pCi/L & 0.0089 & 0.012 & 0.00989 & & $\mathrm{~J}$ \\
\hline 2023 & UISO_IE_PLATE_AEA & STLRL & 0.00976 & pCi/L & 0.0084 & 0.012 & 0.015 & & U \\
\hline 2024 & UISO_IE_PLATE_AEA & STLRL & 0.00685 & $\mathrm{pCi} / \mathrm{L}$ & 0.006 & 0.0092 & 0.0113 & & U \\
\hline 2025 & UISO_IE_PLATE_AEA & STLRL & 0.0129 & $\mathrm{pCi} / \mathrm{L}$ & 0.01 & 0.013 & 0.0195 & & U \\
\hline 2026 & UISO_IE_PLATE_AEA & STLRL & 0.00983 & pCi/L & 0.0076 & 0.011 & 0.0135 & & U \\
\hline 2027 & UISO_IE_PLATE_AEA & STLRL & 0.952 & $\mathrm{pCi} / \mathrm{L}$ & 0.35 & 0.42 & 0.232 & & \\
\hline 2028 & UISO_IE_PLATE_AEA & STLRL & 46.6 & $\mathrm{pCi} / \mathrm{L}$ & 2.7 & 8.6 & 0.408 & & \\
\hline 2029 & UISO_IE_PLATE_AEA & STLRL & 49.2 & pCi/L & 2.7 & 9 & 0.21 & & \\
\hline 2030 & UISO_IE_PLATE_AEA & STLRL & 0.00761 & $\mathrm{pCi} / \mathrm{L}$ & 0.007 & 0.01 & 0.0147 & & $\mathrm{U}$ \\
\hline 2031 & UISO_IE_PLATE_AEA & STLRL & 0.00275 & $\mathrm{pCi} / \mathrm{L}$ & 0.004 & 0.0091 & 0.0168 & & U \\
\hline 2032 & UISO_IE_PLATE_AEA & STLRL & 0.00874 & pCi/L & 0.007 & 0.01 & 0.0115 & & U \\
\hline 2033 & UISO_IE_PLATE_AEA & STLRL & 0.00484 & $\mathrm{pCi} / \mathrm{L}$ & 0.0073 & 0.012 & 0.0226 & & U \\
\hline 2034 & UISO_IE_PLATE_AEA & STLRL & -0.00113 & pCi/L & 0.0029 & 0.0059 & 0.0126 & & $U$ \\
\hline 2035 & UISO_IE_PLATE_AEA & STLRL & 0.00474 & pCi/L & 0.005 & 0.0088 & 0.0131 & & U \\
\hline 2036 & UISO_IE_PLATE_AEA & STLRL & 0.0101 & $\mathrm{pCi} / \mathrm{L}$ & 0.007 & 0.0097 & 0.00995 & & $\mathrm{~J}$ \\
\hline 2037 & UISO_IE_PLATE_AEA & STLRL & 0.0016 & $\mathrm{pCi} / \mathrm{L}$ & 0.0029 & 0.009 & 0.0176 & & $U$ \\
\hline 2038 & UISO_IE_PLATE_AEA & STLRL & 0.00872 & pCi/L & 0.0076 & 0.011 & 0.0134 & & U \\
\hline 2039 & UISO_IE_PLATE_AEA & STLRL & 0.00927 & $\mathrm{pCi} / \mathrm{L}$ & 0.0087 & 0.012 & 0.017 & & U \\
\hline 2040 & UISO_IE_PLATE_AEA & STLRL & 0.00353 & $\mathrm{pCi} / \mathrm{L}$ & 0.006 & 0.012 & 0.0233 & & U \\
\hline 2041 & UISO_IE_PLATE_AEA & STLRL & 0.011 & pCi/L & 0.0073 & 0.0099 & 0.00429 & & $\mathrm{~J}$ \\
\hline 2042 & UISO_IE_PLATE_AEA & STLRL & 0.00458 & $\mathrm{pCi} / \mathrm{L}$ & 0.0054 & 0.0095 & 0.0154 & & U \\
\hline 2043 & UISO_IE_PLATE_AEA & STLRL & -0.000224 & $\mathrm{pCi} / \mathrm{L}$ & 0.00061 & 0.0085 & 0.0188 & & $U$ \\
\hline 2044 & UISO_IE_PLATE_AEA & STLRL & 0.00119 & pCi/L & 0.002 & 0.0078 & 0.0142 & & U \\
\hline 2045 & UISO_IE_PLATE_AEA & STLRL & -0.00209 & pCi/L & 0.0049 & 0.0049 & 0.0118 & & U \\
\hline 2046 & UISO_IE_PLATE_AEA & STLRL & 85.7 & $\mathrm{pCi} / \mathrm{L}$ & 3.6 & 15 & 0.21 & & \\
\hline 2047 & UISO_IE_PLATE_AEA & STLRL & 0.00993 & pCi/L & 0.0074 & 0.01 & 0.0111 & & U \\
\hline 2048 & UISO_IE_PLATE_AEA & STLRL & 0.00791 & $\mathrm{pCi} / \mathrm{L}$ & 0.0087 & 0.013 & 0.0184 & & U \\
\hline 2049 & UISO_IE_PLATE_AEA & STLRL & 0.00897 & $\mathrm{pCi} / \mathrm{L}$ & 0.0087 & 0.012 & 0.0163 & & $U$ \\
\hline 2050 & UISO_IE_PLATE_AEA & STLRL & -0.0000967 & $\mathrm{pCi} / \mathrm{L}$ & 0.00018 & 0.0062 & 0.0115 & & U \\
\hline 2051 & UISO_IE_PLATE_AEA & STLRL & 0.011 & pCi/L & 0.0088 & 0.012 & 0.0136 & & U \\
\hline 2052 & UISO_IE_PLATE_AEA & STLRL & 0.00204 & $\mathrm{pCi} / \mathrm{L}$ & 0.002 & 0.0057 & 0.0066 & & U \\
\hline 2053 & UISO_IE_PLATE_AEA & STLRL & 0.00953 & $\mathrm{pCi} / \mathrm{L}$ & 0.0057 & 0.0082 & 0.00317 & & $\mathrm{~J}$ \\
\hline
\end{tabular}




\begin{tabular}{|c|c|c|c|c|c|c|c|c|c|}
\hline & $\mathrm{K}$ & $\mathrm{L}$ & $\mathrm{M}$ & $\mathrm{N}$ & $\mathrm{O}$ & $\mathrm{P}$ & $\mathrm{Q}$ & $\mathrm{R}$ & $\mathrm{S}$ \\
\hline 1 & METHOD_NAME & LAB_CODE & VALUE_RPTD & ANAL_UNITS_RPTD & COUNTING_ERROR & TOTAL_ANAL_ERROR & MIN_DETECTABLE_ACTIVITY & PCNT_MOISTURE & LAB_QUALIFIER \\
\hline 2054 & UISO_IE_PLATE_AEA & STLRL & 0.000215 & $\mathrm{pCi} / \mathrm{L}$ & 0.00025 & 0.0045 & 0.00321 & & $U$ \\
\hline 2055 & UISO_IE_PLATE_AEA & STLRL & -0.00117 & $\mathrm{pCi} / \mathrm{L}$ & 0.0022 & 0.0044 & 0.00765 & & $\mathrm{U}$ \\
\hline 2056 & UISO_IE_PLATE_AEA & STLRL & 0.00653 & $\mathrm{pCi} / \mathrm{L}$ & 0.0053 & 0.0083 & 0.00845 & & $\mathrm{U}$ \\
\hline 2057 & UISO_IE_PLATE_AEA & STLRL & 0.00356 & $\mathrm{pCi} / \mathrm{L}$ & 0.0044 & 0.0087 & 0.0142 & & $U$ \\
\hline 2058 & UISO_IE_PLATE_AEA & STLRL & 0.00664 & $\mathrm{pCi} / \mathrm{L}$ & 0.0066 & 0.01 & 0.0123 & & $U$ \\
\hline 2059 & UISO_IE_PLATE_AEA & STLRL & 0.00411 & $\mathrm{pCi} / \mathrm{L}$ & 0.0045 & 0.0085 & 0.0122 & & $U$ \\
\hline 2060 & UISO_IE_PLATE_AEA & STLRL & 0.00922 & $\mathrm{pCi} / \mathrm{L}$ & 0.0087 & 0.012 & 0.0169 & & $U$ \\
\hline 2061 & UISO_IE_PLATE_AEA & STLRL & 0.00506 & $\mathrm{pCi} / \mathrm{L}$ & 0.0043 & 0.0075 & 0.00964 & & $U$ \\
\hline 2062 & UISO_IE_PLATE_AEA & STLRL & 0.0113 & $\mathrm{pCi} / \mathrm{L}$ & 0.011 & 0.014 & 0.0197 & & $U$ \\
\hline 2063 & UISO_IE_PLATE_AEA & STLRL & 0.00584 & $\mathrm{pCi} / \mathrm{L}$ & 0.0052 & 0.0085 & 0.00927 & & $U$ \\
\hline 2064 & UISO_IE_PLATE_AEA & STLRL & 0.00662 & $\mathrm{pCi} / \mathrm{L}$ & 0.0058 & 0.009 & 0.011 & & $\mathrm{U}$ \\
\hline 2065 & UISO_IE_PLATE_AEA & STLRL & 0.00617 & $\mathrm{pCi} / \mathrm{L}$ & 0.0044 & 0.0071 & 0.00322 & & $\mathrm{U}$ \\
\hline 2066 & UISO_IE_PLATE_AEA & STLRL & 0.00377 & $\mathrm{pCi} / \mathrm{L}$ & 0.0034 & 0.0067 & 0.00386 & & U \\
\hline 2067 & UISO_IE_PLATE_AEA & STLRL & 0.00321 & $\mathrm{pCi} / \mathrm{L}$ & 0.0032 & 0.0069 & 0.00444 & & $U$ \\
\hline 2068 & UISO_IE_PLATE_AEA & STLRL & -0.00141 & $\mathrm{pCi} / \mathrm{L}$ & 0.0056 & 0.0078 & 0.0188 & & U \\
\hline 2069 & UISO_IE_PLATE_AEA & STLRL & 0.00388 & $\mathrm{pCi} / \mathrm{L}$ & 0.0038 & 0.0074 & 0.00887 & & $U$ \\
\hline 2070 & UISO_IE_PLATE_AEA & STLRL & 0.00818 & $\mathrm{pCi} / \mathrm{L}$ & 0.0073 & 0.011 & 0.00625 & & $U$ \\
\hline 2071 & UISO_IE_PLATE_AEA & STLRL & 0.000504 & $\mathrm{pCi} / \mathrm{L}$ & 0.00093 & 0.0073 & 0.0136 & & $U$ \\
\hline 2072 & UISO_IE_PLATE_AEA & STLRL & 0.00474 & $\mathrm{pCi} / \mathrm{L}$ & 0.0058 & 0.01 & 0.0166 & & $U$ \\
\hline 2073 & UISO_IE_PLATE_AEA & STLRL & 0.00396 & $\mathrm{pCi} / \mathrm{L}$ & 0.0046 & 0.0088 & 0.0143 & & $U$ \\
\hline 2074 & UISO_IE_PLATE_AEA & STLRL & 0.004 & $\mathrm{pCi} / \mathrm{L}$ & 0.0045 & 0.0086 & 0.0115 & & $\mathrm{U}$ \\
\hline 2075 & UISO_IE_PLATE_AEA & STLRL & 0.00167 & $\mathrm{pCi} / \mathrm{L}$ & 0.0027 & 0.0083 & 0.0155 & & $\mathrm{U}$ \\
\hline 2076 & UISO_IE_PLATE_AEA & STLRL & 0.00158 & $\mathrm{pCi} / \mathrm{L}$ & 0.002 & 0.0064 & 0.0101 & & $U$ \\
\hline 2077 & UISO_IE_PLATE_AEA & STLRL & 0.00452 & $\mathrm{pCi} / \mathrm{L}$ & 0.0037 & 0.0068 & 0.00355 & & $\mathrm{U}$ \\
\hline 2078 & UISO_IE_PLATE_AEA & STLRL & -0.002 & $\mathrm{pCi} / \mathrm{L}$ & 0.0056 & 0.0056 & 0.013 & & U \\
\hline 2079 & UISO_IE_PLATE_AEA & STLRL & 0.00874 & $\mathrm{pCi} / \mathrm{L}$ & 0.0071 & 0.01 & 0.0103 & & $U$ \\
\hline 2080 & UISO_IE_PLATE_AEA & STLRL & 0.0136 & $\mathrm{pCi} / \mathrm{L}$ & 0.0094 & 0.012 & 0.0135 & & $\mathrm{~J}$ \\
\hline 2081 & UISO_IE_PLATE_AEA & STLRL & 0.00632 & $\mathrm{pCi} / \mathrm{L}$ & 0.0056 & 0.0089 & 0.00975 & & $U$ \\
\hline 2082 & UISO_IE_PLATE_AEA & STLRL & 0.00504 & $\mathrm{pCi} / \mathrm{L}$ & 0.0054 & 0.0092 & 0.0136 & & $U$ \\
\hline 2083 & UISO_IE_PLATE_AEA & STLRL & 0.00922 & $\mathrm{pCi} / \mathrm{L}$ & 0.0065 & 0.0093 & 0.00425 & & $U$ \\
\hline 2084 & UISO_IE_PLATE_AEA & STLRL & 0.00993 & $\mathrm{pCi} / \mathrm{L}$ & 0.0081 & 0.011 & 0.00599 & & $U$ \\
\hline 2085 & UISO_IE_PLATE_AEA & STLRL & 0.00276 & $\mathrm{pCi} / \mathrm{L}$ & 0.0042 & 0.0094 & 0.017 & & $U$ \\
\hline 2086 & UISO_IE_PLATE_AEA & STLRL & 0.00385 & $\mathrm{pCi} / \mathrm{L}$ & 0.0031 & 0.0062 & 0.00325 & & $U$ \\
\hline 2087 & UISO_IE_PLATE_AEA & STLRL & 0.00875 & $\mathrm{pCi} / \mathrm{L}$ & 0.0058 & 0.0085 & 0.00364 & & $\mathrm{~J}$ \\
\hline 2088 & UISO_IE_PLATE_AEA & STLRL & -0.00178 & $\mathrm{pCi} / \mathrm{L}$ & 0.0044 & 0.0044 & 0.00893 & & $U$ \\
\hline 2089 & UISO_IE_PLATE_AEA & STLRL & 0.0114 & $\mathrm{pCi} / \mathrm{L}$ & 0.0096 & 0.013 & 0.0172 & & $U$ \\
\hline 2090 & UISO_IE_PLATE_AEA & STLRL & 0.011 & $\mathrm{pCi} / \mathrm{L}$ & 0.0087 & 0.012 & 0.0144 & & U \\
\hline 2091 & UISO_IE_PLATE_AEA & STLRL & 0.00209 & $\mathrm{pCi} / \mathrm{L}$ & 0.0027 & 0.0073 & 0.0117 & & $U$ \\
\hline
\end{tabular}




\begin{tabular}{|c|c|c|c|c|c|c|c|c|c|}
\hline & $\mathrm{K}$ & $\mathrm{L}$ & $\bar{M}$ & $\mathrm{~N}$ & $\mathrm{O}$ & $P$ & $\mathrm{Q}$ & $\mathrm{R}$ & $\mathrm{S}$ \\
\hline 1 & METHOD_NAME & LAB_CODE & VALUE_RPTD & ANAL_UNITS_RPTD & COUNTING_ERROR & TOTAL_ANAL_ERROR & MIN_DETECTABLE_ACTIVITY & PCNT_MOISTURE & LAB_QUALIFIER \\
\hline 2092 & UISO_IE_PLATE_AEA & STLRL & 0.000331 & $\mathrm{pCi} / \mathrm{L}$ & 0.00045 & 0.0054 & 0.00798 & & $U$ \\
\hline 2093 & UISO_IE_PLATE_AEA & STLRL & 0.00433 & $\mathrm{pCi} / \mathrm{L}$ & 0.0035 & 0.0066 & 0.00347 & & $U$ \\
\hline 2094 & UISO_IE_PLATE_AEA & STLRL & 0.0015 & $\mathrm{pCi} / \mathrm{L}$ & 0.0015 & 0.0052 & 0.00328 & & $\mathrm{U}$ \\
\hline 2095 & UISO_IE_PLATE_AEA & STLRL & -0.000213 & $\mathrm{pCi} / \mathrm{L}$ & 0.00058 & 0.0087 & 0.0192 & & $U$ \\
\hline 2096 & UISO_IE_PLATE_AEA & STLRL & -0.0000493 & $\mathrm{pCi} / \mathrm{L}$ & 0.0001 & 0.0071 & 0.0143 & & $U$ \\
\hline 2097 & UISO_IE_PLATE_AEA & STLRL & 0.00888 & $\mathrm{pCi} / \mathrm{L}$ & 0.0076 & 0.011 & 0.014 & & $U$ \\
\hline 2098 & UISO_IE_PLATE_AEA & STLRL & 0.0028 & $\mathrm{pCi} / \mathrm{L}$ & 0.0028 & 0.0064 & 0.00755 & & 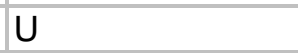 \\
\hline 2099 & UISO_IE_PLATE_AEA & STLRL & -0.00135 & $\mathrm{pCi} / \mathrm{L}$ & 0.0034 & 0.0054 & 0.0114 & & 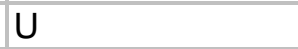 \\
\hline 2100 & UISO_IE_PLATE_AEA & STLRL & 0.00249 & $\mathrm{pCi} / \mathrm{L}$ & 0.0032 & 0.0078 & 0.0125 & & $\mathrm{U}$ \\
\hline 2101 & UISO_IE_PLATE_AEA & STLRL & 0.008 & $\mathrm{pCi} / \mathrm{L}$ & 0.0079 & 0.011 & 0.0176 & & $\mathrm{U}$ \\
\hline 2102 & UISO_IE_PLATE_AEA & STLRL & 0.00198 & $\mathrm{pCi} / \mathrm{L}$ & 0.0032 & 0.0088 & 0.0165 & & $U$ \\
\hline 2103 & UISO_IE_PLATE_AEA & STLRL & 0.0014 & $\mathrm{pCi} / \mathrm{L}$ & 0.0014 & 0.0051 & 0.00321 & & $U$ \\
\hline 2104 & UISO_IE_PLATE_AEA & STLRL & 0.00674 & $\mathrm{pCi} / \mathrm{L}$ & 0.0051 & 0.0079 & 0.00749 & & $\mathrm{U}$ \\
\hline 2105 & UISO_IE_PLATE_AEA & STLRL & 0.00264 & $\mathrm{pCi} / \mathrm{L}$ & 0.0026 & 0.0063 & 0.00405 & & $U$ \\
\hline 2106 & UISO_IE_PLATE_AEA & STLRL & 0.028 & $\mathrm{pCi} / \mathrm{L}$ & 0.012 & 0.014 & 0.00387 & & $\mathrm{~J}$ \\
\hline 2107 & UISO_IE_PLATE_AEA & STLRL & 1.35 & $\mathrm{pCi} / \mathrm{L}$ & 0.087 & 0.25 & 0.00384 & & \\
\hline 2108 & UISO_IE_PLATE_AEA & STLRL & 0.0336 & $\mathrm{pCi} / \mathrm{L}$ & 0.013 & 0.016 & 0.00757 & & $\mathrm{~J}$ \\
\hline 2109 & UISO_IE_PLATE_AEA & STLRL & 0.0514 & $\mathrm{pCi} / \mathrm{L}$ & 0.016 & 0.02 & 0.00744 & & $\mathrm{~J}$ \\
\hline 2110 & UISO_IE_PLATE_AEA & STLRL & 2.51 & $\mathrm{pCi} / \mathrm{L}$ & 1.2 & 1.4 & 0.429 & & \\
\hline 2111 & UISO_IE_PLATE_AEA & STLRL & 0.0319 & $\mathrm{pCi} / \mathrm{L}$ & 0.013 & 0.016 & 0.00415 & & $\mathrm{~J}$ \\
\hline 2112 & UISO_IE_PLATE_AEA & STLRL & 0.00112 & $\mathrm{pCi} / \mathrm{L}$ & 0.0021 & 0.0084 & 0.0158 & & $U$ \\
\hline 2113 & UISO_IE_PLATE_AEA & STLRL & 0.00215 & $\mathrm{pCi} / \mathrm{L}$ & 0.0024 & 0.0063 & 0.00899 & & $\mathrm{U}$ \\
\hline 2114 & UISO_IE_PLATE_AEA & STLRL & 132 & $\mathrm{pCi} / \mathrm{L}$ & 4.2 & 23 & 0.091 & & \\
\hline 2115 & UISO_IE_PLATE_AEA & STLRL & 0.00938 & $\mathrm{pCi} / \mathrm{L}$ & 0.0066 & 0.0094 & 0.00431 & & $U$ \\
\hline 2116 & UISO_IE_PLATE_AEA & STLRL & 0.000669 & $\mathrm{pCi} / \mathrm{L}$ & 0.00077 & 0.005 & 0.00362 & & $\mathrm{U}$ \\
\hline 2117 & UISO_IE_PLATE_AEA & STLRL & -0.000432 & $\mathrm{pCi} / \mathrm{L}$ & 0.00061 & 0.0045 & 0.00393 & & $\mathrm{U}$ \\
\hline 2118 & UISO_IE_PLATE_AEA & STLRL & 0.00567 & $\mathrm{pCi} / \mathrm{L}$ & 0.0043 & 0.0072 & 0.00348 & & U \\
\hline 2119 & UISO_IE_PLATE_AEA & STLRL & 0.0477 & $\mathrm{pCi} / \mathrm{L}$ & 0.016 & 0.019 & 0.00355 & & $\mathrm{~J}$ \\
\hline 2120 & UISO_IE_PLATE_AEA & STLRL & -0.00122 & $\mathrm{pCi} / \mathrm{L}$ & 0.0054 & 0.0057 & 0.0121 & & 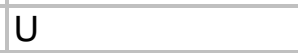 \\
\hline 2121 & UISO_IE_PLATE_AEA & STLRL & 0.0135 & $\mathrm{pCi} / \mathrm{L}$ & 0.013 & 0.013 & 0.00652 & & $\mathrm{~J}$ \\
\hline 2122 & UISO_IE_PLATE_AEA & STLRL & -0.00433 & $\mathrm{pCi} / \mathrm{L}$ & 0.0014 & 0.0023 & 0.00846 & & $\mathrm{U}$ \\
\hline 2123 & UISO_IE_PLATE_AEA & STLRL & 0.000439 & $\mathrm{pCi} / \mathrm{L}$ & 0.0044 & 0.0048 & 0.00341 & & U \\
\hline 2124 & UISO_IE_PLATE_AEA & STLRL & 0.00401 & $\mathrm{pCi} / \mathrm{L}$ & 0.011 & 0.012 & 0.0221 & & $U$ \\
\hline 2125 & UISO_IE_PLATE_AEA & STLRL & 0.0197 & $\mathrm{pCi} / \mathrm{L}$ & 0.013 & 0.014 & 0.0138 & & $\mathrm{~J}$ \\
\hline 2126 & |UISO_IE_PLATE_AEA & STLRL & 0.00338 & $\mathrm{pCi} / \mathrm{L}$ & 0.006 & 0.0064 & 0.00364 & & U \\
\hline 2127 & UISO_IE_PLATE_AEA & STLRL & 0.00199 & $\mathrm{pCi} / \mathrm{L}$ & 0.006 & 0.0063 & 0.00837 & & $U$ \\
\hline 2128 & UISO_IE_PLATE_AEA & STLRL & 0.00849 & $\mathrm{pCi} / \mathrm{L}$ & 0.013 & 0.014 & 0.0237 & & $U$ \\
\hline 2129 & UISO_IE_PLATE_AEA & STLRL & 0.0101 & $\mathrm{pCi} / \mathrm{L}$ & 0.011 & 0.012 & 0.0156 & & U \\
\hline
\end{tabular}




\begin{tabular}{|c|c|c|c|c|c|c|c|c|c|}
\hline & $\mathrm{K}$ & $\mathrm{L}$ & $\bar{M}$ & $\mathrm{~N}$ & $\mathrm{O}$ & $P$ & $\mathrm{Q}$ & $\mathrm{R}$ & $\mathrm{S}$ \\
\hline 1 & METHOD_NAME & LAB_CODE & VALUE_RPTD & ANAL_UNITS_RPTD & COUNTING_ERROR & TOTAL_ANAL_ERROR & MIN_DETECTABLE_ACTIVITY & PCNT_MOISTURE & LAB_QUALIFIER \\
\hline 2130 & UISO_IE_PLATE_AEA & STLRL & 0.00126 & $\mathrm{pCi} / \mathrm{L}$ & 0.0063 & 0.0066 & 0.00999 & & $U$ \\
\hline 2131 & UISO_IE_PLATE_AEA & STLRL & 0.004 & $\mathrm{pCi} / \mathrm{L}$ & 0.0096 & 0.0098 & 0.017 & & $U$ \\
\hline 2132 & UISO_IE_PLATE_AEA & STLRL & 0.0109 & $\mathrm{pCi} / \mathrm{L}$ & 0.011 & 0.011 & 0.0106 & & $\mathrm{U}$ \\
\hline 2133 & UISO_IE_PLATE_AEA & STLRL & 0.0157 & $\mathrm{pCi} / \mathrm{L}$ & 0.013 & 0.013 & 0.0112 & & $\mathrm{~J}$ \\
\hline 2134 & UISO_IE_PLATE_AEA & STLRL & 0.00599 & $\mathrm{pCi} / \mathrm{L}$ & 0.0075 & 0.0078 & 0.0089 & & $U$ \\
\hline 2135 & UISO_IE_PLATE_AEA & STLRL & 0.00145 & $\mathrm{pCi} / \mathrm{L}$ & 0.0054 & 0.0058 & 0.00751 & & $U$ \\
\hline 2136 & UISO_IE_PLATE_AEA & STLRL & 0.0176 & $\mathrm{pCi} / \mathrm{L}$ & 0.016 & 0.016 & 0.0175 & & $\mathrm{~J}$ \\
\hline 2137 & UISO_IE_PLATE_AEA & STLRL & 0.00236 & $\mathrm{pCi} / \mathrm{L}$ & 0.0057 & 0.006 & 0.00386 & & $U$ \\
\hline 2138 & UISO_IE_PLATE_AEA & STLRL & -0.000368 & $\mathrm{pCi} / \mathrm{L}$ & 0.0041 & 0.0045 & 0.00646 & & $\mathrm{U}$ \\
\hline 2139 & UISO_IE_PLATE_AEA & STLRL & 0.00302 & $\mathrm{pCi} / \mathrm{L}$ & 0.0056 & 0.006 & 0.00642 & & $\mathrm{U}$ \\
\hline 2140 & UISO_IE_PLATE_AEA & STLRL & 0.0157 & $\mathrm{pCi} / \mathrm{L}$ & 0.021 & 0.022 & 0.0354 & & $U$ \\
\hline 2141 & UISO_IE_PLATE_AEA & STLRL & 13.5 & $\mathrm{pCi} / \mathrm{L}$ & 1.2 & 2.6 & 0.172 & & \\
\hline 2142 & UISO_IE_PLATE_AEA & STLRL & 0.00542 & $\mathrm{pCi} / \mathrm{L}$ & 0.0066 & 0.007 & 0.00339 & & $\mathrm{U}$ \\
\hline 2143 & UISO_IE_PLATE_AEA & STLRL & 0.00542 & $\mathrm{pCi} / \mathrm{L}$ & 0.0076 & 0.008 & 0.0097 & & $U$ \\
\hline 2144 & UISO_IE_PLATE_AEA & STLRL & 0.00151 & $\mathrm{pCi} / \mathrm{L}$ & 0.0089 & 0.0091 & 0.0171 & & $\mathrm{U}$ \\
\hline 2145 & UISO_IE_PLATE_AEA & STLRL & 0.0115 & $\mathrm{pCi} / \mathrm{L}$ & 0.0094 & 0.0098 & 0.00792 & & $\mathrm{~J}$ \\
\hline 2146 & UISO_IE_PLATE_AEA & STLRL & 0.00805 & $\mathrm{pCi} / \mathrm{L}$ & 0.008 & 0.0084 & 0.00748 & & 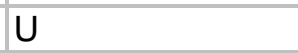 \\
\hline 2147 & UISO_IE_PLATE_AEA & STLRL & 0.00647 & $\mathrm{pCi} / \mathrm{L}$ & 0.0069 & 0.0073 & 0.00332 & & $U$ \\
\hline 2148 & UISO_IE_PLATE_AEA & STLRL & 0.0158 & $\mathrm{pCi} / \mathrm{L}$ & 0.017 & 0.017 & 0.025 & & $\mathrm{U}$ \\
\hline 2149 & UISO_IE_PLATE_AEA & STLRL & -0.00253 & $\mathrm{pCi} / \mathrm{L}$ & 0.0065 & 0.0068 & 0.0173 & & U \\
\hline 2150 & UISO_IE_PLATE_AEA & STLRL & 0.0172 & $\mathrm{pCi} / \mathrm{L}$ & 0.013 & 0.013 & 0.0126 & & $\mathrm{~J}$ \\
\hline 2151 & UISO_IE_PLATE_AEA & STLRL & -0.000493 & $\mathrm{pCi} / \mathrm{L}$ & 0.0072 & 0.0075 & 0.0163 & & $\mathrm{U}$ \\
\hline 2152 & UISO_IE_PLATE_AEA & STLRL & 0.00711 & $\mathrm{pCi} / \mathrm{L}$ & 0.01 & 0.011 & 0.0162 & & $U$ \\
\hline 2153 & UISO_IE_PLATE_AEA & STLRL & 0.00728 & $\mathrm{pCi} / \mathrm{L}$ & 0.018 & 0.018 & 0.0332 & & $U$ \\
\hline 2154 & UISO_IE_PLATE_AEA & STLRL & 0.00483 & $\mathrm{pCi} / \mathrm{L}$ & 0.0087 & 0.0089 & 0.0133 & & $\mathrm{U}$ \\
\hline 2155 & UISO_IE_PLATE_AEA & STLRL & 0.00415 & $\mathrm{pCi} / \mathrm{L}$ & 0.0071 & 0.0074 & 0.0101 & & $\mathrm{U}$ \\
\hline 2156 & UISO_IE_PLATE_AEA & STLRL & 0.00611 & $\mathrm{pCi} / \mathrm{L}$ & 0.0077 & 0.0081 & 0.00427 & & U \\
\hline 2157 & UISO_IE_PLATE_AEA & STLRL & 0.0084 & $\mathrm{pCi} / \mathrm{L}$ & 0.0074 & 0.0079 & 0.00318 & & $\mathrm{~J}$ \\
\hline 2158 & UISO_IE_PLATE_AEA & STLRL & 0.00513 & $\mathrm{pCi} / \mathrm{L}$ & 0.0087 & 0.009 & 0.0135 & & 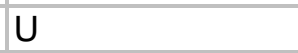 \\
\hline 2159 & UISO_IE_PLATE_AEA & STLRL & 17.5 & $\mathrm{pCi} / \mathrm{L}$ & 1.4 & 3.4 & 0.518 & & \\
\hline 2160 & UISO_IE_PLATE_AEA & STLRL & 0.00521 & $\mathrm{pCi} / \mathrm{L}$ & 0.007 & 0.0073 & 0.00386 & & $\mathrm{~J}$ \\
\hline 2161 & UISO_IE_PLATE_AEA & STLRL & 0.0124 & $\mathrm{pCi} / \mathrm{L}$ & 0.013 & 0.013 & 0.0182 & & U \\
\hline 2162 & UISO_IE_PLATE_AEA & STLRL & 0.0594 & $\mathrm{pCi} / \mathrm{L}$ & 0.023 & 0.025 & 0.0154 & & \\
\hline 2163 & UISO_IE_PLATE_AEA & STLRL & 0.045 & $\mathrm{pCi} / \mathrm{L}$ & 0.016 & 0.018 & 0.00916 & & \\
\hline 2164 & UISO_IE_PLATE_AEA & STLRL & 0.0741 & $\mathrm{pCi} / \mathrm{L}$ & 0.019 & 0.023 & 0.00318 & & \\
\hline 2165 & UISO_IE_PLATE_AEA & STLRL & 0.0586 & $\mathrm{pCi} / \mathrm{L}$ & 0.021 & 0.023 & 0.0128 & & \\
\hline 2166 & UISO_IE_PLATE_AEA & STLRL & 0.017 & $\mathrm{pCi} / \mathrm{L}$ & 0.0098 & 0.011 & 0.00651 & & \\
\hline 2167 & UISO_IE_PLATE_AEA & STLRL & 0.0111 & $\mathrm{pCi} / \mathrm{L}$ & 0.0083 & 0.0088 & 0.00326 & & \\
\hline
\end{tabular}




\begin{tabular}{|c|c|c|c|c|c|c|c|c|c|}
\hline & $\mathrm{K}$ & $\mathrm{L}$ & $\bar{M}$ & $\mathrm{~N}$ & $\mathrm{O}$ & $P$ & $\mathrm{Q}$ & $\mathrm{R}$ & $\mathrm{S}$ \\
\hline 1 & METHOD_NAME & LAB_CODE & VALUE_RPTD & ANAL_UNITS_RPTD & COUNTING_ERROR & TOTAL_ANAL_ERROR & MIN_DETECTABLE_ACTIVITY & PCNT_MOISTURE & LAB_QUALIFIER \\
\hline 2168 & UISO_IE_PLATE_AEA & STLRL & 0.00346 & $\mathrm{pCi} / \mathrm{L}$ & 0.006 & 0.0064 & 0.00686 & & $U$ \\
\hline 2169 & UISO IE_PLATE_AEA & STLRL & 2.93 & $\mathrm{pCi} / \mathrm{L}$ & 0.12 & 0.52 & 0.0083 & & \\
\hline 2170 & UISO_IE_PLATE_AEA & STLRL & 0.00436 & $\mathrm{pCi} / \mathrm{L}$ & 0.0067 & 0.0071 & 0.00853 & & $\mathrm{U}$ \\
\hline 2171 & UISO_IE_PLATE_AEA & STLRL & 0.0103 & $\mathrm{pCi} / \mathrm{L}$ & 0.0082 & 0.0087 & 0.00667 & & \\
\hline 2172 & UISO_IE_PLATE_AEA & STLRL & 1.17 & $\mathrm{pCi} / \mathrm{L}$ & 0.17 & 0.27 & 0.0237 & & \\
\hline 2173 & UISO_IE_PLATE_AEA & STLRL & 0.0138 & $\mathrm{pCi} / \mathrm{L}$ & 0.014 & 0.015 & 0.00664 & & $U$ \\
\hline 2174 & UISO_IE_PLATE_AEA & STLRL & 0.00638 & $\mathrm{pCi} / \mathrm{L}$ & 0.0062 & 0.0063 & 0.00842 & & 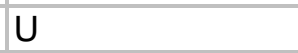 \\
\hline 2175 & UISO_IE_PLATE_AEA & STLRL & 0.0115 & $\mathrm{pCi} / \mathrm{L}$ & 0.0077 & 0.0079 & 0.00347 & & \\
\hline 2176 & UISO_IE_PLATE_AEA & STLRL & 0.00421 & $\mathrm{pCi} / \mathrm{L}$ & 0.0058 & 0.0058 & 0.00916 & & $\mathrm{U}$ \\
\hline 2177 & UISO_IE_PLATE_AEA & STLRL & 0.00811 & $\mathrm{pCi} / \mathrm{L}$ & 0.0071 & 0.0072 & 0.00899 & & $\mathrm{U}$ \\
\hline 2178 & UISO_IE_PLATE_AEA & STLRL & 0.00833 & $\mathrm{pCi} / \mathrm{L}$ & 0.0073 & 0.0074 & 0.00923 & & U \\
\hline 2179 & UISO_IE_PLATE_AEA & STLRL & 0.00821 & $\mathrm{pCi} / \mathrm{L}$ & 0.0062 & 0.0064 & 0.00318 & & \\
\hline 2180 & UISO_IE_PLATE_AEA & STLRL & 0.00551 & $\mathrm{pCi} / \mathrm{L}$ & 0.006 & 0.0061 & 0.00901 & & $\mathrm{U}$ \\
\hline 2181 & UISO_IE_PLATE_AEA & STLRL & 0.0065 & $\mathrm{pCi} / \mathrm{L}$ & 0.0058 & 0.0059 & 0.00352 & & \\
\hline 2182 & UISO_IE_PLATE_AEA & STLRL & 0.017 & $\mathrm{pCi} / \mathrm{L}$ & 0.0094 & 0.0099 & 0.00353 & & \\
\hline 2183 & UISO_IE_PLATE_AEA & STLRL & 0.016 & $\mathrm{pCi} / \mathrm{L}$ & 0.0093 & 0.0097 & 0.00362 & & \\
\hline 2184 & UISO_IE_PLATE_AEA & STLRL & 0.0151 & $\mathrm{pCi} / \mathrm{L}$ & 0.0094 & 0.0098 & 0.00923 & & \\
\hline 2185 & UISO_IE_PLATE_AEA & STLRL & 0.00732 & $\mathrm{pCi} / \mathrm{L}$ & 0.0073 & 0.0074 & 0.00496 & & $U$ \\
\hline 2186 & UISO_IE_PLATE_AEA & STLRL & 0.019 & $\mathrm{pCi} / \mathrm{L}$ & 0.012 & 0.013 & 0.0137 & & \\
\hline 2187 & UISO_IE_PLATE_AEA & STLRL & 0.00809 & $\mathrm{pCi} / \mathrm{L}$ & 0.011 & 0.011 & 0.0188 & & U \\
\hline 2188 & UISO_IE_PLATE_AEA & STLRL & -0.00558 & $\mathrm{pCi} / \mathrm{L}$ & 0.0096 & 0.0098 & 0.0321 & & $U$ \\
\hline 2189 & UISO_IE_PLATE_AEA & STLRL & 0.0195 & $\mathrm{pCi} / \mathrm{L}$ & 0.022 & 0.022 & 0.0308 & & $\mathrm{U}$ \\
\hline 2190 & UISO_IE_PLATE_AEA & STLRL & 0.00752 & $\mathrm{pCi} / \mathrm{L}$ & 0.0096 & 0.01 & 0.011 & & $U$ \\
\hline 2191 & UISO_IE_PLATE_AEA & STLRL & -0.000926 & $\mathrm{pCi} / \mathrm{L}$ & 0.0061 & 0.0064 & 0.0138 & & $U$ \\
\hline 2192 & UISO_IE_PLATE_AEA & STLRL & 0.0039 & $\mathrm{pCi} / \mathrm{L}$ & 0.0072 & 0.0076 & 0.00491 & & $\mathrm{U}$ \\
\hline 2193 & UISO_IE_PLATE_AEA & STLRL & 0.00981 & $\mathrm{pCi} / \mathrm{L}$ & 0.016 & 0.016 & 0.027 & & $\mathrm{U}$ \\
\hline 2194 & UISO_IE_PLATE_AEA & STLRL & 88.5 & $\mathrm{pCi} / \mathrm{L}$ & 11 & 19 & 3.1 & & \\
\hline 2195 & UISO_IE_PLATE_AEA & STLRL & 0.00362 & $\mathrm{pCi} / \mathrm{L}$ & 0.014 & 0.014 & 0.0337 & & $U$ \\
\hline 2196 & UISO_IE_PLATE_AEA & STLRL & 0.0148 & $\mathrm{pCi} / \mathrm{L}$ & 0.018 & 0.018 & 0.032 & & 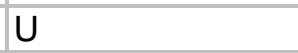 \\
\hline 2197 & UISO_IE_PLATE_AEA & STLRL & 0.00501 & $\mathrm{pCi} / \mathrm{L}$ & 0.0089 & 0.0091 & 0.0136 & & $U$ \\
\hline 2198 & UISO_IE_PLATE_AEA & STLRL & 0.00692 & $\mathrm{pCi} / \mathrm{L}$ & 0.0092 & 0.0095 & 0.00556 & & $\mathrm{U}$ \\
\hline 2199 & UISO_IE_PLATE_AEA & STLRL & 0.615 & $\mathrm{pCi} / \mathrm{L}$ & 0.062 & 0.13 & 0.00417 & & \\
\hline 2200 & UISO_IE_PLATE_AEA & STLRL & 2.24 & $\mathrm{pCi} / \mathrm{L}$ & 0.11 & 0.42 & 0.0089 & & \\
\hline 2201 & RADISOTOPES_ICPMS & PNL329 & 0.004908648 & $\mathrm{pCi} / \mathrm{L}$ & & & & & \\
\hline 2202 & RADISOTOPES_ICPMS & PNL329 & 0.47075448 & $\mathrm{pCi} / \mathrm{L}$ & & & & & \\
\hline 2203 & RADISOTOPES_ICPMS & PNL329 & 1.77727656 & $\mathrm{pCi} / \mathrm{L}$ & & & & & \\
\hline 2204 & RADISOTOPES_ICPMS & PNL329 & 0.006314208 & $\mathrm{pCi} / \mathrm{L}$ & & & & & \\
\hline 2205 & UISO_IE_PLATE_AEA & STLRL & 0.00374 & $\mathrm{pCi} / \mathrm{L}$ & 0.0068 & 0.0072 & 0.00935 & & $\mathrm{U}$ \\
\hline
\end{tabular}




\begin{tabular}{|c|c|c|c|c|c|c|c|c|c|}
\hline & $\mathrm{K}$ & $\mathrm{L}$ & $\mathrm{M}$ & $\mathrm{N}$ & $\mathrm{O}$ & $\mathrm{P}$ & $\mathrm{Q}$ & $\mathrm{R}$ & $\mathrm{S}$ \\
\hline 1 & METHOD_NAME & LAB_CODE & VALUE_RPTD & ANAL_UNITS_RPTD & COUNTING_ERROR & TOTAL_ANAL_ERROR & MIN_DETECTABLE_ACTIVITY & PCNT_MOISTURE & LAB_QUALIFIER \\
\hline 2206 & UISO_IE_PLATE_AEA & STLRL & 0.000984 & $\mathrm{pCi} / \mathrm{L}$ & 0.005 & 0.0053 & 0.00391 & & $U$ \\
\hline 2207 & UISO_IE_PLATE_AEA & STLRL & 0.0124 & $\mathrm{pCi} / \mathrm{L}$ & 0.01 & 0.01 & 0.00427 & & \\
\hline 2208 & UISO_IE_PLATE_AEA & STLRL & 0.184 & $\mathrm{pCi} / \mathrm{L}$ & 0.033 & 0.047 & 0.00979 & & \\
\hline 2209 & UISO_IE_PLATE_AEA & STLRL & 0.101 & $\mathrm{pCi} / \mathrm{L}$ & 0.025 & 0.031 & 0.00822 & & \\
\hline 2210 & UISO_IE_PLATE_AEA & STLRL & 0.00849 & $\mathrm{pCi} / \mathrm{L}$ & 0.0083 & 0.0087 & 0.00883 & & $U$ \\
\hline 2211 & UISO_IE_PLATE_AEA & STLRL & 0.0617 & $\mathrm{pCi} / \mathrm{L}$ & 0.019 & 0.022 & 0.00947 & & \\
\hline 2212 & UISO_IE_PLATE_AEA & STLRL & 0.0141 & $\mathrm{pCi} / \mathrm{L}$ & 0.01 & 0.011 & 0.00907 & & \\
\hline 2213 & UISO_IE_PLATE_AEA & STLRL & 0.0132 & $\mathrm{pCi} / \mathrm{L}$ & 0.0095 & 0.01 & 0.00373 & & \\
\hline 2214 & UISO_IE_PLATE_AEA & STLRL & 0.0107 & $\mathrm{pCi} / \mathrm{L}$ & 0.0094 & 0.0098 & 0.00424 & & \\
\hline 2215 & UISO_IE_PLATE_AEA & STLRL & 1.14 & $\mathrm{pCi} / \mathrm{L}$ & 0.082 & 0.22 & 0.00828 & & \\
\hline 2216 & UISO_IE_PLATE_AEA & STLRL & 0.0607 & $\mathrm{pCi} / \mathrm{L}$ & 0.023 & 0.026 & 0.00578 & & \\
\hline 2217 & UISO_IE_PLATE_AEA & STLRL & 0.0139 & $\mathrm{pCi} / \mathrm{L}$ & 0.011 & 0.012 & 0.0101 & & \\
\hline 2218 & UISO_IE_PLATE_AEA & STLRL & 0.288 & $\mathrm{pCi} / \mathrm{L}$ & 0.041 & 0.066 & 0.00797 & & \\
\hline 2219 & UISO_IE_PLATE_AEA & STLRL & 0.374 & $\mathrm{pCi} / \mathrm{L}$ & 0.047 & 0.083 & 0.0102 & & \\
\hline 2220 & UISO_IE_PLATE_AEA & STLRL & 0.0782 & $\mathrm{pCi} / \mathrm{L}$ & 0.021 & 0.026 & 0.0076 & & \\
\hline 2221 & UISO_IE_PLATE_AEA & STLRL & 0.0187 & $\mathrm{pCi} / \mathrm{L}$ & 0.011 & 0.012 & 0.00373 & & \\
\hline 2222 & UISO_IE_PLATE_AEA & STLRL & 0.248 & $\mathrm{pCi} / \mathrm{L}$ & 0.036 & 0.058 & 0.00889 & & \\
\hline 2223 & UISO_IE_PLATE_AEA & STLRL & 0.0274 & $\mathrm{pCi} / \mathrm{L}$ & 0.013 & 0.014 & 0.00909 & & \\
\hline 2224 & UISO_IE_PLATE_AEA & STLRL & 0.058 & $\mathrm{pCi} / \mathrm{L}$ & 0.018 & 0.021 & 0.00353 & & \\
\hline 2225 & UISO_IE_PLATE_AEA & STLRL & 0.0937 & $\mathrm{pCi} / \mathrm{L}$ & 0.024 & 0.03 & 0.00404 & & \\
\hline 2226 & UISO_IE_PLATE_AEA & STLRL & 0.0251 & $\mathrm{pCi} / \mathrm{L}$ & 0.013 & 0.014 & 0.00385 & & \\
\hline 2227 & UISO_IE_PLATE_AEA & STLRL & 0.0307 & $\mathrm{pCi} / \mathrm{L}$ & 0.016 & 0.018 & 0.00541 & & \\
\hline 2228 & UISO_IE_PLATE_AEA & STLRL & 0.00885 & $\mathrm{pCi} / \mathrm{L}$ & 0.01 & 0.011 & 0.014 & & $U$ \\
\hline 2229 & UISO_IE_PLATE_AEA & STLRL & 0.0223 & $\mathrm{pCi} / \mathrm{L}$ & 0.012 & 0.013 & 0.00969 & & \\
\hline 2230 & UISO_IE_PLATE_AEA & STLRL & 0.0116 & $\mathrm{pCi} / \mathrm{L}$ & 0.0098 & 0.01 & 0.01 & & \\
\hline 2231 & UISO_IE_PLATE_AEA & STLRL & 0.00979 & $\mathrm{pCi} / \mathrm{L}$ & 0.0099 & 0.01 & 0.00508 & & $U$ \\
\hline 2232 & UISO_IE_PLATE_AEA & STLRL & 0.0085 & $\mathrm{pCi} / \mathrm{L}$ & 0.0084 & 0.0088 & 0.00401 & & $\mathrm{U}$ \\
\hline 2233 & UISO_IE_PLATE_AEA & STLRL & 0.00739 & $\mathrm{pCi} / \mathrm{L}$ & 0.0081 & 0.0085 & 0.00415 & & $U$ \\
\hline 2234 & UISO_IE_PLATE_AEA & STLRL & 0.00187 & $\mathrm{pCi} / \mathrm{L}$ & 0.0079 & 0.0081 & 0.0145 & & $U$ \\
\hline 2235 & UISO_IE_PLATE_AEA & STLRL & 0.0103 & $\mathrm{pCi} / \mathrm{L}$ & 0.009 & 0.0095 & 0.00919 & & \\
\hline 2236 & UISO_IE_PLATE_AEA & STLRL & 0.0322 & $\mathrm{pCi} / \mathrm{L}$ & 0.014 & 0.016 & 0.00385 & & \\
\hline 2237 & UISO_IE_PLATE_AEA & STLRL & 0.017 & $\mathrm{pCi} / \mathrm{L}$ & 0.012 & 0.013 & 0.00499 & & \\
\hline 2238 & UISO_IE_PLATE_AEA & STLRL & 0.0066 & $\mathrm{pCi} / \mathrm{L}$ & 0.0075 & 0.0079 & 0.00384 & & U \\
\hline 2239 & UISO_IE_PLATE_AEA & STLRL & 0.00776 & $\mathrm{pCi} / \mathrm{L}$ & 0.009 & 0.0093 & 0.00497 & & $U$ \\
\hline 2240 & UISO_IE_PLATE_AEA & STLRL & 0.00411 & $\mathrm{pCi} / \mathrm{L}$ & 0.0066 & 0.0069 & 0.00398 & & $U$ \\
\hline 2241 & UISO_IE_PLATE_AEA & STLRL & 0.0155 & $\mathrm{pCi} / \mathrm{L}$ & 0.012 & 0.012 & 0.0115 & & \\
\hline 2242 & UISO_IE_PLATE_AEA & STLRL & 0.00617 & $\mathrm{pCi} / \mathrm{L}$ & 0.0084 & 0.0087 & 0.00957 & & U \\
\hline 2243 & UISO_IE_PLATE_AEA & STLRL & 0.000155 & $\mathrm{pCi} / \mathrm{L}$ & 0.0049 & 0.0052 & 0.00469 & & $U$ \\
\hline
\end{tabular}




\begin{tabular}{|c|c|c|c|c|c|c|c|c|c|}
\hline & $\mathrm{K}$ & $\mathrm{L}$ & $M$ & $\mathrm{~N}$ & $\mathrm{O}$ & $P$ & $\mathrm{Q}$ & $\mathrm{R}$ & $\mathrm{S}$ \\
\hline 1 & METHOD_NAME & LAB_CODE & VALUE_RPTD & ANAL_UNITS_RPTD & COUNTING_ERROR & \begin{tabular}{|l|} 
TOTAL_ANAL_ERROR \\
\end{tabular} & MIN_DETECTABLE_ACTIVITY & PCNT_MOISTURE & LAB_QUALIFIER \\
\hline 2244 & UISO_IE_PLATE_AEA & STLRL & 0.00625 & $\mathrm{pCi} / \mathrm{L}$ & 0.0096 & 0.0099 & 0.00648 & & $U$ \\
\hline 2245 & UISO_IE_PLATE_AEA & STLRL & 0.007 & $\mathrm{pCi} / \mathrm{L}$ & 0.0084 & 0.0088 & 0.00466 & & $U$ \\
\hline 2246 & UISO_IE_PLATE_AEA & STLRL & 0.00691 & $\mathrm{pCi} / \mathrm{L}$ & 0.0083 & 0.0087 & 0.00878 & & $U$ \\
\hline 2247 & UISO_IE_PLATE_AEA & STLRL & 0.00671 & $\mathrm{pCi} / \mathrm{L}$ & 0.0076 & 0.008 & 0.00389 & & $U$ \\
\hline 2248 & UISO_IE_PLATE_AEA & STLRL & 0.0112 & $\mathrm{pCi} / \mathrm{L}$ & 0.011 & 0.011 & 0.00563 & & $U$ \\
\hline 2249 & UISO_IE_PLATE_AEA & STLRL & 0.00442 & $\mathrm{pCi} / \mathrm{L}$ & 0.0069 & 0.0073 & 0.0042 & & $U$ \\
\hline 2250 & UISO_IE_PLATE_AEA & STLRL & 0.00616 & $\mathrm{pCi} / \mathrm{L}$ & 0.0085 & 0.0088 & 0.00514 & & U \\
\hline 2251 & UISO_IE_PLATE_AEA & STLRL & -0.00168 & $\mathrm{pCi} / \mathrm{L}$ & 0.0059 & 0.0062 & 0.0155 & & $U$ \\
\hline 2252 & UISO_IE_PLATE_AEA & STLRL & 0.00306 & $\mathrm{pCi} / \mathrm{L}$ & 0.0074 & 0.0077 & 0.00578 & & $\mathrm{U}$ \\
\hline 2253 & UISO_IE_PLATE_AEA & STLRL & 0.00718 & $\mathrm{pCi} / \mathrm{L}$ & 0.0086 & 0.0089 & 0.00475 & & U \\
\hline 2254 & UISO_IE_PLATE_AEA & STLRL & 0.0127 & $\mathrm{pCi} / \mathrm{L}$ & 0.011 & 0.012 & 0.0105 & & \\
\hline 2255 & UISO_IE_PLATE_AEA & STLRL & 0.00217 & $\mathrm{pCi} / \mathrm{L}$ & 0.0071 & 0.0074 & 0.0118 & & $U$ \\
\hline 2256 & UISO_IE_PLATE_AEA & STLRL & 0.00756 & $\mathrm{pCi} / \mathrm{L}$ & 0.0082 & 0.0086 & 0.00809 & & $U$ \\
\hline 2257 & UISO_IE_PLATE_AEA & STLRL & 0.0071 & $\mathrm{pCi} / \mathrm{L}$ & 0.0079 & 0.0083 & 0.00404 & & $U$ \\
\hline 2258 & UISO_IE_PLATE_AEA & STLRL & 0.0128 & $\mathrm{pCi} / \mathrm{L}$ & 0.012 & 0.013 & 0.012 & & \\
\hline 2259 & UISO_IE_PLATE_AEA & STLRL & 0.00732 & $\mathrm{pCi} / \mathrm{L}$ & 0.0081 & 0.0084 & 0.00412 & & $U$ \\
\hline 2260 & UISO_IE_PLATE_AEA & STLRL & 0.00176 & $\mathrm{pCi} / \mathrm{L}$ & 0.0058 & 0.0061 & 0.00801 & & U \\
\hline 2261 & UISO_IE_PLATE_AEA & STLRL & 0.0072 & $\mathrm{pCi} / \mathrm{L}$ & 0.0094 & 0.0098 & 0.00571 & & $U$ \\
\hline 2262 & UISO_IE_PLATE_AEA & STLRL & 0.000725 & $\mathrm{pCi} / \mathrm{L}$ & 0.0047 & 0.0051 & 0.00367 & & $U$ \\
\hline 2263 & UISO_IE_PLATE_AEA & STLRL & -0.0019 & $\mathrm{pCi} / \mathrm{L}$ & 0.0051 & 0.0054 & 0.0135 & & $U$ \\
\hline 2264 & UISO_IE_PLATE_AEA & STLRL & 0.00111 & $\mathrm{pCi} / \mathrm{L}$ & 0.0051 & 0.0055 & 0.00401 & & $U$ \\
\hline 2265 & UISO_IE_PLATE_AEA & STLRL & 0.00772 & $\mathrm{pCi} / \mathrm{L}$ & 0.0084 & 0.0087 & 0.00428 & & $\mathrm{U}$ \\
\hline 2266 & UISO_IE_PLATE_AEA & STLRL & 0.0143 & $\mathrm{pCi} / \mathrm{L}$ & 0.0098 & 0.01 & 0.00367 & & \\
\hline 2267 & UISO_IE_PLATE_AEA & STLRL & 0.000948 & $\mathrm{pCi} / \mathrm{L}$ & 0.005 & 0.0053 & 0.00387 & & $U$ \\
\hline 2268 & UISO_IE_PLATE_AEA & STLRL & 0.00303 & $\mathrm{pCi} / \mathrm{L}$ & 0.0063 & 0.0066 & 0.00782 & & $U$ \\
\hline 2269 & UISO_IE_PLATE_AEA & STLRL & 0.012 & $\mathrm{pCi} / \mathrm{L}$ & 0.0097 & 0.01 & 0.00415 & & \\
\hline 2270 & UISO_IE_PLATE_AEA & STLRL & 0.00916 & $\mathrm{pCi} / \mathrm{L}$ & 0.0094 & 0.0098 & 0.00484 & & $U$ \\
\hline 2271 & UISO_IE_PLATE_AEA & STLRL & 0.00768 & $\mathrm{pCi} / \mathrm{L}$ & 0.0078 & 0.0082 & 0.00373 & & $U$ \\
\hline 2272 & UISO_IE_PLATE_AEA & STLRL & 0.00893 & $\mathrm{pCi} / \mathrm{L}$ & 0.0087 & 0.0091 & 0.00415 & & U \\
\hline 2273 & UISO_IE_PLATE_AEA & STLRL & 0.00892 & $\mathrm{pCi} / \mathrm{L}$ & 0.0086 & 0.009 & 0.00805 & & $U$ \\
\hline 2274 & UISO_IE_PLATE_AEA & STLRL & 0.00652 & $\mathrm{pCi} / \mathrm{L}$ & 0.0074 & 0.0078 & 0.00731 & & $U$ \\
\hline 2275 & UISO_IE_PLATE_AEA & STLRL & 0.00779 & $\mathrm{pCi} / \mathrm{L}$ & 0.0083 & 0.0087 & 0.00932 & & U \\
\hline 2276 & UISO_IE_PLATE_AEA & STLRL & 0.0126 & $\mathrm{pCi} / \mathrm{L}$ & 0.0092 & 0.0098 & 0.0036 & & \\
\hline 2277 & UISO_IE_PLATE_AEA & STLRL & -0.000425 & $\mathrm{pCi} / \mathrm{L}$ & 0.0041 & 0.0045 & 0.00393 & & $U$ \\
\hline 2278 & UISO_IE_PLATE_AEA & STLRL & -0.00101 & $\mathrm{pCi} / \mathrm{L}$ & 0.0043 & 0.0047 & 0.00822 & & U \\
\hline 2279 & UISO_IE_PLATE_AEA & STLRL & 0.0191 & $\mathrm{pCi} / \mathrm{L}$ & 0.012 & 0.012 & 0.00405 & & \\
\hline 2280 & UISO_IE_PLATE_AEA & STLRL & 0.00759 & $\mathrm{pCi} / \mathrm{L}$ & 0.0077 & 0.0081 & 0.0037 & & $U$ \\
\hline 2281 & UISO_IE_PLATE_AEA & STLRL & 0.00331 & $\mathrm{pCi} / \mathrm{L}$ & 0.0059 & 0.0063 & 0.0036 & & $\mathrm{U}$ \\
\hline
\end{tabular}




\begin{tabular}{|c|c|c|c|c|c|c|c|c|c|}
\hline & $\mathrm{K}$ & $\mathrm{L}$ & $\bar{M}$ & $\mathrm{~N}$ & $\mathrm{O}$ & $P$ & $\mathrm{Q}$ & $\mathrm{R}$ & $\mathrm{S}$ \\
\hline 1 & METHOD_NAME & LAB_CODE & VALUE_RPTD & ANAL_UNITS_RPTD & COUNTING_ERROR & TOTAL_ANAL_ERROR & \begin{tabular}{|l|} 
MIN_DETECTABLE_ACTIVITY \\
\end{tabular} & PCNT_MOISTURE & LAB_QUALIFIER \\
\hline 2282 & UISO_IE_PLATE_AEA & STLRL & 0.00131 & $\mathrm{pCi} / \mathrm{L}$ & 0.0053 & 0.0056 & 0.0073 & & $U$ \\
\hline 2283 & UISO IE_PLATE_AEA & STLRL & 0.00421 & $\mathrm{pCi} / \mathrm{L}$ & 0.0067 & 0.007 & 0.00762 & & $\mathrm{U}$ \\
\hline 2284 & UISO_IE_PLATE_AEA & STLRL & 0.0033 & $\mathrm{pCi} / \mathrm{L}$ & 0.0059 & 0.0063 & 0.0036 & & U \\
\hline 2285 & UISO_IE_PLATE_AEA & STLRL & 0.0123 & $\mathrm{pCi} / \mathrm{L}$ & 0.0095 & 0.01 & 0.00386 & & \\
\hline 2286 & UISO_IE_PLATE_AEA & STLRL & 0.00546 & $\mathrm{pCi} / \mathrm{L}$ & 0.0072 & 0.0075 & 0.00396 & & $\mathrm{U}$ \\
\hline 2287 & UISO_IE_PLATE_AEA & STLRL & 0.00638 & $\mathrm{pCi} / \mathrm{L}$ & 0.0079 & 0.0083 & 0.00439 & & U \\
\hline 2288 & UISO_IE_PLATE_AEA & STLRL & 0.00247 & $\mathrm{pCi} / \mathrm{L}$ & 0.0058 & 0.0061 & 0.00393 & & $U$ \\
\hline 2289 & UISO_IE_PLATE_AEA & STLRL & -0.00235 & pCi/L & 0.0035 & 0.004 & 0.00928 & & U \\
\hline 2290 & UISO_IE_PLATE_AEA & STLRL & 0.000713 & $\mathrm{pCi} / \mathrm{L}$ & 0.0057 & 0.006 & 0.00548 & & U \\
\hline 2291 & UISO_IE_PLATE_AEA & STLRL & 0.0117 & $\mathrm{pCi} / \mathrm{L}$ & 0.0098 & 0.01 & 0.0109 & & \\
\hline 2292 & |UISO_IE_PLATE_AEA & STLRL & 0.000331 & $\mathrm{pCi} / \mathrm{L}$ & 0.0061 & 0.0064 & 0.0115 & & U \\
\hline 2293 & UISO_IE_PLATE_AEA & STLRL & 0.00777 & $\mathrm{pCi} / \mathrm{L}$ & 0.0079 & 0.0083 & 0.00376 & & U \\
\hline 2294 & UISO_IE_PLATE_AEA & STLRL & 1.5 & $\mathrm{pCi} / \mathrm{L}$ & 0.09 & 0.29 & 0.0116 & & \\
\hline 2295 & UISO_IE_PLATE_AEA & STLRL & 1.7 & $\mathrm{pCi} / \mathrm{L}$ & 0.11 & 0.33 & 0.00515 & & \\
\hline 2296 & UISO_IE_PLATE_AEA & STLRL & 1.13 & $\mathrm{pCi} / \mathrm{L}$ & 0.087 & 0.22 & 0.0045 & & \\
\hline 2297 & UISO_IE_PLATE_AEA & STLRL & 2.38 & $\mathrm{pCi} / \mathrm{L}$ & 0.12 & 0.45 & 0.00432 & & \\
\hline 2298 & UISO_IE_PLATE_AEA & STLRL & 2.32 & $\mathrm{pCi} / \mathrm{L}$ & 0.13 & 0.44 & 0.0116 & & \\
\hline 2299 & UISO_IE_PLATE_AEA & STLRL & 4.25 & $\mathrm{pCi} / \mathrm{L}$ & 0.16 & 0.79 & 0.00414 & & \\
\hline 2300 & UISO_IE_PLATE_AEA & STLRL & 0.655 & $\mathrm{pCi} / \mathrm{L}$ & 0.061 & 0.13 & 0.011 & & \\
\hline 2301 & UISO_IE_PLATE_AEA & STLRL & 2.88 & pCi/L & 0.13 & 0.54 & 0.00878 & & \\
\hline 2302 & UISO_IE_PLATE_AEA & STLRL & 1.21 & $\mathrm{pCi} / \mathrm{L}$ & 0.088 & 0.24 & 0.00435 & & \\
\hline 2303 & UISO_IE_PLATE_AEA & STLRL & 1.43 & $\mathrm{pCi} / \mathrm{L}$ & 0.12 & 0.29 & 0.00653 & & \\
\hline 2304 & UISO_IE_PLATE_AEA & STLRL & 1.43 & $\mathrm{pCi} / \mathrm{L}$ & 0.11 & 0.28 & 0.00573 & & \\
\hline 2305 & UISO_IE_PLATE_AEA & STLRL & 0.292 & $\mathrm{pCi} / \mathrm{L}$ & 0.045 & 0.07 & 0.00461 & & \\
\hline 2306 & UISO_IE_PLATE_AEA & STLRL & 0.00815 & $\mathrm{pCi} / \mathrm{L}$ & 0.01 & 0.011 & 0.00622 & & U \\
\hline 2307 & UISO_IE_PLATE_AEA & STLRL & 0.0113 & $\mathrm{pCi} / \mathrm{L}$ & 0.011 & 0.011 & 0.00568 & & $\mathrm{U}$ \\
\hline 2308 & UISO_IE_PLATE_AEA & STLRL & 0.0783 & $\mathrm{pCi} / \mathrm{L}$ & 0.027 & 0.031 & 0.0188 & & \\
\hline 2309 & UISO_IE_PLATE_AEA & STLRL & 0.0329 & $\mathrm{pCi} / \mathrm{L}$ & 0.014 & 0.015 & 0.00883 & & \\
\hline 2310 & UISO_IE_PLATE_AEA & STLRL & 0.056 & $\mathrm{pCi} / \mathrm{L}$ & 0.018 & 0.021 & 0.00357 & & \\
\hline 2311 & UISO_IE_PLATE_AEA & STLRL & 0.00873 & pCi/L & 0.0084 & 0.0088 & 0.00901 & & $U$ \\
\hline 2312 & UISO_IE_PLATE_AEA & STLRL & 0.00175 & $\mathrm{pCi} / \mathrm{L}$ & 0.0063 & 0.0066 & 0.0104 & & U \\
\hline 2313 & UISO_IE_PLATE_AEA & STLRL & 0.00255 & $\mathrm{pCi} / \mathrm{L}$ & 0.0053 & 0.0053 & 0.011 & & U \\
\hline 2314 & UISO_IE_PLATE_AEA & STLRL & 0.0141 & $\mathrm{pCi} / \mathrm{L}$ & 0.011 & 0.011 & 0.0105 & & \\
\hline 2315 & UISO_IE_PLATE_AEA & STLRL & 0.0119 & $\mathrm{pCi} / \mathrm{L}$ & 0.0089 & 0.0091 & 0.01 & & \\
\hline 2316 & UISO_IE_PLATE_AEA & STLRL & 0.00779 & $\mathrm{pCi} / \mathrm{L}$ & 0.0079 & 0.0083 & 0.00377 & & $\mathrm{U}$ \\
\hline 2317 & UISO_IE_PLATE_AEA & STLRL & 0.00246 & pCi/L & 0.0071 & 0.0074 & 0.0118 & & $U$ \\
\hline 2318 & UISO_IE_PLATE_AEA & STLRL & -0.00117 & $\mathrm{pCi} / \mathrm{L}$ & 0.0039 & 0.0043 & 0.00754 & & U \\
\hline 2319 & UISO_IE_PLATE_AEA & STLRL & 0.00502 & $\mathrm{pCi} / \mathrm{L}$ & 0.0068 & 0.0072 & 0.00377 & & U \\
\hline
\end{tabular}




\begin{tabular}{|c|c|c|c|c|c|c|c|c|c|}
\hline & $\mathrm{K}$ & $\mathrm{L}$ & $\bar{M}$ & $\mathrm{~N}$ & $\mathrm{O}$ & $P$ & $\mathrm{Q}$ & $\mathrm{R}$ & $\mathrm{S}$ \\
\hline 1 & METHOD_NAME & LAB_CODE & VALUE_RPTD & ANAL_UNITS_RPTD & $\mid$ COUNTING_ERROR & TOTAL_ANAL_ERROR & MIN_DETECTABLE_ACTIVITY & PCNT_MOISTURE & LAB_QUALIFIER \\
\hline 2320 & UISO_IE_PLATE_AEA & STLRL & 0.00698 & $\mathrm{pCi} / \mathrm{L}$ & 0.0078 & 0.0082 & 0.00399 & & $U$ \\
\hline 2321 & UISO IE PLATE AEA & STLRL & 0.00974 & $\mathrm{pCi} / \mathrm{L}$ & 0.0087 & 0.0091 & 0.00768 & & \\
\hline 2322 & UISO IE PLATE AEA & STLRL & 0.00557 & $\mathrm{pCi} / \mathrm{L}$ & 0.0088 & 0.0091 & 0.0109 & & $u$ \\
\hline 2323 & UISO IE PLATE AEA & STLRL & 0.00948 & $\mathrm{pCi} / \mathrm{L}$ & 0.0089 & 0.0094 & 0.00955 & & $\mathrm{U}$ \\
\hline 2324 & UISO_IE_PLATE_AEA & STLRL & 0.00192 & $\mathrm{pCi} / \mathrm{L}$ & 0.0059 & 0.0063 & 0.00823 & & $U$ \\
\hline 2325 & UISO_IE_PLATE_AEA & STLRL & 0.0041 & $\mathrm{pCi} / \mathrm{L}$ & 0.0066 & 0.007 & 0.00401 & & $U$ \\
\hline 2326 & UISO_IE_PLATE_AEA & STLRL & 0.014 & $\mathrm{pCi} / \mathrm{L}$ & 0.011 & 0.011 & 0.0047 & & \\
\hline 2327 & UISO_IE_PLATE_AEA & STLRL & 0.00431 & $\mathrm{pCi} / \mathrm{L}$ & 0.0068 & 0.0071 & 0.00413 & & $U$ \\
\hline 2328 & UISO_IE_PLATE_AEA & STLRL & 0.004 & $\mathrm{pCi} / \mathrm{L}$ & 0.0065 & 0.0069 & 0.00397 & & $u$ \\
\hline 2329 & UISO_IE_PLATE_AEA & STLRL & 0.00124 & $\mathrm{pCi} / \mathrm{L}$ & 0.0059 & 0.0062 & 0.00977 & & $U$ \\
\hline 2330 & UISO_IE_PLATE_AEA & STLRL & -0.000581 & $\mathrm{pCi} / \mathrm{L}$ & 0.0057 & 0.006 & 0.0119 & & U \\
\hline 2331 & UISO_IE_PLATE_AEA & STLRL & 0.0121 & $\mathrm{pCi} / \mathrm{L}$ & 0.0097 & 0.01 & 0.00822 & & \\
\hline 2332 & UISO_IE_PLATE_AEA & STLRL & 0.0073 & $\mathrm{pCi} / \mathrm{L}$ & 0.0086 & 0.009 & 0.0091 & & $U$ \\
\hline 2333 & UISO_IE_PLATE_AEA & STLRL & 0.00442 & $\mathrm{pCi} / \mathrm{L}$ & 0.0063 & 0.0067 & 0.0035 & & $U$ \\
\hline 2334 & UISO_IE_PLATE_AEA & STLRL & 0.00994 & $\mathrm{pCi} / \mathrm{L}$ & 0.0094 & 0.0098 & 0.0045 & & \\
\hline 2335 & UISO_IE_PLATE_AEA & STLRL & 0.00659 & $\mathrm{pCi} / \mathrm{L}$ & 0.0081 & 0.0085 & 0.00448 & & $U$ \\
\hline \begin{tabular}{|l|}
2336 \\
\end{tabular} & UISO IE PLATE AEA & STLRL & 0.00183 & $\mathrm{pCi} / \mathrm{L}$ & 0.006 & 0.0063 & 0.00467 & & U \\
\hline 2337 & UISO_IE_PLATE_AEA & STLRL & 0.00543 & $\mathrm{pCi} / \mathrm{L}$ & 0.0072 & 0.0075 & 0.00396 & & $u$ \\
\hline 2338 & UISO_IE_PLATE_AEA & STLRL & 0.00838 & $\mathrm{pCi} / \mathrm{L}$ & 0.0078 & 0.0082 & 0.00353 & & \\
\hline 2339 & UISO_IE_PLATE_AEA & STLRL & 0.00225 & $\mathrm{pCi} / \mathrm{L}$ & 0.0066 & 0.0069 & 0.0109 & & $U$ \\
\hline 2340 & UISO_IE_PLATE_AEA & STLRL & 0.0072 & $\mathrm{pCi} / \mathrm{L}$ & 0.0078 & 0.0082 & 0.00883 & & $U$ \\
\hline 2341 & UISO_IE_PLATE_AEA & STLRL & 0.00687 & $\mathrm{pCi} / \mathrm{L}$ & 0.0077 & 0.0081 & 0.00395 & & $U$ \\
\hline 2342 & UISO_IE_PLATE_AEA & STLRL & 0.00934 & $\mathrm{pCi} / \mathrm{L}$ & 0.0085 & 0.0089 & 0.00382 & & \\
\hline 2343 & UISO_IE_PLATE_AEA & STLRL & 0.00726 & $\mathrm{pCi} / \mathrm{L}$ & 0.0075 & 0.0079 & 0.00359 & & $\mathrm{U}$ \\
\hline 2344 & UISO_IE_PLATE_AEA & STLRL & 0.00312 & $\mathrm{pCi} / \mathrm{L}$ & 0.01 & 0.011 & 0.02 & & $U$ \\
\hline 2345 & UISO_IE_PLATE_AEA & STLRL & 0.00945 & $\mathrm{pCi} / \mathrm{L}$ & 0.009 & 0.0094 & 0.00841 & & \\
\hline 2346 & UISO_IE_PLATE_AEA & STLRL & 0.00398 & $\mathrm{pCi} / \mathrm{L}$ & 0.0065 & 0.0069 & 0.00397 & & $U$ \\
\hline 2347 & UISO_IE_PLATE_AEA & STLRL & 0.0213 & $\mathrm{pCi} / \mathrm{L}$ & 0.012 & 0.013 & 0.00371 & & \\
\hline 2348 & UISO_IE_PLATE_AEA & STLRL & 0.0191 & $\mathrm{pCi} / \mathrm{L}$ & 0.012 & 0.012 & 0.00405 & & \\
\hline 2349 & UISO_IE_PLATE_AEA & STLRL & 0.0181 & $\mathrm{pCi} / \mathrm{L}$ & 0.012 & 0.013 & 0.00448 & & \\
\hline 2350 & UISO_IE_PLATE_AEA & STLRL & 0.0142 & $\mathrm{pCi} / \mathrm{L}$ & 0.013 & 0.013 & 0.016 & & $U$ \\
\hline \begin{tabular}{|l|}
2351 \\
\end{tabular} & UISO IE PLATE AEA & STLRL & 0.0223 & $\mathrm{pCi} / \mathrm{L}$ & 0.013 & 0.014 & 0.012 & & \\
\hline 2352 & UISO_IE_PLATE_AEA & STLRL & -0.000429 & $\mathrm{pCi} / \mathrm{L}$ & 0.0041 & 0.0045 & 0.00391 & & $U$ \\
\hline 2353 & UISO_IE_PLATE_AEA & STLRL & 0.0106 & $\mathrm{pCi} / \mathrm{L}$ & 0.0093 & 0.0097 & 0.00419 & & \\
\hline 2354 & UISO_IE_PLATE_AEA & STLRL & 0.00297 & $\mathrm{pCi} / \mathrm{L}$ & 0.0071 & 0.0074 & 0.00981 & & $u$ \\
\hline 2355 & UISO_IE_PLATE_AEA & STLRL & 0.0121 & $\mathrm{pCi} / \mathrm{L}$ & 0.014 & 0.014 & 0.0154 & & $U$ \\
\hline 2356 & UISO_IE_PLATE_AEA & STLRL & 0.0000525 & $\mathrm{pCi} / \mathrm{L}$ & 0.0046 & 0.005 & 0.00737 & & U \\
\hline 2357 & UISO_IE_PLATE_AEA & STLRL & -0.000831 & $\mathrm{pCi} / \mathrm{L}$ & 0.0035 & 0.004 & 0.0034 & & U \\
\hline
\end{tabular}




\begin{tabular}{|c|c|c|c|c|c|c|c|c|c|}
\hline & $\mathrm{K}$ & $\mathrm{L}$ & $\bar{M}$ & $\mathrm{~N}$ & $\mathrm{O}$ & $P$ & $\mathrm{Q}$ & $\mathrm{R}$ & $\mathrm{S}$ \\
\hline 1 & METHOD_NAME & LAB_CODE & VALUE_RPTD & ANAL_UNITS_RPTD & COUNTING_ERROR & TOTAL_ANAL_ERROR & MIN_DETECTABLE_ACTIVITY & PCNT_MOISTURE & LAB_QUALIFIER \\
\hline 2358 & UISO_IE_PLATE_AEA & STLRL & 0.0142 & $\mathrm{pCi} / \mathrm{L}$ & 0.01 & 0.011 & 0.00787 & & \\
\hline 2359 & UISO_IE_PLATE_AEA & STLRL & 0.0017 & $\mathrm{pCi} / \mathrm{L}$ & 0.005 & 0.0054 & 0.00341 & & $U$ \\
\hline 2360 & UISO_IE_PLATE_AEA & STLRL & 0.00206 & $\mathrm{pCi} / \mathrm{L}$ & 0.007 & 0.0073 & 0.0116 & & $\mathrm{U}$ \\
\hline 2361 & UISO_IE_PLATE_AEA & STLRL & 0.00272 & $\mathrm{pCi} / \mathrm{L}$ & 0.006 & 0.0063 & 0.00745 & & $\mathrm{U}$ \\
\hline 2362 & UISO_IE_PLATE_AEA & STLRL & 0.00668 & $\mathrm{pCi} / \mathrm{L}$ & 0.0097 & 0.01 & 0.0132 & & $U$ \\
\hline 2363 & UISO_IE_PLATE_AEA & STLRL & -0.00217 & $\mathrm{pCi} / \mathrm{L}$ & 0.0046 & 0.005 & 0.0114 & & $U$ \\
\hline 2364 & UISO_IE_PLATE_AEA & STLRL & 0.000467 & $\mathrm{pCi} / \mathrm{L}$ & 0.0044 & 0.0048 & 0.00344 & & $\mathrm{U}$ \\
\hline 2365 & UISO_IE_PLATE_AEA & STLRL & 0.0089 & $\mathrm{pCi} / \mathrm{L}$ & 0.009 & 0.0094 & 0.01 & & $U$ \\
\hline 2366 & UISO_IE_PLATE_AEA & STLRL & 0.00207 & $\mathrm{pCi} / \mathrm{L}$ & 0.0066 & 0.0069 & 0.00996 & & $\mathrm{U}$ \\
\hline 2367 & UISO_IE_PLATE_AEA & STLRL & 0.0106 & $\mathrm{pCi} / \mathrm{L}$ & 0.011 & 0.012 & 0.0133 & & $\mathrm{U}$ \\
\hline 2368 & UISO_IE_PLATE_AEA & STLRL & 0.01 & $\mathrm{pCi} / \mathrm{L}$ & 0.0092 & 0.0097 & 0.0106 & & U \\
\hline 2369 & UISO_IE_PLATE_AEA & STLRL & 0.00755 & $\mathrm{pCi} / \mathrm{L}$ & 0.0077 & 0.0081 & 0.00369 & & $U$ \\
\hline 2370 & UISO_IE_PLATE_AEA & STLRL & 0.000584 & $\mathrm{pCi} / \mathrm{L}$ & 0.0058 & 0.0061 & 0.00963 & & $\mathrm{U}$ \\
\hline 2371 & UISO_IE_PLATE_AEA & STLRL & 0.00348 & $\mathrm{pCi} / \mathrm{L}$ & 0.0072 & 0.0075 & 0.01 & & $U$ \\
\hline 2372 & UISO_IE_PLATE_AEA & STLRL & 0.00739 & $\mathrm{pCi} / \mathrm{L}$ & 0.0085 & 0.0088 & 0.00987 & & $\mathrm{U}$ \\
\hline 2373 & UISO_IE_PLATE_AEA & STLRL & 0.00513 & $\mathrm{pCi} / \mathrm{L}$ & 0.0069 & 0.0073 & 0.00382 & & $\mathrm{U}$ \\
\hline 2374 & UISO_IE_PLATE_AEA & STLRL & 0.00395 & $\mathrm{pCi} / \mathrm{L}$ & 0.0064 & 0.0067 & 0.00388 & & 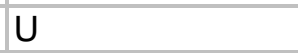 \\
\hline 2375 & UISO_IE_PLATE_AEA & STLRL & 0.00205 & $\mathrm{pCi} / \mathrm{L}$ & 0.008 & 0.0082 & 0.0131 & & $U$ \\
\hline 2376 & UISO_IE_PLATE_AEA & STLRL & 0.00693 & $\mathrm{pCi} / \mathrm{L}$ & 0.0078 & 0.0081 & 0.00397 & & $\mathrm{U}$ \\
\hline 2377 & UISO_IE_PLATE_AEA & STLRL & 0.0078 & $\mathrm{pCi} / \mathrm{L}$ & 0.012 & 0.012 & 0.0164 & & U \\
\hline 2378 & UISO_IE_PLATE_AEA & STLRL & 0.00233 & $\mathrm{pCi} / \mathrm{L}$ & 0.01 & 0.01 & 0.0176 & & $U$ \\
\hline 2379 & UISO_IE_PLATE_AEA & STLRL & 0.00584 & $\mathrm{pCi} / \mathrm{L}$ & 0.0087 & 0.009 & 0.0113 & & $\mathrm{U}$ \\
\hline 2380 & UISO_IE_PLATE_AEA & STLRL & 0.0034 & $\mathrm{pCi} / \mathrm{L}$ & 0.006 & 0.0064 & 0.00365 & & $U$ \\
\hline 2381 & UISO_IE_PLATE_AEA & STLRL & 0.00251 & $\mathrm{pCi} / \mathrm{L}$ & 0.0064 & 0.0067 & 0.00957 & & $U$ \\
\hline 2382 & UISO_IE_PLATE_AEA & STLRL & -0.00194 & $\mathrm{pCi} / \mathrm{L}$ & 0.0063 & 0.0065 & 0.013 & & $\mathrm{U}$ \\
\hline 2383 & UISO_IE_PLATE_AEA & STLRL & 0.00959 & $\mathrm{pCi} / \mathrm{L}$ & 0.0086 & 0.0091 & 0.00389 & & \\
\hline 2384 & UISO_IE_PLATE_AEA & STLRL & 0.00559 & $\mathrm{pCi} / \mathrm{L}$ & 0.0082 & 0.0085 & 0.012 & & $U$ \\
\hline 2385 & UISO_IE_PLATE_AEA & STLRL & 0.00353 & $\mathrm{pCi} / \mathrm{L}$ & 0.0085 & 0.0087 & 0.0141 & & $U$ \\
\hline 2386 & UISO_IE_PLATE_AEA & STLRL & 0.00424 & $\mathrm{pCi} / \mathrm{L}$ & 0.0062 & 0.0066 & 0.00343 & & 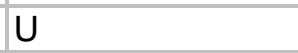 \\
\hline 2387 & UISO_IE_PLATE_AEA & STLRL & 0.00616 & $\mathrm{pCi} / \mathrm{L}$ & 0.0094 & 0.0097 & 0.0154 & & $U$ \\
\hline 2388 & UISO_IE_PLATE_AEA & STLRL & 0.00572 & $\mathrm{pCi} / \mathrm{L}$ & 0.0069 & 0.0072 & 0.00351 & & $\mathrm{U}$ \\
\hline 2389 & UISO_IE_PLATE_AEA & STLRL & 0.00374 & $\mathrm{pCi} / \mathrm{L}$ & 0.0071 & 0.0074 & 0.00479 & & U \\
\hline 2390 & UISO_IE_PLATE_AEA & STLRL & 0.00451 & $\mathrm{pCi} / \mathrm{L}$ & 0.0064 & 0.0068 & 0.00355 & & $U$ \\
\hline 2391 & UISO_IE_PLATE_AEA & STLRL & 0.00882 & $\mathrm{pCi} / \mathrm{L}$ & 0.0077 & 0.0082 & 0.0033 & & \\
\hline 2392 & UISO_IE_PLATE_AEA & STLRL & 0.00551 & $\mathrm{pCi} / \mathrm{L}$ & 0.0067 & 0.0071 & 0.00342 & & U \\
\hline 2393 & UISO_IE_PLATE_AEA & STLRL & 0.00175 & $\mathrm{pCi} / \mathrm{L}$ & 0.0051 & 0.0055 & 0.00345 & & $U$ \\
\hline 2394 & UISO_IE_PLATE_AEA & STLRL & 0.00236 & $\mathrm{pCi} / \mathrm{L}$ & 0.0057 & 0.006 & 0.00386 & & $U$ \\
\hline 2395 & UISO_IE_PLATE_AEA & STLRL & 0.00147 & $\mathrm{pCi} / \mathrm{L}$ & 0.0048 & 0.0052 & 0.00326 & & $\mathrm{U}$ \\
\hline
\end{tabular}




\begin{tabular}{|c|c|c|c|c|c|c|c|c|c|}
\hline & $\mathrm{K}$ & $\mathrm{L}$ & $\bar{M}$ & $\mathrm{~N}$ & $\mathrm{O}$ & $P$ & $\mathrm{Q}$ & $\mathrm{R}$ & $\mathrm{S}$ \\
\hline 1 & METHOD_NAME & LAB_CODE & VALUE_RPTD & ANAL_UNITS_RPTD & COUNTING_ERROR & TOTAL_ANAL_ERROR & \begin{tabular}{|l|} 
MIN_DETECTABLE_ACTIVITY \\
\end{tabular} & PCNT_MOISTURE & LAB_QUALIFIER \\
\hline 2396 & UISO_IE_PLATE_AEA & STLRL & 0.0101 & $\mathrm{pCi} / \mathrm{L}$ & 0.0085 & 0.0089 & 0.00363 & & \\
\hline 2397 & UISO IE_PLATE_AEA & STLRL & 0.000455 & $\mathrm{pCi} / \mathrm{L}$ & 0.0057 & 0.006 & 0.00931 & & $\mathrm{U}$ \\
\hline 2398 & UISO_IE_PLATE_AEA & STLRL & 0.000259 & $\mathrm{pCi} / \mathrm{L}$ & 0.0054 & 0.0058 & 0.01 & & U \\
\hline 2399 & UISO_IE_PLATE_AEA & STLRL & 0.00236 & $\mathrm{pCi} / \mathrm{L}$ & 0.0057 & 0.006 & 0.00386 & & U \\
\hline 2400 & UISO_IE_PLATE_AEA & STLRL & -0.00141 & $\mathrm{pCi} / \mathrm{L}$ & 0.0039 & 0.0043 & 0.00523 & & U \\
\hline 2401 & UISO_IE_PLATE_AEA & STLRL & 0.00199 & $\mathrm{pCi} / \mathrm{L}$ & 0.0053 & 0.0057 & 0.00361 & & U \\
\hline 2402 & UISO_IE_PLATE_AEA & STLRL & 0.00609 & $\mathrm{pCi} / \mathrm{L}$ & 0.0077 & 0.0081 & 0.00426 & & $U$ \\
\hline 2403 & UISO_IE_PLATE_AEA & STLRL & 0.00336 & pCi/L & 0.0071 & 0.0074 & 0.00986 & & U \\
\hline 2404 & UISO_IE_PLATE_AEA & STLRL & 0.00428 & $\mathrm{pCi} / \mathrm{L}$ & 0.0089 & 0.0092 & 0.0149 & & U \\
\hline 2405 & UISO_IE_PLATE_AEA & STLRL & 0.0109 & $\mathrm{pCi} / \mathrm{L}$ & 0.017 & 0.017 & 0.0319 & & U \\
\hline 2406 & |UISO_IE_PLATE_AEA & STLRL & 0.0071 & $\mathrm{pCi} / \mathrm{L}$ & 0.0079 & 0.0083 & 0.00404 & & U \\
\hline 2407 & UISO_IE_PLATE_AEA & STLRL & 0.00214 & $\mathrm{pCi} / \mathrm{L}$ & 0.0055 & 0.0058 & 0.00371 & & U \\
\hline 2408 & UISO_IE_PLATE_AEA & STLRL & 0.00439 & $\mathrm{pCi} / \mathrm{L}$ & 0.0063 & 0.0067 & 0.00349 & & U \\
\hline 2409 & UISO_IE_PLATE_AEA & STLRL & 0.0053 & $\mathrm{pCi} / \mathrm{L}$ & 0.0065 & 0.0069 & 0.00334 & & $U$ \\
\hline 2410 & UISO_IE_PLATE_AEA & STLRL & 0.00538 & $\mathrm{pCi} / \mathrm{L}$ & 0.0071 & 0.0075 & 0.00394 & & $\mathrm{U}$ \\
\hline 2411 & UISO_IE_PLATE_AEA & STLRL & 0.000669 & $\mathrm{pCi} / \mathrm{L}$ & 0.0046 & 0.005 & 0.00362 & & U \\
\hline 2412 & UISO_IE_PLATE_AEA & STLRL & 0.00271 & $\mathrm{pCi} / \mathrm{L}$ & 0.0064 & 0.0067 & 0.00891 & & $U$ \\
\hline 2413 & UISO_IE_PLATE_AEA & STLRL & 0.00543 & $\mathrm{pCi} / \mathrm{L}$ & 0.0081 & 0.0084 & 0.0118 & & U \\
\hline 2414 & UISO_IE_PLATE_AEA & STLRL & 0.0136 & $\mathrm{pCi} / \mathrm{L}$ & 0.011 & 0.012 & 0.0143 & & U \\
\hline 2415 & UISO_IE_PLATE_AEA & STLRL & -0.0000818 & pCi/L & 0.0046 & 0.005 & 0.00441 & & U \\
\hline 2416 & UISO_IE_PLATE_AEA & STLRL & -0.000975 & $\mathrm{pCi} / \mathrm{L}$ & 0.0043 & 0.0047 & 0.00835 & & U \\
\hline 2417 & |UISO_IE_PLATE_AEA & STLRL & -0.00334 & $\mathrm{pCi} / \mathrm{L}$ & 0.0041 & 0.0041 & 0.00404 & & U \\
\hline 2418 & UISO_IE_PLATE_AEA & STLRL & 0.00339 & $\mathrm{pCi} / \mathrm{L}$ & 0.006 & 0.0064 & 0.00365 & & U \\
\hline 2419 & UISO_IE_PLATE_AEA & STLRL & 0.00249 & $\mathrm{pCi} / \mathrm{L}$ & 0.0058 & 0.0061 & 0.00716 & & U \\
\hline 2420 & UISO_IE_PLATE_AEA & STLRL & 0.00333 & $\mathrm{pCi} / \mathrm{L}$ & 0.006 & 0.0063 & 0.00362 & & $\mathrm{U}$ \\
\hline 2421 & UISO_IE_PLATE_AEA & STLRL & -0.00062 & $\mathrm{pCi} / \mathrm{L}$ & 0.0076 & 0.0078 & 0.0167 & & $\mathrm{U}$ \\
\hline 2422 & UISO_IE_PLATE_AEA & STLRL & 0.00866 & $\mathrm{pCi} / \mathrm{L}$ & 0.0098 & 0.01 & 0.00542 & & U \\
\hline 2423 & UISO_IE_PLATE_AEA & STLRL & 0.0117 & $\mathrm{pCi} / \mathrm{L}$ & 0.012 & 0.012 & 0.0151 & & U \\
\hline 2424 & UISO_IE_PLATE_AEA & STLRL & 0.00475 & $\mathrm{pCi} / \mathrm{L}$ & 0.0066 & 0.007 & 0.00366 & & U \\
\hline 2425 & UISO_IE_PLATE_AEA & STLRL & -0.00088 & pCi/L & 0.0035 & 0.0039 & 0.00333 & & U \\
\hline 2426 & UISO_IE_PLATE_AEA & STLRL & 0.00866 & $\mathrm{pCi} / \mathrm{L}$ & 0.008 & 0.0084 & 0.00361 & & \\
\hline 2427 & UISO_IE_PLATE_AEA & STLRL & 0.00401 & $\mathrm{pCi} / \mathrm{L}$ & 0.0069 & 0.0072 & 0.00901 & & $U$ \\
\hline 2428 & UISO_IE_PLATE_AEA & STLRL & 0.0106 & $\mathrm{pCi} / \mathrm{L}$ & 0.0099 & 0.01 & 0.0129 & & $\mathrm{U}$ \\
\hline 2429 & UISO_IE_PLATE_AEA & STLRL & 0.0213 & $\mathrm{pCi} / \mathrm{L}$ & 0.018 & 0.019 & 0.031 & & U \\
\hline 2430 & UISO_IE_PLATE_AEA & STLRL & 0.0126 & $\mathrm{pCi} / \mathrm{L}$ & 0.013 & 0.014 & 0.02 & & U \\
\hline 2431 & UISO_IE_PLATE_AEA & STLRL & 0.00728 & pCi/L & 0.0075 & 0.0079 & 0.0036 & & $U$ \\
\hline 2432 & UISO_IE_PLATE_AEA & STLRL & 0.00194 & $\mathrm{pCi} / \mathrm{L}$ & 0.0058 & 0.0061 & 0.00863 & & U \\
\hline 2433 & UISO_IE_PLATE_AEA & STLRL & 0.00221 & $\mathrm{pCi} / \mathrm{L}$ & 0.0055 & 0.0059 & 0.00376 & & U \\
\hline
\end{tabular}




\begin{tabular}{|c|c|c|c|c|c|c|c|c|c|}
\hline & $\mathrm{K}$ & $\mathrm{L}$ & $\bar{M}$ & $\mathrm{~N}$ & $\mathrm{O}$ & $P$ & $\mathrm{Q}$ & $\mathrm{R}$ & $\mathrm{S}$ \\
\hline 1 & METHOD_NAME & LAB_CODE & VALUE_RPTD & ANAL_UNITS_RPTD & $\mid$ COUNTING_ERROR & TOTAL_ANAL_ERROR & MIN_DETECTABLE_ACTIVITY & PCNT_MOISTURE & LAB_QUALIFIER \\
\hline 2434 & UISO_IE_PLATE_AEA & STLRL & 0.0106 & $\mathrm{pCi} / \mathrm{L}$ & 0.0099 & 0.01 & 0.00472 & & \\
\hline 2435 & UISO IE PLATE AEA & STLRL & 0.00452 & $\mathrm{pCi} / \mathrm{L}$ & 0.008 & 0.0083 & 0.0125 & & $\mathrm{U}$ \\
\hline 2436 & UISO_IE_PLATE_AEA & STLRL & -0.000699 & $\mathrm{pCi} / \mathrm{L}$ & 0.0037 & 0.0042 & 0.00358 & & U \\
\hline 2437 & UISO IE PLATE AEA & STLRL & -0.000357 & $\mathrm{pCi} / \mathrm{L}$ & 0.006 & 0.0063 & 0.00808 & & $\mathrm{U}$ \\
\hline 2438 & UISO_IE_PLATE_AEA & STLRL & 0.0109 & $\mathrm{pCi} / \mathrm{L}$ & 0.0077 & 0.008 & 0.0037 & & \\
\hline 2439 & UISO_IE_PLATE_AEA & STLRL & 0.00411 & $\mathrm{pCi} / \mathrm{L}$ & 0.0047 & 0.0048 & 0.00371 & & $U$ \\
\hline 2440 & UISO_IE_PLATE_AEA & STLRL & 0.0325 & $\mathrm{pCi} / \mathrm{L}$ & 0.014 & 0.016 & 0.00388 & & \\
\hline 2441 & UISO_IE_PLATE_AEA & STLRL & 0.726 & $\mathrm{pCi} / \mathrm{L}$ & 0.062 & 0.15 & 0.00358 & & \\
\hline 2442 & UISO_IE_PLATE_AEA & STLRL & 0.0351 & $\mathrm{pCi} / \mathrm{L}$ & 0.014 & 0.016 & 0.00347 & & \\
\hline 2443 & UISO_IE_PLATE_AEA & STLRL & 0.052 & $\mathrm{pCi} / \mathrm{L}$ & 0.017 & 0.019 & 0.0034 & & \\
\hline 2444 & UISO_IE_PLATE_AEA & STLRL & 0.0629 & $\mathrm{pCi} / \mathrm{L}$ & 0.018 & 0.022 & 0.00345 & & \\
\hline 2445 & UISO_IE_PLATE_AEA & STLRL & 0.00456 & $\mathrm{pCi} / \mathrm{L}$ & 0.0078 & 0.0081 & 0.00527 & & U \\
\hline 2446 & UISO_IE_PLATE_AEA & STLRL & 0.00419 & $\mathrm{pCi} / \mathrm{L}$ & 0.0067 & 0.0071 & 0.00408 & & $U$ \\
\hline 2447 & UISO_IE_PLATE_AEA & STLRL & 0.00367 & $\mathrm{pCi} / \mathrm{L}$ & 0.0062 & 0.0065 & 0.00373 & & $U$ \\
\hline 2448 & UISO_IE_PLATE_AEA & STLRL & 0.00182 & $\mathrm{pCi} / \mathrm{L}$ & 0.0051 & 0.0055 & 0.00346 & & $U$ \\
\hline 2449 & UISO_IE_PLATE_AEA & STLRL & 0.00278 & $\mathrm{pCi} / \mathrm{L}$ & 0.0075 & 0.0077 & 0.0112 & & $U$ \\
\hline 2450 & UISO IE PLATE AEA & STLRL & -0.00096 & $\mathrm{pCi} / \mathrm{L}$ & 0.0081 & 0.0083 & 0.0173 & & $\mathrm{U}$ \\
\hline 2451 & UISO_IE_PLATE_AEA & STLRL & 0.00412 & $\mathrm{pCi} / \mathrm{L}$ & 0.018 & 0.018 & 0.0324 & & $u$ \\
\hline 2452 & UISO_IE_PLATE_AEA & STLRL & -0.00332 & $\mathrm{pCi} / \mathrm{L}$ & 0.012 & 0.013 & 0.0263 & & U \\
\hline 2453 & UISO_IE_PLATE_AEA & STLRL & -0.00117 & $\mathrm{pCi} / \mathrm{L}$ & 0.0074 & 0.0076 & 0.0157 & & $U$ \\
\hline 2454 & UISO_IE_PLATE_AEA & STLRL & 0.00689 & $\mathrm{pCi} / \mathrm{L}$ & 0.0083 & 0.0087 & 0.00462 & & $U$ \\
\hline 2455 & UISO_IE_PLATE_AEA & STLRL & 0.00694 & $\mathrm{pCi} / \mathrm{L}$ & 0.0091 & 0.0095 & 0.00554 & & $U$ \\
\hline 2456 & UISO IE PLATE AEA & STLRL & 0.0056 & $\mathrm{pCi} / \mathrm{L}$ & 0.0089 & 0.0092 & 0.00603 & & $U$ \\
\hline 2457 & UISO_IE_PLATE_AEA & STLRL & 0.000551 & $\mathrm{pCi} / \mathrm{L}$ & 0.0077 & 0.0079 & 0.0142 & & $\mathrm{U}$ \\
\hline 2458 & UISO_IE_PLATE_AEA & STLRL & 0.000723 & $\mathrm{pCi} / \mathrm{L}$ & 0.0057 & 0.006 & 0.0055 & & $U$ \\
\hline 2459 & UISO_IE_PLATE_AEA & STLRL & 0.0032 & $\mathrm{pCi} / \mathrm{L}$ & 0.0099 & 0.01 & 0.0182 & & $U$ \\
\hline 2460 & UISO_IE_PLATE_AEA & STLRL & 0.00508 & $\mathrm{pCi} / \mathrm{L}$ & 0.0079 & 0.0082 & 0.0102 & & $U$ \\
\hline 2461 & UISO_IE_PLATE_AEA & STLRL & 0.00286 & $\mathrm{pCi} / \mathrm{L}$ & 0.0076 & 0.0078 & 0.0114 & & $U$ \\
\hline 2462 & UISO_IE_PLATE_AEA & STLRL & 0.0025 & $\mathrm{pCi} / \mathrm{L}$ & 0.0057 & 0.0061 & 0.00389 & & U \\
\hline 2463 & UISO_IE_PLATE_AEA & STLRL & 0.0013 & $\mathrm{pCi} / \mathrm{L}$ & 0.0076 & 0.0079 & 0.0144 & & $U$ \\
\hline 2464 & UISO_IE_PLATE_AEA & QTESRL & 754 & $\mathrm{pCi} / \mathrm{L}$ & 10 & 120 & 0.323 & & \\
\hline 2465 & UISO IE PLATE AEA & QTESRL & 0.189 & $\mathrm{pCi} / \mathrm{L}$ & 0.032 & 0.044 & 0.00941 & & \\
\hline 2466 & UISO_IE_PLATE_AEA & QTESRL & 0.155 & $\mathrm{pCi} / \mathrm{L}$ & 0.034 & 0.043 & 0.0151 & & \\
\hline 2467 & UISO_IE_PLATE_AEA & QTESRL & 0.23 & $\mathrm{pCi} / \mathrm{L}$ & 0.047 & 0.061 & 0.0168 & & \\
\hline 2468 & UISO_IE_PLATE_AEA & QTESRL & 0.161 & $\mathrm{pCi} / \mathrm{L}$ & 0.035 & 0.044 & 0.0184 & & \\
\hline 2469 & UISO_IE_PLATE_AEA & QTESRL & 0.162 & $\mathrm{pCi} / \mathrm{L}$ & 0.037 & 0.046 & 0.0181 & & \\
\hline 2470 & UISO_IE_PLATE_AEA & QTESRL & 0.2 & $\mathrm{pCi} / \mathrm{L}$ & 0.038 & 0.051 & 0.0172 & & \\
\hline 2471 & UISO_IE_PLATE_AEA & QTESRL & 0.183 & $\mathrm{pCi} / \mathrm{L}$ & 0.04 & 0.05 & 0.0397 & & \\
\hline
\end{tabular}




\begin{tabular}{|c|c|c|c|c|c|c|c|c|c|}
\hline & $\mathrm{K}$ & $\mathrm{L}$ & $\mathrm{M}$ & $\mathrm{N}$ & $\mathrm{O}$ & $P$ & $\mathrm{Q}$ & $\mathrm{R}$ & $S$ \\
\hline 1 & METHOD_NAME & LAB_CODE & VALUE_RPTD & ANAL_UNITS_RPTD & COUNTING_ERROR & TOTAL_ANAL_ERROR & MIN_DETECTABLE_ACTIVITY & PCNT_MOISTURE & LAB_QUALIFIER \\
\hline 2472 & UISO_IE_PLATE_AEA & QTESRL & 0.203 & $\mathrm{pCi} / \mathrm{L}$ & 0.039 & 0.052 & 0.0216 & & \\
\hline 2473 & UISO_IE_PLATE_AEA & QTESRL & 0.222 & $\mathrm{pCi} / \mathrm{L}$ & 0.041 & 0.055 & 0.0389 & & \\
\hline 2474 & UISO_IE_PLATE_AEA & QTESRL & 0.212 & $\mathrm{pCi} / \mathrm{L}$ & 0.038 & 0.051 & 0.0159 & & \\
\hline 2475 & UISO_IE_PLATE_AEA & QTESRL & 0.147 & $\mathrm{pCi} / \mathrm{L}$ & 0.033 & 0.041 & 0.0215 & & \\
\hline 2476 & UISO_IE_PLATE_AEA & QTESRL & 0.213 & $\mathrm{pCi} / \mathrm{L}$ & 0.039 & 0.052 & 0.0188 & & \\
\hline 2477 & UISO_IE_PLATE_AEA & QTESRL & 0.177 & $\mathrm{pCi} / \mathrm{L}$ & 0.036 & 0.047 & 0.0186 & & \\
\hline 2478 & UISO_IE_PLATE_AEA & QTESRL & 0.185 & $\mathrm{pCi} / \mathrm{L}$ & 0.037 & 0.048 & 0.0156 & & \\
\hline 2479 & UISO_IE_PLATE_AEA & QTESRL & 0.39 & $\mathrm{pCi} / \mathrm{L}$ & 0.051 & 0.08 & 0.0156 & & \\
\hline 2480 & UISO_IE_PLATE_AEA & QTESRL & 0.184 & $\mathrm{pCi} / \mathrm{L}$ & 0.036 & 0.047 & 0.0167 & & \\
\hline 2481 & UISO_IE_PLATE_AEA & QTESRL & 0.228 & $\mathrm{pCi} / \mathrm{L}$ & 0.039 & 0.054 & 0.0119 & & \\
\hline 2482 & UISO_IE_PLATE_AEA & QTESRL & 0.21 & $\mathrm{pCi} / \mathrm{L}$ & 0.037 & 0.05 & 0.0141 & & \\
\hline 2483 & UISO_IE_PLATE_AEA & QTESRL & 0.189 & $\mathrm{pCi} / \mathrm{L}$ & 0.031 & 0.044 & 0.00745 & & \\
\hline 2484 & UISO_IE_PLATE_AEA & QTESRL & 491 & $\mathrm{pCi} / \mathrm{L}$ & 9.7 & 78 & 0.997 & & \\
\hline 2485 & UISO_IE_PLATE_AEA & QTESRL & 0.231 & $\mathrm{pCi} / \mathrm{L}$ & 0.04 & 0.054 & 0.0134 & & \\
\hline 2486 & UISO_IE_PLATE_AEA & QTESRL & 0.177 & $\mathrm{pCi} / \mathrm{L}$ & 0.034 & 0.045 & 0.0172 & & \\
\hline 2487 & UISO_IE_PLATE_AEA & STLRL & 0.173 & $\mathrm{pCi} / \mathrm{L}$ & 0.035 & 0.045 & 0.0141 & & \\
\hline 2488 & UISO_IE_PLATE_AEA & STLRL & 0.187 & $\mathrm{pCi} / \mathrm{L}$ & 0.036 & 0.048 & 0.0193 & & \\
\hline 2489 & UISO_IE_PLATE_AEA & STLRL & 0.238 & $\mathrm{pCi} / \mathrm{L}$ & 0.042 & 0.057 & 0.0132 & & \\
\hline 2490 & UISO_IE_PLATE_AEA & STLRL & 0.142 & $\mathrm{pCi} / \mathrm{L}$ & 0.031 & 0.041 & 0.0163 & & \\
\hline 2491 & UISO_IE_PLATE_AEA & STLRL & 0.181 & $\mathrm{pCi} / \mathrm{L}$ & 0.034 & 0.048 & 0.0151 & & \\
\hline 2492 & UISO_IE_PLATE_AEA & STLRL & 0.216 & $\mathrm{pCi} / \mathrm{L}$ & 0.038 & 0.055 & 0.0273 & & \\
\hline 2493 & UISO_IE_PLATE_AEA & STLRL & 27.7 & $\mathrm{pCi} / \mathrm{L}$ & 1.9 & 5.2 & 0.389 & & \\
\hline 2494 & UISO_IE_PLATE_AEA & STLRL & 1120 & $\mathrm{pCi} / \mathrm{L}$ & 13 & 200 & 0.562 & & \\
\hline 2495 & UISO_IE_PLATE_AEA & STLRL & 1280 & $\mathrm{pCi} / \mathrm{L}$ & 14 & 220 & 0.551 & & \\
\hline 2496 & UISO_IE_PLATE_AEA & STLRL & 0.157 & $\mathrm{pCi} / \mathrm{L}$ & 0.035 & 0.046 & 0.0344 & & \\
\hline 2497 & UISO_IE_PLATE_AEA & STLRL & 0.152 & $\mathrm{pCi} / \mathrm{L}$ & 0.033 & 0.044 & 0.0257 & & \\
\hline 2498 & UISO_IE_PLATE_AEA & STLRL & 0.144 & $\mathrm{pCi} / \mathrm{L}$ & 0.031 & 0.041 & 0.0175 & & \\
\hline 2499 & UISO_IE_PLATE_AEA & STLRL & 0.178 & $\mathrm{pCi} / \mathrm{L}$ & 0.046 & 0.057 & 0.0304 & & \\
\hline 2500 & UISO_IE_PLATE_AEA & STLRL & 0.17 & $\mathrm{pCi} / \mathrm{L}$ & 0.035 & 0.047 & 0.0126 & & \\
\hline 2501 & UISO_IE_PLATE_AEA & STLRL & 0.156 & $\mathrm{pCi} / \mathrm{L}$ & 0.032 & 0.043 & 0.0116 & & \\
\hline 2502 & UISO_IE_PLATE_AEA & STLRL & 0.166 & $\mathrm{pCi} / \mathrm{L}$ & 0.031 & 0.043 & 0.00995 & & \\
\hline 2503 & UISO_IE_PLATE_AEA & STLRL & 0.15 & $\mathrm{pCi} / \mathrm{L}$ & 0.034 & 0.045 & 0.00555 & & \\
\hline 2504 & UISO_IE_PLATE_AEA & STLRL & 0.152 & $\mathrm{pCi} / \mathrm{L}$ & 0.034 & 0.045 & 0.0134 & & \\
\hline 2505 & UISO_IE_PLATE_AEA & STLRL & 0.258 & $\mathrm{pCi} / \mathrm{L}$ & 0.047 & 0.066 & 0.0149 & & \\
\hline 2506 & UISO_IE_PLATE_AEA & STLRL & 0.163 & $\mathrm{pCi} / \mathrm{L}$ & 0.036 & 0.048 & 0.02 & & \\
\hline 2507 & UISO_IE_PLATE_AEA & STLRL & 0.156 & $\mathrm{pCi} / \mathrm{L}$ & 0.031 & 0.042 & 0.00429 & & \\
\hline 2508 & UISO_IE_PLATE_AEA & STLRL & 0.157 & $\mathrm{pCi} / \mathrm{L}$ & 0.033 & 0.044 & 0.0154 & & \\
\hline 2509 & UISO_IE_PLATE_AEA & STLRL & 0.198 & $\mathrm{pCi} / \mathrm{L}$ & 0.041 & 0.055 & 0.00592 & & \\
\hline
\end{tabular}




\begin{tabular}{|c|c|c|c|c|c|c|c|c|c|}
\hline & $\mathrm{K}$ & $\mathrm{L}$ & $\mathrm{M}$ & $\mathrm{N}$ & $\mathrm{O}$ & $P$ & $\mathrm{Q}$ & $\mathrm{R}$ & $\mathrm{S}$ \\
\hline 1 & METHOD_NAME & LAB_CODE & VALUE_RPTD & ANAL_UNITS_RPTD & COUNTING_ERROR & TOTAL_ANAL_ERROR & MIN_DETECTABLE_ACTIVITY & PCNT_MOISTURE & LAB_QUALIFIER \\
\hline 2510 & UISO_IE_PLATE_AEA & STLRL & 0.193 & $\mathrm{pCi} / \mathrm{L}$ & 0.04 & 0.054 & 0.0177 & & \\
\hline 2511 & UISO_IE_PLATE_AEA & STLRL & 0.166 & $\mathrm{pCi} / \mathrm{L}$ & 0.037 & 0.048 & 0.00565 & & \\
\hline 2512 & UISO_IE_PLATE_AEA & STLRL & 1880 & $\mathrm{pCi} / \mathrm{L}$ & 17 & 330 & 0.39 & & \\
\hline 2513 & UISO_IE_PLATE_AEA & STLRL & 0.149 & $\mathrm{pCi} / \mathrm{L}$ & 0.034 & 0.044 & 0.0341 & & \\
\hline 2514 & UISO_IE_PLATE_AEA & STLRL & 0.176 & $\mathrm{pCi} / \mathrm{L}$ & 0.044 & 0.056 & 0.0295 & & \\
\hline 2515 & UISO_IE_PLATE_AEA & STLRL & 0.163 & $\mathrm{pCi} / \mathrm{L}$ & 0.039 & 0.05 & 0.022 & & \\
\hline 2516 & UISO_IE_PLATE_AEA & STLRL & 0.149 & $\mathrm{pCi} / \mathrm{L}$ & 0.035 & 0.045 & 0.0174 & & \\
\hline 2517 & UISO_IE_PLATE_AEA & STLRL & 0.229 & $\mathrm{pCi} / \mathrm{L}$ & 0.042 & 0.059 & 0.0136 & & \\
\hline 2518 & UISO_IE_PLATE_AEA & STLRL & 0.141 & $\mathrm{pCi} / \mathrm{L}$ & 0.025 & 0.036 & 0.00317 & & \\
\hline 2519 & UISO_IE_PLATE_AEA & STLRL & 0.177 & $\mathrm{pCi} / \mathrm{L}$ & 0.028 & 0.043 & 0.00317 & & \\
\hline 2520 & UISO_IE_PLATE_AEA & STLRL & 0.154 & $\mathrm{pCi} / \mathrm{L}$ & 0.027 & 0.039 & 0.00321 & & \\
\hline 2521 & UISO_IE_PLATE_AEA & STLRL & 0.157 & $\mathrm{pCi} / \mathrm{L}$ & 0.029 & 0.041 & 0.0093 & & \\
\hline 2522 & UISO_IE_PLATE_AEA & STLRL & 0.156 & $\mathrm{pCi} / \mathrm{L}$ & 0.03 & 0.042 & 0.00405 & & \\
\hline 2523 & UISO_IE_PLATE_AEA & STLRL & 0.146 & $\mathrm{pCi} / \mathrm{L}$ & 0.032 & 0.042 & 0.0103 & & \\
\hline 2524 & UISO_IE_PLATE_AEA & STLRL & 0.166 & $\mathrm{pCi} / \mathrm{L}$ & 0.038 & 0.049 & 0.0149 & & \\
\hline 2525 & UISO_IE_PLATE_AEA & STLRL & 0.153 & $\mathrm{pCi} / \mathrm{L}$ & 0.033 & 0.043 & 0.01 & & \\
\hline 2526 & UISO_IE_PLATE_AEA & STLRL & 0.173 & $\mathrm{pCi} / \mathrm{L}$ & 0.039 & 0.051 & 0.025 & & \\
\hline 2527 & UISO_IE_PLATE_AEA & STLRL & 0.148 & $\mathrm{pCi} / \mathrm{L}$ & 0.027 & 0.038 & 0.00335 & & \\
\hline 2528 & UISO_IE_PLATE_AEA & STLRL & 0.135 & $\mathrm{pCi} / \mathrm{L}$ & 0.037 & 0.046 & 0.025 & & \\
\hline 2529 & UISO_IE_PLATE_AEA & STLRL & 0.137 & $\mathrm{pCi} / \mathrm{L}$ & 0.029 & 0.039 & 0.00927 & & \\
\hline 2530 & UISO_IE_PLATE_AEA & STLRL & 0.16 & $\mathrm{pCi} / \mathrm{L}$ & 0.032 & 0.043 & 0.00435 & & \\
\hline 2531 & UISO_IE_PLATE_AEA & STLRL & 0.16 & $\mathrm{pCi} / \mathrm{L}$ & 0.027 & 0.04 & 0.00322 & & \\
\hline 2532 & UISO_IE_PLATE_AEA & STLRL & 0.165 & $\mathrm{pCi} / \mathrm{L}$ & 0.03 & 0.043 & 0.00386 & & \\
\hline 2533 & UISO_IE_PLATE_AEA & STLRL & 0.149 & $\mathrm{pCi} / \mathrm{L}$ & 0.031 & 0.042 & 0.00925 & & \\
\hline 2534 & UISO_IE_PLATE_AEA & STLRL & 0.189 & $\mathrm{pCi} / \mathrm{L}$ & 0.042 & 0.055 & 0.0136 & & \\
\hline 2535 & UISO_IE_PLATE_AEA & STLRL & 0.174 & $\mathrm{pCi} / \mathrm{L}$ & 0.033 & 0.046 & 0.0108 & & \\
\hline 2536 & UISO_IE_PLATE_AEA & STLRL & 0.155 & $\mathrm{pCi} / \mathrm{L}$ & 0.038 & 0.048 & 0.018 & & \\
\hline 2537 & UISO_IE_PLATE_AEA & STLRL & 0.161 & $\mathrm{pCi} / \mathrm{L}$ & 0.039 & 0.05 & 0.0165 & & \\
\hline 2538 & UISO_IE_PLATE_AEA & STLRL & 0.163 & $\mathrm{pCi} / \mathrm{L}$ & 0.037 & 0.048 & 0.0146 & & \\
\hline 2539 & UISO_IE_PLATE_AEA & STLRL & 0.182 & $\mathrm{pCi} / \mathrm{L}$ & 0.034 & 0.048 & 0.0114 & & \\
\hline 2540 & UISO_IE_PLATE_AEA & STLRL & 0.174 & $\mathrm{pCi} / \mathrm{L}$ & 0.037 & 0.05 & 0.014 & & \\
\hline 2541 & UISO_IE_PLATE_AEA & STLRL & 0.164 & $\mathrm{pCi} / \mathrm{L}$ & 0.033 & 0.045 & 0.00944 & & \\
\hline 2542 & UISO_IE_PLATE_AEA & STLRL & 0.162 & $\mathrm{pCi} / \mathrm{L}$ & 0.029 & 0.041 & 0.0089 & & \\
\hline 2543 & UISO_IE_PLATE_AEA & STLRL & 0.147 & $\mathrm{pCi} / \mathrm{L}$ & 0.027 & 0.039 & 0.0074 & & \\
\hline 2544 & UISO_IE_PLATE_AEA & STLRL & 0.156 & $\mathrm{pCi} / \mathrm{L}$ & 0.032 & 0.043 & 0.00944 & & \\
\hline 2545 & UISO_IE_PLATE_AEA & STLRL & 0.167 & $\mathrm{pCi} / \mathrm{L}$ & 0.034 & 0.046 & 0.00496 & & \\
\hline 2546 & UISO_IE_PLATE_AEA & STLRL & 0.139 & $\mathrm{pCi} / \mathrm{L}$ & 0.03 & 0.04 & 0.00975 & & \\
\hline 2547 & UISO_IE_PLATE_AEA & STLRL & 0.13 & $\mathrm{pCi} / \mathrm{L}$ & 0.029 & 0.039 & 0.0119 & & \\
\hline
\end{tabular}




\begin{tabular}{|c|c|c|c|c|c|c|c|c|c|}
\hline & $\mathrm{K}$ & $\mathrm{L}$ & $\bar{M}$ & $\mathrm{~N}$ & $\mathrm{O}$ & $P$ & $\mathrm{Q}$ & $\mathrm{R}$ & $\mathrm{S}$ \\
\hline 1 & METHOD_NAME & LAB_CODE & VALUE_RPTD & ANAL_UNITS_RPTD & COUNTING_ERROR & TOTAL_ANAL_ERROR & MIN_DETECTABLE_ACTIVITY & PCNT_MOISTURE & LAB_QUALIFIER \\
\hline 2548 & UISO_IE_PLATE_AEA & STLRL & 0.162 & $\mathrm{pCi} / \mathrm{L}$ & 0.033 & 0.045 & 0.00987 & & \\
\hline 2549 & UISO_IE_PLATE_AEA & STLRL & 0.157 & $\mathrm{pCi} / \mathrm{L}$ & 0.031 & 0.042 & 0.0108 & & \\
\hline 2550 & UISO_IE_PLATE_AEA & STLRL & 0.16 & $\mathrm{pCi} / \mathrm{L}$ & 0.037 & 0.048 & 0.0173 & & \\
\hline 2551 & UISO_IE_PLATE_AEA & STLRL & 0.169 & $\mathrm{pCi} / \mathrm{L}$ & 0.038 & 0.049 & 0.00591 & & \\
\hline 2552 & UISO_IE_PLATE_AEA & STLRL & 0.168 & $\mathrm{pCi} / \mathrm{L}$ & 0.028 & 0.041 & 0.00325 & & \\
\hline 2553 & UISO_IE_PLATE_AEA & STLRL & 0.174 & $\mathrm{pCi} / \mathrm{L}$ & 0.03 & 0.044 & 0.00364 & & \\
\hline 2554 & UISO_IE_PLATE_AEA & STLRL & 0.161 & $\mathrm{pCi} / \mathrm{L}$ & 0.028 & 0.041 & 0.00352 & & \\
\hline 2555 & UISO_IE_PLATE_AEA & STLRL & 0.165 & $\mathrm{pCi} / \mathrm{L}$ & 0.036 & 0.048 & 0.0172 & & \\
\hline 2556 & UISO_IE_PLATE_AEA & STLRL & 0.202 & $\mathrm{pCi} / \mathrm{L}$ & 0.038 & 0.053 & 0.0158 & & \\
\hline 2557 & UISO_IE_PLATE_AEA & STLRL & 0.246 & $\mathrm{pCi} / \mathrm{L}$ & 0.04 & 0.06 & 0.0046 & & \\
\hline 2558 & UISO_IE_PLATE_AEA & STLRL & 0.166 & $\mathrm{pCi} / \mathrm{L}$ & 0.03 & 0.043 & 0.0097 & & \\
\hline 2559 & UISO_IE_PLATE_AEA & STLRL & 0.144 & $\mathrm{pCi} / \mathrm{L}$ & 0.027 & 0.038 & 0.00347 & & \\
\hline 2560 & UISO_IE_PLATE_AEA & STLRL & 0.134 & $\mathrm{pCi} / \mathrm{L}$ & 0.025 & 0.035 & 0.00328 & & \\
\hline 2561 & UISO_IE_PLATE_AEA & STLRL & 0.165 & $\mathrm{pCi} / \mathrm{L}$ & 0.038 & 0.049 & 0.0192 & & \\
\hline 2562 & UISO_IE_PLATE_AEA & STLRL & 0.155 & $\mathrm{pCi} / \mathrm{L}$ & 0.034 & 0.045 & 0.017 & & \\
\hline 2563 & UISO_IE_PLATE_AEA & STLRL & 0.311 & $\mathrm{pCi} / \mathrm{L}$ & 0.047 & 0.073 & 0.00487 & & \\
\hline 2564 & UISO_IE_PLATE_AEA & STLRL & 0.171 & $\mathrm{pCi} / \mathrm{L}$ & 0.03 & 0.043 & 0.0124 & & \\
\hline 2565 & UISO_IE_PLATE_AEA & STLRL & 0.144 & $\mathrm{pCi} / \mathrm{L}$ & 0.03 & 0.041 & 0.00935 & & \\
\hline 2566 & UISO_IE_PLATE_AEA & STLRL & 0.182 & $\mathrm{pCi} / \mathrm{L}$ & 0.036 & 0.049 & 0.0103 & & \\
\hline 2567 & UISO_IE_PLATE_AEA & STLRL & 0.162 & $\mathrm{pCi} / \mathrm{L}$ & 0.035 & 0.046 & 0.0107 & & \\
\hline 2568 & UISO_IE_PLATE_AEA & STLRL & 0.162 & $\mathrm{pCi} / \mathrm{L}$ & 0.033 & 0.045 & 0.01 & & \\
\hline 2569 & UISO_IE_PLATE_AEA & STLRL & 0.244 & $\mathrm{pCi} / \mathrm{L}$ & 0.034 & 0.055 & 0.00669 & & \\
\hline 2570 & UISO_IE_PLATE_AEA & STLRL & 0.187 & $\mathrm{pCi} / \mathrm{L}$ & 0.031 & 0.046 & 0.00911 & & \\
\hline 2571 & UISO_IE_PLATE_AEA & STLRL & 0.21 & $\mathrm{pCi} / \mathrm{L}$ & 0.035 & 0.052 & 0.00405 & & \\
\hline 2572 & UISO_IE_PLATE_AEA & STLRL & 0.797 & $\mathrm{pCi} / \mathrm{L}$ & 0.067 & 0.15 & 0.0098 & & \\
\hline 2573 & UISO_IE_PLATE_AEA & STLRL & 23.6 & $\mathrm{pCi} / \mathrm{L}$ & 0.37 & 4.1 & 0.00384 & & \\
\hline 2574 & UISO_IE_PLATE_AEA & STLRL & 0.993 & $\mathrm{pCi} / \mathrm{L}$ & 0.073 & 0.19 & 0.00363 & & \\
\hline 2575 & UISO_IE_PLATE_AEA & STLRL & 1.3 & $\mathrm{pCi} / \mathrm{L}$ & 0.082 & 0.24 & 0.00357 & & \\
\hline 2576 & UISO_IE_PLATE_AEA & STLRL & 61 & $\mathrm{pCi} / \mathrm{L}$ & 6.2 & 12 & 0.429 & & \\
\hline 2577 & UISO_IE_PLATE_AEA & STLRL & 0.53 & $\mathrm{pCi} / \mathrm{L}$ & 0.057 & 0.11 & 0.00866 & & \\
\hline 2578 & UISO_IE_PLATE_AEA & STLRL & 0.193 & $\mathrm{pCi} / \mathrm{L}$ & 0.046 & 0.058 & 0.00756 & & \\
\hline 2579 & UISO_IE_PLATE_AEA & STLRL & 0.206 & $\mathrm{pCi} / \mathrm{L}$ & 0.032 & 0.049 & 0.00739 & & \\
\hline 2580 & UISO_IE_PLATE_AEA & STLRL & 2500 & $\mathrm{pCi} / \mathrm{L}$ & 18 & 430 & 0.19 & & \\
\hline 2581 & UISO_IE_PLATE_AEA & STLRL & 0.236 & $\mathrm{pCi} / \mathrm{L}$ & 0.038 & 0.057 & 0.00431 & & \\
\hline 2582 & UISO_IE_PLATE_AEA & STLRL & 0.138 & $\mathrm{pCi} / \mathrm{L}$ & 0.027 & 0.037 & 0.00755 & & \\
\hline 2583 & UISO_IE_PLATE_AEA & STLRL & 0.177 & $\mathrm{pCi} / \mathrm{L}$ & 0.032 & 0.045 & 0.00393 & & \\
\hline 2584 & UISO_IE_PLATE_AEA & STLRL & 0.195 & $\mathrm{pCi} / \mathrm{L}$ & 0.031 & 0.047 & 0.00726 & & \\
\hline 2585 & UISO_IE_PLATE_AEA & STLRL & 1.12 & $\mathrm{pCi} / \mathrm{L}$ & 0.077 & 0.21 & 0.00355 & & \\
\hline
\end{tabular}




\begin{tabular}{|c|c|c|c|c|c|c|c|c|c|}
\hline & $\mathrm{K}$ & $\mathrm{L}$ & $\mathrm{M}$ & $\mathrm{N}$ & $\mathrm{O}$ & $P$ & $\mathrm{Q}$ & $\mathrm{R}$ & $\mathrm{S}$ \\
\hline 1 & METHOD_NAME & LAB_CODE & VALUE_RPTD & ANAL_UNITS_RPTD & COUNTING_ERROR & TOTAL_ANAL_ERROR & MIN_DETECTABLE_ACTIVITY & PCNT_MOISTURE & LAB_QUALIFIER \\
\hline 2586 & UISO_IE_PLATE_AEA & STLRL & 0.192 & $2 \mathrm{pCi} / \mathrm{L}$ & 0.037 & 0.051 & 0.00479 & & \\
\hline 2587 & UISO_IE_PLATE_AEA & STLRL & 0.175 & $5 \mathrm{pCi} / \mathrm{L}$ & 0.042 & 0.052 & 0.00652 & & \\
\hline 2588 & UISO_IE_PLATE_AEA & STLRL & 0.156 & $6 \mathrm{pCi} / \mathrm{L}$ & 0.028 & 0.039 & 0.00334 & & \\
\hline 2589 & UISO_IE_PLATE_AEA & STLRL & 0.162 & $2 \mathrm{pCi} / \mathrm{L}$ & 0.029 & 0.041 & 0.00341 & & \\
\hline 2590 & UISO_IE_PLATE_AEA & STLRL & 0.178 & $8 \mathrm{pCi} / \mathrm{L}$ & 0.037 & 0.049 & 0.0171 & & \\
\hline 2591 & UISO_IE_PLATE_AEA & STLRL & 0.151 & $1 \mathrm{pCi} / \mathrm{L}$ & 0.032 & 0.042 & 0.0125 & & \\
\hline 2592 & UISO_IE_PLATE_AEA & STLRL & 0.156 & $6 \mathrm{pCi} / \mathrm{L}$ & 0.029 & 0.04 & 0.00364 & & \\
\hline 2593 & UISO_IE_PLATE_AEA & STLRL & 0.201 & $1 \mathrm{pCi} / \mathrm{L}$ & 0.035 & 0.05 & 0.00837 & & \\
\hline 2594 & UISO_IE_PLATE_AEA & STLRL & 0.17 & $7 \mathrm{pCi} / \mathrm{L}$ & 0.038 & 0.048 & 0.0154 & & \\
\hline 2595 & UISO_IE_PLATE_AEA & STLRL & 0.192 & $2 \mathrm{pCi} / \mathrm{L}$ & 0.038 & 0.051 & 0.0141 & & \\
\hline 2596 & UISO_IE_PLATE_AEA & STLRL & 0.185 & $5 \mathrm{pCi} / \mathrm{L}$ & 0.037 & 0.05 & 0.0138 & & \\
\hline 2597 & UISO_IE_PLATE_AEA & STLRL & 0.186 & $6 \mathrm{pCi} / \mathrm{L}$ & 0.038 & 0.05 & 0.0104 & & \\
\hline 2598 & UISO_IE_PLATE_AEA & STLRL & 0.336 & $6 \mathrm{pCi} / \mathrm{L}$ & 0.051 & 0.078 & 0.0147 & & \\
\hline 2599 & UISO_IE_PLATE_AEA & STLRL & 0.162 & $2 \mathrm{pCi} / \mathrm{L}$ & 0.037 & 0.047 & 0.0154 & & \\
\hline 2600 & UISO_IE_PLATE_AEA & STLRL & 0.172 & $2 \mathrm{pCi} / \mathrm{L}$ & 0.03 & 0.043 & 0.00351 & & \\
\hline 2601 & UISO_IE_PLATE_AEA & STLRL & 0.175 & $5 \mathrm{pCi} / \mathrm{L}$ & 0.031 & 0.044 & 0.00751 & & \\
\hline 2602 & UISO_IE_PLATE_AEA & STLRL & 0.171 & $1 \mathrm{pCi} / \mathrm{L}$ & 0.042 & 0.052 & 0.00692 & & \\
\hline 2603 & UISO_IE_PLATE_AEA & STLRL & 0.177 & $7 \mathrm{pCi} / \mathrm{L}$ & 0.032 & 0.045 & 0.0098 & & \\
\hline 2604 & UISO_IE_PLATE_AEA & STLRL & 0.171 & $1 \mathrm{pCi} / \mathrm{L}$ & 0.028 & 0.042 & 0.00646 & & \\
\hline 2605 & UISO_IE_PLATE_AEA & STLRL & 0.174 & $4 \mathrm{pCi} / \mathrm{L}$ & 0.029 & 0.042 & 0.00308 & & \\
\hline 2606 & UISO_IE_PLATE_AEA & STLRL & 0.23 & $3 \mathrm{pCi} / \mathrm{L}$ & 0.061 & 0.074 & 0.0297 & & \\
\hline 2607 & UISO_IE_PLATE_AEA & STLRL & 326 & $6 \mathrm{pCi} / \mathrm{L}$ & 5.7 & 57 & 0.141 & & \\
\hline 2608 & UISO_IE_PLATE_AEA & STLRL & 0.226 & $6 \mathrm{pCi} / \mathrm{L}$ & 0.034 & 0.052 & 0.00339 & & \\
\hline 2609 & UISO_IE_PLATE_AEA & STLRL & 0.232 & $2 \mathrm{pCi} / \mathrm{L}$ & 0.037 & 0.055 & 0.00383 & & \\
\hline 2610 & UISO_IE_PLATE_AEA & STLRL & 0.161 & $1 \mathrm{pCi} / \mathrm{L}$ & 0.046 & 0.054 & 0.0237 & & \\
\hline 2611 & UISO_IE_PLATE_AEA & STLRL & 0.207 & $7 \mathrm{pCi} / \mathrm{L}$ & 0.035 & 0.05 & 0.00792 & & \\
\hline 2612 & UISO_IE_PLATE_AEA & STLRL & 0.22 & $2 \mathrm{pCi} / \mathrm{L}$ & 0.035 & 0.052 & 0.00748 & & \\
\hline 2613 & UISO_IE_PLATE_AEA & STLRL & 0.209 & $9 \mathrm{pCi} / \mathrm{L}$ & 0.032 & 0.049 & 0.00332 & & \\
\hline 2614 & UISO_IE_PLATE_AEA & STLRL & 0.291 & $1 \mathrm{pCi} / \mathrm{L}$ & 0.056 & 0.076 & 0.0291 & & \\
\hline 2615 & UISO_IE_PLATE_AEA & STLRL & 0.207 & $7 \mathrm{pCi} / \mathrm{L}$ & 0.042 & 0.056 & 0.0173 & & \\
\hline 2616 & UISO_IE_PLATE_AEA & STLRL & 0.255 & $5 \mathrm{pCi} / \mathrm{L}$ & 0.044 & 0.063 & 0.0126 & & \\
\hline 2617 & UISO_IE_PLATE_AEA & STLRL & 0.212 & $2 \mathrm{pCi} / \mathrm{L}$ & 0.046 & 0.059 & 0.0134 & & \\
\hline 2618 & UISO_IE_PLATE_AEA & STLRL & 0.187 & $7 \mathrm{pCi} / \mathrm{L}$ & 0.037 & 0.05 & 0.0173 & & \\
\hline 2619 & UISO_IE_PLATE_AEA & STLRL & 0.187 & $7 \mathrm{pCi} / \mathrm{L}$ & 0.062 & 0.071 & 0.0377 & & \\
\hline 2620 & UISO_IE_PLATE_AEA & STLRL & 0.204 & $4 \mathrm{pCi} / \mathrm{L}$ & 0.038 & 0.052 & 0.00461 & & \\
\hline 2621 & UISO_IE_PLATE_AEA & STLRL & 0.238 & $8 \mathrm{pCi} / \mathrm{L}$ & 0.036 & 0.055 & 0.0073 & & \\
\hline 2622 & UISO_IE_PLATE_AEA & STLRL & 0.233 & $3 \mathrm{pCi} / \mathrm{L}$ & 0.039 & 0.057 & 0.0108 & & \\
\hline 2623 & UISO_IE_PLATE_AEA & STLRL & 0.201 & $1 \mathrm{pCi} / \mathrm{L}$ & 0.031 & 0.047 & 0.00318 & & \\
\hline
\end{tabular}




\begin{tabular}{|c|c|c|c|c|c|c|c|c|c|}
\hline & $\mathrm{K}$ & $\mathrm{L}$ & $\mathrm{M}$ & $\mathrm{N}$ & $\mathrm{O}$ & $\mathrm{P}$ & $\mathrm{Q}$ & $\mathrm{R}$ & $\mathrm{S}$ \\
\hline 1 & METHOD_NAME & LAB_CODE & VALUE_RPTD & ANAL_UNITS_RPTD & COUNTING_ERROR & TOTAL_ANAL_ERROR & MIN_DETECTABLE_ACTIVITY & PCNT_MOISTURE & LAB_QUALIFIER \\
\hline 2624 & UISO_IE_PLATE_AEA & STLRL & 0.25 & $\mathrm{pCi} / \mathrm{L}$ & 0.04 & 0.06 & 0.0155 & & \\
\hline 2625 & UISO_IE_PLATE_AEA & STLRL & 425 & $\mathrm{pCi} / \mathrm{L}$ & 6.9 & 74 & 0.679 & & \\
\hline 2626 & UISO_IE_PLATE_AEA & STLRL & 0.22 & $\mathrm{pCi} / \mathrm{L}$ & 0.036 & 0.053 & 0.00805 & & \\
\hline 2627 & UISO_IE_PLATE_AEA & STLRL & 0.24 & $\mathrm{pCi} / \mathrm{L}$ & 0.044 & 0.062 & 0.0182 & & \\
\hline 2628 & UISO_IE_PLATE_AEA & STLRL & 1.58 & $\mathrm{pCi} / \mathrm{L}$ & 0.11 & 0.3 & 0.0217 & & \\
\hline 2629 & UISO_IE_PLATE_AEA & STLRL & 1.11 & $\mathrm{pCi} / \mathrm{L}$ & 0.077 & 0.21 & 0.00754 & & \\
\hline 2630 & UISO_IE_PLATE_AEA & STLRL & 1.96 & $\mathrm{pCi} / \mathrm{L}$ & 0.096 & 0.35 & 0.00806 & & \\
\hline 2631 & UISO_IE_PLATE_AEA & STLRL & 1.4 & $\mathrm{pCi} / \mathrm{L}$ & 0.096 & 0.26 & 0.0152 & & \\
\hline 2632 & UISO_IE_PLATE_AEA & STLRL & 0.467 & $\mathrm{pCi} / \mathrm{L}$ & 0.047 & 0.094 & 0.00792 & & \\
\hline 2633 & UISO_IE_PLATE_AEA & STLRL & 0.305 & $\mathrm{pCi} / \mathrm{L}$ & 0.039 & 0.066 & 0.00679 & & \\
\hline 2634 & UISO_IE_PLATE_AEA & STLRL & 0.171 & $\mathrm{pCi} / \mathrm{L}$ & 0.029 & 0.042 & 0.00686 & & \\
\hline 2635 & UISO_IE_PLATE_AEA & STLRL & 34.2 & $\mathrm{pCi} / \mathrm{L}$ & 0.41 & 6 & 0.0083 & & \\
\hline 2636 & UISO_IE_PLATE_AEA & STLRL & 0.425 & $\mathrm{pCi} / \mathrm{L}$ & 0.046 & 0.088 & 0.00336 & & \\
\hline 2637 & UISO_IE_PLATE_AEA & STLRL & 0.227 & $\mathrm{pCi} / \mathrm{L}$ & 0.033 & 0.052 & 0.0032 & & \\
\hline 2638 & UISO_IE_PLATE_AEA & STLRL & 25.3 & $\mathrm{pCi} / \mathrm{L}$ & 0.77 & 4.7 & 0.027 & & \\
\hline 2639 & UISO_IE_PLATE_AEA & STLRL & 0.729 & $\mathrm{pCi} / \mathrm{L}$ & 0.085 & 0.16 & 0.0138 & & \\
\hline 2640 & UISO_IE_PLATE_AEA & STLRL & 0.239 & $\mathrm{pCi} / \mathrm{L}$ & 0.034 & 0.054 & 0.00332 & & \\
\hline 2641 & UISO_IE_PLATE_AEA & STLRL & 0.202 & $\mathrm{pCi} / \mathrm{L}$ & 0.032 & 0.048 & 0.00347 & & \\
\hline 2642 & UISO_IE_PLATE_AEA & STLRL & 0.16 & $\mathrm{pCi} / \mathrm{L}$ & 0.032 & 0.043 & 0.00916 & & \\
\hline 2643 & UISO_IE_PLATE_AEA & STLRL & 0.258 & $\mathrm{pCi} / \mathrm{L}$ & 0.037 & 0.058 & 0.00354 & & \\
\hline 2644 & UISO_IE_PLATE_AEA & STLRL & 0.223 & $\mathrm{pCi} / \mathrm{L}$ & 0.035 & 0.052 & 0.0105 & & \\
\hline 2645 & UISO_IE_PLATE_AEA & STLRL & 0.221 & $\mathrm{pCi} / \mathrm{L}$ & 0.032 & 0.05 & 0.00318 & & \\
\hline 2646 & UISO_IE_PLATE_AEA & STLRL & 0.206 & $\mathrm{pCi} / \mathrm{L}$ & 0.033 & 0.048 & 0.00355 & & \\
\hline 2647 & UISO_IE_PLATE_AEA & STLRL & 0.187 & $\mathrm{pCi} / \mathrm{L}$ & 0.031 & 0.045 & 0.00352 & & \\
\hline 2648 & UISO_IE_PLATE_AEA & STLRL & 0.196 & $\mathrm{pCi} / \mathrm{L}$ & 0.032 & 0.047 & 0.00353 & & \\
\hline 2649 & UISO_IE_PLATE_AEA & STLRL & 0.278 & $\mathrm{pCi} / \mathrm{L}$ & 0.039 & 0.062 & 0.00362 & & \\
\hline 2650 & UISO_IE_PLATE_AEA & STLRL & 0.206 & $\mathrm{pCi} / \mathrm{L}$ & 0.034 & 0.049 & 0.0116 & & \\
\hline 2651 & UISO_IE_PLATE_AEA & STLRL & 0.19 & $\mathrm{pCi} / \mathrm{L}$ & 0.037 & 0.05 & 0.0103 & & \\
\hline 2652 & UISO_IE_PLATE_AEA & STLRL & 0.199 & $\mathrm{pCi} / \mathrm{L}$ & 0.038 & 0.051 & 0.0121 & & \\
\hline 2653 & UISO_IE_PLATE_AEA & STLRL & 0.244 & $\mathrm{pCi} / \mathrm{L}$ & 0.045 & 0.062 & 0.0223 & & \\
\hline 2654 & UISO_IE_PLATE_AEA & STLRL & 0.23 & $\mathrm{pCi} / \mathrm{L}$ & 0.061 & 0.075 & 0.0347 & & \\
\hline 2655 & UISO_IE_PLATE_AEA & STLRL & 0.228 & $\mathrm{pCi} / \mathrm{L}$ & 0.061 & 0.075 & 0.0271 & & \\
\hline 2656 & UISO_IE_PLATE_AEA & STLRL & 0.235 & $\mathrm{pCi} / \mathrm{L}$ & 0.043 & 0.061 & 0.0151 & & \\
\hline 2657 & UISO_IE_PLATE_AEA & STLRL & 0.208 & $\mathrm{pCi} / \mathrm{L}$ & 0.042 & 0.057 & 0.0138 & & \\
\hline 2658 & UISO_IE_PLATE_AEA & STLRL & 0.189 & $\mathrm{pCi} / \mathrm{L}$ & 0.038 & 0.051 & 0.0102 & & \\
\hline 2659 & UISO_IE_PLATE_AEA & STLRL & 0.175 & $\mathrm{pCi} / \mathrm{L}$ & 0.051 & 0.06 & 0.0238 & & \\
\hline 2660 & UISO_IE_PLATE_AEA & STLRL & 2240 & $\mathrm{pCi} / \mathrm{L}$ & 55 & 410 & 3.31 & & \\
\hline 2661 & UISO_IE_PLATE_AEA & STLRL & 0.22 & $\mathrm{pCi} / \mathrm{L}$ & 0.057 & 0.07 & 0.0283 & & \\
\hline
\end{tabular}




\begin{tabular}{|c|c|c|c|c|c|c|c|c|c|}
\hline & $\mathrm{K}$ & $\mathrm{L}$ & $\bar{M}$ & $\mathrm{~N}$ & $\mathrm{O}$ & $P$ & $\mathrm{Q}$ & $\mathrm{R}$ & $\mathrm{S}$ \\
\hline 1 & METHOD_NAME & LAB_CODE & VALUE_RPTD & ANAL_UNITS_RPTD & COUNTING_ERROR & TOTAL_ANAL_ERROR & MIN_DETECTABLE_ACTIVITY & PCNT_MOISTURE & LAB_QUALIFIER \\
\hline 2662 & UISO_IE_PLATE_AEA & STLRL & 0.259 & $\mathrm{pCi} / \mathrm{L}$ & 0.059 & 0.076 & 0.0277 & & \\
\hline 2663 & UISO IE_PLATE_AEA & STLRL & 0.215 & $\mathrm{pCi} / \mathrm{L}$ & 0.039 & 0.056 & 0.0136 & & \\
\hline 2664 & UISO_IE_PLATE_AEA & STLRL & 0.13 & $\mathrm{pCi} / \mathrm{L}$ & 0.034 & 0.042 & 0.0141 & & \\
\hline 2665 & UISO_IE_PLATE_AEA & STLRL & 13.4 & $\mathrm{pCi} / \mathrm{L}$ & 0.29 & 2.4 & 0.00417 & & \\
\hline 2666 & UISO_IE_PLATE_AEA & STLRL & 47.5 & $\mathrm{pCi} / \mathrm{L}$ & 0.5 & 8.6 & 0.0101 & & \\
\hline 2667 & RADISOTOTOPES_ICPMS & PNL329 & 0.102897804 & $\mathrm{pCi} / \mathrm{L}$ & & & & & \\
\hline 2668 & RADISOTOPES_ICPMS & PNL329 & 9.21723 & $\mathrm{pCi} / \mathrm{L}$ & & & & & \\
\hline 2669 & RADISOTOPES_ICPMS & PNL329 & 34.019958 & $\mathrm{pCi} / \mathrm{L}$ & & & & & \\
\hline 2670 & RADISOTOPES_ICPMS & PNL329 & 0.131957216 & $\mathrm{pCi} / \mathrm{L}$ & & & & & \\
\hline 2671 & UISO_IE_PLATE_AEA & STLRL & 0.22 & $\mathrm{pCi} / \mathrm{L}$ & 0.035 & 0.053 & 0.00769 & & \\
\hline 2672 & UISO_IE_PLATE_AEA & STLRL & 0.156 & $\mathrm{pCi} / \mathrm{L}$ & 0.031 & 0.042 & 0.0099 & & \\
\hline 2673 & UISO_IE_PLATE_AEA & STLRL & 0.343 & $\mathrm{pCi} / \mathrm{L}$ & 0.047 & 0.078 & 0.00427 & & \\
\hline 2674 & UISO_IE_PLATE_AEA & STLRL & 4.85 & $\mathrm{pCi} / \mathrm{L}$ & 0.17 & 0.89 & 0.0123 & & \\
\hline 2675 & UISO_IE_PLATE_AEA & STLRL & 1.56 & $\mathrm{pCi} / \mathrm{L}$ & 0.096 & 0.3 & 0.00394 & & \\
\hline 2676 & UISO_IE_PLATE_AEA & STLRL & 0.442 & $\mathrm{pCi} / \mathrm{L}$ & 0.048 & 0.093 & 0.00727 & & \\
\hline 2677 & UISO_IE_PLATE_AEA & STLRL & 1.27 & $\mathrm{pCi} / \mathrm{L}$ & 0.084 & 0.25 & 0.00947 & & \\
\hline 2678 & UISO_IE_PLATE_AEA & STLRL & 0.482 & $\mathrm{pCi} / \mathrm{L}$ & 0.051 & 0.1 & 0.00746 & & \\
\hline 2679 & UISO_IE_PLATE_AEA & STLRL & 0.354 & $\mathrm{pCi} / \mathrm{L}$ & 0.045 & 0.078 & 0.00946 & & \\
\hline 2680 & UISO_IE_PLATE_AEA & STLRL & 0.374 & $\mathrm{pCi} / \mathrm{L}$ & 0.049 & 0.084 & 0.00424 & & \\
\hline 2681 & UISO_IE_PLATE_AEA & STLRL & 27.8 & $\mathrm{pCi} / \mathrm{L}$ & 0.4 & 5 & 0.00828 & & \\
\hline 2682 & UISO_IE_PLATE_AEA & STLRL & 1.17 & $\mathrm{pCi} / \mathrm{L}$ & 0.1 & 0.24 & 0.0121 & & \\
\hline 2683 & UISO_IE_PLATE_AEA & STLRL & 0.348 & $\mathrm{pCi} / \mathrm{L}$ & 0.05 & 0.081 & 0.00486 & & \\
\hline 2684 & UISO_IE_PLATE_AEA & STLRL & 4.26 & $\mathrm{pCi} / \mathrm{L}$ & 0.16 & 0.78 & 0.00797 & & \\
\hline 2685 & UISO_IE_PLATE_AEA & STLRL & 4.62 & $\mathrm{pCi} / \mathrm{L}$ & 0.17 & 0.85 & 0.0128 & & \\
\hline 2686 & UISO_IE_PLATE_AEA & STLRL & 1.57 & $\mathrm{pCi} / \mathrm{L}$ & 0.092 & 0.3 & 0.0076 & & \\
\hline 2687 & UISO_IE_PLATE_AEA & STLRL & 0.542 & $\mathrm{pCi} / \mathrm{L}$ & 0.055 & 0.11 & 0.00373 & & \\
\hline 2688 & UISO_IE_PLATE_AEA & STLRL & 4.48 & $\mathrm{pCi} / \mathrm{L}$ & 0.15 & 0.82 & 0.0101 & & \\
\hline 2689 & UISO_IE_PLATE_AEA & STLRL & 0.627 & $\mathrm{pCi} / \mathrm{L}$ & 0.058 & 0.13 & 0.00748 & & \\
\hline 2690 & UISO_IE_PLATE_AEA & STLRL & 1.28 & $\mathrm{pCi} / \mathrm{L}$ & 0.082 & 0.24 & 0.00896 & & \\
\hline 2691 & UISO_IE_PLATE_AEA & STLRL & 2.48 & $\mathrm{pCi} / \mathrm{L}$ & 0.12 & 0.47 & 0.00404 & & \\
\hline 2692 & UISO_IE_PLATE_AEA & STLRL & 0.609 & $\mathrm{pCi} / \mathrm{L}$ & 0.059 & 0.13 & 0.00385 & & \\
\hline 2693 & UISO_IE_PLATE_AEA & STLRL & 0.437 & $\mathrm{pCi} / \mathrm{L}$ & 0.06 & 0.1 & 0.0113 & & \\
\hline 2694 & UISO_IE_PLATE_AEA & STLRL & 0.454 & $\mathrm{pCi} / \mathrm{L}$ & 0.058 & 0.1 & 0.0178 & & \\
\hline 2695 & UISO_IE_PLATE_AEA & STLRL & 0.433 & $\mathrm{pCi} / \mathrm{L}$ & 0.05 & 0.093 & 0.00797 & & \\
\hline 2696 & UISO_IE_PLATE_AEA & STLRL & 0.407 & $\mathrm{pCi} / \mathrm{L}$ & 0.049 & 0.089 & 0.0125 & & \\
\hline 2697 & UISO_IE_PLATE_AEA & STLRL & 0.149 & $\mathrm{pCi} / \mathrm{L}$ & 0.034 & 0.044 & 0.0106 & & \\
\hline 2698 & UISO_IE_PLATE_AEA & STLRL & 0.378 & $\mathrm{pCi} / \mathrm{L}$ & 0.048 & 0.084 & 0.00401 & & \\
\hline 2699 & UISO_IE_PLATE_AEA & STLRL & 0.255 & $\mathrm{pCi} / \mathrm{L}$ & 0.04 & 0.062 & 0.00415 & & \\
\hline
\end{tabular}




\begin{tabular}{|c|c|c|c|c|c|c|c|c|c|}
\hline & $\mathrm{K}$ & $\mathrm{L}$ & $\bar{M}$ & $\mathrm{~N}$ & $\mathrm{O}$ & $P$ & $\mathrm{Q}$ & $\mathrm{R}$ & $\mathrm{S}$ \\
\hline 1 & METHOD_NAME & LAB_CODE & VALUE_RPTD & ANAL_UNITS_RPTD & COUNTING_ERROR & TOTAL_ANAL_ERROR & MIN_DETECTABLE_ACTIVITY & PCNT_MOISTURE & LAB_QUALIFIER \\
\hline 2700 & UISO_IE_PLATE_AEA & STLRL & 0.222 & $\mathrm{pCi} / \mathrm{L}$ & 0.041 & 0.058 & 0.016 & & \\
\hline 2701 & UISO_IE_PLATE_AEA & STLRL & 0.254 & $\mathrm{pCi} / \mathrm{L}$ & 0.037 & 0.06 & 0.00919 & & \\
\hline 2702 & UISO_IE_PLATE_AEA & STLRL & 0.639 & $\mathrm{pCi} / \mathrm{L}$ & 0.061 & 0.13 & 0.00385 & & \\
\hline 2703 & UISO_IE_PLATE_AEA & STLRL & 0.287 & $\mathrm{pCi} / \mathrm{L}$ & 0.046 & 0.07 & 0.0104 & & \\
\hline 2704 & UISO_IE_PLATE_AEA & STLRL & 0.278 & $\mathrm{pCi} / \mathrm{L}$ & 0.04 & 0.065 & 0.00384 & & \\
\hline 2705 & UISO_IE_PLATE_AEA & STLRL & 0.172 & $\mathrm{pCi} / \mathrm{L}$ & 0.036 & 0.048 & 0.0104 & & \\
\hline 2706 & UISO_IE_PLATE_AEA & STLRL & 0.152 & $\mathrm{pCi} / \mathrm{L}$ & 0.03 & 0.041 & 0.00398 & & \\
\hline 2707 & UISO_IE_PLATE_AEA & STLRL & 0.189 & $\mathrm{pCi} / \mathrm{L}$ & 0.036 & 0.05 & 0.0095 & & \\
\hline 2708 & UISO_IE_PLATE_AEA & STLRL & 0.184 & $\mathrm{pCi} / \mathrm{L}$ & 0.036 & 0.049 & 0.00459 & & \\
\hline 2709 & UISO_IE_PLATE_AEA & STLRL & 0.148 & $\mathrm{pCi} / \mathrm{L}$ & 0.033 & 0.043 & 0.00979 & & \\
\hline 2710 & UISO_IE_PLATE_AEA & STLRL & 0.21 & $\mathrm{pCi} / \mathrm{L}$ & 0.046 & 0.06 & 0.0164 & & \\
\hline 2711 & UISO_IE_PLATE_AEA & STLRL & 0.204 & $\mathrm{pCi} / \mathrm{L}$ & 0.038 & 0.053 & 0.00466 & & \\
\hline 2712 & UISO_IE_PLATE_AEA & STLRL & 0.192 & $\mathrm{pCi} / \mathrm{L}$ & 0.035 & 0.05 & 0.00421 & & \\
\hline 2713 & UISO_IE_PLATE_AEA & STLRL & 0.164 & $\mathrm{pCi} / \mathrm{L}$ & 0.031 & 0.043 & 0.00389 & & \\
\hline 2714 & UISO_IE_PLATE_AEA & STLRL & 0.197 & $\mathrm{pCi} / \mathrm{L}$ & 0.041 & 0.055 & 0.0143 & & \\
\hline 2715 & UISO_IE_PLATE_AEA & STLRL & 0.245 & $\mathrm{pCi} / \mathrm{L}$ & 0.039 & 0.06 & 0.00876 & & \\
\hline 2716 & UISO_IE_PLATE_AEA & STLRL & 0.167 & $\mathrm{pCi} / \mathrm{L}$ & 0.036 & 0.048 & 0.00514 & & \\
\hline 2717 & UISO_IE_PLATE_AEA & STLRL & 0.197 & $\mathrm{pCi} / \mathrm{L}$ & 0.047 & 0.06 & 0.00741 & & \\
\hline 2718 & UISO_IE_PLATE_AEA & STLRL & 0.144 & $\mathrm{pCi} / \mathrm{L}$ & 0.036 & 0.045 & 0.0146 & & \\
\hline 2719 & UISO_IE_PLATE_AEA & STLRL & 0.186 & $\mathrm{pCi} / \mathrm{L}$ & 0.037 & 0.05 & 0.00475 & & \\
\hline 2720 & UISO_IE_PLATE_AEA & STLRL & 0.165 & $\mathrm{pCi} / \mathrm{L}$ & 0.036 & 0.047 & 0.00503 & & \\
\hline 2721 & UISO_IE_PLATE_AEA & STLRL & 0.157 & $\mathrm{pCi} / \mathrm{L}$ & 0.034 & 0.044 & 0.0097 & & \\
\hline 2722 & UISO_IE_PLATE_AEA & STLRL & 0.152 & $\mathrm{pCi} / \mathrm{L}$ & 0.03 & 0.041 & 0.00388 & & \\
\hline 2723 & UISO_IE_PLATE_AEA & STLRL & 0.16 & $\mathrm{pCi} / \mathrm{L}$ & 0.031 & 0.043 & 0.00843 & & \\
\hline 2724 & UISO_IE_PLATE_AEA & STLRL & 0.16 & $\mathrm{pCi} / \mathrm{L}$ & 0.037 & 0.048 & 0.00573 & & \\
\hline 2725 & UISO_IE_PLATE_AEA & STLRL & 0.198 & $\mathrm{pCi} / \mathrm{L}$ & 0.035 & 0.051 & 0.0086 & & \\
\hline 2726 & UISO_IE_PLATE_AEA & STLRL & 0.173 & $\mathrm{pCi} / \mathrm{L}$ & 0.032 & 0.045 & 0.00801 & & \\
\hline 2727 & UISO_IE_PLATE_AEA & STLRL & 0.193 & $\mathrm{pCi} / \mathrm{L}$ & 0.041 & 0.055 & 0.0145 & & \\
\hline 2728 & UISO_IE_PLATE_AEA & STLRL & 0.218 & $\mathrm{pCi} / \mathrm{L}$ & 0.035 & 0.053 & 0.00367 & & \\
\hline 2729 & UISO_IE_PLATE_AEA & STLRL & 0.215 & $\mathrm{pCi} / \mathrm{L}$ & 0.046 & 0.061 & 0.0135 & & \\
\hline 2730 & UISO_IE_PLATE_AEA & STLRL & 0.196 & $\mathrm{pCi} / \mathrm{L}$ & 0.035 & 0.05 & 0.00401 & & \\
\hline 2731 & UISO_IE_PLATE_AEA & STLRL & 0.194 & $\mathrm{pCi} / \mathrm{L}$ & 0.036 & 0.051 & 0.00892 & & \\
\hline 2732 & UISO_IE_PLATE_AEA & STLRL & 0.198 & $\mathrm{pCi} / \mathrm{L}$ & 0.033 & 0.049 & 0.00765 & & \\
\hline 2733 & UISO_IE_PLATE_AEA & STLRL & 0.166 & $\mathrm{pCi} / \mathrm{L}$ & 0.031 & 0.044 & 0.00387 & & \\
\hline 2734 & UISO_IE_PLATE_AEA & STLRL & 0.173 & $\mathrm{pCi} / \mathrm{L}$ & 0.031 & 0.045 & 0.00375 & & \\
\hline 2735 & UISO_IE_PLATE_AEA & STLRL & 0.175 & $\mathrm{pCi} / \mathrm{L}$ & 0.033 & 0.046 & 0.00866 & & \\
\hline 2736 & UISO_IE_PLATE_AEA & STLRL & 0.211 & $\mathrm{pCi} / \mathrm{L}$ & 0.039 & 0.055 & 0.0123 & & \\
\hline 2737 & UISO_IE_PLATE_AEA & STLRL & 0.261 & $\mathrm{pCi} / \mathrm{L}$ & 0.038 & 0.061 & 0.00373 & & \\
\hline
\end{tabular}




\begin{tabular}{|c|c|c|c|c|c|c|c|c|c|}
\hline & $\mathrm{K}$ & $\mathrm{L}$ & $\bar{M}$ & $\mathrm{~N}$ & $\mathrm{O}$ & $P$ & $\mathrm{Q}$ & $\mathrm{R}$ & $\mathrm{S}$ \\
\hline 1 & METHOD_NAME & LAB_CODE & VALUE_RPTD & ANAL_UNITS_RPTD & COUNTING_ERROR & TOTAL_ANAL_ERROR & MIN_DETECTABLE_ACTIVITY & PCNT_MOISTURE & LAB_QUALIFIER \\
\hline 2738 & UISO_IE_PLATE_AEA & STLRL & 0.222 & $\mathrm{pCi} / \mathrm{L}$ & 0.037 & 0.055 & 0.0105 & & \\
\hline 2739 & UISO_IE_PLATE_AEA & STLRL & 0.187 & $\mathrm{pCi} / \mathrm{L}$ & 0.033 & 0.048 & 0.00386 & & \\
\hline 2740 & UISO_IE_PLATE_AEA & STLRL & 0.216 & $\mathrm{pCi} / \mathrm{L}$ & 0.034 & 0.052 & 0.00351 & & \\
\hline 2741 & UISO_IE_PLATE_AEA & STLRL & 0.191 & $\mathrm{pCi} / \mathrm{L}$ & 0.033 & 0.048 & 0.00767 & & \\
\hline 2742 & UISO_IE_PLATE_AEA & STLRL & 0.161 & $\mathrm{pCi} / \mathrm{L}$ & 0.03 & 0.042 & 0.0036 & & \\
\hline 2743 & UISO_IE_PLATE_AEA & STLRL & 0.213 & $\mathrm{pCi} / \mathrm{L}$ & 0.036 & 0.053 & 0.00393 & & \\
\hline 2744 & UISO_IE_PLATE_AEA & STLRL & 0.184 & $\mathrm{pCi} / \mathrm{L}$ & 0.033 & 0.047 & 0.00394 & & \\
\hline 2745 & UISO_IE_PLATE_AEA & STLRL & 0.787 & $\mathrm{pCi} / \mathrm{L}$ & 0.069 & 0.16 & 0.00844 & & \\
\hline 2746 & UISO_IE_PLATE_AEA & STLRL & 0.183 & $\mathrm{pCi} / \mathrm{L}$ & 0.032 & 0.046 & 0.00772 & & \\
\hline 2747 & UISO_IE_PLATE_AEA & STLRL & 0.22 & $\mathrm{pCi} / \mathrm{L}$ & 0.035 & 0.053 & 0.00752 & & \\
\hline 2748 & UISO_IE_PLATE_AEA & STLRL & 0.19 & $\mathrm{pCi} / \mathrm{L}$ & 0.032 & 0.047 & 0.0035 & & \\
\hline 2749 & UISO_IE_PLATE_AEA & STLRL & 0.16 & $\mathrm{pCi} / \mathrm{L}$ & 0.03 & 0.042 & 0.00365 & & \\
\hline 2750 & UISO_IE_PLATE_AEA & STLRL & 0.191 & $\mathrm{pCi} / \mathrm{L}$ & 0.032 & 0.048 & 0.0036 & & \\
\hline 2751 & UISO_IE_PLATE_AEA & STLRL & 0.359 & $\mathrm{pCi} / \mathrm{L}$ & 0.046 & 0.08 & 0.00386 & & \\
\hline 2752 & UISO_IE_PLATE_AEA & STLRL & 0.202 & $\mathrm{pCi} / \mathrm{L}$ & 0.035 & 0.051 & 0.00396 & & \\
\hline 2753 & UISO_IE_PLATE_AEA & STLRL & 0.167 & $\mathrm{pCi} / \mathrm{L}$ & 0.034 & 0.046 & 0.0111 & & \\
\hline 2754 & UISO_IE_PLATE_AEA & STLRL & 0.168 & $\mathrm{pCi} / \mathrm{L}$ & 0.032 & 0.044 & 0.0082 & & \\
\hline 2755 & UISO_IE_PLATE_AEA & STLRL & 0.21 & $\mathrm{pCi} / \mathrm{L}$ & 0.038 & 0.054 & 0.00445 & & \\
\hline 2756 & UISO_IE_PLATE_AEA & STLRL & 0.197 & $\mathrm{pCi} / \mathrm{L}$ & 0.041 & 0.055 & 0.0139 & & \\
\hline 2757 & UISO_IE_PLATE_AEA & STLRL & 0.293 & $\mathrm{pCi} / \mathrm{L}$ & 0.041 & 0.067 & 0.00787 & & \\
\hline 2758 & UISO_IE_PLATE_AEA & STLRL & 0.2 & $\mathrm{pCi} / \mathrm{L}$ & 0.037 & 0.052 & 0.00452 & & \\
\hline 2759 & UISO_IE_PLATE_AEA & STLRL & 0.213 & $\mathrm{pCi} / \mathrm{L}$ & 0.035 & 0.052 & 0.00954 & & \\
\hline 2760 & UISO_IE_PLATE_AEA & STLRL & 33.8 & $\mathrm{pCi} / \mathrm{L}$ & 0.43 & 6.1 & 0.00929 & & \\
\hline 2761 & UISO_IE_PLATE_AEA & STLRL & 31.6 & $\mathrm{pCi} / \mathrm{L}$ & 0.49 & 5.8 & 0.0107 & & \\
\hline 2762 & UISO_IE_PLATE_AEA & STLRL & 46.8 & $\mathrm{pCi} / \mathrm{L}$ & 0.56 & 8.5 & 0.0045 & & \\
\hline 2763 & UISO_IE_PLATE_AEA & STLRL & 51.8 & $\mathrm{pCi} / \mathrm{L}$ & 0.57 & 9.4 & 0.00901 & & \\
\hline 2764 & UISO_IE_PLATE_AEA & STLRL & 51.8 & $\mathrm{pCi} / \mathrm{L}$ & 0.59 & 9.4 & 0.00459 & & \\
\hline 2765 & UISO_IE_PLATE_AEA & STLRL & 67.9 & $\mathrm{pCi} / \mathrm{L}$ & 0.64 & 12 & 0.0142 & & \\
\hline 2766 & UISO_IE_PLATE_AEA & STLRL & 16.1 & $\mathrm{pCi} / \mathrm{L}$ & 0.3 & 2.9 & 0.00794 & & \\
\hline 2767 & UISO_IE_PLATE_AEA & STLRL & 64.7 & $\mathrm{pCi} / \mathrm{L}$ & 0.63 & 12 & 0.0121 & & \\
\hline 2768 & UISO_IE_PLATE_AEA & STLRL & 31.2 & $\mathrm{pCi} / \mathrm{L}$ & 0.45 & 5.7 & 0.00435 & & \\
\hline 2769 & UISO_IE_PLATE_AEA & STLRL & 31.5 & $\mathrm{pCi} / \mathrm{L}$ & 0.55 & 5.8 & 0.0188 & & \\
\hline 2770 & UISO_IE_PLATE_AEA & STLRL & 30.9 & $\mathrm{pCi} / \mathrm{L}$ & 0.51 & 5.6 & 0.012 & & \\
\hline 2771 & UISO_IE_PLATE_AEA & STLRL & 34.6 & $\mathrm{pCi} / \mathrm{L}$ & 0.49 & 6.3 & 0.0188 & & \\
\hline 2772 & UISO_IE_PLATE_AEA & STLRL & 0.142 & $\mathrm{pCi} / \mathrm{L}$ & 0.037 & 0.046 & 0.0158 & & \\
\hline 2773 & UISO_IE_PLATE_AEA & STLRL & 0.223 & $\mathrm{pCi} / \mathrm{L}$ & 0.044 & 0.06 & 0.00568 & & \\
\hline 2774 & UISO_IE_PLATE_AEA & STLRL & 2.41 & $\mathrm{pCi} / \mathrm{L}$ & 0.15 & 0.46 & 0.0188 & & \\
\hline 2775 & UISO_IE_PLATE_AEA & STLRL & 0.39 & $\mathrm{pCi} / \mathrm{L}$ & 0.045 & 0.084 & 0.00726 & & \\
\hline
\end{tabular}




\begin{tabular}{|c|c|c|c|c|c|c|c|c|c|}
\hline & $\mathrm{K}$ & $\mathrm{L}$ & $\bar{M}$ & $\mathrm{~N}$ & $\mathrm{O}$ & $P$ & $\mathrm{Q}$ & $\mathrm{R}$ & $\mathrm{S}$ \\
\hline 1 & METHOD_NAME & LAB_CODE & VALUE_RPTD & ANAL_UNITS_RPTD & COUNTING_ERROR & TOTAL_ANAL_ERROR & MIN_DETECTABLE_ACTIVITY & PCNT_MOISTURE & LAB_QUALIFIER \\
\hline 2776 & UISO_IE_PLATE_AEA & STLRL & 0.668 & $\mathrm{pCi} / \mathrm{L}$ & 0.06 & 0.13 & 0.00357 & & \\
\hline 2777 & UISO_IE_PLATE_AEA & STLRL & 0.185 & $\mathrm{pCi} / \mathrm{L}$ & 0.032 & 0.047 & 0.0113 & & \\
\hline 2778 & UISO_IE_PLATE_AEA & STLRL & 0.149 & $\mathrm{pCi} / \mathrm{L}$ & 0.029 & 0.04 & 0.00756 & & \\
\hline 2779 & UISO_IE_PLATE_AEA & STLRL & 0.188 & $\mathrm{pCi} / \mathrm{L}$ & 0.033 & 0.047 & 0.00972 & & \\
\hline 2780 & UISO_IE_PLATE_AEA & STLRL & 0.185 & $\mathrm{pCi} / \mathrm{L}$ & 0.037 & 0.05 & 0.0127 & & \\
\hline 2781 & UISO_IE_PLATE_AEA & STLRL & 0.221 & $\mathrm{pCi} / \mathrm{L}$ & 0.036 & 0.054 & 0.0114 & & \\
\hline 2782 & UISO_IE_PLATE_AEA & STLRL & 0.202 & $\mathrm{pCi} / \mathrm{L}$ & 0.034 & 0.05 & 0.00955 & & \\
\hline 2783 & UISO_IE_PLATE_AEA & STLRL & 0.178 & $\mathrm{pCi} / \mathrm{L}$ & 0.034 & 0.047 & 0.0104 & & \\
\hline 2784 & UISO_IE_PLATE_AEA & STLRL & 0.153 & $\mathrm{pCi} / \mathrm{L}$ & 0.029 & 0.041 & 0.00362 & & \\
\hline 2785 & UISO_IE_PLATE_AEA & STLRL & 0.205 & $\mathrm{pCi} / \mathrm{L}$ & 0.034 & 0.051 & 0.00377 & & \\
\hline 2786 & UISO_IE_PLATE_AEA & STLRL & 0.205 & $\mathrm{pCi} / \mathrm{L}$ & 0.035 & 0.052 & 0.00833 & & \\
\hline 2787 & UISO_IE_PLATE_AEA & STLRL & 0.227 & $\mathrm{pCi} / \mathrm{L}$ & 0.036 & 0.055 & 0.00368 & & \\
\hline 2788 & UISO_IE_PLATE_AEA & STLRL & 0.171 & $\mathrm{pCi} / \mathrm{L}$ & 0.037 & 0.049 & 0.0133 & & \\
\hline 2789 & UISO_IE_PLATE_AEA & STLRL & 0.186 & $\mathrm{pCi} / \mathrm{L}$ & 0.033 & 0.047 & 0.0108 & & \\
\hline 2790 & UISO_IE_PLATE_AEA & STLRL & 0.211 & $\mathrm{pCi} / \mathrm{L}$ & 0.036 & 0.053 & 0.00823 & & \\
\hline 2791 & UISO_IE_PLATE_AEA & STLRL & 0.232 & $\mathrm{pCi} / \mathrm{L}$ & 0.038 & 0.057 & 0.00837 & & \\
\hline 2792 & UISO_IE_PLATE_AEA & STLRL & 0.394 & $\mathrm{pCi} / \mathrm{L}$ & 0.053 & 0.089 & 0.0119 & & \\
\hline 2793 & UISO_IE_PLATE_AEA & STLRL & 0.218 & $\mathrm{pCi} / \mathrm{L}$ & 0.037 & 0.054 & 0.0086 & & \\
\hline 2794 & UISO_IE_PLATE_AEA & STLRL & 0.195 & $\mathrm{pCi} / \mathrm{L}$ & 0.034 & 0.05 & 0.00397 & & \\
\hline 2795 & UISO_IE_PLATE_AEA & STLRL & 0.197 & $\mathrm{pCi} / \mathrm{L}$ & 0.034 & 0.05 & 0.00385 & & \\
\hline 2796 & UISO_IE_PLATE_AEA & STLRL & 0.249 & $\mathrm{pCi} / \mathrm{L}$ & 0.04 & 0.06 & 0.0105 & & \\
\hline 2797 & UISO_IE_PLATE_AEA & STLRL & 0.225 & $\mathrm{pCi} / \mathrm{L}$ & 0.037 & 0.055 & 0.00822 & & \\
\hline 2798 & UISO_IE_PLATE_AEA & STLRL & 0.203 & $\mathrm{pCi} / \mathrm{L}$ & 0.037 & 0.052 & 0.0091 & & \\
\hline 2799 & UISO_IE_PLATE_AEA & STLRL & 0.195 & $\mathrm{pCi} / \mathrm{L}$ & 0.032 & 0.048 & 0.0035 & & \\
\hline 2800 & UISO_IE_PLATE_AEA & STLRL & 0.235 & $\mathrm{pCi} / \mathrm{L}$ & 0.04 & 0.059 & 0.0129 & & \\
\hline 2801 & UISO_IE_PLATE_AEA & STLRL & 0.182 & $\mathrm{pCi} / \mathrm{L}$ & 0.035 & 0.049 & 0.00448 & & \\
\hline 2802 & UISO_IE_PLATE_AEA & STLRL & 0.239 & $\mathrm{pCi} / \mathrm{L}$ & 0.041 & 0.06 & 0.0135 & & \\
\hline 2803 & UISO_IE_PLATE_AEA & STLRL & 0.216 & $\mathrm{pCi} / \mathrm{L}$ & 0.036 & 0.054 & 0.00826 & & \\
\hline 2804 & UISO_IE_PLATE_AEA & STLRL & 0.24 & $\mathrm{pCi} / \mathrm{L}$ & 0.036 & 0.057 & 0.00736 & & \\
\hline 2805 & UISO_IE_PLATE_AEA & STLRL & 0.217 & $\mathrm{pCi} / \mathrm{L}$ & 0.034 & 0.052 & 0.00345 & & \\
\hline 2806 & UISO_IE_PLATE_AEA & STLRL & 0.231 & $\mathrm{pCi} / \mathrm{L}$ & 0.035 & 0.055 & 0.00348 & & \\
\hline 2807 & UISO_IE_PLATE_AEA & STLRL & 0.385 & $\mathrm{pCi} / \mathrm{L}$ & 0.048 & 0.085 & 0.00395 & & \\
\hline 2808 & UISO_IE_PLATE_AEA & STLRL & 0.373 & $\mathrm{pCi} / \mathrm{L}$ & 0.046 & 0.082 & 0.011 & & \\
\hline 2809 & UISO_IE_PLATE_AEA & STLRL & 0.232 & $\mathrm{pCi} / \mathrm{L}$ & 0.035 & 0.055 & 0.00359 & & \\
\hline 2810 & UISO_IE_PLATE_AEA & STLRL & 0.229 & $\mathrm{pCi} / \mathrm{L}$ & 0.045 & 0.062 & 0.0122 & & \\
\hline 2811 & UISO_IE_PLATE_AEA & STLRL & 0.23 & $\mathrm{pCi} / \mathrm{L}$ & 0.038 & 0.057 & 0.0138 & & \\
\hline 2812 & UISO_IE_PLATE_AEA & STLRL & 0.255 & $\mathrm{pCi} / \mathrm{L}$ & 0.039 & 0.061 & 0.00828 & & \\
\hline 2813 & UISO_IE_PLATE_AEA & STLRL & 0.66 & $\mathrm{pCi} / \mathrm{L}$ & 0.06 & 0.13 & 0.00774 & & \\
\hline
\end{tabular}




\begin{tabular}{|c|c|c|c|c|c|c|c|c|c|}
\hline & $\mathrm{K}$ & $\mathrm{L}$ & $\mathrm{M}$ & $\mathrm{N}$ & $\mathrm{O}$ & $\mathrm{P}$ & $\mathrm{Q}$ & $\mathrm{R}$ & $\mathrm{S}$ \\
\hline 1 & METHOD_NAME & LAB_CODE & VALUE_RPTD & ANAL_UNITS_RPTD & COUNTING_ERROR & TOTAL_ANAL_ERROR & MIN_DETECTABLE_ACTIVITY & PCNT_MOISTURE & LAB_QUALIFIER \\
\hline 2814 & UISO_IE_PLATE_AEA & STLRL & 0.335 & $\mathrm{pCi} / \mathrm{L}$ & 0.045 & 0.076 & 0.0103 & & \\
\hline 2815 & UISO_IE_PLATE_AEA & STLRL & 0.325 & $\mathrm{pCi} / \mathrm{L}$ & 0.047 & 0.076 & 0.00448 & & \\
\hline 2816 & UISO_IE_PLATE_AEA & STLRL & 0.417 & $\mathrm{pCi} / \mathrm{L}$ & 0.056 & 0.095 & 0.0105 & & \\
\hline 2817 & UISO_IE_PLATE_AEA & STLRL & 0.395 & $\mathrm{pCi} / \mathrm{L}$ & 0.047 & 0.086 & 0.00377 & & \\
\hline 2818 & UISO_IE_PLATE_AEA & STLRL & 0.184 & $\mathrm{pCi} / \mathrm{L}$ & 0.033 & 0.047 & 0.00391 & & \\
\hline 2819 & UISO_IE_PLATE_AEA & STLRL & 0.26 & $\mathrm{pCi} / \mathrm{L}$ & 0.041 & 0.063 & 0.0121 & & \\
\hline 2820 & UISO_IE_PLATE_AEA & STLRL & 0.223 & $\mathrm{pCi} / \mathrm{L}$ & 0.04 & 0.057 & 0.00981 & & \\
\hline 2821 & UISO_IE_PLATE_AEA & STLRL & 0.232 & $\mathrm{pCi} / \mathrm{L}$ & 0.051 & 0.067 & 0.0213 & & \\
\hline 2822 & UISO_IE_PLATE_AEA & STLRL & 0.22 & $\mathrm{pCi} / \mathrm{L}$ & 0.034 & 0.053 & 0.00737 & & \\
\hline 2823 & UISO_IE_PLATE_AEA & STLRL & 0.191 & $\mathrm{pCi} / \mathrm{L}$ & 0.031 & 0.047 & 0.00709 & & \\
\hline 2824 & UISO_IE_PLATE_AEA & STLRL & 0.188 & $\mathrm{pCi} / \mathrm{L}$ & 0.033 & 0.048 & 0.00787 & & \\
\hline 2825 & UISO_IE_PLATE_AEA & STLRL & 0.195 & $\mathrm{pCi} / \mathrm{L}$ & 0.032 & 0.048 & 0.00983 & & \\
\hline 2826 & UISO_IE_PLATE_AEA & STLRL & 0.154 & $\mathrm{pCi} / \mathrm{L}$ & 0.033 & 0.044 & 0.0116 & & \\
\hline 2827 & UISO_IE_PLATE_AEA & STLRL & 0.162 & $\mathrm{pCi} / \mathrm{L}$ & 0.03 & 0.042 & 0.0113 & & \\
\hline 2828 & UISO_IE_PLATE_AEA & STLRL & 0.153 & $\mathrm{pCi} / \mathrm{L}$ & 0.035 & 0.045 & 0.0132 & & \\
\hline 2829 & UISO_IE_PLATE_AEA & STLRL & 0.15 & $\mathrm{pCi} / \mathrm{L}$ & 0.03 & 0.041 & 0.01 & & \\
\hline 2830 & UISO_IE_PLATE_AEA & STLRL & 0.161 & $\mathrm{pCi} / \mathrm{L}$ & 0.029 & 0.042 & 0.00934 & & \\
\hline 2831 & UISO_IE_PLATE_AEA & STLRL & 0.194 & $\mathrm{pCi} / \mathrm{L}$ & 0.033 & 0.049 & 0.01 & & \\
\hline 2832 & UISO_IE_PLATE_AEA & STLRL & 0.146 & $\mathrm{pCi} / \mathrm{L}$ & 0.029 & 0.04 & 0.00996 & & \\
\hline 2833 & UISO_IE_PLATE_AEA & STLRL & 0.198 & $\mathrm{pCi} / \mathrm{L}$ & 0.04 & 0.054 & 0.0133 & & \\
\hline 2834 & UISO_IE_PLATE_AEA & STLRL & 0.171 & $\mathrm{pCi} / \mathrm{L}$ & 0.031 & 0.044 & 0.00936 & & \\
\hline 2835 & UISO_IE_PLATE_AEA & STLRL & 0.222 & $\mathrm{pCi} / \mathrm{L}$ & 0.036 & 0.054 & 0.0126 & & \\
\hline 2836 & UISO_IE_PLATE_AEA & STLRL & 0.163 & $\mathrm{pCi} / \mathrm{L}$ & 0.03 & 0.042 & 0.00963 & & \\
\hline 2837 & UISO_IE_PLATE_AEA & STLRL & 0.184 & $\mathrm{pCi} / \mathrm{L}$ & 0.032 & 0.047 & 0.01 & & \\
\hline 2838 & UISO_IE_PLATE_AEA & STLRL & 0.154 & $\mathrm{pCi} / \mathrm{L}$ & 0.029 & 0.041 & 0.00363 & & \\
\hline 2839 & UISO_IE_PLATE_AEA & STLRL & 0.178 & $\mathrm{pCi} / \mathrm{L}$ & 0.032 & 0.046 & 0.00382 & & \\
\hline 2840 & UISO_IE_PLATE_AEA & STLRL & 0.168 & $\mathrm{pCi} / \mathrm{L}$ & 0.032 & 0.044 & 0.00388 & & \\
\hline 2841 & UISO_IE_PLATE_AEA & STLRL & 0.189 & $\mathrm{pCi} / \mathrm{L}$ & 0.038 & 0.051 & 0.0165 & & \\
\hline 2842 & UISO_IE_PLATE_AEA & STLRL & 0.182 & $\mathrm{pCi} / \mathrm{L}$ & 0.033 & 0.047 & 0.00397 & & \\
\hline 2843 & UISO_IE_PLATE_AEA & STLRL & 0.193 & $\mathrm{pCi} / \mathrm{L}$ & 0.043 & 0.056 & 0.0207 & & \\
\hline 2844 & UISO_IE_PLATE_AEA & STLRL & 0.212 & $\mathrm{pCi} / \mathrm{L}$ & 0.041 & 0.056 & 0.00512 & & \\
\hline 2845 & UISO_IE_PLATE_AEA & STLRL & 0.102 & $\mathrm{pCi} / \mathrm{L}$ & 0.027 & 0.033 & 0.0165 & & \\
\hline 2846 & UISO_IE_PLATE_AEA & STLRL & 0.155 & $\mathrm{pCi} / \mathrm{L}$ & 0.029 & 0.041 & 0.00365 & & \\
\hline 2847 & UISO_IE_PLATE_AEA & STLRL & 0.195 & $\mathrm{pCi} / \mathrm{L}$ & 0.033 & 0.049 & 0.00787 & & \\
\hline 2848 & UISO_IE_PLATE_AEA & STLRL & 0.174 & $\mathrm{pCi} / \mathrm{L}$ & 0.032 & 0.045 & 0.00379 & & \\
\hline 2849 & UISO_IE_PLATE_AEA & STLRL & 0.292 & $\mathrm{pCi} / \mathrm{L}$ & 0.041 & 0.067 & 0.00389 & & \\
\hline 2850 & UISO_IE_PLATE_AEA & STLRL & 0.155 & $\mathrm{pCi} / \mathrm{L}$ & 0.03 & 0.042 & 0.013 & & \\
\hline 2851 & UISO_IE_PLATE_AEA & STLRL & 0.175 & $\mathrm{pCi} / \mathrm{L}$ & 0.036 & 0.049 & 0.0141 & & \\
\hline
\end{tabular}




\begin{tabular}{|c|c|c|c|c|c|c|c|c|c|}
\hline & $\mathrm{K}$ & $\mathrm{L}$ & $\bar{M}$ & $\mathrm{~N}$ & $\mathrm{O}$ & $P$ & $\mathrm{Q}$ & $\mathrm{R}$ & $\mathrm{S}$ \\
\hline 1 & METHOD_NAME & LAB_CODE & VALUE_RPTD & ANAL_UNITS_RPTD & COUNTING_ERROR & TOTAL_ANAL_ERROR & MIN_DETECTABLE_ACTIVITY & PCNT_MOISTURE & LAB_QUALIFIER \\
\hline 2852 & UISO_IE_PLATE_AEA & STLRL & 0.175 & $\mathrm{pCi} / \mathrm{L}$ & 0.03 & 0.044 & 0.00714 & & \\
\hline 2853 & UISO_IE_PLATE_AEA & STLRL & 0.144 & $\mathrm{pCi} / \mathrm{L}$ & 0.03 & 0.04 & 0.018 & & \\
\hline 2854 & UISO_IE_PLATE_AEA & STLRL & 0.143 & $\mathrm{pCi} / \mathrm{L}$ & 0.028 & 0.038 & 0.00351 & & \\
\hline 2855 & UISO_IE_PLATE_AEA & STLRL & 0.152 & $\mathrm{pCi} / \mathrm{L}$ & 0.034 & 0.044 & 0.013 & & \\
\hline 2856 & UISO_IE_PLATE_AEA & STLRL & 0.152 & $\mathrm{pCi} / \mathrm{L}$ & 0.029 & 0.04 & 0.00355 & & \\
\hline 2857 & UISO_IE_PLATE_AEA & STLRL & 0.151 & $\mathrm{pCi} / \mathrm{L}$ & 0.028 & 0.039 & 0.00895 & & \\
\hline 2858 & UISO_IE_PLATE_AEA & STLRL & 0.19 & $\mathrm{pCi} / \mathrm{L}$ & 0.031 & 0.047 & 0.00342 & & \\
\hline 2859 & UISO_IE_PLATE_AEA & STLRL & 0.152 & $\mathrm{pCi} / \mathrm{L}$ & 0.029 & 0.04 & 0.0118 & & \\
\hline 2860 & UISO_IE_PLATE_AEA & STLRL & 0.146 & $\mathrm{pCi} / \mathrm{L}$ & 0.029 & 0.04 & 0.00386 & & \\
\hline 2861 & UISO_IE_PLATE_AEA & STLRL & 0.152 & $\mathrm{pCi} / \mathrm{L}$ & 0.028 & 0.039 & 0.00326 & & \\
\hline 2862 & UISO_IE_PLATE_AEA & STLRL & 0.157 & $\mathrm{pCi} / \mathrm{L}$ & 0.029 & 0.041 & 0.00363 & & \\
\hline 2863 & UISO_IE_PLATE_AEA & STLRL & 0.14 & $\mathrm{pCi} / \mathrm{L}$ & 0.027 & 0.037 & 0.00343 & & \\
\hline 2864 & UISO_IE_PLATE_AEA & STLRL & 0.153 & $\mathrm{pCi} / \mathrm{L}$ & 0.029 & 0.04 & 0.00348 & & \\
\hline 2865 & UISO_IE_PLATE_AEA & STLRL & 0.15 & $\mathrm{pCi} / \mathrm{L}$ & 0.03 & 0.041 & 0.00386 & & \\
\hline 2866 & UISO_IE_PLATE_AEA & STLRL & 0.153 & $\mathrm{pCi} / \mathrm{L}$ & 0.035 & 0.045 & 0.00523 & & \\
\hline 2867 & UISO_IE_PLATE_AEA & STLRL & 0.158 & $\mathrm{pCi} / \mathrm{L}$ & 0.03 & 0.042 & 0.00361 & & \\
\hline 2868 & UISO_IE_PLATE_AEA & STLRL & 0.166 & $\mathrm{pCi} / \mathrm{L}$ & 0.033 & 0.045 & 0.00426 & & \\
\hline 2869 & UISO_IE_PLATE_AEA & STLRL & 0.166 & $\mathrm{pCi} / \mathrm{L}$ & 0.03 & 0.043 & 0.00363 & & \\
\hline 2870 & UISO_IE_PLATE_AEA & STLRL & 0.168 & $\mathrm{pCi} / \mathrm{L}$ & 0.035 & 0.047 & 0.0119 & & \\
\hline 2871 & UISO_IE_PLATE_AEA & STLRL & 0.211 & $\mathrm{pCi} / \mathrm{L}$ & 0.049 & 0.063 & 0.0721 & & \\
\hline 2872 & UISO_IE_PLATE_AEA & STLRL & 0.221 & $\mathrm{pCi} / \mathrm{L}$ & 0.037 & 0.055 & 0.00404 & & \\
\hline 2873 & UISO_IE_PLATE_AEA & STLRL & 0.192 & $\mathrm{pCi} / \mathrm{L}$ & 0.033 & 0.048 & 0.0127 & & \\
\hline 2874 & UISO_IE_PLATE_AEA & STLRL & 0.159 & $\mathrm{pCi} / \mathrm{L}$ & 0.029 & 0.041 & 0.00349 & & \\
\hline 2875 & UISO_IE_PLATE_AEA & STLRL & 0.178 & $\mathrm{pCi} / \mathrm{L}$ & 0.03 & 0.045 & 0.00334 & & \\
\hline 2876 & UISO_IE_PLATE_AEA & STLRL & 0.197 & $\mathrm{pCi} / \mathrm{L}$ & 0.035 & 0.05 & 0.0107 & & \\
\hline 2877 & UISO_IE_PLATE_AEA & STLRL & 0.16 & $\mathrm{pCi} / \mathrm{L}$ & 0.03 & 0.042 & 0.00362 & & \\
\hline 2878 & UISO_IE_PLATE_AEA & STLRL & 0.165 & $\mathrm{pCi} / \mathrm{L}$ & 0.029 & 0.042 & 0.00328 & & \\
\hline 2879 & UISO_IE_PLATE_AEA & STLRL & 0.193 & $\mathrm{pCi} / \mathrm{L}$ & 0.033 & 0.049 & 0.0127 & & \\
\hline 2880 & UISO_IE_PLATE_AEA & STLRL & 0.422 & $\mathrm{pCi} / \mathrm{L}$ & 0.051 & 0.092 & 0.0106 & & \\
\hline 2881 & UISO_IE_PLATE_AEA & STLRL & 0.156 & $\mathrm{pCi} / \mathrm{L}$ & 0.033 & 0.044 & 0.0112 & & \\
\hline 2882 & UISO_IE_PLATE_AEA & STLRL & 0.167 & $\mathrm{pCi} / \mathrm{L}$ & 0.032 & 0.044 & 0.00835 & & \\
\hline 2883 & UISO_IE_PLATE_AEA & STLRL & 0.15 & $\mathrm{pCi} / \mathrm{L}$ & 0.031 & 0.041 & 0.0102 & & \\
\hline 2884 & UISO_IE_PLATE_AEA & STLRL & 0.154 & $\mathrm{pCi} / \mathrm{L}$ & 0.029 & 0.041 & 0.00925 & & \\
\hline 2885 & UISO_IE_PLATE_AEA & STLRL & 0.166 & $\mathrm{pCi} / \mathrm{L}$ & 0.03 & 0.043 & 0.00871 & & \\
\hline 2886 & UISO_IE_PLATE_AEA & STLRL & 0.158 & $\mathrm{pCi} / \mathrm{L}$ & 0.03 & 0.042 & 0.00917 & & \\
\hline 2887 & UISO_IE_PLATE_AEA & STLRL & 0.167 & $\mathrm{pCi} / \mathrm{L}$ & 0.033 & 0.045 & 0.0213 & & \\
\hline 2888 & UISO_IE_PLATE_AEA & STLRL & 0.201 & $\mathrm{pCi} / \mathrm{L}$ & 0.041 & 0.055 & 0.00542 & & \\
\hline 2889 & UISO_IE_PLATE_AEA & STLRL & 0.3 & $\mathrm{pCi} / \mathrm{L}$ & 0.049 & 0.074 & 0.0151 & & \\
\hline
\end{tabular}




\begin{tabular}{|c|c|c|c|c|c|c|c|c|c|}
\hline & $\mathrm{K}$ & $\mathrm{L}$ & $\bar{M}$ & $\mathrm{~N}$ & $\mathrm{O}$ & $P$ & $\mathrm{Q}$ & $\mathrm{R}$ & $\mathrm{S}$ \\
\hline 1 & METHOD_NAME & LAB_CODE & VALUE_RPTD & ANAL_UNITS_RPTD & COUNTING_ERROR & TOTAL_ANAL_ERROR & MIN_DETECTABLE_ACTIVITY & PCNT_MOISTURE & LAB_QUALIFIER \\
\hline 2890 & UISO_IE_PLATE_AEA & STLRL & 0.162 & $\mathrm{pCi} / \mathrm{L}$ & 0.03 & 0.042 & 0.00366 & & \\
\hline 2891 & UISO_IE_PLATE_AEA & STLRL & 0.149 & $\mathrm{pCi} / \mathrm{L}$ & 0.028 & 0.039 & 0.00333 & & \\
\hline 2892 & UISO_IE_PLATE_AEA & STLRL & 0.163 & $\mathrm{pCi} / \mathrm{L}$ & 0.03 & 0.042 & 0.00361 & & \\
\hline 2893 & UISO_IE_PLATE_AEA & STLRL & 0.171 & $\mathrm{pCi} / \mathrm{L}$ & 0.029 & 0.043 & 0.00332 & & \\
\hline 2894 & UISO_IE_PLATE_AEA & STLRL & 0.194 & $\mathrm{pCi} / \mathrm{L}$ & 0.033 & 0.049 & 0.00955 & & \\
\hline 2895 & UISO_IE_PLATE_AEA & STLRL & 0.268 & $\mathrm{pCi} / \mathrm{L}$ & 0.045 & 0.066 & 0.0482 & & \\
\hline 2896 & UISO_IE_PLATE_AEA & STLRL & 0.165 & $\mathrm{pCi} / \mathrm{L}$ & 0.037 & 0.048 & 0.0256 & & \\
\hline 2897 & UISO_IE_PLATE_AEA & STLRL & 0.203 & $\mathrm{pCi} / \mathrm{L}$ & 0.033 & 0.05 & 0.00912 & & \\
\hline 2898 & UISO_IE_PLATE_AEA & STLRL & 0.157 & $\mathrm{pCi} / \mathrm{L}$ & 0.029 & 0.041 & 0.00863 & & \\
\hline 2899 & UISO_IE_PLATE_AEA & STLRL & 0.165 & $\mathrm{pCi} / \mathrm{L}$ & 0.031 & 0.043 & 0.00952 & & \\
\hline 2900 & UISO_IE_PLATE_AEA & STLRL & 0.151 & $\mathrm{pCi} / \mathrm{L}$ & 0.033 & 0.044 & 0.0128 & & \\
\hline 2901 & UISO_IE_PLATE_AEA & STLRL & 0.162 & $\mathrm{pCi} / \mathrm{L}$ & 0.032 & 0.044 & 0.0135 & & \\
\hline 2902 & UISO_IE_PLATE_AEA & STLRL & 0.183 & $\mathrm{pCi} / \mathrm{L}$ & 0.032 & 0.046 & 0.00358 & & \\
\hline 2903 & UISO_IE_PLATE_AEA & STLRL & 0.177 & $\mathrm{pCi} / \mathrm{L}$ & 0.048 & 0.058 & 0.0277 & & \\
\hline 2904 & UISO_IE_PLATE_AEA & STLRL & 0.146 & $\mathrm{pCi} / \mathrm{L}$ & 0.028 & 0.039 & 0.01 & & \\
\hline 2905 & UISO_IE_PLATE_AEA & STLRL & 0.23 & $\mathrm{pCi} / \mathrm{L}$ & 0.036 & 0.055 & 0.0127 & & \\
\hline 2906 & UISO_IE_PLATE_AEA & STLRL & 0.975 & $\mathrm{pCi} / \mathrm{L}$ & 0.075 & 0.19 & 0.0105 & & \\
\hline 2907 & UISO_IE_PLATE_AEA & STLRL & 16.8 & $\mathrm{pCi} / \mathrm{L}$ & 0.3 & 3.1 & 0.00358 & & \\
\hline 2908 & UISO_IE_PLATE_AEA & STLRL & 0.94 & $\mathrm{pCi} / \mathrm{L}$ & 0.07 & 0.18 & 0.00943 & & \\
\hline 2909 & UISO_IE_PLATE_AEA & STLRL & 0.801 & $\mathrm{pCi} / \mathrm{L}$ & 0.064 & 0.16 & 0.0151 & & \\
\hline 2910 & UISO_IE_PLATE_AEA & STLRL & 2.03 & $\mathrm{pCi} / \mathrm{L}$ & 0.1 & 0.38 & 0.00345 & & \\
\hline 2911 & UISO_IE_PLATE_AEA & STLRL & 0.238 & $\mathrm{pCi} / \mathrm{L}$ & 0.044 & 0.062 & 0.0143 & & \\
\hline 2912 & UISO_IE_PLATE_AEA & STLRL & 0.196 & $\mathrm{pCi} / \mathrm{L}$ & 0.035 & 0.051 & 0.014 & & \\
\hline 2913 & UISO_IE_PLATE_AEA & STLRL & 0.171 & $\mathrm{pCi} / \mathrm{L}$ & 0.031 & 0.044 & 0.00373 & & \\
\hline 2914 & UISO_IE_PLATE_AEA & STLRL & 0.166 & $\mathrm{pCi} / \mathrm{L}$ & 0.03 & 0.042 & 0.00346 & & \\
\hline 2915 & UISO_IE_PLATE_AEA & STLRL & 0.181 & $\mathrm{pCi} / \mathrm{L}$ & 0.035 & 0.048 & 0.0183 & & \\
\hline 2916 & UISO_IE_PLATE_AEA & STLRL & 0.182 & $\mathrm{pCi} / \mathrm{L}$ & 0.042 & 0.054 & 0.00636 & & \\
\hline 2917 & UISO_IE_PLATE_AEA & STLRL & 0.182 & $\mathrm{pCi} / \mathrm{L}$ & 0.048 & 0.059 & 0.0412 & & \\
\hline 2918 & UISO_IE_PLATE_AEA & STLRL & 0.168 & $\mathrm{pCi} / \mathrm{L}$ & 0.04 & 0.051 & 0.0203 & & \\
\hline 2919 & UISO_IE_PLATE_AEA & STLRL & 0.187 & $\mathrm{pCi} / \mathrm{L}$ & 0.041 & 0.053 & 0.00579 & & \\
\hline 2920 & UISO_IE_PLATE_AEA & STLRL & 0.19 & $\mathrm{pCi} / \mathrm{L}$ & 0.037 & 0.051 & 0.00462 & & \\
\hline 2921 & UISO_IE_PLATE_AEA & STLRL & 0.148 & $\mathrm{pCi} / \mathrm{L}$ & 0.035 & 0.045 & 0.00554 & & \\
\hline 2922 & UISO_IE_PLATE_AEA & STLRL & 0.179 & $\mathrm{pCi} / \mathrm{L}$ & 0.041 & 0.053 & 0.00603 & & \\
\hline 2923 & UISO_IE_PLATE_AEA & STLRL & 0.133 & $\mathrm{pCi} / \mathrm{L}$ & 0.033 & 0.041 & 0.00521 & & \\
\hline 2924 & UISO_IE_PLATE_AEA & STLRL & 0.137 & $\mathrm{pCi} / \mathrm{L}$ & 0.034 & 0.042 & 0.0055 & & \\
\hline 2925 & UISO_IE_PLATE_AEA & STLRL & 0.291 & $\mathrm{pCi} / \mathrm{L}$ & 0.053 & 0.076 & 0.0244 & & \\
\hline 2926 & UISO_IE_PLATE_AEA & STLRL & 0.166 & $\mathrm{pCi} / \mathrm{L}$ & 0.031 & 0.043 & 0.00376 & & \\
\hline 2927 & UISO_IE_PLATE_AEA & STLRL & 0.157 & $\mathrm{pCi} / \mathrm{L}$ & 0.033 & 0.044 & 0.0186 & & \\
\hline
\end{tabular}




\begin{tabular}{|c|c|c|c|c|c|c|c|c|}
\hline & $\mathrm{T}$ & $U$ & $\mathrm{~V}$ & $\mathrm{~W}$ & $\mathrm{X}$ & $\mathrm{Y}$ & $Z$ & $\mathrm{AA}$ \\
\hline 1 & REVIEW QUALIFIER & SAMP FROM & SAMP ITEM & SAMP MTHD & COLL MTHD & SAMP SITE ID & SAMP SITE NAME & WELL NAME \\
\hline 2 & & RIVER & & RAW GRAB & TRANSECT & 317 & VERNITA-1 HRM 0.3 & \\
\hline 3 & & RIVER & & RAW GRAB & TRANSECT & 570 & VERNITA-2 HRM 0.3 & \\
\hline 4 & & RIVER & & RAW GRAB & TRANSECT & 318 & VERNITA-3 HRM 0.3 & \\
\hline 5 & & RIVER & & RAW GRAB & TRANSECT & 319 & VERNITA-4 HRM 0.3 & \\
\hline 6 & & RIVER & & RAW GRAB & TRANSECT & 327 & RICH.PMPHS-10 HRM46.4 & \\
\hline 7 & & RIVER & & RAW GRAB & TRANSECT & 571 & RICH.PMPHS-1 HRM46.4 & \\
\hline 8 & & RIVER & & RAW GRAB & TRANSECT & 321 & RICH.PMPHS-2 HRM46.4 & \\
\hline 9 & & RIVER & & RAW GRAB & TRANSECT & 322 & RICH.PMPHS-3 HRM46.4 & \\
\hline 10 & & RIVER & & RAW GRAB & TRANSECT & 323 & RICH.PMPHS-5 HRM46.4 & \\
\hline 11 & & RIVER & & RAW GRAB & TRANSECT & 325 & RICH.PMPHS-7 HRM46.4 & \\
\hline 12 & & RIVER & & RAW GRAB & TRANSECT & 8223 & RICH.PMPHS HRM 43.5 & \\
\hline 13 & & RIVER & & RAW GRAB & TRANSECT & 8222 & RICH.PMPHS HRM 43.9 & \\
\hline 14 & & RIVER & & RAW GRAB & TRANSECT & 8221 & RICH.PMPHS HRM 45.0 & \\
\hline 15 & & RIVER & & RAW GRAB & TRANSECT & 8220 & RICH.PMPHS HRM 45.8 & \\
\hline 16 & & SEEP & & RAW GRAB & UNFILTERED & 8144 & 300 AREA SPR DR 42-2 & \\
\hline 17 & & SEEP & & RAW GRAB & UNFILTERED & 488 & 300 AREA SPRING 42-2 & \\
\hline 18 & & SEEP & & RAW GRAB & UNFILTERED & 8145 & 100-K SPRING 63-1 & \\
\hline 19 & & SEEP & & RAW GRAB & UNFILTERED & 8140 & 100-B SPRING 38-3 & \\
\hline 20 & & SEEP & & RAW GRAB & UNFILTERED & 8146 & 100-K SPRING 77-1 & \\
\hline 21 & & SEEP & & RAW GRAB & & 14056 & 100-H SPRING 150-1 & \\
\hline 22 & $\mathrm{H}$ & SEEP & & & & 8147 & 100-F SPRING 207-1 & \\
\hline 23 & & SEEP & & RAW GRAB & UNFILTERED & 488 & 300 AREA SPRING $42-2$ & \\
\hline 24 & & SEEP & & RAW GRAB & UNFILTERED & 8144 & 300 AREA SPR DR 42-2 & \\
\hline 25 & & RIVER & & RAW GRAB & UNFILTERED & 9236 & VERNITA BRIDGE -1 & \\
\hline 26 & & RIVER & & RAW GRAB & UNFILTERED & 9237 & VERNITA BRIDGE -2 & \\
\hline 27 & & RIVER & & RAW GRAB & TRANSECT & 319 & VERNITA-4 HRM 0.3 & \\
\hline 28 & & RIVER & & RAW GRAB & TRANSECT & 318 & VERNITA-3 HRM 0.3 & \\
\hline 29 & & RIVER & & RAW GRAB & TRANSECT & 570 & VERNITA-2 HRM 0.3 & \\
\hline 30 & & RIVER & & RAW GRAB & TRANSECT & 317 & VERNITA-1 HRM 0.3 & \\
\hline 31 & & RIVER & & RAW GRAB & TRANSECT & 8223 & RICH.PMPHS HRM 43.5 & \\
\hline 32 & & RIVER & & RAW GRAB & TRANSECT & 8222 & RICH.PMPHS HRM 43.9 & \\
\hline 33 & & RIVER & & RAW GRAB & TRANSECT & 8221 & RICH.PMPHS HRM 45.0 & \\
\hline 34 & & RIVER & & RAW GRAB & TRANSECT & 8220 & RICH.PMPHS HRM 45.8 & \\
\hline 35 & & RIVER & & RAW GRAB & TRANSECT & 325 & RICH.PMPHS-7 HRM46.4 & \\
\hline 36 & & RIVER & & RAW GRAB & TRANSECT & 323 & RICH.PMPHS-5 HRM46.4 & \\
\hline 37 & & RIVER & & RAW GRAB & TRANSECT & 322 & RICH.PMPHS-3 HRM46.4 & \\
\hline 38 & & RIVER & & RAW GRAB & TRANSECT & 321 & RICH.PMPHS-2 HRM46.4 & \\
\hline 39 & & RIVER & & RAW GRAB & TRANSECT & 571 & RICH.PMPHS-1 HRM46.4 & \\
\hline
\end{tabular}




\begin{tabular}{|c|c|c|c|c|c|c|c|c|}
\hline & $\mathrm{T}$ & $\mathrm{U}$ & $\mathrm{V}$ & $\mathrm{W}$ & $\mathrm{X}$ & $\mathrm{Y}$ & $Z$ & $\overline{\mathrm{AA}}$ \\
\hline 1 & REVIEW_QUALIFIER & SAMP_FROM & SAMP_ITEM & SAMP_MTHD & COLL_MTHD & SAMP_SITE_ID & SAMP_SITE_NAME & WELL_NAME \\
\hline 40 & & RIVER & & RAW GRAB & TRANSECT & 327 & RICH.PMPHS-10 HRM46.4 & \\
\hline 41 & & SEEP & & RAW GRAB & UNFILTERED & 8140 & 100-B SPRING 38-3 & \\
\hline 42 & & SEEP & & RAW GRAB & UNFILTERED & 8145 & 100-K SPRING 63-1 & \\
\hline 43 & & SEEP & & RAW GRAB & UNFILTERED & 8146 & 100-K SPRING 77-1 & \\
\hline 44 & & RIVER & & RAW GRAB & TRANSECT & 319 & VERNITA-4 HRM 0.3 & \\
\hline 45 & & RIVER & & RAW GRAB & TRANSECT & 318 & VERNITA-3 HRM 0.3 & \\
\hline 46 & & RIVER & & RAW GRAB & TRANSECT & 570 & VERNITA-2 HRM 0.3 & \\
\hline 47 & & RIVER & & RAW GRAB & TRANSECT & 317 & VERNITA-1 HRM 0.3 & \\
\hline 48 & & RIVER & & RAW GRAB & TRANSECT & 8223 & RICH.PMPHS HRM 43.5 & \\
\hline 49 & & RIVER & & RAW GRAB & TRANSECT & 8222 & RICH.PMPHS HRM 43.9 & \\
\hline 50 & & RIVER & & RAW GRAB & TRANSECT & 8221 & RICH.PMPHS HRM 45.0 & \\
\hline 51 & & RIVER & & RAW GRAB & TRANSECT & 8220 & RICH.PMPHS HRM 45.8 & \\
\hline 52 & & RIVER & & RAW GRAB & TRANSECT & 327 & RICH.PMPHS-10 HRM46.4 & \\
\hline 53 & & RIVER & & RAW GRAB & TRANSECT & 325 & RICH.PMPHS-7 HRM46.4 & \\
\hline 54 & & RIVER & & RAW GRAB & TRANSECT & 323 & RICH.PMPHS-5 HRM46.4 & \\
\hline 55 & & RIVER & & RAW GRAB & TRANSECT & 322 & RICH.PMPHS-3 HRM46.4 & \\
\hline 56 & & RIVER & & RAW GRAB & TRANSECT & 321 & RICH.PMPHS-2 HRM46.4 & \\
\hline 57 & & RIVER & & RAW GRAB & TRANSECT & 571 & RICH.PMPHS-1 HRM46.4 & \\
\hline 58 & & SEEP & & RAW GRAB & UNFILTERED & 9106 & 100-B SPRING 37-1 & \\
\hline 59 & & SEEP & & RAW GRAB & UNFILTERED & 13792 & 100-K SPRING 63-2 & \\
\hline 60 & & SEEP & & RAW GRAB & & 488 & 300 AREA SPRING 42-2 & \\
\hline 61 & & SEEP & & RAW GRAB & & 8146 & 100-K SPRING 77-1 & \\
\hline 62 & & SEEP & & & & 8162 & 100-D SPRING 110-1 & \\
\hline 63 & & SEEP & & & & 8162 & 100-D SPRING 110-1 & \\
\hline 64 & & SEEP & & & & 8162 & 100-D SPRING $110-1$ & \\
\hline 65 & & SEEP & & & & 8162 & 100-D SPRING 110-1 & \\
\hline 66 & & SEEP & & & & 8163 & 100-D SPRING 102-1 & \\
\hline 67 & & SEEP & & & & 8163 & 100-D SPRING 102-1 & \\
\hline 68 & & SEEP & & & & 8140 & 100-B SPRING 38-3 & \\
\hline 69 & & SEEP & & & & 8165 & 100-H SPRING 145-1 & \\
\hline 70 & & SEEP & & & & 8165 & 100-H SPRING 145-1 & \\
\hline 71 & & SEEP & & & & 8147 & $100-F$ SPRING $207-1$ & \\
\hline 72 & & BASIN & & & & 13420 & 100-HR-3 ISRM & \\
\hline 73 & & BASIN & & & & 13421 & 100-HR-3 ISRM & \\
\hline 74 & & BASIN & & & & 13936 & 100-HR-3 ISRM & \\
\hline 75 & & BASIN & WATER & & & 13937 & 100-HR-3 ISRM & \\
\hline 76 & $\mathrm{~F}$ & SURFACE & & RAW GRAB & UNFILTERED & 147 & WEST LAKE & \\
\hline 77 & & SURFACE & & RAW GRAB & UNFILTERED & 136 & FFTF POND & \\
\hline
\end{tabular}

Page 158 


\begin{tabular}{|c|c|c|c|c|c|c|c|c|}
\hline & $\mathrm{T}$ & $U$ & $\mathrm{~V}$ & $\mathrm{~W}$ & $\mathrm{X}$ & $\mathrm{Y}$ & $Z$ & $\mathrm{AA}$ \\
\hline 1 & REVIEW QUALIFIER & SAMP FROM & SAMP ITEM & SAMP MTHD & COLL MTHD & SAMP SITE ID & SAMP SITE NAME & WELL NAME \\
\hline 78 & & RIVER & & CONTINUOUS & RESIN & 463 & RICH.PMPHS HRM 46.4 & \\
\hline 79 & & RIVER & & CONTINUOUS & FILTER & 463 & RICH.PMPHS HRM 46.4 & \\
\hline 80 & & RIVER & & CONTINUOUS & FILTER & 252 & PRIEST RAPIDS-RIVER & \\
\hline 81 & & RIVER & & CONTINUOUS & RESIN & 252 & PRIEST RAPIDS-RIVER & \\
\hline 82 & & RIVER & & CONTINUOUS & RESIN & 463 & RICH.PMPHS HRM 46.4 & \\
\hline 83 & $F$ & RIVER & & CONTINUOUS & FILTER & 463 & RICH.PMPHS HRM 46.4 & \\
\hline 84 & $\mathrm{~F}$ & RIVER & & CONTINUOUS & FILTER & 252 & PRIEST RAPIDS-RIVER & \\
\hline 85 & & RIVER & & CONTINUOUS & RESIN & 252 & PRIEST RAPIDS-RIVER & \\
\hline 86 & $F$ & SURFACE & & RAW GRAB & UNFILTERED & 147 & WEST LAKE & \\
\hline 87 & & SURFACE & & RAW GRAB & UNFILTERED & 136 & FFTF POND & \\
\hline 88 & & SURFACE & & RAW GRAB & UNFILTERED & 136 & FFTF POND & \\
\hline 89 & & RIVER & & CONTINUOUS & RESIN & 463 & RICH.PMPHS HRM 46.4 & \\
\hline 90 & & RIVER & & CONTINUOUS & FILTER & 463 & RICH.PMPHS HRM 46.4 & \\
\hline 91 & & RIVER & & CONTINUOUS & FILTER & 252 & PRIEST RAPIDS-RIVER & \\
\hline 92 & & RIVER & & CONTINUOUS & RESIN & 252 & PRIEST RAPIDS-RIVER & \\
\hline 93 & $F$ & RIVER & & CONTINUOUS & FILTER & 252 & PRIEST RAPIDS-RIVER & \\
\hline 94 & & RIVER & & CONTINUOUS & RESIN & 252 & PRIEST RAPIDS-RIVER & \\
\hline 95 & & RIVER & & CONTINUOUS & RESIN & 463 & RICH.PMPHS HRM 46.4 & \\
\hline 96 & & RIVER & & CONTINUOUS & FILTER & 463 & RICH.PMPHS HRM 46.4 & \\
\hline 97 & & IRRIGATION & & RAW GRAB & UNFILTERED & 440 & RIVERVIEW CANAL & \\
\hline 98 & & RIVER & & CONTINUOUS & FILTER & 252 & PRIEST RAPIDS-RIVER & \\
\hline 99 & & RIVER & & CONTINUOUS & RESIN & 252 & PRIEST RAPIDS-RIVER & \\
\hline 100 & & RIVER & & CONTINUOUS & RESIN & 463 & RICH.PMPHS HRM 46.4 & \\
\hline 101 & & RIVER & & CONTINUOUS & FILTER & 463 & RICH.PMPHS HRM 46.4 & \\
\hline 102 & & IRRIGATION & & RAW GRAB & UNFILTERED & 440 & RIVERVIEW CANAL & \\
\hline 103 & & SEEP & & RAW GRAB & & 8626 & WEST LAKE SPRING & \\
\hline 104 & & SURFACE & & RAW GRAB & UNFILTERED & 147 & WEST LAKE & \\
\hline 105 & & RIVER & & CONTINUOUS & FILTER & 252 & PRIEST RAPIDS-RIVER & \\
\hline 106 & & RIVER & & CONTINUOUS & RESIN & 252 & PRIEST RAPIDS-RIVER & \\
\hline 107 & & RIVER & & CONTINUOUS & RESIN & 463 & RICH.PMPHS HRM 46.4 & \\
\hline 108 & & RIVER & & CONTINUOUS & FILTER & 463 & RICH.PMPHS HRM 46.4 & \\
\hline 109 & $F$ & SURFACE & & RAW GRAB & UNFILTERED & 147 & WEST LAKE & \\
\hline 110 & & SURFACE & & RAW GRAB & UNFILTERED & 136 & FFTF POND & \\
\hline 111 & & IRRIGATION & & RAW GRAB & UNFILTERED & 440 & RIVERVIEW CANAL & \\
\hline 112 & & RIVER & & CONTINUOUS & FILTER & 252 & PRIEST RAPIDS-RIVER & \\
\hline 113 & & RIVER & & CONTINUOUS & RESIN & 252 & PRIEST RAPIDS-RIVER & \\
\hline 114 & & RIVER & & CONTINUOUS & RESIN & 463 & RICH.PMPHS HRM 46.4 & \\
\hline 115 & & RIVER & & CONTINUOUS & FILTER & 463 & RICH.PMPHS HRM 46.4 & \\
\hline
\end{tabular}




\begin{tabular}{|c|c|c|c|c|c|c|c|c|}
\hline & $\mathrm{T}$ & $U$ & $\mathrm{~V}$ & $\mathrm{~W}$ & $\mathrm{X}$ & $\mathrm{Y}$ & $Z$ & $\mathrm{AA}$ \\
\hline 1 & REVIEW QUALIFIER & SAMP FROM & SAMP ITEM & SAMP MTHD & COLL MTHD & SAMP SITE ID & SAMP SITE NAME & WELL NAME \\
\hline 116 & & RIVER & & CONTINUOUS & FILTER & 252 & PRIEST RAPIDS-RIVER & \\
\hline 117 & & RIVER & & CONTINUOUS & RESIN & 252 & PRIEST RAPIDS-RIVER & \\
\hline 118 & & RIVER & & CONTINUOUS & RESIN & 463 & RICH.PMPHS HRM 46.4 & \\
\hline 119 & & RIVER & & CONTINUOUS & FILTER & 463 & RICH.PMPHS HRM 46.4 & \\
\hline 120 & & SEEP & & RAW GRAB & UNFILTERED & 151 & HANFORD SPRING 28-2 & \\
\hline 121 & & SEEP & & RAW GRAB & UNFILTERED & 488 & 300 AREA SPRING 42-2 & \\
\hline 122 & & SEEP & & RAW GRAB & UNFILTERED & 8142 & HANFORD SPR UR 28-2 & \\
\hline 123 & & SEEP & & RAW GRAB & UNFILTERED & 8143 & HANFORD SPR DR 28-2 & \\
\hline 124 & & SEEP & & RAW GRAB & UNFILTERED & 8144 & 300 AREA SPR DR 42-2 & \\
\hline 125 & & IRRIGATION & & RAW GRAB & UNFILTERED & 605 & HORN RAPIDS AREA & \\
\hline 126 & & RIVER & & CONTINUOUS & FILTER & 252 & PRIEST RAPIDS-RIVER & \\
\hline 127 & & RIVER & & CONTINUOUS & RESIN & 252 & PRIEST RAPIDS-RIVER & \\
\hline 128 & & RIVER & & CONTINUOUS & RESIN & 463 & RICH.PMPHS HRM 46.4 & \\
\hline 129 & & RIVER & & CONTINUOUS & FILTER & 463 & RICH.PMPHS HRM 46.4 & \\
\hline 130 & $\mathrm{~F}$ & SURFACE & & RAW GRAB & UNFILTERED & 147 & WEST LAKE & \\
\hline 131 & & SURFACE & & RAW GRAB & UNFILTERED & 136 & FFTF POND & \\
\hline 132 & $\mathrm{~F}$ & SEEP & & RAW GRAB & UNFILTERED & 202 & 100-N SPRING 8-13 & \\
\hline 133 & & SEEP & & RAW GRAB & UNFILTERED & 8145 & 100-K SPRING 63-1 & \\
\hline 134 & & SEEP & & RAW GRAB & UNFILTERED & 8162 & 100-D SPRING 110-1 & \\
\hline 135 & & SEEP & & RAW GRAB & UNFILTERED & 8163 & 100-D SPRING 102-1 & \\
\hline 136 & & SEEP & & RAW GRAB & UNFILTERED & 8140 & 100-B SPRING 38-3 & \\
\hline 137 & & SEEP & & RAW GRAB & UNFILTERED & 8146 & 100-K SPRING 77-1 & \\
\hline 138 & & SEEP & & RAW GRAB & UNFILTERED & 8165 & 100-H SPRING 145-1 & \\
\hline 139 & & SEEP & & RAW GRAB & UNFILTERED & 8164 & 100-H SPRING 153-1 & \\
\hline 140 & & RIVER & & CONTINUOUS & FILTER & 252 & PRIEST RAPIDS-RIVER & \\
\hline 141 & & RIVER & & CONTINUOUS & RESIN & 252 & PRIEST RAPIDS-RIVER & \\
\hline 142 & $F$ & RIVER & & CONTINUOUS & FILTER & 463 & RICH.PMPHS HRM 46.4 & \\
\hline 143 & $\mathrm{~F}$ & RIVER & & CONTINUOUS & RESIN & 463 & RICH.PMPHS HRM 46.4 & \\
\hline 144 & & SEEP & & RAW GRAB & UNFILTERED & 8147 & 100-F SPRING 207-1 & \\
\hline 145 & & RIVER & & CONTINUOUS & FILTER & 252 & PRIEST RAPIDS-RIVER & \\
\hline 146 & & RIVER & & CONTINUOUS & RESIN & 252 & PRIEST RAPIDS-RIVER & \\
\hline 147 & & RIVER & & CONTINUOUS & RESIN & 463 & RICH.PMPHS HRM 46.4 & \\
\hline 148 & & RIVER & & CONTINUOUS & FILTER & 463 & RICH.PMPHS HRM 46.4 & \\
\hline 149 & & RIVER & & CONTINUOUS & FILTER & 252 & PRIEST RAPIDS-RIVER & \\
\hline 150 & $F$ & RIVER & & CONTINUOUS & RESIN & 252 & PRIEST RAPIDS-RIVER & \\
\hline 151 & $\mathrm{~F}$ & RIVER & & CONTINUOUS & RESIN & 463 & RICH.PMPHS HRM 46.4 & \\
\hline 152 & & RIVER & & CONTINUOUS & FILTER & 463 & RICH.PMPHS HRM 46.4 & \\
\hline 153 & $\mathrm{~F}$ & RIVER & & CONTINUOUS & FILTER & 252 & PRIEST RAPIDS-RIVER & \\
\hline
\end{tabular}




\begin{tabular}{|c|c|c|c|c|c|c|c|c|}
\hline & $\mathrm{T}$ & $\mathrm{U}$ & $\mathrm{V}$ & $\mathrm{W}$ & $\mathrm{X}$ & $\mathrm{Y}$ & $Z$ & $\overline{\mathrm{AA}}$ \\
\hline 1 & REVIEW_QUALIFIER & SAMP_FROM & SAMP_ITEM & SAMP_MTHD & COLL_MTHD & SAMP_SITE_ID & SAMP_SITE_NAME & WELL_NAME \\
\hline 154 & & RIVER & & CONTINUOUS & RESIN & 252 & PRIEST RAPIDS-RIVER & \\
\hline 155 & & RIVER & & CONTINUOUS & RESIN & 463 & RICH.PMPHS HRM 46.4 & \\
\hline 156 & & RIVER & & CONTINUOUS & FILTER & 463 & RICH.PMPHS HRM 46.4 & \\
\hline 157 & $F$ & SURFACE & & RAW GRAB & UNFILTERED & 147 & WEST LAKE & \\
\hline 158 & & SURFACE & & RAW GRAB & UNFILTERED & 136 & FFTF POND & \\
\hline 159 & & RIVER & & CONTINUOUS & FILTER & 252 & PRIEST RAPIDS-RIVER & \\
\hline 160 & & RIVER & & CONTINUOUS & RESIN & 252 & PRIEST RAPIDS-RIVER & \\
\hline 161 & & RIVER & & CONTINUOUS & RESIN & 463 & RICH.PMPHS HRM 46.4 & \\
\hline 162 & $F$ & RIVER & & CONTINUOUS & FILTER & 463 & RICH.PMPHS HRM 46.4 & \\
\hline 163 & $F$ & SURFACE & & RAW GRAB & UNFILTERED & 147 & WEST LAKE & \\
\hline 164 & & SURFACE & & RAW GRAB & UNFILTERED & 136 & FFTF POND & \\
\hline 165 & & SURFACE & & RAW GRAB & UNFILTERED & 136 & FFTF POND & \\
\hline 166 & & RIVER & & CONTINUOUS & FILTER & 252 & PRIEST RAPIDS-RIVER & \\
\hline 167 & & RIVER & & CONTINUOUS & RESIN & 252 & PRIEST RAPIDS-RIVER & \\
\hline 168 & & RIVER & & CONTINUOUS & RESIN & 463 & RICH.PMPHS HRM 46.4 & \\
\hline 169 & & RIVER & & CONTINUOUS & FILTER & 463 & RICH.PMPHS HRM 46.4 & \\
\hline 170 & & SEEP & & RAW GRAB & UNFILTERED & 151 & HANFORD SPRING 28-2 & \\
\hline 171 & & SEEP & & RAW GRAB & UNFILTERED & 8165 & 100-H SPRING 145-1 & \\
\hline 172 & & SEEP & & RAW GRAB & UNFILTERED & 8147 & 100-F SPRING 207-1 & \\
\hline 173 & & SEEP & & RAW GRAB & UNFILTERED & 8143 & HANFORD SPR DR 28-2 & \\
\hline 174 & & SEEP & & RAW GRAB & UNFILTERED & 8164 & 100-H SPRING 153-1 & \\
\hline 175 & & RIVER & & CONTINUOUS & RESIN & 463 & RICH.PMPHS HRM 46.4 & \\
\hline 176 & & RIVER & & CONTINUOUS & FILTER & 463 & RICH.PMPHS HRM 46.4 & \\
\hline 177 & & RIVER & & CONTINUOUS & FILTER & 252 & PRIEST RAPIDS-RIVER & \\
\hline 178 & & RIVER & & CONTINUOUS & RESIN & 252 & PRIEST RAPIDS-RIVER & \\
\hline 179 & & SEEP & & RAW GRAB & UNFILTERED & 8144 & 300 AREA SPR DR 42-2 & \\
\hline 180 & & SEEP & & RAW GRAB & UNFILTERED & 202 & 100-N SPRING 8-13 & \\
\hline 181 & & SEEP & & RAW GRAB & UNFILTERED & 9106 & 100-B SPRING 37-1 & \\
\hline 182 & & SEEP & & RAW GRAB & UNFILTERED & 9110 & 100-K SPRING 82-2 & \\
\hline 183 & & SEEP & & RAW GRAB & UNFILTERED & 8162 & 100-D SPRING 110-1 & \\
\hline 184 & & IRRIGATION & & RAW GRAB & UNFILTERED & 440 & RIVERVIEW CANAL & \\
\hline 185 & & IRRIGATION & & RAW GRAB & UNFILTERED & 605 & HORN RAPIDS AREA & \\
\hline 186 & & SEEP & & RAW GRAB & UNFILTERED & 488 & 300 AREA SPRING 42-2 & \\
\hline 187 & & RIVER & & CONTINUOUS & RESIN & 463 & RICH.PMPHS HRM 46.4 & \\
\hline 188 & & RIVER & & CONTINUOUS & FILTER & 463 & RICH.PMPHS HRM 46.4 & \\
\hline 189 & & RIVER & & CONTINUOUS & FILTER & 252 & PRIEST RAPIDS-RIVER & \\
\hline 190 & & RIVER & & CONTINUOUS & RESIN & 252 & PRIEST RAPIDS-RIVER & \\
\hline 191 & & IRRIGATION & & RAW GRAB & UNFILTERED & 440 & RIVERVIEW CANAL & \\
\hline
\end{tabular}




\begin{tabular}{|c|c|c|c|c|c|c|c|c|}
\hline & $\mathrm{T}$ & $U$ & $\mathrm{~V}$ & $\mathrm{~W}$ & $\mathrm{X}$ & $\mathrm{Y}$ & Z & AA \\
\hline 1 & REVIEW_QUALIFIER & SAMP_FROM & SAMP_ITEM & SAMP_MTHD & COLL_MTHD & SAMP_SITE_ID & SAMP_SITE_NAME & WELL_NAME \\
\hline 192 & & IRRIGATION & & RAW GRAB & UNFILTERED & 605 & HORN RAPIDS AREA & \\
\hline 193 & & RIVER & & CONTINUOUS & RESIN & 463 & RICH.PMPHS HRM 46.4 & \\
\hline 194 & & RIVER & & CONTINUOUS & FILTER & 463 & RICH.PMPHS HRM 46.4 & \\
\hline 195 & & RIVER & & CONTINUOUS & FILTER & 252 & PRIEST RAPIDS-RIVER & \\
\hline 196 & & RIVER & & CONTINUOUS & RESIN & 252 & PRIEST RAPIDS-RIVER & \\
\hline 197 & $F$ & SURFACE & & RAW GRAB & UNFILTERED & 147 & WEST LAKE & \\
\hline 198 & & SURFACE & & RAW GRAB & UNFILTERED & 136 & FFTF POND & \\
\hline 199 & & IRRIGATION & & RAW GRAB & UNFILTERED & 440 & RIVERVIEW CANAL & \\
\hline 200 & & IRRIGATION & & RAW GRAB & UNFILTERED & 605 & HORN RAPIDS AREA & \\
\hline 201 & & RIVER & & CONTINUOUS & RESIN & 463 & RICH.PMPHS HRM 46.4 & \\
\hline 202 & & RIVER & & CONTINUOUS & FILTER & 463 & RICH.PMPHS HRM 46.4 & \\
\hline 203 & $F$ & RIVER & & CONTINUOUS & FILTER & 252 & PRIEST RAPIDS-RIVER & \\
\hline 204 & & RIVER & & CONTINUOUS & RESIN & 252 & PRIEST RAPIDS-RIVER & \\
\hline 205 & & SEEP & & RAW GRAB & UNFILTERED & 488 & 300 AREA SPRING 42-2 & \\
\hline 206 & & SEEP & & RAW GRAB & UNFILTERED & 8144 & 300 AREA SPR DR 42-2 & \\
\hline 207 & & RIVER & & RAW GRAB & UNFILTERED & 9236 & VERNITA BRIDGE -1 & \\
\hline 208 & & RIVER & & RAW GRAB & UNFILTERED & 9237 & VERNITA BRIDGE -2 & \\
\hline 209 & $F$ & RIVER & & RAW GRAB & UNFILTERED & 9238 & VERNITA BRIDGE -3 & \\
\hline 210 & & RIVER & & RAW GRAB & UNFILTERED & 9210 & 300 SPR $7-1$ & \\
\hline 211 & & RIVER & & RAW GRAB & UNFILTERED & 9211 & 300 SPR $7-2$ & \\
\hline 212 & & RIVER & & RAW GRAB & UNFILTERED & 9213 & 300 SPR $7-3$ & \\
\hline 213 & & RIVER & & RAW GRAB & UNFILTERED & 9214 & 300 SPR DR $7-1$ & \\
\hline 214 & $F$ & RIVER & & RAW GRAB & UNFILTERED & 9215 & 300 SPR DR 7 -2 & \\
\hline 215 & & RIVER & & RAW GRAB & UNFILTERED & 9216 & 300 SPR DR $7-3$ & \\
\hline 216 & & RIVER & & RAW GRAB & UNFILTERED & 9217 & 300 SPR 7 THRU SPR 9 & \\
\hline 217 & & RIVER & & RAW GRAB & UNFILTERED & 9218 & 300 SPR 9 -1 & \\
\hline 218 & & RIVER & & RAW GRAB & UNFILTERED & 9219 & 300 SPR 9 -2 & \\
\hline 219 & & RIVER & & RAW GRAB & UNFILTERED & 9220 & 300 SPR $9-3$ & \\
\hline 220 & & RIVER & & RAW GRAB & UNFILTERED & 9221 & 300 SPR DR $9-1$ & \\
\hline 221 & $F$ & RIVER & & RAW GRAB & UNFILTERED & 9222 & 300 SPR DR $9-2$ & \\
\hline 222 & & RIVER & & RAW GRAB & UNFILTERED & 9223 & 300 SPR DR $9-3$ & \\
\hline 223 & & RIVER & & RAW GRAB & UNFILTERED & 9224 & 300 SPR 9 THRU SPR 11 & \\
\hline 224 & & RIVER & & RAW GRAB & UNFILTERED & 9226 & 300 SPR $11-1$ & \\
\hline 225 & & RIVER & & RAW GRAB & UNFILTERED & 9227 & 300 SPR $11-2$ & \\
\hline 226 & & RIVER & & RAW GRAB & UNFILTERED & 9228 & 300 SPR $11-3$ & \\
\hline 227 & & RIVER & & RAW GRAB & UNFILTERED & 9229 & 300 SPR DR $11-1$ & \\
\hline 228 & & RIVER & & RAW GRAB & UNFILTERED & 9230 & 300 SPR DR $11-2$ & \\
\hline 229 & & RIVER & & RAW GRAB & UNFILTERED & 9231 & 300 SPR DR $11-3$ & \\
\hline
\end{tabular}




\begin{tabular}{|c|c|c|c|c|c|c|c|c|}
\hline & $\mathrm{T}$ & $U$ & $\mathrm{~V}$ & $\mathrm{~W}$ & $\mathrm{x}$ & $\mathrm{Y}$ & $Z$ & $\mathrm{AA}$ \\
\hline 1 & REVIEW_QUALIFIER & SAMP_FROM & SAMP_ITEM & SAMP_MTHD & COLL_MTHD & SAMP_SITE_ID & SAMP_SITE_NAME & WELL_NAME \\
\hline 230 & & RIVER & & RAW GRAB & UNFILTERED & 9233 & 3300 SPR 14 -1 & \\
\hline 231 & & RIVER & & RAW GRAB & UNFILTERED & 9234 & 4300 SPR 14 -2 & \\
\hline 232 & & RIVER & & RAW GRAB & UNFILTERED & 9235 & 5300 SPR 14 -3 & \\
\hline 233 & & RIVER & & RAW GRAB & UNFILTERED & 9280 & VERNITA BRIDGE -4 & \\
\hline 234 & & RIVER & & RAW GRAB & UNFILTERED & 9273 & 300 SPR $7-4$ & \\
\hline 235 & & RIVER & & RAW GRAB & UNFILTERED & 9274 & 4300 SPR DR $7-4$ & \\
\hline 236 & & RIVER & & RAW GRAB & UNFILTERED & 9275 & 5300 SPR $9-4$ & \\
\hline 237 & & RIVER & & RAW GRAB & UNFILTERED & 9276 & 300 SPR DR $9-4$ & \\
\hline 238 & & RIVER & & RAW GRAB & UNFILTERED & 9277 & 7300 SPR $11-4$ & \\
\hline 239 & & RIVER & & RAW GRAB & UNFILTERED & 9278 & 300 SPR DR $11-4$ & \\
\hline 240 & & RIVER & & RAW GRAB & UNFILTERED & 9279 & 9 300 SPR $14-4$ & \\
\hline 241 & & RIVER & & CONTINUOUS & RESIN & 463 & 3 RICH.PMPHS HRM 46.4 & \\
\hline 242 & $F$ & RIVER & & CONTINUOUS & FILTER & 463 & 3 RICH.PMPHS HRM 46.4 & \\
\hline 243 & & RIVER & & CONTINUOUS & FILTER & 252 & 2 PRIEST RAPIDS-RIVER & \\
\hline 244 & & RIVER & & CONTINUOUS & RESIN & 252 & PRIEST RAPIDS-RIVER & \\
\hline 245 & & SEEP & & RAW GRAB & UNFILTERED & 8144 & 4300 AREA SPR DR 42-2 & \\
\hline 246 & & DRIVE POINT & & RAW GRAB & UNFILTERED & 9539 & 9300 SPR 9 -1 (2 FT) & \\
\hline 247 & & DRIVE POINT & & RAW GRAB & UNFILTERED & 9540 & 300 SPR 9 -1 (4 FT) & \\
\hline 248 & & DRIVE POINT & & RAW GRAB & UNFILTERED & 9541 & 300 SPR 9 -2 (2 FT) & \\
\hline 249 & & DRIVE POINT & & RAW GRAB & UNFILTERED & 9542 & 2300 SPR $9-2$ (4.5FT) & \\
\hline 250 & & DRIVE POINT & & RAW GRAB & UNFILTERED & 9543 & 300 SPR 9 -3 (5.5FT) & \\
\hline 251 & & SEEP & & RAW GRAB & UNFILTERED & 488 & 300 AREA SPRING 42-2 & \\
\hline 252 & & DRIVE POINT & & RAW GRAB & UNFILTERED & 9533 & 300 SPR 7 -1 (2.5FT) & \\
\hline 253 & & DRIVE POINT & & RAW GRAB & UNFILTERED & 9534 & 400 SPR 7 -1 (4 FT) & \\
\hline 254 & & DRIVE POINT & & RAW GRAB & UNFILTERED & 9535 & 5300 SPR 7 -1 (6 FT) & \\
\hline 255 & & DRIVE POINT & & RAW GRAB & UNFILTERED & 9536 & 300 SPR 7 -2 (2 FT) & \\
\hline 256 & & DRIVE POINT & & RAW GRAB & UNFILTERED & 9537 & 7300 SPR 7 -2 (4.2FT) & \\
\hline 257 & & RIVER & & CONTINUOUS & FILTER & 252 & PRIEST RAPIDS-RIVER & \\
\hline 258 & & RIVER & & CONTINUOUS & RESIN & 252 & PRIEST RAPIDS-RIVER & \\
\hline 259 & $F$ & RIVER & & CONTINUOUS & RESIN & 463 & 3 RICH.PMPHS HRM 46.4 & \\
\hline 260 & & RIVER & & CONTINUOUS & FILTER & 463 & 3 RICH.PMPHS HRM 46.4 & \\
\hline 261 & & SURFACE & & RAW GRAB & UNFILTERED & 136 & FFTF POND & \\
\hline 262 & & SEEP & & RAW GRAB & UNFILTERED & 8140 & 100-B SPRING 38-3 & \\
\hline 263 & & SEEP & & RAW GRAB & UNFILTERED & 8147 & 7 100-F SPRING 207-1 & \\
\hline 264 & & SEEP & & RAW GRAB & UNFILTERED & 202 & 100-N SPRING 8-13 & \\
\hline 265 & & SEEP & & RAW GRAB & UNFILTERED & 8145 & 5 100-K SPRING 63-1 & \\
\hline 266 & & SEEP & & RAW GRAB & UNFILTERED & 8146 & 100-K SPRING 77-1 & \\
\hline 267 & & SEEP & & RAW GRAB & UNFILTERED & 8165 & 5 100-H SPRING 145-1 & \\
\hline
\end{tabular}




\begin{tabular}{|c|c|c|c|c|c|c|c|c|}
\hline & $\mathrm{T}$ & $U$ & $\mathrm{~V}$ & $\mathrm{~W}$ & $\mathrm{X}$ & $\mathrm{Y}$ & $Z$ & $\mathrm{AA}$ \\
\hline 1 & REVIEW QUALIFIER & SAMP FROM & SAMP ITEM & SAMP MTHD & COLL MTHD & SAMP SITE ID & SAMP SITE NAME & WELL NAME \\
\hline 268 & & SEEP & & RAW GRAB & UNFILTERED & 8846 & 100-H SPRING 152-2 & \\
\hline 269 & $\mathrm{~F}$ & RIVER & & CONTINUOUS & RESIN & 463 & RICH.PMPHS HRM 46.4 & \\
\hline 270 & & RIVER & & CONTINUOUS & FILTER & 463 & RICH.PMPHS HRM 46.4 & \\
\hline 271 & & RIVER & & CONTINUOUS & FILTER & 252 & PRIEST RAPIDS-RIVER & \\
\hline 272 & & RIVER & & CONTINUOUS & RESIN & 252 & PRIEST RAPIDS-RIVER & \\
\hline 273 & & SEEP & & RAW GRAB & UNFILTERED & 9236 & VERNITA BRIDGE -1 & \\
\hline 274 & & RIVER & & CONTINUOUS & FILTER & 252 & PRIEST RAPIDS-RIVER & \\
\hline 275 & & RIVER & & CONTINUOUS & RESIN & 252 & PRIEST RAPIDS-RIVER & \\
\hline 276 & & RIVER & & CONTINUOUS & RESIN & 463 & RICH.PMPHS HRM 46.4 & \\
\hline 277 & & RIVER & & CONTINUOUS & FILTER & 463 & RICH.PMPHS HRM 46.4 & \\
\hline 278 & & RIVER & & CONTINUOUS & FILTER & 252 & PRIEST RAPIDS-RIVER & \\
\hline 279 & & RIVER & & CONTINUOUS & RESIN & 252 & PRIEST RAPIDS-RIVER & \\
\hline 280 & & RIVER & & CONTINUOUS & RESIN & 463 & RICH.PMPHS HRM 46.4 & \\
\hline 281 & & RIVER & & CONTINUOUS & FILTER & 463 & RICH.PMPHS HRM 46.4 & \\
\hline 282 & & RIVER & & CONTINUOUS & RESIN & 463 & RICH.PMPHS HRM 46.4 & \\
\hline 283 & & RIVER & & CONTINUOUS & FILTER & 463 & RICH.PMPHS HRM 46.4 & \\
\hline 284 & $F$ & RIVER & & CONTINUOUS & FILTER & 252 & PRIEST RAPIDS-RIVER & \\
\hline 285 & & RIVER & & CONTINUOUS & RESIN & 252 & PRIEST RAPIDS-RIVER & \\
\hline 286 & & SURFACE & & RAW GRAB & UNFILTERED & 136 & FFTF POND & \\
\hline 287 & & RIVER & & CONTINUOUS & FILTER & 252 & PRIEST RAPIDS-RIVER & \\
\hline 288 & & RIVER & & CONTINUOUS & RESIN & 252 & PRIEST RAPIDS-RIVER & \\
\hline 289 & & RIVER & & CONTINUOUS & RESIN & 463 & RICH.PMPHS HRM 46.4 & \\
\hline 290 & & RIVER & & CONTINUOUS & FILTER & 463 & RICH.PMPHS HRM 46.4 & \\
\hline 291 & & RIVER & & CONTINUOUS & FILTER & 252 & PRIEST RAPIDS-RIVER & \\
\hline 292 & $F$ & RIVER & & CONTINUOUS & RESIN & 252 & PRIEST RAPIDS-RIVER & \\
\hline 293 & & RIVER & & CONTINUOUS & RESIN & 463 & RICH.PMPHS HRM 46.4 & \\
\hline 294 & & RIVER & & CONTINUOUS & FILTER & 463 & RICH.PMPHS HRM 46.4 & \\
\hline 295 & & SURFACE & & RAW GRAB & UNFILTERED & 136 & FFTF POND & \\
\hline 296 & & SURFACE & & RAW GRAB & UNFILTERED & 136 & FFTF POND & \\
\hline 297 & $F$ & RIVER & & CONTINUOUS & FILTER & 252 & PRIEST RAPIDS-RIVER & \\
\hline 298 & & RIVER & & CONTINUOUS & RESIN & 252 & PRIEST RAPIDS-RIVER & \\
\hline 299 & & RIVER & & CONTINUOUS & RESIN & 463 & RICH.PMPHS HRM 46.4 & \\
\hline 300 & & RIVER & & CONTINUOUS & FILTER & 463 & RICH.PMPHS HRM 46.4 & \\
\hline 301 & & IRRIGATION & & RAW GRAB & UNFILTERED & 440 & RIVERVIEW CANAL & \\
\hline 302 & & IRRIGATION & & RAW GRAB & UNFILTERED & 605 & HORN RAPIDS AREA & \\
\hline 303 & & RIVER & & CONTINUOUS & FILTER & 252 & PRIEST RAPIDS-RIVER & \\
\hline 304 & & RIVER & & CONTINUOUS & RESIN & 252 & PRIEST RAPIDS-RIVER & \\
\hline 305 & & RIVER & & CONTINUOUS & RESIN & 463 & RICH.PMPHS HRM 46.4 & \\
\hline
\end{tabular}




\begin{tabular}{|c|c|c|c|c|c|c|c|c|}
\hline & $\mathrm{T}$ & $U$ & $\mathrm{~V}$ & $\mathrm{~W}$ & $\mathrm{X}$ & $\mathrm{Y}$ & $Z$ & $\mathrm{AA}$ \\
\hline 1 & REVIEW QUALIFIER & SAMP FROM & SAMP ITEM & SAMP MTHD & COLL MTHD & SAMP SITE ID & SAMP SITE NAME & WELL NAME \\
\hline 306 & & RIVER & & CONTINUOUS & FILTER & 463 & RICH.PMPHS HRM 46.4 & \\
\hline 307 & & RIVER & & CONTINUOUS & FILTER & 252 & PRIEST RAPIDS-RIVER & \\
\hline 308 & & RIVER & & CONTINUOUS & RESIN & 252 & PRIEST RAPIDS-RIVER & \\
\hline 309 & & RIVER & & CONTINUOUS & RESIN & 463 & RICH.PMPHS HRM 46.4 & \\
\hline 310 & $F$ & RIVER & & CONTINUOUS & FILTER & 463 & RICH.PMPHS HRM 46.4 & \\
\hline 311 & & IRRIGATION & & RAW GRAB & UNFILTERED & 440 & RIVERVIEW CANAL & \\
\hline 312 & & IRRIGATION & & RAW GRAB & UNFILTERED & 605 & HORN RAPIDS AREA & \\
\hline 313 & & SURFACE & & RAW GRAB & UNFILTERED & 136 & FFTF POND & \\
\hline 314 & & IRRIGATION & & RAW GRAB & UNFILTERED & 440 & RIVERVIEW CANAL & \\
\hline 315 & & IRRIGATION & & RAW GRAB & UNFILTERED & 605 & HORN RAPIDS AREA & \\
\hline 316 & & RIVER & & CONTINUOUS & FILTER & 252 & PRIEST RAPIDS-RIVER & \\
\hline 317 & $F$ & RIVER & & CONTINUOUS & RESIN & 252 & PRIEST RAPIDS-RIVER & \\
\hline 318 & & RIVER & & CONTINUOUS & RESIN & 463 & RICH.PMPHS HRM 46.4 & \\
\hline 319 & & RIVER & & CONTINUOUS & FILTER & 463 & RICH.PMPHS HRM 46.4 & \\
\hline 320 & & RIVER & & CONTINUOUS & FILTER & 252 & PRIEST RAPIDS-RIVER & \\
\hline 321 & $\mathrm{~F}$ & RIVER & & CONTINUOUS & RESIN & 252 & PRIEST RAPIDS-RIVER & \\
\hline 322 & & RIVER & & CONTINUOUS & RESIN & 463 & RICH.PMPHS HRM 46.4 & \\
\hline 323 & $F$ & RIVER & & CONTINUOUS & FILTER & 463 & RICH.PMPHS HRM 46.4 & \\
\hline 324 & & SEEP & & RAW GRAB & UNFILTERED & 202 & 100-N SPRING 8-13 & \\
\hline 325 & & SEEP & & RAW GRAB & UNFILTERED & 9106 & 100-B SPRING 37-1 & \\
\hline 326 & & SEEP & & RAW GRAB & UNFILTERED & 13792 & 100-K SPRING 63-2 & \\
\hline 327 & & RIVER & & CONTINUOUS & FILTER & 252 & PRIEST RAPIDS-RIVER & \\
\hline 328 & $F$ & RIVER & & CONTINUOUS & RESIN & 252 & PRIEST RAPIDS-RIVER & \\
\hline 329 & & RIVER & & CONTINUOUS & RESIN & 463 & RICH.PMPHS HRM 46.4 & \\
\hline 330 & & RIVER & & CONTINUOUS & FILTER & 463 & RICH.PMPHS HRM 46.4 & \\
\hline 331 & & SEEP & & RAW GRAB & UNFILTERED & 151 & HANFORD SPRING 28-2 & \\
\hline 332 & & SEEP & & RAW GRAB & UNFILTERED & 488 & 300 AREA SPRING 42-2 & \\
\hline 333 & & SEEP & & RAW GRAB & UNFILTERED & 8143 & HANFORD SPR DR 28-2 & \\
\hline 334 & & SURFACE & & RAW GRAB & UNFILTERED & 136 & FFTF POND & \\
\hline 335 & & SEEP & & RAW GRAB & UNFILTERED & 8146 & 100-K SPRING 77-1 & \\
\hline 336 & & SEEP & & RAW GRAB & UNFILTERED & 8162 & 100-D SPRING 110-1 & \\
\hline 337 & & SEEP & & RAW GRAB & UNFILTERED & 8846 & 100-H SPRING 152-2 & \\
\hline 338 & & SEEP & & RAW GRAB & UNFILTERED & 8147 & 100-F SPRING 207-1 & \\
\hline 339 & & RIVER & & CONTINUOUS & RESIN & 463 & RICH.PMPHS HRM 46.4 & \\
\hline 340 & & RIVER & & CONTINUOUS & FILTER & 463 & RICH.PMPHS HRM 46.4 & \\
\hline 341 & & RIVER & & CONTINUOUS & FILTER & 252 & PRIEST RAPIDS-RIVER & \\
\hline 342 & & RIVER & & CONTINUOUS & RESIN & 252 & PRIEST RAPIDS-RIVER & \\
\hline 343 & & RIVER & & CONTINUOUS & FILTER & 252 & PRIEST RAPIDS-RIVER & \\
\hline
\end{tabular}




\begin{tabular}{|c|c|c|c|c|c|c|c|c|}
\hline & $T$ & $\bar{U}$ & $\mathrm{~V}$ & $\mathrm{~W}$ & $\bar{x}$ & $\bar{Y}$ & $\bar{Z}$ & $\overline{A A}$ \\
\hline 1 & REVIEW_QUALIFIER & SAMP_FROM & SAMP_ITEM & SAMP_MTHD & COLL_MTHD & SAMP_SITE_ID & SAMP_SITE_NAME & WELL_NAME \\
\hline 344 & & RIVER & & CONTINUOUS & RESIN & 252 & PRIEST RAPIDS-RIVER & \\
\hline 345 & & RIVER & & CONTINUOUS & RESIN & 463 & RICH.PMPHS HRM 46.4 & \\
\hline 346 & & RIVER & & CONTINUOUS & FILTER & 463 & RICH.PMPHS HRM 46.4 & \\
\hline 347 & & RIVER & & CUMULATIVE & UNFILTERED & 463 & RICH.PMPHS HRM 46.4 & \\
\hline 348 & & RIVER & & CUMULATIVE & UNFILTERED & 252 & PRIEST RAPIDS-RIVER & \\
\hline 349 & & RIVER & & CUMULATIVE & UNFILTERED & 252 & PRIEST RAPIDS-RIVER & \\
\hline 350 & & RIVER & & CUMULATIVE & UNFILTERED & 463 & RICH.PMPHS HRM 46.4 & \\
\hline 351 & & SEEP & & RAW GRAB & UNFILTERED & 151 & HANFORD SPRING 28-2 & \\
\hline 352 & & SEEP & & RAW GRAB & UNFILTERED & 488 & 300 AREA SPRING $42-2$ & \\
\hline 353 & & SEEP & & RAW GRAB & UNFILTERED & 8142 & HANFORD SPR UR 28-2 & \\
\hline 354 & & SEEP & & RAW GRAB & UNFILTERED & 8143 & HANFORD SPR DR 28-2 & \\
\hline 355 & & SEEP & & RAW GRAB & UNFILTERED & 8144 & 300 AREA SPR DR 42-2 & \\
\hline 356 & & RIVER & & CUMULATIVE & UNFILTERED & 252 & PRIEST RAPIDS-RIVER & \\
\hline 357 & & RIVER & & CUMULATIVE & UNFILTERED & 463 & RICH.PMPHS HRM 46.4 & \\
\hline 358 & & RIVER & & CUMULATIVE & UNFILTERED & 252 & PRIEST RAPIDS-RIVER & \\
\hline 359 & & RIVER & & CUMULATIVE & UNFILTERED & 463 & RICH.PMPHS HRM 46.4 & \\
\hline 360 & & RIVER & & CUMULATIVE & UNFILTERED & 252 & PRIEST RAPIDS-RIVER & \\
\hline 361 & & RIVER & & CUMULATIVE & UNFILTERED & 463 & RICH.PMPHS HRM 46.4 & \\
\hline 362 & & SEEP & & RAW GRAB & UNFILTERED & 151 & HANFORD SPRING 28-2 & \\
\hline 363 & & SEEP & & RAW GRAB & UNFILTERED & 8143 & HANFORD SPR DR 28-2 & \\
\hline 364 & & SEEP & & RAW GRAB & UNFILTERED & 8144 & 300 AREA SPR DR 42-2 & \\
\hline 365 & & SEEP & & RAW GRAB & UNFILTERED & 488 & 300 AREA SPRING 42-2 & \\
\hline 366 & & RIVER & & CUMULATIVE & UNFILTERED & 463 & RICH.PMPHS HRM 46.4 & \\
\hline 367 & & RIVER & & CUMULATIVE & UNFILTERED & 252 & PRIEST RAPIDS-RIVER & \\
\hline 368 & & SEEP & & RAW GRAB & UNFILTERED & 488 & 300 AREA SPRING 42-2 & \\
\hline 369 & & SEEP & & RAW GRAB & UNFILTERED & 8144 & 300 AREA SPR DR 42-2 & \\
\hline 370 & & RIVER & & CUMULATIVE & UNFILTERED & 252 & PRIEST RAPIDS-RIVER & \\
\hline 371 & & RIVER & & CUMULATIVE & UNFILTERED & 463 & RICH.PMPHS HRM 46.4 & \\
\hline 372 & & RIVER & & CUMULATIVE & UNFILTERED & 463 & RICH.PMPHS HRM 46.4 & \\
\hline 373 & & RIVER & & CUMULATIVE & UNFILTERED & 252 & PRIEST RAPIDS-RIVER & \\
\hline 374 & & RIVER & & CONTINUOUS & RESIN & 463 & RICH.PMPHS HRM 46.4 & \\
\hline 375 & $\mathrm{~F}$ & RIVER & & CONTINUOUS & FILTER & 463 & RICH.PMPHS HRM 46.4 & \\
\hline 376 & & RIVER & & CONTINUOUS & FILTER & 252 & PRIEST RAPIDS-RIVER & \\
\hline 377 & & RIVER & & CONTINUOUS & RESIN & 252 & PRIEST RAPIDS-RIVER & \\
\hline 378 & & RIVER & & CONTINUOUS & FILTER & 252 & PRIEST RAPIDS-RIVER & \\
\hline 379 & & RIVER & & CONTINUOUS & RESIN & 252 & PRIEST RAPIDS-RIVER & \\
\hline 380 & & RIVER & & CONTINUOUS & RESIN & 463 & RICH.PMPHS HRM 46.4 & \\
\hline 381 & & RIVER & & CONTINUOUS & FILTER & 463 & RICH.PMPHS HRM 46.4 & \\
\hline
\end{tabular}




\begin{tabular}{|c|c|c|c|c|c|c|c|c|}
\hline & $T$ & $\mathrm{U}$ & $\mathrm{V}$ & $\mathrm{W}$ & $X$ & $\mathrm{Y}$ & $Z$ & $\mathrm{AA}$ \\
\hline 1 & REVIEW_QUALIFIER & SAMP_FROM & SAMP_ITEM & SAMP_MTHD & COLL_MTHD & SAMP_SITE_ID & SAMP_SITE_NAME & WELL_NAME \\
\hline 382 & & RIVER & & CONTINUOUS & FILTER & 252 & PRIEST RAPIDS-RIVER & \\
\hline 383 & & RIVER & & CONTINUOUS & RESIN & 252 & PRIEST RAPIDS-RIVER & \\
\hline 384 & & RIVER & & CONTINUOUS & RESIN & 463 & RICH.PMPHS HRM 46.4 & \\
\hline 385 & & RIVER & & CONTINUOUS & FILTER & 463 & RICH.PMPHS HRM 46.4 & \\
\hline 386 & & RIVER & & CONTINUOUS & FILTER & 252 & PRIEST RAPIDS-RIVER & \\
\hline 387 & & RIVER & & CONTINUOUS & RESIN & 252 & PRIEST RAPIDS-RIVER & \\
\hline 388 & & RIVER & & CONTINUOUS & RESIN & 463 & RICH.PMPHS HRM 46.4 & \\
\hline 389 & & RIVER & & CONTINUOUS & FILTER & 463 & RICH.PMPHS HRM 46.4 & \\
\hline 390 & & RIVER & & CONTINUOUS & FILTER & 252 & PRIEST RAPIDS-RIVER & \\
\hline 391 & & RIVER & & CONTINUOUS & RESIN & 252 & PRIEST RAPIDS-RIVER & \\
\hline 392 & & RIVER & & CONTINUOUS & RESIN & 463 & RICH.PMPHS HRM 46.4 & \\
\hline 393 & & RIVER & & CONTINUOUS & FILTER & 463 & RICH.PMPHS HRM 46.4 & \\
\hline 394 & & RIVER & & CONTINUOUS & RESIN & 463 & RICH.PMPHS HRM 46.4 & \\
\hline 395 & & RIVER & & CONTINUOUS & FILTER & 463 & RICH.PMPHS HRM 46.4 & \\
\hline 396 & & RIVER & & CONTINUOUS & FILTER & 252 & PRIEST RAPIDS-RIVER & \\
\hline 397 & & RIVER & & CONTINUOUS & RESIN & 252 & PRIEST RAPIDS-RIVER & \\
\hline 398 & $F$ & RIVER & & CONTINUOUS & FILTER & 252 & PRIEST RAPIDS-RIVER & \\
\hline 399 & & RIVER & & CONTINUOUS & RESIN & 252 & PRIEST RAPIDS-RIVER & \\
\hline 400 & $F$ & RIVER & & CONTINUOUS & RESIN & 463 & RICH.PMPHS HRM 46.4 & \\
\hline 401 & $F$ & RIVER & & CONTINUOUS & FILTER & 463 & RICH.PMPHS HRM 46.4 & \\
\hline 402 & & RIVER & & CONTINUOUS & FILTER & 252 & PRIEST RAPIDS-RIVER & \\
\hline 403 & & RIVER & & CONTINUOUS & RESIN & 252 & PRIEST RAPIDS-RIVER & \\
\hline 404 & & RIVER & & CONTINUOUS & RESIN & 463 & RICH.PMPHS HRM 46.4 & \\
\hline 405 & & RIVER & & CONTINUOUS & FILTER & 463 & RICH.PMPHS HRM 46.4 & \\
\hline 406 & & RIVER & & CONTINUOUS & FILTER & 252 & PRIEST RAPIDS-RIVER & \\
\hline 407 & & RIVER & & CONTINUOUS & RESIN & 252 & PRIEST RAPIDS-RIVER & \\
\hline 408 & & RIVER & & CONTINUOUS & RESIN & 463 & RICH.PMPHS HRM 46.4 & \\
\hline 409 & $F$ & RIVER & & CONTINUOUS & FILTER & 463 & RICH.PMPHS HRM 46.4 & \\
\hline 410 & & RIVER & & CONTINUOUS & FILTER & 252 & PRIEST RAPIDS-RIVER & \\
\hline 411 & & RIVER & & CONTINUOUS & RESIN & 252 & PRIEST RAPIDS-RIVER & \\
\hline 412 & & RIVER & & CONTINUOUS & RESIN & 463 & RICH.PMPHS HRM 46.4 & \\
\hline 413 & & RIVER & & CONTINUOUS & FILTER & 463 & RICH.PMPHS HRM 46.4 & \\
\hline 414 & & RIVER & & CONTINUOUS & FILTER & 252 & PRIEST RAPIDS-RIVER & \\
\hline 415 & & RIVER & & CONTINUOUS & RESIN & 252 & PRIEST RAPIDS-RIVER & \\
\hline 416 & & RIVER & & CONTINUOUS & RESIN & 463 & RICH.PMPHS HRM 46.4 & \\
\hline 417 & & RIVER & & CONTINUOUS & FILTER & 463 & RICH.PMPHS HRM 46.4 & \\
\hline 418 & & RIVER & & CUMULATIVE & UNFILTERED & 463 & RICH.PMPHS HRM 46.4 & \\
\hline 419 & & RIVER & & CUMULATIVE & UNFILTERED & 252 & PRIEST RAPIDS-RIVER & \\
\hline
\end{tabular}




\begin{tabular}{|c|c|c|c|c|c|c|c|c|}
\hline & $\mathrm{T}$ & $\bar{U}$ & $\mathrm{~V}$ & $\mathrm{~W}$ & $\mathrm{X}$ & $\bar{Y}$ & $\mathrm{Z}$ & $\mathrm{AA}$ \\
\hline 1 & REVIEW_QUALIFIER & SAMP_FROM & SAMP_ITEM & SAMP_MTHD & COLL_MTHD & SAMP_SITE_ID & SAMP_SITE_NAME & WELL_NAME \\
\hline 420 & & DRINKING & & RAW GRAB & UNFILTERED & 553 & 100 B AREA-RIVER & \\
\hline 421 & & DRINKING & & RAW GRAB & UNFILTERED & 530 & 100 D AREA & \\
\hline 422 & & DRINKING & & SAN. GRAB & UNFILTERED & 512 & FFTF & \\
\hline 423 & & DRINKING & & RAW GRAB & UNFILTERED & 200 & $100 \mathrm{~K}$ AREA & \\
\hline 424 & & RIVER & & CUMULATIVE & UNFILTERED & 463 & RICH.PMPHS HRM 46.4 & \\
\hline 425 & & RIVER & & CUMULATIVE & UNFILTERED & 252 & PRIEST RAPIDS-RIVER & \\
\hline 426 & & RIVER & & RAW GRAB & TRANSECT & 317 & VERNITA-1 HRM 0.3 & \\
\hline 427 & & RIVER & & RAW GRAB & TRANSECT & 570 & VERNITA-2 HRM 0.3 & \\
\hline 428 & & RIVER & & RAW GRAB & TRANSECT & 318 & VERNITA-3 HRM 0.3 & \\
\hline 429 & & RIVER & & RAW GRAB & TRANSECT & 319 & VERNITA-4 HRM 0.3 & \\
\hline 430 & & RIVER & & RAW GRAB & TRANSECT & 571 & RICH.PMPHS-1 HRM46.4 & \\
\hline 431 & & RIVER & & RAW GRAB & TRANSECT & 571 & RICH.PMPHS-1 HRM46.4 & \\
\hline 432 & & RIVER & & RAW GRAB & TRANSECT & 321 & RICH.PMPHS-2 HRM46.4 & \\
\hline 433 & & RIVER & & RAW GRAB & TRANSECT & 322 & RICH.PMPHS-3 HRM46.4 & \\
\hline 434 & & RIVER & & RAW GRAB & TRANSECT & 323 & RICH.PMPHS-5 HRM46.4 & \\
\hline 435 & & RIVER & & RAW GRAB & TRANSECT & 325 & RICH.PMPHS-7 HRM46.4 & \\
\hline 436 & & RIVER & & RAW GRAB & TRANSECT & 327 & RICH.PMPHS-10 HRM46.4 & \\
\hline 437 & & RIVER & & RAW GRAB & TRANSECT & 8220 & RICH.PMPHS HRM 45.8 & \\
\hline 438 & & RIVER & & RAW GRAB & TRANSECT & 8221 & RICH.PMPHS HRM 45.0 & \\
\hline 439 & & RIVER & & RAW GRAB & TRANSECT & 8222 & RICH.PMPHS HRM 43.9 & \\
\hline 440 & & RIVER & & RAW GRAB & TRANSECT & 8223 & RICH.PMPHS HRM 43.5 & \\
\hline 441 & & RIVER & & CUMULATIVE & UNFILTERED & 463 & RICH.PMPHS HRM 46.4 & \\
\hline 442 & & RIVER & & CUMULATIVE & UNFILTERED & 252 & PRIEST RAPIDS-RIVER & \\
\hline 443 & & DRINKING & & RAW GRAB & UNFILTERED & 553 & 100 B AREA-RIVER & \\
\hline 444 & & DRINKING & & RAW GRAB & UNFILTERED & 530 & 100 D AREA & \\
\hline 445 & & DRINKING & & SAN. GRAB & UNFILTERED & 512 & FFTF & \\
\hline 446 & & DRINKING & & RAW GRAB & UNFILTERED & 200 & $100 \mathrm{~K}$ AREA & \\
\hline 447 & & RIVER & & CUMULATIVE & UNFILTERED & 252 & PRIEST RAPIDS-RIVER & \\
\hline 448 & & RIVER & & CUMULATIVE & UNFILTERED & 463 & RICH.PMPHS HRM 46.4 & \\
\hline 449 & & IRRIGATION & & RAW GRAB & UNFILTERED & 440 & RIVERVIEW CANAL & \\
\hline 450 & & RIVER & & CUMULATIVE & UNFILTERED & 252 & PRIEST RAPIDS-RIVER & \\
\hline 451 & & RIVER & & CUMULATIVE & UNFILTERED & 463 & RICH.PMPHS HRM 46.4 & \\
\hline 452 & & IRRIGATION & & RAW GRAB & UNFILTERED & 440 & RIVERVIEW CANAL & \\
\hline 453 & & RIVER & & RAW GRAB & TRANSECT & 317 & VERNITA-1 HRM 0.3 & \\
\hline 454 & & RIVER & & RAW GRAB & TRANSECT & 570 & VERNITA-2 HRM 0.3 & \\
\hline 455 & & RIVER & & RAW GRAB & TRANSECT & 318 & VERNITA-3 HRM 0.3 & \\
\hline 456 & & RIVER & & RAW GRAB & TRANSECT & 319 & VERNITA-4 HRM 0.3 & \\
\hline 457 & & RIVER & & RAW GRAB & TRANSECT & 571 & RICH.PMPHS-1 HRM46.4 & \\
\hline
\end{tabular}




\begin{tabular}{|c|c|c|c|c|c|c|c|c|}
\hline & $\mathrm{T}$ & $\bar{U}$ & $\mathrm{~V}$ & $\mathrm{~W}$ & $\mathrm{X}$ & $\bar{Y}$ & $\mathrm{Z}$ & $\mathrm{AA}$ \\
\hline 1 & REVIEW_QUALIFIER & SAMP_FROM & SAMP_ITEM & SAMP_MTHD & COLL_MTHD & SAMP_SITE_ID & SAMP_SITE_NAME & WELL_NAME \\
\hline 458 & & RIVER & & RAW GRAB & TRANSECT & 321 & RICH.PMPHS-2 HRM46.4 & \\
\hline 459 & & RIVER & & RAW GRAB & TRANSECT & 322 & RICH.PMPHS-3 HRM46.4 & \\
\hline 460 & & RIVER & & RAW GRAB & TRANSECT & 323 & RICH.PMPHS-5 HRM46.4 & \\
\hline 461 & & RIVER & & RAW GRAB & TRANSECT & 325 & RICH.PMPHS-7 HRM46.4 & \\
\hline 462 & & RIVER & & RAW GRAB & TRANSECT & 327 & RICH.PMPHS-10 HRM46.4 & \\
\hline 463 & & RIVER & & RAW GRAB & TRANSECT & 8220 & RICH.PMPHS HRM 45.8 & \\
\hline 464 & & RIVER & & RAW GRAB & TRANSECT & 8221 & RICH.PMPHS HRM 45.0 & \\
\hline 465 & & RIVER & & RAW GRAB & TRANSECT & 8222 & RICH.PMPHS HRM 43.9 & \\
\hline 466 & & RIVER & & RAW GRAB & TRANSECT & 8223 & RICH.PMPHS HRM 43.5 & \\
\hline 467 & & RIVER & & CUMULATIVE & UNFILTERED & 252 & PRIEST RAPIDS-RIVER & \\
\hline 468 & & RIVER & & CUMULATIVE & UNFILTERED & 463 & RICH.PMPHS HRM 46.4 & \\
\hline 469 & & IRRIGATION & & RAW GRAB & UNFILTERED & 440 & RIVERVIEW CANAL & \\
\hline 470 & & DRINKING & & RAW GRAB & UNFILTERED & 553 & 100 B AREA-RIVER & \\
\hline 471 & & DRINKING & & RAW GRAB & UNFILTERED & 530 & 100 D AREA & \\
\hline 472 & & DRINKING & & SAN. GRAB & UNFILTERED & 512 & FFTF & \\
\hline 473 & & DRINKING & & RAW GRAB & UNFILTERED & 200 & $100 \mathrm{~K}$ AREA & \\
\hline 474 & & RIVER & & CUMULATIVE & UNFILTERED & 252 & PRIEST RAPIDS-RIVER & \\
\hline 475 & & RIVER & & CUMULATIVE & UNFILTERED & 463 & RICH.PMPHS HRM 46.4 & \\
\hline 476 & & RIVER & & CUMULATIVE & UNFILTERED & 252 & PRIEST RAPIDS-RIVER & \\
\hline 477 & & RIVER & & CUMULATIVE & UNFILTERED & 463 & RICH.PMPHS HRM 46.4 & \\
\hline 478 & & RIVER & & RAW GRAB & TRANSECT & 328 & 100 N -1 HRM 9.5 & \\
\hline 479 & & RIVER & & RAW GRAB & TRANSECT & 317 & VERNITA-1 HRM 0.3 & \\
\hline 480 & & RIVER & & RAW GRAB & TRANSECT & 570 & VERNITA-2 HRM 0.3 & \\
\hline 481 & & RIVER & & RAW GRAB & TRANSECT & 318 & VERNITA-3 HRM 0.3 & \\
\hline 482 & & RIVER & & RAW GRAB & TRANSECT & 319 & VERNITA-4 HRM 0.3 & \\
\hline 483 & & RIVER & & RAW GRAB & TRANSECT & 328 & 100 N -1 HRM 9.5 & \\
\hline 484 & & RIVER & & RAW GRAB & TRANSECT & 329 & 100 N -2 HRM 9.5 & \\
\hline 485 & & RIVER & & RAW GRAB & TRANSECT & 331 & 100 N -5 HRM 9.5 & \\
\hline 486 & & RIVER & & RAW GRAB & TRANSECT & 335 & 100 N -10 HRM 9.5 & \\
\hline 487 & & RIVER & & RAW GRAB & TRANSECT & 330 & 100 N -3 HRM 9.5 & \\
\hline 488 & & RIVER & & RAW GRAB & TRANSECT & 333 & 100 N -7 HRM 9.5 & \\
\hline 489 & & RIVER & & RAW GRAB & TRANSECT & 8244 & 100 N SHORE HRM 8.4 & \\
\hline 490 & & RIVER & & RAW GRAB & TRANSECT & 8245 & 100 N SHORE HRM 8.9 & \\
\hline 491 & & RIVER & & RAW GRAB & TRANSECT & 8246 & 100 N SHORE HRM 9.2 & \\
\hline 492 & & RIVER & & RAW GRAB & TRANSECT & 8247 & 100 N SHORE HRM 9.8 & \\
\hline 493 & & RIVER & & RAW GRAB & TRANSECT & 336 & 100 F -1 HRM 19.0 & \\
\hline 494 & & RIVER & & RAW GRAB & TRANSECT & 337 & 100 F -3 HRM 19.0 & \\
\hline 495 & & RIVER & & RAW GRAB & TRANSECT & 344 & HANFRD TS-5 HRM 28.7 & \\
\hline
\end{tabular}




\begin{tabular}{|c|c|c|c|c|c|c|c|c|}
\hline & $T$ & $U$ & $\mathrm{~V}$ & W & $x$ & $\mathrm{Y}$ & Z & AA \\
\hline 1 & REVIEW_QUALIFIER & SAMP_FROM & SAMP_ITEM & SAMP_MTHD & COLL_MTHD & SAMP_SITE_ID & SAMP_SITE_NAME & WELL_NAME \\
\hline 496 & & RIVER & & RAW GRAB & TRANSECT & 576 & 100 F -2 HRM 19.0 & \\
\hline 497 & & RIVER & & RAW GRAB & TRANSECT & 577 & 100 F -5 HRM 19.0 & \\
\hline 498 & & RIVER & & RAW GRAB & TRANSECT & 339 & 100 F -7 HRM 19.0 & \\
\hline 499 & & RIVER & & RAW GRAB & TRANSECT & 341 & $100 \mathrm{~F}-10 \mathrm{HRM} 19.0$ & \\
\hline 500 & & RIVER & & RAW GRAB & TRANSECT & 578 & HANFRD TS-1 HRM 28.7 & \\
\hline 501 & & RIVER & & RAW GRAB & TRANSECT & 342 & HANFRD TS-2 HRM 28.7 & \\
\hline 502 & & RIVER & & RAW GRAB & TRANSECT & 469 & HANFRD TS-7 HRM 28.7 & \\
\hline 503 & & RIVER & & RAW GRAB & TRANSECT & 347 & HANFRD TS-10 HRM 28.7 & \\
\hline 504 & & RIVER & & RAW GRAB & TRANSECT & 468 & HANFRD TS-3 HRM 28.7 & \\
\hline 505 & & RIVER & & RAW GRAB & TRANSECT & 8100 & HANFRD TWNSITE HRM26 & \\
\hline 506 & & RIVER & & RAW GRAB & TRANSECT & 8101 & HANFRD TWNSITE HRM27 & \\
\hline 507 & & RIVER & & RAW GRAB & TRANSECT & 8103 & HANFRD TWNSITE HRM30 & \\
\hline 508 & & RIVER & & RAW GRAB & TRANSECT & 274 & HANFRD TWNSITE HRM28 & \\
\hline 509 & & RIVER & & RAW GRAB & TRANSECT & 8726 & 100 F SHORE HRM 18 & \\
\hline 510 & & RIVER & & RAW GRAB & TRANSECT & 8727 & 100 F SHORE HRM 22 & \\
\hline 511 & & RIVER & & RAW GRAB & TRANSECT & 8728 & 100 F SHORE HRM 23 & \\
\hline 512 & & RIVER & & RAW GRAB & TRANSECT & 348 & 300 AREA -1 HRM 43.1 & \\
\hline 513 & & RIVER & & RAW GRAB & TRANSECT & 470 & 300 AREA -2 HRM 43.1 & \\
\hline 514 & & RIVER & & RAW GRAB & TRANSECT & 580 & 300 AREA -3 HRM 43.1 & \\
\hline 515 & & RIVER & & RAW GRAB & TRANSECT & 607 & 300 AREA -5 HRM 43.1 & \\
\hline 516 & & RIVER & & RAW GRAB & TRANSECT & 351 & 300 AREA -7 HRM 43.1 & \\
\hline 517 & & RIVER & & RAW GRAB & TRANSECT & 352 & 300 AREA-10 HRM 43.1 & \\
\hline 518 & & RIVER & & RAW GRAB & TRANSECT & 571 & RICH.PMPHS-1 HRM46.4 & \\
\hline 519 & & RIVER & & RAW GRAB & TRANSECT & 321 & RICH.PMPHS-2 HRM46.4 & \\
\hline 520 & & RIVER & & RAW GRAB & TRANSECT & 322 & RICH.PMPHS-3 HRM46.4 & \\
\hline 521 & & RIVER & & RAW GRAB & TRANSECT & 323 & RICH.PMPHS-5 HRM46.4 & \\
\hline 522 & & RIVER & & RAW GRAB & TRANSECT & 325 & RICH.PMPHS-7 HRM46.4 & \\
\hline 523 & & RIVER & & RAW GRAB & TRANSECT & 327 & RICH.PMPHS-10 HRM46.4 & \\
\hline 524 & & RIVER & & RAW GRAB & TRANSECT & 8220 & RICH.PMPHS HRM 45.8 & \\
\hline 525 & & RIVER & & RAW GRAB & TRANSECT & 8221 & RICH.PMPHS HRM 45.0 & \\
\hline 526 & & RIVER & & RAW GRAB & TRANSECT & 8222 & RICH.PMPHS HRM 43.9 & \\
\hline 527 & & RIVER & & RAW GRAB & TRANSECT & 8223 & RICH.PMPHS HRM 43.5 & \\
\hline 528 & & RIVER & & RAW GRAB & TRANSECT & 8240 & 300 AREA SHR HRM41.5 & \\
\hline 529 & & RIVER & & RAW GRAB & TRANSECT & 8241 & 300 AREA SHR HRM42.1 & \\
\hline 530 & & RIVER & & RAW GRAB & TRANSECT & 8242 & 300 AREA SHR HRM42.5 & \\
\hline 531 & & RIVER & & RAW GRAB & TRANSECT & 8243 & 300 AREA SHR HRM42.9 & \\
\hline 532 & $\mathrm{~F}$ & SEEP & & RAW GRAB & UNFILTERED & 488 & 300 AREA SPRING 42-2 & \\
\hline 533 & $\mathrm{~F}$ & SEEP & & RAW GRAB & UNFILTERED & 8144 & 300 AREA SPR DR 42-2 & \\
\hline
\end{tabular}




\begin{tabular}{|c|c|c|c|c|c|c|c|c|}
\hline & $\mathrm{T}$ & $\mathrm{U}$ & $\mathrm{V}$ & $\mathrm{W}$ & $\mathrm{X}$ & $\mathrm{Y}$ & $Z$ & $\overline{\mathrm{AA}}$ \\
\hline 1 & REVIEW_QUALIFIER & SAMP_FROM & SAMP_ITEM & SAMP_MTHD & COLL_MTHD & SAMP_SITE_ID & SAMP_SITE_NAME & WELL_NAME \\
\hline 534 & & IRRIGATION & & RAW GRAB & UNFILTERED & 605 & HORN RAPIDS AREA & \\
\hline 535 & & RIVER & & CUMULATIVE & UNFILTERED & 252 & PRIEST RAPIDS-RIVER & \\
\hline 536 & & RIVER & & CUMULATIVE & UNFILTERED & 463 & RICH.PMPHS HRM 46.4 & \\
\hline 537 & & SEEP & & RAW GRAB & UNFILTERED & 202 & 100-N SPRING 8-13 & \\
\hline 538 & & SEEP & & RAW GRAB & UNFILTERED & 8145 & 100-K SPRING 63-1 & \\
\hline 539 & $F$ & SEEP & & RAW GRAB & UNFILTERED & 8162 & 100-D SPRING 110-1 & \\
\hline 540 & $F$ & SEEP & & RAW GRAB & UNFILTERED & 8163 & 100-D SPRING 102-1 & \\
\hline 541 & & DRINKING & & RAW GRAB & UNFILTERED & 553 & 100 B AREA-RIVER & \\
\hline 542 & & DRINKING & & RAW GRAB & UNFILTERED & 530 & 100 D AREA & \\
\hline 543 & & DRINKING & & SAN. GRAB & UNFILTERED & 512 & FFTF & \\
\hline 544 & & DRINKING & & RAW GRAB & UNFILTERED & 200 & $100 \mathrm{~K}$ AREA & \\
\hline 545 & $F$ & SEEP & & RAW GRAB & UNFILTERED & 8140 & 100-B SPRING 38-3 & \\
\hline 546 & $F$ & SEEP & & RAW GRAB & UNFILTERED & 8146 & 100-K SPRING 77-1 & \\
\hline 547 & & SEEP & & & & 8140 & 100-B SPRING 38-3 & \\
\hline 548 & & SEEP & & RAW GRAB & UNFILTERED & 8165 & 100-H SPRING 145-1 & \\
\hline 549 & $\mathrm{~F}$ & SEEP & & RAW GRAB & UNFILTERED & 8164 & 100-H SPRING 153-1 & \\
\hline 550 & & RIVER & & CUMULATIVE & UNFILTERED & 252 & PRIEST RAPIDS-RIVER & \\
\hline 551 & & RIVER & & CUMULATIVE & UNFILTERED & 463 & RICH.PMPHS HRM 46.4 & \\
\hline 552 & & SEEP & & RAW GRAB & UNFILTERED & 8147 & 100-F SPRING 207-1 & \\
\hline 553 & & SEEP & & & & 8147 & 100-F SPRING 207-1 & \\
\hline 554 & & RIVER & & CUMULATIVE & UNFILTERED & 252 & PRIEST RAPIDS-RIVER & \\
\hline 555 & & RIVER & & CUMULATIVE & UNFILTERED & 463 & RICH.PMPHS HRM 46.4 & \\
\hline 556 & & RIVER & & RAW GRAB & TRANSECT & 317 & VERNITA-1 HRM 0.3 & \\
\hline 557 & & RIVER & & RAW GRAB & TRANSECT & 570 & VERNITA-2 HRM 0.3 & \\
\hline 558 & & RIVER & & RAW GRAB & TRANSECT & 318 & VERNITA-3 HRM 0.3 & \\
\hline 559 & & RIVER & & RAW GRAB & TRANSECT & 319 & VERNITA-4 HRM 0.3 & \\
\hline 560 & & RIVER & & RAW GRAB & TRANSECT & 571 & RICH.PMPHS-1 HRM46.4 & \\
\hline 561 & & RIVER & & RAW GRAB & TRANSECT & 571 & RICH.PMPHS-1 HRM46.4 & \\
\hline 562 & & RIVER & & RAW GRAB & TRANSECT & 321 & RICH.PMPHS-2 HRM46.4 & \\
\hline 563 & & RIVER & & RAW GRAB & TRANSECT & 322 & RICH.PMPHS-3 HRM46.4 & \\
\hline 564 & & RIVER & & RAW GRAB & TRANSECT & 323 & RICH.PMPHS-5 HRM46.4 & \\
\hline 565 & & RIVER & & RAW GRAB & TRANSECT & 325 & RICH.PMPHS-7 HRM46.4 & \\
\hline 566 & & RIVER & & RAW GRAB & TRANSECT & 327 & RICH.PMPHS-10 HRM46.4 & \\
\hline 567 & & RIVER & & RAW GRAB & TRANSECT & 8220 & RICH.PMPHS HRM 45.8 & \\
\hline 568 & & RIVER & & RAW GRAB & TRANSECT & 8221 & RICH.PMPHS HRM 45.0 & \\
\hline 569 & & RIVER & & RAW GRAB & TRANSECT & 8222 & RICH.PMPHS HRM 43.9 & \\
\hline 570 & & RIVER & & RAW GRAB & TRANSECT & 8223 & RICH.PMPHS HRM 43.5 & \\
\hline 571 & & RIVER & & CUMULATIVE & UNFILTERED & 252 & PRIEST RAPIDS-RIVER & \\
\hline
\end{tabular}




\begin{tabular}{|c|c|c|c|c|c|c|c|c|}
\hline & $\mathrm{T}$ & $U$ & $\mathrm{~V}$ & $\mathrm{~W}$ & $\mathrm{X}$ & $\mathrm{Y}$ & Z & AA \\
\hline 1 & REVIEW_QUALIFIER & SAMP_FROM & SAMP_ITEM & SAMP_MTHD & COLL_MTHD & SAMP_SITE_ID & SAMP_SITE_NAME & WELL_NAME \\
\hline 572 & & RIVER & & CUMULATIVE & UNFILTERED & 463 & RICH.PMPHS HRM 46.4 & \\
\hline 573 & & RIVER & & CUMULATIVE & UNFILTERED & 252 & PRIEST RAPIDS-RIVER & \\
\hline 574 & & RIVER & & CUMULATIVE & UNFILTERED & 463 & RICH.PMPHS HRM 46.4 & \\
\hline 575 & & DRINKING & & RAW GRAB & UNFILTERED & 553 & 100 B AREA-RIVER & \\
\hline 576 & & DRINKING & & RAW GRAB & UNFILTERED & 530 & 100 D AREA & \\
\hline 577 & & DRINKING & & SAN. GRAB & UNFILTERED & 512 & FFTF & \\
\hline 578 & & DRINKING & & RAW GRAB & UNFILTERED & 200 & $100 \mathrm{~K}$ AREA & \\
\hline 579 & & RIVER & & RAW GRAB & TRANSECT & 571 & RICH.PMPHS-1 HRM46.4 & \\
\hline 580 & & RIVER & & RAW GRAB & TRANSECT & 571 & RICH.PMPHS-1 HRM46.4 & \\
\hline 581 & & RIVER & & RAW GRAB & TRANSECT & 321 & RICH.PMPHS-2 HRM46.4 & \\
\hline 582 & & RIVER & & RAW GRAB & TRANSECT & 322 & RICH.PMPHS-3 HRM46.4 & \\
\hline 583 & & RIVER & & RAW GRAB & TRANSECT & 323 & RICH.PMPHS-5 HRM46.4 & \\
\hline 584 & & RIVER & & RAW GRAB & TRANSECT & 325 & RICH.PMPHS-7 HRM46.4 & \\
\hline 585 & & RIVER & & RAW GRAB & TRANSECT & 327 & RICH.PMPHS-10 HRM46.4 & \\
\hline 586 & & RIVER & & RAW GRAB & TRANSECT & 8220 & RICH.PMPHS HRM 45.8 & \\
\hline 587 & & RIVER & & RAW GRAB & TRANSECT & 8221 & RICH.PMPHS HRM 45.0 & \\
\hline 588 & & RIVER & & RAW GRAB & TRANSECT & 8222 & RICH.PMPHS HRM 43.9 & \\
\hline 589 & & RIVER & & RAW GRAB & TRANSECT & 8223 & RICH.PMPHS HRM 43.5 & \\
\hline 590 & & RIVER & & RAW GRAB & TRANSECT & 317 & VERNITA-1 HRM 0.3 & \\
\hline 591 & & RIVER & & RAW GRAB & TRANSECT & 570 & VERNITA-2 HRM 0.3 & \\
\hline 592 & & RIVER & & RAW GRAB & TRANSECT & 318 & VERNITA-3 HRM 0.3 & \\
\hline 593 & & RIVER & & RAW GRAB & TRANSECT & 319 & VERNITA-4 HRM 0.3 & \\
\hline 594 & & RIVER & & CUMULATIVE & UNFILTERED & 252 & PRIEST RAPIDS-RIVER & \\
\hline 595 & & RIVER & & CUMULATIVE & UNFILTERED & 463 & RICH.PMPHS HRM 46.4 & \\
\hline 596 & & RIVER & & CUMULATIVE & UNFILTERED & 252 & PRIEST RAPIDS-RIVER & \\
\hline 597 & & RIVER & & CUMULATIVE & UNFILTERED & 463 & RICH.PMPHS HRM 46.4 & \\
\hline 598 & & DRINKING & & RAW GRAB & UNFILTERED & 553 & 100 B AREA-RIVER & \\
\hline 599 & & DRINKING & & RAW GRAB & UNFILTERED & 530 & 100 D AREA & \\
\hline 600 & & DRINKING & & SAN. GRAB & UNFILTERED & 512 & FFTF & \\
\hline 601 & & DRINKING & & RAW GRAB & UNFILTERED & 200 & $100 \mathrm{~K}$ AREA & \\
\hline 602 & $F$ & SEEP & & RAW GRAB & UNFILTERED & 8165 & 100-H SPRING 145-1 & \\
\hline 603 & & SEEP & & RAW GRAB & UNFILTERED & 8147 & 100-F SPRING 207-1 & \\
\hline 604 & $F$ & SEEP & & RAW GRAB & UNFILTERED & 8164 & 100-H SPRING 153-1 & \\
\hline 605 & & RIVER & & CUMULATIVE & UNFILTERED & 463 & RICH.PMPHS HRM 46.4 & \\
\hline 606 & & RIVER & & CUMULATIVE & UNFILTERED & 252 & PRIEST RAPIDS-RIVER & \\
\hline 607 & $F$ & SEEP & & RAW GRAB & UNFILTERED & 8144 & 300 AREA SPR DR 42-2 & \\
\hline 608 & & SEEP & & RAW GRAB & UNFILTERED & 202 & 100-N SPRING 8-13 & \\
\hline 609 & & SEEP & & RAW GRAB & UNFILTERED & 9106 & 100-B SPRING 37-1 & \\
\hline
\end{tabular}




\begin{tabular}{|c|c|c|c|c|c|c|c|c|}
\hline & $T$ & $\bar{U}$ & $\mathrm{~V}$ & $\mathrm{~W}$ & $\bar{x}$ & $\bar{Y}$ & $\bar{Z}$ & $\overline{A A}$ \\
\hline 1 & \begin{tabular}{|l|} 
REVIEW_QUALIFIER \\
\end{tabular} & SAMP_FROM & SAMP_ITEM & SAMP_MTHD & COLL_MTHD & SAMP_SITE_ID & SAMP_SITE_NAME & WELL_NAME \\
\hline 610 & & SEEP & & RAW GRAB & UNFILTERED & 9110 & 100-K SPRING 82-2 & \\
\hline 611 & $F$ & SEEP & & RAW GRAB & UNFILTERED & 8162 & 100-D SPRING 110-1 & \\
\hline 612 & & IRRIGATION & & RAW GRAB & UNFILTERED & 440 & RIVERVIEW CANAL & \\
\hline 613 & & IRRIGATION & & RAW GRAB & UNFILTERED & 605 & HORN RAPIDS AREA & \\
\hline 614 & $F$ & SEEP & & RAW GRAB & UNFILTERED & 488 & 300 AREA SPRING 42-2 & \\
\hline 615 & & RIVER & & CUMULATIVE & UNFILTERED & 463 & RICH.PMPHS HRM 46.4 & \\
\hline 616 & & RIVER & & CUMULATIVE & UNFILTERED & 252 & PRIEST RAPIDS-RIVER & \\
\hline 617 & & IRRIGATION & & RAW GRAB & UNFILTERED & 440 & RIVERVIEW CANAL & \\
\hline 618 & & IRRIGATION & & RAW GRAB & UNFILTERED & 605 & HORN RAPIDS AREA & \\
\hline 619 & & RIVER & & RAW GRAB & TRANSECT & 571 & RICH.PMPHS-1 HRM46.4 & \\
\hline 620 & & RIVER & & RAW GRAB & TRANSECT & 321 & RICH.PMPHS-2 HRM46.4 & \\
\hline 621 & & RIVER & & RAW GRAB & TRANSECT & 322 & RICH.PMPHS-3 HRM46.4 & \\
\hline 622 & & RIVER & & RAW GRAB & TRANSECT & 323 & RICH.PMPHS-5 HRM46.4 & \\
\hline 623 & & RIVER & & RAW GRAB & TRANSECT & 325 & RICH.PMPHS-7 HRM46.4 & \\
\hline 624 & & RIVER & & RAW GRAB & TRANSECT & 327 & RICH.PMPHS-10 HRM46.4 & \\
\hline 625 & & RIVER & & RAW GRAB & TRANSECT & 8220 & RICH.PMPHS HRM 45.8 & \\
\hline 626 & & RIVER & & RAW GRAB & TRANSECT & 8221 & RICH.PMPHS HRM 45.0 & \\
\hline 627 & & RIVER & & RAW GRAB & TRANSECT & 8222 & RICH.PMPHS HRM 43.9 & \\
\hline 628 & & RIVER & & RAW GRAB & TRANSECT & 8223 & RICH.PMPHS HRM 43.5 & \\
\hline 629 & & RIVER & & RAW GRAB & TRANSECT & 317 & VERNITA-1 HRM 0.3 & \\
\hline 630 & & RIVER & & RAW GRAB & TRANSECT & 570 & VERNITA-2 HRM 0.3 & \\
\hline 631 & & RIVER & & RAW GRAB & TRANSECT & 318 & VERNITA-3 HRM 0.3 & \\
\hline 632 & & RIVER & & RAW GRAB & TRANSECT & 319 & VERNITA-4 HRM 0.3 & \\
\hline 633 & & RIVER & & CUMULATIVE & UNFILTERED & 463 & RICH.PMPHS HRM 46.4 & \\
\hline 634 & & RIVER & & CUMULATIVE & UNFILTERED & 252 & PRIEST RAPIDS-RIVER & \\
\hline 635 & & IRRIGATION & & RAW GRAB & UNFILTERED & 440 & RIVERVIEW CANAL & \\
\hline 636 & & IRRIGATION & & RAW GRAB & UNFILTERED & 605 & HORN RAPIDS AREA & \\
\hline 637 & & DRINKING & & RAW GRAB & UNFILTERED & 553 & 100 B AREA-RIVER & \\
\hline 638 & & DRINKING & & RAW GRAB & UNFILTERED & 530 & 100 D AREA & \\
\hline 639 & & DRINKING & & SAN. GRAB & UNFILTERED & 512 & FFTF & \\
\hline 640 & & DRINKING & & RAW GRAB & UNFILTERED & 200 & $100 \mathrm{~K}$ AREA & \\
\hline 641 & & RIVER & & CUMULATIVE & UNFILTERED & 463 & RICH.PMPHS HRM 46.4 & \\
\hline 642 & & RIVER & & CUMULATIVE & UNFILTERED & 252 & PRIEST RAPIDS-RIVER & \\
\hline 643 & $F$ & SEEP & & RAW GRAB & UNFILTERED & 488 & 300 AREA SPRING 42-2 & \\
\hline 644 & $F$ & SEEP & & RAW GRAB & UNFILTERED & 8144 & 300 AREA SPR DR 42-2 & \\
\hline 645 & & RIVER & & CUMULATIVE & UNFILTERED & 463 & RICH.PMPHS HRM 46.4 & \\
\hline 646 & & RIVER & & CUMULATIVE & UNFILTERED & 252 & PRIEST RAPIDS-RIVER & \\
\hline 647 & & RIVER & & RAW GRAB & TRANSECT & 328 & 100 N -1 HRM 9.5 & \\
\hline
\end{tabular}




\begin{tabular}{|c|c|c|c|c|c|c|c|c|}
\hline & $\mathrm{T}$ & $U$ & $\mathrm{~V}$ & $\mathrm{~W}$ & $\mathrm{X}$ & $\mathrm{Y}$ & $Z$ & $\mathrm{AA}$ \\
\hline 1 & REVIEW QUALIFIER & SAMP FROM & SAMP ITEM & SAMP MTHD & COLL MTHD & SAMP SITE ID & SAMP SITE NAME & WELL NAME \\
\hline 648 & & RIVER & & RAW GRAB & TRANSECT & 317 & VERNITA-1 HRM 0.3 & \\
\hline 649 & & RIVER & & RAW GRAB & TRANSECT & 570 & VERNITA-2 HRM 0.3 & \\
\hline 650 & & RIVER & & RAW GRAB & TRANSECT & 318 & VERNITA-3 HRM 0.3 & \\
\hline 651 & & RIVER & & RAW GRAB & TRANSECT & 319 & VERNITA-4 HRM 0.3 & \\
\hline 652 & & RIVER & & RAW GRAB & TRANSECT & 328 & 100 N -1 HRM 9.5 & \\
\hline 653 & & RIVER & & RAW GRAB & TRANSECT & 329 & 100 N -2 HRM 9.5 & \\
\hline 654 & & RIVER & & RAW GRAB & TRANSECT & 331 & 100 N -5 HRM 9.5 & \\
\hline 655 & & RIVER & & RAW GRAB & TRANSECT & 335 & 100 N -10 HRM 9.5 & \\
\hline 656 & & RIVER & & RAW GRAB & TRANSECT & 330 & 100 N -3 HRM 9.5 & \\
\hline 657 & & RIVER & & RAW GRAB & TRANSECT & 333 & 100 N -7 HRM 9.5 & \\
\hline 658 & & RIVER & & RAW GRAB & TRANSECT & 8244 & 100 N SHORE HRM 8.4 & \\
\hline 659 & & RIVER & & RAW GRAB & TRANSECT & 8245 & 100 N SHORE HRM 8.9 & \\
\hline 660 & & RIVER & & RAW GRAB & TRANSECT & 8246 & 100 N SHORE HRM 9.2 & \\
\hline 661 & & RIVER & & RAW GRAB & TRANSECT & 8247 & 100 N SHORE HRM 9.8 & \\
\hline 662 & & RIVER & & RAW GRAB & TRANSECT & 336 & 100 F -1 HRM 19.0 & \\
\hline 663 & & RIVER & & RAW GRAB & TRANSECT & 337 & 100 F -3 HRM 19.0 & \\
\hline 664 & & RIVER & & RAW GRAB & TRANSECT & 344 & HANFRD TS-5 HRM 28.7 & \\
\hline 665 & & RIVER & & RAW GRAB & TRANSECT & 576 & 100 F -2 HRM 19.0 & \\
\hline 666 & & RIVER & & RAW GRAB & TRANSECT & 577 & 100 F -5 HRM 19.0 & \\
\hline 667 & & RIVER & & RAW GRAB & TRANSECT & 339 & 100 F -7 HRM 19.0 & \\
\hline 668 & & RIVER & & RAW GRAB & TRANSECT & 341 & $100 \mathrm{~F}-10 \mathrm{HRM} 19.0$ & \\
\hline 669 & & RIVER & & RAW GRAB & TRANSECT & 578 & HANFRD TS-1 HRM 28.7 & \\
\hline 670 & & RIVER & & RAW GRAB & TRANSECT & 342 & HANFRD TS-2 HRM 28.7 & \\
\hline 671 & & RIVER & & RAW GRAB & TRANSECT & 469 & HANFRD TS-7 HRM 28.7 & \\
\hline 672 & & RIVER & & RAW GRAB & TRANSECT & 347 & HANFRD TS-10 HRM 28.7 & \\
\hline 673 & & RIVER & & RAW GRAB & TRANSECT & 468 & HANFRD TS-3 HRM 28.7 & \\
\hline 674 & & RIVER & & RAW GRAB & TRANSECT & 8100 & HANFRD TWNSITE HRM26 & \\
\hline 675 & & RIVER & & RAW GRAB & TRANSECT & 8101 & HANFRD TWNSITE HRM27 & \\
\hline 676 & & RIVER & & RAW GRAB & TRANSECT & 8103 & HANFRD TWNSITE HRM30 & \\
\hline 677 & & RIVER & & RAW GRAB & TRANSECT & 274 & HANFRD TWNSITE HRM28 & \\
\hline 678 & & RIVER & & RAW GRAB & TRANSECT & 8726 & 100 F SHORE HRM 18 & \\
\hline 679 & & RIVER & & RAW GRAB & TRANSECT & 8728 & 100 F SHORE HRM 23 & \\
\hline 680 & & RIVER & & RAW GRAB & TRANSECT & 348 & 300 AREA -1 HRM 43.1 & \\
\hline 681 & & RIVER & & RAW GRAB & TRANSECT & 470 & 300 AREA -2 HRM 43.1 & \\
\hline 682 & & RIVER & & RAW GRAB & TRANSECT & 580 & 300 AREA -3 HRM 43.1 & \\
\hline 683 & & RIVER & & RAW GRAB & TRANSECT & 607 & 300 AREA -5 HRM 43.1 & \\
\hline 684 & & RIVER & & RAW GRAB & TRANSECT & 351 & 300 AREA -7 HRM 43.1 & \\
\hline 685 & & RIVER & & RAW GRAB & TRANSECT & 352 & 300 AREA-10 HRM 43.1 & \\
\hline
\end{tabular}




\begin{tabular}{|c|c|c|c|c|c|c|c|c|}
\hline & $\mathrm{T}$ & $\mathrm{U}$ & $\mathrm{V}$ & $\mathrm{W}$ & $\mathrm{X}$ & $\mathrm{Y}$ & $Z$ & $\overline{\mathrm{AA}}$ \\
\hline 1 & REVIEW_QUALIFIER & SAMP_FROM & SAMP_ITEM & SAMP_MTHD & COLL_MTHD & SAMP_SITE_ID & SAMP_SITE_NAME & WELL_NAME \\
\hline 686 & & RIVER & & RAW GRAB & TRANSECT & 571 & RICH.PMPHS-1 HRM46.4 & \\
\hline 687 & & RIVER & & RAW GRAB & TRANSECT & 321 & RICH.PMPHS-2 HRM46.4 & \\
\hline 688 & & RIVER & & RAW GRAB & TRANSECT & 322 & RICH.PMPHS-3 HRM46.4 & \\
\hline 689 & & RIVER & & RAW GRAB & TRANSECT & 323 & RICH.PMPHS-5 HRM46.4 & \\
\hline 690 & & RIVER & & RAW GRAB & TRANSECT & 325 & RICH.PMPHS-7 HRM46.4 & \\
\hline 691 & & RIVER & & RAW GRAB & TRANSECT & 327 & RICH.PMPHS-10 HRM46.4 & \\
\hline 692 & & RIVER & & RAW GRAB & TRANSECT & 8220 & RICH.PMPHS HRM 45.8 & \\
\hline 693 & & RIVER & & RAW GRAB & TRANSECT & 8221 & RICH.PMPHS HRM 45.0 & \\
\hline 694 & & RIVER & & RAW GRAB & TRANSECT & 8222 & RICH.PMPHS HRM 43.9 & \\
\hline 695 & & RIVER & & RAW GRAB & TRANSECT & 8223 & RICH.PMPHS HRM 43.5 & \\
\hline 696 & & RIVER & & RAW GRAB & TRANSECT & 8240 & 300 AREA SHR HRM41.5 & \\
\hline 697 & & RIVER & & RAW GRAB & TRANSECT & 8241 & 300 AREA SHR HRM42.1 & \\
\hline 698 & & RIVER & & RAW GRAB & TRANSECT & 8242 & 300 AREA SHR HRM42.5 & \\
\hline 699 & & RIVER & & RAW GRAB & TRANSECT & 8243 & 300 AREA SHR HRM42.9 & \\
\hline 700 & & RIVER & & CUMULATIVE & UNFILTERED & 252 & PRIEST RAPIDS-RIVER & \\
\hline 701 & & RIVER & & CUMULATIVE & UNFILTERED & 463 & RICH.PMPHS HRM 46.4 & \\
\hline 702 & $F$ & DRINKING & & RAW GRAB & UNFILTERED & 553 & 100 B AREA-RIVER & \\
\hline 703 & & DRINKING & & RAW GRAB & UNFILTERED & 530 & 100 D AREA & \\
\hline 704 & & DRINKING & & SAN. GRAB & UNFILTERED & 512 & FFTF & \\
\hline 705 & & DRINKING & & RAW GRAB & UNFILTERED & 200 & $100 \mathrm{~K}$ AREA & \\
\hline 706 & & SEEP & & RAW GRAB & UNFILTERED & 8140 & 100-B SPRING 38-3 & \\
\hline 707 & & SEEP & & RAW GRAB & UNFILTERED & 8147 & 100-F SPRING 207-1 & \\
\hline 708 & & SEEP & & RAW GRAB & UNFILTERED & 202 & $100-N$ SPRING 8-13 & \\
\hline 709 & $F$ & SEEP & & RAW GRAB & UNFILTERED & 8145 & 100-K SPRING 63-1 & \\
\hline 710 & $F$ & SEEP & & RAW GRAB & UNFILTERED & 8146 & 100-K SPRING 77-1 & \\
\hline 711 & $\mathrm{~F}$ & SEEP & & RAW GRAB & UNFILTERED & 8165 & 100-H SPRING 145-1 & \\
\hline 712 & $F$ & SEEP & & RAW GRAB & UNFILTERED & 8846 & 100-H SPRING 152-2 & \\
\hline 713 & & RIVER & & CUMULATIVE & UNFILTERED & 463 & RICH.PMPHS HRM 46.4 & \\
\hline 714 & & RIVER & & CUMULATIVE & UNFILTERED & 252 & PRIEST RAPIDS-RIVER & \\
\hline 715 & $F$ & SEEP & & RAW GRAB & UNFILTERED & 9236 & VERNITA BRIDGE -1 & \\
\hline 716 & & RIVER & & CUMULATIVE & UNFILTERED & 252 & PRIEST RAPIDS-RIVER & \\
\hline 717 & & RIVER & & CUMULATIVE & UNFILTERED & 463 & RICH.PMPHS HRM 46.4 & \\
\hline 718 & $F$ & RIVER & & RAW GRAB & TRANSECT & 317 & VERNITA-1 HRM 0.3 & \\
\hline 719 & & RIVER & & RAW GRAB & TRANSECT & 570 & VERNITA-2 HRM 0.3 & \\
\hline 720 & & RIVER & & RAW GRAB & TRANSECT & 318 & VERNITA-3 HRM 0.3 & \\
\hline 721 & & RIVER & & RAW GRAB & TRANSECT & 319 & VERNITA-4 HRM 0.3 & \\
\hline 722 & & RIVER & & RAW GRAB & TRANSECT & 571 & RICH.PMPHS-1 HRM46.4 & \\
\hline 723 & & RIVER & & RAW GRAB & TRANSECT & 571 & RICH.PMPHS-1 HRM46.4 & \\
\hline
\end{tabular}




\begin{tabular}{|c|c|c|c|c|c|c|c|c|}
\hline & $T$ & $\mathrm{U}$ & $\mathrm{V}$ & $\mathrm{W}$ & $X$ & $\mathrm{Y}$ & $Z$ & $\mathrm{AA}$ \\
\hline 1 & REVIEW_QUALIFIER & SAMP_FROM & SAMP_ITEM & SAMP_MTHD & COLL_MTHD & SAMP_SITE_ID & SAMP_SITE_NAME & WELL_NAME \\
\hline 724 & & RIVER & & RAW GRAB & TRANSECT & 321 & RICH.PMPHS-2 HRM46.4 & \\
\hline 725 & & RIVER & & RAW GRAB & TRANSECT & 322 & RICH.PMPHS-3 HRM46.4 & \\
\hline 726 & & RIVER & & RAW GRAB & TRANSECT & 323 & RICH.PMPHS-5 HRM46.4 & \\
\hline 727 & & RIVER & & RAW GRAB & TRANSECT & 325 & RICH.PMPHS-7 HRM46.4 & \\
\hline 728 & & RIVER & & RAW GRAB & TRANSECT & 327 & RICH.PMPHS-10 HRM46.4 & \\
\hline 729 & & RIVER & & RAW GRAB & TRANSECT & 8220 & RICH.PMPHS HRM 45.8 & \\
\hline 730 & & RIVER & & RAW GRAB & TRANSECT & 8221 & RICH.PMPHS HRM 45.0 & \\
\hline 731 & & RIVER & & RAW GRAB & TRANSECT & 8222 & RICH.PMPHS HRM 43.9 & \\
\hline 732 & & RIVER & & RAW GRAB & TRANSECT & 8223 & RICH.PMPHS HRM 43.5 & \\
\hline 733 & & RIVER & & CUMULATIVE & UNFILTERED & 463 & RICH.PMPHS HRM 46.4 & \\
\hline 734 & & RIVER & & CUMULATIVE & UNFILTERED & 252 & PRIEST RAPIDS-RIVER & \\
\hline 735 & & RIVER & & CUMULATIVE & UNFILTERED & 463 & RICH.PMPHS HRM 46.4 & \\
\hline 736 & & RIVER & & CUMULATIVE & UNFILTERED & 252 & PRIEST RAPIDS-RIVER & \\
\hline 737 & & DRINKING & & SAN. GRAB & UNFILTERED & 200 & $100 \mathrm{~K}$ AREA & \\
\hline 738 & & DRINKING & & SAN. GRAB & UNFILTERED & 201 & 100 N AREA & \\
\hline 739 & & DRINKING & & SAN. GRAB & UNFILTERED & 404 & 200 W AREA & \\
\hline 740 & & DRINKING & & SAN. GRAB & UNFILTERED & 134 & 400 AREA & \\
\hline 741 & & RIVER & & CUMULATIVE & UNFILTERED & 252 & PRIEST RAPIDS-RIVER & \\
\hline 742 & & RIVER & & CUMULATIVE & UNFILTERED & 463 & RICH.PMPHS HRM 46.4 & \\
\hline 743 & & RIVER & & RAW GRAB & TRANSECT & 317 & VERNITA-1 HRM 0.3 & \\
\hline 744 & & RIVER & & RAW GRAB & TRANSECT & 570 & VERNITA-2 HRM 0.3 & \\
\hline 745 & & RIVER & & RAW GRAB & TRANSECT & 318 & VERNITA-3 HRM 0.3 & \\
\hline 746 & & RIVER & & RAW GRAB & TRANSECT & 319 & VERNITA-4 HRM 0.3 & \\
\hline 747 & & RIVER & & RAW GRAB & TRANSECT & 571 & RICH.PMPHS-1 HRM46.4 & \\
\hline 748 & & RIVER & & RAW GRAB & TRANSECT & 571 & RICH.PMPHS-1 HRM46.4 & \\
\hline 749 & & RIVER & & RAW GRAB & TRANSECT & 321 & RICH.PMPHS-2 HRM46.4 & \\
\hline 750 & & RIVER & & RAW GRAB & TRANSECT & 322 & RICH.PMPHS-3 HRM46.4 & \\
\hline 751 & & RIVER & & RAW GRAB & TRANSECT & 323 & RICH.PMPHS-5 HRM46.4 & \\
\hline 752 & & RIVER & & RAW GRAB & TRANSECT & 325 & RICH.PMPHS-7 HRM46.4 & \\
\hline 753 & & RIVER & & RAW GRAB & TRANSECT & 327 & RICH.PMPHS-10 HRM46.4 & \\
\hline 754 & & RIVER & & RAW GRAB & TRANSECT & 8220 & RICH.PMPHS HRM 45.8 & \\
\hline 755 & & RIVER & & RAW GRAB & TRANSECT & 8221 & RICH.PMPHS HRM 45.0 & \\
\hline 756 & & RIVER & & RAW GRAB & TRANSECT & 8222 & RICH.PMPHS HRM 43.9 & \\
\hline 757 & & RIVER & & RAW GRAB & TRANSECT & 8223 & RICH.PMPHS HRM 43.5 & \\
\hline 758 & & RIVER & & CUMULATIVE & UNFILTERED & 252 & PRIEST RAPIDS-RIVER & \\
\hline 759 & & RIVER & & CUMULATIVE & UNFILTERED & 463 & RICH.PMPHS HRM 46.4 & \\
\hline 760 & & DRINKING & & SAN. GRAB & UNFILTERED & 201 & 100 N AREA & \\
\hline 761 & & DRINKING & & SAN. GRAB & UNFILTERED & 134 & 400 AREA & \\
\hline
\end{tabular}

Page 176 


\begin{tabular}{|c|c|c|c|c|c|c|c|c|}
\hline & $\mathrm{T}$ & $\mathrm{U}$ & $\mathrm{V}$ & $\mathrm{W}$ & $\mathrm{X}$ & $\mathrm{Y}$ & $Z$ & $\overline{\mathrm{AA}}$ \\
\hline 1 & REVIEW_QUALIFIER & SAMP_FROM & SAMP_ITEM & SAMP_MTHD & COLL_MTHD & SAMP_SITE_ID & SAMP_SITE_NAME & WELL_NAME \\
\hline 762 & & DRINKING & & SAN. GRAB & UNFILTERED & 200 & $100 \mathrm{~K}$ AREA & \\
\hline 763 & & DRINKING & & SAN. GRAB & UNFILTERED & 404 & 200 W AREA & \\
\hline 764 & & RIVER & & CUMULATIVE & UNFILTERED & 252 & PRIEST RAPIDS-RIVER & \\
\hline 765 & & RIVER & & CUMULATIVE & UNFILTERED & 463 & RICH.PMPHS HRM 46.4 & \\
\hline 766 & & IRRIGATION & & RAW GRAB & UNFILTERED & 440 & RIVERVIEW CANAL & \\
\hline 767 & & IRRIGATION & & RAW GRAB & UNFILTERED & 605 & HORN RAPIDS AREA & \\
\hline 768 & & RIVER & & CUMULATIVE & UNFILTERED & 252 & PRIEST RAPIDS-RIVER & \\
\hline 769 & & RIVER & & CUMULATIVE & UNFILTERED & 463 & RICH.PMPHS HRM 46.4 & \\
\hline 770 & & RIVER & & RAW GRAB & TRANSECT & 317 & VERNITA-1 HRM 0.3 & \\
\hline 771 & & RIVER & & RAW GRAB & TRANSECT & 570 & VERNITA-2 HRM 0.3 & \\
\hline 772 & & RIVER & & RAW GRAB & TRANSECT & 318 & VERNITA-3 HRM 0.3 & \\
\hline 773 & & RIVER & & RAW GRAB & TRANSECT & 319 & VERNITA-4 HRM 0.3 & \\
\hline 774 & & RIVER & & RAW GRAB & TRANSECT & 571 & RICH.PMPHS-1 HRM46.4 & \\
\hline 775 & & RIVER & & RAW GRAB & TRANSECT & 321 & RICH.PMPHS-2 HRM46.4 & \\
\hline 776 & & RIVER & & RAW GRAB & TRANSECT & 322 & RICH.PMPHS-3 HRM46.4 & \\
\hline 777 & & RIVER & & RAW GRAB & TRANSECT & 323 & RICH.PMPHS-5 HRM46.4 & \\
\hline 778 & & RIVER & & RAW GRAB & TRANSECT & 325 & RICH.PMPHS-7 HRM46.4 & \\
\hline 779 & & RIVER & & RAW GRAB & TRANSECT & 327 & RICH.PMPHS-10 HRM46.4 & \\
\hline 780 & & RIVER & & RAW GRAB & TRANSECT & 8220 & RICH.PMPHS HRM 45.8 & \\
\hline 781 & & RIVER & & RAW GRAB & TRANSECT & 8221 & RICH.PMPHS HRM 45.0 & \\
\hline 782 & & RIVER & & RAW GRAB & TRANSECT & 8222 & RICH.PMPHS HRM 43.9 & \\
\hline 783 & & RIVER & & RAW GRAB & TRANSECT & 8223 & RICH.PMPHS HRM 43.5 & \\
\hline 784 & & RIVER & & CUMULATIVE & UNFILTERED & 252 & PRIEST RAPIDS-RIVER & \\
\hline 785 & & RIVER & & CUMULATIVE & UNFILTERED & 463 & RICH.PMPHS HRM 46.4 & \\
\hline 786 & & IRRIGATION & & RAW GRAB & UNFILTERED & 440 & RIVERVIEW CANAL & \\
\hline 787 & & IRRIGATION & & RAW GRAB & UNFILTERED & 605 & HORN RAPIDS AREA & \\
\hline 788 & & IRRIGATION & & RAW GRAB & UNFILTERED & 440 & RIVERVIEW CANAL & \\
\hline 789 & & DRINKING & & SAN. GRAB & UNFILTERED & 200 & $100 \mathrm{~K}$ AREA & \\
\hline 790 & & IRRIGATION & & RAW GRAB & UNFILTERED & 605 & HORN RAPIDS AREA & \\
\hline 791 & & DRINKING & & SAN. GRAB & UNFILTERED & 201 & 100 N AREA & \\
\hline 792 & & DRINKING & & SAN. GRAB & UNFILTERED & 404 & 200 W AREA & \\
\hline 793 & & DRINKING & & SAN. GRAB & UNFILTERED & 134 & 400 AREA & \\
\hline 794 & & RIVER & & CUMULATIVE & UNFILTERED & 252 & PRIEST RAPIDS-RIVER & \\
\hline 795 & & RIVER & & CUMULATIVE & UNFILTERED & 463 & RICH.PMPHS HRM 46.4 & \\
\hline 796 & & RIVER & & CUMULATIVE & UNFILTERED & 252 & PRIEST RAPIDS-RIVER & \\
\hline 797 & & RIVER & & CUMULATIVE & UNFILTERED & 463 & RICH.PMPHS HRM 46.4 & \\
\hline 798 & & RIVER & & RAW GRAB & TRANSECT & 328 & $100 \mathrm{~N}-1$ HRM 9.5 & \\
\hline 799 & & RIVER & & RAW GRAB & TRANSECT & 319 & VERNITA-4 HRM 0.3 & \\
\hline
\end{tabular}




\begin{tabular}{|c|c|c|c|c|c|c|c|c|}
\hline & $\mathrm{T}$ & $U$ & $\mathrm{~V}$ & $\mathrm{~W}$ & $\mathrm{X}$ & $\mathrm{Y}$ & $Z$ & $\mathrm{AA}$ \\
\hline 1 & REVIEW QUALIFIER & SAMP FROM & SAMP ITEM & SAMP MTHD & COLL MTHD & SAMP SITE ID & SAMP SITE NAME & WELL NAME \\
\hline 800 & & RIVER & & RAW GRAB & TRANSECT & 570 & VERNITA-2 HRM 0.3 & \\
\hline 801 & & RIVER & & RAW GRAB & TRANSECT & 318 & VERNITA-3 HRM 0.3 & \\
\hline 802 & & RIVER & & RAW GRAB & TRANSECT & 317 & VERNITA-1 HRM 0.3 & \\
\hline 803 & & RIVER & & RAW GRAB & TRANSECT & 328 & 100 N -1 HRM 9.5 & \\
\hline 804 & & RIVER & & RAW GRAB & TRANSECT & 329 & 100 N -2 HRM 9.5 & \\
\hline 805 & & RIVER & & RAW GRAB & TRANSECT & 331 & 100 N -5 HRM 9.5 & \\
\hline 806 & & RIVER & & RAW GRAB & TRANSECT & 335 & 100 N -10 HRM 9.5 & \\
\hline 807 & & RIVER & & RAW GRAB & TRANSECT & 330 & 100 N -3 HRM 9.5 & \\
\hline 808 & & RIVER & & RAW GRAB & TRANSECT & 333 & 100 N -7 HRM 9.5 & \\
\hline 809 & & RIVER & & RAW GRAB & TRANSECT & 8244 & 100 N SHORE HRM 8.4 & \\
\hline 810 & & RIVER & & RAW GRAB & TRANSECT & 8245 & 100 N SHORE HRM 8.9 & \\
\hline 811 & & RIVER & & RAW GRAB & TRANSECT & 8246 & 100 N SHORE HRM 9.2 & \\
\hline 812 & & RIVER & & RAW GRAB & TRANSECT & 8247 & 100 N SHORE HRM 9.8 & \\
\hline 813 & & RIVER & & RAW GRAB & TRANSECT & 336 & 100 F -1 HRM 19.0 & \\
\hline 814 & & RIVER & & RAW GRAB & TRANSECT & 337 & 100 F -3 HRM 19.0 & \\
\hline 815 & & RIVER & & RAW GRAB & TRANSECT & 344 & HANFRD TS-5 HRM 28.7 & \\
\hline 816 & & RIVER & & RAW GRAB & TRANSECT & 576 & 100 F -2 HRM 19.0 & \\
\hline 817 & & RIVER & & RAW GRAB & TRANSECT & 577 & 100 F -5 HRM 19.0 & \\
\hline 818 & & RIVER & & RAW GRAB & TRANSECT & 339 & $100 \mathrm{~F}-7 \mathrm{HRM} 19.0$ & \\
\hline 819 & & RIVER & & RAW GRAB & TRANSECT & 341 & $100 \mathrm{~F}-10 \mathrm{HRM} 19.0$ & \\
\hline 820 & & RIVER & & RAW GRAB & TRANSECT & 578 & HANFRD TS-1 HRM 28.7 & \\
\hline 821 & & RIVER & & RAW GRAB & TRANSECT & 342 & HANFRD TS-2 HRM 28.7 & \\
\hline 822 & & RIVER & & RAW GRAB & TRANSECT & 469 & HANFRD TS-7 HRM 28.7 & \\
\hline 823 & & RIVER & & RAW GRAB & TRANSECT & 347 & HANFRD TS-10 HRM 28.7 & \\
\hline 824 & & RIVER & & RAW GRAB & TRANSECT & 468 & HANFRD TS-3 HRM 28.7 & \\
\hline 825 & & RIVER & & RAW GRAB & TRANSECT & 8100 & HANFRD TWNSITE HRM26 & \\
\hline 826 & & RIVER & & RAW GRAB & TRANSECT & 8101 & HANFRD TWNSITE HRM27 & \\
\hline 827 & & RIVER & & RAW GRAB & TRANSECT & 8103 & HANFRD TWNSITE HRM30 & \\
\hline 828 & & RIVER & & RAW GRAB & TRANSECT & 274 & HANFRD TWNSITE HRM28 & \\
\hline 829 & & RIVER & & RAW GRAB & TRANSECT & 8726 & 100 F SHORE HRM 18 & \\
\hline 830 & & RIVER & & RAW GRAB & TRANSECT & 13656 & 100 F SHORE HRM 24 & \\
\hline 831 & & RIVER & & RAW GRAB & TRANSECT & 8728 & 100 F SHORE HRM 23 & \\
\hline 832 & & RIVER & & RAW GRAB & TRANSECT & 348 & 300 AREA -1 HRM 43.1 & \\
\hline 833 & & RIVER & & RAW GRAB & TRANSECT & 470 & 300 AREA -2 HRM 43.1 & \\
\hline 834 & & RIVER & & RAW GRAB & TRANSECT & 580 & 300 AREA -3 HRM 43.1 & \\
\hline 835 & & RIVER & & RAW GRAB & TRANSECT & 607 & 300 AREA -5 HRM 43.1 & \\
\hline 836 & & RIVER & & RAW GRAB & TRANSECT & 351 & 300 AREA -7 HRM 43.1 & \\
\hline 837 & & RIVER & & RAW GRAB & TRANSECT & 352 & 300 AREA-10 HRM 43.1 & \\
\hline
\end{tabular}




\begin{tabular}{|c|c|c|c|c|c|c|c|c|}
\hline & $\mathrm{T}$ & $\mathrm{U}$ & $\mathrm{V}$ & $\mathrm{W}$ & $\mathrm{X}$ & $\mathrm{Y}$ & $Z$ & $\overline{\mathrm{AA}}$ \\
\hline 1 & REVIEW_QUALIFIER & SAMP_FROM & SAMP_ITEM & SAMP_MTHD & COLL_MTHD & SAMP_SITE_ID & SAMP_SITE_NAME & WELL_NAME \\
\hline 838 & & RIVER & & RAW GRAB & TRANSECT & 571 & RICH.PMPHS-1 HRM46.4 & \\
\hline 839 & & RIVER & & RAW GRAB & TRANSECT & 321 & RICH.PMPHS-2 HRM46.4 & \\
\hline 840 & & RIVER & & RAW GRAB & TRANSECT & 322 & RICH.PMPHS-3 HRM46.4 & \\
\hline 841 & & RIVER & & RAW GRAB & TRANSECT & 323 & RICH.PMPHS-5 HRM46.4 & \\
\hline 842 & & RIVER & & RAW GRAB & TRANSECT & 325 & RICH.PMPHS-7 HRM46.4 & \\
\hline 843 & & RIVER & & RAW GRAB & TRANSECT & 327 & RICH.PMPHS-10 HRM46.4 & \\
\hline 844 & & RIVER & & RAW GRAB & TRANSECT & 8220 & RICH.PMPHS HRM 45.8 & \\
\hline 845 & & RIVER & & RAW GRAB & TRANSECT & 8221 & RICH.PMPHS HRM 45.0 & \\
\hline 846 & & RIVER & & RAW GRAB & TRANSECT & 8222 & RICH.PMPHS HRM 43.9 & \\
\hline 847 & & RIVER & & RAW GRAB & TRANSECT & 8223 & RICH.PMPHS HRM 43.5 & \\
\hline 848 & & RIVER & & RAW GRAB & TRANSECT & 8240 & 300 AREA SHR HRM41.5 & \\
\hline 849 & & RIVER & & RAW GRAB & TRANSECT & 8241 & 300 AREA SHR HRM42.1 & \\
\hline 850 & & RIVER & & RAW GRAB & TRANSECT & 8242 & 300 AREA SHR HRM42.5 & \\
\hline 851 & & RIVER & & RAW GRAB & TRANSECT & 8243 & 300 AREA SHR HRM42.9 & \\
\hline 852 & $\mathrm{~F}$ & SEEP & & RAW GRAB & UNFILTERED & 202 & 100-N SPRING 8-13 & \\
\hline 853 & & SEEP & & RAW GRAB & UNFILTERED & 9106 & 100-B SPRING 37-1 & \\
\hline 854 & $F$ & SEEP & & RAW GRAB & UNFILTERED & 13792 & 100-K SPRING 63-2 & \\
\hline 855 & & RIVER & & CUMULATIVE & UNFILTERED & 252 & PRIEST RAPIDS-RIVER & \\
\hline 856 & & RIVER & & CUMULATIVE & UNFILTERED & 463 & RICH.PMPHS HRM 46.4 & \\
\hline 857 & & SEEP & & RAW GRAB & UNFILTERED & 488 & 300 AREA SPRING 42-2 & \\
\hline 858 & $F$ & SEEP & & RAW GRAB & UNFILTERED & 8146 & 100-K SPRING 77-1 & \\
\hline 859 & $F$ & SEEP & & RAW GRAB & UNFILTERED & 8846 & 100-H SPRING 152-2 & \\
\hline 860 & & SEEP & & RAW GRAB & UNFILTERED & 8147 & 100-F SPRING 207-1 & \\
\hline 861 & & RIVER & & CUMULATIVE & UNFILTERED & 252 & PRIEST RAPIDS-RIVER & \\
\hline 862 & & RIVER & & CUMULATIVE & UNFILTERED & 463 & RICH.PMPHS HRM 46.4 & \\
\hline 863 & & RIVER & & CUMULATIVE & UNFILTERED & 252 & PRIEST RAPIDS-RIVER & \\
\hline 864 & & RIVER & & CUMULATIVE & UNFILTERED & 463 & RICH.PMPHS HRM 46.4 & \\
\hline 865 & & RIVER & & RAW GRAB & TRANSECT & 317 & VERNITA-1 HRM 0.3 & \\
\hline 866 & & RIVER & & RAW GRAB & TRANSECT & 570 & VERNITA-2 HRM 0.3 & \\
\hline 867 & & RIVER & & RAW GRAB & TRANSECT & 318 & VERNITA-3 HRM 0.3 & \\
\hline 868 & & RIVER & & RAW GRAB & TRANSECT & 319 & VERNITA-4 HRM 0.3 & \\
\hline 869 & & RIVER & & RAW GRAB & TRANSECT & 571 & RICH.PMPHS-1 HRM46.4 & \\
\hline 870 & & RIVER & & RAW GRAB & TRANSECT & 571 & RICH.PMPHS-1 HRM46.4 & \\
\hline 871 & & RIVER & & RAW GRAB & TRANSECT & 321 & RICH.PMPHS-2 HRM46.4 & \\
\hline 872 & & RIVER & & RAW GRAB & TRANSECT & 322 & RICH.PMPHS-3 HRM46.4 & \\
\hline 873 & & RIVER & & RAW GRAB & TRANSECT & 323 & RICH.PMPHS-5 HRM46.4 & \\
\hline 874 & & RIVER & & RAW GRAB & TRANSECT & 325 & RICH.PMPHS-7 HRM46.4 & \\
\hline 875 & & RIVER & & RAW GRAB & TRANSECT & 327 & RICH.PMPHS-10 HRM46.4 & \\
\hline
\end{tabular}




\begin{tabular}{|c|c|c|c|c|c|c|c|c|}
\hline & $T$ & $\bar{U}$ & $\mathrm{~V}$ & $\mathrm{~W}$ & $\bar{x}$ & $\bar{Y}$ & $\bar{Z}$ & $\overline{A A}$ \\
\hline 1 & REVIEW_QUALIFIER & SAMP_FROM & SAMP_ITEM & SAMP_MTHD & COLL_MTHD & SAMP_SITE_ID & SAMP_SITE_NAME & WELL_NAME \\
\hline 876 & & RIVER & & RAW GRAB & TRANSECT & 8220 & RICH.PMPHS HRM 45.8 & \\
\hline 877 & & RIVER & & RAW GRAB & TRANSECT & 8221 & RICH.PMPHS HRM 45.0 & \\
\hline 878 & & RIVER & & RAW GRAB & TRANSECT & 8222 & RICH.PMPHS HRM 43.9 & \\
\hline 879 & & RIVER & & RAW GRAB & TRANSECT & 8223 & RICH.PMPHS HRM 43.5 & \\
\hline 880 & & SURFACE & & RAW GRAB & UNFILTERED & 147 & WEST LAKE & \\
\hline 881 & & RIVER & & CUMULATIVE & UNFILTERED & 463 & RICH.PMPHS HRM 46.4 & \\
\hline 882 & & RIVER & & CUMULATIVE & UNFILTERED & 252 & PRIEST RAPIDS-RIVER & \\
\hline 883 & & RIVER & & CUMULATIVE & UNFILTERED & 463 & RICH.PMPHS HRM 46.4 & \\
\hline 884 & & RIVER & & CUMULATIVE & UNFILTERED & 252 & PRIEST RAPIDS-RIVER & \\
\hline 885 & & SURFACE & & RAW GRAB & UNFILTERED & 147 & WEST LAKE & \\
\hline 886 & & RIVER & & CUMULATIVE & UNFILTERED & 463 & RICH.PMPHS HRM 46.4 & \\
\hline 887 & & RIVER & & CUMULATIVE & UNFILTERED & 252 & PRIEST RAPIDS-RIVER & \\
\hline 888 & & RIVER & & CUMULATIVE & UNFILTERED & 252 & PRIEST RAPIDS-RIVER & \\
\hline 889 & & RIVER & & CUMULATIVE & UNFILTERED & 463 & RICH.PMPHS HRM 46.4 & \\
\hline 890 & & RIVER & & CUMULATIVE & UNFILTERED & 252 & PRIEST RAPIDS-RIVER & \\
\hline 891 & & RIVER & & CUMULATIVE & UNFILTERED & 463 & RICH.PMPHS HRM 46.4 & \\
\hline 892 & & RIVER & & CUMULATIVE & UNFILTERED & 252 & PRIEST RAPIDS-RIVER & \\
\hline 893 & & RIVER & & CUMULATIVE & UNFILTERED & 463 & RICH.PMPHS HRM 46.4 & \\
\hline 894 & & SURFACE & & RAW GRAB & UNFILTERED & 147 & WEST LAKE & \\
\hline 895 & & RIVER & & CUMULATIVE & UNFILTERED & 252 & PRIEST RAPIDS-RIVER & \\
\hline 896 & & RIVER & & CUMULATIVE & UNFILTERED & 463 & RICH.PMPHS HRM 46.4 & \\
\hline 897 & & RIVER & & CUMULATIVE & UNFILTERED & 252 & PRIEST RAPIDS-RIVER & \\
\hline 898 & & RIVER & & CUMULATIVE & UNFILTERED & 463 & RICH.PMPHS HRM 46.4 & \\
\hline 899 & $\mathrm{~F}$ & SEEP & & RAW GRAB & UNFILTERED & 151 & HANFORD SPRING 28-2 & \\
\hline 900 & $\mathrm{~F}$ & SEEP & & RAW GRAB & UNFILTERED & 488 & 300 AREA SPRING $42-2$ & \\
\hline 901 & $\mathrm{~F}$ & SEEP & & RAW GRAB & UNFILTERED & 8142 & HANFORD SPR UR 28-2 & \\
\hline 902 & $\mathrm{~F}$ & SEEP & & RAW GRAB & UNFILTERED & 8143 & HANFORD SPR DR 28-2 & \\
\hline 903 & $\mathrm{~F}$ & SEEP & & RAW GRAB & UNFILTERED & 8144 & 300 AREA SPR DR 42-2 & \\
\hline 904 & & RIVER & & CUMULATIVE & UNFILTERED & 252 & PRIEST RAPIDS-RIVER & \\
\hline 905 & & RIVER & & CUMULATIVE & UNFILTERED & 463 & RICH.PMPHS HRM 46.4 & \\
\hline 906 & & SURFACE & & RAW GRAB & UNFILTERED & 147 & WEST LAKE & \\
\hline 907 & & SEEP & & RAW GRAB & UNFILTERED & 8140 & 100-B SPRING 38-3 & \\
\hline 908 & & SEEP & & RAW GRAB & UNFILTERED & 8146 & 100-K SPRING 77-1 & \\
\hline 909 & & SEEP & & RAW GRAB & UNFILTERED & 8165 & $100-H$ SPRING $145-1$ & \\
\hline 910 & & SEEP & & RAW GRAB & UNFILTERED & 8164 & 100-H SPRING 153-1 & \\
\hline 911 & & RIVER & & CUMULATIVE & UNFILTERED & 252 & PRIEST RAPIDS-RIVER & \\
\hline 912 & & RIVER & & CUMULATIVE & UNFILTERED & 463 & RICH.PMPHS HRM 46.4 & \\
\hline 913 & & RIVER & & CUMULATIVE & UNFILTERED & 252 & PRIEST RAPIDS-RIVER & \\
\hline
\end{tabular}




\begin{tabular}{|c|c|c|c|c|c|c|c|c|}
\hline & $\mathrm{T}$ & $\mathrm{U}$ & $\mathrm{V}$ & W & $\mathrm{x}$ & $\mathrm{Y}$ & Z & $A A$ \\
\hline 1 & REVIEW_QUALIFIER & SAMP_FROM & SAMP_ITEM & SAMP_MTHD & COLL_MTHD & SAMP_SITE_ID & SAMP_SITE_NAME & WELL_NAME \\
\hline 914 & & RIVER & & CUMULATIVE & UNFILTERED & 463 & RICH.PMPHS HRM 46.4 & \\
\hline 915 & & RIVER & & CUMULATIVE & UNFILTERED & 252 & PRIEST RAPIDS-RIVER & \\
\hline 916 & & RIVER & & CUMULATIVE & UNFILTERED & 463 & RICH.PMPHS HRM 46.4 & \\
\hline 917 & & RIVER & & CUMULATIVE & UNFILTERED & 252 & PRIEST RAPIDS-RIVER & \\
\hline 918 & & RIVER & & CUMULATIVE & UNFILTERED & 463 & RICH.PMPHS HRM 46.4 & \\
\hline 919 & & SURFACE & & RAW GRAB & UNFILTERED & 147 & WEST LAKE & \\
\hline 920 & & RIVER & & CUMULATIVE & UNFILTERED & 252 & PRIEST RAPIDS-RIVER & \\
\hline 921 & & RIVER & & CUMULATIVE & UNFILTERED & 463 & RICH.PMPHS HRM 46.4 & \\
\hline 922 & & SURFACE & & RAW GRAB & UNFILTERED & 147 & WEST LAKE & \\
\hline 923 & & RIVER & & CUMULATIVE & UNFILTERED & 252 & PRIEST RAPIDS-RIVER & \\
\hline 924 & & RIVER & & CUMULATIVE & UNFILTERED & 463 & RICH.PMPHS HRM 46.4 & \\
\hline 925 & $\mathrm{~F}$ & SEEP & & RAW GRAB & UNFILTERED & 151 & HANFORD SPRING 28-2 & \\
\hline 926 & & SEEP & & RAW GRAB & UNFILTERED & 8165 & 100-H SPRING 145-1 & \\
\hline 927 & $F$ & SEEP & & RAW GRAB & UNFILTERED & 8143 & HANFORD SPR DR 28-2 & \\
\hline 928 & & SEEP & & RAW GRAB & UNFILTERED & 8164 & 100-H SPRING 153-1 & \\
\hline 929 & & RIVER & & CUMULATIVE & UNFILTERED & 463 & RICH.PMPHS HRM 46.4 & \\
\hline 930 & & RIVER & & CUMULATIVE & UNFILTERED & 252 & PRIEST RAPIDS-RIVER & \\
\hline 931 & & SEEP & & RAW GRAB & UNFILTERED & 9106 & 100-B SPRING 37-1 & \\
\hline 932 & & SEEP & & RAW GRAB & UNFILTERED & 9110 & 100-K SPRING 82-2 & \\
\hline 933 & & RIVER & & CUMULATIVE & UNFILTERED & 463 & RICH.PMPHS HRM 46.4 & \\
\hline 934 & & RIVER & & CUMULATIVE & UNFILTERED & 252 & PRIEST RAPIDS-RIVER & \\
\hline 935 & & RIVER & & CUMULATIVE & UNFILTERED & 463 & RICH.PMPHS HRM 46.4 & \\
\hline 936 & & RIVER & & CUMULATIVE & UNFILTERED & 252 & PRIEST RAPIDS-RIVER & \\
\hline 937 & & SURFACE & & RAW GRAB & UNFILTERED & 147 & WEST LAKE & \\
\hline 938 & & RIVER & & CUMULATIVE & UNFILTERED & 463 & RICH.PMPHS HRM 46.4 & \\
\hline 939 & & RIVER & & CUMULATIVE & UNFILTERED & 252 & PRIEST RAPIDS-RIVER & \\
\hline 940 & & RIVER & & CUMULATIVE & UNFILTERED & 463 & RICH.PMPHS HRM 46.4 & \\
\hline 941 & & RIVER & & CUMULATIVE & UNFILTERED & 252 & PRIEST RAPIDS-RIVER & \\
\hline 942 & $F$ & SEEP & & RAW GRAB & UNFILTERED & 8144 & 300 AREA SPR DR 42-2 & \\
\hline 943 & $F$ & DRIVE POINT & & RAW GRAB & UNFILTERED & 9539 & 300 SPR 9 -1 (2 FT) & \\
\hline 944 & $F$ & DRIVE POINT & & RAW GRAB & UNFILTERED & 9540 & 300 SPR 9 -1 (4 FT) & \\
\hline 945 & $F$ & DRIVE POINT & & RAW GRAB & UNFILTERED & 9541 & 300 SPR $9-2$ (2 FT) & \\
\hline 946 & $\mathrm{~F}$ & DRIVE POINT & & RAW GRAB & UNFILTERED & 9542 & 300 SPR 9 -2 (4.5FT) & \\
\hline 947 & $F$ & DRIVE POINT & & RAW GRAB & UNFILTERED & 9543 & 300 SPR 9 -3 (5.5FT) & \\
\hline 948 & $F$ & SEEP & & RAW GRAB & UNFILTERED & 488 & 300 AREA SPRING $42-2$ & \\
\hline 949 & & DRIVE POINT & & RAW GRAB & UNFILTERED & 9533 & 300 SPR 7 -1 (2.5FT) & \\
\hline 950 & $\mathrm{~F}$ & DRIVE POINT & & RAW GRAB & UNFILTERED & 9534 & 300 SPR 7 -1 (4 FT) & \\
\hline 951 & $\mathrm{~F}$ & DRIVE POINT & & RAW GRAB & UNFILTERED & 9535 & 300 SPR 7 -1 (6 FT) & \\
\hline
\end{tabular}




\begin{tabular}{|c|c|c|c|c|c|c|c|c|}
\hline & $\mathrm{T}$ & $\mathrm{U}$ & $\mathrm{V}$ & $\mathrm{W}$ & $\mathrm{X}$ & $\mathrm{Y}$ & $Z$ & $\overline{\mathrm{AA}}$ \\
\hline 1 & REVIEW_QUALIFIER & SAMP_FROM & SAMP_ITEM & SAMP_MTHD & COLL_MTHD & SAMP_SITE_ID & SAMP_SITE_NAME & WELL_NAME \\
\hline 952 & $F$ & DRIVE POINT & & RAW GRAB & UNFILTERED & 9536 & 300 SPR 7 -2 (2 FT) & \\
\hline 953 & & DRIVE POINT & & RAW GRAB & UNFILTERED & 9537 & 300 SPR 7 -2 (4.2FT) & \\
\hline 954 & & RIVER & & CUMULATIVE & UNFILTERED & 252 & PRIEST RAPIDS-RIVER & \\
\hline 955 & & RIVER & & CUMULATIVE & UNFILTERED & 463 & RICH.PMPHS HRM 46.4 & \\
\hline 956 & & SEEP & & RAW GRAB & UNFILTERED & 8140 & 100-B SPRING 38-3 & \\
\hline 957 & & SEEP & & RAW GRAB & UNFILTERED & 8145 & 100-K SPRING 63-1 & \\
\hline 958 & & SEEP & & RAW GRAB & UNFILTERED & 8146 & 100-K SPRING 77-1 & \\
\hline 959 & & SEEP & & RAW GRAB & UNFILTERED & 8165 & 100-H SPRING 145-1 & \\
\hline 960 & & SEEP & & RAW GRAB & UNFILTERED & 8846 & 100-H SPRING 152-2 & \\
\hline 961 & & RIVER & & CUMULATIVE & UNFILTERED & 463 & RICH.PMPHS HRM 46.4 & \\
\hline 962 & & RIVER & & CUMULATIVE & UNFILTERED & 252 & PRIEST RAPIDS-RIVER & \\
\hline 963 & & RIVER & & CUMULATIVE & UNFILTERED & 252 & PRIEST RAPIDS-RIVER & \\
\hline 964 & & RIVER & & CUMULATIVE & UNFILTERED & 463 & RICH.PMPHS HRM 46.4 & \\
\hline 965 & & RIVER & & CUMULATIVE & UNFILTERED & 463 & RICH.PMPHS HRM 46.4 & \\
\hline 966 & & RIVER & & CUMULATIVE & UNFILTERED & 252 & PRIEST RAPIDS-RIVER & \\
\hline 967 & & RIVER & & CUMULATIVE & UNFILTERED & 463 & RICH.PMPHS HRM 46.4 & \\
\hline 968 & & RIVER & & CUMULATIVE & UNFILTERED & 252 & PRIEST RAPIDS-RIVER & \\
\hline 969 & & RIVER & & CUMULATIVE & UNFILTERED & 252 & PRIEST RAPIDS-RIVER & \\
\hline 970 & & RIVER & & CUMULATIVE & UNFILTERED & 463 & RICH.PMPHS HRM 46.4 & \\
\hline 971 & & RIVER & & CUMULATIVE & UNFILTERED & 252 & PRIEST RAPIDS-RIVER & \\
\hline 972 & & RIVER & & CUMULATIVE & UNFILTERED & 463 & RICH.PMPHS HRM 46.4 & \\
\hline 973 & & RIVER & & CUMULATIVE & UNFILTERED & 252 & PRIEST RAPIDS-RIVER & \\
\hline 974 & & RIVER & & CUMULATIVE & UNFILTERED & 463 & RICH.PMPHS HRM 46.4 & \\
\hline 975 & & RIVER & & CUMULATIVE & UNFILTERED & 252 & PRIEST RAPIDS-RIVER & \\
\hline 976 & & RIVER & & CUMULATIVE & UNFILTERED & 463 & RICH.PMPHS HRM 46.4 & \\
\hline 977 & & RIVER & & CUMULATIVE & UNFILTERED & 252 & PRIEST RAPIDS-RIVER & \\
\hline 978 & & RIVER & & CUMULATIVE & UNFILTERED & 463 & RICH.PMPHS HRM 46.4 & \\
\hline 979 & & RIVER & & CUMULATIVE & UNFILTERED & 252 & PRIEST RAPIDS-RIVER & \\
\hline 980 & & RIVER & & CUMULATIVE & UNFILTERED & 463 & RICH.PMPHS HRM 46.4 & \\
\hline 981 & & RIVER & & CUMULATIVE & UNFILTERED & 252 & PRIEST RAPIDS-RIVER & \\
\hline 982 & & RIVER & & CUMULATIVE & UNFILTERED & 463 & RICH.PMPHS HRM 46.4 & \\
\hline 983 & & SEEP & & RAW GRAB & UNFILTERED & 9106 & 100-B SPRING 37-1 & \\
\hline 984 & & SEEP & & RAW GRAB & UNFILTERED & 13792 & 100-K SPRING 63-2 & \\
\hline 985 & $F$ & RIVER & & CUMULATIVE & UNFILTERED & 252 & PRIEST RAPIDS-RIVER & \\
\hline 986 & $F$ & RIVER & & CUMULATIVE & UNFILTERED & 463 & RICH.PMPHS HRM 46.4 & \\
\hline 987 & $F$ & SEEP & & RAW GRAB & UNFILTERED & 151 & HANFORD SPRING 28-2 & \\
\hline 988 & $\mathrm{~F}$ & SEEP & & RAW GRAB & UNFILTERED & 8143 & HANFORD SPR DR 28-2 & \\
\hline 989 & & SEEP & & RAW GRAB & UNFILTERED & 8146 & 100-K SPRING 77-1 & \\
\hline
\end{tabular}




\begin{tabular}{|c|c|c|c|c|c|c|c|c|}
\hline & $T$ & $\bar{U}$ & $\mathrm{~V}$ & $\mathrm{~W}$ & $\bar{X}$ & $\bar{Y}$ & $\bar{Z}$ & AA \\
\hline 1 & REVIEW_QUALIFIER & SAMP_FROM & SAMP_ITEM & SAMP_MTHD & COLL_MTHD & SAMP_SITE_ID & SAMP_SITE_NAME & WELL_NAME \\
\hline 990 & & SEEP & & RAW GRAB & UNFILTERED & 8846 & 100-H SPRING 152-2 & \\
\hline 991 & & RIVER & & CUMULATIVE & UNFILTERED & 252 & PRIEST RAPIDS-RIVER & \\
\hline 992 & & RIVER & & CUMULATIVE & UNFILTERED & 463 & RICH.PMPHS HRM 46.4 & \\
\hline $993 \mathrm{~F}$ & $\mathrm{~F}$ & RIVER & & CUMULATIVE & UNFILTERED & 252 & PRIEST RAPIDS-RIVER & \\
\hline $994 \mathrm{~F}$ & $\mathrm{~F}$ & RIVER & & CUMULATIVE & UNFILTERED & 463 & RICH.PMPHS HRM 46.4 & \\
\hline 995 & & SURFACE & & RAW GRAB & UNFILTERED & 147 & WEST LAKE & \\
\hline $996 \mathrm{~F}$ & $\mathrm{~F}$ & SURFACE & & RAW GRAB & UNFILTERED & 136 & FFTF POND & \\
\hline 997 & & RIVER & & CUMULATIVE & UNFILTERED & 463 & RICH.PMPHS HRM 46.4 & \\
\hline 998 & & RIVER & & CUMULATIVE & UNFILTERED & 252 & PRIEST RAPIDS-RIVER & \\
\hline 999 & & RIVER & & RAW GRAB & UNFILTERED & 8466 & ENERGY NW INTAKE & \\
\hline 1000 & & RIVER & & RAW GRAB & UNFILTERED & 8467 & DR ENERGY NW POWERLINE INTAKE & \\
\hline 1001 & & RIVER & & RAW GRAB & UNFILTERED & 8468 & NEARSHORE WOODED ISL & \\
\hline 1002 & & DRINKING & & RAW GRAB & UNFILTERED & 553 & 100 B AREA-RIVER & \\
\hline 1003 & & DRINKING & & RAW GRAB & UNFILTERED & 530 & 100 D AREA & \\
\hline 1004 & & DRINKING & & SAN. GRAB & UNFILTERED & 512 & FFTF & \\
\hline $1005 \mathrm{~F}$ & $\mathrm{~F}$ & DRINKING & & RAW GRAB & UNFILTERED & 200 & $100 \mathrm{~K}$ AREA & \\
\hline 1006 & & RIVER & & CUMULATIVE & UNFILTERED & 463 & RICH.PMPHS HRM 46.4 & \\
\hline 1007 & & RIVER & & CUMULATIVE & UNFILTERED & 252 & PRIEST RAPIDS-RIVER & \\
\hline 1008 & & RIVER & & RAW GRAB & TRANSECT & 317 & VERNITA-1 HRM 0.3 & \\
\hline 1009 & & RIVER & & RAW GRAB & TRANSECT & 570 & VERNITA-2 HRM 0.3 & \\
\hline 1010 & & RIVER & & RAW GRAB & TRANSECT & 318 & VERNITA-3 HRM 0.3 & \\
\hline 1011 & & RIVER & & RAW GRAB & TRANSECT & 319 & VERNITA-4 HRM 0.3 & \\
\hline 1012 & & RIVER & & RAW GRAB & TRANSECT & 571 & RICH.PMPHS-1 HRM46.4 & \\
\hline 1013 & & RIVER & & RAW GRAB & TRANSECT & 571 & RICH.PMPHS-1 HRM46.4 & \\
\hline 1014 & & RIVER & & RAW GRAB & TRANSECT & 321 & RICH.PMPHS-2 HRM46.4 & \\
\hline 1015 & & RIVER & & RAW GRAB & TRANSECT & 322 & RICH.PMPHS-3 HRM46.4 & \\
\hline 1016 & & RIVER & & RAW GRAB & TRANSECT & 323 & RICH.PMPHS-5 HRM46.4 & \\
\hline 1017 & & RIVER & & RAW GRAB & TRANSECT & 325 & RICH.PMPHS-7 HRM46.4 & \\
\hline 1018 & & RIVER & & RAW GRAB & TRANSECT & 327 & RICH.PMPHS-10 HRM46.4 & \\
\hline 1019 & & RIVER & & RAW GRAB & TRANSECT & 8220 & RICH.PMPHS HRM 45.8 & \\
\hline 1020 & & RIVER & & RAW GRAB & TRANSECT & 8221 & RICH.PMPHS HRM 45.0 & \\
\hline 1021 & & RIVER & & RAW GRAB & TRANSECT & 8222 & RICH.PMPHS HRM 43.9 & \\
\hline 1022 & & RIVER & & RAW GRAB & TRANSECT & 8223 & RICH.PMPHS HRM 43.5 & \\
\hline $1023 \mathrm{~F}$ & $\mathrm{~F}$ & SURFACE & & RAW GRAB & UNFILTERED & 147 & WEST LAKE & \\
\hline $1024 \mathrm{~F}$ & $\mathrm{~F}$ & SURFACE & & RAW GRAB & UNFILTERED & 136 & FFTF POND & \\
\hline $1025 \mathrm{~F}$ & $\mathrm{~F}$ & SURFACE & & RAW GRAB & UNFILTERED & 136 & FFTF POND & \\
\hline 1026 & & RIVER & & CUMULATIVE & UNFILTERED & 463 & RICH.PMPHS HRM 46.4 & \\
\hline 1027 & & RIVER & & CUMULATIVE & UNFILTERED & 252 & PRIEST RAPIDS-RIVER & \\
\hline
\end{tabular}




\begin{tabular}{|c|c|c|c|c|c|c|c|c|}
\hline & $\mathrm{T}$ & $U$ & $\mathrm{~V}$ & $\mathrm{~W}$ & $\mathrm{X}$ & $\mathrm{Y}$ & $Z$ & $\mathrm{AA}$ \\
\hline 1 & REVIEW QUALIFIER & SAMP FROM & SAMP ITEM & SAMP MTHD & COLL MTHD & SAMP SITE ID & SAMP SITE NAME & WELL NAME \\
\hline 1028 & & DRINKING & & RAW GRAB & UNFILTERED & 553 & 100 B AREA-RIVER & \\
\hline 1029 & & DRINKING & & RAW GRAB & UNFILTERED & 530 & 100 D AREA & \\
\hline 1030 & & DRINKING & & SAN. GRAB & UNFILTERED & 512 & FFTF & \\
\hline $1031 \mathrm{~F}$ & $\mathrm{~F}$ & DRINKING & & RAW GRAB & UNFILTERED & 200 & $100 \mathrm{~K}$ AREA & \\
\hline 1032 & & RIVER & & CUMULATIVE & UNFILTERED & 252 & PRIEST RAPIDS-RIVER & \\
\hline 1033 & & RIVER & & CUMULATIVE & UNFILTERED & 463 & RICH.PMPHS HRM 46.4 & \\
\hline 1034 & & IRRIGATION & & RAW GRAB & UNFILTERED & 440 & RIVERVIEW CANAL & \\
\hline 1035 & & RIVER & & CUMULATIVE & UNFILTERED & 252 & PRIEST RAPIDS-RIVER & \\
\hline 1036 & & RIVER & & CUMULATIVE & UNFILTERED & 463 & RICH.PMPHS HRM 46.4 & \\
\hline 1037 & & IRRIGATION & & RAW GRAB & UNFILTERED & 440 & RIVERVIEW CANAL & \\
\hline 1038 & & RIVER & & RAW GRAB & TRANSECT & 317 & VERNITA-1 HRM 0.3 & \\
\hline 1039 & & RIVER & & RAW GRAB & TRANSECT & 570 & VERNITA-2 HRM 0.3 & \\
\hline 1040 & & RIVER & & RAW GRAB & TRANSECT & 318 & VERNITA-3 HRM 0.3 & \\
\hline 1041 & & RIVER & & RAW GRAB & TRANSECT & 319 & VERNITA-4 HRM 0.3 & \\
\hline 1042 & & RIVER & & RAW GRAB & TRANSECT & 571 & RICH.PMPHS-1 HRM46.4 & \\
\hline 1043 & & RIVER & & RAW GRAB & TRANSECT & 321 & RICH.PMPHS-2 HRM46.4 & \\
\hline 1044 & & RIVER & & RAW GRAB & TRANSECT & 322 & RICH.PMPHS-3 HRM46.4 & \\
\hline 1045 & & RIVER & & RAW GRAB & TRANSECT & 323 & RICH.PMPHS-5 HRM46.4 & \\
\hline 1046 & & RIVER & & RAW GRAB & TRANSECT & 325 & RICH.PMPHS-7 HRM46.4 & \\
\hline 1047 & & RIVER & & RAW GRAB & TRANSECT & 327 & RICH.PMPHS-10 HRM46.4 & \\
\hline 1048 & & RIVER & & RAW GRAB & TRANSECT & 8220 & RICH.PMPHS HRM 45.8 & \\
\hline 1049 & & RIVER & & RAW GRAB & TRANSECT & 8221 & RICH.PMPHS HRM 45.0 & \\
\hline 1050 & & RIVER & & RAW GRAB & TRANSECT & 8222 & RICH.PMPHS HRM 43.9 & \\
\hline 1051 & & RIVER & & RAW GRAB & TRANSECT & 8223 & RICH.PMPHS HRM 43.5 & \\
\hline 1052 & & RIVER & & CUMULATIVE & UNFILTERED & 252 & PRIEST RAPIDS-RIVER & \\
\hline 1053 & & RIVER & & CUMULATIVE & UNFILTERED & 463 & RICH.PMPHS HRM 46.4 & \\
\hline $1054 \mathrm{~F}$ & $\mathrm{~F}$ & SURFACE & & RAW GRAB & UNFILTERED & 147 & WEST LAKE & \\
\hline $1055 \mathrm{~F}$ & $\mathrm{~F}$ & SURFACE & & RAW GRAB & UNFILTERED & 136 & FFTF POND & \\
\hline 1056 & & SURFACE & & RAW GRAB & UNFILTERED & 8606 & S OF HANFORD TOWNSITE & \\
\hline 1057 & & IRRIGATION & & RAW GRAB & UNFILTERED & 440 & RIVERVIEW CANAL & \\
\hline 1058 & & DRINKING & & RAW GRAB & UNFILTERED & 553 & 100 B AREA-RIVER & \\
\hline 1059 & & DRINKING & & RAW GRAB & UNFILTERED & 530 & 100 D AREA & \\
\hline 1060 & & DRINKING & & SAN. GRAB & UNFILTERED & 512 & FFTF & \\
\hline $1061 \mathrm{~F}$ & $\mathrm{~F}$ & DRINKING & & RAW GRAB & UNFILTERED & 200 & $100 \mathrm{~K}$ AREA & \\
\hline 1062 & & RIVER & & CUMULATIVE & UNFILTERED & 252 & PRIEST RAPIDS-RIVER & \\
\hline 1063 & & RIVER & & CUMULATIVE & UNFILTERED & 463 & RICH.PMPHS HRM 46.4 & \\
\hline 1064 & & RIVER & & CUMULATIVE & UNFILTERED & 252 & PRIEST RAPIDS-RIVER & \\
\hline 1065 & & RIVER & & CUMULATIVE & UNFILTERED & 463 & RICH.PMPHS HRM 46.4 & \\
\hline
\end{tabular}




\begin{tabular}{|c|c|c|c|c|c|c|c|c|}
\hline & $T$ & $\bar{U}$ & $\mathrm{~V}$ & $\mathrm{~W}$ & $\bar{x}$ & $\bar{Y}$ & $\mathrm{Z}$ & AA \\
\hline 1 & REVIEW_QUALIFIER & SAMP_FROM & SAMP ITEM & SAMP_MTHD & COLL_MTHD & SAMP_SITE_ID & SAMP_SITE_NAME & WELL_NAME \\
\hline 1066 & & RIVER & & RAW GRAB & TRANSECT & 328 & $100 \mathrm{~N}-1$ HRM 9.5 & \\
\hline 1067 & & RIVER & & RAW GRAB & TRANSECT & 317 & 7 VERNITA-1 HRM 0.3 & \\
\hline 1068 & & RIVER & & RAW GRAB & TRANSECT & 570 & VERNITA-2 HRM 0.3 & \\
\hline 1069 & & RIVER & & RAW GRAB & TRANSECT & 318 & 3 VERNITA-3 HRM 0.3 & \\
\hline 1070 & & RIVER & & RAW GRAB & TRANSECT & 319 & 9 VERNITA-4 HRM 0.3 & \\
\hline 1071 & & RIVER & & RAW GRAB & TRANSECT & 328 & 3100 N -1 HRM 9.5 & \\
\hline 1072 & & RIVER & & RAW GRAB & TRANSECT & 329 & 9100 N -2 HRM 9.5 & \\
\hline 1073 & & RIVER & & RAW GRAB & TRANSECT & 331 & 100 N -5 HRM 9.5 & \\
\hline 1074 & & RIVER & & RAW GRAB & TRANSECT & 335 & 100 N -10 HRM 9.5 & \\
\hline 1075 & & RIVER & & RAW GRAB & TRANSECT & 330 & 100 N -3 HRM 9.5 & \\
\hline 1076 & & RIVER & & RAW GRAB & TRANSECT & 333 & 3100 N -7 HRM 9.5 & \\
\hline 1077 & & RIVER & & RAW GRAB & TRANSECT & 8244 & 4100 N SHORE HRM 8.4 & \\
\hline 1078 & & RIVER & & RAW GRAB & TRANSECT & 8245 & 5100 N SHORE HRM 8.9 & \\
\hline 1079 & & RIVER & & RAW GRAB & TRANSECT & 8246 & 100 N SHORE HRM 9.2 & \\
\hline 1080 & & RIVER & & RAW GRAB & TRANSECT & 8247 & 7100 N SHORE HRM 9.8 & \\
\hline 1081 & & RIVER & & RAW GRAB & TRANSECT & 328 & 3100 N -1 HRM 9.5 & \\
\hline 1082 & & RIVER & & RAW GRAB & TRANSECT & 329 & 9100 N -2 HRM 9.5 & \\
\hline 1083 & & RIVER & & RAW GRAB & TRANSECT & 8245 & 5100 N SHORE HRM 8.9 & \\
\hline 1084 & & RIVER & & RAW GRAB & TRANSECT & 8244 & 4100 N SHORE HRM 8.4 & \\
\hline 1085 & & RIVER & & RAW GRAB & TRANSECT & 8246 & 100 N SHORE HRM 9.2 & \\
\hline 1086 & & RIVER & & RAW GRAB & TRANSECT & 8247 & 7100 N SHORE HRM 9.8 & \\
\hline 1087 & & RIVER & & RAW GRAB & TRANSECT & 336 & 100 F -1 HRM 19.0 & \\
\hline 1088 & & RIVER & & RAW GRAB & TRANSECT & 337 & 7100 F -3 HRM 19.0 & \\
\hline 1089 & & RIVER & & RAW GRAB & TRANSECT & 344 & 4 HANFRD TS-5 HRM 28.7 & \\
\hline 1090 & & RIVER & & RAW GRAB & TRANSECT & 576 & 100 F -2 HRM 19.0 & \\
\hline 1091 & & RIVER & & RAW GRAB & TRANSECT & 577 & 7100 F -5 HRM 19.0 & \\
\hline 1092 & & RIVER & & RAW GRAB & TRANSECT & 339 & 9100 F -7 HRM 19.0 & \\
\hline 1093 & $F$ & RIVER & & RAW GRAB & TRANSECT & 341 & 100 F - 10 HRM 19.0 & \\
\hline 1094 & & RIVER & & RAW GRAB & TRANSECT & 578 & 3 HANFRD TS-1 HRM 28.7 & \\
\hline 1095 & & RIVER & & RAW GRAB & TRANSECT & 342 & 2 HANFRD TS-2 HRM 28.7 & \\
\hline 1096 & & RIVER & & RAW GRAB & TRANSECT & 469 & HANFRD TS-7 HRM 28.7 & \\
\hline 1097 & & RIVER & & RAW GRAB & TRANSECT & 347 & 7 HANFRD TS-10 HRM 28.7 & \\
\hline 1098 & & RIVER & & RAW GRAB & TRANSECT & 468 & 3 HANFRD TS-3 HRM 28.7 & \\
\hline 1099 & & RIVER & & RAW GRAB & TRANSECT & 8100 & HANFRD TWNSITE HRM26 & \\
\hline 1100 & & RIVER & & RAW GRAB & TRANSECT & 8101 & 1 HANFRD TWNSITE HRM27 & \\
\hline 1101 & & RIVER & & RAW GRAB & TRANSECT & 8103 & 3 HANFRD TWNSITE HRM30 & \\
\hline 1102 & $F$ & RIVER & & RAW GRAB & TRANSECT & 274 & 4 HANFRD TWNSITE HRM28 & \\
\hline 1103 & & RIVER & & RAW GRAB & TRANSECT & 8726 & 100 F SHORE HRM 18 & \\
\hline
\end{tabular}




\begin{tabular}{|c|c|c|c|c|c|c|c|c|}
\hline & $\mathrm{T}$ & $\bar{U}$ & $\mathrm{~V}$ & $\mathrm{~W}$ & $\mathrm{x}$ & $\mathrm{Y}$ & $Z$ & $\mathrm{AA}$ \\
\hline 1 & REVIEW_QUALIFIER & SAMP_FROM & SAMP_ITEM & SAMP_MTHD & COLL_MTHD & SAMP_SITE_ID & SAMP_SITE_NAME & WELL_NAME \\
\hline 1104 & & RIVER & & RAW GRAB & TRANSECT & 8727 & 7100 F SHORE HRM 22 & \\
\hline 1105 & & RIVER & & RAW GRAB & TRANSECT & 8728 & 3100 F SHORE HRM 23 & \\
\hline 1106 & & RIVER & & RAW GRAB & TRANSECT & 336 & 100 F -1 HRM 19.0 & \\
\hline 1107 & & RIVER & & RAW GRAB & TRANSECT & 8726 & 100 F SHORE HRM 18 & \\
\hline 1108 & & RIVER & & RAW GRAB & TRANSECT & 578 & 3 HANFRD TS-1 HRM 28.7 & \\
\hline 1109 & & RIVER & & RAW GRAB & TRANSECT & 342 & 2 HANFRD TS-2 HRM 28.7 & \\
\hline $1110 \mathrm{~F}$ & $F$ & RIVER & & RAW GRAB & TRANSECT & 8101 & 1 HANFRD TWNSITE HRM27 & \\
\hline $1111 \mathrm{~F}$ & $\mathrm{~F}$ & RIVER & & RAW GRAB & TRANSECT & 274 & 4 HANFRD TWNSITE HRM28 & \\
\hline 1112 & & RIVER & & RAW GRAB & TRANSECT & 8103 & 3 HANFRD TWNSITE HRM30 & \\
\hline 1113 & & RIVER & & RAW GRAB & TRANSECT & 348 & 300 AREA -1 HRM 43.1 & \\
\hline 1114 & & RIVER & & RAW GRAB & TRANSECT & 470 & 300 AREA -2 HRM 43.1 & \\
\hline 1115 & & RIVER & & RAW GRAB & TRANSECT & 580 & 300 AREA -3 HRM 43.1 & \\
\hline 1116 & & RIVER & & RAW GRAB & TRANSECT & 607 & 7300 AREA -5 HRM 43.1 & \\
\hline 1117 & & RIVER & & RAW GRAB & TRANSECT & 351 & 300 AREA -7 HRM 43.1 & \\
\hline 1118 & & RIVER & & RAW GRAB & TRANSECT & 352 & 300 AREA-10 HRM 43.1 & \\
\hline 1119 & & RIVER & & RAW GRAB & TRANSECT & 571 & RICH.PMPHS-1 HRM46.4 & \\
\hline 1120 & & RIVER & & RAW GRAB & TRANSECT & 321 & RICH.PMPHS-2 HRM46.4 & \\
\hline 1121 & & RIVER & & RAW GRAB & TRANSECT & 322 & 2 RICH.PMPHS-3 HRM46.4 & \\
\hline 1122 & & RIVER & & RAW GRAB & TRANSECT & 323 & RICH.PMPHS-5 HRM46.4 & \\
\hline 1123 & & RIVER & & RAW GRAB & TRANSECT & 325 & RICH.PMPHS-7 HRM46.4 & \\
\hline 1124 & & RIVER & & RAW GRAB & TRANSECT & 327 & 7 RICH.PMPHS-10 HRM46.4 & \\
\hline 1125 & & RIVER & & RAW GRAB & TRANSECT & 8220 & RICH.PMPHS HRM 45.8 & \\
\hline 1126 & & RIVER & & RAW GRAB & TRANSECT & 8221 & RICH.PMPHS HRM 45.0 & \\
\hline 1127 & & RIVER & & RAW GRAB & TRANSECT & 8222 & 2 RICH.PMPHS HRM 43.9 & \\
\hline 1128 & & RIVER & & RAW GRAB & TRANSECT & 8223 & 3 RICH.PMPHS HRM 43.5 & \\
\hline 1129 & & RIVER & & RAW GRAB & TRANSECT & 8240 & 300 AREA SHR HRM41.5 & \\
\hline $1130 \mathrm{~F}$ & $\mathrm{~F}$ & RIVER & & RAW GRAB & TRANSECT & 8241 & 300 AREA SHR HRM42.1 & \\
\hline 1131 & & RIVER & & RAW GRAB & TRANSECT & 8242 & 2300 AREA SHR HRM42.5 & \\
\hline 1132 & & RIVER & & RAW GRAB & TRANSECT & 8243 & 300 AREA SHR HRM42.9 & \\
\hline 1133 & & RIVER & & RAW GRAB & TRANSECT & 348 & 300 AREA -1 HRM 43.1 & \\
\hline 1134 & & RIVER & & RAW GRAB & TRANSECT & 470 & 300 AREA -2 HRM 43.1 & \\
\hline 1135 & & RIVER & & RAW GRAB & TRANSECT & 8240 & 300 AREA SHR HRM41.5 & \\
\hline $1136 \mathrm{~F}$ & $F$ & RIVER & & RAW GRAB & TRANSECT & 8241 & 300 AREA SHR HRM42.1 & \\
\hline 1137 & & RIVER & & RAW GRAB & TRANSECT & 8242 & 2300 AREA SHR HRM42.5 & \\
\hline 1138 & & RIVER & & RAW GRAB & TRANSECT & 8243 & 3300 AREA SHR HRM42.9 & \\
\hline 1139 & & RIVER & & RAW GRAB & TRANSECT & 571 & RICH.PMPHS-1 HRM46.4 & \\
\hline 1140 & & RIVER & & RAW GRAB & TRANSECT & 321 & RICH.PMPHS-2 HRM46.4 & \\
\hline 1141 & & RIVER & & RAW GRAB & TRANSECT & 8223 & $3 \mathrm{RICH} . \mathrm{PMPHS}$ HRM 43.5 & \\
\hline
\end{tabular}




\begin{tabular}{|c|c|c|c|c|c|c|c|c|}
\hline & $\mathrm{T}$ & $U$ & $\mathrm{~V}$ & $\mathrm{~W}$ & $\mathrm{X}$ & $\mathrm{Y}$ & $Z$ & $\mathrm{AA}$ \\
\hline 1 & REVIEW QUALIFIER & SAMP FROM & SAMP ITEM & SAMP MTHD & COLL MTHD & SAMP SITE ID & SAMP SITE NAME & WELL NAME \\
\hline 1142 & & RIVER & & RAW GRAB & TRANSECT & 8221 & RICH.PMPHS HRM 45.0 & \\
\hline 1143 & & RIVER & & RAW GRAB & TRANSECT & 8220 & RICH.PMPHS HRM 45.8 & \\
\hline 1144 & $\mathrm{~F}$ & SEEP & & RAW GRAB & UNFILTERED & 151 & HANFORD SPRING $28-2$ & \\
\hline 1145 & $\mathrm{~F}$ & SEEP & & RAW GRAB & UNFILTERED & 488 & 300 AREA SPRING 42-2 & \\
\hline 1146 & $\mathrm{~F}$ & SEEP & & RAW GRAB & UNFILTERED & 8142 & HANFORD SPR UR 28-2 & \\
\hline 1147 & $\mathrm{~F}$ & SEEP & & RAW GRAB & UNFILTERED & 8143 & HANFORD SPR DR 28-2 & \\
\hline 1148 & $\mathrm{~F}$ & SEEP & & RAW GRAB & UNFILTERED & 8144 & 300 AREA SPR DR 42-2 & \\
\hline 1149 & & RIVER & & CUMULATIVE & UNFILTERED & 252 & PRIEST RAPIDS-RIVER & \\
\hline 1150 & & RIVER & & CUMULATIVE & UNFILTERED & 463 & RICH.PMPHS HRM 46.4 & \\
\hline 1151 & $\mathrm{~F}$ & SURFACE & & RAW GRAB & UNFILTERED & 147 & WEST LAKE & \\
\hline 1152 & $\mathrm{~F}$ & SURFACE & & RAW GRAB & UNFILTERED & 136 & FFTF POND & \\
\hline 1153 & $\mathrm{~F}$ & SEEP & & RAW GRAB & UNFILTERED & 202 & 100-N SPRING 8-13 & \\
\hline 1154 & $\mathrm{~F}$ & SEEP & & RAW GRAB & UNFILTERED & 8145 & 100-K SPRING 63-1 & \\
\hline 1155 & $\mathrm{~F}$ & SEEP & & RAW GRAB & UNFILTERED & 8162 & 100-D SPRING 110-1 & \\
\hline 1156 & $\mathrm{~F}$ & SEEP & & RAW GRAB & UNFILTERED & 8163 & 100-D SPRING 102-1 & \\
\hline 1157 & & SEEP & & & & 8162 & 100-D SPRING 110-1 & \\
\hline 1158 & & SEEP & & & & 8162 & 100-D SPRING 110-1 & \\
\hline 1159 & & SEEP & & & & 8163 & 100-D SPRING 102-1 & \\
\hline 1160 & & DRINKING & & RAW GRAB & UNFILTERED & 553 & 100 B AREA-RIVER & \\
\hline 1161 & & DRINKING & & RAW GRAB & UNFILTERED & 530 & 100 D AREA & \\
\hline 1162 & & DRINKING & & SAN. GRAB & UNFILTERED & 512 & FFTF & \\
\hline 1163 & & DRINKING & & RAW GRAB & UNFILTERED & 200 & $100 \mathrm{~K}$ AREA & \\
\hline 1164 & $\mathrm{~F}$ & SEEP & & RAW GRAB & UNFILTERED & 8140 & 100-B SPRING 38-3 & \\
\hline 1165 & $\mathrm{~F}$ & SEEP & & RAW GRAB & UNFILTERED & 8146 & 100-K SPRING 77-1 & \\
\hline 1166 & & SEEP & & & & 8140 & 100-B SPRING 38-3 & \\
\hline 1167 & & SEEP & & RAW GRAB & UNFILTERED & 8165 & 100-H SPRING 145-1 & \\
\hline 1168 & $\mathrm{~F}$ & SEEP & & RAW GRAB & UNFILTERED & 8164 & 100-H SPRING 153-1 & \\
\hline 1169 & & SEEP & & & & 8165 & 100-H SPRING 145-1 & \\
\hline 1170 & $\mathrm{~F}$ & RIVER & & CUMULATIVE & UNFILTERED & 252 & PRIEST RAPIDS-RIVER & \\
\hline 1171 & & RIVER & & CUMULATIVE & UNFILTERED & 463 & RICH.PMPHS HRM 46.4 & \\
\hline 1172 & & SEEP & & RAW GRAB & UNFILTERED & 8147 & 100-F SPRING 207-1 & \\
\hline 1173 & & SEEP & & & & 8147 & 100-F SPRING 207-1 & \\
\hline 1174 & & RIVER & & CUMULATIVE & UNFILTERED & 252 & PRIEST RAPIDS-RIVER & \\
\hline 1175 & & RIVER & & CUMULATIVE & UNFILTERED & 463 & RICH.PMPHS HRM 46.4 & \\
\hline 1176 & & RIVER & & RAW GRAB & TRANSECT & 317 & VERNITA-1 HRM 0.3 & \\
\hline 1177 & & RIVER & & RAW GRAB & TRANSECT & 570 & VERNITA-2 HRM 0.3 & \\
\hline 1178 & & RIVER & & RAW GRAB & TRANSECT & 318 & VERNITA-3 HRM 0.3 & \\
\hline 1179 & & RIVER & & RAW GRAB & TRANSECT & 319 & VERNITA-4 HRM 0.3 & \\
\hline
\end{tabular}

Page 187 


\begin{tabular}{|c|c|c|c|c|c|c|c|c|}
\hline & $T$ & $\bar{U}$ & $\mathrm{~V}$ & $\mathrm{~W}$ & $\bar{X}$ & $\bar{Y}$ & $\bar{Z}$ & $\overline{A A}$ \\
\hline 1 & REVIEW_QUALIFIER & SAMP_FROM & SAMP_ITEM & SAMP_MTHD & COLL_MTHD & SAMP_SITE_ID & SAMP_SITE_NAME & WELL_NAME \\
\hline 1180 & & RIVER & & RAW GRAB & TRANSECT & 571 & RICH.PMPHS-1 HRM46.4 & \\
\hline 1181 & & RIVER & & RAW GRAB & TRANSECT & 571 & RICH.PMPHS-1 HRM46.4 & \\
\hline 1182 & & RIVER & & RAW GRAB & TRANSECT & 321 & RICH.PMPHS-2 HRM46.4 & \\
\hline 1183 & $\mathrm{~F}$ & RIVER & & RAW GRAB & TRANSECT & 322 & RICH.PMPHS-3 HRM46.4 & \\
\hline 1184 & & RIVER & & RAW GRAB & TRANSECT & 323 & RICH.PMPHS-5 HRM46.4 & \\
\hline 1185 & & RIVER & & RAW GRAB & TRANSECT & 325 & RICH.PMPHS-7 HRM46.4 & \\
\hline 1186 & & RIVER & & RAW GRAB & TRANSECT & 327 & RICH.PMPHS-10 HRM46.4 & \\
\hline 1187 & & RIVER & & RAW GRAB & TRANSECT & 8220 & RICH.PMPHS HRM 45.8 & \\
\hline 1188 & & RIVER & & RAW GRAB & TRANSECT & 8221 & RICH.PMPHS HRM 45.0 & \\
\hline 1189 & & RIVER & & RAW GRAB & TRANSECT & 8222 & RICH.PMPHS HRM 43.9 & \\
\hline 1190 & & RIVER & & RAW GRAB & TRANSECT & 8223 & RICH.PMPHS HRM 43.5 & \\
\hline 1191 & & RIVER & & CUMULATIVE & UNFILTERED & 252 & PRIEST RAPIDS-RIVER & \\
\hline 1192 & & RIVER & & CUMULATIVE & UNFILTERED & 463 & RICH.PMPHS HRM 46.4 & \\
\hline 1193 & & RIVER & & CUMULATIVE & UNFILTERED & 252 & PRIEST RAPIDS-RIVER & \\
\hline 1194 & & RIVER & & CUMULATIVE & UNFILTERED & 463 & RICH.PMPHS HRM 46.4 & \\
\hline 1195 & $\mathrm{~F}$ & SURFACE & & RAW GRAB & UNFILTERED & 147 & WEST LAKE & \\
\hline 1196 & $\mathrm{~F}$ & SURFACE & & RAW GRAB & UNFILTERED & 136 & FFTF POND & \\
\hline 1197 & & DRINKING & & RAW GRAB & UNFILTERED & 553 & 100 B AREA-RIVER & \\
\hline 1198 & & DRINKING & & RAW GRAB & UNFILTERED & 530 & 100 D AREA & \\
\hline 1199 & & DRINKING & & SAN. GRAB & UNFILTERED & 512 & FFTF & \\
\hline 1200 & $\mathrm{~F}$ & DRINKING & & RAW GRAB & UNFILTERED & 200 & $100 \mathrm{~K}$ AREA & \\
\hline 1201 & & RIVER & & RAW GRAB & TRANSECT & 571 & RICH.PMPHS-1 HRM46.4 & \\
\hline 1202 & & RIVER & & RAW GRAB & TRANSECT & 571 & RICH.PMPHS-1 HRM46.4 & \\
\hline 1203 & & RIVER & & RAW GRAB & TRANSECT & 321 & RICH.PMPHS-2 HRM46.4 & \\
\hline 1204 & & RIVER & & RAW GRAB & TRANSECT & 322 & RICH.PMPHS-3 HRM46.4 & \\
\hline 1205 & & RIVER & & RAW GRAB & TRANSECT & 323 & RICH.PMPHS-5 HRM46.4 & \\
\hline 1206 & & RIVER & & RAW GRAB & TRANSECT & 325 & RICH.PMPHS-7 HRM46.4 & \\
\hline 1207 & & RIVER & & RAW GRAB & TRANSECT & 327 & RICH.PMPHS-10 HRM46.4 & \\
\hline 1208 & & RIVER & & RAW GRAB & TRANSECT & 8220 & RICH.PMPHS HRM 45.8 & \\
\hline 1209 & & RIVER & & RAW GRAB & TRANSECT & 8221 & RICH.PMPHS HRM 45.0 & \\
\hline 1210 & & RIVER & & RAW GRAB & TRANSECT & 8222 & RICH.PMPHS HRM 43.9 & \\
\hline 1211 & & RIVER & & RAW GRAB & TRANSECT & 8223 & RICH.PMPHS HRM 43.5 & \\
\hline 1212 & & RIVER & & RAW GRAB & TRANSECT & 317 & VERNITA-1 HRM 0.3 & \\
\hline 1213 & & RIVER & & RAW GRAB & TRANSECT & 570 & VERNITA-2 HRM 0.3 & \\
\hline 1214 & & RIVER & & RAW GRAB & TRANSECT & 318 & VERNITA-3 HRM 0.3 & \\
\hline 1215 & & RIVER & & RAW GRAB & TRANSECT & 319 & VERNITA-4 HRM 0.3 & \\
\hline 1216 & & RIVER & & CUMULATIVE & UNFILTERED & 252 & PRIEST RAPIDS-RIVER & \\
\hline 1217 & & RIVER & & CUMULATIVE & UNFILTERED & 463 & RICH.PMPHS HRM 46.4 & \\
\hline
\end{tabular}




\begin{tabular}{|c|c|c|c|c|c|c|c|c|}
\hline & $T$ & $\bar{U}$ & $\mathrm{~V}$ & $\mathrm{~W}$ & $\bar{X}$ & $\bar{Y}$ & $\bar{Z}$ & $\overline{A A}$ \\
\hline 1 & REVIEW_QUALIFIER & SAMP_FROM & SAMP_ITEM & SAMP_MTHD & COLL_MTHD & SAMP_SITE_ID & SAMP_SITE_NAME & WELL_NAME \\
\hline 1218 & & SURFACE & & RAW GRAB & UNFILTERED & 147 & WEST LAKE & \\
\hline $1219 \mathrm{~F}$ & $\mathrm{~F}$ & SURFACE & & RAW GRAB & UNFILTERED & 136 & FFTF POND & \\
\hline $1220 \mathrm{~F}$ & $\mathrm{~F}$ & SURFACE & & RAW GRAB & UNFILTERED & 136 & FFTF POND & \\
\hline 1221 & & RIVER & & CUMULATIVE & UNFILTERED & 252 & PRIEST RAPIDS-RIVER & \\
\hline 1222 & & RIVER & & CUMULATIVE & UNFILTERED & 463 & RICH.PMPHS HRM 46.4 & \\
\hline 1223 & & DRINKING & & RAW GRAB & UNFILTERED & 553 & 100 B AREA-RIVER & \\
\hline 1224 & & DRINKING & & RAW GRAB & UNFILTERED & 530 & 100 D AREA & \\
\hline 1225 & & DRINKING & & SAN. GRAB & UNFILTERED & 512 & FFTF & \\
\hline 1226 & & DRINKING & & RAW GRAB & UNFILTERED & 200 & $100 \mathrm{~K}$ AREA & \\
\hline $1227 \mathrm{~F}$ & $\mathrm{~F}$ & SEEP & & RAW GRAB & UNFILTERED & 151 & HANFORD SPRING 28-2 & \\
\hline 1228 & & SEEP & & RAW GRAB & UNFILTERED & 8165 & 100-H SPRING 145-1 & \\
\hline 1229 & & SEEP & & RAW GRAB & UNFILTERED & 8147 & 100-F SPRING 207-1 & \\
\hline $1230 \mathrm{~F}$ & $\mathrm{~F}$ & SEEP & & RAW GRAB & UNFILTERED & 8143 & HANFORD SPR DR 28-2 & \\
\hline 1231 & & SEEP & & RAW GRAB & UNFILTERED & 8164 & 100-H SPRING 153-1 & \\
\hline 1232 & & RIVER & & CUMULATIVE & UNFILTERED & 463 & RICH.PMPHS HRM 46.4 & \\
\hline 1233 & & RIVER & & CUMULATIVE & UNFILTERED & 252 & PRIEST RAPIDS-RIVER & \\
\hline 1234 & & SEEP & & RAW GRAB & UNFILTERED & 8144 & 300 AREA SPR DR 42-2 & \\
\hline $1235 \mathrm{~F}$ & $\mathrm{~F}$ & SEEP & & RAW GRAB & UNFILTERED & 202 & 100-N SPRING 8-13 & \\
\hline $1236 \mathrm{~F}$ & $\mathrm{~F}$ & SEEP & & RAW GRAB & UNFILTERED & 9106 & 100-B SPRING 37-1 & \\
\hline 1237 & & SEEP & & RAW GRAB & UNFILTERED & 9110 & 100-K SPRING 82-2 & \\
\hline 1238 & & SEEP & & RAW GRAB & UNFILTERED & 8162 & 100-D SPRING 110-1 & \\
\hline 1239 & & IRRIGATION & & RAW GRAB & UNFILTERED & 440 & RIVERVIEW CANAL & \\
\hline 1240 & & IRRIGATION & & RAW GRAB & UNFILTERED & 605 & HORN RAPIDS AREA & \\
\hline $1241 \mathrm{~F}$ & $\mathrm{~F}$ & SEEP & & RAW GRAB & UNFILTERED & 488 & 300 AREA SPRING 42-2 & \\
\hline 1242 & & RIVER & & RAW GRAB & TRANSECT & 571 & RICH.PMPHS-1 HRM46.4 & \\
\hline 1243 & & RIVER & & RAW GRAB & TRANSECT & 321 & RICH.PMPHS-2 HRM46.4 & \\
\hline 1244 & & RIVER & & RAW GRAB & TRANSECT & 322 & RICH.PMPHS-3 HRM46.4 & \\
\hline 1245 & & RIVER & & RAW GRAB & TRANSECT & 323 & RICH.PMPHS-5 HRM46.4 & \\
\hline 1246 & & SEEP & & RAW GRAB & UNFILTERED & 9147 & RICHLAND SPR HRM44.4 & \\
\hline 1247 & & RIVER & & CUMULATIVE & UNFILTERED & 463 & RICH.PMPHS HRM 46.4 & \\
\hline 1248 & & RIVER & & CUMULATIVE & UNFILTERED & 252 & PRIEST RAPIDS-RIVER & \\
\hline 1249 & & IRRIGATION & & RAW GRAB & UNFILTERED & 440 & RIVERVIEW CANAL & \\
\hline 1250 & & IRRIGATION & & RAW GRAB & UNFILTERED & 440 & RIVERVIEW CANAL & \\
\hline 1251 & & IRRIGATION & & RAW GRAB & UNFILTERED & 605 & HORN RAPIDS AREA & \\
\hline 1252 & & IRRIGATION & & RAW GRAB & UNFILTERED & 605 & HORN RAPIDS AREA & \\
\hline 1253 & & RIVER & & RAW GRAB & TRANSECT & 571 & RICH.PMPHS-1 HRM46.4 & \\
\hline 1254 & & RIVER & & RAW GRAB & TRANSECT & 321 & RICH.PMPHS-2 HRM46.4 & \\
\hline 1255 & & RIVER & & RAW GRAB & TRANSECT & 322 & RICH.PMPHS-3 HRM46.4 & \\
\hline
\end{tabular}




\begin{tabular}{|c|c|c|c|c|c|c|c|c|}
\hline & $T$ & $\bar{U}$ & $\mathrm{~V}$ & $\mathrm{~W}$ & $\bar{X}$ & $\bar{Y}$ & $\bar{Z}$ & $\overline{A A}$ \\
\hline 1 & REVIEW_QUALIFIER & SAMP_FROM & SAMP_ITEM & SAMP_MTHD & COLL_MTHD & SAMP_SITE_ID & SAMP_SITE_NAME & WELL_NAME \\
\hline 1256 & & RIVER & & RAW GRAB & TRANSECT & 323 & RICH.PMPHS-5 HRM46.4 & \\
\hline 1257 & & RIVER & & RAW GRAB & TRANSECT & 325 & RICH.PMPHS-7 HRM46.4 & \\
\hline 1258 & & RIVER & & RAW GRAB & TRANSECT & 327 & RICH.PMPHS-10 HRM46.4 & \\
\hline 1259 & & RIVER & & RAW GRAB & TRANSECT & 8220 & RICH.PMPHS HRM 45.8 & \\
\hline 1260 & & RIVER & & RAW GRAB & TRANSECT & 8221 & RICH.PMPHS HRM 45.0 & \\
\hline 1261 & & RIVER & & RAW GRAB & TRANSECT & 8222 & RICH.PMPHS HRM 43.9 & \\
\hline 1262 & & RIVER & & RAW GRAB & TRANSECT & 8223 & RICH.PMPHS HRM 43.5 & \\
\hline 1263 & & RIVER & & RAW GRAB & TRANSECT & 317 & VERNITA-1 HRM 0.3 & \\
\hline 1264 & & RIVER & & RAW GRAB & TRANSECT & 570 & VERNITA-2 HRM 0.3 & \\
\hline 1265 & & RIVER & & RAW GRAB & TRANSECT & 318 & VERNITA-3 HRM 0.3 & \\
\hline 1266 & & RIVER & & RAW GRAB & TRANSECT & 319 & VERNITA-4 HRM 0.3 & \\
\hline 1267 & & RIVER & & CUMULATIVE & UNFILTERED & 463 & RICH.PMPHS HRM 46.4 & \\
\hline $1268 \mathrm{~F}$ & $\mathrm{~F}$ & RIVER & & CUMULATIVE & UNFILTERED & 252 & PRIEST RAPIDS-RIVER & \\
\hline 1269 & & SURFACE & & RAW GRAB & UNFILTERED & 136 & FFTF POND & \\
\hline 1270 & & IRRIGATION & & RAW GRAB & UNFILTERED & 440 & RIVERVIEW CANAL & \\
\hline 1271 & & IRRIGATION & & RAW GRAB & UNFILTERED & 605 & HORN RAPIDS AREA & \\
\hline 1272 & & DRINKING & & RAW GRAB & UNFILTERED & 553 & 100 B AREA-RIVER & \\
\hline 1273 & & DRINKING & & RAW GRAB & UNFILTERED & 530 & 100 D AREA & \\
\hline 1274 & & DRINKING & & SAN. GRAB & UNFILTERED & 512 & FFTF & \\
\hline 1275 & & DRINKING & & RAW GRAB & UNFILTERED & 200 & $100 \mathrm{~K}$ AREA & \\
\hline 1276 & & RIVER & & CUMULATIVE & UNFILTERED & 463 & RICH.PMPHS HRM 46.4 & \\
\hline 1277 & & RIVER & & CUMULATIVE & UNFILTERED & 252 & PRIEST RAPIDS-RIVER & \\
\hline 1278 & & SURFACE & & RAW GRAB & UNFILTERED & 9190 & HRM 36 SHORELINE & \\
\hline $1279 \mathrm{~F}$ & $\mathrm{~F}$ & SEEP & & RAW GRAB & UNFILTERED & 488 & 300 AREA SPRING 42-2 & \\
\hline $1280 \mathrm{~F}$ & $\mathrm{~F}$ & SEEP & & RAW GRAB & UNFILTERED & 8144 & 300 AREA SPR DR 42-2 & \\
\hline 1281 & & RIVER & & CUMULATIVE & UNFILTERED & 463 & RICH.PMPHS HRM 46.4 & \\
\hline 1282 & & RIVER & & CUMULATIVE & UNFILTERED & 252 & PRIEST RAPIDS-RIVER & \\
\hline 1283 & & RIVER & & RAW GRAB & TRANSECT & 328 & 100 N -1 HRM 9.5 & \\
\hline 1284 & & RIVER & & RAW GRAB & TRANSECT & 317 & VERNITA-1 HRM 0.3 & \\
\hline $1285 \mathrm{~F}$ & $\mathrm{~F}$ & RIVER & & RAW GRAB & TRANSECT & 570 & VERNITA-2 HRM 0.3 & \\
\hline $1286 \mathrm{~F}$ & $\mathrm{~F}$ & RIVER & & RAW GRAB & TRANSECT & 318 & VERNITA-3 HRM 0.3 & \\
\hline $1287 \mathrm{~F}$ & $\mathrm{~F}$ & RIVER & & RAW GRAB & TRANSECT & 319 & VERNITA-4 HRM 0.3 & \\
\hline 1288 & & RIVER & & RAW GRAB & TRANSECT & 328 & 100 N -1 HRM 9.5 & \\
\hline 1289 & & RIVER & & RAW GRAB & TRANSECT & 329 & 100 N -2 HRM 9.5 & \\
\hline $1290 \mathrm{~F}$ & $\mathrm{~F}$ & RIVER & & RAW GRAB & TRANSECT & 331 & 100 N -5 HRM 9.5 & \\
\hline 1291 & & RIVER & & RAW GRAB & TRANSECT & 335 & 100 N -10 HRM 9.5 & \\
\hline 1292 & & RIVER & & RAW GRAB & TRANSECT & 330 & 100 N -3 HRM 9.5 & \\
\hline 1293 & & RIVER & & RAW GRAB & TRANSECT & 333 & 100 N -7 HRM 9.5 & \\
\hline
\end{tabular}




\begin{tabular}{|c|c|c|c|c|c|c|c|c|}
\hline & $\mathrm{T}$ & $U$ & $\mathrm{~V}$ & $\mathrm{~W}$ & $\mathrm{x}$ & $\bar{Y}$ & $Z$ & AA \\
\hline 1 & REVIEW_QUALIFIER & SAMP_FROM & SAMP ITEM & SAMP_MTHD & COLL_MTHD & SAMP_SITE_ID & SAMP_SITE_NAME & WELL_NAME \\
\hline 1294 & & RIVER & & RAW GRAB & TRANSECT & 8244 & 4100 N SHORE HRM 8.4 & \\
\hline 1295 & & RIVER & & RAW GRAB & TRANSECT & 8245 & 5100 N SHORE HRM 8.9 & \\
\hline 1296 & & RIVER & & RAW GRAB & TRANSECT & 8246 & 100 N SHORE HRM 9.2 & \\
\hline 1297 & & RIVER & & RAW GRAB & TRANSECT & 8247 & 7100 N SHORE HRM 9.8 & \\
\hline 1298 & & RIVER & & RAW GRAB & TRANSECT & 336 & 100 F -1 HRM 19.0 & \\
\hline 1299 & & RIVER & & RAW GRAB & TRANSECT & 337 & 7100 F -3 HRM 19.0 & \\
\hline 1300 & & RIVER & & RAW GRAB & TRANSECT & 344 & 4 HANFRD TS-5 HRM 28.7 & \\
\hline 1301 & & RIVER & & RAW GRAB & TRANSECT & 576 & 100 F -2 HRM 19.0 & \\
\hline 1302 & & RIVER & & RAW GRAB & TRANSECT & 577 & 7100 F -5 HRM 19.0 & \\
\hline 1303 & & RIVER & & RAW GRAB & TRANSECT & 339 & 9100 F -7 HRM 19.0 & \\
\hline 1304 & & RIVER & & RAW GRAB & TRANSECT & 341 & 100 F -10 HRM 19.0 & \\
\hline 1305 & $F$ & RIVER & & RAW GRAB & TRANSECT & 578 & 3 HANFRD TS-1 HRM 28.7 & \\
\hline 1306 & & RIVER & & RAW GRAB & TRANSECT & 342 & 2 HANFRD TS-2 HRM 28.7 & \\
\hline 1307 & & RIVER & & RAW GRAB & TRANSECT & 469 & 9 HANFRD TS-7 HRM 28.7 & \\
\hline 1308 & & RIVER & & RAW GRAB & TRANSECT & 347 & 7 HANFRD TS-10 HRM 28.7 & \\
\hline 1309 & & RIVER & & RAW GRAB & TRANSECT & 468 & 3 HANFRD TS-3 HRM 28.7 & \\
\hline 1310 & & RIVER & & RAW GRAB & TRANSECT & 8100 & HANFRD TWNSITE HRM26 & \\
\hline 1311 & & RIVER & & RAW GRAB & TRANSECT & 8101 & 1 HANFRD TWNSITE HRM27 & \\
\hline 1312 & $F$ & RIVER & & RAW GRAB & TRANSECT & 8103 & 3 HANFRD TWNSITE HRM30 & \\
\hline 1313 & $F$ & RIVER & & RAW GRAB & TRANSECT & 274 & 4 HANFRD TWNSITE HRM28 & \\
\hline 1314 & & RIVER & & RAW GRAB & TRANSECT & 8726 & 100 F SHORE HRM 18 & \\
\hline 1315 & & RIVER & & RAW GRAB & TRANSECT & 8728 & 3100 F SHORE HRM 23 & \\
\hline 1316 & & RIVER & & RAW GRAB & TRANSECT & 348 & 300 AREA -1 HRM 43.1 & \\
\hline 1317 & & RIVER & & RAW GRAB & TRANSECT & 470 & 300 AREA -2 HRM 43.1 & \\
\hline 1318 & & RIVER & & RAW GRAB & TRANSECT & 580 & 300 AREA -3 HRM 43.1 & \\
\hline 1319 & & RIVER & & RAW GRAB & TRANSECT & 607 & 7300 AREA -5 HRM 43.1 & \\
\hline 1320 & & RIVER & & RAW GRAB & TRANSECT & 351 & 300 AREA -7 HRM 43.1 & \\
\hline \begin{tabular}{|l|}
1321 \\
1321 \\
\end{tabular} & & RIVER & & RAW GRAB & TRANSECT & 352 & 300 AREA-10 HRM 43.1 & \\
\hline 1322 & & RIVER & & RAW GRAB & TRANSECT & 571 & RICH.PMPHS-1 HRM46.4 & \\
\hline 1323 & & RIVER & & RAW GRAB & TRANSECT & 321 & RICH.PMPHS-2 HRM46.4 & \\
\hline 1324 & & RIVER & & RAW GRAB & TRANSECT & 322 & 2 RICH.PMPHS-3 HRM46.4 & \\
\hline 1325 & & RIVER & & RAW GRAB & TRANSECT & 323 & 3 RICH.PMPHS-5 HRM46.4 & \\
\hline 1326 & & RIVER & & RAW GRAB & TRANSECT & 325 & 5 RICH.PMPHS-7 HRM46.4 & \\
\hline 1327 & $F$ & RIVER & & RAW GRAB & TRANSECT & 327 & 7 RICH.PMPHS-10 HRM46.4 & \\
\hline 1328 & & RIVER & & RAW GRAB & TRANSECT & 8220 & RICH.PMPHS HRM 45.8 & \\
\hline 1329 & & RIVER & & RAW GRAB & TRANSECT & 8221 & RICH.PMPHS HRM 45.0 & \\
\hline 1330 & & RIVER & & RAW GRAB & TRANSECT & 8222 & 2 RICH.PMPHS HRM 43.9 & \\
\hline 1331 & & RIVER & & RAW GRAB & TRANSECT & 8223 & 3 RICH.PMPHS HRM 43.5 & \\
\hline
\end{tabular}




\begin{tabular}{|c|c|c|c|c|c|c|c|c|}
\hline & $\mathrm{T}$ & $U$ & $\mathrm{~V}$ & $\mathrm{~W}$ & $\mathrm{X}$ & $\mathrm{Y}$ & $Z$ & $\mathrm{AA}$ \\
\hline 1 & REVIEW QUALIFIER & SAMP FROM & SAMP ITEM & SAMP MTHD & COLL MTHD & SAMP SITE ID & SAMP SITE NAME & WELL NAME \\
\hline 1332 & & RIVER & & RAW GRAB & TRANSECT & 8240 & 300 AREA SHR HRM41.5 & \\
\hline $1333 \mathrm{~F}$ & $\mathrm{~F}$ & RIVER & & RAW GRAB & TRANSECT & 8241 & 300 AREA SHR HRM42.1 & \\
\hline 1334 & & RIVER & & RAW GRAB & TRANSECT & 8242 & 300 AREA SHR HRM42.5 & \\
\hline 1335 & & RIVER & & RAW GRAB & TRANSECT & 8243 & 300 AREA SHR HRM42.9 & \\
\hline $1336 \mathrm{~F}$ & $\mathrm{~F}$ & SEEP & & RAW GRAB & UNFILTERED & 8144 & 300 AREA SPR DR 42-2 & \\
\hline 1337 & & DRIVE POINT & & RAW GRAB & UNFILTERED & 9539 & 300 SPR 9 -1 (2 FT) & \\
\hline 1338 & & DRIVE POINT & & RAW GRAB & UNFILTERED & 9540 & 300 SPR 9 -1 (4 FT) & \\
\hline 1339 & & DRIVE POINT & & RAW GRAB & UNFILTERED & 9541 & 300 SPR 9 -2 (2 FT) & \\
\hline 1340 & & DRIVE POINT & & RAW GRAB & UNFILTERED & 9542 & 300 SPR 9 -2 (4.5FT) & \\
\hline 1341 & & DRIVE POINT & & RAW GRAB & UNFILTERED & 9543 & 300 SPR 9 -3 (5.5FT) & \\
\hline $1342 \mathrm{~F}$ & $\mathrm{~F}$ & SEEP & & RAW GRAB & UNFILTERED & 488 & 300 AREA SPRING 42-2 & \\
\hline 1343 & & DRIVE POINT & & RAW GRAB & UNFILTERED & 9533 & 300 SPR 7 -1 (2.5FT) & \\
\hline 1344 & & DRIVE POINT & & RAW GRAB & UNFILTERED & 9534 & 300 SPR 7 -1 (4 FT) & \\
\hline 1345 & & DRIVE POINT & & RAW GRAB & UNFILTERED & 9535 & 300 SPR 7 -1 (6 FT) & \\
\hline 1346 & & DRIVE POINT & & RAW GRAB & UNFILTERED & 9536 & 300 SPR 7 -2 (2 FT) & \\
\hline 1347 & & DRIVE POINT & & RAW GRAB & UNFILTERED & 9537 & 300 SPR 7 -2 (4.2FT) & \\
\hline 1348 & & RIVER & & CUMULATIVE & UNFILTERED & 252 & PRIEST RAPIDS-RIVER & \\
\hline 1349 & & RIVER & & CUMULATIVE & UNFILTERED & 463 & RICH.PMPHS HRM 46.4 & \\
\hline $1350 \mathrm{~F}$ & $\mathrm{~F}$ & SURFACE & & RAW GRAB & UNFILTERED & 147 & WEST LAKE & \\
\hline 1351 & & SURFACE & & RAW GRAB & UNFILTERED & 136 & FFTF POND & \\
\hline 1352 & & DRINKING & & RAW GRAB & UNFILTERED & 553 & 100 B AREA-RIVER & \\
\hline 1353 & & DRINKING & & RAW GRAB & UNFILTERED & 530 & 100 D AREA & \\
\hline 1354 & & DRINKING & & SAN. GRAB & UNFILTERED & 512 & FFTF & \\
\hline 1355 & & DRINKING & & RAW GRAB & UNFILTERED & 200 & $100 \mathrm{~K}$ AREA & \\
\hline $1356 \mathrm{~F}$ & $\mathrm{~F}$ & SEEP & & RAW GRAB & UNFILTERED & 8140 & 100-B SPRING 38-3 & \\
\hline 1357 & & SEEP & & RAW GRAB & UNFILTERED & 8147 & 100-F SPRING 207-1 & \\
\hline $1358 \mathrm{~F}$ & $\mathrm{~F}$ & SEEP & & RAW GRAB & UNFILTERED & 202 & 100-N SPRING 8-13 & \\
\hline 1359 & & SEEP & & RAW GRAB & UNFILTERED & 8145 & 100-K SPRING 63-1 & \\
\hline 1360 & & SEEP & & RAW GRAB & UNFILTERED & 8146 & 100-K SPRING 77-1 & \\
\hline 1361 & & SEEP & & RAW GRAB & UNFILTERED & 8165 & 100-H SPRING 145-1 & \\
\hline 1362 & & SEEP & & RAW GRAB & UNFILTERED & 8846 & 100-H SPRING 152-2 & \\
\hline 1363 & & RIVER & & CUMULATIVE & UNFILTERED & 463 & RICH.PMPHS HRM 46.4 & \\
\hline 1364 & & RIVER & & CUMULATIVE & UNFILTERED & 252 & PRIEST RAPIDS-RIVER & \\
\hline 1365 & & SEEP & & RAW GRAB & UNFILTERED & 9236 & VERNITA BRIDGE -1 & \\
\hline 1366 & & RIVER & & CUMULATIVE & UNFILTERED & 252 & PRIEST RAPIDS-RIVER & \\
\hline 1367 & & RIVER & & CUMULATIVE & UNFILTERED & 463 & RICH.PMPHS HRM 46.4 & \\
\hline 1368 & & RIVER & & RAW GRAB & TRANSECT & 317 & VERNITA-1 HRM 0.3 & \\
\hline 1369 & & RIVER & & RAW GRAB & TRANSECT & 570 & VERNITA-2 HRM 0.3 & \\
\hline
\end{tabular}

Page 192 


\begin{tabular}{|c|c|c|c|c|c|c|c|c|}
\hline & $\mathrm{T}$ & $U$ & $\mathrm{~V}$ & $\mathrm{~W}$ & $\mathrm{x}$ & $\bar{Y}$ & $Z$ & AA \\
\hline 1 & REVIEW_QUALIFIER & SAMP_FROM & SAMP ITEM & SAMP_MTHD & COLL_MTHD & SAMP_SITE_ID & SAMP_SITE_NAME & WELL_NAME \\
\hline 1370 & & RIVER & & RAW GRAB & TRANSECT & 318 & VERNITA-3 HRM 0.3 & \\
\hline 1371 & & RIVER & & RAW GRAB & TRANSECT & 319 & VERNITA-4 HRM 0.3 & \\
\hline 1372 & & RIVER & & RAW GRAB & TRANSECT & 571 & RICH.PMPHS-1 HRM46.4 & \\
\hline 1373 & & RIVER & & RAW GRAB & TRANSECT & 571 & RICH.PMPHS-1 HRM46.4 & \\
\hline 1374 & & RIVER & & RAW GRAB & TRANSECT & 321 & RICH.PMPHS-2 HRM46.4 & \\
\hline 1375 & & RIVER & & RAW GRAB & TRANSECT & 322 & RICH.PMPHS-3 HRM46.4 & \\
\hline 1376 & & RIVER & & RAW GRAB & TRANSECT & 323 & RICH.PMPHS-5 HRM46.4 & \\
\hline 1377 & & RIVER & & RAW GRAB & TRANSECT & 325 & RICH.PMPHS-7 HRM46.4 & \\
\hline 1378 & & RIVER & & RAW GRAB & TRANSECT & 327 & RICH.PMPHS-10 HRM46.4 & \\
\hline 1379 & & RIVER & & RAW GRAB & TRANSECT & 8220 & RICH.PMPHS HRM 45.8 & \\
\hline 1380 & & RIVER & & RAW GRAB & TRANSECT & 8221 & RICH.PMPHS HRM 45.0 & \\
\hline 1381 & & RIVER & & RAW GRAB & TRANSECT & 8222 & RICH.PMPHS HRM 43.9 & \\
\hline 1382 & & RIVER & & RAW GRAB & TRANSECT & 8223 & RICH.PMPHS HRM 43.5 & \\
\hline 1383 & & RIVER & & CUMULATIVE & UNFILTERED & 463 & RICH.PMPHS HRM 46.4 & \\
\hline 1384 & & RIVER & & CUMULATIVE & UNFILTERED & 252 & PRIEST RAPIDS-RIVER & \\
\hline 1385 & & RIVER & & CUMULATIVE & UNFILTERED & 463 & RICH.PMPHS HRM 46.4 & \\
\hline 1386 & & RIVER & & CUMULATIVE & UNFILTERED & 252 & PRIEST RAPIDS-RIVER & \\
\hline 1387 & & SURFACE & & RAW GRAB & UNFILTERED & 147 & WEST LAKE & \\
\hline 1388 & $F$ & SURFACE & & RAW GRAB & UNFILTERED & 136 & FFTF POND & \\
\hline 1389 & & DRINKING & & SAN. GRAB & UNFILTERED & 200 & $100 \mathrm{~K}$ AREA & \\
\hline 1390 & & DRINKING & & SAN. GRAB & UNFILTERED & 201 & 100 N AREA & \\
\hline 1391 & & DRINKING & & SAN. GRAB & UNFILTERED & 404 & 200 W AREA & \\
\hline 1392 & & DRINKING & & SAN. GRAB & UNFILTERED & 134 & 400 AREA & \\
\hline 1393 & & RIVER & & CUMULATIVE & UNFILTERED & 252 & PRIEST RAPIDS-RIVER & \\
\hline 1394 & & RIVER & & CUMULATIVE & UNFILTERED & 463 & RICH.PMPHS HRM 46.4 & \\
\hline 1395 & & RIVER & & RAW GRAB & TRANSECT & 317 & VERNITA-1 HRM 0.3 & \\
\hline 1396 & & RIVER & & RAW GRAB & TRANSECT & 570 & VERNITA-2 HRM 0.3 & \\
\hline 1397 & & RIVER & & RAW GRAB & TRANSECT & 318 & VERNITA-3 HRM 0.3 & \\
\hline 1398 & & RIVER & & RAW GRAB & TRANSECT & 319 & VERNITA-4 HRM 0.3 & \\
\hline 1399 & & RIVER & & RAW GRAB & TRANSECT & 571 & RICH.PMPHS-1 HRM46.4 & \\
\hline 1400 & & RIVER & & RAW GRAB & TRANSECT & 571 & RICH.PMPHS-1 HRM46.4 & \\
\hline 1401 & & RIVER & & RAW GRAB & TRANSECT & 321 & RICH.PMPHS-2 HRM46.4 & \\
\hline 1402 & & RIVER & & RAW GRAB & TRANSECT & 322 & RICH.PMPHS-3 HRM46.4 & \\
\hline 1403 & & RIVER & & RAW GRAB & TRANSECT & 323 & RICH.PMPHS-5 HRM46.4 & \\
\hline 1404 & & RIVER & & RAW GRAB & TRANSECT & 325 & RICH.PMPHS-7 HRM46.4 & \\
\hline 1405 & & RIVER & & RAW GRAB & TRANSECT & 327 & RICH.PMPHS-10 HRM46.4 & \\
\hline 1406 & & RIVER & & RAW GRAB & TRANSECT & 8220 & RICH.PMPHS HRM 45.8 & \\
\hline 1407 & & RIVER & & RAW GRAB & TRANSECT & 8221 & RICH.PMPHS HRM 45.0 & \\
\hline
\end{tabular}




\begin{tabular}{|c|c|c|c|c|c|c|c|c|}
\hline & $T$ & $\bar{U}$ & $\mathrm{~V}$ & $\mathrm{~W}$ & $\bar{X}$ & $\bar{Y}$ & $\bar{Z}$ & $\overline{A A}$ \\
\hline 1 & REVIEW_QUALIFIER & SAMP_FROM & SAMP_ITEM & SAMP_MTHD & COLL_MTHD & SAMP_SITE_ID & SAMP_SITE_NAME & WELL_NAME \\
\hline 1408 & & RIVER & & RAW GRAB & TRANSECT & 8222 & RICH.PMPHS HRM 43.9 & \\
\hline 1409 & & RIVER & & RAW GRAB & TRANSECT & 8223 & RICH.PMPHS HRM 43.5 & \\
\hline 1410 & & RIVER & & CUMULATIVE & UNFILTERED & 252 & PRIEST RAPIDS-RIVER & \\
\hline 1411 & & RIVER & & CUMULATIVE & UNFILTERED & 463 & RICH.PMPHS HRM 46.4 & \\
\hline 1412 & & SURFACE & & RAW GRAB & UNFILTERED & 147 & WEST LAKE & \\
\hline 1413 & $\mathrm{~F}$ & SURFACE & & RAW GRAB & UNFILTERED & 136 & FFTF POND & \\
\hline 1414 & $\mathrm{~F}$ & SURFACE & & RAW GRAB & UNFILTERED & 136 & FFTF POND & \\
\hline 1415 & & DRINKING & & SAN. GRAB & UNFILTERED & 201 & 100 N AREA & \\
\hline 1416 & & DRINKING & & SAN. GRAB & UNFILTERED & 134 & 400 AREA & \\
\hline 1417 & & DRINKING & & SAN. GRAB & UNFILTERED & 200 & $100 \mathrm{~K}$ AREA & \\
\hline 1418 & & DRINKING & & SAN. GRAB & UNFILTERED & 404 & 200 W AREA & \\
\hline 1419 & & RIVER & & CUMULATIVE & UNFILTERED & 252 & PRIEST RAPIDS-RIVER & \\
\hline 1420 & & RIVER & & CUMULATIVE & UNFILTERED & 463 & RICH.PMPHS HRM 46.4 & \\
\hline 1421 & & BASIN & & & & 13420 & 100-HR-3 ISRM & \\
\hline 1422 & & BASIN & & & & 13421 & 100-HR-3 ISRM & \\
\hline 1423 & & IRRIGATION & & RAW GRAB & UNFILTERED & 440 & RIVERVIEW CANAL & \\
\hline 1424 & & IRRIGATION & & RAW GRAB & UNFILTERED & 605 & HORN RAPIDS AREA & \\
\hline 1425 & & RIVER & & CUMULATIVE & UNFILTERED & 252 & PRIEST RAPIDS-RIVER & \\
\hline 1426 & & RIVER & & CUMULATIVE & UNFILTERED & 463 & RICH.PMPHS HRM 46.4 & \\
\hline 1427 & & RIVER & & RAW GRAB & TRANSECT & 317 & VERNITA-1 HRM 0.3 & \\
\hline 1428 & & RIVER & & RAW GRAB & TRANSECT & 570 & VERNITA-2 HRM 0.3 & \\
\hline 1429 & & RIVER & & RAW GRAB & TRANSECT & 318 & VERNITA-3 HRM 0.3 & \\
\hline 1430 & & RIVER & & RAW GRAB & TRANSECT & 319 & VERNITA-4 HRM 0.3 & \\
\hline 1431 & & RIVER & & RAW GRAB & TRANSECT & 571 & RICH.PMPHS-1 HRM46.4 & \\
\hline 1432 & $\mathrm{~F}$ & RIVER & & RAW GRAB & TRANSECT & 321 & RICH.PMPHS-2 HRM46.4 & \\
\hline 1433 & $\mathrm{~F}$ & RIVER & & RAW GRAB & TRANSECT & 322 & RICH.PMPHS-3 HRM46.4 & \\
\hline 1434 & & RIVER & & RAW GRAB & TRANSECT & 323 & RICH.PMPHS-5 HRM46.4 & \\
\hline 1435 & $\mathrm{~F}$ & RIVER & & RAW GRAB & TRANSECT & 325 & RICH.PMPHS-7 HRM46.4 & \\
\hline 1436 & $\mathrm{~F}$ & RIVER & & RAW GRAB & TRANSECT & 327 & RICH.PMPHS-10 HRM46.4 & \\
\hline 1437 & $\mathrm{~F}$ & RIVER & & RAW GRAB & TRANSECT & 8220 & RICH.PMPHS HRM 45.8 & \\
\hline 1438 & $\mathrm{~F}$ & RIVER & & RAW GRAB & TRANSECT & 8221 & RICH.PMPHS HRM 45.0 & \\
\hline 1439 & $\mathrm{~F}$ & RIVER & & RAW GRAB & TRANSECT & 8222 & RICH.PMPHS HRM 43.9 & \\
\hline 1440 & $\mathrm{~F}$ & RIVER & & RAW GRAB & TRANSECT & 8223 & RICH.PMPHS HRM 43.5 & \\
\hline 1441 & $\mathrm{~F}$ & RIVER & & CUMULATIVE & UNFILTERED & 252 & PRIEST RAPIDS-RIVER & \\
\hline 1442 & $\mathrm{~F}$ & RIVER & & CUMULATIVE & UNFILTERED & 463 & RICH.PMPHS HRM 46.4 & \\
\hline 1443 & $\mathrm{~F}$ & IRRIGATION & & RAW GRAB & UNFILTERED & 440 & RIVERVIEW CANAL & \\
\hline 1444 & $\mathrm{~F}$ & IRRIGATION & & RAW GRAB & UNFILTERED & 605 & HORN RAPIDS AREA & \\
\hline 1445 & & SURFACE & & RAW GRAB & UNFILTERED & 147 & WEST LAKE & \\
\hline
\end{tabular}

Page 194 


\begin{tabular}{|c|c|c|c|c|c|c|c|c|}
\hline & $T$ & $\bar{U}$ & $\mathrm{~V}$ & $\mathrm{~W}$ & $\bar{X}$ & $\bar{Y}$ & $\bar{Z}$ & $\overline{A A}$ \\
\hline 1 & REVIEW_QUALIFIER & SAMP_FROM & SAMP_ITEM & SAMP_MTHD & COLL_MTHD & SAMP_SITE_ID & SAMP_SITE_NAME & WELL_NAME \\
\hline 1446 & $\mathrm{~F}$ & SURFACE & & RAW GRAB & UNFILTERED & 136 & FFTF POND & \\
\hline 1447 & $\mathrm{~F}$ & IRRIGATION & & RAW GRAB & UNFILTERED & 440 & RIVERVIEW CANAL & \\
\hline 1448 & & DRINKING & & SAN. GRAB & UNFILTERED & 200 & $100 \mathrm{~K}$ AREA & \\
\hline 1449 & & IRRIGATION & & RAW GRAB & UNFILTERED & 605 & HORN RAPIDS AREA & \\
\hline 1450 & & DRINKING & & SAN. GRAB & UNFILTERED & 201 & 100 N AREA & \\
\hline 1451 & & DRINKING & & SAN. GRAB & UNFILTERED & 404 & 200 W AREA & \\
\hline 1452 & & DRINKING & & SAN. GRAB & UNFILTERED & 134 & 400 AREA & \\
\hline 1453 & $\mathrm{~F}$ & RIVER & & CUMULATIVE & UNFILTERED & 252 & PRIEST RAPIDS-RIVER & \\
\hline 1454 & & RIVER & & CUMULATIVE & UNFILTERED & 463 & RICH.PMPHS HRM 46.4 & \\
\hline 1455 & & BASIN & & & & 13936 & 100-HR-3 ISRM & \\
\hline 1456 & & BASIN & WATER & & & 13937 & 100-HR-3 ISRM & \\
\hline 1457 & $\mathrm{~F}$ & RIVER & & CUMULATIVE & UNFILTERED & 252 & PRIEST RAPIDS-RIVER & \\
\hline 1458 & & RIVER & & CUMULATIVE & UNFILTERED & 463 & RICH.PMPHS HRM 46.4 & \\
\hline 1459 & & RIVER & & RAW GRAB & TRANSECT & 328 & 100 N -1 HRM 9.5 & \\
\hline 1460 & $\mathrm{~F}$ & RIVER & & RAW GRAB & TRANSECT & 319 & VERNITA-4 HRM 0.3 & \\
\hline 1461 & & RIVER & & RAW GRAB & TRANSECT & 570 & VERNITA-2 HRM 0.3 & \\
\hline 1462 & $\mathrm{~F}$ & RIVER & & RAW GRAB & TRANSECT & 318 & VERNITA-3 HRM 0.3 & \\
\hline 1463 & & RIVER & & RAW GRAB & TRANSECT & 317 & VERNITA-1 HRM 0.3 & \\
\hline 1464 & & RIVER & & RAW GRAB & TRANSECT & 328 & 100 N -1 HRM 9.5 & \\
\hline 1465 & & RIVER & & RAW GRAB & TRANSECT & 329 & 100 N -2 HRM 9.5 & \\
\hline 1466 & $\mathrm{~F}$ & RIVER & & RAW GRAB & TRANSECT & 331 & 100 N -5 HRM 9.5 & \\
\hline 1467 & $\mathrm{~F}$ & RIVER & & RAW GRAB & TRANSECT & 335 & 100 N -10 HRM 9.5 & \\
\hline 1468 & $\mathrm{~F}$ & RIVER & & RAW GRAB & TRANSECT & 330 & 100 N -3 HRM 9.5 & \\
\hline 1469 & $\mathrm{~F}$ & RIVER & & RAW GRAB & TRANSECT & 333 & 100 N -7 HRM 9.5 & \\
\hline 1470 & & RIVER & & RAW GRAB & TRANSECT & 8244 & 100 N SHORE HRM 8.4 & \\
\hline 1471 & $\mathrm{~F}$ & RIVER & & RAW GRAB & TRANSECT & 8245 & 100 N SHORE HRM 8.9 & \\
\hline 1472 & & RIVER & & RAW GRAB & TRANSECT & 8246 & 100 N SHORE HRM 9.2 & \\
\hline 1473 & $\mathrm{~F}$ & RIVER & & RAW GRAB & TRANSECT & 8247 & 100 N SHORE HRM 9.8 & \\
\hline 1474 & $\mathrm{~F}$ & RIVER & & RAW GRAB & TRANSECT & 336 & 100 F -1 HRM 19.0 & \\
\hline 1475 & $\mathrm{~F}$ & RIVER & & RAW GRAB & TRANSECT & 337 & 100 F -3 HRM 19.0 & \\
\hline 1476 & $\mathrm{~F}$ & RIVER & & RAW GRAB & TRANSECT & 344 & HANFRD TS-5 HRM 28.7 & \\
\hline 1477 & $\mathrm{~F}$ & RIVER & & RAW GRAB & TRANSECT & 576 & 100 F -2 HRM 19.0 & \\
\hline 1478 & $\mathrm{~F}$ & RIVER & & RAW GRAB & TRANSECT & 577 & 100 F -5 HRM 19.0 & \\
\hline 1479 & $\mathrm{~F}$ & RIVER & & RAW GRAB & TRANSECT & 339 & 100 F -7 HRM 19.0 & \\
\hline 1480 & & RIVER & & RAW GRAB & TRANSECT & 341 & $100 \mathrm{~F}-10 \mathrm{HRM} 19.0$ & \\
\hline 1481 & & RIVER & & RAW GRAB & TRANSECT & 578 & HANFRD TS-1 HRM 28.7 & \\
\hline 1482 & & RIVER & & RAW GRAB & TRANSECT & 342 & HANFRD TS-2 HRM 28.7 & \\
\hline 1483 & $\mathrm{~F}$ & RIVER & & RAW GRAB & TRANSECT & 469 & HANFRD TS-7 HRM 28.7 & \\
\hline
\end{tabular}




\begin{tabular}{|c|c|c|c|c|c|c|c|c|}
\hline & $T$ & $\bar{U}$ & $\mathrm{~V}$ & $\mathrm{~W}$ & $\bar{X}$ & $\bar{Y}$ & $\bar{Z}$ & $\overline{A A}$ \\
\hline 1 & REVIEW_QUALIFIER & SAMP_FROM & SAMP_ITEM & SAMP_MTHD & COLL_MTHD & SAMP_SITE_ID & SAMP_SITE_NAME & WELL_NAME \\
\hline 1484 & $\mathrm{~F}$ & RIVER & & RAW GRAB & TRANSECT & 347 & HANFRD TS-10 HRM 28.7 & \\
\hline 1485 & & RIVER & & RAW GRAB & TRANSECT & 468 & HANFRD TS-3 HRM 28.7 & \\
\hline 1486 & $\mathrm{~F}$ & RIVER & & RAW GRAB & TRANSECT & 8100 & HANFRD TWNSITE HRM26 & \\
\hline 1487 & & RIVER & & RAW GRAB & TRANSECT & 8101 & HANFRD TWNSITE HRM27 & \\
\hline 1488 & $\mathrm{~F}$ & RIVER & & RAW GRAB & TRANSECT & 8103 & HANFRD TWNSITE HRM30 & \\
\hline 1489 & & RIVER & & RAW GRAB & TRANSECT & 274 & HANFRD TWNSITE HRM28 & \\
\hline 1490 & & RIVER & & RAW GRAB & TRANSECT & 8726 & 100 F SHORE HRM 18 & \\
\hline 1491 & $\mathrm{~F}$ & RIVER & & RAW GRAB & TRANSECT & 13656 & 100 F SHORE HRM 24 & \\
\hline 1492 & $\mathrm{~F}$ & RIVER & & RAW GRAB & TRANSECT & 8728 & 100 F SHORE HRM 23 & \\
\hline 1493 & & RIVER & & RAW GRAB & TRANSECT & 348 & 300 AREA -1 HRM 43.1 & \\
\hline 1494 & & RIVER & & RAW GRAB & TRANSECT & 470 & 300 AREA -2 HRM 43.1 & \\
\hline 1495 & & RIVER & & RAW GRAB & TRANSECT & 580 & 300 AREA -3 HRM 43.1 & \\
\hline 1496 & & RIVER & & RAW GRAB & TRANSECT & 607 & 300 AREA -5 HRM 43.1 & \\
\hline 1497 & & RIVER & & RAW GRAB & TRANSECT & 351 & 300 AREA -7 HRM 43.1 & \\
\hline 1498 & & RIVER & & RAW GRAB & TRANSECT & 352 & 300 AREA-10 HRM 43.1 & \\
\hline 1499 & & RIVER & & RAW GRAB & TRANSECT & 571 & RICH.PMPHS-1 HRM46.4 & \\
\hline 1500 & & RIVER & & RAW GRAB & TRANSECT & 321 & RICH.PMPHS-2 HRM46.4 & \\
\hline 1501 & & RIVER & & RAW GRAB & TRANSECT & 322 & RICH.PMPHS-3 HRM46.4 & \\
\hline 1502 & $\mathrm{~F}$ & RIVER & & RAW GRAB & TRANSECT & 323 & RICH.PMPHS-5 HRM46.4 & \\
\hline 1503 & & RIVER & & RAW GRAB & TRANSECT & 325 & RICH.PMPHS-7 HRM46.4 & \\
\hline 1504 & & RIVER & & RAW GRAB & TRANSECT & 327 & RICH.PMPHS-10 HRM46.4 & \\
\hline 1505 & & RIVER & & RAW GRAB & TRANSECT & 8220 & RICH.PMPHS HRM 45.8 & \\
\hline 1506 & & RIVER & & RAW GRAB & TRANSECT & 8221 & RICH.PMPHS HRM 45.0 & \\
\hline 1507 & & RIVER & & RAW GRAB & TRANSECT & 8222 & RICH.PMPHS HRM 43.9 & \\
\hline 1508 & & RIVER & & RAW GRAB & TRANSECT & 8223 & RICH.PMPHS HRM 43.5 & \\
\hline 1509 & & RIVER & & RAW GRAB & TRANSECT & 8240 & 300 AREA SHR HRM41.5 & \\
\hline 1510 & & RIVER & & RAW GRAB & TRANSECT & 8241 & 300 AREA SHR HRM42.1 & \\
\hline 1511 & & RIVER & & RAW GRAB & TRANSECT & 8242 & 300 AREA SHR HRM42.5 & \\
\hline 1512 & & RIVER & & RAW GRAB & TRANSECT & 8243 & 300 AREA SHR HRM42.9 & \\
\hline 1513 & $\mathrm{~F}$ & SEEP & & RAW GRAB & UNFILTERED & 202 & $100-N$ SPRING 8-13 & \\
\hline 1514 & $\mathrm{~F}$ & SEEP & & RAW GRAB & UNFILTERED & 9106 & 100-B SPRING 37-1 & \\
\hline 1515 & $\mathrm{~F}$ & SEEP & & RAW GRAB & UNFILTERED & 13792 & 100-K SPRING 63-2 & \\
\hline 1516 & & RIVER & & CUMULATIVE & UNFILTERED & 252 & PRIEST RAPIDS-RIVER & \\
\hline 1517 & & RIVER & & CUMULATIVE & UNFILTERED & 463 & RICH.PMPHS HRM 46.4 & \\
\hline 1518 & $\mathrm{~F}$ & SEEP & & RAW GRAB & UNFILTERED & 151 & HANFORD SPRING 28-2 & \\
\hline 1519 & $\mathrm{~F}$ & SEEP & & RAW GRAB & UNFILTERED & 488 & 300 AREA SPRING 42-2 & \\
\hline 1520 & $\mathrm{~F}$ & SEEP & & RAW GRAB & UNFILTERED & 8143 & HANFORD SPR DR 28-2 & \\
\hline 1521 & & DRINKING & & SAN. GRAB & UNFILTERED & 134 & 400 AREA & \\
\hline
\end{tabular}

Page 196 


\begin{tabular}{|c|c|c|c|c|c|c|c|c|}
\hline & $\mathrm{T}$ & $U$ & $\mathrm{~V}$ & $\mathrm{~W}$ & $\mathrm{X}$ & $\mathrm{Y}$ & $Z$ & $\mathrm{AA}$ \\
\hline 1 & REVIEW QUALIFIER & SAMP FROM & SAMP ITEM & SAMP MTHD & COLL MTHD & SAMP SITE ID & SAMP SITE NAME & WELL NAME \\
\hline 1522 & & SURFACE & & RAW GRAB & UNFILTERED & 136 & FFTF POND & \\
\hline 1523 & & SURFACE & & RAW GRAB & UNFILTERED & 147 & WEST LAKE & \\
\hline 1524 & & SEEP & & RAW GRAB & UNFILTERED & 8146 & 100-K SPRING 77-1 & \\
\hline 1525 & & SEEP & & RAW GRAB & UNFILTERED & 8162 & 100-D SPRING 110-1 & \\
\hline 1526 & & SEEP & & RAW GRAB & UNFILTERED & 8846 & 100-H SPRING 152-2 & \\
\hline 1527 & & SEEP & & RAW GRAB & UNFILTERED & 8147 & 100-F SPRING 207-1 & \\
\hline 1528 & & RIVER & & CUMULATIVE & UNFILTERED & 252 & PRIEST RAPIDS-RIVER & \\
\hline 1529 & & RIVER & & CUMULATIVE & UNFILTERED & 463 & RICH.PMPHS HRM 46.4 & \\
\hline 1530 & & RIVER & & CUMULATIVE & UNFILTERED & 252 & PRIEST RAPIDS-RIVER & \\
\hline 1531 & & RIVER & & CUMULATIVE & UNFILTERED & 463 & RICH.PMPHS HRM 46.4 & \\
\hline 1532 & $\mathrm{~F}$ & SURFACE & & RAW GRAB & UNFILTERED & 147 & WEST LAKE & \\
\hline 1533 & & RIVER & & CUMULATIVE & UNFILTERED & 463 & RICH.PMPHS HRM 46.4 & \\
\hline 1534 & & RIVER & & CUMULATIVE & UNFILTERED & 252 & PRIEST RAPIDS-RIVER & \\
\hline 1535 & & RIVER & & CUMULATIVE & UNFILTERED & 463 & RICH.PMPHS HRM 46.4 & \\
\hline 1536 & & RIVER & & CUMULATIVE & UNFILTERED & 252 & PRIEST RAPIDS-RIVER & \\
\hline 1537 & & RIVER & & RAW GRAB & TRANSECT & 317 & VERNITA-1 HRM 0.3 & \\
\hline 1538 & & RIVER & & RAW GRAB & TRANSECT & 570 & VERNITA-2 HRM 0.3 & \\
\hline 1539 & & RIVER & & RAW GRAB & TRANSECT & 318 & VERNITA-3 HRM 0.3 & \\
\hline 1540 & & RIVER & & RAW GRAB & TRANSECT & 319 & VERNITA-4 HRM 0.3 & \\
\hline 1541 & & RIVER & & RAW GRAB & TRANSECT & 571 & RICH.PMPHS-1 HRM46.4 & \\
\hline 1542 & & RIVER & & RAW GRAB & TRANSECT & 571 & RICH.PMPHS-1 HRM46.4 & \\
\hline 1543 & & RIVER & & RAW GRAB & TRANSECT & 321 & RICH.PMPHS-2 HRM46.4 & \\
\hline 1544 & & RIVER & & RAW GRAB & TRANSECT & 322 & RICH.PMPHS-3 HRM46.4 & \\
\hline 1545 & & RIVER & & RAW GRAB & TRANSECT & 323 & RICH.PMPHS-5 HRM46.4 & \\
\hline 1546 & & RIVER & & RAW GRAB & TRANSECT & 325 & RICH.PMPHS-7 HRM46.4 & \\
\hline 1547 & $\mathrm{~F}$ & RIVER & & RAW GRAB & TRANSECT & 327 & RICH.PMPHS-10 HRM46.4 & \\
\hline 1548 & & RIVER & & RAW GRAB & TRANSECT & 8220 & RICH.PMPHS HRM 45.8 & \\
\hline 1549 & & RIVER & & RAW GRAB & TRANSECT & 8221 & RICH.PMPHS HRM 45.0 & \\
\hline 1550 & & RIVER & & RAW GRAB & TRANSECT & 8222 & RICH.PMPHS HRM 43.9 & \\
\hline 1551 & & RIVER & & RAW GRAB & TRANSECT & 8223 & RICH.PMPHS HRM 43.5 & \\
\hline 1552 & $\mathrm{~F}$ & SURFACE & & RAW GRAB & UNFILTERED & 147 & WEST LAKE & \\
\hline 1553 & & RIVER & & CUMULATIVE & UNFILTERED & 463 & RICH.PMPHS HRM 46.4 & \\
\hline 1554 & & RIVER & & CUMULATIVE & UNFILTERED & 252 & PRIEST RAPIDS-RIVER & \\
\hline 1555 & & RIVER & & CUMULATIVE & UNFILTERED & 252 & PRIEST RAPIDS-RIVER & \\
\hline 1556 & & RIVER & & CUMULATIVE & UNFILTERED & 463 & RICH.PMPHS HRM 46.4 & \\
\hline 1557 & & IRRIGATION & & RAW GRAB & UNFILTERED & 440 & RIVERVIEW CANAL & \\
\hline 1558 & & RIVER & & CUMULATIVE & UNFILTERED & 252 & PRIEST RAPIDS-RIVER & \\
\hline 1559 & & RIVER & & CUMULATIVE & UNFILTERED & 463 & RICH.PMPHS HRM 46.4 & \\
\hline
\end{tabular}




\begin{tabular}{|c|c|c|c|c|c|c|c|c|}
\hline & $\mathrm{T}$ & $U$ & $\mathrm{~V}$ & $\mathrm{~W}$ & $\mathrm{X}$ & $\mathrm{Y}$ & Z & AA \\
\hline 1 & REVIEW_QUALIFIER & SAMP_FROM & SAMP_ITEM & SAMP_MTHD & COLL_MTHD & SAMP_SITE_ID & SAMP_SITE_NAME & WELL_NAME \\
\hline 1560 & & IRRIGATION & & RAW GRAB & UNFILTERED & 440 & RIVERVIEW CANAL & \\
\hline 1561 & $\mathrm{~F}$ & SEEP & & RAW GRAB & & 8626 & WEST LAKE SPRING & \\
\hline 1562 & $\mathrm{~F}$ & SURFACE & & RAW GRAB & UNFILTERED & 147 & WEST LAKE & \\
\hline 1563 & $\mathrm{~F}$ & SURFACE & & RAW GRAB & & 147 & WEST LAKE & \\
\hline 1564 & & RIVER & & RAW GRAB & TRANSECT & 317 & VERNITA-1 HRM 0.3 & \\
\hline 1565 & & RIVER & & RAW GRAB & TRANSECT & 570 & VERNITA-2 HRM 0.3 & \\
\hline 1566 & & RIVER & & RAW GRAB & TRANSECT & 318 & VERNITA-3 HRM 0.3 & \\
\hline 1567 & & RIVER & & RAW GRAB & TRANSECT & 319 & VERNITA-4 HRM 0.3 & \\
\hline 1568 & & RIVER & & RAW GRAB & TRANSECT & 571 & RICH.PMPHS-1 HRM46.4 & \\
\hline 1569 & & RIVER & & RAW GRAB & TRANSECT & 321 & RICH.PMPHS-2 HRM46.4 & \\
\hline 1570 & & RIVER & & RAW GRAB & TRANSECT & 322 & RICH.PMPHS-3 HRM46.4 & \\
\hline 1571 & & RIVER & & RAW GRAB & TRANSECT & 323 & RICH.PMPHS-5 HRM46.4 & \\
\hline 1572 & & RIVER & & RAW GRAB & TRANSECT & 325 & RICH.PMPHS-7 HRM46.4 & \\
\hline 1573 & & RIVER & & RAW GRAB & TRANSECT & 327 & RICH.PMPHS-10 HRM46.4 & \\
\hline 1574 & & RIVER & & RAW GRAB & TRANSECT & 8220 & RICH.PMPHS HRM 45.8 & \\
\hline 1575 & & RIVER & & RAW GRAB & TRANSECT & 8221 & RICH.PMPHS HRM 45.0 & \\
\hline 1576 & & RIVER & & RAW GRAB & TRANSECT & 8222 & RICH.PMPHS HRM 43.9 & \\
\hline 1577 & & RIVER & & RAW GRAB & TRANSECT & 8223 & RICH.PMPHS HRM 43.5 & \\
\hline 1578 & & RIVER & & CUMULATIVE & UNFILTERED & 252 & PRIEST RAPIDS-RIVER & \\
\hline 1579 & & RIVER & & CUMULATIVE & UNFILTERED & 463 & RICH.PMPHS HRM 46.4 & \\
\hline 1580 & $\mathrm{~F}$ & SURFACE & & RAW GRAB & UNFILTERED & 147 & WEST LAKE & \\
\hline 1581 & & IRRIGATION & & RAW GRAB & UNFILTERED & 440 & RIVERVIEW CANAL & \\
\hline 1582 & & RIVER & & CUMULATIVE & UNFILTERED & 252 & PRIEST RAPIDS-RIVER & \\
\hline 1583 & & RIVER & & CUMULATIVE & UNFILTERED & 463 & RICH.PMPHS HRM 46.4 & \\
\hline 1584 & & RIVER & & CUMULATIVE & UNFILTERED & 252 & PRIEST RAPIDS-RIVER & \\
\hline 1585 & & RIVER & & CUMULATIVE & UNFILTERED & 463 & RICH.PMPHS HRM 46.4 & \\
\hline 1586 & & RIVER & & RAW GRAB & TRANSECT & 328 & 100 N -1 HRM 9.5 & \\
\hline 1587 & & RIVER & & RAW GRAB & TRANSECT & 317 & VERNITA-1 HRM 0.3 & \\
\hline 1588 & & RIVER & & RAW GRAB & TRANSECT & 570 & VERNITA-2 HRM 0.3 & \\
\hline 1589 & & RIVER & & RAW GRAB & TRANSECT & 318 & VERNITA-3 HRM 0.3 & \\
\hline 1590 & & RIVER & & RAW GRAB & TRANSECT & 319 & VERNITA-4 HRM 0.3 & \\
\hline 1591 & & RIVER & & RAW GRAB & TRANSECT & 328 & 100 N -1 HRM 9.5 & \\
\hline 1592 & & RIVER & & RAW GRAB & TRANSECT & 329 & 100 N -2 HRM 9.5 & \\
\hline 1593 & & RIVER & & RAW GRAB & TRANSECT & 331 & 100 N -5 HRM 9.5 & \\
\hline 1594 & & RIVER & & RAW GRAB & TRANSECT & 335 & 100 N -10 HRM 9.5 & \\
\hline 1595 & & RIVER & & RAW GRAB & TRANSECT & 330 & 100 N -3 HRM 9.5 & \\
\hline 1596 & & RIVER & & RAW GRAB & TRANSECT & 333 & 100 N -7 HRM 9.5 & \\
\hline 1597 & & RIVER & & RAW GRAB & TRANSECT & 8244 & 100 N SHORE HRM 8.4 & \\
\hline
\end{tabular}




\begin{tabular}{|c|c|c|c|c|c|c|c|c|}
\hline & $T$ & $\bar{U}$ & $\mathrm{~V}$ & $\mathrm{~W}$ & $\bar{x}$ & $\bar{Y}$ & $\mathrm{Z}$ & AA \\
\hline 1 & REVIEW_QUALIFIER & SAMP_FROM & SAMP ITEM & SAMP_MTHD & COLL_MTHD & SAMP_SITE_ID & SAMP_SITE_NAME & WELL_NAME \\
\hline 1598 & & RIVER & & RAW GRAB & TRANSECT & 8245 & 5100 N SHORE HRM 8.9 & \\
\hline 1599 & & RIVER & & RAW GRAB & TRANSECT & 8246 & 100 N SHORE HRM 9.2 & \\
\hline 1600 & & RIVER & & RAW GRAB & TRANSECT & 8247 & 7100 N SHORE HRM 9.8 & \\
\hline 1601 & & RIVER & & RAW GRAB & TRANSECT & 336 & 100 F -1 HRM 19.0 & \\
\hline 1602 & & RIVER & & RAW GRAB & TRANSECT & 337 & 7100 F -3 HRM 19.0 & \\
\hline 1603 & & RIVER & & RAW GRAB & TRANSECT & 344 & 4 HANFRD TS-5 HRM 28.7 & \\
\hline 1604 & & RIVER & & RAW GRAB & TRANSECT & 576 & 100 F -2 HRM 19.0 & \\
\hline 1605 & & RIVER & & RAW GRAB & TRANSECT & 577 & 7100 F -5 HRM 19.0 & \\
\hline 1606 & & RIVER & & RAW GRAB & TRANSECT & 339 & 100 F -7 HRM 19.0 & \\
\hline 1607 & & RIVER & & RAW GRAB & TRANSECT & 341 & 100 F -10 HRM 19.0 & \\
\hline 1608 & & RIVER & & RAW GRAB & TRANSECT & 578 & 3 HANFRD TS-1 HRM 28.7 & \\
\hline 1609 & & RIVER & & RAW GRAB & TRANSECT & 342 & 2 HANFRD TS-2 HRM 28.7 & \\
\hline 1610 & & RIVER & & RAW GRAB & TRANSECT & 469 & HANFRD TS-7 HRM 28.7 & \\
\hline 1611 & & RIVER & & RAW GRAB & TRANSECT & 347 & 7 HANFRD TS-10 HRM 28.7 & \\
\hline 1612 & & RIVER & & RAW GRAB & TRANSECT & 468 & 3 HANFRD TS-3 HRM 28.7 & \\
\hline 1613 & & RIVER & & RAW GRAB & TRANSECT & 8100 & HANFRD TWNSITE HRM26 & \\
\hline 1614 & & RIVER & & RAW GRAB & TRANSECT & 8101 & 1 HANFRD TWNSITE HRM27 & \\
\hline 1615 & & RIVER & & RAW GRAB & TRANSECT & 8103 & 3 HANFRD TWNSITE HRM30 & \\
\hline 1616 & & RIVER & & RAW GRAB & TRANSECT & 274 & 4 HANFRD TWNSITE HRM28 & \\
\hline 1617 & & RIVER & & RAW GRAB & TRANSECT & 8726 & 100 F SHORE HRM 18 & \\
\hline 1618 & & RIVER & & RAW GRAB & TRANSECT & 8727 & 7100 F SHORE HRM 22 & \\
\hline 1619 & & RIVER & & RAW GRAB & TRANSECT & 8728 & 3100 F SHORE HRM 23 & \\
\hline 1620 & & RIVER & & RAW GRAB & TRANSECT & 348 & 300 AREA -1 HRM 43.1 & \\
\hline 1621 & & RIVER & & RAW GRAB & TRANSECT & 470 & 300 AREA -2 HRM 43.1 & \\
\hline 1622 & & RIVER & & RAW GRAB & TRANSECT & 580 & 300 AREA -3 HRM 43.1 & \\
\hline 1623 & & RIVER & & RAW GRAB & TRANSECT & 607 & 7300 AREA -5 HRM 43.1 & \\
\hline 1624 & & RIVER & & RAW GRAB & TRANSECT & 351 & 300 AREA -7 HRM 43.1 & \\
\hline 1625 & & RIVER & & RAW GRAB & TRANSECT & 352 & 300 AREA-10 HRM 43.1 & \\
\hline 1626 & & RIVER & & RAW GRAB & TRANSECT & 571 & RICH.PMPHS-1 HRM46.4 & \\
\hline 1627 & & RIVER & & RAW GRAB & TRANSECT & 321 & RICH.PMPHS-2 HRM46.4 & \\
\hline 1628 & & RIVER & & RAW GRAB & TRANSECT & 322 & 2 RICH.PMPHS-3 HRM46.4 & \\
\hline 1629 & & RIVER & & RAW GRAB & TRANSECT & 323 & 3 RICH.PMPHS-5 HRM46.4 & \\
\hline 1630 & & RIVER & & RAW GRAB & TRANSECT & 325 & RICH.PMPHS-7 HRM46.4 & \\
\hline 1631 & & RIVER & & RAW GRAB & TRANSECT & 327 & 7 RICH.PMPHS-10 HRM46.4 & \\
\hline 1632 & & RIVER & & RAW GRAB & TRANSECT & 8220 & RICH.PMPHS HRM 45.8 & \\
\hline 1633 & & RIVER & & RAW GRAB & TRANSECT & 8221 & RICH.PMPHS HRM 45.0 & \\
\hline 1634 & & RIVER & & RAW GRAB & TRANSECT & 8222 & 2 RICH.PMPHS HRM 43.9 & \\
\hline 1635 & & RIVER & & RAW GRAB & TRANSECT & 8223 & 3 RICH.PMPHS HRM 43.5 & \\
\hline
\end{tabular}




\begin{tabular}{|c|c|c|c|c|c|c|c|c|}
\hline & $T$ & $\bar{U}$ & $\mathrm{~V}$ & $\mathrm{~W}$ & $\bar{X}$ & $\bar{Y}$ & $\bar{Z}$ & $\overline{A A}$ \\
\hline 1 & REVIEW_QUALIFIER & SAMP_FROM & SAMP_ITEM & SAMP_MTHD & COLL_MTHD & SAMP_SITE_ID & SAMP_SITE_NAME & WELL_NAME \\
\hline 1636 & & RIVER & & RAW GRAB & TRANSECT & 8240 & 300 AREA SHR HRM41.5 & \\
\hline 1637 & & RIVER & & RAW GRAB & TRANSECT & 8241 & 300 AREA SHR HRM42.1 & \\
\hline 1638 & & RIVER & & RAW GRAB & TRANSECT & 8242 & 300 AREA SHR HRM42.5 & \\
\hline 1639 & & RIVER & & RAW GRAB & TRANSECT & 8243 & 300 AREA SHR HRM42.9 & \\
\hline 1640 & $\mathrm{~F}$ & SEEP & & RAW GRAB & UNFILTERED & 151 & HANFORD SPRING $28-2$ & \\
\hline 1641 & $\mathrm{~F}$ & SEEP & & RAW GRAB & UNFILTERED & 488 & 300 AREA SPRING 42-2 & \\
\hline 1642 & $\mathrm{~F}$ & SEEP & & RAW GRAB & UNFILTERED & 8142 & HANFORD SPR UR 28-2 & \\
\hline 1643 & $\mathrm{~F}$ & SEEP & & RAW GRAB & UNFILTERED & 8143 & HANFORD SPR DR 28-2 & \\
\hline 1644 & $\mathrm{~F}$ & SEEP & & RAW GRAB & UNFILTERED & 8144 & 300 AREA SPR DR 42-2 & \\
\hline 1645 & $\mathrm{~F}$ & IRRIGATION & & RAW GRAB & UNFILTERED & 605 & HORN RAPIDS AREA & \\
\hline 1646 & & RIVER & & CUMULATIVE & UNFILTERED & 252 & PRIEST RAPIDS-RIVER & \\
\hline 1647 & & RIVER & & CUMULATIVE & UNFILTERED & 463 & RICH.PMPHS HRM 46.4 & \\
\hline 1648 & $\mathrm{~F}$ & SURFACE & & RAW GRAB & UNFILTERED & 147 & WEST LAKE & \\
\hline 1649 & $\mathrm{~F}$ & SEEP & & RAW GRAB & UNFILTERED & 8165 & 100-H SPRING 145-1 & \\
\hline 1650 & $\mathrm{~F}$ & SEEP & & RAW GRAB & UNFILTERED & 8164 & 100-H SPRING 153-1 & \\
\hline 1651 & & RIVER & & CUMULATIVE & UNFILTERED & 252 & PRIEST RAPIDS-RIVER & \\
\hline 1652 & & RIVER & & CUMULATIVE & UNFILTERED & 463 & RICH.PMPHS HRM 46.4 & \\
\hline 1653 & $\mathrm{~F}$ & SEEP & & RAW GRAB & UNFILTERED & 8147 & 100-F SPRING 207-1 & \\
\hline 1654 & & RIVER & & CUMULATIVE & UNFILTERED & 252 & PRIEST RAPIDS-RIVER & \\
\hline 1655 & & RIVER & & CUMULATIVE & UNFILTERED & 463 & RICH.PMPHS HRM 46.4 & \\
\hline 1656 & & RIVER & & RAW GRAB & TRANSECT & 317 & VERNITA-1 HRM 0.3 & \\
\hline 1657 & & RIVER & & RAW GRAB & TRANSECT & 570 & VERNITA-2 HRM 0.3 & \\
\hline 1658 & & RIVER & & RAW GRAB & TRANSECT & 318 & VERNITA-3 HRM 0.3 & \\
\hline 1659 & & RIVER & & RAW GRAB & TRANSECT & 319 & VERNITA-4 HRM 0.3 & \\
\hline 1660 & & RIVER & & RAW GRAB & TRANSECT & 571 & RICH.PMPHS-1 HRM46.4 & \\
\hline 1661 & & RIVER & & RAW GRAB & TRANSECT & 571 & RICH.PMPHS-1 HRM46.4 & \\
\hline 1662 & & RIVER & & RAW GRAB & TRANSECT & 321 & RICH.PMPHS-2 HRM46.4 & \\
\hline 1663 & & RIVER & & RAW GRAB & TRANSECT & 322 & RICH.PMPHS-3 HRM46.4 & \\
\hline 1664 & & RIVER & & RAW GRAB & TRANSECT & 323 & RICH.PMPHS-5 HRM46.4 & \\
\hline 1665 & & RIVER & & RAW GRAB & TRANSECT & 325 & RICH.PMPHS-7 HRM46.4 & \\
\hline 1666 & & RIVER & & RAW GRAB & TRANSECT & 327 & RICH.PMPHS-10 HRM46.4 & \\
\hline 1667 & & RIVER & & RAW GRAB & TRANSECT & 8220 & RICH.PMPHS HRM 45.8 & \\
\hline 1668 & & RIVER & & RAW GRAB & TRANSECT & 8221 & RICH.PMPHS HRM 45.0 & \\
\hline 1669 & & RIVER & & RAW GRAB & TRANSECT & 8222 & RICH.PMPHS HRM 43.9 & \\
\hline 1670 & & RIVER & & RAW GRAB & TRANSECT & 8223 & RICH.PMPHS HRM 43.5 & \\
\hline 1671 & & RIVER & & CUMULATIVE & UNFILTERED & 252 & PRIEST RAPIDS-RIVER & \\
\hline 1672 & & RIVER & & CUMULATIVE & UNFILTERED & 463 & RICH.PMPHS HRM 46.4 & \\
\hline 1673 & & RIVER & & CUMULATIVE & UNFILTERED & 252 & PRIEST RAPIDS-RIVER & \\
\hline
\end{tabular}




\begin{tabular}{|c|c|c|c|c|c|c|c|c|}
\hline & $T$ & $\bar{U}$ & $\mathrm{~V}$ & $\mathrm{~W}$ & $\bar{x}$ & $\bar{Y}$ & $\mathrm{Z}$ & AA \\
\hline 1 & REVIEW_QUALIFIER & SAMP_FROM & SAMP ITEM & SAMP_MTHD & COLL_MTHD & SAMP_SITE_ID & SAMP_SITE_NAME & WELL_NAME \\
\hline 1674 & & RIVER & & CUMULATIVE & UNFILTERED & 463 & RICH.PMPHS HRM 46.4 & \\
\hline 1675 & $F$ & SURFACE & & RAW GRAB & UNFILTERED & 147 & WEST LAKE & \\
\hline 1676 & & RIVER & & RAW GRAB & TRANSECT & 571 & RICH.PMPHS-1 HRM46.4 & \\
\hline 1677 & & RIVER & & RAW GRAB & TRANSECT & 571 & RICH.PMPHS-1 HRM46.4 & \\
\hline 1678 & & RIVER & & RAW GRAB & TRANSECT & 321 & RICH.PMPHS-2 HRM46.4 & \\
\hline 1679 & & RIVER & & RAW GRAB & TRANSECT & 322 & RICH.PMPHS-3 HRM46.4 & \\
\hline 1680 & & RIVER & & RAW GRAB & TRANSECT & 323 & RICH.PMPHS-5 HRM46.4 & \\
\hline 1681 & & RIVER & & RAW GRAB & TRANSECT & 325 & RICH.PMPHS-7 HRM46.4 & \\
\hline 1682 & $\mathrm{~F}$ & RIVER & & RAW GRAB & TRANSECT & 327 & RICH.PMPHS-10 HRM46.4 & \\
\hline 1683 & & RIVER & & RAW GRAB & TRANSECT & 8220 & RICH.PMPHS HRM 45.8 & \\
\hline 1684 & & RIVER & & RAW GRAB & TRANSECT & 8221 & RICH.PMPHS HRM 45.0 & \\
\hline 1685 & & RIVER & & RAW GRAB & TRANSECT & 8222 & RICH.PMPHS HRM 43.9 & \\
\hline 1686 & & RIVER & & RAW GRAB & TRANSECT & 8223 & RICH.PMPHS HRM 43.5 & \\
\hline 1687 & & RIVER & & RAW GRAB & TRANSECT & 317 & VERNITA-1 HRM 0.3 & \\
\hline 1688 & & RIVER & & RAW GRAB & TRANSECT & 570 & VERNITA-2 HRM 0.3 & \\
\hline 1689 & & RIVER & & RAW GRAB & TRANSECT & 318 & VERNITA-3 HRM 0.3 & \\
\hline 1690 & & RIVER & & RAW GRAB & TRANSECT & 319 & VERNITA-4 HRM 0.3 & \\
\hline 1691 & & RIVER & & CUMULATIVE & UNFILTERED & 252 & PRIEST RAPIDS-RIVER & \\
\hline 1692 & & RIVER & & CUMULATIVE & UNFILTERED & 463 & RICH.PMPHS HRM 46.4 & \\
\hline 1693 & $F$ & SURFACE & & RAW GRAB & UNFILTERED & 147 & WEST LAKE & \\
\hline 1694 & & RIVER & & CUMULATIVE & UNFILTERED & 252 & PRIEST RAPIDS-RIVER & \\
\hline 1695 & & RIVER & & CUMULATIVE & UNFILTERED & 463 & 3 RICH.PMPHS HRM 46.4 & \\
\hline 1696 & $F$ & SEEP & & RAW GRAB & UNFILTERED & 151 & HANFORD SPRING 28-2 & \\
\hline 1697 & $F$ & SEEP & & RAW GRAB & UNFILTERED & 8165 & 100-H SPRING 145-1 & \\
\hline 1698 & $F$ & SEEP & & RAW GRAB & UNFILTERED & 8147 & 100-F SPRING 207-1 & \\
\hline 1699 & $F$ & SEEP & & RAW GRAB & UNFILTERED & 8143 & 3 HANFORD SPR DR 28-2 & \\
\hline 1700 & $F$ & SEEP & & RAW GRAB & UNFILTERED & 8164 & 100-H SPRING 153-1 & \\
\hline 1701 & & RIVER & & CUMULATIVE & UNFILTERED & 463 & RICH.PMPHS HRM 46.4 & \\
\hline 1702 & & RIVER & & CUMULATIVE & UNFILTERED & 252 & PRIEST RAPIDS-RIVER & \\
\hline 1703 & $F$ & SEEP & & RAW GRAB & UNFILTERED & 8144 & 300 AREA SPR DR 42-2 & \\
\hline 1704 & $F$ & IRRIGATION & & RAW GRAB & UNFILTERED & 440 & RIVERVIEW CANAL & \\
\hline 1705 & & IRRIGATION & & RAW GRAB & UNFILTERED & 605 & HORN RAPIDS AREA & \\
\hline 1706 & $F$ & SEEP & & RAW GRAB & UNFILTERED & 488 & 300 AREA SPRING 42-2 & \\
\hline 1707 & & SEEP & & RAW GRAB & UNFILTERED & 9147 & RICHLAND SPR HRM44.4 & \\
\hline 1708 & & RIVER & & CUMULATIVE & UNFILTERED & 463 & RICH.PMPHS HRM 46.4 & \\
\hline 1709 & & RIVER & & CUMULATIVE & UNFILTERED & 252 & PRIEST RAPIDS-RIVER & \\
\hline 1710 & & IRRIGATION & & RAW GRAB & UNFILTERED & 440 & RIVERVIEW CANAL & \\
\hline 1711 & & IRRIGATION & & RAW GRAB & UNFILTERED & 605 & HORN RAPIDS AREA & \\
\hline
\end{tabular}




\begin{tabular}{|c|c|c|c|c|c|c|c|c|}
\hline & $T$ & $\bar{U}$ & $\mathrm{~V}$ & $\mathrm{~W}$ & $\bar{x}$ & $\bar{Y}$ & $\mathrm{Z}$ & AA \\
\hline 1 & REVIEW_QUALIFIER & SAMP_FROM & SAMP ITEM & SAMP_MTHD & COLL_MTHD & SAMP_SITE_ID & SAMP_SITE_NAME & WELL_NAME \\
\hline 1712 & & RIVER & & RAW GRAB & TRANSECT & 571 & RICH.PMPHS-1 HRM46.4 & \\
\hline 1713 & & RIVER & & RAW GRAB & TRANSECT & 321 & RICH.PMPHS-2 HRM46.4 & \\
\hline 1714 & & RIVER & & RAW GRAB & TRANSECT & 322 & 2 RICH.PMPHS-3 HRM46.4 & \\
\hline 1715 & & RIVER & & RAW GRAB & TRANSECT & 323 & 3 RICH.PMPHS-5 HRM46.4 & \\
\hline 1716 & & RIVER & & RAW GRAB & TRANSECT & 325 & 5 RICH.PMPHS-7 HRM46.4 & \\
\hline 1717 & & RIVER & & RAW GRAB & TRANSECT & 327 & 7 RICH.PMPHS-10 HRM46.4 & \\
\hline 1718 & & RIVER & & RAW GRAB & TRANSECT & 8220 & RICH.PMPHS HRM 45.8 & \\
\hline 1719 & & RIVER & & RAW GRAB & TRANSECT & 8221 & RICH.PMPHS HRM 45.0 & \\
\hline 1720 & & RIVER & & RAW GRAB & TRANSECT & 8222 & 2 RICH.PMPHS HRM 43.9 & \\
\hline 1721 & & RIVER & & RAW GRAB & TRANSECT & 8223 & 3 RICH.PMPHS HRM 43.5 & \\
\hline 1722 & & RIVER & & RAW GRAB & TRANSECT & 317 & 7 VERNITA-1 HRM 0.3 & \\
\hline 1723 & & RIVER & & RAW GRAB & TRANSECT & 570 & VERNITA-2 HRM 0.3 & \\
\hline 1724 & & RIVER & & RAW GRAB & TRANSECT & 318 & 3 VERNITA-3 HRM 0.3 & \\
\hline 1725 & & RIVER & & RAW GRAB & TRANSECT & 319 & 9 VERNITA-4 HRM 0.3 & \\
\hline 1726 & & RIVER & & CUMULATIVE & UNFILTERED & 463 & 3 RICH.PMPHS HRM 46.4 & \\
\hline 1727 & & RIVER & & CUMULATIVE & UNFILTERED & 252 & PRIEST RAPIDS-RIVER & \\
\hline 1728 & $F$ & SURFACE & & RAW GRAB & UNFILTERED & 147 & 7 WEST LAKE & \\
\hline 1729 & & IRRIGATION & & RAW GRAB & UNFILTERED & 440 & RIVERVIEW CANAL & \\
\hline 1730 & & IRRIGATION & & RAW GRAB & UNFILTERED & 605 & 5 HORN RAPIDS AREA & \\
\hline 1731 & & RIVER & & CUMULATIVE & UNFILTERED & 463 & 3 RICH.PMPHS HRM 46.4 & \\
\hline 1732 & & RIVER & & CUMULATIVE & UNFILTERED & 252 & PRIEST RAPIDS-RIVER & \\
\hline 1733 & $F$ & SEEP & & RAW GRAB & UNFILTERED & 488 & 300 AREA SPRING 42-2 & \\
\hline 1734 & $F$ & SEEP & & RAW GRAB & UNFILTERED & 8144 & 300 AREA SPR DR 42-2 & \\
\hline 1735 & & RIVER & & RAW GRAB & & 9236 & VERNITA BRIDGE -1 & \\
\hline 1736 & & SEEP & & RAW GRAB & & 488 & 300 AREA SPRING 42-2 & \\
\hline 1737 & & SEEP & & RAW GRAB & & 8144 & 300 AREA SPR DR 42-2 & \\
\hline 1738 & & RIVER & & RAW GRAB & & 9237 & 7 VERNITA BRIDGE -2 & \\
\hline 1739 & & RIVER & & RAW GRAB & UNFILTERED & 9236 & VERNITA BRIDGE -1 & \\
\hline 1740 & & RIVER & & RAW GRAB & UNFILTERED & 9237 & 7 VERNITA BRIDGE -2 & \\
\hline 1741 & & RIVER & & RAW GRAB & UNFILTERED & 9238 & 3 VERNITA BRIDGE -3 & \\
\hline 1742 & $F$ & RIVER & & RAW GRAB & UNFILTERED & 9210 & 300 SPR $7-1$ & \\
\hline 1743 & $F$ & RIVER & & RAW GRAB & UNFILTERED & 9211 & 300 SPR $7-2$ & \\
\hline 1744 & $F$ & RIVER & & RAW GRAB & UNFILTERED & 9213 & 300 SPR $7-3$ & \\
\hline 1745 & $F$ & RIVER & & RAW GRAB & UNFILTERED & 9214 & 4300 SPR DR $7-1$ & \\
\hline 1746 & $F$ & RIVER & & RAW GRAB & UNFILTERED & 9215 & 5300 SPR DR 7 -2 & \\
\hline 1747 & $F$ & RIVER & & RAW GRAB & UNFILTERED & 9216 & 300 SPR DR $7-3$ & \\
\hline 1748 & $F$ & RIVER & & RAW GRAB & UNFILTERED & 9217 & 7300 SPR 7 THRU SPR 9 & \\
\hline 1749 & $F$ & RIVER & & RAW GRAB & UNFILTERED & 9218 & 300 SPR $9-1$ & \\
\hline
\end{tabular}




\begin{tabular}{|c|c|c|c|c|c|c|c|c|}
\hline & $\mathrm{T}$ & $U$ & $\mathrm{~V}$ & $\mathrm{~W}$ & $\mathrm{X}$ & $\mathrm{Y}$ & Z & AA \\
\hline 1 & REVIEW_QUALIFIER & SAMP_FROM & SAMP_ITEM & SAMP_MTHD & COLL_MTHD & SAMP_SITE_ID & SAMP_SITE_NAME & WELL_NAME \\
\hline 1750 & $\mathrm{~F}$ & RIVER & & RAW GRAB & UNFILTERED & 9219 & 300 SPR $9-2$ & \\
\hline 1751 & & RIVER & & RAW GRAB & UNFILTERED & 9220 & 300 SPR $9-3$ & \\
\hline 1752 & $\mathrm{~F}$ & RIVER & & RAW GRAB & UNFILTERED & 9221 & 300 SPR DR 9 -1 & \\
\hline 1753 & $\mathrm{~F}$ & RIVER & & RAW GRAB & UNFILTERED & 9222 & 300 SPR DR $9-2$ & \\
\hline 1754 & $\mathrm{~F}$ & RIVER & & RAW GRAB & UNFILTERED & 9223 & 300 SPR DR $9-3$ & \\
\hline 1755 & $\mathrm{~F}$ & RIVER & & RAW GRAB & UNFILTERED & 9224 & 300 SPR 9 THRU SPR 11 & \\
\hline 1756 & $\mathrm{~F}$ & RIVER & & RAW GRAB & UNFILTERED & 9226 & 300 SPR $11-1$ & \\
\hline 1757 & $\mathrm{~F}$ & RIVER & & RAW GRAB & UNFILTERED & 9227 & 300 SPR $11-2$ & \\
\hline 1758 & $\mathrm{~F}$ & RIVER & & RAW GRAB & UNFILTERED & 9228 & 300 SPR $11-3$ & \\
\hline 1759 & $\mathrm{~F}$ & RIVER & & RAW GRAB & UNFILTERED & 9229 & 300 SPR DR $11-1$ & \\
\hline 1760 & $\mathrm{~F}$ & RIVER & & RAW GRAB & UNFILTERED & 9230 & 300 SPR DR $11-2$ & \\
\hline 1761 & $\mathrm{~F}$ & RIVER & & RAW GRAB & UNFILTERED & 9231 & 300 SPR DR $11-3$ & \\
\hline 1762 & $\mathrm{~F}$ & RIVER & & RAW GRAB & UNFILTERED & 9233 & 300 SPR $14-1$ & \\
\hline 1763 & $\mathrm{~F}$ & RIVER & & RAW GRAB & UNFILTERED & 9234 & 300 SPR $14-2$ & \\
\hline 1764 & $\mathrm{~F}$ & RIVER & & RAW GRAB & UNFILTERED & 9235 & 300 SPR $14-3$ & \\
\hline 1765 & & RIVER & & RAW GRAB & UNFILTERED & 9280 & VERNITA BRIDGE -4 & \\
\hline 1766 & $\mathrm{~F}$ & RIVER & & RAW GRAB & UNFILTERED & 9273 & 300 SPR $7-4$ & \\
\hline 1767 & & RIVER & & RAW GRAB & UNFILTERED & 9274 & 300 SPR DR $7-4$ & \\
\hline 1768 & & RIVER & & RAW GRAB & UNFILTERED & 9275 & 300 SPR $9-4$ & \\
\hline 1769 & & RIVER & & RAW GRAB & UNFILTERED & 9276 & 300 SPR DR $9-4$ & \\
\hline 1770 & $\mathrm{~F}$ & RIVER & & RAW GRAB & UNFILTERED & 9277 & 300 SPR $11-4$ & \\
\hline 1771 & & RIVER & & RAW GRAB & UNFILTERED & 9278 & 300 SPR DR $11-4$ & \\
\hline 1772 & & RIVER & & RAW GRAB & UNFILTERED & 9279 & 300 SPR $14-4$ & \\
\hline 1773 & & RIVER & & CUMULATIVE & UNFILTERED & 463 & RICH.PMPHS HRM 46.4 & \\
\hline 1774 & & RIVER & & CUMULATIVE & UNFILTERED & 252 & PRIEST RAPIDS-RIVER & \\
\hline 1775 & & RIVER & & RAW GRAB & TRANSECT & 328 & 100 N -1 HRM 9.5 & \\
\hline 1776 & & RIVER & & RAW GRAB & TRANSECT & 317 & VERNITA-1 HRM 0.3 & \\
\hline 1777 & & RIVER & & RAW GRAB & TRANSECT & 570 & VERNITA-2 HRM 0.3 & \\
\hline 1778 & & RIVER & & RAW GRAB & TRANSECT & 318 & VERNITA-3 HRM 0.3 & \\
\hline 1779 & & RIVER & & RAW GRAB & TRANSECT & 319 & VERNITA-4 HRM 0.3 & \\
\hline 1780 & & RIVER & & RAW GRAB & TRANSECT & 328 & 100 N -1 HRM 9.5 & \\
\hline 1781 & & RIVER & & RAW GRAB & TRANSECT & 329 & 100 N -2 HRM 9.5 & \\
\hline 1782 & & RIVER & & RAW GRAB & TRANSECT & 331 & 100 N -5 HRM 9.5 & \\
\hline 1783 & & RIVER & & RAW GRAB & TRANSECT & 335 & 100 N -10 HRM 9.5 & \\
\hline 1784 & & RIVER & & RAW GRAB & TRANSECT & 330 & 100 N -3 HRM 9.5 & \\
\hline 1785 & & RIVER & & RAW GRAB & TRANSECT & 333 & 100 N -7 HRM 9.5 & \\
\hline 1786 & & RIVER & & RAW GRAB & TRANSECT & 8244 & 100 N SHORE HRM 8.4 & \\
\hline 1787 & & RIVER & & RAW GRAB & TRANSECT & 8245 & 100 N SHORE HRM 8.9 & \\
\hline
\end{tabular}




\begin{tabular}{|c|c|c|c|c|c|c|c|c|}
\hline & $T$ & $\bar{U}$ & $\overline{\mathrm{V}}$ & $\mathrm{W}$ & $\bar{x}$ & $\bar{Y}$ & Z & AA \\
\hline 1 & REVIEW_QUALIFIER & SAMP_FROM & SAMP_ITEM & SAMP_MTHD & COLL_MTHD & SAMP_SITE_ID & SAMP_SITE_NAME & WELL_NAME \\
\hline 1788 & & RIVER & & RAW GRAB & TRANSECT & $\overline{8246}$ & 100 N SHORE HRM 9.2 & \\
\hline 1789 & & RIVER & & RAW GRAB & TRANSECT & 8247 & 100 N SHORE HRM 9.8 & \\
\hline 1790 & & RIVER & & RAW GRAB & TRANSECT & 336 & 100 F -1 HRM 19.0 & \\
\hline 1791 & & RIVER & & RAW GRAB & TRANSECT & 337 & 100 F -3 HRM 19.0 & \\
\hline 1792 & & RIVER & & RAW GRAB & TRANSECT & 344 & HANFRD TS-5 HRM 28.7 & \\
\hline 1793 & & RIVER & & RAW GRAB & TRANSECT & 576 & 100 F -2 HRM 19.0 & \\
\hline 1794 & & RIVER & & RAW GRAB & TRANSECT & 577 & 100 F -5 HRM 19.0 & \\
\hline 1795 & & RIVER & & RAW GRAB & TRANSECT & 339 & 100 F -7 HRM 19.0 & \\
\hline 1796 & & RIVER & & RAW GRAB & TRANSECT & 341 & 100 F - 10 HRM 19.0 & \\
\hline 1797 & & RIVER & & RAW GRAB & TRANSECT & 578 & HANFRD TS-1 HRM 28.7 & \\
\hline 1798 & & RIVER & & RAW GRAB & TRANSECT & 342 & HANFRD TS-2 HRM 28.7 & \\
\hline 1799 & & RIVER & & RAW GRAB & TRANSECT & 469 & HANFRD TS-7 HRM 28.7 & \\
\hline 1800 & & RIVER & & RAW GRAB & TRANSECT & 347 & HANFRD TS-10 HRM 28.7 & \\
\hline 1801 & & RIVER & & RAW GRAB & TRANSECT & 468 & 3 HANFRD TS-3 HRM 28.7 & \\
\hline 1802 & & RIVER & & RAW GRAB & TRANSECT & 8100 & HANFRD TWNSITE HRM26 & \\
\hline 1803 & & RIVER & & RAW GRAB & TRANSECT & 8101 & HANFRD TWNSITE HRM27 & \\
\hline 1804 & & RIVER & & RAW GRAB & TRANSECT & 8103 & 3 HANFRD TWNSITE HRM30 & \\
\hline 1805 & & RIVER & & RAW GRAB & TRANSECT & 274 & HANFRD TWNSITE HRM28 & \\
\hline 1806 & & RIVER & & RAW GRAB & TRANSECT & 8726 & 100 F SHORE HRM 18 & \\
\hline 1807 & & RIVER & & RAW GRAB & TRANSECT & 8728 & 100 F SHORE HRM 23 & \\
\hline 1808 & & RIVER & & RAW GRAB & TRANSECT & 348 & 300 AREA -1 HRM 43.1 & \\
\hline 1809 & & RIVER & & RAW GRAB & TRANSECT & 470 & 300 AREA -2 HRM 43.1 & \\
\hline 1810 & & RIVER & & RAW GRAB & TRANSECT & 580 & 300 AREA -3 HRM 43.1 & \\
\hline 1811 & & RIVER & & RAW GRAB & TRANSECT & 607 & 300 AREA -5 HRM 43.1 & \\
\hline 1812 & & RIVER & & RAW GRAB & TRANSECT & 351 & 300 AREA -7 HRM 43.1 & \\
\hline 1813 & $\mathrm{~F}$ & RIVER & & RAW GRAB & TRANSECT & 352 & 300 AREA-10 HRM 43.1 & \\
\hline 1814 & & RIVER & & RAW GRAB & TRANSECT & 571 & RICH.PMPHS-1 HRM46.4 & \\
\hline 1815 & & RIVER & & RAW GRAB & TRANSECT & 321 & RICH.PMPHS-2 HRM46.4 & \\
\hline 1816 & & RIVER & & RAW GRAB & TRANSECT & 322 & RICH.PMPHS-3 HRM46.4 & \\
\hline 1817 & & RIVER & & RAW GRAB & TRANSECT & 323 & RICH.PMPHS-5 HRM46.4 & \\
\hline 1818 & & RIVER & & RAW GRAB & TRANSECT & 325 & RICH.PMPHS-7 HRM46.4 & \\
\hline 1819 & $\mathrm{~F}$ & RIVER & & RAW GRAB & TRANSECT & 327 & RICH.PMPHS-10 HRM46.4 & \\
\hline 1820 & & RIVER & & RAW GRAB & TRANSECT & 8220 & RICH.PMPHS HRM 45.8 & \\
\hline 1821 & & RIVER & & RAW GRAB & TRANSECT & 8221 & RICH.PMPHS HRM 45.0 & \\
\hline 1822 & & RIVER & & RAW GRAB & TRANSECT & 8222 & RICH.PMPHS HRM 43.9 & \\
\hline 1823 & & RIVER & & RAW GRAB & TRANSECT & 8223 & RICH.PMPHS HRM 43.5 & \\
\hline 1824 & & RIVER & & RAW GRAB & TRANSECT & 8240 & 300 AREA SHR HRM41.5 & \\
\hline 1825 & & RIVER & & RAW GRAB & TRANSECT & 8241 & 300 AREA SHR HRM42.1 & \\
\hline
\end{tabular}




\begin{tabular}{|c|c|c|c|c|c|c|c|c|}
\hline & $\mathrm{T}$ & $U$ & $\mathrm{~V}$ & $\mathrm{~W}$ & $\mathrm{X}$ & $\mathrm{Y}$ & Z & AA \\
\hline 1 & REVIEW_QUALIFIER & SAMP_FROM & SAMP_ITEM & SAMP_MTHD & COLL_MTHD & SAMP_SITE_ID & SAMP_SITE_NAME & WELL_NAME \\
\hline 1826 & & RIVER & & RAW GRAB & TRANSECT & 8242 & 300 AREA SHR HRM42.5 & \\
\hline 1827 & & RIVER & & RAW GRAB & TRANSECT & 8243 & 300 AREA SHR HRM42.9 & \\
\hline 1828 & $\mathrm{~F}$ & SEEP & & RAW GRAB & UNFILTERED & 8144 & 300 AREA SPR DR 42-2 & \\
\hline 1829 & $\mathrm{~F}$ & DRIVE POINT & & RAW GRAB & UNFILTERED & 9539 & 300 SPR 9 -1 (2 FT) & \\
\hline 1830 & $\mathrm{~F}$ & DRIVE POINT & & RAW GRAB & UNFILTERED & 9540 & 300 SPR 9 -1 (4 FT) & \\
\hline 1831 & $\mathrm{~F}$ & DRIVE POINT & & RAW GRAB & UNFILTERED & 9541 & 300 SPR $9-2$ (2 FT) & \\
\hline 1832 & $\mathrm{~F}$ & DRIVE POINT & & RAW GRAB & UNFILTERED & 9542 & 300 SPR $9-2$ (4.5FT) & \\
\hline 1833 & $\mathrm{~F}$ & DRIVE POINT & & RAW GRAB & UNFILTERED & 9543 & 300 SPR 9 -3 (5.5FT) & \\
\hline 1834 & $\mathrm{~F}$ & SEEP & & RAW GRAB & UNFILTERED & 488 & 300 AREA SPRING $42-2$ & \\
\hline 1835 & $\mathrm{~F}$ & DRIVE POINT & & RAW GRAB & UNFILTERED & 9533 & 300 SPR 7 -1 (2.5FT) & \\
\hline 1836 & $\mathrm{~F}$ & DRIVE POINT & & RAW GRAB & UNFILTERED & 9534 & 300 SPR 7 -1 (4 FT) & \\
\hline 1837 & $\mathrm{~F}$ & DRIVE POINT & & RAW GRAB & UNFILTERED & 9535 & 300 SPR 7 -1 (6 FT) & \\
\hline 1838 & $\mathrm{~F}$ & DRIVE POINT & & RAW GRAB & UNFILTERED & 9536 & 300 SPR 7 -2 (2 FT) & \\
\hline 1839 & $\mathrm{~F}$ & DRIVE POINT & & RAW GRAB & UNFILTERED & 9537 & 300 SPR 7 -2 (4.2FT) & \\
\hline 1840 & & RIVER & & CUMULATIVE & UNFILTERED & 252 & PRIEST RAPIDS-RIVER & \\
\hline 1841 & & RIVER & & CUMULATIVE & UNFILTERED & 463 & RICH.PMPHS HRM 46.4 & \\
\hline 1842 & & SEEP & & RAW GRAB & UNFILTERED & 8147 & 100-F SPRING 207-1 & \\
\hline 1843 & & SEEP & & RAW GRAB & UNFILTERED & 8165 & 100-H SPRING 145-1 & \\
\hline 1844 & & SEEP & & RAW GRAB & UNFILTERED & 8846 & 100-H SPRING 152-2 & \\
\hline 1845 & & RIVER & & CUMULATIVE & UNFILTERED & 463 & RICH.PMPHS HRM 46.4 & \\
\hline 1846 & & RIVER & & CUMULATIVE & UNFILTERED & 252 & PRIEST RAPIDS-RIVER & \\
\hline 1847 & & SEEP & & RAW GRAB & UNFILTERED & 9236 & VERNITA BRIDGE -1 & \\
\hline 1848 & & RIVER & & CUMULATIVE & UNFILTERED & 252 & PRIEST RAPIDS-RIVER & \\
\hline 1849 & & RIVER & & CUMULATIVE & UNFILTERED & 463 & RICH.PMPHS HRM 46.4 & \\
\hline 1850 & & RIVER & & RAW GRAB & TRANSECT & 317 & VERNITA-1 HRM 0.3 & \\
\hline 1851 & & RIVER & & RAW GRAB & TRANSECT & 570 & VERNITA-2 HRM 0.3 & \\
\hline 1852 & & RIVER & & RAW GRAB & TRANSECT & 318 & VERNITA-3 HRM 0.3 & \\
\hline 1853 & & RIVER & & RAW GRAB & TRANSECT & 319 & VERNITA-4 HRM 0.3 & \\
\hline 1854 & & RIVER & & RAW GRAB & TRANSECT & 571 & RICH.PMPHS-1 HRM46.4 & \\
\hline 1855 & & RIVER & & RAW GRAB & TRANSECT & 571 & RICH.PMPHS-1 HRM46.4 & \\
\hline 1856 & & RIVER & & RAW GRAB & TRANSECT & 321 & RICH.PMPHS-2 HRM46.4 & \\
\hline 1857 & & RIVER & & RAW GRAB & TRANSECT & 322 & RICH.PMPHS-3 HRM46.4 & \\
\hline 1858 & & RIVER & & RAW GRAB & TRANSECT & 323 & RICH.PMPHS-5 HRM46.4 & \\
\hline 1859 & & RIVER & & RAW GRAB & TRANSECT & 325 & RICH.PMPHS-7 HRM46.4 & \\
\hline 1860 & $\mathrm{~F}$ & RIVER & & RAW GRAB & TRANSECT & 327 & RICH.PMPHS-10 HRM46.4 & \\
\hline 1861 & & RIVER & & RAW GRAB & TRANSECT & 8220 & RICH.PMPHS HRM 45.8 & \\
\hline 1862 & & RIVER & & RAW GRAB & TRANSECT & 8221 & RICH.PMPHS HRM 45.0 & \\
\hline 1863 & & RIVER & & RAW GRAB & TRANSECT & 8222 & RICH.PMPHS HRM 43.9 & \\
\hline
\end{tabular}




\begin{tabular}{|c|c|c|c|c|c|c|c|c|}
\hline & $\mathrm{T}$ & $U$ & $\mathrm{~V}$ & $\mathrm{~W}$ & $\mathrm{X}$ & $\mathrm{Y}$ & Z & AA \\
\hline 1 & REVIEW_QUALIFIER & SAMP_FROM & SAMP_ITEM & SAMP_MTHD & COLL_MTHD & SAMP_SITE_ID & SAMP_SITE_NAME & WELL_NAME \\
\hline 1864 & & RIVER & & RAW GRAB & TRANSECT & 8223 & RICH.PMPHS HRM 43.5 & \\
\hline 1865 & & RIVER & & CUMULATIVE & UNFILTERED & 463 & RICH.PMPHS HRM 46.4 & \\
\hline 1866 & & RIVER & & CUMULATIVE & UNFILTERED & 252 & PRIEST RAPIDS-RIVER & \\
\hline 1867 & & RIVER & & CUMULATIVE & UNFILTERED & 463 & RICH.PMPHS HRM 46.4 & \\
\hline 1868 & & RIVER & & CUMULATIVE & UNFILTERED & 252 & PRIEST RAPIDS-RIVER & \\
\hline 1869 & & RIVER & & CUMULATIVE & UNFILTERED & 252 & PRIEST RAPIDS-RIVER & \\
\hline 1870 & & RIVER & & CUMULATIVE & UNFILTERED & 463 & RICH.PMPHS HRM 46.4 & \\
\hline 1871 & & RIVER & & RAW GRAB & TRANSECT & 317 & VERNITA-1 HRM 0.3 & \\
\hline 1872 & & RIVER & & RAW GRAB & TRANSECT & 570 & VERNITA-2 HRM 0.3 & \\
\hline 1873 & & RIVER & & RAW GRAB & TRANSECT & 318 & VERNITA-3 HRM 0.3 & \\
\hline 1874 & & RIVER & & RAW GRAB & TRANSECT & 319 & VERNITA-4 HRM 0.3 & \\
\hline 1875 & $\mathrm{~F}$ & RIVER & & RAW GRAB & TRANSECT & 571 & RICH.PMPHS-1 HRM46.4 & \\
\hline 1876 & $\mathrm{~F}$ & RIVER & & RAW GRAB & TRANSECT & 571 & RICH.PMPHS-1 HRM46.4 & \\
\hline 1877 & & RIVER & & RAW GRAB & TRANSECT & 321 & RICH.PMPHS-2 HRM46.4 & \\
\hline 1878 & & RIVER & & RAW GRAB & TRANSECT & 322 & RICH.PMPHS-3 HRM46.4 & \\
\hline 1879 & & RIVER & & RAW GRAB & TRANSECT & 323 & RICH.PMPHS-5 HRM46.4 & \\
\hline 1880 & & RIVER & & RAW GRAB & TRANSECT & 325 & RICH.PMPHS-7 HRM46.4 & \\
\hline 1881 & $\mathrm{~F}$ & RIVER & & RAW GRAB & TRANSECT & 327 & RICH.PMPHS-10 HRM46.4 & \\
\hline 1882 & $\mathrm{~F}$ & RIVER & & RAW GRAB & TRANSECT & 8220 & RICH.PMPHS HRM 45.8 & \\
\hline 1883 & $\mathrm{~F}$ & RIVER & & RAW GRAB & TRANSECT & 8221 & RICH.PMPHS HRM 45.0 & \\
\hline 1884 & $\mathrm{~F}$ & RIVER & & RAW GRAB & TRANSECT & 8222 & RICH.PMPHS HRM 43.9 & \\
\hline 1885 & $\mathrm{~F}$ & RIVER & & RAW GRAB & TRANSECT & 8223 & RICH.PMPHS HRM 43.5 & \\
\hline 1886 & & RIVER & & CUMULATIVE & UNFILTERED & 252 & PRIEST RAPIDS-RIVER & \\
\hline 1887 & & RIVER & & CUMULATIVE & UNFILTERED & 463 & RICH.PMPHS HRM 46.4 & \\
\hline 1888 & & RIVER & & CUMULATIVE & UNFILTERED & 252 & PRIEST RAPIDS-RIVER & \\
\hline 1889 & & RIVER & & CUMULATIVE & UNFILTERED & 463 & RICH.PMPHS HRM 46.4 & \\
\hline 1890 & & IRRIGATION & & RAW GRAB & UNFILTERED & 440 & RIVERVIEW CANAL & \\
\hline 1891 & & IRRIGATION & & RAW GRAB & UNFILTERED & 605 & HORN RAPIDS AREA & \\
\hline 1892 & & RIVER & & CUMULATIVE & UNFILTERED & 252 & PRIEST RAPIDS-RIVER & \\
\hline 1893 & & RIVER & & CUMULATIVE & UNFILTERED & 463 & RICH.PMPHS HRM 46.4 & \\
\hline 1894 & & RIVER & & RAW GRAB & TRANSECT & 317 & VERNITA-1 HRM 0.3 & \\
\hline 1895 & & RIVER & & RAW GRAB & TRANSECT & 570 & VERNITA-2 HRM 0.3 & \\
\hline 1896 & & RIVER & & RAW GRAB & TRANSECT & 318 & VERNITA-3 HRM 0.3 & \\
\hline 1897 & & RIVER & & RAW GRAB & TRANSECT & 319 & VERNITA-4 HRM 0.3 & \\
\hline 1898 & & RIVER & & RAW GRAB & TRANSECT & 571 & RICH.PMPHS-1 HRM46.4 & \\
\hline 1899 & & RIVER & & RAW GRAB & TRANSECT & 321 & RICH.PMPHS-2 HRM46.4 & \\
\hline 1900 & & RIVER & & RAW GRAB & TRANSECT & 322 & RICH.PMPHS-3 HRM46.4 & \\
\hline 1901 & & RIVER & & RAW GRAB & TRANSECT & 323 & RICH.PMPHS-5 HRM46.4 & \\
\hline
\end{tabular}




\begin{tabular}{|c|c|c|c|c|c|c|c|c|}
\hline & $\mathrm{T}$ & $\bar{U}$ & $\mathrm{~V}$ & $\mathrm{~W}$ & $\mathrm{X}$ & $\bar{Y}$ & $Z$ & $\overline{A A}$ \\
\hline 1 & REVIEW_QUALIFIER & SAMP_FROM & SAMP_ITEM & SAMP_MTHD & COLL_MTHD & SAMP_SITE_ID & SAMP_SITE_NAME & WELL_NAME \\
\hline 1902 & & RIVER & & RAW GRAB & TRANSECT & 325 & RICH.PMPHS-7 HRM46.4 & \\
\hline 1903 & & RIVER & & RAW GRAB & TRANSECT & 327 & RICH.PMPHS-10 HRM46.4 & \\
\hline 1904 & & RIVER & & RAW GRAB & TRANSECT & 8220 & RICH.PMPHS HRM 45.8 & \\
\hline 1905 & & RIVER & & RAW GRAB & TRANSECT & 8221 & RICH.PMPHS HRM 45.0 & \\
\hline 1906 & & RIVER & & RAW GRAB & TRANSECT & 8222 & RICH.PMPHS HRM 43.9 & \\
\hline 1907 & & RIVER & & RAW GRAB & TRANSECT & 8223 & RICH.PMPHS HRM 43.5 & \\
\hline 1908 & & RIVER & & CUMULATIVE & UNFILTERED & 252 & PRIEST RAPIDS-RIVER & \\
\hline 1909 & & RIVER & & CUMULATIVE & UNFILTERED & 463 & RICH.PMPHS HRM 46.4 & \\
\hline 1910 & & IRRIGATION & & RAW GRAB & UNFILTERED & 440 & RIVERVIEW CANAL & \\
\hline 1911 & & IRRIGATION & & RAW GRAB & UNFILTERED & 605 & HORN RAPIDS AREA & \\
\hline 1912 & & IRRIGATION & & RAW GRAB & UNFILTERED & 440 & RIVERVIEW CANAL & \\
\hline 1913 & & IRRIGATION & & RAW GRAB & UNFILTERED & 605 & HORN RAPIDS AREA & \\
\hline 1914 & & RIVER & & CUMULATIVE & UNFILTERED & 252 & PRIEST RAPIDS-RIVER & \\
\hline 1915 & & RIVER & & CUMULATIVE & UNFILTERED & 463 & RICH.PMPHS HRM 46.4 & \\
\hline 1916 & & RIVER & & CUMULATIVE & UNFILTERED & 252 & PRIEST RAPIDS-RIVER & \\
\hline 1917 & & RIVER & & CUMULATIVE & UNFILTERED & 463 & RICH.PMPHS HRM 46.4 & \\
\hline 1918 & & RIVER & & RAW GRAB & TRANSECT & 328 & 100 N -1 HRM 9.5 & \\
\hline 1919 & & RIVER & & RAW GRAB & TRANSECT & 319 & VERNITA-4 HRM 0.3 & \\
\hline 1920 & & RIVER & & RAW GRAB & TRANSECT & 570 & VERNITA-2 HRM 0.3 & \\
\hline 1921 & & RIVER & & RAW GRAB & TRANSECT & 318 & VERNITA-3 HRM 0.3 & \\
\hline 1922 & & RIVER & & RAW GRAB & TRANSECT & 317 & VERNITA-1 HRM 0.3 & \\
\hline 1923 & & RIVER & & RAW GRAB & TRANSECT & 328 & 100 N -1 HRM 9.5 & \\
\hline 1924 & & RIVER & & RAW GRAB & TRANSECT & 329 & 100 N -2 HRM 9.5 & \\
\hline 1925 & & RIVER & & RAW GRAB & TRANSECT & 331 & 100 N -5 HRM 9.5 & \\
\hline 1926 & & RIVER & & RAW GRAB & TRANSECT & 335 & 100 N -10 HRM 9.5 & \\
\hline \begin{tabular}{|l|}
1927 \\
\end{tabular} & & RIVER & & RAW GRAB & TRANSECT & 330 & 100 N -3 HRM 9.5 & \\
\hline 1928 & & RIVER & & RAW GRAB & TRANSECT & 333 & 100 N -7 HRM 9.5 & \\
\hline 1929 & & RIVER & & RAW GRAB & TRANSECT & 8244 & 100 N SHORE HRM 8.4 & \\
\hline 1930 & & RIVER & & RAW GRAB & TRANSECT & 8245 & 100 N SHORE HRM 8.9 & \\
\hline \begin{tabular}{|l|}
1931 \\
\end{tabular} & & RIVER & & RAW GRAB & TRANSECT & 8246 & 100 N SHORE HRM 9.2 & \\
\hline 1932 & & RIVER & & RAW GRAB & TRANSECT & 8247 & 100 N SHORE HRM 9.8 & \\
\hline 1933 & & RIVER & & RAW GRAB & TRANSECT & 336 & 100 F -1 HRM 19.0 & \\
\hline 1934 & & RIVER & & RAW GRAB & TRANSECT & 337 & 100 F -3 HRM 19.0 & \\
\hline 1935 & & RIVER & & RAW GRAB & TRANSECT & 344 & HANFRD TS-5 HRM 28.7 & \\
\hline 1936 & & RIVER & & RAW GRAB & TRANSECT & 576 & 100 F -2 HRM 19.0 & \\
\hline 1937 & & RIVER & & RAW GRAB & TRANSECT & 577 & 100 F -5 HRM 19.0 & \\
\hline 1938 & & RIVER & & RAW GRAB & TRANSECT & 339 & 100 F -7 HRM 19.0 & \\
\hline \begin{tabular}{|l|l|}
1939 \\
\end{tabular} & & RIVER & & RAW GRAB & TRANSECT & 341 & $100 \mathrm{~F}-10 \mathrm{HRM} 19.0$ & \\
\hline
\end{tabular}




\begin{tabular}{|c|c|c|c|c|c|c|c|c|}
\hline & $\mathrm{T}$ & $U$ & $\mathrm{~V}$ & $\mathrm{~W}$ & $\mathrm{x}$ & $\bar{Y}$ & Z & AA \\
\hline 1 & REVIEW_QUALIFIER & SAMP_FROM & SAMP ITEM & SAMP_MTHD & COLL_MTHD & SAMP_SITE_ID & SAMP_SITE_NAME & WELL_NAME \\
\hline 1940 & & RIVER & & RAW GRAB & TRANSECT & 578 & 3 HANFRD TS-1 HRM 28.7 & \\
\hline 1941 & & RIVER & & RAW GRAB & TRANSECT & 342 & 2 HANFRD TS-2 HRM 28.7 & \\
\hline 1942 & & RIVER & & RAW GRAB & TRANSECT & 469 & 9 HANFRD TS-7 HRM 28.7 & \\
\hline 1943 & & RIVER & & RAW GRAB & TRANSECT & 347 & 7 HANFRD TS-10 HRM 28.7 & \\
\hline 1944 & & RIVER & & RAW GRAB & TRANSECT & 468 & 3 HANFRD TS-3 HRM 28.7 & \\
\hline 1945 & & RIVER & & RAW GRAB & TRANSECT & 8100 & HANFRD TWNSITE HRM26 & \\
\hline 1946 & & RIVER & & RAW GRAB & TRANSECT & 8101 & 1 HANFRD TWNSITE HRM27 & \\
\hline 1947 & & RIVER & & RAW GRAB & TRANSECT & 8103 & 3 HANFRD TWNSITE HRM30 & \\
\hline 1948 & $\mathrm{~F}$ & RIVER & & RAW GRAB & TRANSECT & 274 & 4 HANFRD TWNSITE HRM28 & \\
\hline 1949 & & RIVER & & RAW GRAB & TRANSECT & 8726 & 5100 F SHORE HRM 18 & \\
\hline 1950 & & RIVER & & RAW GRAB & TRANSECT & 13656 & 5100 F SHORE HRM 24 & \\
\hline 1951 & & RIVER & & RAW GRAB & TRANSECT & 8728 & 3100 F SHORE HRM 23 & \\
\hline 1952 & & RIVER & & RAW GRAB & TRANSECT & 348 & 300 AREA -1 HRM 43.1 & \\
\hline 1953 & & RIVER & & RAW GRAB & TRANSECT & 470 & 300 AREA -2 HRM 43.1 & \\
\hline 1954 & & RIVER & & RAW GRAB & TRANSECT & 580 & 300 AREA -3 HRM 43.1 & \\
\hline 1955 & & RIVER & & RAW GRAB & TRANSECT & 607 & 7300 AREA -5 HRM 43.1 & \\
\hline 1956 & & RIVER & & RAW GRAB & TRANSECT & 351 & 300 AREA - 7 HRM 43.1 & \\
\hline 1957 & $F$ & RIVER & & RAW GRAB & TRANSECT & 352 & 2300 AREA-10 HRM 43.1 & \\
\hline 1958 & & RIVER & & RAW GRAB & TRANSECT & 571 & RICH.PMPHS-1 HRM46.4 & \\
\hline 1959 & & RIVER & & RAW GRAB & TRANSECT & 321 & RICH.PMPHS-2 HRM46.4 & \\
\hline 1960 & & RIVER & & RAW GRAB & TRANSECT & 322 & 2 RICH.PMPHS-3 HRM46.4 & \\
\hline 1961 & & RIVER & & RAW GRAB & TRANSECT & 323 & 3 RICH.PMPHS-5 HRM46.4 & \\
\hline 1962 & & RIVER & & RAW GRAB & TRANSECT & 325 & 5 RICH.PMPHS-7 HRM46.4 & \\
\hline 1963 & & RIVER & & RAW GRAB & TRANSECT & 327 & 7 RICH.PMPHS-10 HRM46.4 & \\
\hline 1964 & & RIVER & & RAW GRAB & TRANSECT & 8220 & RICH.PMPHS HRM 45.8 & \\
\hline 1965 & & RIVER & & RAW GRAB & TRANSECT & 8221 & RICH.PMPHS HRM 45.0 & \\
\hline 1966 & & RIVER & & RAW GRAB & TRANSECT & 8222 & 2 RICH.PMPHS HRM 43.9 & \\
\hline 1967 & & RIVER & & RAW GRAB & TRANSECT & 8223 & 3 RICH.PMPHS HRM 43.5 & \\
\hline 1968 & & RIVER & & RAW GRAB & TRANSECT & 8240 & 300 AREA SHR HRM41.5 & \\
\hline 1969 & & RIVER & & RAW GRAB & TRANSECT & 8241 & 300 AREA SHR HRM42.1 & \\
\hline 1970 & & RIVER & & RAW GRAB & TRANSECT & 8242 & 2300 AREA SHR HRM42.5 & \\
\hline 1971 & & RIVER & & RAW GRAB & TRANSECT & 8243 & 3300 AREA SHR HRM42.9 & \\
\hline 1972 & & RIVER & & CUMULATIVE & UNFILTERED & 252 & PRIEST RAPIDS-RIVER & \\
\hline 1973 & & RIVER & & CUMULATIVE & UNFILTERED & 463 & 3 RICH.PMPHS HRM 46.4 & \\
\hline 1974 & & SEEP & & RAW GRAB & UNFILTERED & 151 & HANFORD SPRING 28-2 & \\
\hline 1975 & $\mathrm{~F}$ & SEEP & & RAW GRAB & UNFILTERED & 488 & 300 AREA SPRING 42-2 & \\
\hline 1976 & & SEEP & & RAW GRAB & UNFILTERED & 8143 & 3 HANFORD SPR DR 28-2 & \\
\hline 1977 & & SEEP & & RAW GRAB & UNFILTERED & 8846 & 100-H SPRING 152-2 & \\
\hline
\end{tabular}




\begin{tabular}{|c|c|c|c|c|c|c|c|c|}
\hline & $\mathrm{T}$ & $U$ & $\mathrm{~V}$ & $\mathrm{~W}$ & $\mathrm{x}$ & $\bar{Y}$ & $Z$ & AA \\
\hline 1 & REVIEW_QUALIFIER & SAMP_FROM & SAMP ITEM & SAMP_MTHD & COLL_MTHD & SAMP_SITE_ID & SAMP_SITE_NAME & WELL_NAME \\
\hline 1978 & & SEEP & & RAW GRAB & UNFILTERED & 8147 & $100-F$ SPRING 207-1 & \\
\hline 1979 & & RIVER & & CUMULATIVE & UNFILTERED & 252 & PRIEST RAPIDS-RIVER & \\
\hline 1980 & & RIVER & & CUMULATIVE & UNFILTERED & 463 & RICH.PMPHS HRM 46.4 & \\
\hline 1981 & & RIVER & & CUMULATIVE & UNFILTERED & 252 & PRIEST RAPIDS-RIVER & \\
\hline 1982 & & RIVER & & CUMULATIVE & UNFILTERED & 463 & RICH.PMPHS HRM 46.4 & \\
\hline 1983 & & RIVER & & RAW GRAB & TRANSECT & 317 & VERNITA-1 HRM 0.3 & \\
\hline 1984 & & RIVER & & RAW GRAB & TRANSECT & 570 & VERNITA-2 HRM 0.3 & \\
\hline 1985 & & RIVER & & RAW GRAB & TRANSECT & 318 & VERNITA-3 HRM 0.3 & \\
\hline 1986 & & RIVER & & RAW GRAB & TRANSECT & 319 & VERNITA-4 HRM 0.3 & \\
\hline 1987 & & RIVER & & RAW GRAB & TRANSECT & 571 & RICH.PMPHS-1 HRM46.4 & \\
\hline 1988 & & RIVER & & RAW GRAB & TRANSECT & 571 & RICH.PMPHS-1 HRM46.4 & \\
\hline 1989 & & RIVER & & RAW GRAB & TRANSECT & 321 & RICH.PMPHS-2 HRM46.4 & \\
\hline 1990 & & RIVER & & RAW GRAB & TRANSECT & 322 & RICH.PMPHS-3 HRM46.4 & \\
\hline 1991 & & RIVER & & RAW GRAB & TRANSECT & 323 & RICH.PMPHS-5 HRM46.4 & \\
\hline 1992 & & RIVER & & RAW GRAB & TRANSECT & 325 & RICH.PMPHS-7 HRM46.4 & \\
\hline 1993 & & RIVER & & RAW GRAB & TRANSECT & 327 & RICH.PMPHS-10 HRM46.4 & \\
\hline 1994 & & RIVER & & RAW GRAB & TRANSECT & 8220 & RICH.PMPHS HRM 45.8 & \\
\hline 1995 & & RIVER & & RAW GRAB & TRANSECT & 8221 & RICH.PMPHS HRM 45.0 & \\
\hline 1996 & & RIVER & & RAW GRAB & TRANSECT & 8222 & RICH.PMPHS HRM 43.9 & \\
\hline 1997 & & RIVER & & RAW GRAB & TRANSECT & 8223 & RICH.PMPHS HRM 43.5 & \\
\hline $1998 \mathrm{~F}$ & $\mathrm{~F}$ & SURFACE & & RAW GRAB & UNFILTERED & 147 & WEST LAKE & \\
\hline $1999 \mathrm{~F}$ & $F$ & RIVER & & CUMULATIVE & UNFILTERED & 463 & RICH.PMPHS HRM 46.4 & \\
\hline $2000 \mathrm{~F}$ & $F$ & RIVER & & CUMULATIVE & UNFILTERED & 252 & PRIEST RAPIDS-RIVER & \\
\hline 2001 & & RIVER & & CUMULATIVE & UNFILTERED & 463 & RICH.PMPHS HRM 46.4 & \\
\hline 2002 & & RIVER & & CUMULATIVE & UNFILTERED & 252 & PRIEST RAPIDS-RIVER & \\
\hline 2003 & & RIVER & & RAW GRAB & TRANSECT & 317 & VERNITA-1 HRM 0.3 & \\
\hline $2004 \mathrm{~F}$ & $F$ & RIVER & & RAW GRAB & TRANSECT & 570 & VERNITA-2 HRM 0.3 & \\
\hline 2005 & & RIVER & & RAW GRAB & TRANSECT & 318 & VERNITA-3 HRM 0.3 & \\
\hline 2006 & & RIVER & & RAW GRAB & TRANSECT & 319 & VERNITA-4 HRM 0.3 & \\
\hline $2007 \mathrm{~F}$ & $F$ & RIVER & & RAW GRAB & TRANSECT & 571 & RICH.PMPHS-1 HRM46.4 & \\
\hline 2008 & & RIVER & & RAW GRAB & TRANSECT & 571 & RICH.PMPHS-1 HRM46.4 & \\
\hline 2009 & & RIVER & & RAW GRAB & TRANSECT & 321 & RICH.PMPHS-2 HRM46.4 & \\
\hline $2010 \mathrm{~F}$ & $F$ & RIVER & & RAW GRAB & TRANSECT & 322 & RICH.PMPHS-3 HRM46.4 & \\
\hline 2011 & & RIVER & & RAW GRAB & TRANSECT & 323 & RICH.PMPHS-5 HRM46.4 & \\
\hline 2012 & & RIVER & & RAW GRAB & TRANSECT & 325 & RICH.PMPHS-7 HRM46.4 & \\
\hline 2013 & & RIVER & & RAW GRAB & TRANSECT & 327 & RICH.PMPHS-10 HRM46.4 & \\
\hline 2014 & & RIVER & & RAW GRAB & TRANSECT & 8220 & RICH.PMPHS HRM 45.8 & \\
\hline 2015 & & RIVER & & RAW GRAB & TRANSECT & 8221 & RICH.PMPHS HRM 45.0 & \\
\hline
\end{tabular}




\begin{tabular}{|c|c|c|c|c|c|c|c|c|}
\hline & $\mathrm{T}$ & $\bar{U}$ & $\mathrm{~V}$ & $\mathrm{~W}$ & $\mathrm{X}$ & $\bar{Y}$ & $Z$ & $\overline{A A}$ \\
\hline 1 & REVIEW_QUALIFIER & SAMP_FROM & SAMP_ITEM & SAMP_MTHD & COLL_MTHD & SAMP_SITE_ID & SAMP_SITE_NAME & WELL_NAME \\
\hline 2016 & & RIVER & & RAW GRAB & TRANSECT & 8222 & RICH.PMPHS HRM 43.9 & \\
\hline 2017 & & RIVER & & RAW GRAB & TRANSECT & 8223 & RICH.PMPHS HRM 43.5 & \\
\hline 2018 & $F$ & SURFACE & & RAW GRAB & UNFILTERED & 147 & WEST LAKE & \\
\hline 2019 & & RIVER & & CUMULATIVE & UNFILTERED & 463 & RICH.PMPHS HRM 46.4 & \\
\hline 2020 & & RIVER & & CUMULATIVE & UNFILTERED & 252 & PRIEST RAPIDS-RIVER & \\
\hline 2021 & & RIVER & & CUMULATIVE & UNFILTERED & 252 & PRIEST RAPIDS-RIVER & \\
\hline 2022 & & RIVER & & CUMULATIVE & UNFILTERED & 463 & RICH.PMPHS HRM 46.4 & \\
\hline 2023 & $F$ & IRRIGATION & & RAW GRAB & UNFILTERED & 440 & RIVERVIEW CANAL & \\
\hline 2024 & $F$ & RIVER & & CUMULATIVE & UNFILTERED & 252 & PRIEST RAPIDS-RIVER & \\
\hline 2025 & & RIVER & & CUMULATIVE & UNFILTERED & 463 & RICH.PMPHS HRM 46.4 & \\
\hline 2026 & $F$ & IRRIGATION & & RAW GRAB & UNFILTERED & 440 & RIVERVIEW CANAL & \\
\hline 2027 & $F$ & SEEP & & RAW GRAB & & 8626 & WEST LAKE SPRING & \\
\hline 2028 & $F$ & SURFACE & & RAW GRAB & UNFILTERED & 147 & WEST LAKE & \\
\hline 2029 & $F$ & SURFACE & & RAW GRAB & & 147 & WEST LAKE & \\
\hline 2030 & $F$ & RIVER & & RAW GRAB & TRANSECT & 317 & VERNITA-1 HRM 0.3 & \\
\hline 2031 & & RIVER & & RAW GRAB & TRANSECT & 570 & VERNITA-2 HRM 0.3 & \\
\hline 2032 & $F$ & RIVER & & RAW GRAB & TRANSECT & 318 & VERNITA-3 HRM 0.3 & \\
\hline 2033 & & RIVER & & RAW GRAB & TRANSECT & 319 & VERNITA-4 HRM 0.3 & \\
\hline 2034 & & RIVER & & RAW GRAB & TRANSECT & 571 & RICH.PMPHS-1 HRM46.4 & \\
\hline 2035 & & RIVER & & RAW GRAB & TRANSECT & 321 & RICH.PMPHS-2 HRM46.4 & \\
\hline 2036 & & RIVER & & RAW GRAB & TRANSECT & 322 & RICH.PMPHS-3 HRM46.4 & \\
\hline 2037 & & RIVER & & RAW GRAB & TRANSECT & 323 & RICH.PMPHS-5 HRM46.4 & \\
\hline 2038 & $F$ & RIVER & & RAW GRAB & TRANSECT & 325 & RICH.PMPHS-7 HRM46.4 & \\
\hline 2039 & $\mathrm{~F}$ & RIVER & & RAW GRAB & TRANSECT & 327 & RICH.PMPHS-10 HRM46.4 & \\
\hline 2040 & & RIVER & & RAW GRAB & TRANSECT & 8220 & RICH.PMPHS HRM 45.8 & \\
\hline 2041 & & RIVER & & RAW GRAB & TRANSECT & 8221 & RICH.PMPHS HRM 45.0 & \\
\hline 2042 & & RIVER & & RAW GRAB & TRANSECT & 8222 & RICH.PMPHS HRM 43.9 & \\
\hline 2043 & & RIVER & & RAW GRAB & TRANSECT & 8223 & RICH.PMPHS HRM 43.5 & \\
\hline 2044 & & RIVER & & CUMULATIVE & UNFILTERED & 252 & PRIEST RAPIDS-RIVER & \\
\hline 2045 & & RIVER & & CUMULATIVE & UNFILTERED & 463 & RICH.PMPHS HRM 46.4 & \\
\hline 2046 & $\mathrm{~F}$ & SURFACE & & RAW GRAB & UNFILTERED & 147 & WEST LAKE & \\
\hline 2047 & $F$ & IRRIGATION & & RAW GRAB & UNFILTERED & 440 & RIVERVIEW CANAL & \\
\hline 2048 & & RIVER & & CUMULATIVE & UNFILTERED & 252 & PRIEST RAPIDS-RIVER & \\
\hline 2049 & $\mathrm{~F}$ & RIVER & & CUMULATIVE & UNFILTERED & 463 & RICH.PMPHS HRM 46.4 & \\
\hline 2050 & & RIVER & & CUMULATIVE & UNFILTERED & 252 & PRIEST RAPIDS-RIVER & \\
\hline 2051 & & RIVER & & CUMULATIVE & UNFILTERED & 463 & RICH.PMPHS HRM 46.4 & \\
\hline 2052 & $F$ & RIVER & & RAW GRAB & TRANSECT & 328 & 100 N -1 HRM 9.5 & \\
\hline 2053 & $F$ & RIVER & & RAW GRAB & TRANSECT & 317 & VERNITA-1 HRM 0.3 & \\
\hline
\end{tabular}




\begin{tabular}{|c|c|c|c|c|c|c|c|c|}
\hline & $T$ & $\bar{U}$ & $\mathrm{~V}$ & $\mathrm{~W}$ & $\bar{X}$ & $\bar{Y}$ & $\bar{Z}$ & $\overline{A A}$ \\
\hline 1 & REVIEW_QUALIFIER & SAMP_FROM & SAMP_ITEM & SAMP_MTHD & COLL_MTHD & SAMP_SITE_ID & SAMP_SITE_NAME & WELL_NAME \\
\hline 2054 & & RIVER & & RAW GRAB & TRANSECT & 570 & VERNITA-2 HRM 0.3 & \\
\hline 2055 & & RIVER & & RAW GRAB & TRANSECT & 318 & VERNITA-3 HRM 0.3 & \\
\hline 2056 & $\mathrm{~F}$ & RIVER & & RAW GRAB & TRANSECT & 319 & VERNITA-4 HRM 0.3 & \\
\hline 2057 & & RIVER & & RAW GRAB & TRANSECT & 328 & 100 N -1 HRM 9.5 & \\
\hline 2058 & $\mathrm{~F}$ & RIVER & & RAW GRAB & TRANSECT & 329 & 100 N -2 HRM 9.5 & \\
\hline 2059 & & RIVER & & RAW GRAB & TRANSECT & 331 & 100 N -5 HRM 9.5 & \\
\hline 2060 & $\mathrm{~F}$ & RIVER & & RAW GRAB & TRANSECT & 335 & 100 N -10 HRM 9.5 & \\
\hline 2061 & $\mathrm{~F}$ & RIVER & & RAW GRAB & TRANSECT & 330 & 100 N -3 HRM 9.5 & \\
\hline 2062 & & RIVER & & RAW GRAB & TRANSECT & 333 & 100 N -7 HRM 9.5 & \\
\hline 2063 & $\mathrm{~F}$ & RIVER & & RAW GRAB & TRANSECT & 8244 & 100 N SHORE HRM 8.4 & \\
\hline 2064 & $\mathrm{~F}$ & RIVER & & RAW GRAB & TRANSECT & 8245 & 100 N SHORE HRM 8.9 & \\
\hline 2065 & $\mathrm{~F}$ & RIVER & & RAW GRAB & TRANSECT & 8246 & 100 N SHORE HRM 9.2 & \\
\hline 2066 & $\mathrm{~F}$ & RIVER & & RAW GRAB & TRANSECT & 8247 & 100 N SHORE HRM 9.8 & \\
\hline 2067 & $\mathrm{~F}$ & RIVER & & RAW GRAB & TRANSECT & 336 & 100 F -1 HRM 19.0 & \\
\hline 2068 & & RIVER & & RAW GRAB & TRANSECT & 337 & 100 F -3 HRM 19.0 & \\
\hline 2069 & $\mathrm{~F}$ & RIVER & & RAW GRAB & TRANSECT & 344 & HANFRD TS-5 HRM 28.7 & \\
\hline 2070 & $\mathrm{~F}$ & RIVER & & RAW GRAB & TRANSECT & 576 & 100 F -2 HRM 19.0 & \\
\hline 2071 & & RIVER & & RAW GRAB & TRANSECT & 577 & 100 F -5 HRM 19.0 & \\
\hline 2072 & & RIVER & & RAW GRAB & TRANSECT & 339 & 100 F -7 HRM 19.0 & \\
\hline 2073 & & RIVER & & RAW GRAB & TRANSECT & 341 & $100 \mathrm{~F}-10 \mathrm{HRM} 19.0$ & \\
\hline 2074 & & RIVER & & RAW GRAB & TRANSECT & 578 & HANFRD TS-1 HRM 28.7 & \\
\hline 2075 & & RIVER & & RAW GRAB & TRANSECT & 342 & HANFRD TS-2 HRM 28.7 & \\
\hline 2076 & & RIVER & & RAW GRAB & TRANSECT & 469 & HANFRD TS-7 HRM 28.7 & \\
\hline 2077 & $\mathrm{~F}$ & RIVER & & RAW GRAB & TRANSECT & 347 & HANFRD TS-10 HRM 28.7 & \\
\hline 2078 & & RIVER & & RAW GRAB & TRANSECT & 468 & HANFRD TS-3 HRM 28.7 & \\
\hline 2079 & $\mathrm{~F}$ & RIVER & & RAW GRAB & TRANSECT & 8100 & HANFRD TWNSITE HRM26 & \\
\hline 2080 & & RIVER & & RAW GRAB & TRANSECT & 8101 & HANFRD TWNSITE HRM 27 & \\
\hline 2081 & $\mathrm{~F}$ & RIVER & & RAW GRAB & TRANSECT & 8103 & HANFRD TWNSITE HRM30 & \\
\hline 2082 & & RIVER & & RAW GRAB & TRANSECT & 274 & HANFRD TWNSITE HRM28 & \\
\hline 2083 & $\mathrm{~F}$ & RIVER & & RAW GRAB & TRANSECT & 8726 & 100 F SHORE HRM 18 & \\
\hline 2084 & $\mathrm{~F}$ & RIVER & & RAW GRAB & TRANSECT & 8727 & 100 F SHORE HRM 22 & \\
\hline 2085 & & RIVER & & RAW GRAB & TRANSECT & 8728 & 100 F SHORE HRM 23 & \\
\hline 2086 & $\mathrm{~F}$ & RIVER & & RAW GRAB & TRANSECT & 348 & 300 AREA -1 HRM 43.1 & \\
\hline 2087 & $\mathrm{~F}$ & RIVER & & RAW GRAB & TRANSECT & 470 & 300 AREA -2 HRM 43.1 & \\
\hline 2088 & & RIVER & & RAW GRAB & TRANSECT & 580 & 300 AREA -3 HRM 43.1 & \\
\hline 2089 & & RIVER & & RAW GRAB & TRANSECT & 607 & 300 AREA -5 HRM 43.1 & \\
\hline 2090 & & RIVER & & RAW GRAB & TRANSECT & 351 & 300 AREA -7 HRM 43.1 & \\
\hline 2091 & & RIVER & & RAW GRAB & TRANSECT & 352 & 300 AREA-10 HRM 43.1 & \\
\hline
\end{tabular}




\begin{tabular}{|c|c|c|c|c|c|c|c|c|}
\hline & $T$ & $\bar{U}$ & $\mathrm{~V}$ & $\mathrm{~W}$ & $\bar{X}$ & $\bar{Y}$ & $\bar{Z}$ & $\overline{A A}$ \\
\hline 1 & REVIEW_QUALIFIER & SAMP_FROM & SAMP_ITEM & SAMP_MTHD & COLL_MTHD & SAMP_SITE_ID & SAMP_SITE_NAME & WELL_NAME \\
\hline 2092 & & RIVER & & RAW GRAB & TRANSECT & 571 & RICH.PMPHS-1 HRM46.4 & \\
\hline 2093 & $\mathrm{~F}$ & RIVER & & RAW GRAB & TRANSECT & 321 & RICH.PMPHS-2 HRM46.4 & \\
\hline 2094 & $\mathrm{~F}$ & RIVER & & RAW GRAB & TRANSECT & 322 & RICH.PMPHS-3 HRM46.4 & \\
\hline 2095 & & RIVER & & RAW GRAB & TRANSECT & 323 & RICH.PMPHS-5 HRM46.4 & \\
\hline 2096 & & RIVER & & RAW GRAB & TRANSECT & 325 & RICH.PMPHS-7 HRM46.4 & \\
\hline 2097 & $\mathrm{~F}$ & RIVER & & RAW GRAB & TRANSECT & 327 & RICH.PMPHS-10 HRM46.4 & \\
\hline 2098 & $\mathrm{~F}$ & RIVER & & RAW GRAB & TRANSECT & 8220 & RICH.PMPHS HRM 45.8 & \\
\hline 2099 & & RIVER & & RAW GRAB & TRANSECT & 8221 & RICH.PMPHS HRM 45.0 & \\
\hline 2100 & & RIVER & & RAW GRAB & TRANSECT & 8222 & RICH.PMPHS HRM 43.9 & \\
\hline 2101 & $\mathrm{~F}$ & RIVER & & RAW GRAB & TRANSECT & 8223 & RICH.PMPHS HRM 43.5 & \\
\hline 2102 & & RIVER & & RAW GRAB & TRANSECT & 8240 & 300 AREA SHR HRM41.5 & \\
\hline 2103 & $\mathrm{~F}$ & RIVER & & RAW GRAB & TRANSECT & 8241 & 300 AREA SHR HRM42.1 & \\
\hline 2104 & $\mathrm{~F}$ & RIVER & & RAW GRAB & TRANSECT & 8242 & 300 AREA SHR HRM42.5 & \\
\hline 2105 & $\mathrm{~F}$ & RIVER & & RAW GRAB & TRANSECT & 8243 & 300 AREA SHR HRM42.9 & \\
\hline 2106 & $\mathrm{~F}$ & SEEP & & RAW GRAB & UNFILTERED & 151 & HANFORD SPRING 28-2 & \\
\hline 2107 & & SEEP & & RAW GRAB & UNFILTERED & 488 & 300 AREA SPRING 42-2 & \\
\hline 2108 & $\mathrm{~F}$ & SEEP & & RAW GRAB & UNFILTERED & 8142 & HANFORD SPR UR 28-2 & \\
\hline 2109 & $\mathrm{~F}$ & SEEP & & RAW GRAB & UNFILTERED & 8143 & HANFORD SPR DR 28-2 & \\
\hline 2110 & & SEEP & & RAW GRAB & UNFILTERED & 8144 & 300 AREA SPR DR 42-2 & \\
\hline 2111 & & IRRIGATION & & RAW GRAB & UNFILTERED & 605 & HORN RAPIDS AREA & \\
\hline 2112 & & RIVER & & CUMULATIVE & UNFILTERED & 252 & PRIEST RAPIDS-RIVER & \\
\hline 2113 & & RIVER & & CUMULATIVE & UNFILTERED & 463 & RICH.PMPHS HRM 46.4 & \\
\hline 2114 & $\mathrm{~F}$ & SURFACE & & RAW GRAB & UNFILTERED & 147 & WEST LAKE & \\
\hline 2115 & $\mathrm{~F}$ & SEEP & & RAW GRAB & UNFILTERED & 8165 & 100-H SPRING 145-1 & \\
\hline 2116 & & SEEP & & RAW GRAB & UNFILTERED & 8164 & 100-H SPRING 153-1 & \\
\hline 2117 & & RIVER & & CUMULATIVE & UNFILTERED & 252 & PRIEST RAPIDS-RIVER & \\
\hline 2118 & $\mathrm{~F}$ & RIVER & & CUMULATIVE & UNFILTERED & 463 & RICH.PMPHS HRM 46.4 & \\
\hline 2119 & $\mathrm{~F}$ & SEEP & & RAW GRAB & UNFILTERED & 8147 & 100-F SPRING 207-1 & \\
\hline 2120 & & RIVER & & CUMULATIVE & UNFILTERED & 252 & PRIEST RAPIDS-RIVER & \\
\hline 2121 & & RIVER & & CUMULATIVE & UNFILTERED & 463 & RICH.PMPHS HRM 46.4 & \\
\hline 2122 & & RIVER & & RAW GRAB & TRANSECT & 317 & VERNITA-1 HRM 0.3 & \\
\hline 2123 & & RIVER & & RAW GRAB & TRANSECT & 570 & VERNITA-2 HRM 0.3 & \\
\hline 2124 & & RIVER & & RAW GRAB & TRANSECT & 318 & VERNITA-3 HRM 0.3 & \\
\hline 2125 & & RIVER & & RAW GRAB & TRANSECT & 319 & VERNITA-4 HRM 0.3 & \\
\hline 2126 & & RIVER & & RAW GRAB & TRANSECT & 571 & RICH.PMPHS-1 HRM46.4 & \\
\hline 2127 & & RIVER & & RAW GRAB & TRANSECT & 571 & RICH.PMPHS-1 HRM46.4 & \\
\hline 2128 & & RIVER & & RAW GRAB & TRANSECT & 321 & RICH.PMPHS-2 HRM46.4 & \\
\hline 2129 & & RIVER & & RAW GRAB & TRANSECT & 322 & RICH.PMPHS-3 HRM46.4 & \\
\hline
\end{tabular}

Page 212 


\begin{tabular}{|c|c|c|c|c|c|c|c|c|}
\hline & $\mathrm{T}$ & $\bar{U}$ & $\mathrm{~V}$ & $\mathrm{~W}$ & $\mathrm{X}$ & $\bar{Y}$ & $\mathrm{Z}$ & $\mathrm{AA}$ \\
\hline 1 & REVIEW_QUALIFIER & SAMP_FROM & SAMP_ITEM & SAMP_MTHD & COLL_MTHD & SAMP_SITE_ID & SAMP_SITE_NAME & WELL_NAME \\
\hline 2130 & & RIVER & & RAW GRAB & TRANSECT & 323 & RICH.PMPHS-5 HRM46.4 & \\
\hline 2131 & & RIVER & & RAW GRAB & TRANSECT & 325 & RICH.PMPHS-7 HRM46.4 & \\
\hline 2132 & & RIVER & & RAW GRAB & TRANSECT & 327 & RICH.PMPHS-10 HRM46.4 & \\
\hline 2133 & & RIVER & & RAW GRAB & TRANSECT & 8220 & RICH.PMPHS HRM 45.8 & \\
\hline 2134 & & RIVER & & RAW GRAB & TRANSECT & 8221 & RICH.PMPHS HRM 45.0 & \\
\hline 2135 & & RIVER & & RAW GRAB & TRANSECT & 8222 & RICH.PMPHS HRM 43.9 & \\
\hline 2136 & & RIVER & & RAW GRAB & TRANSECT & 8223 & RICH.PMPHS HRM 43.5 & \\
\hline 2137 & & RIVER & & CUMULATIVE & UNFILTERED & 252 & PRIEST RAPIDS-RIVER & \\
\hline 2138 & & RIVER & & CUMULATIVE & UNFILTERED & 463 & RICH.PMPHS HRM 46.4 & \\
\hline 2139 & & RIVER & & CUMULATIVE & UNFILTERED & 252 & PRIEST RAPIDS-RIVER & \\
\hline 2140 & & RIVER & & CUMULATIVE & UNFILTERED & 463 & RICH.PMPHS HRM 46.4 & \\
\hline 2141 & $\mathrm{~F}$ & SURFACE & & RAW GRAB & UNFILTERED & 147 & WEST LAKE & \\
\hline 2142 & & RIVER & & RAW GRAB & TRANSECT & 571 & RICH.PMPHS-1 HRM46.4 & \\
\hline 2143 & & RIVER & & RAW GRAB & TRANSECT & 571 & RICH.PMPHS-1 HRM46.4 & \\
\hline 2144 & & RIVER & & RAW GRAB & TRANSECT & 321 & RICH.PMPHS-2 HRM46.4 & \\
\hline 2145 & & RIVER & & RAW GRAB & TRANSECT & 322 & RICH.PMPHS-3 HRM46.4 & \\
\hline 2146 & $\mathrm{~F}$ & RIVER & & RAW GRAB & TRANSECT & 323 & RICH.PMPHS-5 HRM46.4 & \\
\hline 2147 & & RIVER & & RAW GRAB & TRANSECT & 325 & RICH.PMPHS-7 HRM46.4 & \\
\hline 2148 & & RIVER & & RAW GRAB & TRANSECT & 327 & RICH.PMPHS-10 HRM46.4 & \\
\hline 2149 & & RIVER & & RAW GRAB & TRANSECT & 8220 & RICH.PMPHS HRM 45.8 & \\
\hline 2150 & & RIVER & & RAW GRAB & TRANSECT & 8221 & RICH.PMPHS HRM 45.0 & \\
\hline 2151 & & RIVER & & RAW GRAB & TRANSECT & 8222 & RICH.PMPHS HRM 43.9 & \\
\hline 2152 & & RIVER & & RAW GRAB & TRANSECT & 8223 & RICH.PMPHS HRM 43.5 & \\
\hline 2153 & & RIVER & & RAW GRAB & TRANSECT & 317 & VERNITA-1 HRM 0.3 & \\
\hline 2154 & & RIVER & & RAW GRAB & TRANSECT & 570 & VERNITA-2 HRM 0.3 & \\
\hline 2155 & & RIVER & & RAW GRAB & TRANSECT & 318 & VERNITA-3 HRM 0.3 & \\
\hline 2156 & & RIVER & & RAW GRAB & TRANSECT & 319 & VERNITA-4 HRM 0.3 & \\
\hline 2157 & $\mathrm{~F}$ & RIVER & & CUMULATIVE & UNFILTERED & 252 & PRIEST RAPIDS-RIVER & \\
\hline 2158 & & RIVER & & CUMULATIVE & UNFILTERED & 463 & RICH.PMPHS HRM 46.4 & \\
\hline 2159 & $\mathrm{~F}$ & SURFACE & & RAW GRAB & UNFILTERED & 147 & WEST LAKE & \\
\hline 2160 & & RIVER & & CUMULATIVE & UNFILTERED & 252 & PRIEST RAPIDS-RIVER & \\
\hline 2161 & & RIVER & & CUMULATIVE & UNFILTERED & 463 & RICH.PMPHS HRM 46.4 & \\
\hline 2162 & $\mathrm{~F}$ & SEEP & & RAW GRAB & UNFILTERED & 151 & HANFORD SPRING 28-2 & \\
\hline 2163 & $\mathrm{~F}$ & SEEP & & RAW GRAB & UNFILTERED & 8165 & 100-H SPRING 145-1 & \\
\hline 2164 & $\mathrm{~F}$ & SEEP & & RAW GRAB & UNFILTERED & 8147 & 100-F SPRING 207-1 & \\
\hline 2165 & $\mathrm{~F}$ & SEEP & & RAW GRAB & UNFILTERED & 8143 & HANFORD SPR DR $28-2$ & \\
\hline 2166 & $\mathrm{~F}$ & SEEP & & RAW GRAB & UNFILTERED & 8164 & 100-H SPRING 153-1 & \\
\hline 2167 & & RIVER & & CUMULATIVE & UNFILTERED & 463 & RICH.PMPHS HRM 46.4 & \\
\hline
\end{tabular}




\begin{tabular}{|c|c|c|c|c|c|c|c|c|}
\hline & $\mathrm{T}$ & $U$ & $\mathrm{~V}$ & $\mathrm{~W}$ & $\mathrm{X}$ & $\mathrm{Y}$ & Z & AA \\
\hline 1 & REVIEW_QUALIFIER & SAMP_FROM & SAMP_ITEM & SAMP_MTHD & COLL_MTHD & SAMP_SITE_ID & SAMP_SITE_NAME & WELL_NAME \\
\hline 2168 & & RIVER & & CUMULATIVE & UNFILTERED & 252 & PRIEST RAPIDS-RIVER & \\
\hline 2169 & & SEEP & & RAW GRAB & UNFILTERED & 8144 & 300 AREA SPR DR 42-2 & \\
\hline 2170 & & IRRIGATION & & RAW GRAB & UNFILTERED & 440 & RIVERVIEW CANAL & \\
\hline 2171 & & IRRIGATION & & RAW GRAB & UNFILTERED & 605 & HORN RAPIDS AREA & \\
\hline 2172 & & SEEP & & RAW GRAB & UNFILTERED & 488 & 300 AREA SPRING 42-2 & \\
\hline 2173 & & SEEP & & RAW GRAB & UNFILTERED & 9147 & RICHLAND SPR HRM44.4 & \\
\hline 2174 & $\mathrm{~F}$ & RIVER & & CUMULATIVE & UNFILTERED & 463 & RICH.PMPHS HRM 46.4 & \\
\hline 2175 & & RIVER & & CUMULATIVE & UNFILTERED & 252 & PRIEST RAPIDS-RIVER & \\
\hline 2176 & & IRRIGATION & & RAW GRAB & UNFILTERED & 440 & RIVERVIEW CANAL & \\
\hline 2177 & $\mathrm{~F}$ & IRRIGATION & & RAW GRAB & UNFILTERED & 605 & HORN RAPIDS AREA & \\
\hline 2178 & $\mathrm{~F}$ & RIVER & & RAW GRAB & TRANSECT & 571 & RICH.PMPHS-1 HRM46.4 & \\
\hline 2179 & $\mathrm{~F}$ & RIVER & & RAW GRAB & TRANSECT & 321 & RICH.PMPHS-2 HRM46.4 & \\
\hline 2180 & & RIVER & & RAW GRAB & TRANSECT & 322 & RICH.PMPHS-3 HRM46.4 & \\
\hline 2181 & $\mathrm{~F}$ & RIVER & & RAW GRAB & TRANSECT & 323 & RICH.PMPHS-5 HRM46.4 & \\
\hline 2182 & & RIVER & & RAW GRAB & TRANSECT & 325 & RICH.PMPHS-7 HRM46.4 & \\
\hline 2183 & & RIVER & & RAW GRAB & TRANSECT & 327 & RICH.PMPHS-10 HRM46.4 & \\
\hline 2184 & & RIVER & & RAW GRAB & TRANSECT & 8220 & RICH.PMPHS HRM 45.8 & \\
\hline 2185 & $\mathrm{~F}$ & RIVER & & RAW GRAB & TRANSECT & 8221 & RICH.PMPHS HRM 45.0 & \\
\hline 2186 & & RIVER & & RAW GRAB & TRANSECT & 8222 & RICH.PMPHS HRM 43.9 & \\
\hline 2187 & & RIVER & & RAW GRAB & TRANSECT & 8223 & RICH.PMPHS HRM 43.5 & \\
\hline 2188 & & RIVER & & RAW GRAB & TRANSECT & 317 & VERNITA-1 HRM 0.3 & \\
\hline 2189 & & RIVER & & RAW GRAB & TRANSECT & 570 & VERNITA-2 HRM 0.3 & \\
\hline 2190 & & RIVER & & RAW GRAB & TRANSECT & 318 & VERNITA-3 HRM 0.3 & \\
\hline 2191 & & RIVER & & RAW GRAB & TRANSECT & 319 & VERNITA-4 HRM 0.3 & \\
\hline 2192 & & RIVER & & CUMULATIVE & UNFILTERED & 463 & RICH.PMPHS HRM 46.4 & \\
\hline 2193 & & RIVER & & CUMULATIVE & UNFILTERED & 252 & PRIEST RAPIDS-RIVER & \\
\hline 2194 & $\mathrm{~F}$ & SURFACE & & RAW GRAB & UNFILTERED & 147 & WEST LAKE & \\
\hline 2195 & & IRRIGATION & & RAW GRAB & UNFILTERED & 440 & RIVERVIEW CANAL & \\
\hline 2196 & & IRRIGATION & & RAW GRAB & UNFILTERED & 605 & HORN RAPIDS AREA & \\
\hline 2197 & & RIVER & & CUMULATIVE & UNFILTERED & 463 & RICH.PMPHS HRM 46.4 & \\
\hline 2198 & & RIVER & & CUMULATIVE & UNFILTERED & 252 & PRIEST RAPIDS-RIVER & \\
\hline 2199 & $\mathrm{~F}$ & SEEP & & RAW GRAB & UNFILTERED & 488 & 300 AREA SPRING 42-2 & \\
\hline 2200 & & SEEP & & RAW GRAB & UNFILTERED & 8144 & 300 AREA SPR DR 42-2 & \\
\hline 2201 & & RIVER & & RAW GRAB & & 9236 & VERNITA BRIDGE -1 & \\
\hline 2202 & & SEEP & & RAW GRAB & & 488 & 300 AREA SPRING 42-2 & \\
\hline 2203 & & SEEP & & RAW GRAB & & 8144 & 300 AREA SPR DR 42-2 & \\
\hline 2204 & & RIVER & & RAW GRAB & & 9237 & VERNITA BRIDGE -2 & \\
\hline 2205 & & RIVER & & RAW GRAB & UNFILTERED & 9236 & VERNITA BRIDGE -1 & \\
\hline
\end{tabular}




\begin{tabular}{|c|c|c|c|c|c|c|c|c|}
\hline & $T$ & $\bar{U}$ & $\mathrm{~V}$ & $\mathrm{~W}$ & $\bar{X}$ & $\bar{Y}$ & $\bar{Z}$ & $\overline{A A}$ \\
\hline 1 & REVIEW_QUALIFIER & SAMP_FROM & SAMP_ITEM & SAMP_MTHD & COLL_MTHD & SAMP_SITE_ID & SAMP_SITE_NAME & WELL_NAME \\
\hline 2206 & & RIVER & & RAW GRAB & UNFILTERED & 9237 & VERNITA BRIDGE -2 & \\
\hline 2207 & & RIVER & & RAW GRAB & UNFILTERED & 9238 & VERNITA BRIDGE -3 & \\
\hline 2208 & $\mathrm{~F}$ & RIVER & & RAW GRAB & UNFILTERED & 9210 & 300 SPR $7-1$ & \\
\hline 2209 & $\mathrm{~F}$ & RIVER & & RAW GRAB & UNFILTERED & 9211 & 300 SPR $7-2$ & \\
\hline 2210 & $\mathrm{~F}$ & RIVER & & RAW GRAB & UNFILTERED & 9213 & 300 SPR $7-3$ & \\
\hline 2211 & $\mathrm{~F}$ & RIVER & & RAW GRAB & UNFILTERED & 9214 & 300 SPR DR $7-1$ & \\
\hline 2212 & & RIVER & & RAW GRAB & UNFILTERED & 9215 & 300 SPR DR $7-2$ & \\
\hline 2213 & & RIVER & & RAW GRAB & UNFILTERED & 9216 & 300 SPR DR $7-3$ & \\
\hline 2214 & & RIVER & & RAW GRAB & UNFILTERED & 9217 & 300 SPR 7 THRU SPR 9 & \\
\hline 2215 & $\mathrm{~F}$ & RIVER & & RAW GRAB & UNFILTERED & 9218 & 300 SPR 9 -1 & \\
\hline 2216 & $\mathrm{~F}$ & RIVER & & RAW GRAB & UNFILTERED & 9219 & 300 SPR $9-2$ & \\
\hline 2217 & & RIVER & & RAW GRAB & UNFILTERED & 9220 & 300 SPR $9-3$ & \\
\hline 2218 & $\mathrm{~F}$ & RIVER & & RAW GRAB & UNFILTERED & 9221 & 300 SPR DR 9 -1 & \\
\hline 2219 & $\mathrm{~F}$ & RIVER & & RAW GRAB & UNFILTERED & 9222 & 300 SPR DR 9 -2 & \\
\hline 2220 & $\mathrm{~F}$ & RIVER & & RAW GRAB & UNFILTERED & 9223 & 300 SPR DR $9-3$ & \\
\hline 2221 & & RIVER & & RAW GRAB & UNFILTERED & 9224 & 300 SPR 9 THRU SPR 11 & \\
\hline 2222 & $\mathrm{~F}$ & RIVER & & RAW GRAB & UNFILTERED & 9226 & 300 SPR $11-1$ & \\
\hline 2223 & & RIVER & & RAW GRAB & UNFILTERED & 9227 & 300 SPR $11-2$ & \\
\hline 2224 & $\mathrm{~F}$ & RIVER & & RAW GRAB & UNFILTERED & 9228 & 300 SPR $11-3$ & \\
\hline 2225 & $\mathrm{~F}$ & RIVER & & RAW GRAB & UNFILTERED & 9229 & 300 SPR DR $11-1$ & \\
\hline 2226 & & RIVER & & RAW GRAB & UNFILTERED & 9230 & 300 SPR DR $11-2$ & \\
\hline 2227 & & RIVER & & RAW GRAB & UNFILTERED & 9231 & 300 SPR DR $11-3$ & \\
\hline 2228 & & RIVER & & RAW GRAB & UNFILTERED & 9233 & 300 SPR $14-1$ & \\
\hline 2229 & & RIVER & & RAW GRAB & UNFILTERED & 9234 & 300 SPR $14-2$ & \\
\hline 2230 & & RIVER & & RAW GRAB & UNFILTERED & 9235 & 300 SPR $14-3$ & \\
\hline 2231 & & RIVER & & RAW GRAB & UNFILTERED & 9280 & VERNITA BRIDGE -4 & \\
\hline 2232 & $\mathrm{~F}$ & RIVER & & RAW GRAB & UNFILTERED & 9273 & 300 SPR $7-4$ & \\
\hline 2233 & & RIVER & & RAW GRAB & UNFILTERED & 9274 & 300 SPR DR $7-4$ & \\
\hline 2234 & & RIVER & & RAW GRAB & UNFILTERED & 9275 & 300 SPR $9-4$ & \\
\hline 2235 & & RIVER & & RAW GRAB & UNFILTERED & 9276 & 300 SPR DR $9-4$ & \\
\hline 2236 & & RIVER & & RAW GRAB & UNFILTERED & 9277 & 300 SPR $11-4$ & \\
\hline 2237 & & RIVER & & RAW GRAB & UNFILTERED & 9278 & 300 SPR DR $11-4$ & \\
\hline 2238 & & RIVER & & RAW GRAB & UNFILTERED & 9279 & 300 SPR $14-4$ & \\
\hline 2239 & & RIVER & & CUMULATIVE & UNFILTERED & 463 & RICH.PMPHS HRM 46.4 & \\
\hline 2240 & & RIVER & & CUMULATIVE & UNFILTERED & 252 & PRIEST RAPIDS-RIVER & \\
\hline 2241 & & RIVER & & RAW GRAB & TRANSECT & 328 & 100 N -1 HRM 9.5 & \\
\hline 2242 & & RIVER & & RAW GRAB & TRANSECT & 317 & VERNITA-1 HRM 0.3 & \\
\hline 2243 & & RIVER & & RAW GRAB & TRANSECT & 570 & VERNITA-2 HRM 0.3 & \\
\hline
\end{tabular}




\begin{tabular}{|c|c|c|c|c|c|c|c|c|}
\hline & $T$ & $\bar{U}$ & $\mathrm{~V}$ & $\mathrm{~W}$ & $\bar{X}$ & $\bar{Y}$ & $\bar{Z}$ & $\overline{A A}$ \\
\hline 1 & REVIEW_QUALIFIER & SAMP_FROM & SAMP_ITEM & SAMP_MTHD & COLL_MTHD & SAMP_SITE_ID & SAMP_SITE_NAME & WELL_NAME \\
\hline 2244 & & RIVER & & RAW GRAB & TRANSECT & 318 & VERNITA-3 HRM 0.3 & \\
\hline 2245 & & RIVER & & RAW GRAB & TRANSECT & 319 & VERNITA-4 HRM 0.3 & \\
\hline 2246 & & RIVER & & RAW GRAB & TRANSECT & 328 & 100 N -1 HRM 9.5 & \\
\hline 2247 & & RIVER & & RAW GRAB & TRANSECT & 329 & 100 N -2 HRM 9.5 & \\
\hline 2248 & & RIVER & & RAW GRAB & TRANSECT & 331 & 100 N -5 HRM 9.5 & \\
\hline 2249 & & RIVER & & RAW GRAB & TRANSECT & 335 & 100 N -10 HRM 9.5 & \\
\hline 2250 & & RIVER & & RAW GRAB & TRANSECT & 330 & 100 N -3 HRM 9.5 & \\
\hline 2251 & & RIVER & & RAW GRAB & TRANSECT & 333 & 100 N -7 HRM 9.5 & \\
\hline 2252 & & RIVER & & RAW GRAB & TRANSECT & 8244 & 100 N SHORE HRM 8.4 & \\
\hline 2253 & & RIVER & & RAW GRAB & TRANSECT & 8245 & 100 N SHORE HRM 8.9 & \\
\hline 2254 & & RIVER & & RAW GRAB & TRANSECT & 8246 & 100 N SHORE HRM 9.2 & \\
\hline 2255 & & RIVER & & RAW GRAB & TRANSECT & 8247 & 100 N SHORE HRM 9.8 & \\
\hline 2256 & & RIVER & & RAW GRAB & TRANSECT & 336 & 100 F -1 HRM 19.0 & \\
\hline 2257 & & RIVER & & RAW GRAB & TRANSECT & 337 & 100 F -3 HRM 19.0 & \\
\hline 2258 & & RIVER & & RAW GRAB & TRANSECT & 344 & HANFRD TS-5 HRM 28.7 & \\
\hline 2259 & & RIVER & & RAW GRAB & TRANSECT & 576 & 100 F -2 HRM 19.0 & \\
\hline 2260 & & RIVER & & RAW GRAB & TRANSECT & 577 & 100 F -5 HRM 19.0 & \\
\hline 2261 & & RIVER & & RAW GRAB & TRANSECT & 339 & 100 F -7 HRM 19.0 & \\
\hline 2262 & & RIVER & & RAW GRAB & TRANSECT & 341 & $100 \mathrm{~F}-10 \mathrm{HRM} 19.0$ & \\
\hline 2263 & & RIVER & & RAW GRAB & TRANSECT & 578 & HANFRD TS-1 HRM 28.7 & \\
\hline 2264 & & RIVER & & RAW GRAB & TRANSECT & 342 & HANFRD TS-2 HRM 28.7 & \\
\hline 2265 & & RIVER & & RAW GRAB & TRANSECT & 469 & HANFRD TS-7 HRM 28.7 & \\
\hline 2266 & & RIVER & & RAW GRAB & TRANSECT & 347 & HANFRD TS-10 HRM 28.7 & \\
\hline 2267 & & RIVER & & RAW GRAB & TRANSECT & 468 & HANFRD TS-3 HRM 28.7 & \\
\hline 2268 & & RIVER & & RAW GRAB & TRANSECT & 8100 & HANFRD TWNSITE HRM26 & \\
\hline 2269 & & RIVER & & RAW GRAB & TRANSECT & 8101 & HANFRD TWNSITE HRM27 & \\
\hline 2270 & & RIVER & & RAW GRAB & TRANSECT & 8103 & HANFRD TWNSITE HRM30 & \\
\hline 2271 & & RIVER & & RAW GRAB & TRANSECT & 274 & HANFRD TWNSITE HRM28 & \\
\hline 2272 & $\mathrm{~F}$ & RIVER & & RAW GRAB & TRANSECT & 8726 & 100 F SHORE HRM 18 & \\
\hline 2273 & $\mathrm{~F}$ & RIVER & & RAW GRAB & TRANSECT & 8728 & 100 F SHORE HRM 23 & \\
\hline 2274 & & RIVER & & RAW GRAB & TRANSECT & 348 & 300 AREA -1 HRM 43.1 & \\
\hline 2275 & & RIVER & & RAW GRAB & TRANSECT & 470 & 300 AREA -2 HRM 43.1 & \\
\hline 2276 & & RIVER & & RAW GRAB & TRANSECT & 580 & 300 AREA -3 HRM 43.1 & \\
\hline 2277 & & RIVER & & RAW GRAB & TRANSECT & 607 & 300 AREA -5 HRM 43.1 & \\
\hline 2278 & & RIVER & & RAW GRAB & TRANSECT & 351 & 300 AREA -7 HRM 43.1 & \\
\hline 2279 & & RIVER & & RAW GRAB & TRANSECT & 352 & 300 AREA-10 HRM 43.1 & \\
\hline 2280 & & RIVER & & RAW GRAB & TRANSECT & 571 & RICH.PMPHS-1 HRM46.4 & \\
\hline 2281 & & RIVER & & RAW GRAB & TRANSECT & 321 & RICH.PMPHS-2 HRM46.4 & \\
\hline
\end{tabular}




\begin{tabular}{|c|c|c|c|c|c|c|c|c|}
\hline & $T$ & $\bar{U}$ & $\mathrm{~V}$ & $\mathrm{~W}$ & $\bar{X}$ & $\bar{Y}$ & $\bar{Z}$ & $\overline{A A}$ \\
\hline 1 & REVIEW_QUALIFIER & SAMP_FROM & SAMP_ITEM & SAMP_MTHD & COLL_MTHD & SAMP_SITE_ID & SAMP_SITE_NAME & WELL_NAME \\
\hline 2282 & & RIVER & & RAW GRAB & TRANSECT & 322 & RICH.PMPHS-3 HRM46.4 & \\
\hline 2283 & & RIVER & & RAW GRAB & TRANSECT & 323 & RICH.PMPHS-5 HRM46.4 & \\
\hline 2284 & & RIVER & & RAW GRAB & TRANSECT & 325 & RICH.PMPHS-7 HRM46.4 & \\
\hline 2285 & & RIVER & & RAW GRAB & TRANSECT & 327 & RICH.PMPHS-10 HRM46.4 & \\
\hline 2286 & & RIVER & & RAW GRAB & TRANSECT & 8220 & RICH.PMPHS HRM 45.8 & \\
\hline 2287 & & RIVER & & RAW GRAB & TRANSECT & 8221 & RICH.PMPHS HRM 45.0 & \\
\hline 2288 & & RIVER & & RAW GRAB & TRANSECT & 8222 & RICH.PMPHS HRM 43.9 & \\
\hline 2289 & & RIVER & & RAW GRAB & TRANSECT & 8223 & RICH.PMPHS HRM 43.5 & \\
\hline 2290 & & RIVER & & RAW GRAB & TRANSECT & 8240 & 300 AREA SHR HRM41.5 & \\
\hline 2291 & & RIVER & & RAW GRAB & TRANSECT & 8241 & 300 AREA SHR HRM42.1 & \\
\hline 2292 & & RIVER & & RAW GRAB & TRANSECT & 8242 & 300 AREA SHR HRM42.5 & \\
\hline 2293 & & RIVER & & RAW GRAB & TRANSECT & 8243 & 300 AREA SHR HRM42.9 & \\
\hline 2294 & & SEEP & & RAW GRAB & UNFILTERED & 8144 & 300 AREA SPR DR 42-2 & \\
\hline 2295 & & DRIVE POINT & & RAW GRAB & UNFILTERED & 9539 & 300 SPR 9 -1 (2 FT) & \\
\hline 2296 & & DRIVE POINT & & RAW GRAB & UNFILTERED & 9540 & 300 SPR 9 -1 (4 FT) & \\
\hline 2297 & & DRIVE POINT & & RAW GRAB & UNFILTERED & 9541 & 300 SPR 9 -2 (2 FT) & \\
\hline 2298 & & DRIVE POINT & & RAW GRAB & UNFILTERED & 9542 & 300 SPR $9-2(4.5 F T)$ & \\
\hline 2299 & $\mathrm{~F}$ & DRIVE POINT & & RAW GRAB & UNFILTERED & 9543 & 300 SPR 9 -3 (5.5FT) & \\
\hline 2300 & $\mathrm{~F}$ & SEEP & & RAW GRAB & UNFILTERED & 488 & 300 AREA SPRING 42-2 & \\
\hline 2301 & & DRIVE POINT & & RAW GRAB & UNFILTERED & 9533 & 300 SPR 7 -1 (2.5FT) & \\
\hline 2302 & & DRIVE POINT & & RAW GRAB & UNFILTERED & 9534 & 300 SPR 7 -1 (4 FT) & \\
\hline 2303 & & DRIVE POINT & & RAW GRAB & UNFILTERED & 9535 & 300 SPR 7 -1 (6 FT) & \\
\hline 2304 & & DRIVE POINT & & RAW GRAB & UNFILTERED & 9536 & 300 SPR 7 -2 (2 FT) & \\
\hline 2305 & & DRIVE POINT & & RAW GRAB & UNFILTERED & 9537 & 300 SPR 7 -2 (4.2FT) & \\
\hline 2306 & & RIVER & & CUMULATIVE & UNFILTERED & 252 & PRIEST RAPIDS-RIVER & \\
\hline 2307 & & RIVER & & CUMULATIVE & UNFILTERED & 463 & RICH.PMPHS HRM 46.4 & \\
\hline 2308 & $\mathrm{~F}$ & SEEP & & RAW GRAB & UNFILTERED & 8147 & 100-F SPRING 207-1 & \\
\hline 2309 & $\mathrm{~F}$ & SEEP & & RAW GRAB & UNFILTERED & 8165 & 100-H SPRING 145-1 & \\
\hline 2310 & $\mathrm{~F}$ & SEEP & & RAW GRAB & UNFILTERED & 8846 & 100-H SPRING 152-2 & \\
\hline 2311 & $\mathrm{~F}$ & RIVER & & CUMULATIVE & UNFILTERED & 463 & RICH.PMPHS HRM 46.4 & \\
\hline 2312 & & RIVER & & CUMULATIVE & UNFILTERED & 252 & PRIEST RAPIDS-RIVER & \\
\hline 2313 & & SEEP & & RAW GRAB & UNFILTERED & 9236 & VERNITA BRIDGE -1 & \\
\hline 2314 & & RIVER & & CUMULATIVE & UNFILTERED & 252 & PRIEST RAPIDS-RIVER & \\
\hline 2315 & & RIVER & & CUMULATIVE & UNFILTERED & 463 & RICH.PMPHS HRM 46.4 & \\
\hline 2316 & & RIVER & & RAW GRAB & TRANSECT & 317 & VERNITA-1 HRM 0.3 & \\
\hline 2317 & & RIVER & & RAW GRAB & TRANSECT & 570 & VERNITA-2 HRM 0.3 & \\
\hline 2318 & & RIVER & & RAW GRAB & TRANSECT & 318 & VERNITA-3 HRM 0.3 & \\
\hline 2319 & & RIVER & & RAW GRAB & TRANSECT & 319 & VERNITA-4 HRM 0.3 & \\
\hline
\end{tabular}




\begin{tabular}{|c|c|c|c|c|c|c|c|c|}
\hline & $T$ & $\bar{U}$ & $\mathrm{~V}$ & $\mathrm{~W}$ & $\bar{X}$ & $\bar{Y}$ & $\bar{Z}$ & $\overline{A A}$ \\
\hline 1 & REVIEW_QUALIFIER & SAMP_FROM & SAMP_ITEM & SAMP_MTHD & COLL_MTHD & SAMP_SITE_ID & SAMP_SITE_NAME & WELL_NAME \\
\hline 2320 & & RIVER & & RAW GRAB & TRANSECT & 571 & RICH.PMPHS-1 HRM46.4 & \\
\hline 2321 & $\mathrm{~F}$ & RIVER & & RAW GRAB & TRANSECT & 571 & RICH.PMPHS-1 HRM46.4 & \\
\hline 2322 & & RIVER & & RAW GRAB & TRANSECT & 321 & RICH.PMPHS-2 HRM46.4 & \\
\hline 2323 & $\mathrm{~F}$ & RIVER & & RAW GRAB & TRANSECT & 322 & RICH.PMPHS-3 HRM46.4 & \\
\hline 2324 & & RIVER & & RAW GRAB & TRANSECT & 323 & RICH.PMPHS-5 HRM46.4 & \\
\hline 2325 & & RIVER & & RAW GRAB & TRANSECT & 325 & RICH.PMPHS-7 HRM46.4 & \\
\hline 2326 & & RIVER & & RAW GRAB & TRANSECT & 327 & RICH.PMPHS-10 HRM46.4 & \\
\hline 2327 & & RIVER & & RAW GRAB & TRANSECT & 8220 & RICH.PMPHS HRM 45.8 & \\
\hline 2328 & & RIVER & & RAW GRAB & TRANSECT & 8221 & RICH.PMPHS HRM 45.0 & \\
\hline 2329 & & RIVER & & RAW GRAB & TRANSECT & 8222 & RICH.PMPHS HRM 43.9 & \\
\hline 2330 & & RIVER & & RAW GRAB & TRANSECT & 8223 & RICH.PMPHS HRM 43.5 & \\
\hline 2331 & & RIVER & & CUMULATIVE & UNFILTERED & 463 & RICH.PMPHS HRM 46.4 & \\
\hline 2332 & & RIVER & & CUMULATIVE & UNFILTERED & 252 & PRIEST RAPIDS-RIVER & \\
\hline 2333 & & RIVER & & CUMULATIVE & UNFILTERED & 463 & RICH.PMPHS HRM 46.4 & \\
\hline 2334 & $\mathrm{~F}$ & RIVER & & CUMULATIVE & UNFILTERED & 252 & PRIEST RAPIDS-RIVER & \\
\hline 2335 & & RIVER & & CUMULATIVE & UNFILTERED & 252 & PRIEST RAPIDS-RIVER & \\
\hline 2336 & & RIVER & & CUMULATIVE & UNFILTERED & 463 & RICH.PMPHS HRM 46.4 & \\
\hline 2337 & & RIVER & & RAW GRAB & TRANSECT & 317 & VERNITA-1 HRM 0.3 & \\
\hline 2338 & $\mathrm{~F}$ & RIVER & & RAW GRAB & TRANSECT & 570 & VERNITA-2 HRM 0.3 & \\
\hline 2339 & & RIVER & & RAW GRAB & TRANSECT & 318 & VERNITA-3 HRM 0.3 & \\
\hline 2340 & & RIVER & & RAW GRAB & TRANSECT & 319 & VERNITA-4 HRM 0.3 & \\
\hline 2341 & & RIVER & & RAW GRAB & TRANSECT & 571 & RICH.PMPHS-1 HRM46.4 & \\
\hline 2342 & $\mathrm{~F}$ & RIVER & & RAW GRAB & TRANSECT & 571 & RICH.PMPHS-1 HRM46.4 & \\
\hline 2343 & & RIVER & & RAW GRAB & TRANSECT & 321 & RICH.PMPHS-2 HRM46.4 & \\
\hline 2344 & & RIVER & & RAW GRAB & TRANSECT & 322 & RICH.PMPHS-3 HRM46.4 & \\
\hline 2345 & $\mathrm{~F}$ & RIVER & & RAW GRAB & TRANSECT & 323 & RICH.PMPHS-5 HRM46.4 & \\
\hline 2346 & & RIVER & & RAW GRAB & TRANSECT & 325 & RICH.PMPHS-7 HRM46.4 & \\
\hline 2347 & & RIVER & & RAW GRAB & TRANSECT & 327 & RICH.PMPHS-10 HRM46.4 & \\
\hline 2348 & & RIVER & & RAW GRAB & TRANSECT & 8220 & RICH.PMPHS HRM 45.8 & \\
\hline 2349 & & RIVER & & RAW GRAB & TRANSECT & 8221 & RICH.PMPHS HRM 45.0 & \\
\hline 2350 & & RIVER & & RAW GRAB & TRANSECT & 8222 & RICH.PMPHS HRM 43.9 & \\
\hline 2351 & & RIVER & & RAW GRAB & TRANSECT & 8223 & RICH.PMPHS HRM 43.5 & \\
\hline 2352 & & RIVER & & CUMULATIVE & UNFILTERED & 252 & PRIEST RAPIDS-RIVER & \\
\hline 2353 & & RIVER & & CUMULATIVE & UNFILTERED & 463 & RICH.PMPHS HRM 46.4 & \\
\hline 2354 & & RIVER & & CUMULATIVE & UNFILTERED & 252 & PRIEST RAPIDS-RIVER & \\
\hline 2355 & & RIVER & & CUMULATIVE & UNFILTERED & 463 & RICH.PMPHS HRM 46.4 & \\
\hline 2356 & & IRRIGATION & & RAW GRAB & UNFILTERED & 440 & RIVERVIEW CANAL & \\
\hline 2357 & & IRRIGATION & & RAW GRAB & UNFILTERED & 605 & HORN RAPIDS AREA & \\
\hline
\end{tabular}




\begin{tabular}{|c|c|c|c|c|c|c|c|c|}
\hline & $\mathrm{T}$ & $\mathrm{U}$ & $\mathrm{V}$ & W & $\mathrm{X}$ & $\mathrm{Y}$ & Z & AA \\
\hline 1 & REVIEW_QUALIFIER & SAMP_FROM & SAMP_ITEM & SAMP_MTHD & COLL_MTHD & SAMP_SITE_ID & SAMP_SITE_NAME & WELL_NAME \\
\hline 2358 & & RIVER & & CUMULATIVE & UNFILTERED & 252 & PRIEST RAPIDS-RIVER & \\
\hline 2359 & & RIVER & & CUMULATIVE & UNFILTERED & 463 & RICH.PMPHS HRM 46.4 & \\
\hline 2360 & & RIVER & & RAW GRAB & TRANSECT & 317 & VERNITA-1 HRM 0.3 & \\
\hline 2361 & & RIVER & & RAW GRAB & TRANSECT & 570 & VERNITA-2 HRM 0.3 & \\
\hline 2362 & & RIVER & & RAW GRAB & TRANSECT & 318 & VERNITA-3 HRM 0.3 & \\
\hline 2363 & & RIVER & & RAW GRAB & TRANSECT & 319 & VERNITA-4 HRM 0.3 & \\
\hline 2364 & & RIVER & & RAW GRAB & TRANSECT & 571 & RICH.PMPHS-1 HRM46.4 & \\
\hline 2365 & & RIVER & & RAW GRAB & TRANSECT & 321 & RICH.PMPHS-2 HRM46.4 & \\
\hline 2366 & & RIVER & & RAW GRAB & TRANSECT & 322 & RICH.PMPHS-3 HRM46.4 & \\
\hline 2367 & & RIVER & & RAW GRAB & TRANSECT & 323 & RICH.PMPHS-5 HRM46.4 & \\
\hline 2368 & & RIVER & & RAW GRAB & TRANSECT & 325 & RICH.PMPHS-7 HRM46.4 & \\
\hline 2369 & & RIVER & & RAW GRAB & TRANSECT & 327 & RICH.PMPHS-10 HRM46.4 & \\
\hline 2370 & & RIVER & & RAW GRAB & TRANSECT & 8220 & RICH.PMPHS HRM 45.8 & \\
\hline 2371 & & RIVER & & RAW GRAB & TRANSECT & 8221 & RICH.PMPHS HRM 45.0 & \\
\hline 2372 & & RIVER & & RAW GRAB & TRANSECT & 8222 & RICH.PMPHS HRM 43.9 & \\
\hline 2373 & & RIVER & & RAW GRAB & TRANSECT & 8223 & RICH.PMPHS HRM 43.5 & \\
\hline 2374 & & RIVER & & CUMULATIVE & UNFILTERED & 252 & PRIEST RAPIDS-RIVER & \\
\hline 2375 & & RIVER & & CUMULATIVE & UNFILTERED & 463 & RICH.PMPHS HRM 46.4 & \\
\hline 2376 & & IRRIGATION & & RAW GRAB & UNFILTERED & 440 & RIVERVIEW CANAL & \\
\hline 2377 & & IRRIGATION & & RAW GRAB & UNFILTERED & 605 & HORN RAPIDS AREA & \\
\hline 2378 & & IRRIGATION & & RAW GRAB & UNFILTERED & 440 & RIVERVIEW CANAL & \\
\hline 2379 & & IRRIGATION & & RAW GRAB & UNFILTERED & 605 & HORN RAPIDS AREA & \\
\hline 2380 & & RIVER & & CUMULATIVE & UNFILTERED & 252 & PRIEST RAPIDS-RIVER & \\
\hline 2381 & & RIVER & & CUMULATIVE & UNFILTERED & 463 & RICH.PMPHS HRM 46.4 & \\
\hline 2382 & & RIVER & & CUMULATIVE & UNFILTERED & 252 & PRIEST RAPIDS-RIVER & \\
\hline 2383 & $\mathrm{~F}$ & RIVER & & CUMULATIVE & UNFILTERED & 463 & RICH.PMPHS HRM 46.4 & \\
\hline 2384 & & RIVER & & RAW GRAB & TRANSECT & 328 & 100 N -1 HRM 9.5 & \\
\hline 2385 & & RIVER & & RAW GRAB & TRANSECT & 319 & VERNITA-4 HRM 0.3 & \\
\hline 2386 & & RIVER & & RAW GRAB & TRANSECT & 570 & VERNITA-2 HRM 0.3 & \\
\hline 2387 & & RIVER & & RAW GRAB & TRANSECT & 318 & VERNITA-3 HRM 0.3 & \\
\hline 2388 & & RIVER & & RAW GRAB & TRANSECT & 317 & VERNITA-1 HRM 0.3 & \\
\hline 2389 & & RIVER & & RAW GRAB & TRANSECT & 328 & 100 N -1 HRM 9.5 & \\
\hline 2390 & & RIVER & & RAW GRAB & TRANSECT & 329 & 100 N -2 HRM 9.5 & \\
\hline 2391 & $\mathrm{~F}$ & RIVER & & RAW GRAB & TRANSECT & 331 & 100 N -5 HRM 9.5 & \\
\hline 2392 & & RIVER & & RAW GRAB & TRANSECT & 335 & 100 N -10 HRM 9.5 & \\
\hline 2393 & & RIVER & & RAW GRAB & TRANSECT & 330 & 100 N -3 HRM 9.5 & \\
\hline 2394 & & RIVER & & RAW GRAB & TRANSECT & 333 & 100 N -7 HRM 9.5 & \\
\hline 2395 & & RIVER & & RAW GRAB & TRANSECT & 8244 & 100 N SHORE HRM 8.4 & \\
\hline
\end{tabular}




\begin{tabular}{|c|c|c|c|c|c|c|c|c|}
\hline & $\mathrm{T}$ & $U$ & $\mathrm{~V}$ & $\mathrm{~W}$ & $\mathrm{X}$ & $\mathrm{Y}$ & Z & AA \\
\hline 1 & REVIEW_QUALIFIER & SAMP_FROM & SAMP_ITEM & SAMP_MTHD & COLL_MTHD & SAMP_SITE_ID & SAMP_SITE_NAME & WELL_NAME \\
\hline 2396 & & RIVER & & RAW GRAB & TRANSECT & 8245 & 100 N SHORE HRM 8.9 & \\
\hline 2397 & & RIVER & & RAW GRAB & TRANSECT & 8246 & 100 N SHORE HRM 9.2 & \\
\hline 2398 & & RIVER & & RAW GRAB & TRANSECT & 8247 & 100 N SHORE HRM 9.8 & \\
\hline 2399 & & RIVER & & RAW GRAB & TRANSECT & 336 & 100 F -1 HRM 19.0 & \\
\hline 2400 & & RIVER & & RAW GRAB & TRANSECT & 337 & 100 F -3 HRM 19.0 & \\
\hline 2401 & & RIVER & & RAW GRAB & TRANSECT & 344 & HANFRD TS-5 HRM 28.7 & \\
\hline 2402 & & RIVER & & RAW GRAB & TRANSECT & 576 & 100 F -2 HRM 19.0 & \\
\hline 2403 & & RIVER & & RAW GRAB & TRANSECT & 577 & 100 F -5 HRM 19.0 & \\
\hline 2404 & & RIVER & & RAW GRAB & TRANSECT & 339 & 100 F -7 HRM 19.0 & \\
\hline 2405 & & RIVER & & RAW GRAB & TRANSECT & 341 & $100 \mathrm{~F}-10 \mathrm{HRM} 19.0$ & \\
\hline 2406 & & RIVER & & RAW GRAB & TRANSECT & 578 & HANFRD TS-1 HRM 28.7 & \\
\hline 2407 & & RIVER & & RAW GRAB & TRANSECT & 342 & HANFRD TS-2 HRM 28.7 & \\
\hline 2408 & & RIVER & & RAW GRAB & TRANSECT & 469 & HANFRD TS-7 HRM 28.7 & \\
\hline 2409 & & RIVER & & RAW GRAB & TRANSECT & 347 & HANFRD TS-10 HRM 28.7 & \\
\hline 2410 & & RIVER & & RAW GRAB & TRANSECT & 468 & HANFRD TS-3 HRM 28.7 & \\
\hline 2411 & & RIVER & & RAW GRAB & TRANSECT & 8100 & HANFRD TWNSITE HRM26 & \\
\hline 2412 & & RIVER & & RAW GRAB & TRANSECT & 8101 & HANFRD TWNSITE HRM27 & \\
\hline 2413 & & RIVER & & RAW GRAB & TRANSECT & 8103 & HANFRD TWNSITE HRM30 & \\
\hline 2414 & & RIVER & & RAW GRAB & TRANSECT & 274 & HANFRD TWNSITE HRM28 & \\
\hline 2415 & & RIVER & & RAW GRAB & TRANSECT & 8726 & 100 F SHORE HRM 18 & \\
\hline 2416 & & RIVER & & RAW GRAB & TRANSECT & 13656 & 100 F SHORE HRM 24 & \\
\hline 2417 & & RIVER & & RAW GRAB & TRANSECT & 8728 & 100 F SHORE HRM 23 & \\
\hline 2418 & & RIVER & & RAW GRAB & TRANSECT & 348 & 300 AREA -1 HRM 43.1 & \\
\hline 2419 & & RIVER & & RAW GRAB & TRANSECT & 470 & 300 AREA -2 HRM 43.1 & \\
\hline 2420 & & RIVER & & RAW GRAB & TRANSECT & 580 & 300 AREA -3 HRM 43.1 & \\
\hline 2421 & & RIVER & & RAW GRAB & TRANSECT & 607 & 300 AREA -5 HRM 43.1 & \\
\hline 2422 & & RIVER & & RAW GRAB & TRANSECT & 351 & 300 AREA -7 HRM 43.1 & \\
\hline 2423 & & RIVER & & RAW GRAB & TRANSECT & 352 & 300 AREA-10 HRM 43.1 & \\
\hline 2424 & & RIVER & & RAW GRAB & TRANSECT & 571 & RICH.PMPHS-1 HRM46.4 & \\
\hline 2425 & & RIVER & & RAW GRAB & TRANSECT & 321 & RICH.PMPHS-2 HRM46.4 & \\
\hline 2426 & $\mathrm{~F}$ & RIVER & & RAW GRAB & TRANSECT & 322 & RICH.PMPHS-3 HRM46.4 & \\
\hline 2427 & & RIVER & & RAW GRAB & TRANSECT & 323 & RICH.PMPHS-5 HRM46.4 & \\
\hline 2428 & & RIVER & & RAW GRAB & TRANSECT & 325 & RICH.PMPHS-7 HRM46.4 & \\
\hline 2429 & & RIVER & & RAW GRAB & TRANSECT & 327 & RICH.PMPHS-10 HRM46.4 & \\
\hline 2430 & & RIVER & & RAW GRAB & TRANSECT & 8220 & RICH.PMPHS HRM 45.8 & \\
\hline 2431 & & RIVER & & RAW GRAB & TRANSECT & 8221 & RICH.PMPHS HRM 45.0 & \\
\hline 2432 & & RIVER & & RAW GRAB & TRANSECT & 8222 & RICH.PMPHS HRM 43.9 & \\
\hline 2433 & & RIVER & & RAW GRAB & TRANSECT & 8223 & RICH.PMPHS HRM 43.5 & \\
\hline
\end{tabular}




\begin{tabular}{|c|c|c|c|c|c|c|c|c|}
\hline & $T$ & $\bar{U}$ & $\mathrm{~V}$ & $\mathrm{~W}$ & $\bar{X}$ & $\bar{Y}$ & $\bar{Z}$ & $\overline{A A}$ \\
\hline 1 & REVIEW_QUALIFIER & SAMP_FROM & SAMP_ITEM & SAMP_MTHD & COLL_MTHD & SAMP_SITE_ID & SAMP_SITE_NAME & WELL_NAME \\
\hline 2434 & & RIVER & & RAW GRAB & TRANSECT & 8240 & 300 AREA SHR HRM41.5 & \\
\hline 2435 & & RIVER & & RAW GRAB & TRANSECT & 8241 & 300 AREA SHR HRM42.1 & \\
\hline 2436 & & RIVER & & RAW GRAB & TRANSECT & 8242 & 300 AREA SHR HRM42.5 & \\
\hline 2437 & & RIVER & & RAW GRAB & TRANSECT & 8243 & 300 AREA SHR HRM42.9 & \\
\hline 2438 & & RIVER & & CUMULATIVE & UNFILTERED & 252 & PRIEST RAPIDS-RIVER & \\
\hline 2439 & & RIVER & & CUMULATIVE & UNFILTERED & 463 & RICH.PMPHS HRM 46.4 & \\
\hline $2440 \mathrm{~F}$ & $\mathrm{~F}$ & SEEP & & RAW GRAB & UNFILTERED & 151 & HANFORD SPRING 28-2 & \\
\hline 2441 F & $\mathrm{F}$ & SEEP & & RAW GRAB & UNFILTERED & 488 & 300 AREA SPRING 42-2 & \\
\hline $2442 \mathrm{~F}$ & $\mathrm{~F}$ & SEEP & & RAW GRAB & UNFILTERED & 8143 & HANFORD SPR DR 28-2 & \\
\hline $2443 \mathrm{~F}$ & $\mathrm{~F}$ & SEEP & & RAW GRAB & UNFILTERED & 8846 & 100-H SPRING 152-2 & \\
\hline $2444 \mathrm{~F}$ & $\mathrm{~F}$ & SEEP & & RAW GRAB & UNFILTERED & 8147 & 100-F SPRING 207-1 & \\
\hline 2445 & & RIVER & & CUMULATIVE & UNFILTERED & 252 & PRIEST RAPIDS-RIVER & \\
\hline 2446 & & RIVER & & CUMULATIVE & UNFILTERED & 463 & RICH.PMPHS HRM 46.4 & \\
\hline 2447 & & RIVER & & CUMULATIVE & UNFILTERED & 252 & PRIEST RAPIDS-RIVER & \\
\hline 2448 & & RIVER & & CUMULATIVE & UNFILTERED & 463 & RICH.PMPHS HRM 46.4 & \\
\hline 2449 & & RIVER & & RAW GRAB & TRANSECT & 317 & VERNITA-1 HRM 0.3 & \\
\hline 2450 & & RIVER & & RAW GRAB & TRANSECT & 570 & VERNITA-2 HRM 0.3 & \\
\hline 2451 & & RIVER & & RAW GRAB & TRANSECT & 318 & VERNITA-3 HRM 0.3 & \\
\hline 2452 & & RIVER & & RAW GRAB & TRANSECT & 319 & VERNITA-4 HRM 0.3 & \\
\hline 2453 & & RIVER & & RAW GRAB & TRANSECT & 571 & RICH.PMPHS-1 HRM46.4 & \\
\hline 2454 & & RIVER & & RAW GRAB & TRANSECT & 571 & RICH.PMPHS-1 HRM46.4 & \\
\hline 2455 & & RIVER & & RAW GRAB & TRANSECT & 321 & RICH.PMPHS-2 HRM46.4 & \\
\hline 2456 & & RIVER & & RAW GRAB & TRANSECT & 322 & RICH.PMPHS-3 HRM46.4 & \\
\hline 2457 & & RIVER & & RAW GRAB & TRANSECT & 323 & RICH.PMPHS-5 HRM46.4 & \\
\hline 2458 & & RIVER & & RAW GRAB & TRANSECT & 325 & RICH.PMPHS-7 HRM46.4 & \\
\hline 2459 & & RIVER & & RAW GRAB & TRANSECT & 327 & RICH.PMPHS-10 HRM46.4 & \\
\hline 2460 & & RIVER & & RAW GRAB & TRANSECT & 8220 & RICH.PMPHS HRM 45.8 & \\
\hline 2461 & & RIVER & & RAW GRAB & TRANSECT & 8221 & RICH.PMPHS HRM 45.0 & \\
\hline 2462 & & RIVER & & RAW GRAB & TRANSECT & 8222 & RICH.PMPHS HRM 43.9 & \\
\hline 2463 & & RIVER & & RAW GRAB & TRANSECT & 8223 & RICH.PMPHS HRM 43.5 & \\
\hline $2464 \mathrm{~F}$ & $\mathrm{~F}$ & SURFACE & & RAW GRAB & UNFILTERED & 147 & WEST LAKE & \\
\hline 2465 & & RIVER & & CUMULATIVE & UNFILTERED & 463 & RICH.PMPHS HRM 46.4 & \\
\hline 2466 & & RIVER & & CUMULATIVE & UNFILTERED & 252 & PRIEST RAPIDS-RIVER & \\
\hline 2467 & & RIVER & & CUMULATIVE & UNFILTERED & 463 & RICH.PMPHS HRM 46.4 & \\
\hline 2468 & & RIVER & & CUMULATIVE & UNFILTERED & 252 & PRIEST RAPIDS-RIVER & \\
\hline 2469 & & RIVER & & RAW GRAB & TRANSECT & 317 & VERNITA-1 HRM 0.3 & \\
\hline 2470 & & RIVER & & RAW GRAB & TRANSECT & 570 & VERNITA-2 HRM 0.3 & \\
\hline 2471 & & RIVER & & RAW GRAB & TRANSECT & 318 & VERNITA-3 HRM 0.3 & \\
\hline
\end{tabular}




\begin{tabular}{|c|c|c|c|c|c|c|c|c|}
\hline & $T$ & $\bar{U}$ & $\mathrm{~V}$ & $\mathrm{~W}$ & $\bar{x}$ & $\bar{Y}$ & $\mathrm{Z}$ & AA \\
\hline 1 & REVIEW_QUALIFIER & SAMP_FROM & SAMP ITEM & SAMP_MTHD & COLL_MTHD & SAMP_SITE_ID & SAMP_SITE_NAME & WELL_NAME \\
\hline 2472 & & RIVER & & RAW GRAB & TRANSECT & 319 & VERNITA-4 HRM 0.3 & \\
\hline 2473 & & RIVER & & RAW GRAB & TRANSECT & 571 & RICH.PMPHS-1 HRM46.4 & \\
\hline 2474 & & RIVER & & RAW GRAB & TRANSECT & 571 & RICH.PMPHS-1 HRM46.4 & \\
\hline 2475 & & RIVER & & RAW GRAB & TRANSECT & 321 & RICH.PMPHS-2 HRM46.4 & \\
\hline 2476 & & RIVER & & RAW GRAB & TRANSECT & 322 & RICH.PMPHS-3 HRM46.4 & \\
\hline 2477 & & RIVER & & RAW GRAB & TRANSECT & 323 & RICH.PMPHS-5 HRM46.4 & \\
\hline 2478 & & RIVER & & RAW GRAB & TRANSECT & 325 & RICH.PMPHS-7 HRM46.4 & \\
\hline 2479 & & RIVER & & RAW GRAB & TRANSECT & 327 & RICH.PMPHS-10 HRM46.4 & \\
\hline 2480 & & RIVER & & RAW GRAB & TRANSECT & 8220 & RICH.PMPHS HRM 45.8 & \\
\hline 2481 & & RIVER & & RAW GRAB & TRANSECT & 8221 & RICH.PMPHS HRM 45.0 & \\
\hline 2482 & & RIVER & & RAW GRAB & TRANSECT & 8222 & RICH.PMPHS HRM 43.9 & \\
\hline 2483 & & RIVER & & RAW GRAB & TRANSECT & 8223 & RICH.PMPHS HRM 43.5 & \\
\hline 2484 & $F$ & SURFACE & & RAW GRAB & UNFILTERED & 147 & WEST LAKE & \\
\hline 2485 & & RIVER & & CUMULATIVE & UNFILTERED & 463 & RICH.PMPHS HRM 46.4 & \\
\hline 2486 & & RIVER & & CUMULATIVE & UNFILTERED & 252 & PRIEST RAPIDS-RIVER & \\
\hline 2487 & & RIVER & & CUMULATIVE & UNFILTERED & 252 & PRIEST RAPIDS-RIVER & \\
\hline 2488 & & RIVER & & CUMULATIVE & UNFILTERED & 463 & RICH.PMPHS HRM 46.4 & \\
\hline 2489 & & IRRIGATION & & RAW GRAB & UNFILTERED & 440 & RIVERVIEW CANAL & \\
\hline 2490 & & RIVER & & CUMULATIVE & UNFILTERED & 252 & PRIEST RAPIDS-RIVER & \\
\hline 2491 & & RIVER & & CUMULATIVE & UNFILTERED & 463 & RICH.PMPHS HRM 46.4 & \\
\hline 2492 & & IRRIGATION & & RAW GRAB & UNFILTERED & 440 & RIVERVIEW CANAL & \\
\hline 2493 & $F$ & SEEP & & RAW GRAB & & 8626 & WEST LAKE SPRING & \\
\hline 2494 & $F$ & SURFACE & & RAW GRAB & UNFILTERED & 147 & WEST LAKE & \\
\hline 2495 & $F$ & SURFACE & & RAW GRAB & & 147 & WEST LAKE & \\
\hline 2496 & & RIVER & & RAW GRAB & TRANSECT & 317 & VERNITA-1 HRM 0.3 & \\
\hline 2497 & & RIVER & & RAW GRAB & TRANSECT & 570 & VERNITA-2 HRM 0.3 & \\
\hline 2498 & & RIVER & & RAW GRAB & TRANSECT & 318 & VERNITA-3 HRM 0.3 & \\
\hline 2499 & & RIVER & & RAW GRAB & TRANSECT & 319 & VERNITA-4 HRM 0.3 & \\
\hline 2500 & & RIVER & & RAW GRAB & TRANSECT & 571 & RICH.PMPHS-1 HRM46.4 & \\
\hline 2501 & & RIVER & & RAW GRAB & TRANSECT & 321 & RICH.PMPHS-2 HRM46.4 & \\
\hline 2502 & & RIVER & & RAW GRAB & TRANSECT & 322 & RICH.PMPHS-3 HRM46.4 & \\
\hline 2503 & & RIVER & & RAW GRAB & TRANSECT & 323 & RICH.PMPHS-5 HRM46.4 & \\
\hline 2504 & & RIVER & & RAW GRAB & TRANSECT & 325 & RICH.PMPHS-7 HRM46.4 & \\
\hline 2505 & & RIVER & & RAW GRAB & TRANSECT & 327 & RICH.PMPHS-10 HRM46.4 & \\
\hline 2506 & & RIVER & & RAW GRAB & TRANSECT & 8220 & RICH.PMPHS HRM 45.8 & \\
\hline 2507 & & RIVER & & RAW GRAB & TRANSECT & 8221 & RICH.PMPHS HRM 45.0 & \\
\hline 2508 & & RIVER & & RAW GRAB & TRANSECT & 8222 & RICH.PMPHS HRM 43.9 & \\
\hline 2509 & & RIVER & & RAW GRAB & TRANSECT & 8223 & RICH.PMPHS HRM 43.5 & \\
\hline
\end{tabular}




\begin{tabular}{|c|c|c|c|c|c|c|c|c|}
\hline & $\mathrm{T}$ & $U$ & $\mathrm{~V}$ & $\mathrm{~W}$ & $\mathrm{X}$ & $\mathrm{Y}$ & Z & AA \\
\hline 1 & REVIEW_QUALIFIER & SAMP_FROM & SAMP_ITEM & SAMP_MTHD & COLL_MTHD & SAMP_SITE_ID & SAMP_SITE_NAME & WELL_NAME \\
\hline 2510 & & RIVER & & CUMULATIVE & UNFILTERED & 252 & PRIEST RAPIDS-RIVER & \\
\hline 2511 & & RIVER & & CUMULATIVE & UNFILTERED & 463 & RICH.PMPHS HRM 46.4 & \\
\hline 2512 & $\mathrm{~F}$ & SURFACE & & RAW GRAB & UNFILTERED & 147 & WEST LAKE & \\
\hline 2513 & & IRRIGATION & & RAW GRAB & UNFILTERED & 440 & RIVERVIEW CANAL & \\
\hline 2514 & & RIVER & & CUMULATIVE & UNFILTERED & 252 & PRIEST RAPIDS-RIVER & \\
\hline 2515 & & RIVER & & CUMULATIVE & UNFILTERED & 463 & RICH.PMPHS HRM 46.4 & \\
\hline 2516 & & RIVER & & CUMULATIVE & UNFILTERED & 252 & PRIEST RAPIDS-RIVER & \\
\hline 2517 & & RIVER & & CUMULATIVE & UNFILTERED & 463 & RICH.PMPHS HRM 46.4 & \\
\hline 2518 & & RIVER & & RAW GRAB & TRANSECT & 328 & 100 N -1 HRM 9.5 & \\
\hline 2519 & & RIVER & & RAW GRAB & TRANSECT & 317 & VERNITA-1 HRM 0.3 & \\
\hline 2520 & & RIVER & & RAW GRAB & TRANSECT & 570 & VERNITA-2 HRM 0.3 & \\
\hline 2521 & & RIVER & & RAW GRAB & TRANSECT & 318 & VERNITA-3 HRM 0.3 & \\
\hline 2522 & & RIVER & & RAW GRAB & TRANSECT & 319 & VERNITA-4 HRM 0.3 & \\
\hline 2523 & & RIVER & & RAW GRAB & TRANSECT & 328 & 100 N -1 HRM 9.5 & \\
\hline 2524 & & RIVER & & RAW GRAB & TRANSECT & 329 & 100 N -2 HRM 9.5 & \\
\hline 2525 & & RIVER & & RAW GRAB & TRANSECT & 331 & 100 N -5 HRM 9.5 & \\
\hline 2526 & & RIVER & & RAW GRAB & TRANSECT & 335 & 100 N -10 HRM 9.5 & \\
\hline 2527 & & RIVER & & RAW GRAB & TRANSECT & 330 & 100 N -3 HRM 9.5 & \\
\hline 2528 & & RIVER & & RAW GRAB & TRANSECT & 333 & 100 N -7 HRM 9.5 & \\
\hline 2529 & & RIVER & & RAW GRAB & TRANSECT & 8244 & 100 N SHORE HRM 8.4 & \\
\hline 2530 & & RIVER & & RAW GRAB & TRANSECT & 8245 & 100 N SHORE HRM 8.9 & \\
\hline 2531 & & RIVER & & RAW GRAB & TRANSECT & 8246 & 100 N SHORE HRM 9.2 & \\
\hline 2532 & & RIVER & & RAW GRAB & TRANSECT & 8247 & 100 N SHORE HRM 9.8 & \\
\hline 2533 & & RIVER & & RAW GRAB & TRANSECT & 336 & 100 F -1 HRM 19.0 & \\
\hline 2534 & & RIVER & & RAW GRAB & TRANSECT & 337 & 100 F -3 HRM 19.0 & \\
\hline 2535 & & RIVER & & RAW GRAB & TRANSECT & 344 & HANFRD TS-5 HRM 28.7 & \\
\hline 2536 & & RIVER & & RAW GRAB & TRANSECT & 576 & 100 F -2 HRM 19.0 & \\
\hline 2537 & & RIVER & & RAW GRAB & TRANSECT & 577 & 100 F -5 HRM 19.0 & \\
\hline 2538 & & RIVER & & RAW GRAB & TRANSECT & 339 & 100 F -7 HRM 19.0 & \\
\hline 2539 & & RIVER & & RAW GRAB & TRANSECT & 341 & $100 \mathrm{~F}-10 \mathrm{HRM} 19.0$ & \\
\hline 2540 & & RIVER & & RAW GRAB & TRANSECT & 578 & HANFRD TS-1 HRM 28.7 & \\
\hline 2541 & & RIVER & & RAW GRAB & TRANSECT & 342 & HANFRD TS-2 HRM 28.7 & \\
\hline 2542 & & RIVER & & RAW GRAB & TRANSECT & 469 & HANFRD TS-7 HRM 28.7 & \\
\hline 2543 & & RIVER & & RAW GRAB & TRANSECT & 347 & HANFRD TS-10 HRM 28.7 & \\
\hline 2544 & & RIVER & & RAW GRAB & TRANSECT & 468 & HANFRD TS-3 HRM 28.7 & \\
\hline 2545 & & RIVER & & RAW GRAB & TRANSECT & 8100 & HANFRD TWNSITE HRM26 & \\
\hline 2546 & & RIVER & & RAW GRAB & TRANSECT & 8101 & HANFRD TWNSITE HRM27 & \\
\hline 2547 & & RIVER & & RAW GRAB & TRANSECT & 8103 & HANFRD TWNSITE HRM30 & \\
\hline
\end{tabular}




\begin{tabular}{|c|c|c|c|c|c|c|c|c|}
\hline & $T$ & $\bar{U}$ & $\mathrm{~V}$ & $\mathrm{~W}$ & $\bar{X}$ & $\bar{Y}$ & $\bar{Z}$ & $\overline{A A}$ \\
\hline 1 & REVIEW_QUALIFIER & SAMP_FROM & SAMP_ITEM & SAMP_MTHD & COLL_MTHD & SAMP_SITE_ID & SAMP_SITE_NAME & WELL_NAME \\
\hline 2548 & & RIVER & & RAW GRAB & TRANSECT & 274 & HANFRD TWNSITE HRM28 & \\
\hline 2549 & & RIVER & & RAW GRAB & TRANSECT & 8726 & 100 F SHORE HRM 18 & \\
\hline 2550 & & RIVER & & RAW GRAB & TRANSECT & 8727 & 100 F SHORE HRM 22 & \\
\hline 2551 & & RIVER & & RAW GRAB & TRANSECT & 8728 & 100 F SHORE HRM 23 & \\
\hline 2552 & & RIVER & & RAW GRAB & TRANSECT & 348 & 300 AREA -1 HRM 43.1 & \\
\hline 2553 & & RIVER & & RAW GRAB & TRANSECT & 470 & 300 AREA -2 HRM 43.1 & \\
\hline 2554 & & RIVER & & RAW GRAB & TRANSECT & 580 & 300 AREA -3 HRM 43.1 & \\
\hline 2555 & & RIVER & & RAW GRAB & TRANSECT & 607 & 300 AREA -5 HRM 43.1 & \\
\hline 2556 & & RIVER & & RAW GRAB & TRANSECT & 351 & 300 AREA -7 HRM 43.1 & \\
\hline 2557 & & RIVER & & RAW GRAB & TRANSECT & 352 & 300 AREA-10 HRM 43.1 & \\
\hline 2558 & & RIVER & & RAW GRAB & TRANSECT & 571 & RICH.PMPHS-1 HRM46.4 & \\
\hline 2559 & & RIVER & & RAW GRAB & TRANSECT & 321 & RICH.PMPHS-2 HRM46.4 & \\
\hline 2560 & & RIVER & & RAW GRAB & TRANSECT & 322 & RICH.PMPHS-3 HRM46.4 & \\
\hline 2561 & & RIVER & & RAW GRAB & TRANSECT & 323 & RICH.PMPHS-5 HRM46.4 & \\
\hline 2562 & & RIVER & & RAW GRAB & TRANSECT & 325 & RICH.PMPHS-7 HRM46.4 & \\
\hline 2563 & & RIVER & & RAW GRAB & TRANSECT & 327 & RICH.PMPHS-10 HRM46.4 & \\
\hline 2564 & & RIVER & & RAW GRAB & TRANSECT & 8220 & RICH.PMPHS HRM 45.8 & \\
\hline 2565 & & RIVER & & RAW GRAB & TRANSECT & 8221 & RICH.PMPHS HRM 45.0 & \\
\hline 2566 & & RIVER & & RAW GRAB & TRANSECT & 8222 & RICH.PMPHS HRM 43.9 & \\
\hline 2567 & & RIVER & & RAW GRAB & TRANSECT & 8223 & RICH.PMPHS HRM 43.5 & \\
\hline 2568 & & RIVER & & RAW GRAB & TRANSECT & 8240 & 300 AREA SHR HRM41.5 & \\
\hline 2569 & & RIVER & & RAW GRAB & TRANSECT & 8241 & 300 AREA SHR HRM42.1 & \\
\hline 2570 & & RIVER & & RAW GRAB & TRANSECT & 8242 & 300 AREA SHR HRM42.5 & \\
\hline 2571 & & RIVER & & RAW GRAB & TRANSECT & 8243 & 300 AREA SHR HRM42.9 & \\
\hline 2572 & $\mathrm{~F}$ & SEEP & & RAW GRAB & UNFILTERED & 151 & HANFORD SPRING 28-2 & \\
\hline 2573 & $\mathrm{~F}$ & SEEP & & RAW GRAB & UNFILTERED & 488 & 300 AREA SPRING 42-2 & \\
\hline 2574 & $\mathrm{~F}$ & SEEP & & RAW GRAB & UNFILTERED & 8142 & HANFORD SPR UR 28-2 & \\
\hline 2575 & $\mathrm{~F}$ & SEEP & & RAW GRAB & UNFILTERED & 8143 & HANFORD SPR DR 28-2 & \\
\hline 2576 & $\mathrm{~F}$ & SEEP & & RAW GRAB & UNFILTERED & 8144 & 300 AREA SPR DR 42-2 & \\
\hline 2577 & $\mathrm{~F}$ & IRRIGATION & & RAW GRAB & UNFILTERED & 605 & HORN RAPIDS AREA & \\
\hline 2578 & & RIVER & & CUMULATIVE & UNFILTERED & 252 & PRIEST RAPIDS-RIVER & \\
\hline 2579 & & RIVER & & CUMULATIVE & UNFILTERED & 463 & RICH.PMPHS HRM 46.4 & \\
\hline 2580 & $\mathrm{~F}$ & SURFACE & & RAW GRAB & UNFILTERED & 147 & WEST LAKE & \\
\hline 2581 & $\mathrm{~F}$ & SEEP & & RAW GRAB & UNFILTERED & 8165 & 100-H SPRING 145-1 & \\
\hline 2582 & $\mathrm{~F}$ & SEEP & & RAW GRAB & UNFILTERED & 8164 & 100-H SPRING 153-1 & \\
\hline 2583 & & RIVER & & CUMULATIVE & UNFILTERED & 252 & PRIEST RAPIDS-RIVER & \\
\hline 2584 & & RIVER & & CUMULATIVE & UNFILTERED & 463 & RICH.PMPHS HRM 46.4 & \\
\hline 2585 & $\mathrm{~F}$ & SEEP & & RAW GRAB & UNFILTERED & 8147 & 100-F SPRING 207-1 & \\
\hline
\end{tabular}




\begin{tabular}{|c|c|c|c|c|c|c|c|c|}
\hline & $T$ & $\bar{U}$ & $\mathrm{~V}$ & $\mathrm{~W}$ & $\bar{X}$ & $\bar{Y}$ & $\bar{Z}$ & $\overline{A A}$ \\
\hline 1 & REVIEW_QUALIFIER & SAMP_FROM & SAMP_ITEM & SAMP_MTHD & COLL_MTHD & SAMP_SITE_ID & SAMP_SITE_NAME & WELL_NAME \\
\hline 2586 & & RIVER & & CUMULATIVE & UNFILTERED & 252 & PRIEST RAPIDS-RIVER & \\
\hline 2587 & & RIVER & & CUMULATIVE & UNFILTERED & 463 & RICH.PMPHS HRM 46.4 & \\
\hline 2588 & & RIVER & & RAW GRAB & TRANSECT & 317 & VERNITA-1 HRM 0.3 & \\
\hline 2589 & & RIVER & & RAW GRAB & TRANSECT & 570 & VERNITA-2 HRM 0.3 & \\
\hline 2590 & & RIVER & & RAW GRAB & TRANSECT & 318 & VERNITA-3 HRM 0.3 & \\
\hline 2591 & & RIVER & & RAW GRAB & TRANSECT & 319 & VERNITA-4 HRM 0.3 & \\
\hline 2592 & & RIVER & & RAW GRAB & TRANSECT & 571 & RICH.PMPHS-1 HRM46.4 & \\
\hline 2593 & & RIVER & & RAW GRAB & TRANSECT & 571 & RICH.PMPHS-1 HRM46.4 & \\
\hline 2594 & & RIVER & & RAW GRAB & TRANSECT & 321 & RICH.PMPHS-2 HRM46.4 & \\
\hline 2595 & & RIVER & & RAW GRAB & TRANSECT & 322 & RICH.PMPHS-3 HRM46.4 & \\
\hline 2596 & & RIVER & & RAW GRAB & TRANSECT & 323 & RICH.PMPHS-5 HRM46.4 & \\
\hline 2597 & & RIVER & & RAW GRAB & TRANSECT & 325 & RICH.PMPHS-7 HRM46.4 & \\
\hline 2598 & & RIVER & & RAW GRAB & TRANSECT & 327 & RICH.PMPHS-10 HRM46.4 & \\
\hline 2599 & & RIVER & & RAW GRAB & TRANSECT & 8220 & RICH.PMPHS HRM 45.8 & \\
\hline 2600 & & RIVER & & RAW GRAB & TRANSECT & 8221 & RICH.PMPHS HRM 45.0 & \\
\hline 2601 & & RIVER & & RAW GRAB & TRANSECT & 8222 & RICH.PMPHS HRM 43.9 & \\
\hline 2602 & & RIVER & & RAW GRAB & TRANSECT & 8223 & RICH.PMPHS HRM 43.5 & \\
\hline 2603 & & RIVER & & CUMULATIVE & UNFILTERED & 252 & PRIEST RAPIDS-RIVER & \\
\hline 2604 & & RIVER & & CUMULATIVE & UNFILTERED & 463 & RICH.PMPHS HRM 46.4 & \\
\hline 2605 & & RIVER & & CUMULATIVE & UNFILTERED & 252 & PRIEST RAPIDS-RIVER & \\
\hline 2606 & & RIVER & & CUMULATIVE & UNFILTERED & 463 & RICH.PMPHS HRM 46.4 & \\
\hline $2607 \mathrm{~F}$ & $\mathrm{~F}$ & SURFACE & & RAW GRAB & UNFILTERED & 147 & WEST LAKE & \\
\hline 2608 & & RIVER & & RAW GRAB & TRANSECT & 571 & RICH.PMPHS-1 HRM46.4 & \\
\hline 2609 & & RIVER & & RAW GRAB & TRANSECT & 571 & RICH.PMPHS-1 HRM46.4 & \\
\hline 2610 & & RIVER & & RAW GRAB & TRANSECT & 321 & RICH.PMPHS-2 HRM46.4 & \\
\hline 2611 & & RIVER & & RAW GRAB & TRANSECT & 322 & RICH.PMPHS-3 HRM46.4 & \\
\hline 2612 & & RIVER & & RAW GRAB & TRANSECT & 323 & RICH.PMPHS-5 HRM46.4 & \\
\hline 2613 & & RIVER & & RAW GRAB & TRANSECT & 325 & RICH.PMPHS-7 HRM46.4 & \\
\hline 2614 & & RIVER & & RAW GRAB & TRANSECT & 327 & RICH.PMPHS-10 HRM46.4 & \\
\hline 2615 & & RIVER & & RAW GRAB & TRANSECT & 8220 & RICH.PMPHS HRM 45.8 & \\
\hline 2616 & & RIVER & & RAW GRAB & TRANSECT & 8221 & RICH.PMPHS HRM 45.0 & \\
\hline 2617 & & RIVER & & RAW GRAB & TRANSECT & 8222 & RICH.PMPHS HRM 43.9 & \\
\hline 2618 & & RIVER & & RAW GRAB & TRANSECT & 8223 & RICH.PMPHS HRM 43.5 & \\
\hline 2619 & & RIVER & & RAW GRAB & TRANSECT & 317 & VERNITA-1 HRM 0.3 & \\
\hline 2620 & & RIVER & & RAW GRAB & TRANSECT & 570 & VERNITA-2 HRM 0.3 & \\
\hline 2621 & & RIVER & & RAW GRAB & TRANSECT & 318 & VERNITA-3 HRM 0.3 & \\
\hline 2622 & & RIVER & & RAW GRAB & TRANSECT & 319 & VERNITA-4 HRM 0.3 & \\
\hline 2623 & & RIVER & & CUMULATIVE & UNFILTERED & 252 & PRIEST RAPIDS-RIVER & \\
\hline
\end{tabular}




\begin{tabular}{|c|c|c|c|c|c|c|c|c|}
\hline & $T$ & $\bar{U}$ & $\mathrm{~V}$ & $\mathrm{~W}$ & $\bar{X}$ & $\bar{Y}$ & $\bar{Z}$ & $\overline{A A}$ \\
\hline 1 & REVIEW_QUALIFIER & SAMP_FROM & SAMP_ITEM & SAMP_MTHD & COLL_MTHD & SAMP_SITE_ID & SAMP_SITE_NAME & WELL_NAME \\
\hline 2624 & & RIVER & & CUMULATIVE & UNFILTERED & 463 & RICH.PMPHS HRM 46.4 & \\
\hline 2625 & $\mathrm{~F}$ & SURFACE & & RAW GRAB & UNFILTERED & 147 & WEST LAKE & \\
\hline 2626 & & RIVER & & CUMULATIVE & UNFILTERED & 252 & PRIEST RAPIDS-RIVER & \\
\hline 2627 & & RIVER & & CUMULATIVE & UNFILTERED & 463 & RICH.PMPHS HRM 46.4 & \\
\hline 2628 & $\mathrm{~F}$ & SEEP & & RAW GRAB & UNFILTERED & 151 & HANFORD SPRING $28-2$ & \\
\hline 2629 & $\mathrm{~F}$ & SEEP & & RAW GRAB & UNFILTERED & 8165 & 100-H SPRING 145-1 & \\
\hline 2630 & $\mathrm{~F}$ & SEEP & & RAW GRAB & UNFILTERED & 8147 & 100-F SPRING 207-1 & \\
\hline 2631 & $\mathrm{~F}$ & SEEP & & RAW GRAB & UNFILTERED & 8143 & HANFORD SPR DR 28-2 & \\
\hline 2632 & $\mathrm{~F}$ & SEEP & & RAW GRAB & UNFILTERED & 8164 & 100-H SPRING 153-1 & \\
\hline 2633 & & RIVER & & CUMULATIVE & UNFILTERED & 463 & RICH.PMPHS HRM 46.4 & \\
\hline 2634 & & RIVER & & CUMULATIVE & UNFILTERED & 252 & PRIEST RAPIDS-RIVER & \\
\hline 2635 & $\mathrm{~F}$ & SEEP & & RAW GRAB & UNFILTERED & 8144 & 300 AREA SPR DR 42-2 & \\
\hline 2636 & $\mathrm{~F}$ & IRRIGATION & & RAW GRAB & UNFILTERED & 440 & RIVERVIEW CANAL & \\
\hline 2637 & & IRRIGATION & & RAW GRAB & UNFILTERED & 605 & HORN RAPIDS AREA & \\
\hline 2638 & $\mathrm{~F}$ & SEEP & & RAW GRAB & UNFILTERED & 488 & 300 AREA SPRING 42-2 & \\
\hline 2639 & & SEEP & & RAW GRAB & UNFILTERED & 9147 & RICHLAND SPR HRM44.4 & \\
\hline 2640 & & RIVER & & CUMULATIVE & UNFILTERED & 463 & RICH.PMPHS HRM 46.4 & \\
\hline 2641 & & RIVER & & CUMULATIVE & UNFILTERED & 252 & PRIEST RAPIDS-RIVER & \\
\hline 2642 & & IRRIGATION & & RAW GRAB & UNFILTERED & 440 & RIVERVIEW CANAL & \\
\hline 2643 & & IRRIGATION & & RAW GRAB & UNFILTERED & 605 & HORN RAPIDS AREA & \\
\hline 2644 & & RIVER & & RAW GRAB & TRANSECT & 571 & RICH.PMPHS-1 HRM46.4 & \\
\hline 2645 & & RIVER & & RAW GRAB & TRANSECT & 321 & RICH.PMPHS-2 HRM46.4 & \\
\hline 2646 & & RIVER & & RAW GRAB & TRANSECT & 322 & RICH.PMPHS-3 HRM46.4 & \\
\hline 2647 & & RIVER & & RAW GRAB & TRANSECT & 323 & RICH.PMPHS-5 HRM46.4 & \\
\hline 2648 & & RIVER & & RAW GRAB & TRANSECT & 325 & RICH.PMPHS-7 HRM46.4 & \\
\hline 2649 & & RIVER & & RAW GRAB & TRANSECT & 327 & RICH.PMPHS-10 HRM46.4 & \\
\hline 2650 & & RIVER & & RAW GRAB & TRANSECT & 8220 & RICH.PMPHS HRM 45.8 & \\
\hline 2651 & & RIVER & & RAW GRAB & TRANSECT & 8221 & RICH.PMPHS HRM 45.0 & \\
\hline 2652 & & RIVER & & RAW GRAB & TRANSECT & 8222 & RICH.PMPHS HRM 43.9 & \\
\hline 2653 & & RIVER & & RAW GRAB & TRANSECT & 8223 & RICH.PMPHS HRM 43.5 & \\
\hline 2654 & & RIVER & & RAW GRAB & TRANSECT & 317 & VERNITA-1 HRM 0.3 & \\
\hline 2655 & & RIVER & & RAW GRAB & TRANSECT & 570 & VERNITA-2 HRM 0.3 & \\
\hline 2656 & & RIVER & & RAW GRAB & TRANSECT & 318 & VERNITA-3 HRM 0.3 & \\
\hline 2657 & & RIVER & & RAW GRAB & TRANSECT & 319 & VERNITA-4 HRM 0.3 & \\
\hline 2658 & & RIVER & & CUMULATIVE & UNFILTERED & 463 & RICH.PMPHS HRM 46.4 & \\
\hline 2659 & & RIVER & & CUMULATIVE & UNFILTERED & 252 & PRIEST RAPIDS-RIVER & \\
\hline 2660 & $\mathrm{~F}$ & SURFACE & & RAW GRAB & UNFILTERED & 147 & WEST LAKE & \\
\hline 2661 & & IRRIGATION & & RAW GRAB & UNFILTERED & 440 & RIVERVIEW CANAL & \\
\hline
\end{tabular}




\begin{tabular}{|c|c|c|c|c|c|c|c|c|}
\hline & $\mathrm{T}$ & $U$ & $\mathrm{~V}$ & $\mathrm{~W}$ & $\mathrm{X}$ & $\mathrm{Y}$ & Z & AA \\
\hline 1 & REVIEW_QUALIFIER & SAMP_FROM & SAMP_ITEM & SAMP_MTHD & COLL_MTHD & SAMP_SITE_ID & SAMP_SITE_NAME & WELL_NAME \\
\hline 2662 & & IRRIGATION & & RAW GRAB & UNFILTERED & 605 & HORN RAPIDS AREA & \\
\hline 2663 & & RIVER & & CUMULATIVE & UNFILTERED & 463 & RICH.PMPHS HRM 46.4 & \\
\hline 2664 & & RIVER & & CUMULATIVE & UNFILTERED & 252 & PRIEST RAPIDS-RIVER & \\
\hline 2665 & $\mathrm{~F}$ & SEEP & & RAW GRAB & UNFILTERED & 488 & 300 AREA SPRING 42-2 & \\
\hline 2666 & $\mathrm{~F}$ & SEEP & & RAW GRAB & UNFILTERED & 8144 & 300 AREA SPR DR 42-2 & \\
\hline 2667 & & RIVER & & RAW GRAB & & 9236 & VERNITA BRIDGE -1 & \\
\hline 2668 & & SEEP & & RAW GRAB & & 488 & 300 AREA SPRING 42-2 & \\
\hline 2669 & & SEEP & & RAW GRAB & & 8144 & 300 AREA SPR DR 42-2 & \\
\hline 2670 & & RIVER & & RAW GRAB & & 9237 & VERNITA BRIDGE -2 & \\
\hline 2671 & & RIVER & & RAW GRAB & UNFILTERED & 9236 & VERNITA BRIDGE -1 & \\
\hline 2672 & & RIVER & & RAW GRAB & UNFILTERED & 9237 & VERNITA BRIDGE -2 & \\
\hline 2673 & & RIVER & & RAW GRAB & UNFILTERED & 9238 & VERNITA BRIDGE -3 & \\
\hline 2674 & $\mathrm{~F}$ & RIVER & & RAW GRAB & UNFILTERED & 9210 & 300 SPR 7 -1 & \\
\hline 2675 & $\mathrm{~F}$ & RIVER & & RAW GRAB & UNFILTERED & 9211 & 300 SPR $7-2$ & \\
\hline 2676 & $\mathrm{~F}$ & RIVER & & RAW GRAB & UNFILTERED & 9213 & 300 SPR $7-3$ & \\
\hline 2677 & $\mathrm{~F}$ & RIVER & & RAW GRAB & UNFILTERED & 9214 & 300 SPR DR $7-1$ & \\
\hline 2678 & $\mathrm{~F}$ & RIVER & & RAW GRAB & UNFILTERED & 9215 & 300 SPR DR $7-2$ & \\
\hline 2679 & & RIVER & & RAW GRAB & UNFILTERED & 9216 & 300 SPR DR $7-3$ & \\
\hline 2680 & & RIVER & & RAW GRAB & UNFILTERED & 9217 & 300 SPR 7 THRU SPR 9 & \\
\hline 2681 & $\mathrm{~F}$ & RIVER & & RAW GRAB & UNFILTERED & 9218 & 300 SPR 9 -1 & \\
\hline 2682 & $\mathrm{~F}$ & RIVER & & RAW GRAB & UNFILTERED & 9219 & 300 SPR $9-2$ & \\
\hline 2683 & & RIVER & & RAW GRAB & UNFILTERED & 9220 & 300 SPR $9-3$ & \\
\hline 2684 & $\mathrm{~F}$ & RIVER & & RAW GRAB & UNFILTERED & 9221 & 300 SPR DR 9 -1 & \\
\hline 2685 & $\mathrm{~F}$ & RIVER & & RAW GRAB & UNFILTERED & 9222 & 300 SPR DR $9-2$ & \\
\hline 2686 & $\mathrm{~F}$ & RIVER & & RAW GRAB & UNFILTERED & 9223 & 300 SPR DR 9 -3 & \\
\hline 2687 & $\mathrm{~F}$ & RIVER & & RAW GRAB & UNFILTERED & 9224 & 300 SPR 9 THRU SPR 11 & \\
\hline 2688 & $\mathrm{~F}$ & RIVER & & RAW GRAB & UNFILTERED & 9226 & 300 SPR $11-1$ & \\
\hline 2689 & $\mathrm{~F}$ & RIVER & & RAW GRAB & UNFILTERED & 9227 & 300 SPR $11-2$ & \\
\hline 2690 & $\mathrm{~F}$ & RIVER & & RAW GRAB & UNFILTERED & 9228 & 300 SPR $11-3$ & \\
\hline 2691 & $\mathrm{~F}$ & RIVER & & RAW GRAB & UNFILTERED & 9229 & 300 SPR DR $11-1$ & \\
\hline 2692 & $\mathrm{~F}$ & RIVER & & RAW GRAB & UNFILTERED & 9230 & 300 SPR DR $11-2$ & \\
\hline 2693 & $\mathrm{~F}$ & RIVER & & RAW GRAB & UNFILTERED & 9231 & 300 SPR DR $11-3$ & \\
\hline 2694 & $\mathrm{~F}$ & RIVER & & RAW GRAB & UNFILTERED & 9233 & 300 SPR $14-1$ & \\
\hline 2695 & $\mathrm{~F}$ & RIVER & & RAW GRAB & UNFILTERED & 9234 & 300 SPR $14-2$ & \\
\hline 2696 & $\mathrm{~F}$ & RIVER & & RAW GRAB & UNFILTERED & 9235 & 300 SPR $14-3$ & \\
\hline 2697 & & RIVER & & RAW GRAB & UNFILTERED & 9280 & VERNITA BRIDGE -4 & \\
\hline 2698 & & RIVER & & RAW GRAB & UNFILTERED & 9273 & 300 SPR $7-4$ & \\
\hline 2699 & & RIVER & & RAW GRAB & UNFILTERED & 9274 & 300 SPR DR $7-4$ & \\
\hline
\end{tabular}




\begin{tabular}{|c|c|c|c|c|c|c|c|c|}
\hline & $\mathrm{T}$ & $\mathrm{U}$ & $\mathrm{V}$ & $\mathrm{W}$ & $\mathrm{x}$ & $\mathrm{Y}$ & $Z$ & $\mathrm{AA}$ \\
\hline 1 & REVIEW_QUALIFIER & SAMP_FROM & SAMP_ITEM & SAMP_MTHD & COLL_MTHD & SAMP_SITE_ID & SAMP_SITE_NAME & WELL_NAME \\
\hline 2700 & & RIVER & & RAW GRAB & UNFILTERED & 9275 & 500 SPR $9-4$ & \\
\hline 2701 & & RIVER & & RAW GRAB & UNFILTERED & 9276 & 300 SPR DR $9-4$ & \\
\hline 2702 & $F$ & RIVER & & RAW GRAB & UNFILTERED & 9277 & 7300 SPR $11-4$ & \\
\hline 2703 & & RIVER & & RAW GRAB & UNFILTERED & 9278 & 300 SPR DR 11 -4 & \\
\hline 2704 & & RIVER & & RAW GRAB & UNFILTERED & 9279 & 9 300 SPR $14-4$ & \\
\hline 2705 & & RIVER & & CUMULATIVE & UNFILTERED & 463 & 3 RICH.PMPHS HRM 46.4 & \\
\hline 2706 & & RIVER & & CUMULATIVE & UNFILTERED & 252 & 2 PRIEST RAPIDS-RIVER & \\
\hline 2707 & & RIVER & & RAW GRAB & TRANSECT & 328 & 3100 N -1 HRM 9.5 & \\
\hline 2708 & & RIVER & & RAW GRAB & TRANSECT & 317 & 7 VERNITA-1 HRM 0.3 & \\
\hline 2709 & & RIVER & & RAW GRAB & TRANSECT & 570 & VERNITA-2 HRM 0.3 & \\
\hline 2710 & & RIVER & & RAW GRAB & TRANSECT & 318 & 3 VERNITA-3 HRM 0.3 & \\
\hline 2711 & & RIVER & & RAW GRAB & TRANSECT & 319 & VERNITA-4 HRM 0.3 & \\
\hline 2712 & & RIVER & & RAW GRAB & TRANSECT & 328 & 3100 N -1 HRM 9.5 & \\
\hline 2713 & & RIVER & & RAW GRAB & TRANSECT & 329 & $9100 \mathrm{~N}-2$ HRM 9.5 & \\
\hline 2714 & & RIVER & & RAW GRAB & TRANSECT & 331 & 100 N -5 HRM 9.5 & \\
\hline 2715 & & RIVER & & RAW GRAB & TRANSECT & 335 & $5100 \mathrm{~N}-10$ HRM 9.5 & \\
\hline 2716 & & RIVER & & RAW GRAB & TRANSECT & 330 & 100 N -3 HRM 9.5 & \\
\hline 2717 & & RIVER & & RAW GRAB & TRANSECT & 333 & 3100 N -7 HRM 9.5 & \\
\hline 2718 & & RIVER & & RAW GRAB & TRANSECT & 8244 & 4100 N SHORE HRM 8.4 & \\
\hline 2719 & & RIVER & & RAW GRAB & TRANSECT & 8245 & 5100 N SHORE HRM 8.9 & \\
\hline 2720 & & RIVER & & RAW GRAB & TRANSECT & 8246 & 100 N SHORE HRM 9.2 & \\
\hline 2721 & & RIVER & & RAW GRAB & TRANSECT & 8247 & 7100 N SHORE HRM 9.8 & \\
\hline 2722 & & RIVER & & RAW GRAB & TRANSECT & 336 & 100 F -1 HRM 19.0 & \\
\hline 2723 & & RIVER & & RAW GRAB & TRANSECT & 337 & 7100 F -3 HRM 19.0 & \\
\hline 2724 & & RIVER & & RAW GRAB & TRANSECT & 344 & 4 HANFRD TS-5 HRM 28.7 & \\
\hline 2725 & & RIVER & & RAW GRAB & TRANSECT & 576 & 100 F -2 HRM 19.0 & \\
\hline 2726 & & RIVER & & RAW GRAB & TRANSECT & 577 & 7100 F -5 HRM 19.0 & \\
\hline 2727 & & RIVER & & RAW GRAB & TRANSECT & 339 & 100 F -7 HRM 19.0 & \\
\hline 2728 & & RIVER & & RAW GRAB & TRANSECT & 341 & 100 F -10 HRM 19.0 & \\
\hline 2729 & & RIVER & & RAW GRAB & TRANSECT & 578 & 3 HANFRD TS-1 HRM 28.7 & \\
\hline 2730 & & RIVER & & RAW GRAB & TRANSECT & 342 & 2 HANFRD TS-2 HRM 28.7 & \\
\hline 2731 & & RIVER & & RAW GRAB & TRANSECT & 469 & 9 HANFRD TS-7 HRM 28.7 & \\
\hline 2732 & & RIVER & & RAW GRAB & TRANSECT & 347 & 7 HANFRD TS-10 HRM 28.7 & \\
\hline 2733 & & RIVER & & RAW GRAB & TRANSECT & 468 & 3 HANFRD TS-3 HRM 28.7 & \\
\hline 2734 & & RIVER & & RAW GRAB & TRANSECT & 8100 & HANFRD TWNSITE HRM26 & \\
\hline 2735 & & RIVER & & RAW GRAB & TRANSECT & 8101 & 1 HANFRD TWNSITE HRM27 & \\
\hline 2736 & & RIVER & & RAW GRAB & TRANSECT & 8103 & 3 HANFRD TWNSITE HRM30 & \\
\hline 2737 & & RIVER & & RAW GRAB & TRANSECT & 274 & 4 HANFRD TWNSITE HRM28 & \\
\hline
\end{tabular}




\begin{tabular}{|c|c|c|c|c|c|c|c|c|}
\hline & $\mathrm{T}$ & $U$ & $\mathrm{~V}$ & $\mathrm{~W}$ & $\mathrm{X}$ & $\mathrm{Y}$ & Z & AA \\
\hline 1 & REVIEW_QUALIFIER & SAMP_FROM & SAMP_ITEM & SAMP_MTHD & COLL_MTHD & SAMP_SITE_ID & SAMP_SITE_NAME & WELL_NAME \\
\hline 2738 & & RIVER & & RAW GRAB & TRANSECT & 8726 & 100 F SHORE HRM 18 & \\
\hline 2739 & & RIVER & & RAW GRAB & TRANSECT & 8728 & 100 F SHORE HRM 23 & \\
\hline 2740 & & RIVER & & RAW GRAB & TRANSECT & 348 & 300 AREA -1 HRM 43.1 & \\
\hline 2741 & & RIVER & & RAW GRAB & TRANSECT & 470 & 300 AREA -2 HRM 43.1 & \\
\hline 2742 & & RIVER & & RAW GRAB & TRANSECT & 580 & 300 AREA -3 HRM 43.1 & \\
\hline 2743 & & RIVER & & RAW GRAB & TRANSECT & 607 & 300 AREA -5 HRM 43.1 & \\
\hline 2744 & & RIVER & & RAW GRAB & TRANSECT & 351 & 300 AREA -7 HRM 43.1 & \\
\hline 2745 & $\mathrm{~F}$ & RIVER & & RAW GRAB & TRANSECT & 352 & 300 AREA-10 HRM 43.1 & \\
\hline 2746 & & RIVER & & RAW GRAB & TRANSECT & 571 & RICH.PMPHS-1 HRM46.4 & \\
\hline 2747 & & RIVER & & RAW GRAB & TRANSECT & 321 & RICH.PMPHS-2 HRM46.4 & \\
\hline 2748 & & RIVER & & RAW GRAB & TRANSECT & 322 & RICH.PMPHS-3 HRM46.4 & \\
\hline 2749 & & RIVER & & RAW GRAB & TRANSECT & 323 & RICH.PMPHS-5 HRM46.4 & \\
\hline 2750 & & RIVER & & RAW GRAB & TRANSECT & 325 & RICH.PMPHS-7 HRM46.4 & \\
\hline 2751 & & RIVER & & RAW GRAB & TRANSECT & 327 & RICH.PMPHS-10 HRM46.4 & \\
\hline 2752 & & RIVER & & RAW GRAB & TRANSECT & 8220 & RICH.PMPHS HRM 45.8 & \\
\hline 2753 & & RIVER & & RAW GRAB & TRANSECT & 8221 & RICH.PMPHS HRM 45.0 & \\
\hline 2754 & & RIVER & & RAW GRAB & TRANSECT & 8222 & RICH.PMPHS HRM 43.9 & \\
\hline 2755 & & RIVER & & RAW GRAB & TRANSECT & 8223 & RICH.PMPHS HRM 43.5 & \\
\hline 2756 & & RIVER & & RAW GRAB & TRANSECT & 8240 & 300 AREA SHR HRM41.5 & \\
\hline 2757 & & RIVER & & RAW GRAB & TRANSECT & 8241 & 300 AREA SHR HRM42.1 & \\
\hline 2758 & & RIVER & & RAW GRAB & TRANSECT & 8242 & 300 AREA SHR HRM42.5 & \\
\hline 2759 & & RIVER & & RAW GRAB & TRANSECT & 8243 & 300 AREA SHR HRM42.9 & \\
\hline 2760 & $\mathrm{~F}$ & SEEP & & RAW GRAB & UNFILTERED & 8144 & 300 AREA SPR DR 42-2 & \\
\hline 2761 & $\mathrm{~F}$ & DRIVE POINT & & RAW GRAB & UNFILTERED & 9539 & 300 SPR 9 -1 (2 FT) & \\
\hline 2762 & $\mathrm{~F}$ & DRIVE POINT & & RAW GRAB & UNFILTERED & 9540 & 300 SPR 9 -1 (4 FT) & \\
\hline 2763 & $\mathrm{~F}$ & DRIVE POINT & & RAW GRAB & UNFILTERED & 9541 & 300 SPR $9-2$ (2 FT) & \\
\hline 2764 & $\mathrm{~F}$ & DRIVE POINT & & RAW GRAB & UNFILTERED & 9542 & 300 SPR 9 -2 (4.5FT) & \\
\hline 2765 & $\mathrm{~F}$ & DRIVE POINT & & RAW GRAB & UNFILTERED & 9543 & 300 SPR 9 -3 (5.5FT) & \\
\hline 2766 & $\mathrm{~F}$ & SEEP & & RAW GRAB & UNFILTERED & 488 & 300 AREA SPRING 42-2 & \\
\hline 2767 & $\mathrm{~F}$ & DRIVE POINT & & RAW GRAB & UNFILTERED & 9533 & 300 SPR 7 -1 (2.5FT) & \\
\hline 2768 & $\mathrm{~F}$ & DRIVE POINT & & RAW GRAB & UNFILTERED & 9534 & 300 SPR 7 -1 (4 FT) & \\
\hline 2769 & $\mathrm{~F}$ & DRIVE POINT & & RAW GRAB & UNFILTERED & 9535 & 300 SPR 7 -1 (6 FT) & \\
\hline 2770 & $\mathrm{~F}$ & DRIVE POINT & & RAW GRAB & UNFILTERED & 9536 & 300 SPR 7 -2 (2 FT) & \\
\hline 2771 & $\mathrm{~F}$ & DRIVE POINT & & RAW GRAB & UNFILTERED & 9537 & 300 SPR 7 -2 (4.2FT) & \\
\hline 2772 & & RIVER & & CUMULATIVE & UNFILTERED & 252 & PRIEST RAPIDS-RIVER & \\
\hline 2773 & & RIVER & & CUMULATIVE & UNFILTERED & 463 & RICH.PMPHS HRM 46.4 & \\
\hline 2774 & & SEEP & & RAW GRAB & UNFILTERED & 8147 & 100-F SPRING 207-1 & \\
\hline 2775 & & SEEP & & RAW GRAB & UNFILTERED & 8165 & 100-H SPRING 145-1 & \\
\hline
\end{tabular}




\begin{tabular}{|c|c|c|c|c|c|c|c|c|}
\hline & $\mathrm{T}$ & $\bar{U}$ & $\mathrm{~V}$ & $\mathrm{~W}$ & $\mathrm{X}$ & $\bar{Y}$ & $Z$ & $\overline{A A}$ \\
\hline 1 & REVIEW_QUALIFIER & SAMP_FROM & SAMP_ITEM & SAMP_MTHD & COLL_MTHD & SAMP_SITE_ID & SAMP_SITE_NAME & WELL_NAME \\
\hline 2776 & & SEEP & & RAW GRAB & UNFILTERED & 8846 & 100-H SPRING 152-2 & \\
\hline 2777 & & RIVER & & CUMULATIVE & UNFILTERED & 463 & RICH.PMPHS HRM 46.4 & \\
\hline 2778 & & RIVER & & CUMULATIVE & UNFILTERED & 252 & PRIEST RAPIDS-RIVER & \\
\hline 2779 & & SEEP & & RAW GRAB & UNFILTERED & 9236 & VERNITA BRIDGE -1 & \\
\hline 2780 & & RIVER & & CUMULATIVE & UNFILTERED & 252 & PRIEST RAPIDS-RIVER & \\
\hline 2781 & & RIVER & & CUMULATIVE & UNFILTERED & 463 & RICH.PMPHS HRM 46.4 & \\
\hline 2782 & & RIVER & & RAW GRAB & TRANSECT & 317 & VERNITA-1 HRM 0.3 & \\
\hline 2783 & & RIVER & & RAW GRAB & TRANSECT & 570 & VERNITA-2 HRM 0.3 & \\
\hline 2784 & & RIVER & & RAW GRAB & TRANSECT & 318 & VERNITA-3 HRM 0.3 & \\
\hline 2785 & & RIVER & & RAW GRAB & TRANSECT & 319 & VERNITA-4 HRM 0.3 & \\
\hline 2786 & & RIVER & & RAW GRAB & TRANSECT & 571 & RICH.PMPHS-1 HRM46.4 & \\
\hline 2787 & & RIVER & & RAW GRAB & TRANSECT & 571 & RICH.PMPHS-1 HRM46.4 & \\
\hline 2788 & & RIVER & & RAW GRAB & TRANSECT & 321 & RICH.PMPHS-2 HRM46.4 & \\
\hline 2789 & & RIVER & & RAW GRAB & TRANSECT & 322 & RICH.PMPHS-3 HRM46.4 & \\
\hline 2790 & & RIVER & & RAW GRAB & TRANSECT & 323 & RICH.PMPHS-5 HRM46.4 & \\
\hline 2791 & & RIVER & & RAW GRAB & TRANSECT & 325 & RICH.PMPHS-7 HRM46.4 & \\
\hline 2792 & & RIVER & & RAW GRAB & TRANSECT & 327 & RICH.PMPHS-10 HRM46.4 & \\
\hline 2793 & & RIVER & & RAW GRAB & TRANSECT & 8220 & RICH.PMPHS HRM 45.8 & \\
\hline 2794 & & RIVER & & RAW GRAB & TRANSECT & 8221 & RICH.PMPHS HRM 45.0 & \\
\hline 2795 & & RIVER & & RAW GRAB & TRANSECT & 8222 & RICH.PMPHS HRM 43.9 & \\
\hline 2796 & & RIVER & & RAW GRAB & TRANSECT & 8223 & RICH.PMPHS HRM 43.5 & \\
\hline 2797 & & RIVER & & CUMULATIVE & UNFILTERED & 463 & RICH.PMPHS HRM 46.4 & \\
\hline 2798 & & RIVER & & CUMULATIVE & UNFILTERED & 252 & PRIEST RAPIDS-RIVER & \\
\hline 2799 & & RIVER & & CUMULATIVE & UNFILTERED & 463 & RICH.PMPHS HRM 46.4 & \\
\hline 2800 & & RIVER & & CUMULATIVE & UNFILTERED & 252 & PRIEST RAPIDS-RIVER & \\
\hline 2801 & & RIVER & & CUMULATIVE & UNFILTERED & 252 & PRIEST RAPIDS-RIVER & \\
\hline 2802 & & RIVER & & CUMULATIVE & UNFILTERED & 463 & RICH.PMPHS HRM 46.4 & \\
\hline 2803 & & RIVER & & RAW GRAB & TRANSECT & 317 & VERNITA-1 HRM 0.3 & \\
\hline 2804 & & RIVER & & RAW GRAB & TRANSECT & 570 & VERNITA-2 HRM 0.3 & \\
\hline 2805 & & RIVER & & RAW GRAB & TRANSECT & 318 & VERNITA-3 HRM 0.3 & \\
\hline 2806 & & RIVER & & RAW GRAB & TRANSECT & 319 & VERNITA-4 HRM 0.3 & \\
\hline 2807 & & RIVER & & RAW GRAB & TRANSECT & 571 & RICH.PMPHS-1 HRM46.4 & \\
\hline 2808 & & RIVER & & RAW GRAB & TRANSECT & 571 & RICH.PMPHS-1 HRM46.4 & \\
\hline 2809 & & RIVER & & RAW GRAB & TRANSECT & 321 & RICH.PMPHS-2 HRM46.4 & \\
\hline 2810 & & RIVER & & RAW GRAB & TRANSECT & 322 & RICH.PMPHS-3 HRM46.4 & \\
\hline 2811 & & RIVER & & RAW GRAB & TRANSECT & 323 & RICH.PMPHS-5 HRM46.4 & \\
\hline 2812 & & RIVER & & RAW GRAB & TRANSECT & 325 & RICH.PMPHS-7 HRM46.4 & \\
\hline \begin{tabular}{|l|l|}
2813 \\
\end{tabular} & $\mathrm{~F}$ & RIVER & & RAW GRAB & TRANSECT & 327 & RICH.PMPHS-10 HRM46.4 & \\
\hline
\end{tabular}




\begin{tabular}{|c|c|c|c|c|c|c|c|c|}
\hline & $T$ & $\bar{U}$ & $\mathrm{~V}$ & $\mathrm{~W}$ & $\bar{X}$ & $\bar{Y}$ & $\bar{Z}$ & $\overline{A A}$ \\
\hline 1 & REVIEW_QUALIFIER & SAMP_FROM & SAMP_ITEM & SAMP_MTHD & COLL_MTHD & SAMP_SITE_ID & SAMP_SITE_NAME & WELL_NAME \\
\hline 2814 & & RIVER & & RAW GRAB & TRANSECT & 8220 & RICH.PMPHS HRM 45.8 & \\
\hline 2815 & & RIVER & & RAW GRAB & TRANSECT & 8221 & RICH.PMPHS HRM 45.0 & \\
\hline 2816 & $\mathrm{~F}$ & RIVER & & RAW GRAB & TRANSECT & 8222 & RICH.PMPHS HRM 43.9 & \\
\hline 2817 & & RIVER & & RAW GRAB & TRANSECT & 8223 & RICH.PMPHS HRM 43.5 & \\
\hline 2818 & & RIVER & & CUMULATIVE & UNFILTERED & 252 & PRIEST RAPIDS-RIVER & \\
\hline 2819 & & RIVER & & CUMULATIVE & UNFILTERED & 463 & RICH.PMPHS HRM 46.4 & \\
\hline 2820 & & RIVER & & CUMULATIVE & UNFILTERED & 252 & PRIEST RAPIDS-RIVER & \\
\hline 2821 & & RIVER & & CUMULATIVE & UNFILTERED & 463 & RICH.PMPHS HRM 46.4 & \\
\hline 2822 & & IRRIGATION & & RAW GRAB & UNFILTERED & 440 & RIVERVIEW CANAL & \\
\hline 2823 & & IRRIGATION & & RAW GRAB & UNFILTERED & 605 & HORN RAPIDS AREA & \\
\hline 2824 & & RIVER & & CUMULATIVE & UNFILTERED & 252 & PRIEST RAPIDS-RIVER & \\
\hline 2825 & & RIVER & & CUMULATIVE & UNFILTERED & 463 & RICH.PMPHS HRM 46.4 & \\
\hline 2826 & & RIVER & & RAW GRAB & TRANSECT & 317 & VERNITA-1 HRM 0.3 & \\
\hline 2827 & & RIVER & & RAW GRAB & TRANSECT & 570 & VERNITA-2 HRM 0.3 & \\
\hline 2828 & & RIVER & & RAW GRAB & TRANSECT & 318 & VERNITA-3 HRM 0.3 & \\
\hline 2829 & & RIVER & & RAW GRAB & TRANSECT & 319 & VERNITA-4 HRM 0.3 & \\
\hline 2830 & & RIVER & & RAW GRAB & TRANSECT & 571 & RICH.PMPHS-1 HRM46.4 & \\
\hline 2831 & & RIVER & & RAW GRAB & TRANSECT & 321 & RICH.PMPHS-2 HRM46.4 & \\
\hline 2832 & & RIVER & & RAW GRAB & TRANSECT & 322 & RICH.PMPHS-3 HRM46.4 & \\
\hline 2833 & & RIVER & & RAW GRAB & TRANSECT & 323 & RICH.PMPHS-5 HRM46.4 & \\
\hline 2834 & & RIVER & & RAW GRAB & TRANSECT & 325 & RICH.PMPHS-7 HRM46.4 & \\
\hline 2835 & & RIVER & & RAW GRAB & TRANSECT & 327 & RICH.PMPHS-10 HRM46.4 & \\
\hline 2836 & & RIVER & & RAW GRAB & TRANSECT & 8220 & RICH.PMPHS HRM 45.8 & \\
\hline 2837 & & RIVER & & RAW GRAB & TRANSECT & 8221 & RICH.PMPHS HRM 45.0 & \\
\hline 2838 & & RIVER & & RAW GRAB & TRANSECT & 8222 & RICH.PMPHS HRM 43.9 & \\
\hline 2839 & & RIVER & & RAW GRAB & TRANSECT & 8223 & RICH.PMPHS HRM 43.5 & \\
\hline 2840 & & RIVER & & CUMULATIVE & UNFILTERED & 252 & PRIEST RAPIDS-RIVER & \\
\hline 2841 & & RIVER & & CUMULATIVE & UNFILTERED & 463 & RICH.PMPHS HRM 46.4 & \\
\hline 2842 & & IRRIGATION & & RAW GRAB & UNFILTERED & 440 & RIVERVIEW CANAL & \\
\hline 2843 & & IRRIGATION & & RAW GRAB & UNFILTERED & 605 & HORN RAPIDS AREA & \\
\hline 2844 & & IRRIGATION & & RAW GRAB & UNFILTERED & 440 & RIVERVIEW CANAL & \\
\hline 2845 & & IRRIGATION & & RAW GRAB & UNFILTERED & 605 & HORN RAPIDS AREA & \\
\hline 2846 & & RIVER & & CUMULATIVE & UNFILTERED & 252 & PRIEST RAPIDS-RIVER & \\
\hline 2847 & & RIVER & & CUMULATIVE & UNFILTERED & 463 & RICH.PMPHS HRM 46.4 & \\
\hline 2848 & & RIVER & & CUMULATIVE & UNFILTERED & 252 & PRIEST RAPIDS-RIVER & \\
\hline 2849 & & RIVER & & CUMULATIVE & UNFILTERED & 463 & RICH.PMPHS HRM 46.4 & \\
\hline 2850 & & RIVER & & RAW GRAB & TRANSECT & 328 & 100 N -1 HRM 9.5 & \\
\hline 2851 & & RIVER & & RAW GRAB & TRANSECT & 319 & VERNITA-4 HRM 0.3 & \\
\hline
\end{tabular}




\begin{tabular}{|c|c|c|c|c|c|c|c|c|}
\hline & $\mathrm{T}$ & $\mathrm{U}$ & $\mathrm{V}$ & $\mathrm{W}$ & $\mathrm{X}$ & $\mathrm{Y}$ & $Z$ & $\overline{\mathrm{AA}}$ \\
\hline 1 & REVIEW_QUALIFIER & SAMP_FROM & SAMP_ITEM & SAMP_MTHD & COLL_MTHD & SAMP_SITE_ID | & SAMP_SITE_NAME & WELL_NAME \\
\hline 2852 & & RIVER & & RAW GRAB & TRANSECT & 570 & VERNITA-2 HRM 0.3 & \\
\hline 2853 & & RIVER & & RAW GRAB & TRANSECT & 318 & VERNITA-3 HRM 0.3 & \\
\hline 2854 & & RIVER & & RAW GRAB & TRANSECT & 317 & VERNITA-1 HRM 0.3 & \\
\hline 2855 & & RIVER & & RAW GRAB & TRANSECT & 328 & 100 N -1 HRM 9.5 & \\
\hline 2856 & & RIVER & & RAW GRAB & TRANSECT & 329 & 100 N -2 HRM 9.5 & \\
\hline 2857 & & RIVER & & RAW GRAB & TRANSECT & 331 & 100 N -5 HRM 9.5 & \\
\hline 2858 & & RIVER & & RAW GRAB & TRANSECT & 335 & 100 N -10 HRM 9.5 & \\
\hline 2859 & & RIVER & & RAW GRAB & TRANSECT & 330 & 100 N -3 HRM 9.5 & \\
\hline 2860 & & RIVER & & RAW GRAB & TRANSECT & 333 & 100 N -7 HRM 9.5 & \\
\hline 2861 & & RIVER & & RAW GRAB & TRANSECT & 8244 & 100 N SHORE HRM 8.4 & \\
\hline 2862 & & RIVER & & RAW GRAB & TRANSECT & 8245 & 100 N SHORE HRM 8.9 & \\
\hline 2863 & & RIVER & & RAW GRAB & TRANSECT & 8246 & 100 N SHORE HRM 9.2 & \\
\hline 2864 & & RIVER & & RAW GRAB & TRANSECT & 8247 & 100 N SHORE HRM 9.8 & \\
\hline 2865 & & RIVER & & RAW GRAB & TRANSECT & 336 & 100 F -1 HRM 19.0 & \\
\hline 2866 & & RIVER & & RAW GRAB & TRANSECT & 337 & 100 F -3 HRM 19.0 & \\
\hline 2867 & & RIVER & & RAW GRAB & TRANSECT & 344 & HANFRD TS-5 HRM 28.7 & \\
\hline 2868 & & RIVER & & RAW GRAB & TRANSECT & 576 & 100 F -2 HRM 19.0 & \\
\hline 2869 & & RIVER & & RAW GRAB & TRANSECT & 577 & 100 F -5 HRM 19.0 & \\
\hline 2870 & & RIVER & & RAW GRAB & TRANSECT & 339 & 100 F -7 HRM 19.0 & \\
\hline 2871 & & RIVER & & RAW GRAB & TRANSECT & 341 & $100 \mathrm{~F}-10 \mathrm{HRM} 19.0$ & \\
\hline 2872 & & RIVER & & RAW GRAB & TRANSECT & 578 & HANFRD TS-1 HRM 28.7 & \\
\hline 2873 & & RIVER & & RAW GRAB & TRANSECT & 342 & HANFRD TS-2 HRM 28.7 & \\
\hline 2874 & & RIVER & & RAW GRAB & TRANSECT & 469 & HANFRD TS-7 HRM 28.7 & \\
\hline 2875 & & RIVER & & RAW GRAB & TRANSECT & 347 & HANFRD TS-10 HRM 28.7 & \\
\hline 2876 & & RIVER & & RAW GRAB & TRANSECT & 468 & HANFRD TS-3 HRM 28.7 & \\
\hline 2877 & & RIVER & & RAW GRAB & TRANSECT & 8100 & HANFRD TWNSITE HRM26 & \\
\hline 2878 & & RIVER & & RAW GRAB & TRANSECT & 8101 & HANFRD TWNSITE HRM27 & \\
\hline 2879 & & RIVER & & RAW GRAB & TRANSECT & 8103 & HANFRD TWNSITE HRM30 & \\
\hline 2880 & $\mathrm{~F}$ & RIVER & & RAW GRAB & TRANSECT & 274 & HANFRD TWNSITE HRM28 & \\
\hline 2881 & & RIVER & & RAW GRAB & TRANSECT & 8726 & 100 F SHORE HRM 18 & \\
\hline 2882 & & RIVER & & RAW GRAB & TRANSECT & 13656 & 100 F SHORE HRM 24 & \\
\hline 2883 & & RIVER & & RAW GRAB & TRANSECT & 8728 & 100 F SHORE HRM 23 & \\
\hline 2884 & & RIVER & & RAW GRAB & TRANSECT & 348 & 300 AREA -1 HRM 43.1 & \\
\hline 2885 & & RIVER & & RAW GRAB & TRANSECT & 470 & 300 AREA -2 HRM 43.1 & \\
\hline 2886 & & RIVER & & RAW GRAB & TRANSECT & 580 & 300 AREA -3 HRM 43.1 & \\
\hline 2887 & & RIVER & & RAW GRAB & TRANSECT & 607 & 300 AREA -5 HRM 43.1 & \\
\hline 2888 & & RIVER & & RAW GRAB & TRANSECT & 351 & 300 AREA -7 HRM 43.1 & \\
\hline 2889 & & RIVER & & RAW GRAB & TRANSECT & 352 & 300 AREA-10 HRM 43.1 & \\
\hline
\end{tabular}




\begin{tabular}{|c|c|c|c|c|c|c|c|c|}
\hline & $\mathrm{T}$ & $U$ & $\mathrm{~V}$ & $\mathrm{~W}$ & $\mathrm{X}$ & $\mathrm{Y}$ & Z & AA \\
\hline 1 & REVIEW_QUALIFIER & SAMP_FROM & SAMP_ITEM & SAMP_MTHD & COLL_MTHD & SAMP_SITE_ID & SAMP_SITE_NAME & WELL_NAME \\
\hline 2890 & & RIVER & & RAW GRAB & TRANSECT & 571 & RICH.PMPHS-1 HRM46.4 & \\
\hline 2891 & & RIVER & & RAW GRAB & TRANSECT & 321 & RICH.PMPHS-2 HRM46.4 & \\
\hline 2892 & & RIVER & & RAW GRAB & TRANSECT & 322 & RICH.PMPHS-3 HRM46.4 & \\
\hline 2893 & & RIVER & & RAW GRAB & TRANSECT & 323 & RICH.PMPHS-5 HRM46.4 & \\
\hline 2894 & & RIVER & & RAW GRAB & TRANSECT & 325 & RICH.PMPHS-7 HRM46.4 & \\
\hline 2895 & & RIVER & & RAW GRAB & TRANSECT & 327 & RICH.PMPHS-10 HRM46.4 & \\
\hline 2896 & & RIVER & & RAW GRAB & TRANSECT & 8220 & RICH.PMPHS HRM 45.8 & \\
\hline 2897 & & RIVER & & RAW GRAB & TRANSECT & 8221 & RICH.PMPHS HRM 45.0 & \\
\hline 2898 & & RIVER & & RAW GRAB & TRANSECT & 8222 & RICH.PMPHS HRM 43.9 & \\
\hline 2899 & & RIVER & & RAW GRAB & TRANSECT & 8223 & RICH.PMPHS HRM 43.5 & \\
\hline 2900 & & RIVER & & RAW GRAB & TRANSECT & 8240 & 300 AREA SHR HRM41.5 & \\
\hline 2901 & & RIVER & & RAW GRAB & TRANSECT & 8241 & 300 AREA SHR HRM42.1 & \\
\hline 2902 & & RIVER & & RAW GRAB & TRANSECT & 8242 & 300 AREA SHR HRM42.5 & \\
\hline 2903 & & RIVER & & RAW GRAB & TRANSECT & 8243 & 300 AREA SHR HRM42.9 & \\
\hline 2904 & & RIVER & & CUMULATIVE & UNFILTERED & 252 & PRIEST RAPIDS-RIVER & \\
\hline 2905 & & RIVER & & CUMULATIVE & UNFILTERED & 463 & RICH.PMPHS HRM 46.4 & \\
\hline 2906 & & SEEP & & RAW GRAB & UNFILTERED & 151 & HANFORD SPRING 28-2 & \\
\hline 2907 & $\mathrm{~F}$ & SEEP & & RAW GRAB & UNFILTERED & 488 & 300 AREA SPRING 42-2 & \\
\hline 2908 & & SEEP & & RAW GRAB & UNFILTERED & 8143 & HANFORD SPR DR 28-2 & \\
\hline 2909 & & SEEP & & RAW GRAB & UNFILTERED & 8846 & 100-H SPRING 152-2 & \\
\hline 2910 & & SEEP & & RAW GRAB & UNFILTERED & 8147 & 100-F SPRING 207-1 & \\
\hline 2911 & & RIVER & & CUMULATIVE & UNFILTERED & 252 & PRIEST RAPIDS-RIVER & \\
\hline 2912 & & RIVER & & CUMULATIVE & UNFILTERED & 463 & RICH.PMPHS HRM 46.4 & \\
\hline 2913 & & RIVER & & CUMULATIVE & UNFILTERED & 252 & PRIEST RAPIDS-RIVER & \\
\hline 2914 & & RIVER & & CUMULATIVE & UNFILTERED & 463 & RICH.PMPHS HRM 46.4 & \\
\hline 2915 & & RIVER & & RAW GRAB & TRANSECT & 317 & VERNITA-1 HRM 0.3 & \\
\hline 2916 & & RIVER & & RAW GRAB & TRANSECT & 570 & VERNITA-2 HRM 0.3 & \\
\hline 2917 & & RIVER & & RAW GRAB & TRANSECT & 318 & VERNITA-3 HRM 0.3 & \\
\hline 2918 & & RIVER & & RAW GRAB & TRANSECT & 319 & VERNITA-4 HRM 0.3 & \\
\hline 2919 & & RIVER & & RAW GRAB & TRANSECT & 571 & RICH.PMPHS-1 HRM46.4 & \\
\hline 2920 & & RIVER & & RAW GRAB & TRANSECT & 571 & RICH.PMPHS-1 HRM46.4 & \\
\hline 2921 & & RIVER & & RAW GRAB & TRANSECT & 321 & RICH.PMPHS-2 HRM46.4 & \\
\hline 2922 & & RIVER & & RAW GRAB & TRANSECT & 322 & RICH.PMPHS-3 HRM46.4 & \\
\hline 2923 & & RIVER & & RAW GRAB & TRANSECT & 323 & RICH.PMPHS-5 HRM46.4 & \\
\hline 2924 & & RIVER & & RAW GRAB & TRANSECT & 325 & RICH.PMPHS-7 HRM46.4 & \\
\hline 2925 & & RIVER & & RAW GRAB & TRANSECT & 327 & RICH.PMPHS-10 HRM46.4 & \\
\hline 2926 & & RIVER & & RAW GRAB & TRANSECT & 8220 & RICH.PMPHS HRM 45.8 & \\
\hline 2927 & & RIVER & & RAW GRAB & TRANSECT & 8221 & RICH.PMPHS HRM 45.0 & \\
\hline
\end{tabular}




\begin{tabular}{|c|c|c|c|c|c|c|c|c|}
\hline & T & $U$ & $V$ & $W$ & $X$ & $Y$ & AA \\
\hline 1 & REVIEW_QUALIFIER & SAMP_FROM & SAMP_ITEM & SAMP_MTHD & COLL_MTHD & SAMP_SITE_ID & SAMP_SITE_NAME & WELL_NAME \\
\hline 2928 & & RIVER & RAW GRAB & TRANSECT & 8222 RICH.PMPHS HRM 43.9 & \\
\hline 2929 & & RIVER & & RAW GRAB & TRANSECT & 8223 RICH.PMPHS HRM 43.5 \\
\hline
\end{tabular}




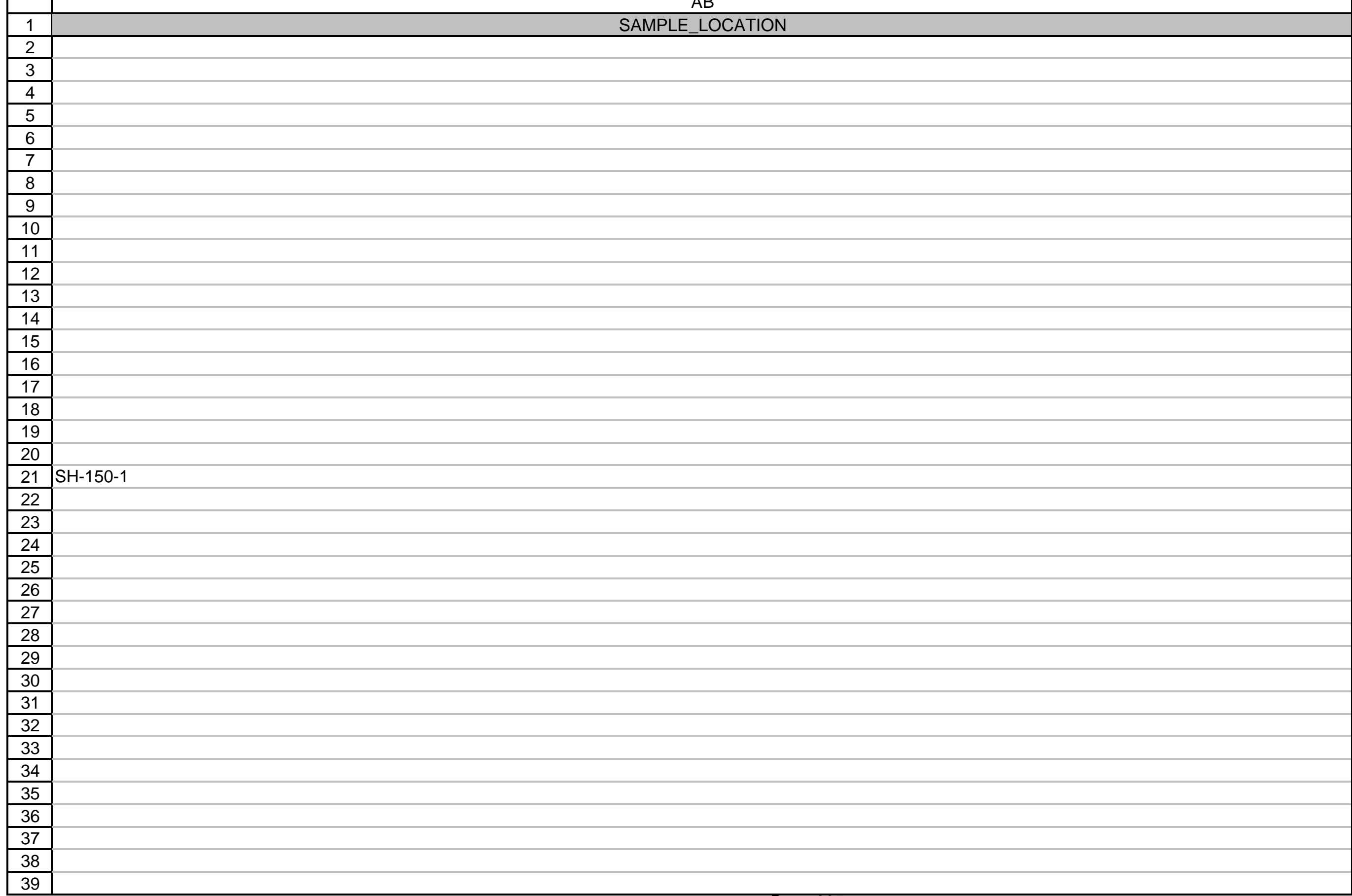




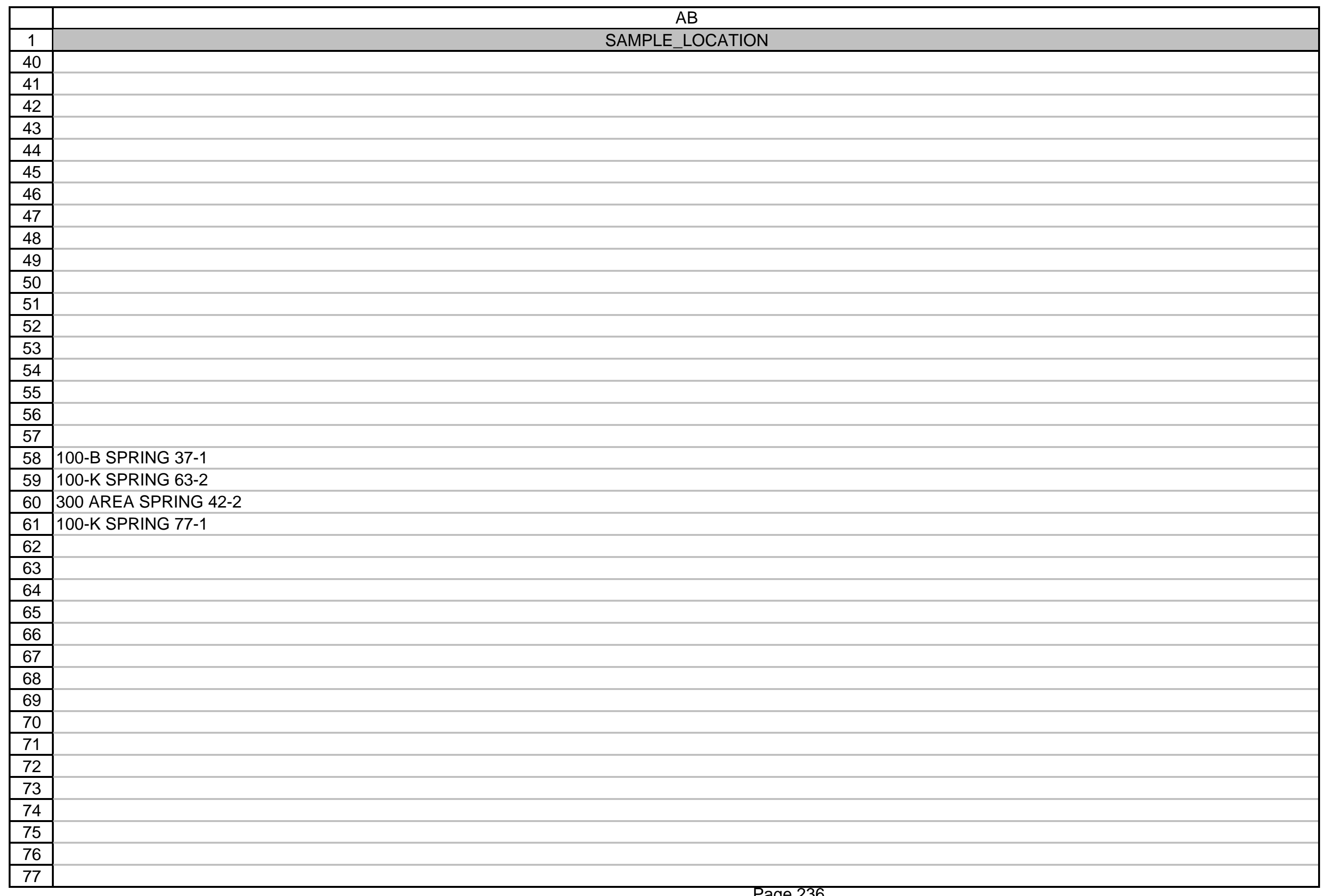


AB

SAMPLE LOCATION

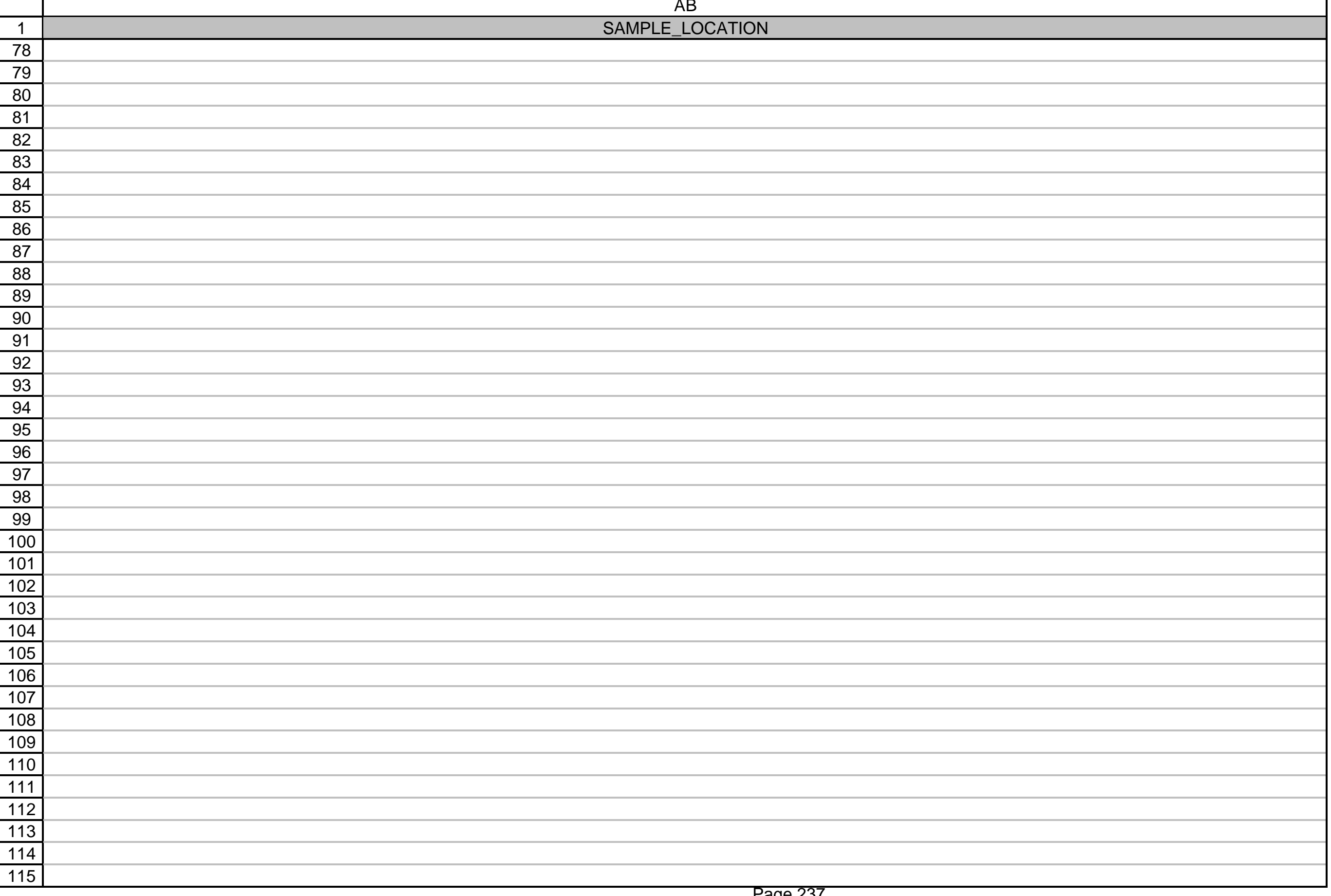

Page 237 
AB

SAMPLE LOCATION

\begin{tabular}{|c|}
\hline 116 \\
\hline 117 \\
\hline 118
\end{tabular}

\begin{tabular}{|l|}
118 \\
\hline 119 \\
\hline
\end{tabular}

\begin{tabular}{|l|}
\hline 119 \\
\hline 120 \\
\hline
\end{tabular}

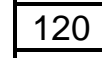

\begin{tabular}{|l|}
\hline 121 \\
\hline 122 \\
\hline
\end{tabular}

122

123

\begin{tabular}{|l|}
\hline 124 \\
\hline 125 \\
\hline 126
\end{tabular}

126

\begin{tabular}{|l|}
\hline 127 \\
\hline 128 \\
\hline 129 \\
\hline
\end{tabular}

129

130

131

132

133

134

\begin{tabular}{|l|}
\hline 135 \\
\hline 136 \\
\hline
\end{tabular}

\begin{tabular}{|l|}
\hline 136 \\
\hline 137 \\
\hline
\end{tabular}

137

138

\begin{tabular}{|l|}
\hline 138 \\
\hline 140 \\
\hline 141 \\
\hline
\end{tabular}

141

142

143

144

145

146

\begin{tabular}{|l|}
146 \\
\hline 147 \\
\hline 148 \\
\hline
\end{tabular}

148

149

150

151

152

\begin{tabular}{|l|}
152 \\
\hline 153 \\
\hline
\end{tabular} 
AB

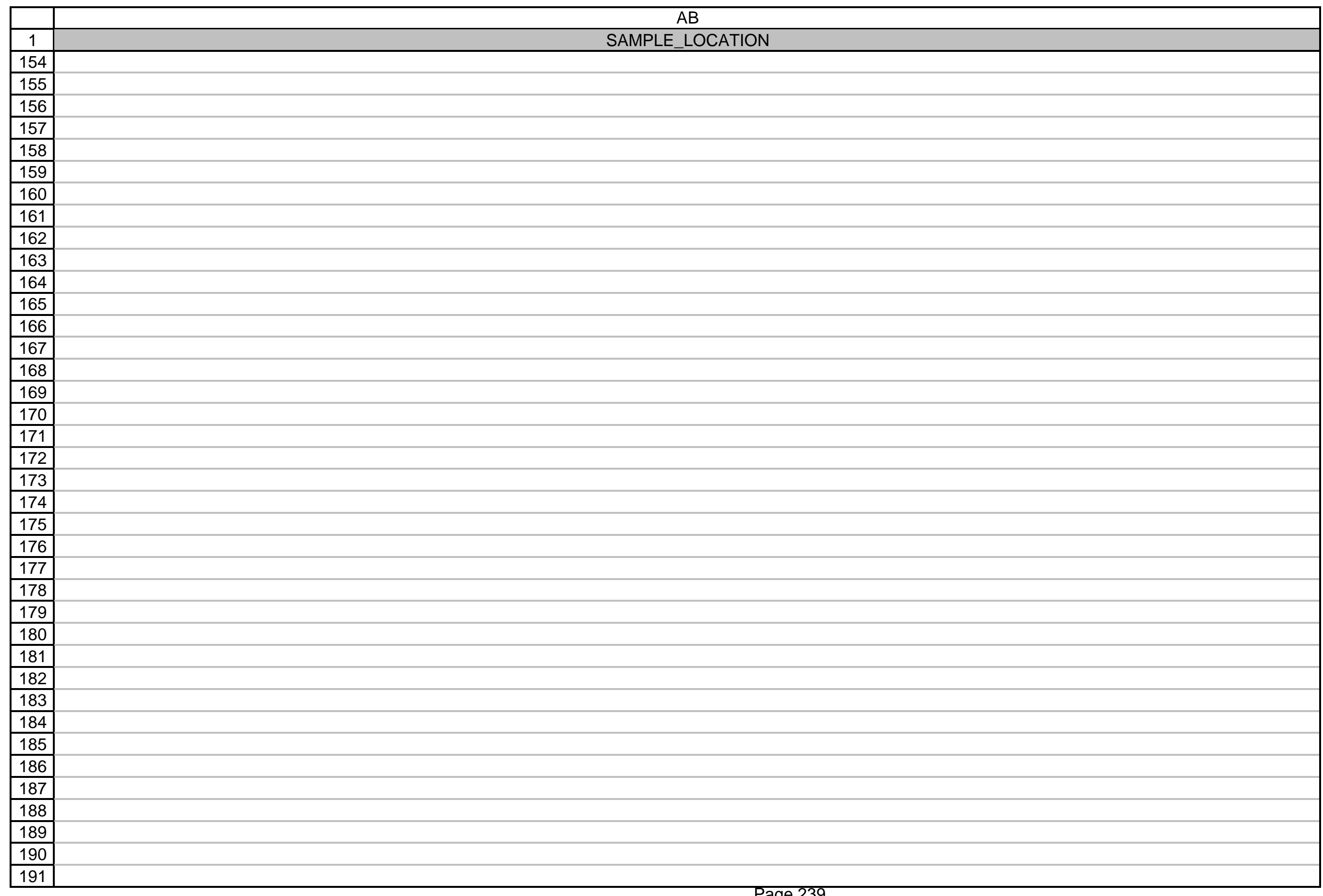

Page 239 
AB

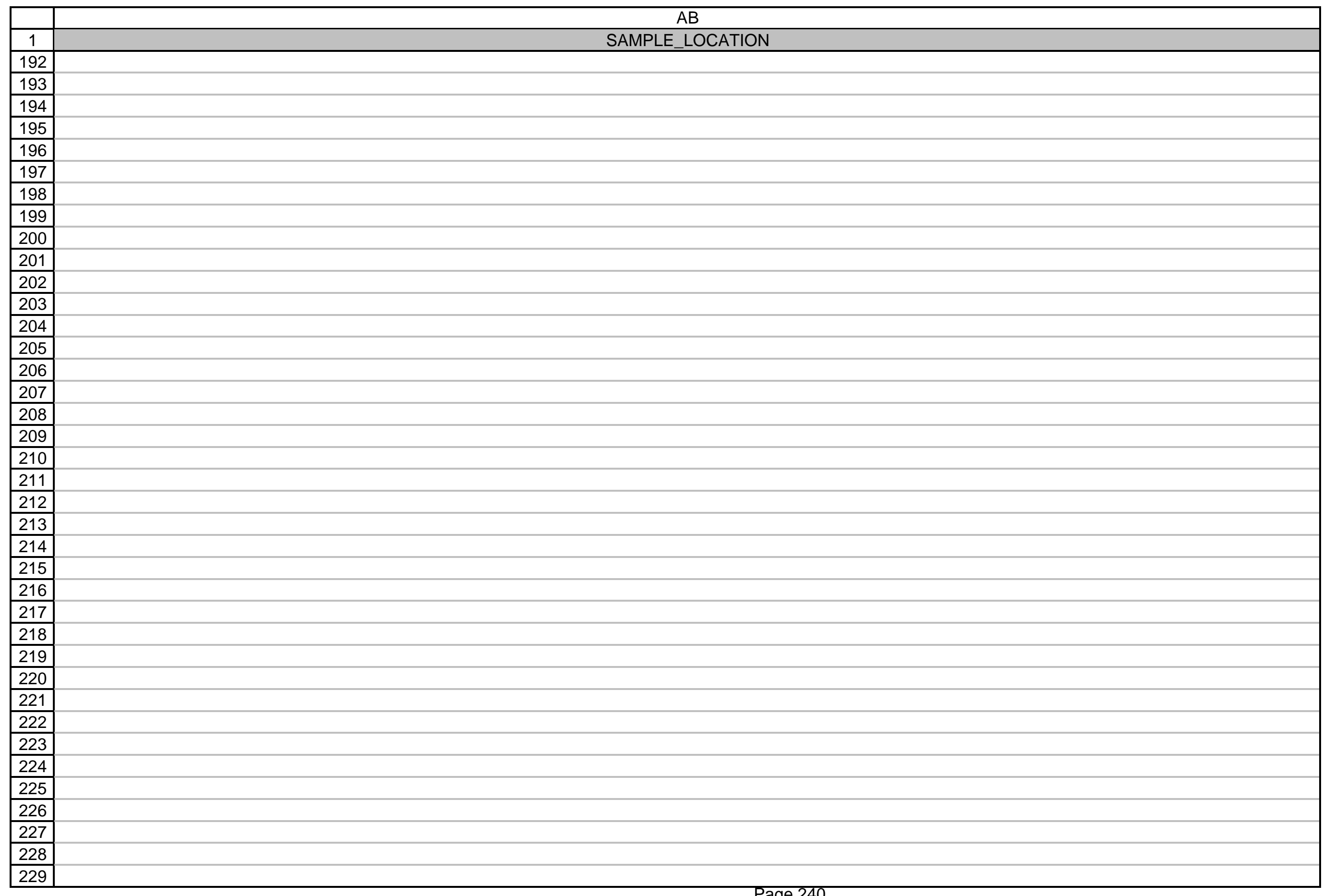

Page 240 
AB

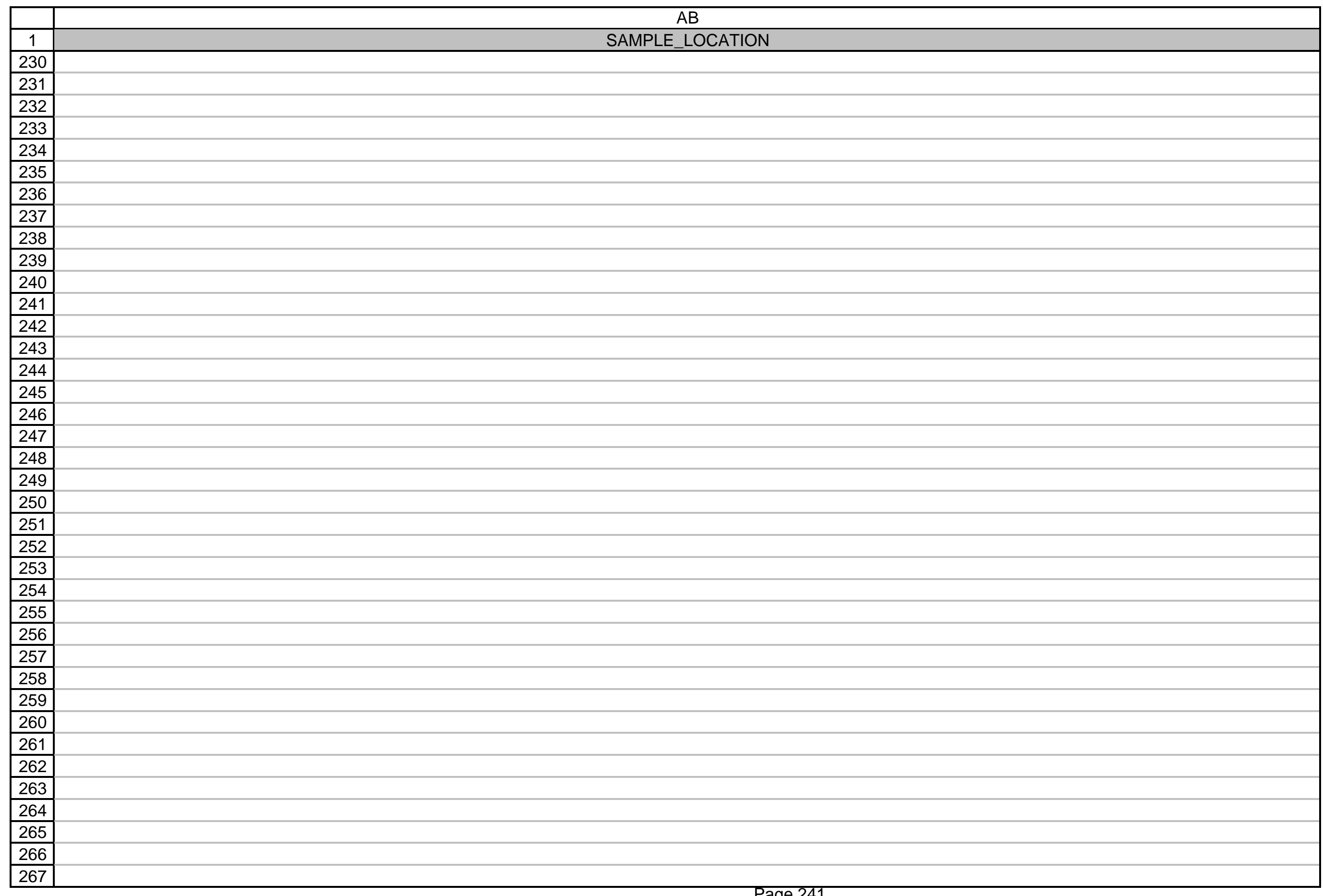

Page 241 
AB

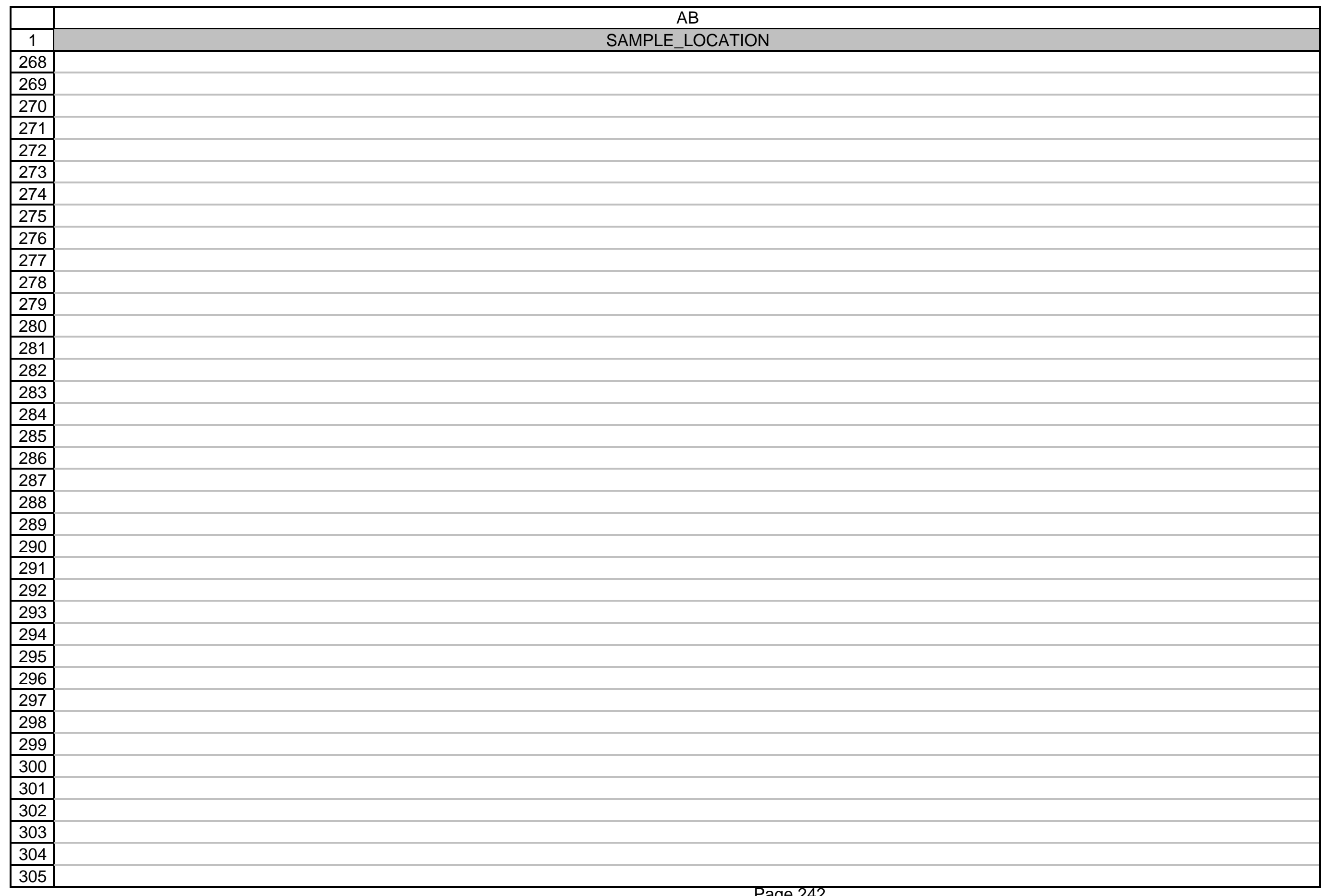

Page 242 
AB

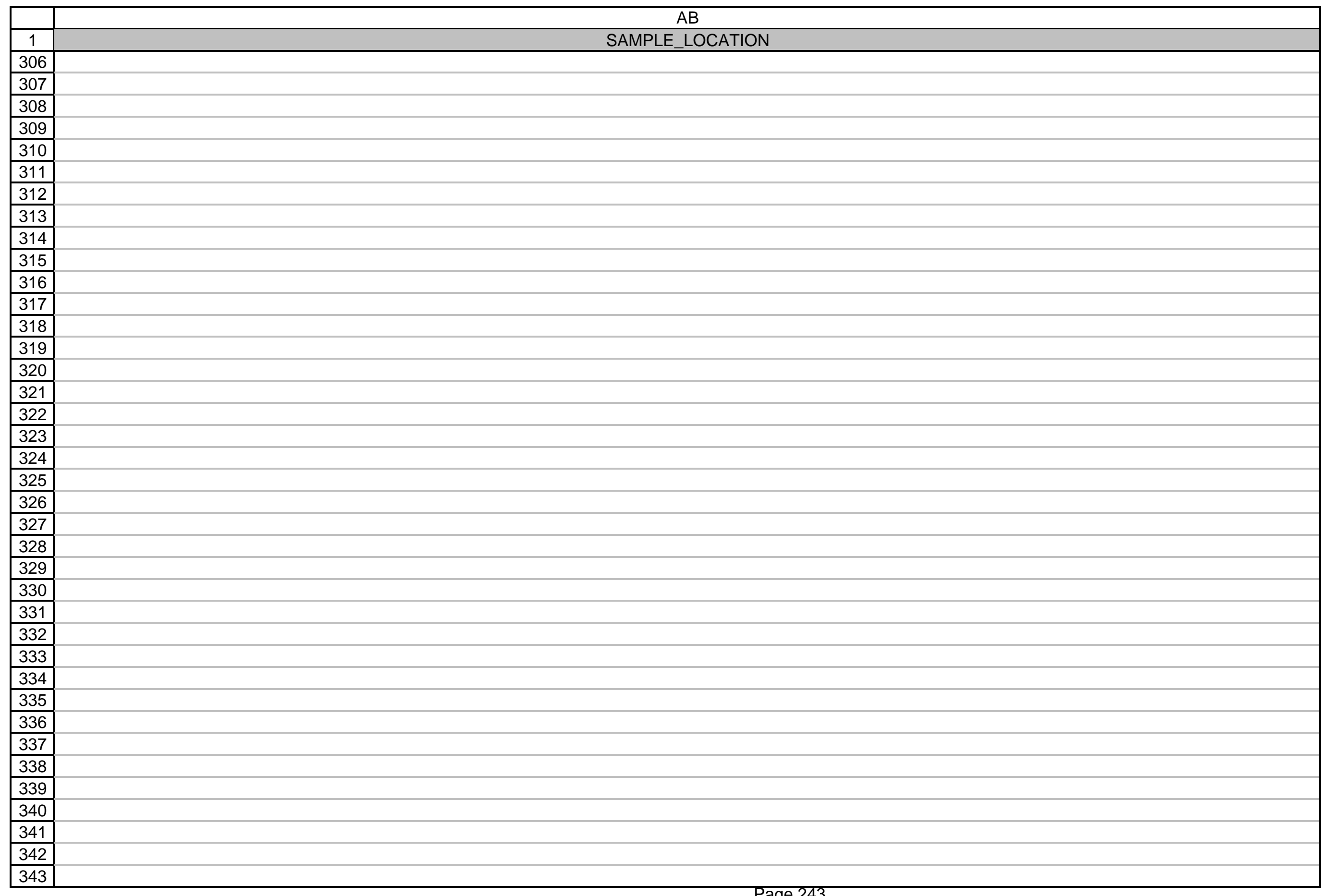

Page 243 
AB

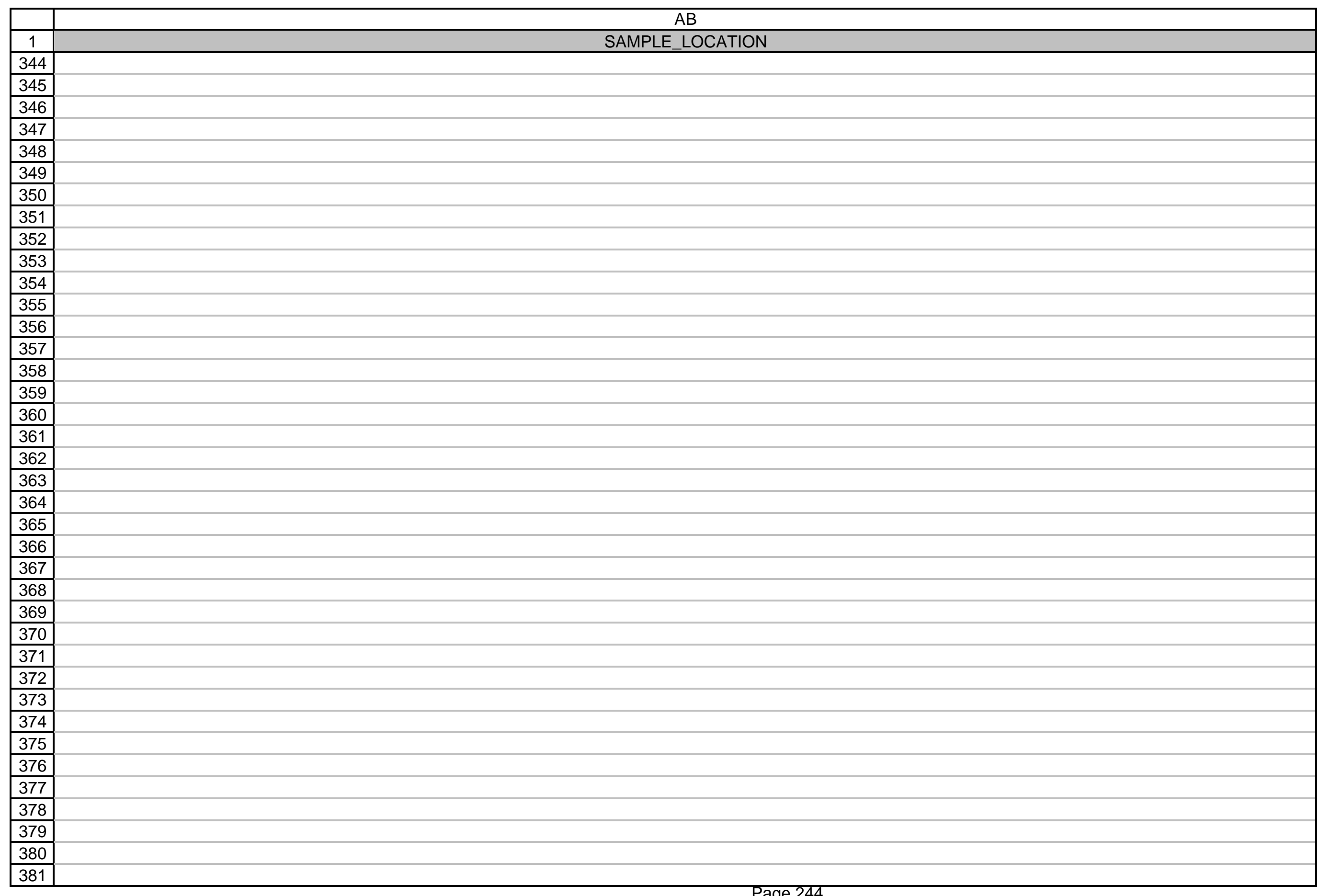

Page 244 
AB

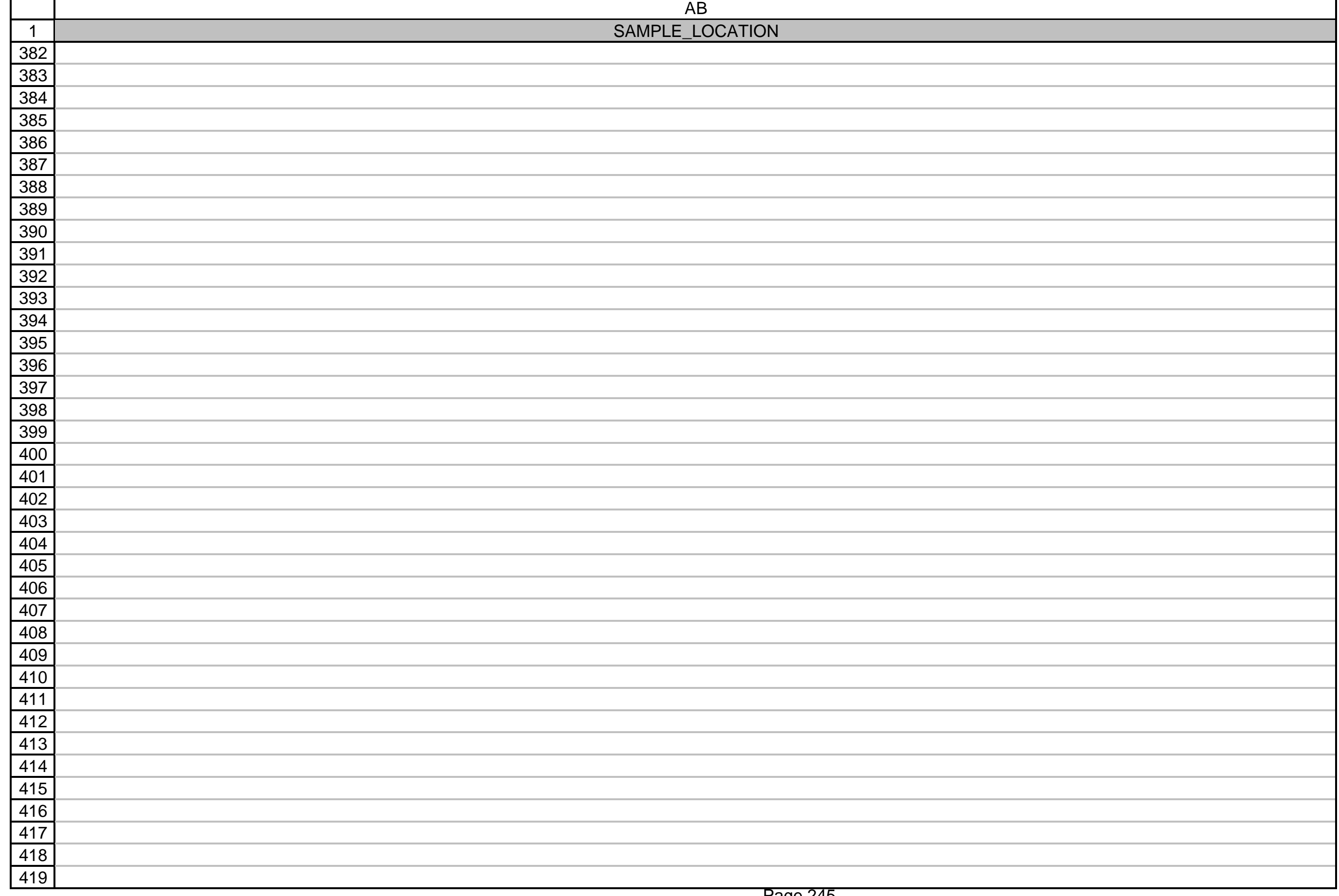

Page 245 
AB

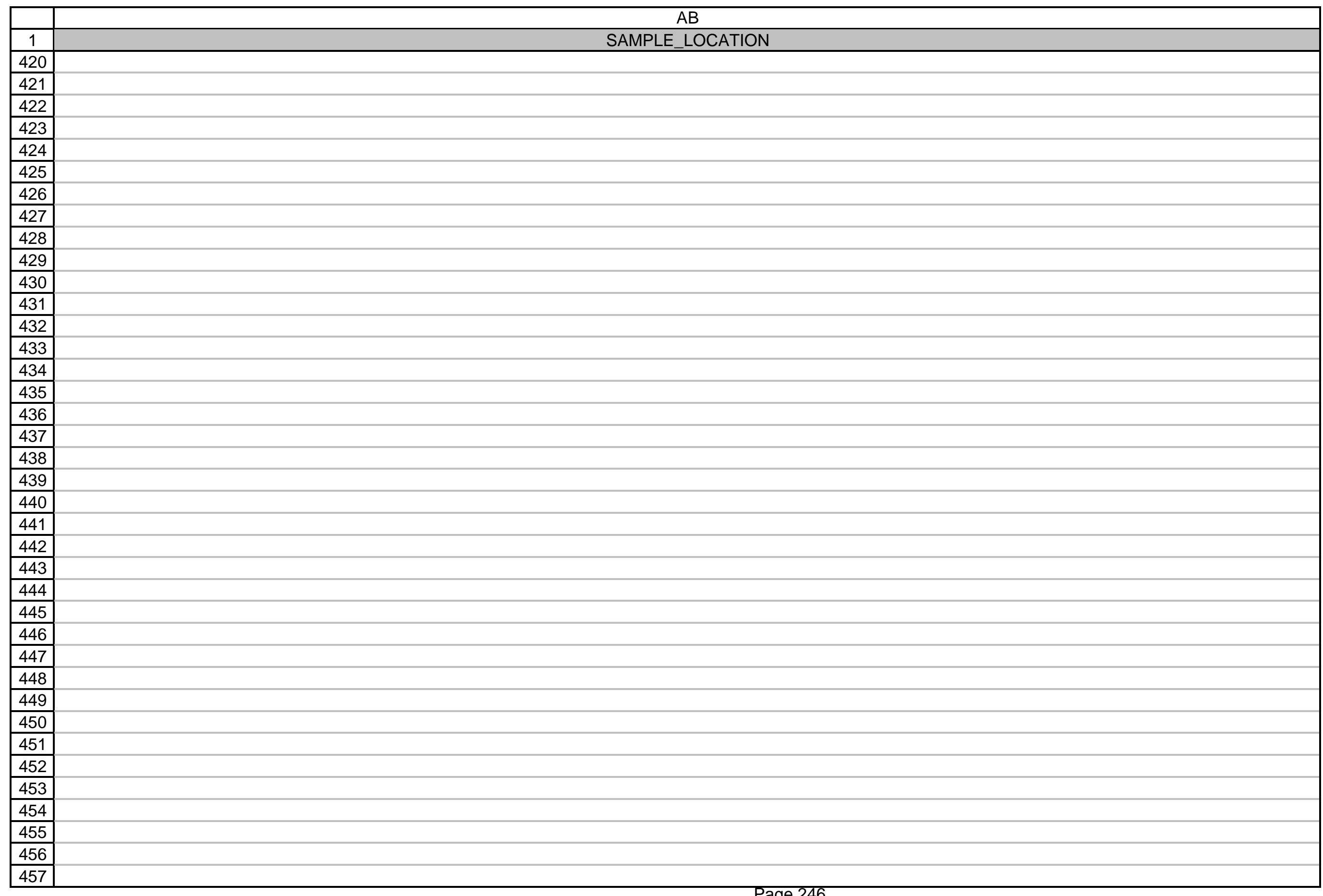

Page 246 
AB

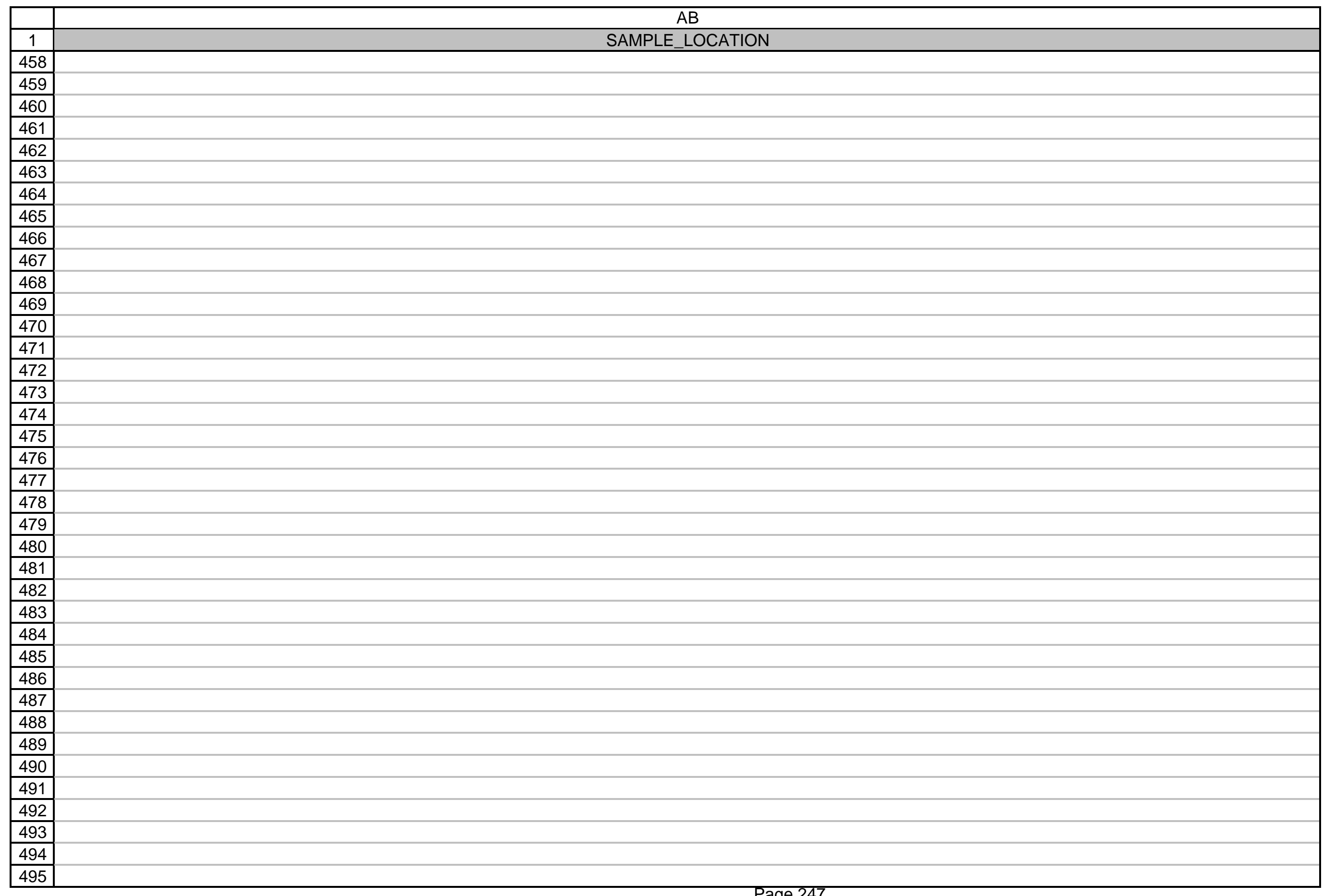

Page 247 


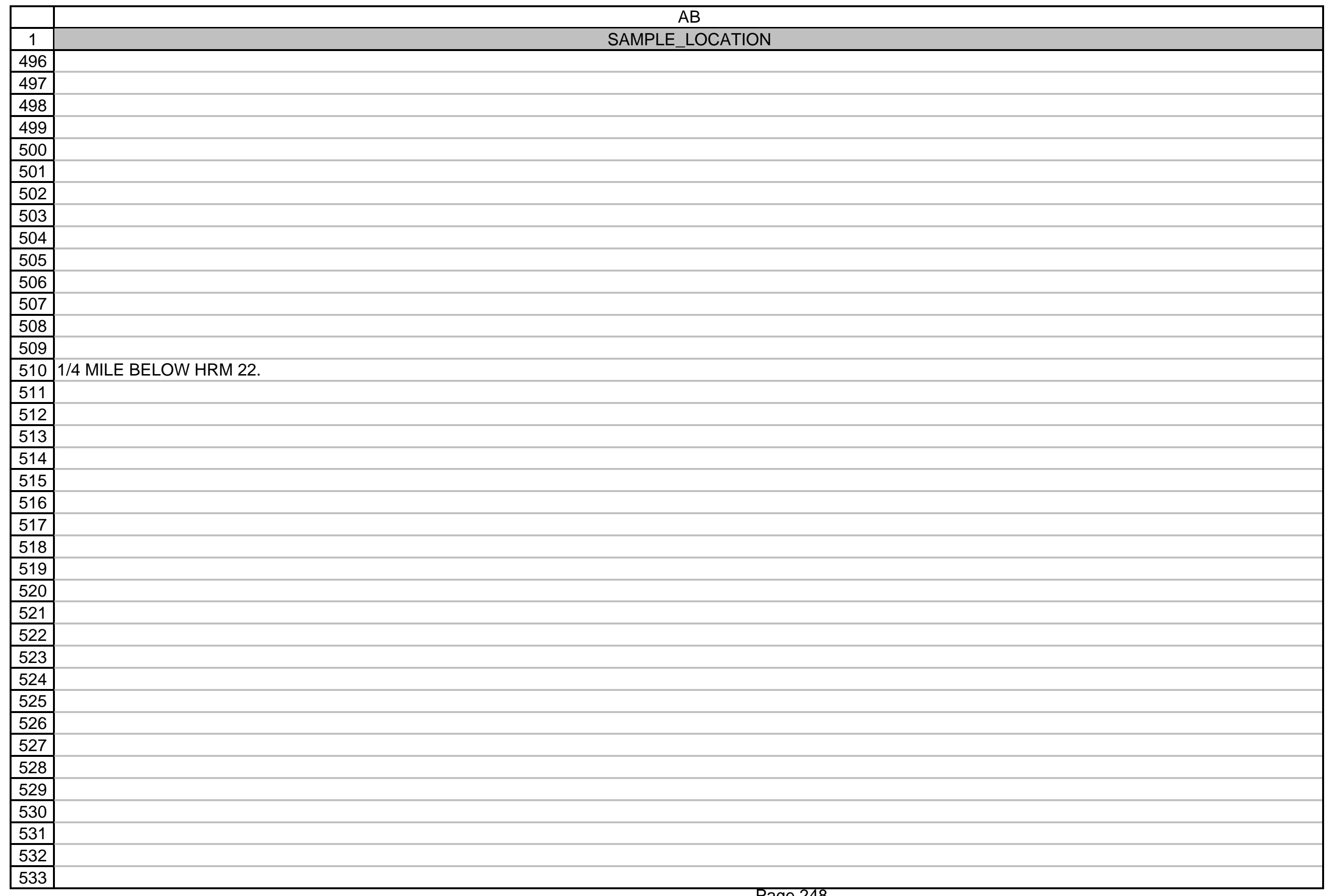


$A B$

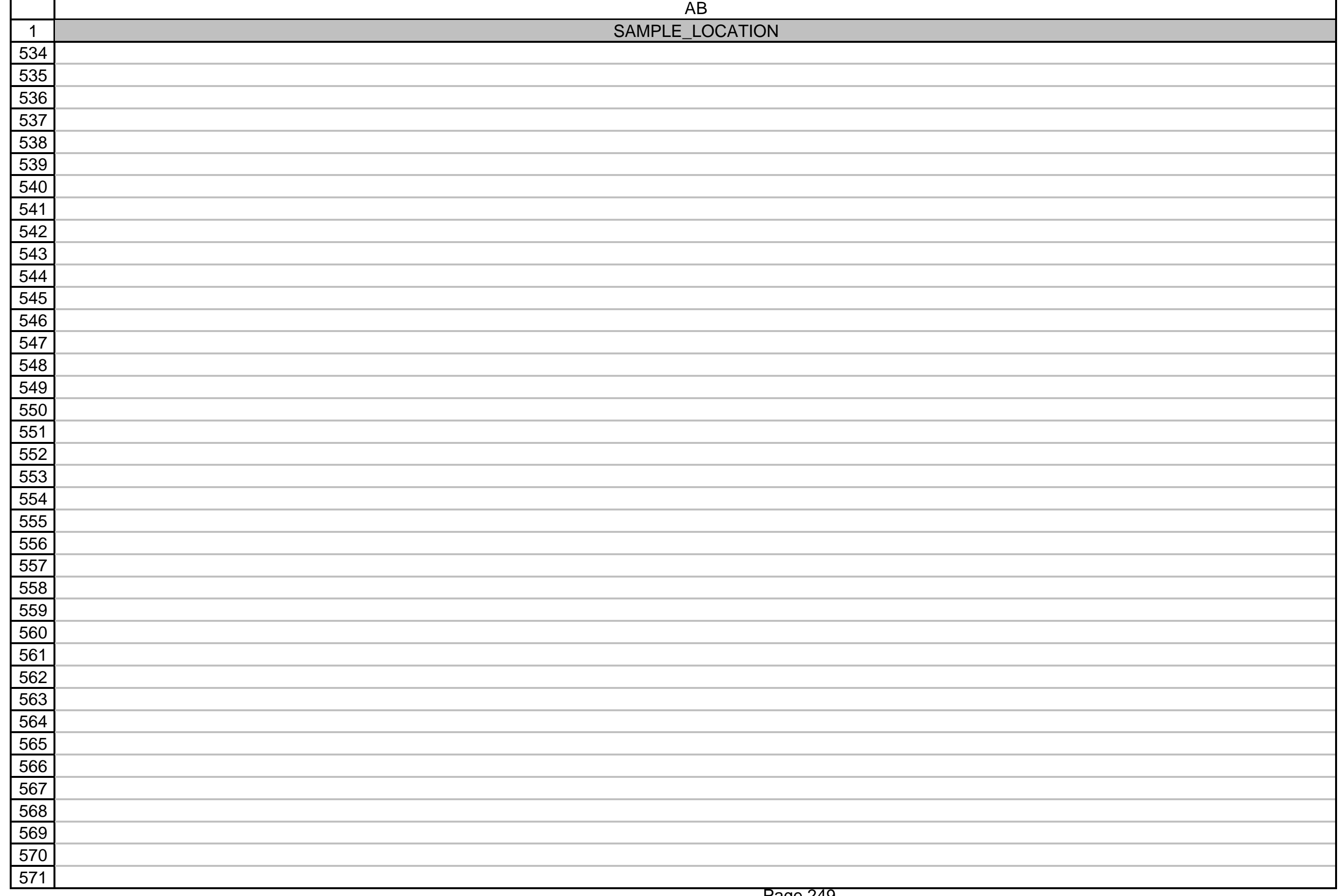

Page 249 
AB

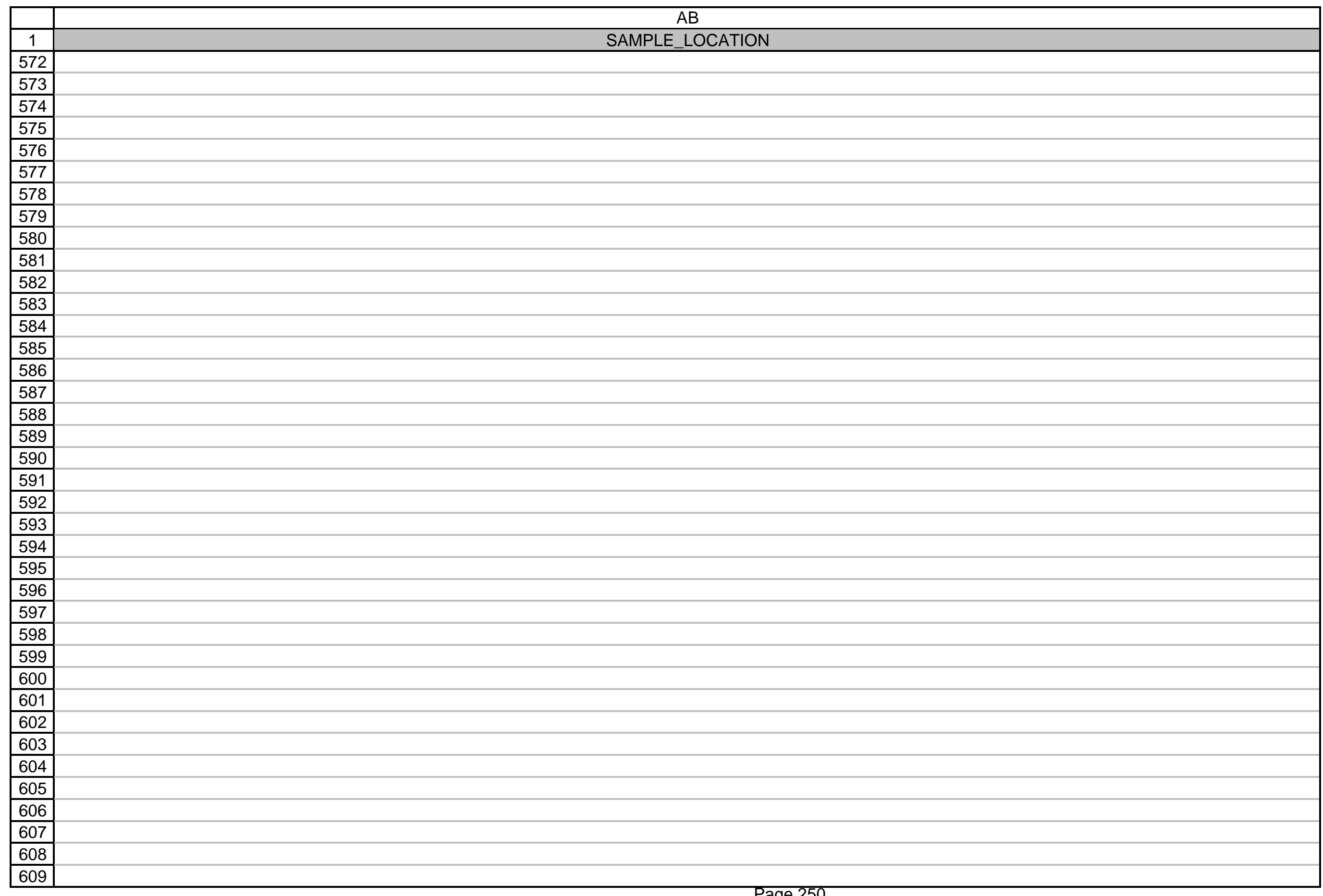

Page 250 
$A B$

SAMPLE_LOCATION

\begin{tabular}{|l|}
\hline 610 \\
\hline 611 \\
\hline 612 \\
\hline
\end{tabular}

\begin{tabular}{|l|}
\hline 611 \\
\hline 612 \\
\hline 613
\end{tabular}

\begin{tabular}{|l|}
612 \\
613 \\
614
\end{tabular}

\begin{tabular}{|l|}
\hline 614 \\
\hline 615 \\
\hline
\end{tabular}

615

616

617

618

619

621

\begin{tabular}{|l|}
\hline 622 \\
\hline 623 \\
\hline
\end{tabular}

623

624

625

626

\begin{tabular}{l}
627 \\
\hline 628
\end{tabular}

628

629

630

631

632

633

634

635

636

637

638

639

640

\begin{tabular}{|l|}
640 \\
\hline 641 \\
\hline 642 \\
\hline
\end{tabular}

\begin{tabular}{|l|}
641 \\
\hline 643 \\
\hline
\end{tabular}

\begin{tabular}{|l|}
643 \\
\hline 644 \\
\hline
\end{tabular}

\begin{tabular}{|l|}
\hline 644 \\
\hline 645 \\
\hline 646 \\
\hline
\end{tabular}

646

\begin{tabular}{|l|}
\hline 647 \\
\hline
\end{tabular}

Page 251 
AB

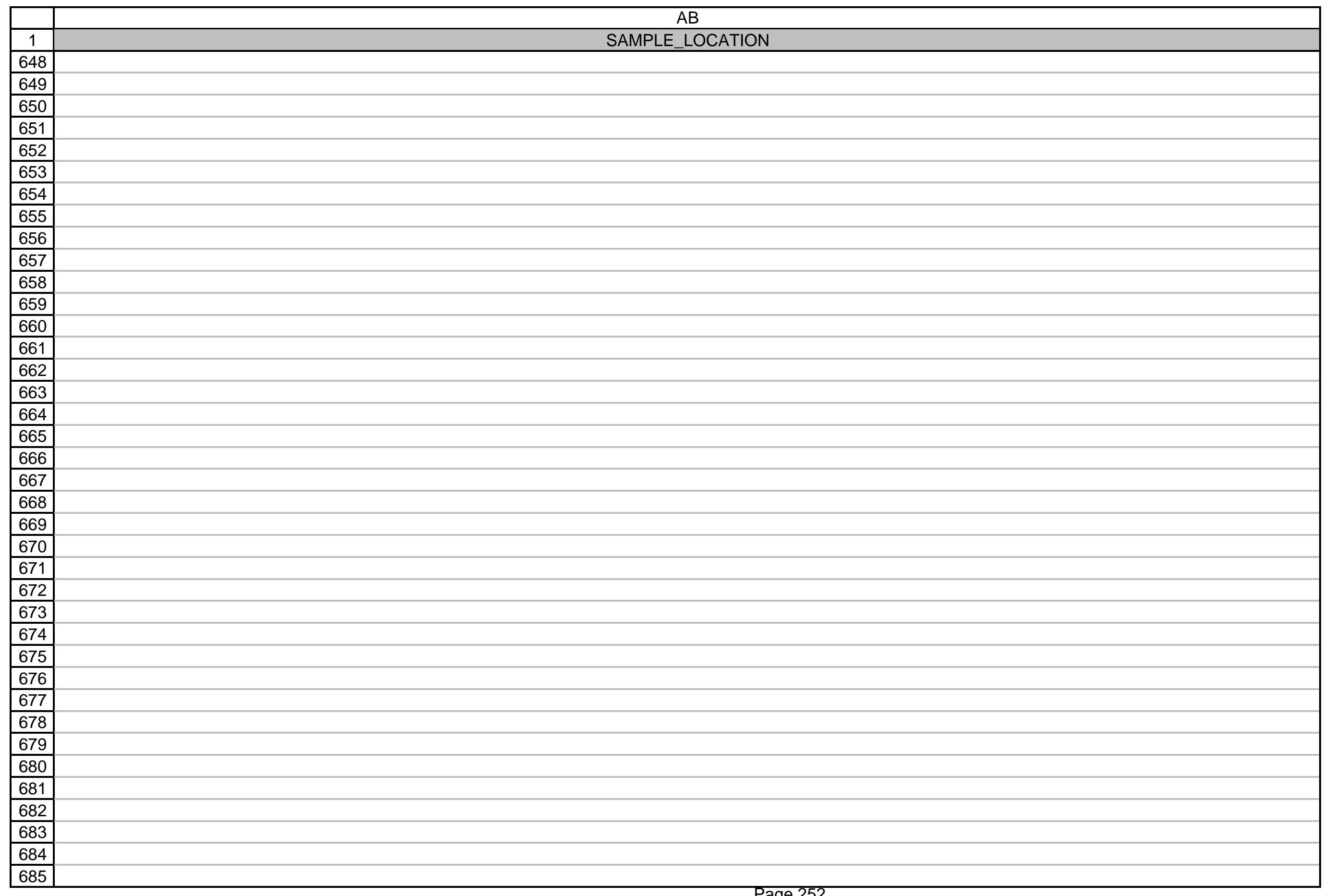

Page 252 
AB

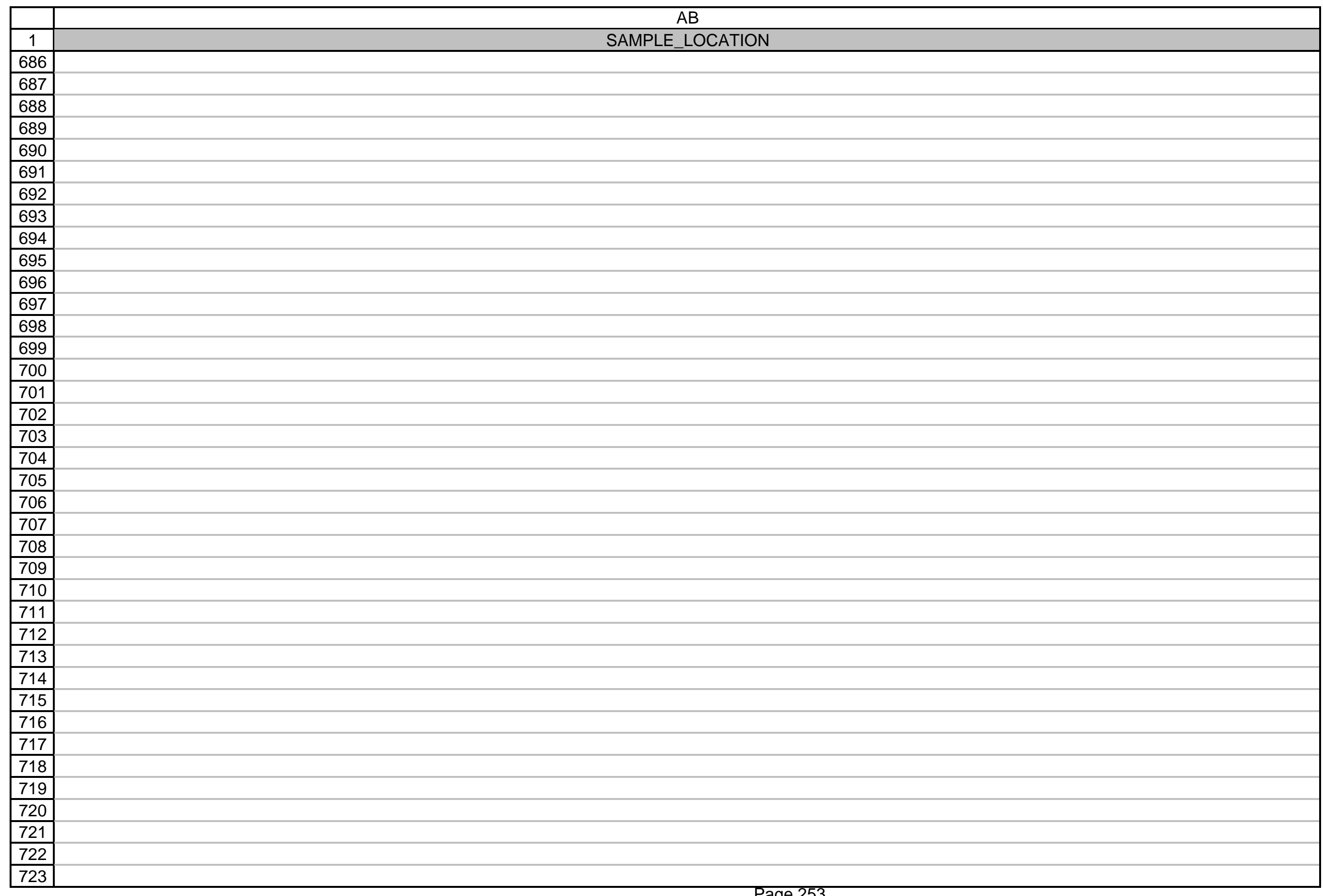

Page 253 
AB

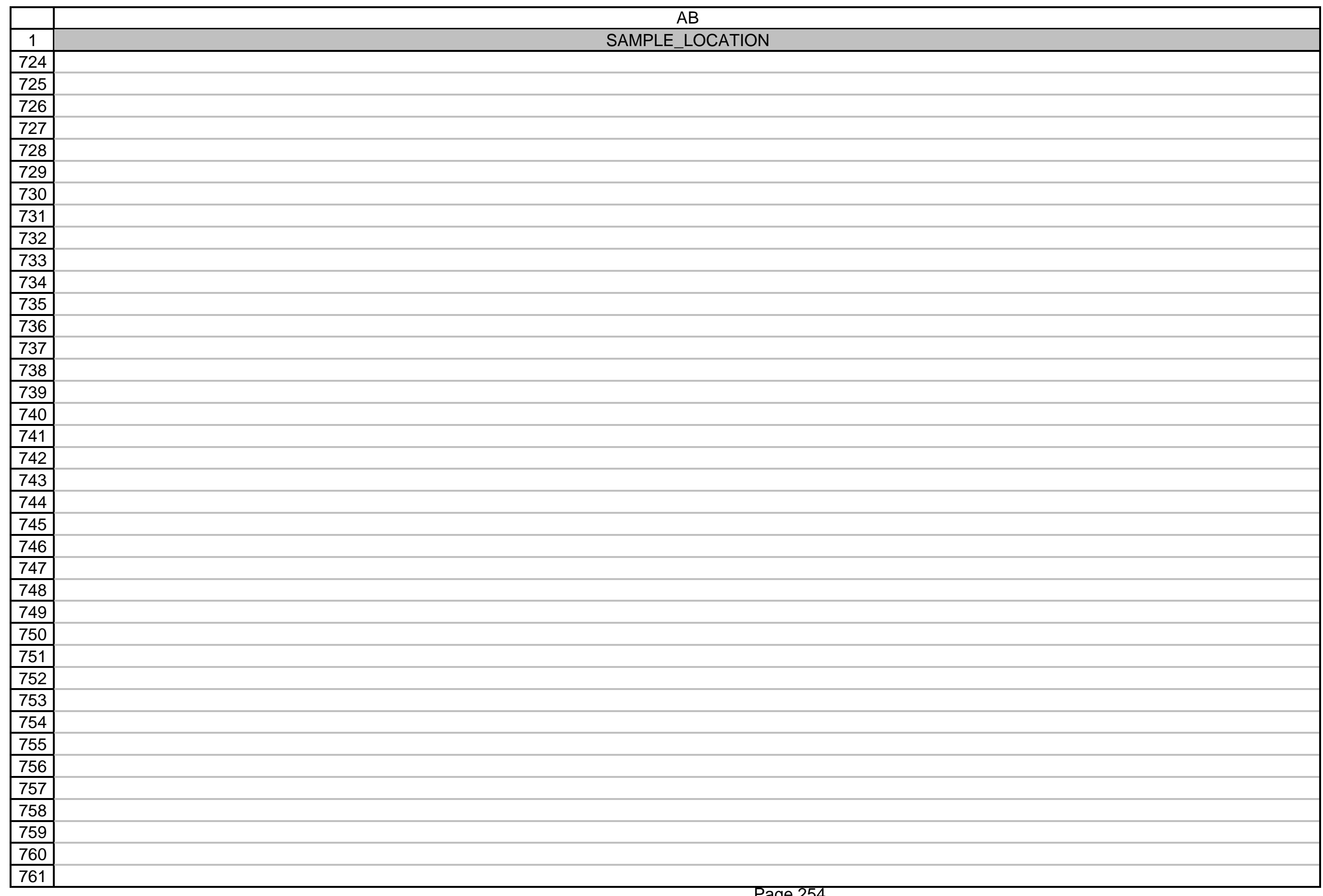

Page 254 
AB

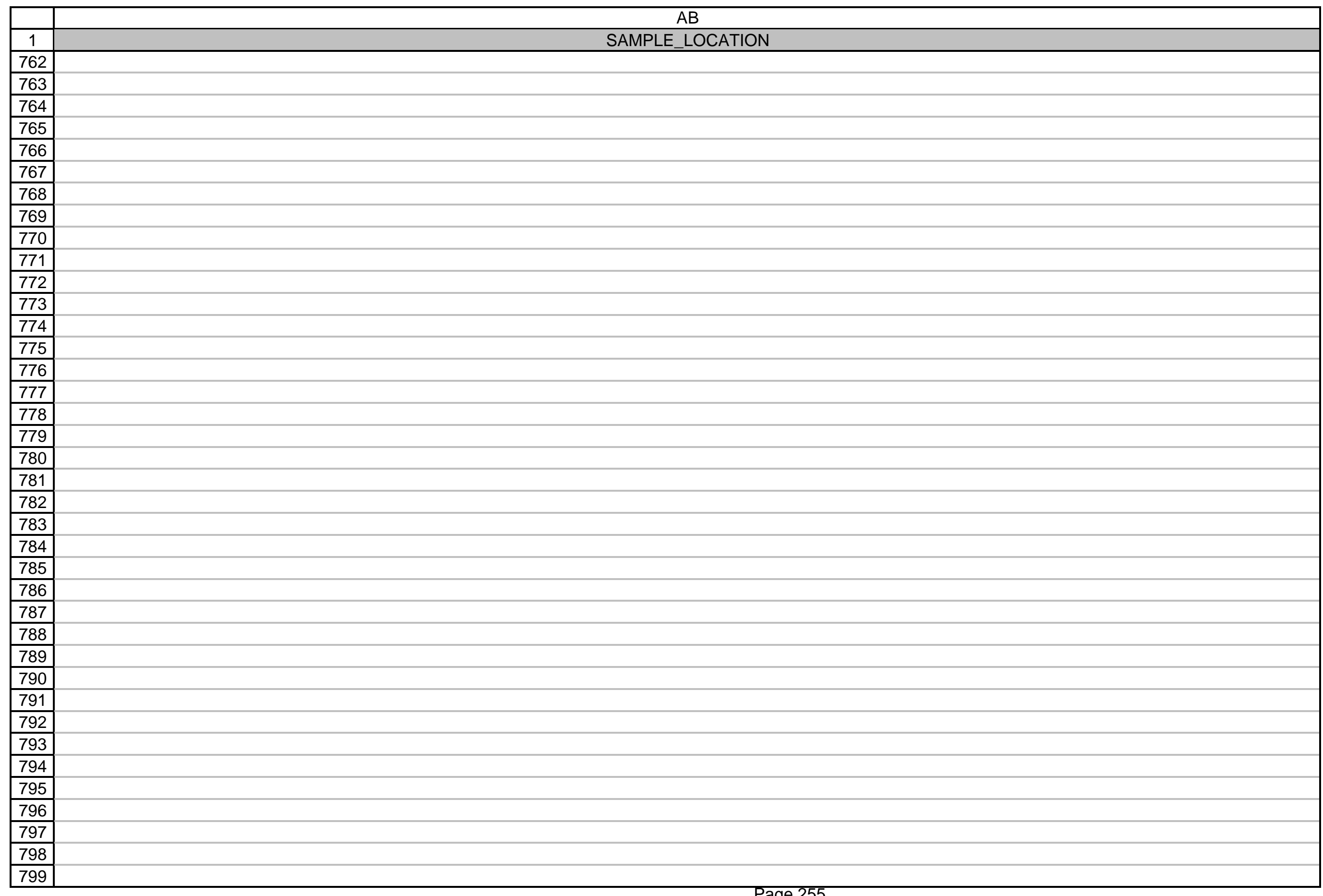

Page 255 
AB

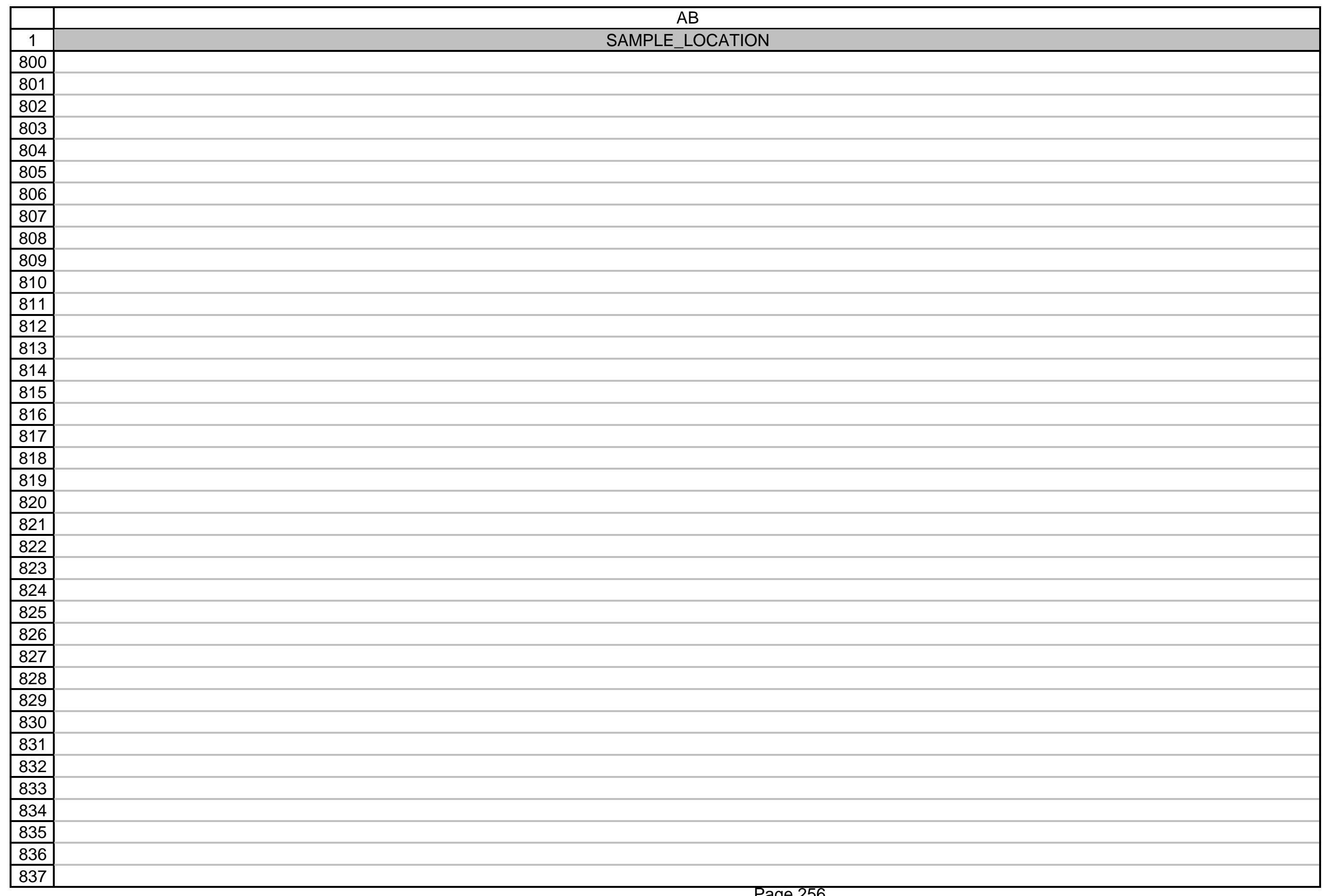

Page 256 
AB

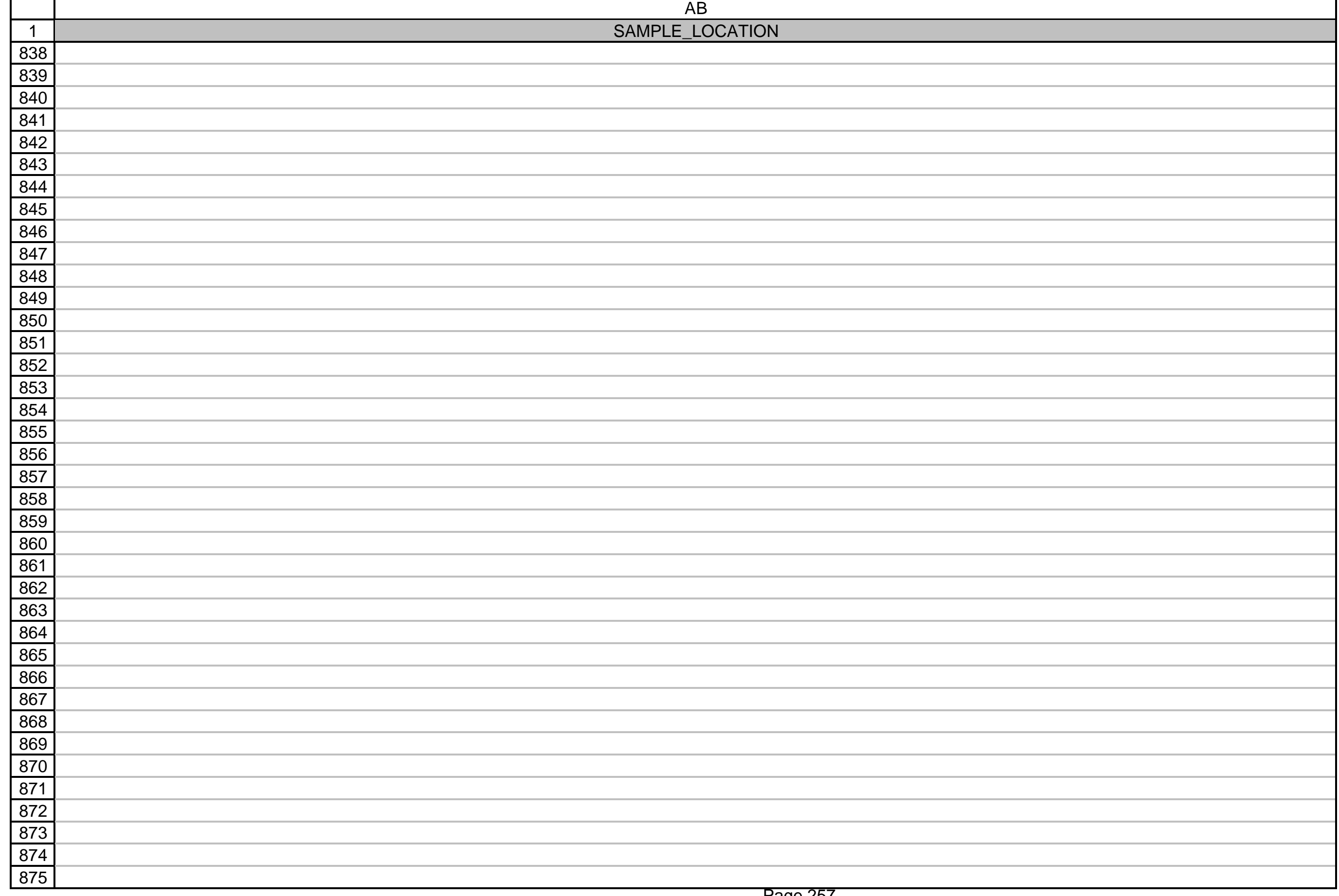

Page 257 
AB

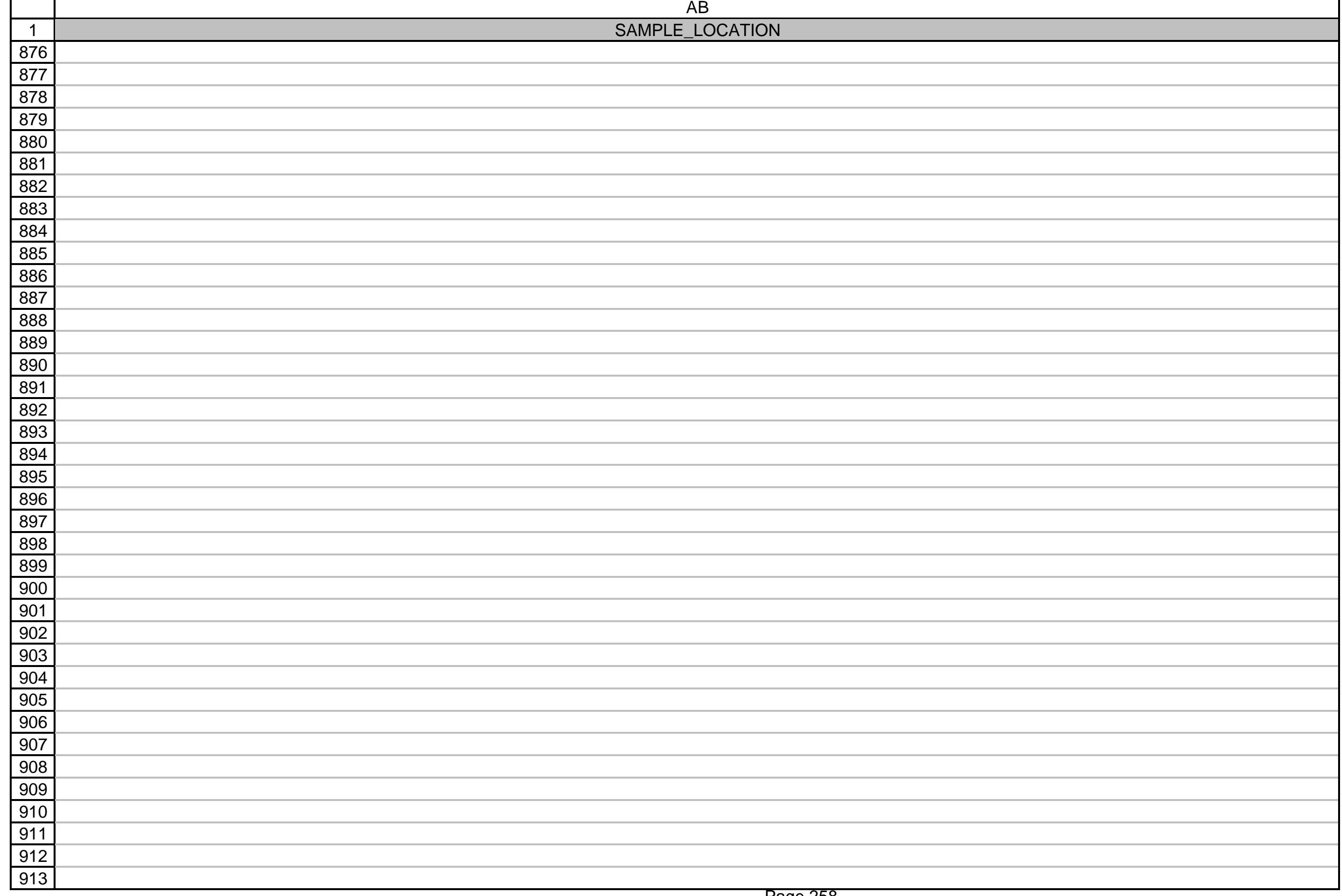

Page 258 
AB

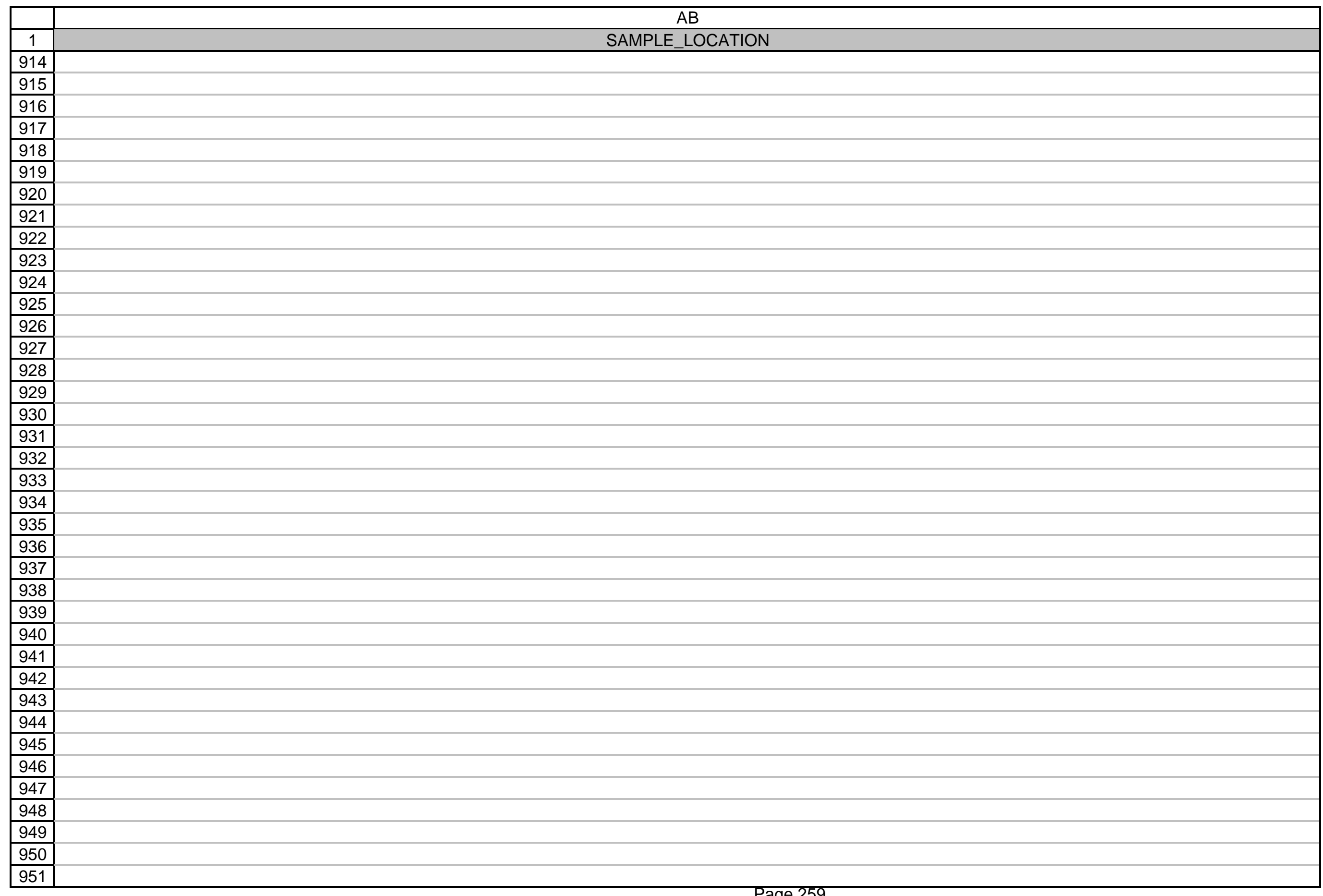

Page 259 
AB

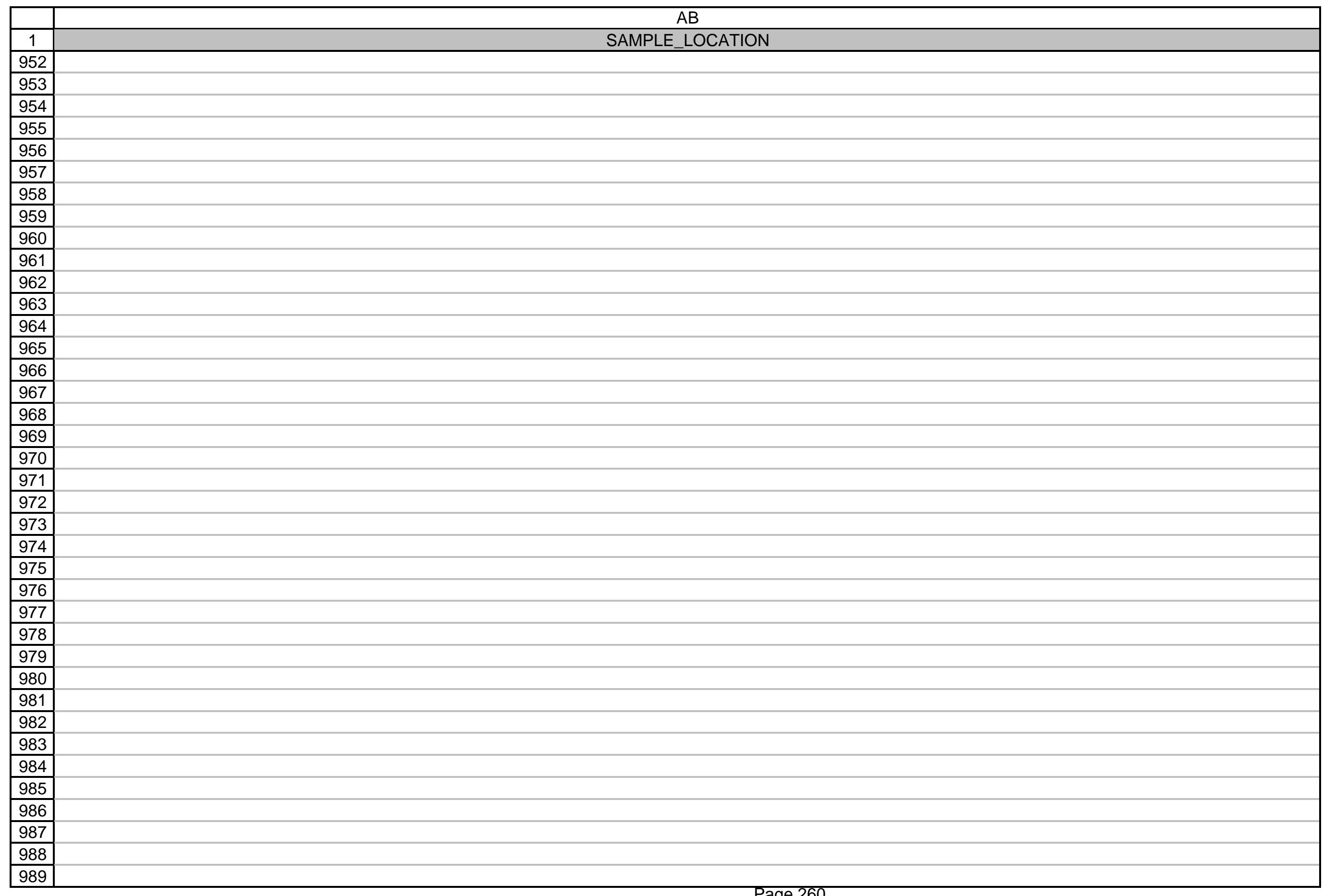

Page 260 
AB

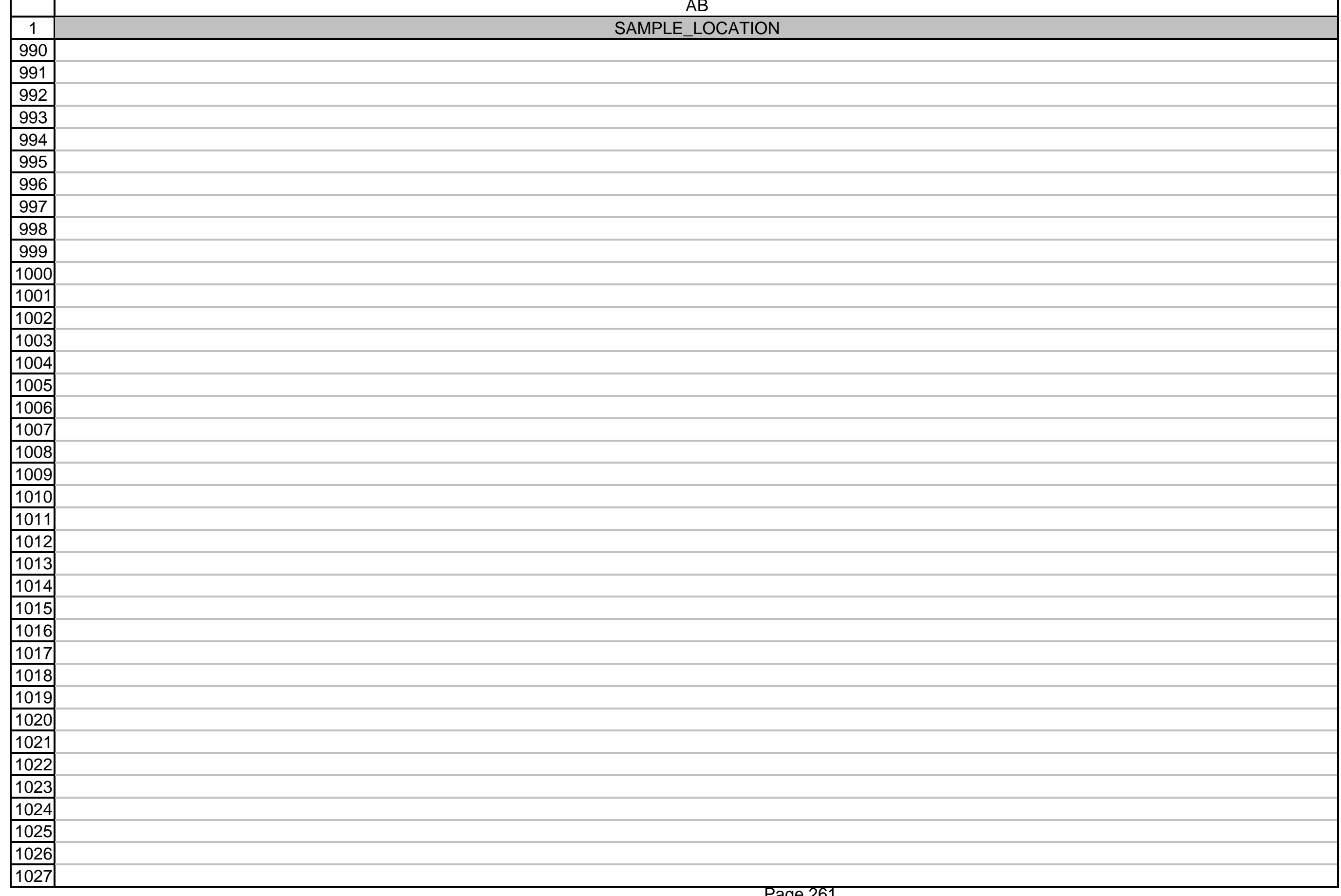

Page 261 
AB

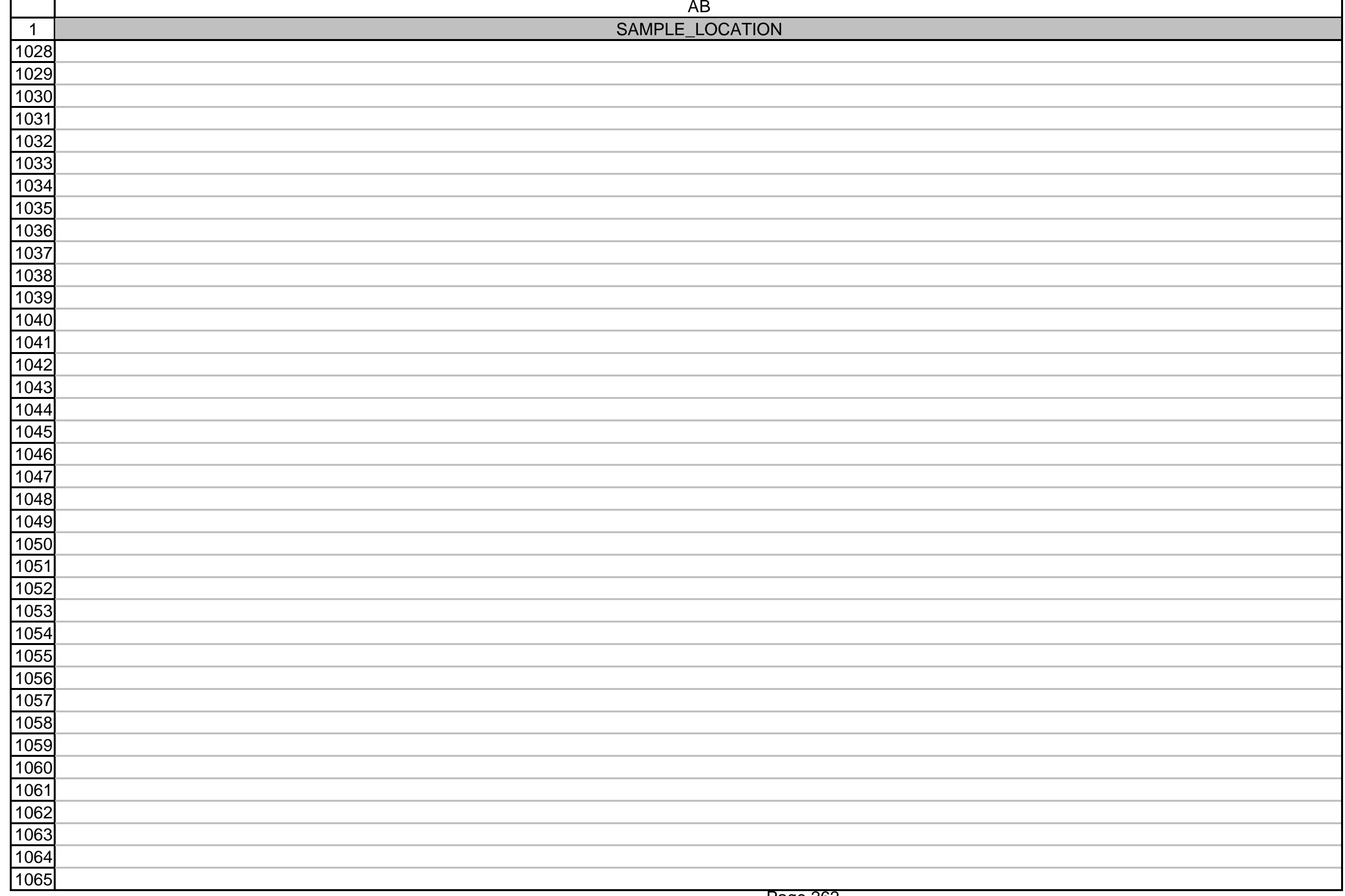

Page 262 


\begin{tabular}{|c|c|}
\hline & 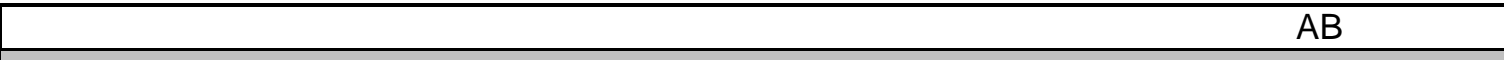 \\
\hline 1 & SAMPLE_LOCATION \\
\hline 1066 & \\
\hline 1067 & \\
\hline 1068 & \\
\hline 1069 & \\
\hline 1070 & \\
\hline 1071 & \\
\hline 1072 & \\
\hline 1073 & \\
\hline 1074 & \\
\hline 1075 & \\
\hline 1076 & \\
\hline 1077 & \\
\hline 1078 & \\
\hline 1079 & \\
\hline 1080 & \\
\hline 1081 & WATER COLLECTED AT RIVERBOTTOM. \\
\hline \begin{tabular}{|l|l|}
1082 \\
\end{tabular} & WATER COLLECTED AT RIVERBOTTOM. \\
\hline 1083 & WATER COLLECTED AT RIVERBOTTOM. \\
\hline 1084 & WATER COLLECTED AT RIVERBOTTOM. \\
\hline 1085 & WATER COLLECTED AT RIVERBOTTOM. \\
\hline 1086 & WATER COLLECTED AT RIVERBOTTOM. \\
\hline 1087 & \\
\hline 1088 & \\
\hline 1089 & \\
\hline 1090 & \\
\hline 1091 & \\
\hline 1092 & \\
\hline 1093 & \\
\hline 1094 & \\
\hline 1095 & \\
\hline 1096 & \\
\hline 1097 & \\
\hline 1098 & \\
\hline 1099 & \\
\hline 1100 & \\
\hline 1101 & \\
\hline 1102 & \\
\hline 1103 & \\
\hline
\end{tabular}


1106 WATER COLLECTED AT RIVERBOTTOM.

1107 WATER COLLECTED AT RIVERBOTTOM

1108 WATER COLLECTED AT RIVERBOTTOM

1109 WATER COLLECTED AT RIVERBOTTOM.

1110 WATER COLLECTED AT RIVERBOTTOM.

1111 WATER COLLECTED AT RIVERBOTTOM.

\begin{tabular}{|l|l}
1112 & WATER COLLECTED AT RIVERBOTTOM \\
\hline 1113 &
\end{tabular}

1113 .

1114

1115

1116

1117

1118

1119

1120

1121

1122

1123

1124

1125

1126

1127

1128

1129

1130

1131

1132

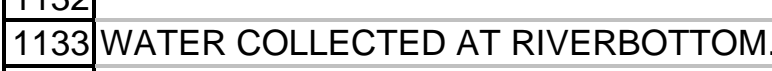

1134 WATER COLLECTED AT RIVERBOTTOM.

1135 WATER COLLECTED AT RIVERBOTTOM.

1136 WATER COLLECTED AT RIVERBOTTOM.

1137 WATER COLLECTED AT RIVERBOTTOM.

1138 WATER COLLECTED AT RIVERBOTTOM.

1139 WATER COLLECTED AT RIVERBOTTOM

1140 WATER COLLECTED AT RIVERBOTTOM

\begin{tabular}{|l|l|}
11141 & WATER COLLECTED AT RIVERBOTTOM \\
\hline
\end{tabular} 


\begin{tabular}{|c|c|}
\hline & $\mathrm{AB}$ \\
\hline 1 & SAMPLE_LOCATION \\
\hline 1142 & WATER COLLECTED AT RIVERBOTTOM. \\
\hline 1143 & WATER COLLECTED AT RIVERBOTTOM. \\
\hline 1144 & \\
\hline 1145 & \\
\hline 1146 & \\
\hline 1147 & \\
\hline 1148 & \\
\hline 1149 & \\
\hline 1151 & \\
\hline 1152 & \\
\hline 1153 & \\
\hline 1154 & \\
\hline 1155 & \\
\hline 1156 & \\
\hline 1157 & \\
\hline 1158 & \\
\hline 1159 & \\
\hline 1161 & \\
\hline 1162 & \\
\hline 1163 & \\
\hline 1164 & \\
\hline 1165 & \\
\hline 1166 & \\
\hline 1167 & \\
\hline 1168 & \\
\hline 1169 & \\
\hline 1170 & \\
\hline 1171 & \\
\hline 1172 & \\
\hline 1173 & \\
\hline 1174 & \\
\hline 1175 & \\
\hline 1176 & \\
\hline 1177 & \\
\hline 1178 & \\
\hline
\end{tabular}


AB

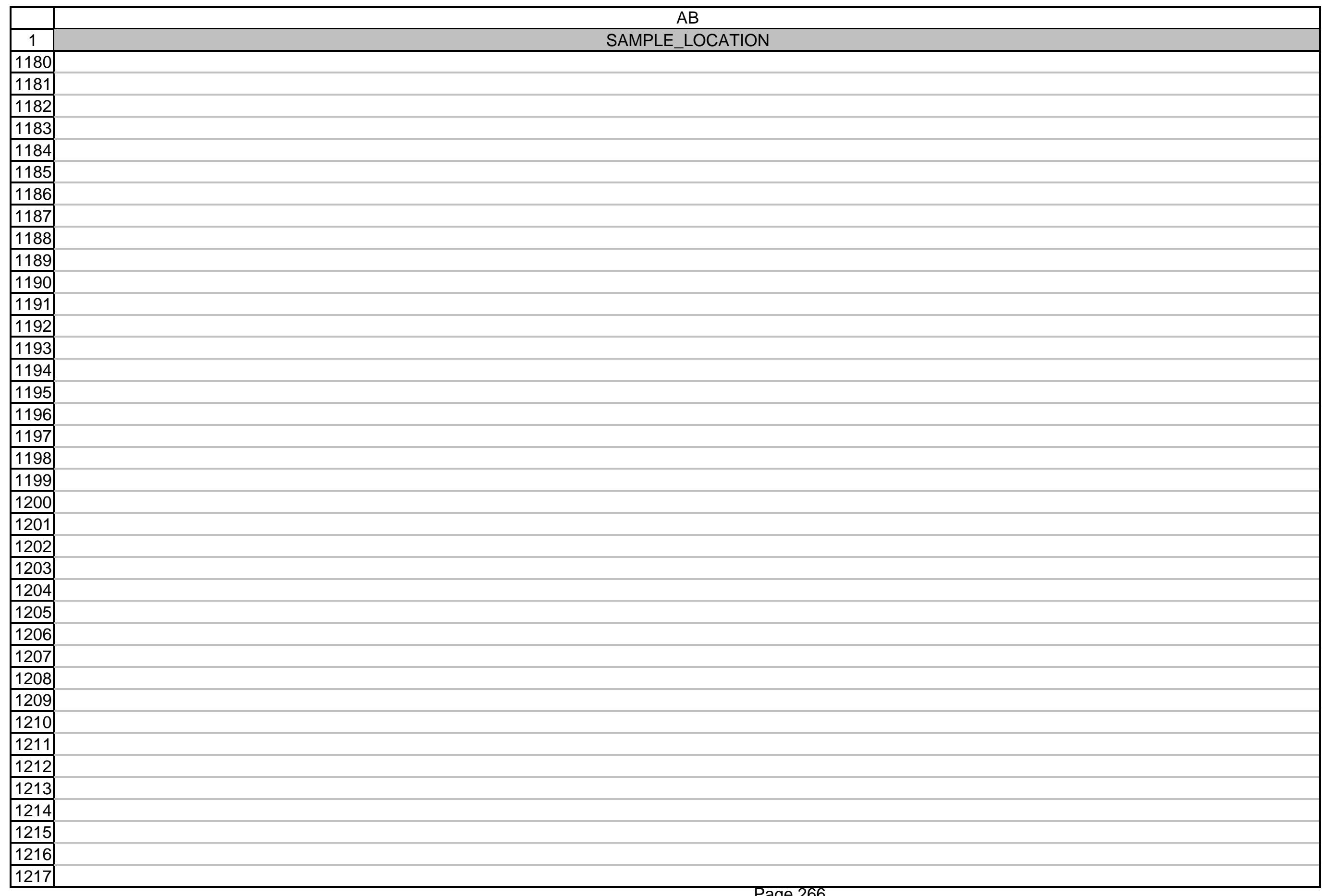

Page 266 
AB

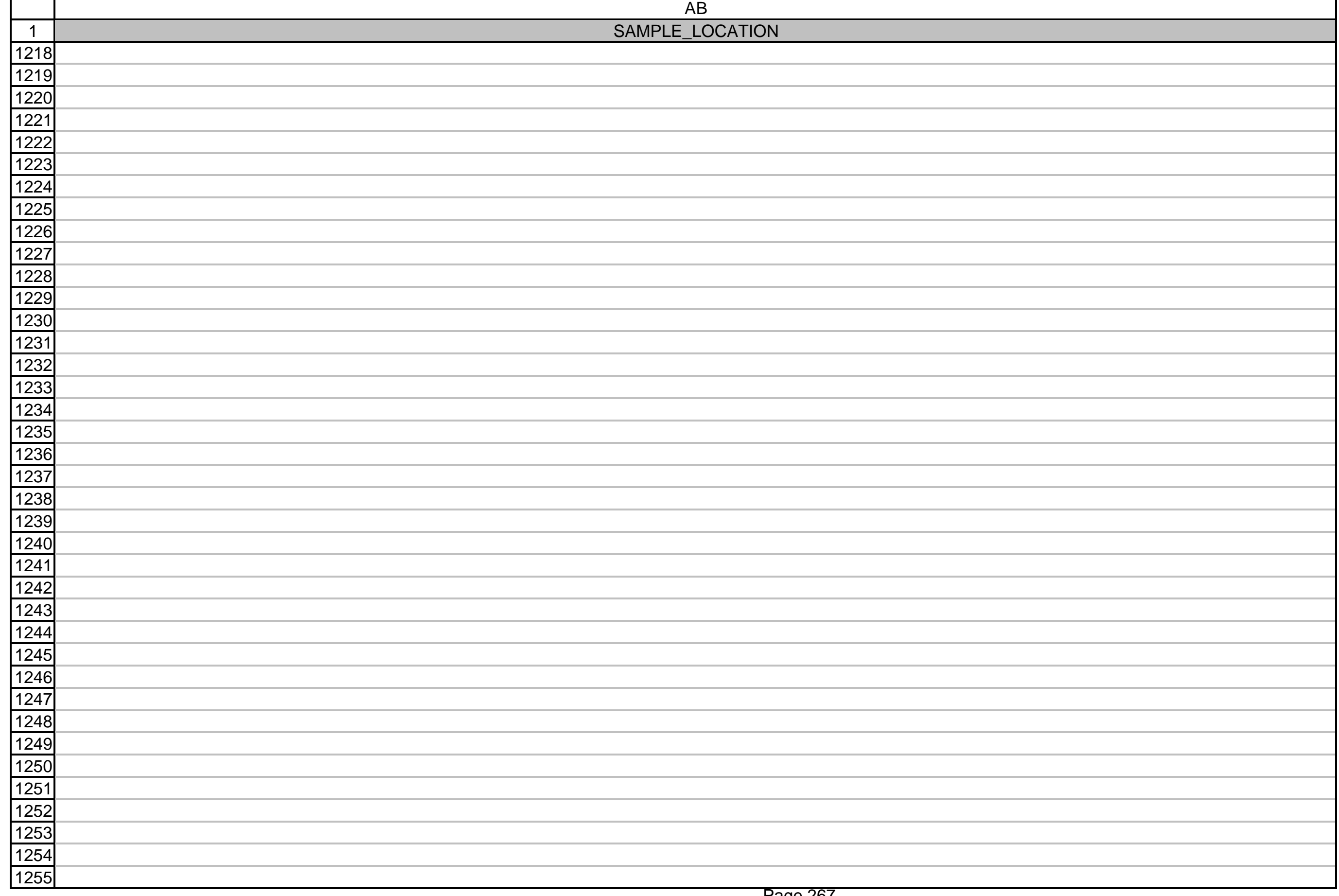

Page 267 


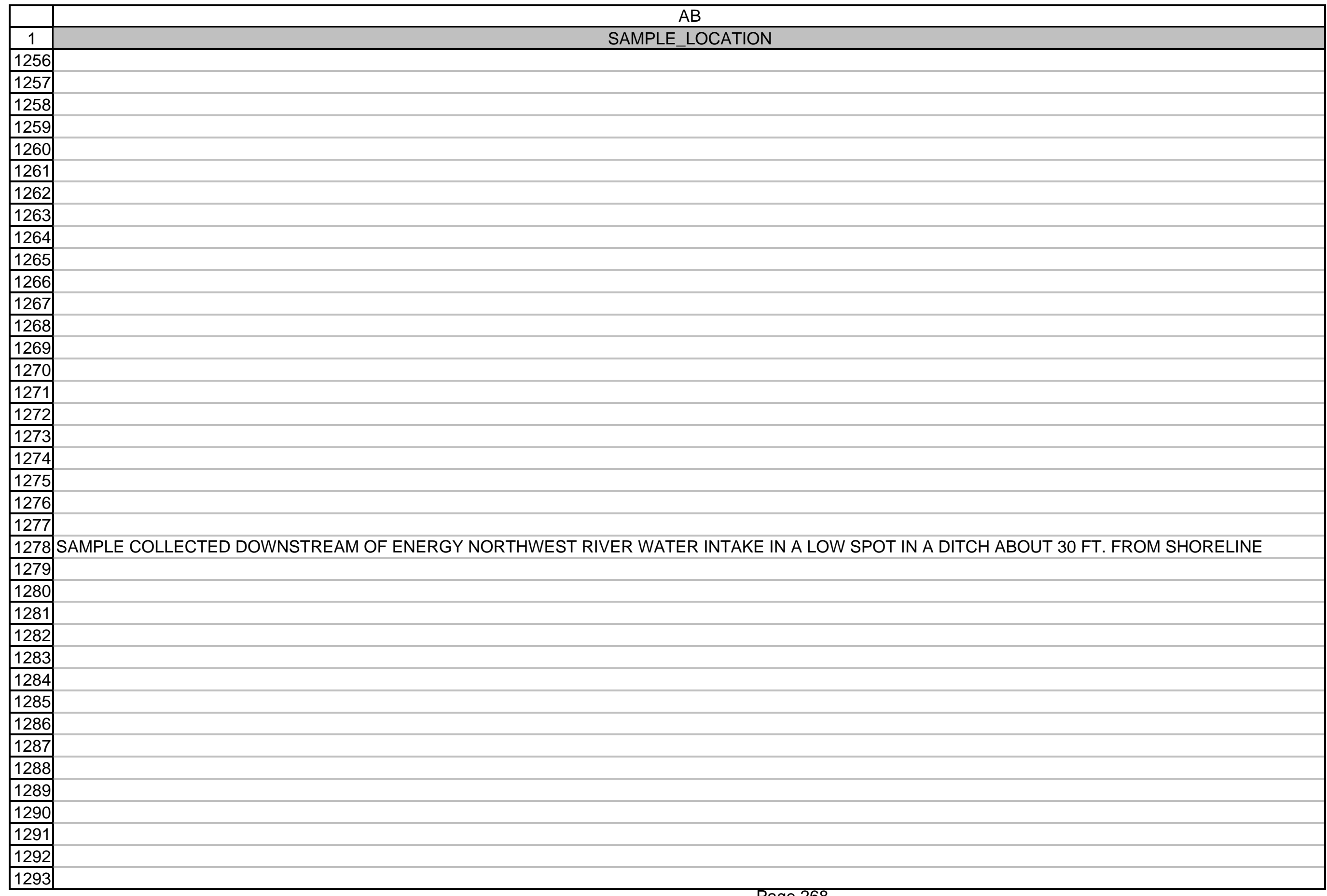


AB

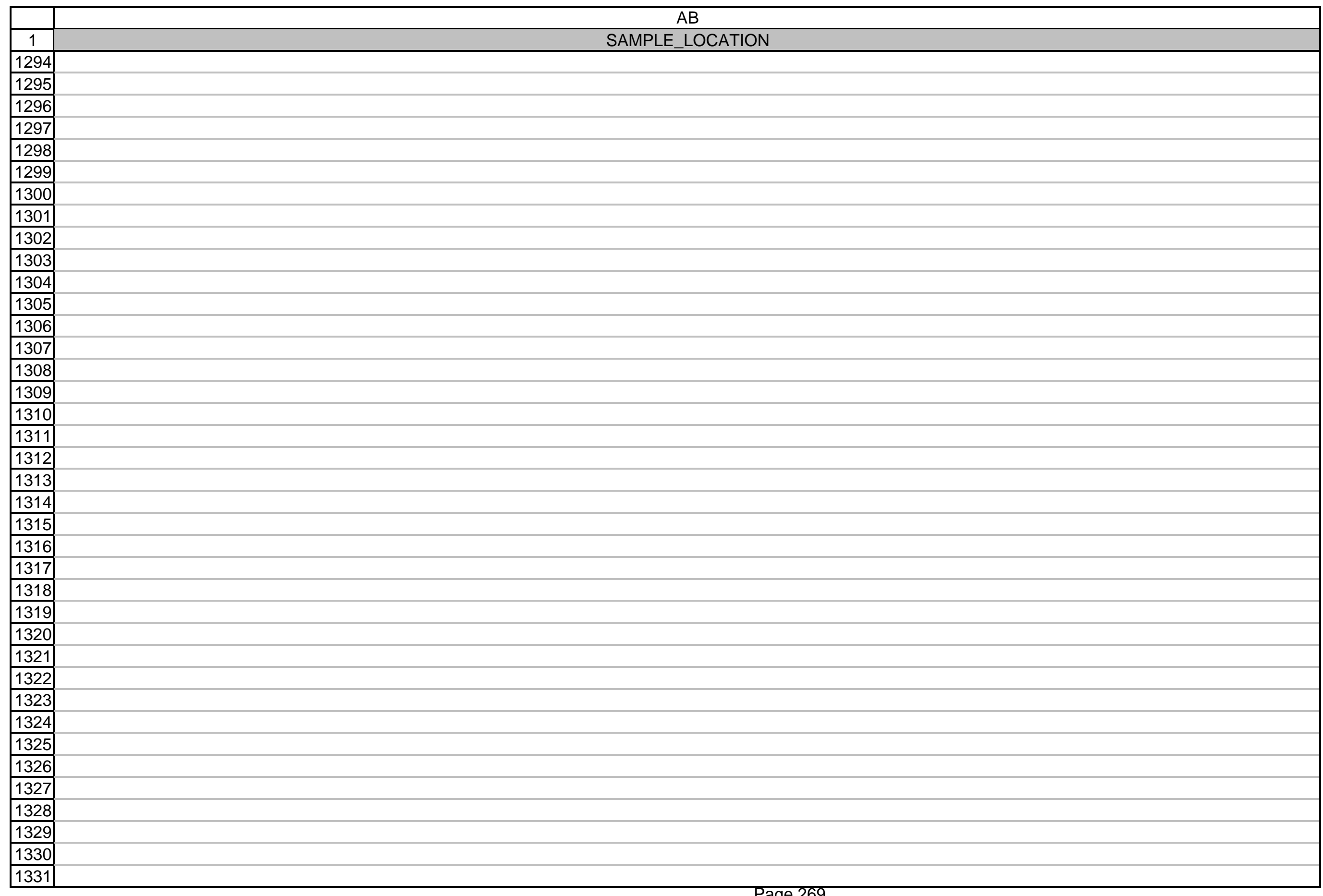

Page 269 
$A B$

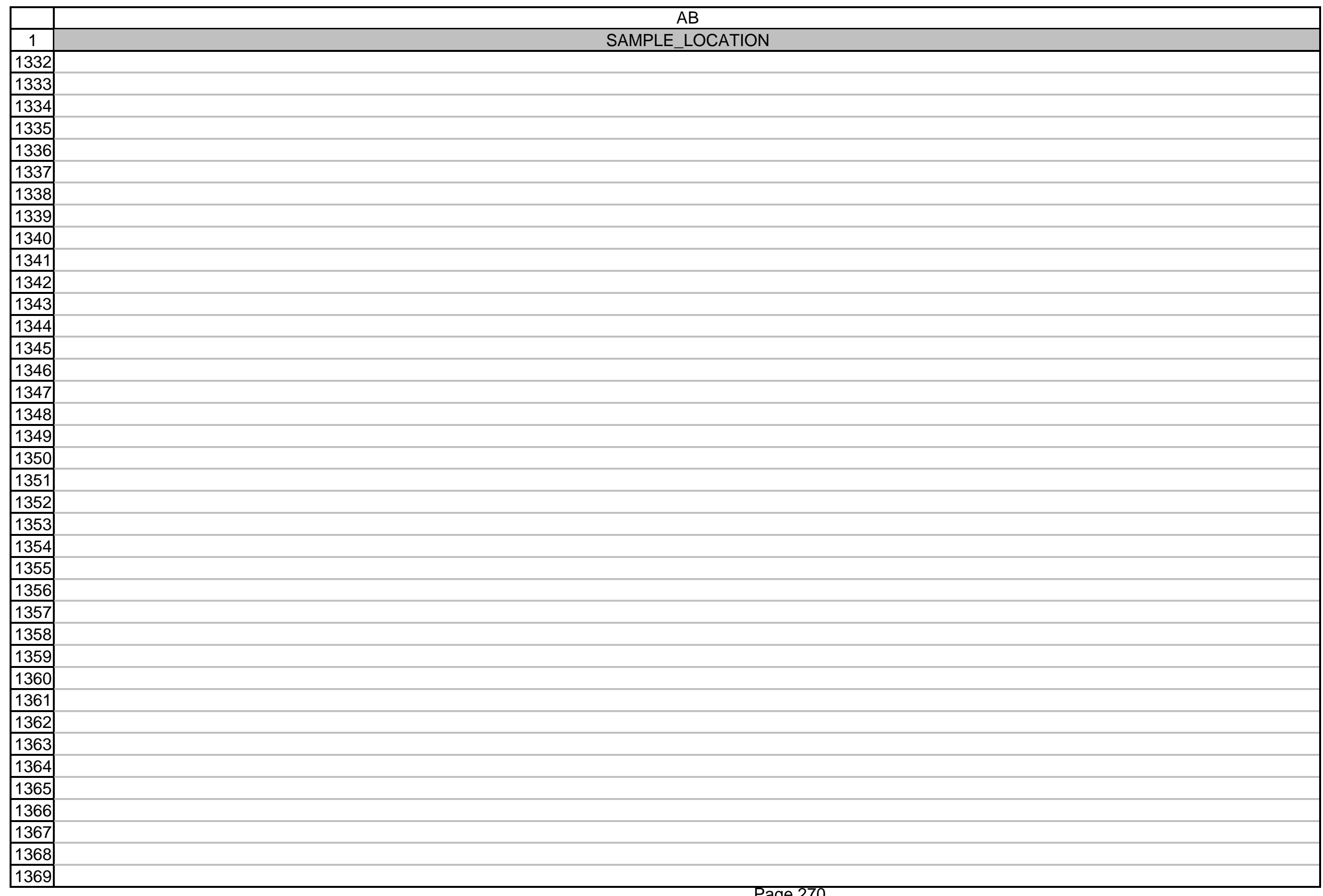


AB

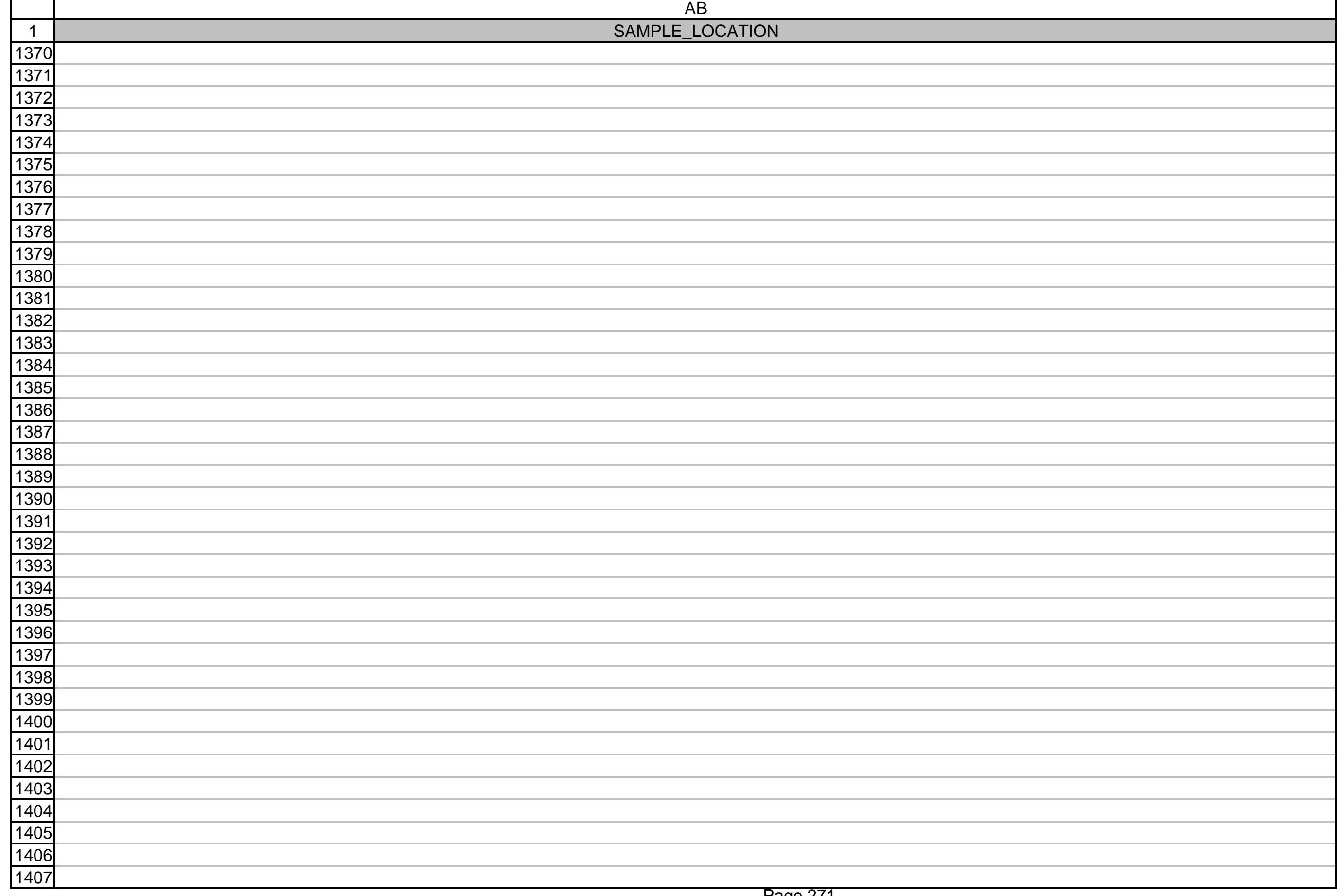

Page 271 
AB

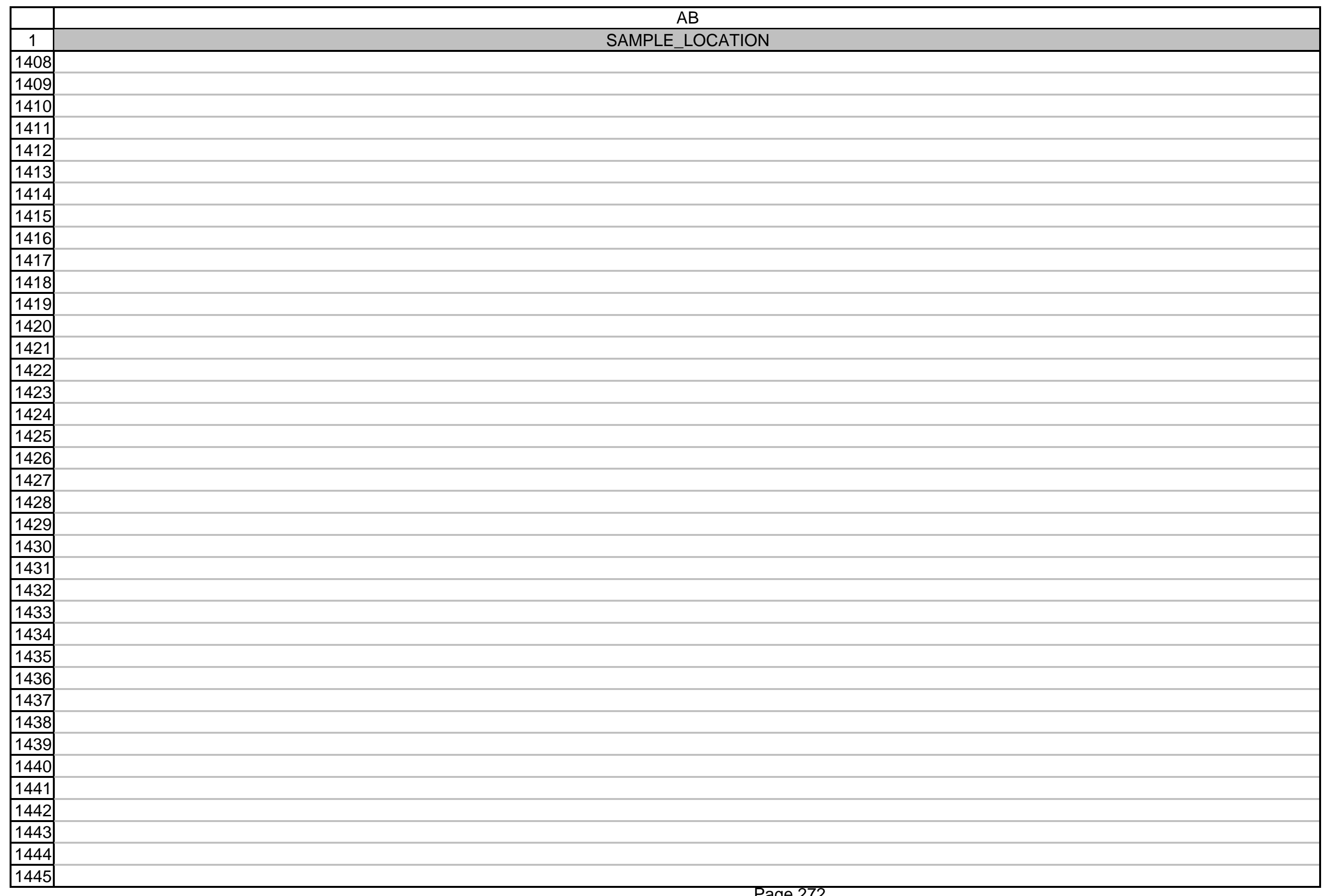

Page 272 
AB

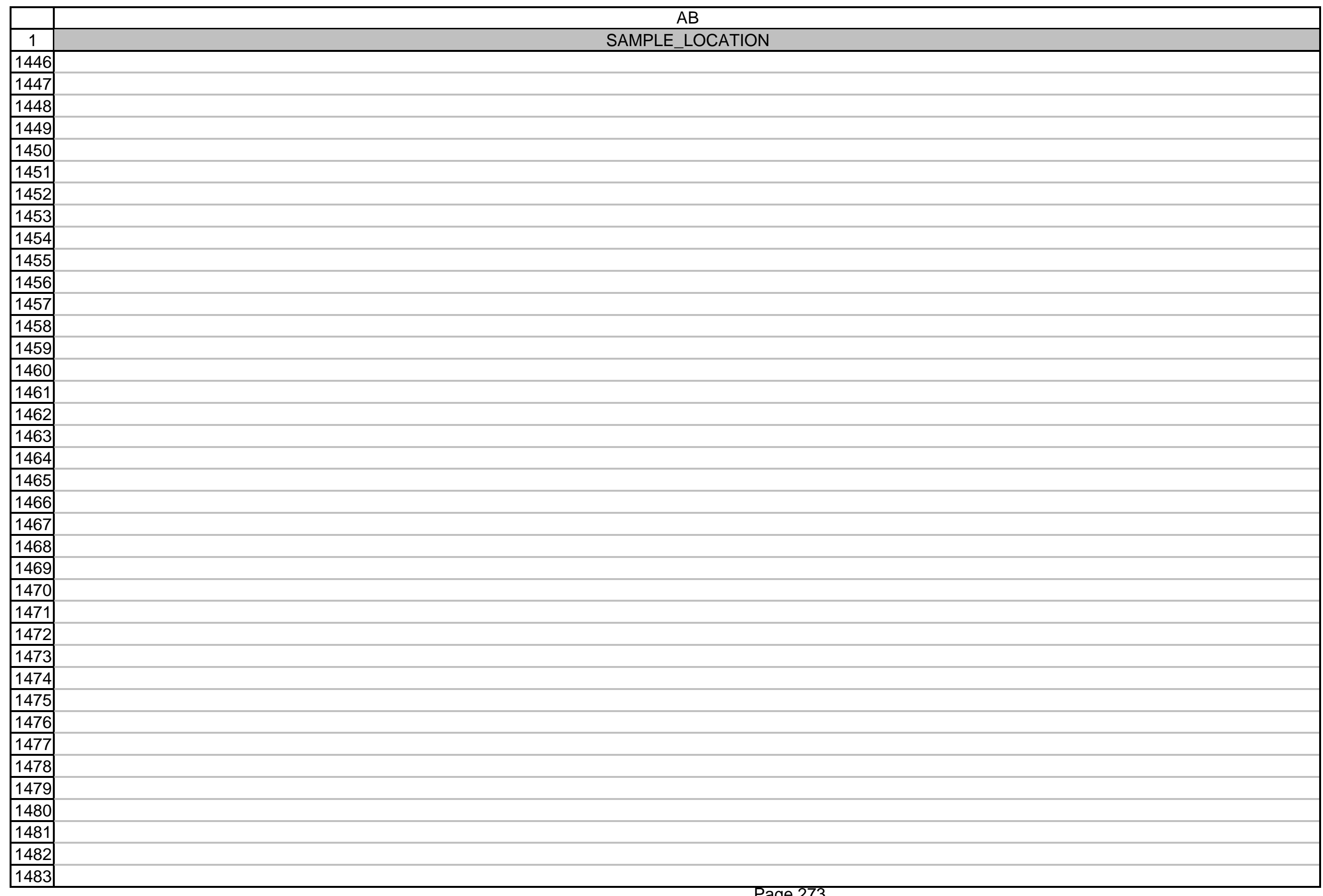

Page 273 
AB

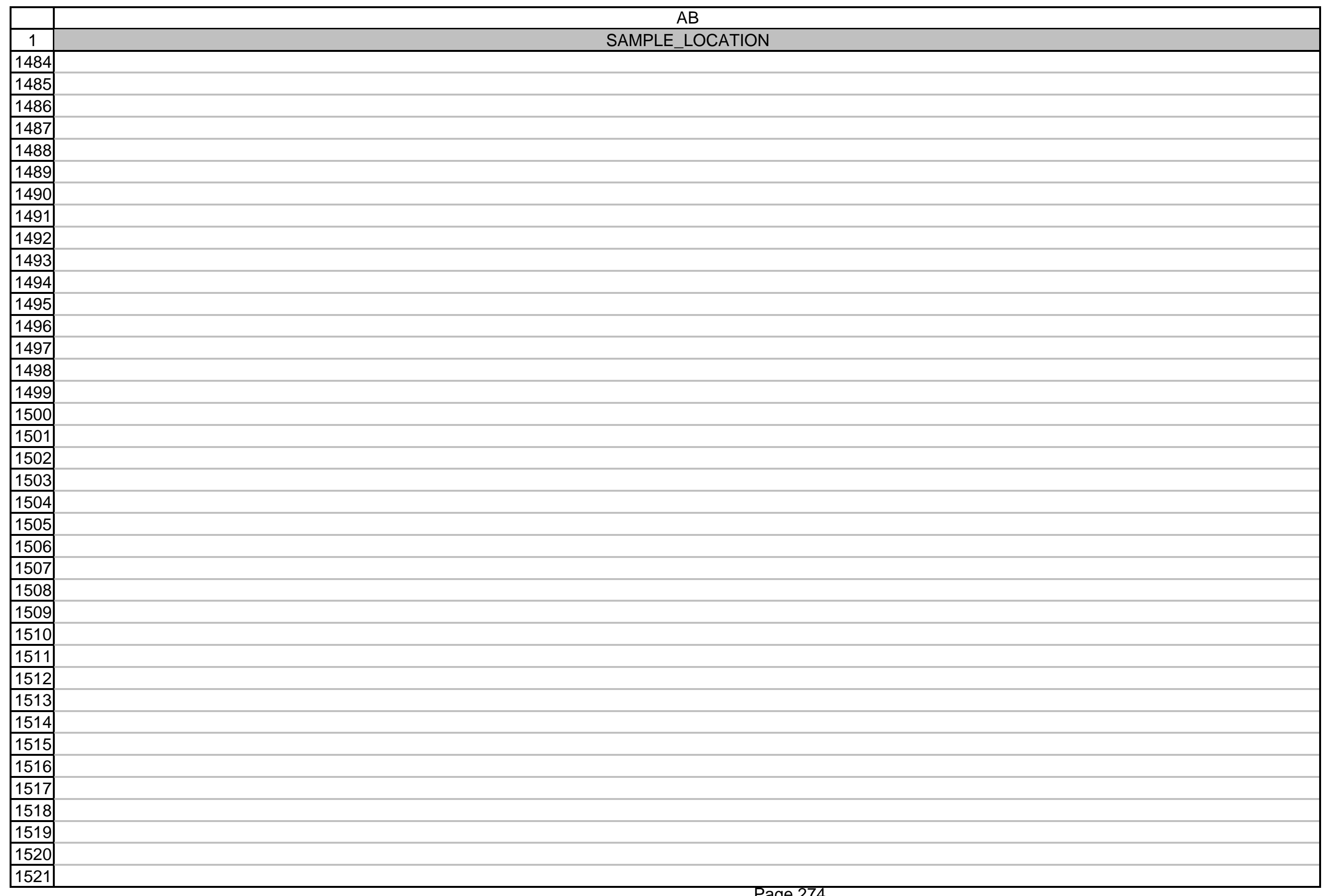

Page 274 
AB

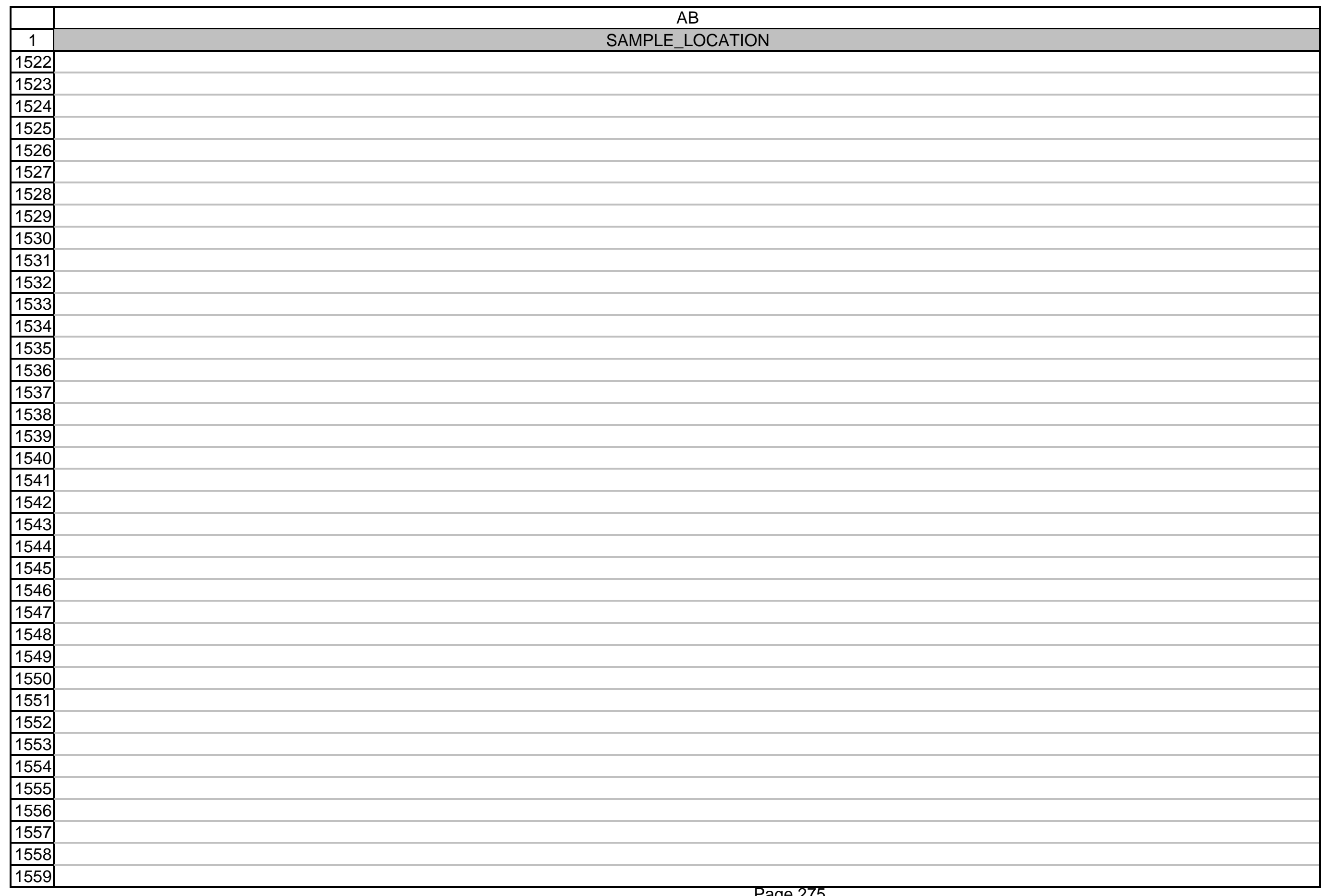


AB

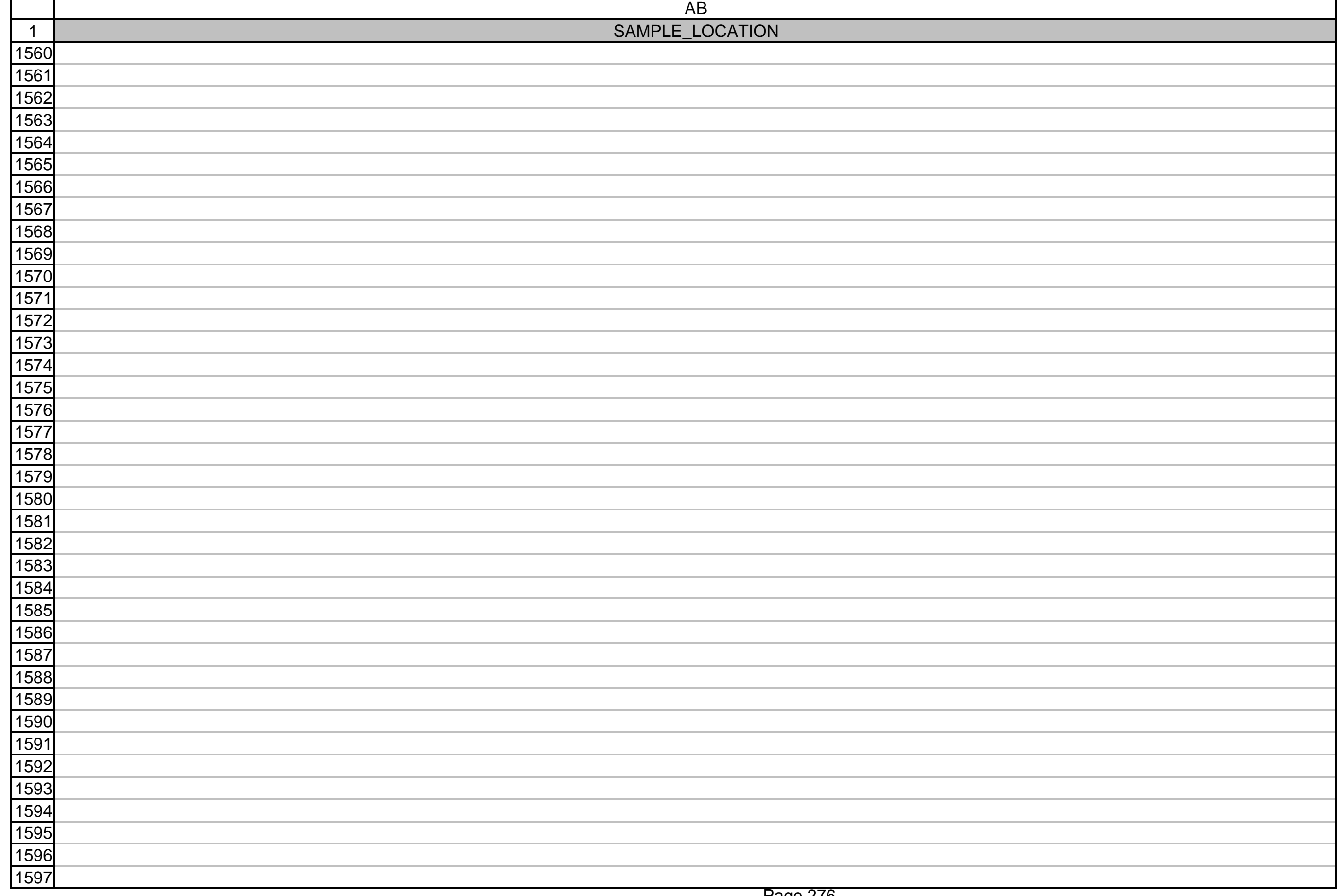




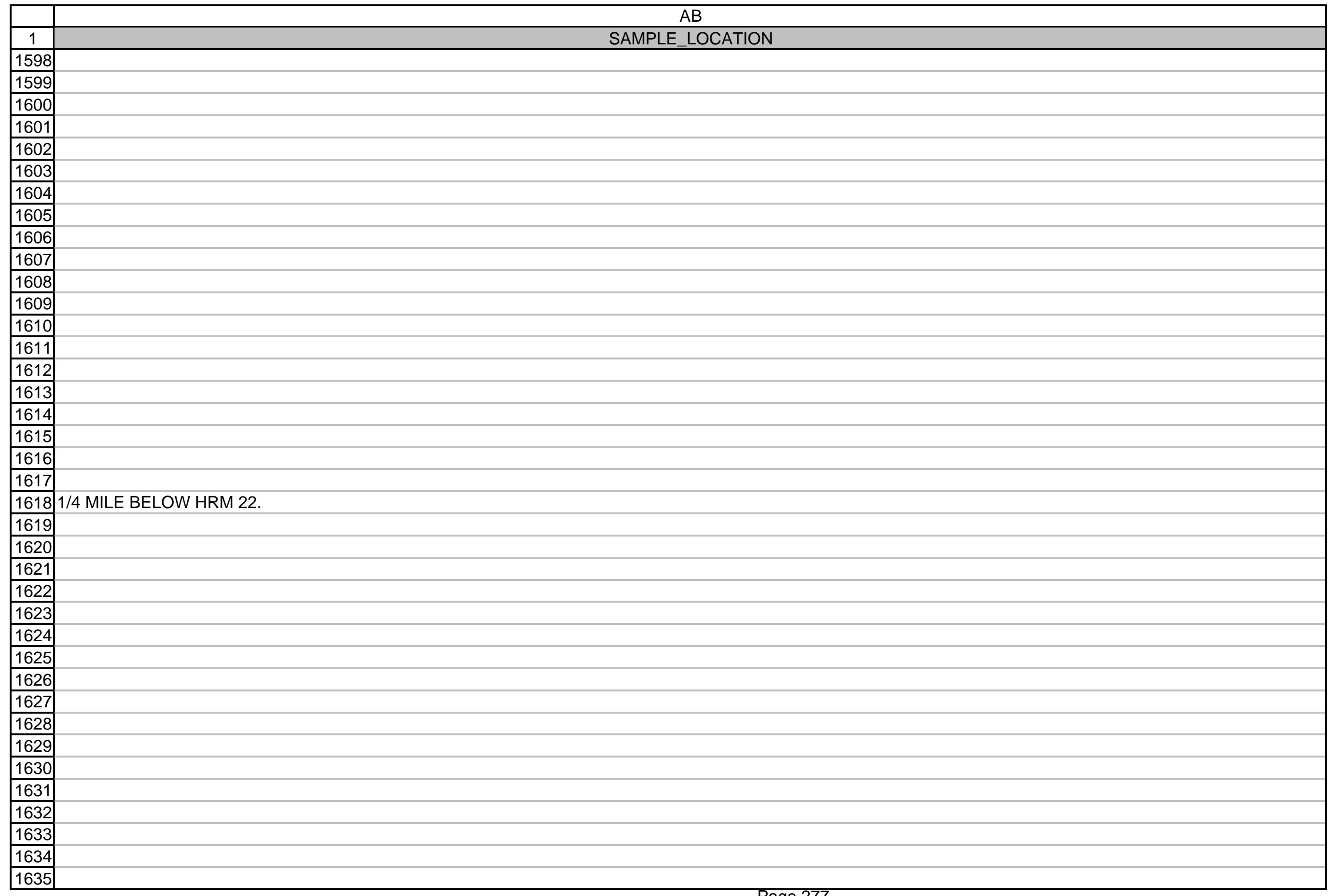


AB

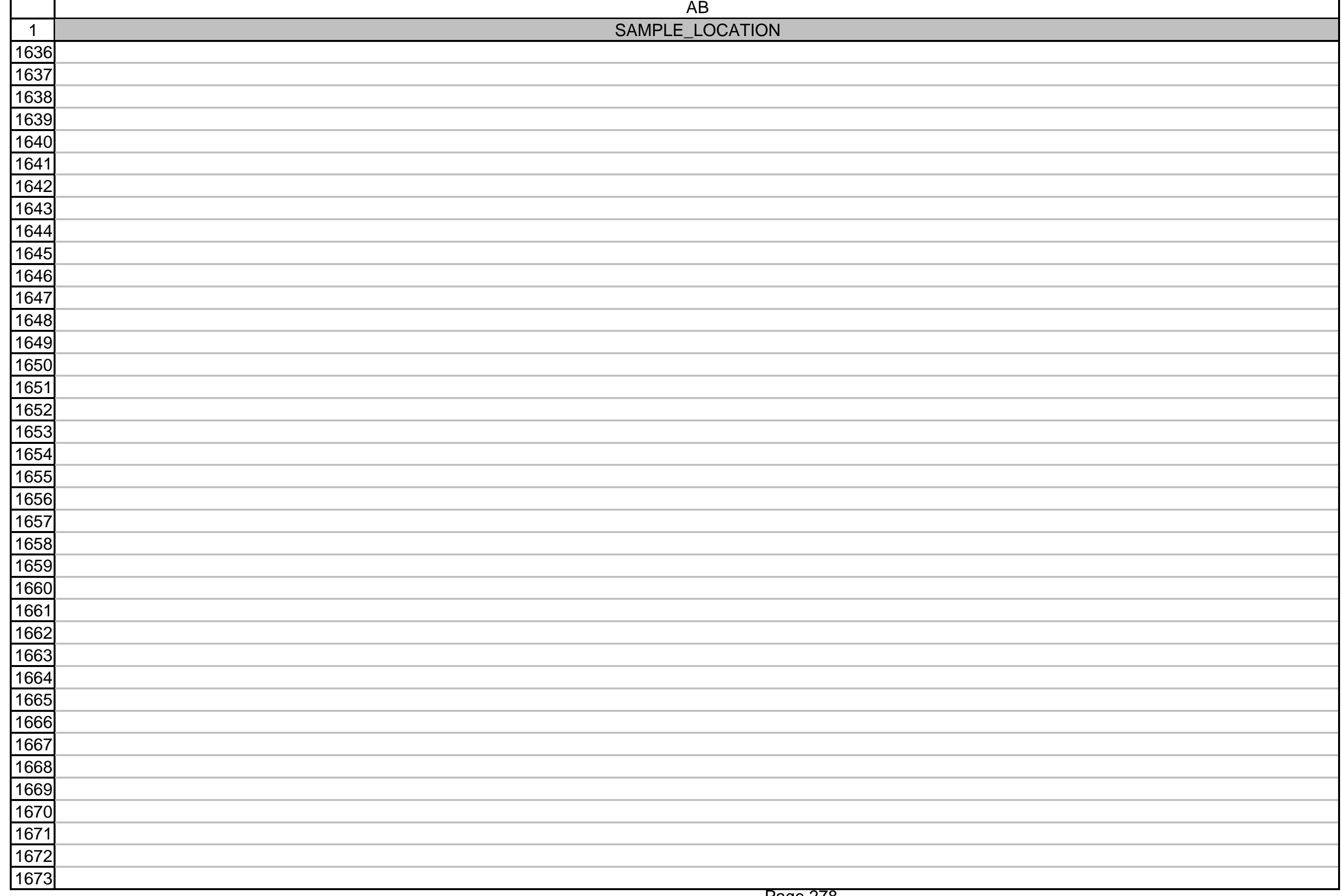


AB

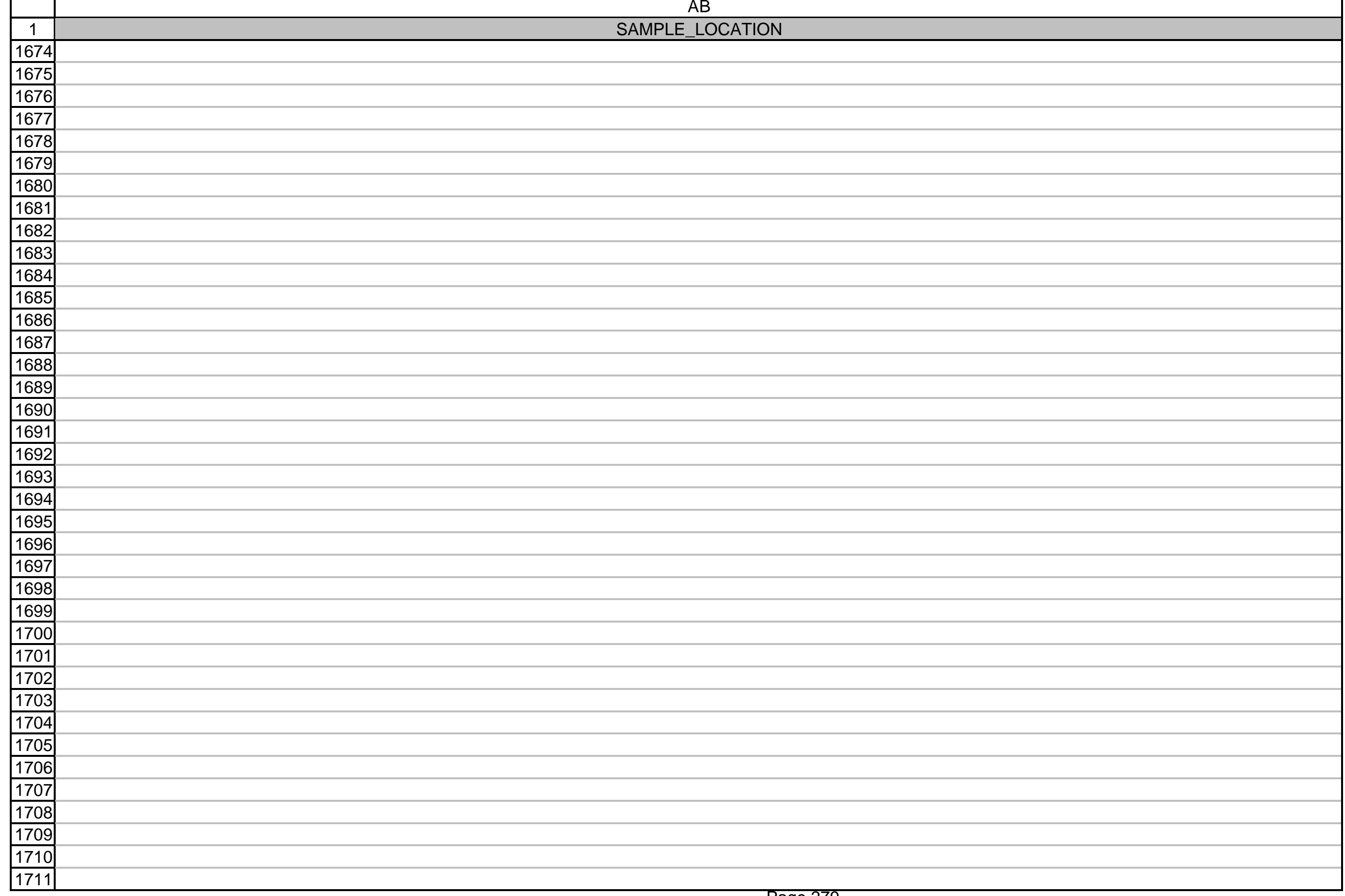


AB

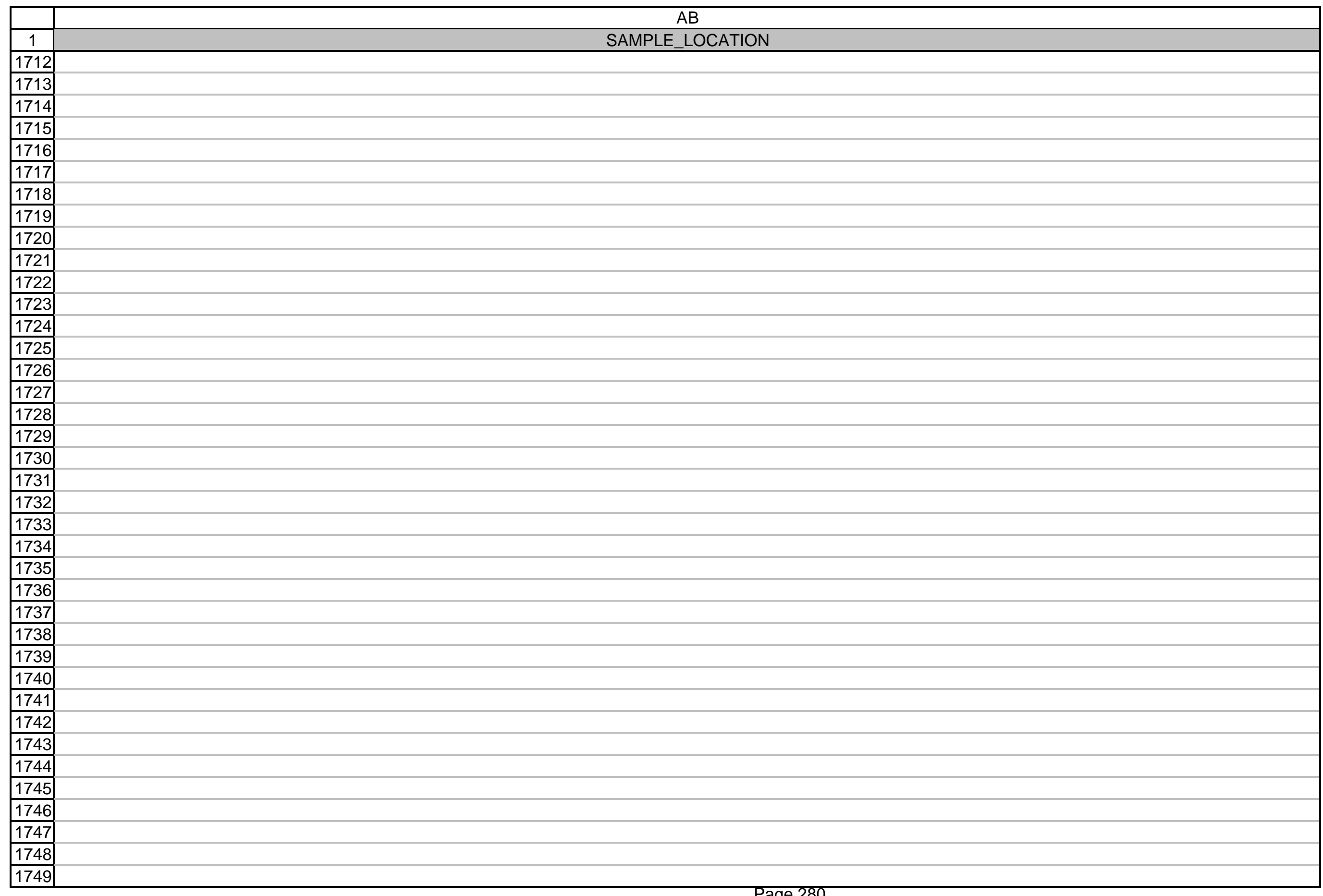

Page 280 
AB

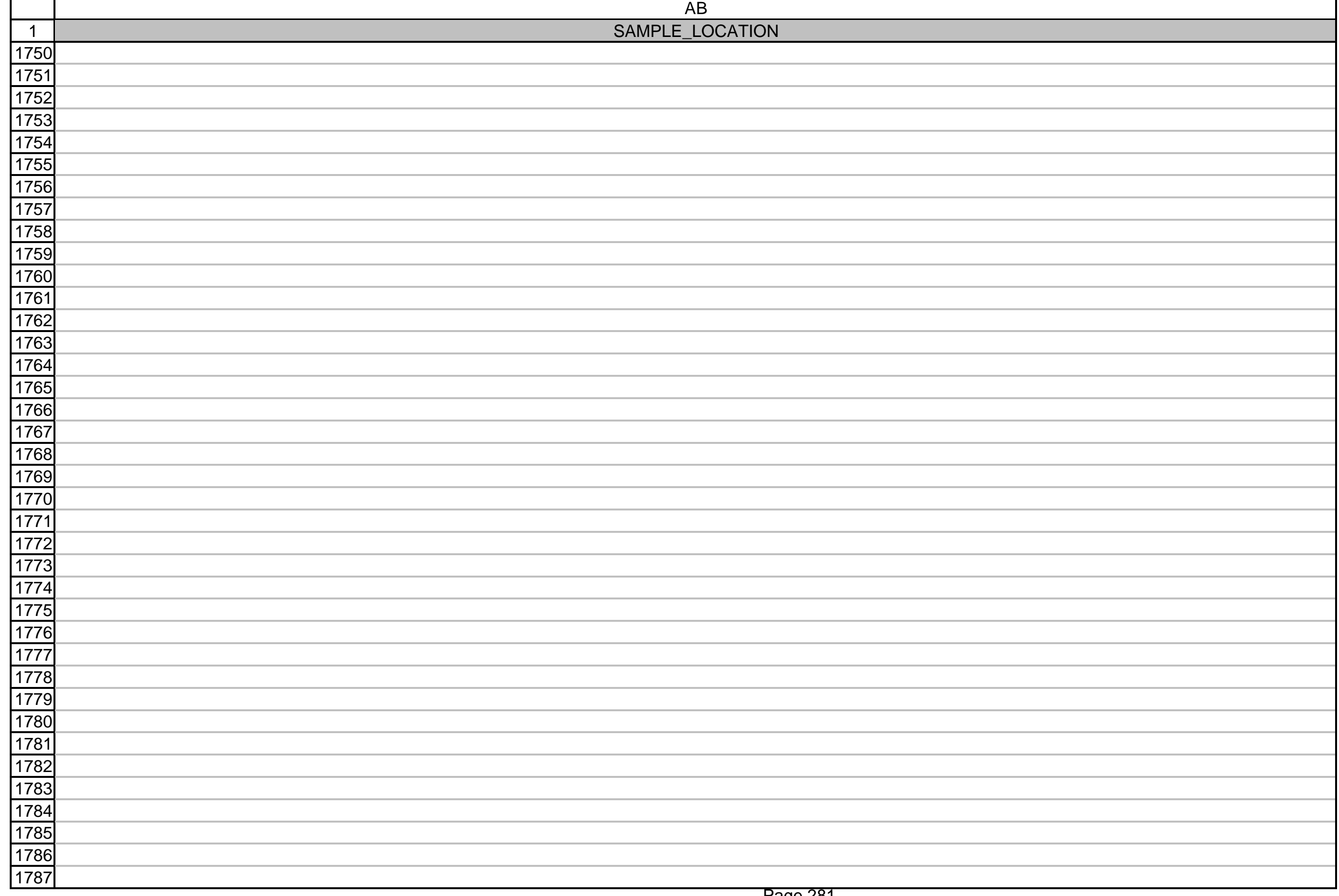

Page 281 
AB

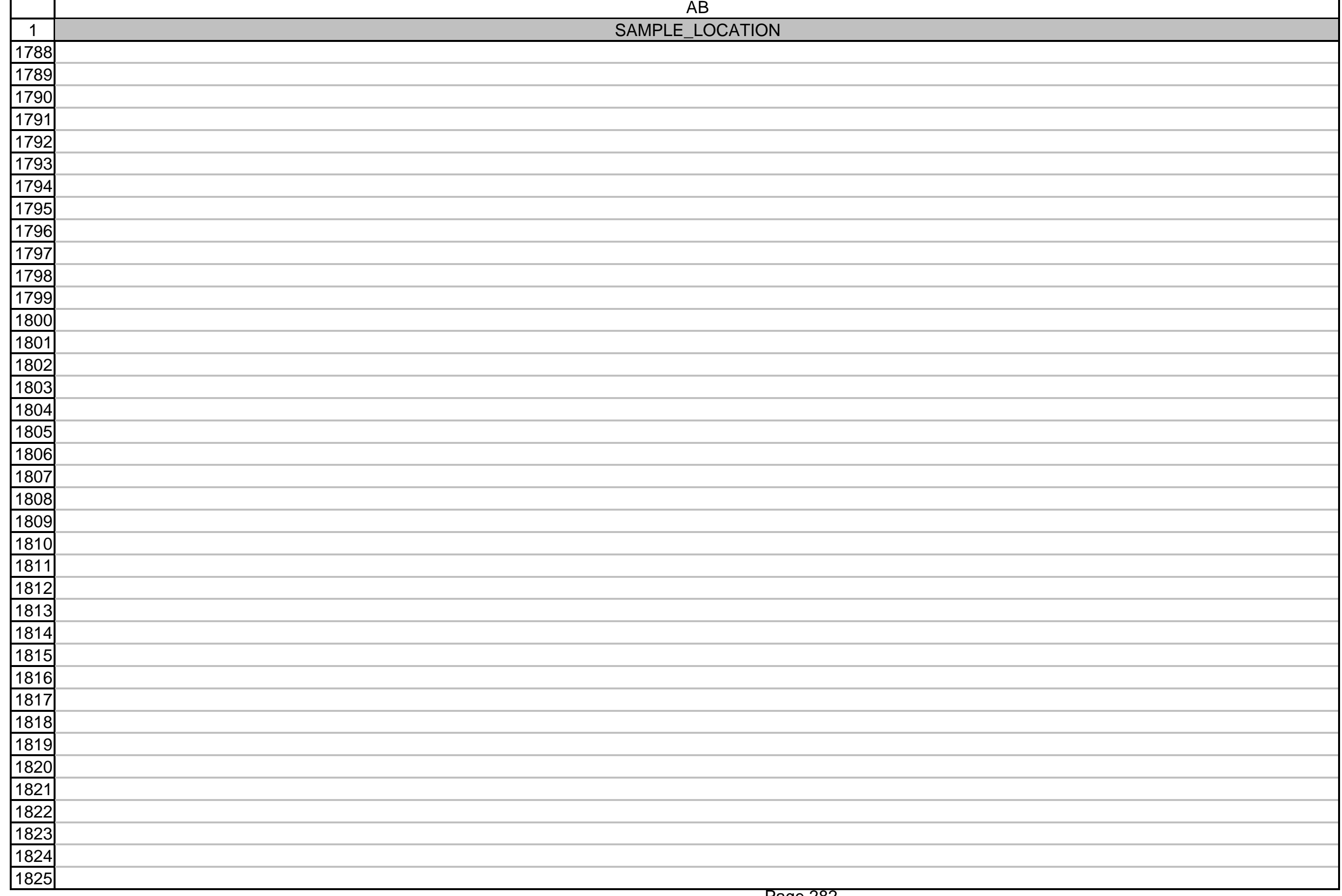

Page 282 
AB

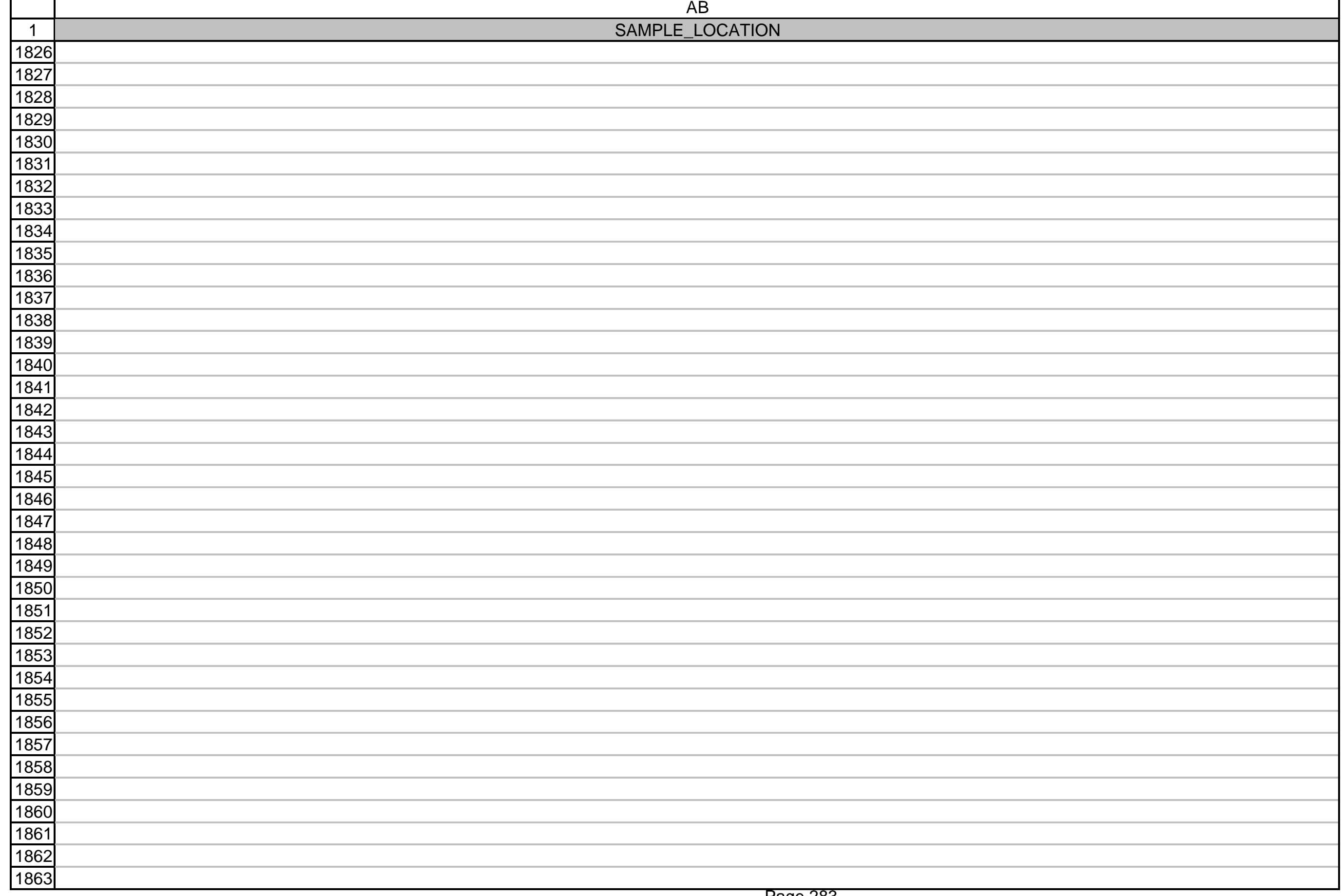

Page 283 
AB

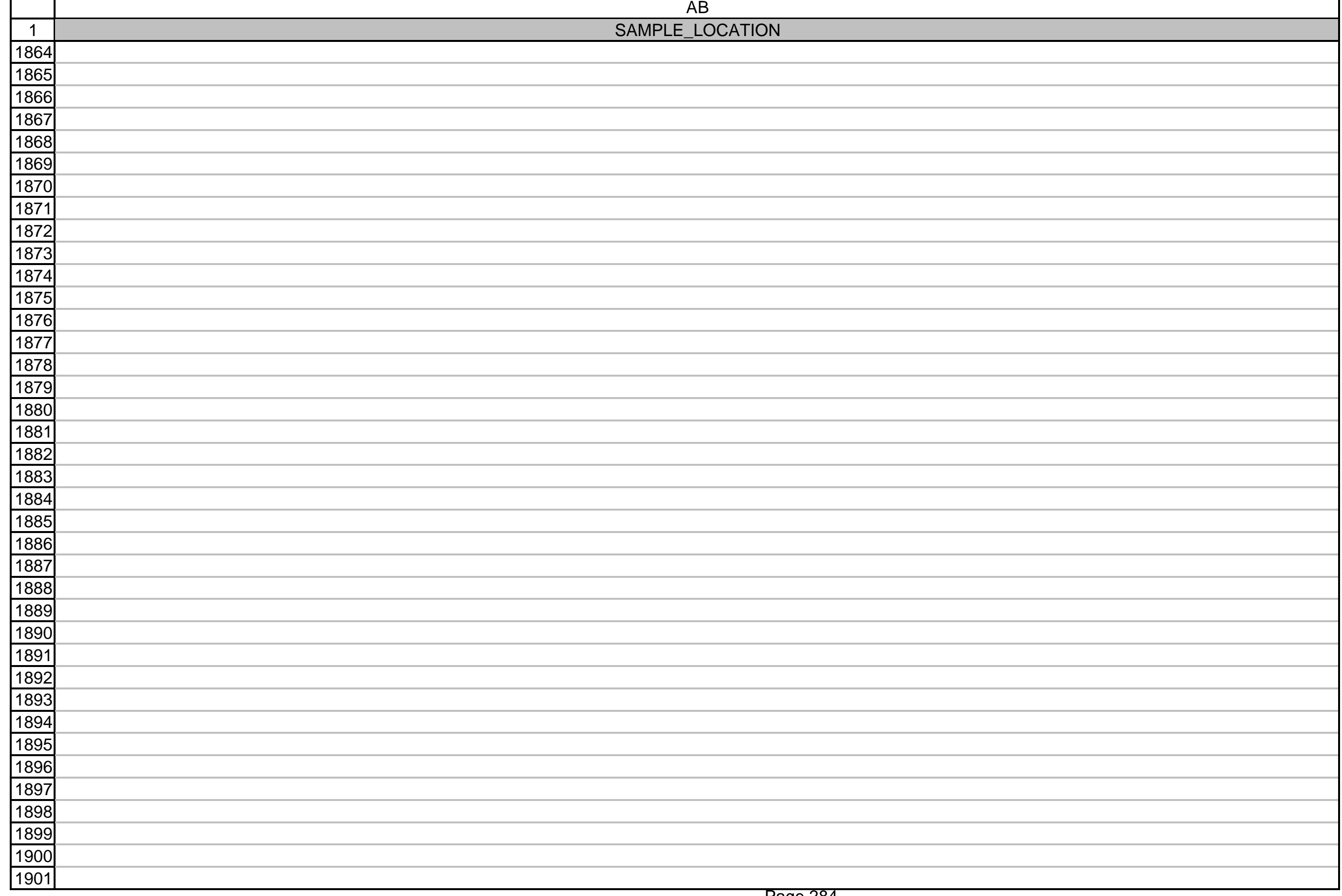

Page 284 
AB

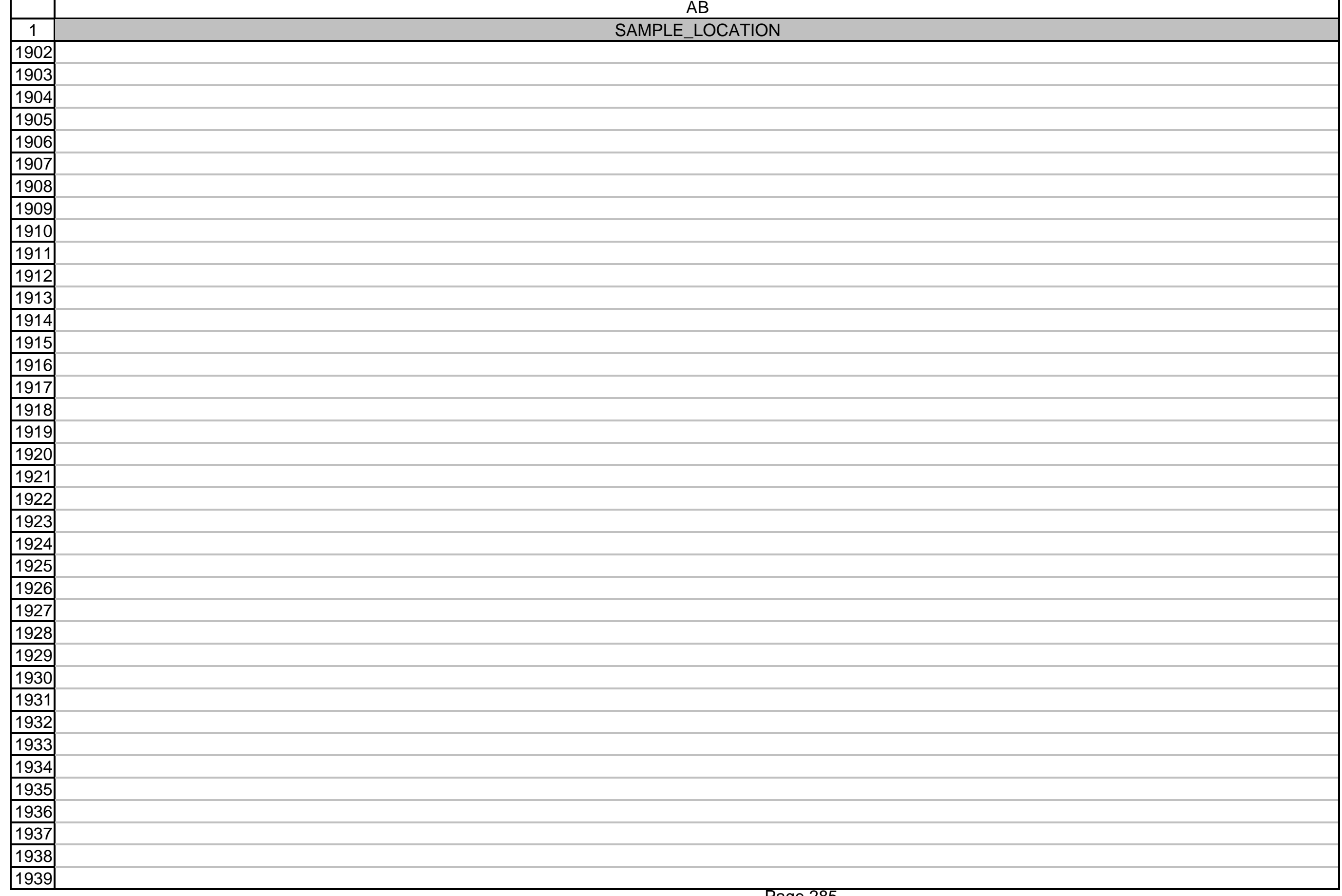


AB

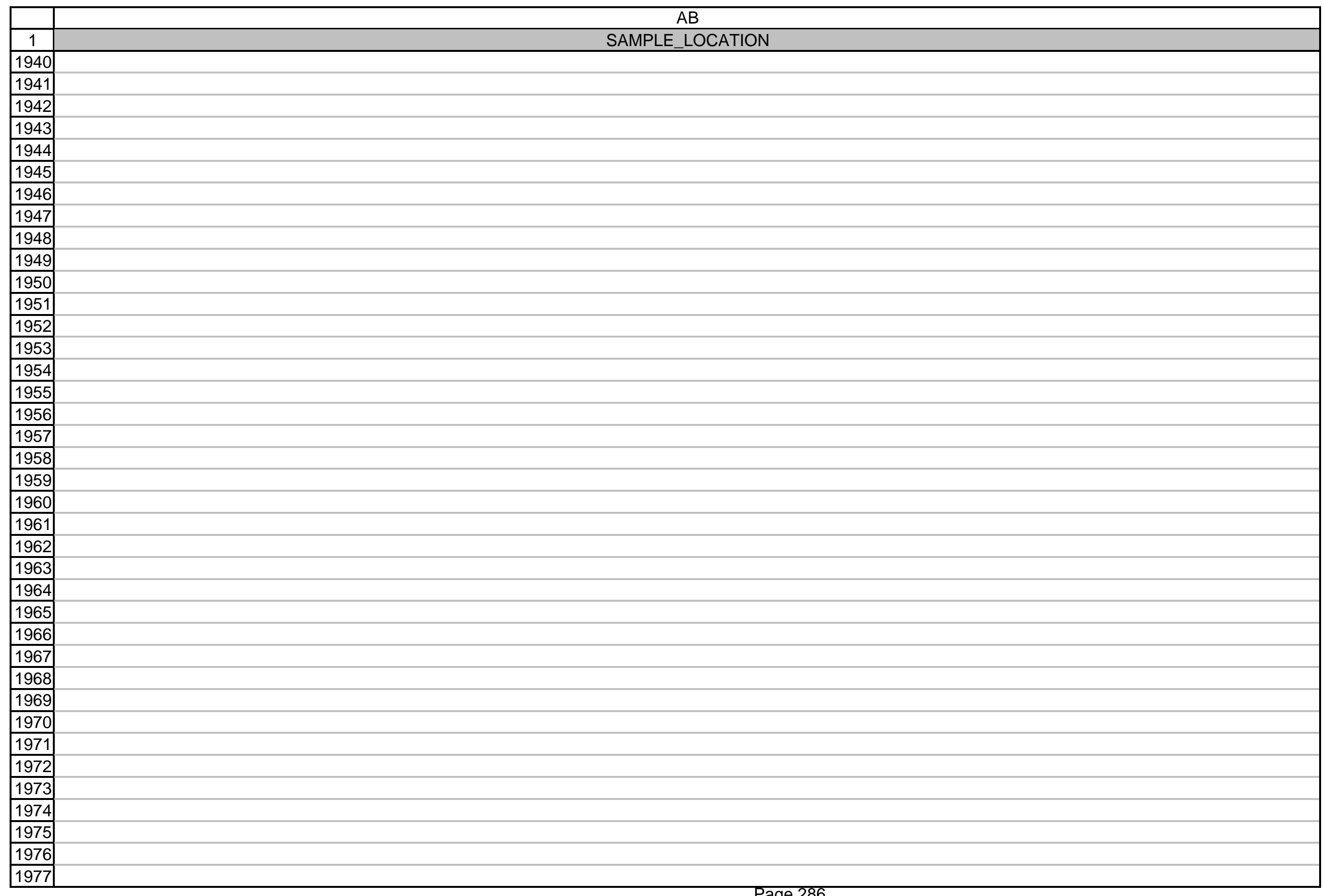

Page 286 
AB

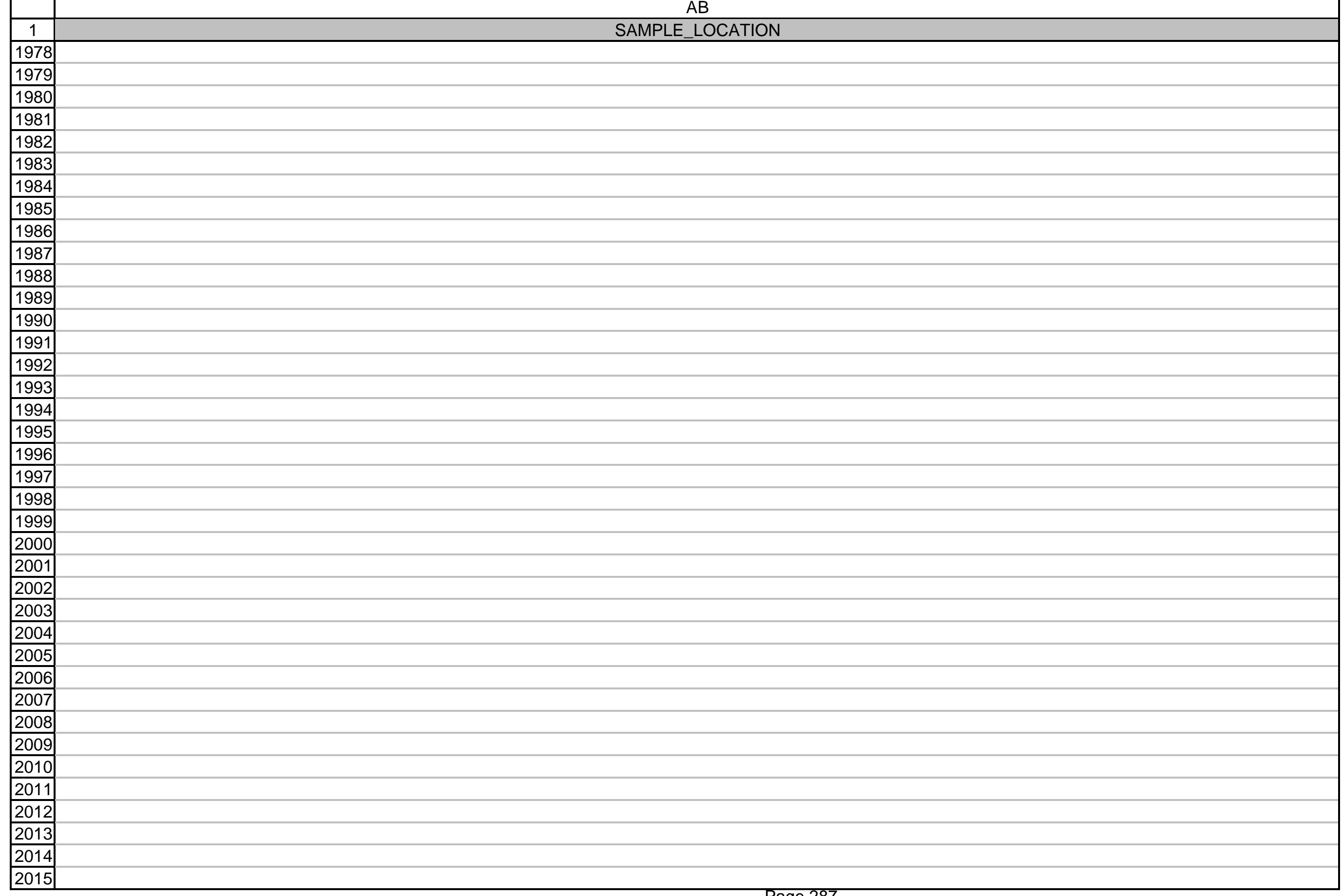

Page 287 


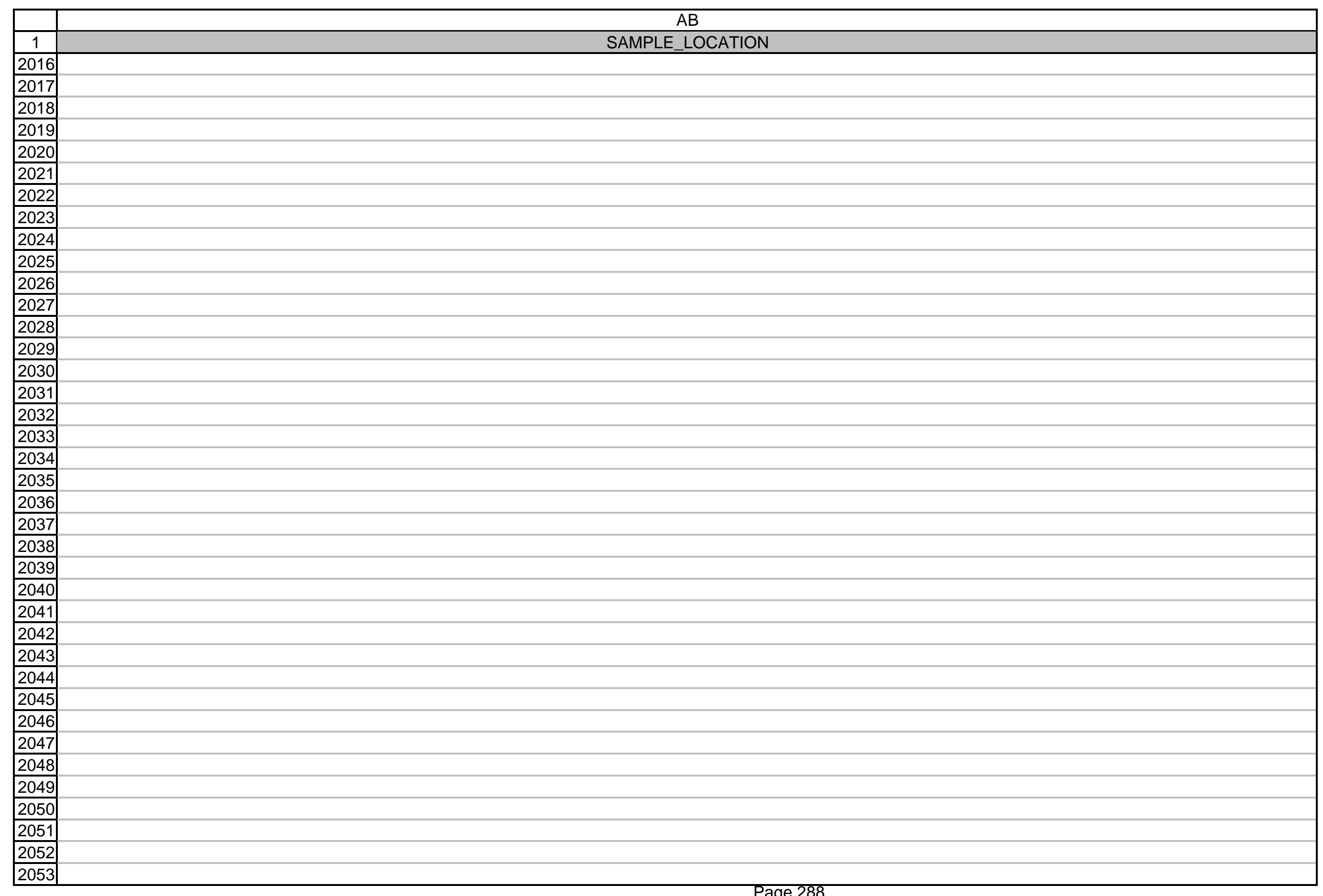


AB

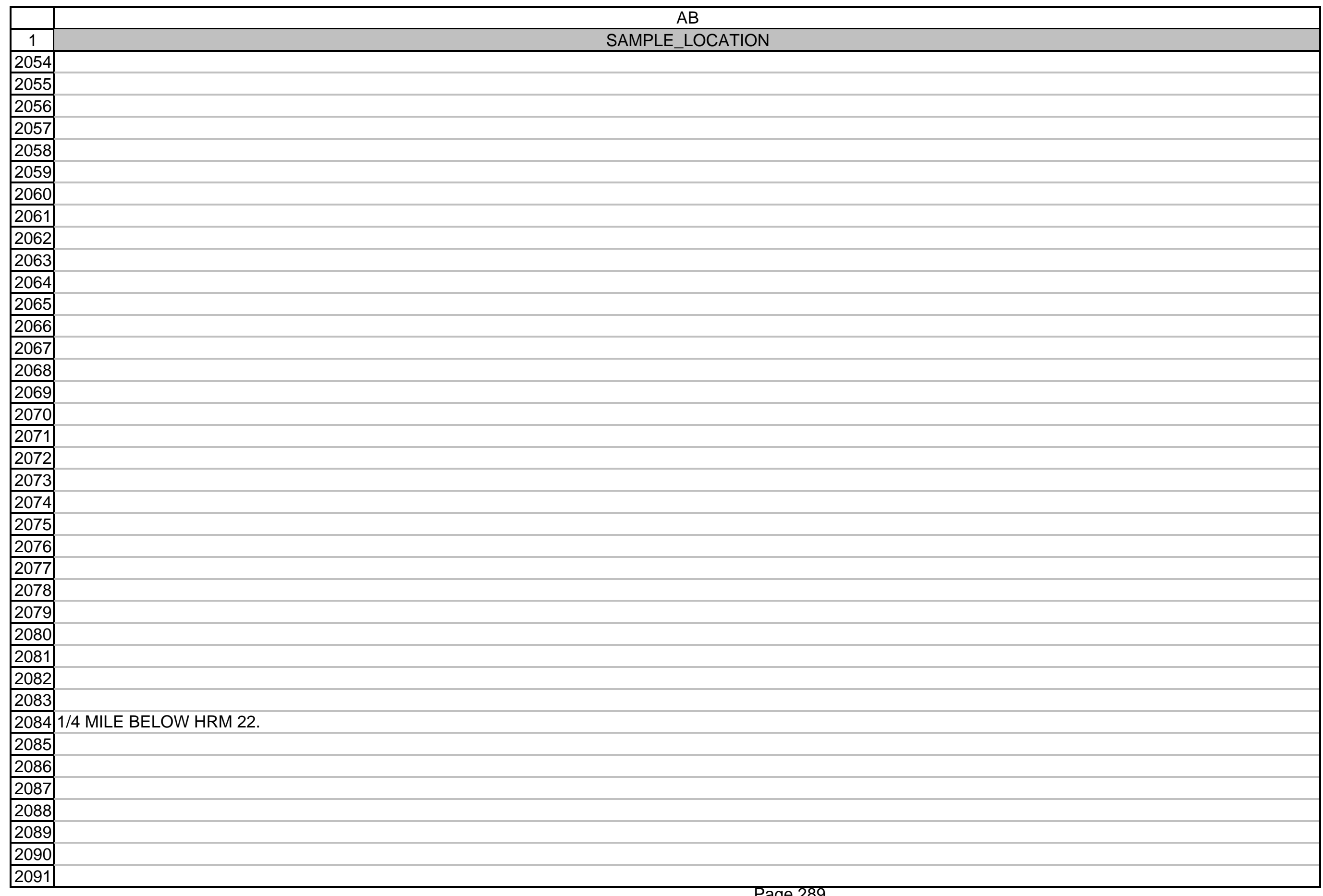

Page 289 
$A B$

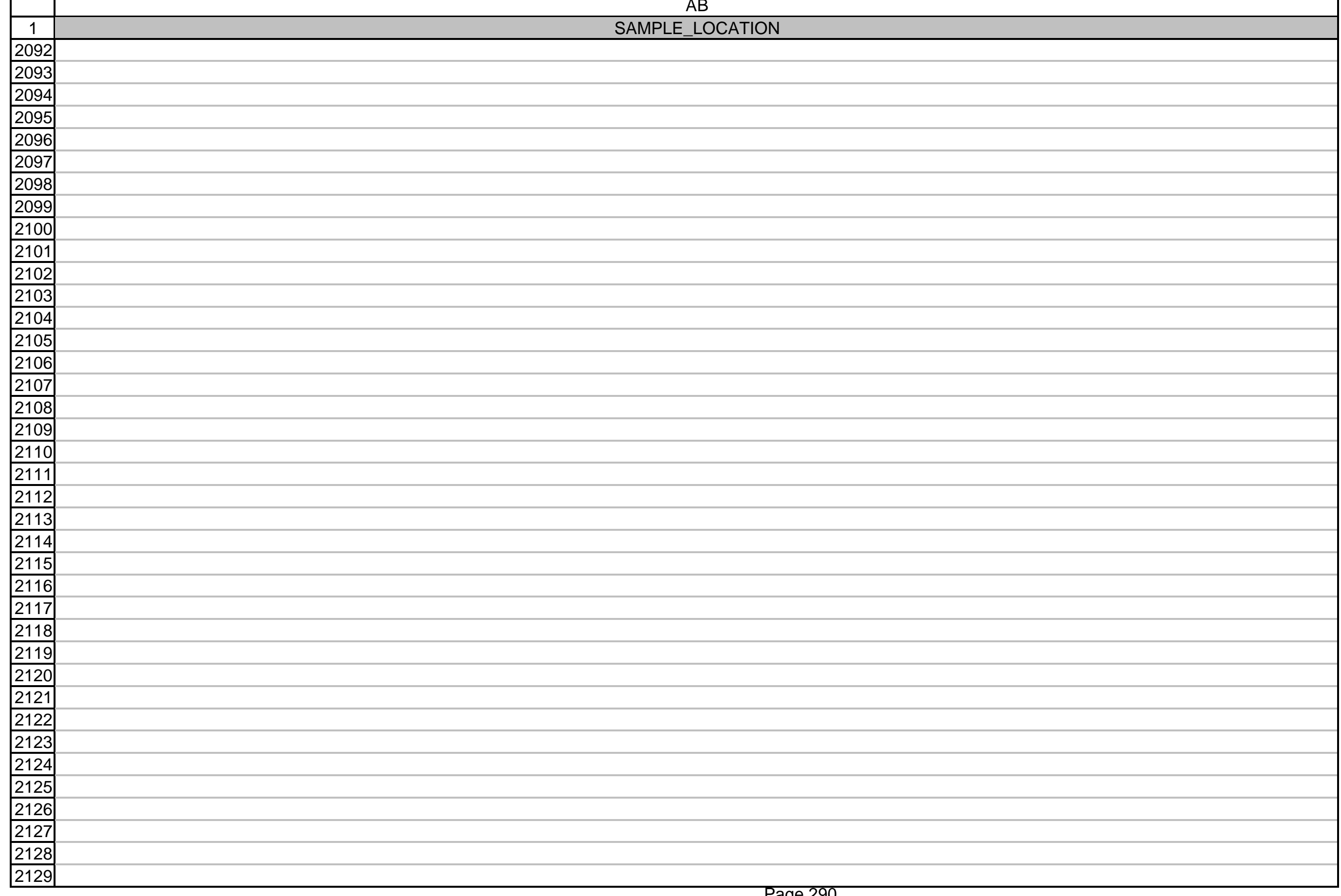

Page 290 
AB

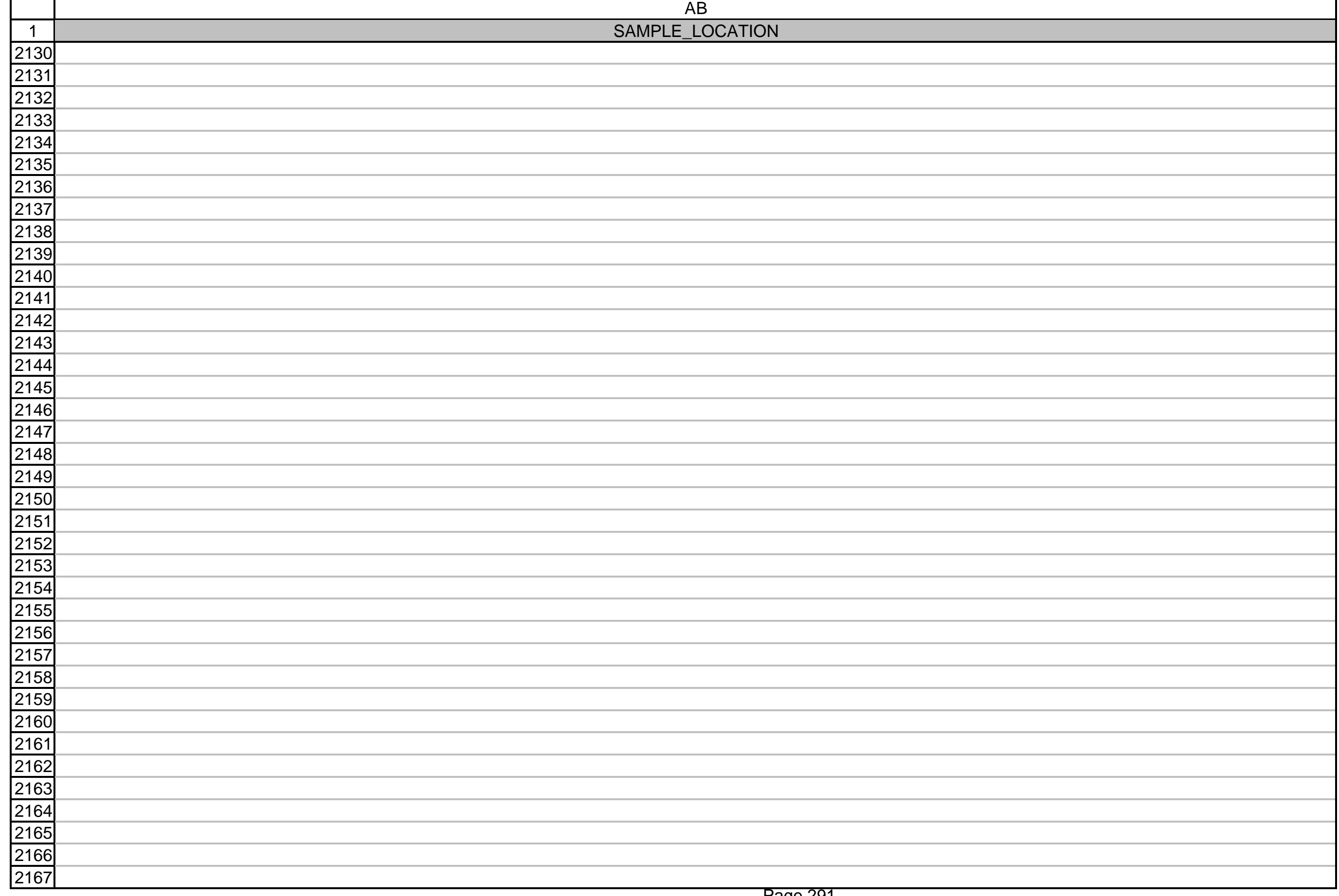

Page 291 


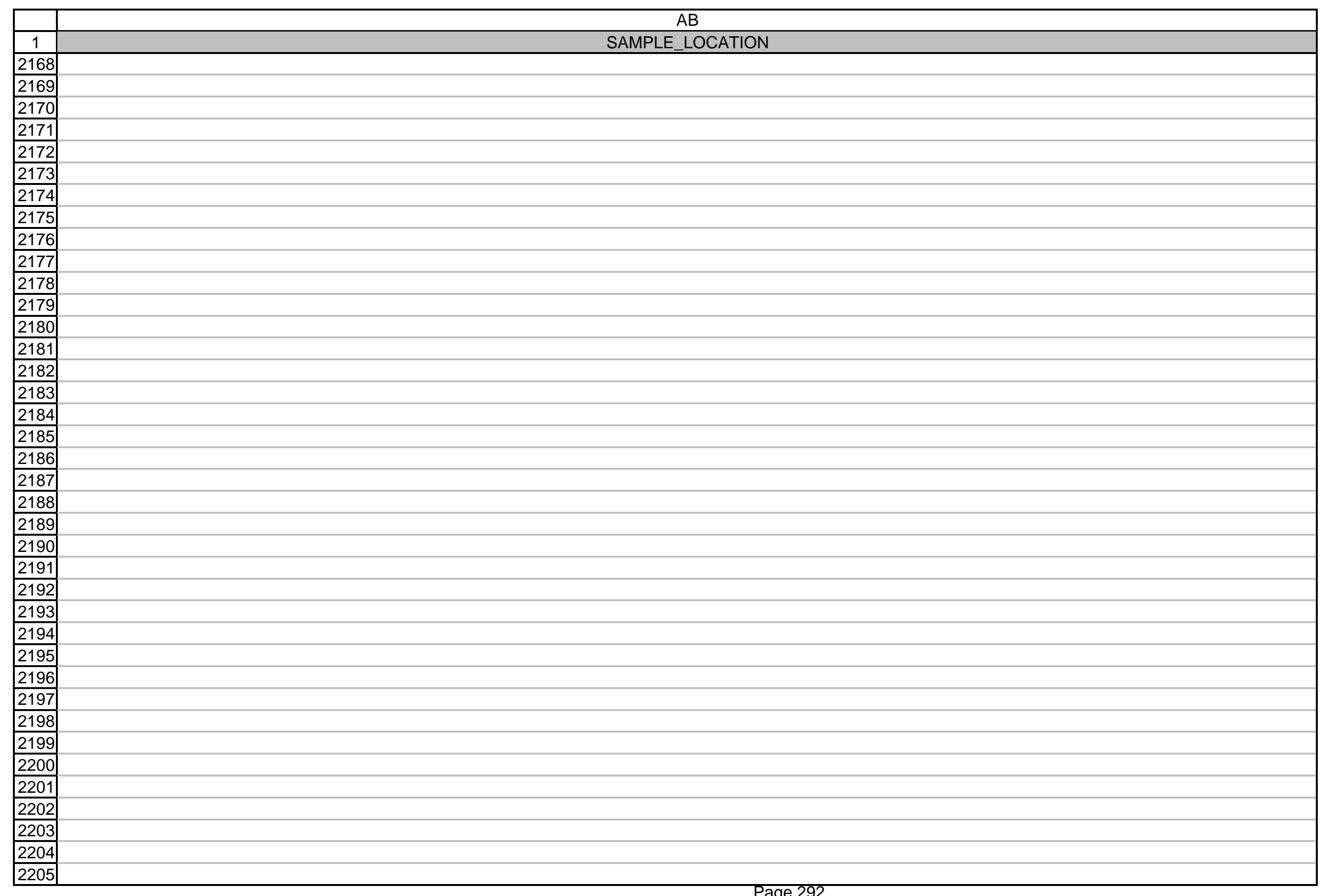




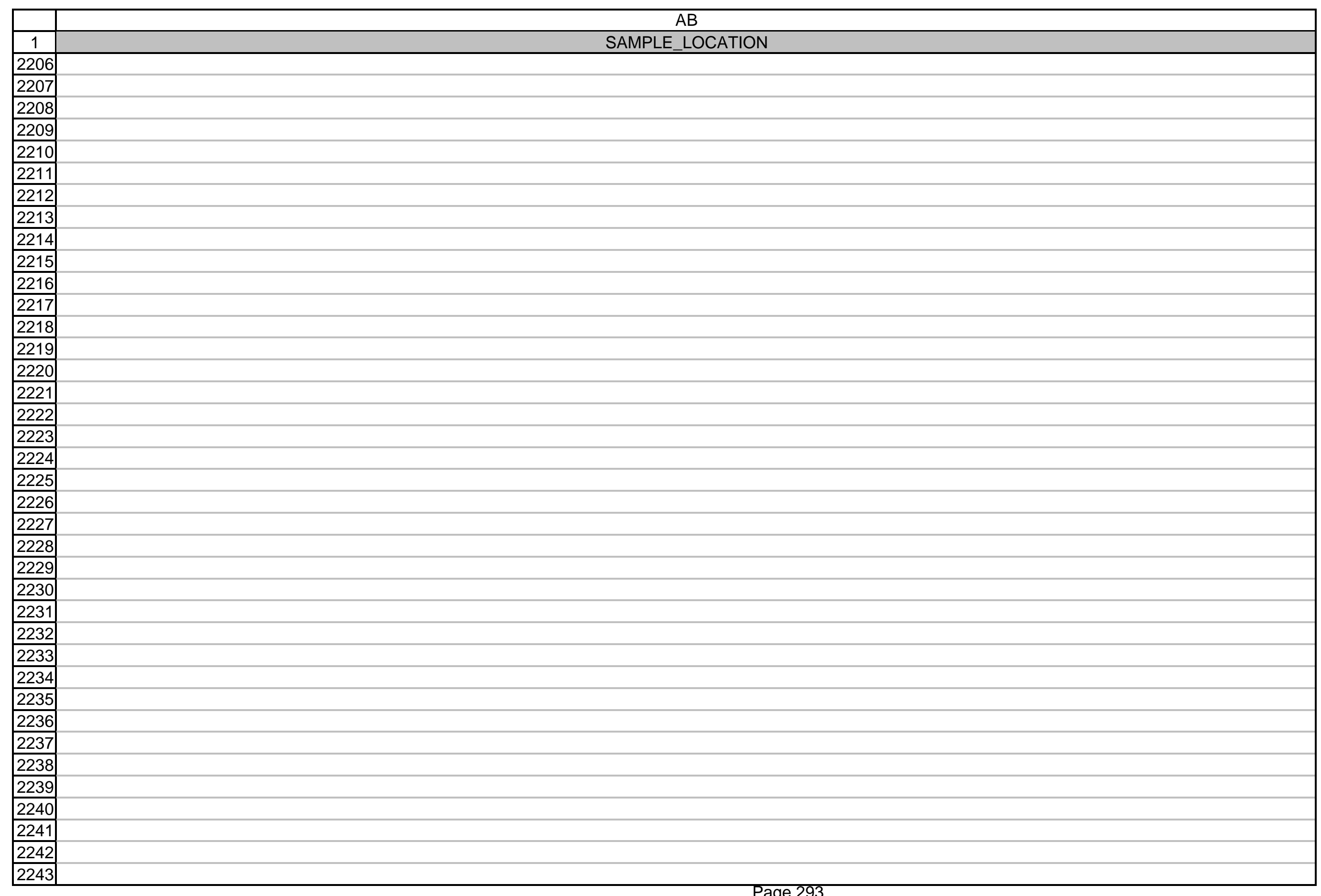




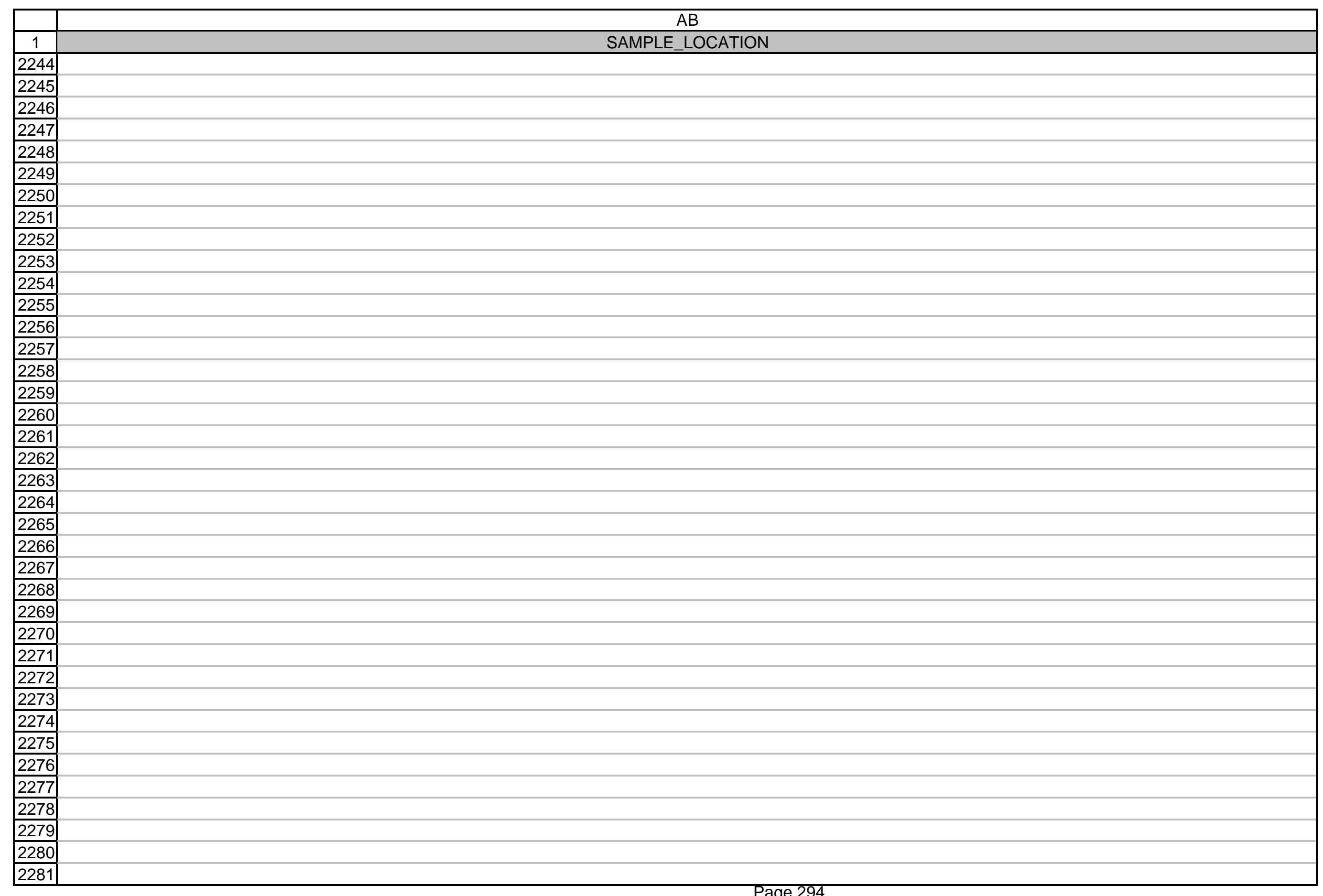




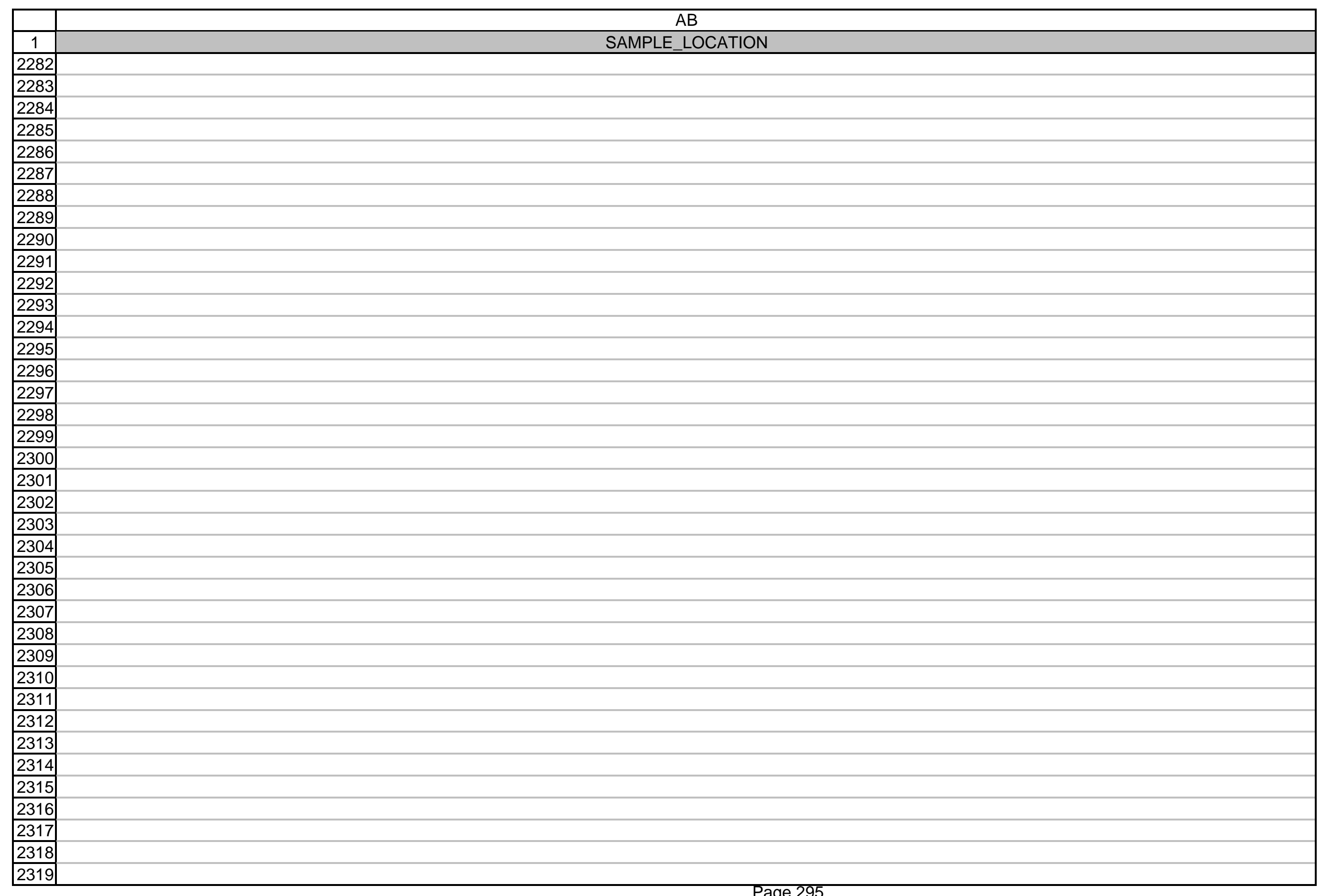




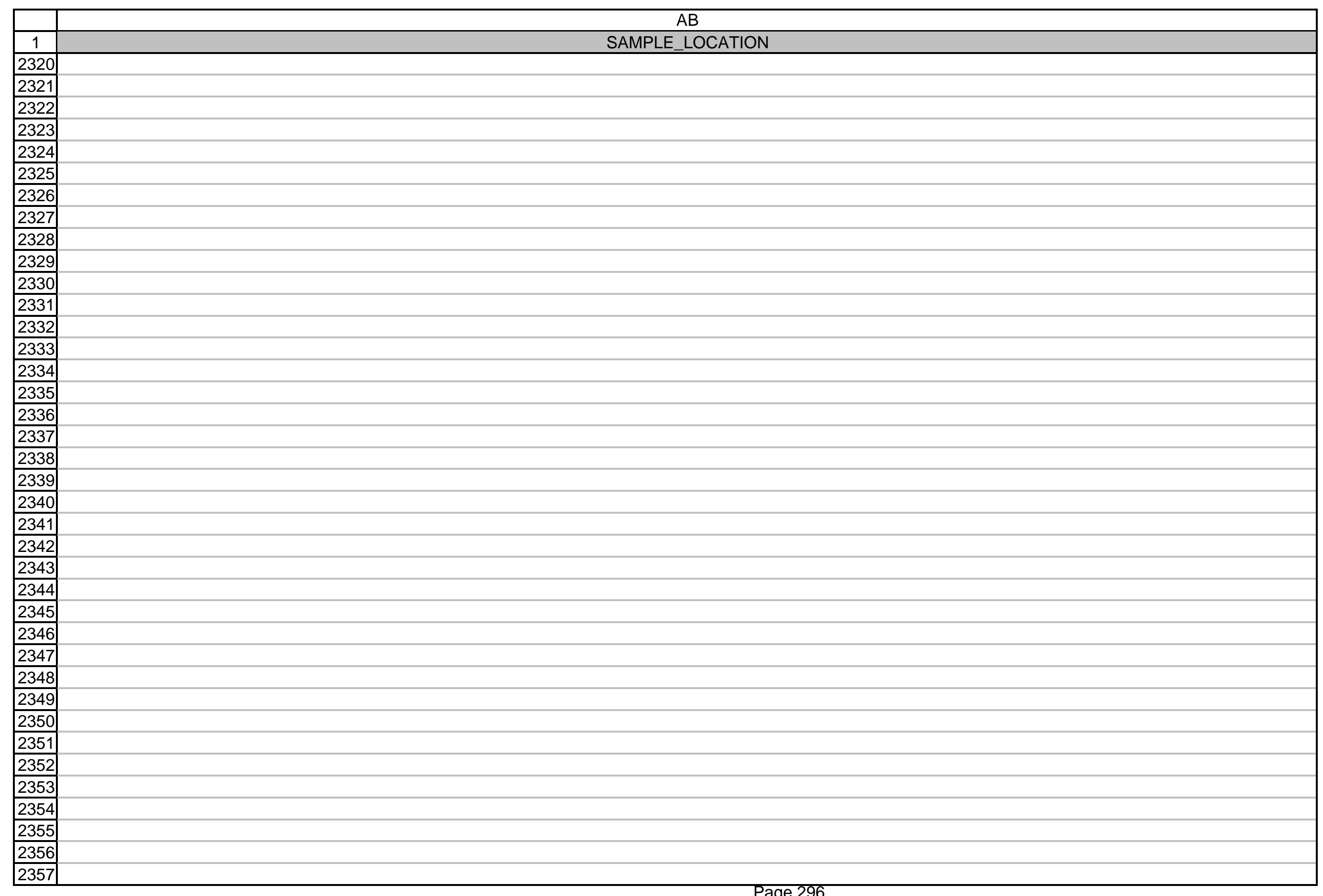




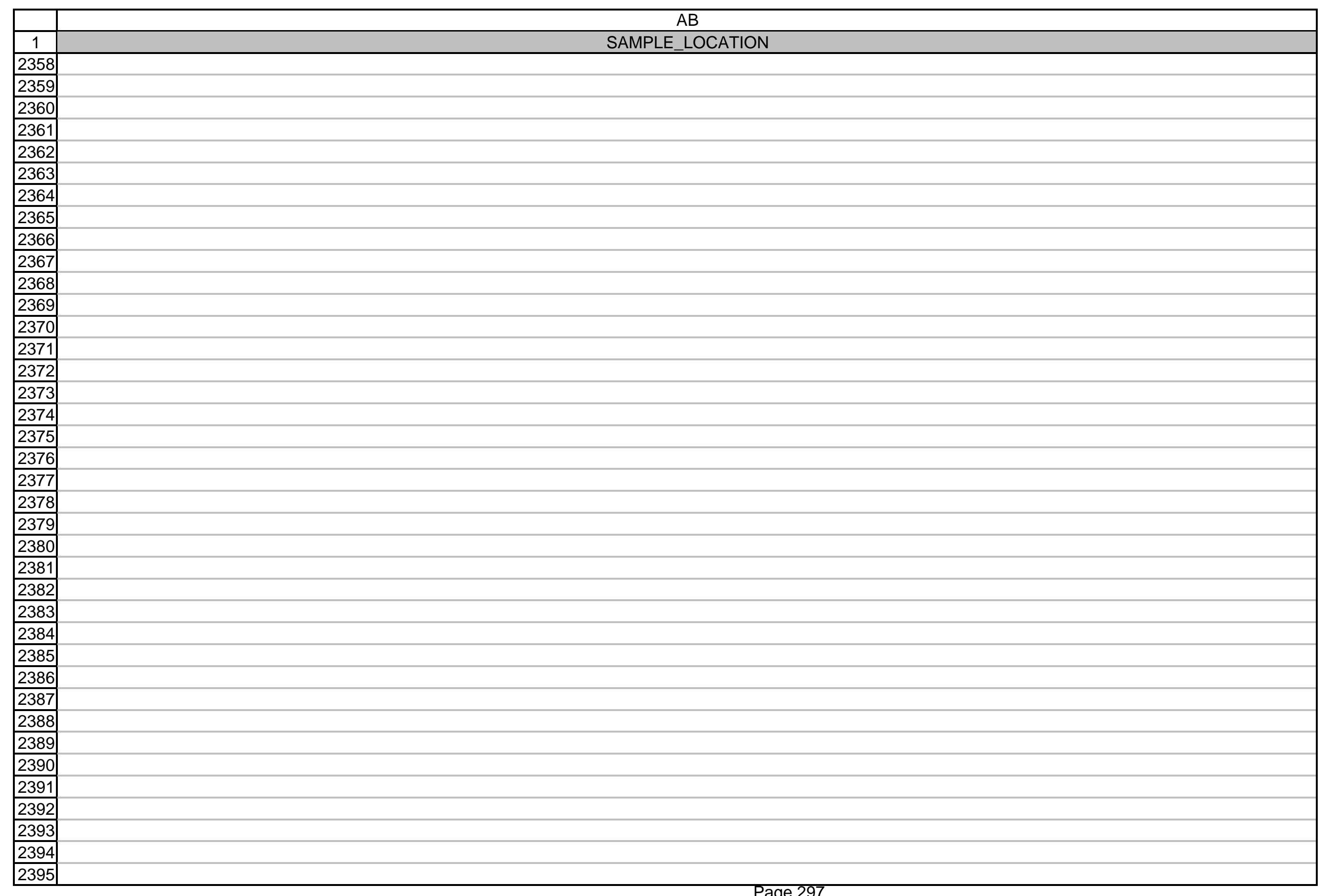


$A B$

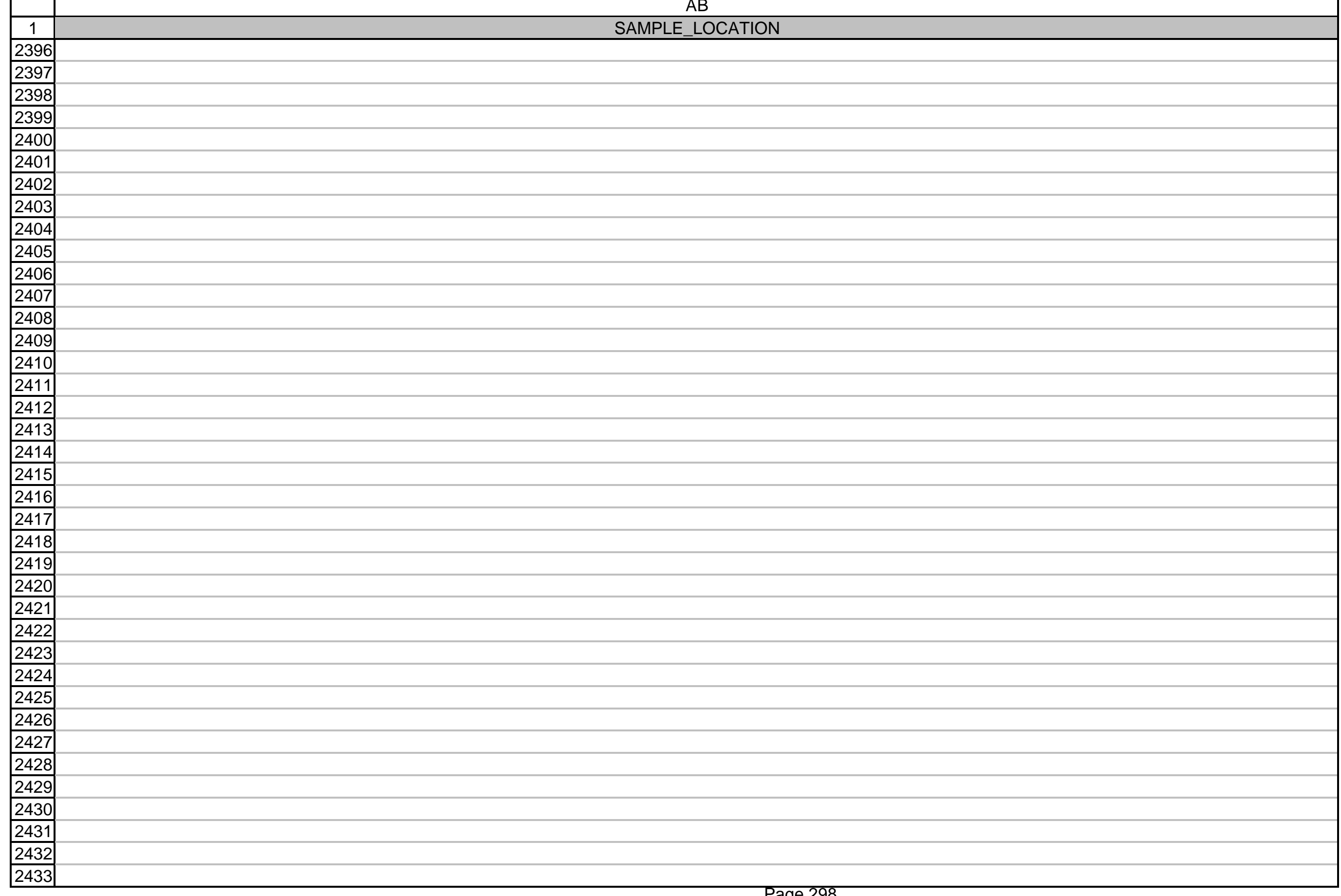

Page 298 
$A B$

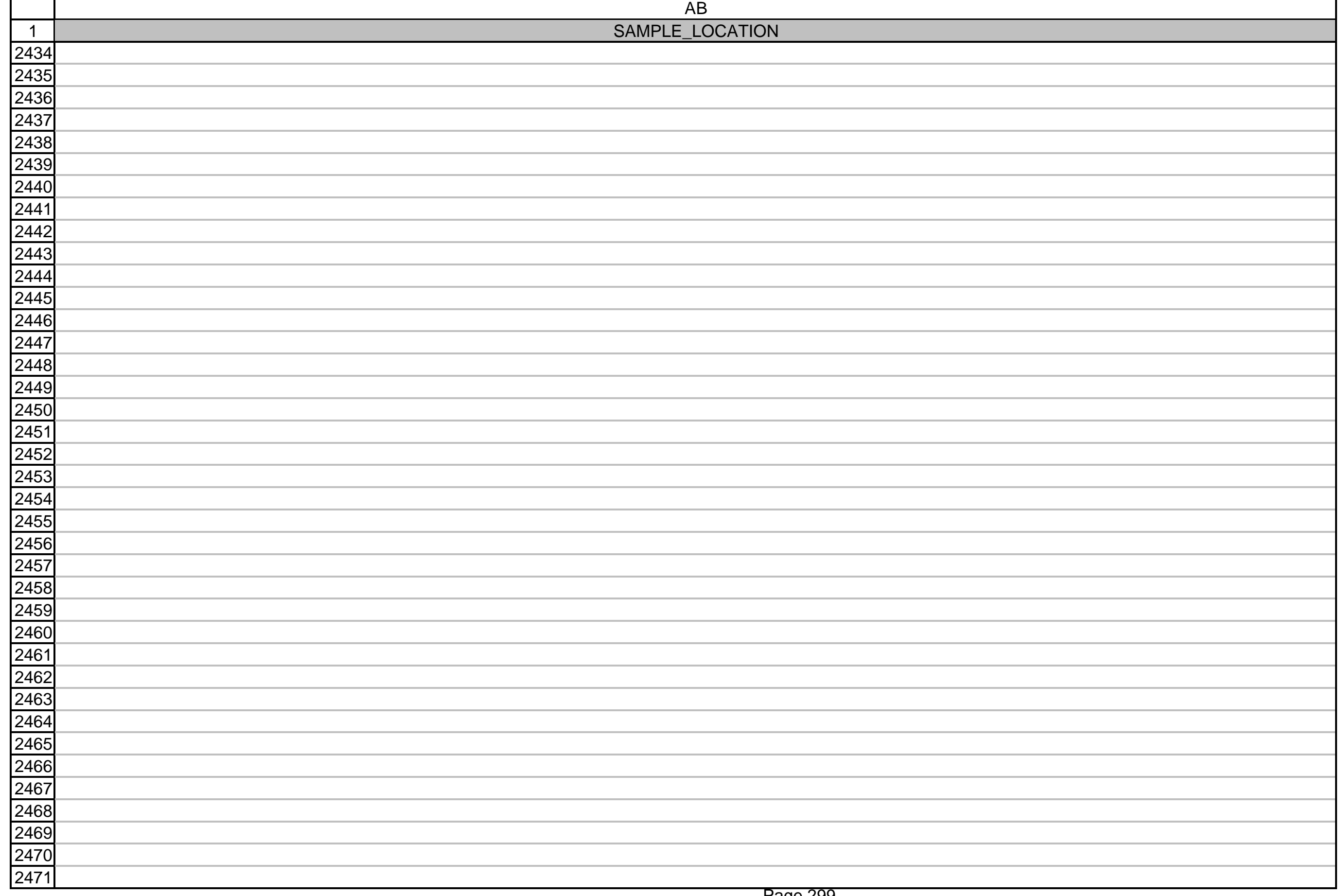

Page 299 
$A B$

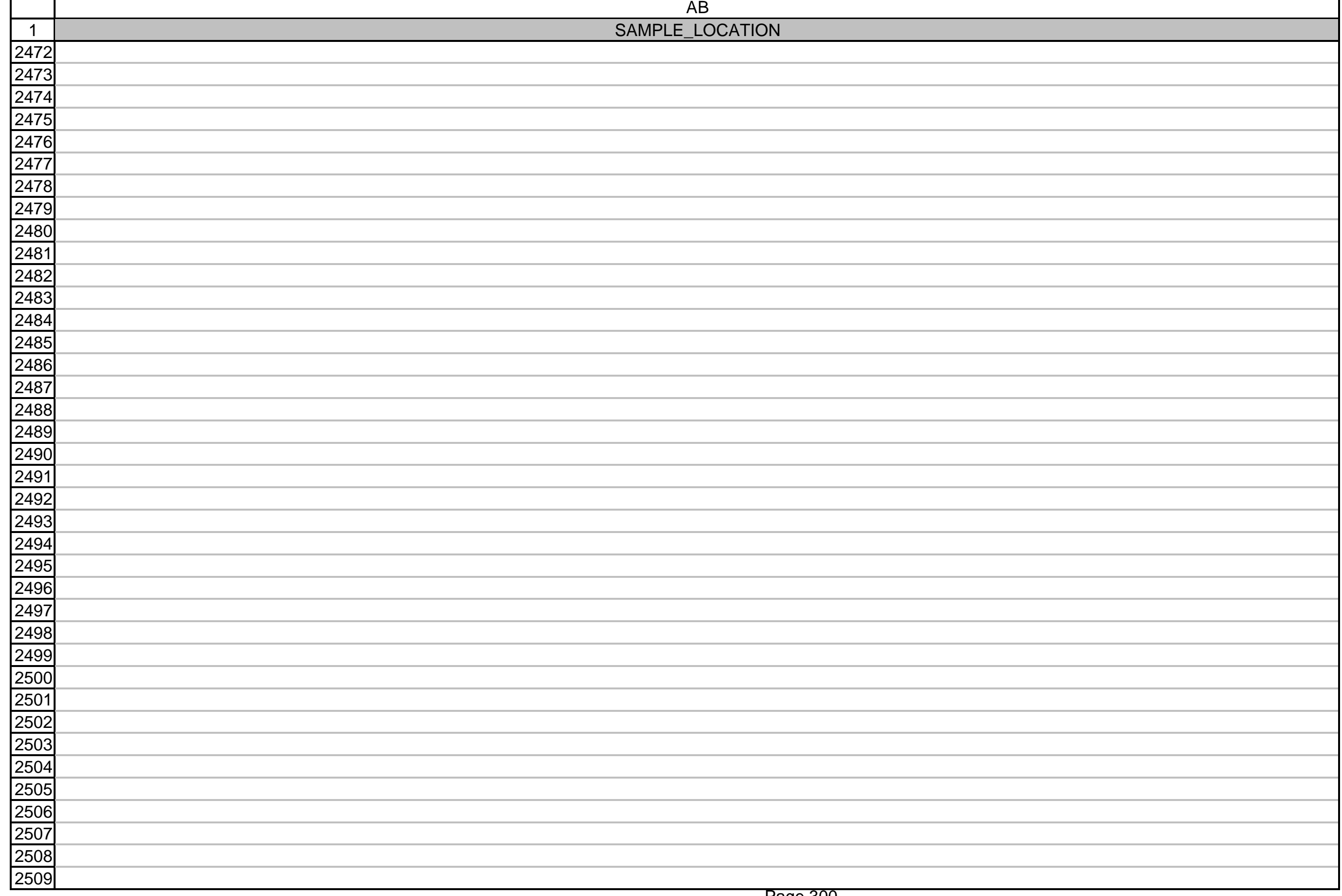

Page 300 


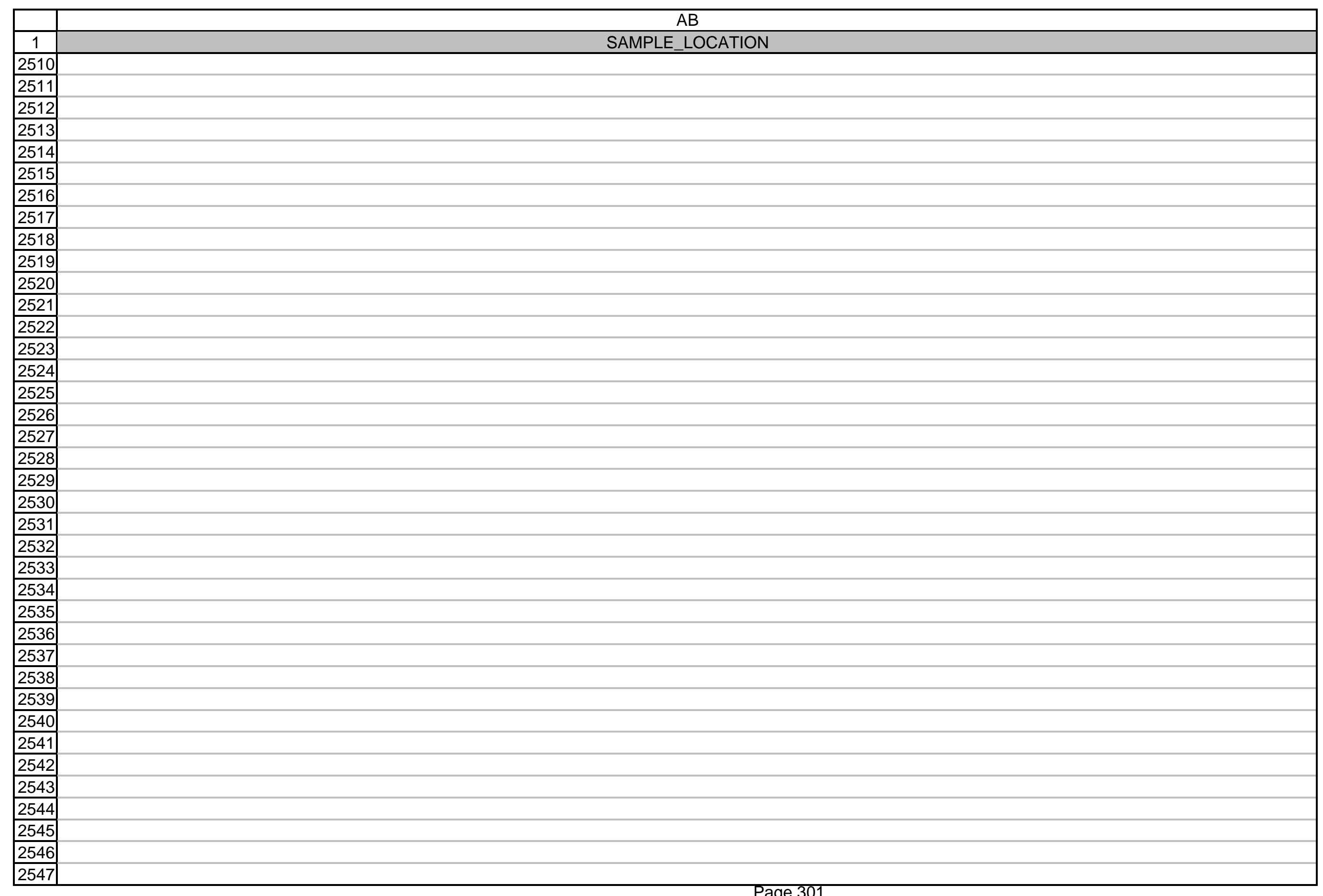


AB

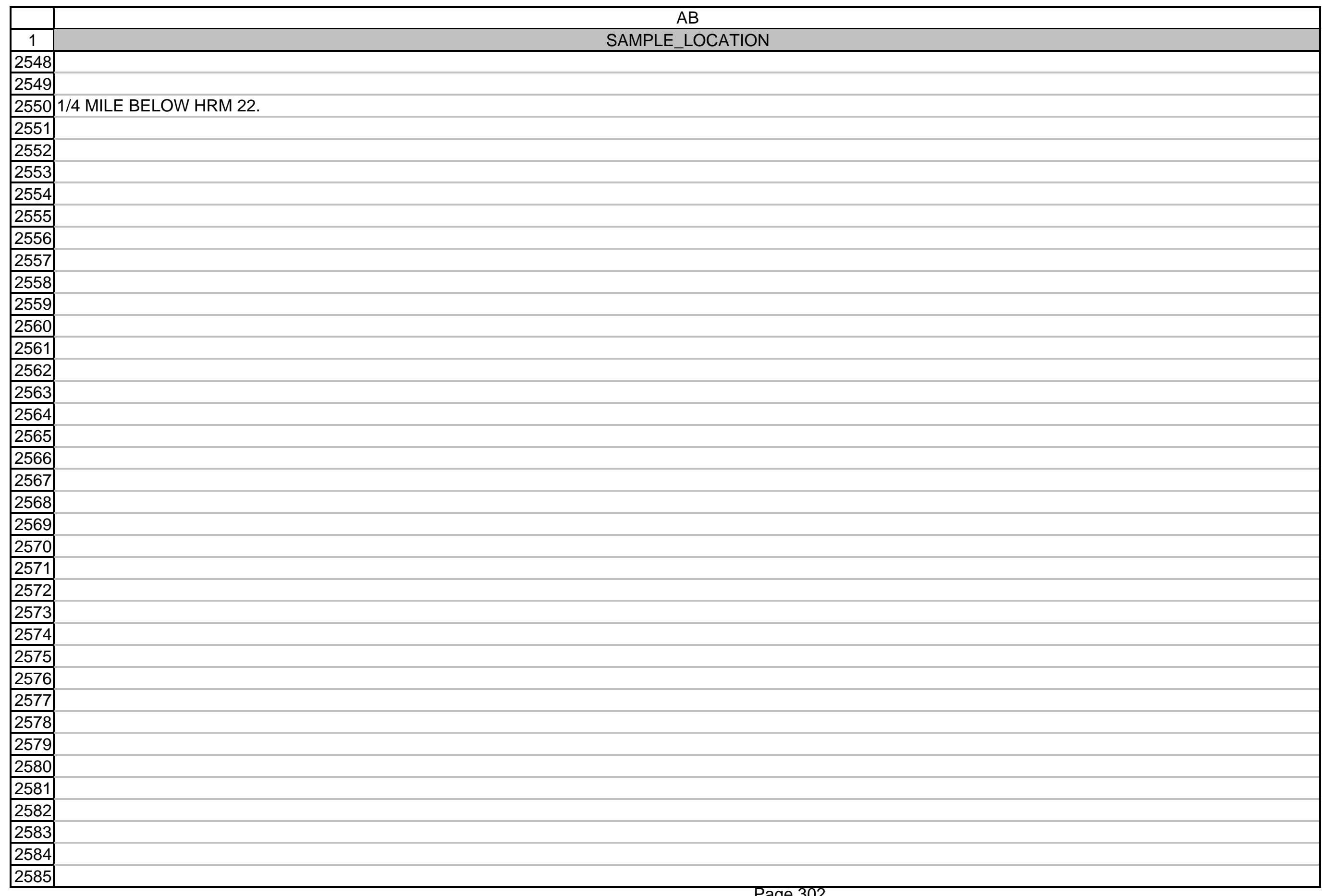


$A B$

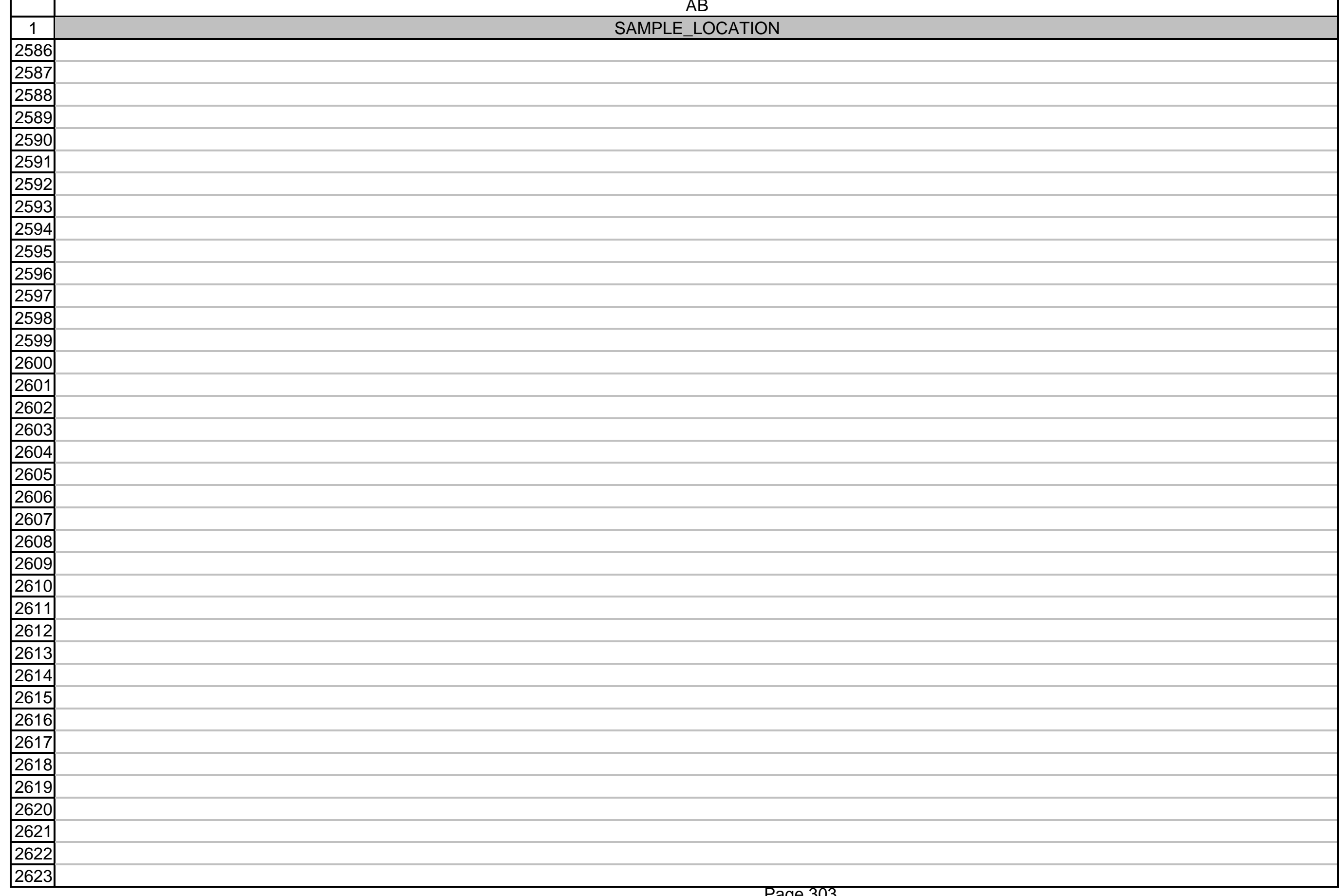

Page 303 
$A B$

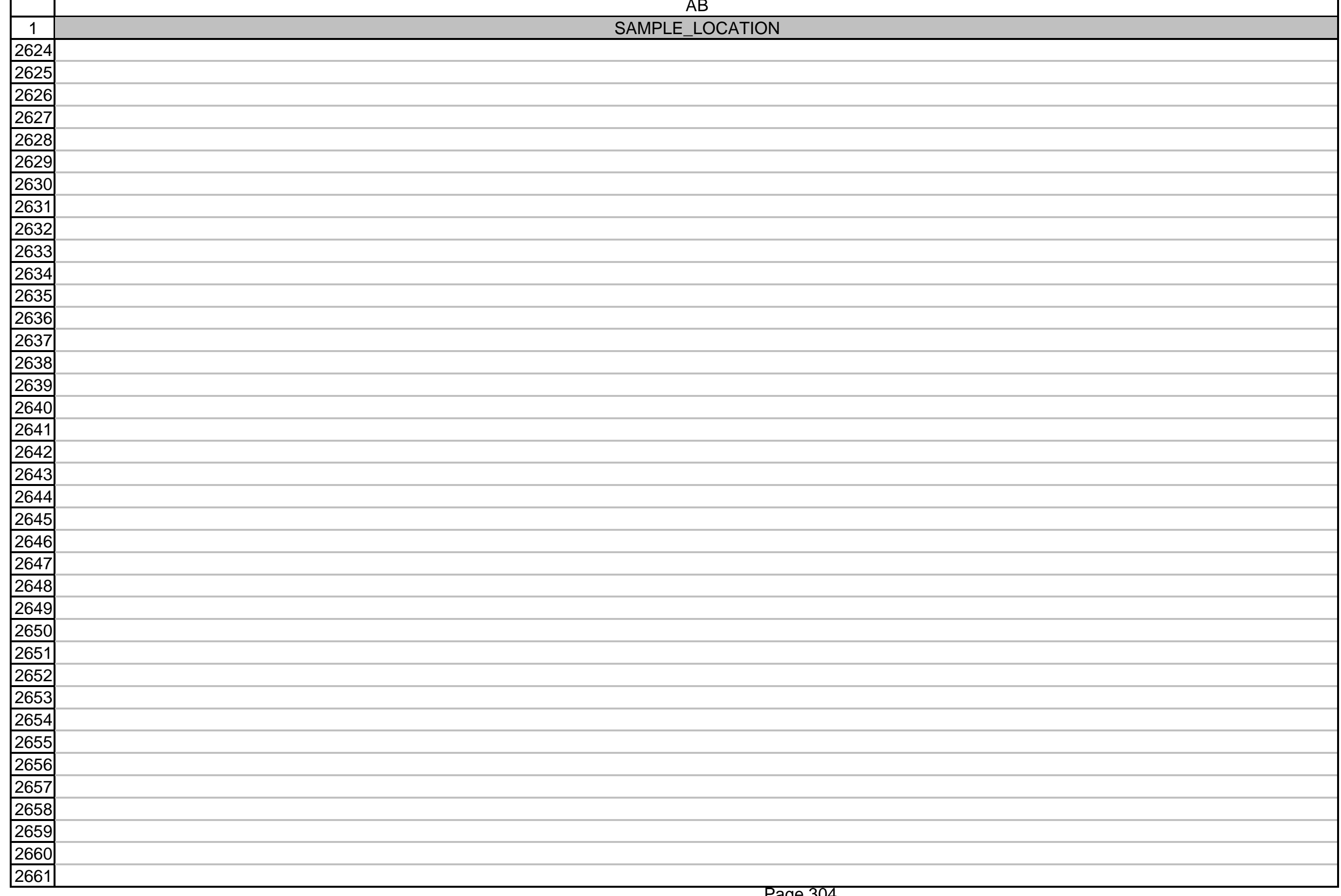

Page 304 


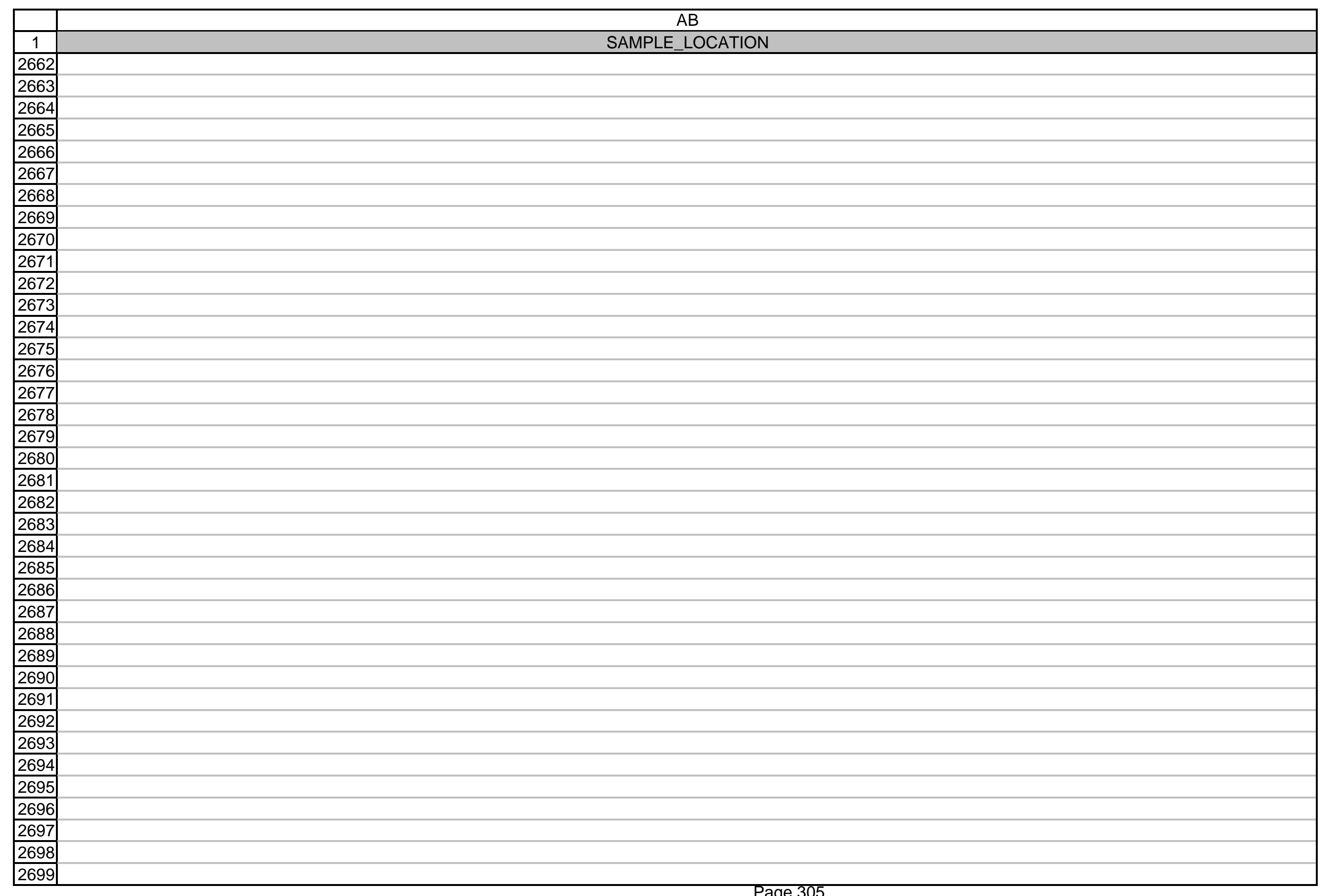


AB

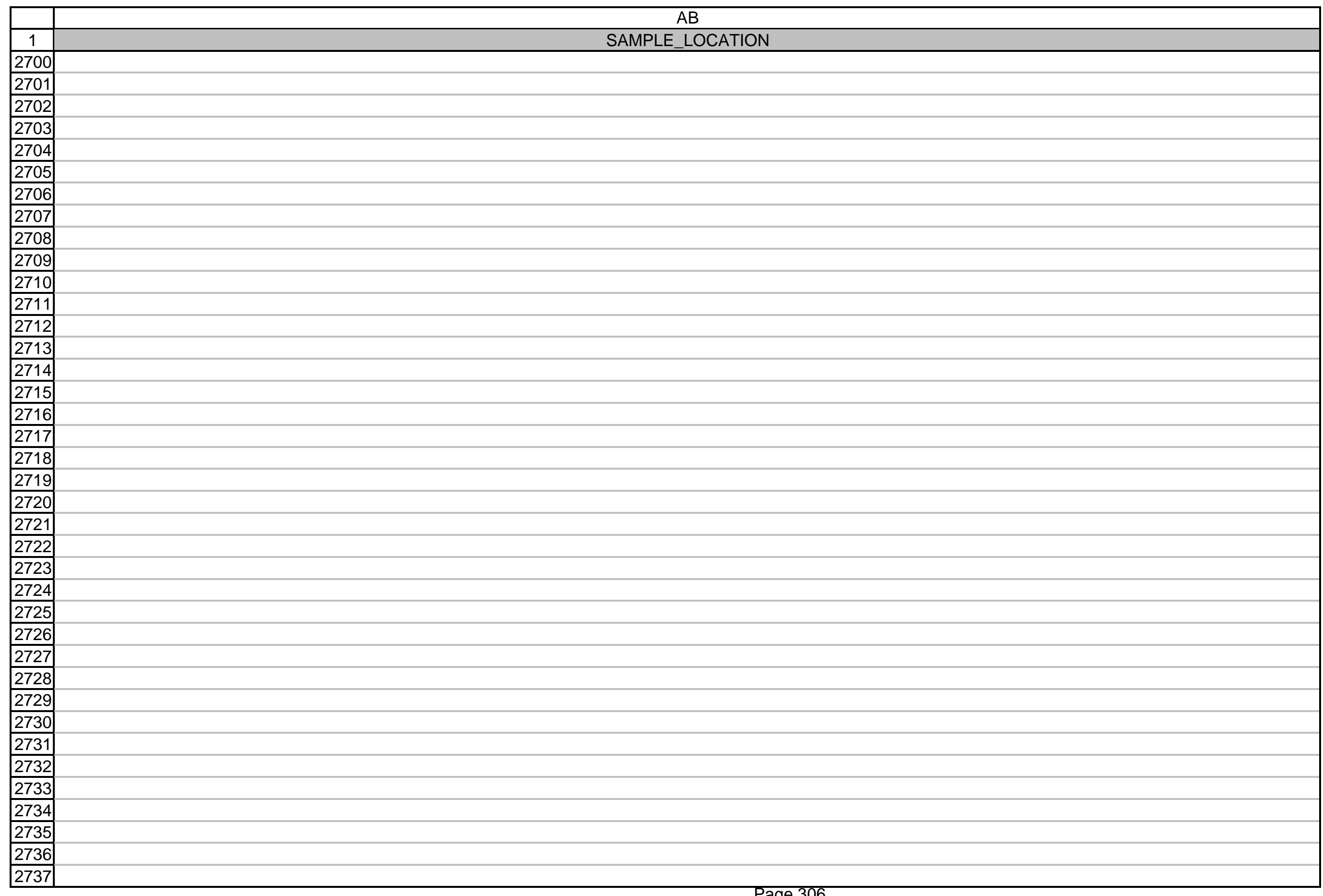

Page 306 
$A B$

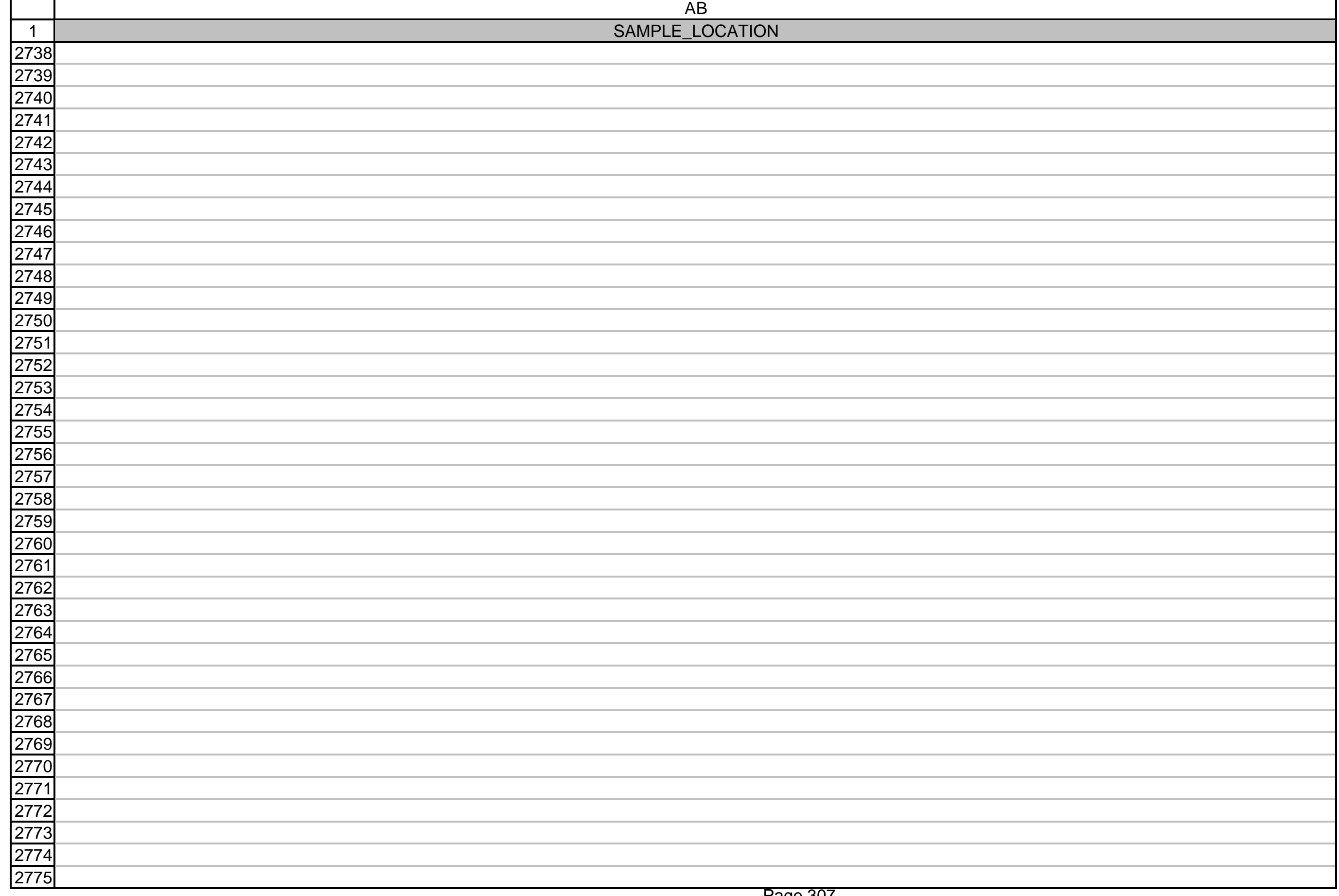

Page 307 


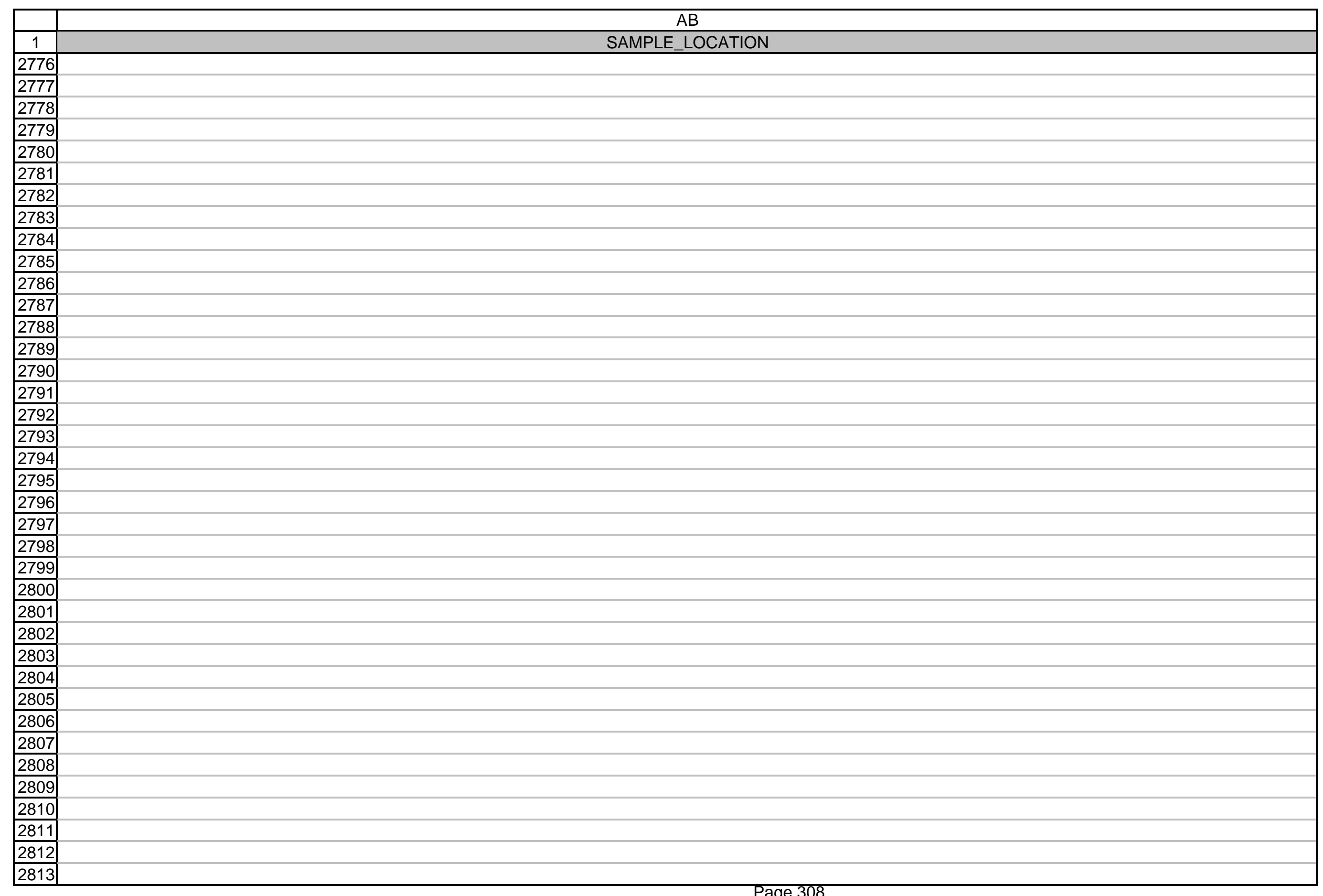




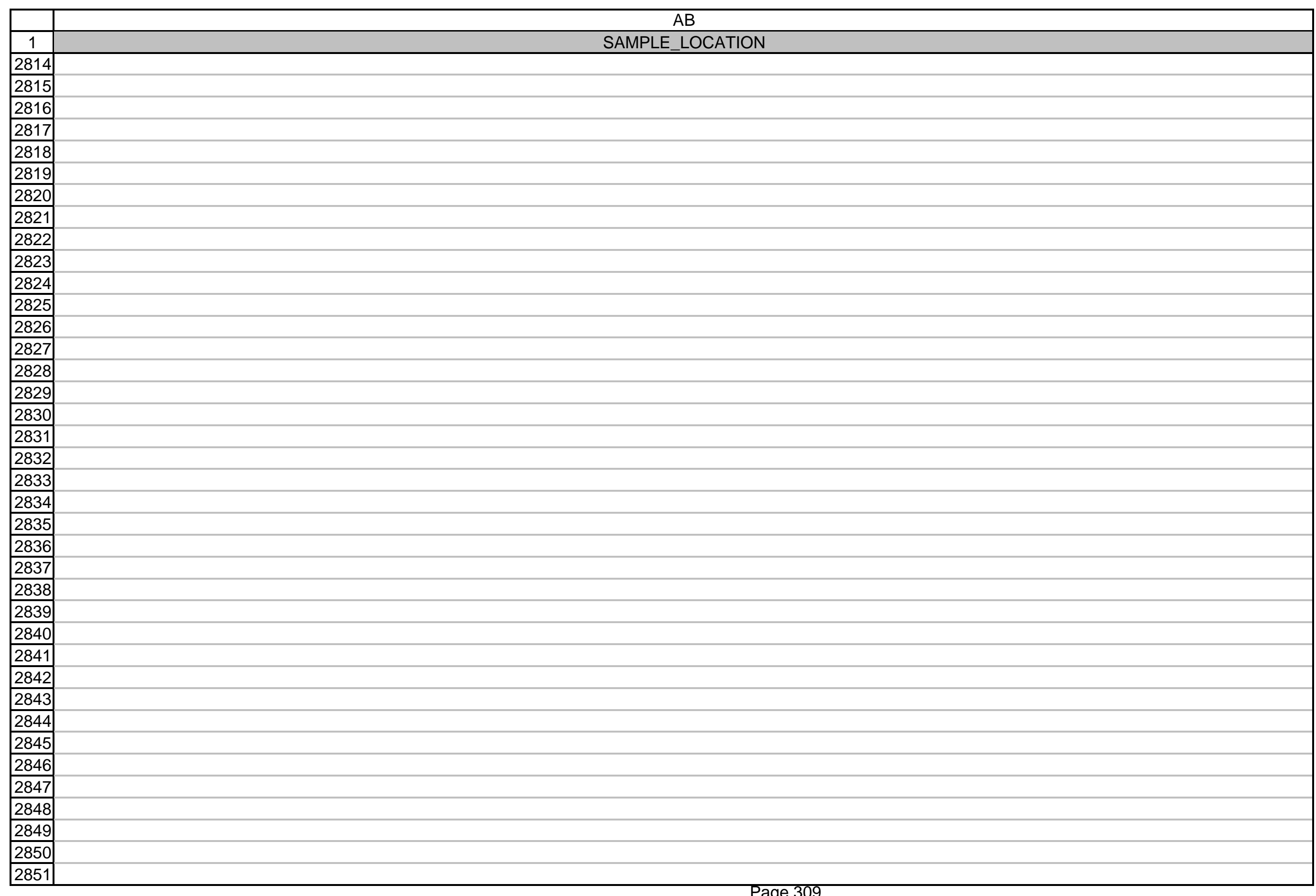

Page 309 


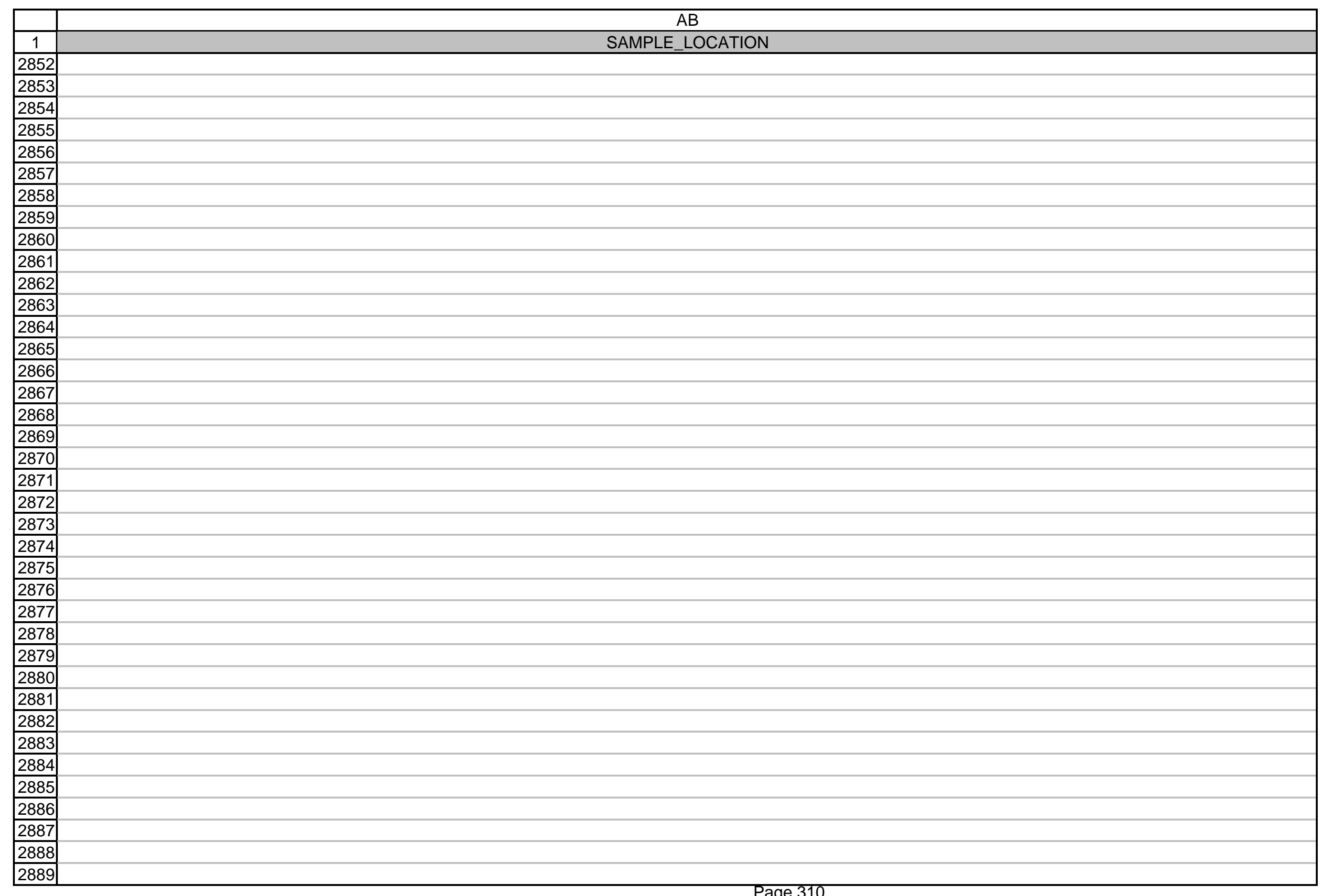




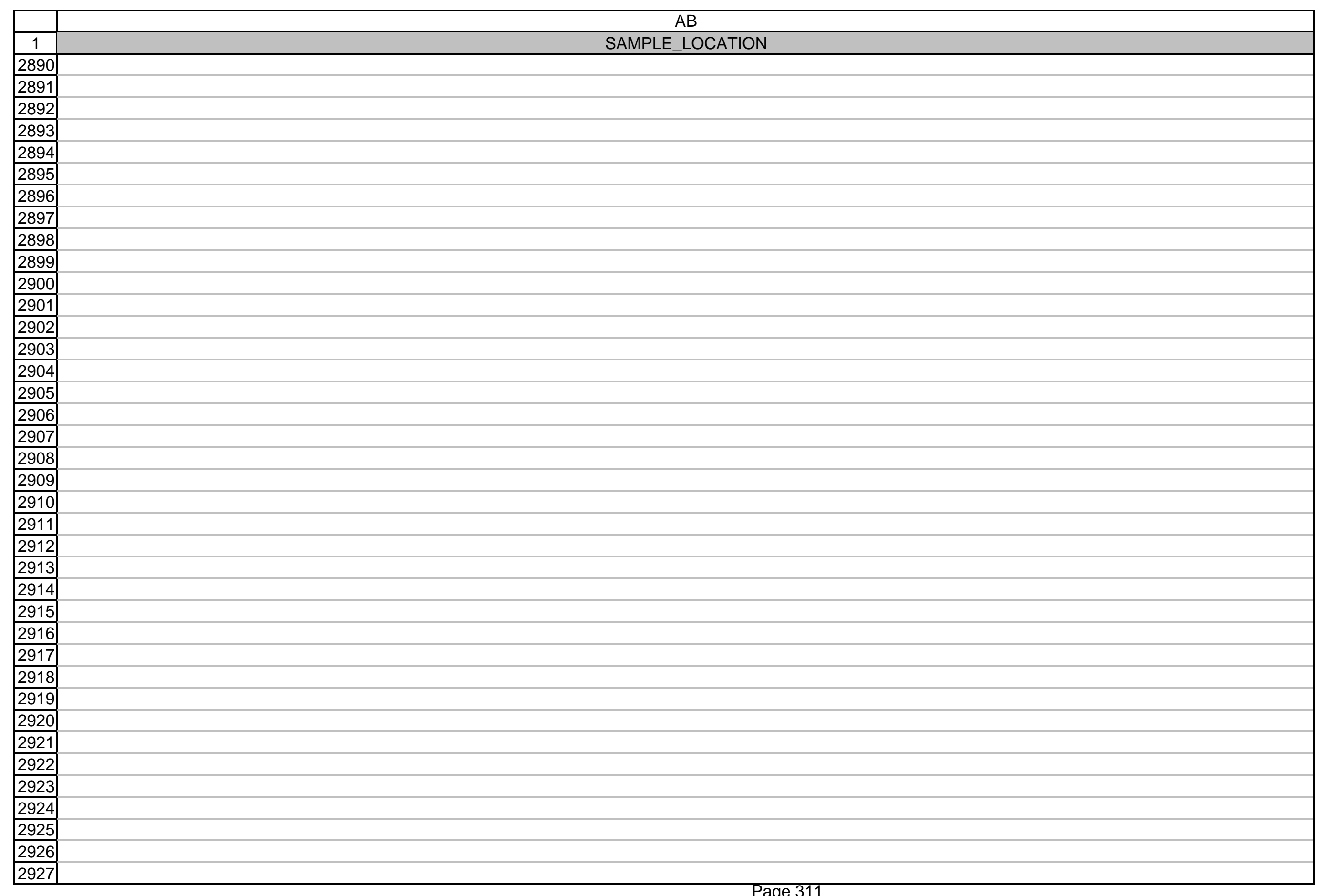


$A B$ 


$$
\square
$$


AC

\begin{tabular}{|c|c|}
\hline & $\mathrm{AC}$ \\
\hline 1 & SAMP_COMMENT \\
\hline 40 & \\
\hline 41 & \\
\hline 42 & \\
\hline 43 & \\
\hline 44 & \\
\hline 45 & \\
\hline 46 & \\
\hline 47 & \\
\hline 48 & \\
\hline 49 & \\
\hline 50 & \\
\hline 51 & \\
\hline 52 & \\
\hline 53 & \\
\hline 54 & \\
\hline 55 & \\
\hline 56 & \\
\hline 57 & \\
\hline 58 & \\
\hline 59 & \\
\hline 60 & \\
\hline 61 & \\
\hline 62 & Samples shipped to St. Louis on $10 / 18 / 00$ and received on $10 / 19 / 00$ at $08: 35$ \\
\hline 63 & Samples shipped to St. Louis on $10 / 18 / 00$ and received on $10 / 19 / 00$ at $08: 35$ \\
\hline 64 & Samples shipped to St. Louis on $10 / 18 / 00$ and received on $10 / 19 / 00$ at $08: 35$ \\
\hline 65 & Samples shipped to St. Louis on $10 / 18 / 00$ and received on $10 / 19 / 00$ at $08: 35$ \\
\hline 66 & Samples shipped to St. Louis on $10 / 18 / 00$ and received on $10 / 19 / 00$ at $08: 35$ \\
\hline 67 & Samples shipped to St. Louis on $10 / 18 / 00$ and received on $10 / 19 / 00$ at $08: 35$ \\
\hline 68 & \\
\hline 69 & Sample shipped from Richland to St. Louis on $11 / 02 / 00$ and received on $11 / 03 / 00$ at 09:00 \\
\hline 70 & Chemical only/Sample shipped from Richland to St. Louis on $11 / 02 / 00$ and received on $11 / 03 / 00$ at 09:00 \\
\hline 71 & \\
\hline 72 & Chemical only \\
\hline 73 & Chemical only \\
\hline 74 & RECRA RECVD 8/21/02 09:20 \\
\hline 75 & RCRA RCVD 08/21/02 09:20 \\
\hline 76 & \\
\hline 77 & \\
\hline
\end{tabular}




$$
\square
$$




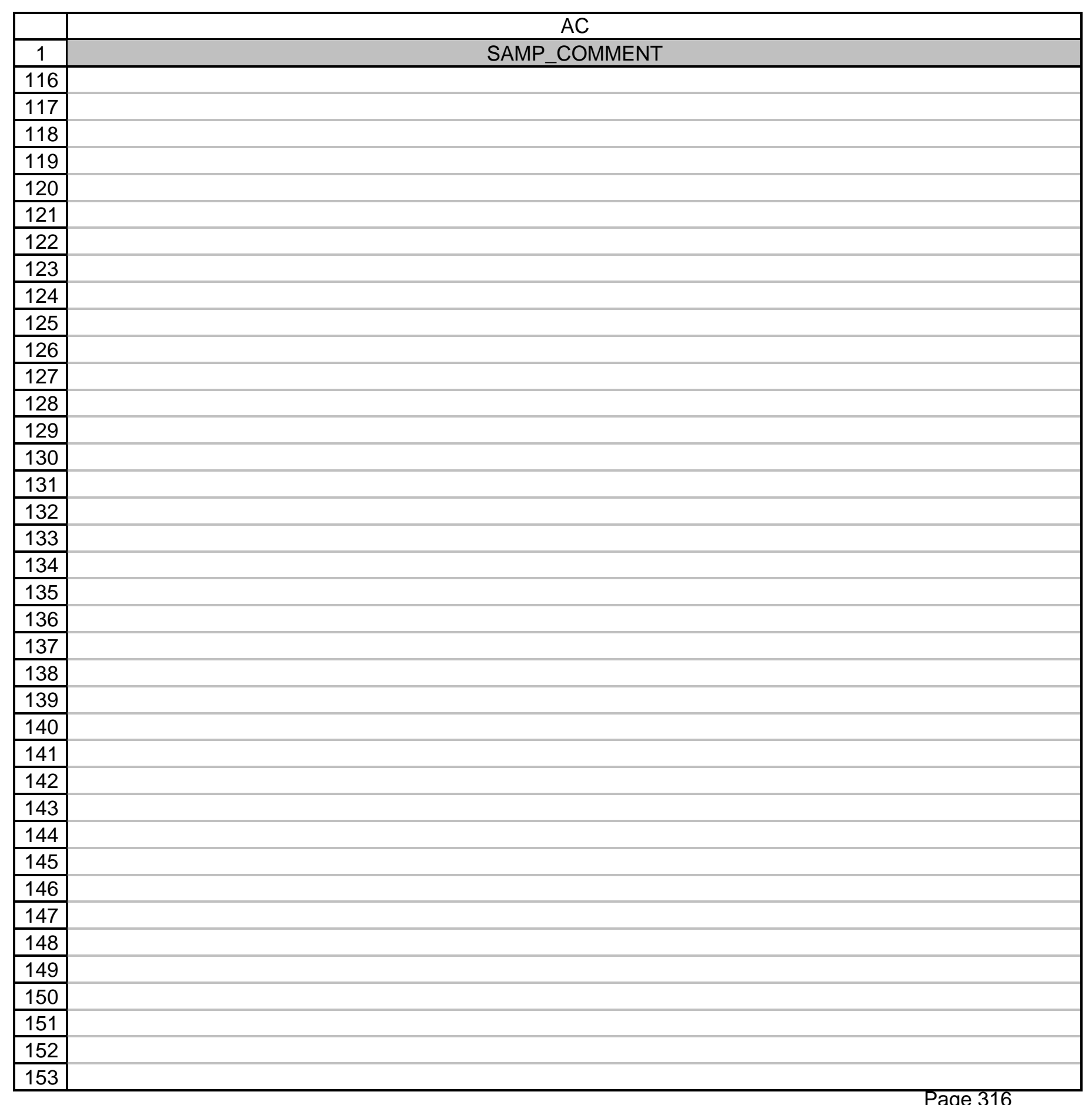




$$
F
$$


AC

\begin{tabular}{|c|c|}
\hline 1 & SAMP_COMMENT \\
\hline 192 & \\
\hline 193 & \\
\hline 194 & \\
\hline 195 & \\
\hline 196 & \\
\hline 197 & \\
\hline 198 & \\
\hline 199 & \\
\hline 200 & \\
\hline 201 & \\
\hline 202 & \\
\hline 203 & \\
\hline 204 & \\
\hline 205 & \\
\hline 206 & \\
\hline 207 & WATER CAME UP 2-3 FEET INTO RIPARIAN VEGETATION. 1 METER DEPTH AT GPS FLAG. \\
\hline 208 & WATER CAME UP 2-3 FEET INTO RIPARIAN VEGETATION. 1 METER DEPTH AT GPS FLAG. \\
\hline 209 & WATER CAME UP 2-3 FEET INTO RIPARIAN VEGETATION. 1 METER DEPTH AT GPS FLAG. \\
\hline 210 & \\
\hline 211 & \\
\hline 212 & \\
\hline 213 & \\
\hline 214 & \\
\hline 215 & \\
\hline 216 & \\
\hline 217 & \\
\hline 218 & \\
\hline 219 & \\
\hline 220 & \\
\hline 221 & \\
\hline 222 & \\
\hline 223 & \\
\hline 224 & \\
\hline 225 & \\
\hline 226 & \\
\hline 227 & \\
\hline 228 & \\
\hline 229 & \\
\hline
\end{tabular}


AC

\begin{tabular}{|c|c|}
\hline 1 & SAMP COMMENT \\
\hline 230 & \\
\hline 231 & \\
\hline 232 & \\
\hline 233 & WATER CAME UP 2-3 FEET INTO RIPARIAN VEGETATION. 1 METER DEPTH AT GPS FLAG. \\
\hline 234 & \\
\hline 235 & \\
\hline 236 & \\
\hline 237 & \\
\hline 238 & \\
\hline 239 & \\
\hline 240 & \\
\hline 241 & \\
\hline 242 & \\
\hline 243 & \\
\hline 244 & \\
\hline 245 & \\
\hline 246 & \\
\hline 247 & \\
\hline 248 & \\
\hline 249 & \\
\hline 250 & \\
\hline 251 & \\
\hline 252 & \\
\hline 253 & \\
\hline 254 & \\
\hline 255 & \\
\hline 256 & \\
\hline 257 & \\
\hline 258 & \\
\hline 259 & \\
\hline 260 & \\
\hline 261 & \\
\hline 262 & \\
\hline 263 & \\
\hline 264 & \\
\hline 265 & \\
\hline 266 & \\
\hline 267 & \\
\hline
\end{tabular}




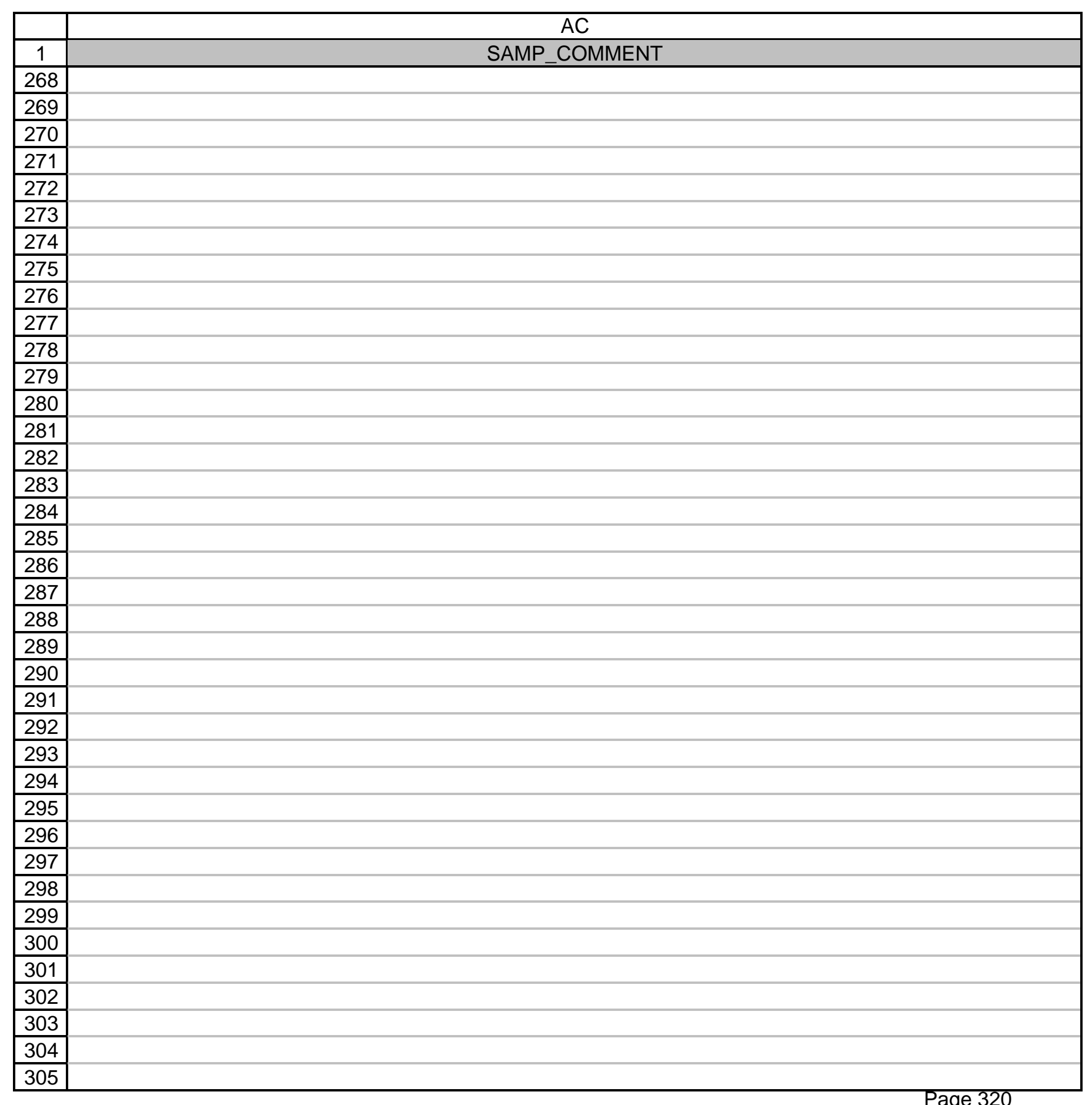




$$
F
$$


AC

\begin{tabular}{|c|c|}
\hline & 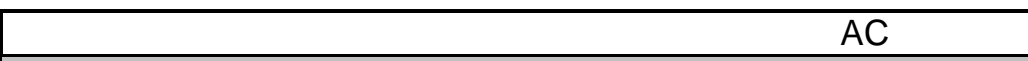 \\
\hline 1 & SAMP_COMMENT \\
\hline 344 & \\
\hline 345 & \\
\hline 346 & \\
\hline 347 & \\
\hline 348 & \\
\hline 349 & \\
\hline 350 & \\
\hline 351 & RESULTS NOT REPORTED FOR I-127 OR I-129/127 RATIO. \\
\hline 352 & \\
\hline 353 & RESULTS NOT REPORTED FOR I-127 OR I-129/127 RATIO. \\
\hline 354 & RESULTS NOT REPORTED FOR I-127 OR I-129/127 RATIO. \\
\hline 355 & \\
\hline 356 & \\
\hline 357 & \\
\hline 358 & \\
\hline 359 & \\
\hline 360 & \\
\hline 361 & \\
\hline 362 & \\
\hline 363 & \\
\hline 364 & \\
\hline 365 & \\
\hline 366 & \\
\hline 367 & \\
\hline 368 & \\
\hline 369 & \\
\hline 370 & \\
\hline 371 & \\
\hline 372 & \\
\hline 373 & \\
\hline 374 & \\
\hline 375 & \\
\hline 376 & \\
\hline 377 & \\
\hline 378 & \\
\hline 379 & \\
\hline 380 & \\
\hline 381 & \\
\hline
\end{tabular}




$$
\square
$$


AC

\begin{tabular}{|c|c|}
\hline 1 & SAMP_COMMENT \\
\hline 420 & \\
\hline 421 & \\
\hline 422 & \\
\hline 423 & \\
\hline 424 & \\
\hline 425 & \\
\hline 426 & \\
\hline 427 & \\
\hline 428 & \\
\hline 429 & \\
\hline 430 & \\
\hline 431 & \\
\hline 432 & \\
\hline 433 & \\
\hline 434 & \\
\hline 435 & \\
\hline 436 & \\
\hline 437 & \\
\hline 438 & \\
\hline 439 & \\
\hline 440 & \\
\hline 441 & \\
\hline 442 & \\
\hline 443 & PUMP OFF, SAMPLE COLLECTED AT SETTLING POND. \\
\hline 444 & PUMP OFF, SAMPLE COLLECTED AT SETTLING POND. \\
\hline 445 & \\
\hline 446 & PUMP OFF, SAMPLE COLLECTED AT SETTLING POND. \\
\hline 447 & \\
\hline 448 & \\
\hline 449 & \\
\hline 450 & \\
\hline 451 & \\
\hline 452 & \\
\hline 453 & \\
\hline 454 & \\
\hline 455 & \\
\hline 456 & \\
\hline 457 & \\
\hline
\end{tabular}




$$
\square
$$




$$
\square
$$




$$
\square
$$




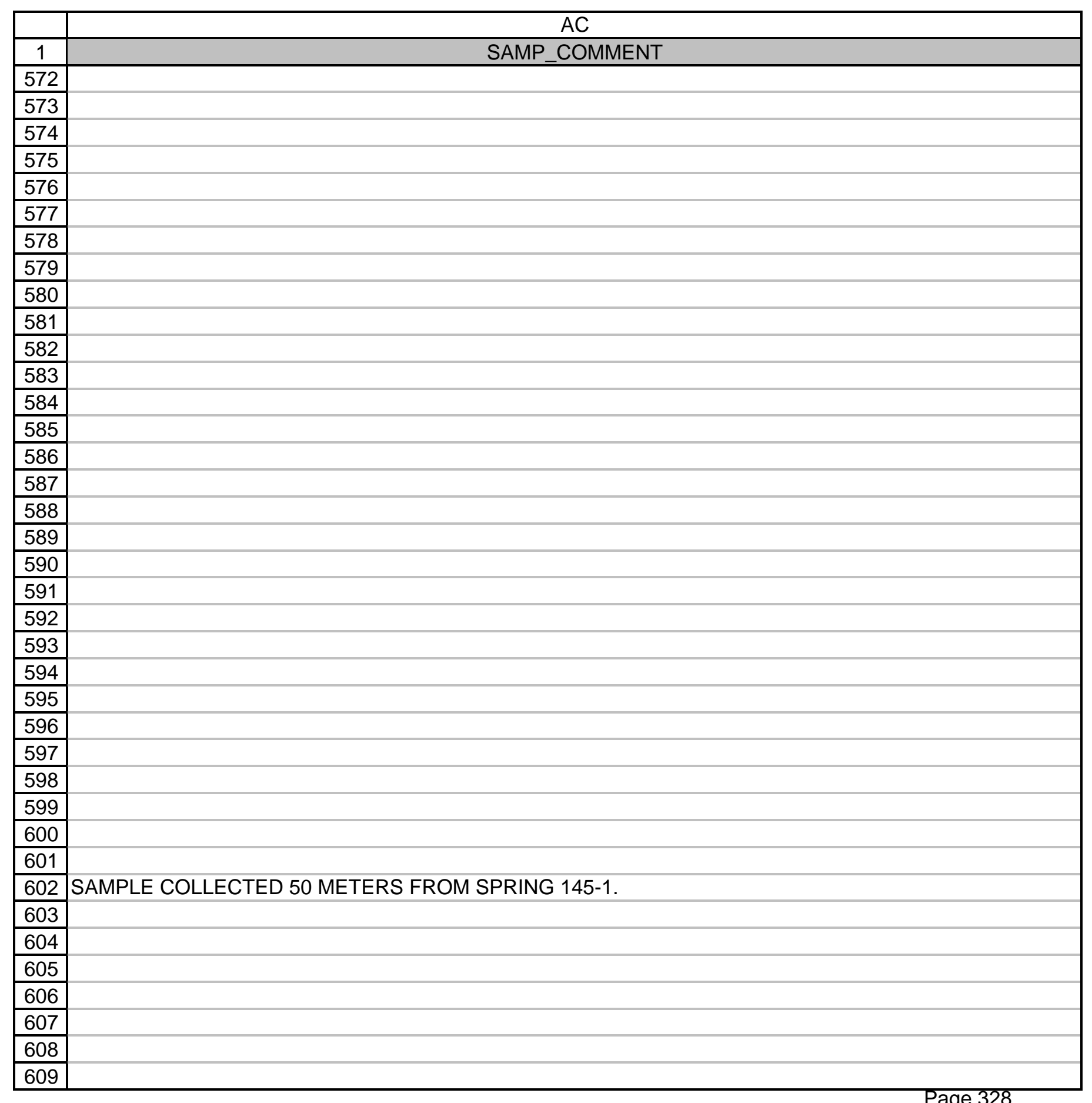




$$
\square
$$




$$
\square
$$




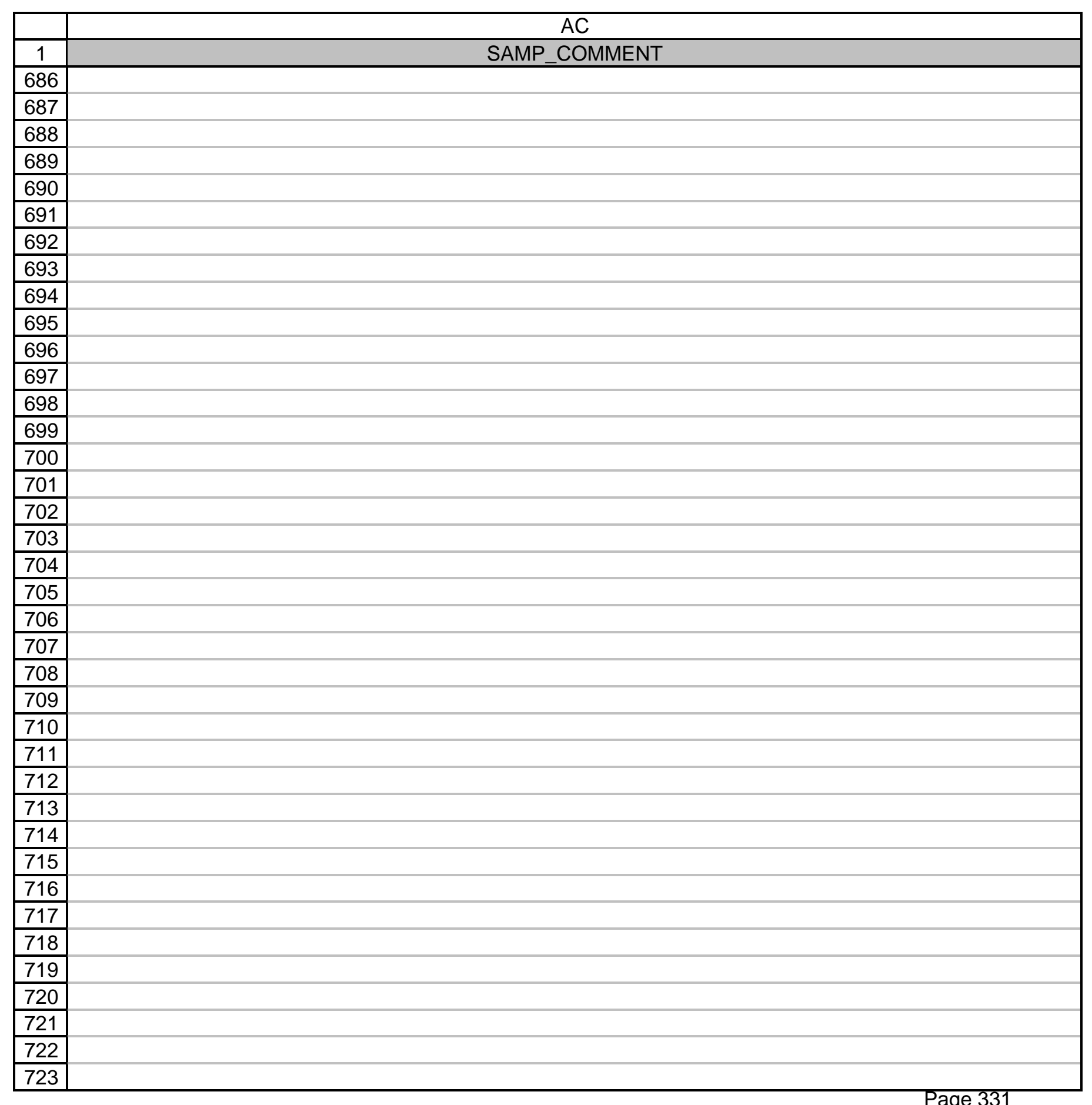




$$
\square
$$




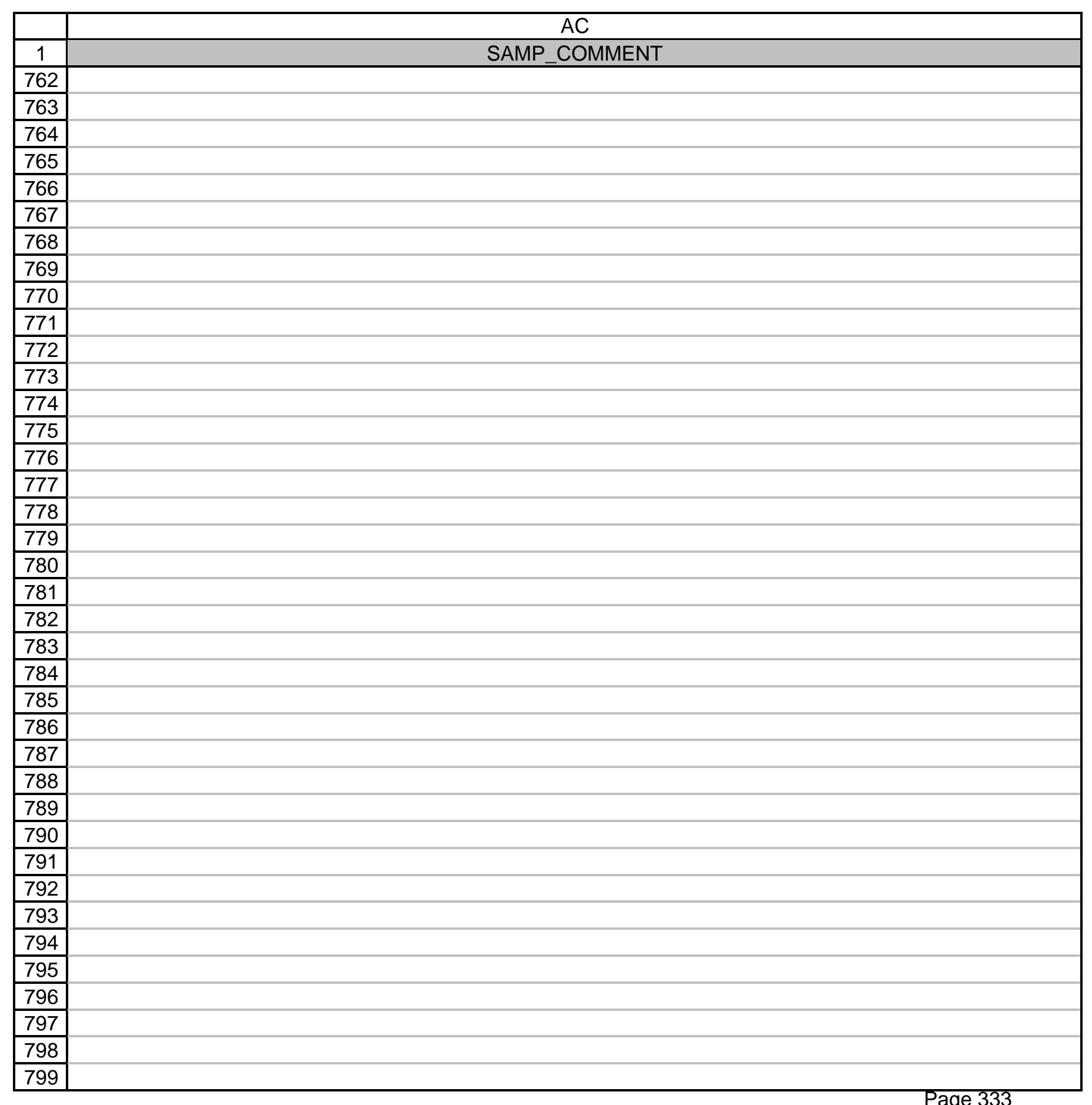




$$
\square
$$




$$
F
$$




$$
\square
$$




\begin{tabular}{|c|c|c|c|}
\hline & & & $\mathrm{AC}$ \\
\hline & & 1 & SAMP_COMMENT \\
\hline & & 914 & \\
\hline & & 915 & \\
\hline & & 916 & \\
\hline & & 917 & \\
\hline & & 918 & \\
\hline & & 919 & \\
\hline & & 920 & \\
\hline & & 921 & \\
\hline & & 922 & \\
\hline & & 923 & \\
\hline & & 924 & \\
\hline & & 925 & \\
\hline & & 926 & SAMPLE COLLECTED 50 METERS FROM SPRING 145-1. \\
\hline & & 927 & \\
\hline & & 928 & \\
\hline & & 929 & \\
\hline & & 930 & \\
\hline & & 931 & \\
\hline & & 932 & \\
\hline & & 933 & \\
\hline & & 934 & \\
\hline & & 935 & \\
\hline & & 936 & \\
\hline & & 937 & \\
\hline & & 938 & \\
\hline & & 939 & \\
\hline & & 940 & \\
\hline & & 941 & \\
\hline & & 942 & \\
\hline & & 943 & \\
\hline & & 944 & \\
\hline & & 945 & \\
\hline & & 946 & \\
\hline & & 947 & \\
\hline & & 948 & \\
\hline & & 949 & \\
\hline & & 950 & \\
\hline & & 951 & \\
\hline
\end{tabular}




$$
\square
$$




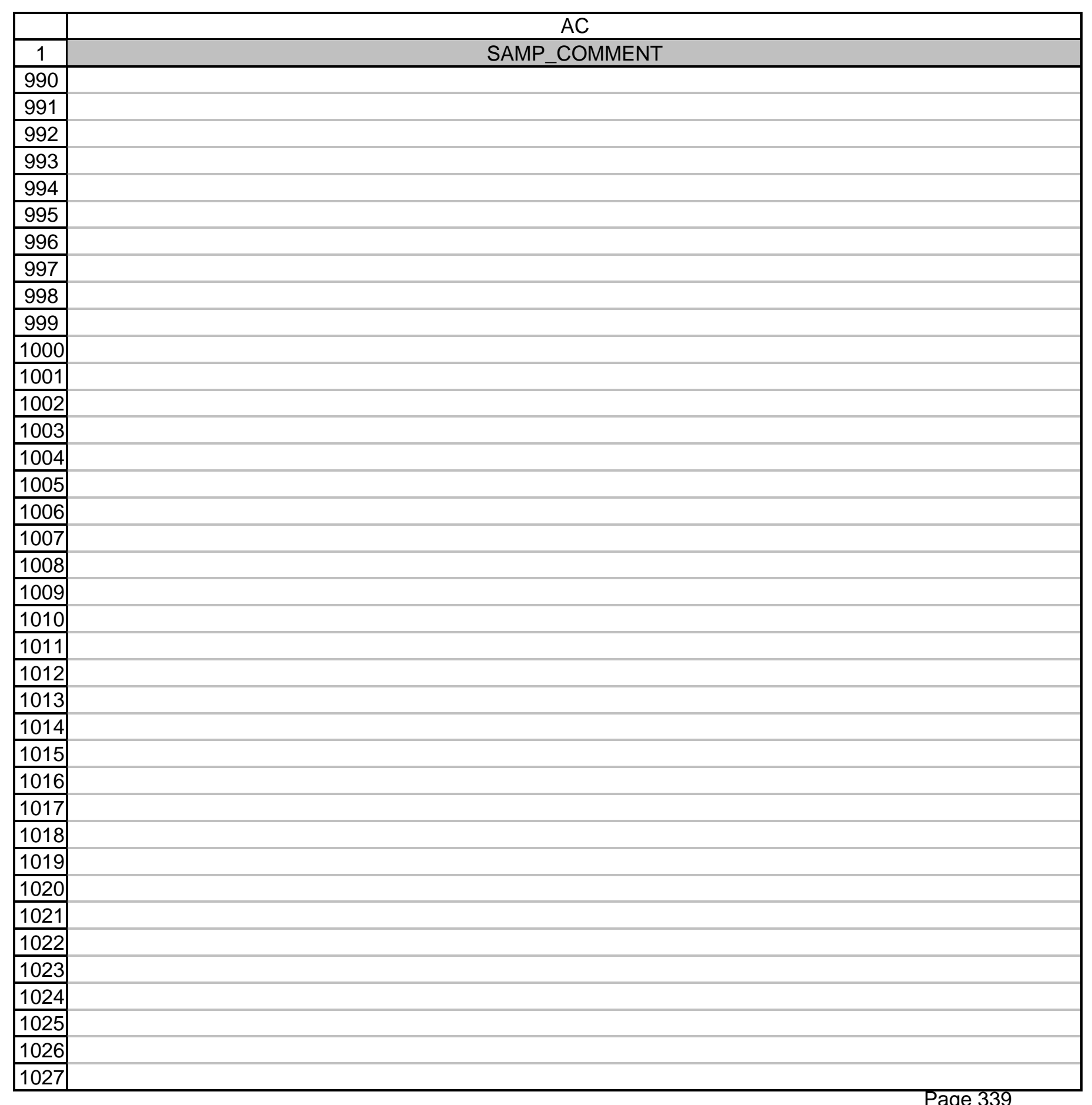


1028 PUMP OFF, SAMPLE COLLECTED AT SETTLING POND.

11029 PUMP OFF, SAMPLE COLLECTED AT SETTLING POND.

1030

1031 PUMP OFF, SAMPLE COLLECTED AT SETTLING POND.

1032

1033

1034

1035

1036

1037

1038

1039

1040

1041

1042

1043

1044

1045

1046

1047

1048

1049

1050

1051

\begin{tabular}{|l|}
1051 \\
\hline 1053 \\
\hline
\end{tabular}

1053

1054

1055

1056 APRICOT LEAVES/CONDENSATION.

1057

1058

1059

1060

1061

1062

1063

1064

1065 


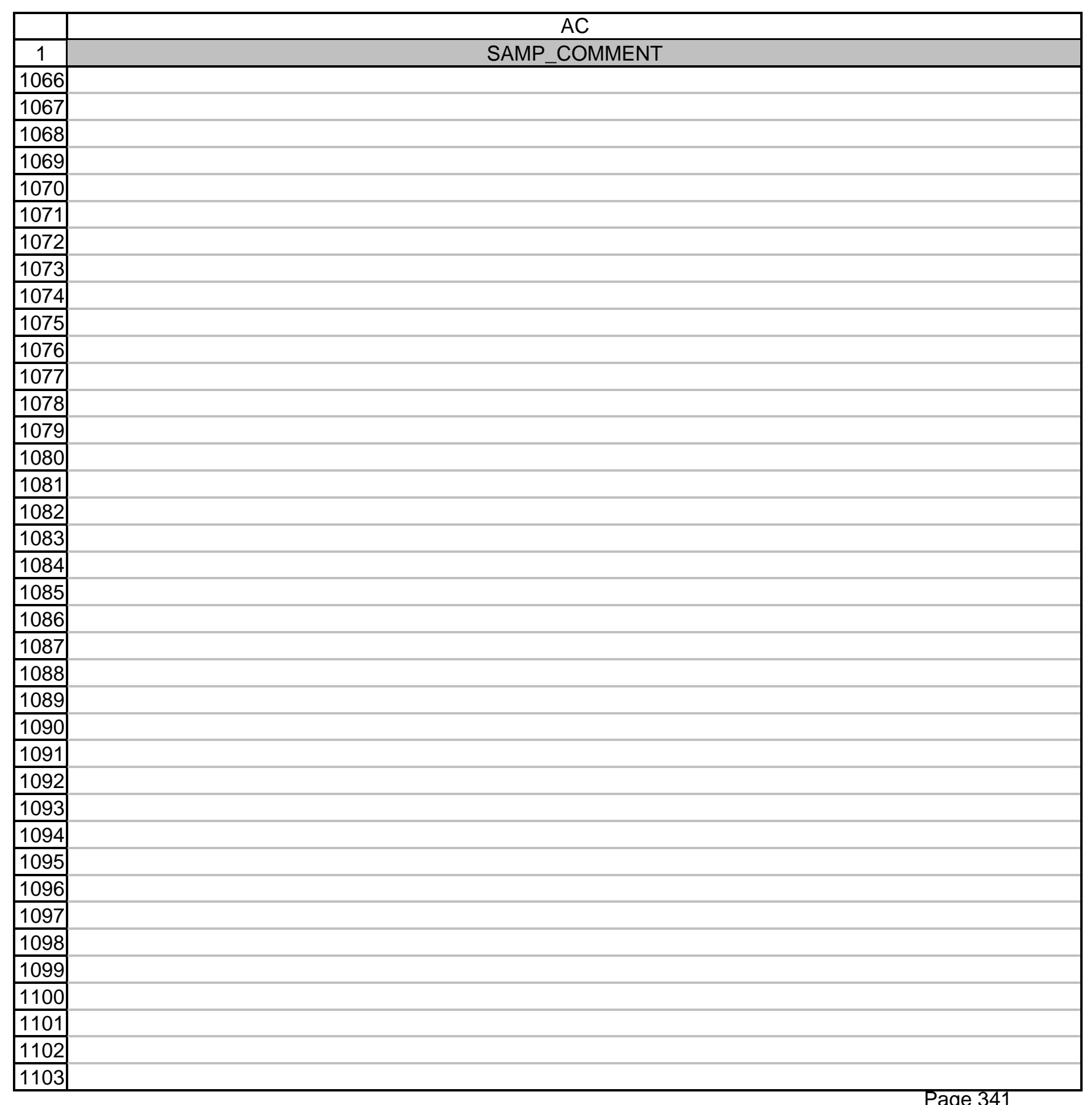




$$
\square
$$


AC

\begin{tabular}{|c|c|}
\hline & 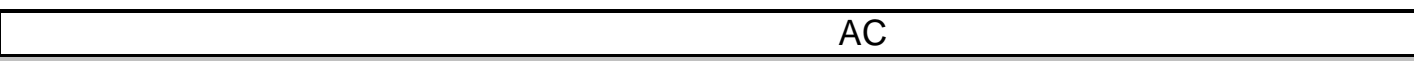 \\
\hline 1 & 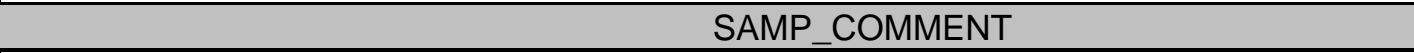 \\
\hline 1142 & \\
\hline 1143 & \\
\hline 1144 & \\
\hline 1145 & \\
\hline 1146 & \\
\hline 1147 & \\
\hline 1148 & \\
\hline 1149 & \\
\hline 1150 & \\
\hline 1151 & \\
\hline 1152 & \\
\hline 1153 & \\
\hline 1154 & \\
\hline 1155 & \\
\hline 1156 & \\
\hline 1157 & Samples shipped to St. Louis on $10 / 18 / 00$ and received on $10 / 19 / 00$ at $08: 35$ \\
\hline 1158 & Samples shipped to St. Louis on $10 / 18 / 00$ and received on $10 / 19 / 00$ at $08: 35$ \\
\hline 1159 & Samples shipped to St. Louis on $10 / 18 / 00$ and received on $10 / 19 / 00$ at $08: 35$ \\
\hline 1160 & \\
\hline 1161 & \\
\hline 1162 & \\
\hline 1163 & \\
\hline 1164 & \\
\hline 1165 & \\
\hline 1166 & \\
\hline 1167 & \\
\hline 1168 & \\
\hline 1169 & Sample shipped from Richland to St. Louis on $11 / 02 / 00$ and received on $11 / 03 / 00$ at 09:00 \\
\hline 1170 & \\
\hline 1171 & \\
\hline 1172 & \\
\hline 1173 & \\
\hline 1174 & \\
\hline 1175 & \\
\hline 1176 & \\
\hline 1177 & \\
\hline 1178 & \\
\hline 1179 & \\
\hline
\end{tabular}




$$
\square
$$




\begin{tabular}{|c|c|}
\hline & $\mathrm{AC}$ \\
\hline 1 & SAMP_COMMENT \\
\hline 1218 & \\
\hline 1219 & \\
\hline 1220 & \\
\hline 1221 & \\
\hline 1222 & \\
\hline 1223 & \\
\hline 1224 & \\
\hline 1225 & \\
\hline 1226 & \\
\hline 1227 & \\
\hline 1228 & SAMPLE COLLECTED 50 METERS FROM SPRING 145-1. \\
\hline 1229 & \\
\hline 1230 & \\
\hline 1231 & \\
\hline 1232 & \\
\hline 1233 & \\
\hline 1234 & \\
\hline 1235 & \\
\hline 1236 & \\
\hline 1237 & \\
\hline 1238 & \\
\hline 1239 & \\
\hline 1240 & \\
\hline 1241 & \\
\hline 1242 & \\
\hline 1243 & \\
\hline 1244 & \\
\hline 1245 & \\
\hline 1246 & \\
\hline 1247 & \\
\hline 1248 & \\
\hline 1249 & \\
\hline 1250 & \\
\hline 1251 & \\
\hline 1252 & \\
\hline 1253 & \\
\hline 1254 & \\
\hline 1255 & \\
\hline
\end{tabular}




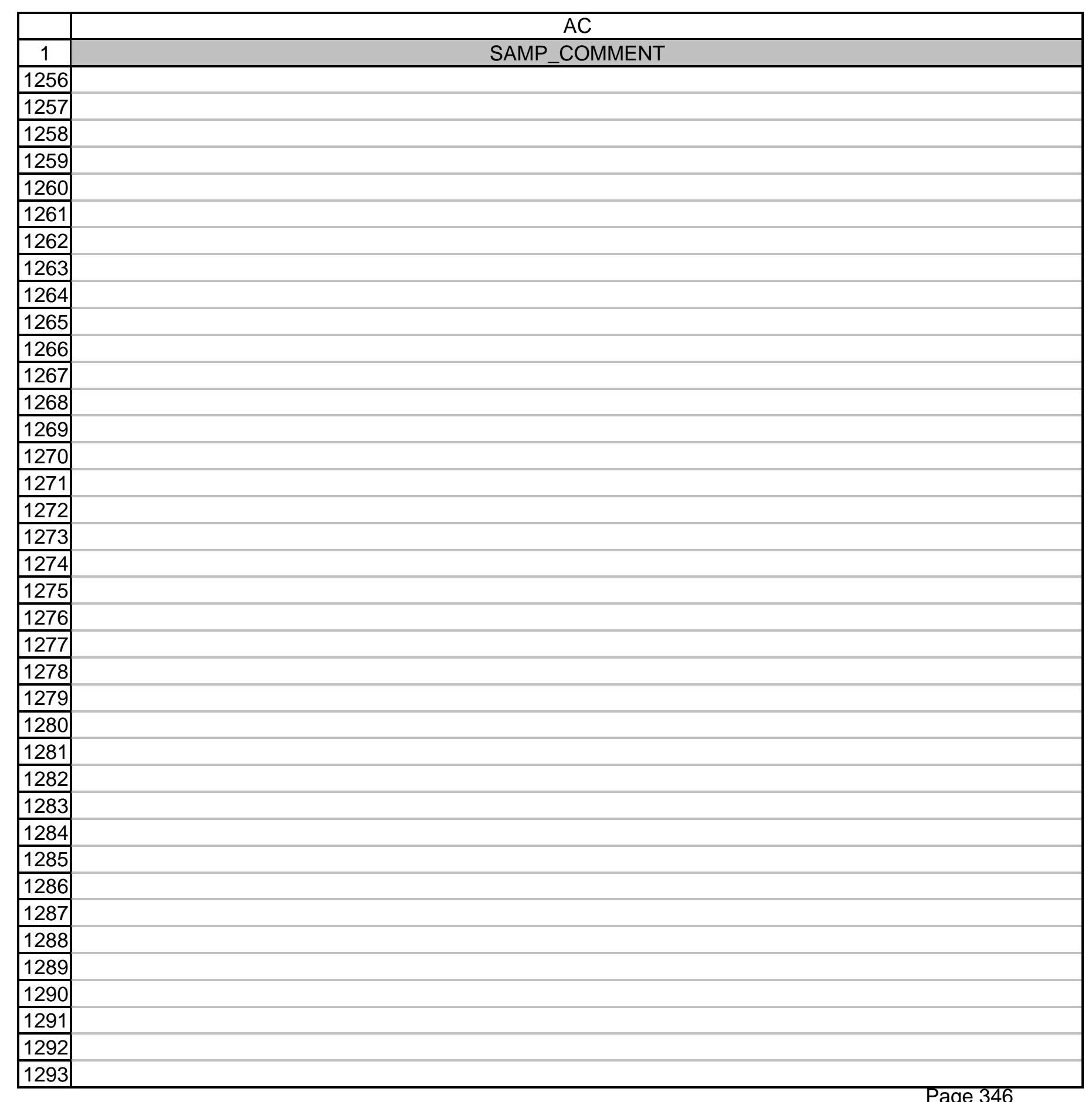




$$
\square
$$




$$
\square
$$




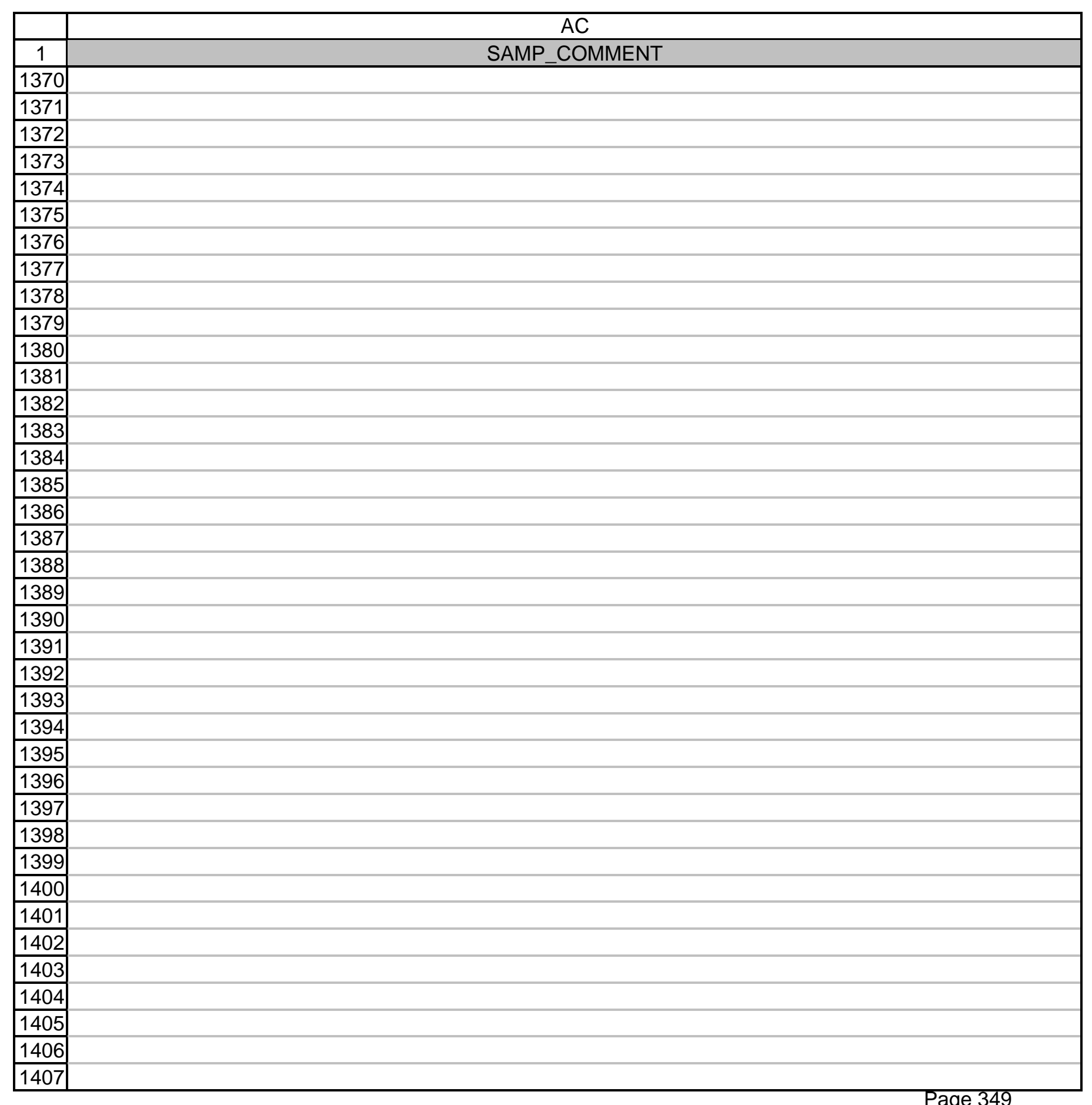




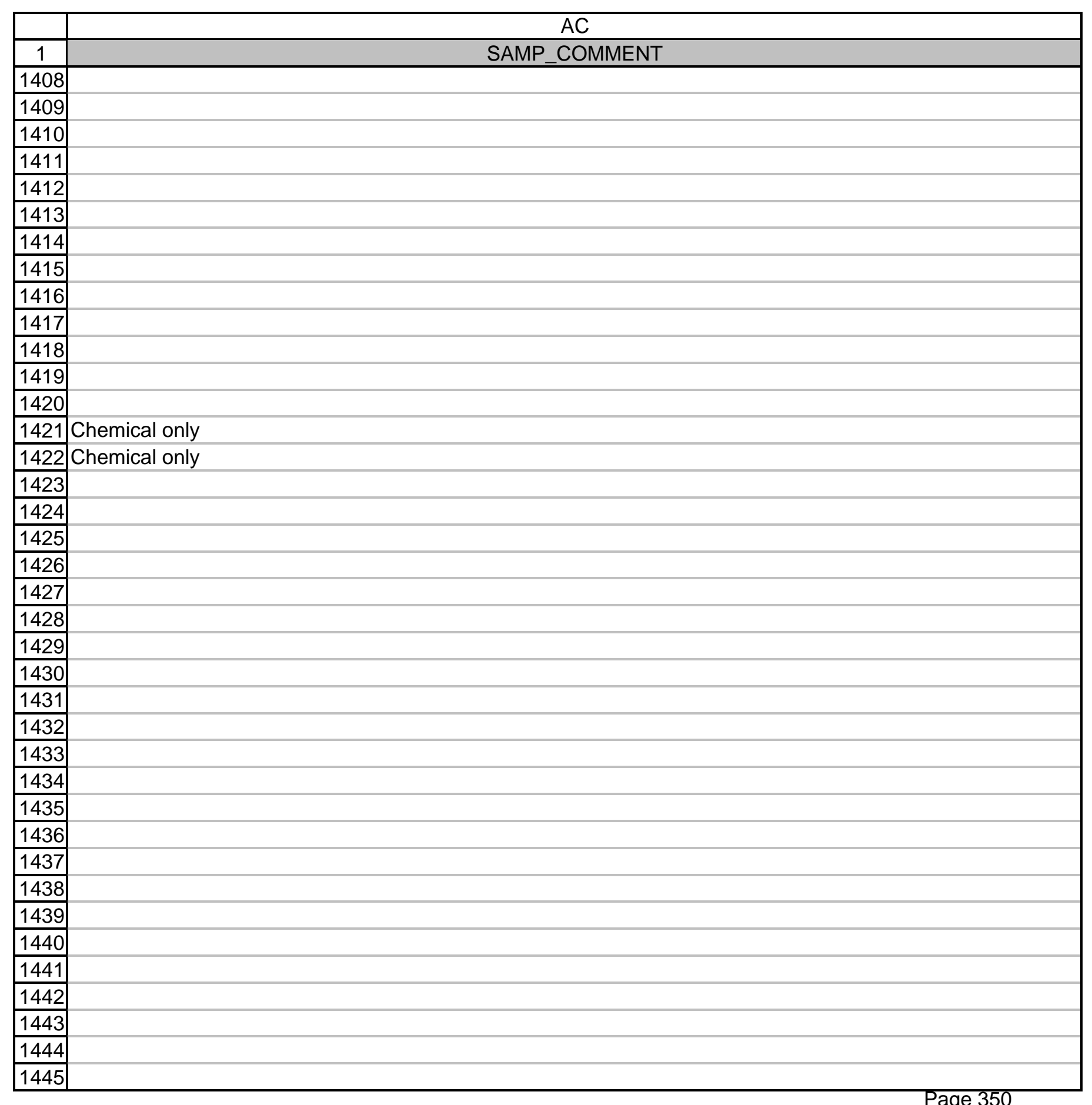




$$
F
$$




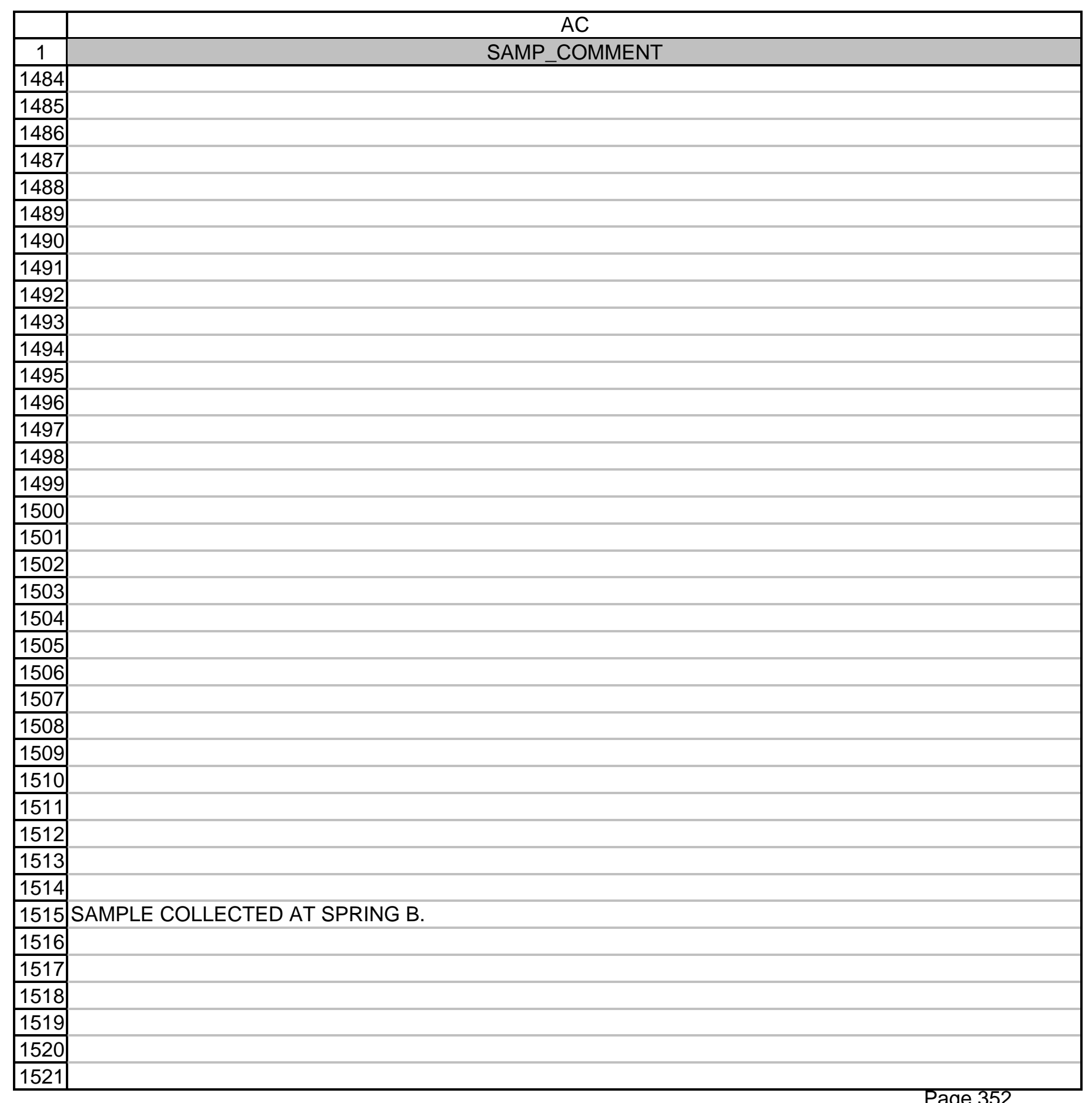




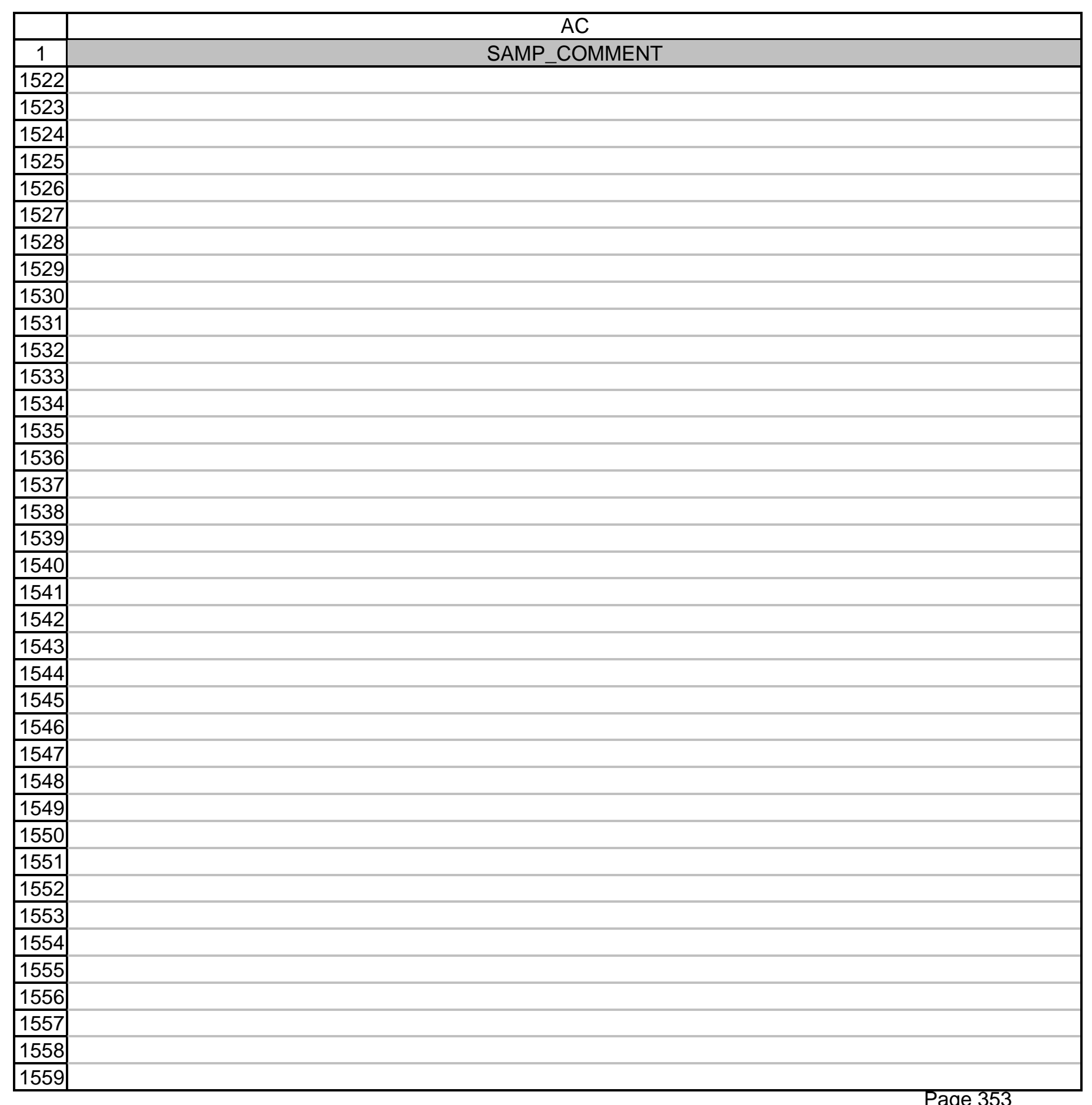




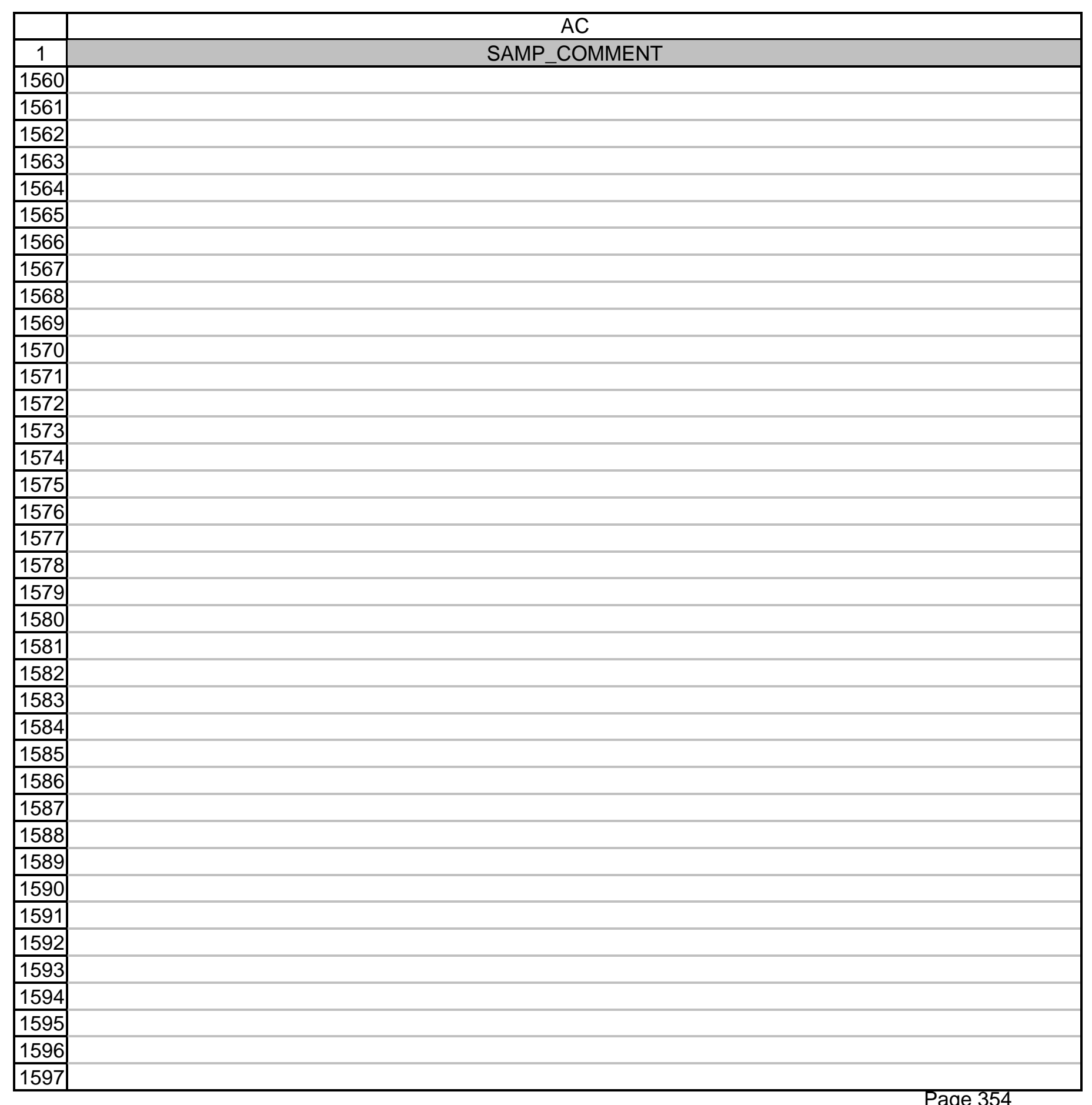




$$
\square
$$




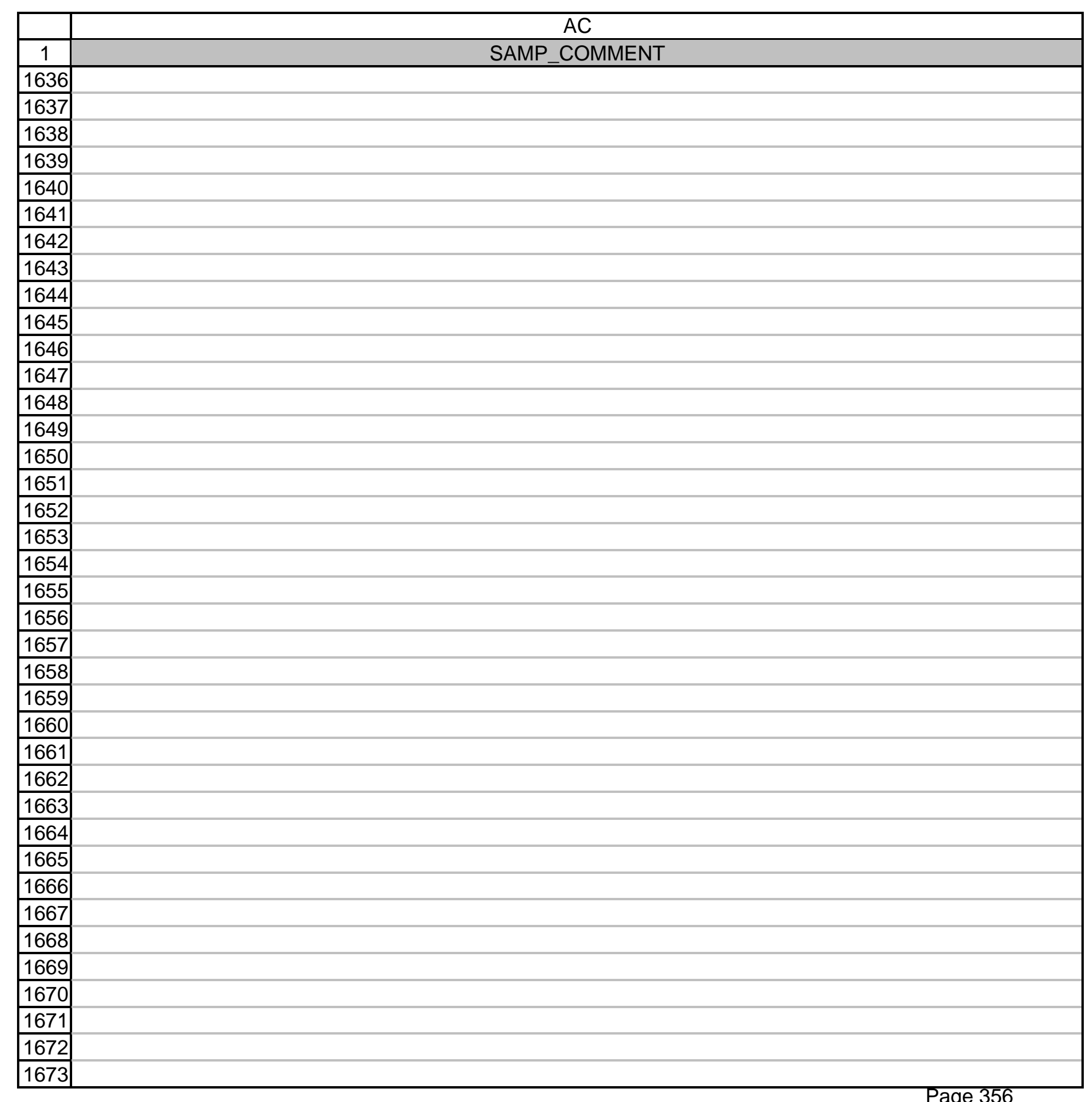




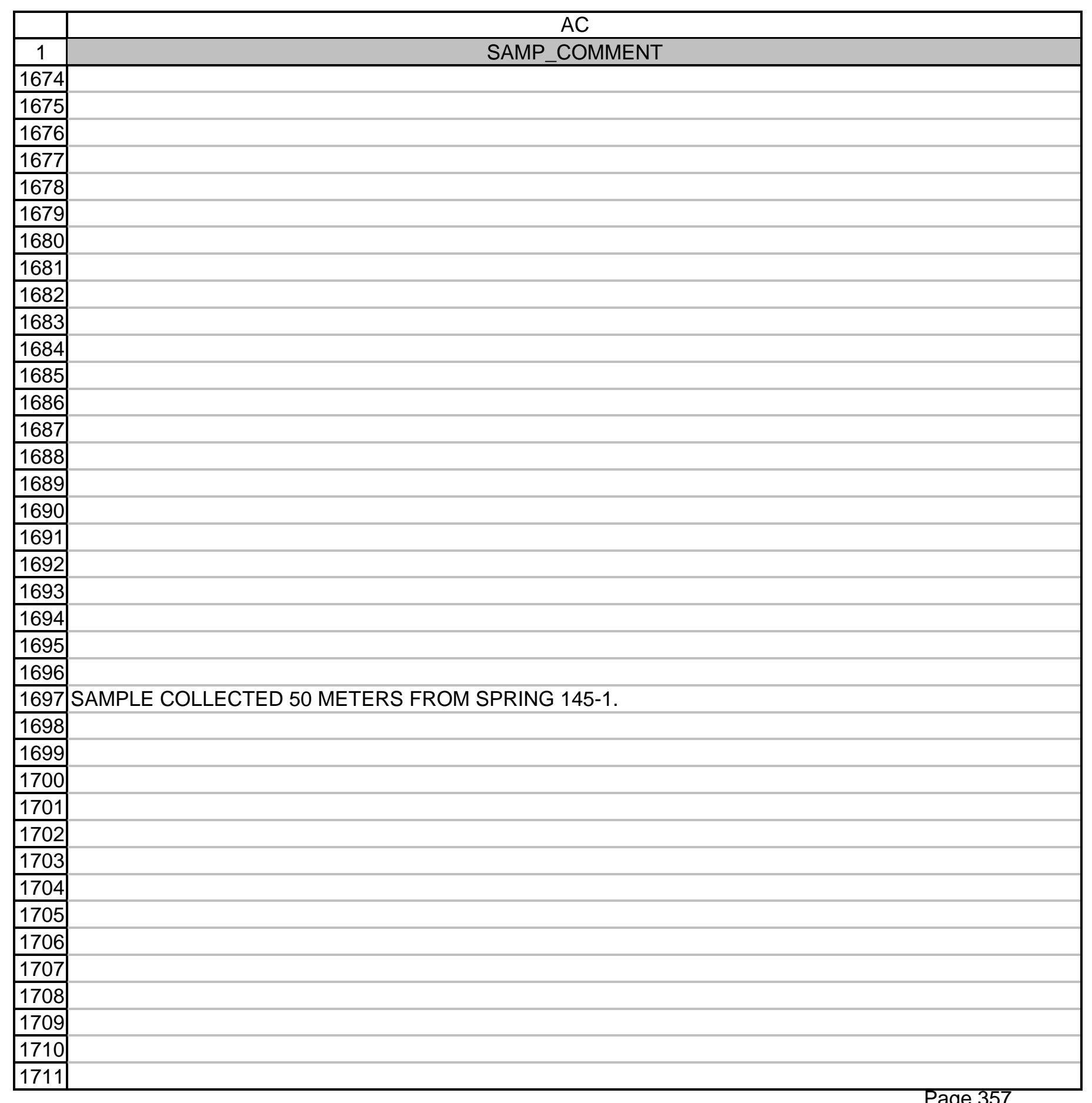


AC

\begin{tabular}{|c|c|}
\hline 1 & SAMP COMMENT \\
\hline 1712 & \\
\hline 1713 & \\
\hline 1714 & \\
\hline \begin{tabular}{|l|}
1715 \\
\end{tabular} & \\
\hline 1716 & \\
\hline \begin{tabular}{|l|}
1717 \\
\end{tabular} & \\
\hline 1718 & \\
\hline 1719 & \\
\hline 1720 & \\
\hline 1721 & \\
\hline 1722 & \\
\hline 1723 & \\
\hline 1724 & \\
\hline 1725 & \\
\hline 1726 & \\
\hline 1727 & \\
\hline 1728 & \\
\hline 1729 & \\
\hline 1730 & \\
\hline 1731 & \\
\hline 1732 & \\
\hline 1733 & \\
\hline 1734 & \\
\hline 1735 & \\
\hline 1736 & \\
\hline 1737 & \\
\hline 1738 & \\
\hline 1739 & WATER CAME UP 2-3 FEET INTO RIPARIAN VEGETATION. 1 METER DEPTH AT GPS FLAG. \\
\hline 1740 & WATER CAME UP 2-3 FEET INTO RIPARIAN VEGETATION. 1 METER DEPTH AT GPS FLAG. \\
\hline 1741 & WATER CAME UP 2-3 FEET INTO RIPARIAN VEGETATION. 1 METER DEPTH AT GPS FLAG. \\
\hline 1742 & \\
\hline 1743 & \\
\hline 1744 & \\
\hline 1745 & \\
\hline 1746 & \\
\hline 1747 & \\
\hline 1748 & \\
\hline 1749 & \\
\hline
\end{tabular}




\begin{tabular}{|c|c|}
\hline & $\mathrm{AC}$ \\
\hline 1 & SAMP_COMMENT \\
\hline 1750 & \\
\hline 1751 & \\
\hline 1752 & \\
\hline 1753 & \\
\hline 1754 & \\
\hline 1755 & \\
\hline 1756 & \\
\hline 1757 & \\
\hline 1758 & \\
\hline 1759 & \\
\hline 1760 & \\
\hline 1761 & \\
\hline 1762 & \\
\hline 1763 & \\
\hline 1764 & \\
\hline 1765 & WATER CAME UP 2-3 FEET INTO RIPARIAN VEGETATION. 1 METER DEPTH AT GPS FLAG. \\
\hline 1766 & \\
\hline 1767 & \\
\hline 1768 & \\
\hline 1769 & \\
\hline 1770 & \\
\hline 1771 & \\
\hline 1772 & \\
\hline 1773 & \\
\hline 1774 & \\
\hline 1775 & \\
\hline 1776 & \\
\hline 1777 & \\
\hline 1778 & \\
\hline 1779 & \\
\hline 1780 & \\
\hline 1781 & \\
\hline 1782 & \\
\hline 1783 & \\
\hline 1784 & \\
\hline 1785 & \\
\hline 1786 & \\
\hline 1787 & \\
\hline
\end{tabular}




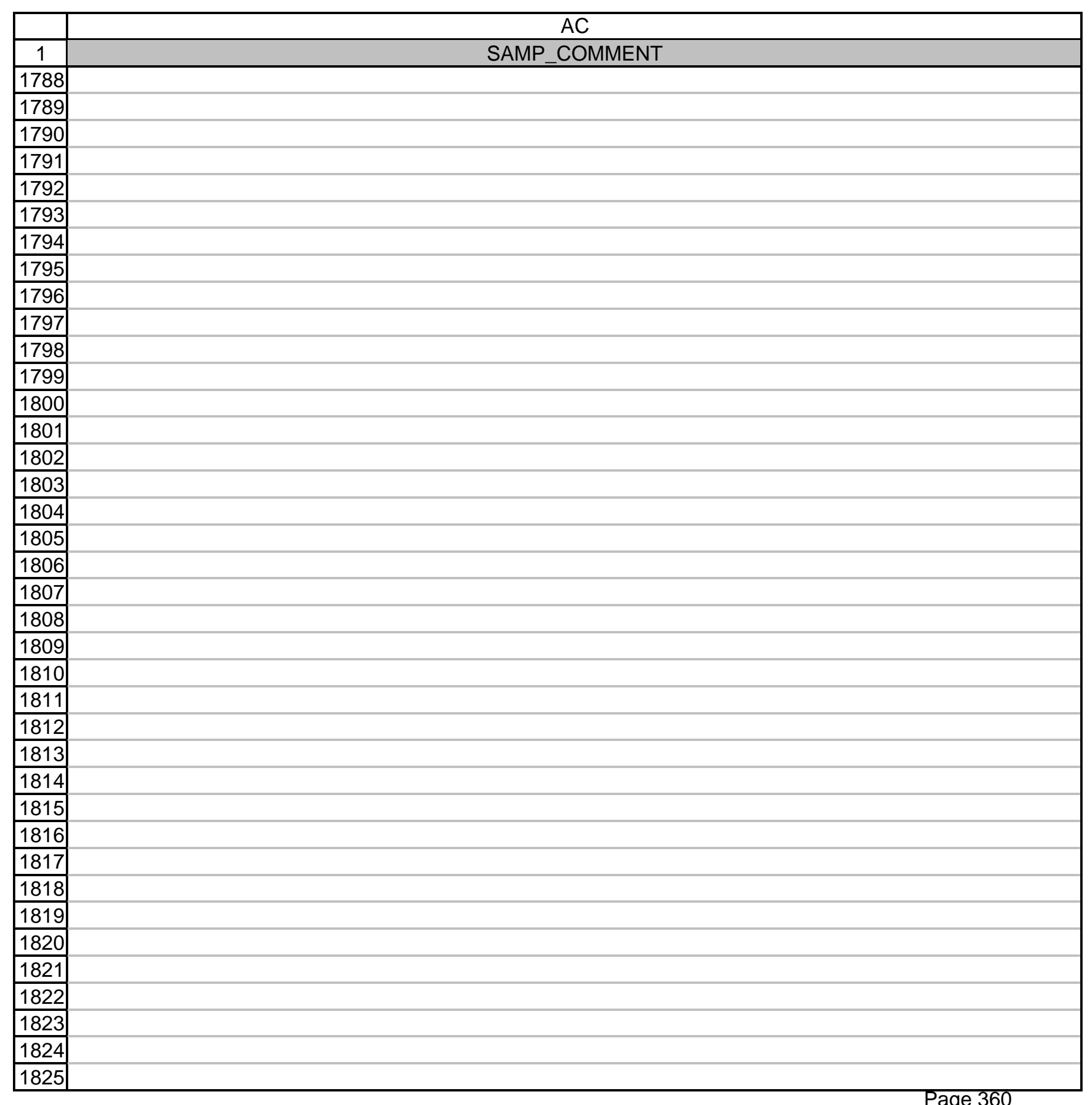




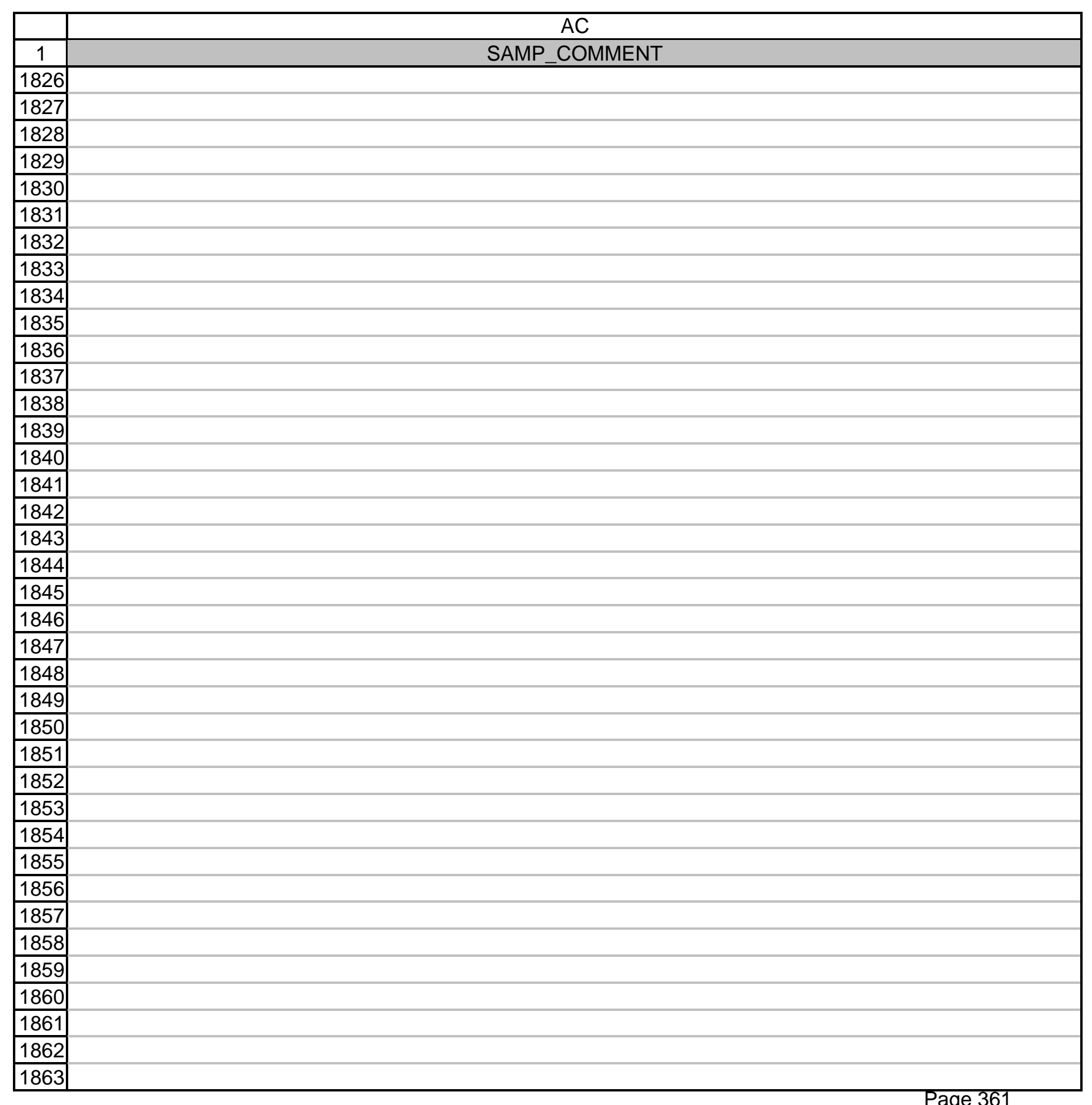




$$
\square
$$




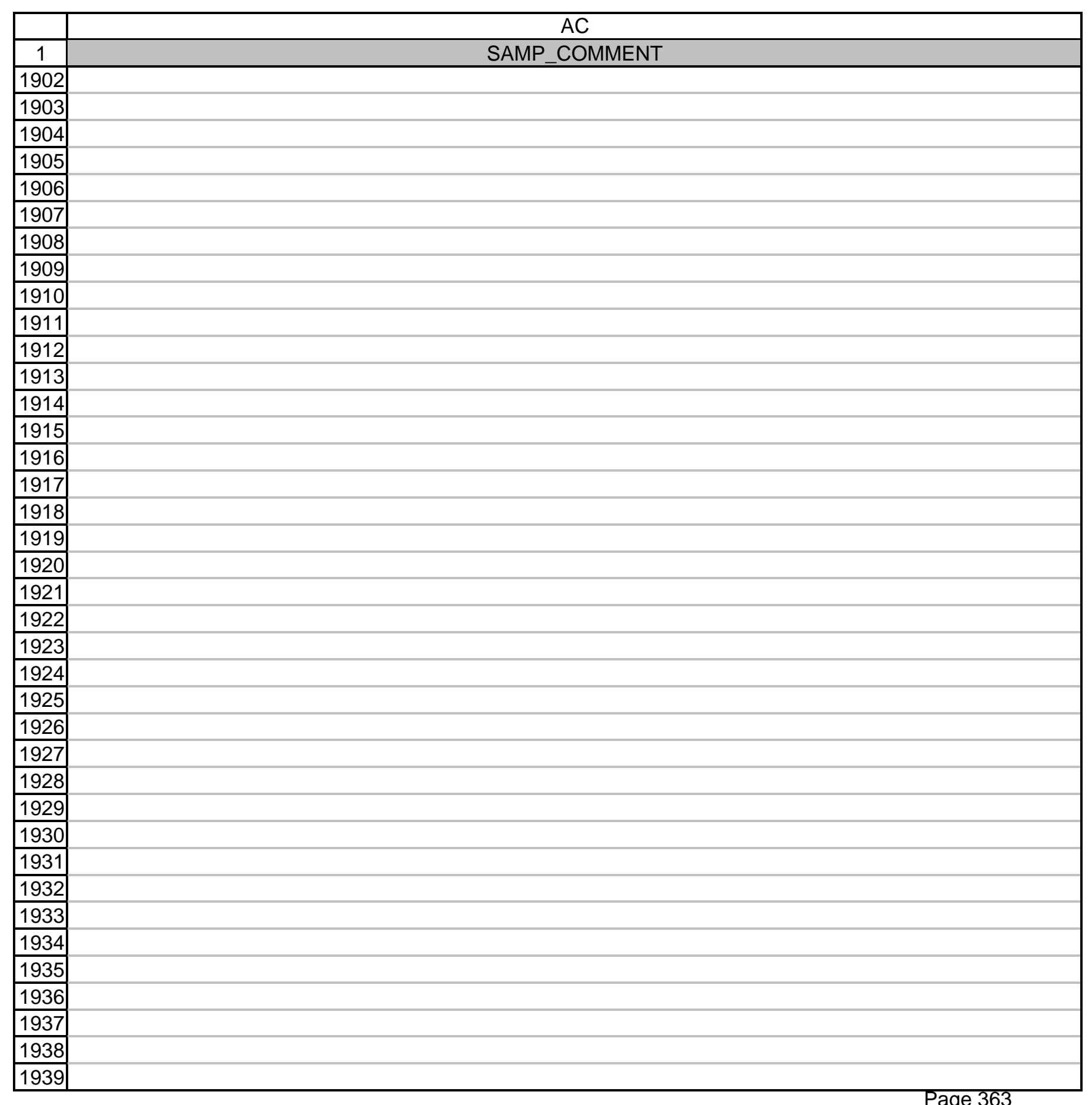




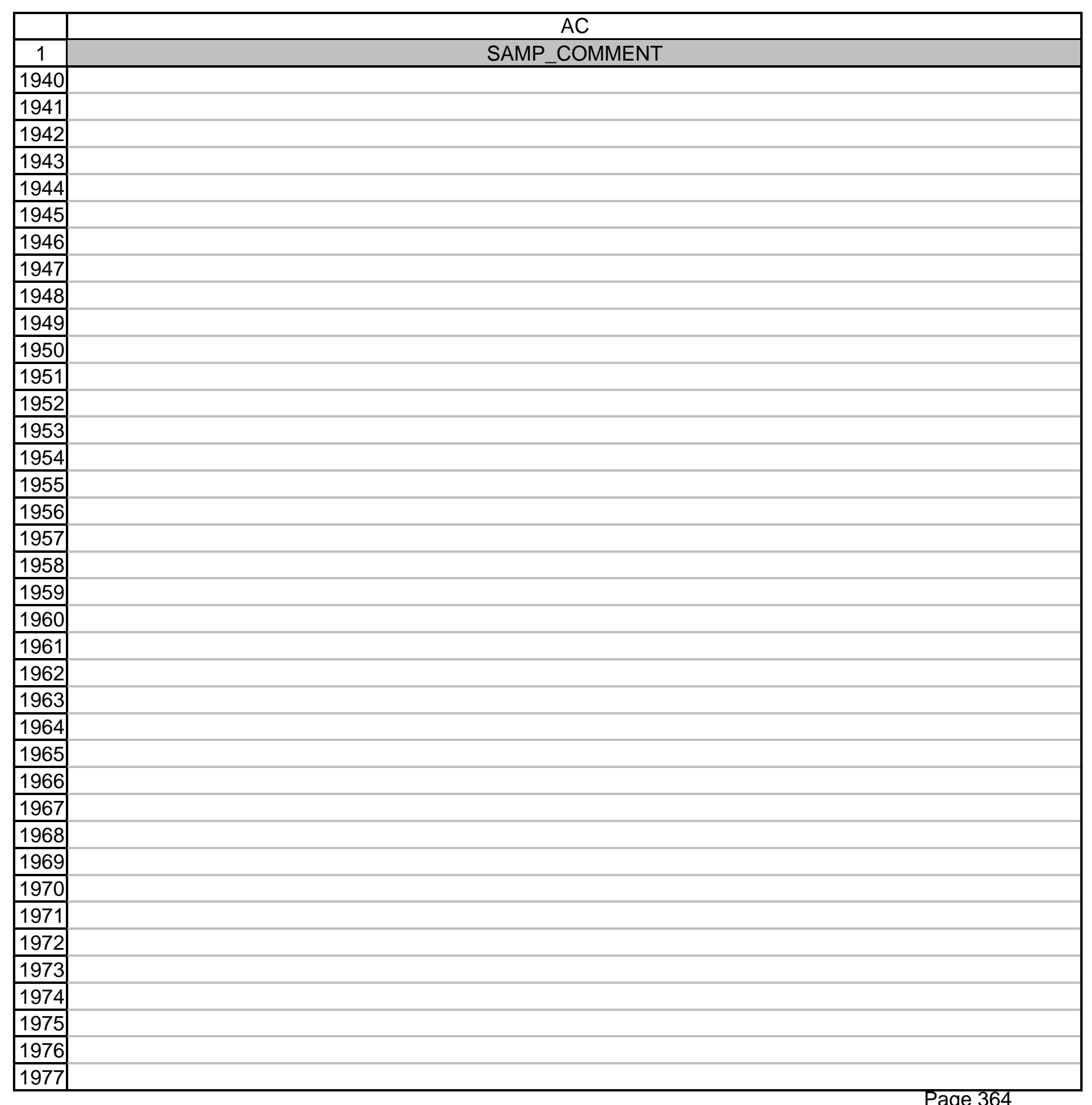




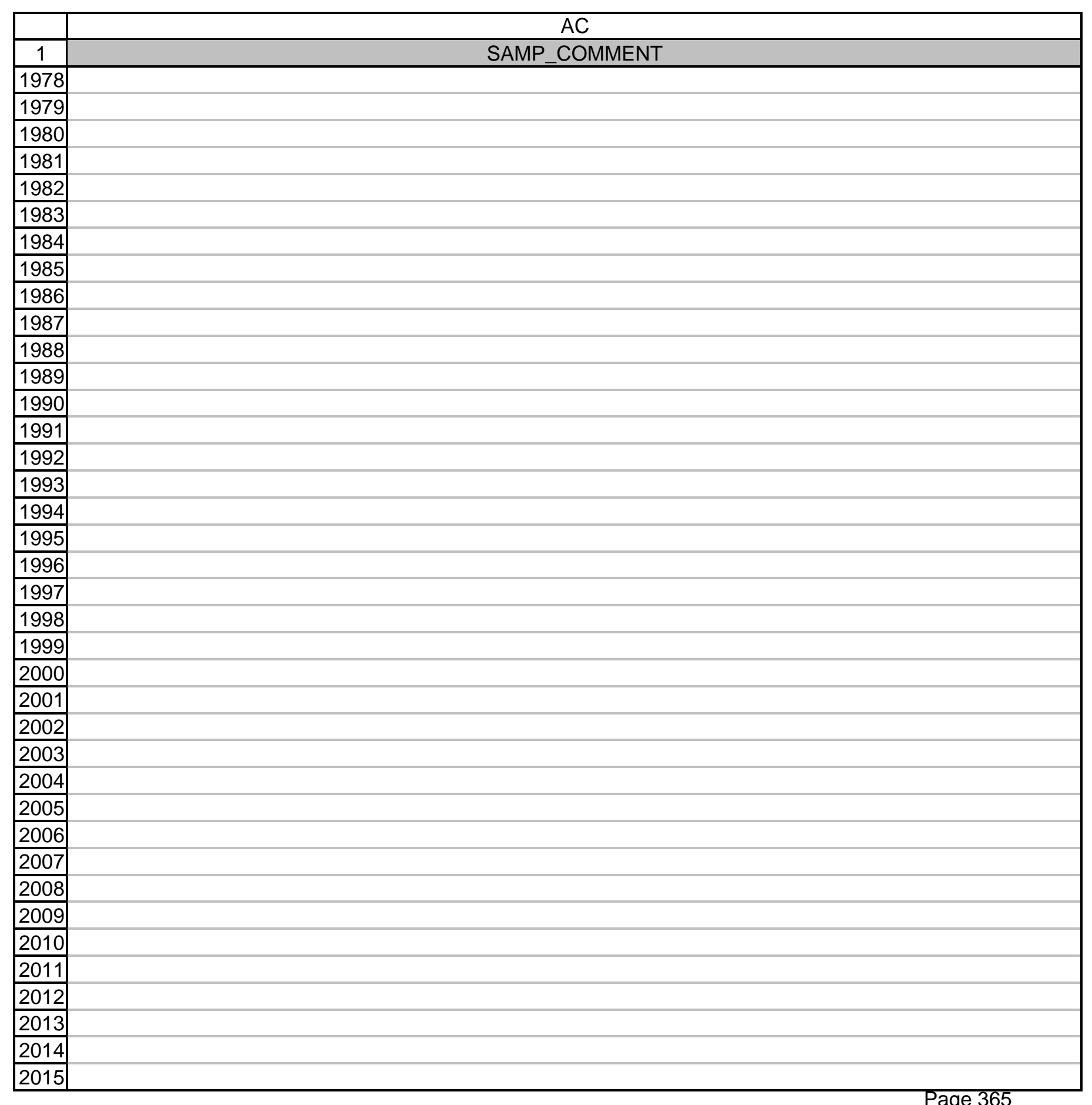




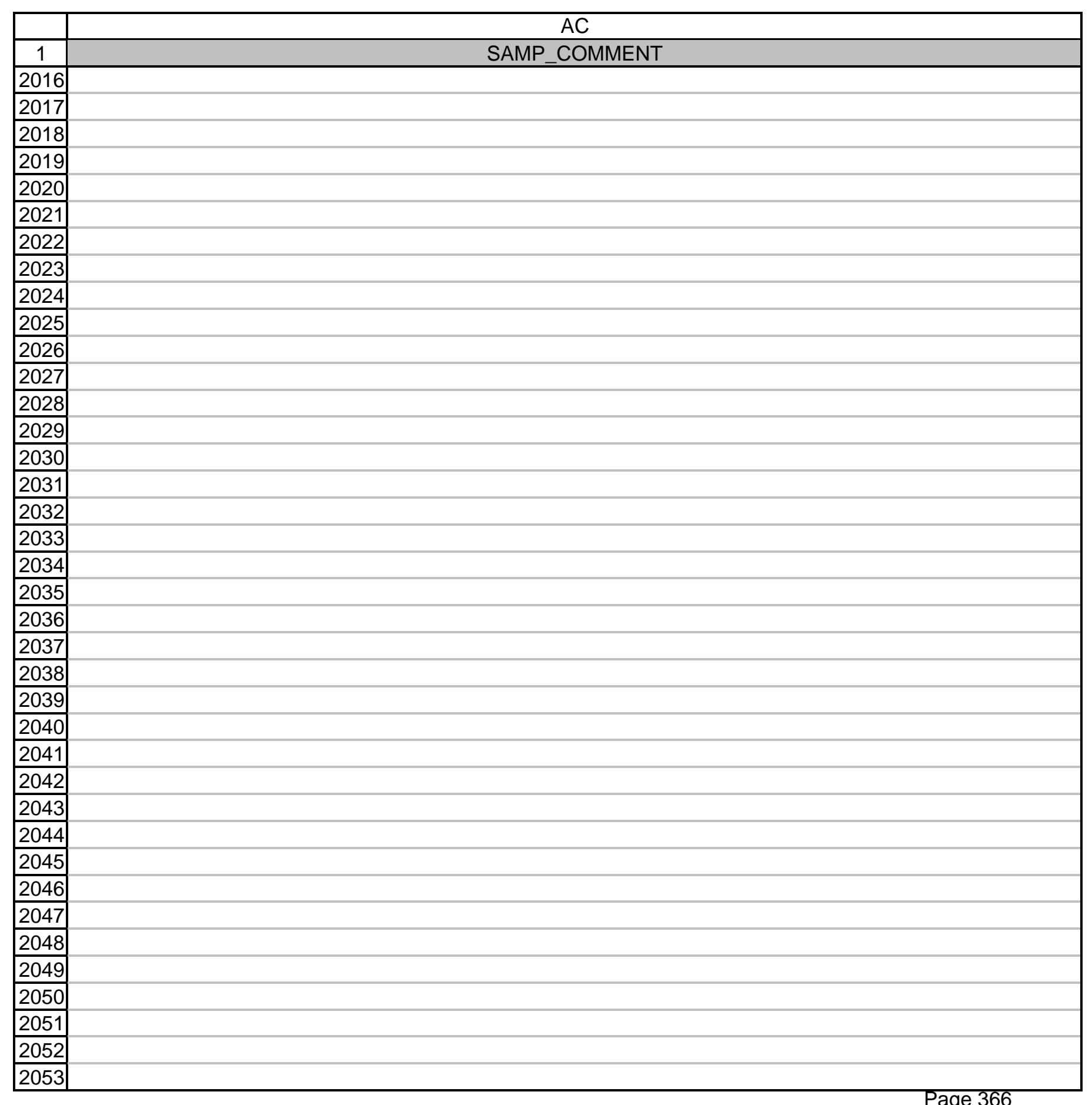




$$
\square
$$




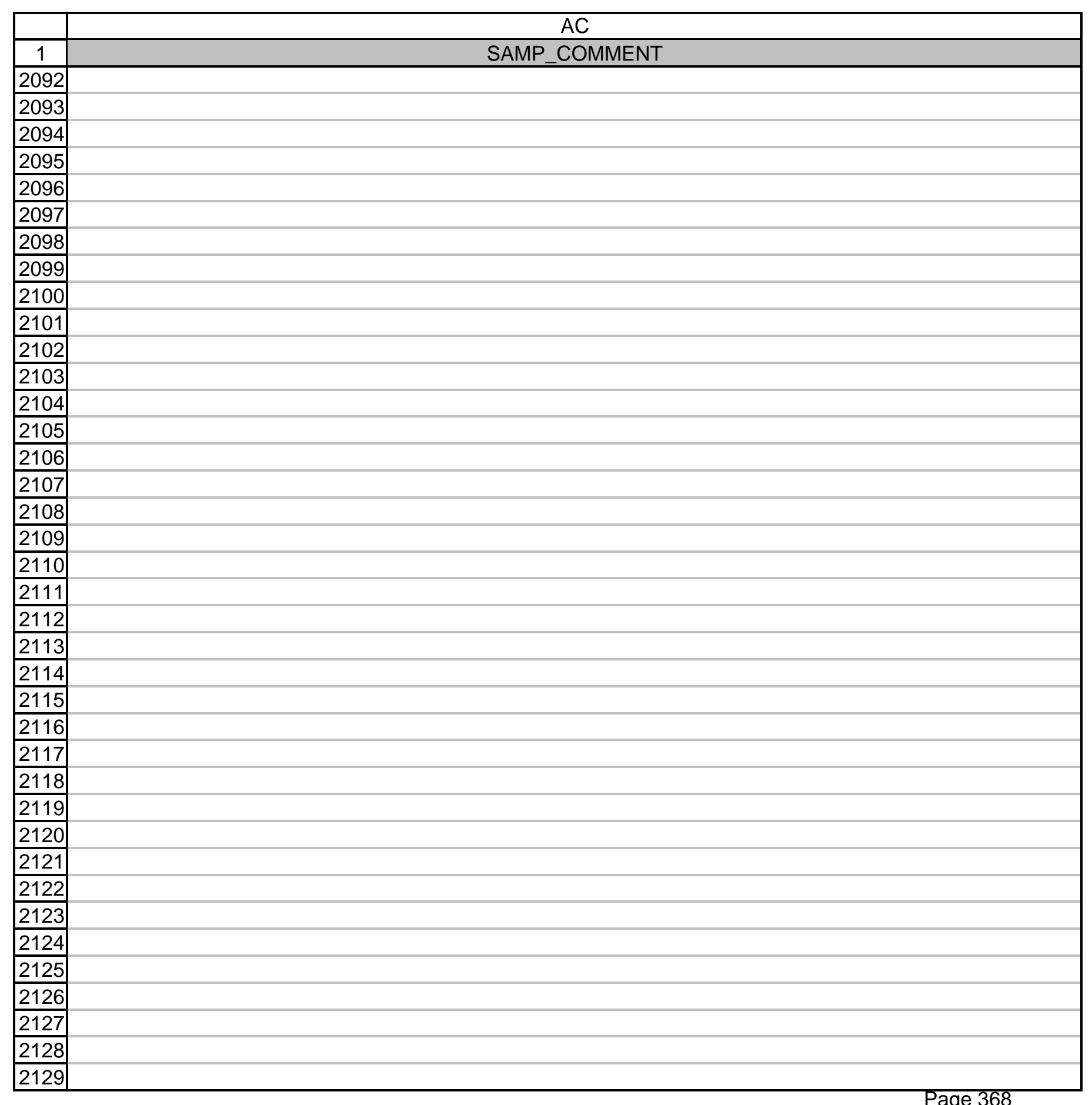




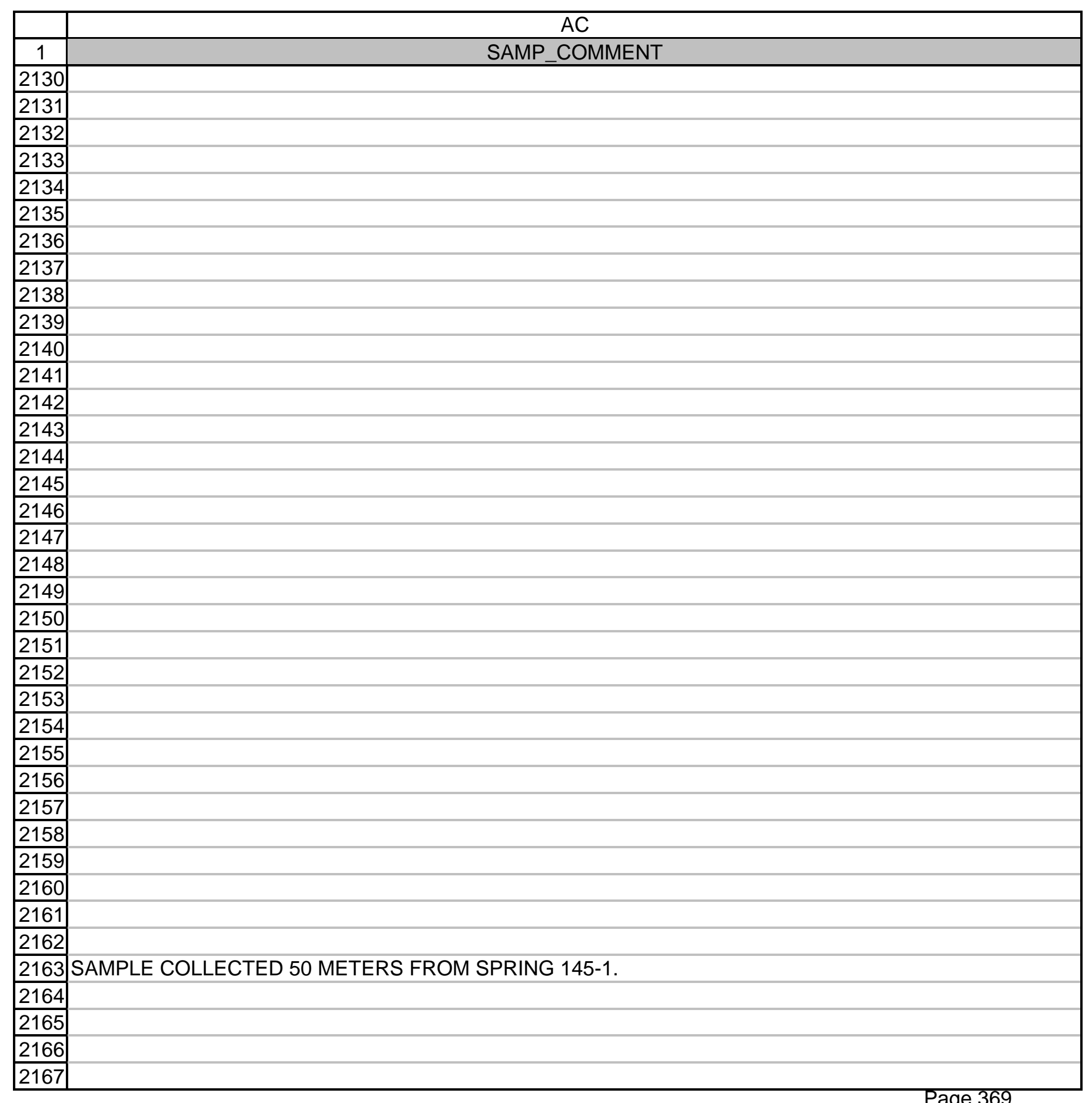




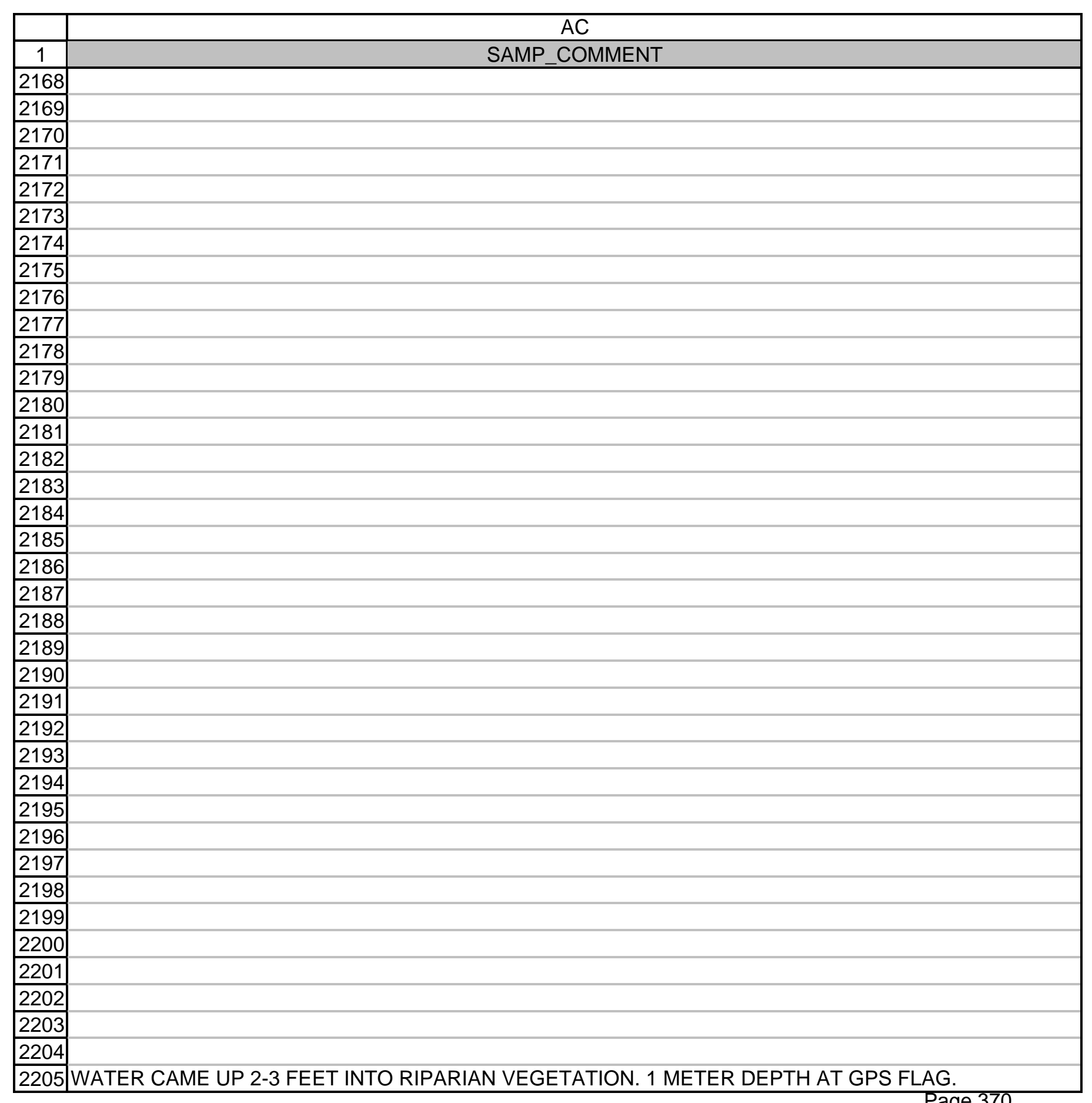


2206 WATER CAME UP 2-3 FEET INTO RIPARIAN VEGETATION. 1 METER DEPTH AT GPS FLAG.

2207 WATER CAME UP 2-3 FEET INTO RIPARIAN VEGETATION. 1 METER DEPTH AT GPS FLAG.

2208

2209

2210

2211

2212

2213

2214

2215

2216

2217

2218

2219

2220

2221

2222

2223

2224

2225

2226

2227

2228

2229

2230

2231 WATER CAME UP 2-3 FEET INTO RIPARIAN VEGETATION. 1 METER DEPTH AT GPS FLAG.

2232

2233

2234

2235

2236

2237

2238

2239

2240

2241

2242

\begin{tabular}{|l}
2242 \\
\hline 2243 \\
\hline
\end{tabular} 


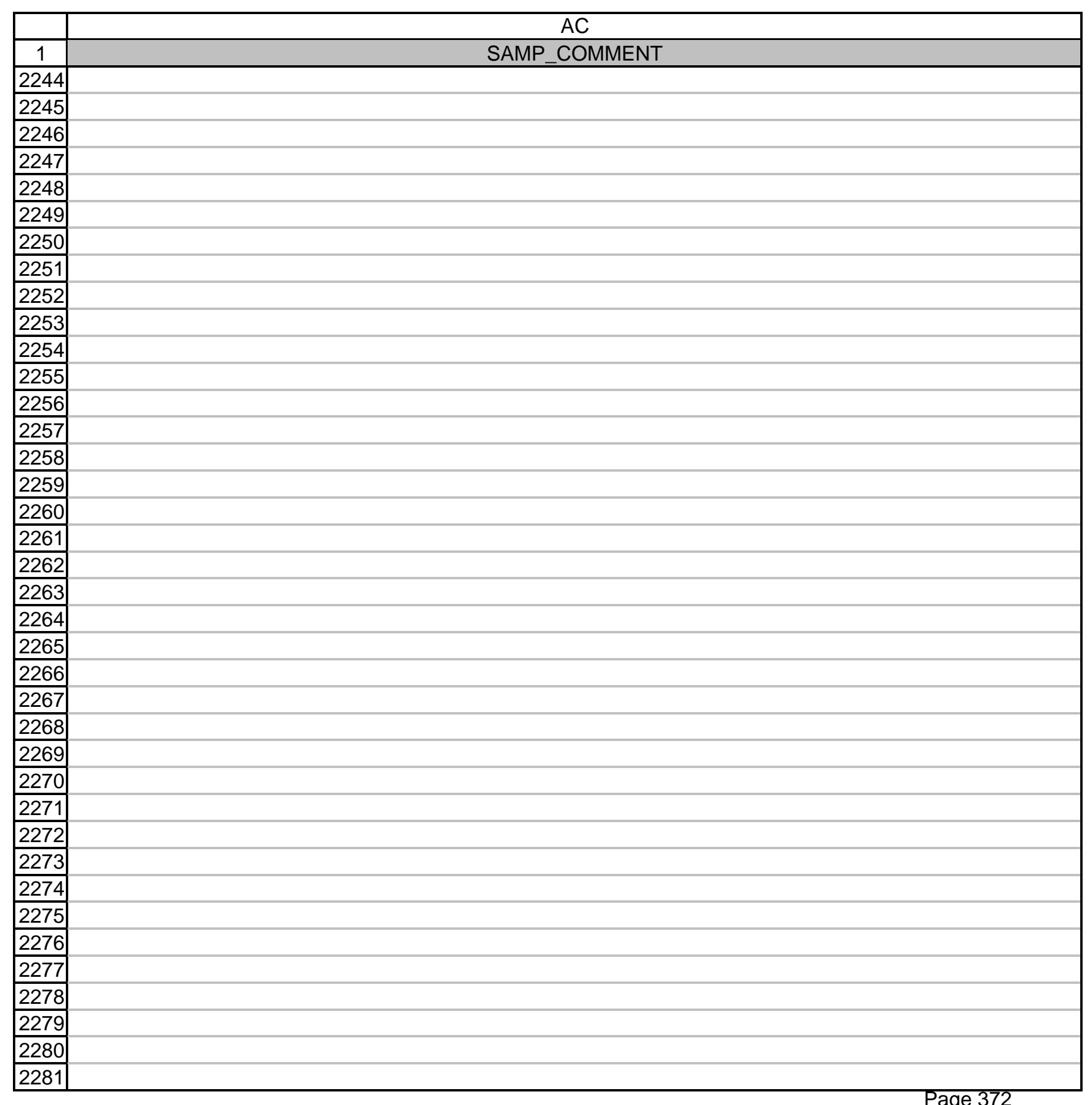




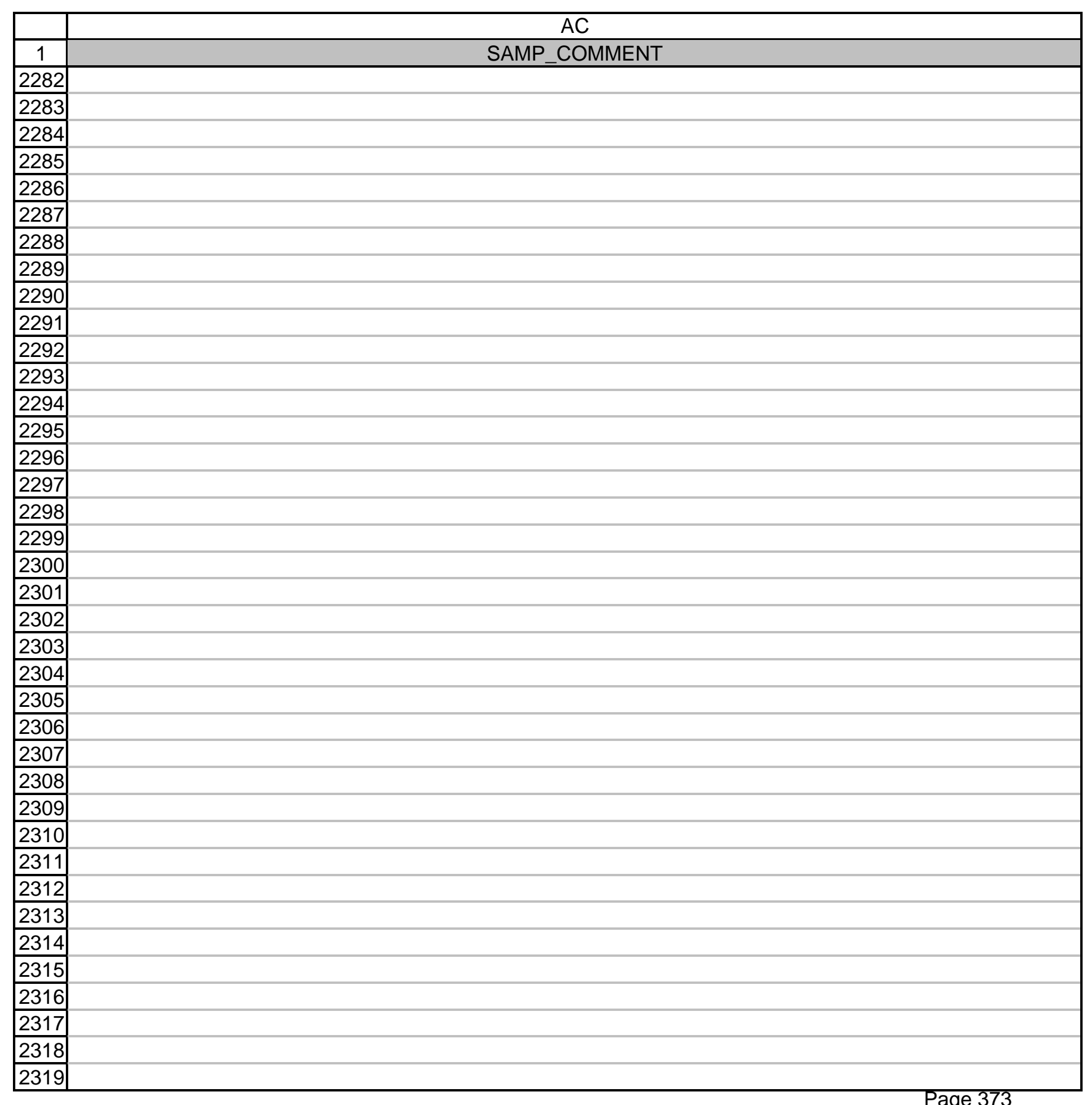




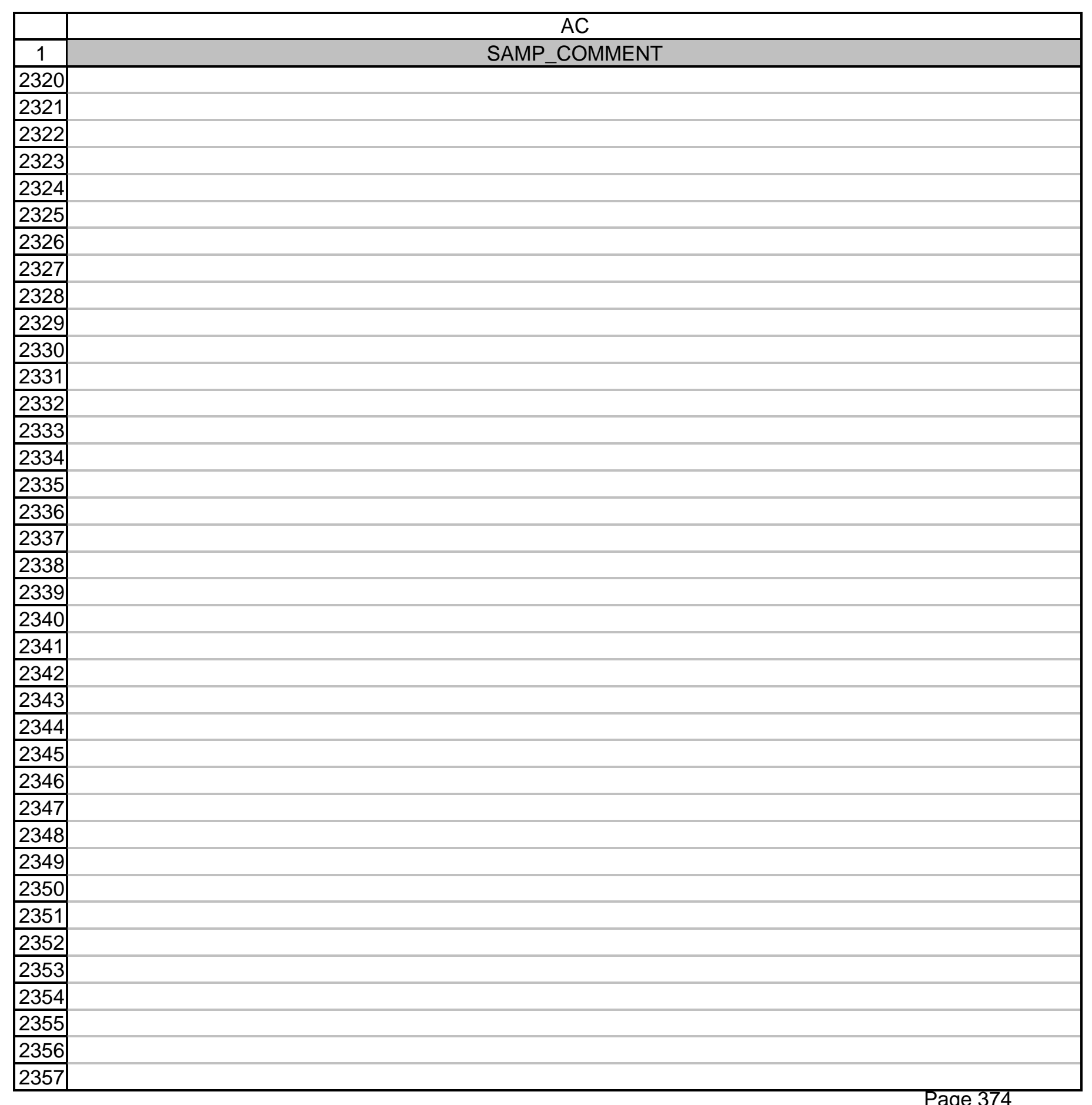




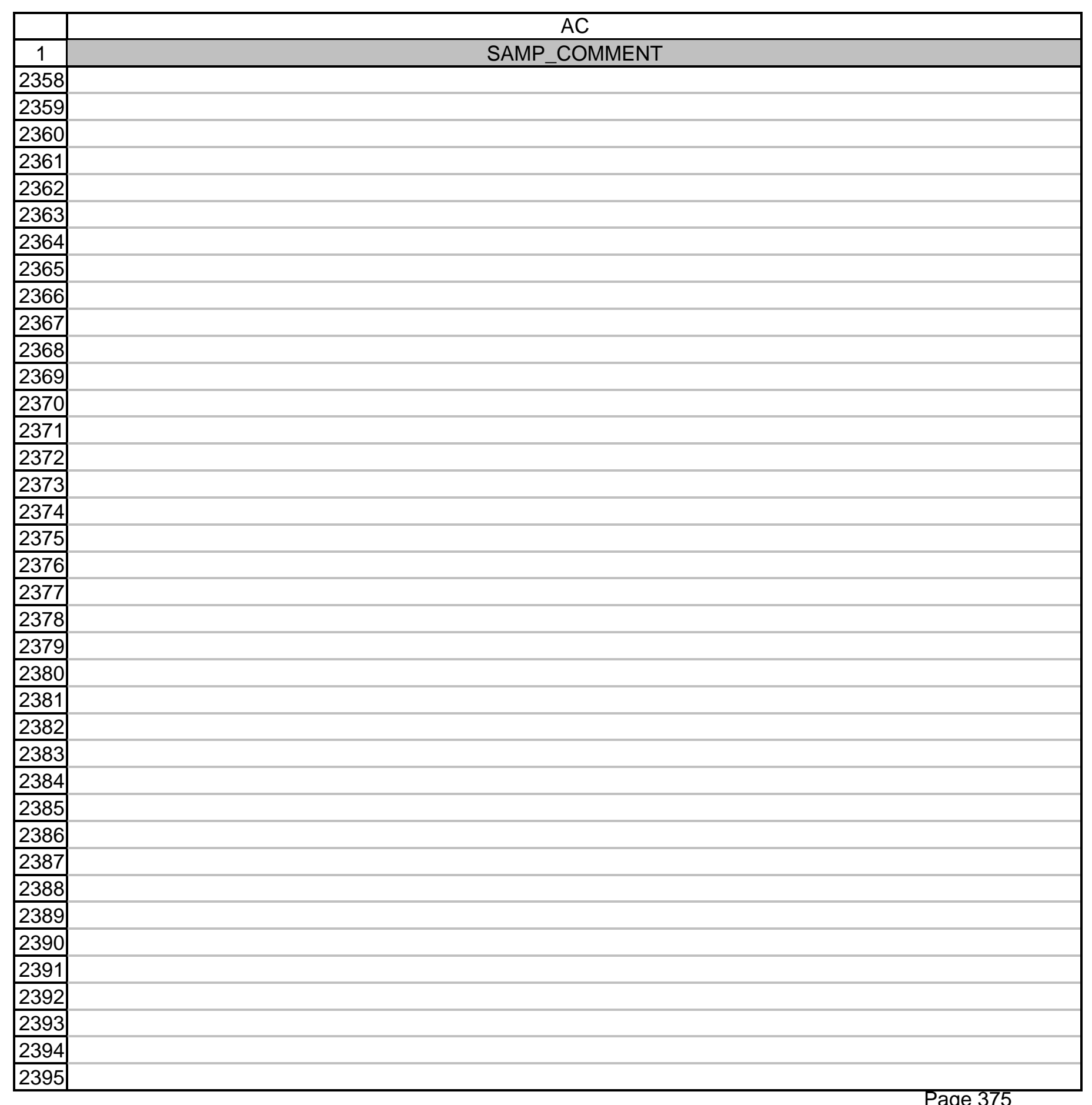




$$
\square
$$




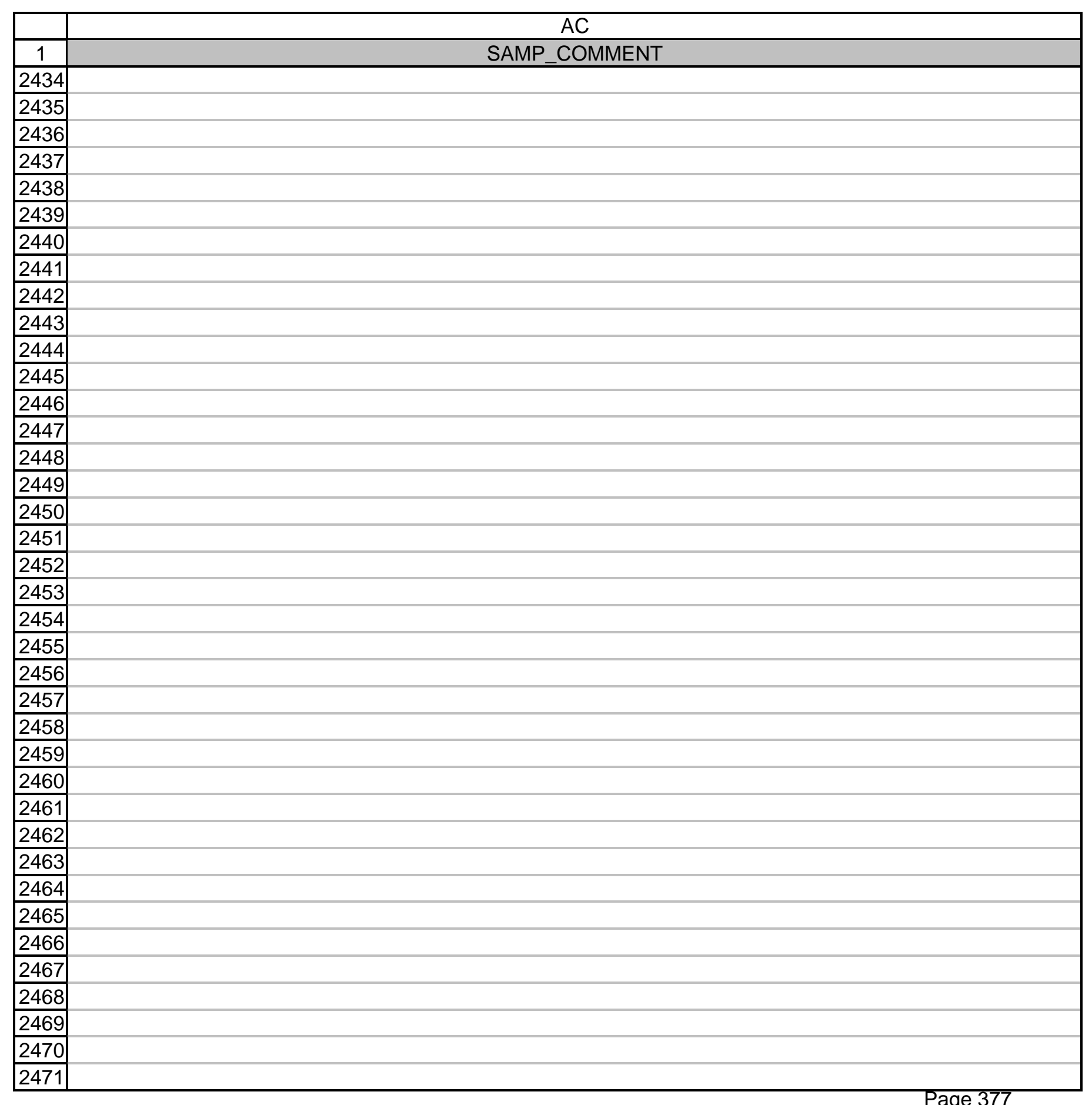




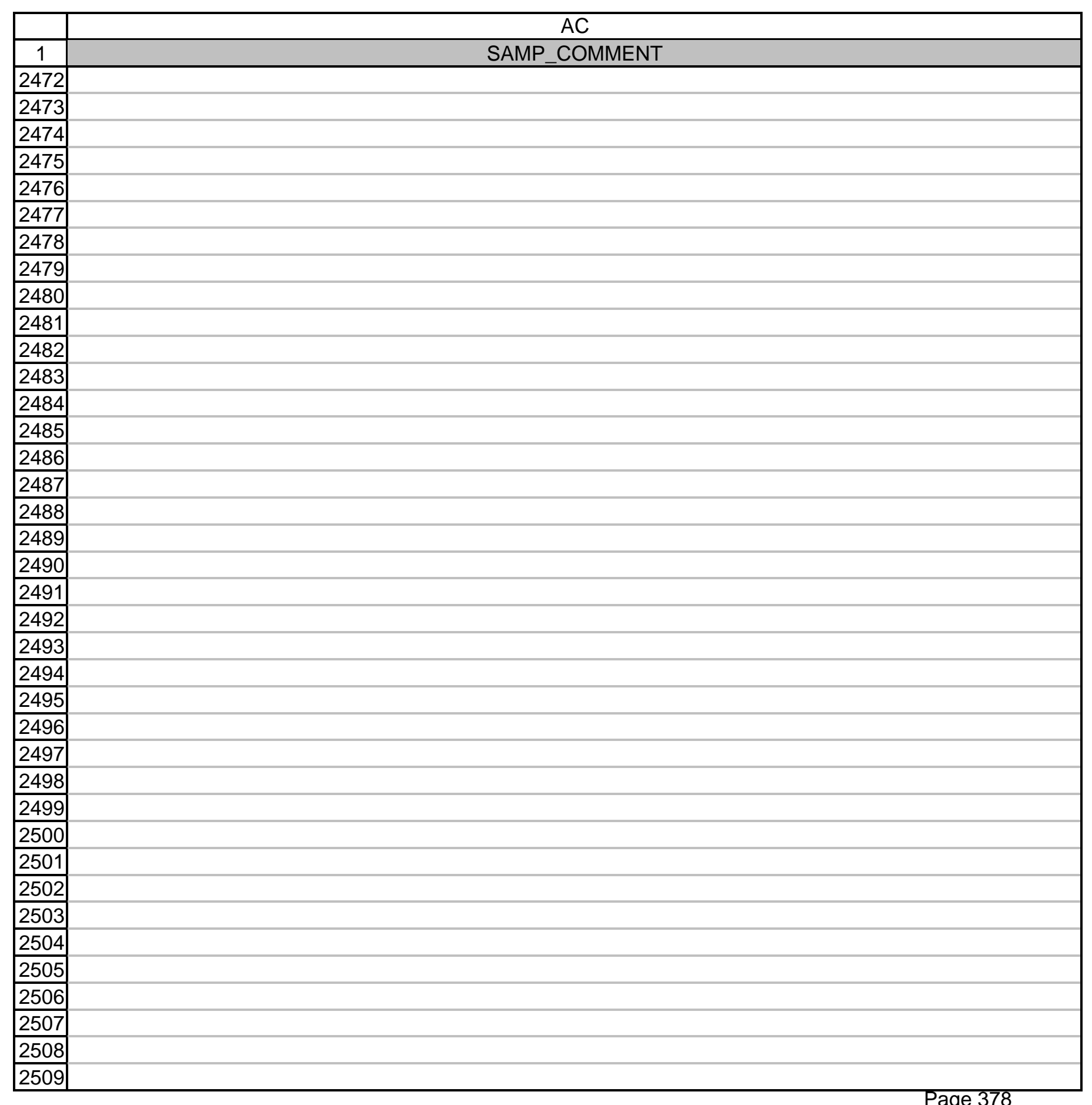




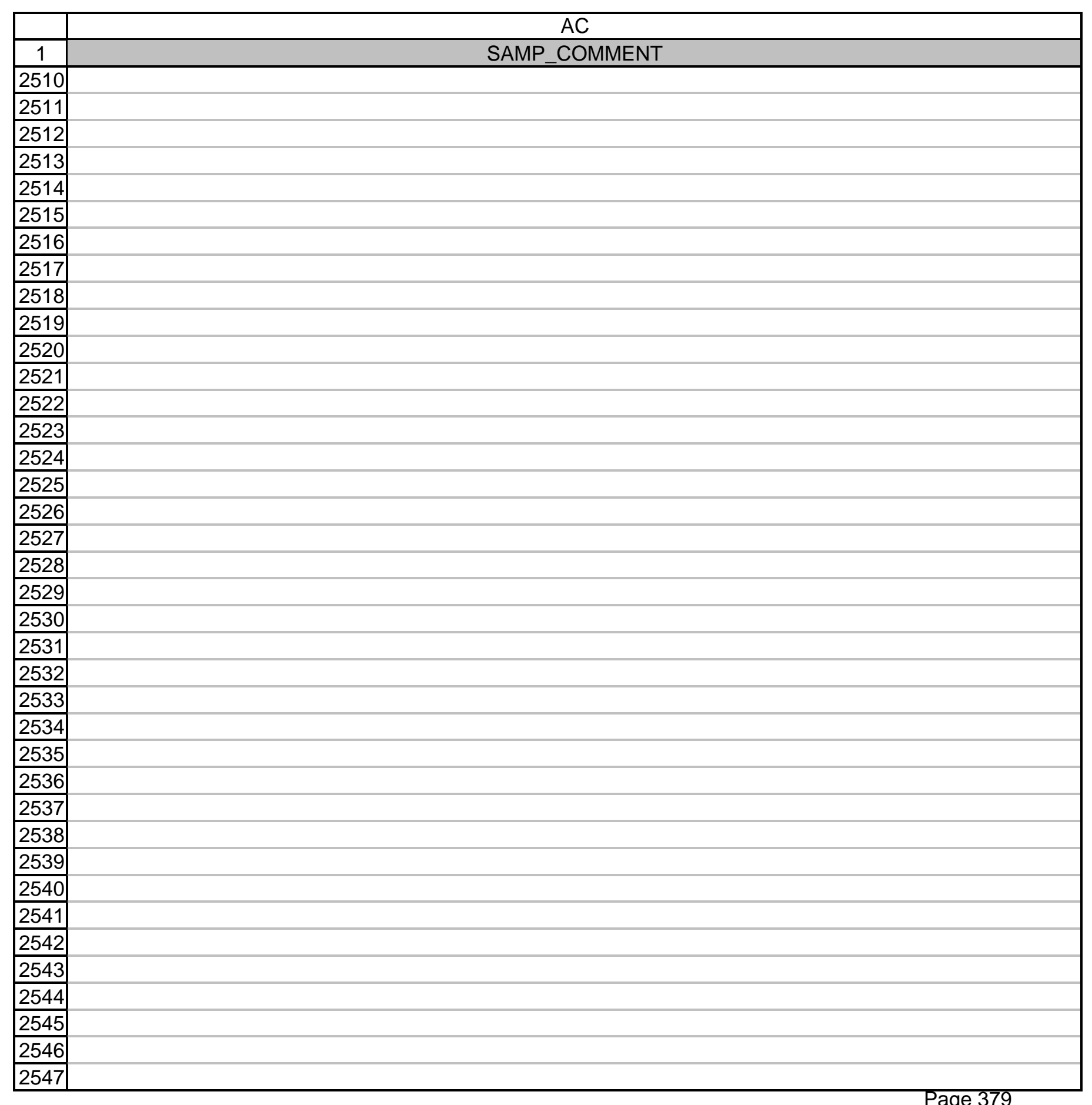




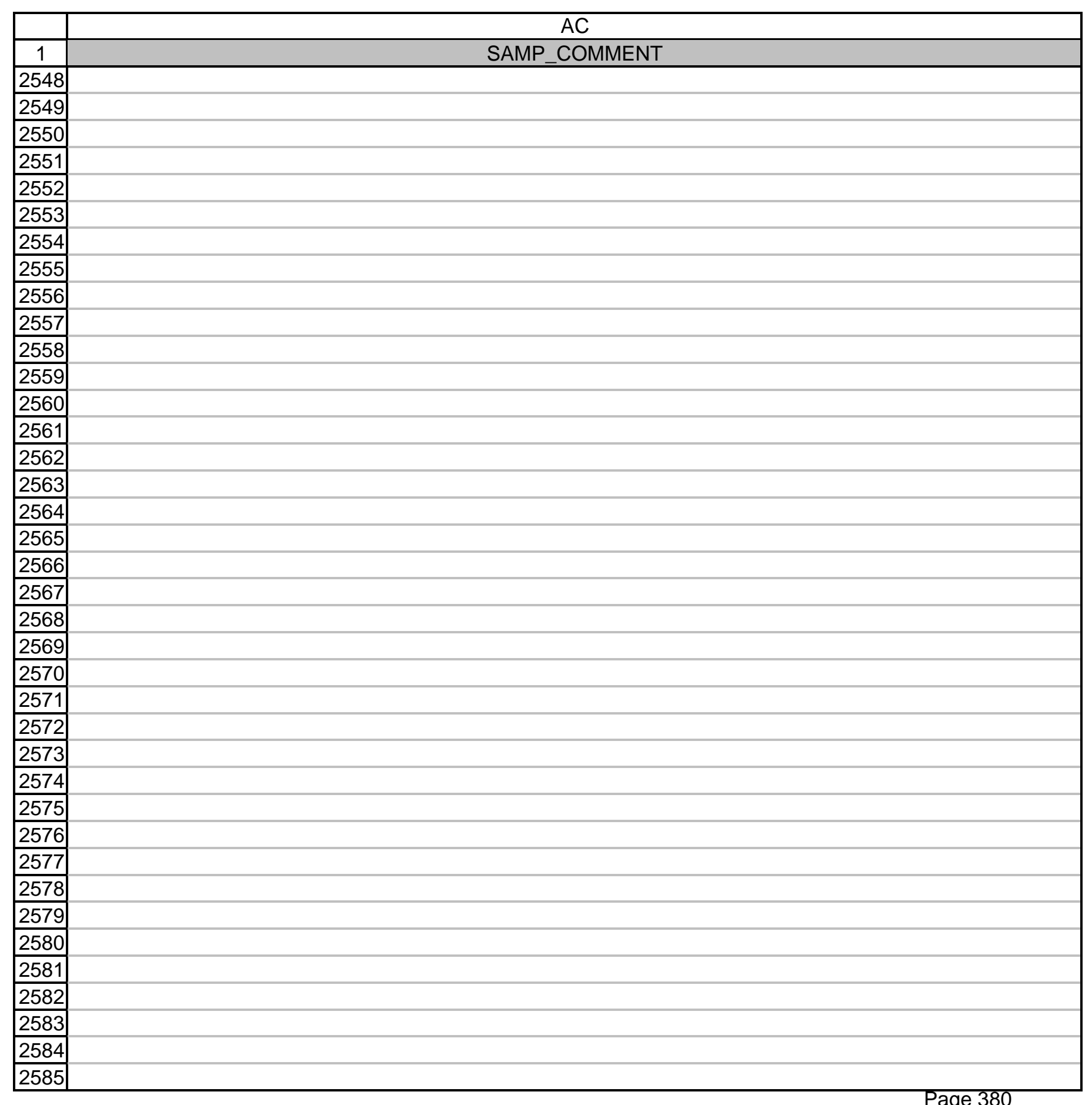




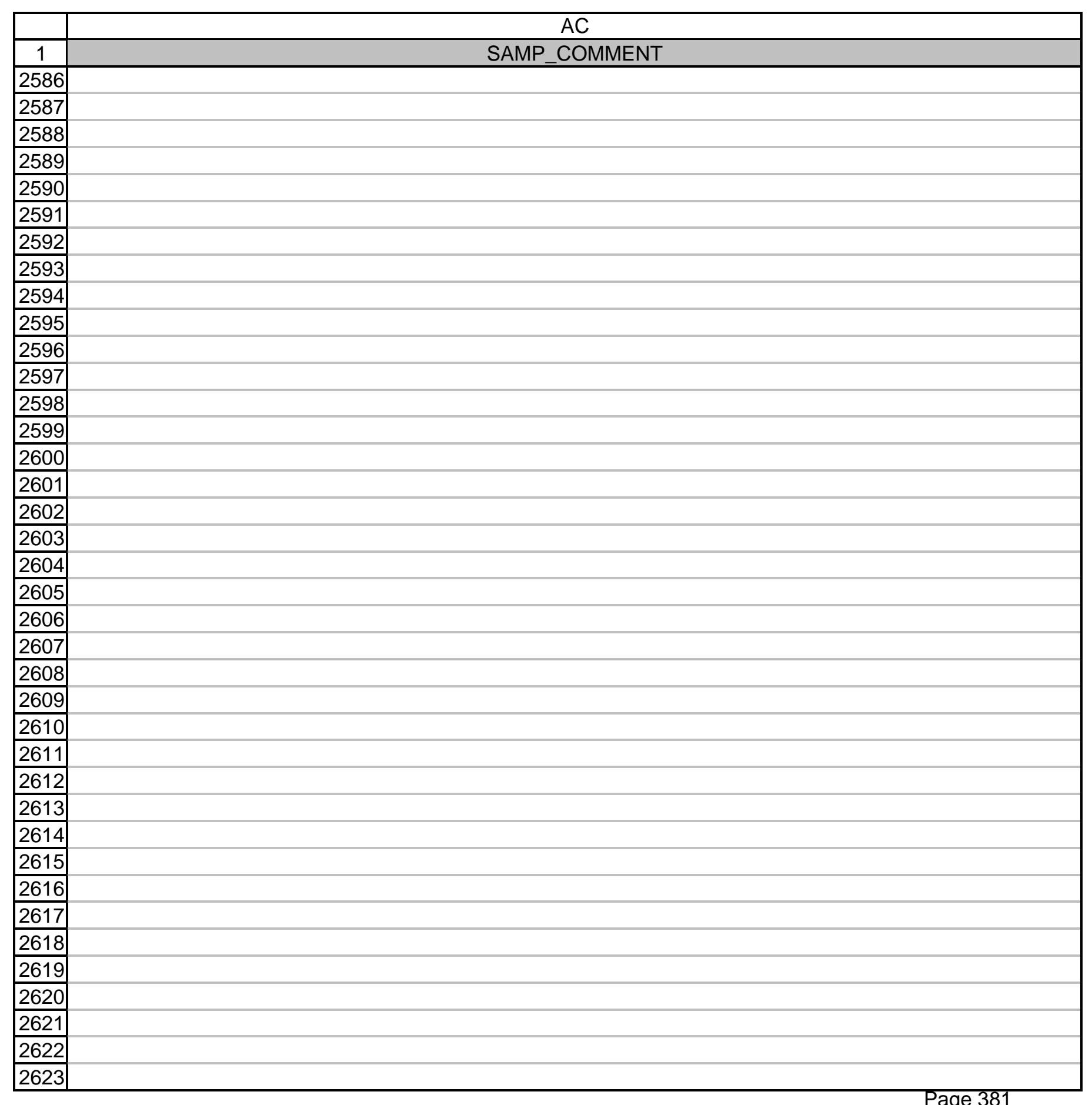




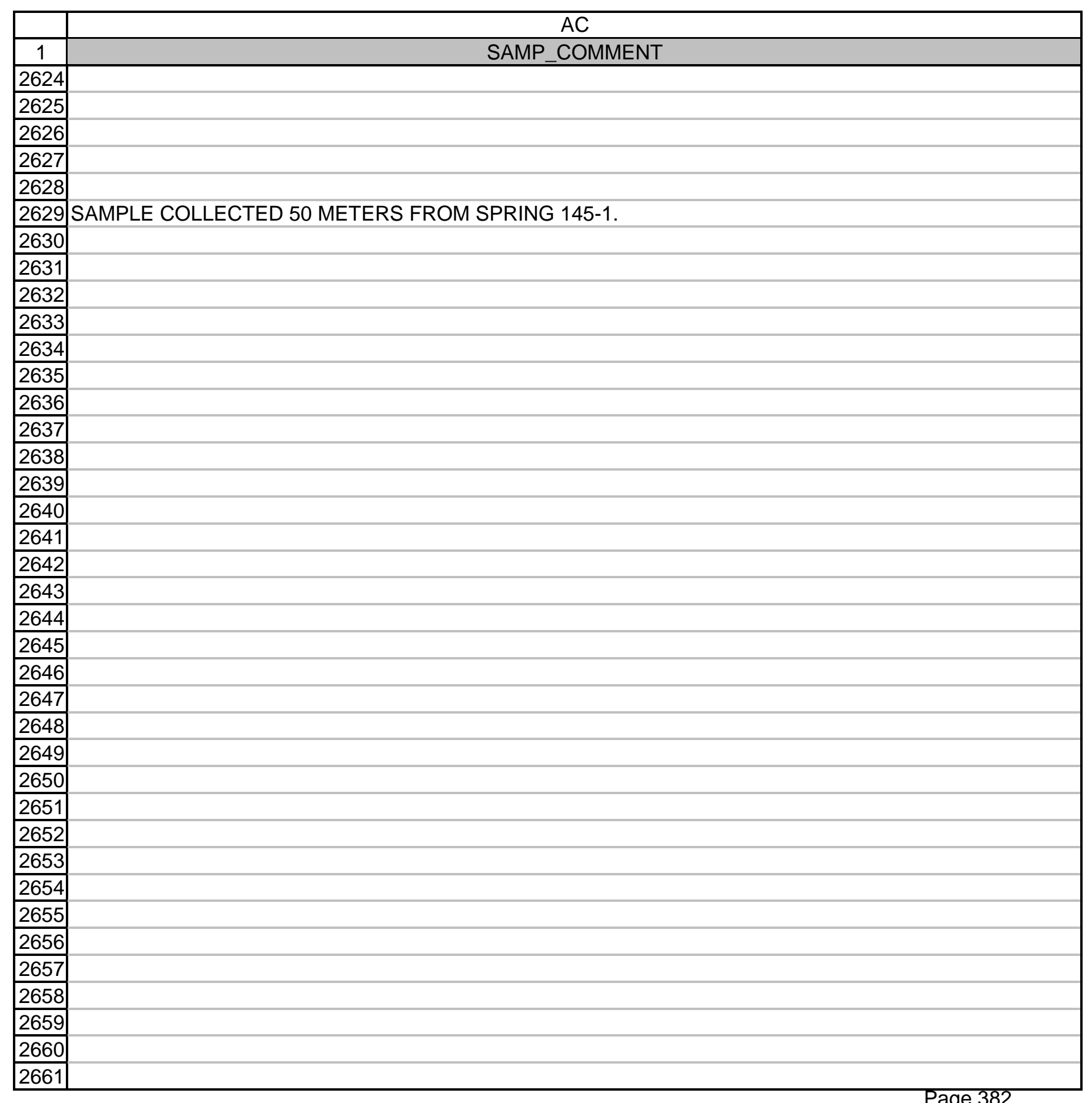


AC

\begin{tabular}{|c|c|}
\hline 1 & SAMP_COMMENT \\
\hline \multicolumn{2}{|l|}{2662} \\
\hline \multicolumn{2}{|l|}{\begin{tabular}{|l|}
2663 \\
\end{tabular}} \\
\hline \multicolumn{2}{|l|}{2664} \\
\hline \multicolumn{2}{|l|}{2665} \\
\hline \multicolumn{2}{|l|}{2666} \\
\hline \multicolumn{2}{|l|}{2667} \\
\hline \multicolumn{2}{|l|}{2668} \\
\hline \multicolumn{2}{|l|}{2669} \\
\hline \multicolumn{2}{|l|}{2670} \\
\hline \multicolumn{2}{|r|}{ 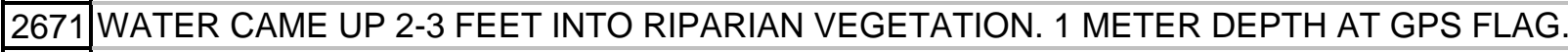 } \\
\hline \multicolumn{2}{|r|}{2672 WATER CAME UP 2-3 FEET INTO RIPARIAN VEGETATION. 1 METER DEPTH AT GPS FLAG } \\
\hline \multirow{2}{*}{\multicolumn{2}{|c|}{\begin{tabular}{|l|l|}
2673 & WATER CAME UP 2-3 FEET INTO RIPARIAN VEGETATION. 1 METER DEPTH AT GPS FLAG \\
2674 &
\end{tabular}}} \\
\hline \multirow{2}{*}{\multicolumn{2}{|c|}{$\frac{2664}{2675}$}} \\
\hline & \\
\hline \multicolumn{2}{|l|}{2676} \\
\hline \multicolumn{2}{|l|}{2677} \\
\hline \multicolumn{2}{|l|}{2678} \\
\hline \multicolumn{2}{|l|}{2679} \\
\hline \multicolumn{2}{|l|}{2680} \\
\hline \multicolumn{2}{|l|}{2681} \\
\hline \multicolumn{2}{|l|}{2682} \\
\hline \multicolumn{2}{|l|}{2683} \\
\hline \multicolumn{2}{|l|}{2684} \\
\hline \multicolumn{2}{|l|}{2685} \\
\hline \multicolumn{2}{|l|}{2686} \\
\hline \multicolumn{2}{|l|}{2687} \\
\hline \multicolumn{2}{|l|}{2688} \\
\hline \multicolumn{2}{|l|}{2689} \\
\hline \multicolumn{2}{|l|}{2690} \\
\hline \multicolumn{2}{|l|}{2691} \\
\hline \begin{tabular}{|l|}
2692 \\
\end{tabular} & \\
\hline \begin{tabular}{|l|}
2693 \\
\end{tabular} & \\
\hline 2694 & \\
\hline \begin{tabular}{|l|}
2695 \\
\end{tabular} & \\
\hline \begin{tabular}{|l|}
2696 \\
\end{tabular} & \\
\hline 2697 & WATER CAME UP 2-3 FEET INTO RIPARIAN VEGETATION. 1 METER DEPTH AT GPS FLAG. \\
\hline \begin{tabular}{|l|}
2698 \\
\end{tabular} & \\
\hline \begin{tabular}{|l|}
2699 \\
\end{tabular} & 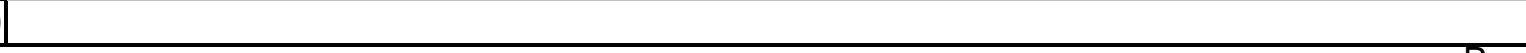 \\
\hline
\end{tabular}




$$
\square
$$




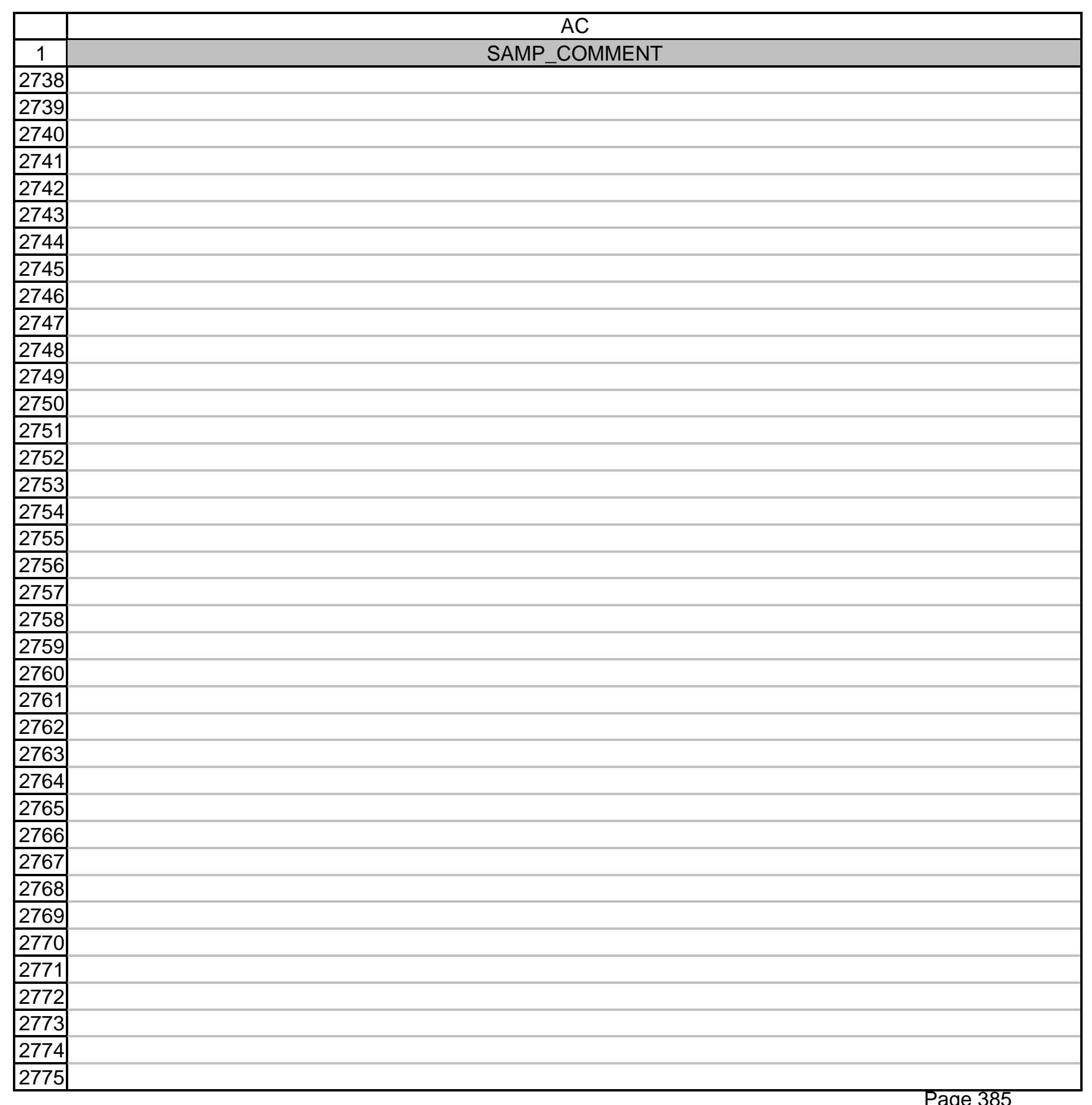




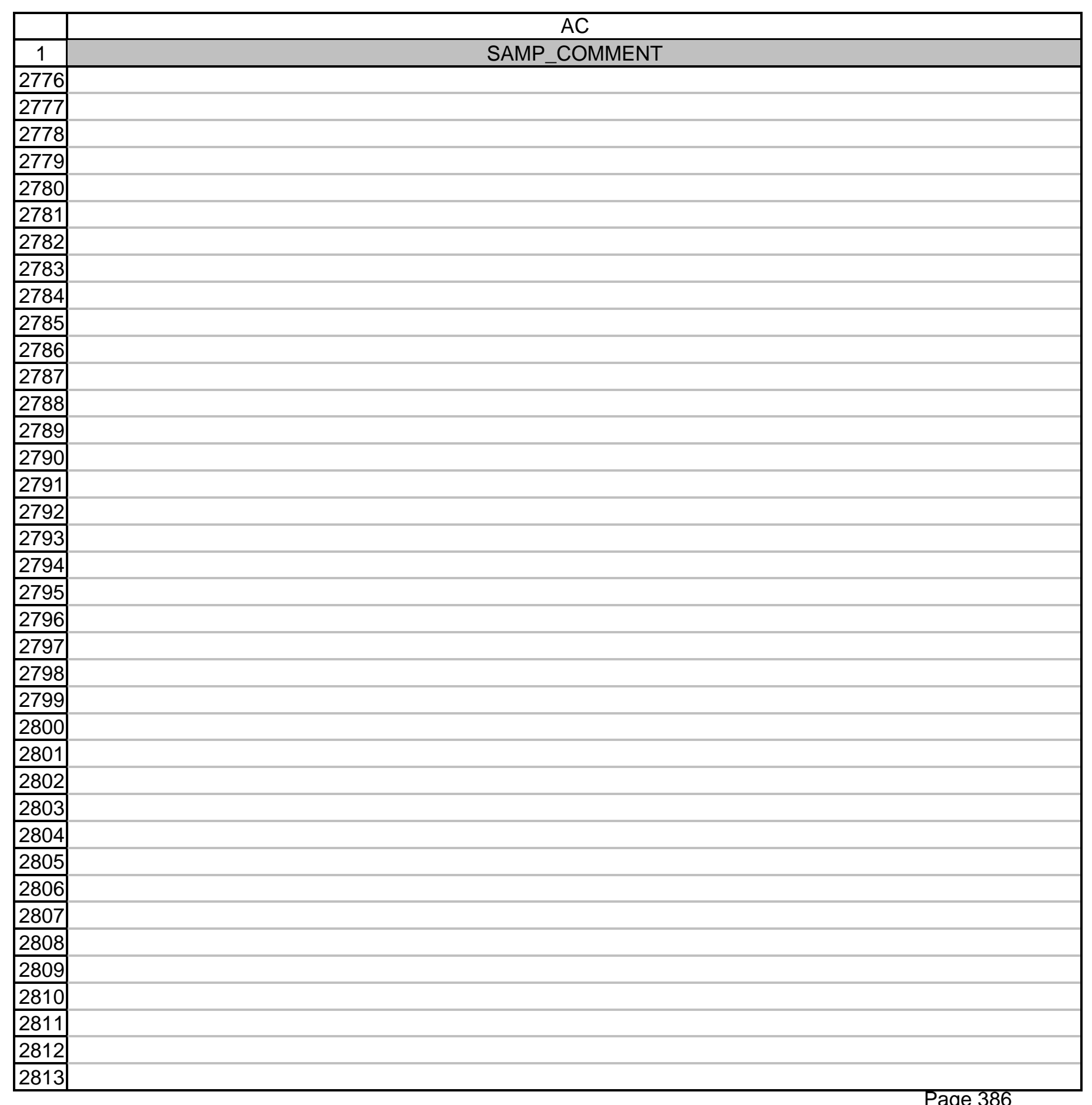




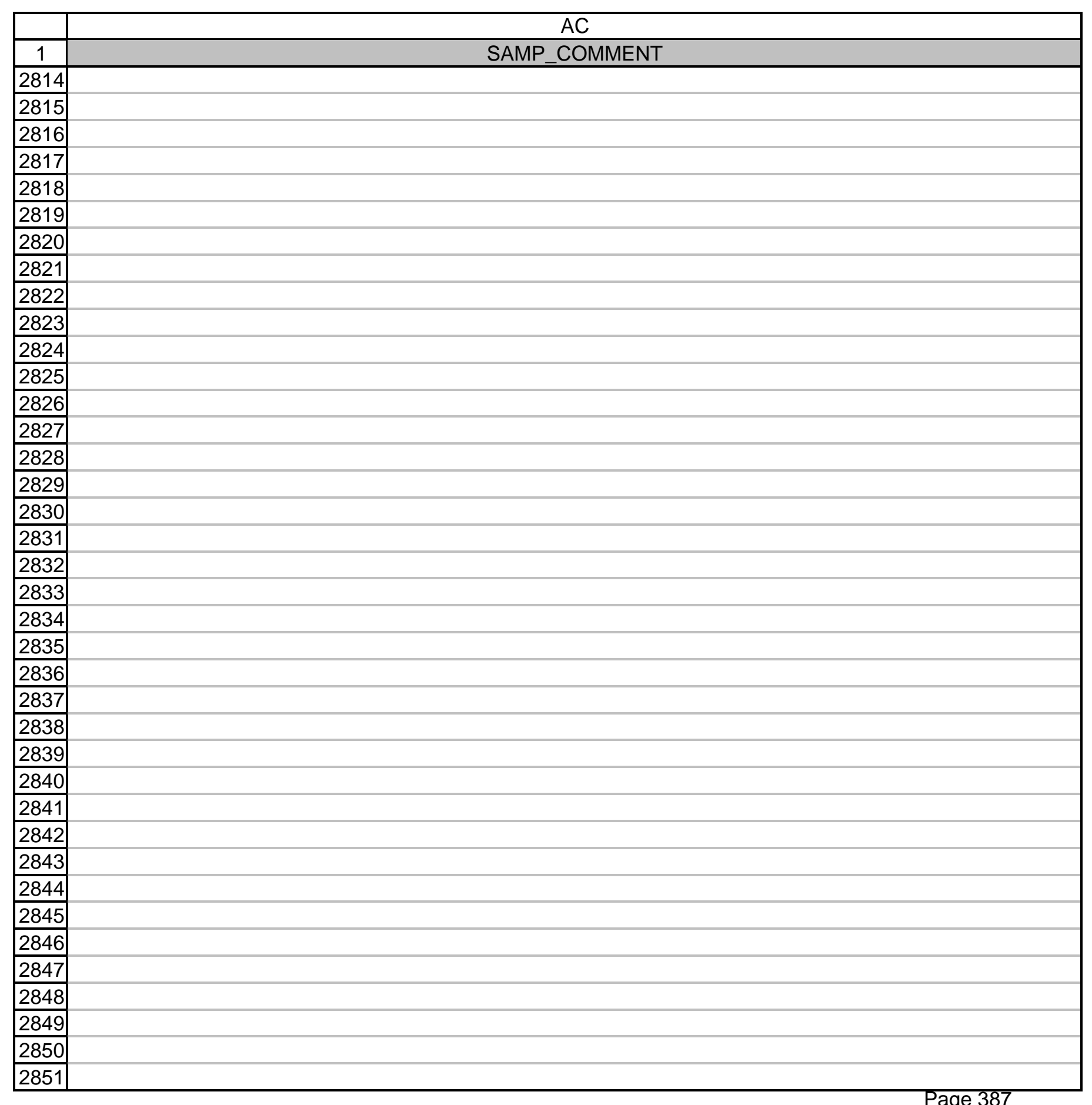




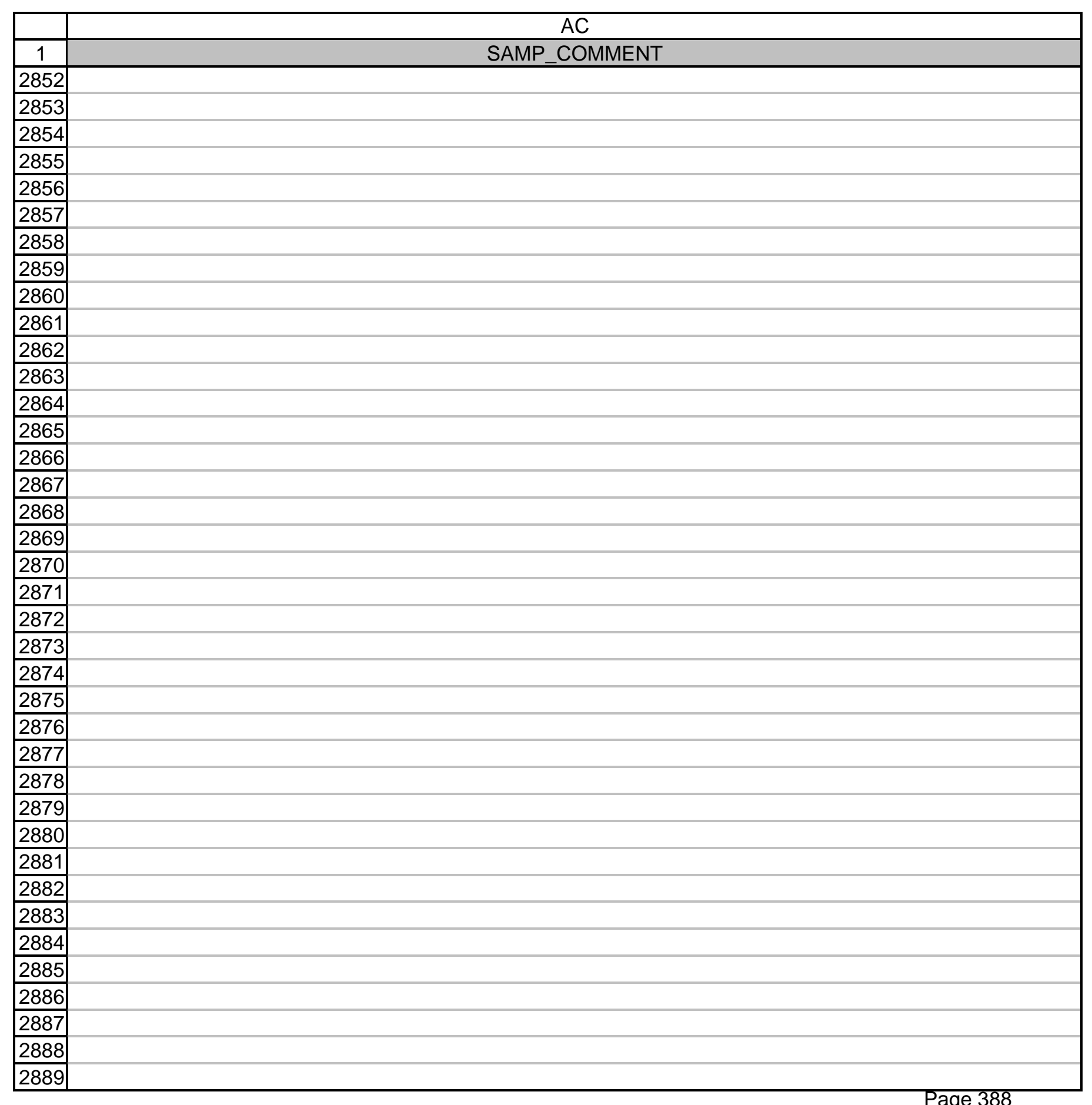




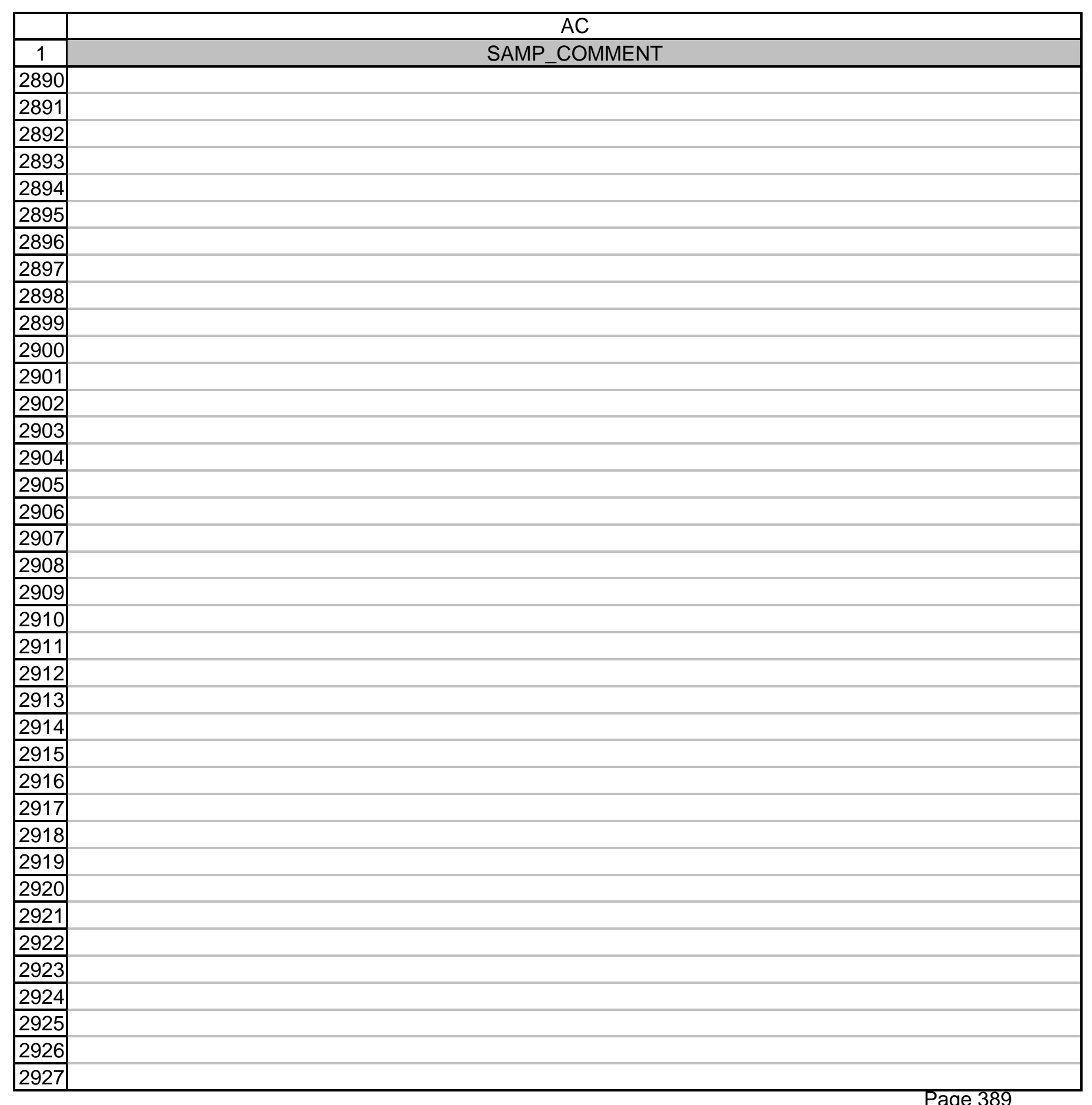


AC 


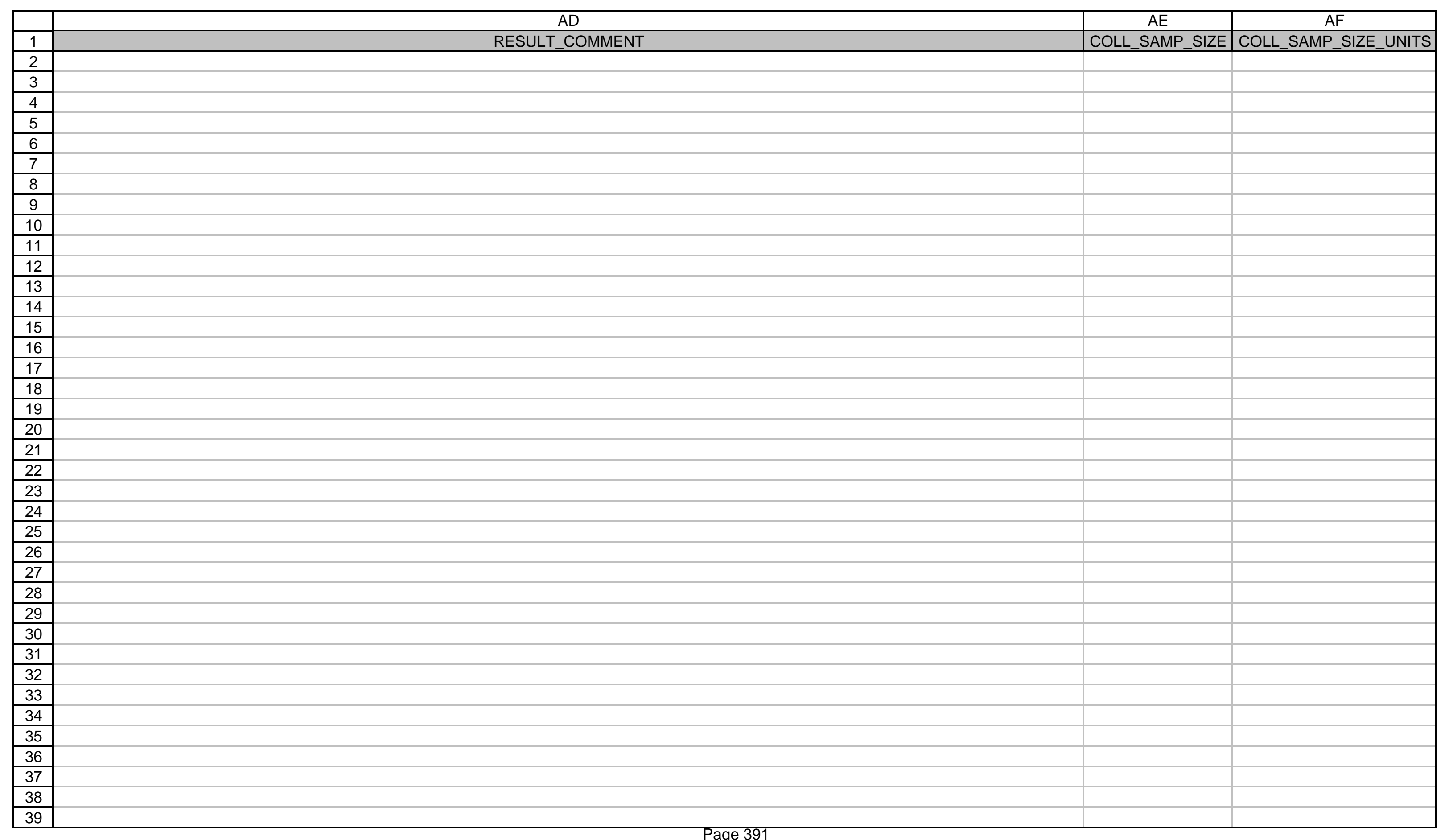




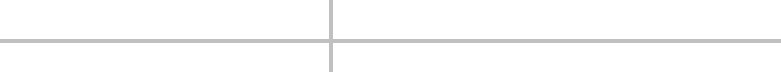




\begin{tabular}{|c|c|c|c|}
\hline & $A D$ & $\mathrm{AE}$ & $\mathrm{AF}$ \\
\hline 1 & RESULT COMMENT & COLL SAMP SIZE & COLL SAMP SIZE UNITS \\
\hline \multicolumn{4}{|l|}{78} \\
\hline \multicolumn{4}{|l|}{79} \\
\hline \multicolumn{4}{|l|}{80} \\
\hline \multicolumn{4}{|l|}{81} \\
\hline \multicolumn{4}{|l|}{82} \\
\hline \multicolumn{4}{|l|}{83} \\
\hline \multicolumn{4}{|l|}{84} \\
\hline \multicolumn{4}{|l|}{85} \\
\hline 87 & & 10 & $\mathrm{~L}$ \\
\hline 88 & & 10 & L \\
\hline \multicolumn{4}{|l|}{89} \\
\hline \multicolumn{4}{|l|}{90} \\
\hline \multicolumn{4}{|l|}{91} \\
\hline \multicolumn{4}{|l|}{92} \\
\hline \multicolumn{4}{|l|}{93} \\
\hline \multicolumn{4}{|l|}{94} \\
\hline \multicolumn{4}{|l|}{95} \\
\hline 97 & \# IR-0051; LCS RECOVERY BELOW LIMIT. & 19 & L \\
\hline \multicolumn{4}{|l|}{98} \\
\hline \multicolumn{4}{|l|}{99} \\
\hline \multicolumn{4}{|l|}{100} \\
\hline \multicolumn{4}{|l|}{101} \\
\hline 102 & & 19 & L \\
\hline 103 & & 4 & L \\
\hline 104 & & 3 & L \\
\hline \multicolumn{4}{|l|}{$\frac{105}{105}$} \\
\hline \multicolumn{4}{|l|}{106} \\
\hline \multicolumn{4}{|l|}{107} \\
\hline \multicolumn{4}{|l|}{108} \\
\hline 109 & \#IR-0054 THE ACHIEVED MDA DOES NOT MEET THE CRDL & 10 & L \\
\hline 110 & & 10 & L \\
\hline 111 & & 19 & L \\
\hline 112 & & & \\
\hline 113 & & & \\
\hline 114 & & & \\
\hline
\end{tabular}




\begin{tabular}{|c|c|c|c|}
\hline & 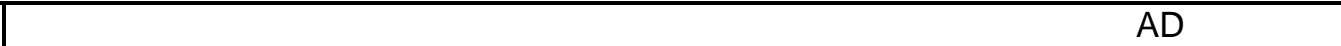 & $\mathrm{AE}$ & $\mathrm{AF}$ \\
\hline 1 & $\begin{array}{lr} & \text { RESULT_COMMENT } \\
\end{array}$ & COLL_SAMP_SIZE & COLL_SAMP_SIZE_UNITS \\
\hline \multicolumn{4}{|c|}{ (2) } \\
\hline \multicolumn{4}{|l|}{117} \\
\hline \multicolumn{4}{|l|}{118} \\
\hline \multicolumn{4}{|l|}{119} \\
\hline 120 & & 8 & $\mathrm{~L}$ \\
\hline 121 & & 14 & L \\
\hline 122 & & 8 & L \\
\hline 123 & & 8 & L \\
\hline 124 & & 14 & L \\
\hline 125 & & 16 & L \\
\hline \multicolumn{4}{|l|}{126} \\
\hline \multicolumn{4}{|l|}{127} \\
\hline \multicolumn{4}{|l|}{128} \\
\hline \multicolumn{4}{|l|}{129} \\
\hline 130 & & 9 & L \\
\hline 131 & & 4 & L \\
\hline 132 & REFER TO FOLLOW-UP ANALYSIS ORDER \#W053. & 13 & L \\
\hline 133 & & 13 & L \\
\hline 134 & & 12 & L \\
\hline 135 & & 12 & L \\
\hline 136 & & 16 & L \\
\hline 137 & & 12 & L \\
\hline 138 & & 16 & L \\
\hline 139 & & 16 & L \\
\hline \multicolumn{4}{|l|}{140} \\
\hline \multicolumn{4}{|l|}{141} \\
\hline \multicolumn{4}{|c|}{142} \\
\hline \multicolumn{4}{|l|}{143} \\
\hline 144 & & 16 & L \\
\hline \multicolumn{4}{|l|}{145} \\
\hline \multicolumn{4}{|l|}{146} \\
\hline \multicolumn{4}{|l|}{147} \\
\hline \multicolumn{4}{|l|}{148} \\
\hline \multicolumn{4}{|l|}{149} \\
\hline \multicolumn{4}{|c|}{150} \\
\hline \multicolumn{4}{|l|}{151} \\
\hline \multicolumn{4}{|l|}{152} \\
\hline 153 & & & \\
\hline
\end{tabular}




\begin{tabular}{|c|c|c|c|}
\hline & $A D$ & $\mathrm{AE}$ & $\mathrm{AF}$ \\
\hline 1 & RESULT_COMMENT & COLL_SAMP_SIZE & COLL_SAMP_SIZE_UNITS \\
\hline \multicolumn{4}{|r|}{ 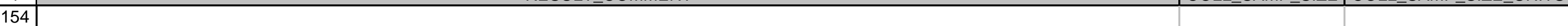 } \\
\hline \multicolumn{4}{|l|}{155} \\
\hline \multicolumn{4}{|l|}{156} \\
\hline 157 & IR-0104; MDA > CRDL & 9 & $\mathrm{~L}$ \\
\hline 158 & & 4 & $\mathrm{~L}$ \\
\hline \multicolumn{4}{|l|}{159} \\
\hline \multicolumn{4}{|l|}{160} \\
\hline \multicolumn{4}{|l|}{161} \\
\hline \multicolumn{4}{|l|}{162} \\
\hline 163 & \#IR-0115 The achieved MDA does not meet the CRDL & 9 & $\mathrm{~L}$ \\
\hline 164 & & 4 & $L$ \\
\hline 165 & IR-0115; MDA > CRDL & 4 & $\mathrm{~L}$ \\
\hline \multicolumn{4}{|l|}{166} \\
\hline \multicolumn{4}{|l|}{167} \\
\hline \multicolumn{4}{|l|}{168} \\
\hline \multicolumn{4}{|l|}{169} \\
\hline 170 & & 8 & $\mathrm{~L}$ \\
\hline 171 & & 16 & $\mathrm{~L}$ \\
\hline 172 & & 12 & $\mathrm{~L}$ \\
\hline 173 & & 8 & $\mathrm{~L}$ \\
\hline 174 & & 16 & $\mathrm{~L}$ \\
\hline \multicolumn{4}{|l|}{175} \\
\hline \multicolumn{4}{|l|}{176} \\
\hline \multicolumn{4}{|l|}{177} \\
\hline \multicolumn{4}{|l|}{178} \\
\hline 179 & & 12 & $\mathrm{~L}$ \\
\hline 180 & & 12 & $\mathrm{~L}$ \\
\hline 181 & & 16 & $\mathrm{~L}$ \\
\hline 182 & & 12 & $\mathrm{~L}$ \\
\hline$\frac{1}{183}$ & & 12 & $L$ \\
\hline 184 & & 15 & $\mathrm{~L}$ \\
\hline 185 & & 15 & $\bar{L}$ \\
\hline 186 & & 16 & $\mathrm{~L}$ \\
\hline \multicolumn{4}{|l|}{187} \\
\hline \multicolumn{4}{|l|}{188} \\
\hline \multicolumn{4}{|l|}{189} \\
\hline \multicolumn{4}{|l|}{190} \\
\hline 191 & 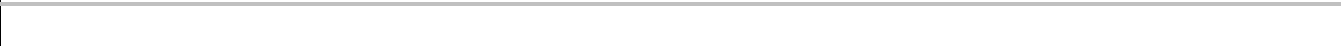 & 15 & $\mathrm{~L}$ \\
\hline
\end{tabular}




\begin{tabular}{|c|c|c|c|}
\hline & - the & $\mathrm{AE}$ & $\mathrm{AF}$ \\
\hline 1 & RESULT_COMMENT & COLL_SAMP_SIZE & COLL_SAMP_SIZE_UNITS \\
\hline 192 & & 15 & L \\
\hline 193 & & & \\
\hline 194 & & & \\
\hline 195 & & & \\
\hline 196 & & & \\
\hline 197 & IR-0143; MDA > CRDL & 10 & $\mathrm{~L}$ \\
\hline 198 & & 4 & $\mathrm{~L}$ \\
\hline 199 & & 15 & $\mathrm{~L}$ \\
\hline 200 & & 15 & $\mathrm{~L}$ \\
\hline 201 & & & \\
\hline 202 & & & \\
\hline 203 & & & \\
\hline 204 & & & \\
\hline 205 & & 15 & $\mathrm{~L}$ \\
\hline 206 & & 15 & $\mathrm{~L}$ \\
\hline 207 & & 5 & L \\
\hline 208 & & 3 & $\mathrm{~L}$ \\
\hline 209 & & 3 & $\mathrm{~L}$ \\
\hline 210 & & 3 & L \\
\hline 211 & & 3 & L \\
\hline 212 & & 3 & L \\
\hline 213 & & 3 & $\mathrm{~L}$ \\
\hline 214 & & 3 & $\mathrm{~L}$ \\
\hline 215 & & 3 & L \\
\hline 216 & & 3 & L \\
\hline 217 & & 3 & L \\
\hline 218 & & 3 & $\mathrm{~L}$ \\
\hline 219 & & 3 & $\mathrm{~L}$ \\
\hline 220 & & 3 & $L$ \\
\hline 221 & & 3 & $\mathrm{~L}$ \\
\hline 222 & & 3 & L \\
\hline 223 & & 3 & L \\
\hline 224 & & 3 & L \\
\hline 225 & & 3 & $\mathrm{~L}$ \\
\hline 226 & & 3 & L \\
\hline 227 & & 3 & L \\
\hline 228 & & 3 & L \\
\hline 229 & & 3 & $\mathrm{~L}$ \\
\hline
\end{tabular}




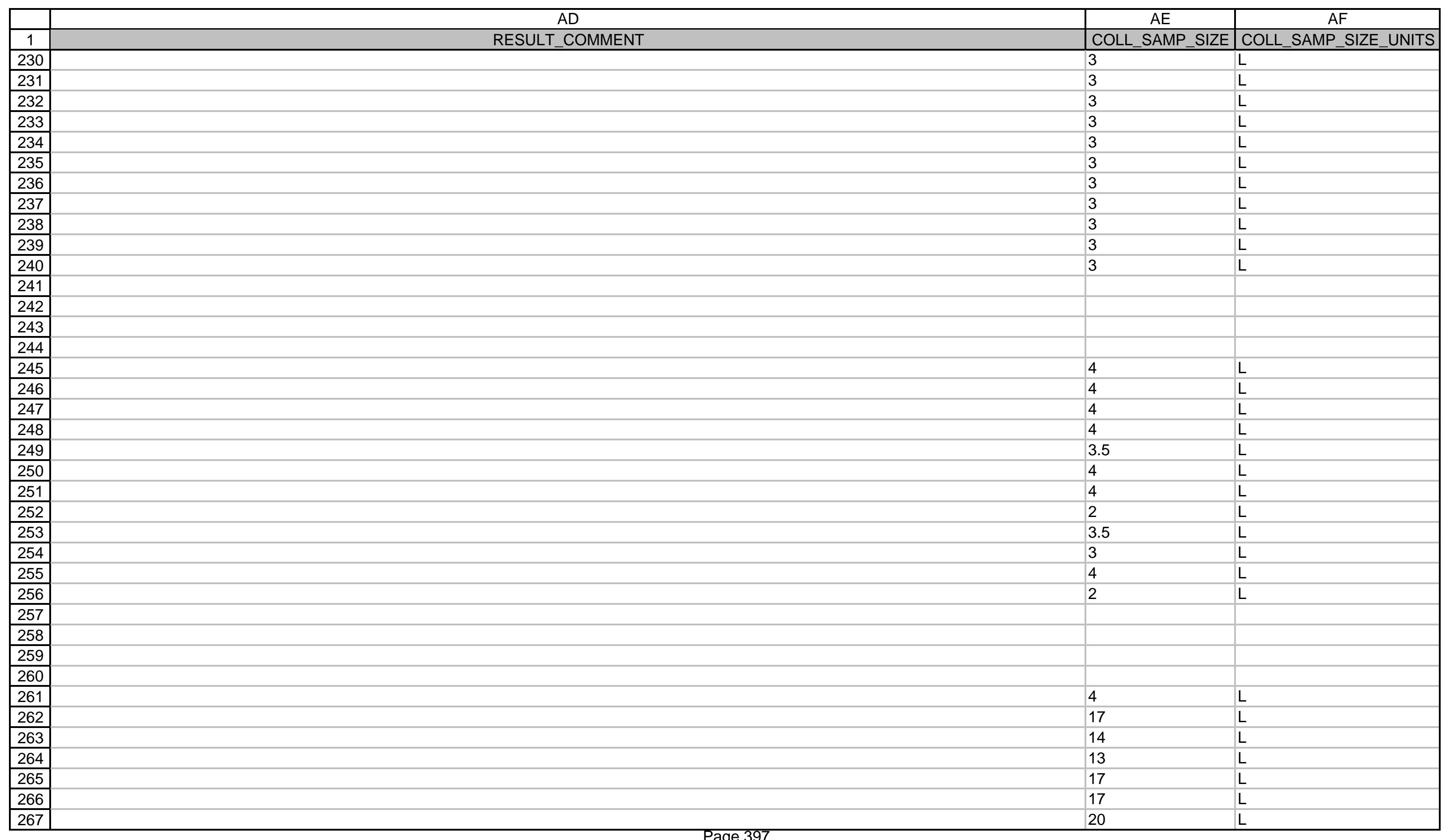




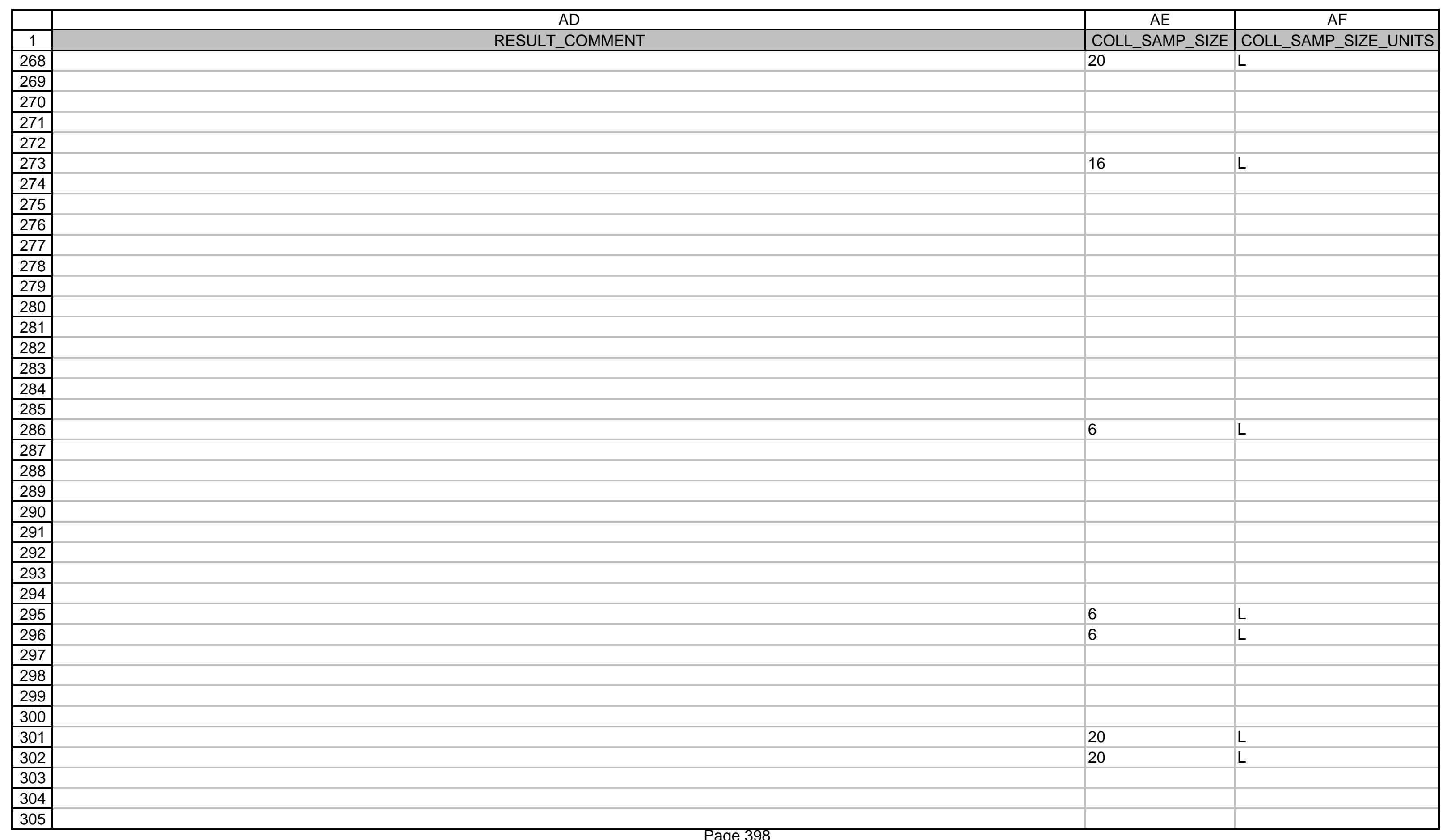




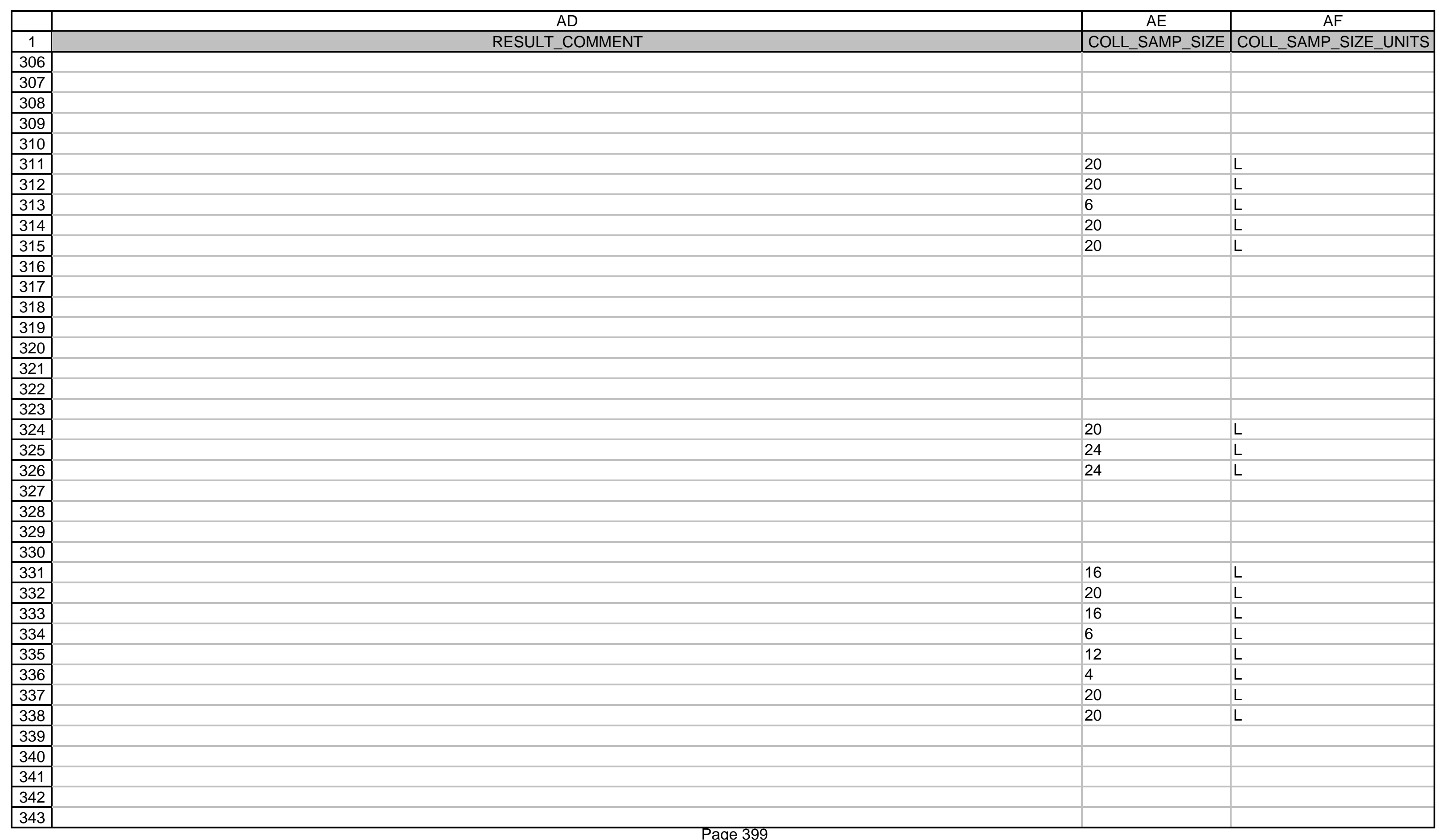




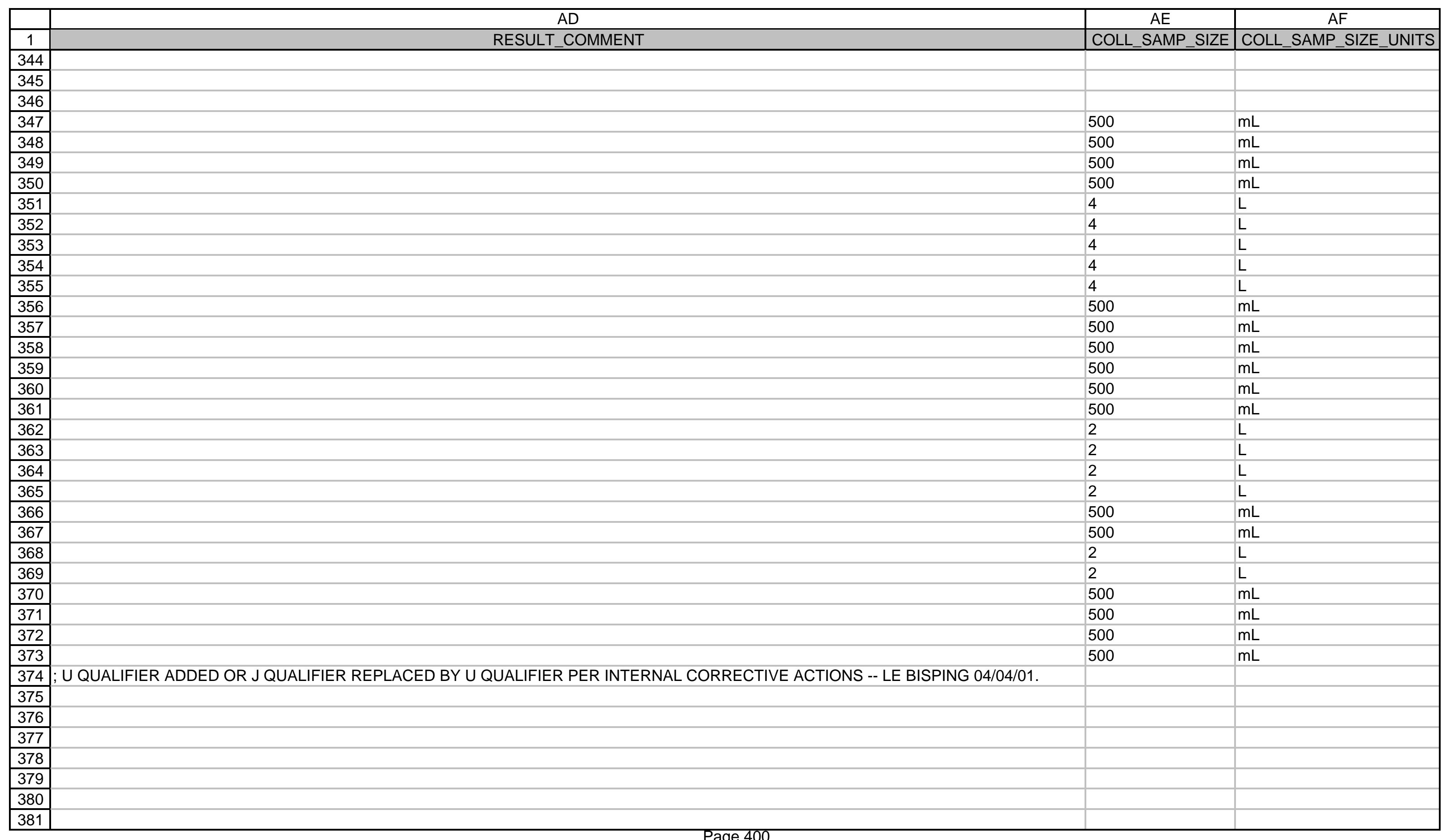




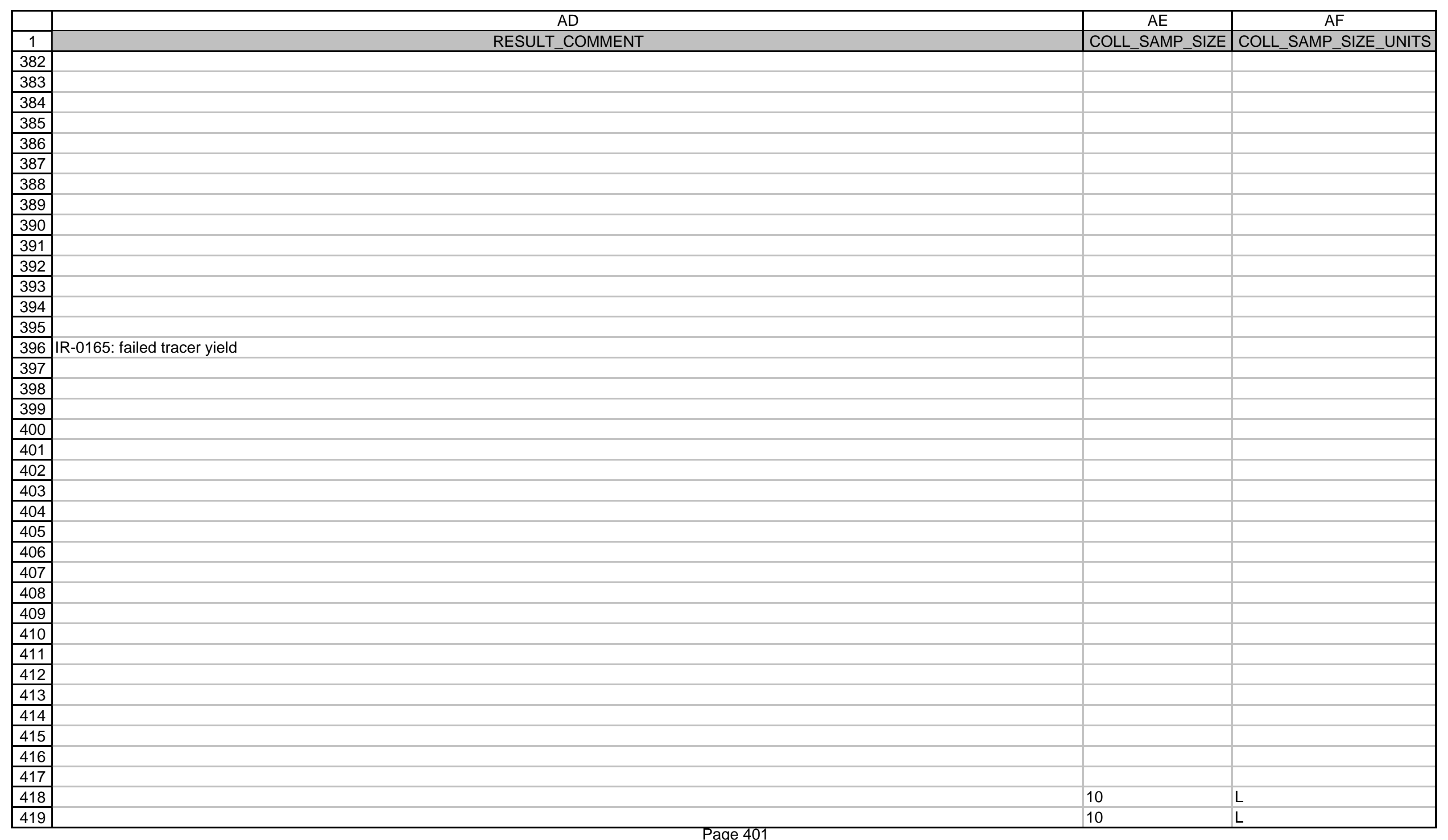




\begin{tabular}{|c|c|c|c|}
\hline & 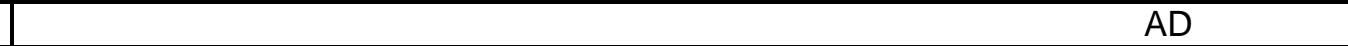 & $\mathrm{AE}$ & $\mathrm{AF}$ \\
\hline 1 & \begin{tabular}{|r|r|} 
& RESULT_COMMENT \\
\end{tabular} & COLL_SAMP_SIZE & COLL_SAMP_SIZE_UNITS \\
\hline 420 & & 19 & $\mathrm{~L}$ \\
\hline 421 & & 19 & L \\
\hline 422 & & 19 & L \\
\hline 423 & & 19 & L \\
\hline 424 & . & 10 & L \\
\hline 425 & t & 10 & $\mathrm{~L}$ \\
\hline 426 & . & 20 & L \\
\hline 427 & & 20 & L \\
\hline 428 & & 20 & L \\
\hline 429 & & 20 & L \\
\hline 430 & & 20 & L \\
\hline 431 & & 20 & L \\
\hline 432 & & 20 & L \\
\hline 433 & & 20 & L \\
\hline 434 & & 20 & L \\
\hline 435 & & 20 & L \\
\hline 436 & & 20 & L \\
\hline 437 & & 20 & L \\
\hline 439 & & 20 & L \\
\hline 440 & & 20 & L \\
\hline 441 & & 10 & L \\
\hline 442 & & 10 & L \\
\hline 443 & & 19 & L \\
\hline 444 & & 19 & L \\
\hline 445 & & 19 & L \\
\hline 446 & & 19 & $\mathrm{~L}$ \\
\hline 447 & & 10 & $\mathrm{~L}$ \\
\hline 448 & & 10 & L \\
\hline 449 & & 19 & L \\
\hline 450 & & 10 & L \\
\hline 451 & & 10 & L \\
\hline 452 & & 19 & L \\
\hline 453 & & 20 & L \\
\hline 454 & & 20 & L \\
\hline 455 & & 20 & L \\
\hline 456 & & 20 & L \\
\hline
\end{tabular}




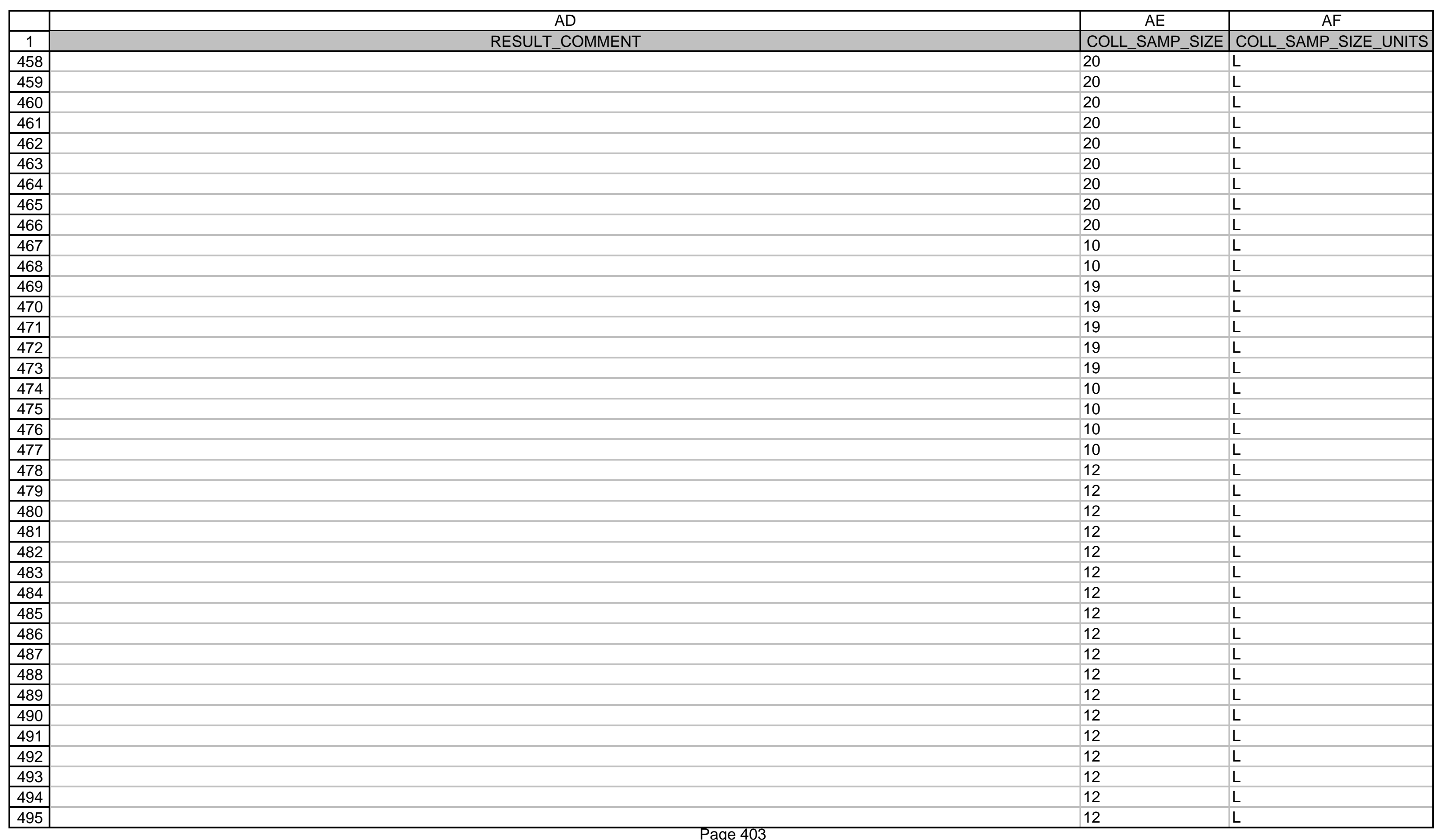




\begin{tabular}{|c|c|c|c|}
\hline & 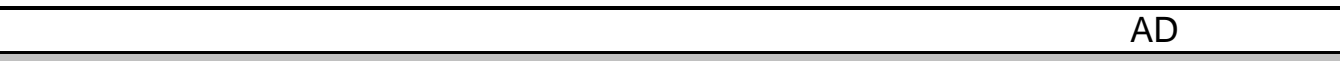 & $\mathrm{AE}$ & $\mathrm{AF}$ \\
\hline 1 & RESULT_COMMENT & COLL_SAMP_SIZE & COLL_SAMP_SIZE_UNITS \\
\hline 496 & & 12 & $\mathrm{~L}$ \\
\hline 497 & & 12 & $\mathrm{~L}$ \\
\hline 498 & & 12 & $L$ \\
\hline 499 & & 12 & $L$ \\
\hline 500 & & 12 & $\mathrm{~L}$ \\
\hline 501 & & 12 & $\mathrm{~L}$ \\
\hline 502 & & 12 & L \\
\hline 503 & & 12 & L \\
\hline 504 & & 12 & $\mathrm{~L}$ \\
\hline 505 & & 12 & $\mathrm{~L}$ \\
\hline 506 & & 12 & L \\
\hline 507 & & 12 & $\mathrm{~L}$ \\
\hline 508 & & 12 & L \\
\hline 509 & & 12 & $\mathrm{~L}$ \\
\hline 510 & & 12 & $\mathrm{~L}$ \\
\hline 511 & & 12 & $\mathrm{~L}$ \\
\hline 512 & & 12 & $\mathrm{~L}$ \\
\hline 513 & & 12 & $\mathrm{~L}$ \\
\hline 514 & & 12 & $\mathrm{~L}$ \\
\hline 515 & & 12 & L \\
\hline 516 & & 12 & $\mathrm{~L}$ \\
\hline 517 & & 12 & $\mathrm{~L}$ \\
\hline 518 & & 12 & L \\
\hline 519 & & 12 & L \\
\hline 520 & & 12 & L \\
\hline 521 & & 12 & L \\
\hline 522 & & 12 & L \\
\hline 523 & & 12 & $\mathrm{~L}$ \\
\hline 524 & & 12 & $\mathrm{~L}$ \\
\hline 525 & & 12 & $\mathrm{~L}$ \\
\hline 526 & & 12 & L \\
\hline 527 & & 12 & $\mathrm{~L}$ \\
\hline 528 & & 12 & $\mathrm{~L}$ \\
\hline 529 & & 12 & $\mathrm{~L}$ \\
\hline 530 & & 12 & $\mathrm{~L}$ \\
\hline 531 & & 12 & L \\
\hline 532 & IR-0107; MDA > CRDL & 14 & L \\
\hline 533 & & 14 & $\mathrm{~L}$ \\
\hline
\end{tabular}


AD

\begin{tabular}{|c|c|c|c|}
\hline & 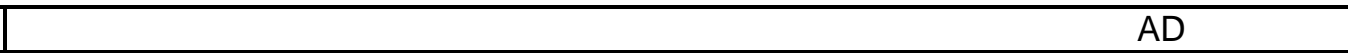 & $\mathrm{AE}$ & $\mathrm{AF}$ \\
\hline 1 & +2 & $\mid$ COLL_SAMP_SIZE & COLL_SAMP_SIZE_UNITS \\
\hline 534 & & 16 & $\mathrm{~L}$ \\
\hline 535 & & 10 & $\mathrm{~L}$ \\
\hline 536 & 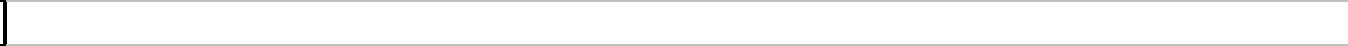 & 10 & L \\
\hline 537 & 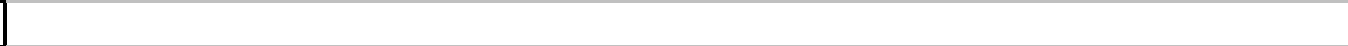 & 13 & $\mathrm{~L}$ \\
\hline 538 & & 13 & $\mathrm{~L}$ \\
\hline 539 & & 12 & $\mathrm{~L}$ \\
\hline 540 & & 12 & $\mathrm{~L}$ \\
\hline 541 & & 12 & $\mathrm{~L}$ \\
\hline 543 & & 12 & $\mathrm{~L}$ \\
\hline 544 & & 12 & $L$ \\
\hline 545 & & 16 & $L$ \\
\hline 546 & & 12 & $\mathrm{~L}$ \\
\hline 547 & & & \\
\hline 548 & & 16 & $L$ \\
\hline 549 & & 16 & $\mathrm{~L}$ \\
\hline 550 & & 10 & $\mathrm{~L}$ \\
\hline 551 & & 10 & $\mathrm{~L}$ \\
\hline 553 & & & \\
\hline 554 & & 10 & $\mathrm{~L}$ \\
\hline 555 & & 10 & L \\
\hline 556 & & 12 & $\mathrm{~L}$ \\
\hline 557 & & 12 & $L$ \\
\hline 558 & & 12 & $\mathrm{~L}$ \\
\hline 559 & & 12 & $L$ \\
\hline 560 & & 12 & $\mathrm{~L}$ \\
\hline 561 & & 12 & L \\
\hline 562 & & 12 & $\mathrm{~L}$ \\
\hline 563 & & 12 & $\mathrm{~L}$ \\
\hline 564 & & 12 & $\mathrm{~L}$ \\
\hline 565 & & 12 & $\mathrm{~L}$ \\
\hline 566 & & 12 & $L$ \\
\hline 567 & & 12 & $L$ \\
\hline 568 & & 12 & $\mathrm{~L}$ \\
\hline 569 & & 12 & L \\
\hline 570 & & 12 & $\mathrm{~L}$ \\
\hline
\end{tabular}




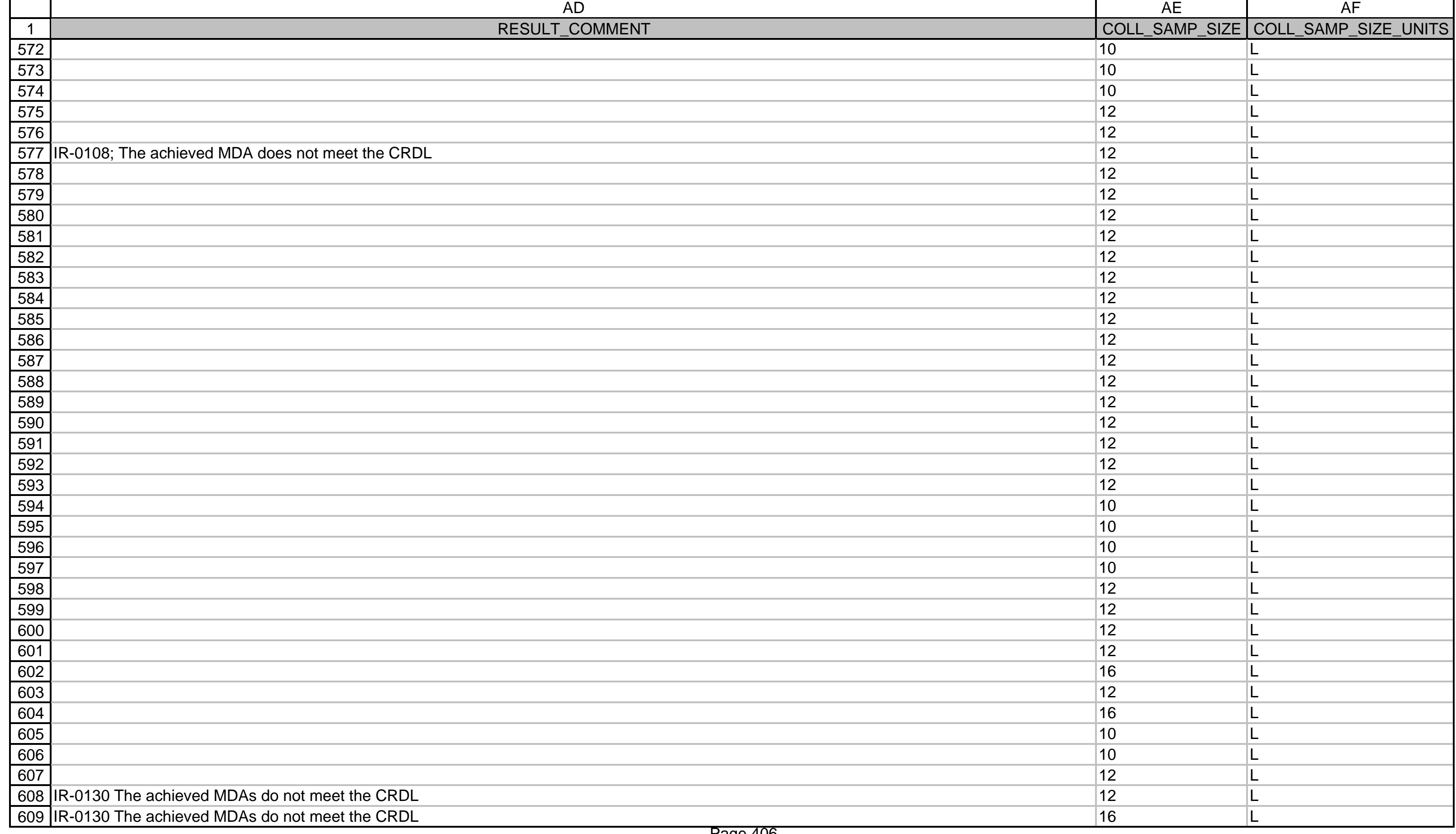


\begin{tabular}{|l|l|}
\hline 610 & IR-0130 The achieved MDAs do not meet the CRDL \\
\hline 611 & REFER TO FOLLOW-UPANALYSIS HWO55. DATABA
\end{tabular}

RESULT COMMENT

611 REFER TO FOLLOW-UP ANALYSIS \#W055. DATABASE MODIFIED PER TASK MANAGER

612

613

614

615

616

617

618

619

620

621

\begin{tabular}{|l|}
\hline 622 \\
\hline 623 \\
\hline
\end{tabular}

624 IR-0141; MDA > CRDL

625

626

627

\begin{tabular}{|l|}
\hline 628 \\
\hline 629 \\
\hline 630
\end{tabular}

629

631

\begin{tabular}{|l|}
631 \\
\hline 632 \\
\hline 633 \\
\hline
\end{tabular}

633

634

635

636

637

638

639

640

641

642

643 IR-0169; MDA > CRDL

644

645

646

\begin{tabular}{l}
646 \\
\hline 647 \\
\hline
\end{tabular} \begin{tabular}{c|c} 
AE & \multicolumn{2}{|c}{ AF } \\
\hline COLL_SAMP_SIZE & COLL_SAMP_SIZE_UNITS \\
\hline
\end{tabular}

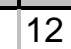

12

15

16

10

15

15

12

12

12

12

12

12

12

12

10

10

15

2

12

12

12

10

10

15
15

10

10

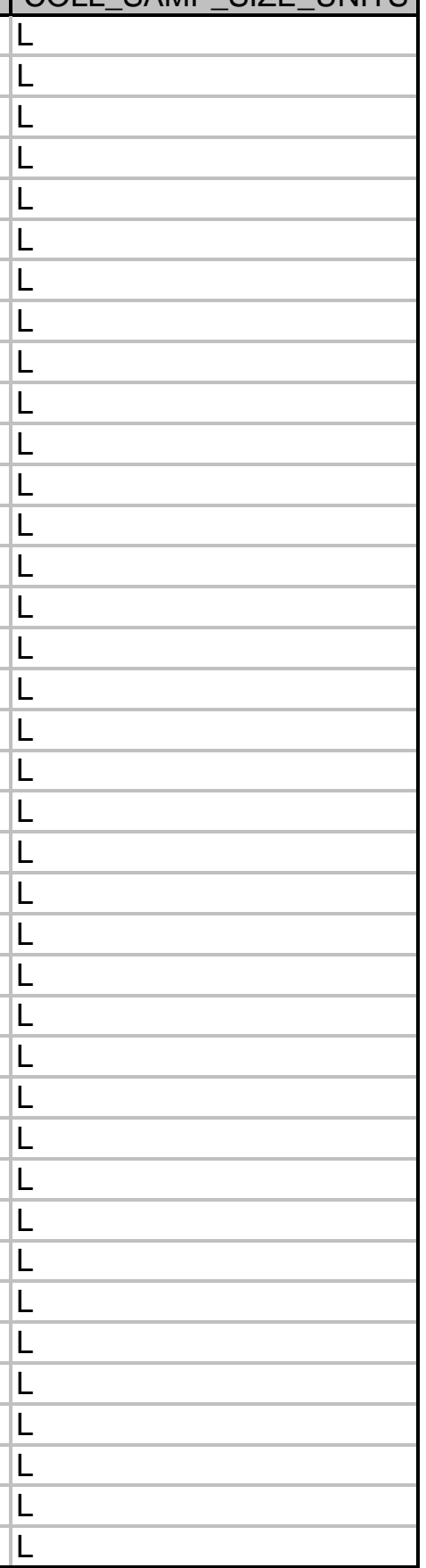




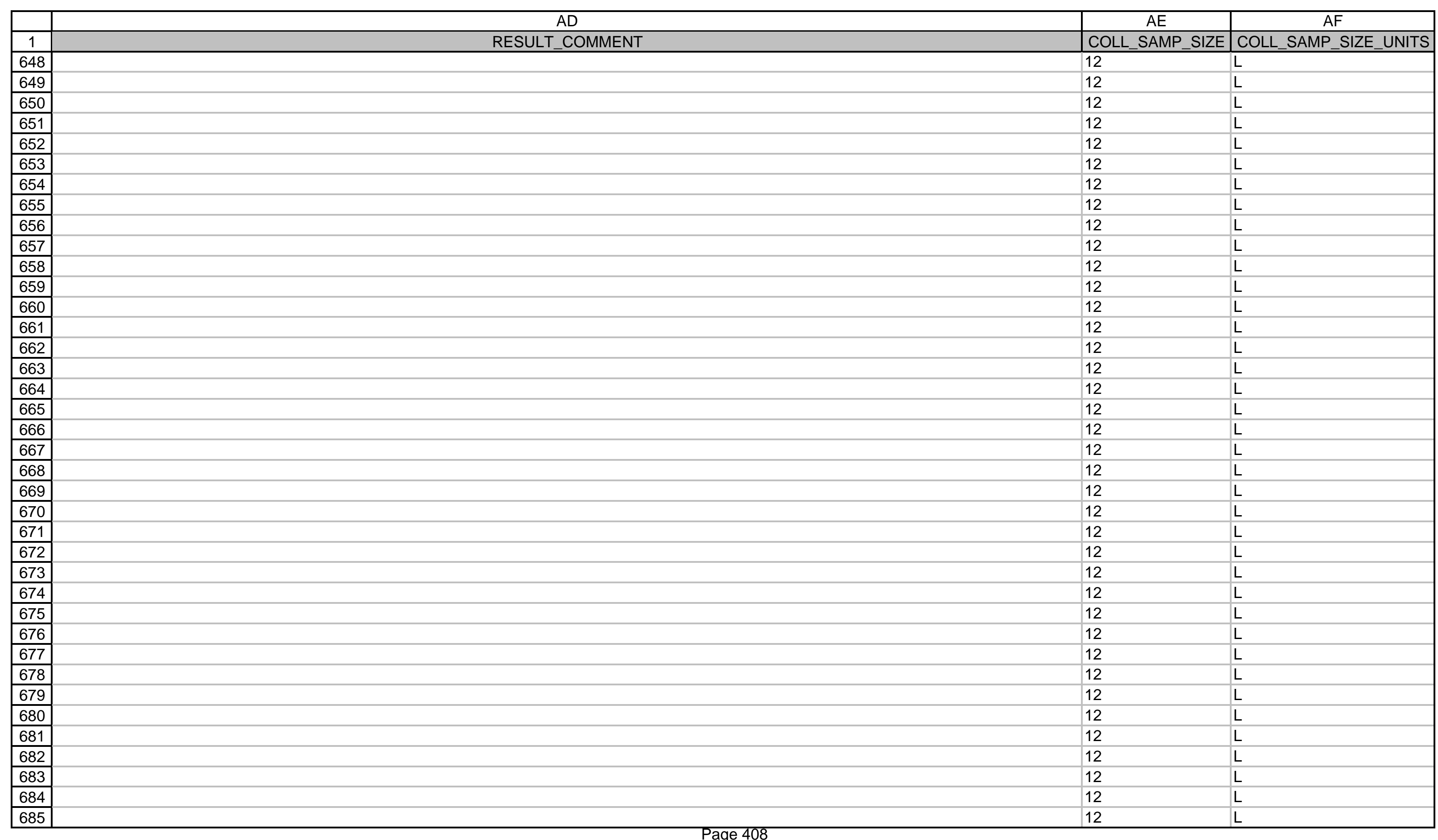




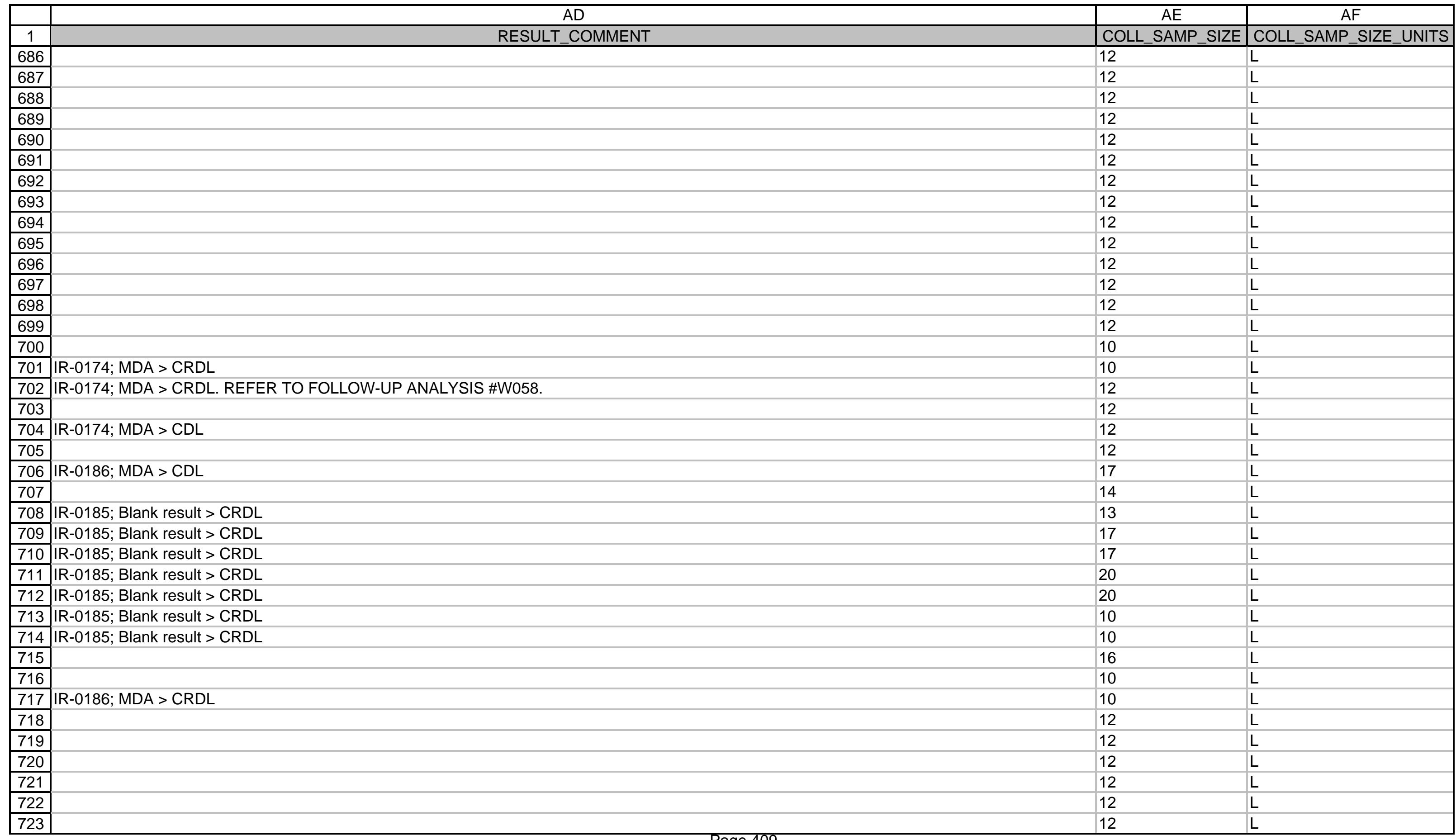




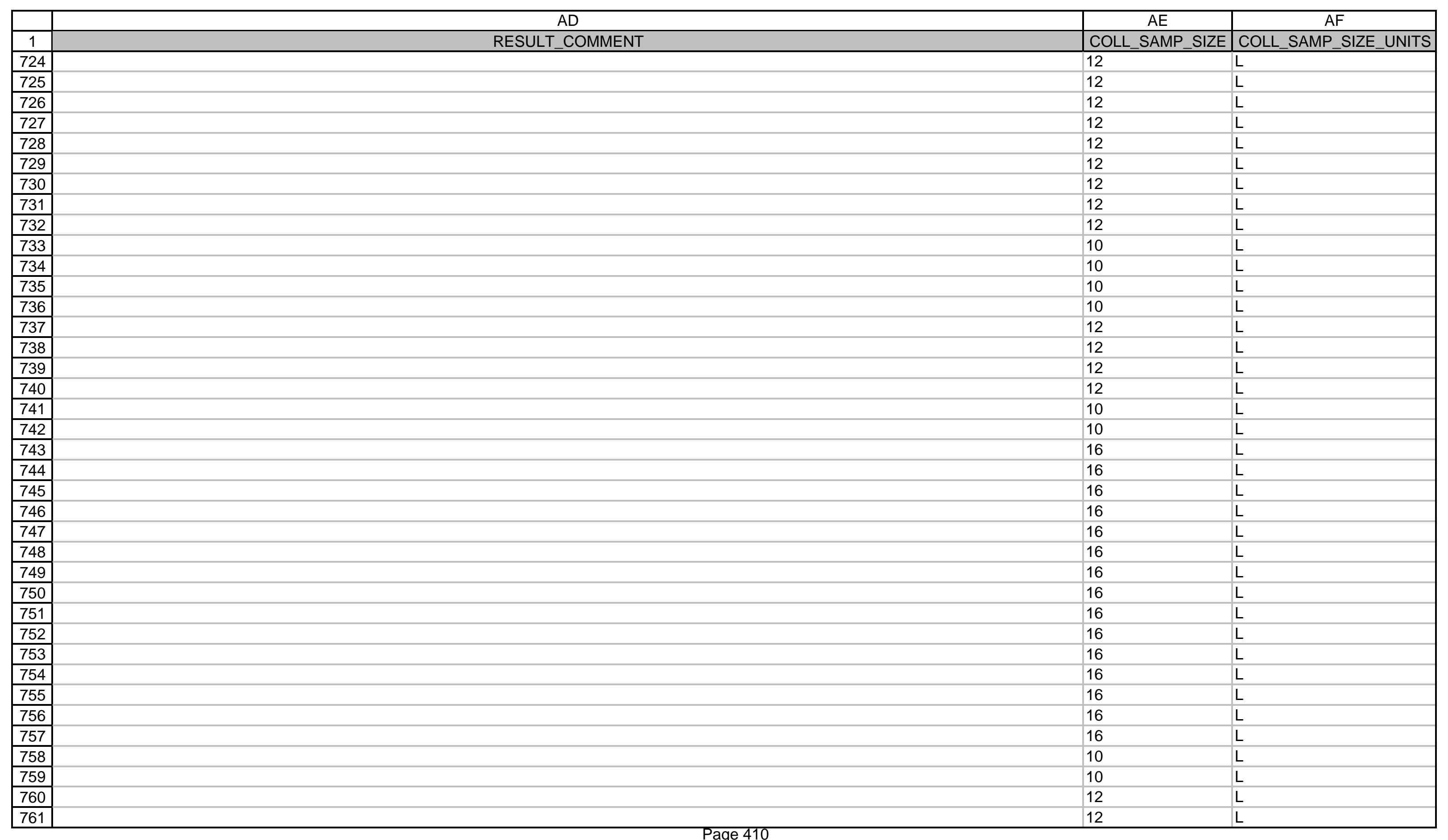




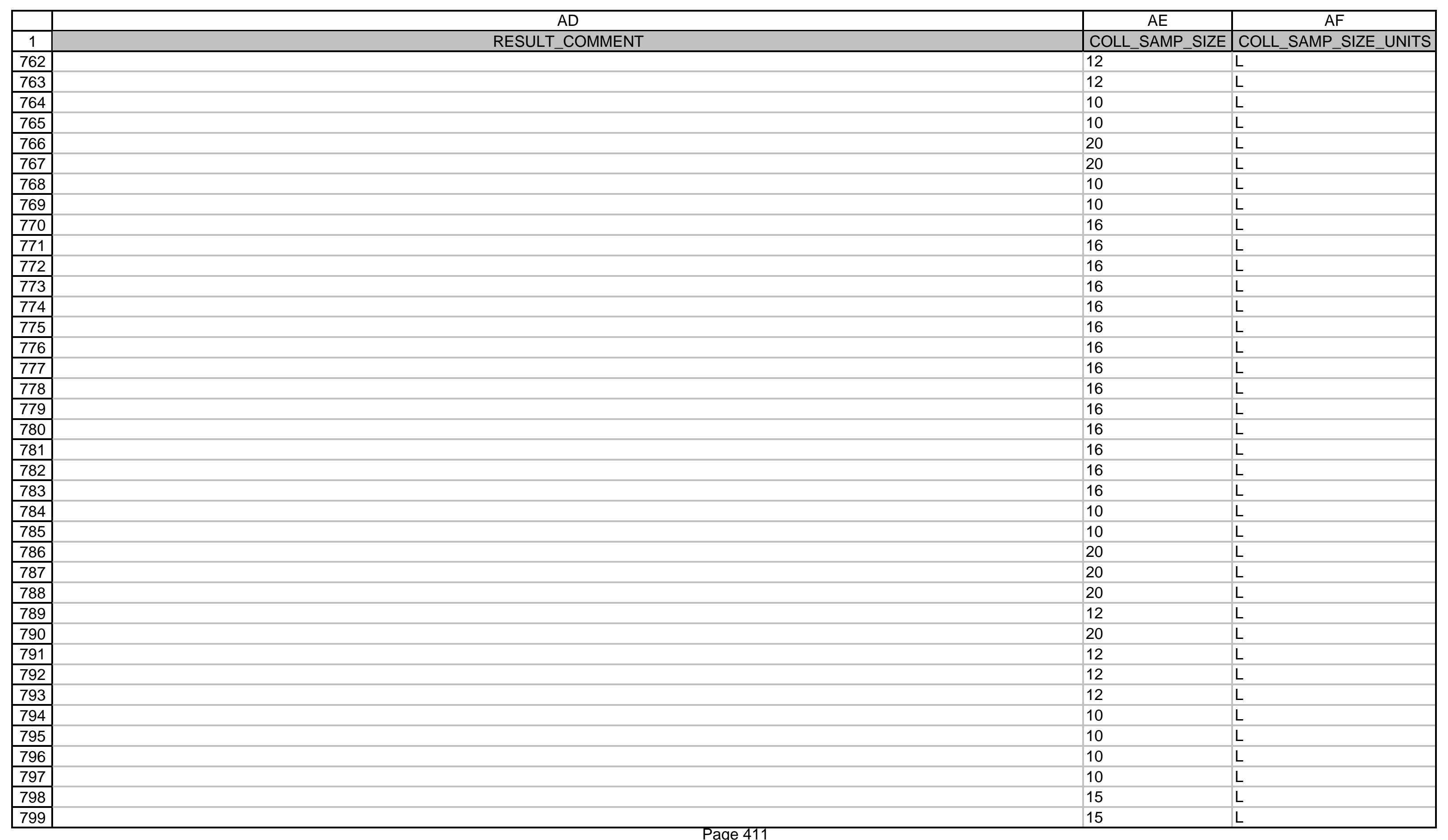




\begin{tabular}{|c|c|c|c|}
\hline & 20 & $\mathrm{AE}$ & $\mathrm{AF}$ \\
\hline 1 & RESULT_COMMENT & COLL_SAMP_SIZE & COLL_SAMP_SIZE_UNITS \\
\hline 800 & & 15 & $\mathrm{~L}$ \\
\hline 801 & & 15 & $\mathrm{~L}$ \\
\hline 802 & & 15 & $\mathrm{~L}$ \\
\hline 803 & & 15 & L \\
\hline 804 & & 15 & L \\
\hline 805 & & 15 & $\mathrm{~L}$ \\
\hline 806 & & 15 & L \\
\hline 807 & & 15 & L \\
\hline 809 & & 15 & $\mathrm{~L}$ \\
\hline 810 & & 15 & L \\
\hline 811 & IR-0245: MDA>CRDL. & 15 & $\mathrm{~L}$ \\
\hline 812 & IR-0245: MDA>CRDL. & 15 & L \\
\hline 813 & & 15 & L \\
\hline 814 & & 15 & $\mathrm{~L}$ \\
\hline 815 & & 15 & L \\
\hline 816 & & 15 & $\mathrm{~L}$ \\
\hline 817 & & 15 & L \\
\hline 819 & & 15 & L \\
\hline 820 & & 15 & $\mathrm{~L}$ \\
\hline 821 & & 15 & $\mathrm{~L}$ \\
\hline 822 & & 15 & L \\
\hline 823 & & 15 & L \\
\hline 824 & & 15 & L \\
\hline 825 & & 15 & L \\
\hline 826 & & 15 & L \\
\hline 827 & & 15 & $\mathrm{~L}$ \\
\hline 828 & & 15 & L \\
\hline 829 & & 15 & L \\
\hline 830 & & 15 & L \\
\hline 831 & & 15 & L \\
\hline 832 & & 15 & $\mathrm{~L}$ \\
\hline 833 & & 15 & $L$ \\
\hline 834 & & 15 & L \\
\hline 835 & & 15 & L \\
\hline 836 & & 15 & L \\
\hline
\end{tabular}




\begin{tabular}{|c|c|c|c|}
\hline & 20 & $\mathrm{AE}$ & $\mathrm{AF}$ \\
\hline 1 & RESULT_COMMENT & COLL_SAMP_SIZE & COLL_SAMP_SIZE_UNITS \\
\hline 838 & & 15 & L \\
\hline 839 & & 15 & $\mathrm{~L}$ \\
\hline 840 & & 15 & $\mathrm{~L}$ \\
\hline 841 & & 15 & L \\
\hline 842 & & 15 & L \\
\hline 843 & & 15 & $\mathrm{~L}$ \\
\hline 844 & & 15 & $\mathrm{~L}$ \\
\hline 845 & & 15 & L \\
\hline 847 & & 15 & L \\
\hline 848 & & 15 & L \\
\hline 849 & & 15 & $\mathrm{~L}$ \\
\hline 850 & & 15 & L \\
\hline 851 & & 15 & $\mathrm{~L}$ \\
\hline 852 & & 20 & $\mathrm{~L}$ \\
\hline 853 & & 24 & L \\
\hline 854 & & 24 & $\mathrm{~L}$ \\
\hline 855 & & 10 & L \\
\hline 857 & IR-0259; Lost in Lab & 20 & L \\
\hline 858 & & 12 & $\mathrm{~L}$ \\
\hline 859 & & 20 & $\mathrm{~L}$ \\
\hline 860 & & 20 & L \\
\hline 861 & & 10 & L \\
\hline 862 & & 10 & L \\
\hline 863 & & 10 & L \\
\hline 864 & & 10 & L \\
\hline 865 & & 15 & $\mathrm{~L}$ \\
\hline 866 & & 15 & L \\
\hline 867 & & 15 & L \\
\hline 868 & & 15 & L \\
\hline 869 & & 15 & L \\
\hline 870 & & 15 & $\mathrm{~L}$ \\
\hline 871 & & 15 & $\mathrm{~L}$ \\
\hline 872 & & 15 & L \\
\hline 873 & & 15 & L \\
\hline 874 & & 15 & L \\
\hline
\end{tabular}




\begin{tabular}{|c|c|c|c|}
\hline & $A D$ & $\mathrm{AE}$ & $\mathrm{AF}$ \\
\hline 1 & RESULT_COMMENT & COLL_SAMP_SIZE & COLL_SAMP_SIZE_UNITS \\
\hline 876 & & 15 & L \\
\hline 877 & & 15 & L \\
\hline 878 & & 15 & L \\
\hline 879 & & 15 & L \\
\hline 880 & & 10 & L \\
\hline 881 & & 10 & $\mathrm{~L}$ \\
\hline 882 & & 10 & L \\
\hline 883 & & 10 & L \\
\hline 885 & IR-0052; MDA > CRDL & 10 & $L$ \\
\hline 886 & & 10 & L \\
\hline 887 & & 10 & L \\
\hline 888 & & 10 & L \\
\hline 889 & & 10 & L \\
\hline 890 & & 10 & L \\
\hline 891 & & 10 & $\mathrm{~L}$ \\
\hline 892 & & 10 & L \\
\hline 893 & & 10 & L \\
\hline 895 & & 10 & $\mathrm{~L}$ \\
\hline 896 & & 10 & $\mathrm{~L}$ \\
\hline 897 & & 10 & $L$ \\
\hline 898 & & 10 & L \\
\hline 899 & & 8 & $\mathrm{~L}$ \\
\hline 900 & & 14 & $\mathrm{~L}$ \\
\hline 901 & & 8 & L \\
\hline 902 & & 8 & L \\
\hline 903 & & 14 & $\mathrm{~L}$ \\
\hline 904 & & 10 & L \\
\hline 905 & & 10 & L \\
\hline 906 & IR-0082 MDA > CRDL & 9 & $\mathrm{~L}$ \\
\hline 907 & & 16 & $\mathrm{~L}$ \\
\hline 908 & & 12 & L \\
\hline 909 & & 16 & $\mathrm{~L}$ \\
\hline 910 & & 16 & $\mathrm{~L}$ \\
\hline 911 & & 10 & $\mathrm{~L}$ \\
\hline 912 & & 10 & $\mathrm{~L}$ \\
\hline
\end{tabular}




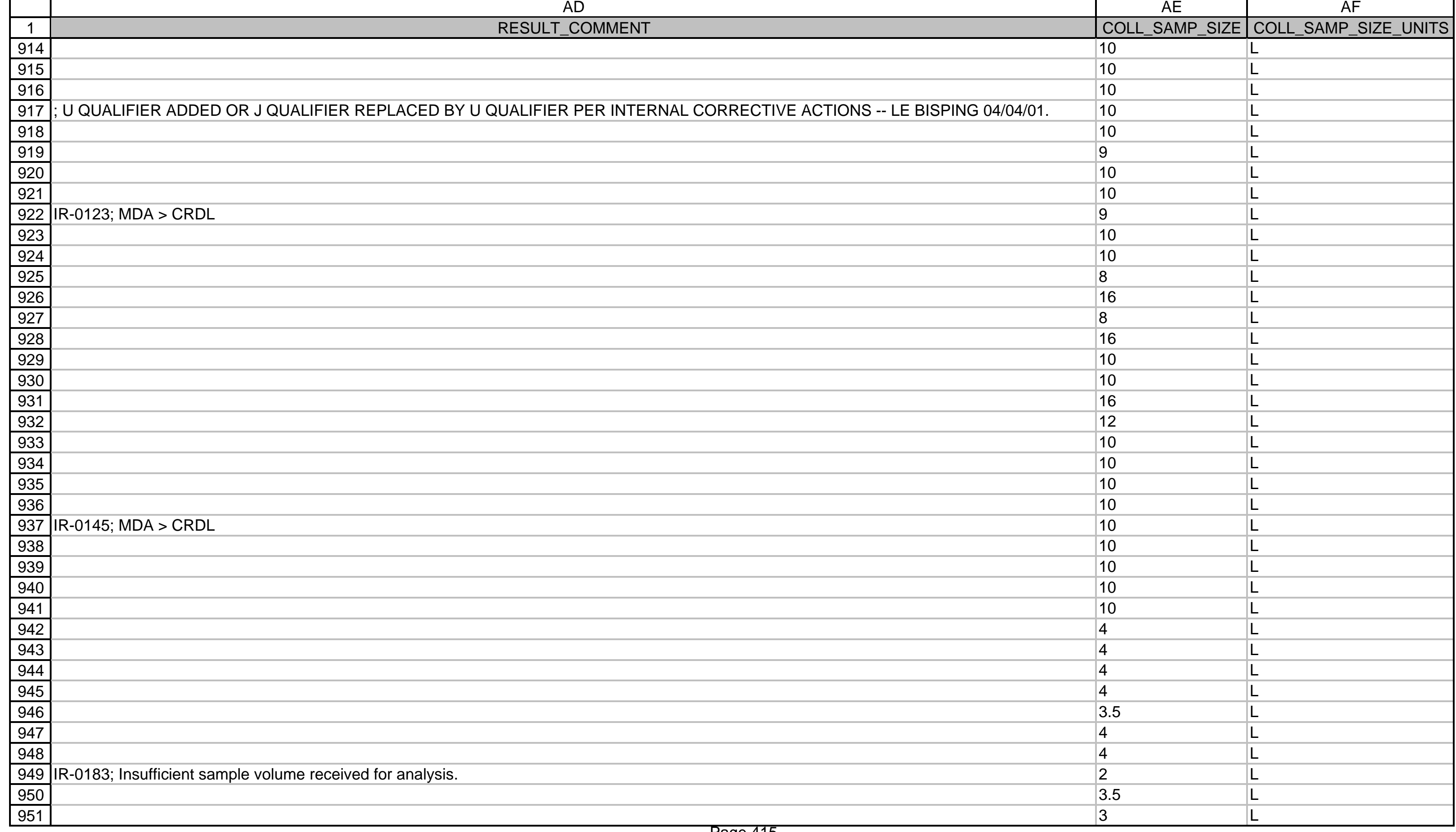




\begin{tabular}{|c|c|c|c|}
\hline & $\begin{array}{ll}-1 D \\
\end{array}$ & AE & AF \\
\hline 1 & RESULT_COMMENT & COLL_SAMP_SIZE & COLL_SAMP_SIZE_UNITS \\
\hline 952 & & 4 & $\mathrm{~L}$ \\
\hline 953 & IR-0183; Insufficient sample volume received for analysis & 2 & $\mathrm{~L}$ \\
\hline 954 & & 10 & $\mathrm{~L}$ \\
\hline 955 & & 10 & L \\
\hline 956 & & 17 & L \\
\hline 957 & & 17 & $\mathrm{~L}$ \\
\hline 958 & & 17 & $\mathrm{~L}$ \\
\hline 959 & & 20 & L \\
\hline 960 & & 20 & $\mathrm{~L}$ \\
\hline 961 & & 10 & $\mathrm{~L}$ \\
\hline 962 & & 10 & $\mathrm{~L}$ \\
\hline 963 & & 10 & L \\
\hline 964 & & 10 & L \\
\hline 965 & & 10 & L \\
\hline 966 & & 10 & $\mathrm{~L}$ \\
\hline 967 & & 10 & $\mathrm{~L}$ \\
\hline 968 & & 10 & L \\
\hline 969 & & 10 & L \\
\hline 972 & & 10 & $\mathrm{~L}$ \\
\hline 973 & & 10 & $\mathrm{~L}$ \\
\hline 974 & & 10 & $\mathrm{~L}$ \\
\hline 975 & & 10 & $\mathrm{~L}$ \\
\hline 976 & & 10 & $\mathrm{~L}$ \\
\hline \begin{tabular}{|l|}
977 \\
\end{tabular} & & 10 & L \\
\hline 978 & & 10 & L \\
\hline 979 & & 10 & $\mathrm{~L}$ \\
\hline 980 & & 10 & L \\
\hline 981 & & 10 & L \\
\hline 982 & & 10 & $\mathrm{~L}$ \\
\hline 983 & & 24 & $\mathrm{~L}$ \\
\hline 984 & & 24 & $\mathrm{~L}$ \\
\hline 985 & IR-0242: Blank > CRDL. REFER TO FOLLOW-UP ANALYSIS \#W063. & 10 & $\mathrm{~L}$ \\
\hline 986 & IR-0242: Blank > CRDL. REFER TO FOLLOW-UP ANALYSIS \#W063 & 10 & $\mathrm{~L}$ \\
\hline 987 & & 16 & $\mathrm{~L}$ \\
\hline 988 & & 16 & $\mathrm{~L}$ \\
\hline 989 & & 12 & $\mathrm{~L}$ \\
\hline
\end{tabular}




\begin{tabular}{|c|c|c|c|}
\hline & $A D$ & $\mathrm{AE}$ & $\mathrm{AF}$ \\
\hline 1 & $\begin{array}{lc} & \text { RESULT_COMMENT } \\
\end{array}$ & COLL_SAMP_SIZE & COLL_SAMP_SIZE_UNITS \\
\hline 990 & & 20 & L \\
\hline 991 & & 10 & L \\
\hline 992 & & 10 & $\mathrm{~L}$ \\
\hline 993 & & 10 & L \\
\hline 994 & & 10 & L \\
\hline 995 & & 10 & L \\
\hline 996 & 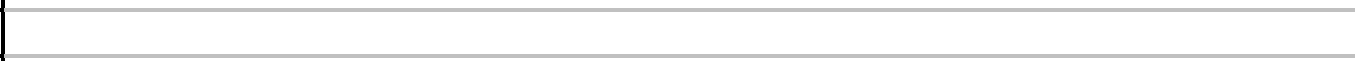 & 10 & $\mathrm{~L}$ \\
\hline 997 & & 10 & $\mathrm{~L}$ \\
\hline \begin{tabular}{|l|}
998 \\
\end{tabular} & & 10 & L \\
\hline \begin{tabular}{|l|}
999 \\
\end{tabular} & 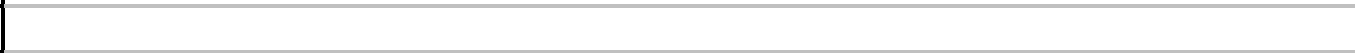 & 10 & L \\
\hline 1000 & & 10 & $\mathrm{~L}$ \\
\hline 1001 & & 10 & $\mathrm{~L}$ \\
\hline 1002 & & 19 & L \\
\hline 1003 & & 19 & L \\
\hline 1004 & & 19 & $\mathrm{~L}$ \\
\hline 1005 & & 19 & L \\
\hline 1006 & & 10 & L \\
\hline 1007 & & 10 & $\mathrm{~L}$ \\
\hline 1009 & & 20 & $\mathrm{~L}$ \\
\hline 1010 & & 20 & $\mathrm{~L}$ \\
\hline 1011 & & 20 & L \\
\hline 1012 & & 20 & L \\
\hline 1013 & & 20 & L \\
\hline 1014 & & 20 & L \\
\hline 1015 & & 20 & L \\
\hline \begin{tabular}{|l|}
1016 \\
\end{tabular} & & 20 & $\mathrm{~L}$ \\
\hline \begin{tabular}{|l|}
1017 \\
\end{tabular} & & 20 & L \\
\hline 1018 & & 20 & L \\
\hline 1019 & & 20 & L \\
\hline 1020 & & 20 & L \\
\hline 1021 & & 20 & $\mathrm{~L}$ \\
\hline 1022 & & 20 & L \\
\hline 1023 & & 10 & L \\
\hline 1024 & & 10 & L \\
\hline 1025 & & 10 & $\mathrm{~L}$ \\
\hline 1026 & & 10 & L \\
\hline
\end{tabular}




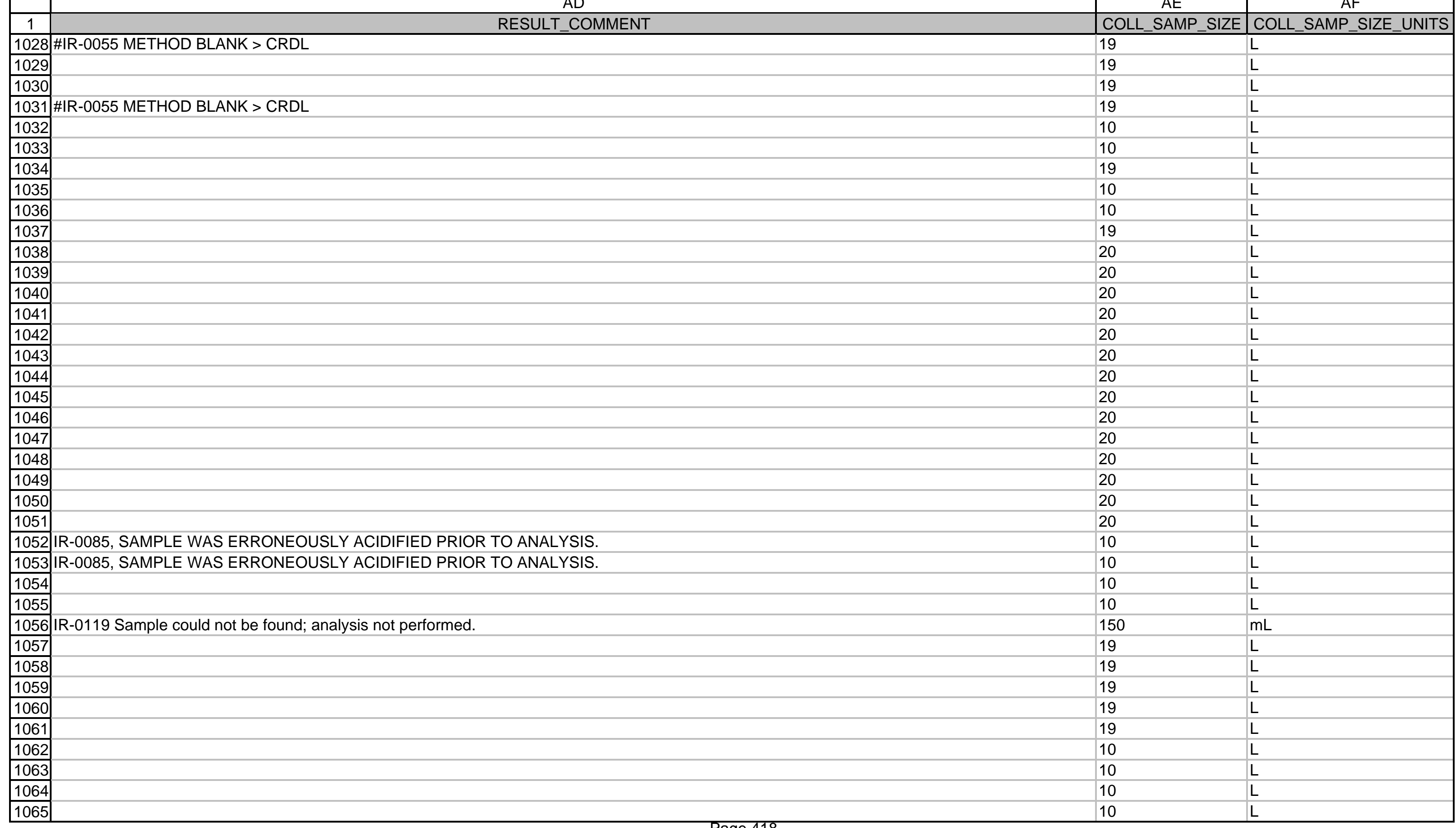




\begin{tabular}{|c|c|c|c|}
\hline & 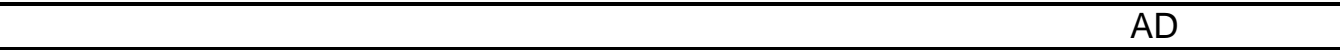 & $\mathrm{AE}$ & $\mathrm{AF}$ \\
\hline 1 & RESULT_COMMENT & COLL_SAMP_SIZE & COLL_SAMP_SIZE_UNITS \\
\hline 1066 & & 12 & $\mathrm{~L}$ \\
\hline 1067 & \#IR-0080 Dups do not agree within limits. & 12 & L \\
\hline 1068 & & 12 & $\mathrm{~L}$ \\
\hline 1069 & & 12 & $\mathrm{~L}$ \\
\hline 1070 & & 12 & L \\
\hline 1071 & & 12 & L \\
\hline 1072 & & 12 & L \\
\hline 1073 & & 12 & $\mathrm{~L}$ \\
\hline 1074 & & 12 & $\mathrm{~L}$ \\
\hline 1075 & & 12 & L \\
\hline 1076 & & 12 & L \\
\hline 1077 & & 12 & $\mathrm{~L}$ \\
\hline 1078 & & 12 & L \\
\hline 1079 & & 12 & $\mathrm{~L}$ \\
\hline 1080 & & 12 & $\mathrm{~L}$ \\
\hline 1081 & & 500 & $\mathrm{~mL}$ \\
\hline 1082 & & 500 & $\mathrm{~mL}$ \\
\hline 1083 & & 500 & $\mathrm{~mL}$ \\
\hline 1084 & & 500 & $\mathrm{~mL}$ \\
\hline 1085 & & 500 & $\mathrm{~mL}$ \\
\hline 1086 & & 500 & $\mathrm{~mL}$ \\
\hline 1087 & & 12 & $\mathrm{~L}$ \\
\hline 1088 & & 12 & L \\
\hline 1089 & & 12 & L \\
\hline 1090 & & 12 & $\mathrm{~L}$ \\
\hline 1091 & & 12 & L \\
\hline 1092 & & 12 & L \\
\hline 1093 & & 12 & $\mathrm{~L}$ \\
\hline 1094 & & 12 & L \\
\hline 1095 & & 12 & L \\
\hline 1096 & & 12 & L \\
\hline 1097 & & 12 & L \\
\hline 1098 & & 12 & L \\
\hline 1099 & & 12 & $\mathrm{~L}$ \\
\hline 1100 & & 12 & L \\
\hline 1101 & & 12 & L \\
\hline 1102 & & 12 & $\mathrm{~L}$ \\
\hline 1103 & & 12 & $\mathrm{~L}$ \\
\hline
\end{tabular}




\begin{tabular}{|c|c|c|c|}
\hline & $A D$ & $\mathrm{AE}$ & $\mathrm{AF}$ \\
\hline 1 & RESULT_COMMENT & COLL_SAMP_SIZE & COLL_SAMP_SIZE_UNITS \\
\hline 1104 & & 12 & $\mathrm{~L}$ \\
\hline \begin{tabular}{|l|}
1105 \\
\end{tabular} & & 12 & L \\
\hline \begin{tabular}{|l|}
1106 \\
\end{tabular} & & 500 & $\mathrm{~mL}$ \\
\hline \begin{tabular}{|l|}
1107 \\
\end{tabular} & & 500 & $\mathrm{~mL}$ \\
\hline \begin{tabular}{|l|}
1108 \\
\end{tabular} & & 500 & $\mathrm{~mL}$ \\
\hline \begin{tabular}{|l|}
1109 \\
\end{tabular} & 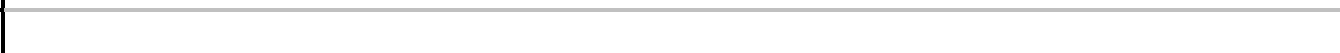 & 500 & $\mathrm{~mL}$ \\
\hline \begin{tabular}{|l|}
1110 \\
\end{tabular} & 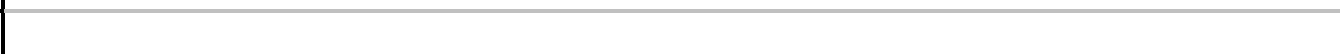 & 500 & $\mathrm{~mL}$ \\
\hline \begin{tabular}{|l|}
1111 \\
\end{tabular} & 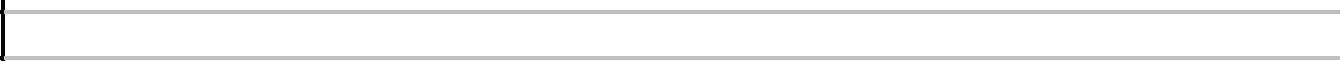 & 500 & $\mathrm{~mL}$ \\
\hline \begin{tabular}{|l|}
11113 \\
\end{tabular} & 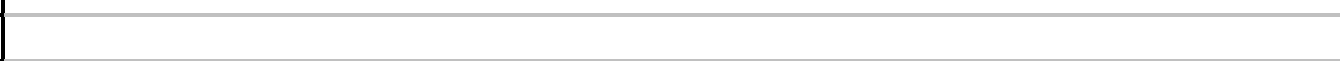 & 12 & $\mathrm{~L}$ \\
\hline \begin{tabular}{|l|}
11114 \\
\end{tabular} & & 12 & $\mathrm{~L}$ \\
\hline \begin{tabular}{|l|}
1115 \\
\end{tabular} & & 12 & $\mathrm{~L}$ \\
\hline \begin{tabular}{|l|}
1116 \\
\end{tabular} & & 12 & $\mathrm{~L}$ \\
\hline \begin{tabular}{|l|}
1117 \\
\end{tabular} & & 12 & L \\
\hline \begin{tabular}{|l|}
1118 \\
\end{tabular} & & 12 & $\mathrm{~L}$ \\
\hline \begin{tabular}{|l|}
1119 \\
\end{tabular} & & 12 & $\mathrm{~L}$ \\
\hline \begin{tabular}{|l|}
1120 \\
\end{tabular} & & 12 & $\mathrm{~L}$ \\
\hline \begin{tabular}{|l|}
1121 \\
1121 \\
\end{tabular} & & 12 & $\mathrm{~L}$ \\
\hline \begin{tabular}{|l|}
1123 \\
\end{tabular} & & 12 & $\mathrm{~L}$ \\
\hline \begin{tabular}{|l|}
1124 \\
\end{tabular} & & 12 & $\mathrm{~L}$ \\
\hline \begin{tabular}{|l|}
1125 \\
\end{tabular} & & 12 & $\mathrm{~L}$ \\
\hline \begin{tabular}{|l|}
1126 \\
\end{tabular} & & 12 & $\mathrm{~L}$ \\
\hline \begin{tabular}{|l|}
1127 \\
\end{tabular} & & 12 & L \\
\hline \begin{tabular}{|l|}
1128 \\
\end{tabular} & & 12 & $\mathrm{~L}$ \\
\hline \begin{tabular}{|l|}
1129 \\
\end{tabular} & & 12 & $\mathrm{~L}$ \\
\hline \begin{tabular}{|l|}
1130 \\
\end{tabular} & & 12 & $\mathrm{~L}$ \\
\hline \begin{tabular}{|l|}
1131 \\
\end{tabular} & & 12 & $\mathrm{~L}$ \\
\hline \begin{tabular}{|l|}
1132 \\
\end{tabular} & & 12 & $\mathrm{~L}$ \\
\hline \begin{tabular}{|l|}
1133 \\
\end{tabular} & & 500 & $\mathrm{~mL}$ \\
\hline \begin{tabular}{|l|}
1104 \\
1134 \\
\end{tabular} & & 500 & $\mathrm{~mL}$ \\
\hline \begin{tabular}{|l|}
1135 \\
\end{tabular} & & 500 & $\mathrm{~mL}$ \\
\hline \begin{tabular}{|l|}
1136 \\
\end{tabular} & & 500 & $\mathrm{~mL}$ \\
\hline \begin{tabular}{|l|}
1137 \\
\end{tabular} & & 500 & $\mathrm{~mL}$ \\
\hline \begin{tabular}{|l|}
1138 \\
\end{tabular} & & 500 & $\mathrm{~mL}$ \\
\hline \begin{tabular}{|l|}
1139 \\
113
\end{tabular} & & 500 & $\mathrm{~mL}$ \\
\hline \begin{tabular}{|l|}
1140 \\
\end{tabular} & & 500 & $\mathrm{~mL}$ \\
\hline
\end{tabular}




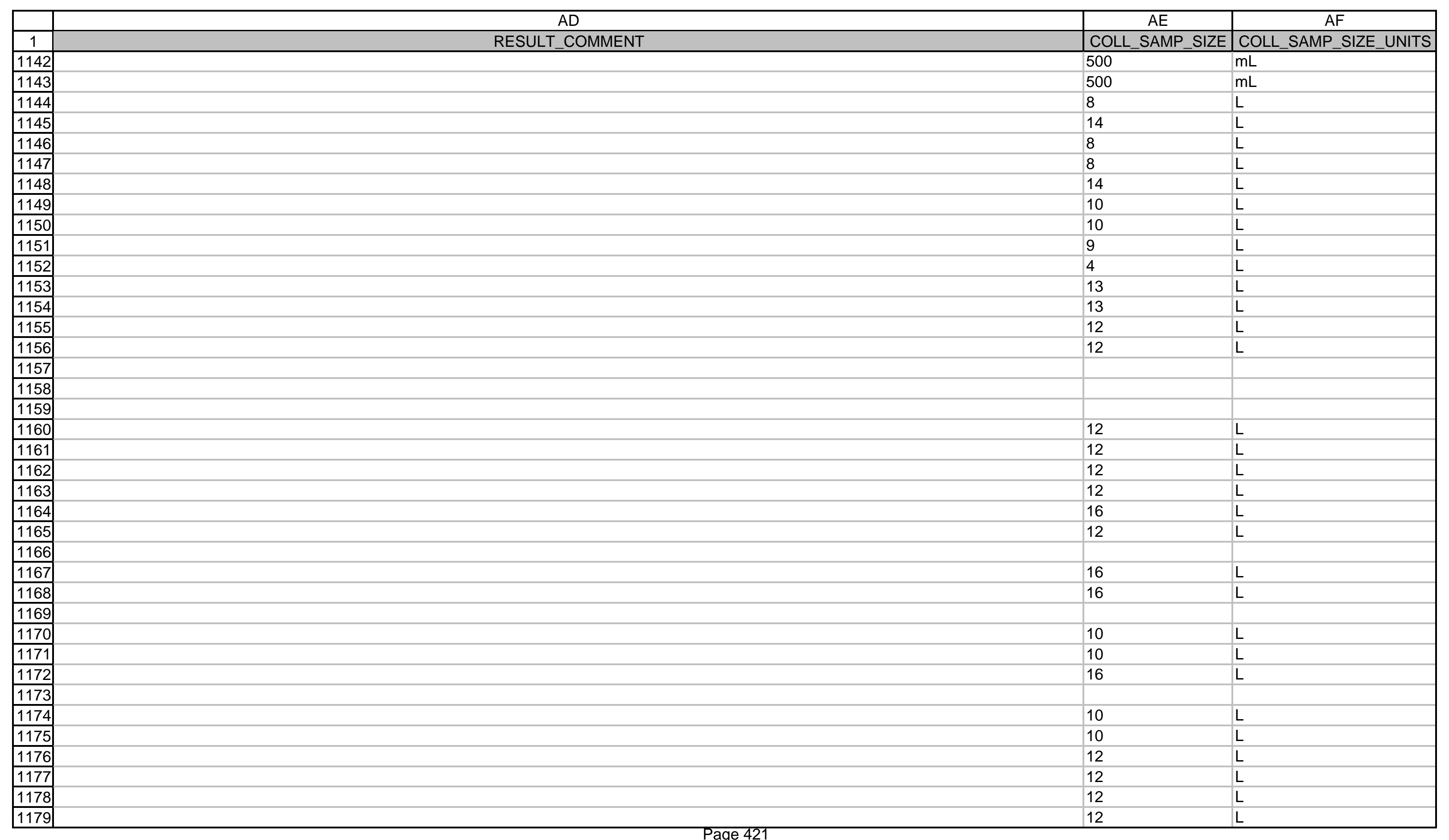


1183 IR-0101; MDA > CRDL

1184 IR-0101; MDA > CRDL

\begin{tabular}{|l|l|l}
1185 & IR-0101; MDA $>$ CRDL \\
\hline 1186 & IR-0101; MDA $>$ CRDL \\
\hline
\end{tabular}

1186 IR-0101; MDA > CRDL

1187 IR-0101; MDA > CRDL

\begin{tabular}{|l|l}
1188 & IR-0101; MDA $>$ CRDL \\
\hline 1189 &
\end{tabular}

1189 IR-0101; MDA > CRDL

1190 IR-0101; MDA > CRDL

\begin{tabular}{|r|l}
1191 & IR-0105 - Sample was inadvertently acidified
\end{tabular}

1192 IR-0105 - Sample was inadvertently acidified

1193 IR-0105 - Sample was inadvertently acidified

1194 IR-0105 - Sample was inadvertently acidified

1195

1196

1197 IR-0109; Blank Result > CRDL

1198

1199

1200 IR-0109; Blank Result > CRDL

1201 IR-0117; LCS Result Out Of Limit

\begin{tabular}{|l|l|}
1202 & IR-0117; LCS Result Out Of Limit \\
\hline
\end{tabular}

1203 IR-0117; LCS Result Out Of Limit

1204 IR-0117; LCS Result Out Of Limit

1205 IR-0117; LCS Result Out Of Limit

\begin{tabular}{|l|l}
1206 & IR-0117; LCS Result Out Of Limit \\
\hline 1207 & IR-0117; LCS Result Out Of Limit
\end{tabular}

1207 IR-0117; LCS Result Out Of Limit

1208 IR0121; Duplicate do not agree within limits

1209

1210

1211 IR-0117; LCS Result Out Of Limit

12

12

12

12

12

12

12

12

10

10

0

0

9

4

12

12

12

12

12

12

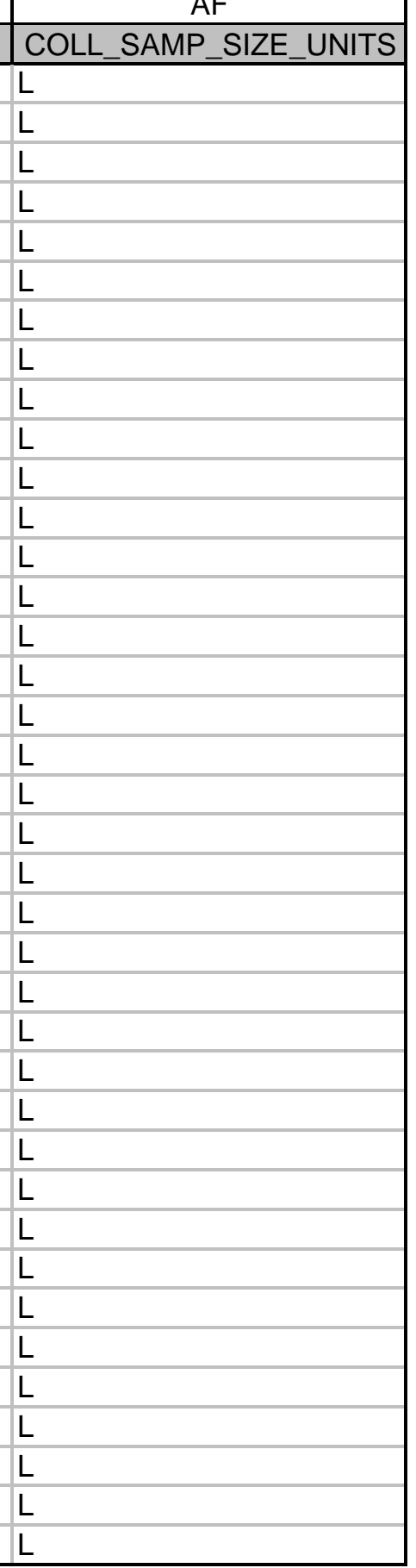




\begin{tabular}{|c|c|c|c|}
\hline & $A D$ & $\mathrm{AE}$ & $\mathrm{AF}$ \\
\hline 1 & RESULT_COMMENT & COLL_SAMP_SIZE & COLL_SAMP_SIZE_UNITS \\
\hline 1218 & & 9 & $\mathrm{~L}$ \\
\hline 1219 & & 4 & $\mathrm{~L}$ \\
\hline 1220 & & 4 & L \\
\hline 1221 & & 10 & $L$ \\
\hline 1222 & & 10 & $\mathrm{~L}$ \\
\hline 1223 & IR-0132; Sample spilled during preparation. & 12 & $\mathrm{~L}$ \\
\hline 1224 & & 12 & L \\
\hline 1225 & & 12 & $L$ \\
\hline 1227 & & 8 & $L$ \\
\hline 1228 & & 16 & $\mathrm{~L}$ \\
\hline 1229 & & 12 & $\mathrm{~L}$ \\
\hline 1230 & & 8 & $\mathrm{~L}$ \\
\hline 1231 & & 16 & $\mathrm{~L}$ \\
\hline 1232 & IR-0131; Blank result > CRDL & 10 & L \\
\hline 1233 & IR-0131; Blank result > CRDL & 10 & $\mathrm{~L}$ \\
\hline 1234 & & 12 & $\mathrm{~L}$ \\
\hline 1235 & & 12 & $L$ \\
\hline 1237 & & 12 & L \\
\hline 1238 & & 12 & $L$ \\
\hline 1239 & & 15 & $\mathrm{~L}$ \\
\hline 1240 & & 15 & $L$ \\
\hline 1241 & & 16 & L \\
\hline 1242 & & 500 & $\mathrm{~mL}$ \\
\hline 1243 & & 500 & $\mathrm{~mL}$ \\
\hline 1244 & & 500 & $\mathrm{~mL}$ \\
\hline 1245 & & 500 & $\mathrm{~mL}$ \\
\hline 1246 & & 2 & L \\
\hline 1247 & IR-0147; Blank result > CRDL & 10 & $L$ \\
\hline 1248 & IR-0147; Blank result > CRDL & 10 & $\mathrm{~L}$ \\
\hline 1249 & 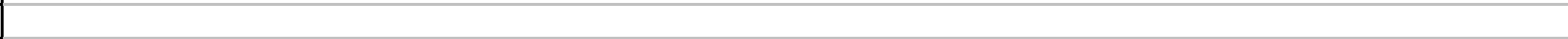 & 15 & $\mathrm{~L}$ \\
\hline 1250 & IR-0150; Blank result > CRDL & 15 & $L$ \\
\hline 1251 & & 15 & $L$ \\
\hline 1252 & IR-0168; Blank exceeds CRDL; LCS high. REFER TO FOLLOW-UP ANALYSIS \#W056, DATABASE MODIFIED PER TASK MANAGER. & 15 & $\mathrm{~L}$ \\
\hline 1253 & & 12 & $\mathrm{~L}$ \\
\hline 1254 & & 12 & $\mathrm{~L}$ \\
\hline
\end{tabular}




\begin{tabular}{|c|c|c|c|}
\hline & 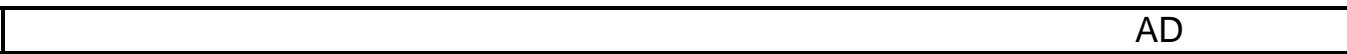 & $\mathrm{AE}$ & $\mathrm{AF}$ \\
\hline 1 & $\begin{array}{l}\text { RESULT_COMMENT } \\
\end{array}$ & COLL_SAMP_SIZE & COLL_SAMP_SIZE_UNITS \\
\hline 1256 & & 12 & L \\
\hline 1257 & & 12 & $\mathrm{~L}$ \\
\hline 1258 & & 12 & $\mathrm{~L}$ \\
\hline 1259 & & 12 & $\mathrm{~L}$ \\
\hline 1260 & & 12 & $\mathrm{~L}$ \\
\hline 1261 & & 12 & $\mathrm{~L}$ \\
\hline 1262 & & 12 & $\mathrm{~L}$ \\
\hline 1263 & & 12 & $\mathrm{~L}$ \\
\hline 1265 & & 12 & L \\
\hline 1266 & & 12 & $\mathrm{~L}$ \\
\hline 1267 & & 10 & $\mathrm{~L}$ \\
\hline 1268 & & 10 & $\mathrm{~L}$ \\
\hline 1269 & & 4 & $\mathrm{~L}$ \\
\hline 1270 & IR-0150; Blank result > CRDL & 15 & $L$ \\
\hline 1271 & IR-0150; Blank result > CRDL & 15 & $\mathrm{~L}$ \\
\hline 1272 & IR-0150; Blank result > CRDL & 12 & $\mathrm{~L}$ \\
\hline 1273 & & 12 & $\mathrm{~L}$ \\
\hline 1275 & IR-0150; Blank result > CRDL & 12 & L \\
\hline 1276 & & 10 & $\mathrm{~L}$ \\
\hline 1277 & & 10 & L \\
\hline 1278 & IR-0172; Blank result > CRDL & 500 & $\mathrm{~mL}$ \\
\hline 1279 & & 15 & L \\
\hline 1280 & & 15 & L \\
\hline 1281 & IR-0172; Blank result > CRDL & 10 & L \\
\hline 1282 & IR-0172; Blank result > CRDL & 10 & L \\
\hline 1283 & & 12 & L \\
\hline 1284 & & 12 & $\mathrm{~L}$ \\
\hline 1285 & & 12 & $\mathrm{~L}$ \\
\hline 1286 & & 12 & $L$ \\
\hline 1287 & & 12 & L \\
\hline 1288 & & 12 & L \\
\hline 1289 & & 12 & $L$ \\
\hline 1290 & & 12 & L \\
\hline 1291 & & 12 & $\mathrm{~L}$ \\
\hline 1292 & & 12 & L \\
\hline
\end{tabular}




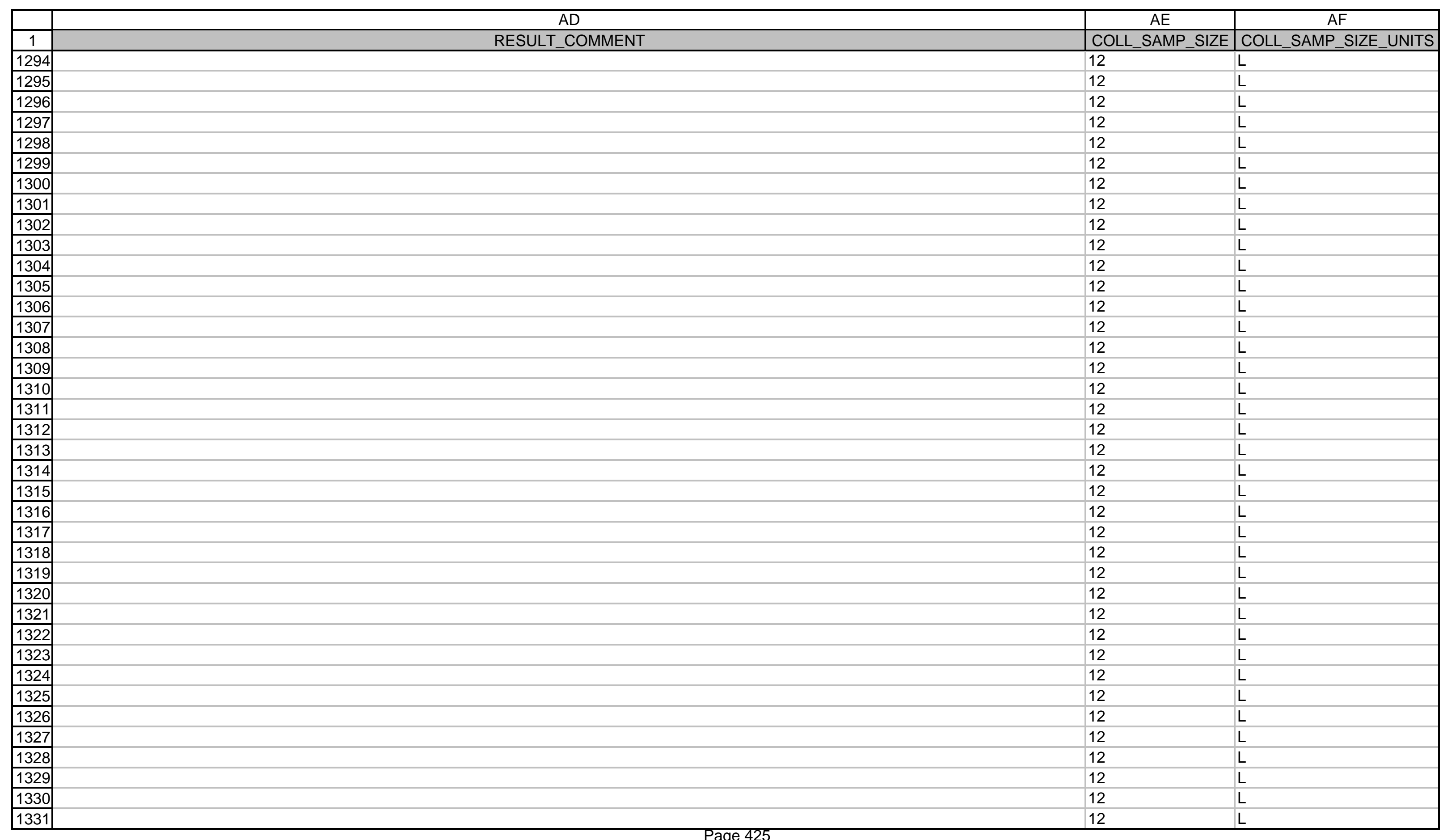




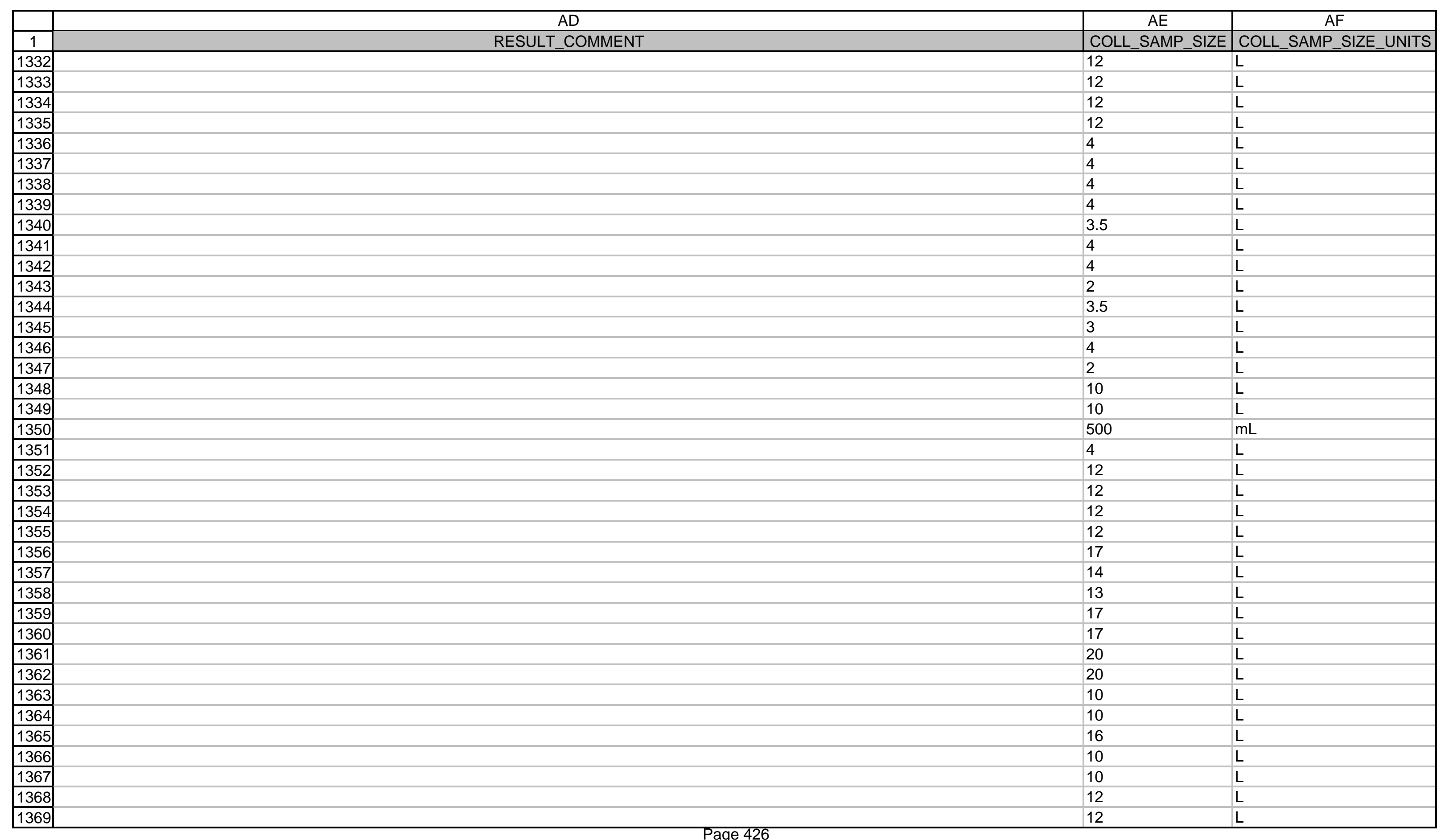




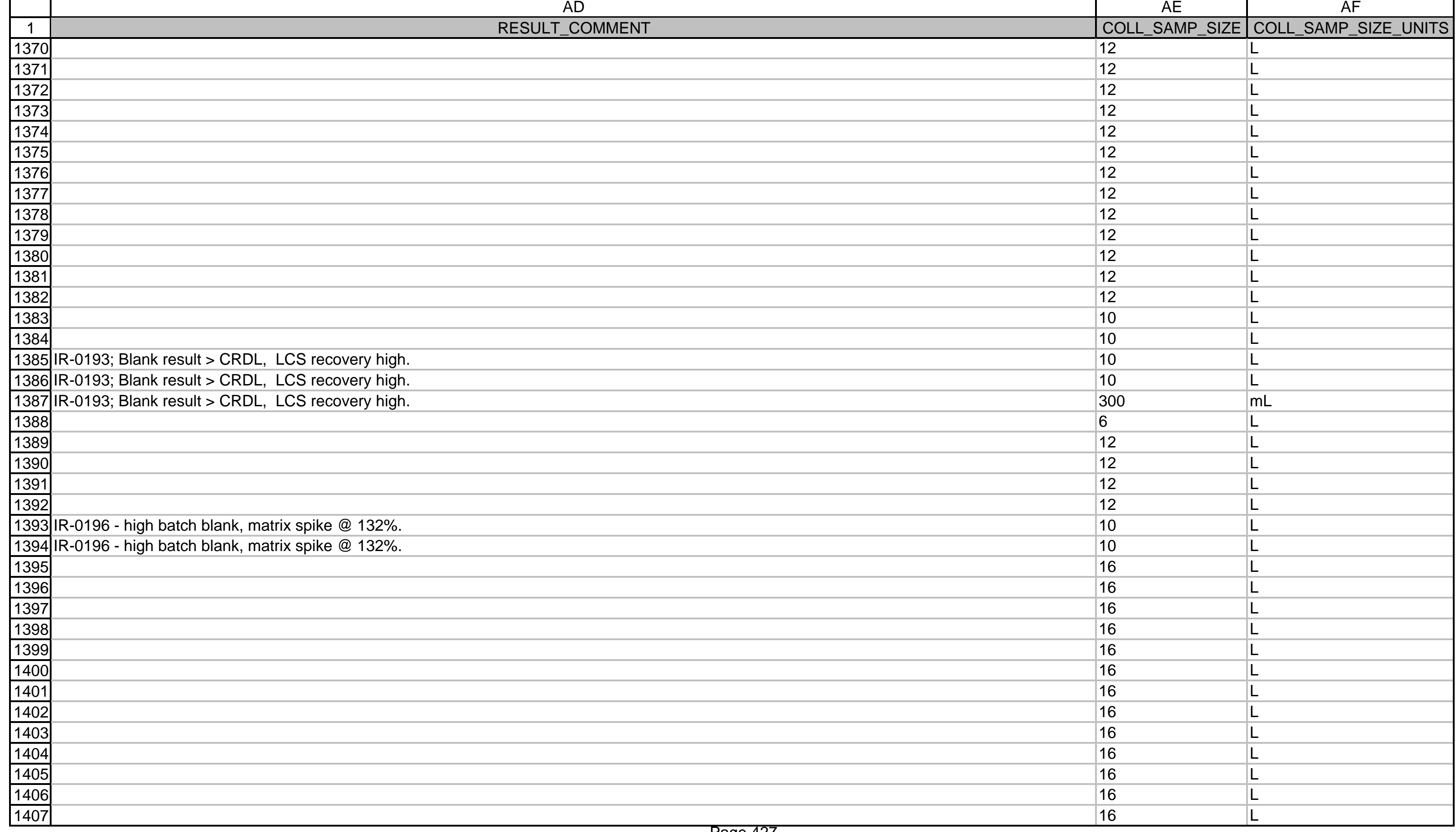


1410 IR-0196 - high batch blank, matrix spike @ 132\%.

1411 IR-0196 - high batch blank, matrix spike @ 132\%.

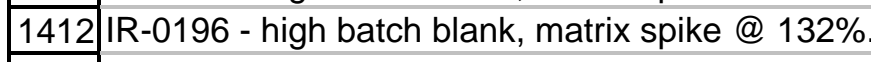

1413

1414

1415

1416

1427 IR-0203: Batch blanks high at 16 and $13 \mathrm{pCi} / \mathrm{L}$.

1428 IR-0203: Batch blanks high at 16 and $13 \mathrm{pCi} / \mathrm{L}$.

1429 IR-0203: Batch blanks high at 16 and $13 \mathrm{pCi} / \mathrm{L}$.

1430 IR-0203: Batch blanks high at 16 and $13 \mathrm{pCi} / \mathrm{L}$.

1431 IR-0209: Matrix blank > RDL.

1432 IR-0209: Matrix blank > RDL.

1433 IR-0209: Matrix blank > RDL.

1434 IR-0209: Matrix blank > RDL.

1435 IR-0209: Matrix blank > RDL.

1436 IR-0209: Matrix blank > RDL.

1437 IR-0209: Matrix blank > RDL.

1438 IR-0209: Matrix blank > RDL.

1439 IR-0209: Matrix blank > RDL.

1440 IR-0209: Matrix blank > RDL.

16

16

10

300

6

12

12

10

$\mathrm{L}$

$L$

L

L

\begin{tabular}{l|l}
\hline $\mathrm{L}$ \\
$\mathrm{L}$
\end{tabular}

$$
\begin{array}{|l}
\hline \text { L } \\
\hline \text { L } \\
\hline
\end{array}
$$$$
\text { L }
$$

L 
1446 IR-0211: Matrix blank (325) >CRDL. Sample results a magnitude higher.

6

1447

1448

1449

1450

1451

1452

1453 IR-0223: Direct spike low at $68 \%$ recovery.

1454 IR-0223: Direct spike low at $68 \%$ recovery.

1455

1456

\begin{tabular}{|l|}
\hline 1457 \\
\hline 1458 \\
\hline
\end{tabular}

\begin{tabular}{|l|}
1458 \\
\hline 1459 \\
\hline
\end{tabular}

1459

1460

1461

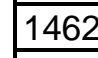

\begin{tabular}{|l|}
1463 \\
\hline 1464 \\
\hline
\end{tabular}

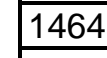

\begin{tabular}{|l}
1465 \\
\hline 1466 \\
\hline
\end{tabular}

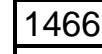

1467

1468

1469

1470

\begin{tabular}{|l|}
\hline 1471 \\
\hline 1472 \\
\hline 1473 \\
\hline
\end{tabular}

\begin{tabular}{|l|}
\hline 1472 \\
\hline 1473 \\
\hline 1474 \\
\hline
\end{tabular}

\begin{tabular}{|l|}
\hline 1474 \\
\hline 1475 \\
\hline
\end{tabular}

\begin{tabular}{|l|}
\hline 1474 \\
\hline 1475 \\
\hline 1476 \\
\hline
\end{tabular}

\begin{tabular}{|l|}
\hline 1476 \\
\hline 1477 \\
\hline
\end{tabular}

1477

\begin{tabular}{|l|}
\hline 1478 \\
\hline 1479 \\
\hline
\end{tabular}

1479

1480

1481

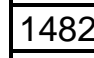

1483

RESULT COMMENT

COLL SAMP SIZE COLL SAMP SIZE_UNITS

12

12

12

10

15

15

15

15

15

15

15

15

5

15

15

15

15

15

15

15

15

15

15

15

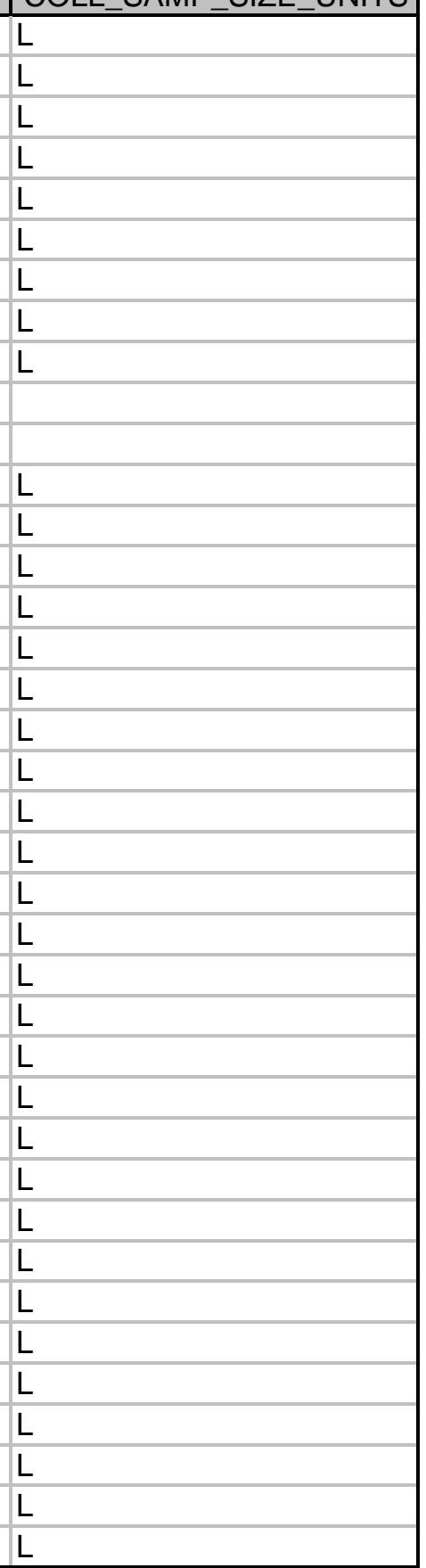




\begin{tabular}{|c|c|c|c|}
\hline & & & \\
\hline 1 & $\begin{array}{r}\text { RESULT_COMMENT } \\
\end{array}$ & COLL_SAMP_SIZE & COLL_SAMP_SIZE_UNITS \\
\hline 1484 & & $15=$ & $\mathrm{L}=-2$ \\
\hline 1485 & & 15 & L \\
\hline 1486 & & 15 & L \\
\hline 1487 & & 15 & L \\
\hline 1488 & & 15 & L \\
\hline 1489 & & 15 & L \\
\hline 1490 & & 15 & L \\
\hline 1491 & & 15 & L \\
\hline 1492 & & 15 & L \\
\hline $\mid 1493$ & IR-0253: Both blanks >CRDL. & 15 & L \\
\hline 1494 | & IR-0253: Both blanks >CRDL. & 15 & L \\
\hline 1495 & IR-0253: Both blanks >CRDL. & 15 & L \\
\hline 1496 & IR-0253: Both blanks >CRDL. & 15 & L \\
\hline 1497 I & IR-0253: Both blanks >CRDL. & 15 & L \\
\hline 1498 | & IR-0253: Both blanks >CRDL. & 15 & L \\
\hline 1499 & IR-0254: One blank > CRDL. & 15 & L \\
\hline 1500 & IR-0254: One blank > CRDL. & 15 & L \\
\hline 1501 & IR-0254: One blank > CRDL. & 15 & L \\
\hline 1502 & IR-0254: One blank > CRDL. & 15 & L \\
\hline 1503 & IR-0254: One blank > CRDL. & 15 & L \\
\hline 1504 I & IR-0254: One blank > CRDL. & 15 & L \\
\hline 1505 & IR-0254: One blank > CRDL. & 15 & L \\
\hline 1506 & IR-0254: One blank > CRDL. & 15 & L \\
\hline 1507 I & IR-0254: One blank > CRDL. & 15 & L \\
\hline 1508 & IR-0254: One blank > CRDL. & 15 & L \\
\hline 1509 & IR-0253: Both blanks >CRDL. & 15 & L \\
\hline 1510 & IR-0253: Both blanks >CRDL. & 15 & L \\
\hline 1511 & IR-0253: Both blanks >CRDL. & 15 & L \\
\hline 1512 & IR-0253: Both blanks >CRDL. & 15 & L \\
\hline 1513 & & 20 & L \\
\hline 1514 & & 24 & L \\
\hline 1515 & & 24 & L \\
\hline 1516 I & IR-0257: Matrix and reagent blank results>CRDL. & 10 & L \\
\hline 1517 & IR-0257: Matrix and reagent blank results>CRDL. & 10 & L \\
\hline 1518 & & 16 & L \\
\hline 1519 & & 20 & $\mathrm{~L}$ \\
\hline 1520 & & 16 & L \\
\hline 1521 & & 19 & $\mathrm{~L}$ \\
\hline
\end{tabular}


1524 IR-0257: Matrix and reagent blank results>CRDL.

1525 IR-0257: Matrix and reagent blank results>CRDL.

1526 IR-0261: First Blank > CRDL. Second blank < CRDL.

1527 IR-0257: Matrix and reagent blank results>CRDL.

1528 IR-0257: Matrix and reagent blank results>CRDL.

1529 IR-0257: Matrix and reagent blank results>CRDL.

1530 IR-0261: First Blank > CRDL. Second blank < CRDL.

1531 IR-0261: First Blank > CRDL. Second blank < CRDL.

1532

L

L

L

L

L

L

L

L

L

L

L

L

L

L

L

L

L

L

L

L

L

L

L

L

L 


\begin{tabular}{|c|c|c|c|}
\hline & AD & $\mathrm{AE}$ & $\mathrm{AF}$ \\
\hline 1 & $\begin{aligned} \text { RESULT_COMMENT } \\
\end{aligned}$ & COLL_SAMP_SIZE & COLL_SAMP_SIZE_UNITS \\
\hline 1560 & & 19 & L \\
\hline $1561 \neq \neq$ & \#IR-0062 MDA > CRDL & 4 & $\mathrm{~L}$ \\
\hline $1562 \neq$ & \#IR-0062 MDA > CRDL & 3 & L \\
\hline 1563 & \#IR-0062 MDA > CRDL & 1 & L \\
\hline 1564 & & 20 & L \\
\hline 1565 & & 20 & L \\
\hline 1566 & & 20 & L \\
\hline 1567 & & 20 & L \\
\hline 1568 & & 20 & L \\
\hline 1569 & & 20 & L \\
\hline 1570 & & 20 & L \\
\hline 1571 & & 20 & $\mathrm{~L}$ \\
\hline 1572 & & 20 & L \\
\hline 1573 & & 20 & L \\
\hline 1574 & & 20 & L \\
\hline 1575 & & 20 & L \\
\hline 1576 & & 20 & L \\
\hline 1577 & & 20 & L \\
\hline $\mid$ & \#IR-0059 Blank Result > CRDL & 10 & L \\
\hline 1581 & & 19 & L \\
\hline 1582 & & 10 & L \\
\hline 1583 & & 10 & L \\
\hline 1584 & & 10 & $\mathrm{~L}$ \\
\hline 1585 & & 10 & L \\
\hline 1586 & & 12 & L \\
\hline 1587 & & 12 & $\mathrm{~L}$ \\
\hline 1588 & & 12 & L \\
\hline 1589 & & 12 & L \\
\hline 1590 & & 12 & $\mathrm{~L}$ \\
\hline 1591 & & 12 & L \\
\hline 1592 & & 12 & L \\
\hline \begin{tabular}{|l|l|}
1593 \\
\end{tabular} & & 12 & $L$ \\
\hline 1594 & & 12 & L \\
\hline 1595 & & 12 & L \\
\hline 1596 & & 12 & $\mathrm{~L}$ \\
\hline 1597 & & 12 & $L$ \\
\hline
\end{tabular}




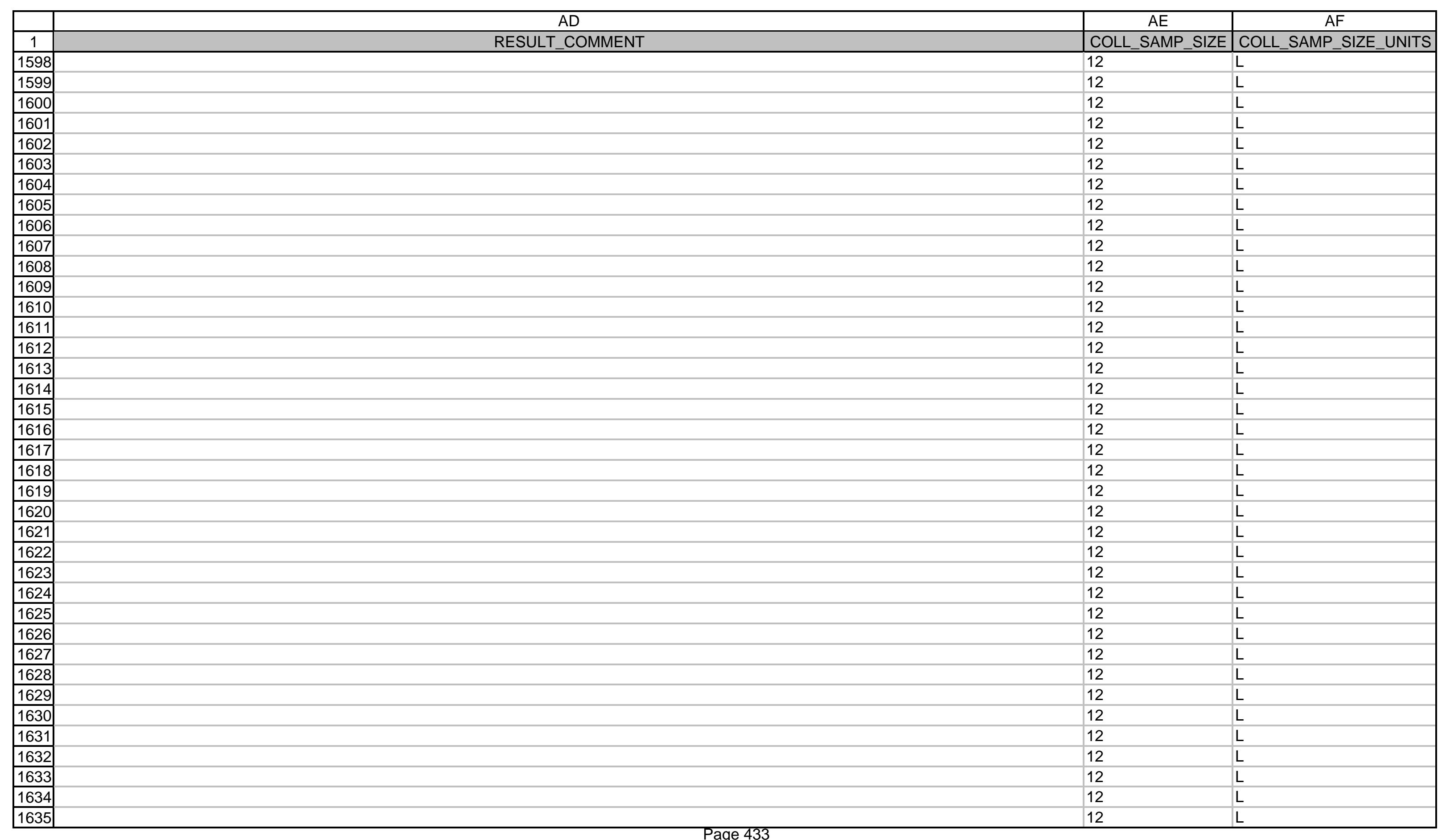




\begin{tabular}{|c|c|c|c|}
\hline & $A D$ & $\mathrm{AE}$ & $\mathrm{AF}$ \\
\hline 1 & RESULT_COMMENT & COLL_SAMP_SIZE & COLL_SAMP_SIZE_UNITS \\
\hline 1636 & & 12 & $\mathrm{~L}$ \\
\hline 1637 & & 12 & L \\
\hline 1638 & & 12 & $\mathrm{~L}$ \\
\hline 1639 & & 12 & $\bar{L}$ \\
\hline 1640 & & 8 & $\mathrm{~L}$ \\
\hline 1641 & & 14 & L \\
\hline 1642 & & 8 & $\mathrm{~L}$ \\
\hline 1643 & & 8 & $\mathrm{~L}$ \\
\hline 1645 & & 16 & L \\
\hline 1646 & & 10 & $\mathrm{~L}$ \\
\hline 1647 & & 10 & L \\
\hline 1648 & MDAs DO NOT MEET THE CRDL & 9 & $\mathrm{~L}$ \\
\hline 1649 & & 16 & $\mathrm{~L}$ \\
\hline 1650 & & 16 & L \\
\hline 1651 & & 10 & L \\
\hline 1652 & & 10 & $\mathrm{~L}$ \\
\hline \begin{tabular}{|c|}
1653 \\
\end{tabular} & & 16 & $\mathrm{~L}$ \\
\hline 1655 & & 10 & $\mathrm{~L}$ \\
\hline 1656 & & 12 & $\mathrm{~L}$ \\
\hline 1657 & & 12 & L \\
\hline 1658 & & 12 & $\bar{L}$ \\
\hline 1659 & & 12 & L \\
\hline 1660 & & 12 & L \\
\hline 1661 & & 12 & $\bar{L}$ \\
\hline 1662 & & 12 & L \\
\hline 1663 & & 12 & L \\
\hline 1664 & & 12 & L \\
\hline \begin{tabular}{|l|}
1665 \\
\end{tabular} & & 12 & $L$ \\
\hline 1666 & & 12 & L \\
\hline 1667 & & 12 & $\mathrm{~L}$ \\
\hline 1668 & & 12 & L \\
\hline 1669 & & 12 & L \\
\hline 1670 & & 12 & $\mathrm{~L}$ \\
\hline \begin{tabular}{|l|}
1671 \\
\end{tabular} & & 10 & $L$ \\
\hline 1672 & & 10 & $\mathrm{~L}$ \\
\hline
\end{tabular}




\begin{tabular}{|c|c|c|c|}
\hline & $A D$ & $\mathrm{AE}$ & $\mathrm{AF}$ \\
\hline 1 & RESULT_COMMENT & COLL_SAMP_SIZE & COLL_SAMP_SIZE_UNITS \\
\hline 1674 & & 10 & $L$ \\
\hline 1675 & \#IR-0103 - The achieved MDAs do not meet the CRDL. & 9 & $\mathrm{~L}$ \\
\hline 1676 & & 12 & $\mathrm{~L}$ \\
\hline 1677 & & 12 & L \\
\hline 1678 & & 12 & $\mathrm{~L}$ \\
\hline 1679 & & 12 & $\mathrm{~L}$ \\
\hline 1680 & & 12 & $\mathrm{~L}$ \\
\hline 1681 & & 12 & L \\
\hline 1682 & & 12 & $\mathrm{~L}$ \\
\hline 1683 & & 12 & $\mathrm{~L}$ \\
\hline 1684 & & 12 & L \\
\hline 1685 & & 12 & $\mathrm{~L}$ \\
\hline 1686 & & 12 & $\mathrm{~L}$ \\
\hline \begin{tabular}{|l|l|}
1687 \\
\end{tabular} & & 12 & $\mathrm{~L}$ \\
\hline 1688 & & 12 & $\mathrm{~L}$ \\
\hline 1689 & & 12 & L \\
\hline 1690 & & 12 & $\mathrm{~L}$ \\
\hline 1691 & & 10 & $\mathrm{~L}$ \\
\hline 1693 & IR-0116; MDA > CRDL & 9 & L \\
\hline 1694 & & 10 & $\mathrm{~L}$ \\
\hline 1695 & & 10 & $\mathrm{~L}$ \\
\hline \begin{tabular}{|l|l|}
1696 \\
\end{tabular} & & 8 & $\mathrm{~L}$ \\
\hline 1697 & & 16 & $\mathrm{~L}$ \\
\hline 1698 & & 12 & $\mathrm{~L}$ \\
\hline 1699 & & 8 & $\mathrm{~L}$ \\
\hline \begin{tabular}{|l|l|}
1700 \\
\end{tabular} & & 16 & $\mathrm{~L}$ \\
\hline 1701 & & 10 & $\mathrm{~L}$ \\
\hline \begin{tabular}{|l|}
1702 \\
\end{tabular} & & 10 & $\mathrm{~L}$ \\
\hline 1703 & & 12 & $\mathrm{~L}$ \\
\hline 1704 & & 15 & $\mathrm{~L}$ \\
\hline \begin{tabular}{|l|l|}
1705 \\
\end{tabular} & & 15 & $\mathrm{~L}$ \\
\hline 1706 & & 16 & $\mathrm{~L}$ \\
\hline \begin{tabular}{|l|}
1707 \\
\end{tabular} & & 2 & $\mathrm{~L}$ \\
\hline 1708 & & 10 & $\mathrm{~L}$ \\
\hline 1709 & & 10 & $\mathrm{~L}$ \\
\hline 1710 & & 15 & $\mathrm{~L}$ \\
\hline
\end{tabular}




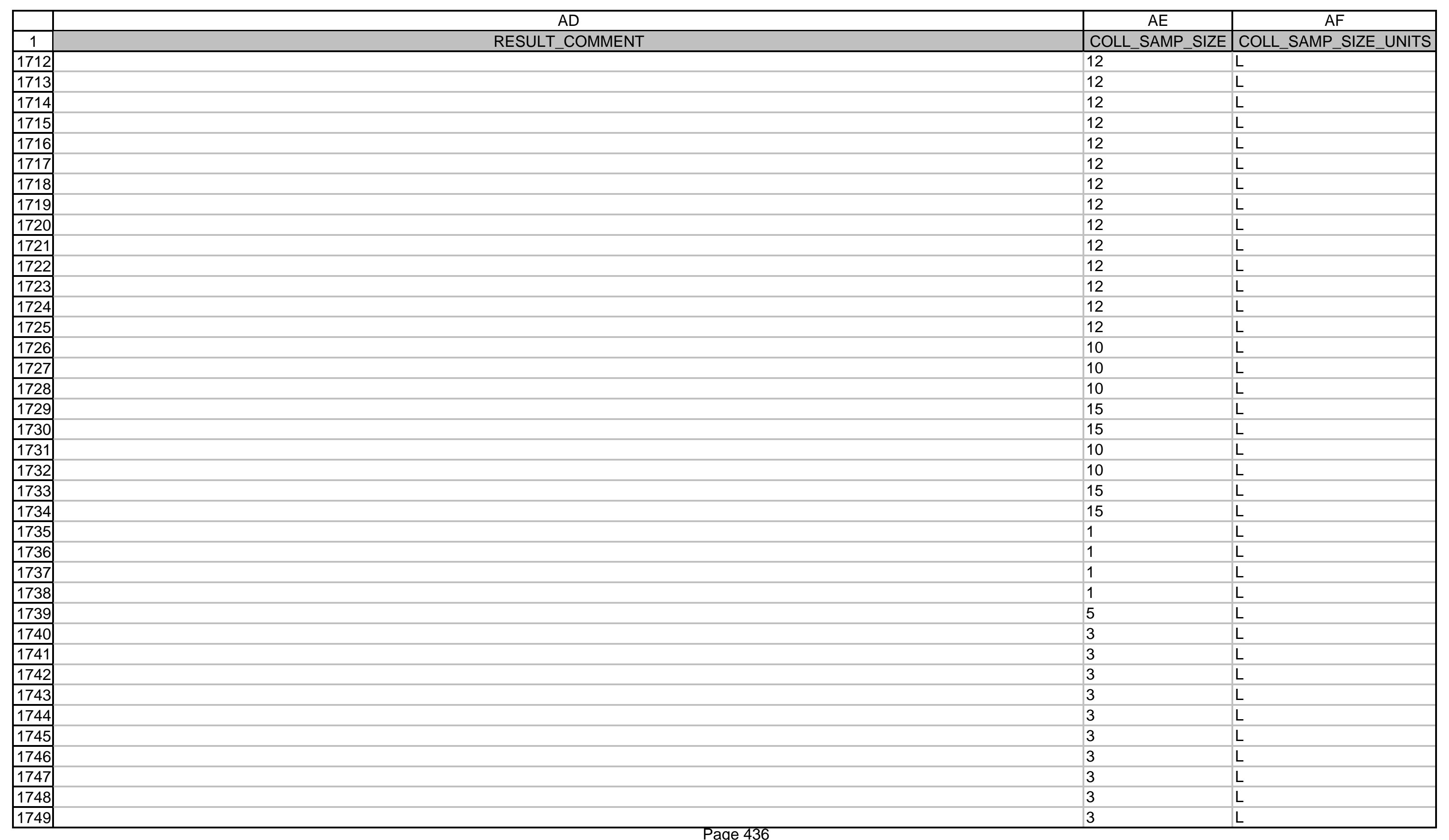




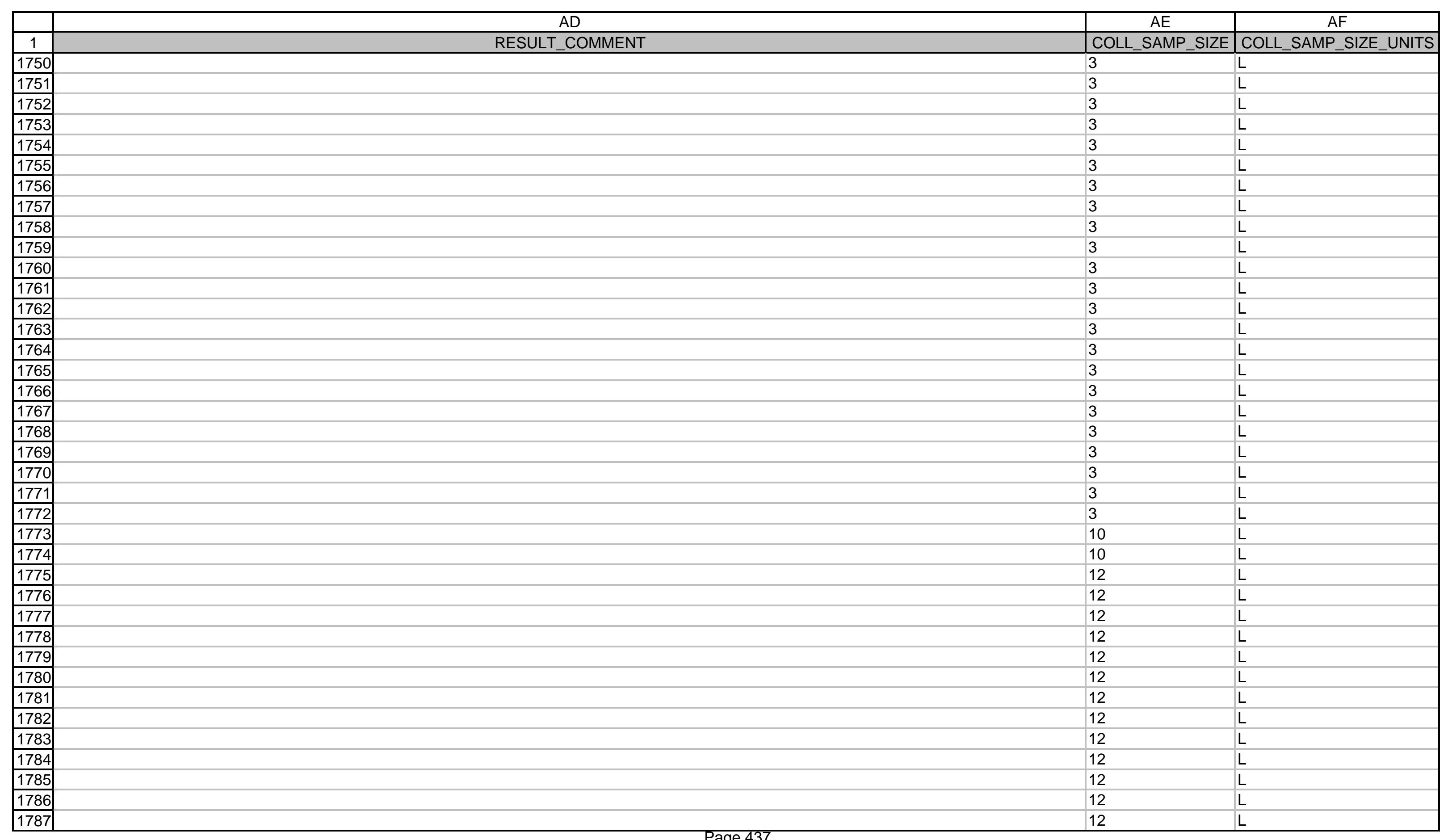




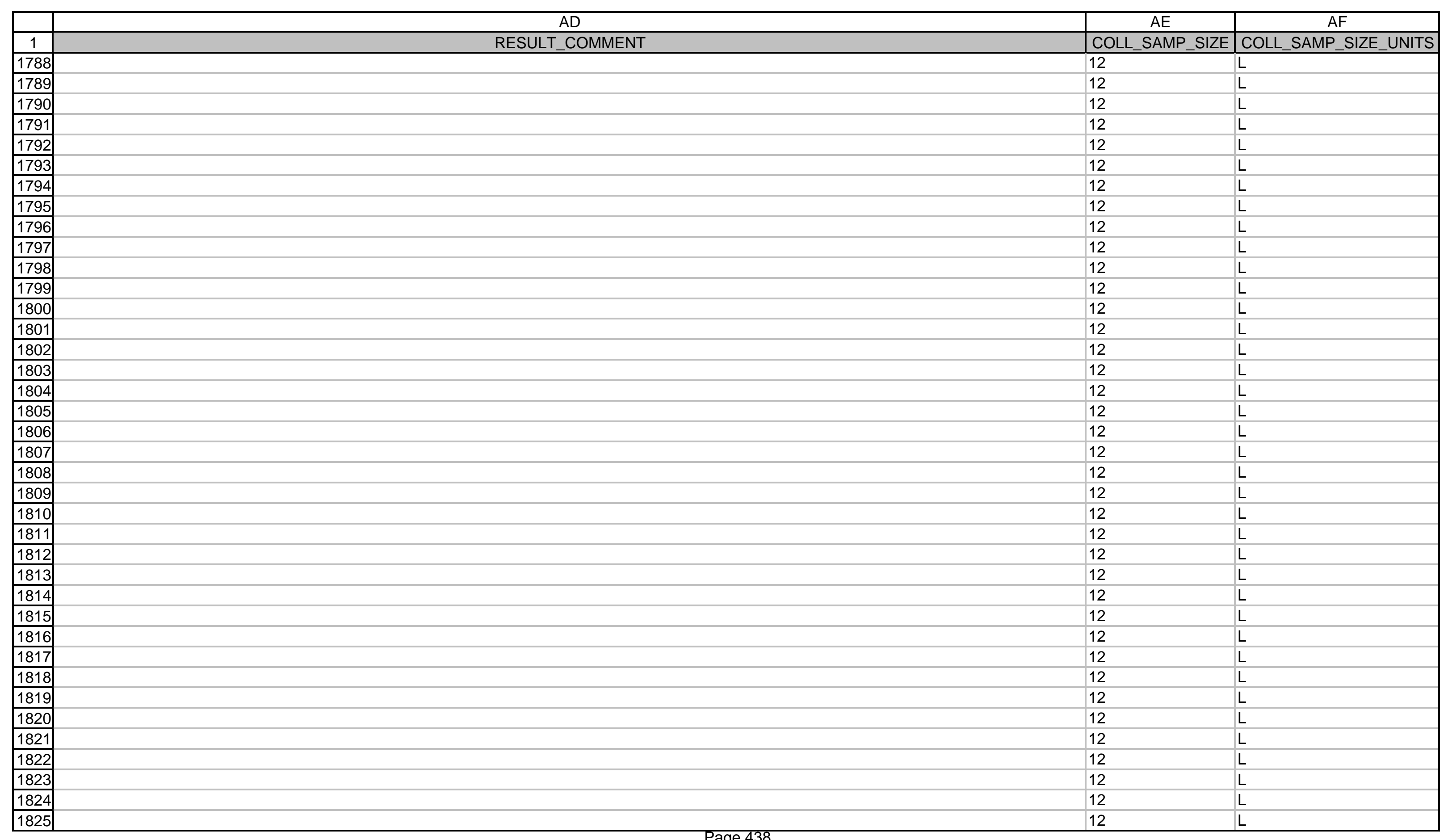




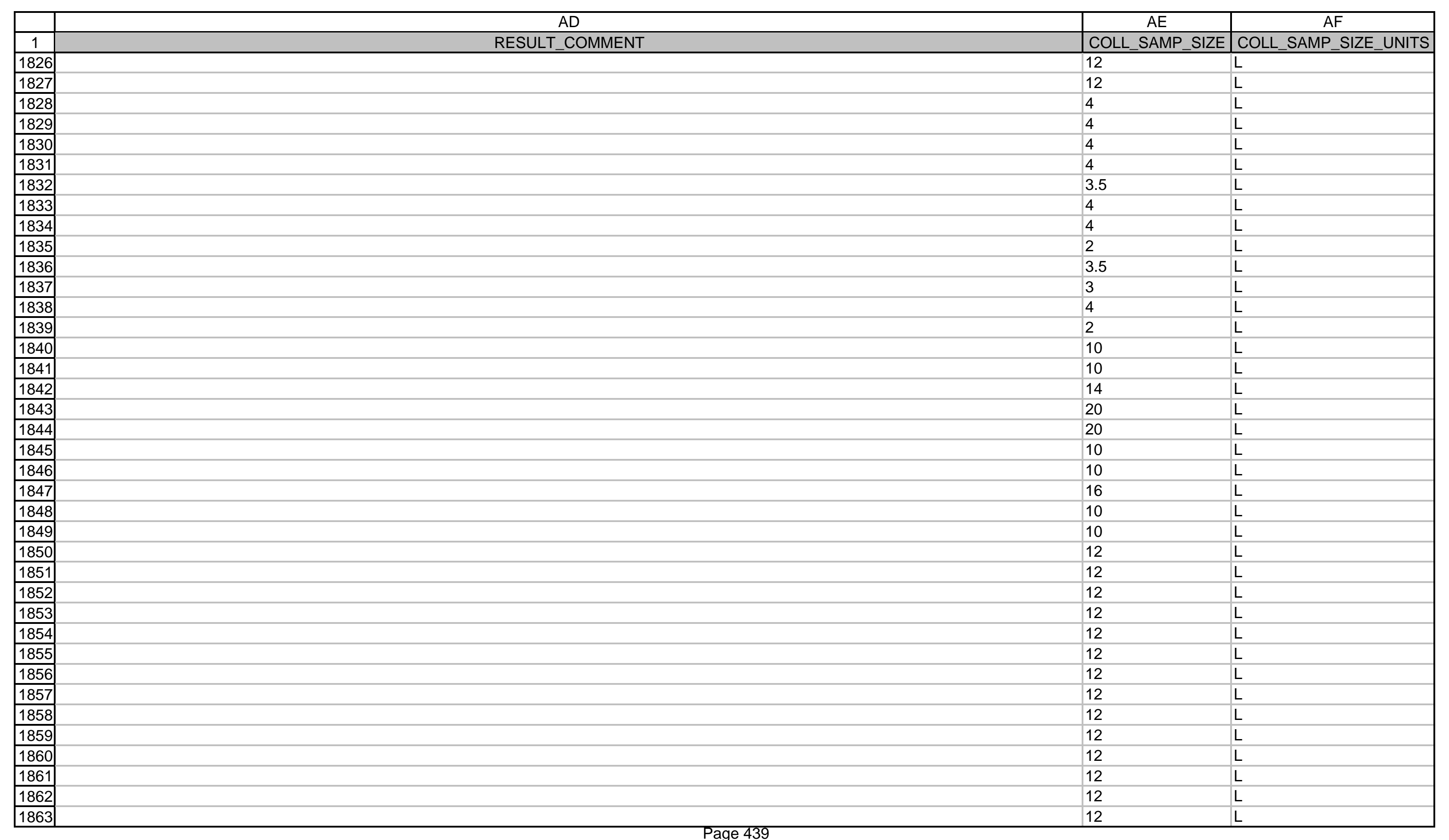




\begin{tabular}{|c|c|c|c|}
\hline & $A D$ & $\mathrm{AE}$ & $\mathrm{AF}$ \\
\hline 1 & RESULT_COMMENT & $\mid$\begin{tabular}{|l|} 
COLL_SAMP_SIZE \\
\end{tabular} & COLL_SAMP_SIZE_UNITS \\
\hline 1864 & & 12 & $\mathrm{~L}$ \\
\hline 1865 & & 10 & L \\
\hline 1866 & & 10 & L \\
\hline \begin{tabular}{|l|}
1867 \\
\end{tabular} & & 10 & $\mathrm{~L}$ \\
\hline 1868 & & 10 & L \\
\hline 1869 & & 10 & L \\
\hline \begin{tabular}{|l|}
1870 \\
\end{tabular} & & 10 & $\mathrm{~L}$ \\
\hline 1871 & & 16 & L \\
\hline 1872 & & 16 & L \\
\hline 1873 & & 16 & $\mathrm{~L}$ \\
\hline 1874 & & 16 & L \\
\hline 1875 & & 16 & $\mathrm{~L}$ \\
\hline 1876 & & 16 & $\mathrm{~L}$ \\
\hline 1877 & & 16 & L \\
\hline 1878 & & 16 & L \\
\hline \begin{tabular}{|l|}
1879 \\
\end{tabular} & & 16 & $\mathrm{~L}$ \\
\hline \begin{tabular}{|l|}
1880 \\
\end{tabular} & & 16 & $\mathrm{~L}$ \\
\hline 1881 & & 16 & L \\
\hline 1882 & & 16 & L \\
\hline \begin{tabular}{|l|}
1883 \\
\end{tabular} & & 16 & $\mathrm{~L}$ \\
\hline 1884 & & 16 & L \\
\hline 1885 & & 16 & L \\
\hline \begin{tabular}{|l|}
1886 \\
\end{tabular} & & 10 & $\mathrm{~L}$ \\
\hline 1887 & & 10 & L \\
\hline 1888 & & 10 & $\mathrm{~L}$ \\
\hline \begin{tabular}{|l|}
1889 \\
\end{tabular} & & 10 & $\mathrm{~L}$ \\
\hline 1890 & & 20 & L \\
\hline 1891 & & 20 & L \\
\hline \begin{tabular}{|l|}
1892 \\
\end{tabular} & & 10 & $\mathrm{~L}$ \\
\hline \begin{tabular}{|l|}
1893 \\
\end{tabular} & & 10 & $\mathrm{~L}$ \\
\hline 1894 & & 16 & L \\
\hline \begin{tabular}{|l|}
1895 \\
\end{tabular} & & 16 & $\mathrm{~L}$ \\
\hline \begin{tabular}{|l|}
1896 \\
\end{tabular} & & 16 & $\mathrm{~L}$ \\
\hline 1897 & & 16 & L \\
\hline \begin{tabular}{|l|}
1898 \\
\end{tabular} & & 16 & L \\
\hline \begin{tabular}{|l|}
1899 \\
\end{tabular} & & 16 & $\mathrm{~L}$ \\
\hline 1900 & & 16 & L \\
\hline \begin{tabular}{|l|}
1901 \\
\end{tabular} & & 16 & L \\
\hline
\end{tabular}




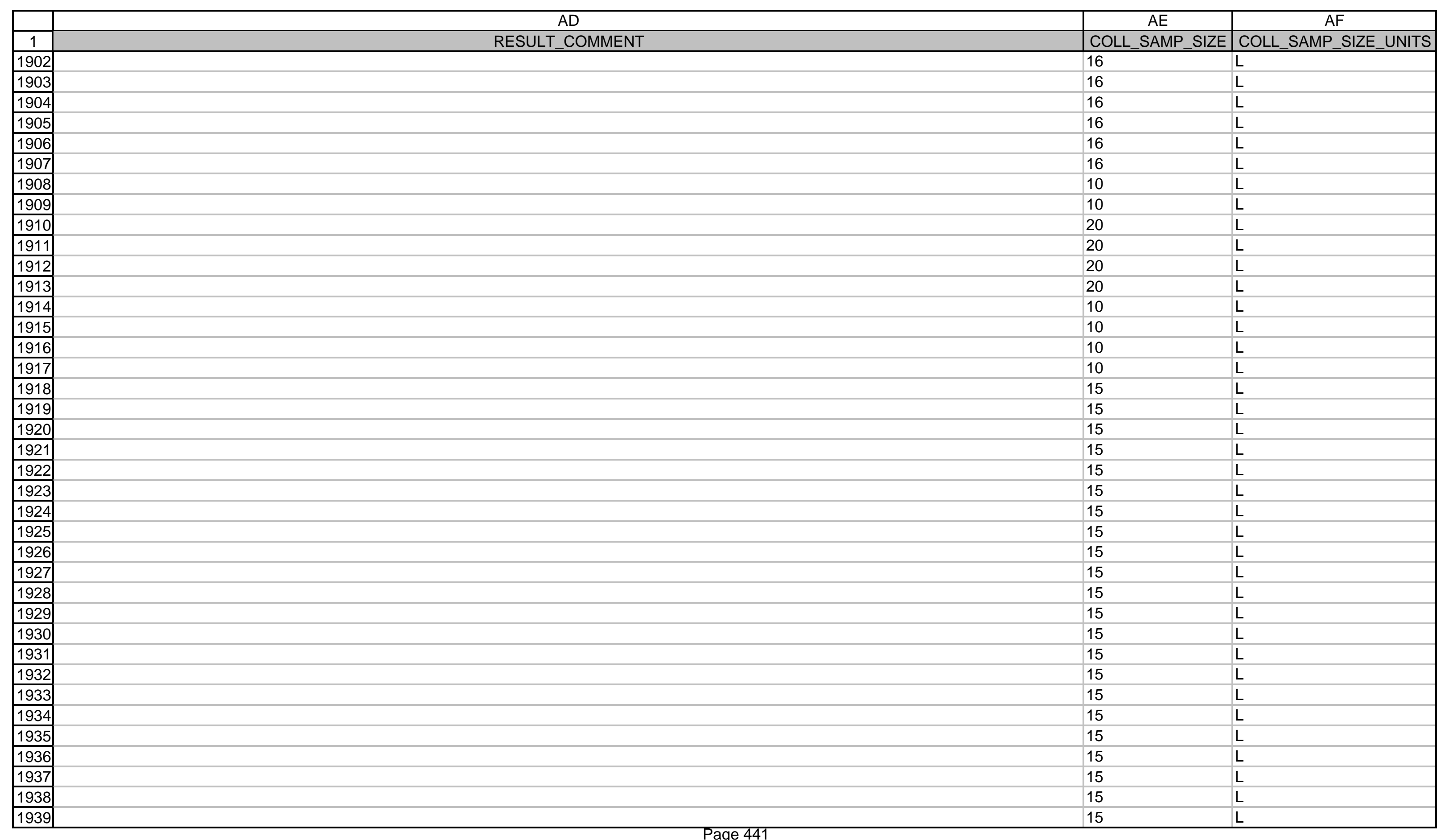




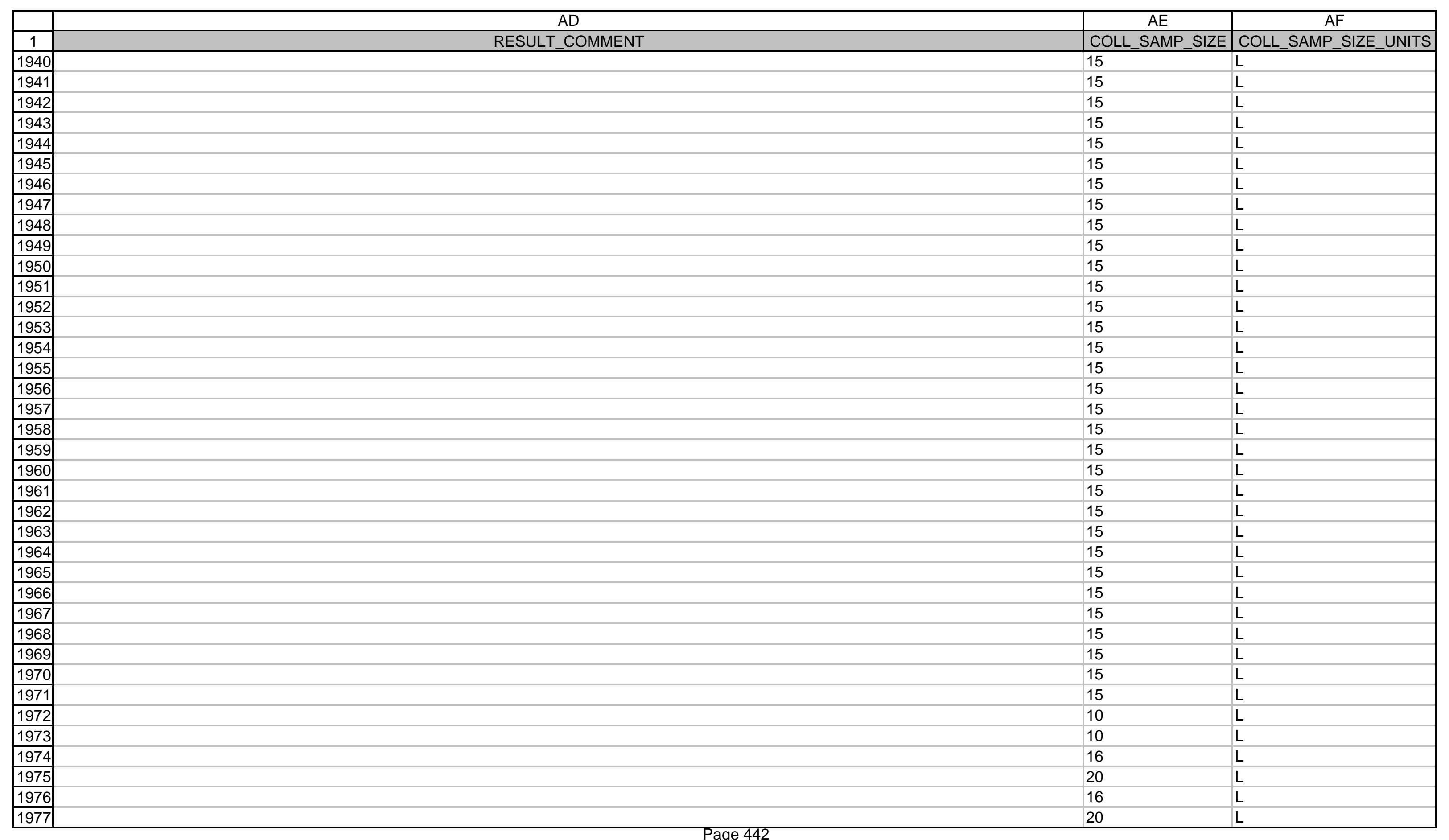




\begin{tabular}{|c|c|c|c|}
\hline & $A D$ & $\mathrm{AE}$ & $\mathrm{AF}$ \\
\hline 1 & RESULT_COMMENT & COLL_SAMP_SIZE & COLL_SAMP_SIZE_UNITS \\
\hline 1978 & & 20 & $\mathrm{~L}$ \\
\hline 1979 & & 10 & $\mathrm{~L}$ \\
\hline 1980 & & 10 & $\mathrm{~L}$ \\
\hline 1981 & & 10 & $\bar{L}$ \\
\hline 1982 & & 10 & $\mathrm{~L}$ \\
\hline 1983 & & 15 & $\mathrm{~L}$ \\
\hline 1984 & & 15 & $\mathrm{~L}$ \\
\hline 1985 & & 15 & $L$ \\
\hline 1987 & & 15 & L \\
\hline 1988 & & 15 & $\mathrm{~L}$ \\
\hline 1989 & & 15 & L \\
\hline 1990 & & 15 & $\mathrm{~L}$ \\
\hline 1991 & & 15 & $\mathrm{~L}$ \\
\hline 1992 & & 15 & L \\
\hline 1993 & & 15 & L \\
\hline 1994 & & 15 & $\mathrm{~L}$ \\
\hline 1995 & & 15 & L \\
\hline 1997 & & 15 & $\mathrm{~L}$ \\
\hline 1998 & & 10 & L \\
\hline 1999 & & 10 & L \\
\hline 2000 & & 10 & $\bar{L}$ \\
\hline 2001 & & 10 & $\mathrm{~L}$ \\
\hline 2002 & & 10 & L \\
\hline 2003 & & 20 & $\bar{L}$ \\
\hline 2004 & ; U QUALIFIER ADDED OR J QUALIFIER REPLACED BY U QUALIFIER PER INTERNAL CORRECTIVE ACTIONS -- LE BISPING 04/04/01. & 20 & $\bar{L}$ \\
\hline 2005 & & 20 & L \\
\hline 2006 & & 20 & $\mathrm{~L}$ \\
\hline 2007 & & 20 & $\mathrm{~L}$ \\
\hline 2008 & & 20 & L \\
\hline 2009 & & 20 & $\mathrm{~L}$ \\
\hline 2010 & & 20 & $\mathrm{~L}$ \\
\hline 2011 & & 20 & $\mathrm{~L}$ \\
\hline 2012 & & 20 & $\mathrm{~L}$ \\
\hline 2013 & & 20 & $\mathrm{~L}$ \\
\hline 2014 & & 20 & $\mathrm{~L}$ \\
\hline
\end{tabular}




\begin{tabular}{|c|c|c|c|}
\hline & 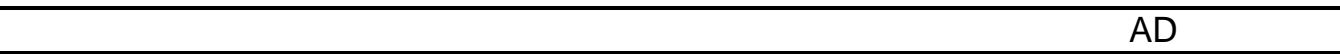 & $\mathrm{AE}$ & $\mathrm{AF}$ \\
\hline 1 & 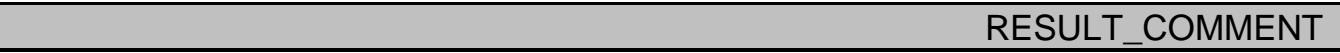 & COLL_SAMP_SIZE & COLL_SAMP_SIZE_UNITS \\
\hline 2016 & & 20 & $\mathrm{~L}$ \\
\hline 2017 & & 20 & $\mathrm{~L}$ \\
\hline 2018 & & 10 & $\mathrm{~L}$ \\
\hline 2019 & & 10 & $\mathrm{~L}$ \\
\hline 2020 & & 10 & $L$ \\
\hline 2021 & & 10 & $\mathrm{~L}$ \\
\hline 2022 & & 10 & $\mathrm{~L}$ \\
\hline 2023 & & 19 & $\mathrm{~L}$ \\
\hline 2025 & & 10 & L \\
\hline 2026 & & 19 & $\mathrm{~L}$ \\
\hline 2027 & \#IR-0062 MDA > CRDL & 4 & $L$ \\
\hline 2028 & \#IR-0062 MDA > CRDL & 3 & L \\
\hline 2029 & \#IR-0062 MDA > CRDL & 1 & $\mathrm{~L}$ \\
\hline 2030 & & 20 & $\mathrm{~L}$ \\
\hline 2031 & & 20 & $\mathrm{~L}$ \\
\hline 2032 & & 20 & $\mathrm{~L}$ \\
\hline 2033 & & 20 & L \\
\hline 2035 & & 20 & L \\
\hline 2036 & & 20 & $\mathrm{~L}$ \\
\hline 2037 & & 20 & $\mathrm{~L}$ \\
\hline 2038 & & 20 & L \\
\hline 2039 & & 20 & $L$ \\
\hline 2040 & & 20 & $\mathrm{~L}$ \\
\hline 2041 & & 20 & L \\
\hline 2042 & & 20 & $\mathrm{~L}$ \\
\hline 2043 & & 20 & $\mathrm{~L}$ \\
\hline 2044 & & 10 & $\mathrm{~L}$ \\
\hline 2045 & & 10 & L \\
\hline 2046 & & 10 & L \\
\hline 2047 & & 19 & L \\
\hline 2048 & & 10 & $\mathrm{~L}$ \\
\hline 2049 & & 10 & $\mathrm{~L}$ \\
\hline 2050 & & 10 & L \\
\hline 2051 & & 10 & $\mathrm{~L}$ \\
\hline 2052 & & 12 & $\mathrm{~L}$ \\
\hline
\end{tabular}




\begin{tabular}{|c|c|c|c|}
\hline & $A D$ & $\mathrm{AE}$ & AF \\
\hline 1 & RESULT_COMMENT & COLL_SAMP_SIZE & COLL_SAMP_SIZE_UNITS \\
\hline 2054 & & 12 & $\mathrm{~L}$ \\
\hline 2055 & & 12 & L \\
\hline 2056 & & 12 & $\mathrm{~L}$ \\
\hline 2057 & & 12 & $\mathrm{~L}$ \\
\hline 2058 & & 12 & $\mathrm{~L}$ \\
\hline 2059 & & 12 & L \\
\hline 2060 & & 12 & $L$ \\
\hline 2061 & & 12 & L \\
\hline 2062 & & 12 & L \\
\hline 2063 & & 12 & L \\
\hline 2064 & & 12 & L \\
\hline 2065; & ; U QUALIFIER ADDED OR J QUALIFIER REPLACED BY U QUALIFIER PER INTERNAL CORRECTIVE ACTIONS -- LE BISPING 04/04/01. & 12 & L \\
\hline 2066 & & 12 & L \\
\hline 2067 & & 12 & $\mathrm{~L}$ \\
\hline 2068 & & 12 & L \\
\hline 2069 & & 12 & L \\
\hline 2070 ; & ; U QUALIFIER ADDED OR J QUALIFIER REPLACED BY U QUALIFIER PER INTERNAL CORRECTIVE ACTIONS -- LE BISPING 04/04/01. & 12 & L \\
\hline 2071 & & 12 & L \\
\hline 2072 & & 12 & L \\
\hline 2073 & & 12 & L \\
\hline 2074 & & 12 & L \\
\hline 2075 & & 12 & L \\
\hline 2076 & & 12 & L \\
\hline 2077 ; & ; U QUALIFIER ADDED OR J QUALIFIER REPLACED BY U QUALIFIER PER INTERNAL CORRECTIVE ACTIONS -- LE BISPING 04/04/01. & 12 & L \\
\hline 2078 & & 12 & L \\
\hline 2079 & & 12 & L \\
\hline 2080 & & 12 & L \\
\hline 2081 & & 12 & L \\
\hline 2082 & & 12 & L \\
\hline 2083 ; & ; U QUALIFIER ADDED OR J QUALIFIER REPLACED BY U QUALIFIER PER INTERNAL CORRECTIVE ACTIONS -- LE BISPING 04/04/01. & 12 & L \\
\hline 2084 ; & ; U QUALIFIER ADDED OR J QUALIFIER REPLACED BY U QUALIFIER PER INTERNAL CORRECTIVE ACTIONS -- LE BISPING 04/04/01. & 12 & L \\
\hline 2085 & & 12 & L \\
\hline 2086 ; & ; U QUALIFIER ADDED OR J QUALIFIER REPLACED BY U QUALIFIER PER INTERNAL CORRECTIVE ACTIONS -- LE BISPING 04/04/01. & 12 & L \\
\hline 2087 & & 12 & L \\
\hline 2088 & & 12 & L \\
\hline 2089 & & 12 & $\mathrm{~L}$ \\
\hline 2090 & & 12 & L \\
\hline 2091 & & 12 & L \\
\hline
\end{tabular}




\begin{tabular}{|c|c|c|c|}
\hline & & & \\
\hline 1 & RESULT_COMMENT & COLL_SAMP_SIZE & COLL_SAMP_SIZE_UNITS \\
\hline 2092 & & 12 & L \\
\hline 2093 & ; U QUALIFIER ADDED OR J QUALIFIER REPLACED BY U QUALIFIER PER INTERNAL CORRECTIVE ACTIONS -- LE BISPING 04/04/01. & 12 & L \\
\hline 2094 & & 12 & L \\
\hline 2095 & & 12 & L \\
\hline 2096 & & 12 & L \\
\hline 2097 & & 12 & $\mathrm{~L}$ \\
\hline 2098 & & 12 & $\mathrm{~L}$ \\
\hline 2099 & & 12 & L \\
\hline 2100 & & 12 & L \\
\hline 2101 & & 12 & $\mathrm{~L}$ \\
\hline 2102 & & 12 & L \\
\hline 2103 & & 12 & L \\
\hline 2104 & & 12 & $\mathrm{~L}$ \\
\hline 2105 & & 12 & L \\
\hline 2106 & & 8 & L \\
\hline 2107 & & 14 & $\mathrm{~L}$ \\
\hline 2108 & & 8 & $\mathrm{~L}$ \\
\hline 2109 & & 8 & L \\
\hline 2112 & & 10 & L \\
\hline 2113 & & 10 & L \\
\hline 2114 & MDAs DO NOT MEET THE CRDL & 9 & $\mathrm{~L}$ \\
\hline 2115 & ; U QUALIFIER ADDED OR J QUALIFIER REPLACED BY U QUALIFIER PER INTERNAL CORRECTIVE ACTIONS -- LE BISPING 04/04/01. & 16 & L \\
\hline 2116 & & 16 & $\mathrm{~L}$ \\
\hline 2117 & & 10 & $\mathrm{~L}$ \\
\hline 2118 & ; U QUALIFIER ADDED OR J QUALIFIER REPLACED BY U QUALIFIER PER INTERNAL CORRECTIVE ACTIONS -- LE BISPING 04/04/01. & 10 & L \\
\hline 2119 & & 16 & $\mathrm{~L}$ \\
\hline 2120 & & 10 & $\mathrm{~L}$ \\
\hline 2121 & & 10 & L \\
\hline 2122 & & 12 & $\mathrm{~L}$ \\
\hline 2123 & & 12 & $\mathrm{~L}$ \\
\hline 2124 & & 12 & L \\
\hline 2125 & & 12 & L \\
\hline 2126 & & 12 & L \\
\hline 2127 & & 12 & L \\
\hline 2128 & & 12 & L \\
\hline 2129 & & 12 & L \\
\hline
\end{tabular}




\begin{tabular}{|c|c|c|c|}
\hline & $A D$ & $\mathrm{AE}$ & $\mathrm{AF}$ \\
\hline 1 & RESULT_COMMENT & COLL_SAMP_SIZE & COLL_SAMP_SIZE_UNITS \\
\hline 2130 & & 12 & $\mathrm{~L}$ \\
\hline 2131 & & 12 & L \\
\hline 2132 & ; U QUALIFIER ADDED OR J QUALIFIER REPLACED BY U QUALIFIER PER INTERNAL CORRECTIVE ACTIONS -- LE BISPING 04/04/01. & 12 & $\mathrm{~L}$ \\
\hline 2133 & & 12 & L \\
\hline 2134 & & 12 & L \\
\hline 2135 & & 12 & L \\
\hline 2136 & & 12 & $\mathrm{~L}$ \\
\hline 2137 & & 10 & L \\
\hline 2139 & & 10 & L \\
\hline 2140 & & 10 & L \\
\hline 2141 & \#IR-0103 - The achieved MDAs do not meet the CRDL. & 9 & L \\
\hline 2142 & ; U QUALIFIER ADDED OR J QUALIFIER REPLACED BY U QUALIFIER PER INTERNAL CORRECTIVE ACTIONS -- LE BISPING 04/04/01. & 12 & $\mathrm{~L}$ \\
\hline 2143 & & 12 & L \\
\hline 2144 & & 12 & L \\
\hline 2145 & & 12 & $\mathrm{~L}$ \\
\hline 2146 & ; U QUALIFIER ADDED OR J QUALIFIER REPLACED BY U QUALIFIER PER INTERNAL CORRECTIVE ACTIONS -- LE BISPING 04/04/01. & 12 & L \\
\hline 2147 & ; U QUALIFIER ADDED OR J QUALIFIER REPLACED BY U QUALIFIER PER INTERNAL CORRECTIVE ACTIONS -- LE BISPING 04/04/01. & 12 & L \\
\hline 2149 & & 12 & L \\
\hline 2150 & & 12 & $\mathrm{~L}$ \\
\hline 2151 & & 12 & L \\
\hline 2152 & & 12 & $\mathrm{~L}$ \\
\hline 2153 & & 12 & L \\
\hline 2154 & & 12 & L \\
\hline 2155 & & 12 & L \\
\hline 2156 & ; U QUALIFIER ADDED OR J QUALIFIER REPLACED BY U QUALIFIER PER INTERNAL CORRECTIVE ACTIONS -- LE BISPING 04/04/01. & 12 & L \\
\hline 2157 & & 10 & L \\
\hline 2158 & & 10 & L \\
\hline 2159 & IR-0116; MDA > CRDL & 9 & L \\
\hline 2160 & & 10 & $L$ \\
\hline 2161 & & 10 & L \\
\hline 2162 & & 8 & L \\
\hline 2163 & & 16 & L \\
\hline 2164 & & 12 & L \\
\hline 2165 & & 8 & $L$ \\
\hline 2166 & & 16 & $L$ \\
\hline
\end{tabular}




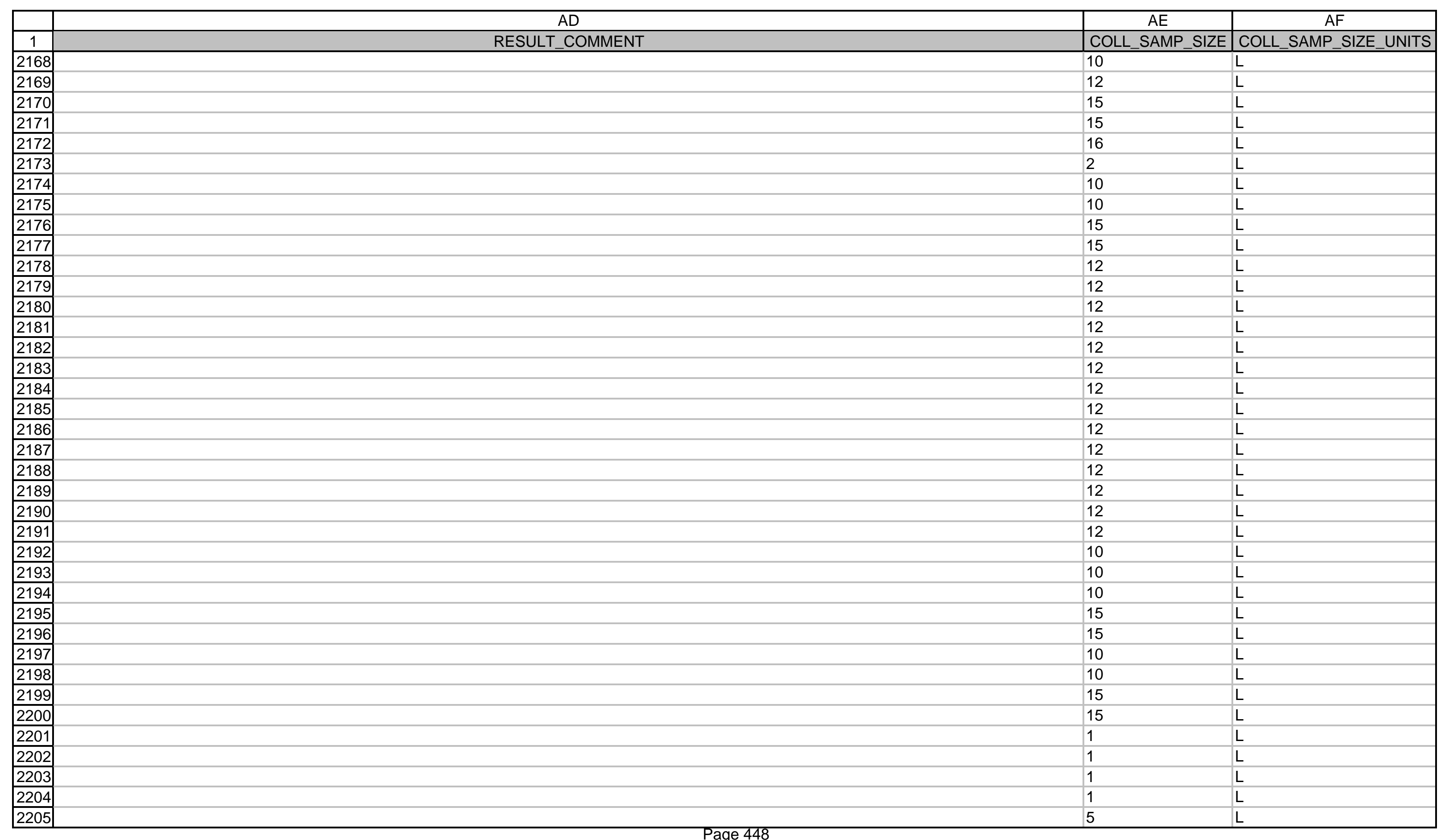




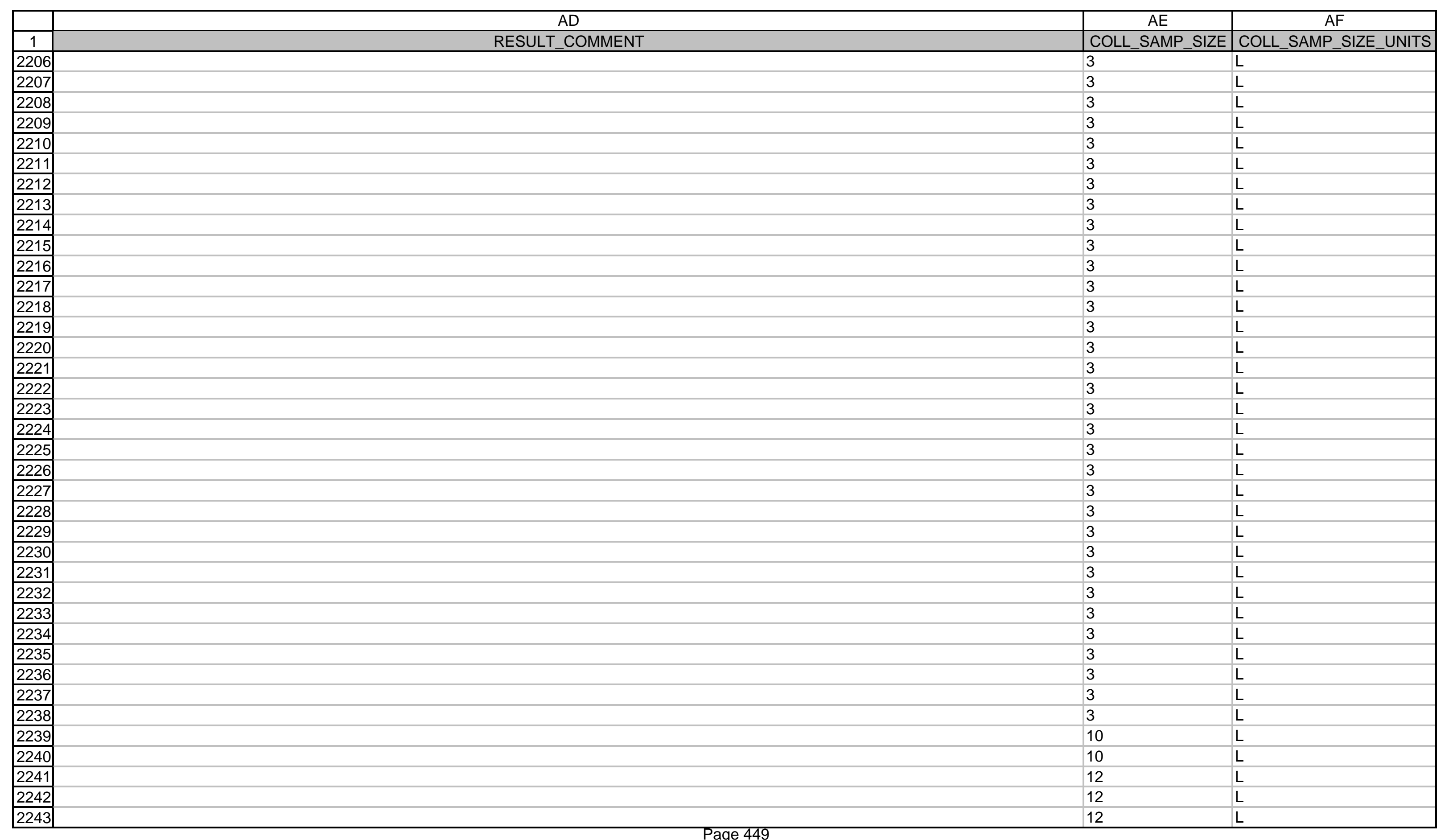




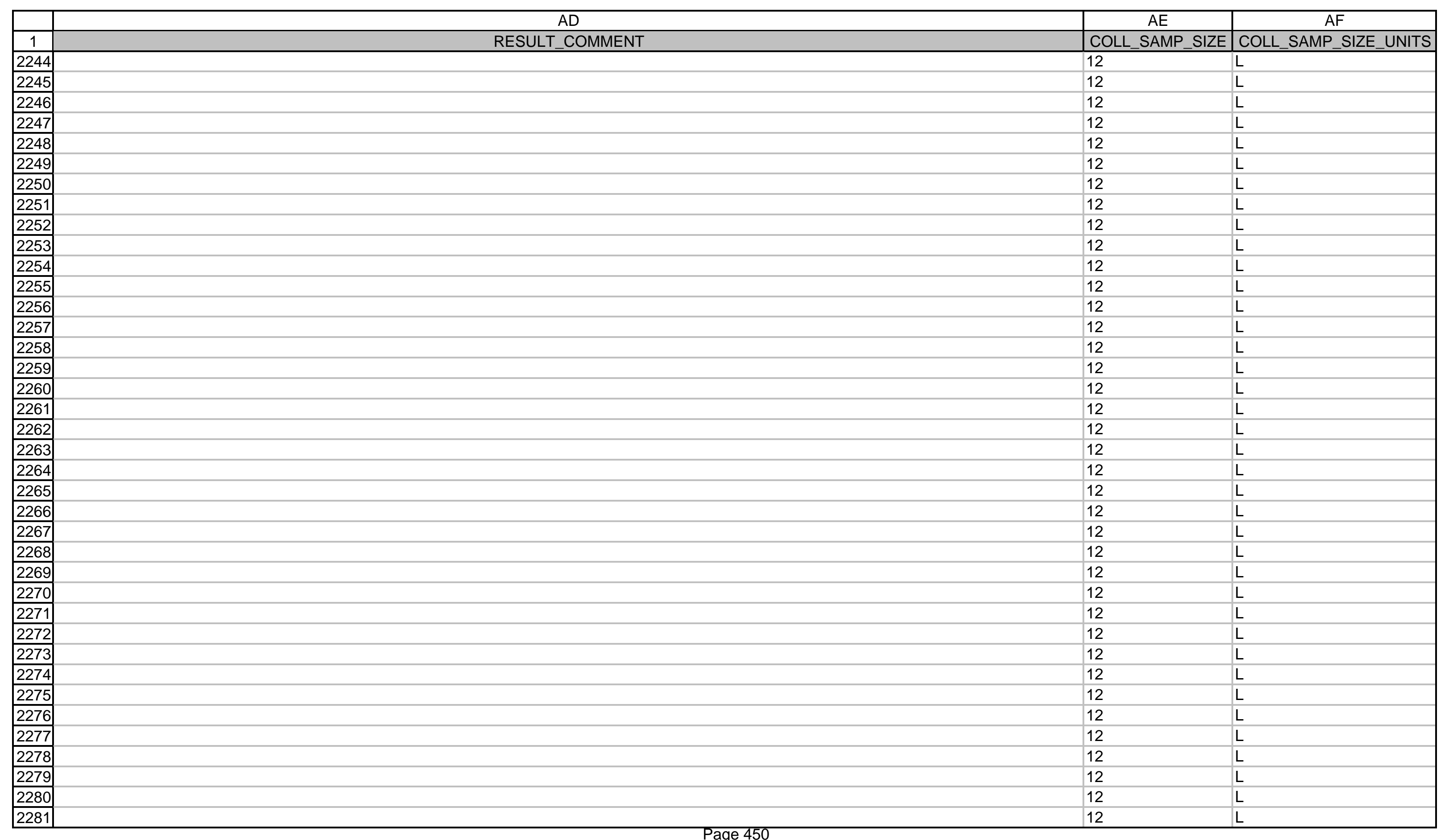




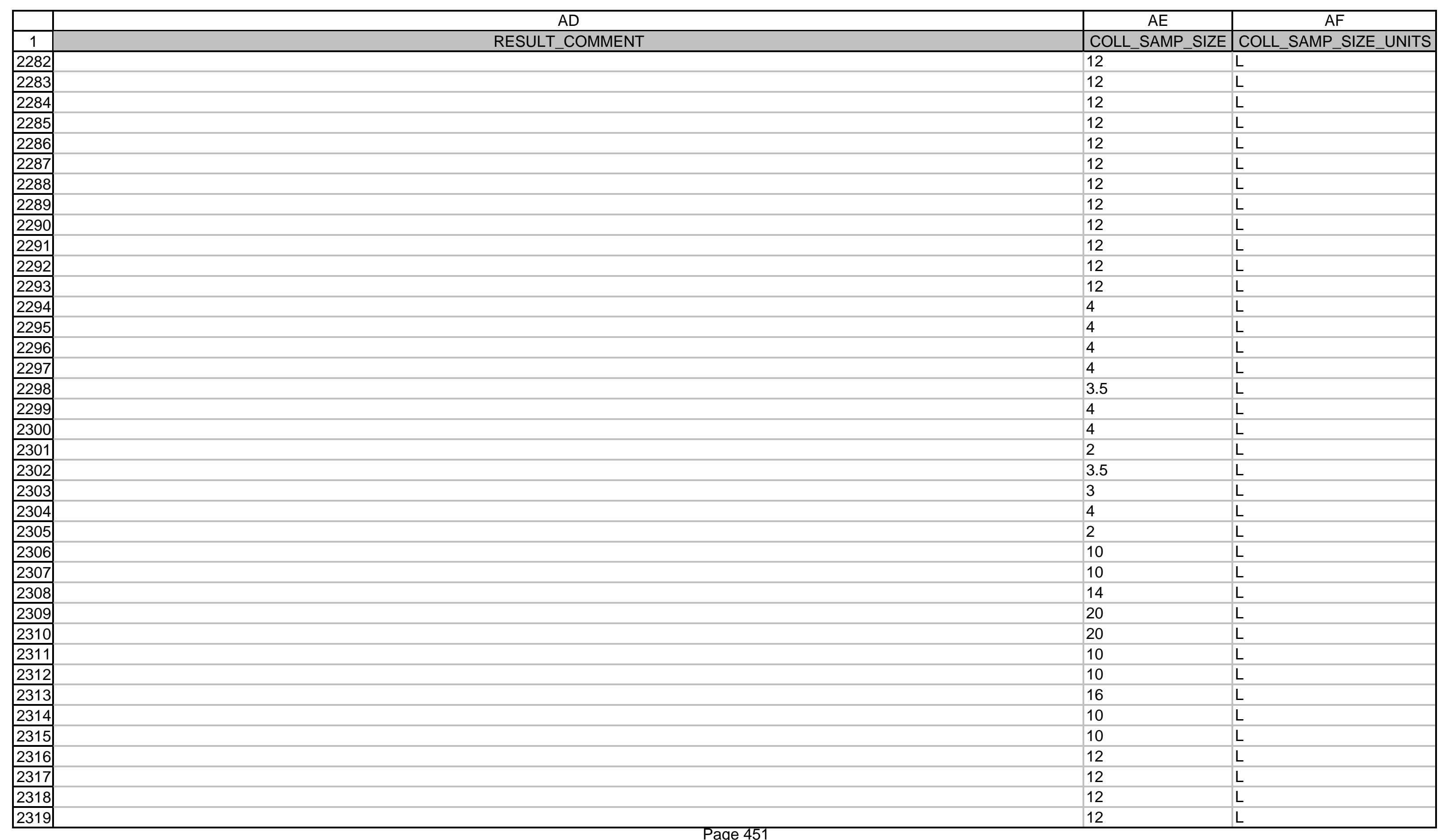




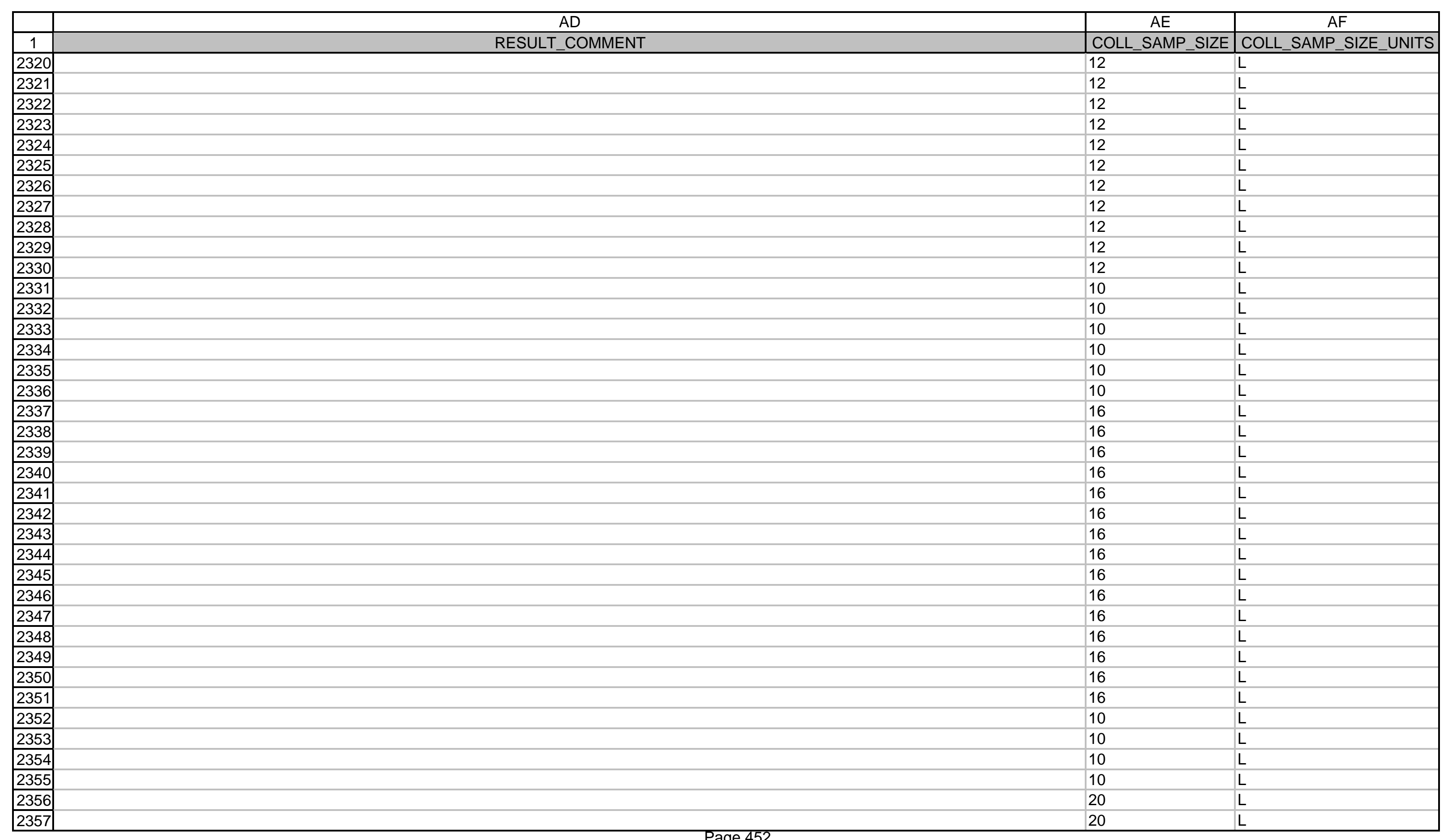




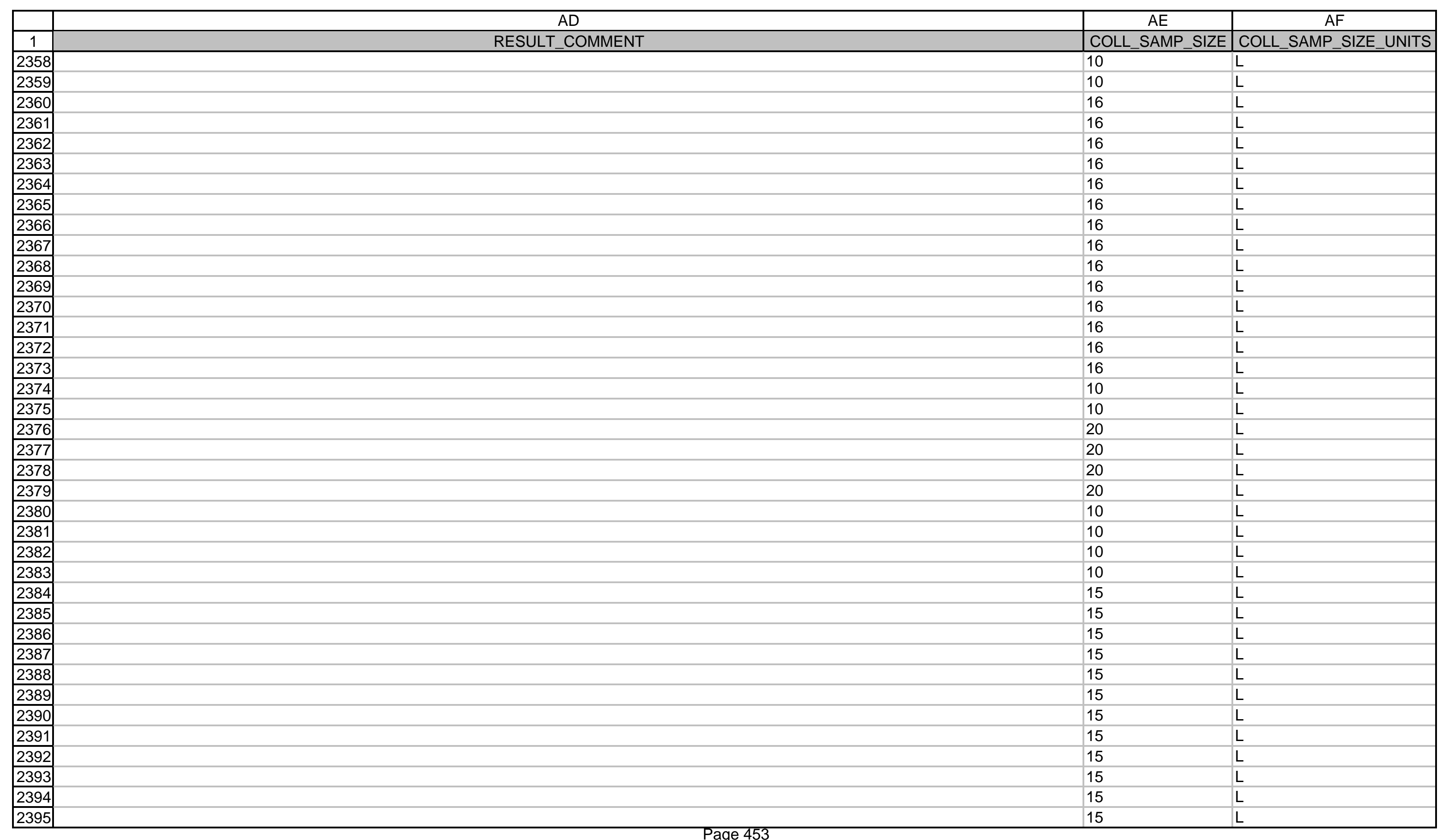




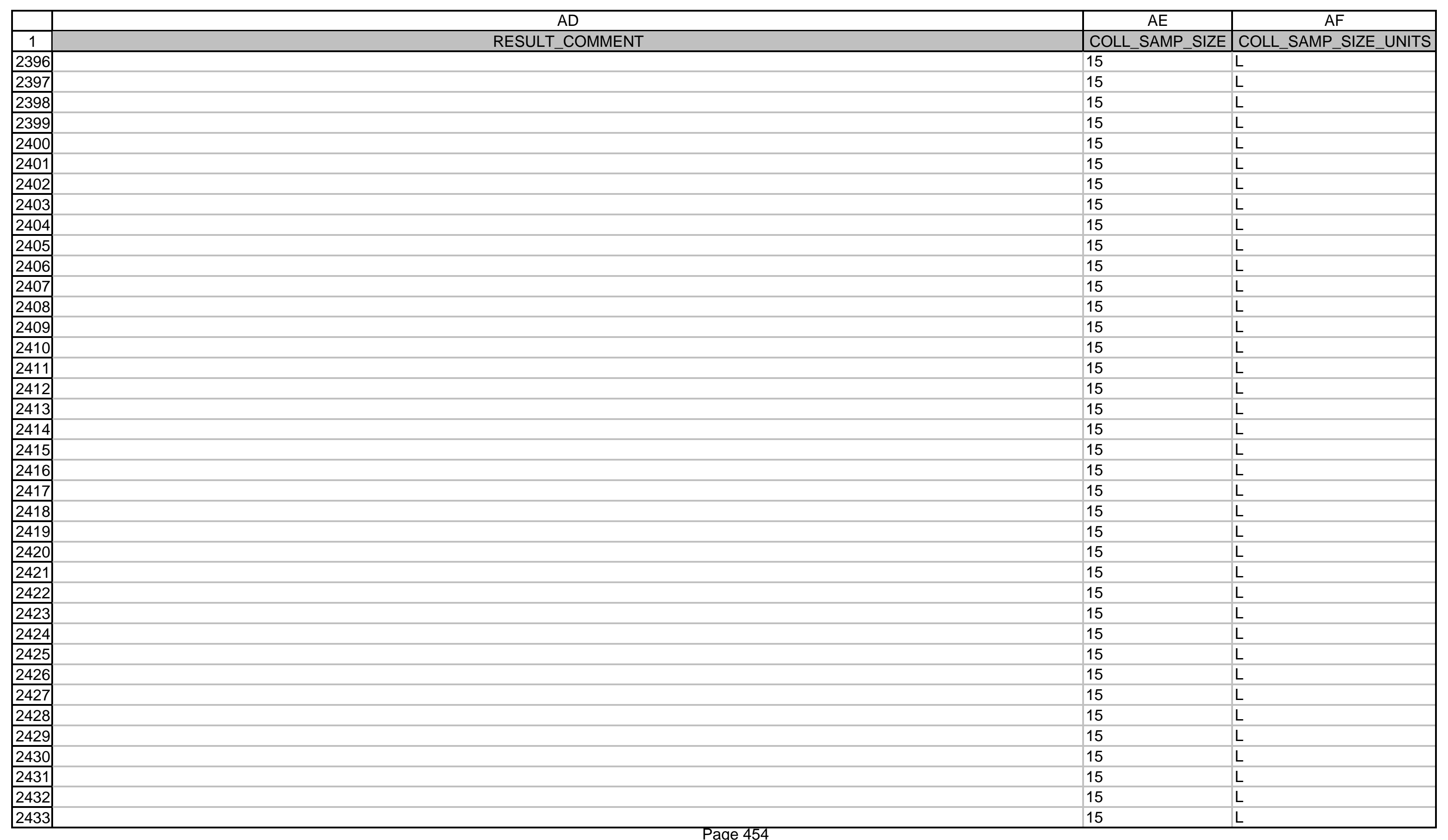




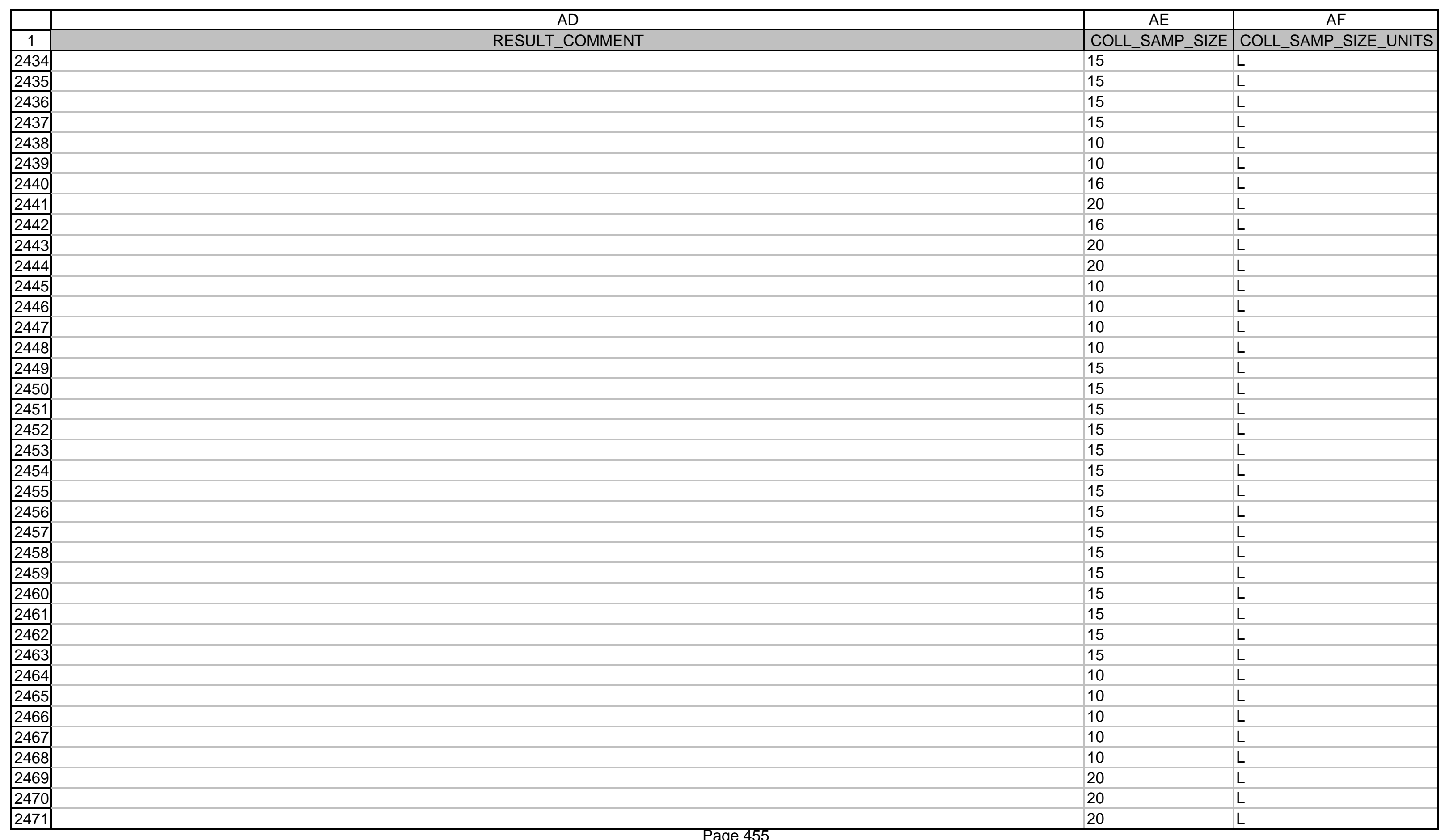




\begin{tabular}{|c|c|c|c|}
\hline & $A D$ & $\mathrm{AE}$ & $\mathrm{AF}$ \\
\hline 1 & RESULT_COMMENT & COLL_SAMP_SIZE & COLL_SAMP_SIZE_UNITS \\
\hline 2472 & & 20 & $\mathrm{~L}$ \\
\hline 24 & & 20 & L \\
\hline \begin{tabular}{|l|}
2474 \\
\end{tabular} & & 20 & $\mathrm{~L}$ \\
\hline \begin{tabular}{|l|}
2475 \\
\end{tabular} & & 20 & $\mathrm{~L}$ \\
\hline \begin{tabular}{|l|}
2476 \\
\end{tabular} & & 20 & $\mathrm{~L}$ \\
\hline \begin{tabular}{|l|}
2477 \\
\end{tabular} & & 20 & $\mathrm{~L}$ \\
\hline \begin{tabular}{|l|}
2478 \\
\end{tabular} & & 20 & $\mathrm{~L}$ \\
\hline \begin{tabular}{|l|}
2479 \\
\end{tabular} & & 20 & $\mathrm{~L}$ \\
\hline \begin{tabular}{|l|}
2481 \\
\end{tabular} & & 20 & $\mathrm{~L}$ \\
\hline \begin{tabular}{|l|}
2482 \\
2482 \\
\end{tabular} & & 20 & L \\
\hline 2483 & & 20 & $\mathrm{~L}$ \\
\hline 2484 & & 10 & $\mathrm{~L}$ \\
\hline 2485 & & 10 & L \\
\hline \begin{tabular}{|l|}
2486 \\
\end{tabular} & & 10 & $\mathrm{~L}$ \\
\hline \begin{tabular}{|l|}
2487 \\
\end{tabular} & & 10 & $\mathrm{~L}$ \\
\hline \begin{tabular}{|l|}
2488 \\
\end{tabular} & & 10 & $\mathrm{~L}$ \\
\hline 2489 & & 19 & L \\
\hline \begin{tabular}{|l|}
2491 \\
\end{tabular} & & 10 & $\mathrm{~L}$ \\
\hline \begin{tabular}{|l|}
2492 \\
240 \\
\end{tabular} & & 19 & $\mathrm{~L}$ \\
\hline 2493 & \#IR-0062 MDA > CRDL & 4 & $\mathrm{~L}$ \\
\hline \begin{tabular}{|l|}
2494 \\
\end{tabular} & \#IR-0062 MDA > CRDL & 3 & $\mathrm{~L}$ \\
\hline 2495 & \#IR-0062 MDA > CRDL & 1 & L \\
\hline \begin{tabular}{|l|}
2496 \\
\end{tabular} & & 20 & $\mathrm{~L}$ \\
\hline 2497 & & 20 & $\mathrm{~L}$ \\
\hline 2498 & & 20 & $\mathrm{~L}$ \\
\hline \begin{tabular}{|l|}
2499 \\
\end{tabular} & & 20 & $\mathrm{~L}$ \\
\hline \begin{tabular}{|l|}
2500 \\
\end{tabular} & & 20 & $\mathrm{~L}$ \\
\hline \begin{tabular}{|l|}
2501 \\
2501
\end{tabular} & & 20 & $\mathrm{~L}$ \\
\hline \begin{tabular}{|l|}
2502 \\
\end{tabular} & & 20 & $\mathrm{~L}$ \\
\hline \begin{tabular}{|l|}
2503 \\
\end{tabular} & & 20 & $\mathrm{~L}$ \\
\hline \begin{tabular}{|l|}
2504 \\
\end{tabular} & & 20 & $\mathrm{~L}$ \\
\hline \begin{tabular}{|l|}
2505 \\
\end{tabular} & & 20 & $\mathrm{~L}$ \\
\hline \begin{tabular}{|l|}
2506 \\
\end{tabular} & & 20 & $\mathrm{~L}$ \\
\hline \begin{tabular}{|l|}
2507 \\
\end{tabular} & & 20 & L \\
\hline \begin{tabular}{|l|}
2508 \\
\end{tabular} & & 20 & L \\
\hline
\end{tabular}




\begin{tabular}{|c|c|c|c|}
\hline & & $\mathrm{AE}$ & \\
\hline 1 & $\begin{array}{ll}\text { RESULT COMMENT } \\
\end{array}$ & COLL SAMP SIZE & COLL SAMP SIZE UNITS \\
\hline 2510 & \#IR-0059 Blank Result > CRDL & 10 & $\mathrm{~L}$ \\
\hline 2511 & \#IR-0059 Blank Result > CRDL & 10 & L \\
\hline 2512 & \#IR-0059 Blank Result > CRDL & 10 & $\mathrm{~L}$ \\
\hline 2513 & & 19 & $\mathrm{~L}$ \\
\hline 2514 & & 10 & $\mathrm{~L}$ \\
\hline 2515 & & 10 & L \\
\hline 2516 & & 10 & L \\
\hline 2517 & & 10 & L \\
\hline 2518 & & 12 & L \\
\hline 2519 & & 12 & L \\
\hline 2520 & & 12 & $\mathrm{~L}$ \\
\hline 2521 & & 12 & L \\
\hline 2522 & & 12 & $\mathrm{~L}$ \\
\hline 2523 & & 12 & L \\
\hline 2524 & & 12 & L \\
\hline 2525 & & 12 & L \\
\hline 2526 & & 12 & L \\
\hline 2527 & & 12 & L \\
\hline 2530 & & 12 & $\mathrm{~L}$ \\
\hline 2531 & & 12 & L \\
\hline 2532 & & 12 & L \\
\hline 2533 & & 12 & L \\
\hline 2534 & & 12 & L \\
\hline 2535 & & 12 & L \\
\hline 2536 & & 12 & L \\
\hline 2537 & & 12 & $\mathrm{~L}$ \\
\hline 2538 & & 12 & L \\
\hline 2539 & & 12 & L \\
\hline 2540 & & 12 & L \\
\hline 2541 & & 12 & $\mathrm{~L}$ \\
\hline 2542 & & 12 & L \\
\hline 2543 & & 12 & $\mathrm{~L}$ \\
\hline 2544 & & 12 & $\mathrm{~L}$ \\
\hline 2545 & & 12 & $\mathrm{~L}$ \\
\hline 2546 & & 12 & L \\
\hline 2547 & 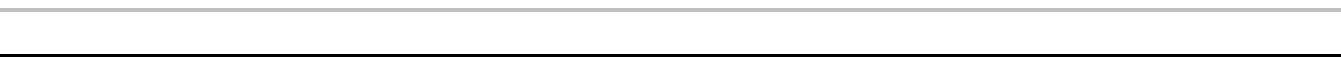 & 12 & $\mathrm{~L}$ \\
\hline
\end{tabular}




\begin{tabular}{|c|c|c|c|}
\hline & $A D$ & $\mathrm{AE}$ & $\mathrm{AF}$ \\
\hline 1 & RESULT_COMMENT & COLL_SAMP_SIZE & COLL_SAMP_SIZE_UNITS \\
\hline 2548 & & 12 & $\mathrm{~L}$ \\
\hline 2549 & & 12 & L \\
\hline \begin{tabular}{|l|}
2550 \\
\end{tabular} & & 12 & $\mathrm{~L}$ \\
\hline 2551 & & 12 & $\bar{L}$ \\
\hline 2552 & & 12 & L \\
\hline 2553 & & 12 & L \\
\hline \begin{tabular}{|l|}
2554 \\
\end{tabular} & & 12 & $\mathrm{~L}$ \\
\hline 2555 & & 12 & $\mathrm{~L}$ \\
\hline \begin{tabular}{|l|}
2557 \\
\end{tabular} & & 12 & L \\
\hline 2558 & & 12 & $\mathrm{~L}$ \\
\hline 2559 & & 12 & $\mathrm{~L}$ \\
\hline 2560 & & 12 & $\mathrm{~L}$ \\
\hline 2561 & & 12 & $L$ \\
\hline 2562 & & 12 & L \\
\hline \begin{tabular}{|l|}
2563 \\
\end{tabular} & & 12 & L \\
\hline 2564 & & 12 & L \\
\hline 2565 & & 12 & L \\
\hline \begin{tabular}{|l|}
2567 \\
\end{tabular} & & 12 & $\mathrm{~L}$ \\
\hline \begin{tabular}{|l|}
2568 \\
\end{tabular} & & 12 & $\mathrm{~L}$ \\
\hline 2569 & & 12 & L \\
\hline \begin{tabular}{|l|}
2570 \\
\end{tabular} & & 12 & $\mathrm{~L}$ \\
\hline 2571 & & 12 & L \\
\hline 2572 & & 8 & $\mathrm{~L}$ \\
\hline 2573 & & 14 & $\mathrm{~L}$ \\
\hline 2574 & & 8 & L \\
\hline 2575 & & 8 & L \\
\hline 2576 & & 14 & L \\
\hline \begin{tabular}{|l|}
2577 \\
\end{tabular} & & 16 & $L$ \\
\hline 2578 & & 10 & L \\
\hline \begin{tabular}{|l|}
2579 \\
\end{tabular} & & 10 & $\mathrm{~L}$ \\
\hline 2580 & MDAs DO NOT MEET THE CRDL & 9 & L \\
\hline 2581 & & 16 & L \\
\hline 2582 & & 16 & $\mathrm{~L}$ \\
\hline 2583 & & 10 & $\mathrm{~L}$ \\
\hline 2584 & & 10 & $\mathrm{~L}$ \\
\hline
\end{tabular}




\begin{tabular}{|c|c|c|c|}
\hline & $A D$ & $\mathrm{AE}$ & $\mathrm{AF}$ \\
\hline 1 & RESULT_COMMENT & COLL_SAMP_SIZE & COLL_SAMP_SIZE_UNITS \\
\hline 2586 & & 10 & $\mathrm{~L}$ \\
\hline 2587 & & 10 & L \\
\hline 2588 & & 12 & $\mathrm{~L}$ \\
\hline 2589 & & 12 & $\mathrm{~L}$ \\
\hline 2590 & & 12 & $\mathrm{~L}$ \\
\hline 2591 & & 12 & L \\
\hline \begin{tabular}{|l|}
2592 \\
\end{tabular} & & 12 & $\mathrm{~L}$ \\
\hline \begin{tabular}{|l|}
2593 \\
\end{tabular} & & 12 & L \\
\hline 2595 & & 12 & $\mathrm{~L}$ \\
\hline \begin{tabular}{|l|}
2596 \\
\end{tabular} & & 12 & L \\
\hline 2597 & & 12 & $\mathrm{~L}$ \\
\hline 2598 & & 12 & $\mathrm{~L}$ \\
\hline 2599 & & 12 & L \\
\hline 2600 & & 12 & $\mathrm{~L}$ \\
\hline 2601 & & 12 & $\mathrm{~L}$ \\
\hline \begin{tabular}{|l|}
2602 \\
\end{tabular} & & 12 & $\mathrm{~L}$ \\
\hline 2603 & & 10 & L \\
\hline 2605 & & 10 & $\mathrm{~L}$ \\
\hline 2606 & & 10 & $\mathrm{~L}$ \\
\hline 2607 & \#IR-0103 - The achieved MDAs do not meet the CRDL. & 9 & $\mathrm{~L}$ \\
\hline 2608 & & 12 & $\mathrm{~L}$ \\
\hline 2609 & & 12 & L \\
\hline 2610 & & 12 & $\mathrm{~L}$ \\
\hline 2611 & & 12 & L \\
\hline 2612 & & 12 & $\mathrm{~L}$ \\
\hline 2613 & & 12 & $\mathrm{~L}$ \\
\hline 2614 & & 12 & $\mathrm{~L}$ \\
\hline 2615 & & 12 & L \\
\hline 2616 & & 12 & L \\
\hline 2617 & & 12 & $\mathrm{~L}$ \\
\hline 2618 & & 12 & L \\
\hline 2619 & & 12 & $\mathrm{~L}$ \\
\hline 2620 & & 12 & $\mathrm{~L}$ \\
\hline 2621 & & 12 & L \\
\hline 2622 & & 12 & L \\
\hline
\end{tabular}




\begin{tabular}{|c|c|c|c|}
\hline & $A D$ & $\mathrm{AE}$ & $\mathrm{AF}$ \\
\hline 1 & $\begin{array}{ll}\text { RESULT_COMMENT } \\
\end{array}$ & COLL_SAMP_SIZE & COLL_SAMP_SIZE_UNITS \\
\hline 2624 & & 10 & $\mathrm{~L}$ \\
\hline 2625 & IR-0116; MDA > CRDL & 9 & L \\
\hline 2626 & & 10 & L \\
\hline 2628 & & 8 & L \\
\hline 2629 & & 16 & L \\
\hline 2630 & & 12 & L \\
\hline 2631 & & 8 & $\mathrm{~L}$ \\
\hline 2633 & & 10 & L \\
\hline 2634 & & 10 & L \\
\hline 2635 & & 12 & $\mathrm{~L}$ \\
\hline 2636 & & 15 & L \\
\hline 2637 & & 15 & L \\
\hline 2638 & & 16 & L \\
\hline 2639 & & 2 & L \\
\hline 2640 & & 10 & L \\
\hline 2641 & & 10 & L \\
\hline 2643 & & 15 & L \\
\hline 2644 & & 12 & $\mathrm{~L}$ \\
\hline 2645 & & 12 & $\mathrm{~L}$ \\
\hline 2646 & & 12 & L \\
\hline 2647 & & 12 & $\mathrm{~L}$ \\
\hline 2648 & & 12 & $\mathrm{~L}$ \\
\hline 2649 & & 12 & L \\
\hline 2650 & & 12 & L \\
\hline 2651 & & 12 & L \\
\hline 2652 & & 12 & L \\
\hline 2653 & & 12 & L \\
\hline 2654 & & 12 & $\mathrm{~L}$ \\
\hline 2655 & & 12 & L \\
\hline 2656 & & 12 & $\mathrm{~L}$ \\
\hline 2657 & & 12 & L \\
\hline 2658 & & 10 & L \\
\hline 2659 & & 10 & L \\
\hline 2660 & & 10 & L \\
\hline 2661 & 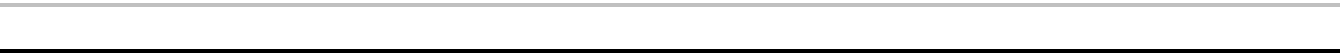 & 15 & $\mathrm{~L}$ \\
\hline
\end{tabular}




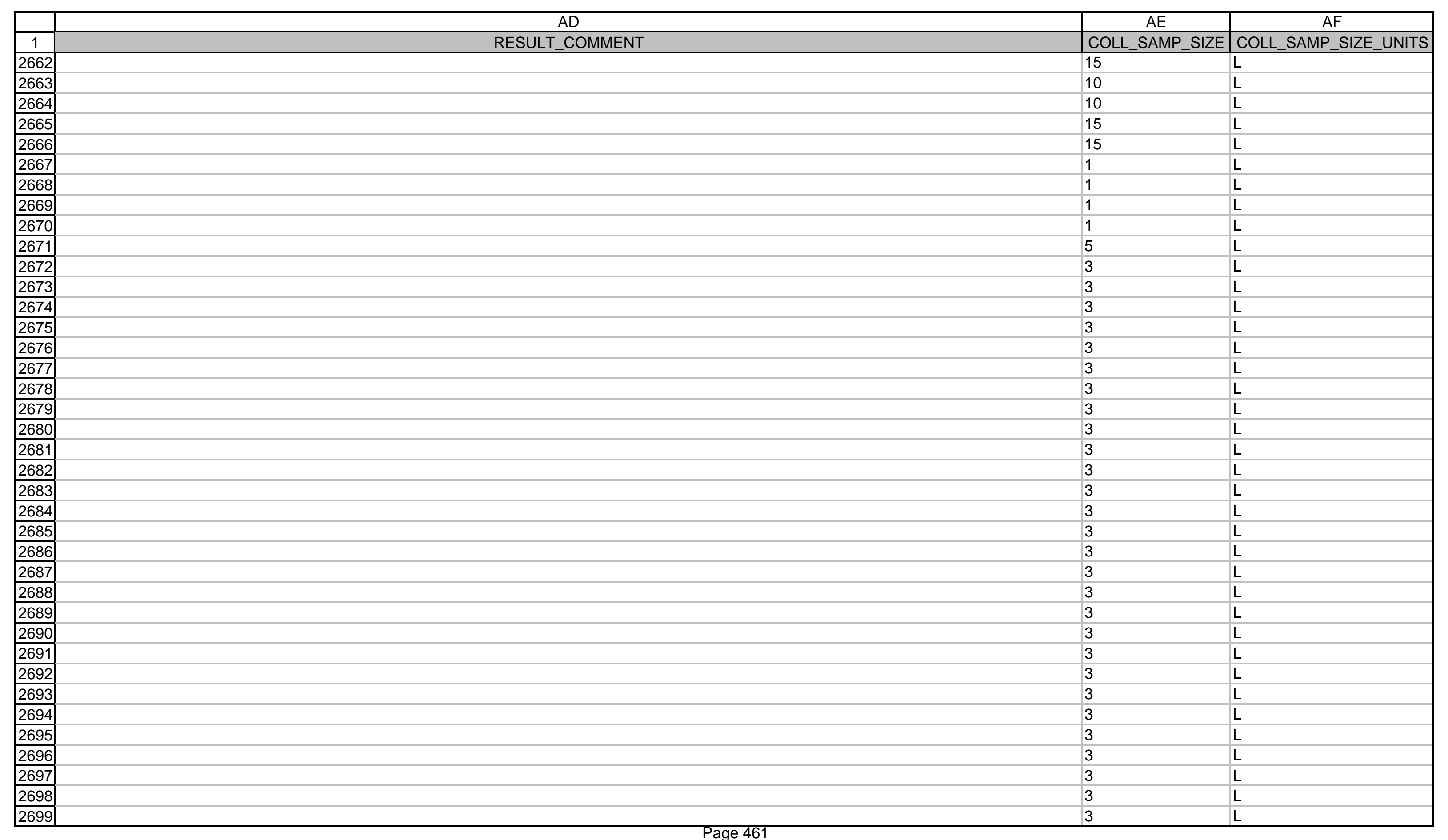




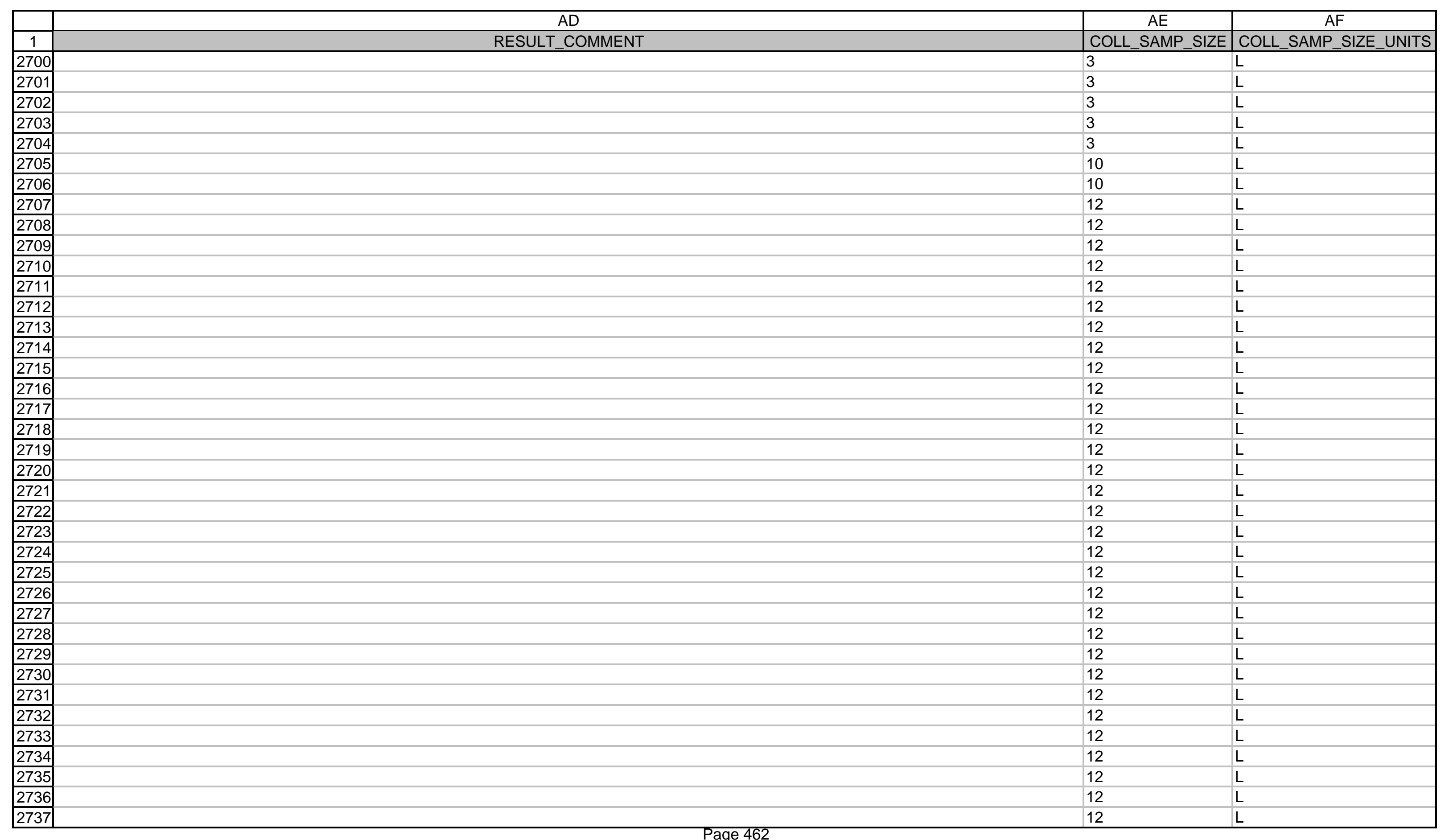




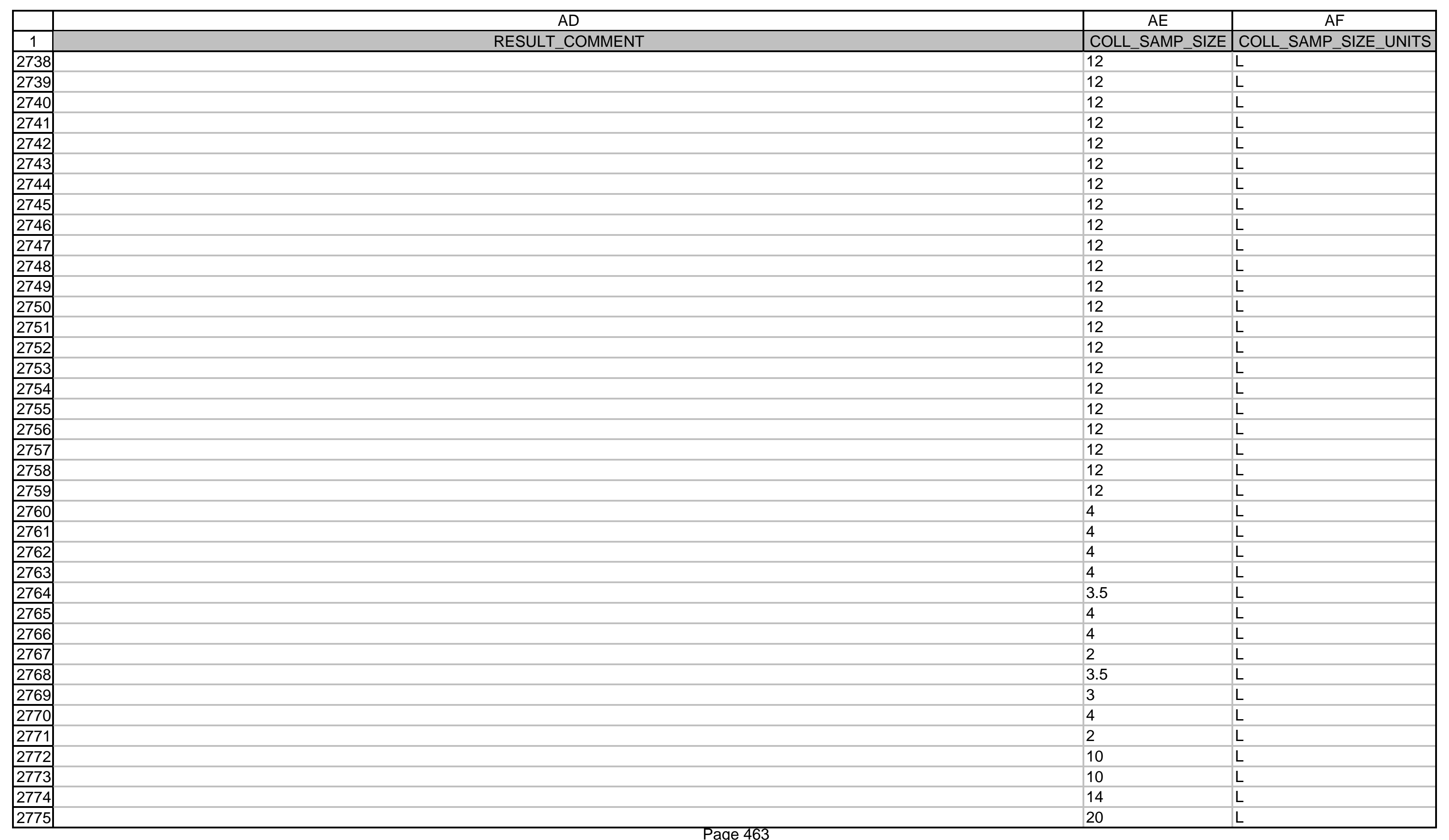




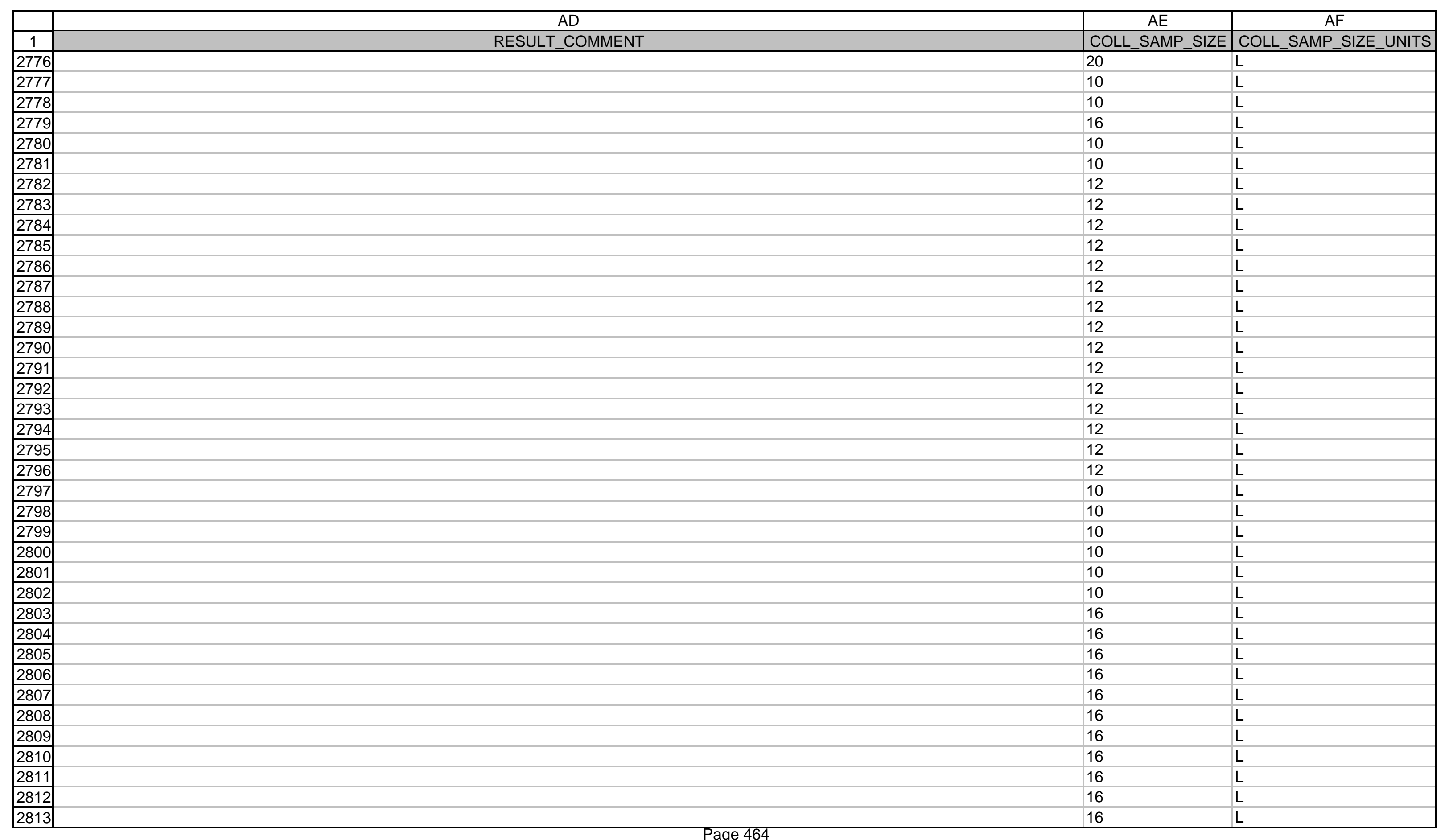




\begin{tabular}{|c|c|c|c|}
\hline & 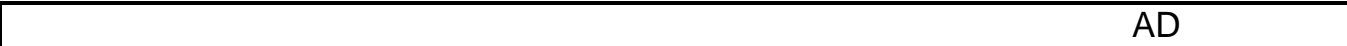 & $\mathrm{AE}$ & $\mathrm{AF}$ \\
\hline 1 & $\begin{array}{lc} & \text { RESULT_COMMENT } \\
\end{array}$ & COLL_SAMP_SIZE & COLL_SAMP_SIZE_UNITS \\
\hline 2814 & & 16 & $\mathrm{~L}$ \\
\hline 2815 & & 16 & $\mathrm{~L}$ \\
\hline 2816 & & 16 & L \\
\hline 2817 & & 16 & L \\
\hline 2818 & . & 10 & $\mathrm{~L}$ \\
\hline 2819 & 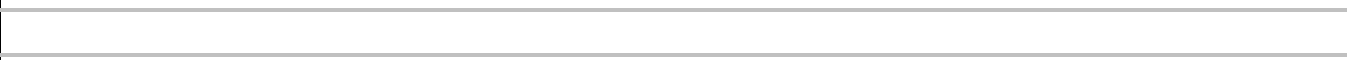 & 10 & $\mathrm{~L}$ \\
\hline 2820 & & 10 & $\mathrm{~L}$ \\
\hline 2821 & & 10 & L \\
\hline 2822 & & 20 & L \\
\hline 2823 & & 20 & L \\
\hline 2824 & & 10 & L \\
\hline 2825 & & 10 & L \\
\hline 2826 & & 16 & L \\
\hline 2827 & & 16 & L \\
\hline 2828 & & 16 & L \\
\hline 2829 & & 16 & L \\
\hline 2830 & & 16 & L \\
\hline \begin{tabular}{|l|}
2831 \\
\end{tabular} & & 16 & L \\
\hline 2833 & & 16 & L \\
\hline 2834 & & 16 & L \\
\hline 2835 & & 16 & L \\
\hline 2836 & & 16 & L \\
\hline 2837 & & 16 & L \\
\hline 2838 & & 16 & L \\
\hline 2839 & & 16 & L \\
\hline \begin{tabular}{|l|}
2840 \\
\end{tabular} & & 10 & $\mathrm{~L}$ \\
\hline 2841 & & 10 & L \\
\hline 2842 & & 20 & L \\
\hline 2843 & & 20 & L \\
\hline 2844 & & 20 & $\mathrm{~L}$ \\
\hline 2845 & & 20 & L \\
\hline 2846 & & 10 & L \\
\hline 2847 & & 10 & L \\
\hline 2848 & & 10 & $\mathrm{~L}$ \\
\hline 2849 & & 10 & L \\
\hline 2850 & & 15 & L \\
\hline
\end{tabular}




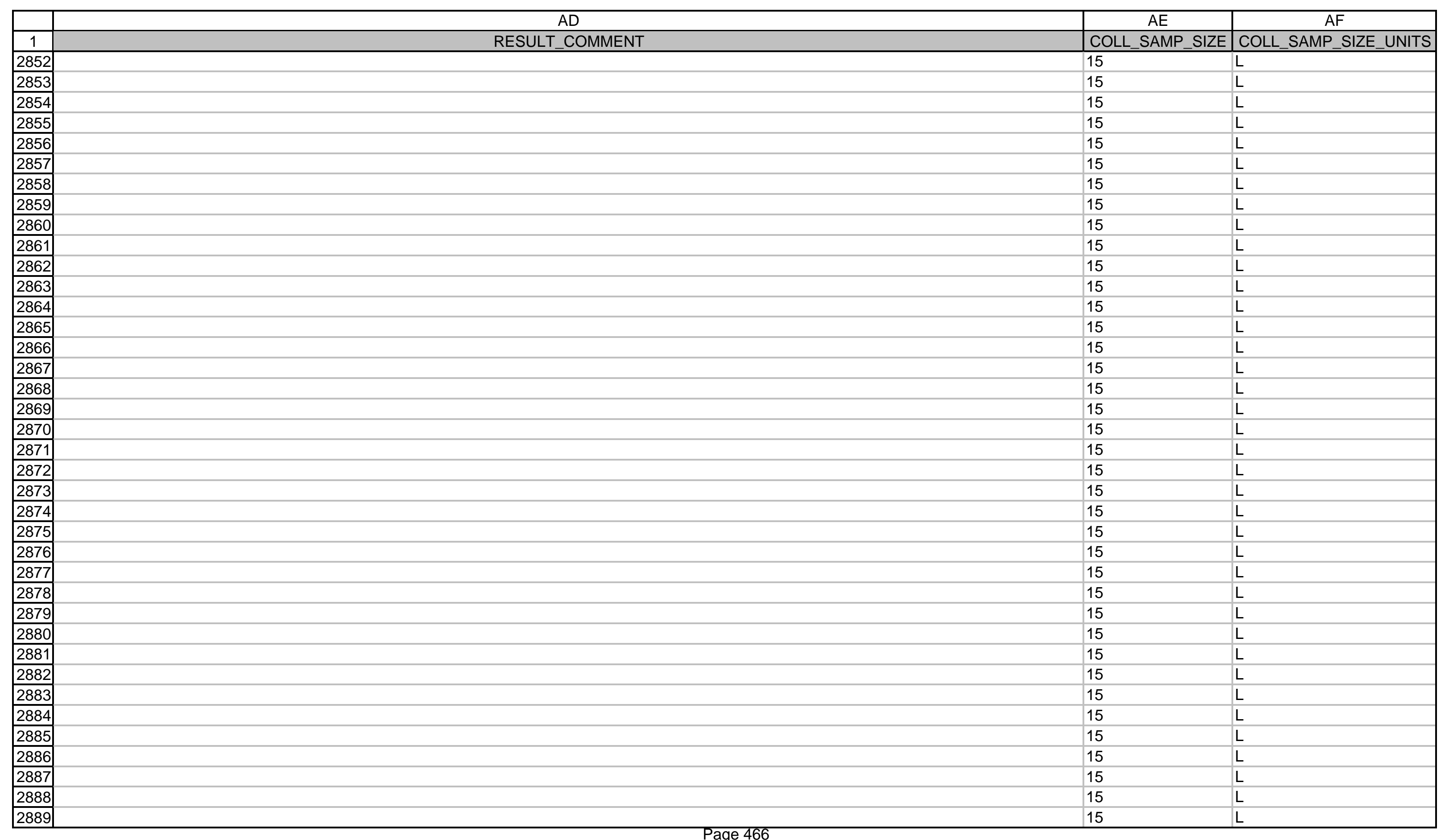




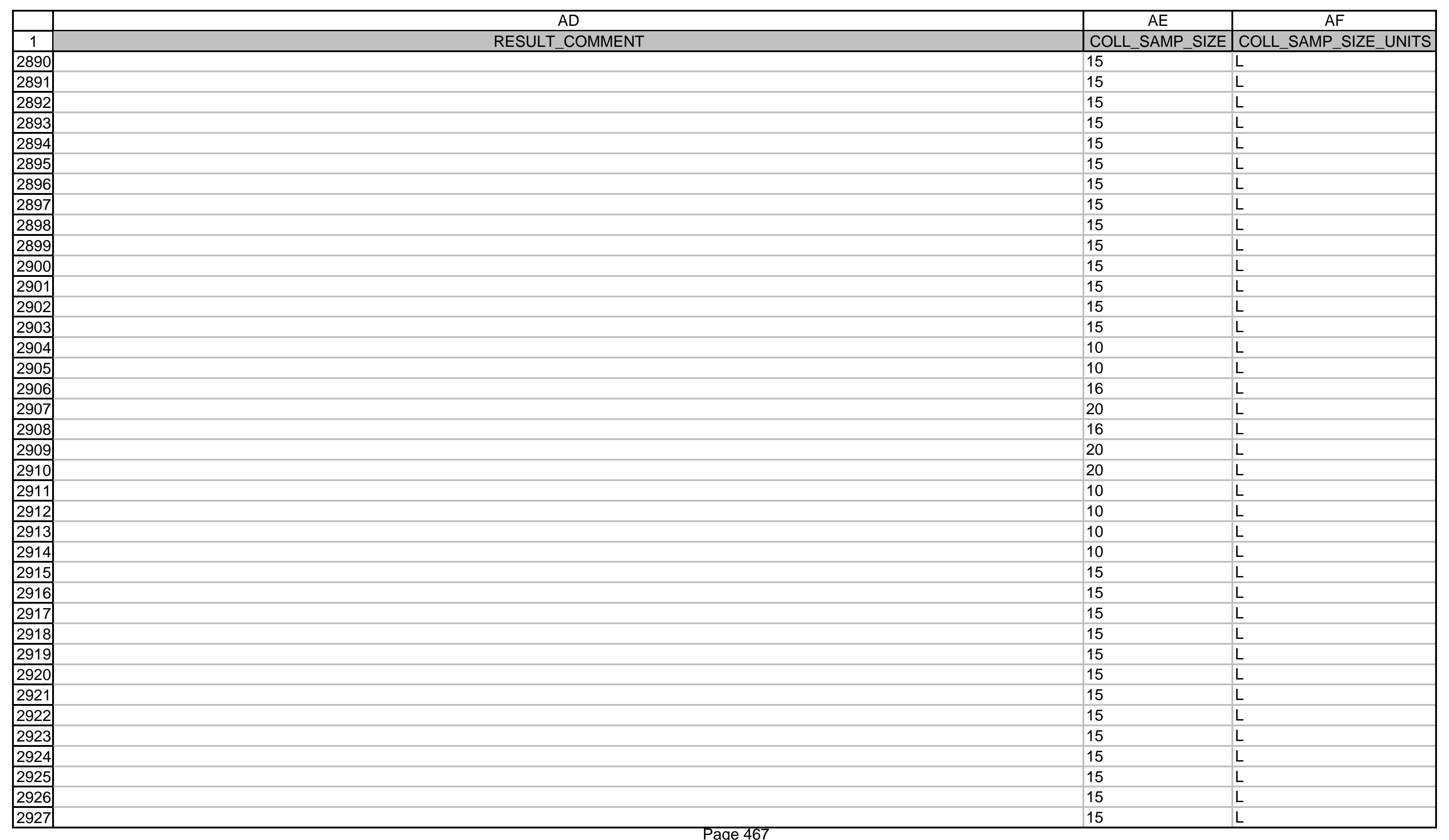




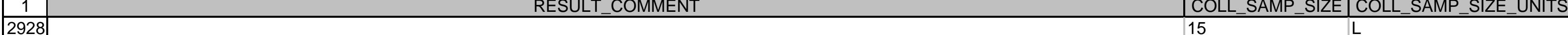




\begin{tabular}{|c|c|c|c|c|c|c|c|c|c|}
\hline & $\overline{A G}$ & $\mathrm{AH}$ & $\overline{\mathrm{Al}}$ & $\overline{A J}$ & AK & $\mathrm{AL}$ & AM & AN & $\mathrm{AO}$ \\
\hline 1 & DIST_CLASS & OWNER_ID & COLLECTION_PURPOSE & X_Y_COORD_FINAL.SAMP_NUM & COORDINATE SYSTEM & DATUM & LATITUDE ${ }^{\circ} \mathrm{N}$ & LATITUDE MIN_MIN & LATITUDE MIN_SEC \\
\hline 2 & OFFSITE & SESPMNT & $\mathrm{R}$ & & & & & & \\
\hline 3 & OFFSITE & SESPMNT & $\mathrm{R}$ & & & & & & \\
\hline 4 & OFFSITE & SESPMNT & $\mathrm{R}$ & & & & & & \\
\hline 5 & OFFSITE & SESPMNT & $\mathrm{R}$ & & & & & & \\
\hline 6 & OFFSITE & SESPMNT & $\mathrm{R}$ & & & & & & \\
\hline 7 & OFFSITE & SESPMNT & $\mathrm{R}$ & & & & & & \\
\hline 8 & OFFSITE & SESPMNT & $\mathrm{R}$ & & & & & & \\
\hline 9 & OFFSITE & SESPMNT & $\mathrm{R}$ & & & & & & \\
\hline 10 & OFFSITE & SESPMNT & $\mathrm{R}$ & & & & & & \\
\hline 11 & OFFSITE & SESPMNT & $\mathrm{R}$ & & & & & & \\
\hline 12 & OFFSITE & SESPMNT & $\mathrm{R}$ & & & & & & \\
\hline 13 & OFFSITE & SESPMNT & $\mathrm{R}$ & & & & & & \\
\hline 14 & OFFSITE & SESPMNT & $\mathrm{R}$ & & & & & & \\
\hline 15 & OFFSITE & SESPMNT & $\mathrm{R}$ & & & & & & \\
\hline 16 & ONSITE & SESPMNT & $\mathrm{R}$ & & & & & & \\
\hline 17 & ONSITE & SESPMNT & $\mathrm{R}$ & & & & & & \\
\hline 18 & ONSITE & SESPMNT & $\mathrm{R}$ & & & & & & \\
\hline 19 & ONSITE & SESPMNT & $\mathrm{R}$ & & & & & & \\
\hline 20 & ONSITE & SESPMNT & $\mathrm{R}$ & & & & & & \\
\hline 21 & ONSITE & SESPMNT & $\mathrm{R}$ & & & & & & \\
\hline 22 & ONSITE & PNLGW & & & & & & & \\
\hline 23 & ONSITE & SESPMNT & $\mathrm{R}$ & & & & & & \\
\hline 24 & ONSITE & SESPMNT & $\mathrm{R}$ & & & & & & \\
\hline 25 & ONSITE & SESPMNT & $\mathrm{R}$ & & & & & & \\
\hline 26 & ONSITE & SESPMNT & $\mathrm{R}$ & & & & & & \\
\hline 27 & OFFSITE & SESPMNT & $\mathrm{R}$ & & & & & & \\
\hline 28 & OFFSITE & SESPMNT & $\mathrm{R}$ & & & & & & \\
\hline 29 & OFFSITE & SESPMNT & $\mathrm{R}$ & & & & & & \\
\hline 30 & OFFSITE & SESPMNT & $\mathrm{R}$ & & & & & & \\
\hline 31 & OFFSITE & SESPMNT & $\mathrm{R}$ & & & & & & \\
\hline 32 & OFFSITE & SESPMNT & $\mathrm{R}$ & & & & & & \\
\hline 33 & OFFSITE & SESPMNT & $\mathrm{R}$ & & & & & & \\
\hline 34 & OFFSITE & SESPMNT & $\mathrm{R}$ & & & & & & \\
\hline 35 & OFFSITE & SESPMNT & $\mathrm{R}$ & & & & & & \\
\hline 36 & OFFSITE & SESPMNT & $\mathrm{R}$ & & & & & & \\
\hline 37 & OFFSITE & SESPMNT & $\mathrm{R}$ & & & & & & \\
\hline 38 & OFFSITE & SESPMNT & $\mathrm{R}$ & & & & & & \\
\hline 39 & OFFSITE & SESPMNT & $\mathrm{R}$ & & & & & & \\
\hline
\end{tabular}




\begin{tabular}{|c|c|c|c|c|c|c|c|c|c|}
\hline & $A G$ & $\mathrm{AH}$ & $\mathrm{Al}$ & $\mathrm{AJ}$ & $A K$ & $\mathrm{AL}$ & $\mathrm{AM}$ & $\mathrm{AN}$ & $\mathrm{AO}$ \\
\hline 1 & DIST_CLASS & OWNER_ID & COLLECTION_PURPOSE & X_Y_COORD_FINAL.SAMP_NUM & COORDINATE SYSTEM & DATUM & LATITUDE ${ }^{\circ} \mathrm{N}$ & LATITUDE MIN_MIN & LATITUDE MIN_SEC \\
\hline 40 & OFFSITE & SESPMNT & $\mathrm{R}$ & & & & & & \\
\hline 41 & ONSITE & SESPMNT & $\mathrm{R}$ & & & & & & \\
\hline 42 & ONSITE & SESPMNT & $\mathrm{R}$ & & & & & & \\
\hline 43 & ONSITE & SESPMNT & $\mathrm{R}$ & & & & & & \\
\hline 44 & OFFSITE & SESPMNT & & & & & & & \\
\hline 45 & OFFSITE & SESPMNT & & & & & & & \\
\hline 46 & OFFSITE & SESPMNT & & & & & & & \\
\hline 47 & OFFSITE & SESPMNT & & & & & & & \\
\hline 48 & OFFSITE & SESPMNT & & & & & & & \\
\hline 49 & OFFSITE & SESPMNT & & & & & & & \\
\hline 50 & OFFSITE & SESPMNT & & & & & & & \\
\hline 51 & OFFSITE & SESPMNT & & & & & & & \\
\hline 52 & OFFSITE & SESPMNT & & & & & & & \\
\hline 53 & OFFSITE & SESPMNT & & & & & & & \\
\hline 54 & OFFSITE & SESPMNT & & & & & & & \\
\hline 55 & OFFSITE & SESPMNT & & & & & & & \\
\hline 56 & OFFSITE & SESPMNT & & & & & & & \\
\hline 57 & OFFSITE & SESPMNT & & & & & & & \\
\hline 58 & ONSITE & SESPMNT & & & & & & & \\
\hline 59 & ONSITE & SESPMNT & & & & & & & \\
\hline 60 & ONSITE & SESPMNT & $\mathrm{R}$ & & & & & & \\
\hline 61 & ONSITE & SESPMNT & $\mathrm{R}$ & & & & & & \\
\hline 62 & ONSITE & CENTPLAT & & & & & & & \\
\hline 63 & ONSITE & CENTPLAT & & & & & & & \\
\hline 64 & ONSITE & CENTPLAT & & & & & & & \\
\hline 65 & ONSITE & CENTPLAT & & & & & & & \\
\hline 66 & ONSITE & CENTPLAT & & & & & & & \\
\hline 67 & ONSITE & CENTPLAT & & & & & & & \\
\hline 68 & ONSITE & PNLGW & & & & & & & \\
\hline 69 & ONSITE & CENTPLAT & & & & & & & \\
\hline 70 & ONSITE & CENTPLAT & & & & & & & \\
\hline 71 & ONSITE & PNLGW & & & & & & & \\
\hline 72 & & CENTPLAT & IP & & & & & & \\
\hline 73 & & CENTPLAT & IP & & & & & & \\
\hline 74 & & CENTPLAT & IP & & & & & & \\
\hline 75 & & CENTPLAT & IP & & & & & & \\
\hline 76 & ONSITE & SESPMNT & $\mathrm{R}$ & & & & & & \\
\hline 77 & ONSITE & SESPMNT & $\mathrm{R}$ & & & & & & \\
\hline
\end{tabular}




\begin{tabular}{|c|c|c|c|c|c|c|c|c|c|}
\hline & $A G$ & $\mathrm{AH}$ & $\mathrm{Al}$ & $\mathrm{AJ}$ & $A K$ & $\mathrm{AL}$ & $\mathrm{AM}$ & $\mathrm{AN}$ & $\mathrm{AO}$ \\
\hline 1 & DIST_CLASS & OWNER_ID & COLLECTION_PURPOSE & X_Y_COORD_FINAL.SAMP_NUM & COORDINATE SYSTEM & DATUM & LATITUDE ${ }^{\circ} \mathrm{N}$ & LATITUDE MIN_MIN & LATITUDE MIN_SEC \\
\hline 78 & OFFSITE & SESPMNT & $\mathrm{R}$ & & & & & & \\
\hline 79 & OFFSITE & SESPMNT & $\mathrm{R}$ & & & & & & \\
\hline 80 & OFFSITE & SESPMNT & $\mathrm{R}$ & & & & & & \\
\hline 81 & OFFSITE & SESPMNT & $\mathrm{R}$ & & & & & & \\
\hline 82 & OFFSITE & SESPMNT & $\mathrm{R}$ & & & & & & \\
\hline 83 & OFFSITE & SESPMNT & $\mathrm{R}$ & & & & & & \\
\hline 84 & OFFSITE & SESPMNT & $\mathrm{R}$ & & & & & & \\
\hline 85 & OFFSITE & SESPMNT & $\mathrm{R}$ & & & & & & \\
\hline 86 & ONSITE & SESPMNT & $\mathrm{R}$ & & & & & & \\
\hline 87 & ONSITE & SESPMNT & $\mathrm{R}$ & & & & & & \\
\hline 88 & ONSITE & SESPSPEC & S & & & & & & \\
\hline 89 & OFFSITE & SESPMNT & $\mathrm{R}$ & & & & & & \\
\hline 90 & OFFSITE & SESPMNT & $\mathrm{R}$ & & & & & & \\
\hline 91 & OFFSITE & SESPMNT & $\mathrm{R}$ & & & & & & \\
\hline 92 & OFFSITE & SESPMNT & $\mathrm{R}$ & & & & & & \\
\hline 93 & OFFSITE & SESPMNT & $\mathrm{R}$ & & & & & & \\
\hline 94 & OFFSITE & SESPMNT & $\mathrm{R}$ & & & & & & \\
\hline 95 & OFFSITE & SESPMNT & $\mathrm{R}$ & & & & & & \\
\hline 96 & OFFSITE & SESPMNT & $\mathrm{R}$ & & & & & & \\
\hline 97 & OFFSITE & SESPMNT & $\mathrm{R}$ & & & & & & \\
\hline 98 & OFFSITE & SESPMNT & $\mathrm{R}$ & & & & & & \\
\hline 99 & OFFSITE & SESPMNT & $\mathrm{R}$ & & & & & & \\
\hline 100 & OFFSITE & SESPMNT & $\mathrm{R}$ & & & & & & \\
\hline 101 & OFFSITE & SESPMNT & $\mathrm{R}$ & & & & & & \\
\hline 102 & OFFSITE & SESPMNT & $\mathrm{R}$ & & & & & & \\
\hline 103 & ONSITE & SESPSPEC & S & & & & & & \\
\hline 104 & ONSITE & SESPSPEC & S & & & & & & \\
\hline 105 & OFFSITE & SESPMNT & $\mathrm{R}$ & & & & & & \\
\hline 106 & OFFSITE & SESPMNT & $\mathrm{R}$ & & & & & & \\
\hline 107 & OFFSITE & SESPMNT & $\mathrm{R}$ & & & & & & \\
\hline 108 & OFFSITE & SESPMNT & $\mathrm{R}$ & & & & & & \\
\hline 109 & ONSITE & SESPMNT & $\mathrm{R}$ & & & & & & \\
\hline 110 & ONSITE & SESPMNT & $\mathrm{R}$ & & & & & & \\
\hline 111 & OFFSITE & SESPMNT & $\mathrm{R}$ & & & & & & \\
\hline 112 & OFFSITE & SESPMNT & $\mathrm{R}$ & & & & & & \\
\hline 113 & OFFSITE & SESPMNT & $\mathrm{R}$ & & & & & & \\
\hline 114 & OFFSITE & SESPMNT & $\mathrm{R}$ & & & & & & \\
\hline 115 & OFFSITE & SESPMNT & $\mathrm{R}$ & & & & & & \\
\hline
\end{tabular}




\begin{tabular}{|c|c|c|c|c|c|c|c|c|c|}
\hline & $A G$ & $\mathrm{AH}$ & $\mathrm{Al}$ & $\mathrm{AJ}$ & AK & $\mathrm{AL}$ & $\mathrm{AM}$ & AN & $\mathrm{AO}$ \\
\hline 1 & DIST_CLASS & OWNER_ID & COLLECTION_PURPOSE & X_Y_COORD_FINAL.SAMP_NUM & COORDINATE SYSTEM & DATUM & LATITUDE ${ }^{\circ} \mathrm{N}$ & LATITUDE MIN_MIN & LATITUDE MIN_SEC \\
\hline 116 & OFFSITE & SESPMNT & $\mathrm{R}$ & & & & & & \\
\hline 117 & OFFSITE & SESPMNT & $\mathrm{R}$ & & & & & & \\
\hline 118 & OFFSITE & SESPMNT & $\mathrm{R}$ & & & & & & \\
\hline 119 & OFFSITE & SESPMNT & $\mathrm{R}$ & & & & & & \\
\hline 120 & ONSITE & SESPMNT & $\mathrm{R}$ & B10912 & STATE PLANE & & & & \\
\hline 121 & ONSITE & SESPMNT & $\mathrm{R}$ & B10913 & STATE PLANE & & & & \\
\hline 122 & ONSITE & SESPMNT & $\mathrm{R}$ & B10945 & STATE PLANE & & & & \\
\hline 123 & ONSITE & SESPMNT & $\mathrm{R}$ & B10946 & STATE PLANE & & & & \\
\hline 124 & ONSITE & SESPMNT & $\mathrm{R}$ & B10949 & STATE PLANE & & & & \\
\hline 125 & PERIMETER & SESPMNT & $\mathrm{R}$ & B109J0 & STATE PLANE & & & & \\
\hline 126 & OFFSITE & SESPMNT & $\mathrm{R}$ & & & & & & \\
\hline 127 & OFFSITE & SESPMNT & $\mathrm{R}$ & & & & & & \\
\hline 128 & OFFSITE & SESPMNT & $\mathrm{R}$ & & & & & & \\
\hline 129 & OFFSITE & SESPMNT & $\mathrm{R}$ & & & & & & \\
\hline 130 & ONSITE & SESPMNT & $\mathrm{R}$ & & & & & & \\
\hline 131 & ONSITE & SESPMNT & $\mathrm{R}$ & & & & & & \\
\hline 132 & ONSITE & SESPMNT & $\mathrm{R}$ & & & & & & \\
\hline 133 & ONSITE & SESPMNT & $\mathrm{R}$ & & & & & & \\
\hline 134 & ONSITE & SESPMNT & $\mathrm{R}$ & & & & & & \\
\hline 135 & ONSITE & SESPMNT & $\mathrm{R}$ & & & & & & \\
\hline 136 & ONSITE & SESPMNT & $\mathrm{R}$ & & & & & & \\
\hline 137 & ONSITE & SESPMNT & $\mathrm{R}$ & & & & & & \\
\hline 138 & ONSITE & SESPMNT & $\mathrm{R}$ & & & & & & \\
\hline 139 & ONSITE & SESPMNT & $\mathrm{R}$ & & & & & & \\
\hline 140 & OFFSITE & SESPMNT & $\mathrm{R}$ & & & & & & \\
\hline 141 & OFFSITE & SESPMNT & $\mathrm{R}$ & & & & & & \\
\hline 142 & OFFSITE & SESPMNT & $\mathrm{R}$ & & & & & & \\
\hline 143 & OFFSITE & SESPMNT & $\mathrm{R}$ & & & & & & \\
\hline 144 & ONSITE & SESPMNT & $\mathrm{R}$ & & & & & & \\
\hline 145 & OFFSITE & SESPMNT & $\mathrm{R}$ & & & & & & \\
\hline 146 & OFFSITE & SESPMNT & $\mathrm{R}$ & & & & & & \\
\hline 147 & OFFSITE & SESPMNT & $\mathrm{R}$ & & & & & & \\
\hline 148 & OFFSITE & SESPMNT & $\mathrm{R}$ & & & & & & \\
\hline 149 & OFFSITE & SESPMNT & $\mathrm{R}$ & & & & & & \\
\hline 150 & OFFSITE & SESPMNT & $\mathrm{R}$ & & & & & & \\
\hline 151 & OFFSITE & SESPMNT & $\mathrm{R}$ & & & & & & \\
\hline 152 & OFFSITE & SESPMNT & $\mathrm{R}$ & & & & & & \\
\hline 153 & OFFSITE & SESPMNT & $\mathrm{R}$ & & & & & & \\
\hline
\end{tabular}




\begin{tabular}{|c|c|c|c|c|c|c|c|c|c|}
\hline & $A G$ & $\mathrm{AH}$ & $\mathrm{Al}$ & $\mathrm{AJ}$ & AK & $\mathrm{AL}$ & $\mathrm{AM}$ & AN & $\mathrm{AO}$ \\
\hline 1 & DIST_CLASS & OWNER_ID & COLLECTION_PURPOSE & X_Y_COORD_FINAL.SAMP_NUM & COORDINATE SYSTEM & DATUM & LATITUDE ${ }^{\circ} \mathrm{N}$ & LATITUDE MIN_MIN & LATITUDE MIN_SEC \\
\hline 154 & OFFSITE & SESPMNT & $\mathrm{R}$ & & & & & & \\
\hline 155 & OFFSITE & SESPMNT & $\mathrm{R}$ & & & & & & \\
\hline 156 & OFFSITE & SESPMNT & $\mathrm{R}$ & & & & & & \\
\hline 157 & ONSITE & SESPMNT & $\mathrm{R}$ & & & & & & \\
\hline 158 & ONSITE & SESPMNT & $\mathrm{R}$ & & & & & & \\
\hline 159 & OFFSITE & SESPMNT & $\mathrm{R}$ & & & & & & \\
\hline 160 & OFFSITE & SESPMNT & $\mathrm{R}$ & & & & & & \\
\hline 161 & OFFSITE & SESPMNT & $\mathrm{R}$ & & & & & & \\
\hline 162 & OFFSITE & SESPMNT & $\mathrm{R}$ & & & & & & \\
\hline 163 & ONSITE & SESPMNT & $\mathrm{R}$ & & & & & & \\
\hline 164 & ONSITE & SESPMNT & $\mathrm{R}$ & & & & & & \\
\hline 165 & ONSITE & SESPSPEC & S & & & & & & \\
\hline 166 & OFFSITE & SESPMNT & $\mathrm{R}$ & & & & & & \\
\hline 167 & OFFSITE & SESPMNT & $\mathrm{R}$ & & & & & & \\
\hline 168 & OFFSITE & SESPMNT & $\mathrm{R}$ & & & & & & \\
\hline 169 & OFFSITE & SESPMNT & $\mathrm{R}$ & & & & & & \\
\hline 170 & ONSITE & SESPMNT & $\mathrm{R}$ & & & & & & \\
\hline 171 & ONSITE & SESPMNT & $\mathrm{R}$ & & & & & & \\
\hline 172 & ONSITE & SESPMNT & $\mathrm{R}$ & & & & & & \\
\hline 173 & ONSITE & SESPMNT & $\mathrm{R}$ & & & & & & \\
\hline 174 & ONSITE & SESPMNT & $\mathrm{R}$ & & & & & & \\
\hline 175 & OFFSITE & SESPMNT & $\mathrm{R}$ & & & & & & \\
\hline 176 & OFFSITE & SESPMNT & $\mathrm{R}$ & & & & & & \\
\hline 177 & OFFSITE & SESPMNT & $\mathrm{R}$ & & & & & & \\
\hline 178 & OFFSITE & SESPMNT & $\mathrm{R}$ & & & & & & \\
\hline 179 & ONSITE & SESPMNT & $\mathrm{R}$ & & & & & & \\
\hline 180 & ONSITE & SESPMNT & $\mathrm{R}$ & B11W35 & LAT/LONG & & 46 & & 41.0.717 \\
\hline 181 & ONSITE & SESPMNT & $\mathrm{R}$ & B11W40 & LAT/LONG & & 46 & & 38.14 .823 \\
\hline 182 & ONSITE & SESPMNT & $\mathrm{R}$ & B11W43 & LAT/LONG & & 46 & & 40.05 .774 \\
\hline 183 & ONSITE & SESPMNT & $\mathrm{R}$ & & & & & & \\
\hline 184 & OFFSITE & SESPMNT & $\mathrm{R}$ & & & & & & \\
\hline 185 & PERIMETER & SESPMNT & $\mathrm{R}$ & & & & & & \\
\hline 186 & ONSITE & SESPMNT & $\mathrm{R}$ & & & & & & \\
\hline 187 & OFFSITE & SESPMNT & $\mathrm{R}$ & & & & & & \\
\hline 188 & OFFSITE & SESPMNT & $\mathrm{R}$ & & & & & & \\
\hline 189 & OFFSITE & SESPMNT & $\mathrm{R}$ & & & & & & \\
\hline 190 & OFFSITE & SESPMNT & $\mathrm{R}$ & & & & & & \\
\hline 191 & OFFSITE & SESPMNT & $\mathrm{R}$ & & & & & & \\
\hline
\end{tabular}




\begin{tabular}{|c|c|c|c|c|c|c|c|c|c|}
\hline & $A G$ & $\mathrm{AH}$ & $\mathrm{Al}$ & $\mathrm{AJ}$ & AK & $\mathrm{AL}$ & $\mathrm{AM}$ & AN & $\mathrm{AO}$ \\
\hline 1 & DIST_CLASS & OWNER_ID & COLLECTION_PURPOSE & X_Y_COORD_FINAL.SAMP_NUM & COORDINATE SYSTEM & DATUM & LATITUDE ${ }^{\circ} \mathrm{N}$ & LATITUDE MIN_MIN & LATITUDE MIN_SEC \\
\hline 192 & PERIMETER & SESPMNT & $\mathrm{R}$ & & & & & & \\
\hline 193 & OFFSITE & SESPMNT & $\mathrm{R}$ & & & & & & \\
\hline 194 & OFFSITE & SESPMNT & $\mathrm{R}$ & & & & & & \\
\hline 195 & OFFSITE & SESPMNT & $\mathrm{R}$ & & & & & & \\
\hline 196 & OFFSITE & SESPMNT & $\mathrm{R}$ & & & & & & \\
\hline 197 & ONSITE & SESPMNT & $\mathrm{R}$ & & & & & & \\
\hline 198 & ONSITE & SESPMNT & $\mathrm{R}$ & & & & & & \\
\hline 199 & OFFSITE & SESPMNT & $\mathrm{R}$ & & & & & & \\
\hline 200 & PERIMETER & SESPMNT & $\mathrm{R}$ & & & & & & \\
\hline 201 & OFFSITE & SESPMNT & $\mathrm{R}$ & & & & & & \\
\hline 202 & OFFSITE & SESPMNT & $\mathrm{R}$ & & & & & & \\
\hline 203 & OFFSITE & SESPMNT & $\mathrm{R}$ & & & & & & \\
\hline 204 & OFFSITE & SESPMNT & $\mathrm{R}$ & & & & & & \\
\hline 205 & ONSITE & SESPMNT & $\mathrm{R}$ & B12RM1 & LAT/LONG & WGS84 & 46 & 37664 & \\
\hline 206 & ONSITE & SESPMNT & $\mathrm{R}$ & B12RM6 & LAT/LONG & WGS84 & 46 & 37218 & \\
\hline 207 & ONSITE & SESPSPEC & S & B12RP9 & LAT/LONG & WGS84 & 46 & 63453 & \\
\hline 208 & ONSITE & SESPSPEC & S & B12RR1 & LAT/LONG & WGS84 & 46 & 63453 & \\
\hline 209 & ONSITE & SESPSPEC & S & B12RR3 & LAT/LONG & WGS84 & 46 & 63453 & \\
\hline 210 & ONSITE & SESPSPEC & S & B12RR5 & LAT/LONG & WGS84 & 46 & 37663 & \\
\hline 211 & ONSITE & SESPSPEC & S & B12RR7 & LAT/LONG & WGS84 & 46 & 37662 & \\
\hline 212 & ONSITE & SESPSPEC & S & B12RR9 & LAT/LONG & WGS84 & 46 & 37663 & \\
\hline 213 & ONSITE & SESPSPEC & S & B12RT1 & LAT/LONG & WGS84 & 46 & 37547 & \\
\hline 214 & ONSITE & SESPSPEC & S & B12RT3 & LAT/LONG & WGS84 & 46 & 37547 & \\
\hline 215 & ONSITE & SESPSPEC & S & B12RT5 & LAT/LONG & WGS84 & 46 & 37547 & \\
\hline 216 & ONSITE & SESPSPEC & S & B12RT7 & LAT/LONG & WGS84 & 46 & 37362 & \\
\hline 217 & ONSITE & SESPSPEC & $S$ & B12RT9 & LAT/LONG & WGS84 & 46 & 3722 & \\
\hline 218 & ONSITE & SESPSPEC & S & B12RV1 & LAT/LONG & WGS84 & 46 & 37218 & \\
\hline 219 & ONSITE & SESPSPEC & S & B12RV3 & LAT/LONG & WGS84 & 46 & 37223 & \\
\hline 220 & ONSITE & SESPSPEC & S & B12RV5 & LAT/LONG & WGS84 & 46 & 37166 & \\
\hline 221 & ONSITE & SESPSPEC & S & B12RV7 & LAT/LONG & WGS84 & 46 & 37168 & \\
\hline 222 & ONSITE & SESPSPEC & S & B12RV9 & LAT/LONG & WGS84 & 46 & 37166 & \\
\hline 223 & ONSITE & SESPSPEC & S & B12RW1 & LAT/LONG & WGS84 & 46 & 36953 & \\
\hline 224 & ONSITE & SESPSPEC & S & B12RW5 & LAT/LONG & WGS84 & 46 & 36714 & \\
\hline 225 & ONSITE & SESPSPEC & $\mathrm{S}$ & B12RW7 & LAT/LONG & WGS84 & 46 & 36715 & \\
\hline 226 & ONSITE & SESPSPEC & S & B12RW9 & LAT/LONG & WGS84 & 46 & 36714 & \\
\hline 227 & ONSITE & SESPSPEC & $S$ & & & & & & \\
\hline 228 & ONSITE & SESPSPEC & $S$ & & & & & & \\
\hline 229 & ONSITE & SESPSPEC & S & & & & & & \\
\hline
\end{tabular}




\begin{tabular}{|c|c|c|c|c|c|c|c|c|c|}
\hline & $A G$ & $\mathrm{AH}$ & $\mathrm{Al}$ & $\mathrm{AJ}$ & $\mathrm{AK}$ & $\mathrm{AL}$ & $\mathrm{AM}$ & AN & $\mathrm{AO}$ \\
\hline 1 & DIST_CLASS & OWNER_ID & COLLECTION_PURPOSE & X_Y_COORD_FINAL.SAMP_NUM & COORDINATE SYSTEM & DATUM & LATITUDE ${ }^{\circ} \mathrm{N}$ & LATITUDE MIN_MIN & LATITUDE MIN_SEC \\
\hline 230 & ONSITE & SESPSPEC & S & B12RX9 & LAT/LONG & WGS84 & 46 & 35556 & \\
\hline 231 & ONSITE & SESPSPEC & $\mathrm{S}$ & B12RY1 & LAT/LONG & WGS84 & 46 & 35556 & \\
\hline 232 & ONSITE & SESPSPEC & S & B12RY3 & LAT/LONG & WGS84 & 46 & 35557 & \\
\hline 233 & ONSITE & SESPSPEC & S & B12T11 & LAT/LONG & WGS84 & 46 & 63453 & \\
\hline 234 & ONSITE & SESPSPEC & S & B12T13 & LAT/LONG & WGS84 & 46 & 37661 & \\
\hline 235 & ONSITE & SESPSPEC & S & B12T15 & LAT/LONG & WGS84 & 46 & 37546 & \\
\hline 236 & ONSITE & SESPSPEC & S & B12T17 & LAT/LONG & WGS84 & 46 & 37223 & \\
\hline 237 & ONSITE & SESPSPEC & S & B12T19 & LAT/LONG & WGS84 & 46 & 37166 & \\
\hline 238 & ONSITE & SESPSPEC & S & B12T21 & LAT/LONG & WGS84 & 46 & 36716 & \\
\hline 239 & ONSITE & SESPSPEC & S & & & & & & \\
\hline 240 & ONSITE & SESPSPEC & S & B12T25 & LAT/LONG & WGS84 & 46 & 35558 & \\
\hline 241 & OFFSITE & SESPMNT & $\mathrm{R}$ & & & & & & \\
\hline 242 & OFFSITE & SESPMNT & $\mathrm{R}$ & & & & & & \\
\hline 243 & OFFSITE & SESPMNT & $\mathrm{R}$ & & & & & & \\
\hline 244 & OFFSITE & SESPMNT & $\mathrm{R}$ & & & & & & \\
\hline 245 & ONSITE & SESPMNT & $\mathrm{R}$ & B12XK2 & LAT/LONG & & 46 & & 22.20 .036 \\
\hline 246 & ONSITE & SESPSPEC & S & B12XK8 & LAT/LONG & & 46 & & 22.20 .059 \\
\hline 247 & ONSITE & SESPSPEC & S & B12XK9 & LAT/LONG & & 46 & & 22.20 .059 \\
\hline 248 & ONSITE & SESPSPEC & S & B12XL0 & LAT/LONG & & 46 & & 22.20 .084 \\
\hline 249 & ONSITE & SESPSPEC & S & B12XL1 & LAT/LONG & & 46 & & 22.20 .084 \\
\hline 250 & ONSITE & SESPSPEC & S & B12XL2 & LAT/LONG & & 46 & & 22.20 .031 \\
\hline 251 & ONSITE & SESPMNT & $\mathrm{R}$ & B12XK1 & LAT/LONG & & 46 & & 22.36 .037 \\
\hline 252 & ONSITE & SESPSPEC & S & B12XK3 & LAT/LONG & & 46 & & 22.35.976 \\
\hline 253 & ONSITE & SESPSPEC & S & B12XK4 & LAT/LONG & & 46 & & 22.35.976 \\
\hline 254 & ONSITE & SESPSPEC & S & B12XK5 & LAT/LONG & & 46 & & 22.35 .976 \\
\hline 255 & ONSITE & SESPSPEC & $S$ & B12XK6 & LAT/LONG & & 46 & & 22.36 .045 \\
\hline 256 & ONSITE & SESPSPEC & S & B12XK7 & LAT/LONG & & 46 & & 22.36.045 \\
\hline 257 & OFFSITE & SESPMNT & $\mathrm{R}$ & & & & & & \\
\hline 258 & OFFSITE & SESPMNT & $\mathrm{R}$ & & & & & & \\
\hline 259 & OFFSITE & SESPMNT & $\mathrm{R}$ & & & & & & \\
\hline 260 & OFFSITE & SESPMNT & $\mathrm{R}$ & & & & & & \\
\hline 261 & ONSITE & SESPMNT & $\mathrm{R}$ & & & & & & \\
\hline 262 & ONSITE & SESPMNT & $\mathrm{R}$ & & & & & & \\
\hline 263 & ONSITE & SESPMNT & $\mathrm{R}$ & & & & & & \\
\hline 264 & ONSITE & SESPMNT & $\mathrm{R}$ & B12WY1 & LAT/LONG & & 46 & & 40.59 .99 \\
\hline 265 & ONSITE & SESPMNT & $\mathrm{R}$ & B12X41 & LAT/LONG & & 46 & & 39.3 .62 \\
\hline 266 & ONSITE & SESPMNT & $\mathrm{R}$ & B12X42 & LAT/LONG & & 46 & & 39.40 .61 \\
\hline 267 & ONSITE & SESPMNT & $\mathrm{R}$ & B12X48 & LAT/LONG & & 46 & & 42.41 .7 \\
\hline
\end{tabular}

Page 475 


\begin{tabular}{|c|c|c|c|c|c|c|c|c|c|}
\hline & $A G$ & $\mathrm{AH}$ & $\mathrm{Al}$ & $\mathrm{AJ}$ & $A K$ & $\mathrm{AL}$ & $\mathrm{AM}$ & $\mathrm{AN}$ & $\mathrm{AO}$ \\
\hline 1 & DIST_CLASS & OWNER_ID & COLLECTION_PURPOSE & X_Y_COORD_FINAL.SAMP_NUM & COORDINATE SYSTEM & DATUM & LATITUDE ${ }^{\circ} \mathrm{N}$ & LATITUDE MIN_MIN & LATITUDE MIN_SEC \\
\hline 268 & ONSITE & SESPMNT & $\mathrm{R}$ & B12X85 & LAT/LONG & & 46 & & 42.12 .8 \\
\hline 269 & OFFSITE & SESPMNT & $\mathrm{R}$ & & & & & & \\
\hline 270 & OFFSITE & SESPMNT & $\mathrm{R}$ & & & & & & \\
\hline 271 & OFFSITE & SESPMNT & $\mathrm{R}$ & & & & & & \\
\hline 272 & OFFSITE & SESPMNT & $\mathrm{R}$ & & & & & & \\
\hline 273 & ONSITE & SESPSPEC & S & & & & & & \\
\hline 274 & OFFSITE & SESPMNT & $\mathrm{R}$ & & & & & & \\
\hline 275 & OFFSITE & SESPMNT & $\mathrm{R}$ & & & & & & \\
\hline 276 & OFFSITE & SESPMNT & $\mathrm{R}$ & & & & & & \\
\hline 277 & OFFSITE & SESPMNT & $\mathrm{R}$ & & & & & & \\
\hline 278 & OFFSITE & SESPMNT & $\mathrm{R}$ & & & & & & \\
\hline 279 & OFFSITE & SESPMNT & $\mathrm{R}$ & & & & & & \\
\hline 280 & OFFSITE & SESPMNT & $\mathrm{R}$ & & & & & & \\
\hline 281 & OFFSITE & SESPMNT & $\mathrm{R}$ & & & & & & \\
\hline 282 & OFFSITE & SESPMNT & $\mathrm{R}$ & & & & & & \\
\hline 283 & OFFSITE & SESPMNT & $\mathrm{R}$ & & & & & & \\
\hline 284 & OFFSITE & SESPMNT & $\mathrm{R}$ & & & & & & \\
\hline 285 & OFFSITE & SESPMNT & $\mathrm{R}$ & & & & & & \\
\hline 286 & ONSITE & SESPMNT & $\mathrm{R}$ & & & & & & \\
\hline 287 & OFFSITE & SESPMNT & $\mathrm{R}$ & & & & & & \\
\hline 288 & OFFSITE & SESPMNT & $\mathrm{R}$ & & & & & & \\
\hline 289 & OFFSITE & SESPMNT & $\mathrm{R}$ & & & & & & \\
\hline 290 & OFFSITE & SESPMNT & $\mathrm{R}$ & & & & & & \\
\hline 291 & OFFSITE & SESPMNT & $\mathrm{R}$ & & & & & & \\
\hline 292 & OFFSITE & SESPMNT & $\mathrm{R}$ & & & & & & \\
\hline 293 & OFFSITE & SESPMNT & $\mathrm{R}$ & & & & & & \\
\hline 294 & OFFSITE & SESPMNT & $\mathrm{R}$ & & & & & & \\
\hline 295 & ONSITE & SESPMNT & $\mathrm{R}$ & & & & & & \\
\hline 296 & ONSITE & SESPSPEC & S & & & & & & \\
\hline 297 & OFFSITE & SESPMNT & $\mathrm{R}$ & & & & & & \\
\hline 298 & OFFSITE & SESPMNT & $\mathrm{R}$ & & & & & & \\
\hline 299 & OFFSITE & SESPMNT & $\mathrm{R}$ & & & & & & \\
\hline 300 & OFFSITE & SESPMNT & $\mathrm{R}$ & & & & & & \\
\hline 301 & OFFSITE & SESPMNT & $\mathrm{R}$ & B14LJ0 & LAT/LONG & & 46 & 25832 & \\
\hline 302 & PERIMETER & SESPMNT & $\mathrm{R}$ & B14LJ2 & LAT/LONG & & 46 & 34120 & \\
\hline 303 & OFFSITE & SESPMNT & $\mathrm{R}$ & & & & & & \\
\hline 304 & OFFSITE & SESPMNT & $\mathrm{R}$ & & & & & & \\
\hline 305 & OFFSITE & SESPMNT & $\mathrm{R}$ & & & & & & \\
\hline
\end{tabular}




\begin{tabular}{|c|c|c|c|c|c|c|c|c|c|}
\hline & $A G$ & $\mathrm{AH}$ & $\mathrm{Al}$ & $\mathrm{AJ}$ & $\mathrm{AK}$ & $\overline{\mathrm{AL}}$ & AM & AN & $\mathrm{AO}$ \\
\hline 1 & DIST_CLASS & OWNER_ID & COLLECTION_PURPOSE & X_Y_COORD_FINAL.SAMP_NUM & COORDINATE SYSTEM & DATUM & LATITUDE ${ }^{\circ} \mathrm{N}$ & LATITUDE MIN_MIN & LATITUDE MIN_SEC \\
\hline 306 & OFFSITE & SESPMNT & $\mathrm{R}$ & & & & & & \\
\hline 307 & OFFSITE & SESPMNT & $\mathrm{R}$ & & & & & & \\
\hline 308 & OFFSITE & SESPMNT & $\mathrm{R}$ & & & & & & \\
\hline 309 & OFFSITE & SESPMNT & $\mathrm{R}$ & & & & & & \\
\hline 310 & OFFSITE & SESPMNT & $\mathrm{R}$ & & & & & & \\
\hline 311 & OFFSITE & SESPMNT & $\mathrm{R}$ & & & & & & \\
\hline 312 & PERIMETER & SESPMNT & $\mathrm{R}$ & & & & & & \\
\hline 313 & ONSITE & SESPMNT & $\mathrm{R}$ & & & & & & \\
\hline 314 & OFFSITE & SESPMNT & $\mathrm{R}$ & & & & & & \\
\hline 315 & PERIMETER & SESPMNT & $\mathrm{R}$ & & & & & & \\
\hline 316 & OFFSITE & SESPMNT & $\mathrm{R}$ & & & & & & \\
\hline 317 & OFFSITE & SESPMNT & $\mathrm{R}$ & & & & & & \\
\hline 318 & OFFSITE & SESPMNT & $\mathrm{R}$ & & & & & & \\
\hline 319 & OFFSITE & SESPMNT & $\mathrm{R}$ & & & & & & \\
\hline 320 & OFFSITE & SESPMNT & $\mathrm{R}$ & & & & & & \\
\hline 321 & OFFSITE & SESPMNT & $\mathrm{R}$ & & & & & & \\
\hline 322 & OFFSITE & SESPMNT & $\mathrm{R}$ & & & & & & \\
\hline 323 & OFFSITE & SESPMNT & $\mathrm{R}$ & & & & & & \\
\hline 324 & ONSITE & SESPMNT & $\mathrm{R}$ & & & & & & \\
\hline 325 & ONSITE & SESPMNT & $\mathrm{R}$ & & & & & & \\
\hline 326 & ONSITE & SESPMNT & $\mathrm{R}$ & & & & & & \\
\hline 327 & OFFSITE & SESPMNT & $\mathrm{R}$ & & & & & & \\
\hline 328 & OFFSITE & SESPMNT & $\mathrm{R}$ & & & & & & \\
\hline 329 & OFFSITE & SESPMNT & $\mathrm{R}$ & & & & & & \\
\hline 330 & OFFSITE & SESPMNT & $\mathrm{R}$ & & & & & & \\
\hline 331 & ONSITE & SESPMNT & $\mathrm{R}$ & B15C09 & LAT/LONG & & 46 & & 33.55.313 \\
\hline 332 & ONSITE & SESPMNT & $\mathrm{R}$ & & & & & & \\
\hline 333 & ONSITE & SESPMNT & $\mathrm{R}$ & B15C73 & LAT/LONG & & 46 & & 33.50 .499 \\
\hline 334 & ONSITE & SESPMNT & $\mathrm{R}$ & & & & & & \\
\hline 335 & ONSITE & SESPMNT & $\mathrm{R}$ & B15C60 & LAT/LONG & & 46 & & 39.40 .613 \\
\hline 336 & ONSITE & SESPMNT & $\mathrm{R}$ & B15C65 & LAT/LONG & & 46 & & 42.04 .2747 \\
\hline 337 & ONSITE & SESPMNT & $\mathrm{R}$ & B15CB3 & LAT/LONG & & 46 & & 42.12 .884 \\
\hline 338 & ONSITE & SESPMNT & $\mathrm{R}$ & B15C69 & LAT/LONG & & 46 & & 38.14 .440 \\
\hline 339 & OFFSITE & SESPMNT & $\mathrm{R}$ & & & & & & \\
\hline 340 & OFFSITE & SESPMNT & $\mathrm{R}$ & & & & & & \\
\hline 341 & OFFSITE & SESPMNT & $\mathrm{R}$ & & & & & & \\
\hline 342 & OFFSITE & SESPMNT & $\mathrm{R}$ & & & & & & \\
\hline 343 & OFFSITE & SESPMNT & $\mathrm{R}$ & & & & & & \\
\hline
\end{tabular}




\begin{tabular}{|c|c|c|c|c|c|c|c|c|c|}
\hline & $A G$ & $\mathrm{AH}$ & $\mathrm{Al}$ & $\mathrm{AJ}$ & $\mathrm{AK}$ & $\mathrm{AL}$ & $\mathrm{AM}$ & $\mathrm{AN}$ & $\mathrm{AO}$ \\
\hline 1 & DIST_CLASS & OWNER_ID & COLLECTION_PURPOSE & X_Y_COORD_FINAL.SAMP_NUM & COORDINATE SYSTEM & DATUM & LATITUDE ${ }^{\circ} \mathrm{N}$ & LATITUDE MIN_MIN & LATITUDE MIN_SEC \\
\hline 344 & OFFSITE & SESPMNT & $\mathrm{R}$ & & & & & & \\
\hline 345 & OFFSITE & SESPMNT & $\mathrm{R}$ & & & & & & \\
\hline 346 & OFFSITE & SESPMNT & $\mathrm{R}$ & & & & & & \\
\hline 347 & OFFSITE & SESPMNT & $\mathrm{R}$ & & & & & & \\
\hline 348 & OFFSITE & SESPMNT & $\mathrm{R}$ & & & & & & \\
\hline 349 & OFFSITE & SESPMNT & $\mathrm{R}$ & & & & & & \\
\hline 350 & OFFSITE & SESPMNT & $\mathrm{R}$ & & & & & & \\
\hline 351 & ONSITE & SESPMNT & $\mathrm{R}$ & B10906 & STATE PLANE & & & & \\
\hline 352 & ONSITE & SESPMNT & $\mathrm{R}$ & B10907 & STATE PLANE & & & & \\
\hline 353 & ONSITE & SESPMNT & $\mathrm{R}$ & B10951 & STATE PLANE & & & & \\
\hline 354 & ONSITE & SESPMNT & $\mathrm{R}$ & B10952 & STATE PLANE & & & & \\
\hline 355 & ONSITE & SESPMNT & $\mathrm{R}$ & B10976 & STATE PLANE & & & & \\
\hline 356 & OFFSITE & SESPMNT & $\mathrm{R}$ & & & & & & \\
\hline 357 & OFFSITE & SESPMNT & $\mathrm{R}$ & & & & & & \\
\hline 358 & JOFFSITE & SESPMNT & $\mathrm{R}$ & & & & & & \\
\hline 359 & OFFSITE & SESPMNT & $\mathrm{R}$ & & & & & & \\
\hline 360 & OFFSITE & SESPMNT & $\mathrm{R}$ & & & & & & \\
\hline 361 & OFFSITE & SESPMNT & $\mathrm{R}$ & & & & & & \\
\hline 362 & ONSITE & SESPMNT & $\mathrm{R}$ & & & & & & \\
\hline 363 & ONSITE & SESPMNT & $\mathrm{R}$ & & & & & & \\
\hline 364 & ONSITE & SESPMNT & $\mathrm{R}$ & & & & & & \\
\hline 365 & ONSITE & SESPMNT & $\mathrm{R}$ & & & & & & \\
\hline 366 & OFFSITE & SESPMNT & $\mathrm{R}$ & & & & & & \\
\hline 367 & OFFSITE & SESPMNT & $\mathrm{R}$ & & & & & & \\
\hline 368 & ONSITE & SESPMNT & $\mathrm{R}$ & B12RL8 & LAT/LONG & WGS84 & 46 & 37664 & \\
\hline 369 & ONSITE & SESPMNT & $\mathrm{R}$ & B12RN1 & LAT/LONG & WGS84 & 46 & 37218 & \\
\hline 370 & OFFSITE & SESPMNT & $\mathrm{R}$ & & & & & & \\
\hline 371 & OFFSITE & SESPMNT & $\mathrm{R}$ & & & & & & \\
\hline 372 & OFFSITE & SESPMNT & $\mathrm{R}$ & & & & & & \\
\hline 373 & OFFSITE & SESPMNT & $\mathrm{R}$ & & & & & & \\
\hline 374 & OFFSITE & SESPMNT & $\mathrm{R}$ & & & & & & \\
\hline 375 & OFFSITE & SESPMNT & $\mathrm{R}$ & & & & & & \\
\hline 376 & OFFSITE & SESPMNT & $\mathrm{R}$ & & & & & & \\
\hline 377 & OFFSITE & SESPMNT & $\mathrm{R}$ & & & & & & \\
\hline 378 & OFFSITE & SESPMNT & $\mathrm{R}$ & & & & & & \\
\hline 379 & OFFSITE & SESPMNT & $\mathrm{R}$ & & & & & & \\
\hline 380 & OFFSITE & SESPMNT & $\mathrm{R}$ & & & & & & \\
\hline 381 & OFFSITE & SESPMNT & $\mathrm{R}$ & & & & & & \\
\hline
\end{tabular}




\begin{tabular}{|c|c|c|c|c|c|c|c|c|c|}
\hline & $A G$ & $\mathrm{AH}$ & $\mathrm{Al}$ & $\mathrm{AJ}$ & $A K$ & $\mathrm{AL}$ & $\mathrm{AM}$ & $\mathrm{AN}$ & $\mathrm{AO}$ \\
\hline 1 & DIST_CLASS & OWNER_ID & COLLECTION_PURPOSE & X_Y_COORD_FINAL.SAMP_NUM & COORDINATE SYSTEM & DATUM & LATITUDE ${ }^{\circ} \mathrm{N}$ & LATITUDE MIN_MIN & LATITUDE MIN_SEC \\
\hline 382 & OFFSITE & SESPMNT & $\mathrm{R}$ & & & & & & \\
\hline 383 & OFFSITE & SESPMNT & $\mathrm{R}$ & & & & & & \\
\hline 384 & OFFSITE & SESPMNT & $\mathrm{R}$ & & & & & & \\
\hline 385 & OFFSITE & SESPMNT & $\mathrm{R}$ & & & & & & \\
\hline 386 & OFFSITE & SESPMNT & $\mathrm{R}$ & & & & & & \\
\hline 387 & OFFSITE & SESPMNT & $\mathrm{R}$ & & & & & & \\
\hline 388 & OFFSITE & SESPMNT & $\mathrm{R}$ & & & & & & \\
\hline 389 & OFFSITE & SESPMNT & $\mathrm{R}$ & & & & & & \\
\hline 390 & OFFSITE & SESPMNT & $\mathrm{R}$ & & & & & & \\
\hline 391 & OFFSITE & SESPMNT & $\mathrm{R}$ & & & & & & \\
\hline 392 & OFFSITE & SESPMNT & $\mathrm{R}$ & & & & & & \\
\hline 393 & OFFSITE & SESPMNT & $\mathrm{R}$ & & & & & & \\
\hline 394 & OFFSITE & SESPMNT & $\mathrm{R}$ & & & & & & \\
\hline 395 & OFFSITE & SESPMNT & $\mathrm{R}$ & & & & & & \\
\hline 396 & OFFSITE & SESPMNT & $\mathrm{R}$ & & & & & & \\
\hline 397 & OFFSITE & SESPMNT & $\mathrm{R}$ & & & & & & \\
\hline 398 & OFFSITE & SESPMNT & $\mathrm{R}$ & & & & & & \\
\hline 399 & OFFSITE & SESPMNT & $\mathrm{R}$ & & & & & & \\
\hline 400 & OFFSITE & SESPMNT & $\mathrm{R}$ & & & & & & \\
\hline 401 & OFFSITE & SESPMNT & $\mathrm{R}$ & & & & & & \\
\hline 402 & OFFSITE & SESPMNT & $\mathrm{R}$ & & & & & & \\
\hline 403 & OFFSITE & SESPMNT & $\mathrm{R}$ & & & & & & \\
\hline 404 & OFFSITE & SESPMNT & $\mathrm{R}$ & & & & & & \\
\hline 405 & OFFSITE & SESPMNT & $\mathrm{R}$ & & & & & & \\
\hline 406 & OFFSITE & SESPMNT & $\mathrm{R}$ & & & & & & \\
\hline 407 & OFFSITE & SESPMNT & $\mathrm{R}$ & & & & & & \\
\hline 408 & OFFSITE & SESPMNT & $\mathrm{R}$ & & & & & & \\
\hline 409 & OFFSITE & SESPMNT & $\mathrm{R}$ & & & & & & \\
\hline 410 & OFFSITE & SESPMNT & $\mathrm{R}$ & & & & & & \\
\hline 411 & OFFSITE & SESPMNT & $\mathrm{R}$ & & & & & & \\
\hline 412 & OFFSITE & SESPMNT & $\mathrm{R}$ & & & & & & \\
\hline 413 & OFFSITE & SESPMNT & $\mathrm{R}$ & & & & & & \\
\hline 414 & OFFSITE & SESPMNT & $\mathrm{R}$ & & & & & & \\
\hline 415 & OFFSITE & SESPMNT & $\mathrm{R}$ & & & & & & \\
\hline 416 & OFFSITE & SESPMNT & $\mathrm{R}$ & & & & & & \\
\hline 417 & OFFSITE & SESPMNT & $\mathrm{R}$ & & & & & & \\
\hline 418 & OFFSITE & SESPMNT & $\mathrm{R}$ & & & & & & \\
\hline 419 & OFFSITE & SESPMNT & $\mathrm{R}$ & & & & & & \\
\hline
\end{tabular}




\begin{tabular}{|c|c|c|c|c|c|c|c|c|c|}
\hline & $A G$ & $\mathrm{AH}$ & $\mathrm{Al}$ & $\mathrm{AJ}$ & AK & $\mathrm{AL}$ & $\mathrm{AM}$ & AN & $\mathrm{AO}$ \\
\hline 1 & DIST_CLASS & OWNER_ID & COLLECTION_PURPOSE & X_Y_COORD_FINAL.SAMP_NUM & COORDINATE SYSTEM & DATUM & LATITUDE ${ }^{\circ} \mathrm{N}$ & LATITUDE MIN_MIN & LATITUDE MIN_SEC \\
\hline 420 & ONSITE & SESPMNT & $\mathrm{R}$ & & & & & & \\
\hline 421 & ONSITE & SESPMNT & $\mathrm{R}$ & & & & & & \\
\hline 422 & ONSITE & SESPMNT & $\mathrm{R}$ & & & & & & \\
\hline 423 & ONSITE & SESPMNT & $\mathrm{R}$ & & & & & & \\
\hline 424 & OFFSITE & SESPMNT & $\mathrm{R}$ & & & & & & \\
\hline 425 & OFFSITE & SESPMNT & $\mathrm{R}$ & & & & & & \\
\hline 426 & OFFSITE & SESPMNT & $\mathrm{R}$ & & & & & & \\
\hline 427 & OFFSITE & SESPMNT & $\mathrm{R}$ & & & & & & \\
\hline 428 & OFFSITE & SESPMNT & $\mathrm{R}$ & & & & & & \\
\hline 429 & OFFSITE & SESPMNT & $\mathrm{R}$ & & & & & & \\
\hline 430 & OFFSITE & SESPSPEC & S & & & & & & \\
\hline 431 & OFFSITE & SESPMNT & $\mathrm{R}$ & & & & & & \\
\hline 432 & OFFSITE & SESPMNT & $\mathrm{R}$ & & & & & & \\
\hline 433 & OFFSITE & SESPMNT & $\mathrm{R}$ & & & & & & \\
\hline 434 & OFFSITE & SESPMNT & $\mathrm{R}$ & & & & & & \\
\hline 435 & OFFSITE & SESPMNT & $\mathrm{R}$ & & & & & & \\
\hline 436 & OFFSITE & SESPMNT & $\mathrm{R}$ & & & & & & \\
\hline 437 & OFFSITE & SESPMNT & $\mathrm{R}$ & & & & & & \\
\hline 438 & OFFSITE & SESPMNT & $\mathrm{R}$ & & & & & & \\
\hline 439 & OFFSITE & SESPMNT & $\mathrm{R}$ & & & & & & \\
\hline 440 & OFFSITE & SESPMNT & $\mathrm{R}$ & & & & & & \\
\hline 441 & OFFSITE & SESPMNT & $\mathrm{R}$ & & & & & & \\
\hline 442 & OFFSITE & SESPMNT & $\mathrm{R}$ & & & & & & \\
\hline 443 & ONSITE & SESPMNT & $\mathrm{R}$ & & & & & & \\
\hline 444 & ONSITE & SESPMNT & $\mathrm{R}$ & & & & & & \\
\hline 445 & ONSITE & SESPMNT & $\mathrm{R}$ & & & & & & \\
\hline 446 & ONSITE & SESPMNT & $\mathrm{R}$ & & & & & & \\
\hline 447 & OFFSITE & SESPMNT & $\mathrm{R}$ & & & & & & \\
\hline 448 & OFFSITE & SESPMNT & $\mathrm{R}$ & & & & & & \\
\hline 449 & OFFSITE & SESPMNT & $\mathrm{R}$ & & & & & & \\
\hline 450 & OFFSITE & SESPMNT & $\mathrm{R}$ & & & & & & \\
\hline 451 & OFFSITE & SESPMNT & $\mathrm{R}$ & & & & & & \\
\hline 452 & OFFSITE & SESPMNT & $\mathrm{R}$ & & & & & & \\
\hline 453 & OFFSITE & SESPMNT & $\mathrm{R}$ & BOYCD7 & STATE PLANE & & & & \\
\hline 454 & OFFSITE & SESPMNT & $\mathrm{R}$ & B0YCD8 & STATE PLANE & & & & \\
\hline 455 & OFFSITE & SESPMNT & $\mathrm{R}$ & BOYCD9 & STATE PLANE & & & & \\
\hline 456 & OFFSITE & SESPMNT & $\mathrm{R}$ & BOYCFO & STATE PLANE & & & & \\
\hline 457 & OFFSITE & SESPMNT & $\mathrm{R}$ & BOYCF1 & STATE PLANE & & & & \\
\hline
\end{tabular}




\begin{tabular}{|c|c|c|c|c|c|c|c|c|c|}
\hline & $A G$ & $\mathrm{AH}$ & $\mathrm{Al}$ & $\mathrm{AJ}$ & $\mathrm{AK}$ & $\mathrm{AL}$ & $\mathrm{AM}$ & AN & $\mathrm{AO}$ \\
\hline 1 & DIST_CLASS & OWNER_ID & COLLECTION_PURPOSE & X_Y_COORD_FINAL.SAMP_NUM & COORDINATE SYSTEM & DATUM & LATITUDE ${ }^{\circ} \mathrm{N}$ & LATITUDE MIN_MIN & LATITUDE MIN_SEC \\
\hline 458 & OFFSITE & SESPMNT & $\mathrm{R}$ & BOYCF2 & STATE PLANE & & & & \\
\hline 459 & OFFSITE & SESPMNT & $\mathrm{R}$ & BOYCF3 & STATE PLANE & & & & \\
\hline 460 & OFFSITE & SESPMNT & $\mathrm{R}$ & BOYCF4 & STATE PLANE & & & & \\
\hline 461 & OFFSITE & SESPMNT & $\mathrm{R}$ & B0YCF5 & STATE PLANE & & & & \\
\hline 462 & OFFSITE & SESPMNT & $\mathrm{R}$ & BOYCF6 & STATE PLANE & & & & \\
\hline 463 & OFFSITE & SESPMNT & $\mathrm{R}$ & BOYCK3 & STATE PLANE & & & & \\
\hline 464 & OFFSITE & SESPMNT & $\mathrm{R}$ & BOYCK4 & STATE PLANE & & & & \\
\hline 465 & OFFSITE & SESPMNT & $\mathrm{R}$ & B0YCK5 & STATE PLANE & & & & \\
\hline 466 & OFFSITE & SESPMNT & $\mathrm{R}$ & BOYCK6 & STATE PLANE & & & & \\
\hline 467 & OFFSITE & SESPMNT & $\mathrm{R}$ & & & & & & \\
\hline 468 & OFFSITE & SESPMNT & $\mathrm{R}$ & & & & & & \\
\hline 469 & OFFSITE & SESPMNT & $\mathrm{R}$ & & & & & & \\
\hline 470 & ONSITE & SESPMNT & $\mathrm{R}$ & & & & & & \\
\hline 471 & ONSITE & SESPMNT & $\mathrm{R}$ & & & & & & \\
\hline 472 & ONSITE & SESPMNT & $\mathrm{R}$ & & & & & & \\
\hline 473 & ONSITE & SESPMNT & $\mathrm{R}$ & & & & & & \\
\hline 474 & OFFSITE & SESPMNT & $\mathrm{R}$ & & & & & & \\
\hline 475 & OFFSITE & SESPMNT & $\mathrm{R}$ & & & & & & \\
\hline 476 & OFFSITE & SESPMNT & $\mathrm{R}$ & & & & & & \\
\hline 477 & OFFSITE & SESPMNT & $\mathrm{R}$ & & & & & & \\
\hline 478 & ONSITE & SESPSPEC & S & B10672 & STATE PLANE & & & & \\
\hline 479 & OFFSITE & SESPMNT & $\mathrm{R}$ & B10673 & STATE PLANE & & & & \\
\hline 480 & OFFSITE & SESPMNT & $\mathrm{R}$ & B10674 & STATE PLANE & & & & \\
\hline 481 & OFFSITE & SESPMNT & $\mathrm{R}$ & B10675 & STATE PLANE & & & & \\
\hline 482 & OFFSITE & SESPMNT & $\mathrm{R}$ & B10676 & STATE PLANE & & & & \\
\hline 483 & ONSITE & SESPMNT & $\mathrm{R}$ & B10677 & STATE PLANE & & & & \\
\hline 484 & ONSITE & SESPMNT & $\mathrm{R}$ & B10679 & STATE PLANE & & & & \\
\hline 485 & ONSITE & SESPMNT & $\mathrm{R}$ & & & & & & \\
\hline 486 & ONSITE & SESPMNT & $\mathrm{R}$ & B10683 & STATE PLANE & & & & \\
\hline 487 & ONSITE & SESPMNT & $\mathrm{R}$ & B10684 & STATE PLANE & & & & \\
\hline 488 & ONSITE & SESPMNT & $\mathrm{R}$ & & & & & & \\
\hline 489 & ONSITE & SESPMNT & $\mathrm{R}$ & & & & & & \\
\hline 490 & ONSITE & SESPMNT & $\mathrm{R}$ & B106B1 & STATE PLANE & & & & \\
\hline 491 & ONSITE & SESPMNT & $\mathrm{R}$ & & & & & & \\
\hline 492 & ONSITE & SESPMNT & $\mathrm{R}$ & B106B7 & STATE PLANE & & & & \\
\hline 493 & ONSITE & SESPMNT & $\mathrm{R}$ & B106W1 & STATE PLANE & & & & \\
\hline 494 & ONSITE & SESPMNT & $\mathrm{R}$ & B106W2 & STATE PLANE & & & & \\
\hline 495 & ONSITE & SESPMNT & $\mathrm{R}$ & B106W3 & STATE PLANE & & & & \\
\hline
\end{tabular}




\begin{tabular}{|c|c|c|c|c|c|c|c|c|c|}
\hline & $A G$ & $\mathrm{AH}$ & $\mathrm{Al}$ & $\mathrm{AJ}$ & AK & $\mathrm{AL}$ & $\mathrm{AM}$ & AN & $\mathrm{AO}$ \\
\hline 1 & DIST_CLASS & OWNER_ID & COLLECTION_PURPOSE & X_Y_COORD_FINAL.SAMP_NUM & COORDINATE SYSTEM & DATUM & LATITUDE ${ }^{\circ} \mathrm{N}$ & LATITUDE MIN_MIN & LATITUDE MIN_SEC \\
\hline 496 & ONSITE & SESPMNT & $\mathrm{R}$ & B106W4 & STATE PLANE & & & & \\
\hline 497 & ONSITE & SESPMNT & $\mathrm{R}$ & B106W5 & STATE PLANE & & & & \\
\hline 498 & ONSITE & SESPMNT & $\mathrm{R}$ & B106W6 & STATE PLANE & & & & \\
\hline 499 & ONSITE & SESPMNT & $\mathrm{R}$ & B106W7 & STATE PLANE & & & & \\
\hline 500 & ONSITE & SESPMNT & $\mathrm{R}$ & B106W8 & STATE PLANE & & & & \\
\hline 501 & ONSITE & SESPMNT & $\mathrm{R}$ & B106W9 & STATE PLANE & & & & \\
\hline 502 & ONSITE & SESPMNT & $\mathrm{R}$ & B106X0 & STATE PLANE & & & & \\
\hline 503 & ONSITE & SESPMNT & $\mathrm{R}$ & B106X1 & STATE PLANE & & & & \\
\hline 504 & ONSITE & SESPMNT & $\mathrm{R}$ & B106X2 & STATE PLANE & & & & \\
\hline 505 & ONSITE & SESPMNT & $\mathrm{R}$ & B10728 & STATE PLANE & & & & \\
\hline 506 & ONSITE & SESPMNT & $\mathrm{R}$ & B10729 & STATE PLANE & & & & \\
\hline 507 & ONSITE & SESPMNT & $\mathrm{R}$ & B10730 & STATE PLANE & & & & \\
\hline 508 & ONSITE & SESPMNT & $\mathrm{R}$ & B10785 & STATE PLANE & & & & \\
\hline 509 & ONSITE & SESPMNT & $\mathrm{R}$ & B10788 & STATE PLANE & & & & \\
\hline 510 & ONSITE & SESPMNT & $\mathrm{R}$ & B10791 & STATE PLANE & & & & \\
\hline 511 & ONSITE & SESPMNT & $\mathrm{R}$ & B10794 & STATE PLANE & & & & \\
\hline 512 & ONSITE & SESPMNT & $\mathrm{R}$ & & & & & & \\
\hline 513 & ONSITE & SESPMNT & $\mathrm{R}$ & & & & & & \\
\hline 514 & ONSITE & SESPMNT & $\mathrm{R}$ & B106X7 & STATE PLANE & & & & \\
\hline 515 & ONSITE & SESPMNT & $\mathrm{R}$ & B106X9 & STATE PLANE & & & & \\
\hline 516 & ONSITE & SESPMNT & $\mathrm{R}$ & B106Y1 & STATE PLANE & & & & \\
\hline 517 & ONSITE & SESPMNT & $\mathrm{R}$ & B106Y3 & STATE PLANE & & & & \\
\hline 518 & OFFSITE & SESPMNT & $\mathrm{R}$ & B106Y4 & STATE PLANE & & & & \\
\hline 519 & OFFSITE & SESPMNT & $\mathrm{R}$ & B106Y5 & STATE PLANE & & & & \\
\hline 520 & OFFSITE & SESPMNT & $\mathrm{R}$ & B106Y6 & STATE PLANE & & & & \\
\hline 521 & OFFSITE & SESPMNT & $\mathrm{R}$ & B106Y7 & STATE PLANE & & & & \\
\hline 522 & OFFSITE & SESPMNT & $\mathrm{R}$ & B106Y8 & STATE PLANE & & & & \\
\hline 523 & OFFSITE & SESPMNT & $\mathrm{R}$ & B106Y9 & STATE PLANE & & & & \\
\hline 524 & OFFSITE & SESPMNT & $\mathrm{R}$ & B10761 & STATE PLANE & & & & \\
\hline 525 & OFFSITE & SESPMNT & $\mathrm{R}$ & B10762 & STATE PLANE & & & & \\
\hline 526 & OFFSITE & SESPMNT & $\mathrm{R}$ & B10763 & STATE PLANE & & & & \\
\hline 527 & OFFSITE & SESPMNT & $\mathrm{R}$ & B10764 & STATE PLANE & & & & \\
\hline 528 & ONSITE & SESPMNT & $\mathrm{R}$ & B10773 & STATE PLANE & & & & \\
\hline 529 & ONSITE & SESPMNT & $\mathrm{R}$ & B10776 & STATE PLANE & & & & \\
\hline 530 & ONSITE & SESPMNT & $\mathrm{R}$ & B10779 & STATE PLANE & & & & \\
\hline 531 & ONSITE & SESPMNT & $\mathrm{R}$ & B10782 & STATE PLANE & & & & \\
\hline 532 & ONSITE & SESPMNT & $\mathrm{R}$ & B10913 & STATE PLANE & & & & \\
\hline 533 & ONSITE & SESPMNT & $\mathrm{R}$ & B10949 & STATE PLANE & & & & \\
\hline
\end{tabular}




\begin{tabular}{|c|c|c|c|c|c|c|c|c|c|}
\hline & $A G$ & $\mathrm{AH}$ & $\mathrm{Al}$ & $\mathrm{AJ}$ & AK & $\mathrm{AL}$ & AM & AN & $\mathrm{AO}$ \\
\hline 1 & DIST_CLASS & OWNER_ID & COLLECTION_PURPOSE & X_Y_COORD_FINAL.SAMP_NUM & COORDINATE SYSTEM & DATUM & LATITUDE ${ }^{\circ} \mathrm{N}$ & LATITUDE MIN_MIN & LATITUDE MIN_SEC \\
\hline 534 & PERIMETER & SESPMNT & $\mathrm{R}$ & B109J0 & STATE PLANE & & & & \\
\hline 535 & OFFSITE & SESPMNT & $\mathrm{R}$ & & & & & & \\
\hline 536 & OFFSITE & SESPMNT & $\mathrm{R}$ & & & & & & \\
\hline 537 & ONSITE & SESPMNT & $\mathrm{R}$ & & & & & & \\
\hline 538 & ONSITE & SESPMNT & $\mathrm{R}$ & & & & & & \\
\hline 539 & ONSITE & SESPMNT & $\mathrm{R}$ & & & & & & \\
\hline 540 & ONSITE & SESPMNT & $\mathrm{R}$ & & & & & & \\
\hline 541 & ONSITE & SESPMNT & $\mathrm{R}$ & & & & & & \\
\hline 542 & ONSITE & SESPMNT & $\mathrm{R}$ & & & & & & \\
\hline 543 & ONSITE & SESPMNT & $\mathrm{R}$ & & & & & & \\
\hline 544 & ONSITE & SESPMNT & $\mathrm{R}$ & & & & & & \\
\hline 545 & ONSITE & SESPMNT & $\mathrm{R}$ & & & & & & \\
\hline 546 & ONSITE & SESPMNT & $\mathrm{R}$ & & & & & & \\
\hline 547 & ONSITE & PNLGW & & & & & & & \\
\hline 548 & ONSITE & SESPMNT & $\mathrm{R}$ & & & & & & \\
\hline 549 & ONSITE & SESPMNT & $\mathrm{R}$ & & & & & & \\
\hline 550 & OFFSITE & SESPMNT & $\mathrm{R}$ & & & & & & \\
\hline 551 & OFFSITE & SESPMNT & $\mathrm{R}$ & & & & & & \\
\hline 552 & ONSITE & SESPMNT & $R$ & & & & & & \\
\hline 553 & ONSITE & PNLGW & & & & & & & \\
\hline 554 & OFFSITE & SESPMNT & $\mathrm{R}$ & & & & & & \\
\hline 555 & OFFSITE & SESPMNT & $\mathrm{R}$ & & & & & & \\
\hline 556 & OFFSITE & SESPMNT & $\mathrm{R}$ & B11125 & STATE PLANE & & & & \\
\hline 557 & OFFSITE & SESPMNT & $\mathrm{R}$ & B11126 & STATE PLANE & & & & \\
\hline 558 & OFFSITE & SESPMNT & $\mathrm{R}$ & B11127 & STATE PLANE & & & & \\
\hline 559 & OFFSITE & SESPMNT & $\mathrm{R}$ & B11128 & STATE PLANE & & & & \\
\hline 560 & OFFSITE & SESPSPEC & S & B11124 & STATE PLANE & & & & \\
\hline 561 & OFFSITE & SESPMNT & $\mathrm{R}$ & B11129 & STATE PLANE & & & & \\
\hline 562 & OFFSITE & SESPMNT & $\mathrm{R}$ & B11130 & STATE PLANE & & & & \\
\hline 563 & OFFSITE & SESPMNT & $\mathrm{R}$ & B11131 & STATE PLANE & & & & \\
\hline 564 & OFFSITE & SESPMNT & $\mathrm{R}$ & B11132 & STATE PLANE & & & & \\
\hline 565 & OFFSITE & SESPMNT & $\mathrm{R}$ & B11133 & STATE PLANE & & & & \\
\hline 566 & OFFSITE & SESPMNT & $\mathrm{R}$ & B11134 & STATE PLANE & & & & \\
\hline 567 & OFFSITE & SESPMNT & $\mathrm{R}$ & B11145 & STATE PLANE & & & & \\
\hline 568 & OFFSITE & SESPMNT & $\mathrm{R}$ & B11146 & STATE PLANE & & & & \\
\hline 569 & OFFSITE & SESPMNT & $\mathrm{R}$ & B11147 & STATE PLANE & & & & \\
\hline 570 & OFFSITE & SESPMNT & $\mathrm{R}$ & B11148 & STATE PLANE & & & & \\
\hline 571 & OFFSITE & SESPMNT & $\mathrm{R}$ & & & & & & \\
\hline
\end{tabular}




\begin{tabular}{|c|c|c|c|c|c|c|c|c|c|}
\hline & $A G$ & $\mathrm{AH}$ & $\mathrm{Al}$ & $\mathrm{AJ}$ & $\mathrm{AK}$ & $\mathrm{AL}$ & AM & $\mathrm{AN}$ & $\mathrm{AO}$ \\
\hline 1 & DIST_CLASS & OWNER_ID & COLLECTION_PURPOSE & X_Y_COORD_FINAL.SAMP_NUM & COORDINATE SYSTEM & \begin{tabular}{|l|} 
DATUM \\
\end{tabular} & LATITUDE ${ }^{\circ} \mathrm{N}$ & LATITUDE MIN_MIN & LATITUDE MIN_SEC \\
\hline 572 & OFFSITE & SESPMNT & $\mathrm{R}$ & & & & & & \\
\hline 573 & OFFSITE & SESPMNT & $\mathrm{R}$ & & & & & & \\
\hline 574 & OFFSITE & SESPMNT & $\mathrm{R}$ & & & & & & \\
\hline 575 & ONSITE & SESPMNT & $\mathrm{R}$ & & & & & & \\
\hline 576 & ONSITE & SESPMNT & $\mathrm{R}$ & & & & & & \\
\hline 577 & ONSITE & SESPMNT & $\mathrm{R}$ & & & & & & \\
\hline 578 & ONSITE & SESPMNT & $\mathrm{R}$ & & & & & & \\
\hline 579 & OFFSITE & SESPSPEC & S & B11H38 & LAT/LONG & & 46 & & 18.54 .68 \\
\hline 580 & OFFSITE & SESPMNT & $\mathrm{R}$ & B11H43 & LAT/LONG & & 46 & & 18.54 .68 \\
\hline 581 & OFFSITE & SESPMNT & $\mathrm{R}$ & B11H44 & LAT/LONG & & 46 & & 18.52 .97 \\
\hline 582 & OFFSITE & SESPMNT & $\mathrm{R}$ & B11H45 & LAT/LONG & & 46 & & 18.51 .22 \\
\hline 583 & OFFSITE & SESPMNT & $\mathrm{R}$ & B11H46 & LAT/LONG & & 46 & & 18.54 .18 \\
\hline 584 & OFFSITE & SESPMNT & $\mathrm{R}$ & B11H47 & LAT/LONG & & 46 & & 18.46.53 \\
\hline 585 & OFFSITE & SESPMNT & $\mathrm{R}$ & B11H48 & LAT/LONG & & NONE & & \\
\hline 586 & OFFSITE & SESPMNT & $\mathrm{R}$ & B11H59 & LAT/LONG & & 46 & & 19.25 .05 \\
\hline 587 & OFFSITE & SESPMNT & $\mathrm{R}$ & B11H60 & LAT/LONG & & No GPS sig. & & \\
\hline 588 & OFFSITE & SESPMNT & $\mathrm{R}$ & B11H61 & LAT/LONG & & 46 & & 20.59 .09 \\
\hline 589 & OFFSITE & SESPMNT & $\mathrm{R}$ & B11H62 & LAT/LONG & & 46 & & 21.27.18 \\
\hline 590 & OFFSITE & SESPMNT & $R$ & B11H39 & LAT/LONG & & 46 & & 38.30 .72 \\
\hline 591 & OFFSITE & SESPMNT & $\mathrm{R}$ & B11H40 & LAT/LONG & & 46 & & 38.34 .81 \\
\hline 592 & OFFSITE & SESPMNT & $\mathrm{R}$ & B11H41 & LAT/LONG & & 46 & & 38.40 .47 \\
\hline 593 & OFFSITE & SESPMNT & $\mathrm{R}$ & B11H42 & LAT/LONG & & 46 & & 38.42 .31 \\
\hline 594 & OFFSITE & SESPMNT & $\mathrm{R}$ & & & & & & \\
\hline 595 & OFFSITE & SESPMNT & $\mathrm{R}$ & & & & & & \\
\hline 596 & OFFSITE & SESPMNT & $\mathrm{R}$ & & & & & & \\
\hline 597 & OFFSITE & SESPMNT & $\mathrm{R}$ & & & & & & \\
\hline 598 & ONSITE & SESPMNT & $\mathrm{R}$ & & & & & & \\
\hline 599 & ONSITE & SESPMNT & $\mathrm{R}$ & & & & & & \\
\hline 600 & ONSITE & SESPMNT & $\mathrm{R}$ & & & & & & \\
\hline 601 & ONSITE & SESPMNT & $R$ & & & & & & \\
\hline 602 & ONSITE & SESPMNT & $R$ & & & & & & \\
\hline 603 & ONSITE & SESPMNT & $\mathrm{R}$ & & & & & & \\
\hline 604 & ONSITE & SESPMNT & $\mathrm{R}$ & & & & & & \\
\hline 605 & OFFSITE & SESPMNT & $\mathrm{R}$ & & & & & & \\
\hline 606 & OFFSITE & SESPMNT & $\mathrm{R}$ & & & & & & \\
\hline 607 & ONSITE & SESPMNT & $\mathrm{R}$ & & & & & & \\
\hline 608 & ONSITE & SESPMNT & $\mathrm{R}$ & B11W35 & LAT/LONG & & 46 & & 41.0 .717 \\
\hline 609 & ONSITE & SESPMNT & $\mathrm{R}$ & B11W40 & LAT/LONG & & 46 & & 38.14 .823 \\
\hline
\end{tabular}




\begin{tabular}{|c|c|c|c|c|c|c|c|c|c|}
\hline & $A G$ & $\mathrm{AH}$ & $\mathrm{Al}$ & $\mathrm{AJ}$ & AK & $\mathrm{AL}$ & $\mathrm{AM}$ & AN & $\mathrm{AO}$ \\
\hline 1 & DIST_CLASS & OWNER_ID & COLLECTION_PURPOSE & X_Y_COORD_FINAL.SAMP_NUM & COORDINATE SYSTEM & DATUM & LATITUDE ${ }^{\circ} \mathrm{N}$ & LATITUDE MIN_MIN & LATITUDE MIN_SEC \\
\hline 610 & ONSITE & SESPMNT & $\mathrm{R}$ & B11W43 & LAT/LONG & & 46 & & 40.05 .774 \\
\hline 611 & ONSITE & SESPMNT & $\mathrm{R}$ & & & & & & \\
\hline 612 & OFFSITE & SESPMNT & $\mathrm{R}$ & & & & & & \\
\hline 613 & PERIMETER & SESPMNT & $\mathrm{R}$ & & & & & & \\
\hline 614 & ONSITE & SESPMNT & $\mathrm{R}$ & & & & & & \\
\hline 615 & OFFSITE & SESPMNT & $\mathrm{R}$ & & & & & & \\
\hline 616 & OFFSITE & SESPMNT & $\mathrm{R}$ & & & & & & \\
\hline 617 & OFFSITE & SESPMNT & $\mathrm{R}$ & & & & & & \\
\hline 618 & PERIMETER & SESPMNT & $\mathrm{R}$ & & & & & & \\
\hline 619 & OFFSITE & SESPMNT & $\mathrm{R}$ & B12505 & LAT/LONG & & 46 & & 18.54 .400 \\
\hline 620 & OFFSITE & SESPMNT & $\mathrm{R}$ & B12506 & LAT/LONG & & 46 & & 18.53 .114 \\
\hline 621 & OFFSITE & SESPMNT & $\mathrm{R}$ & B12507 & LAT/LONG & & 46 & & 18.50 .753 \\
\hline 622 & OFFSITE & SESPMNT & $\mathrm{R}$ & B12508 & LAT/LONG & & 46 & & 18.50 .6000 \\
\hline 623 & OFFSITE & SESPMNT & $\mathrm{R}$ & B12509 & LAT/LONG & & 46 & & 18.44.67124 \\
\hline 624 & OFFSITE & SESPMNT & $\mathrm{R}$ & B12510 & LAT/LONG & & 46 & & 18.51 .627 \\
\hline 625 & OFFSITE & SESPMNT & $\mathrm{R}$ & B12531 & LAT/LONG & & 46 & & 19.24.674 \\
\hline 626 & OFFSITE & SESPMNT & $\mathrm{R}$ & B12532 & LAT/LONG & & 46 & & 20.07.051 \\
\hline 627 & OFFSITE & SESPMNT & $\mathrm{R}$ & B12533 & LAT/LONG & & 46 & & 20.58.719 \\
\hline 628 & OFFSITE & SESPMNT & $\mathrm{R}$ & B12534 & LAT/LONG & & 46 & & 21.20 .830 \\
\hline 629 & OFFSITE & SESPMNT & $\mathrm{R}$ & B12501 & LAT/LONG & & 46 & & 38.33.187 \\
\hline 630 & OFFSITE & SESPMNT & $\mathrm{R}$ & B12502 & LAT/LONG & & 46 & & 38.36 .25143 \\
\hline 631 & OFFSITE & SESPMNT & $\mathrm{R}$ & B12503 & LAT/LONG & & 46 & & 38.39.361 \\
\hline 632 & OFFSITE & SESPMNT & $\mathrm{R}$ & B12504 & LAT/LONG & & 46 & & 38.42 .047 \\
\hline 633 & OFFSITE & SESPMNT & $\mathrm{R}$ & & & & & & \\
\hline 634 & OFFSITE & SESPMNT & $\mathrm{R}$ & & & & & & \\
\hline 635 & OFFSITE & SESPMNT & $\mathrm{R}$ & & & & & & \\
\hline 636 & PERIMETER & SESPMNT & $\mathrm{R}$ & & & & & & \\
\hline 637 & ONSITE & SESPMNT & $\mathrm{R}$ & & & & & & \\
\hline 638 & ONSITE & SESPMNT & $\mathrm{R}$ & & & & & & \\
\hline 639 & ONSITE & SESPMNT & $\mathrm{R}$ & & & & & & \\
\hline 640 & ONSITE & SESPMNT & $\mathrm{R}$ & & & & & & \\
\hline 641 & OFFSITE & SESPMNT & $\mathrm{R}$ & & & & & & \\
\hline 642 & OFFSITE & SESPMNT & $\mathrm{R}$ & & & & & & \\
\hline 643 & ONSITE & SESPMNT & $\mathrm{R}$ & B12RM1 & LAT/LONG & WGS84 & 46 & 37664 & \\
\hline 644 & ONSITE & SESPMNT & $\mathrm{R}$ & B12RM6 & LAT/LONG & WGS84 & 46 & 37218 & \\
\hline 645 & OFFSITE & SESPMNT & $\mathrm{R}$ & & & & & & \\
\hline 646 & OFFSITE & SESPMNT & $\mathrm{R}$ & & & & & & \\
\hline 647 & ONSITE & SESPSPEC & S & B12T85 & LAT/LONG & & 46 & & 41.01 .827 \\
\hline
\end{tabular}




\begin{tabular}{|c|c|c|c|c|c|c|c|c|c|}
\hline & $A G$ & $\mathrm{AH}$ & $\mathrm{Al}$ & $\mathrm{AJ}$ & AK & $\mathrm{AL}$ & AM & $\mathrm{AN}$ & $\mathrm{AO}$ \\
\hline 1 & DIST_CLASS & OWNER_ID & COLLECTION_PURPOSE & X_Y_COORD_FINAL.SAMP_NUM & COORDINATE SYSTEM & \begin{tabular}{|l|} 
DATUM \\
\end{tabular} & LATITUDE ${ }^{\circ} \mathrm{N}$ & LATITUDE MIN_MIN & LATITUDE MIN_SEC \\
\hline 648 & OFFSITE & SESPMNT & $R$ & В12T87 & LAT/LONG & & 46 & & 38.32 .516 \\
\hline 649 & OFFSITE & SESPMNT & $\mathrm{R}$ & B12T88 & LAT/LONG & & 46 & & 38.36 .290 \\
\hline 650 & OFFSITE & SESPMNT & $\mathrm{R}$ & B12T89 & LAT/LONG & & 46 & & 38.40 .091 \\
\hline 651 & OFFSITE & SESPMNT & $\mathrm{R}$ & B12T90 & LAT/LONG & & 46 & & 38.42 .087 \\
\hline 652 & ONSITE & SESPMNT & $\mathrm{R}$ & B12T91 & LAT/LONG & & 46 & & 41.01 .827 \\
\hline 653 & ONSITE & SESPMNT & $\mathrm{R}$ & B12T92 & LAT/LONG & & 46 & & 41.02.142 \\
\hline 654 & ONSITE & SESPMNT & $\mathrm{R}$ & B12T93 & LAT/LONG & & 46 & & 41.8 .044 \\
\hline 655 & ONSITE & SESPMNT & $\mathrm{R}$ & B12TD4 & LAT/LONG & & 46 & & 41.8.658 \\
\hline 656 & ONSITE & SESPMNT & $\mathrm{R}$ & B12TD5 & LAT/LONG & & 46 & & 41.03 .989 \\
\hline 657 & ONSITE & SESPMNT & $\mathrm{R}$ & B12TD6 & LAT/LONG & & 46 & & 41.8.070 \\
\hline 658 & ONSITE & SESPMNT & $\mathrm{R}$ & B12TN2 & LAT/LONG & & 46 & & 40.14 .547 \\
\hline 659 & ONSITE & SESPMNT & $\mathrm{R}$ & B12TN5 & LAT/LONG & & 46 & & 40.43 .042 \\
\hline 660 & ONSITE & SESPMNT & $\mathrm{R}$ & B12TN8 & LAT/LONG & & 46 & & 40.52 .424 \\
\hline 661 & ONSITE & SESPMNT & $\mathrm{R}$ & B12TP1 & LAT/LONG & & 46 & & 41.17.965 \\
\hline 662 & ONSITE & SESPMNT & $\mathrm{R}$ & B12T94 & LAT/LONG & & 46 & & 39.35 .60 \\
\hline 663 & ONSITE & SESPMNT & $\mathrm{R}$ & B12T95 & LAT/LONG & & 46 & & 39.39 .25 \\
\hline 664 & ONSITE & SESPMNT & $\mathrm{R}$ & B12T96 & LAT/LONG & & 46 & & 33.38.38 \\
\hline 665 & ONSITE & SESPMNT & $\mathrm{R}$ & В12T97 & LAT/LONG & & 46 & & 39.36 .77 \\
\hline 666 & ONSITE & SESPMNT & $\mathrm{R}$ & B12T98 & LAT/LONG & & 46 & & 39.45 .22 \\
\hline 667 & ONSITE & SESPMNT & $\mathrm{R}$ & B12T99 & LAT/LONG & & 46 & & 39.49 .98 \\
\hline 668 & ONSITE & SESPMNT & $\mathrm{R}$ & B12TB0 & LAT/LONG & & 46 & & 39.51 .88 \\
\hline 669 & ONSITE & SESPMNT & $\mathrm{R}$ & B12TB1 & LAT/LONG & & 46 & & 33.38 .28 \\
\hline 670 & ONSITE & SESPMNT & $\mathrm{R}$ & B12TB2 & LAT/LONG & & 46 & & 33.38 .08 \\
\hline 671 & ONSITE & SESPMNT & $\mathrm{R}$ & B12TB3 & LAT/LONG & & 46 & & 33.41 .45 \\
\hline 672 & ONSITE & SESPMNT & $\mathrm{R}$ & B12TB4 & LAT/LONG & & 46 & & 33.42 .84 \\
\hline 673 & ONSITE & SESPMNT & $\mathrm{R}$ & B12TB5 & LAT/LONG & & 46 & & 33.37.95 \\
\hline 674 & ONSITE & SESPMNT & $\mathrm{R}$ & B12TF7 & LAT/LONG & & 46 & & 34.50 .68 \\
\hline 675 & ONSITE & SESPMNT & $\mathrm{R}$ & B12TF8 & LAT/LONG & & 46 & & 34.21 .43 \\
\hline 676 & ONSITE & SESPMNT & $\mathrm{R}$ & B12TF9 & LAT/LONG & & 46 & & 32.44 .93 \\
\hline 677 & ONSITE & SESPMNT & $\mathrm{R}$ & В12TT0 & LAT/LONG & & 46 & & 33.57 .87 \\
\hline 678 & ONSITE & SESPMNT & $\mathrm{R}$ & B12TT3 & LAT/LONG & & 46 & & 40.10 .94 \\
\hline 679 & ONSITE & SESPMNT & $\mathrm{R}$ & B12TT9 & LAT/LONG & & 46 & & 37.2.31 \\
\hline 680 & ONSITE & SESPMNT & $\mathrm{R}$ & B12TB6 & LAT/LONG & & 46 & & 21.38.24 \\
\hline 681 & ONSITE & SESPMNT & $\mathrm{R}$ & B12TB8 & LAT/LONG & & 46 & & 21.38.21 \\
\hline 682 & ONSITE & SESPMNT & $\mathrm{R}$ & B12TC0 & LAT/LONG & & 46 & & 21.37.47 \\
\hline 683 & ONSITE & SESPMNT & $\mathrm{R}$ & B12TC2 & LAT/LONG & & 46 & & 21.38.45 \\
\hline 684 & ONSITE & SESPMNT & $\mathrm{R}$ & B12TC4 & LAT/LONG & & 46 & & 21.37 .97 \\
\hline 685 & ONSITE & SESPMNT & $\mathrm{R}$ & B12TC6 & LAT/LONG & & 46 & & 21.38.57 \\
\hline
\end{tabular}

Page 486 


\begin{tabular}{|c|c|c|c|c|c|c|c|c|c|}
\hline & $A G$ & $\mathrm{AH}$ & $\mathrm{Al}$ & $\mathrm{AJ}$ & $\mathrm{AK}$ & $\mathrm{AL}$ & $\mathrm{AM}$ & AN & $\mathrm{AO}$ \\
\hline 1 & DIST_CLASS & OWNER_ID & COLLECTION_PURPOSE & X_Y_COORD_FINAL.SAMP_NUM & COORDINATE SYSTEM & DATUM & LATITUDE ${ }^{\circ} \mathrm{N}$ & LATITUDE MIN_MIN & LATITUDE MIN_SEC \\
\hline 686 & OFFSITE & SESPMNT & $\mathrm{R}$ & & & & & & \\
\hline 687 & OFFSITE & SESPMNT & $\mathrm{R}$ & B12TC9 & LAT/LONG & & 46 & & 18.53 .55 \\
\hline 688 & OFFSITE & SESPMNT & $\mathrm{R}$ & B12TD0 & LAT/LONG & & 46 & & 18.52 .78 \\
\hline 689 & OFFSITE & SESPMNT & $\mathrm{R}$ & B12TD1 & LAT/LONG & & 46 & & 18.52 .99 \\
\hline 690 & OFFSITE & SESPMNT & $\mathrm{R}$ & B12TD2 & LAT/LONG & & 46 & & 18.51.17 \\
\hline 691 & OFFSITE & SESPMNT & $\mathrm{R}$ & B12TD3 & LAT/LONG & & 46 & & 18.52 .48 \\
\hline 692 & OFFSITE & SESPMNT & $\mathrm{R}$ & B12TM0 & LAT/LONG & & 46 & & 19.25 .20 \\
\hline 693 & OFFSITE & SESPMNT & $\mathrm{R}$ & B12TM1 & LAT/LONG & & 46 & & 20.7.76 \\
\hline 694 & OFFSITE & SESPMNT & $\mathrm{R}$ & B12TM2 & LAT/LONG & & 46 & & 20.59 .083 \\
\hline 695 & OFFSITE & SESPMNT & $\mathrm{R}$ & B12TM3 & LAT/LONG & & 46 & & 21.20 .88 \\
\hline 696 & ONSITE & SESPMNT & $\mathrm{R}$ & B12TP4 & LAT/LONG & & 46 & & 23.2.95 \\
\hline 697 & ONSITE & SESPMNT & $\mathrm{R}$ & B12TP8 & LAT/LONG & & 46 & & 22.35.97 \\
\hline 698 & ONSITE & SESPMNT & $\mathrm{R}$ & B12TR2 & LAT/LONG & & 46 & & 22.15 .61 \\
\hline 699 & ONSITE & SESPMNT & $\mathrm{R}$ & B12TR6 & LAT/LONG & & 46 & & 21.48.54 \\
\hline 700 & OFFSITE & SESPMNT & $\mathrm{R}$ & & & & & & \\
\hline 701 & OFFSITE & SESPMNT & $\mathrm{R}$ & & & & & & \\
\hline 702 & ONSITE & SESPMNT & $\mathrm{R}$ & & & & & & \\
\hline 703 & ONSITE & SESPMNT & $\mathrm{R}$ & & & & & & \\
\hline 704 & ONSITE & SESPMNT & $\mathrm{R}$ & & & & & & \\
\hline 705 & ONSITE & SESPMNT & $\mathrm{R}$ & & & & & & \\
\hline 706 & ONSITE & SESPMNT & $\mathrm{R}$ & & & & & & \\
\hline 707 & ONSITE & SESPMNT & $\mathrm{R}$ & & & & & & \\
\hline 708 & ONSITE & SESPMNT & $\mathrm{R}$ & B12WY1 & LAT/LONG & & 46 & & 40.59 .99 \\
\hline 709 & ONSITE & SESPMNT & $\mathrm{R}$ & B12X41 & LAT/LONG & & 46 & & 39.3 .62 \\
\hline 710 & ONSITE & SESPMNT & $\mathrm{R}$ & B12X42 & LAT/LONG & & 46 & & 39.40 .61 \\
\hline 711 & ONSITE & SESPMNT & $\mathrm{R}$ & B12X48 & LAT/LONG & & 46 & & 42.41 .7 \\
\hline 712 & ONSITE & SESPMNT & $R$ & B12X85 & LAT/LONG & & 46 & & 42.12 .8 \\
\hline 713 & OFFSITE & SESPMNT & $\mathrm{R}$ & & & & & & \\
\hline 714 & OFFSITE & SESPMNT & $\mathrm{R}$ & & & & & & \\
\hline 715 & ONSITE & SESPSPEC & $S$ & & & & & & \\
\hline 716 & OFFSITE & SESPMNT & $\mathrm{R}$ & & & & & & \\
\hline 717 & OFFSITE & SESPMNT & $\mathrm{R}$ & & & & & & \\
\hline 718 & OFFSITE & SESPMNT & $\mathrm{R}$ & B13LF3 & LAT/LONG & & 46 & & 38.33.72 \\
\hline 719 & OFFSITE & SESPMNT & $\mathrm{R}$ & B13LF4 & LAT/LONG & & 46 & & 38.36.24 \\
\hline 720 & OFFSITE & SESPMNT & $\mathrm{R}$ & B13LF5 & LAT/LONG & & 46 & & 38.40 .19 \\
\hline 721 & OFFSITE & SESPMNT & $\mathrm{R}$ & B13LF6 & LAT/LONG & & 46 & & 38.42 .52 \\
\hline 722 & OFFSITE & SESPSPEC & $S$ & B13LF2 & LAT/LONG & & 46 & & 18.54.62 \\
\hline 723 & OFFSITE & SESPMNT & $\mathrm{R}$ & B13LF7 & LAT/LONG & & 46 & & 18.54 .62 \\
\hline
\end{tabular}

Page 487 


\begin{tabular}{|c|c|c|c|c|c|c|c|c|c|}
\hline & $A G$ & $\mathrm{AH}$ & $\mathrm{Al}$ & $\mathrm{AJ}$ & AK & $\mathrm{AL}$ & $\mathrm{AM}$ & AN & $\mathrm{AO}$ \\
\hline 1 & DIST_CLASS & OWNER_ID & COLLECTION_PURPOSE & X_Y_COORD_FINAL.SAMP_NUM & COORDINATE SYSTEM & DATUM & LATITUDE ${ }^{\circ} \mathrm{N}$ & LATITUDE MIN_MIN & LATITUDE MIN_SEC \\
\hline 724 & OFFSITE & SESPMNT & $\mathrm{R}$ & B13LF8 & LAT/LONG & & 46 & & 18.52 .90 \\
\hline 725 & OFFSITE & SESPMNT & $\mathrm{R}$ & B13LF9 & LAT/LONG & & 46 & & 18.52 .68 \\
\hline 726 & OFFSITE & SESPMNT & $\mathrm{R}$ & B13LHO & LAT/LONG & & 46 & & 18.53 .97 \\
\hline 727 & OFFSITE & SESPMNT & $\mathrm{R}$ & B13LH1 & LAT/LONG & & 46 & & 18.53 .50 \\
\hline 728 & OFFSITE & SESPMNT & $\mathrm{R}$ & B13LH2 & LAT/LONG & & 46 & & 19.52.07 \\
\hline 729 & OFFSITE & SESPMNT & $\mathrm{R}$ & B13LJ3 & LAT/LONG & & 46 & & 19.24.77 \\
\hline 730 & OFFSITE & SESPMNT & $\mathrm{R}$ & B13LJ4 & LAT/LONG & & 46 & & 20.07 .46233 \\
\hline 731 & OFFSITE & SESPMNT & $\mathrm{R}$ & B13LJ5 & LAT/LONG & & 46 & & 20.59 .29 \\
\hline 732 & OFFSITE & SESPMNT & $\mathrm{R}$ & B13LJ6 & LAT/LONG & & 46 & & 21.21 .83 \\
\hline 733 & OFFSITE & SESPMNT & $\mathrm{R}$ & & & & & & \\
\hline 734 & OFFSITE & SESPMNT & $\mathrm{R}$ & & & & & & \\
\hline 735 & OFFSITE & SESPMNT & $\mathrm{R}$ & & & & & & \\
\hline 736 & OFFSITE & SESPMNT & $\mathrm{R}$ & & & & & & \\
\hline 737 & ONSITE & SESPMNT & $\mathrm{R}$ & & & & & & \\
\hline 738 & ONSITE & SESPMNT & $\mathrm{R}$ & & & & & & \\
\hline 739 & ONSITE & SESPMNT & $\mathrm{R}$ & & & & & & \\
\hline 740 & ONSITE & SESPMNT & $\mathrm{R}$ & & & & & & \\
\hline 741 & OFFSITE & SESPMNT & $\mathrm{R}$ & & & & & & \\
\hline 742 & OFFSITE & SESPMNT & $\mathrm{R}$ & & & & & & \\
\hline 743 & OFFSITE & SESPMNT & $\mathrm{R}$ & B147T3 & LAT/LONG & & 46 & & 38.32.125 \\
\hline 744 & OFFSITE & SESPMNT & $\mathrm{R}$ & B147T4 & LAT/LONG & & 46 & & 38.33 .902 \\
\hline 745 & OFFSITE & SESPMNT & $\mathrm{R}$ & B147T5 & LAT/LONG & & 46 & & 38.40 .586 \\
\hline 746 & OFFSITE & SESPMNT & $\mathrm{R}$ & B147T6 & LAT/LONG & & 46 & & 38.41 .856 \\
\hline 747 & OFFSITE & SESPSPEC & S & B147T2 & LAT/LONG & & 46 & & 18.54.797 \\
\hline 748 & OFFSITE & SESPMNT & $\mathrm{R}$ & B147T7 & LAT/LONG & & 46 & & 18.54 .797 \\
\hline 749 & OFFSITE & SESPMNT & $\mathrm{R}$ & B147T8 & LAT/LONG & & 46 & & 18.53.211 \\
\hline 750 & OFFSITE & SESPMNT & $\mathrm{R}$ & B147T9 & LAT/LONG & & 46 & & 18.52 .338 \\
\hline 751 & OFFSITE & SESPMNT & $\mathrm{R}$ & B147V0 & LAT/LONG & & 46 & & 18.52 .299 \\
\hline 752 & OFFSITE & SESPMNT & $\mathrm{R}$ & B147V1 & LAT/LONG & & 46 & & 18.51 .346 \\
\hline 753 & OFFSITE & SESPMNT & $\mathrm{R}$ & B147V2 & LAT/LONG & & 46 & & 18.50 .697 \\
\hline 754 & OFFSITE & SESPMNT & $\mathrm{R}$ & B147W3 & LAT/LONG & & 46 & & 19.24 .739 \\
\hline 755 & OFFSITE & SESPMNT & $\mathrm{R}$ & B147W4 & LAT/LONG & & 46 & & 20.7.138 \\
\hline 756 & OFFSITE & SESPMNT & $\mathrm{R}$ & B147W5 & LAT/LONG & & 46 & & 20.59 .129 \\
\hline 757 & OFFSITE & SESPMNT & $\mathrm{R}$ & B147W6 & LAT/LONG & & 46 & & 21.20 .938 \\
\hline 758 & OFFSITE & SESPMNT & $\mathrm{R}$ & & & & & & \\
\hline 759 & OFFSITE & SESPMNT & $\mathrm{R}$ & & & & & & \\
\hline 760 & ONSITE & SESPMNT & $\mathrm{R}$ & & & & & & \\
\hline 761 & ONSITE & SESPMNT & $\mathrm{R}$ & & & & & & \\
\hline
\end{tabular}




\begin{tabular}{|c|c|c|c|c|c|c|c|c|c|}
\hline & $A G$ & $\mathrm{AH}$ & $\mathrm{Al}$ & $\mathrm{AJ}$ & AK & $\mathrm{AL}$ & $\mathrm{AM}$ & AN & $\mathrm{AO}$ \\
\hline 1 & DIST_CLASS & OWNER_ID & COLLECTION_PURPOSE & X_Y_COORD_FINAL.SAMP_NUM & COORDINATE SYSTEM & DATUM & LATITUDE ${ }^{\circ} \mathrm{N}$ & LATITUDE MIN_MIN & LATITUDE MIN_SEC \\
\hline 762 & ONSITE & SESPMNT & $\mathrm{R}$ & & & & & & \\
\hline 763 & ONSITE & SESPMNT & $\mathrm{R}$ & & & & & & \\
\hline 764 & OFFSITE & SESPMNT & $\mathrm{R}$ & & & & & & \\
\hline 765 & OFFSITE & SESPMNT & $\mathrm{R}$ & & & & & & \\
\hline 766 & OFFSITE & SESPMNT & $\mathrm{R}$ & B14LJ0 & LAT/LONG & & 46 & 25832 & \\
\hline 767 & PERIMETER & SESPMNT & $\mathrm{R}$ & B14LJ2 & LAT/LONG & & 46 & 34120 & \\
\hline 768 & OFFSITE & SESPMNT & $\mathrm{R}$ & & & & & & \\
\hline 769 & OFFSITE & SESPMNT & $\mathrm{R}$ & & & & & & \\
\hline 770 & OFFSITE & SESPMNT & $\mathrm{R}$ & B14RV4 & LAT/LONG & & 46 & & 38.32.346 \\
\hline 771 & OFFSITE & SESPMNT & $\mathrm{R}$ & B14RV5 & LAT/LONG & & 46 & & 38.35 .710 \\
\hline 772 & OFFSITE & SESPMNT & $\mathrm{R}$ & B14RV6 & LAT/LONG & & 46 & & 38.40 .744 \\
\hline 773 & OFFSITE & SESPMNT & $\mathrm{R}$ & B14RV7 & LAT/LONG & & 46 & & 38.43 .263 \\
\hline 774 & OFFSITE & SESPMNT & $\mathrm{R}$ & B14RV8 & LAT/LONG & & 46 & & 18.54 .564 \\
\hline 775 & OFFSITE & SESPMNT & $\mathrm{R}$ & B14RV9 & LAT/LONG & & 46 & & 18.50 .434 \\
\hline 776 & OFFSITE & SESPMNT & $\mathrm{R}$ & B14RW0 & LAT/LONG & & 46 & & 18.51 .872 \\
\hline 777 & OFFSITE & SESPMNT & $\mathrm{R}$ & B14RW1 & LAT/LONG & & 46 & & 18.52.771 \\
\hline 778 & OFFSITE & SESPMNT & $\mathrm{R}$ & B14RW2 & LAT/LONG & & 46 & & 18.48.011 \\
\hline 779 & OFFSITE & SESPMNT & $\mathrm{R}$ & B14RW3 & LAT/LONG & & 46 & & 18.52 .018 \\
\hline 780 & OFFSITE & SESPMNT & $\mathrm{R}$ & B14RX4 & LAT/LONG & & 46 & & 19.24 .503 \\
\hline 781 & OFFSITE & SESPMNT & $\mathrm{R}$ & B14RX5 & LAT/LONG & & 46 & & 20.06.731 \\
\hline 782 & OFFSITE & SESPMNT & $\mathrm{R}$ & B14RX6 & LAT/LONG & & 46 & & 20.59 .569 \\
\hline 783 & OFFSITE & SESPMNT & $\mathrm{R}$ & B14RX7 & LAT/LONG & & 46 & & 21.21 .521 \\
\hline 784 & OFFSITE & SESPMNT & $\mathrm{R}$ & & & & & & \\
\hline 785 & OFFSITE & SESPMNT & $\mathrm{R}$ & & & & & & \\
\hline 786 & OFFSITE & SESPMNT & $\mathrm{R}$ & & & & & & \\
\hline 787 & PERIMETER & SESPMNT & $\mathrm{R}$ & & & & & & \\
\hline 788 & OFFSITE & SESPMNT & $\mathrm{R}$ & & & & & & \\
\hline 789 & ONSITE & SESPMNT & $\mathrm{R}$ & & & & & & \\
\hline 790 & PERIMETER & SESPMNT & $\mathrm{R}$ & & & & & & \\
\hline 791 & ONSITE & SESPMNT & $\mathrm{R}$ & & & & & & \\
\hline 792 & ONSITE & SESPMNT & $\mathrm{R}$ & & & & & & \\
\hline 793 & ONSITE & SESPMNT & $\mathrm{R}$ & & & & & & \\
\hline 794 & OFFSITE & SESPMNT & $\mathrm{R}$ & & & & & & \\
\hline 795 & OFFSITE & SESPMNT & $\mathrm{R}$ & & & & & & \\
\hline 796 & OFFSITE & SESPMNT & $\mathrm{R}$ & & & & & & \\
\hline 797 & OFFSITE & SESPMNT & $\mathrm{R}$ & & & & & & \\
\hline 798 & ONSITE & SESPSPEC & S & B158H3 & LAT/LONG & & 46 & & 41.02 .29 \\
\hline 799 & OFFSITE & SESPMNT & $\mathrm{R}$ & $\mathrm{B} 158 \mathrm{H} 4$ & LAT/LONG & & 46 & & 38.42 .82 \\
\hline
\end{tabular}




\begin{tabular}{|c|c|c|c|c|c|c|c|c|c|}
\hline & $A G$ & $\mathrm{AH}$ & $\mathrm{Al}$ & $\mathrm{AJ}$ & AK & $\mathrm{AL}$ & $\mathrm{AM}$ & AN & $\mathrm{AO}$ \\
\hline 1 & DIST_CLASS & OWNER_ID & COLLECTION_PURPOSE & X_Y_COORD_FINAL.SAMP_NUM & COORDINATE SYSTEM & DATUM & LATITUDE ${ }^{\circ} \mathrm{N}$ & LATITUDE MIN_MIN & LATITUDE MIN_SEC \\
\hline 800 & OFFSITE & SESPMNT & $\mathrm{R}$ & B158H5 & LAT/LONG & & 46 & & 38.35 .52 \\
\hline 801 & OFFSITE & SESPMNT & $\mathrm{R}$ & B158H6 & LAT/LONG & & 46 & & 38.39.47 \\
\hline 802 & OFFSITE & SESPMNT & $\mathrm{R}$ & B158H7 & LAT/LONG & & 46 & & 38.31 .26 \\
\hline 803 & ONSITE & SESPMNT & $\mathrm{R}$ & B158H8 & LAT/LONG & & 46 & & 41.02 .29 \\
\hline 804 & ONSITE & SESPMNT & $\mathrm{R}$ & B158H9 & LAT/LONG & & 46 & & 41.03 .77 \\
\hline 805 & ONSITE & SESPMNT & $\mathrm{R}$ & B158J0 & LAT/LONG & & 46 & & 41.11 .21 \\
\hline 806 & ONSITE & SESPMNT & $\mathrm{R}$ & B158M7 & LAT/LONG & & 46 & & 41.10 .20 \\
\hline 807 & ONSITE & SESPMNT & $\mathrm{R}$ & B158M8 & LAT/LONG & & 46 & & 41.06 .12 \\
\hline 808 & ONSITE & SESPMNT & $\mathrm{R}$ & B158M9 & LAT/LONG & & 46 & & 41.09 .52 \\
\hline 809 & ONSITE & SESPMNT & $\mathrm{R}$ & B158W8 & LAT/LONG & & 46 & & 40.14 .09 \\
\hline 810 & ONSITE & SESPMNT & $\mathrm{R}$ & B158X1 & LAT/LONG & & 46 & & 40.43 .18 \\
\hline 811 & ONSITE & SESPMNT & $\mathrm{R}$ & B158X4 & LAT/LONG & & 46 & & 40.51 .95 \\
\hline 812 & ONSITE & SESPMNT & $\mathrm{R}$ & B158X7 & LAT/LONG & & 46 & & 41.18 .54 \\
\hline 813 & ONSITE & SESPMNT & $\mathrm{R}$ & B158J1 & LAT/LONG & & 46 & & 39.35.36 \\
\hline 814 & ONSITE & SESPMNT & $\mathrm{R}$ & B158J3 & LAT/LONG & & 46 & & 39.39 .60 \\
\hline 815 & ONSITE & SESPMNT & $\mathrm{R}$ & B158J5 & LAT/LONG & & 46 & & 33.39 .50 \\
\hline 816 & ONSITE & SESPMNT & $\mathrm{R}$ & B158J7 & LAT/LONG & & 46 & & 39.37 .16 \\
\hline 817 & ONSITE & SESPMNT & $\mathrm{R}$ & B158J9 & LAT/LONG & & 46 & & 39.44 .48 \\
\hline 818 & ONSITE & SESPMNT & $\mathrm{R}$ & B158K1 & LAT/LONG & & 46 & & 39.48 .58 \\
\hline 819 & ONSITE & SESPMNT & $\mathrm{R}$ & B158K3 & LAT/LONG & & 46 & & 39.51 .94 \\
\hline 820 & ONSITE & SESPMNT & $\mathrm{R}$ & B158K5 & LAT/LONG & & 46 & & 33.40 .64 \\
\hline 821 & ONSITE & SESPMNT & $\mathrm{R}$ & B158K7 & LAT/LONG & & 46 & & 33.40 .63 \\
\hline 822 & ONSITE & SESPMNT & $\mathrm{R}$ & B158K9 & LAT/LONG & & 46 & & 33.42 .44 \\
\hline 823 & ONSITE & SESPMNT & $\mathrm{R}$ & B158L1 & LAT/LONG & & 46 & & 33.43 .598 \\
\hline 824 & ONSITE & SESPMNT & $\mathrm{R}$ & B158L3 & LAT/LONG & & 46 & & 33.40 .47 \\
\hline 825 & ONSITE & SESPMNT & $\mathrm{R}$ & B158N0 & LAT/LONG & & 46 & & 34.50 .32 \\
\hline 826 & ONSITE & SESPMNT & $R$ & B158N2 & LAT/LONG & & 46 & & 34.21 .43 \\
\hline 827 & ONSITE & SESPMNT & $\mathrm{R}$ & B158N4 & LAT/LONG & & 46 & & 32.44 .22 \\
\hline 828 & ONSITE & SESPMNT & $\mathrm{R}$ & B15902 & LAT/LONG & & 46 & & 33.57 .46 \\
\hline 829 & ONSITE & SESPMNT & $\mathrm{R}$ & B15906 & LAT/LONG & & 46 & & 40.10 .84 \\
\hline 830 & ONSITE & SESPMNT & $\mathrm{R}$ & B15910 & LAT/LONG & & 46 & & 36.22.01 \\
\hline 831 & ONSITE & SESPMNT & $\mathrm{R}$ & B15914 & LAT/LONG & & 46 & & 37.02 .66 \\
\hline 832 & ONSITE & SESPMNT & $\mathrm{R}$ & B158L5 & LAT/LONG & & 46 & & 21.37.20 \\
\hline 833 & ONSITE & SESPMNT & $\mathrm{R}$ & B158L6 & LAT/LONG & & 46 & & 21.36 .55 \\
\hline 834 & ONSITE & SESPMNT & $\mathrm{R}$ & B158L7 & LAT/LONG & & 46 & & 21.35 .70 \\
\hline 835 & ONSITE & SESPMNT & $\mathrm{R}$ & B158L8 & LAT/LONG & & 46 & & 21.37.67 \\
\hline 836 & ONSITE & SESPMNT & $\mathrm{R}$ & B158L9 & LAT/LONG & & 46 & & 21.38.28 \\
\hline 837 & ONSITE & SESPMNT & $\mathrm{R}$ & B158M0 & LAT/LONG & & 46 & & 21.38 .48 \\
\hline
\end{tabular}




\begin{tabular}{|c|c|c|c|c|c|c|c|c|c|}
\hline & $A G$ & $\mathrm{AH}$ & $\mathrm{Al}$ & $\mathrm{AJ}$ & $\mathrm{AK}$ & $\mathrm{AL}$ & $\mathrm{AM}$ & AN & $\mathrm{AO}$ \\
\hline 1 & DIST_CLASS & OWNER_ID & COLLECTION_PURPOSE & X_Y_COORD_FINAL.SAMP_NUM & COORDINATE SYSTEM & DATUM & LATITUDE ${ }^{\circ} \mathrm{N}$ & LATITUDE MIN_MIN & LATITUDE MIN_SEC \\
\hline 838 & OFFSITE & SESPMNT & $\mathrm{R}$ & B158M1 & LAT/LONG & & 46 & & 18.54 .5 \\
\hline 839 & OFFSITE & SESPMNT & $\mathrm{R}$ & B158M2 & LAT/LONG & & 46 & & 18.52.7 \\
\hline 840 & OFFSITE & SESPMNT & $\mathrm{R}$ & B158M3 & LAT/LONG & & 46 & & 18.85 .0 \\
\hline 841 & OFFSITE & SESPMNT & $\mathrm{R}$ & B158M4 & LAT/LONG & & 46 & & 18.51 .50 \\
\hline 842 & OFFSITE & SESPMNT & $\mathrm{R}$ & B158M5 & LAT/LONG & & 46 & & 18.51.37 \\
\hline 843 & OFFSITE & SESPMNT & $\mathrm{R}$ & B158M6 & LAT/LONG & & 46 & & 18.52.36 \\
\hline 844 & OFFSITE & SESPMNT & $\mathrm{R}$ & B158V6 & LAT/LONG & & 46 & & 19.24 .75 \\
\hline 845 & OFFSITE & SESPMNT & $\mathrm{R}$ & B158V7 & LAT/LONG & & 46 & & 20.07.17 \\
\hline 846 & OFFSITE & SESPMNT & $\mathrm{R}$ & B158V8 & LAT/LONG & & 46 & & 20.58 .56 \\
\hline 847 & OFFSITE & SESPMNT & $\mathrm{R}$ & B158V9 & LAT/LONG & & 46 & & 21.21 .49 \\
\hline 848 & ONSITE & SESPMNT & $\mathrm{R}$ & B158Y0 & LAT/LONG & & 46 & & 23.03 .00 \\
\hline 849 & ONSITE & SESPMNT & $\mathrm{R}$ & B158Y3 & LAT/LONG & & 46 & & 22.35 .44 \\
\hline 850 & ONSITE & SESPMNT & $\mathrm{R}$ & B158Y6 & LAT/LONG & & 46 & & 22.19 .60 \\
\hline 851 & ONSITE & SESPMNT & $\mathrm{R}$ & B158Y9 & LAT/LONG & & 46 & & 21.48.39 \\
\hline 852 & ONSITE & SESPMNT & $\mathrm{R}$ & & & & & & \\
\hline 853 & ONSITE & SESPMNT & $\mathrm{R}$ & & & & & & \\
\hline 854 & ONSITE & SESPMNT & $\mathrm{R}$ & & & & & & \\
\hline 855 & OFFSITE & SESPMNT & $\mathrm{R}$ & & & & & & \\
\hline 856 & OFFSITE & SESPMNT & $\mathrm{R}$ & & & & & & \\
\hline 857 & ONSITE & SESPMNT & $\mathrm{R}$ & & & & & & \\
\hline 858 & ONSITE & SESPMNT & $\mathrm{R}$ & B15C60 & LAT/LONG & & 46 & & 39.40 .613 \\
\hline 859 & ONSITE & SESPMNT & $\mathrm{R}$ & B15CB3 & LAT/LONG & & 46 & & 42.12 .884 \\
\hline 860 & ONSITE & SESPMNT & $\mathrm{R}$ & B15C69 & LAT/LONG & & 46 & & 38.14 .440 \\
\hline 861 & OFFSITE & SESPMNT & $\mathrm{R}$ & & & & & & \\
\hline 862 & OFFSITE & SESPMNT & $\mathrm{R}$ & & & & & & \\
\hline 863 & OFFSITE & SESPMNT & $\mathrm{R}$ & & & & & & \\
\hline 864 & OFFSITE & SESPMNT & $\mathrm{R}$ & & & & & & \\
\hline 865 & OFFSITE & SESPMNT & $\mathrm{R}$ & B16342 & LAT/LONG & & 46 & 38544193 & \\
\hline 866 & OFFSITE & SESPMNT & $\mathrm{R}$ & B16343 & LAT/LONG & & 46 & 3861303 & \\
\hline 867 & OFFSITE & SESPMNT & $\mathrm{R}$ & B16344 & LAT/LONG & & 46 & 384081025 & \\
\hline 868 & OFFSITE & SESPMNT & $\mathrm{R}$ & B16345 & LAT/LONG & & 46 & 384290077 & \\
\hline 869 & OFFSITE & SESPSPEC & S & B16341 & LAT/LONG & & 46 & 185442945 & \\
\hline 870 & OFFSITE & SESPMNT & $\mathrm{R}$ & B16346 & LAT/LONG & & 46 & 185442945 & \\
\hline 871 & OFFSITE & SESPMNT & $\mathrm{R}$ & B16347 & LAT/LONG & & 46 & 18519365 & \\
\hline 872 & OFFSITE & SESPMNT & $\mathrm{R}$ & B16348 & LAT/LONG & & 46 & 184989755 & \\
\hline 873 & OFFSITE & SESPMNT & $\mathrm{R}$ & B16349 & LAT/LONG & & 46 & 185086976 & \\
\hline 874 & OFFSITE & SESPMNT & $\mathrm{R}$ & B16350 & LAT/LONG & & 46 & 185163798 & \\
\hline 875 & OFFSITE & SESPMNT & $\mathrm{R}$ & B16351 & LAT/LONG & & 46 & 185184158 & \\
\hline
\end{tabular}




\begin{tabular}{|c|c|c|c|c|c|c|c|c|c|}
\hline & $A G$ & $\mathrm{AH}$ & $\mathrm{Al}$ & $\mathrm{AJ}$ & $\mathrm{AK}$ & $\mathrm{AL}$ & $\mathrm{AM}$ & $\mathrm{AN}$ & $\mathrm{AO}$ \\
\hline 1 & DIST_CLASS & OWNER_ID & COLLECTION_PURPOSE & X_Y_COORD_FINAL.SAMP_NUM & COORDINATE SYSTEM & DATUM & LATITUDE ${ }^{\circ} \mathrm{N}$ & LATITUDE MIN_MIN & LATITUDE MIN_SEC \\
\hline 876 & OFFSITE & SESPMNT & $\mathrm{R}$ & B16362 & LAT/LONG & & 46 & 192473133 & \\
\hline 877 & OFFSITE & SESPMNT & $\mathrm{R}$ & B16363 & LAT/LONG & & 46 & 200718556 & \\
\hline 878 & OFFSITE & SESPMNT & $\mathrm{R}$ & B16364 & LAT/LONG & & 46 & 205947829 & \\
\hline 879 & OFFSITE & SESPMNT & $\mathrm{R}$ & B16365 & LAT/LONG & & 46 & 212135241 & \\
\hline 880 & ONSITE & SESPMNT & $\mathrm{R}$ & & & & & & \\
\hline 881 & OFFSITE & SESPMNT & $\mathrm{R}$ & & & & & & \\
\hline 882 & OFFSITE & SESPMNT & $\mathrm{R}$ & & & & & & \\
\hline 883 & OFFSITE & SESPMNT & $\mathrm{R}$ & & & & & & \\
\hline 884 & OFFSITE & SESPMNT & $\mathrm{R}$ & & & & & & \\
\hline 885 & ONSITE & SESPMNT & $\mathrm{R}$ & & & & & & \\
\hline 886 & OFFSITE & SESPMNT & $\mathrm{R}$ & & & & & & \\
\hline 887 & OFFSITE & SESPMNT & $\mathrm{R}$ & & & & & & \\
\hline 888 & OFFSITE & SESPMNT & $\mathrm{R}$ & & & & & & \\
\hline 889 & OFFSITE & SESPMNT & $\mathrm{R}$ & & & & & & \\
\hline 890 & OFFSITE & SESPMNT & $\mathrm{R}$ & & & & & & \\
\hline 891 & OFFSITE & SESPMNT & $\mathrm{R}$ & & & & & & \\
\hline 892 & OFFSITE & SESPMNT & $\mathrm{R}$ & & & & & & \\
\hline 893 & OFFSITE & SESPMNT & $\mathrm{R}$ & & & & & & \\
\hline 894 & ONSITE & SESPMNT & $\mathrm{R}$ & & & & & & \\
\hline 895 & OFFSITE & SESPMNT & $\mathrm{R}$ & & & & & & \\
\hline 896 & OFFSITE & SESPMNT & $\mathrm{R}$ & & & & & & \\
\hline 897 & OFFSITE & SESPMNT & $\mathrm{R}$ & & & & & & \\
\hline 898 & OFFSITE & SESPMNT & $\mathrm{R}$ & & & & & & \\
\hline 899 & ONSITE & SESPMNT & $\mathrm{R}$ & B10912 & STATE PLANE & & & & \\
\hline 900 & ONSITE & SESPMNT & $\mathrm{R}$ & B10913 & STATE PLANE & & & & \\
\hline 901 & ONSITE & SESPMNT & $\mathrm{R}$ & B10945 & STATE PLANE & & & & \\
\hline 902 & ONSITE & SESPMNT & $\mathrm{R}$ & B10946 & STATE PLANE & & & & \\
\hline 903 & ONSITE & SESPMNT & $\mathrm{R}$ & B10949 & STATE PLANE & & & & \\
\hline 904 & OFFSITE & SESPMNT & $\mathrm{R}$ & & & & & & \\
\hline 905 & OFFSITE & SESPMNT & $\mathrm{R}$ & & & & & & \\
\hline 906 & ONSITE & SESPMNT & $\mathrm{R}$ & & & & & & \\
\hline 907 & ONSITE & SESPMNT & $\mathrm{R}$ & & & & & & \\
\hline 908 & ONSITE & SESPMNT & $\mathrm{R}$ & & & & & & \\
\hline 909 & ONSITE & SESPMNT & $\mathrm{R}$ & & & & & & \\
\hline 910 & ONSITE & SESPMNT & $\mathrm{R}$ & & & & & & \\
\hline 911 & OFFSITE & SESPMNT & $\mathrm{R}$ & & & & & & \\
\hline 912 & OFFSITE & SESPMNT & $\mathrm{R}$ & & & & & & \\
\hline 913 & OFFSITE & SESPMNT & $\mathrm{R}$ & & & & & & \\
\hline
\end{tabular}




\begin{tabular}{|c|c|c|c|c|c|c|c|c|c|}
\hline & $A G$ & $\mathrm{AH}$ & $\mathrm{Al}$ & $\mathrm{AJ}$ & AK & $\mathrm{AL}$ & $\mathrm{AM}$ & AN & $\mathrm{AO}$ \\
\hline 1 & DIST_CLASS & OWNER_ID & COLLECTION_PURPOSE & X_Y_COORD_FINAL.SAMP_NUM & COORDINATE SYSTEM & DATUM & LATITUDE ${ }^{\circ} \mathrm{N}$ & LATITUDE MIN_MIN & LATITUDE MIN_SEC \\
\hline 914 & OFFSITE & SESPMNT & $\mathrm{R}$ & & & & & & \\
\hline 915 & OFFSITE & SESPMNT & $\mathrm{R}$ & & & & & & \\
\hline 916 & OFFSITE & SESPMNT & $\mathrm{R}$ & & & & & & \\
\hline 917 & OFFSITE & SESPMNT & $\mathrm{R}$ & & & & & & \\
\hline 918 & OFFSITE & SESPMNT & $\mathrm{R}$ & & & & & & \\
\hline 919 & ONSITE & SESPMNT & $\mathrm{R}$ & & & & & & \\
\hline 920 & OFFSITE & SESPMNT & $\mathrm{R}$ & & & & & & \\
\hline 921 & OFFSITE & SESPMNT & $\mathrm{R}$ & & & & & & \\
\hline 922 & ONSITE & SESPMNT & $\mathrm{R}$ & & & & & & \\
\hline 923 & OFFSITE & SESPMNT & $\mathrm{R}$ & & & & & & \\
\hline 924 & OFFSITE & SESPMNT & $\mathrm{R}$ & & & & & & \\
\hline 925 & ONSITE & SESPMNT & $\mathrm{R}$ & & & & & & \\
\hline 926 & ONSITE & SESPMNT & $\mathrm{R}$ & & & & & & \\
\hline 927 & ONSITE & SESPMNT & $\mathrm{R}$ & & & & & & \\
\hline 928 & ONSITE & SESPMNT & $\mathrm{R}$ & & & & & & \\
\hline 929 & OFFSITE & SESPMNT & $\mathrm{R}$ & & & & & & \\
\hline 930 & OFFSITE & SESPMNT & $\mathrm{R}$ & & & & & & \\
\hline 931 & ONSITE & SESPMNT & $\mathrm{R}$ & B11W40 & LAT/LONG & & 46 & & 38.14 .823 \\
\hline 932 & ONSITE & SESPMNT & $\mathrm{R}$ & B11W43 & LAT/LONG & & 46 & & 40.05 .774 \\
\hline 933 & OFFSITE & SESPMNT & $\mathrm{R}$ & & & & & & \\
\hline 934 & OFFSITE & SESPMNT & $\mathrm{R}$ & & & & & & \\
\hline 935 & OFFSITE & SESPMNT & $\mathrm{R}$ & & & & & & \\
\hline 936 & OFFSITE & SESPMNT & $\mathrm{R}$ & & & & & & \\
\hline 937 & ONSITE & SESPMNT & $\mathrm{R}$ & & & & & & \\
\hline 938 & OFFSITE & SESPMNT & $\mathrm{R}$ & & & & & & \\
\hline 939 & OFFSITE & SESPMNT & $\mathrm{R}$ & & & & & & \\
\hline 940 & OFFSITE & SESPMNT & $\mathrm{R}$ & & & & & & \\
\hline 941 & OFFSITE & SESPMNT & $\mathrm{R}$ & & & & & & \\
\hline 942 & ONSITE & SESPMNT & $\mathrm{R}$ & B12XK2 & LAT/LONG & & 46 & & 22.20 .036 \\
\hline 943 & ONSITE & SESPSPEC & S & B12XK8 & LAT/LONG & & 46 & & 22.20 .059 \\
\hline 944 & ONSITE & SESPSPEC & S & B12XK9 & LAT/LONG & & 46 & & 22.20 .059 \\
\hline 945 & ONSITE & SESPSPEC & S & B12XL0 & LAT/LONG & & 46 & & 22.20 .084 \\
\hline 946 & ONSITE & SESPSPEC & S & B12XL1 & LAT/LONG & & 46 & & 22.20 .084 \\
\hline 947 & ONSITE & SESPSPEC & S & B12XL2 & LAT/LONG & & 46 & & 22.20.031 \\
\hline 948 & ONSITE & SESPMNT & $\mathrm{R}$ & B12XK1 & LAT/LONG & & 46 & & 22.36 .037 \\
\hline 949 & ONSITE & SESPSPEC & S & B12XK3 & LAT/LONG & & 46 & & 22.35.976 \\
\hline 950 & ONSITE & SESPSPEC & S & B12XK4 & LAT/LONG & & 46 & & 22.35.976 \\
\hline 951 & ONSITE & SESPSPEC & S & B12XK5 & LAT/LONG & & 46 & & 22.35.976 \\
\hline
\end{tabular}




\begin{tabular}{|c|c|c|c|c|c|c|c|c|c|}
\hline & $A G$ & $\mathrm{AH}$ & $\mathrm{Al}$ & $\mathrm{AJ}$ & $\mathrm{AK}$ & $\overline{\mathrm{AL}}$ & AM & AN & $\mathrm{AO}$ \\
\hline 1 & DIST_CLASS & OWNER_ID & COLLECTION_PURPOSE & X_Y_COORD_FINAL.SAMP_NUM & COORDINATE SYSTEM & DATUM & LATITUDE ${ }^{\circ} \mathrm{N}$ & LATITUDE MIN_MIN & LATITUDE MIN_SEC \\
\hline 952 & ONSITE & SESPSPEC & $S$ & B12XK6 & LAT/LONG & & 46 & & 22.36.045 \\
\hline 953 & ONSITE & SESPSPEC & $\mathrm{S}$ & B12XK7 & LAT/LONG & & 46 & & 22.36.045 \\
\hline 954 & OFFSITE & SESPMNT & $\mathrm{R}$ & & & & & & \\
\hline 955 & OFFSITE & SESPMNT & $\mathrm{R}$ & & & & & & \\
\hline 956 & ONSITE & SESPMNT & $\mathrm{R}$ & & & & & & \\
\hline 957 & ONSITE & SESPMNT & $\mathrm{R}$ & B12X41 & LAT/LONG & & 46 & & 39.3 .62 \\
\hline 958 & ONSITE & SESPMNT & $\mathrm{R}$ & B12X42 & LAT/LONG & & 46 & & 39.40 .61 \\
\hline 959 & ONSITE & SESPMNT & $\mathrm{R}$ & B12X48 & LAT/LONG & & 46 & & 42.41 .7 \\
\hline 960 & ONSITE & SESPMNT & $\mathrm{R}$ & B12X85 & LAT/LONG & & 46 & & 42.12 .8 \\
\hline 961 & OFFSITE & SESPMNT & $\mathrm{R}$ & & & & & & \\
\hline 962 & OFFSITE & SESPMNT & $\mathrm{R}$ & & & & & & \\
\hline 963 & OFFSITE & SESPMNT & $\mathrm{R}$ & & & & & & \\
\hline 964 & OFFSITE & SESPMNT & $\mathrm{R}$ & & & & & & \\
\hline 965 & OFFSITE & SESPMNT & $\mathrm{R}$ & & & & & & \\
\hline 966 & OFFSITE & SESPMNT & $\mathrm{R}$ & & & & & & \\
\hline 967 & OFFSITE & SESPMNT & $\mathrm{R}$ & & & & & & \\
\hline 968 & OFFSITE & SESPMNT & $\mathrm{R}$ & & & & & & \\
\hline 969 & OFFSITE & SESPMNT & $\mathrm{R}$ & & & & & & \\
\hline 970 & OFFSITE & SESPMNT & $\mathrm{R}$ & & & & & & \\
\hline 971 & OFFSITE & SESPMNT & $\mathrm{R}$ & & & & & & \\
\hline 972 & OFFSITE & SESPMNT & $\mathrm{R}$ & & & & & & \\
\hline 973 & OFFSITE & SESPMNT & $\mathrm{R}$ & & & & & & \\
\hline 974 & OFFSITE & SESPMNT & $\mathrm{R}$ & & & & & & \\
\hline 975 & OFFSITE & SESPMNT & $\mathrm{R}$ & & & & & & \\
\hline 976 & OFFSITE & SESPMNT & $\mathrm{R}$ & & & & & & \\
\hline 977 & OFFSITE & SESPMNT & $\mathrm{R}$ & & & & & & \\
\hline 978 & OFFSITE & SESPMNT & $\mathrm{R}$ & & & & & & \\
\hline 979 & OFFSITE & SESPMNT & $\mathrm{R}$ & & & & & & \\
\hline 980 & OFFSITE & SESPMNT & $\mathrm{R}$ & & & & & & \\
\hline 981 & OFFSITE & SESPMNT & $\mathrm{R}$ & & & & & & \\
\hline 982 & OFFSITE & SESPMNT & $\mathrm{R}$ & & & & & & \\
\hline 983 & ONSITE & SESPMNT & $\mathrm{R}$ & & & & & & \\
\hline 984 & ONSITE & SESPMNT & $\mathrm{R}$ & & & & & & \\
\hline 985 & OFFSITE & SESPMNT & $\mathrm{R}$ & & & & & & \\
\hline 986 & OFFSITE & SESPMNT & $\mathrm{R}$ & & & & & & \\
\hline 987 & ONSITE & SESPMNT & $\mathrm{R}$ & B15C09 & LAT/LONG & & 46 & & 33.55 .313 \\
\hline 988 & ONSITE & SESPMNT & $\mathrm{R}$ & B15C73 & LAT/LONG & & 46 & & 33.50 .499 \\
\hline 989 & ONSITE & SESPMNT & $\mathrm{R}$ & B15C60 & LAT/LONG & & 46 & & 39.40 .613 \\
\hline
\end{tabular}




\begin{tabular}{|c|c|c|c|c|c|c|c|c|c|}
\hline & $A G$ & $\mathrm{AH}$ & $\mathrm{Al}$ & $\mathrm{AJ}$ & $\mathrm{AK}$ & $\mathrm{AL}$ & $\mathrm{AM}$ & $\mathrm{AN}$ & $\mathrm{AO}$ \\
\hline 1 & DIST_CLASS & OWNER_ID & COLLECTION_PURPOSE & X_Y_COORD_FINAL.SAMP_NUM & COORDINATE SYSTEM & DATUM & LATITUDE ${ }^{\circ} \mathrm{N}$ & LATITUDE MIN_MIN & LATITUDE MIN_SEC \\
\hline 990 & ONSITE & SESPMNT & $\mathrm{R}$ & B15CB3 & LAT/LONG & & 46 & & 42.12 .884 \\
\hline 991 & OFFSITE & SESPMNT & $\mathrm{R}$ & & & & & & \\
\hline 992 & OFFSITE & SESPMNT & $\mathrm{R}$ & & & & & & \\
\hline 993 & OFFSITE & SESPMNT & $\mathrm{R}$ & & & & & & \\
\hline 994 & OFFSITE & SESPMNT & $\mathrm{R}$ & & & & & & \\
\hline 995 & ONSITE & SESPMNT & $\mathrm{R}$ & & & & & & \\
\hline 996 & ONSITE & SESPMNT & $\mathrm{R}$ & & & & & & \\
\hline 997 & OFFSITE & SESPMNT & $\mathrm{R}$ & & & & & & \\
\hline 998 & OFFSITE & SESPMNT & $\mathrm{R}$ & & & & & & \\
\hline 999 & RIVER_SHORELINE & SESPSPEC & S & BOXJP2 & UTM & & & & \\
\hline 1000 & RIVER_SHORELINE & SESPSPEC & $\mathrm{S}$ & B0XJP4 & UTM & & & & \\
\hline 1001 & RIVER_SHORELINE & SESPSPEC & $\mathrm{S}$ & B0XJP6 & UTM & & & & \\
\hline 1002 & ONSITE & SESPMNT & $\mathrm{R}$ & & & & & & \\
\hline 1003 & ONSITE & SESPMNT & $\mathrm{R}$ & & & & & & \\
\hline 1004 & ONSITE & SESPMNT & $\mathrm{R}$ & & & & & & \\
\hline 1005 & ONSITE & SESPMNT & $\mathrm{R}$ & & & & & & \\
\hline 1006 & OFFSITE & SESPMNT & $\mathrm{R}$ & & & & & & \\
\hline 1007 & OFFSITE & SESPMNT & $\mathrm{R}$ & & & & & & \\
\hline 1008 & OFFSITE & SESPMNT & $\mathrm{R}$ & & & & & & \\
\hline 1009 & OFFSITE & SESPMNT & $\mathrm{R}$ & & & & & & \\
\hline 1010 & OFFSITE & SESPMNT & $\mathrm{R}$ & & & & & & \\
\hline 1011 & OFFSITE & SESPMNT & $\mathrm{R}$ & & & & & & \\
\hline 1012 & OFFSITE & SESPSPEC & $S$ & & & & & & \\
\hline 1013 & OFFSITE & SESPMNT & $\mathrm{R}$ & & & & & & \\
\hline 1014 & OFFSITE & SESPMNT & $\mathrm{R}$ & & & & & & \\
\hline 1015 & OFFSITE & SESPMNT & $\mathrm{R}$ & & & & & & \\
\hline 1016 & OFFSITE & SESPMNT & $\mathrm{R}$ & & & & & & \\
\hline 1017 & 7 OFFSITE & SESPMNT & $\mathrm{R}$ & & & & & & \\
\hline 1018 & OFFSITE & SESPMNT & $\mathrm{R}$ & & & & & & \\
\hline 1019 & OFFSITE & SESPMNT & $\mathrm{R}$ & & & & & & \\
\hline 1020 & OFFSITE & SESPMNT & $\mathrm{R}$ & & & & & & \\
\hline 1021 & OFFSITE & SESPMNT & $\mathrm{R}$ & & & & & & \\
\hline 1022 & OFFSITE & SESPMNT & $\mathrm{R}$ & & & & & & \\
\hline 1023 & ONSITE & SESPMNT & $\mathrm{R}$ & & & & & & \\
\hline 1024 & ONSITE & SESPMNT & $\mathrm{R}$ & & & & & & \\
\hline 1025 & ONSITE & SESPSPEC & $S$ & & & & & & \\
\hline 1026 & OFFSITE & SESPMNT & $\mathrm{R}$ & & & & & & \\
\hline 1027 & OFFSITE & SESPMNT & $\mathrm{R}$ & & & & & & \\
\hline
\end{tabular}




\begin{tabular}{|c|c|c|c|c|c|c|c|c|c|}
\hline & $A G$ & $\mathrm{AH}$ & $\mathrm{Al}$ & AJ & AK & $A L$ & AM & AN & $\mathrm{AO}$ \\
\hline 1 & DIST_CLASS & OWNER_ID & COLLECTION_PURPOSE & X_Y_COORD_FINAL.SAMP_NUM & COORDINATE SYSTEM & DATUM & LATITUDE ${ }^{\circ} \mathrm{N}$ & LATITUDE MIN_MIN & LATITUDE MIN_SEC \\
\hline 1028 & ONSITE & SESPMNT & $\mathrm{R}$ & & & & & & \\
\hline 1029 & ONSITE & SESPMNT & $\mathrm{R}$ & & & & & & \\
\hline 1030 & ONSITE & SESPMNT & $\mathrm{R}$ & & & & & & \\
\hline 1031 & ONSITE & SESPMNT & $\mathrm{R}$ & & & & & & \\
\hline 1032 & OFFSITE & SESPMNT & $\mathrm{R}$ & & & & & & \\
\hline 1033 & OFFSITE & SESPMNT & $\mathrm{R}$ & & & & & & \\
\hline 1034 & OFFSITE & SESPMNT & $\mathrm{R}$ & & & & & & \\
\hline 1035 & OFFSITE & SESPMNT & $\mathrm{R}$ & & & & & & \\
\hline 1036 & OFFSITE & SESPMNT & $\mathrm{R}$ & & & & & & \\
\hline 1037 & OFFSITE & SESPMNT & $\mathrm{R}$ & & & & & & \\
\hline 1038 & OFFSITE & SESPMNT & $\mathrm{R}$ & BOYCD7 & STATE PLANE & & & & \\
\hline 1039 & OFFSITE & SESPMNT & $\mathrm{R}$ & B0YCD8 & STATE PLANE & & & & \\
\hline 1040 & OFFSITE & SESPMNT & $\mathrm{R}$ & BOYCD9 & STATE PLANE & & & & \\
\hline 1041 & OFFSITE & SESPMNT & $\mathrm{R}$ & BOYCFO & STATE PLANE & & & & \\
\hline 1042 & OFFSITE & SESPMNT & $\mathrm{R}$ & B0YCF1 & STATE PLANE & & & & \\
\hline 1043 & OFFSITE & SESPMNT & $\mathrm{R}$ & BOYCF2 & STATE PLANE & & & & \\
\hline 1044 & OFFSITE & SESPMNT & $\mathrm{R}$ & BOYCF3 & STATE PLANE & & & & \\
\hline 1045 & OFFSITE & SESPMNT & $\mathrm{R}$ & BOYCF4 & STATE PLANE & & & & \\
\hline 1046 & OFFSITE & SESPMNT & $\mathrm{R}$ & B0YCF5 & STATE PLANE & & & & \\
\hline 1047 & OFFSITE & SESPMNT & $\mathrm{R}$ & BOYCF6 & STATE PLANE & & & & \\
\hline 1048 & OFFSITE & SESPMNT & $\mathrm{R}$ & BOYCK3 & STATE PLANE & & & & \\
\hline 1049 & OFFSITE & SESPMNT & $\mathrm{R}$ & BOYCK4 & STATE PLANE & & & & \\
\hline 1050 & OFFSITE & SESPMNT & $\mathrm{R}$ & B0YCK5 & STATE PLANE & & & & \\
\hline 1051 & OFFSITE & SESPMNT & $\mathrm{R}$ & BOYCK6 & STATE PLANE & & & & \\
\hline 1052 & OFFSITE & SESPMNT & $\mathrm{R}$ & & & & & & \\
\hline 1053 & OFFSITE & SESPMNT & $\mathrm{R}$ & & & & & & \\
\hline 1054 & ONSITE & SESPMNT & $\mathrm{R}$ & & & & & & \\
\hline 1055 & ONSITE & SESPMNT & $\mathrm{R}$ & & & & & & \\
\hline 1056 & ONSITE & SESPSPEC & S & BOYVM2 & STATE PLANE & & & & \\
\hline 1057 & OFFSITE & SESPMNT & $\mathrm{R}$ & & & & & & \\
\hline 1058 & ONSITE & SESPMNT & $\mathrm{R}$ & & & & & & \\
\hline 1059 & ONSITE & SESPMNT & $\mathrm{R}$ & & & & & & \\
\hline 1060 & ONSITE & SESPMNT & $\mathrm{R}$ & & & & & & \\
\hline 1061 & ONSITE & SESPMNT & $\mathrm{R}$ & & & & & & \\
\hline 1062 & OFFSITE & SESPMNT & $\mathrm{R}$ & & & & & & \\
\hline 1063 & OFFSITE & SESPMNT & $\mathrm{R}$ & & & & & & \\
\hline 1064 & OFFSITE & SESPMNT & $\mathrm{R}$ & & & & & & \\
\hline 1065 & OFFSITE & SESPMNT & $\mathrm{R}$ & & & & & & \\
\hline
\end{tabular}




\begin{tabular}{|c|c|c|c|c|c|c|c|c|c|}
\hline & $A G$ & $\mathrm{AH}$ & $\mathrm{Al}$ & $\mathrm{AJ}$ & $\mathrm{AK}$ & $\mathrm{AL}$ & $\mathrm{AM}$ & AN & $\mathrm{AO}$ \\
\hline 1 & DIST_CLASS & OWNER_ID & COLLECTION_PURPOSE & X_Y_COORD_FINAL.SAMP_NUM & COORDINATE SYSTEM & DATUM & LATITUDE ${ }^{\circ} \mathrm{N}$ & LATITUDE MIN_MIN & LATITUDE MIN_SEC \\
\hline 1066 & ONSITE & SESPSPEC & S & B10672 & STATE PLANE & & & & \\
\hline 1067 & OFFSITE & SESPMNT & $\mathrm{R}$ & B10673 & STATE PLANE & & & & \\
\hline 1068 & OFFSITE & SESPMNT & $\mathrm{R}$ & B10674 & STATE PLANE & & & & \\
\hline 1069 & OFFSITE & SESPMNT & $\mathrm{R}$ & B10675 & STATE PLANE & & & & \\
\hline 1070 & OFFSITE & SESPMNT & $\mathrm{R}$ & B10676 & STATE PLANE & & & & \\
\hline 1071 & ONSITE & SESPMNT & $\mathrm{R}$ & B10677 & STATE PLANE & & & & \\
\hline 1072 & ONSITE & SESPMNT & $\mathrm{R}$ & B10679 & STATE PLANE & & & & \\
\hline 1073 & ONSITE & SESPMNT & $\mathrm{R}$ & & & & & & \\
\hline 1074 & ONSITE & SESPMNT & $\mathrm{R}$ & B10683 & STATE PLANE & & & & \\
\hline 1075 & ONSITE & SESPMNT & $\mathrm{R}$ & B10684 & STATE PLANE & & & & \\
\hline 1076 & ONSITE & SESPMNT & $\mathrm{R}$ & & & & & & \\
\hline 1077 & ONSITE & SESPMNT & $\mathrm{R}$ & & & & & & \\
\hline 1078 & ONSITE & SESPMNT & $\mathrm{R}$ & B106B1 & STATE PLANE & & & & \\
\hline 1079 & ONSITE & SESPMNT & $\mathrm{R}$ & & & & & & \\
\hline 1080 & ONSITE & SESPMNT & $\mathrm{R}$ & B106B7 & STATE PLANE & & & & \\
\hline 1081 & ONSITE & SESPSPEC & S & & & & & & \\
\hline 1082 & ONSITE & SESPSPEC & S & & & & & & \\
\hline 1083 & ONSITE & SESPSPEC & S & & & & & & \\
\hline 1084 & ONSITE & SESPSPEC & S & & & & & & \\
\hline 1085 & ONSITE & SESPSPEC & S & & & & & & \\
\hline 1086 & ONSITE & SESPSPEC & S & & & & & & \\
\hline 1087 & ONSITE & SESPMNT & $\mathrm{R}$ & B106W1 & STATE PLANE & & & & \\
\hline 1088 & ONSITE & SESPMNT & $\mathrm{R}$ & B106W2 & STATE PLANE & & & & \\
\hline 1089 & ONSITE & SESPMNT & $\mathrm{R}$ & B106W3 & STATE PLANE & & & & \\
\hline 1090 & ONSITE & SESPMNT & $\mathrm{R}$ & B106W4 & STATE PLANE & & & & \\
\hline 1091 & ONSITE & SESPMNT & $\mathrm{R}$ & B106W5 & STATE PLANE & & & & \\
\hline 1092 & ONSITE & SESPMNT & $\mathrm{R}$ & B106W6 & STATE PLANE & & & & \\
\hline 1093 & ONSITE & SESPMNT & $\mathrm{R}$ & B106W7 & STATE PLANE & & & & \\
\hline 1094 & ONSITE & SESPMNT & $\mathrm{R}$ & B106W8 & STATE PLANE & & & & \\
\hline 1095 & ONSITE & SESPMNT & $\mathrm{R}$ & B106W9 & STATE PLANE & & & & \\
\hline 1096 & ONSITE & SESPMNT & $\mathrm{R}$ & B106X0 & STATE PLANE & & & & \\
\hline 1097 & ONSITE & SESPMNT & $\mathrm{R}$ & B106X1 & STATE PLANE & & & & \\
\hline 1098 & ONSITE & SESPMNT & $\mathrm{R}$ & B106X2 & STATE PLANE & & & & \\
\hline 1099 & ONSITE & SESPMNT & $\mathrm{R}$ & B10728 & STATE PLANE & & & & \\
\hline 1100 & ONSITE & SESPMNT & $\mathrm{R}$ & B10729 & STATE PLANE & & & & \\
\hline 1101 & ONSITE & SESPMNT & $\mathrm{R}$ & B10730 & STATE PLANE & & & & \\
\hline 1102 & ONSITE & SESPMNT & $\mathrm{R}$ & B10785 & STATE PLANE & & & & \\
\hline 1103 & ONSITE & SESPMNT & $\mathrm{R}$ & B10788 & STATE PLANE & & & & \\
\hline
\end{tabular}




\begin{tabular}{|c|c|c|c|c|c|c|c|c|c|}
\hline & $A G$ & $\mathrm{AH}$ & $\mathrm{Al}$ & $\mathrm{AJ}$ & $\mathrm{AK}$ & $\mathrm{AL}$ & AM & AN & $\mathrm{AO}$ \\
\hline 1 & DIST_CLASS & OWNER_ID & |COLLECTION_PURPOSE & X_Y_COORD_FINAL.SAMP_NUM & COORDINATE SYSTEM & DATUM & LATITUDE ${ }^{\circ} \mathrm{N}$ & LATITUDE MIN_MIN & LATITUDE MIN_SEC \\
\hline 1104 & ONSITE & SESPMNT & $\mathrm{R}$ & B10791 & STATE PLANE & & & & \\
\hline 1105 & ONSITE & SESPMNT & $\mathrm{R}$ & B10794 & STATE PLANE & & & & \\
\hline 1106 & ONSITE & SESPSPEC & $\mathrm{S}$ & & & & & & \\
\hline 1107 & ONSITE & SESPSPEC & S & & & & & & \\
\hline 1108 & ONSITE & SESPSPEC & S & B109C2 & STATE PLANE & & & & \\
\hline 1109 & ONSITE & SESPSPEC & S & B109C3 & STATE PLANE & & & & \\
\hline 1110 & ONSITE & SESPSPEC & S & B109C4 & STATE PLANE & & & & \\
\hline 1111 & ONSITE & SESPSPEC & $S$ & B109C5 & STATE PLANE & & & & \\
\hline 1112 & ONSITE & SESPSPEC & S & B109C6 & STATE PLANE & & & & \\
\hline 1113 & ONSITE & SESPMNT & $\mathrm{R}$ & & & & & & \\
\hline 1114 & ONSITE & SESPMNT & $\mathrm{R}$ & & & & & & \\
\hline 1115 & ONSITE & SESPMNT & $\mathrm{R}$ & B106X7 & STATE PLANE & & & & \\
\hline 1116 & ONSITE & SESPMNT & $\mathrm{R}$ & B106X9 & STATE PLANE & & & & \\
\hline 1117 & ONSITE & SESPMNT & $\mathrm{R}$ & B106Y1 & STATE PLANE & & & & \\
\hline 1118 & ONSITE & SESPMNT & $\mathrm{R}$ & B106Y3 & STATE PLANE & & & & \\
\hline 1119 & OFFSITE & SESPMNT & $\mathrm{R}$ & B106Y4 & STATE PLANE & & & & \\
\hline 1120 & OFFSITE & SESPMNT & $\mathrm{R}$ & B106Y5 & STATE PLANE & & & & \\
\hline 1121 & OFFSITE & SESPMNT & $\mathrm{R}$ & B106Y6 & STATE PLANE & & & & \\
\hline 1122 & OFFSITE & SESPMNT & $\mathrm{R}$ & B106Y7 & STATE PLANE & & & & \\
\hline 1123 & OFFSITE & SESPMNT & $\mathrm{R}$ & B106Y8 & STATE PLANE & & & & \\
\hline 1124 & OFFSITE & SESPMNT & $\mathrm{R}$ & B106Y9 & STATE PLANE & & & & \\
\hline 1125 & OFFSITE & SESPMNT & $\mathrm{R}$ & B10761 & STATE PLANE & & & & \\
\hline 1126 & OFFSITE & SESPMNT & $\mathrm{R}$ & B10762 & STATE PLANE & & & & \\
\hline 1127 & OFFSITE & SESPMNT & $\mathrm{R}$ & B10763 & STATE PLANE & & & & \\
\hline 1128 & OFFSITE & SESPMNT & $\mathrm{R}$ & B10764 & STATE PLANE & & & & \\
\hline 1129 & ONSITE & SESPMNT & $\mathrm{R}$ & B10773 & STATE PLANE & & & & \\
\hline 1130 & ONSITE & SESPMNT & $\mathrm{R}$ & B10776 & STATE PLANE & & & & \\
\hline 1131 & ONSITE & SESPMNT & $\mathrm{R}$ & B10779 & STATE PLANE & & & & \\
\hline 1132 & ONSITE & SESPMNT & $\mathrm{R}$ & B10782 & STATE PLANE & & & & \\
\hline 1133 & ONSITE & SESPSPEC & $\mathrm{S}$ & & & & & & \\
\hline 1134 & ONSITE & SESPSPEC & S & & & & & & \\
\hline 1135 & ONSITE & SESPSPEC & S & & & & & & \\
\hline 1136 & ONSITE & SESPSPEC & S & & & & & & \\
\hline 1137 & ONSITE & SESPSPEC & S & & & & & & \\
\hline 1138 & ONSITE & SESPSPEC & S & & & & & & \\
\hline 1139 & OFFSITE & SESPSPEC & S & & & & & & \\
\hline 1140 & OFFSITE & SESPSPEC & $\mathrm{S}$ & & & & & & \\
\hline 1141 & OFFSITE & SESPSPEC & $S$ & & & & & & \\
\hline
\end{tabular}




\begin{tabular}{|c|c|c|c|c|c|c|c|c|c|}
\hline & $A G$ & $\mathrm{AH}$ & $\mathrm{Al}$ & $\mathrm{AJ}$ & $\mathrm{AK}$ & $\mathrm{AL}$ & $\mathrm{AM}$ & AN & $\mathrm{AO}$ \\
\hline 1 & DIST_CLASS & OWNER_ID & COLLECTION_PURPOSE & X_Y_COORD_FINAL.SAMP_NUM & COORDINATE SYSTEM & DATUM & LATITUDE ${ }^{\circ} \mathrm{N}$ & LATITUDE MIN_MIN & LATITUDE MIN_SEC \\
\hline 1142 & OFFSITE & SESPSPEC & S & & & & & & \\
\hline 1143 & OFFSITE & SESPSPEC & S & & & & & & \\
\hline 1144 & ONSITE & SESPMNT & $\mathrm{R}$ & B10912 & STATE PLANE & & & & \\
\hline 1145 & ONSITE & SESPMNT & $\mathrm{R}$ & B10913 & STATE PLANE & & & & \\
\hline 1146 & ONSITE & SESPMNT & $\mathrm{R}$ & B10945 & STATE PLANE & & & & \\
\hline 1147 & ONSITE & SESPMNT & $\mathrm{R}$ & B10946 & STATE PLANE & & & & \\
\hline 1148 & ONSITE & SESPMNT & $\mathrm{R}$ & B10949 & STATE PLANE & & & & \\
\hline 1149 & OFFSITE & SESPMNT & $\mathrm{R}$ & & & & & & \\
\hline 1150 & OFFSITE & SESPMNT & $\mathrm{R}$ & & & & & & \\
\hline 1151 & ONSITE & SESPMNT & $\mathrm{R}$ & & & & & & \\
\hline 1152 & ONSITE & SESPMNT & $\mathrm{R}$ & & & & & & \\
\hline 1153 & ONSITE & SESPMNT & $\mathrm{R}$ & & & & & & \\
\hline 1154 & ONSITE & SESPMNT & $\mathrm{R}$ & & & & & & \\
\hline 1155 & ONSITE & SESPMNT & $\mathrm{R}$ & & & & & & \\
\hline 1156 & ONSITE & SESPMNT & $\mathrm{R}$ & & & & & & \\
\hline 1157 & ONSITE & CENTPLAT & & & & & & & \\
\hline 1158 & ONSITE & CENTPLAT & & & & & & & \\
\hline 1159 & ONSITE & CENTPLAT & & & & & & & \\
\hline 1160 & ONSITE & SESPMNT & $\mathrm{R}$ & & & & & & \\
\hline 1161 & ONSITE & SESPMNT & $\mathrm{R}$ & & & & & & \\
\hline 1162 & ONSITE & SESPMNT & $\mathrm{R}$ & & & & & & \\
\hline 1163 & ONSITE & SESPMNT & $\mathrm{R}$ & & & & & & \\
\hline 1164 & ONSITE & SESPMNT & $\mathrm{R}$ & & & & & & \\
\hline 1165 & ONSITE & SESPMNT & $\mathrm{R}$ & & & & & & \\
\hline 1166 & ONSITE & PNLGW & & & & & & & \\
\hline 1167 & ONSITE & SESPMNT & $\mathrm{R}$ & & & & & & \\
\hline 1168 & ONSITE & SESPMNT & $\mathrm{R}$ & & & & & & \\
\hline 1169 & ONSITE & CENTPLAT & & & & & & & \\
\hline 1170 & OFFSITE & SESPMNT & $\mathrm{R}$ & & & & & & \\
\hline 1171 & OFFSITE & SESPMNT & $\mathrm{R}$ & & & & & & \\
\hline 1172 & ONSITE & SESPMNT & $\mathrm{R}$ & & & & & & \\
\hline 1173 & ONSITE & PNLGW & & & & & & & \\
\hline 1174 & OFFSITE & SESPMNT & $\mathrm{R}$ & & & & & & \\
\hline 1175 & OFFSITE & SESPMNT & $\mathrm{R}$ & & & & & & \\
\hline 1176 & OFFSITE & SESPMNT & $\mathrm{R}$ & B11125 & STATE PLANE & & & & \\
\hline 1177 & OFFSITE & SESPMNT & $\mathrm{R}$ & B11126 & STATE PLANE & & & & \\
\hline 1178 & OFFSITE & SESPMNT & $\mathrm{R}$ & B11127 & STATE PLANE & & & & \\
\hline 1179 & OFFSITE & SESPMNT & $\mathrm{R}$ & B11128 & STATE PLANE & & & & \\
\hline
\end{tabular}




\begin{tabular}{|c|c|c|c|c|c|c|c|c|c|}
\hline & $A G$ & $\mathrm{AH}$ & $\mathrm{Al}$ & $\mathrm{AJ}$ & AK & $\mathrm{AL}$ & AM & $\mathrm{AN}$ & $\mathrm{AO}$ \\
\hline 1 & DIST_CLASS & OWNER_ID & COLLECTION_PURPOSE & X_Y_COORD_FINAL.SAMP_NUM & COORDINATE SYSTEM & \begin{tabular}{|l|} 
DATUM \\
\end{tabular} & LATITUDE ${ }^{\circ} \mathrm{N}$ & LATITUDE MIN_MIN & LATITUDE MIN_SEC \\
\hline 1180 & OFFSITE & SESPSPEC & S & B11124 & STATE PLANE & & & & \\
\hline 1181 & OFFSITE & SESPMNT & $\mathrm{R}$ & B11129 & STATE PLANE & & & & \\
\hline 1182 & OFFSITE & SESPMNT & $\mathrm{R}$ & B11130 & STATE PLANE & & & & \\
\hline 1183 & OFFSITE & SESPMNT & $\mathrm{R}$ & B11131 & STATE PLANE & & & & \\
\hline 1184 & OFFSITE & SESPMNT & $\mathrm{R}$ & B11132 & STATE PLANE & & & & \\
\hline 1185 & OFFSITE & SESPMNT & $\mathrm{R}$ & B11133 & STATE PLANE & & & & \\
\hline 1186 & OFFSITE & SESPMNT & $\mathrm{R}$ & B11134 & STATE PLANE & & & & \\
\hline 1187 & OFFSITE & SESPMNT & $\mathrm{R}$ & B11145 & STATE PLANE & & & & \\
\hline 1188 & OFFSITE & SESPMNT & $\mathrm{R}$ & B11146 & STATE PLANE & & & & \\
\hline 1189 & OFFSITE & SESPMNT & $\mathrm{R}$ & B11147 & STATE PLANE & & & & \\
\hline 1190 & OFFSITE & SESPMNT & $\mathrm{R}$ & B11148 & STATE PLANE & & & & \\
\hline 1191 & OFFSITE & SESPMNT & $\mathrm{R}$ & & & & & & \\
\hline 1192 & OFFSITE & SESPMNT & $\mathrm{R}$ & & & & & & \\
\hline 1193 & OFFSITE & SESPMNT & $\mathrm{R}$ & & & & & & \\
\hline 1194 & OFFSITE & SESPMNT & $\mathrm{R}$ & & & & & & \\
\hline 1195 & ONSITE & SESPMNT & $\mathrm{R}$ & & & & & & \\
\hline 1196 & ONSITE & SESPMNT & $\mathrm{R}$ & & & & & & \\
\hline 1197 & ONSITE & SESPMNT & $\mathrm{R}$ & & & & & & \\
\hline 1198 & ONSITE & SESPMNT & $\mathrm{R}$ & & & & & & \\
\hline 1199 & ONSITE & SESPMNT & $\mathrm{R}$ & & & & & & \\
\hline 1200 & ONSITE & SESPMNT & $\mathrm{R}$ & & & & & & \\
\hline 1201 & OFFSITE & SESPSPEC & S & B11H38 & LAT/LONG & & 46 & & 18.54 .68 \\
\hline 1202 & OFFSITE & SESPMNT & $\mathrm{R}$ & B11H43 & LAT/LONG & & 46 & & 18.54.68 \\
\hline 1203 & OFFSITE & SESPMNT & $\mathrm{R}$ & B11H44 & LAT/LONG & & 46 & & 18.52 .97 \\
\hline 1204 & OFFSITE & SESPMNT & $\mathrm{R}$ & B11H45 & LAT/LONG & & 46 & & 18.51 .22 \\
\hline 1205 & OFFSITE & SESPMNT & $\mathrm{R}$ & B11H46 & LAT/LONG & & 46 & & 18.54.18 \\
\hline 1206 & OFFSITE & SESPMNT & $\mathrm{R}$ & B11H47 & LAT/LONG & & 46 & & 18.46 .53 \\
\hline 1207 & OFFSITE & SESPMNT & $\mathrm{R}$ & B11H48 & LAT/LONG & & NONE & & \\
\hline 1208 & OFFSITE & SESPMNT & $\mathrm{R}$ & B11H59 & LAT/LONG & & 46 & & 19.25 .05 \\
\hline 1209 & OFFSITE & SESPMNT & $\mathrm{R}$ & B11H60 & LAT/LONG & & No GPS sig. & & \\
\hline 1210 & OFFSITE & SESPMNT & $\mathrm{R}$ & B11H61 & LAT/LONG & & 46 & & 20.59 .09 \\
\hline 1211 & OFFSITE & SESPMNT & $\mathrm{R}$ & B11H62 & LAT/LONG & & 46 & & 21.27.18 \\
\hline 1212 & OFFSITE & SESPMNT & $\mathrm{R}$ & B11H39 & LAT/LONG & & 46 & & 38.30 .72 \\
\hline 1213 & OFFSITE & SESPMNT & $\mathrm{R}$ & B11H40 & LAT/LONG & & 46 & & 38.34.81 \\
\hline 1214 & OFFSITE & SESPMNT & $\mathrm{R}$ & B11H41 & LAT/LONG & & 46 & & 38.40 .47 \\
\hline 1215 & OFFSITE & SESPMNT & $\mathrm{R}$ & B11H42 & LAT/LONG & & 46 & & 38.42 .31 \\
\hline 1216 & OFFSITE & SESPMNT & $\mathrm{R}$ & & & & & & \\
\hline 1217 & OFFSITE & SESPMNT & $\mathrm{R}$ & & & & & & \\
\hline
\end{tabular}




\begin{tabular}{|c|c|c|c|c|c|c|c|c|c|}
\hline & $A G$ & $\mathrm{AH}$ & $\mathrm{Al}$ & $\mathrm{AJ}$ & $\mathrm{AK}$ & $\mathrm{AL}$ & AM & AN & $\mathrm{AO}$ \\
\hline 1 & DIST_CLASS & OWNER_ID & COLLECTION_PURPOSE & X_Y_COORD_FINAL.SAMP_NUM & COORDINATE SYSTEM & DATUM & LATITUDE ${ }^{\circ} \mathrm{N}$ & LATITUDE MIN_MIN & LATITUDE MIN_SEC \\
\hline 1218 & ONSITE & SESPMNT & $\mathrm{R}$ & & & & & & \\
\hline 1219 & ONSITE & SESPMNT & $\mathrm{R}$ & & & & & & \\
\hline 1220 & ONSITE & SESPSPEC & $\mathrm{S}$ & & & & & & \\
\hline 1221 & OFFSITE & SESPMNT & $\mathrm{R}$ & & & & & & \\
\hline 1222 & OFFSITE & SESPMNT & $\mathrm{R}$ & & & & & & \\
\hline 1223 & ONSITE & SESPMNT & $\mathrm{R}$ & & & & & & \\
\hline 1224 & ONSITE & SESPMNT & $\mathrm{R}$ & & & & & & \\
\hline 1225 & ONSITE & SESPMNT & $\mathrm{R}$ & & & & & & \\
\hline 1226 & ONSITE & SESPMNT & $\mathrm{R}$ & & & & & & \\
\hline 1227 & ONSITE & SESPMNT & $\mathrm{R}$ & & & & & & \\
\hline 1228 & ONSITE & SESPMNT & $\mathrm{R}$ & & & & & & \\
\hline 1229 & ONSITE & SESPMNT & $\mathrm{R}$ & & & & & & \\
\hline 1230 & ONSITE & SESPMNT & $\mathrm{R}$ & & & & & & \\
\hline 1231 & ONSITE & SESPMNT & $\mathrm{R}$ & & & & & & \\
\hline 1232 & OFFSITE & SESPMNT & $\mathrm{R}$ & & & & & & \\
\hline 1233 & OFFSITE & SESPMNT & $\mathrm{R}$ & & & & & & \\
\hline 1234 & ONSITE & SESPMNT & $\mathrm{R}$ & & & & & & \\
\hline 1235 & ONSITE & SESPMNT & $\mathrm{R}$ & B11W35 & LAT/LONG & & 46 & & 41.0.717 \\
\hline 1236 & ONSITE & SESPMNT & $\mathrm{R}$ & B11W40 & LAT/LONG & & 46 & & 38.14 .823 \\
\hline 1237 & ONSITE & SESPMNT & $\mathrm{R}$ & B11W43 & LAT/LONG & & 46 & & 40.05 .774 \\
\hline 1238 & ONSITE & SESPMNT & $\mathrm{R}$ & & & & & & \\
\hline 1239 & OFFSITE & SESPMNT & $\mathrm{R}$ & & & & & & \\
\hline 1240 & PERIMETER & SESPMNT & $\mathrm{R}$ & & & & & & \\
\hline 1241 & ONSITE & SESPMNT & $\mathrm{R}$ & & & & & & \\
\hline 1242 & OFFSITE & SESPSPEC & $\mathrm{S}$ & B121R8 & LAT/LONG & & 46 & & 18.54.33709 \\
\hline 1243 & OFFSITE & SESPSPEC & $\mathrm{S}$ & B121R9 & LAT/LONG & & 46 & & 18.53.27905 \\
\hline 1244 & OFFSITE & SESPSPEC & $\mathrm{S}$ & B121T0 & LAT/LONG & & 46 & & 18.52 .7344 \\
\hline 1245 & OFFSITE & SESPSPEC & S & B121T1 & LAT/LONG & & 46 & & 18.51 .48525 \\
\hline 1246 & OFFSITE & SESPSPEC & $\mathrm{S}$ & & & & & & \\
\hline 1247 & OFFSITE & SESPMNT & $\mathrm{R}$ & & & & & & \\
\hline 1248 & OFFSITE & SESPMNT & $\mathrm{R}$ & & & & & & \\
\hline 1249 & OFFSITE & SESPMNT & $\mathrm{R}$ & & & & & & \\
\hline 1250 & OFFSITE & SESPMNT & $\mathrm{R}$ & & & & & & \\
\hline 1251 & PERIMETER & SESPMNT & $\mathrm{R}$ & & & & & & \\
\hline 1252 & PERIMETER & SESPMNT & $\mathrm{R}$ & & & & & & \\
\hline 1253 & OFFSITE & SESPMNT & $\mathrm{R}$ & B12505 & LAT/LONG & & 46 & & 18.54 .400 \\
\hline 1254 & OFFSITE & SESPMNT & $\mathrm{R}$ & B12506 & LAT/LONG & & 46 & & 18.53 .114 \\
\hline 1255 & OFFSITE & SESPMNT & $\mathrm{R}$ & B12507 & LAT/LONG & & 46 & & 18.50 .753 \\
\hline
\end{tabular}




\begin{tabular}{|c|c|c|c|c|c|c|c|c|c|}
\hline & $A G$ & $\mathrm{AH}$ & $\mathrm{Al}$ & $\mathrm{AJ}$ & $\mathrm{AK}$ & $\mathrm{AL}$ & $\mathrm{AM}$ & AN & $\mathrm{AO}$ \\
\hline 1 & DIST_CLASS & OWNER_ID & COLLECTION_PURPOSE & X_Y_COORD_FINAL.SAMP_NUM & COORDINATE SYSTEM & DATUM & LATITUDE ${ }^{\circ} \mathrm{N}$ & LATITUDE MIN_MIN & LATITUDE MIN_SEC \\
\hline 1256 & OFFSITE & SESPMNT & $\mathrm{R}$ & B12508 & LAT/LONG & & 46 & & 18.50 .6000 \\
\hline 1257 & OFFSITE & SESPMNT & $\mathrm{R}$ & B12509 & LAT/LONG & & 46 & & 18.44.67124 \\
\hline 1258 & OFFSITE & SESPMNT & $\mathrm{R}$ & B12510 & LAT/LONG & & 46 & & 18.51 .627 \\
\hline 1259 & OFFSITE & SESPMNT & $\mathrm{R}$ & B12531 & LAT/LONG & & 46 & & 19.24.674 \\
\hline 1260 & OFFSITE & SESPMNT & $\mathrm{R}$ & B12532 & LAT/LONG & & 46 & & 20.07.051 \\
\hline 1261 & OFFSITE & SESPMNT & $\mathrm{R}$ & B12533 & LAT/LONG & & 46 & & 20.58 .719 \\
\hline 1262 & OFFSITE & SESPMNT & $\mathrm{R}$ & B12534 & LAT/LONG & & 46 & & 21.20 .830 \\
\hline 1263 & OFFSITE & SESPMNT & $\mathrm{R}$ & B12501 & LAT/LONG & & 46 & & 38.33.187 \\
\hline 1264 & OFFSITE & SESPMNT & $\mathrm{R}$ & B12502 & LAT/LONG & & 46 & & 38.36 .25143 \\
\hline 1265 & OFFSITE & SESPMNT & $\mathrm{R}$ & B12503 & LAT/LONG & & 46 & & 38.39 .361 \\
\hline 1266 & OFFSITE & SESPMNT & $\mathrm{R}$ & B12504 & LAT/LONG & & 46 & & 38.42 .047 \\
\hline 1267 & OFFSITE & SESPMNT & $\mathrm{R}$ & & & & & & \\
\hline 1268 & OFFSITE & SESPMNT & $\mathrm{R}$ & & & & & & \\
\hline 1269 & ONSITE & SESPMNT & $\mathrm{R}$ & & & & & & \\
\hline 1270 & OFFSITE & SESPMNT & $\mathrm{R}$ & & & & & & \\
\hline 1271 & PERIMETER & SESPMNT & $\mathrm{R}$ & & & & & & \\
\hline 1272 & ONSITE & SESPMNT & $\mathrm{R}$ & & & & & & \\
\hline 1273 & ONSITE & SESPMNT & $\mathrm{R}$ & & & & & & \\
\hline \begin{tabular}{|l}
1274 \\
\end{tabular} & ONSITE & SESPMNT & $\mathrm{R}$ & & & & & & \\
\hline 1275 & ONSITE & SESPMNT & $\mathrm{R}$ & & & & & & \\
\hline 1276 & OFFSITE & SESPMNT & $\mathrm{R}$ & & & & & & \\
\hline \begin{tabular}{|l}
1277 \\
\end{tabular} & OFFSITE & SESPMNT & $\mathrm{R}$ & & & & & & \\
\hline 1278 & ONSITE & SESPSPEC & S & B12MM7 & LAT/LONG & & 46 & 27997 & \\
\hline 1279 & ONSITE & SESPMNT & $\mathrm{R}$ & B12RM1 & LAT/LONG & WGS84 & 46 & 37664 & \\
\hline \begin{tabular}{|l}
1280 \\
\end{tabular} & ONSITE & SESPMNT & $\mathrm{R}$ & B12RM6 & LAT/LONG & WGS84 & 46 & 37218 & \\
\hline 1281 & OFFSITE & SESPMNT & $\mathrm{R}$ & & & & & & \\
\hline 1282 & OFFSITE & SESPMNT & $\mathrm{R}$ & & & & & & \\
\hline 1283 & ONSITE & SESPSPEC & S & B12T85 & LAT/LONG & & 46 & & 41.01 .827 \\
\hline 1284 & OFFSITE & SESPMNT & $\mathrm{R}$ & B12T87 & LAT/LONG & & 46 & & 38.32 .516 \\
\hline 1285 & OFFSITE & SESPMNT & $\mathrm{R}$ & B12T88 & LAT/LONG & & 46 & & 38.36.290 \\
\hline 1286 & OFFSITE & SESPMNT & $\mathrm{R}$ & B12T89 & LAT/LONG & & 46 & & 38.40 .091 \\
\hline \begin{tabular}{|l}
1287 \\
\end{tabular} & OFFSITE & SESPMNT & $\mathrm{R}$ & B12T90 & LAT/LONG & & 46 & & 38.42 .087 \\
\hline 1288 & ONSITE & SESPMNT & $\mathrm{R}$ & B12T91 & LAT/LONG & & 46 & & 41.01 .827 \\
\hline 1289 & ONSITE & SESPMNT & $\mathrm{R}$ & B12T92 & LAT/LONG & & 46 & & 41.02 .142 \\
\hline \begin{tabular}{|l}
1290 \\
\end{tabular} & ONSITE & SESPMNT & $\mathrm{R}$ & B12T93 & LAT/LONG & & 46 & & 41.8 .044 \\
\hline \begin{tabular}{|l}
1291 \\
\end{tabular} & ONSITE & SESPMNT & $\mathrm{R}$ & B12TD4 & LAT/LONG & & 46 & & 41.8 .658 \\
\hline 1292 & ONSITE & SESPMNT & $\mathrm{R}$ & B12TD5 & LAT/LONG & & 46 & & 41.03 .989 \\
\hline \begin{tabular}{|l}
1293 \\
\end{tabular} & ONSITE & SESPMNT & $\mathrm{R}$ & B12TD6 & LAT/LONG & & 46 & & 41.8 .070 \\
\hline
\end{tabular}




\begin{tabular}{|c|c|c|c|c|c|c|c|c|c|}
\hline & $A G$ & $\mathrm{AH}$ & $\mathrm{Al}$ & $\mathrm{AJ}$ & $\mathrm{AK}$ & $\mathrm{AL}$ & $\mathrm{AM}$ & AN & $\mathrm{AO}$ \\
\hline 1 & DIST_CLASS & OWNER_ID & COLLECTION_PURPOSE & X_Y_COORD_FINAL.SAMP_NUM & COORDINATE SYSTEM & DATUM & LATITUDE ${ }^{\circ} \mathrm{N}$ & LATITUDE MIN_MIN & LATITUDE MIN_SEC \\
\hline 1294 & ONSITE & SESPMNT & $\mathrm{R}$ & B12TN2 & LAT/LONG & & 46 & & 40.14 .547 \\
\hline 1295 & ONSITE & SESPMNT & $\mathrm{R}$ & B12TN5 & LAT/LONG & & 46 & & 40.43 .042 \\
\hline 1296 & ONSITE & SESPMNT & $\mathrm{R}$ & B12TN8 & LAT/LONG & & 46 & & 40.52 .424 \\
\hline 1297 & ONSITE & SESPMNT & $\mathrm{R}$ & B12TP1 & LAT/LONG & & 46 & & 41.17.965 \\
\hline 1298 & ONSITE & SESPMNT & $\mathrm{R}$ & B12T94 & LAT/LONG & & 46 & & 39.35 .60 \\
\hline 1299 & ONSITE & SESPMNT & $\mathrm{R}$ & B12T95 & LAT/LONG & & 46 & & 39.39 .25 \\
\hline 1300 & ONSITE & SESPMNT & $\mathrm{R}$ & B12T96 & LAT/LONG & & 46 & & 33.38 .38 \\
\hline 1301 & ONSITE & SESPMNT & $\mathrm{R}$ & B12T97 & LAT/LONG & & 46 & & 39.36 .77 \\
\hline 1302 & ONSITE & SESPMNT & $\mathrm{R}$ & B12T98 & LAT/LONG & & 46 & & 39.45 .22 \\
\hline 1303 & ONSITE & SESPMNT & $\mathrm{R}$ & B12T99 & LAT/LONG & & 46 & & 39.49 .98 \\
\hline 1304 & ONSITE & SESPMNT & $\mathrm{R}$ & B12TB0 & LAT/LONG & & 46 & & 39.51 .88 \\
\hline 1305 & ONSITE & SESPMNT & $\mathrm{R}$ & B12TB1 & LAT/LONG & & 46 & & 33.38 .28 \\
\hline 1306 & ONSITE & SESPMNT & $\mathrm{R}$ & B12TB2 & LAT/LONG & & 46 & & 33.38 .08 \\
\hline 1307 & ONSITE & SESPMNT & $\mathrm{R}$ & B12TB3 & LAT/LONG & & 46 & & 33.41 .45 \\
\hline 1308 & ONSITE & SESPMNT & $\mathrm{R}$ & B12TB4 & LAT/LONG & & 46 & & 33.42 .84 \\
\hline 1309 & ONSITE & SESPMNT & $\mathrm{R}$ & B12TB5 & LAT/LONG & & 46 & & 33.37 .95 \\
\hline 1310 & ONSITE & SESPMNT & $\mathrm{R}$ & B12TF7 & LAT/LONG & & 46 & & 34.50 .68 \\
\hline 1311 & ONSITE & SESPMNT & $\mathrm{R}$ & B12TF8 & LAT/LONG & & 46 & & 34.21 .43 \\
\hline \begin{tabular}{|l}
1312 \\
\end{tabular} & ONSITE & SESPMNT & $\mathrm{R}$ & B12TF9 & LAT/LONG & & 46 & & 32.44 .93 \\
\hline \begin{tabular}{|l}
1313 \\
\end{tabular} & ONSITE & SESPMNT & $\mathrm{R}$ & B12TT0 & LAT/LONG & & 46 & & 33.57 .87 \\
\hline 1314 & ONSITE & SESPMNT & $\mathrm{R}$ & B12TT3 & LAT/LONG & & 46 & & 40.10 .94 \\
\hline $\begin{array}{l}1315 \\
\end{array}$ & ONSITE & SESPMNT & $\mathrm{R}$ & B12TT9 & LAT/LONG & & 46 & & 37.2 .31 \\
\hline 1316 & ONSITE & SESPMNT & $\mathrm{R}$ & B12TB6 & LAT/LONG & & 46 & & 21.38.24 \\
\hline \begin{tabular}{|l}
1317 \\
\end{tabular} & ONSITE & SESPMNT & $\mathrm{R}$ & B12TB8 & LAT/LONG & & 46 & & 21.38.21 \\
\hline 1318 & ONSITE & SESPMNT & $\mathrm{R}$ & B12TCO & LAT/LONG & & 46 & & 21.37 .47 \\
\hline 1319 & ONSITE & SESPMNT & $\mathrm{R}$ & B12TC2 & LAT/LONG & & 46 & & 21.38 .45 \\
\hline 1320 & ONSITE & SESPMNT & $\mathrm{R}$ & B12TC4 & LAT/LONG & & 46 & & 21.37.97 \\
\hline 1321 & ONSITE & SESPMNT & $\mathrm{R}$ & B12TC6 & LAT/LONG & & 46 & & 21.38 .57 \\
\hline 1322 & OFFSITE & SESPMNT & $\mathrm{R}$ & & & & & & \\
\hline $\begin{array}{l}1323 \\
\end{array}$ & OFFSITE & SESPMNT & $\mathrm{R}$ & B12TC9 & LAT/LONG & & 46 & & 18.53 .55 \\
\hline \begin{tabular}{|l}
1324 \\
\end{tabular} & OFFSITE & SESPMNT & $\mathrm{R}$ & B12TD0 & LAT/LONG & & 46 & & 18.52.78 \\
\hline \begin{tabular}{|l}
1325 \\
\end{tabular} & OFFSITE & SESPMNT & $\mathrm{R}$ & B12TD1 & LAT/LONG & & 46 & & 18.52 .99 \\
\hline 1326 & OFFSITE & SESPMNT & $\mathrm{R}$ & B12TD2 & LAT/LONG & & 46 & & 18.51.17 \\
\hline $\begin{array}{l}1327 \\
\end{array}$ & OFFSITE & SESPMNT & $\mathrm{R}$ & B12TD3 & LAT/LONG & & 46 & & 18.52 .48 \\
\hline \begin{tabular}{|l}
1328 \\
\end{tabular} & OFFSITE & SESPMNT & $\mathrm{R}$ & B12TM0 & LAT/LONG & & 46 & & 19.25 .20 \\
\hline \begin{tabular}{|l}
1329 \\
\end{tabular} & OFFSITE & SESPMNT & $\mathrm{R}$ & B12TM1 & LAT/LONG & & 46 & & 20.7.76 \\
\hline 1330 & OFFSITE & SESPMNT & $\mathrm{R}$ & B12TM2 & LAT/LONG & & 46 & & 20.59 .083 \\
\hline \begin{tabular}{|l}
1331 \\
\end{tabular} & OFFSITE & SESPMNT & $\mathrm{R}$ & B12TM3 & LAT/LONG & & 46 & & 21.20 .88 \\
\hline
\end{tabular}




\begin{tabular}{|c|c|c|c|c|c|c|c|c|c|}
\hline & $A G$ & $\mathrm{AH}$ & $\mathrm{Al}$ & $\mathrm{AJ}$ & AK & $\mathrm{AL}$ & $\mathrm{AM}$ & AN & $\mathrm{AO}$ \\
\hline 1 & DIST_CLASS & OWNER_ID & COLLECTION_PURPOSE & X_Y_COORD_FINAL.SAMP_NUM & COORDINATE SYSTEM & DATUM & LATITUDE ${ }^{\circ} \mathrm{N}$ & LATITUDE MIN_MIN & LATITUDE MIN_SEC \\
\hline 1332 & ONSITE & SESPMNT & $\mathrm{R}$ & B12TP4 & LAT/LONG & & 46 & & 23.2.95 \\
\hline 1333 & ONSITE & SESPMNT & $\mathrm{R}$ & B12TP8 & LAT/LONG & & 46 & & 22.35 .97 \\
\hline 1334 & ONSITE & SESPMNT & $\mathrm{R}$ & B12TR2 & LAT/LONG & & 46 & & 22.15 .61 \\
\hline 1335 & ONSITE & SESPMNT & $\mathrm{R}$ & B12TR6 & LAT/LONG & & 46 & & 21.48 .54 \\
\hline 1336 & ONSITE & SESPMNT & $\mathrm{R}$ & B12XK2 & LAT/LONG & & 46 & & 22.20 .036 \\
\hline 1337 & ONSITE & SESPSPEC & S & B12XK8 & LAT/LONG & & 46 & & 22.20 .059 \\
\hline 1338 & ONSITE & SESPSPEC & S & B12XK9 & LAT/LONG & & 46 & & 22.20 .059 \\
\hline 1339 & ONSITE & SESPSPEC & S & B12XLO & LAT/LONG & & 46 & & 22.20 .084 \\
\hline 1340 & ONSITE & SESPSPEC & S & B12XL1 & LAT/LONG & & 46 & & 22.20 .084 \\
\hline 1341 & ONSITE & SESPSPEC & S & B12XL2 & LAT/LONG & & 46 & & 22.20 .031 \\
\hline 1342 & ONSITE & SESPMNT & $\mathrm{R}$ & B12XK1 & LAT/LONG & & 46 & & 22.36 .037 \\
\hline 1343 & ONSITE & SESPSPEC & S & B12XK3 & LAT/LONG & & 46 & & 22.35 .976 \\
\hline 1344 & ONSITE & SESPSPEC & S & B12XK4 & LAT/LONG & & 46 & & 22.35.976 \\
\hline 1345 & ONSITE & SESPSPEC & S & B12XK5 & LAT/LONG & & 46 & & 22.35.976 \\
\hline 1346 & ONSITE & SESPSPEC & S & B12XK6 & LAT/LONG & & 46 & & 22.36 .045 \\
\hline 1347 & ONSITE & SESPSPEC & S & B12XK7 & LAT/LONG & & 46 & & 22.36.045 \\
\hline 1348 & OFFSITE & SESPMNT & $\mathrm{R}$ & & & & & & \\
\hline 1349 & OFFSITE & SESPMNT & $\mathrm{R}$ & & & & & & \\
\hline 1350 & ONSITE & SESPMNT & $\mathrm{R}$ & & & & & & \\
\hline 1351 & ONSITE & SESPMNT & $\mathrm{R}$ & & & & & & \\
\hline 1352 & ONSITE & SESPMNT & $\mathrm{R}$ & & & & & & \\
\hline 1353 & ONSITE & SESPMNT & $\mathrm{R}$ & & & & & & \\
\hline 1354 & ONSITE & SESPMNT & $\mathrm{R}$ & & & & & & \\
\hline 1355 & ONSITE & SESPMNT & $\mathrm{R}$ & & & & & & \\
\hline 1356 & ONSITE & SESPMNT & $\mathrm{R}$ & & & & & & \\
\hline 1357 & ONSITE & SESPMNT & $\mathrm{R}$ & & & & & & \\
\hline 1358 & ONSITE & SESPMNT & $\mathrm{R}$ & B12WY1 & LAT/LONG & & 46 & & 40.59 .99 \\
\hline 1359 & ONSITE & SESPMNT & $\mathrm{R}$ & B12X41 & LAT/LONG & & 46 & & 39.3 .62 \\
\hline 1360 & ONSITE & SESPMNT & $\mathrm{R}$ & B12X42 & LAT/LONG & & 46 & & 39.40 .61 \\
\hline 1361 & ONSITE & SESPMNT & $\mathrm{R}$ & B12X48 & LAT/LONG & & 46 & & 42.41 .7 \\
\hline 1362 & ONSITE & SESPMNT & $\mathrm{R}$ & B12X85 & LAT/LONG & & 46 & & 42.12 .8 \\
\hline 1363 & OFFSITE & SESPMNT & $\mathrm{R}$ & & & & & & \\
\hline 1364 & OFFSITE & SESPMNT & $\mathrm{R}$ & & & & & & \\
\hline 1365 & ONSITE & SESPSPEC & S & & & & & & \\
\hline 1366 & OFFSITE & SESPMNT & $\mathrm{R}$ & & & & & & \\
\hline 1367 & OFFSITE & SESPMNT & $\mathrm{R}$ & & & & & & \\
\hline 1368 & OFFSITE & SESPMNT & $\mathrm{R}$ & B13LF3 & LAT/LONG & & 46 & & 38.33 .72 \\
\hline 1369 & OFFSITE & SESPMNT & $\mathrm{R}$ & B13LF4 & LAT/LONG & & 46 & & 38.36 .24 \\
\hline
\end{tabular}




\begin{tabular}{|c|c|c|c|c|c|c|c|c|c|}
\hline & $A G$ & $\mathrm{AH}$ & $\mathrm{Al}$ & $\mathrm{AJ}$ & $\mathrm{AK}$ & $\mathrm{AL}$ & $\mathrm{AM}$ & AN & $\mathrm{AO}$ \\
\hline 1 & DIST_CLASS & OWNER_ID & COLLECTION_PURPOSE & X_Y_COORD_FINAL.SAMP_NUM & COORDINATE SYSTEM & DATUM & LATITUDE ${ }^{\circ} \mathrm{N}$ & LATITUDE MIN_MIN & LATITUDE MIN_SEC \\
\hline 1370 & OFFSITE & SESPMNT & $\mathrm{R}$ & B13LF5 & LAT/LONG & & 46 & & 38.40 .19 \\
\hline 1371 & OFFSITE & SESPMNT & $\mathrm{R}$ & B13LF6 & LAT/LONG & & 46 & & 38.42 .52 \\
\hline 1372 & OFFSITE & SESPSPEC & S & B13LF2 & LAT/LONG & & 46 & & 18.54 .62 \\
\hline 1373 & OFFSITE & SESPMNT & $\mathrm{R}$ & B13LF7 & LAT/LONG & & 46 & & 18.54 .62 \\
\hline 1374 & OFFSITE & SESPMNT & $\mathrm{R}$ & B13LF8 & LAT/LONG & & 46 & & 18.52 .90 \\
\hline 1375 & OFFSITE & SESPMNT & $\mathrm{R}$ & B13LF9 & LAT/LONG & & 46 & & 18.52 .68 \\
\hline 1376 & OFFSITE & SESPMNT & $\mathrm{R}$ & B13LH0 & LAT/LONG & & 46 & & 18.53.97 \\
\hline 1377 & OFFSITE & SESPMNT & $\mathrm{R}$ & B13LH1 & LAT/LONG & & 46 & & 18.53 .50 \\
\hline 1378 & OFFSITE & SESPMNT & $\mathrm{R}$ & B13LH2 & LAT/LONG & & 46 & & 19.52.07 \\
\hline \begin{tabular}{|l}
1379 \\
\end{tabular} & OFFSITE & SESPMNT & $\mathrm{R}$ & B13LJ3 & LAT/LONG & & 46 & & 19.24.77 \\
\hline 1380 & OFFSITE & SESPMNT & $\mathrm{R}$ & B13LJ4 & LAT/LONG & & 46 & & 20.07 .46233 \\
\hline 1381 & OFFSITE & SESPMNT & $\mathrm{R}$ & B13LJ5 & LAT/LONG & & 46 & & 20.59 .29 \\
\hline 1382 & OFFSITE & SESPMNT & $\mathrm{R}$ & B13LJ6 & LAT/LONG & & 46 & & 21.21 .83 \\
\hline 1383 & OFFSITE & SESPMNT & $\mathrm{R}$ & & & & & & \\
\hline 1384 & OFFSITE & SESPMNT & $\mathrm{R}$ & & & & & & \\
\hline 1385 & OFFSITE & SESPMNT & $\mathrm{R}$ & & & & & & \\
\hline 1386 & OFFSITE & SESPMNT & $\mathrm{R}$ & & & & & & \\
\hline $\begin{array}{l}1387 \\
\end{array}$ & ONSITE & SESPMNT & $\mathrm{R}$ & & & & & & \\
\hline \begin{tabular}{|l}
1388 \\
\end{tabular} & ONSITE & SESPMNT & $\mathrm{R}$ & & & & & & \\
\hline 1389 & ONSITE & SESPMNT & $\mathrm{R}$ & & & & & & \\
\hline 1390 & ONSITE & SESPMNT & $\mathrm{R}$ & & & & & & \\
\hline \begin{tabular}{|l}
1391 \\
\end{tabular} & ONSITE & SESPMNT & $\mathrm{R}$ & & & & & & \\
\hline 1392 & ONSITE & SESPMNT & $\mathrm{R}$ & & & & & & \\
\hline $\begin{array}{l}1393 \\
\end{array}$ & OFFSITE & SESPMNT & $\mathrm{R}$ & & & & & & \\
\hline 1394 & OFFSITE & SESPMNT & $\mathrm{R}$ & & & & & & \\
\hline 1395 & OFFSITE & SESPMNT & $\mathrm{R}$ & B147T3 & LAT/LONG & & 46 & & 38.32 .125 \\
\hline 1396 & OFFSITE & SESPMNT & $\mathrm{R}$ & B147T4 & LAT/LONG & & 46 & & 38.33 .902 \\
\hline 1397 & OFFSITE & SESPMNT & $\mathrm{R}$ & B147T5 & LAT/LONG & & 46 & & 38.40 .586 \\
\hline 1398 & OFFSITE & SESPMNT & $\mathrm{R}$ & B147T6 & LAT/LONG & & 46 & & 38.41 .856 \\
\hline 1399 & OFFSITE & SESPSPEC & S & B147T2 & LAT/LONG & & 46 & & 18.54 .797 \\
\hline 1400 & OFFSITE & SESPMNT & $\mathrm{R}$ & B147T7 & LAT/LONG & & 46 & & 18.54 .797 \\
\hline \begin{tabular}{|l}
1401 \\
\end{tabular} & OFFSITE & SESPMNT & $\mathrm{R}$ & B147T8 & LAT/LONG & & 46 & & 18.53.211 \\
\hline \begin{tabular}{|l|}
1402 \\
\end{tabular} & OFFSITE & SESPMNT & $\mathrm{R}$ & B147T9 & LAT/LONG & & 46 & & 18.52 .338 \\
\hline $\begin{array}{l}1403 \\
\end{array}$ & OFFSITE & SESPMNT & $\mathrm{R}$ & B147V0 & LAT/LONG & & 46 & & 18.52 .299 \\
\hline \begin{tabular}{|l}
1404 \\
\end{tabular} & OFFSITE & SESPMNT & $\mathrm{R}$ & B147V1 & LAT/LONG & & 46 & & 18.51 .346 \\
\hline 1405 & OFFSITE & SESPMNT & $\mathrm{R}$ & B147V2 & LAT/LONG & & 46 & & 18.50 .697 \\
\hline 1406 & OFFSITE & SESPMNT & $\mathrm{R}$ & B147W3 & LAT/LONG & & 46 & & 19.24 .739 \\
\hline 1407 & OFFSITE & SESPMNT & $\mathrm{R}$ & B147W4 & LAT/LONG & & 46 & & 20.7.138 \\
\hline
\end{tabular}




\begin{tabular}{|c|c|c|c|c|c|c|c|c|c|}
\hline & $A G$ & $\mathrm{AH}$ & $\mathrm{Al}$ & $\mathrm{AJ}$ & $\mathrm{AK}$ & $\mathrm{AL}$ & $\mathrm{AM}$ & AN & $\mathrm{AO}$ \\
\hline 1 & DIST_CLASS & OWNER_ID & COLLECTION_PURPOSE & X_Y_COORD_FINAL.SAMP_NUM & COORDINATE SYSTEM & DATUM & LATITUDE ${ }^{\circ} \mathrm{N}$ & LATITUDE MIN_MIN & LATITUDE MIN_SEC \\
\hline 1408 & OFFSITE & SESPMNT & $\mathrm{R}$ & B147W5 & LAT/LONG & & 46 & & 20.59.129 \\
\hline 1409 & OFFSITE & SESPMNT & $\mathrm{R}$ & B147W6 & LAT/LONG & & 46 & & 21.20.938 \\
\hline 1410 & OFFSITE & SESPMNT & $\mathrm{R}$ & & & & & & \\
\hline 1411 & OFFSITE & SESPMNT & $\mathrm{R}$ & & & & & & \\
\hline 1412 & ONSITE & SESPMNT & $\mathrm{R}$ & & & & & & \\
\hline 1413 & ONSITE & SESPMNT & $\mathrm{R}$ & & & & & & \\
\hline 1414 & ONSITE & SESPSPEC & S & & & & & & \\
\hline 1415 & ONSITE & SESPMNT & $\mathrm{R}$ & & & & & & \\
\hline 1416 & ONSITE & SESPMNT & $\mathrm{R}$ & & & & & & \\
\hline 1417 & ONSITE & SESPMNT & $\mathrm{R}$ & & & & & & \\
\hline 1418 & ONSITE & SESPMNT & $\mathrm{R}$ & & & & & & \\
\hline 1419 & OFFSITE & SESPMNT & $\mathrm{R}$ & & & & & & \\
\hline 1420 & OFFSITE & SESPMNT & $\mathrm{R}$ & & & & & & \\
\hline 1421 & & CENTPLAT & IP & & & & & & \\
\hline 1422 & & CENTPLAT & IP & & & & & & \\
\hline 1423 & OFFSITE & SESPMNT & $\mathrm{R}$ & B14LJ0 & LAT/LONG & & 46 & 25832 & \\
\hline 1424 & PERIMETER & SESPMNT & $\mathrm{R}$ & B14LJ2 & LAT/LONG & & 46 & 34120 & \\
\hline 1425 & OFFSITE & SESPMNT & $\mathrm{R}$ & & & & & & \\
\hline 1426 & OFFSITE & SESPMNT & $\mathrm{R}$ & & & & & & \\
\hline 1427 & OFFSITE & SESPMNT & $\mathrm{R}$ & B14RV4 & LAT/LONG & & 46 & & 38.32 .346 \\
\hline 1428 & OFFSITE & SESPMNT & $\mathrm{R}$ & B14RV5 & LAT/LONG & & 46 & & 38.35 .710 \\
\hline 1429 & OFFSITE & SESPMNT & $\mathrm{R}$ & B14RV6 & LAT/LONG & & 46 & & 38.40 .744 \\
\hline 1430 & OFFSITE & SESPMNT & $\mathrm{R}$ & B14RV7 & LAT/LONG & & 46 & & 38.43 .263 \\
\hline 1431 & OFFSITE & SESPMNT & $\mathrm{R}$ & B14RV8 & LAT/LONG & & 46 & & 18.54 .564 \\
\hline 1432 & OFFSITE & SESPMNT & $\mathrm{R}$ & B14RV9 & LAT/LONG & & 46 & & 18.50 .434 \\
\hline 1433 & OFFSITE & SESPMNT & $\mathrm{R}$ & B14RW0 & LAT/LONG & & 46 & & 18.51.872 \\
\hline 1434 & OFFSITE & SESPMNT & $\mathrm{R}$ & B14RW1 & LAT/LONG & & 46 & & 18.52.771 \\
\hline 1435 & OFFSITE & SESPMNT & $\mathrm{R}$ & B14RW2 & LAT/LONG & & 46 & & 18.48.011 \\
\hline 1436 & OFFSITE & SESPMNT & $\mathrm{R}$ & B14RW3 & LAT/LONG & & 46 & & 18.52 .018 \\
\hline 1437 & OFFSITE & SESPMNT & $\mathrm{R}$ & B14RX4 & LAT/LONG & & 46 & & 19.24 .503 \\
\hline 1438 & OFFSITE & SESPMNT & $\mathrm{R}$ & B14RX5 & LAT/LONG & & 46 & & 20.06.731 \\
\hline 1439 & OFFSITE & SESPMNT & $\mathrm{R}$ & B14RX6 & LAT/LONG & & 46 & & 20.59 .569 \\
\hline 1440 & OFFSITE & SESPMNT & $\mathrm{R}$ & B14RX7 & LAT/LONG & & 46 & & 21.21.521 \\
\hline 1441 & OFFSITE & SESPMNT & $\mathrm{R}$ & & & & & & \\
\hline 1442 & OFFSITE & SESPMNT & $\mathrm{R}$ & & & & & & \\
\hline 1443 & OFFSITE & SESPMNT & $\mathrm{R}$ & & & & & & \\
\hline 1444 & PERIMETER & SESPMNT & $\mathrm{R}$ & & & & & & \\
\hline 1445 & ONSITE & SESPMNT & $\mathrm{R}$ & & & & & & \\
\hline
\end{tabular}




\begin{tabular}{|c|c|c|c|c|c|c|c|c|c|}
\hline & $A G$ & $\mathrm{AH}$ & $\mathrm{Al}$ & $\mathrm{AJ}$ & $\mathrm{AK}$ & $\mathrm{AL}$ & $\mathrm{AM}$ & AN & $\mathrm{AO}$ \\
\hline 1 & DIST_CLASS & OWNER_ID & COLLECTION_PURPOSE & X_Y_COORD_FINAL.SAMP_NUM & COORDINATE SYSTEM & DATUM & LATITUDE ${ }^{\circ} \mathrm{N}$ & LATITUDE MIN_MIN & LATITUDE MIN_SEC \\
\hline 1446 & ONSITE & SESPMNT & $\mathrm{R}$ & & & & & & \\
\hline 1447 & OFFSITE & SESPMNT & $\mathrm{R}$ & & & & & & \\
\hline 1448 & ONSITE & SESPMNT & $\mathrm{R}$ & & & & & & \\
\hline 1449 & PERIMETER & SESPMNT & $\mathrm{R}$ & & & & & & \\
\hline 1450 & ONSITE & SESPMNT & $\mathrm{R}$ & & & & & & \\
\hline $\mid 1451$ & ONSITE & SESPMNT & $\mathrm{R}$ & & & & & & \\
\hline 1452 & ONSITE & SESPMNT & $\mathrm{R}$ & & & & & & \\
\hline 1453 & OFFSITE & SESPMNT & $\mathrm{R}$ & & & & & & \\
\hline 1454 & OFFSITE & SESPMNT & $\mathrm{R}$ & & & & & & \\
\hline 1455 & & CENTPLAT & IP & & & & & & \\
\hline \begin{tabular}{|l|}
1456 \\
\end{tabular} & & CENTPLAT & IP & & & & & & \\
\hline 1457 & OFFSITE & SESPMNT & $\mathrm{R}$ & & & & & & \\
\hline 1458 & OFFSITE & SESPMNT & $\mathrm{R}$ & & & & & & \\
\hline 1459 & ONSITE & SESPSPEC & S & B158H3 & LAT/LONG & & 46 & & 41.02 .29 \\
\hline 1460 & OFFSITE & SESPMNT & $\mathrm{R}$ & B158H4 & LAT/LONG & & 46 & & 38.42 .82 \\
\hline 1461 & OFFSITE & SESPMNT & $\mathrm{R}$ & B158H5 & LAT/LONG & & 46 & & 38.35 .52 \\
\hline 1462 & OFFSITE & SESPMNT & $\mathrm{R}$ & B158H6 & LAT/LONG & & 46 & & 38.39 .47 \\
\hline 1463 & OFFSITE & SESPMNT & $\mathrm{R}$ & B158H7 & LAT/LONG & & 46 & & 38.31 .26 \\
\hline \begin{tabular}{|l|}
1464 \\
\end{tabular} & ONSITE & SESPMNT & $\mathrm{R}$ & B158H8 & LAT/LONG & & 46 & & 41.02 .29 \\
\hline 1465 & ONSITE & SESPMNT & $\mathrm{R}$ & В158H9 & LAT/LONG & & 46 & & 41.03 .77 \\
\hline 1466 & ONSITE & SESPMNT & $\mathrm{R}$ & B158J0 & LAT/LONG & & 46 & & 41.11 .21 \\
\hline 1467 & ONSITE & SESPMNT & $\mathrm{R}$ & B158M7 & LAT/LONG & & 46 & & 41.10 .20 \\
\hline \begin{tabular}{|l|}
1468 \\
\end{tabular} & ONSITE & SESPMNT & $\mathrm{R}$ & B158M8 & LAT/LONG & & 46 & & 41.06 .12 \\
\hline 1469 & ONSITE & SESPMNT & $\mathrm{R}$ & B158M9 & LAT/LONG & & 46 & & 41.09 .52 \\
\hline 1470 & ONSITE & SESPMNT & $\mathrm{R}$ & B158W8 & LAT/LONG & & 46 & & 40.14 .09 \\
\hline 1471 & ONSITE & SESPMNT & $\mathrm{R}$ & B158X1 & LAT/LONG & & 46 & & 40.43 .18 \\
\hline 1472 & ONSITE & SESPMNT & $\mathrm{R}$ & B158X4 & LAT/LONG & & 46 & & 40.51 .95 \\
\hline \begin{tabular}{|l|}
1473 \\
\end{tabular} & ONSITE & SESPMNT & $\mathrm{R}$ & B158X7 & LAT/LONG & & 46 & & 41.18 .54 \\
\hline 1474 & ONSITE & SESPMNT & $\mathrm{R}$ & B158J1 & LAT/LONG & & 46 & & 39.35 .36 \\
\hline 1475 & ONSITE & SESPMNT & $\mathrm{R}$ & B158J3 & LAT/LONG & & 46 & & 39.39 .60 \\
\hline \begin{tabular}{|l|}
1476 \\
\end{tabular} & ONSITE & SESPMNT & $\mathrm{R}$ & B158J5 & LAT/LONG & & 46 & & 33.39 .50 \\
\hline 1477 & ONSITE & SESPMNT & $\mathrm{R}$ & B158J7 & LAT/LONG & & 46 & & 39.37 .16 \\
\hline \begin{tabular}{|l|}
1478 \\
\end{tabular} & ONSITE & SESPMNT & $\mathrm{R}$ & B158J9 & LAT/LONG & & 46 & & 39.44 .48 \\
\hline 1479 & ONSITE & SESPMNT & $\mathrm{R}$ & B158K1 & LAT/LONG & & 46 & & 39.48 .58 \\
\hline 1480 & ONSITE & SESPMNT & $\mathrm{R}$ & B158K3 & LAT/LONG & & 46 & & 39.51 .94 \\
\hline \begin{tabular}{|l|}
1481 \\
\end{tabular} & ONSITE & SESPMNT & $\mathrm{R}$ & B158K5 & LAT/LONG & & 46 & & 33.40 .64 \\
\hline \begin{tabular}{|l|}
1482 \\
\end{tabular} & ONSITE & SESPMNT & $\mathrm{R}$ & B158K7 & LAT/LONG & & 46 & & 33.40 .63 \\
\hline 1483 & ONSITE & SESPMNT & $\mathrm{R}$ & B158K9 & LAT/LONG & & 46 & & 33.42 .44 \\
\hline
\end{tabular}




\begin{tabular}{|c|c|c|c|c|c|c|c|c|c|}
\hline & $A G$ & $\mathrm{AH}$ & $\mathrm{Al}$ & $\mathrm{AJ}$ & $A K$ & $\mathrm{AL}$ & $\mathrm{AM}$ & AN & $\mathrm{AO}$ \\
\hline 1 & DIST_CLASS & OWNER_ID & COLLECTION_PURPOSE & X_Y_COORD_FINAL.SAMP_NUM & COORDINATE SYSTEM & DATUM & LATITUDE ${ }^{\circ} \mathrm{N}$ & LATITUDE MIN_MIN & LATITUDE MIN_SEC \\
\hline 1484 & ONSITE & SESPMNT & $\mathrm{R}$ & B158L1 & LAT/LONG & & 46 & & 33.43.598 \\
\hline 1485 & ONSITE & SESPMNT & $\mathrm{R}$ & B158L3 & LAT/LONG & & 46 & & 33.40 .47 \\
\hline 1486 & ONSITE & SESPMNT & $\mathrm{R}$ & B158NO & LAT/LONG & & 46 & & 34.50 .32 \\
\hline \begin{tabular}{|l|}
1487 \\
\end{tabular} & ONSITE & SESPMNT & $\mathrm{R}$ & B158N2 & LAT/LONG & & 46 & & 34.21 .43 \\
\hline 1488 & ONSITE & SESPMNT & $\mathrm{R}$ & B158N4 & LAT/LONG & & 46 & & 32.44 .22 \\
\hline 1489 & ONSITE & SESPMNT & $\mathrm{R}$ & B15902 & LAT/LONG & & 46 & & 33.57 .46 \\
\hline 1490 & ONSITE & SESPMNT & $\mathrm{R}$ & B15906 & LAT/LONG & & 46 & & 40.10 .84 \\
\hline \begin{tabular}{|l|}
1491 \\
\end{tabular} & ONSITE & SESPMNT & $\mathrm{R}$ & B15910 & LAT/LONG & & 46 & & 36.22 .01 \\
\hline 1492 & ONSITE & SESPMNT & $\mathrm{R}$ & B15914 & LAT/LONG & & 46 & & 37.02 .66 \\
\hline 1493 & ONSITE & SESPMNT & $\mathrm{R}$ & B158L5 & LAT/LONG & & 46 & & 21.37.20 \\
\hline 1494 & ONSITE & SESPMNT & $\mathrm{R}$ & B158L6 & LAT/LONG & & 46 & & 21.36 .55 \\
\hline 1495 & ONSITE & SESPMNT & $\mathrm{R}$ & B158L7 & LAT/LONG & & 46 & & 21.35 .70 \\
\hline 1496 & ONSITE & SESPMNT & $\mathrm{R}$ & B158L8 & LAT/LONG & & 46 & & 21.37 .67 \\
\hline \begin{tabular}{|l|}
1497 \\
\end{tabular} & ONSITE & SESPMNT & $\mathrm{R}$ & B158L9 & LAT/LONG & & 46 & & 21.38.28 \\
\hline 1498 & ONSITE & SESPMNT & $\mathrm{R}$ & B158M0 & LAT/LONG & & 46 & & 21.38 .48 \\
\hline 1499 & OFFSITE & SESPMNT & $\mathrm{R}$ & B158M1 & LAT/LONG & & 46 & & 18.54 .5 \\
\hline 1500 & OFFSITE & SESPMNT & $\mathrm{R}$ & B158M2 & LAT/LONG & & 46 & & 18.52 .7 \\
\hline 1501 & OFFSITE & SESPMNT & $\mathrm{R}$ & B158M3 & LAT/LONG & & 46 & & 18.85 .0 \\
\hline 1502 & OFFSITE & SESPMNT & $\mathrm{R}$ & B158M4 & LAT/LONG & & 46 & & 18.51 .50 \\
\hline 1503 & OFFSITE & SESPMNT & $\mathrm{R}$ & B158M5 & LAT/LONG & & 46 & & 18.51.37 \\
\hline 1504 & OFFSITE & SESPMNT & $\mathrm{R}$ & B158M6 & LAT/LONG & & 46 & & 18.52.36 \\
\hline 1505 & OFFSITE & SESPMNT & $\mathrm{R}$ & B158V6 & LAT/LONG & & 46 & & 19.24.75 \\
\hline 1506 & OFFSITE & SESPMNT & $\mathrm{R}$ & B158V7 & LAT/LONG & & 46 & & 20.07.17 \\
\hline \begin{tabular}{|l|}
1507 \\
\end{tabular} & OFFSITE & SESPMNT & $\mathrm{R}$ & B158V8 & LAT/LONG & & 46 & & 20.58 .56 \\
\hline 1508 & OFFSITE & SESPMNT & $\mathrm{R}$ & B158V9 & LAT/LONG & & 46 & & 21.21 .49 \\
\hline 1509 & ONSITE & SESPMNT & $\mathrm{R}$ & B158Y0 & LAT/LONG & & 46 & & 23.03 .00 \\
\hline 1510 & ONSITE & SESPMNT & $\mathrm{R}$ & B158Y3 & LAT/LONG & & 46 & & 22.35 .44 \\
\hline 1511 & ONSITE & SESPMNT & $\mathrm{R}$ & B158Y6 & LAT/LONG & & 46 & & 22.19 .60 \\
\hline 1512 & ONSITE & SESPMNT & $\mathrm{R}$ & B158Y9 & LAT/LONG & & 46 & & 21.48.39 \\
\hline \begin{tabular}{|l|}
1513 \\
\end{tabular} & ONSITE & SESPMNT & $\mathrm{R}$ & & & & & & \\
\hline 1514 & ONSITE & SESPMNT & $\mathrm{R}$ & & & & & & \\
\hline 1515 & ONSITE & SESPMNT & $\mathrm{R}$ & & & & & & \\
\hline 1516 & OFFSITE & SESPMNT & $\mathrm{R}$ & & & & & & \\
\hline \begin{tabular}{|l|}
1517 \\
\end{tabular} & OFFSITE & SESPMNT & $\mathrm{R}$ & & & & & & \\
\hline 1518 & ONSITE & SESPMNT & $\mathrm{R}$ & B15C09 & LAT/LONG & & 46 & & 33.55 .313 \\
\hline 1519 & ONSITE & SESPMNT & $\mathrm{R}$ & & & & & & \\
\hline 1520 & ONSITE & SESPMNT & $\mathrm{R}$ & B15C73 & LAT/LONG & & 46 & & 33.50 .499 \\
\hline \begin{tabular}{|l|}
1521 \\
\end{tabular} & ONSITE & SESPMNT & $\mathrm{R}$ & & & & & & \\
\hline
\end{tabular}

Page 508 


\begin{tabular}{|c|c|c|c|c|c|c|c|c|c|}
\hline & AG & $\mathrm{AH}$ & $\mathrm{Al}$ & AJ & AK & $\mathrm{AL}$ & AM & AN & $\mathrm{AO}$ \\
\hline 1 & DIST_CLASS & OWNER_ID & COLLECTION_PURPOSE & X_Y_COORD_FINAL.SAMP_NUM & COORDINATE SYSTEM & DATUM & LATITUDE ${ }^{\circ} \mathrm{N}$ & LATITUDE MIN_MIN & LATITUDE MIN_SEC \\
\hline 1522 & ONSITE & SESPMNT & $\mathrm{R}$ & & & & & & \\
\hline 1523 & ONSITE & SESPMNT & $\mathrm{R}$ & & & & & & \\
\hline 1524 & ONSITE & SESPMNT & $\mathrm{R}$ & B15C60 & LAT/LONG & & 46 & & 39.40 .613 \\
\hline 1525 & ONSITE & SESPMNT & $\mathrm{R}$ & B15C65 & LAT/LONG & & 46 & & 42.04 .2747 \\
\hline 1526 & ONSITE & SESPMNT & $\mathrm{R}$ & B15CB3 & LAT/LONG & & 46 & & 42.12 .884 \\
\hline 1527 & ONSITE & SESPMNT & $\mathrm{R}$ & B15C69 & LAT/LONG & & 46 & & 38.14 .440 \\
\hline 1528 & OFFSITE & SESPMNT & $\mathrm{R}$ & & & & & & \\
\hline 1529 & OFFSITE & SESPMNT & $\mathrm{R}$ & & & & & & \\
\hline 1530 & OFFSITE & SESPMNT & $\mathrm{R}$ & & & & & & \\
\hline 1531 & OFFSITE & SESPMNT & $\mathrm{R}$ & & & & & & \\
\hline 1532 & ONSITE & SESPMNT & $\mathrm{R}$ & & & & & & \\
\hline 1533 & OFFSITE & SESPMNT & $\mathrm{R}$ & & & & & & \\
\hline 1534 & OFFSITE & SESPMNT & $\mathrm{R}$ & & & & & & \\
\hline 1535 & OFFSITE & SESPMNT & $\mathrm{R}$ & & & & & & \\
\hline 1536 & OFFSITE & SESPMNT & $\mathrm{R}$ & & & & & & \\
\hline 1537 & OFFSITE & SESPMNT & $\mathrm{R}$ & & & & & & \\
\hline 1538 & OFFSITE & SESPMNT & $\mathrm{R}$ & & & & & & \\
\hline 1539 & OFFSITE & SESPMNT & $\mathrm{R}$ & & & & & & \\
\hline 1540 & OFFSITE & SESPMNT & $\mathrm{R}$ & & & & & & \\
\hline 1541 & OFFSITE & SESPSPEC & S & & & & & & \\
\hline 1542 & OFFSITE & SESPMNT & $\mathrm{R}$ & & & & & & \\
\hline 1543 & OFFSITE & SESPMNT & $\mathrm{R}$ & & & & & & \\
\hline 1544 & OFFSITE & SESPMNT & $\mathrm{R}$ & & & & & & \\
\hline 1545 & OFFSITE & SESPMNT & $\mathrm{R}$ & & & & & & \\
\hline 1546 & OFFSITE & SESPMNT & $\mathrm{R}$ & & & & & & \\
\hline 1547 & OFFSITE & SESPMNT & $\mathrm{R}$ & & & & & & \\
\hline 1548 & OFFSITE & SESPMNT & $\mathrm{R}$ & & & & & & \\
\hline 1549 & OFFSITE & SESPMNT & $\mathrm{R}$ & & & & & & \\
\hline 1550 & OFFSITE & SESPMNT & $\mathrm{R}$ & & & & & & \\
\hline 1551 & OFFSITE & SESPMNT & $\mathrm{R}$ & & & & & & \\
\hline 1552 & ONSITE & SESPMNT & $\mathrm{R}$ & & & & & & \\
\hline 1553 & OFFSITE & SESPMNT & $\mathrm{R}$ & & & & & & \\
\hline 1554 & OFFSITE & SESPMNT & $\mathrm{R}$ & & & & & & \\
\hline 1555 & OFFSITE & SESPMNT & $\mathrm{R}$ & & & & & & \\
\hline 1556 & OFFSITE & SESPMNT & $\mathrm{R}$ & & & & & & \\
\hline 1557 & OFFSITE & SESPMNT & $\mathrm{R}$ & & & & & & \\
\hline 1558 & OFFSITE & SESPMNT & $\mathrm{R}$ & & & & & & \\
\hline 1559 & OFFSITE & SESPMNT & $\mathrm{R}$ & & & & & & \\
\hline
\end{tabular}




\begin{tabular}{|c|c|c|c|c|c|c|c|c|c|}
\hline & AG & $\mathrm{AH}$ & $\mathrm{Al}$ & $\overline{\mathrm{AJ}}$ & AK & $\overline{\mathrm{AL}}$ & AM & AN & $\mathrm{AO}$ \\
\hline 1 & DIST_CLASS & OWNER_ID & COLLECTION_PURPOSE & X_Y_COORD_FINAL.SAMP_NUM & COORDINATE SYSTEM & DATUM & LATITUDE ${ }^{\circ} \mathrm{N}$ & LATITUDE MIN_MIN & LATITUDE MIN_SEC \\
\hline 1560 & OFFSITE & SESPMNT & $\mathrm{R}$ & & & & & & \\
\hline 1561 & ONSITE & SESPSPEC & S & & & & & & \\
\hline 1562 & ONSITE & SESPSPEC & S & & & & & & \\
\hline 1563 & ONSITE & SESPSPEC & $\mathrm{S}$ & & & & & & \\
\hline \begin{tabular}{|l|}
1564 \\
\end{tabular} & OFFSITE & SESPMNT & $\mathrm{R}$ & BOYCD7 & STATE PLANE & & & & \\
\hline 1565 & OFFSITE & SESPMNT & $\mathrm{R}$ & BOYCD8 & STATE PLANE & & & & \\
\hline 1566 & OFFSITE & SESPMNT & $\mathrm{R}$ & BOYCD9 & STATE PLANE & & & & \\
\hline 1567 & OFFSITE & SESPMNT & $\mathrm{R}$ & BOYCFO & STATE PLANE & & & & \\
\hline 1568 & OFFSITE & SESPMNT & $\mathrm{R}$ & BOYCF1 & STATE PLANE & & & & \\
\hline 1569 & OFFSITE & SESPMNT & $\mathrm{R}$ & BOYCF2 & STATE PLANE & & & & \\
\hline 1570 & OFFSITE & SESPMNT & $\mathrm{R}$ & BOYCF3 & STATE PLANE & & & & \\
\hline 1571 & OFFSITE & SESPMNT & $\mathrm{R}$ & BOYCF4 & STATE PLANE & & & & \\
\hline 1572 & OFFSITE & SESPMNT & $\mathrm{R}$ & BOYCF5 & STATE PLANE & & & & \\
\hline 1573 & OFFSITE & SESPMNT & $\mathrm{R}$ & B0YCF6 & STATE PLANE & & & & \\
\hline 1574 & OFFSITE & SESPMNT & $\mathrm{R}$ & BOYCK3 & STATE PLANE & & & & \\
\hline \begin{tabular}{|l|}
1575 \\
\end{tabular} & OFFSITE & SESPMNT & $\mathrm{R}$ & BOYCK4 & STATE PLANE & & & & \\
\hline \begin{tabular}{|l|}
1576 \\
\end{tabular} & OFFSITE & SESPMNT & $\mathrm{R}$ & BOYCK5 & STATE PLANE & & & & \\
\hline 1577 & OFFSITE & SESPMNT & $\mathrm{R}$ & BOYCK6 & STATE PLANE & & & & \\
\hline \begin{tabular}{|l|}
1578 \\
\end{tabular} & OFFSITE & SESPMNT & $\mathrm{R}$ & & & & & & \\
\hline \begin{tabular}{|l|}
1579 \\
\end{tabular} & OFFSITE & SESPMNT & $\mathrm{R}$ & & & & & & \\
\hline 1580 & ONSITE & SESPMNT & $\mathrm{R}$ & & & & & & \\
\hline 1581 & OFFSITE & SESPMNT & $\mathrm{R}$ & & & & & & \\
\hline 1582 & OFFSITE & SESPMNT & $\mathrm{R}$ & & & & & & \\
\hline 1583 & OFFSITE & SESPMNT & $\mathrm{R}$ & & & & & & \\
\hline 1584 & OFFSITE & SESPMNT & $\mathrm{R}$ & & & & & & \\
\hline 1585 & OFFSITE & SESPMNT & $\mathrm{R}$ & & & & & & \\
\hline 1586 & ONSITE & SESPSPEC & S & B10672 & STATE PLANE & & & & \\
\hline \begin{tabular}{|l|}
1587 \\
\end{tabular} & OFFSITE & SESPMNT & $\mathrm{R}$ & B10673 & STATE PLANE & & & & \\
\hline \begin{tabular}{|l|}
1588 \\
\end{tabular} & OFFSITE & SESPMNT & $\mathrm{R}$ & B10674 & STATE PLANE & & & & \\
\hline \begin{tabular}{|l|}
1589 \\
\end{tabular} & OFFSITE & SESPMNT & $\mathrm{R}$ & B10675 & STATE PLANE & & & & \\
\hline 1590 & OFFSITE & SESPMNT & $\mathrm{R}$ & B10676 & STATE PLANE & & & & \\
\hline 1591 & ONSITE & SESPMNT & $\mathrm{R}$ & B10677 & STATE PLANE & & & & \\
\hline 1592 & ONSITE & SESPMNT & $\mathrm{R}$ & B10679 & STATE PLANE & & & & \\
\hline 1593 & ONSITE & SESPMNT & $\mathrm{R}$ & & & & & & \\
\hline 1594 & ONSITE & SESPMNT & $\mathrm{R}$ & B10683 & STATE PLANE & & & & \\
\hline 1595 & ONSITE & SESPMNT & $\mathrm{R}$ & B10684 & STATE PLANE & & & & \\
\hline 1596 & ONSITE & SESPMNT & $\mathrm{R}$ & & & & & & \\
\hline 1597 & ONSITE & SESPMNT & $\mathrm{R}$ & & & & & & \\
\hline
\end{tabular}




\begin{tabular}{|c|c|c|c|c|c|c|c|c|c|}
\hline & $A G$ & $\mathrm{AH}$ & $\mathrm{Al}$ & $\mathrm{AJ}$ & AK & $\mathrm{AL}$ & $\mathrm{AM}$ & AN & $\mathrm{AO}$ \\
\hline 1 & DIST_CLASS & OWNER_ID & COLLECTION_PURPOSE & X_Y_COORD_FINAL.SAMP_NUM & COORDINATE SYSTEM & DATUM & LATITUDE $^{\circ} \mathrm{N}$ & LATITUDE MIN_MIN & LATITUDE MIN_SEC \\
\hline 1598 & ONSITE & SESPMNT & $\mathrm{R}$ & B106B1 & STATE PLANE & & & & \\
\hline 1599 & ONSITE & SESPMNT & $\mathrm{R}$ & & & & & & \\
\hline 1600 & ONSITE & SESPMNT & $\mathrm{R}$ & B106B7 & STATE PLANE & & & & \\
\hline 1601 & ONSITE & SESPMNT & $\mathrm{R}$ & B106W1 & STATE PLANE & & & & \\
\hline 1602 & ONSITE & SESPMNT & $\mathrm{R}$ & B106W2 & STATE PLANE & & & & \\
\hline 1603 & ONSITE & SESPMNT & $\mathrm{R}$ & B106W3 & STATE PLANE & & & & \\
\hline 1604 & ONSITE & SESPMNT & $\mathrm{R}$ & B106W4 & STATE PLANE & & & & \\
\hline 1605 & ONSITE & SESPMNT & $\mathrm{R}$ & B106W5 & STATE PLANE & & & & \\
\hline 1606 & ONSITE & SESPMNT & $\mathrm{R}$ & B106W6 & STATE PLANE & & & & \\
\hline \begin{tabular}{|l|l|}
1607 \\
\end{tabular} & ONSITE & SESPMNT & $\mathrm{R}$ & B106W7 & STATE PLANE & & & & \\
\hline 1608 & ONSITE & SESPMNT & $\mathrm{R}$ & B106W8 & STATE PLANE & & & & \\
\hline 1609 & ONSITE & SESPMNT & $\mathrm{R}$ & B106W9 & STATE PLANE & & & & \\
\hline 1610 & ONSITE & SESPMNT & $\mathrm{R}$ & B106X0 & STATE PLANE & & & & \\
\hline 1611 & ONSITE & SESPMNT & $\mathrm{R}$ & B106X1 & STATE PLANE & & & & \\
\hline 1612 & ONSITE & SESPMNT & $\mathrm{R}$ & B106X2 & STATE PLANE & & & & \\
\hline 1613 & ONSITE & SESPMNT & $\mathrm{R}$ & B10728 & STATE PLANE & & & & \\
\hline 1614 & ONSITE & SESPMNT & $\mathrm{R}$ & B10729 & STATE PLANE & & & & \\
\hline 1615 & ONSITE & SESPMNT & $\mathrm{R}$ & B10730 & STATE PLANE & & & & \\
\hline \begin{tabular}{|l|l|}
1616 \\
\end{tabular} & ONSITE & SESPMNT & $\mathrm{R}$ & B10785 & STATE PLANE & & & & \\
\hline 1617 & ONSITE & SESPMNT & $\mathrm{R}$ & B10788 & STATE PLANE & & & & \\
\hline 1618 & ONSITE & SESPMNT & $\mathrm{R}$ & B10791 & STATE PLANE & & & & \\
\hline 1619 & ONSITE & SESPMNT & $\mathrm{R}$ & B10794 & STATE PLANE & & & & \\
\hline 1620 & ONSITE & SESPMNT & $\mathrm{R}$ & & & & & & \\
\hline 1621 & ONSITE & SESPMNT & $\mathrm{R}$ & & & & & & \\
\hline 1622 & ONSITE & SESPMNT & $\mathrm{R}$ & B106X7 & STATE PLANE & & & & \\
\hline 1623 & ONSITE & SESPMNT & $\mathrm{R}$ & B106X9 & STATE PLANE & & & & \\
\hline 1624 & ONSITE & SESPMNT & $\mathrm{R}$ & B106Y1 & STATE PLANE & & & & \\
\hline 1625 & ONSITE & SESPMNT & $\mathrm{R}$ & B106Y3 & STATE PLANE & & & & \\
\hline 1626 & OFFSITE & SESPMNT & $\mathrm{R}$ & B106Y4 & STATE PLANE & & & & \\
\hline 1627 & OFFSITE & SESPMNT & $\mathrm{R}$ & B106Y5 & STATE PLANE & & & & \\
\hline \begin{tabular}{|l|}
1628 \\
\end{tabular} & OFFSITE & SESPMNT & $\mathrm{R}$ & B106Y6 & STATE PLANE & & & & \\
\hline \begin{tabular}{|l|}
1629 \\
\end{tabular} & OFFSITE & SESPMNT & $\mathrm{R}$ & B106Y7 & STATE PLANE & & & & \\
\hline 1630 & OFFSITE & SESPMNT & $\mathrm{R}$ & B106Y8 & STATE PLANE & & & & \\
\hline 1631 & OFFSITE & SESPMNT & $\mathrm{R}$ & B106Y9 & STATE PLANE & & & & \\
\hline \begin{tabular}{|l|}
1632 \\
\end{tabular} & OFFSITE & SESPMNT & $\mathrm{R}$ & B10761 & STATE PLANE & & & & \\
\hline 1633 & OFFSITE & SESPMNT & $\mathrm{R}$ & B10762 & STATE PLANE & & & & \\
\hline \begin{tabular}{|l|}
1634 \\
\end{tabular} & OFFSITE & SESPMNT & $\mathrm{R}$ & B10763 & STATE PLANE & & & & \\
\hline 1635 & OFFSITE & SESPMNT & $\mathrm{R}$ & B10764 & STATE PLANE & & & & \\
\hline
\end{tabular}




\begin{tabular}{|c|c|c|c|c|c|c|c|c|c|}
\hline & $A G$ & $\mathrm{AH}$ & $\mathrm{Al}$ & $\mathrm{AJ}$ & AK & $\mathrm{AL}$ & $\mathrm{AM}$ & AN & $\mathrm{AO}$ \\
\hline 1 & DIST_CLASS & OWNER_ID & COLLECTION_PURPOSE & X_Y_COORD_FINAL.SAMP_NUM & COORDINATE SYSTEM & DATUM & LATITUDE ${ }^{\circ} \mathrm{N}$ & LATITUDE MIN_MIN & LATITUDE MIN_SEC \\
\hline 1636 & ONSITE & SESPMNT & $\mathrm{R}$ & B10773 & STATE PLANE & & & & \\
\hline 1637 & ONSITE & SESPMNT & $\mathrm{R}$ & B10776 & STATE PLANE & & & & \\
\hline 1638 & ONSITE & SESPMNT & $\mathrm{R}$ & B10779 & STATE PLANE & & & & \\
\hline 1639 & ONSITE & SESPMNT & $\mathrm{R}$ & B10782 & STATE PLANE & & & & \\
\hline 1640 & ONSITE & SESPMNT & $\mathrm{R}$ & B10912 & STATE PLANE & & & & \\
\hline 1641 & ONSITE & SESPMNT & $\mathrm{R}$ & B10913 & STATE PLANE & & & & \\
\hline 1642 & ONSITE & SESPMNT & $\mathrm{R}$ & B10945 & STATE PLANE & & & & \\
\hline 1643 & ONSITE & SESPMNT & $\mathrm{R}$ & B10946 & STATE PLANE & & & & \\
\hline 1644 & ONSITE & SESPMNT & $\mathrm{R}$ & B10949 & STATE PLANE & & & & \\
\hline 1645 & PERIMETER & SESPMNT & $\mathrm{R}$ & B109J0 & STATE PLANE & & & & \\
\hline$\overline{1646}$ & OFFSITE & SESPMNT & $\mathrm{R}$ & & & & & & \\
\hline 1647 & OFFSITE & SESPMNT & $\mathrm{R}$ & & & & & & \\
\hline 1648 & ONSITE & SESPMNT & $\mathrm{R}$ & & & & & & \\
\hline 1649 & ONSITE & SESPMNT & $\mathrm{R}$ & & & & & & \\
\hline 1650 & ONSITE & SESPMNT & $\mathrm{R}$ & & & & & & \\
\hline 1651 & OFFSITE & SESPMNT & $\mathrm{R}$ & & & & & & \\
\hline 1652 & OFFSITE & SESPMNT & $\mathrm{R}$ & & & & & & \\
\hline 1653 & ONSITE & SESPMNT & $\mathrm{R}$ & & & & & & \\
\hline \begin{tabular}{|l}
1654 \\
\end{tabular} & OFFSITE & SESPMNT & $\mathrm{R}$ & & & & & & \\
\hline 1655 & OFFSITE & SESPMNT & $\mathrm{R}$ & & & & & & \\
\hline 1656 & OFFSITE & SESPMNT & $\mathrm{R}$ & B11125 & STATE PLANE & & & & \\
\hline \begin{tabular}{|l}
1657 \\
\end{tabular} & OFFSITE & SESPMNT & $\mathrm{R}$ & B11126 & STATE PLANE & & & & \\
\hline 1658 & OFFSITE & SESPMNT & $\mathrm{R}$ & B11127 & STATE PLANE & & & & \\
\hline 1659 & OFFSITE & SESPMNT & $\mathrm{R}$ & B11128 & STATE PLANE & & & & \\
\hline 1660 & OFFSITE & SESPSPEC & S & B11124 & STATE PLANE & & & & \\
\hline 1661 & OFFSITE & SESPMNT & $\mathrm{R}$ & B11129 & STATE PLANE & & & & \\
\hline 1662 & OFFSITE & SESPMNT & $\mathrm{R}$ & B11130 & STATE PLANE & & & & \\
\hline 1663 & OFFSITE & SESPMNT & $\mathrm{R}$ & B11131 & STATE PLANE & & & & \\
\hline 1664 & OFFSITE & SESPMNT & $\mathrm{R}$ & B11132 & STATE PLANE & & & & \\
\hline 1665 & OFFSITE & SESPMNT & $\mathrm{R}$ & B11133 & STATE PLANE & & & & \\
\hline 1666 & OFFSITE & SESPMNT & $\mathrm{R}$ & B11134 & STATE PLANE & & & & \\
\hline \begin{tabular}{|l}
1667 \\
\end{tabular} & OFFSITE & SESPMNT & $\mathrm{R}$ & B11145 & STATE PLANE & & & & \\
\hline 1668 & OFFSITE & SESPMNT & $\mathrm{R}$ & B11146 & STATE PLANE & & & & \\
\hline 1669 & OFFSITE & SESPMNT & $\mathrm{R}$ & B11147 & STATE PLANE & & & & \\
\hline 1670 & OFFSITE & SESPMNT & $\mathrm{R}$ & B11148 & STATE PLANE & & & & \\
\hline 1671 & OFFSITE & SESPMNT & $\mathrm{R}$ & & & & & & \\
\hline 1672 & OFFSITE & SESPMNT & $\mathrm{R}$ & & & & & & \\
\hline 1673 & OFFSITE & SESPMNT & $\mathrm{R}$ & & & & & & \\
\hline
\end{tabular}




\begin{tabular}{|c|c|c|c|c|c|c|c|c|c|}
\hline & $A G$ & $\mathrm{AH}$ & $\mathrm{Al}$ & $\mathrm{AJ}$ & $\mathrm{AK}$ & $\mathrm{AL}$ & AM & AN & $\mathrm{AO}$ \\
\hline 1 & DIST_CLASS & OWNER_ID & COLLECTION_PURPOSE & X_Y_COORD_FINAL.SAMP_NUM & COORDINATE SYSTEM & DATUM & LATITUDE ${ }^{\circ} \mathrm{N}$ & LATITUDE MIN_MIN & LATITUDE MIN_SEC \\
\hline 1674 & OFFSITE & SESPMNT & $\mathrm{R}$ & & & & & & \\
\hline 1675 & ONSITE & SESPMNT & $\mathrm{R}$ & & & & & & \\
\hline 1676 & OFFSITE & SESPSPEC & $S$ & B11H38 & LAT/LONG & & 46 & & 18.54 .68 \\
\hline 1677 & OFFSITE & SESPMNT & $\mathrm{R}$ & B11H43 & LAT/LONG & & 46 & & 18.54 .68 \\
\hline 1678 & OFFSITE & SESPMNT & $\mathrm{R}$ & B11H44 & LAT/LONG & & 46 & & 18.52.97 \\
\hline 1679 & OFFSITE & SESPMNT & $\mathrm{R}$ & B11H45 & LAT/LONG & & 46 & & 18.51.22 \\
\hline 1680 & OFFSITE & SESPMNT & $\mathrm{R}$ & B11H46 & LAT/LONG & & 46 & & 18.54 .18 \\
\hline 1681 & OFFSITE & SESPMNT & $\mathrm{R}$ & $\mathrm{B} 11 \mathrm{H} 47$ & LAT/LONG & & 46 & & 18.46.53 \\
\hline 1682 & OFFSITE & SESPMNT & $\mathrm{R}$ & B11H48 & LAT/LONG & & NONE & & \\
\hline 1683 & OFFSITE & SESPMNT & $\mathrm{R}$ & B11H59 & LAT/LONG & & 46 & & 19.25 .05 \\
\hline 1684 & OFFSITE & SESPMNT & $\mathrm{R}$ & B11H60 & LAT/LONG & & No GPS sig. & & \\
\hline 1685 & OFFSITE & SESPMNT & $\mathrm{R}$ & B11H61 & LAT/LONG & & 46 & & 20.59.09 \\
\hline 1686 & OFFSITE & SESPMNT & $\mathrm{R}$ & B11H62 & LAT/LONG & & 46 & & 21.27.18 \\
\hline 1687 & OFFSITE & SESPMNT & $\mathrm{R}$ & B11H39 & LAT/LONG & & 46 & & 38.30 .72 \\
\hline 1688 & OFFSITE & SESPMNT & $\mathrm{R}$ & B11H40 & LAT/LONG & & 46 & & 38.34 .81 \\
\hline 1689 & OFFSITE & SESPMNT & $\mathrm{R}$ & B11H41 & LAT/LONG & & 46 & & 38.40 .47 \\
\hline 1690 & OFFSITE & SESPMNT & $\mathrm{R}$ & B11H42 & LAT/LONG & & 46 & & 38.42 .31 \\
\hline 1691 & OFFSITE & SESPMNT & $\mathrm{R}$ & & & & & & \\
\hline 1692 & OFFSITE & SESPMNT & $\mathrm{R}$ & & & & & & \\
\hline 1693 & ONSITE & SESPMNT & $\mathrm{R}$ & & & & & & \\
\hline 1694 & OFFSITE & SESPMNT & $\mathrm{R}$ & & & & & & \\
\hline 1695 & OFFSITE & SESPMNT & $\mathrm{R}$ & & & & & & \\
\hline 1696 & ONSITE & SESPMNT & $\mathrm{R}$ & & & & & & \\
\hline 1697 & ONSITE & SESPMNT & $\mathrm{R}$ & & & & & & \\
\hline 1698 & ONSITE & SESPMNT & $\mathrm{R}$ & & & & & & \\
\hline 1699 & ONSITE & SESPMNT & $\mathrm{R}$ & & & & & & \\
\hline 1700 & ONSITE & SESPMNT & $\mathrm{R}$ & & & & & & \\
\hline 1701 & OFFSITE & SESPMNT & $\mathrm{R}$ & & & & & & \\
\hline 1702 & OFFSITE & SESPMNT & $\mathrm{R}$ & & & & & & \\
\hline 1703 & ONSITE & SESPMNT & $\mathrm{R}$ & & & & & & \\
\hline 1704 & OFFSITE & SESPMNT & $\mathrm{R}$ & & & & & & \\
\hline 1705 & PERIMETER & SESPMNT & $\mathrm{R}$ & & & & & & \\
\hline 1706 & ONSITE & SESPMNT & $\mathrm{R}$ & & & & & & \\
\hline 1707 & OFFSITE & SESPSPEC & $S$ & & & & & & \\
\hline 1708 & OFFSITE & SESPMNT & $\mathrm{R}$ & & & & & & \\
\hline 1709 & OFFSITE & SESPMNT & $\mathrm{R}$ & & & & & & \\
\hline 1710 & OFFSITE & SESPMNT & $\mathrm{R}$ & & & & & & \\
\hline 1711 & PERIMETER & SESPMNT & $\mathrm{R}$ & & & & & & \\
\hline
\end{tabular}




\begin{tabular}{|c|c|c|c|c|c|c|c|c|c|}
\hline & $A G$ & $\mathrm{AH}$ & $\mathrm{Al}$ & $\mathrm{AJ}$ & $\mathrm{AK}$ & $\mathrm{AL}$ & AM & $\mathrm{AN}$ & $\mathrm{AO}$ \\
\hline 1 & DIST_CLASS & OWNER_ID & COLLECTION_PURPOSE & X_Y_COORD_FINAL.SAMP_NUM & COORDINATE SYSTEM & DATUM & LATITUDE ${ }^{\circ} \mathrm{N}$ & LATITUDE MIN_MIN & LATITUDE MIN_SEC \\
\hline 1712 & OFFSITE & SESPMNT & $\mathrm{R}$ & B12505 & LAT/LONG & & 46 & & 18.54 .400 \\
\hline 1713 & OFFSITE & SESPMNT & $\mathrm{R}$ & B12506 & LAT/LONG & & 46 & & 18.53.114 \\
\hline 1714 & OFFSITE & SESPMNT & $\mathrm{R}$ & B12507 & LAT/LONG & & 46 & & 18.50 .753 \\
\hline 1715 & OFFSITE & SESPMNT & $\mathrm{R}$ & B12508 & LAT/LONG & & 46 & & 18.50 .6000 \\
\hline 1716 & OFFSITE & SESPMNT & $\mathrm{R}$ & B12509 & LAT/LONG & & 46 & & 18.44 .67124 \\
\hline 1717 & OFFSITE & SESPMNT & $\mathrm{R}$ & B12510 & LAT/LONG & & 46 & & 18.51 .627 \\
\hline 1718 & OFFSITE & SESPMNT & $\mathrm{R}$ & B12531 & LAT/LONG & & 46 & & 19.24.674 \\
\hline 1719 & OFFSITE & SESPMNT & $\mathrm{R}$ & B12532 & LAT/LONG & & 46 & & 20.07 .051 \\
\hline 1720 & OFFSITE & SESPMNT & $\mathrm{R}$ & B12533 & LAT/LONG & & 46 & & 20.58 .719 \\
\hline 1721 & OFFSITE & SESPMNT & $\mathrm{R}$ & B12534 & LAT/LONG & & 46 & & 21.20 .830 \\
\hline 1722 & OFFSITE & SESPMNT & $\mathrm{R}$ & B12501 & LAT/LONG & & 46 & & 38.33 .187 \\
\hline 1723 & OFFSITE & SESPMNT & $\mathrm{R}$ & B12502 & LAT/LONG & & 46 & & 38.36 .25143 \\
\hline 1724 & OFFSITE & SESPMNT & $\mathrm{R}$ & B12503 & LAT/LONG & & 46 & & 38.39 .361 \\
\hline 1725 & OFFSITE & SESPMNT & $\mathrm{R}$ & B12504 & LAT/LONG & & 46 & & 38.42 .047 \\
\hline 1726 & OFFSITE & SESPMNT & $\mathrm{R}$ & & & & & & \\
\hline 1727 & OFFSITE & SESPMNT & $\mathrm{R}$ & & & & & & \\
\hline 1728 & ONSITE & SESPMNT & $\mathrm{R}$ & & & & & & \\
\hline 1729 & OFFSITE & SESPMNT & $\mathrm{R}$ & & & & & & \\
\hline 1730 & PERIMETER & SESPMNT & $\mathrm{R}$ & & & & & & \\
\hline 1731 & OFFSITE & SESPMNT & $\mathrm{R}$ & & & & & & \\
\hline 1732 & OFFSITE & SESPMNT & $\mathrm{R}$ & & & & & & \\
\hline 1733 & ONSITE & SESPMNT & $\mathrm{R}$ & B12RM1 & LAT/LONG & WGS84 & 46 & 37664 & \\
\hline 1734 & ONSITE & SESPMNT & $\mathrm{R}$ & B12RM6 & LAT/LONG & WGS84 & 46 & 37218 & \\
\hline 1735 & ONSITE & SESPSPEC & $S$ & B12RN4 & LAT/LONG & WGS84 & 46 & 63453 & \\
\hline 1736 & ONSITE & SESPSPEC & $S$ & B12RN5 & LAT/LONG & WGS84 & 46 & 37664 & \\
\hline 1737 & ONSITE & SESPSPEC & S & B12RN6 & LAT/LONG & WGS84 & 46 & 37218 & \\
\hline 1738 & ONSITE & SESPSPEC & S & B12RN7 & LAT/LONG & WGS84 & 46 & 63453 & \\
\hline 1739 & ONSITE & SESPSPEC & S & B12RP9 & LAT/LONG & WGS84 & 46 & 63453 & \\
\hline 1740 & ONSITE & SESPSPEC & S & B12RR1 & LAT/LONG & WGS84 & 46 & 63453 & \\
\hline 1741 & ONSITE & SESPSPEC & S & B12RR3 & LAT/LONG & WGS84 & 46 & 63453 & \\
\hline 1742 & ONSITE & SESPSPEC & S & B12RR5 & LAT/LONG & WGS84 & 46 & 37663 & \\
\hline 1743 & ONSITE & SESPSPEC & S & B12RR7 & LAT/LONG & WGS84 & 46 & 37662 & \\
\hline 1744 & ONSITE & SESPSPEC & S & B12RR9 & LAT/LONG & WGS84 & 46 & 37663 & \\
\hline 1745 & ONSITE & SESPSPEC & S & B12RT1 & LAT/LONG & WGS84 & 46 & 37547 & \\
\hline 1746 & ONSITE & SESPSPEC & S & B12RT3 & LAT/LONG & WGS84 & 46 & 37547 & \\
\hline 1747 & ONSITE & SESPSPEC & S & B12RT5 & LAT/LONG & WGS84 & 46 & 37547 & \\
\hline 1748 & ONSITE & SESPSPEC & S & B12RT7 & LAT/LONG & WGS84 & 46 & 37362 & \\
\hline 1749 & ONSITE & SESPSPEC & $S$ & B12RT9 & LAT/LONG & WGS84 & 46 & 3722 & \\
\hline
\end{tabular}




\begin{tabular}{|c|c|c|c|c|c|c|c|c|c|}
\hline & $A G$ & $\mathrm{AH}$ & $\mathrm{Al}$ & $\mathrm{AJ}$ & $\mathrm{AK}$ & $\mathrm{AL}$ & $\mathrm{AM}$ & AN & $\mathrm{AO}$ \\
\hline 1 & DIST_CLASS & OWNER_ID & COLLECTION_PURPOSE & X_Y_COORD_FINAL.SAMP_NUM & COORDINATE SYSTEM & DATUM & LATITUDE ${ }^{\circ} \mathrm{N}$ & LATITUDE MIN_MIN & LATITUDE MIN_SEC \\
\hline 1750 & ONSITE & SESPSPEC & S & B12RV1 & LAT/LONG & WGS84 & 46 & 37218 & \\
\hline 1751 & ONSITE & SESPSPEC & S & B12RV3 & LAT/LONG & WGS84 & 46 & 37223 & \\
\hline 1752 & ONSITE & SESPSPEC & S & B12RV5 & LAT/LONG & WGS84 & 46 & 37166 & \\
\hline 1753 & ONSITE & SESPSPEC & S & B12RV7 & LAT/LONG & WGS84 & 46 & 37168 & \\
\hline 1754 & ONSITE & SESPSPEC & S & B12RV9 & LAT/LONG & WGS84 & 46 & 37166 & \\
\hline 1755 & ONSITE & SESPSPEC & S & B12RW1 & LAT/LONG & WGS84 & 46 & 36953 & \\
\hline $1756 \mid$ & ONSITE & SESPSPEC & S & B12RW5 & LAT/LONG & WGS84 & 46 & 36714 & \\
\hline 1757 & ONSITE & SESPSPEC & S & B12RW7 & LAT/LONG & WGS84 & 46 & 36715 & \\
\hline 1758 & ONSITE & SESPSPEC & S & B12RW9 & LAT/LONG & WGS84 & 46 & 36714 & \\
\hline 1759 & ONSITE & SESPSPEC & S & & & & & & \\
\hline 1760 & ONSITE & SESPSPEC & S & & & & & & \\
\hline \begin{tabular}{|l|}
1761 \\
\end{tabular} & ONSITE & SESPSPEC & S & & & & & & \\
\hline 1762 & ONSITE & SESPSPEC & S & B12RX9 & LAT/LONG & WGS84 & 46 & 35556 & \\
\hline 1763 & ONSITE & SESPSPEC & S & B12RY1 & LAT/LONG & WGS84 & 46 & 35556 & \\
\hline 1764 & ONSITE & SESPSPEC & S & B12RY3 & LAT/LONG & WGS84 & 46 & 35557 & \\
\hline 1765 & ONSITE & SESPSPEC & S & B12T11 & LAT/LONG & WGS84 & 46 & 63453 & \\
\hline 1766 & ONSITE & SESPSPEC & S & B12T13 & LAT/LONG & WGS84 & 46 & 37661 & \\
\hline 1767 & ONSITE & SESPSPEC & S & B12T15 & LAT/LONG & WGS84 & 46 & 37546 & \\
\hline \begin{tabular}{|l|}
1768 \\
\end{tabular} & ONSITE & SESPSPEC & S & B12T17 & LAT/LONG & WGS84 & 46 & 37223 & \\
\hline 1769 & ONSITE & SESPSPEC & S & B12T19 & LAT/LONG & WGS84 & 46 & 37166 & \\
\hline 1770 & ONSITE & SESPSPEC & S & B12T21 & LAT/LONG & WGS84 & 46 & 36716 & \\
\hline \begin{tabular}{|l|}
1771 \\
\end{tabular} & ONSITE & SESPSPEC & S & & & & & & \\
\hline 1772 & ONSITE & SESPSPEC & S & B12T25 & LAT/LONG & WGS84 & 46 & 35558 & \\
\hline 1773 & OFFSITE & SESPMNT & $\mathrm{R}$ & & & & & & \\
\hline \begin{tabular}{|l|}
1774 \\
\end{tabular} & OFFSITE & SESPMNT & $\mathrm{R}$ & & & & & & \\
\hline 1775 & ONSITE & SESPSPEC & S & B12T85 & LAT/LONG & & 46 & & 41.01 .827 \\
\hline 1776 & OFFSITE & SESPMNT & $\mathrm{R}$ & B12T87 & LAT/LONG & & 46 & & 38.32 .516 \\
\hline 1777 & OFFSITE & SESPMNT & $\mathrm{R}$ & B12T88 & LAT/LONG & & 46 & & 38.36 .290 \\
\hline 1778 & OFFSITE & SESPMNT & $\mathrm{R}$ & B12T89 & LAT/LONG & & 46 & & 38.40 .091 \\
\hline 1779 & OFFSITE & SESPMNT & $\mathrm{R}$ & B12T90 & LAT/LONG & & 46 & & 38.42 .087 \\
\hline 1780 & ONSITE & SESPMNT & $\mathrm{R}$ & B12T91 & LAT/LONG & & 46 & & 41.01 .827 \\
\hline \begin{tabular}{|l|}
1781 \\
\end{tabular} & ONSITE & SESPMNT & $\mathrm{R}$ & B12T92 & LAT/LONG & & 46 & & 41.02 .142 \\
\hline \begin{tabular}{|l|}
1782 \\
\end{tabular} & ONSITE & SESPMNT & $\mathrm{R}$ & B12T93 & LAT/LONG & & 46 & & 41.8 .044 \\
\hline 1783 & ONSITE & SESPMNT & $\mathrm{R}$ & B12TD4 & LAT/LONG & & 46 & & 41.8.658 \\
\hline \begin{tabular}{|l|}
1784 \\
\end{tabular} & ONSITE & SESPMNT & $\mathrm{R}$ & B12TD5 & LAT/LONG & & 46 & & 41.03 .989 \\
\hline \begin{tabular}{|l|}
1785 \\
\end{tabular} & ONSITE & SESPMNT & $\mathrm{R}$ & B12TD6 & LAT/LONG & & 46 & & 41.8.070 \\
\hline \begin{tabular}{|l|}
1786 \\
\end{tabular} & ONSITE & SESPMNT & $\mathrm{R}$ & B12TN2 & LAT/LONG & & 46 & & 40.14 .547 \\
\hline \begin{tabular}{|l|}
1787 \\
\end{tabular} & ONSITE & SESPMNT & $\mathrm{R}$ & B12TN5 & LAT/LONG & & 46 & & 40.43 .042 \\
\hline
\end{tabular}




\begin{tabular}{|c|c|c|c|c|c|c|c|c|c|}
\hline & $A G$ & $\mathrm{AH}$ & $\mathrm{Al}$ & $\mathrm{AJ}$ & $\mathrm{AK}$ & $\mathrm{AL}$ & $\mathrm{AM}$ & AN & $\mathrm{AO}$ \\
\hline 1 & DIST_CLASS & OWNER_ID & COLLECTION_PURPOSE & X_Y_COORD_FINAL.SAMP_NUM & COORDINATE SYSTEM & DATUM & LATITUDE ${ }^{\circ} \mathrm{N}$ & LATITUDE MIN_MIN & LATITUDE MIN_SEC \\
\hline 1788 & ONSITE & SESPMNT & $\mathrm{R}$ & B12TN8 & LAT/LONG & & 46 & & 40.52 .424 \\
\hline 1789 & ONSITE & SESPMNT & $\mathrm{R}$ & B12TP1 & LAT/LONG & & 46 & & 41.17 .965 \\
\hline 1790 & ONSITE & SESPMNT & $\mathrm{R}$ & B12T94 & LAT/LONG & & 46 & & 39.35 .60 \\
\hline 1791 & ONSITE & SESPMNT & $\mathrm{R}$ & B12T95 & LAT/LONG & & 46 & & 39.39 .25 \\
\hline 1792 & ONSITE & SESPMNT & $\mathrm{R}$ & B12T96 & LAT/LONG & & 46 & & 33.38.38 \\
\hline 1793 & ONSITE & SESPMNT & $\mathrm{R}$ & B12T97 & LAT/LONG & & 46 & & 39.36.77 \\
\hline \begin{tabular}{|l|l|}
1794 \\
\end{tabular} & ONSITE & SESPMNT & $\mathrm{R}$ & B12T98 & LAT/LONG & & 46 & & 39.45 .22 \\
\hline 1795 & ONSITE & SESPMNT & $\mathrm{R}$ & B12T99 & LAT/LONG & & 46 & & 39.49 .98 \\
\hline 1796 & ONSITE & SESPMNT & $\mathrm{R}$ & B12TB0 & LAT/LONG & & 46 & & 39.51 .88 \\
\hline \begin{tabular}{|l|}
1797 \\
\end{tabular} & ONSITE & SESPMNT & $\mathrm{R}$ & B12TB1 & LAT/LONG & & 46 & & 33.38 .28 \\
\hline \begin{tabular}{|l|}
1798 \\
\end{tabular} & ONSITE & SESPMNT & $\mathrm{R}$ & B12TB2 & LAT/LONG & & 46 & & 33.38.08 \\
\hline 1799 & ONSITE & SESPMNT & $\mathrm{R}$ & B12TB3 & LAT/LONG & & 46 & & 33.41 .45 \\
\hline 1800 & ONSITE & SESPMNT & $\mathrm{R}$ & B12TB4 & LAT/LONG & & 46 & & 33.42 .84 \\
\hline 1801 & ONSITE & SESPMNT & $\mathrm{R}$ & B12TB5 & LAT/LONG & & 46 & & 33.37 .95 \\
\hline 1802 & ONSITE & SESPMNT & $\mathrm{R}$ & B12TF7 & LAT/LONG & & 46 & & 34.50 .68 \\
\hline 1803 & ONSITE & SESPMNT & $\mathrm{R}$ & B12TF8 & LAT/LONG & & 46 & & 34.21 .43 \\
\hline 1804 & ONSITE & SESPMNT & $\mathrm{R}$ & B12TF9 & LAT/LONG & & 46 & & 32.44 .93 \\
\hline 1805 & ONSITE & SESPMNT & $\mathrm{R}$ & B12TT0 & LAT/LONG & & 46 & & 33.57 .87 \\
\hline \begin{tabular}{|l|}
1806 \\
\end{tabular} & ONSITE & SESPMNT & $\mathrm{R}$ & B12TT3 & LAT/LONG & & 46 & & 40.10 .94 \\
\hline \begin{tabular}{|l|}
1807 \\
\end{tabular} & ONSITE & SESPMNT & $\mathrm{R}$ & B12TT9 & LAT/LONG & & 46 & & 37.2 .31 \\
\hline 1808 & ONSITE & SESPMNT & $\mathrm{R}$ & B12TB6 & LAT/LONG & & 46 & & 21.38.24 \\
\hline 1809 & ONSITE & SESPMNT & $\mathrm{R}$ & B12TB8 & LAT/LONG & & 46 & & 21.38.21 \\
\hline \begin{tabular}{|l|}
1810 \\
\end{tabular} & ONSITE & SESPMNT & $\mathrm{R}$ & B12TC0 & LAT/LONG & & 46 & & 21.37.47 \\
\hline 1811 & ONSITE & SESPMNT & $\mathrm{R}$ & B12TC2 & LAT/LONG & & 46 & & 21.38.45 \\
\hline \begin{tabular}{|l|}
1812 \\
\end{tabular} & ONSITE & SESPMNT & $\mathrm{R}$ & B12TC4 & LAT/LONG & & 46 & & 21.37.97 \\
\hline 1813 & ONSITE & SESPMNT & $\mathrm{R}$ & B12TC6 & LAT/LONG & & 46 & & 21.38.57 \\
\hline 1814 & OFFSITE & SESPMNT & $\mathrm{R}$ & & & & & & \\
\hline \begin{tabular}{|l|}
1815 \\
\end{tabular} & OFFSITE & SESPMNT & $\mathrm{R}$ & В12TC9 & LAT/LONG & & 46 & & 18.53.55 \\
\hline \begin{tabular}{|l|}
1816 \\
\end{tabular} & OFFSITE & SESPMNT & $\mathrm{R}$ & B12TD0 & LAT/LONG & & 46 & & 18.52 .78 \\
\hline 1817 & OFFSITE & SESPMNT & $\mathrm{R}$ & B12TD1 & LAT/LONG & & 46 & & 18.52 .99 \\
\hline \begin{tabular}{|l|}
1818 \\
\end{tabular} & OFFSITE & SESPMNT & $\mathrm{R}$ & B12TD2 & LAT/LONG & & 46 & & 18.51.17 \\
\hline \begin{tabular}{|l|}
1819 \\
\end{tabular} & OFFSITE & SESPMNT & $\mathrm{R}$ & B12TD3 & LAT/LONG & & 46 & & 18.52.48 \\
\hline \begin{tabular}{|l|}
1820 \\
\end{tabular} & OFFSITE & SESPMNT & $\mathrm{R}$ & B12TM0 & LAT/LONG & & 46 & & 19.25 .20 \\
\hline \begin{tabular}{|l|}
1821 \\
\end{tabular} & OFFSITE & SESPMNT & $\mathrm{R}$ & B12TM1 & LAT/LONG & & 46 & & 20.7.76 \\
\hline \begin{tabular}{|l|}
1822 \\
\end{tabular} & OFFSITE & SESPMNT & $\mathrm{R}$ & B12TM2 & LAT/LONG & & 46 & & 20.59 .083 \\
\hline 1823 & OFFSITE & SESPMNT & $\mathrm{R}$ & B12TM3 & LAT/LONG & & 46 & & 21.20 .88 \\
\hline \begin{tabular}{|l|}
1824 \\
\end{tabular} & ONSITE & SESPMNT & $\mathrm{R}$ & B12TP4 & LAT/LONG & & 46 & & 23.2.95 \\
\hline \begin{tabular}{|l|}
1825 \\
\end{tabular} & ONSITE & SESPMNT & $\mathrm{R}$ & B12TP8 & LAT/LONG & & 46 & & 22.35 .97 \\
\hline
\end{tabular}




\begin{tabular}{|c|c|c|c|c|c|c|c|c|c|}
\hline & $A G$ & $\mathrm{AH}$ & $\mathrm{Al}$ & $\mathrm{AJ}$ & $\mathrm{AK}$ & $\mathrm{AL}$ & $\mathrm{AM}$ & AN & $\mathrm{AO}$ \\
\hline 1 & DIST_CLASS & OWNER_ID & COLLECTION_PURPOSE & X_Y_COORD_FINAL.SAMP_NUM & COORDINATE SYSTEM & DATUM & LATITUDE ${ }^{\circ} \mathrm{N}$ & LATITUDE MIN_MIN & LATITUDE MIN_SEC \\
\hline 1826 & ONSITE & SESPMNT & $\mathrm{R}$ & B12TR2 & LAT/LONG & & 46 & & 22.15 .61 \\
\hline 1827 & ONSITE & SESPMNT & $\mathrm{R}$ & B12TR6 & LAT/LONG & & 46 & & 21.48 .54 \\
\hline 1828 & ONSITE & SESPMNT & $\mathrm{R}$ & B12XK2 & LAT/LONG & & 46 & & 22.20 .036 \\
\hline 1829 & ONSITE & SESPSPEC & S & B12XK8 & LAT/LONG & & 46 & & 22.20 .059 \\
\hline 1830 & ONSITE & SESPSPEC & S & B12XK9 & LAT/LONG & & 46 & & 22.20 .059 \\
\hline 1831 & ONSITE & SESPSPEC & S & B12XL0 & LAT/LONG & & 46 & & 22.20 .084 \\
\hline \begin{tabular}{|l|l|}
1832 \\
\end{tabular} & ONSITE & SESPSPEC & S & B12XL1 & LAT/LONG & & 46 & & 22.20 .084 \\
\hline 1833 & ONSITE & SESPSPEC & S & B12XL2 & LAT/LONG & & 46 & & 22.20 .031 \\
\hline 1834 & ONSITE & SESPMNT & $\mathrm{R}$ & B12XK1 & LAT/LONG & & 46 & & 22.36 .037 \\
\hline \begin{tabular}{|l|}
1835 \\
\end{tabular} & ONSITE & SESPSPEC & S & B12XK3 & LAT/LONG & & 46 & & 22.35 .976 \\
\hline 1836 & ONSITE & SESPSPEC & S & B12XK4 & LAT/LONG & & 46 & & 22.35.976 \\
\hline \begin{tabular}{|l|}
1837 \\
\end{tabular} & ONSITE & SESPSPEC & S & B12XK5 & LAT/LONG & & 46 & & 22.35.976 \\
\hline 1838 & ONSITE & SESPSPEC & $S$ & B12XK6 & LAT/LONG & & 46 & & 22.36 .045 \\
\hline 1839 & ONSITE & SESPSPEC & S & B12XK7 & LAT/LONG & & 46 & & 22.36.045 \\
\hline 1840 & OFFSITE & SESPMNT & $\mathrm{R}$ & & & & & & \\
\hline 1841 & OFFSITE & SESPMNT & $\mathrm{R}$ & & & & & & \\
\hline \begin{tabular}{|l|}
1842 \\
\end{tabular} & ONSITE & SESPMNT & $\mathrm{R}$ & & & & & & \\
\hline 1843 & ONSITE & SESPMNT & $\mathrm{R}$ & B12X48 & LAT/LONG & & 46 & & 42.41 .7 \\
\hline \begin{tabular}{|l|}
1844 \\
\end{tabular} & ONSITE & SESPMNT & $\mathrm{R}$ & B12X85 & LAT/LONG & & 46 & & 42.12 .8 \\
\hline \begin{tabular}{|l|}
1845 \\
\end{tabular} & OFFSITE & SESPMNT & $\mathrm{R}$ & & & & & & \\
\hline \begin{tabular}{|l|}
1846 \\
\end{tabular} & OFFSITE & SESPMNT & $\mathrm{R}$ & & & & & & \\
\hline \begin{tabular}{|l|}
1847 \\
\end{tabular} & ONSITE & SESPSPEC & S & & & & & & \\
\hline \begin{tabular}{|l|}
1848 \\
\end{tabular} & OFFSITE & SESPMNT & $\mathrm{R}$ & & & & & & \\
\hline 1849 & OFFSITE & SESPMNT & $\mathrm{R}$ & & & & & & \\
\hline 1850 & OFFSITE & SESPMNT & $\mathrm{R}$ & B13LF3 & LAT/LONG & & 46 & & 38.33.72 \\
\hline \begin{tabular}{|l|}
1851 \\
\end{tabular} & OFFSITE & SESPMNT & $\mathrm{R}$ & B13LF4 & LAT/LONG & & 46 & & 38.36.24 \\
\hline 1852 & OFFSITE & SESPMNT & $\mathrm{R}$ & B13LF5 & LAT/LONG & & 46 & & 38.40 .19 \\
\hline 1853 & OFFSITE & SESPMNT & $\mathrm{R}$ & B13LF6 & LAT/LONG & & 46 & & 38.42 .52 \\
\hline 1854 & OFFSITE & SESPSPEC & S & B13LF2 & LAT/LONG & & 46 & & 18.54 .62 \\
\hline 1855 & OFFSITE & SESPMNT & $\mathrm{R}$ & B13LF7 & LAT/LONG & & 46 & & 18.54 .62 \\
\hline \begin{tabular}{|l|}
1856 \\
\end{tabular} & OFFSITE & SESPMNT & $\mathrm{R}$ & B13LF8 & LAT/LONG & & 46 & & 18.52 .90 \\
\hline \begin{tabular}{|l|}
1857 \\
\end{tabular} & OFFSITE & SESPMNT & $\mathrm{R}$ & B13LF9 & LAT/LONG & & 46 & & 18.52 .68 \\
\hline \begin{tabular}{|l|}
1858 \\
\end{tabular} & OFFSITE & SESPMNT & $\mathrm{R}$ & B13LH0 & LAT/LONG & & 46 & & 18.53.97 \\
\hline 1859 & OFFSITE & SESPMNT & $\mathrm{R}$ & B13LH1 & LAT/LONG & & 46 & & 18.53 .50 \\
\hline \begin{tabular}{|l|}
1860 \\
\end{tabular} & OFFSITE & SESPMNT & $\mathrm{R}$ & B13LH2 & LAT/LONG & & 46 & & 19.52.07 \\
\hline \begin{tabular}{|l|}
1861 \\
\end{tabular} & OFFSITE & SESPMNT & $\mathrm{R}$ & B13LJ3 & LAT/LONG & & 46 & & 19.24.77 \\
\hline \begin{tabular}{|l|}
1862 \\
\end{tabular} & OFFSITE & SESPMNT & $\mathrm{R}$ & B13LJ4 & LAT/LONG & & 46 & & 20.07 .46233 \\
\hline 1863 & OFFSITE & SESPMNT & $\mathrm{R}$ & B13LJ5 & LAT/LONG & & 46 & & 20.59 .29 \\
\hline
\end{tabular}




\begin{tabular}{|c|c|c|c|c|c|c|c|c|c|}
\hline & $A G$ & $\mathrm{AH}$ & $\mathrm{Al}$ & $\mathrm{AJ}$ & $\mathrm{AK}$ & $\mathrm{AL}$ & $\mathrm{AM}$ & AN & $\mathrm{AO}$ \\
\hline 1 & DIST_CLASS & OWNER_ID & COLLECTION_PURPOSE & X_Y_COORD_FINAL.SAMP_NUM & COORDINATE SYSTEM & DATUM & LATITUDE ${ }^{\circ} \mathrm{N}$ & LATITUDE MIN_MIN & LATITUDE MIN_SEC \\
\hline 1864 & OFFSITE & SESPMNT & $\mathrm{R}$ & B13LJ6 & LAT/LONG & & 46 & & 21.21 .83 \\
\hline 1865 & OFFSITE & SESPMNT & $\mathrm{R}$ & & & & & & \\
\hline 1866 & OFFSITE & SESPMNT & $\mathrm{R}$ & & & & & & \\
\hline 1867 & OFFSITE & SESPMNT & $\mathrm{R}$ & & & & & & \\
\hline 1868 & OFFSITE & SESPMNT & $\mathrm{R}$ & & & & & & \\
\hline 1869 & OFFSITE & SESPMNT & $\mathrm{R}$ & & & & & & \\
\hline 1870 & OFFSITE & SESPMNT & $\mathrm{R}$ & & & & & & \\
\hline 1871 & OFFSITE & SESPMNT & $\mathrm{R}$ & B147T3 & LAT/LONG & & 46 & & 38.32 .125 \\
\hline 1872 & OFFSITE & SESPMNT & $\mathrm{R}$ & B147T4 & LAT/LONG & & 46 & & 38.33.902 \\
\hline 1873 & OFFSITE & SESPMNT & $\mathrm{R}$ & B147T5 & LAT/LONG & & 46 & & 38.40 .586 \\
\hline 1874 & OFFSITE & SESPMNT & $\mathrm{R}$ & B147T6 & LAT/LONG & & 46 & & 38.41 .856 \\
\hline 1875 & OFFSITE & SESPSPEC & S & B147T2 & LAT/LONG & & 46 & & 18.54 .797 \\
\hline 1876 & OFFSITE & SESPMNT & $\mathrm{R}$ & B147T7 & LAT/LONG & & 46 & & 18.54.797 \\
\hline 1877 & OFFSITE & SESPMNT & $\mathrm{R}$ & B147T8 & LAT/LONG & & 46 & & 18.53.211 \\
\hline 1878 & OFFSITE & SESPMNT & $\mathrm{R}$ & B147T9 & LAT/LONG & & 46 & & 18.52.338 \\
\hline 1879 & OFFSITE & SESPMNT & $\mathrm{R}$ & B147V0 & LAT/LONG & & 46 & & 18.52 .299 \\
\hline 1880 & OFFSITE & SESPMNT & $\mathrm{R}$ & B147V1 & LAT/LONG & & 46 & & 18.51 .346 \\
\hline 1881 & OFFSITE & SESPMNT & $\mathrm{R}$ & B147V2 & LAT/LONG & & 46 & & 18.50 .697 \\
\hline \begin{tabular}{|l}
1882 \\
\end{tabular} & OFFSITE & SESPMNT & $\mathrm{R}$ & B147W3 & LAT/LONG & & 46 & & 19.24 .739 \\
\hline 1883 & OFFSITE & SESPMNT & $\mathrm{R}$ & B147W4 & LAT/LONG & & 46 & & 20.7 .138 \\
\hline 1884 & OFFSITE & SESPMNT & $\mathrm{R}$ & B147W5 & LAT/LONG & & 46 & & 20.59 .129 \\
\hline 1885 & OFFSITE & SESPMNT & $\mathrm{R}$ & B147W6 & LAT/LONG & & 46 & & 21.20.938 \\
\hline 1886 & OFFSITE & SESPMNT & $\mathrm{R}$ & & & & & & \\
\hline 1887 & OFFSITE & SESPMNT & $\mathrm{R}$ & & & & & & \\
\hline 1888 & OFFSITE & SESPMNT & $\mathrm{R}$ & & & & & & \\
\hline 1889 & OFFSITE & SESPMNT & $\mathrm{R}$ & & & & & & \\
\hline 1890 & OFFSITE & SESPMNT & $\mathrm{R}$ & B14LJ0 & LAT/LONG & & 46 & 25832 & \\
\hline 1891 & PERIMETER & SESPMNT & $\mathrm{R}$ & B14LJ2 & LAT/LONG & & 46 & 34120 & \\
\hline 1892 & OFFSITE & SESPMNT & $\mathrm{R}$ & & & & & & \\
\hline 1893 & OFFSITE & SESPMNT & $\mathrm{R}$ & & & & & & \\
\hline 1894 & OFFSITE & SESPMNT & $\mathrm{R}$ & B14RV4 & LAT/LONG & & 46 & & 38.32.346 \\
\hline 1895 & OFFSITE & SESPMNT & $\mathrm{R}$ & B14RV5 & LAT/LONG & & 46 & & 38.35 .710 \\
\hline 1896 & OFFSITE & SESPMNT & $\mathrm{R}$ & B14RV6 & LAT/LONG & & 46 & & 38.40 .744 \\
\hline 1897 & OFFSITE & SESPMNT & $\mathrm{R}$ & B14RV7 & LAT/LONG & & 46 & & 38.43 .263 \\
\hline 1898 & OFFSITE & SESPMNT & $\mathrm{R}$ & B14RV8 & LAT/LONG & & 46 & & 18.54 .564 \\
\hline 1899 & OFFSITE & SESPMNT & $\mathrm{R}$ & B14RV9 & LAT/LONG & & 46 & & 18.50 .434 \\
\hline 1900 & OFFSITE & SESPMNT & $\mathrm{R}$ & B14RW0 & LAT/LONG & & 46 & & 18.51 .872 \\
\hline 1901 & OFFSITE & SESPMNT & $\mathrm{R}$ & B14RW1 & LAT/LONG & & 46 & & 18.52.771 \\
\hline
\end{tabular}

Page 518 


\begin{tabular}{|c|c|c|c|c|c|c|c|c|c|}
\hline & $A G$ & $\mathrm{AH}$ & $\mathrm{Al}$ & $\mathrm{AJ}$ & AK & $\mathrm{AL}$ & AM & $\mathrm{AN}$ & $\mathrm{AO}$ \\
\hline 1 & DIST_CLASS & OWNER_ID & COLLECTION_PURPOSE & X_Y_COORD_FINAL.SAMP_NUM & COORDINATE SYSTEM & \begin{tabular}{|l|} 
DATUM \\
\end{tabular} & LATITUDE ${ }^{\circ} \mathrm{N}$ & LATITUDE MIN_MIN & LATITUDE MIN_SEC \\
\hline 1902 & OFFSITE & SESPMNT & $\mathrm{R}$ & B14RW2 & LAT/LONG & & 46 & & 18.48 .011 \\
\hline 1903 & OFFSITE & SESPMNT & $\mathrm{R}$ & B14RW3 & LAT/LONG & & 46 & & 18.52 .018 \\
\hline 1904 & OFFSITE & SESPMNT & $\mathrm{R}$ & B14RX4 & LAT/LONG & & 46 & & 19.24 .503 \\
\hline 1905 & OFFSITE & SESPMNT & $\mathrm{R}$ & B14RX5 & LAT/LONG & & 46 & & 20.06.731 \\
\hline 1906 & OFFSITE & SESPMNT & $\mathrm{R}$ & B14RX6 & LAT/LONG & & 46 & & 20.59 .569 \\
\hline 1907 & OFFSITE & SESPMNT & $\mathrm{R}$ & B14RX7 & LAT/LONG & & 46 & & 21.21.521 \\
\hline 1908 & OFFSITE & SESPMNT & $\mathrm{R}$ & & & & & & \\
\hline 1909 & OFFSITE & SESPMNT & $\mathrm{R}$ & & & & & & \\
\hline 1910 & OFFSITE & SESPMNT & $\mathrm{R}$ & & & & & & \\
\hline 1911 & PERIMETER & SESPMNT & $\mathrm{R}$ & & & & & & \\
\hline 1912 & OFFSITE & SESPMNT & $\mathrm{R}$ & & & & & & \\
\hline 1913 & PERIMETER & SESPMNT & $\mathrm{R}$ & & & & & & \\
\hline 1914 & OFFSITE & SESPMNT & $\mathrm{R}$ & & & & & & \\
\hline 1915 & OFFSITE & SESPMNT & $\mathrm{R}$ & & & & & & \\
\hline 1916 & OFFSITE & SESPMNT & $\mathrm{R}$ & & & & & & \\
\hline 1917 & OFFSITE & SESPMNT & $\mathrm{R}$ & & & & & & \\
\hline 1918 & ONSITE & SESPSPEC & S & B158H3 & LAT/LONG & & 46 & & 41.02 .29 \\
\hline 1919 & OFFSITE & SESPMNT & $\mathrm{R}$ & B158H4 & LAT/LONG & & 46 & & 38.42 .82 \\
\hline 1920 & OFFSITE & SESPMNT & $R$ & B158H5 & LAT/LONG & & 46 & & 38.35 .52 \\
\hline 1921 & OFFSITE & SESPMNT & $\mathrm{R}$ & B158H6 & LAT/LONG & & 46 & & 38.39 .47 \\
\hline 1922 & OFFSITE & SESPMNT & $\mathrm{R}$ & B158H7 & LAT/LONG & & 46 & & 38.31 .26 \\
\hline 1923 & ONSITE & SESPMNT & $\mathrm{R}$ & B158H8 & LAT/LONG & & 46 & & 41.02 .29 \\
\hline 1924 & ONSITE & SESPMNT & $\mathrm{R}$ & B158H9 & LAT/LONG & & 46 & & 41.03 .77 \\
\hline 1925 & ONSITE & SESPMNT & $\mathrm{R}$ & B158J0 & LAT/LONG & & 46 & & 41.11 .21 \\
\hline 1926 & ONSITE & SESPMNT & $\mathrm{R}$ & B158M7 & LAT/LONG & & 46 & & 41.10 .20 \\
\hline 1927 & ONSITE & SESPMNT & $\mathrm{R}$ & B158M8 & LAT/LONG & & 46 & & 41.06 .12 \\
\hline 1928 & ONSITE & SESPMNT & $\mathrm{R}$ & B158M9 & LAT/LONG & & 46 & & 41.09 .52 \\
\hline 1929 & ONSITE & SESPMNT & $\mathrm{R}$ & B158W8 & LAT/LONG & & 46 & & 40.14 .09 \\
\hline 1930 & ONSITE & SESPMNT & $\mathrm{R}$ & B158X1 & LAT/LONG & & 46 & & 40.43 .18 \\
\hline 1931 & ONSITE & SESPMNT & $\mathrm{R}$ & B158X4 & LAT/LONG & & 46 & & 40.51 .95 \\
\hline 1932 & ONSITE & SESPMNT & $\mathrm{R}$ & B158X7 & LAT/LONG & & 46 & & 41.18 .54 \\
\hline 1933 & ONSITE & SESPMNT & $\mathrm{R}$ & B158J1 & LAT/LONG & & 46 & & 39.35 .36 \\
\hline 1934 & ONSITE & SESPMNT & $\mathrm{R}$ & B158J3 & LAT/LONG & & 46 & & 39.39 .60 \\
\hline 1935 & ONSITE & SESPMNT & $\mathrm{R}$ & B158J5 & LAT/LONG & & 46 & & 33.39 .50 \\
\hline 1936 & ONSITE & SESPMNT & $\mathrm{R}$ & B158J7 & LAT/LONG & & 46 & & 39.37 .16 \\
\hline 1937 & ONSITE & SESPMNT & $\mathrm{R}$ & B158J9 & LAT/LONG & & 46 & & 39.44 .48 \\
\hline 1938 & ONSITE & SESPMNT & $\mathrm{R}$ & B158K1 & LAT/LONG & & 46 & & 39.48 .58 \\
\hline 1939 & ONSITE & SESPMNT & $\mathrm{R}$ & B158K3 & LAT/LONG & & 46 & & 39.51 .94 \\
\hline
\end{tabular}

Page 519 


\begin{tabular}{|c|c|c|c|c|c|c|c|c|c|}
\hline & $A G$ & $\mathrm{AH}$ & $\mathrm{Al}$ & $\mathrm{AJ}$ & AK & $\mathrm{AL}$ & AM & $\mathrm{AN}$ & $\mathrm{AO}$ \\
\hline 1 & DIST_CLASS & OWNER_ID & COLLECTION_PURPOSE & X_Y_COORD_FINAL.SAMP_NUM & COORDINATE SYSTEM & \begin{tabular}{|l|} 
DATUM \\
\end{tabular} & LATITUDE ${ }^{\circ} \mathrm{N}$ & LATITUDE MIN_MIN & LATITUDE MIN_SEC \\
\hline 1940 & ONSITE & SESPMNT & $\mathrm{R}$ & B158K5 & LAT/LONG & & 46 & & 33.40 .64 \\
\hline 1941 & ONSITE & SESPMNT & $\mathrm{R}$ & B158K7 & LAT/LONG & & 46 & & 33.40 .63 \\
\hline 1942 & ONSITE & SESPMNT & $\mathrm{R}$ & B158K9 & LAT/LONG & & 46 & & 33.42 .44 \\
\hline 1943 & ONSITE & SESPMNT & $\mathrm{R}$ & B158L1 & LAT/LONG & & 46 & & 33.43 .598 \\
\hline 1944 & ONSITE & SESPMNT & $\mathrm{R}$ & B158L3 & LAT/LONG & & 46 & & 33.40 .47 \\
\hline 1945 & ONSITE & SESPMNT & $\mathrm{R}$ & B158N0 & LAT/LONG & & 46 & & 34.50 .32 \\
\hline 1946 & ONSITE & SESPMNT & $\mathrm{R}$ & B158N2 & LAT/LONG & & 46 & & 34.21 .43 \\
\hline 1947 & ONSITE & SESPMNT & $\mathrm{R}$ & B158N4 & LAT/LONG & & 46 & & 32.44 .22 \\
\hline 1948 & ONSITE & SESPMNT & $\mathrm{R}$ & B15902 & LAT/LONG & & 46 & & 33.57 .46 \\
\hline 1949 & ONSITE & SESPMNT & $\mathrm{R}$ & B15906 & LAT/LONG & & 46 & & 40.10 .84 \\
\hline 1950 & ONSITE & SESPMNT & $\mathrm{R}$ & B15910 & LAT/LONG & & 46 & & 36.22 .01 \\
\hline 1951 & ONSITE & SESPMNT & $\mathrm{R}$ & B15914 & LAT/LONG & & 46 & & 37.02 .66 \\
\hline 1952 & ONSITE & SESPMNT & $\mathrm{R}$ & B158L5 & LAT/LONG & & 46 & & 21.37 .20 \\
\hline 1953 & ONSITE & SESPMNT & $\mathrm{R}$ & B158L6 & LAT/LONG & & 46 & & 21.36 .55 \\
\hline 1954 & ONSITE & SESPMNT & $\mathrm{R}$ & B158L7 & LAT/LONG & & 46 & & 21.35 .70 \\
\hline 1955 & ONSITE & SESPMNT & $\mathrm{R}$ & B158L8 & LAT/LONG & & 46 & & 21.37 .67 \\
\hline 1956 & ONSITE & SESPMNT & $\mathrm{R}$ & B158L9 & LAT/LONG & & 46 & & 21.38.28 \\
\hline 1957 & ONSITE & SESPMNT & $\mathrm{R}$ & B158M0 & LAT/LONG & & 46 & & 21.38 .48 \\
\hline 1958 & OFFSITE & SESPMNT & $\mathrm{R}$ & B158M1 & LAT/LONG & & 46 & & 18.54 .5 \\
\hline 1959 & OFFSITE & SESPMNT & $\mathrm{R}$ & B158M2 & LAT/LONG & & 46 & & 18.52 .7 \\
\hline 1960 & OFFSITE & SESPMNT & $\mathrm{R}$ & B158M3 & LAT/LONG & & 46 & & 18.85 .0 \\
\hline 1961 & OFFSITE & SESPMNT & $\mathrm{R}$ & B158M4 & LAT/LONG & & 46 & & 18.51 .50 \\
\hline 1962 & OFFSITE & SESPMNT & $\mathrm{R}$ & B158M5 & LAT/LONG & & 46 & & 18.51 .37 \\
\hline 1963 & OFFSITE & SESPMNT & $\mathrm{R}$ & B158M6 & LAT/LONG & & 46 & & 18.52 .36 \\
\hline 1964 & OFFSITE & SESPMNT & $\mathrm{R}$ & B158V6 & LAT/LONG & & 46 & & 19.24.75 \\
\hline 1965 & OFFSITE & SESPMNT & $\mathrm{R}$ & B158V7 & LAT/LONG & & 46 & & 20.07.17 \\
\hline 1966 & OFFSITE & SESPMNT & $\mathrm{R}$ & B158V8 & LAT/LONG & & 46 & & 20.58 .56 \\
\hline 1967 & OFFSITE & SESPMNT & $\mathrm{R}$ & B158V9 & LAT/LONG & & 46 & & 21.21 .49 \\
\hline 1968 & ONSITE & SESPMNT & $\mathrm{R}$ & B158Y0 & LAT/LONG & & 46 & & 23.03 .00 \\
\hline 1969 & ONSITE & SESPMNT & $\mathrm{R}$ & B158Y3 & LAT/LONG & & 46 & & 22.35 .44 \\
\hline 1970 & ONSITE & SESPMNT & $\mathrm{R}$ & B158Y6 & LAT/LONG & & 46 & & 22.19 .60 \\
\hline 1971 & ONSITE & SESPMNT & $\mathrm{R}$ & B158Y9 & LAT/LONG & & 46 & & 21.48 .39 \\
\hline 1972 & OFFSITE & SESPMNT & $\mathrm{R}$ & & & & & & \\
\hline 1973 & OFFSITE & SESPMNT & $\mathrm{R}$ & & & & & & \\
\hline 1974 & ONSITE & SESPMNT & $\mathrm{R}$ & B15C09 & LAT/LONG & & 46 & & 33.55 .313 \\
\hline 1975 & ONSITE & SESPMNT & $\mathrm{R}$ & & & & & & \\
\hline 1976 & ONSITE & SESPMNT & $\mathrm{R}$ & B15C73 & LAT/LONG & & 46 & & 33.50 .499 \\
\hline 1977 & ONSITE & SESPMNT & $\mathrm{R}$ & B15CB3 & LAT/LONG & & 46 & & 42.12 .884 \\
\hline
\end{tabular}

Page 520 


\begin{tabular}{|c|c|c|c|c|c|c|c|c|c|}
\hline & $\mathrm{AG}$ & $\mathrm{AH}$ & $\mathrm{Al}$ & $\mathrm{AJ}$ & $\mathrm{AK}$ & $\mathrm{AL}$ & $\mathrm{AM}$ & AN & $\mathrm{AO}$ \\
\hline 1 & DIST_CLASS & OWNER_ID & COLLECTION_PURPOSE & X_Y_COORD_FINAL.SAMP_NUM & COORDINATE SYSTEM & DATUM & LATITUDE ${ }^{\circ} \mathrm{N}$ & LATITUDE MIN_MIN & LATITUDE MIN_SEC \\
\hline 1978 & ONSITE & SESPMNT & $\mathrm{R}$ & B15C69 & LAT/LONG & & 46 & & 38.14 .440 \\
\hline 1979 & OFFSITE & SESPMNT & $\mathrm{R}$ & & & & & & \\
\hline 1980 & OFFSITE & SESPMNT & $\mathrm{R}$ & & & & & & \\
\hline 1981 & OFFSITE & SESPMNT & $\mathrm{R}$ & & & & & & \\
\hline 1982 & OFFSITE & SESPMNT & $\mathrm{R}$ & & & & & & \\
\hline \begin{tabular}{|l|}
1983 \\
\end{tabular} & OFFSITE & SESPMNT & $\mathrm{R}$ & B16342 & LAT/LONG & & 46 & 38544193 & \\
\hline 1984 & OFFSITE & SESPMNT & $\mathrm{R}$ & B16343 & LAT/LONG & & 46 & 3861303 & \\
\hline 1985 & OFFSITE & SESPMNT & $\mathrm{R}$ & B16344 & LAT/LONG & & 46 & 384081025 & \\
\hline 1986 & OFFSITE & SESPMNT & $\mathrm{R}$ & B16345 & LAT/LONG & & 46 & 384290077 & \\
\hline 1987 & OFFSITE & SESPSPEC & S & B16341 & LAT/LONG & & 46 & 185442945 & \\
\hline 1988 & OFFSITE & SESPMNT & $\mathrm{R}$ & B16346 & LAT/LONG & & 46 & 185442945 & \\
\hline 1989 & OFFSITE & SESPMNT & $\mathrm{R}$ & B16347 & LAT/LONG & & 46 & 18519365 & \\
\hline 1990 & OFFSITE & SESPMNT & $\mathrm{R}$ & B16348 & LAT/LONG & & 46 & 184989755 & \\
\hline 1991 & OFFSITE & SESPMNT & $\mathrm{R}$ & B16349 & LAT/LONG & & 46 & 185086976 & \\
\hline 1992 & OFFSITE & SESPMNT & $\mathrm{R}$ & B16350 & LAT/LONG & & 46 & 185163798 & \\
\hline 1993 & OFFSITE & SESPMNT & $\mathrm{R}$ & B16351 & LAT/LONG & & 46 & 185184158 & \\
\hline 1994 & OFFSITE & SESPMNT & $\mathrm{R}$ & B16362 & LAT/LONG & & 46 & 192473133 & \\
\hline 1995 & OFFSITE & SESPMNT & $\mathrm{R}$ & B16363 & LAT/LONG & & 46 & 200718556 & \\
\hline 1996 & OFFSITE & SESPMNT & $\mathrm{R}$ & B16364 & LAT/LONG & & 46 & 205947829 & \\
\hline 1997 & OFFSITE & SESPMNT & $\mathrm{R}$ & B16365 & LAT/LONG & & 46 & 212135241 & \\
\hline 1998 & ONSITE & SESPMNT & $\mathrm{R}$ & & & & & & \\
\hline 1999 & OFFSITE & SESPMNT & $\mathrm{R}$ & & & & & & \\
\hline 2000 & OFFSITE & SESPMNT & $\mathrm{R}$ & & & & & & \\
\hline 2001 & OFFSITE & SESPMNT & $\mathrm{R}$ & & & & & & \\
\hline 2002 & OFFSITE & SESPMNT & $\mathrm{R}$ & & & & & & \\
\hline 2003 & OFFSITE & SESPMNT & $\mathrm{R}$ & & & & & & \\
\hline 2004 & OFFSITE & SESPMNT & $\mathrm{R}$ & & & & & & \\
\hline 2005 & OFFSITE & SESPMNT & $\mathrm{R}$ & & & & & & \\
\hline 2006 & OFFSITE & SESPMNT & $\mathrm{R}$ & & & & & & \\
\hline 2007 & OFFSITE & SESPSPEC & S & & & & & & \\
\hline 2008 & OFFSITE & SESPMNT & $\mathrm{R}$ & & & & & & \\
\hline 2009 & OFFSITE & SESPMNT & $\mathrm{R}$ & & & & & & \\
\hline 2010 & OFFSITE & SESPMNT & $\mathrm{R}$ & & & & & & \\
\hline 2011 & OFFSITE & SESPMNT & $\mathrm{R}$ & & & & & & \\
\hline 2012 & OFFSITE & SESPMNT & $\mathrm{R}$ & & & & & & \\
\hline 2013 & OFFSITE & SESPMNT & $\mathrm{R}$ & & & & & & \\
\hline 2014 & OFFSITE & SESPMNT & $\mathrm{R}$ & & & & & & \\
\hline 2015 & OFFSITE & SESPMNT & $\mathrm{R}$ & & & & & & \\
\hline
\end{tabular}




\begin{tabular}{|c|c|c|c|c|c|c|c|c|c|}
\hline & $A G$ & $\mathrm{AH}$ & $\mathrm{Al}$ & $\mathrm{AJ}$ & $\mathrm{AK}$ & $\mathrm{AL}$ & $\mathrm{AM}$ & AN & $\mathrm{AO}$ \\
\hline 1 & DIST_CLASS & OWNER_ID & COLLECTION_PURPOSE & X_Y_COORD_FINAL.SAMP_NUM & COORDINATE SYSTEM & DATUM & LATITUDE ${ }^{\circ} \mathrm{N}$ & LATITUDE MIN_MIN & LATITUDE MIN_SEC \\
\hline 2016 & OFFSITE & SESPMNT & $\mathrm{R}$ & & & & & & \\
\hline 2017 & OFFSITE & SESPMNT & $\mathrm{R}$ & & & & & & \\
\hline 2018 & ONSITE & SESPMNT & $\mathrm{R}$ & & & & & & \\
\hline 2019 & OFFSITE & SESPMNT & $\mathrm{R}$ & & & & & & \\
\hline 2020 & OFFSITE & SESPMNT & $\mathrm{R}$ & & & & & & \\
\hline 2021 & OFFSITE & SESPMNT & $\mathrm{R}$ & & & & & & \\
\hline 2022 & OFFSITE & SESPMNT & $\mathrm{R}$ & & & & & & \\
\hline 2023 & OFFSITE & SESPMNT & $\mathrm{R}$ & & & & & & \\
\hline 2024 & OFFSITE & SESPMNT & $\mathrm{R}$ & & & & & & \\
\hline 2025 & OFFSITE & SESPMNT & $\mathrm{R}$ & & & & & & \\
\hline 2026 & OFFSITE & SESPMNT & $\mathrm{R}$ & & & & & & \\
\hline 2027 & ONSITE & SESPSPEC & S & & & & & & \\
\hline 2028 & ONSITE & SESPSPEC & S & & & & & & \\
\hline 2029 & ONSITE & SESPSPEC & S & & & & & & \\
\hline 2030 & OFFSITE & SESPMNT & $\mathrm{R}$ & BOYCD7 & STATE PLANE & & & & \\
\hline 2031 & OFFSITE & SESPMNT & $\mathrm{R}$ & B0YCD8 & STATE PLANE & & & & \\
\hline 2032 & OFFSITE & SESPMNT & $\mathrm{R}$ & BOYCD9 & STATE PLANE & & & & \\
\hline 2033 & OFFSITE & SESPMNT & $\mathrm{R}$ & BOYCFO & STATE PLANE & & & & \\
\hline \begin{tabular}{|l}
2034 \\
\end{tabular} & OFFSITE & SESPMNT & $\mathrm{R}$ & BOYCF1 & STATE PLANE & & & & \\
\hline 2035 & OFFSITE & SESPMNT & $\mathrm{R}$ & BOYCF2 & STATE PLANE & & & & \\
\hline 2036 & OFFSITE & SESPMNT & $\mathrm{R}$ & BOYCF3 & STATE PLANE & & & & \\
\hline 2037 & OFFSITE & SESPMNT & $\mathrm{R}$ & BOYCF4 & STATE PLANE & & & & \\
\hline 2038 & OFFSITE & SESPMNT & $\mathrm{R}$ & B0YCF5 & STATE PLANE & & & & \\
\hline 2039 & OFFSITE & SESPMNT & $\mathrm{R}$ & BOYCF6 & STATE PLANE & & & & \\
\hline 2040 & OFFSITE & SESPMNT & $\mathrm{R}$ & BOYCK3 & STATE PLANE & & & & \\
\hline 2041 & OFFSITE & SESPMNT & $\mathrm{R}$ & BOYCK4 & STATE PLANE & & & & \\
\hline 2042 & OFFSITE & SESPMNT & $\mathrm{R}$ & BOYCK5 & STATE PLANE & & & & \\
\hline 2043 & OFFSITE & SESPMNT & $\mathrm{R}$ & BOYCK6 & STATE PLANE & & & & \\
\hline 2044 & OFFSITE & SESPMNT & $\mathrm{R}$ & & & & & & \\
\hline 2045 & OFFSITE & SESPMNT & $\mathrm{R}$ & & & & & & \\
\hline 2046 & ONSITE & SESPMNT & $\mathrm{R}$ & & & & & & \\
\hline 2047 & OFFSITE & SESPMNT & $\mathrm{R}$ & & & & & & \\
\hline 2048 & OFFSITE & SESPMNT & $\mathrm{R}$ & & & & & & \\
\hline 2049 & OFFSITE & SESPMNT & $\mathrm{R}$ & & & & & & \\
\hline 2050 & OFFSITE & SESPMNT & $\mathrm{R}$ & & & & & & \\
\hline 2051 & OFFSITE & SESPMNT & $\mathrm{R}$ & & & & & & \\
\hline 2052 & ONSITE & SESPSPEC & S & B10672 & STATE PLANE & & & & \\
\hline 2053 & OFFSITE & SESPMNT & $\mathrm{R}$ & B10673 & STATE PLANE & & & & \\
\hline
\end{tabular}




\begin{tabular}{|c|c|c|c|c|c|c|c|c|c|}
\hline & $A G$ & $\mathrm{AH}$ & $\mathrm{Al}$ & $\mathrm{AJ}$ & AK & $\mathrm{AL}$ & $\mathrm{AM}$ & AN & $\mathrm{AO}$ \\
\hline 1 & DIST_CLASS & OWNER_ID & COLLECTION_PURPOSE & X_Y_COORD_FINAL.SAMP_NUM & COORDINATE SYSTEM & DATUM & LATITUDE ${ }^{\circ} \mathrm{N}$ & LATITUDE MIN_MIN & LATITUDE MIN_SEC \\
\hline 2054 & OFFSITE & SESPMNT & $\mathrm{R}$ & B10674 & STATE PLANE & & & & \\
\hline 2055 & OFFSITE & SESPMNT & $\mathrm{R}$ & B10675 & STATE PLANE & & & & \\
\hline 2056 & OFFSITE & SESPMNT & $\mathrm{R}$ & B10676 & STATE PLANE & & & & \\
\hline 2057 & ONSITE & SESPMNT & $\mathrm{R}$ & B10677 & STATE PLANE & & & & \\
\hline 2058 & ONSITE & SESPMNT & $\mathrm{R}$ & B10679 & STATE PLANE & & & & \\
\hline 2059 & ONSITE & SESPMNT & $\mathrm{R}$ & & & & & & \\
\hline 2060 & ONSITE & SESPMNT & $\mathrm{R}$ & B10683 & STATE PLANE & & & & \\
\hline 2061 & ONSITE & SESPMNT & $\mathrm{R}$ & B10684 & STATE PLANE & & & & \\
\hline \begin{tabular}{|l|l|}
2062 \\
2062
\end{tabular} & ONSITE & SESPMNT & $\mathrm{R}$ & & & & & & \\
\hline 2063 & ONSITE & SESPMNT & $\mathrm{R}$ & & & & & & \\
\hline 2064 & ONSITE & SESPMNT & $\mathrm{R}$ & B106B1 & STATE PLANE & & & & \\
\hline 2065 & ONSITE & SESPMNT & $\mathrm{R}$ & & & & & & \\
\hline 2066 & ONSITE & SESPMNT & $\mathrm{R}$ & B106B7 & STATE PLANE & & & & \\
\hline 2067 & ONSITE & SESPMNT & $\mathrm{R}$ & B106W1 & STATE PLANE & & & & \\
\hline 2068 & ONSITE & SESPMNT & $\mathrm{R}$ & B106W2 & STATE PLANE & & & & \\
\hline 2069 & ONSITE & SESPMNT & $\mathrm{R}$ & B106W3 & STATE PLANE & & & & \\
\hline 2070 & ONSITE & SESPMNT & $\mathrm{R}$ & B106W4 & STATE PLANE & & & & \\
\hline 2071 & ONSITE & SESPMNT & $\mathrm{R}$ & B106W5 & STATE PLANE & & & & \\
\hline 2072 & ONSITE & SESPMNT & $\mathrm{R}$ & B106W6 & STATE PLANE & & & & \\
\hline 2073 & ONSITE & SESPMNT & $\mathrm{R}$ & B106W7 & STATE PLANE & & & & \\
\hline 2074 & ONSITE & SESPMNT & $\mathrm{R}$ & B106W8 & STATE PLANE & & & & \\
\hline 2075 & ONSITE & SESPMNT & $\mathrm{R}$ & B106W9 & STATE PLANE & & & & \\
\hline 2076 & ONSITE & SESPMNT & $\mathrm{R}$ & B106X0 & STATE PLANE & & & & \\
\hline 2077 & ONSITE & SESPMNT & $\mathrm{R}$ & B106X1 & STATE PLANE & & & & \\
\hline 2078 & ONSITE & SESPMNT & $\mathrm{R}$ & B106X2 & STATE PLANE & & & & \\
\hline 2079 & ONSITE & SESPMNT & $\mathrm{R}$ & B10728 & STATE PLANE & & & & \\
\hline 2080 & ONSITE & SESPMNT & $\mathrm{R}$ & B10729 & STATE PLANE & & & & \\
\hline 2081 & ONSITE & SESPMNT & $\mathrm{R}$ & B10730 & STATE PLANE & & & & \\
\hline 2082 & ONSITE & SESPMNT & $\mathrm{R}$ & B10785 & STATE PLANE & & & & \\
\hline 2083 & ONSITE & SESPMNT & $\mathrm{R}$ & B10788 & STATE PLANE & & & & \\
\hline 2084 & ONSITE & SESPMNT & $\mathrm{R}$ & B10791 & STATE PLANE & & & & \\
\hline 2085 & ONSITE & SESPMNT & $\mathrm{R}$ & B10794 & STATE PLANE & & & & \\
\hline 2086 & ONSITE & SESPMNT & $\mathrm{R}$ & & & & & & \\
\hline 2087 & ONSITE & SESPMNT & $\mathrm{R}$ & & & & & & \\
\hline 2088 & ONSITE & SESPMNT & $\mathrm{R}$ & B106X7 & STATE PLANE & & & & \\
\hline 2089 & ONSITE & SESPMNT & $\mathrm{R}$ & B106X9 & STATE PLANE & & & & \\
\hline 2090 & ONSITE & SESPMNT & $\mathrm{R}$ & B106Y1 & STATE PLANE & & & & \\
\hline 2091 & ONSITE & SESPMNT & $\mathrm{R}$ & B106Y3 & STATE PLANE & & & & \\
\hline
\end{tabular}




\begin{tabular}{|c|c|c|c|c|c|c|c|c|c|}
\hline & $A G$ & $\mathrm{AH}$ & $\mathrm{Al}$ & $\mathrm{AJ}$ & AK & $\mathrm{AL}$ & $\mathrm{AM}$ & AN & $\mathrm{AO}$ \\
\hline 1 & DIST_CLASS & OWNER_ID & COLLECTION_PURPOSE & X_Y_COORD_FINAL.SAMP_NUM & COORDINATE SYSTEM & DATUM & LATITUDE ${ }^{\circ} \mathrm{N}$ & LATITUDE MIN_MIN & LATITUDE MIN_SEC \\
\hline 2092 & OFFSITE & SESPMNT & $\mathrm{R}$ & B106Y4 & STATE PLANE & & & & \\
\hline 2093 & OFFSITE & SESPMNT & $\mathrm{R}$ & B106Y5 & STATE PLANE & & & & \\
\hline 2094 & OFFSITE & SESPMNT & $\mathrm{R}$ & B106Y6 & STATE PLANE & & & & \\
\hline 2095 & OFFSITE & SESPMNT & $\mathrm{R}$ & B106Y7 & STATE PLANE & & & & \\
\hline 2096 & OFFSITE & SESPMNT & $\mathrm{R}$ & B106Y8 & STATE PLANE & & & & \\
\hline 2097 & OFFSITE & SESPMNT & $\mathrm{R}$ & B106Y9 & STATE PLANE & & & & \\
\hline 2098 & OFFSITE & SESPMNT & $\mathrm{R}$ & B10761 & STATE PLANE & & & & \\
\hline 2099 & OFFSITE & SESPMNT & $\mathrm{R}$ & B10762 & STATE PLANE & & & & \\
\hline 2100 & OFFSITE & SESPMNT & $\mathrm{R}$ & B10763 & STATE PLANE & & & & \\
\hline 2101 & OFFSITE & SESPMNT & $\mathrm{R}$ & B10764 & STATE PLANE & & & & \\
\hline 2102 & ONSITE & SESPMNT & $\mathrm{R}$ & B10773 & STATE PLANE & & & & \\
\hline 2103 & ONSITE & SESPMNT & $\mathrm{R}$ & B10776 & STATE PLANE & & & & \\
\hline 2104 & ONSITE & SESPMNT & $\mathrm{R}$ & B10779 & STATE PLANE & & & & \\
\hline 2105 & ONSITE & SESPMNT & $\mathrm{R}$ & B10782 & STATE PLANE & & & & \\
\hline 2106 & ONSITE & SESPMNT & $\mathrm{R}$ & B10912 & STATE PLANE & & & & \\
\hline 2107 & ONSITE & SESPMNT & $\mathrm{R}$ & B10913 & STATE PLANE & & & & \\
\hline 2108 & ONSITE & SESPMNT & $\mathrm{R}$ & B10945 & STATE PLANE & & & & \\
\hline 2109 & ONSITE & SESPMNT & $\mathrm{R}$ & B10946 & STATE PLANE & & & & \\
\hline 2110 & ONSITE & SESPMNT & $\mathrm{R}$ & B10949 & STATE PLANE & & & & \\
\hline 2111 & PERIMETER & SESPMNT & $\mathrm{R}$ & B109J0 & STATE PLANE & & & & \\
\hline 2112 & OFFSITE & SESPMNT & $\mathrm{R}$ & & & & & & \\
\hline 2113 & OFFSITE & SESPMNT & $\mathrm{R}$ & & & & & & \\
\hline 2114 & ONSITE & SESPMNT & $\mathrm{R}$ & & & & & & \\
\hline 2115 & ONSITE & SESPMNT & $\mathrm{R}$ & & & & & & \\
\hline 2116 & ONSITE & SESPMNT & $\mathrm{R}$ & & & & & & \\
\hline 2117 & OFFSITE & SESPMNT & $\mathrm{R}$ & & & & & & \\
\hline 2118 & OFFSITE & SESPMNT & $\mathrm{R}$ & & & & & & \\
\hline 2119 & ONSITE & SESPMNT & $\mathrm{R}$ & & & & & & \\
\hline 2120 & OFFSITE & SESPMNT & $\mathrm{R}$ & & & & & & \\
\hline 2121 & OFFSITE & SESPMNT & $\mathrm{R}$ & & & & & & \\
\hline 2122 & OFFSITE & SESPMNT & $\mathrm{R}$ & B11125 & STATE PLANE & & & & \\
\hline \begin{tabular}{|l}
2123 \\
\end{tabular} & OFFSITE & SESPMNT & $\mathrm{R}$ & B11126 & STATE PLANE & & & & \\
\hline 2124 & OFFSITE & SESPMNT & $\mathrm{R}$ & B11127 & STATE PLANE & & & & \\
\hline 2125 & OFFSITE & SESPMNT & $\mathrm{R}$ & B11128 & STATE PLANE & & & & \\
\hline 2126 & OFFSITE & SESPSPEC & S & B11124 & STATE PLANE & & & & \\
\hline 2127 & OFFSITE & SESPMNT & $\mathrm{R}$ & B11129 & STATE PLANE & & & & \\
\hline 2128 & OFFSITE & SESPMNT & $\mathrm{R}$ & B11130 & STATE PLANE & & & & \\
\hline \begin{tabular}{|l}
2129 \\
2129 \\
\end{tabular} & OFFSITE & SESPMNT & $\mathrm{R}$ & B11131 & STATE PLANE & & & & \\
\hline
\end{tabular}




\begin{tabular}{|c|c|c|c|c|c|c|c|c|c|}
\hline & $A G$ & $\mathrm{AH}$ & $\mathrm{Al}$ & $\mathrm{AJ}$ & AK & $\mathrm{AL}$ & AM & $\mathrm{AN}$ & $\mathrm{AO}$ \\
\hline 1 & DIST_CLASS & OWNER_ID & COLLECTION_PURPOSE & X_Y_COORD_FINAL.SAMP_NUM & COORDINATE SYSTEM & \begin{tabular}{|l|} 
DATUM \\
\end{tabular} & LATITUDE ${ }^{\circ} \mathrm{N}$ & LATITUDE MIN_MIN & LATITUDE MIN_SEC \\
\hline 2130 & OFFSITE & SESPMNT & $\mathrm{R}$ & B11132 & STATE PLANE & & & & \\
\hline 2131 & OFFSITE & SESPMNT & $\mathrm{R}$ & B11133 & STATE PLANE & & & & \\
\hline 2132 & OFFSITE & SESPMNT & $\mathrm{R}$ & B11134 & STATE PLANE & & & & \\
\hline 2133 & OFFSITE & SESPMNT & $\mathrm{R}$ & B11145 & STATE PLANE & & & & \\
\hline 2134 & OFFSITE & SESPMNT & $\mathrm{R}$ & B11146 & STATE PLANE & & & & \\
\hline 2135 & OFFSITE & SESPMNT & $\mathrm{R}$ & B11147 & STATE PLANE & & & & \\
\hline 2136 & OFFSITE & SESPMNT & $\mathrm{R}$ & B11148 & STATE PLANE & & & & \\
\hline 2137 & OFFSITE & SESPMNT & $\mathrm{R}$ & & & & & & \\
\hline 2138 & OFFSITE & SESPMNT & $\mathrm{R}$ & & & & & & \\
\hline 2139 & OFFSITE & SESPMNT & $\mathrm{R}$ & & & & & & \\
\hline 2140 & OFFSITE & SESPMNT & $\mathrm{R}$ & & & & & & \\
\hline 2141 & ONSITE & SESPMNT & $\mathrm{R}$ & & & & & & \\
\hline 2142 & OFFSITE & SESPSPEC & S & B11H38 & LAT/LONG & & 46 & & 18.54 .68 \\
\hline 2143 & OFFSITE & SESPMNT & $\mathrm{R}$ & B11H43 & LAT/LONG & & 46 & & 18.54 .68 \\
\hline 2144 & OFFSITE & SESPMNT & $\mathrm{R}$ & B11H44 & LAT/LONG & & 46 & & 18.52 .97 \\
\hline 2145 & OFFSITE & SESPMNT & $\mathrm{R}$ & B11H45 & LAT/LONG & & 46 & & 18.51 .22 \\
\hline 2146 & OFFSITE & SESPMNT & $\mathrm{R}$ & B11H46 & LAT/LONG & & 46 & & 18.54 .18 \\
\hline 2147 & OFFSITE & SESPMNT & $\mathrm{R}$ & B11H47 & LAT/LONG & & 46 & & 18.46 .53 \\
\hline 2148 & OFFSITE & SESPMNT & $\mathrm{R}$ & B11H48 & LAT/LONG & & NONE & & \\
\hline 2149 & OFFSITE & SESPMNT & $\mathrm{R}$ & B11H59 & LAT/LONG & & 46 & & 19.25.05 \\
\hline 2150 & OFFSITE & SESPMNT & $\mathrm{R}$ & B11H60 & LAT/LONG & & No GPS sig. & & \\
\hline 2151 & OFFSITE & SESPMNT & $\mathrm{R}$ & B11H61 & LAT/LONG & & 46 & & 20.59 .09 \\
\hline 2152 & OFFSITE & SESPMNT & $\mathrm{R}$ & B11H62 & LAT/LONG & & 46 & & 21.27.18 \\
\hline 2153 & OFFSITE & SESPMNT & $\mathrm{R}$ & B11H39 & LAT/LONG & & 46 & & 38.30 .72 \\
\hline 2154 & OFFSITE & SESPMNT & $\mathrm{R}$ & B11H40 & LAT/LONG & & 46 & & 38.34 .81 \\
\hline 2155 & OFFSITE & SESPMNT & $\mathrm{R}$ & B11H41 & LAT/LONG & & 46 & & 38.40 .47 \\
\hline 2156 & OFFSITE & SESPMNT & $\mathrm{R}$ & B11H42 & LAT/LONG & & 46 & & 38.42 .31 \\
\hline 2157 & OFFSITE & SESPMNT & $\mathrm{R}$ & & & & & & \\
\hline 2158 & OFFSITE & SESPMNT & $\mathrm{R}$ & & & & & & \\
\hline 2159 & ONSITE & SESPMNT & $\mathrm{R}$ & & & & & & \\
\hline 2160 & OFFSITE & SESPMNT & $R$ & & & & & & \\
\hline 2161 & OFFSITE & SESPMNT & $\mathrm{R}$ & & & & & & \\
\hline 2162 & ONSITE & SESPMNT & $\mathrm{R}$ & & & & & & \\
\hline 2163 & ONSITE & SESPMNT & $\mathrm{R}$ & & & & & & \\
\hline 2164 & ONSITE & SESPMNT & $\mathrm{R}$ & & & & & & \\
\hline 2165 & ONSITE & SESPMNT & $\mathrm{R}$ & & & & & & \\
\hline 2166 & ONSITE & SESPMNT & $\mathrm{R}$ & & & & & & \\
\hline 2167 & OFFSITE & SESPMNT & $\mathrm{R}$ & & & & & & \\
\hline
\end{tabular}




\begin{tabular}{|c|c|c|c|c|c|c|c|c|c|}
\hline & $A G$ & $\mathrm{AH}$ & $\mathrm{Al}$ & AJ & AK & $A L$ & AM & AN & $\mathrm{AO}$ \\
\hline 1 & DIST_CLASS & OWNER_ID & COLLECTION_PURPOSE & X_Y_COORD_FINAL.SAMP_NUM & COORDINATE SYSTEM & DATUM & LATITUDE ${ }^{\circ} \mathrm{N}$ & LATITUDE MIN_MIN & LATITUDE MIN_SEC \\
\hline 2168 & OFFSITE & SESPMNT & $\mathrm{R}$ & & & & & & \\
\hline 2169 & ONSITE & SESPMNT & $\mathrm{R}$ & & & & & & \\
\hline 2170 & OFFSITE & SESPMNT & $\mathrm{R}$ & & & & & & \\
\hline 2171 & PERIMETER & SESPMNT & $\mathrm{R}$ & & & & & & \\
\hline 2172 & ONSITE & SESPMNT & $\mathrm{R}$ & & & & & & \\
\hline 2173 & OFFSITE & SESPSPEC & S & & & & & & \\
\hline 2174 & OFFSITE & SESPMNT & $\mathrm{R}$ & & & & & & \\
\hline 2175 & OFFSITE & SESPMNT & $\mathrm{R}$ & & & & & & \\
\hline 2176 & OFFSITE & SESPMNT & $\mathrm{R}$ & & & & & & \\
\hline 2177 & PERIMETER & SESPMNT & $\mathrm{R}$ & & & & & & \\
\hline 2178 & OFFSITE & SESPMNT & $\mathrm{R}$ & B12505 & LAT/LONG & & 46 & & 18.54 .400 \\
\hline 2179 & OFFSITE & SESPMNT & $\mathrm{R}$ & B12506 & LAT/LONG & & 46 & & 18.53.114 \\
\hline 2180 & OFFSITE & SESPMNT & $\mathrm{R}$ & B12507 & LAT/LONG & & 46 & & 18.50 .753 \\
\hline 2181 & OFFSITE & SESPMNT & $\mathrm{R}$ & B12508 & LAT/LONG & & 46 & & 18.50 .6000 \\
\hline 2182 & OFFSITE & SESPMNT & $\mathrm{R}$ & B12509 & LAT/LONG & & 46 & & 18.44.67124 \\
\hline 2183 & OFFSITE & SESPMNT & $\mathrm{R}$ & B12510 & LAT/LONG & & 46 & & 18.51 .627 \\
\hline 2184 & OFFSITE & SESPMNT & $\mathrm{R}$ & B12531 & LAT/LONG & & 46 & & 19.24.674 \\
\hline 2185 & OFFSITE & SESPMNT & $\mathrm{R}$ & B12532 & LAT/LONG & & 46 & & 20.07.051 \\
\hline 2186 & OFFSITE & SESPMNT & $\mathrm{R}$ & B12533 & LAT/LONG & & 46 & & 20.58.719 \\
\hline 2187 & OFFSITE & SESPMNT & $\mathrm{R}$ & B12534 & LAT/LONG & & 46 & & 21.20 .830 \\
\hline 2188 & OFFSITE & SESPMNT & $\mathrm{R}$ & B12501 & LAT/LONG & & 46 & & 38.33.187 \\
\hline 2189 & OFFSITE & SESPMNT & $\mathrm{R}$ & B12502 & LAT/LONG & & 46 & & 38.36 .25143 \\
\hline 2190 & OFFSITE & SESPMNT & $\mathrm{R}$ & B12503 & LAT/LONG & & 46 & & 38.39 .361 \\
\hline 2191 & OFFSITE & SESPMNT & $\mathrm{R}$ & B12504 & LAT/LONG & & 46 & & 38.42.047 \\
\hline 2192 & OFFSITE & SESPMNT & $\mathrm{R}$ & & & & & & \\
\hline 2193 & OFFSITE & SESPMNT & $\mathrm{R}$ & & & & & & \\
\hline 2194 & ONSITE & SESPMNT & $\mathrm{R}$ & & & & & & \\
\hline 2195 & OFFSITE & SESPMNT & $\mathrm{R}$ & & & & & & \\
\hline 2196 & PERIMETER & SESPMNT & $\mathrm{R}$ & & & & & & \\
\hline 2197 & OFFSITE & SESPMNT & $\mathrm{R}$ & & & & & & \\
\hline 2198 & OFFSITE & SESPMNT & $\mathrm{R}$ & & & & & & \\
\hline 2199 & ONSITE & SESPMNT & $\mathrm{R}$ & B12RM1 & LAT/LONG & WGS84 & 46 & 37664 & \\
\hline 2200 & ONSITE & SESPMNT & $\mathrm{R}$ & B12RM6 & LAT/LONG & WGS84 & 46 & 37218 & \\
\hline 2201 & ONSITE & SESPSPEC & $S$ & B12RN4 & LAT/LONG & WGS84 & 46 & 63453 & \\
\hline 2202 & ONSITE & SESPSPEC & $S$ & B12RN5 & LAT/LONG & WGS84 & 46 & 37664 & \\
\hline 2203 & ONSITE & SESPSPEC & $S$ & B12RN6 & LAT/LONG & WGS84 & 46 & 37218 & \\
\hline 2204 & ONSITE & SESPSPEC & S & B12RN7 & LAT/LONG & WGS84 & 46 & 63453 & \\
\hline 2205 & ONSITE & SESPSPEC & $S$ & B12RP9 & LAT/LONG & WGS84 & 46 & 63453 & \\
\hline
\end{tabular}




\begin{tabular}{|c|c|c|c|c|c|c|c|c|c|}
\hline & AG & $\mathrm{AH}$ & $\mathrm{Al}$ & $\mathrm{AJ}$ & AK & $\mathrm{AL}$ & AM & AN & $\mathrm{AO}$ \\
\hline 1 & DIST_CLASS & OWNER_ID & COLLECTION_PURPOSE & X_Y_COORD_FINAL.SAMP_NUM & COORDINATE SYSTEM & DATUM & LATITUDE ${ }^{\circ} \mathrm{N}$ & LATITUDE MIN_MIN & LATITUDE MIN_SEC \\
\hline 2206 & ONSITE & SESPSPEC & S & B12RR1 & LAT/LONG & WGS84 & 46 & 63453 & \\
\hline 2207 & ONSITE & SESPSPEC & S & B12RR3 & LAT/LONG & WGS84 & 46 & 63453 & \\
\hline 2208 & ONSITE & SESPSPEC & S & B12RR5 & LAT/LONG & WGS84 & 46 & 37663 & \\
\hline 2209 & ONSITE & SESPSPEC & S & B12RR7 & LAT/LONG & WGS84 & 46 & 37662 & \\
\hline 2210 & ONSITE & SESPSPEC & $\mathrm{S}$ & B12RR9 & LAT/LONG & WGS84 & 46 & 37663 & \\
\hline 2211 & ONSITE & SESPSPEC & $\mathrm{S}$ & B12RT1 & LAT/LONG & WGS84 & 46 & 37547 & \\
\hline 2212 & ONSITE & SESPSPEC & S & B12RT3 & LAT/LONG & WGS84 & 46 & 37547 & \\
\hline 2213 & ONSITE & SESPSPEC & S & B12RT5 & LAT/LONG & WGS84 & 46 & 37547 & \\
\hline 2214 & ONSITE & SESPSPEC & S & B12RT7 & LAT/LONG & WGS84 & 46 & 37362 & \\
\hline 2215 & ONSITE & SESPSPEC & S & B12RT9 & LAT/LONG & WGS84 & 46 & 3722 & \\
\hline 2216 & ONSITE & SESPSPEC & S & B12RV1 & LAT/LONG & WGS84 & 46 & 37218 & \\
\hline 2217 & ONSITE & SESPSPEC & S & B12RV3 & LAT/LONG & WGS84 & 46 & 37223 & \\
\hline 2218 & ONSITE & SESPSPEC & S & B12RV5 & LAT/LONG & WGS84 & 46 & 37166 & \\
\hline 2219 & ONSITE & SESPSPEC & S & B12RV7 & LAT/LONG & WGS84 & 46 & 37168 & \\
\hline 2220 & ONSITE & SESPSPEC & S & B12RV9 & LAT/LONG & WGS84 & 46 & 37166 & \\
\hline 2221 & ONSITE & SESPSPEC & S & B12RW1 & LAT/LONG & WGS84 & 46 & 36953 & \\
\hline 2222 & ONSITE & SESPSPEC & S & B12RW5 & LAT/LONG & WGS84 & 46 & 36714 & \\
\hline 2223 & ONSITE & SESPSPEC & $\mathrm{S}$ & B12RW7 & LAT/LONG & WGS84 & 46 & 36715 & \\
\hline 2224 & ONSITE & SESPSPEC & S & B12RW9 & LAT/LONG & WGS84 & 46 & 36714 & \\
\hline 2225 & ONSITE & SESPSPEC & S & & & & & & \\
\hline 2226 & ONSITE & SESPSPEC & S & & & & & & \\
\hline 2227 & ONSITE & SESPSPEC & S & & & & & & \\
\hline 2228 & ONSITE & SESPSPEC & S & B12RX9 & LAT/LONG & WGS84 & 46 & 35556 & \\
\hline 2229 & ONSITE & SESPSPEC & S & B12RY1 & LAT/LONG & WGS84 & 46 & 35556 & \\
\hline 2230 & ONSITE & SESPSPEC & S & B12RY3 & LAT/LONG & WGS84 & 46 & 35557 & \\
\hline 2231 & ONSITE & SESPSPEC & S & B12T11 & LAT/LONG & WGS84 & 46 & 63453 & \\
\hline 2232 & ONSITE & SESPSPEC & S & B12T13 & LAT/LONG & WGS84 & 46 & 37661 & \\
\hline 2233 & ONSITE & SESPSPEC & S & B12T15 & LAT/LONG & WGS84 & 46 & 37546 & \\
\hline 2234 & ONSITE & SESPSPEC & S & B12T17 & LAT/LONG & WGS84 & 46 & 37223 & \\
\hline 2235 & ONSITE & SESPSPEC & S & B12T19 & LAT/LONG & WGS84 & 46 & 37166 & \\
\hline 2236 & ONSITE & SESPSPEC & S & B12T21 & LAT/LONG & WGS84 & 46 & 36716 & \\
\hline 2237 & ONSITE & SESPSPEC & S & & & & & & \\
\hline 2238 & ONSITE & SESPSPEC & S & B12T25 & LAT/LONG & WGS84 & 46 & 35558 & \\
\hline 2239 & OFFSITE & SESPMNT & $\mathrm{R}$ & & & & & & \\
\hline 2240 & OFFSITE & SESPMNT & $\mathrm{R}$ & & & & & & \\
\hline 2241 & ONSITE & SESPSPEC & S & B12T85 & LAT/LONG & & 46 & & 41.01 .827 \\
\hline 2242 & OFFSITE & SESPMNT & $\mathrm{R}$ & B12T87 & LAT/LONG & & 46 & & 38.32.516 \\
\hline 2243 & OFFSITE & SESPMNT & $\mathrm{R}$ & B12T88 & LAT/LONG & & 46 & & 38.36.290 \\
\hline
\end{tabular}

Page 527 


\begin{tabular}{|c|c|c|c|c|c|c|c|c|c|}
\hline & $A G$ & $\mathrm{AH}$ & $\mathrm{Al}$ & $\mathrm{AJ}$ & $\mathrm{AK}$ & $\mathrm{AL}$ & $\mathrm{AM}$ & AN & $\mathrm{AO}$ \\
\hline 1 & DIST_CLASS & OWNER_ID & COLLECTION_PURPOSE & X_Y_COORD_FINAL.SAMP_NUM & COORDINATE SYSTEM & DATUM & LATITUDE ${ }^{\circ} \mathrm{N}$ & LATITUDE MIN_MIN & LATITUDE MIN_SEC \\
\hline 2244 & OFFSITE & SESPMNT & $\mathrm{R}$ & B12T89 & LAT/LONG & & 46 & & 38.40 .091 \\
\hline 2245 & OFFSITE & SESPMNT & $\mathrm{R}$ & B12T90 & LAT/LONG & & 46 & & 38.42 .087 \\
\hline 2246 & ONSITE & SESPMNT & $\mathrm{R}$ & B12T91 & LAT/LONG & & 46 & & 41.01 .827 \\
\hline 2247 & ONSITE & SESPMNT & $\mathrm{R}$ & B12T92 & LAT/LONG & & 46 & & 41.02 .142 \\
\hline 2248 & ONSITE & SESPMNT & $\mathrm{R}$ & B12T93 & LAT/LONG & & 46 & & 41.8 .044 \\
\hline 2249 & ONSITE & SESPMNT & $\mathrm{R}$ & B12TD4 & LAT/LONG & & 46 & & 41.8 .658 \\
\hline 2250 & ONSITE & SESPMNT & $\mathrm{R}$ & B12TD5 & LAT/LONG & & 46 & & 41.03 .989 \\
\hline 2251 & ONSITE & SESPMNT & $\mathrm{R}$ & B12TD6 & LAT/LONG & & 46 & & 41.8 .070 \\
\hline 2252 & ONSITE & SESPMNT & $\mathrm{R}$ & B12TN2 & LAT/LONG & & 46 & & 40.14 .547 \\
\hline 2253 & ONSITE & SESPMNT & $\mathrm{R}$ & B12TN5 & LAT/LONG & & 46 & & 40.43 .042 \\
\hline 2254 & ONSITE & SESPMNT & $\mathrm{R}$ & B12TN8 & LAT/LONG & & 46 & & 40.52 .424 \\
\hline 2255 & ONSITE & SESPMNT & $\mathrm{R}$ & B12TP1 & LAT/LONG & & 46 & & 41.17.965 \\
\hline 2256 & ONSITE & SESPMNT & $\mathrm{R}$ & B12T94 & LAT/LONG & & 46 & & 39.35 .60 \\
\hline 2257 & ONSITE & SESPMNT & $\mathrm{R}$ & B12T95 & LAT/LONG & & 46 & & 39.39 .25 \\
\hline 2258 & ONSITE & SESPMNT & $\mathrm{R}$ & B12T96 & LAT/LONG & & 46 & & 33.38 .38 \\
\hline 2259 & ONSITE & SESPMNT & $\mathrm{R}$ & B12T97 & LAT/LONG & & 46 & & 39.36.77 \\
\hline 2260 & ONSITE & SESPMNT & $\mathrm{R}$ & B12T98 & LAT/LONG & & 46 & & 39.45 .22 \\
\hline 2261 & ONSITE & SESPMNT & $\mathrm{R}$ & B12T99 & LAT/LONG & & 46 & & 39.49 .98 \\
\hline \begin{tabular}{|l}
2262 \\
\end{tabular} & ONSITE & SESPMNT & $\mathrm{R}$ & В12ТВ0 & LAT/LONG & & 46 & & 39.51 .88 \\
\hline \begin{tabular}{|l}
2263 \\
\end{tabular} & ONSITE & SESPMNT & $\mathrm{R}$ & B12TB1 & LAT/LONG & & 46 & & 33.38 .28 \\
\hline 2264 & ONSITE & SESPMNT & $\mathrm{R}$ & B12TB2 & LAT/LONG & & 46 & & 33.38.08 \\
\hline 2265 & ONSITE & SESPMNT & $\mathrm{R}$ & B12TB3 & LAT/LONG & & 46 & & 33.41 .45 \\
\hline \begin{tabular}{|l}
2266 \\
\end{tabular} & ONSITE & SESPMNT & $\mathrm{R}$ & B12TB4 & LAT/LONG & & 46 & & 33.42 .84 \\
\hline 2267 & ONSITE & SESPMNT & $\mathrm{R}$ & B12TB5 & LAT/LONG & & 46 & & 33.37 .95 \\
\hline 2268 & ONSITE & SESPMNT & $\mathrm{R}$ & B12TF7 & LAT/LONG & & 46 & & 34.50 .68 \\
\hline 2269 & ONSITE & SESPMNT & $\mathrm{R}$ & B12TF8 & LAT/LONG & & 46 & & 34.21 .43 \\
\hline 2270 & ONSITE & SESPMNT & $\mathrm{R}$ & B12TF9 & LAT/LONG & & 46 & & 32.44 .93 \\
\hline 2271 & ONSITE & SESPMNT & $\mathrm{R}$ & B12TT0 & LAT/LONG & & 46 & & 33.57 .87 \\
\hline 2272 & ONSITE & SESPMNT & $\mathrm{R}$ & B12TT3 & LAT/LONG & & 46 & & 40.10 .94 \\
\hline 22273 & ONSITE & SESPMNT & $\mathrm{R}$ & B12TT9 & LAT/LONG & & 46 & & 37.2.31 \\
\hline \begin{tabular}{|l}
2274 \\
\end{tabular} & ONSITE & SESPMNT & $\mathrm{R}$ & B12TB6 & LAT/LONG & & 46 & & 21.38.24 \\
\hline \begin{tabular}{|l}
2275 \\
\end{tabular} & ONSITE & SESPMNT & $\mathrm{R}$ & B12TB8 & LAT/LONG & & 46 & & 21.38.21 \\
\hline \begin{tabular}{|l}
2276 \\
\end{tabular} & ONSITE & SESPMNT & $\mathrm{R}$ & B12TC0 & LAT/LONG & & 46 & & 21.37.47 \\
\hline \begin{tabular}{|l|}
2277 \\
\end{tabular} & ONSITE & SESPMNT & $\mathrm{R}$ & B12TC2 & LAT/LONG & & 46 & & 21.38.45 \\
\hline \begin{tabular}{|l}
2278 \\
\end{tabular} & ONSITE & SESPMNT & $\mathrm{R}$ & B12TC4 & LAT/LONG & & 46 & & 21.37.97 \\
\hline 2279 & ONSITE & SESPMNT & $\mathrm{R}$ & B12TC6 & LAT/LONG & & 46 & & 21.38.57 \\
\hline 2280 & OFFSITE & SESPMNT & $\mathrm{R}$ & & & & & & \\
\hline \begin{tabular}{|l}
2281 \\
228 \\
\end{tabular} & OFFSITE & SESPMNT & $\mathrm{R}$ & B12TC9 & LAT/LONG & & 46 & & 18.53 .55 \\
\hline
\end{tabular}




\begin{tabular}{|c|c|c|c|c|c|c|c|c|c|}
\hline & $A G$ & $\mathrm{AH}$ & $\mathrm{Al}$ & $\mathrm{AJ}$ & $\mathrm{AK}$ & $\mathrm{AL}$ & $\mathrm{AM}$ & AN & $\mathrm{AO}$ \\
\hline 1 & DIST_CLASS & OWNER_ID & COLLECTION_PURPOSE & X_Y_COORD_FINAL.SAMP_NUM & COORDINATE SYSTEM & DATUM & LATITUDE ${ }^{\circ} \mathrm{N}$ & LATITUDE MIN_MIN & LATITUDE MIN_SEC \\
\hline 2282 & OFFSITE & SESPMNT & $\mathrm{R}$ & B12TD0 & LAT/LONG & & 46 & & 18.52 .78 \\
\hline 2283 & OFFSITE & SESPMNT & $\mathrm{R}$ & B12TD1 & LAT/LONG & & 46 & & 18.52 .99 \\
\hline 2284 & OFFSITE & SESPMNT & $\mathrm{R}$ & B12TD2 & LAT/LONG & & 46 & & 18.51.17 \\
\hline 2285 & OFFSITE & SESPMNT & $\mathrm{R}$ & B12TD3 & LAT/LONG & & 46 & & 18.52 .48 \\
\hline 2286 & OFFSITE & SESPMNT & $\mathrm{R}$ & B12TM0 & LAT/LONG & & 46 & & 19.25 .20 \\
\hline 2287 & OFFSITE & SESPMNT & $\mathrm{R}$ & B12TM1 & LAT/LONG & & 46 & & 20.7.76 \\
\hline 2288 & OFFSITE & SESPMNT & $\mathrm{R}$ & B12TM2 & LAT/LONG & & 46 & & 20.59 .083 \\
\hline 2289 & OFFSITE & SESPMNT & $\mathrm{R}$ & B12TM3 & LAT/LONG & & 46 & & 21.20 .88 \\
\hline 2290 & ONSITE & SESPMNT & $\mathrm{R}$ & B12TP4 & LAT/LONG & & 46 & & 23.2.95 \\
\hline 2291 & ONSITE & SESPMNT & $\mathrm{R}$ & B12TP8 & LAT/LONG & & 46 & & 22.35 .97 \\
\hline 2292 & ONSITE & SESPMNT & $\mathrm{R}$ & B12TR2 & LAT/LONG & & 46 & & 22.15.61 \\
\hline 2293 & ONSITE & SESPMNT & $\mathrm{R}$ & B12TR6 & LAT/LONG & & 46 & & 21.48.54 \\
\hline 2294 & ONSITE & SESPMNT & $\mathrm{R}$ & B12XK2 & LAT/LONG & & 46 & & 22.20.036 \\
\hline 2295 & ONSITE & SESPSPEC & S & B12XK8 & LAT/LONG & & 46 & & 22.20 .059 \\
\hline 2296 & ONSITE & SESPSPEC & S & B12XK9 & LAT/LONG & & 46 & & 22.20 .059 \\
\hline 2297 & ONSITE & SESPSPEC & S & B12XL0 & LAT/LONG & & 46 & & 22.20.084 \\
\hline 2298 & ONSITE & SESPSPEC & S & B12XL1 & LAT/LONG & & 46 & & 22.20 .084 \\
\hline 2299 & ONSITE & SESPSPEC & S & B12XL2 & LAT/LONG & & 46 & & 22.20.031 \\
\hline \begin{tabular}{|l}
2300 \\
\end{tabular} & ONSITE & SESPMNT & $\mathrm{R}$ & B12XK1 & LAT/LONG & & 46 & & 22.36 .037 \\
\hline 2301 & ONSITE & SESPSPEC & S & B12XK3 & LAT/LONG & & 46 & & 22.35 .976 \\
\hline 2302 & ONSITE & SESPSPEC & S & B12XK4 & LAT/LONG & & 46 & & 22.35 .976 \\
\hline 2303 & ONSITE & SESPSPEC & S & B12XK5 & LAT/LONG & & 46 & & 22.35 .976 \\
\hline \begin{tabular}{|l}
2304 \\
\end{tabular} & ONSITE & SESPSPEC & S & B12XK6 & LAT/LONG & & 46 & & 22.36 .045 \\
\hline 2305 & ONSITE & SESPSPEC & S & B12XK7 & LAT/LONG & & 46 & & 22.36.045 \\
\hline 2306 & OFFSITE & SESPMNT & $\mathrm{R}$ & & & & & & \\
\hline 2307 & OFFSITE & SESPMNT & $\mathrm{R}$ & & & & & & \\
\hline 2308 & ONSITE & SESPMNT & $\mathrm{R}$ & & & & & & \\
\hline 2309 & ONSITE & SESPMNT & $\mathrm{R}$ & B12X48 & LAT/LONG & & 46 & & 42.41 .7 \\
\hline 2310 & ONSITE & SESPMNT & $\mathrm{R}$ & B12X85 & LAT/LONG & & 46 & & 42.12 .8 \\
\hline 2311 & OFFSITE & SESPMNT & $\mathrm{R}$ & & & & & & \\
\hline 2312 & OFFSITE & SESPMNT & $\mathrm{R}$ & & & & & & \\
\hline \begin{tabular}{|l}
2313 \\
\end{tabular} & ONSITE & SESPSPEC & S & & & & & & \\
\hline 2314 & OFFSITE & SESPMNT & $\mathrm{R}$ & & & & & & \\
\hline 2315 & OFFSITE & SESPMNT & $\mathrm{R}$ & & & & & & \\
\hline \begin{tabular}{|l}
2316 \\
\end{tabular} & OFFSITE & SESPMNT & $\mathrm{R}$ & B13LF3 & LAT/LONG & & 46 & & 38.33 .72 \\
\hline 2317 & OFFSITE & SESPMNT & $\mathrm{R}$ & B13LF4 & LAT/LONG & & 46 & & 38.36.24 \\
\hline 2318 & OFFSITE & SESPMNT & $\mathrm{R}$ & B13LF5 & LAT/LONG & & 46 & & 38.40 .19 \\
\hline \begin{tabular}{|l}
2319 \\
2319 \\
\end{tabular} & OFFSITE & SESPMNT & $\mathrm{R}$ & B13LF6 & LAT/LONG & & 46 & & 38.42 .52 \\
\hline
\end{tabular}

Page 529 


\begin{tabular}{|c|c|c|c|c|c|c|c|c|c|}
\hline & $A G$ & $\mathrm{AH}$ & $\mathrm{Al}$ & $\mathrm{AJ}$ & $\mathrm{AK}$ & $\mathrm{AL}$ & $\mathrm{AM}$ & AN & $\mathrm{AO}$ \\
\hline 1 & DIST_CLASS & OWNER_ID & COLLECTION_PURPOSE & X_Y_COORD_FINAL.SAMP_NUM & COORDINATE SYSTEM & DATUM & LATITUDE ${ }^{\circ} \mathrm{N}$ & LATITUDE MIN_MIN & LATITUDE MIN_SEC \\
\hline 2320 & OFFSITE & SESPSPEC & S & B13LF2 & LAT/LONG & & 46 & & 18.54 .62 \\
\hline 2321 & OFFSITE & SESPMNT & $\mathrm{R}$ & B13LF7 & LAT/LONG & & 46 & & 18.54 .62 \\
\hline 2322 & OFFSITE & SESPMNT & $\mathrm{R}$ & B13LF8 & LAT/LONG & & 46 & & 18.52 .90 \\
\hline 2323 & OFFSITE & SESPMNT & $\mathrm{R}$ & B13LF9 & LAT/LONG & & 46 & & 18.52 .68 \\
\hline 2324 & OFFSITE & SESPMNT & $\mathrm{R}$ & B13LH0 & LAT/LONG & & 46 & & 18.53.97 \\
\hline 2325 & OFFSITE & SESPMNT & $\mathrm{R}$ & B13LH1 & LAT/LONG & & 46 & & 18.53 .50 \\
\hline 2326 & OFFSITE & SESPMNT & $\mathrm{R}$ & B13LH2 & LAT/LONG & & 46 & & 19.52.07 \\
\hline 2327 & OFFSITE & SESPMNT & $\mathrm{R}$ & B13LJ3 & LAT/LONG & & 46 & & 19.24.77 \\
\hline 2328 & OFFSITE & SESPMNT & $\mathrm{R}$ & B13LJ4 & LAT/LONG & & 46 & & 20.07 .46233 \\
\hline 2329 & OFFSITE & SESPMNT & $\mathrm{R}$ & B13LJ5 & LAT/LONG & & 46 & & 20.59 .29 \\
\hline 2330 & OFFSITE & SESPMNT & $\mathrm{R}$ & B13LJ6 & LAT/LONG & & 46 & & 21.21.83 \\
\hline 2331 & OFFSITE & SESPMNT & $\mathrm{R}$ & & & & & & \\
\hline 2332 & OFFSITE & SESPMNT & $\mathrm{R}$ & & & & & & \\
\hline 2333 & OFFSITE & SESPMNT & $\mathrm{R}$ & & & & & & \\
\hline 2334 & OFFSITE & SESPMNT & $\mathrm{R}$ & & & & & & \\
\hline 2335 & OFFSITE & SESPMNT & $\mathrm{R}$ & & & & & & \\
\hline 2336 & OFFSITE & SESPMNT & $\mathrm{R}$ & & & & & & \\
\hline 2337 & OFFSITE & SESPMNT & $\mathrm{R}$ & B147T3 & LAT/LONG & & 46 & & 38.32 .125 \\
\hline 2338 & OFFSITE & SESPMNT & $\mathrm{R}$ & B147T4 & LAT/LONG & & 46 & & 38.33.902 \\
\hline 2339 & OFFSITE & SESPMNT & $\mathrm{R}$ & B147T5 & LAT/LONG & & 46 & & 38.40 .586 \\
\hline 2340 & OFFSITE & SESPMNT & $\mathrm{R}$ & B147T6 & LAT/LONG & & 46 & & 38.41 .856 \\
\hline 2341 & OFFSITE & SESPSPEC & S & B147T2 & LAT/LONG & & 46 & & 18.54 .797 \\
\hline 2342 & OFFSITE & SESPMNT & $\mathrm{R}$ & B147T7 & LAT/LONG & & 46 & & 18.54 .797 \\
\hline 2343 & OFFSITE & SESPMNT & $\mathrm{R}$ & B147T8 & LAT/LONG & & 46 & & 18.53.211 \\
\hline 2344 & OFFSITE & SESPMNT & $\mathrm{R}$ & B147T9 & LAT/LONG & & 46 & & 18.52 .338 \\
\hline 2345 & OFFSITE & SESPMNT & $\mathrm{R}$ & B147V0 & LAT/LONG & & 46 & & 18.52 .299 \\
\hline 2346 & OFFSITE & SESPMNT & $\mathrm{R}$ & B147V1 & LAT/LONG & & 46 & & 18.51.346 \\
\hline 2347 & OFFSITE & SESPMNT & $\mathrm{R}$ & B147V2 & LAT/LONG & & 46 & & 18.50 .697 \\
\hline 2348 & OFFSITE & SESPMNT & $\mathrm{R}$ & B147W3 & LAT/LONG & & 46 & & 19.24 .739 \\
\hline 2349 & OFFSITE & SESPMNT & $\mathrm{R}$ & B147W4 & LAT/LONG & & 46 & & 20.7 .138 \\
\hline 2350 & OFFSITE & SESPMNT & $\mathrm{R}$ & B147W5 & LAT/LONG & & 46 & & 20.59 .129 \\
\hline 2351 & OFFSITE & SESPMNT & $\mathrm{R}$ & B147W6 & LAT/LONG & & 46 & & 21.20 .938 \\
\hline 2352 & OFFSITE & SESPMNT & $\mathrm{R}$ & & & & & & \\
\hline 2353 & OFFSITE & SESPMNT & $\mathrm{R}$ & & & & & & \\
\hline 2354 & OFFSITE & SESPMNT & $\mathrm{R}$ & & & & & & \\
\hline 2355 & OFFSITE & SESPMNT & $\mathrm{R}$ & & & & & & \\
\hline 2356 & OFFSITE & SESPMNT & $\mathrm{R}$ & B14LJ0 & LAT/LONG & & 46 & 25832 & \\
\hline 2357 & PERIMETER & SESPMNT & $\mathrm{R}$ & B14LJ2 & LAT/LONG & & 46 & 34120 & \\
\hline
\end{tabular}




\begin{tabular}{|c|c|c|c|c|c|c|c|c|c|}
\hline & $A G$ & $\mathrm{AH}$ & $\mathrm{Al}$ & $\mathrm{AJ}$ & $\mathrm{AK}$ & $\mathrm{AL}$ & AM & $\mathrm{AN}$ & $\mathrm{AO}$ \\
\hline 1 & DIST_CLASS & OWNER_ID & COLLECTION_PURPOSE & X_Y_COORD_FINAL.SAMP_NUM & COORDINATE SYSTEM & \begin{tabular}{|l|} 
DATUM \\
\end{tabular} & LATITUDE ${ }^{\circ} \mathrm{N}$ & LATITUDE MIN_MIN & LATITUDE MIN_SEC \\
\hline 2358 & OFFSITE & SESPMNT & $\mathrm{R}$ & & & & & & \\
\hline 2359 & OFFSITE & SESPMNT & $\mathrm{R}$ & & & & & & \\
\hline 2360 & OFFSITE & SESPMNT & $\mathrm{R}$ & B14RV4 & LAT/LONG & & 46 & & 38.32 .346 \\
\hline 2361 & OFFSITE & SESPMNT & $\mathrm{R}$ & B14RV5 & LAT/LONG & & 46 & & 38.35 .710 \\
\hline 2362 & OFFSITE & SESPMNT & $\mathrm{R}$ & B14RV6 & LAT/LONG & & 46 & & 38.40 .744 \\
\hline 2363 & OFFSITE & SESPMNT & $\mathrm{R}$ & B14RV7 & LAT/LONG & & 46 & & 38.43 .263 \\
\hline 2364 & OFFSITE & SESPMNT & $\mathrm{R}$ & B14RV8 & LAT/LONG & & 46 & & 18.54 .564 \\
\hline 2365 & OFFSITE & SESPMNT & $\mathrm{R}$ & B14RV9 & LAT/LONG & & 46 & & 18.50 .434 \\
\hline 2366 & OFFSITE & SESPMNT & $\mathrm{R}$ & B14RW0 & LAT/LONG & & 46 & & 18.51.872 \\
\hline 2367 & OFFSITE & SESPMNT & $\mathrm{R}$ & B14RW1 & LAT/LONG & & 46 & & 18.52.771 \\
\hline 2368 & OFFSITE & SESPMNT & $\mathrm{R}$ & B14RW2 & LAT/LONG & & 46 & & 18.48.011 \\
\hline 2369 & OFFSITE & SESPMNT & $\mathrm{R}$ & B14RW3 & LAT/LONG & & 46 & & 18.52.018 \\
\hline 2370 & OFFSITE & SESPMNT & $\mathrm{R}$ & B14RX4 & LAT/LONG & & 46 & & 19.24.503 \\
\hline 2371 & OFFSITE & SESPMNT & $\mathrm{R}$ & B14RX5 & LAT/LONG & & 46 & & 20.06.731 \\
\hline 2372 & OFFSITE & SESPMNT & $\mathrm{R}$ & B14RX6 & LAT/LONG & & 46 & & 20.59 .569 \\
\hline 2373 & OFFSITE & SESPMNT & $\mathrm{R}$ & B14RX7 & LAT/LONG & & 46 & & 21.21.521 \\
\hline 2374 & OFFSITE & SESPMNT & $\mathrm{R}$ & & & & & & \\
\hline 2375 & OFFSITE & SESPMNT & $\mathrm{R}$ & & & & & & \\
\hline 2376 & OFFSITE & SESPMNT & $\mathrm{R}$ & & & & & & \\
\hline 2377 & PERIMETER & SESPMNT & $\mathrm{R}$ & & & & & & \\
\hline 2378 & OFFSITE & SESPMNT & $\mathrm{R}$ & & & & & & \\
\hline 2379 & PERIMETER & SESPMNT & $\mathrm{R}$ & & & & & & \\
\hline 2380 & OFFSITE & SESPMNT & $\mathrm{R}$ & & & & & & \\
\hline 2381 & OFFSITE & SESPMNT & $\mathrm{R}$ & & & & & & \\
\hline 2382 & OFFSITE & SESPMNT & $\mathrm{R}$ & & & & & & \\
\hline 2383 & OFFSITE & SESPMNT & $\mathrm{R}$ & & & & & & \\
\hline 2384 & ONSITE & SESPSPEC & S & B158H3 & LAT/LONG & & 46 & & 41.02 .29 \\
\hline 2385 & OFFSITE & SESPMNT & $\mathrm{R}$ & B158H4 & LAT/LONG & & 46 & & 38.42 .82 \\
\hline 2386 & OFFSITE & SESPMNT & $\mathrm{R}$ & B158H5 & LAT/LONG & & 46 & & 38.35 .52 \\
\hline 2387 & OFFSITE & SESPMNT & $\mathrm{R}$ & B158H6 & LAT/LONG & & 46 & & 38.39 .47 \\
\hline 2388 & OFFSITE & SESPMNT & $R$ & B158H7 & LAT/LONG & & 46 & & 38.31 .26 \\
\hline 2389 & ONSITE & SESPMNT & $\mathrm{R}$ & B158H8 & LAT/LONG & & 46 & & 41.02 .29 \\
\hline 2390 & ONSITE & SESPMNT & $\mathrm{R}$ & В158H9 & LAT/LONG & & 46 & & 41.03.77 \\
\hline 2391 & ONSITE & SESPMNT & $\mathrm{R}$ & B158J0 & LAT/LONG & & 46 & & 41.11 .21 \\
\hline 2392 & ONSITE & SESPMNT & $\mathrm{R}$ & B158M7 & LAT/LONG & & 46 & & 41.10 .20 \\
\hline 2393 & ONSITE & SESPMNT & $\mathrm{R}$ & B158M8 & LAT/LONG & & 46 & & 41.06 .12 \\
\hline 2394 & ONSITE & SESPMNT & $\mathrm{R}$ & B158M9 & LAT/LONG & & 46 & & 41.09 .52 \\
\hline 2395 & ONSITE & SESPMNT & $\mathrm{R}$ & B158W8 & LAT/LONG & & 46 & & 40.14 .09 \\
\hline
\end{tabular}

Page 531 


\begin{tabular}{|c|c|c|c|c|c|c|c|c|c|}
\hline & $A G$ & $\mathrm{AH}$ & $\mathrm{Al}$ & $\mathrm{AJ}$ & $\mathrm{AK}$ & $\mathrm{AL}$ & $\mathrm{AM}$ & AN & $\mathrm{AO}$ \\
\hline 1 & DIST_CLASS & OWNER_ID & COLLECTION_PURPOSE & X_Y_COORD_FINAL.SAMP_NUM & COORDINATE SYSTEM & DATUM & LATITUDE ${ }^{\circ} \mathrm{N}$ & LATITUDE MIN_MIN & LATITUDE MIN_SEC \\
\hline 2396 & ONSITE & SESPMNT & $\mathrm{R}$ & $\mathrm{B} 158 \times 1$ & LAT/LONG & & 46 & & 40.43 .18 \\
\hline 2397 & ONSITE & SESPMNT & $\mathrm{R}$ & B158X4 & LAT/LONG & & 46 & & 40.51 .95 \\
\hline 2398 & ONSITE & SESPMNT & $\mathrm{R}$ & B158X7 & LAT/LONG & & 46 & & 41.18 .54 \\
\hline 2399 & ONSITE & SESPMNT & $\mathrm{R}$ & B158J1 & LAT/LONG & & 46 & & 39.35 .36 \\
\hline 2400 & ONSITE & SESPMNT & $\mathrm{R}$ & B158J3 & LAT/LONG & & 46 & & 39.39 .60 \\
\hline 2401 & ONSITE & SESPMNT & $\mathrm{R}$ & B158J5 & LAT/LONG & & 46 & & 33.39 .50 \\
\hline 2402 & ONSITE & SESPMNT & $\mathrm{R}$ & B158J7 & LAT/LONG & & 46 & & 39.37 .16 \\
\hline 2403 & ONSITE & SESPMNT & $\mathrm{R}$ & B158J9 & LAT/LONG & & 46 & & 39.44 .48 \\
\hline 2404 & ONSITE & SESPMNT & $\mathrm{R}$ & B158K1 & LAT/LONG & & 46 & & 39.48 .58 \\
\hline 2405 & ONSITE & SESPMNT & $\mathrm{R}$ & B158K3 & LAT/LONG & & 46 & & 39.51 .94 \\
\hline 2406 & ONSITE & SESPMNT & $\mathrm{R}$ & B158K5 & LAT/LONG & & 46 & & 33.40 .64 \\
\hline 2407 & ONSITE & SESPMNT & $\mathrm{R}$ & B158K7 & LAT/LONG & & 46 & & 33.40 .63 \\
\hline 2408 & ONSITE & SESPMNT & $\mathrm{R}$ & B158K9 & LAT/LONG & & 46 & & 33.42 .44 \\
\hline 2409 & ONSITE & SESPMNT & $\mathrm{R}$ & B158L1 & LAT/LONG & & 46 & & 33.43 .598 \\
\hline 2410 & ONSITE & SESPMNT & $\mathrm{R}$ & B158L3 & LAT/LONG & & 46 & & 33.40 .47 \\
\hline 2411 & ONSITE & SESPMNT & $\mathrm{R}$ & B158N0 & LAT/LONG & & 46 & & 34.50 .32 \\
\hline 2412 & ONSITE & SESPMNT & $\mathrm{R}$ & B158N2 & LAT/LONG & & 46 & & 34.21 .43 \\
\hline 2413 & ONSITE & SESPMNT & $\mathrm{R}$ & B158N4 & LAT/LONG & & 46 & & 32.44 .22 \\
\hline \begin{tabular}{|l}
2414 \\
\end{tabular} & ONSITE & SESPMNT & $\mathrm{R}$ & B15902 & LAT/LONG & & 46 & & 33.57 .46 \\
\hline \begin{tabular}{|l|}
2415 \\
\end{tabular} & ONSITE & SESPMNT & $\mathrm{R}$ & B15906 & LAT/LONG & & 46 & & 40.10 .84 \\
\hline 2416 & ONSITE & SESPMNT & $\mathrm{R}$ & B15910 & LAT/LONG & & 46 & & 36.22.01 \\
\hline \begin{tabular}{|l}
2417 \\
\end{tabular} & ONSITE & SESPMNT & $\mathrm{R}$ & B15914 & LAT/LONG & & 46 & & 37.02 .66 \\
\hline \begin{tabular}{|l|}
2418 \\
\end{tabular} & ONSITE & SESPMNT & $\mathrm{R}$ & B158L5 & LAT/LONG & & 46 & & 21.37 .20 \\
\hline 2419 & ONSITE & SESPMNT & $\mathrm{R}$ & B158L6 & LAT/LONG & & 46 & & 21.36.55 \\
\hline 2420 & ONSITE & SESPMNT & $\mathrm{R}$ & B158L7 & LAT/LONG & & 46 & & 21.35 .70 \\
\hline 2421 & ONSITE & SESPMNT & $\mathrm{R}$ & B158L8 & LAT/LONG & & 46 & & 21.37 .67 \\
\hline 2422 & ONSITE & SESPMNT & $\mathrm{R}$ & B158L9 & LAT/LONG & & 46 & & 21.38.28 \\
\hline 2423 & ONSITE & SESPMNT & $\mathrm{R}$ & B158M0 & LAT/LONG & & 46 & & 21.38 .48 \\
\hline 2424 & OFFSITE & SESPMNT & $\mathrm{R}$ & B158M1 & LAT/LONG & & 46 & & 18.54 .5 \\
\hline 2425 & OFFSITE & SESPMNT & $\mathrm{R}$ & B158M2 & LAT/LONG & & 46 & & 18.52 .7 \\
\hline 2426 & OFFSITE & SESPMNT & $\mathrm{R}$ & B158M3 & LAT/LONG & & 46 & & 18.85 .0 \\
\hline \begin{tabular}{|l}
2427 \\
\end{tabular} & OFFSITE & SESPMNT & $\mathrm{R}$ & B158M4 & LAT/LONG & & 46 & & 18.51 .50 \\
\hline 2428 & OFFSITE & SESPMNT & $\mathrm{R}$ & B158M5 & LAT/LONG & & 46 & & 18.51.37 \\
\hline 2429 & OFFSITE & SESPMNT & $\mathrm{R}$ & B158M6 & LAT/LONG & & 46 & & 18.52.36 \\
\hline 2430 & OFFSITE & SESPMNT & $\mathrm{R}$ & B158V6 & LAT/LONG & & 46 & & 19.24 .75 \\
\hline \begin{tabular}{|l}
2431 \\
\end{tabular} & OFFSITE & SESPMNT & $\mathrm{R}$ & B158V7 & LAT/LONG & & 46 & & 20.07 .17 \\
\hline 2432 & OFFSITE & SESPMNT & $\mathrm{R}$ & B158V8 & LAT/LONG & & 46 & & 20.58 .56 \\
\hline \begin{tabular}{|l}
2433 \\
\end{tabular} & OFFSITE & SESPMNT & $\mathrm{R}$ & B158V9 & LAT/LONG & & 46 & & 21.21.49 \\
\hline
\end{tabular}

Page 532 


\begin{tabular}{|c|c|c|c|c|c|c|c|c|c|}
\hline & $A G$ & $\mathrm{AH}$ & $\mathrm{Al}$ & $\mathrm{AJ}$ & $\mathrm{AK}$ & $\mathrm{AL}$ & $\mathrm{AM}$ & AN & $\mathrm{AO}$ \\
\hline 1 & DIST_CLASS & OWNER_ID & COLLECTION_PURPOSE & X_Y_COORD_FINAL.SAMP_NUM & COORDINATE SYSTEM & DATUM & LATITUDE ${ }^{\circ} \mathrm{N}$ & LATITUDE MIN_MIN & LATITUDE MIN_SEC \\
\hline 2434 & ONSITE & SESPMNT & $\mathrm{R}$ & B158Y0 & LAT/LONG & & 46 & & 23.03 .00 \\
\hline 2435 & ONSITE & SESPMNT & $\mathrm{R}$ & B158Y3 & LAT/LONG & & 46 & & 22.35 .44 \\
\hline 2436 & ONSITE & SESPMNT & $\mathrm{R}$ & B158Y6 & LAT/LONG & & 46 & & 22.19 .60 \\
\hline 2437 & ONSITE & SESPMNT & $\mathrm{R}$ & B158Y9 & LAT/LONG & & 46 & & 21.48.39 \\
\hline 2438 & OFFSITE & SESPMNT & $\mathrm{R}$ & & & & & & \\
\hline 2439 & OFFSITE & SESPMNT & $\mathrm{R}$ & & & & & & \\
\hline 2440 & ONSITE & SESPMNT & $\mathrm{R}$ & B15C09 & LAT/LONG & & 46 & & 33.55 .313 \\
\hline 2441 & ONSITE & SESPMNT & $\mathrm{R}$ & & & & & & \\
\hline 2442 & ONSITE & SESPMNT & $\mathrm{R}$ & B15C73 & LAT/LONG & & 46 & & 33.50 .499 \\
\hline 2443 & ONSITE & SESPMNT & $\mathrm{R}$ & B15CB3 & LAT/LONG & & 46 & & 42.12 .884 \\
\hline 2444 & ONSITE & SESPMNT & $\mathrm{R}$ & B15C69 & LAT/LONG & & 46 & & 38.14 .440 \\
\hline 2445 & OFFSITE & SESPMNT & $\mathrm{R}$ & & & & & & \\
\hline 2446 & OFFSITE & SESPMNT & $\mathrm{R}$ & & & & & & \\
\hline 2447 & OFFSITE & SESPMNT & $\mathrm{R}$ & & & & & & \\
\hline 2448 & OFFSITE & SESPMNT & $\mathrm{R}$ & & & & & & \\
\hline 2449 & OFFSITE & SESPMNT & $\mathrm{R}$ & B16342 & LAT/LONG & & 46 & 38544193 & \\
\hline 2450 & OFFSITE & SESPMNT & $\mathrm{R}$ & B16343 & LAT/LONG & & 46 & 3861303 & \\
\hline 2451 & OFFSITE & SESPMNT & $\mathrm{R}$ & B16344 & LAT/LONG & & 46 & 384081025 & \\
\hline \begin{tabular}{|l}
2452 \\
\end{tabular} & OFFSITE & SESPMNT & $\mathrm{R}$ & B16345 & LAT/LONG & & 46 & 384290077 & \\
\hline 2453 & OFFSITE & SESPSPEC & S & B16341 & LAT/LONG & & 46 & 185442945 & \\
\hline 2454 & OFFSITE & SESPMNT & $\mathrm{R}$ & B16346 & LAT/LONG & & 46 & 185442945 & \\
\hline 2455 & OFFSITE & SESPMNT & $\mathrm{R}$ & B16347 & LAT/LONG & & 46 & 18519365 & \\
\hline 2456 & OFFSITE & SESPMNT & $\mathrm{R}$ & B16348 & LAT/LONG & & 46 & 184989755 & \\
\hline 2457 & OFFSITE & SESPMNT & $\mathrm{R}$ & B16349 & LAT/LONG & & 46 & 185086976 & \\
\hline 2458 & OFFSITE & SESPMNT & $\mathrm{R}$ & B16350 & LAT/LONG & & 46 & 185163798 & \\
\hline 2459 & OFFSITE & SESPMNT & $\mathrm{R}$ & B16351 & LAT/LONG & & 46 & 185184158 & \\
\hline 2460 & OFFSITE & SESPMNT & $\mathrm{R}$ & B16362 & LAT/LONG & & 46 & 192473133 & \\
\hline 2461 & OFFSITE & SESPMNT & $\mathrm{R}$ & B16363 & LAT/LONG & & 46 & 200718556 & \\
\hline 2462 & OFFSITE & SESPMNT & $\mathrm{R}$ & B16364 & LAT/LONG & & 46 & 205947829 & \\
\hline 2463 & OFFSITE & SESPMNT & $\mathrm{R}$ & B16365 & LAT/LONG & & 46 & 212135241 & \\
\hline 2464 & ONSITE & SESPMNT & $\mathrm{R}$ & & & & & & \\
\hline 2465 & OFFSITE & SESPMNT & $\mathrm{R}$ & & & & & & \\
\hline 2466 & OFFSITE & SESPMNT & $\mathrm{R}$ & & & & & & \\
\hline 2467 & OFFSITE & SESPMNT & $\mathrm{R}$ & & & & & & \\
\hline 2468 & OFFSITE & SESPMNT & $\mathrm{R}$ & & & & & & \\
\hline 2469 & OFFSITE & SESPMNT & $\mathrm{R}$ & & & & & & \\
\hline 2470 & OFFSITE & SESPMNT & $\mathrm{R}$ & & & & & & \\
\hline 2471 & OFFSITE & SESPMNT & $\mathrm{R}$ & & & & & & \\
\hline
\end{tabular}




\begin{tabular}{|c|c|c|c|c|c|c|c|c|c|}
\hline & $A G$ & $\mathrm{AH}$ & $\mathrm{Al}$ & $\mathrm{AJ}$ & $\mathrm{AK}$ & $\overline{\mathrm{AL}}$ & AM & AN & $\mathrm{AO}$ \\
\hline 1 & DIST_CLASS & OWNER_ID & COLLECTION_PURPOSE & X_Y_COORD_FINAL.SAMP_NUM & COORDINATE SYSTEM & DATUM & LATITUDE ${ }^{\circ} \mathrm{N}$ & LATITUDE MIN_MIN & LATITUDE MIN_SEC \\
\hline 2472 & OFFSITE & SESPMNT & $\mathrm{R}$ & & & & & & \\
\hline 2473 & OFFSITE & SESPSPEC & $\mathrm{S}$ & & & & & & \\
\hline 2474 & OFFSITE & SESPMNT & $\mathrm{R}$ & & & & & & \\
\hline 2475 & OFFSITE & SESPMNT & $\mathrm{R}$ & & & & & & \\
\hline 2476 & OFFSITE & SESPMNT & $\mathrm{R}$ & & & & & & \\
\hline 2477 & OFFSITE & SESPMNT & $\mathrm{R}$ & & & & & & \\
\hline 2478 & OFFSITE & SESPMNT & $\mathrm{R}$ & & & & & & \\
\hline 2479 & OFFSITE & SESPMNT & $\mathrm{R}$ & & & & & & \\
\hline 2480 & OFFSITE & SESPMNT & $\mathrm{R}$ & & & & & & \\
\hline 2481 & OFFSITE & SESPMNT & $\mathrm{R}$ & & & & & & \\
\hline 2482 & OFFSITE & SESPMNT & $\mathrm{R}$ & & & & & & \\
\hline 2483 & OFFSITE & SESPMNT & $\mathrm{R}$ & & & & & & \\
\hline 2484 & ONSITE & SESPMNT & $\mathrm{R}$ & & & & & & \\
\hline 2485 & OFFSITE & SESPMNT & $\mathrm{R}$ & & & & & & \\
\hline 2486 & OFFSITE & SESPMNT & $\mathrm{R}$ & & & & & & \\
\hline 2487 & OFFSITE & SESPMNT & $\mathrm{R}$ & & & & & & \\
\hline 2488 & OFFSITE & SESPMNT & $\mathrm{R}$ & & & & & & \\
\hline 2489 & OFFSITE & SESPMNT & $\mathrm{R}$ & & & & & & \\
\hline 2490 & OFFSITE & SESPMNT & $\mathrm{R}$ & & & & & & \\
\hline 2491 & OFFSITE & SESPMNT & $\mathrm{R}$ & & & & & & \\
\hline 2492 & OFFSITE & SESPMNT & $\mathrm{R}$ & & & & & & \\
\hline 2493 & ONSITE & SESPSPEC & S & & & & & & \\
\hline 2494 & ONSITE & SESPSPEC & $\mathrm{S}$ & & & & & & \\
\hline 2495 & ONSITE & SESPSPEC & S & & & & & & \\
\hline 2496 & OFFSITE & SESPMNT & $\mathrm{R}$ & B0YCD7 & STATE PLANE & & & & \\
\hline 2497 & OFFSITE & SESPMNT & $\mathrm{R}$ & B0YCD8 & STATE PLANE & & & & \\
\hline 2498 & OFFSITE & SESPMNT & $\mathrm{R}$ & B0YCD9 & STATE PLANE & & & & \\
\hline 2499 & OFFSITE & SESPMNT & $\mathrm{R}$ & BOYCFO & STATE PLANE & & & & \\
\hline 2500 & OFFSITE & SESPMNT & $\mathrm{R}$ & B0YCF1 & STATE PLANE & & & & \\
\hline 2501 & OFFSITE & SESPMNT & $\mathrm{R}$ & BOYCF2 & STATE PLANE & & & & \\
\hline 2502 & OFFSITE & SESPMNT & $\mathrm{R}$ & BOYCF3 & STATE PLANE & & & & \\
\hline 2503 & OFFSITE & SESPMNT & $\mathrm{R}$ & BOYCF4 & STATE PLANE & & & & \\
\hline 2504 & OFFSITE & SESPMNT & $\mathrm{R}$ & B0YCF5 & STATE PLANE & & & & \\
\hline 2505 & OFFSITE & SESPMNT & $\mathrm{R}$ & BOYCF6 & STATE PLANE & & & & \\
\hline 2506 & OFFSITE & SESPMNT & $\mathrm{R}$ & BOYCK3 & STATE PLANE & & & & \\
\hline 2507 & OFFSITE & SESPMNT & $\mathrm{R}$ & BOYCK4 & STATE PLANE & & & & \\
\hline 2508 & OFFSITE & SESPMNT & $\mathrm{R}$ & B0YCK5 & STATE PLANE & & & & \\
\hline 2509 & OFFSITE & SESPMNT & $\mathrm{R}$ & BOYCK6 & STATE PLANE & & & & \\
\hline
\end{tabular}




\begin{tabular}{|c|c|c|c|c|c|c|c|c|c|}
\hline & $A G$ & $\mathrm{AH}$ & $\mathrm{Al}$ & AJ & AK & $A L$ & AM & AN & $\mathrm{AO}$ \\
\hline 1 & DIST_CLASS & OWNER_ID & COLLECTION_PURPOSE & X_Y_COORD_FINAL.SAMP_NUM & COORDINATE SYSTEM & DATUM & LATITUDE ${ }^{\circ} \mathrm{N}$ & LATITUDE MIN_MIN & LATITUDE MIN_SEC \\
\hline 2510 & OFFSITE & SESPMNT & $\mathrm{R}$ & & & & & & \\
\hline 2511 & OFFSITE & SESPMNT & $\mathrm{R}$ & & & & & & \\
\hline 2512 & ONSITE & SESPMNT & $\mathrm{R}$ & & & & & & \\
\hline 2513 & OFFSITE & SESPMNT & $\mathrm{R}$ & & & & & & \\
\hline 2514 & OFFSITE & SESPMNT & $\mathrm{R}$ & & & & & & \\
\hline 2515 & OFFSITE & SESPMNT & $\mathrm{R}$ & & & & & & \\
\hline 2516 & OFFSITE & SESPMNT & $\mathrm{R}$ & & & & & & \\
\hline 2517 & OFFSITE & SESPMNT & $\mathrm{R}$ & & & & & & \\
\hline 2518 & ONSITE & SESPSPEC & S & B10672 & STATE PLANE & & & & \\
\hline 2519 & OFFSITE & SESPMNT & $\mathrm{R}$ & B10673 & STATE PLANE & & & & \\
\hline 2520 & OFFSITE & SESPMNT & $\mathrm{R}$ & B10674 & STATE PLANE & & & & \\
\hline 2521 & OFFSITE & SESPMNT & $\mathrm{R}$ & B10675 & STATE PLANE & & & & \\
\hline 2522 & OFFSITE & SESPMNT & $\mathrm{R}$ & B10676 & STATE PLANE & & & & \\
\hline 2523 & ONSITE & SESPMNT & $\mathrm{R}$ & B10677 & STATE PLANE & & & & \\
\hline 2524 & ONSITE & SESPMNT & $\mathrm{R}$ & B10679 & STATE PLANE & & & & \\
\hline 2525 & ONSITE & SESPMNT & $\mathrm{R}$ & & & & & & \\
\hline 2526 & ONSITE & SESPMNT & $\mathrm{R}$ & B10683 & STATE PLANE & & & & \\
\hline 2527 & ONSITE & SESPMNT & $\mathrm{R}$ & B10684 & STATE PLANE & & & & \\
\hline 2528 & ONSITE & SESPMNT & $\mathrm{R}$ & & & & & & \\
\hline 2529 & ONSITE & SESPMNT & $\mathrm{R}$ & & & & & & \\
\hline 2530 & ONSITE & SESPMNT & $\mathrm{R}$ & B106B1 & STATE PLANE & & & & \\
\hline 2531 & ONSITE & SESPMNT & $\mathrm{R}$ & & & & & & \\
\hline 2532 & ONSITE & SESPMNT & $\mathrm{R}$ & B106B7 & STATE PLANE & & & & \\
\hline 2533 & ONSITE & SESPMNT & $\mathrm{R}$ & B106W1 & STATE PLANE & & & & \\
\hline 2534 & ONSITE & SESPMNT & $\mathrm{R}$ & B106W2 & STATE PLANE & & & & \\
\hline 2535 & ONSITE & SESPMNT & $\mathrm{R}$ & B106W3 & STATE PLANE & & & & \\
\hline 2536 & ONSITE & SESPMNT & $\mathrm{R}$ & B106W4 & STATE PLANE & & & & \\
\hline 2537 & ONSITE & SESPMNT & $\mathrm{R}$ & B106W5 & STATE PLANE & & & & \\
\hline 2538 & ONSITE & SESPMNT & $\mathrm{R}$ & B106W6 & STATE PLANE & & & & \\
\hline 2539 & ONSITE & SESPMNT & $\mathrm{R}$ & B106W7 & STATE PLANE & & & & \\
\hline 2540 & ONSITE & SESPMNT & $\mathrm{R}$ & B106W8 & STATE PLANE & & & & \\
\hline 2541 & ONSITE & SESPMNT & $\mathrm{R}$ & B106W9 & STATE PLANE & & & & \\
\hline 2542 & ONSITE & SESPMNT & $\mathrm{R}$ & B106X0 & STATE PLANE & & & & \\
\hline 2543 & ONSITE & SESPMNT & $\mathrm{R}$ & B106X1 & STATE PLANE & & & & \\
\hline 2544 & ONSITE & SESPMNT & $\mathrm{R}$ & B106X2 & STATE PLANE & & & & \\
\hline 2545 & ONSITE & SESPMNT & $\mathrm{R}$ & B10728 & STATE PLANE & & & & \\
\hline 2546 & ONSITE & SESPMNT & $\mathrm{R}$ & B10729 & STATE PLANE & & & & \\
\hline 2547 & ONSITE & SESPMNT & $\mathrm{R}$ & B10730 & STATE PLANE & & & & \\
\hline
\end{tabular}




\begin{tabular}{|c|c|c|c|c|c|c|c|c|c|}
\hline & $A G$ & $\mathrm{AH}$ & $\mathrm{Al}$ & $\mathrm{AJ}$ & AK & $\mathrm{AL}$ & $\mathrm{AM}$ & AN & $\mathrm{AO}$ \\
\hline 1 & DIST_CLASS & OWNER_ID & COLLECTION_PURPOSE & X_Y_COORD_FINAL.SAMP_NUM & COORDINATE SYSTEM & DATUM & LATITUDE ${ }^{\circ} \mathrm{N}$ & LATITUDE MIN_MIN & LATITUDE MIN_SEC \\
\hline 2548 & ONSITE & SESPMNT & $\mathrm{R}$ & B10785 & STATE PLANE & & & & \\
\hline 2549 & ONSITE & SESPMNT & $\mathrm{R}$ & B10788 & STATE PLANE & & & & \\
\hline 2550 & ONSITE & SESPMNT & $\mathrm{R}$ & B10791 & STATE PLANE & & & & \\
\hline 2551 & ONSITE & SESPMNT & $\mathrm{R}$ & B10794 & STATE PLANE & & & & \\
\hline 2552 & ONSITE & SESPMNT & $\mathrm{R}$ & & & & & & \\
\hline 2553 & ONSITE & SESPMNT & $\mathrm{R}$ & & & & & & \\
\hline 2554 & ONSITE & SESPMNT & $\mathrm{R}$ & B106X7 & STATE PLANE & & & & \\
\hline 2555 & ONSITE & SESPMNT & $\mathrm{R}$ & B106X9 & STATE PLANE & & & & \\
\hline 2556 & ONSITE & SESPMNT & $\mathrm{R}$ & B106Y1 & STATE PLANE & & & & \\
\hline 2557 & ONSITE & SESPMNT & $\mathrm{R}$ & B106Y3 & STATE PLANE & & & & \\
\hline 2558 & OFFSITE & SESPMNT & $\mathrm{R}$ & B106Y4 & STATE PLANE & & & & \\
\hline 2559 & OFFSITE & SESPMNT & $\mathrm{R}$ & B106Y5 & STATE PLANE & & & & \\
\hline 2560 & OFFSITE & SESPMNT & $\mathrm{R}$ & B106Y6 & STATE PLANE & & & & \\
\hline 2561 & OFFSITE & SESPMNT & $\mathrm{R}$ & B106Y7 & STATE PLANE & & & & \\
\hline 2562 & OFFSITE & SESPMNT & $\mathrm{R}$ & B106Y8 & STATE PLANE & & & & \\
\hline 2563 & OFFSITE & SESPMNT & $\mathrm{R}$ & B106Y9 & STATE PLANE & & & & \\
\hline 2564 & OFFSITE & SESPMNT & $\mathrm{R}$ & B10761 & STATE PLANE & & & & \\
\hline 2565 & OFFSITE & SESPMNT & $\mathrm{R}$ & B10762 & STATE PLANE & & & & \\
\hline 2566 & OFFSITE & SESPMNT & $\mathrm{R}$ & B10763 & STATE PLANE & & & & \\
\hline 2567 & OFFSITE & SESPMNT & $\mathrm{R}$ & B10764 & STATE PLANE & & & & \\
\hline 2568 & ONSITE & SESPMNT & $\mathrm{R}$ & B10773 & STATE PLANE & & & & \\
\hline 2569 & ONSITE & SESPMNT & $\mathrm{R}$ & B10776 & STATE PLANE & & & & \\
\hline 2570 & ONSITE & SESPMNT & $\mathrm{R}$ & B10779 & STATE PLANE & & & & \\
\hline 2571 & ONSITE & SESPMNT & $\mathrm{R}$ & B10782 & STATE PLANE & & & & \\
\hline 2572 & ONSITE & SESPMNT & $\mathrm{R}$ & B10912 & STATE PLANE & & & & \\
\hline 2573 & ONSITE & SESPMNT & $\mathrm{R}$ & B10913 & STATE PLANE & & & & \\
\hline 2574 & ONSITE & SESPMNT & $\mathrm{R}$ & B10945 & STATE PLANE & & & & \\
\hline 2575 & ONSITE & SESPMNT & $\mathrm{R}$ & B10946 & STATE PLANE & & & & \\
\hline 2576 & ONSITE & SESPMNT & $\mathrm{R}$ & B10949 & STATE PLANE & & & & \\
\hline 2577 & PERIMETER & SESPMNT & $\mathrm{R}$ & B109J0 & STATE PLANE & & & & \\
\hline 2578 & OFFSITE & SESPMNT & $\mathrm{R}$ & & & & & & \\
\hline 2579 & OFFSITE & SESPMNT & $\mathrm{R}$ & & & & & & \\
\hline 2580 & ONSITE & SESPMNT & $\mathrm{R}$ & & & & & & \\
\hline 2581 & ONSITE & SESPMNT & $\mathrm{R}$ & & & & & & \\
\hline 2582 & ONSITE & SESPMNT & $\mathrm{R}$ & & & & & & \\
\hline 2583 & OFFSITE & SESPMNT & $\mathrm{R}$ & & & & & & \\
\hline 2584 & OFFSITE & SESPMNT & $\mathrm{R}$ & & & & & & \\
\hline 2585 & ONSITE & SESPMNT & $\mathrm{R}$ & & & & & & \\
\hline
\end{tabular}




\begin{tabular}{|c|c|c|c|c|c|c|c|c|c|}
\hline & $A G$ & $\mathrm{AH}$ & $\mathrm{Al}$ & $\mathrm{AJ}$ & $\mathrm{AK}$ & $\mathrm{AL}$ & AM & $\mathrm{AN}$ & $\mathrm{AO}$ \\
\hline 1 & DIST_CLASS & OWNER_ID & COLLECTION_PURPOSE & X_Y_COORD_FINAL.SAMP_NUM & COORDINATE SYSTEM & \begin{tabular}{|l|} 
DATUM \\
\end{tabular} & LATITUDE ${ }^{\circ} \mathrm{N}$ & LATITUDE MIN_MIN & LATITUDE MIN_SEC \\
\hline 2586 & OFFSITE & SESPMNT & $\mathrm{R}$ & & & & & & \\
\hline 2587 & OFFSITE & SESPMNT & $\mathrm{R}$ & & & & & & \\
\hline 2588 & OFFSITE & SESPMNT & $\mathrm{R}$ & B11125 & STATE PLANE & & & & \\
\hline 2589 & OFFSITE & SESPMNT & $\mathrm{R}$ & B11126 & STATE PLANE & & & & \\
\hline 2590 & OFFSITE & SESPMNT & $\mathrm{R}$ & B11127 & STATE PLANE & & & & \\
\hline 2591 & OFFSITE & SESPMNT & $\mathrm{R}$ & B11128 & STATE PLANE & & & & \\
\hline 2592 & OFFSITE & SESPSPEC & S & B11124 & STATE PLANE & & & & \\
\hline 2593 & OFFSITE & SESPMNT & $\mathrm{R}$ & B11129 & STATE PLANE & & & & \\
\hline 2594 & OFFSITE & SESPMNT & $\mathrm{R}$ & B11130 & STATE PLANE & & & & \\
\hline 2595 & OFFSITE & SESPMNT & $\mathrm{R}$ & B11131 & STATE PLANE & & & & \\
\hline 2596 & OFFSITE & SESPMNT & $\mathrm{R}$ & B11132 & STATE PLANE & & & & \\
\hline 2597 & OFFSITE & SESPMNT & $\mathrm{R}$ & B11133 & STATE PLANE & & & & \\
\hline 2598 & OFFSITE & SESPMNT & $\mathrm{R}$ & B11134 & STATE PLANE & & & & \\
\hline 2599 & OFFSITE & SESPMNT & $\mathrm{R}$ & B11145 & STATE PLANE & & & & \\
\hline 2600 & OFFSITE & SESPMNT & $\mathrm{R}$ & B11146 & STATE PLANE & & & & \\
\hline 2601 & OFFSITE & SESPMNT & $\mathrm{R}$ & B11147 & STATE PLANE & & & & \\
\hline 2602 & OFFSITE & SESPMNT & $\mathrm{R}$ & B11148 & STATE PLANE & & & & \\
\hline 2603 & OFFSITE & SESPMNT & $\mathrm{R}$ & & & & & & \\
\hline 2604 & OFFSITE & SESPMNT & $R$ & & & & & & \\
\hline 2605 & OFFSITE & SESPMNT & $\mathrm{R}$ & & & & & & \\
\hline 2606 & OFFSITE & SESPMNT & $\mathrm{R}$ & & & & & & \\
\hline 2607 & ONSITE & SESPMNT & $\mathrm{R}$ & & & & & & \\
\hline 2608 & OFFSITE & SESPSPEC & S & B11H38 & LAT/LONG & & 46 & & 18.54.68 \\
\hline 2609 & OFFSITE & SESPMNT & $\mathrm{R}$ & B11H43 & LAT/LONG & & 46 & & 18.54 .68 \\
\hline 2610 & OFFSITE & SESPMNT & $\mathrm{R}$ & B11H44 & LAT/LONG & & 46 & & 18.52 .97 \\
\hline 2611 & OFFSITE & SESPMNT & $\mathrm{R}$ & B11H45 & LAT/LONG & & 46 & & 18.51.22 \\
\hline 2612 & OFFSITE & SESPMNT & $\mathrm{R}$ & B11H46 & LAT/LONG & & 46 & & 18.54.18 \\
\hline 2613 & OFFSITE & SESPMNT & $\mathrm{R}$ & B11H47 & LAT/LONG & & 46 & & 18.46 .53 \\
\hline 2614 & OFFSITE & SESPMNT & $\mathrm{R}$ & B11H48 & LAT/LONG & & NONE & & \\
\hline 2615 & OFFSITE & SESPMNT & $\mathrm{R}$ & B11H59 & LAT/LONG & & 46 & & 19.25 .05 \\
\hline 2616 & OFFSITE & SESPMNT & $\mathrm{R}$ & B11H60 & LAT/LONG & & No GPS sig. & & \\
\hline 2617 & OFFSITE & SESPMNT & $\mathrm{R}$ & B11H61 & LAT/LONG & & 46 & & 20.59.09 \\
\hline 2618 & OFFSITE & SESPMNT & $\mathrm{R}$ & B11H62 & LAT/LONG & & 46 & & 21.27.18 \\
\hline 2619 & OFFSITE & SESPMNT & $\mathrm{R}$ & B11H39 & LAT/LONG & & 46 & & 38.30 .72 \\
\hline 2620 & OFFSITE & SESPMNT & $\mathrm{R}$ & B11H40 & LAT/LONG & & 46 & & 38.34 .81 \\
\hline 2621 & OFFSITE & SESPMNT & $\mathrm{R}$ & B11H41 & LAT/LONG & & 46 & & 38.40 .47 \\
\hline 2622 & OFFSITE & SESPMNT & $\mathrm{R}$ & B11H42 & LAT/LONG & & 46 & & 38.42 .31 \\
\hline 2623 & OFFSITE & SESPMNT & $\mathrm{R}$ & & & & & & \\
\hline
\end{tabular}

Page 537 


\begin{tabular}{|c|c|c|c|c|c|c|c|c|c|}
\hline & $A G$ & $\mathrm{AH}$ & $\mathrm{Al}$ & $\mathrm{AJ}$ & $\mathrm{AK}$ & $\mathrm{AL}$ & AM & $\mathrm{AN}$ & $\mathrm{AO}$ \\
\hline 1 & DIST_CLASS & OWNER_ID & COLLECTION_PURPOSE & X_Y_COORD_FINAL.SAMP_NUM & COORDINATE SYSTEM & \begin{tabular}{|l|} 
DATUM \\
\end{tabular} & LATITUDE ${ }^{\circ} \mathrm{N}$ & LATITUDE MIN_MIN & LATITUDE MIN_SEC \\
\hline 2624 & OFFSITE & SESPMNT & $\mathrm{R}$ & & & & & & \\
\hline 2625 & ONSITE & SESPMNT & $\mathrm{R}$ & & & & & & \\
\hline 2626 & OFFSITE & SESPMNT & $\mathrm{R}$ & & & & & & \\
\hline 2627 & OFFSITE & SESPMNT & $\mathrm{R}$ & & & & & & \\
\hline 2628 & ONSITE & SESPMNT & $\mathrm{R}$ & & & & & & \\
\hline 2629 & ONSITE & SESPMNT & $\mathrm{R}$ & & & & & & \\
\hline 2630 & ONSITE & SESPMNT & $\mathrm{R}$ & & & & & & \\
\hline 2631 & ONSITE & SESPMNT & $\mathrm{R}$ & & & & & & \\
\hline 2632 & ONSITE & SESPMNT & $\mathrm{R}$ & & & & & & \\
\hline 2633 & OFFSITE & SESPMNT & $\mathrm{R}$ & & & & & & \\
\hline 2634 & OFFSITE & SESPMNT & $\mathrm{R}$ & & & & & & \\
\hline 2635 & ONSITE & SESPMNT & $\mathrm{R}$ & & & & & & \\
\hline 2636 & OFFSITE & SESPMNT & $\mathrm{R}$ & & & & & & \\
\hline 2637 & PERIMETER & SESPMNT & $\mathrm{R}$ & & & & & & \\
\hline 2638 & ONSITE & SESPMNT & $\mathrm{R}$ & & & & & & \\
\hline 2639 & OFFSITE & SESPSPEC & $\mathrm{S}$ & & & & & & \\
\hline 2640 & OFFSITE & SESPMNT & $\mathrm{R}$ & & & & & & \\
\hline 2641 & OFFSITE & SESPMNT & $\mathrm{R}$ & & & & & & \\
\hline 2642 & OFFSITE & SESPMNT & $\mathrm{R}$ & & & & & & \\
\hline 2643 & PERIMETER & SESPMNT & $\mathrm{R}$ & & & & & & \\
\hline 2644 & OFFSITE & SESPMNT & $\mathrm{R}$ & B12505 & LAT/LONG & & 46 & & 18.54 .400 \\
\hline 2645 & OFFSITE & SESPMNT & $\mathrm{R}$ & B12506 & LAT/LONG & & 46 & & 18.53 .114 \\
\hline 2646 & OFFSITE & SESPMNT & $\mathrm{R}$ & B12507 & LAT/LONG & & 46 & & 18.50 .753 \\
\hline 2647 & OFFSITE & SESPMNT & $\mathrm{R}$ & B12508 & LAT/LONG & & 46 & & 18.50 .6000 \\
\hline 2648 & OFFSITE & SESPMNT & $\mathrm{R}$ & B12509 & LAT/LONG & & 46 & & 18.44 .67124 \\
\hline 2649 & OFFSITE & SESPMNT & $\mathrm{R}$ & B12510 & LAT/LONG & & 46 & & 18.51.627 \\
\hline 2650 & OFFSITE & SESPMNT & $\mathrm{R}$ & B12531 & LAT/LONG & & 46 & & 19.24.674 \\
\hline 2651 & OFFSITE & SESPMNT & $\mathrm{R}$ & B12532 & LAT/LONG & & 46 & & 20.07.051 \\
\hline 2652 & OFFSITE & SESPMNT & $\mathrm{R}$ & B12533 & LAT/LONG & & 46 & & 20.58.719 \\
\hline 2653 & OFFSITE & SESPMNT & $\mathrm{R}$ & B12534 & LAT/LONG & & 46 & & 21.20 .830 \\
\hline 2654 & OFFSITE & SESPMNT & $\mathrm{R}$ & B12501 & LAT/LONG & & 46 & & 38.33 .187 \\
\hline 2655 & OFFSITE & SESPMNT & $\mathrm{R}$ & B12502 & LAT/LONG & & 46 & & 38.36 .25143 \\
\hline 2656 & OFFSITE & SESPMNT & $\mathrm{R}$ & B12503 & LAT/LONG & & 46 & & 38.39 .361 \\
\hline 2657 & OFFSITE & SESPMNT & $\mathrm{R}$ & B12504 & LAT/LONG & & 46 & & 38.42 .047 \\
\hline 2658 & OFFSITE & SESPMNT & $\mathrm{R}$ & & & & & & \\
\hline 2659 & OFFSITE & SESPMNT & $\mathrm{R}$ & & & & & & \\
\hline 2660 & ONSITE & SESPMNT & $\mathrm{R}$ & & & & & & \\
\hline 2661 & OFFSITE & SESPMNT & $\mathrm{R}$ & & & & & & \\
\hline
\end{tabular}




\begin{tabular}{|c|c|c|c|c|c|c|c|c|c|}
\hline & $A G$ & $\mathrm{AH}$ & $\mathrm{Al}$ & $\mathrm{AJ}$ & $\mathrm{AK}$ & $\mathrm{AL}$ & $\mathrm{AM}$ & AN & $\mathrm{AO}$ \\
\hline 1 & DIST_CLASS & OWNER_ID & COLLECTION_PURPOSE & X_Y_COORD_FINAL.SAMP_NUM & COORDINATE SYSTEM & DATUM & LATITUDE ${ }^{\circ} \mathrm{N}$ & LATITUDE MIN_MIN & LATITUDE MIN_SEC \\
\hline 2662 & PERIMETER & SESPMNT & $\mathrm{R}$ & & & & & & \\
\hline 2663 & OFFSITE & SESPMNT & $\mathrm{R}$ & & & & & & \\
\hline 2664 & OFFSITE & SESPMNT & $\mathrm{R}$ & & & & & & \\
\hline 2665 & ONSITE & SESPMNT & $\mathrm{R}$ & B12RM1 & LAT/LONG & WGS84 & 46 & 37664 & \\
\hline 2666 & ONSITE & SESPMNT & $\mathrm{R}$ & B12RM6 & LAT/LONG & WGS84 & 46 & 37218 & \\
\hline 2667 & ONSITE & SESPSPEC & S & B12RN4 & LAT/LONG & WGS84 & 46 & 63453 & \\
\hline \begin{tabular}{|l|}
2668 \\
\end{tabular} & ONSITE & SESPSPEC & S & B12RN5 & LAT/LONG & WGS84 & 46 & 37664 & \\
\hline 2669 & ONSITE & SESPSPEC & S & B12RN6 & LAT/LONG & WGS84 & 46 & 37218 & \\
\hline 2670 & ONSITE & SESPSPEC & S & B12RN7 & LAT/LONG & WGS84 & 46 & 63453 & \\
\hline \begin{tabular}{|l|}
2671 \\
\end{tabular} & ONSITE & SESPSPEC & S & B12RP9 & LAT/LONG & WGS84 & 46 & 63453 & \\
\hline \begin{tabular}{|l|}
2672 \\
\end{tabular} & ONSITE & SESPSPEC & S & B12RR1 & LAT/LONG & WGS84 & 46 & 63453 & \\
\hline \begin{tabular}{|l|}
2673 \\
\end{tabular} & ONSITE & SESPSPEC & S & B12RR3 & LAT/LONG & WGS84 & 46 & 63453 & \\
\hline 2674 & ONSITE & SESPSPEC & $S$ & B12RR5 & LAT/LONG & WGS84 & 46 & 37663 & \\
\hline 2675 & ONSITE & SESPSPEC & S & B12RR7 & LAT/LONG & WGS84 & 46 & 37662 & \\
\hline 2676 & ONSITE & SESPSPEC & S & B12RR9 & LAT/LONG & WGS84 & 46 & 37663 & \\
\hline \begin{tabular}{|l|}
2677 \\
\end{tabular} & ONSITE & SESPSPEC & S & B12RT1 & LAT/LONG & WGS84 & 46 & 37547 & \\
\hline \begin{tabular}{|l|}
2678 \\
\end{tabular} & ONSITE & SESPSPEC & S & B12RT3 & LAT/LONG & WGS84 & 46 & 37547 & \\
\hline 2679 & ONSITE & SESPSPEC & S & B12RT5 & LAT/LONG & WGS84 & 46 & 37547 & \\
\hline \begin{tabular}{|l|}
2680 \\
\end{tabular} & ONSITE & SESPSPEC & S & B12RT7 & LAT/LONG & WGS84 & 46 & 37362 & \\
\hline \begin{tabular}{|l|}
2681 \\
\end{tabular} & ONSITE & SESPSPEC & S & B12RT9 & LAT/LONG & WGS84 & 46 & 3722 & \\
\hline \begin{tabular}{|l|}
2682 \\
\end{tabular} & ONSITE & SESPSPEC & S & B12RV1 & LAT/LONG & WGS84 & 46 & 37218 & \\
\hline \begin{tabular}{|l|}
2683 \\
\end{tabular} & ONSITE & SESPSPEC & S & B12RV3 & LAT/LONG & WGS84 & 46 & 37223 & \\
\hline \begin{tabular}{|l|}
2684 \\
\end{tabular} & ONSITE & SESPSPEC & S & B12RV5 & LAT/LONG & WGS84 & 46 & 37166 & \\
\hline 2685 & ONSITE & SESPSPEC & S & B12RV7 & LAT/LONG & WGS84 & 46 & 37168 & \\
\hline \begin{tabular}{|l|}
2686 \\
\end{tabular} & ONSITE & SESPSPEC & S & B12RV9 & LAT/LONG & WGS84 & 46 & 37166 & \\
\hline \begin{tabular}{|l|}
2687 \\
\end{tabular} & ONSITE & SESPSPEC & $\mathrm{S}$ & B12RW1 & LAT/LONG & WGS84 & 46 & 36953 & \\
\hline 2688 & ONSITE & SESPSPEC & S & B12RW5 & LAT/LONG & WGS84 & 46 & 36714 & \\
\hline \begin{tabular}{|l|}
2689 \\
\end{tabular} & ONSITE & SESPSPEC & S & B12RW7 & LAT/LONG & WGS84 & 46 & 36715 & \\
\hline 2690 & ONSITE & SESPSPEC & S & B12RW9 & LAT/LONG & WGS84 & 46 & 36714 & \\
\hline 2691 & ONSITE & SESPSPEC & S & & & & & & \\
\hline \begin{tabular}{|l|}
2692 \\
\end{tabular} & ONSITE & SESPSPEC & S & & & & & & \\
\hline \begin{tabular}{|l|}
2693 \\
\end{tabular} & ONSITE & SESPSPEC & S & & & & & & \\
\hline 2694 & ONSITE & SESPSPEC & S & B12RX9 & LAT/LONG & WGS84 & 46 & 35556 & \\
\hline 2695 & ONSITE & SESPSPEC & S & B12RY1 & LAT/LONG & WGS84 & 46 & 35556 & \\
\hline \begin{tabular}{|l|}
2696 \\
\end{tabular} & ONSITE & SESPSPEC & S & B12RY3 & LAT/LONG & WGS84 & 46 & 35557 & \\
\hline \begin{tabular}{|l|}
2697 \\
\end{tabular} & ONSITE & SESPSPEC & S & B12T11 & LAT/LONG & WGS84 & 46 & 63453 & \\
\hline \begin{tabular}{|l|}
2698 \\
\end{tabular} & ONSITE & SESPSPEC & S & B12T13 & LAT/LONG & WGS84 & 46 & 37661 & \\
\hline \begin{tabular}{|l|}
2699 \\
\end{tabular} & ONSITE & SESPSPEC & S & B12T15 & LAT/LONG & WGS84 & 46 & 37546 & \\
\hline
\end{tabular}




\begin{tabular}{|c|c|c|c|c|c|c|c|c|c|}
\hline & $A G$ & $\mathrm{AH}$ & $\mathrm{Al}$ & $\mathrm{AJ}$ & $\mathrm{AK}$ & $\mathrm{AL}$ & $\mathrm{AM}$ & AN & $\mathrm{AO}$ \\
\hline 1 & DIST_CLASS & OWNER_ID & COLLECTION_PURPOSE & X_Y_COORD_FINAL.SAMP_NUM & COORDINATE SYSTEM & DATUM & LATITUDE ${ }^{\circ} \mathrm{N}$ & LATITUDE MIN_MIN & LATITUDE MIN_SEC \\
\hline 2700 & ONSITE & SESPSPEC & S & B12T17 & LAT/LONG & WGS84 & 46 & 37223 & \\
\hline 2701 & ONSITE & SESPSPEC & S & B12T19 & LAT/LONG & WGS84 & 46 & 37166 & \\
\hline 2702 & ONSITE & SESPSPEC & S & B12T21 & LAT/LONG & WGS84 & 46 & 36716 & \\
\hline 2703 & ONSITE & SESPSPEC & S & & & & & & \\
\hline 2704 & ONSITE & SESPSPEC & S & B12T25 & LAT/LONG & WGS84 & 46 & 35558 & \\
\hline 2705 & OFFSITE & SESPMNT & $\mathrm{R}$ & & & & & & \\
\hline 2706 & OFFSITE & SESPMNT & $\mathrm{R}$ & & & & & & \\
\hline 2707 & ONSITE & SESPSPEC & S & B12T85 & LAT/LONG & & 46 & & 41.01 .827 \\
\hline 2708 & OFFSITE & SESPMNT & $\mathrm{R}$ & B12T87 & LAT/LONG & & 46 & & 38.32 .516 \\
\hline 2709 & OFFSITE & SESPMNT & $\mathrm{R}$ & B12T88 & LAT/LONG & & 46 & & 38.36 .290 \\
\hline 2710 & OFFSITE & SESPMNT & $\mathrm{R}$ & B12T89 & LAT/LONG & & 46 & & 38.40 .091 \\
\hline \begin{tabular}{|l|}
2711 \\
\end{tabular} & OFFSITE & SESPMNT & $\mathrm{R}$ & B12T90 & LAT/LONG & & 46 & & 38.42 .087 \\
\hline 2712 & ONSITE & SESPMNT & $\mathrm{R}$ & B12T91 & LAT/LONG & & 46 & & 41.01 .827 \\
\hline 2713 & ONSITE & SESPMNT & $\mathrm{R}$ & B12T92 & LAT/LONG & & 46 & & 41.02.142 \\
\hline 2714 & ONSITE & SESPMNT & $\mathrm{R}$ & B12T93 & LAT/LONG & & 46 & & 41.8 .044 \\
\hline 2715 & ONSITE & SESPMNT & $\mathrm{R}$ & B12TD4 & LAT/LONG & & 46 & & 41.8 .658 \\
\hline 2716 & ONSITE & SESPMNT & $\mathrm{R}$ & B12TD5 & LAT/LONG & & 46 & & 41.03 .989 \\
\hline 2717 & ONSITE & SESPMNT & $\mathrm{R}$ & B12TD6 & LAT/LONG & & 46 & & 41.8 .070 \\
\hline \begin{tabular}{|l|}
2718 \\
\end{tabular} & ONSITE & SESPMNT & $\mathrm{R}$ & B12TN2 & LAT/LONG & & 46 & & 40.14 .547 \\
\hline 2719 & ONSITE & SESPMNT & $\mathrm{R}$ & B12TN5 & LAT/LONG & & 46 & & 40.43 .042 \\
\hline \begin{tabular}{|l|}
2720 \\
\end{tabular} & ONSITE & SESPMNT & $\mathrm{R}$ & B12TN8 & LAT/LONG & & 46 & & 40.52 .424 \\
\hline \begin{tabular}{|l|}
2721 \\
\end{tabular} & ONSITE & SESPMNT & $\mathrm{R}$ & B12TP1 & LAT/LONG & & 46 & & 41.17 .965 \\
\hline 2722 & ONSITE & SESPMNT & $\mathrm{R}$ & B12T94 & LAT/LONG & & 46 & & 39.35 .60 \\
\hline 2723 & ONSITE & SESPMNT & $\mathrm{R}$ & B12T95 & LAT/LONG & & 46 & & 39.39 .25 \\
\hline \begin{tabular}{|l|}
2724 \\
\end{tabular} & ONSITE & SESPMNT & $\mathrm{R}$ & B12T96 & LAT/LONG & & 46 & & 33.38.38 \\
\hline 2725 & ONSITE & SESPMNT & $\mathrm{R}$ & B12T97 & LAT/LONG & & 46 & & 39.36.77 \\
\hline 2726 & ONSITE & SESPMNT & $\mathrm{R}$ & B12T98 & LAT/LONG & & 46 & & 39.45 .22 \\
\hline \begin{tabular}{|l|}
2727 \\
\end{tabular} & ONSITE & SESPMNT & $\mathrm{R}$ & B12T99 & LAT/LONG & & 46 & & 39.49 .98 \\
\hline \begin{tabular}{|l|}
2728 \\
\end{tabular} & ONSITE & SESPMNT & $\mathrm{R}$ & B12TB0 & LAT/LONG & & 46 & & 39.51 .88 \\
\hline 2729 & ONSITE & SESPMNT & $\mathrm{R}$ & B12TB1 & LAT/LONG & & 46 & & 33.38 .28 \\
\hline \begin{tabular}{|l|}
2730 \\
\end{tabular} & ONSITE & SESPMNT & $\mathrm{R}$ & B12TB2 & LAT/LONG & & 46 & & 33.38.08 \\
\hline \begin{tabular}{|l|}
2731 \\
\end{tabular} & ONSITE & SESPMNT & $\mathrm{R}$ & B12TB3 & LAT/LONG & & 46 & & 33.41 .45 \\
\hline \begin{tabular}{|l|}
2732 \\
\end{tabular} & ONSITE & SESPMNT & $\mathrm{R}$ & B12TB4 & LAT/LONG & & 46 & & 33.42 .84 \\
\hline 2733 & ONSITE & SESPMNT & $\mathrm{R}$ & B12TB5 & LAT/LONG & & 46 & & 33.37 .95 \\
\hline \begin{tabular}{|l|}
2734 \\
\end{tabular} & ONSITE & SESPMNT & $\mathrm{R}$ & B12TF7 & LAT/LONG & & 46 & & 34.50 .68 \\
\hline 2735 & ONSITE & SESPMNT & $\mathrm{R}$ & B12TF8 & LAT/LONG & & 46 & & 34.21 .43 \\
\hline \begin{tabular}{|l|}
2736 \\
\end{tabular} & ONSITE & SESPMNT & $\mathrm{R}$ & B12TF9 & LAT/LONG & & 46 & & 32.44 .93 \\
\hline \begin{tabular}{|l|}
2737 \\
\end{tabular} & ONSITE & SESPMNT & $\mathrm{R}$ & B12TT0 & LAT/LONG & & 46 & & 33.57 .87 \\
\hline
\end{tabular}

Page 540 


\begin{tabular}{|c|c|c|c|c|c|c|c|c|c|}
\hline & AG & $\mathrm{AH}$ & Al & AJ & AK & $\mathrm{AL}$ & AM & AN & $\mathrm{AO}$ \\
\hline 1 & DIST_CLASS & OWNER_ID & COLLECTION_PURPOSE & X_Y_COORD_FINAL.SAMP_NUM & COORDINATE SYSTEM & DATUM & LATITUDE ${ }^{\circ} \mathrm{N}$ & LATITUDE MIN_MIN & LATITUDE MIN_SEC \\
\hline 2738 & ONSITE & SESPMNT & $\mathrm{R}$ & B12TT3 & LAT/LONG & & 46 & & 40.10 .94 \\
\hline 2739 & ONSITE & SESPMNT & $\mathrm{R}$ & B12TT9 & LAT/LONG & & 46 & & 37.2 .31 \\
\hline 2740 & ONSITE & SESPMNT & $\mathrm{R}$ & B12TB6 & LAT/LONG & & 46 & & 21.38 .24 \\
\hline 2741 & ONSITE & SESPMNT & $\mathrm{R}$ & B12TB8 & LAT/LONG & & 46 & & 21.38 .21 \\
\hline 2742 & ONSITE & SESPMNT & $\mathrm{R}$ & B12TC0 & LAT/LONG & & 46 & & 21.37.47 \\
\hline 2743 & ONSITE & SESPMNT & $\mathrm{R}$ & B12TC2 & LAT/LONG & & 46 & & 21.38 .45 \\
\hline 2744 & ONSITE & SESPMNT & $\mathrm{R}$ & B12TC4 & LAT/LONG & & 46 & & 21.37 .97 \\
\hline 2745 & ONSITE & SESPMNT & $\mathrm{R}$ & B12TC6 & LAT/LONG & & 46 & & 21.38 .57 \\
\hline 2746 & OFFSITE & SESPMNT & $\mathrm{R}$ & & & & & & \\
\hline 2747 & OFFSITE & SESPMNT & $\mathrm{R}$ & В12TC9 & LAT/LONG & & 46 & & 18.53 .55 \\
\hline 2748 & OFFSITE & SESPMNT & $\mathrm{R}$ & B12TD0 & LAT/LONG & & 46 & & 18.52 .78 \\
\hline 2749 & OFFSITE & SESPMNT & $\mathrm{R}$ & B12TD1 & LAT/LONG & & 46 & & 18.52 .99 \\
\hline 2750 & OFFSITE & SESPMNT & $\mathrm{R}$ & B12TD2 & LAT/LONG & & 46 & & 18.51.17 \\
\hline 2751 & OFFSITE & SESPMNT & $\mathrm{R}$ & B12TD3 & LAT/LONG & & 46 & & 18.52 .48 \\
\hline 2752 & OFFSITE & SESPMNT & $\mathrm{R}$ & B12TM0 & LAT/LONG & & 46 & & 19.25 .20 \\
\hline 2753 & OFFSITE & SESPMNT & $\mathrm{R}$ & B12TM1 & LAT/LONG & & 46 & & 20.7 .76 \\
\hline 2754 & OFFSITE & SESPMNT & $\mathrm{R}$ & B12TM2 & LAT/LONG & & 46 & & 20.59 .083 \\
\hline 2755 & OFFSITE & SESPMNT & $\mathrm{R}$ & B12TM3 & LAT/LONG & & 46 & & 21.20 .88 \\
\hline 2756 & ONSITE & SESPMNT & $\mathrm{R}$ & B12TP4 & LAT/LONG & & 46 & & 23.2.95 \\
\hline 2757 & ONSITE & SESPMNT & $\mathrm{R}$ & B12TP8 & LAT/LONG & & 46 & & 22.35 .97 \\
\hline 2758 & ONSITE & SESPMNT & $\mathrm{R}$ & B12TR2 & LAT/LONG & & 46 & & 22.15.61 \\
\hline 2759 & ONSITE & SESPMNT & $\mathrm{R}$ & B12TR6 & LAT/LONG & & 46 & & 21.48 .54 \\
\hline 2760 & ONSITE & SESPMNT & $\mathrm{R}$ & B12XK2 & LAT/LONG & & 46 & & 22.20 .036 \\
\hline 2761 & ONSITE & SESPSPEC & S & B12XK8 & LAT/LONG & & 46 & & 22.20 .059 \\
\hline 2762 & ONSITE & SESPSPEC & $\mathrm{S}$ & B12XK9 & LAT/LONG & & 46 & & 22.20 .059 \\
\hline 2763 & ONSITE & SESPSPEC & $\mathrm{S}$ & B12XL0 & LAT/LONG & & 46 & & 22.20 .084 \\
\hline 2764 & ONSITE & SESPSPEC & $S$ & B12XL1 & LAT/LONG & & 46 & & 22.20 .084 \\
\hline 2765 & ONSITE & SESPSPEC & $S$ & B12XL2 & LAT/LONG & & 46 & & 22.20 .031 \\
\hline 2766 & ONSITE & SESPMNT & $\mathrm{R}$ & B12XK1 & LAT/LONG & & 46 & & 22.36 .037 \\
\hline 2767 & ONSITE & SESPSPEC & S & B12XK3 & LAT/LONG & & 46 & & 22.35 .976 \\
\hline 2768 & ONSITE & SESPSPEC & S & B12XK4 & LAT/LONG & & 46 & & 22.35 .976 \\
\hline 2769 & ONSITE & SESPSPEC & S & B12XK5 & LAT/LONG & & 46 & & 22.35 .976 \\
\hline 2770 & ONSITE & SESPSPEC & S & B12XK6 & LAT/LONG & & 46 & & 22.36 .045 \\
\hline 2771 & ONSITE & SESPSPEC & S & B12XK7 & LAT/LONG & & 46 & & 22.36 .045 \\
\hline 2772 & OFFSITE & SESPMNT & $\mathrm{R}$ & & & & & & \\
\hline 2773 & OFFSITE & SESPMNT & $\mathrm{R}$ & & & & & & \\
\hline 2774 & ONSITE & SESPMNT & $\mathrm{R}$ & & & & & & \\
\hline 2775 & ONSITE & SESPMNT & $\mathrm{R}$ & B12X48 & LAT/LONG & & 46 & & 42.41 .7 \\
\hline
\end{tabular}




\begin{tabular}{|c|c|c|c|c|c|c|c|c|c|}
\hline & $A G$ & $\mathrm{AH}$ & $\mathrm{Al}$ & $\mathrm{AJ}$ & AK & $\mathrm{AL}$ & AM & $\mathrm{AN}$ & $\mathrm{AO}$ \\
\hline 1 & DIST_CLASS & OWNER_ID & COLLECTION_PURPOSE & X_Y_COORD_FINAL.SAMP_NUM & COORDINATE SYSTEM & \begin{tabular}{|l|} 
DATUM \\
\end{tabular} & LATITUDE ${ }^{\circ} \mathrm{N}$ & LATITUDE MIN_MIN & LATITUDE MIN_SEC \\
\hline 2776 & ONSITE & SESPMNT & $\mathrm{R}$ & B12X85 & LAT/LONG & & 46 & & 42.12 .8 \\
\hline 2777 & OFFSITE & SESPMNT & $\mathrm{R}$ & & & & & & \\
\hline 2778 & OFFSITE & SESPMNT & $\mathrm{R}$ & & & & & & \\
\hline 2779 & ONSITE & SESPSPEC & S & & & & & & \\
\hline 2780 & OFFSITE & SESPMNT & $\mathrm{R}$ & & & & & & \\
\hline 2781 & OFFSITE & SESPMNT & $\mathrm{R}$ & & & & & & \\
\hline 2782 & OFFSITE & SESPMNT & $\mathrm{R}$ & B13LF3 & LAT/LONG & & 46 & & 38.33.72 \\
\hline 2783 & OFFSITE & SESPMNT & $\mathrm{R}$ & B13LF4 & LAT/LONG & & 46 & & 38.36 .24 \\
\hline 2784 & OFFSITE & SESPMNT & $\mathrm{R}$ & B13LF5 & LAT/LONG & & 46 & & 38.40 .19 \\
\hline 2785 & OFFSITE & SESPMNT & $\mathrm{R}$ & B13LF6 & LAT/LONG & & 46 & & 38.42 .52 \\
\hline 2786 & OFFSITE & SESPSPEC & $S$ & B13LF2 & LAT/LONG & & 46 & & 18.54 .62 \\
\hline 2787 & OFFSITE & SESPMNT & $\mathrm{R}$ & B13LF7 & LAT/LONG & & 46 & & 18.54 .62 \\
\hline 2788 & OFFSITE & SESPMNT & $\mathrm{R}$ & B13LF8 & LAT/LONG & & 46 & & 18.52 .90 \\
\hline 2789 & OFFSITE & SESPMNT & $\mathrm{R}$ & B13LF9 & LAT/LONG & & 46 & & 18.52 .68 \\
\hline 2790 & OFFSITE & SESPMNT & $\mathrm{R}$ & B13LH0 & LAT/LONG & & 46 & & 18.53 .97 \\
\hline 2791 & OFFSITE & SESPMNT & $\mathrm{R}$ & B13LH1 & LAT/LONG & & 46 & & 18.53 .50 \\
\hline 2792 & OFFSITE & SESPMNT & $\mathrm{R}$ & B13LH2 & LAT/LONG & & 46 & & 19.52 .07 \\
\hline 2793 & OFFSITE & SESPMNT & $\mathrm{R}$ & B13LJ3 & LAT/LONG & & 46 & & 19.24 .77 \\
\hline 2794 & OFFSITE & SESPMNT & $R$ & B13LJ4 & LAT/LONG & & 46 & & 20.07 .46233 \\
\hline 2795 & OFFSITE & SESPMNT & $\mathrm{R}$ & B13LJ5 & LAT/LONG & & 46 & & 20.59 .29 \\
\hline 2796 & OFFSITE & SESPMNT & $\mathrm{R}$ & B13LJ6 & LAT/LONG & & 46 & & 21.21.83 \\
\hline 2797 & OFFSITE & SESPMNT & $\mathrm{R}$ & & & & & & \\
\hline 2798 & OFFSITE & SESPMNT & $\mathrm{R}$ & & & & & & \\
\hline 2799 & OFFSITE & SESPMNT & $\mathrm{R}$ & & & & & & \\
\hline 2800 & OFFSITE & SESPMNT & $\mathrm{R}$ & & & & & & \\
\hline 2801 & OFFSITE & SESPMNT & $\mathrm{R}$ & & & & & & \\
\hline 2802 & OFFSITE & SESPMNT & $\mathrm{R}$ & & & & & & \\
\hline 2803 & OFFSITE & SESPMNT & $\mathrm{R}$ & B147T3 & LAT/LONG & & 46 & & 38.32 .125 \\
\hline 2804 & OFFSITE & SESPMNT & $\mathrm{R}$ & B147T4 & LAT/LONG & & 46 & & 38.33 .902 \\
\hline 2805 & OFFSITE & SESPMNT & $\mathrm{R}$ & B147T5 & LAT/LONG & & 46 & & 38.40 .586 \\
\hline 2806 & OFFSITE & SESPMNT & $\mathrm{R}$ & B147T6 & LAT/LONG & & 46 & & 38.41 .856 \\
\hline 2807 & OFFSITE & SESPSPEC & $S$ & B147T2 & LAT/LONG & & 46 & & 18.54 .797 \\
\hline 2808 & OFFSITE & SESPMNT & $\mathrm{R}$ & B147T7 & LAT/LONG & & 46 & & 18.54 .797 \\
\hline 2809 & OFFSITE & SESPMNT & $\mathrm{R}$ & B147T8 & LAT/LONG & & 46 & & 18.53 .211 \\
\hline 2810 & OFFSITE & SESPMNT & $\mathrm{R}$ & B147T9 & LAT/LONG & & 46 & & 18.52 .338 \\
\hline 2811 & OFFSITE & SESPMNT & $\mathrm{R}$ & B147V0 & LAT/LONG & & 46 & & 18.52.299 \\
\hline 2812 & OFFSITE & SESPMNT & $\mathrm{R}$ & B147V1 & LAT/LONG & & 46 & & 18.51 .346 \\
\hline 2813 & OFFSITE & SESPMNT & $\mathrm{R}$ & B147V2 & LAT/LONG & & 46 & & 18.50 .697 \\
\hline
\end{tabular}




\begin{tabular}{|c|c|c|c|c|c|c|c|c|c|}
\hline & $A G$ & $\mathrm{AH}$ & $\mathrm{Al}$ & $\mathrm{AJ}$ & AK & $\mathrm{AL}$ & AM & $\mathrm{AN}$ & $\mathrm{AO}$ \\
\hline 1 & DIST_CLASS & OWNER_ID & COLLECTION_PURPOSE & X_Y_COORD_FINAL.SAMP_NUM & COORDINATE SYSTEM & \begin{tabular}{|l|} 
DATUM \\
\end{tabular} & LATITUDE ${ }^{\circ} \mathrm{N}$ & LATITUDE MIN_MIN & LATITUDE MIN_SEC \\
\hline 2814 & OFFSITE & SESPMNT & $\mathrm{R}$ & B147W3 & LAT/LONG & & 46 & & 19.24 .739 \\
\hline 2815 & OFFSITE & SESPMNT & $\mathrm{R}$ & B147W4 & LAT/LONG & & 46 & & 20.7 .138 \\
\hline 2816 & OFFSITE & SESPMNT & $\mathrm{R}$ & B147W5 & LAT/LONG & & 46 & & 20.59 .129 \\
\hline 2817 & OFFSITE & SESPMNT & $\mathrm{R}$ & B147W6 & LAT/LONG & & 46 & & 21.20 .938 \\
\hline 2818 & OFFSITE & SESPMNT & $\mathrm{R}$ & & & & & & \\
\hline 2819 & OFFSITE & SESPMNT & $\mathrm{R}$ & & & & & & \\
\hline 2820 & OFFSITE & SESPMNT & $\mathrm{R}$ & & & & & & \\
\hline 2821 & OFFSITE & SESPMNT & $\mathrm{R}$ & & & & & & \\
\hline 2822 & OFFSITE & SESPMNT & $\mathrm{R}$ & B14LJ0 & LAT/LONG & & 46 & 25832 & \\
\hline 2823 & PERIMETER & SESPMNT & $\mathrm{R}$ & B14LJ2 & LAT/LONG & & 46 & 34120 & \\
\hline 2824 & OFFSITE & SESPMNT & $\mathrm{R}$ & & & & & & \\
\hline 2825 & OFFSITE & SESPMNT & $\mathrm{R}$ & & & & & & \\
\hline 2826 & OFFSITE & SESPMNT & $\mathrm{R}$ & B14RV4 & LAT/LONG & & 46 & & 38.32.346 \\
\hline 2827 & OFFSITE & SESPMNT & $\mathrm{R}$ & B14RV5 & LAT/LONG & & 46 & & 38.35 .710 \\
\hline 2828 & OFFSITE & SESPMNT & $\mathrm{R}$ & B14RV6 & LAT/LONG & & 46 & & 38.40 .744 \\
\hline 2829 & OFFSITE & SESPMNT & $\mathrm{R}$ & B14RV7 & LAT/LONG & & 46 & & 38.43 .263 \\
\hline 2830 & OFFSITE & SESPMNT & $\mathrm{R}$ & B14RV8 & LAT/LONG & & 46 & & 18.54 .564 \\
\hline 2831 & OFFSITE & SESPMNT & $\mathrm{R}$ & B14RV9 & LAT/LONG & & 46 & & 18.50 .434 \\
\hline 2832 & OFFSITE & SESPMNT & $R$ & B14RW0 & LAT/LONG & & 46 & & 18.51 .872 \\
\hline 2833 & OFFSITE & SESPMNT & $\mathrm{R}$ & B14RW1 & LAT/LONG & & 46 & & 18.52 .771 \\
\hline 2834 & OFFSITE & SESPMNT & $\mathrm{R}$ & B14RW2 & LAT/LONG & & 46 & & 18.48 .011 \\
\hline 2835 & OFFSITE & SESPMNT & $\mathrm{R}$ & B14RW3 & LAT/LONG & & 46 & & 18.52 .018 \\
\hline 2836 & OFFSITE & SESPMNT & $\mathrm{R}$ & B14RX4 & LAT/LONG & & 46 & & 19.24.503 \\
\hline 2837 & OFFSITE & SESPMNT & $\mathrm{R}$ & B14RX5 & LAT/LONG & & 46 & & 20.06.731 \\
\hline 2838 & OFFSITE & SESPMNT & $\mathrm{R}$ & B14RX6 & LAT/LONG & & 46 & & 20.59 .569 \\
\hline 2839 & OFFSITE & SESPMNT & $\mathrm{R}$ & B14RX7 & LAT/LONG & & 46 & & 21.21.521 \\
\hline 2840 & OFFSITE & SESPMNT & $\mathrm{R}$ & & & & & & \\
\hline 2841 & OFFSITE & SESPMNT & $\mathrm{R}$ & & & & & & \\
\hline 2842 & OFFSITE & SESPMNT & $\mathrm{R}$ & & & & & & \\
\hline 2843 & PERIMETER & SESPMNT & $\mathrm{R}$ & & & & & & \\
\hline 2844 & OFFSITE & SESPMNT & $\mathrm{R}$ & & & & & & \\
\hline 2845 & PERIMETER & SESPMNT & $\mathrm{R}$ & & & & & & \\
\hline 2846 & OFFSITE & SESPMNT & $\mathrm{R}$ & & & & & & \\
\hline 2847 & OFFSITE & SESPMNT & $\mathrm{R}$ & & & & & & \\
\hline 2848 & OFFSITE & SESPMNT & $\mathrm{R}$ & & & & & & \\
\hline 2849 & OFFSITE & SESPMNT & $\mathrm{R}$ & & & & & & \\
\hline 2850 & ONSITE & SESPSPEC & S & B158H3 & LAT/LONG & & 46 & & 41.02 .29 \\
\hline 2851 & OFFSITE & SESPMNT & $\mathrm{R}$ & B158H4 & LAT/LONG & & 46 & & 38.42 .82 \\
\hline
\end{tabular}




\begin{tabular}{|c|c|c|c|c|c|c|c|c|c|}
\hline & $A G$ & $\mathrm{AH}$ & $\mathrm{Al}$ & $\mathrm{AJ}$ & $\mathrm{AK}$ & $\mathrm{AL}$ & $\mathrm{AM}$ & AN & $\mathrm{AO}$ \\
\hline 1 & DIST_CLASS & OWNER_ID & COLLECTION_PURPOSE & X_Y_COORD_FINAL.SAMP_NUM & COORDINATE SYSTEM & DATUM & LATITUDE ${ }^{\circ} \mathrm{N}$ & LATITUDE MIN_MIN & LATITUDE MIN_SEC \\
\hline 2852 & OFFSITE & SESPMNT & $\mathrm{R}$ & B158H5 & LAT/LONG & & 46 & & 38.35 .52 \\
\hline 2853 & OFFSITE & SESPMNT & $\mathrm{R}$ & B158H6 & LAT/LONG & & 46 & & 38.39 .47 \\
\hline 2854 & OFFSITE & SESPMNT & $\mathrm{R}$ & B158H7 & LAT/LONG & & 46 & & 38.31 .26 \\
\hline 2855 & ONSITE & SESPMNT & $\mathrm{R}$ & B158H8 & LAT/LONG & & 46 & & 41.02 .29 \\
\hline 2856 & ONSITE & SESPMNT & $\mathrm{R}$ & B158H9 & LAT/LONG & & 46 & & 41.03 .77 \\
\hline 2857 & ONSITE & SESPMNT & $\mathrm{R}$ & B158J0 & LAT/LONG & & 46 & & 41.11 .21 \\
\hline 2858 & ONSITE & SESPMNT & $\mathrm{R}$ & B158M7 & LAT/LONG & & 46 & & 41.10 .20 \\
\hline 2859 & ONSITE & SESPMNT & $\mathrm{R}$ & B158M8 & LAT/LONG & & 46 & & 41.06 .12 \\
\hline 2860 & ONSITE & SESPMNT & $\mathrm{R}$ & B158M9 & LAT/LONG & & 46 & & 41.09 .52 \\
\hline 2861 & ONSITE & SESPMNT & $\mathrm{R}$ & B158W8 & LAT/LONG & & 46 & & 40.14 .09 \\
\hline 2862 & ONSITE & SESPMNT & $\mathrm{R}$ & B158X1 & LAT/LONG & & 46 & & 40.43 .18 \\
\hline 2863 & ONSITE & SESPMNT & $\mathrm{R}$ & B158X4 & LAT/LONG & & 46 & & 40.51 .95 \\
\hline 2864 & ONSITE & SESPMNT & $\mathrm{R}$ & B158X7 & LAT/LONG & & 46 & & 41.18 .54 \\
\hline 2865 & ONSITE & SESPMNT & $\mathrm{R}$ & B158J1 & LAT/LONG & & 46 & & 39.35 .36 \\
\hline 2866 & ONSITE & SESPMNT & $\mathrm{R}$ & B158J3 & LAT/LONG & & 46 & & 39.39 .60 \\
\hline 2867 & ONSITE & SESPMNT & $\mathrm{R}$ & B158J5 & LAT/LONG & & 46 & & 33.39 .50 \\
\hline 2868 & ONSITE & SESPMNT & $\mathrm{R}$ & B158J7 & LAT/LONG & & 46 & & 39.37 .16 \\
\hline 2869 & ONSITE & SESPMNT & $\mathrm{R}$ & B158J9 & LAT/LONG & & 46 & & 39.44 .48 \\
\hline 2870 & ONSITE & SESPMNT & $\mathrm{R}$ & B158K1 & LAT/LONG & & 46 & & 39.48 .58 \\
\hline 2871 & ONSITE & SESPMNT & $\mathrm{R}$ & B158K3 & LAT/LONG & & 46 & & 39.51 .94 \\
\hline 2872 & ONSITE & SESPMNT & $\mathrm{R}$ & B158K5 & LAT/LONG & & 46 & & 33.40 .64 \\
\hline 2873 & ONSITE & SESPMNT & $\mathrm{R}$ & B158K7 & LAT/LONG & & 46 & & 33.40 .63 \\
\hline 2874 & ONSITE & SESPMNT & $\mathrm{R}$ & B158K9 & LAT/LONG & & 46 & & 33.42 .44 \\
\hline 2875 & ONSITE & SESPMNT & $\mathrm{R}$ & B158L1 & LAT/LONG & & 46 & & 33.43 .598 \\
\hline 2876 & ONSITE & SESPMNT & $\mathrm{R}$ & B158L3 & LAT/LONG & & 46 & & 33.40 .47 \\
\hline 2877 & ONSITE & SESPMNT & $\mathrm{R}$ & B158N0 & LAT/LONG & & 46 & & 34.50 .32 \\
\hline 2878 & ONSITE & SESPMNT & $\mathrm{R}$ & B158N2 & LAT/LONG & & 46 & & 34.21 .43 \\
\hline 2879 & ONSITE & SESPMNT & $\mathrm{R}$ & B158N4 & LAT/LONG & & 46 & & 32.44 .22 \\
\hline 2880 & ONSITE & SESPMNT & $\mathrm{R}$ & B15902 & LAT/LONG & & 46 & & 33.57 .46 \\
\hline 2881 & ONSITE & SESPMNT & $\mathrm{R}$ & B15906 & LAT/LONG & & 46 & & 40.10 .84 \\
\hline 2882 & ONSITE & SESPMNT & $\mathrm{R}$ & B15910 & LAT/LONG & & 46 & & 36.22 .01 \\
\hline 2883 & ONSITE & SESPMNT & $\mathrm{R}$ & B15914 & LAT/LONG & & 46 & & 37.02 .66 \\
\hline 2884 & ONSITE & SESPMNT & $\mathrm{R}$ & B158L5 & LAT/LONG & & 46 & & 21.37.20 \\
\hline 2885 & ONSITE & SESPMNT & $\mathrm{R}$ & B158L6 & LAT/LONG & & 46 & & 21.36.55 \\
\hline 2886 & ONSITE & SESPMNT & $\mathrm{R}$ & B158L7 & LAT/LONG & & 46 & & 21.35 .70 \\
\hline 2887 & ONSITE & SESPMNT & $\mathrm{R}$ & B158L8 & LAT/LONG & & 46 & & 21.37 .67 \\
\hline 2888 & ONSITE & SESPMNT & $\mathrm{R}$ & B158L9 & LAT/LONG & & 46 & & 21.38.28 \\
\hline 2889 & ONSITE & SESPMNT & $\mathrm{R}$ & B158M0 & LAT/LONG & & 46 & & 21.38.48 \\
\hline
\end{tabular}




\begin{tabular}{|c|c|c|c|c|c|c|c|c|c|}
\hline & $A G$ & $\mathrm{AH}$ & $\mathrm{Al}$ & $\mathrm{AJ}$ & $\mathrm{AK}$ & $\mathrm{AL}$ & $\mathrm{AM}$ & AN & $\mathrm{AO}$ \\
\hline 1 & DIST_CLASS & OWNER_ID & COLLECTION_PURPOSE & X_Y_COORD_FINAL.SAMP_NUM & COORDINATE SYSTEM & DATUM & LATITUDE ${ }^{\circ} \mathrm{N}$ & LATITUDE MIN_MIN & LATITUDE MIN_SEC \\
\hline 2890 & OFFSITE & SESPMNT & $\mathrm{R}$ & B158M1 & LAT/LONG & & 46 & & 18.54 .5 \\
\hline 2891 & OFFSITE & SESPMNT & $\mathrm{R}$ & B158M2 & LAT/LONG & & 46 & & 18.52.7 \\
\hline 2892 & OFFSITE & SESPMNT & $\mathrm{R}$ & B158M3 & LAT/LONG & & 46 & & 18.85 .0 \\
\hline 2893 & OFFSITE & SESPMNT & $\mathrm{R}$ & B158M4 & LAT/LONG & & 46 & & 18.51 .50 \\
\hline 2894 & OFFSITE & SESPMNT & $\mathrm{R}$ & B158M5 & LAT/LONG & & 46 & & 18.51.37 \\
\hline 2895 & OFFSITE & SESPMNT & $\mathrm{R}$ & B158M6 & LAT/LONG & & 46 & & 18.52.36 \\
\hline 2896 & OFFSITE & SESPMNT & $\mathrm{R}$ & B158V6 & LAT/LONG & & 46 & & 19.24 .75 \\
\hline 2897 & OFFSITE & SESPMNT & $\mathrm{R}$ & B158V7 & LAT/LONG & & 46 & & 20.07 .17 \\
\hline 2898 & OFFSITE & SESPMNT & $\mathrm{R}$ & B158V8 & LAT/LONG & & 46 & & 20.58 .56 \\
\hline 2899 & OFFSITE & SESPMNT & $\mathrm{R}$ & B158V9 & LAT/LONG & & 46 & & 21.21 .49 \\
\hline 2900 & ONSITE & SESPMNT & $\mathrm{R}$ & B158Y0 & LAT/LONG & & 46 & & 23.03 .00 \\
\hline 2901 & ONSITE & SESPMNT & $\mathrm{R}$ & B158Y3 & LAT/LONG & & 46 & & 22.35 .44 \\
\hline 2902 & ONSITE & SESPMNT & $\mathrm{R}$ & B158Y6 & LAT/LONG & & 46 & & 22.19 .60 \\
\hline 2903 & ONSITE & SESPMNT & $\mathrm{R}$ & B158Y9 & LAT/LONG & & 46 & & 21.48 .39 \\
\hline 2904 & OFFSITE & SESPMNT & $\mathrm{R}$ & & & & & & \\
\hline 2905 & OFFSITE & SESPMNT & $\mathrm{R}$ & & & & & & \\
\hline 2906 & ONSITE & SESPMNT & $\mathrm{R}$ & B15C09 & LAT/LONG & & 46 & & 33.55 .313 \\
\hline 2907 & ONSITE & SESPMNT & $\mathrm{R}$ & & & & & & \\
\hline 2908 & ONSITE & SESPMNT & $\mathrm{R}$ & B15C73 & LAT/LONG & & 46 & & 33.50 .499 \\
\hline 2909 & ONSITE & SESPMNT & $\mathrm{R}$ & B15CB3 & LAT/LONG & & 46 & & 42.12 .884 \\
\hline 2910 & ONSITE & SESPMNT & $\mathrm{R}$ & B15C69 & LAT/LONG & & 46 & & 38.14 .440 \\
\hline 2911 & OFFSITE & SESPMNT & $\mathrm{R}$ & & & & & & \\
\hline 2912 & OFFSITE & SESPMNT & $\mathrm{R}$ & & & & & & \\
\hline 2913 & OFFSITE & SESPMNT & $\mathrm{R}$ & & & & & & \\
\hline 2914 & OFFSITE & SESPMNT & $\mathrm{R}$ & & & & & & \\
\hline 2915 & OFFSITE & SESPMNT & $\mathrm{R}$ & B16342 & LAT/LONG & & 46 & 38544193 & \\
\hline 2916 & OFFSITE & SESPMNT & $\mathrm{R}$ & B16343 & LAT/LONG & & 46 & 3861303 & \\
\hline 2917 & OFFSITE & SESPMNT & $\mathrm{R}$ & B16344 & LAT/LONG & & 46 & 384081025 & \\
\hline 2918 & OFFSITE & SESPMNT & $\mathrm{R}$ & B16345 & LAT/LONG & & 46 & 384290077 & \\
\hline 2919 & OFFSITE & SESPSPEC & S & B16341 & LAT/LONG & & 46 & 185442945 & \\
\hline 2920 & OFFSITE & SESPMNT & $\mathrm{R}$ & B16346 & LAT/LONG & & 46 & 185442945 & \\
\hline 2921 & OFFSITE & SESPMNT & $\mathrm{R}$ & B16347 & LAT/LONG & & 46 & 18519365 & \\
\hline 2922 & OFFSITE & SESPMNT & $\mathrm{R}$ & B16348 & LAT/LONG & & 46 & 184989755 & \\
\hline 2923 & OFFSITE & SESPMNT & $\mathrm{R}$ & B16349 & LAT/LONG & & 46 & 185086976 & \\
\hline 2924 & OFFSITE & SESPMNT & $\mathrm{R}$ & B16350 & LAT/LONG & & 46 & 185163798 & \\
\hline 2925 & OFFSITE & SESPMNT & $\mathrm{R}$ & B16351 & LAT/LONG & & 46 & 185184158 & \\
\hline 2926 & OFFSITE & SESPMNT & $\mathrm{R}$ & B16362 & LAT/LONG & & 46 & 192473133 & \\
\hline 2927 & OFFSITE & SESPMNT & $\mathrm{R}$ & B16363 & LAT/LONG & & 46 & 200718556 & \\
\hline
\end{tabular}




\begin{tabular}{|c|c|c|c|c|c|c|c|c|c|}
\hline & $A G$ & $\mathrm{AH}$ & $\mathrm{Al}$ & AJ & AK & $\overline{A L}$ & AM & AN & $\mathrm{AO}$ \\
\hline 1 & DIST_CLASS & OWNER_ID & COLLECTION_PURPOSE & X_Y_COORD_FINAL.SAMP_NUM & COORDINATE SYSTEM & DATUM & LATITUDE ${ }^{\circ} \mathrm{N}$ & LATITUDE MIN_MIN & LATITUDE MIN_SEC \\
\hline 2928 & OFFSITE & SESPMNT & $\mathrm{R}$ & B16364 & LAT/LONG & & 46 & 205947829 & \\
\hline 2929 & OFFSITE & SESPMNT & $\mathrm{R}$ & B16365 & LAT/LONG & & 46 & 212135241 & \\
\hline
\end{tabular}




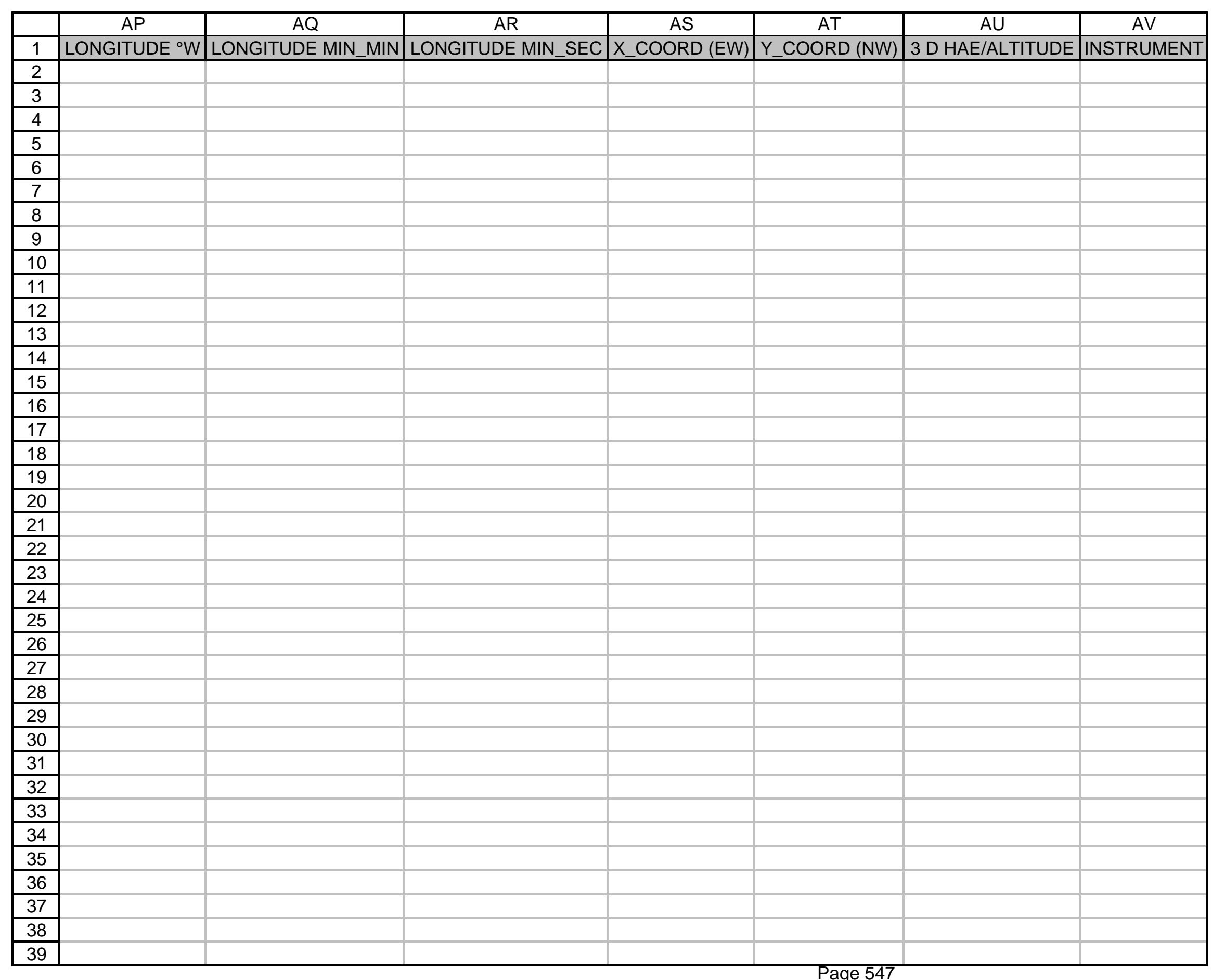




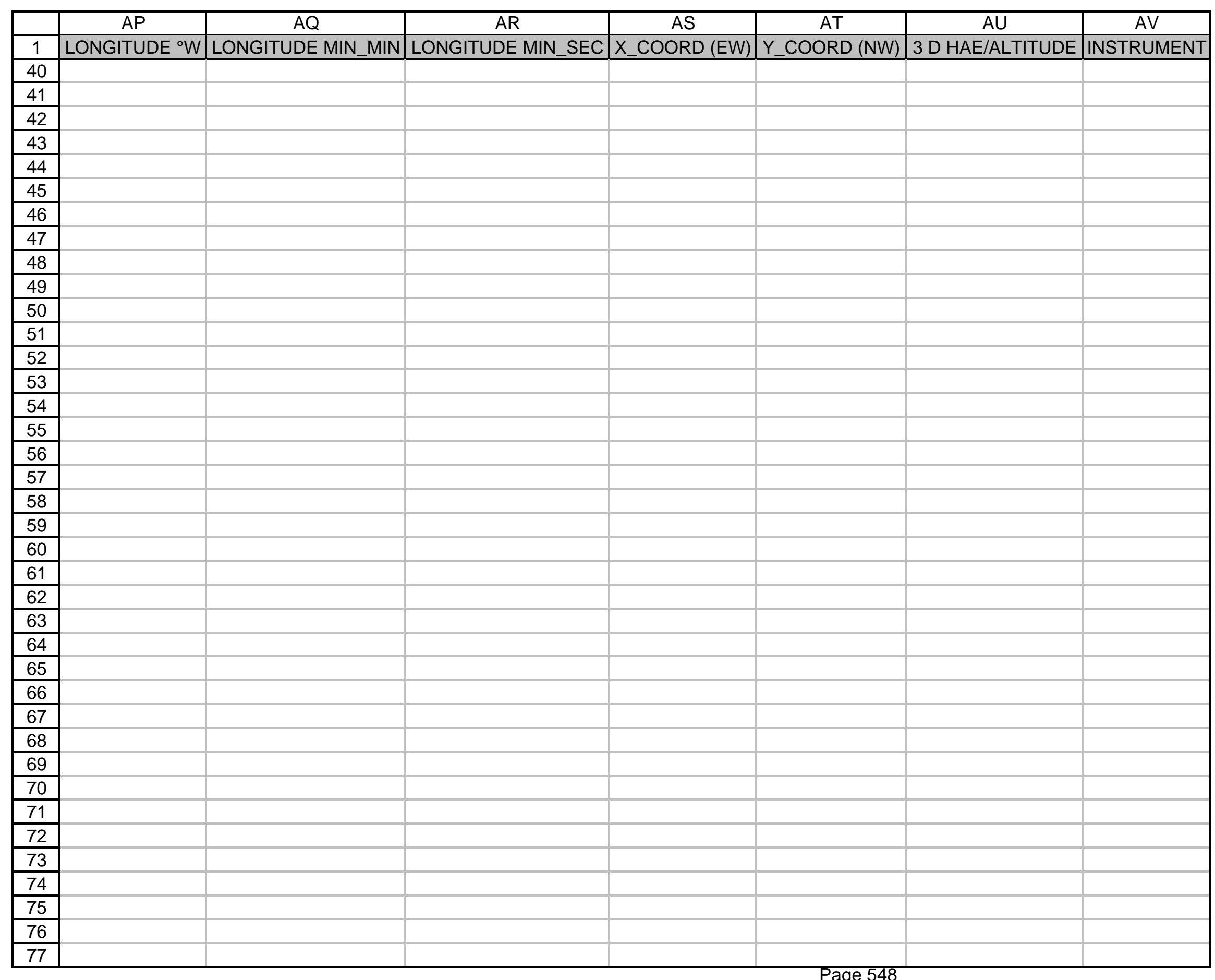




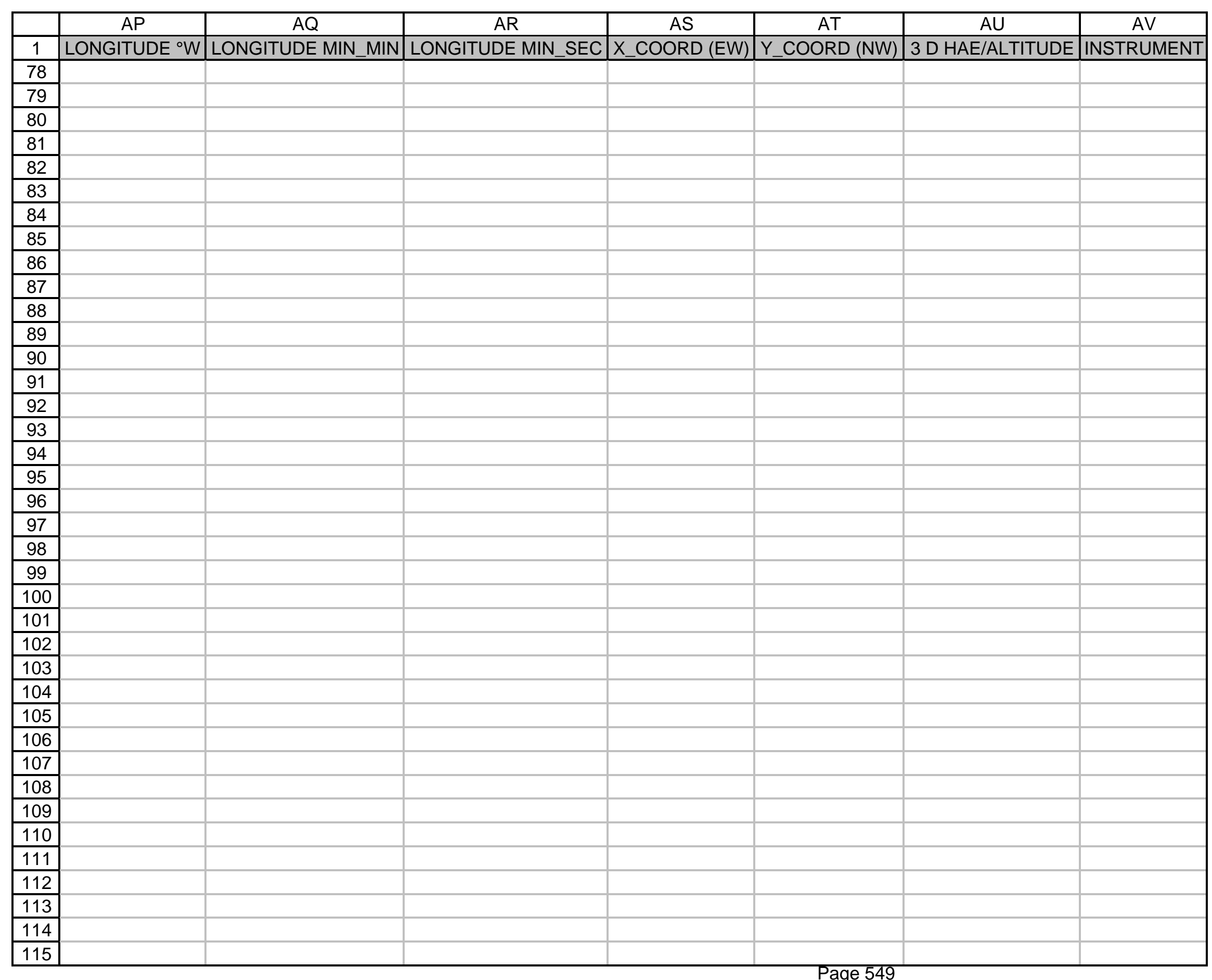




\begin{tabular}{|c|c|c|c|c|c|c|c|}
\hline & $\mathrm{AP}$ & $\mathrm{AQ}$ & AR & AS & AT & $\mathrm{AU}$ & $\mathrm{AV}$ \\
\hline 1 & $\mid$ LONGITUDE $^{\circ} \mathrm{W}$ & LONGITUDE MIN_MIN & LONGITUDE MIN_SEC & X_COORD (EW) & Y_COORD (NW) & 3 D HAE/ALTITUDE & INSTRUMENT \\
\hline \multicolumn{8}{|c|}{116} \\
\hline \multicolumn{8}{|l|}{117} \\
\hline \multicolumn{8}{|l|}{118} \\
\hline \multicolumn{8}{|l|}{119} \\
\hline 120 & & & & 589172 & 137602 & & \\
\hline 121 & & & & 594392 & 116700 & 82 & \\
\hline 122 & & & & 588808 & 137808 & 94 & \\
\hline 123 & & & & 589438 & 137451 & & \\
\hline 124 & & & & 594489 & 116208 & 82.5 & \\
\hline 125 & & & & 593883 & 112747 & 100 & \\
\hline \multicolumn{8}{|l|}{126} \\
\hline \multicolumn{8}{|l|}{127} \\
\hline \multicolumn{8}{|c|}{128} \\
\hline \multicolumn{8}{|l|}{129} \\
\hline \multicolumn{8}{|l|}{130} \\
\hline \multicolumn{8}{|l|}{131} \\
\hline \multicolumn{8}{|l|}{$\begin{array}{l}+01 \\
132 \\
\end{array}$} \\
\hline \multicolumn{8}{|l|}{133} \\
\hline \multicolumn{8}{|l|}{134} \\
\hline \multicolumn{8}{|l|}{135} \\
\hline \multicolumn{8}{|l|}{136} \\
\hline \multicolumn{8}{|l|}{137} \\
\hline \multicolumn{8}{|l|}{138} \\
\hline \multicolumn{8}{|l|}{139} \\
\hline \multicolumn{8}{|l|}{140} \\
\hline \multicolumn{8}{|l|}{141} \\
\hline \multicolumn{8}{|l|}{142} \\
\hline \multicolumn{8}{|l|}{143} \\
\hline \multicolumn{8}{|l|}{144} \\
\hline \multicolumn{8}{|l|}{145} \\
\hline \multicolumn{8}{|l|}{146} \\
\hline 147 & & & & & & & \\
\hline 148 & & & & & & & \\
\hline 149 & & & & & & & \\
\hline 150 & & & & & & & \\
\hline 151 & & & & & & & \\
\hline 152 & & & & & & & \\
\hline 153 & & & & & & & \\
\hline
\end{tabular}




\begin{tabular}{|c|c|c|c|c|c|c|c|}
\hline & $\mathrm{AP}$ & $\mathrm{AQ}$ & AR & AS & AT & $\mathrm{AU}$ & $\mathrm{AV}$ \\
\hline 1 & LONGITUDE ${ }^{\circ} \mathrm{W}$ & LONGITUDE MIN_MIN & LONGITUDE MIN_SEC & X_COORD (EW) & Y_COORD (NW) & 3 D HAE/ALTITUDE & INSTRUMENT \\
\hline \multicolumn{8}{|l|}{154} \\
\hline \multicolumn{8}{|l|}{155} \\
\hline \multicolumn{8}{|l|}{156} \\
\hline \multicolumn{8}{|l|}{157} \\
\hline \multicolumn{8}{|l|}{158} \\
\hline \multicolumn{8}{|l|}{159} \\
\hline \multicolumn{8}{|l|}{160} \\
\hline \multicolumn{8}{|l|}{161} \\
\hline \multicolumn{8}{|l|}{162} \\
\hline \multicolumn{8}{|l|}{163} \\
\hline \multicolumn{8}{|l|}{164} \\
\hline \multicolumn{8}{|l|}{165} \\
\hline \multicolumn{8}{|l|}{166} \\
\hline \multicolumn{8}{|l|}{167} \\
\hline \multicolumn{8}{|l|}{168} \\
\hline \multicolumn{8}{|l|}{169} \\
\hline \multicolumn{8}{|l|}{170} \\
\hline \multicolumn{8}{|l|}{171} \\
\hline \multicolumn{8}{|l|}{172} \\
\hline \multicolumn{8}{|l|}{173} \\
\hline \multicolumn{8}{|l|}{174} \\
\hline \multicolumn{8}{|l|}{175} \\
\hline \multicolumn{8}{|l|}{176} \\
\hline \multicolumn{8}{|l|}{177} \\
\hline \multicolumn{8}{|l|}{178} \\
\hline 179 & & & & & & & \\
\hline 180 & 119 & & 33.46 .410 & & & & \\
\hline 181 & 119 & & 39.21 .806 & & & & \\
\hline 182 & 119 & & 34.48 .990 & & & & \\
\hline 183 & & & & & & & \\
\hline 184 & & & & & & & \\
\hline 185 & & & & & & & \\
\hline 186 & & & & & & & \\
\hline 187 & & & & & & & \\
\hline 188 & & & & & & & \\
\hline 189 & & & & & & & \\
\hline 190 & & & & & & & \\
\hline 191 & & & & & & & \\
\hline
\end{tabular}




\begin{tabular}{|c|c|c|c|c|c|c|c|}
\hline & AP & $\overline{\mathrm{AQ}}$ & AR & AS & AT & $A U$ & AV \\
\hline 1 & LONGITUDE ${ }^{\circ} \mathrm{W}$ & LONGITUDE MIN_MIN & LONGITUDE MIN_SEC & X_COORD (EW) & Y_COORD (NW) & 3 D HAE/ALTITUDE & INSTRUMENT \\
\hline \multicolumn{8}{|c|}{ 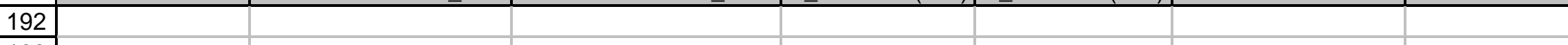 } \\
\hline \multicolumn{8}{|l|}{193} \\
\hline \multicolumn{8}{|l|}{194} \\
\hline \multicolumn{8}{|l|}{195} \\
\hline \multicolumn{8}{|l|}{196} \\
\hline \multicolumn{8}{|l|}{197} \\
\hline \multicolumn{8}{|l|}{198} \\
\hline \multicolumn{8}{|l|}{199} \\
\hline \multicolumn{8}{|l|}{200} \\
\hline \multicolumn{8}{|l|}{201} \\
\hline \multicolumn{8}{|l|}{202} \\
\hline \multicolumn{8}{|l|}{203} \\
\hline \multicolumn{8}{|l|}{204} \\
\hline 205 & 119 & 27295 & & 594392.9328 & 116697.2814 & & GPS \\
\hline 206 & 119 & 27173 & & 594494.7346 & 116202.6015 & & GPS \\
\hline 207 & 119 & 74824 & & 557558.6994 & 144902.4606 & & GPS \\
\hline 208 & 119 & 74824 & & 557558.6994 & 144902.4606 & & GPS \\
\hline 209 & 119 & 74824 & & 557558.6994 & 144902.4606 & & GPS \\
\hline 210 & 119 & 27287 & & 594399.2531 & 116695.838 & & GPS \\
\hline 211 & 119 & 27283 & & 594402.1182 & 116695.2311 & & GPS \\
\hline 212 & 119 & 2728 & & 594404.9572 & 116696.5481 & & GPS \\
\hline 213 & 119 & 27265 & & 594418.067 & 116567.4607 & & GPS \\
\hline 214 & 119 & 27262 & & 594420.161 & 116567.6005 & & GPS \\
\hline 215 & 119 & 27255 & & 594425.6435 & 116567.8565 & & GPS \\
\hline 216 & 119 & 27227 & & 594450.3417 & 116362.2444 & & GPS \\
\hline 217 & 119 & 27172 & & 594495.7216 & 116205.7327 & & GPS \\
\hline 218 & 119 & 27167 & & 594499.4158 & 116203.1668 & & GPS \\
\hline 219 & 119 & 27163 & & 594501.9964 & 116209.2038 & & GPS \\
\hline 220 & 119 & 27154 & & 594510.2381 & 116145.7579 & & GPS \\
\hline 221 & 119 & 27152 & & 594511.5832 & 116147.8569 & & GPS \\
\hline 222 & 119 & 27143 & & 594518.7508 & 116146.0857 & & GPS \\
\hline 223 & 119 & 27079 & & 594571.7032 & 115909.9801 & & GPS \\
\hline 224 & 119 & 2699 & & 594644.1671 & 115645.5062 & & GPS \\
\hline 225 & 119 & 26987 & & 594646.1043 & 115645.9576 & & GPS \\
\hline 226 & 119 & 26986 & & 594647.3591 & 115645.6143 & & GPS \\
\hline \multicolumn{8}{|l|}{227} \\
\hline \multicolumn{8}{|l|}{228} \\
\hline 229 & & & & & & & \\
\hline
\end{tabular}




\begin{tabular}{|c|c|c|c|c|c|c|c|}
\hline & $\mathrm{AP}$ & $\mathrm{AQ}$ & $\overline{A R}$ & AS & AT & $\overline{A U}$ & $\mathrm{AV}$ \\
\hline 1 & LONGITUDE ${ }^{\circ} \mathrm{W}$ & LONGITUDE MIN_MIN & LONGITUDE MIN_SEC & X_COORD (EW) & Y_COORD (NW) & 3 D HAE/ALTITUDE & INSTRUMENT \\
\hline 230 & 119 & 26697 & & 594889.7354 & 114361.6456 & & GPS \\
\hline 231 & 119 & 26693 & & 594892.5316 & 114361.8727 & & GPS \\
\hline 232 & 119 & 26692 & & 594893.2853 & 114362.3731 & & GPS \\
\hline 233 & 119 & 74824 & & 557558.6994 & 144902.4606 & & GPS \\
\hline 234 & 119 & 27276 & & 594407.8154 & 116693.7483 & & GPS \\
\hline 235 & 119 & 27252 & & 594428.029 & 116566.4842 & & GPS \\
\hline 236 & 119 & 27154 & & 594508.882 & 116209.1859 & & GPS \\
\hline 237 & 119 & 27137 & & 594523.0808 & 116145.8184 & & GPS \\
\hline 238 & 119 & 26982 & & 594650.1201 & 115647.6407 & & GPS \\
\hline 239 & & & & & & & \\
\hline 240 & 119 & 26688 & & 594896.5766 & 114364.2648 & & GPS \\
\hline 241 & & & & & & & \\
\hline 242 & & & & & & & \\
\hline 243 & & & & & & & \\
\hline 244 & & & & & & & \\
\hline 245 & 119 & & 16.18.498 & & & & \\
\hline 246 & 119 & & 16.18.450 & & & & \\
\hline 247 & 119 & & 16.18.450 & & & & \\
\hline 248 & 119 & & 16.18.119 & & & & \\
\hline 249 & 119 & & 16.18.119 & & & & \\
\hline 250 & 119 & & 16.17.828 & & & & \\
\hline 251 & 119 & & 16.22 .585 & & & & \\
\hline 252 & 119 & & 16.22.369 & & & & \\
\hline 253 & 119 & & 16.22.369 & & & & \\
\hline 254 & 119 & & 16.22.369 & & & & \\
\hline 255 & 119 & & 16.22.143 & & & & \\
\hline 256 & 119 & & 16.22.143 & & & & \\
\hline 257 & & & & & & & \\
\hline 258 & & & & & & & \\
\hline 259 & & & & & & & \\
\hline 260 & & & & & & & \\
\hline 261 & & & & & & & \\
\hline 262 & & & & & & & \\
\hline 263 & & & & & & & \\
\hline 264 & 119 & & 33.46 .90 & & & & \\
\hline 265 & 119 & & 36.23 .99 & & & & \\
\hline 266 & 119 & & 35.28 .29 & & & & \\
\hline 267 & 119 & & 29.23 .4 & & & & \\
\hline
\end{tabular}

Page 553 


\begin{tabular}{|c|c|c|c|c|c|c|c|}
\hline & AP & $\mathrm{AQ}$ & AR & AS & AT & $A U$ & $\mathrm{AV}$ \\
\hline 1 & LONGITUDE ${ }^{\circ} \mathrm{W}$ & LONGITUDE MIN_MIN & LONGITUDE MIN_SEC & X_COORD (EW) & Y_COORD (NW) & 3 D HAE/ALTITUDE & INSTRUMENT \\
\hline 268 & 119 & & 28.38 .8 & & & & \\
\hline \multicolumn{8}{|l|}{269} \\
\hline \multicolumn{8}{|l|}{270} \\
\hline \multicolumn{8}{|l|}{271} \\
\hline \multicolumn{8}{|l|}{272} \\
\hline \multicolumn{8}{|l|}{273} \\
\hline \multicolumn{8}{|l|}{274} \\
\hline \multicolumn{8}{|l|}{275} \\
\hline \multicolumn{8}{|l|}{276} \\
\hline \multicolumn{8}{|l|}{277} \\
\hline \multicolumn{8}{|l|}{278} \\
\hline \multicolumn{8}{|l|}{279} \\
\hline \multicolumn{8}{|l|}{280} \\
\hline \multicolumn{8}{|l|}{281} \\
\hline \multicolumn{8}{|l|}{282} \\
\hline \multicolumn{8}{|l|}{283} \\
\hline \multicolumn{8}{|l|}{284} \\
\hline \multicolumn{8}{|l|}{285} \\
\hline \multicolumn{8}{|l|}{286} \\
\hline \multicolumn{8}{|l|}{287} \\
\hline \multicolumn{8}{|l|}{288} \\
\hline \multicolumn{8}{|l|}{289} \\
\hline \multicolumn{8}{|l|}{290} \\
\hline \multicolumn{8}{|c|}{291} \\
\hline \multicolumn{8}{|l|}{292} \\
\hline \multicolumn{8}{|l|}{293} \\
\hline 294 & & & & & & & \\
\hline 295 & & & & & & & \\
\hline 296 & & & & & & & \\
\hline 297 & & & & & & & \\
\hline 298 & & & & & & & \\
\hline 299 & & & & & & & \\
\hline 300 & & & & & & & \\
\hline 301 & 119 & 18039 & & & & & \\
\hline 302 & 119 & 28027 & & & & & \\
\hline 303 & & & & & & & \\
\hline 304 & & & & & & & \\
\hline 305 & & & & & & & \\
\hline
\end{tabular}




\begin{tabular}{|c|c|c|c|c|c|c|c|}
\hline & $\mathrm{AP}$ & $\mathrm{AQ}$ & AR & AS & AT & $\mathrm{AU}$ & AV \\
\hline 1 & LONGITUDE ${ }^{\circ} \mathrm{W}$ & LONGITUDE MIN_MIN & LONGITUDE MIN_SEC & X_COORD (EW) & Y_COORD (NW) & 3 D HAE/ALTITUDE & INSTRUMENT \\
\hline \multicolumn{8}{|l|}{306} \\
\hline \multicolumn{8}{|l|}{307} \\
\hline \multicolumn{8}{|l|}{308} \\
\hline \multicolumn{8}{|l|}{309} \\
\hline \multicolumn{8}{|l|}{310} \\
\hline \multicolumn{8}{|l|}{311} \\
\hline \multicolumn{8}{|l|}{312} \\
\hline \multicolumn{8}{|l|}{313} \\
\hline \multicolumn{8}{|l|}{314} \\
\hline \multicolumn{8}{|l|}{315} \\
\hline \multicolumn{8}{|l|}{316} \\
\hline \multicolumn{8}{|l|}{317} \\
\hline \multicolumn{8}{|l|}{318} \\
\hline \multicolumn{8}{|l|}{319} \\
\hline \multicolumn{8}{|c|}{320} \\
\hline \multicolumn{8}{|c|}{321} \\
\hline \multicolumn{8}{|l|}{322} \\
\hline \multicolumn{8}{|l|}{323} \\
\hline \multicolumn{8}{|l|}{324} \\
\hline \multicolumn{8}{|l|}{325} \\
\hline \multicolumn{8}{|l|}{326} \\
\hline \multicolumn{8}{|l|}{327} \\
\hline \multicolumn{8}{|l|}{328} \\
\hline \multicolumn{8}{|l|}{329} \\
\hline \multicolumn{8}{|l|}{330} \\
\hline 331 & 119 & & 20.10 .491 & & & & \\
\hline 332 & & & & & & & \\
\hline 333 & 119 & & 20.00 .067 & & & & \\
\hline 334 & & & & & & & \\
\hline 335 & 119 & & 35.28 .175 & & & & \\
\hline 336 & 119 & & 32.14 .9785 & & & & \\
\hline 337 & 119 & & 28.38.784 & & & & \\
\hline 338 & 119 & & 25.12.798 & & & & \\
\hline 339 & & & & & & & \\
\hline 340 & & & & & & & \\
\hline 341 & & & & & & & \\
\hline 342 & & & & & & & \\
\hline 343 & & & & & & & \\
\hline
\end{tabular}




\begin{tabular}{|c|c|c|c|c|c|c|c|}
\hline & $\mathrm{AP}$ & $\mathrm{AQ}$ & AR & AS & AT & $\mathrm{AU}$ & $\mathrm{AV}$ \\
\hline 1 & LONGITUDE ${ }^{\circ} \mathrm{W}$ & LONGITUDE MIN_MIN & LONGITUDE MIN_SEC & X_COORD (EW) & Y_COORD (NW) & 3 D HAE/ALTITUDE & INSTRUMENT \\
\hline \multicolumn{8}{|l|}{344} \\
\hline \multicolumn{8}{|l|}{345} \\
\hline \multicolumn{8}{|l|}{346} \\
\hline \multicolumn{8}{|l|}{347} \\
\hline \multicolumn{8}{|l|}{348} \\
\hline \multicolumn{8}{|l|}{349} \\
\hline \multicolumn{8}{|l|}{350} \\
\hline 351 & & & & 589172 & 137602 & & \\
\hline 352 & & & & 594392 & 116700 & 82 & \\
\hline 353 & & & & 588808 & 137808 & 94 & \\
\hline 354 & & & & 589438 & 137451 & & \\
\hline 355 & & & & 594489 & 116208 & 82.5 & \\
\hline \multicolumn{8}{|l|}{356} \\
\hline \multicolumn{8}{|c|}{357} \\
\hline \multicolumn{8}{|c|}{358} \\
\hline \multicolumn{8}{|c|}{359} \\
\hline \multicolumn{8}{|c|}{360} \\
\hline \multicolumn{8}{|c|}{361} \\
\hline \multicolumn{8}{|l|}{362} \\
\hline \multicolumn{8}{|l|}{363} \\
\hline \multicolumn{8}{|l|}{364} \\
\hline \multicolumn{8}{|l|}{365} \\
\hline \multicolumn{8}{|l|}{366} \\
\hline \multicolumn{8}{|l|}{367} \\
\hline 368 & 119 & 27295 & & 594392.9328 & 116697.2814 & & GPS \\
\hline 369 & 119 & 27173 & & 594494.7346 & 116202.6015 & & GPS \\
\hline \multicolumn{8}{|c|}{370} \\
\hline \multicolumn{8}{|l|}{371} \\
\hline \multicolumn{8}{|l|}{372} \\
\hline \multicolumn{8}{|l|}{373} \\
\hline \multicolumn{8}{|l|}{374} \\
\hline \multicolumn{8}{|c|}{375} \\
\hline 376 & & & & & & & \\
\hline 377 & & & & & & & \\
\hline 378 & & & & & & & \\
\hline 379 & & & & & & & \\
\hline 380 & & & & & & & \\
\hline 381 & & & & & & & \\
\hline
\end{tabular}




\begin{tabular}{|c|c|c|c|c|c|c|c|}
\hline & $\mathrm{AP}$ & $\mathrm{AQ}$ & AR & AS & AT & $A U$ & AV \\
\hline 1 & LONGITUDE ${ }^{\circ} \mathrm{W}$ & LONGITUDE MIN_MIN & LONGITUDE MIN_SEC & X_COORD (EW) & Y_COORD (NW) & 3 D HAE/ALTITUDE & INSTRUMENT \\
\hline 382 & & & & & & & \\
\hline 383 & & & & & & & \\
\hline 384 & & & & & & & \\
\hline 385 & & & & & & & \\
\hline 386 & & & & & & & \\
\hline 387 & & & & & & & \\
\hline 388 & & & & & & & \\
\hline 389 & & & & & & & \\
\hline 390 & & & & & & & \\
\hline 391 & & & & & & & \\
\hline 392 & & & & & & & \\
\hline 393 & & & & & & & \\
\hline 394 & & & & & & & \\
\hline 395 & & & & & & & \\
\hline 396 & & & & & & & \\
\hline 397 & & & & & & & \\
\hline 398 & & & & & & & \\
\hline 399 & & & & & & & \\
\hline 400 & & & & & & & \\
\hline 401 & & & & & & & \\
\hline 402 & & & & & & & \\
\hline 403 & & & & & & & \\
\hline 404 & & & & & & & \\
\hline 405 & & & & & & & \\
\hline 406 & & & & & & & \\
\hline 407 & & & & & & & \\
\hline 408 & & & & & & & \\
\hline 409 & & & & & & & \\
\hline 410 & & & & & & & \\
\hline 411 & & & & & & & \\
\hline 412 & & & & & & & \\
\hline 413 & & & & & & & \\
\hline 414 & & & & & & & \\
\hline 415 & & & & & & & \\
\hline 416 & & & & & & & \\
\hline 417 & & & & & & & \\
\hline 418 & & & & & & & \\
\hline 419 & & & & & & & \\
\hline
\end{tabular}




\begin{tabular}{|c|c|c|c|c|c|c|c|}
\hline & AP & $\mathrm{AQ}$ & AR & AS & AT & $A U$ & AV \\
\hline 1 & LONGITUDE ${ }^{\circ} \mathrm{W}$ & LONGITUDE MIN_MIN & LONGITUDE MIN_SEC & X_COORD (EW) & Y_COORD (NW) & 3 D HAE/ALTITUDE & INSTRUMENT \\
\hline \multicolumn{8}{|l|}{420} \\
\hline \multicolumn{8}{|l|}{421} \\
\hline \multicolumn{8}{|l|}{422} \\
\hline \multicolumn{8}{|l|}{423} \\
\hline \multicolumn{8}{|l|}{424} \\
\hline \multicolumn{8}{|l|}{425} \\
\hline \multicolumn{8}{|l|}{426} \\
\hline \multicolumn{8}{|l|}{427} \\
\hline \multicolumn{8}{|l|}{428} \\
\hline \multicolumn{8}{|l|}{429} \\
\hline \multicolumn{8}{|l|}{430} \\
\hline \multicolumn{8}{|l|}{431} \\
\hline \multicolumn{8}{|l|}{432} \\
\hline \multicolumn{8}{|l|}{433} \\
\hline \multicolumn{8}{|l|}{434} \\
\hline \multicolumn{8}{|l|}{435} \\
\hline \multicolumn{8}{|l|}{436} \\
\hline \multicolumn{8}{|l|}{437} \\
\hline \multicolumn{8}{|l|}{438} \\
\hline \multicolumn{8}{|l|}{439} \\
\hline \multicolumn{8}{|l|}{440} \\
\hline \multicolumn{8}{|l|}{441} \\
\hline \multicolumn{8}{|l|}{442} \\
\hline \multicolumn{8}{|l|}{443} \\
\hline \multicolumn{8}{|l|}{444} \\
\hline 445 & & & & & & & \\
\hline 446 & & & & & & & \\
\hline 447 & & & & & & & \\
\hline 448 & & & & & & & \\
\hline 449 & & & & & & & \\
\hline 450 & & & & & & & \\
\hline 451 & & & & & & & \\
\hline 452 & & & & & & & \\
\hline 453 & & & & 145775 & 559128 & & \\
\hline 454 & & & & 145888 & 559110 & & \\
\hline 455 & & & & 146030 & 559077 & & \\
\hline 456 & & & & 146118 & 559037 & & \\
\hline 457 & & & & 109886 & 595516 & & \\
\hline
\end{tabular}




\begin{tabular}{|c|c|c|c|c|c|c|c|}
\hline & $\mathrm{AP}$ & $\mathrm{AQ}$ & AR & AS & AT & $\mathrm{AU}$ & $\mathrm{AV}$ \\
\hline 1 & LONGITUDE ${ }^{\circ} \mathrm{W}$ & LONGITUDE MIN_MIN & LONGITUDE MIN_SEC & X_COORD (EW) & Y_COORD (NW) & 3 D HAE/ALTITUDE & INSTRUMENT \\
\hline 458 & & & & 109861 & 595700 & & \\
\hline 459 & & & & 109840 & 595880 & & \\
\hline 460 & & & & 109811 & 596091 & & \\
\hline 461 & & & & 109824 & 596212 & & \\
\hline 462 & & & & 109822 & 596358 & & \\
\hline 463 & & & & 111001.9 & 595469.9 & & \\
\hline 464 & & & & 112119.9 & 595260.9 & & \\
\hline 465 & & & & 113946.4 & 594945.8 & & \\
\hline 466 & & & & 114400.8 & 594886.3 & & \\
\hline \multicolumn{8}{|l|}{467} \\
\hline \multicolumn{8}{|l|}{468} \\
\hline \multicolumn{8}{|l|}{469} \\
\hline \multicolumn{8}{|l|}{470} \\
\hline \multicolumn{8}{|l|}{471} \\
\hline \multicolumn{8}{|l|}{472} \\
\hline \multicolumn{8}{|l|}{473} \\
\hline \multicolumn{8}{|l|}{474} \\
\hline \multicolumn{8}{|l|}{475} \\
\hline \multicolumn{8}{|l|}{476} \\
\hline \multicolumn{8}{|l|}{477} \\
\hline 478 & & & & 571726 & 150552 & 95.5 & \\
\hline 479 & & & & 559152 & 145816 & 100 & \\
\hline 480 & & & & 559123 & 145934 & 101 & \\
\hline 481 & & & & 558979 & 145994 & 99.4 & \\
\hline 482 & & & & 559021 & 146105 & 101 & \\
\hline 483 & & & & 571726 & 150552 & 95.5 & \\
\hline 484 & & & & 571712 & 150603 & 95.1 & \\
\hline \multicolumn{8}{|l|}{485} \\
\hline 486 & & & & 571385 & 150788 & 97.2 & \\
\hline 487 & & & & 571652 & 150596 & 96.1 & \\
\hline \multicolumn{8}{|l|}{488} \\
\hline \multicolumn{8}{|l|}{489} \\
\hline 490 & & & & 571313 & 149952 & 98 & \\
\hline \multicolumn{8}{|l|}{491} \\
\hline 492 & & & & 572099 & 151070 & 95.2 & \\
\hline 493 & & & & 581213 & 147964 & 92.8 & GPS \\
\hline 494 & & & & 581313 & 148169 & 92 & GPS \\
\hline 495 & & & & 590188 & 137123 & 87.2 & GPS \\
\hline
\end{tabular}




\begin{tabular}{|c|c|c|c|c|c|c|c|}
\hline & $\mathrm{AP}$ & $\overline{A Q}$ & AR & AS & AT & $\overline{A U}$ & AV \\
\hline 1 & LONGITUDE ${ }^{\circ} \mathrm{W}$ & LONGITUDE MIN_MIN & LONGITUDE MIN_SEC & X_COORD (EW) & Y_COORD (NW) & 3 D HAE/ALTITUDE & INSTRUMENT \\
\hline 496 & & & & 581253 & 148028 & 91.7 & GPS \\
\hline 497 & & & & 581443 & 148274 & 92.6 & GPS \\
\hline 498 & & & & 581502 & 148393 & 90 & GPS \\
\hline 499 & & & & 581543 & 148471 & 88 & GPS \\
\hline 500 & & & & 589999 & 137077 & 87.5 & GPS \\
\hline 501 & & & & 590039 & 137078 & 86.9 & GPS \\
\hline 502 & & & & 590732 & 137195 & 86.5 & GPS \\
\hline 503 & & & & 590268 & 137247 & 86.8 & GPS \\
\hline 504 & & & & 590087 & 137068 & 86.6 & GPS \\
\hline 505 & & & & 586752 & 139253 & 87.2 & GPS \\
\hline 506 & & & & 587946 & 138371 & 86.9 & GPS \\
\hline 507 & & & & 591113 & 135398 & 85 & GPS \\
\hline 508 & & & & 589105 & 137662 & 74.9 & GPS \\
\hline 509 & & & & 579910 & 149070 & 92 & GPS \\
\hline 510 & & & & 583186 & 144426 & 88.5 & GPS \\
\hline 511 & & & & 583498 & 143300 & 85.9 & GPS \\
\hline \multicolumn{8}{|l|}{512} \\
\hline \multicolumn{8}{|l|}{513} \\
\hline 514 & & & & 594916 & 114856 & 81.6 & GPS \\
\hline 515 & & & & 595098 & 114923 & 81.6 & GPS \\
\hline 516 & & & & 595395 & 114927 & 83 & GPS \\
\hline 517 & & & & 595472 & 114951 & 83 & GPS \\
\hline 518 & & & & 595518 & 109870 & 82.2 & GPS \\
\hline 519 & & & & 595563 & 109836 & 82.7 & GPS \\
\hline 520 & & & & 595677 & 109831 & 83.4 & GPS \\
\hline 521 & & & & 595869 & 109857 & 82.3 & GPS \\
\hline 522 & & & & 596226 & 109758 & 83.3 & GPS \\
\hline 523 & & & & 596363 & 109808 & 82.8 & GPS \\
\hline 524 & & & & 595483 & 110804 & 82.4 & GPS \\
\hline 525 & & & & 595278 & 112118 & 82.2 & GPS \\
\hline 526 & & & & 954956 & 113692 & 83 & GPS \\
\hline 527 & & & & 594889 & 114400 & 83.4 & GPS \\
\hline 528 & & & & 594314 & 117539 & 82.8 & GPS \\
\hline 529 & & & & 594396 & 116699 & 82.8 & GPS \\
\hline 530 & & & & 594540 & 116076 & 82.6 & GPS \\
\hline 531 & & & & 594738 & 115238 & 83.2 & GPS \\
\hline 532 & & & & 594392 & 116700 & 82 & \\
\hline 533 & & & & 594489 & 116208 & 82.5 & \\
\hline
\end{tabular}




\begin{tabular}{|c|c|c|c|c|c|c|c|}
\hline & AP & AQ & AR & AS & AT & $A U$ & AV \\
\hline 1 & LONGITUDE ${ }^{\circ} \mathrm{W}$ & LONGITUDE MIN_MIN & LONGITUDE MIN_SEC & X_COORD (EW) & Y_COORD (NW) & 3 D HAE/ALTITUDE & INSTRUMENT \\
\hline 534 & & & & 593883 & 112747 & 100 & \\
\hline 535 & & & & & & & \\
\hline 536 & & & & & & & \\
\hline 537 & & & & & & & \\
\hline 538 & & & & & & & \\
\hline 539 & & & & & & & \\
\hline 540 & & & & & & & \\
\hline 541 & & & & & & & \\
\hline 542 & & & & & & & \\
\hline 543 & & & & & & & \\
\hline 544 & & & & & & & \\
\hline 545 & & & & & & & \\
\hline 546 & & & & & & & \\
\hline 547 & & & & & & & \\
\hline 548 & & & & & & & \\
\hline 549 & & & & & & & \\
\hline 550 & & & & & & & \\
\hline 551 & & & & & & & \\
\hline 552 & & & & & & & \\
\hline 553 & & & & & & & \\
\hline 554 & & & & & & & \\
\hline 555 & & & & & & & \\
\hline 556 & & & & 559150 & 145822 & 99.7 & GPS \\
\hline 557 & & & & 559160 & 145921 & 100.9 & GPS \\
\hline 558 & & & & 559069 & 146021 & 99 & GPS \\
\hline 559 & & & & 559036 & 146104.449 & 98 & GPS \\
\hline 560 & & & & 595517 & 109875 & 83 & GPS \\
\hline 561 & & & & 595517 & 109875 & 83 & GPS \\
\hline 562 & & & & 595577 & 109840 & 84 & GPS \\
\hline 563 & & & & 595726 & 109770 & 81 & GPS \\
\hline 564 & & & & 595872 & 109798 & 84 & GPS \\
\hline 565 & & & & 596159 & 109608 & 80.7 & GPS \\
\hline 566 & & & & 596362 & 109803 & 89.1 & GPS \\
\hline 567 & & & & 595484 & 110809 & 83 & GPS \\
\hline 568 & & & & 595270 & 112116 & 84 & GPS \\
\hline 569 & & & & 594955 & 113780 & 83 & GPS \\
\hline 570 & & & & 594887 & 114396 & 84 & GPS \\
\hline 571 & & & & & & & \\
\hline
\end{tabular}

Page 561 


\begin{tabular}{|c|c|c|c|c|c|c|c|}
\hline & AP & $\overline{A Q}$ & AR & AS & AT & $\overline{A U}$ & AV \\
\hline 1 & LONGITUDE ${ }^{\circ} \mathrm{W}$ & LONGITUDE MIN_MIN & LONGITUDE MIN_SEC & X_COORD (EW) & Y_COORD (NW) & 3 D HAE/ALTITUDE & INSTRUMENT \\
\hline \multicolumn{8}{|l|}{572} \\
\hline \multicolumn{8}{|l|}{573} \\
\hline \multicolumn{8}{|l|}{574} \\
\hline \multicolumn{8}{|l|}{575} \\
\hline \multicolumn{8}{|l|}{576} \\
\hline \multicolumn{8}{|l|}{577} \\
\hline \multicolumn{8}{|l|}{578} \\
\hline 579 & 119 & & 15.35 .53 & & & & \\
\hline 580 & 119 & & 15.35 .53 & & & & \\
\hline 581 & 119 & & 15.32 .09 & & & & \\
\hline 582 & 119 & & 15.28 .44 & & & & \\
\hline 583 & 119 & & 15.17.99 & & & & \\
\hline 584 & 119 & & 15.05.39 & & & & \\
\hline \multicolumn{8}{|l|}{585} \\
\hline 586 & 119 & & 15.35 .50 & & & & \\
\hline \multicolumn{8}{|c|}{587} \\
\hline 588 & 119 & & 15.58 .34 & & & & \\
\hline 589 & 119 & & 16.02.37 & & & & \\
\hline 590 & 119 & & 43.41 .36 & & & & \\
\hline 591 & 119 & & 43.42 .62 & & & & \\
\hline 592 & 119 & & 43.42 .99 & & & & \\
\hline 593 & 119 & & 43.46 .54 & & & & \\
\hline \multicolumn{8}{|l|}{594} \\
\hline \multicolumn{8}{|l|}{595} \\
\hline \multicolumn{8}{|l|}{596} \\
\hline \multicolumn{8}{|l|}{597} \\
\hline \multicolumn{8}{|l|}{598} \\
\hline \multicolumn{8}{|l|}{599} \\
\hline \multicolumn{8}{|l|}{600} \\
\hline \multicolumn{8}{|l|}{601} \\
\hline \multicolumn{8}{|l|}{602} \\
\hline \multicolumn{8}{|l|}{603} \\
\hline \multicolumn{8}{|l|}{604} \\
\hline \multicolumn{8}{|l|}{605} \\
\hline \multicolumn{8}{|l|}{606} \\
\hline \multicolumn{8}{|l|}{607} \\
\hline 608 & 119 & & 33.46 .410 & & & & \\
\hline 609 & 119 & & 39.21 .806 & & & & \\
\hline
\end{tabular}




\begin{tabular}{|c|c|c|c|c|c|c|c|}
\hline & $\mathrm{AP}$ & $\mathrm{AQ}$ & AR & AS & AT & $\mathrm{AU}$ & $\mathrm{AV}$ \\
\hline 1 & LONGITUDE ${ }^{\circ} \mathrm{W}$ & LONGITUDE MIN_MIN & LONGITUDE MIN_SEC & X_COORD (EW) & Y_COORD (NW) & 3 D HAE/ALTITUDE & INSTRUMENT \\
\hline 610 & 119 & & 34.48 .990 & & & & \\
\hline \multicolumn{8}{|c|}{611} \\
\hline \multicolumn{8}{|l|}{612} \\
\hline \multicolumn{8}{|l|}{613} \\
\hline \multicolumn{8}{|l|}{614} \\
\hline \multicolumn{8}{|l|}{615} \\
\hline \multicolumn{8}{|l|}{616} \\
\hline \multicolumn{8}{|l|}{617} \\
\hline \multicolumn{8}{|l|}{618} \\
\hline 619 & 119 & & 15.35.167 & & & & \\
\hline 620 & 119 & & 15.32.854 & & & & \\
\hline 621 & 119 & & 15.25 .556 & & & & \\
\hline 622 & 119 & & 15.18.321 & & & & \\
\hline 623 & 119 & & 15.05 .22609 & & & & \\
\hline 624 & 119 & & 14.55 .469 & & & & \\
\hline 625 & 119 & & 15.35.841 & & & & \\
\hline 626 & 119 & & 15.45 .231 & & & & \\
\hline 627 & 119 & & 15.58 .444 & & & & \\
\hline 628 & 119 & & 16.01.079 & & & & \\
\hline 629 & 119 & & 43.38 .457 & & & & \\
\hline 630 & 119 & & 43.39 .10128 & & & & \\
\hline 631 & 119 & & 43.45 .782 & & & & \\
\hline 632 & 119 & & 43.45 .852 & & & & \\
\hline \multicolumn{8}{|c|}{633} \\
\hline \multicolumn{8}{|l|}{634} \\
\hline \multicolumn{8}{|l|}{635} \\
\hline \multicolumn{8}{|c|}{636} \\
\hline \multicolumn{8}{|l|}{637} \\
\hline \multicolumn{8}{|l|}{638} \\
\hline \multicolumn{8}{|l|}{639} \\
\hline \multicolumn{8}{|l|}{640} \\
\hline \multicolumn{8}{|l|}{641} \\
\hline \multicolumn{8}{|l|}{642} \\
\hline 643 & 119 & 27295 & & 594392.9328 & 116697.2814 & & GPS \\
\hline 644 & 119 & 27173 & & 594494.7346 & 116202.6015 & & GPS \\
\hline \multicolumn{8}{|c|}{645} \\
\hline \multicolumn{8}{|l|}{646} \\
\hline 647 & 119 & & 33.46 .160 & & & & \\
\hline
\end{tabular}




\begin{tabular}{|c|c|c|c|c|c|c|c|}
\hline & $\mathrm{AP}$ & $\mathrm{AQ}$ & AR & AS & AT & $\mathrm{AU}$ & AV \\
\hline 1 & LONGITUDE ${ }^{\circ} \mathrm{W}$ & LONGITUDE MIN MIN & LONGITUDE MIN SEC & X COORD (EW) & Y COORD (NW) & 3 D HAE/ALTITUDE & INSTRUMENT \\
\hline 648 & 119 & & 43.42 .160 & & & & \\
\hline 649 & 119 & & 43.44 .211 & & & & \\
\hline 650 & 119 & & 43.43 .646 & & & & \\
\hline 651 & 119 & & 43.45 .184 & & & & \\
\hline 652 & 119 & & 33.46 .160 & & & & \\
\hline 653 & 119 & & 33.47 .037 & & & & \\
\hline 654 & 119 & & 33.52 .522 & & & & \\
\hline 655 & 119 & & 33.59 .834 & & & & \\
\hline 656 & 119 & & 33.47 .707 & & & & \\
\hline 657 & 119 & & 33.56 .914 & & & & \\
\hline 658 & 119 & & 34.37.401 & & & & \\
\hline 659 & 119 & & 34.4 .571 & & & & \\
\hline 660 & 119 & & 33.54 .646 & & & & \\
\hline 661 & 119 & & 33.27 .425 & & & & \\
\hline 662 & 119 & & 26.20 .61 & & & & \\
\hline 663 & 119 & & 26.15 .64 & & & & \\
\hline 664 & 119 & & 19.25 .34 & & & & \\
\hline 665 & 119 & & 26.19 .26 & & & & \\
\hline 666 & 119 & & 26.12 .37 & & & & \\
\hline 667 & 119 & & 26.9 .25 & & & & \\
\hline 668 & 119 & & 26.7 .82 & & & & \\
\hline 669 & 119 & & 19.34.19 & & & & \\
\hline 670 & 119 & & 19.32.37 & & & & \\
\hline 671 & 119 & & 19.24 .78 & & & & \\
\hline 672 & 119 & & 19.21.99 & & & & \\
\hline 673 & 119 & & 19.29 .69 & & & & \\
\hline 674 & 119 & & 22.5 .67 & & & & \\
\hline 675 & 119 & & 21.10 .49 & & & & \\
\hline 676 & 119 & & 18.44.07 & & & & \\
\hline 677 & 119 & & 20.16 .74 & & & & \\
\hline 678 & 119 & & 27.20 .00 & & & & \\
\hline 679 & 119 & & 24.34 .94 & & & & \\
\hline 680 & 119 & & 16.4 .62 & & & & \\
\hline 681 & 119 & & 16.4 .10 & & & & \\
\hline 682 & 119 & & 15.58 .71 & & & & \\
\hline 683 & 119 & & 15.50 .94 & & & & \\
\hline 684 & 119 & & 15.36 .76 & & & & \\
\hline 685 & 119 & & 15.32 .79 & & & & \\
\hline
\end{tabular}

Page 564 


\begin{tabular}{|c|c|c|c|c|c|c|c|}
\hline & $\mathrm{AP}$ & $\mathrm{AQ}$ & AR & AS & AT & $A U$ & $\mathrm{AV}$ \\
\hline 1 & LONGITUDE ${ }^{\circ} \mathrm{W}$ & LONGITUDE MIN MIN & LONGITUDE MIN SEC & X COORD (EW) & Y COORD (NW) & 3 D HAE/ALTITUDE & INSTRUMENT \\
\hline \multicolumn{8}{|c|}{686} \\
\hline 687 & 119 & & 15.34 .15 & & & & \\
\hline 688 & 119 & & 15.27 .44 & & & & \\
\hline 689 & 119 & & 15.18.26 & & & & \\
\hline 690 & 119 & & 15.1 .78 & & & & \\
\hline 691 & 119 & & 14.55 .21 & & & & \\
\hline 692 & 119 & & 15.36.010 & & & & \\
\hline 693 & 119 & & 15.45 .053 & & & & \\
\hline 694 & 119 & & 15.58 .45 & & & & \\
\hline 695 & 119 & & 16.1.057 & & & & \\
\hline 696 & 119 & & 16.25 .78 & & & & \\
\hline 697 & 119 & & 16.22 .25 & & & & \\
\hline 698 & 119 & & 16.16 .39 & & & & \\
\hline 699 & 119 & & 16.7 .69 & & & & \\
\hline \multicolumn{8}{|c|}{700} \\
\hline \multicolumn{8}{|c|}{701} \\
\hline \multicolumn{8}{|l|}{702} \\
\hline \multicolumn{8}{|l|}{703} \\
\hline \multicolumn{8}{|c|}{704} \\
\hline \multicolumn{8}{|c|}{705} \\
\hline \multicolumn{8}{|c|}{706} \\
\hline \multicolumn{8}{|l|}{707} \\
\hline 708 & 119 & & 33.46 .90 & & & & \\
\hline 709 & 119 & & 36.23 .99 & & & & \\
\hline 710 & 119 & & 35.28 .29 & & & & \\
\hline 711 & 119 & & 29.23 .4 & & & & \\
\hline 712 & 119 & & 28.38 .8 & & & & \\
\hline \multicolumn{8}{|c|}{713} \\
\hline \multicolumn{8}{|c|}{714} \\
\hline \multicolumn{8}{|c|}{715} \\
\hline \multicolumn{8}{|c|}{716} \\
\hline \multicolumn{8}{|c|}{717} \\
\hline 718 & 119 & & 43.32 .98 & & & & \\
\hline 719 & 119 & & 43.39 .75 & & & & \\
\hline 720 & 119 & & 43.41 .72 & & & & \\
\hline 721 & 119 & & 43.44 .28 & & & & \\
\hline 722 & 119 & & 15.35 .75 & & & & \\
\hline 723 & 119 & & 15.35 .75 & & & & \\
\hline
\end{tabular}

Page 565 


\begin{tabular}{|c|c|c|c|c|c|c|c|}
\hline & $\mathrm{AP}$ & $\mathrm{AQ}$ & AR & AS & AT & $\mathrm{AU}$ & $\mathrm{AV}$ \\
\hline 1 & LONGITUDE ${ }^{\circ} \mathrm{W}$ & LONGITUDE MIN_MIN & LONGITUDE MIN_SEC & X_COORD (EW) & $\mathrm{Y}_{\mathrm{Z}} \mathrm{COORD}(\mathrm{NW})$ & 3 D HAE/ALTITUDE & INSTRUMENT \\
\hline 724 & 119 & & 15.31 .17 & & & & \\
\hline 725 & 119 & & 15.24 .39 & & & & \\
\hline 726 & 119 & & 15.18 .12 & & & & \\
\hline 727 & 119 & & 15.5 .25 & & & & \\
\hline 728 & 119 & & 14.56 .18 & & & & \\
\hline 729 & 119 & & 15.36 .06 & & & & \\
\hline 730 & 119 & & 15.44 .45557 & & & & \\
\hline 731 & 119 & & 15.58 .49 & & & & \\
\hline 732 & 119 & & 16.1.47 & & & & \\
\hline 733 & & & & & & & \\
\hline 734 & & & & & & & \\
\hline 735 & & & & & & & \\
\hline 736 & & & & & & & \\
\hline 737 & & & & & & & \\
\hline 738 & & & & & & & \\
\hline 739 & & & & & & & \\
\hline 740 & & & & & & & \\
\hline 741 & & & & & & & \\
\hline 742 & & & & & & & \\
\hline 743 & 119 & & 43.41 .968 & & & & \\
\hline 744 & 119 & & 43.45 .470 & & & & \\
\hline 745 & 119 & & 43.38 .87 & & & & \\
\hline 746 & 119 & & 43.45 .970 & & & & \\
\hline 747 & 119 & & 15.35 .173 & & & & \\
\hline 748 & 119 & & 15.35 .173 & & & & \\
\hline 749 & 119 & & 15.32 .280 & & & & \\
\hline 750 & 119 & & 15.27 .450 & & & & \\
\hline 751 & 119 & & 15.18 .880 & & & & \\
\hline 752 & 119 & & 15.2 .305 & & & & \\
\hline 753 & 119 & & 14.55 .643 & & & & \\
\hline 754 & 119 & & 15.35 .894 & & & & \\
\hline 755 & 119 & & 15.44 .136 & & & & \\
\hline 756 & 119 & & 15.58 .274 & & & & \\
\hline 757 & 119 & & 16.1.155 & & & & \\
\hline 758 & & & & & & & \\
\hline 759 & & & & & & & \\
\hline 760 & & & & & & & \\
\hline 761 & & & & & & & \\
\hline
\end{tabular}




\begin{tabular}{|c|c|c|c|c|c|c|c|}
\hline & $\mathrm{AP}$ & $\overline{A Q}$ & AR & AS & AT & $\overline{A U}$ & AV \\
\hline 1 & LONGITUDE ${ }^{\circ} \mathrm{W}$ & LONGITUDE MIN_MIN & LONGITUDE MIN_SEC & X_COORD (EW) & Y_COORD (NW) & 3 D HAE/ALTITUDE & INSTRUMENT \\
\hline \multicolumn{8}{|c|}{\begin{tabular}{l|l|l|l|l|}
762 & & $=$ & $=$ \\
\end{tabular}} \\
\hline \multicolumn{8}{|l|}{763} \\
\hline \multicolumn{8}{|l|}{764} \\
\hline \multicolumn{8}{|l|}{765} \\
\hline 766 & 119 & 18039 & & & & & \\
\hline 767 & 119 & 28027 & & & & & \\
\hline \multicolumn{8}{|l|}{768} \\
\hline \multicolumn{8}{|l|}{769} \\
\hline 770 & 119 & & 43.38.216 & & & & \\
\hline 771 & 119 & & 43.40 .351 & & & & \\
\hline 772 & 119 & & 43.42 .623 & & & & \\
\hline 773 & 119 & & 43.45 .564 & & & & \\
\hline 774 & 119 & & 15.35 .664 & & & & \\
\hline 775 & 119 & & 15.31 .280 & & & & \\
\hline 776 & 119 & & 15.26 .899 & & & & \\
\hline 777 & 119 & & 15.17.787 & & & & \\
\hline 778 & 119 & & 15.04.289 & & & & \\
\hline 779 & 119 & & 14.55 .302 & & & & \\
\hline 780 & 119 & & 15.35.921 & & & & \\
\hline 781 & 119 & & 15.46 .088 & & & & \\
\hline 782 & 119 & & 15.58 .751 & & & & \\
\hline 783 & 119 & & 16.01.371 & & & & \\
\hline \multicolumn{8}{|l|}{784} \\
\hline \multicolumn{8}{|l|}{785} \\
\hline \multicolumn{8}{|l|}{786} \\
\hline \multicolumn{8}{|l|}{787} \\
\hline \multicolumn{8}{|l|}{788} \\
\hline \multicolumn{8}{|l|}{789} \\
\hline \multicolumn{8}{|l|}{790} \\
\hline \multicolumn{8}{|l|}{791} \\
\hline \multicolumn{8}{|l|}{792} \\
\hline \multicolumn{8}{|l|}{793} \\
\hline \multicolumn{8}{|l|}{794} \\
\hline \multicolumn{8}{|l|}{795} \\
\hline \multicolumn{8}{|l|}{796} \\
\hline \multicolumn{8}{|l|}{797} \\
\hline 798 & 119 & & 33.43.61 & & & & \\
\hline 799 & 119 & & 43.45 .22 & & & & \\
\hline
\end{tabular}




\begin{tabular}{|c|c|c|c|c|c|c|c|}
\hline & $\mathrm{AP}$ & $\mathrm{AQ}$ & $\mathrm{AR}$ & AS & AT & $\mathrm{AU}$ & $\mathrm{AV}$ \\
\hline 1 & LONGITUDE ${ }^{\circ} \mathrm{W}$ & LONGITUDE MIN_MIN & LONGITUDE MIN_SEC & X_COORD (EW) & Y_COORD (NW) & 3 D HAE/ALTITUDE & INSTRUMENT \\
\hline 800 & 119 & & 43.42.11 & & & & \\
\hline 801 & 119 & & 43.44 .59 & & & & \\
\hline 802 & 119 & & 43.40 .35 & & & & \\
\hline 803 & 119 & & 33.43 .61 & & & & \\
\hline 804 & 119 & & 33.44 .32 & & & & \\
\hline 805 & 119 & & 33.50 .75 & & & & \\
\hline 806 & 119 & & 34.00 .50 & & & & \\
\hline 807 & 119 & & 33.46 .14 & & & & \\
\hline 808 & 119 & & 33.57 .27 & & & & \\
\hline 809 & 119 & & 34.37 .60 & & & & \\
\hline 810 & 119 & & 34.03 .81 & & & & \\
\hline 811 & 119 & & 33.54 .88 & & & & \\
\hline 812 & 119 & & 33.26.71 & & & & \\
\hline 813 & 119 & & 26.20 .44 & & & & \\
\hline 814 & 119 & & 26.11 .61 & & & & \\
\hline 815 & 119 & & 19.27.34 & & & & \\
\hline 816 & 119 & & 26.17 .75 & & & & \\
\hline 817 & 119 & & 26.10.71 & & & & \\
\hline 818 & 119 & & 26.09.29 & & & & \\
\hline 819 & 119 & & 26.08.28 & & & & \\
\hline 820 & 119 & & 19.36.81 & & & & \\
\hline 821 & 119 & & 19.35.13 & & & & \\
\hline 822 & 119 & & 19.24 .58 & & & & \\
\hline 823 & 119 & & 19.22.285 & & & & \\
\hline 824 & 119 & & 19.32.76 & & & & \\
\hline 825 & 119 & & 22.04 .98 & & & & \\
\hline 826 & 119 & & 21.10 .15 & & & & \\
\hline 827 & 119 & & 18.43.71 & & & & \\
\hline 828 & 119 & & 20.15 .46 & & & & \\
\hline 829 & 119 & & 27.19 .87 & & & & \\
\hline 830 & 119 & & 23.52 .66 & & & & \\
\hline 831 & 119 & & 24.35 .08 & & & & \\
\hline 832 & 119 & & 16.04.61 & & & & \\
\hline 833 & 119 & & 16.02.71 & & & & \\
\hline 834 & 119 & & 15.56 .24 & & & & \\
\hline 835 & 119 & & 15.50 .83 & & & & \\
\hline 836 & 119 & & 15.37 .57 & & & & \\
\hline 837 & 119 & & 15.33 .16 & & & & \\
\hline
\end{tabular}




\begin{tabular}{|c|c|c|c|c|c|c|c|}
\hline & $\mathrm{AP}$ & $\mathrm{AQ}$ & AR & AS & AT & $A U$ & $\mathrm{AV}$ \\
\hline 1 & LONGITUDE ${ }^{\circ} \mathrm{W}$ & LONGITUDE MIN MIN & LONGITUDE MIN SEC & X COORD (EW) & Y COORD (NW) & 3 D HAE/ALTITUDE & INSTRUMENT \\
\hline 838 & 119 & & 15.34 .9 & & & & \\
\hline 839 & 119 & & 15.31 .3 & & & & \\
\hline 840 & 119 & & 15.41 .5 & & & & \\
\hline 841 & 119 & & 15.18 .35 & & & & \\
\hline 842 & 119 & & 15.01 .42 & & & & \\
\hline 843 & 119 & & 14.55 .48 & & & & \\
\hline 844 & 119 & & 15.35 .90 & & & & \\
\hline 845 & 119 & & 15.44 .92 & & & & \\
\hline 846 & 119 & & 15.58 .55 & & & & \\
\hline 847 & 119 & & 16.01 .26 & & & & \\
\hline 848 & 119 & & 16.25 .66 & & & & \\
\hline 849 & 119 & & 16.21 .89 & & & & \\
\hline 850 & 119 & & 16.18 .03 & & & & \\
\hline 851 & 119 & & 16.07.38 & & & & \\
\hline 852 & & & & & & & \\
\hline 853 & & & & & & & \\
\hline 854 & & & & & & & \\
\hline 855 & & & & & & & \\
\hline 856 & & & & & & & \\
\hline 857 & & & & & & & \\
\hline 858 & 119 & & 35.28 .175 & & & & \\
\hline 859 & 119 & & 28.38 .784 & & & & \\
\hline 860 & 119 & & 25.12.798 & & & & \\
\hline 861 & & & & & & & \\
\hline 862 & & & & & & & \\
\hline 863 & & & & & & & \\
\hline 864 & & & & & & & \\
\hline 865 & 119 & 4363830 & & & & & \\
\hline 866 & 119 & 4365845 & & & & & \\
\hline 867 & 119 & 434070431 & & & & & \\
\hline 868 & 119 & 434589260 & & & & & \\
\hline 869 & 119 & 153521977 & & & & & \\
\hline 870 & 119 & 153521977 & & & & & \\
\hline 871 & 119 & 15290058 & & & & & \\
\hline 872 & 119 & 152340608 & & & & & \\
\hline 873 & 119 & 151897178 & & & & & \\
\hline 874 & 119 & 150525588 & & & & & \\
\hline 875 & 119 & 145567351 & & & & & \\
\hline
\end{tabular}




\begin{tabular}{|c|c|c|c|c|c|c|c|}
\hline & $\mathrm{AP}$ & $\mathrm{AQ}$ & AR & AS & AT & $A U$ & $\mathrm{AV}$ \\
\hline 1 & LONGITUDE ${ }^{\circ} \mathrm{W}$ & LONGITUDE MIN_MIN & LONGITUDE MIN_SEC & X_COORD (EW) & Y_COORD (NW) & 3 D HAE/ALTITUDE & INSTRUMENT \\
\hline 876 & 119 & 153598423 & & & & & \\
\hline 877 & 119 & 154392604 & & & & & \\
\hline 878 & 119 & 155819461 & & & & & \\
\hline 879 & 119 & 16009196 & & & & & \\
\hline \multicolumn{8}{|l|}{880} \\
\hline \multicolumn{8}{|l|}{881} \\
\hline \multicolumn{8}{|l|}{882} \\
\hline \multicolumn{8}{|l|}{883} \\
\hline \multicolumn{8}{|l|}{884} \\
\hline \multicolumn{8}{|l|}{885} \\
\hline \multicolumn{8}{|l|}{886} \\
\hline \multicolumn{8}{|l|}{887} \\
\hline \multicolumn{8}{|l|}{888} \\
\hline \multicolumn{8}{|l|}{889} \\
\hline \multicolumn{8}{|l|}{890} \\
\hline \multicolumn{8}{|l|}{891} \\
\hline \multicolumn{8}{|l|}{892} \\
\hline \multicolumn{8}{|l|}{893} \\
\hline \multicolumn{8}{|l|}{894} \\
\hline \multicolumn{8}{|l|}{895} \\
\hline \multicolumn{8}{|l|}{896} \\
\hline \multicolumn{8}{|l|}{897} \\
\hline \multicolumn{8}{|l|}{898} \\
\hline 899 & & & & 589172 & 137602 & & \\
\hline 900 & & & & 594392 & 116700 & 82 & \\
\hline 901 & & & & 588808 & 137808 & 94 & \\
\hline 902 & & & & 589438 & 137451 & & \\
\hline 903 & & & & 594489 & 116208 & 82.5 & \\
\hline \multicolumn{8}{|l|}{904} \\
\hline \multicolumn{8}{|l|}{905} \\
\hline \multicolumn{8}{|l|}{906} \\
\hline \multicolumn{8}{|l|}{907} \\
\hline \multicolumn{8}{|l|}{908} \\
\hline \multicolumn{8}{|l|}{909} \\
\hline 910 & & & & & & & \\
\hline 911 & & & & & & & \\
\hline 912 & & & & & & & \\
\hline 913 & & & & & & & \\
\hline
\end{tabular}




\begin{tabular}{|c|c|c|c|c|c|c|c|}
\hline & $\mathrm{AP}$ & $\mathrm{AQ}$ & AR & AS & AT & $A U$ & $\mathrm{AV}$ \\
\hline 1 & LONGITUDE ${ }^{\circ} \mathrm{W}$ & LONGITUDE MIN MIN & LONGITUDE MIN SEC & X COORD (EW) & Y COORD (NW) & 3 D HAE/ALTITUDE & INSTRUMENT \\
\hline \multicolumn{8}{|l|}{914} \\
\hline \multicolumn{8}{|c|}{915} \\
\hline \multicolumn{8}{|l|}{916} \\
\hline \multicolumn{8}{|l|}{917} \\
\hline \multicolumn{8}{|l|}{918} \\
\hline \multicolumn{8}{|l|}{919} \\
\hline \multicolumn{8}{|l|}{920} \\
\hline \multicolumn{8}{|l|}{921} \\
\hline \multicolumn{8}{|l|}{922} \\
\hline \multicolumn{8}{|c|}{923} \\
\hline \multicolumn{8}{|c|}{924} \\
\hline \multicolumn{8}{|c|}{925} \\
\hline \multicolumn{8}{|c|}{926} \\
\hline \multicolumn{8}{|c|}{927} \\
\hline \multicolumn{8}{|c|}{928} \\
\hline \multicolumn{8}{|c|}{929} \\
\hline \multicolumn{8}{|c|}{930} \\
\hline 931 & 119 & & 39.21 .806 & & & & \\
\hline 932 & 119 & & 34.48 .990 & & & & \\
\hline \multicolumn{8}{|l|}{933} \\
\hline \multicolumn{8}{|c|}{934} \\
\hline \multicolumn{8}{|c|}{935} \\
\hline \multicolumn{8}{|c|}{936} \\
\hline \multicolumn{8}{|c|}{937} \\
\hline \multicolumn{8}{|c|}{938} \\
\hline \multicolumn{8}{|c|}{939} \\
\hline \multicolumn{8}{|c|}{940} \\
\hline 941 & & & & & & & \\
\hline 942 & 119 & & 16.18.498 & & & & \\
\hline 943 & 119 & & 16.18 .450 & & & & \\
\hline 944 & 119 & & 16.18 .450 & & & & \\
\hline 945 & 119 & & 16.18.119 & & & & \\
\hline 946 & 119 & & 16.18.119 & & & & \\
\hline 947 & 119 & & 16.17.828 & & & & \\
\hline 948 & 119 & & 16.22 .585 & & & & \\
\hline 949 & 119 & & 16.22.369 & & & & \\
\hline 950 & 119 & & 16.22 .369 & & & & \\
\hline 951 & 119 & & 16.22.369 & & & & \\
\hline
\end{tabular}




\begin{tabular}{|c|c|c|c|c|c|c|c|}
\hline & $\mathrm{AP}$ & $\mathrm{AQ}$ & AR & AS & AT & $A U$ & $\mathrm{AV}$ \\
\hline 1 & LONGITUDE ${ }^{\circ} \mathrm{W}$ & LONGITUDE MIN_MIN & LONGITUDE MIN_SEC & X_COORD (EW) & Y_COORD (NW) & 3 D HAE/ALTITUDE & INSTRUMENT \\
\hline 952 & 119 & & 16.22 .143 & & & & \\
\hline 953 & 119 & & 16.22 .143 & & & & \\
\hline \multicolumn{8}{|l|}{954} \\
\hline \multicolumn{8}{|l|}{955} \\
\hline \multicolumn{8}{|l|}{956} \\
\hline 957 & 119 & & 36.23 .99 & & & & \\
\hline 958 & 119 & & 35.28 .29 & & & & \\
\hline 959 & 119 & & 29.23 .4 & & & & \\
\hline 960 & 119 & & 28.38 .8 & & & & \\
\hline \multicolumn{8}{|l|}{961} \\
\hline \multicolumn{8}{|l|}{962} \\
\hline \multicolumn{8}{|l|}{963} \\
\hline \multicolumn{8}{|l|}{964} \\
\hline \multicolumn{8}{|l|}{965} \\
\hline \multicolumn{8}{|l|}{966} \\
\hline \multicolumn{8}{|l|}{967} \\
\hline \multicolumn{8}{|l|}{968} \\
\hline \multicolumn{8}{|l|}{969} \\
\hline \multicolumn{8}{|l|}{970} \\
\hline \multicolumn{8}{|l|}{971} \\
\hline \multicolumn{8}{|l|}{972} \\
\hline \multicolumn{8}{|l|}{973} \\
\hline \multicolumn{8}{|l|}{974} \\
\hline \multicolumn{8}{|c|}{975} \\
\hline \multicolumn{8}{|l|}{976} \\
\hline \multicolumn{8}{|l|}{977} \\
\hline \multicolumn{8}{|l|}{978} \\
\hline \multicolumn{8}{|l|}{979} \\
\hline \multicolumn{8}{|l|}{980} \\
\hline \multicolumn{8}{|l|}{981} \\
\hline \multicolumn{8}{|l|}{982} \\
\hline 983 & & & & & & & \\
\hline 984 & & & & & & & \\
\hline 985 & & & & & & & \\
\hline 986 & & & & & & & \\
\hline 987 & 119 & & 20.10 .491 & & & & \\
\hline 988 & 119 & & 20.00 .067 & & & & \\
\hline 989 & 119 & & 35.28 .175 & & & & \\
\hline
\end{tabular}




\begin{tabular}{|c|c|c|c|c|c|c|c|}
\hline & $\mathrm{AP}$ & $\mathrm{AQ}$ & AR & AS & AT & $A U$ & AV \\
\hline 1 & LONGITUDE ${ }^{\circ} \mathrm{W}$ & LONGITUDE MIN_MIN & LONGITUDE MIN_SEC & X_COORD (EW) & Y_COORD (NW) & 3 D HAE/ALTITUDE & INSTRUMENT \\
\hline 990 & 119 & & 28.38 .784 & & & & \\
\hline \multicolumn{8}{|l|}{991} \\
\hline \multicolumn{8}{|l|}{992} \\
\hline \multicolumn{8}{|l|}{993} \\
\hline \multicolumn{8}{|l|}{994} \\
\hline \multicolumn{8}{|l|}{995} \\
\hline \multicolumn{8}{|l|}{996} \\
\hline \multicolumn{8}{|l|}{997} \\
\hline \multicolumn{8}{|l|}{998} \\
\hline 999 & & & & 127181.6 & 594833.6 & & \\
\hline 1000 & & & & 125754.8 & 594814.8 & & \\
\hline 1001 & & & & 123765.9 & 594459.8 & & \\
\hline \multicolumn{8}{|l|}{1002} \\
\hline \multicolumn{8}{|l|}{1003} \\
\hline \multicolumn{8}{|l|}{1004} \\
\hline \multicolumn{8}{|l|}{\begin{tabular}{|l|}
1005 \\
\end{tabular}} \\
\hline \multicolumn{8}{|l|}{1006} \\
\hline \multicolumn{8}{|l|}{1007} \\
\hline \multicolumn{8}{|l|}{1008} \\
\hline \multicolumn{8}{|l|}{1009} \\
\hline \multicolumn{8}{|l|}{1010} \\
\hline \multicolumn{8}{|l|}{1011} \\
\hline \multicolumn{8}{|l|}{\begin{tabular}{|l|}
1012 \\
\end{tabular}} \\
\hline \multicolumn{8}{|l|}{1013} \\
\hline \multicolumn{8}{|l|}{1014} \\
\hline \multicolumn{8}{|l|}{1015} \\
\hline \multicolumn{8}{|l|}{1016} \\
\hline \multicolumn{8}{|l|}{1017} \\
\hline \multicolumn{8}{|l|}{\begin{tabular}{|l|}
1018 \\
\end{tabular}} \\
\hline \begin{tabular}{|l|}
1019 \\
\end{tabular} & & & & & & & \\
\hline 1020 & & & & & & & \\
\hline \begin{tabular}{|l|}
1021 \\
\end{tabular} & & & & & & & \\
\hline \begin{tabular}{|l|}
1022 \\
\end{tabular} & & & & & & & \\
\hline \begin{tabular}{|l|}
1023 \\
\end{tabular} & & & & & & & \\
\hline 1024 & & & & & & & \\
\hline 1025 & & & & & & & \\
\hline 1026 & & & & & & & \\
\hline \begin{tabular}{|l|}
1027 \\
\end{tabular} & & & & & & & \\
\hline
\end{tabular}




\begin{tabular}{|c|c|c|c|c|c|c|c|}
\hline & $\mathrm{AP}$ & $\mathrm{AQ}$ & AR & AS & AT & $A U$ & AV \\
\hline 1 & LONGITUDE ${ }^{\circ} \mathrm{W}$ & LONGITUDE MIN_MIN & LONGITUDE MIN_SEC & X_COORD (EW) & Y_COORD (NW) & 3 D HAE/ALTITUDE & INSTRUMENT \\
\hline \multicolumn{8}{|l|}{1028} \\
\hline \multicolumn{8}{|l|}{1029} \\
\hline \multicolumn{8}{|l|}{1030} \\
\hline \multicolumn{8}{|l|}{1031} \\
\hline \multicolumn{8}{|l|}{1032} \\
\hline \multicolumn{8}{|l|}{1033} \\
\hline \multicolumn{8}{|l|}{1034} \\
\hline \multicolumn{8}{|l|}{1035} \\
\hline \multicolumn{8}{|l|}{1036} \\
\hline \multicolumn{8}{|l|}{1037} \\
\hline 1038 & & & & 145775 & 559128 & & \\
\hline 1039 & & & & 145888 & 559110 & & \\
\hline 1040 & & & & 146030 & 559077 & & \\
\hline \begin{tabular}{|c|}
1041 \\
\end{tabular} & & & & 146118 & 559037 & & \\
\hline $\mid 1042$ & & & & 109886 & 595516 & & \\
\hline 1043 & & & & 109861 & 595700 & & \\
\hline 1044 & & & & 109840 & 595880 & & \\
\hline \begin{tabular}{|l|}
1045 \\
\end{tabular} & & & & 109811 & 596091 & & \\
\hline \begin{tabular}{|l|}
1046 \\
\end{tabular} & & & & 109824 & 596212 & & \\
\hline \begin{tabular}{|l|}
1047 \\
\end{tabular} & & & & 109822 & 596358 & & \\
\hline 1048 & & & & 111001.9 & 595469.9 & & \\
\hline 1049 & & & & 112119.9 & 595260.9 & & \\
\hline 1050 & & & & 113946.4 & 594945.8 & & \\
\hline 1051 & & & & 114400.8 & 594886.3 & & \\
\hline \multicolumn{8}{|l|}{1052} \\
\hline \multicolumn{8}{|l|}{1053} \\
\hline \multicolumn{8}{|l|}{1054} \\
\hline \multicolumn{8}{|l|}{1055} \\
\hline \begin{tabular}{|l|}
1056 \\
\end{tabular} & & & & 587434.122 & 138556.3 & & GPS \\
\hline \multicolumn{8}{|l|}{1057} \\
\hline \multicolumn{8}{|l|}{1058} \\
\hline \multicolumn{8}{|l|}{1059} \\
\hline \multicolumn{8}{|l|}{1060} \\
\hline \multicolumn{8}{|l|}{1061} \\
\hline \multicolumn{8}{|l|}{1062} \\
\hline \multicolumn{8}{|l|}{1063} \\
\hline \multicolumn{8}{|l|}{1064} \\
\hline \begin{tabular}{|l|}
1065 \\
\end{tabular} & & & & & & & \\
\hline
\end{tabular}




\begin{tabular}{|c|c|c|c|c|c|c|c|}
\hline & $\mathrm{AP}$ & $\overline{A Q}$ & AR & AS & AT & $\mathrm{AU}$ & AV \\
\hline 1 & LONGITUDE ${ }^{\circ} \mathrm{W}$ & LONGITUDE MIN_MIN & LONGITUDE MIN_SEC & X_COORD (EW) & Y_COORD (NW) & 3 D HAE/ALTITUDE & INSTRUMENT \\
\hline 1066 & & & & 571726 & 150552 & 95.5 & \\
\hline 1067 & & & & 559152 & 145816 & 100 & \\
\hline 1068 & & & & 559123 & 145934 & 101 & \\
\hline 1069 & & & & 558979 & 145994 & 99.4 & \\
\hline 1070 & & & & 559021 & 146105 & 101 & \\
\hline 1071 & & & & 571726 & 150552 & 95.5 & \\
\hline 1072 & & & & 571712 & 150603 & 95.1 & \\
\hline 1073 & & & & & & & \\
\hline 1074 & & & & 571385 & 150788 & 97.2 & \\
\hline 1075 & & & & 571652 & 150596 & 96.1 & \\
\hline 1076 & & & & & & & \\
\hline 1077 & & & & & & & \\
\hline 1078 & & & & 571313 & 149952 & 98 & \\
\hline 1079 & & & & & & & \\
\hline 1080 & & & & 572099 & 151070 & 95.2 & \\
\hline 1081 & & & & & & & \\
\hline 1082 & & & & & & & \\
\hline 1083 & & & & & & & \\
\hline 1084 & & & & & & & \\
\hline 1085 & & & & & & & \\
\hline 1086 & & & & & & & \\
\hline 1087 & & & & 581213 & 147964 & 92.8 & GPS \\
\hline 1088 & & & & 581313 & 148169 & 92 & GPS \\
\hline 1089 & & & & 590188 & 137123 & 87.2 & GPS \\
\hline 1090 & & & & 581253 & 148028 & 91.7 & GPS \\
\hline 1091 & & & & 581443 & 148274 & 92.6 & GPS \\
\hline 1092 & & & & 581502 & 148393 & 90 & GPS \\
\hline 1093 & & & & 581543 & 148471 & 88 & GPS \\
\hline 1094 & & & & 589999 & 137077 & 87.5 & GPS \\
\hline 1095 & & & & 590039 & 137078 & 86.9 & GPS \\
\hline 1096 & & & & 590732 & 137195 & 86.5 & GPS \\
\hline 1097 & & & & 590268 & 137247 & 86.8 & GPS \\
\hline 1098 & & & & 590087 & 137068 & 86.6 & GPS \\
\hline 1099 & & & & 586752 & 139253 & 87.2 & GPS \\
\hline 1100 & & & & 587946 & 138371 & 86.9 & GPS \\
\hline 1101 & & & & 591113 & 135398 & 85 & GPS \\
\hline 1102 & & & & 589105 & 137662 & 74.9 & GPS \\
\hline 1103 & & & & 579910 & 149070 & 92 & GPS \\
\hline
\end{tabular}




\begin{tabular}{|c|c|c|c|c|c|c|c|}
\hline & AP & $\mathrm{AQ}$ & AR & AS & AT & $A U$ & AV \\
\hline 1 & LONGITUDE ${ }^{\circ} \mathrm{W}$ & LONGITUDE MIN MIN & LONGITUDE MIN SEC & X COORD (EW) & Y COORD (NW) & 3 D HAE/ALTITUDE & INSTRUMENT \\
\hline 1104 & & & & 583186 & 144426 & 88.5 & GPS \\
\hline 1105 & & & & 583498 & 143300 & 85.9 & GPS \\
\hline \multicolumn{8}{|l|}{1106} \\
\hline \multicolumn{8}{|l|}{1107} \\
\hline 1108 & & & & 589999 & 137077 & 87.5 & GPS \\
\hline 1109 & & & & 590039 & 137078 & 86.9 & GPS \\
\hline 1110 & & & & 587946 & 138371 & 86.9 & GPS \\
\hline 1111 & & & & 589105 & 137662 & 74.9 & GPS \\
\hline 1112 & & & & 591113 & 135398 & 85 & GPS \\
\hline \multicolumn{8}{|l|}{1113} \\
\hline \multicolumn{8}{|l|}{1114} \\
\hline 1115 & & & & 594916 & 114856 & 81.6 & GPS \\
\hline 1116 & & & & 595098 & 114923 & 81.6 & GPS \\
\hline 1117 & & & & 595395 & 114927 & 83 & GPS \\
\hline 1118 & & & & 595472 & 114951 & 83 & GPS \\
\hline 1119 & & & & 595518 & 109870 & 82.2 & GPS \\
\hline 1120 & & & & 595563 & 109836 & 82.7 & GPS \\
\hline 1121 & & & & 595677 & 109831 & 83.4 & GPS \\
\hline 1122 & & & & 595869 & 109857 & 82.3 & GPS \\
\hline 1123 & & & & 596226 & 109758 & 83.3 & GPS \\
\hline 1124 & & & & 596363 & 109808 & 82.8 & GPS \\
\hline 1125 & & & & 595483 & 110804 & 82.4 & GPS \\
\hline 1126 & & & & 595278 & 112118 & 82.2 & GPS \\
\hline 1127 & & & & 954956 & 113692 & 83 & GPS \\
\hline 1128 & & & & 594889 & 114400 & 83.4 & GPS \\
\hline 1129 & & & & 594314 & 117539 & 82.8 & GPS \\
\hline 1130 & & & & 594396 & 116699 & 82.8 & GPS \\
\hline 1131 & & & & 594540 & 116076 & 82.6 & GPS \\
\hline 1132 & & & & 594738 & 115238 & 83.2 & GPS \\
\hline \multicolumn{8}{|l|}{1133} \\
\hline \multicolumn{8}{|c|}{1134} \\
\hline \multicolumn{8}{|c|}{1135} \\
\hline \multicolumn{8}{|l|}{\begin{tabular}{|l|l|}
1136 \\
113
\end{tabular}} \\
\hline \multicolumn{8}{|l|}{1137} \\
\hline \multicolumn{8}{|l|}{1138} \\
\hline \multicolumn{8}{|l|}{1139} \\
\hline \multicolumn{8}{|l|}{1140} \\
\hline 1141 & & & & & & & \\
\hline
\end{tabular}




\begin{tabular}{|c|c|c|c|c|c|c|c|}
\hline & $\mathrm{AP}$ & $\mathrm{AQ}$ & AR & AS & AT & $A U$ & AV \\
\hline 1 & LONGITUDE ${ }^{\circ} \mathrm{W}$ & LONGITUDE MIN_MIN & LONGITUDE MIN_SEC & X_COORD (EW) & Y_COORD (NW) & 3 D HAE/ALTITUDE & INSTRUMENT \\
\hline \multicolumn{8}{|l|}{1142} \\
\hline \multicolumn{8}{|l|}{1143} \\
\hline \begin{tabular}{|l|}
1144 \\
\end{tabular} & & & & 589172 & 137602 & & \\
\hline \begin{tabular}{|l|}
1145 \\
\end{tabular} & & & & 594392 & 116700 & 82 & \\
\hline \begin{tabular}{|l|}
1146 \\
\end{tabular} & & & & 588808 & 137808 & 94 & \\
\hline \begin{tabular}{|l|}
1147 \\
\end{tabular} & & & & 589438 & 137451 & & \\
\hline \begin{tabular}{|l|}
1148 \\
\end{tabular} & & & & 594489 & 116208 & 82.5 & \\
\hline \multicolumn{8}{|l|}{1149} \\
\hline \multicolumn{8}{|l|}{1150} \\
\hline \multicolumn{8}{|l|}{1151} \\
\hline \multicolumn{8}{|l|}{1152} \\
\hline \multicolumn{8}{|l|}{1153} \\
\hline \multicolumn{8}{|l|}{1154} \\
\hline \multicolumn{8}{|l|}{1155} \\
\hline \multicolumn{8}{|l|}{1156} \\
\hline \multicolumn{8}{|l|}{1157} \\
\hline \multicolumn{8}{|l|}{1158} \\
\hline \multicolumn{8}{|l|}{1159} \\
\hline \multicolumn{8}{|l|}{1160} \\
\hline \multicolumn{8}{|l|}{1161} \\
\hline \multicolumn{8}{|l|}{1162} \\
\hline \multicolumn{8}{|l|}{1163} \\
\hline \multicolumn{8}{|l|}{1164} \\
\hline \multicolumn{8}{|l|}{1165} \\
\hline \multicolumn{8}{|l|}{1166} \\
\hline \multicolumn{8}{|l|}{1167} \\
\hline \multicolumn{8}{|l|}{1168} \\
\hline \multicolumn{8}{|l|}{1169} \\
\hline \multicolumn{8}{|l|}{1170} \\
\hline \multicolumn{8}{|l|}{1171} \\
\hline \begin{tabular}{|l|}
1172 \\
\end{tabular} & & & & & & & \\
\hline \begin{tabular}{|l|}
1173 \\
\end{tabular} & & & & & & & \\
\hline \begin{tabular}{|l|}
1174 \\
\end{tabular} & & & & & & & \\
\hline \begin{tabular}{|l|}
1175 \\
\end{tabular} & & & & & & & \\
\hline \begin{tabular}{|l|}
1176 \\
1176
\end{tabular} & & & & 559150 & 145822 & 99.7 & GPS \\
\hline 1177 & & & & 559160 & 145921 & 100.9 & GPS \\
\hline \begin{tabular}{|l|}
1178 \\
\end{tabular} & & & & 559069 & 146021 & 99 & GPS \\
\hline \begin{tabular}{|l|}
1179 \\
\end{tabular} & & & & 559036 & 146104.449 & 98 & GPS \\
\hline
\end{tabular}




\begin{tabular}{|c|c|c|c|c|c|c|c|}
\hline & $\mathrm{AP}$ & $\mathrm{AQ}$ & AR & AS & AT & $A U$ & $\mathrm{AV}$ \\
\hline 1 & LONGITUDE ${ }^{\circ} \mathrm{W}$ & LONGITUDE MIN_MIN & LONGITUDE MIN_SEC & X_COORD (EW) & Y_COORD (NW) & 3 D HAE/ALTITUDE & INSTRUMENT \\
\hline 1180 & & & & 595517 & 109875 & 83 & GPS \\
\hline 1181 & & & & 595517 & 109875 & 83 & GPS \\
\hline 1182 & & & & 595577 & 109840 & 84 & GPS \\
\hline 1183 & & & & 595726 & 109770 & 81 & GPS \\
\hline 1184 & & & & 595872 & 109798 & 84 & GPS \\
\hline 1185 & & & & 596159 & 109608 & 80.7 & GPS \\
\hline 1186 & & & & 596362 & 109803 & 89.1 & GPS \\
\hline 1187 & & & & 595484 & 110809 & 83 & GPS \\
\hline 1188 & & & & 595270 & 112116 & 84 & GPS \\
\hline 1189 & & & & 594955 & 113780 & 83 & GPS \\
\hline 1190 & & & & 594887 & 114396 & 84 & GPS \\
\hline \multicolumn{8}{|l|}{\begin{tabular}{|l|}
1191 \\
\end{tabular}} \\
\hline \multicolumn{8}{|l|}{1192} \\
\hline \multicolumn{8}{|l|}{\begin{tabular}{|l|}
1193 \\
\end{tabular}} \\
\hline \multicolumn{8}{|l|}{\begin{tabular}{|l|}
1194 \\
\end{tabular}} \\
\hline \multicolumn{8}{|l|}{\begin{tabular}{|l|}
1195 \\
\end{tabular}} \\
\hline \multicolumn{8}{|l|}{\begin{tabular}{|l|}
1196 \\
\end{tabular}} \\
\hline \multicolumn{8}{|l|}{\begin{tabular}{|l|}
1197 \\
\end{tabular}} \\
\hline \multicolumn{8}{|c|}{1198} \\
\hline \multicolumn{8}{|l|}{1199} \\
\hline \multicolumn{8}{|l|}{1200} \\
\hline 1201 & 119 & & 15.35 .53 & & & & \\
\hline 1202 & 119 & & 15.35 .53 & & & & \\
\hline 1203 & 119 & & 15.32.09 & & & & \\
\hline 1204 & 119 & & 15.28 .44 & & & & \\
\hline 1205 & 119 & & 15.17 .99 & & & & \\
\hline 1206 & 119 & & 15.05 .39 & & & & \\
\hline \multicolumn{8}{|c|}{\begin{tabular}{|l|}
1207 \\
\end{tabular}} \\
\hline 1208 & 119 & & 15.35 .50 & & & & \\
\hline \multicolumn{8}{|c|}{\begin{tabular}{|l|l}
1209 \\
\end{tabular}} \\
\hline 1210 & 119 & & 15.58 .34 & & & & \\
\hline 1211 & 119 & & 16.02 .37 & & & & \\
\hline 1212 & 119 & & 43.41 .36 & & & & \\
\hline 1213 & 119 & & 43.42 .62 & & & & \\
\hline 1214 & 119 & & 43.42 .99 & & & & \\
\hline 1215 & 119 & & 43.46 .54 & & & & \\
\hline \multicolumn{8}{|c|}{\begin{tabular}{|l|}
1216 \\
\end{tabular}} \\
\hline 1217 & & & & & & & \\
\hline
\end{tabular}




\begin{tabular}{|c|c|c|c|c|c|c|c|}
\hline & $\mathrm{AP}$ & $\mathrm{AQ}$ & AR & AS & AT & $A U$ & AV \\
\hline 1 & LONGITUDE ${ }^{\circ} \mathrm{W}$ & LONGITUDE MIN_MIN & LONGITUDE MIN_SEC & X_COORD (EW) & Y_COORD (NW) & 3 D HAE/ALTITUDE & INSTRUMENT \\
\hline \multicolumn{8}{|l|}{1218} \\
\hline \multicolumn{8}{|l|}{1219} \\
\hline \multicolumn{8}{|c|}{1220} \\
\hline \multicolumn{8}{|c|}{1221} \\
\hline \multicolumn{8}{|l|}{\begin{tabular}{|l|}
1222 \\
122
\end{tabular}} \\
\hline \multicolumn{8}{|l|}{1223} \\
\hline \multicolumn{8}{|l|}{1224} \\
\hline \multicolumn{8}{|l|}{1225} \\
\hline \multicolumn{8}{|l|}{1226} \\
\hline \multicolumn{8}{|l|}{1227} \\
\hline \multicolumn{8}{|l|}{1228} \\
\hline \multicolumn{8}{|l|}{1229} \\
\hline \multicolumn{8}{|l|}{1230} \\
\hline \multicolumn{8}{|l|}{1231} \\
\hline \multicolumn{8}{|l|}{1232} \\
\hline \multicolumn{8}{|l|}{1233} \\
\hline \multicolumn{8}{|l|}{1234} \\
\hline 1235 & 119 & & 33.46 .410 & & & & \\
\hline \begin{tabular}{|l|}
1236 \\
\end{tabular} & 119 & & 39.21 .806 & & & & \\
\hline \begin{tabular}{|l|}
1237 \\
\end{tabular} & 119 & & 34.48 .990 & & & & \\
\hline \multicolumn{8}{|l|}{1238} \\
\hline \multicolumn{8}{|l|}{1239} \\
\hline \multicolumn{8}{|l|}{1240} \\
\hline \multicolumn{8}{|c|}{1241} \\
\hline 1242 & 119 & & 15.35.16202 & & & & \\
\hline 1243 & 119 & & 15.32 .8451 & & & & \\
\hline \begin{tabular}{|l|}
1244 \\
\end{tabular} & 119 & & 15.27 .2786 & & & & \\
\hline \begin{tabular}{|l|}
1245 \\
\end{tabular} & 119 & & 15.19 .05643 & & & & \\
\hline \multicolumn{8}{|c|}{1246} \\
\hline \multicolumn{8}{|l|}{1247} \\
\hline \multicolumn{8}{|l|}{1248} \\
\hline \multicolumn{8}{|l|}{1249} \\
\hline 1250 & & & & & & & \\
\hline \begin{tabular}{|l|}
1251 \\
\end{tabular} & & & & & & & \\
\hline 1252 & & & & & & & \\
\hline 1253 & 119 & & 15.35.167 & & & & \\
\hline 1254 & 119 & & 15.32 .854 & & & & \\
\hline 1255 & 119 & & 15.25 .556 & & & & \\
\hline
\end{tabular}




\begin{tabular}{|c|c|c|c|c|c|c|c|}
\hline & $\mathrm{AP}$ & $\mathrm{AQ}$ & AR & AS & AT & $\mathrm{AU}$ & AV \\
\hline 1 & LONGITUDE ${ }^{\circ} \mathrm{W}$ & LONGITUDE MIN_MIN & LONGITUDE MIN_SEC & X_COORD (EW) & Y_COORD (NW) & 3 D HAE/ALTITUDE & INSTRUMENT \\
\hline 1256 & 119 & & 15.18 .321 & & & & \\
\hline 1257 & 119 & & 15.05.22609 & & & & \\
\hline 1258 & 119 & & 14.55 .469 & & & & \\
\hline 1259 & 119 & & 15.35 .841 & & & & \\
\hline 1260 & 119 & & 15.45 .231 & & & & \\
\hline 1261 & 119 & & 15.58 .444 & & & & \\
\hline 1262 & 119 & & 16.01.079 & & & & \\
\hline 1263 & 119 & & 43.38 .457 & & & & \\
\hline 1264 & 119 & & 43.39.10128 & & & & \\
\hline 1265 & 119 & & 43.45 .782 & & & & \\
\hline 1266 & 119 & & 43.45 .852 & & & & \\
\hline \multicolumn{8}{|l|}{1267} \\
\hline \multicolumn{8}{|l|}{1268} \\
\hline \multicolumn{8}{|l|}{1269} \\
\hline \multicolumn{8}{|l|}{1270} \\
\hline \multicolumn{8}{|l|}{1271} \\
\hline \multicolumn{8}{|l|}{1272} \\
\hline \multicolumn{8}{|l|}{1273} \\
\hline \multicolumn{8}{|c|}{1274} \\
\hline \multicolumn{8}{|c|}{1275} \\
\hline \multicolumn{8}{|l|}{1276} \\
\hline \multicolumn{8}{|l|}{1277} \\
\hline 1278 & 119 & 15871 & & & & & \\
\hline 1279 & 119 & 27295 & & 594392.9328 & 116697.2814 & & GPS \\
\hline 1280 & 119 & 27173 & & 594494.7346 & 116202.6015 & & GPS \\
\hline \multicolumn{8}{|l|}{1281} \\
\hline \multicolumn{8}{|l|}{1282} \\
\hline 1283 & 119 & & 33.46 .160 & & & & \\
\hline 1284 & 119 & & 43.42 .160 & & & & \\
\hline 1285 & 119 & & 43.44.211 & & & & \\
\hline 1286 & 119 & & 43.43 .646 & & & & \\
\hline 1287 & 119 & & 43.45 .184 & & & & \\
\hline 1288 & 119 & & 33.46 .160 & & & & \\
\hline 1289 & 119 & & 33.47 .037 & & & & \\
\hline 1290 & 119 & & 33.52 .522 & & & & \\
\hline 1291 & 119 & & 33.59.834 & & & & \\
\hline 1292 & 119 & & 33.47 .707 & & & & \\
\hline 1293 & 119 & & 33.56 .914 & & & & \\
\hline
\end{tabular}




\begin{tabular}{|c|c|c|c|c|c|c|c|}
\hline & $\mathrm{AP}$ & $\mathrm{AQ}$ & AR & AS & AT & $\mathrm{AU}$ & AV \\
\hline 1 & LONGITUDE ${ }^{\circ} \mathrm{W}$ & LONGITUDE MIN_MIN & LONGITUDE MIN_SEC & X_COORD (EW) & Y_COORD (NW) & 3 D HAE/ALTITUDE & INSTRUMENT \\
\hline 1294 & 119 & & 34.37.401 & & & & \\
\hline 1295 & 119 & & 34.4 .571 & & & & \\
\hline 1296 & 119 & & 33.54 .646 & & & & \\
\hline 1297 & 119 & & 33.27 .425 & & & & \\
\hline 1298 & 119 & & 26.20.61 & & & & \\
\hline 1299 & 119 & & 26.15 .64 & & & & \\
\hline 1300 & 119 & & 19.25 .34 & & & & \\
\hline 1301 & 119 & & 26.19.26 & & & & \\
\hline 1302 & 119 & & 26.12.37 & & & & \\
\hline 1303 & 119 & & 26.9 .25 & & & & \\
\hline 1304 & 119 & & 26.7 .82 & & & & \\
\hline 1305 & 119 & & 19.34.19 & & & & \\
\hline 1306 & 119 & & 19.32.37 & & & & \\
\hline 1307 & 119 & & 19.24.78 & & & & \\
\hline 1308 & 119 & & 19.21.99 & & & & \\
\hline 1309 & 119 & & 19.29.69 & & & & \\
\hline 1310 & 119 & & 22.5 .67 & & & & \\
\hline 1311 & 119 & & 21.10 .49 & & & & \\
\hline 1312 & 119 & & 18.44 .07 & & & & \\
\hline 1313 & 119 & & 20.16.74 & & & & \\
\hline 1314 & 119 & & 27.20.00 & & & & \\
\hline 1315 & 119 & & 24.34.94 & & & & \\
\hline 1316 & 119 & & 16.4 .62 & & & & \\
\hline 1317 & 119 & & 16.4 .10 & & & & \\
\hline 1318 & 119 & & 15.58 .71 & & & & \\
\hline 1319 & 119 & & 15.50 .94 & & & & \\
\hline 1320 & 119 & & 15.36 .76 & & & & \\
\hline 1321 & 119 & & 15.32 .79 & & & & \\
\hline \multicolumn{8}{|c|}{1322} \\
\hline 1323 & 119 & & 15.34 .15 & & & & \\
\hline 1324 & 119 & & 15.27 .44 & & & & \\
\hline 1325 & 119 & & 15.18 .26 & & & & \\
\hline 1326 & 119 & & 15.1.78 & & & & \\
\hline 1327 & 119 & & 14.55 .21 & & & & \\
\hline \begin{tabular}{|l|}
1328 \\
\end{tabular} & 119 & & 15.36 .010 & & & & \\
\hline 1329 & 119 & & 15.45 .053 & & & & \\
\hline 1330 & 119 & & 15.58 .45 & & & & \\
\hline 1331 & 119 & & 16.1 .057 & & & & \\
\hline
\end{tabular}




\begin{tabular}{|c|c|c|c|c|c|c|c|}
\hline & $\mathrm{AP}$ & $\mathrm{AQ}$ & AR & AS & AT & $\mathrm{AU}$ & AV \\
\hline 1 & LONGITUDE ${ }^{\circ} \mathrm{W}$ & LONGITUDE MIN_MIN & LONGITUDE MIN_SEC & X_COORD (EW) & Y_COORD (NW) & 3 D HAE/ALTITUDE & INSTRUMENT \\
\hline 1332 & 119 & & 16.25.78 & & & & \\
\hline 1333 & 119 & & 16.22 .25 & & & & \\
\hline 1334 & 119 & & 16.16.39 & & & & \\
\hline 1335 & 119 & & 16.7 .69 & & & & \\
\hline 1336 & 119 & & 16.18.498 & & & & \\
\hline 1337 & 119 & & 16.18 .450 & & & & \\
\hline 1338 & 119 & & 16.18 .450 & & & & \\
\hline 1339 & 119 & & 16.18.119 & & & & \\
\hline 1340 & 119 & & 16.18.119 & & & & \\
\hline 1341 & 119 & & 16.17.828 & & & & \\
\hline 1342 & 119 & & 16.22 .585 & & & & \\
\hline 1343 & 119 & & 16.22.369 & & & & \\
\hline 1344 & 119 & & 16.22.369 & & & & \\
\hline 1345 & 119 & & 16.22.369 & & & & \\
\hline 1346 & 119 & & 16.22.143 & & & & \\
\hline 1347 & 119 & & 16.22.143 & & & & \\
\hline \multicolumn{8}{|l|}{1348} \\
\hline \multicolumn{8}{|l|}{1349} \\
\hline \multicolumn{8}{|l|}{1350} \\
\hline \multicolumn{8}{|l|}{1351} \\
\hline \multicolumn{8}{|l|}{1352} \\
\hline \multicolumn{8}{|l|}{1353} \\
\hline \multicolumn{8}{|c|}{1354} \\
\hline \multicolumn{8}{|l|}{1355} \\
\hline \multicolumn{8}{|l|}{1356} \\
\hline \multicolumn{8}{|l|}{1357} \\
\hline 1358 & 119 & & 33.46 .90 & & & & \\
\hline 1359 & 119 & & 36.23 .99 & & & & \\
\hline 1360 & 119 & & 35.28 .29 & & & & \\
\hline 1361 & 119 & & 29.23 .4 & & & & \\
\hline 1362 & 119 & & 28.38 .8 & & & & \\
\hline \multicolumn{8}{|l|}{1363} \\
\hline \multicolumn{8}{|l|}{1364} \\
\hline \multicolumn{8}{|c|}{1365} \\
\hline \multicolumn{8}{|l|}{1366} \\
\hline \multicolumn{8}{|l|}{1367} \\
\hline 1368 & 119 & & 43.32 .98 & & & & \\
\hline 1369 & 119 & & 43.39.75 & & & & \\
\hline
\end{tabular}




\begin{tabular}{|c|c|c|c|c|c|c|c|}
\hline & $\mathrm{AP}$ & $\mathrm{AQ}$ & AR & AS & AT & $\mathrm{AU}$ & AV \\
\hline 1 & LONGITUDE ${ }^{\circ} \mathrm{W}$ & LONGITUDE MIN_MIN & LONGITUDE MIN_SEC & X_COORD (EW) & Y_COORD (NW) & 3 D HAE/ALTITUDE & INSTRUMENT \\
\hline 1370 & 119 & & 43.41 .72 & & & & \\
\hline 1371 & 119 & & 43.44 .28 & & & & \\
\hline 1372 & 119 & & 15.35 .75 & & & & \\
\hline 1373 & 119 & & 15.35 .75 & & & & \\
\hline 1374 & 119 & & 15.31.17 & & & & \\
\hline 1375 & 119 & & 15.24 .39 & & & & \\
\hline 1376 & 119 & & 15.18 .12 & & & & \\
\hline 1377 & 119 & & 15.5 .25 & & & & \\
\hline 1378 & 119 & & 14.56.18 & & & & \\
\hline 1379 & 119 & & 15.36.06 & & & & \\
\hline 1380 & 119 & & 15.44 .45557 & & & & \\
\hline 1381 & 119 & & 15.58 .49 & & & & \\
\hline 1382 & 119 & & 16.1.47 & & & & \\
\hline 1383 & & & & & & & \\
\hline 1384 & & & & & & & \\
\hline 1385 & & & & & & & \\
\hline 1386 & & & & & & & \\
\hline 1387 & & & & & & & \\
\hline 1388 & & & & & & & \\
\hline 1389 & & & & & & & \\
\hline 1390 & & & & & & & \\
\hline 1391 & & & & & & & \\
\hline 1392 & & & & & & & \\
\hline 1393 & & & & & & & \\
\hline 1394 & & & & & & & \\
\hline 1395 & 119 & & 43.41 .968 & & & & \\
\hline 1396 & 119 & & 43.45 .470 & & & & \\
\hline 1397 & 119 & & 43.38.87 & & & & \\
\hline 1398 & 119 & & 43.45 .970 & & & & \\
\hline 1399 & 119 & & 15.35 .173 & & & & \\
\hline 1400 & 119 & & 15.35 .173 & & & & \\
\hline 1401 & 119 & & 15.32 .280 & & & & \\
\hline 1402 & 119 & & 15.27 .450 & & & & \\
\hline 1403 & 119 & & 15.18 .880 & & & & \\
\hline 1404 & 119 & & 15.2.305 & & & & \\
\hline 1405 & 119 & & 14.55 .643 & & & & \\
\hline 1406 & 119 & & 15.35.894 & & & & \\
\hline 1407 & 119 & & 15.44.136 & & & & \\
\hline
\end{tabular}




\begin{tabular}{|c|c|c|c|c|c|c|c|}
\hline & $\mathrm{AP}$ & $\mathrm{AQ}$ & AR & AS & AT & $A U$ & AV \\
\hline 1 & LONGITUDE ${ }^{\circ} \mathrm{W}$ & LONGITUDE MIN_MIN & LONGITUDE MIN_SEC & X_COORD (EW) & Y_COORD (NW) & 3 D HAE/ALTITUDE & INSTRUMENT \\
\hline 1408 & 119 & & 15.58 .274 & & & & \\
\hline 1409 & 119 & & 16.1.155 & & & & \\
\hline \multicolumn{8}{|l|}{1410} \\
\hline \multicolumn{8}{|l|}{1411} \\
\hline \multicolumn{8}{|l|}{1412} \\
\hline \multicolumn{8}{|l|}{1413} \\
\hline \multicolumn{8}{|l|}{1414} \\
\hline \multicolumn{8}{|l|}{1415} \\
\hline \multicolumn{8}{|l|}{1416} \\
\hline \multicolumn{8}{|l|}{1417} \\
\hline \multicolumn{8}{|c|}{1418} \\
\hline \multicolumn{8}{|c|}{1419} \\
\hline \multicolumn{8}{|l|}{1420} \\
\hline \multicolumn{8}{|l|}{1421} \\
\hline \multicolumn{8}{|l|}{1422} \\
\hline 1423 & 119 & 18039 & & & & & \\
\hline \begin{tabular}{|l|}
1424 \\
\end{tabular} & 119 & 28027 & & & & & \\
\hline \multicolumn{8}{|l|}{1425} \\
\hline \multicolumn{8}{|l|}{1426} \\
\hline \begin{tabular}{|l|}
1427 \\
\end{tabular} & 119 & & 43.38.216 & & & & \\
\hline \begin{tabular}{|l|}
1428 \\
\end{tabular} & 119 & & 43.40.351 & & & & \\
\hline 1429 & 119 & & 43.42 .623 & & & & \\
\hline 1430 & 119 & & 43.45 .564 & & & & \\
\hline 1431 & 119 & & 15.35 .664 & & & & \\
\hline 1432 & 119 & & 15.31 .280 & & & & \\
\hline 1433 & 119 & & 15.26.899 & & & & \\
\hline 1434 & 119 & & 15.17 .787 & & & & \\
\hline 1435 & 119 & & 15.04.289 & & & & \\
\hline 1436 & 119 & & 14.55.302 & & & & \\
\hline \begin{tabular}{|l|}
1437 \\
\end{tabular} & 119 & & 15.35 .921 & & & & \\
\hline 1438 & 119 & & 15.46 .088 & & & & \\
\hline 1439 & 119 & & 15.58.751 & & & & \\
\hline 1440 & 119 & & 16.01.371 & & & & \\
\hline \multicolumn{8}{|c|}{1441} \\
\hline \multicolumn{8}{|l|}{1442} \\
\hline \multicolumn{8}{|c|}{1443} \\
\hline \multicolumn{8}{|c|}{1444} \\
\hline 1445 & & & & & & & \\
\hline
\end{tabular}




\begin{tabular}{|c|c|c|c|c|c|c|c|}
\hline & AP & $\mathrm{AQ}$ & AR & AS & AT & $A U$ & AV \\
\hline 1 & LONGITUDE ${ }^{\circ} \mathrm{W}$ & LONGITUDE MIN_MIN & LONGITUDE MIN_SEC & X_COORD (EW) & Y_COORD (NW) & 3 D HAE/ALTITUDE & INSTRUMENT \\
\hline 1446 & & & & & & & \\
\hline 1447 & & & & & & & \\
\hline 1448 & & & & & & & \\
\hline 1449 & & & & & & & \\
\hline 1450 & & & & & & & \\
\hline 1451 & & & & & & & \\
\hline 1452 & & & & & & & \\
\hline 1453 & & & & & & & \\
\hline 1454 & & & & & & & \\
\hline 1455 & & & & & & & \\
\hline 1456 & & & & & & & \\
\hline 1457 & & & & & & & \\
\hline 1458 & & & & & & & \\
\hline 1459 & 119 & & 33.43 .61 & & & & \\
\hline 1460 & 119 & & 43.45 .22 & & & & \\
\hline 1461 & 119 & & 43.42 .11 & & & & \\
\hline 1462 & 119 & & 43.44 .59 & & & & \\
\hline 1463 & 119 & & 43.40 .35 & & & & \\
\hline 1464 & 119 & & 33.43.61 & & & & \\
\hline 1465 & 119 & & 33.44 .32 & & & & \\
\hline 1466 & 119 & & 33.50 .75 & & & & \\
\hline 1467 & 119 & & 34.00 .50 & & & & \\
\hline 1468 & 119 & & 33.46 .14 & & & & \\
\hline 1469 & 119 & & 33.57 .27 & & & & \\
\hline 1470 & 119 & & 34.37 .60 & & & & \\
\hline 1471 & 119 & & 34.03 .81 & & & & \\
\hline 1472 & 119 & & 33.54 .88 & & & & \\
\hline 1473 & 119 & & 33.26.71 & & & & \\
\hline 1474 & 119 & & 26.20 .44 & & & & \\
\hline 1475 & 119 & & 26.11.61 & & & & \\
\hline 1476 & 119 & & 19.27.34 & & & & \\
\hline 1477 & 119 & & 26.17 .75 & & & & \\
\hline 1478 & 119 & & 26.10.71 & & & & \\
\hline 1479 & 119 & & 26.09 .29 & & & & \\
\hline 1480 & 119 & & 26.08 .28 & & & & \\
\hline 1481 & 119 & & 19.36.81 & & & & \\
\hline 1482 & 119 & & 19.35.13 & & & & \\
\hline 1483 & 119 & & 19.24 .58 & & & & \\
\hline
\end{tabular}




\begin{tabular}{|c|c|c|c|c|c|c|c|}
\hline & $\mathrm{AP}$ & $\mathrm{AQ}$ & AR & AS & AT & $A U$ & AV \\
\hline 1 & LONGITUDE ${ }^{\circ} \mathrm{W}$ & LONGITUDE MIN MIN & LONGITUDE MIN SEC & $X$ COORD (EW) & Y COORD (NW) & 3 D HAE/ALTITUDE & \begin{tabular}{|l} 
INSTRUMENT \\
\end{tabular} \\
\hline 1484 & 119 & & 19.22 .285 & & & & \\
\hline 1485 & 119 & & 19.32 .76 & & & & \\
\hline 1486 & 119 & & 22.04 .98 & & & & \\
\hline 1487 & 119 & & 21.10 .15 & & & & \\
\hline 1488 & 119 & & 18.43.71 & & & & \\
\hline 1489 & 119 & & 20.15 .46 & & & & \\
\hline 1490 & 119 & & 27.19 .87 & & & & \\
\hline 1491 & 119 & & 23.52 .66 & & & & \\
\hline 1492 & 119 & & 24.35 .08 & & & & \\
\hline 1493 & 119 & & 16.04.61 & & & & \\
\hline 1494 & 119 & & 16.02.71 & & & & \\
\hline 1495 & 119 & & 15.56 .24 & & & & \\
\hline 1496 & 119 & & 15.50 .83 & & & & \\
\hline 1497 & 119 & & 15.37 .57 & & & & \\
\hline 1498 & 119 & & 15.33 .16 & & & & \\
\hline 1499 & 119 & & 15.34 .9 & & & & \\
\hline 1500 & 119 & & 15.31 .3 & & & & \\
\hline 1501 & 119 & & 15.41 .5 & & & & \\
\hline \begin{tabular}{|l|}
1502 \\
\end{tabular} & 119 & & 15.18 .35 & & & & \\
\hline 1503 & 119 & & 15.01 .42 & & & & \\
\hline 1504 & 119 & & 14.55 .48 & & & & \\
\hline 1505 & 119 & & 15.35 .90 & & & & \\
\hline 1506 & 119 & & 15.44 .92 & & & & \\
\hline \begin{tabular}{|l|}
1507 \\
\end{tabular} & 119 & & 15.58 .55 & & & & \\
\hline 1508 & 119 & & 16.01.26 & & & & \\
\hline 1509 & 119 & & 16.25 .66 & & & & \\
\hline 1510 & 119 & & 16.21 .89 & & & & \\
\hline 1511 & 119 & & 16.18 .03 & & & & \\
\hline 1512 & 119 & & 16.07.38 & & & & \\
\hline \multicolumn{8}{|l|}{1513} \\
\hline \multicolumn{8}{|l|}{1514} \\
\hline \multicolumn{8}{|l|}{1515} \\
\hline \multicolumn{8}{|l|}{1516} \\
\hline \multicolumn{8}{|l|}{1517} \\
\hline 1518 & 119 & & 20.10.491 & & & & \\
\hline \multicolumn{8}{|l|}{1519} \\
\hline 1520 & 119 & & 20.00 .067 & & & & \\
\hline 1521 & & & & & & & \\
\hline
\end{tabular}




\begin{tabular}{|c|c|c|c|c|c|c|c|}
\hline & $\mathrm{AP}$ & $\mathrm{AQ}$ & $\mathrm{AR}$ & AS & AT & $\mathrm{AU}$ & AV \\
\hline 1 & LONGITUDE ${ }^{\circ} \mathrm{W}$ & LONGITUDE MIN_MIN & LONGITUDE MIN_SEC & X_COORD (EW) & Y_COORD (NW) & 3 D HAE/ALTITUDE & INSTRUMENT \\
\hline \multicolumn{8}{|l|}{1522} \\
\hline \multicolumn{8}{|l|}{1523} \\
\hline 1524 & 119 & & 35.28 .175 & & & & \\
\hline 1525 & 119 & & 32.14 .9785 & & & & \\
\hline 1526 & 119 & & 28.38 .784 & & & & \\
\hline 1527 & 119 & & 25.12 .798 & & & & \\
\hline \multicolumn{8}{|l|}{1528} \\
\hline \multicolumn{8}{|l|}{1529} \\
\hline \multicolumn{8}{|l|}{1530} \\
\hline \multicolumn{8}{|l|}{1531} \\
\hline \multicolumn{8}{|l|}{1532} \\
\hline \multicolumn{8}{|l|}{1533} \\
\hline \multicolumn{8}{|l|}{1534} \\
\hline \multicolumn{8}{|l|}{1535} \\
\hline \multicolumn{8}{|l|}{1536} \\
\hline \multicolumn{8}{|l|}{\begin{tabular}{|l|}
1537 \\
\end{tabular}} \\
\hline \multicolumn{8}{|l|}{\begin{tabular}{|l|}
1538 \\
153
\end{tabular}} \\
\hline \multicolumn{8}{|l|}{1539} \\
\hline \multicolumn{8}{|l|}{1540} \\
\hline \multicolumn{8}{|l|}{1541} \\
\hline \multicolumn{8}{|l|}{1542} \\
\hline \multicolumn{8}{|l|}{1543} \\
\hline \multicolumn{8}{|c|}{\begin{tabular}{|l|}
1544 \\
\end{tabular}} \\
\hline \multicolumn{8}{|l|}{1545} \\
\hline \multicolumn{8}{|l|}{1546} \\
\hline \multicolumn{8}{|l|}{1547} \\
\hline \multicolumn{8}{|l|}{1548} \\
\hline \multicolumn{8}{|l|}{1549} \\
\hline \multicolumn{8}{|l|}{\begin{tabular}{|l|}
1550 \\
\end{tabular}} \\
\hline 1551 & & & & & & & \\
\hline 1552 & & & & & & & \\
\hline 1553 & & & & & & & \\
\hline 1554 & & & & & & & \\
\hline 1555 & & & & & & & \\
\hline \begin{tabular}{|l|}
1556 \\
\end{tabular} & & & & & & & \\
\hline 1557 & & & & & & & \\
\hline 1558 & & & & & & & \\
\hline 1559 & & & & & & & \\
\hline
\end{tabular}




\begin{tabular}{|c|c|c|c|c|c|c|c|}
\hline & AP & $\mathrm{AQ}$ & AR & AS & AT & $A U$ & $\mathrm{AV}$ \\
\hline 1 & LONGITUDE ${ }^{\circ} \mathrm{W}$ & LONGITUDE MIN_MIN & LONGITUDE MIN_SEC & X_COORD (EW) & Y_COORD (NW) & 3 D HAE/ALTITUDE & INSTRUMENT \\
\hline \multicolumn{8}{|c|}{\begin{tabular}{|l|}
1560 \\
\end{tabular}} \\
\hline \multicolumn{8}{|l|}{1561} \\
\hline \multicolumn{8}{|l|}{1562} \\
\hline \multicolumn{8}{|l|}{1563} \\
\hline 1564 & & & & 145775 & 559128 & & \\
\hline 1565 & & & & 145888 & 559110 & & \\
\hline 1566 & & & & 146030 & 559077 & & \\
\hline 1567 & & & & 146118 & 559037 & & \\
\hline 1568 & & & & 109886 & 595516 & & \\
\hline 1569 & & & & 109861 & 595700 & & \\
\hline 1570 & & & & 109840 & 595880 & & \\
\hline 1571 & & & & 109811 & 596091 & & \\
\hline 1572 & & & & 109824 & 596212 & & \\
\hline 1573 & & & & 109822 & 596358 & & \\
\hline 1574 & & & & 111001.9 & 595469.9 & & \\
\hline 1575 & & & & 112119.9 & 595260.9 & & \\
\hline 1576 & & & & 113946.4 & 594945.8 & & \\
\hline 1577 & & & & 114400.8 & 594886.3 & & \\
\hline \multicolumn{8}{|l|}{\begin{tabular}{|l|}
1578 \\
\end{tabular}} \\
\hline \multicolumn{8}{|l|}{1579} \\
\hline \multicolumn{8}{|l|}{1580} \\
\hline \multicolumn{8}{|l|}{1581} \\
\hline \multicolumn{8}{|l|}{1582} \\
\hline \multicolumn{8}{|l|}{1583} \\
\hline \multicolumn{8}{|c|}{\begin{tabular}{|l|}
1584 \\
\end{tabular}} \\
\hline \multicolumn{8}{|c|}{\begin{tabular}{|l|}
1585 \\
\end{tabular}} \\
\hline 1586 & & & & 571726 & 150552 & 95.5 & \\
\hline 1587 & & & & 559152 & 145816 & 100 & \\
\hline 1588 & & & & 559123 & 145934 & 101 & \\
\hline 1589 & & & & 558979 & 145994 & 99.4 & \\
\hline 1590 & & & & 559021 & 146105 & 101 & \\
\hline 1591 & & & & 571726 & 150552 & 95.5 & \\
\hline 1592 & & & & 571712 & 150603 & 95.1 & \\
\hline \multicolumn{8}{|c|}{1593} \\
\hline 1594 & & & & 571385 & 150788 & 97.2 & \\
\hline 1595 & & & & 571652 & 150596 & 96.1 & \\
\hline \multicolumn{8}{|c|}{\begin{tabular}{|l|}
1596 \\
\end{tabular}} \\
\hline 1597 & & & & & & & \\
\hline
\end{tabular}




\begin{tabular}{|c|c|c|c|c|c|c|c|}
\hline & $\mathrm{AP}$ & $\mathrm{AQ}$ & AR & AS & AT & $\mathrm{AU}$ & $\mathrm{AV}$ \\
\hline 1 & LONGITUDE ${ }^{\circ} \mathrm{W}$ & LONGITUDE MIN_MIN & LONGITUDE MIN_SEC & X_COORD (EW) & $\mathrm{Y}_{2} \mathrm{COORD}(\mathrm{NW})$ & 3 D HAE/ALTITUDE & INSTRUMENT \\
\hline 1598 & & & & 571313 & 149952 & 98 & \\
\hline 1599 & & & & & & & \\
\hline 1600 & & & & 572099 & 151070 & 95.2 & \\
\hline 1601 & & & & 581213 & 147964 & 92.8 & GPS \\
\hline 1602 & & & & 581313 & 148169 & 92 & GPS \\
\hline 1603 & & & & 590188 & 137123 & 87.2 & GPS \\
\hline 1604 & & & & 581253 & 148028 & 91.7 & GPS \\
\hline 1605 & & & & 581443 & 148274 & 92.6 & GPS \\
\hline 1606 & & & & 581502 & 148393 & 90 & GPS \\
\hline 1607 & & & & 581543 & 148471 & 88 & GPS \\
\hline 1608 & & & & 589999 & 137077 & 87.5 & GPS \\
\hline 1609 & & & & 590039 & 137078 & 86.9 & GPS \\
\hline 1610 & & & & 590732 & 137195 & 86.5 & GPS \\
\hline 1611 & & & & 590268 & 137247 & 86.8 & GPS \\
\hline 1612 & & & & 590087 & 137068 & 86.6 & GPS \\
\hline 1613 & & & & 586752 & 139253 & 87.2 & GPS \\
\hline 1614 & & & & 587946 & 138371 & 86.9 & GPS \\
\hline 1615 & & & & 591113 & 135398 & 85 & GPS \\
\hline 1616 & & & & 589105 & 137662 & 74.9 & GPS \\
\hline 1617 & & & & 579910 & 149070 & 92 & GPS \\
\hline 1618 & & & & 583186 & 144426 & 88.5 & GPS \\
\hline 1619 & & & & 583498 & 143300 & 85.9 & GPS \\
\hline 1620 & & & & & & & \\
\hline 1621 & & & & & & & \\
\hline 1622 & & & & 594916 & 114856 & 81.6 & GPS \\
\hline 1623 & & & & 595098 & 114923 & 81.6 & GPS \\
\hline 1624 & & & & 595395 & 114927 & 83 & GPS \\
\hline 1625 & & & & 595472 & 114951 & 83 & GPS \\
\hline 1626 & & & & 595518 & 109870 & 82.2 & GPS \\
\hline 1627 & & & & 595563 & 109836 & 82.7 & GPS \\
\hline 1628 & & & & 595677 & 109831 & 83.4 & GPS \\
\hline 1629 & & & & 595869 & 109857 & 82.3 & GPS \\
\hline 1630 & & & & 596226 & 109758 & 83.3 & GPS \\
\hline 1631 & & & & 596363 & 109808 & 82.8 & GPS \\
\hline 1632 & & & & 595483 & 110804 & 82.4 & GPS \\
\hline 1633 & & & & 595278 & 112118 & 82.2 & GPS \\
\hline 1634 & & & & 954956 & 113692 & 83 & GPS \\
\hline 1635 & & & & 594889 & 114400 & 83.4 & GPS \\
\hline
\end{tabular}




\begin{tabular}{|c|c|c|c|c|c|c|c|}
\hline & $\mathrm{AP}$ & $\mathrm{AQ}$ & AR & AS & AT & $\mathrm{AU}$ & $\mathrm{AV}$ \\
\hline 1 & LONGITUDE ${ }^{\circ} \mathrm{W}$ & LONGITUDE MIN_MIN & LONGITUDE MIN_SEC & X_COORD (EW) & $\mathrm{Y}_{\mathrm{Z}} \mathrm{COORD}(\mathrm{NW})$ & 3 D HAE/ALTITUDE & INSTRUMENT \\
\hline 1636 & & & & 594314 & 117539 & 82.8 & GPS \\
\hline 1637 & & & & 594396 & 116699 & 82.8 & GPS \\
\hline 1638 & & & & 594540 & 116076 & 82.6 & GPS \\
\hline 1639 & & & & 594738 & 115238 & 83.2 & GPS \\
\hline 1640 & & & & 589172 & 137602 & & \\
\hline 1641 & & & & 594392 & 116700 & 82 & \\
\hline 1642 & & & & 588808 & 137808 & 94 & \\
\hline 1643 & & & & 589438 & 137451 & & \\
\hline 1644 & & & & 594489 & 116208 & 82.5 & \\
\hline 1645 & & & & 593883 & 112747 & 100 & \\
\hline \multicolumn{8}{|l|}{\begin{tabular}{|l|}
1646 \\
\end{tabular}} \\
\hline \multicolumn{8}{|l|}{\begin{tabular}{|l|}
1647 \\
\end{tabular}} \\
\hline \multicolumn{8}{|l|}{1648} \\
\hline \multicolumn{8}{|l|}{\begin{tabular}{|l|}
1649 \\
\end{tabular}} \\
\hline \multicolumn{8}{|l|}{1650} \\
\hline \multicolumn{8}{|l|}{1651} \\
\hline \multicolumn{8}{|l|}{\begin{tabular}{|l|}
1652 \\
\end{tabular}} \\
\hline \multicolumn{8}{|l|}{\begin{tabular}{|l|}
1653 \\
\end{tabular}} \\
\hline \multicolumn{8}{|l|}{\begin{tabular}{|l|}
1654 \\
\end{tabular}} \\
\hline \multicolumn{8}{|l|}{\begin{tabular}{|l|l|}
1655 \\
\end{tabular}} \\
\hline 1656 & & & & 559150 & 145822 & 99.7 & GPS \\
\hline 1657 & & & & 559160 & 145921 & 100.9 & GPS \\
\hline 1658 & & & & 559069 & 146021 & 99 & GPS \\
\hline 1659 & & & & 559036 & 146104.449 & 98 & GPS \\
\hline 1660 & & & & 595517 & 109875 & 83 & GPS \\
\hline 1661 & & & & 595517 & 109875 & 83 & GPS \\
\hline 1662 & & & & 595577 & 109840 & 84 & GPS \\
\hline 1663 & & & & 595726 & 109770 & 81 & GPS \\
\hline 1664 & & & & 595872 & 109798 & 84 & GPS \\
\hline 1665 & & & & 596159 & 109608 & 80.7 & GPS \\
\hline 1666 & & & & 596362 & 109803 & 89.1 & GPS \\
\hline 1667 & & & & 595484 & 110809 & 83 & GPS \\
\hline 1668 & & & & 595270 & 112116 & 84 & GPS \\
\hline 1669 & & & & 594955 & 113780 & 83 & GPS \\
\hline 1670 & & & & 594887 & 114396 & 84 & GPS \\
\hline \multicolumn{8}{|l|}{\begin{tabular}{|l|}
1671 \\
\end{tabular}} \\
\hline \multicolumn{8}{|c|}{\begin{tabular}{|l|}
1672 \\
\end{tabular}} \\
\hline 1673 & & & & & & & \\
\hline
\end{tabular}




\begin{tabular}{|c|c|c|c|c|c|c|c|}
\hline & $\mathrm{AP}$ & $\mathrm{AQ}$ & AR & AS & AT & $A U$ & AV \\
\hline 1 & LONGITUDE ${ }^{\circ} \mathrm{W}$ & LONGITUDE MIN_MIN & LONGITUDE MIN_SEC & X_COORD (EW) & Y_COORD (NW) & 3 D HAE/ALTITUDE & INSTRUMENT \\
\hline \multicolumn{8}{|l|}{1674} \\
\hline \multicolumn{8}{|l|}{1675} \\
\hline 1676 & 119 & & 15.35 .53 & & & & \\
\hline \begin{tabular}{|l|}
1677 \\
\end{tabular} & 119 & & 15.35 .53 & & & & \\
\hline \begin{tabular}{|l|}
1678 \\
\end{tabular} & 119 & & 15.32 .09 & & & & \\
\hline \begin{tabular}{|l|}
1679 \\
\end{tabular} & 119 & & 15.28 .44 & & & & \\
\hline 1680 & 119 & & 15.17.99 & & & & \\
\hline 1681 & 119 & & 15.05 .39 & & & & \\
\hline \multicolumn{8}{|c|}{1682} \\
\hline \begin{tabular}{|l|}
1683 \\
\end{tabular} & 119 & & 15.35 .50 & & & & \\
\hline \multicolumn{8}{|l|}{1684} \\
\hline 1685 & 119 & & 15.58.34 & & & & \\
\hline 1686 & 119 & & 16.02 .37 & & & & \\
\hline 1687 & 119 & & 43.41 .36 & & & & \\
\hline \begin{tabular}{|l|}
1688 \\
\end{tabular} & 119 & & 43.42 .62 & & & & \\
\hline \begin{tabular}{|l|}
1689 \\
\end{tabular} & 119 & & 43.42 .99 & & & & \\
\hline 1690 & 119 & & 43.46 .54 & & & & \\
\hline \multicolumn{8}{|l|}{1691} \\
\hline \multicolumn{8}{|l|}{1692} \\
\hline \multicolumn{8}{|l|}{1693} \\
\hline \multicolumn{8}{|l|}{1694} \\
\hline \multicolumn{8}{|l|}{1695} \\
\hline \multicolumn{8}{|l|}{1696} \\
\hline \multicolumn{8}{|l|}{1697} \\
\hline \multicolumn{8}{|l|}{1698} \\
\hline \multicolumn{8}{|l|}{1699} \\
\hline \multicolumn{8}{|l|}{1700} \\
\hline \multicolumn{8}{|l|}{1701} \\
\hline \multicolumn{8}{|l|}{\begin{tabular}{|l|}
1702 \\
\end{tabular}} \\
\hline \multicolumn{8}{|l|}{1703} \\
\hline \multicolumn{8}{|l|}{1704} \\
\hline \multicolumn{8}{|l|}{1705} \\
\hline \multicolumn{8}{|l|}{1706} \\
\hline \multicolumn{8}{|l|}{1707} \\
\hline \multicolumn{8}{|l|}{1708} \\
\hline \multicolumn{8}{|l|}{1709} \\
\hline \multicolumn{8}{|l|}{1710} \\
\hline \begin{tabular}{|l|}
1711 \\
\end{tabular} & & & & & & & \\
\hline
\end{tabular}




\begin{tabular}{|c|c|c|c|c|c|c|c|}
\hline & $\mathrm{AP}$ & $\mathrm{AQ}$ & AR & AS & AT & $\mathrm{AU}$ & AV \\
\hline 1 & LONGITUDE ${ }^{\circ} \mathrm{W}$ & LONGITUDE MIN_MIN & LONGITUDE MIN_SEC & X_COORD (EW) & Y_COORD (NW) & 3 D HAE/ALTITUDE & INSTRUMENT \\
\hline 1712 & 119 & & 15.35.167 & & & & \\
\hline 1713 & 119 & & 15.32.854 & & & & \\
\hline 1714 & 119 & & 15.25 .556 & & & & \\
\hline 1715 & 119 & & 15.18.321 & & & & \\
\hline 1716 & 119 & & 15.05 .22609 & & & & \\
\hline 1717 & 119 & & 14.55 .469 & & & & \\
\hline 1718 & 119 & & 15.35 .841 & & & & \\
\hline 1719 & 119 & & 15.45.231 & & & & \\
\hline 1720 & 119 & & 15.58 .444 & & & & \\
\hline 1721 & 119 & & 16.01.079 & & & & \\
\hline 1722 & 119 & & 43.38 .457 & & & & \\
\hline 1723 & 119 & & 43.39.10128 & & & & \\
\hline 1724 & 119 & & 43.45 .782 & & & & \\
\hline 1725 & 119 & & 43.45 .852 & & & & \\
\hline \multicolumn{8}{|l|}{1726} \\
\hline \multicolumn{8}{|l|}{1727} \\
\hline \multicolumn{8}{|l|}{1728} \\
\hline \multicolumn{8}{|l|}{1729} \\
\hline \multicolumn{8}{|l|}{1730} \\
\hline \multicolumn{8}{|l|}{1731} \\
\hline \multicolumn{8}{|l|}{1732} \\
\hline 1733 & 119 & 27295 & & 594392.9328 & 116697.2814 & & GPS \\
\hline 1734 & 119 & 27173 & & 594494.7346 & 116202.6015 & & GPS \\
\hline 1735 & 119 & 74824 & & 557558.6994 & 144902.4606 & & GPS \\
\hline 1736 & 119 & 27295 & & 594392.9328 & 116697.2814 & & GPS \\
\hline 1737 & 119 & 27173 & & 594494.7346 & 116202.6015 & & GPS \\
\hline 1738 & 119 & 74824 & & 557558.6994 & 144902.4606 & & GPS \\
\hline 1739 & 119 & 74824 & & 557558.6994 & 144902.4606 & & GPS \\
\hline 1740 & 119 & 74824 & & 557558.6994 & 144902.4606 & & GPS \\
\hline 1741 & 119 & 74824 & & 557558.6994 & 144902.4606 & & GPS \\
\hline 1742 & 119 & 27287 & & 594399.2531 & 116695.838 & & GPS \\
\hline 1743 & 119 & 27283 & & 594402.1182 & 116695.2311 & & GPS \\
\hline 1744 & 119 & 2728 & & 594404.9572 & 116696.5481 & & GPS \\
\hline 1745 & 119 & 27265 & & 594418.067 & 116567.4607 & & GPS \\
\hline 1746 & 119 & 27262 & & 594420.161 & 116567.6005 & & GPS \\
\hline 1747 & 119 & 27255 & & 594425.6435 & 116567.8565 & & GPS \\
\hline 1748 & 119 & 27227 & & 594450.3417 & 116362.2444 & & GPS \\
\hline 1749 & 119 & 27172 & & 594495.7216 & 116205.7327 & & GPS \\
\hline
\end{tabular}




\begin{tabular}{|c|c|c|c|c|c|c|c|}
\hline & $\mathrm{AP}$ & $\mathrm{AQ}$ & AR & AS & AT & $A U$ & $\mathrm{AV}$ \\
\hline 1 & LONGITUDE ${ }^{\circ} \mathrm{W}$ & LONGITUDE MIN_MIN & LONGITUDE MIN_SEC & X_COORD (EW) & Y_COORD (NW) & 3 D HAE/ALTITUDE & INSTRUMENT \\
\hline 1750 & 119 & 27167 & & 594499.4158 & 116203.1668 & & GPS \\
\hline 1751 & 119 & 27163 & & 594501.9964 & 116209.2038 & & GPS \\
\hline 1752 & 119 & 27154 & & 594510.2381 & 116145.7579 & & GPS \\
\hline 1753 & 119 & 27152 & & 594511.5832 & 116147.8569 & & GPS \\
\hline 1754 & 119 & 27143 & & 594518.7508 & 116146.0857 & & GPS \\
\hline 1755 & 119 & 27079 & & 594571.7032 & 115909.9801 & & GPS \\
\hline 1756 & 119 & 2699 & & 594644.1671 & 115645.5062 & & GPS \\
\hline 1757 & 119 & 26987 & & 594646.1043 & 115645.9576 & & GPS \\
\hline 1758 & 119 & 26986 & & 594647.3591 & 115645.6143 & & GPS \\
\hline \multicolumn{8}{|l|}{1759} \\
\hline \multicolumn{8}{|l|}{1760} \\
\hline \multicolumn{8}{|l|}{1761} \\
\hline 1762 & 119 & 26697 & & 594889.7354 & 114361.6456 & & GPS \\
\hline 1763 & 119 & 26693 & & 594892.5316 & 114361.8727 & & GPS \\
\hline 1764 & 119 & 26692 & & 594893.2853 & 114362.3731 & & GPS \\
\hline 1765 & 119 & 74824 & & 557558.6994 & 144902.4606 & & GPS \\
\hline 1766 & 119 & 27276 & & 594407.8154 & 116693.7483 & & GPS \\
\hline 1767 & 119 & 27252 & & 594428.029 & 116566.4842 & & GPS \\
\hline 1768 & 119 & 27154 & & 594508.882 & 116209.1859 & & GPS \\
\hline 1769 & 119 & 27137 & & 594523.0808 & 116145.8184 & & GPS \\
\hline 1770 & 119 & 26982 & & 594650.1201 & 115647.6407 & & GPS \\
\hline \multicolumn{8}{|c|}{\begin{tabular}{|l|}
1771 \\
\end{tabular}} \\
\hline 1772 & 119 & 26688 & & 594896.5766 & 114364.2648 & & GPS \\
\hline \multicolumn{8}{|c|}{\begin{tabular}{|l|}
1773 \\
\end{tabular}} \\
\hline \multicolumn{8}{|c|}{\begin{tabular}{|l|}
1774 \\
\end{tabular}} \\
\hline 1775 & 119 & & 33.46 .160 & & & & \\
\hline 1776 & 119 & & 43.42 .160 & & & & \\
\hline 1777 & 119 & & 43.44 .211 & & & & \\
\hline 1778 & 119 & & 43.43 .646 & & & & \\
\hline 1779 & 119 & & 43.45 .184 & & & & \\
\hline 1780 & 119 & & 33.46 .160 & & & & \\
\hline 1781 & 119 & & 33.47 .037 & & & & \\
\hline 1782 & 119 & & 33.52 .522 & & & & \\
\hline 1783 & 119 & & 33.59 .834 & & & & \\
\hline 1784 & 119 & & 33.47 .707 & & & & \\
\hline 1785 & 119 & & 33.56.914 & & & & \\
\hline 1786 & 119 & & 34.37 .401 & & & & \\
\hline 1787 & 119 & & 34.4 .571 & & & & \\
\hline
\end{tabular}

Page 593 


\begin{tabular}{|c|c|c|c|c|c|c|c|}
\hline & $\mathrm{AP}$ & $\mathrm{AQ}$ & AR & AS & AT & $\mathrm{AU}$ & AV \\
\hline 1 & LONGITUDE ${ }^{\circ} \mathrm{W}$ & LONGITUDE MIN_MIN & LONGITUDE MIN_SEC & X_COORD (EW) & Y_COORD (NW) & 3 D HAE/ALTITUDE & INSTRUMENT \\
\hline 1788 & 119 & & 33.54 .646 & & & & \\
\hline 1789 & 119 & & 33.27 .425 & & & & \\
\hline 1790 & 119 & & 26.20 .61 & & & & \\
\hline 1791 & 119 & & 26.15 .64 & & & & \\
\hline 1792 & 119 & & 19.25.34 & & & & \\
\hline 1793 & 119 & & 26.19 .26 & & & & \\
\hline 1794 & 119 & & 26.12 .37 & & & & \\
\hline 1795 & 119 & & 26.9 .25 & & & & \\
\hline 1796 & 119 & & 26.7 .82 & & & & \\
\hline \begin{tabular}{|l|}
1797 \\
\end{tabular} & 119 & & 19.34.19 & & & & \\
\hline 1798 & 119 & & 19.32.37 & & & & \\
\hline 1799 & 119 & & 19.24.78 & & & & \\
\hline 1800 & 119 & & 19.21.99 & & & & \\
\hline 1801 & 119 & & 19.29.69 & & & & \\
\hline 1802 & 119 & & 22.5 .67 & & & & \\
\hline 1803 & 119 & & 21.10 .49 & & & & \\
\hline 1804 & 119 & & 18.44 .07 & & & & \\
\hline 1805 & 119 & & 20.16 .74 & & & & \\
\hline 1806 & 119 & & 27.20.00 & & & & \\
\hline 1807 & 119 & & 24.34 .94 & & & & \\
\hline 1808 & 119 & & 16.4 .62 & & & & \\
\hline 1809 & 119 & & 16.4 .10 & & & & \\
\hline 1810 & 119 & & 15.58 .71 & & & & \\
\hline 1811 & 119 & & 15.50 .94 & & & & \\
\hline 1812 & 119 & & 15.36 .76 & & & & \\
\hline 1813 & 119 & & 15.32 .79 & & & & \\
\hline \multicolumn{8}{|c|}{1814} \\
\hline 1815 & 119 & & 15.34.15 & & & & \\
\hline 1816 & 119 & & 15.27 .44 & & & & \\
\hline 1817 & 119 & & 15.18.26 & & & & \\
\hline 1818 & 119 & & 15.1.78 & & & & \\
\hline 1819 & 119 & & 14.55 .21 & & & & \\
\hline 1820 & 119 & & 15.36 .010 & & & & \\
\hline 1821 & 119 & & 15.45 .053 & & & & \\
\hline \begin{tabular}{|l|}
1822 \\
\end{tabular} & 119 & & 15.58 .45 & & & & \\
\hline 1823 & 119 & & 16.1.057 & & & & \\
\hline 1824 & 119 & & 16.25 .78 & & & & \\
\hline 1825 & 119 & & 16.22 .25 & & & & \\
\hline
\end{tabular}




\begin{tabular}{|c|c|c|c|c|c|c|c|}
\hline & $\mathrm{AP}$ & $\mathrm{AQ}$ & AR & AS & AT & $\mathrm{AU}$ & $\mathrm{AV}$ \\
\hline 1 & LONGITUDE ${ }^{\circ} \mathrm{W}$ & LONGITUDE MIN_MIN & LONGITUDE MIN_SEC & X_COORD (EW) & $\mathrm{Y}_{\mathrm{Z}} \mathrm{COORD}(\mathrm{NW})$ & 3 D HAE/ALTITUDE & INSTRUMENT \\
\hline 1826 & 119 & & 16.16 .39 & & & & \\
\hline 1827 & 119 & & 16.7 .69 & & & & \\
\hline 1828 & 119 & & 16.18.498 & & & & \\
\hline 1829 & 119 & & 16.18 .450 & & & & \\
\hline 1830 & 119 & & 16.18 .450 & & & & \\
\hline 1831 & 119 & & 16.18.119 & & & & \\
\hline 1832 & 119 & & 16.18.119 & & & & \\
\hline 1833 & 119 & & 16.17.828 & & & & \\
\hline 1834 & 119 & & 16.22 .585 & & & & \\
\hline 1835 & 119 & & 16.22 .369 & & & & \\
\hline 1836 & 119 & & 16.22.369 & & & & \\
\hline 1837 & 119 & & 16.22.369 & & & & \\
\hline 1838 & 119 & & 16.22 .143 & & & & \\
\hline 1839 & 119 & & 16.22 .143 & & & & \\
\hline 1840 & & & & & & & \\
\hline 1841 & & & & & & & \\
\hline 1842 & & & & & & & \\
\hline 1843 & 119 & & 29.23 .4 & & & & \\
\hline 1844 & 119 & & 28.38 .8 & & & & \\
\hline 1845 & & & & & & & \\
\hline 1846 & & & & & & & \\
\hline 1847 & & & & & & & \\
\hline 1848 & & & & & & & \\
\hline 1849 & & & & & & & \\
\hline 1850 & 119 & & 43.32 .98 & & & & \\
\hline 1851 & 119 & & 43.39 .75 & & & & \\
\hline 1852 & 119 & & 43.41 .72 & & & & \\
\hline 1853 & 119 & & 43.44 .28 & & & & \\
\hline 1854 & 119 & & 15.35 .75 & & & & \\
\hline 1855 & 119 & & 15.35 .75 & & & & \\
\hline 1856 & 119 & & 15.31.17 & & & & \\
\hline 1857 & 119 & & 15.24 .39 & & & & \\
\hline 1858 & 119 & & 15.18 .12 & & & & \\
\hline 1859 & 119 & & 15.5 .25 & & & & \\
\hline 1860 & 119 & & 14.56 .18 & & & & \\
\hline 1861 & 119 & & 15.36 .06 & & & & \\
\hline 1862 & 119 & & 15.44 .45557 & & & & \\
\hline 1863 & 119 & & 15.58 .49 & & & & \\
\hline
\end{tabular}

Page 595 


\begin{tabular}{|c|c|c|c|c|c|c|c|}
\hline & $\mathrm{AP}$ & $\mathrm{AQ}$ & AR & AS & AT & $\mathrm{AU}$ & AV \\
\hline 1 & LONGITUDE ${ }^{\circ} \mathrm{W}$ & LONGITUDE MIN_MIN & LONGITUDE MIN_SEC & X_COORD (EW) & Y_COORD (NW) & 3 D HAE/ALTITUDE & INSTRUMENT \\
\hline 1864 & 119 & & 16.1.47 & & & & \\
\hline \multicolumn{8}{|l|}{1865} \\
\hline \multicolumn{8}{|l|}{1866} \\
\hline \multicolumn{8}{|l|}{1867} \\
\hline \multicolumn{8}{|c|}{1868} \\
\hline \multicolumn{8}{|c|}{1869} \\
\hline \multicolumn{8}{|l|}{1870} \\
\hline 1871 & 119 & & 43.41 .968 & & & & \\
\hline 1872 & 119 & & 43.45 .470 & & & & \\
\hline 1873 & 119 & & 43.38.87 & & & & \\
\hline 1874 & 119 & & 43.45 .970 & & & & \\
\hline 1875 & 119 & & 15.35.173 & & & & \\
\hline 1876 & 119 & & 15.35.173 & & & & \\
\hline 1877 & 119 & & 15.32 .280 & & & & \\
\hline 1878 & 119 & & 15.27 .450 & & & & \\
\hline 1879 & 119 & & 15.18 .880 & & & & \\
\hline 1880 & 119 & & 15.2 .305 & & & & \\
\hline 1881 & 119 & & 14.55 .643 & & & & \\
\hline 1882 & 119 & & 15.35.894 & & & & \\
\hline 1883 & 119 & & 15.44 .136 & & & & \\
\hline 1884 & 119 & & 15.58.274 & & & & \\
\hline 1885 & 119 & & 16.1.155 & & & & \\
\hline \multicolumn{8}{|l|}{1886} \\
\hline \multicolumn{8}{|l|}{1887} \\
\hline \multicolumn{8}{|l|}{1888} \\
\hline \multicolumn{8}{|l|}{1889} \\
\hline 1890 & 119 & 18039 & & & & & \\
\hline 1891 & 119 & 28027 & & & & & \\
\hline \multicolumn{8}{|c|}{1892} \\
\hline \multicolumn{8}{|l|}{1893} \\
\hline 1894 & 119 & & 43.38 .216 & & & & \\
\hline 1895 & 119 & & 43.40 .351 & & & & \\
\hline 1896 & 119 & & 43.42 .623 & & & & \\
\hline 1897 & 119 & & 43.45 .564 & & & & \\
\hline 1898 & 119 & & 15.35 .664 & & & & \\
\hline 1899 & 119 & & 15.31 .280 & & & & \\
\hline 1900 & 119 & & 15.26.899 & & & & \\
\hline 1901 & 119 & & 15.17 .787 & & & & \\
\hline
\end{tabular}




\begin{tabular}{|c|c|c|c|c|c|c|c|}
\hline & $\mathrm{AP}$ & $\mathrm{AQ}$ & AR & AS & AT & $\mathrm{AU}$ & AV \\
\hline 1 & LONGITUDE ${ }^{\circ} \mathrm{W}$ & LONGITUDE MIN_MIN & LONGITUDE MIN_SEC & X_COORD (EW) & Y_COORD (NW) & 3 D HAE/ALTITUDE & INSTRUMENT \\
\hline 1902 & 119 & & 15.04.289 & & & & \\
\hline 1903 & 119 & & 14.55.302 & & & & \\
\hline 1904 & 119 & & 15.35 .921 & & & & \\
\hline 1905 & 119 & & 15.46 .088 & & & & \\
\hline 1906 & 119 & & 15.58 .751 & & & & \\
\hline 1907 & 119 & & 16.01.371 & & & & \\
\hline 1908 & & & & & & & \\
\hline 1909 & & & & & & & \\
\hline 1910 & & & & & & & \\
\hline 1911 & & & & & & & \\
\hline 1912 & & & & & & & \\
\hline 1913 & & & & & & & \\
\hline 1914 & & & & & & & \\
\hline 1915 & & & & & & & \\
\hline 1916 & & & & & & & \\
\hline 1917 & & & & & & & \\
\hline 1918 & 119 & & 33.43.61 & & & & \\
\hline 1919 & 119 & & 43.45 .22 & & & & \\
\hline 1920 & 119 & & 43.42 .11 & & & & \\
\hline 1921 & 119 & & 43.44 .59 & & & & \\
\hline 1922 & 119 & & 43.40 .35 & & & & \\
\hline 1923 & 119 & & 33.43.61 & & & & \\
\hline 1924 & 119 & & 33.44.32 & & & & \\
\hline 1925 & 119 & & 33.50 .75 & & & & \\
\hline 1926 & 119 & & 34.00 .50 & & & & \\
\hline 1927 & 119 & & 33.46 .14 & & & & \\
\hline 1928 & 119 & & 33.57 .27 & & & & \\
\hline 1929 & 119 & & 34.37 .60 & & & & \\
\hline 1930 & 119 & & 34.03 .81 & & & & \\
\hline 1931 & 119 & & 33.54 .88 & & & & \\
\hline 1932 & 119 & & 33.26.71 & & & & \\
\hline 1933 & 119 & & 26.20 .44 & & & & \\
\hline 1934 & 119 & & 26.11.61 & & & & \\
\hline 1935 & 119 & & 19.27.34 & & & & \\
\hline 1936 & 119 & & 26.17 .75 & & & & \\
\hline 1937 & 119 & & 26.10.71 & & & & \\
\hline 1938 & 119 & & 26.09 .29 & & & & \\
\hline 1939 & 119 & & 26.08.28 & & & & \\
\hline
\end{tabular}




\begin{tabular}{|c|c|c|c|c|c|c|c|}
\hline & $\mathrm{AP}$ & $\mathrm{AQ}$ & AR & AS & AT & $\mathrm{AU}$ & AV \\
\hline 1 & LONGITUDE ${ }^{\circ} \mathrm{W}$ & LONGITUDE MIN_MIN & LONGITUDE MIN_SEC & X_COORD (EW) & Y_COORD (NW) & 3 D HAE/ALTITUDE & INSTRUMENT \\
\hline 1940 & 119 & & 19.36.81 & & & & \\
\hline 1941 & 119 & & 19.35.13 & & & & \\
\hline 1942 & 119 & & 19.24.58 & & & & \\
\hline 1943 & 119 & & 19.22.285 & & & & \\
\hline 1944 & 119 & & 19.32.76 & & & & \\
\hline 1945 & 119 & & 22.04 .98 & & & & \\
\hline 1946 & 119 & & 21.10 .15 & & & & \\
\hline 1947 & 119 & & 18.43.71 & & & & \\
\hline 1948 & 119 & & 20.15 .46 & & & & \\
\hline 1949 & 119 & & 27.19 .87 & & & & \\
\hline 1950 & 119 & & 23.52 .66 & & & & \\
\hline 1951 & 119 & & 24.35 .08 & & & & \\
\hline 1952 & 119 & & 16.04 .61 & & & & \\
\hline 1953 & 119 & & 16.02 .71 & & & & \\
\hline 1954 & 119 & & 15.56 .24 & & & & \\
\hline 1955 & 119 & & 15.50 .83 & & & & \\
\hline 1956 & 119 & & 15.37 .57 & & & & \\
\hline 1957 & 119 & & 15.33.16 & & & & \\
\hline 1958 & 119 & & 15.34 .9 & & & & \\
\hline 1959 & 119 & & 15.31 .3 & & & & \\
\hline 1960 & 119 & & 15.41 .5 & & & & \\
\hline 1961 & 119 & & 15.18.35 & & & & \\
\hline 1962 & 119 & & 15.01 .42 & & & & \\
\hline 1963 & 119 & & 14.55 .48 & & & & \\
\hline 1964 & 119 & & 15.35 .90 & & & & \\
\hline 1965 & 119 & & 15.44 .92 & & & & \\
\hline 1966 & 119 & & 15.58 .55 & & & & \\
\hline 1967 & 119 & & 16.01 .26 & & & & \\
\hline 1968 & 119 & & 16.25 .66 & & & & \\
\hline 1969 & 119 & & 16.21 .89 & & & & \\
\hline 1970 & 119 & & 16.18.03 & & & & \\
\hline 1971 & 119 & & 16.07.38 & & & & \\
\hline \multicolumn{8}{|l|}{1972} \\
\hline \multicolumn{8}{|l|}{1973} \\
\hline 1974 & 119 & & 20.10.491 & & & & \\
\hline \multicolumn{8}{|l|}{1975} \\
\hline 1976 & 119 & & 20.00.067 & & & & \\
\hline 1977 & 119 & & 28.38.784 & & & & \\
\hline
\end{tabular}




\begin{tabular}{|c|c|c|c|c|c|c|c|}
\hline & $\mathrm{AP}$ & $\mathrm{AQ}$ & AR & AS & AT & $A U$ & $\mathrm{AV}$ \\
\hline 1 & LONGITUDE ${ }^{\circ} \mathrm{W}$ & LONGITUDE MIN MIN & LONGITUDE MIN SEC & $X$ COORD (EW) & Y COORD (NW) & 3 D HAE/ALTITUDE & \begin{tabular}{|l} 
INSTRUMENT \\
\end{tabular} \\
\hline 1978 & 119 & & 25.12 .798 & & & & \\
\hline \multicolumn{8}{|l|}{1979} \\
\hline \multicolumn{8}{|l|}{1980} \\
\hline \multicolumn{8}{|l|}{1981} \\
\hline \multicolumn{8}{|l|}{1982} \\
\hline \begin{tabular}{|l|}
1983 \\
\end{tabular} & 119 & 4363830 & & & & & \\
\hline 1984 & 119 & 4365845 & & & & & \\
\hline 1985 & 119 & 434070431 & & & & & \\
\hline \begin{tabular}{|l|}
1986 \\
\end{tabular} & 119 & 434589260 & & & & & \\
\hline \begin{tabular}{|l|}
1987 \\
\end{tabular} & 119 & 153521977 & & & & & \\
\hline 1988 & 119 & 153521977 & & & & & \\
\hline \begin{tabular}{|l|}
1989 \\
\end{tabular} & 119 & 15290058 & & & & & \\
\hline 1990 & 119 & 152340608 & & & & & \\
\hline 1991 & 119 & 151897178 & & & & & \\
\hline 1992 & 119 & 150525588 & & & & & \\
\hline 1993 & 119 & 145567351 & & & & & \\
\hline 1994 & 119 & 153598423 & & & & & \\
\hline \begin{tabular}{|l|}
1995 \\
\end{tabular} & 119 & 154392604 & & & & & \\
\hline \begin{tabular}{|l|}
1996 \\
\end{tabular} & 119 & 155819461 & & & & & \\
\hline 1997. & 119 & 16009196 & & & & & \\
\hline \multicolumn{8}{|l|}{1998} \\
\hline \multicolumn{8}{|l|}{1999} \\
\hline \multicolumn{8}{|l|}{2000} \\
\hline \multicolumn{8}{|l|}{2001} \\
\hline \multicolumn{8}{|l|}{2002} \\
\hline \multicolumn{8}{|l|}{2003} \\
\hline \multicolumn{8}{|l|}{2004} \\
\hline \multicolumn{8}{|l|}{2005} \\
\hline \multicolumn{8}{|l|}{2006} \\
\hline \multicolumn{8}{|l|}{2007} \\
\hline \multicolumn{8}{|l|}{2008} \\
\hline \multicolumn{8}{|l|}{2009} \\
\hline \multicolumn{8}{|l|}{2010} \\
\hline \multicolumn{8}{|l|}{2011} \\
\hline \multicolumn{8}{|l|}{2012} \\
\hline \multicolumn{8}{|l|}{2013} \\
\hline \multicolumn{8}{|l|}{2014} \\
\hline 2015 & & & & & & & \\
\hline
\end{tabular}




\begin{tabular}{|c|c|c|c|c|c|c|c|}
\hline & AP & $\mathrm{AQ}$ & AR & AS & AT & $A U$ & AV \\
\hline 1 & LONGITUDE ${ }^{\circ} \mathrm{W}$ & LONGITUDE MIN_MIN & LONGITUDE MIN_SEC & X_COORD (EW) & Y_COORD (NW) & 3 D HAE/ALTITUDE & INSTRUMENT \\
\hline \multicolumn{8}{|l|}{2016} \\
\hline \multicolumn{8}{|l|}{2017} \\
\hline \multicolumn{8}{|l|}{2018} \\
\hline \multicolumn{8}{|l|}{2019} \\
\hline \multicolumn{8}{|l|}{2020} \\
\hline \multicolumn{8}{|l|}{2021} \\
\hline \multicolumn{8}{|l|}{2022} \\
\hline \multicolumn{8}{|l|}{2023} \\
\hline \multicolumn{8}{|l|}{2024} \\
\hline \multicolumn{8}{|l|}{2025} \\
\hline \multicolumn{8}{|l|}{2026} \\
\hline \multicolumn{8}{|l|}{2027} \\
\hline \multicolumn{8}{|l|}{2028} \\
\hline \multicolumn{8}{|l|}{2029} \\
\hline 2030 & & & & 145775 & 559128 & & \\
\hline 2031 & & & & 145888 & 559110 & & \\
\hline 2032 & & & & 146030 & 559077 & & \\
\hline 2033 & & & & 146118 & 559037 & & \\
\hline 2034 & & & & 109886 & 595516 & & \\
\hline 2035 & & & & 109861 & 595700 & & \\
\hline 2036 & & & & 109840 & 595880 & & \\
\hline 2037 & & & & 109811 & 596091 & & \\
\hline 2038 & & & & 109824 & 596212 & & \\
\hline 2039 & & & & 109822 & 596358 & & \\
\hline 2040 & & & & 111001.9 & 595469.9 & & \\
\hline 2041 & & & & 112119.9 & 595260.9 & & \\
\hline 2042 & & & & 113946.4 & 594945.8 & & \\
\hline 2043 & & & & 114400.8 & 594886.3 & & \\
\hline \multicolumn{8}{|l|}{2044} \\
\hline \multicolumn{8}{|l|}{2045} \\
\hline \multicolumn{8}{|l|}{2046} \\
\hline \multicolumn{8}{|l|}{2047} \\
\hline \multicolumn{8}{|l|}{2048} \\
\hline \multicolumn{8}{|l|}{2049} \\
\hline \multicolumn{8}{|l|}{2050} \\
\hline \multicolumn{8}{|l|}{2051} \\
\hline 2052 & & & & 571726 & 150552 & 95.5 & \\
\hline 2053 & & & & 559152 & 145816 & 100 & \\
\hline
\end{tabular}




\begin{tabular}{|c|c|c|c|c|c|c|c|}
\hline & $\mathrm{AP}$ & $\overline{A Q}$ & AR & AS & AT & $\mathrm{AU}$ & AV \\
\hline 1 & LONGITUDE ${ }^{\circ} \mathrm{W}$ & LONGITUDE MIN_MIN & LONGITUDE MIN_SEC & X_COORD (EW) & $\mathrm{Y}_{-} \mathrm{COORD}(\mathrm{NW})$ & 3 D HAE/ALTITUDE & INSTRUMENT \\
\hline 2054 & & & & 559123 & 145934 & 101 & \\
\hline 2055 & & & & 558979 & 145994 & 99.4 & \\
\hline 2056 & & & & 559021 & 146105 & 101 & \\
\hline 2057 & & & & 571726 & 150552 & 95.5 & \\
\hline 2058 & & & & 571712 & 150603 & 95.1 & \\
\hline \multicolumn{8}{|l|}{2059} \\
\hline 2060 & & & & 571385 & 150788 & 97.2 & \\
\hline 2061 & & & & 571652 & 150596 & 96.1 & \\
\hline \multicolumn{8}{|l|}{2062} \\
\hline \multicolumn{8}{|l|}{2063} \\
\hline 2064 & & & & 571313 & 149952 & 98 & \\
\hline \multicolumn{8}{|l|}{2065} \\
\hline 2066 & & & & 572099 & 151070 & 95.2 & \\
\hline 2067 & & & & 581213 & 147964 & 92.8 & GPS \\
\hline 2068 & & & & 581313 & 148169 & 92 & GPS \\
\hline 2069 & & & & 590188 & 137123 & 87.2 & GPS \\
\hline 2070 & & & & 581253 & 148028 & 91.7 & GPS \\
\hline 2071 & & & & 581443 & 148274 & 92.6 & GPS \\
\hline 2072 & & & & 581502 & 148393 & 90 & GPS \\
\hline 2073 & & & & 581543 & 148471 & 88 & GPS \\
\hline 2074 & & & & 589999 & 137077 & 87.5 & GPS \\
\hline 2075 & & & & 590039 & 137078 & 86.9 & GPS \\
\hline 2076 & & & & 590732 & 137195 & 86.5 & GPS \\
\hline 2077 & & & & 590268 & 137247 & 86.8 & GPS \\
\hline 2078 & & & & 590087 & 137068 & 86.6 & GPS \\
\hline 2079 & & & & 586752 & 139253 & 87.2 & GPS \\
\hline 2080 & & & & 587946 & 138371 & 86.9 & GPS \\
\hline 2081 & & & & 591113 & 135398 & 85 & GPS \\
\hline 2082 & & & & 589105 & 137662 & 74.9 & GPS \\
\hline 2083 & & & & 579910 & 149070 & 92 & GPS \\
\hline 2084 & & & & 583186 & 144426 & 88.5 & GPS \\
\hline 2085 & & & & 583498 & 143300 & 85.9 & GPS \\
\hline \multicolumn{8}{|l|}{2086} \\
\hline \multicolumn{8}{|l|}{2087} \\
\hline 2088 & & & & 594916 & 114856 & 81.6 & GPS \\
\hline 2089 & & & & 595098 & 114923 & 81.6 & GPS \\
\hline 2090 & & & & 595395 & 114927 & 83 & GPS \\
\hline 2091 & & & & 595472 & 114951 & 83 & GPS \\
\hline
\end{tabular}




\begin{tabular}{|c|c|c|c|c|c|c|c|}
\hline & $\mathrm{AP}$ & AQ & AR & AS & AT & $A U$ & $\mathrm{AV}$ \\
\hline 1 & LONGITUDE ${ }^{\circ} \mathrm{W}$ & LONGITUDE MIN_MIN & LONGITUDE MIN_SEC & X_COORD (EW) & Y_COORD (NW) & 3 D HAE/ALTITUDE & INSTRUMENT \\
\hline 2092 & & & & 595518 & 109870 & 82.2 & GPS \\
\hline 2093 & & & & 595563 & 109836 & 82.7 & GPS \\
\hline 2094 & & & & 595677 & 109831 & 83.4 & GPS \\
\hline 2095 & & & & 595869 & 109857 & 82.3 & GPS \\
\hline 2096 & & & & 596226 & 109758 & 83.3 & GPS \\
\hline 2097 & & & & 596363 & 109808 & 82.8 & GPS \\
\hline 2098 & & & & 595483 & 110804 & 82.4 & GPS \\
\hline 2099 & & & & 595278 & 112118 & 82.2 & GPS \\
\hline 2100 & & & & 954956 & 113692 & 83 & GPS \\
\hline 2101 & & & & 594889 & 114400 & 83.4 & GPS \\
\hline 2102 & & & & 594314 & 117539 & 82.8 & GPS \\
\hline 2103 & & & & 594396 & 116699 & 82.8 & GPS \\
\hline 2104 & & & & 594540 & 116076 & 82.6 & GPS \\
\hline 2105 & & & & 594738 & 115238 & 83.2 & GPS \\
\hline 2106 & & & & 589172 & 137602 & & \\
\hline 2107 & & & & 594392 & 116700 & 82 & \\
\hline 2108 & & & & 588808 & 137808 & 94 & \\
\hline 2109 & & & & 589438 & 137451 & & \\
\hline 2110 & & & & 594489 & 116208 & 82.5 & \\
\hline 2111 & & & & 593883 & 112747 & 100 & \\
\hline \multicolumn{8}{|l|}{\begin{tabular}{|l|}
2112 \\
\end{tabular}} \\
\hline \multicolumn{8}{|l|}{2113} \\
\hline \multicolumn{8}{|c|}{2114} \\
\hline \multicolumn{8}{|l|}{2115} \\
\hline \multicolumn{8}{|l|}{\begin{tabular}{|l|}
2116 \\
\end{tabular}} \\
\hline \multicolumn{8}{|l|}{\begin{tabular}{|l|}
2117 \\
\end{tabular}} \\
\hline \multicolumn{8}{|l|}{\begin{tabular}{|l|}
2118 \\
\end{tabular}} \\
\hline \multicolumn{8}{|l|}{\begin{tabular}{|l|}
2119 \\
\end{tabular}} \\
\hline \multicolumn{8}{|l|}{2120} \\
\hline \multicolumn{8}{|l|}{\begin{tabular}{|l|}
2121 \\
\end{tabular}} \\
\hline 2122 & & & & 559150 & 145822 & 99.7 & GPS \\
\hline 2123 & & & & 559160 & 145921 & 100.9 & GPS \\
\hline 2124 & & & & 559069 & 146021 & 99 & GPS \\
\hline 2125 & & & & 559036 & 146104.449 & 98 & GPS \\
\hline 2126 & & & & 595517 & 109875 & 83 & GPS \\
\hline 2127 & & & & 595517 & 109875 & 83 & GPS \\
\hline 2128 & & & & 595577 & 109840 & 84 & GPS \\
\hline 2129 & & & & 595726 & 109770 & 81 & GPS \\
\hline
\end{tabular}




\begin{tabular}{|c|c|c|c|c|c|c|c|}
\hline & $\mathrm{AP}$ & $\mathrm{AQ}$ & AR & AS & AT & $\overline{A U}$ & AV \\
\hline 1 & LONGITUDE ${ }^{\circ} \mathrm{W}$ & LONGITUDE MIN_MIN & LONGITUDE MIN_SEC & X_COORD (EW) & Y_COORD (NW) & 3 D HAE/ALTITUDE & INSTRUMENT \\
\hline 2130 & & & & 595872 & 109798 & 84 & GPS \\
\hline 2131 & & & & 596159 & 109608 & 80.7 & GPS \\
\hline 2132 & & & & 596362 & 109803 & 89.1 & GPS \\
\hline 2133 & & & & 595484 & 110809 & 83 & GPS \\
\hline 2134 & & & & 595270 & 112116 & 84 & GPS \\
\hline 2135 & & & & 594955 & 113780 & 83 & GPS \\
\hline 2136 & & & & 594887 & 114396 & 84 & GPS \\
\hline \multicolumn{8}{|l|}{2137} \\
\hline \multicolumn{8}{|l|}{2138} \\
\hline \multicolumn{8}{|l|}{2139} \\
\hline \multicolumn{8}{|l|}{2140} \\
\hline \multicolumn{8}{|l|}{\begin{tabular}{|l|}
2141 \\
\end{tabular}} \\
\hline 2142 & 119 & & 15.35 .53 & & & & \\
\hline 2143 & 119 & & 15.35 .53 & & & & \\
\hline 2144 & 119 & & 15.32 .09 & & & & \\
\hline 2145 & 119 & & 15.28 .44 & & & & \\
\hline 2146 & 119 & & 15.17.99 & & & & \\
\hline 2147 & 119 & & 15.05.39 & & & & \\
\hline \multicolumn{8}{|l|}{2148} \\
\hline 2149 & 119 & & 15.35 .50 & & & & \\
\hline \multicolumn{8}{|l|}{2150} \\
\hline 2151 & 119 & & 15.58.34 & & & & \\
\hline 2152 & 119 & & 16.02 .37 & & & & \\
\hline 2153 & 119 & & 43.41 .36 & & & & \\
\hline 2154 & 119 & & 43.42 .62 & & & & \\
\hline 2155 & 119 & & 43.42 .99 & & & & \\
\hline 2156 & 119 & & 43.46 .54 & & & & \\
\hline \multicolumn{8}{|l|}{2157} \\
\hline \multicolumn{8}{|l|}{2158} \\
\hline \multicolumn{8}{|l|}{2159} \\
\hline \multicolumn{8}{|l|}{2160} \\
\hline \multicolumn{8}{|l|}{2161} \\
\hline \multicolumn{8}{|l|}{2162} \\
\hline \multicolumn{8}{|l|}{2163} \\
\hline \multicolumn{8}{|l|}{2164} \\
\hline \multicolumn{8}{|l|}{2165} \\
\hline \multicolumn{8}{|l|}{2166} \\
\hline 2167 & & & & & & & \\
\hline
\end{tabular}




\begin{tabular}{|c|c|c|c|c|c|c|c|}
\hline & $\mathrm{AP}$ & $\mathrm{AQ}$ & AR & AS & AT & $\mathrm{AU}$ & AV \\
\hline 1 & LONGITUDE ${ }^{\circ} \mathrm{W}$ & LONGITUDE MIN_MIN & LONGITUDE MIN_SEC & X_COORD (EW) & Y_COORD (NW) & 3 D HAE/ALTITUDE & INSTRUMENT \\
\hline \multicolumn{8}{|l|}{2168} \\
\hline \multicolumn{8}{|l|}{2169} \\
\hline \multicolumn{8}{|c|}{2170} \\
\hline \multicolumn{8}{|l|}{2171} \\
\hline \multicolumn{8}{|l|}{2172} \\
\hline \multicolumn{8}{|l|}{2173} \\
\hline \multicolumn{8}{|l|}{2174} \\
\hline \multicolumn{8}{|l|}{2175} \\
\hline \multicolumn{8}{|l|}{2176} \\
\hline \multicolumn{8}{|l|}{2177} \\
\hline 2178 & 119 & & 15.35 .167 & & & & \\
\hline 2179 & 119 & & 15.32 .854 & & & & \\
\hline 2180 & 119 & & 15.25 .556 & & & & \\
\hline 2181 & 119 & & 15.18.321 & & & & \\
\hline 2182 & 119 & & 15.05 .22609 & & & & \\
\hline 2183 & 119 & & 14.55 .469 & & & & \\
\hline 2184 & 119 & & 15.35 .841 & & & & \\
\hline 2185 & 119 & & 15.45 .231 & & & & \\
\hline 2186 & 119 & & 15.58 .444 & & & & \\
\hline 2187 & 119 & & 16.01 .079 & & & & \\
\hline 2188 & 119 & & 43.38 .457 & & & & \\
\hline 2189 & 119 & & 43.39 .10128 & & & & \\
\hline 2190 & 119 & & 43.45 .782 & & & & \\
\hline 2191 & 119 & & 43.45 .852 & & & & \\
\hline \multicolumn{8}{|c|}{2192} \\
\hline \multicolumn{8}{|c|}{2193} \\
\hline \multicolumn{8}{|c|}{2194} \\
\hline \multicolumn{8}{|c|}{\begin{tabular}{|l|}
2195 \\
\end{tabular}} \\
\hline \multicolumn{8}{|c|}{2196} \\
\hline \multicolumn{8}{|l|}{\begin{tabular}{|l|}
2197 \\
\end{tabular}} \\
\hline \multicolumn{8}{|l|}{\begin{tabular}{|l|}
2198 \\
\end{tabular}} \\
\hline 2199 & 119 & 27295 & & 594392.9328 & 116697.2814 & & GPS \\
\hline 2200 & 119 & 27173 & & 594494.7346 & 116202.6015 & & GPS \\
\hline 2201 & 119 & 74824 & & 557558.6994 & 144902.4606 & & GPS \\
\hline 2202 & 119 & 27295 & & 594392.9328 & 116697.2814 & & GPS \\
\hline 2203 & 119 & 27173 & & 594494.7346 & 116202.6015 & & GPS \\
\hline 2204 & 119 & 74824 & & 557558.6994 & 144902.4606 & & GPS \\
\hline 2205 & 119 & 74824 & & 557558.6994 & 144902.4606 & & GPS \\
\hline
\end{tabular}




\begin{tabular}{|c|c|c|c|c|c|c|c|}
\hline & $\mathrm{AP}$ & $\mathrm{AQ}$ & AR & AS & AT & $\mathrm{AU}$ & AV \\
\hline 1 & LONGITUDE ${ }^{\circ} \mathrm{W}$ & LONGITUDE MIN MIN & LONGITUDE MIN SEC & X COORD (EW) & Y COORD (NW) & 3 D HAE/ALTITUDE & INSTRUMENT \\
\hline 2206 & 119 & 74824 & & 557558.6994 & 144902.4606 & & GPS \\
\hline 2207 & 119 & 74824 & & 557558.6994 & 144902.4606 & & GPS \\
\hline 2208 & 119 & 27287 & & 594399.2531 & 116695.838 & & GPS \\
\hline 2209 & 119 & 27283 & & 594402.1182 & 116695.2311 & & GPS \\
\hline 2210 & 119 & 2728 & & 594404.9572 & 116696.5481 & & GPS \\
\hline 2211 & 119 & 27265 & & 594418.067 & 116567.4607 & & GPS \\
\hline 2212 & 119 & 27262 & & 594420.161 & 116567.6005 & & GPS \\
\hline 2213 & 119 & 27255 & & 594425.6435 & 116567.8565 & & GPS \\
\hline 2214 & 119 & 27227 & & 594450.3417 & 116362.2444 & & GPS \\
\hline 2215 & 119 & 27172 & & 594495.7216 & 116205.7327 & & GPS \\
\hline 2216 & 119 & 27167 & & 594499.4158 & 116203.1668 & & GPS \\
\hline 2217 & 119 & 27163 & & 594501.9964 & 116209.2038 & & GPS \\
\hline 2218 & 119 & 27154 & & 594510.2381 & 116145.7579 & & GPS \\
\hline 2219 & 119 & 27152 & & 594511.5832 & 116147.8569 & & GPS \\
\hline 2220 & 119 & 27143 & & 594518.7508 & 116146.0857 & & GPS \\
\hline 2221 & 119 & 27079 & & 594571.7032 & 115909.9801 & & GPS \\
\hline 2222 & 119 & 2699 & & 594644.1671 & 115645.5062 & & GPS \\
\hline 2223 & 119 & 26987 & & 594646.1043 & 115645.9576 & & GPS \\
\hline 2224 & 119 & 26986 & & 594647.3591 & 115645.6143 & & GPS \\
\hline \multicolumn{8}{|l|}{2225} \\
\hline \multicolumn{8}{|l|}{2226} \\
\hline \multicolumn{8}{|l|}{2227} \\
\hline 2228 & 119 & 26697 & & 594889.7354 & 114361.6456 & & GPS \\
\hline 2229 & 119 & 26693 & & 594892.5316 & 114361.8727 & & GPS \\
\hline 2230 & 119 & 26692 & & 594893.2853 & 114362.3731 & & GPS \\
\hline 2231 & 119 & 74824 & & 557558.6994 & 144902.4606 & & GPS \\
\hline 2232 & 119 & 27276 & & 594407.8154 & 116693.7483 & & GPS \\
\hline 2233 & 119 & 27252 & & 594428.029 & 116566.4842 & & GPS \\
\hline 2234 & 119 & 27154 & & 594508.882 & 116209.1859 & & GPS \\
\hline 2235 & 119 & 27137 & & 594523.0808 & 116145.8184 & & GPS \\
\hline 2236 & 119 & 26982 & & 594650.1201 & 115647.6407 & & GPS \\
\hline \multicolumn{8}{|l|}{2237} \\
\hline 2238 & 119 & 26688 & & 594896.5766 & 114364.2648 & & GPS \\
\hline \multicolumn{8}{|l|}{2239} \\
\hline \multicolumn{8}{|l|}{2240} \\
\hline 2241 & 119 & & 33.46 .160 & & & & \\
\hline 2242 & 119 & & 43.42 .160 & & & & \\
\hline 2243 & 119 & & 43.44 .211 & & & & \\
\hline
\end{tabular}

Page 605 


\begin{tabular}{|c|c|c|c|c|c|c|c|}
\hline & $\mathrm{AP}$ & $\mathrm{AQ}$ & AR & AS & AT & $\mathrm{AU}$ & AV \\
\hline 1 & LONGITUDE ${ }^{\circ} \mathrm{W}$ & LONGITUDE MIN_MIN & LONGITUDE MIN_SEC & X_COORD (EW) & Y_COORD (NW) & 3 D HAE/ALTITUDE & INSTRUMENT \\
\hline 2244 & 119 & & 43.43 .646 & & & & \\
\hline 2245 & 119 & & 43.45 .184 & & & & \\
\hline 2246 & 119 & & 33.46 .160 & & & & \\
\hline 2247 & 119 & & 33.47.037 & & & & \\
\hline 2248 & 119 & & 33.52 .522 & & & & \\
\hline 2249 & 119 & & 33.59 .834 & & & & \\
\hline 2250 & 119 & & 33.47 .707 & & & & \\
\hline 2251 & 119 & & 33.56 .914 & & & & \\
\hline 2252 & 119 & & 34.37 .401 & & & & \\
\hline 2253 & 119 & & 34.4 .571 & & & & \\
\hline 2254 & 119 & & 33.54 .646 & & & & \\
\hline 2255 & 119 & & 33.27 .425 & & & & \\
\hline 2256 & 119 & & 26.20 .61 & & & & \\
\hline 2257 & 119 & & 26.15 .64 & & & & \\
\hline 2258 & 119 & & 19.25.34 & & & & \\
\hline 2259 & 119 & & 26.19.26 & & & & \\
\hline 2260 & 119 & & 26.12.37 & & & & \\
\hline 2261 & 119 & & 26.9 .25 & & & & \\
\hline 2262 & 119 & & 26.7 .82 & & & & \\
\hline 2263 & 119 & & 19.34.19 & & & & \\
\hline 2264 & 119 & & 19.32.37 & & & & \\
\hline 2265 & 119 & & 19.24.78 & & & & \\
\hline 2266 & 119 & & 19.21.99 & & & & \\
\hline 2267 & 119 & & 19.29.69 & & & & \\
\hline 2268 & 119 & & 22.5 .67 & & & & \\
\hline 2269 & 119 & & 21.10 .49 & & & & \\
\hline 2270 & 119 & & 18.44.07 & & & & \\
\hline 2271 & 119 & & 20.16.74 & & & & \\
\hline 2272 & 119 & & 27.20.00 & & & & \\
\hline 2273 & 119 & & 24.34 .94 & & & & \\
\hline 2274 & 119 & & 16.4 .62 & & & & \\
\hline 2275 & 119 & & 16.4 .10 & & & & \\
\hline 2276 & 119 & & 15.58.71 & & & & \\
\hline 2277 & 119 & & 15.50 .94 & & & & \\
\hline 2278 & 119 & & 15.36 .76 & & & & \\
\hline 2279 & 119 & & 15.32 .79 & & & & \\
\hline \multicolumn{8}{|l|}{2280} \\
\hline 2281 & 119 & & 15.34 .15 & & & & \\
\hline
\end{tabular}




\begin{tabular}{|c|c|c|c|c|c|c|c|}
\hline & $\mathrm{AP}$ & $\mathrm{AQ}$ & AR & AS & AT & $\mathrm{AU}$ & AV \\
\hline 1 & LONGITUDE ${ }^{\circ} \mathrm{W}$ & LONGITUDE MIN_MIN & LONGITUDE MIN_SEC & X_COORD (EW) & Y_COORD (NW) & 3 D HAE/ALTITUDE & INSTRUMENT \\
\hline 2282 & 119 & & 15.27.44 & & & & \\
\hline 2283 & 119 & & 15.18.26 & & & & \\
\hline 2284 & 119 & & 15.1.78 & & & & \\
\hline 2285 & 119 & & 14.55 .21 & & & & \\
\hline 2286 & 119 & & 15.36 .010 & & & & \\
\hline 2287 & 119 & & 15.45 .053 & & & & \\
\hline 2288 & 119 & & 15.58 .45 & & & & \\
\hline 2289 & 119 & & 16.1.057 & & & & \\
\hline 2290 & 119 & & 16.25.78 & & & & \\
\hline 2291 & 119 & & 16.22 .25 & & & & \\
\hline 2292 & 119 & & 16.16 .39 & & & & \\
\hline 2293 & 119 & & 16.7 .69 & & & & \\
\hline 2294 & 119 & & 16.18.498 & & & & \\
\hline 2295 & 119 & & 16.18 .450 & & & & \\
\hline 2296 & 119 & & 16.18 .450 & & & & \\
\hline 2297 & 119 & & 16.18.119 & & & & \\
\hline 2298 & 119 & & 16.18.119 & & & & \\
\hline 2299 & 119 & & 16.17.828 & & & & \\
\hline 2300 & 119 & & 16.22 .585 & & & & \\
\hline 2301 & 119 & & 16.22.369 & & & & \\
\hline 2302 & 119 & & 16.22 .369 & & & & \\
\hline 2303 & 119 & & 16.22.369 & & & & \\
\hline 2304 & 119 & & 16.22.143 & & & & \\
\hline 2305 & 119 & & 16.22.143 & & & & \\
\hline \multicolumn{8}{|l|}{2306} \\
\hline \multicolumn{8}{|l|}{2307} \\
\hline \multicolumn{8}{|l|}{2308} \\
\hline 2309 & 119 & & 29.23 .4 & & & & \\
\hline 2310 & 119 & & 28.38 .8 & & & & \\
\hline \multicolumn{8}{|l|}{2311} \\
\hline \multicolumn{8}{|l|}{2312} \\
\hline \multicolumn{8}{|l|}{2313} \\
\hline \multicolumn{8}{|l|}{2314} \\
\hline \multicolumn{8}{|l|}{2315} \\
\hline 2316 & 119 & & 43.32.98 & & & & \\
\hline 2317 & 119 & & 43.39.75 & & & & \\
\hline 2318 & 119 & & 43.41 .72 & & & & \\
\hline 2319 & 119 & & 43.44 .28 & & & & \\
\hline
\end{tabular}




\begin{tabular}{|c|c|c|c|c|c|c|c|}
\hline & $\mathrm{AP}$ & $\mathrm{AQ}$ & AR & AS & AT & $A U$ & AV \\
\hline 1 & LONGITUDE ${ }^{\circ} \mathrm{W}$ & LONGITUDE MIN_MIN & LONGITUDE MIN_SEC & X_COORD (EW) & Y_COORD (NW) & 3 D HAE/ALTITUDE & INSTRUMENT \\
\hline 2320 & 119 & & 15.35 .75 & & & & \\
\hline 2321 & 119 & & 15.35 .75 & & & & \\
\hline \begin{tabular}{|l|}
2322 \\
\end{tabular} & 119 & & 15.31 .17 & & & & \\
\hline 2323 & 119 & & 15.24 .39 & & & & \\
\hline 2324 & 119 & & 15.18 .12 & & & & \\
\hline 2325 & 119 & & 15.5 .25 & & & & \\
\hline \begin{tabular}{|l|}
2326 \\
\end{tabular} & 119 & & 14.56 .18 & & & & \\
\hline \begin{tabular}{|l|}
2327 \\
\end{tabular} & 119 & & 15.36.06 & & & & \\
\hline \begin{tabular}{|l|}
2328 \\
\end{tabular} & 119 & & 15.44 .45557 & & & & \\
\hline \begin{tabular}{|l|}
2329 \\
\end{tabular} & 119 & & 15.58 .49 & & & & \\
\hline 2330 & 119 & & 16.1.47 & & & & \\
\hline \multicolumn{8}{|l|}{2331} \\
\hline \multicolumn{8}{|l|}{2332} \\
\hline \multicolumn{8}{|l|}{2333} \\
\hline \multicolumn{8}{|l|}{2334} \\
\hline \multicolumn{8}{|l|}{2335} \\
\hline \multicolumn{8}{|l|}{2336} \\
\hline \begin{tabular}{|l|}
2337 \\
\end{tabular} & 119 & & 43.41 .968 & & & & \\
\hline \begin{tabular}{|l|}
2338 \\
\end{tabular} & 119 & & 43.45 .470 & & & & \\
\hline 2339 & 119 & & 43.38 .87 & & & & \\
\hline 2340 & 119 & & 43.45 .970 & & & & \\
\hline \begin{tabular}{|l|}
2341 \\
\end{tabular} & 119 & & 15.35 .173 & & & & \\
\hline 2342 & 119 & & 15.35 .173 & & & & \\
\hline 2343 & 119 & & 15.32 .280 & & & & \\
\hline 2344 & 119 & & 15.27 .450 & & & & \\
\hline 2345 & 119 & & 15.18 .880 & & & & \\
\hline 2346 & 119 & & 15.2 .305 & & & & \\
\hline \begin{tabular}{|l|}
2347 \\
\end{tabular} & 119 & & 14.55 .643 & & & & \\
\hline \begin{tabular}{|l|}
2348 \\
\end{tabular} & 119 & & 15.35 .894 & & & & \\
\hline 2349 & 119 & & 15.44 .136 & & & & \\
\hline 2350 & 119 & & 15.58 .274 & & & & \\
\hline \begin{tabular}{|l|}
2351 \\
\end{tabular} & 119 & & 16.1.155 & & & & \\
\hline \multicolumn{8}{|l|}{2352} \\
\hline \multicolumn{8}{|l|}{2353} \\
\hline \multicolumn{8}{|l|}{2354} \\
\hline \multicolumn{8}{|l|}{2355} \\
\hline 2356 & 119 & 18039 & & & & & \\
\hline \begin{tabular}{|l|}
2357 \\
\end{tabular} & 119 & 28027 & & & & & \\
\hline
\end{tabular}




\begin{tabular}{|c|c|c|c|c|c|c|c|}
\hline & $\mathrm{AP}$ & $\mathrm{AQ}$ & AR & AS & AT & $\mathrm{AU}$ & AV \\
\hline 1 & LONGITUDE ${ }^{\circ} \mathrm{W}$ & LONGITUDE MIN_MIN & LONGITUDE MIN_SEC & X_COORD (EW) & Y_COORD (NW) & 3 D HAE/ALTITUDE & INSTRUMENT \\
\hline \multicolumn{8}{|l|}{2358} \\
\hline \multicolumn{8}{|l|}{2359} \\
\hline 2360 & 119 & & 43.38 .216 & & & & \\
\hline 2361 & 119 & & 43.40 .351 & & & & \\
\hline 2362 & 119 & & 43.42 .623 & & & & \\
\hline 2363 & 119 & & 43.45 .564 & & & & \\
\hline 2364 & 119 & & 15.35 .664 & & & & \\
\hline 2365 & 119 & & 15.31 .280 & & & & \\
\hline 2366 & 119 & & 15.26 .899 & & & & \\
\hline 2367 & 119 & & 15.17.787 & & & & \\
\hline 2368 & 119 & & 15.04 .289 & & & & \\
\hline 2369 & 119 & & 14.55.302 & & & & \\
\hline 2370 & 119 & & 15.35.921 & & & & \\
\hline 2371 & 119 & & 15.46.088 & & & & \\
\hline 2372 & 119 & & 15.58 .751 & & & & \\
\hline 2373 & 119 & & 16.01.371 & & & & \\
\hline \multicolumn{8}{|c|}{2374} \\
\hline \multicolumn{8}{|l|}{2375} \\
\hline \multicolumn{8}{|l|}{2376} \\
\hline \multicolumn{8}{|l|}{2377} \\
\hline \multicolumn{8}{|l|}{2378} \\
\hline \multicolumn{8}{|l|}{2379} \\
\hline \multicolumn{8}{|l|}{2380} \\
\hline \multicolumn{8}{|l|}{2381} \\
\hline \multicolumn{8}{|l|}{2382} \\
\hline \multicolumn{8}{|l|}{2383} \\
\hline 2384 & 119 & & 33.43.61 & & & & \\
\hline 2385 & 119 & & 43.45 .22 & & & & \\
\hline 2386 & 119 & & 43.42 .11 & & & & \\
\hline 2387 & 119 & & 43.44 .59 & & & & \\
\hline 2388 & 119 & & 43.40 .35 & & & & \\
\hline 2389 & 119 & & 33.43.61 & & & & \\
\hline 2390 & 119 & & 33.44 .32 & & & & \\
\hline 2391 & 119 & & 33.50 .75 & & & & \\
\hline 2392 & 119 & & 34.00 .50 & & & & \\
\hline 2393 & 119 & & 33.46 .14 & & & & \\
\hline 2394 & 119 & & 33.57 .27 & & & & \\
\hline 2395 & 119 & & 34.37 .60 & & & & \\
\hline
\end{tabular}




\begin{tabular}{|c|c|c|c|c|c|c|c|}
\hline & $\mathrm{AP}$ & $\mathrm{AQ}$ & AR & AS & AT & $\mathrm{AU}$ & AV \\
\hline 1 & LONGITUDE ${ }^{\circ} \mathrm{W}$ & LONGITUDE MIN_MIN & LONGITUDE MIN_SEC & X_COORD (EW) & Y_COORD (NW) & 3 D HAE/ALTITUDE & INSTRUMENT \\
\hline 2396 & 119 & & 34.03 .81 & & & & \\
\hline 2397 & 119 & & 33.54 .88 & & & & \\
\hline 2398 & 119 & & 33.26 .71 & & & & \\
\hline 2399 & 119 & & 26.20 .44 & & & & \\
\hline 2400 & 119 & & 26.11.61 & & & & \\
\hline 2401 & 119 & & 19.27.34 & & & & \\
\hline 2402 & 119 & & 26.17.75 & & & & \\
\hline 2403 & 119 & & 26.10.71 & & & & \\
\hline 2404 & 119 & & 26.09.29 & & & & \\
\hline 2405 & 119 & & 26.08.28 & & & & \\
\hline 2406 & 119 & & 19.36.81 & & & & \\
\hline 2407 & 119 & & 19.35.13 & & & & \\
\hline 2408 & 119 & & 19.24.58 & & & & \\
\hline 2409 & 119 & & 19.22.285 & & & & \\
\hline 2410 & 119 & & 19.32.76 & & & & \\
\hline 2411 & 119 & & 22.04 .98 & & & & \\
\hline 2412 & 119 & & 21.10 .15 & & & & \\
\hline 2413 & 119 & & 18.43.71 & & & & \\
\hline 2414 & 119 & & 20.15 .46 & & & & \\
\hline 2415 & 119 & & 27.19.87 & & & & \\
\hline 2416 & 119 & & 23.52.66 & & & & \\
\hline 2417 & 119 & & 24.35.08 & & & & \\
\hline 2418 & 119 & & 16.04.61 & & & & \\
\hline 2419 & 119 & & 16.02 .71 & & & & \\
\hline 2420 & 119 & & 15.56 .24 & & & & \\
\hline 2421 & 119 & & 15.50 .83 & & & & \\
\hline 2422 & 119 & & 15.37 .57 & & & & \\
\hline 2423 & 119 & & 15.33 .16 & & & & \\
\hline 2424 & 119 & & 15.34 .9 & & & & \\
\hline 2425 & 119 & & 15.31 .3 & & & & \\
\hline 2426 & 119 & & 15.41 .5 & & & & \\
\hline 2427 & 119 & & 15.18.35 & & & & \\
\hline 2428 & 119 & & 15.01 .42 & & & & \\
\hline 2429 & 119 & & 14.55 .48 & & & & \\
\hline 2430 & 119 & & 15.35 .90 & & & & \\
\hline 2431 & 119 & & 15.44 .92 & & & & \\
\hline 2432 & 119 & & 15.58 .55 & & & & \\
\hline 2433 & 119 & & 16.01 .26 & & & & \\
\hline
\end{tabular}




\begin{tabular}{|c|c|c|c|c|c|c|c|}
\hline & AP & $\mathrm{AQ}$ & AR & AS & AT & $A U$ & AV \\
\hline 1 & LONGITUDE ${ }^{\circ} \mathrm{W}$ & LONGITUDE MIN_MIN & LONGITUDE MIN_SEC & X_COORD (EW) & Y_COORD (NW) & 3 D HAE/ALTITUDE & INSTRUMENT \\
\hline 2434 & 119 & & 16.25 .66 & & & & \\
\hline 2435 & 119 & & 16.21 .89 & & & & \\
\hline 2436 & 119 & & 16.18 .03 & & & & \\
\hline 2437 & 119 & & 16.07 .38 & & & & \\
\hline \multicolumn{8}{|l|}{2438} \\
\hline \multicolumn{8}{|l|}{2439} \\
\hline 2440 & 119 & & 20.10 .491 & & & & \\
\hline \multicolumn{8}{|l|}{2441} \\
\hline 2442 & 119 & & 20.00 .067 & & & & \\
\hline 2443 & 119 & & 28.38.784 & & & & \\
\hline 2444 & 119 & & 25.12.798 & & & & \\
\hline \multicolumn{8}{|l|}{2445} \\
\hline \multicolumn{8}{|l|}{2446} \\
\hline \multicolumn{8}{|l|}{2447} \\
\hline \multicolumn{8}{|l|}{2448} \\
\hline 2449 & 119 & 4363830 & & & & & \\
\hline 2450 & 119 & 4365845 & & & & & \\
\hline 2451 & 119 & 434070431 & & & & & \\
\hline 2452 & 119 & 434589260 & & & & & \\
\hline 2453 & 119 & 153521977 & & & & & \\
\hline 2454 & 119 & 153521977 & & & & & \\
\hline 2455 & 119 & 15290058 & & & & & \\
\hline 2456 & 119 & 152340608 & & & & & \\
\hline 2457 & 119 & 151897178 & & & & & \\
\hline 2458 & 119 & 150525588 & & & & & \\
\hline 2459 & 119 & 145567351 & & & & & \\
\hline 2460 & 119 & 153598423 & & & & & \\
\hline 2461 & 119 & 154392604 & & & & & \\
\hline 2462 & 119 & 155819461 & & & & & \\
\hline 2463 & 119 & 16009196 & & & & & \\
\hline \multicolumn{8}{|l|}{2464} \\
\hline \multicolumn{8}{|l|}{2465} \\
\hline \multicolumn{8}{|l|}{2466} \\
\hline \multicolumn{8}{|l|}{2467} \\
\hline \multicolumn{8}{|l|}{2468} \\
\hline \multicolumn{8}{|l|}{2469} \\
\hline \multicolumn{8}{|l|}{2470} \\
\hline 2471 & & & & & & & \\
\hline
\end{tabular}




\begin{tabular}{|c|c|c|c|c|c|c|c|}
\hline & $\mathrm{AP}$ & $\mathrm{AQ}$ & AR & AS & AT & $A U$ & $\mathrm{AV}$ \\
\hline 1 & LONGITUDE ${ }^{\circ} \mathrm{W}$ & LONGITUDE MIN MIN & LONGITUDE MIN SEC & X COORD (EW) & Y COORD (NW) & 3 D HAE/ALTITUDE & INSTRUMENT \\
\hline 2472 & & & & & & & \\
\hline 2473 & & & & & & & \\
\hline 2474 & & & & & & & \\
\hline 2475 & & & & & & & \\
\hline 2476 & & & & & & & \\
\hline 2477 & & & & & & & \\
\hline 2478 & & & & & & & \\
\hline 2479 & & & & & & & \\
\hline 2480 & & & & & & & \\
\hline 2481 & & & & & & & \\
\hline 2482 & & & & & & & \\
\hline 2483 & & & & & & & \\
\hline 2484 & & & & & & & \\
\hline 2485 & & & & & & & \\
\hline 2486 & & & & & & & \\
\hline 2487 & & & & & & & \\
\hline 2488 & & & & & & & \\
\hline 2489 & & & & & & & \\
\hline 2490 & & & & & & & \\
\hline 2491 & & & & & & & \\
\hline 2492 & & & & & & & \\
\hline 2493 & & & & & & & \\
\hline 2494 & & & & & & & \\
\hline 2495 & & & & & & & \\
\hline 2496 & & & & 145775 & 559128 & & \\
\hline 2497 & & & & 145888 & 559110 & & \\
\hline 2498 & & & & 146030 & 559077 & & \\
\hline 2499 & & & & 146118 & 559037 & & \\
\hline 2500 & & & & 109886 & 595516 & & \\
\hline 2501 & & & & 109861 & 595700 & & \\
\hline 2502 & & & & 109840 & 595880 & & \\
\hline 2503 & & & & 109811 & 596091 & & \\
\hline 2504 & & & & 109824 & 596212 & & \\
\hline 2505 & & & & 109822 & 596358 & & \\
\hline 2506 & & & & 111001.9 & 595469.9 & & \\
\hline 2507 & & & & 112119.9 & 595260.9 & & \\
\hline 2508 & & & & 113946.4 & 594945.8 & & \\
\hline 2509 & & & & 114400.8 & 594886.3 & & \\
\hline
\end{tabular}




\begin{tabular}{|c|c|c|c|c|c|c|c|}
\hline & $\mathrm{AP}$ & $\mathrm{AQ}$ & AR & AS & AT & $\mathrm{AU}$ & AV \\
\hline 1 & LONGITUDE ${ }^{\circ} \mathrm{W}$ & LONGITUDE MIN_MIN & LONGITUDE MIN_SEC & X_COORD (EW) & Y_COORD (NW) & 3 D HAE/ALTITUDE & INSTRUMENT \\
\hline \multicolumn{8}{|l|}{2510} \\
\hline \multicolumn{8}{|l|}{2511} \\
\hline \multicolumn{8}{|l|}{2512} \\
\hline \multicolumn{8}{|l|}{2513} \\
\hline \multicolumn{8}{|l|}{2514} \\
\hline \multicolumn{8}{|l|}{2515} \\
\hline \multicolumn{8}{|l|}{2516} \\
\hline \multicolumn{8}{|l|}{2517} \\
\hline 2518 & & & & 571726 & 150552 & 95.5 & \\
\hline 2519 & & & & 559152 & 145816 & 100 & \\
\hline 2520 & & & & 559123 & 145934 & 101 & \\
\hline 2521 & & & & 558979 & 145994 & 99.4 & \\
\hline 2522 & & & & 559021 & 146105 & 101 & \\
\hline 2523 & & & & 571726 & 150552 & 95.5 & \\
\hline 2524 & & & & 571712 & 150603 & 95.1 & \\
\hline \multicolumn{8}{|c|}{2525} \\
\hline 2526 & & & & 571385 & 150788 & 97.2 & \\
\hline 2527 & & & & 571652 & 150596 & 96.1 & \\
\hline \multicolumn{8}{|c|}{2528} \\
\hline \multicolumn{8}{|l|}{2529} \\
\hline 2530 & & & & 571313 & 149952 & 98 & \\
\hline \multicolumn{8}{|l|}{2531} \\
\hline 2532 & & & & 572099 & 151070 & 95.2 & \\
\hline 2533 & & & & 581213 & 147964 & 92.8 & GPS \\
\hline 2534 & & & & 581313 & 148169 & 92 & GPS \\
\hline 2535 & & & & 590188 & 137123 & 87.2 & GPS \\
\hline 2536 & & & & 581253 & 148028 & 91.7 & GPS \\
\hline 2537 & & & & 581443 & 148274 & 92.6 & GPS \\
\hline 2538 & & & & 581502 & 148393 & 90 & GPS \\
\hline 2539 & & & & 581543 & 148471 & 88 & GPS \\
\hline 2540 & & & & 589999 & 137077 & 87.5 & GPS \\
\hline 2541 & & & & 590039 & 137078 & 86.9 & GPS \\
\hline 2542 & & & & 590732 & 137195 & 86.5 & GPS \\
\hline 2543 & & & & 590268 & 137247 & 86.8 & GPS \\
\hline 2544 & & & & 590087 & 137068 & 86.6 & GPS \\
\hline 2545 & & & & 586752 & 139253 & 87.2 & GPS \\
\hline 2546 & & & & 587946 & 138371 & 86.9 & GPS \\
\hline 2547 & & & & 591113 & 135398 & 85 & GPS \\
\hline
\end{tabular}




\begin{tabular}{|c|c|c|c|c|c|c|c|}
\hline & $\mathrm{AP}$ & $\mathrm{AQ}$ & AR & AS & AT & $\overline{A U}$ & AV \\
\hline 1 & LONGITUDE ${ }^{\circ} \mathrm{W}$ & LONGITUDE MIN_MIN & LONGITUDE MIN_SEC & X_COORD (EW) & Y_COORD (NW) & 3 D HAE/ALTITUDE & INSTRUMENT \\
\hline 2548 & & & & 589105 & 137662 & 74.9 & GPS \\
\hline 2549 & & & & 579910 & 149070 & 92 & GPS \\
\hline 2550 & & & & 583186 & 144426 & 88.5 & GPS \\
\hline 2551 & & & & 583498 & 143300 & 85.9 & GPS \\
\hline \multicolumn{8}{|l|}{2552} \\
\hline \multicolumn{8}{|l|}{2553} \\
\hline 2554 & & & & 594916 & 114856 & 81.6 & GPS \\
\hline 2555 & & & & 595098 & 114923 & 81.6 & GPS \\
\hline 2556 & & & & 595395 & 114927 & 83 & GPS \\
\hline 2557 & & & & 595472 & 114951 & 83 & GPS \\
\hline 2558 & & & & 595518 & 109870 & 82.2 & GPS \\
\hline 2559 & & & & 595563 & 109836 & 82.7 & GPS \\
\hline 2560 & & & & 595677 & 109831 & 83.4 & GPS \\
\hline 2561 & & & & 595869 & 109857 & 82.3 & GPS \\
\hline 2562 & & & & 596226 & 109758 & 83.3 & GPS \\
\hline 2563 & & & & 596363 & 109808 & 82.8 & GPS \\
\hline 2564 & & & & 595483 & 110804 & 82.4 & GPS \\
\hline 2565 & & & & 595278 & 112118 & 82.2 & GPS \\
\hline 2566 & & & & 954956 & 113692 & 83 & GPS \\
\hline 2567 & & & & 594889 & 114400 & 83.4 & GPS \\
\hline 2568 & & & & 594314 & 117539 & 82.8 & GPS \\
\hline 2569 & & & & 594396 & 116699 & 82.8 & GPS \\
\hline 2570 & & & & 594540 & 116076 & 82.6 & GPS \\
\hline 2571 & & & & 594738 & 115238 & 83.2 & GPS \\
\hline 2572 & & & & 589172 & 137602 & & \\
\hline 2573 & & & & 594392 & 116700 & 82 & \\
\hline 2574 & & & & 588808 & 137808 & 94 & \\
\hline 2575 & & & & 589438 & 137451 & & \\
\hline 2576 & & & & 594489 & 116208 & 82.5 & \\
\hline 2577 & & & & 593883 & 112747 & 100 & \\
\hline \\
\hline \multicolumn{8}{|l|}{2579} \\
\hline \multicolumn{8}{|l|}{2580} \\
\hline \multicolumn{8}{|l|}{2581} \\
\hline \multicolumn{8}{|l|}{2582} \\
\hline \multicolumn{8}{|l|}{2583} \\
\hline \multicolumn{8}{|l|}{2584} \\
\hline 2585 & & & & & & & \\
\hline
\end{tabular}




\begin{tabular}{|c|c|c|c|c|c|c|c|}
\hline & $\mathrm{AP}$ & $\mathrm{AQ}$ & AR & AS & AT & $\overline{A U}$ & AV \\
\hline 1 & LONGITUDE ${ }^{\circ} \mathrm{W}$ & LONGITUDE MIN_MIN & LONGITUDE MIN_SEC & X_COORD (EW) & Y_COORD (NW) & 3 D HAE/ALTITUDE & INSTRUMENT \\
\hline \multicolumn{8}{|l|}{2586} \\
\hline \multicolumn{8}{|l|}{2587} \\
\hline 2588 & & & & 559150 & 145822 & 99.7 & GPS \\
\hline 2589 & & & & 559160 & 145921 & 100.9 & GPS \\
\hline 2590 & & & & 559069 & 146021 & 99 & GPS \\
\hline 2591 & & & & 559036 & 146104.449 & 98 & GPS \\
\hline 2592 & & & & 595517 & 109875 & 83 & GPS \\
\hline 2593 & & & & 595517 & 109875 & 83 & GPS \\
\hline 2594 & & & & 595577 & 109840 & 84 & GPS \\
\hline 2595 & & & & 595726 & 109770 & 81 & GPS \\
\hline 2596 & & & & 595872 & 109798 & 84 & GPS \\
\hline 2597 & & & & 596159 & 109608 & 80.7 & GPS \\
\hline 2598 & & & & 596362 & 109803 & 89.1 & GPS \\
\hline 2599 & & & & 595484 & 110809 & 83 & GPS \\
\hline 2600 & & & & 595270 & 112116 & 84 & GPS \\
\hline 2601 & & & & 594955 & 113780 & 83 & GPS \\
\hline 2602 & & & & 594887 & 114396 & 84 & GPS \\
\hline \multicolumn{8}{|l|}{2603} \\
\hline \multicolumn{8}{|l|}{2604} \\
\hline \multicolumn{8}{|c|}{2605} \\
\hline \multicolumn{8}{|c|}{2606} \\
\hline \multicolumn{8}{|l|}{2607} \\
\hline 2608 & 119 & & 15.35 .53 & & & & \\
\hline 2609 & 119 & & 15.35 .53 & & & & \\
\hline 2610 & 119 & & 15.32.09 & & & & \\
\hline 2611 & 119 & & 15.28 .44 & & & & \\
\hline 2612 & 119 & & 15.17.99 & & & & \\
\hline 2613 & 119 & & 15.05.39 & & & & \\
\hline \multicolumn{8}{|c|}{\begin{tabular}{|l|}
2614 \\
\end{tabular}} \\
\hline 2615 & 119 & & 15.35 .50 & & & & \\
\hline \multicolumn{8}{|l|}{2616} \\
\hline 2617 & 119 & & 15.58 .34 & & & & \\
\hline 2618 & 119 & & 16.02 .37 & & & & \\
\hline 2619 & 119 & & 43.41 .36 & & & & \\
\hline 2620 & 119 & & 43.42 .62 & & & & \\
\hline 2621 & 119 & & 43.42 .99 & & & & \\
\hline 2622 & 119 & & 43.46 .54 & & & & \\
\hline 2623 & & & & & & & \\
\hline
\end{tabular}




\begin{tabular}{|c|c|c|c|c|c|c|c|}
\hline & $\mathrm{AP}$ & $\mathrm{AQ}$ & AR & AS & AT & $A U$ & AV \\
\hline 1 & LONGITUDE ${ }^{\circ} \mathrm{W}$ & LONGITUDE MIN_MIN & LONGITUDE MIN_SEC & X_COORD (EW) & Y_COORD (NW) & 3 D HAE/ALTITUDE & INSTRUMENT \\
\hline \multicolumn{8}{|c|}{2624} \\
\hline \multicolumn{8}{|l|}{2625} \\
\hline \multicolumn{8}{|l|}{2626} \\
\hline \multicolumn{8}{|l|}{2627} \\
\hline \multicolumn{8}{|c|}{2628} \\
\hline \multicolumn{8}{|l|}{2629} \\
\hline \multicolumn{8}{|l|}{2630} \\
\hline \multicolumn{8}{|l|}{\begin{tabular}{|l|}
2631 \\
\end{tabular}} \\
\hline \multicolumn{8}{|c|}{2632} \\
\hline \multicolumn{8}{|c|}{2633} \\
\hline \multicolumn{8}{|c|}{2634} \\
\hline \multicolumn{8}{|c|}{2635} \\
\hline \multicolumn{8}{|c|}{2636} \\
\hline \multicolumn{8}{|l|}{2637} \\
\hline \multicolumn{8}{|l|}{2638} \\
\hline \multicolumn{8}{|l|}{2639} \\
\hline \multicolumn{8}{|l|}{2640} \\
\hline \multicolumn{8}{|l|}{2641} \\
\hline \multicolumn{8}{|l|}{2642} \\
\hline \multicolumn{8}{|l|}{2643} \\
\hline 2644 & 119 & & 15.35 .167 & & & & \\
\hline 2645 & 119 & & 15.32.854 & & & & \\
\hline 2646 & 119 & & 15.25 .556 & & & & \\
\hline 2647 & 119 & & 15.18.321 & & & & \\
\hline 2648 & 119 & & 15.05 .22609 & & & & \\
\hline 2649 & 119 & & 14.55 .469 & & & & \\
\hline 2650 & 119 & & 15.35 .841 & & & & \\
\hline 2651 & 119 & & 15.45 .231 & & & & \\
\hline 2652 & 119 & & 15.58 .444 & & & & \\
\hline 2653 & 119 & & 16.01.079 & & & & \\
\hline 2654 & 119 & & 43.38 .457 & & & & \\
\hline 2655 & 119 & & 43.39.10128 & & & & \\
\hline 2656 & 119 & & 43.45 .782 & & & & \\
\hline 2657 & 119 & & 43.45 .852 & & & & \\
\hline \multicolumn{8}{|l|}{2658} \\
\hline \multicolumn{8}{|c|}{2659} \\
\hline \multicolumn{8}{|c|}{2660} \\
\hline 2661 & & & & & & & \\
\hline
\end{tabular}




\begin{tabular}{|c|c|c|c|c|c|c|c|}
\hline & AP & $\mathrm{AQ}$ & AR & AS & AT & $A U$ & AV \\
\hline 1 & LONGITUDE ${ }^{\circ} \mathrm{W}$ & LONGITUDE MIN_MIN & LONGITUDE MIN_SEC & X_COORD (EW) & Y_COORD (NW) & 3 D HAE/ALTITUDE & INSTRUMENT \\
\hline \multicolumn{8}{|l|}{\begin{tabular}{|l|}
2662 \\
\end{tabular}} \\
\hline \multicolumn{8}{|l|}{2663} \\
\hline \multicolumn{8}{|l|}{2664} \\
\hline 2665 & 119 & 27295 & & 594392.9328 & 116697.2814 & & GPS \\
\hline 2666 & 119 & 27173 & & 594494.7346 & 116202.6015 & & GPS \\
\hline 2667 & 119 & 74824 & & 557558.6994 & 144902.4606 & & GPS \\
\hline 2668 & 119 & 27295 & & 594392.9328 & 116697.2814 & & GPS \\
\hline 2669 & 119 & 27173 & & 594494.7346 & 116202.6015 & & GPS \\
\hline 2670 & 119 & 74824 & & 557558.6994 & 144902.4606 & & GPS \\
\hline 2671 & 119 & 74824 & & 557558.6994 & 144902.4606 & & GPS \\
\hline 2672 & 119 & 74824 & & 557558.6994 & 144902.4606 & & GPS \\
\hline 2673 & 119 & 74824 & & 557558.6994 & 144902.4606 & & GPS \\
\hline 2674 & 119 & 27287 & & 594399.2531 & 116695.838 & & GPS \\
\hline 2675 & 119 & 27283 & & 594402.1182 & 116695.2311 & & GPS \\
\hline 2676 & 119 & 2728 & & 594404.9572 & 116696.5481 & & GPS \\
\hline 2677 & 119 & 27265 & & 594418.067 & 116567.4607 & & GPS \\
\hline 2678 & 119 & 27262 & & 594420.161 & 116567.6005 & & GPS \\
\hline 2679 & 119 & 27255 & & 594425.6435 & 116567.8565 & & GPS \\
\hline 2680 & 119 & 27227 & & 594450.3417 & 116362.2444 & & GPS \\
\hline 2681 & 119 & 27172 & & 594495.7216 & 116205.7327 & & GPS \\
\hline 2682 & 119 & 27167 & & 594499.4158 & 116203.1668 & & GPS \\
\hline 2683 & 119 & 27163 & & 594501.9964 & 116209.2038 & & GPS \\
\hline 2684 & 119 & 27154 & & 594510.2381 & 116145.7579 & & GPS \\
\hline 2685 & 119 & 27152 & & 594511.5832 & 116147.8569 & & GPS \\
\hline 2686 & 119 & 27143 & & 594518.7508 & 116146.0857 & & GPS \\
\hline 2687 & 119 & 27079 & & 594571.7032 & 115909.9801 & & GPS \\
\hline 2688 & 119 & 2699 & & 594644.1671 & 115645.5062 & & GPS \\
\hline 2689 & 119 & 26987 & & 594646.1043 & 115645.9576 & & GPS \\
\hline 2690 & 119 & 26986 & & 594647.3591 & 115645.6143 & & GPS \\
\hline \multicolumn{8}{|l|}{2691} \\
\hline \multicolumn{8}{|c|}{2692} \\
\hline \multicolumn{8}{|l|}{2693} \\
\hline 2694 & 119 & 26697 & & 594889.7354 & 114361.6456 & & GPS \\
\hline 2695 & 119 & 26693 & & 594892.5316 & 114361.8727 & & GPS \\
\hline 2696 & 119 & 26692 & & 594893.2853 & 114362.3731 & & GPS \\
\hline 2697 & 119 & 74824 & & 557558.6994 & 144902.4606 & & GPS \\
\hline 2698 & 119 & 27276 & & 594407.8154 & 116693.7483 & & GPS \\
\hline 2699 & 119 & 27252 & & 594428.029 & 116566.4842 & & GPS \\
\hline
\end{tabular}

$$
\text { Page } 617
$$




\begin{tabular}{|c|c|c|c|c|c|c|c|}
\hline & $\mathrm{AP}$ & $\mathrm{AQ}$ & AR & AS & AT & $A U$ & AV \\
\hline 1 & $\mid$ LONGITUDE $^{\circ} \mathrm{W}$ & LONGITUDE MIN_MIN & LONGITUDE MIN_SEC & X_COORD (EW) & Y_COORD (NW) & 3 D HAE/ALTITUDE & INSTRUMENT \\
\hline 2700 & 119 & 27154 & & 594508.882 & 116209.1859 & & GPS \\
\hline 2701 & 119 & 27137 & & 594523.0808 & 116145.8184 & & GPS \\
\hline 2702 & 119 & 26982 & & 594650.1201 & 115647.6407 & & GPS \\
\hline \multicolumn{8}{|l|}{2703} \\
\hline 2704 & 119 & 26688 & & 594896.5766 & 114364.2648 & & GPS \\
\hline \multicolumn{8}{|l|}{2705} \\
\hline \multicolumn{8}{|l|}{2706} \\
\hline 2707 & 119 & & 33.46 .160 & & & & \\
\hline 2708 & 119 & & 43.42 .160 & & & & \\
\hline 2709 & 119 & & 43.44 .211 & & & & \\
\hline 2710 & 119 & & 43.43 .646 & & & & \\
\hline 2711 & 119 & & 43.45 .184 & & & & \\
\hline 2712 & 119 & & 33.46 .160 & & & & \\
\hline 2713 & 119 & & 33.47.037 & & & & \\
\hline 2714 & 119 & & 33.52 .522 & & & & \\
\hline \begin{tabular}{|l|}
2715 \\
\end{tabular} & 119 & & 33.59 .834 & & & & \\
\hline \begin{tabular}{|l|}
2716 \\
\end{tabular} & 119 & & 33.47 .707 & & & & \\
\hline 2717 & 119 & & 33.56 .914 & & & & \\
\hline \begin{tabular}{|l|}
2718 \\
\end{tabular} & 119 & & 34.37 .401 & & & & \\
\hline 2719 & 119 & & 34.4 .571 & & & & \\
\hline 2720 & 119 & & 33.54 .646 & & & & \\
\hline 2721 & 119 & & 33.27 .425 & & & & \\
\hline 2722 & 119 & & 26.20 .61 & & & & \\
\hline 2723 & 119 & & 26.15 .64 & & & & \\
\hline 2724 & 119 & & 19.25.34 & & & & \\
\hline 2725 & 119 & & 26.19.26 & & & & \\
\hline 2726 & 119 & & 26.12.37 & & & & \\
\hline 2727 & 119 & & 26.9 .25 & & & & \\
\hline 2728 & 119 & & 26.7 .82 & & & & \\
\hline 2729 & 119 & & 19.34.19 & & & & \\
\hline 2730 & 119 & & 19.32.37 & & & & \\
\hline 2731 & 119 & & 19.24.78 & & & & \\
\hline 2732 & 119 & & 19.21.99 & & & & \\
\hline 2733 & 119 & & 19.29 .69 & & & & \\
\hline 2734 & 119 & & 22.5 .67 & & & & \\
\hline 2735 & 119 & & 21.10 .49 & & & & \\
\hline 2736 & 119 & & 18.44.07 & & & & \\
\hline 2737 & 119 & & 20.16 .74 & & & & \\
\hline
\end{tabular}

Page 618 


\begin{tabular}{|c|c|c|c|c|c|c|c|}
\hline & $\mathrm{AP}$ & $\mathrm{AQ}$ & AR & AS & AT & $\mathrm{AU}$ & AV \\
\hline 1 & LONGITUDE ${ }^{\circ} \mathrm{W}$ & LONGITUDE MIN_MIN & LONGITUDE MIN_SEC & X_COORD (EW) & Y_COORD (NW) & 3 D HAE/ALTITUDE & INSTRUMENT \\
\hline 2738 & 119 & & 27.20 .00 & & & & \\
\hline 2739 & 119 & & 24.34 .94 & & & & \\
\hline 2740 & 119 & & 16.4 .62 & & & & \\
\hline 2741 & 119 & & 16.4.10 & & & & \\
\hline 2742 & 119 & & 15.58.71 & & & & \\
\hline 2743 & 119 & & 15.50 .94 & & & & \\
\hline 2744 & 119 & & 15.36 .76 & & & & \\
\hline 2745 & 119 & & 15.32 .79 & & & & \\
\hline \multicolumn{8}{|l|}{2746} \\
\hline 2747 & 119 & & 15.34.15 & & & & \\
\hline 2748 & 119 & & 15.27 .44 & & & & \\
\hline 2749 & 119 & & 15.18.26 & & & & \\
\hline 2750 & 119 & & 15.1.78 & & & & \\
\hline 2751 & 119 & & 14.55 .21 & & & & \\
\hline 2752 & 119 & & 15.36 .010 & & & & \\
\hline 2753 & 119 & & 15.45 .053 & & & & \\
\hline 2754 & 119 & & 15.58 .45 & & & & \\
\hline 2755 & 119 & & 16.1.057 & & & & \\
\hline 2756 & 119 & & 16.25.78 & & & & \\
\hline 2757 & 119 & & 16.22 .25 & & & & \\
\hline 2758 & 119 & & 16.16.39 & & & & \\
\hline 2759 & 119 & & 16.7 .69 & & & & \\
\hline 2760 & 119 & & 16.18.498 & & & & \\
\hline 2761 & 119 & & 16.18 .450 & & & & \\
\hline 2762 & 119 & & 16.18 .450 & & & & \\
\hline 2763 & 119 & & 16.18.119 & & & & \\
\hline 2764 & 119 & & 16.18.119 & & & & \\
\hline 2765 & 119 & & 16.17.828 & & & & \\
\hline 2766 & 119 & & 16.22 .585 & & & & \\
\hline 2767 & 119 & & 16.22 .369 & & & & \\
\hline 2768 & 119 & & 16.22 .369 & & & & \\
\hline 2769 & 119 & & 16.22.369 & & & & \\
\hline 2770 & 119 & & 16.22.143 & & & & \\
\hline 2771 & 119 & & 16.22.143 & & & & \\
\hline \multicolumn{8}{|l|}{2772} \\
\hline \multicolumn{8}{|l|}{2773} \\
\hline \multicolumn{8}{|c|}{\begin{tabular}{|l|}
2774 \\
\end{tabular}} \\
\hline 2775 & 119 & & 29.23 .4 & & & & \\
\hline
\end{tabular}




\begin{tabular}{|c|c|c|c|c|c|c|c|}
\hline & $\mathrm{AP}$ & $\mathrm{AQ}$ & AR & AS & AT & $\mathrm{AU}$ & AV \\
\hline 1 & LONGITUDE ${ }^{\circ} \mathrm{W}$ & LONGITUDE MIN_MIN & LONGITUDE MIN_SEC & X_COORD (EW) & Y_COORD (NW) & 3 D HAE/ALTITUDE & INSTRUMENT \\
\hline 2776 & 119 & & 28.38 .8 & & & & \\
\hline \multicolumn{8}{|l|}{2777} \\
\hline \multicolumn{8}{|l|}{2778} \\
\hline \multicolumn{8}{|l|}{2779} \\
\hline \multicolumn{8}{|l|}{2780} \\
\hline \multicolumn{8}{|l|}{2781} \\
\hline 2782 & 119 & & 43.32 .98 & & & & \\
\hline 2783 & 119 & & 43.39.75 & & & & \\
\hline 2784 & 119 & & 43.41 .72 & & & & \\
\hline 2785 & 119 & & 43.44 .28 & & & & \\
\hline 2786 & 119 & & 15.35 .75 & & & & \\
\hline 2787 & 119 & & 15.35.75 & & & & \\
\hline 2788 & 119 & & 15.31 .17 & & & & \\
\hline 2789 & 119 & & 15.24.39 & & & & \\
\hline 2790 & 119 & & 15.18.12 & & & & \\
\hline 2791 & 119 & & 15.5 .25 & & & & \\
\hline 2792 & 119 & & 14.56 .18 & & & & \\
\hline 2793 & 119 & & 15.36 .06 & & & & \\
\hline 2794 & 119 & & 15.44 .45557 & & & & \\
\hline 2795 & 119 & & 15.58 .49 & & & & \\
\hline 2796 & 119 & & 16.1.47 & & & & \\
\hline \multicolumn{8}{|l|}{2797} \\
\hline \multicolumn{8}{|l|}{2798} \\
\hline \multicolumn{8}{|l|}{2799} \\
\hline \multicolumn{8}{|l|}{2800} \\
\hline \multicolumn{8}{|l|}{2801} \\
\hline \multicolumn{8}{|l|}{2802} \\
\hline 2803 & 119 & & 43.41 .968 & & & & \\
\hline 2804 & 119 & & 43.45 .470 & & & & \\
\hline 2805 & 119 & & 43.38 .87 & & & & \\
\hline 2806 & 119 & & 43.45 .970 & & & & \\
\hline 2807 & 119 & & 15.35.173 & & & & \\
\hline 2808 & 119 & & 15.35.173 & & & & \\
\hline 2809 & 119 & & 15.32 .280 & & & & \\
\hline 2810 & 119 & & 15.27 .450 & & & & \\
\hline 2811 & 119 & & 15.18 .880 & & & & \\
\hline 2812 & 119 & & 15.2.305 & & & & \\
\hline 2813 & 119 & & 14.55 .643 & & & & \\
\hline
\end{tabular}




\begin{tabular}{|c|c|c|c|c|c|c|c|}
\hline & $\mathrm{AP}$ & $\mathrm{AQ}$ & AR & AS & AT & $\mathrm{AU}$ & AV \\
\hline 1 & LONGITUDE ${ }^{\circ} \mathrm{W}$ & LONGITUDE MIN_MIN & LONGITUDE MIN_SEC & X_COORD (EW) & Y_COORD (NW) & 3 D HAE/ALTITUDE & INSTRUMENT \\
\hline 2814 & 119 & & 15.35.894 & & & & \\
\hline 2815 & 119 & & 15.44 .136 & & & & \\
\hline 2816 & 119 & & 15.58.274 & & & & \\
\hline 2817 & 119 & & 16.1.155 & & & & \\
\hline \multicolumn{8}{|l|}{2818} \\
\hline \multicolumn{8}{|l|}{2819} \\
\hline \multicolumn{8}{|l|}{2820} \\
\hline \multicolumn{8}{|l|}{2821} \\
\hline 2822 & 119 & 18039 & & & & & \\
\hline 2823 & 119 & 28027 & & & & & \\
\hline \multicolumn{8}{|l|}{2824} \\
\hline \multicolumn{8}{|l|}{2825} \\
\hline 2826 & 119 & & 43.38.216 & & & & \\
\hline 2827 & 119 & & 43.40 .351 & & & & \\
\hline 2828 & 119 & & 43.42 .623 & & & & \\
\hline 2829 & 119 & & 43.45 .564 & & & & \\
\hline 2830 & 119 & & 15.35 .664 & & & & \\
\hline 2831 & 119 & & 15.31 .280 & & & & \\
\hline 2832 & 119 & & 15.26.899 & & & & \\
\hline 2833 & 119 & & 15.17.787 & & & & \\
\hline 2834 & 119 & & 15.04.289 & & & & \\
\hline 2835 & 119 & & 14.55 .302 & & & & \\
\hline 2836 & 119 & & 15.35 .921 & & & & \\
\hline 2837 & 119 & & 15.46 .088 & & & & \\
\hline 2838 & 119 & & 15.58.751 & & & & \\
\hline 2839 & 119 & & 16.01.371 & & & & \\
\hline \multicolumn{8}{|c|}{2840} \\
\hline \multicolumn{8}{|l|}{2841} \\
\hline \multicolumn{8}{|l|}{2842} \\
\hline \multicolumn{8}{|l|}{2843} \\
\hline \multicolumn{8}{|l|}{2844} \\
\hline \multicolumn{8}{|l|}{2845} \\
\hline \multicolumn{8}{|l|}{2846} \\
\hline \multicolumn{8}{|l|}{2847} \\
\hline \multicolumn{8}{|l|}{2848} \\
\hline \multicolumn{8}{|l|}{2849} \\
\hline 2850 & 119 & & 33.43 .61 & & & & \\
\hline 2851 & 119 & & 43.45 .22 & & & & \\
\hline
\end{tabular}




\begin{tabular}{|c|c|c|c|c|c|c|c|}
\hline & $\mathrm{AP}$ & $\mathrm{AQ}$ & AR & AS & AT & $\mathrm{AU}$ & AV \\
\hline 1 & LONGITUDE ${ }^{\circ} \mathrm{W}$ & LONGITUDE MIN_MIN & LONGITUDE MIN_SEC & X_COORD (EW) & Y_COORD (NW) & 3 D HAE/ALTITUDE & INSTRUMENT \\
\hline 2852 & 119 & & 43.42.11 & & & & \\
\hline 2853 & 119 & & 43.44 .59 & & & & \\
\hline 2854 & 119 & & 43.40 .35 & & & & \\
\hline 2855 & 119 & & 33.43 .61 & & & & \\
\hline 2856 & 119 & & 33.44 .32 & & & & \\
\hline 2857 & 119 & & 33.50 .75 & & & & \\
\hline 2858 & 119 & & 34.00 .50 & & & & \\
\hline 2859 & 119 & & 33.46 .14 & & & & \\
\hline 2860 & 119 & & 33.57 .27 & & & & \\
\hline 2861 & 119 & & 34.37 .60 & & & & \\
\hline 2862 & 119 & & 34.03 .81 & & & & \\
\hline 2863 & 119 & & 33.54 .88 & & & & \\
\hline 2864 & 119 & & 33.26 .71 & & & & \\
\hline 2865 & 119 & & 26.20 .44 & & & & \\
\hline 2866 & 119 & & 26.11.61 & & & & \\
\hline 2867 & 119 & & 19.27.34 & & & & \\
\hline 2868 & 119 & & 26.17 .75 & & & & \\
\hline 2869 & 119 & & 26.10 .71 & & & & \\
\hline 2870 & 119 & & 26.09.29 & & & & \\
\hline 2871 & 119 & & 26.08.28 & & & & \\
\hline 2872 & 119 & & 19.36.81 & & & & \\
\hline 2873 & 119 & & 19.35.13 & & & & \\
\hline 2874 & 119 & & 19.24 .58 & & & & \\
\hline 2875 & 119 & & 19.22.285 & & & & \\
\hline 2876 & 119 & & 19.32.76 & & & & \\
\hline 2877 & 119 & & 22.04 .98 & & & & \\
\hline 2878 & 119 & & 21.10 .15 & & & & \\
\hline 2879 & 119 & & 18.43.71 & & & & \\
\hline 2880 & 119 & & 20.15 .46 & & & & \\
\hline 2881 & 119 & & 27.19 .87 & & & & \\
\hline 2882 & 119 & & 23.52.66 & & & & \\
\hline 2883 & 119 & & 24.35 .08 & & & & \\
\hline 2884 & 119 & & 16.04.61 & & & & \\
\hline 2885 & 119 & & 16.02.71 & & & & \\
\hline 2886 & 119 & & 15.56 .24 & & & & \\
\hline 2887 & 119 & & 15.50 .83 & & & & \\
\hline 2888 & 119 & & 15.37 .57 & & & & \\
\hline 2889 & 119 & & 15.33 .16 & & & & \\
\hline
\end{tabular}




\begin{tabular}{|c|c|c|c|c|c|c|c|}
\hline & $\mathrm{AP}$ & $\mathrm{AQ}$ & AR & AS & AT & $\mathrm{AU}$ & AV \\
\hline 1 & LONGITUDE ${ }^{\circ} \mathrm{W}$ & LONGITUDE MIN_MIN & LONGITUDE MIN_SEC & X_COORD (EW) & Y_COORD (NW) & 3 D HAE/ALTITUDE & INSTRUMENT \\
\hline 2890 & 119 & & 15.34 .9 & & & & \\
\hline 2891 & 119 & & 15.31 .3 & & & & \\
\hline 2892 & 119 & & 15.41 .5 & & & & \\
\hline 2893 & 119 & & 15.18.35 & & & & \\
\hline 2894 & 119 & & 15.01 .42 & & & & \\
\hline 2895 & 119 & & 14.55 .48 & & & & \\
\hline 2896 & 119 & & 15.35 .90 & & & & \\
\hline 2897 & 119 & & 15.44 .92 & & & & \\
\hline 2898 & 119 & & 15.58 .55 & & & & \\
\hline 2899 & 119 & & 16.01.26 & & & & \\
\hline 2900 & 119 & & 16.25 .66 & & & & \\
\hline 2901 & 119 & & 16.21 .89 & & & & \\
\hline 2902 & 119 & & 16.18.03 & & & & \\
\hline 2903 & 119 & & 16.07.38 & & & & \\
\hline \multicolumn{8}{|l|}{2904} \\
\hline \multicolumn{8}{|l|}{2905} \\
\hline 2906 & 119 & & 20.10.491 & & & & \\
\hline \multicolumn{8}{|l|}{2907} \\
\hline 2908 & 119 & & 20.00 .067 & & & & \\
\hline 2909 & 119 & & 28.38.784 & & & & \\
\hline 2910 & 119 & & 25.12.798 & & & & \\
\hline \multicolumn{8}{|l|}{2911} \\
\hline \multicolumn{8}{|l|}{2912} \\
\hline \multicolumn{8}{|l|}{2913} \\
\hline \multicolumn{8}{|c|}{2914} \\
\hline 2915 & 119 & 4363830 & & & & & \\
\hline 2916 & 119 & 4365845 & & & & & \\
\hline 2917 & 119 & 434070431 & & & & & \\
\hline 2918 & 119 & 434589260 & & & & & \\
\hline 2919 & 119 & 153521977 & & & & & \\
\hline 2920 & 119 & 153521977 & & & & & \\
\hline 2921 & 119 & 15290058 & & & & & \\
\hline 2922 & 119 & 152340608 & & & & & \\
\hline 2923 & 119 & 151897178 & & & & & \\
\hline 2924 & 119 & 150525588 & & & & & \\
\hline 2925 & 119 & 145567351 & & & & & \\
\hline 2926 & 119 & 153598423 & & & & & \\
\hline 2927 & 119 & 154392604 & & & & & \\
\hline
\end{tabular}




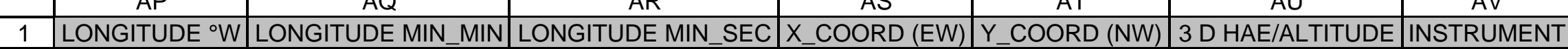

\begin{tabular}{l|l|l}
2928 & 119 & 155819461
\end{tabular}

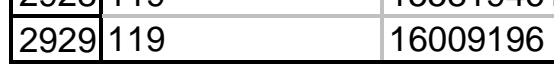




\section{SED_SANDS}




\begin{tabular}{|c|c|c|c|c|c|c|c|c|c|c|}
\hline & A & $\mathrm{B}$ & $\mathrm{C}$ & $\mathrm{D}$ & $E$ & $\mathrm{~F}$ & $G$ & $\mathrm{H}$ & $\mathrm{I}$ & $\mathrm{J}$ \\
\hline 1 & SAMPLE_NUM_ASSIGNED_TO & SAMPLER & MEDIA & BIOTA_MEDIA & TAG_ID & SAMP_NUM & SAMP_DATE_TIME & SAMP_DATE_TIME_ON & CON_ID & CON_SHORT_NAME \\
\hline 2 & SESP SCHEDULING & & SO & & & BOWFL6 & 02-Aug-99 & & $10045-97-3$ & CS-137 \\
\hline 3 & SESP SCHEDULING & & SO & & & BOWFL7 & 02-Aug-99 & & $10045-97-3$ & CS-137 \\
\hline 4 & SESP SCHEDULING & & SO & & & BOWFL8 & 02-Aug-99 & & $10045-97-3$ & CS-137 \\
\hline 5 & SESP SCHEDULING & & SO & & & BOWFK9 & 03-Aug-99 & & $10045-97-3$ & CS-137 \\
\hline 6 & SESP SCHEDULING & & SO & & & BOWFLO & 03-Aug-99 & & $10045-97-3$ & CS-137 \\
\hline 7 & SESP SCHEDULING & & SO & & & B0WFL1 & 03-Aug-99 & & $10045-97-3$ & CS-137 \\
\hline 8 & SESP SCHEDULING & & SO & & & B0WFL2 & 03-Aug-99 & & $10045-97-3$ & CS-137 \\
\hline 9 & SESP SCHEDULING & & SO & & & B0WFL3 & 03-Aug-99 & & $10045-97-3$ & CS-137 \\
\hline 10 & SESP SCHEDULING & & SO & & & BOWFMO & 09-Aug-99 & & $10045-97-3$ & CS-137 \\
\hline 11 & SESP SCHEDULING & & SO & & & B0WFM1 & 09-Aug-99 & & $10045-97-3$ & CS-137 \\
\hline 12 & SESP SCHEDULING & & SO & & & BOWFM2 & 09-Aug-99 & & $10045-97-3$ & CS-137 \\
\hline 13 & SESP SCHEDULING & & SO & & & BOWFM3 & 09-Aug-99 & & $10045-97-3$ & CS-137 \\
\hline 14 & SESP SCHEDULING & & SO & & & B0WFM5 & 09-Aug-99 & & $10045-97-3$ & CS-137 \\
\hline 15 & SESP SCHEDULING & & SO & & & B0WFM6 & 09-Aug-99 & & $10045-97-3$ & CS-137 \\
\hline 16 & SESP SCHEDULING & & SO & & & BOWFM7 & 09-Aug-99 & & $10045-97-3$ & CS-137 \\
\hline 17 & SESP SCHEDULING & & SO & & & B0WFM8 & 09-Aug-99 & & $10045-97-3$ & CS-137 \\
\hline 18 & SESP SCHEDULING & & SO & & & BOWFNO & 09-Aug-99 & & $10045-97-3$ & CS-137 \\
\hline 19 & SESP SCHEDULING & & SO & & & B0WFN1 & 09-Aug-99 & & $10045-97-3$ & CS-137 \\
\hline 20 & SESP SCHEDULING & & SO & & & BOWFN2 & 09-Aug-99 & & $10045-97-3$ & CS-137 \\
\hline 21 & SESP SCHEDULING & & SO & & & BOWFN3 & 09-Aug-99 & & $10045-97-3$ & CS-137 \\
\hline 22 & SESP SCHEDULING & & SO & & & B0WFK5 & 16-Aug-99 & & $10045-97-3$ & CS-137 \\
\hline 23 & SESP SCHEDULING & & SO & & & BOWFK6 & 16-Aug-99 & & $10045-97-3$ & CS-137 \\
\hline 24 & SESP SCHEDULING & & SO & & & BOWFK7 & 16-Aug-99 & & $10045-97-3$ & CS-137 \\
\hline 25 & SESP SCHEDULING & & SO & & & BOWFK8 & 16-Aug-99 & & $10045-97-3$ & CS-137 \\
\hline 26 & SESP SCHEDULING & & SO & & & BOWFN5 & 03-Sep-99 & & $10045-97-3$ & CS-137 \\
\hline 27 & SESP SCHEDULING & & SO & & & B0WFN6 & 03-Sep-99 & & $10045-97-3$ & CS-137 \\
\hline 28 & SESP SCHEDULING & & SO & & & BOWFN7 & 03-Sep-99 & & $10045-97-3$ & CS-137 \\
\hline 29 & SESP SCHEDULING & & SO & & & BOWFN8 & 03-Sep-99 & & $10045-97-3$ & CS-137 \\
\hline 30 & SESP SCHEDULING & & SO & & & B0WFL6 & 02-Aug-99 & & $10098-97-2$ & SR-90 \\
\hline 31 & SESP SCHEDULING & & SO & & & BOWFL7 & 02-Aug-99 & & 10098-97-2 & SR-90 \\
\hline 32 & SESP SCHEDULING & & SO & & & B0WFL8 & 02-Aug-99 & & 10098-97-2 & SR-90 \\
\hline 33 & SESP SCHEDULING & & SO & & & BOWFK9 & 03-Aug-99 & & $10098-97-2$ & SR-90 \\
\hline 34 & SESP SCHEDULING & & SO & & & BOWFLO & 03-Aug-99 & & 10098-97-2 & SR-90 \\
\hline 35 & SESP SCHEDULING & & SO & & & B0WFL1 & 03-Aug-99 & & 10098-97-2 & SR-90 \\
\hline 36 & SESP SCHEDULING & & SO & & & BOWFL2 & 03-Aug-99 & & 10098-97-2 & SR-90 \\
\hline 37 & SESP SCHEDULING & & SO & & & B0WFL3 & 03-Aug-99 & & $10098-97-2$ & SR-90 \\
\hline 38 & SESP SCHEDULING & & SO & & & BOWFMO & 09-Aug-99 & & $10098-97-2$ & SR-90 \\
\hline 39 & SESP SCHEDULING & & SO & & & B0WFM1 & 09-Aug-99 & & 10098-97-2 & SR-90 \\
\hline
\end{tabular}




\begin{tabular}{|c|c|c|c|c|c|c|c|c|c|c|}
\hline & A & $\mathrm{B}$ & C & $\mathrm{D}$ & $E$ & $\mathrm{~F}$ & $G$ & $\mathrm{H}$ & $\mathrm{I}$ & $\mathrm{J}$ \\
\hline 1 & SAMPLE_NUM_ASSIGNED_TO & SAMPLER & MEDIA & BIOTA_MEDIA & TAG_ID & SAMP_NUM & SAMP_DATE_TIME & SAMP_DATE_TIME_ON & CON_ID & CON_SHORT_NAME \\
\hline 40 & SESP SCHEDULING & & SO & & & BOWFM2 & 09-Aug-99 & & $10098-97-2$ & SR-90 \\
\hline 41 & SESP SCHEDULING & & SO & & & BOWFM3 & 09-Aug-99 & & $10098-97-2$ & SR-90 \\
\hline 42 & SESP SCHEDULING & & SO & & & B0WFM5 & 09-Aug-99 & & 10098-97-2 & SR-90 \\
\hline 43 & SESP SCHEDULING & & SO & & & B0WFM6 & 09-Aug-99 & & 10098-97-2 & SR-90 \\
\hline 44 & SESP SCHEDULING & & SO & & & B0WFM7 & 09-Aug-99 & & 10098-97-2 & SR-90 \\
\hline 45 & SESP SCHEDULING & & SO & & & B0WFM8 & 09-Aug-99 & & $10098-97-2$ & SR-90 \\
\hline 46 & SESP SCHEDULING & & SO & & & BOWFNO & 09-Aug-99 & & $10098-97-2$ & SR-90 \\
\hline 47 & SESP SCHEDULING & & SO & & & B0WFN1 & 09-Aug-99 & & $10098-97-2$ & SR-90 \\
\hline 48 & SESP SCHEDULING & & SO & & & BOWFN2 & 09-Aug-99 & & 10098-97-2 & SR-90 \\
\hline 49 & SESP SCHEDULING & & SO & & & BOWFN3 & 09-Aug-99 & & 10098-97-2 & SR-90 \\
\hline 50 & SESP SCHEDULING & & SO & & & B0WFK5 & 16-Aug-99 & & $10098-97-2$ & SR-90 \\
\hline 51 & SESP SCHEDULING & & SO & & & BOWFK6 & 16-Aug-99 & & $10098-97-2$ & SR-90 \\
\hline 52 & SESP SCHEDULING & & SO & & & BOWFK7 & 16-Aug-99 & & $10098-97-2$ & SR-90 \\
\hline 53 & SESP SCHEDULING & & SO & & & BOWFK8 & 16-Aug-99 & & $10098-97-2$ & SR-90 \\
\hline 54 & SESP SCHEDULING & & SO & & & B0WFN5 & 03-Sep-99 & & $10098-97-2$ & SR-90 \\
\hline 55 & SESP SCHEDULING & & SO & & & B0WFN6 & 03-Sep-99 & & 10098-97-2 & SR-90 \\
\hline 56 & SESP SCHEDULING & & SO & & & BOWFN7 & 03-Sep-99 & & 10098-97-2 & SR-90 \\
\hline 57 & SESP SCHEDULING & & SO & & & BOWFN8 & 03-Sep-99 & & $10098-97-2$ & SR-90 \\
\hline 58 & SESP SCHEDULING & & SO & & & BoWFL6 & 02-Aug-99 & & 13966-29-5 & U-234 \\
\hline 59 & SESP SCHEDULING & & SO & & & BOWFL7 & 02-Aug-99 & & 13966-29-5 & U-234 \\
\hline 60 & SESP SCHEDULING & & SO & & & B0WFL8 & 02-Aug-99 & & $13966-29-5$ & U-234 \\
\hline 61 & SESP SCHEDULING & & SO & & & BOWFK9 & 03-Aug-99 & & $13966-29-5$ & U-234 \\
\hline 62 & SESP SCHEDULING & & SO & & & BOWFLO & 03-Aug-99 & & $13966-29-5$ & U-234 \\
\hline 63 & SESP SCHEDULING & & SO & & & B0WFL1 & 03-Aug-99 & & $13966-29-5$ & $\mathrm{U}-234$ \\
\hline 64 & SESP SCHEDULING & & SO & & & BOWFL2 & 03-Aug-99 & & 13966-29-5 & U-234 \\
\hline 65 & SESP SCHEDULING & & SO & & & B0WFL3 & 03-Aug-99 & & $13966-29-5$ & U-234 \\
\hline 66 & SESP SCHEDULING & & SO & & & BOWFMO & 09-Aug-99 & & $13966-29-5$ & U-234 \\
\hline 67 & SESP SCHEDULING & & SO & & & B0WFM1 & 09-Aug-99 & & $13966-29-5$ & U-234 \\
\hline 68 & SESP SCHEDULING & & SO & & & BOWFM2 & 09-Aug-99 & & 13966-29-5 & U-234 \\
\hline 69 & SESP SCHEDULING & & SO & & & BOWFM3 & 09-Aug-99 & & $13966-29-5$ & $\mathrm{U}-234$ \\
\hline 70 & SESP SCHEDULING & & SO & & & B0WFM5 & 09-Aug-99 & & $13966-29-5$ & U-234 \\
\hline 71 & SESP SCHEDULING & & SO & & & B0WFM6 & 09-Aug-99 & & $13966-29-5$ & $\mathrm{U}-234$ \\
\hline 72 & SESP SCHEDULING & & SO & & & B0WFM7 & 09-Aug-99 & & $13966-29-5$ & $\mathrm{U}-234$ \\
\hline 73 & SESP SCHEDULING & & SO & & & B0WFM8 & 09-Aug-99 & & $13966-29-5$ & U-234 \\
\hline 74 & SESP SCHEDULING & & SO & & & BOWFNO & 09-Aug-99 & & $13966-29-5$ & $\mathrm{U}-234$ \\
\hline 75 & SESP SCHEDULING & & SO & & & B0WFN1 & 09-Aug-99 & & $13966-29-5$ & $\mathrm{U}-234$ \\
\hline 76 & SESP SCHEDULING & & SO & & & BOWFN2 & 09-Aug-99 & & $13966-29-5$ & U-234 \\
\hline 77 & SESP SCHEDULING & & SO & & & BOWFN3 & 09-Aug-99 & & $13966-29-5$ & U-234 \\
\hline
\end{tabular}




\begin{tabular}{|c|c|c|c|c|c|c|c|c|c|c|}
\hline & $A$ & $\mathrm{~B}$ & $\mathrm{C}$ & $\mathrm{D}$ & $E$ & $\mathrm{~F}$ & $G$ & $\mathrm{H}$ & $\mathrm{I}$ & $\mathrm{J}$ \\
\hline 1 & SAMPLE_NUM_ASSIGNED_TO & SAMPLER & MEDIA & BIOTA_MEDIA & TAG_ID & SAMP_NUM & SAMP_DATE_TIME & SAMP_DATE_TIME_ON & CON_ID & CON_SHORT_NAME \\
\hline 78 & SESP SCHEDULING & & SO & & & B0WFK5 & 16-Aug-99 & & $13966-29-5$ & U-234 \\
\hline 79 & SESP SCHEDULING & & SO & & & BOWFK6 & 16-Aug-99 & & $13966-29-5$ & U-234 \\
\hline 80 & SESP SCHEDULING & & SO & & & BOWFK7 & 16-Aug-99 & & $13966-29-5$ & U-234 \\
\hline 81 & SESP SCHEDULING & & SO & & & BOWFK8 & 16-Aug-99 & & 13966-29-5 & U-234 \\
\hline 82 & SESP SCHEDULING & & SO & & & BOWFN5 & 03-Sep-99 & & 13966-29-5 & U-234 \\
\hline 83 & SESP SCHEDULING & & SO & & & BOWFN6 & 03-Sep-99 & & $13966-29-5$ & U-234 \\
\hline 84 & SESP SCHEDULING & & SO & & & BOWFN7 & 03-Sep-99 & & $13966-29-5$ & U-234 \\
\hline 85 & SESP SCHEDULING & & SO & & & BOWFN8 & 03-Sep-99 & & $13966-29-5$ & U-234 \\
\hline 86 & SESP SCHEDULING & & SO & & & B0WFL6 & 02-Aug-99 & & 15117-96-1 & U-235 \\
\hline 87 & SESP SCHEDULING & & SO & & & BOWFL7 & 02-Aug-99 & & $15117-96-1$ & U-235 \\
\hline 88 & SESP SCHEDULING & & SO & & & B0WFL8 & 02-Aug-99 & & 15117-96-1 & U-235 \\
\hline 89 & SESP SCHEDULING & & SO & & & BOWFK9 & 03-Aug-99 & & 15117-96-1 & U-235 \\
\hline 90 & SESP SCHEDULING & & SO & & & BOWFLO & 03-Aug-99 & & $15117-96-1$ & U-235 \\
\hline 91 & SESP SCHEDULING & & SO & & & BOWFL1 & 03-Aug-99 & & $15117-96-1$ & U-235 \\
\hline 92 & SESP SCHEDULING & & SO & & & BOWFL2 & 03-Aug-99 & & $15117-96-1$ & U-235 \\
\hline 93 & SESP SCHEDULING & & SO & & & BOWFL3 & 03-Aug-99 & & $15117-96-1$ & U-235 \\
\hline 94 & SESP SCHEDULING & & SO & & & BOWFMO & 09-Aug-99 & & 15117-96-1 & U-235 \\
\hline 95 & SESP SCHEDULING & & SO & & & BOWFM1 & 09-Aug-99 & & 15117-96-1 & U-235 \\
\hline 96 & SESP SCHEDULING & & SO & & & BOWFM2 & 09-Aug-99 & & $15117-96-1$ & U-235 \\
\hline 97 & SESP SCHEDULING & & SO & & & BOWFM3 & 09-Aug-99 & & $15117-96-1$ & U-235 \\
\hline 98 & SESP SCHEDULING & & SO & & & B0WFM5 & 09-Aug-99 & & 15117-96-1 & U-235 \\
\hline 99 & SESP SCHEDULING & & SO & & & B0WFM6 & 09-Aug-99 & & 15117-96-1 & U-235 \\
\hline 100 & SESP SCHEDULING & & SO & & & BOWFM7 & 09-Aug-99 & & $15117-96-1$ & U-235 \\
\hline 101 & SESP SCHEDULING & & SO & & & B0WFM8 & 09-Aug-99 & & 15117-96-1 & U-235 \\
\hline 102 & SESP SCHEDULING & & SO & & & BOWFNO & 09-Aug-99 & & 15117-96-1 & U-235 \\
\hline 103 & SESP SCHEDULING & & SO & & & B0WFN1 & 09-Aug-99 & & $15117-96-1$ & U-235 \\
\hline 104 & SESP SCHEDULING & & SO & & & BOWFN2 & 09-Aug-99 & & $15117-96-1$ & U-235 \\
\hline 105 & SESP SCHEDULING & & SO & & & BOWFN3 & 09-Aug-99 & & $15117-96-1$ & U-235 \\
\hline 106 & SESP SCHEDULING & & SO & & & BOWFK5 & 16-Aug-99 & & $15117-96-1$ & U-235 \\
\hline 107 & SESP SCHEDULING & & SO & & & BOWFK6 & 16-Aug-99 & & 15117-96-1 & U-235 \\
\hline 108 & SESP SCHEDULING & & SO & & & BOWFK7 & 16-Aug-99 & & 15117-96-1 & U-235 \\
\hline 109 & SESP SCHEDULING & & SO & & & BOWFK8 & 16-Aug-99 & & $15117-96-1$ & U-235 \\
\hline 110 & SESP SCHEDULING & & SO & & & BOWFN5 & 03-Sep-99 & & $15117-96-1$ & U-235 \\
\hline 111 & SESP SCHEDULING & & SO & & & BOWFN6 & 03-Sep-99 & & 15117-96-1 & U-235 \\
\hline 112 & SESP SCHEDULING & & SO & & & BOWFN7 & 03-Sep-99 & & $15117-96-1$ & U-235 \\
\hline 113 & SESP SCHEDULING & & SO & & & BOWFN8 & 03-Sep-99 & & 15117-96-1 & U-235 \\
\hline 114 & SESP SCHEDULING & & SO & & & B0WFL6 & 02-Aug-99 & & U-238 & U-238 \\
\hline 115 & SESP SCHEDULING & & SO & & & BOWFL7 & 02-Aug-99 & & U-238 & U-238 \\
\hline
\end{tabular}




\begin{tabular}{|c|c|c|c|c|c|c|c|c|c|c|}
\hline & A & $B$ & $\mathrm{C}$ & $\mathrm{D}$ & $\mathrm{E}$ & $\mathrm{F}$ & G & $\mathrm{H}$ & $\mathrm{I}$ & $\mathrm{J}$ \\
\hline 1 & SAMPLE_NUM_ASSIGNED_TO & SAMPLER & MEDIA & BIOTA_MEDIA & TAG_ID & SAMP_NUM & SAMP_DATE_TIME & SAMP_DATE_TIME_ON & CON_ID & CON_SHORT_NAME \\
\hline 116 & SESP SCHEDULING & & SO & & & B0WFL8 & 02-Aug-99 & & U-238 & U-238 \\
\hline 117 & SESP SCHEDULING & & SO & & & BOWFK9 & 03-Aug-99 & & U-238 & U-238 \\
\hline 118 & SESP SCHEDULING & & SO & & & BOWFLO & 03-Aug-99 & & U-238 & U-238 \\
\hline 119 & SESP SCHEDULING & & so & & & B0WFL1 & 03-Aug-99 & & U-238 & U-238 \\
\hline 120 & SESP SCHEDULING & & so & & & BOWFL2 & 03-Aug-99 & & U-238 & U-238 \\
\hline 121 & SESP SCHEDULING & & SO & & & BOWFL3 & 03-Aug-99 & & U-238 & U-238 \\
\hline 122 & SESP SCHEDULING & & SO & & & BOWFMO & 09-Aug-99 & & U-238 & U-238 \\
\hline 123 & SESP SCHEDULING & & SO & & & BOWFM1 & 09-Aug-99 & & U-238 & U-238 \\
\hline 124 & SESP SCHEDULING & & SO & & & BOWFM2 & 09-Aug-99 & & U-238 & U-238 \\
\hline 125 & SESP SCHEDULING & & SO & & & BOWFM3 & 09-Aug-99 & & U-238 & U-238 \\
\hline 126 & SESP SCHEDULING & & SO & & & B0WFM5 & 09-Aug-99 & & U-238 & U-238 \\
\hline 127 & SESP SCHEDULING & & SO & & & B0WFM6 & 09-Aug-99 & & U-238 & U-238 \\
\hline 128 & SESP SCHEDULING & & so & & & BOWFM7 & 09-Aug-99 & & U-238 & U-238 \\
\hline 129 & SESP SCHEDULING & & so & & & B0WFM8 & 09-Aug-99 & & U-238 & U-238 \\
\hline 130 & SESP SCHEDULING & & SO & & & BOWFNO & 09-Aug-99 & & U-238 & U-238 \\
\hline 131 & SESP SCHEDULING & & SO & & & BoWFN1 & 09-Aug-99 & & U-238 & $\mathrm{U}-238$ \\
\hline 132 & SESP SCHEDULING & & so & & & BOWFN2 & 09-Aug-99 & & U-238 & U-238 \\
\hline 133 & SESP SCHEDULING & & SO & & & BOWFN3 & 09-Aug-99 & & U-238 & U-238 \\
\hline 134 & SESP SCHEDULING & & SO & & & B0WFK5 & 16-Aug-99 & & U-238 & U-238 \\
\hline 135 & SESP SCHEDULING & & SO & & & BOWFK6 & 16-Aug-99 & & U-238 & U-238 \\
\hline 136 & SESP SCHEDULING & & SO & & & BOWFK7 & 16-Aug-99 & & U-238 & U-238 \\
\hline 137 & SESP SCHEDULING & & SO & & & BOWFK8 & 16-Aug-99 & & U-238 & U-238 \\
\hline 138 & SESP SCHEDULING & & so & & & B0WFN5 & 03-Sep-99 & & U-238 & U-238 \\
\hline 139 & SESP SCHEDULING & & SO & & & B0WFN6 & 03-Sep-99 & & U-238 & U-238 \\
\hline 140 & SESP SCHEDULING & & SO & & & BOWFN7 & 03-Sep-99 & & $\mathrm{U}-238$ & U-238 \\
\hline 141 & SESP SCHEDULING & & SO & & & BOWFN8 & 03-Sep-99 & & $\mathrm{U}-238$ & $\mathrm{U}-238$ \\
\hline
\end{tabular}




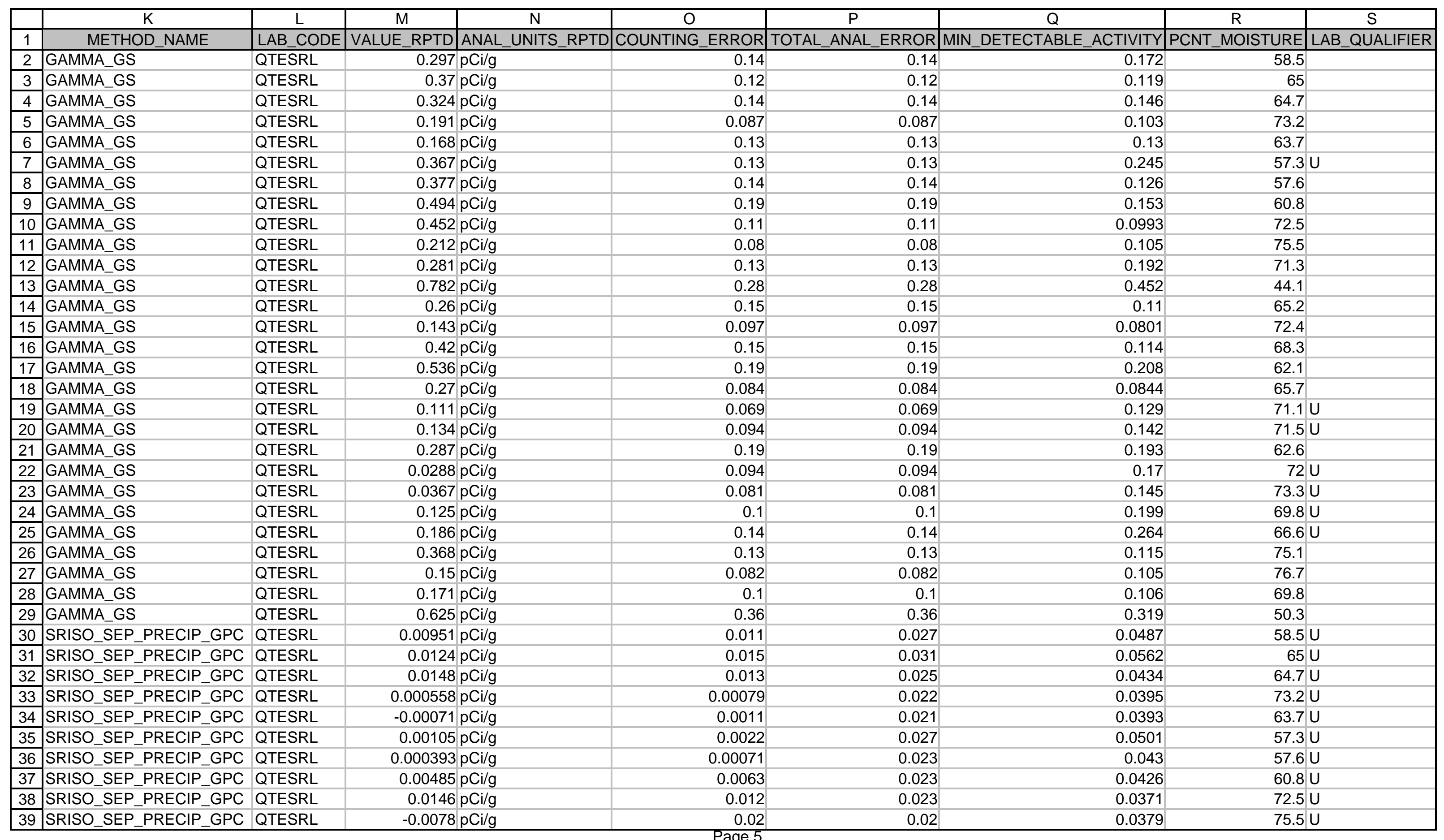




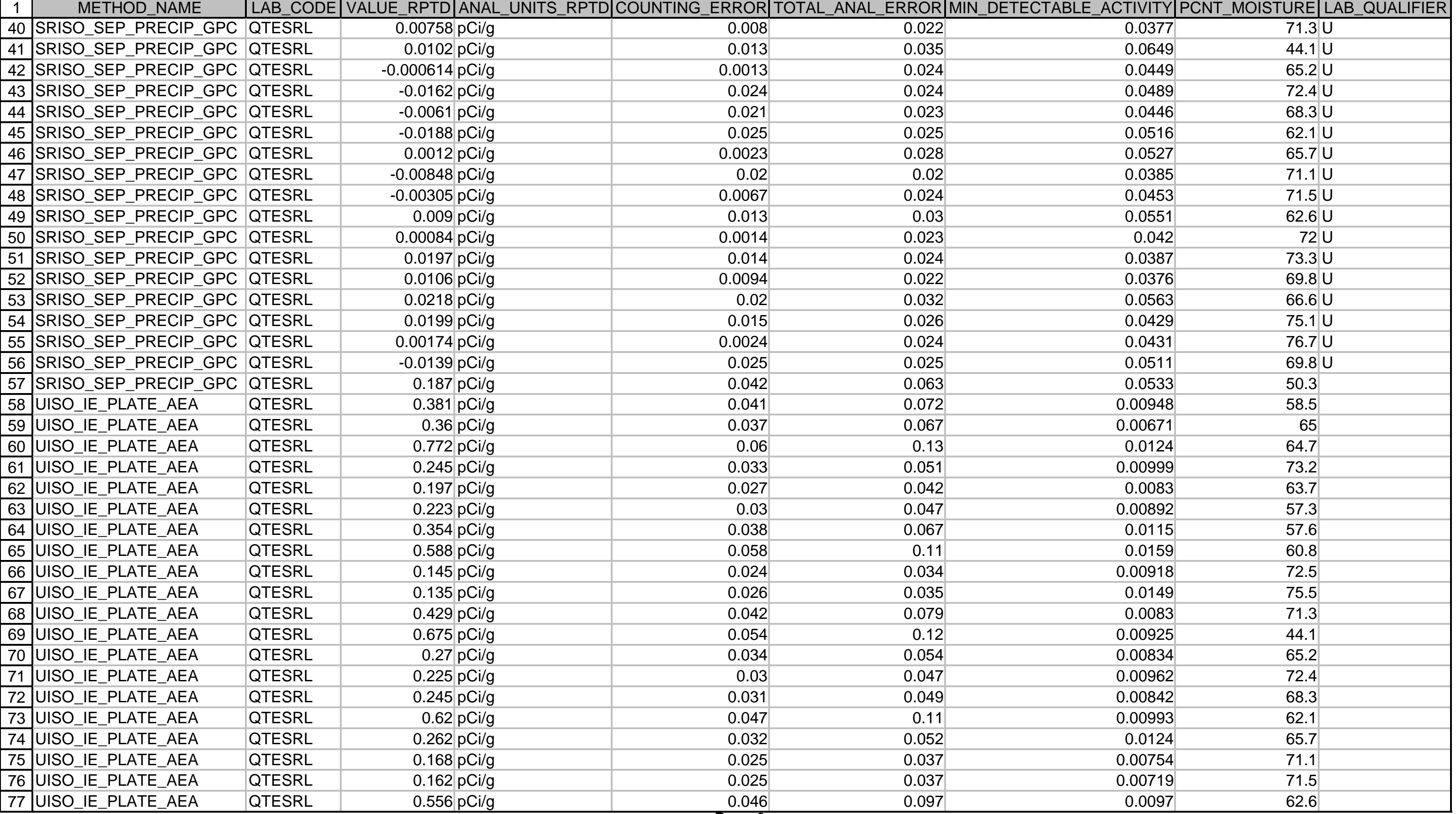




\begin{tabular}{|c|c|c|c|c|c|c|c|c|c|}
\hline & $\mathrm{K}$ & $L$ & $\mathrm{M}$ & $\mathrm{N}$ & $\mathrm{O}$ & $\mathrm{P}$ & $\mathrm{Q}$ & $\mathrm{R}$ & $\mathrm{S}$ \\
\hline 1 & METHOD_NAME & LAB_CODE & VALUE_RPTD & ANAL_UNITS_RPTD & COUNTING_ERROR & TOTAL_ANAL_ERROR & MIN_DETECTABLE_ACTIVITY & PCNT_MOISTURE|I & LAB_QUALIFIER \\
\hline 78 & UISO_IE_PLATE_AEA & QTESRL & 0.191 & $\mathrm{pCi} / \mathrm{g}$ & 0.03 & 0.043 & 0.015 & 72 & \\
\hline 79 & ]UISO_IE_PLATE_AEA & QTESRL & 0.234 & $\mathrm{pCi} / \mathrm{g}$ & 0.037 & 0.053 & 0.0182 & 73.3 & \\
\hline 80 & UISO_IE_PLATE_AEA & QTESRL & 0.28 & $\mathrm{pCi} / \mathrm{g}$ & 0.038 & 0.059 & 0.0105 & 69.8 & \\
\hline 81 & UISO_IE_PLATE_AEA & QTESRL & 0.744 & $\mathrm{pCi} / \mathrm{g}$ & 0.056 & 0.13 & 0.0115 & 66.6 & \\
\hline 82 & UISO_IE_PLATE_AEA & QTESRL & 0.138 & $\mathrm{pCi} / \mathrm{g}$ & 0.02 & 0.03 & 0.00427 & 75.1 & \\
\hline 83 & UISO_IE_PLATE_AEA & QTESRL & 0.106 & $\mathrm{pCi} / \mathrm{g}$ & 0.02 & 0.027 & 0.0102 & 76.7 & \\
\hline 84 & UISO_IE_PLATE_AEA & QTESRL & 0.3 & $\mathrm{pCi} / \mathrm{g}$ & 0.034 & 0.058 & 0.0105 & 69.8 & \\
\hline 85 & UISO_IE_PLATE_AEA & QTESRL & 0.661 & $\mathrm{pCi} / \mathrm{g}$ & 0.055 & 0.12 & 0.0137 & 50.3 & \\
\hline \begin{tabular}{|l|}
87 \\
\end{tabular} & UISO_IE_PLATE_AEA & QTESRL & 0.0148 & $\mathrm{pCi} / \mathrm{g}$ & 0.0076 & 0.0088 & 0.00762 & 65 & \\
\hline 88 & UISO_IE_PLATE_AEA & QTESRL & 0.0215 & $\mathrm{pCi} / \mathrm{g}$ & 0.0099 & 0.011 & 0.00667 & 64.7 & \\
\hline 89 & UISO_IE_PLATE_AEA & QTESRL & 0.0109 & $\mathrm{pCi} / \mathrm{g}$ & 0.0071 & 0.0084 & 0.00907 & 73.2 & \\
\hline 90 & UISO_IE_PLATE_AEA & QTESRL & 0.00304 & $\mathrm{pCi} / \mathrm{g}$ & 0.0032 & 0.005 & 0.00754 & $63.7 \mathrm{C}$ & \\
\hline 91 & UISO_IE_PLATE_AEA & QTESRL & 0.0121 & $\mathrm{pCi} / \mathrm{g}$ & 0.0069 & 0.0081 & 0.00713 & 57.3 & \\
\hline 92 & UISO_IE_PLATE_AEA & QTESRL & 0.013 & $\mathrm{pCi} / \mathrm{g}$ & 0.0076 & 0.0088 & 0.00967 & 57.6 & \\
\hline 93 & UISO_IE_PLATE_AEA & QTESRL & 0.0342 & $\mathrm{pCi} / \mathrm{g}$ & 0.014 & 0.016 & 0.0113 & 60.8 & \\
\hline 94 & UISO_IE_PLATE_AEA & QTESRL & 0.00747 & $\mathrm{pCi} / \mathrm{g}$ & 0.0052 & 0.0065 & 0.00679 & 72.5 & \\
\hline 95 & UISO_IE_PLATE_AEA & QTESRL & -0.000373 & $\mathrm{pCi} / \mathrm{g}$ & 0.0015 & 0.0049 & 0.0115 & $75.5 \mathrm{C}$ & \\
\hline \begin{tabular}{|l|}
97 \\
\end{tabular} & UISO_IE_PLATE_AEA & QTESRL & 0.0229 & $\mathrm{pCi} / \mathrm{g}$ & 0.01 & 0.011 & 0.0084 & 44.1 & \\
\hline 98 & UISO_IE_PLATE_AEA & QTESRL & 0.0125 & $\mathrm{pCi} / \mathrm{g}$ & 0.0072 & 0.0084 & 0.00734 & 65.2 & \\
\hline 99 & UISO_IE_PLATE_AEA & QTESRL & 0.00458 & $\mathrm{pCi} / \mathrm{g}$ & 0.0037 & 0.0052 & 0.00281 & 72.4 & \\
\hline 100 & UISO_IE_PLATE_AEA & QTESRL & 0.00838 & $\mathrm{pCi} / \mathrm{g}$ & 0.0055 & 0.0068 & 0.00673 & 68.3 & \\
\hline 101 & UISO_IE_PLATE_AEA & QTESRL & 0.02 & $\mathrm{pCi} / \mathrm{g}$ & 0.0086 & 0.0099 & 0.00836 & 62.1 & \\
\hline 102 & UISO_IE_PLATE_AEA & QTESRL & 0.0165 & $\mathrm{pCi} / \mathrm{g}$ & 0.0079 & 0.0091 & 0.00752 & 65.7 & \\
\hline 103 & UISO_IE_PLATE_AEA & QTESRL & 0.00496 & $\mathrm{pCi} / \mathrm{g}$ & 0.0042 & 0.0057 & 0.00754 & $71.1 \mathrm{l}$ & \\
\hline 104 & UISO_IE_PLATE_AEA & QTESRL & 0.0158 & $\mathrm{pCi} / \mathrm{g}$ & 0.0079 & 0.0091 & 0.00591 & 71.5 & \\
\hline 105 & UISO_IE_PLATE_AEA & QTESRL & 0.0181 & $\mathrm{pCi} / \mathrm{g}$ & 0.0083 & 0.0095 & 0.00672 & 62.6 & \\
\hline 106 & UISO_IE_PLATE_AEA & QTESRL & 0.00798 & $\mathrm{pCi} / \mathrm{g}$ & 0.0066 & 0.008 & 0.0112 & 72 & $U$ \\
\hline 107 & UISO_IE_PLATE_AEA & QTESRL & 0.00131 & $\mathrm{pCi} / \mathrm{g}$ & 0.0029 & 0.0064 & 0.0135 & 73.31 & \\
\hline 108 & UISO_IE_PLATE_AEA & QTESRL & 0.0121 & $\mathrm{pCi} / \mathrm{g}$ & 0.008 & 0.0093 & 0.00921 & 69.8 & \\
\hline 109 & UISO_IE_PLATE_AEA & QTESRL & 0.0217 & $\mathrm{pCi} / \mathrm{g}$ & 0.0096 & 0.011 & 0.00895 & 66.6 & \\
\hline 110 & UISO_IE_PLATE_AEA & QTESRL & 0.000338 & $\mathrm{pCi} / \mathrm{g}$ & 0.00046 & 0.0028 & 0.00427 & $75.1 \mathrm{C}$ & \\
\hline 111 & UISO_IE_PLATE_AEA & QTESRL & 0.000529 & $\mathrm{pCi} / \mathrm{g}$ & 0.00088 & 0.0036 & 0.0067 & $76.7 \mathrm{C}$ & $U$ \\
\hline 112 & UISO_IE_PLATE_AEA & QTESRL & 0.0183 & $\mathrm{pCi} / \mathrm{g}$ & 0.0085 & 0.0097 & 0.00883 & 69.8 & \\
\hline 113 & UISO_IE_PLATE_AEA & QTESRL & 0.0382 & $\mathrm{pCi} / \mathrm{g}$ & 0.013 & 0.015 & 0.00893 & 50.3 & \\
\hline 114 & UISO_IE_PLATE_AEA & QTESRL & 0.259 & $\mathrm{pCi} / \mathrm{g}$ & 0.034 & 0.053 & 0.0109 & 58.5 & \\
\hline
\end{tabular}




\begin{tabular}{|c|c|c|c|c|c|c|c|c|c|}
\hline & $\mathrm{K}$ & $\mathrm{L}$ & $\mathrm{M}$ & $\mathrm{N}$ & $\mathrm{O}$ & $\mathrm{P}$ & $\mathrm{Q}$ & $\mathrm{R}$ & $\mathrm{S}$ \\
\hline 1 & METHOD_NAME & LAB_CODE & VALUE_RPTD & ANAL_UNITS_RPTD & COUNTING_ERROR & TOTAL_ANAL_ERROR & MIN_DETECTABLE_ACTIVITY & PCNT_MOISTURE & LAB_QUALIFIER \\
\hline 116 & UISO_IE_PLATE_AEA & QTESRL & 0.633 & $\mathrm{pCi} / \mathrm{g}$ & 0.055 & 0.11 & 0.0202 & 64.7 & \\
\hline 117 & UISO_IE_PLATE_AEA & QTESRL & 0.217 & $\mathrm{pCi} / \mathrm{g}$ & 0.032 & 0.047 & 0.0115 & 73.2 & \\
\hline 118 & UISO_IE_PLATE_AEA & QTESRL & 0.163 & $\mathrm{pCi} / \mathrm{g}$ & 0.025 & 0.036 & 0.00754 & 63.7 & \\
\hline 119 & UISO_IE_PLATE_AEA & QTESRL & 0.197 & $\mathrm{pCi} / \mathrm{g}$ & 0.028 & 0.042 & 0.0081 & 57.3 & \\
\hline 120 & UISO_IE_PLATE_AEA & QTESRL & 0.317 & $\mathrm{pCi} / \mathrm{g}$ & 0.037 & 0.061 & 0.0175 & 57.6 & \\
\hline 121 & UISO_IE_PLATE_AEA & QTESRL & 0.504 & $\mathrm{pCi} / \mathrm{g}$ & 0.054 & 0.095 & 0.00992 & 60.8 & \\
\hline 122 & UISO_IE_PLATE_AEA & QTESRL & 0.2 & $\mathrm{pCi} / \mathrm{g}$ & 0.028 & 0.042 & 0.0109 & 72.5 & \\
\hline 123 & UISO_IE_PLATE_AEA & QTESRL & 0.127 & $\mathrm{pCi} / \mathrm{g}$ & 0.026 & 0.033 & 0.0165 & 75.5 & \\
\hline 125 & UISO_IE_PLATE_AEA & QTESRL & 0.597 & $\mathrm{pCi} / \mathrm{g}$ & 0.051 & 0.1 & 0.0084 & 44.1 & \\
\hline 126 & UISO_IE_PLATE_AEA & QTESRL & 0.272 & $\mathrm{pCi} / \mathrm{g}$ & 0.034 & 0.054 & 0.0123 & 65.2 & \\
\hline 127 & UISO_IE_PLATE_AEA & QTESRL & 0.208 & $\mathrm{pCi} / \mathrm{g}$ & 0.029 & 0.044 & 0.00808 & 72.4 & \\
\hline 128 & UISO_IE_PLATE_AEA & QTESRL & 0.231 & $\mathrm{pCi} / \mathrm{g}$ & 0.03 & 0.047 & 0.00765 & 68.3 & \\
\hline 129 & |UISO_IE_PLATE_AEA & QTESRL & 0.579 & $\mathrm{pCi} / \mathrm{g}$ & 0.046 & 0.099 & 0.0157 & 62.1 & \\
\hline 130 & UISO_IE_PLATE_AEA & QTESRL & 0.231 & $\mathrm{pCi} / \mathrm{g}$ & 0.03 & 0.047 & 0.00662 & 65.7 & \\
\hline 131 & UISO_IE_PLATE_AEA & QTESRL & 0.142 & $\mathrm{pCi} / \mathrm{g}$ & 0.024 & 0.033 & 0.0132 & 71.1 & \\
\hline 132 & UISO_IE_PLATE_AEA & QTESRL & 0.21 & $\mathrm{pCi} / \mathrm{g}$ & 0.03 & 0.045 & 0.0173 & 71.5 & \\
\hline 133 & UISO_IE_PLATE_AEA & QTESRL & 0.46 & $\mathrm{pCi} / \mathrm{g}$ & 0.042 & 0.082 & 0.0108 & 62.6 & \\
\hline 135 & UISO_IE_PLATE_AEA & QTESRL & 0.206 & $\mathrm{pCi} / \mathrm{g}$ & 0.035 & 0.048 & 0.0188 & 73.3 & \\
\hline 136 & UISO_IE_PLATE_AEA & QTESRL & 0.219 & $\mathrm{pCi} / \mathrm{g}$ & 0.034 & 0.049 & 0.0133 & 69.8 & \\
\hline 137 & UISO_IE_PLATE_AEA & QTESRL & 0.572 & $\mathrm{pCi} / \mathrm{g}$ & 0.049 & 0.1 & 0.00588 & 66.6 & \\
\hline 138 & UISO_IE_PLATE_AEA & QTESRL & 0.118 & $\mathrm{pCi} / \mathrm{g}$ & 0.019 & 0.027 & 0.00519 & 75.1 & \\
\hline 139 & UISO_IE_PLATE_AEA & QTESRL & 0.102 & $\mathrm{pCi} / \mathrm{g}$ & 0.02 & 0.026 & 0.00761 & 76.7 & \\
\hline 140 & UISO_IE_PLATE_AEA & QTESRL & 0.29 & $\mathrm{pCi} / \mathrm{g}$ & 0.034 & 0.056 & 0.0203 & 69.8 & \\
\hline 141 & UISO_IE_PLATE_AEA & QTESRL & 0.623 & $\mathrm{pCi} / \mathrm{g}$ & 0.053 & 0.11 & 0.00786 & 50.3 & \\
\hline
\end{tabular}




\begin{tabular}{|c|c|c|c|c|c|c|c|c|c|c|}
\hline & $T$ & $\mathrm{U}$ & $\mathrm{V}$ & $\mathrm{W}$ & $\mathrm{X}$ & $\mathrm{Y}$ & $Z$ & $\overline{A A}$ & $A B$ & $\mathrm{AC}$ \\
\hline 1 & REVIEW_QUALIFIER & SAMP_FROM & SAMP_ITEM & SAMP_MTHD & COLL_MTHD & SAMP_SITE_ID & SAMP_SITE_NAME & WELL_NAME & SAMPLE_LOCATION & SAMP_COMMENT \\
\hline 2 & & SUB_SURFACE & FINE SAND & & & 500 & MC NARY DAM & & & \\
\hline 3 & & SUB_SURFACE & VERY FINE SAND & & & 500 & MC NARY DAM & & & \\
\hline 4 & & SUB_SURFACE & SILT_CLAY & & & 500 & MC NARY DAM & & & \\
\hline 5 & & SUB_SURFACE & COARSE SAND & & & 454 & PRIEST RAPIDS DAM & & & \\
\hline 6 & & SUB_SURFACE & MEDIUM SAND & & & 454 & PRIEST RAPIDS DAM & & & \\
\hline 7 & & SUB_SURFACE & FINE SAND & & & 454 & PRIEST RAPIDS DAM & & & \\
\hline 8 & & SUB_SURFACE & VERY FINE SAND & & & 454 & PRIEST RAPIDS DAM & & & \\
\hline 9 & & SUB_SURFACE & SILT_CLAY & & & 454 & PRIEST RAPIDS DAM & & & \\
\hline 10 & & SUB_SURFACE & MEDIUM SAND & & & 561 & WHITE BLUFFS SLOUGH & & & \\
\hline 11 & & SUB_SURFACE & FINE SAND & & & 561 & WHITE BLUFFS SLOUGH & & & \\
\hline 12 & & SUB_SURFACE & VERY FINE SAND & & & 561 & WHITE BLUFFS SLOUGH & & & \\
\hline 13 & & SUB_SURFACE & SILT_CLAY & & & 561 & WHITE BLUFFS SLOUGH & & & \\
\hline 14 & & SUB_SURFACE & MEDIUM SAND & & & 195 & 100 F SLOUGH & & & \\
\hline 15 & & SUB_SURFACE & FINE SAND & & & 195 & 100 F SLOUGH & & & \\
\hline 16 & & SUB_SURFACE & VERY FINE SAND & & & 195 & 100 F SLOUGH & & & \\
\hline 17 & & SUB_SURFACE & SILT_CLAY & & & 195 & 100 F SLOUGH & & & \\
\hline 18 & & SUB_SURFACE & MEDIUM SAND & & & 563 & HANFORD SLOUGH & & & \\
\hline 19 & & SUB_SURFACE & FINE SAND & & & 563 & HANFORD SLOUGH & & & \\
\hline 20 & & SUB_SURFACE & VERY FINE SAND & & & 563 & HANFORD SLOUGH & & & \\
\hline 21 & & SUB_SURFACE & SILT_CLAY & & & 563 & HANFORD SLOUGH & & & \\
\hline 22 & $F$ & SUB_SURFACE & MEDIUM SAND & & & 7754 & ICE HARBOR DAM & & & \\
\hline 23 & $F$ & SUB_SURFACE & FINE SAND & & & 7754 & ICE HARBOR DAM & & & \\
\hline 24 & & SUB_SURFACE & VERY FINE SAND & & & 7754 & ICE HARBOR DAM & & & \\
\hline 25 & & SUB_SURFACE & SILT_CLAY & & & 7754 & ICE HARBOR DAM & & & \\
\hline 26 & & SUB_SURFACE & MEDIUM SAND & & & 311 & RICHLAND-RIVER & & & \\
\hline 27 & & SUB_SURFACE & FINE SAND & & & 311 & RICHLAND-RIVER & & & \\
\hline 28 & & SUB_SURFACE & VERY FINE SAND & & & 311 & RICHLAND-RIVER & & & \\
\hline 29 & & SUB_SURFACE & SILT_CLAY & & & 311 & RICHLAND-RIVER & & & \\
\hline 30 & & SUB_SURFACE & FINE SAND & & & 500 & MC NARY DAM & & & \\
\hline 31 & & SUB_SURFACE & VERY FINE SAND & & & 500 & MC NARY DAM & & & \\
\hline 32 & & SUB_SURFACE & SILT_CLAY & & & 500 & MC NARY DAM & & & \\
\hline 33 & & SUB_SURFACE & COARSE SAND & & & 454 & PRIEST RAPIDS DAM & & & \\
\hline 34 & & SUB_SURFACE & MEDIUM SAND & & & 454 & PRIEST RAPIDS DAM & & & \\
\hline 35 & & SUB_SURFACE & FINE SAND & & & 454 & PRIEST RAPIDS DAM & & & \\
\hline 36 & & SUB_SURFACE & VERY FINE SAND & & & 454 & PRIEST RAPIDS DAM & & & \\
\hline 37 & & SUB_SURFACE & SILT_CLAY & & & 454 & PRIEST RAPIDS DAM & & & \\
\hline 38 & & SUB_SURFACE & MEDIUM SAND & & & 561 & WHITE BLUFFS SLOUGH & & & \\
\hline 39 & & SUB_SURFACE & FINE SAND & & & 561 & WHITE BLUFFS SLOUGH & & & \\
\hline
\end{tabular}




\begin{tabular}{|c|c|c|c|c|c|c|c|c|c|c|}
\hline & $\mathrm{T}$ & $U$ & $\mathrm{~V}$ & $\mathrm{~W}$ & $x$ & $Y$ & $Z$ & $\mathrm{AA}$ & $A B$ & $A C$ \\
\hline 1 & REVIEW_QUALIFIER & SAMP_FROM & SAMP_ITEM & SAMP_MTHD & COLL_MTHD & SAMP_SITE_ID & SAMP_SITE_NAME & WELL_NAME & SAMPLE_LOCATION & SAMP_COMMENT \\
\hline 40 & & SUB_SURFACE & VERY FINE SAND & & & 561 & WHITE BLUFFS SLOUGH & & & \\
\hline 41 & & SUB_SURFACE & SILT_CLAY & & & 561 & WHITE BLUFFS SLOUGH & & & \\
\hline 42 & & SUB_SURFACE & MEDIUM SAND & & & 195 & 100 F SLOUGH & & & \\
\hline 43 & & SUB_SURFACE & FINE SAND & & & 195 & 100 F SLOUGH & & & \\
\hline 44 & & SUB_SURFACE & VERY FINE SAND & & & 195 & 100 F SLOUGH & & & \\
\hline 45 & & SUB_SURFACE & SILT_CLAY & & & 195 & 100 F SLOUGH & & & \\
\hline 46 & & SUB SURFACE & MEDIUM SAND & & & 563 & HANFORD SLOUGH & & & \\
\hline 47 & & SUB_SURFACE & FINE SAND & & & 563 & HANFORD SLOUGH & & & \\
\hline 48 & & SUB_SURFACE & VERY FINE SAND & & & 563 & HANFORD SLOUGH & & & \\
\hline 49 & & SUB_SURFACE & SILT_CLAY & & & 563 & HANFORD SLOUGH & & & \\
\hline 50 & & SUB_SURFACE & MEDIUM SAND & & & 7754 & ICE HARBOR DAM & & & \\
\hline 51 & & SUB_SURFACE & FINE SAND & & & 7754 & ICE HARBOR DAM & & & \\
\hline 52 & & SUB_SURFACE & VERY FINE SAND & & & 7754 & ICE HARBOR DAM & & & \\
\hline 53 & & SUB_SURFACE & SILT_CLAY & & & 7754 & ICE HARBOR DAM & & & \\
\hline 54 & & SUB_SURFACE & MEDIUM SAND & & & 311 & RICHLAND-RIVER & & & \\
\hline 55 & & SUB_SURFACE & FINE SAND & & & 311 & RICHLAND-RIVER & & & \\
\hline 56 & & SUB_SURFACE & VERY FINE SAND & & & 311 & RICHLAND-RIVER & & & \\
\hline 57 & & SUB_SURFACE & SILT_CLAY & & & 311 & RICHLAND-RIVER & & & \\
\hline 58 & & SUB_SURFACE & FINE SAND & & & 500 & MC NARY DAM & & & \\
\hline 59 & & SUB_SURFACE & VERY FINE SAND & & & 500 & MC NARY DAM & & & \\
\hline 60 & & SUB_SURFACE & SILT_CLAY & & & 500 & MC NARY DAM & & & \\
\hline 61 & & SUB_SURFACE & COARSE SAND & & & 454 & PRIEST RAPIDS DAM & & & \\
\hline 62 & & SUB_SURFACE & MEDIUM SAND & & & 454 & PRIEST RAPIDS DAM & & & \\
\hline 63 & & SUB_SURFACE & FINE SAND & & & 454 & PRIEST RAPIDS DAM & & & \\
\hline 64 & & SUB_SURFACE & VERY FINE SAND & & & 454 & PRIEST RAPIDS DAM & & & \\
\hline 65 & & SUB_SURFACE & SILT_CLAY & & & 454 & PRIEST RAPIDS DAM & & & \\
\hline 66 & & SUB_SURFACE & MEDIUM SAND & & & 561 & WHITE BLUFFS SLOUGH & & & \\
\hline 67 & & SUB_SURFACE & FINE SAND & & & 561 & WHITE BLUFFS SLOUGH & & & \\
\hline 68 & & SUB_SURFACE & VERY FINE SAND & & & 561 & WHITE BLUFFS SLOUGH & & & \\
\hline 69 & & SUB_SURFACE & SILT_CLAY & & & 561 & WHITE BLUFFS SLOUGH & & & \\
\hline 70 & & SUB_SURFACE & MEDIUM SAND & & & 195 & 100 F SLOUGH & & & \\
\hline 71 & & SUB_SURFACE & FINE SAND & & & 195 & $100 \mathrm{~F}$ SLOUGH & & & \\
\hline 72 & & SUB_SURFACE & VERY FINE SAND & & & 195 & $100 \mathrm{~F}$ SLOUGH & & & \\
\hline 73 & & SUB_SURFACE & SILT_CLAY & & & 195 & 100 F SLOUGH & & & \\
\hline 74 & & SUB_SURFACE & MEDIUM SAND & & & 563 & HANFORD SLOUGH & & & \\
\hline 75 & & SUB_SURFACE & FINE SAND & & & 563 & HANFORD SLOUGH & & & \\
\hline 76 & & SUB_SURFACE & VERY FINE SAND & & & 563 & HANFORD SLOUGH & & & \\
\hline 77 & & SUB_SURFACE & SILT_CLAY & & & 563 & HANFORD SLOUGH & & & \\
\hline
\end{tabular}




\begin{tabular}{|c|c|c|c|c|c|c|c|c|c|c|}
\hline & $T$ & $\mathrm{U}$ & $\mathrm{V}$ & $\mathrm{W}$ & $\mathrm{X}$ & $\mathrm{Y}$ & $Z$ & $\mathrm{AA}$ & $A B$ & $\mathrm{AC}$ \\
\hline 1 & REVIEW_QUALIFIER & SAMP_FROM & SAMP_ITEM & SAMP_MTHD & COLL_MTHD & SAMP_SITE_ID & SAMP_SITE_NAME & WELL_NAME & SAMPLE_LOCATION & SAMP_COMMENT \\
\hline 78 & & SUB_SURFACE & MEDIUM SAND & & & 7754 & ICE HARBOR DAM & & & \\
\hline 79 & & SUB_SURFACE & FINE SAND & & & 7754 & ICE HARBOR DAM & & & \\
\hline 80 & & SUB_SURFACE & VERY FINE SAND & & & 7754 & ICE HARBOR DAM & & & \\
\hline 81 & & SUB_SURFACE & SILT_CLAY & & & 7754 & ICE HARBOR DAM & & & \\
\hline 82 & & SUB_SURFACE & MEDIUM SAND & & & 311 & RICHLAND-RIVER & & & \\
\hline 83 & & SUB_SURFACE & FINE SAND & & & 311 & RICHLAND-RIVER & & & \\
\hline 84 & & SUB_SURFACE & VERY FINE SAND & & & 311 & RICHLAND-RIVER & & & \\
\hline 85 & & SUB_SURFACE & SILT_CLAY & & & 311 & RICHLAND-RIVER & & & \\
\hline 86 & & SUB_SURFACE & FINE SAND & & & 500 & MC NARY DAM & & & \\
\hline 87 & & SUB_SURFACE & VERY FINE SAND & & & 500 & MC NARY DAM & & & \\
\hline 88 & & SUB_SURFACE & SILT_CLAY & & & 500 & MC NARY DAM & & & \\
\hline 89 & & SUB_SURFACE & COARSE SAND & & & 454 & PRIEST RAPIDS DAM & & & \\
\hline 90 & & SUB_SURFACE & MEDIUM SAND & & & 454 & PRIEST RAPIDS DAM & & & \\
\hline 91 & & SUB_SURFACE & FINE SAND & & & 454 & PRIEST RAPIDS DAM & & & \\
\hline 92 & & SUB_SURFACE & VERY FINE SAND & & & 454 & PRIEST RAPIDS DAM & & & \\
\hline 93 & & SUB_SURFACE & SILT_CLAY & & & 454 & PRIEST RAPIDS DAM & & & \\
\hline 94 & & SUB_SURFACE & MEDIUM SAND & & & 561 & WHITE BLUFFS SLOUGH & & & \\
\hline 95 & & SUB_SURFACE & FINE SAND & & & 561 & WHITE BLUFFS SLOUGH & & & \\
\hline 96 & & SUB_SURFACE & VERY FINE SAND & & & 561 & WHITE BLUFFS SLOUGH & & & \\
\hline 97 & & SUB_SURFACE & SILT_CLAY & & & 561 & WHITE BLUFFS SLOUGH & & & \\
\hline 98 & & SUB_SURFACE & MEDIUM SAND & & & 195 & 100 F SLOUGH & & & \\
\hline 99 & & SUB_SURFACE & FINE SAND & & & 195 & 100 F SLOUGH & & & \\
\hline 100 & & SUB_SURFACE & VERY FINE SAND & & & 195 & 100 F SLOUGH & & & \\
\hline 101 & & SUB_SURFACE & SILT_CLAY & & & 195 & $100 \mathrm{~F}$ SLOUGH & & & \\
\hline 102 & & SUB_SURFACE & MEDIUM SAND & & & 563 & HANFORD SLOUGH & & & \\
\hline 103 & & SUB_SURFACE & FINE SAND & & & 563 & HANFORD SLOUGH & & & \\
\hline 104 & & SUB_SURFACE & VERY FINE SAND & & & 563 & HANFORD SLOUGH & & & \\
\hline 105 & & SUB_SURFACE & SILT_CLAY & & & 563 & HANFORD SLOUGH & & & \\
\hline 106 & & SUB_SURFACE & MEDIUM SAND & & & 7754 & ICE HARBOR DAM & & & \\
\hline 107 & & SUB_SURFACE & FINE SAND & & & 7754 & ICE HARBOR DAM & & & \\
\hline 108 & & SUB_SURFACE & VERY FINE SAND & & & 7754 & ICE HARBOR DAM & & & \\
\hline 109 & & SUB_SURFACE & SILT_CLAY & & & 7754 & ICE HARBOR DAM & & & \\
\hline 110 & & SUB_SURFACE & MEDIUM SAND & & & 311 & RICHLAND-RIVER & & & \\
\hline 111 & & SUB_SURFACE & FINE SAND & & & 311 & RICHLAND-RIVER & & & \\
\hline 112 & & SUB_SURFACE & VERY FINE SAND & & & 311 & RICHLAND-RIVER & & & \\
\hline 113 & & SUB_SURFACE & SILT_CLAY & & & 311 & RICHLAND-RIVER & & & \\
\hline 114 & & SUB_SURFACE & FINE SAND & & & 500 & MC NARY DAM & & & \\
\hline 115 & & SUB_SURFACE & VERY FINE SAND & & & 500 & MC NARY DAM & & & \\
\hline
\end{tabular}




\begin{tabular}{|c|c|c|c|c|c|c|c|c|c|c|}
\hline & $T$ & $\mathrm{U}$ & $\mathrm{V}$ & $\mathrm{W}$ & $\mathrm{X}$ & $\mathrm{Y}$ & $Z$ & $\overline{A A}$ & $A B$ & $\mathrm{AC}$ \\
\hline 1 & REVIEW QUALIFIER & SAMP FROM & SAMP ITEM & SAMP MTHD & COLL MTHD & SAMP SITE ID & SAMP SITE NAME & WELL NAME & SAMPLE LOCATION & SAMP COMMENT \\
\hline 116 & & SUB_SURFACE & SILT_CLAY & & & 500 & MC NARY DAM & & & \\
\hline 117 & & SUB_SURFACE & COARSE SAND & & & 454 & PRIEST RAPIDS DAM & & & \\
\hline 118 & & SUB_SURFACE & MEDIUM SAND & & & 454 & PRIEST RAPIDS DAM & & & \\
\hline 119 & & SUB_SURFACE & FINE SAND & & & 454 & PRIEST RAPIDS DAM & & & \\
\hline 120 & & SUB_SURFACE & VERY FINE SAND & & & 454 & PRIEST RAPIDS DAM & & & \\
\hline 121 & & SUB_SURFACE & SILT_CLAY & & & 454 & PRIEST RAPIDS DAM & & & \\
\hline 122 & & SUB SURFACE & MEDIUM SAND & & & 561 & WHITE BLUFFS SLOUGH & & & \\
\hline 123 & & SUB_SURFACE & FINE SAND & & & 561 & WHITE BLUFFS SLOUGH & & & \\
\hline $\mid 124$ & & SUB SURFACE & VERY FINE SAND & & & 561 & WHITE BLUFFS SLOUGH & & & \\
\hline 125 & & SUB_SURFACE & SILT_CLAY & & & 561 & WHITE BLUFFS SLOUGH & & & \\
\hline 126 & & SUB_SURFACE & MEDIUM SAND & & & 195 & 100 F SLOUGH & & & \\
\hline 127 & & SUB_SURFACE & FINE SAND & & & 195 & $100 \mathrm{~F}$ SLOUGH & & & \\
\hline 128 & & SUB SURFACE & VERY FINE SAND & & & 195 & 100 F SLOUGH & & & \\
\hline 129 & & SUB_SURFACE & SILT_CLAY & & & 195 & 100 F SLOUGH & & & \\
\hline 130 & & SUB_SURFACE & MEDIUM SAND & & & 563 & HANFORD SLOUGH & & & \\
\hline 131 & & SUB_SURFACE & FINE SAND & & & 563 & HANFORD SLOUGH & & & \\
\hline 132 & & SUB_SURFACE & VERY FINE SAND & & & 563 & HANFORD SLOUGH & & & \\
\hline 133 & & SUB_SURFACE & SILT_CLAY & & & 563 & HANFORD SLOUGH & & & \\
\hline 134 & & SUB_SURFACE & MEDIUM SAND & & & 7754 & ICE HARBOR DAM & & & \\
\hline 135 & & SUB_SURFACE & FINE SAND & & & 7754 & ICE HARBOR DAM & & & \\
\hline 136 & & SUB_SURFACE & VERY FINE SAND & & & 7754 & ICE HARBOR DAM & & & \\
\hline 137 & & SUB_SURFACE & SILT_CLAY & & & 7754 & ICE HARBOR DAM & & & \\
\hline 138 & & SUB_SURFACE & MEDIUM SAND & & & 311 & RICHLAND-RIVER & & & \\
\hline 139 & & SUB_SURFACE & FINE SAND & & & 311 & RICHLAND-RIVER & & & \\
\hline 140 & & SUB_SURFACE & VERY FINE SAND & & & 311 & RICHLAND-RIVER & & & \\
\hline 141 & & SUB_SURFACE & SILT_CLAY & & & 311 & RICHLAND-RIVER & & & \\
\hline
\end{tabular}




\begin{tabular}{|c|c|c|c|c|c|c|}
\hline & $A D$ & $\mathrm{AE}$ & $\mathrm{AF}$ & $A G$ & $\mathrm{AH}$ & $\mathrm{Al}$ \\
\hline 1 & RESULT_COMMENT & COLL_SAMP_SIZE & COLL_SAMP_SIZE_UNITS & DIST_CLASS & OWNER ID & COLLECTION_PURPOSE \\
\hline 2 & & 500 & g & DISTANT & SESPSPEC & $S$ \\
\hline 3 & & 500 & g & DISTANT & SESPSPEC & $S$ \\
\hline 4 & & 500 & g & DISTANT & SESPSPEC & $\mathrm{S}$ \\
\hline 5 & & 500 & g & OFFSITE & SESPSPEC & S \\
\hline 6 & & 500 & g & OFFSITE & SESPSPEC & $S$ \\
\hline 7 & & 500 & g & OFFSITE & SESPSPEC & S \\
\hline 8 & & 500 & g & OFFSITE & SESPSPEC & $S$ \\
\hline 9 & & 500 & g & OFFSITE & SESPSPEC & $S$ \\
\hline 10 & & 500 & g & RIVER_SHORELINE & SESPSPEC & S \\
\hline 11 & & 500 & g & RIVER_SHORELINE & SESPSPEC & $\mathrm{S}$ \\
\hline 12 & & 500 & g & RIVER_SHORELINE & SESPSPEC & $S$ \\
\hline 13 & & 500 & g & RIVER_SHORELINE & SESPSPEC & S \\
\hline 14 & & 500 & g & ONSITE & SESPSPEC & $\mathrm{S}$ \\
\hline 15 & & 500 & g & ONSITE & SESPSPEC & $S$ \\
\hline 16 & & 500 & g & ONSITE & SESPSPEC & $S$ \\
\hline 17 & & 500 & g & ONSITE & SESPSPEC & S \\
\hline 18 & & 500 & g & RIVER_SHORELINE & SESPSPEC & S \\
\hline 19 & & 500 & g & RIVER_SHORELINE & SESPSPEC & S \\
\hline 20 & & 500 & g & RIVER_SHORELINE & SESPSPEC & S \\
\hline 21 & & 500 & g & RIVER_SHORELINE & SESPSPEC & S \\
\hline 22 & & 500 & g & OFFSITE & SESPSPEC & $S$ \\
\hline 23 & & 500 & g & OFFSITE & SESPSPEC & S \\
\hline 24 & & 500 & g & OFFSITE & SESPSPEC & $S$ \\
\hline 25 & & 500 & g & OFFSITE & SESPSPEC & $S$ \\
\hline 26 & & 500 & g & RIVER_SHORELINE & SESPSPEC & $S$ \\
\hline 27 & & 500 & g & RIVER_SHORELINE & SESPSPEC & $\mathrm{S}$ \\
\hline 28 & & 500 & g & RIVER_SHORELINE & SESPSPEC & S \\
\hline 29 & & 500 & g & RIVER_SHORELINE & SESPSPEC & S \\
\hline 30 & & 500 & g & DISTANTT & SESPSPEC & $S$ \\
\hline 31 & IR-0022 MDA > CRDL & 500 & g & DISTANT & SESPSPEC & $\mathrm{S}$ \\
\hline 32 & & 500 & g & DISTANT & SESPSPEC & S \\
\hline 33 & & 500 & g & OFFSITE & SESPSPEC & $S$ \\
\hline 34 & & 500 & g & OFFSITE & SESPSPEC & $S$ \\
\hline 35 & & 500 & g & OFFSITE & SESPSPEC & S \\
\hline 36 & & 500 & g & OFFSITE & SESPSPEC & $\mathrm{S}$ \\
\hline 37 & & 500 & g & OFFSITE & SESPSPEC & $S$ \\
\hline 38 & & 500 & g & RIVER_SHORELINE & SESPSPEC & S \\
\hline 39 & & 500 & g & RIVER_SHORELINE & SESPSPEC & $\mathrm{S}$ \\
\hline
\end{tabular}




\begin{tabular}{|c|c|c|c|c|c|c|}
\hline & $A D$ & $\mathrm{AE}$ & $\mathrm{AF}$ & $\mathrm{AG}$ & $\mathrm{AH}$ & $\mathrm{Al}$ \\
\hline 1 & RESULT_COMMENT & COLL_SAMP_SIZE & COLL_SAMP_SIZE_UNITS & DIST_CLASS & OWNER_ID & COLLECTION_PURPOSE \\
\hline 40 & & 500 & g & RIVER_SHORELINE & SESPSPEC & $\mathrm{S}$ \\
\hline 41 & IR-0022 MDA > CRDL & 500 & g & RIVER_SHORELINE & SESPSPEC & $\mathrm{S}$ \\
\hline 42 & & 500 & g & ONSITE & SESPSPEC & $S$ \\
\hline 43 & & 500 & g & ONSITE & SESPSPEC & $S$ \\
\hline 44 & & 500 & g & ONSITE & SESPSPEC & $S$ \\
\hline 45 & & 500 & g & ONSITE & SESPSPEC & S \\
\hline 46 & & 500 & g & RIVER_SHORELINE & SESPSPEC & S \\
\hline 47 & & 500 & g & RIVER_SHORELINE & SESPSPEC & S \\
\hline 48 & & 500 & g & RIVER_SHORELINE & SESPSPEC & S \\
\hline 49 & & 500 & g & RIVER_SHORELINE & SESPSPEC & $S$ \\
\hline 50 & & 500 & g & OFFSITE & SESPSPEC & S \\
\hline 51 & & 500 & g & OFFSITE & SESPSPEC & $S$ \\
\hline 52 & & 500 & g & OFFSITE & SESPSPEC & $\mathrm{S}$ \\
\hline 53 & IR-0022 MDA > CRDL & 500 & g & OFFSITE & SESPSPEC & $\mathrm{S}$ \\
\hline 54 & & 500 & g & RIVER_SHORELINE & SESPSPEC & $\mathrm{S}$ \\
\hline 55 & & 500 & g & RIVER_SHORELINE & SESPSPEC & $\mathrm{S}$ \\
\hline 56 & & 500 & g & RIVER_SHORELINE & SESPSPEC & S \\
\hline 57 & & 500 & g & RIVER_SHORELINE & SESPSPEC & $S$ \\
\hline 58 & & 500 & $\mathrm{~g}$ & DISTAN̄T & SESPSPEC & $S$ \\
\hline 59 & & 500 & g & DISTANT & SESPSPEC & S \\
\hline 60 & & 500 & g & DISTANT & SESPSPEC & S \\
\hline 61 & IR-0015 Matrix Blank > CRDL & 500 & g & OFFSITE & SESPSPEC & S \\
\hline 62 & IR-0015 Matrix Blank > CRDL & 500 & g & OFFSITE & SESPSPEC & $S$ \\
\hline 63 & IR-0015 Matrix Blank > CRDL & 500 & g & OFFSITE & SESPSPEC & S \\
\hline 64 & IR-0015 Matrix Blank > CRDL & 500 & g & OFFSITE & SESPSPEC & $\mathrm{S}$ \\
\hline 65 & IR-0015 Matrix Blank > CRDL & 500 & g & OFFSITE & SESPSPEC & S \\
\hline 66 & & 500 & g & RIVER_SHORELINE & SESPSPEC & S \\
\hline 67 & & 500 & g & RIVER_SHORELINE & SESPSPEC & S \\
\hline 68 & & 500 & g & RIVER_SHORELINE & SESPSPEC & S \\
\hline 69 & & 500 & g & RIVER_SHORELINE & SESPSPEC & S \\
\hline 70 & IR-0015 Matrix Blank > CRDL & 500 & g & ONSITE & SESPSPEC & S \\
\hline 71 & IR-0015 Matrix Blank > CRDL & 500 & g & ONSITE & SESPSPEC & $\mathrm{S}$ \\
\hline 72 & IR-0015 Matrix Blank > CRDL & 500 & g & ONSITE & SESPSPEC & S \\
\hline 73 & IR-0015 Matrix Blank > CRDL & 500 & g & ONSITE & SESPSPEC & S \\
\hline 74 & IR-0015 Matrix Blank > CRDL & 500 & g & RIVER_SHORELINE & SESPSPEC & $\mathrm{S}$ \\
\hline 75 & IR-0015 Matrix Blank > CRDL & 500 & g & RIVER_SHORELINE & SESPSPEC & S \\
\hline 76 & IR-0015 Matrix Blank > CRDL & 500 & g & RIVER_SHORELINE & SESPSPEC & S \\
\hline 77 & IR-0015 Matrix Blank > CRDL & 500 & g & RIVER_SHORELINE & SESPSPEC & $S$ \\
\hline
\end{tabular}

Page 14 


\begin{tabular}{|c|c|c|c|c|c|c|}
\hline & $\overline{A D}$ & $\overline{A E}$ & $\mathrm{AF}$ & $A G$ & $\mathrm{AH}$ & $\mathrm{Al}$ \\
\hline 1 & RESULT COMMENT & COLL SAMP SIZE & COLL SAMP SIZE UNITS & DIST CLASS & OWNER ID & COLLECTION PURPOSE \\
\hline 78 & IR-0015 Matrix Blank > CRDL & 500 & g & OFFSITE & SESPSPEC & $\mathrm{S}$ \\
\hline 79 & & 500 & $\mathrm{~g}$ & OFFSITE & SESPSPEC & $\mathrm{S}$ \\
\hline 80 & & 500 & g & OFFSITE & SESPSPEC & $\mathrm{S}$ \\
\hline 81 & & 500 & g & OFFSITE & SESPSPEC & S \\
\hline 82 & & 500 & g & RIVER_SHORELINE & SESPSPEC & S \\
\hline 83 & & 500 & g & RIVER_SHORELINE & SESPSPEC & S \\
\hline 84 & & 500 & $\mathrm{~g}$ & RIVER SHORELINE & SESPSPEC & $\mathrm{S}$ \\
\hline 85 & & 500 & $\mathrm{~g}$ & RIVER_SHORELINE & SESPSPEC & S \\
\hline 86 & & 500 & $\mathrm{~g}$ & DISTAN̄T & SESPSPEC & S \\
\hline 87 & & 500 & g & DISTANT & SESPSPEC & $\mathrm{S}$ \\
\hline 88 & & 500 & g & DISTANT & SESPSPEC & S \\
\hline 89 & IR-0015 Matrix Blank > CRDL & 500 & g & OFFSITE & SESPSPEC & S \\
\hline 90 & IR-0015 Matrix Blank > CRDL & 500 & g & OFFSITE & SESPSPEC & $\mathrm{S}$ \\
\hline 91 & IR-0015 Matrix Blank > CRDL & 500 & g & OFFSITE & SESPSPEC & S \\
\hline 92 & IR-0015 Matrix Blank > CRDL & 500 & g & OFFSITE & SESPSPEC & $\mathrm{S}$ \\
\hline 93 & IR-0015 Matrix Blank > CRDL & 500 & g & OFFSITE & SESPSPEC & $\mathrm{S}$ \\
\hline 94 & & 500 & g & RIVER_SHORELINE & SESPSPEC & S \\
\hline 95 & & 500 & $\mathrm{~g}$ & RIVER_SHORELINE & SESPSPEC & S \\
\hline 96 & & 500 & g & RIVER_SHORELINE & SESPSPEC & $\mathrm{S}$ \\
\hline 97 & & 500 & g & RIVER_SHORELINE & SESPSPEC & $\mathrm{S}$ \\
\hline 98 & IR-0015 Matrix Blank > CRDL & 500 & g & ONSITE & SESPSPEC & S \\
\hline 99 & IR-0015 Matrix Blank > CRDL & 500 & g & ONSITE & SESPSPEC & $\mathrm{S}$ \\
\hline 100 & IR-0015 Matrix Blank > CRDL & 500 & g & ONSITE & SESPSPEC & $\mathrm{S}$ \\
\hline 101 & IR-0015 Matrix Blank > CRDL & 500 & g & ONSITE & SESPSPEC & $\mathrm{S}$ \\
\hline 102 & IR-0015 Matrix Blank > CRDL & 500 & g & RIVER_SHORELINE & SESPSPEC & S \\
\hline 103 & IR-0015 Matrix Blank > CRDL & 500 & g & RIVER_SHORELINE & SESPSPEC & $\mathrm{S}$ \\
\hline 104 & IR-0015 Matrix Blank > CRDL & 500 & $\mathrm{~g}$ & RIVER_SHORELINE & SESPSPEC & S \\
\hline 105 & |IR-0015 Matrix Blank > CRDL & 500 & g & RIVER_SHORELINE & SESPSPEC & $\mathrm{S}$ \\
\hline 106 & IR-0015 Matrix Blank > CRDL & 500 & g & OFFSITE & SESPSPEC & S \\
\hline 107 & & 500 & $\mathrm{~g}$ & OFFSITE & SESPSPEC & S \\
\hline 108 & & 500 & g & OFFSITE & SESPSPEC & S \\
\hline 109 & & 500 & g & OFFSITE & SESPSPEC & $\mathrm{S}$ \\
\hline 110 & & 500 & $\mathrm{~g}$ & RIVER_SHORELINE & SESPSPEC & S \\
\hline 111 & & 500 & $\mathrm{~g}$ & RIVER_SHORELINE & SESPSPEC & S \\
\hline 112 & & 500 & g & RIVER_SHORELINE & SESPSPEC & $\mathrm{S}$ \\
\hline 113 & & 500 & g & RIVER_SHORELINE & SESPSPEC & S \\
\hline 114 & & 500 & g & DISTAN̄T & SESPSPEC & S \\
\hline 115 & & 500 & g & DISTANT & SESPSPEC & $\mathrm{S}$ \\
\hline
\end{tabular}

Page 15 


\begin{tabular}{|c|c|c|c|c|c|c|}
\hline & $A D$ & $\mathrm{AE}$ & $\mathrm{AF}$ & AG & $\mathrm{AH}$ & $\mathrm{Al}$ \\
\hline 1 & RESULT_COMMENT & COLL_SAMP_SIZE & COLL_SAMP_SIZE_UNITS & DIST_CLASS & OWNER_ID & COLLECTION_PURPOSE \\
\hline 116 & & 500 & $\mathrm{~g}$ & DISTANT & SESPSPEC & $\mathrm{S}$ \\
\hline 117 & IR-0015 Matrix Blank > CRDL & 500 & g & OFFSITE & SESPSPEC & $\mathrm{S}$ \\
\hline 118 & IR-0015 Matrix Blank > CRDL & 500 & g & OFFSITE & SESPSPEC & $\mathrm{S}$ \\
\hline 119 & IR-0015 Matrix Blank > CRDL & 500 & g & OFFSITE & SESPSPEC & $\mathrm{S}$ \\
\hline 120 & IR-0015 Matrix Blank > CRDL & 500 & $\mathrm{~g}$ & OFFSITE & SESPSPEC & $\mathrm{S}$ \\
\hline 121 & IR-0015 Matrix Blank > CRDL & 500 & g & OFFSITE & SESPSPEC & $\mathrm{S}$ \\
\hline 122 & & 500 & g & RIVER_SHORELINE & SESPSPEC & $\mathrm{S}$ \\
\hline 123 & & 500 & $\mathrm{~g}$ & RIVER_SHORELINE & SESPSPEC & $\mathrm{S}$ \\
\hline 124 & & 500 & g & RIVER_SHORELINE & SESPSPEC & $\mathrm{S}$ \\
\hline 125 & & 500 & g & RIVER_SHORELINE & SESPSPEC & S \\
\hline 126 & IR-0015 Matrix Blank > CRDL & 500 & $\mathrm{~g}$ & ONSITE & SESPSPEC & $\mathrm{S}$ \\
\hline 127 & IR-0015 Matrix Blank > CRDL & 500 & g & ONSITE & SESPSPEC & $\mathrm{S}$ \\
\hline 128 & IR-0015 Matrix Blank > CRDL & 500 & g & ONSITE & SESPSPEC & $\mathrm{S}$ \\
\hline 129 & IR-0015 Matrix Blank > CRDL & 500 & $\mathrm{~g}$ & ONSITE & SESPSPEC & $\mathrm{S}$ \\
\hline 130 & IR-0015 Matrix Blank > CRDL & 500 & g & RIVER_SHORELINE & SESPSPEC & S \\
\hline 131 & IR-0015 Matrix Blank > CRDL & 500 & g & RIVER_SHORELINE & SESPSPEC & $\mathrm{S}$ \\
\hline 132 & IR-0015 Matrix Blank > CRDL & 500 & g & RIVER_SHORELINE & SESPSPEC & $\mathrm{S}$ \\
\hline 133 & IR-0015 Matrix Blank > CRDL & 500 & g & RIVER_SHORELINE & SESPSPEC & S \\
\hline 134 & IR-0015 Matrix Blank > CRDL & 500 & g & OFFSITE & SESPSPEC & S \\
\hline 135 & & 500 & g & OFFSITE & SESPSPEC & S \\
\hline 136 & & 500 & g & OFFSITE & SESPSPEC & $\mathrm{S}$ \\
\hline 137 & & 500 & g & OFFSITE & SESPSPEC & S \\
\hline 138 & & 500 & g & RIVER_SHORELINE & SESPSPEC & $\mathrm{S}$ \\
\hline 139 & & 500 & g & RIVER_SHORELINE & SESPSPEC & $\mathrm{S}$ \\
\hline 140 & & 500 & $g$ & RIVER_SHORELINE & SESPSPEC & $\mathrm{S}$ \\
\hline 141 & & 500 & $\mathrm{~g}$ & RIVER_SHORELINE & SESPSPEC & $\mathrm{S}$ \\
\hline
\end{tabular}




\section{HEIS Sampling Sites}




\begin{tabular}{|c|c|c|c|c|c|c|c|c|c|}
\hline & A & $\mathrm{B}$ & $\mathrm{C}$ & $\mathrm{D}$ & $E$ & $\mathrm{~F}$ & $\mathrm{G}$ & $\mathrm{H}$ & $\mathrm{I}$ \\
\hline 1 & SAMP_SITE_ID & HORIZ_COORD_TYPE & EW_COORD & NS_COORD & OWNER_ID & COORD_SOURCE & DIST_CLASS & RAD_LEVEL & SAMP_SITE_NAME \\
\hline 2 & 50 & & & & SESPMNT & & & & FFTF (699 S 00-07) \\
\hline 3 & 51 & & & & SESPMNT & & & & 100 B AREA \\
\hline 4 & 52 & & & & SESPMNT & & ONSITE & LOW & 100-B SPRING \\
\hline 5 & 53 & & & & SESPMNT & & & & RT. 4N MI. 2 \\
\hline 6 & 54 & & & & SESPMNT & & & & RT. 11A MI. 8 \\
\hline 7 & 55 & & & & SESPMNT & & ONSITE & & RT. 11A MI. 9 \\
\hline 8 & 56 & & & & SESPMNT & & & & RT. 11A MI. 15 \\
\hline 9 & 57 & & & & SESPMNT & & ONSITE & & 200 NORTH \\
\hline 10 & 58 & & & & SESPMNT & & & & NORTHWEST \\
\hline 11 & 60 & & & & SESPMNT & & & & 300 POND \\
\hline 12 & 61 & 1 NAD83 & 594057.63 & 116441.33 & SESPMNT & GPS & ONSITE & LOW & 300 TRENCH \\
\hline 13 & 62 & & & & SESPMNT & & & & 300 TRENCH NO.2 \\
\hline 14 & 63 & & & & SESPMNT & & & & 300 LEACH TRENCH \\
\hline 15 & 64 & & & & SESPMNT & & & & 320 PROCESS SEWER \\
\hline 16 & 65 & & & & SESPMNT & & ONSITE & & 331 BLDG. 300 AREA \\
\hline 17 & 66 & & & & SESPMNT & & & & 331 BLDG. CP 50 \\
\hline 18 & 67 & & & & SESPMNT & & & & 331 POND 300 AREA \\
\hline 19 & 68 & & & & SESPMNT & & & & 377 BLDG. 300 AREA \\
\hline 20 & 69 & & & & SESPMNT & & & & 377 BLDG. SO. FENCE \\
\hline 21 & 70 & NAD83 & 593637.46 & 116047.67 & SESPMNT & GPS & ONSITE & & 3705 BLDG. 300 AREA \\
\hline 22 & 71 & & & & SESPMNT & & & & 3614-A BLDG. \\
\hline 23 & 72 & & & & SESPMNT & & & & 3614A BLDG.NO.2 \\
\hline 24 & 73 & 3 NAD83 & 594351.99 & 115177.49 & SESPMNT & GPS & ONSITE & LOW & 300 SOUTH GATE \\
\hline 25 & 74 & 4 NAD83 & 593748.38 & 115484.74 & SESPMNT & GPS & ONSITE & & 300 SOUTHWEST GATE \\
\hline 26 & 75 & 5 NAD83 & 594395 & 116312 & SESPMNT & SURVEYED & ONSITE & LOW & $300 \mathrm{NE}$ \\
\hline 27 & 76 & & & & SESPMNT & & ONSITE & LOW & NORTH 300 AREA \\
\hline 28 & 77 & & & & SESPMNT & & ONSITE & LOW & SOUTH 300 AREA \\
\hline 29 & 79 & & & & SESPMNT & & & & COOKE BROS \\
\hline 30 & 80 & & & & SESPMNT & & COMMUNITY & LOW & COLUMBIA BASIN \\
\hline 31 & 81 & & & & SESPMNT & & & & COL BASIN COMP NO. 2 \\
\hline 32 & 82 & 2 NAD83 & 601978.81 & 166978.59 & SESPMNT & GPS & COMMUNITY & LOW & OTHELLO \\
\hline 33 & 83 & & & & SESPMNT & & & & WAHLUKE CP 20 \\
\hline 34 & 84 & & & & SESPMNT & & & & WAHLUKE CP 21 \\
\hline 35 & 85 & & & & SESPMNT & & & & WAHLUKE CP 23 \\
\hline 36 & 86 & & & & SESPMNT & & & & WAHLUKE CP 24 \\
\hline 37 & 87 & & & & SESPMNT & & & & WAHLUKE CP 46 \\
\hline 38 & 88 & & & & SESPMNT & & & & INNER NE SECTOR \\
\hline 39 & 90 & & & & SESPMNT & & COMMUNITY & LOW & WAHLUKE AREA COMP \\
\hline
\end{tabular}




\begin{tabular}{|c|c|c|c|c|c|c|c|c|c|}
\hline & A & $\mathrm{B}$ & $\mathrm{C}$ & $\mathrm{D}$ & $E$ & $\mathrm{~F}$ & $\mathrm{G}$ & $\mathrm{H}$ & $\mathrm{I}$ \\
\hline 1 & SAMP_SITE_ID & HORIZ_COORD_TYPE & EW_COORD & NS_COORD & OWNER_ID & COORD_SOURCE & DIST_CLASS & RAD_LEVEL & SAMP_SITE_NAME \\
\hline 40 & 91 & & & & SESPMNT & & COMMUNITY & LOW & EAST WAHLUKE AREA \\
\hline 41 & 92 & & & & SESPMNT & & COMMUNITY & & NE COMMUNITIES \\
\hline 42 & 93 & & & & SESPMNT & & PERIMETER & & SAGEHILL MET TOWER \\
\hline 43 & & NAD83 & 596840 & 135448 & 3 SESPMNT & & PERIMETER & LOW & RINGOLD MET TOWER \\
\hline 44 & 95 & & & & SESPMNT & & & & WEBBER RANCH \\
\hline 45 & 96 & & & & SESPMNT & & & & BACKGROUND \\
\hline 46 & 97 & & & & SESPMNT & & DISTANT & & OUTER NE SECTOR \\
\hline 47 & 98 & & & & SESPMNT & & DISTANT & & OUTER SE SECTOR \\
\hline 48 & 99 & & & & SESPMNT & & DISTANT & & MOSES LAKE \\
\hline 49 & 100 & & & & SESPMNT & & DISTANT & & MOSES LAKE AREA \\
\hline 50 & 101 & & & & SESPMNT & & DISTANT & LOW & WASHTUCNA \\
\hline 51 & 102 & & & & SESPMNT & & OFFSITE & LOW & MCNARY-OREGON SHORE \\
\hline 52 & 103 & & & & SESPMNT & & OFFSITE & LOW & MCNARY-1/3 OR. SHORE \\
\hline 53 & 104 & & & & SESPMNT & & OFFSITE & LOW & MCNARY-WASH. SHORE \\
\hline 54 & 105 & & & & SESPMNT & & & & SUNNYSIDE NO. 2 \\
\hline 55 & 106 & & & & SESPMNT & & & & J MOON-SUNNYSIDE \\
\hline 56 & 107 & & & & SESPMNT & & DISTANT & LOW & SUNNYSIDE AREA \\
\hline 57 & 108 & & & & SESPMNT & & & & SEQUIM BAY \\
\hline 58 & 109 & & & & SESPMNT & & DISTANT & LOW & YAKIMA VALLEY \\
\hline 59 & 110 & & & & SESPMNT & & DISTANT & LOW & YAKIMA COUNTY \\
\hline 60 & 111 & & & & SESPMNT & & DISTANT & LOW & VANTAGE \\
\hline 61 & 112 & & & & SESPMNT & & & & WAPATO CANAL \\
\hline 62 & 113 & & & & SESPMNT & & DISTANT & LOW & HARRAH/WAPATO AREA \\
\hline 63 & 114 & & & & SESPMNT & & & & CASEYS POND \\
\hline 64 & 115 & & & & SESPMNT & & & & RIMROCK LAKE SOUTH \\
\hline 65 & 116 & & & & SESPMNT & & & & RIMROCK LAKE NORTH \\
\hline 66 & 117 & & & & SESPMNT & & ONSITE & LOW & 200 E AREA \\
\hline 67 & 118 & & & & SESPMNT & & & & 200 E AREA CP 59 \\
\hline 68 & 119 & NAD83 & 575242.59 & 131770.46 & SESPMNT & GPS & ONSITE & LOW & S OF $200 \mathrm{E}$ \\
\hline 69 & 120 & & & & SESPMNT & & ONSITE & & BC CRIB 200 E AREA \\
\hline 70 & 121 & & & & SESPMNT & & & & 216 B 63 DITCH 200 E \\
\hline 71 & 122 & & & & SESPMNT & & ONSITE & LOW & 200 ENC \\
\hline 72 & 123 & NAD83 & 575122.86 & 138052.27 & 7 SESPMNT & GPS & ONSITE & LOW & N OF $200 \mathrm{E}$ \\
\hline 73 & 124 & & & & SESPMNT & & & & 200 EEC \\
\hline 74 & 125 & & & & SESPMNT & & & & 200 EWC \\
\hline 75 & 126 & & & & SESPMNT & & ONSITE & LOW & B POND \\
\hline 76 & 127 & & & & SESPMNT & & & & B POND NO. 2 \\
\hline 77 & 131 & & & & SESPMNT & & & & ACTIVE AREA NO. 2 \\
\hline
\end{tabular}




\begin{tabular}{|c|c|c|c|c|c|c|c|c|c|}
\hline & $\mathrm{A}$ & $\mathrm{B}$ & $\mathrm{C}$ & $\mathrm{D}$ & $E$ & $\mathrm{~F}$ & $G$ & $\mathrm{H}$ & $\mathrm{I}$ \\
\hline 1 & SAMP_SITE_ID & HORIZ_COORD_TYPE & EW_COORD & NS_COORD & OWNER_ID & COORD_SOURCE & DIST_CLASS & RAD_LEVEL & SAMP_SITE_NAME \\
\hline 78 & 132 & & & & SESPMNT & & ONSITE & LOW & 200 PONDS \\
\hline 79 & 133 & & & & SESPMNT & & ONSITE & LOW & EAST \\
\hline 80 & 134 & & & & SESPMNT & & ONSITE & LOW & 400 AREA \\
\hline 81 & 135 & NAD83 & 587673.07 & 122479.88 & SESPMNT & GPS & ONSITE & LOW & $400 \mathrm{~S}$ \\
\hline 82 & 136 & & & & SESPMNT & & ONSITE & LOW & FFTF POND \\
\hline 83 & 137 & & & & SESPMNT & & & & P-15 PUMP \\
\hline 84 & 138 & & & & SESPMNT & & & & P-16 PUMP \\
\hline 85 & 139 & & & & SESPMNT & & & & FFTF SOUTHEAST \\
\hline 86 & 140 & & & & SESPMNT & & ONSITE & LOW & SE SIDE OF FFTF \\
\hline 87 & 141 & & & & SESPMNT & & & & HORN RAPIDS RD \\
\hline 88 & 142 & NAD83 & 587613 & 114926 & SESPMNT & & PERIMETER & LOW & HORN RAPIDS SUBSTA \\
\hline 89 & 143 & & & & SESPMNT & & & & HORN RAPIDS RD MI 12 \\
\hline 90 & 144 & & & & SESPMNT & & ONSITE & LOW & FIRING RANGE \\
\hline 91 & 145 & & & & SESPMNT & & & & GABLE POND INLET \\
\hline 92 & 146 & & & & SESPMNT & & & & GABLE POND N END \\
\hline 93 & 147 & & & & SESPMNT & & ONSITE & LOW & WEST LAKE \\
\hline 94 & 148 & & & & SESPMNT & & & & GABLE MOUNTAIN DUNES \\
\hline 95 & 149 & & & & SESPMNT & & ONSITE & LOW & HANFORD TOWNSITE \\
\hline 96 & 150 & & & & SESPMNT & & & & HANFORD NO. 2 \\
\hline 97 & 151 & & & & SESPMNT & & ONSITE & LOW & HANFORD SPRING 28-2 \\
\hline 98 & 152 & & & & SESPMNT & & & & B-Y PHONE EXCHANGE \\
\hline 99 & 153 & & & & SESPMNT & & ONSITE & LOW & NORTHEAST \\
\hline 100 & 154 & & & & SESPMNT & & & & COL BASIN COMP NO. 3 \\
\hline 101 & 155 & & & & SESPMNT & & PERIMETER & & WPPSS 8; FIR ROAD \\
\hline 102 & 156 & & & & SESPMNT & & PERIMETER & LOW & RINGOLD AREA \\
\hline 103 & 157 & & & & SESPMNT & & & & KINNE FARM-RINGOLD \\
\hline 104 & 158 & & & & SESPMNT & & COMMUNITY & & ELTOPIA \\
\hline 105 & 159 & & & & SESPMNT & & & & ELTOPIA NO. 2 \\
\hline 106 & 160 & & & & SESPMNT & & PERIMETER & & SAGEMOOR-BLEAZARD FARM \\
\hline 107 & 161 & & & & SESPMNT & & PERIMETER & & PETTETT FARM \\
\hline 108 & 162 & & & & SESPMNT & & PERIMETER & LOW & SAGEMOOR AREA \\
\hline 109 & 163 & & & & SESPMNT & & PERIMETER & LOW & SAGEMOOR COMPOSITE \\
\hline 110 & 164 & & & & SESPMNT & & & & TAYLOR FLATS NO. 1 \\
\hline 111 & 165 & & & & SESPMNT & & & & BYERS LND PUMP HOUSE \\
\hline 112 & 166 & NAD83 & 596370.54 & 114721.18 & SESPMNT & GPS & PERIMETER & LOW & BYERS LANDING \\
\hline 113 & 167 & & & & SESPMNT & & & & RIVERVIEW CP 55 \\
\hline 114 & 168 & & & & SESPMNT & & PERIMETER & LOW & RIVRVIEW-HARRIS \\
\hline 115 & 169 & & & & SESPMNT & & COMMUNITY & LOW & RIVERVIEW AREA \\
\hline
\end{tabular}




\begin{tabular}{|c|c|c|c|c|c|c|c|c|c|}
\hline & A & $\mathrm{B}$ & $\mathrm{C}$ & $\mathrm{D}$ & $E$ & $\mathrm{~F}$ & G & $\mathrm{H}$ & $\mathrm{I}$ \\
\hline 1 & SAMP_SITE_ID & |HORIZ_COORD_TYPE & EW_COORD & NS_COORD & OWNER_ID & COORD_SOURCE & DIST_CLASS & RAD_LEVEL & SAMP_SITE_NAME \\
\hline 116 & 170 & & & & SESPMNT & & & & HARVEY OLSEN FARM \\
\hline 117 & 171 & & & & SESPMNT & & & & PASCO MEAT PACKERS \\
\hline 118 & 172 & & & & SESPMNT & & & & WHITE BLUFFS ASSOC \\
\hline 119 & 173 & & & & SESPMNT & & OFFSITE & LOW & WHITE BLUFFS-SHALLOW \\
\hline 120 & 174 & & & & SESPMNT & & OFFSITE & LOW & WHITE BLUFFS-DEEP \\
\hline 121 & 175 & NAD83 & 600157.25 & 122785.61 & SESPMNT & GPS & COMMUNITY & LOW & EDWIN MARKHAM SCHOOL \\
\hline 122 & 176 & NAD83 & 603134.64 & 141087.42 & SESPMNT & GPS & COMMUNITY & LOW & BASIN CITY SCHOOL \\
\hline 123 & 177 & NAD83 & 577137 & 105235 & SESPMNT & SURVEYED & COMMUNITY & LOW & BENTON CITY \\
\hline 124 & 178 & & & & SESPMNT & & COMMUNITY & & BENTON CITY COMP \\
\hline 125 & 179 & & & & SESPMNT & & & & KELLY-BENTON CITY \\
\hline 126 & 180 & & & & SESPMNT & & & & RHOADES-BENTON CITY \\
\hline 127 & 181 & & & & SESPMNT & & & & TOWLE-BENTON CITY \\
\hline 128 & 182 & & & & SESPMNT & & COMMUNITY & LOW & BENTON CITY AREA \\
\hline 129 & 183 & & & & SESPMNT & & & & INNER SW SECTOR \\
\hline 130 & 184 & & & & SESPMNT & & & & ERC \\
\hline 131 & 185 & & & & SESPMNT & & & & ERC NO.2 \\
\hline 132 & 186 & & & & SESPMNT & & ONSITE & LOW & ALE HDQTRS \\
\hline 133 & 187 & & & & SESPMNT & & ONSITE & LOW & OBSEVATORY-RTLSNK MT \\
\hline 134 & 188 & NAD83 & 562783.692 & 131376.289 & SESPMNT & GPS & PERIMETER & LOW & RATTLESNAKE SPRINGS \\
\hline 135 & 189 & & & & SESPMNT & & PERIMETER & LOW & COLD CREEK AREA \\
\hline 136 & 190 & & & & SESPMNT & & & & PROSSER \\
\hline 137 & 191 & & & & SESPMNT & & PERIMETER & & WEST PERIMETER \\
\hline 138 & 192 & & & & SESPMNT & & ONSITE & LOW & 100 AREA FIRE STAT \\
\hline 139 & 193 & & & & SESPMNT & & ONSITE & LOW & 100-D SPRING \\
\hline 140 & 194 & & & & SESPMNT & & & & $100 \mathrm{D}$ TO $100 \mathrm{~F}$ \\
\hline 141 & 195 & & & & SESPMNT & & ONSITE & LOW & 100 F SLOUGH \\
\hline 142 & 196 & & & & SESPMNT & & ONSITE & LOW & 100-F SPRING \\
\hline 143 & 197 & & & & SESPMNT & & & & $141 \mathrm{~F}$ BLDG 100F AREA \\
\hline 144 & 199 & & & & SESPMNT & & ONSITE & LOW & 100-H SPRING \\
\hline 145 & 200 & & & & SESPMNT & & ONSITE & LOW & $100 \mathrm{~K}$ AREA \\
\hline 146 & 201 & & & & SESPMNT & & ONSITE & LOW & 100 N AREA \\
\hline 147 & 202 & & & & SESPMNT & & ONSITE & LOW & $100-N$ SPRING 8-13 \\
\hline 148 & 203 & & & & SESPMNT & & & & 181 N BLDG 100N AREA \\
\hline 149 & 204 & & & & SESPMNT & & & & NE CORNER 100 N AREA \\
\hline 150 & 205 & & & & SESPMNT & & ONSITE & LOW & E OF 100 N AREA \\
\hline 151 & 206 & & & & SESPMNT & & & & $100 \mathrm{~N}$ TRENCH \\
\hline 152 & 207 & & & & SESPMNT & & ONSITE & LOW & NEAR $100-N$ \\
\hline 153 & 208 & & & & SESPMNT & & ONSITE & & 100 N-WPPSS AREA \\
\hline
\end{tabular}




\begin{tabular}{|c|c|c|c|c|c|c|c|c|c|}
\hline & A & $\mathrm{B}$ & $\mathrm{C}$ & $\mathrm{D}$ & $E$ & $\mathrm{~F}$ & G & $\mathrm{H}$ & $\mathrm{I}$ \\
\hline 1 & SAMP_SITE_ID & HORIZ_COORD_TYPE & EW_COORD & NS_COORD & OWNER_ID & COORD_SOURCE & DIST_CLASS & RAD_LEVEL & SAMP_SITE_NAME \\
\hline 154 & 209 & NAD83 & 572008 & 149597 & SESPMNT & & ONSITE & LOW & $100 \mathrm{~N}-1325$ CRIB \\
\hline 155 & 210 & & & & SESPMNT & & & & SE OF $100 \mathrm{~N}$ TRENCH \\
\hline 156 & 211 & & & & SESPMNT & & ONSITE & & 100 N SPRING \\
\hline 157 & 212 & & & & SESPMNT & & & & RT. 2N MI. 8 \\
\hline 158 & 213 & & & & SESPMNT & & & & INNER EASTERN SECTOR \\
\hline 159 & 214 & NAD83 & 572387.57 & 134747.83 & SESPMNT & GPS & ONSITE & & US ECOLOGY NE CORNER \\
\hline 160 & 215 & NAD83 & 571942.28 & 134750.42 & SESPMNT & GPS & ONSITE & & US ECOLOGY NW CORNER \\
\hline 161 & 216 & NAD83 & 571944.74 & 133836.57 & SESPMNT & GPS & ONSITE & & US ECOLOGY SW CORNER \\
\hline 162 & 217 & & & & SESPMNT & & & & E SIDE US ECOLOGY BG \\
\hline 163 & 218 & NAD83 & 576339.63 & 134592.47 & SESPMNT & GPS & ONSITE & LOW & 200 ESE \\
\hline 164 & 219 & & & & SESPMNT & & ONSITE & LOW & WYE BARRICADE \\
\hline 165 & 220 & & & & SESPMNT & & & & WYE BARR. NO. 2 \\
\hline 166 & 221 & & & & SESPMNT & & & & RT. 10 MILE 1.6 \\
\hline 167 & 222 & & & & SESPMNT & & & & 331 FARMS-300 AREA \\
\hline 168 & 223 & & & & SESPMNT & & & & MERRILL FARM-W RICH. \\
\hline 169 & 224 & & & & SESPMNT & & & & RIDERS MEAT-RICHLAND \\
\hline 170 & 225 & & & & SESPMNT & & & & GABLE POND A \\
\hline 171 & 226 & & & & SESPMNT & & & & WEST LAKE A \\
\hline 172 & 227 & & & & SESPMNT & & & & FFTF POND A \\
\hline 173 & 228 & & & & SESPMNT & & & & FFTF POND B \\
\hline 174 & 229 & & & & SESPMNT & & & & $600 \mathrm{~A}$ \\
\hline 175 & 230 & & & & SESPMNT & & & & $600 \mathrm{~B}$ \\
\hline 176 & 231 & & & & SESPMNT & & & & $600 \mathrm{D}$ \\
\hline 177 & 232 & & & & SESPMNT & & & & $600 \mathrm{G}$ \\
\hline 178 & 233 & & & & SESPMNT & & ONSITE & LOW & $600 \mathrm{H}$ \\
\hline 179 & 234 & & & & SESPMNT & & ONSITE & LOW & 6001 \\
\hline 180 & 235 & & & & SESPMNT & & PERIMETER & LOW & BASIN FARM B \\
\hline 181 & 236 & & & & SESPMNT & & & & VALLEY FARM A \\
\hline 182 & 237 & & & & SESPMNT & & & & VALLEY FARM B \\
\hline 183 & 238 & & & & SESPMNT & & & & FARM B \\
\hline 184 & 239 & & & & SESPMNT & & & & FARM C \\
\hline 185 & 240 & & & & SESPMNT & & & & FARM E \\
\hline 186 & 241 & & & & SESPMNT & & & & FARM F \\
\hline 187 & 242 & & & & SESPMNT & & & & FARM G \\
\hline 188 & 243 & & & & SESPMNT & & & & $600 \mathrm{~W}$ \\
\hline 189 & 246 & & & & SESPMNT & & & & $600 \mathrm{Y}$ \\
\hline 190 & 247 & & & & SESPMNT & & OFFSITE & LOW & UPPER REACH RIVER \\
\hline 191 & 248 & & & & SESPMNT & & & & 600 Y NO. 2 \\
\hline
\end{tabular}




\begin{tabular}{|c|c|c|c|c|c|c|c|c|c|}
\hline & $\mathrm{A}$ & $\mathrm{B}$ & $\mathrm{C}$ & $\mathrm{D}$ & $E$ & $\mathrm{~F}$ & $G$ & $\mathrm{H}$ & $\mathrm{I}$ \\
\hline 1 & SAMP_SITE_ID & HORIZ_COORD_TYPE & EW_COORD & NS_COORD & OWNER_ID & COORD_SOURCE & DIST_CLASS & RAD_LEVEL & SAMP_SITE_NAME \\
\hline 192 & 249 & & & & SESPMNT & & PERIMETER & LOW & FRANKLIN FARM B \\
\hline 193 & 250 & & & & SESPMNT & & OFFSITE & & PRIEST RAPIDS HATCH. \\
\hline 194 & 251 & & & & SESPMNT & & & & ABOVE PRIEST RAPIDS \\
\hline 195 & 252 & & & & SESPMNT & & OFFSITE & LOW & PRIEST RAPIDS-RIVER \\
\hline 196 & 253 & & & & SESPMNT & & & & BELOW PRIEST RAPIDS \\
\hline 197 & 254 & & & & SESPMNT & & OFFSITE & LOW & PRD-GRANT COUNTY \\
\hline 198 & 255 & & & & SESPMNT & & OFFSITE & LOW & PRD-2/3 GRANT SHORE \\
\hline 199 & 256 & & & & SESPMNT & & OFFSITE & LOW & PRD-YAKIMA CNTY SHOR \\
\hline 200 & 257 & NAD83 & 563649.77 & 145601.8 & SESPMNT & GPS & RIVER_SHORELINE & & ABOVE 100 B AREA \\
\hline 201 & 258 & & & & SESPMNT & & RIVER_SHORELINE & & COYOTE RAPIDS \\
\hline 202 & 259 & NAD83 & 568226.88 & 146651.39 & SESPMNT & GPS & RIVER_SHORELINE & & ABOVE 1K BOAT RAMP \\
\hline 203 & 260 & & & & SESPMNT & & & & ABOVE $181 \mathrm{KW}-\mathrm{RIV}$ \\
\hline 204 & 261 & & & & SESPMNT & & & & K-AREA PONDS \\
\hline 205 & 262 & & & & SESPMNT & & & & BELOW 181KE FAR SHOR \\
\hline 206 & 263 & & & & SESPMNT & & & & BELOW 181 KE-RIV \\
\hline 207 & 264 & & & & SESPMNT & & & & 100 N AREA-RIVER \\
\hline 208 & 265 & & & & SESPMNT & & ONSITE & LOW & 100N SHORE ABOVE HGP \\
\hline 209 & 266 & & & & SESPMNT & & ONSITE & LOW & 100N SPRING SHORELIN \\
\hline 210 & 267 & NAD83 & 571059.35 & 149586.25 & SESPMNT & GPS & RIVER_SHORELINE & LOW & BELOW 100N OUTFALL \\
\hline 211 & 268 & & & & SESPMNT & & ONSITE & LOW & ABOVE 100D PUMPHOUSE \\
\hline 212 & 269 & & & & SESPMNT & & & & BELOW 100N TRENCH \\
\hline 213 & 270 & & & & SESPMNT & & ONSITE & LOW & 300 AREA SHORELINE \\
\hline 214 & 271 & NAD83 & 571147.61 & 149704.58 & SESPMNT & GPS & RIVER_SHORELINE & LOW & BELOW 100N STACK \\
\hline 215 & 272 & NAD83 & 571168.09 & 149739.03 & SESPMNT & GPS & RIVER_SHORELINE & LOW & ABOVE TIP 100N BERM \\
\hline 216 & 273 & & & & SESPMNT & & & & BELOW $181 \mathrm{~N}$ \\
\hline 217 & 274 & & & & SESPMNT & & ONSITE & LOW & HANFRD TWNSITE HRM28 \\
\hline 218 & 275 & & & & SESPMNT & & & & ABOVE 100 D AREA \\
\hline 219 & 276 & & & & SESPMNT & & ONSITE & LOW & ABOVE 181D FAR SHORE \\
\hline 220 & 277 & & & & SESPMNT & & & & 100 D RESERVOIR \\
\hline 221 & 278 & & & & SESPMNT & & ONSITE & & 100 D AREA-RIVER \\
\hline 222 & 279 & NAD83 & 573465.13 & 153322.77 & SESPMNT & GPS & RIVER_SHORELINE & & BELOW OPP $100 \mathrm{D}$ \\
\hline 223 & 280 & NAD83 & 573497.81 & 152739.19 & SESPMNT & GPS & RIVER_SHORELINE & & 100-D ISLAND \\
\hline 224 & 281 & & & & SESPMNT & & & & BELOW 100-D ISLAND \\
\hline 225 & 282 & & & & SESPMNT & & RIVER_SHORELINE & LOW & $100 \mathrm{H}$ AREA \\
\hline 226 & 283 & & & & SESPMNT & & & & LOCKE ISLAND P SHORE \\
\hline 227 & 284 & NAD83 & 580220.26 & 151412.93 & SESPMNT & GPS & RIVER_SHORELINE & & LO END LOCKE ISL \\
\hline 228 & 285 & & & & SESPMNT & & & & WHITE BLUFFS-RIVER \\
\hline 229 & 286 & NAD83 & 579595.07 & 149417.16 & SESPMNT & GPS & RIVER_SHORELINE & & WHITE BLUFFS FY LND. \\
\hline
\end{tabular}




\begin{tabular}{|c|c|c|c|c|c|c|c|c|c|}
\hline & A & $\mathrm{B}$ & $\mathrm{C}$ & $\mathrm{D}$ & $E$ & $\mathrm{~F}$ & $\mathrm{G}$ & $\mathrm{H}$ & $\mathrm{I}$ \\
\hline 1 & SAMP_SITE_ID & HORIZ_COORD_TYPE & EW_COORD & NS_COORD & OWNER_ID & COORD_SOURCE & DIST_CLASS & RAD_LEVEL & SAMP_SITE_NAME \\
\hline 230 & 287 & & & & SESPMNT & & & & WHITE BLUFFS F SHORE \\
\hline 231 & 288 & & & & SESPMNT & & & & $100 \mathrm{~F}$ BARGE \\
\hline 232 & 289 & NAD83 & 581155.94 & 147998.52 & SESPMNT & GPS & RIVER_SHORELINE & & BELOW $100 \mathrm{~F}$ \\
\hline 233 & 290 & & & & SESPMNT & & & & HANFORD TOWNSITE-RIV \\
\hline 234 & 291 & & & & SESPMNT & & & & HANFORD FAR SHORE \\
\hline 235 & 292 & NAD83 & 586721.84 & 139881.4 & SESPMNT & GPS & RIVER_SHORELINE & & HANFORD FERRY LND. \\
\hline 236 & 293 & NAD83 & 586846 & 139098 & SESPMNT & SURVEYED & RIVER SHORELINE & & HANFORD RR TRACK \\
\hline 237 & 294 & NAD83 & 590292.12 & 137256.32 & SESPMNT & GPS & & & SAVAGE ISLAND \\
\hline 238 & 295 & & & & SESPMNT & & RIVER_SHORELINE & & SAVAGE ISL SLOUGH \\
\hline 239 & 296 & NAD83 & 594369.07 & 132003.26 & SESPMNT & GPS & RIVER_SHORELINE & & RINGOLD ISLAND \\
\hline 240 & 297 & & & & SESPMNT & & & & RINGOLD-RIVER \\
\hline 241 & 298 & & & & SESPMNT & & & & LOWER RIVER \\
\hline 242 & 299 & & & & SESPMNT & & & & POWER LINE FAR SHORE \\
\hline 243 & 300 & NAD83 & 594815.25 & 124643.79 & SESPMNT & GPS & RIVER_SHORELINE & & N END WOODED ISLAND \\
\hline 244 & 301 & NAD83 & 594892.43 & 121902.41 & SESPMNT & GPS & RIVER_SHORELINE & & S END WOODED ISLAND \\
\hline 245 & 302 & & & & SESPMNT & & & & SOUTH OF WOODED ISL \\
\hline 246 & 303 & & & & SESPMNT & & & & SHOT ROCK ISL. OLD \\
\hline 247 & 304 & & & & SESPMNT & & & & SHOT ROCK ISL. DEEP \\
\hline 248 & 305 & & & & SESPMNT & & & & ABOVE 300 TRAVERSE \\
\hline 249 & 306 & & & & SESPMNT & & & & 300 RIVERBANK SPR 1 \\
\hline 250 & 307 & & & & SESPMNT & & ONSITE & LOW & 300 AREA \\
\hline 251 & 308 & NAD83 & 594718.82 & 117290.44 & SESPMNT & GPS & RIVER_SHORELINE & & ISLND ABOVE 300 AREA \\
\hline 252 & 309 & NAD83 & 595169.52 & 115109.53 & SESPMNT & GPS & RIVER_SHORELINE & & ISLAND NEAR 300 AREA \\
\hline 253 & 310 & & & & SESPMNT & & & & PORT OF BENTON SPR \\
\hline 254 & 311 & & & & SESPMNT & & RIVER_SHORELINE & LOW & RICHLAND-RIVER \\
\hline 255 & 312 & & & & SESPMNT & & OFFSITE & LOW & RICHLAND NO. 2 \\
\hline 256 & 313 & & & & SESPMNT & & & & ISLAND 340-RICH-RIV \\
\hline 257 & 314 & & & & SESPMNT & & & & SACAJAWEA-RIVER \\
\hline 258 & 315 & & & & SESPMNT & & & & BURBANK-RIVER \\
\hline 259 & 316 & & & & SESPMNT & & & & HOVER-RIVER \\
\hline 260 & 317 & & & & SESPMNT & & OFFSITE & LOW & VERNITA-1 HRM 0.3 \\
\hline 261 & 318 & & & & SESPMNT & & OFFSITE & LOW & VERNITA-3 HRM 0.3 \\
\hline 262 & 319 & & & & SESPMNT & & OFFSITE & LOW & VERNITA-4 HRM 0.3 \\
\hline 263 & 320 & & & & SESPMNT & & & & RICH. PUMPHOUSE -2B \\
\hline 264 & 321 & & & & SESPMNT & & OFFSITE & LOW & RICH.PMPHS-2 HRM46.4 \\
\hline 265 & 322 & & & & SESPMNT & & OFFSITE & LOW & RICH.PMPHS-3 HRM46.4 \\
\hline 266 & 323 & & & & SESPMNT & & OFFSITE & LOW & RICH.PMPHS-5 HRM46.4 \\
\hline 267 & 324 & & & & SESPMNT & & OFFSITE & LOW & RICH.PMPHS-6 HRM46.4 \\
\hline
\end{tabular}




\begin{tabular}{|c|c|c|c|c|c|c|c|c|c|}
\hline & $\mathrm{A}$ & $\mathrm{B}$ & $\mathrm{C}$ & $\mathrm{D}$ & $E$ & $\mathrm{~F}$ & $\mathrm{G}$ & $\mathrm{H}$ & $\mathrm{I}$ \\
\hline 1 & SAMP_SITE_ID & HORIZ_COORD_TYPE & EW_COORD & NS_COORD & OWNER_ID & COORD_SOURCE & DIST_CLASS & RAD_LEVEL & SAMP_SITE_NAME \\
\hline 268 & 325 & & & & SESPMNT & & OFFSITE & LOW & RICH.PMPHS-7 HRM46.4 \\
\hline 269 & 326 & & & & SESPMNT & & OFFSITE & LOW & RICH.PMPHS-8 HRM46.4 \\
\hline 270 & 327 & & & & SESPMNT & & OFFSITE & LOW & RICH.PMPHS-10 HRM46.4 \\
\hline 271 & 328 & & & & SESPMNT & & ONSITE & LOW & 100 N -1 HRM 9.5 \\
\hline 272 & 329 & & & & SESPMNT & & ONSITE & LOW & 100 N -2 HRM 9.5 \\
\hline 273 & 330 & & & & SESPMNT & & ONSITE & LOW & 100 N -3 HRM 9.5 \\
\hline 274 & 331 & & & & SESPMNT & & ONSITE & LOW & 100 N -5 HRM 9.5 \\
\hline 275 & 332 & & & & SESPMNT & & ONSITE & LOW & 100 N -6 HRM 9.5 \\
\hline 276 & 333 & & & & SESPMNT & & ONSITE & LOW & 100 N -7 HRM 9.5 \\
\hline 277 & 334 & & & & SESPMNT & & ONSITE & LOW & 100 N -9 HRM 9.5 \\
\hline 278 & 335 & & & & SESPMNT & & ONSITE & LOW & $100 \mathrm{~N}-10$ HRM 9.5 \\
\hline 279 & 336 & & & & SESPMNT & & ONSITE & LOW & 100 F -1 HRM 19.0 \\
\hline 280 & 337 & & & & SESPMNT & & ONSITE & LOW & 100 F -3 HRM 19.0 \\
\hline 281 & 338 & & & & SESPMNT & & ONSITE & LOW & 100 F -4 HRM 19.0 \\
\hline 282 & 339 & & & & SESPMNT & & ONSITE & LOW & $100 \mathrm{~F}-7$ HRM 19.0 \\
\hline 283 & 340 & & & & SESPMNT & & ONSITE & LOW & 100 F -8 HRM 19.0 \\
\hline 284 & 341 & & & & SESPMNT & & ONSITE & LOW & 100 F -10 HRM 19.0 \\
\hline 285 & 342 & & & & SESPMNT & & ONSITE & LOW & HANFRD TS-2 HRM 28.7 \\
\hline 286 & 343 & & & & SESPMNT & & ONSITE & LOW & HANFRD TS-4 HRM 28.7 \\
\hline 287 & 344 & & & & SESPMNT & & ONSITE & LOW & HANFRD TS-5 HRM 28.7 \\
\hline 288 & 345 & & & & SESPMNT & & ONSITE & LOW & HANFRD TS-6 HRM 28.7 \\
\hline 289 & 346 & & & & SESPMNT & & ONSITE & LOW & HANFRD TS-9 HRM 28.7 \\
\hline 290 & 347 & & & & SESPMNT & & ONSITE & LOW & HANFRD TS-10 HRM 28.7 \\
\hline 291 & 348 & & & & SESPMNT & & ONSITE & LOW & 300 AREA -1 HRM 43.1 \\
\hline 292 & 349 & & & & SESPMNT & & ONSITE & LOW & 300 AREA -4 HRM 43.1 \\
\hline 293 & 350 & & & & SESPMNT & & ONSITE & LOW & 300 AREA -6 HRM 43.1 \\
\hline 294 & 351 & & & & SESPMNT & & ONSITE & LOW & 300 AREA -7 HRM 43.1 \\
\hline 295 & 352 & & & & SESPMNT & & ONSITE & LOW & 300 AREA-10 HRM 43.1 \\
\hline 296 & 353 & & & & SESPMNT & & & & $100 \mathrm{~N}-0 \mathrm{~B}$ \\
\hline 297 & 354 & & & & SESPMNT & & & & $100 \mathrm{~N}-2 \mathrm{~B}$ \\
\hline 298 & 355 & & & & SESPMNT & & & & $100 \mathrm{~N}-3 \mathrm{~B}$ \\
\hline 299 & 356 & & & & SESPMNT & & & & $100 \mathrm{~N}-4 \mathrm{~B}$ \\
\hline 300 & 357 & & & & SESPMNT & & & & $100 \mathrm{~N}-6 \mathrm{~B}$ \\
\hline 301 & 358 & & & & SESPMNT & & & & $100 \mathrm{~N}-7 \mathrm{~B}$ \\
\hline 302 & 359 & & & & SESPMNT & & & & $100 \mathrm{~N}-8 \mathrm{~B}$ \\
\hline 303 & 360 & & & & SESPMNT & & & & $100 N-10 B$ \\
\hline 304 & 361 & & & & SESPMNT & & & & $100 N-11 B$ \\
\hline 305 & 362 & & & & SESPMNT & & & & HANFORD TOWNSITE-OB \\
\hline
\end{tabular}




\begin{tabular}{|c|c|c|c|c|c|c|c|c|c|}
\hline & A & $\mathrm{B}$ & $\mathrm{C}$ & $\mathrm{D}$ & $E$ & $\mathrm{~F}$ & $\mathrm{G}$ & $\mathrm{H}$ & $\mathrm{I}$ \\
\hline 1 & SAMP_SITE_ID & |HORIZ_COORD_TYPE & EW_COORD & NS_COORD & OWNER_ID & COORD_SOURCE & DIST_CLASS & RAD_LEVEL & SAMP_SITE_NAME \\
\hline 306 & 363 & & & & SESPMNT & & & & HANFORD TOWNSITE-2B \\
\hline 307 & 364 & & & & SESPMNT & & & & HANFORD TOWNSITE-3B \\
\hline 308 & 365 & & & & SESPMNT & & & & HANFORD TOWNSITE-4B \\
\hline 309 & 366 & & & & SESPMNT & & & & HANFORD TOWNSITE-6B \\
\hline 310 & 367 & & & & SESPMNT & & & & HANFORD TOWNSITE-7B \\
\hline 311 & $36 \varepsilon$ & & & & SESPMNT & & & & HANFORD TOWNSITE-8B \\
\hline 312 & 369 & & & & SESPMNT & & & & HANFORD TOWNSITE-9B \\
\hline 313 & 370 & & & & SESPMNT & & & & RT. 2S MI. 5 \\
\hline 314 & 371 & & & & SESPMNT & & & & RT. 4S MI. 7 \\
\hline 315 & 372 & & & & SESPMNT & & ONSITE & LOW & RT. 4S MI. 13 \\
\hline 316 & 373 & & & & SESPMNT & & & & RT. 4S MI. 19 \\
\hline 317 & 374 & & & & SESPMNT & & & & WPPSS 2; N OF WNP 2 \\
\hline 318 & 375 & & & & SESPMNT & & & & WPPSS 3; SE OF WNP 2 \\
\hline 319 & 376 & & & & SESPMNT & & & & N 1 MI. WPPSS NO. 2 \\
\hline 320 & 377 & & & & SESPMNT & & & & N 3 MI. WPPSS NO.2 \\
\hline 321 & 378 & & & & SESPMNT & & ONSITE & LOW & SPECIAL \\
\hline 322 & 379 & & & & SESPMNT & & PERIMETER & & 1100 AREA \\
\hline 323 & 380 & & & & SESPMNT & & & & NE CORNER EXXON SITE \\
\hline 324 & 381 & & & & SESPMNT & & & & RRC CP 64 \\
\hline 325 & 382 & & & & SESPMNT & & & & RRC CP 66 \\
\hline 326 & 383 & & & & SESPMNT & & ONSITE & LOW & BATTELLE FIELD \\
\hline 327 & 384 & & & & SESPMNT & & & & NORTH RICHLAND CP 56 \\
\hline 328 & 385 & & & & SESPMNT & & & & RICHLAND SAN PLANT \\
\hline 329 & 386 & NAD83 & 595399.47 & 109822.91 & SESPMNT & GPS & COMMUNITY & LOW & LESLIE GROVES-RCHLND \\
\hline 330 & 387 & & & & SESPMNT & & & & W RICH-BENTON CITY \\
\hline 331 & 388 & & & & SESPMNT & & & & DINNEEN-WEST RICH \\
\hline 332 & 389 & NAD83 & 603513.23 & 96444.79 & SESPMNT & GPS & COMMUNITY & LOW & KENNEWICK-ELY STREET \\
\hline 333 & 390 & & & & SESPMNT & & & & SAFEWAY \\
\hline 334 & 391 & & & & SESPMNT & & & & LUCERNE \\
\hline 335 & 392 & & & & SESPMNT & & & & DARIGOLD-ARDEN \\
\hline 336 & 393 & & & & SESPMNT & & & & TRI CITY \\
\hline 337 & 394 & & & & SESPMNT & & & & INNER NW SECTOR \\
\hline 338 & 395 & & & & SESPMNT & & PERIMETER & LOW & N END VERNITA BRIDGE \\
\hline 339 & 396 & NAD83 & 558790.136 & 145412.504 & SESPMNT & GPS & PERIMETER & & S END VERNITA BRIDGE \\
\hline 340 & 397 & & & & SESPMNT & & & & WAHLUKE CP 18 \\
\hline 341 & 398 & & & & SESPMNT & & & & WAHLUKE CP 19 \\
\hline 342 & 399 & & & & SESPMNT & & & & WAHLUKE OPP $100 \mathrm{~N}$ \\
\hline 343 & 400 & NAD83 & 546087.94 & 156108.63 & SESPMNT & GPS & COMMUNITY & LOW & MATTAWA \\
\hline
\end{tabular}




\begin{tabular}{|c|c|c|c|c|c|c|c|c|c|}
\hline & A & $\mathrm{B}$ & $\mathrm{C}$ & $\mathrm{D}$ & $\mathrm{E}$ & $\mathrm{F}$ & $\mathrm{G}$ & $\mathrm{H}$ & $\mathrm{I}$ \\
\hline 1 & SAMP_SITE_ID & HORIZ_COORD_TYPE & EW_COORD & NS_COORD & OWNER_ID & COORD_SOURCE & DIST_CLASS & RAD_LEVEL & SAMP_SITE_NAME \\
\hline 344 & 401 & & & & SESPMNT & & & & MATTAWA NO. 2 \\
\hline 345 & 402 & & & & SESPMNT & & COMMUNITY & LOW & MATTAWA AREA \\
\hline 346 & 403 & NAD83 & 571133.84 & 135791.27 & 7 SESPMNT & GPS & ONSITE & LOW & 200 TEL. EXCHANGE \\
\hline 347 & 404 & & & & SESPMNT & & ONSITE & LOW & 200 W AREA \\
\hline 348 & 405 & & & & SESPMNT & & ONSITE & LOW & 200 W SOUTH EAST \\
\hline 349 & 406 & & & & SESPMNT & & & & 200 W GUARDHOUSE \\
\hline 350 & 407 & & & & SESPMNT & & & & 200 WNE \\
\hline 351 & 408 & & & & SESPMNT & & & & 200 WWC \\
\hline 352 & 409 & & & & SESPMNT & & ONSITE & LOW & E OF 200 W GATE \\
\hline 353 & 410 & & & & SESPMNT & & & & 242-S BLDG. $200 \mathrm{~W}$ \\
\hline 354 & 411 & & & & SESPMNT & & & & METEOROLOGY STATION \\
\hline 355 & 412 & & & & SESPMNT & & & & U POND \\
\hline 356 & 413 & & & & SESPMNT & & & & T POND \\
\hline 357 & 414 & & & & SESPMNT & & & & 231 DITCH OUTLT 200W \\
\hline 358 & 415 & & & & SESPMNT & & & & 200W LDRY DTCH OUTLT \\
\hline 359 & 416 & & & & SESPMNT & & & & RT. 3 MI. 4 \\
\hline 360 & 417 & & & & SESPMNT & & ONSITE & LOW & S OF $200 \mathrm{~W}$ \\
\hline 361 & 418 & NAD83 & 568520.05 & 132116.22 & SESPMNT & GPS & ONSITE & LOW & ARMY LOOP CAMP \\
\hline 362 & 419 & & & & SESPMNT & & ONSITE & LOW & SW OF B/C CRIBS \\
\hline 363 & 420 & & & & SESPMNT & & & & 331 ANIMAL WSTE SEWR \\
\hline 364 & 421 & NAD83 & 594549.68 & 115798.52 & 2 SESPMNT & GPS & ONSITE & LOW & 300 WATER INTAKE \\
\hline 365 & 422 & NAD83 & 594351.67 & 115183.71 & SESPMNT & GPS & & & 300 S GATE NO.2 \\
\hline 366 & 423 & & & & SESPMNT & & & & 300 NE NO. 2 \\
\hline 367 & 424 & & & & SESPMNT & & PERIMETER & LOW & BERG RANCH \\
\hline 368 & 425 & & & & SESPMNT & & & & OUTER WESTERN SECTOR \\
\hline 369 & 426 & & & & SESPMNT & & DISTANT & LOW & WALLA WALLA \\
\hline 370 & 427 & & & & SESPMNT & & OFFSITE & LOW & MCNARY-2/3 OR. SHORE \\
\hline 371 & 428 & & & & SESPMNT & & & & ROSA IRR D. SUNNYSID \\
\hline 372 & 429 & & & & SESPMNT & & DISTANT & LOW & YAKIMA \\
\hline 373 & 430 & & & & SESPMNT & & & & $600 \mathrm{~N}$ \\
\hline 374 & 431 & & & & SESPMNT & & ONSITE & & 600 AREA \\
\hline 375 & 432 & & & & SESPMNT & & & & RT. 4S MI. 9 \\
\hline 376 & 433 & NAD83 & 586943.19 & 123071.24 & 4 SESPMNT & GPS & ONSITE & LOW & $400 \mathrm{~W}$ \\
\hline 377 & 434 & & & & SESPMNT & & & & P-14 PUMP \\
\hline 378 & 435 & & & & SESPMNT & & & & FFTF NORTH \\
\hline 379 & 436 & & & & SESPMNT & & ONSITE & LOW & SOUTHEAST \\
\hline 380 & 437 & NAD83 & 597052.45 & 121212.99 & 9 SESPMNT & GPS & PERIMETER & LOW & DOGWOOD MET TOWER \\
\hline 381 & 438 & & & & SESPMNT & & PERIMETER & LOW & TAYLOR FLATS NO. 2 \\
\hline
\end{tabular}




\begin{tabular}{|c|c|c|c|c|c|c|c|c|c|}
\hline & A & $\mathrm{B}$ & $\mathrm{C}$ & $\mathrm{D}$ & $E$ & $\mathrm{~F}$ & G & $\mathrm{H}$ & $\mathrm{I}$ \\
\hline 1 & SAMP_SITE_ID & HORIZ_COORD_TYPE & EW_COORD & NS_COORD & OWNER_ID & COORD_SOURCE & DIST_CLASS & RAD_LEVEL & SAMP_SITE_NAME \\
\hline 382 & 439 & & & & SESPMNT & & & & EVA HALL FARM \\
\hline 383 & 440 & & & & SESPMNT & & OFFSITE & LOW & RIVERVIEW CANAL \\
\hline 384 & 441 & & & & SESPMNT & & PERIMETER & & EAST PERIMETER \\
\hline 385 & 442 & & & & SESPMNT & & ONSITE & LOW & 100 AREAS \\
\hline 386 & 443 & & & & SESPMNT & & ONSITE & LOW & 100-D TO 100-H \\
\hline 387 & 444 & & & & SESPMNT & & & & $100 \mathrm{~F}$ TRENCH \\
\hline 388 & 445 & & & & SESPMNT & & & & 100 F CP 58 \\
\hline 389 & 446 & & & & SESPMNT & & ONSITE & LOW & $100-$ H TO $100-F$ \\
\hline 390 & 447 & & & & SESPMNT & & & & ARMY LOOP ROAD CP 40 \\
\hline 391 & 448 & & & & SESPMNT & & & & PROSSER BARR COMP \\
\hline 392 & 449 & & & & SESPMNT & & COMMUNITY & LOW & TRI CITIES \\
\hline 393 & 450 & & & & SESPMNT & & & & WEST LAKE B \\
\hline 394 & 451 & & & & SESPMNT & & & & B POND A \\
\hline 395 & 452 & & & & SESPMNT & & & & $600 \mathrm{C}$ \\
\hline 396 & 453 & & & & SESPMNT & & PERIMETER & LOW & FRANKLIN FARM A \\
\hline 397 & 454 & & & & SESPMNT & & OFFSITE & & PRIEST RAPIDS DAM \\
\hline 398 & 455 & & & & SESPMNT & & & & PR DAM-VERNITA BRIDG \\
\hline 399 & 456 & & & & SESPMNT & & OFFSITE & LOW & VERNITA \\
\hline 400 & 457 & NAD83 & 565481.4 & 145456.03 & SESPMNT & GPS & RIVER_SHORELINE & & BELOW 100B RET BASIN \\
\hline 401 & 458 & & & & SESPMNT & & & & $100 \mathrm{~K}$ BARGE \\
\hline 402 & 450 & & & & SESPMNT & & & & E ISLAND FAR SHORE \\
\hline 403 & 460 & & & & SESPMNT & & & & ISLAND ADJ LOCKE ISL \\
\hline 404 & 461 & & & & SESPMNT & & & & 100 F AREA-RIVER \\
\hline 405 & 462 & NAD83 & 585631.83 & 140698.61 & SESPMNT & GPS & RIVER_SHORELINE & & HANF POWERLINE XING \\
\hline 406 & 463 & & & & SESPMNT & & OFFSITE & LOW & RICH.PMPHS HRM 46.4 \\
\hline 407 & 464 & NAD83 & 600367.63 & 101184.44 & SESPMNT & GPS & RIVER_SHORELINE & & ISL DS BATEMAN ISL \\
\hline 408 & 465 & & & & SESPMNT & & & & BONNEVILLE DAM-RIVER \\
\hline 409 & 466 & & & & SESPMNT & & & & RICH. PUMPHOUSE -1B \\
\hline 410 & 467 & & & & SESPMNT & & ONSITE & LOW & 100 F -9 HRM 19.0 \\
\hline 411 & 468 & & & & SESPMNT & & ONSITE & LOW & HANFRD TS-3 HRM 28.7 \\
\hline 412 & 469 & & & & SESPMNT & & ONSITE & LOW & HANFRD TS-7 HRM 28.7 \\
\hline 413 & 470 & & & & SESPMNT & & ONSITE & LOW & 300 AREA -2 HRM 43.1 \\
\hline 414 & 471 & & & & SESPMNT & & ONSITE & LOW & 300 AREA -9 HRM 43.1 \\
\hline 415 & 472 & & & & SESPMNT & & & & HANFORD TOWNSITE-11B \\
\hline 416 & 473 & & & & SESPMNT & & & & RT. 4S MI. 20 \\
\hline 417 & 474 & NAD83 & 594709.33 & 112689.75 & SESPMNT & GPS & & & WPPSS \\
\hline 418 & 475 & & & & SESPMNT & & & & S 1 MI. WPPSS NO. 2 \\
\hline 419 & 476 & & & & SESPMNT & & PERIMETER & & WPPSS 4; WPS WAREHSE \\
\hline
\end{tabular}




\begin{tabular}{|c|c|c|c|c|c|c|c|c|c|}
\hline & A & $\mathrm{B}$ & $\mathrm{C}$ & $\mathrm{D}$ & $\mathrm{E}$ & $\mathrm{F}$ & $G$ & $\mathrm{H}$ & $\mathrm{I}$ \\
\hline 1 & SAMP_SITE_ID & HORIZ_COORD_TYPE & EW_COORD & NS_COORD & OWNER_ID & COORD_SOURCE & DIST_CLASS & RAD_LEVEL & SAMP_SITE_NAME \\
\hline 420 & $\overline{477}$ & & & & SESPMNT & & & & RRC CP 67 \\
\hline 421 & 478 & & & & SESPMNT & & PERIMETER & & NORTHWEST PERIMETER \\
\hline 422 & 479 & & & & SESPMNT & & & & 200 AREA FIRE STAT \\
\hline 423 & 480 & NAD83 & 568361 & 134353 & SESPMNT & & ONSITE & LOW & 200 W SE \\
\hline 424 & 481 & & & & SESPMNT & & & & 200 WEC \\
\hline 425 & 482 & & & & SESPMNT & & & & 200 W AREA CP 60 \\
\hline 426 & 483 & & & & SESPMNT & & & & MET TOWER 622R BLDG. \\
\hline 427 & 484 & & & & SESPMNT & & & & 222-S POND $200 \mathrm{~W}$ \\
\hline 428 & 485 & & & & SESPMNT & & & & 309 WELL (399 04-05) \\
\hline 429 & 486 & & & & SESPMNT & & & & 251 BLDG. RT. 11A \\
\hline 430 & 487 & NAD83 & 559316.38 & 138632.94 & SESPMNT & GPS & PERIMETER & LOW & YAKIMA BARRICADE \\
\hline 431 & 488 & & & & SESPMNT & & ONSITE & LOW & 300 AREA SPRING 42-2 \\
\hline 432 & 489 & & & & SESPMNT & & & & 320 BLDG. 300 AREA \\
\hline 433 & 490 & & & & SESPMNT & & & & 331 PROCESS SEWER \\
\hline 434 & 491 & & & & SESPMNT & & & & 300 SAN WATER PLANT \\
\hline 435 & 492 & & & & SESPMNT & & & & N CENTER 300 FENCE \\
\hline 436 & 493 & & & & SESPMNT & & & & LAZY D FARMS,INC. \\
\hline 437 & 494 & & & & SESPMNT & & COMMUNITY & & CONNELL \\
\hline 438 & 495 & & & & SESPMNT & & & & WAHLUKE CP 22 \\
\hline 439 & 496 & & & & SESPMNT & & & & WAHLUKE WATERMASTER \\
\hline 440 & 497 & & & & SESPMNT & & PERIMETER & & NORTHEAST PERIMETER \\
\hline 441 & 498 & & & & SESPMNT & & & & RINGOLD MET NO. 2 \\
\hline 442 & 499 & & & & SESPMNT & & & & ELLENSBURG \\
\hline 443 & 500 & & & & SESPMNT & & DISTANT & LOW & MC NARY DAM \\
\hline 444 & 501 & & & & SESPMNT & & DISTANT & LOW & SUNNYSIDE \\
\hline 445 & 502 & & & & SESPMNT & & & & WILLAPA BAY \\
\hline 446 & 503 & & & & SESPMNT & & & & YAKIMA NO. 2 \\
\hline 447 & 504 & & & & SESPMNT & & & & KETTLE RIVER \\
\hline 448 & 505 & & & & SESPMNT & & OFFSITE & LOW & BOARDMAN,OREGON \\
\hline 449 & 506 & & & & SESPMNT & & & & 200 E GUARDHOUSE \\
\hline 450 & 507 & & & & SESPMNT & & & & PUREX CHEM SEWR 200E \\
\hline 451 & 508 & NAD83 & 576869.35 & 135738.65 & SESPMNT & GPS & ONSITE & LOW & E OF $200 \mathrm{E}$ \\
\hline 452 & 509 & & & & SESPMNT & & ONSITE & LOW & B POND NORTH SIDE \\
\hline 453 & 510 & & & & SESPMNT & & & & RT. 4 S MI. 5 \\
\hline 454 & 511 & NAD83 & 587294.71 & 123531.23 & SESPMNT & GPS & ONSITE & LOW & $400 \mathrm{~N}$ \\
\hline 455 & 512 & & & & SESPMNT & & ONSITE & LOW & FFTF \\
\hline 456 & 513 & & & & SESPMNT & & & & FFTF CP 62 \\
\hline 457 & 514 & & & & SESPMNT & & & & HORN RAPIDS RD COMP \\
\hline
\end{tabular}




\begin{tabular}{|c|c|c|c|c|c|c|c|c|c|}
\hline & A & $\mathrm{B}$ & C & $\mathrm{D}$ & $E$ & $\mathrm{~F}$ & G & $\mathrm{H}$ & $\mathrm{I}$ \\
\hline 1 & SAMP_SITE_ID & HORIZ_COORD_TYPE & EW_COORD & NS_COORD & OWNER_ID & COORD_SOURCE & DIST_CLASS & RAD_LEVEL & SAMP_SITE_NAME \\
\hline 458 & 515 & & & & SESPMNT & & & & GABLE POND \\
\hline 459 & 516 & & & & SESPMNT & & & & GABLE POND CP 43 \\
\hline 460 & 517 & & & & SESPMNT & & & & HANFORD \\
\hline 461 & 518 & & & & SESPMNT & & & & INNER SE SECTOR \\
\hline 462 & 519 & & & & SESPMNT & & PERIMETER & LOW & RINGOLD HATCHERY \\
\hline 463 & 520 & & & & SESPMNT & & & & W END OF FIR RD NO.2 \\
\hline 464 & 521 & & & & SESPMNT & & PERIMETER & LOW & SAGEMOOR FARM \\
\hline 465 & 522 & & & & SESPMNT & & & & BAXTER SUBSTATION \\
\hline 466 & 523 & NAD83 & 606442.34 & 103006.15 & SESPMNT & GPS & COMMUNITY & LOW & PASCO \\
\hline 467 & 524 & & & & SESPMNT & & OFFSITE & LOW & MATHEWS CORNER \\
\hline 468 & 525 & & & & SESPMNT & & OFFSITE & LOW & ALEXANDER FARM \\
\hline 469 & 526 & & & & SESPMNT & & & & COURNEYA-BENTON CITY \\
\hline 470 & 527 & & & & SESPMNT & & & & TRUHLICKA-BENTON CTY \\
\hline 471 & 528 & & & & SESPMNT & & PERIMETER & LOW & ALE FIELD LAB \\
\hline 472 & 529 & & & & SESPMNT & & & & RT. 240 CP 54 \\
\hline 473 & 530 & & & & SESPMNT & & ONSITE & LOW & 100 D AREA \\
\hline 474 & 531 & & & & SESPMNT & & ONSITE & LOW & $100 \mathrm{~F}$ AREA \\
\hline 475 & 532 & & & & SESPMNT & & & & WHITE BLUFFS \\
\hline 476 & 533 & & & & SESPMNT & & ONSITE & LOW & 100-K SPRING \\
\hline 477 & 534 & & & & SESPMNT & & ONSITE & LOW & $100-N-100-D$ \\
\hline 478 & 535 & & & & SESPMNT & & ONSITE & LOW & NE OF 100 N AREA \\
\hline 479 & 536 & & & & SESPMNT & & & & 100 N SHORELINE \\
\hline 480 & 537 & & & & SESPMNT & & & & RT. 4N MI. 6 \\
\hline 481 & 538 & NAD83 & 572401.81 & 133836.47 & SESPMNT & GPS & ONSITE & & US ECOLOGY SE CORNER \\
\hline 482 & 539 & & & & SESPMNT & & & & 4S-ARMY LOOP INTER \\
\hline 483 & 540 & & & & SESPMNT & & PERIMETER & LOW & PROSSER BARRICADE \\
\hline 484 & 541 & & & & SESPMNT & & & & GABLE POND B \\
\hline 485 & 542 & & & & SESPMNT & & & & B POND B \\
\hline 486 & 544 & & & & SESPMNT & & & & $600 \mathrm{~F}$ \\
\hline 487 & 545 & & & & SESPMNT & & PERIMETER & LOW & BASIN FARM A \\
\hline 488 & 546 & & & & SESPMNT & & & & FARM A \\
\hline 489 & 547 & & & & SESPMNT & & & & FARM D \\
\hline 490 & 548 & & & & SESPMNT & & & & $600 \mathrm{E}$ \\
\hline 491 & 549 & & & & SESPMNT & & & & $600 \mathrm{~S}$ \\
\hline 492 & 550 & & & & SESPMNT & & OFFSITE & LOW & LOWER REACH RIVER \\
\hline 493 & 551 & & & & SESPMNT & & OFFSITE & & WANAPUM DAM-RIVER \\
\hline 494 & 552 & & & & SESPMNT & & OFFSITE & LOW & PRD-1/3 GRANT SHORE \\
\hline 495 & 553 & & & & SESPMNT & & ONSITE & LOW & 100 B AREA-RIVER \\
\hline
\end{tabular}




\begin{tabular}{|c|c|c|c|c|c|c|c|c|c|}
\hline & A & $\mathrm{B}$ & $\mathrm{C}$ & $\mathrm{D}$ & $E$ & $\mathrm{~F}$ & G & $\mathrm{H}$ & $\mathrm{I}$ \\
\hline 1 & SAMP_SITE_ID & HORIZ_COORD_TYPE & EW_COORD & NS_COORD & OWNER_ID & COORD_SOURCE & DIST_CLASS & RAD_LEVEL & SAMP_SITE_NAME \\
\hline 496 & 554 & & & & SESPMNT & & & & $100 \mathrm{~K}$ AREA-RIVER \\
\hline 497 & 555 & & & & SESPMNT & & & & BELOW 100K RET BASIN \\
\hline 498 & 556 & 6 NAD83 & 571326.98 & 149919.43 & 3 SESPMNT & GPS & RIVER SHORELINE & LOW & 100 N TRENCH SPRING \\
\hline 499 & 557 & & & & SESPMNT & & & & BELOW 100 NE-RIVER \\
\hline 500 & 558 & & & & SESPMNT & & & & ABOVE HANF.FRRY LNDG \\
\hline 501 & 559 & 9 NAD83 & 573475.69 & 152366.3 & 3 SESPMNT & GPS & RIVER_SHORELINE & & BELOW 100 D AREA \\
\hline 502 & 560 & & & & SESPMNT & & & & ABOVE 100D SHORE \\
\hline 503 & 561 & 1 NAD83 & 579441.42 & 149995.67 & 7 SESPMNT & GPS & RIVER SHORELINE & LOW & WHITE BLUFFS SLOUGH \\
\hline 504 & 562 & 2 NAD83 & 581402.12 & 147740.53 & 3 SESPMNT & GPS & RIVER_SHORELINE & & 100 F FLOOD PLAIN \\
\hline 505 & 563 & 3 NAD83 & 584319.83 & 141587.01 & SESPMNT & GPS & RIVER_SHORELINE & LOW & HANFORD SLOUGH \\
\hline 506 & 564 & & & & SESPMNT & & & & RINGOLD FAR SHORE \\
\hline 507 & 565 & 5 NAD83 & 594798.03 & 125730.92 & SESPMNT & GPS & RIVER_SHORELINE & & POWERLINE CROSSING \\
\hline 508 & 566 & & & & SESPMNT & & & & SHOT ROCK ISL. COMP. \\
\hline 509 & 567 & & & & SESPMNT & & & & ABOVE 300 AREA SPR \\
\hline 510 & 568 & & & & SESPMNT & & RIVER_SHORELINE & & PORT OF BENTON-RIVER \\
\hline 511 & 569 & & & & SESPMNT & & & & ISLAND VIEW-RIVER \\
\hline 512 & 570 & & & & SESPMNT & & OFFSITE & LOW & VERNITA-2 HRM 0.3 \\
\hline 513 & 571 & & & & SESPMNT & & OFFSITE & LOW & RICH.PMPHS-1 HRM46.4 \\
\hline 514 & 572 & & & & SESPMNT & & OFFSITE & LOW & RICH.PMPHS-4 HRM46.4 \\
\hline 515 & 573 & & & & SESPMNT & & OFFSITE & LOW & RICH.PMPHS-9 HRM46.4 \\
\hline 516 & 574 & & & & SESPMNT & & ONSITE & LOW & 100 N -4 HRM 9.5 \\
\hline 517 & 575 & & & & SESPMNT & & ONSITE & LOW & 100 N -8 HRM 9.5 \\
\hline 518 & 576 & & & & SESPMNT & & ONSITE & & 100 F -2 HRM 19.0 \\
\hline 519 & 577 & & & & SESPMNT & & ONSITE & LOW & 100 F -5 HRM 19.0 \\
\hline 520 & 578 & & & & SESPMNT & & ONSITE & LOW & HANFRD TS-1 HRM 28.7 \\
\hline 521 & 579 & & & & SESPMNT & & ONSITE & LOW & HANFRD TS-8 HRM 28.7 \\
\hline 522 & 580 & & & & SESPMNT & & ONSITE & LOW & 300 AREA -3 HRM 43.1 \\
\hline 523 & 581 & & & & SESPMNT & & ONSITE & LOW & 300 AREA -8 HRM 43.1 \\
\hline 524 & 582 & & & & SESPMNT & & & & $100 N-1 B$ \\
\hline 525 & 583 & & & & SESPMNT & & & & $100 N-5 B$ \\
\hline 526 & 584 & & & & SESPMNT & & & & $100 N-9 B$ \\
\hline 527 & 585 & & & & SESPMNT & & & & HANFORD TOWNSITE-1B \\
\hline 528 & 586 & & & & SESPMNT & & & & HANFORD TOWNSITE-5B \\
\hline 529 & 587 & & & & SESPMNT & & & & HANFORD TOWNSITE-10B \\
\hline 530 & 588 & 8 NAD83 & 589478.21 & 125180.46 & SESPMNT & GPS & ONSITE & & WPPSS 1 ; S OF WNP 2 \\
\hline 531 & 589 & & & & SESPMNT & & & & WPPSS NO. 2 AREA \\
\hline 532 & 590 & & & & SESPMNT & & & & RRC CP 63 \\
\hline 533 & 591 & 1 NAD83 & 593733.85 & 112661.28 & 3 SESPMNT & GPS & PERIMETER & LOW & BATTELLE COMPLEX \\
\hline
\end{tabular}




\begin{tabular}{|c|c|c|c|c|c|c|c|c|c|}
\hline & A & $\mathrm{B}$ & $\mathrm{C}$ & $\mathrm{D}$ & $\mathrm{E}$ & $\mathrm{F}$ & $G$ & $\mathrm{H}$ & $\mathrm{I}$ \\
\hline 1 & SAMP_SITE_ID & HORIZ_COORD_TYPE & EW_COORD & NS_COORD & OWNER_ID & COORD_SOURCE & DIST_CLASS & RAD_LEVEL & SAMP_SITE_NAME \\
\hline 534 & 592 & & & & SESPMNT & & COMMUNITY & & RICHLAND 700 AREA \\
\hline 535 & 593 & & & & SESPMNT & & & & BLACKBURN-FINLEY \\
\hline 536 & 594 & & & & SESPMNT & & PERIMETER & & SOUTHEAST PERIMETER \\
\hline 537 & 595 & & & & SESPMNT & & & & WAHLUKE CP 17 \\
\hline 538 & 596 & NAD83 & 565651.14 & 156362.85 & SESPMNT & GPS & PERIMETER & LOW & WAHLUKE SLOPE \\
\hline 539 & 597 & & & & SESPMNT & & & & WEST \\
\hline 540 & 598 & & & & SESPMNT & & & & 200 W SE NO. 2 \\
\hline 541 & 599 & & & & SESPMNT & & & & REDOX \\
\hline 542 & 600 & & & & SESPMNT & & & & U PLANT \\
\hline 543 & 601 & & & & SESPMNT & & & & REDOX POND \\
\hline 544 & 602 & & & & SESPMNT & & & & UMATILLA \\
\hline 545 & 603 & NAD83 & 587814.58 & 123087.47 & SESPMNT & GPS & ONSITE & LOW & $400 \mathrm{E}$ \\
\hline 546 & 604 & & & & SESPMNT & & PERIMETER & LOW & W END OF FIR ROAD \\
\hline 547 & 605 & NAD83 & 587612.88 & 114926.41 & SESPMNT & GPS & PERIMETER & LOW & HORN RAPIDS AREA \\
\hline 548 & 606 & & & & SESPMNT & & ONSITE & LOW & 100 F -6 HRM 19.0 \\
\hline 549 & 607 & & & & SESPMNT & & ONSITE & LOW & 300 AREA -5 HRM 43.1 \\
\hline 550 & 1944 & NAD83 & 594463.906 & 118383.339 & HEISPROD & ESTIMATED & & & 300-FF-5 RI|B01832 \\
\hline 551 & 1945 & NAD83 & 594426.022 & 116715.284 & HEISPROD & ESTIMATED & & & 300-FF-5 RI|B01834 \\
\hline 552 & 1946 & NAD83 & 594524.858 & 115853.078 & HEISPROD & ESTIMATED & & & 300-FF-5 RI|B01836 \\
\hline 553 & 1947 & NAD83 & 594551.698 & 115513.335 & HEISPROD & ESTIMATED & & & 300-FF-5 RI|B01838 \\
\hline 554 & 1948 & NAD83 & 594794.837 & 115023.561 & HEISPROD & ESTIMATED & & & 300-FF-5 RI|B01840 \\
\hline 555 & 1949 & NAD83 & 562497 & 146017 & HEISPROD & ESTIMATED & & & 100 AREA BIO 91|B00V88 \\
\hline 556 & 1950 & NAD83 & 554754.2 & 146410.3 & HEISPROD & GPS & & & 100 AREA BIO 91|B00VT3 \\
\hline 557 & 1951 & NAD83 & 567384 & 146094 & HEISPROD & GPS & & & *WHITE BLUFF* \\
\hline 558 & 1952 & NAD83 & 579920 & 148947 & HEISPROD & GPS & & & 100 AREA BIO 91|B00VZ1 \\
\hline 559 & 1953 & NAD83 & 581871.8 & 146715.1 & HEISPROD & ESTIMATED & & & 100 AREA BIO 92|B06N41 \\
\hline 560 & 1954 & NAD83 & 580774.5 & 148726.8 & HEISPROD & ESTIMATED & & & 100 AREA BIO 92|B06N42 \\
\hline 561 & 1955 & NAD83 & 570715.9 & 148787.7 & HEISPROD & ESTIMATED & & & 100 AREA BIO 92|B06N43 \\
\hline 562 & 1956 & NAD83 & 569740.5 & 147781.9 & HEISPROD & ESTIMATED & & & 100 AREA BIO 92|B06N44 \\
\hline 563 & 1957 & NAD83 & 568003.1 & 146318.8 & HEISPROD & ESTIMATED & & & 100 AREA BIO 92|B06N45 \\
\hline 564 & 1958 & NAD83 & 584172 & 116051.7 & HEISPROD & ESTIMATED & & & 100 AREA BIO 91|B00V91 \\
\hline 565 & 1960 & NAD83 & 580905 & 148129 & HEISPROD & ESTIMATED & & & 100 AREA BIO 92|B06N49 \\
\hline 566 & 1962 & NAD83 & 579536 & 149497 & HEISPROD & ESTIMATED & & & 100 AREA BIO 92|B06N51 \\
\hline 567 & 1967 & NAD83 & 572390 & 151342 & HEISPROD & ESTIMATED & & & 100 AREA BIO 91|B00VZ8 \\
\hline 568 & 1968 & NAD83 & 569649 & 147989 & HEISPROD & ESTIMATED & & & 100 AREA BIO 92|B06N58 \\
\hline 569 & 1970 & NAD83 & 566305 & 145632 & HEISPROD & ESTIMATED & & & 100 AREA BIO 92|B06N60 \\
\hline 570 & 1971 & NAD83 & 564194 & 145230 & HEISPROD & ESTIMATED & & & 100 AREA BIO 92|B06N61 \\
\hline 571 & 1972 & NAD83 & 558355 & 145798 & HEISPROD & ESTIMATED & & & 100 AREA BIO 92|B06N62 \\
\hline
\end{tabular}




\begin{tabular}{|c|c|c|c|c|c|c|c|c|c|}
\hline & $\mathrm{A}$ & $\mathrm{B}$ & $\mathrm{C}$ & $\mathrm{D}$ & $E$ & $\mathrm{~F}$ & $\mathrm{G}$ & $\mathrm{H}$ & $\mathrm{I}$ \\
\hline 1 & SAMP_SITE_ID & HORIZ_COORD_TYPE & EW_COORD & NS_COORD & OWNER_ID & COORD_SOURCE & DIST_CLASS & RAD_LEVEL & SAMP_SITE_NAME \\
\hline 572 & 1973 & NAD83 & 580670 & 147450 & O HEISPROD & ESTIMATED & & & 100 AREA BIO 92|B06N95 \\
\hline 573 & 1974 & NAD83 & 580700 & 147730 & 0 HEISPROD & ESTIMATED & & & 100 AREA BIO 92|B06N96 \\
\hline 574 & 1975 & NAD83 & 580165 & 147700 & 0 HEISPROD & ESTIMATED & & & 100 AREA BIO 92|B06N99 \\
\hline 575 & 1976 & NAD83 & 580090 & 147643 & 3 HEISPROD & ESTIMATED & & & 100 AREA BIO 92|B06NB1 \\
\hline 576 & 1977 & NAD83 & 579930 & 147547 & 7 HEISPROD & ESTIMATED & & & 100 AREA BIO 92|B06NB2 \\
\hline 577 & 1978 & NAD83 & 580020 & 147450 & HEISPROD & ESTIMATED & & & 100 AREA BIO 92|B06NB3 \\
\hline 578 & 1979 & NAD83 & 577750 & 152300 & HEISPROD & ESTIMATED & & & 100 AREA BIO 92|B06NB4 \\
\hline 579 & 1980 & NAD83 & 577655 & 152110 & HEISPROD & ESTIMATED & & & 100 AREA BIO 92|B06NB6 \\
\hline 580 & 1981 & NAD83 & 577980 & 152515 & 5 HEISPROD & ESTIMATED & & & 100 AREA BIO 92|B06NB8 \\
\hline 581 & 1982 & NAD83 & 577510 & 152523 & 3 HEISPROD & ESTIMATED & & & 100 AREA BIO 92|B06NC1 \\
\hline 582 & 1983 & NAD83 & 573565 & 151750 & HEISPROD & ESTIMATED & & & 100 AREA BIO 92|B06NC2 \\
\hline 583 & 1984 & NAD83 & 573820 & 151818 & 8 HEISPROD & ESTIMATED & & & 100 AREA BIO 92|B06NC3 \\
\hline 584 & 1986 & NAD83 & 573560 & 151285 & 5 HEISPROD & ESTIMATED & & & 100 AREA BIO 92|B06NC6 \\
\hline 585 & 1987 & NAD83 & 573923.1 & 152756.3 & 3 HEISPROD & ESTIMATED & & & 100 AREA BIO 92|B06ND0 \\
\hline 586 & 1988 & NAD83 & 562129.6 & 151716.9 & 9 HEISPROD & ESTIMATED & & & 100 AREA BIO 92|B06ND2 \\
\hline 587 & 1989 & NAD83 & 578938.8 & 157695.7 & 7 HEISPROD & ESTIMATED & & & 100 AREA BIO 92|B06ND4 \\
\hline 588 & 1990 & NAD83 & 580405.8 & 162670.1 & 1 HEISPROD & ESTIMATED & & & 100 AREA BIO 92|B06ND6 \\
\hline 589 & 1991 & NAD83 & 583145.8 & 142341.1 & 1 HEISPROD & ESTIMATED & & & 100 AREA BIO 92|B06ND8 \\
\hline 590 & 1992 & NAD83 & 580770.8 & 147702 & 2 HEISPROD & ESTIMATED & & & 100 AREA BIO 92|B06NF0 \\
\hline 591 & 1993 & NAD83 & 564620 & 144050 & HEISPROD & ESTIMATED & & & 100 AREA BIO 92|B078Z0 \\
\hline 592 & 1994 & NAD83 & 566068 & $14417 \varepsilon$ & 8 HEISPROD & ESTIMATED & & & 100 AREA BIO 92|B078Z4 \\
\hline 593 & 1995 & NAD83 & 569185 & 146907 & 7 HEISPROD & ESTIMATED & & & 100 AREA BIO 92|B078Z9 \\
\hline 594 & 1996 & NAD83 & 571586 & 149530 & HEISPROD & ESTIMATED & & & 100 AREA BIO 92|B07908 \\
\hline 595 & 1997 & NAD83 & 577433 & 151950 & HEISPROD & ESTIMATED & & & 100 AREA BIO 92|B07913 \\
\hline 596 & 1998 & NAD83 & 579550 & 149398 & 8 HEISPROD & ESTIMATED & & & 100 AREA BIO 92|B07915 \\
\hline 597 & 1999 & NAD83 & 566928 & 156753 & 3 HEISPROD & ESTIMATED & & & 100 AREA BIO 92|B07918 \\
\hline 598 & 2000 & NAD83 & 571395 & 149985 & 5 HEISPROD & ESTIMATED & & & 100 AREA BIO 92|B07921 \\
\hline 599 & 2001 & NAD83 & 556050 & 144500 & O HEISPROD & ESTIMATED & & & 100 AREA BIO 91|B00VT9 \\
\hline 600 & 2002 & NAD83 & 566025 & 145375 & 5 HEISPROD & ESTIMATED & & & 100 AREA BIO 92|B07927 \\
\hline 601 & 2003 & NAD83 & 569250 & 147585 & 5 HEISPROD & ESTIMATED & & & 100 AREA BIO 92|B07929 \\
\hline 602 & 2004 & NAD83 & 567370 & 145965 & 5 HEISPROD & ESTIMATED & & & 100 AREA BIO 91|B00VV8 \\
\hline 603 & 2005 & NAD83 & 571970 & 149475 & 5 HEISPROD & ESTIMATED & & & 100 AREA BIO 92|B07906 \\
\hline 604 & 2006 & NAD83 & 573796 & 152505 & 5 HEISPROD & ESTIMATED & & & 100 AREA BIO 91|B00V83 \\
\hline 605 & 2007 & NAD83 & 473654 & 152438 & 8 HEISPROD & ESTIMATED & & & 100 AREA BIO 91|B00V84 \\
\hline 606 & 2008 & NAD83 & 573516 & 152338 & 8 HEISPROD & ESTIMATED & & & 100 AREA BIO 91|B00V85 \\
\hline 607 & 2010 & NAD83 & 572934 & 151723 & 3 HEISPROD & ESTIMATED & & & 100 AREA BIO 91|B00V87 \\
\hline 608 & 2011 & NAD83 & 573952 & 152763 & 3 HEISPROD & GPS & & & 100 AREA BIO 91|B00VV9 \\
\hline 609 & 2027 & NAD83 & 578458 & 152125 & 5 HEISPROD & ESTIMATED & & & 100 AREA BIO 91|B00V89 \\
\hline
\end{tabular}




\begin{tabular}{|c|c|c|c|c|c|c|c|c|c|}
\hline & A & $\mathrm{B}$ & C & $\mathrm{D}$ & $\mathrm{E}$ & $\mathrm{F}$ & $\mathrm{G}$ & $\mathrm{H}$ & $\mathrm{I}$ \\
\hline 1 & SAMP_SITE_ID & HORIZ_COORD_TYPE & EW_COORD & NS_COORD & OWNER_ID & COORD_SOURCE & DIST_CLASS & RAD_LEVEL & SAMP_SITE_NAME \\
\hline 610 & 2028 & NAD83 & 578450 & 152350 & HEISPROD & ESTIMATED & & & 100 AREA BIO 91|B00VY3 \\
\hline 611 & 2034 & NAD83 & 578334 & 152500 & HEISPROD & ESTIMATED & & & 100 AREA BIO 91|B00VY9 \\
\hline 612 & 2036 & NAD83 & 577463 & 153456 & HEISPROD & ESTIMATED & & & 100 AREA BIO 91|B00V78 \\
\hline 613 & 2038 & NAD83 & 576434 & 154104 & HEISPROD & ESTIMATED & & & 100 AREA BIO 91|B00V80 \\
\hline 614 & 2039 & NAD83 & 576110 & 154261 & HEISPROD & ESTIMATED & & & 100 AREA BIO 91|B00V81 \\
\hline 615 & 2040 & NAD83 & 575719 & 153322 & HEISPROD & ESTIMATED & & & 100 AREA BIO 91|B00V82 \\
\hline 616 & 2043 & NAD83 & 559224.7 & 145678.7 & HEISPROD & ESTIMATED & & & 100 AREA AQ BIO|B00V95 \\
\hline 617 & 2044 & NAD83 & 573367.8 & 151805.3 & HEISPROD & ESTIMATED & & & 100 AREA AQ BIO|B00V97 \\
\hline 618 & 2045 & NAD83 & 578747.6 & 152390.5 & HEISPROD & ESTIMATED & & & 100 AREA AQ BIO|B00V99 \\
\hline 619 & 2046 & NAD83 & 575379.5 & 154213.3 & HEISPROD & ESTIMATED & & & 100 AREA AQ BIO|B00VB0 \\
\hline 620 & 2047 & NAD83 & 578488.5 & 149342.5 & HEISPROD & ESTIMATED & & & 100 AREA AQ BIO|B00VB3 \\
\hline 621 & 2048 & NAD83 & 574720 & 154520 & HEISPROD & ESTIMATED & & & 100 AREA BIO 91|B00VW7 \\
\hline 622 & 2054 & NAD83 & 574636 & 154465 & HEISPROD & ESTIMATED & & & 100 AREA BIO 91|B00VX3 \\
\hline 623 & 2056 & NAD83 & 576898 & 153851 & HEISPROD & GPS & & & 100 AREA BIO 91|B00VX5 \\
\hline 624 & 2065 & NAD83 & 571508.4 & 149732.6 & HEISPROD & ESTIMATED & & & 100 AREA AQ BIO|B00VC5 \\
\hline 625 & 2066 & NAD83 & 571904.7 & 150220.3 & HEISPROD & ESTIMATED & & & 100 AREA AQ BIO|B00VC9 \\
\hline 626 & 2067 & NAD83 & 571386.5 & 149549.7 & HEISPROD & ESTIMATED & & & 100 AREA AQ BIO|B00VD1 \\
\hline 627 & 2068 & NAD83 & 571721.8 & 150006.9 & HEISPROD & ESTIMATED & & & 100 AREA AQ BIO|B00VF3 \\
\hline 628 & 2070 & NAD83(91) & 594568.75 & 115846.41 & HEISPROD & MEASURED & & & 300-FF-1 RI|ASPG01 \\
\hline 629 & 2071 & NAD83(91) & 594576.35 & 115805.41 & HEISPROD & MEASURED & & & 300-FF-1 RI|ASPG02 \\
\hline 630 & 2072 & NAD83(91) & 594535.49 & 115976.43 & HEISPROD & MEASURED & & & 300-FF-1 RI|ASPG03 \\
\hline 631 & 2073 & NAD83(91) & 594491.51 & 116162.07 & HEISPROD & MEASURED & & & 300-FF-1 RI|ASPG04 \\
\hline 632 & 2074 & NAD83(91) & 594443.25 & 116327.98 & HEISPROD & MEASURED & & & 300-FF-1 RI|ASPG05 \\
\hline 633 & 2075 & NAD83(91) & 594353.04 & 116920.4 & HEISPROD & MEASURED & & & 300-FF-1 RI|ASPG06 \\
\hline 634 & 2076 & NAD83(91) & 594846.6 & 114515.18 & HEISPROD & MEASURED & & & 300-FF-1 RI|ASPG07 \\
\hline 635 & 2077 & NAD83(91) & 594829.74 & 114652.17 & HEISPROD & MEASURED & & & 300-FF-1 RI|ASPG08 \\
\hline 636 & 2078 & NAD83(91) & 594797.15 & 114831.92 & HEISPROD & MEASURED & & & 300-FF-1 RI|ASPG09 \\
\hline 637 & 2079 & NAD83(91) & 594775.38 & 115034.4 & HEISPROD & MEASURED & & & 300-FF-1 RI|ASPG10 \\
\hline 638 & 2080 & NAD83(91) & 594732.77 & 114426 & HEISPROD & MEASURED & & & 300-FF-1 RI|ASPG11 \\
\hline 639 & 2081 & NAD83(91) & 594743.61 & 114535.4 & HEISPROD & MEASURED & & & 300-FF-1 RI|ASPG12 \\
\hline 640 & 2082 & NAD83(91) & 594323.16 & 117170.16 & HEISPROD & MEASURED & & & 300-FF-1 RI|ASPG13 \\
\hline 641 & 2083 & NAD83(91) & 594318.57 & 117221.78 & HEISPROD & MEASURED & & & 300-FF-1 RI|ASPG14 \\
\hline 642 & 2084 & NAD83(91) & 594305.32 & 117343.9 & HEISPROD & MEASURED & & & 300-FF-1 RI|ASPG15 \\
\hline 643 & 2085 & NAD83(91) & 594320.55 & 117740.47 & HEISPROD & MEASURED & & & 300-FF-1 RI|ASPG16 \\
\hline 644 & 2086 & NAD83(91) & 594352.37 & 117822.85 & HEISPROD & MEASURED & & & 300-FF-1 RI|ASPG17 \\
\hline 645 & 2087 & NAD83(91) & 594378.45 & 118042.92 & HEISPROD & MEASURED & & & 300-FF-1 RI|ASPG18 \\
\hline 646 & 2088 & NAD83 & 594729.8 & 114396.1 & HEISPROD & MEASURED & & & 300-FF-1 RI|ASPG19 \\
\hline 647 & 2089 & NAD83 & 594682.7 & 114447 & HEISPROD & MEASURED & & & 300-FF-1 RI|ASPG20 \\
\hline
\end{tabular}




\begin{tabular}{|c|c|c|c|c|c|c|c|c|c|}
\hline & $A$ & $\mathrm{~B}$ & $\mathrm{C}$ & $\mathrm{D}$ & $\mathrm{E}$ & $\mathrm{F}$ & $G$ & $\mathrm{H}$ & $\mathrm{I}$ \\
\hline 1 & SAMP_SITE_ID & HORIZ_COORD_TYPE & EW_COORD & NS_COORD & OWNER_ID & COORD_SOURCE & DIST_CLASS & RAD_LEVEL & SAMP_SITE_NAME \\
\hline 648 & 2090 & NAD83 & 594715 & 114456.5 & HEISPROD & MEASURED & & & 300-FF-1 RI|ASPG22 \\
\hline 649 & 2091 & NAD83 & 587010 & 136900 & HEISPROD & ESTIMATED & & & 300-FF-1 RI|ASPG23 \\
\hline 650 & 2092 & NAD83 & 584580 & 138260 & HEISPROD & ESTIMATED & & & 300-FF-1 RI|ASPG24 \\
\hline 651 & 2093 & NAD83 & 585340 & 138730 & HEISPROD & ESTIMATED & & & 300-FF-1 RI|ASPG25 \\
\hline 652 & 2094 & NAD83 & 585440 & 139220 & HEISPROD & ESTIMATED & & & 300-FF-1 RI|ASPG26 \\
\hline 653 & 2095 & NAD83 & 582110 & 142660 & HEISPROD & ESTIMATED & & & 300-FF-1 RI|ASPG27 \\
\hline 654 & 2096 & NAD83 & 585600 & 138570 & HEISPROD & ESTIMATED & & & 300-FF-1 RI|ASPG28 \\
\hline 655 & 2097 & NAD83 & 603335 & 114625 & HEISPROD & ESTIMATED & & & 300-FF-1 RI|ASPG29 \\
\hline 656 & 2098 & NAD83 & 597563 & 124304 & HEISPROD & ESTIMATED & & & 300-FF-1 RI|ASPG35 \\
\hline 657 & 2099 & NAD83 & 608710 & 125970 & HEISPROD & ESTIMATED & & & 300-FF-1 RI|ASPG41 \\
\hline 658 & 2100 & NAD83 & 594787.8 & 114482.1 & HEISPROD & MEASURED & & & 300-FF-1 RI|ASPG48 \\
\hline 659 & 2101 & NAD83 & 594280 & 118580 & HEISPROD & ESTIMATED & & & 300-FF-1 RI|B00975 \\
\hline 660 & 2102 & NAD83 & 594330 & 118240 & HEISPROD & ESTIMATED & & & 300-FF-1 RI|B00976 \\
\hline 661 & 2103 & NAD83 & 594792.6 & 114954.4 & HEISPROD & MEASURED & & & 300-FF-1 RI|B00982 \\
\hline 662 & 2104 & NAD83 & 594792.3 & 114946 & HEISPROD & MEASURED & & & 300-FF-1 RI|B00983 \\
\hline 663 & 2105 & NAD83 & 593482.4 & 115026.4 & HEISPROD & MEASURED & & & 300-FF-1 RI|B00985 \\
\hline 664 & 2106 & NAD83 & 593528.5 & 115034.3 & HEISPROD & MEASURED & & & 300-FF-1 RI|B00986 \\
\hline 665 & 2107 & NAD83 & 593471.7 & 114885.5 & HEISPROD & MEASURED & & & 300-FF-1 RI|B00987 \\
\hline 666 & 2108 & NAD83 & 593429.4 & 115015 & HEISPROD & MEASURED & & & 300-FF-1 RI|B00988 \\
\hline 667 & 2109 & NAD83 & 549140 & 102300 & HEISPROD & ESTIMATED & & & 300-FF-1 RI|B00989 \\
\hline 668 & 2110 & NAD83 & 586000 & 111000 & HEISPROD & ESTIMATED & & & 300-FF-1 RI|B00991 \\
\hline 669 & 2112 & NAD83 & 580000 & 110000 & HEISPROD & ESTIMATED & & & 300-FF-1 RI|B00998 \\
\hline 670 & 2113 & NAD83 & 577900 & 104940 & HEISPROD & ESTIMATED & & & 300-FF-1 RI|B009B4 \\
\hline 671 & 2114 & NAD83 & 594394.2 & 116542.4 & HEISPROD & MEASURED & & & 300-FF-1 RI|B009C3 \\
\hline 672 & 2115 & NAD83 & 594760.8 & 115112.8 & HEISPROD & MEASURED & & & 300-FF-1 RI|B009C4 \\
\hline 673 & 2116 & NAD83 & 594732.6 & 115189 & HEISPROD & MEASURED & & & 300-FF-1 RI|B009C5 \\
\hline 674 & 2117 & NAD83 & 594706.6 & 115298.6 & HEISPROD & MEASURED & & & 300-FF-1 RI|B009C6 \\
\hline 675 & 2118 & NAD83 & 594689.6 & 115401.8 & HEISPROD & MEASURED & & & 300-FF-1 RI|B009C7 \\
\hline 676 & 2119 & NAD83 & 594671.5 & 115507.5 & HEISPROD & MEASURED & & & 300-FF-1 RI|B009C8 \\
\hline 677 & 2120 & NAD83 & 594544 & 115916.6 & HEISPROD & MEASURED & & & 300-FF-1 RI|B009D2 \\
\hline 678 & 2121 & NAD83 & 594357.3 & 116583.1 & HEISPROD & MEASURED & & & 300-FF-1 RI|B009N7 \\
\hline 679 & 2122 & NAD83 & 594323.1 & 116705.5 & HEISPROD & MEASURED & & & 300-FF-1 RI|B009P0 \\
\hline 680 & 2123 & NAD83 & 594141.3 & 116677.3 & HEISPROD & MEASURED & & & 300-FF-1 RI|B009P1 \\
\hline 681 & 2124 & NAD83 & 594135.2 & 116910.3 & HEISPROD & MEASURED & & & 300-FF-1 RI|B009P3 \\
\hline 682 & 2125 & NAD83 & 594009.9 & 117021.4 & HEISPROD & MEASURED & & & 300-FF-1 RI|B009P5 \\
\hline 683 & 2126 & NAD83 & 593996.3 & 117080 & HEISPROD & MEASURED & & & 300-FF-1 RI|B009P7 \\
\hline 684 & 2128 & NAD83 & 593870.2 & 117602.2 & HEISPROD & MEASURED & & & 300-FF-1 RI|B009Q1 \\
\hline 685 & 2131 & NAD83 & 594480 & 119660 & HEISPROD & ESTIMATED & & & 300-FF-5 RI|B01842 \\
\hline
\end{tabular}




\begin{tabular}{|c|c|c|c|c|c|c|c|c|c|}
\hline & $A$ & $\mathrm{~B}$ & $\mathrm{C}$ & $\mathrm{D}$ & $E$ & $\mathrm{~F}$ & $G$ & $\mathrm{H}$ & $\mathrm{I}$ \\
\hline 1 & SAMP_SITE_ID & HORIZ_COORD_TYPE & EW_COORD & NS_COORD & OWNER_ID & COORD_SOURCE & DIST_CLASS & RAD_LEVEL & SAMP_SITE_NAME \\
\hline 686 & 2132 & NAD83 & 594420 & 116130 & HEISPROD & ESTIMATED & & & 300-FF-5 RI|B07G52 \\
\hline 687 & 2133 & NAD83 & 594530 & 115720 & HEISPROD & ESTIMATED & & & 300-FF-5 RI|B07G86 \\
\hline 688 & 2134 & NAD83 & 594358.805 & 116931.008 & HEISPROD & ESTIMATED & & & 300-FF-5 RI|B01824 \\
\hline 689 & 2135 & NAD83 & 594429.377 & 116499.678 & HEISPROD & ESTIMATED & & & 300-FF-5 RI|B017Z8 \\
\hline 690 & 2136 & NAD83 & 594633.252 & 115761.391 & HEISPROD & ESTIMATED & & & 300-FF-5 RI|B017Z2 \\
\hline 691 & 2137 & NAD83 & 594679.7 & 115546.457 & HEISPROD & ESTIMATED & & & 300-FF-5 RI|B017Z3 \\
\hline 692 & 2138 & NAD83 & 594818.997 & 114807.169 & HEISPROD & ESTIMATED & & & 300-FF-5 RI|B017Z5 \\
\hline 693 & 2139 & NAD83 & 593561.153 & 114571.979 & HEISPROD & ESTIMATED & & & 300-FF-5 RI|B017Z6 \\
\hline 694 & 2140 & NAD83 & 594500.235 & 118816.335 & HEISPROD & ESTIMATED & & & 300-FF-5 RI|B01823 \\
\hline 695 & 2141 & NAD83 & 594521.984 & 116037.566 & HEISPROD & ESTIMATED & & & 300-FF-5 RI|B017Z1 \\
\hline 696 & 2142 & NAD83 & 594814.657 & 115085.011 & HEISPROD & ESTIMATED & & & 300-FF-5 RI|B017Z4 \\
\hline 697 & 2143 & NAD83 & 594691 & 118695 & HEISPROD & GPS & & & 300-FF-5 RI|B012L5 \\
\hline 698 & 2144 & NAD83 & 594564 & 118851 & HEISPROD & GPS & & & 300-FF-5 RI|B01358 \\
\hline 699 & 2145 & NAD83 & 594412 & 117885 & HEISPROD & GPS & & & 300-FF-5 RI|B012M7 \\
\hline 700 & 2146 & NAD83 & 594394 & 117422 & HEISPROD & GPS & & & 300-FF-5 RI|B012R8 \\
\hline 701 & 2147 & NAD83 & 594468 & 116775 & HEISPROD & GPS & & & 300-FF-5 RI|B012V2 \\
\hline 702 & 2148 & NAD83 & 594567 & 116183 & HEISPROD & GPS & & & 300-FF-5 RI|B01337 \\
\hline 703 & 2149 & NAD83 & 594683 & 116167 & HEISPROD & GPS & & & 300-FF-5 RI|B012W5 \\
\hline 704 & 2150 & NAD83 & 594635 & 115925 & HEISPROD & GPS & & & 300-FF-5 RI|B012V8 \\
\hline 705 & 2151 & NAD83 & 594728 & 115704 & HEISPROD & GPS & & & 300-FF-5 RI|B012Z4 \\
\hline 706 & 2152 & NAD83 & 594786 & 115260 & HEISPROD & GPS & & & 300-FF-5 RI|B012K9 \\
\hline 707 & 2153 & NAD83 & 594868 & 114965 & HEISPROD & ESTIMATED & & & 300-FF-5 RI|B012J9 \\
\hline 708 & 2154 & NAD83 & 594953 & 114448 & HEISPROD & GPS & & & 300-FF-5 RI|B012H6 \\
\hline 709 & 2155 & NAD83 & 594528 & 118665 & HEISPROD & GPS & & & 300-FF-5 RI|B017N6 \\
\hline 710 & 2156 & NAD83 & 594544 & 118443 & HEISPROD & GPS & & & 300-FF-5 RI|B017P3 \\
\hline 711 & 2157 & NAD83 & 594415 & 117700 & HEISPROD & GPS & & & 300-FF-5 RI|B017Q5 \\
\hline 712 & 2158 & NAD83 & 594448 & 117256 & HEISPROD & GPS & & & 300-FF-5 RI|B017P9 \\
\hline 713 & 2159 & NAD83 & 594437 & 117108 & HEISPROD & GPS & & & 300-FF-5 RI|B017R1 \\
\hline 714 & 2160 & NAD83 & 594427 & 116922 & HEISPROD & GPS & & & 300-FF-5 RI|B017R7 \\
\hline 715 & 2161 & NAD83 & 594632 & 116110 & HEISPROD & GPS & & & 300-FF-5 RI|B017W5 \\
\hline 716 & 2162 & NAD83 & 594922 & 114818 & HEISPROD & GPS & & & 300-FF-5 RI|B017V7 \\
\hline 717 & 2163 & NAD83 & 594980 & 114374 & HEISPROD & GPS & & & 300-FF-5 RI|B017V1 \\
\hline 718 & 2173 & & & & SESPMNT & & & & GARBAGE SITE \\
\hline 719 & 2174 & & & & SESPMNT & & & & GARBAGE SITE2 \\
\hline 720 & 2175 & & & & SESPMNT & & OFFSITE & LOW & SILVERLAKE,OR - MOUNTAINS \\
\hline 721 & 2176 & & & & SESPMNT & & OFFSITE & LOW & SILVER LAKE, OR - WINTER AREA \\
\hline 722 & 2177 & & & & SESPMNT & & OFFSITE & LOW & SILVER LAKE, OR \\
\hline 723 & 2178 & & & & SESPMNT & & ONSITE & LOW & EAST AREA (DUNES NEAR COLUMBIA RIVER) \\
\hline
\end{tabular}




\begin{tabular}{|c|c|c|c|c|c|c|c|c|c|}
\hline & A & $\mathrm{B}$ & $\mathrm{C}$ & $\mathrm{D}$ & $\mathrm{E}$ & $\mathrm{F}$ & G & $\mathrm{H}$ & $\mathrm{I}$ \\
\hline 1 & SAMP_SITE_ID & HORIZ_COORD_TYPE & EW_COORD & NS_COORD & OWNER_ID & COORD_SOURCE & DIST_CLASS & RAD_LEVEL & SAMP_SITE_NAME \\
\hline 724 & 2179 & & & & SESPMNT & & ONSITE & LOW & $100 \mathrm{~K}$ TO $100 \mathrm{~N}$ \\
\hline 725 & 2180 & & & & SESPMNT & & OFFSITE & LOW & SILVER LAKE, OR - MOUNTAINS \\
\hline 726 & 2181 & & & & SESPMNT & & ONSITE & LOW & ALE 1200 FT. RD. \\
\hline 727 & 2182 & & & & SESPMNT & & ONSITE & LOW & 200 AREA PLATEAU \\
\hline 728 & 2183 & & & & SESPMNT & & ONSITE & LOW & 100 KE TO $100 \mathrm{~N}$ \\
\hline 729 & 2184 & NAD83 & 567197 & 152541 & HEISPROD & MATH_TRANS & & & SADDLE MTN MAMMAL TRANSECT LINE \# 1 \\
\hline 730 & 2190 & NAD83 & 567338 & 152457 & HEISPROD & MATH_TRANS & & & SADDLE MTN MAMMAL TRANSECT LINE \# 2 \\
\hline 731 & 2192 & NAD83 & 567260 & 152573 & HEISPROD & MATH_TRANS & & & SADDLE MTN INSECT PIT-CAN TRANSECT \#1 \\
\hline 732 & 2193 & NAD83 & 567196 & 152552 & HEISPROD & MATH_TRANS & & & SADDLE MTN INSECT PIT-CAN TRANSECT \#2 \\
\hline 733 & 2198 & NAD83 & 567228 & 152562 & HEISPROD & MATH_TRANS & & & SADDLE MTN LAKE INSECT PIT-CANS \\
\hline 734 & 2199 & NAD83 & 576586 & 136830 & HEISPROD & MATH_TRANS & & & 216-B-3 MAMMAL TRANSECT LINE \# 1 \\
\hline 735 & 2202 & NAD83 & 576613 & 136709 & HEISPROD & MATH_TRANS & & & 216-B-3 MAMMAL TRANSECT LINE \#2 \\
\hline 736 & 2203 & NAD83 & 575626 & 136393 & HEISPROD & MATH_TRANS & & & 216-A-24 MAMMAL TRANSECT LINE \#1 \\
\hline 737 & 2204 & NAD83 & 575645 & 136388 & HEISPROD & MATH_TRANS & & & 216-A-24 MAMMAL TRANSECT LINE \#2 \\
\hline 738 & 2205 & NAD83 & 566842 & 136921 & HEISPROD & MATH_TRANS & & & 216-T-4 MAMMAL TRANSECT LINE \#1 \\
\hline 739 & 2206 & NAD83 & 566818 & 136973 & HEISPROD & MATH_TRANS & & & 216-T-4 MAMMAL TRANSECT LINE \#2 \\
\hline 740 & 2207 & NAD83 & 565882 & 134946 & HEISPROD & MATH_TRANS & & & 216-U-11 MAMMAL TRANSECT LINE \#1 \\
\hline 741 & 2208 & NAD83 & 565832 & 134910 & HEISPROD & MATH_TRANS & & & 216-U-11 MAMMAL TRANSECT LINE \#2 \\
\hline 742 & 2210 & NAD83 & 567268 & 152599 & HEISPROD & MATH_TRANS & & & SADDLE MTN MAMMAL TRANSECTS \\
\hline 743 & 2211 & NAD83 & 576600 & 136769 & HEISPROD & MATH_TRANS & & & 216-B-3 MAMMAL TRANSECTS \\
\hline 744 & 2212 & NAD83 & 575636 & 136390 & HEISPROD & MATH_TRANS & & & 216-A-24 MAMMAL TRANSECTS \\
\hline 745 & 2213 & NAD83 & 576830 & 136947 & HEISPROD & MATH_TRANS & & & 216-T-4 MAMMAL TRANSECTS \\
\hline 746 & 2214 & NAD83 & 565857 & 134928 & HEISPROD & MATH_TRANS & & & 216-U-11 MAMMAL TRANSECTS LINES \\
\hline 747 & 2216 & NAD83 & 564540 & 145275 & CENTPLAT & ESTIMATED & & & SEEP 037-1 \\
\hline 748 & 2217 & NAD83 & 564675 & 145275 & HEISPROD & ESTIMATED & & & SEEP 038-3 \\
\hline 749 & 2218 & NAD83 & 564940 & 145350 & CENTPLAT & ESTIMATED & & & SEEP 039-2 \\
\hline 750 & 2219 & NAD83 & 565260 & 145400 & HEISPROD & ESTIMATED & & & SEEP 041-2 \\
\hline 751 & 2220 & NAD83 & 567585 & 146210 & HEISPROD & ESTIMATED & & & SEEP 056-3 \\
\hline 752 & 2221 & NAD83 & 569680 & 148070 & HEISPROD & ESTIMATED & & & SEEP 074-1 \\
\hline 753 & 2222 & NAD83 & 570415 & 148780 & HEISPROD & ESTIMATED & & & SEEP 080-2 \\
\hline 754 & 2223 & NAD83 & 571300 & 149920 & HEISPROD & ESTIMATED & & & SEEP 090-1 \\
\hline 755 & 2224 & NAD83 & 571465 & 150150 & HEISPROD & ESTIMATED & & & SEEP 091-2 \\
\hline 756 & 2225 & NAD83 & 571480 & 150170 & HEISPROD & ESTIMATED & & & SEEP 092-1 \\
\hline 757 & 2226 & NAD83 & 571500 & 150185 & HEISPROD & ESTIMATED & & & SEEP 092-2 \\
\hline 758 & 2227 & NAD83 & 571680 & 150465 & HEISPROD & ESTIMATED & & & SEEP 094-1 \\
\hline 759 & 2228 & NAD83 & 573480 & 152375 & CENTPLAT & ESTIMATED & & & SEEP 110-1 \\
\hline 760 & 2229 & NAD83 & 571597 & 152470 & CENTPLAT & ESTIMATED & & & SEEP $110-2$ \\
\hline 761 & 2230 & NAD83 & 573597 & 152470 & HEISPROD & ESTIMATED & & & SEEP $110-5$ \\
\hline
\end{tabular}




\begin{tabular}{|c|c|c|c|c|c|c|c|c|c|}
\hline & $A$ & $\mathrm{~B}$ & $\mathrm{C}$ & $\mathrm{D}$ & $\mathrm{E}$ & $\mathrm{F}$ & $G$ & $\mathrm{H}$ & $\mathrm{I}$ \\
\hline 1 & SAMP_SITE_ID & HORIZ_COORD_TYPE & EW_COORD & NS_COORD & OWNER_ID & COORD_SOURCE & DIST_CLASS & RAD_LEVEL & SAMP_SITE_NAME \\
\hline 762 & 2231 & NAD83 & 577080 & 153770 & HEISPROD & ESTIMATED & & & SEEP 143-1 \\
\hline 763 & 2232 & NAD83 & 577255 & 153660 & CENTPLAT & ESTIMATED & & & SEEP 144-1 \\
\hline 764 & 2233 & NAD83 & 577330 & 153615 & HEISPROD & ESTIMATED & & & SEEP 146-1 \\
\hline 765 & 2234 & NAD83 & 577885 & 153160 & HEISPROD & ESTIMATED & & & SEEP 149-1 \\
\hline 766 & 2235 & NAD83 & 578235 & 152660 & HEISPROD & ESTIMATED & & & SEEP 154-1 \\
\hline 767 & 2236 & NAD83 & 580820 & 148275 & HEISPROD & ESTIMATED & & & SEEP $187-1$ \\
\hline 768 & 2237 & NAD83 & 581230 & 147940 & HEISPROD & ESTIMATED & & & SEEP 190-4 \\
\hline 769 & 2238 & NAD83 & 582621 & 145597 & HEISPROD & ESTIMATED & & & SEEP 208-1 \\
\hline 770 & 2239 & NAD83 & 582864 & 145130 & HEISPROD & ESTIMATED & & & SEEP 216-1 \\
\hline 771 & 2240 & NAD83 & 582962 & 144813 & HEISPROD & ESTIMATED & & & SEEP 218-1 \\
\hline 772 & 2241 & NAD83 & 583132 & 144317 & HEISPROD & ESTIMATED & & & SEEP 221-1 \\
\hline 773 & 2242 & NAD83 & 584986 & 140838 & HEISPROD & ESTIMATED & & & SEEP 246-1 \\
\hline 774 & 2243 & NAD83 & 585729 & 140070 & HEISPROD & ESTIMATED & & & SEEP 252-1 \\
\hline 775 & 2244 & & & & SESPMNT & & ONSITE & LOW & B POND EXTENSION \\
\hline 776 & 2245 & NAD83 & 578165 & 152813 & CENTPLAT & MEASURED & & & SEEP $152-2$ \\
\hline 777 & 2246 & NAD83 & 578160 & 152813 & HEISPROD & MEASURED & & & SEEP 152-3 \\
\hline 778 & 2247 & NAD83(91) & 582629.289 & 145474.592 & HEISPROD & GPS & & & SEEP 205-1 \\
\hline 779 & 2248 & NAD83(91) & 580508.504 & 148521.186 & HEISPROD & GPS & & & SEEP 189-1 \\
\hline 780 & 2249 & & & & SESPMNT & & OFFSITE & LOW & STEVENS COUNTY \\
\hline 781 & 2250 & & & & SESPMNT & & OFFSITE & LOW & ROCKY REACH \\
\hline 782 & 2252 & & & & SESPMNT & & ONSITE & LOW & HIGHWAY 240 \\
\hline 783 & 2254 & NAD83 & 508224 & 115680 & SESPMNT & SURVEYED & DISTANT & LOW & TOPPENISH \\
\hline 784 & 2255 & NAD83 & 573843 & 151589 & HEISPROD & MEASURED & & & 116-D-1A \\
\hline 785 & 2256 & NAD83 & 573820 & 151120 & HEISPROD & MEASURED & & & 100 AREA BIO 92|B06NC7 \\
\hline 786 & 2257 & NAD83 & 593837 & 117627.7 & HEISPROD & & & & 300-FF-1 RI|B009P9 \\
\hline 787 & 2259 & NAD83(91) & 594389.11 & 117702.96 & HEISPROD & GPS & & & 300-FF-5 RI|B01800 \\
\hline 788 & 2260 & NAD83(91) & 594399.593 & 118413.463 & HEISPROD & GPS & & & 300-FF-5 RI|B01825 \\
\hline 789 & 2262 & NAD83 & 594651.8 & 115568.9 & HEISPROD & ESTIMATED & & & 30-FF-1 RI|B00B88 \\
\hline 790 & 2263 & NAD83 & 565900 & 134969 & HEISPROD & ESTIMATED & & & 200 AREA BIOTA|B08YF7 \\
\hline 791 & 2264 & NAD83 & 565891 & 134963 & HEISPROD & ESTIMATED & & & 200 AREA BIOTA|B08YF9 \\
\hline 792 & 2265 & NAD83 & 565852 & 134935 & HEISPROD & ESTIMATED & & & 200 AREA BIOTA|B08YG1 \\
\hline 793 & 2266 & NAD83 & 565840 & 134927 & HEISPROD & ESTIMATED & & & 200 AREA BIOTA|B08YG3 \\
\hline 794 & 2267 & NAD83 & 576611 & 136844 & HEISPROD & ESTIMATED & & & 200 AREA BIOTA|B08YG9 \\
\hline 795 & 2268 & NAD83 & 576599 & 136827 & HEISPROD & ESTIMATED & & & 200 AREA BIOTA|B08YH0 \\
\hline 796 & 2269 & NAD83 & 576617 & 136728 & HEISPROD & ESTIMATED & & & 200 AREA BIOTA|B08YH1 \\
\hline 797 & 2270 & NAD83 & 576619 & 136713 & HEISPROD & ESTIMATED & & & 200 AREA BIOTA|B08YH2 \\
\hline 798 & 2271 & NAD83 & 575608 & 136388 & HEISPROD & ESTIMATED & & & 200 AREA BIOTA|B08YH7 \\
\hline 799 & 2272 & NAD83 & 575627 & 136386 & HEISPROD & ESTIMATED & & & 200 AREA BIOTA|B08YH8 \\
\hline
\end{tabular}




\begin{tabular}{|c|c|c|c|c|c|c|c|c|c|}
\hline & $A$ & $\mathrm{~B}$ & $\mathrm{C}$ & $\mathrm{D}$ & $E$ & $\mathrm{~F}$ & G & $\mathrm{H}$ & $\mathrm{I}$ \\
\hline 1 & SAMP_SITE_ID & HORIZ_COORD_TYPE & EW_COORD & NS_COORD & OWNER_ID & COORD_SOURCE & DIST_CLASS & RAD_LEVEL & SAMP_SITE_NAME \\
\hline 800 & 2273 & NAD83 & 575649 & 136388 & 8 HEISPROD & ESTIMATED & & & 200 AREA BIOTA|B08YH9 \\
\hline 801 & 2274 & NAD83 & 575576 & 136406 & 6 HEISPROD & ESTIMATED & & & 200 AREA BIOTA|B08YJ0 \\
\hline 802 & 2275 & NAD83 & 566837 & 136903 & 3 HEISPROD & ESTIMATED & & & 200 AREA BIOTA|B08YJ5 \\
\hline 803 & 2276 & NAD83 & 566833 & 136927 & 7 HEISPROD & ESTIMATED & & & 200 AREA BIOTA|B08YJ6 \\
\hline 804 & 2277 & NAD83 & 566819 & 136952 & 2 HEISPROD & ESTIMATED & & & 200 AREA BIOTA|B08YJ7 \\
\hline 805 & 2278 & NAD83 & 566818 & 136956 & 6 HEISPROD & ESTIMATED & & & 200 AREA BIOTA|B08YJ8 \\
\hline 806 & 2279 & NAD83 & 567201 & 152563 & 3 HEISPROD & ESTIMATED & & & 200 AREA BIOTA|B08YL9 \\
\hline 807 & 2280 & NAD83 & 567246 & 152525 & 5 HEISPROD & ESTIMATED & & & 200 AREA BIOTA|B08YM0 \\
\hline 808 & 2281 & NAD83 & 567198 & 152536 & 6 HEISPROD & ESTIMATED & & & 200 AREA BIOTA|B08YM1 \\
\hline 809 & 2282 & NAD83 & 567187 & 152576 & 6 HEISPROD & ESTIMATED & & & 200 AREA BIOTA|B08YM2 \\
\hline 810 & 2284 & NAD83 & 567617 & 134905 & 5 HEISPROD & ESTIMATED & & & 200-UP-2 LFI|B0BFL8 \\
\hline 811 & 2285 & NAD83 & 567617 & 134870 & HEISPROD & ESTIMATED & & & 200-UP-2 LFI|BOBFL9 \\
\hline 812 & 2286 & NAD83 & 567617 & 134815 & 5 HEISPROD & ESTIMATED & & & 200-UP-2 LFI|BOBFM0 \\
\hline 813 & 2287 & NAD83 & 567617 & 134750 & 0 HEISPROD & ESTIMATED & & & 200-UP-2 LFI|B0BFM2 \\
\hline 814 & 2288 & NAD83 & 567600 & 134850 & 0 HEISPROD & ESTIMATED & & & 200-UP-2 LFI|B0BKN1 \\
\hline 815 & 2289 & NAD83 & 567627 & 134880 & 0 HEISPROD & ESTIMATED & & & 200-UP-2 LFI|B0BKN3 \\
\hline 816 & 2290 & NAD83 & 566115 & 134439 & 9 HEISPROD & ESTIMATED & & & 200-UP-2 LFI|B0BP32 \\
\hline 817 & 2291 & NAD83 & 566133 & $13454 \varepsilon$ & 8 HEISPROD & ESTIMATED & & & 200-UP-2 LFI|B0BP33 \\
\hline 818 & 2292 & NAD83 & 565888 & 134958 & 8 HEISPROD & MATH_TRANS & COMMUNITY & & 216-U-11 INSECT PIT-CAN TRANSECTS \\
\hline 819 & 2294 & NAD83 & 567835 & 134923 & 3 HEISPROD & & & & 200-UP-1 PUMP AND TREAT SYSTEM \\
\hline 820 & 2297 & NAD83 & 573730 & 151721 & 1 CENTPLAT & & & & 100-HR-3 PUMP \& TREAT \\
\hline 821 & 2298 & NAD83 & 578234 & 152733 & 3 HEISPROD & & & & TRANSECT TH1-A \\
\hline 822 & 2299 & NAD83 & 578246 & 152740 & HEISPROD & & & & TRANSECT TH1-B \\
\hline 823 & 2300 & NAD83 & 578195 & 152775 & 5 HEISPROD & & & & TRANSECT TH2-A \\
\hline 824 & 2301 & NAD83 & 578210 & 152780 & HEISPROD & & & & TRANSECT TH2-B \\
\hline 825 & 2302 & NAD83 & 578215 & 152851 & 1 HEISPROD & & & & TRANSECT TH3-A \\
\hline 826 & 2303 & NAD83 & 578227 & 152860 & 0 HEISPROD & & & & TRANSECT TH3-B \\
\hline 827 & 2304 & NAD83 & 578176 & 152912 & 2 HEISPROD & & & & TRANSECT TH4-A \\
\hline 828 & 2305 & NAD83 & 578188 & 152921 & 1 HEISPROD & & & & TRANSECT TH-B \\
\hline 829 & 2306 & NAD83 & 578160 & 152952 & 2 HEISPROD & & & & TRANSECT TH-A \\
\hline 830 & 2307 & NAD83 & 578171 & 152961 & 1 HEISPROD & & & & TRANSECT TH5-B \\
\hline 831 & 2308 & NAD83 & 578114 & 153012 & 2 HEISPROD & & & & TRANSECT TH6-A \\
\hline 832 & 2309 & NAD83 & 578128 & 153023 & 3 HEISPROD & & & & TRANSECT TH6-B \\
\hline 833 & 2310 & NAD83 & 578081 & 153065 & 5 HEISPROD & & & & TRANSECT TH7-A \\
\hline 834 & 2311 & NAD83 & 578092 & 153073 & 3 HEISPROD & & & & TRANSECT TH7-B \\
\hline 835 & 2312 & NAD83 & 578039 & 153125 & 5 HEISPROD & & & & TRANSECT TH8-A \\
\hline 836 & 2314 & NAD83 & 578007 & 153163 & 3 HEISPROD & & & & TRANSECT TH9-A \\
\hline 837 & 2315 & NAD83 & 578018 & 153171 & 1 HEISPROD & & & & TRANSECT TH9-B \\
\hline
\end{tabular}




\begin{tabular}{|c|c|c|c|c|c|c|c|c|c|}
\hline & A & $\mathrm{B}$ & $\mathrm{C}$ & $\mathrm{D}$ & $E$ & $\mathrm{~F}$ & $G$ & $\mathrm{H}$ & $\mathrm{I}$ \\
\hline 1 & SAMP_SITE_ID & HORIZ_COORD_TYPE & EW_COORD & NS_COORD & OWNER_ID & COORD_SOURCE & DIST_CLASS & RAD_LEVEL & SAMP_SITE_NAME \\
\hline 838 & 2316 & NAD83 & 577959 & 153217 & HEISPROD & & & & TRANSECT TH10-A \\
\hline 839 & 2317 & NAD83 & 577969 & 153226 & HEISPROD & & & & TRANSECT TH10-B \\
\hline 840 & 2318 & NAD83 & 577912 & 153268 & HEISPROD & & & & TRANSECT TH11-A \\
\hline 841 & 2319 & NAD83 & 577924 & 153278 & HEISPROD & & & & TRANSECT TH11-B \\
\hline 842 & 2320 & NAD83 & 577869 & 153315 & HEISPROD & & & & TRANSECT TH12-A \\
\hline 843 & 2321 & NAD83 & 577879 & 153325 & HEISPROD & & & & TRANSECT TH12-B \\
\hline 844 & 2322 & NAD83 & 577818 & 153368 & HEISPROD & & & & TRANSECT TH13-A \\
\hline 845 & 2323 & NAD83 & 577827 & 153379 & HEISPROD & & & & TRANSECT TH13-B \\
\hline 846 & 2324 & NAD83 & 577784 & 153392 & HEISPROD & & & & TRANSECT TH14-A \\
\hline 847 & 2325 & NAD83 & 577794 & 153403 & HEISPROD & & & & TRANSECT TH14-B \\
\hline 848 & 2326 & NAD83 & 577722 & 153439 & HEISPROD & & & & TRANSECT TH15-A \\
\hline 849 & 2327 & NAD83 & 577732 & 153449 & HEISPROD & & & & TRANSECT TH15-B \\
\hline 850 & 2328 & NAD83 & 577687 & 153465 & HEISPROD & & & & TRANSECT TH16-A \\
\hline 851 & 2329 & NAD83 & 577696 & 153475 & HEISPROD & & & & TRANSECT TH16-B \\
\hline 852 & 2330 & NAD83 & 577634 & 153503 & HEISPROD & & & & TRANSECT TH17-A \\
\hline 853 & 2331 & NAD83 & 577643 & 153513 & HEISPROD & & & & TRANSECT TH17-B \\
\hline 854 & 2332 & NAD83 & 566385 & 135429 & CENTPLAT & ESTIMATED & & & 200-ZP-1 PUMP/TREAT LOCATION \\
\hline 855 & 2334 & & & & SESPMNT & & ONSITE & LOW & 100-K EAST BASIN \\
\hline 856 & 2335 & & & & SESPMNT & & ONSITE & LOW & 100-K WEST BASIN \\
\hline 857 & 2337 & NAD83 & 571542.1 & 150251.9 & HEISPROD & GPS & & & NS-13 SEEP \\
\hline 858 & 2338 & NAD83 & 571513.3 & 150216.3 & HEISPROD & GPS & & & NS-12A SEEP \\
\hline 859 & 2339 & NAD83 & 571519.3 & 150214.7 & HEISPROD & GPS & & & NS-12B SEEP \\
\hline 860 & 2340 & NAD83 & 571495.5 & 150190.4 & CENTPLAT & GPS & & & NS-11 SEEP \\
\hline 861 & 2341 & NAD83 & 571430.5 & 150109.5 & CENTPLAT & GPS & & & NS-10 SEEP \\
\hline 862 & 2342 & NAD83 & 571412.4 & 150080.8 & HEISPROD & GPS & & & NS-9 SEEP \\
\hline 863 & 2343 & NAD83 & 571396.5 & 150051.8 & CENTPLAT & GPS & & & NS-8A SEEP \\
\hline 864 & 2344 & NAD83 & 571399.8 & 150050.3 & CENTPLAT & GPS & & & NS-8B SEEP \\
\hline 865 & 2345 & NAD83 & 571384 & 150027.1 & CENTPLAT & GPS & & & NS-7 SEEP \\
\hline 866 & 2346 & NAD83 & 571370.2 & 150003.5 & CENTPLAT & GPS & & & NS-6 SEEP \\
\hline 867 & 2347 & NAD83 & 571351.7 & 149978.4 & CENTPLAT & GPS & & & NS-5 SEEP \\
\hline 868 & 2348 & NAD83 & 571335.7 & 149954.5 & CENTPLAT & GPS & & & NS-4 SEEP \\
\hline 869 & 2349 & NAD83 & 571312.5 & 149934.3 & CENTPLAT & GPS & & & NS-3 SEEP \\
\hline 870 & 2350 & NAD83 & 571290.4 & 149911.8 & CENTPLAT & GPS & & & NS-2 SEEP \\
\hline 871 & 2351 & NAD83 & 571270.6 & 149888.6 & CENTPLAT & GPS & & & NS-1 SEEP \\
\hline 872 & 2357 & NAD83 & 548400 & 144300 & HEISPROD & GPS & & & COL RIVER BELOW PRIEST RAPID DAM \\
\hline 873 & 2358 & NAD83 & 573500 & 152400 & HEISPROD & ESTIMATED & & & 100-D COMPOSITE \\
\hline 874 & 2359 & NAD83 & 594154 & 116249 & HEISPROD & GPS & & & 300-FF-1 TEDF L-045 \\
\hline 875 & 2367 & NAD83 & 567615 & 134910 & HEISPROD & GPS & & & 200WEST0001 \\
\hline
\end{tabular}




\begin{tabular}{|c|c|c|c|c|c|c|c|c|c|}
\hline & $\mathrm{A}$ & $B$ & $\mathrm{C}$ & $\mathrm{D}$ & $E$ & $\mathrm{~F}$ & $G$ & $\mathrm{H}$ & $\mathrm{I}$ \\
\hline 1 & SAMP_SITE_ID & HORIZ_COORD_TYPE & EW_COORD & NS_COORD & OWNER_ID & COORD_SOURCE & DIST_CLASS & RAD_LEVEL & SAMP_SITE_NAME \\
\hline 876 & 2368 & NAD83 & 567615 & 134790 & HEISPROD & GPS & & & 200WEST0002 \\
\hline 877 & 2369 & NAD83 & 567373 & 135039 & HEISPROD & GPS & & & 200WEST0003 \\
\hline 878 & 2370 & NAD83 & 567387 & 135060 & HEISPROD & GPS & & & 200WEST0004 \\
\hline 879 & 2371 & NAD83 & 567342 & 135062 & HEISPROD & GPS & & & 200WEST0005 \\
\hline 880 & 2372 & NAD83 & 567369 & 135025 & HEISPROD & GPS & & & 200WEST0006 \\
\hline 881 & 2373 & NAD83 & 567369 & 135046 & HEISPROD & GPS & & & 200WEST0007 \\
\hline 882 & 2374 & NAD83 & 566930 & 135028 & HEISPROD & GPS & & & 200WEST0008 \\
\hline 883 & 2375 & NAD83 & 566922 & 135019 & HEISPROD & GPS & & & 200WEST0009 \\
\hline 884 & 2376 & NAD83 & 566965 & 135195 & HEISPROD & GPS & & & 200WEST0010 \\
\hline 885 & 2377 & NAD83 & 566470 & 134745 & HEISPROD & GPS & & & 200WEST0011 \\
\hline 886 & 2378 & NAD83 & 566554 & 134822 & HEISPROD & GPS & & & 200WEST0012 \\
\hline 887 & 2379 & NAD83 & 565650 & 134630 & HEISPROD & GPS & & & 200WEST0013 \\
\hline 888 & 2380 & NAD83 & 566605 & 134552 & HEISPROD & GPS & & & 200WEST0014 \\
\hline 889 & 2381 & NAD83 & 566574 & 134735 & HEISPROD & GPS & & & 200WEST0015 \\
\hline 890 & 2382 & NAD83 & 566414 & 134692 & HEISPROD & GPS & & & 200WEST0016 \\
\hline 891 & 2383 & NAD83 & 566186 & 134450 & HEISPROD & GPS & & & 200WEST0017 \\
\hline 892 & 2384 & NAD83 & 566216 & 134675 & HEISPROD & GPS & & & 200WEST0018 \\
\hline 893 & 2385 & NAD83 & 566133 & 134450 & HEISPROD & GPS & & & 200WEST0019 \\
\hline 894 & 2386 & NAD83 & 565875 & 134638 & HEISPROD & GPS & & & 200WEST0020 \\
\hline 895 & 2388 & NAD83(91) & 564341.45 & 143967.77 & HEISPROD & GPS & & & 118-B EXCAVATION TREAT TEST 1 \\
\hline 896 & 2389 & NAD83(91) & 564386.4 & 143941.86 & HEISPROD & GPS & & & 118-B EXCAVATION TREAT TEST 2 \\
\hline 897 & 2390 & NAD83(91) & 564374.06 & 143897.67 & HEISPROD & GPS & & & 118-B EXCAVATION TREAT TEST 3 \\
\hline 898 & 2391 & NAD83(91) & 564374.06 & 143906.81 & HEISPROD & GPS & & & 118-B EXCAVATION TREAT TEST 4 \\
\hline 899 & 2392 & NAD83(91) & 564384.88 & 143879.38 & HEISPROD & GPS & & & 118-B EXCAVATION TREAT TEST 5 \\
\hline 900 & 2393 & NAD83(91) & 564394.02 & 143870.24 & HEISPROD & GPS & & & 118-B EXCAVATION TREAT TEST 6 \\
\hline 901 & 2394 & NAD83(91) & 564371.16 & 143855 & HEISPROD & GPS & & & 118-B EXCAVATION TREAT TEST 7 \\
\hline 902 & 2395 & NAD83(91) & 564368.12 & 143870.24 & HEISPROD & GPS & & & 118-B EXCAVATION TREAT TEST 8 \\
\hline 903 & 2398 & NAD83 & 594513 & 121353 & HEISPROD & MEASURED & & & 300 WEST - SOUTH SIDE \\
\hline 904 & 2399 & NAD83 & 594513 & 121359 & HEISPROD & MEASURED & & & 300 WEST - CENTRAL SIDE \\
\hline 905 & 2401 & NAD83 & 594513 & 121366 & HEISPROD & MEASURED & & & 300 WEST - NORTH TRENCH \\
\hline 906 & 2402 & NAD83 & 555610 & 138269 & HEISPROD & GPS & & & RIVERLAND \\
\hline 907 & 2403 & NAD83 & 568720 & 134276 & HEISPROD & GPS & & & ERDF SOIL AND VEGETATION - 1 \\
\hline 908 & 2404 & NAD83 & 568647 & 134105 & HEISPROD & GPS & & & ERDF SOIL AND VEGETATION - 2 \\
\hline 909 & 2405 & NAD83 & 568803 & 134006 & HEISPROD & GPS & & & ERDF SOIL AND VEGETATION - 3 \\
\hline 910 & 2406 & NAD83 & 569180 & 134259 & HEISPROD & GPS & & & ERDF SOIL AND VEGETATION - 4 \\
\hline 911 & 2407 & NAD83 & 569756 & 133974 & HEISPROD & GPS & & & ERDF SOIL AND VEGETATION - 5 \\
\hline 912 & 2408 & NAD83 & 569869 & 134248 & HEISPROD & GPS & & & ERDF SOIL AND VEGETATION - 6 \\
\hline 913 & 2409 & NAD83 & 570215 & 134176 & HEISPROD & GPS & & & ERDF SOIL AND VEGETATION - 7 \\
\hline
\end{tabular}




\begin{tabular}{|c|c|c|c|c|c|c|c|c|c|}
\hline & $\mathrm{A}$ & $B$ & $\mathrm{C}$ & $\mathrm{D}$ & $E$ & $\mathrm{~F}$ & $G$ & $\mathrm{H}$ & $\mathrm{I}$ \\
\hline 1 & SAMP_SITE_ID & HORIZ_COORD_TYPE & EW_COORD & NS_COORD & OWNER_ID & COORD_SOURCE & DIST_CLASS & RAD_LEVEL & SAMP_SITE_NAME \\
\hline 914 & 2410 & NAD83 & 570608 & 134289 & HEISPROD & GPS & & & ERDF SOIL AND VEGETATION - 8 \\
\hline 915 & 2411 & NAD83 & 571377 & 133954 & HEISPROD & GPS & & & ERDF SOIL AND VEGETATION - 9 \\
\hline 916 & 2412 & NAD83 & 571403 & 134183 & HEISPROD & GPS & & & ERDF SOIL AND VEGETATION - 10 \\
\hline 917 & 2413 & NAD83 & 571398 & 134333 & HEISPROD & GPS & & & ERDF SOIL AND VEGETATION - 11 \\
\hline 918 & 2414 & NAD83 & 565045 & 143983 & HEISPROD & GPS & & & RIVERLAND ERA - 1 \\
\hline 919 & 2415 & NAD83 & 565042 & 143971 & HEISPROD & GPS & & & RIVERLAND ERA - 2 \\
\hline 920 & 2416 & NAD83 & 565030 & 143964 & HEISPROD & GPS & & & RIVERLAND ERA - 3 \\
\hline 921 & 2417 & NAD83 & 565048 & 143955 & HEISPROD & GPS & & & RIVERLAND ERA - 4 \\
\hline 922 & 2418 & NAD83 & 565059 & 143969 & HEISPROD & GPS & & & RIVERLAND ERA - 5 \\
\hline 923 & 2419 & NAD83 & 565050 & 143968 & HEISPROD & GPS & & & RIVERLAND ERA - 6 \\
\hline 924 & 2420 & NAD83 & 565028 & 143978 & HEISPROD & GPS & & & RIVERLAND ERA - 7 \\
\hline 925 & 2421 & NAD83 & 565055 & 143976 & HEISPROD & GPS & & & RIVERLAND ERA - 8 \\
\hline 926 & 2422 & NAD83(91) & 566779.792 & 133648.12 & HEISPROD & GPS & & & 216-S-10D \\
\hline 927 & 2423 & NAD83 & 565370 & 144000 & HEISPROD & GPS & & & 105-C REACTOR \\
\hline 928 & 2424 & NAD83 & 558564 & 143500 & HEISPROD & GPS & & & RIVERLAND ERA 1 \\
\hline 929 & 2425 & NAD83 & 558620 & 143473 & HEISPROD & GPS & & & RIVERLAND ERA 2 \\
\hline 930 & 2426 & NAD83 & 558650 & 143473 & HEISPROD & GPS & & & RIVERLAND ERA 3 \\
\hline 931 & 2427 & NAD83 & 558696 & 143473 & HEISPROD & GPS & & & RIVERLAND ERA 4 \\
\hline 932 & 2428 & NAD83 & 558539 & 143568 & HEISPROD & GPS & & & RIVERLAND ERA 5 \\
\hline 933 & 2429 & NAD83 & 577876 & 152849 & HEISPROD & & & & 183-H BUILDING -CENTER \\
\hline 934 & 2433 & NAD83(91) & 580387.637 & 147522.477 & HEISPROD & & & & 100 AREA EXC TS|B097C7 \\
\hline 935 & 2438 & NAD83(91) & 580385.808 & 147523.391 & HEISPROD & & & & 100 AREA EXC TS|B09788 \\
\hline 936 & 2439 & NAD83(91) & 580386.723 & 147523.391 & HEISPROD & & & & 100 AREA EXC TS|B09791 \\
\hline 937 & 2441 & NAD83(91) & 580388.856 & 147523.391 & HEISPROD & & & & 100 AREA EXC TS|B09F24 \\
\hline 938 & 2442 & NAD83(91) & 580389.466 & 147523.391 & HEISPROD & & & & 100 AREA EXC TS|B09793 \\
\hline 939 & 2443 & NAD83(91) & 580387.637 & 147523.848 & HEISPROD & & & & 100 AREA EXC TS|B09792 \\
\hline 940 & 2444 & NAD83(91) & 580386.723 & 147524.305 & HEISPROD & & & & 100 AREA EXC TS|B097B9 \\
\hline 941 & 2445 & NAD83(91) & 580387.637 & 147524.305 & HEISPROD & & & & 100 AREA EXC TS|B097B3 \\
\hline 942 & 2446 & NAD83(91) & 580387.637 & 147526.134 & HEISPROD & & & & 100 AREA EXC TS|B097B5 \\
\hline 943 & 2447 & NAD83(91) & 580385.808 & 147527.049 & HEISPROD & & & & 100 AREA EXC TS|B09790 \\
\hline 944 & 2448 & NAD83(91) & 580387.637 & 147527.049 & HEISPROD & & & & 100 AREA EXC TS|B09787 \\
\hline 945 & 2449 & NAD83(91) & 580387.637 & 147527.963 & HEISPROD & & & & 100 AREA EXC TS|B097B2 \\
\hline 946 & 2450 & NAD83(91) & 580387.637 & 147529.487 & HEISPROD & & & & 100 AREA EXC TS|B09F23 \\
\hline 947 & 2452 & NAD83(91) & 580387.18 & 147526.363 & HEISPROD & GPS & & & 100 AREA EXC TS|B09796 \\
\hline 948 & 2453 & NAD83(91) & 580384.894 & 147523.391 & HEISPROD & GPS & & & 100 AREA EXC TS|B09797 \\
\hline 949 & 2461 & NAD83 & 571764.5 & 150141.5 & CENTPLAT & ESTIMATED & & & INFLUENT POINT AT 100-NR-2 PUMP/TREAT \\
\hline 950 & 2462 & NAD83 & 571773.5 & 150129 & HEISPROD & ESTIMATED & & & EFFLUENT POINT A 100-NR-2, PUMP/TREAT \\
\hline 951 & 2464 & NAD83(91) & 572763.103 & 136186.772 & HEISPROD & & & & HWVP BASELINE|B01FH7 \\
\hline
\end{tabular}




\begin{tabular}{|c|c|c|c|c|c|c|c|c|c|}
\hline & A & $\mathrm{B}$ & $\mathrm{C}$ & $\mathrm{D}$ & $\mathrm{E}$ & $\mathrm{F}$ & $G$ & $\mathrm{H}$ & $\mathrm{I}$ \\
\hline 1 & SAMP_SITE_ID & HORIZ_COORD_TYPE & EW_COORD & NS_COORD & OWNER_ID & COORD_SOURCE & DIST_CLASS & RAD_LEVEL & SAMP_SITE_NAME \\
\hline 952 & 2465 & NAD83(91) & 572915.494 & 136187.206 & HEISPROD & & & & HWVP BASELINE|B01FH8 \\
\hline 953 & 2466 & NAD83(91) & 572884.845 & 136248.071 & HEISPROD & & & & HWVP BASELINE|B01FH9 \\
\hline 954 & 2467 & NAD83(91) & 572793.235 & 136308.761 & HEISPROD & & & & HWVP BASELINE|B01FJ0 \\
\hline 955 & 2468 & NAD83(91) & 572762.759 & 136308.675 & HEISPROD & & & & HWVP BASELINE|B01FJ1 \\
\hline 956 & 2469 & NAD83(91) & 572701.892 & 136278.027 & HEISPROD & & & & HWVP BASELINE|B01FJ2 \\
\hline 957 & 2470 & NAD83(91) & 572976.021 & 136339.758 & HEISPROD & & & & HWVP BASELINE|B01FJ3 \\
\hline 958 & 2471 & NAD83(91) & 572640.681 & 136369.282 & HEISPROD & & & & HWVP BASELINE|B01FJ6 \\
\hline 959 & 2472 & NAD83(91) & 572609.809 & 136521.592 & HEISPROD & & & & HWVP BASELINE|B01FJ5 \\
\hline 960 & 2473 & NAD83(91) & 572791.732 & 136918.156 & HEISPROD & & & & HWVP BASELINE|B01FJ7 \\
\hline 961 & 2474 & NAD83(91) & 572822.22 & 136918.245 & HEISPROD & & & & HWVP BASELINE|B01FK4 \\
\hline 962 & 2475 & NAD83(91) & 572913.896 & 136857.514 & HEISPROD & & & & HWVP BASELINE|B01FKO \\
\hline 963 & 2476 & NAD83(91) & 572883.618 & 136796.426 & HEISPROD & & & & HWVP BASELINE|B01FJ9 \\
\hline 964 & 2479 & NAD83(91) & 572761.741 & 136704.673 & HEISPROD & & & & HWVP BASELINE|B01FK1 \\
\hline 965 & 2480 & NAD83(91) & 572822.725 & 136704.848 & HEISPROD & & & & HWVP BASELINE|B01FK5 \\
\hline 966 & 2482 & NAD83(91) & 572883.691 & 136674.724 & HEISPROD & & & & HWVP BASELINE|B01FK6 \\
\hline 967 & 2483 & NAD83(91) & 572639.686 & 136734.815 & HEISPROD & & & & HWVP BASELINE|B01FK7 \\
\hline 968 & 2489 & NAD83(91) & 572731.884 & 136430.968 & HEISPROD & & & & HWVP BASELINE|B01FL0 \\
\hline 969 & 2492 & NAD83(91) & 572914.582 & 136522.448 & HEISPROD & & & & HWVP BASELINE|B01FL3 \\
\hline 970 & 2493 & NAD83(91) & 572731.884 & 136460.968 & HEISPROD & & & & HWVP BASELINE|B01FL8 \\
\hline 971 & 2501 & NAD83 & 573239.7 & 135514.5 & HEISPROD & ESTIMATED & & & 2101-M POND SAMPLE POINT "L" \\
\hline 972 & 2502 & NAD83 & 573241.8 & 135306.9 & HEISPROD & ESTIMATED & & & 2101-M POND SAMPLE POINT "K" \\
\hline 973 & 2503 & NAD83 & 573241.8 & 135091 & HEISPROD & ESTIMATED & & & 2101-M POND SAMPLE POINT "J" \\
\hline 974 & 2504 & NAD83 & 573239.7 & 134879 & HEISPROD & ESTIMATED & & & 2101-M POND SAMPLE POINT "I" \\
\hline 975 & 2505 & NAD83 & 573262.5 & 134847.2 & HEISPROD & ESTIMATED & & & 2101-M POND SAMPLE POINT "H" \\
\hline 976 & 2506 & NAD83 & 573289.8 & 134838 & HEISPROD & ESTIMATED & & & 2101-M POND SAMPLE POINT "G" \\
\hline 977 & 2507 & NAD83 & 573312.5 & 134845 & HEISPROD & ESTIMATED & & & 2101-M POND SAMPLE POINT "F" \\
\hline 978 & 2508 & NAD83 & 573355.5 & 134845 & HEISPROD & ESTIMATED & & & 2101-M POND SAMPLE POINT "E" \\
\hline 979 & 2509 & NAD83 & 573487.8 & 134854 & HEISPROD & ESTIMATED & & & 2101-M POND SAMPLE POINT "D" \\
\hline 980 & 2510 & NAD83 & 573492 & 135054 & HEISPROD & ESTIMATED & & & 2101-M POND SAMPLE POINT "C" \\
\hline 981 & 2511 & NAD83 & 573489 & 135254 & HEISPROD & ESTIMATED & & & 2101-M POND SAMPLE POINT "B" \\
\hline 982 & 2512 & NAD83 & 573492 & 135462 & HEISPROD & ESTIMATED & & & 2101-M POND SAMPLE POINT "A" \\
\hline 983 & 2513 & NAD83 & 572671.553 & 136918.156 & HEISPROD & ESTIMATED & & & HWVP BASELINE|B01FJ8 \\
\hline 984 & 2514 & NAD83 & 594162 & 116268 & HEISPROD & ESTIMATED & & & 300-FF-1 RI|B00YR1 \\
\hline 985 & 2516 & NAD83 & 594235 & 116294 & HEISPROD & ESTIMATED & & & 300-FF-1 RI|B00YR3 \\
\hline 986 & 2517 & NAD83 & 594304 & 116324 & HEISPROD & ESTIMATED & & & 300-FF-1 RI|B00YR8 \\
\hline 987 & 2519 & NAD83 & 573440 & 135530 & HEISPROD & ESTIMATED & & & WET BACKGROUD SAMPLE AREA \\
\hline 988 & 2520 & NAD83 & 573340 & 135530 & HEISPROD & ESTIMATED & & & DRY AREA SAMPLING POINT \\
\hline 989 & 2521 & NAD83(91) & 577787.816 & 147477.76 & HEISPROD & GPS & & & PICKLING ACID CRIB|B07Q14 \\
\hline
\end{tabular}




\begin{tabular}{|c|c|c|c|c|c|c|c|c|c|}
\hline & $\mathrm{A}$ & $B$ & $\mathrm{C}$ & $\mathrm{D}$ & $E$ & $\mathrm{~F}$ & $G$ & $\mathrm{H}$ & I \\
\hline 1 & SAMP_SITE_ID & HORIZ_COORD_TYPE & EW_COORD & NS_COORD & OWNER_ID & COORD_SOURCE & DIST_CLASS & RAD_LEVEL & SAMP_SITE_NAME \\
\hline 990 & 2522 & NAD83(91) & 577787.816 & 147493 & HEISPROD & GPS & & & PICKLING ACID CRIBS|B07Q15 \\
\hline 991 & 2523 & NAD83(91) & 577787.816 & 147507.33 & HEISPROD & GPS & & & PICLING ACID CRIBS|B07Q16 \\
\hline 992 & 2524 & NAD83 & 580910 & 147915 & HEISPROD & GPS & & & 100-FR-1 LFI|B07201 \\
\hline 993 & 2526 & NAD83 & 580915 & 147915 & HEISPROD & GPS & & & 100-FR-1 LFI|B07203 \\
\hline 994 & 2528 & NAD83 & 577552.8 & 153433.3 & HEISPROD & ESTIMATED & & & 100-HR-1 LFI|B07206 \\
\hline 995 & 2536 & NAD83 & 567617 & 134935 & HEISPROD & ESTIMATED & & & 200-UP-2|BOBFH2 \\
\hline 996 & 2537 & NAD83 & 567617 & 134915 & HEISPROD & ESTIMATED & & & 200-UP-2|B0BFF5 \\
\hline 997 & 2538 & NAD83 & 567617 & 134856 & HEISPROD & ESTIMATED & & & 200-UP-2|BOBFG0 \\
\hline 998 & 2539 & NAD83 & 567617 & 134833 & HEISPROD & ESTIMATED & & & 200-UP-2|BOBFH1 \\
\hline 999 & 2540 & NAD83 & 567617 & 134797 & HEISPROD & ESTIMATED & & & 200-UP-2|BOBFH3 \\
\hline 1000 & 2541 & NAD83 & 567617 & 134788 & HEISPROD & ESTIMATED & & & 200-UP-2|BOBFH4 \\
\hline 1001 & 2542 & NAD83 & 567617 & 134746 & HEISPROD & ESTIMATED & & & 200-UP-2|BOBFH8 \\
\hline 1002 & 2543 & NAD83 & 567617 & 134796 & HEISPROD & ESTIMATED & & & 200-UP-2|B0BFM8 \\
\hline 1003 & 2545 & NAD83 & 594365 & 111400 & HEISPROD & ESTIMATED & & & PIT \#1|1100-EM-1 RI|6157A \\
\hline 1004 & 2546 & NAD83 & 593386 & 110761 & HEISPROD & ESTIMATED & & & EPHEMERAL POOL|1100-EM-1 RI|6164A \\
\hline 1005 & 2548 & NAD83 & 592811 & 116323 & HEISPROD & ESTIMATED & & & 618-9 BURIAL GD|B00YC9 \\
\hline 1006 & 2550 & NAD83 & 592828 & 116323 & HEISPROD & ESTIMATED & & & 618-9 BURIAL GD|B00YC1 \\
\hline 1007 & 2551 & NAD83 & 592829 & 116324 & HEISPROD & ESTIMATED & & & 618-9 BURIAL GD|B00YC3 \\
\hline 1008 & 2552 & NAD83 & 592827 & 116325 & HEISPROD & ESTIMATED & & & 618-9 BURIAL GD|B00YC4 \\
\hline 1009 & 2553 & NAD83 & 592822 & 116326 & HEISPROD & ESTIMATED & & & 618-9 BURIAL GD|B00YC6 \\
\hline 1010 & 2554 & NAD83 & 592819 & 116322 & HEISPROD & ESTIMATED & & & 618-9 BURIAL GD|B00YC7 \\
\hline 1011 & 2556 & NAD83 & 592797 & 116313 & HEISPROD & ESTIMATED & & & 618-9 BURIAL GD|B00Y98 \\
\hline 1012 & 2557 & NAD83 & 592807 & 116337 & HEISPROD & ESTIMATED & & & 618-9 BURIAL GD|B00Y99 \\
\hline 1013 & 2558 & NAD83 & 592797 & 116337 & HEISPROD & ESTIMATED & & & 618-9 BURIAL GD|B00YB3 \\
\hline 1014 & 2559 & NAD83 & 592838 & 116326 & HEISPROD & ESTIMATED & & & 618-9 BURIAL GD|B00YB4 \\
\hline 1015 & 2560 & NAD83 & 592833 & 116323 & HEISPROD & ESTIMATED & & & 618-9 BURIAL GD|B00YB5 \\
\hline 1016 & 2561 & NAD83 & 592836 & 116324 & HEISPROD & ESTIMATED & & & 618-9 BURIAL GD|B00YB6 \\
\hline 1017 & 2562 & NAD83 & 592831 & 116324 & HEISPROD & ESTIMATED & & & 618-9 BURIAL GD|B00YB8 \\
\hline 1018 & 2564 & NAD83 & 592799 & 116324 & HEISPROD & ESTIMATED & & & 618-9 BURIAL GD|B00Y87 \\
\hline 1019 & 2565 & NAD83 & 592803 & 116326 & HEISPROD & ESTIMATED & & & 618-9 BURIAL GD|B00Y88 \\
\hline 1020 & 2566 & NAD83 & 592803 & 116325 & HEISPROD & ESTIMATED & & & 618-9 BURIAL GD|B00Y90 \\
\hline 1021 & 2567 & NAD83 & 592804 & 116322 & HEISPROD & ESTIMATED & & & 618-9 BURIAL GD|B00Y92 \\
\hline 1022 & 2568 & NAD83 & 592807 & 116313 & HEISPROD & ESTIMATED & & & 618-9 BURIAL GD|B00Y97 \\
\hline 1023 & 2570 & NAD83 & 592807 & 116326 & HEISPROD & ESTIMATED & & & 618-9 BURIAL GD|B00Y80 \\
\hline 1024 & 2571 & NAD83 & 592808 & 116327 & HEISPROD & ESTIMATED & & & 618-9 BURIAL GD|B00Y83 \\
\hline 1025 & 2572 & NAD83 & 592804 & 116324 & HEISPROD & ESTIMATED & & & 618-9 BURIAL GD|B00Y84 \\
\hline 1026 & 2573 & NAD83 & 592799 & 116323 & HEISPROD & ESTIMATED & & & 618-9 BURIAL GD|B00Y85 \\
\hline 1027 & 2574 & NAD83 & 566900 & 156840 & HEISPROD & ESTIMATED & & & NORTH SLOPE|B07KQ4 \\
\hline
\end{tabular}




\begin{tabular}{|c|c|c|c|c|c|c|c|c|c|}
\hline & A & $\mathrm{B}$ & $\mathrm{C}$ & $\mathrm{D}$ & $\mathrm{E}$ & $\mathrm{F}$ & G & $\mathrm{H}$ & $\mathrm{I}$ \\
\hline 1 & SAMP_SITE_ID & HORIZ_COORD_TYPE & EW_COORD & NS_COORD & OWNER_ID & COORD_SOURCE & DIST_CLASS & RAD_LEVEL & SAMP_SITE_NAME \\
\hline 1028 & 2575 & NAD83 & 588400 & 150200 & HEISPROD & ESTIMATED & & & NORTH SLOPE|B07KR9 \\
\hline 1029 & 2576 & NAD83 & 580498 & 147524 & HEISPROD & ESTIMATED & & & 100 AREA EXC TS|B09769 \\
\hline 1030 & 2577 & NAD83 & 580388 & 147494 & HEISPROD & ESTIMATED & & & 100 AREA EXC TS|B09785 \\
\hline 1031 & 2578 & NAD83 & 573770 & 151686 & HEISPROD & ESTIMATED & & & 100-DR-1 LFI|B07263 \\
\hline 1032 & 2581 & NAD83 & 594016.6 & 116224.9 & HEISPROD & GPS & & & 300-FF-2 SE|B0BQQ2 \\
\hline 1033 & 2582 & NAD83 & 594356 & 116079 & HEISPROD & ESTIMATED & & & 300-FF-1|B0GLN2 \\
\hline 1034 & 2583 & NAD83 & 594306 & 116020 & HEISPROD & ESTIMATED & & & 300-FF-1|B0GLG2 \\
\hline 1035 & 2584 & NAD83 & 594306 & 116028 & HEISPROD & ESTIMATED & & & 300-FF-1|B0GLG3 \\
\hline 1036 & 2585 & NAD83 & 594306 & 116066 & HEISPROD & ESTIMATED & & & 300-FF-1|B0GLG4 \\
\hline 1037 & 2586 & NAD83 & 594306 & 116133 & HEISPROD & ESTIMATED & & & 300-FF-1|B0GLG5 \\
\hline 1038 & 2587 & NAD83 & 594306 & 116146 & HEISPROD & ESTIMATED & & & 300-FF-1|B0GLG6 \\
\hline 1039 & 2588 & NAD83 & 594306 & 116154 & HEISPROD & ESTIMATED & & & 300-FF-1|B0GLG7 \\
\hline 1040 & 2589 & NAD83 & 594297 & 116154 & HEISPROD & ESTIMATED & & & 300-FF-1|B0GLG8 \\
\hline 1041 & 2590 & NAD83 & 594297 & 116121 & HEISPROD & ESTIMATED & & & 300-FF-1|B0GLG9 \\
\hline 1042 & 2591 & NAD83 & 594297 & 116112 & HEISPROD & ESTIMATED & & & 300-FF-1|B0GLH0 \\
\hline 1043 & 2592 & NAD83 & 594297 & 116100 & HEISPROD & ESTIMATED & & & 300-FF-1|B0GLH1 \\
\hline 1044 & 2593 & NAD83 & 594297 & 116087 & HEISPROD & ESTIMATED & & & 300-FF-1|B0GLH2 \\
\hline 1045 & 2594 & NAD83 & 594297 & 116049 & HEISPROD & ESTIMATED & & & 300-FF-1|B0GLH3 \\
\hline 1047 & 2596 & NAD83 & 594297 & 116028 & HEISPROD & ESTIMATED & & & 300-FF-1|B0GLH5 \\
\hline 1048 & 2597 & NAD83 & 594289 & 116041 & HEISPROD & ESTIMATED & & & 300-FF-1|B0GLH6 \\
\hline 1049 & 2598 & NAD83 & 594289 & 116049 & HEISPROD & ESTIMATED & & & 300-FF-1|B0GLH7 \\
\hline 1050 & 2599 & NAD83 & 594289 & 116058 & HEISPROD & ESTIMATED & & & 300-FF-1|B0GLH8 \\
\hline 1051 & 2600 & NAD83 & 594289 & 116087 & HEISPROD & ESTIMATED & & & 300-FF-1|B0GLH9 \\
\hline 1052 & 2601 & NAD83 & 594289 & 116146 & HEISPROD & ESTIMATED & & & 300-FF-1|B0GLJ0 \\
\hline 1053 & 2602 & NAD83 & 594289 & 116162 & HEISPROD & ESTIMATED & & & 300-FF-1|BOGLJ1 \\
\hline 1054 & 2603 & NAD83 & 594281 & 116162 & HEISPROD & ESTIMATED & & & 300-FF-1|B0GLJ2 \\
\hline 1055 & 2604 & NAD83 & 594281 & 116146 & HEISPROD & ESTIMATED & & & 300-FF-1|B0GLJ3 \\
\hline 1056 & 2605 & NAD83 & 594281 & 116121 & HEISPROD & ESTIMATED & & & 300-FF-1|B0GLJ4 \\
\hline 1057 & 2606 & NAD83 & 594281 & 116079 & HEISPROD & ESTIMATED & & & 300-FF-1|B0GLJ5 \\
\hline 1058 & 2607 & NAD83 & 594281 & 116066 & HEISPROD & ESTIMATED & & & 300-FF-1|B0GLJ6 \\
\hline 1059 & 2608 & NAD83 & 594281 & 116041 & HEISPROD & ESTIMATED & & & 300-FF-1|B0GLJ7 \\
\hline 1060 & 2609 & NAD83 & 594281 & 116028 & HEISPROD & ESTIMATED & & & 300-FF-1|B0GLJ8 \\
\hline 1061 & 2610 & NAD83 & 594272 & 116041 & HEISPROD & ESTIMATED & & & 300-FF-1|B0GLJ9 \\
\hline 1062 & 2611 & NAD83 & 594272 & 116049 & HEISPROD & ESTIMATED & & & 300-FF-1|BOGLK0 \\
\hline 1063 & 2612 & NAD83 & 594272 & 116058 & HEISPROD & ESTIMATED & & & 300-FF-1|B0GLK1 \\
\hline 1064 & 2613 & NAD83 & 594272 & 116100 & HEISPROD & ESTIMATED & & & 300-FF-1|B0GLK2 \\
\hline
\end{tabular}




\begin{tabular}{|c|c|c|c|c|c|c|c|c|c|}
\hline & $\mathrm{A}$ & $B$ & $\mathrm{C}$ & $\mathrm{D}$ & $\mathrm{E}$ & $\mathrm{F}$ & $G$ & $\mathrm{H}$ & $\mathrm{I}$ \\
\hline 1 & SAMP_SITE_ID & HORIZ_COORD_TYPE & EW_COORD & NS_COORD & OWNER_ID & COORD_SOURCE & DIST_CLASS & RAD_LEVEL & SAMP_SITE_NAME \\
\hline 1066 & 2616 & NAD83 & 594272 & 116162 & HEISPROD & ESTIMATED & & & 300-FF-1|B0GLK5 \\
\hline 1067 & 2617 & NAD83 & 594272 & 116196 & HEISPROD & ESTIMATED & & & 300-FF-1|B0GLK6 \\
\hline 1068 & 2618 & NAD83 & 594264 & 116066 & HEISPROD & ESTIMATED & & & 300-FF-1|B0GLK7 \\
\hline 1069 & 2619 & NAD83 & 594264 & 116100 & HEISPROD & ESTIMATED & & & 300-FF-1|B0GLK9 \\
\hline 1070 & 2620 & NAD83 & 594247 & 116146 & HEISPROD & ESTIMATED & & & 300-FF-1|B0GLLO \\
\hline 1071 & 2621 & NAD83 & 594314 & 116058 & HEISPROD & ESTIMATED & & & 300-FF-1|B0GLL1 \\
\hline 1072 & 2622 & NAD83 & 594314 & 116079 & HEISPROD & ESTIMATED & & & 300-FF-1|B0GLL2 \\
\hline 1073 & 2623 & NAD83 & 594314 & 116087 & HEISPROD & ESTIMATED & & & 300-FF-1|B0GLL3 \\
\hline 1074 & 2624 & NAD83 & 594314 & 116162 & HEISPROD & ESTIMATED & & & 300-FF-1|B0GLL4 \\
\hline 1075 & 2625 & NAD83 & 594327 & 116187 & HEISPROD & ESTIMATED & & & 300-FF-1|B0GLL5 \\
\hline 1076 & 2626 & NAD83 & 594327 & 116100 & HEISPROD & ESTIMATED & & & 300-FF-1|B0GLL6 \\
\hline 1077 & 2627 & NAD83 & 594327 & 116087 & HEISPROD & ESTIMATED & & & 300-FF-1|B0GLL7 \\
\hline 1078 & 2628 & NAD83 & 594327 & 116070 & HEISPROD & ESTIMATED & & & 300-FF-1|B0GLL8 \\
\hline 1079 & 2629 & NAD83 & 594327 & 116058 & HEISPROD & ESTIMATED & & & 300-FF-1|B0GLL9 \\
\hline 1080 & 2630 & NAD83 & 594327 & 116049 & HEISPROD & ESTIMATED & & & 300-FF-1|B0GLM0 \\
\hline 1081 & 2631 & NAD83 & 594335 & 116070 & HEISPROD & ESTIMATED & & & 300-FF-1|B0GLM1 \\
\hline 1082 & 2632 & NAD83 & 594335 & 116087 & HEISPROD & ESTIMATED & & & 300-FF-1|B0GLM3 \\
\hline 1083 & 2633 & NAD83 & 594335 & 116121 & HEISPROD & ESTIMATED & & & 300-FF-1|B0GLM4 \\
\hline 1084 & 2634 & NAD83 & 594343 & 116187 & HEISPROD & ESTIMATED & & & 300-FF-1|B0GLM5 \\
\hline 1085 & 2635 & NAD83 & 594343 & 116146 & HEISPROD & ESTIMATED & & & 300-FF-1|B0GLM6 \\
\hline 1086 & 2636 & NAD83 & 594343 & 116121 & HEISPROD & ESTIMATED & & & 300-FF-1|B0GLM7 \\
\hline 1087 & 2637 & NAD83 & 594343 & 116070 & HEISPROD & ESTIMATED & & & 300-FF-1|B0GLM8 \\
\hline 1088 & 2638 & NAD83 & 594343 & 116049 & HEISPROD & ESTIMATED & & & 300-FF-1|B0GLM9 \\
\hline 1089 & 2639 & NAD83 & 594356 & 116041 & HEISPROD & ESTIMATED & & & 300-FF-1|B0GLN0 \\
\hline 1090 & 2640 & NAD83 & 594364 & 116049 & HEISPROD & ESTIMATED & & & 300-FF-1|B0GLN3 \\
\hline 1091 & 2641 & NAD83 & 594364 & 116104 & HEISPROD & ESTIMATED & & & 300-FF-1|BOGLN4 \\
\hline 1092 & 2642 & NAD83 & 594364 & 116121 & HEISPROD & ESTIMATED & & & 300-FF-1|B0GLN5 \\
\hline 1093 & 2643 & NAD83 & 594373 & 116146 & HEISPROD & ESTIMATED & & & 300-FF-1|B0GLN6 \\
\hline 1094 & 2644 & NAD83 & 594373 & 116062 & HEISPROD & ESTIMATED & & & 300-FF-1|B0GLN8 \\
\hline 1095 & 2645 & NAD83 & 594373 & 116028 & HEISPROD & ESTIMATED & & & 300-FF-1|B0GLN9 \\
\hline 1096 & 2646 & NAD83 & 594385 & 116028 & HEISPROD & ESTIMATED & & & 300-FF-1|B0GLP0 \\
\hline 1097 & 2647 & NAD83 & 594385 & 116070 & HEISPROD & ESTIMATED & & & 300-FF-1|B0GLP1 \\
\hline 1098 & 2648 & NAD83 & 594385 & 116095 & HEISPROD & ESTIMATED & & & 300-FF-1|B0GLP2 \\
\hline 1099 & 2649 & NAD83 & 594385 & 116167 & HEISPROD & ESTIMATED & & & 300-FF-1|B0GLP4 \\
\hline 1100 & 2650 & NAD83 & 594394 & 116028 & HEISPROD & ESTIMATED & & & 300-FF-1|B0GLP5 \\
\hline 1101 & 2651 & NAD83 & 594394 & 116054 & HEISPROD & ESTIMATED & & & 300-FF-1|B0GLP6 \\
\hline 1102 & 2652 & NAD83 & 594394 & 116104 & HEISPROD & ESTIMATED & & & 300-FF-1|B0GLP7 \\
\hline 1103 & 2653 & NAD83 & 594394 & 116133 & HEISPROD & ESTIMATED & & & 300-FF-1|B0GLP9 \\
\hline
\end{tabular}




\begin{tabular}{|c|c|c|c|c|c|c|c|c|c|}
\hline & A & $\mathrm{B}$ & $\mathrm{C}$ & $\mathrm{D}$ & $\mathrm{E}$ & $\mathrm{F}$ & G & $\mathrm{H}$ & $\mathrm{I}$ \\
\hline 1 & SAMP_SITE_ID & HORIZ_COORD_TYPE & EW_COORD & NS_COORD & OWNER_ID & COORD_SOURCE & DIST_CLASS & RAD_LEVEL & SAMP_SITE_NAME \\
\hline 1104 & 2654 & NAD83 & 594402 & 116154 & HEISPROD & ESTIMATED & & & 300-FF-1|BOGLQ0 \\
\hline 1105 & 2655 & NAD83 & 594402 & 116125 & HEISPROD & ESTIMATED & & & 300-FF-1|B0GLQ1 \\
\hline 1106 & 2656 & NAD83 & 594402 & 116112 & HEISPROD & ESTIMATED & & & 300-FF-1|B0GLQ2 \\
\hline 1107 & 2657 & NAD83 & 594402 & 116079 & HEISPROD & ESTIMATED & & & 300-FF-1|B0GLQ3 \\
\hline 1108 & 2658 & NAD83 & 594410 & 116146 & HEISPROD & ESTIMATED & & & 300-FF-1|B0GLQ4 \\
\hline 1109 & 2660 & NAD83 & 594201 & 116126 & HEISPROD & ESTIMATED & & & 300-FF-1|B0GLR2 \\
\hline 1110 & 2661 & NAD83 & 594193 & 116138 & HEISPROD & ESTIMATED & & & 300-FF-1|B0GLR3 \\
\hline 1111 & 2662 & NAD83 & 594207 & 116084 & HEISPROD & ESTIMATED & & & 300-FF-1|B0GLR4 \\
\hline 1112 & 2663 & NAD83 & 594227 & 116027 & HEISPROD & ESTIMATED & & & 300-FF-1|B0GLR5 \\
\hline 1113 & 2664 & NAD83 & 577908 & 152841 & HEISPROD & ESTIMATED & & & 183-H BASIN|B0GNX5 \\
\hline 1114 & 2665 & NAD83 & 577892 & 152841 & HEISPROD & ESTIMATED & & & 183-H BASIN|B0GNX9 \\
\hline 1115 & 2666 & NAD83 & 577875 & 152843 & HEISPROD & ESTIMATED & & & 183-H BASIN|B0GNX2 \\
\hline 1116 & 2667 & NAD83 & 577858 & 152842 & HEISPROD & ESTIMATED & & & 183-H BASIN|BOGNYO \\
\hline 1117 & 2668 & NAD83 & 577879.4 & 152851.3 & HEISPROD & ESTIMATED & & & 183-H BASIN|BOGSH2 \\
\hline 1118 & 2669 & NAD83 & 565770.8 & 145274.6 & HEISPROD & ESTIMATED & & & 116-C-1 TRENCH EXCAVATION|B0GJ11 \\
\hline 1119 & 2670 & NAD83 & 565773.7 & 145263.7 & HEISPROD & ESTIMATED & & & 116-C-1 TRENCH EXCAVATION|B0GJ12 \\
\hline 1120 & 2671 & NAD83 & 565776.4 & 145254 & HEISPROD & ESTIMATED & & & 116-C-1 TRENCH EXCAVATION|BOGJ13 \\
\hline 1121 & 2672 & NAD83 & 565777.7 & 145243.3 & HEISPROD & ESTIMATED & & & 116-C-1 TRENCH EXCAVATION|BOGJ14 \\
\hline 1122 & 2673 & NAD83 & 565803.5 & 145247.2 & HEISPROD & ESTIMATED & & & 116-C-1 TRENCH EXCAVATION|B0GJ15 \\
\hline 1123 & 2674 & NAD83 & 565802.8 & 145258 & HEISPROD & ESTIMATED & & & 116-C-1 TRENCH EXCAVATION|B0GJ16 \\
\hline 1124 & 2675 & NAD83 & 565802.2 & 145267 & HEISPROD & ESTIMATED & & & 116-C-1 TRENCH EXCAVATION|BOGJ17 \\
\hline 1125 & 2676 & NAD83 & 565782.5 & 145277.8 & HEISPROD & ESTIMATED & & & 116-C-1 TRENCH EXCAVATION|B0GJ18 \\
\hline 1126 & 2677 & NAD83 & 565791.1 & 145280.1 & HEISPROD & ESTIMATED & & & 116-C-1 TRENCH EXCAVATION|BOGJ19 \\
\hline 1127 & 2678 & NAD83 & 565799.6 & 145282.4 & HEISPROD & ESTIMATED & & & 116-C-1 TRENCH EXCAVATION|B0GJ20 \\
\hline 1128 & 2679 & NAD83 & 565807.5 & 145284.6 & HEISPROD & ESTIMATED & & & 116-C-1 TRENCH EXCAVATION|B0GJ21 \\
\hline 1129 & 2680 & NAD83 & 565809.7 & 145276.6 & HEISPROD & ESTIMATED & & & 116-C-1 TRENCH EXCAVATION|B0GJ22 \\
\hline 1130 & 2681 & NAD83 & 565809.9 & 145276 & HEISPROD & ESTIMATED & & & 116-C-1 TRENCH EXCAVATION|B0GJ23 \\
\hline 1131 & 2682 & NAD83 & 565814.3 & 145259.6 & HEISPROD & ESTIMATED & & & 116-C-1 TRENCH EXCAVATION|BOGJ24 \\
\hline 1132 & 2683 & NAD83 & 565815 & 145251.2 & HEISPROD & ESTIMATED & & & 116-C-1 TRENCH EXCAVATION|B0GJ25 \\
\hline 1133 & 2684 & NAD83 & 565769.5 & 145267.6 & HEISPROD & ESTIMATED & & & 116-C-1 TRENCH EXCAVATION|B0GJ26 \\
\hline 1134 & 2685 & NAD83 & 565767.8 & 145256.4 & HEISPROD & ESTIMATED & & & 116-C-1 TRENCH EXCAVATION|BOGJ27 \\
\hline 1135 & 2686 & NAD83 & 565768.1 & 145243.8 & HEISPROD & ESTIMATED & & & 116-C-1 TRENCH EXCAVATION|B0GJ28 \\
\hline 1136 & 2687 & NAD83 & 565770.1 & 145236.5 & HEISPROD & ESTIMATED & & & 116-C-1 TRENCH EXCAVATION|B0GJ29 \\
\hline 1137 & 2688 & NAD83 & 565802.8 & 145250.8 & HEISPROD & ESTIMATED & & & 116-C-1 TRENCH EXCAVATION|B0GJ30 \\
\hline 1138 & 2689 & NAD83 & 565799.6 & 145255.6 & HEISPROD & ESTIMATED & & & 116-C-1 TRENCH EXCAVATION|B0GJ32 \\
\hline 1139 & 2690 & NAD83 & 565802.8 & 145261.2 & HEISPROD & ESTIMATED & & & 116-C-1 TRENCH EXCAVATION|B0GJ34 \\
\hline 1140 & 2691 & NAD83 & 565785.6 & 145272.3 & HEISPROD & ESTIMATED & & & 116-C-1 TRENCH EXCAVATION|B0GJ36 \\
\hline 1141 & 2693 & NAD83 & 565808.6 & 145251.7 & HEISPROD & ESTIMATED & & & 116-C-1 TRENCH EXCAVATION|B0GJ38 \\
\hline
\end{tabular}




\begin{tabular}{|c|c|c|c|c|c|c|c|c|c|}
\hline & A & $\mathrm{B}$ & $\mathrm{C}$ & $\mathrm{D}$ & $\mathrm{E}$ & $\mathrm{F}$ & G & $\mathrm{H}$ & $\mathrm{I}$ \\
\hline 1 & SAMP_SITE_ID & HORIZ_COORD_TYPE & EW_COORD & NS_COORD & OWNER_ID & COORD_SOURCE & DIST_CLASS & RAD_LEVEL & SAMP_SITE_NAME \\
\hline 1142 & 2694 & NAD83 & 565809.8 & 145247.3 & HEISPROD & ESTIMATED & & & 116-C-1 TRENCH EXCAVATION|B0GJ40 \\
\hline 1143 & 2695 & NAD83 & 565805.4 & 145246.1 & HEISPROD & ESTIMATED & & & 116-C-1 TRENCH EXCAVATION|B0GJ42 \\
\hline 1144 & 2697 & NAD83 & 565804.9 & 145257 & HEISPROD & ESTIMATED & & & 116-C-1 TRENCH EXCAVATION|B0GJ44 \\
\hline 1145 & 2698 & NAD83 & 565807.4 & 145256.1 & HEISPROD & ESTIMATED & & & 116-C-1 TRENCH EXCAVATION|B0GJ46 \\
\hline 1146 & 2699 & NAD83 & 565803 & 145254.9 & HEISPROD & ESTIMATED & & & 116-C-1 TRENCH EXCAVATION|B0GJ47 \\
\hline 1147 & 2700 & NAD83 & 565802.6 & 145256.4 & HEISPROD & ESTIMATED & & & 116-C-1 TRENCH EXCAVATION|B0GJ50 \\
\hline 1148 & 2701 & NAD83 & 565800.6 & 145249.5 & HEISPROD & ESTIMATED & & & 116-C-1 TRENCH EXCAVATION|B0GJ52 \\
\hline 1149 & 2702 & NAD83 & 565801.4 & 145246.6 & HEISPROD & ESTIMATED & & & 116-C-1 TRENCH EXCAVATION|B0GJ54 \\
\hline 1150 & 2703 & NAD83 & 565799.1 & 145246 & HEISPROD & ESTIMATED & & & 116-C-1 TRENCH EXCAVATION|B0GJ56 \\
\hline 1151 & 2704 & NAD83 & 565798.3 & 145248.9 & HEISPROD & ESTIMATED & & & 116-C-1 TRENCH EXCAVATION|B0GJ58 \\
\hline 1152 & 2705 & NAD83 & 565796.3 & 145256.2 & HEISPROD & ESTIMATED & & & 116-C-1 TRENCH EXCAVATION|B0GJ60 \\
\hline 1153 & 2706 & NAD83 & 565799.8 & 145252.5 & HEISPROD & ESTIMATED & & & 116-C-1 TRENCH EXCAVATION|B0GJ62 \\
\hline 1154 & 2707 & NAD83 & 565797.5 & 145251.8 & HEISPROD & ESTIMATED & & & 116-C-1 TRENCH EXCAVATION|B0GJ64 \\
\hline 1155 & 2708 & NAD83 & 565794 & 145258.8 & HEISPROD & ESTIMATED & & & 116-C-1 TRENCH EXCAVATION|B0GJ66 \\
\hline 1156 & 2709 & NAD83 & 565793 & 145262.6 & HEISPROD & ESTIMATED & & & 116-C-1 TRENCH EXCAVATION|B0GJ72 \\
\hline 1157 & 2710 & NAD83 & 565797.4 & 145263.8 & HEISPROD & ESTIMATED & & & 116-C-1 TRENCH EXCAVATION|B0GJ68 \\
\hline 1158 & 2711 & NAD83 & 566798.4 & 145260 & HEISPROD & ESTIMATED & & & 116-C-1 TRENCH EXCAVATION|B0GJ70 \\
\hline 1159 & 2712 & NAD83 & 565786.7 & 145256.8 & HEISPROD & ESTIMATED & & & 116-C-1 TRENCH EXCAVATION|B0GJ80 \\
\hline 1160 & 2713 & NAD83 & 565791.1 & 145258 & HEISPROD & ESTIMATED & & & 116-C-1 TRENCH EXCAVATION|B0GJ78 \\
\hline 1161 & 2714 & NAD83 & 565787.5 & 145253.8 & HEISPROD & ESTIMATED & & & 116-C-1 TRENCH EXCAVATION|B0GJ82 \\
\hline 1162 & 2715 & NAD83 & 565791.9 & 145255 & HEISPROD & ESTIMATED & & & 116-C-1 TRENCH EXCAVATION|B0GJ84 \\
\hline 1163 & 2716 & NAD83 & 565788.7 & 145249.4 & HEISPROD & ESTIMATED & & & 116-C-1 TRENCH EXCAVATION|B0GJ88 \\
\hline 1164 & 2717 & NAD83 & 565793.1 & 145250.6 & HEISPROD & ESTIMATED & & & 116-C-1 TRENCH EXCAVATION|B0GJ86 \\
\hline 1165 & 2718 & NAD83 & 565777.9 & 145254.1 & HEISPROD & ESTIMATED & & & 116-C-1 TRENCH EXCAVATION|B0GJ76 \\
\hline 1166 & 2719 & NAD83 & 565780.6 & 145244.4 & HEISPROD & ESTIMATED & & & 116-C-1 TRENCH EXCAVATION|B0GJ92 \\
\hline 1167 & 2720 & NAD83 & 565794.7 & 145244.8 & HEISPROD & ESTIMATED & & & 116-C-1 TRENCH EXCAVATION|B0GJ94 \\
\hline 1168 & 2721 & NAD83 & 565785.6 & 145260.6 & HEISPROD & ESTIMATED & & & 116-C-1 TRENCH EXCAVATION|BOGJB4 \\
\hline 1169 & 2722 & NAD83 & 565791.2 & 145262.1 & HEISPROD & ESTIMATED & & & 116-C-1 TRENCH EXCAVATION|B0GJB6 \\
\hline 1170 & 2723 & NAD83 & 565775.3 & 145263.8 & HEISPROD & ESTIMATED & & & 116-C-1 TRENCH EXCAVATION|B0GJ96 \\
\hline 1171 & 2724 & NAD83 & 565772.7 & 145273.2 & HEISPROD & ESTIMATED & & & 116-C-1 TRENCH EXCAVATION|B0GJB2 \\
\hline 1172 & 2725 & NAD83 & 565680.6 & 145147.4 & HEISPROD & & & & 116-C-1 TRENCH EXCAVATION|B0GJG7 \\
\hline 1173 & 2726 & NAD83 & 565784.7 & 145264.1 & HEISPROD & ESTIMATED & & & 116-C-1 TRENCH EXCAVATION \\
\hline 1174 & 2727 & NAD83 & 565798.6 & 145271.1 & HEISPROD & ESTIMATED & & & 116-C-1 TRENCH EXCAVATION|B0GJB9 \\
\hline 1175 & 2728 & NAD83 & 565797.4 & 145258.1 & HEISPROD & ESTIMATED & & & 116-C-1 TRENCH EXCAVATION|B0GJC0 \\
\hline 1176 & 2729 & NAD83 & 565791.5 & 145256.5 & HEISPROD & ESTIMATED & & & 116-C-1 TRENCH EXCAVATION|B0GJC1 \\
\hline 1177 & 2730 & NAD83 & 565774.5 & 145272.4 & HEISPROD & ESTIMATED & & & 116-C-1 TRENCH EXCAVATION|B0GJC3 \\
\hline 1178 & 2731 & NAD83 & 565779.3 & 145254.8 & HEISPROD & ESTIMATED & & & 116-C-1 TRENCH EXCAVATION|B0GJC4 \\
\hline 1179 & 2732 & NAD83 & 565802.3 & 145268.9 & HEISPROD & ESTIMATED & & & 116-C-1 TRENCH EXCAVATION|B0GJC5 \\
\hline
\end{tabular}




\begin{tabular}{|c|c|c|c|c|c|c|c|c|c|}
\hline & $A$ & $B$ & $\mathrm{C}$ & $D$ & $E$ & $F$ & $G$ & $\mathrm{H}$ & $\mathrm{I}$ \\
\hline 1 & SAMP_SITE_ID & HORIZ_COORD_TYPE & EW_COORD & NS_COORD & OWNER_ID & COORD_SOURCE & DIST_CLASS & RAD_LEVEL & SAMP_SITE_NAME \\
\hline 1180 & 2733 & NAD83 & 565801.3 & 145261.6 & HEISPROD & ESTIMATED & & & 116-C-1 TRENCH EXCAVATION|B0GJC6 \\
\hline 1181 & 2734 & NAD83 & 565811.7 & 145263.6 & HEISPROD & ESTIMATED & & & 116-C-1 TRENCH EXCAVATION|B0GJC7 \\
\hline 1182 & 2735 & NAD83 & 565824.8 & 145273.5 & HEISPROD & ESTIMATED & & & 116-C-1 TRENCH EXCAVATION|B0GJD0 \\
\hline 1183 & 2736 & NAD83 & 565851.4 & 145274.4 & HEISPROD & ESTIMATED & & & 116-C-1 TRENCH EXCAVATION|B0GJD1 \\
\hline 1184 & 2737 & NAD83 & 565797 & 145259.6 & HEISPROD & ESTIMATED & & & 116-C-1 TRENCH EXCAVATION|B0GJD8 \\
\hline 1185 & 2738 & NAD83 & 565795 & 145266.9 & HEISPROD & ESTIMATED & & & 116-C-1 TRENCH EXCAVATION|B0GJD7 \\
\hline 1186 & 2739 & NAD83 & 565789.6 & 145257.6 & HEISPROD & ESTIMATED & & & 116-C-1 TRENCH EXCAVATION|B0GJD6 \\
\hline 1187 & 2740 & NAD83 & 565787.6 & 145264.9 & HEISPROD & ESTIMATED & & & 116-C-1 TRENCH EXCAVATION|B0GJD5 \\
\hline 1188 & 2742 & NAD83 & 565804.3 & 145261.6 & HEISPROD & ESTIMATED & & & 116-C-1 TRENCH EXCAVATION|B0GJF2 \\
\hline 1189 & 2744 & NAD83 & 565825.8 & 145275.3 & HEISPROD & ESTIMATED & & & 116-C-1 TRENCH EXCAVATION|B0GJF5 \\
\hline 1190 & 2745 & NAD83 & 565817.9 & 145263.7 & HEISPROD & ESTIMATED & & & 116-C-1 TRENCH EXCAVATION|B0GJF4 \\
\hline 1191 & 2746 & NAD83 & 565848.8 & 145272.1 & HEISPROD & ESTIMATED & & & 116-C-1 TRENCH EXCAVATION|B0GJF6 \\
\hline 1192 & 2749 & NAD83 & 565788.4 & 145262 & HEISPROD & ESTIMATED & & & 116-C-1 TRENCH EXCAVATION|B0GJF7 \\
\hline 1193 & 2750 & NAD83 & 565797.2 & 145264.4 & HEISPROD & ESTIMATED & & & 116-C-1 TRENCH EXCAVATION|BOGJF8 \\
\hline 1194 & 2751 & NAD83 & 565789.5 & 145263.9 & HEISPROD & ESTIMATED & & & 116-C-1 TRENCH EXCAVATION|B0GJF9 \\
\hline 1195 & 2752 & NAD83 & 565798.7 & 145264.8 & HEISPROD & ESTIMATED & & & 116-C-1 TRENCH EXCAVATION|B0GJG0 \\
\hline 1196 & 2753 & NAD83 & 565794 & 145276.2 & HEISPROD & ESTIMATED & & & 116-C-1 TRENCH EXCAVATION|B0GJG1 \\
\hline 1197 & 2754 & NAD83 & 565782.4 & 145318.8 & HEISPROD & ESTIMATED & & & 116-C-1 TRENCH EXCAVATION|B0GJG2 \\
\hline 1198 & 2756 & NAD83 & 565787.9 & 145252.4 & HEISPROD & ESTIMATED & & & 116-C-1 TRENCH EXCAVATION|B0GJG4 \\
\hline 1199 & 2758 & NAD83 & 565776.4 & 145271.4 & HEISPROD & ESTIMATED & & & 116-C-1 TRENCH EXCAVATION|B0GJG6 \\
\hline 1200 & 2761 & NAD83(91) & 587486.82 & 123554.59 & HEISPROD & GPS & & & 300-FF-2 LFI|B0GG05 \\
\hline 1201 & 2765 & NAD83(91) & 592659.88 & 115852.16 & HEISPROD & GPS & & & 300-FF-2 LFI|B0GG07 \\
\hline 1202 & 2766 & NAD83(91) & 592414.24 & 115863.51 & HEISPROD & GPS & & & 300-FF-2 LFI|B0GG09 \\
\hline 1203 & 2768 & NAD83 & 565367.7 & 144507.9 & HEISPROD & ESTIMATED & & & 100-BC-1 EXC PN|B0G8H1 \\
\hline 1204 & 2769 & NAD83 & 565368.1 & 144508.8 & HEISPROD & ESTIMATED & & & 100-BC-1 EXC PN|B0G8H3 \\
\hline 1205 & 2771 & NAD83 & 565367.3 & 144508.8 & HEISPROD & ESTIMATED & & & 100-BC-1 EXC PN|B0G8H5 \\
\hline 1206 & 2772 & NAD83 & 565365.1 & 144514.9 & HEISPROD & ESTIMATED & & & 100-BC-1 EXC PN|B0G8H7 \\
\hline 1207 & 2773 & NAD83 & 565365.1 & 144502.7 & HEISPROD & ESTIMATED & & & 100-BC-1 EXC PN|B0G8H9 \\
\hline 1208 & 2775 & NAD83 & 565363.6 & 144508.8 & HEISPROD & ESTIMATED & & & 100-BC-1 EXC PN|B0G8J5 \\
\hline 1209 & 2776 & NAD83 & 565367.7 & 144508.4 & HEISPROD & ESTIMATED & & & 100-BC-1 EXC PN|B0G8K1 \\
\hline 1210 & 2777 & NAD83 & 565347.6 & 144515.6 & HEISPROD & ESTIMATED & & & 100-BC-1 EXC PN|B0G8M9 \\
\hline 1211 & 2778 & NAD83 & 565349.9 & 144514.8 & HEISPROD & ESTIMATED & & & 100-BC-1 EXC PN|B0G8N1 \\
\hline 1212 & 2779 & NAD83 & 565352.2 & 144514.1 & HEISPROD & ESTIMATED & & & 100-BC-1 EXC PN|B0G8N3 \\
\hline 1213 & 2780 & NAD83 & 565354.6 & 144513.3 & HEISPROD & ESTIMATED & & & 100-BC-1 EXC PN|B0G8N5 \\
\hline 1214 & 2781 & NAD83 & 565356.9 & 144512.5 & HEISPROD & ESTIMATED & & & 100-BC-1 EXC PN|B0G8N7 \\
\hline 1215 & 2782 & NAD83 & 565369.2 & 144511.8 & HEISPROD & ESTIMATED & & & 100-BC-1 EXC PN|B0G8N9 \\
\hline 1216 & 2783 & NAD83 & 565361.5 & 144511 & HEISPROD & ESTIMATED & & & 100-BC-1 EXC PN|B0G8P1 \\
\hline 1217 & 2784 & NAD83 & 565369.7 & 144512.5 & HEISPROD & ESTIMATED & & & 100-BC-1 EXC PN|B0G8P3 \\
\hline
\end{tabular}




\begin{tabular}{|c|c|c|c|c|c|c|c|c|c|}
\hline & A & $\mathrm{B}$ & $\mathrm{C}$ & $\mathrm{D}$ & $E$ & $\mathrm{~F}$ & G & $\mathrm{H}$ & $\mathrm{I}$ \\
\hline 1 & SAMP_SITE_ID & HORIZ_COORD_TYPE & EW_COORD & NS_COORD & OWNER_ID & COORD_SOURCE & DIST_CLASS & RAD_LEVEL & SAMP_SITE_NAME \\
\hline 1218 & 2786 & NAD83 & 565365.7 & 144512.5 & HEISPROD & ESTIMATED & & & 100-BC-1 EXC PN|B0G8P5 \\
\hline 1219 & 2787 & NAD83 & 565369.7 & 144514 & HEISPROD & ESTIMATED & & & 100-BC-1 EXC PN|B0G8Q5 \\
\hline 1220 & 2788 & NAD83 & 565365.7 & 144514 & HEISPROD & ESTIMATED & & & 100-BC-1 EXC PN|B0G8Q6 \\
\hline 1221 & 2789 & NAD83 & 565367.7 & 144512.5 & HEISPROD & ESTIMATED & & & 100-BC-1 EXC PN|B0G8Q7 \\
\hline 1222 & 2790 & NAD83 & 565367.7 & 144514 & HEISPROD & ESTIMATED & & & 100-BC-1 EXC PN|B0G8Q9 \\
\hline 1223 & 2791 & NAD83 & 565357.5 & 144510.7 & HEISPROD & ESTIMATED & & & 100-BC-1 EXC PN|B0G8R1 \\
\hline 1224 & 2792 & NAD83 & 565320.9 & 144469.2 & HEISPROD & ESTIMATED & & & 100-BC-1 EXC PN|B0G8R2 \\
\hline 1225 & 2793 & NAD83 & 571208.4 & 149240.1 & HEISPROD & & & & 100N 90-DAY STORAGE PAD \\
\hline 1226 & 2794 & NAD83 & 571399.32 & 149510.61 & HEISPROD & ESTIMATED & & & 100N-SS-28-UST \\
\hline 1227 & 2795 & NAD83 & 587552 & 123180 & HEISPROD & ESTIMATED & & & 400-FFTF-T-18/400 AREA \\
\hline 1228 & 2796 & NAD83 & 571769 & 150135.25 & HEISPROD & ESTIMATED & & & 100-NR-2 EAL PROCESS WASTE SAMPLES \\
\hline 1229 & 2797 & NAD83 & 565294.4 & 144768 & HEISPROD & ESTIMATED & & & $116-B-5$ \\
\hline 1230 & 2801 & NAD83 & 565289.8 & 144780 & HEISPROD & ESTIMATED & & & 116-B-5|1 \\
\hline 1231 & 2802 & NAD83 & 565289.8 & 144777.8 & HEISPROD & ESTIMATED & & & $116-B-5 \mid 2$ \\
\hline 1232 & 2803 & NAD83 & 565289.8 & 144775.6 & HEISPROD & ESTIMATED & & & $116-B-5 \mid 3$ \\
\hline 1233 & 2804 & NAD83 & 565289.8 & 144773.3 & HEISPROD & ESTIMATED & & & 116-B-5|4 \\
\hline 1234 & 2805 & NAD83 & 565289.8 & 144771.1 & HEISPROD & ESTIMATED & & & 116-B-5|5 \\
\hline 1235 & 2806 & NAD83 & 565289.8 & 144768.8 & HEISPROD & ESTIMATED & & & $116-B-5 \mid 6$ \\
\hline 1237 & 2808 & NAD83 & 565289.8 & 144764.4 & HEISPROD & ESTIMATED & & & $116-B-5 \mid 8$ \\
\hline 1238 & 2809 & NAD83 & 565289.8 & 144762.1 & HEISPROD & ESTIMATED & & & 116-B-5|9 \\
\hline 1239 & 2810 & NAD83 & 565289.8 & 144759.9 & HEISPROD & ESTIMATED & & & 116-B-5|10 \\
\hline 1240 & 2811 & NAD83 & 565289.8 & 144757.7 & HEISPROD & ESTIMATED & & & 116-B-5|11 \\
\hline 1241 & 2812 & NAD83 & 565289.8 & 144755.4 & HEISPROD & ESTIMATED & & & 116-B-5|12 \\
\hline 1242 & 2813 & NAD83 & 565289.8 & 144753.1 & HEISPROD & ESTIMATED & & & 116-B-5|13 \\
\hline 1243 & 2814 & NAD83 & 565289.8 & 144750.1 & HEISPROD & ESTIMATED & & & 116-B-5|14 \\
\hline 1244 & 2815 & NAD83 & 565289.8 & 144747 & HEISPROD & ESTIMATED & & & 116-B-5|15 \\
\hline 1245 & 2816 & NAD83 & 565289.8 & 144744 & HEISPROD & ESTIMATED & & & $116-B-5 \mid 16$ \\
\hline 1246 & 2817 & NAD83 & 565289.8 & 144740 & HEISPROD & ESTIMATED & & & 116-B-5|17 \\
\hline 1247 & 2818 & NAD83 & 565289.8 & 144737.9 & HEISPROD & ESTIMATED & & & 116-B-5|18 \\
\hline 1248 & 2819 & NAD83 & 565289.8 & 144735.7 & HEISPROD & ESTIMATED & & & 116-B-5|19 \\
\hline 1249 & 2821 & NAD83 & 594248.6 & 116433.6 & HEISPROD & ESTIMATED & & & 618-12 \\
\hline 1250 & 2822 & NAD83 & 594154.5 & 116283.1 & HEISPROD & ESTIMATED & & & $628-4$ \\
\hline 1251 & 2824 & NAD83 & 594184.1 & 116830 & HEISPROD & ESTIMATED & & & $618-5$ \\
\hline 1252 & 2825 & NAD83 & 593929.3 & 117011.7 & HEISPROD & ESTIMATED & & & $618-4$ \\
\hline 1253 & 2826 & NAD83 & 594238.7 & 116566.4 & HEISPROD & ESTIMATED & & & $316-2$ \\
\hline 1254 & 2827 & NAD83 & 565793.2 & 145261.7 & HEISPROD & ESTIMATED & & & $116-C-1 \mid 1$ \\
\hline
\end{tabular}




\begin{tabular}{|c|c|c|c|c|c|c|c|c|c|}
\hline & $A$ & $\mathrm{~B}$ & $\mathrm{C}$ & $\mathrm{D}$ & $E$ & $\mathrm{~F}$ & $G$ & $\mathrm{H}$ & $\mathrm{I}$ \\
\hline 1 & SAMP_SITE_ID & HORIZ_COORD_TYPE & EW_COORD & NS_COORD & OWNER_ID & COORD_SOURCE & DIST_CLASS & RAD_LEVEL & SAMP_SITE_NAME \\
\hline 1256 & 2829 & NAD83 & 565779.6 & 145259.6 & HEISPROD & ESTIMATED & & & $116-C-1 \mid 3$ \\
\hline 1257 & 2830 & NAD83 & 565793.5 & 145266.5 & HEISPROD & ESTIMATED & & & $|116-C-1| 4$ \\
\hline 1258 & 2837 & NAD83 & 594333.3 & 117092 & HEISPROD & ESTIMATED & & & 300-FF-5 RIF|3 \\
\hline 1259 & 2838 & NAD83 & 594336.2 & 117092 & HEISPROD & ESTIMATED & & & 300-FF-5 Rl:4 \\
\hline 1260 & 2839 & NAD83 & 594340.4 & 117092 & HEISPROD & ESTIMATED & & & 300-FF-5 RI:5 \\
\hline 1261 & 2840 & NAD83 & 594501.3 & 116203 & HEISPROD & ESTIMATED & & & 300-FF-5 RI:6 \\
\hline 1262 & 2841 & NAD83 & 594503.2 & 116203 & HEISPROD & ESTIMATED & & & 300-FF-5 RI:7 \\
\hline 1263 & 2842 & NAD83 & 594507.4 & 116203 & HEISPROD & ESTIMATED & & & 300-FF-5 RI:8 \\
\hline 1264 & 2843 & NAD83 & 594593.3 & 115831 & HEISPROD & ESTIMATED & & & 300-FF-5 RI:9 \\
\hline 1265 & 2844 & NAD83 & 594596.2 & 115831 & HEISPROD & ESTIMATED & & & 300-FF-5 RI:11 \\
\hline 1266 & 2845 & NAD83 & 594600.4 & 115831 & HEISPROD & ESTIMATED & & & 300-FF-5 Rl:12 \\
\hline 1267 & 2847 & NAD83 & 566758 & 135610.6 & CENTPLAT & ESTIMATED & & & 216-Z-9 TRENCH SITE, 200-ZP-2 \\
\hline 1268 & 3002 & NAD83 & 565367.7 & 144515.5 & HEISPROD & ESTIMATED & & & 116-B-4 FRENCH DRAIN|1 \\
\hline 1269 & 3003 & NAD83 & 565367.7 & 144503.7 & HEISPROD & ESTIMATED & & & 116-B-4 FRENCH DRAIN|2 \\
\hline 1270 & 3004 & NAD83 & 565361.1 & 144514 & HEISPROD & ESTIMATED & & & 116-B-4 FRENCH DRAIN|3 \\
\hline 1271 & 3005 & NAD83 & 565361.4 & 144508.2 & HEISPROD & ESTIMATED & & & 116-B-4 FRENCH DRAIN|4 \\
\hline 1272 & 3006 & NAD83 & 565350.8 & 144508.2 & HEISPROD & ESTIMATED & & & 116-B-4 FRENCH DRAIN|5 \\
\hline 1273 & 3007 & NAD83 & 565375.2 & 144515.9 & HEISPROD & ESTIMATED & & & 116-B-4 FRENCH DRAIN|6 \\
\hline 1274 & 3008 & NAD83 & 565365.1 & 144509.9 & HEISPROD & ESTIMATED & & & 116-B-4 FRENCH DRAIN|7 \\
\hline 1275 & 3009 & NAD83 & 565359.9 & 144515.9 & HEISPROD & ESTIMATED & & & 116-B-4 FRENCH DRAIN|8 \\
\hline 1276 & 3010 & NAD83 & 565375.2 & 144500.6 & HEISPROD & ESTIMATED & & & 116-B-4 FRENCH DRAIN|9 \\
\hline 1277 & 3026 & NAD83(91) & 573796.938 & 152220.484 & HEISPROD & HGIS & & & 1607-D2 SEPTIC SYSTEM SAMPLING 1 \\
\hline 1278 & 3027 & NAD83(91) & 573798.375 & 152407.081 & HEISPROD & HGIS & & & 1607-D2 SEPTIC SYSTEM SAMPLING 2 \\
\hline 1279 & 3053 & NAD83 & 577904 & 152863 & HEISPROD & ESTIMATED & & & 183H BASINS|1 \\
\hline 1280 & 3054 & NAD83 & 577847 & 152829 & HEISPROD & ESTIMATED & & & 183-H BASIN|2 \\
\hline 1281 & 3055 & NAD83 & 577850 & 152841 & HEISPROD & ESTIMATED & & & 183-H BASIN|3 \\
\hline 1282 & 3056 & NAD83 & 577850 & 152847 & HEISPROD & ESTIMATED & & & 183-H BASIN|4 \\
\hline 1283 & 3057 & NAD83 & 577853 & 152865 & HEISPROD & ESTIMATED & & & 183-H BASIN|5 \\
\hline 1284 & 3058 & NAD83 & 577856 & 152835 & HEISPROD & ESTIMATED & & & 183-H BASIN|6 \\
\hline 1285 & 3059 & NAD83 & 577856 & 152841 & HEISPROD & ESTIMATED & & & 183-H BASIN|7 \\
\hline 1286 & 3060 & NAD83 & 577859 & 152841 & HEISPROD & ESTIMATED & & & 183-H BASIN|8 \\
\hline 1287 & 3061 & NAD83 & 577859 & 152853 & HEISPROD & ESTIMATED & & & 183-H BASIN|9 \\
\hline 1288 & 3062 & NAD83 & 577865 & 152850 & HEISPROD & ESTIMATED & & & 183-H BASIN|10 \\
\hline 1289 & 3063 & NAD83 & 577865 & 152865 & HEISPROD & ESTIMATED & & & 183-H BASIN|11 \\
\hline 1290 & 3064 & NAD83 & 577871 & 152838 & HEISPROD & ESTIMATED & & & 183-H BASIN|12 \\
\hline 1291 & 3065 & NAD83 & 577880 & 152847 & HEISPROD & ESTIMATED & & & 183-H BASIN|13 \\
\hline 1292 & 3066 & NAD83 & 577880 & 152856 & HEISPROD & ESTIMATED & & & 183-H BASIN|14 \\
\hline 1293 & 3067 & NAD83 & 577883 & 152835 & HEISPROD & ESTIMATED & & & 183-H BASIN|15 \\
\hline
\end{tabular}




\begin{tabular}{|c|c|c|c|c|c|c|c|c|c|}
\hline & A & $B$ & $\mathrm{C}$ & $\mathrm{D}$ & $\mathrm{E}$ & $\mathrm{F}$ & G & $\mathrm{H}$ & $\mathrm{I}$ \\
\hline 1 & SAMP_SITE_ID & HORIZ_COORD_TYPE & EW_COORD & NS_COORD & OWNER_ID & COORD_SOURCE & DIST_CLASS & RAD_LEVEL & SAMP_SITE_NAME \\
\hline 1294 & 3068 & NAD83 & 577883 & 152841 & HEISPROD & ESTIMATED & & & 183-H BASIN|16 \\
\hline 1295 & 3069 & NAD83 & 577886 & 152841 & HEISPROD & ESTIMATED & & & 183-H BASIN|17 \\
\hline 1296 & 3070 & NAD83 & 577892 & 152835 & HEISPROD & ESTIMATED & & & 183-H BASIN|18 \\
\hline 1297 & 3071 & NAD83 & 577895 & 152844 & HEISPROD & ESTIMATED & & & 183-H BASIN|19 \\
\hline 1298 & 3072 & NAD83 & 577895 & 152853 & HEISPROD & ESTIMATED & & & 183-H BASIN|20 \\
\hline 1299 & 3073 & NAD83 & 577899 & 152835 & HEISPROD & ESTIMATED & & & 183-H BASIN|21 \\
\hline 1300 & 3074 & NAD83 & 577899 & 152850 & HEISPROD & ESTIMATED & & & 183-H BASIN|22 \\
\hline 1301 & 3075 & NAD83 & 577899 & 152865 & HEISPROD & ESTIMATED & & & 183-H BASIN|23 \\
\hline 1302 & 3076 & NAD83 & 577902 & 152841 & HEISPROD & ESTIMATED & & & 183-H BASIN|24 \\
\hline 1303 & 3077 & NAD83 & 577902 & 152865 & HEISPROD & ESTIMATED & & & 183-H BASIN|25 \\
\hline 1304 & 3078 & NAD83 & 577903 & 152855 & HEISPROD & ESTIMATED & & & 183-H BASIN|26 \\
\hline 1305 & 3079 & NAD83 & 577905 & 152838 & HEISPROD & ESTIMATED & & & 183-H BASIN|27 \\
\hline 1306 & 3080 & NAD83 & 577905 & 152844 & HEISPROD & ESTIMATED & & & 183-H BASIN|28 \\
\hline 1307 & 3081 & NAD83 & 577905 & 152850 & HEISPROD & ESTIMATED & & & 183-H BASIN|29 \\
\hline 1308 & 3082 & NAD83 & 577905 & 152853 & HEISPROD & ESTIMATED & & & 183-H BASIN|30 \\
\hline 1309 & 3083 & NAD83 & 577905 & 152865 & HEISPROD & ESTIMATED & & & 183-H BASIN|31 \\
\hline 1310 & 3084 & NAD83 & 577908 & 152832 & HEISPROD & ESTIMATED & & & 183-H BASIN|32 \\
\hline 1311 & 3085 & NAD83 & 577908 & 152838 & HEISPROD & ESTIMATED & & & 183-H BASIN|33 \\
\hline 1312 & 3086 & NAD83 & 577908 & 152850 & HEISPROD & ESTIMATED & & & 183-H BASIN|34 \\
\hline 1313 & 3087 & NAD83 & 577908 & 152853 & HEISPROD & ESTIMATED & & & 183-H BASIN|35 \\
\hline 1314 & 3088 & NAD83 & 577908 & 152856 & HEISPROD & ESTIMATED & & & 183-H BASIN|36 \\
\hline 1315 & 3089 & NAD83 & 577911 & 152847 & HEISPROD & ESTIMATED & & & 183-H BASIN|37 \\
\hline 1316 & 3090 & NAD83 & 577914 & 152856 & HEISPROD & ESTIMATED & & & 183-H BASIN|38 \\
\hline 1317 & 3093 & NAD83 & 571234 & 149315 & HEISPROD & ESTIMATED & & & 183N Water Treatment Plant \\
\hline 1318 & 3095 & NAD83 & 565444.3 & 145058.2 & HEISPROD & ESTIMATED & & & 116-C-5 DIVERSION BOX|100-BC-1 \\
\hline 1319 & 3096 & NAD83 & 565498 & 145110 & HEISPROD & ESTIMATED & & & 116-C-5 BASIN EAST|100-BC-1 \\
\hline 1320 & 3097 & NAD83 & 565390 & 145109.5 & HEISPROD & ESTIMATED & & & 116-C-5 BASIN WEST|100-BC-1 \\
\hline 1321 & 3098 & NAD83 & 565428.8 & 145343.7 & HEISPROD & ESTIMATED & & & 116-B-14 SLUDGE TRENCH|100-BC-1 \\
\hline 1322 & 3103 & & & & CENTPLAT & & & & 200-UP-1|BV21 INFLUENT \\
\hline 1323 & 3104 & & & & CENTPLAT & & & & 200-UP-1|BV107 EFFLUENT \\
\hline 1324 & 3105 & NAD83 & 573770 & 151680 & CENTPLAT & ESTIMATED & & & 100-DR-1|SAMPLES FOR 91-106 \\
\hline 1325 & 3106 & NAD83 & 577903 & 152883 & HEISPROD & ESTIMATED & & & 183-H SOIL|LOCATION 1 \\
\hline 1326 & 3107 & NAD83 & 577879 & 152884 & HEISPROD & ESTIMATED & & & 183-H SOIL|LOCATION 2 \\
\hline 1327 & 3108 & NAD83 & 577837 & 152890 & HEISPROD & ESTIMATED & & & 183-H SOIL|LOCATION 3 \\
\hline 1328 & 3109 & NAD83 & 577925 & 152846 & HEISPROD & ESTIMATED & & & 183-H SOIL|LOCATION 4 \\
\hline 1329 & 3110 & NAD83 & 577925 & 152831 & HEISPROD & ESTIMATED & & & 183-H SOIL|LOCATION 5 \\
\hline 1330 & 3112 & NAD83 & 577925 & 152814 & HEISPROD & ESTIMATED & & & 183-H SOIL|LOCATION 7 \\
\hline 1331 & 3909 & NAD83 & 571291.5 & 149439.2 & HEISPROD & ESTIMATED & & & 184NTANK \\
\hline
\end{tabular}




\begin{tabular}{|c|c|c|c|c|c|c|c|c|c|}
\hline & A & $\mathrm{B}$ & $\mathrm{C}$ & $\mathrm{D}$ & $\mathrm{E}$ & $\mathrm{F}$ & G & $\mathrm{H}$ & $\mathrm{I}$ \\
\hline 1 & SAMP_SITE_ID & HORIZ_COORD_TYPE & EW_COORD & NS_COORD & OWNER_ID & COORD_SOURCE & DIST_CLASS & RAD_LEVEL & SAMP_SITE_NAME \\
\hline 1332 & 3910 & NAD83 & 571182.2 & 149521.2 & CENTPLAT & ESTIMATED & & & N REACTOR FUEL STORAGE \\
\hline 1333 & 3911 & NAD83 & 566832 & 135526 & CENTPLAT & ESTIMATED & & & VAPOR EXTRACTION LAY DOWN YARD 200-ZP-2 \\
\hline 1334 & 3912 & NAD83 & 573799.8 & 152472.6 & HEISPROD & ESTIMATED & & & 1607-D2 TILE FIELD \\
\hline 1335 & 3913 & NAD83 & 572838.1 & 151748.8 & HEISPROD & ESTIMATED & & & 1607-D5 TILE FIELD \\
\hline 1336 & 3914 & NAD83 & 571213 & 149516 & HEISPROD & ESTIMATED & & & CENTRAL LOCATION FOR 105N REACTOR \\
\hline 1337 & 3915 & NAD83 & 565812.867 & 145276.508 & HEISPROD & ESTIMATED & & & 100-BC-1 REMEDIAL ACTION-1 \\
\hline 1338 & 3916 & NAD83 & 565809.211 & 145276.319 & HEISPROD & ESTIMATED & & & 100-BC-1 REMEDIAL ACTION-2 \\
\hline 1339 & 3917 & NAD83 & 565821.364 & 145276.206 & HEISPROD & ESTIMATED & & & 100-BC-1 REMEDIAL ACTION-3 \\
\hline 1340 & 3918 & NAD83 & 565884.314 & 145311.936 & HEISPROD & ESTIMATED & & & 100-BC-1 REMEDIAL ACTION-4 \\
\hline 1341 & 3919 & NAD83 & 565902.807 & 145317.769 & HEISPROD & ESTIMATED & & & 100-BC-1 REMEDIAL ACTION-5 \\
\hline 1342 & 3920 & NAD83 & 571207 & 149326 & HEISPROD & ESTIMATED & & & $183 \mathrm{~N}$ building control room sink \\
\hline 1343 & 3922 & NAD83 & 572625 & 151600 & CENTPLAT & SURVEYED & & & SD-009-1 D AREA \\
\hline 1344 & 4718 & NAD83 & 572125 & 151945 & CENTPLAT & ESTIMATED & & & SD-104-1 \\
\hline 1345 & 4719 & NAD83 & 572155 & 152008 & CENTPLAT & ESTIMATED & & & SD-105-1 \\
\hline 1346 & 4721 & NAD83 & 572200 & 151150 & CENTPLAT & ESTIMATED & & & SD-098-1 \\
\hline 1347 & 4723 & NAD83 & 572250 & 151210 & CENTPLAT & ESTIMATED & & & SD-098-2 \\
\hline 1348 & 4724 & NAD83 & 572295 & 151265 & CENTPLAT & ESTIMATED & & & SD-098-3 \\
\hline 1349 & 4732 & NAD83 & 573340 & 152280 & CENTPLAT & ESTIMATED & & & SD-107-1 \\
\hline 1350 & 4733 & NAD83 & 573380 & 152300 & CENTPLAT & & & & SD-108-1 \\
\hline 1351 & 4734 & NAD83 & 573455 & 152345 & CENTPLAT & ESTIMATED & & & SD-109-1 \\
\hline 1352 & 4738 & NAD83 & 574015 & 153260 & CENTPLAT & ESTIMATED & & & TDP-01A \\
\hline 1353 & 4739 & NAD83 & 574000 & 153215 & CENTPLAT & ESTIMATED & & & TDP-02A \\
\hline 1354 & 4740 & NAD83 & 573980 & 153175 & CENTPLAT & ESTIMATED & & & TDP-03A \\
\hline 1355 & 4741 & NAD83 & 573940 & 153070 & CENTPLAT & ESTIMATED & & & TDP-05A \\
\hline 1356 & 4742 & NAD83 & 573980 & 153025 & CENTPLAT & ESTIMATED & & & TDP-06A \\
\hline 1357 & 4743 & NAD83 & 573910 & 152975 & CENTPLAT & ESTIMATED & & & TDP-07A \\
\hline 1358 & 4744 & NAD83 & 573900 & 152920 & CENTPLAT & ESTIMATED & & & TDP-08A \\
\hline 1359 & 4745 & NAD83 & 573880 & 152850 & CENTPLAT & ESTIMATED & & & TDP-09A \\
\hline 1360 & 4746 & NAD83 & 573860 & 152790 & CENTPLAT & ESTIMATED & & & TDP-10A \\
\hline 1361 & 4747 & NAD83 & 573830 & 157760 & CENTPLAT & ESTIMATED & & & TDP-11A \\
\hline 1362 & 4748 & NAD83 & 573820 & 152700 & CENTPLAT & ESTIMATED & & & TDP-12A \\
\hline 1363 & 4749 & NAD83 & 573800 & 152635 & CENTPLAT & ESTIMATED & & & TDP-13A \\
\hline 1364 & 4750 & NAD83 & 573750 & 152595 & CENTPLAT & ESTIMATED & & & TDP-14A \\
\hline 1365 & 4751 & NAD83 & 573700 & 152555 & CENTPLAT & ESTIMATED & & & TDP-15A \\
\hline 1366 & 4752 & NAD83 & 573645 & 152520 & CENTPLAT & ESTIMATED & & & TDP-16A \\
\hline 1367 & 4753 & NAD83 & 573600 & 152470 & CENTPLAT & ESTIMATED & & & TDP-17A \\
\hline 1368 & 4754 & NAD83 & 573535 & 152420 & CENTPLAT & ESTIMATED & & & TDP-18A \\
\hline 1369 & 4755 & NAD83 & 573490 & 152380 & CENTPLAT & ESTIMATED & & & TDP-19A \\
\hline
\end{tabular}




\begin{tabular}{|c|c|c|c|c|c|c|c|c|c|}
\hline & A & $\mathrm{B}$ & $\mathrm{C}$ & $\mathrm{D}$ & $E$ & $\mathrm{~F}$ & G & $\mathrm{H}$ & $\mathrm{I}$ \\
\hline 1 & SAMP_SITE_ID & \begin{tabular}{|l} 
HORIZ_COORD_TYPE \\
\end{tabular} & EW_COORD & NS_COORD & OWNER_ID & COORD_SOURCE & DIST_CLASS & RAD_LEVEL & SAMP_SITE_NAME \\
\hline 1370 & $\overline{4756}$ & NAD83 & 573445 & 152340 & CENTPLAT & ESTIMATED & & & TDP-20A \\
\hline 1371 & 4757 & NAD83 & 573380 & 152300 & CENTPLAT & & & & TDP-21A \\
\hline 1372 & 4758 & NAD83 & 573310 & 152270 & CENTPLAT & ESTIMATED & & & TDP-22A \\
\hline 1373 & 4759 & NAD83 & 573255 & 152235 & CENTPLAT & ESTIMATED & & & TDP-23A \\
\hline 1374 & 4760 & NAD83 & 573205 & 152205 & CENTPLAT & ESTIMATED & & & TDP-24A \\
\hline 1375 & 4761 & NAD83 & 573155 & 152170 & CENTPLAT & ESTIMATED & & & TDP-25A \\
\hline 1376 & 4762 & NAD83 & 573100 & 152135 & CENTPLAT & ESTIMATED & & & TDP-26A \\
\hline 1377 & 4763 & NAD83 & 573055 & 152100 & CENTPLAT & ESTIMATED & & & TDP-27A \\
\hline 1378 & 4764 & NAD83 & 573035 & 152065 & CENTPLAT & ESTIMATED & & & TDP-28A \\
\hline 1379 & 4765 & NAD83 & 572975 & 152025 & CENTPLAT & ESTIMATED & & & TDP-29A \\
\hline 1380 & 4766 & NAD83 & 572940 & 151985 & CENTPLAT & ESTIMATED & & & TDP-30A \\
\hline 1381 & 4767 & NAD83 & 572915 & 151970 & CENTPLAT & ESTIMATED & & & TDP-31B \\
\hline 1382 & 4768 & NAD83 & 572910 & 151910 & CENTPLAT & ESTIMATED & & & TDP-32A \\
\hline 1383 & 4769 & NAD83 & 572875 & 151870 & CENTPLAT & ESTIMATED & & & TDP-33A \\
\hline 1384 & 4772 & NAD83 & 572800 & 151800 & CENTPLAT & ESTIMATED & & & TDP-34A \\
\hline 1385 & 4773 & NAD83 & 572630 & 151675 & CENTPLAT & ESTIMATED & & & TDP-38A \\
\hline 1386 & 4774 & NAD83 & 572610 & 151615 & CENTPLAT & ESTIMATED & & & TDP-39A \\
\hline 1387 & 4775 & NAD83 & 572570 & 151580 & CENTPLAT & ESTIMATED & & & TDP-40A \\
\hline 1389 & 4777 & NAD83 & 572485 & 151495 & CENTPLAT & ESTIMATED & & & TDP-42A \\
\hline 1390 & 4778 & NAD83 & 572450 & 151440 & CENTPLAT & ESTIMATED & & & TDP-43A \\
\hline 1391 & 4779 & NAD83 & 572415 & 151395 & CENTPLAT & ESTIMATED & & & TDP-44A \\
\hline 1392 & 4780 & NAD83 & 572365 & 151355 & CENTPLAT & ESTIMATED & & & TDP-45A \\
\hline 1393 & 4781 & NAD83 & 572350 & 151370 & CENTPLAT & ESTIMATED & & & TDP-45B \\
\hline 1394 & 4782 & NAD83 & 572400 & 151410 & CENTPLAT & ESTIMATED & & & TDP-44B \\
\hline 1395 & 4783 & NAD83 & 572435 & 151455 & CENTPLAT & ESTIMATED & & & TDP-43B \\
\hline 1396 & 4784 & NAD83 & 572470 & 151510 & CENTPLAT & ESTIMATED & & & TDP-42B \\
\hline 1397 & 4785 & NAD83 & 572505 & 151545 & CENTPLAT & ESTIMATED & & & TDP-41B \\
\hline 1398 & 4786 & NAD83 & 572555 & 151595 & CENTPLAT & ESTIMATED & & & TDP-40B \\
\hline 1399 & 4787 & NAD83 & 572595 & 151630 & CENTPLAT & ESTIMATED & & & TDP-39B \\
\hline 1400 & 4788 & NAD83 & 572325 & 151305 & CENTPLAT & ESTIMATED & & & TDP-46A \\
\hline 1401 & 4789 & NAD83 & 572290 & 151260 & CENTPLAT & ESTIMATED & & & TDP-47A \\
\hline 1402 & 4790 & NAD83 & 572260 & 151223 & CENTPLAT & ESTIMATED & & & TDP-48A \\
\hline 1403 & 4791 & NAD83 & 572895 & 151925 & CENTPLAT & ESTIMATED & & & TDP-32B \\
\hline 1404 & 4792 & NAD83 & 572930 & 151955 & CENTPLAT & ESTIMATED & & & TDP-31A \\
\hline 1405 & 4793 & NAD83 & 572925 & 152000 & CENTPLAT & ESTIMATED & & & TDP-30B \\
\hline 1406 & 4794 & NAD83 & 573020 & 152080 & CENTPLAT & ESTIMATED & & & TDP-28B \\
\hline
\end{tabular}




\begin{tabular}{|c|c|c|c|c|c|c|c|c|c|}
\hline & $\mathrm{A}$ & $B$ & $\mathrm{C}$ & $\mathrm{D}$ & $\mathrm{E}$ & $\mathrm{F}$ & $G$ & $\mathrm{H}$ & $\mathrm{I}$ \\
\hline 1 & SAMP_SITE_ID & HORIZ_COORD_TYPE & EW_COORD & NS_COORD & OWNER_ID & COORD_SOURCE & DIST_CLASS & RAD_LEVEL & SAMP_SITE_NAME \\
\hline 1408 & 4796 & NAD83 & 573085 & 152150 & CENTPLAT & ESTIMATED & & & TDP-26B \\
\hline 1409 & 4797 & NAD83 & 573140 & 152185 & CENTPLAT & ESTIMATED & & & TDP-25B \\
\hline 1410 & 4798 & NAD83 & 573190 & 152220 & CENTPLAT & ESTIMATED & & & TDP-24B \\
\hline 1411 & 4799 & NAD83 & 573240 & 152250 & CENTPLAT & ESTIMATED & & & TDP-23B \\
\hline 1412 & 4800 & NAD83 & 572615 & 151680 & CENTPLAT & ESTIMATED & & & TDP-38B \\
\hline 1413 & 4801 & NAD83 & 572785 & 151815 & CENTPLAT & ESTIMATED & & & TDP-34B \\
\hline 1414 & 4802 & NAD83 & 572860 & 151885 & CENTPLAT & ESTIMATED & & & TDP-33B \\
\hline 1415 & 4803 & NAD83 & 572310 & 151320 & CENTPLAT & ESTIMATED & & & TDP-46B \\
\hline 1416 & 4804 & NAD83 & 572275 & 151275 & CENTPLAT & ESTIMATED & & & TDP-47B \\
\hline 1417 & 4805 & NAD83 & 572245 & 151238 & CENTPLAT & ESTIMATED & & & TDP-48B \\
\hline 1418 & 4806 & NAD83 & 573295 & 152285 & CENTPLAT & ESTIMATED & & & TDP-22B \\
\hline 1419 & 4807 & NAD83 & 573365 & 152315 & CENTPLAT & ESTIMATED & & & TDP-21B \\
\hline 1420 & 4808 & NAD83 & 573340 & 152355 & CENTPLAT & ESTIMATED & & & TDP-20B \\
\hline 1421 & 4809 & NAD83 & 573475 & 152395 & CENTPLAT & ESTIMATED & & & TDP-19B \\
\hline 1422 & 4810 & NAD83 & 573520 & 152435 & CENTPLAT & ESTIMATED & & & TDP-18B \\
\hline 1423 & 4811 & NAD83 & 573585 & 152485 & CENTPLAT & ESTIMATED & & & TDP-17B \\
\hline 1424 & 4812 & NAD83 & 573630 & 152535 & CENTPLAT & ESTIMATED & & & TDP-16B \\
\hline 1425 & 4813 & NAD83 & 573685 & 152570 & CENTPLAT & ESTIMATED & & & TDP-15B \\
\hline 1426 & 4814 & NAD83 & 573735 & 152610 & CENTPLAT & ESTIMATED & & & TDP-14B \\
\hline 1427 & 4815 & NAD83 & 573785 & 152640 & CENTPLAT & ESTIMATED & & & TDP-13B \\
\hline 1428 & 4816 & NAD83 & 573805 & 152715 & CENTPLAT & ESTIMATED & & & TDP-12B \\
\hline 1429 & 4817 & NAD83 & 573815 & 157775 & CENTPLAT & ESTIMATED & & & TDP-11B \\
\hline 1430 & 4818 & NAD83 & 573845 & 152805 & CENTPLAT & ESTIMATED & & & TDP-10B \\
\hline 1431 & 4819 & NAD83 & 573865 & 152865 & CENTPLAT & ESTIMATED & & & TDP-09B \\
\hline 1432 & 4820 & NAD83 & 573885 & 152935 & CENTPLAT & ESTIMATED & & & TDP-08B \\
\hline 1433 & 4821 & NAD83 & 573895 & 152990 & CENTPLAT & ESTIMATED & & & TDP-07B \\
\hline 1434 & 4822 & NAD83 & 573905 & 153040 & CENTPLAT & ESTIMATED & & & TDP-06B \\
\hline 1435 & 4823 & NAD83 & 572220 & 151175 & CENTPLAT & ESTIMATED & & & TDP-49A \\
\hline 1436 & 4824 & NAD83 & 572185 & 151124 & CENTPLAT & ESTIMATED & & & TDP-50A \\
\hline 1437 & 4825 & NAD83 & 572135 & 151075 & CENTPLAT & ESTIMATED & & & TDP-51A \\
\hline 1438 & 4826 & NAD83 & 572120 & 151090 & CENTPLAT & ESTIMATED & & & TDP-51B \\
\hline 1439 & 4827 & NAD83 & 572170 & 151139 & CENTPLAT & ESTIMATED & & & TDP-50B \\
\hline 1440 & 4828 & NAD83 & 572205 & 151190 & CENTPLAT & ESTIMATED & & & TDP-49B \\
\hline 1441 & 4829 & NAD83 & 554285 & 139315 & HEISPROD & & & & RIVERLAND ERA|B08NQ6 \\
\hline 1442 & 4831 & NAD83 & 554285 & 139415 & HEISPROD & & & & RIVERLAND ERA|B08NQ9 \\
\hline 1443 & 4833 & NAD83 & 556975.5 & 144852.6 & HEISPROD & & & & COLUMBIA RIVER|B07NF8 \\
\hline 1444 & 4835 & NAD83 & 557066.3 & 144898.2 & HEISPROD & & & & COLUMBIA RIVER|B07NF7 \\
\hline 1445 & 4836 & NAD83 & 557603.1 & 144860.7 & HEISPROD & & & & COLUMBIA RIVER|B07NF5 \\
\hline
\end{tabular}


A

$\mathrm{B}$

C

$\mathrm{D}$

E

\begin{tabular}{|c|c|c|}
\hline F & G & H \\
\hline
\end{tabular}
4837 NAD83

4838 NAD83

4839 NAD83

4840 NAD83

4841 NAD83

4843 NAD83

4845 NAD83

4847 NAD83

4849 NAD83

4851 NAD83

4853 NAD83

4855 NAD83

4856 NAD83

4858 NAD83

4859 NAD83

4861 NAD83

4862 NAD83

4864 NAD83

4865 NAD83

4866 NAD83

4868 NAD83

4869 NAD83

4871 NAD83

4872 NAD83

4874 NAD83

4876 NAD83

4877 NAD83

4878 NAD83

4879 NAD83

4881 NAD83(91)

4883 NAD83(91)

4884 NAD83

4886 NAD83(91)

4888 NAD83

4890 NAD83

4892 NAD83

4894 NAD83

4895 NAD83

558584

558655

558858.6

564535.5

564635.7

564824

564890

564913

564920

565115

565116

565136

565155

565163

565180

565188

565190

565206

565390

565499.7

565903

566590

567619.7

568105.9

568519.7

570348.6

572030

572658.7

572830

573329.833

573358.789

573399.297

143454.6 HEISPROD

143475 HEISPROD

143475 HEISPROD

145279.2 HEISPROD

145303.4 HEISPROD

145176 HEISPROD

144500 HEISPROD

114144 HEISPROD

114358 HEISPROD

144577 HEISPROD

144420 HEISPROD

144775 HEISPROD

144046 HEISPROD

143970 HEISPROD

144465 HEISPROD

143977 HEISPROD

144043 HEISPROD

144494 HEISPROD

145110 HEISPROD

144031.5 HEISPROD

134967 HEISPROD

144760 HEISPROD

146308.7 HEISPROD

146712.5 HEISPROD

146960.5 HEISPROD

148672.4 HEISPROD

151690 HEISPROD

151684.5 HEISPROD

151750 HEISPROD

137603.389 HEISPROD

151590 HEISPROD

$573459 \quad 152075$ HEISPROD

$573460 \quad 152016$ HEISPROD

$573467 \quad 152035$ HEISPROD

$573470 \quad 152037$ HEISPROD

573471

152085 HEISPROD

I

RIVERLAND ERA|B08NN7

RIVERLAND ERA|B08NP6

RIVERLAND ERA|B08NP1

COLUMBIA RIVER|B07NF3

COLUMBIA RIVER|B07ND1

100-BC-1 LFI|B018Q6

100-BC-1 LFI|B018Q7

100-BC-1 LFI|B018S2

100-BC-1 LFI|B018S3

100-BC-1 LFI|B018Q8

100-BC-1 LFI|B018Q9

100-BC-1 LFI|B018S4

100-BC-1 LFI|B018R3

100-BC-1 LFI|B018R8

100-BC-1 LFI|B018R0

100-BC-1 LFI|B018R9

100-BC-1 LFI|B018R5

100-BC-1 LFI|B018R2

100-BC-1 LFI|B018V4

100-BC-2 LFI|B08RB5

200 AREA BIOTA|B08YF8

100-BC-1 LFI|B05XZ4

COLUMBIA RIVER|B07NG2

COLUMBIA RIVER|B07NF9

COLUMBIA RIVER|B07NG1

COLUMBIA RIVER|B07NG4

100-DR-1 LFI|B00ZR7

COLUMBIA RIVER|B07NF1

100-DR-1 LFI|B018W5

200-BP-1 RI|B00J59

200-BP-1 RI|B00J57

100-DR-1 LFI|B00ZR4

200-BP-1 RI|B00J58

100-D PONDS|B06D42

100-D PONDS II|BODMS7

100-D PONDS II|BODMT0

100-D PONDS|B06D43

Page 39

100-D PONDS|B06D26 
A

C

$\mathrm{D}$

E

$\mathrm{F}$ G $\mathrm{H}$ I

\begin{tabular}{|c|c|c|c|c|c|c|c|c|c|}
\hline & $\mathrm{A}$ & $\mathrm{B}$ & $\mathrm{C}$ & $\mathrm{D}$ & $E$ & $\mathrm{~F}$ & $G$ & $\mathrm{H}$ & $\mathrm{I}$ \\
\hline 1 & SAMP_SITE_ID & HORIZ_COORD_TYPE & EW_COORD & NS_COORD & OWNER_ID & COORD_SOURCE & DIST_CLASS & RAD_LEVEL & SAMP_SITE_NAME \\
\hline 1484 & 4897 & NAD83 & 573472 & 152066 & HEISPROD & & & & 100-D PONDS|B06D41 \\
\hline 1485 & 4899 & NAD83 & 573473 & 152041 & HEISPROD & & & & 100-D PONDS II|B0DMS8 \\
\hline 1486 & 4901 & NAD83(91) & 573475.623 & 137559.272 & HEISPROD & & & & 200-BP-1 RI|B00J68 \\
\hline 1487 & 4903 & NAD83 & 573477 & 152067 & HEISPROD & & & & 100-D PONDS|B06D40 \\
\hline 1488 & 4904 & NAD83 & 573480 & 152020 & HEISPROD & & & & 100-D PONDS|B06D27 \\
\hline 1489 & 4906 & NAD83 & 573482 & 152065 & HEISPROD & & & & 100-D PONDS|B06D39 \\
\hline 1490 & 4907 & NAD83 & 573483 & 152078 & HEISPROD & & & & 100-D PONDS|B06D25 \\
\hline 1491 & 4908 & NAD83 & 573485 & 151565 & HEISPROD & & & & 100-DR-1 LFI|B00ZS1 \\
\hline 1492 & 4910 & NAD83 & 573485 & 152063 & HEISPROD & & & & 100-D PONDS|B06D38 \\
\hline 1493 & 4912 & NAD83 & 573486 & 152023 & HEISPROD & & & & 100-D PONDS II|BODMT1 \\
\hline 1494 & 4913 & NAD83 & 573488 & 152020 & HEISPROD & & & & 100-D PONDS|B06D30 \\
\hline 1495 & 4915 & NAD83 & 573488 & 152034 & HEISPROD & & & & 100-D PONDS II|BODMT7 \\
\hline 1496 & 4917 & NAD83 & 573488 & 152037 & HEISPROD & & & & 100-D PONDS|B06D47 \\
\hline 1497 & 4919 & NAD83 & 573493 & 152041 & HEISPROD & & & & 100-D PONDS II|BODMS9 \\
\hline 1498 & 4921 & NAD83 & 573496 & 152020 & HEISPROD & & & & 100-D PONDS|B06D51 \\
\hline 1499 & 4923 & NAD83 & 573496 & 152037 & HEISPROD & & & & 100-D PONDS|B06D48 \\
\hline 1500 & 4925 & NAD83 & 573498 & 152028 & HEISPROD & & & & 100-D PONDS II|BODMS6 \\
\hline 1501 & 4927 & NAD83(91) & 573498.296 & 137533.388 & HEISPROD & & & & 200-BP-1 RI|B00J69 \\
\hline 1502 & 4928 & NAD83(91) & 573498.442 & 137618.481 & HEISPROD & & & & 200-BP-1 RI|B00J66 \\
\hline 1503 & 4930 & NAD83(91) & 573498.778 & 137523.168 & HEISPROD & & & & 200-BP-1 RI|B00J72 \\
\hline 1504 & 4932 & NAD83 & 573501 & 152025 & HEISPROD & & & & 100-D PONDS|B06D46 \\
\hline 1505 & 4934 & NAD83 & 573501 & 152027 & HEISPROD & & & & 100-D PONDS|B06D45 \\
\hline 1506 & 4936 & NAD83(91) & 573502.104 & 137520.236 & HEISPROD & & & & 200-BP-1 R||B00J73 \\
\hline 1507 & 4938 & NAD83(91) & 573503.479 & 137523.308 & HEISPROD & & & & 200-BP-1 RI|B00J71 \\
\hline 1508 & 4940 & NAD83(91) & 573503.63 & 137527.656 & HEISPROD & & & & 200-BP-1 RI|B00J70 \\
\hline 1509 & 4942 & NAD83 & 573540 & 151090 & HEISPROD & & & & 100-DR-1 LFI|B00ZQ7 \\
\hline 1510 & 4944 & NAD83 & 573540 & 151205 & HEISPROD & & & & 100-DR-1 LFI|B00ZQ6 \\
\hline 1511 & 4945 & NAD83 & 573555 & 151580 & HEISPROD & & & & 100-DR-1 LFI|B00ZR1 \\
\hline 1512 & 4947 & NAD83 & 573555 & 151620 & HEISPROD & & & & 100-DR-1 LFI|B00ZS0 \\
\hline 1513 & 4949 & NAD83 & 573560 & 151340 & HEISPROD & & & & 100-DR-1 LFI|B00ZQ5 \\
\hline 1514 & 4951 & NAD83(91) & 573572.803 & 137725.962 & HEISPROD & & & & 200-BP-1 RI|B00J65 \\
\hline 1515 & 4953 & NAD83(91) & 573597.297 & 137577.262 & HEISPROD & & & & 200-BP-1 RI|B00J52 \\
\hline 1516 & 4955 & NAD83(91) & 573604.65 & 137587.814 & HEISPROD & & & & 200-BP-1 RI|B00J51 \\
\hline 1517 & 4957 & NAD83(91) & 573604.993 & 137612.625 & HEISPROD & & & & 200-BP-1 RI|B00J53 \\
\hline 1518 & 4959 & NAD83(91) & 573607.314 & 137611.161 & HEISPROD & & & & 200-BP-1 RI|B00J55 \\
\hline 1519 & 4961 & NAD83(91) & 573608.363 & 137581.749 & HEISPROD & & & & 200-BP-1 RI|B00J50 \\
\hline 1520 & 4963 & NAD83(91) & 573609.522 & 137617.479 & HEISPROD & & & & 200-BP-1 RI|B00J54 \\
\hline 1521 & 4965 & NAD83(91) & 573612.736 & 137611.07 & HEISPROD & & & & 200-BP-1 RI|B00J56 \\
\hline
\end{tabular}

Page 40 200-BP-1 RI|B00J56 
A

$\mathrm{B}$

C

$\mathrm{D}$

E

\begin{tabular}{r|r}
573613.03 & 137590.774 HEISPROD \\
\hline 573613.23 & 137580.927 HEISPROD
\end{tabular}

4967 NAD83(91)

4969 NAD83(91)

4971 NAD83(91)

4973 NAD83(91)

4974 NAD83(91)

4975 NAD83

4977 NAD83(91)

4979 NAD83

4981 NAD83(91)

4982 NAD83

4984 NAD83(91)

4985 NAD83

4987 NAD83

4988 NAD83

4989 NAD83

573651.439137656 .579 HEISPROD

573655.159137662 .888 HEISPROD

573656.267 137653.438 HEISPROD

\begin{tabular}{r|r}
573660 & 151505 HEISPROD
\end{tabular}

573661.673137655 .713 HEISPROD

$573675 \quad 151585$ HEISPROD

\begin{tabular}{r|r}
573684.67 & 137582.316 HEISPROD
\end{tabular}

$573687.5 \quad 152981.9$ HEISPROD

$573692.301 \quad 137682.174$ HEISPROD

$573711.3 \quad 153053.2$ HEISPROD

$573730 \quad 151610$ HEISPROD

573793.6153078 HEISPROD

$573800 \quad 152460$ HEISPROD

\begin{tabular}{|l|r|r|}
\hline 4990 NAD83 & 573860 & 151525 HEISPROD \\
\hline
\end{tabular}

\begin{tabular}{|l|r|r|}
\hline 4991 NAD83 & 574306.1 & 153825.1 HEISPROD \\
\hline
\end{tabular}

\begin{tabular}{|l|r|r}
4992 NAD83 & 574430 & 151185 HEISPROD
\end{tabular}

\begin{tabular}{|l|r|r|}
\hline 4993 NAD83 & 575174.1 & 154444.1 HEISPROD \\
\hline
\end{tabular}

\begin{tabular}{|l|r|r|}
\hline 4995 NAD83 & 575580.4 & 155101.1 HEISPROD \\
\hline
\end{tabular}

\begin{tabular}{l|r|r}
4996 NAD83 & 575820 & 151071.5 HEISPROD
\end{tabular}

\begin{tabular}{|l|l|l|}
\hline 4998 NAD83 & 575820 & 151071.7 HEISPROD
\end{tabular}

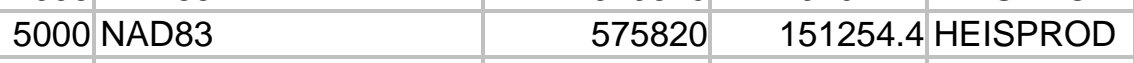

\begin{tabular}{|l|r|r|}
\hline 5002 NAD83 & 575820 & 151412.9 HEISPROD \\
\hline 5003 AAD83
\end{tabular}

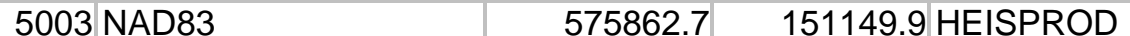

\begin{tabular}{|l|l|l|}
\hline 5005 NAD83 & 575912.5 & 151253.7 HEISPROD \\
\hline
\end{tabular}

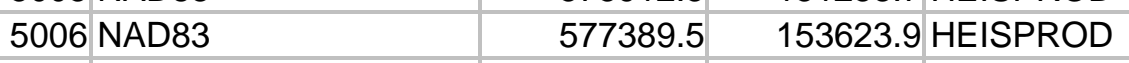

\begin{tabular}{|l|r|r|}
5008 NAD83 & 577475 & 152570 HEISPROD
\end{tabular}

\begin{tabular}{|l|l|l|}
5010 NAD83 & 577487 & 152594 HEISPROD
\end{tabular}

\begin{tabular}{|l|l|l|}
\hline 5012 NAD83 & 577495 & 152570 HEISPROD \\
\hline
\end{tabular}

\begin{tabular}{|l|l|l|}
5013 NAD83 & 577510 & 152592 HEISPROD
\end{tabular}

\begin{tabular}{l|l|l}
5015 NAD83 & 577510 & 152614 HEISPROD
\end{tabular}

\begin{tabular}{|l|l|l|}
\hline 5017 NAD83 & 577770 & 152560 HEISPROD
\end{tabular}

\begin{tabular}{|l|l|l|}
\hline 5019 NAD83 & 577857 & 147443 HEISPROD \\
\hline
\end{tabular}

\begin{tabular}{|l|l|l}
5021 NAD83 & 577859 & 147432 HEISPROD
\end{tabular}

\begin{tabular}{l|l|l}
5022 NAD83 & 577861 & 147420 HEISPROD
\end{tabular}

\begin{tabular}{|l|r|r|}
\hline 5023 NAD83 & 578263 & 152108.2 HEISPROD \\
\hline
\end{tabular}

5025 NAD83

578910.9

151754.4 HEISPROD

G

$\mathrm{H}$

I

200-BP-1 Rा|B00J48

200-BP-1 RI|B00J49

200-BP-1 RI|B00J60

200-BP-1 RI|B00J61

200-BP-1 RI|B00J62

100-DR-1 LFI|B00ZQ8

200-BP-1 RI|B00J63

100-DR-1 LFI|B00ZR9

200-BP-1 RI|B00J67

COLUMBIA RIVER|B07ND6

200-BP-1 RI|B00J64

COLUMBIA RIVER|B07NH5

100-DR-1 LFI|B00ZS3

COLUMBIA RIVER|B07ND4

100-DR-1 LFI|B018W4

100-DR-1 LFI|B018Q4

COLUMBIA RIVER|B07NH1

100-DR-1 LFI|B00ZS4

COLUMBIA RIVER|B07NC9

COLUMBIA RIVER|B07NF0

SODIUM DICHROM|B018Z6

SODIUM DICHROM|B01900

SODIUM DICHROM|B018Z7

SODIUM DICHROM|B018Z8

SODIUM DICHROM|B018Z1

SODIUM DICHROM|B018Z5

COLUMBIA RIVER|B07NG3

100-HR-1 LFI|B018T0

100-HR-1 LFI|B018T1

100-HR-1 LFI|B018S9

100-HR-1 LFI|B018S6

100-HR-1 LFI|B018T2

100-HR-1 LFI|B018S5

PKLNG ACID CRIB|B07Q10

PKLNG ACID CRIB|B07Q11

PKLNG ACID CRIB|B07Q12

COLUMBIA RIVER|B07NC0

COLUMBIA RIVER|B07NC2

Page 41 


\begin{tabular}{|c|c|c|c|c|c|c|c|c|c|}
\hline & $A$ & $B$ & $\mathrm{C}$ & $D$ & $E$ & $F$ & $G$ & $\mathrm{H}$ & $\mathrm{I}$ \\
\hline 1 & SAMP_SITE_ID & HORIZ_COORD_TYPE & EW_COORD & NS_COORD & OWNER_ID & COORD_SOURCE & DIST_CLASS & RAD_LEVEL & SAMP_SITE_NAME \\
\hline 1560 & 5027 & NAD83 & 579164 & 150833.8 & HEISPROD & & & & COLUMBIA RIVER|B07NC3 \\
\hline 1561 & 5028 & NAD83 & 580200 & 148400 & HEISPROD & & & & 100-FR-1 LFI|B080R1 \\
\hline 1562 & 5033 & NAD83 & 580615 & 147615 & HEISPROD & & & & 100-FR-1 LFI|B080R4 \\
\hline 1563 & 5034 & NAD83 & 582032.7 & 146961.3 & HEISPROD & & & & COLUMBIA RIVER|B07NC4 \\
\hline 1564 & 5036 & NAD83 & 582149.9 & 146304.3 & HEISPROD & & & & COLUMBIA RIVER|B07NB7 \\
\hline 1565 & 5038 & NAD83 & 582262.2 & 146193.7 & HEISPROD & & & & COLUMBIA RIVER|B07NB6 \\
\hline 1566 & 5039 & NAD83 & 582692.1 & 145485 & HEISPROD & & & & COLUMBIA RIVER|B07NB8 \\
\hline 1567 & 5040 & NAD83 & 582731.8 & 146871.7 & HEISPROD & & & & COLUMBIA RIVER|B07NG9 \\
\hline 1568 & 5041 & NAD83 & 584739.5 & 141117.5 & HEISPROD & & & & COLUMBIA RIVER|B07ND2 \\
\hline 1569 & 5042 & NAD83 & 585380.6 & 141598.9 & HEISPROD & & & & COLUMBIA RIVER|B07NH3 \\
\hline 1570 & 5043 & NAD83 & 585736.8 & 140407.7 & HEISPROD & & & & COLUMBIA RIVER|B07NC6 \\
\hline 1571 & 5044 & NAD83 & 592245.8 & 113994.7 & HEISPROD & & & & 1100-EM-1 RI|AH224 \\
\hline 1572 & 5045 & NAD83 & 592501.2 & 113866.3 & HEISPROD & & & & 1100-EM-1 RI|AH197 \\
\hline 1573 & 5047 & NAD83 & 592501.2 & 113943.7 & HEISPROD & & & & 1100-EM-1 RI|AH213 \\
\hline 1574 & 5049 & NAD83 & 592501.2 & 113985.5 & HEISPROD & & & & 1100-EM-1 RI|AH205 \\
\hline 1575 & 5051 & NAD83 & 592560 & 114010.5 & HEISPROD & & & & 1100-EM-1 RI|AH195 \\
\hline 1576 & 5053 & NAD83 & 592570.6 & 113899.3 & HEISPROD & & & & 1100-EM-1 RI|AH196 \\
\hline 1577 & 5055 & NAD83 & 592609 & 113894 & HEISPROD & & & & 1100-EM-1 RI|AH203 \\
\hline 1578 & 5057 & NAD83 & 592621.6 & 114257.9 & HEISPROD & & & & 1100-EM-1 RI|AH192 \\
\hline 1579 & 5059 & NAD83 & 592623.2 & 113863.5 & HEISPROD & & & & 1100-EM-1 RI|AH198 \\
\hline 1580 & 5061 & NAD83 & 592648.2 & 114097 & HEISPROD & & & & 1100-EM-1 RI|AH194 \\
\hline 1581 & 5063 & NAD83 & 592654.2 & 114016.1 & HEISPROD & & & & 1100-EM-1 RI|AH208 \\
\hline 1582 & 5065 & NAD83 & 592659.3 & 114281 & HEISPROD & & & & 1100-EM-1 RI|AH191 \\
\hline 1583 & 5067 & NAD83 & 592677.6 & 114167.8 & HEISPROD & & & & 1100-EM-1 RI|AH193 \\
\hline 1584 & 5068 & NAD83 & 592706.7 & 114312.9 & HEISPROD & & & & 1100-EM-1 RI|AH211 \\
\hline 1585 & 5070 & NAD83 & 592707 & 114313.1 & HEISPROD & & & & 1100-EM-1 RI|AH215 \\
\hline 1586 & 5072 & NAD83 & 592714.6 & 113967.6 & HEISPROD & & & & 1100-EM-1 RI|AH199 \\
\hline 1587 & 5074 & NAD83 & 592714.6 & 114153.5 & HEISPROD & & & & 1100-EM-1 RI|AH200 \\
\hline 1588 & 5076 & NAD83 & 592714.9 & 114043.4 & HEISPROD & & & & 1100-EM-1 RI|AH207 \\
\hline 1589 & 5078 & NAD83 & 592723.2 & 113902.6 & HEISPROD & & & & 1100-EM-1 RI|AH206 \\
\hline 1590 & 5080 & NAD83 & 592725.4 & 114117.5 & HEISPROD & & & & 1100-EM-1 RI|AH210 \\
\hline 1591 & 5082 & NAD83 & 592730.7 & 114315.1 & HEISPROD & & & & 1100-EM-1 R\|AH190 \\
\hline 1592 & 5084 & NAD83 & 592734.5 & 114175.5 & HEISPROD & & & & 1100-EM-1 RI|AH187 \\
\hline 1593 & 5086 & NAD83 & 592737.7 & 113989.9 & HEISPROD & & & & 1100-EM-1 RI|AH185 \\
\hline 1594 & 5088 & NAD83 & 592741.8 & 114251.6 & HEISPROD & & & & 1100-EM-1 RI|AH189 \\
\hline 1595 & 5090 & NAD83 & 592744.9 & 114235.2 & HEISPROD & & & & 1100-EM-1 RI|AH188 \\
\hline 1596 & 5092 & NAD83 & 592769 & 114163.3 & HEISPROD & & & & 1100-EM-1 RI|AH186 \\
\hline 1597 & 5094 & NAD83 & 592781.3 & 114022.6 & HEISPROD & & & & 1100-EM-1 RI|AH209 \\
\hline
\end{tabular}




\begin{tabular}{|c|c|c|c|c|c|c|c|c|c|}
\hline & $A$ & $\mathrm{~B}$ & C & $\mathrm{D}$ & $E$ & $\mathrm{~F}$ & $G$ & $\mathrm{H}$ & $\mathrm{I}$ \\
\hline 1 & SAMP_SITE_ID & HORIZ_COORD_TYPE & EW_COORD & NS_COORD & OWNER_ID & COORD_SOURCE & DIST_CLASS & RAD_LEVEL & SAMP_SITE_NAME \\
\hline 1598 & 5096 & NAD83 & 592784.5 & 114115.7 & HEISPROD & & & & 1100-EM-1 RI|AH184 \\
\hline 1599 & 5097 & NAD83 & 592787.6 & 114128.3 & HEISPROD & & & & 1100-EM-1 RI|AH181 \\
\hline 1600 & 5099 & NAD83 & 592808.6 & 113956.5 & HEISPROD & & & & 1100-EM-1 RI|AH169 \\
\hline 1601 & 5101 & NAD83 & 592819.9 & 113961.4 & HEISPROD & & & & 1100-EM-1 RI|AH168 \\
\hline \begin{tabular}{|l|}
1602 \\
\end{tabular} & 5103 & NAD83 & 592820.1 & 114127.6 & HEISPROD & & & & 1100-EM-1 RI|AH178 \\
\hline \begin{tabular}{|l|}
1603 \\
\end{tabular} & 5105 & NAD83 & 592824.2 & 114131.6 & HEISPROD & & & & 1100-EM-1 RI|AH179 \\
\hline 1604 & 5107 & NAD83 & 592826.6 & 114120 & HEISPROD & & & & 1100-EM-1 RI|AH180 \\
\hline 1605 & 5109 & NAD83 & 592833.9 & 114021.7 & HEISPROD & & & & 1100-EM-1 RI|AH173 \\
\hline 1606 & 5111 & NAD83 & 592837.3 & 114093 & HEISPROD & & & & 1100-EM-1 RI|AH175 \\
\hline 1607 & 5113 & NAD83 & 592858.9 & 114140.6 & HEISPROD & & & & 1100-EM-1 RI|AH176 \\
\hline 1608 & 5115 & NAD83 & 592868.1 & 114050.4 & HEISPROD & & & & 1100-EM-1 RI|AH174 \\
\hline 1609 & 5117 & NAD83 & 592868.3 & 114005 & HEISPROD & & & & 1100-EM-1 RI|AH170 \\
\hline 1610 & 5119 & NAD83 & 592873.5 & 114159.2 & HEISPROD & & & & 1100-EM-1 RI|AH177 \\
\hline 1611 & 5121 & NAD83 & 592874 & 114012.4 & HEISPROD & & & & 1100-EM-1 RI|AH171 \\
\hline \begin{tabular}{|l|}
1612 \\
\end{tabular} & 5123 & NAD83 & 592885 & 114011.1 & HEISPROD & & & & 1100-EM-1 RI|AH172 \\
\hline 1613 & 5125 & NAD83 & 592897.5 & 114046.4 & HEISPROD & & & & 1100-EM-1 RI|AH202 \\
\hline 1614 & 5126 & NAD83 & 592974.1 & 112126.5 & HEISPROD & & & & 1100-EM-1 RI|AH222 \\
\hline 1615 & 5128 & NAD83 & 593032.7 & 112133.3 & HEISPROD & & & & 1100-EM-1 RI|A3107 \\
\hline 1616 & 5130 & NAD83 & 593225.8 & 111394.7 & HEISPROD & & & & 1100-EM-1 RI|6142A \\
\hline \begin{tabular}{|l|}
1617 \\
\end{tabular} & 5132 & NAD83 & 593243.3 & 111407.2 & HEISPROD & & & & 1100-EM-1 RI|6144A \\
\hline 1618 & 5134 & NAD83 & 593246.1 & 112108.9 & HEISPROD & & & & 1100-EM-1 RI|A3101 \\
\hline 1619 & 5136 & NAD83 & 593254.2 & 111413.5 & HEISPROD & & & & 1100-EM-1 RI|6141A \\
\hline 1620 & 5138 & NAD83 & 593254.4 & 111422.1 & HEISPROD & & & & 1100-EM-1 RI|6143A \\
\hline 1621 & 5140 & NAD83 & 593258.3 & 112121.1 & HEISPROD & & & & 1100-EM-1 RI|A3102 \\
\hline 1622 & 5142 & NAD83 & 593269.7 & 111432.5 & HEISPROD & & & & 1100-EM-1 RI|6145A \\
\hline \begin{tabular}{|l|}
1623 \\
\end{tabular} & 5144 & NAD83 & 593270.5 & 112133.3 & HEISPROD & & & & 1100-EM-1 RI|A3103 \\
\hline 1624 & 5146 & NAD83 & 593274.1 & 111432.4 & HEISPROD & & & & 1100-EM-1 RI|6146A \\
\hline 1625 & 5148 & NAD83 & 593274.1 & 111436.7 & HEISPROD & & & & 1100-EM-1 RI|6147A \\
\hline 1626 & 5150 & NAD83 & 593278.2 & 111429.2 & HEISPROD & & & & 1100-EM-1 RI|6150A \\
\hline \begin{tabular}{|l|}
1627 \\
\end{tabular} & 5152 & NAD83 & 593278.3 & 111431 & HEISPROD & & & & 1100-EM-1 RI|6154A \\
\hline 1628 & 5154 & NAD83 & 593279.6 & 111429.4 & HEISPROD & & & & 1100-EM-1 RI|6152A \\
\hline \begin{tabular}{|l|}
1629 \\
\end{tabular} & 5156 & NAD83 & 593280.3 & 111425.8 & HEISPROD & & & & 1100-EM-1 RI|6149A \\
\hline 1630 & 5158 & NAD83 & 593283.7 & 111435.5 & HEISPROD & & & & 1100-EM-1 RI|6153A \\
\hline 1631 & 5160 & NAD83 & 593283.8 & 111432.3 & HEISPROD & & & & 1100-EM-1 RI|6151A \\
\hline 1632 & 5161 & NAD83 & 593284.8 & 111435.6 & HEISPROD & & & & 1100-EM-1 RI|6155A \\
\hline 1633 & 5163 & NAD83 & 593289.5 & 111451.5 & HEISPROD & & & & 1100-EM-1 RI|6148A \\
\hline 1634 & 5165 & NAD83 & 593294.8 & 112145.5 & HEISPROD & & & & 1100-EM-1 RI|A3104 \\
\hline \begin{tabular}{|l|}
1635 \\
\end{tabular} & 5167 & NAD83 & 593294.8 & 112157.7 & HEISPROD & & & & 1100-EM-1 RI|A3105 \\
\hline
\end{tabular}




\begin{tabular}{|c|c|c|c|c|c|c|c|c|c|}
\hline & A & $\mathrm{B}$ & $\mathrm{C}$ & $\mathrm{D}$ & $\mathrm{E}$ & $\mathrm{F}$ & $G$ & $\mathrm{H}$ & I \\
\hline 1 & SAMP_SITE_ID & HORIZ_COORD_TYPE & EW_COORD & NS_COORD & OWNER_ID & COORD_SOURCE & DIST_CLASS & RAD_LEVEL & SAMP_SITE_NAME \\
\hline 1636 & 5168 & NAD83 & 593302.9 & 110925.3 & HEISPROD & & & & 1100-EM-1 R\||AH217 \\
\hline 1637 & 5170 & NAD83 & 593307 & 112072.3 & HEISPROD & & & & 1100-EM-1 RI|A3112 \\
\hline 1638 & 5172 & NAD83 & 593307 & 112145.5 & HEISPROD & & & & 1100-EM-1 RI|A3106 \\
\hline 1639 & 5174 & NAD83 & 593331.4 & 112096.7 & HEISPROD & & & & 1100-EM-1 RI|A3109 \\
\hline 1640 & 5175 & NAD83 & 593331.4 & 112145.5 & HEISPROD & & & & 1100-EM-1 RI|2139A \\
\hline 1641 & 5177 & NAD83 & 593514.3 & 112092.6 & HEISPROD & & & & 1100-EM-1 RI|A2127 \\
\hline 1642 & 5179 & NAD83 & 593518 & 112075.8 & HEISPROD & & & & 1100-EM-1 RI|A2126 \\
\hline 1643 & 5181 & NAD83 & 593519 & 112031.9 & HEISPROD & & & & 1100-EM-1 RI|A2122 \\
\hline 1644 & 5183 & NAD83 & 593519.3 & 112031.9 & HEISPROD & & & & 1100-EM-1 RI|3136A \\
\hline 1645 & 5185 & NAD83 & 593520.8 & 112102.9 & HEISPROD & & & & 1100-EM-1 RI|A2128 \\
\hline 1646 & 5186 & NAD83 & 593522.8 & 112015 & HEISPROD & & & & 1100-EM-1 R||3140A \\
\hline 1647 & 5188 & NAD83 & 593522.8 & 112018.8 & HEISPROD & & & & 1100-EM-1 RI|2162A \\
\hline 1648 & 5190 & NAD83 & 593524.6 & 112086.1 & HEISPROD & & & & 1100-EM-1 RI|A2125 \\
\hline 1649 & 5192 & NAD83 & 593525.5 & 112042.1 & HEISPROD & & & & 1100-EM-1 R\||A2115 \\
\hline 1650 & 5193 & NAD83 & 593529.3 & 112025.3 & HEISPROD & & & & 1100-EM-1 RI|3137A \\
\hline 1651 & 5195 & NAD83 & 593535.8 & 112035.6 & HEISPROD & & & & 1100-EM-1 RI|A2114 \\
\hline 1652 & 5196 & NAD83 & 593539.6 & 112018.8 & HEISPROD & & & & 1100-EM-1 RI|3138A \\
\hline 1653 & 5198 & NAD83 & 593542.4 & 112045.9 & HEISPROD & & & & 1100-EM-1 RI|A2113 \\
\hline 1654 & 5200 & NAD83 & 593545.1 & 112073.1 & HEISPROD & & & & 1100-EM-1 RI|A2129 \\
\hline 1655 & 5202 & NAD83 & 593546.2 & 112029.1 & HEISPROD & & & & 1100-EM-1 RI|A2116 \\
\hline 1656 & 5204 & NAD83 & 593556.5 & 112022.6 & HEISPROD & & & & 1100-EM-1 RI|A2117 \\
\hline 1657 & 5206 & NAD83 & 593564.7 & 112103.9 & HEISPROD & & & & 1100-EM-1 RI|A2130 \\
\hline 1658 & 5208 & NAD83 & 593566.7 & 112016 & HEISPROD & & & & 1100-EM-1 RI|A2118 \\
\hline 1659 & 5210 & NAD83 & 593578.8 & 112080.4 & HEISPROD & & & & 1100-EM-1 RI|A2134 \\
\hline 1660 & 5212 & NAD83 & 593585.3 & 112090.9 & HEISPROD & & & & 1100-EM-1 RI|A2131 \\
\hline 1661 & 5214 & NAD83 & 593595.6 & 112084.3 & HEISPROD & & & & 1100-EM-1 RI|A2132 \\
\hline 1662 & 5216 & NAD83 & 593608.7 & 112104.9 & HEISPROD & & & & 1100-EM-1 RI|A2133 \\
\hline 1663 & 5218 & NAD83 & 593894.1 & 116611.8 & HEISPROD & & & & 300-FF-2 LFI|B0DP96 \\
\hline 1664 & 5219 & NAD83 & 594048.6 & 116455.9 & HEISPROD & & & & 300-FF-2 LFI|B0DP95 \\
\hline 1665 & 5220 & NAD83 & 594211 & 116130 & HEISPROD & & & & 300-FF-1 RI|B00H44 \\
\hline 1666 & 5222 & NAD83 & 594270 & 115972 & HEISPROD & & & & 300-FF-1 RI|B0OHY9 \\
\hline 1667 & 5223 & NAD83 & 594288 & 115962 & HEISPROD & & & & 300-FF-1 RI|B0OHY7 \\
\hline 1668 & 5225 & NAD83 & 594304 & 115962 & HEISPROD & & & & 300-FF-1 RI|B0OHY6 \\
\hline 1669 & 5226 & NAD83 & 594327.7 & 117249.8 & HEISPROD & & & & 300-FF-5 RI|B01318 \\
\hline 1670 & 5227 & NAD83 & 594365.4 & 117861.6 & HEISPROD & & & & 300-FF-5 RI|B01312 \\
\hline 1671 & 5228 & NAD83 & 594392.1 & 116696.5 & HEISPROD & & & & 300-FF-5 RI|B01322 \\
\hline 1672 & 5230 & NAD83 & 594400 & 115970 & HEISPROD & & & & 300-FF-1 RI|B0OHY3 \\
\hline 1673 & 5232 & NAD83 & 594409 & 115680 & HEISPROD & & & & 300-FF-1 RI|BOOHZ0 \\
\hline
\end{tabular}




\begin{tabular}{|c|c|c|c|c|c|c|c|c|c|}
\hline & A & $\mathrm{B}$ & $\mathrm{C}$ & $\mathrm{D}$ & $\mathrm{E}$ & $\mathrm{F}$ & G & $\mathrm{H}$ & $\mathrm{I}$ \\
\hline 1 & SAMP_SITE_ID & HORIZ_COORD_TYPE & EW_COORD & NS_COORD & OWNER_ID & COORD_SOURCE & DIST_CLASS & RAD_LEVEL & SAMP_SITE_NAME \\
\hline 1674 & 5234 & NAD83 & 594409 & 115972 & HEISPROD & & & & 300-FF-1 RI|B0OHZ1 \\
\hline 1675 & 5235 & NAD83(91) & 594420.938 & 117132.899 & HEISPROD & MEASURED & & & 300-FF-5 RI|B0C2R3 \\
\hline 1676 & 5237 & NAD83 & 594425 & 115970 & HEISPROD & & & & 300-FF-1 RI|B0OHY4 \\
\hline 1677 & 5238 & NAD83 & 594430 & 115977 & HEISPROD & & & & 300-FF-1 RI|B00HZ2 \\
\hline 1678 & 5240 & NAD83 & 594434 & 115984 & HEISPROD & & & & 300-FF-1 RI|B00HY5 \\
\hline 1679 & 5241 & NAD83 & 594450.2 & 118401 & HEISPROD & & & & 300-FF-5 RI|B01311 \\
\hline 1680 & 5242 & NAD83 & 594467.5 & 116289.9 & HEISPROD & & & & 300-FF-5 RI|B01381 \\
\hline 1681 & 5243 & NAD83(91) & 594584.932 & 116209.092 & HEISPROD & MEASURED & & & 300-FF-5 RI|B0C2S2 \\
\hline 1682 & 5244 & NAD83(91) & 594721.335 & 115686.282 & HEISPROD & MEASURED & & & 300-FF-5 RI|B0C2T5 \\
\hline 1683 & 5254 & NAD83 & 573486.848 & 152067.942 & HEISPROD & ESTIMATED & & & 120-D-1|P-1 \\
\hline 1684 & 5255 & NAD83 & 573484.65 & 152058.942 & HEISPROD & ESTIMATED & & & 120-D-1|P-3 \\
\hline 1685 & 5256 & NAD83 & 573475.594 & 152058.847 & HEISPROD & ESTIMATED & & & 120-D-1|P-4 \\
\hline 1686 & 5257 & NAD83 & 573474.035 & 152063.915 & HEISPROD & ESTIMATED & & & 120-D-1|P-5A \\
\hline 1687 & 5258 & NAD83 & 573468.575 & 152064.987 & HEISPROD & ESTIMATED & & & 120-D-1|PB-1 \\
\hline 1688 & 5259 & NAD83 & 573466.462 & 152034.863 & HEISPROD & ESTIMATED & & & 120-D-1|S-5 \\
\hline 1689 & 5260 & NAD83 & 573475.969 & 152034.503 & HEISPROD & ESTIMATED & & & 120-D-1|SR-1 \\
\hline 1690 & 5261 & NAD83 & 573481.993 & 152034.479 & HEISPROD & ESTIMATED & & & 120-D-1|SR-2 \\
\hline 1691 & 5262 & NAD83 & 573500.042 & 152038.326 & HEISPROD & ESTIMATED & & & 120-D-1|SR-7 \\
\hline 1692 & 5264 & NAD83 & 573497.55 & 152025.277 & HEISPROD & ESTIMATED & & & 120-D-1|S-1 \\
\hline 1693 & 5265 & NAD83 & 573487.934 & 152021.471 & HEISPROD & ESTIMATED & & & 120-D-1|S-6 \\
\hline 1694 & 5266 & NAD83 & 573465.225 & 152016.436 & HEISPROD & ESTIMATED & & & 120-D-1|SB-1 \\
\hline 1695 & 5267 & NAD83 & 573466.094 & 152023.463 & HEISPROD & ESTIMATED & & & 120-D-1|TP-1 \\
\hline 1696 & 5268 & NAD83 & 573473.05 & 152025.88 & HEISPROD & ESTIMATED & & & 120-D-1|SR-3 \\
\hline 1697 & 5269 & NAD83 & 571315.6 & 149653.6 & HEISPROD & ESTIMATED & & & 100N BUNKER TANK|1715-N5 \\
\hline 1698 & 5271 & NAD83 & 567536.6 & 135171.8 & CENTPLAT & ESTIMATED & & & $271-U$ \\
\hline 1699 & 5273 & NAD83 & 571104.11 & 149571.07 & HEISPROD & ESTIMATED & & & 107N STACK \\
\hline 1700 & 5275 & NAD83 & 571676.6 & 149253.1 & HEISPROD & ESTIMATED & & & EAL (MO425)|EAL WASTE CHARACTERIZATION \\
\hline 1701 & 5277 & NAD83 & 571070.7 & 149379.4 & HEISPROD & ESTIMATED & & & 181N AND 182N OIL SAMPLING|182N FACILITY \\
\hline 1702 & 5278 & NAD83 & 567235.6 & 134861.3 & CENTPLAT & ESTIMATED & & & 200-UP-2, 216-U-16 U03 CRIB \\
\hline 1703 & 5280 & NAD83 & 565405 & 145120 & HEISPROD & ESTIMATED & & & 116-C-5 RET. BASIN|\#1 BLACK ABRAISIVE \\
\hline 1704 & 5281 & NAD83 & 565398 & 145120 & HEISPROD & ESTIMATED & & & 116-C-5 RET. BASIN|\#2 BLACK ABRAISIVE \\
\hline 1705 & 5282 & NAD83 & 565408 & 145130 & HEISPROD & ESTIMATED & & & 116-C-5 RET. BASIN|\#3 BLACK ABRAISIVE \\
\hline 1706 & 5283 & NAD83 & 565411 & 145133 & HEISPROD & ESTIMATED & & & 116-C-5 RET. BASIN|\#4 BLACK ABRAISIVE \\
\hline 1707 & 5284 & NAD83 & 565485 & 145065 & HEISPROD & ESTIMATED & & & 116-C-5 RETENTION BASIN|\#1 PIT \\
\hline 1708 & 5285 & NAD83 & 565455 & 145085 & HEISPROD & ESTIMATED & & & 116-C-5 RETENTION BASIN|\#2 PIT \\
\hline 1709 & 5286 & NAD83 & 565455 & 145125 & HEISPROD & ESTIMATED & & & 116-C-5 RETENTION BASIN|\#3 PIT \\
\hline 1710 & 5287 & NAD83 & 565475 & 145150 & HEISPROD & ESTIMATED & & & 116-C-5 RETENTION BASIN|\#4 PIT \\
\hline 1711 & 5288 & NAD83 & 565520 & 145150 & HEISPROD & ESTIMATED & & & 116-C-5 RETENTION BASIN|\#5 PIT \\
\hline
\end{tabular}




\begin{tabular}{|c|c|c|c|c|c|c|c|c|c|}
\hline & A & $\mathrm{B}$ & $\mathrm{C}$ & $\mathrm{D}$ & $\mathrm{E}$ & $\mathrm{F}$ & G & $\mathrm{H}$ & $\mathrm{I}$ \\
\hline 1 & SAMP_SITE_ID & HORIZ_COORD_TYPE & EW_COORD & NS_COORD & OWNER_ID & COORD_SOURCE & DIST_CLASS & RAD_LEVEL & SAMP_SITE_NAME \\
\hline 1712 & 5289 & NAD83 & 565540 & 145115 & HEISPROD & ESTIMATED & & & 116-C-5 RETENTION BASIN|\#6 PIT \\
\hline 1713 & 5290 & NAD83 & 565530 & 145080 & HEISPROD & ESTIMATED & & & 116-C-5 RETENTION BASIN|\#7 PIT \\
\hline 1714 & 5292 & NAD83 & 571184.8 & 149458 & CENTPLAT & ESTIMATED & & & 100N 90 DAY PAD \\
\hline 1715 & 5293 & & & & HEISPROD & & & & 1713H BUILDING \\
\hline 1716 & 5295 & NAD83 & 571079.312 & 149536.078 & CENTPLAT & ESTIMATED & & & 1304N EMERGENCY DUMP TANK \\
\hline 1717 & 5297 & NAD83 & 566352 & 135928 & CENTPLAT & ESTIMATED & & & 200-ZP-1|CENTRAL POINT OF TREATMENT BLDG \\
\hline 1718 & 5298 & NAD83 & 565839 & 145093 & HEISPROD & ESTIMATED & & & 100-BC-1 TRENCH|B0J315 \\
\hline 1719 & 5299 & NAD83 & 565848 & 145063 & HEISPROD & ESTIMATED & & & 100-BC-1 TRENCH|B0J317,B0J318, B0J319 \\
\hline 1720 & 5300 & NAD83 & 565927 & 145117 & HEISPROD & ESTIMATED & & & 100-BC-1 TRENCH|B0J320,B0J321 \\
\hline 1721 & 5302 & NAD83 & 567530 & 135000 & HEISPROD & ESTIMATED & & & U0-3 PLANT/PIPELINE \\
\hline 1722 & 5303 & NAD83 & 567588 & 146210 & CENTPLAT & ESTIMATED & & & SEEP 057-3 \\
\hline 1723 & 5304 & NAD83 & 570375 & 148782 & CENTPLAT & ESTIMATED & & & SEEP 082-2 \\
\hline 1724 & 5306 & NAD83 & 577257 & 153666 & CENTPLAT & ESTIMATED & & & SEEP 145-2 \\
\hline 1725 & 5307 & NAD83 & 577958 & 153090 & CENTPLAT & ESTIMATED & & & SEEP $150-3$ \\
\hline 1726 & 5308 & NAD83 & 594123 & 115620 & HEISPROD & ESTIMATED & & & TANK 309-1 SAMPLING|SITE 6 \\
\hline 1727 & 5309 & NAD83 & 594124 & 115623 & HEISPROD & ESTIMATED & & & TANK 309-1 SAMPLING|SITES 5 AND 7 \\
\hline 1728 & 5310 & NAD83 & 594128 & 115620 & HEISPROD & ESTIMATED & & & TANK 309-1 SAMPLING|SITE 4 \\
\hline 1729 & 5312 & NAD83 & 594127 & 115613 & HEISPROD & ESTIMATED & & & TANK 309-1 SAMPLING|SITE 8 \\
\hline 1730 & 5313 & NAD83 & 594126 & 115613 & HEISPROD & ESTIMATED & & & TANK 309-1 SAMPLING|SITE 9 \\
\hline 1731 & 5324 & NAD83 & 569532 & 147984 & CENTPLAT & ESTIMATED & & & SEEP 077-1 \\
\hline 1732 & 5325 & NAD83 & 578244.733 & 152673.473 & CENTPLAT & ESTIMATED & & & SEEP 153-1 \\
\hline 1733 & 5326 & NAD83 & 572632.2 & 151680.1 & CENTPLAT & ESTIMATED & & & SEEP 102-1 \\
\hline 1734 & 5327 & NAD83 & 594290 & 115648 & HEISPROD & ESTIMATED & & & 3765 BUILDING|CENTRAL POINT \\
\hline 1735 & 5328 & NAD83 & 578086.5 & 152649.4 & HEISPROD & ESTIMATED & & & 116-H-7 RETENTION BASIN (107-H) \\
\hline 1736 & 5329 & NAD83 & 574013.6 & 152328.5 & HEISPROD & ESTIMATED & & & 116-DR-1 \& 2 \\
\hline 1737 & 5330 & NAD83 & 573861.3 & 151588.9 & HEISPROD & ESTIMATED & & & 116-D1-A \\
\hline 1738 & 5331 & NAD83 & 578088.7 & 152305 & HEISPROD & ESTIMATED & & & $116-\mathrm{H}-1$ \\
\hline 1739 & 5333 & NAD83 & 569075 & 147045.7 & CENTPLAT & ESTIMATED & & & 116-K-1\&2, 116-KW-3\&4 CENTROID \\
\hline 1740 & 5334 & NAD83 & 569376.3 & 146383.9 & HEISPROD & ESTIMATED & & & HPT COUNTING LAB \\
\hline 1741 & 5335 & NAD83 & 594283.6 & 116106.1 & HEISPROD & ESTIMATED & & & SOUTH PROCESS POND(SP-1)|300-FF-1 OU \\
\hline 1742 & 5336 & NAD83 & 569797.5 & 147715.9 & HEISPROD & ESTIMATED & & & $116-\mathrm{K}-2$ \\
\hline 1743 & 5337 & NAD83 & 577644.3 & 152438.1 & HEISPROD & ESTIMATED & & & $116-\mathrm{H}-9$ \\
\hline 1744 & 5339 & NAD83 & 565390.2 & 145109.8 & HEISPROD & ESTIMATED & & & 116-C-5 SLUDGE TRENCH|100-BC-1 \\
\hline 1745 & 5340 & NAD83 & 580600 & 147615 & HEISPROD & ESTIMATED & & & 108-F BIOLOGICAL LAB \\
\hline 1746 & 5341 & NAD83 & 566619 & 135471 & CENTPLAT & ESTIMATED & & & COMPOSITE|200-ZP-2 SOIL VAPOR EXT SYSTEM \\
\hline 1747 & 5342 & NAD83 & 565368 & 145102 & HEISPROD & ESTIMATED & & & 100-BC-1 C5 TRENCH|B0JCV6 \\
\hline 1748 & 5343 & NAD83 & 565378 & 145098 & HEISPROD & ESTIMATED & & & 100-BC-1 C5 TRENCH|B0JCV7 \\
\hline 1749 & 5344 & NAD83 & 565481 & 145088 & HEISPROD & ESTIMATED & & & 100-BC-1 C5 TRENCH|B0JCV8 \\
\hline
\end{tabular}




\begin{tabular}{|c|c|c|c|c|c|c|c|c|c|}
\hline & A & $\mathrm{B}$ & $\mathrm{C}$ & $\mathrm{D}$ & $E$ & $\mathrm{~F}$ & $\mathrm{G}$ & $\mathrm{H}$ & $\mathrm{I}$ \\
\hline 1 & SAMP_SITE_ID & HORIZ_COORD_TYPE & EW_COORD & NS_COORD & OWNER_ID & COORD_SOURCE & DIST_CLASS & RAD_LEVEL & SAMP_SITE_NAME \\
\hline 1750 & 5345 & NAD83 & 565782 & 145265 & HEISPROD & ESTIMATED & & & N. PIPELINE RUST/SCALE SAMPLING|B0J395 \\
\hline 1751 & 5346 & NAD83 & 565782 & 145263 & HEISPROD & ESTIMATED & & & N. PIPELINE RUST/SCALE SAMPLING|B0J396 \\
\hline 1752 & 5347 & NAD83 & 565781 & 145261 & HEISPROD & ESTIMATED & & & N. PIPELINE RUST/SCALE SAMPLING|B0J397\&8 \\
\hline 1753 & 5349 & & & & SESPMNT & & OFFSITE & & ACROSS RIVER $100 \mathrm{~N}$ \\
\hline 1754 & 5371 & NAD83 & 565446 & 145069 & HEISPROD & ESTIMATED & & & 100-BC-1 C5 TRENCH|B0JCV9 \\
\hline 1755 & 5372 & NAD83 & 565358 & 145114 & HEISPROD & ESTIMATED & & & 100-BC-1 C5 TRENCH|B0JCW2 \\
\hline 1756 & 5373 & NAD83 & 593682.4 & 116068.3 & HEISPROD & ESTIMATED & & & 3734 BUILDING \\
\hline 1757 & 5374 & NAD83 & 573352 & 151406 & HEISPROD & ESTIMATED & & & 100-D-12 TP 1 \\
\hline 1758 & 5375 & NAD83 & 573803.875 & 151747.875 & HEISPROD & ESTIMATED & & & 116-D-4 \\
\hline 1759 & 5376 & NAD83 & 565267.1 & 144713 & HEISPROD & ESTIMATED & & & 116-B-9 \\
\hline 1760 & 5377 & NAD83 & 565268.9 & 144691.9 & HEISPROD & ESTIMATED & & & 116-B-10 \\
\hline 1761 & 5378 & NAD83 & 565383.6 & 144365.5 & HEISPROD & ESTIMATED & & & $116-B-16$ \\
\hline 1762 & 5379 & NAD83 & 593027 & 113521 & HEISPROD & & & & 300 AREA RAMP [1SWB] \\
\hline 1763 & 5380 & NAD83 & 590541 & 126923 & HEISPROD & & & & WPSS SOLID WASTE PIT [2SWB] \\
\hline 1764 & 5381 & NAD83 & 585821 & 129866 & HEISPROD & & & & HANFORD PIT\#9 [3SWB] \\
\hline 1765 & 5382 & NAD83 & 589892 & 136951 & HEISPROD & & & & RIVER, HANFORD TOWN [4SWB] \\
\hline 1766 & 5383 & NAD83 & 574625 & 150583 & HEISPROD & & & & HANFORD PIT \#21 [5SWB] \\
\hline 1767 & 5384 & NAD83 & 576396 & 135754 & HEISPROD & & & & GROUT PIT [6SWB] \\
\hline 1768 & 5385 & NAD83 & 575775 & 137558 & HEISPROD & & & & 218-E-128, TRENCH 94 [7SWB] \\
\hline 1769 & 5386 & NAD83 & 571415 & 136705 & HEISPROD & & & & BATCH PLANT [9SWB] \\
\hline 1770 & 5387 & NAD83 & 559449 & 141788 & HEISPROD & & & & VERNITA GRADE [10SWB] \\
\hline 1771 & 5388 & NAD83 & 558513 & 135554 & HEISPROD & & & & COLD CREEK [11SWB] \\
\hline 1772 & 5389 & NAD83 & 560751 & 130363 & HEISPROD & & & & DRY CREEK [12SWB] \\
\hline 1773 & 5390 & NAD83 & 595783 & 115965 & HEISPROD & & & & E OF 300 AREA [13SWB] \\
\hline 1774 & 5391 & NAD83 & 596217 & 126410 & HEISPROD & & & & RINGOLD COULEE [14SWB] \\
\hline 1775 & 5392 & NAD83 & 560085 & 137948 & HEISPROD & & & & YAKIMA BARRICADE [15SWB] \\
\hline 1776 & 5393 & NAD83 & 569900 & 118020 & HEISPROD & & & & RATTLESNAKE MOUNTAIN [E-1] \\
\hline 1777 & 5394 & NAD83 & 560670 & 130520 & HEISPROD & & & & RATTLESNAKE SPRING GREASEWOOD [E-2] \\
\hline 1778 & 5395 & NAD83 & 562600 & 145900 & HEISPROD & & & & E OF VERNITA BR., RIPARIAN JUNIPER [E-4] \\
\hline 1779 & 5396 & NAD83 & 570150 & 128750 & HEISPROD & & & & W. OF GOOSE EGG HILL, PLAYA[E-9] \\
\hline 1780 & 5397 & & & & HEISPROD & & & & MID.RINGOLD FM., E. OF RIVER [RINGOLD] \\
\hline 1781 & 5398 & NAD83 & 563000 & 145800 & HEISPROD & & & & RIPARIAN GRASS [E-3] \\
\hline 1782 & 5399 & NAD83 & 577600 & 138700 & HEISPROD & & & & NE OF 200-E BWIP ECA BIG SAGE [E-5] \\
\hline 1783 & 5400 & NAD83 & 584300 & 140500 & HEISPROD & & & & OLD FIELD CONTROLLED AREA [E-6] \\
\hline 1784 & 5401 & NAD83 & 571900 & 129900 & HEISPROD & & & & COLUMBIA RIVER PLAIN SAGEBRUSH [E-7] \\
\hline 1785 & 5402 & NAD83 & 571900 & 129900 & HEISPROD & & & & COLUMBIA RIVER PLAIN RABBITBRUSH [E-8] \\
\hline 1786 & 5404 & NAD83 & 562900 & 134100 & HEISPROD & & & & COLUMBIA RIVER PLAIN BWIP ECA [E-11] \\
\hline 1787 & 5405 & NAD83 & 563800 & 137800 & HEISPROD & & & & 200 AREA PLAT BWIP ECA CTRL AREA [E-12] \\
\hline
\end{tabular}




\begin{tabular}{|c|c|c|c|c|c|c|c|c|c|}
\hline & A & $\mathrm{B}$ & $\mathrm{C}$ & $\mathrm{D}$ & $\mathrm{E}$ & $\mathrm{F}$ & G & $\mathrm{H}$ & $\mathrm{I}$ \\
\hline 1 & SAMP_SITE_ID & HORIZ_COORD_TYPE & EW_COORD & NS_COORD & OWNER_ID & COORD_SOURCE & DIST_CLASS & RAD_LEVEL & SAMP_SITE_NAME \\
\hline 1788 & $\overline{5407}$ & NAD83 & 572395 & 145209 & HEISPROD & & & & 100N FIRE [1DOH] \\
\hline 1789 & 5408 & NAD83 & 496974 & 137361 & HEISPROD & & & & ACI-YAKIMA [12DOH] \\
\hline 1790 & 5409 & NAD83 & 568071 & 131394 & HEISPROD & & & & ARMY LOOP [13DOH] \\
\hline 1791 & 5410 & NAD83 & 496716 & 138304 & HEISPROD & & & & CEMETERY [14DOH] \\
\hline 1792 & 5411 & NAD83 & 624888 & 149477 & HEISPROD & & & & CONNELL [15DOH] \\
\hline 1793 & 5412 & NAD83 & 584875 & 139836 & HEISPROD & & & & HANFORD TOWN [19DOH] \\
\hline 1794 & 5413 & NAD83 & 538418 & 109402 & HEISPROD & & & & INDEPENDENCE [22DOH] \\
\hline 1795 & 5414 & NAD83 & 594261 & 199910 & HEISPROD & & & & MOSES LAKE [24DOH] \\
\hline 1796 & 5415 & NAD83 & 589486 & 125040 & HEISPROD & & & & S1 [34DOH] \\
\hline 1797 & 5416 & NAD83 & 591895 & 127321 & HEISPROD & & & & $\mathrm{S} 2[35 \mathrm{DOH}]$ \\
\hline 1798 & 5417 & NAD83 & 538395 & 113107 & HEISPROD & & & & SUNNYSIDE [41DOH] \\
\hline 1799 & 5418 & NAD83 & 584606 & 127841 & HEISPROD & & & & WYE [45DOH] \\
\hline 1800 & 5419 & NAD83 & 581721 & 138436 & HEISPROD & & & & YAKIMA B. [46DOH] \\
\hline 1801 & 5420 & NAD83 & 593500 & 125040 & HEISPROD & & & & $\mathrm{S} 23$ [49DOH] \\
\hline 1802 & 5422 & NAD83 & 572806 & 137121 & HEISPROD & & & & EAST SIDE OF PIT \#9, WEST OF 200-E AREA \\
\hline 1803 & 5424 & NAD83 & 571106 & 149568 & HEISPROD & ESTIMATED & & & $107 \mathrm{~N}$ \\
\hline 1804 & 5426 & NAD83 & 571379 & 149637 & HEISPROD & ESTIMATED & & & $1310 N^{*}$ \\
\hline 1805 & 5429 & NAD83 & 565288.188 & 144512.219 & HEISPROD & ESTIMATED & & & 118-B-8,105-B REACTOR BUILDING \\
\hline 1806 & 5430 & NAD83 & 567343.875 & 134010.734 & CENTPLAT & ESTIMATED & & & 233-S PLUTONIUM CONCENTRATION FACITLITY \\
\hline 1807 & 5431 & NAD83 & 565478.125 & 144022.312 & HEISPROD & ESTIMATED & & & 116-C-2C,105-C PLUTO CRIB SAND FILTER \\
\hline 1808 & 5432 & NAD83 & 571201.438 & 149489.484 & CENTPLAT & ESTIMATED & & & 100-N-66, 105-N/109-N REACTOR BLDG CMPLX \\
\hline 1809 & 5433 & NAD83 & 573854.375 & 151290.828 & HEISPROD & ESTIMATED & & & 116-DR-6 TP 1 \\
\hline 1810 & 5436 & NAD83 & 565504 & 145076 & HEISPROD & ESTIMATED & & & 100-BC-1 C5 \\
\hline 1811 & 5437 & NAD83 & 565359 & 145126 & HEISPROD & ESTIMATED & & & 116-C-5 TRNCH REM-RED GRVL UNDR METAL PL \\
\hline 1812 & 5439 & NAD83 & 565359 & 145121 & HEISPROD & ESTIMATED & & & 116-C-5 WEST BASIN ASPHAULTIC LAYER \\
\hline 1813 & 5440 & NAD83 & 565374 & 145149 & HEISPROD & ESTIMATED & & & 116-C-5 WEST BASIN ASPHALTIC LAYER \\
\hline 1814 & 5441 & NAD83 & 565509 & 145160 & HEISPROD & ESTIMATED & & & 116-C-5,EAST BASN-WEST SIDE OF SPOILS PI \\
\hline 1815 & 5442 & NAD83 & 565528 & 145150 & HEISPROD & ESTIMATED & & & 116-C-5,EAST BASN-EAST SIDE OF SPOILS PI \\
\hline 1816 & 5443 & NAD83 & 565933.6 & 145296.4 & HEISPROD & ESTIMATED & & & 116-C-1,SOUTHERN PORTION,NODE A2,15 \\
\hline 1817 & 5444 & & & & HEISPROD & & & & 200E-96-0014 WASTE CONTAINER \\
\hline 1818 & 5445 & & & & HEISPROD & & & & 100N-96-0607 WASTE CONTAINER \\
\hline 1819 & 5446 & & & & HEISPROD & & & & 100N-95-0254 WASTE CONTAINER SAMPLING \\
\hline 1820 & 5447 & & & & HEISPROD & & & & 100N-95-0279 DRUM CONTAINER \\
\hline 1821 & 5448 & NAD83 & 573960 & 152347 & HEISPROD & ESTIMATED & & & 100-DR-1,N=152347,E=573960,A=133.9 \\
\hline 1822 & 5452 & NAD83 & 573960 & 152334 & HEISPROD & ESTIMATED & & & 100-DR-1,N=152334,E=573960. $A=130.4$ \\
\hline 1823 & 5453 & NAD83 & 573957 & 152316 & HEISPROD & ESTIMATED & & & $100-D R-1, N=152316, E=573957 . A=130.4$ \\
\hline 1824 & 5454 & NAD83 & 573957 & 152329 & HEISPROD & ESTIMATED & & & 100-DR-1,N=152329,E=573957. $A=130.4$ \\
\hline 1825 & 5455 & NAD83 & 573957 & 152339 & HEISPROD & ESTIMATED & & & 100-DR-1,N=152339,E=573957, $A=130.4$ \\
\hline
\end{tabular}




\begin{tabular}{|c|c|c|c|c|c|c|c|c|c|}
\hline & A & $\mathrm{B}$ & C & $\mathrm{D}$ & $\mathrm{E}$ & $\mathrm{F}$ & $\mathrm{G}$ & $\mathrm{H}$ & $\mathrm{I}$ \\
\hline 1 & SAMP_SITE_ID & HORIZ_COORD_TYPE & EW_COORD & NS_COORD & OWNER_ID & COORD_SOURCE & DIST_CLASS & RAD_LEVEL & SAMP_SITE_NAME \\
\hline 1826 & 5456 & NAD83 & 573957 & 152349 & HEISPROD & ESTIMATED & & & 100-DR-1,N=152349,E=573957, $A=130.1$ \\
\hline 1827 & 5457 & NAD83 & 573967 & 152349 & HEISPROD & ESTIMATED & & & 100-DR-1,N=152349,E=573967, $A=130.1$ \\
\hline 1828 & 5458 & NAD83 & 573967 & 152339 & HEISPROD & ESTIMATED & & & $100-D R-1, N=152339, E=573967, A=130.1$ \\
\hline 1829 & 5459 & NAD83 & 573967 & 152316 & HEISPROD & ESTIMATED & & & 100-DR-1,N=152316,E=573967, $A=130.1$ \\
\hline 1830 & 5460 & NAD83 & 573967 & 152329 & HEISPROD & ESTIMATED & & & 100-DR-1,N=152329,E=573967, $A=130.1$ \\
\hline 1831 & 5461 & NAD83 & 573977 & 152349 & HEISPROD & ESTIMATED & & & 100-DR-1,N=152349,E=573977, $A=130.1$ \\
\hline 1832 & 5462 & NAD83 & 573977 & 152338 & HEISPROD & ESTIMATED & & & 100-DR-1,N=152338,E=573977, $A=130.1$ \\
\hline 1833 & 5463 & NAD83 & 573977 & 152315 & HEISPROD & ESTIMATED & & & 100-DR-1,N=152315,E=573977, $A=130.1$ \\
\hline 1834 & 5464 & NAD83 & 573977 & 152328 & HEISPROD & ESTIMATED & & & 100-DR-1,N=152328,E=573977, $A=130.1$ \\
\hline 1835 & 5465 & NAD83 & 573977 & 152348 & HEISPROD & ESTIMATED & & & 100-DR-1,N=152348,E=573977, $A=130.1$ \\
\hline 1836 & 5467 & NAD83 & 573987 & 152315 & HEISPROD & ESTIMATED & & & $100-\mathrm{DR}-1, \mathrm{~N}=152315, \mathrm{E}=573987, \mathrm{~A}=130.1$ \\
\hline 1837 & 5468 & NAD83 & 573987 & 152328 & HEISPROD & ESTIMATED & & & $100-D R-1, N=152328, E=573987, A=130.1$ \\
\hline 1838 & 5469 & NAD83 & 573997 & 152346 & HEISPROD & ESTIMATED & & & $100-D R-1, N=152346, E=573997, A=130.1$ \\
\hline 1839 & 5470 & NAD83 & 573999 & 152328 & HEISPROD & ESTIMATED & & & $100-\mathrm{DR}-1, \mathrm{~N}=152328, \mathrm{E}=573999, \mathrm{~A}=133.5$ \\
\hline 1840 & 5471 & NAD83 & 573999 & 152318 & HEISPROD & ESTIMATED & & & 100-DR-1,N=152318,E=573999,A=130.1 \\
\hline 1841 & 5472 & NAD83 & 573999 & 152338 & HEISPROD & ESTIMATED & & & 100-DR-1,N=152338,E=573999,A=130.1 \\
\hline 1842 & 5473 & NAD83 & 574007 & 152328 & HEISPROD & ESTIMATED & & & 100-DR-1,N=152328,E=574007,A=133.5 \\
\hline 1843 & 5474 & NAD83 & 574007 & 152328 & HEISPROD & ESTIMATED & & & 100-DR-1,N=152328,E=574007,A=132.0 \\
\hline 1844 & 5475 & NAD83 & 574007 & 152328 & HEISPROD & ESTIMATED & & & 100-DR-1,N=152328,E=574007,A=130.1 \\
\hline 1845 & 5476 & NAD83 & 574005 & 152314 & HEISPROD & ESTIMATED & & & 100-DR-1,N=152314,E=574005,A=133.5 \\
\hline 1846 & 5477 & NAD83 & 574005 & 152314 & HEISPROD & ESTIMATED & & & 100-DR-1,N=152314,E=574005,A=132.0 \\
\hline 1847 & 5478 & NAD83 & 574005 & 152314 & HEISPROD & ESTIMATED & & & $100-D R-1, N=152314, E=574005, A=130.1$ \\
\hline 1848 & 5479 & NAD83 & 574006 & 152341 & HEISPROD & ESTIMATED & & & 100-DR-1,N=152341,E=574006,A=130.1 \\
\hline 1849 & 5480 & NAD83 & 574006 & 152341 & HEISPROD & ESTIMATED & & & $100-\mathrm{DR}-1, \mathrm{~N}=152341, \mathrm{E}=574006, \mathrm{~A}=132.0$ \\
\hline 1850 & 5481 & NAD83 & 574006 & 152341 & HEISPROD & ESTIMATED & & & $100-D R-1, N=152341, E=574006, A=133.5$ \\
\hline 1851 & 5482 & NAD83 & 574019 & 152322 & HEISPROD & ESTIMATED & & & $100-D R-1, N=152322, E=574019, A=130.1$ \\
\hline 1852 & 5483 & NAD83 & 574019 & 152322 & HEISPROD & ESTIMATED & & & 100-DR-1,N=152322,E=574019,A=132.0 \\
\hline 1853 & 5484 & NAD83 & 574019 & 152322 & HEISPROD & ESTIMATED & & & $100-D R-1, N=152322, E=574019, A=133.5$ \\
\hline 1854 & 5485 & NAD83 & 574019 & 152332 & HEISPROD & ESTIMATED & & & 100-DR-1,N=152332,E=574019,A=130.1 \\
\hline 1855 & 5486 & NAD83 & 574019 & 152332 & HEISPROD & ESTIMATED & & & $100-D R-1, N=152332, E=574019, A=132.0$ \\
\hline 1856 & 5487 & NAD83 & 574019 & 152332 & HEISPROD & ESTIMATED & & & $100-D R-1, N=152332, E=574019, A=133.5$ \\
\hline 1857 & 5488 & NAD83 & 574019 & 152312 & HEISPROD & ESTIMATED & & & 100-DR-1,N=152312,E=574019,A=130.1 \\
\hline 1858 & 5489 & NAD83 & 574019 & 152312 & HEISPROD & ESTIMATED & & & $100-D R-1, N=152312, E=574019, A=132.0$ \\
\hline 1859 & 5490 & NAD83 & 574019 & 152312 & HEISPROD & ESTIMATED & & & $100-\mathrm{DR}-1, \mathrm{~N}=152312, \mathrm{E}=574019, \mathrm{~A}=133.5$ \\
\hline 1860 & 5491 & & & & HEISPROD & & & & IDW SAMPLING DRUM \# EFSG-93-0549 \\
\hline 1861 & 5492 & NAD83 & 573977 & 152338 & HEISPROD & ESTIMATED & & & 100-DR-1, N=152338,E=573977,A=130.1 \\
\hline 1862 & 5495 & NAD83 & 573782.938 & 151612.047 & HEISPROD & ESTIMATED & & & 116-D-6 TEST PIT \\
\hline 1863 & 5496 & NAD83 & 571229.81 & 149664.24 & HEISPROD & ESTIMATED & & & 116N MAIN STACK \\
\hline
\end{tabular}




\begin{tabular}{|c|c|c|c|c|c|c|c|c|c|}
\hline & A & $\mathrm{B}$ & $\mathrm{C}$ & $\mathrm{D}$ & $\mathrm{E}$ & $\mathrm{F}$ & G & $\mathrm{H}$ & $\mathrm{I}$ \\
\hline 1 & SAMP_SITE_ID & HORIZ_COORD_TYPE & EW_COORD & NS_COORD & OWNER_ID & COORD_SOURCE & DIST_CLASS & RAD_LEVEL & SAMP_SITE_NAME \\
\hline 1864 & 5497 & NAD83 & 571452 & 149667 & HEISPROD & ESTIMATED & & & EAST OF 1301-N CRIB \\
\hline 1865 & 5498 & NAD83 & 571257.7 & 149870.3 & CENTPLAT & ESTIMATED & RIVER_SHORELINE & & N SPRINGS SEEPAGE SAMPLE LOCATION \#1 \\
\hline 1866 & 5499 & NAD83 & 571299 & 149920.8 & CENTPLAT & ESTIMATED & RIVER_SHORELINE & & N SPRINGS SEEPAGE SAMPLE LOCATION \#2 \\
\hline 1867 & 5500 & NAD83 & 571323.1 & 149941.4 & CENTPLAT & ESTIMATED & RIVER_SHORELINE & & N SPRINGS SEEPAGE SAMPLE LOCATION \#3 \\
\hline 1868 & 5501 & NAD83 & 571350.7 & 149977 & CENTPLAT & ESTIMATED & RIVER_SHORELINE & & N SPRINGS SEEPAGE SAMPLE LOCATION \#4 \\
\hline 1869 & 5502 & NAD83 & 571370.2 & 150003.4 & CENTPLAT & ESTIMATED & RIVER_SHORELINE & & N SPRINGS SEEPAGE SAMPLE LOCATION \#5 \\
\hline 1870 & 5503 & NAD83 & 571386.3 & 150028.7 & CENTPLAT & ESTIMATED & RIVER_SHORELINE & & N SPRINGS SEEPAGE SAMPLE LOCATION \#6 \\
\hline 1871 & 5504 & NAD83 & 571398.9 & 150050.5 & CENTPLAT & ESTIMATED & RIVER_SHORELINE & & N SPRINGS SEEPAGE SAMPLE LOCATION \#7 \\
\hline 1872 & 5505 & NAD83 & 571401.2 & 150062 & CENTPLAT & ESTIMATED & RIVER_SHORELINE & & N SPRINGS SEEPAGE SAMPLE LOCATION \#8 \\
\hline 1873 & 5506 & NAD83 & 571427.6 & 150080.3 & CENTPLAT & ESTIMATED & RIVER_SHORELINE & & N SPRINGS SEEPAGE SAMPLE LOCATION \#9 \\
\hline 1874 & 5507 & NAD83 & 571572.2 & 149772.7 & HEISPROD & ESTIMATED & & & 100-N SOIL/VEG. SAMP SITES Y702 \& Y602 \\
\hline 1875 & 5508 & NAD83 & 571482.7 & 149804.8 & HEISPROD & ESTIMATED & & & 100-N SOIL/VEG. SAMP SITES Y703 \& Y603 \\
\hline 1876 & 5509 & NAD83 & 571718 & 149977 & HEISPROD & ESTIMATED & & & 100-N SOIL/VEG. SAMP SITES Y704 \& Y604 \\
\hline 1877 & 5510 & NAD83 & 571704.3 & 150039 & HEISPROD & ESTIMATED & & & 100-N SOIL/VEG. SAMP SITES Y705 \& Y605 \\
\hline 1878 & 5511 & NAD83 & 571858.1 & 149727.9 & HEISPROD & ESTIMATED & & & 100-N SOIL/VEG. SAMP SITES Y708 \& Y608 \\
\hline 1879 & 5512 & NAD83 & 571907.4 & 149420.3 & HEISPROD & ESTIMATED & & & 100-N SOIL/VEG. SAMP SITES Y710 \& Y610 \\
\hline 1880 & 5513 & NAD83 & 572079.6 & 149608.5 & HEISPROD & ESTIMATED & & & 100-N SOIL/VEG. SAMP SITES Y711 \& Y611 \\
\hline 1883 & 5516 & NAD83 & 571273.2 & 149875.7 & HEISPROD & ESTIMATED & & & 100-N SOIL VEGITATION SITE Y719 \\
\hline 1884 & 5517 & & & & SESPMNT & & ONSITE & & N. 300 AREA CLEANUP SITE \\
\hline 1885 & 5518 & & & & SESPMNT & & ONSITE & & W. 300 AREA CLEANUP \\
\hline 1886 & 5519 & & & & SESPMNT & & PERIMETER & & RIVERSHORE ROAD \\
\hline 1887 & 5520 & & & & SESPMNT & & PERIMETER & & SAGEMOOR SPUR/COL.RIV. RD INTERSECTION \\
\hline 1888 & 5521 & & & & SESPMNT & & PERIMETER & & POTHOLES CANAL RD/COL.RIV. RD. INTERSECT \\
\hline 1889 & 5522 & & & & SESPMNT & & PERIMETER & & GULLY E. OF COL.RIV. RD. \\
\hline 1890 & 5523 & & & & SESPMNT & & ONSITE & & 100 N SPRING-1 \\
\hline 1891 & 5524 & & & & SESPMNT & & ONSITE & & 100 N SPRING-2 \\
\hline 1892 & 5525 & & & & SESPMNT & & ONSITE & & 100 N SPRING-3 \\
\hline 1893 & 5526 & & & & SESPMNT & & ONSITE & & 100 N SPRING-4 \\
\hline 1894 & 5527 & & & & SESPMNT & & ONSITE & & 100 N SPRING-1A \\
\hline 1895 & 5528 & & & & SESPMNT & & ONSITE & & 100 N SPRING-1B \\
\hline 1896 & 5529 & & & & SESPMNT & & ONSITE & & 100 N SPRING-1C \\
\hline 1897 & 5530 & & & & SESPMNT & & ONSITE & & 100 N SPRING-1D \\
\hline 1898 & 5531 & & & & SESPMNT & & ONSITE & & 100 N SPRING-3A \\
\hline 1899 & 5532 & & & & SESPMNT & & ONSITE & & 100 N SPRING-3B \\
\hline 1900 & 5533 & & & & SESPMNT & & ONSITE & & 100 N SPRING-3C \\
\hline
\end{tabular}




\begin{tabular}{|c|c|c|c|c|c|c|c|c|c|}
\hline & $A$ & $\mathrm{~B}$ & C & $\mathrm{D}$ & $E$ & $\mathrm{~F}$ & $G$ & $\mathrm{H}$ & $\mathrm{I}$ \\
\hline 1 & SAMP_SITE_ID & HORIZ_COORD_TYPE & EW_COORD & NS_COORD & OWNER_ID & COORD_SOURCE & DIST_CLASS & RAD_LEVEL & SAMP_SITE_NAME \\
\hline 1902 & 5535 & & & & SESPMNT & & ONSITE & & 100 N SPRING-4A \\
\hline 1903 & 5536 & & & & SESPMNT & & ONSITE & & 100 N SPRING-4B \\
\hline 1904 & 5537 & & & & SESPMNT & & ONSITE & & 100 N SPRING-4C \\
\hline 1905 & 5538 & & & & SESPMNT & & ONSITE & & 100 N SPRING-4D \\
\hline 1906 & 5539 & & & & SESPMNT & & ONSITE & & 100 N SPRING-5 \\
\hline 1907 & 7001 & NAD83 & 573405 & 151405 & HEISPROD & ESTIMATED & & & 100-D-12 TP 2 \\
\hline 1908 & 7002 & NAD83 & 566227.1 & 134672 & HEISPROD & & & & 216-U-10-TP \\
\hline 1909 & 7003 & NAD83 & 577893 & 147534 & HEISPROD & & & & IU-5 TP-C3 \\
\hline 1910 & 7004 & NAD83 & 577897 & 147542 & HEISPROD & & & & IU-5 TP-C4 \\
\hline 1911 & 7005 & NAD83 & 573370 & 151405 & HEISPROD & ESTIMATED & & & 100-D-12 TP 3 \\
\hline 1912 & 7006 & NAD83 & 573784 & 151200 & HEISPROD & ESTIMATED & & & 118-D-5 TP \\
\hline 1913 & 7007 & NAD83 & 573820 & 151205 & HEISPROD & ESTIMATED & & & 116-DR-3 TP \\
\hline 1914 & 7008 & NAD83(91) & 575886.74 & 151184.31 & HEISPROD & & & & IU-4 TESTPIT G6 \\
\hline 1915 & 7009 & NAD83(91) & 575880.64 & 151184.31 & HEISPROD & & & & IU-4 TESTPIT G7 \\
\hline 1916 & 7010 & NAD83 & 577858 & 147510 & HEISPROD & & & & IU-5 TP-A1 \\
\hline \begin{tabular}{|c|}
1917 \\
\end{tabular} & 7011 & NAD83 & 577858 & 147510 & HEISPROD & & & & IU-5 TP-A2 \\
\hline 1918 & 7012 & NAD83 & 577854 & 147512 & HEISPROD & & & & IU-5 TP-A3 \\
\hline 1919 & 7013 & NAD83 & 577861 & 147508 & HEISPROD & & & & IU-5 TP-A4 \\
\hline 1920 & 7014 & NAD83 & 577885 & 147520 & HEISPROD & & & & IU-5 TP-E1 \\
\hline \begin{tabular}{|l|}
1921 \\
\end{tabular} & 7015 & NAD83 & 577885 & 147520 & HEISPROD & & & & IU-5 TP-E2 \\
\hline 1922 & 7016 & NAD83 & 577843 & 147493 & HEISPROD & & & & IU-5 TP-B1 \\
\hline 1923 & 7017 & NAD83 & 577847 & 147491 & HEISPROD & & & & IU-5 TP-B2 \\
\hline 1924 & 7018 & NAD83 & 577847 & 147491 & HEISPROD & & & & IU-5 TP-B3 \\
\hline 1925 & 7019 & NAD83 & 577850 & 147489 & HEISPROD & & & & IU-5 TP-B4 \\
\hline 1926 & 7020 & NAD83 & 577859 & 147484 & HEISPROD & & & & IU-5 TP-B5 \\
\hline 1927 & 7021 & NAD83 & 577859 & 147484 & HEISPROD & & & & IU-5 TP-B6 \\
\hline 1928 & 7022 & NAD83 & 577862 & 147482 & HEISPROD & & & & IU-5 TP-B7 \\
\hline 1929 & 7023 & NAD83 & 577862 & 147482 & HEISPROD & & & & IU-5 TP-B8 \\
\hline 1930 & 7024 & NAD83 & 577866 & 147479 & HEISPROD & & & & IU-5 TP-B9 \\
\hline 1931 & 7025 & NAD83 & 577866 & 147479 & HEISPROD & & & & IU-5 TP-B10 \\
\hline 1932 & 7026 & NAD83 & 577885 & 147520 & HEISPROD & & & & IU-5 TP-C1 \\
\hline 1933 & 7027 & NAD83 & 577888 & 147526 & HEISPROD & & & & IU-5 TP-C2 \\
\hline 1934 & 7028 & NAD83(91) & 575848.62 & 151156.86 & HEISPROD & & & & IU-4 TESTPIT E1 \\
\hline 1935 & 7029 & NAD83(91) & 575848.62 & 151166.01 & HEISPROD & & & & IU-4 TESTPIT E2 \\
\hline 1936 & 7030 & NAD83(91) & 575845.57 & 151162.96 & HEISPROD & & & & IU-4 TESTPIT E3 \\
\hline 1937 & 7031 & NAD83(91) & 575851.67 & 151162.96 & HEISPROD & & & & IU-4 TESTPIT E4 \\
\hline 1938 & 7032 & NAD83(91) & 575845.57 & 151169.06 & HEISPROD & & & & IU-4 TESTPIT E5 \\
\hline 1939 & 7033 & NAD83(91) & 575851.67 & 151169.06 & HEISPROD & & & & IU-4 TESTPIT E6 \\
\hline
\end{tabular}




\begin{tabular}{|c|c|c|c|c|c|c|c|c|c|}
\hline & $\bar{A}$ & $\mathrm{~B}$ & $\mathrm{C}$ & $\mathrm{D}$ & $E$ & $\mathrm{~F}$ & G & $\mathrm{H}$ & $\mathrm{I}$ \\
\hline 1 & SAMP_SITE_ID & HORIZ_COORD_TYPE & EW_COORD & NS_COORD & OWNER_ID & COORD_SOURCE & DIST_CLASS & RAD_LEVEL & SAMP_SITE_NAME \\
\hline 1940 & 7034 & NAD83(91) & 575873.02 & 151112.06 & HEISPROD & & & & IU-4 TESTPIT D1 \\
\hline 1941 & 7035 & NAD83(91) & 575873.02 & 151108.06 & HEISPROD & & & & IU-4 TESTPIT D2 \\
\hline 1942 & 7036 & NAD83(91) & 575856.24 & 151101.96 & HEISPROD & & & & IU-4 TESTPIT C1 \\
\hline 1943 & 7037 & NAD83(91) & 575859.29 & 151108.06 & HEISPROD & & & & IU-4 TESTPIT C2 \\
\hline 1944 & 7038 & NAD83(91) & 575853.19 & 151108.06 & HEISPROD & & & & IU-4 TESTPIT C3 \\
\hline 1945 & 7039 & NAD83(91) & 575848.62 & 151108.06 & HEISPROD & & & & IU-4 TESTPIT C4 \\
\hline 1946 & 7040 & NAD83(91) & 575851.67 & 151111.11 & HEISPROD & & & & IU-4 TESTPIT C5 \\
\hline 1947 & 7041 & NAD83(91) & 575854.72 & 151114.16 & HEISPROD & & & & IU-4 TESTPIT C6 \\
\hline 1948 & 7042 & NAD83(91) & 575848.62 & 151114.16 & HEISPROD & & & & IU-4 TESTPIT C7 \\
\hline 1949 & 7043 & NAD83(91) & 575850.14 & 151117.21 & HEISPROD & & & & IU-4 TESTPIT C8 \\
\hline 1950 & 7044 & NAD83(91) & 575892.84 & 151172.11 & HEISPROD & & & & IU-4 TESTPIT G1 \\
\hline 1951 & 7045 & NAD83(91) & 575886.74 & 151172.11 & HEISPROD & & & & IU-4 TESTPIT G2 \\
\hline 1952 & 7046 & NAD83(91) & 575892.84 & 151178.21 & HEISPROD & & & & IU-4 TESTPIT G3 \\
\hline 1953 & 7047 & NAD83(91) & 575886.74 & 151178.21 & HEISPROD & & & & IU-4 TESTPIT G4 \\
\hline 1954 & 7048 & NAD83(91) & 575892.84 & 151184.31 & HEISPROD & & & & IU-4 TESTPIT G5 \\
\hline 1955 & 7049 & NAD83 & 580010 & 147960 & HEISPROD & & & & 116-F-1B-TP1 \\
\hline 1956 & 7050 & NAD83 & 581080 & 147900 & HEISPROD & & & & 116-F-9D-TP1 \\
\hline 1957 & 7051 & NAD83 & 580425 & 147530 & HEISPROD & & & & 116-F-3-TP1 \\
\hline 1958 & 7052 & NAD83 & 580010 & 148160 & HEISPROD & & & & 116-F-1C-TP1 \\
\hline 1959 & 7053 & NAD83 & 571320 & 149180 & HEISPROD & & & & 100-NR-1-TP1 \\
\hline 1960 & 7054 & NAD83(91) & 594207.07 & 116503.93 & HEISPROD & & & & 316-2-TP1 \\
\hline 1961 & 7055 & NAD83(91) & 594204.44 & 116622.4 & HEISPROD & & & & 316-2-TP2 \\
\hline 1962 & 7056 & NAD83(91) & 594285.49 & 116620.18 & HEISPROD & & & & 316-2-TP3 \\
\hline 1963 & 7057 & NAD83 & 593948 & 117030 & HEISPROD & & & & 618-4-TP1 \\
\hline 1964 & 7058 & NAD83 & 593880 & 116960 & HEISPROD & & & & 618-4-TP2 \\
\hline 1965 & 7059 & NAD83(91) & 594227.92 & 116875.05 & HEISPROD & & & & 618-5-TP1 \\
\hline 1966 & 7060 & NAD83(91) & 594189.44 & 116834.37 & HEISPROD & & & & 618-5-TP2 \\
\hline 1967 & 7061 & NAD83(91) & 594215.15 & 116119.38 & HEISPROD & & & & 316-1-TP3 \\
\hline 1968 & 7062 & NAD83 & 565415 & 145138 & HEISPROD & & & & 116-C-5-TP02 \\
\hline 1969 & 7063 & NAD83 & 565404 & 145075 & HEISPROD & & & & 116-C-5-TP01 \\
\hline 1970 & 7064 & NAD83 & 565360 & 145112 & HEISPROD & & & & 116-C-5-TP03 \\
\hline 1971 & 7065 & NAD83 & 565480 & 145080 & HEISPROD & & & & 116-C-5-TP04 \\
\hline 1972 & 7066 & NAD83 & 565480 & 145136 & HEISPROD & & & & 116-C-5-TP05 \\
\hline 1973 & 7067 & NAD83 & 565542 & 145108 & HEISPROD & & & & 116-C-5-TP06 \\
\hline 1974 & 7068 & NAD83 & 594090 & 116862 & HEISPROD & & & & 316-5-TP1 \\
\hline 1975 & 7069 & NAD83 & 594090 & 116557 & HEISPROD & & & & 316-5-TP2 \\
\hline 1976 & 7070 & NAD83 & 594090 & 116475 & HEISPROD & & & & 316-5-TP3 \\
\hline 1977 & 7071 & NAD83 & 594090 & 116455 & HEISPROD & & & & 316-5-TP4 \\
\hline
\end{tabular}

Page 52 


\begin{tabular}{|c|c|c|c|c|c|c|c|c|c|}
\hline & A & $\mathrm{B}$ & C & $\mathrm{D}$ & $E$ & $\mathrm{~F}$ & $G$ & $\mathrm{H}$ & $\mathrm{I}$ \\
\hline 1 & SAMP_SITE_ID & HORIZ_COORD_TYPE & EW_COORD & NS_COORD & OWNER_ID & COORD_SOURCE & DIST_CLASS & RAD_LEVEL & SAMP_SITE_NAME \\
\hline 1978 & 7072 & NAD83 & 594090 & 116458 & HEISPROD & & & & 316-5-TP5 \\
\hline 1979 & 7073 & NAD83 & 594090 & 116478 & HEISPROD & & & & 316-5-TP6 \\
\hline 1980 & 7074 & NAD83 & 594090 & 116560 & HEISPROD & & & & 316-5-TP7 \\
\hline 1981 & 7075 & NAD83 & 594090 & 116832 & HEISPROD & & & & 316-5-TP8 \\
\hline 1982 & 7076 & NAD83 & 594076 & 116458 & HEISPROD & & & & 316-5-TP9 \\
\hline 1983 & 7077 & NAD83 & 594076 & 116478 & HEISPROD & & & & 316-5-TP10 \\
\hline 1984 & 7078 & NAD83(91) & 594280.75 & 116110.18 & HEISPROD & & & & 316-1-TP1 \\
\hline 1985 & 7079 & NAD83(91) & 594243.36 & 116032.19 & HEISPROD & & & & 316-1-TP2 \\
\hline 1986 & 7080 & NAD83 & 592746.7 & 114291.9 & HEISPROD & & & & HRD-TP11 \\
\hline 1987 & 7081 & NAD83 & 592675 & 114076.9 & HEISPROD & & & & HRD-TP1 \\
\hline 1988 & 7082 & NAD83 & 592673.7 & 113956.5 & HEISPROD & & & & HRD-TP7 \\
\hline 1989 & 7083 & NAD83 & 592696.4 & 114022.1 & HEISPROD & & & & HRD-TP3B \\
\hline 1990 & 7084 & NAD83 & 592702.5 & 114022 & HEISPROD & & & & HRD-TP3A \\
\hline 1991 & 7085 & NAD83 & 592673.8 & 114005.3 & HEISPROD & & & & HRD-TP4/5 \\
\hline 1992 & 7086 & NAD83 & 592601.3 & 114027.1 & HEISPROD & & & & HRD-TP8 \\
\hline 1993 & 7087 & NAD83 & 592604.8 & 113889.3 & 3 HEISPROD & & & & HRD-TPHRL-2A \\
\hline 1994 & 7088 & NAD83 & 592612.9 & 113890.3 & 3 HEISPROD & & & & HRD-TPHRL-3A \\
\hline 1997 & 7091 & NAD83 & 573468 & 152023 & HEISPROD & MEASURED & & & D-POND-TP1 \\
\hline 1998 & 7092 & NAD83 & 573490 & 152041 & HEISPROD & MEASURED & & & D-POND-TP2 \\
\hline 1999 & 7093 & NAD83 & 573488 & 152057 & HEISPROD & MEASURED & & & D-POND-TP3 \\
\hline 2000 & 7094 & NAD83 & 573475 & 152049 & HEISPROD & MEASURED & & & D-POND-TP4 \\
\hline 2001 & 7095 & NAD83 & 573492 & 152063 & HEISPROD & MEASURED & & & D-POND-TP5 \\
\hline 2002 & 7096 & NAD83 & 573727 & 152259 & HEISPROD & ESTIMATED & & & 107-D3-TP-D \\
\hline 2003 & 7097 & NAD83(91) & 573740 & 151780 & HEISPROD & HGIS & & & 108-D-TNKS-TP-1 \\
\hline 2004 & 7098 & NAD83(91) & 573760 & 151785 & HEISPROD & HGIS & & & 108-D-TP-1 \\
\hline 2005 & 7099 & NAD83 & 572610.15 & ; 151602.824 & HEISPROD & ESTIMATED & & & 1907-DR-TP-1 \\
\hline 2006 & 7100 & NAD83 & 572614.29 & 151597.637 & HEISPROD & ESTIMATED & & & 1907-DR-TP-2 \\
\hline 2007 & 7101 & NAD83 & 572616.366 & 151591.425 & HEISPROD & ESTIMATED & & & 1907-DR-TP-3 \\
\hline 2008 & 7102 & NAD83 & 572619.5 & 151597.637 & HEISPROD & ESTIMATED & & & 1907-DR-TP-4 \\
\hline 2009 & 7103 & NAD83 & 569130 & 147110 & HEISPROD & MEASURED & & & 100-KR-1-TP1 \\
\hline 2010 & 7104 & NAD83 & 569055 & 147085 & HEISPROD & ESTIMATED & & & 100-KR-1-TP2 \\
\hline 2011 & 7105 & NAD83 & 568550 & 146750 & HEISPROD & ESTIMATED & & & 100-KR-1-TP3 \\
\hline 2012 & 7106 & NAD83 & 568390 & 146660 & HEISPROD & ESTIMATED & & & 100-KR-1-TP4 \\
\hline 2013 & 7107 & NAD83 & 573820 & 151500 & HEISPROD & ESTIMATED & & & 1607-D4-TP1 \\
\hline 2014 & 7108 & NAD83(91) & 565388.7 & 145262.4 & HEISPROD & GPS & & & 116-B-11-TP1 \\
\hline
\end{tabular}




\begin{tabular}{|c|c|c|c|c|c|c|c|c|c|}
\hline & A & $\mathrm{B}$ & $\mathrm{C}$ & $\mathrm{D}$ & $E$ & $\mathrm{~F}$ & $\mathrm{G}$ & $\mathrm{H}$ & $\mathrm{I}$ \\
\hline 1 & SAMP_SITE_ID & HORIZ_COORD_TYPE & EW_COORD & NS_COORD & OWNER_ID & COORD_SOURCE & DIST_CLASS & RAD_LEVEL & SAMP_SITE_NAME \\
\hline 2016 & $\overline{7110}$ & NAD83(91) & 573460.7 & 151970.6 & HEISPROD & GPS & & & 126-D-3-TP1 \\
\hline 2017 & 7111 & NAD83 & 592604.8 & 113889.3 & HEISPROD & ESTIMATED & & & HRD-TPB4 \\
\hline 2018 & 7112 & NAD83 & 592600 & 113890 & HEISPROD & ESTIMATED & & & HRD-TPB5 \\
\hline 2019 & 7113 & NAD83(91) & 573846.65 & 151286.5 & HEISPROD & GPS & & & 116-DR-6 TP 2 \\
\hline 2020 & 7215 & NAD83(91) & 580378.812 & 147504.562 & HEISPROD & HGIS & & & 116-F-5-TP \\
\hline 2021 & 7217 & NAD83(91) & 580450.875 & 147576 & HEISPROD & HGIS & & & 116-F-11 French Drain \\
\hline 2022 & 7218 & NAD83(91) & 568868.188 & 146071.578 & HEISPROD & HGIS & & & 100-K-17-TP Acid Neutralization Pit \\
\hline 2023 & 7219 & NAD83(91) & 578088.688 & 152305.016 & HEISPROD & HGIS & & & 116-H-1-TP \\
\hline 2024 & 7234 & & & & HEISPROD & & & & 1607-F2 SEPTIC SYSTEM \\
\hline 2025 & 7235 & & & & HEISPROD & & & & 1607-H2 SEPTIC SYSTEM \\
\hline 2026 & 7254 & NAD83 & 571155 & 149346 & HEISPROD & ESTIMATED & & & 183N Water Treatment Plant - Intake H2O \\
\hline 2027 & 7255 & NAD83 & 571487 & 148787 & HEISPROD & ESTIMATED & & & 183N Backwash End of Pipe \\
\hline 2028 & 7357 & NAD83(91) & 582488.312 & 142072.094 & HEISPROD & HGIS & & & UPR-600-19 \\
\hline 2029 & 7375 & NAD83 & 574086.4 & 137181.3 & CENTPLAT & & & & 216-B-2-2 TRENCH, AT BORING \#B8079 \\
\hline 2030 & 7395 & & & & CENTPLAT & & & & \\
\hline 2031 & 7396 & & & & CENTPLAT & & & & 100-HR-3 PUMP \& TREAT \\
\hline 2032 & 7414 & & & & CENTPLAT & & & & 244-AR UST \\
\hline 2033 & 7434 & NAD83 & 572806 & 137121 & HEISPROD & & & & E side of $P \# 9, W$ of $200-E$ Area [8SWB] \\
\hline 2035 & 7455 & NAD83 & 571141.4 & 149298.6 & HEISPROD & ESTIMATED & & & Potable water pump outlet, $183 \mathrm{~N}$ clearwell \\
\hline 2036 & 7456 & NAD83 & 571236.1 & 149323.3 & HEISPROD & ESTIMATED & & & $183 \mathrm{~N}$ control room sample point \\
\hline 2037 & 7457 & NAD83 & 571181.8 & 149327.9 & HEISPROD & ESTIMATED & & & $183 \mathrm{~N}$ pipe gallery \\
\hline 2038 & 7474 & NAD83 & 571197.4 & 149329.6 & HEISPROD & ESTIMATED & & & $183 \mathrm{~N}$ control room sample point \\
\hline 2039 & 7494 & NAD83 & 565836 & 145278 & HEISPROD & ESTIMATED & & & 116-C-1 VADOSE TEST PIT \\
\hline 2040 & 7514 & & & & CENTPLAT & & & & 221-U CANYON \\
\hline 2041 & 7534 & & & & HEISPROD & & & & 183N Backwash Sump \\
\hline 2042 & 7535 & & & & HEISPROD & & & & 183N Sump \#2 \\
\hline 2043 & 7536 & & & & HEISPROD & & & & 183N Sludge Sump \\
\hline 2044 & 7537 & & & & HEISPROD & & & & 183N Influent Flume Filter Bed 2 \\
\hline 2045 & 7538 & & & & HEISPROD & & & & $183 \mathrm{~N}$ cntrl rm sink filter 1 \\
\hline 2046 & 7539 & & & & HEISPROD & & & & $183 \mathrm{~N}$ cntrl rm sink filter 2 \\
\hline 2047 & 7574 & & & & SESPMNT & & OFFSITE & & STATE HIGHWAY 24 \\
\hline 2048 & 7595 & NAD83 & 583818.159 & 148037.761 & HEISPROD & & & & 2,4-D BURIAL \\
\hline 2049 & 7596 & NAD83 & 578965.311 & 157415.025 & HEISPROD & & & & $\mathrm{H}-04(\mathrm{E}) / \mathrm{A}-1-1(\mathrm{TP})$ \\
\hline 2050 & 7597 & NAD83 & 578992.148 & 157424.332 & HEISPROD & & & & $\mathrm{H}-04(\mathrm{E}) / \mathrm{A}-1-2(\mathrm{TP})$ \\
\hline 2051 & 7598 & NAD83 & 578938.806 & 157510.096 & HEISPROD & & & & $\mathrm{H}-04(\mathrm{~W}) / \mathrm{A}-1-2(\mathrm{TP})$ \\
\hline 2052 & 7599 & NAD83 & 578943.313 & 157490.083 & HEISPROD & & & & $\mathrm{H}-04(\mathrm{~W}) / \mathrm{A}-1-3$ (TP) \\
\hline
\end{tabular}

Page 54 


\begin{tabular}{|c|c|c|c|c|c|c|c|c|c|}
\hline & $A$ & $\mathrm{~B}$ & $\mathrm{C}$ & $\mathrm{D}$ & $E$ & $\mathrm{~F}$ & G & $\mathrm{H}$ & $\mathrm{I}$ \\
\hline 1 & SAMP_SITE_ID & HORIZ_COORD_TYPE & EW_COORD & NS_COORD & OWNER_ID & COORD_SOURCE & DIST_CLASS & RAD_LEVEL & SAMP_SITE_NAME \\
\hline 2054 & 7602 & NAD83 & 578910.708 & 157499.846 & HEISPROD & & & & $\mathrm{H}-04(\mathrm{~W}) / \mathrm{A}-3-1$ (TP) \\
\hline 2055 & 7603 & NAD83 & 581726.987 & 157800.025 & HEISPROD & & & & H-06-L (TP) \\
\hline 2056 & 7604 & NAD83 & 581763.994 & 157804.105 & HEISPROD & & & & H-06-L ROCK PIT (TP) \\
\hline 2057 & 7607 & NAD83 & 582869.048 & 157118.807 & HEISPROD & & & & $\mathrm{H}-06 \mathrm{H}(\mathrm{E}) / \mathrm{A}-11-1$ (TP) \\
\hline 2058 & 7608 & NAD83 & 582858.495 & 157114.339 & HEISPROD & & & & $\mathrm{H}-06 \mathrm{H}(\mathrm{E}) / \mathrm{A}-11-2(\mathrm{TP})$ \\
\hline 2059 & 7609 & NAD83 & 582874.248 & 157126.599 & HEISPROD & & & & $\mathrm{H}-06 \mathrm{H}(\mathrm{E}) / \mathrm{A}-12-1$ (TP) \\
\hline 2060 & 7610 & NAD83 & 582946.158 & 157161.559 & HEISPROD & & & & $\mathrm{H}-06 \mathrm{H}(\mathrm{E}) / \mathrm{A}-12-2(\mathrm{TP})$ \\
\hline 2061 & 7611 & NAD83 & 582800.538 & 157037.27 & HEISPROD & & & & $\mathrm{H}-06 \mathrm{H}(\mathrm{E}) / \mathrm{A}-2-1$ (TP) \\
\hline 2062 & 7612 & NAD83 & 582863.818 & 157066.857 & HEISPROD & & & & $\mathrm{H}-06 \mathrm{H}(\mathrm{E}) / \mathrm{A}-6-4$ (TP) \\
\hline 2063 & 7613 & NAD83 & 582877.622 & 157143.629 & HEISPROD & & & & $\mathrm{H}-06 \mathrm{H}(\mathrm{E}) / \mathrm{A}-7-1$ (TP) \\
\hline 2064 & 7614 & NAD83 & 582475.902 & 157040.847 & HEISPROD & & & & $\mathrm{H}-06 \mathrm{H}(\mathrm{W}) / \mathrm{A}-16-1$ (TP) \\
\hline 2065 & 7615 & NAD83 & 582523.272 & 157054.156 & HEISPROD & & & & $\mathrm{H}-06 \mathrm{H}(\mathrm{W}) / \mathrm{A}-19-2(\mathrm{TP})$ \\
\hline 2066 & 7616 & NAD83 & 582506.802 & 157063.195 & HEISPROD & & & & $\mathrm{H}-06 \mathrm{H}(\mathrm{W}) / \mathrm{A}-19-3(\mathrm{TP})$ \\
\hline 2067 & 7617 & NAD83 & 582269.941 & 156997.271 & HEISPROD & & & & $\mathrm{H}-06 \mathrm{H}(\mathrm{W}) / \mathrm{A}-2-2(\mathrm{TP})$ \\
\hline 2068 & 7618 & NAD83 & 582347.691 & 156993.392 & HEISPROD & & & & $\mathrm{H}-06 \mathrm{H}(\mathrm{W}) / \mathrm{A}-5-2$ (TP) \\
\hline 2069 & 7619 & NAD83 & 582339.469 & 156989.265 & HEISPROD & & & & $\mathrm{H}-06 \mathrm{H}(\mathrm{W}) / \mathrm{A}-5-5$ (TP) \\
\hline 2070 & 7620 & NAD83 & 582402.179 & 157029.648 & HEISPROD & & & & $\mathrm{H}-06 \mathrm{H}(\mathrm{W}) / \mathrm{A}-7-1$ (TP) \\
\hline 2071 & 7621 & NAD83 & 582136 & 156977 & HEISPROD & & & & $\mathrm{H}-07-\mathrm{H}(\mathrm{TP})$ \\
\hline 2072 & 7622 & NAD83 & 583519.289 & 150955.476 & HEISPROD & & & & $\mathrm{H}-12-\mathrm{L} \# 2$ (TP) \\
\hline 2073 & 7623 & NAD83 & 583520.3 & 150965.2 & HEISPROD & & & & $\mathrm{H}-12-\mathrm{L} \# 3$ (TP) \\
\hline 2074 & 7624 & NAD83 & 561059.787 & 148977.257 & HEISPROD & & & & H-81-R (TP) \\
\hline 2075 & 7625 & NAD83 & 561605 & 151755 & HEISPROD & & & & H-83-L/A-1-3 (TP) \\
\hline 2076 & 7626 & NAD83 & 561550 & 151798 & HEISPROD & & & & H-83-L/A-2-2 (TP) \\
\hline 2077 & 7627 & NAD83 & 561555 & 151780 & HEISPROD & & & & H-83-L/A-2-3 (TP) \\
\hline 2078 & 7628 & NAD83 & 561590 & 151835 & HEISPROD & & & & H-83-L/A-3-2 (TP) \\
\hline 2079 & 7629 & NAD83 & 561590 & 151845 & HEISPROD & & & & H-83-L/A-3-3 (TP) \\
\hline 2080 & 7630 & NAD83 & 561620 & 151830 & HEISPROD & & & & H-83-L/A-4-1 (TP) \\
\hline 2081 & 7631 & NAD83 & 575896.22 & 151132.48 & HEISPROD & & & & IU-4 TEST PIT 1 (TP) \\
\hline 2082 & 7632 & NAD83 & 575852.53 & 151116.65 & HEISPROD & & & & IU-4 TRENCH1 N (TP) \\
\hline 2083 & 7633 & NAD83 & 575853.55 & 151102 & HEISPROD & & & & IU-4 TRENCH1 S (TP) \\
\hline 2084 & 7634 & NAD83 & 575896.22 & 151169.05 & HEISPROD & & & & IU-4 TRENCH2 E (TP) \\
\hline 2085 & 7635 & NAD83 & 575880.98 & 151169.05 & HEISPROD & & & & IU-4 TRENCH2 W (TP) \\
\hline 2086 & 7655 & & & & HEISPROD & & & & 105C FSB FLOOR LOC 1, X24;Y47 \\
\hline 2087 & 7656 & & & & HEISPROD & & & & 105C FSB FLOOR LOC 2, X23; Y24 \\
\hline 2088 & 7657 & & & & HEISPROD & & & & 105C FSB FLOOR LOC 3, X19; Y70 \\
\hline 2089 & 7658 & & & & HEISPROD & & & & 105C FSB FLOOR LOC 4, X19;Y57 \\
\hline 2090 & 7659 & & & & HEISPROD & & & & 105C FSB FLOOR LOC 5, X15:Y55 \\
\hline 2091 & 7674 & & & & HEISPROD & & & & 233SA Exhaust Facility \\
\hline
\end{tabular}




\begin{tabular}{|c|c|c|c|c|c|c|c|c|c|}
\hline & A & $\mathrm{B}$ & C & $\mathrm{D}$ & $\mathrm{E}$ & $\mathrm{F}$ & $\mathrm{G}$ & $\mathrm{H}$ & $\mathrm{I}$ \\
\hline 1 & SAMP_SITE_ID & HORIZ_COORD_TYPE & EW_COORD & NS_COORD & OWNER_ID & COORD_SOURCE & DIST_CLASS & RAD_LEVEL & SAMP_SITE_NAME \\
\hline 2092 & 7675 & & & & HEISPROD & & & & 223-S Pipe Gallery Steam Line \\
\hline 2093 & 7696 & & & & HEISPROD & & & & Effluent Filter B, 200-ZP-1 Pump / Treat \\
\hline 2094 & 7714 & & & & CENTPLAT & & & & SEEP 190-D \\
\hline 2095 & 7734 & & & & SESPMNT & & OFFSITE & LOW & ICE HARBOR-FRANKLIN SHORE \\
\hline 2096 & 7735 & & & & SESPMNT & & OFFSITE & LOW & ICE HARBOR-MID RIVER \\
\hline 2097 & 7736 & & & & SESPMNT & & OFFSITE & LOW & ICE HARBOR-WALLA WALLA SHORE \\
\hline 2098 & 7737 & & & & SESPMNT & & OFFSITE & LOW & PRD-YAKIMA SIDE NEAR DAM \\
\hline 2099 & 7738 & & & & SESPMNT & & OFFSITE & LOW & PRD-GRANT SIDE NEAR DAM \\
\hline 2100 & 7739 & & & & SESPMNT & & OFFSITE & LOW & MCNARY-OR.SIDE NEAR DAM \\
\hline 2101 & 7740 & & & & SESPMNT & & OFFSITE & LOW & MCNARY-WASH.SIDE NEAR DAM \\
\hline 2102 & 7741 & & & & SESPMNT & & OFFSITE & LOW & FRANKLIN CO. SHORE \\
\hline 2103 & 7754 & & & & SESPMNT & & OFFSITE & LOW & ICE HARBOR DAM \\
\hline 2104 & 7774 & & & & SESPMNT & & ONSITE & LOW & HANFORD REACH \\
\hline 2105 & 7794 & NAD83 & 573890 & 152246 & 5 HEISPROD & ESTIMATED & & & 116-DR-9 \\
\hline 2106 & 7818 & & & & SESPMNT & & PERIMETER & & SAGEMOOR-VAN BATAVIA FARM \\
\hline 2107 & 7819 & & & & SESPMNT & & COMMUNITY & & WAHLUKE-RADAR HILL FARM \\
\hline 2108 & 7820 & & & & SESPMNT & & COMMUNITY & & WAHLUKE-BAGINSKI FARM \\
\hline 2109 & 7821 & & & & SESPMNT & & COMMUNITY & & WAHLUKE-SCHEENSTRA FARM \\
\hline 2110 & 7822 & & & & SESPMNT & & DISTANT & & SUNNYSIDE-MEEKER FARM \\
\hline 2111 & 7840 & NAD83(91) & 575657.688 & 135885.031 & 1 CENTPLAT & & & & 216-A-29 DITCH \\
\hline 2112 & 7861 & NAD83 & 573696 & 152307 & 7 HEISPROD & & & & Center of $116-D-7$ \\
\hline 2113 & 7862 & NAD83 & 573777 & 151255 & 5 HEISPROD & & & & Center of 116-DR-7 \\
\hline 2114 & 7880 & NAD83 & 580204.9 & 145217.3 & 3 SESPMNT & GPS & ONSITE & & $100 \mathrm{~F}$ MET TOWER \\
\hline 2115 & 7900 & & & & SESPMNT & & COMMUNITY & & KENNEWICK \\
\hline 2116 & 7920 & & & & SESPMNT & & DISTANT & LOW & DESERT AIRE \\
\hline 2117 & 7960 & NAD83 & 593721 & 115222 & SESPMNT & GPS & ONSITE & LOW & 300 SOUTH WEST \\
\hline 2118 & 7980 & & & & HEISPROD & & & & ERDF Leachate Waste Storage/Loadout Tank \\
\hline 2119 & 8000 & & & & SESPMNT & & ONSITE & & $100 \mathrm{~B} / \mathrm{C}$ AREA \\
\hline 2120 & 8001 & & & & SESPMNT & & OFFSITE & & WSU-RICHLAND \\
\hline 2121 & 8040 & & & & CENTPLAT & & & & DIVE RIVER SAMPLE 39P-D \\
\hline 2122 & 8041 & & & & CENTPLAT & & & & DIVE RIVER SAMPLE 39P-S \\
\hline 2123 & 8060 & NAD83(91) & 566051.2 & 145321.8 & 3 HEISPROD & GPS & & & 116-C-1 Overburden, Unit 1, A1 \\
\hline 2124 & 8061 & NAD83(91) & 556018.1 & 145293.4 & 4 HEISPROD & GPS & & & 116-C-1 Overburden, Unit 1, A2 \\
\hline 2125 & 8062 & NAD83(91) & 566037.9 & 145294 & 4 HEISPROD & GPS & & & 116-C-1 Overburden, Unit 1, A3 \\
\hline 2126 & 8063 & NAD83(91) & 566057.9 & 145290 & HEISPROD & GPS & & & 116-C-1 Overburden, Unit 1, A4 \\
\hline 2127 & 8064 & NAD83(91) & 566017 & 145253.1 & HEISPROD & GPS & & & 116-C-1 Overburden, Unit 2, B1 \\
\hline 2128 & 8065 & NAD83(91) & 566041.7 & 145242.6 & HEISPROD & GPS & & & 116-C-1 Overburden, Unit 2, B2 \\
\hline 2129 & 8066 & NAD83(91) & 566064.3 & 145252.6 & HEISPROD & GPS & & & 116-C-1 Overburden, Unit 2, B3 \\
\hline
\end{tabular}




\begin{tabular}{|c|c|c|c|c|c|c|c|c|c|}
\hline & A & $\mathrm{B}$ & C & $\mathrm{D}$ & $E$ & $\mathrm{~F}$ & G & $\mathrm{H}$ & $\mathrm{I}$ \\
\hline 1 & SAMP_SITE_ID & HORIZ_COORD_TYPE & EW_COORD & NS_COORD & OWNER_ID & COORD_SOURCE & DIST_CLASS & RAD_LEVEL & SAMP_SITE_NAME \\
\hline 2130 & 8067 & NAD83(91) & 565925.4 & 145123.9 & 9 HEISPROD & GPS & & & 116-C-1 Overburden, Unit 2, B4 \\
\hline 2131 & 8068 & NAD83(91) & 566006.2 & 145122.5 & 5 HEISPROD & GPS & & & 116-C-1 Overburden, Unit 3, C1 \\
\hline 2132 & 8069 & NAD83(91) & 565951.9 & 145118.2 & 2 HEISPROD & GPS & & & 116-C-1 Overburden, Unit 3, C2 \\
\hline 2133 & 8070 & NAD83(91) & 565991.9 & 145117.2 & 2 HEISPROD & GPS & & & 116-C-1 Overburden, Unit 3, C3 \\
\hline 2134 & 8071 & NAD83(91) & 566021.7 & 145117.4 & 4 HEISPROD & GPS & & & 116-C-1 Overburden, Unit 3, C4 \\
\hline 2135 & 8072 & NAD83(91) & 565899.4 & 145086.6 & HEISPROD & GPS & & & 116-C-1 Overburden, Unit 4, D1 \\
\hline 2136 & 8073 & NAD83(91) & 565915.6 & 145084.4 & 4 HEISPROD & GPS & & & 116-C-1 Overburden, Unit 4, D2 \\
\hline 2137 & 8074 & NAD83(91) & 565941.4 & 145078.5 & 5 HEISPROD & GPS & & & 116-C-1 Overburden, Unit 4, D3 \\
\hline 2138 & 8075 & NAD83(91) & 565956.9 & 145097.9 & HEISPROD & GPS & & & 116-C-1 Overburden, Unit 4, D4 \\
\hline 2139 & 8076 & NAD83(91) & 565798.2 & 145299.1 & HEISPROD & GPS & & & 116-C-1 S-Zone, Side Walls, Unit 1, A1 \\
\hline 2140 & 8077 & NAD83(91) & 565846.9 & 145328.7 & HEISPROD & GPS & & & 116-C-1 S-Zone, Side Walls, Unit 1, A2 \\
\hline 2141 & 8078 & NAD83(91) & 565888.3 & 145332.1 & HEISPROD & GPS & & & 116-C-1 S-Zone, Side Walls, Unit 1, A3 \\
\hline 2142 & 8079 & NAD83(91) & 565950.3 & 145340.7 & HEISPROD & GPS & & & 116-C-1 S-Zone, Side Walls, Unit 1, A4 \\
\hline 2143 & 8080 & NAD83(91) & 565812.9 & 145239.4 & HEISPROD & GPS & & & 116-C-1 S-Zone, Side Walls, Unit 2, B1 \\
\hline 2144 & 8081 & NAD83(91) & 565874.6 & 145247.4 & HEISPROD & GPS & & & 116-C-1 S-Zone, Side Walls, Unit 2, B2 \\
\hline 2145 & 8082 & NAD83(91) & 565922.4 & 145271.8 & HEISPROD & GPS & & & 116-C-1 S-Zone, Side Walls, Unit 2, B3 \\
\hline 2146 & 8083 & NAD83(91) & 565956.4 & 145294.6 & HEISPROD & GPS & & & 116-C-1 S-Zone, Side Walls, Unit 2, B4 \\
\hline 2149 & 8086 & NAD83(91) & 565829.5 & 145259.4 & HEISPROD & GPS & & & 116-C-1 D-Zone, Floor Verif, Unit 1, A3 \\
\hline 2150 & 8087 & NAD83(91) & 565809.7 & 145290.6 & HEISPROD & GPS & & & 116-C-1 D-Zone, Floor Verif, Unit 2, B1 \\
\hline 2151 & 8088 & NAD83(91) & 565835 & 145303.8 & HEISPROD & GPS & & & 116-C-1 D-Zone, Floor Verif, Unit 2, B2 \\
\hline 2152 & 8089 & NAD83(91) & 565863.2 & 145318.6 & HEISPROD & GPS & & & 116-C-1 D-Zone, Floor Verif, Unit 2, B3 \\
\hline 2153 & 8090 & NAD83(91) & 565910 & 145323.2 & HEISPROD & GPS & & & 116-C-1 D-Zone, Floor Verif, Unit 3, C1 \\
\hline 2154 & 8091 & NAD83(91) & 565929.4 & 145313.7 & HEISPROD & GPS & & & 116-C-1 D-Zone, Floor Verif, Unit 3, C2 \\
\hline 2155 & 8092 & NAD83(91) & 565836.6 & 145259.2 & HEISPROD & GPS & & & 116-C-1 D-Zone, Floor Verif, Unit 3, C3 \\
\hline 2156 & 8100 & & & & SESPMNT & & ONSITE & LOW & HANFRD TWNSITE HRM26 \\
\hline 2157 & 8101 & & & & SESPMNT & & ONSITE & LOW & HANFRD TWNSITE HRM27 \\
\hline 2158 & 8102 & & & & SESPMNT & & ONSITE & LOW & HANFRD TWNSITE HRM29 \\
\hline 2159 & 8103 & & & & SESPMNT & & ONSITE & LOW & HANFRD TWNSITE HRM30 \\
\hline 2160 & 8120 & NAD83(91) & 593951.1 & 116027.9 & HEISPROD & HGIS & & & 300-26, Powerhouse Fuel Oil Spill \\
\hline 2161 & 8121 & NAD83(91) & 593951.1 & 116027.9 & HEISPROD & HGIS & & & 366 Building French Drain \\
\hline 2162 & 8122 & & & & SESPMNT & & ONSITE & & DRIVE POINT 1 \\
\hline 2163 & 8123 & & & & SESPMNT & & ONSITE & & DRIVE POINT 2 \\
\hline 2164 & 8124 & & & & SESPMNT & & OFFSITE & & DRIVE POINT 3 \\
\hline 2165 & 8125 & & & & SESPMNT & & OFFSITE & & DRIVE POINT 4 \\
\hline 2166 & 8126 & & & & SESPMNT & & OFFSITE & & DRIVE POINT 5 \\
\hline
\end{tabular}




\begin{tabular}{|c|c|c|c|c|c|c|c|c|c|}
\hline & $\bar{A}$ & $\mathrm{~B}$ & $\mathrm{C}$ & $\mathrm{D}$ & $E$ & $\mathrm{~F}$ & G & $\mathrm{H}$ & $\mathrm{I}$ \\
\hline 1 & SAMP_SITE_ID & HORIZ_COORD_TYPE & EW_COORD & NS_COORD & OWNER_ID & COORD_SOURCE & DIST_CLASS & RAD_LEVEL & SAMP_SITE_NAME \\
\hline 2168 & 8128 & & & & SESPMNT & & OFFSITE & & PORT OF BENTON PUMPHOUSE \\
\hline 2169 & 8140 & & & & SESPMNT & & ONSITE & & 100-B SPRING 38-3 \\
\hline 2170 & 8141 & & & & SESPMNT & & ONSITE & & 100-B SPRING 39-2 \\
\hline 2171 & 8142 & & & & SESPMNT & & ONSITE & & HANFORD SPR UR 28-2 \\
\hline 2172 & 8143 & & & & SESPMNT & & ONSITE & & HANFORD SPR DR 28-2 \\
\hline 2173 & 8144 & & & & SESPMNT & & ONSITE & & 300 AREA SPR DR 42-2 \\
\hline 2174 & 8145 & & & & SESPMNT & & ONSITE & & 100-K SPRING 63-1 \\
\hline 2175 & 8146 & & & & SESPMNT & & ONSITE & & 100-K SPRING 77-1 \\
\hline 2176 & 8147 & & & & SESPMNT & & ONSITE & & 100-F SPRING 207-1 \\
\hline 2177 & 8161 & & & & SESPMNT & & ONSITE & & 100-N SPRING 199N-46 \\
\hline 2178 & 8162 & & & & SESPMNT & & ONSITE & & 100-D SPRING 110-1 \\
\hline 2179 & 8163 & & & & SESPMNT & & ONSITE & & 100-D SPRING 102-1 \\
\hline 2180 & 8164 & & & & SESPMNT & & ONSITE & & 100-H SPRING 153-1 \\
\hline 2181 & 8165 & & & & SESPMNT & & ONSITE & & 100-H SPRING 145-1 \\
\hline 2182 & 8181 & & & & SESPMNT & & DISTANT & & IDAHO \\
\hline 2183 & 8182 & & & & SESPMNT & & PERIMETER & & ALE \\
\hline 2184 & 8220 & NAD83 & 595485.3 & 110803 & SESPMNT & GPS & OFFSITE & & RICH.PMPHS HRM 45.8 \\
\hline 2185 & 8221 & NAD83 & 595256.9 & 112138.9 & SESPMNT & GPS & OFFSITE & & RICH.PMPHS HRM 45.0 \\
\hline 2186 & 8222 & NAD83 & 594954.4 & 113710 & SESPMNT & GPS & OFFSITE & & RICH.PMPHS HRM 43.9 \\
\hline 2187 & 8223 & NAD83 & 594886.8 & 114383.9 & SESPMNT & GPS & OFFSITE & & RICH.PMPHS HRM 43.5 \\
\hline 2188 & 8240 & NAD83 & 594728 & 115279 & SESPMNT & GPS & ONSITE & LOW & 300 AREA SHR HRM41.5 \\
\hline 2189 & 8241 & NAD83 & 594539 & 116069 & SESPMNT & GPS & ONSITE & LOW & 300 AREA SHR HRM42.1 \\
\hline 2190 & 8242 & NAD83 & 594398 & 116702 & SESPMNT & GPS & ONSITE & LOW & 300 AREA SHR HRM42.5 \\
\hline 2191 & 8243 & NAD83 & 594314 & 117537 & SESPMNT & GPS & ONSITE & LOW & 300 AREA SHR HRM42.9 \\
\hline 2192 & 8244 & & & & SESPMNT & & ONSITE & LOW & 100 N SHORE HRM 8.4 \\
\hline 2193 & 8245 & & & & SESPMNT & & ONSITE & LOW & 100 N SHORE HRM 8.9 \\
\hline 2194 & 8246 & & & & SESPMNT & & ONSITE & LOW & 100 N SHORE HRM 9.2 \\
\hline 2195 & 8247 & & & & SESPMNT & & ONSITE & LOW & 100 N SHORE HRM 9.8 \\
\hline 2196 & 8260 & & & & SESPMNT & & ONSITE & & 200 AREAS \\
\hline 2197 & 8280 & & & & SESPMNT & & DISTANT & & WAHA \\
\hline 2198 & 8302 & NAD83(91) & 565828.49 & 156055.95 & HEISPROD & & & & North Slope PSN-90, D1 \\
\hline 2199 & 8303 & NAD83(91) & 565827.79 & 156081.83 & HEISPROD & & & & North Slope PSN-90, D2 \\
\hline 2200 & 8304 & NAD83(91) & 565823.46 & 156125.02 & HEISPROD & & & & North Slope PSN-90, D3 \\
\hline 2201 & 8305 & NAD83(91) & 565854.43 & 156058.85 & HEISPROD & & & & North Slope PSN-90, E1 \\
\hline 2202 & 8306 & NAD83(91) & 565852.44 & 156081.85 & HEISPROD & & & & North Slope PSN-90, E2 \\
\hline 2203 & 8307 & NAD83(91) & 565849.59 & 156128.1 & HEISPROD & & & & North Slope PSN-90, E3 \\
\hline 2204 & 8308 & NAD83(91) & 565884.77 & 156060.92 & HEISPROD & & & & North Slope PSN-90, F1 \\
\hline 2205 & 8309 & NAD83(91) & 565876.74 & 156082.38 & HEISPROD & & & & North Slope PSN-90, F2 \\
\hline
\end{tabular}


C

$\mathrm{D}$

E $\mathrm{F}$ G 8310 NAD83(91) 8311 NAD83(91) 565876.27
571337.37 156127.98 HEISPROD 8312 NAD83(91) \begin{tabular}{|l|l|}
571337.37 & 158364.12 \\
\hline
\end{tabular} 8313 NAD83(91)

$571398.38 \quad 158408.37$ HEISPROD 8314 NAD83(91) 8315 NAD83(91) 8316 NAD83(91) \begin{tabular}{|l|l}
582786.95 & 157107.88 HEISPROD
\end{tabular} \begin{tabular}{|r|r|}
582812.31 & 157124 HEISPROD
\end{tabular} \begin{tabular}{|l|l|l|}
8317 NAD83(91) & 582863.66 & 157156.91 \\
\hline
\end{tabular} \begin{tabular}{|l|l|l|}
\hline 8318 NAD83(91) & 582801.13 & 157079.93 HEISPROD \\
\hline
\end{tabular} \begin{tabular}{|l|l|l|}
\hline 8319 NAD83(91) & 582828.15 & 157097.46 HEISPROD \\
\hline
\end{tabular} \begin{tabular}{|l|r|r|}
\hline 8320 NAD83(91) & 582852.89 & 157113.47 HEISPROD
\end{tabular} \begin{tabular}{|l|r|r|}
\hline 8321 NAD83(91) & 582878.83 & 157130.05 HEISPROD \\
\hline
\end{tabular} 8322 NAD83(91) 8323 NAD83(91) 8324 NAD83(91) 8325 NAD83(91) 8326 NAD83(91) 8327 NAD83(91) 8328 NAD83(91) 8329 NAD83(91) 8330 NAD83(91) 8331 NAD83(91) 8332 NAD83(91) 8333 NAD83(91) 8334 NAD83(91) 8335 NAD83(91) 8336 NAD83(91) 8337 NAD83(91) 8338 NAD83(91) 8339 NAD83(91) 8340 NAD83(91) 8341 NAD83(91) 8342 NAD83(91) 8343 NAD83(91) 8344 NAD83(91) 8345 NAD83(91) 8346 NAD83(91) 8347 NAD83(91)

\begin{tabular}{|l|l|l|}
582878.83 & 157130.05 & HEISPROD \\
\hline 582815.23 & 157055.16 HEISPROD
\end{tabular}

$582841.53 \quad 157071.24$ HEISPROD

$582867.08 \quad 157086.7$ HEISPROD

\begin{tabular}{|l|r|}
582894.14 & 157102.76 HEISPROD
\end{tabular}

$582829.41 \quad 157029.28$ HEISPROD

582855.12157044 .16 HEISPROD

$582882.9 \quad 157059.54$ HEISPROD

\begin{tabular}{|l|l|}
582909.49 & 157076.09 HEISPROD
\end{tabular}

$574476.95 \quad 118491.49$ HEISPROD

\begin{tabular}{|l|l}
574504.24 & $118477.62 \mathrm{HEISPROD}$
\end{tabular}

\begin{tabular}{|l|r|}
574529.44 & 118459.5 HEISPROD
\end{tabular}

$574551.07 \quad 118441.63$ HEISPROD

$574575.38 \quad 118424.9$ HEISPROD

$574509.02 \quad 118535.18$ HEISPROD

\begin{tabular}{|l|l|}
574530.21 & 118513.42 HEISPROD
\end{tabular}

$574552 \quad 118491.72$ HEISPROD

$574573.42 \quad 118470.49$ HEISPROD

\begin{tabular}{|l|l|}
574594.19 & 118448.27 HEISPROD
\end{tabular}

574528.5118560 .13 HEISPROD

$574549.89 \quad 118538.19$ HEISPROD

$574570.27 \quad 118516.35$ HEISPROD

\begin{tabular}{|l|l|}
574590.78 & 118494.56 HEISPROD
\end{tabular}

$574612.3 \quad 118471.34$ HEISPROD

$574567.48 \quad 118559.93$ HEISPROD

\begin{tabular}{|l|l|l|}
574587.85 & 118541.01 HEISPROD
\end{tabular}

$574608.65 \quad 118518.84$ HEISPROD
$\mathrm{H}$ $\mathrm{I}$

North Slope PSN-90, F3

North Slope PSN-01, G1

North Slope PSN-01, G2

North Slope PSN-01, G3

H-06-LE, H1

H-06-LE, H2

$\mathrm{H}-06-\mathrm{LE}, \mathrm{H} 3$

H-06-LE, H4

H-06-LE, I1

H-06-LE, 12

$\mathrm{H}-06$-LE, 13

H-06-LE, 14

H-06-LE, J1

H-06-LE, J2

H-06-LE, J3

H-06-LE, J4

H-06-LE, K1

H-06-LE, K2

H-06-LE, K3

H-06-LE, K4

600 Area Horseshoe Landfill, A1

600 Area Horseshoe Landfill, A2

600 Area Horseshoe Landfill, A3

600 Area Horseshoe Landfill, A4

600 Area Horseshoe Landfill, A5

600 Area Horseshoe Landfill, B1

600 Area Horseshoe Landfill, B2

600 Area Horseshoe Landfill, B3

600 Area Horseshoe Landfill, B4

600 Area Horseshoe Landfill, B5

600 Area Horseshoe Landfill, C1

600 Area Horseshoe Landfill, C2

600 Area Horseshoe Landfill, C3

600 Area Horseshoe Landfill, C4

600 Area Horseshoe Landfill, C5

600 Area Horseshoe Landfill, $\mathrm{DH} 2$

600 Area Horseshoe Landfill, DH3

600 Area Horseshoe Landfill, DH4 
C

$\mathrm{D}$ $\mathrm{F}$ G 8348 NAD83(91) \begin{tabular}{l|l} 
COORD & NS_COORD OWNER \\
\hline
\end{tabular} 8349 NAD83(91) $574631.37 \quad 118495.71 \mathrm{HEISPROD}$ 8350 NAD83(91) $\begin{array}{ll}574622.02 & 118537.53 \mathrm{HEISPROD}\end{array}$ 8351 NAD83(91)

$573742.64 \quad 118963.99$ HEISPROD

573760.35118986 .75 HEISPROD

\begin{tabular}{|l|l|}
573750.87 & 118923.74 HEISPROD
\end{tabular} 8353 NAD83(91) 8354 NAD83(91)

$573767.38 \quad 118946.62$ HEISPROD 8355 NAD83(91) $573784.65 \quad 118970.53$ HEISPROD $573775.15 \quad 118905.13$ HEISPROD $573791.8 \quad 118929.99$ HEISPROD $573809.86 \quad 118954.9$ HEISPROD $573798.72 \quad 118886.31$ HEISPROD \begin{tabular}{|r|r|}
573817.14 & $118912.8 \mathrm{HEISPROD}$
\end{tabular} $573833.67 \quad 118938.98$ HEISPROD $573822.79 \quad 118867.04$ HEISPROD $573842.38 \quad 118896$ HEISPROD $573859.23 \quad 118921.26$ HEISPROD $571369.11 \quad 158387.86$ HEISPROD $582825.09 \quad 157132.03$ HEISPROD \begin{tabular}{|l|l|}
582794.04 & 157093.91 HEISPROD
\end{tabular} $582822.32 \quad 157042.22$ HEISPROD $582896.2 \quad 157067.81$ HEISPROD $574622.33 \quad 118429.93$ HEISPROD $563198.628 \quad 153186.935$ HEISPROD 563573.913153252 .643 HEISPROD $563858.575 \quad 153851.611$ HEISPROD 564211.343154232 .082 HEISPROD $565296.781 \quad 155392.446$ HEISPROD 565530.84155543 .208 HEISPROD $567583.161 \quad 156387.283$ HEISPROD $568017.152 \quad 156497.161$ HEISPROD 568595.002156463 .577 HEISPROD $568911.474 \quad 156451.753$ HEISPROD $\begin{array}{ll}570227.312 & 156491.668 \text { HEISPROD }\end{array}$ 570230.802156556 .555 HEISPROD 570862.566156452 .795 HEISPROD \begin{tabular}{|l|r|}
571811.991 & 156596.83 HEISPROD
\end{tabular} \begin{tabular}{l|l}
571730.895 & 156453.818 HEISPROD
\end{tabular}

\begin{tabular}{|c|c|c|c|}
\hline \multirow{2}{*}{\begin{tabular}{|c|} 
F \\
COORD_SOURCE
\end{tabular}} & G & $\mathrm{H}$ & $\mathrm{I}$ \\
\hline & DIST_CLASS & RAD_LEVEL & SAMP_SITE_NAME \\
\hline & & & 600 Area Horseshoe Landfill, DH5 \\
\hline & & & 600 Area Horseshoe Landfill, DH6 \\
\hline & & & ALE Control Site, ZA1 \\
\hline & & & ALE Control Site, ZA2 \\
\hline & & & ALE Control Site, ZA3 \\
\hline & & & ALE Control Site, ZB1 \\
\hline & & & ALE Control Site, ZB2 \\
\hline & & & ALE Control Site, ZB3 \\
\hline & & & ALE Control Site, ZC1 \\
\hline & & & ALE Control Site, ZC2 \\
\hline & & & ALE Control Site, ZC3 \\
\hline & & & ALE Control Site, ZD1 \\
\hline & & & ALE Control Site, ZD2 \\
\hline & & & ALE Control Site, ZD3 \\
\hline & & & ALE Control Site, ZE1 \\
\hline & & & ALE Control Site, ZE2 \\
\hline & & & ALE Control Site, ZE3 \\
\hline & & & COMPOSITE OF PSN-01, G1, G2, and G3 \\
\hline & & & COMPOSITE OF H-06-LE, H2 AND H3 \\
\hline & & & COMPOSITE OF H-06-LE, H1 AND II \\
\hline & & & COMPOSITE OF H-06-LE, J1 AND K1 \\
\hline & & & COMPOSITE OF H-06-LE, K3 AND K4 \\
\hline & & & 600 Area Horseshoe LF Meadowlark Nest \\
\hline & & & Hwy24, MP50.0, \#1 \\
\hline & & & Hwy24, MP50.3, \#2 \\
\hline & & & Hwy24, MP50.6, \#3 \\
\hline & & & Hwy24, MP51, \#4 \\
\hline & & & Hwy24, MP52 , \#5 \\
\hline & & & Hwy24, MP52.2, \#6 \\
\hline & & & Hwy24, MP53.7, South side, \#7 \\
\hline & & & Hwy24, MP53.7, At willow tree, \#8 \\
\hline & & & Hwy24, At E Rd SW, So side, \#9 \\
\hline & & & Hwy24, .2 mi past E Rd SW, \#10 \\
\hline & & & Hwy24, MP55.2 Pwrline, So side, \#11 \\
\hline & & & Hwy24, MP55.2 Pwrline, No side, \#12 \\
\hline & & & Hwy24, .4 mi past Pwrline, So side, $\# 13$ \\
\hline & & & Hwy24, MP56.2, Gravel Piles No, Trap \#14 \\
\hline & & & Hwy24, MP56.2, Gravel Piles So, \#15 \\
\hline
\end{tabular}




\begin{tabular}{|c|c|c|c|c|c|c|c|c|c|}
\hline & A & $\mathrm{B}$ & $\mathrm{C}$ & $\mathrm{D}$ & $E$ & $\mathrm{~F}$ & $\mathrm{G}$ & $\mathrm{H}$ & $\mathrm{I}$ \\
\hline 1 & SAMP_SITE_ID & HORIZ_COORD_TYPE & EW_COORD & NS_COORD & OWNER_ID & COORD_SOURCE & DIST_CLASS & RAD_LEVEL & SAMP_SITE_NAME \\
\hline 2282 & 8421 & NAD83(91) & 572273.477 & 156531.332 & HEISPROD & & & & Hwy24, MP56.3, South side, \#16 \\
\hline 2283 & 8422 & NAD83(91) & 574086.082 & 156571.899 & HEISPROD & & & & Hwy24, MP57.5 Access Rd No side, \#17 \\
\hline 2284 & 8423 & NAD83(91) & 574087.674 & 156615.15 & HEISPROD & & & & Hwy24, MP57.5 Access Rd No side, \#18 \\
\hline 2285 & 8424 & NAD83(91) & 576746.226 & 156577.439 & HEISPROD & & & & Hwy24, MP59.2, South side, \#19 \\
\hline 2286 & 8425 & NAD83(91) & 578357.691 & 156737.159 & HEISPROD & & & & Hwy24, MP60.3 Access Rd No side \#20 \\
\hline 2287 & 8426 & NAD83(91) & 583775 & 157105 & HEISPROD & & & & H-06-LE Meadowlark Nest \\
\hline 2288 & 8447 & & & & HEISPROD & & & & H-06-LE, East half of trap grid \\
\hline 2289 & 8448 & & & & HEISPROD & & & & H-06-LE, West half of trap grid \\
\hline 2290 & 8466 & NAD83 & 594833.6 & 127181.6 & SESPMNT & GPS & RIVER_SHORELINE & & ENERGY NW INTAKE \\
\hline 2291 & 8467 & NAD83 & 594814.8 & 125754.8 & SESPMNT & GPS & RIVER_SHORELINE & & DR ENERGY NW POWERLINE INTAKE \\
\hline 2292 & 8468 & NAD83 & 594459.8 & 123765.9 & SESPMNT & GPS & RIVER_SHORELINE & & NEARSHORE WOODED ISL \\
\hline 2293 & 8486 & & & & SESPMNT & & & & QC_SAMPLE_SESP \\
\hline 2294 & 8508 & NAD83(91) & 574551.923 & 118492.684 & HEISPROD & & & & Dpt. of Ecol. Horseshoe Landfill, A1 \\
\hline 2295 & 8509 & NAD83(91) & 574591.679 & 118493.866 & HEISPROD & & & & Dpt. of Ecol. Horseshoe Landfill, A2 \\
\hline 2296 & 8510 & NAD83(91) & 574626.701 & 118480.069 & HEISPROD & & & & Dpt. of Ecol. Horseshoe Landfill, A3 \\
\hline 2297 & 8546 & NAD83 & 595484.516 & 109903.226 & SESPMNT & GPS & COMMUNITY & LOW & LESLIE GROVES-RCHLND \\
\hline 2298 & 8566 & NAD83 & 567727.559 & 133160.099 & SESPMNT & GPS & ONSITE & & 216-S-19 POND \\
\hline 2299 & 8567 & NAD83 & 572448.282 & 130675.917 & SESPMNT & GPS & ONSITE & & SW OF B/C CRIB POWERLINE \\
\hline 2300 & 8586 & NAD83 & 579785.998 & 131107.087 & SESPMNT & GPS & ONSITE & & ARMY LOOP CENTRAL LANDFILL \\
\hline 2301 & 8606 & NAD83 & 587434.122 & 138556.3 & SESPMNT & GPS & ONSITE & LOW & S OF HANFORD TOWNSITE \\
\hline 2302 & 8626 & & & & SESPMNT & & ONSITE & & WEST LAKE SPRING \\
\hline 2303 & 8646 & & & & SESPMNT & & OFFSITE & & PASCO-RIVER \\
\hline 2304 & 8706 & & & & SESPMNT & & & & FINLEY \\
\hline 2305 & 8707 & & & & SESPMNT & & OFFSITE & & N. RICHLAND \\
\hline 2306 & 8726 & & & & SESPMNT & & ONSITE & & 100 F SHORE HRM 18 \\
\hline 2307 & 8727 & & & & SESPMNT & & ONSITE & & 100 F SHORE HRM 22 \\
\hline 2308 & 8728 & & & & SESPMNT & & ONSITE & & 100 F SHORE HRM 23 \\
\hline 2309 & 8747 & NAD83 & 344571 & 5111469 & SESPMNT & GPS & OFFSITE & & FINLEY RR TRACK \\
\hline 2310 & 8748 & NAD83 & 281206 & 5194601 & SESPMNT & GPS & OFFSITE & & WANAPUM \\
\hline 2311 & 8749 & NAD83 & 273486 & 5217507 & SESPMNT & GPS & OFFSITE & & GEORGE \\
\hline 2312 & 8750 & NAD83 & 599772.6 & 163648.9 & SESPMNT & GPS & COMMUNITY & & OTHELLO \\
\hline 2313 & 8751 & NAD83 & 601121.8 & 142481.2 & SESPMNT & GPS & COMMUNITY & & BASIN CITY \\
\hline 2314 & 8752 & NAD83 & 344817.7 & 5145885.6 & SESPMNT & GPS & OFFSITE & & MESA \\
\hline 2315 & 8753 & NAD83 & 605782 & 102334.2 & SESPMNT & GPS & COMMUNITY & & PASCO \\
\hline 2316 & 8754 & NAD83 & 606984.6 & 103728.8 & SESPMNT & GPS & COMMUNITY & & PASCO AIRPORT \\
\hline 2317 & 8755 & NAD83 & 598083.6 & 106086.6 & SESPMNT & GPS & COMMUNITY & & BROADMOOR, PASCO \\
\hline 2318 & 8766 & NAD83 & 595548.1 & 103420.9 & SESPMNT & GPS & OFFSITE & & YAKIMA DELTA \\
\hline 2319 & 8767 & NAD83 & 328960 & 5120733 & SESPMNT & GPS & OFFSITE & & TRI-CITY COLISEUM \\
\hline
\end{tabular}




\begin{tabular}{|c|c|c|c|c|c|c|c|c|c|}
\hline & A & $\mathrm{B}$ & C & $\mathrm{D}$ & $\mathrm{E}$ & $\mathrm{F}$ & $\mathrm{G}$ & $\mathrm{H}$ & $\mathrm{I}$ \\
\hline 1 & SAMP_SITE_ID & HORIZ_COORD_TYPE & EW_COORD & NS_COORD & OWNER_ID & COORD_SOURCE & DIST_CLASS & RAD_LEVEL & SAMP_SITE_NAME \\
\hline 2320 & 8786 & NAD83 & 604128.8 & 97528.4 & SESPMNT & GPS & COMMUNITY & & KENNEWICK \\
\hline 2321 & 8806 & & & & SESPMNT & & OFFSITE & & MULE DRY FIRE-MABTON \\
\hline 2322 & 8826 & & & & SESPMNT & & ONSITE & LOW & 200 ESE \\
\hline 2323 & 8846 & & & & SESPMNT & & ONSITE & & 100-H SPRING 152-2 \\
\hline 2324 & 8866 & & & & SESPMNT & & & & MOXEE \\
\hline 2325 & 8886 & NAD83 & 539988.9 & 113447.3 & 3 SESPMNT & GPS & DISTANT & & SUNNYSIDE \\
\hline 2326 & 8906 & NAD83 & 325649 & 5133622 & 2 SESPMNT & GPS & OFFSITE & & WSU \\
\hline 2327 & 8926 & NAD83 & 558778.469 & 145492.112 & 2 SESPMNT & GPS & RIVER_SHORELINE & & S END VERNITA BRIDGE \\
\hline 2328 & 8927 & NAD83 & 600423 & 101167 & 7 SESPMNT & GPS & RIVER_SHORELINE & & ISL DS BATEMAN ISL \\
\hline 2329 & 8946 & & & & SESPMNT & & OFFSITE & & BRAZIL \\
\hline 2330 & 8966 & NAD83(91) & 581100.941 & 147786.379 & 9 HEISPROD & GPS & & & $116-F-14$ \\
\hline 2331 & 8968 & NAD83(91) & 581170.063 & 147594.174 & 4 HEISPROD & GPS & & & $116-F-2$ \\
\hline 2332 & 8970 & NAD83(91) & 581169.534 & 147590.868 & 3 HEISPROD & GPS & & & $116-\mathrm{F}-2$ \\
\hline 2333 & 8971 & NAD83(91) & 581213.165 & 147931.619 & 9 HEISPROD & GPS & & & UPR-100-F-2 \\
\hline 2334 & 8972 & NAD83(91) & 581213.165 & 147931.619 & 9 HEISPROD & GPS & & & UPR-100-F-2 \\
\hline 2335 & 9603 & UNKNOWN & 580968.03 & 147818.44 & 4 HEISPROD & ESTIMATED & ONSITE & & 116-F-14 Shallow Zone \\
\hline 2336 & 9604 & UNKNOWN & 580965.92 & 147814.15 & 5 HEISPROD & ESTIMATED & ONSITE & & 116-F-14 Shallow Zone \\
\hline 2337 & 9605 & UNKNOWN & 580966.8 & 147810.3 & 3 HEISPROD & ESTIMATED & ONSITE & & 116-F-14 Shallow Zone \\
\hline 2338 & 9606 & UNKNOWN & 580972.03 & 147809.75 & 5 HEISPROD & ESTIMATED & ONSITE & & 116-F-14 Shallow Zone \\
\hline 2339 & 9607 & UNKNOWN & 580972.28 & 147805.4 & 4 HEISPROD & ESTIMATED & ONSITE & & 116-F-14 Shallow Zone \\
\hline 2340 & 9608 & UNKNOWN & 580969.2 & 147800.47 & 7 HEISPROD & ESTIMATED & ONSITE & & 116-F-14 Shallow Zone \\
\hline 2341 & 9609 & & & & HEISPROD & & & & 100-F-19 Overburden Zone \\
\hline 2342 & 9610 & & & & HEISPROD & & & & 100-F-19 Overburden Zone \\
\hline 2343 & 9611 & UNKNOWN & 580971.06 & 147790.84 & 4 HEISPROD & ESTIMATED & ONSITE & & 116-F-14 Shallow Zone \\
\hline 2344 & 9612 & & & & HEISPROD & & & & 100-F-19 Overburden Zone \\
\hline 2345 & 9613 & UNKNOWN & 580970.82 & 147785.4 & 4 HEISPROD & ESTIMATED & ONSITE & & 116-F-14 Shallow Zone \\
\hline 2346 & 9614 & UNKNOWN & 580968.73 & 147771.79 & 9 HEISPROD & ESTIMATED & ONSITE & & 116-F-14 Shallow Zone \\
\hline 2347 & 9615 & UNKNOWN & 580971.8 & 147771.92 & 2 HEISPROD & ESTIMATED & ONSITE & & 116-F-14 Shallow Zone \\
\hline 2348 & 9616 & & & & HEISPROD & & & & 100-F-19 Overburden Zone \\
\hline 2349 & 9617 & UNKNOWN & 580968.63 & 147765.62 & 2 HEISPROD & ESTIMATED & ONSITE & & 116-F-14 Shallow Zone \\
\hline 2350 & 9618 & UNKNOWN & 580962.64 & 147759.32 & 2 HEISPROD & ESTIMATED & ONSITE & & 116-F-14 Shallow Zone \\
\hline 2351 & 9619 & & & & HEISPROD & & & & 100-F-19 Overburden Zone \\
\hline 2352 & 9620 & UNKNOWN & 580971.44 & 147759.52 & 2 HEISPROD & ESTIMATED & ONSITE & & 116-F-14 Shallow Zone \\
\hline 2353 & 9621 & UNKNOWN & 580962.43 & 147752.93 & 3 HEISPROD & ESTIMATED & ONSITE & & 116-F-14 Shallow Zone \\
\hline 2354 & 9622 & & & & HEISPROD & & & & 100-F-19 Overburden Zone \\
\hline 2355 & 9623 & & & & HEISPROD & & & & 100-F-19 Overburden Zone \\
\hline 2356 & 9633 & UNKNOWN & 580962.37 & 147746.67 & 7 HEISPROD & ESTIMATED & ONSITE & & 116-F-14 Shallow Zone \\
\hline 2357 & 9634 & UNKNOWN & 580965.16 & 147746.72 & 2 HEISPROD & ESTIMATED & ONSITE & & 116-F-14 Shallow Zone \\
\hline
\end{tabular}


A

$\mathrm{B}$

C 1 D

\begin{tabular}{|c|c|c|}
\hline & $\mathrm{E}$ & $\mathrm{F}$ \\
\hline
\end{tabular} F 9635 UNKNOWN 9636 UNKNOWN 9637 UNKNOWN

9638 UNKNOWN 9639 UNKNOWN 9640 UNKNOWN 9641 UNKNOWN 9642 UNKNOWN 9643 UNKNOWN 9644 UNKNOWN 9645 UNKNOWN 9646 UNKNOWN 9647 UNKNOWN 9648 UNKNOWN 9649 UNKNOWN 9650 UNKNOWN 9651 UNKNOWN 9652 UNKNOWN

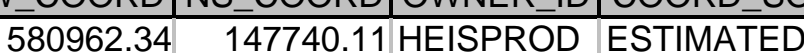
\begin{tabular}{|l|l|l}
580967.94 & 147740.27 HEISPROD & ESTIMATED
\end{tabular} 580962.34 147727.32 HEISPROD ESTIMATED $580967.94 \quad 147727.31$ HEISPROD ESTIMATED 580963.11 147721.63 HEISPROD ESTIMATED \begin{tabular}{r|l|l}
580977.9 & 147711.55 HEISPROD & ESTIMATED
\end{tabular} $580983.48 \quad 147711.36$ HEISPROD ESTIMATED 580988.24 147714.87 HEISPROD ESTIMATED \begin{tabular}{|r|r|}
580993.4 & 147714.96 HEISPROD \\
\hline
\end{tabular} $580993.42 \quad 147711.2$ HEISPROD ESTIMATED $580998.14 \quad 147714.87$ HEISPROD ESTIMATED 581088.72 147743.67 HEISPROD ESTIMATED $581090.33 \quad 147750.8$ HEISPROD ESTIMATED

$581093 \quad 147741.35$ HEISPROD ESTIMATED $581093.92 \quad 147752.68$ HEISPROD ESTIMATED $\begin{array}{lll}581092.14 & 147762.66 \text { HEISPROD } & \text { ESTIMATED }\end{array}$ $581095.33 \quad 147779.06$ HEISPROD ESTIMATED 581088.71 147783.24 HEISPROD ESTIMATED

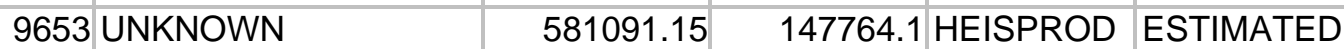
\begin{tabular}{|l|r|r|r|}
\hline 9654 & UNKNOWN & 581092.91 & 147794.52 \\
\hline
\end{tabular} 9655 UNKNOWN \begin{tabular}{|l|l|l|}
581092.91 & 147794.52 HEISPROD & ESTIMATED \\
\hline 581093.29 & 147805.14 HEISPROD & ESTIMATED \\
\hline
\end{tabular} 9656 UNKNOWN \begin{tabular}{ll|l|l}
581089.45 & $147809.66 \mathrm{HEISPROD}$ & ESTIMATED
\end{tabular} G $\mathrm{H}$ I DIST_CLASS $\quad$ RAD_LEVEL ONSITE ONSITE ONSITE ONSITE ONSITE ONSITE ONSITE ONSITE ONSITE ONSITE ONSITE ONSITE ONSITE ONSITE ONSITE ONSITE ONSITE ONSITE ONSITE ONSITE ONSITE

ONSITE \begin{tabular}{l|l|l}
9658 & HEISPROD \\
\hline 9659 & HEISPROD
\end{tabular} \begin{tabular}{|l|l|}
\hline 9660 & HEISPROD \\
\hline 9661 & HEISPROD
\end{tabular} \begin{tabular}{|l|l|l|}
\hline 9661 & HEISPROD \\
\hline 9662 & HEISPROD
\end{tabular} \begin{tabular}{l|l|l}
9662 & HEISPROD
\end{tabular}

\begin{tabular}{|l|l|l|}
\hline 9663 & HEISPROD
\end{tabular}

\begin{tabular}{|l|l|l}
\hline 9664 & HEISPROD
\end{tabular}

\begin{tabular}{|l|l|l|}
\hline 9665 & HEISPROD
\end{tabular}

\begin{tabular}{|l|l|l}
\hline 9666 & HEISPROD
\end{tabular}

\begin{tabular}{|l|l|l|}
\hline 9667 & HEISPROD \\
\hline 9668 & HEISPROD
\end{tabular}

\begin{tabular}{|l|l|l|}
9668 & HEISPROD \\
\hline 9669 & & HEISPROD
\end{tabular}

9670 HEISPROD

\begin{tabular}{|l|l|}
\hline 9670 & HEISPROD \\
\hline 9671 & HEISPROD \\
\hline
\end{tabular}

9672 HEISPROD

116-F-14 Shallow Zone

116-F-14 Shallow Zone

116-F-14 Shallow Zone

116-F-14 Shallow Zone

116-F-14 Shallow Zone

116-F-14 Shallow Zone

116-F-14 Shallow Zone

116-F-14 Shallow Zone

116-F-14 Shallow Zone

116-F-14 Shallow Zone

116-F-14 Shallow Zone

116-F-14 Shallow Zone

116-F-14 Shallow Zone

116-F-14 Shallow Zone

116-F-14 Shallow Zone

116-F-14 Shallow Zone

116-F-14 Shallow Zone

116-F-14 Shallow Zone

116-F-14 Shallow Zone

116-F-14 Shallow Zone

116-F-14 Shallow Zone

116-F-14 Shallow Zone

100-F-19 Overburden Zone

100-F-19 Overburden Zone

100-F-19 Overburden Zone

100-F-19 Overburden Zone

100-F-19 Overburden Zone

100-F-19 Overburden Zone

100-F-19 Shallow Zone

100-F-19 Shallow Zone

100-F-19 Shallow Zone

100-F-19 Shallow Zone

100-F-19 Shallow Zone

100-F-19 Shallow Zone

100-F-19 Shallow Zone

100-F-19 Shallow Zone

100-F-19 Shallow Zone

100-F-19 Shallow Zone

SITE_NAME

Page 63 


\begin{tabular}{|c|c|c|c|c|c|c|c|c|c|}
\hline & $A$ & $\mathrm{~B}$ & $\mathrm{C}$ & $\mathrm{D}$ & $E$ & $\mathrm{~F}$ & $\mathrm{G}$ & $\mathrm{H}$ & $\mathrm{I}$ \\
\hline 1 & SAMP_SITE_ID & HORIZ_COORD_TYPE & EW_COORD & NS_COORD & OWNER_ID & COORD_SOURCE & DIST_CLASS & RAD_LEVEL & SAMP_SITE_NAME \\
\hline 2396 & 9673 & & & & HEISPROD & & & & 100-F-19 Shallow Zone \\
\hline 2397 & 9674 & & & & HEISPROD & & & & 100-F-19 Shallow Zone \\
\hline 2398 & 9675 & & & & HEISPROD & & & & 100-F-19 Shallow Zone \\
\hline 2399 & 9676 & & & & HEISPROD & & & & 100-F-19 Shallow Zone \\
\hline 2400 & 9677 & NAD83 & 580239.77 & 147960.82 & 2 HEISPROD & GPS & & & $100-F-19$ \\
\hline 2401 & 9678 & NAD83 & 580243.45 & 147953.46 & S HEISPROD & GPS & & & $100-F-19$ \\
\hline 2402 & 9679 & NAD83 & 580248.04 & 147960.89 & 9 HEISPROD & GPS & & & $100-F-19$ \\
\hline 2403 & 9680 & & & & HEISPROD & & & & 116-F-14 Deep Zone \\
\hline 2404 & 9681 & NAD83 & 580269.53 & 147953.2 & 2 HEISPROD & GPS & & & 100-F-19 \\
\hline 2405 & 9682 & NAD83 & 580284.3 & 147951.04 & 4 HEISPROD & GPS & & & 100-F-19 \\
\hline 2406 & 9683 & NAD83 & 580289.64 & 147949.98 & 3 HEISPROD & GPS & & & 100-F-19 \\
\hline 2407 & 9684 & NAD83 & 580297.63 & 147950.72 & 2 HEISPROD & GPS & & & 100-F-19 \\
\hline 2408 & 9685 & NAD83 & 580298.17 & 147945.4 & 4 HEISPROD & GPS & ONSITE & & $100-F-19$ \\
\hline 2409 & 9686 & NAD83 & 580308.94 & 147945.42 & 2 HEISPROD & GPS & ONSITE & & $100-F-19$ \\
\hline 2410 & 9687 & NAD83 & 580319.96 & 147956.82 & 2 HEISPROD & GPS & ONSITE & & 100-F-19 \\
\hline 2411 & 9688 & NAD83 & 580331.23 & 147957.68 & 3 HEISPROD & GPS & ONSITE & & 100-F-19 \\
\hline 2412 & 9689 & NAD83 & 580331.48 & 147946.6 & HEISPROD & GPS & ONSITE & & 100-F-19 \\
\hline 2413 & 9690 & NAD83 & 580342.54 & 147957.87 & 7 HEISPROD & GPS & ONSITE & & $100-F-19$ \\
\hline 2414 & 9691 & NAD83 & 580342.61 & 147952.43 & 3 HEISPROD & GPS & ONSITE & & 100-F-19 \\
\hline 2415 & 9692 & NAD83 & 580342.68 & 147946.96 & HEISPROD & GPS & ONSITE & & 100-F-19 \\
\hline 2416 & 9693 & NAD83 & 580365.72 & 147957.86 & 5 HEISPROD & GPS & ONSITE & & 100-F-19 \\
\hline 2417 & 9694 & NAD83 & 580377.32 & 147962.91 & 1 HEISPROD & GPS & ONSITE & & 100-F-19 \\
\hline 2418 & 9713 & & & & HEISPROD & & ONSITE & & 116-F-14 Deep Zone \\
\hline 2419 & 9714 & & & & HEISPROD & & ONSITE & & 116-F-14 Deep Zone \\
\hline 2420 & 9715 & & & & HEISPROD & & ONSITE & & 116-F-14 Deep Zone \\
\hline 2421 & 9716 & & & & HEISPROD & & ONSITE & & 116-F-14 Deep Zone \\
\hline 2422 & 9717 & & & & HEISPROD & & ONSITE & & 116-F-14 Deep Zone \\
\hline 2423 & 9718 & & & & HEISPROD & & ONSITE & & 116-F-14 Deep Zone \\
\hline 2424 & 9719 & & & & HEISPROD & & ONSITE & & 116-F-14 Deep Zone \\
\hline 2425 & 9720 & & & & HEISPROD & & ONSITE & & 116-F-14 Deep Zone \\
\hline 2426 & 9721 & & & & HEISPROD & & ONSITE & & 116-F-14 Deep Zone \\
\hline 2427 & 9722 & & & & HEISPROD & & ONSITE & & 116-F-14 Deep Zone \\
\hline 2428 & 9723 & & & & HEISPROD & & ONSITE & & 116-F-14 Deep Zone \\
\hline 2429 & 9724 & & & & HEISPROD & & ONSITE & & 116-F-14 Deep Zone \\
\hline 2430 & 9725 & & & & HEISPROD & & ONSITE & & 116-F-14 Deep Zone \\
\hline 2431 & 9726 & NAD83 & 580377.71 & 147945.41 & 1 HEISPROD & GPS & ONSITE & & 100-F-19 \\
\hline 2432 & 9727 & & & & HEISPROD & & ONSITE & & 116-F-14 Deep Zone \\
\hline 2433 & 9728 & & & & HEISPROD & & ONSITE & & 116-F-14 Deep Zone \\
\hline
\end{tabular}

Page 64 


\begin{tabular}{|c|c|c|c|c|c|c|c|c|c|}
\hline & A & $\mathrm{B}$ & $\mathrm{C}$ & $\mathrm{D}$ & $E$ & $\mathrm{~F}$ & G & $\mathrm{H}$ & $\mathrm{I}$ \\
\hline 1 & SAMP_SITE_ID & HORIZ_COORD_TYPE & EW_COORD & NS_COORD & OWNER_ID & COORD_SOURCE & DIST_CLASS & RAD_LEVEL & SAMP_SITE_NAME \\
\hline 2434 & 9729 & & & & HEISPROD & & ONSITE & & 116-F-14 Deep Zone \\
\hline 2435 & 9730 & & & & HEISPROD & & ONSITE & & 116-F-14 Deep Zone \\
\hline 2436 & 9731 & NAD83 & 580388.4 & 147949.8 & HEISPROD & GPS & ONSITE & & 100-F-19 \\
\hline 2437 & 9732 & NAD83 & 580175.54 & 147953.15 & HEISPROD & GPS & ONSITE & & 100-F-19 Pipe Trench Shallow Zone \\
\hline 2438 & 9733 & NAD83 & 580399.41 & 147957.41 & HEISPROD & GPS & ONSITE & & 100-F-19 \\
\hline 2439 & 9734 & NAD83 & 580193.71 & 147953.02 & HEISPROD & GPS & ONSITE & & 100-F-19 Pipe Trench Shallow Zone \\
\hline 2440 & 9735 & NAD83 & 580399.36 & 147952.26 & HEISPROD & GPS & ONSITE & & $100-F-19$ \\
\hline 2441 & 9736 & NAD83 & 580411.52 & 147957.53 & HEISPROD & GPS & ONSITE & & $100-F-19$ \\
\hline 2442 & 9737 & NAD83 & 580210.99 & 147953 & HEISPROD & GPS & ONSITE & & 100-F-19 Pipe Trench Shallow Zone \\
\hline 2443 & 9738 & NAD83 & 580423.79 & 147957.12 & HEISPROD & GPS & ONSITE & & 100-F-19 \\
\hline 2444 & 9739 & NAD83 & 580424.05 & 147952.08 & HEISPROD & GPS & ONSITE & & 100-F-19 \\
\hline 2445 & 9740 & NAD83 & 580214.81 & 147960.45 & HEISPROD & GPS & ONSITE & & 100-F-19 Pipe Trench Shallow Zone \\
\hline 2446 & 9741 & NAD83 & 580431.87 & 147959.63 & HEISPROD & GPS & ONSITE & & 100-F-19 Shallow Zone \\
\hline 2447 & 9742 & NAD83 & 580433.57 & 147949.29 & HEISPROD & GPS & ONSITE & & 100-F-19 Shallow Zone \\
\hline 2448 & 9743 & NAD83 & 580440.25 & 147949.15 & HEISPROD & GPS & ONSITE & & 100-F-19 Shallow Zone \\
\hline 2449 & 9744 & NAD83 & 580443.45 & 147959.63 & HEISPROD & GPS & ONSITE & & 100-F-19 Shallow Zone \\
\hline 2450 & 9745 & NAD83 & 580454.13 & 147959.63 & HEISPROD & GPS & ONSITE & & 100-F-19 Shallow Zone \\
\hline 2451 & 9746 & NAD83 & 580458.74 & 147958.96 & HEISPROD & GPS & ONSITE & & 100-F-19 Shallow Zone \\
\hline 2452 & 9747 & NAD83 & 580471.52 & 147984.86 & HEISPROD & GPS & ONSITE & & 100-F-19 Shallow Zone \\
\hline 2453 & 9748 & NAD83 & 580475.71 & 147984.26 & HEISPROD & GPS & ONSITE & & 100-F-19 Shallow Zone \\
\hline 2454 & 9749 & NAD83 & 580465.21 & 147955.52 & HEISPROD & GPS & ONSITE & & 100-F-19 Shallow Zone \\
\hline 2455 & 9750 & NAD83 & 580465.31 & 147947.57 & HEISPROD & GPS & ONSITE & & 100-F-19 Shallow Zone \\
\hline 2456 & 9751 & NAD83 & 580478.52 & 147959.01 & HEISPROD & GPS & ONSITE & & 100-F-19 Shallow Zone \\
\hline 2457 & 9752 & NAD83 & 580481.91 & 147948.83 & HEISPROD & GPS & ONSITE & & $100-F-19$ \\
\hline 2458 & 9753 & NAD83 & 580499.26 & 147957.3 & HEISPROD & GPS & ONSITE & & 100-F-19 Shallow Zone \\
\hline 2459 & 9754 & NAD83 & 580481.91 & 147948.83 & HEISPROD & GPS & ONSITE & & $100-F-19$ \\
\hline 2460 & 9755 & & & & HEISPROD & & ONSITE & & 100-F-19 Shallow Zone \\
\hline 2461 & 9756 & NAD83 & 580481.91 & 147948.83 & HEISPROD & GPS & ONSITE & & 100-F-19 Shallow Zone \\
\hline 2462 & 9757 & & & & HEISPROD & & ONSITE & & 126-F-1 \\
\hline 2463 & 9758 & & & & HEISPROD & & ONSITE & & 126-F-1 \\
\hline 2464 & 9759 & & & & HEISPROD & & ONSITE & & 126-F-1 \\
\hline 2465 & 9760 & & & & HEISPROD & & ONSITE & & 126-F-1 \\
\hline 2466 & 9761 & NAD83 & 580963.55 & 148076.41 & HEISPROD & GPS & ONSITE & & 100-F-19 Shallow Zone \\
\hline 2467 & 9762 & NAD83 & 580962.37 & 148063.52 & HEISPROD & GPS & ONSITE & & 100-F-19 Shallow Zone \\
\hline 2468 & 9763 & NAD83 & 580971.83 & 148035.87 & HEISPROD & GPS & ONSITE & & 100-F-19 Shallow Zone \\
\hline 2469 & 9764 & NAD83 & 580977.69 & 148038.18 & HEISPROD & GPS & ONSITE & & 100-F-19 Shallow Zone \\
\hline 2470 & 9765 & NAD83 & 580975.27 & 148026.72 & HEISPROD & GPS & ONSITE & & 100-F-19 Shallow Zone \\
\hline 2471 & 9766 & UNKNOWN & 565713 & 145470 & HEISPROD & ESTIMATED & ONSITE & & $100-C-6$ \\
\hline
\end{tabular}




\begin{tabular}{|c|c|c|c|c|c|c|c|c|c|}
\hline & $\bar{A}$ & $\mathrm{~B}$ & $\mathrm{C}$ & $\mathrm{D}$ & $E$ & $\mathrm{~F}$ & G & $\mathrm{H}$ & $\mathrm{I}$ \\
\hline 1 & SAMP_SITE_ID & HORIZ_COORD_TYPE & EW_COORD & NS_COORD & OWNER_ID & COORD_SOURCE & DIST_CLASS & RAD_LEVEL & SAMP_SITE_NAME \\
\hline 2472 & 9767 & UNKNOWN & 565716 & 145470 & HEISPROD & ESTIMATED & ONSITE & & $100-C-6$ \\
\hline 2473 & 9768 & NAD83 & 580987.65 & 148011.04 & HEISPROD & GPS & ONSITE & & 100-F-19 Shallow Zone \\
\hline 2474 & 9769 & UNKNOWN & 571562 & 149649 & HEISPROD & ESTIMATED & ONSITE & & $116-\mathrm{N}-3$ \\
\hline 2475 & 9770 & UNKNOWN & 571559 & 149651 & HEISPROD & ESTIMATED & ONSITE & & $116-N-3$ \\
\hline 2476 & 9771 & NAD83 & 580993.76 & 147993.42 & HEISPROD & GPS & ONSITE & & 100-F-19 Shallow Zone \\
\hline 2477 & 9772 & NAD83 & 580996.95 & 147983.68 & HEISPROD & GPS & ONSITE & & 100-F-19 Shallow Zone \\
\hline 2478 & 9773 & NAD83 & 580913.48 & 148041.12 & HEISPROD & GPS & ONSITE & & 100-F-19 Shallow Zone \\
\hline 2479 & 9774 & & & & HEISPROD & & & & 100-F-19 Shallow Zone \\
\hline 2480 & 9775 & NAD83 & 580923.56 & 147996.89 & HEISPROD & GPS & ONSITE & & 100-F-19 Shallow Zone \\
\hline 2481 & 9776 & NAD83 & 580900.44 & 147998.56 & HEISPROD & GPS & ONSITE & & 100-F-19 Shallow Zone \\
\hline 2482 & 9777 & NAD83 & 580836.78 & 148077.25 & HEISPROD & GPS & ONSITE & & 100-F-19 \\
\hline 2483 & 9778 & NAD83 & 580834.89 & 148065.53 & HEISPROD & GPS & ONSITE & & 100-F-19 \\
\hline 2484 & 9779 & NAD83 & 580834.28 & 148061.05 & HEISPROD & GPS & ONSITE & & $100-F-19$ \\
\hline 2485 & 9780 & NAD83 & 580844.58 & 148075.72 & HEISPROD & GPS & ONSITE & & 100-F-19 \\
\hline 2486 & 9781 & & & & HEISPROD & & & & 100-IU-6 \\
\hline 2487 & 9782 & NAD83 & 580861.58 & 148076.42 & HEISPROD & GPS & ONSITE & & 100-F-19 \\
\hline 2488 & 9783 & NAD83 & 580856.17 & 148065.32 & HEISPROD & GPS & ONSITE & & 100-F-19 \\
\hline 2489 & 9784 & NAD83 & 580846.9 & 148057.26 & HEISPROD & GPS & ONSITE & & $100-F-19$ \\
\hline 2490 & 9785 & NAD83 & 580860.84 & 148057.87 & HEISPROD & GPS & ONSITE & & 100-F-19 \\
\hline 2491 & 9786 & NAD83 & 580874.81 & 148058.48 & HEISPROD & GPS & ONSITE & & 100-F-19 \\
\hline 2492 & 9787 & NAD83 & 580874.96 & 148054.07 & HEISPROD & GPS & ONSITE & & 100-F-19 \\
\hline 2493 & 9788 & NAD83 & 580875.18 & 148049.75 & HEISPROD & GPS & ONSITE & & 100-F-19 \\
\hline 2494 & 9789 & NAD83 & 580832.77 & 148043.58 & HEISPROD & GPS & ONSITE & & 100-F-19 \\
\hline 2495 & 9790 & NAD83 & 580861.89 & 148040.43 & HEISPROD & GPS & ONSITE & & 100-F-19 \\
\hline 2496 & 9791 & NAD83 & 580876.07 & 148041.09 & HEISPROD & GPS & ONSITE & & 100-F-19 \\
\hline 2497 & 9792 & NAD83 & 580836.52 & 148034.66 & HEISPROD & GPS & ONSITE & & $100-F-19$ \\
\hline 2498 & 9793 & NAD83 & 580851.57 & 148030.6 & HEISPROD & GPS & ONSITE & & 100-F-19 \\
\hline 2499 & 9794 & NAD83 & 580853.42 & 148025.7 & HEISPROD & GPS & ONSITE & & 100-F-19 \\
\hline 2500 & 9795 & NAD83 & 580877.43 & 148026.93 & HEISPROD & GPS & ONSITE & & 100-F-19 \\
\hline 2501 & 9796 & NAD83 & 580851.07 & 148017.2 & HEISPROD & GPS & ONSITE & & 100-F-19 \\
\hline 2502 & 9797 & NAD83 & 580856.41 & 148017.49 & HEISPROD & GPS & ONSITE & & 100-F-19 \\
\hline 2503 & 9798 & NAD83 & 580879.51 & 148019.95 & HEISPROD & GPS & ONSITE & & 100-F-19 \\
\hline 2504 & 9799 & NAD83 & 580846.11 & 148005.26 & HEISPROD & GPS & ONSITE & & 100-F-19 \\
\hline 2505 & 9800 & NAD83 & 580867.57 & 148006.65 & HEISPROD & GPS & ONSITE & & 100-F-19 \\
\hline 2506 & 9801 & NAD83 & 580878.61 & 148008.27 & HEISPROD & GPS & ONSITE & & 100-F-19 \\
\hline 2507 & 9802 & NAD83 & 580852.55 & 147996.64 & HEISPROD & GPS & ONSITE & & 100-F-19 \\
\hline 2508 & 9803 & NAD83 & 580862.57 & 147997.42 & HEISPROD & GPS & ONSITE & & 100-F-19 \\
\hline 2509 & 9804 & NAD83 & 580873.16 & 147998.35 & HEISPROD & GPS & ONSITE & & $100-F-19$ \\
\hline
\end{tabular}




\begin{tabular}{|c|c|c|c|c|c|c|c|c|c|}
\hline & $A$ & $\mathrm{~B}$ & $\mathrm{C}$ & $\mathrm{D}$ & $E$ & $\mathrm{~F}$ & $\mathrm{G}$ & $\mathrm{H}$ & $\mathrm{I}$ \\
\hline 1 & SAMP_SITE_ID & HORIZ_COORD_TYPE & EW_COORD & NS_COORD & OWNER_ID & COORD_SOURCE & DIST_CLASS & RAD_LEVEL & SAMP_SITE_NAME \\
\hline 2510 & 9805 & NAD83 & 580871.78 & 147984.09 & HEISPROD & GPS & ONSITE & & 100-F-19 \\
\hline \begin{tabular}{|l|}
2511 \\
\end{tabular} & 9807 & NAD83 & 580857.17 & 147969.82 & HEISPROD & GPS & ONSITE & & 100-F-19 \\
\hline 2512 & 9808 & NAD83 & 580878.59 & 147972.09 & HEISPROD & GPS & ONSITE & & 100-F-19 \\
\hline 2513 & 9809 & NAD83 & 580863.55 & 147964.83 & HEISPROD & GPS & ONSITE & & $100-F-19$ \\
\hline 2514 & 9810 & NAD83 & 580850.93 & 147958.89 & HEISPROD & GPS & ONSITE & & $100-F-19$ \\
\hline 2515 & 9811 & NAD83 & 580859.02 & 147958.3 & HEISPROD & GPS & ONSITE & & 100-F-19 \\
\hline 2516 & 9812 & NAD83 & 580850.88 & 147950.6 & HEISPROD & GPS & ONSITE & & 100-F-19 \\
\hline 2517 & 9813 & NAD83 & 580854.67 & 147940.01 & HEISPROD & GPS & ONSITE & & $100-F-19$ \\
\hline \begin{tabular}{|l|}
2518 \\
\end{tabular} & 9814 & NAD83 & 580859.86 & 147940.49 & HEISPROD & GPS & ONSITE & & 100-F-19 \\
\hline 2519 & 9815 & NAD83 & 580901.57 & 147982.12 & HEISPROD & GPS & ONSITE & & 100-F-19 \\
\hline 2520 & 9816 & NAD83 & 580903.2 & 147977.29 & HEISPROD & GPS & ONSITE & & 100-F-19 \\
\hline \begin{tabular}{|l|}
2521 \\
\end{tabular} & 9817 & NAD83 & 580914.02 & 147978.88 & HEISPROD & GPS & ONSITE & & 100-F-19 \\
\hline 2522 & 9818 & NAD83 & 580921.78 & 147989.28 & HEISPROD & GPS & ONSITE & & $100-F-19$ \\
\hline 2523 & 9819 & NAD83 & 580988.2 & 148038.64 & HEISPROD & GPS & ONSITE & & $100-F-19$ \\
\hline \begin{tabular}{|l|}
2524 \\
\end{tabular} & 9820 & NAD83 & 580998.21 & 148041.93 & HEISPROD & GPS & ONSITE & & 100-F-19 \\
\hline \begin{tabular}{|l|}
2525 \\
\end{tabular} & 9821 & NAD83 & 580997.23 & 148015.81 & HEISPROD & GPS & ONSITE & & 100-F-19 \\
\hline 2526 & 9822 & NAD83 & 581007.48 & 148021.44 & HEISPROD & GPS & ONSITE & & 100-F-19 \\
\hline 2527 & 9823 & NAD83 & 581001.94 & 148001.84 & HEISPROD & GPS & ONSITE & & $100-F-19$ \\
\hline \begin{tabular}{|l|}
2528 \\
\end{tabular} & 9824 & NAD83 & 581004.57 & 147994.04 & HEISPROD & GPS & ONSITE & & 100-F-19 \\
\hline 2529 & 9825 & NAD83 & 581012.43 & 147999.87 & HEISPROD & GPS & ONSITE & & 100-F-19 \\
\hline 2530 & 9826 & NAD83 & 581010.03 & 147976.81 & HEISPROD & GPS & ONSITE & & 100-F-19 \\
\hline \begin{tabular}{|l|}
2531 \\
\end{tabular} & 9827 & NAD83 & 580336.35 & 148161.14 & HEISPROD & GPS & ONSITE & & 100-F-19 Pipe Trench Shallow Zone \\
\hline 2532 & 9828 & NAD83 & 580342.95 & 148162.39 & HEISPROD & GPS & ONSITE & & 100-F-19 Pipe Trench Shallow Zone \\
\hline 2533 & 9829 & NAD83 & 580352.28 & 148162.78 & HEISPROD & GPS & ONSITE & & 100-F-19 Pipe Trench Shallow Zone \\
\hline 2534 & 9830 & NAD83 & 580352.15 & 148155.4 & HEISPROD & GPS & ONSITE & & 100-F-19 Pipe Trench Shallow Zone \\
\hline 2535 & 9831 & NAD83 & 580350.45 & 148138.96 & HEISPROD & GPS & ONSITE & & 100-F-19 Pipe Trench Shallow Zone \\
\hline 2536 & 9832 & NAD83 & 580347.37 & 148119.8 & HEISPROD & GPS & ONSITE & & 100-F-19 Pipe Trench Shallow Zone \\
\hline 2537 & 9833 & NAD83 & 580369.58 & 148163.57 & HEISPROD & GPS & ONSITE & & 100-F-19 Pipe Trench Shallow Zone \\
\hline 2538 & 9834 & NAD83 & 580369.9 & 148150.61 & HEISPROD & GPS & ONSITE & & 100-F-19 Pipe Trench Shallow Zone \\
\hline 2539 & 9835 & NAD83 & 580379.66 & 148162.01 & HEISPROD & GPS & ONSITE & & 100-F-19 Pipe Trench Shallow Zone \\
\hline 2540 & 9836 & NAD83 & 580400.47 & 148154.8 & HEISPROD & GPS & ONSITE & & 100-F-19 Pipe Trench Shallow Zone \\
\hline 2541 & 9837 & NAD83 & 580400.4 & 148143.08 & HEISPROD & GPS & ONSITE & & 100-F-19 Pipe Trench Shallow Zone \\
\hline 2542 & 9838 & NAD83 & 580411.57 & 148161.1 & HEISPROD & GPS & ONSITE & & 100-F-19 Pipe Trench Shallow Zone \\
\hline 2543 & 9839 & NAD83 & 580411.07 & 148149.73 & HEISPROD & GPS & ONSITE & & 100-F-19 Pipe Trench Shallow Zone \\
\hline 2544 & 9840 & NAD83 & 580410.86 & 148143.67 & HEISPROD & GPS & ONSITE & & 100-F-19 Pipe Trench Shallow Zone \\
\hline 2545 & 9847 & NAD83 & 580419.13 & 148157.83 & HEISPROD & GPS & ONSITE & & 100-F-19 Pipe Trench Shallow Zone \\
\hline 2546 & 9848 & NAD83 & 580424.89 & 148158.13 & HEISPROD & GPS & ONSITE & & 100-F-19 Pipe Trench Shallow Zone \\
\hline 2547 & 9849 & NAD83 & 580425.14 & 148147.08 & HEISPROD & GPS & ONSITE & & 100-F-19 Pipe Trench Shallow Zone \\
\hline
\end{tabular}




\begin{tabular}{|c|c|c|c|c|c|c|c|c|c|}
\hline & A & $\mathrm{B}$ & C & $\mathrm{D}$ & $\mathrm{E}$ & $\mathrm{F}$ & $\mathrm{G}$ & $\mathrm{H}$ & $\mathrm{I}$ \\
\hline 1 & SAMP_SITE_ID & HORIZ_COORD_TYPE & EW_COORD & NS_COORD & OWNER_ID & COORD_SOURCE & DIST_CLASS & RAD_LEVEL & SAMP_SITE_NAME \\
\hline 2548 & 9850 & NAD83 & 580430.52 & 148154.85 & HEISPROD & GPS & ONSITE & & 100-F-19 Pipe Trench Shallow Zone \\
\hline 2549 & 9869 & NAD83 & 580939.27 & 148045.66 & HEISPROD & GPS & ONSITE & & 100-F-19 Shallow Zone \\
\hline 2550 & 9887 & NAD83 & 580939.27 & 148045.66 & HEISPROD & GPS & ONSITE & & 100-F-19 \\
\hline 2551 & 9888 & UNKNOWN & 565465 & 145413 & HEISPROD & ESTIMATED & ONSITE & & 132-B-6 Outfall Structure \\
\hline 2552 & 9889 & UNKNOWN & 565454 & 145425 & HEISPROD & ESTIMATED & ONSITE & & 132-B-6 Outfall Structure \\
\hline 2553 & 9890 & UNKNOWN & 565454 & 145425 & HEISPROD & ESTIMATED & ONSITE & & 132-B-6 Outfall Structure \\
\hline 2554 & 9891 & & & & HEISPROD & & & & 132-B-6 Outfall Structure \\
\hline 2555 & 9892 & NAD83 & 580941.6 & 148033.29 & HEISPROD & GPS & ONSITE & & 100-F-19 Shallow Zone \\
\hline 2556 & 9893 & NAD83 & 580949.31 & 148037.66 & HEISPROD & GPS & ONSITE & & 100-F-19 Shallow Zone \\
\hline 2557 & 9894 & NAD83 & 580944.32 & 148024.6 & HEISPROD & GPS & ONSITE & & 100-F-19 Shallow Zone \\
\hline 2558 & 9895 & NAD83 & 580952.82 & 148026.41 & HEISPROD & GPS & ONSITE & & 100-F-19 Shallow Zone \\
\hline 2559 & 9896 & NAD83 & 580954.65 & 148003.53 & HEISPROD & GPS & ONSITE & & 100-F-19 Shallow Zone \\
\hline 2560 & 9897 & NAD83 & 580949.17 & 148105.51 & HEISPROD & GPS & ONSITE & & 100-F-19 Shallow Zone \\
\hline 2561 & 9898 & NAD83 & 580948.99 & 148096.28 & HEISPROD & GPS & ONSITE & & 100-F-19 Shallow Zone \\
\hline 2562 & 9899 & NAD83 & 580946.36 & 148089.94 & HEISPROD & GPS & ONSITE & & 100-F-19 Shallow Zone \\
\hline 2563 & 9900 & NAD83 & 580953.54 & 148080.64 & HEISPROD & GPS & ONSITE & & 100-F-19 Shallow Zone \\
\hline 2564 & 9901 & NAD83 & 581003.62 & 147959.87 & HEISPROD & GPS & ONSITE & & 100-F-19 Shallow Zone \\
\hline 2565 & 9902 & NAD83 & 581006.08 & 147952.86 & HEISPROD & GPS & ONSITE & & 100-F-19 Shallow Zone \\
\hline 2566 & 9903 & NAD83 & 581016.39 & 147922.81 & HEISPROD & GPS & ONSITE & & 100-F-19 Shallow Zone \\
\hline 2567 & 9904 & NAD83 & 581023.94 & 147910.36 & HEISPROD & GPS & ONSITE & & 100-F-19 Shallow Zone \\
\hline 2568 & 9905 & NAD83 & 581045.99 & 147893.21 & HEISPROD & GPS & ONSITE & & 100-F-19 Shallow Zone \\
\hline 2569 & 9906 & NAD83 & 581052.67 & 147882.41 & HEISPROD & GPS & ONSITE & & 100-F-19 Shallow Zone \\
\hline 2570 & 9907 & NAD83 & 580939.94 & 147994.3 & HEISPROD & GPS & ONSITE & & 100-F-19 \\
\hline 2571 & 9908 & NAD83 & 580956.99 & 147933.02 & HEISPROD & GPS & ONSITE & & $100-F-19$ \\
\hline 2572 & 9909 & NAD83 & 580957 & 147916.27 & HEISPROD & GPS & ONSITE & & $100-F-19$ \\
\hline 2573 & 9910 & NAD83 & 580956.65 & 147906.16 & HEISPROD & GPS & ONSITE & & 100-F-19 \\
\hline 2574 & 9911 & NAD83 & 580953.51 & 147889.65 & HEISPROD & GPS & ONSITE & & 100-F-19 \\
\hline 2575 & 9912 & NAD83 & 580953.86 & 147881.92 & HEISPROD & GPS & ONSITE & & 100-F-19 \\
\hline 2576 & 9913 & & & & HEISPROD & & & & 100-F-19 Pipe Trench Deep Zone \\
\hline 2577 & 9914 & & & & HEISPROD & & & & 100-F-19 Pipe Trench Deep Zone \\
\hline 2578 & 9915 & & & & HEISPROD & & & & 100-F-19 Pipe Trench Deep Zone \\
\hline 2579 & 9916 & & & & HEISPROD & & & & 100-F-19 Pipe Trench Deep Zone \\
\hline 2580 & 9917 & & & & HEISPROD & & & & 100-F-19 Pipe Trench Deep Zone \\
\hline 2581 & 9918 & & & & HEISPROD & & & & 100-F-19 Pipe Trench Deep Zone \\
\hline 2582 & 9919 & & & & HEISPROD & & & & 100-F-19 Pipe Trench Deep Zone \\
\hline 2583 & 9920 & & & & HEISPROD & & & & 100-F-19 Pipe Trench Deep Zone \\
\hline 2584 & 9921 & & & & HEISPROD & & & & 100-F-19 Pipe Trench Deep Zone \\
\hline 2585 & 9922 & NAD83 & 580175.07 & 148151.31 & HEISPROD & GPS & ONSITE & & 100-F-19 Pipe Trench Shallow Zone \\
\hline
\end{tabular}




\begin{tabular}{|c|c|c|c|c|c|c|c|c|c|}
\hline & A & $\mathrm{B}$ & $\mathrm{C}$ & $\mathrm{D}$ & $\mathrm{E}$ & $\mathrm{F}$ & G & $\mathrm{H}$ & $\mathrm{I}$ \\
\hline 1 & SAMP_SITE_ID & HORIZ_COORD_TYPE & EW_COORD & NS_COORD & OWNER_ID & COORD_SOURCE & DIST_CLASS & RAD_LEVEL & SAMP_SITE_NAME \\
\hline 2586 & 9923 & NAD83 & 580183.41 & 148158.3 & HEISPROD & GPS & ONSITE & & 100-F-19 Pipe Trench Shallow Zone \\
\hline 2587 & 9924 & NAD83 & 580184.67 & 148151.54 & HEISPROD & GPS & ONSITE & & 100-F-19 Pipe Trench Shallow Zone \\
\hline 2588 & 9925 & NAD83 & 580214 & 148151.88 & HEISPROD & GPS & ONSITE & & 100-F-19 Pipe Trench Shallow Zone \\
\hline 2589 & 9926 & NAD83 & 580235.13 & 148158.89 & HEISPROD & GPS & ONSITE & & 100-F-19 Pipe Trench Shallow Zone \\
\hline 2590 & 9927 & NAD83 & 580242.87 & 148152.03 & HEISPROD & GPS & ONSITE & & 100-F-19 Pipe Trench Shallow Zone \\
\hline 2591 & 9928 & NAD83 & 580259.01 & 148158.78 & HEISPROD & GPS & ONSITE & & 100-F-19 Pipe Trench Shallow Zone \\
\hline 2592 & 9929 & NAD83 & 580271.5 & 148151.03 & HEISPROD & GPS & ONSITE & & 100-F-19 Pipe Trench Shallow Zone \\
\hline 2593 & 9930 & NAD83 & 580274.06 & 148159.37 & HEISPROD & GPS & ONSITE & & 100-F-19 Pipe Trench Shallow Zone \\
\hline 2594 & 9931 & NAD83 & 580285.42 & 148149.67 & HEISPROD & GPS & ONSITE & & 100-F-19 Pipe Trench Shallow Zone \\
\hline 2595 & 9932 & NAD83 & 580297.48 & 148150.11 & HEISPROD & GPS & ONSITE & & 100-F-19 Pipe Trench Shallow Zone \\
\hline 2596 & 9933 & NAD83 & 580302.37 & 148159.57 & HEISPROD & GPS & ONSITE & & 100-F-19 Pipe Trench Shallow Zone \\
\hline 2597 & 9934 & NAD83 & 580309.14 & 148159.4 & HEISPROD & GPS & ONSITE & & 100-F-19 Pipe Trench Shallow Zone \\
\hline 2598 & 9935 & NAD83 & 580309.33 & 148149.42 & HEISPROD & GPS & ONSITE & & 100-F-19 Pipe Trench Shallow Zone \\
\hline 2599 & 9936 & NAD83 & 580315.36 & 148149.33 & HEISPROD & GPS & ONSITE & & 100-F-19 Pipe Trench Shallow Zone \\
\hline 2600 & 9937 & NAD83 & 580321.59 & 148159.51 & HEISPROD & GPS & ONSITE & & 100-F-19 Pipe Trench Shallow Zone \\
\hline 2601 & 9938 & NAD83 & 580337.8 & 148112.68 & HEISPROD & GPS & ONSITE & & 100-F-19 Pipe Trench Shallow Zone \\
\hline 2602 & 9939 & NAD83 & 580343.35 & 148113.72 & HEISPROD & GPS & ONSITE & & 100-F-19 Pipe Trench Shallow Zone \\
\hline 2603 & 9940 & NAD83 & 580339.41 & 148089.75 & HEISPROD & GPS & ONSITE & & 100-F-19 Pipe Trench Shallow Zone \\
\hline 2604 & 9941 & NAD83 & 580339.67 & 148077.86 & HEISPROD & GPS & ONSITE & & 100-F-19 Pipe Trench Shallow Zone \\
\hline 2605 & 9942 & NAD83 & 580346.37 & 148078.36 & HEISPROD & GPS & ONSITE & & 100-F-19 Pipe Trench Shallow Zone \\
\hline 2606 & 9943 & NAD83 & 580340.93 & 148058.8 & HEISPROD & GPS & ONSITE & & 100-F-19 Pipe Trench Shallow Zone \\
\hline 2607 & 9944 & UNKNOWN & 505306 & 145093 & HEISPROD & ESTIMATED & ONSITE & & 100-B-8 \\
\hline 2608 & 9945 & UNKNOWN & 656256 & 145328 & HEISPROD & ESTIMATED & & & 116-B-7 \\
\hline 2609 & 9946 & UNKNOWN & 565322 & 144851 & HEISPROD & ESTIMATED & & & 100-B-8 \\
\hline 2610 & 9947 & UNKNOWN & 565311 & 145190 & HEISPROD & ESTIMATED & & & 100-B-8 \\
\hline 2611 & 9948 & UNKNOWN & 565824 & 144821 & HEISPROD & ESTIMATED & ONSITE & & 100-B-8 \\
\hline 2612 & 9949 & UNKNOWN & 505457 & 145400 & HEISPROD & ESTIMATED & & & 132-B-6 \\
\hline 2613 & 9950 & UNKNOWN & 565268 & 144819 & HEISPROD & ESTIMATED & & & 100-B-8 \\
\hline 2614 & 9951 & UNKNOWN & 565259 & 145329 & HEISPROD & ESTIMATED & & & 116-B-7 \\
\hline 2615 & 9952 & UNKNOWN & 565458 & 145402 & HEISPROD & ESTIMATED & & & 132-B-6 \\
\hline 2616 & 9953 & UNKNOWN & 565458 & 145402 & HEISPROD & ESTIMATED & & & 132-B-6 \\
\hline 2617 & 9954 & UNKNOWN & 565457 & 145400 & HEISPROD & ESTIMATED & & & 132-B-6 \\
\hline 2618 & 9967 & UNKNOWN & 565311 & 145170 & HEISPROD & ESTIMATED & & & 100-B-8 \\
\hline 2619 & 9968 & UNKNOWN & 565311 & 145133 & HEISPROD & ESTIMATED & & & 100-B-8 \\
\hline 2620 & 9970 & UNKNOWN & 565302 & 145261 & HEISPROD & ESTIMATED & & & 100-B-8 \\
\hline 2621 & 9987 & UNKNOWN & 565302 & 145251 & HEISPROD & ESTIMATED & & & 100-B-8 \\
\hline 2622 & 9988 & UNKNOWN & 565302 & 145241 & HEISPROD & ESTIMATED & & & 100-B-8 \\
\hline 2623 & 9989 & UNKNOWN & 565302 & 145231 & HEISPROD & ESTIMATED & & & 100-B-8 \\
\hline
\end{tabular}


A

$\mathrm{B}$

C 1 D

$\mathrm{D} \quad \mathrm{E}$

\begin{tabular}{c|c|c|} 
& G & H \\
\hline
\end{tabular}
\begin{tabular}{|r|rr|r} 
& & \\
\hline 9990 UNKNOWN & 565302 & 145221 HEISPROD & ESTIMATED
\end{tabular} \begin{tabular}{|l|l|l|l|}
\hline 9991 UNKNOWN & 565302 & 145211 HEISPROD & ESTIMATED \\
\hline
\end{tabular}

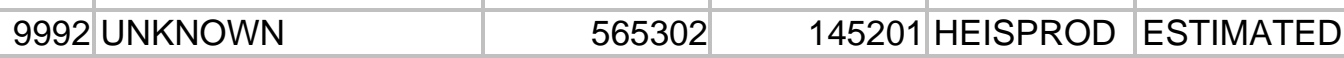

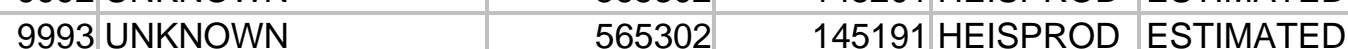

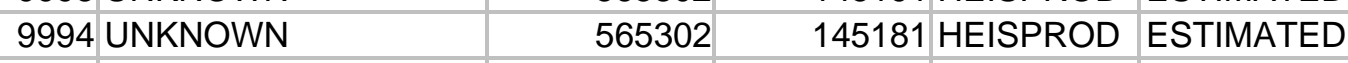
\begin{tabular}{|l|l|l|l|}
\hline 9995 UNKNOWN & 565302 & 145171 HEISPROD & ESTIMATED \\
\hline
\end{tabular} \begin{tabular}{|l|l|l|l}
\hline 9996 UNKNOWN & 565302 & 145161 HEISPROD & ESTIMATED \\
\hline
\end{tabular} \begin{tabular}{|l|l|l|l}
\hline 10001 & $5 N K N O W N$ & 144778 HEISPROD & ESTIMATED \\
\hline
\end{tabular}

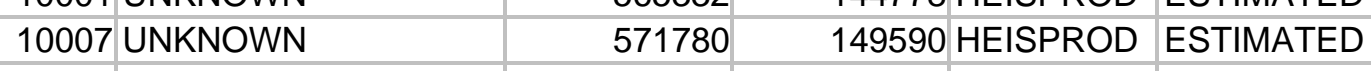
\begin{tabular}{|l|l|l|l}
\hline 10008 & 571592 & 149662 HEISPROD & ESTIMATED
\end{tabular} \begin{tabular}{|l|l|l|l}
\hline 10027 & 565479 & 145249 HEISPROD & ESTIMATED
\end{tabular} \begin{tabular}{|l|l|l|l}
\hline 10028 & 565496 & 145182 HEISPROD & ESTIMATED \\
\hline
\end{tabular} 10029 HEISPROD 10031

10031

10032

10033

10034

10035

10047

10048

10049

10068

10069

10088 UNKNOWN

HEISPROD

HEISPROD

HEISPROD

HEISPROD

HEISPROD

HEISPROD

HEISPROD

HEISPROD

HEISPROD

HEISPROD

10128

10129

10130

10131

10132

10133

10134

10135

10136

10137

10138

10139

10140

\begin{tabular}{|l|}
\hline CENTPLAT \\
\hline CENTPLAT \\
\hline CENTPLAT \\
\hline CENTPLAT \\
\hline CENTPLAT \\
\hline CENTPLAT \\
\hline CENTPLAT \\
\hline CENTPLAT \\
\hline CENTPLAT \\
\hline CENTPLAT \\
\hline CENTPLAT \\
\hline CENTPLAT \\
\hline CENTPLAT \\
\hline
\end{tabular}

ONSITE

ONSITE

ONSITE

ONSITE

100-B-8

100-B-8

100-B-8

100-B-8

100-B-8

100-B-8

100-B-8

$116-\mathrm{N}-3$

$116-\mathrm{N}-3$

$100 \mathrm{~B} / \mathrm{C}$ Pipeline 12 (South)

100 B/C Pipeline 16

$100 \mathrm{~B} / \mathrm{C}$ Pipeline 16

$116-\mathrm{N}-1$

$116-\mathrm{N}-1$

$116-\mathrm{N}-1$

$116-\mathrm{N}-1$

$116-\mathrm{N}-1$

$116-\mathrm{N}-1$

116-F-14 Shallow Zone

$116-\mathrm{N}-3$

$116-\mathrm{N}-3$

$116-\mathrm{N}-3$

$116-\mathrm{N}-3$

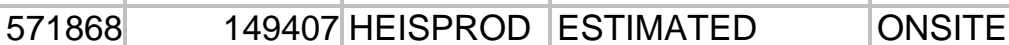

$116-\mathrm{N}-3$

216-A-29

216-A-29

216-A-29

216-A-29

216-A-29

216-A-29

216-A-29

216-A-29

216-A-29

216-A-29

216-A-29

216-A-29

216-A-29 


\begin{tabular}{|c|c|c|c|c|c|c|c|c|c|}
\hline & A & $\mathrm{B}$ & C & $\mathrm{D}$ & $\mathrm{E}$ & $\mathrm{F}$ & $G$ & $\mathrm{H}$ & $\mathrm{I}$ \\
\hline 1 & SAMP_SITE_ID & HORIZ_COORD_TYPE & EW_COORD & NS_COORD & OWNER_ID & COORD_SOURCE & DIST_CLASS & RAD_LEVEL & SAMP_SITE_NAME \\
\hline \begin{tabular}{|l|}
2662 \\
\end{tabular} & 10141 & & & & CENTPLAT & & & & $216-A-29$ \\
\hline 2663 & 10142 & & & & CENTPLAT & & & & 216-B-63 \\
\hline 2664 & 10143 & & & & CENTPLAT & & & & 216-B-63 \\
\hline 2665 & 10144 & & & & CENTPLAT & & & & 216-A-29 \\
\hline 2666 & 10145 & & & & CENTPLAT & & & & $216-A-29$ \\
\hline 2667 & 10146 & & & & CENTPLAT & & & & 216-A-29 \\
\hline 2668 & 10147 & & & & CENTPLAT & & & & 216-B-63 \\
\hline 2669 & 10148 & & & & CENTPLAT & & & & $216-A-29$ \\
\hline 2670 & 10149 & & & & CENTPLAT & & & & 216-A-29 \\
\hline 2671 & 10150 & & & & CENTPLAT & & & & 216-A-29 \\
\hline 2672 & 10151 & & & & CENTPLAT & & & & $216-B-63$ \\
\hline 2673 & 10168 & & & & HEISPROD & & & & 126-F-1 Ash Pit \\
\hline 2674 & 10169 & & & & HEISPROD & & & & 126-F-1 Ash Pit \\
\hline 2675 & 10170 & & & & HEISPROD & & & & 126-F-1 Ash Pit \\
\hline 2676 & 10171 & & & & HEISPROD & & & & 126-F-1 Ash Pit \\
\hline 2677 & 10172 & & & & HEISPROD & & & & 126-F-1 Ash Pit \\
\hline 2678 & 10173 & & & & HEISPROD & & & & 126-F-1 Ash Pit \\
\hline 2679 & 10174 & & & & HEISPROD & & & & 126-F-1 Ash Pit \\
\hline 2680 & 10175 & & & & HEISPROD & & & & 126-F-1 Ash Pit \\
\hline \begin{tabular}{|l|}
2681 \\
\end{tabular} & 10176 & & & & HEISPROD & & & & 126-F-1 Ash Pit \\
\hline 2682 & 10177 & & & & HEISPROD & & & & 126-F-1 Ash Pit \\
\hline 2683 & 10178 & & & & HEISPROD & & & & 116-F-9 \\
\hline 2684 & 10179 & & & & HEISPROD & & & & 100-F-19 \\
\hline 2685 & 10191 & UNKNOWN & 571591 & 149804 & 4 HEISPROD & ESTIMATED & ONSITE & & $116-\mathrm{N}-1$ \\
\hline 2686 & 10192 & UNKNOWN & 571591 & 149804 & 4 HEISPROD & ESTIMATED & ONSITE & & $116-\mathrm{N}-1$ \\
\hline \begin{tabular}{|l|}
2687 \\
\end{tabular} & 10193 & UNKNOWN & 571591 & 149804 & 4 HEISPROD & ESTIMATED & ONSITE & & $116-\mathrm{N}-1$ \\
\hline 2688 & 10194 & UNKNOWN & 571591 & 149804 & 4 HEISPROD & ESTIMATED & ONSITE & & $116-\mathrm{N}-1$ \\
\hline 2689 & 10195 & UNKNOWN & 571591 & 149804 & 4 HEISPROD & ESTIMATED & ONSITE & & $116-\mathrm{N}-1$ \\
\hline 2690 & 10196 & UNKNOWN & 571591 & 149804 & 4 HEISPROD & ESTIMATED & ONSITE & & $116-\mathrm{N}-1$ \\
\hline 2691 & 10197 & UNKNOWN & 571594 & 149840 & HEISPROD & ESTIMATED & ONSITE & & $116-\mathrm{N}-1$ \\
\hline 2692 & 10199 & UNKNOWN & 571594 & 149840 & HEISPROD & ESTIMATED & ONSITE & & $116-\mathrm{N}-1$ \\
\hline 2693 & 10200 & UNKNOWN & 571518 & 149721 & 1 HEISPROD & ESTIMATED & ONSITE & & $116-\mathrm{N}-1$ \\
\hline 2694 & 10201 & UNKNOWN & 571594 & 149840 & HEISPROD & ESTIMATED & ONSITE & & $116-\mathrm{N}-1$ \\
\hline 2695 & 10202 & UNKNOWN & 571594 & 149840 & HEISPROD & ESTIMATED & ONSITE & & $116-\mathrm{N}-1$ \\
\hline 2696 & 10203 & UNKNOWN & 571594 & 149840 & HEISPROD & ESTIMATED & ONSITE & & $116-\mathrm{N}-1$ \\
\hline \begin{tabular}{|l|}
2697 \\
\end{tabular} & 10204 & UNKNOWN & 571594 & 149840 & HEISPROD & ESTIMATED & ONSITE & & $116-\mathrm{N}-1$ \\
\hline 2698 & 10205 & UNKNOWN & 571535 & 149745 & 5 HEISPROD & ESTIMATED & ONSITE & & 116-N-1 \\
\hline \begin{tabular}{|l|}
2699 \\
\end{tabular} & 10206 & UNKNOWN & 571535 & 149745 & 5 HEISPROD & ESTIMATED & ONSITE & & $116-\mathrm{N}-1$ \\
\hline
\end{tabular}




\begin{tabular}{|c|c|c|c|c|c|c|c|c|c|}
\hline & A & $\mathrm{B}$ & C & $\mathrm{D}$ & $\mathrm{E}$ & $\mathrm{F}$ & $G$ & $\mathrm{H}$ & $\mathrm{I}$ \\
\hline 1 & SAMP_SITE_ID & HORIZ_COORD_TYPE & EW_COORD & NS_COORD & OWNER_ID & COORD_SOURCE & DIST_CLASS & RAD_LEVEL & SAMP_SITE_NAME \\
\hline 2700 & 10207 & UNKNOWN & 571535 & 149745 & HEISPROD & ESTIMATED & ONSITE & & $116-\mathrm{N}-1$ \\
\hline 2701 & 10208 & UNKNOWN & 571535 & 149745 & HEISPROD & ESTIMATED & ONSITE & & $116-\mathrm{N}-1$ \\
\hline 2702 & 10209 & UNKNOWN & 571535 & 149745 & HEISPROD & ESTIMATED & ONSITE & & $116-\mathrm{N}-1$ \\
\hline 2703 & 10210 & UNKNOWN & 571518 & 149721 & HEISPROD & ESTIMATED & ONSITE & & $116-\mathrm{N}-1$ \\
\hline 2704 & 10211 & UNKNOWN & 571518 & 149721 & HEISPROD & ESTIMATED & ONSITE & & $116-\mathrm{N}-1$ \\
\hline 2705 & 10212 & UNKNOWN & 571518 & 149721 & HEISPROD & ESTIMATED & ONSITE & & $116-\mathrm{N}-1$ \\
\hline 2706 & 10213 & UNKNOWN & 571518 & 149721 & HEISPROD & ESTIMATED & ONSITE & & $116-\mathrm{N}-1$ \\
\hline 2707 & 10214 & UNKNOWN & 571518 & 149721 & HEISPROD & ESTIMATED & ONSITE & & $116-\mathrm{N}-1$ \\
\hline 2708 & 10215 & & & & HEISPROD & & & & $100 \mathrm{~B} / \mathrm{C}$ \\
\hline 2709 & 10216 & & & & HEISPROD & & & & $100 \mathrm{~B} / \mathrm{C}$ \\
\hline 2710 & 10217 & & & & HEISPROD & & & & $100-B-8$ \\
\hline 2711 & 10218 & UNKNOWN & 565305 & 145237 & HEISPROD & ESTIMATED & ONSITE & & 100-B-8 \\
\hline 2712 & 10220 & UNKNOWN & 571427 & 149709 & HEISPROD & ESTIMATED & ONSITE & & $116-\mathrm{N}-1$ \\
\hline 2713 & 10221 & UNKNOWN & 571427 & 149709 & HEISPROD & ESTIMATED & ONSITE & & $116-\mathrm{N}-1$ \\
\hline 2714 & 10222 & UNKNOWN & 571427 & 149709 & HEISPROD & ESTIMATED & ONSITE & & 116-N-1 \\
\hline 2715 & 10223 & UNKNOWN & 571427 & 149709 & HEISPROD & ESTIMATED & ONSITE & & $116-\mathrm{N}-1$ \\
\hline \begin{tabular}{|l|}
2716 \\
\end{tabular} & 10224 & UNKNOWN & 571427 & 149709 & HEISPROD & ESTIMATED & ONSITE & & $116-\mathrm{N}-1$ \\
\hline \begin{tabular}{|l|}
2717 \\
\end{tabular} & 10225 & UNKNOWN & 571427 & 149709 & HEISPROD & ESTIMATED & ONSITE & & $116-\mathrm{N}-1$ \\
\hline 2718 & 10226 & UNKNOWN & 571427 & 149709 & HEISPROD & ESTIMATED & ONSITE & & $116-\mathrm{N}-1$ \\
\hline \begin{tabular}{|l|}
2719 \\
\end{tabular} & 10227 & UNKNOWN & 571427 & 149709 & HEISPROD & ESTIMATED & ONSITE & & $116-\mathrm{N}-1$ \\
\hline 2720 & 10228 & UNKNOWN & 571427 & 149709 & HEISPROD & ESTIMATED & ONSITE & & 116-N-1 \\
\hline 2721 & 10229 & UNKNOWN & 571427 & 149709 & HEISPROD & ESTIMATED & ONSITE & & 116-N-1 \\
\hline \begin{tabular}{|l|}
2722 \\
\end{tabular} & 10230 & UNKNOWN & 571427 & 149709 & HEISPROD & ESTIMATED & ONSITE & & $116-\mathrm{N}-1$ \\
\hline 2723 & 10231 & UNKNOWN & 571510 & 149783 & HEISPROD & ESTIMATED & ONSITE & & 116-N-1 \\
\hline 2724 & 10232 & UNKNOWN & 571780 & 149590 & HEISPROD & ESTIMATED & ONSITE & & $116-\mathrm{N}-3$ \\
\hline \begin{tabular}{|l|}
2725 \\
\end{tabular} & 10233 & UNKNOWN & 565457 & 145406 & HEISPROD & ESTIMATED & ONSITE & & 100-B-8 \\
\hline 2726 & 10234 & UNKNOWN & 565714 & 145490 & HEISPROD & ESTIMATED & ONSITE & & 100-B-8 \\
\hline \begin{tabular}{|l|}
2727 \\
\end{tabular} & 10235 & UNKNOWN & 565716 & 145490 & HEISPROD & ESTIMATED & ONSITE & & 100-B-8 \\
\hline \begin{tabular}{|l|}
2728 \\
\end{tabular} & 10236 & UNKNOWN & 565305 & 145237 & HEISPROD & ESTIMATED & ONSITE & & 100-B-8 \\
\hline \begin{tabular}{|l|}
2729 \\
\end{tabular} & 10237 & UNKNOWN & 571457 & 149758 & HEISPROD & ESTIMATED & ONSITE & & $116-\mathrm{N}-1$ \\
\hline 2730 & 10238 & UNKNOWN & 571510 & 149783 & HEISPROD & ESTIMATED & ONSITE & & 116-N-1 \\
\hline \begin{tabular}{|l|}
2731 \\
\end{tabular} & 10239 & UNKNOWN & 571510 & 149783 & HEISPROD & ESTIMATED & ONSITE & & $116-\mathrm{N}-1$ \\
\hline \begin{tabular}{|l|}
2732 \\
\end{tabular} & 10240 & UNKNOWN & 571510 & 149783 & HEISPROD & ESTIMATED & ONSITE & & 116-N-1 \\
\hline 2733 & 10241 & UNKNOWN & 571510 & 149783 & HEISPROD & ESTIMATED & ONSITE & & $116-\mathrm{N}-1$ \\
\hline 2734 & 10242 & UNKNOWN & 571510 & 149783 & HEISPROD & ESTIMATED & ONSITE & & $116-\mathrm{N}-1$ \\
\hline 2735 & 10243 & UNKNOWN & 571549 & 149826 & HEISPROD & ESTIMATED & ONSITE & & $116-\mathrm{N}-1$ \\
\hline 2736 & 10244 & UNKNOWN & 571549 & 149826 & HEISPROD & ESTIMATED & ONSITE & & 116-N-1 \\
\hline \begin{tabular}{|l|}
2737 \\
\end{tabular} & 10245 & UNKNOWN & 571549 & 149826 & HEISPROD & ESTIMATED & ONSITE & & $116-\mathrm{N}-1$ \\
\hline
\end{tabular}




\begin{tabular}{|c|c|c|c|c|c|c|c|c|c|}
\hline & $A$ & $\mathrm{~B}$ & $\mathrm{C}$ & $\mathrm{D}$ & $E$ & $\mathrm{~F}$ & $\mathrm{G}$ & $\mathrm{H}$ & $\mathrm{I}$ \\
\hline 1 & SAMP_SITE_ID & HORIZ_COORD_TYPE & EW_COORD & NS_COORD & OWNER_ID & COORD_SOURCE & DIST_CLASS & RAD_LEVEL & SAMP_SITE_NAME \\
\hline 2738 & 10246 & UNKNOWN & 571549 & 149826 & HEISPROD & ESTIMATED & ONSITE & & $116-\mathrm{N}-1$ \\
\hline \begin{tabular}{|l|}
2739 \\
\end{tabular} & 10247 & UNKNOWN & 571549 & 149826 & HEISPROD & ESTIMATED & ONSITE & & $116-\mathrm{N}-1$ \\
\hline 2740 & 10248 & UNKNOWN & 571549 & 149826 & HEISPROD & ESTIMATED & ONSITE & & $116-\mathrm{N}-1$ \\
\hline 2741 & 10249 & & & & HEISPROD & & & & $116-\mathrm{N}-3$ \\
\hline 2742 & 10250 & NAD83(91) & 565450.155 & 145433.001 & HEISPROD & GPS & ONSITE & & 132-B-6 \\
\hline \begin{tabular}{|l|}
2743 \\
\end{tabular} & 10251 & UNKNOWN & 571868 & 149407 & HEISPROD & ESTIMATED & ONSITE & & $116-\mathrm{N}-3$ \\
\hline 2744 & 10252 & NAD83(91) & 565446.546 & 145432.604 & HEISPROD & GPS & ONSITE & & 132-B-6 \\
\hline 2745 & 10253 & NAD83(91) & 565448.88 & 145428.587 & HEISPROD & GPS & ONSITE & & 132-B-6 \\
\hline \begin{tabular}{|l|}
2746 \\
\end{tabular} & 10254 & NAD83(91) & 565458.078 & 145427.256 & HEISPROD & GPS & ONSITE & & 132-B-6 \\
\hline \begin{tabular}{|l|}
2747 \\
\end{tabular} & 10255 & NAD83(91) & 565460.123 & 145423.787 & HEISPROD & GPS & ONSITE & & 132-B-6 \\
\hline 2748 & 10256 & NAD83(91) & 565453.724 & 145421.115 & HEISPROD & GPS & ONSITE & & 132-B-6 \\
\hline 2749 & 10257 & NAD83(91) & 565447.627 & 145410.679 & HEISPROD & GPS & ONSITE & & 132-B-6 \\
\hline 2750 & 10258 & NAD83(91) & 565449.493 & 145407.581 & HEISPROD & GPS & ONSITE & & 132-B-6 \\
\hline \begin{tabular}{|l|}
2751 \\
\end{tabular} & 10259 & NAD83(91) & 565451.343 & 145404.23 & HEISPROD & GPS & ONSITE & & 132-B-6 \\
\hline \begin{tabular}{|l|}
2752 \\
\end{tabular} & 10260 & NAD83(91) & 565456.106 & 145412.21 & HEISPROD & GPS & ONSITE & & 132-B-6 \\
\hline 2753 & 10261 & NAD83(91) & 565456.804 & 145418.565 & HEISPROD & GPS & ONSITE & & 132-B-6 \\
\hline 2754 & 10262 & NAD83(91) & 565462.449 & 145405.401 & HEISPROD & GPS & ONSITE & & 132-B-6 \\
\hline 2755 & 10263 & NAD83(91) & 565463.77 & 145409.359 & HEISPROD & GPS & ONSITE & & 132-B-6 \\
\hline \begin{tabular}{|l|}
2756 \\
\end{tabular} & 10264 & NAD83(91) & 565464.771 & 145415.371 & HEISPROD & GPS & ONSITE & & 132-B-6 \\
\hline \begin{tabular}{|l|}
2757 \\
\end{tabular} & 10265 & NAD83(91) & 565466.383 & 145410.282 & HEISPROD & GPS & ONSITE & & 132-B-6 \\
\hline \begin{tabular}{|l|}
2758 \\
\end{tabular} & 10266 & NAD83(91) & 565467.336 & 145416.341 & HEISPROD & GPS & ONSITE & & 132-B-6 \\
\hline 2759 & 10267 & NAD83(91) & 565470.016 & 145421.543 & HEISPROD & GPS & ONSITE & & 132-B-6 \\
\hline 2760 & 10268 & NAD83(91) & 565469.805 & 145416.496 & HEISPROD & GPS & ONSITE & & 132-B-6 \\
\hline \begin{tabular}{|l|}
2761 \\
\end{tabular} & 10269 & NAD83(91) & 565457.037 & 145394.017 & HEISPROD & GPS & ONSITE & & 132-B-6 \\
\hline 2762 & 10270 & NAD83(91) & 565461.401 & 145397.49 & HEISPROD & GPS & ONSITE & & 132-B-6 \\
\hline 2763 & 10271 & NAD83(91) & 565463.262 & 145391.435 & HEISPROD & GPS & ONSITE & & 132-B-6 \\
\hline \begin{tabular}{|l|}
2764 \\
\end{tabular} & 10272 & NAD83(91) & 565465.923 & 145398.575 & HEISPROD & GPS & ONSITE & & 132-B-6 \\
\hline \begin{tabular}{|l|}
2765 \\
\end{tabular} & 10273 & NAD83(91) & 565470.509 & 145400.225 & HEISPROD & GPS & ONSITE & & 132-B-6 \\
\hline \begin{tabular}{|l|}
2766 \\
\end{tabular} & 10274 & NAD83(91) & 565473.188 & 145401.949 & HEISPROD & GPS & ONSITE & & 132-B-6 \\
\hline \begin{tabular}{|l|}
2767 \\
\end{tabular} & 10275 & NAD83(91) & 565697.23 & 145481.926 & HEISPROD & GPS & ONSITE & & 132-C-2 \\
\hline 2768 & 10276 & NAD83(91) & 565697.023 & 145478.471 & HEISPROD & GPS & ONSITE & & $132-C-2$ \\
\hline 2769 & 10277 & NAD83(91) & 565701.968 & 145480.335 & HEISPROD & GPS & ONSITE & & 132-C-2 \\
\hline 2770 & 10278 & NAD83(91) & 565702.723 & 145489.877 & HEISPROD & GPS & ONSITE & & 132-C-2 \\
\hline \begin{tabular}{|l|}
2771 \\
\end{tabular} & 10279 & NAD83(91) & 565707.932 & 145486.302 & HEISPROD & GPS & ONSITE & & $132-C-2$ \\
\hline 2772 & 10280 & NAD83(91) & 565711.158 & 145491.754 & HEISPROD & GPS & ONSITE & & $132-C-2$ \\
\hline 2773 & 10281 & NAD83(91) & 565718.826 & 145495.139 & HEISPROD & GPS & ONSITE & & $132-C-2$ \\
\hline \begin{tabular}{|l|}
2774 \\
\end{tabular} & 10282 & NAD83(91) & 565721.978 & 145495.562 & HEISPROD & GPS & ONSITE & & $132-C-2$ \\
\hline 2775 & 10283 & NAD83(91) & 565718.939 & 145490.394 & HEISPROD & GPS & ONSITE & & $132-C-2$ \\
\hline
\end{tabular}




\begin{tabular}{|c|c|c|c|c|c|c|c|c|c|}
\hline & $A$ & $\mathrm{~B}$ & $\mathrm{C}$ & $\mathrm{D}$ & $E$ & $\mathrm{~F}$ & $\mathrm{G}$ & $\mathrm{H}$ & $\mathrm{I}$ \\
\hline 1 & SAMP_SITE_ID & HORIZ_COORD_TYPE & EW_COORD & NS_COORD & OWNER_ID & COORD_SOURCE & DIST_CLASS & RAD_LEVEL & SAMP_SITE_NAME \\
\hline 2776 & 10284 & NAD83(91) & 565724.799 & 145490.562 & HEISPROD & GPS & ONSITE & & $132-C-2$ \\
\hline \begin{tabular}{|l|}
2777 \\
\end{tabular} & 10285 & NAD83(91) & 565738.541 & 145493.773 & HEISPROD & GPS & ONSITE & & $132-C-2$ \\
\hline 2778 & 10286 & NAD83(91) & 565734.813 & 145488.815 & HEISPROD & GPS & ONSITE & & $132-C-2$ \\
\hline \begin{tabular}{|l|}
2779 \\
\end{tabular} & 10287 & NAD83(91) & 565734.114 & 145482.913 & HEISPROD & GPS & ONSITE & & $132-C-2$ \\
\hline 2780 & 10288 & NAD83(91) & 565741.659 & 145483.924 & HEISPROD & GPS & ONSITE & & $132-C-2$ \\
\hline \begin{tabular}{|l|}
2781 \\
\end{tabular} & 10289 & NAD83(91) & 565746.84 & 145475.543 & HEISPROD & GPS & ONSITE & & $132-C-2$ \\
\hline \begin{tabular}{|l|}
2782 \\
\end{tabular} & 10290 & NAD83(91) & 565742.115 & 145489.768 & HEISPROD & GPS & ONSITE & & $132-C-2$ \\
\hline \begin{tabular}{|l|}
2783 \\
\end{tabular} & 10291 & NAD83(91) & 565746.482 & 145481.701 & HEISPROD & GPS & ONSITE & & $132-C-2$ \\
\hline \begin{tabular}{|l|}
2784 \\
\end{tabular} & 10292 & NAD83(91) & 565748.501 & 145476.346 & HEISPROD & GPS & ONSITE & & $132-C-2$ \\
\hline \begin{tabular}{|l|}
2785 \\
\end{tabular} & 10293 & NAD83(91) & 565262.509 & 145313.767 & HEISPROD & ESTIMATED & ONSITE & & 116-B-7 \\
\hline \begin{tabular}{|l|}
2786 \\
\end{tabular} & 10294 & NAD83(91) & 565259.004 & 145308.106 & HEISPROD & ESTIMATED & ONSITE & & 116-B-7 \\
\hline 2787 & 10295 & NAD83(91) & 565260.635 & 145303.331 & HEISPROD & ESTIMATED & ONSITE & & 116-B-7 \\
\hline 2788 & 10296 & NAD83(91) & 565263.554 & 145303.719 & HEISPROD & ESTIMATED & ONSITE & & 116-B-7 \\
\hline \begin{tabular}{|l|}
2789 \\
\end{tabular} & 10297 & NAD83(91) & 565263.88 & 145299.655 & HEISPROD & ESTIMATED & ONSITE & & 116-B-7 \\
\hline 2790 & 10298 & NAD83(91) & 565428.184 & 145423.102 & HEISPROD & ESTIMATED & ONSITE & & 132-B-6 \\
\hline \begin{tabular}{|l|}
2791 \\
\end{tabular} & 10299 & NAD83(91) & 565429.448 & 145420.393 & HEISPROD & ESTIMATED & ONSITE & & 132-B-6 \\
\hline 2792 & 10300 & NAD83(91) & 565430.782 & 145417.57 & HEISPROD & ESTIMATED & ONSITE & & 132-B-6 \\
\hline 2793 & 10301 & NAD83(91) & 565436.623 & 145410.769 & HEISPROD & ESTIMATED & ONSITE & & 132-B-6 \\
\hline 2794 & 10302 & NAD83(91) & 565433.208 & 145423.243 & HEISPROD & ESTIMATED & ONSITE & & 132-B-6 \\
\hline 2795 & 10303 & NAD83(91) & 565440.739 & 145396.257 & HEISPROD & ESTIMATED & ONSITE & & 132-B-6 \\
\hline \begin{tabular}{|l|}
2796 \\
\end{tabular} & 10304 & NAD83(91) & 565443.044 & 145394.476 & HEISPROD & ESTIMATED & ONSITE & & 132-B-6 \\
\hline \begin{tabular}{|l|}
2797 \\
\end{tabular} & 10305 & NAD83(91) & 565446.197 & 145391.628 & HEISPROD & ESTIMATED & ONSITE & & 132-B-6 \\
\hline \begin{tabular}{|l|}
2798 \\
\end{tabular} & 10306 & NAD83(91) & 565449.881 & 145391.622 & HEISPROD & ESTIMATED & ONSITE & & 132-B-6 \\
\hline \begin{tabular}{|l|}
2799 \\
\end{tabular} & 10307 & NAD83(91) & 565451.406 & 145393.776 & HEISPROD & ESTIMATED & ONSITE & & 132-B-6 \\
\hline 2800 & 10308 & NAD83(91) & 565444.781 & 145408.133 & HEISPROD & ESTIMATED & ONSITE & & 132-B-6 \\
\hline 2801 & 10309 & NAD83(91) & 565439.446 & 145420.849 & HEISPROD & ESTIMATED & ONSITE & & 132-B-6 \\
\hline \begin{tabular}{|l|}
2802 \\
\end{tabular} & 10310 & NAD83(91) & 565442.491 & 145416.269 & HEISPROD & ESTIMATED & ONSITE & & 132-B-6 \\
\hline 2803 & 10311 & NAD83(91) & 565437.294 & 145429.354 & HEISPROD & ESTIMATED & ONSITE & & 132-B-6 \\
\hline 2804 & 10312 & NAD83(91) & 565442.851 & 145426.642 & HEISPROD & ESTIMATED & ONSITE & & 132-B-6 \\
\hline 2805 & 10313 & NAD83(91) & 565445.756 & 145427.732 & HEISPROD & ESTIMATED & ONSITE & & 132-B-6 \\
\hline 2806 & 10314 & NAD83(91) & 565450.688 & 145419.711 & HEISPROD & ESTIMATED & ONSITE & & 132-B-6 \\
\hline \begin{tabular}{|l|}
2807 \\
\end{tabular} & 10315 & NAD83(91) & 565268.895 & 145338.906 & HEISPROD & ESTIMATED & ONSITE & & 116-B-7 \\
\hline 2808 & 10316 & NAD83(91) & 565263.88 & 145299.655 & HEISPROD & ESTIMATED & ONSITE & & 116-B-7 \\
\hline 2809 & 10317 & NAD83(91) & 565267.044 & 145336.674 & HEISPROD & ESTIMATED & ONSITE & & 116-B-7 \\
\hline 2810 & 10318 & NAD83(91) & 565263.404 & 145333.625 & HEISPROD & ESTIMATED & ONSITE & & 116-B-7 \\
\hline 2811 & 10319 & NAD83(91) & 565257.981 & 145341.251 & HEISPROD & ESTIMATED & ONSITE & & 116-B-7 \\
\hline \begin{tabular}{|l|}
2812 \\
\end{tabular} & 10320 & NAD83(91) & 565252.479 & 145343.709 & HEISPROD & ESTIMATED & ONSITE & & 116-B-7 \\
\hline 2813 & 10321 & NAD83(91) & 565253.745 & 145334.246 & HEISPROD & ESTIMATED & ONSITE & & 116-B-7 \\
\hline
\end{tabular}




\begin{tabular}{|c|c|c|c|c|c|c|c|c|c|}
\hline & $\bar{A}$ & $\mathrm{~B}$ & $\mathrm{C}$ & $\mathrm{D}$ & $E$ & $\mathrm{~F}$ & G & $\mathrm{H}$ & $\mathrm{I}$ \\
\hline 1 & SAMP_SITE_ID & HORIZ_COORD_TYPE & EW_COORD & NS_COORD & OWNER_ID & COORD_SOURCE & DIST_CLASS & RAD_LEVEL & SAMP_SITE_NAME \\
\hline 2814 & 10322 & NAD83(91) & 565248.419 & 145338.216 & HEISPROD & ESTIMATED & ONSITE & & $116-B-7$ \\
\hline 2815 & 10323 & NAD83(91) & 565242.369 & 145338.19 & HEISPROD & ESTIMATED & ONSITE & & 116-B-7 \\
\hline 2816 & 10324 & NAD83(91) & 565244.2 & 145336.375 & HEISPROD & ESTIMATED & ONSITE & & 116-B-7 \\
\hline 2817 & 10325 & NAD83(91) & 565239.24 & 145333.826 & HEISPROD & ESTIMATED & ONSITE & & 116-B-7 \\
\hline 2818 & 10326 & NAD83(91) & 565239.824 & 145328.911 & HEISPROD & ESTIMATED & ONSITE & & 116-B-7 \\
\hline 2819 & 10327 & NAD83(91) & 565243.255 & 145328.443 & HEISPROD & ESTIMATED & ONSITE & & 116-B-7 \\
\hline 2820 & 10328 & NAD83(91) & 565235.992 & 145324.453 & HEISPROD & ESTIMATED & ONSITE & & 116-B-7 \\
\hline 2821 & 10329 & NAD83(91) & 565238.867 & 145324.664 & HEISPROD & ESTIMATED & ONSITE & & 116-B-7 \\
\hline 2822 & 10330 & NAD83(91) & 565247.179 & 145324.776 & HEISPROD & ESTIMATED & ONSITE & & 116-B-7 \\
\hline 2823 & 10331 & NAD83(91) & 565237.413 & 145320.486 & HEISPROD & ESTIMATED & ONSITE & & 116-B-7 \\
\hline 2824 & 10332 & NAD83(91) & 565239.188 & 145316.815 & HEISPROD & ESTIMATED & ONSITE & & 116-B-7 \\
\hline 2825 & 10333 & NAD83(91) & 565247.385 & 145318.261 & HEISPROD & ESTIMATED & ONSITE & & 116-B-7 \\
\hline 2826 & 10334 & NAD83(91) & 565247.248 & 145305.624 & HEISPROD & ESTIMATED & ONSITE & & $116-B-7$ \\
\hline 2827 & 10335 & NAD83(91) & 565248.644 & 145302.13 & HEISPROD & ESTIMATED & ONSITE & & 116-B-7 \\
\hline 2828 & 10336 & NAD83(91) & 565251.707 & 145307.011 & HEISPROD & ESTIMATED & ONSITE & & 116-B-7 \\
\hline 2829 & 10337 & NAD83(91) & 565250.632 & 145298.138 & HEISPROD & ESTIMATED & ONSITE & & 116-B-7 \\
\hline 2830 & 10338 & NAD83(91) & 565258.634 & 145301.114 & HEISPROD & ESTIMATED & ONSITE & & 116-B-7 \\
\hline 2831 & 10339 & NAD83(91) & 565253.685 & 145295.021 & HEISPROD & ESTIMATED & ONSITE & & 116-B-7 \\
\hline 2832 & 10340 & NAD83(91) & 565264.267 & 145331.521 & HEISPROD & ESTIMATED & ONSITE & & 116-B-7 \\
\hline 2833 & 10341 & NAD83(91) & 565268.494 & 145332.584 & HEISPROD & ESTIMATED & ONSITE & & 116-B-7 \\
\hline 2834 & 10342 & NAD83(91) & 565265.154 & 145327.732 & HEISPROD & ESTIMATED & ONSITE & & 116-B-7 \\
\hline 2835 & 10343 & NAD83(91) & 565271.586 & 145326.658 & HEISPROD & ESTIMATED & ONSITE & & 116-B-7 \\
\hline 2836 & 10344 & NAD83(91) & 565278.733 & 145335.613 & HEISPROD & ESTIMATED & ONSITE & & 116-B-7 \\
\hline 2837 & 10345 & NAD83(91) & 565279.191 & 145325.624 & HEISPROD & ESTIMATED & ONSITE & & 116-B-7 \\
\hline 2838 & 10346 & NAD83(91) & 565263.388 & 145326.89 & HEISPROD & ESTIMATED & ONSITE & & 116-B-7 \\
\hline 2839 & 10347 & NAD83(91) & 565266.324 & 145315.673 & HEISPROD & ESTIMATED & ONSITE & & $116-B-7$ \\
\hline 2840 & 10353 & & & & HEISPROD & & & & 116-F-14 Test Pit \\
\hline 2841 & 10354 & & & & HEISPROD & & & & 116-F-14 Test Pit \\
\hline 2842 & 10355 & UNKNOWN & 565671 & 145272 & HEISPROD & ESTIMATED & ONSITE & & $100-C-6$ \\
\hline 2843 & 10373 & UNKNOWN & 565674 & 145280 & HEISPROD & ESTIMATED & ONSITE & & $100-C-6$ \\
\hline 2844 & 10374 & UNKNOWN & 565693 & 145271 & HEISPROD & ESTIMATED & ONSITE & & $100-C-6$ \\
\hline 2845 & 10375 & UNKNOWN & 565714 & 145262 & HEISPROD & ESTIMATED & ONSITE & & $100-C-6$ \\
\hline 2846 & 10376 & UNKNOWN & 565794 & 145368 & HEISPROD & ESTIMATED & ONSITE & & $100-C-6$ \\
\hline 2847 & 10377 & UNKNOWN & 565312 & 145250 & HEISPROD & ESTIMATED & ONSITE & & 100-B-8 \\
\hline 2848 & 10378 & UNKNOWN & 565313 & 145177 & HEISPROD & ESTIMATED & ONSITE & & $100-B-8$ \\
\hline 2849 & 10393 & UNKNOWN & 571535 & 149745 & HEISPROD & ESTIMATED & ONSITE & & $116-\mathrm{N}-1$ \\
\hline 2850 & 10396 & UNKNOWN & 571576 & 149628 & HEISPROD & ESTIMATED & & & $116-N-3$ \\
\hline 2851 & 10397 & UNKNOWN & 571656 & 149949 & HEISPROD & ESTIMATED & ONSITE & & $116-\mathrm{N}-1$ \\
\hline
\end{tabular}




\begin{tabular}{|c|c|c|c|c|c|c|c|c|c|}
\hline & A & $\mathrm{B}$ & $\mathrm{C}$ & $\mathrm{D}$ & $E$ & $\mathrm{~F}$ & G & $\mathrm{H}$ & $\mathrm{I}$ \\
\hline 1 & SAMP_SITE_ID & HORIZ_COORD_TYPE & EW_COORD & NS_COORD & OWNER_ID & COORD_SOURCE & DIST_CLASS & RAD_LEVEL & SAMP_SITE_NAME \\
\hline \begin{tabular}{|l|}
2852 \\
\end{tabular} & 10398 & UNKNOWN & 571743 & 150030 & HEISPROD & ESTIMATED & ONSITE & & $116-\mathrm{N}-1$ \\
\hline \begin{tabular}{|l|}
2853 \\
\end{tabular} & 10399 & & & & HEISPROD & & & & 116-F-14 Test Pit \\
\hline 2854 & 10401 & UNKNOWN & 571728 & 150055 & HEISPROD & ESTIMATED & ONSITE & & $116-\mathrm{N}-1$ \\
\hline 2855 & 10402 & UNKNOWN & 571728 & 150055 & HEISPROD & ESTIMATED & ONSITE & & $116-\mathrm{N}-1$ \\
\hline \begin{tabular}{|l|}
2856 \\
\end{tabular} & 10403 & UNKNOWN & 571728 & 150055 & HEISPROD & ESTIMATED & ONSITE & & $116-\mathrm{N}-1$ \\
\hline \begin{tabular}{|l|}
2857 \\
\end{tabular} & 10405 & UNKNOWN & 571652 & 149918.5 & HEISPROD & ESTIMATED & & & $116-\mathrm{N}-1$ \\
\hline \begin{tabular}{|l|}
2858 \\
\end{tabular} & 10406 & UNKNOWN & 571652 & 149918.5 & HEISPROD & ESTIMATED & ONSITE & & $116-\mathrm{N}-1$ \\
\hline 2859 & 10407 & UNKNOWN & 571652 & 149918.5 & HEISPROD & ESTIMATED & ONSITE & & $116-\mathrm{N}-1$ \\
\hline 2860 & 10408 & & & & HEISPROD & & & & 116-F-14 Test Pit \\
\hline 2861 & 10409 & & & & HEISPROD & & & & 116-F-14 Test Pit \\
\hline 2862 & 10410 & & & & HEISPROD & & & & 116-F-14 Test Pit \\
\hline 2863 & 10411 & & & & HEISPROD & & & & 116-F-14 Test Pit \\
\hline 2864 & 10412 & & & & HEISPROD & & & & 116-F-14 Test Pit \\
\hline 2865 & 10414 & & & & HEISPROD & & & & \\
\hline \begin{tabular}{|l|}
2866 \\
\end{tabular} & 10415 & UNKNOWN & 571576 & 149628 & HEISPROD & ESTIMATED & & & $116-\mathrm{N}-3$ \\
\hline \begin{tabular}{|l|}
2867 \\
\end{tabular} & 10416 & UNKNOWN & 541654 & 149949 & HEISPROD & ESTIMATED & ONSITE & & $116-\mathrm{N}-1$ \\
\hline 2868 & 10417 & UNKNOWN & 541654 & 149949 & HEISPROD & ESTIMATED & & & $116-\mathrm{N}-1$ \\
\hline 2869 & 10418 & UNKNOWN & 541654 & 149949 & HEISPROD & ESTIMATED & & & $116-\mathrm{N}-1$ \\
\hline 2870 & 10419 & UNKNOWN & 541654 & 149949 & HEISPROD & ESTIMATED & & & $116-\mathrm{N}-1$ \\
\hline 2871 & 10420 & UNKNOWN & 571743 & 150030 & HEISPROD & ESTIMATED & & & $116-\mathrm{N}-1$ \\
\hline 2872 & 10421 & UNKNOWN & 571743 & 150030 & HEISPROD & ESTIMATED & & & $116-\mathrm{N}-1$ \\
\hline 2873 & 8973 & NAD83(91) & 581219.766 & 147917.749 & HEISPROD & GPS & & & UPR-100-F-2 \\
\hline 2874 & 8974 & NAD83(91) & 581175.423 & 147917.901 & HEISPROD & GPS & & & UPR-100-F-2 \\
\hline 2875 & 8975 & NAD83(91) & 581145.118 & 147942.116 & HEISPROD & GPS & & & UPR-100-F-2 \\
\hline 2876 & 8976 & NAD83(91) & 581106.47 & 147967.427 & HEISPROD & GPS & & & UPR-100-F-2 \\
\hline 2877 & 9046 & & & & HEISPROD & & & & 100-B-1 \\
\hline 2878 & 9048 & & & & HEISPROD & & & & $100-D-49: 1$ \\
\hline 2879 & 9066 & & & & HEISPROD & & & & Gravel Pit \#21 \\
\hline 2880 & 9067 & & & & HEISPROD & & & & \\
\hline 2881 & 9086 & NAD83 & 573629.71 & 152347.79 & HEISPROD & SURVEYED & & & North Pipelines from 116-D-7 to the Outfalls \\
\hline 2882 & 9106 & & & & SESPMNT & & ONSITE & & 100-B SPRING 37-1 \\
\hline 2883 & 9110 & & & & SESPMNT & & ONSITE & LOW & 100-K SPRING 82-2 \\
\hline 2884 & 9131 & UNKNOWN & 565269 & 144852 & HEISPROD & ESTIMATED & ONSITE & & $100-B-8$ \\
\hline 2885 & 9132 & UNKNOWN & 565320 & 144980 & HEISPROD & ESTIMATED & ONSITE & & $100-B-8$ \\
\hline 2886 & 9147 & & & & SESPMNT & & OFFSITE & & RICHLAND SPR HRM44.4 \\
\hline 2887 & 9170 & UNKNOWN & 572016 & 149652 & HEISPROD & ESTIMATED & ONSITE & & $116-N-3$ \\
\hline 2888 & 9190 & & & & SESPMNT & & ONSITE & & HRM 36 SHORELINE \\
\hline 2889 & 9191 & & & & SESPMNT & & ONSITE & & HRM 28 SHORELINE \\
\hline
\end{tabular}




\begin{tabular}{|c|c|c|c|c|c|c|c|c|c|}
\hline & $A$ & $\mathrm{~B}$ & $\mathrm{C}$ & $\mathrm{D}$ & $E$ & $\mathrm{~F}$ & $\mathrm{G}$ & $\mathrm{H}$ & $\mathrm{I}$ \\
\hline 1 & SAMP_SITE_ID & HORIZ_COORD_TYPE & EW_COORD & NS_COORD & OWNER_ID & COORD_SOURCE & DIST_CLASS & RAD_LEVEL & SAMP_SITE_NAME \\
\hline 2890 & 9210 & & & & SESPMNT & & ONSITE & & 300 SPR $7-1$ \\
\hline 2891 & 9211 & & & & SESPMNT & & ONSITE & & 300 SPR $7-2$ \\
\hline 2892 & 9213 & & & & SESPMNT & & ONSITE & & 300 SPR $7-3$ \\
\hline 2893 & 9214 & & & & SESPMNT & & ONSITE & & 300 SPR DR $7-1$ \\
\hline 2894 & 9215 & & & & SESPMNT & & ONSITE & & 300 SPR DR 7 -2 \\
\hline 2895 & 9216 & & & & SESPMNT & & ONSITE & & 300 SPR DR $7-3$ \\
\hline 2896 & 9217 & & & & SESPMNT & & ONSITE & & 300 SPR 7 THRU SPR 9 \\
\hline 2897 & 9218 & & & & SESPMNT & & ONSITE & & 300 SPR 9 -1 \\
\hline 2898 & 9219 & & & & SESPMNT & & ONSITE & & 300 SPR $9-2$ \\
\hline 2899 & 9220 & & & & SESPMNT & & ONSITE & & 300 SPR $9-3$ \\
\hline 2900 & 9221 & & & & SESPMNT & & ONSITE & & 300 SPR DR $9-1$ \\
\hline 2901 & 9222 & & & & SESPMNT & & ONSITE & & 300 SPR DR $9-2$ \\
\hline 2902 & 9223 & & & & SESPMNT & & ONSITE & & 300 SPR DR $9-3$ \\
\hline 2903 & 9224 & & & & SESPMNT & & ONSITE & & 300 SPR 9 THRU SPR 11 \\
\hline 2904 & 9225 & & & & SESPMNT & & ONSITE & & 300 SPR 11 \\
\hline 2905 & 9226 & & & & SESPMNT & & ONSITE & & 300 SPR $11-1$ \\
\hline 2906 & 9227 & & & & SESPMNT & & ONSITE & & 300 SPR $11-2$ \\
\hline 2907 & 9228 & & & & SESPMNT & & ONSITE & & 300 SPR $11-3$ \\
\hline 2908 & 9229 & & & & SESPMNT & & ONSITE & & 300 SPR DR $11-1$ \\
\hline 2909 & 9230 & & & & SESPMNT & & ONSITE & & 300 SPR DR $11-2$ \\
\hline 2910 & 9231 & & & & SESPMNT & & ONSITE & & 300 SPR DR $11-3$ \\
\hline 2911 & 9232 & & & & SESPMNT & & ONSITE & & 300 SPR 14 \\
\hline 2912 & 9233 & & & & SESPMNT & & ONSITE & & 300 SPR $14-1$ \\
\hline 2913 & 9234 & & & & SESPMNT & & ONSITE & & 300 SPR $14-2$ \\
\hline 2914 & 9235 & & & & SESPMNT & & ONSITE & & 300 SPR $14-3$ \\
\hline 2915 & 9236 & & & & SESPMNT & & ONSITE & & VERNITA BRIDGE -1 \\
\hline 2916 & 9237 & & & & SESPMNT & & ONSITE & & VERNITA BRIDGE -2 \\
\hline 2917 & 9238 & & & & SESPMNT & & ONSITE & & VERNITA BRIDGE -3 \\
\hline 2918 & 9250 & UNKNOWN & 565457 & 145417 & HEISPROD & ESTIMATED & ONSITE & & 132-B-6 Outfall \\
\hline 2919 & 9251 & UNKNOWN & 565457 & 145409 & HEISPROD & ESTIMATED & ONSITE & & 132-B-6 Outfall \\
\hline 2920 & 9252 & UNKNOWN & 565726 & 145475 & HEISPROD & ESTIMATED & ONSITE & & $132-C-2$ \\
\hline 2921 & 9253 & UNKNOWN & 565720 & 145482 & HEISPROD & ESTIMATED & ONSITE & & 132-C-2 \\
\hline 2922 & 9254 & UNKNOWN & 565441 & 145418 & HEISPROD & ESTIMATED & ONSITE & & 132-B-6 \\
\hline 2923 & 9255 & UNKNOWN & 565446 & 145419 & HEISPROD & ESTIMATED & ONSITE & & 132-B-6 \\
\hline 2924 & 9256 & UNKNOWN & 565457 & 145423 & HEISPROD & ESTIMATED & ONSITE & & 132-B-6 \\
\hline 2925 & 9257 & & & & HEISPROD & & & & UPR-100-F-2 \\
\hline 2926 & 9258 & & & & HEISPROD & & & & UPR-100-F-2 \\
\hline 2927 & 9259 & & & & HEISPROD & & & & UPR-100-F-2 \\
\hline
\end{tabular}

Page 77 


\begin{tabular}{|c|c|c|c|c|c|c|c|c|c|}
\hline & A & $\mathrm{B}$ & $\mathrm{C}$ & $\mathrm{D}$ & $E$ & $\mathrm{~F}$ & $G$ & $\mathrm{H}$ & $\mathrm{I}$ \\
\hline 1 & SAMP_SITE_ID & HORIZ_COORD_TYPE & EW_COORD & NS_COORD & OWNER_ID & COORD_SOURCE & DIST_CLASS & RAD_LEVEL & SAMP_SITE_NAME \\
\hline 2928 & 9260 & & & & HEISPROD & & & & UPR-100-F-2 \\
\hline 2929 & 9261 & & & & HEISPROD & & & & UPR-100-F-2 \\
\hline 2930 & 9262 & & & & HEISPROD & & & & UPR-100-F-2 \\
\hline 2931 & 9263 & & & & HEISPROD & & & & UPR-100-F-2 \\
\hline 2932 & 9264 & & & & HEISPROD & & & & UPR-100-F-2 \\
\hline 2933 & 9265 & & & & HEISPROD & & & & UPR-100-F-2 \\
\hline 2934 & 9266 & & & & HEISPROD & & & & UPR-100-F-2 \\
\hline 2935 & 9267 & & & & HEISPROD & & & & UPR-100-F-2 \\
\hline 2936 & 9268 & & & & HEISPROD & & & & UPR-100-F-2 \\
\hline \begin{tabular}{|l|}
2937 \\
\end{tabular} & 9269 & & & & HEISPROD & & & & UPR-100-F-2 \\
\hline 2938 & 9270 & & & & HEISPROD & & & & UPR-100-F-2 \\
\hline 2939 & 9271 & & & & HEISPROD & & & & 116-F-14 Retention Basin \\
\hline 2940 & 9272 & & & & HEISPROD & & & & 116-F-14 Retention Basin \\
\hline 2941 & 9273 & & & & SESPMNT & & ONSITE & & 300 SPR $7-4$ \\
\hline 2942 & 9274 & & & & SESPMNT & & ONSITE & & 300 SPR DR 7 -4 \\
\hline 2943 & 9275 & & & & SESPMNT & & ONSITE & & 300 SPR 9 -4 \\
\hline 2944 & 9276 & & & & SESPMNT & & ONSITE & & 300 SPR DR $9-4$ \\
\hline 2945 & 9277 & & & & SESPMNT & & ONSITE & & 300 SPR $11-4$ \\
\hline \begin{tabular}{|l|}
2946 \\
\end{tabular} & 9278 & & & & SESPMNT & & ONSITE & & 300 SPR DR $11-4$ \\
\hline \begin{tabular}{|l|}
2947 \\
\end{tabular} & 9279 & & & & SESPMNT & & ONSITE & & 300 SPR $14-4$ \\
\hline 2948 & 9280 & & & & SESPMNT & & ONSITE & & VERNITA BRIDGE -4 \\
\hline 2949 & 9290 & NAD83 & 580196.87 & 148135.04 & 4 HEISPROD & GPS & ONSITE & & $100-F-19$ \\
\hline \begin{tabular}{|l|}
2950 \\
\end{tabular} & 9291 & NAD83 & 580205.69 & 148141.02 & 2 HEISPROD & GPS & ONSITE & & 100-F-19 \\
\hline \begin{tabular}{|l|}
2951 \\
\end{tabular} & 9292 & NAD83 & 580209.45 & 148134.86 & HEISPROD & GPS & ONSITE & & 100-F-19 \\
\hline \begin{tabular}{|l|}
2952 \\
\end{tabular} & 9293 & NAD83 & 580241.02 & 148133.62 & 2 HEISPROD & GPS & ONSITE & & $100-F-19$ \\
\hline \begin{tabular}{|l|}
2953 \\
\end{tabular} & 9294 & NAD83 & 580253.19 & 148140.45 & 5 HEISPROD & GPS & ONSITE & & 100-F-19 \\
\hline 2954 & 9295 & NAD83 & 580263.73 & 148133 & 3 HEISPROD & GPS & ONSITE & & 100-F-19 \\
\hline \begin{tabular}{|l|}
2955 \\
\end{tabular} & 9296 & NAD83 & 580288.44 & 148142.12 & 2 HEISPROD & GPS & ONSITE & & $100-F-19$ \\
\hline 2956 & 9297 & NAD83 & 580296.75 & 148134.09 & 9 HEISPROD & GPS & ONSITE & & 100-F-19 \\
\hline 2957 & 9298 & NAD83 & 580306.65 & 148140.91 & 1 HEISPROD & GPS & ONSITE & & 100-F-19 \\
\hline \begin{tabular}{|l|}
2958 \\
\end{tabular} & 9299 & NAD83 & 580313.29 & 148132.8 & 3 HEISPROD & GPS & ONSITE & & 100-F-19 \\
\hline \begin{tabular}{|l|}
2959 \\
\end{tabular} & 9300 & NAD83 & 580289.85 & 148119.02 & 2 HEISPROD & GPS & ONSITE & & 100-F-19 \\
\hline \begin{tabular}{|l|}
2960 \\
\end{tabular} & 9301 & NAD83 & 580284.4 & 148119.03 & 3 HEISPROD & GPS & ONSITE & & $100-F-19$ \\
\hline \begin{tabular}{|l|}
2961 \\
\end{tabular} & 9302 & NAD83 & 580276.63 & 148108.45 & 5 HEISPROD & GPS & ONSITE & & 100-F-19 \\
\hline \begin{tabular}{|l|}
2962 \\
\end{tabular} & 9303 & NAD83 & 580276.67 & 148098.63 & 3 HEISPROD & GPS & ONSITE & & $100-F-19$ \\
\hline \begin{tabular}{|l|}
2963 \\
\end{tabular} & 9304 & NAD83 & 580281.96 & 148097.84 & 4 HEISPROD & GPS & ONSITE & & $100-F-19$ \\
\hline \begin{tabular}{|l|}
2964 \\
\end{tabular} & 9305 & NAD83 & 580288.91 & 148108.48 & 3 HEISPROD & GPS & ONSITE & & 100-F-19 \\
\hline \begin{tabular}{|l|}
2965 \\
\end{tabular} & 9306 & NAD83 & 580302.91 & 148108.97 & 7 HEISPROD & GPS & ONSITE & & 100-F-19 \\
\hline
\end{tabular}




\begin{tabular}{|c|c|c|c|c|c|c|c|c|c|}
\hline & $\bar{A}$ & $\mathrm{~B}$ & $\mathrm{C}$ & $\mathrm{D}$ & $E$ & $\mathrm{~F}$ & G & $\mathrm{H}$ & $\mathrm{I}$ \\
\hline 1 & SAMP_SITE_ID & HORIZ_COORD_TYPE & EW_COORD & NS_COORD & OWNER_ID & COORD_SOURCE & DIST_CLASS & RAD_LEVEL & SAMP_SITE_NAME \\
\hline 2966 & 9307 & NAD83 & 580309.17 & 148107.44 & HEISPROD & GPS & ONSITE & & 100-F-19 \\
\hline 2967 & 9308 & NAD83 & 580356.96 & 148115.14 & HEISPROD & GPS & ONSITE & & 100-F-19 \\
\hline 2968 & 9309 & NAD83 & 580366.94 & 148113.94 & HEISPROD & GPS & ONSITE & & 100-F-19 \\
\hline 2969 & 9310 & NAD83 & 580356.47 & 148101.18 & HEISPROD & GPS & ONSITE & & 100-F-19 \\
\hline 2970 & 9311 & NAD83 & 580354.87 & 148093.03 & HEISPROD & GPS & ONSITE & & 100-F-19 \\
\hline 2971 & 9312 & NAD83 & 580361.62 & 148084.08 & HEISPROD & GPS & ONSITE & & 100-F-19 \\
\hline 2972 & 9313 & NAD83 & 580356.7 & 148077.19 & HEISPROD & GPS & ONSITE & & 100-F-19 \\
\hline 2973 & 9314 & NAD83 & 580383.47 & 148117.13 & HEISPROD & GPS & ONSITE & & 100-F-19 \\
\hline 2974 & 9315 & NAD83 & 580382.93 & 148109.11 & HEISPROD & GPS & ONSITE & & 100-F-19 \\
\hline 2975 & 9316 & NAD83 & 580391.45 & 148117.8 & HEISPROD & GPS & ONSITE & & 100-F-19 \\
\hline 2976 & 9317 & NAD83 & 580391.99 & 148102.1 & HEISPROD & GPS & ONSITE & & 100-F-19 \\
\hline 2977 & 9318 & NAD83 & 580406.91 & 148118.92 & HEISPROD & GPS & ONSITE & & 100-F-19 \\
\hline 2978 & 9319 & NAD83 & 580407.32 & 148102.48 & HEISPROD & GPS & ONSITE & & $100-F-19$ \\
\hline 2979 & 9320 & NAD83 & 580414.51 & 148121.69 & HEISPROD & GPS & ONSITE & & 100-F-19 \\
\hline 2980 & 9321 & NAD83 & 580428.1 & 148113.64 & HEISPROD & GPS & ONSITE & & 100-F-19 \\
\hline 2981 & 9322 & NAD83 & 580428.05 & 148094.69 & HEISPROD & GPS & ONSITE & & 100-F-19 \\
\hline 2982 & 9323 & NAD83 & 580434.45 & 148122.83 & HEISPROD & GPS & ONSITE & & 100-F-19 \\
\hline 2983 & 9324 & NAD83 & 580434.5 & 148102.81 & HEISPROD & GPS & ONSITE & & $100-F-19$ \\
\hline 2984 & 9325 & NAD83 & 580434.69 & 148092.99 & HEISPROD & GPS & ONSITE & & 100-F-19 \\
\hline 2985 & 9326 & NAD83 & 580441.94 & 148124.42 & HEISPROD & GPS & ONSITE & & 100-F-19 \\
\hline 2986 & 9327 & NAD83 & 580443.16 & 148118.61 & HEISPROD & GPS & ONSITE & & 100-F-19 \\
\hline 2987 & 9328 & NAD83 & 580454.59 & 148119.31 & HEISPROD & GPS & ONSITE & & 100-F-19 \\
\hline 2988 & 9329 & NAD83 & 580442.96 & 148112.97 & HEISPROD & GPS & ONSITE & & 100-F-19 \\
\hline 2989 & 9330 & NAD83 & 580452.66 & 148107.44 & HEISPROD & GPS & ONSITE & & 100-F-19 \\
\hline 2990 & 9331 & NAD83 & 580444.29 & 148094.97 & HEISPROD & GPS & ONSITE & & 100-F-19 \\
\hline 2991 & 9335 & NAD83 & 580126.61 & 147943.21 & HEISPROD & GPS & ONSITE & & $100-F-19$ \\
\hline 2992 & 9336 & NAD83 & 580127.28 & 147939.4 & HEISPROD & GPS & ONSITE & & 100-F-19 \\
\hline 2993 & 9337 & NAD83 & 580140.29 & 147942.55 & HEISPROD & GPS & ONSITE & & 100-F-19 \\
\hline 2994 & 9338 & NAD83 & 580163.41 & 147941.4 & HEISPROD & GPS & ONSITE & & 100-F-19 \\
\hline 2995 & 9339 & NAD83 & 580181.13 & 147941.18 & HEISPROD & GPS & ONSITE & & 100-F-19 \\
\hline 2996 & 9340 & NAD83 & 580180.79 & 147932.18 & HEISPROD & GPS & ONSITE & & 100-F-19 \\
\hline 2997 & 9341 & NAD83 & 580204.47 & 147939.9 & HEISPROD & GPS & ONSITE & & 100-F-19 \\
\hline 2998 & 9342 & NAD83 & 580204.55 & 147930.44 & HEISPROD & GPS & ONSITE & & 100-F-19 \\
\hline 2999 & 9343 & NAD83 & 580212.93 & 147942.09 & HEISPROD & GPS & ONSITE & & 100-F-19 \\
\hline 3000 & 9344 & NAD83 & 580222.48 & 147933.89 & HEISPROD & GPS & ONSITE & & 100-F-19 \\
\hline 3001 & 9345 & NAD83 & 580230.9 & 147933.75 & HEISPROD & GPS & ONSITE & & 100-F-19 \\
\hline 3002 & 9346 & NAD83 & 580231.18 & 147918.52 & HEISPROD & GPS & ONSITE & & 100-F-19 \\
\hline 3003 & 9347 & NAD83 & 580239.25 & 147926.23 & HEISPROD & GPS & ONSITE & & $100-F-19$ \\
\hline
\end{tabular}




\begin{tabular}{|c|c|c|c|c|c|c|c|c|c|}
\hline & $\bar{A}$ & $\mathrm{~B}$ & $\mathrm{C}$ & $\mathrm{D}$ & $E$ & $\mathrm{~F}$ & G & $\mathrm{H}$ & $\mathrm{I}$ \\
\hline 1 & SAMP_SITE_ID & HORIZ_COORD_TYPE & EW_COORD & NS_COORD & OWNER_ID & COORD_SOURCE & DIST_CLASS & RAD_LEVEL & SAMP_SITE_NAME \\
\hline 3004 & 9348 & NAD83 & 580239.3 & 147918.53 & HEISPROD & GPS & ONSITE & & 100-F-19 \\
\hline 3005 & 9349 & NAD83 & 580247.49 & 147919.03 & HEISPROD & GPS & ONSITE & & 100-F-19 \\
\hline 3006 & 9350 & NAD83 & 580255.5 & 147936.26 & HEISPROD & GPS & ONSITE & & 100-F-19 \\
\hline 3007 & 9351 & NAD83 & 580263.47 & 147937.03 & HEISPROD & GPS & ONSITE & & 100-F-19 \\
\hline 3008 & 9352 & NAD83 & 580263.38 & 147921.17 & HEISPROD & GPS & ONSITE & & 100-F-19 \\
\hline 3009 & 9353 & NAD83 & 580271.75 & 147936.72 & HEISPROD & GPS & ONSITE & & 100-F-19 \\
\hline 3010 & 9354 & NAD83 & 580271.38 & 147929.53 & HEISPROD & GPS & ONSITE & & 100-F-19 \\
\hline 3011 & 9355 & NAD83 & 580270.95 & 147921.12 & HEISPROD & GPS & ONSITE & & 100-F-19 \\
\hline 3012 & 9356 & NAD83 & 580287.78 & 147921.14 & HEISPROD & GPS & ONSITE & & 100-F-19 \\
\hline 3013 & 9357 & & & & HEISPROD & & ONSITE & & 100-F-19 \\
\hline 3014 & 9358 & & & & HEISPROD & & ONSITE & & 100-F-19 \\
\hline 3015 & 9359 & NAD83 & 580313.93 & 147932.18 & HEISPROD & GPS & ONSITE & & 100-F-19 \\
\hline 3016 & 9360 & NAD83 & 580343.67 & 147934.97 & HEISPROD & GPS & ONSITE & & $100-F-19$ \\
\hline 3017 & 9361 & NAD83 & 580348.03 & 147927.24 & HEISPROD & GPS & ONSITE & & $100-F-19$ \\
\hline 3018 & 9362 & NAD83 & 580345.67 & 147919.49 & HEISPROD & GPS & ONSITE & & 100-F-19 \\
\hline 3019 & 9363 & NAD83 & 580354.78 & 147925.4 & HEISPROD & GPS & ONSITE & & 100-F-19 \\
\hline 3020 & 9364 & NAD83 & 580360.42 & 147923.64 & HEISPROD & GPS & ONSITE & & 100-F-19 \\
\hline 3021 & 9365 & NAD83 & 580357.68 & 147913.19 & HEISPROD & GPS & ONSITE & & $100-F-19$ \\
\hline 3022 & 9366 & NAD83 & 580368.92 & 147934.45 & HEISPROD & GPS & ONSITE & & 100-F-19 \\
\hline 3023 & 9367 & NAD83 & 580365.94 & 147922.94 & HEISPROD & GPS & ONSITE & & 100-F-19 \\
\hline 3024 & 9368 & NAD83 & 580360.73 & 147901.25 & HEISPROD & GPS & ONSITE & & 100-F-19 \\
\hline 3025 & 9369 & NAD83 & 580373.65 & 147933.3 & HEISPROD & GPS & ONSITE & & 100-F-19 \\
\hline 3026 & 9370 & NAD83 & 580378.53 & 147928.1 & HEISPROD & GPS & ONSITE & & 100-F-19 \\
\hline 3027 & 9371 & NAD83 & 580374.4 & 147908.44 & HEISPROD & GPS & ONSITE & & 100-F-19 \\
\hline 3028 & 9372 & NAD83 & 580388.62 & 147905.79 & HEISPROD & GPS & ONSITE & & $100-F-19$ \\
\hline 3029 & 9373 & NAD83 & 580387.36 & 147897.96 & HEISPROD & GPS & ONSITE & & $100-F-19$ \\
\hline 3030 & 9374 & NAD83 & 580396.38 & 147905.1 & HEISPROD & GPS & ONSITE & & 100-F-19 \\
\hline 3031 & 9375 & NAD83 & 580406.02 & 147920.27 & HEISPROD & GPS & ONSITE & & 100-F-19 \\
\hline 3032 & 9376 & NAD83 & 580403.51 & 147896.97 & HEISPROD & GPS & ONSITE & & 100-F-19 \\
\hline 3033 & 9377 & NAD83 & 580410.74 & 147900.03 & HEISPROD & GPS & ONSITE & & 100-F-19 \\
\hline 3034 & 9378 & NAD83 & 580448.01 & 147927.79 & HEISPROD & GPS & ONSITE & & 100-F-19 \\
\hline 3035 & 9379 & NAD83 & 580451.07 & 147916.68 & HEISPROD & GPS & ONSITE & & 100-F-19 \\
\hline 3036 & 9380 & NAD83 & 580456.72 & 147933.91 & HEISPROD & GPS & ONSITE & & 100-F-19 \\
\hline 3037 & 9381 & NAD83 & 580462.29 & 147935.31 & HEISPROD & GPS & ONSITE & & 100-F-19 \\
\hline 3038 & 9382 & NAD83 & 580488.78 & 147934.83 & HEISPROD & GPS & ONSITE & & 100-F-19 \\
\hline 3039 & 9383 & NAD83 & 580455.26 & 147971.17 & HEISPROD & GPS & ONSITE & & 100-F-19 \\
\hline 3040 & 9384 & NAD83 & 580448.07 & 147979.49 & HEISPROD & GPS & ONSITE & & 100-F-19 \\
\hline 3041 & 9385 & NAD83 & 580483.94 & 147979.47 & HEISPROD & GPS & ONSITE & & $100-F-19$ \\
\hline
\end{tabular}




\begin{tabular}{|c|c|c|c|c|c|c|c|c|c|}
\hline & $A$ & $\mathrm{~B}$ & $\mathrm{C}$ & $\mathrm{D}$ & $E$ & $\mathrm{~F}$ & $\mathrm{G}$ & $\mathrm{H}$ & $\mathrm{I}$ \\
\hline 1 & SAMP_SITE_ID & HORIZ_COORD_TYPE & EW_COORD & NS_COORD & OWNER_ID & COORD_SOURCE & DIST_CLASS & RAD_LEVEL & SAMP_SITE_NAME \\
\hline 3042 & 9387 & NAD83 & 580486.52 & 147985.54 & HEISPROD & GPS & ONSITE & & $100-F-19$ \\
\hline 3043 & 9388 & NAD83 & 580848.5 & 148085.73 & HEISPROD & GPS & ONSITE & & 100-F-19 \\
\hline 3044 & 9389 & NAD83 & 580856.87 & 148086.07 & HEISPROD & GPS & ONSITE & & 100-F-19 \\
\hline 3045 & 9390 & UNKNOWN & 572110 & 149801 & HEISPROD & GPS & ONSITE & & 116-N-3 Trench \\
\hline 3046 & 9391 & UNKNOWN & 572110 & 149801 & HEISPROD & GPS & ONSITE & & 116-N-3 Trench \\
\hline 3047 & 9392 & UNKNOWN & 572064 & 149733 & HEISPROD & GPS & ONSITE & & 116-N-3 Trench \\
\hline 3048 & 9393 & UNKNOWN & 572054 & 149729 & HEISPROD & GPS & & & 116-N-3 Trench \\
\hline 3049 & 9394 & UNKNOWN & 572039 & 149699 & HEISPROD & GPS & & & 116-N-3 Trench \\
\hline 3050 & 9395 & UNKNOWN & 572029 & 149700 & HEISPROD & GPS & & & 116-N-3 Trench \\
\hline 3051 & 9396 & UNKNOWN & 572097 & 149802 & HEISPROD & GPS & & & 116-N-3 Trench \\
\hline 3052 & 9397 & UNKNOWN & 572068 & 149767 & HEISPROD & GPS & & & 116-N-3 Trench \\
\hline 3053 & 9398 & UNKNOWN & 572064 & 149733 & HEISPROD & GPS & & & 116-N-3 Trench \\
\hline 3054 & 9399 & UNKNOWN & 572064 & 149733 & HEISPROD & GPS & & & 116-N-3 Trench \\
\hline 3055 & 9400 & UNKNOWN & 572054 & 149729 & HEISPROD & GPS & & & 116-N-3 Trench \\
\hline 3056 & 9401 & UNKNOWN & 572039 & 149699 & HEISPROD & GPS & & & 116-N-3 Trench \\
\hline 3057 & 9402 & UNKNOWN & 572029 & 149700 & HEISPROD & GPS & & & 116-N-3 Trench \\
\hline 3058 & 9403 & UNKNOWN & 572097 & 149802 & HEISPROD & GPS & & & 116-N-3 Trench \\
\hline 3059 & 9404 & UNKNOWN & 572068 & 149767 & HEISPROD & GPS & & & 116-N-3 Trench \\
\hline 3060 & 9405 & UNKNOWN & 572070 & 149763 & HEISPROD & GPS & & & 116-N-3 Trench \\
\hline 3061 & 9406 & UNKNOWN & 572020 & 149707 & HEISPROD & GPS & & & 116-N-3 Trench \\
\hline 3062 & 9407 & UNKNOWN & 572011 & 149695 & HEISPROD & GPS & & & 116-N-3 Trench \\
\hline 3063 & 9408 & UNKNOWN & 572010 & 149686 & HEISPROD & GPS & & & 116-N-3 Trench \\
\hline 3064 & 9409 & UNKNOWN & 571992 & 149671 & HEISPROD & GPS & & & 116-N-3 Trench \\
\hline 3065 & 9410 & UNKNOWN & 571994 & 149668 & HEISPROD & GPS & & & 116-N-3 Trench \\
\hline 3066 & 9411 & UNKNOWN & 572011 & 149695 & HEISPROD & GPS & & & 116-N-3 Trench \\
\hline 3067 & 9412 & UNKNOWN & 572070 & 149763 & HEISPROD & GPS & & & 116-N-3 Trench \\
\hline 3068 & 9413 & UNKNOWN & 572020 & 149707 & HEISPROD & GPS & & & 116-N-3 Trench \\
\hline 3069 & 9414 & UNKNOWN & 572011 & 149695 & HEISPROD & GPS & & & 116-N-3 Trench \\
\hline 3070 & 9415 & UNKNOWN & 572010 & 149686 & HEISPROD & GPS & & & 116-N-3 Trench \\
\hline 3071 & 9416 & UNKNOWN & 571992 & 149671 & HEISPROD & GPS & & & 116-N-3 Trench \\
\hline 3072 & 9417 & UNKNOWN & 571994 & 149668 & HEISPROD & GPS & & & 116-N-3 Trench \\
\hline 3073 & 9418 & UNKNOWN & 572003 & 149630 & HEISPROD & GPS & & & 116-N-3 Trench \\
\hline 3074 & 9419 & UNKNOWN & 572003 & 149630 & HEISPROD & GPS & & & 116-N-3 Trench \\
\hline 3075 & 9420 & UNKNOWN & 572003 & 149630 & HEISPROD & GPS & & & 116-N-3 Trench \\
\hline 3076 & 9421 & UNKNOWN & 572003 & 149630 & HEISPROD & GPS & & & 116-N-3 Trench \\
\hline 3077 & 9422 & UNKNOWN & 572003 & 149630 & HEISPROD & GPS & & & 116-N-3 Trench \\
\hline 3078 & 9423 & UNKNOWN & 571986 & 149613 & HEISPROD & GPS & & & 116-N-3 Trench \\
\hline 3079 & 9424 & UNKNOWN & 571966 & 149619 & HEISPROD & GPS & & & 116-N-3 Trench \\
\hline
\end{tabular}




\begin{tabular}{|c|c|c|c|c|c|c|c|c|c|}
\hline & A & $\mathrm{B}$ & $\mathrm{C}$ & $\mathrm{D}$ & $E$ & $\mathrm{~F}$ & G & $\mathrm{H}$ & $\mathrm{I}$ \\
\hline 1 & SAMP_SITE_ID & HORIZ_COORD_TYPE & EW_COORD & NS_COORD & OWNER_ID & COORD_SOURCE & DIST_CLASS & RAD_LEVEL & SAMP_SITE_NAME \\
\hline 3080 & 9433 & UNKNOWN & 571966 & 149619 & HEISPROD & GPS & & & 116-N-3 Trench \\
\hline 3081 & 9434 & UNKNOWN & 571988 & 149623 & HEISPROD & GPS & & & 116-N-3 Trench \\
\hline 3082 & 9435 & UNKNOWN & 572001 & 149656 & HEISPROD & GPS & & & 116-N-3 Trench \\
\hline 3083 & 9436 & UNKNOWN & 572012 & 149641 & HEISPROD & GPS & & & 116-N-3 Trench \\
\hline 3084 & 9437 & UNKNOWN & 571988 & 149623 & HEISPROD & GPS & & & 116-N-3 Trench \\
\hline 3085 & 9438 & UNKNOWN & 571988 & 149623 & HEISPROD & GPS & & & 116-N-3 Trench \\
\hline 3086 & 9439 & UNKNOWN & 571988 & 149623 & HEISPROD & GPS & & & 116-N-3 Trench \\
\hline 3087 & 9440 & UNKNOWN & 572001 & 149656 & HEISPROD & GPS & & & 116-N-3 Trench \\
\hline 3088 & 9441 & UNKNOWN & 572012 & 149641 & HEISPROD & GPS & & & 116-N-3 Trench \\
\hline 3089 & 9453 & NAD83 & 580116.88 & 147953.03 & HEISPROD & GPS & ONSITE & & 100-F-19 Pipe Trench Shallow Zone \\
\hline 3090 & 9454 & NAD83 & 580116.88 & 147953.03 & HEISPROD & GPS & ONSITE & & 100-F-19 Pipe Trench Shallow Zone \\
\hline 3091 & 9455 & NAD83 & 580166.08 & 147953.1 & HEISPROD & GPS & ONSITE & & 100-F-19 Pipe Trench Shallow Zone \\
\hline 3092 & 9456 & NAD83 & 580173.76 & 147960.3 & HEISPROD & GPS & & & 100-F-19 Pipe Trench Shallow Zone \\
\hline 3093 & 9473 & UNKNOWN & 565280 & 145304 & HEISPROD & ESTIMATED & ONSITE & & 100-B-8 \\
\hline 3094 & 9474 & UNKNOWN & 565256 & 145333 & HEISPROD & ESTIMATED & ONSITE & & $100-B-8$ \\
\hline 3095 & 9475 & UNKNOWN & 565256 & 145333 & HEISPROD & ESTIMATED & ONSITE & & $100-B-8$ \\
\hline 3096 & 9476 & UNKNOWN & 565256 & 145312 & HEISPROD & ESTIMATED & ONSITE & & 116-B-7 \\
\hline 3097 & 9477 & UNKNOWN & 565457 & 145393 & HEISPROD & ESTIMATED & ONSITE & & 132-B-6 \\
\hline 3098 & 9478 & UNKNOWN & 565256 & 145366 & HEISPROD & ESTIMATED & ONSITE & & $100-B-8$ \\
\hline 3099 & 9479 & UNKNOWN & 565256 & 145299 & HEISPROD & ESTIMATED & ONSITE & & 100-B-14 \\
\hline 3100 & 9480 & UNKNOWN & 565259 & 145316 & HEISPROD & ESTIMATED & ONSITE & & $100-B-8$ \\
\hline 3101 & 9481 & UNKNOWN & 565273 & 145305 & HEISPROD & ESTIMATED & ONSITE & & 100-B-8 \\
\hline 3102 & 9482 & UNKNOWN & 565271 & 145315 & HEISPROD & ESTIMATED & ONSITE & & 100-B-8 \\
\hline 3103 & 9483 & UNKNOWN & 565277 & 145299 & HEISPROD & ESTIMATED & ONSITE & & 100-B-8 \\
\hline 3104 & 9484 & UNKNOWN & 565452 & 145392 & HEISPROD & ESTIMATED & ONSITE & & 132-B-6 \\
\hline 3105 & 9493 & UNKNOWN & 565457 & 145393 & HEISPROD & ESTIMATED & ONSITE & & 132-B-6 \\
\hline 3106 & 9494 & UNKNOWN & 565460 & 145391 & HEISPROD & ESTIMATED & ONSITE & & 132-B-6 \\
\hline 3107 & 9495 & UNKNOWN & 565475 & 145412 & HEISPROD & ESTIMATED & ONSITE & & 132-B-6 \\
\hline 3108 & 9496 & UNKNOWN & 565475 & 145420 & HEISPROD & ESTIMATED & ONSITE & & 132-B-6 \\
\hline 3109 & 9497 & UNKNOWN & 565300 & 145261 & HEISPROD & ESTIMATED & ONSITE & & 100-B-8 \\
\hline 3110 & 9498 & UNKNOWN & 565300 & 145241 & HEISPROD & ESTIMATED & ONSITE & & 100-B-8 \\
\hline 3111 & 9499 & UNKNOWN & 565300 & 145232 & HEISPROD & ESTIMATED & ONSITE & & 100-B-8 \\
\hline 3112 & 9500 & UNKNOWN & 565300 & 145213 & HEISPROD & ESTIMATED & ONSITE & & 100-B-8 \\
\hline 3113 & 9501 & UNKNOWN & 565300 & 145192 & HEISPROD & ESTIMATED & ONSITE & & 100-B-8 \\
\hline 3114 & 9502 & UNKNOWN & 565300 & 145176 & HEISPROD & ESTIMATED & ONSITE & & $100-B-8$ \\
\hline 3115 & 9503 & UNKNOWN & 565300 & 145166 & HEISPROD & ESTIMATED & ONSITE & & $100-B-8$ \\
\hline 3116 & 9504 & UNKNOWN & 565300 & 145154 & HEISPROD & ESTIMATED & ONSITE & & 100-B-8 \\
\hline 3117 & 9505 & UNKNOWN & 565300 & 145131 & HEISPROD & ESTIMATED & ONSITE & & 100-B-8 \\
\hline
\end{tabular}




\begin{tabular}{|c|c|c|c|c|c|c|c|c|c|}
\hline & A & $\mathrm{B}$ & C & $\mathrm{D}$ & $\mathrm{E}$ & $\mathrm{F}$ & $\mathrm{G}$ & $\mathrm{H}$ & $\mathrm{I}$ \\
\hline 1 & SAMP_SITE_ID & HORIZ_COORD_TYPE & EW_COORD & NS_COORD & OWNER_ID & COORD_SOURCE & DIST_CLASS & RAD_LEVEL & SAMP_SITE_NAME \\
\hline 3118 & 9513 & UNKNOWN & 565300 & 145116 & 5 HEISPROD & ESTIMATED & ONSITE & & $100-B-8$ \\
\hline 3119 & 9533 & & & & SESPMNT & & ONSITE & & 300 SPR 7 -1 (2.5FT) \\
\hline 3120 & 9534 & & & & SESPMNT & & ONSITE & & 300 SPR 7 -1 (4 FT) \\
\hline 3121 & 9535 & & & & SESPMNT & & ONSITE & & 300 SPR 7 -1 (6 FT) \\
\hline 3122 & 9536 & & & & SESPMNT & & ONSITE & & 300 SPR 7 -2 (2 FT) \\
\hline 3123 & 9537 & & & & SESPMNT & & ONSITE & & 300 SPR 7 -2 (4.2FT) \\
\hline 3124 & 9539 & & & & SESPMNT & & ONSITE & & 300 SPR 9 -1 (2 FT) \\
\hline 3125 & 9540 & & & & SESPMNT & & ONSITE & & 300 SPR 9 -1 (4 FT) \\
\hline 3126 & 9541 & & & & SESPMNT & & ONSITE & & 300 SPR $9-2$ (2 FT) \\
\hline 3127 & 9542 & & & & SESPMNT & & ONSITE & & 300 SPR $9-2$ (4.5FT) \\
\hline 3128 & 9543 & & & & SESPMNT & & ONSITE & & 300 SPR 9 -3 (5.5FT) \\
\hline 3129 & 9545 & & & & SESPMNT & & RIVER_SHORELINE & & N. RICHLAND \\
\hline 3130 & 9546 & & & & SESPMNT & & ONSITE & & 100 B REACTOR MUSEUM \\
\hline 3131 & 9547 & & & & SESPMNT & & ONSITE & & LIGO \\
\hline 3132 & 9548 & & & & SESPMNT & & ONSITE & & 313 BLDG. \\
\hline 3133 & 9553 & & & & SESPMNT & & RIVER_SHORELINE & & ISLAND \#1 \\
\hline 3134 & 9554 & & & & SESPMNT & & RIVER_SHORELINE & & ISLAND \#5 \\
\hline 3135 & 9555 & & & & SESPMNT & & RIVER_SHORELINE & & ISLAND \#10 \\
\hline 3136 & 9556 & & & & SESPMNT & & RIVER_SHORELINE & & ISLAND \#15 \\
\hline 3137 & 9557 & & & & SESPMNT & & RIVER_SHORELINE & & ISLAND \#17 \\
\hline 3138 & 9558 & & & & SESPMNT & & RIVER_SHORELINE & & ISLAND \#18 \\
\hline 3139 & 9559 & & & & SESPMNT & & RIVER_SHORELINE & & ISLAND \#19 \\
\hline 3140 & 9560 & & & & SESPMNT & & RIVER_SHORELINE & & ISLAND \#20 \\
\hline 3141 & 9561 & & & & SESPMNT & & RIVER_SHORELINE & & PRIEST RAPIDS RESERVOIR \\
\hline 3142 & 9573 & & & & SESPMNT & & ONSITE & & N OF VIT PLANT \\
\hline 3143 & 9593 & UNKNOWN & 580956.08 & 147876.62 & 2 HEISPROD & ESTIMATED & ONSITE & & 116-F-14 Shallow Zone \\
\hline 3144 & 9594 & UNKNOWN & 580951.67 & 147872.13 & 3 HEISPROD & ESTIMATED & ONSITE & & 116-F-14 Shallow Zone \\
\hline 3145 & 9595 & UNKNOWN & 580956.01 & 147872.14 & 4 HEISPROD & ESTIMATED & ONSITE & & 116-F-14 Shallow Zone \\
\hline 3146 & 9596 & UNKNOWN & 580951.94 & 147861.09 & 9 HEISPROD & ESTIMATED & ONSITE & & 116-F-14 Shallow Zone \\
\hline 3147 & 9597 & UNKNOWN & 580948.47 & 147852.18 & 3 HEISPROD & ESTIMATED & ONSITE & & 116-F-14 Shallow Zone \\
\hline 3148 & 9598 & UNKNOWN & 580956.09 & 147847.56 & 6 HEISPROD & ESTIMATED & ONSITE & & 116-F-14 Shallow Zone \\
\hline 3149 & 9599 & UNKNOWN & 580954.76 & 147840.43 & 3 HEISPROD & ESTIMATED & ONSITE & & 116-F-14 Shallow Zone \\
\hline 3150 & 9600 & UNKNOWN & 580960.52 & 147835.2 & 2 HEISPROD & ESTIMATED & ONSITE & & 116-F-14 Shallow Zone \\
\hline 3151 & 9601 & UNKNOWN & 580956.66 & 147831.41 & 1 HEISPROD & ESTIMATED & ONSITE & & 116-F-14 Shallow Zone \\
\hline 3152 & 9602 & UNKNOWN & 580963.44 & 147826.87 & 7 HEISPROD & ESTIMATED & ONSITE & & 116-F-14 Shallow Zone \\
\hline 3153 & 10422 & UNKNOWN & 571743 & 150030 & HEISPROD & ESTIMATED & & & $116-\mathrm{N}-1$ \\
\hline 3154 & 10423 & UNKNOWN & 571517 & 149653 & 3 HEISPROD & ESTIMATED & ONSITE & & $116-N-3$ \\
\hline 3155 & 10424 & UNKNOWN & 571531 & 149646 & 5 HEISPROD & ESTIMATED & & & $116-N-3$ \\
\hline
\end{tabular}




\begin{tabular}{|c|c|c|c|c|c|c|c|c|c|}
\hline & A & $\mathrm{B}$ & $\mathrm{C}$ & $\mathrm{D}$ & $\mathrm{E}$ & $\mathrm{F}$ & G & $\mathrm{H}$ & $\mathrm{I}$ \\
\hline 1 & SAMP_SITE_ID & HORIZ_COORD_TYPE & EW_COORD & NS_COORD & OWNER_ID & COORD_SOURCE & DIST_CLASS & RAD_LEVEL & SAMP_SITE_NAME \\
\hline 3156 & 10425 & UNKNOWN & 571546 & 149649 & HEISPROD & ESTIMATED & ONSITE & & $116-N-3$ \\
\hline 3157 & 10427 & UNKNOWN & 571562 & 149636 & HEISPROD & ESTIMATED & ONSITE & & $116-N-3$ \\
\hline 3158 & 10428 & & & & HEISPROD & & & & 116-N-3 Pipeline \& By-Pass Trench \\
\hline 3159 & 10429 & UNKNOWN & 571817 & 149587 & HEISPROD & GPS & ONSITE & & 116-N-3 Pipeline \& By-Pass Trench \\
\hline 3160 & 10430 & UNKNOWN & 571604 & 149659 & HEISPROD & GPS & ONSITE & & 116-N-3 Pipeline \& By-Pass Trench \\
\hline 3161 & 10431 & UNKNOWN & 571586 & 149667 & HEISPROD & GPS & ONSITE & & 116-N-3 Pipeline \& By-Pass Trench \\
\hline 3162 & 10432 & UNKNOWN & 571619 & 149656 & HEISPROD & GPS & ONSITE & & 116-N-3 Pipeline \& By-Pass Trench \\
\hline 3163 & 10433 & UNKNOWN & 571757 & 149602 & HEISPROD & GPS & ONSITE & & 116-N-3 Pipeline \& By-Pass Trench \\
\hline 3164 & 10434 & UNKNOWN & 571649 & 149657 & HEISPROD & GPS & ONSITE & & 116-N-3 Pipeline \& By-Pass Trench \\
\hline 3165 & 10435 & UNKNOWN & 571630 & 149678 & HEISPROD & GPS & ONSITE & & 116-N-3 Pipeline \& By-Pass Trench \\
\hline 3166 & 10436 & UNKNOWN & 571633 & 149665 & HEISPROD & GPS & ONSITE & & 116-N-3 Pipeline \& By-Pass Trench \\
\hline 3167 & 10437 & UNKNOWN & 571689 & 149626 & HEISPROD & GPS & ONSITE & & 116-N-3 Pipeline \& By-Pass Trench \\
\hline 3168 & 10438 & UNKNOWN & 571604 & 149659 & HEISPROD & GPS & ONSITE & & 116-N-3 Pipeline \& By-Pass Trench \\
\hline 3169 & 10439 & & & & HEISPROD & & & & 116-N-3 Pipeline \& By-Pass Trench \\
\hline 3170 & 10449 & UNKNOWN & 571744 & 149619 & HEISPROD & ESTIMATED & & & $116-\mathrm{N}-3$ \\
\hline 3171 & 10450 & UNKNOWN & 571699 & 149636 & HEISPROD & ESTIMATED & & & $116-\mathrm{N}-3$ \\
\hline 3172 & 10451 & UNKNOWN & 571721 & 149628 & HEISPROD & ESTIMATED & & & $116-N-3$ \\
\hline 3173 & 10468 & UNKNOWN & 571744 & 149619 & HEISPROD & ESTIMATED & & & $116-\mathrm{N}-3$ \\
\hline 3174 & 10488 & UNKNOWN & 571576 & 149626 & HEISPROD & GPS & ONSITE & & 116-N-3 Pipeline \& By-Pass Trench \\
\hline 3175 & 10489 & UNKNOWN & 571573 & 149625 & HEISPROD & GPS & ONSITE & & 116-N-3 Pipeline \& By-Pass Trench \\
\hline 3176 & 10490 & UNKNOWN & 571572 & 149626 & HEISPROD & GPS & ONSITE & & 116-N-3 Pipeline \& By-Pass Trench \\
\hline 3177 & 10491 & UNKNOWN & 571569 & 149627 & HEISPROD & GPS & ONSITE & & 116-N-3 Pipeline \& By-Pass Trench \\
\hline 3178 & 10492 & UNKNOWN & 571570 & 149626 & HEISPROD & GPS & ONSITE & & 116-N-3 Pipeline \& By-Pass Trench \\
\hline 3179 & 10493 & UNKNOWN & 571579 & 149632 & HEISPROD & GPS & ONSITE & & 116-N-3 Pipeline \& By-Pass Trench \\
\hline 3180 & 10494 & UNKNOWN & 571579 & 149630 & HEISPROD & GPS & ONSITE & & 116-N-3 Pipeline \& By-Pass Trench \\
\hline 3181 & 10495 & UNKNOWN & 571973 & 149563 & HEISPROD & GPS & ONSITE & & 116-N-3 Pipeline \& By-Pass Trench \\
\hline 3182 & 10496 & UNKNOWN & 571977 & 149565 & HEISPROD & GPS & ONSITE & & 116-N-3 Pipeline \& By-Pass Trench \\
\hline 3183 & 10497 & UNKNOWN & 571981 & 149567 & HEISPROD & GPS & ONSITE & & 116-N-3 Pipeline \& By-Pass Trench \\
\hline 3184 & 10498 & UNKNOWN & 571577 & 149627 & HEISPROD & GPS & ONSITE & & 116-N-3 Pipeline \& By-Pass Trench \\
\hline 3185 & 10508 & UNKNOWN & 571721 & 149628 & HEISPROD & ESTIMATED & & & $116-\mathrm{N}-3$ \\
\hline 3186 & 10509 & UNKNOWN & 571721 & 149628 & HEISPROD & ESTIMATED & & & $116-N-3$ \\
\hline 3187 & 10510 & UNKNOWN & 571699 & 149636 & HEISPROD & ESTIMATED & & & $116-N-3$ \\
\hline 3188 & 10511 & UNKNOWN & 571851 & 149605 & HEISPROD & ESTIMATED & & & $116-\mathrm{N}-3$ \\
\hline 3189 & 10512 & UNKNOWN & 571917 & 149654 & HEISPROD & ESTIMATED & & & $116-N-3$ \\
\hline 3190 & 10513 & UNKNOWN & 571953 & 149648 & HEISPROD & ESTIMATED & & & $116-N-3$ \\
\hline 3191 & 10514 & UNKNOWN & 571862 & 149628 & HEISPROD & ESTIMATED & & & $116-N-3$ \\
\hline 3192 & 10515 & UNKNOWN & 571978 & 149586 & HEISPROD & ESTIMATED & & & $116-N-3$ \\
\hline 3193 & 10516 & UNKNOWN & 571979 & 149581 & HEISPROD & ESTIMATED & & & $116-\mathrm{N}-3$ \\
\hline
\end{tabular}


A

B

C

D

E

$\mathrm{F}$ G $\mathrm{H}$ I

\begin{tabular}{|c|c|c|c|c|c|c|c|c|c|}
\hline & $\mathrm{A}$ & $\mathrm{B}$ & $\mathrm{C}$ & $\mathrm{D}$ & $E$ & $\mathrm{~F}$ & $G$ & $\mathrm{H}$ & $\mathrm{I}$ \\
\hline 1 & SAMP_SITE_ID & HORIZ_COORD_TYPE & EW_COORD & NS_COORD & OWNER_ID & COORD_SOURCE & DIST_CLASS & RAD_LEVEL & SAMP_SITE_NAME \\
\hline 3194 & 10517 & UNKNOWN & 571975 & 149576 & HEISPROD & ESTIMATED & & & $116-\mathrm{N}-3$ \\
\hline 3195 & 10518 & UNKNOWN & 571957 & 149578 & HEISPROD & ESTIMATED & & & $116-\mathrm{N}-3$ \\
\hline 3196 & 10519 & UNKNOWN & 571865 & 149585 & HEISPROD & ESTIMATED & & & $116-\mathrm{N}-3$ \\
\hline \begin{tabular}{|l|}
3197 \\
\end{tabular} & 10520 & UNKNOWN & 571860 & 149594 & HEISPROD & ESTIMATED & & & $116-\mathrm{N}-3$ \\
\hline \begin{tabular}{|l|}
3198 \\
\end{tabular} & 10521 & UNKNOWN & 571856 & 149603 & HEISPROD & ESTIMATED & & & $116-\mathrm{N}-3$ \\
\hline 3199 & 10522 & UNKNOWN & 571852 & 149612 & HEISPROD & ESTIMATED & & & $116-\mathrm{N}-3$ \\
\hline 3200 & 10528 & & & & HEISPROD & & & & 126-F-1 \\
\hline 3201 & 10529 & & & & HEISPROD & & & & $100-F-2$ \\
\hline 3202 & 10530 & & & & HEISPROD & & & & $116-F-2$ \\
\hline 3203 & 10531 & & & & HEISPROD & & & & 126-F-1 Ash Pit \\
\hline 3204 & 10532 & & & & HEISPROD & & & & 126-F-1 Ash Pit \\
\hline 3205 & 10533 & & & & HEISPROD & & & & 126-F-1 Ash Pit \\
\hline 3206 & 10534 & & & & HEISPROD & & & & 126-F-1 Ash Pit \\
\hline 3207 & 10535 & & & & HEISPROD & & & & 126-F-1 Ash Pit \\
\hline 3208 & 10536 & & & & HEISPROD & & & & $100 \mathrm{~F}$ Area \\
\hline 3209 & 10556 & UNKNOWN & 565432 & 145384 & HEISPROD & ESTIMATED & ONSITE & & 132-B-6 Outfall \\
\hline 3210 & 10557 & UNKNOWN & 565432 & 145384 & HEISPROD & ESTIMATED & ONSITE & & 132-B-6 Outfall \\
\hline 3211 & 10558 & UNKNOWN & 565432 & 145325 & HEISPROD & ESTIMATED & ONSITE & & 132-B-6 Outfall \\
\hline 3212 & 10559 & UNKNOWN & 565437 & 145325 & HEISPROD & ESTIMATED & ONSITE & & 132-B-6 Outfall \\
\hline 3213 & 10560 & & & & HEISPROD & & & & 116-B-7 Outfall \\
\hline 3214 & 10561 & & & & HEISPROD & & & & 116-B-7 Outfall \\
\hline 3215 & 10562 & & & & HEISPROD & & & & 116-B-7 Outfall \\
\hline 3216 & 10563 & & & & HEISPROD & & & & 116-B-7 Outfall \\
\hline \begin{tabular}{|l|}
3217 \\
\end{tabular} & 10564 & & & & HEISPROD & & & & 116-B-7 Outfall \\
\hline 3218 & 10565 & & & & HEISPROD & & & & 116-B-7 Outfall \\
\hline 3219 & 10566 & & & & HEISPROD & & & & 132-B-6 Outfall \\
\hline 3220 & 10567 & & & & HEISPROD & & & & 132-B-6 Outfall \\
\hline 3221 & 10568 & & & & HEISPROD & & & & 132-B-6 Outfall \\
\hline 3222 & 10569 & & & & HEISPROD & & & & 132-B-6 Outfall \\
\hline 3223 & 10570 & & & & HEISPROD & & & & 132-B-6 Outfall \\
\hline \begin{tabular}{|l|}
3224 \\
\end{tabular} & 10571 & & & & HEISPROD & & & & 132-B-6 Outfall \\
\hline 3225 & 10572 & & & & HEISPROD & & & & 132-B-6 Outfall \\
\hline \begin{tabular}{|l|}
3226 \\
\end{tabular} & 10573 & & & & HEISPROD & & & & 132-C-2 Outfall \\
\hline \begin{tabular}{|l|}
3227 \\
\end{tabular} & 10574 & & & & HEISPROD & & & & 132-C-2 Outfall \\
\hline 3228 & 10575 & & & & HEISPROD & & & & 132-C-2 Outfall \\
\hline 3229 & 10576 & & & & HEISPROD & & & & 116-B-7 Outfall \\
\hline 3230 & 10577 & & & & HEISPROD & & & & 132-B-6 Outfall \\
\hline 3231 & 10578 & & & & HEISPROD & & & & 116-B-7 Outfall \\
\hline
\end{tabular}




\begin{tabular}{|c|c|c|c|c|c|c|c|c|c|}
\hline & $A$ & $\mathrm{~B}$ & $\mathrm{C}$ & $\mathrm{D}$ & $E$ & $\mathrm{~F}$ & $\mathrm{G}$ & $\mathrm{H}$ & $\mathrm{I}$ \\
\hline 1 & SAMP_SITE_ID & HORIZ_COORD_TYPE & EW_COORD & NS_COORD & OWNER_ID & COORD_SOURCE & DIST_CLASS & RAD_LEVEL & SAMP_SITE_NAME \\
\hline 3232 & 10579 & & & & HEISPROD & & & & 132-B-6 Outfall \\
\hline 3233 & 10580 & & & & HEISPROD & & & & 132-C-2 Outfall \\
\hline 3234 & 10581 & & & & HEISPROD & & & & 116-B-7 Outfall \\
\hline 3235 & 10582 & & & & HEISPROD & & & & 116-B-7 Outfall \\
\hline 3236 & 10596 & & & & HEISPROD & & & & 116-F-14 Test Pit \\
\hline 3237 & 10597 & & & & HEISPROD & & & & 116-F-14 Test Pit \\
\hline 3238 & 10598 & & & & HEISPROD & & & & 116-F-14 Shallow Zone \\
\hline 3239 & 10599 & & & & HEISPROD & & & & 116-F-14 Shallow Zone \\
\hline 3240 & 10600 & & & & HEISPROD & & & & 116-F-14 Shallow Zone \\
\hline 3241 & 10601 & & & & HEISPROD & & & & 116-F-14 Shallow Zone \\
\hline 3242 & 10602 & & & & HEISPROD & & & & 116-F-14 Shallow Zone \\
\hline 3243 & 10603 & & & & HEISPROD & & & & 116-F-14 Shallow Zone \\
\hline 3244 & 10604 & & & & HEISPROD & & & & 116-F-14 Shallow Zone \\
\hline 3245 & 10605 & & & & HEISPROD & & & & 116-F-14 Shallow Zone \\
\hline 3246 & 10606 & & & & HEISPROD & & & & 116-F-14 Test Pit \\
\hline 3247 & 10607 & & & & HEISPROD & & & & 116-F-14 Test Pit \\
\hline 3248 & 10608 & & & & HEISPROD & & & & 116-F-14 Test Pit \\
\hline 3249 & 10609 & & & & HEISPROD & & & & 116-F-14 Test Pit \\
\hline 3250 & 10610 & & & & HEISPROD & & & & 116-F-14 Test Pit \\
\hline 3251 & 10611 & UNKNOWN & 571848 & 149621 & HEISPROD & ESTIMATED & & & $116-\mathrm{N}-3$ \\
\hline 3252 & 10612 & UNKNOWN & 571858 & 149630 & HEISPROD & ESTIMATED & & & $116-\mathrm{N}-3$ \\
\hline 3253 & 10613 & UNKNOWN & 571868 & 149634 & HEISPROD & ESTIMATED & & & $116-N-3$ \\
\hline 3254 & 10615 & UNKNOWN & 571878 & 149638 & HEISPROD & ESTIMATED & & & $116-N-3$ \\
\hline 3255 & 10616 & UNKNOWN & 571886 & 149643 & HEISPROD & ESTIMATED & & & $116-N-3$ \\
\hline 3256 & 10617 & UNKNOWN & 571895 & 149647 & HEISPROD & ESTIMATED & & & $116-N-3$ \\
\hline 3257 & 10618 & UNKNOWN & 571905 & 149650 & HEISPROD & ESTIMATED & & & $116-\mathrm{N}-3$ \\
\hline 3258 & 10619 & UNKNOWN & 571914 & 149653 & HEISPROD & ESTIMATED & & & $116-\mathrm{N}-3$ \\
\hline 3259 & 10620 & UNKNOWN & 571923 & 149657 & HEISPROD & ESTIMATED & & & $116-N-3$ \\
\hline 3260 & 10621 & UNKNOWN & 571932 & 149660 & HEISPROD & ESTIMATED & & & $116-N-3$ \\
\hline 3261 & 10622 & UNKNOWN & 571940 & 149664 & HEISPROD & ESTIMATED & & & $116-N-3$ \\
\hline 3262 & 10623 & UNKNOWN & 571951 & 149659 & HEISPROD & ESTIMATED & & & $116-\mathrm{N}-3$ \\
\hline 3263 & 10624 & UNKNOWN & 571955 & 149649 & HEISPROD & ESTIMATED & & & $116-N-3$ \\
\hline 3264 & 10625 & UNKNOWN & 571959 & 149640 & HEISPROD & ESTIMATED & & & $116-N-3$ \\
\hline 3265 & 10626 & & & & HEISPROD & & & & 100-NR-1 \\
\hline 3266 & 10627 & UNKNOWN & 572097 & 149802 & HEISPROD & ESTIMATED & ONSITE & & $116-N-3$ \\
\hline 3267 & 10628 & & & & HEISPROD & & & & 116-NR-1 \\
\hline 3268 & 10646 & & & & HEISPROD & & & & 116-F-9 \\
\hline 3269 & 10647 & & & & HEISPROD & & & & 116-F-9 \\
\hline
\end{tabular}




\begin{tabular}{|c|c|c|c|c|c|c|c|c|c|}
\hline & A & $\mathrm{B}$ & $\mathrm{C}$ & $\mathrm{D}$ & $E$ & $\mathrm{~F}$ & G & $\mathrm{H}$ & $\mathrm{I}$ \\
\hline 1 & SAMP_SITE_ID & HORIZ_COORD_TYPE & EW_COORD & NS_COORD & OWNER_ID & COORD_SOURCE & DIST_CLASS & RAD_LEVEL & SAMP_SITE_NAME \\
\hline 3270 & 10648 & & & & HEISPROD & & & & $116-F-9$ \\
\hline 3271 & 10649 & & & & HEISPROD & & & & $116-\mathrm{F}-9$ \\
\hline 3272 & 10650 & UNKNOWN & 571796.7 & 149582.6 & HEISPROD & GPS & ONSITE & & 116-N-3 Pipeline \& By-Pass Trench \\
\hline 3273 & 10666 & UNKNOWN & 571969 & 149561 & 1 HEISPROD & GPS & ONSITE & & 116-N-3 Crib \\
\hline 3274 & 10667 & UNKNOWN & 571973 & 149563 & 3 HEISPROD & GPS & ONSITE & & 116-N-3 Crib \\
\hline 3275 & 10668 & UNKNOWN & 571977 & 149565 & 5 HEISPROD & GPS & ONSITE & & 116-N-3 Crib \\
\hline 3276 & 10669 & UNKNOWN & 571981 & 149567 & 7 HEISPROD & GPS & ONSITE & & 116-N-3 Crib \\
\hline 3277 & 10670 & & & & HEISPROD & & & & 100-F-15 Stockpile \\
\hline 3278 & 10671 & & & & HEISPROD & & & & 100-F-15 Stockpile \\
\hline 3279 & 10690 & UNKNOWN & 571680 & 149678 & 3 HEISPROD & GPS & ONSITE & & 116-N-3 Crib Overburden Stockpiles \\
\hline 3280 & 10691 & UNKNOWN & 571553 & 149699 & HEISPROD & GPS & ONSITE & & 116-N-3 Crib Overburden Stockpiles \\
\hline 3281 & 10692 & UNKNOWN & 571562 & 149696 & HEISPROD & GPS & ONSITE & & 116-N-3 Crib Overburden Stockpiles \\
\hline 3282 & 10693 & UNKNOWN & 571548 & 149683 & 3 HEISPROD & GPS & ONSITE & & 116-N-3 Crib Overburden Stockpiles \\
\hline 3283 & 10694 & UNKNOWN & 571646 & 149690 & HEISPROD & GPS & ONSITE & & 116-N-3 Crib Overburden Stockpiles \\
\hline 3284 & 10695 & UNKNOWN & 571680 & 149694 & 4 HEISPROD & GPS & ONSITE & & 116-N-3 Crib Overburden Stockpiles \\
\hline 3285 & 10696 & UNKNOWN & 571821 & 149619 & HEISPROD & GPS & ONSITE & & 116-N-3 Crib Overburden Stockpiles \\
\hline 3286 & 10697 & UNKNOWN & 571803 & 149617 & 7 HEISPROD & GPS & ONSITE & & 116-N-3 Crib Overburden Stockpiles \\
\hline 3287 & 10698 & UNKNOWN & 571824 & 149608 & 3 HEISPROD & GPS & ONSITE & & 116-N-3 Crib Overburden Stockpiles \\
\hline 3288 & 10710 & & & & HEISPROD & & & & $100 \mathrm{~F}$ Area \\
\hline 3289 & 10730 & & & & HEISPROD & & & & 100-F-11Test Pit \\
\hline 3290 & 10731 & & & & HEISPROD & & & & 100-F-16 Test Pit \\
\hline 3291 & 10732 & & & & HEISPROD & & & & 100-F-4 Test Pit \\
\hline 3292 & 10750 & UNKNOWN & 579688.57 & 146952.23 & 3 HEISPROD & ESTIMATED & ONSITE & & 100-F-2 Shallow Zone \\
\hline 3293 & 10751 & UNKNOWN & 579688.51 & 146957.82 & 2 HEISPROD & ESTIMATED & ONSITE & & 100-F-2 Shallow Zone \\
\hline 3294 & 10752 & UNKNOWN & 579688.66 & 146962.97 & 7 HEISPROD & ESTIMATED & ONSITE & & 100-F-2 Shallow Zone \\
\hline 3295 & 10753 & UNKNOWN & 579688.73 & 146972.09 & 9 HEISPROD & ESTIMATED & ONSITE & & 100-F-2 Shallow Zone \\
\hline 3296 & 10754 & UNKNOWN & 579688.01 & 146975.91 & 1 HEISPROD & ESTIMATED & ONSITE & & 100-F-2 Shallow Zone \\
\hline 3297 & 10755 & UNKNOWN & 579688.96 & 146973.98 & 3 HEISPROD & ESTIMATED & ONSITE & & 100-F-2 Shallow Zone \\
\hline 3298 & 10756 & UNKNOWN & 579691.81 & 146953.4 & 4 HEISPROD & ESTIMATED & ONSITE & & 100-F-2 Shallow Zone \\
\hline 3299 & 10757 & UNKNOWN & 579691.84 & 146955.19 & 9 HEISPROD & ESTIMATED & ONSITE & & 100-F-2 Shallow Zone \\
\hline 3300 & 10758 & UNKNOWN & 579691.95 & 146962.34 & 4 HEISPROD & ESTIMATED & ONSITE & & 100-F-2 Shallow Zone \\
\hline 3301 & 10759 & UNKNOWN & 579692.03 & 146967.71 & 1 HEISPROD & ESTIMATED & ONSITE & & 100-F-2 Shallow Zone \\
\hline 3302 & 10760 & UNKNOWN & 579692.05 & 146969.5 & 5 HEISPROD & ESTIMATED & ONSITE & & 100-F-2 Shallow Zone \\
\hline 3303 & 10761 & UNKNOWN & 579695.37 & 146972.99 & 9 HEISPROD & ESTIMATED & ONSITE & & 100-F-2 Shallow Zone \\
\hline 3304 & 10762 & UNKNOWN & 579695.23 & 146964.05 & 5 HEISPROD & ESTIMATED & ONSITE & & 100-F-2 Shallow Zone \\
\hline 3305 & 10763 & UNKNOWN & 579695.34 & 146971.2 & 2 HEISPROD & ESTIMATED & ONSITE & & 100-F-2 Shallow Zone \\
\hline 3306 & 10764 & UNKNOWN & 579695.18 & 146960.48 & 3 HEISPROD & ESTIMATED & ONSITE & & 100-F-2 Shallow Zone \\
\hline 3307 & 10765 & UNKNOWN & 579695.37 & 146972.99 & 9 HEISPROD & ESTIMATED & ONSITE & & 100-F-2 Shallow Zone \\
\hline
\end{tabular}


A

$\mathrm{B}$

$\mathrm{C}$

$\mathrm{D}$

$\mathrm{E}$

$\mathrm{F}$

G

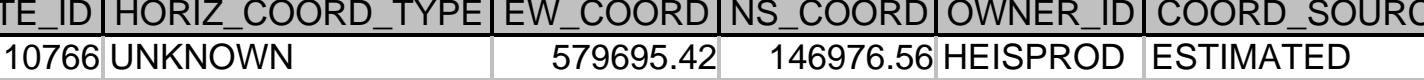

DIST_CLASS

ONSITE

ONSITE

10768 UNKNOWN

\begin{tabular}{l|l|l}
579695.45 & 146978.37 HEISPROD & ESTIMATED
\end{tabular}

ONSITE

ONSITE

$579698.55 \quad 146959.49$ HEISPROD ESTIMATED

10770 UNKNOWN

579698.71 146964.3 HEISPROD ESTIMATED

10771 UNKNOWN

\begin{tabular}{l|l|l}
579698.75 & 146967.46 \\
\end{tabular}

10772 UNKNOWN

579698.75146972 .33 HEISPROD ESTIMATED

ONSITE

ONSITE

579698.77 146973.98 HEISPROD ESTIMATED

ONSITE

10773 UNKNOWN

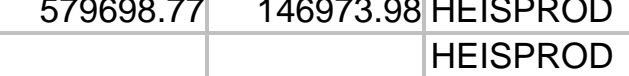

10791

10792

10793

10794

10795

10796

10797

10798

10799

10800

10801

10802

10803

10804

10805

10806

10807

10808

10809

10810

10811

10812

10813

HEISPROD

HEISPROD

HEISPROD

HEISPROD

HEISPROD

HEISPROD

HEISPROD

HEISPROD

HEISPROD

HEISPROD

HEISPROD

HEISPROD

HEISPROD

HEISPROD

HEISPROD

HEISPROD

HEISPROD

HEISPROD

HEISPROD

HEISPROD

HEISPROD

HEISPROD

HEISPROD

HEISPROD

HEISPROD

HEISPROD

HEISPROD

10816

10817

10818

10819

HEISPROD

HEISPROD

ONSITE

\begin{tabular}{|l|l|l|}
\hline ESTIMATED & ONSITE \\
\hline & \\
\hline & \\
\hline & \\
\hline & \\
\hline & \\
\hline & \\
\hline & \\
\hline & \\
\hline & \\
\hline & \\
\hline & \\
\hline & \\
\hline & \\
\hline & \\
\hline & \\
\hline
\end{tabular}

RAD_LEVEL

100-F-2 Shallow Zone

$100-F-2$ Shallow Zone

100-F-2 Shallow Zone

100-F-2 Shallow Zone

100-F-2 Shallow Zone

100-F-2 Shallow Zone

100-F-2 Shallow Zone

100-F-2 Shallow Zone

100-F-15 Stockpile

100-F-15 Stockpile

100-F-15 Stockpile

100-F-15 Stockpile

100-F-15 Stockpile

100-F-15 Stockpile

100-F-15 Stockpile

100-F-15 Stockpile

100-F-15 Stockpile

100-F-15 Stockpile

116-F-9 Trench

100-F-2

100-F-2

$100-\mathrm{F}-2$

100-F-2

100-F-2

100-F-2

100-F-2

100-F-2

$100-\mathrm{F}-2$

$100-\mathrm{F}-2$

116-F-9

116-F-9

116-F-9

116-F-9

116-F-9

116-F-14

116-F-14

Page 88

100-F-2

100-F-2

I

SITE NAME 


\begin{tabular}{|c|c|c|c|c|c|c|c|c|c|}
\hline & $\bar{A}$ & $\mathrm{~B}$ & $\mathrm{C}$ & $\mathrm{D}$ & $E$ & $\mathrm{~F}$ & G & $\mathrm{H}$ & $\mathrm{I}$ \\
\hline 1 & SAMP_SITE_ID & HORIZ_COORD_TYPE & EW_COORD & NS_COORD & OWNER_ID & COORD_SOURCE & DIST_CLASS & RAD_LEVEL & SAMP_SITE_NAME \\
\hline 3346 & 10820 & & & & HEISPROD & & & & 116-F-1 Test Pit \\
\hline 3347 & 10821 & & & & HEISPROD & & & & 116-F-1 Test Pit \\
\hline 3348 & 10822 & & & & HEISPROD & & & & 116-F-1 Test Pit \\
\hline 3349 & 10823 & & & & HEISPROD & & & & 116-F-1 Test Pit \\
\hline 3350 & 10824 & & & & HEISPROD & & & & 116-F-1 Test Pit \\
\hline 3351 & 10825 & & & & HEISPROD & & & & 116-F-1 Test Pit \\
\hline 3352 & 10826 & & & & HEISPROD & & & & 116-F-1 Test Pit \\
\hline 3353 & 10830 & & & & HEISPROD & & & & 100-F-2 Shallow Zone \\
\hline 3354 & 10831 & & & & HEISPROD & & & & 100-F-2 Shallow Zone \\
\hline 3355 & 10832 & & & & HEISPROD & & & & 100-F-2 Shallow Zone \\
\hline 3356 & 10833 & & & & HEISPROD & & & & 100-F-2 Shallow Zone \\
\hline 3357 & 10834 & & & & HEISPROD & & & & 100-F-2 Shallow Zone \\
\hline 3358 & 10835 & & & & HEISPROD & & & & 100-F-2 Shallow Zone \\
\hline 3359 & 10836 & UNKNOWN & 571577 & 149626 & HEISPROD & GPS & ONSITE & & 116-N-3 Pipeline/By-pass \\
\hline 3360 & 10837 & UNKNOWN & 571577 & 149626 & HEISPROD & GPS & ONSITE & & 116-N-3 Pipeline/By-pass \\
\hline 3361 & 10850 & & & & HEISPROD & & & & 116-F-9 Trench \\
\hline 3362 & 10851 & & & & HEISPROD & & & & 116-F-9 Trench \\
\hline 3363 & 10852 & & & & HEISPROD & & & & 116-F-9 Trench \\
\hline 3364 & 10853 & & & & HEISPROD & & & & 116-F-9 Trench \\
\hline 3365 & 10854 & & & & HEISPROD & & & & 116-F-9 Trench \\
\hline 3366 & 10855 & & & & HEISPROD & & & & 100-F-19 Deep Zone \\
\hline 3367 & 10856 & & & & HEISPROD & & & & 100-F-19 \\
\hline 3368 & 10857 & & & & HEISPROD & & & & 100-F-19 \\
\hline 3369 & 10858 & & & & HEISPROD & & & & 116-F-14 Phase II \\
\hline 3370 & 10859 & & & & HEISPROD & & & & 116-F-2 South By-Pass Southeast Plume \\
\hline 3371 & 10860 & & & & HEISPROD & & & & 116-F-2 South By-Pass Southeast Plume \\
\hline 3372 & 10861 & & & & HEISPROD & & & & 116-F-2 South By-Pass Southeast Plume \\
\hline 3373 & 10862 & & & & HEISPROD & & & & 116-F-2 South By-Pass Southeast Plume \\
\hline 3374 & 10863 & & & & HEISPROD & & & & 116-F-14 Shallow Zone \\
\hline 3375 & 10864 & & & & HEISPROD & & & & 116-F-14 Shallow Zone \\
\hline 3376 & 10865 & & & & HEISPROD & & & & 116-F-14 Shallow Zone \\
\hline 3377 & 10866 & & & & HEISPROD & & & & 116-F-14 Shallow Zone \\
\hline 3378 & 10867 & & & & HEISPROD & & & & 116-F-14 Shallow Zone \\
\hline 3379 & 10868 & & & & HEISPROD & & & & 116-F-14 Shallow Zone \\
\hline 3380 & 10869 & & & & HEISPROD & & & & 116-F-14 Shallow Zone \\
\hline 3381 & 10870 & & & & HEISPROD & & & & 116-F-14 Shallow Zone \\
\hline 3382 & 10871 & & & & HEISPROD & & & & 116-F-14 Shallow Zone \\
\hline 3383 & 10890 & & & & HEISPROD & & & & 116-F-1 Test Pit \\
\hline
\end{tabular}

Page 89 


\begin{tabular}{|c|c|c|c|c|c|c|c|c|c|}
\hline & $A$ & $\mathrm{~B}$ & $\mathrm{C}$ & $\mathrm{D}$ & $E$ & $\mathrm{~F}$ & $\mathrm{G}$ & $\mathrm{H}$ & $\mathrm{I}$ \\
\hline 1 & SAMP_SITE_ID & HORIZ_COORD_TYPE & EW_COORD & NS_COORD & OWNER_ID & COORD_SOURCE & DIST_CLASS & RAD_LEVEL & SAMP_SITE_NAME \\
\hline 3384 & 10891 & & & & HEISPROD & & & & 116-F-1 Test Pit \\
\hline 3385 & 10892 & & & & HEISPROD & & & & 116-F-14 Shallow Zone \\
\hline 3386 & 10893 & & & & HEISPROD & & & & 116-F-14 Shallow Zone \\
\hline 3387 & 10894 & & & & HEISPROD & & & & 116-F-14 Shallow Zone \\
\hline 3388 & 10895 & & & & HEISPROD & & & & 116-F-14 Shallow Zone \\
\hline 3389 & 10896 & & & & HEISPROD & & & & 116-F-14 Shallow Zone \\
\hline 3390 & 10897 & & & & HEISPROD & & & & 116-F-14 Shallow Zone \\
\hline 3391 & 10898 & & & & HEISPROD & & & & 116-F-14 Shallow Zone \\
\hline 3392 & 10899 & & & & HEISPROD & & & & $116-\mathrm{F}-9$ \\
\hline 3393 & 10900 & & & & HEISPROD & & & & 100-F-19 \\
\hline 3394 & 10901 & & & & HEISPROD & & & & 100-F-19 \\
\hline 3395 & 10902 & UNKNOWN & 571890 & 149532 & HEISPROD & GPS & ONSITE & & 116-N-3 Crib \\
\hline 3396 & 10903 & UNKNOWN & 571891 & 149532.5 & HEISPROD & GPS & ONSITE & & 116-N-3 Crib \\
\hline 3397 & 10904 & UNKNOWN & 571892.5 & 149532.5 & HEISPROD & GPS & ONSITE & & 116-N-3 Crib \\
\hline 3398 & 10905 & UNKNOWN & 571916 & 149541 & HEISPROD & GPS & ONSITE & & 116-N-3 Crib \\
\hline 3399 & 10906 & UNKNOWN & 571922 & 149543 & HEISPROD & GPS & ONSITE & & 116-N-3 Crib \\
\hline 3400 & 10907 & UNKNOWN & 571926 & 149544 & HEISPROD & GPS & ONSITE & & 116-N-3 Crib \\
\hline 3401 & 10908 & UNKNOWN & 571797 & 149583 & HEISPROD & GPS & ONSITE & & 116-N-3 Crib \\
\hline 3402 & 10909 & UNKNOWN & 571795 & 149584 & HEISPROD & GPS & ONSITE & & 116-N-3 Crib \\
\hline 3403 & 10910 & UNKNOWN & 571573 & 149624.5 & HEISPROD & GPS & ONSITE & & 116-N-3 Pipeline/By-pass \\
\hline 3404 & 10911 & UNKNOWN & 571571 & 149625.5 & HEISPROD & GPS & ONSITE & & 116-N-3 Pipeline/By-pass \\
\hline 3405 & 10912 & UNKNOWN & 571569 & 149626.7 & HEISPROD & GPS & ONSITE & & 116-N-3 Pipeline/By-pass \\
\hline 3406 & 10930 & UNKNOWN & 565299 & 145303 & HEISPROD & ESTIMATED & ONSITE & & 100-B-8 \\
\hline 3407 & 10931 & UNKNOWN & 565285 & 145299 & HEISPROD & ESTIMATED & ONSITE & & 100-B-8 \\
\hline 3408 & 10932 & UNKNOWN & 565277 & 145298 & HEISPROD & ESTIMATED & ONSITE & & 100-B-8 \\
\hline 3409 & 10933 & UNKNOWN & 565260 & 145301 & HEISPROD & ESTIMATED & ONSITE & & 100-B-8 \\
\hline 3410 & 10934 & UNKNOWN & 565265 & 145313 & HEISPROD & ESTIMATED & ONSITE & & 100-B-8 \\
\hline 3411 & 10935 & UNKNOWN & 565274 & 145314 & HEISPROD & ESTIMATED & ONSITE & & 100-B-8 \\
\hline 3412 & 10936 & UNKNOWN & 565283 & 145315 & HEISPROD & ESTIMATED & ONSITE & & 100-B-8 \\
\hline 3413 & 10937 & UNKNOWN & 565293 & 145314 & HEISPROD & ESTIMATED & ONSITE & & 100-B-8 \\
\hline 3414 & 10938 & UNKNOWN & 565271 & 145320 & HEISPROD & ESTIMATED & ONSITE & & 100-B-8 \\
\hline 3415 & 10939 & & & & HEISPROD & & & & 100-B-8 \\
\hline 3416 & 10940 & & & & HEISPROD & & & & 100-B-8 \\
\hline 3417 & 10941 & & & & HEISPROD & & & & 132-B-6 \\
\hline 3418 & 10942 & & & & HEISPROD & & & & 100-B-8 \\
\hline 3419 & 10943 & & & & HEISPROD & & & & 100-B-8 \\
\hline 3420 & 10944 & & & & HEISPROD & & & & 100-B-8 \\
\hline 3421 & 10945 & & & & HEISPROD & & & & 100-B-8 \\
\hline
\end{tabular}


A

$\mathrm{B}$

\begin{tabular}{l|l}
$\mathrm{C}$ & $\mathrm{D}$ \\
\hline
\end{tabular}

\begin{tabular}{l|l|l}
$\mathrm{D}$ & $\mathrm{E}$ & $\mathrm{F}$
\end{tabular}

F
10946 UNKNOWN 565715 \begin{tabular}{c|c|c|}
145288 HEISPROD & ESTIMATED \\
145288 HEISPROD & ESTIMATED
\end{tabular} 10947 UNKNOWN 10948

10949

10950

10951 UNKNOWN

10952

10953 UNKNOWN

10954 UNKNOWN

10955 UNKNOWN

10956

10957 UNKNOWN

10958 UNKNOWN

10959 UNKNOWN

10960 UNKNOWN

HEISPROD

HEISPROD

HEISPROD

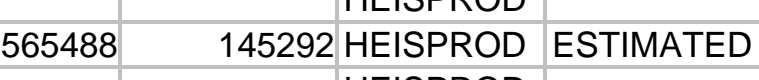
HEISPROD

$565478 \quad 145328$ HEISPROD ESTIMATED

145256 HEISPROD ESTIMATED

145264 HEISPROD ESTIMATED

$$
\text { HEISPROD }
$$

145272 HEISPROD ESTIMATED

145272 HEISPROD ESTIMATED

145266 HEISPROD ESTIMATED

145266 HEISPROD ESTIMATED

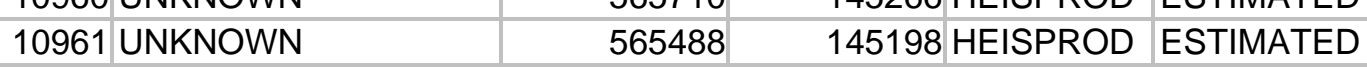

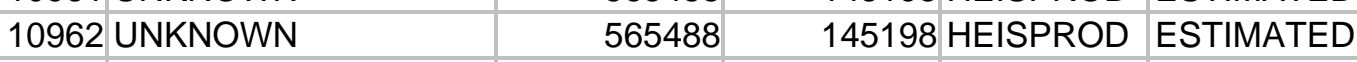

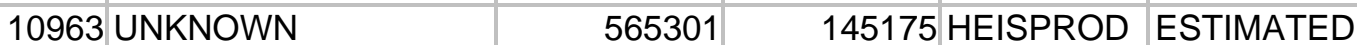

\begin{tabular}{|l|l|l|l}
\hline 10964 UNKNOWN & 565300 & 145138 HEISPROD & ESTIMATED \\
\hline
\end{tabular}

10965 UNKNOWN

565324

144999 HEISPROD ESTIMATED

G

$\mathrm{H}$

I

10971

HEISPROD

HEISPROD

HEISPROD

HEISPROD

HEISPROD

HEISPROD

HEISPROD

HEISPROD

HEISPROD

HEISPROD

HEISPROD

HEISPROD

HEISPROD

HEISPROD

HEISPROD

HEISPROD

HEISPROD

HEISPROD

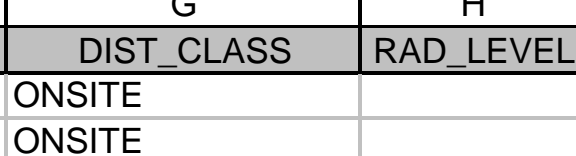

$100-\mathrm{C}-6$

SAMP SITE NAME

$100-\mathrm{C}-6$

100-B-8

$100-B-8$

ONSITE

100-B-8

$100-C-6$

\begin{tabular}{|l|l|}
\hline ONSITE & $100-\mathrm{C}-6$ \\
\hline
\end{tabular}

ONSITE

ONSITE

ONSITE

ONSITE

ONSITE

ONSITE

ONSITE

ONSITE

ONSITE

$100-\mathrm{C}-6$

$100-\mathrm{C}-6$

100-C-9

100-C-9

$100-\mathrm{C}-6$

$100-\mathrm{C}-6$

100-C-6

$100-\mathrm{C}-6$

100-B-8

100-B-8

100-B-8

100-F-19 Overburden Zone

100-F-19 Overburden Zone

100-F-19 Overburden Zone

100-F-19 Overburden Zone

100-F-19 Overburden Zone

100-F-19 Overburden Zone

100-F-19 Overburden Zone

100-F-19 Overburden Zone

100-F-19 Overburden Zone

100-F-19 Overburden Zone

100-F-19 Overburden Zone

100-F-19 Overburden Zone

100-F-19 Overburden Zone

100-F-19 Overburden Zone

100-F-19 Overburden Zone

100-F-19 Shallow Zone

100-F-19 Shallow Zone

10996

10997

Page 91

100-F-19 Shallow Zone 


\begin{tabular}{|c|c|c|c|c|c|c|c|c|c|}
\hline & $A$ & $\mathrm{~B}$ & $\mathrm{C}$ & $\mathrm{D}$ & $E$ & $\mathrm{~F}$ & $\mathrm{G}$ & $\mathrm{H}$ & $\mathrm{I}$ \\
\hline 1 & SAMP_SITE_ID & HORIZ_COORD_TYPE & EW_COORD & NS_COORD & OWNER_ID & COORD_SOURCE & DIST_CLASS & RAD_LEVEL & SAMP_SITE_NAME \\
\hline 3460 & 10998 & & & & HEISPROD & & & & 100-F-19 Shallow Zone \\
\hline 3461 & 10999 & & & & HEISPROD & & & & 100-F-19 Shallow Zone \\
\hline 3462 & 11000 & & & & HEISPROD & & & & 100-F-19 Shallow Zone \\
\hline 3463 & 11001 & & & & HEISPROD & & & & 100-F-19 Shallow Zone \\
\hline 3464 & 11002 & & & & HEISPROD & & & & 100-F-19 Shallow Zone \\
\hline 3465 & 11003 & & & & HEISPROD & & & & 100-F-19 \\
\hline 3466 & 11004 & & & & HEISPROD & & & & 100-F-19 \\
\hline 3467 & 11005 & & & & HEISPROD & & & & $100-F-19$ \\
\hline 3468 & 11006 & & & & HEISPROD & & & & 100-F-19 \\
\hline 3469 & 11007 & & & & HEISPROD & & & & 100-F-19 \\
\hline 3470 & 11008 & & & & HEISPROD & & & & $100-F-19$ \\
\hline 3471 & 11009 & & & & HEISPROD & & & & 100-F-19 \\
\hline 3472 & 11010 & & & & HEISPROD & & & & $100-F-19$ \\
\hline 3473 & 11011 & & & & HEISPROD & & & & $116-F-9$ \\
\hline 3474 & 11012 & & & & HEISPROD & & & & $100-F-2$ \\
\hline 3475 & 11023 & UNKNOWN & 565320 & 144980 & HEISPROD & ESTIMATED & ONSITE & & $100-B-8$ \\
\hline 3476 & 11024 & UNKNOWN & 565320 & 144980 & HEISPROD & ESTIMATED & & & $100-B-8$ \\
\hline 3477 & 11044 & UNKNOWN & 580397.96 & 147671.62 & 2 HEISPROD & ESTIMATED & ONSITE & & $100-F-19$ \\
\hline 3478 & 11064 & NAD83(91) & 554347.44 & 139925.47 & 7 HEISPROD & SURVEYED & ONSITE & & McGee Ranch \\
\hline 3479 & 11065 & NAD83(91) & 554357.64 & 139305.18 & 3 HEISPROD & SURVEYED & ONSITE & & McGee Ranch \\
\hline 3480 & 11066 & NAD83(91) & 554369.97 & 138752.79 & 9 HEISPROD & SURVEYED & ONSITE & & McGee Ranch \\
\hline 3481 & 11067 & NAD83(91) & 554348.84 & 139144.82 & 2 HEISPROD & SURVEYED & ONSITE & & McGee Ranch \\
\hline 3482 & 11068 & NAD83(91) & 554955.26 & 139938.14 & 4 HEISPROD & SURVEYED & ONSITE & & McGee Ranch \\
\hline 3483 & 11069 & NAD83(91) & 554967 & 139316.77 & 7 HEISPROD & SURVEYED & ONSITE & & McGee Ranch \\
\hline 3484 & 11070 & NAD83(91) & 554973.17 & 138732.8 & 3 HEISPROD & SURVEYED & ONSITE & & McGee Ranch \\
\hline 3485 & 11071 & NAD83(91) & 554973.17 & 138732.8 & 3 HEISPROD & SURVEYED & ONSITE & & McGee Ranch \\
\hline 3486 & 11072 & NAD83(91) & 554960.84 & 138119.34 & 4 HEISPROD & SURVEYED & ONSITE & & McGee Ranch \\
\hline 3487 & 11073 & NAD83(91) & 554960.84 & 138119.34 & 4 HEISPROD & SURVEYED & ONSITE & & McGee Ranch \\
\hline 3488 & 11074 & NAD83(91) & 555568.9 & 139927.45 & 5 HEISPROD & SURVEYED & ONSITE & & McGee Ranch \\
\hline 3489 & 11075 & NAD83(91) & 555568.9 & 139927.45 & 5 HEISPROD & SURVEYED & ONSITE & & McGee Ranch \\
\hline 3490 & 11076 & NAD83(91) & 555573.33 & 139318.71 & 1 HEISPROD & SURVEYED & ONSITE & & McGee Ranch \\
\hline 3491 & 11077 & NAD83(91) & 555573.33 & 139318.71 & 1 HEISPROD & SURVEYED & ONSITE & & McGee Ranch \\
\hline 3492 & 11078 & NAD83(91) & 555580.62 & 138739.69 & 9 HEISPROD & SURVEYED & ONSITE & & McGee Ranch \\
\hline 3493 & 11079 & NAD83(91) & 555575.27 & 138090.64 & 4 HEISPROD & SURVEYED & ONSITE & & McGee Ranch \\
\hline 3494 & 11080 & NAD83(91) & 555575.27 & 138090.64 & 4 HEISPROD & SURVEYED & ONSITE & & McGee Ranch \\
\hline 3495 & 11081 & NAD83(91) & 556181.26 & 139927.37 & 7 HEISPROD & SURVEYED & ONSITE & & McGee Ranch \\
\hline 3496 & 11082 & NAD83(91) & 556181.26 & 139927.37 & 7 HEISPROD & SURVEYED & ONSITE & & McGee Ranch \\
\hline 3497 & 11083 & NAD83(91) & 556187.23 & 139320.43 & 3 HEISPROD & SURVEYED & ONSITE & & McGee Ranch \\
\hline
\end{tabular}




\begin{tabular}{|c|c|c|c|c|c|c|c|c|c|}
\hline & $\bar{A}$ & $B$ & $\mathrm{C}$ & $\mathrm{D}$ & $E$ & $\mathrm{~F}$ & G & $\mathrm{H}$ & $\mathrm{I}$ \\
\hline 1 & SAMP_SITE_ID & HORIZ_COORD_TYPE & EW_COORD & NS_COORD & OWNER_ID & COORD_SOURCE & DIST_CLASS & RAD_LEVEL & SAMP_SITE_NAME \\
\hline 3498 & 11084 & NAD83(91) & 556187.23 & 139320.43 & HEISPROD & SURVEYED & ONSITE & & McGee Ranch \\
\hline 3499 & 11085 & NAD83(91) & 556191.79 & 138740.91 & HEISPROD & SURVEYED & ONSITE & & McGee Ranch \\
\hline 3500 & 11086 & NAD83(91) & 556191.79 & 138740.91 & HEISPROD & SURVEYED & ONSITE & & McGee Ranch \\
\hline 3501 & 11087 & NAD83(91) & 556183.99 & 138063.35 & HEISPROD & SURVEYED & ONSITE & & McGee Ranch \\
\hline 3502 & 11088 & NAD83(91) & 556183.99 & 138063.35 & HEISPROD & SURVEYED & ONSITE & & McGee Ranch \\
\hline 3503 & 11089 & NAD83(91) & 556183.99 & 138063.35 & HEISPROD & SURVEYED & ONSITE & & McGee Ranch \\
\hline 3504 & 11090 & NAD83(91) & 556790 & 139936.24 & HEISPROD & SURVEYED & ONSITE & & McGee Ranch \\
\hline 3505 & 11091 & NAD83(91) & 556790 & 139936.24 & HEISPROD & SURVEYED & ONSITE & & McGee Ranch \\
\hline 3506 & 11092 & NAD83(91) & 556796.24 & 139323.71 & HEISPROD & SURVEYED & ONSITE & & McGee Ranch \\
\hline 3507 & 11093 & NAD83(91) & 556796.24 & 139323.71 & HEISPROD & SURVEYED & ONSITE & & McGee Ranch \\
\hline 3508 & 11094 & NAD83(91) & 556799.75 & 138741.3 & HEISPROD & SURVEYED & ONSITE & & McGee Ranch \\
\hline 3509 & 11095 & NAD83(91) & 556799.75 & 138741.3 & HEISPROD & SURVEYED & ONSITE & & McGee Ranch \\
\hline 3510 & 11096 & NAD83(91) & 556772.5 & 138238.19 & HEISPROD & SURVEYED & ONSITE & & McGee Ranch \\
\hline 3511 & 11097 & NAD83(91) & 556772.5 & 138238.19 & HEISPROD & SURVEYED & ONSITE & & McGee Ranch \\
\hline 3512 & 11098 & NAD83(91) & 556772.5 & 138238.19 & HEISPROD & SURVEYED & ONSITE & & McGee Ranch \\
\hline 3513 & 11099 & NAD83(91) & 557398.98 & 139943.5 & HEISPROD & SURVEYED & ONSITE & & McGee Ranch \\
\hline 3514 & 11100 & NAD83(91) & 557398.98 & 139943.5 & HEISPROD & SURVEYED & ONSITE & & McGee Ranch \\
\hline 3515 & 11101 & NAD83(91) & 557403.88 & 139329.5 & HEISPROD & SURVEYED & ONSITE & & McGee Ranch \\
\hline 3516 & 11102 & NAD83(91) & 557403.88 & 139329.5 & HEISPROD & SURVEYED & ONSITE & & McGee Ranch \\
\hline 3517 & 11103 & NAD83(91) & 557403.88 & 139329.5 & HEISPROD & SURVEYED & ONSITE & & McGee Ranch \\
\hline 3518 & 11104 & NAD83(91) & 557410.88 & 138744.59 & HEISPROD & SURVEYED & ONSITE & & McGee Ranch \\
\hline 3519 & 11105 & NAD83(91) & 557410.88 & 138744.59 & HEISPROD & SURVEYED & ONSITE & & McGee Ranch \\
\hline 3520 & 11106 & NAD83(91) & 557410.88 & 138744.59 & HEISPROD & SURVEYED & ONSITE & & McGee Ranch \\
\hline 3521 & 11107 & NAD83(91) & 557884.81 & 138748.36 & HEISPROD & SURVEYED & ONSITE & & McGee Ranch \\
\hline 3522 & 11108 & NAD83(91) & 557884.81 & 138748.36 & HEISPROD & SURVEYED & ONSITE & & McGee Ranch \\
\hline 3523 & 11109 & NAD83(91) & 557884.81 & 138748.36 & HEISPROD & SURVEYED & ONSITE & & McGee Ranch \\
\hline 3524 & 11110 & NAD83(91) & 557884.81 & 138748.36 & HEISPROD & SURVEYED & ONSITE & & McGee Ranch \\
\hline 3525 & 11111 & NAD83(91) & 554646.9 & 139624.66 & HEISPROD & SURVEYED & ONSITE & & McGee Ranch \\
\hline 3526 & 11112 & NAD83(91) & 554666.9 & 139010.9 & HEISPROD & SURVEYED & ONSITE & & McGee Ranch \\
\hline 3527 & 11113 & NAD83(91) & 554660.18 & 138450.48 & HEISPROD & SURVEYED & ONSITE & & McGee Ranch \\
\hline 3528 & 11114 & NAD83(91) & 555276.46 & 139630.67 & HEISPROD & SURVEYED & ONSITE & & McGee Ranch \\
\hline 3529 & 11115 & NAD83(91) & 555276.46 & 139630.67 & HEISPROD & SURVEYED & ONSITE & & McGee Ranch \\
\hline 3530 & 11116 & NAD83(91) & 555277.86 & 139016.84 & HEISPROD & SURVEYED & ONSITE & & McGee Ranch \\
\hline 3531 & 11117 & NAD83(91) & 555277.86 & 139016.84 & HEISPROD & SURVEYED & ONSITE & & McGee Ranch \\
\hline 3532 & 11118 & NAD83(91) & 555273.8 & 138439.08 & HEISPROD & SURVEYED & ONSITE & & McGee Ranch \\
\hline 3533 & 11119 & NAD83(91) & 555273.8 & 138439.08 & HEISPROD & SURVEYED & ONSITE & & McGee Ranch \\
\hline 3534 & 11120 & NAD83(91) & 555213.73 & 137840.14 & HEISPROD & SURVEYED & ONSITE & & McGee Ranch \\
\hline 3535 & 11121 & NAD83(91) & 555878.86 & 139622.14 & HEISPROD & SURVEYED & ONSITE & & McGee Ranch \\
\hline
\end{tabular}




\begin{tabular}{|c|c|c|c|c|c|c|c|c|c|}
\hline & $\bar{A}$ & $B$ & $\mathrm{C}$ & $\mathrm{D}$ & $E$ & $\mathrm{~F}$ & G & $\mathrm{H}$ & $\mathrm{I}$ \\
\hline 1 & SAMP_SITE_ID & HORIZ_COORD_TYPE & EW_COORD & NS_COORD & OWNER_ID & COORD_SOURCE & DIST_CLASS & RAD_LEVEL & SAMP_SITE_NAME \\
\hline 3536 & 11122 & NAD83(91) & 555878.86 & 139622.14 & HEISPROD & SURVEYED & ONSITE & & McGee Ranch \\
\hline 3537 & 11123 & NAD83(91) & 555884.4 & 139013.85 & HEISPROD & SURVEYED & ONSITE & & McGee Ranch \\
\hline 3538 & 11124 & NAD83(91) & 555884.4 & 139013.85 & HEISPROD & SURVEYED & ONSITE & & McGee Ranch \\
\hline 3539 & 11125 & NAD83(91) & 555922.17 & 138541.15 & HEISPROD & SURVEYED & ONSITE & & McGee Ranch \\
\hline 3540 & 11126 & NAD83(91) & 555922.17 & 138541.15 & HEISPROD & SURVEYED & ONSITE & & McGee Ranch \\
\hline 3541 & 11127 & NAD83(91) & 556488 & 139628 & HEISPROD & SURVEYED & ONSITE & & McGee Ranch \\
\hline 3542 & 11128 & NAD83(91) & 556488 & 139628 & HEISPROD & SURVEYED & ONSITE & & McGee Ranch \\
\hline 3543 & 11129 & NAD83(91) & 556495.63 & 139020.52 & HEISPROD & SURVEYED & ONSITE & & McGee Ranch \\
\hline 3544 & 11130 & NAD83(91) & 556495.63 & 139020.52 & HEISPROD & SURVEYED & ONSITE & & McGee Ranch \\
\hline 3545 & 11131 & NAD83(91) & 556522.29 & 138399.14 & HEISPROD & SURVEYED & ONSITE & & McGee Ranch \\
\hline 3546 & 11132 & NAD83(91) & 557097.99 & 139631.83 & HEISPROD & SURVEYED & ONSITE & & McGee Ranch \\
\hline 3547 & 11133 & NAD83(91) & 557100.93 & 139023.06 & HEISPROD & SURVEYED & ONSITE & & McGee Ranch \\
\hline 3548 & 11134 & NAD83(91) & 557126.71 & 138425.63 & HEISPROD & SURVEYED & ONSITE & & McGee Ranch \\
\hline 3549 & 11135 & NAD83(91) & 557703.09 & 139636.94 & HEISPROD & SURVEYED & ONSITE & & McGee Ranch \\
\hline 3550 & 11136 & NAD83(91) & 557703.09 & 139636.94 & HEISPROD & SURVEYED & ONSITE & & McGee Ranch \\
\hline 3551 & 11137 & NAD83(91) & 557693.29 & 139070.61 & HEISPROD & SURVEYED & ONSITE & & McGee Ranch \\
\hline 3552 & 11138 & NAD83(91) & 557693.29 & 139070.61 & HEISPROD & SURVEYED & ONSITE & & McGee Ranch \\
\hline 3553 & 11139 & NAD83(91) & 557733.19 & 138457.16 & HEISPROD & SURVEYED & ONSITE & & McGee Ranch \\
\hline 3554 & 11140 & NAD83(91) & 557733.19 & 138457.16 & HEISPROD & SURVEYED & ONSITE & & McGee Ranch \\
\hline 3555 & 11164 & UNKNOWN & 580920.66 & 147668.48 & HEISPROD & ESTIMATED & ONSITE & & 126-F-1 Ash Pit \\
\hline 3556 & 11165 & UNKNOWN & 580921.15 & 147657.15 & HEISPROD & ESTIMATED & ONSITE & & 126-F-1 Ash Pit \\
\hline 3557 & 11166 & UNKNOWN & 580923.03 & 147623.83 & HEISPROD & ESTIMATED & ONSITE & & 126-F-1 Ash Pit \\
\hline 3558 & 11167 & UNKNOWN & 580924.23 & 147602.49 & HEISPROD & ESTIMATED & ONSITE & & 126-F-1 Ash Pit \\
\hline 3559 & 11168 & UNKNOWN & 580928 & 147627.08 & HEISPROD & ESTIMATED & ONSITE & & 126-F-1 Ash Pit \\
\hline 3560 & 11169 & UNKNOWN & 580929.28 & 147604.75 & HEISPROD & ESTIMATED & ONSITE & & 126-F-1 Ash Pit \\
\hline 3561 & 11170 & UNKNOWN & 580936.48 & 147660.28 & HEISPROD & ESTIMATED & ONSITE & & 126-F-1 Ash Pit \\
\hline 3562 & 11171 & UNKNOWN & 580936.48 & 147660.28 & HEISPROD & ESTIMATED & ONSITE & & 126-F-1 Ash Pit \\
\hline 3563 & 11172 & UNKNOWN & 580937.71 & 147639.46 & HEISPROD & ESTIMATED & ONSITE & & 126-F-1 Ash Pit \\
\hline 3564 & 11173 & UNKNOWN & 580938.35 & 147628.71 & HEISPROD & ESTIMATED & ONSITE & & 126-F-1 Ash Pit \\
\hline 3565 & 11174 & UNKNOWN & 580939.67 & 147606.48 & HEISPROD & ESTIMATED & ONSITE & & 126-F-1 Ash Pit \\
\hline 3566 & 11175 & UNKNOWN & 580940.2 & 147595.09 & HEISPROD & ESTIMATED & ONSITE & & 126-F-1 Ash Pit \\
\hline 3567 & 11176 & UNKNOWN & 580941.36 & 147668.71 & HEISPROD & ESTIMATED & ONSITE & & 126-F-1 Ash Pit \\
\hline 3568 & 11177 & UNKNOWN & 580942.71 & 147646.28 & HEISPROD & ESTIMATED & ONSITE & & 126-F-1 Ash Pit \\
\hline 3569 & 11178 & UNKNOWN & 580943.33 & 147635.59 & HEISPROD & ESTIMATED & ONSITE & & 126-F-1 Ash Pit \\
\hline 3570 & 11179 & UNKNOWN & 580943.95 & 147625.1 & HEISPROD & ESTIMATED & ONSITE & & 126-F-1 Ash Pit \\
\hline 3571 & 11180 & UNKNOWN & 580945.52 & 147594.94 & HEISPROD & ESTIMATED & ONSITE & & 126-F-1 Ash Pit \\
\hline 3572 & 11181 & UNKNOWN & 580948.04 & 147648.04 & HEISPROD & ESTIMATED & ONSITE & & 126-F-1 Ash Pit \\
\hline 3573 & 11182 & UNKNOWN & 580953.32 & 147654.76 & HEISPROD & ESTIMATED & ONSITE & & 126-F-1 Ash Pit \\
\hline
\end{tabular}




\begin{tabular}{|c|c|c|c|c|c|c|c|c|c|}
\hline & $\bar{A}$ & $\mathrm{~B}$ & $\mathrm{C}$ & $\mathrm{D}$ & $E$ & $\mathrm{~F}$ & G & $\mathrm{H}$ & $\mathrm{I}$ \\
\hline 1 & SAMP_SITE_ID & HORIZ_COORD_TYPE & EW_COORD & NS_COORD & OWNER_ID & COORD_SOURCE & DIST_CLASS & RAD_LEVEL & SAMP_SITE_NAME \\
\hline 3574 & 11183 & UNKNOWN & 580953.94 & 147644.42 & HEISPROD & ESTIMATED & ONSITE & & 126-F-1 Ash Pit \\
\hline 3575 & 11184 & UNKNOWN & 580954.55 & 147634.32 & HEISPROD & ESTIMATED & ONSITE & & 126-F-1 Ash Pit \\
\hline 3576 & 11185 & UNKNOWN & 580956.59 & 147596.24 & HEISPROD & ESTIMATED & ONSITE & & 126-F-1 Ash Pit \\
\hline 3577 & 11186 & UNKNOWN & 580960.63 & 147638.23 & HEISPROD & ESTIMATED & ONSITE & & 126-F-1 Ash Pit \\
\hline 3578 & 11187 & UNKNOWN & 580961.31 & 147630.21 & HEISPROD & ESTIMATED & ONSITE & & 126-F-1 Ash Pit \\
\hline 3579 & 11188 & UNKNOWN & 580929.27 & 147581.92 & HEISPROD & ESTIMATED & ONSITE & & 126-F-1 Ash Pit \\
\hline 3580 & 11189 & UNKNOWN & 580929.64 & 147571.27 & HEISPROD & ESTIMATED & ONSITE & & 126-F-1 Ash Pit \\
\hline 3581 & 11190 & UNKNOWN & 580930.57 & 147550.09 & HEISPROD & ESTIMATED & ONSITE & & 126-F-1 Ash Pit \\
\hline 3582 & 11191 & UNKNOWN & 580931.03 & 147539.66 & HEISPROD & ESTIMATED & ONSITE & & 126-F-1 Ash Pit \\
\hline 3583 & 11192 & UNKNOWN & 580934.35 & 147582.36 & HEISPROD & ESTIMATED & ONSITE & & 126-F-1 Ash Pit \\
\hline 3584 & 11193 & UNKNOWN & 580935.44 & 147560.1 & HEISPROD & ESTIMATED & ONSITE & & 126-F-1 Ash Pit \\
\hline 3585 & 11194 & UNKNOWN & 580940.57 & 147561.16 & HEISPROD & ESTIMATED & ONSITE & & 126-F-1 Ash Pit \\
\hline 3586 & 11195 & UNKNOWN & 580941.22 & 147550.3 & HEISPROD & ESTIMATED & ONSITE & & 126-F-1 Ash Pit \\
\hline 3587 & 11196 & UNKNOWN & 580943.13 & 147518.42 & HEISPROD & ESTIMATED & ONSITE & & 126-F-1 Ash Pit \\
\hline 3588 & 11197 & UNKNOWN & 580944.55 & 147584.29 & HEISPROD & ESTIMATED & ONSITE & & 126-F-1 Ash Pit \\
\hline 3589 & 11198 & UNKNOWN & 580946.31 & 147552.61 & HEISPROD & ESTIMATED & ONSITE & & 126-F-1 Ash Pit \\
\hline 3590 & 11199 & UNKNOWN & 580946.96 & 147541.77 & HEISPROD & ESTIMATED & ONSITE & & 126-F-1 Ash Pit \\
\hline 3591 & 11200 & UNKNOWN & 580949.77 & 147584.66 & HEISPROD & ESTIMATED & ONSITE & & 126-F-1 Ash Pit \\
\hline 3592 & 11201 & UNKNOWN & 580950.33 & 147573.58 & HEISPROD & ESTIMATED & ONSITE & & 126-F-1 Ash Pit \\
\hline 3593 & 11202 & UNKNOWN & 580952.28 & 147540.62 & HEISPROD & ESTIMATED & ONSITE & & 126-F-1 Ash Pit \\
\hline 3594 & 11203 & UNKNOWN & 580953.55 & 147519.12 & HEISPROD & ESTIMATED & ONSITE & & 126-F-1 Ash Pit \\
\hline 3595 & 11204 & UNKNOWN & 580957.34 & 147542.84 & HEISPROD & ESTIMATED & ONSITE & & 126-F-1 Ash Pit \\
\hline 3596 & 11205 & UNKNOWN & 580958.66 & 147520.78 & HEISPROD & ESTIMATED & ONSITE & & 126-F-1 Ash Pit \\
\hline 3597 & 11206 & UNKNOWN & 580960.48 & 147586.62 & HEISPROD & ESTIMATED & ONSITE & & 126-F-1 Ash Pit \\
\hline 3598 & 11207 & UNKNOWN & 580965.09 & 147520.96 & HEISPROD & ESTIMATED & ONSITE & & 126-F-1 Ash Pit \\
\hline 3599 & 11208 & UNKNOWN & 580966.59 & 147508 & HEISPROD & ESTIMATED & ONSITE & & 126-F-1 Ash Pit \\
\hline 3600 & 11209 & UNKNOWN & 580971.86 & 147529.28 & HEISPROD & ESTIMATED & ONSITE & & 126-F-1 Ash Pit \\
\hline 3601 & 11210 & UNKNOWN & 580975.44 & 147515.86 & HEISPROD & ESTIMATED & ONSITE & & 126-F-1 Ash Pit \\
\hline 3602 & 11211 & UNKNOWN & 580976.82 & 147509.98 & HEISPROD & ESTIMATED & ONSITE & & 126-F-1 Ash Pit \\
\hline 3603 & 11224 & UNKNOWN & 571882.21 & 149534.77 & HEISPROD & GPS & ONSITE & & 116-N-3 Crib \\
\hline 3604 & 11225 & UNKNOWN & 571952.44 & 149667.95 & HEISPROD & GPS & ONSITE & & 116-N-3 Crib \\
\hline 3605 & 11226 & UNKNOWN & 571848.79 & 149615.1 & HEISPROD & GPS & ONSITE & & 116-N-3 Crib \\
\hline 3606 & 11227 & UNKNOWN & 571990 & 149569.85 & HEISPROD & GPS & ONSITE & & 116-N-3 Crib \\
\hline 3607 & 11228 & UNKNOWN & 571861.35 & 149634.4 & HEISPROD & GPS & ONSITE & & 116-N-3 Crib \\
\hline 3608 & 11229 & UNKNOWN & 571858.09 & 149587.55 & HEISPROD & GPS & ONSITE & & 116-N-3 Crib \\
\hline 3609 & 11230 & UNKNOWN & 571956.2 & 149651.11 & HEISPROD & GPS & ONSITE & & 116-N-3 Crib \\
\hline 3610 & 11231 & UNKNOWN & 571944.29 & 149666.73 & HEISPROD & GPS & ONSITE & & 116-N-3 Crib \\
\hline 3611 & 11232 & UNKNOWN & 571878.42 & 149635 & HEISPROD & GPS & ONSITE & & 116-N-3 Crib \\
\hline
\end{tabular}




\begin{tabular}{|c|c|c|c|c|c|c|c|c|c|}
\hline & A & $\mathrm{B}$ & C & $\mathrm{D}$ & $\mathrm{E}$ & $\mathrm{F}$ & $G$ & $\mathrm{H}$ & $\mathrm{I}$ \\
\hline 1 & SAMP_SITE_ID & HORIZ_COORD_TYPE & EW_COORD & NS_COORD & OWNER_ID & COORD_SOURCE & DIST_CLASS & RAD_LEVEL & SAMP_SITE_NAME \\
\hline 3612 & 11233 & UNKNOWN & 571883.9 & 149533.31 & HEISPROD & GPS & ONSITE & & 116-N-3 Crib \\
\hline 3613 & 11234 & UNKNOWN & 571907.51 & 149653.6 & HEISPROD & GPS & ONSITE & & 116-N-3 Crib \\
\hline 3614 & 11235 & UNKNOWN & 571919.2 & 149654.13 & HEISPROD & GPS & ONSITE & & 116-N-3 Crib \\
\hline 3615 & 11236 & UNKNOWN & 571957.5 & 149637.57 & HEISPROD & GPS & ONSITE & & 116-N-3 Crib \\
\hline 3616 & 11237 & UNKNOWN & 571905.66 & 149652.83 & HEISPROD & GPS & ONSITE & & 116-N-3 Crib \\
\hline 3617 & 11238 & UNKNOWN & 571955.51 & 149647.57 & HEISPROD & GPS & ONSITE & & 116-N-3 Crib \\
\hline 3618 & 11239 & UNKNOWN & 571858.62 & 149588.86 & HEISPROD & GPS & ONSITE & & 116-N-3 Crib \\
\hline 3619 & 11240 & UNKNOWN & 571853.26 & 149627.79 & HEISPROD & GPS & ONSITE & & 116-N-3 Crib \\
\hline 3620 & 11241 & UNKNOWN & 571883.82 & 149538.69 & HEISPROD & GPS & ONSITE & & 116-N-3 Crib \\
\hline 3621 & 11242 & UNKNOWN & 571990.15 & 149572.08 & HEISPROD & GPS & ONSITE & & 116-N-3 Crib \\
\hline 3622 & 11243 & UNKNOWN & 571890.51 & 149525.22 & HEISPROD & GPS & ONSITE & & 116-N-3 Crib \\
\hline 3623 & 11244 & UNKNOWN & 571938.37 & 149542.96 & HEISPROD & GPS & ONSITE & & 116-N-3 Crib \\
\hline 3624 & 11245 & UNKNOWN & 571871.14 & 149556.16 & HEISPROD & GPS & ONSITE & & 116-N-3 Crib \\
\hline 3625 & 11246 & UNKNOWN & 571932.44 & 149541.58 & HEISPROD & GPS & ONSITE & & 116-N-3 Crib \\
\hline 3626 & 11247 & UNKNOWN & 571932.6 & 149543.81 & HEISPROD & GPS & ONSITE & & 116-N-3 Crib \\
\hline \begin{tabular}{|l|}
3627 \\
\end{tabular} & 11248 & UNKNOWN & 571500 & 149661 & HEISPROD & GPS & ONSITE & & $116-N-3$ \\
\hline \begin{tabular}{|l|}
3628 \\
\end{tabular} & 11249 & UNKNOWN & 571523 & 149652 & HEISPROD & GPS & ONSITE & & $116-N-3$ \\
\hline 3629 & 11250 & UNKNOWN & 571869.84 & 149569.7 & HEISPROD & GPS & ONSITE & & 116-N-3 Crib \\
\hline 3630 & 11251 & UNKNOWN & 571868.45 & 149562.62 & HEISPROD & GPS & ONSITE & & 116-N-3 Crib \\
\hline 3631 & 11252 & UNKNOWN & 571868.08 & 149576.55 & HEISPROD & GPS & ONSITE & & 116-N-3 Crib \\
\hline 3632 & 11253 & UNKNOWN & 571870.23 & 149568.78 & HEISPROD & GPS & ONSITE & & 116-N-3 Crib \\
\hline 3633 & 11254 & UNKNOWN & 571865.77 & 149582.09 & HEISPROD & GPS & ONSITE & & 116-N-3 Crib \\
\hline 3634 & 11255 & UNKNOWN & 571982.55 & 149585.17 & HEISPROD & GPS & ONSITE & & 116-N-3 Crib \\
\hline 3635 & 11256 & UNKNOWN & 571501 & 149660 & HEISPROD & GPS & ONSITE & & $116-N-3$ \\
\hline 3636 & 11257 & UNKNOWN & 571501 & 149660 & HEISPROD & GPS & ONSITE & & $116-N-3$ \\
\hline 3637 & 11258 & UNKNOWN & 571534 & 149675 & HEISPROD & GPS & ONSITE & & $116-N-3$ \\
\hline 3638 & 11259 & UNKNOWN & 571806 & 149610 & HEISPROD & GPS & ONSITE & & $116-N-3$ \\
\hline 3639 & 11260 & UNKNOWN & 571806 & 149610 & HEISPROD & GPS & ONSITE & & $116-\mathrm{N}-3$ \\
\hline 3640 & 11261 & UNKNOWN & 571806 & 149610 & HEISPROD & GPS & ONSITE & & $116-\mathrm{N}-3$ \\
\hline 3641 & 11262 & UNKNOWN & 571552 & 149688 & HEISPROD & GPS & ONSITE & & $116-N-3$ \\
\hline 3642 & 11263 & UNKNOWN & 571542 & 149704 & HEISPROD & GPS & ONSITE & & $116-N-3$ \\
\hline \begin{tabular}{|l|}
3643 \\
\end{tabular} & 11264 & UNKNOWN & 571568 & 149687 & HEISPROD & GPS & ONSITE & & $116-N-3$ \\
\hline 3644 & 11265 & UNKNOWN & 571647 & 149694 & HEISPROD & GPS & ONSITE & & $116-\mathrm{N}-3$ \\
\hline 3645 & 11266 & UNKNOWN & 571823 & 149614 & HEISPROD & GPS & ONSITE & & $116-N-3$ \\
\hline 3646 & 11267 & UNKNOWN & 571657 & 149692 & HEISPROD & GPS & ONSITE & & $116-N-3$ \\
\hline \begin{tabular}{|l|}
3647 \\
\end{tabular} & 11268 & UNKNOWN & 571695 & 149689 & HEISPROD & GPS & ONSITE & & $116-N-3$ \\
\hline 3648 & 11269 & UNKNOWN & 571645 & 149692 & HEISPROD & GPS & ONSITE & & $116-N-3$ \\
\hline 3649 & 11270 & UNKNOWN & 571806 & 149610 & HEISPROD & GPS & ONSITE & & $116-N-3$ \\
\hline
\end{tabular}




\begin{tabular}{|c|c|c|c|c|c|c|c|c|c|}
\hline & $\bar{A}$ & $B$ & $\mathrm{C}$ & $\mathrm{D}$ & $E$ & $\mathrm{~F}$ & G & $\mathrm{H}$ & $\mathrm{I}$ \\
\hline 1 & SAMP_SITE_ID & HORIZ_COORD_TYPE & EW_COORD & NS_COORD & OWNER_ID & COORD_SOURCE & DIST_CLASS & RAD_LEVEL & SAMP_SITE_NAME \\
\hline 3650 & 11271 & UNKNOWN & 580697.77 & 147561.86 & HEISPROD & ESTIMATED & ONSITE & & 126-F-1 Ash Pit \\
\hline 3651 & 11272 & UNKNOWN & 580686.77 & 147553.33 & HEISPROD & ESTIMATED & ONSITE & & 126-F-1 Ash Pit \\
\hline 3652 & 11273 & UNKNOWN & 580697.2 & 147556.05 & HEISPROD & ESTIMATED & ONSITE & & 126-F-1 Ash Pit \\
\hline 3653 & 11274 & UNKNOWN & 580686.26 & 147547.86 & HEISPROD & ESTIMATED & ONSITE & & 126-F-1 Ash Pit \\
\hline 3654 & 11275 & UNKNOWN & 580685.01 & 147542.33 & HEISPROD & ESTIMATED & ONSITE & & 126-F-1 Ash Pit \\
\hline 3655 & 11276 & UNKNOWN & 580696.33 & 147545.36 & HEISPROD & ESTIMATED & ONSITE & & 126-F-1 Ash Pit \\
\hline 3656 & 11277 & UNKNOWN & 580673.07 & 147534.19 & HEISPROD & ESTIMATED & ONSITE & & 126-F-1 Ash Pit \\
\hline 3657 & 11278 & UNKNOWN & 580685.79 & 147537.71 & HEISPROD & ESTIMATED & ONSITE & & 126-F-1 Ash Pit \\
\hline 3658 & 11279 & UNKNOWN & 580708.95 & 147543.87 & HEISPROD & ESTIMATED & ONSITE & & 126-F-1 Ash Pit \\
\hline 3659 & 11280 & UNKNOWN & 580671.69 & 147529.26 & HEISPROD & ESTIMATED & ONSITE & & 126-F-1 Ash Pit \\
\hline 3660 & 11281 & UNKNOWN & 580670.73 & 147524.52 & HEISPROD & ESTIMATED & ONSITE & & 126-F-1 Ash Pit \\
\hline 3661 & 11282 & UNKNOWN & 580696.53 & 147531.25 & HEISPROD & ESTIMATED & ONSITE & & 126-F-1 Ash Pit \\
\hline 3662 & 11283 & UNKNOWN & 580695.5 & 147522.43 & HEISPROD & ESTIMATED & ONSITE & & 126-F-1 Ash Pit \\
\hline 3663 & 11284 & UNKNOWN & 580709.45 & 147526.34 & HEISPROD & ESTIMATED & ONSITE & & 126-F-1 Ash Pit \\
\hline 3664 & 11285 & UNKNOWN & 580696.49 & 147518.58 & HEISPROD & ESTIMATED & ONSITE & & 126-F-1 Ash Pit \\
\hline 3665 & 11286 & UNKNOWN & 580671.35 & 147507.42 & HEISPROD & ESTIMATED & ONSITE & & 126-F-1 Ash Pit \\
\hline 3666 & 11287 & UNKNOWN & 580710.91 & 147518.55 & HEISPROD & ESTIMATED & ONSITE & & 126-F-1 Ash Pit \\
\hline 3667 & 11288 & UNKNOWN & 580679.78 & 147507.07 & HEISPROD & ESTIMATED & ONSITE & & 126-F-1 Ash Pit \\
\hline 3668 & 11289 & UNKNOWN & 580734.31 & 147526.82 & HEISPROD & ESTIMATED & ONSITE & & 126-F-1 Ash Pit \\
\hline 3669 & 11290 & UNKNOWN & 580735.82 & 147515.19 & HEISPROD & ESTIMATED & ONSITE & & 126-F-1 Ash Pit \\
\hline 3670 & 11291 & UNKNOWN & 580740.23 & 147516.1 & HEISPROD & ESTIMATED & ONSITE & & 126-F-1 Ash Pit \\
\hline 3671 & 11292 & UNKNOWN & 580749.73 & 147516.6 & HEISPROD & ESTIMATED & ONSITE & & 126-F-1 Ash Pit \\
\hline 3672 & 11293 & UNKNOWN & 580759.01 & 147525.44 & HEISPROD & ESTIMATED & ONSITE & & 126-F-1 Ash Pit \\
\hline 3673 & 11294 & UNKNOWN & 580759.89 & 147512.86 & HEISPROD & ESTIMATED & ONSITE & & 126-F-1 Ash Pit \\
\hline 3674 & 11295 & UNKNOWN & 580767.86 & 147525.39 & HEISPROD & ESTIMATED & ONSITE & & 126-F-1 Ash Pit \\
\hline 3675 & 11296 & UNKNOWN & 580769.16 & 147512.06 & HEISPROD & ESTIMATED & ONSITE & & 126-F-1 Ash Pit \\
\hline 3676 & 11297 & UNKNOWN & 580775.95 & 147532.07 & HEISPROD & ESTIMATED & ONSITE & & 126-F-1 Ash Pit \\
\hline 3677 & 11298 & UNKNOWN & 580777.88 & 147511.32 & HEISPROD & ESTIMATED & ONSITE & & 126-F-1 Ash Pit \\
\hline 3678 & 11299 & UNKNOWN & 580784.72 & 147524.95 & HEISPROD & ESTIMATED & ONSITE & & 126-F-1 Ash Pit \\
\hline 3679 & 11300 & UNKNOWN & 580785.2 & 147518.03 & HEISPROD & ESTIMATED & ONSITE & & 126-F-1 Ash Pit \\
\hline 3680 & 11301 & UNKNOWN & 580800.05 & 147533.82 & HEISPROD & ESTIMATED & ONSITE & & 126-F-1 Ash Pit \\
\hline 3681 & 11302 & UNKNOWN & 580807.61 & 147534.53 & HEISPROD & ESTIMATED & ONSITE & & 126-F-1 Ash Pit \\
\hline 3682 & 11303 & UNKNOWN & 580808.74 & 147519.43 & HEISPROD & ESTIMATED & ONSITE & & 126-F-1 Ash Pit \\
\hline 3683 & 11304 & UNKNOWN & 580814.86 & 147535.89 & HEISPROD & ESTIMATED & ONSITE & & 126-F-1 Ash Pit \\
\hline 3684 & 11305 & UNKNOWN & 580816.02 & 147520.06 & HEISPROD & ESTIMATED & ONSITE & & 126-F-1 Ash Pit \\
\hline 3685 & 11306 & UNKNOWN & 580816.81 & 147511.99 & HEISPROD & ESTIMATED & ONSITE & & 126-F-1 Ash Pit \\
\hline 3686 & 11307 & UNKNOWN & 580835.51 & 147527.61 & HEISPROD & ESTIMATED & ONSITE & & 126-F-1 Ash Pit \\
\hline 3687 & 11308 & UNKNOWN & 580836.28 & 147511.95 & HEISPROD & ESTIMATED & ONSITE & & 126-F-1 Ash Pit \\
\hline
\end{tabular}




\begin{tabular}{|c|c|c|c|c|c|c|c|c|c|}
\hline & $\bar{A}$ & $B$ & $\mathrm{C}$ & $\mathrm{D}$ & $E$ & $\mathrm{~F}$ & G & $\mathrm{H}$ & $\mathrm{I}$ \\
\hline 1 & SAMP_SITE_ID & HORIZ_COORD_TYPE & EW_COORD & NS_COORD & OWNER_ID & COORD_SOURCE & DIST_CLASS & RAD_LEVEL & SAMP_SITE_NAME \\
\hline 3688 & 11309 & UNKNOWN & 580918.25 & 147580.99 & HEISPROD & ESTIMATED & ONSITE & & 126-F-1 Ash Pit \\
\hline 3689 & 11310 & UNKNOWN & 580923.98 & 147581.33 & HEISPROD & ESTIMATED & ONSITE & & 126-F-1 Ash Pit \\
\hline 3690 & 11311 & UNKNOWN & 580908.89 & 147562.09 & HEISPROD & ESTIMATED & ONSITE & & 126-F-1 Ash Pit \\
\hline 3691 & 11312 & UNKNOWN & 580906.34 & 147555.21 & HEISPROD & ESTIMATED & ONSITE & & 126-F-1 Ash Pit \\
\hline 3692 & 11313 & UNKNOWN & 580923.5 & 147555.47 & HEISPROD & ESTIMATED & ONSITE & & 126-F-1 Ash Pit \\
\hline 3693 & 11314 & UNKNOWN & 580911.96 & 147549.29 & HEISPROD & ESTIMATED & ONSITE & & 126-F-1 Ash Pit \\
\hline 3694 & 11315 & UNKNOWN & 580922.68 & 147549.23 & HEISPROD & ESTIMATED & ONSITE & & 126-F-1 Ash Pit \\
\hline 3695 & 11316 & UNKNOWN & 580922.3 & 147544.15 & HEISPROD & ESTIMATED & ONSITE & & 126-F-1 Ash Pit \\
\hline 3696 & 11317 & UNKNOWN & 580895.94 & 147539.58 & HEISPROD & ESTIMATED & ONSITE & & 126-F-1 Ash Pit \\
\hline 3697 & 11318 & UNKNOWN & 580894 & 147535.38 & HEISPROD & ESTIMATED & ONSITE & & 126-F-1 Ash Pit \\
\hline 3698 & 11319 & UNKNOWN & 580921.73 & 147535.25 & HEISPROD & ESTIMATED & ONSITE & & 126-F-1 Ash Pit \\
\hline 3699 & 11320 & UNKNOWN & 580878.33 & 147529.7 & HEISPROD & ESTIMATED & ONSITE & & 126-F-1 Ash Pit \\
\hline 3700 & 11321 & UNKNOWN & 580885.97 & 147529.69 & HEISPROD & ESTIMATED & ONSITE & & 126-F-1 Ash Pit \\
\hline 3701 & 11322 & UNKNOWN & 580893.65 & 147529.67 & HEISPROD & ESTIMATED & ONSITE & & 126-F-1 Ash Pit \\
\hline 3702 & 11323 & UNKNOWN & 580873.36 & 147522.72 & HEISPROD & ESTIMATED & ONSITE & & 126-F-1 Ash Pit \\
\hline 3703 & 11331 & UNKNOWN & 580899.33 & 147522.66 & HEISPROD & ESTIMATED & ONSITE & & 126-F-1 Ash Pit \\
\hline 3704 & 11332 & UNKNOWN & 580924.87 & 147522.54 & HEISPROD & ESTIMATED & ONSITE & & 126-F-1 Ash Pit \\
\hline 3705 & 11333 & UNKNOWN & 580876.47 & 147516.38 & HEISPROD & ESTIMATED & ONSITE & & 126-F-1 Ash Pit \\
\hline 3706 & 11334 & UNKNOWN & 580904.66 & 147516.38 & HEISPROD & ESTIMATED & ONSITE & & 126-F-1 Ash Pit \\
\hline 3707 & 11335 & UNKNOWN & 580914.36 & 147516.38 & HEISPROD & ESTIMATED & ONSITE & & 126-F-1 Ash Pit \\
\hline 3708 & 11336 & UNKNOWN & 580864.71 & 147510.4 & HEISPROD & ESTIMATED & ONSITE & & 126-F-1 Ash Pit \\
\hline 3709 & 11337 & UNKNOWN & 580910.78 & 147510.04 & HEISPROD & ESTIMATED & ONSITE & & 126-F-1 Ash Pit \\
\hline 3710 & 11338 & UNKNOWN & 580919.17 & 147509.45 & HEISPROD & ESTIMATED & ONSITE & & 126-F-1 Ash Pit \\
\hline 3711 & 11339 & UNKNOWN & 580976.57 & 147604.1 & HEISPROD & ESTIMATED & ONSITE & & 126-F-1 Ash Pit \\
\hline 3712 & 11340 & UNKNOWN & 580981.89 & 147605.96 & HEISPROD & ESTIMATED & ONSITE & & 126-F-1 Ash Pit \\
\hline 3713 & 11341 & UNKNOWN & 580984.41 & 147606.68 & HEISPROD & ESTIMATED & ONSITE & & 126-F-1 Ash Pit \\
\hline 3714 & 11342 & UNKNOWN & 580976.03 & 147601.3 & HEISPROD & ESTIMATED & ONSITE & & 126-F-1 Ash Pit \\
\hline 3715 & 11343 & UNKNOWN & 580986.56 & 147602.16 & HEISPROD & ESTIMATED & ONSITE & & 126-F-1 Ash Pit \\
\hline 3716 & 11344 & UNKNOWN & 580994.53 & 147602.9 & HEISPROD & ESTIMATED & ONSITE & & 126-F-1 Ash Pit \\
\hline 3717 & 11345 & UNKNOWN & 580976.92 & 147598.06 & HEISPROD & ESTIMATED & ONSITE & & 126-F-1 Ash Pit \\
\hline 3718 & 11346 & UNKNOWN & 580987.3 & 147598.26 & HEISPROD & ESTIMATED & ONSITE & & 126-F-1 Ash Pit \\
\hline 3719 & 11347 & UNKNOWN & 580990.69 & 147598.33 & HEISPROD & ESTIMATED & ONSITE & & 126-F-1 Ash Pit \\
\hline 3720 & 11348 & UNKNOWN & 580972.77 & 147594.47 & HEISPROD & ESTIMATED & ONSITE & & 126-F-1 Ash Pit \\
\hline 3721 & 11349 & UNKNOWN & 580986.72 & 147594.75 & HEISPROD & ESTIMATED & ONSITE & & 126-F-1 Ash Pit \\
\hline 3722 & 11350 & UNKNOWN & 580990.3 & 147594.82 & HEISPROD & ESTIMATED & ONSITE & & 126-F-1 Ash Pit \\
\hline 3723 & 11351 & UNKNOWN & 580969.66 & 147590.84 & HEISPROD & ESTIMATED & ONSITE & & 126-F-1 Ash Pit \\
\hline 3724 & 11352 & UNKNOWN & 580973.09 & 147590.93 & HEISPROD & ESTIMATED & ONSITE & & 126-F-1 Ash Pit \\
\hline 3725 & 11353 & UNKNOWN & 580979.95 & 147591.12 & HEISPROD & ESTIMATED & ONSITE & & 126-F-1 Ash Pit \\
\hline
\end{tabular}




\begin{tabular}{|c|c|c|c|c|c|c|c|c|c|}
\hline & $A$ & $\mathrm{~B}$ & $\mathrm{C}$ & $\mathrm{D}$ & $E$ & $\mathrm{~F}$ & $\mathrm{G}$ & $\mathrm{H}$ & $\mathrm{I}$ \\
\hline 1 & SAMP_SITE_ID & HORIZ_COORD_TYPE & EW_COORD & NS_COORD & OWNER_ID & COORD_SOURCE & DIST_CLASS & RAD_LEVEL & SAMP_SITE_NAME \\
\hline 3726 & 11354 & UNKNOWN & 580983.41 & 147591.22 & HEISPROD & ESTIMATED & ONSITE & & 126-F-1 Ash Pit \\
\hline 3727 & 11355 & UNKNOWN & 580970.11 & 147587.3 & HEISPROD & ESTIMATED & ONSITE & & 126-F-1 Ash Pit \\
\hline 3728 & 11356 & UNKNOWN & 580977.09 & 147587.5 & HEISPROD & ESTIMATED & ONSITE & & 126-F-1 Ash Pit \\
\hline 3729 & 11357 & UNKNOWN & 580979.6 & 147584.04 & HEISPROD & ESTIMATED & ONSITE & & 126-F-1 Ash Pit \\
\hline 3730 & 11358 & UNKNOWN & 580983.06 & 147583.96 & HEISPROD & ESTIMATED & ONSITE & & 126-F-1 Ash Pit \\
\hline 3731 & 11359 & UNKNOWN & 580991.91 & 147583.76 & HEISPROD & ESTIMATED & ONSITE & & 126-F-1 Ash Pit \\
\hline 3732 & 11360 & UNKNOWN & 580972.64 & 147581.63 & HEISPROD & ESTIMATED & ONSITE & & 126-F-1 Ash Pit \\
\hline 3733 & 11361 & UNKNOWN & 580985.28 & 147579.72 & HEISPROD & ESTIMATED & ONSITE & & 126-F-1 Ash Pit \\
\hline 3734 & 11362 & UNKNOWN & 580987.96 & 147579.31 & HEISPROD & ESTIMATED & ONSITE & & 126-F-1 Ash Pit \\
\hline 3735 & 11363 & & & & HEISPROD & & & & 100-F-19 Deep Zone \\
\hline \begin{tabular}{|l|}
3736 \\
\end{tabular} & 11364 & & & & HEISPROD & & & & 100-F-19 Deep Zone \\
\hline 3737 & 11365 & UNKNOWN & 580997.05 & 147608.13 & HEISPROD & ESTIMATED & ONSITE & & 126-F-1 Ash Pit \\
\hline 3738 & 11366 & UNKNOWN & 580997.06 & 147603.47 & HEISPROD & ESTIMATED & ONSITE & & 126-F-1 Ash Pit \\
\hline 3739 & 11367 & UNKNOWN & 580997.1 & 147589.99 & HEISPROD & ESTIMATED & ONSITE & & 126-F-1 Ash Pit \\
\hline 3740 & 11368 & UNKNOWN & 580997.12 & 147581.06 & HEISPROD & ESTIMATED & ONSITE & & 126-F-1 Ash Pit \\
\hline 3741 & 11369 & UNKNOWN & 580999.75 & 147589.8 & HEISPROD & ESTIMATED & ONSITE & & 126-F-1 Ash Pit \\
\hline 3742 & 11370 & UNKNOWN & 580999.79 & 147580.4 & HEISPROD & ESTIMATED & ONSITE & & 126-F-1 Ash Pit \\
\hline 3743 & 11371 & UNKNOWN & 581002.23 & 147609.51 & HEISPROD & ESTIMATED & ONSITE & & 126-F-1 Ash Pit \\
\hline 3744 & 11372 & UNKNOWN & 581004.66 & 147604.55 & HEISPROD & ESTIMATED & ONSITE & & 126-F-1 Ash Pit \\
\hline 3745 & 11373 & UNKNOWN & 581004.68 & 147594.23 & HEISPROD & ESTIMATED & ONSITE & & 126-F-1 Ash Pit \\
\hline 3746 & 11374 & UNKNOWN & 581004.7 & 147589.11 & HEISPROD & ESTIMATED & ONSITE & & 126-F-1 Ash Pit \\
\hline \begin{tabular}{|l|}
3747 \\
\end{tabular} & 11375 & UNKNOWN & 581004.73 & 147578.94 & HEISPROD & ESTIMATED & ONSITE & & 126-F-1 Ash Pit \\
\hline \begin{tabular}{|l|}
3748 \\
\end{tabular} & 11376 & UNKNOWN & 581004.76 & 147573.63 & HEISPROD & ESTIMATED & ONSITE & & 126-F-1 Ash Pit \\
\hline 3749 & 11377 & UNKNOWN & 581006.95 & 147610.37 & HEISPROD & ESTIMATED & ONSITE & & 126-F-1 Ash Pit \\
\hline 3750 & 11378 & UNKNOWN & 581006.99 & 147599.61 & HEISPROD & ESTIMATED & ONSITE & & 126-F-1 Ash Pit \\
\hline 3751 & 11379 & UNKNOWN & 581007.02 & 147594.3 & HEISPROD & ESTIMATED & ONSITE & & 126-F-1 Ash Pit \\
\hline 3752 & 11380 & UNKNOWN & 581007.04 & 147588.98 & HEISPROD & ESTIMATED & ONSITE & & 126-F-1 Ash Pit \\
\hline 3753 & 11381 & UNKNOWN & 581007.12 & 147572.97 & HEISPROD & ESTIMATED & ONSITE & & 126-F-1 Ash Pit \\
\hline 3754 & 11382 & UNKNOWN & 581009.26 & 147600.05 & HEISPROD & ESTIMATED & ONSITE & & 126-F-1 Ash Pit \\
\hline 3755 & 11383 & UNKNOWN & 581011.48 & 147606.31 & HEISPROD & ESTIMATED & ONSITE & & 126-F-1 Ash Pit \\
\hline 3756 & 11384 & UNKNOWN & 581011.49 & 147600.85 & HEISPROD & ESTIMATED & ONSITE & & 126-F-1 Ash Pit \\
\hline \begin{tabular}{|l|}
3757 \\
\end{tabular} & 11385 & UNKNOWN & 581011.51 & 147595.35 & HEISPROD & ESTIMATED & ONSITE & & 126-F-1 Ash Pit \\
\hline 3758 & 11386 & UNKNOWN & 581011.57 & 147573 & HEISPROD & ESTIMATED & ONSITE & & 126-F-1 Ash Pit \\
\hline 3759 & 11387 & UNKNOWN & 581013.69 & 147595.73 & HEISPROD & ESTIMATED & ONSITE & & 126-F-1 Ash Pit \\
\hline 3760 & 11388 & UNKNOWN & 581013.7 & 147590.12 & HEISPROD & ESTIMATED & ONSITE & & 126-F-1 Ash Pit \\
\hline 3761 & 11389 & UNKNOWN & 571710 & 150042 & HEISPROD & GPS & ONSITE & & $116-\mathrm{N}-1$ \\
\hline 3762 & 11390 & UNKNOWN & 571710 & 150042 & HEISPROD & GPS & ONSITE & & $116-\mathrm{N}-1$ \\
\hline 3763 & 11391 & UNKNOWN & 571709 & 150043 & HEISPROD & GPS & ONSITE & & $116-\mathrm{N}-1$ \\
\hline
\end{tabular}




\begin{tabular}{|c|c|c|c|c|c|c|c|c|c|}
\hline & $A$ & $\mathrm{~B}$ & $\mathrm{C}$ & $\mathrm{D}$ & $E$ & $\mathrm{~F}$ & G & $\mathrm{H}$ & $\mathrm{I}$ \\
\hline 1 & SAMP_SITE_ID & HORIZ_COORD_TYPE & EW_COORD & NS_COORD & OWNER_ID & COORD_SOURCE & DIST_CLASS & RAD_LEVEL & SAMP_SITE_NAME \\
\hline 3764 & 11392 & & & & HEISPROD & & & & $100-C-6$ \\
\hline 3765 & 11393 & & & & HEISPROD & & & & $100-C-6$ \\
\hline 3766 & 11394 & & & & HEISPROD & & & & 116-B-1 \\
\hline 3767 & 11395 & & & & HEISPROD & & & & 116-B-1 \\
\hline 3768 & 11396 & & & & HEISPROD & & & & 116-B-1 \\
\hline 3769 & 11397 & & & & HEISPROD & & & & 116-B-1 \\
\hline 3770 & 11398 & & & & HEISPROD & & & & 116-B-1 \\
\hline 3771 & 11399 & UNKNOWN & 565341 & 144999 & HEISPROD & ESTIMATED & ONSITE & & $100-C-6$ \\
\hline 3772 & 11400 & UNKNOWN & 565348 & 145049 & 9 HEISPROD & ESTIMATED & ONSITE & & $100-C-6$ \\
\hline 3773 & 11401 & UNKNOWN & 565373 & 145021 & 1 HEISPROD & ESTIMATED & ONSITE & & $100-C-6$ \\
\hline 3774 & 11402 & UNKNOWN & 565432 & 145018 & 3 HEISPROD & ESTIMATED & ONSITE & & $100-C-6$ \\
\hline 3775 & 11403 & UNKNOWN & 565444 & 145012 & 2 HEISPROD & ESTIMATED & ONSITE & & $100-C-6$ \\
\hline 3776 & 11404 & UNKNOWN & 565453 & 145037 & 7 HEISPROD & ESTIMATED & ONSITE & & $100-C-6$ \\
\hline 3777 & 11405 & UNKNOWN & 565477 & 145003 & 3 HEISPROD & ESTIMATED & ONSITE & & $100-C-6$ \\
\hline 3778 & 11406 & UNKNOWN & 565470 & 144982 & 2 HEISPROD & ESTIMATED & ONSITE & & $100-C-6$ \\
\hline 3779 & 11407 & UNKNOWN & 565457 & 144959 & HEISPROD & ESTIMATED & ONSITE & & $100-C-6$ \\
\hline 3780 & 11408 & UNKNOWN & 565436 & 144958 & 3 HEISPROD & ESTIMATED & ONSITE & & $100-C-6$ \\
\hline 3781 & 11409 & & & & HEISPROD & & & & 118-B-3 \\
\hline 3782 & 11410 & & & & HEISPROD & & & & 118-B-3 \\
\hline 3783 & 11420 & UNKNOWN & 581027.6 & 147915.73 & 3 HEISPROD & ESTIMATED & ONSITE & & 116-F-9 Shallow Zone \\
\hline 3784 & 11421 & UNKNOWN & 581029.34 & 147919.04 & 4 HEISPROD & ESTIMATED & ONSITE & & 116-F-9 Shallow Zone \\
\hline 3785 & 11422 & UNKNOWN & 581031.2 & 147916.55 & 5 HEISPROD & ESTIMATED & ONSITE & & 116-F-9 Shallow Zone \\
\hline 3786 & 11423 & UNKNOWN & 581042.28 & 147922.76 & HEISPROD & ESTIMATED & ONSITE & & 116-F-9 Shallow Zone \\
\hline 3787 & 11424 & UNKNOWN & 581050.65 & 147921.21 & 1 HEISPROD & ESTIMATED & ONSITE & & 116-F-9 Shallow Zone \\
\hline 3788 & 11425 & UNKNOWN & 581052.49 & 147917.43 & 3 HEISPROD & ESTIMATED & ONSITE & & 116-F-9 Shallow Zone \\
\hline 3789 & 11426 & UNKNOWN & 581059.44 & 147914.39 & 9 HEISPROD & ESTIMATED & ONSITE & & 116-F-9 Shallow Zone \\
\hline 3790 & 11427 & UNKNOWN & 581059.87 & 147908.58 & 3 HEISPROD & ESTIMATED & ONSITE & & 116-F-9 Shallow Zone \\
\hline 3791 & 11428 & UNKNOWN & 581064.3 & 147907.53 & 3 HEISPROD & ESTIMATED & ONSITE & & 116-F-9 Shallow Zone \\
\hline 3792 & 11429 & UNKNOWN & 581064.92 & 147901.66 & HEISPROD & ESTIMATED & ONSITE & & 116-F-9 Shallow Zone \\
\hline 3793 & 11430 & UNKNOWN & 581070.27 & 147896.72 & 2 HEISPROD & ESTIMATED & ONSITE & & 116-F-9 Shallow Zone \\
\hline 3794 & 11431 & UNKNOWN & 581076.87 & 147895.56 & S HEISPROD & ESTIMATED & ONSITE & & 116-F-9 Shallow Zone \\
\hline 3795 & 11432 & UNKNOWN & 581079.54 & 147893.48 & 3 HEISPROD & ESTIMATED & ONSITE & & 116-F-9 Shallow Zone \\
\hline 3796 & 11433 & UNKNOWN & 581077.2 & 147891.13 & 3 HEISPROD & ESTIMATED & ONSITE & & 116-F-9 Shallow Zone \\
\hline 3797 & 11434 & UNKNOWN & 581079.83 & 147888.57 & 7 HEISPROD & ESTIMATED & ONSITE & & 116-F-9 Shallow Zone \\
\hline 3798 & 11435 & UNKNOWN & 581085.34 & 147887.87 & 7 HEISPROD & ESTIMATED & ONSITE & & 116-F-9 Shallow Zone \\
\hline 3799 & 11436 & UNKNOWN & 581089.94 & 147882.7 & 7 HEISPROD & ESTIMATED & ONSITE & & 116-F-9 Shallow Zone \\
\hline 3800 & 11437 & UNKNOWN & 581092.23 & 147880.28 & 3 HEISPROD & ESTIMATED & ONSITE & & 116-F-9 Shallow Zone \\
\hline 3801 & 11438 & UNKNOWN & 581103.62 & 147874.87 & 7 HEISPROD & ESTIMATED & ONSITE & & 116-F-9 Shallow Zone \\
\hline
\end{tabular}




\begin{tabular}{|c|c|c|c|c|c|c|c|c|c|}
\hline & A & $\mathrm{B}$ & $\mathrm{C}$ & $\mathrm{D}$ & $E$ & $\mathrm{~F}$ & G & $\mathrm{H}$ & $\mathrm{T}$ \\
\hline 1 & SAMP_SITE_ID & HORIZ_COORD_TYPE & EW_COORD & NS_COORD & OWNER_ID & COORD_SOURCE & DIST_CLASS & RAD_LEVEL & SAMP_SITE_NAME \\
\hline 3802 & 11439 & UNKNOWN & 581102.44 & 147871.63 & HEISPROD & ESTIMATED & ONSITE & & 116-F-9 Shallow Zone \\
\hline 3803 & 11440 & UNKNOWN & 581110.43 & 147870.74 & HEISPROD & ESTIMATED & ONSITE & & 116-F-9 Shallow Zone \\
\hline 3804 & 11441 & UNKNOWN & 581113.96 & 147871.87 & HEISPROD & ESTIMATED & ONSITE & & 116-F-9 Shallow Zone \\
\hline 3805 & 11442 & UNKNOWN & 581117.41 & 147871.38 & HEISPROD & ESTIMATED & ONSITE & & 116-F-9 Shallow Zone \\
\hline 3806 & 11443 & UNKNOWN & 581120.63 & 147877.45 & HEISPROD & ESTIMATED & ONSITE & & 116-F-9 Shallow Zone \\
\hline 3807 & 11444 & UNKNOWN & 571726 & 150020 & HEISPROD & GPS & ONSITE & & $116-\mathrm{N}-1$ \\
\hline 3808 & 11445 & UNKNOWN & 571726 & 150020 & HEISPROD & GPS & ONSITE & & $116-\mathrm{N}-1$ \\
\hline 3809 & 11446 & UNKNOWN & 571726 & 150020 & HEISPROD & GPS & ONSITE & & $116-\mathrm{N}-1$ \\
\hline 3810 & 11447 & UNKNOWN & 571726 & 150020 & HEISPROD & GPS & ONSITE & & $116-\mathrm{N}-1$ \\
\hline 3811 & 11448 & UNKNOWN & 580917.95 & 147638.87 & HEISPROD & ESTIMATED & ONSITE & & 126-F-1 Ash Pit \\
\hline 3812 & 11468 & UNKNOWN & 581015.9 & 147603.12 & HEISPROD & ESTIMATED & ONSITE & & 126-F-1 Ash Pit \\
\hline 3813 & 11469 & UNKNOWN & 581015.89 & 147597.65 & HEISPROD & ESTIMATED & ONSITE & & 126-F-1 Ash Pit \\
\hline 3814 & 11470 & UNKNOWN & 581015.87 & 147592.05 & HEISPROD & ESTIMATED & ONSITE & & 126-F-1 Ash Pit \\
\hline 3815 & 11471 & UNKNOWN & 581018.07 & 147608.2 & HEISPROD & ESTIMATED & ONSITE & & 126-F-1 Ash Pit \\
\hline 3816 & 11472 & UNKNOWN & 581017.98 & 147585.56 & HEISPROD & ESTIMATED & ONSITE & & 126-F-1 Ash Pit \\
\hline 3817 & 11473 & UNKNOWN & 581017.93 & 147574.59 & HEISPROD & ESTIMATED & ONSITE & & 126-F-1 Ash Pit \\
\hline 3818 & 11474 & UNKNOWN & 581020.19 & 147603.34 & HEISPROD & ESTIMATED & ONSITE & & 126-F-1 Ash Pit \\
\hline 3819 & 11475 & UNKNOWN & 581020.21 & 147597.74 & HEISPROD & ESTIMATED & ONSITE & & 126-F-1 Ash Pit \\
\hline 3820 & 11476 & UNKNOWN & 581020.24 & 147575.66 & HEISPROD & ESTIMATED & ONSITE & & 126-F-1 Ash Pit \\
\hline 3821 & 11477 & UNKNOWN & 581022.4 & 147609.78 & HEISPROD & ESTIMATED & ONSITE & & 126-F-1 Ash Pit \\
\hline 3822 & 11478 & UNKNOWN & 581022.45 & 147588.24 & HEISPROD & ESTIMATED & ONSITE & & 126-F-1 Ash Pit \\
\hline 3823 & 11479 & UNKNOWN & 581022.47 & 147576.88 & HEISPROD & ESTIMATED & ONSITE & & 126-F-1 Ash Pit \\
\hline 3824 & 11480 & UNKNOWN & 581024.65 & 147609.16 & HEISPROD & ESTIMATED & ONSITE & & 126-F-1 Ash Pit \\
\hline 3825 & 11481 & UNKNOWN & 581024.67 & 147603.67 & HEISPROD & ESTIMATED & ONSITE & & 126-F-1 Ash Pit \\
\hline 3826 & 11482 & UNKNOWN & 581024.7 & 147598.29 & HEISPROD & ESTIMATED & ONSITE & & 126-F-1 Ash Pit \\
\hline 3827 & 11483 & UNKNOWN & 581026.95 & 147610.3 & HEISPROD & ESTIMATED & ONSITE & & 126-F-1 Ash Pit \\
\hline 3828 & 11484 & UNKNOWN & 581027.01 & 147595.14 & HEISPROD & ESTIMATED & ONSITE & & 126-F-1 Ash Pit \\
\hline 3829 & 11485 & UNKNOWN & 581027.09 & 147578.89 & HEISPROD & ESTIMATED & ONSITE & & 126-F-1 Ash Pit \\
\hline 3830 & 11486 & UNKNOWN & 581029.4 & 147604.97 & HEISPROD & ESTIMATED & ONSITE & & 126-F-1 Ash Pit \\
\hline 3831 & 11487 & UNKNOWN & 581029.43 & 147590.11 & HEISPROD & ESTIMATED & ONSITE & & 126-F-1 Ash Pit \\
\hline 3832 & 11488 & UNKNOWN & 581029.44 & 147585.23 & HEISPROD & ESTIMATED & ONSITE & & 126-F-1 Ash Pit \\
\hline 3833 & 11489 & UNKNOWN & 581032.22 & 147610.87 & HEISPROD & ESTIMATED & ONSITE & & 126-F-1 Ash Pit \\
\hline 3834 & 11490 & UNKNOWN & 581031.97 & 147593.3 & HEISPROD & ESTIMATED & ONSITE & & 126-F-1 Ash Pit \\
\hline 3835 & 11491 & UNKNOWN & 581031.94 & 147588.44 & HEISPROD & ESTIMATED & ONSITE & & 126-F-1 Ash Pit \\
\hline 3836 & 11508 & UNKNOWN & 580719.41 & 147525.83 & HEISPROD & ESTIMATED & ONSITE & & 126-F-1 Ash Pit \\
\hline 3837 & 11509 & UNKNOWN & 580724.47 & 147526.38 & HEISPROD & ESTIMATED & ONSITE & & 126-F-1 Ash Pit \\
\hline 3838 & 11528 & & & & HEISPROD & & & & 116-F-9 Deep Zone \\
\hline 3839 & 11529 & & & & HEISPROD & & & & 116-F-9 Deep Zone \\
\hline
\end{tabular}




\begin{tabular}{|c|c|c|c|c|c|c|c|c|c|}
\hline & $A$ & $\mathrm{~B}$ & $\mathrm{C}$ & $\mathrm{D}$ & $E$ & $\mathrm{~F}$ & $\mathrm{G}$ & $\mathrm{H}$ & $\mathrm{I}$ \\
\hline 1 & SAMP_SITE_ID & HORIZ_COORD_TYPE & EW_COORD & NS_COORD & OWNER_ID & COORD_SOURCE & DIST_CLASS & RAD_LEVEL & SAMP_SITE_NAME \\
\hline 3840 & 11530 & & & & HEISPROD & & & & 116-F-9 Deep Zone \\
\hline 3841 & 11531 & & & & HEISPROD & & & & 116-F-9 Deep Zone \\
\hline 3842 & 11532 & & & & HEISPROD & & & & 116-F-9 Deep Zone \\
\hline 3843 & 11533 & & & & HEISPROD & & & & 116-F-9 Deep Zone \\
\hline 3844 & 11534 & & & & HEISPROD & & & & 116-F-9 Deep Zone \\
\hline 3845 & 11535 & & & & HEISPROD & & & & 116-F-9 Deep Zone \\
\hline 3846 & 11536 & & & & HEISPROD & & & & 116-F-9 Deep Zone \\
\hline 3847 & 11537 & & & & HEISPROD & & & & 116-F-9 Deep Zone \\
\hline 3848 & 11538 & & & & HEISPROD & & & & 116-F-9 Deep Zone \\
\hline 3849 & 11539 & & & & HEISPROD & & & & 116-F-9 Deep Zone \\
\hline 3850 & 11540 & & & & HEISPROD & & & & 116-F-9 Deep Zone \\
\hline 3851 & 11541 & & & & HEISPROD & & & & 116-F-9 Deep Zone \\
\hline 3852 & 11548 & UNKNOWN & 571931 & 149550 & HEISPROD & GPS & ONSITE & & 116-N-3 Crib \\
\hline 3853 & 11549 & UNKNOWN & 571878 & 149577 & HEISPROD & GPS & ONSITE & & 116-N-3 Crib \\
\hline 3854 & 11550 & UNKNOWN & 571917 & 149591 & HEISPROD & GPS & ONSITE & & 116-N-3 Crib \\
\hline 3855 & 11551 & UNKNOWN & 571888 & 149627 & HEISPROD & GPS & ONSITE & & 116-N-3 Crib \\
\hline 3856 & 11552 & UNKNOWN & 571946 & 149605 & HEISPROD & GPS & ONSITE & & 116-N-3 Crib \\
\hline 3857 & 11553 & UNKNOWN & 571884 & 149573 & HEISPROD & GPS & ONSITE & & 116-N-3 Crib \\
\hline 3858 & 11554 & UNKNOWN & 571951 & 149612 & HEISPROD & GPS & ONSITE & & 116-N-3 Crib \\
\hline 3859 & 11555 & UNKNOWN & 571906 & 149578 & HEISPROD & GPS & ONSITE & & 116-N-3 Crib \\
\hline 3860 & 11556 & UNKNOWN & 571896 & 149552 & HEISPROD & GPS & ONSITE & & 116-N-3 Crib \\
\hline 3861 & 11557 & UNKNOWN & 571866 & 149594 & HEISPROD & GPS & ONSITE & & 116-N-3 Crib \\
\hline 3862 & 11558 & UNKNOWN & 581127.17 & 147880.97 & HEISPROD & ESTIMATED & ONSITE & & 116-F-9 Shallow Zone \\
\hline 3863 & 11559 & UNKNOWN & 581128.38 & 147877.57 & HEISPROD & ESTIMATED & ONSITE & & 116-F-9 Shallow Zone \\
\hline 3864 & 11560 & UNKNOWN & 581131.93 & 147866.23 & HEISPROD & ESTIMATED & ONSITE & & 116-F-9 Shallow Zone \\
\hline 3865 & 11561 & UNKNOWN & 581137.39 & 147863.11 & HEISPROD & ESTIMATED & ONSITE & & 116-F-9 Shallow Zone \\
\hline 3866 & 11562 & UNKNOWN & 581143.8 & 147846.74 & HEISPROD & ESTIMATED & ONSITE & & 116-F-9 Shallow Zone \\
\hline 3867 & 11563 & UNKNOWN & 581141.91 & 147838.02 & HEISPROD & ESTIMATED & ONSITE & & 116-F-9 Shallow Zone \\
\hline 3868 & 11564 & UNKNOWN & 581136.72 & 147836.35 & HEISPROD & ESTIMATED & ONSITE & & 116-F-9 Shallow Zone \\
\hline 3869 & 11565 & UNKNOWN & 581137.65 & 147821.93 & HEISPROD & ESTIMATED & ONSITE & & 116-F-9 Shallow Zone \\
\hline 3870 & 11566 & UNKNOWN & 581133.89 & 147817.49 & HEISPROD & ESTIMATED & ONSITE & & 116-F-9 Shallow Zone \\
\hline 3871 & 11567 & UNKNOWN & 581129.58 & 147820.5 & HEISPROD & ESTIMATED & ONSITE & & 116-F-9 Shallow Zone \\
\hline 3872 & 11568 & UNKNOWN & 581126.55 & 147824.35 & HEISPROD & ESTIMATED & ONSITE & & 116-F-9 Shallow Zone \\
\hline 3873 & 11569 & UNKNOWN & 581125.14 & 147821.66 & HEISPROD & ESTIMATED & ONSITE & & 116-F-9 Shallow Zone \\
\hline 3874 & 11570 & UNKNOWN & 581124.15 & 147826.98 & HEISPROD & ESTIMATED & ONSITE & & 116-F-9 Shallow Zone \\
\hline 3875 & 11571 & UNKNOWN & 581119.2 & 147827.72 & HEISPROD & ESTIMATED & ONSITE & & 116-F-9 Shallow Zone \\
\hline 3876 & 11572 & UNKNOWN & 581117.06 & 147824.72 & HEISPROD & ESTIMATED & ONSITE & & 116-F-9 Shallow Zone \\
\hline 3877 & 11573 & UNKNOWN & 581115.1 & 147829.2 & HEISPROD & ESTIMATED & ONSITE & & 116-F-9 Shallow Zone \\
\hline
\end{tabular}




\begin{tabular}{|c|c|c|c|c|c|c|c|c|c|}
\hline & A & $\mathrm{B}$ & $\mathrm{C}$ & $\mathrm{D}$ & $E$ & $\mathrm{~F}$ & G & $\mathrm{H}$ & $\mathrm{T}$ \\
\hline 1 & SAMP_SITE_ID & HORIZ_COORD_TYPE & EW_COORD & NS_COORD & OWNER_ID & COORD_SOURCE & DIST_CLASS & RAD_LEVEL & SAMP_SITE_NAME \\
\hline 3878 & 11574 & UNKNOWN & 581111.29 & 147827.19 & HEISPROD & ESTIMATED & ONSITE & & 116-F-9 Shallow Zone \\
\hline 3879 & 11575 & UNKNOWN & 581107.43 & 147838.29 & HEISPROD & ESTIMATED & ONSITE & & 116-F-9 Shallow Zone \\
\hline 3880 & 11576 & UNKNOWN & 581098.59 & 147838.52 & HEISPROD & ESTIMATED & ONSITE & & 116-F-9 Shallow Zone \\
\hline 3881 & 11577 & UNKNOWN & 581098.58 & 147835.62 & HEISPROD & ESTIMATED & ONSITE & & 116-F-9 Shallow Zone \\
\hline 3882 & 11578 & UNKNOWN & 581097.35 & 147831.71 & HEISPROD & ESTIMATED & ONSITE & & 116-F-9 Shallow Zone \\
\hline 3883 & 11579 & UNKNOWN & 581097.15 & 147816.03 & HEISPROD & ESTIMATED & ONSITE & & 116-F-9 Shallow Zone \\
\hline 3884 & 11580 & UNKNOWN & 581093.69 & 147830.12 & HEISPROD & ESTIMATED & ONSITE & & 116-F-9 Shallow Zone \\
\hline 3885 & 11581 & UNKNOWN & 581093.7 & 147827.17 & HEISPROD & ESTIMATED & ONSITE & & 116-F-9 Shallow Zone \\
\hline 3886 & 11588 & UNKNOWN & 565256 & 145292 & HEISPROD & ESTIMATED & ONSITE & & $100-B-14$ \\
\hline 3887 & 11589 & UNKNOWN & 565256 & 145292 & HEISPROD & ESTIMATED & ONSITE & & 100-B-14 \\
\hline 3888 & 11590 & UNKNOWN & 565313 & 144970 & HEISPROD & ESTIMATED & ONSITE & & 100-B-8 \\
\hline 3889 & 11591 & UNKNOWN & 565313 & 144970 & HEISPROD & ESTIMATED & ONSITE & & 100-B-8 \\
\hline 3890 & 11608 & UNKNOWN & 571868 & 149408 & HEISPROD & ESTIMATED & ONSITE & & $116-\mathrm{N}-3$ \\
\hline 3891 & 11628 & UNKNOWN & 565437 & 145324 & HEISPROD & ESTIMATED & ONSITE & & 132-B-6 \\
\hline 3892 & 11629 & UNKNOWN & 565431 & 145384 & HEISPROD & ESTIMATED & ONSITE & & 132-B-6 \\
\hline 3893 & 11630 & & & & HEISPROD & & & & 132-C-2 Outfall \\
\hline 3894 & 11631 & & & & HEISPROD & & & & 132-C-2 Outfall \\
\hline 3895 & 11632 & & & & HEISPROD & & & & 132-C-2 Outfall \\
\hline 3896 & 11633 & UNKNOWN & 565286.6 & 145061.5 & HEISPROD & GPS & ONSITE & & 100-B-8 \\
\hline 3897 & 11634 & UNKNOWN & 565286.6 & 145061.5 & HEISPROD & GPS & ONSITE & & 100-B-8 \\
\hline 3898 & 11635 & UNKNOWN & 565278.9 & 145310.8 & HEISPROD & GPS & ONSITE & & 100-B-8 \\
\hline 3899 & 11636 & UNKNOWN & 565278.9 & 145310.8 & HEISPROD & GPS & ONSITE & & 100-B-8 \\
\hline 3900 & 11637 & UNKNOWN & 565673 & 145265 & HEISPROD & GPS & ONSITE & & 100-C-6 \\
\hline 3901 & 11638 & UNKNOWN & 565673 & 145265 & HEISPROD & GPS & ONSITE & & 100-C-6 \\
\hline 3902 & 11639 & UNKNOWN & 565640 & 145136 & HEISPROD & GPS & ONSITE & & $100-C-6$ \\
\hline 3903 & 11640 & UNKNOWN & 565673 & 145265 & HEISPROD & GPS & ONSITE & & $100-C-6$ \\
\hline 3904 & 11641 & UNKNOWN & 565466.3 & 144874.8 & HEISPROD & GPS & ONSITE & & $100-C-6$ \\
\hline 3905 & 11642 & UNKNOWN & 565466.3 & 144874.8 & HEISPROD & GPS & ONSITE & & 100-C-6 \\
\hline 3906 & 11643 & NAD83(91) & 565620 & 145040 & HEISPROD & ESTIMATED & ONSITE & & 100-C-6 \\
\hline 3907 & 11644 & NAD83(91) & 565600 & 145030 & HEISPROD & ESTIMATED & ONSITE & & $100-C-6$ \\
\hline 3908 & 11645 & UNKNOWN & 565307 & 145262 & HEISPROD & MEASURED & ONSITE & & 100-B-8 \\
\hline 3909 & 11646 & UNKNOWN & 565305 & 145220 & HEISPROD & MEASURED & ONSITE & & 100-B-8 \\
\hline 3910 & 11647 & UNKNOWN & 565308 & 145158 & HEISPROD & MEASURED & ONSITE & & 100-B-8 \\
\hline 3911 & 11648 & UNKNOWN & 565309 & 145057 & HEISPROD & MEASURED & ONSITE & & 100-B-8 \\
\hline 3912 & 11649 & UNKNOWN & 565313 & 145012 & HEISPROD & MEASURED & ONSITE & & 100-B-8 \\
\hline 3913 & 11650 & UNKNOWN & 565560 & 144970 & HEISPROD & ESTIMATED & ONSITE & & 100-C-6 \\
\hline 3914 & 11651 & UNKNOWN & 565557 & 144976 & HEISPROD & ESTIMATED & ONSITE & & 100-C-6 \\
\hline 3915 & 11652 & UNKNOWN & 565559 & 145005 & HEISPROD & ESTIMATED & ONSITE & & $100-C-6$ \\
\hline
\end{tabular}




\begin{tabular}{|c|c|c|c|c|c|c|c|c|c|}
\hline & A & $\mathrm{B}$ & C & $\mathrm{D}$ & $\mathrm{E}$ & $\mathrm{F}$ & $G$ & $\mathrm{H}$ & $\mathrm{I}$ \\
\hline 1 & SAMP_SITE_ID & HORIZ_COORD_TYPE & EW_COORD & NS_COORD & OWNER_ID & COORD_SOURCE & DIST_CLASS & RAD_LEVEL & SAMP_SITE_NAME \\
\hline \begin{tabular}{|l|}
3916 \\
\end{tabular} & 11653 & UNKNOWN & 565620 & 144943 & HEISPROD & ESTIMATED & ONSITE & & $100-C-6$ \\
\hline 3917 & 11654 & UNKNOWN & 565614 & 144943 & HEISPROD & ESTIMATED & ONSITE & & $100-C-6$ \\
\hline 3918 & 11655 & UNKNOWN & 565617 & 144954 & HEISPROD & ESTIMATED & ONSITE & & $100-C-6$ \\
\hline 3919 & 11656 & UNKNOWN & 565520 & 145182 & HEISPROD & ESTIMATED & ONSITE & & $100-C-6$ \\
\hline 3920 & 11657 & UNKNOWN & 565504 & 145186 & HEISPROD & ESTIMATED & ONSITE & & $100-C-6$ \\
\hline 3921 & 11658 & UNKNOWN & 565488 & 145222 & HEISPROD & ESTIMATED & ONSITE & & $100-C-6$ \\
\hline 3922 & 11659 & UNKNOWN & 565477 & 145257 & HEISPROD & ESTIMATED & ONSITE & & $100-C-6$ \\
\hline 3923 & 11660 & UNKNOWN & 565479 & 145327 & HEISPROD & ESTIMATED & ONSITE & & $100-C-6$ \\
\hline 3924 & 11661 & UNKNOWN & 565312 & 145091 & HEISPROD & ESTIMATED & ONSITE & & 100-B-8 \\
\hline 3925 & 11662 & UNKNOWN & 565610 & 144940 & HEISPROD & ESTIMATED & ONSITE & & $100-C-6$ \\
\hline 3926 & 11663 & UNKNOWN & 565620 & 144950 & HEISPROD & ESTIMATED & ONSITE & & $100-C-6$ \\
\hline 3927 & 11664 & UNKNOWN & 565620 & 145040 & HEISPROD & ESTIMATED & ONSITE & & $100-C-6$ \\
\hline 3928 & 11665 & UNKNOWN & 565630 & 145040 & HEISPROD & ESTIMATED & ONSITE & & $100-C-6$ \\
\hline \begin{tabular}{|l|}
3929 \\
\end{tabular} & 11666 & UNKNOWN & 565570 & 144980 & HEISPROD & ESTIMATED & ONSITE & & $100-C-6$ \\
\hline 3930 & 11667 & UNKNOWN & 565570 & 145000 & HEISPROD & ESTIMATED & ONSITE & & $100-C-6$ \\
\hline 3931 & 11668 & UNKNOWN & 565650 & 145081 & HEISPROD & ESTIMATED & ONSITE & & $100-C-6$ \\
\hline \begin{tabular}{|l|}
3932 \\
\end{tabular} & 11669 & UNKNOWN & 565645 & 145064 & HEISPROD & ESTIMATED & ONSITE & & $100-C-6$ \\
\hline 3933 & 11670 & & & & HEISPROD & & & & $100-C-6$ \\
\hline 3934 & 11671 & & & & HEISPROD & & & & $100-C-6$ \\
\hline 3935 & 11672 & & & & HEISPROD & & & & $100-C-6$ \\
\hline 3936 & 11673 & UNKNOWN & 565650 & 145030 & HEISPROD & ESTIMATED & ONSITE & & 100-C-6 \\
\hline \begin{tabular}{|l|}
3937 \\
\end{tabular} & 11674 & UNKNOWN & 565650 & 145040 & HEISPROD & ESTIMATED & ONSITE & & 100-C-6 \\
\hline \begin{tabular}{|l|}
3938 \\
\end{tabular} & 11675 & UNKNOWN & 565640 & 145060 & HEISPROD & ESTIMATED & ONSITE & & $100-C-6$ \\
\hline 3939 & 11676 & UNKNOWN & 565640 & 145070 & HEISPROD & ESTIMATED & ONSITE & & $100-C-6$ \\
\hline 3940 & 11677 & UNKNOWN & 565640 & 145090 & HEISPROD & ESTIMATED & ONSITE & & $100-C-6$ \\
\hline 3941 & 11678 & UNKNOWN & 565670 & 144980 & HEISPROD & ESTIMATED & ONSITE & & $100-C-6$ \\
\hline 3942 & 11679 & UNKNOWN & 565561 & 144970 & HEISPROD & ESTIMATED & ONSITE & & $100-C-6$ \\
\hline 3943 & 11680 & UNKNOWN & 565560 & 144970 & HEISPROD & ESTIMATED & ONSITE & & $100-C-6$ \\
\hline 3944 & 11681 & UNKNOWN & 565560 & 144980 & HEISPROD & ESTIMATED & ONSITE & & $100-C-6$ \\
\hline \begin{tabular}{|l|}
3945 \\
\end{tabular} & 11682 & UNKNOWN & 565564 & 144965 & HEISPROD & ESTIMATED & ONSITE & & $100-C-6$ \\
\hline 3946 & 11683 & UNKNOWN & 565580 & 145010 & HEISPROD & ESTIMATED & ONSITE & & 100-C-6 \\
\hline \begin{tabular}{|l|}
3947 \\
\end{tabular} & 11684 & UNKNOWN & 565560 & 145010 & HEISPROD & ESTIMATED & ONSITE & & 100-C-6 \\
\hline 3948 & 11685 & UNKNOWN & 565550 & 145000 & HEISPROD & ESTIMATED & ONSITE & & $100-C-6$ \\
\hline 3949 & 11686 & UNKNOWN & 565560 & 145000 & HEISPROD & ESTIMATED & ONSITE & & $100-C-6$ \\
\hline 3950 & 11687 & & & & HEISPROD & & & & 100-B-8 \\
\hline 3951 & 11688 & UNKNOWN & 565320 & 144930 & HEISPROD & ESTIMATED & ONSITE & & 100-B-8 \\
\hline 3952 & 11689 & UNKNOWN & 565640 & 145110 & HEISPROD & ESTIMATED & ONSITE & & 100-C-6 \\
\hline 3953 & 11690 & UNKNOWN & 565590 & 145100 & HEISPROD & ESTIMATED & ONSITE & & 100-C-6 \\
\hline
\end{tabular}




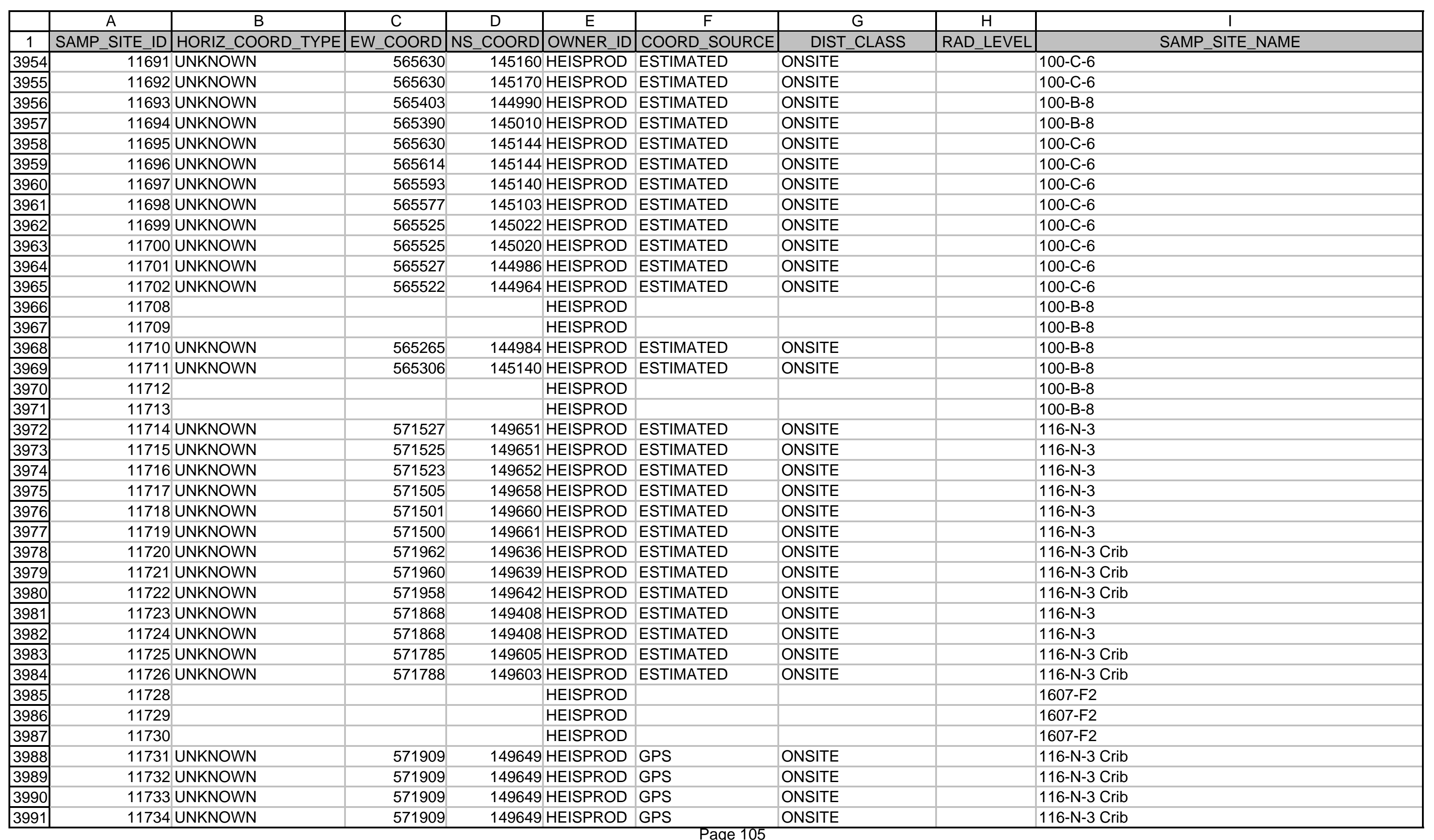




\begin{tabular}{|c|c|c|c|c|c|c|c|c|c|}
\hline & $\bar{A}$ & $\mathrm{~B}$ & $\mathrm{C}$ & $\mathrm{D}$ & $E$ & $\mathrm{~F}$ & G & $\mathrm{H}$ & $\mathrm{I}$ \\
\hline 1 & SAMP_SITE_ID & HORIZ_COORD_TYPE & EW_COORD & NS_COORD & OWNER_ID & COORD_SOURCE & DIST_CLASS & RAD_LEVEL & SAMP_SITE_NAME \\
\hline 3992 & 11735 & UNKNOWN & 571909 & 149649 & HEISPROD & GPS & ONSITE & & 116-N-3 Crib \\
\hline 3993 & 11736 & UNKNOWN & 571901 & 149650 & HEISPROD & GPS & ONSITE & & 116-N-3 Crib \\
\hline 3994 & 11737 & UNKNOWN & 571923 & 149658 & HEISPROD & GPS & ONSITE & & 116-N-3 Crib \\
\hline 3995 & 11738 & UNKNOWN & 571981 & 149560 & HEISPROD & GPS & ONSITE & & 116-N-3 Crib \\
\hline 3996 & 11739 & UNKNOWN & 571918 & 149657 & HEISPROD & GPS & ONSITE & & 116-N-3 Crib \\
\hline 3997 & 11740 & UNKNOWN & 571914 & 149541 & HEISPROD & GPS & ONSITE & & 116-N-3 Crib \\
\hline 3998 & 11741 & UNKNOWN & 571914 & 149541 & HEISPROD & GPS & ONSITE & & 116-N-3 Crib \\
\hline 3999 & 11742 & UNKNOWN & 571855 & 149607 & HEISPROD & GPS & ONSITE & & 116-N-3 Crib \\
\hline 4000 & 11743 & UNKNOWN & 571855 & 149607 & HEISPROD & GPS & ONSITE & & 116-N-3 Crib \\
\hline 4001 & 11744 & UNKNOWN & 571877 & 149547 & HEISPROD & GPS & ONSITE & & 116-N-3 Crib \\
\hline 4002 & 11745 & UNKNOWN & 571987 & 149569 & HEISPROD & GPS & ONSITE & & 116-N-3 Crib \\
\hline 4003 & 11746 & UNKNOWN & 571957 & 149654 & HEISPROD & GPS & ONSITE & & 116-N-3 Crib \\
\hline 4004 & 11747 & & & & HEISPROD & & & & $126-\mathrm{F}-1$ \\
\hline 4005 & 11748 & & & & HEISPROD & & & & $126-\mathrm{F}-1$ \\
\hline 4006 & 11749 & & & & HEISPROD & & & & 126-F-1 \\
\hline 4007 & 11750 & & & & HEISPROD & & & & 126-F-1 \\
\hline 4008 & 11751 & & & & HEISPROD & & & & 126-F-1 \\
\hline 4009 & 11752 & & & & HEISPROD & & & & $100-C-6$ \\
\hline 4010 & 11753 & & & & HEISPROD & & & & 116-F-2 \\
\hline 4011 & 11754 & & & & HEISPROD & & & & 116-F-2 \\
\hline 4012 & 11755 & & & & HEISPROD & & & & 116-F-2 \\
\hline 4013 & 11756 & & & & HEISPROD & & & & 116-F-2 \\
\hline 4014 & 11757 & & & & HEISPROD & & & & 116-F-2 \\
\hline 4015 & 11758 & UNKNOWN & 571931 & 149550 & HEISPROD & GPS & ONSITE & & 116-N-3 Crib \\
\hline 4016 & 11759 & UNKNOWN & 571878 & 149577 & HEISPROD & GPS & ONSITE & & 116-N-3 Crib \\
\hline 4017 & 11768 & UNKNOWN & 571878 & 149577 & HEISPROD & GPS & ONSITE & & 116-N-3 Crib \\
\hline 4018 & 11769 & UNKNOWN & 571878 & 149577 & HEISPROD & GPS & ONSITE & & 116-N-3 Crib \\
\hline 4019 & 11770 & UNKNOWN & 571931 & 149550 & HEISPROD & GPS & ONSITE & & 116-N-3 Crib \\
\hline 4020 & 11772 & UNKNOWN & 571917 & 149591 & HEISPROD & GPS & ONSITE & & 116-N-3 Crib \\
\hline 4021 & 11773 & UNKNOWN & 571888 & 149627 & HEISPROD & GPS & ONSITE & & 116-N-3 Crib \\
\hline 4022 & 11774 & UNKNOWN & 571946 & 149605 & HEISPROD & GPS & ONSITE & & 116-N-3 Crib \\
\hline 4023 & 11775 & UNKNOWN & 571884 & 149573 & HEISPROD & GPS & ONSITE & & 116-N-3 Crib \\
\hline 4024 & 11776 & UNKNOWN & 571951 & 149612 & HEISPROD & GPS & ONSITE & & 116-N-3 Crib \\
\hline 4025 & 11777 & UNKNOWN & 571906 & 149578 & HEISPROD & GPS & ONSITE & & 116-N-3 Crib \\
\hline 4026 & 11778 & UNKNOWN & 571896 & 149552 & HEISPROD & GPS & ONSITE & & 116-N-3 Crib \\
\hline 4027 & 11779 & UNKNOWN & 571866 & 149594 & HEISPROD & GPS & ONSITE & & 116-N-3 Crib \\
\hline 4028 & 11780 & UNKNOWN & 571931 & 149550 & HEISPROD & GPS & ONSITE & & 116-N-3 Crib \\
\hline 4029 & 11781 & UNKNOWN & 571917 & 149591 & HEISPROD & GPS & ONSITE & & 116-N-3 Crib \\
\hline
\end{tabular}




\begin{tabular}{|c|c|c|c|c|c|c|c|c|c|}
\hline & $A$ & $\mathrm{~B}$ & $\mathrm{C}$ & $\mathrm{D}$ & $E$ & $\mathrm{~F}$ & $\mathrm{G}$ & $\mathrm{H}$ & $\mathrm{I}$ \\
\hline 1 & SAMP_SITE_ID & HORIZ_COORD_TYPE & EW_COORD & NS_COORD & OWNER_ID & COORD_SOURCE & DIST_CLASS & RAD_LEVEL & SAMP_SITE_NAME \\
\hline 4030 & 11782 & UNKNOWN & 571906 & 149578 & HEISPROD & GPS & ONSITE & & 116-N-3 Crib \\
\hline 4031 & 11783 & & & & HEISPROD & & & & 116-F-9 Shallow Zone \\
\hline 4032 & 11784 & & & & HEISPROD & & & & 116-F-9 Shallow Zone \\
\hline 4033 & 11785 & & & & HEISPROD & & & & 116-F-9 Shallow Zone \\
\hline 4034 & 11786 & & & & HEISPROD & & & & 116-F-9 Shallow Zone \\
\hline 4035 & 11787 & & & & HEISPROD & & & & 116-F-9 Shallow Zone \\
\hline 4036 & 11788 & & & & HEISPROD & & & & 116-F-9 Shallow Zone \\
\hline 4037 & 11789 & & & & HEISPROD & & & & 116-F-9 Shallow Zone \\
\hline 4038 & 11790 & & & & HEISPROD & & & & 116-F-9 Shallow Zone \\
\hline 4039 & 11791 & & & & HEISPROD & & & & 116-F-9 Shallow Zone \\
\hline 4040 & 11792 & & & & HEISPROD & & & & 116-F-9 Shallow Zone \\
\hline 4041 & 11793 & UNKNOWN & 565322 & 144969 & HEISPROD & ESTIMATED & ONSITE & & 100-B-8 \\
\hline 4042 & 11794 & UNKNOWN & 565313 & 144957 & HEISPROD & ESTIMATED & ONSITE & & 100-B-8 \\
\hline 4043 & 11795 & UNKNOWN & 565319 & 144953 & HEISPROD & ESTIMATED & ONSITE & & 100-B-8 \\
\hline 4044 & 11796 & UNKNOWN & 565319 & 144976 & HEISPROD & ESTIMATED & ONSITE & & $100-B-8$ \\
\hline 4045 & 11797 & UNKNOWN & 565317 & 144982 & HEISPROD & ESTIMATED & ONSITE & & $100-B-8$ \\
\hline 4046 & 11798 & UNKNOWN & 565311 & 144980 & HEISPROD & ESTIMATED & ONSITE & & $100-B-8$ \\
\hline 4047 & 11799 & UNKNOWN & 565315 & 144965 & HEISPROD & ESTIMATED & ONSITE & & 100-B-8 \\
\hline 4048 & 11800 & UNKNOWN & 565309 & 144963 & HEISPROD & ESTIMATED & ONSITE & & $100-B-8$ \\
\hline 4049 & 11801 & UNKNOWN & 565312 & 144977 & HEISPROD & ESTIMATED & ONSITE & & 100-B-8 \\
\hline 4050 & 11802 & UNKNOWN & 565316 & 144972 & HEISPROD & ESTIMATED & ONSITE & & 100-B-8 \\
\hline \begin{tabular}{|l|}
4051 \\
\end{tabular} & 11803 & UNKNOWN & 565287 & 145308 & HEISPROD & ESTIMATED & ONSITE & & 100-B-8 \\
\hline 4052 & 11804 & UNKNOWN & 565275 & 145301 & HEISPROD & ESTIMATED & ONSITE & & 100-B-8 \\
\hline 4053 & 11808 & UNKNOWN & 571950 & 149541 & HEISPROD & ESTIMATED & ONSITE & & $116-\mathrm{N}-3$ \\
\hline 4054 & 11809 & UNKNOWN & 571972 & 149550 & HEISPROD & ESTIMATED & ONSITE & & $116-\mathrm{N}-3$ \\
\hline 4055 & 11810 & UNKNOWN & 571952 & 149539 & HEISPROD & ESTIMATED & ONSITE & & $116-\mathrm{N}-3$ \\
\hline 4056 & 11811 & UNKNOWN & 571969 & 149546 & HEISPROD & ESTIMATED & ONSITE & & $116-\mathrm{N}-3$ \\
\hline \begin{tabular}{|l|}
4057 \\
\end{tabular} & 11812 & UNKNOWN & 571964 & 149537 & HEISPROD & ESTIMATED & ONSITE & & $116-\mathrm{N}-3$ \\
\hline 4058 & 11813 & UNKNOWN & 571977 & 149534 & HEISPROD & ESTIMATED & ONSITE & & $116-\mathrm{N}-3$ \\
\hline 4059 & 11814 & UNKNOWN & 571973 & 149518 & HEISPROD & ESTIMATED & ONSITE & & $116-\mathrm{N}-3$ \\
\hline 4060 & 11815 & UNKNOWN & 571979 & 149506 & HEISPROD & ESTIMATED & ONSITE & & $116-\mathrm{N}-3$ \\
\hline 4061 & 11828 & UNKNOWN & 564402 & 144069 & HEISPROD & ESTIMATED & ONSITE & & 118-B-1 \\
\hline 4062 & 11829 & UNKNOWN & 564401.64 & 144019.58 & HEISPROD & ESTIMATED & ONSITE & & 118-B-1 \\
\hline 4063 & 11830 & UNKNOWN & 564402 & 144069 & HEISPROD & ESTIMATED & ONSITE & & 118-B-1 \\
\hline 4064 & 11831 & & & & HEISPROD & & & & 118-B-3 \\
\hline 4065 & 11832 & UNKNOWN & 564401.64 & 144019.58 & HEISPROD & ESTIMATED & ONSITE & & 118-B-1 \\
\hline 4066 & 11833 & UNKNOWN & 564354.24 & 143987.26 & HEISPROD & ESTIMATED & ONSITE & & 118-B-1 \\
\hline 4067 & 11834 & UNKNOWN & 565349.82 & 144047.01 & HEISPROD & ESTIMATED & ONSITE & & 118-B-1 \\
\hline
\end{tabular}




\begin{tabular}{|c|c|c|c|c|c|c|c|c|c|}
\hline & $A$ & $\mathrm{~B}$ & $\mathrm{C}$ & $\mathrm{D}$ & $E$ & $\mathrm{~F}$ & $\mathrm{G}$ & $\mathrm{H}$ & $\mathrm{I}$ \\
\hline 1 & SAMP_SITE_ID & HORIZ_COORD_TYPE & EW_COORD & NS_COORD & OWNER_ID & COORD_SOURCE & DIST_CLASS & RAD_LEVEL & SAMP_SITE_NAME \\
\hline 4068 & 11848 & UNKNOWN & 565446 & 145419 & HEISPROD & ESTIMATED & ONSITE & & 132-B-6 \\
\hline 4069 & 11849 & UNKNOWN & 565479 & 145289 & HEISPROD & ESTIMATED & ONSITE & & $100-C-6$ \\
\hline 4070 & 11868 & UNKNOWN & 571976 & 149509 & HEISPROD & ESTIMATED & ONSITE & & $116-\mathrm{N}-3$ \\
\hline 4071 & 11869 & UNKNOWN & 571962 & 149523 & HEISPROD & ESTIMATED & ONSITE & & $116-\mathrm{N}-3$ \\
\hline 4072 & 11870 & UNKNOWN & 571976 & 149530 & HEISPROD & ESTIMATED & ONSITE & & $116-\mathrm{N}-3$ \\
\hline \begin{tabular}{|l|}
4073 \\
\end{tabular} & 11871 & UNKNOWN & 571949 & 149540 & HEISPROD & ESTIMATED & ONSITE & & $116-\mathrm{N}-3$ \\
\hline 4074 & 11872 & UNKNOWN & 571961 & 149544 & HEISPROD & ESTIMATED & ONSITE & & $116-N-3$ \\
\hline 4075 & 11873 & UNKNOWN & 571972 & 149549 & HEISPROD & ESTIMATED & ONSITE & & $116-\mathrm{N}-3$ \\
\hline \begin{tabular}{|l|}
4076 \\
\end{tabular} & 11874 & UNKNOWN & 571566 & 149810 & HEISPROD & ESTIMATED & ONSITE & & 116-N-1 Trench \\
\hline \begin{tabular}{|l|}
4077 \\
\end{tabular} & 11875 & UNKNOWN & 571566 & 149810 & HEISPROD & ESTIMATED & ONSITE & & 116-N-1 Trench \\
\hline \begin{tabular}{|l|}
4078 \\
\end{tabular} & 11876 & UNKNOWN & 581083.57 & 147731.61 & HEISPROD & ESTIMATED & ONSITE & & 116-F-2 \\
\hline 4079 & 11877 & UNKNOWN & 581076.95 & 147724.41 & HEISPROD & ESTIMATED & ONSITE & & $116-\mathrm{F}-2$ \\
\hline 4080 & 11878 & UNKNOWN & 581082.66 & 147722.32 & HEISPROD & ESTIMATED & ONSITE & & $116-F-2$ \\
\hline 4081 & 11879 & UNKNOWN & 581113.32 & 147721.2 & HEISPROD & ESTIMATED & ONSITE & & $116-F-2$ \\
\hline 4082 & 11880 & UNKNOWN & 571566 & 149810 & HEISPROD & ESTIMATED & ONSITE & & 116-N-1 Trench \\
\hline 4083 & 11881 & UNKNOWN & 571566 & 149810 & HEISPROD & ESTIMATED & ONSITE & & 116-N-1 Trench \\
\hline 4084 & 11882 & UNKNOWN & 571566 & 149810 & HEISPROD & ESTIMATED & ONSITE & & 116-N-1 Trench \\
\hline 4085 & 11883 & UNKNOWN & 571566 & 149810 & HEISPROD & ESTIMATED & ONSITE & & 116-N-1 Trench \\
\hline 4086 & 11884 & UNKNOWN & 571566 & 149810 & HEISPROD & ESTIMATED & ONSITE & & 116-N-1 Trench \\
\hline 4087 & 11885 & UNKNOWN & 571949 & 149540 & HEISPROD & ESTIMATED & ONSITE & & $116-N-3$ \\
\hline \begin{tabular}{|l|}
4088 \\
\end{tabular} & 11886 & UNKNOWN & 571961 & 149544 & HEISPROD & ESTIMATED & ONSITE & & $116-\mathrm{N}-3$ \\
\hline 4089 & 11887 & UNKNOWN & 581126.32 & 147739.99 & HEISPROD & ESTIMATED & ONSITE & & 116-F-2 \\
\hline 4090 & 11888 & UNKNOWN & 581134.45 & 147732.81 & HEISPROD & ESTIMATED & ONSITE & & 116-F-2 \\
\hline 4091 & 11889 & UNKNOWN & 581147.92 & 147710.87 & HEISPROD & ESTIMATED & ONSITE & & $116-F-2$ \\
\hline 4092 & 11890 & UNKNOWN & 581151.37 & 147697.08 & HEISPROD & ESTIMATED & ONSITE & & $116-F-2$ \\
\hline 4093 & 11891 & UNKNOWN & 581160.78 & 147688.25 & HEISPROD & ESTIMATED & ONSITE & & $116-F-2$ \\
\hline 4094 & 11892 & UNKNOWN & 581163.32 & 147674.96 & HEISPROD & ESTIMATED & ONSITE & & $116-F-2$ \\
\hline 4095 & 11893 & UNKNOWN & 581168.12 & 147653.52 & HEISPROD & ESTIMATED & ONSITE & & $116-\mathrm{F}-2$ \\
\hline 4096 & 11894 & UNKNOWN & 581173.97 & 147644.67 & HEISPROD & ESTIMATED & ONSITE & & $116-\mathrm{F}-2$ \\
\hline 4097 & 11895 & UNKNOWN & 581168.66 & 147633.41 & HEISPROD & ESTIMATED & ONSITE & & 116-F-2 \\
\hline 4098 & 11896 & UNKNOWN & 581174.06 & 147635.07 & HEISPROD & ESTIMATED & ONSITE & & 116-F-2 \\
\hline 4099 & 11897 & UNKNOWN & 581174.17 & 147624.76 & HEISPROD & ESTIMATED & ONSITE & & 116-F-2 \\
\hline 4100 & 11898 & UNKNOWN & 581169.54 & 147613.02 & HEISPROD & ESTIMATED & ONSITE & & 116-F-2 \\
\hline \begin{tabular}{|l|}
4101 \\
\end{tabular} & 11899 & UNKNOWN & 581171.47 & 147590.61 & HEISPROD & ESTIMATED & ONSITE & & $116-\mathrm{F}-2$ \\
\hline 4102 & 11900 & UNKNOWN & 581173.93 & 147576.49 & HEISPROD & ESTIMATED & ONSITE & & $116-\mathrm{F}-2$ \\
\hline 4103 & 11901 & UNKNOWN & 581158.01 & 147618.35 & HEISPROD & ESTIMATED & ONSITE & & 116-F-2 \\
\hline 4104 & 11902 & UNKNOWN & 581154.77 & 147612.47 & HEISPROD & ESTIMATED & ONSITE & & $116-\mathrm{F}-2$ \\
\hline 4105 & 11903 & UNKNOWN & 581156.25 & 147647.37 & HEISPROD & ESTIMATED & ONSITE & & 116-F-2 \\
\hline
\end{tabular}




\begin{tabular}{|c|c|c|c|c|c|c|c|c|c|}
\hline & $\bar{A}$ & $\mathrm{~B}$ & $\mathrm{C}$ & $\mathrm{D}$ & $E$ & $\mathrm{~F}$ & G & $\mathrm{H}$ & $\mathrm{I}$ \\
\hline 1 & SAMP_SITE_ID & HORIZ_COORD_TYPE & EW_COORD & NS_COORD & OWNER_ID & COORD_SOURCE & DIST_CLASS & RAD_LEVEL & SAMP_SITE_NAME \\
\hline 4106 & 11904 & UNKNOWN & 581143.31 & 147650.76 & HEISPROD & ESTIMATED & ONSITE & & $116-\mathrm{F}-2$ \\
\hline 4107 & 11905 & UNKNOWN & 581143.61 & 147658.03 & HEISPROD & ESTIMATED & ONSITE & & $116-\mathrm{F}-2$ \\
\hline 4108 & 11906 & UNKNOWN & 581139.19 & 147657.82 & HEISPROD & ESTIMATED & ONSITE & & 116-F-2 \\
\hline 4109 & 11912 & UNKNOWN & 571868 & 149408 & HEISPROD & ESTIMATED & ONSITE & & $116-\mathrm{N}-3$ \\
\hline 4110 & 11913 & UNKNOWN & 571868 & 149408 & HEISPROD & ESTIMATED & ONSITE & & $116-\mathrm{N}-3$ \\
\hline 4111 & 11914 & UNKNOWN & 565441 & 144751 & HEISPROD & ESTIMATED & ONSITE & & $100-C-6$ \\
\hline 4112 & 11915 & UNKNOWN & 565466 & 144751 & HEISPROD & ESTIMATED & ONSITE & & $100-C-6$ \\
\hline 4113 & 11916 & UNKNOWN & 565317 & 144978 & HEISPROD & ESTIMATED & ONSITE & & 100-B-8 \\
\hline 4114 & 11917 & UNKNOWN & 565310 & 144976 & HEISPROD & ESTIMATED & ONSITE & & $100-B-8$ \\
\hline 4115 & 11918 & UNKNOWN & 565311 & 144970 & HEISPROD & ESTIMATED & ONSITE & & $100-B-8$ \\
\hline 4116 & 11919 & UNKNOWN & 565316 & 144966 & HEISPROD & ESTIMATED & ONSITE & & $100-B-8$ \\
\hline 4117 & 11920 & UNKNOWN & 565313 & 144957 & HEISPROD & ESTIMATED & ONSITE & & 100-B-8 \\
\hline 4118 & 11921 & UNKNOWN & 565315 & 144951 & HEISPROD & ESTIMATED & ONSITE & & 100-B-8 \\
\hline 4119 & 11922 & UNKNOWN & 565319 & 144945 & HEISPROD & ESTIMATED & ONSITE & & $100-B-8$ \\
\hline 4120 & 11923 & UNKNOWN & 565319 & 144945 & HEISPROD & ESTIMATED & ONSITE & & $100-B-8$ \\
\hline 4121 & 11924 & UNKNOWN & 565318 & 144952 & HEISPROD & ESTIMATED & ONSITE & & $100-B-8$ \\
\hline 4122 & 11925 & UNKNOWN & 565318 & 144962 & HEISPROD & ESTIMATED & ONSITE & & 100-B-8 \\
\hline 4123 & 11926 & UNKNOWN & 565318 & 144971 & HEISPROD & ESTIMATED & ONSITE & & 100-B-8 \\
\hline 4124 & 11932 & UNKNOWN & 581136.36 & 147636.9 & HEISPROD & ESTIMATED & ONSITE & & 116-F-2 \\
\hline 4125 & 11933 & UNKNOWN & 581141.11 & 147636.91 & HEISPROD & ESTIMATED & ONSITE & & 116-F-2 \\
\hline 4126 & 11934 & UNKNOWN & 581131 & 147617.68 & HEISPROD & ESTIMATED & ONSITE & & $116-F-2$ \\
\hline 4127 & 11935 & UNKNOWN & 581120.52 & 147616.79 & HEISPROD & ESTIMATED & ONSITE & & 116-F-2 \\
\hline 4128 & 11936 & UNKNOWN & 581099.5 & 147632.2 & HEISPROD & ESTIMATED & ONSITE & & 116-F-2 \\
\hline 4129 & 11937 & UNKNOWN & 581096.09 & 147644.59 & HEISPROD & ESTIMATED & ONSITE & & 116-F-2 \\
\hline 4130 & 11938 & UNKNOWN & 581093.86 & 147656.49 & HEISPROD & ESTIMATED & ONSITE & & 116-F-2 \\
\hline 4131 & 11939 & UNKNOWN & 581051.05 & 147646.91 & HEISPROD & ESTIMATED & ONSITE & & $116-F-2$ \\
\hline 4132 & 11940 & UNKNOWN & 581037.97 & 147644.92 & HEISPROD & ESTIMATED & ONSITE & & 116-F-2 \\
\hline 4133 & 11941 & UNKNOWN & 581035.42 & 147656.66 & HEISPROD & ESTIMATED & ONSITE & & 116-F-2 \\
\hline 4134 & 11942 & UNKNOWN & 581034.24 & 147666.91 & HEISPROD & ESTIMATED & ONSITE & & 116-F-2 \\
\hline 4135 & 11943 & UNKNOWN & 581029.82 & 147662.62 & HEISPROD & ESTIMATED & ONSITE & & $116-F-2$ \\
\hline 4136 & 11944 & UNKNOWN & 581032.17 & 147677.38 & HEISPROD & ESTIMATED & ONSITE & & $116-\mathrm{F}-2$ \\
\hline 4137 & 11945 & UNKNOWN & 581028.21 & 147688.09 & HEISPROD & ESTIMATED & ONSITE & & 116-F-2 \\
\hline 4138 & 11946 & UNKNOWN & 581035.5 & 147687.66 & HEISPROD & ESTIMATED & ONSITE & & 116-F-2 \\
\hline 4139 & 11947 & UNKNOWN & 581034.19 & 147694.75 & HEISPROD & ESTIMATED & ONSITE & & 116-F-2 \\
\hline 4140 & 11948 & UNKNOWN & 581044.24 & 147694.18 & HEISPROD & ESTIMATED & ONSITE & & 116-F-2 \\
\hline 4141 & 11949 & UNKNOWN & 581061.42 & 147683.66 & HEISPROD & ESTIMATED & ONSITE & & 116-F-2 \\
\hline 4142 & 11950 & UNKNOWN & 581088.71 & 147683.69 & HEISPROD & ESTIMATED & ONSITE & & 116-F-2 \\
\hline 4143 & 11951 & UNKNOWN & 581084.51 & 147694.3 & HEISPROD & ESTIMATED & ONSITE & & 116-F-2 \\
\hline
\end{tabular}




\begin{tabular}{|c|c|c|c|c|c|c|c|c|c|}
\hline & $A$ & $\mathrm{~B}$ & $\mathrm{C}$ & $\mathrm{D}$ & $E$ & $\mathrm{~F}$ & $\mathrm{G}$ & $\mathrm{H}$ & $\mathrm{I}$ \\
\hline 1 & SAMP_SITE_ID & HORIZ_COORD_TYPE & EW_COORD & NS_COORD & OWNER_ID & COORD_SOURCE & DIST_CLASS & RAD_LEVEL & SAMP_SITE_NAME \\
\hline 4144 & 11952 & UNKNOWN & 581088.67 & 147696.53 & HEISPROD & ESTIMATED & ONSITE & & $116-F-2$ \\
\hline 4145 & 11953 & UNKNOWN & 581063.42 & 147712.04 & HEISPROD & ESTIMATED & ONSITE & & $116-F-2$ \\
\hline \begin{tabular}{|l|}
4146 \\
\end{tabular} & 11954 & UNKNOWN & 581045.04 & 147706.31 & HEISPROD & ESTIMATED & ONSITE & & $116-\mathrm{F}-2$ \\
\hline 4147 & 11955 & UNKNOWN & 581030.73 & 147712.24 & HEISPROD & ESTIMATED & ONSITE & & $116-F-2$ \\
\hline 4148 & 11956 & UNKNOWN & 580976.33 & 147654.77 & HEISPROD & ESTIMATED & ONSITE & & 116-F-2 \\
\hline 4149 & 11957 & UNKNOWN & 580976.33 & 147651.79 & HEISPROD & ESTIMATED & ONSITE & & 116-F-2 \\
\hline 4150 & 11958 & UNKNOWN & 580976.43 & 147648.71 & HEISPROD & ESTIMATED & ONSITE & & $116-\mathrm{F}-2$ \\
\hline 4151 & 11959 & UNKNOWN & 580980.12 & 147654.24 & HEISPROD & ESTIMATED & ONSITE & & 116-F-2 \\
\hline 4152 & 11960 & UNKNOWN & 580979.98 & 147638.08 & HEISPROD & ESTIMATED & ONSITE & & $116-\mathrm{F}-2$ \\
\hline 4153 & 11961 & UNKNOWN & 580979.86 & 147621.38 & HEISPROD & ESTIMATED & ONSITE & & $116-\mathrm{F}-2$ \\
\hline 4154 & 11962 & UNKNOWN & 580982.64 & 147649.3 & HEISPROD & ESTIMATED & ONSITE & & 116-F-2 \\
\hline 4155 & 11963 & UNKNOWN & 580982.55 & 147631.86 & HEISPROD & ESTIMATED & ONSITE & & $116-\mathrm{F}-2$ \\
\hline 4156 & 11964 & UNKNOWN & 580982.52 & 147626.1 & HEISPROD & ESTIMATED & ONSITE & & $116-F-2$ \\
\hline 4157 & 11965 & UNKNOWN & 580985.1 & 147656.48 & HEISPROD & ESTIMATED & ONSITE & & $116-F-2$ \\
\hline 4158 & 11966 & UNKNOWN & 580984.98 & 147632.43 & HEISPROD & ESTIMATED & ONSITE & & $116-\mathrm{F}-2$ \\
\hline 4159 & 11967 & UNKNOWN & 580984.95 & 147626.35 & HEISPROD & ESTIMATED & ONSITE & & $116-\mathrm{F}-2$ \\
\hline 4160 & 11968 & UNKNOWN & 580987.48 & 147662.8 & HEISPROD & ESTIMATED & ONSITE & & 116-F-2 \\
\hline 4161 & 11969 & UNKNOWN & 580987.44 & 147656.5 & HEISPROD & ESTIMATED & ONSITE & & 116-F-2 \\
\hline 4162 & 11970 & UNKNOWN & 580987.39 & 147644.11 & HEISPROD & ESTIMATED & ONSITE & & $116-\mathrm{F}-2$ \\
\hline 4163 & 11971 & UNKNOWN & 580987.36 & 147638 & HEISPROD & ESTIMATED & ONSITE & & 116-F-2 \\
\hline 4164 & 11972 & UNKNOWN & 580989.81 & 147662.72 & HEISPROD & ESTIMATED & ONSITE & & $116-\mathrm{F}-2$ \\
\hline 4165 & 11973 & UNKNOWN & 580989.77 & 147650.63 & HEISPROD & ESTIMATED & ONSITE & & $116-\mathrm{F}-2$ \\
\hline 4166 & 11974 & UNKNOWN & 580992.11 & 147649.68 & HEISPROD & ESTIMATED & ONSITE & & 116-F-2 \\
\hline 4167 & 11975 & UNKNOWN & 580992.08 & 147643.51 & HEISPROD & ESTIMATED & ONSITE & & $116-F-2$ \\
\hline 4168 & 11976 & UNKNOWN & 580992 & 147625.31 & HEISPROD & ESTIMATED & ONSITE & & $116-F-2$ \\
\hline 4169 & 11977 & UNKNOWN & 580994.41 & 147661.86 & HEISPROD & ESTIMATED & ONSITE & & $116-F-2$ \\
\hline 4170 & 11978 & UNKNOWN & 580994.42 & 147642.97 & HEISPROD & ESTIMATED & ONSITE & & $116-\mathrm{F}-2$ \\
\hline \begin{tabular}{|l|}
4171 \\
\end{tabular} & 11979 & UNKNOWN & 580994.42 & 147636.92 & HEISPROD & ESTIMATED & ONSITE & & $116-\mathrm{F}-2$ \\
\hline 4172 & 11980 & UNKNOWN & 580996.76 & 147661.63 & HEISPROD & ESTIMATED & ONSITE & & $116-\mathrm{F}-2$ \\
\hline 4173 & 11981 & UNKNOWN & 580996.8 & 147655.49 & HEISPROD & ESTIMATED & ONSITE & & 116-F-2 \\
\hline \begin{tabular}{|l|}
4174 \\
\end{tabular} & 11982 & UNKNOWN & 580996.82 & 147638.08 & HEISPROD & ESTIMATED & ONSITE & & 116-F-2 \\
\hline \begin{tabular}{|l|}
4175 \\
\end{tabular} & 11983 & UNKNOWN & 580996.83 & 147626.08 & HEISPROD & ESTIMATED & ONSITE & & 116-F-2 \\
\hline 4176 & 11984 & UNKNOWN & 580999.32 & 147636.02 & HEISPROD & ESTIMATED & ONSITE & & 116-F-2 \\
\hline \begin{tabular}{|l|}
4177 \\
\end{tabular} & 11985 & UNKNOWN & 580999.34 & 147625.15 & HEISPROD & ESTIMATED & ONSITE & & $116-\mathrm{F}-2$ \\
\hline 4178 & 11986 & UNKNOWN & 581001.84 & 147656.91 & HEISPROD & ESTIMATED & ONSITE & & $116-\mathrm{F}-2$ \\
\hline 4179 & 11987 & UNKNOWN & 581004.73 & 147648.96 & HEISPROD & ESTIMATED & ONSITE & & 116-F-2 \\
\hline 4180 & 11988 & UNKNOWN & 581004.85 & 147639.34 & HEISPROD & ESTIMATED & ONSITE & & $116-\mathrm{F}-2$ \\
\hline 4181 & 11989 & UNKNOWN & 581004.92 & 147634.56 & HEISPROD & ESTIMATED & ONSITE & & $116-F-2$ \\
\hline
\end{tabular}




\begin{tabular}{|c|c|c|c|c|c|c|c|c|c|}
\hline & $A$ & $B$ & $\mathrm{C}$ & $\mathrm{D}$ & $E$ & $\mathrm{~F}$ & $G$ & $\mathrm{H}$ & $\mathrm{I}$ \\
\hline 1 & SAMP_SITE_ID & HORIZ_COORD_TYPE & EW_COORD & NS_COORD & OWNER_ID & COORD_SOURCE & DIST_CLASS & RAD_LEVEL & SAMP_SITE_NAME \\
\hline \begin{tabular}{|l|}
4182 \\
\end{tabular} & 11990 & UNKNOWN & 581005.04 & 147625.05 & HEISPROD & ESTIMATED & ONSITE & & $116-F-2$ \\
\hline \begin{tabular}{|l|}
4183 \\
\end{tabular} & 11991 & UNKNOWN & 581005.1 & 147620.22 & HEISPROD & ESTIMATED & ONSITE & & 116-F-2 \\
\hline 4184 & 11992 & UNKNOWN & 581007.51 & 147651.41 & HEISPROD & ESTIMATED & ONSITE & & 116-F-2 \\
\hline \begin{tabular}{|l|}
4185 \\
\end{tabular} & 11993 & UNKNOWN & 581007.66 & 147640.04 & HEISPROD & ESTIMATED & ONSITE & & 116-F-2 \\
\hline \begin{tabular}{|l|}
4186 \\
\end{tabular} & 11994 & UNKNOWN & 581007.74 & 147634.65 & HEISPROD & ESTIMATED & ONSITE & & $116-F-2$ \\
\hline \begin{tabular}{|l|}
4187 \\
\end{tabular} & 11995 & UNKNOWN & 581010.44 & 147648.71 & HEISPROD & ESTIMATED & ONSITE & & $116-F-2$ \\
\hline \begin{tabular}{|l|}
4188 \\
\end{tabular} & 11996 & UNKNOWN & 581010.71 & 147634.17 & HEISPROD & ESTIMATED & ONSITE & & 116-F-2 \\
\hline \begin{tabular}{|l|}
4189 \\
\end{tabular} & 11997 & UNKNOWN & 581014.48 & 147636.76 & HEISPROD & ESTIMATED & ONSITE & & $116-F-2$ \\
\hline 4190 & 11998 & UNKNOWN & 581016.12 & 147630.88 & HEISPROD & ESTIMATED & ONSITE & & 116-F-2 \\
\hline 4191 & 11999 & UNKNOWN & 581022.86 & 147630.93 & HEISPROD & ESTIMATED & ONSITE & & $116-F-2$ \\
\hline 4192 & 12000 & UNKNOWN & 581030.13 & 147631 & HEISPROD & ESTIMATED & ONSITE & & $116-F-2$ \\
\hline 4193 & 12001 & UNKNOWN & 581029.6 & 147628.94 & HEISPROD & ESTIMATED & ONSITE & & 116-F-2 \\
\hline \begin{tabular}{|l|}
4194 \\
\end{tabular} & 12002 & UNKNOWN & 581028.57 & 147626.66 & HEISPROD & ESTIMATED & ONSITE & & 116-F-2 \\
\hline \begin{tabular}{|l|}
4195 \\
\end{tabular} & 12003 & UNKNOWN & 581007.71 & 147621.82 & HEISPROD & ESTIMATED & ONSITE & & $116-F-2$ \\
\hline \begin{tabular}{|l|}
4196 \\
\end{tabular} & 12012 & UNKNOWN & 565719 & 145233 & HEISPROD & ESTIMATED & ONSITE & & $100-C-9$ \\
\hline \begin{tabular}{|l|}
4197 \\
\end{tabular} & 12013 & UNKNOWN & 565723 & 145271 & HEISPROD & ESTIMATED & ONSITE & & $100-C-9$ \\
\hline \begin{tabular}{|l|}
4198 \\
\end{tabular} & 12014 & UNKNOWN & 565709 & 145214 & HEISPROD & ESTIMATED & ONSITE & & $100-C-9$ \\
\hline \begin{tabular}{|l|}
4199 \\
\end{tabular} & 12015 & UNKNOWN & 565707 & 145229 & HEISPROD & ESTIMATED & ONSITE & & 100-C-9 \\
\hline 4200 & 12032 & & & & HEISPROD & & & & $116-F-2$ \\
\hline \begin{tabular}{|l|}
4201 \\
\end{tabular} & 12033 & & & & HEISPROD & & & & 116-F-2 \\
\hline \begin{tabular}{|l|}
4202 \\
\end{tabular} & 12034 & & & & HEISPROD & & & & $116-F-2$ \\
\hline \begin{tabular}{|l|}
4203 \\
\end{tabular} & 12035 & & & & HEISPROD & & & & 116-F-2 \\
\hline 4204 & 12036 & & & & HEISPROD & & & & $116-F-2$ \\
\hline 4205 & 12037 & & & & HEISPROD & & & & $116-\mathrm{F}-2$ \\
\hline \begin{tabular}{|l|}
4206 \\
\end{tabular} & 12038 & & & & HEISPROD & & & & 116-F-2 \\
\hline \begin{tabular}{|l|}
4207 \\
\end{tabular} & 12039 & & & & HEISPROD & & & & 116-F-2 \\
\hline \begin{tabular}{|l|}
4208 \\
\end{tabular} & 12040 & & & & HEISPROD & & & & 116-F-2 \\
\hline \begin{tabular}{|l|}
4209 \\
\end{tabular} & 12041 & & & & HEISPROD & & & & 116-F-2 \\
\hline \begin{tabular}{|l|}
4210 \\
\end{tabular} & 12042 & UNKNOWN & 565707 & 145229 & HEISPROD & ESTIMATED & ONSITE & & $100-C-9$ \\
\hline \begin{tabular}{|l|}
4211 \\
\end{tabular} & 12043 & UNKNOWN & 565707 & 145229 & HEISPROD & ESTIMATED & ONSITE & & $100-C-9$ \\
\hline \begin{tabular}{|l|}
4212 \\
\end{tabular} & 12052 & UNKNOWN & 571560.55 & 149940.42 & HEISPROD & GPS & ONSITE & & $116-\mathrm{N}-1$ \\
\hline \begin{tabular}{|l|}
4213 \\
\end{tabular} & 12072 & UNKNOWN & 571560.55 & 149940.42 & HEISPROD & GPS & ONSITE & & $116-\mathrm{N}-1$ \\
\hline \begin{tabular}{|l|}
4214 \\
\end{tabular} & 12092 & UNKNOWN & 571642.23 & 149984.98 & HEISPROD & GPS & ONSITE & & $116-\mathrm{N}-1$ \\
\hline \begin{tabular}{|l|}
4215 \\
\end{tabular} & 12093 & UNKNOWN & 571677.8 & 149918.48 & HEISPROD & GPS & ONSITE & & $116-\mathrm{N}-1$ \\
\hline \begin{tabular}{|l|}
4216 \\
\end{tabular} & 12094 & UNKNOWN & 571608.9 & 149896.31 & HEISPROD & GPS & ONSITE & & $116-\mathrm{N}-1$ \\
\hline \begin{tabular}{|l|}
4217 \\
\end{tabular} & 12112 & UNKNOWN & 565340 & 145024 & HEISPROD & GPS & ONSITE & & 100-B-8 \\
\hline \begin{tabular}{|l|}
4218 \\
\end{tabular} & 12113 & UNKNOWN & 565346 & 145018 & HEISPROD & GPS & ONSITE & & 100-B-8 \\
\hline \begin{tabular}{|l|}
4219 \\
\end{tabular} & 12152 & & & & HEISPROD & & & & 116-F-2 \\
\hline
\end{tabular}




\begin{tabular}{|c|c|c|c|c|c|c|c|c|c|}
\hline & $A$ & $\mathrm{~B}$ & $\mathrm{C}$ & $\mathrm{D}$ & $E$ & $\mathrm{~F}$ & $\mathrm{G}$ & $\mathrm{H}$ & $\mathrm{I}$ \\
\hline 1 & SAMP_SITE_ID & HORIZ_COORD_TYPE & EW_COORD & NS_COORD & OWNER_ID & COORD_SOURCE & DIST_CLASS & RAD_LEVEL & SAMP_SITE_NAME \\
\hline 4220 & 12172 & & & & HEISPROD & & & & $116-F-2$ \\
\hline 4221 & 12192 & & & & HEISPROD & & & & $116-\mathrm{F}-2$ \\
\hline 4222 & 12193 & & & & HEISPROD & & & & $116-\mathrm{F}-2$ \\
\hline 4223 & 12194 & & & & HEISPROD & & & & $116-F-2$ \\
\hline 4224 & 12195 & & & & HEISPROD & & & & 116-F-2 \\
\hline 4225 & 12196 & & & & HEISPROD & & & & 116-F-2 \\
\hline 4226 & 12197 & & & & HEISPROD & & & & $116-\mathrm{F}-2$ \\
\hline 4227 & 12198 & & & & HEISPROD & & & & 116-F-2 \\
\hline 4228 & 12212 & & & & HEISPROD & & & & $116-\mathrm{F}-2$ \\
\hline 4229 & 12232 & & & & SESPMNT & & ONSITE & & ENERGY NW-1 \\
\hline 4230 & 12252 & & & & SESPMNT & & ONSITE & & ENERGY NW-2 \\
\hline 4231 & 12253 & & & & SESPMNT & & ONSITE & & ENERGY NW-3 \\
\hline 4232 & 12254 & & & & SESPMNT & & ONSITE & & ENERGY NW-4 \\
\hline 4233 & 12255 & & & & SESPMNT & & ONSITE & & ENERGY NW-5 \\
\hline 4234 & 12256 & & & & SESPMNT & & ONSITE & & ENERGY NW-6 \\
\hline 4235 & 12257 & & & & SESPMNT & & ONSITE & & ENERGY NW-7 \\
\hline 4236 & 12258 & & & & SESPMNT & & ONSITE & & ENERGY NW-8 \\
\hline 4237 & 12259 & & & & SESPMNT & & ONSITE & & ENERGY NW-9 \\
\hline 4238 & 12260 & & & & SESPMNT & & ONSITE & & ENERGY NW-10 \\
\hline 4239 & 12272 & UNKNOWN & 571868 & 149408 & HEISPROD & GPS & & & $116-N-3$ \\
\hline 4240 & 12292 & UNKNOWN & 571868 & 149408 & HEISPROD & GPS & & & $116-\mathrm{N}-3$ \\
\hline 4241 & 12293 & & & & HEISPROD & & & & 116-F-2 \\
\hline 4242 & 12313 & & & & CENTPLAT & & & & 276-S HEXONE TANK \#141 \\
\hline 4243 & 12314 & & & & CENTPLAT & & & & 276-S HEXONE TANK \#142 \\
\hline 4244 & 12315 & & & & CENTPLAT & & & & 276-S HEXONE TANK \\
\hline 4245 & 12334 & & & & CENTPLAT & & & & 293-S WIDS SITE \\
\hline 4246 & 12337 & & & & CENTPLAT & & & & 271-U WIDS Site \\
\hline 4247 & 12338 & & & & CENTPLAT & & & & 233-SA EXHAUST FACILITY \\
\hline 4248 & 12352 & & & & CENTPLAT & & & & U PLANT \\
\hline 4249 & 12353 & & & & CENTPLAT & & & & U PLANT \\
\hline 4250 & 12354 & & & & CENTPLAT & & & & U PLANT \\
\hline 4251 & 12355 & & & & CENTPLAT & & & & U PLANT \\
\hline 4252 & 12356 & & & & CENTPLAT & & & & U PLANT \\
\hline 4253 & 12357 & & & & CENTPLAT & & & & U PLANT \\
\hline 4254 & 12358 & & & & CENTPLAT & & & & U PLANT \\
\hline 4255 & 12359 & & & & CENTPLAT & & & & U PLANT \\
\hline 4256 & 12360 & & & & CENTPLAT & & & & U PLANT \\
\hline 4257 & 12361 & & & & CENTPLAT & & & & U PLANT \\
\hline
\end{tabular}




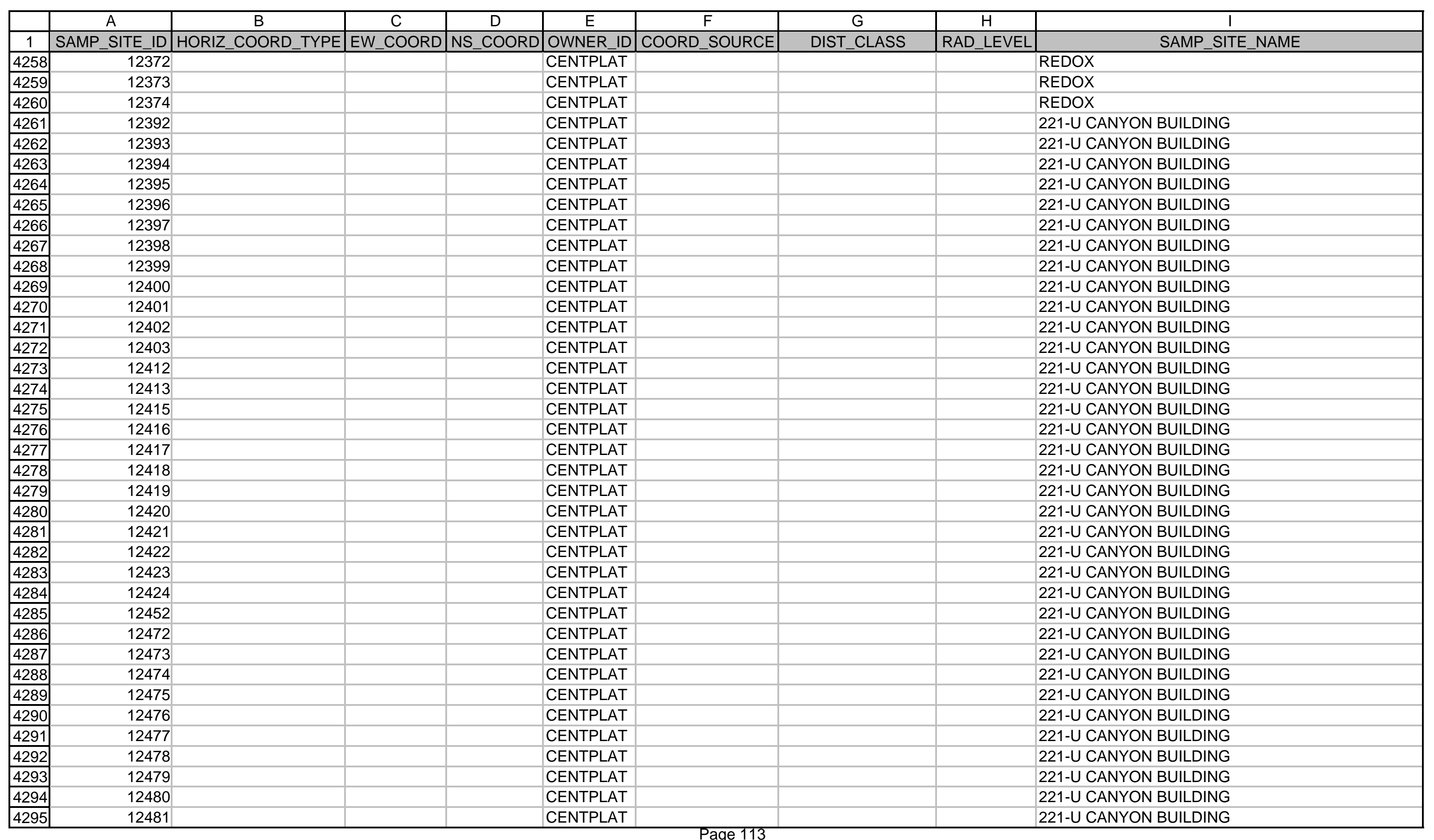




\begin{tabular}{|c|c|c|c|c|c|c|c|c|c|}
\hline & A & $\mathrm{B}$ & $\mathrm{C}$ & $\mathrm{D}$ & $\mathrm{E}$ & $\mathrm{F}$ & $\mathrm{G}$ & $\mathrm{H}$ & $\mathrm{I}$ \\
\hline 1 & SAMP_SITE_ID & HORIZ_COORD_TYPE & EW_COORD & NS_COORD & OWNER_ID & COORD_SOURCE & DIST_CLASS & RAD_LEVEL & SAMP_SITE_NAME \\
\hline 4296 & 12482 & & & & CENTPLAT & & & & 221-U CANYON BUILDING \\
\hline 4297 & 12483 & & & & CENTPLAT & & & & 221-U CANYON BUILDING \\
\hline 4298 & 12484 & & & & CENTPLAT & & & & 221-U CANYON BUILDING \\
\hline 4299 & 12492 & & & & CENTPLAT & & & & 224-B CONCENTRATION FACILITY \\
\hline 4300 & 12493 & & & & CENTPLAT & & & & 181-N PUMPHOUSE \\
\hline 4301 & 12494 & & & & CENTPLAT & & & & 308 BUILDING IN 300 Area \\
\hline 4302 & 12495 & & & & CENTPLAT & & & & $291-U$ \\
\hline 4303 & 12496 & & & & CENTPLAT & & & & $291-U$ \\
\hline 4304 & 12497 & & & & CENTPLAT & & & & $291-U$ \\
\hline 4305 & 12498 & & & & CENTPLAT & & & & $291-U$ \\
\hline 4306 & 12499 & & & & CENTPLAT & & & & COMPOSITE - 291-U, 292-S, \& 293-S \\
\hline 4307 & 12500 & & & & CENTPLAT & & & & COMPOSITE - 291-U, 292-S, \& 293-S \\
\hline 4308 & 12501 & & & & CENTPLAT & & & & $2718-S$ \\
\hline 4309 & 12502 & & & & CENTPLAT & & & & 2718-S \\
\hline 4310 & 12503 & & & & CENTPLAT & & & & 2718-S \\
\hline 4311 & 12504 & & & & CENTPLAT & & & & 202-S \\
\hline 4312 & 12505 & & & & CENTPLAT & & & & 202-S \\
\hline 4313 & 12506 & & & & CENTPLAT & & & & $276-S$ \\
\hline 4314 & 12507 & & & & CENTPLAT & & & & $276-S$ \\
\hline 4315 & 12508 & & & & CENTPLAT & & & & 2715-S \\
\hline 4316 & 12509 & & & & CENTPLAT & & & & 2715-S \\
\hline 4317 & 12510 & & & & CENTPLAT & & & & $224-U$ \\
\hline 4318 & 12511 & & & & CENTPLAT & & & & $224-U$ \\
\hline 4319 & 12512 & & & & CENTPLAT & & & & $276-C$ \\
\hline 4320 & 12513 & & & & CENTPLAT & & & & $276-C$ \\
\hline 4321 & 12514 & & & & CENTPLAT & & & & $215-C$ \\
\hline 4322 & 12515 & & & & CENTPLAT & & & & $215-C$ \\
\hline 4323 & 12516 & & & & CENTPLAT & & & & 224-B \\
\hline 4324 & 12517 & & & & CENTPLAT & & & & 224-B \\
\hline 4325 & 12532 & & & & CENTPLAT & & & & 221-U CANYON BUILDING \\
\hline 4326 & 12533 & & & & CENTPLAT & & & & 221-U CANYON BUILDING \\
\hline 4327 & 12534 & & & & CENTPLAT & & & & 221-U CANYON BUILDING \\
\hline 4328 & 12535 & & & & CENTPLAT & & & & 221-U CANYON BUILDING \\
\hline 4329 & 12536 & & & & CENTPLAT & & & & 221-U CANYON BUILDING \\
\hline 4330 & 12537 & & & & CENTPLAT & & & & 221-U CANYON BUILDING \\
\hline 4331 & 12538 & & & & CENTPLAT & & & & 221-U CANYON BUILDING \\
\hline 4332 & 12539 & & & & CENTPLAT & & & & 221-U CANYON BUILDING \\
\hline 4333 & 12540 & & & & CENTPLAT & & & & 221-U CANYON BUILDING \\
\hline
\end{tabular}




\begin{tabular}{|c|c|c|c|c|c|c|c|c|c|}
\hline & A & $\mathrm{B}$ & $\mathrm{C}$ & $\mathrm{D}$ & $E$ & $\mathrm{~F}$ & G & $\mathrm{H}$ & $\mathrm{I}$ \\
\hline 1 & SAMP_SITE_ID & HORIZ_COORD_TYPE & EW_COORD & NS_COORD & OWNER_ID & COORD_SOURCE & DIST_CLASS & RAD_LEVEL & SAMP_SITE_NAME \\
\hline 4334 & 12541 & & & & CENTPLAT & & & & 221-U CANYON BUILDING \\
\hline 4335 & 12542 & & & & CENTPLAT & & & & 216-T-26 CRIB \\
\hline 4336 & 12543 & & & & CENTPLAT & & & & 216-T-26 CRIB \\
\hline \begin{tabular}{|l|}
4337 \\
\end{tabular} & 12544 & & & & CENTPLAT & & & & 216-T-26 CRIB \\
\hline 4338 & 12545 & & & & CENTPLAT & & & & 216-T-26 CRIB \\
\hline 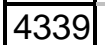 & 12547 & & & & CENTPLAT & & & & 216-T-26 CRIB \\
\hline 4340 & 12548 & & & & CENTPLAT & & & & 216-T-26 CRIB \\
\hline \begin{tabular}{|l|}
4341 \\
\end{tabular} & 12549 & & & & CENTPLAT & & & & 216-B-7A CRIB \\
\hline 4342 & 12550 & & & & CENTPLAT & & & & 216-B-7A CRIB \\
\hline 4343 & 12551 & & & & CENTPLAT & & & & 216-B-7A CRIB \\
\hline \begin{tabular}{|l|}
4344 \\
\end{tabular} & 12552 & & & & CENTPLAT & & & & 216-B-38 \\
\hline 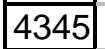 & 12553 & & & & CENTPLAT & & & & 216-B-38 \\
\hline 4346 & 12554 & & & & CENTPLAT & & & & 216-T-26 CRIB \\
\hline 4347 & 12555 & & & & CENTPLAT & & & & 216-B-7A CRIB \\
\hline 4348 & 12556 & & & & CENTPLAT & & & & 216-B-38 \\
\hline \begin{tabular}{|l|}
4349 \\
\end{tabular} & 12557 & & & & CENTPLAT & & & & 216-T-26 CRIB \\
\hline 4350 & 12558 & & & & CENTPLAT & & & & 216-T-26 CRIB \\
\hline \begin{tabular}{|l|}
4351 \\
\end{tabular} & 12559 & & & & CENTPLAT & & & & 216-T-26 CRIB \\
\hline 4352 & 12560 & & & & CENTPLAT & & & & 216-T-26 CRIB \\
\hline 4353 & 12561 & & & & CENTPLAT & & & & 216-T-26 CRIB \\
\hline \begin{tabular}{|l|}
4354 \\
\end{tabular} & 12562 & & & & CENTPLAT & & & & 216-T-26 CRIB \\
\hline \begin{tabular}{|l|}
4355 \\
\end{tabular} & 12563 & & & & CENTPLAT & & & & 216-T-26 CRIB \\
\hline \begin{tabular}{|l|}
4356 \\
\end{tabular} & 12564 & & & & CENTPLAT & & & & 216-T-26 CRIB \\
\hline \begin{tabular}{|l|}
4357 \\
\end{tabular} & 12565 & & & & CENTPLAT & & & & 216-T-26 CRIB \\
\hline 4358 & 12566 & & & & CENTPLAT & & & & 216-T-26 CRIB \\
\hline 4359 & 12567 & & & & CENTPLAT & & & & 216-T-26 CRIB \\
\hline 4360 & 12568 & & & & CENTPLAT & & & & 216-T-26 CRIB \\
\hline 4361 & 12569 & & & & CENTPLAT & & & & 216-B-38 \\
\hline 4362 & 12570 & & & & CENTPLAT & & & & 216-B-38 \\
\hline \begin{tabular}{|l|}
4363 \\
\end{tabular} & 12571 & & & & CENTPLAT & & & & 216-B-38 \\
\hline \begin{tabular}{|l|}
4364 \\
\end{tabular} & 12572 & & & & CENTPLAT & & & & 216-B-38 \\
\hline \begin{tabular}{|l|}
4365 \\
\end{tabular} & 12573 & & & & CENTPLAT & & & & $216-B-38$ \\
\hline \begin{tabular}{|l|}
4366 \\
\end{tabular} & 12574 & & & & CENTPLAT & & & & 216-B-38 \\
\hline \begin{tabular}{|l|}
4367 \\
\end{tabular} & 12575 & & & & CENTPLAT & & & & 216-B-38 \\
\hline 4368 & 12576 & & & & CENTPLAT & & & & 216-B-38 \\
\hline \begin{tabular}{|l|}
4369 \\
\end{tabular} & 12577 & & & & CENTPLAT & & & & 216-B-38 \\
\hline 4370 & 12578 & & & & CENTPLAT & & & & $216-B-38$ \\
\hline \begin{tabular}{|l|}
4371 \\
\end{tabular} & 12579 & & & & CENTPLAT & & & & 216-B-38 \\
\hline
\end{tabular}




\begin{tabular}{|c|c|c|c|c|c|c|c|c|c|}
\hline & $A$ & $\mathrm{~B}$ & $\mathrm{C}$ & $\mathrm{D}$ & $E$ & $\mathrm{~F}$ & G & $\mathrm{H}$ & $\mathrm{I}$ \\
\hline 1 & SAMP_SITE_ID & HORIZ_COORD_TYPE & EW_COORD & NS_COORD & OWNER_ID & COORD_SOURCE & DIST_CLASS & RAD_LEVEL & SAMP_SITE_NAME \\
\hline 4372 & 12580 & & & & CENTPLAT & & & & 216-B-38 \\
\hline 4373 & 12581 & & & & CENTPLAT & & & & 216-B-7A CRIB \\
\hline 4374 & 12582 & & & & CENTPLAT & & & & 216-B-7A CRIB \\
\hline \begin{tabular}{|l|}
4375 \\
\end{tabular} & 12583 & & & & CENTPLAT & & & & 216-B-7A CRIB \\
\hline \begin{tabular}{|l|}
4376 \\
\end{tabular} & 12584 & & & & CENTPLAT & & & & 216-B-7A CRIB \\
\hline \begin{tabular}{|c|}
$4377-2-1$ \\
\end{tabular} & 12585 & & & & CENTPLAT & & & & 216-B-7A CRIB \\
\hline 4378 & 12586 & & & & CENTPLAT & & & & 216-B-7A CRIB \\
\hline \begin{tabular}{|l|}
4379 \\
\end{tabular} & 12587 & & & & CENTPLAT & & & & 216-B-7A CRIB \\
\hline 4380 & 12588 & & & & CENTPLAT & & & & 216-B-7A CRIB \\
\hline 4381 & 12589 & & & & CENTPLAT & & & & 216-B-7A CRIB \\
\hline \begin{tabular}{|l|}
4382 \\
\end{tabular} & 12590 & & & & CENTPLAT & & & & 216-B-7A CRIB \\
\hline 4383 & 12591 & & & & CENTPLAT & & & & 216-B-7A CRIB \\
\hline 4384 & 12592 & & & & CENTPLAT & & & & 216-B-7A CRIB \\
\hline 4385 & 12593 & & & & CENTPLAT & & & & 216-B-7A CRIB \\
\hline 4386 & 12594 & & & & CENTPLAT & & & & 216-B-7A CRIB \\
\hline \begin{tabular}{|l|}
4387 \\
\end{tabular} & 12595 & & & & CENTPLAT & & & & 216-B-7A CRIB \\
\hline \begin{tabular}{|l|}
4388 \\
\end{tabular} & 12596 & & & & CENTPLAT & & & & 216-B-7A CRIB \\
\hline \begin{tabular}{|l|}
4389 \\
\end{tabular} & 12597 & & & & CENTPLAT & & & & 216-B-7A CRIB \\
\hline 4390 & 12598 & & & & CENTPLAT & & & & 216-B-7A CRIB \\
\hline \begin{tabular}{|l|}
4391 \\
\end{tabular} & 12599 & & & & CENTPLAT & & & & 216-T-26 CRIB \\
\hline \begin{tabular}{|l|}
4392 \\
\end{tabular} & 12600 & & & & CENTPLAT & & & & 216-B-38 \\
\hline \begin{tabular}{|l|}
4393 \\
\end{tabular} & 12601 & & & & CENTPLAT & & & & 216-B-38 \\
\hline 4394 & 12602 & & & & CENTPLAT & & & & 216-B-38 \\
\hline \begin{tabular}{|l|}
4395 \\
\end{tabular} & 12603 & & & & CENTPLAT & & & & 216-B-7A CRIB \\
\hline 4396 & 12604 & & & & CENTPLAT & & & & 216-B-38 \\
\hline 4397 & 12612 & & & & CENTPLAT & & & & 216-B-38 \\
\hline 4398 & 12613 & & & & CENTPLAT & & & & 216-B-38 \\
\hline 4399 & 12614 & & & & CENTPLAT & & & & 216-B-7A CRIB \\
\hline 4400 & 12615 & & & & CENTPLAT & & & & 216-B-7A CRIB \\
\hline \begin{tabular}{|l|}
4401 \\
\end{tabular} & 12616 & & & & CENTPLAT & & & & 216-B-7A CRIB \\
\hline \begin{tabular}{|l|}
4402 \\
\end{tabular} & 12617 & & & & CENTPLAT & & & & 216-B-7A CRIB \\
\hline \begin{tabular}{|l|}
4403 \\
\end{tabular} & 12618 & & & & CENTPLAT & & & & 216-B-7A CRIB \\
\hline \begin{tabular}{|l|}
4404 \\
\end{tabular} & 12619 & & & & CENTPLAT & & & & 216-B-7A CRIB \\
\hline \begin{tabular}{|l|}
4405 \\
\end{tabular} & 12620 & & & & CENTPLAT & & & & 216-B-7A CRIB \\
\hline \begin{tabular}{|l|}
4406 \\
\end{tabular} & 12621 & & & & CENTPLAT & & & & 216-B-7A CRIB \\
\hline \begin{tabular}{|l|}
4407 \\
\end{tabular} & 12622 & & & & CENTPLAT & & & & 216-T-26 CRIB \\
\hline \begin{tabular}{|l|}
4408 \\
\end{tabular} & 12623 & & & & CENTPLAT & & & & 216-T-26 CRIB \\
\hline \begin{tabular}{|l|}
4409 \\
\end{tabular} & 12624 & & & & CENTPLAT & & & & 216-T-26 CRIB \\
\hline
\end{tabular}




\begin{tabular}{|c|c|c|c|c|c|c|c|c|c|}
\hline & $A$ & $\mathrm{~B}$ & $\mathrm{C}$ & $\mathrm{D}$ & $E$ & $\mathrm{~F}$ & G & $\mathrm{H}$ & $\mathrm{I}$ \\
\hline 1 & SAMP_SITE_ID & HORIZ_COORD_TYPE & EW_COORD & NS_COORD & OWNER_ID & COORD_SOURCE & DIST_CLASS & RAD_LEVEL & SAMP_SITE_NAME \\
\hline 4410 & 12625 & & & & CENTPLAT & & & & 216-T-26 CRIB \\
\hline 4411 & 12626 & & & & CENTPLAT & & & & 216-T-26 CRIB \\
\hline 4412 & 12627 & & & & CENTPLAT & & & & 216-T-26 CRIB \\
\hline 4413 & 12628 & & & & CENTPLAT & & & & 216-T-26 CRIB \\
\hline 44414 & 12629 & & & & CENTPLAT & & & & 216-T-26 CRIB \\
\hline \begin{tabular}{|l|}
4415 \\
\end{tabular} & 12630 & & & & CENTPLAT & & & & 216-T-26 CRIB \\
\hline 4416 & 12631 & & & & CENTPLAT & & & & 216-T-26 CRIB \\
\hline \begin{tabular}{|l|}
4417 \\
\end{tabular} & 12632 & & & & CENTPLAT & & & & 216-T-26 CRIB \\
\hline 4418 & 12633 & & & & CENTPLAT & & & & 216-B-7A CRIB \\
\hline \begin{tabular}{|l|}
4419 \\
\end{tabular} & 12634 & & & & CENTPLAT & & & & 216-B-38 \\
\hline \begin{tabular}{|l|}
4420 \\
\end{tabular} & 12635 & & & & CENTPLAT & & & & 216-T-26 CRIB \\
\hline \begin{tabular}{|l|}
4421 \\
\end{tabular} & 12636 & & & & CENTPLAT & & & & 216-B-38 \\
\hline 4422 & 12637 & & & & CENTPLAT & & & & 216-B-38 \\
\hline 4423 & 12638 & & & & CENTPLAT & & & & 216-B-38 \\
\hline 4424 & 12639 & & & & CENTPLAT & & & & 216-B-38 \\
\hline \begin{tabular}{|l|}
4425 \\
\end{tabular} & 12640 & & & & CENTPLAT & & & & 216-B-38 \\
\hline \begin{tabular}{|l|}
4426 \\
\end{tabular} & 12641 & & & & CENTPLAT & & & & 216-B-38 \\
\hline \begin{tabular}{|l|}
4427 \\
\end{tabular} & 12642 & & & & CENTPLAT & & & & 216-B-38 \\
\hline \begin{tabular}{|l|}
4428 \\
\end{tabular} & 12643 & & & & CENTPLAT & & & & 216-B-38 \\
\hline \begin{tabular}{|l|}
4429 \\
\end{tabular} & 12644 & & & & CENTPLAT & & & & 216-B-38 \\
\hline \begin{tabular}{|l|}
4430 \\
\end{tabular} & 12645 & & & & CENTPLAT & & & & 216-B-38 \\
\hline \begin{tabular}{|l|}
4431 \\
\end{tabular} & 12646 & & & & CENTPLAT & & & & 216-B-38 \\
\hline \begin{tabular}{|l|}
4432 \\
\end{tabular} & 12647 & & & & CENTPLAT & & & & 216-B-7A CRIB \\
\hline \begin{tabular}{|l|}
4433 \\
\end{tabular} & 12648 & & & & CENTPLAT & & & & 216-B-7A CRIB \\
\hline \begin{tabular}{|l|}
4434 \\
\end{tabular} & 12649 & & & & CENTPLAT & & & & 216-T-26 CRIB \\
\hline 4435 & 12650 & & & & CENTPLAT & & & & 216-B-38 \\
\hline 4436 & 12651 & & & & CENTPLAT & & & & 216-B-38 \\
\hline \begin{tabular}{|l|}
4437 \\
\end{tabular} & 12652 & & & & CENTPLAT & & & & 216-B-38 \\
\hline 44 & 12653 & & & & CENTPLAT & & & & 216-B-7A CRIB \\
\hline \begin{tabular}{|l|}
4439 \\
\end{tabular} & 12654 & & & & CENTPLAT & & & & 216-B-7A CRIB \\
\hline \begin{tabular}{|l|}
4440 \\
\end{tabular} & 12655 & & & & CENTPLAT & & & & 216-B-7A CRIB \\
\hline \begin{tabular}{|l|}
4441 \\
\end{tabular} & 12656 & & & & CENTPLAT & & & & 216-B-7A CRIB \\
\hline \begin{tabular}{|l|}
4442 \\
\end{tabular} & 12657 & & & & CENTPLAT & & & & 216-B-7A CRIB \\
\hline \begin{tabular}{|l|}
4443 \\
\end{tabular} & 12658 & & & & CENTPLAT & & & & 216-B-7A CRIB \\
\hline 4444 & 12659 & & & & CENTPLAT & & & & 216-B-7A CRIB \\
\hline \begin{tabular}{|l|}
4445 \\
\end{tabular} & 12660 & & & & CENTPLAT & & & & 216-B-7A CRIB \\
\hline \begin{tabular}{|l|}
4446 \\
\end{tabular} & 12661 & & & & CENTPLAT & & & & 216-B-7A CRIB \\
\hline \begin{tabular}{|l|}
4447 \\
\end{tabular} & 12662 & & & & CENTPLAT & & & & 216-B-7A CRIB \\
\hline
\end{tabular}




\begin{tabular}{|c|c|c|c|c|c|c|c|c|c|}
\hline & A & $\mathrm{B}$ & $C$ & $\mathrm{D}$ & $E$ & $\mathrm{~F}$ & $\mathrm{G}$ & $\mathrm{H}$ & $\mathrm{I}$ \\
\hline 1 & SAMP_SITE_ID & HORIZ_COORD_TYPE & EW_COORD & NS_COORD & OWNER_ID & COORD_SOURCE & DIST_CLASS & RAD_LEVEL & SAMP_SITE_NAME \\
\hline \begin{tabular}{|l|l|}
4448 \\
\end{tabular} & 12663 & & & & CENTPLAT & & & & 216-B-7A CRIB \\
\hline \begin{tabular}{|l|}
4449 \\
\end{tabular} & 12664 & & & & CENTPLAT & & & & 216-B-7A CRIB \\
\hline \begin{tabular}{|l|}
4450 \\
\end{tabular} & 12665 & & & & CENTPLAT & & & & 216-B-7A CRIB \\
\hline \begin{tabular}{|l|}
4451 \\
\end{tabular} & 12666 & & & & CENTPLAT & & & & 216-B-7A CRIB \\
\hline \begin{tabular}{|l|}
4452 \\
\end{tabular} & 12667 & & & & CENTPLAT & & & & 216-B-7A CRIB \\
\hline \begin{tabular}{|l|}
4453 \\
\end{tabular} & 12668 & & & & CENTPLAT & & & & 216-B-7A CRIB \\
\hline \begin{tabular}{|l|}
4454 \\
\end{tabular} & 12669 & & & & CENTPLAT & & & & 216-B-7A CRIB \\
\hline \begin{tabular}{|l|l|}
4455 \\
\end{tabular} & 12670 & & & & CENTPLAT & & & & 216-B-7A CRIB \\
\hline \begin{tabular}{|l|}
4456 \\
\end{tabular} & 12671 & & & & CENTPLAT & & & & 216-B-7A CRIB \\
\hline \begin{tabular}{|l|}
4457 \\
\end{tabular} & 12672 & & & & CENTPLAT & & & & 216-B-7A CRIB \\
\hline 4458 & 12673 & & & & CENTPLAT & & & & 216-B-7A CRIB \\
\hline 4459 & 12674 & & & & CENTPLAT & & & & 216-B-7A CRIB \\
\hline 44 & 12675 & & & & CENTPLAT & & & & 216-B-7A CRIB \\
\hline \begin{tabular}{|l|}
4461 \\
\end{tabular} & 12676 & & & & CENTPLAT & & & & 216-B-7A CRIB \\
\hline \begin{tabular}{|l|}
4462 \\
\end{tabular} & 12677 & & & & CENTPLAT & & & & 216-B-7A CRIB \\
\hline \begin{tabular}{|l|}
4463 \\
\end{tabular} & 12678 & & & & CENTPLAT & & & & 216-B-7A CRIB \\
\hline \begin{tabular}{|l|}
4464 \\
\end{tabular} & 12679 & & & & CENTPLAT & & & & 216-B-7A CRIB \\
\hline \begin{tabular}{|l|l|}
4465 \\
\end{tabular} & 12680 & & & & CENTPLAT & & & & 216-B-7A CRIB \\
\hline \begin{tabular}{|l|}
4466 \\
\end{tabular} & 12692 & & & & CENTPLAT & & & & 200-ZP-1 \\
\hline \begin{tabular}{|l|}
4467 \\
\end{tabular} & 12693 & & & & CENTPLAT & & & & 200-ZP-1 \\
\hline \begin{tabular}{|l|}
4468 \\
\end{tabular} & 12694 & & & & CENTPLAT & & & & 200-ZP-1 \\
\hline \begin{tabular}{|l|}
4469 \\
\end{tabular} & 12695 & & & & CENTPLAT & & & & 200-ZP-1 \\
\hline \begin{tabular}{|l|}
4470 \\
\end{tabular} & 12696 & & & & CENTPLAT & & & & 200-ZP-1 \\
\hline \begin{tabular}{|l|}
4471 \\
\end{tabular} & 12697 & & & & CENTPLAT & & & & PIT 6/600 AREA \\
\hline \begin{tabular}{|l|}
4472 \\
\end{tabular} & 12698 & & & & CENTPLAT & & & & PIT 6/600 AREA \\
\hline \begin{tabular}{|l|}
4473 \\
\end{tabular} & 12699 & & & & CENTPLAT & & & & PIT 6/600 AREA \\
\hline \begin{tabular}{|l|}
4474 \\
\end{tabular} & 12700 & & & & CENTPLAT & & & & PIT 6/600 AREA \\
\hline \begin{tabular}{|l|l|}
4475 \\
\end{tabular} & 12701 & & & & CENTPLAT & & & & PIT 6/600 AREA \\
\hline \begin{tabular}{|l|l|}
4476 \\
\end{tabular} & 12702 & & & & CENTPLAT & & & & PIT 6/600 AREA \\
\hline \begin{tabular}{|l|}
4477 \\
\end{tabular} & 12712 & & & & CENTPLAT & & & & $271-U$ \\
\hline \begin{tabular}{|l|}
4478 \\
\end{tabular} & 12713 & & & & CENTPLAT & & & & $271-U$ \\
\hline \begin{tabular}{|l|}
4479 \\
\end{tabular} & 12714 & & & & CENTPLAT & & & & $271-U$ \\
\hline \begin{tabular}{|l|}
4480 \\
\end{tabular} & 12715 & & & & CENTPLAT & & & & $271-U$ \\
\hline \begin{tabular}{|l|}
4481 \\
\end{tabular} & 12716 & & & & CENTPLAT & & & & $271-U$ \\
\hline \begin{tabular}{|l|}
4482 \\
\end{tabular} & 12717 & & & & CENTPLAT & & & & $271-U$ \\
\hline \begin{tabular}{|l|}
4483 \\
\end{tabular} & 12718 & & & & CENTPLAT & & & & $271-U$ \\
\hline \begin{tabular}{|l|}
4484 \\
\end{tabular} & 12732 & & & & CENTPLAT & & & & 100-KR-4 PUMP \& TREAT \\
\hline \begin{tabular}{|l|l|}
4485 \\
\end{tabular} & 12733 & & & & CENTPLAT & & & & 300 AREA \\
\hline
\end{tabular}




\begin{tabular}{|c|c|c|c|c|c|c|c|c|c|}
\hline & $A$ & $\mathrm{~B}$ & $\mathrm{C}$ & $\mathrm{D}$ & $E$ & $\mathrm{~F}$ & G & $\mathrm{H}$ & $\mathrm{I}$ \\
\hline 1 & SAMP_SITE_ID & HORIZ_COORD_TYPE & EW_COORD & NS_COORD & OWNER_ID & COORD_SOURCE & DIST_CLASS & RAD_LEVEL & SAMP_SITE_NAME \\
\hline 4486 & 12734 & & & & CENTPLAT & & & & PIT 12 \\
\hline 4487 & 12735 & & & & CENTPLAT & & & & PIT 12 \\
\hline 4488 & 12736 & & & & CENTPLAT & & & & PIT 12 \\
\hline 4489 & 12737 & & & & CENTPLAT & & & & PIT 12 \\
\hline 4490 & 12738 & & & & CENTPLAT & & & & PIT 12 \\
\hline 4491 & 12739 & & & & CENTPLAT & & & & PIT 12 \\
\hline 4492 & 12740 & & & & CENTPLAT & & & & PIT 12 \\
\hline 4493 & 12741 & & & & CENTPLAT & & & & PIT 12 \\
\hline 4494 & 12742 & & & & CENTPLAT & & & & PIT 12 \\
\hline 4495 & 12743 & & & & CENTPLAT & & & & PIT 12 \\
\hline 4496 & 12744 & & & & CENTPLAT & & & & PIT 12 \\
\hline 4497 & 12745 & & & & CENTPLAT & & & & PIT 12 \\
\hline 4498 & 12746 & & & & CENTPLAT & & & & PIT 12 \\
\hline 4499 & 12747 & & & & CENTPLAT & & & & PIT 12 \\
\hline 4500 & 12748 & & & & CENTPLAT & & & & PIT 12 \\
\hline 4501 & 12749 & & & & CENTPLAT & & & & PIT 12 \\
\hline 4502 & 12750 & & & & CENTPLAT & & & & PIT 12 \\
\hline 4503 & 12751 & & & & CENTPLAT & & & & PIT 12 \\
\hline 4504 & 12752 & & & & CENTPLAT & & & & PIT 12 \\
\hline 4505 & 12753 & & & & CENTPLAT & & & & PIT 12 \\
\hline 4506 & 12772 & & & & CENTPLAT & & & & PIT 12 \\
\hline 4507 & 12773 & & & & CENTPLAT & & & & PIT 12 \\
\hline 4508 & 12774 & & & & CENTPLAT & & & & PIT 12 \\
\hline 4509 & 12775 & & & & CENTPLAT & & & & PIT 12 \\
\hline 4510 & 12776 & & & & CENTPLAT & & & & PIT 12 \\
\hline 4511 & 12777 & & & & CENTPLAT & & & & PIT 12 \\
\hline 4512 & 12778 & & & & CENTPLAT & & & & PIT 12 \\
\hline 4513 & 12779 & & & & CENTPLAT & & & & PIT 12 \\
\hline 4514 & 12780 & & & & CENTPLAT & & & & PIT 12 \\
\hline 4515 & 12781 & & & & CENTPLAT & & & & PIT 12 \\
\hline 4516 & 12782 & & & & CENTPLAT & & & & PIT 12 \\
\hline 4517 & 12783 & & & & CENTPLAT & & & & PIT 12 \\
\hline 4518 & 12784 & & & & CENTPLAT & & & & PIT 12 \\
\hline 4519 & 12785 & & & & CENTPLAT & & & & PIT 12 \\
\hline 4520 & 12786 & & & & CENTPLAT & & & & PIT 12 \\
\hline 4521 & 12787 & & & & CENTPLAT & & & & PIT 12 \\
\hline 4522 & 12788 & & & & CENTPLAT & & & & PIT 12 \\
\hline 4523 & 12789 & & & & CENTPLAT & & & & PIT 12 \\
\hline
\end{tabular}




\begin{tabular}{|c|c|c|c|c|c|c|c|c|c|}
\hline & $A$ & $\mathrm{~B}$ & $\mathrm{C}$ & $\mathrm{D}$ & $E$ & $\mathrm{~F}$ & G & $\mathrm{H}$ & $\mathrm{I}$ \\
\hline 1 & SAMP_SITE_ID & HORIZ_COORD_TYPE & EW_COORD & NS_COORD & OWNER_ID & COORD_SOURCE & DIST_CLASS & RAD_LEVEL & SAMP_SITE_NAME \\
\hline 4524 & 12790 & & & & CENTPLAT & & & & PIT 12 \\
\hline 4525 & 12791 & & & & CENTPLAT & & & & PIT 12 \\
\hline 4526 & 12792 & & & & CENTPLAT & & & & PIT 12 \\
\hline \begin{tabular}{|l|}
4527 \\
\end{tabular} & 12793 & & & & CENTPLAT & & & & PIT 12 \\
\hline \begin{tabular}{|c|}
4528 \\
\end{tabular} & 12794 & & & & CENTPLAT & & & & PIT 12 \\
\hline \begin{tabular}{|l|}
4529 \\
\end{tabular} & 12795 & & & & CENTPLAT & & & & PIT 12 \\
\hline 4530 & 12796 & & & & CENTPLAT & & & & PIT 12 \\
\hline \begin{tabular}{|l|}
4531 \\
\end{tabular} & 12797 & & & & CENTPLAT & & & & PIT 12 \\
\hline 4532 & 12812 & & & & CENTPLAT & & & & $271-U$ \\
\hline 4533 & 12813 & & & & CENTPLAT & & & & 100-KR-4 PUMP \& TREAT \\
\hline 4534 & 12814 & & & & CENTPLAT & & & & 100-KR-4 PUMP \& TREAT \\
\hline 4535 & 12815 & & & & CENTPLAT & & & & 100-KR-4 PUMP \& TREAT \\
\hline 4536 & 12816 & & & & CENTPLAT & & & & 100-KR-4 PUMP \& TREAT \\
\hline 4537 & 12817 & & & & CENTPLAT & & & & 100-KR-4 PUMP \& TREAT \\
\hline 4538 & 12818 & & & & CENTPLAT & & & & 100-KR-4 PUMP \& TREAT \\
\hline 4539 & 12819 & & & & CENTPLAT & & & & 100-KR-4 PUMP \& TREAT \\
\hline 4540 & 12820 & & & & CENTPLAT & & & & 100-KR-4 PUMP \& TREAT \\
\hline 4541 & 12821 & & & & CENTPLAT & & & & 100-KR-4 PUMP \& TREAT \\
\hline 4542 & 12822 & & & & CENTPLAT & & & & 100-KR-4 PUMP \& TREAT \\
\hline 4543 & 12823 & & & & CENTPLAT & & & & 100-KR-4 PUMP \& TREAT \\
\hline \begin{tabular}{|l|}
4544 \\
\end{tabular} & 12824 & & & & CENTPLAT & & & & 100-KR-4 PUMP \& TREAT \\
\hline 4545 & 12825 & & & & CENTPLAT & & & & 100-KR-4 PUMP \& TREAT \\
\hline \begin{tabular}{|l|}
4546 \\
\end{tabular} & 12826 & & & & CENTPLAT & & & & 100-KR-4 PUMP \& TREAT \\
\hline \begin{tabular}{|l|}
4547 \\
\end{tabular} & 12827 & & & & CENTPLAT & & & & 100-KR-4 PUMP \& TREAT \\
\hline 4548 & 12828 & & & & CENTPLAT & & & & 100-KR-4 PUMP \& TREAT \\
\hline 4549 & 12829 & & & & CENTPLAT & & & & 100-KR-4 PUMP \& TREAT \\
\hline 4550 & 12830 & & & & CENTPLAT & & & & 100-KR-4 PUMP \& TREAT \\
\hline 4551 & 12831 & & & & CENTPLAT & & & & 100-KR-4 PUMP \& TREAT \\
\hline 4552 & 12832 & & & & CENTPLAT & & & & 100-KR-4 PUMP \& TREAT \\
\hline 4553 & 12833 & & & & CENTPLAT & & & & 100-KR-4 PUMP \& TREAT \\
\hline \begin{tabular}{|l|}
4554 \\
\end{tabular} & 12834 & & & & CENTPLAT & & & & 100-KR-4 PUMP \& TREAT \\
\hline \begin{tabular}{|l|l|}
4555 \\
\end{tabular} & 12835 & & & & CENTPLAT & & & & 100-KR-4 PUMP \& TREAT \\
\hline \begin{tabular}{|l|}
4556 \\
\end{tabular} & 12836 & & & & CENTPLAT & & & & 100-KR-4 PUMP \& TREAT \\
\hline \begin{tabular}{|l|}
4557 \\
\end{tabular} & 12837 & & & & CENTPLAT & & & & 100-KR-4 PUMP \& TREAT \\
\hline 4558 & 12838 & & & & CENTPLAT & & & & 100-KR-4 PUMP \& TREAT \\
\hline 4559 & 12839 & & & & CENTPLAT & & & & 100-KR-4 PUMP \& TREAT \\
\hline 4560 & 12840 & & & & CENTPLAT & & & & 100-KR-4 PUMP \& TREAT \\
\hline \begin{tabular}{|l|}
4561 \\
\end{tabular} & 12841 & & & & CENTPLAT & & & & 100-KR-4 PUMP \& TREAT \\
\hline
\end{tabular}




\begin{tabular}{|c|c|c|c|c|c|c|c|c|c|}
\hline & $A$ & $\mathrm{~B}$ & $\mathrm{C}$ & $\mathrm{D}$ & $E$ & $\mathrm{~F}$ & G & $\mathrm{H}$ & $\mathrm{I}$ \\
\hline 1 & SAMP_SITE_ID & HORIZ_COORD_TYPE & EW_COORD & NS_COORD & OWNER_ID & COORD_SOURCE & DIST_CLASS & RAD_LEVEL & SAMP_SITE_NAME \\
\hline 4562 & 12842 & & & & CENTPLAT & & & & 100-KR-4 PUMP \& TREAT \\
\hline 4563 & 12843 & & & & CENTPLAT & & & & 100-KR-4 PUMP \& TREAT \\
\hline 4564 & 12844 & & & & CENTPLAT & & & & 100-KR-4 PUMP \& TREAT \\
\hline 4565 & 12845 & & & & CENTPLAT & & & & 100-KR-4 PUMP \& TREAT \\
\hline \begin{tabular}{|l|}
4566 \\
\end{tabular} & 12846 & & & & CENTPLAT & & & & 100-KR-4 PUMP \& TREAT \\
\hline \begin{tabular}{|c|}
4567 \\
\end{tabular} & 12847 & & & & CENTPLAT & & & & 100-KR-4 PUMP \& TREAT \\
\hline 4568 & 12848 & & & & CENTPLAT & & & & 100-KR-4 PUMP \& TREAT \\
\hline \begin{tabular}{|l|}
4569 \\
\end{tabular} & 12849 & & & & CENTPLAT & & & & 100-KR-4 PUMP \& TREAT \\
\hline 4570 & 12850 & & & & CENTPLAT & & & & 100-KR-4 PUMP \& TREAT \\
\hline 4571 & 12851 & & & & CENTPLAT & & & & 100-KR-4 PUMP \& TREAT \\
\hline \begin{tabular}{|l|}
4572 \\
\end{tabular} & 12852 & & & & CENTPLAT & & & & 100-KR-4 PUMP \& TREAT \\
\hline 4573 & 12853 & & & & CENTPLAT & & & & 100-KR-4 PUMP \& TREAT \\
\hline 4574 & 12854 & & & & CENTPLAT & & & & 100-KR-4 PUMP \& TREAT \\
\hline 4575 & 12855 & & & & CENTPLAT & & & & 100-KR-4 PUMP \& TREAT \\
\hline 4576 & 12856 & & & & CENTPLAT & & & & 100-KR-4 PUMP \& TREAT \\
\hline 4577 & 12857 & & & & CENTPLAT & & & & 100-KR-4 PUMP \& TREAT \\
\hline \begin{tabular}{|l|}
4578 \\
\end{tabular} & 12858 & & & & CENTPLAT & & & & 100-KR-4 PUMP \& TREAT \\
\hline \begin{tabular}{|l|}
4579 \\
\end{tabular} & 12859 & & & & CENTPLAT & & & & 100-KR-4 PUMP \& TREAT \\
\hline 4580 & 12860 & & & & CENTPLAT & & & & 100-KR-4 PUMP \& TREAT \\
\hline \begin{tabular}{|l|}
4581 \\
\end{tabular} & 12861 & & & & CENTPLAT & & & & 100-KR-4 PUMP \& TREAT \\
\hline \begin{tabular}{|l|}
4582 \\
\end{tabular} & 12862 & & & & CENTPLAT & & & & 100-KR-4 PUMP \& TREAT \\
\hline 4583 & 12863 & & & & CENTPLAT & & & & 100-KR-4 PUMP \& TREAT \\
\hline 4584 & 12864 & & & & CENTPLAT & & & & 100-KR-4 PUMP \& TREAT \\
\hline \begin{tabular}{|l|}
4585 \\
\end{tabular} & 12865 & & & & CENTPLAT & & & & 100-KR-4 PUMP \& TREAT \\
\hline 4586 & 12866 & & & & CENTPLAT & & & & 100-KR-4 PUMP \& TREAT \\
\hline 4587 & 12872 & & & & CENTPLAT & & & & 100-HR-3 PUMP \& TREAT \\
\hline 4588 & 12873 & & & & CENTPLAT & & & & 100-HR-3 PUMP \& TREAT \\
\hline 4589 & 12874 & & & & CENTPLAT & & & & 100-HR-3 PUMP \& TREAT \\
\hline 4590 & 12875 & & & & CENTPLAT & & & & 100-HR-3 PUMP \& TREAT \\
\hline 4591 & 12876 & & & & CENTPLAT & & & & 100-HR-3 PUMP \& TREAT \\
\hline \begin{tabular}{|l|}
4592 \\
\end{tabular} & 12877 & & & & CENTPLAT & & & & 100-HR-3 PUMP \& TREAT \\
\hline 4593 & 12878 & & & & CENTPLAT & & & & 100-HR-3 PUMP \& TREAT \\
\hline 4594 & 12879 & & & & CENTPLAT & & & & 100-HR-3 PUMP \& TREAT \\
\hline \begin{tabular}{|l|}
4595 \\
\end{tabular} & 12880 & & & & CENTPLAT & & & & 100-HR-3 PUMP \& TREAT \\
\hline 4596 & 12881 & & & & CENTPLAT & & & & 100-HR-3 PUMP \& TREAT \\
\hline 4597 & 12882 & & & & CENTPLAT & & & & 100-HR-3 PUMP \& TREAT \\
\hline \begin{tabular}{|l|}
4598 \\
\end{tabular} & 12883 & & & & CENTPLAT & & & & 100-HR-3 PUMP \& TREAT \\
\hline \begin{tabular}{|l|}
4599 \\
\end{tabular} & 12884 & & & & CENTPLAT & & & & 100-HR-3 PUMP \& TREAT \\
\hline
\end{tabular}

Page 121 


\begin{tabular}{|c|c|c|c|c|c|c|c|c|c|}
\hline & $A$ & $\mathrm{~B}$ & $\mathrm{C}$ & $\mathrm{D}$ & $E$ & $\mathrm{~F}$ & G & $\mathrm{H}$ & $\mathrm{I}$ \\
\hline 1 & SAMP_SITE_ID & HORIZ_COORD_TYPE & EW_COORD & NS_COORD & OWNER_ID & COORD_SOURCE & DIST_CLASS & RAD_LEVEL & SAMP_SITE_NAME \\
\hline 4600 & 12885 & & & & CENTPLAT & & & & 100-HR-3 PUMP \& TREAT \\
\hline 4601 & 12886 & & & & CENTPLAT & & & & 100-HR-3 PUMP \& TREAT \\
\hline 4602 & 12887 & & & & CENTPLAT & & & & 100-HR-3 PUMP \& TREAT \\
\hline 4603 & 12888 & & & & CENTPLAT & & & & 100-HR-3 PUMP \& TREAT \\
\hline 4604 & 12889 & & & & CENTPLAT & & & & 100-HR-3 PUMP \& TREAT \\
\hline \begin{tabular}{|c|}
4605 \\
\end{tabular} & 12890 & & & & CENTPLAT & & & & 100-HR-3 PUMP \& TREAT \\
\hline 4606 & 12891 & & & & CENTPLAT & & & & 100-HR-3 PUMP \& TREAT \\
\hline \begin{tabular}{|l|}
4607 \\
\end{tabular} & 12892 & & & & CENTPLAT & & & & 100-HR-3 PUMP \& TREAT \\
\hline 4608 & 12893 & & & & CENTPLAT & & & & 100-HR-3 PUMP \& TREAT \\
\hline 4609 & 12894 & & & & CENTPLAT & & & & 100-HR-3 PUMP \& TREAT \\
\hline 4610 & 12895 & & & & CENTPLAT & & & & 100-HR-3 PUMP \& TREAT \\
\hline 4611 & 12896 & & & & CENTPLAT & & & & 100-HR-3 PUMP \& TREAT \\
\hline 4612 & 12897 & & & & CENTPLAT & & & & 100-HR-3 PUMP \& TREAT \\
\hline 4613 & 12898 & & & & CENTPLAT & & & & 100-HR-3 PUMP \& TREAT \\
\hline 4614 & 12899 & & & & CENTPLAT & & & & 100-HR-3 PUMP \& TREAT \\
\hline 4615 & 12900 & & & & CENTPLAT & & & & 100-HR-3 PUMP \& TREAT \\
\hline \begin{tabular}{|l|}
4616 \\
\end{tabular} & 12901 & & & & CENTPLAT & & & & 100-HR-3 PUMP \& TREAT \\
\hline \begin{tabular}{|l|}
4617 \\
\end{tabular} & 12902 & & & & CENTPLAT & & & & 100-HR-3 PUMP \& TREAT \\
\hline 4618 & 12903 & & & & CENTPLAT & & & & 100-HR-3 PUMP \& TREAT \\
\hline 4619 & 12904 & & & & CENTPLAT & & & & 100-HR-3 PUMP \& TREAT \\
\hline \begin{tabular}{|l|}
4620 \\
\end{tabular} & 12905 & & & & CENTPLAT & & & & 100-HR-3 PUMP \& TREAT \\
\hline 4621 & 12906 & & & & CENTPLAT & & & & 100-HR-3 PUMP \& TREAT \\
\hline \begin{tabular}{|l|}
4622 \\
\end{tabular} & 12907 & & & & CENTPLAT & & & & 100-HR-3 PUMP \& TREAT \\
\hline \begin{tabular}{|l|}
4623 \\
\end{tabular} & 12908 & & & & CENTPLAT & & & & 100-HR-3 PUMP \& TREAT \\
\hline 4624 & 12909 & & & & CENTPLAT & & & & 100-HR-3 PUMP \& TREAT \\
\hline 4625 & 12910 & & & & CENTPLAT & & & & 100-HR-3 PUMP \& TREAT \\
\hline 4626 & 12911 & & & & CENTPLAT & & & & 100-HR-3 PUMP \& TREAT \\
\hline 4627 & 12912 & & & & CENTPLAT & & & & 100-HR-3 PUMP \& TREAT \\
\hline 4628 & 12913 & & & & CENTPLAT & & & & 100-HR-3 PUMP \& TREAT \\
\hline \begin{tabular}{|l|}
4629 \\
\end{tabular} & 12914 & & & & CENTPLAT & & & & 100-HR-3 PUMP \& TREAT \\
\hline \begin{tabular}{|l|}
4630 \\
\end{tabular} & 12915 & & & & CENTPLAT & & & & 100-HR-3 PUMP \& TREAT \\
\hline 4631 & 12916 & & & & CENTPLAT & & & & 100-HR-3 PUMP \& TREAT \\
\hline \begin{tabular}{|l|}
4632 \\
\end{tabular} & 12917 & & & & CENTPLAT & & & & 100-HR-3 PUMP \& TREAT \\
\hline \begin{tabular}{|l|}
4633 \\
\end{tabular} & 12918 & & & & CENTPLAT & & & & 100-HR-3 PUMP \& TREAT \\
\hline 4634 & 12919 & & & & CENTPLAT & & & & 100-HR-3 PUMP \& TREAT \\
\hline 4635 & 12920 & & & & CENTPLAT & & & & 100-HR-3 PUMP \& TREAT \\
\hline 4636 & 12921 & & & & CENTPLAT & & & & 100-HR-3 PUMP \& TREAT \\
\hline \begin{tabular}{|l|}
4637 \\
\end{tabular} & 12922 & & & & CENTPLAT & & & & 100-HR-3 PUMP \& TREAT \\
\hline
\end{tabular}

Page 122 


\begin{tabular}{|c|c|c|c|c|c|c|c|c|c|}
\hline & $A$ & $\mathrm{~B}$ & $\mathrm{C}$ & $\mathrm{D}$ & $E$ & $\mathrm{~F}$ & G & $\mathrm{H}$ & $\mathrm{I}$ \\
\hline 1 & SAMP_SITE_ID & HORIZ_COORD_TYPE & EW_COORD & NS_COORD & OWNER_ID & COORD_SOURCE & DIST_CLASS & RAD_LEVEL & SAMP_SITE_NAME \\
\hline 4638 & 12923 & & & & CENTPLAT & & & & 100-HR-3 PUMP \& TREAT \\
\hline 4639 & 12924 & & & & CENTPLAT & & & & 100-HR-3 PUMP \& TREAT \\
\hline 4640 & 12925 & & & & CENTPLAT & & & & 100-HR-3 PUMP \& TREAT \\
\hline 4641 & 12926 & & & & CENTPLAT & & & & 100-HR-3 PUMP \& TREAT \\
\hline \begin{tabular}{|c|}
4642 \\
\end{tabular} & 12927 & & & & CENTPLAT & & & & 100-HR-3 PUMP \& TREAT \\
\hline \begin{tabular}{|c|}
4643 \\
\end{tabular} & 12928 & & & & CENTPLAT & & & & 100-HR-3 PUMP \& TREAT \\
\hline 4644 & 12929 & & & & CENTPLAT & & & & 100-HR-3 PUMP \& TREAT \\
\hline \begin{tabular}{|l|}
4645 \\
\end{tabular} & 12930 & & & & CENTPLAT & & & & 100-HR-3 PUMP \& TREAT \\
\hline 4646 & 12931 & & & & CENTPLAT & & & & 100-HR-3 PUMP \& TREAT \\
\hline 46 & 12932 & & & & CENTPLAT & & & & 100-HR-3 PUMP \& TREAT \\
\hline \begin{tabular}{|l|}
4648 \\
\end{tabular} & 12933 & & & & CENTPLAT & & & & 100-HR-3 PUMP \& TREAT \\
\hline 4649 & 12934 & & & & CENTPLAT & & & & 100-HR-3 PUMP \& TREAT \\
\hline 4650 & 12935 & & & & CENTPLAT & & & & 100-HR-3 PUMP \& TREAT \\
\hline 4651 & 12952 & & & & CENTPLAT & & & & 202-S \\
\hline 4652 & 12953 & & & & CENTPLAT & & & & 202-S \\
\hline 4653 & 12954 & & & & CENTPLAT & & & & 202-S \\
\hline 4654 & 12955 & & & & CENTPLAT & & & & 202-S \\
\hline \begin{tabular}{|l|}
4655 \\
\end{tabular} & 12956 & & & & CENTPLAT & & & & 202-S \\
\hline 4656 & 12957 & & & & CENTPLAT & & & & 202-S \\
\hline \begin{tabular}{|l|}
4657 \\
\end{tabular} & 12958 & & & & CENTPLAT & & & & 618-11 BURIAL GROUND \\
\hline \begin{tabular}{|l|}
4658 \\
\end{tabular} & 12959 & & & & CENTPLAT & & & & 618-11 BURIAL GROUND \\
\hline 4659 & 12960 & & & & CENTPLAT & & & & 618-11 BURIAL GROUND \\
\hline 4660 & 12961 & & & & CENTPLAT & & & & 618-11 BURIAL GROUND \\
\hline \begin{tabular}{|l|}
4661 \\
\end{tabular} & 12962 & & & & CENTPLAT & & & & 618-11 BURIAL GROUND \\
\hline 4662 & 12972 & & & & CENTPLAT & & & & 1330-N WASTE PAD \\
\hline 4663 & 12973 & & & & CENTPLAT & & & & 1330-N WASTE PAD \\
\hline 4664 & 12974 & & & & CENTPLAT & & & & 1330 N PAD \\
\hline 4665 & 12975 & & & & CENTPLAT & & & & 1330 N PAD \\
\hline 4666 & 12992 & & & & CENTPLAT & & & & 216-B-7A CRIB \\
\hline \begin{tabular}{|l|}
4667 \\
\end{tabular} & 13012 & & & & CENTPLAT & & & & 200 BC CONTROL AREA \\
\hline \begin{tabular}{|l|l|}
4668 \\
\end{tabular} & 13013 & & & & CENTPLAT & & & & 200 BC CONTROL AREA \\
\hline 4669 & 13014 & & & & CENTPLAT & & & & 200 BC CONTROL AREA \\
\hline 4670 & 13015 & & & & CENTPLAT & & & & 200 BC CONTROL AREA \\
\hline \begin{tabular}{|l|}
4671 \\
\end{tabular} & 13016 & & & & CENTPLAT & & & & 200 BC CONTROL AREA \\
\hline 4672 & 13017 & & & & CENTPLAT & & & & 200 BC CONTROL AREA \\
\hline 4673 & 13018 & & & & CENTPLAT & & & & 200 BC CONTROL AREA \\
\hline 4674 & 13019 & & & & CENTPLAT & & & & 200 BC CONTROL AREA \\
\hline 4675 & 13020 & & & & CENTPLAT & & & & 200 BC CONTROL AREA \\
\hline
\end{tabular}




\begin{tabular}{|c|c|c|c|c|c|c|c|c|c|}
\hline & A & $\mathrm{B}$ & $\mathrm{C}$ & $\mathrm{D}$ & $E$ & $\mathrm{~F}$ & G & $\mathrm{H}$ & $\mathrm{I}$ \\
\hline 1 & SAMP_SITE_ID & HORIZ_COORD_TYPE & EW_COORD & NS_COORD & OWNER_ID & COORD_SOURCE & DIST_CLASS & RAD_LEVEL & SAMP_SITE_NAME \\
\hline 4676 & 13021 & & & & CENTPLAT & & & & 200 BC CONTROL AREA \\
\hline 4677 & 13022 & & & & CENTPLAT & & & & 200 BC CONTROL AREA \\
\hline 4678 & 13023 & & & & CENTPLAT & & & & 200 BC CONTROL AREA \\
\hline 4679 & 13024 & & & & CENTPLAT & & & & 200 BC CONTROL AREA \\
\hline 4680 & 13025 & & & & CENTPLAT & & & & 200 BC CONTROL AREA \\
\hline 4681 & 13026 & & & & CENTPLAT & & & & 200 BC CONTROL AREA \\
\hline 4682 & 13027 & & & & CENTPLAT & & & & 200 BC CONTROL AREA \\
\hline 4683 & 13028 & & & & CENTPLAT & & & & 200 BC CONTROL AREA \\
\hline 4684 & 13029 & & & & CENTPLAT & & & & 200 BC CONTROL AREA \\
\hline 4685 & 13030 & & & & CENTPLAT & & & & 200 BC CONTROL AREA \\
\hline 4686 & 13031 & & & & CENTPLAT & & & & 200 BC CONTROL AREA \\
\hline 4687 & 13032 & & & & CENTPLAT & & & & 200 BC CONTROL AREA \\
\hline 4688 & 13033 & & & & CENTPLAT & & & & 200 BC CONTROL AREA \\
\hline 4689 & 13034 & & & & CENTPLAT & & & & 200 BC CONTROL AREA \\
\hline 4690 & 13035 & & & & CENTPLAT & & & & 200 BC CONTROL AREA \\
\hline 4691 & 13036 & & & & CENTPLAT & & & & 200 BC CONTROL AREA \\
\hline 4692 & 13037 & & & & CENTPLAT & & & & 200 BC CONTROL AREA \\
\hline 4693 & 13038 & & & & CENTPLAT & & & & 200 BC CONTROL AREA \\
\hline 4694 & 13039 & & & & CENTPLAT & & & & 200 BC CONTROL AREA \\
\hline 4695 & 13040 & & & & CENTPLAT & & & & 200 BC CONTROL AREA \\
\hline 4696 & 13041 & & & & CENTPLAT & & & & 200 BC CONTROL AREA \\
\hline 4697 & 13042 & & & & CENTPLAT & & & & 200 BC CONTROL AREA \\
\hline 4698 & 13043 & & & & CENTPLAT & & & & 200 BC CONTROL AREA \\
\hline 4699 & 13044 & & & & CENTPLAT & & & & 200 BC CONTROL AREA \\
\hline 4700 & 13045 & & & & CENTPLAT & & & & 200 BC CONTROL AREA \\
\hline 4701 & 13046 & & & & CENTPLAT & & & & 200 BC CONTROL AREA \\
\hline 4702 & 13047 & & & & CENTPLAT & & & & 200 BC CONTROL AREA \\
\hline 4703 & 13048 & & & & CENTPLAT & & & & 100-KR-4 PUMP \& TREAT \\
\hline 4704 & 13049 & & & & CENTPLAT & & & & 100-KR-4 PUMP \& TREAT \\
\hline 4705 & 13050 & & & & CENTPLAT & & & & 100-KR-4 PUMP \& TREAT \\
\hline 4706 & 13051 & & & & CENTPLAT & & & & 100-KR-4 PUMP \& TREAT \\
\hline 4707 & 13052 & & & & CENTPLAT & & & & 100-KR-4 PUMP \& TREAT \\
\hline 4708 & 13053 & & & & CENTPLAT & & & & 100-KR-4 PUMP \& TREAT \\
\hline 4709 & 13054 & & & & CENTPLAT & & & & 100-KR-4 PUMP \& TREAT \\
\hline 4710 & 13055 & & & & CENTPLAT & & & & 100-KR-4 PUMP \& TREAT \\
\hline 4711 & 13056 & & & & CENTPLAT & & & & 100-KR-4 PUMP \& TREAT \\
\hline 4712 & 13057 & & & & CENTPLAT & & & & 100-KR-4 PUMP \& TREAT \\
\hline 4713 & 13058 & & & & CENTPLAT & & & & 100-KR-4 PUMP \& TREAT \\
\hline
\end{tabular}




\begin{tabular}{|c|c|c|c|c|c|c|c|c|c|}
\hline & $A$ & $\mathrm{~B}$ & C & $\mathrm{D}$ & $E$ & $\mathrm{~F}$ & $G$ & $\mathrm{H}$ & $\mathrm{I}$ \\
\hline 1 & SAMP_SITE_ID & HORIZ_COORD_TYPE & EW_COORD & NS_COORD & OWNER_ID & COORD_SOURCE & DIST_CLASS & RAD_LEVEL & SAMP_SITE_NAME \\
\hline \begin{tabular}{|l|}
4714 \\
\end{tabular} & 13059 & & & & CENTPLAT & & & & 100-KR-4 PUMP \& TREAT \\
\hline \begin{tabular}{|l|}
4715 \\
\end{tabular} & 13060 & & & & CENTPLAT & & & & 100-KR-4 PUMP \& TREAT \\
\hline 4716 & 13061 & & & & CENTPLAT & & & & 100-KR-4 PUMP \& TREAT \\
\hline \begin{tabular}{|l|}
4717 \\
\end{tabular} & 13072 & & & & CENTPLAT & & & & 100-HR-3 PUMP \& TREAT \\
\hline \begin{tabular}{|l|}
4718 \\
\end{tabular} & 13073 & & & & CENTPLAT & & & & 100-HR-3 PUMP \& TREAT \\
\hline \begin{tabular}{|l|}
4719 \\
\end{tabular} & 13074 & & & & CENTPLAT & & & & 100-HR-3 PUMP \& TREAT \\
\hline \begin{tabular}{|l|}
4720 \\
\end{tabular} & 13075 & & & & CENTPLAT & & & & 100-HR-3 PUMP \& TREAT \\
\hline \begin{tabular}{|l|}
4721 \\
\end{tabular} & 13076 & & & & CENTPLAT & & & & 100-HR-3 PUMP \& TREAT \\
\hline \begin{tabular}{|l|}
4722 \\
\end{tabular} & 13077 & & & & CENTPLAT & & & & 100-HR-3 PUMP \& TREAT \\
\hline 4723 & 13078 & & & & CENTPLAT & & & & 100-HR-3 PUMP \& TREAT \\
\hline 4724 & 13079 & & & & CENTPLAT & & & & 100-HR-3 PUMP \& TREAT \\
\hline \begin{tabular}{|l|}
4725 \\
\end{tabular} & 13080 & & & & CENTPLAT & & & & 100-HR-3 PUMP \& TREAT \\
\hline \begin{tabular}{|l|}
4726 \\
\end{tabular} & 13081 & & & & CENTPLAT & & & & 100-HR-3 PUMP \& TREAT \\
\hline \begin{tabular}{|l|}
4727 \\
\end{tabular} & 13082 & & & & CENTPLAT & & & & 100-HR-3 PUMP \& TREAT \\
\hline \begin{tabular}{|l|}
4728 \\
\end{tabular} & 13083 & & & & CENTPLAT & & & & 100-HR-3 PUMP \& TREAT \\
\hline \begin{tabular}{|l|}
4729 \\
\end{tabular} & 13084 & & & & CENTPLAT & & & & 100-KR-4 PUMP \& TREAT \\
\hline \begin{tabular}{|l|}
4730 \\
\end{tabular} & 13085 & & & & CENTPLAT & & & & 100-KR-4 PUMP \& TREAT \\
\hline \begin{tabular}{|l|}
4731 \\
\end{tabular} & 13086 & & & & CENTPLAT & & & & 100-KR-4 PUMP \& TREAT \\
\hline \begin{tabular}{|l|}
4732 \\
\end{tabular} & 13087 & & & & CENTPLAT & & & & 100-KR-4 PUMP \& TREAT \\
\hline \begin{tabular}{|l|l|}
4733 \\
\end{tabular} & 13088 & & & & CENTPLAT & & & & 100-KR-4 PUMP \& TREAT \\
\hline 4734 & 13089 & & & & CENTPLAT & & & & 100-KR-4 PUMP \& TREAT \\
\hline 4735 & 13090 & & & & CENTPLAT & & & & 100-KR-4 PUMP \& TREAT \\
\hline 4736 & 13091 & & & & CENTPLAT & & & & 100-KR-4 PUMP \& TREAT \\
\hline 4737 & 13092 & & & & CENTPLAT & & & & 100-KR-4 PUMP \& TREAT \\
\hline \begin{tabular}{|l|}
4738 \\
\end{tabular} & 13093 & & & & CENTPLAT & & & & 100-KR-4 PUMP \& TREAT \\
\hline \begin{tabular}{|l|}
4739 \\
\end{tabular} & 13112 & & & & CENTPLAT & & & & 100-KR-4 PUMP \& TREAT \\
\hline \begin{tabular}{|l|}
4740 \\
\end{tabular} & 13113 & & & & CENTPLAT & & & & 100-KR-4 PUMP \& TREAT \\
\hline \begin{tabular}{|l|}
4741 \\
\end{tabular} & 13114 & & & & CENTPLAT & & & & 100-HR-3 PUMP \& TREAT \\
\hline \begin{tabular}{|l|}
4742 \\
\end{tabular} & 13115 & & & & CENTPLAT & & & & 100-HR-3 PUMP \& TREAT \\
\hline \begin{tabular}{|l|}
4743 \\
\end{tabular} & 13116 & & & & CENTPLAT & & & & 100-HR-3 PUMP \& TREAT \\
\hline \begin{tabular}{|l|}
4744 \\
\end{tabular} & 13117 & & & & CENTPLAT & & & & 100-HR-3 PUMP \& TREAT \\
\hline 4745 & 13118 & & & & CENTPLAT & & & & 100-HR-3 PUMP \& TREAT \\
\hline 4746 & 13119 & & & & CENTPLAT & & & & 100-HR-3 PUMP \& TREAT \\
\hline \begin{tabular}{|l|}
4747 \\
\end{tabular} & 13120 & & & & CENTPLAT & & & & 100-HR-3 PUMP \& TREAT \\
\hline 4748 & 13121 & & & & CENTPLAT & & & & 100-HR-3 PUMP \& TREAT \\
\hline \begin{tabular}{|l|}
4749 \\
\end{tabular} & 13122 & & & & CENTPLAT & & & & 100-HR-3 PUMP \& TREAT \\
\hline 4750 & 13123 & & & & CENTPLAT & & & & 100-HR-3 PUMP \& TREAT \\
\hline \begin{tabular}{|l|}
4751 \\
\end{tabular} & 13124 & & & & CENTPLAT & & & & 100-HR-3 PUMP \& TREAT \\
\hline
\end{tabular}




\begin{tabular}{|c|c|c|c|c|c|c|c|c|c|}
\hline & $A$ & $\mathrm{~B}$ & C & $\mathrm{D}$ & $E$ & $\mathrm{~F}$ & $G$ & $\mathrm{H}$ & $\mathrm{I}$ \\
\hline 1 & SAMP_SITE_ID & HORIZ_COORD_TYPE & EW_COORD & NS_COORD & OWNER_ID & COORD_SOURCE & DIST_CLASS & RAD_LEVEL & SAMP_SITE_NAME \\
\hline 4752 & 13125 & & & & CENTPLAT & & & & 100-HR-3 PUMP \& TREAT \\
\hline 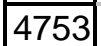 & 13126 & & & & CENTPLAT & & & & 100-HR-3 PUMP \& TREAT \\
\hline 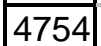 & 13127 & & & & CENTPLAT & & & & 100-HR-3 PUMP \& TREAT \\
\hline 4755 & 13128 & & & & CENTPLAT & & & & 100-HR-3 PUMP \& TREAT \\
\hline \begin{tabular}{|l|}
4756 \\
\end{tabular} & 13129 & & & & CENTPLAT & & & & 100-HR-3 PUMP \& TREAT \\
\hline \begin{tabular}{|l|}
4757 \\
\end{tabular} & 13130 & & & & CENTPLAT & & & & 100-HR-3 PUMP \& TREAT \\
\hline 4758 & 13131 & & & & CENTPLAT & & & & 100-HR-3 PUMP \& TREAT \\
\hline 4759 & 13132 & & & & CENTPLAT & & & & 100-HR-3 PUMP \& TREAT \\
\hline 4760 & 13133 & & & & CENTPLAT & & & & 100-HR-3 PUMP \& TREAT \\
\hline 4761 & 13152 & & & & CENTPLAT & & & & 200-ZP-2 \\
\hline 4762 & 13153 & & & & CENTPLAT & & & & 276-A \\
\hline 4763 & 13154 & & & & CENTPLAT & & & & 276-A \\
\hline 4764 & 13155 & & & & CENTPLAT & & & & $276-A$ \\
\hline 4765 & 13156 & & & & CENTPLAT & & & & 233-S Plutonium Concentration Facility \\
\hline 4766 & 13157 & & & & CENTPLAT & & & & 233-S Plutonium Concentration Facility \\
\hline 4767 & 13158 & & & & CENTPLAT & & & & 233-S Plutonium Concentration Facility \\
\hline 4768 & 13159 & & & & CENTPLAT & & & & 233-S Plutonium Concentration Facility \\
\hline \begin{tabular}{|l|l|}
4769 \\
\end{tabular} & 13160 & & & & CENTPLAT & & & & 233-S Plutonium Concentration Facility \\
\hline 4770 & 13172 & & & & CENTPLAT & & & & 233-S Plutonium Concentration Facility \\
\hline 4771 & 13173 & & & & CENTPLAT & & & & 233-S Plutonium Concentration Facility \\
\hline 4772 & 13174 & & & & CENTPLAT & & & & 233-S Plutonium Concentration Facility \\
\hline 4773 & 13175 & & & & CENTPLAT & & & & 233-S Plutonium Concentration Facility \\
\hline 4774 & 13176 & & & & CENTPLAT & & & & 233-S Plutonium Concentration Facility \\
\hline 4775 & 13177 & & & & CENTPLAT & & & & 233-S Plutonium Concentration Facility \\
\hline 4776 & 13178 & & & & CENTPLAT & & & & 233-S Plutonium Concentration Facility \\
\hline 4777 & 13179 & & & & CENTPLAT & & & & 233-S Plutonium Concentration Facility \\
\hline \begin{tabular}{|l|l|}
4778 \\
\end{tabular} & 13180 & & & & CENTPLAT & & & & 233-S Plutonium Concentration Facility \\
\hline 4779 & 13181 & & & & CENTPLAT & & & & 233-S Plutonium Concentration Facility \\
\hline 4780 & 13182 & & & & CENTPLAT & & & & 233-S Plutonium Concentration Facility \\
\hline 4781 & 13183 & & & & CENTPLAT & & & & 233-S Plutonium Concentration Facility \\
\hline 4782 & 13184 & & & & CENTPLAT & & & & 233-S Plutonium Concentration Facility \\
\hline \begin{tabular}{|l|l|}
4783 \\
\end{tabular} & 13185 & & & & CENTPLAT & & & & 233-S Plutonium Concentration Facility \\
\hline 4784 & 13186 & & & & CENTPLAT & & & & 233-S Plutonium Concentration Facility \\
\hline 4785 & 13187 & & & & CENTPLAT & & & & 233-S Plutonium Concentration Facility \\
\hline 4786 & 13188 & & & & CENTPLAT & & & & 233-S Plutonium Concentration Facility \\
\hline 4787 & 13189 & & & & CENTPLAT & & & & 233-S Plutonium Concentration Facility \\
\hline 4788 & 13190 & & & & CENTPLAT & & & & 233-S Plutonium Concentration Facility \\
\hline \begin{tabular}{|l|l|}
4789 \\
\end{tabular} & 13191 & & & & CENTPLAT & & & & 233-S Plutonium Concentration Facility \\
\hline
\end{tabular}




\begin{tabular}{|c|c|c|c|c|c|c|c|c|c|}
\hline & A & $\mathrm{B}$ & $\mathrm{C}$ & $\mathrm{D}$ & $\mathrm{E}$ & $\mathrm{F}$ & G & $\mathrm{H}$ & $\mathrm{I}$ \\
\hline 1 & SAMP_SITE_ID & HORIZ_COORD_TYPE & EW_COORD & NS_COORD & OWNER_ID & COORD_SOURCE & DIST_CLASS & RAD_LEVEL & SAMP_SITE_NAME \\
\hline 4790 & 13192 & & & & CENTPLAT & & & & 233-S Plutonium Concentration Facility \\
\hline 4791 & 13193 & & & & CENTPLAT & & & & 233-S Plutonium Concentration Facility \\
\hline 4792 & 13194 & & & & CENTPLAT & & & & 233-S Plutonium Concentration Facility \\
\hline 4793 & 13195 & & & & CENTPLAT & & & & 233-S Plutonium Concentration Facility \\
\hline 4794 & 13196 & & & & CENTPLAT & & & & 233-S Plutonium Concentration Facility \\
\hline 4795 & 13197 & & & & CENTPLAT & & & & 233-S Plutonium Concentration Facility \\
\hline 4796 & 13198 & & & & CENTPLAT & & & & 233-S Plutonium Concentration Facility \\
\hline 4797 & 13199 & & & & CENTPLAT & & & & 233-S Plutonium Concentration Facility \\
\hline 4798 & 13200 & & & & CENTPLAT & & & & 233-S Plutonium Concentration Facility \\
\hline 4799 & 13201 & & & & CENTPLAT & & & & 233-S Plutonium Concentration Facility \\
\hline 4800 & 13202 & & & & CENTPLAT & & & & 233-S Plutonium Concentration Facility \\
\hline 4801 & 13203 & & & & CENTPLAT & & & & 233-S Plutonium Concentration Facility \\
\hline 4802 & 13204 & & & & CENTPLAT & & & & 233-S Plutonium Concentration Facility \\
\hline 4803 & 13205 & & & & CENTPLAT & & & & 233-S Plutonium Concentration Facility \\
\hline 4804 & 13206 & & & & CENTPLAT & & & & 233-S Plutonium Concentration Facility \\
\hline 4805 & 13207 & & & & CENTPLAT & & & & 233-S Plutonium Concentration Facility \\
\hline 4806 & 13208 & & & & CENTPLAT & & & & 233-S Plutonium Concentration Facility \\
\hline 4807 & 13209 & & & & CENTPLAT & & & & 233-S Plutonium Concentration Facility \\
\hline 4808 & 13210 & & & & CENTPLAT & & & & 233-S Plutonium Concentration Facility \\
\hline 4809 & 13211 & & & & CENTPLAT & & & & 233-S Plutonium Concentration Facility \\
\hline 4810 & 13212 & & & & CENTPLAT & & & & 233-S Plutonium Concentration Facility \\
\hline 4811 & 13213 & & & & CENTPLAT & & & & 233-S Plutonium Concentration Facility \\
\hline 4812 & 13214 & & & & CENTPLAT & & & & 233-S Plutonium Concentration Facility \\
\hline 4813 & 13215 & & & & CENTPLAT & & & & 233-S Plutonium Concentration Facility \\
\hline 4814 & 13216 & & & & CENTPLAT & & & & 233-S Plutonium Concentration Facility \\
\hline 4815 & 13217 & & & & CENTPLAT & & & & 233-S Plutonium Concentration Facility \\
\hline 4816 & 13218 & & & & CENTPLAT & & & & 233-S Plutonium Concentration Facility \\
\hline 4817 & 13219 & & & & CENTPLAT & & & & 233-S Plutonium Concentration Facility \\
\hline 4818 & 13220 & & & & CENTPLAT & & & & 233-S Plutonium Concentration Facility \\
\hline 4819 & 13221 & & & & CENTPLAT & & & & 233-S Plutonium Concentration Facility \\
\hline 4820 & 13222 & & & & CENTPLAT & & & & 233-S Plutonium Concentration Facility \\
\hline 4821 & 13223 & & & & CENTPLAT & & & & 233-S Plutonium Concentration Facility \\
\hline 4822 & 13224 & & & & CENTPLAT & & & & 233-S Plutonium Concentration Facility \\
\hline 4823 & 13225 & & & & CENTPLAT & & & & 233-S Plutonium Concentration Facility \\
\hline 4824 & 13226 & & & & CENTPLAT & & & & 233-S Plutonium Concentration Facility \\
\hline 4825 & 13227 & & & & CENTPLAT & & & & 233-S Plutonium Concentration Facility \\
\hline 4826 & 13228 & & & & CENTPLAT & & & & 233-S Plutonium Concentration Facility \\
\hline 4827 & 13229 & & & & CENTPLAT & & & & 233-S Plutonium Concentration Facility \\
\hline
\end{tabular}




\begin{tabular}{|c|c|c|c|c|c|c|c|c|c|}
\hline & $\bar{A}$ & $\mathrm{~B}$ & $\mathrm{C}$ & $\mathrm{D}$ & $E$ & $\mathrm{~F}$ & G & $\mathrm{H}$ & $\mathrm{I}$ \\
\hline 1 & SAMP_SITE_ID & HORIZ_COORD_TYPE & EW_COORD & NS_COORD & OWNER_ID & COORD_SOURCE & DIST_CLASS & RAD_LEVEL & SAMP_SITE_NAME \\
\hline 4828 & 13230 & & & & CENTPLAT & & & & 233-S Plutonium Concentration Facility \\
\hline 4829 & 13231 & & & & CENTPLAT & & & & 233-S Plutonium Concentration Facility \\
\hline 4830 & 13232 & & & & CENTPLAT & & & & 233-S Plutonium Concentration Facility \\
\hline 4831 & 13233 & & & & CENTPLAT & & & & 233-S Plutonium Concentration Facility \\
\hline 4832 & 13234 & & & & CENTPLAT & & & & 233-S Plutonium Concentration Facility \\
\hline 4833 & 13235 & & & & CENTPLAT & & & & 233-S Plutonium Concentration Facility \\
\hline 4834 & 13236 & & & & CENTPLAT & & & & 233-S Plutonium Concentration Facility \\
\hline 4835 & 13237 & & & & CENTPLAT & & & & 233-S Plutonium Concentration Facility \\
\hline 4836 & 13238 & & & & CENTPLAT & & & & 233-S Plutonium Concentration Facility \\
\hline 4837 & 13239 & & & & CENTPLAT & & & & 233-S Plutonium Concentration Facility \\
\hline 4838 & 13240 & & & & CENTPLAT & & & & 233-S Plutonium Concentration Facility \\
\hline 4839 & 13241 & & & & CENTPLAT & & & & 233-S Plutonium Concentration Facility \\
\hline 4840 & 13242 & & & & CENTPLAT & & & & 233-S Plutonium Concentration Facility \\
\hline 4841 & 13243 & & & & CENTPLAT & & & & 233-S Plutonium Concentration Facility \\
\hline 4842 & 13244 & & & & CENTPLAT & & & & 233-S Plutonium Concentration Facility \\
\hline 4843 & 13245 & & & & CENTPLAT & & & & 233-S Plutonium Concentration Facility \\
\hline 4844 & 13246 & & & & CENTPLAT & & & & 233-S Plutonium Concentration Facility \\
\hline 4845 & 13247 & & & & CENTPLAT & & & & 233-S Plutonium Concentration Facility \\
\hline 4846 & 13248 & & & & CENTPLAT & & & & 233-S Plutonium Concentration Facility \\
\hline 4847 & 13249 & & & & CENTPLAT & & & & 233-S Plutonium Concentration Facility \\
\hline 4848 & 13250 & & & & CENTPLAT & & & & 233-S Plutonium Concentration Facility \\
\hline 4849 & 13251 & & & & CENTPLAT & & & & 233-S Plutonium Concentration Facility \\
\hline 4850 & 13252 & & & & CENTPLAT & & & & 233-S Plutonium Concentration Facility \\
\hline 4851 & 13253 & & & & CENTPLAT & & & & 233-S Plutonium Concentration Facility \\
\hline 4852 & 13254 & & & & CENTPLAT & & & & 233-S Plutonium Concentration Facility \\
\hline 4853 & 13255 & & & & CENTPLAT & & & & 233-S Plutonium Concentration Facility \\
\hline 4854 & 13256 & & & & CENTPLAT & & & & 233-S Plutonium Concentration Facility \\
\hline 4855 & 13257 & & & & CENTPLAT & & & & 233-S Plutonium Concentration Facility \\
\hline 4856 & 13258 & & & & CENTPLAT & & & & 233-S Plutonium Concentration Facility \\
\hline 4857 & 13259 & & & & CENTPLAT & & & & 233-S Plutonium Concentration Facility \\
\hline 4858 & 13260 & & & & CENTPLAT & & & & 233-S Plutonium Concentration Facility \\
\hline 4859 & 13261 & & & & CENTPLAT & & & & 233-S Plutonium Concentration Facility \\
\hline 4860 & 13262 & & & & CENTPLAT & & & & 233-S Plutonium Concentration Facility \\
\hline 4861 & 13263 & & & & CENTPLAT & & & & 233-S Plutonium Concentration Facility \\
\hline 4862 & 13264 & & & & CENTPLAT & & & & 233-S Plutonium Concentration Facility \\
\hline 4863 & 13265 & & & & CENTPLAT & & & & 233-S Plutonium Concentration Facility \\
\hline 4864 & 13266 & & & & CENTPLAT & & & & 233-S Plutonium Concentration Facility \\
\hline 4865 & 13267 & & & & CENTPLAT & & & & 233-S Plutonium Concentration Facility \\
\hline
\end{tabular}




\begin{tabular}{|c|c|c|c|c|c|c|c|c|c|}
\hline & $\bar{A}$ & $\mathrm{~B}$ & $\mathrm{C}$ & $\mathrm{D}$ & $E$ & $\mathrm{~F}$ & G & $\mathrm{H}$ & $\mathrm{I}$ \\
\hline 1 & SAMP_SITE_ID & HORIZ_COORD_TYPE & EW_COORD & NS_COORD & OWNER_ID & COORD_SOURCE & DIST_CLASS & RAD_LEVEL & SAMP_SITE_NAME \\
\hline 4866 & 13268 & & & & CENTPLAT & & & & 233-S Plutonium Concentration Facility \\
\hline 4867 & 13269 & & & & CENTPLAT & & & & 233-S Plutonium Concentration Facility \\
\hline 4868 & 13270 & & & & CENTPLAT & & & & 233-S Plutonium Concentration Facility \\
\hline 4869 & 13271 & & & & CENTPLAT & & & & 233-S Plutonium Concentration Facility \\
\hline 4870 & 13272 & & & & CENTPLAT & & & & 233-S Plutonium Concentration Facility \\
\hline 4871 & 13273 & & & & CENTPLAT & & & & 233-S Plutonium Concentration Facility \\
\hline 4872 & 13274 & & & & CENTPLAT & & & & 233-S Plutonium Concentration Facility \\
\hline 4873 & 13275 & & & & CENTPLAT & & & & 233-S Plutonium Concentration Facility \\
\hline 4874 & 13276 & & & & CENTPLAT & & & & 233-S Plutonium Concentration Facility \\
\hline 4875 & 13277 & & & & CENTPLAT & & & & 233-S Plutonium Concentration Facility \\
\hline 4876 & 13278 & & & & CENTPLAT & & & & 233-S Plutonium Concentration Facility \\
\hline 4877 & 13279 & & & & CENTPLAT & & & & 233-S Plutonium Concentration Facility \\
\hline 4878 & 13280 & & & & CENTPLAT & & & & 233-S Plutonium Concentration Facility \\
\hline 4879 & 13281 & & & & CENTPLAT & & & & 233-S Plutonium Concentration Facility \\
\hline 4880 & 13282 & & & & CENTPLAT & & & & 233-S Plutonium Concentration Facility \\
\hline 4881 & 13283 & & & & CENTPLAT & & & & 233-S Plutonium Concentration Facility \\
\hline 4882 & 13284 & & & & CENTPLAT & & & & 233-S Plutonium Concentration Facility \\
\hline 4883 & 13285 & & & & CENTPLAT & & & & 233-S Plutonium Concentration Facility \\
\hline 4884 & 13286 & & & & CENTPLAT & & & & 233-S Plutonium Concentration Facility \\
\hline 4885 & 13287 & & & & CENTPLAT & & & & 233-S Plutonium Concentration Facility \\
\hline 4886 & 13288 & & & & CENTPLAT & & & & 233-S Plutonium Concentration Facility \\
\hline 4887 & 13289 & & & & CENTPLAT & & & & 233-S Plutonium Concentration Facility \\
\hline 4888 & 13290 & & & & CENTPLAT & & & & 233-S Plutonium Concentration Facility \\
\hline 4889 & 13291 & & & & CENTPLAT & & & & 233-S Plutonium Concentration Facility \\
\hline 4890 & 13292 & & & & CENTPLAT & & & & 233-S Plutonium Concentration Facility \\
\hline 4891 & 13293 & & & & CENTPLAT & & & & 233-S Plutonium Concentration Facility \\
\hline 4892 & 13294 & & & & CENTPLAT & & & & 233-S Plutonium Concentration Facility \\
\hline 4893 & 13295 & & & & CENTPLAT & & & & 233-S Plutonium Concentration Facility \\
\hline 4894 & 13296 & & & & CENTPLAT & & & & 233-S Plutonium Concentration Facility \\
\hline 4895 & 13297 & & & & CENTPLAT & & & & 233-S Plutonium Concentration Facility \\
\hline 4896 & 13298 & & & & CENTPLAT & & & & 233-S Plutonium Concentration Facility \\
\hline 4897 & 13299 & & & & CENTPLAT & & & & 233-S Plutonium Concentration Facility \\
\hline 4898 & 13300 & & & & CENTPLAT & & & & 233-S Plutonium Concentration Facility \\
\hline 4899 & 13301 & & & & CENTPLAT & & & & 233-S Plutonium Concentration Facility \\
\hline 4900 & 13302 & & & & CENTPLAT & & & & 233-S Plutonium Concentration Facility \\
\hline 4901 & 13303 & & & & CENTPLAT & & & & 233-S Plutonium Concentration Facility \\
\hline 4902 & 13304 & & & & CENTPLAT & & & & 233-S Plutonium Concentration Facility \\
\hline 4903 & 13305 & & & & CENTPLAT & & & & 233-S Plutonium Concentration Facility \\
\hline
\end{tabular}




\begin{tabular}{|c|c|c|c|c|c|c|c|c|c|}
\hline & A & $\mathrm{B}$ & $\mathrm{C}$ & $\mathrm{D}$ & $\mathrm{E}$ & $\mathrm{F}$ & G & $\mathrm{H}$ & $\mathrm{I}$ \\
\hline 1 & SAMP_SITE_ID & HORIZ_COORD_TYPE & EW_COORD & NS_COORD & OWNER_ID & COORD_SOURCE & DIST_CLASS & RAD_LEVEL & SAMP_SITE_NAME \\
\hline 4904 & 13306 & & & & CENTPLAT & & & & 233-S Plutonium Concentration Facility \\
\hline 4905 & 13307 & & & & CENTPLAT & & & & 233-S Plutonium Concentration Facility \\
\hline 4906 & 13308 & & & & CENTPLAT & & & & 233-S Plutonium Concentration Facility \\
\hline 4907 & 13309 & & & & CENTPLAT & & & & 233-S Plutonium Concentration Facility \\
\hline 4908 & 13310 & & & & CENTPLAT & & & & 233-S Plutonium Concentration Facility \\
\hline 4909 & 13311 & & & & CENTPLAT & & & & 233-S Plutonium Concentration Facility \\
\hline 4910 & 13312 & & & & CENTPLAT & & & & 233-S Plutonium Concentration Facility \\
\hline 4911 & 13313 & & & & CENTPLAT & & & & 233-S Plutonium Concentration Facility \\
\hline 4912 & 13314 & & & & CENTPLAT & & & & 233-S Plutonium Concentration Facility \\
\hline 4913 & 13315 & & & & CENTPLAT & & & & 233-S Plutonium Concentration Facility \\
\hline 4914 & 13316 & & & & CENTPLAT & & & & 233-S Plutonium Concentration Facility \\
\hline 4915 & 13317 & & & & CENTPLAT & & & & 233-S Plutonium Concentration Facility \\
\hline 4916 & 13318 & & & & CENTPLAT & & & & 233-S Plutonium Concentration Facility \\
\hline 4917 & 13319 & & & & CENTPLAT & & & & 233-S Plutonium Concentration Facility \\
\hline 4918 & 13320 & & & & CENTPLAT & & & & 233-S Plutonium Concentration Facility \\
\hline 4919 & 13321 & & & & CENTPLAT & & & & 233-S Plutonium Concentration Facility \\
\hline 4920 & 13322 & & & & CENTPLAT & & & & 233-S Plutonium Concentration Facility \\
\hline 4921 & 13323 & & & & CENTPLAT & & & & 233-S Plutonium Concentration Facility \\
\hline 4922 & 13324 & & & & CENTPLAT & & & & 233-S Plutonium Concentration Facility \\
\hline 4923 & 13325 & & & & CENTPLAT & & & & 233-S Plutonium Concentration Facility \\
\hline 4924 & 13326 & & & & CENTPLAT & & & & 233-S Plutonium Concentration Facility \\
\hline 4925 & 13327 & & & & CENTPLAT & & & & 233-S Plutonium Concentration Facility \\
\hline 4926 & 13328 & & & & CENTPLAT & & & & 233-S Plutonium Concentration Facility \\
\hline 4927 & 13329 & & & & CENTPLAT & & & & 233-S Plutonium Concentration Facility \\
\hline 4928 & 13330 & & & & CENTPLAT & & & & 233-S Plutonium Concentration Facility \\
\hline 4929 & 13331 & & & & CENTPLAT & & & & 233-S Plutonium Concentration Facility \\
\hline 4930 & 13332 & & & & CENTPLAT & & & & 233-S Plutonium Concentration Facility \\
\hline 4931 & 13333 & & & & CENTPLAT & & & & 233-S Plutonium Concentration Facility \\
\hline 4932 & 13334 & & & & CENTPLAT & & & & 233-S Plutonium Concentration Facility \\
\hline 4933 & 13335 & & & & CENTPLAT & & & & 233-S Plutonium Concentration Facility \\
\hline 4934 & 13336 & & & & CENTPLAT & & & & 233-S Plutonium Concentration Facility \\
\hline 4935 & 13337 & & & & CENTPLAT & & & & 233-S Plutonium Concentration Facility \\
\hline 4936 & 13338 & & & & CENTPLAT & & & & 233-S Plutonium Concentration Facility \\
\hline 4937 & 13339 & & & & CENTPLAT & & & & 233-S Plutonium Concentration Facility \\
\hline 4938 & 13340 & & & & CENTPLAT & & & & 233-S Plutonium Concentration Facility \\
\hline 4939 & 13341 & & & & CENTPLAT & & & & 233-S Plutonium Concentration Facility \\
\hline 4940 & 13342 & & & & CENTPLAT & & & & 233-S Plutonium Concentration Facility \\
\hline 4941 & 13343 & & & & CENTPLAT & & & & 233-S Plutonium Concentration Facility \\
\hline
\end{tabular}




\begin{tabular}{|c|c|c|c|c|c|c|c|c|c|}
\hline & A & $\mathrm{B}$ & $\mathrm{C}$ & $\mathrm{D}$ & $\mathrm{E}$ & $\mathrm{F}$ & $\bar{G}$ & $\mathrm{H}$ & $\mathrm{I}$ \\
\hline 1 & SAMP_SITE_ID & HORIZ_COORD_TYPE & EW_COORD & NS_COORD & OWNER_ID & COORD_SOURCE & DIST_CLASS & RAD_LEVEL & SAMP_SITE_NAME \\
\hline 4942 & 13344 & & & & CENTPLAT & & & & 233-S Plutonium Concentration Facility \\
\hline 4943 & 13345 & & & & CENTPLAT & & & & 233-S Plutonium Concentration Facility \\
\hline 4944 & 13346 & & & & CENTPLAT & & & & 233-S Plutonium Concentration Facility \\
\hline 4945 & 13347 & & & & CENTPLAT & & & & 233-S Plutonium Concentration Facility \\
\hline 4946 & 13348 & & & & CENTPLAT & & & & 233-S Plutonium Concentration Facility \\
\hline 4947 & 13349 & & & & CENTPLAT & & & & 233-S Plutonium Concentration Facility \\
\hline 4948 & 13350 & & & & CENTPLAT & & & & 233-S Plutonium Concentration Facility \\
\hline 4949 & 13351 & & & & CENTPLAT & & & & 233-S Plutonium Concentration Facility \\
\hline 4950 & 13352 & & & & CENTPLAT & & & & 233-S Plutonium Concentration Facility \\
\hline 4951 & 13353 & & & & CENTPLAT & & & & 233-S Plutonium Concentration Facility \\
\hline 4952 & 13354 & & & & CENTPLAT & & & & 233-S Plutonium Concentration Facility \\
\hline 4953 & 13355 & & & & CENTPLAT & & & & 233-S Plutonium Concentration Facility \\
\hline 4954 & 13356 & & & & CENTPLAT & & & & 233-S Plutonium Concentration Facility \\
\hline 4955 & 13357 & & & & CENTPLAT & & & & 233-S Plutonium Concentration Facility \\
\hline 4956 & 13358 & & & & CENTPLAT & & & & 233-S Plutonium Concentration Facility \\
\hline 4957 & 13359 & & & & CENTPLAT & & & & 233-S Plutonium Concentration Facility \\
\hline 4958 & 13360 & & & & CENTPLAT & & & & 233-S Plutonium Concentration Facility \\
\hline 4959 & 13361 & & & & CENTPLAT & & & & 233-S Plutonium Concentration Facility \\
\hline 4960 & 13362 & & & & CENTPLAT & & & & 233-S Plutonium Concentration Facility \\
\hline 4961 & 13363 & & & & CENTPLAT & & & & 233-S Plutonium Concentration Facility \\
\hline 4962 & 13364 & & & & CENTPLAT & & & & 233-S Plutonium Concentration Facility \\
\hline 4963 & 13365 & & & & CENTPLAT & & & & 233-S Plutonium Concentration Facility \\
\hline 4964 & 13366 & & & & CENTPLAT & & & & 233-S Plutonium Concentration Facility \\
\hline 4965 & 13367 & & & & CENTPLAT & & & & 233-S Plutonium Concentration Facility \\
\hline 4966 & 13368 & & & & CENTPLAT & & & & 233-S Plutonium Concentration Facility \\
\hline 4967 & 13369 & & & & CENTPLAT & & & & 233-S Plutonium Concentration Facility \\
\hline 4968 & 13370 & & & & CENTPLAT & & & & 233-S Plutonium Concentration Facility \\
\hline 4969 & 13371 & & & & CENTPLAT & & & & 233-S Plutonium Concentration Facility \\
\hline 4970 & 13372 & & & & CENTPLAT & & & & 233-S Plutonium Concentration Facility \\
\hline 4971 & 13373 & & & & CENTPLAT & & & & 233-S Plutonium Concentration Facility \\
\hline 4972 & 13374 & & & & CENTPLAT & & & & 233-S Plutonium Concentration Facility \\
\hline 4973 & 13375 & & & & CENTPLAT & & & & 233-S Plutonium Concentration Facility \\
\hline 4974 & 13376 & & & & CENTPLAT & & & & 233-S Plutonium Concentration Facility \\
\hline 4975 & 13377 & & & & CENTPLAT & & & & 233-S Plutonium Concentration Facility \\
\hline 4976 & 13378 & & & & CENTPLAT & & & & 233-S Plutonium Concentration Facility \\
\hline 4977 & 13379 & & & & CENTPLAT & & & & 233-S Plutonium Concentration Facility \\
\hline \begin{tabular}{|l|l|}
4978 \\
\end{tabular} & 13380 & & & & CENTPLAT & & & & 233-S Plutonium Concentration Facility \\
\hline \begin{tabular}{|l|l|}
4979 \\
\end{tabular} & 13381 & & & & CENTPLAT & & & & 233-S Plutonium Concentration Facility \\
\hline
\end{tabular}




\begin{tabular}{|c|c|c|c|c|c|c|c|c|c|}
\hline & A & $\mathrm{B}$ & C & $\mathrm{D}$ & $\mathrm{E}$ & $\mathrm{F}$ & $G$ & $\mathrm{H}$ & $\mathrm{I}$ \\
\hline 1 & SAMP_SITE_ID & HORIZ_COORD_TYPE & EW_COORD & NS_COORD & OWNER_ID & COORD_SOURCE & DIST_CLASS & RAD_LEVEL & SAMP_SITE_NAME \\
\hline 4980 & 13382 & & & & CENTPLAT & & & & 233-S Plutonium Concentration Facility \\
\hline 4981 & 13383 & & & & CENTPLAT & & & & 233-S Plutonium Concentration Facility \\
\hline 4982 & 13384 & & & & CENTPLAT & & & & 233-S Plutonium Concentration Facility \\
\hline 4983 & 13385 & & & & CENTPLAT & & & & 233-S Plutonium Concentration Facility \\
\hline 4984 & 13386 & & & & CENTPLAT & & & & 233-S Plutonium Concentration Facility \\
\hline 4985 & 13387 & & & & CENTPLAT & & & & 233-S Plutonium Concentration Facility \\
\hline 4986 & 13388 & & & & CENTPLAT & & & & 233-S Plutonium Concentration Facility \\
\hline 4987 & 13389 & & & & CENTPLAT & & & & 233-S Plutonium Concentration Facility \\
\hline 4988 & 13390 & & & & CENTPLAT & & & & 233-S Plutonium Concentration Facility \\
\hline 4989 & 13391 & & & & CENTPLAT & & & & 233-S Plutonium Concentration Facility \\
\hline 4990 & 13392 & & & & CENTPLAT & & & & 233-S Plutonium Concentration Facility \\
\hline 4991 & 13393 & & & & CENTPLAT & & & & 233-S Plutonium Concentration Facility \\
\hline 4992 & 13394 & & & & CENTPLAT & & & & 233-S Plutonium Concentration Facility \\
\hline 4993 & 13395 & & & & CENTPLAT & & & & 233-S Plutonium Concentration Facility \\
\hline 4994 & 13396 & & & & CENTPLAT & & & & 233-S Plutonium Concentration Facility \\
\hline 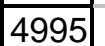 & 13397 & & & & CENTPLAT & & & & 233-S Plutonium Concentration Facility \\
\hline 4996 & 13398 & & & & CENTPLAT & & & & 233-S Plutonium Concentration Facility \\
\hline \begin{tabular}{|l|l|}
4997 \\
\end{tabular} & 13399 & & & & CENTPLAT & & & & 233-S Plutonium Concentration Facility \\
\hline 4998 & 13400 & & & & CENTPLAT & & & & 233-S Plutonium Concentration Facility \\
\hline 4999 & 13401 & & & & CENTPLAT & & & & 233-S Plutonium Concentration Facility \\
\hline 5000 & 13402 & & & & CENTPLAT & & & & 233-S Plutonium Concentration Facility \\
\hline 5001 & 13403 & & & & CENTPLAT & & & & 233-S Plutonium Concentration Facility \\
\hline 5002 & 13404 & & & & CENTPLAT & & & & 233-S Plutonium Concentration Facility \\
\hline 5003 & 13405 & & & & CENTPLAT & & & & 233-S Plutonium Concentration Facility \\
\hline 5004 & 13406 & & & & CENTPLAT & & & & 233-S Plutonium Concentration Facility \\
\hline 5005 & 13407 & & & & CENTPLAT & & & & 233-S Plutonium Concentration Facility \\
\hline 5006 & 13412 & & & & CENTPLAT & & & & $221-U$ \\
\hline 5007 & 13413 & & & & CENTPLAT & & & & 221-U \\
\hline 5008 & 13414 & & & & CENTPLAT & & & & 221-U \\
\hline 5009 & 13415 & & & & CENTPLAT & & & & $221-U$ \\
\hline 5010 & 13416 & & & & CENTPLAT & & & & 100-KR-4 PUMP \& TREAT \\
\hline 5011 & 13417 & & & & CENTPLAT & & & & 100-HR-3 PUMP \& TREAT \\
\hline 5012 & 13418 & & & & CENTPLAT & & & & 221-U CANYON BUILDING \\
\hline 5013 & 13419 & & & & CENTPLAT & & & & 100-HR-3 ISRM \\
\hline 5014 & 13420 & & & & CENTPLAT & & & & 100-HR-3 ISRM \\
\hline 5015 & 13421 & & & & CENTPLAT & & & & 100-HR-3 ISRM \\
\hline 5016 & 13422 & & & & CENTPLAT & & & & 100N WASTE PAD \\
\hline \begin{tabular}{|l|}
5017 \\
\end{tabular} & 13423 & & & & CENTPLAT & & & & 100N WASTE PAD \\
\hline
\end{tabular}




\begin{tabular}{|c|c|c|c|c|c|c|c|c|c|}
\hline & A & $\mathrm{B}$ & $\mathrm{C}$ & $\mathrm{D}$ & $E$ & $\mathrm{~F}$ & G & $\mathrm{H}$ & $\mathrm{I}$ \\
\hline 1 & SAMP_SITE_ID & HORIZ_COORD_TYPE & EW_COORD & NS_COORD & OWNER_ID & COORD_SOURCE & DIST_CLASS & RAD_LEVEL & SAMP_SITE_NAME \\
\hline 5018 & 13424 & & & & CENTPLAT & & & & 100N WASTE PAD \\
\hline 5019 & 13425 & & & & CENTPLAT & & & & 100N WASTE PAD \\
\hline 5020 & 13432 & & & & CENTPLAT & & & & 221-B \\
\hline \begin{tabular}{|l}
5021 \\
\end{tabular} & 13433 & & & & CENTPLAT & & & & 221-B \\
\hline \begin{tabular}{|l|}
5022 \\
\end{tabular} & 13434 & & & & CENTPLAT & & & & 221-B \\
\hline 5023 & 13435 & & & & CENTPLAT & & & & 221-B \\
\hline \begin{tabular}{|l|}
5024 \\
\end{tabular} & 13452 & & & & CENTPLAT & & & & 100-KR-4 PUMP \& TREAT \\
\hline 5025 & 13453 & & & & CENTPLAT & & & & 100-KR-4 PUMP \& TREAT \\
\hline 5026 & 13454 & & & & CENTPLAT & & & & 100-KR-4 PUMP \& TREAT \\
\hline \begin{tabular}{|l|}
5027 \\
\end{tabular} & 13455 & & & & CENTPLAT & & & & 100-KR-4 PUMP \& TREAT \\
\hline 5028 & 13456 & & & & CENTPLAT & & & & 100-KR-4 PUMP \& TREAT \\
\hline 5029 & 13457 & & & & CENTPLAT & & & & 100-KR-4 PUMP \& TREAT \\
\hline 5030 & 13458 & & & & CENTPLAT & & & & 100-KR-4 PUMP \& TREAT \\
\hline 5031 & 13459 & & & & CENTPLAT & & & & 100-KR-4 PUMP \& TREAT \\
\hline 5032 & 13460 & & & & CENTPLAT & & & & 100-KR-4 PUMP \& TREAT \\
\hline 5033 & 13461 & & & & CENTPLAT & & & & 100-KR-4 PUMP \& TREAT \\
\hline \begin{tabular}{|l|}
5034 \\
\end{tabular} & 13462 & & & & CENTPLAT & & & & 100-KR-4 PUMP \& TREAT \\
\hline 5035 & 13463 & & & & CENTPLAT & & & & 100-KR-4 PUMP \& TREAT \\
\hline 5036 & 13464 & & & & CENTPLAT & & & & 100-KR-4 PUMP \& TREAT \\
\hline \begin{tabular}{|l|}
5037 \\
\end{tabular} & 13465 & & & & CENTPLAT & & & & 100-KR-4 PUMP \& TREAT \\
\hline 5038 & 13466 & & & & CENTPLAT & & & & 100-KR-4 PUMP \& TREAT \\
\hline 5039 & 13467 & & & & CENTPLAT & & & & 100-KR-4 PUMP \& TREAT \\
\hline 5040 & 13472 & & & & CENTPLAT & & & & 100-HR-3 PUMP \& TREAT \\
\hline 5041 & 13473 & & & & CENTPLAT & & & & 100-HR-3 PUMP \& TREAT \\
\hline 5042 & 13474 & & & & CENTPLAT & & & & 100-HR-3 PUMP \& TREAT \\
\hline 5043 & 13475 & & & & CENTPLAT & & & & 100-HR-3 PUMP \& TREAT \\
\hline 5044 & 13476 & & & & CENTPLAT & & & & 100-HR-3 PUMP \& TREAT \\
\hline 5045 & 13477 & & & & CENTPLAT & & & & 100-HR-3 PUMP \& TREAT \\
\hline 5046 & 13478 & & & & CENTPLAT & & & & 100-HR-3 PUMP \& TREAT \\
\hline \begin{tabular}{|l|}
5047 \\
\end{tabular} & 13479 & & & & CENTPLAT & & & & 100-HR-3 PUMP \& TREAT \\
\hline 5048 & 13480 & & & & CENTPLAT & & & & 100-HR-3 PUMP \& TREAT \\
\hline \begin{tabular}{|l|}
5049 \\
\end{tabular} & 13481 & & & & CENTPLAT & & & & 100-HR-3 PUMP \& TREAT \\
\hline \begin{tabular}{|l|}
5050 \\
\end{tabular} & 13482 & & & & CENTPLAT & & & & 100-HR-3 PUMP \& TREAT \\
\hline 5051 & 13483 & & & & CENTPLAT & & & & 216-Z-11 Ditch \\
\hline 5052 & 13521 & & & & CENTPLAT & & & & 200-PW-1 \\
\hline 5053 & 13522 & & & & CENTPLAT & & & & 200-PW-1 \\
\hline 5054 & 13523 & & & & CENTPLAT & & & & 200-PW-1 \\
\hline 5055 & 13524 & & & & CENTPLAT & & & & 200-PW-1 \\
\hline
\end{tabular}




\begin{tabular}{|c|c|c|c|c|c|c|c|c|c|}
\hline & A & $\mathrm{B}$ & $C$ & $\mathrm{D}$ & $E$ & $\mathrm{~F}$ & G & $\mathrm{H}$ & $\mathrm{I}$ \\
\hline 1 & SAMP_SITE_ID & HORIZ_COORD_TYPE & EW_COORD & NS_COORD & OWNER_ID & COORD_SOURCE & DIST_CLASS & RAD_LEVEL & SAMP_SITE_NAME \\
\hline 5056 & 13525 & & & & CENTPLAT & & & & 200-PW-1 \\
\hline \begin{tabular}{|l|}
5057 \\
\end{tabular} & 13526 & & & & CENTPLAT & & & & 200-PW-1 \\
\hline 5058 & 13527 & & & & CENTPLAT & & & & 200-PW-1 \\
\hline 5059 & 13528 & & & & CENTPLAT & & & & 200-PW-1 \\
\hline 5060 & 13529 & & & & CENTPLAT & & & & 200-PW-1 \\
\hline 5061 & 13530 & & & & CENTPLAT & & & & 200-PW-1 \\
\hline \begin{tabular}{|l|}
5062 \\
\end{tabular} & 13531 & & & & CENTPLAT & & & & 200-PW-1 \\
\hline 5063 & 13532 & & & & CENTPLAT & & & & 200-PW-1 \\
\hline 5064 & 13533 & & & & CENTPLAT & & & & 200-PW-1 \\
\hline \begin{tabular}{|l|}
5065 \\
\end{tabular} & 13534 & & & & CENTPLAT & & & & 200-PW-1 \\
\hline 5066 & 13535 & & & & CENTPLAT & & & & 200-PW-1 \\
\hline 5067 & 13536 & & & & CENTPLAT & & & & 200-PW-1 \\
\hline \begin{tabular}{|l|}
5068 \\
\end{tabular} & 13537 & & & & CENTPLAT & & & & 200-PW-1 \\
\hline 5069 & 13538 & & & & CENTPLAT & & & & 200-PW-1 \\
\hline 5070 & 13539 & & & & CENTPLAT & & & & 200-PW-1 \\
\hline \begin{tabular}{|l|}
5071 \\
\end{tabular} & 13540 & & & & CENTPLAT & & & & 200-PW-1 \\
\hline \begin{tabular}{|l|}
5072 \\
\end{tabular} & 13541 & & & & CENTPLAT & & & & 200-PW-1 \\
\hline \begin{tabular}{|l|}
5073 \\
\end{tabular} & 13542 & & & & CENTPLAT & & & & 200-PW-1 \\
\hline \begin{tabular}{|l|}
5074 \\
\end{tabular} & 13543 & & & & CENTPLAT & & & & 200-PW-1 \\
\hline \begin{tabular}{|l|}
5075 \\
\end{tabular} & 13552 & & & & CENTPLAT & & & & 200-PW-1 \\
\hline 5076 & 13553 & & & & CENTPLAT & & & & 200-PW-1 \\
\hline \begin{tabular}{|l|}
5077 \\
\end{tabular} & 13554 & & & & CENTPLAT & & & & 200-PW-1 \\
\hline \begin{tabular}{|l|}
5078 \\
\end{tabular} & 13555 & & & & CENTPLAT & & & & 200-PW-1 \\
\hline 5079 & 13556 & & & & CENTPLAT & & & & 200-PW-1 \\
\hline 5080 & 13557 & & & & CENTPLAT & & & & 200-PW-1 \\
\hline \begin{tabular}{|l|}
5081 \\
\end{tabular} & 13558 & & & & CENTPLAT & & & & 216-Z-11 Ditch \\
\hline \begin{tabular}{|l|}
5082 \\
\end{tabular} & 13559 & & & & CENTPLAT & & & & 216-Z-11 Ditch \\
\hline \begin{tabular}{|l|}
5083 \\
\end{tabular} & 13560 & & & & CENTPLAT & & & & 216-Z-11 Ditch \\
\hline \begin{tabular}{|l|}
5084 \\
\end{tabular} & 13561 & & & & CENTPLAT & & & & 216-Z-11 Ditch \\
\hline \begin{tabular}{|l}
5085 \\
\end{tabular} & 13562 & & & & CENTPLAT & & & & 216-Z-11 Ditch \\
\hline \begin{tabular}{|l|}
5080 \\
5086 \\
\end{tabular} & 13563 & & & & CENTPLAT & & & & 216-Z-11 Ditch \\
\hline \begin{tabular}{|l|}
5087 \\
\end{tabular} & 13564 & & & & CENTPLAT & & & & 216-Z-11 Ditch \\
\hline \begin{tabular}{|l|}
5088 \\
\end{tabular} & 13565 & & & & CENTPLAT & & & & 216-Z-11 Ditch \\
\hline 5089 & 13566 & & & & CENTPLAT & & & & 216-Z-11 Ditch \\
\hline \begin{tabular}{|l|}
5090 \\
\end{tabular} & 13567 & & & & CENTPLAT & & & & 216-Z-11 Ditch \\
\hline \begin{tabular}{|l|}
5091 \\
\end{tabular} & 13568 & & & & CENTPLAT & & & & 216-Z-11 Ditch \\
\hline \begin{tabular}{|l|}
5092 \\
\end{tabular} & 13569 & & & & CENTPLAT & & & & 216-Z-11 Ditch \\
\hline 5093 & 13570 & & & & CENTPLAT & & & & 231-Z Manhole \#2 \\
\hline
\end{tabular}




\begin{tabular}{|c|c|c|c|c|c|c|c|c|c|}
\hline & $A$ & $\mathrm{~B}$ & C & $\mathrm{D}$ & $E$ & $\mathrm{~F}$ & $G$ & $\mathrm{H}$ & $\mathrm{I}$ \\
\hline 1 & SAMP_SITE_ID & HORIZ_COORD_TYPE & EW_COORD & NS_COORD & OWNER_ID & COORD_SOURCE & DIST_CLASS & RAD_LEVEL & SAMP_SITE_NAME \\
\hline 5094 & 13571 & & & & CENTPLAT & & & & 231-Z Manhole \#2 \\
\hline 5095 & 13572 & & & & CENTPLAT & & & & 216-T-26 \\
\hline 5096 & 13573 & & & & CENTPLAT & & & & $216-\mathrm{T}-26$ \\
\hline 5097 & 13592 & & & & CENTPLAT & & & & 100-KR-4 PUMP \& TREAT \\
\hline 5098 & 13593 & & & & CENTPLAT & & & & 100-KR-4 PUMP \& TREAT \\
\hline 5099 & 13594 & & & & CENTPLAT & & & & 100-KR-4 PUMP \& TREAT \\
\hline 5100 & 13595 & & & & CENTPLAT & & & & 100-KR-4 PUMP \& TREAT \\
\hline 5101 & 13596 & & & & CENTPLAT & & & & 100-KR-4 PUMP \& TREAT \\
\hline 5102 & 13597 & & & & CENTPLAT & & & & 100-HR-3 PUMP \& TREAT \\
\hline 5103 & 13598 & & & & CENTPLAT & & & & 100-HR-3 PUMP \& TREAT \\
\hline 5104 & 13599 & & & & CENTPLAT & & & & 100-HR-3 PUMP \& TREAT \\
\hline 5105 & 13600 & & & & CENTPLAT & & & & 100-HR-3 PUMP \& TREAT \\
\hline 5106 & 13601 & & & & CENTPLAT & & & & 100-HR-3 PUMP \& TREAT \\
\hline 5107 & 13602 & & & & CENTPLAT & & & & 100-HR-3 PUMP \& TREAT \\
\hline 5108 & 13612 & & & & CENTPLAT & & & & 200-ZP-1 PUMP \& TREAT \\
\hline 5109 & 13613 & & & & CENTPLAT & & & & 200-ZP-1 PUMP \& TREAT \\
\hline 5110 & 13632 & & & & CENTPLAT & & & & 200-ZP-1 \\
\hline 5111 & 13633 & & & & CENTPLAT & & & & 200-ZP-1 \\
\hline 5112 & 13634 & & & & CENTPLAT & & & & 200-ZP-1 \\
\hline \begin{tabular}{|l|l|}
5113 \\
\end{tabular} & 13635 & & & & CENTPLAT & & & & 200-ZP-1 \\
\hline 5114 & 13636 & & & & CENTPLAT & & & & 200-ZP-1 \\
\hline 5115 & 13652 & & & & CENTPLAT & & & & 200-ZP-1 PUMP \& TREAT \\
\hline 5116 & 13653 & & & & CENTPLAT & & & & 200-ZP-1 PUMP \& TREAT \\
\hline 5117 & 13654 & & & & CENTPLAT & & & & 200-ZP-1 PUMP \& TREAT \\
\hline 5118 & 13655 & & & & CENTPLAT & & & & 200-ZP-1 PUMP \& TREAT \\
\hline 5119 & 13656 & & & & SESPMNT & & ONSITE & LOW & 100 F SHORE HRM 24 \\
\hline 5120 & 13672 & & & & CENTPLAT & & & & 100-KR-4 PUMP \& TREAT \\
\hline 5121 & 13673 & & & & CENTPLAT & & & & 100-KR-4 PUMP \& TREAT \\
\hline 5122 & 13674 & & & & CENTPLAT & & & & 100-KR-4 PUMP \& TREAT \\
\hline 5123 & 13675 & & & & CENTPLAT & & & & 100-HR-3 PUMP \& TREAT \\
\hline 5124 & 13676 & & & & CENTPLAT & & & & 100-HR-3 PUMP \& TREAT \\
\hline 5125 & 13677 & & & & CENTPLAT & & & & 100-HR-3 PUMP \& TREAT \\
\hline 5126 & 13678 & & & & CENTPLAT & & & & 100-HR-3 PUMP \& TREAT \\
\hline 5127 & 13679 & & & & CENTPLAT & & & & 100-HR-3 PUMP \& TREAT \\
\hline 5128 & 13680 & & & & CENTPLAT & & & & 100-HR-3 PUMP \& TREAT \\
\hline 5129 & 13692 & & & & SESPMNT & & OFFSITE & & TIETON \\
\hline 5130 & 13693 & & & & SESPMNT & & OFFSITE & & LEECH LAKE \\
\hline 5131 & 13694 & & & & SESPMNT & & OFFSITE & & KNUPPENBURG LAKE \\
\hline
\end{tabular}




\begin{tabular}{|c|c|c|c|c|c|c|c|c|c|}
\hline & A & $B$ & $\mathrm{C}$ & $\mathrm{D}$ & $E$ & $\mathrm{~F}$ & $G$ & $\mathrm{H}$ & $\mathrm{I}$ \\
\hline 1 & SAMP_SITE_ID & HORIZ_COORD_TYPE & EW_COORD & NS_COORD & OWNER_ID & COORD_SOURCE & DIST_CLASS & RAD_LEVEL & SAMP_SITE_NAME \\
\hline 5132 & 13695 & & & & SESPMNT & & OFFSITE & & SUMMIT CREEK \\
\hline 5133 & 13696 & & & & SESPMNT & & OFFSITE & & LA WIS WIS \\
\hline 5134 & 13712 & & & & CENTPLAT & & & & SOLID WASTE LANDFILL \\
\hline 5135 & 13713 & & & & CENTPLAT & & & & SOLID WASTE LANDFILL \\
\hline 5136 & 13714 & & & & CENTPLAT & & & & SOLID WASTE LANDFILL \\
\hline 5137 & 13715 & & & & CENTPLAT & & & & SOLID WASTE LANDFILL \\
\hline 5138 & 13716 & & & & CENTPLAT & & & & SOLID WASTE LANDFILL \\
\hline 5139 & 13717 & & & & CENTPLAT & & & & SOLID WASTE LANDFILL \\
\hline 5140 & 13718 & & & & CENTPLAT & & & & SOLID WASTE LANDFILL \\
\hline 5141 & 13719 & & & & CENTPLAT & & & & SOLID WASTE LANDFILL \\
\hline 5142 & 13720 & & & & CENTPLAT & & & & SOLID WASTE LANDFILL \\
\hline 5143 & 13721 & & & & CENTPLAT & & & & SOLID WASTE LANDFILL \\
\hline 5144 & 13722 & & & & CENTPLAT & & & & SOLID WASTE LANDFILL \\
\hline 5145 & 13723 & & & & CENTPLAT & & & & SOLID WASTE LANDFILL \\
\hline 5146 & 13724 & & & & CENTPLAT & & & & SOLID WASTE LANDFILL \\
\hline 5147 & 13725 & & & & CENTPLAT & & & & SOLID WASTE LANDFILL \\
\hline 5148 & 13726 & & & & CENTPLAT & & & & SOLID WASTE LANDFILL \\
\hline 5149 & 13727 & & & & CENTPLAT & & & & SOLID WASTE LANDFILL \\
\hline 5150 & 13728 & & & & CENTPLAT & & & & SOLID WASTE LANDFILL \\
\hline 5151 & 13729 & & & & CENTPLAT & & & & SOLID WASTE LANDFILL \\
\hline 5152 & 13730 & & & & CENTPLAT & & & & SOLID WASTE LANDFILL \\
\hline 5153 & 13731 & & & & CENTPLAT & & & & SOLID WASTE LANDFILL \\
\hline 5154 & 13732 & & & & CENTPLAT & & & & SOLID WASTE LANDFILL \\
\hline 5155 & 13733 & & & & CENTPLAT & & & & SOLID WASTE LANDFILL \\
\hline 5156 & 13734 & & & & CENTPLAT & & & & SOLID WASTE LANDFILL \\
\hline 5157 & 13735 & & & & CENTPLAT & & & & SOLID WASTE LANDFILL \\
\hline 5158 & 13736 & & & & CENTPLAT & & & & SOLID WASTE LANDFILL \\
\hline 5159 & 13737 & & & & CENTPLAT & & & & 200-ZP-1 PUMP \& TREAT \\
\hline 5160 & 13738 & & & & CENTPLAT & & & & 200-ZP-1 PUMP \& TREAT \\
\hline 5161 & 13739 & & & & CENTPLAT & & & & 200-ZP-1 PUMP \& TREAT \\
\hline 5162 & 13740 & & & & CENTPLAT & & & & 200-ZP-1 PUMP \& TREAT \\
\hline 5163 & 13752 & & & & CENTPLAT & & & & 200-ZP-1 PUMP \& TREAT \\
\hline 5164 & 13753 & & & & CENTPLAT & & & & 200-ZP-1 PUMP \& TREAT \\
\hline 5165 & 13754 & & & & CENTPLAT & & & & 200-ZP-1 PUMP \& TREAT \\
\hline 5166 & 13755 & & & & CENTPLAT & & & & 200-ZP-1 PUMP \& TREAT \\
\hline 5167 & 13756 & & & & CENTPLAT & & & & 200-ZP-1 PUMP \& TREAT \\
\hline 5168 & 13757 & & & & CENTPLAT & & & & 200-ZP-1 PUMP \& TREAT \\
\hline 5169 & 13758 & & & & CENTPLAT & & & & 200-ZP-1 PUMP \& TREAT \\
\hline
\end{tabular}


A B C D \begin{tabular}{l|l}
$\mathrm{E}$ & $\mathrm{F}$
\end{tabular} F $\quad$ G GLASS $\mathrm{H}$ I

\begin{tabular}{|c|c|c|c|c|c|c|c|c|c|}
\hline & $A$ & $\mathrm{~B}$ & $\mathrm{C}$ & $\mathrm{D}$ & $E$ & $\mathrm{~F}$ & $\mathrm{G}$ & $\mathrm{H}$ & $\mathrm{I}$ \\
\hline 1 & SAMP_SITE_ID & HORIZ_COORD_TYPE & EW_COORD & NS_COORD & OWNER_ID & COORD_SOURCE & DIST_CLASS & RAD_LEVEL & SAMP_SITE_NAME \\
\hline 5170 & 13759 & & & & CENTPLAT & & & & 200-ZP-1 PUMP \& TREAT \\
\hline 5171 & 13760 & & & & CENTPLAT & & & & 100-KR-4 PUMP \& TREAT \\
\hline 5172 & 13761 & & & & CENTPLAT & & & & 100-KR-4 PUMP \& TREAT \\
\hline 5173 & 13762 & & & & CENTPLAT & & & & 100-KR-4 PUMP \& TREAT \\
\hline 5174 & 13763 & & & & CENTPLAT & & & & 100-KR-4 PUMP \& TREAT \\
\hline 5175 & 13764 & & & & CENTPLAT & & & & 100-KR-4 PUMP \& TREAT \\
\hline 5176 & 13765 & & & & CENTPLAT & & & & 100-KR-4 PUMP \& TREAT \\
\hline 5177 & 13766 & & & & CENTPLAT & & & & 100-KR-4 PUMP \& TREAT \\
\hline 5178 & 13767 & & & & CENTPLAT & & & & 100-HR-3 PUMP \& TREAT \\
\hline 5179 & 13768 & & & & CENTPLAT & & & & 100-HR-3 PUMP \& TREAT \\
\hline 5180 & 13769 & & & & CENTPLAT & & & & 100-HR-3 PUMP \& TREAT \\
\hline 5181 & 13770 & & & & CENTPLAT & & & & 100-HR-3 PUMP \& TREAT \\
\hline 5182 & 13771 & & & & CENTPLAT & & & & 100-HR-3 PUMP \& TREAT \\
\hline 5183 & 13772 & & & & CENTPLAT & & & & 100-HR-3 PUMP \& TREAT \\
\hline 5184 & 13792 & & & & SESPMNT & & ONSITE & & 100-K SPRING 63-2 \\
\hline 5185 & 13812 & & & & CENTPLAT & & & & 200-ZP-1 PUMP \& TREAT \\
\hline 5186 & 13832 & & & & CENTPLAT & & & & 100-NR-2 PUMP \& TREAT \\
\hline 5187 & 13833 & & & & CENTPLAT & & & & 100-NR-2 PUMP \& TREAT \\
\hline 5188 & 13852 & & & & CENTPLAT & & & & 100-KR-4 PUMP \& TREAT \\
\hline 5189 & 13853 & & & & CENTPLAT & & & & 100-KR-4 PUMP \& TREAT \\
\hline 5190 & 13854 & & & & CENTPLAT & & & & 100-HR-3 PUMP \& TREAT \\
\hline 5191 & 13855 & & & & CENTPLAT & & & & 100-HR-3 PUMP \& TREAT \\
\hline 5192 & 13856 & & & & CENTPLAT & & & & 100-HR-3 PUMP \& TREAT \\
\hline 5193 & 13857 & & & & CENTPLAT & & & & 100-HR-3 PUMP \& TREAT \\
\hline 5194 & 13858 & & & & CENTPLAT & & & & 100-HR-3 PUMP \& TREAT \\
\hline 5195 & 13859 & & & & CENTPLAT & & & & 100-HR-3 PUMP \& TREAT \\
\hline 5196 & 13860 & & & & CENTPLAT & & & & 100-HR-3 PUMP \& TREAT \\
\hline 5197 & 13861 & & & & CENTPLAT & & & & 100-HR-3 PUMP \& TREAT \\
\hline 5198 & 13862 & & & & CENTPLAT & & & & 100-KR-4 PUMP \& TREAT \\
\hline 5199 & 13863 & & & & CENTPLAT & & & & 100-KR-4 PUMP \& TREAT \\
\hline 5200 & 13864 & & & & CENTPLAT & & & & 100-KR-4 PUMP \& TREAT \\
\hline 5201 & 13865 & & & & CENTPLAT & & & & 100-KR-4 PUMP \& TREAT \\
\hline 5202 & 13866 & & & & CENTPLAT & & & & 100-KR-4 PUMP \& TREAT \\
\hline 5203 & 13872 & & & & CENTPLAT & & & & 100-KR-4 PUMP \& TREAT \\
\hline 5204 & 13873 & & & & CENTPLAT & & & & 100-KR-4 PUMP \& TREAT \\
\hline 5205 & 13874 & & & & CENTPLAT & & & & 100-KR-4 PUMP \& TREAT \\
\hline 5206 & 13875 & & & & CENTPLAT & & & & 100-KR-4 PUMP \& TREAT \\
\hline 5207 & 13876 & & & & CENTPLAT & & & & 100-KR-4 PUMP \& TREAT \\
\hline
\end{tabular}




\begin{tabular}{|c|c|c|c|c|c|c|c|c|c|}
\hline & $A$ & $\mathrm{~B}$ & $\mathrm{C}$ & $\mathrm{D}$ & $E$ & $\mathrm{~F}$ & G & $\mathrm{H}$ & $\mathrm{I}$ \\
\hline 1 & SAMP_SITE_ID & HORIZ_COORD_TYPE & EW_COORD & NS_COORD & OWNER_ID & COORD_SOURCE & DIST_CLASS & RAD_LEVEL & SAMP_SITE_NAME \\
\hline 5208 & 13877 & & & & CENTPLAT & & & & 100-KR-4 PUMP \& TREAT \\
\hline 5209 & 13878 & & & & CENTPLAT & & & & 100-KR-4 PUMP \& TREAT \\
\hline 5210 & 13879 & & & & CENTPLAT & & & & 100-KR-4 PUMP \& TREAT \\
\hline 5211 & 13880 & & & & CENTPLAT & & & & 100-KR-4 PUMP \& TREAT \\
\hline 5212 & 13881 & & & & CENTPLAT & & & & 100-KR-4 PUMP \& TREAT \\
\hline 5213 & 13882 & & & & CENTPLAT & & & & 100-KR-4 PUMP \& TREAT \\
\hline 5214 & 13883 & & & & CENTPLAT & & & & 100-KR-4 PUMP \& TREAT \\
\hline 5215 & 13884 & & & & CENTPLAT & & & & 100-KR-4 PUMP \& TREAT \\
\hline 5216 & 13885 & & & & CENTPLAT & & & & 100-KR-4 PUMP \& TREAT \\
\hline 5217 & 13886 & & & & CENTPLAT & & & & 100-HR-3 PUMP \& TREAT \\
\hline 5218 & 13887 & & & & CENTPLAT & & & & 100-HR-3 PUMP \& TREAT \\
\hline 5219 & 13892 & & & & CENTPLAT & & & & 200-ZP-1 PUMP \& TREAT \\
\hline 5220 & 13893 & & & & CENTPLAT & & & & 200-ZP-1 PUMP \& TREAT \\
\hline 5221 & 13894 & & & & CENTPLAT & & & & 200-ZP-1 PUMP \& TREAT \\
\hline 5222 & 13895 & & & & CENTPLAT & & & & 200-ZP-1 PUMP \& TREAT \\
\hline 5223 & 13896 & & & & CENTPLAT & & & & 100-HR-3 PUMP \& TREAT \\
\hline 5224 & 13897 & & & & CENTPLAT & & & & 100-HR-3 PUMP \& TREAT \\
\hline 5225 & 13898 & & & & CENTPLAT & & & & 100-HR-3 PUMP \& TREAT \\
\hline 5226 & 13899 & & & & CENTPLAT & & & & 100-HR-3 PUMP \& TREAT \\
\hline 5227 & 13916 & & & & CENTPLAT & & & & 200-ZP-1 PUMP \& TREAT \\
\hline 5228 & 13917 & & & & CENTPLAT & & & & 200-ZP-1 PUMP \& TREAT \\
\hline 5229 & 13918 & & & & CENTPLAT & & & & 200-ZP-1 PUMP \& TREAT \\
\hline 5230 & 13919 & & & & CENTPLAT & & & & 200-ZP-1 PUMP \& TREAT \\
\hline 5231 & 13920 & & & & CENTPLAT & & & & 200-ZP-1 PUMP \& TREAT \\
\hline 5232 & 13921 & & & & CENTPLAT & & & & 200-ZP-1 PUMP \& TREAT \\
\hline 5233 & 13922 & & & & CENTPLAT & & & & 200-ZP-1 PUMP \& TREAT \\
\hline 5234 & 13923 & & & & CENTPLAT & & & & 200-ZP-1 PUMP \& TREAT \\
\hline 5235 & 13924 & & & & CENTPLAT & & & & 200-ZP-1 PUMP \& TREAT \\
\hline 5236 & 13925 & & & & CENTPLAT & & & & 200-ZP-1 PUMP \& TREAT \\
\hline 5237 & 13926 & & & & CENTPLAT & & & & 200-ZP-1 PUMP \& TREAT \\
\hline 5238 & 13927 & & & & CENTPLAT & & & & 200-ZP-1 PUMP \& TREAT \\
\hline 5239 & 13928 & & & & CENTPLAT & & & & 200-ZP-1 PUMP \& TREAT \\
\hline 5240 & 13936 & & & & CENTPLAT & & & & 100-HR-3 ISRM \\
\hline 5241 & 13937 & & & & CENTPLAT & & & & 100-HR-3 ISRM \\
\hline 5242 & 13956 & & & & CENTPLAT & & & & 100-KR-4 PUMP \& TREAT \\
\hline 5243 & 13957 & & & & CENTPLAT & & & & 100-KR-4 PUMP \& TREAT \\
\hline 5244 & 13958 & & & & CENTPLAT & & & & 100-KR-4 PUMP \& TREAT \\
\hline 5245 & 13959 & & & & CENTPLAT & & & & 100-KR-4 PUMP \& TREAT \\
\hline
\end{tabular}




\begin{tabular}{|c|c|c|c|c|c|c|c|c|c|}
\hline & $A$ & $\mathrm{~B}$ & $\mathrm{C}$ & $\mathrm{D}$ & $E$ & $\mathrm{~F}$ & $\mathrm{G}$ & $\mathrm{H}$ & $\mathrm{I}$ \\
\hline 1 & SAMP_SITE_ID & HORIZ_COORD_TYPE & EW_COORD & NS_COORD & OWNER_ID & COORD_SOURCE & DIST_CLASS & RAD_LEVEL & SAMP_SITE_NAME \\
\hline 5246 & 13960 & & & & CENTPLAT & & & & 100-KR-4 PUMP \& TREAT \\
\hline 5247 & 13961 & & & & CENTPLAT & & & & 100-KR-4 PUMP \& TREAT \\
\hline 5248 & 13962 & & & & CENTPLAT & & & & 100-KR-4 PUMP \& TREAT \\
\hline 5249 & 13963 & & & & CENTPLAT & & & & 100-HR-3 PUMP \& TREAT \\
\hline 5250 & 13964 & & & & CENTPLAT & & & & 100-HR-3 PUMP \& TREAT \\
\hline 5251 & 13965 & & & & CENTPLAT & & & & 100-HR-3 PUMP \& TREAT \\
\hline 5252 & 13966 & & & & CENTPLAT & & & & 100-HR-3 PUMP \& TREAT \\
\hline 5253 & 13967 & & & & CENTPLAT & & & & 100-HR-3 PUMP \& TREAT \\
\hline 5254 & 13968 & & & & CENTPLAT & & & & 100-HR-3 PUMP \& TREAT \\
\hline 5255 & 13976 & & & & CENTPLAT & & & & 100-HR-3 PUMP \& TREAT \\
\hline 5256 & 13977 & & & & CENTPLAT & & & & 100-HR-3 PUMP \& TREAT \\
\hline 5257 & 13978 & & & & CENTPLAT & & & & 100-HR-3 PUMP \& TREAT \\
\hline 5258 & 13996 & & & & CENTPLAT & & & & 200-ZP-1 PUMP \& TREAT \\
\hline 5259 & 13997 & & & & CENTPLAT & & & & 200-ZP-1 PUMP \& TREAT \\
\hline 5260 & 13998 & & & & CENTPLAT & & & & 200-ZP-1 PUMP \& TREAT \\
\hline 5261 & 13999 & & & & CENTPLAT & & & & 200-ZP-1 PUMP \& TREAT \\
\hline 5262 & 14000 & & & & CENTPLAT & & & & 200-ZP-1 PUMP \& TREAT \\
\hline 5263 & 14001 & & & & CENTPLAT & & & & 200-ZP-1 PUMP \& TREAT \\
\hline 5264 & 14016 & & & & CENTPLAT & & & & 200-ZP-1 PUMP \& TREAT \\
\hline 5265 & 14036 & & & & CENTPLAT & & & & 100-HR-3 PUMP \& TREAT \\
\hline 5266 & 14037 & & & & CENTPLAT & & & & 100-HR-3 PUMP \& TREAT \\
\hline 5267 & 14038 & & & & CENTPLAT & & & & 100-HR-3 PUMP \& TREAT \\
\hline 5268 & 14039 & & & & CENTPLAT & & & & 100-HR-3 PUMP \& TREAT \\
\hline 5269 & 14040 & & & & CENTPLAT & & & & 100-HR-3 PUMP \& TREAT \\
\hline 5270 & 14041 & & & & CENTPLAT & & & & 100-KR-4 PUMP \& TREAT \\
\hline 5271 & 14042 & & & & CENTPLAT & & & & 100-KR-4 PUMP \& TREAT \\
\hline 5272 & 14043 & & & & CENTPLAT & & & & 100-KR-4 PUMP \& TREAT \\
\hline 5273 & 14044 & & & & CENTPLAT & & & & 100-KR-4 PUMP \& TREAT \\
\hline 5274 & 14045 & & & & CENTPLAT & & & & 100-KR-4 PUMP \& TREAT \\
\hline 5275 & 14046 & & & & CENTPLAT & & & & 100-KR-4 PUMP \& TREAT \\
\hline 5276 & 14056 & & & & SESPMNT & & ONSITE & & 100-H SPRING 150-1 \\
\hline 5277 & 14076 & & & & CENTPLAT & & & & 216-B-63 \\
\hline 5278 & 14096 & & & & CENTPLAT & & & & 216-S-10P Pond \\
\hline 5279 & 14097 & & & & CENTPLAT & & & & 216-S-10P Pond \\
\hline 5280 & 14098 & & & & CENTPLAT & & & & 216-S-10P Pond \\
\hline 5281 & 14116 & & & & CENTPLAT & & & & 216-S-10P Pond \\
\hline 5282 & 14117 & & & & CENTPLAT & & & & 216-S-10P Pond \\
\hline 5283 & 14118 & & & & CENTPLAT & & & & 216-S-10P Pond \\
\hline
\end{tabular}

Page 139 


\begin{tabular}{|c|c|c|c|c|c|c|c|c|c|}
\hline & $A$ & $\mathrm{~B}$ & $\mathrm{C}$ & $\mathrm{D}$ & $E$ & $\mathrm{~F}$ & $\mathrm{G}$ & $\mathrm{H}$ & $\mathrm{I}$ \\
\hline 1 & SAMP_SITE_ID & HORIZ_COORD_TYPE & EW_COORD & NS_COORD & OWNER_ID & COORD_SOURCE & DIST_CLASS & RAD_LEVEL & SAMP_SITE_NAME \\
\hline 5284 & 14119 & & & & CENTPLAT & & & & 216-S-10P Pond \\
\hline 5285 & 14136 & UNKNOWN & 580698.42 & 147588.68 & HEISPROD & GPS & ONSITE & & 126-F-1 \\
\hline 5286 & 14137 & UNKNOWN & 580698.42 & 147588.68 & HEISPROD & GPS & ONSITE & & 126-F-1 \\
\hline 5287 & 14138 & UNKNOWN & 580705.54 & 147591.51 & HEISPROD & GPS & ONSITE & & 126-F-1 \\
\hline 5288 & 14139 & UNKNOWN & 580688.45 & 147571.02 & HEISPROD & GPS & ONSITE & & $126-F-1$ \\
\hline 5289 & 14140 & UNKNOWN & 580679.39 & 147562.19 & HEISPROD & GPS & ONSITE & & 126-F-1 \\
\hline 5290 & 14141 & UNKNOWN & 580707.61 & 147571.24 & HEISPROD & GPS & ONSITE & & 126-F-1 \\
\hline 5291 & 14142 & UNKNOWN & 580711.68 & 147592.5 & HEISPROD & GPS & ONSITE & & 126-F-1 \\
\hline 5292 & 14143 & UNKNOWN & 580712.97 & 147581.14 & HEISPROD & GPS & ONSITE & & 126-F-1 \\
\hline 5293 & 14144 & UNKNOWN & 580716.86 & 147547.75 & HEISPROD & GPS & ONSITE & & 126-F-1 \\
\hline 5294 & 14145 & UNKNOWN & 580721.8 & 147548.93 & HEISPROD & GPS & ONSITE & & 126-F-1 \\
\hline 5295 & 14146 & UNKNOWN & 580721.23 & 147596.08 & HEISPROD & GPS & ONSITE & & 126-F-1 \\
\hline 5296 & 14147 & UNKNOWN & 580727.41 & 147584.84 & HEISPROD & GPS & ONSITE & & $126-F-1$ \\
\hline 5297 & 14148 & UNKNOWN & 580730.18 & 147561.55 & HEISPROD & GPS & ONSITE & & $126-F-1$ \\
\hline 5298 & 14149 & UNKNOWN & 580731.56 & 147549.94 & HEISPROD & GPS & ONSITE & & $126-F-1$ \\
\hline 5299 & 14150 & UNKNOWN & 580731.04 & 147597.71 & HEISPROD & GPS & ONSITE & & 126-F-1 \\
\hline 5300 & 14151 & UNKNOWN & 580733.4 & 147576.38 & HEISPROD & GPS & ONSITE & & 126-F-1 \\
\hline 5301 & 14152 & UNKNOWN & 580734.65 & 147565.2 & HEISPROD & GPS & ONSITE & & 126-F-1 \\
\hline 5302 & 14153 & UNKNOWN & 580735.95 & 147553.64 & HEISPROD & GPS & ONSITE & & 126-F-1 \\
\hline 5303 & 14154 & UNKNOWN & 580738.91 & 147571.27 & HEISPROD & GPS & ONSITE & & 126-F-1 \\
\hline 5304 & 14155 & UNKNOWN & 580741.65 & 147589.46 & HEISPROD & GPS & ONSITE & & $126-F-1$ \\
\hline 5305 & 14156 & UNKNOWN & 580742.84 & 147578.68 & HEISPROD & GPS & ONSITE & & 126-F-1 \\
\hline 5306 & 14157 & UNKNOWN & 580744.07 & 147567.45 & HEISPROD & GPS & ONSITE & & 126-F-1 \\
\hline 5307 & 14158 & UNKNOWN & 580748.66 & 147571.33 & HEISPROD & GPS & ONSITE & & 126-F-1 \\
\hline 5308 & 14159 & UNKNOWN & 580749.66 & 147559.64 & HEISPROD & GPS & ONSITE & & $126-F-1$ \\
\hline 5309 & 14160 & & & & HEISPROD & & ONSITE & & $116-F-2$ \\
\hline 5310 & 14161 & & & & HEISPROD & & ONSITE & & 116-F-2 \\
\hline 5311 & 14162 & & & & HEISPROD & & ONSITE & & 116-F-2 \\
\hline 5312 & 14163 & & & & HEISPROD & & ONSITE & & 116-F-2 \\
\hline 5313 & 14164 & & & & HEISPROD & & ONSITE & & 116-F-2 \\
\hline \begin{tabular}{|l|}
5314 \\
\end{tabular} & 14165 & & & & HEISPROD & & ONSITE & & 116-F-2 \\
\hline 5315 & 14166 & & & & HEISPROD & & ONSITE & & 116-F-2 \\
\hline 5316 & 14167 & UNKNOWN & 580754.18 & 147594.49 & HEISPROD & GPS & ONSITE & & 126-F-1 \\
\hline 5317 & 14168 & NAD83(91) & 580754.62 & 147588.26 & HEISPROD & GPS & ONSITE & & 126-F-1 \\
\hline 5318 & 14169 & UNKNOWN & 580755.05 & 147582.03 & HEISPROD & GPS & ONSITE & & 126-F-1 \\
\hline 5319 & 14170 & UNKNOWN & 580757.24 & 147550.7 & HEISPROD & GPS & ONSITE & & 126-F-1 \\
\hline 5320 & 14171 & UNKNOWN & 580762.92 & 147597.17 & HEISPROD & GPS & ONSITE & & 126-F-1 \\
\hline 5321 & 14172 & UNKNOWN & 580763.37 & 147590.65 & HEISPROD & GPS & ONSITE & & $126-F-1$ \\
\hline
\end{tabular}




\begin{tabular}{|c|c|c|c|c|c|c|c|c|c|}
\hline & A & $B$ & $C$ & $\mathrm{D}$ & $E$ & $\mathrm{~F}$ & $G$ & $\mathrm{H}$ & $\mathrm{I}$ \\
\hline 1 & SAMP_SITE_ID & HORIZ_COORD_TYPE & EW_COORD & NS_COORD & OWNER_ID & COORD_SOURCE & DIST_CLASS & RAD_LEVEL & SAMP_SITE_NAME \\
\hline 5322 & 14173 & UNKNOWN & 580765.17 & 147564.54 & HEISPROD & GPS & ONSITE & & $126-\mathrm{F}-1$ \\
\hline 5323 & 14174 & UNKNOWN & 580766.06 & 147551.48 & HEISPROD & GPS & ONSITE & & 126-F-1 \\
\hline 5324 & 14175 & UNKNOWN & 580770.82 & 147606.71 & HEISPROD & GPS & ONSITE & & 126-F-1 \\
\hline 5325 & 14176 & UNKNOWN & 580771.28 & 147599.93 & HEISPROD & GPS & ONSITE & & 126-F-1 \\
\hline \begin{tabular}{|l|}
5326 \\
\end{tabular} & 14177 & UNKNOWN & 580771.75 & 147593.14 & HEISPROD & GPS & ONSITE & & 126-F-1 \\
\hline \begin{tabular}{|l|}
5327 \\
\end{tabular} & 14178 & UNKNOWN & 580774.55 & 147552.43 & HEISPROD & GPS & ONSITE & & 126-F-1 \\
\hline \begin{tabular}{|l|}
5328 \\
\end{tabular} & 14179 & UNKNOWN & 580779.32 & 147602.1 & HEISPROD & GPS & ONSITE & & 126-F-1 \\
\hline 5329 & 14180 & UNKNOWN & 580780.81 & 147580.88 & HEISPROD & GPS & ONSITE & & 126-F-1 \\
\hline 5330 & 14181 & UNKNOWN & 580781.3 & 147573.84 & HEISPROD & GPS & ONSITE & & 126-F-1 \\
\hline 5331 & 14182 & UNKNOWN & 580782.77 & 147552.8 & HEISPROD & GPS & ONSITE & & 126-F-1 \\
\hline 5332 & 14183 & NAD83(91) & 580785.92 & 147619.71 & HEISPROD & GPS & ONSITE & & 126-F-1 \\
\hline 53 & 14184 & UNKNOWN & 580786.49 & 147611.83 & HEISPROD & GPS & ONSITE & & 126-F-1 \\
\hline 5334 & 14185 & UNKNOWN & 580787.57 & 147597.14 & HEISPROD & GPS & ONSITE & & 126-F-1 \\
\hline 5335 & 14186 & UNKNOWN & 580788.1 & 147589.84 & HEISPROD & GPS & ONSITE & & 126-F-1 \\
\hline 5336 & 14187 & UNKNOWN & 580790.21 & 147560.88 & HEISPROD & GPS & ONSITE & & 126-F-1 \\
\hline \begin{tabular}{|l|}
5337 \\
\end{tabular} & 14188 & UNKNOWN & 580791.26 & 147546.53 & HEISPROD & GPS & ONSITE & & 126-F-1 \\
\hline 5338 & 14189 & UNKNOWN & 580794.54 & 147607.53 & HEISPROD & GPS & ONSITE & & 126-F-1 \\
\hline 5339 & 14190 & UNKNOWN & 580795.09 & 147600.17 & HEISPROD & GPS & ONSITE & & 126-F-1 \\
\hline \begin{tabular}{|l|}
5340 \\
\end{tabular} & 14191 & NAD83(91) & 580796.74 & 147578.05 & HEISPROD & GPS & ONSITE & & 126-F-1 \\
\hline \begin{tabular}{|l|}
5341 \\
\end{tabular} & 14192 & UNKNOWN & 580797.85 & 147563.31 & HEISPROD & GPS & ONSITE & & 126-F-1 \\
\hline \begin{tabular}{|l|}
5342 \\
\end{tabular} & 14193 & UNKNOWN & 580799.5 & 147541.19 & HEISPROD & GPS & ONSITE & & 126-F-1 \\
\hline \begin{tabular}{|l|}
5343 \\
\end{tabular} & 14194 & UNKNOWN & 580800.79 & 147625.75 & HEISPROD & GPS & ONSITE & & 126-F-1 \\
\hline 5344 & 14195 & UNKNOWN & 580801.39 & 147617.67 & HEISPROD & GPS & ONSITE & & 126-F-1 \\
\hline 5345 & 14196 & UNKNOWN & 580803.09 & 147595.01 & HEISPROD & GPS & ONSITE & & 126-F-1 \\
\hline \begin{tabular}{|l|}
5346 \\
\end{tabular} & 14197 & UNKNOWN & 580804.22 & 147579.88 & HEISPROD & GPS & ONSITE & & 126-F-1 \\
\hline \begin{tabular}{|l|}
5347 \\
\end{tabular} & 14198 & UNKNOWN & 580808.09 & 147628.83 & HEISPROD & GPS & ONSITE & & 126-F-1 \\
\hline 5348 & 14199 & UNKNOWN & 580813.15 & 147559.4 & HEISPROD & GPS & ONSITE & & 126-F-1 \\
\hline 5349 & 14200 & UNKNOWN & 580814.29 & 147543.76 & HEISPROD & GPS & ONSITE & & 126-F-1 \\
\hline 5350 & 14201 & UNKNOWN & 580816.07 & 147632.92 & HEISPROD & GPS & ONSITE & & 126-F-1 \\
\hline 5351 & 14202 & UNKNOWN & 580816.84 & 147619.16 & HEISPROD & GPS & ONSITE & & 126-F-1 \\
\hline \begin{tabular}{|l|}
5352 \\
\end{tabular} & 14203 & UNKNOWN & 580817.23 & 147612.41 & HEISPROD & GPS & ONSITE & & 126-F-1 \\
\hline 5353 & 14204 & UNKNOWN & 580817.62 & 147605.49 & HEISPROD & GPS & ONSITE & & 126-F-1 \\
\hline 5354 & 14205 & UNKNOWN & 580818.87 & 147583.65 & HEISPROD & GPS & ONSITE & & 126-F-1 \\
\hline \begin{tabular}{|l|}
5355 \\
\end{tabular} & 14206 & UNKNOWN & 580820.13 & 147559.65 & HEISPROD & GPS & ONSITE & & 126-F-1 \\
\hline \begin{tabular}{|l|}
5356 \\
\end{tabular} & 14207 & UNKNOWN & 580824.2 & 147626.66 & HEISPROD & GPS & ONSITE & & 126-F-1 \\
\hline \begin{tabular}{|l|}
5357 \\
\end{tabular} & 14208 & UNKNOWN & 580824.55 & 147618.48 & HEISPROD & GPS & ONSITE & & 126-F-1 \\
\hline \begin{tabular}{|l|}
5358 \\
\end{tabular} & 14209 & NAD83(91) & 580824.89 & 147610.3 & HEISPROD & GPS & ONSITE & & 126-F-1 \\
\hline \begin{tabular}{|l|}
5359 \\
\end{tabular} & 14210 & UNKNOWN & 580826.28 & 147577.61 & HEISPROD & GPS & ONSITE & & $126-F-1$ \\
\hline
\end{tabular}




\begin{tabular}{|c|c|c|c|c|c|c|c|c|c|}
\hline & $A$ & $\mathrm{~B}$ & $\mathrm{C}$ & $\mathrm{D}$ & $E$ & $\mathrm{~F}$ & $\mathrm{G}$ & $\mathrm{H}$ & $\mathrm{I}$ \\
\hline 1 & SAMP_SITE_ID & HORIZ_COORD_TYPE & EW_COORD & NS_COORD & OWNER_ID & COORD_SOURCE & DIST_CLASS & RAD_LEVEL & SAMP_SITE_NAME \\
\hline 5360 & 14211 & UNKNOWN & 580827.67 & 147544.89 & HEISPROD & GPS & ONSITE & & 126-F-1 \\
\hline 5361 & 14212 & UNKNOWN & 580828.02 & 147536.71 & HEISPROD & GPS & ONSITE & & 126-F-1 \\
\hline 5362 & 14213 & UNKNOWN & 580831.87 & 147613.43 & HEISPROD & GPS & ONSITE & & 126-F-1 \\
\hline 5363 & 14214 & UNKNOWN & 580832.2 & 147605.63 & HEISPROD & GPS & ONSITE & & $126-F-1$ \\
\hline 5364 & 14215 & UNKNOWN & 580832.53 & 147597.82 & HEISPROD & GPS & ONSITE & & 126-F-1 \\
\hline 5365 & 14216 & UNKNOWN & 580834.19 & 147558.81 & HEISPROD & GPS & ONSITE & & 126-F-1 \\
\hline 5366 & 14217 & UNKNOWN & 580841.19 & 147601.22 & HEISPROD & GPS & ONSITE & & 126-F-1 \\
\hline 5367 & 14218 & UNKNOWN & 580841.31 & 147595.6 & HEISPROD & GPS & ONSITE & & 126-F-1 \\
\hline 5368 & 14219 & NAD83(91) & 580841.83 & 147571.64 & HEISPROD & GPS & ONSITE & & 126-F-1 \\
\hline 5369 & 14220 & UNKNOWN & 580842.11 & 147558.57 & HEISPROD & GPS & ONSITE & & 126-F-1 \\
\hline 5370 & 14221 & UNKNOWN & 580842.58 & 147529.25 & HEISPROD & GPS & ONSITE & & 126-F-1 \\
\hline 5371 & 14222 & UNKNOWN & 580842.86 & 147512.63 & HEISPROD & GPS & ONSITE & & $126-F-1$ \\
\hline 5372 & 14223 & UNKNOWN & 580837.11 & 147627.35 & HEISPROD & GPS & ONSITE & & $126-F-1$ \\
\hline 5373 & 14224 & UNKNOWN & 580841.92 & 147618.91 & HEISPROD & GPS & ONSITE & & $126-F-1$ \\
\hline 5374 & 14225 & UNKNOWN & 580846.24 & 147610.07 & HEISPROD & GPS & ONSITE & & 126-F-1 \\
\hline 5375 & 14226 & UNKNOWN & 580846.6 & 147635 & HEISPROD & GPS & ONSITE & & 126-F-1 \\
\hline 5376 & 14227 & UNKNOWN & 580847.97 & 147645.5 & HEISPROD & GPS & ONSITE & & 126-F-1 \\
\hline 5377 & 14228 & UNKNOWN & 580860.4 & 147623.54 & HEISPROD & GPS & ONSITE & & 126-F-1 \\
\hline 5378 & 14229 & UNKNOWN & 580862.11 & 147635.86 & HEISPROD & GPS & ONSITE & & 126-F-1 \\
\hline 5379 & 14230 & NAD83(91) & 580864.66 & 147647.32 & HEISPROD & GPS & ONSITE & & 126-F-1 \\
\hline 5380 & 14231 & UNKNOWN & 580868.03 & 147641.72 & HEISPROD & GPS & ONSITE & & 126-F-1 \\
\hline 5381 & 14232 & UNKNOWN & 580873.85 & 147644.41 & HEISPROD & GPS & ONSITE & & 126-F-1 \\
\hline 5382 & 14233 & UNKNOWN & 580882.05 & 147653.68 & HEISPROD & GPS & ONSITE & & 126-F-1 \\
\hline 5383 & 14234 & UNKNOWN & 580889.5 & 147666.87 & HEISPROD & GPS & ONSITE & & 126-F-1 \\
\hline 5384 & 14235 & UNKNOWN & 580877.43 & 147636.11 & HEISPROD & GPS & ONSITE & & $126-F-1$ \\
\hline 5385 & 14236 & UNKNOWN & 580886.36 & 147648.51 & HEISPROD & GPS & ONSITE & & $126-F-1$ \\
\hline 5386 & 14237 & UNKNOWN & 580895.71 & 147666.57 & HEISPROD & GPS & ONSITE & & $126-F-1$ \\
\hline 5387 & 14238 & UNKNOWN & 580897.33 & 147657.06 & HEISPROD & GPS & ONSITE & & 126-F-1 \\
\hline 5388 & 14239 & UNKNOWN & 580905.16 & 147643.96 & HEISPROD & GPS & ONSITE & & 126-F-1 \\
\hline 5389 & 14240 & UNKNOWN & 580912.77 & 147660.61 & HEISPROD & GPS & ONSITE & & 126-F-1 \\
\hline 5390 & 14241 & UNKNOWN & 580905.85 & 147597.67 & HEISPROD & GPS & ONSITE & & 126-F-1 \\
\hline 5391 & 14242 & UNKNOWN & 580906.04 & 147588.24 & HEISPROD & GPS & ONSITE & & 126-F-1 \\
\hline 5392 & 14243 & UNKNOWN & 580911.05 & 147602.89 & HEISPROD & GPS & ONSITE & & 126-F-1 \\
\hline 5393 & 14244 & UNKNOWN & 580914.29 & 147633.67 & HEISPROD & GPS & ONSITE & & 126-F-1 \\
\hline 5394 & 14245 & NAD83(91) & 580915.87 & 147592.5 & HEISPROD & GPS & ONSITE & & 126-F-1 \\
\hline 5395 & 14246 & NAD83(91) & 580905.9 & 147571.25 & HEISPROD & GPS & ONSITE & & 126-F-1 \\
\hline 5396 & 14247 & UNKNOWN & 571504 & 149779 & HEISPROD & ESTIMATED & ONSITE & & 116-N-1 \\
\hline 5397 & 14248 & UNKNOWN & 571524 & 149801 & HEISPROD & ESTIMATED & ONSITE & & $116-\mathrm{N}-1$ \\
\hline
\end{tabular}


A

$\mathrm{B}$

\begin{tabular}{l|l}
$\mathrm{C}$ & $\mathrm{D}$ \\
\hline
\end{tabular}

\begin{tabular}{l|l}
$\mathrm{D}$ & $\mathrm{E}$
\end{tabular}

E $\quad \mathrm{F}$ F

G 5715421149823 HEISPROD ESTIMATED 14249 UNKNOWN \begin{tabular}{|c|l|l}
149823 HEISPROD & ESTIMATED \\
\hline 149408 HEISPROD & ESTIMATED \\
\hline 149408 HEISPROD & ESTIMATED
\end{tabular}

ONSITE

149408 HEISPROD ESTIMATED

ONSITE

14251 UNKNOWN

571868

571551

149845 HEISPROD ESTIMATED

\begin{tabular}{|l|l|l|l|}
14252 & UNKNOWN & 571551 & 149845 \\
\hline 14253 UNKNOWN & 571583 & 149825 HEISPROD & ESTIMATED \\
\hline
\end{tabular}

14254 UNKNOWN

571583

14255 UNKNOWN

149810 HEISPROD ESTIMATED

14256 UNKNOWN

149888 HEISPROD ESTIMATED

$571706 \quad 149892$ HEISPROD ESTIMATED

\begin{tabular}{ll}
571496 & 149693 HEISPROD \\
\hline
\end{tabular}

14257 UNKNOWN

$571496 \quad 149693 \mathrm{HEISPROD}$ ESTIMATED

14259 UNKNOWN

$579988.93 \quad 147793.53 \mathrm{HEISPROD}$ ESTIMATED

\begin{tabular}{|l|r|r|r|}
\hline 14260 & UNKNOWN & 579988.93 & 147793.53 \\
\hline
\end{tabular}

14261 UNKNOWN

571868

149408 HEISPROD ESTIMATED

ONSITE

ONSITE

ONSITE

ONSITE

ONSITE

ONSITE

ONSITE

ONSITE

ONSITE

ONSITE

14262

14263

14264

14265

14266

14267

14268

14269

14270

14271

14272

14273

14274

14275

14276

14277

14278

14279

14280

14281

14282

14283

14284

14285

14286

\begin{tabular}{|l|}
\hline HEISPROD \\
\hline HEISPROD \\
\hline HEISPROD \\
\hline HEISPROD \\
\hline HEISPROD \\
\hline HEISPROD \\
\hline HEISPROD \\
\hline HEISPROD \\
\hline HEISPROD \\
\hline HEISPROD \\
\hline HEISPROD \\
\hline HEISPROD \\
\hline HEISPROD \\
\hline HEISPROD \\
\hline HEISPROD \\
\hline HEISPROD \\
\hline HEISPROD \\
\hline HEISPROD \\
\hline HEISPROD \\
\hline HEISPROD \\
\hline HEISPROD \\
\hline HEISPROD \\
\hline HEISPROD \\
\hline HEISPROD \\
\hline
\end{tabular}

ONSITE

\begin{tabular}{c|c} 
H & RAD_LEVEL \\
\end{tabular}

$116-\mathrm{N}-1$

116-N-1

$116-\mathrm{N}-1$

$116-\mathrm{N}-1$

116-N-1

$116-\mathrm{N}-1$

116-N-1

116-N-1

116-N-1

116-N-1

116-F-1

116-F-1

116-N-1

126-F-1

126-F-1

126-F-1

126-F-1

126-F-1

126-F-1

126-F-1

126-F-1

126-F-1

126-F-1

126-F-1

126-F-1

126-F-1

126-F-1

126-F-1

126-F-1

126-F-1

126-F-1

126-F-1

126-F-1

126-F-1

126-F-1

126-F-1

126-F-1

HEISPROD

126-F-1

I

SAMP SITE NAME 


\begin{tabular}{|c|c|c|c|c|c|c|c|c|c|}
\hline & $A$ & $\mathrm{~B}$ & $\mathrm{C}$ & $\mathrm{D}$ & $E$ & $\mathrm{~F}$ & G & $\mathrm{H}$ & $\mathrm{I}$ \\
\hline 1 & SAMP_SITE_ID & HORIZ_COORD_TYPE & EW_COORD & NS_COORD & OWNER_ID & COORD_SOURCE & DIST_CLASS & RAD_LEVEL & SAMP_SITE_NAME \\
\hline 5436 & 14287 & & & & HEISPROD & & & & 126-F-1 \\
\hline 5437 & 14288 & & & & HEISPROD & & & & 126-F-1 \\
\hline 5438 & 14289 & & & & HEISPROD & & & & 126-F-1 \\
\hline 5439 & 14290 & & & & HEISPROD & & & & 126-F-1 \\
\hline 5440 & 14291 & & & & HEISPROD & & & & 126-F-1 \\
\hline 5441 & 14292 & & & & HEISPROD & & & & 126-F-1 \\
\hline 5442 & 14293 & & & & HEISPROD & & & & 126-F-1 \\
\hline 5443 & 14294 & & & & HEISPROD & & & & 126-F-1 \\
\hline 5444 & 14295 & & & & HEISPROD & & & & 126-F-1 \\
\hline 5445 & 14296 & & & & HEISPROD & & & & 126-F-1 \\
\hline \begin{tabular}{|l|}
5446 \\
\end{tabular} & 14297 & & & & HEISPROD & & & & 126-F-1 \\
\hline 5447 & 14298 & & & & HEISPROD & & & & 126-F-1 \\
\hline 5448 & 14299 & & & & HEISPROD & & & & 126-F-1 \\
\hline 5449 & 14300 & & & & HEISPROD & & & & $126-F-1$ \\
\hline 5450 & 14301 & & & & HEISPROD & & & & 126-F-1 \\
\hline \begin{tabular}{|l|}
5451 \\
\end{tabular} & 14302 & UNKNOWN & 565265.072 & 145266.84 & 4 HEISPROD & GPS & ONSITE & & 100-B-8 \\
\hline 5452 & 14303 & UNKNOWN & 565274.656 & 145267.771 & 1 HEISPROD & GPS & ONSITE & & 100-B-8 \\
\hline 5453 & 14304 & UNKNOWN & 565284.751 & 145267.769 & 9 HEISPROD & GPS & ONSITE & & 100-B-8 \\
\hline 5454 & 14305 & UNKNOWN & 565261.504 & 145256.754 & 4 HEISPROD & GPS & ONSITE & & 100-B-8 \\
\hline 5455 & 14306 & UNKNOWN & 565250.67 & 145251.678 & 3 HEISPROD & GPS & ONSITE & & 100-B-8 \\
\hline \begin{tabular}{|l|}
5456 \\
\end{tabular} & 14307 & UNKNOWN & 565284.67 & 145251.857 & 7 HEISPROD & GPS & ONSITE & & 100-B-8 \\
\hline 5457 & 14308 & UNKNOWN & 565273.116 & 145246.606 & S HEISPROD & GPS & ONSITE & & 100-B-8 \\
\hline 5458 & 14309 & UNKNOWN & 565262.434 & 145241.406 & HEISPROD & GPS & ONSITE & & 100-B-8 \\
\hline 5459 & 14310 & UNKNOWN & 565273.261 & 145241.32 & 2 HEISPROD & GPS & ONSITE & & 100-B-8 \\
\hline 5460 & 14311 & UNKNOWN & 565263.027 & 145235.718 & 3 HEISPROD & GPS & ONSITE & & 100-B-8 \\
\hline 5461 & 14312 & UNKNOWN & 565263.537 & 145229.89 & 9 HEISPROD & GPS & ONSITE & & 100-B-8 \\
\hline 5462 & 14313 & UNKNOWN & 565273.639 & 145230.047 & 7 HEISPROD & GPS & ONSITE & & 100-B-8 \\
\hline 5463 & 14314 & UNKNOWN & 565254.073 & 145224.129 & 9 HEISPROD & GPS & ONSITE & & 100-B-8 \\
\hline 5464 & 14315 & UNKNOWN & 565264.215 & 145224.222 & 2 HEISPROD & GPS & ONSITE & & 100-B-8 \\
\hline 5465 & 14316 & UNKNOWN & 565283.827 & 145224.051 & 1 HEISPROD & GPS & ONSITE & & 100-B-8 \\
\hline 5466 & 14317 & UNKNOWN & 565254.861 & 145218.244 & 4 HEISPROD & GPS & ONSITE & & 100-B-8 \\
\hline \begin{tabular}{|l|}
5467 \\
\end{tabular} & 14318 & UNKNOWN & 565255.949 & 145212.204 & 4 HEISPROD & GPS & ONSITE & & 100-B-8 \\
\hline \begin{tabular}{|l|}
5468 \\
\end{tabular} & 14319 & UNKNOWN & 565274.897 & 145212.065 & 5 HEISPROD & GPS & ONSITE & & 100-B-8 \\
\hline 5469 & 14320 & UNKNOWN & 565275.568 & 145199.653 & 3 HEISPROD & GPS & ONSITE & & 100-B-8 \\
\hline 5470 & 14321 & UNKNOWN & 565284.856 & 145199.596 & SEISPROD & GPS & ONSITE & & 100-B-8 \\
\hline \begin{tabular}{|l|}
5471 \\
\end{tabular} & 14322 & UNKNOWN & 565277.342 & 145192.993 & 3 HEISPROD & GPS & ONSITE & & 100-B-8 \\
\hline 5472 & 14323 & UNKNOWN & 565267.006 & 145187.198 & 3 HEISPROD & GPS & ONSITE & & 100-B-8 \\
\hline 5473 & 14324 & UNKNOWN & 565267.006 & 145187.198 & 3 HEISPROD & GPS & ONSITE & & 100-B-8 \\
\hline
\end{tabular}




\begin{tabular}{|c|c|c|c|c|c|c|c|c|c|}
\hline & $A$ & $\mathrm{~B}$ & $\mathrm{C}$ & $\mathrm{D}$ & $E$ & $\mathrm{~F}$ & $\mathrm{G}$ & $\mathrm{H}$ & $\mathrm{I}$ \\
\hline 1 & SAMP_SITE_ID & HORIZ_COORD_TYPE & EW_COORD & NS_COORD & OWNER_ID & COORD_SOURCE & DIST_CLASS & RAD_LEVEL & SAMP_SITE_NAME \\
\hline 5474 & 14325 & UNKNOWN & 565286.532 & 145182.238 & HEISPROD & GPS & ONSITE & & 100-B-8 \\
\hline 5475 & 14326 & UNKNOWN & 565284.215 & 145169.154 & HEISPROD & GPS & ONSITE & & 100-B-8 \\
\hline 5476 & 14327 & UNKNOWN & 565289.789 & 145151.895 & HEISPROD & GPS & ONSITE & & 100-B-8 \\
\hline 5477 & 14328 & UNKNOWN & 565284.893 & 145131.078 & HEISPROD & GPS & ONSITE & & 100-B-8 \\
\hline 5478 & 14329 & UNKNOWN & 565288.398 & 145109.438 & HEISPROD & GPS & ONSITE & & $100-B-8$ \\
\hline 5479 & 14330 & UNKNOWN & 565288.908 & 145091.767 & HEISPROD & GPS & ONSITE & & 100-B-8 \\
\hline 5480 & 14331 & UNKNOWN & 565252.66 & 145023.283 & HEISPROD & GPS & ONSITE & & 100-B-8 \\
\hline 5481 & 14332 & UNKNOWN & 565267.563 & 145022.821 & HEISPROD & GPS & ONSITE & & 100-B-8 \\
\hline 5482 & 14333 & UNKNOWN & 565250.691 & 145017.122 & HEISPROD & GPS & ONSITE & & 100-B-8 \\
\hline 5483 & 14334 & UNKNOWN & 565262.283 & 145005.221 & HEISPROD & GPS & ONSITE & & 100-B-8 \\
\hline 5484 & 14335 & UNKNOWN & 565282.478 & 145004.655 & HEISPROD & GPS & ONSITE & & 100-B-8 \\
\hline 5485 & 14336 & UNKNOWN & 565252.556 & 144999.699 & HEISPROD & GPS & ONSITE & & 100-B-8 \\
\hline 5486 & 14337 & UNKNOWN & 565272.555 & 144999.241 & HEISPROD & GPS & ONSITE & & 100-B-8 \\
\hline 5487 & 14338 & UNKNOWN & 565282.72 & 144998.961 & HEISPROD & GPS & ONSITE & & 100-B-8 \\
\hline 5488 & 14339 & UNKNOWN & 565253.068 & 144993.983 & HEISPROD & GPS & ONSITE & & 100-B-8 \\
\hline 5489 & 14340 & UNKNOWN & 565253.068 & 144993.983 & HEISPROD & GPS & ONSITE & & 100-B-8 \\
\hline 5490 & 14341 & UNKNOWN & 565272.998 & 144993.34 & HEISPROD & GPS & ONSITE & & 100-B-8 \\
\hline 5491 & 14342 & UNKNOWN & 565283.266 & 144993.152 & HEISPROD & GPS & ONSITE & & 100-B-8 \\
\hline 5492 & 14343 & UNKNOWN & 565253.688 & 144987.949 & HEISPROD & GPS & ONSITE & & 100-B-8 \\
\hline 5493 & 14344 & UNKNOWN & 565283.364 & 144987.33 & HEISPROD & GPS & ONSITE & & 100-B-8 \\
\hline 5494 & 14345 & UNKNOWN & 565273.637 & 144981.698 & HEISPROD & GPS & ONSITE & & 100-B-8 \\
\hline 5495 & 14346 & UNKNOWN & 565263.855 & 144969.812 & HEISPROD & GPS & ONSITE & & 100-B-8 \\
\hline 5496 & 14347 & UNKNOWN & 565273.456 & 144969.63 & HEISPROD & GPS & ONSITE & & 100-B-8 \\
\hline 5497 & 14348 & UNKNOWN & 565283.022 & 144969.3 & HEISPROD & GPS & ONSITE & & 100-B-8 \\
\hline 5498 & 14349 & UNKNOWN & 565283.201 & 144963.193 & HEISPROD & GPS & ONSITE & & 100-B-8 \\
\hline 5499 & 14350 & UNKNOWN & 565283.339 & 144956.862 & HEISPROD & GPS & ONSITE & & 100-B-8 \\
\hline 5500 & 14351 & UNKNOWN & 565261.61 & 144951.277 & HEISPROD & GPS & ONSITE & & 100-B-8 \\
\hline 5501 & 14352 & UNKNOWN & 565437 & 144986 & HEISPROD & ESTIMATED & ONSITE & & $100-C-6$ \\
\hline 5502 & 14353 & UNKNOWN & 565437 & 144986 & HEISPROD & ESTIMATED & ONSITE & & $100-C-6$ \\
\hline 5503 & 14354 & UNKNOWN & 565347 & 145015 & HEISPROD & ESTIMATED & ONSITE & & 100-B-8 \\
\hline 5504 & 14355 & UNKNOWN & 565347 & 145015 & HEISPROD & ESTIMATED & ONSITE & & 100-B-8 \\
\hline 5505 & 14356 & UNKNOWN & 580798.64 & 147706.91 & HEISPROD & ESTIMATED & ONSITE & & 1607-F2 \\
\hline 5506 & 14357 & UNKNOWN & 580810.32 & 147712.37 & HEISPROD & ESTIMATED & ONSITE & & 1607-F2 \\
\hline 5507 & 14358 & UNKNOWN & 580808.35 & 147706.65 & HEISPROD & ESTIMATED & ONSITE & & 1607-F2 \\
\hline 5508 & 14359 & UNKNOWN & 580833.08 & 147706.82 & HEISPROD & ESTIMATED & ONSITE & & 1607-F2 \\
\hline 5509 & 14360 & UNKNOWN & 580852.85 & 147716.09 & HEISPROD & ESTIMATED & ONSITE & & 1607-F2 \\
\hline 5510 & 14361 & UNKNOWN & 580857.5 & 147707.13 & HEISPROD & ESTIMATED & ONSITE & & 1607-F2 \\
\hline 5511 & 14362 & UNKNOWN & 580875.97 & 147713.53 & HEISPROD & ESTIMATED & ONSITE & & 1607-F2 \\
\hline
\end{tabular}




\begin{tabular}{|c|c|c|c|c|c|c|c|c|c|}
\hline & $A$ & $\mathrm{~B}$ & $\mathrm{C}$ & $\mathrm{D}$ & $E$ & $\mathrm{~F}$ & $\mathrm{G}$ & $\mathrm{H}$ & $\mathrm{I}$ \\
\hline 1 & SAMP_SITE_ID & HORIZ_COORD_TYPE & EW_COORD & NS_COORD & OWNER_ID & COORD_SOURCE & DIST_CLASS & RAD_LEVEL & SAMP_SITE_NAME \\
\hline 5512 & 14363 & UNKNOWN & 580881.76 & 147706.22 & HEISPROD & ESTIMATED & ONSITE & & 1607-F2 \\
\hline 5513 & 14364 & UNKNOWN & 580891.4 & 147713.88 & HEISPROD & ESTIMATED & ONSITE & & 1607-F2 \\
\hline 5514 & 14365 & UNKNOWN & 580894.22 & 147706.84 & HEISPROD & ESTIMATED & ONSITE & & 1607-F2 \\
\hline 5515 & 14366 & UNKNOWN & 580899.01 & 147686.99 & HEISPROD & ESTIMATED & ONSITE & & 1607-F2 \\
\hline 5516 & 14367 & UNKNOWN & 580904.85 & 147685.04 & HEISPROD & ESTIMATED & ONSITE & & 1607-F2 \\
\hline 5517 & 14368 & UNKNOWN & 580899.88 & 147674.82 & HEISPROD & ESTIMATED & ONSITE & & $1607-F 2$ \\
\hline 5518 & 14369 & UNKNOWN & 580905.67 & 147674.19 & HEISPROD & ESTIMATED & ONSITE & & 1607-F2 \\
\hline 5519 & 14370 & UNKNOWN & 580900.64 & 147639.02 & HEISPROD & ESTIMATED & ONSITE & & 1607-F2 \\
\hline 5520 & 14371 & UNKNOWN & 580896.41 & 147650.26 & HEISPROD & ESTIMATED & ONSITE & & 1607-F2 \\
\hline 5521 & 14372 & UNKNOWN & 580889.43 & 147643.45 & HEISPROD & ESTIMATED & ONSITE & & 1607-F2 \\
\hline 5522 & 14373 & UNKNOWN & 580889.18 & 147626.75 & HEISPROD & ESTIMATED & ONSITE & & 1607-F2 \\
\hline 5523 & 14374 & UNKNOWN & 580874.73 & 147613.22 & HEISPROD & ESTIMATED & ONSITE & & 1607-F2 \\
\hline 5524 & 14375 & UNKNOWN & 580874.71 & 147604.45 & HEISPROD & ESTIMATED & ONSITE & & 1607-F2 \\
\hline 5525 & 14376 & UNKNOWN & 580867.26 & 147606 & HEISPROD & ESTIMATED & ONSITE & & 1607-F2 \\
\hline 5526 & 14377 & UNKNOWN & 580859.53 & 147613.98 & HEISPROD & ESTIMATED & ONSITE & & 1607-F2 \\
\hline 5527 & 14378 & UNKNOWN & 580859.6 & 147590.36 & HEISPROD & ESTIMATED & ONSITE & & 1607-F2 \\
\hline 5528 & 14379 & UNKNOWN & 580851.68 & 147606.22 & HEISPROD & ESTIMATED & ONSITE & & 1607-F2 \\
\hline 5529 & 14380 & UNKNOWN & 580850.77 & 147577.82 & HEISPROD & ESTIMATED & ONSITE & & 1607-F2 \\
\hline 5530 & 14381 & UNKNOWN & 580850.82 & 147568.16 & HEISPROD & ESTIMATED & ONSITE & & 1607-F2 \\
\hline 5531 & 14382 & UNKNOWN & 580850.86 & 147543.94 & HEISPROD & ESTIMATED & ONSITE & & 1607-F2 \\
\hline 5532 & 14383 & UNKNOWN & 580850.89 & 147528.3 & HEISPROD & ESTIMATED & ONSITE & & 1607-F2 \\
\hline 5533 & 14384 & UNKNOWN & 580857.38 & 147551.57 & HEISPROD & ESTIMATED & ONSITE & & 1607-F2 \\
\hline 5534 & 14385 & UNKNOWN & 580857.44 & 147535.61 & HEISPROD & ESTIMATED & ONSITE & & 1607-F2 \\
\hline 5535 & 14386 & UNKNOWN & 580863.84 & 147589.83 & HEISPROD & ESTIMATED & ONSITE & & 1607-F2 \\
\hline 5536 & 14387 & UNKNOWN & 580870.57 & 147587.45 & HEISPROD & ESTIMATED & ONSITE & & 1607-F2 \\
\hline 5537 & 14388 & UNKNOWN & 580870.64 & 147572.21 & HEISPROD & ESTIMATED & ONSITE & & 1607-F2 \\
\hline 5538 & 14389 & UNKNOWN & 580870.68 & 147564.47 & HEISPROD & ESTIMATED & ONSITE & & 1607-F2 \\
\hline 5539 & 14390 & UNKNOWN & 580870.75 & 147548.75 & HEISPROD & ESTIMATED & ONSITE & & 1607-F2 \\
\hline 5540 & 14391 & UNKNOWN & 580870.8 & 147539.27 & HEISPROD & ESTIMATED & ONSITE & & 1607-F2 \\
\hline 5541 & 14392 & UNKNOWN & 580877.26 & 147602.19 & HEISPROD & ESTIMATED & ONSITE & & 1607-F2 \\
\hline 5542 & 14393 & UNKNOWN & 580877.35 & 147585.01 & HEISPROD & ESTIMATED & ONSITE & & 1607-F2 \\
\hline 5543 & 14394 & UNKNOWN & 580877.38 & 147577.27 & HEISPROD & ESTIMATED & ONSITE & & 1607-F2 \\
\hline 5544 & 14395 & UNKNOWN & 580877.42 & 147569.61 & HEISPROD & ESTIMATED & ONSITE & & 1607-F2 \\
\hline 5545 & 14396 & UNKNOWN & 580877.53 & 147545.39 & HEISPROD & ESTIMATED & ONSITE & & 1607-F2 \\
\hline 5546 & 14397 & UNKNOWN & 580884.15 & 147592.6 & HEISPROD & ESTIMATED & ONSITE & & 1607-F2 \\
\hline 5547 & 14398 & UNKNOWN & 580890.99 & 147605.58 & HEISPROD & ESTIMATED & ONSITE & & 1607-F2 \\
\hline 5548 & 14399 & UNKNOWN & 580891.03 & 147597.91 & HEISPROD & ESTIMATED & ONSITE & & 1607-F2 \\
\hline 5549 & 14400 & UNKNOWN & 580891.06 & 147590.32 & HEISPROD & ESTIMATED & ONSITE & & 1607-F2 \\
\hline
\end{tabular}




\begin{tabular}{|c|c|c|c|c|c|c|c|c|c|}
\hline & $A$ & $\mathrm{~B}$ & $\mathrm{C}$ & $\mathrm{D}$ & $E$ & $\mathrm{~F}$ & $\mathrm{G}$ & $\mathrm{H}$ & $\mathrm{I}$ \\
\hline 1 & SAMP_SITE_ID & HORIZ_COORD_TYPE & EW_COORD & NS_COORD & OWNER_ID & COORD_SOURCE & DIST_CLASS & RAD_LEVEL & SAMP_SITE_NAME \\
\hline 5550 & 14401 & NAD83(91) & 580891.21 & 147559.01 & HEISPROD & ESTIMATED & ONSITE & & 1607-F2 \\
\hline 5551 & 14402 & UNKNOWN & 580898.01 & 147598.43 & HEISPROD & ESTIMATED & ONSITE & & 1607-F2 \\
\hline 5552 & 14403 & UNKNOWN & 580898.04 & 147590.99 & HEISPROD & ESTIMATED & ONSITE & & 1607-F2 \\
\hline 5553 & 14404 & UNKNOWN & 565279.113 & 145315.507 & HEISPROD & ESTIMATED & ONSITE & & 100-B-8 \\
\hline 5554 & 14405 & UNKNOWN & 565285.507 & 145316.005 & HEISPROD & ESTIMATED & ONSITE & & $100-B-8$ \\
\hline 5555 & 14406 & UNKNOWN & 565291.696 & 145315.403 & HEISPROD & ESTIMATED & ONSITE & & 100-B-8 \\
\hline 5556 & 14407 & UNKNOWN & 565445.91 & 145388.99 & HEISPROD & ESTIMATED & ONSITE & & 100-B-8 \\
\hline 5557 & 14408 & UNKNOWN & 565472.898 & 145387.915 & HEISPROD & ESTIMATED & ONSITE & & $100-C-6$ \\
\hline 5558 & 14409 & UNKNOWN & 565474.494 & 145383.418 & HEISPROD & ESTIMATED & ONSITE & & $100-C-6$ \\
\hline 5559 & 14410 & UNKNOWN & 565458.088 & 145363 & HEISPROD & ESTIMATED & ONSITE & & $100-C-6$ \\
\hline 5560 & 14411 & UNKNOWN & 565467.408 & 145349.229 & HEISPROD & ESTIMATED & ONSITE & & $100-C-6$ \\
\hline 5561 & 14412 & UNKNOWN & 565464.158 & 145340.251 & HEISPROD & ESTIMATED & ONSITE & & $100-C-6$ \\
\hline 5562 & 14413 & UNKNOWN & 565325.503 & 145325.503 & HEISPROD & ESTIMATED & ONSITE & & $100-C-6$ \\
\hline 5563 & 14414 & UNKNOWN & 565476.191 & 145310.081 & HEISPROD & ESTIMATED & ONSITE & & $100-C-6$ \\
\hline 5564 & 14415 & UNKNOWN & 565469.221 & 145301.034 & HEISPROD & ESTIMATED & ONSITE & & $100-C-6$ \\
\hline 5565 & 14416 & UNKNOWN & 565468.138 & 145371.842 & HEISPROD & ESTIMATED & ONSITE & & $100-C-6$ \\
\hline 5566 & 14417 & UNKNOWN & 565471.816 & 145372.6 & HEISPROD & ESTIMATED & ONSITE & & $100-C-6$ \\
\hline 5567 & 14418 & UNKNOWN & 565476.681 & 145360.704 & HEISPROD & ESTIMATED & ONSITE & & $100-C-6$ \\
\hline 5568 & 14419 & UNKNOWN & 565473.361 & 145352.197 & HEISPROD & ESTIMATED & ONSITE & & $100-C-6$ \\
\hline 5569 & 14420 & UNKNOWN & 565477.945 & 145336.754 & HEISPROD & ESTIMATED & ONSITE & & $100-C-6$ \\
\hline 5570 & 14421 & UNKNOWN & 565480.341 & 145329.552 & HEISPROD & ESTIMATED & ONSITE & & $100-C-6$ \\
\hline \begin{tabular}{|l|}
5571 \\
\end{tabular} & 14422 & UNKNOWN & 565500.281 & 145312.79 & HEISPROD & ESTIMATED & ONSITE & & $100-C-6$ \\
\hline 5572 & 14423 & UNKNOWN & 565500.206 & 145304.729 & HEISPROD & ESTIMATED & ONSITE & & 100-C-6 \\
\hline 5573 & 14424 & UNKNOWN & 565518.057 & 145305.733 & HEISPROD & ESTIMATED & ONSITE & & $100-C-6$ \\
\hline 5574 & 14425 & UNKNOWN & 565482.412 & 145301.534 & HEISPROD & ESTIMATED & ONSITE & & $100-C-6$ \\
\hline 5575 & 14426 & UNKNOWN & 565512.85 & 145295.954 & HEISPROD & ESTIMATED & ONSITE & & $100-C-6$ \\
\hline 5576 & 14427 & UNKNOWN & 565483.09 & 145295.957 & HEISPROD & ESTIMATED & ONSITE & & $100-C-6$ \\
\hline 5577 & 14428 & UNKNOWN & 565469.579 & 145291.862 & HEISPROD & ESTIMATED & ONSITE & & $100-C-6$ \\
\hline 5578 & 14429 & UNKNOWN & 565476.846 & 145290.511 & HEISPROD & ESTIMATED & ONSITE & & $100-C-6$ \\
\hline 5579 & 14430 & UNKNOWN & 565470.088 & 145283.979 & HEISPROD & ESTIMATED & ONSITE & & $100-C-6$ \\
\hline 5580 & 14431 & UNKNOWN & 565488.259 & 145281.332 & HEISPROD & ESTIMATED & ONSITE & & $100-C-6$ \\
\hline 5581 & 14432 & UNKNOWN & 565468.8 & 145271.842 & HEISPROD & ESTIMATED & ONSITE & & $100-C-6$ \\
\hline 5582 & 14433 & UNKNOWN & 565487.033 & 145269.732 & HEISPROD & ESTIMATED & ONSITE & & $100-C-6$ \\
\hline 5583 & 14434 & UNKNOWN & 565495.894 & 145264.946 & HEISPROD & ESTIMATED & ONSITE & & $100-C-6$ \\
\hline 5584 & 14435 & UNKNOWN & 565312.82 & 145225.616 & HEISPROD & ESTIMATED & ONSITE & & 100-B-8 \\
\hline 5585 & 14436 & UNKNOWN & 565312.12 & 145212.671 & HEISPROD & ESTIMATED & ONSITE & & 100-B-8 \\
\hline 5586 & 14437 & UNKNOWN & 565311.966 & 145206.314 & HEISPROD & ESTIMATED & ONSITE & & 100-B-8 \\
\hline 5587 & 14438 & UNKNOWN & 565311.458 & 145193.499 & HEISPROD & ESTIMATED & ONSITE & & 100-B-8 \\
\hline
\end{tabular}




\begin{tabular}{|c|c|c|c|c|c|c|c|c|c|}
\hline & $\mathrm{A}$ & $B$ & $C$ & $\mathrm{D}$ & $E$ & $\mathrm{~F}$ & $G$ & $\mathrm{H}$ & $\mathrm{I}$ \\
\hline 1 & SAMP_SITE_ID & HORIZ_COORD_TYPE & EW_COORD & NS_COORD & OWNER_ID & COORD_SOURCE & DIST_CLASS & RAD_LEVEL & SAMP_SITE_NAME \\
\hline 5588 & 14439 & UNKNOWN & 565311.607 & 145186.817 & HEISPROD & ESTIMATED & ONSITE & & 100-B-8 \\
\hline 5589 & 14440 & UNKNOWN & 565300.057 & 145264.728 & HEISPROD & ESTIMATED & ONSITE & & 100-B-8 \\
\hline 5590 & 14441 & UNKNOWN & 565304.29 & 145252.155 & HEISPROD & ESTIMATED & ONSITE & & 100-B-8 \\
\hline 5591 & 14442 & UNKNOWN & 565299.202 & 145248.025 & HEISPROD & ESTIMATED & ONSITE & & 100-B-8 \\
\hline 5592 & 14443 & UNKNOWN & 565306.083 & 145244.428 & HEISPROD & ESTIMATED & ONSITE & & $100-B-8$ \\
\hline 5593 & 14444 & UNKNOWN & 565299.333 & 145230.679 & HEISPROD & ESTIMATED & ONSITE & & 100-B-8 \\
\hline 5594 & 14445 & UNKNOWN & 565304.56 & 145218.258 & HEISPROD & ESTIMATED & ONSITE & & 100-B-8 \\
\hline 5595 & 14446 & UNKNOWN & 565310.597 & 145182.058 & HEISPROD & ESTIMATED & ONSITE & & 100-B-8 \\
\hline 5596 & 14447 & UNKNOWN & 565301.145 & 145173.281 & HEISPROD & ESTIMATED & ONSITE & & 100-B-8 \\
\hline \begin{tabular}{|l|}
5597 \\
\end{tabular} & 14448 & UNKNOWN & 565311.131 & 145176.501 & HEISPROD & ESTIMATED & ONSITE & & 100-B-8 \\
\hline 5598 & 14449 & UNKNOWN & 565312.37 & 145165.123 & HEISPROD & ESTIMATED & ONSITE & & 100-B-8 \\
\hline 5599 & 14450 & UNKNOWN & 565314.123 & 145155.953 & HEISPROD & ESTIMATED & ONSITE & & 100-B-8 \\
\hline 5600 & 14451 & UNKNOWN & 565302.955 & 145145.481 & HEISPROD & ESTIMATED & ONSITE & & 100-B-8 \\
\hline 5601 & 14452 & UNKNOWN & 565308.978 & 145132.451 & HEISPROD & ESTIMATED & ONSITE & & 100-B-8 \\
\hline 5602 & 14453 & UNKNOWN & 565314.343 & 145133.59 & HEISPROD & ESTIMATED & ONSITE & & 100-B-8 \\
\hline 5603 & 14454 & UNKNOWN & 565299.878 & 145120.873 & HEISPROD & ESTIMATED & ONSITE & & 100-B-8 \\
\hline 5604 & 14455 & UNKNOWN & 565310.235 & 145109.613 & HEISPROD & ESTIMATED & ONSITE & & 100-B-8 \\
\hline 5605 & 14456 & NAD83(91) & 565301.117 & 145094.102 & HEISPROD & ESTIMATED & ONSITE & & 100-B-8 \\
\hline 5606 & 14457 & UNKNOWN & 565301.383 & 145078.024 & HEISPROD & ESTIMATED & ONSITE & & 100-B-8 \\
\hline \begin{tabular}{|l|}
5607 \\
\end{tabular} & 14458 & UNKNOWN & 565299.45 & 145055.587 & HEISPROD & ESTIMATED & ONSITE & & 100-B-8 \\
\hline \begin{tabular}{|l|}
5608 \\
\end{tabular} & 14459 & UNKNOWN & 565304.362 & 145045.48 & HEISPROD & ESTIMATED & ONSITE & & 100-B-8 \\
\hline 5609 & 14460 & UNKNOWN & 565304.966 & 145019.74 & HEISPROD & ESTIMATED & ONSITE & & 100-B-8 \\
\hline 5610 & 14461 & UNKNOWN & 565305.394 & 145010.255 & HEISPROD & ESTIMATED & ONSITE & & 100-B-8 \\
\hline 5611 & 14462 & UNKNOWN & 565305.658 & 144993.357 & HEISPROD & ESTIMATED & ONSITE & & 100-B-8 \\
\hline 5612 & 14463 & UNKNOWN & 565306.282 & 144985.378 & HEISPROD & ESTIMATED & ONSITE & & 100-B-8 \\
\hline 5613 & 14464 & UNKNOWN & 565299.575 & 144975.153 & HEISPROD & ESTIMATED & ONSITE & & 100-B-8 \\
\hline 5614 & 14465 & UNKNOWN & 565299.944 & 144968.23 & HEISPROD & ESTIMATED & ONSITE & & 100-B-8 \\
\hline 5615 & 14466 & UNKNOWN & 565300.01 & 144961.052 & HEISPROD & ESTIMATED & ONSITE & & 100-B-8 \\
\hline 5616 & 14467 & UNKNOWN & 565299.475 & 144914.385 & HEISPROD & ESTIMATED & ONSITE & & 100-B-8 \\
\hline 5617 & 14468 & UNKNOWN & 565313.574 & 144931.739 & HEISPROD & ESTIMATED & ONSITE & & 100-B-8 \\
\hline 5618 & 14469 & UNKNOWN & 565312.542 & 144906.553 & HEISPROD & ESTIMATED & ONSITE & & 100-B-8 \\
\hline 5619 & 14470 & UNKNOWN & 565310.762 & 144889.714 & HEISPROD & ESTIMATED & ONSITE & & 100-B-8 \\
\hline 5620 & 14471 & UNKNOWN & 565301.953 & 144886.585 & HEISPROD & ESTIMATED & ONSITE & & 100-B-8 \\
\hline 5621 & 14472 & UNKNOWN & 565300.032 & 144880.158 & HEISPROD & ESTIMATED & ONSITE & & 100-B-8 \\
\hline 5622 & 14473 & UNKNOWN & 565300.189 & 144860.839 & HEISPROD & ESTIMATED & ONSITE & & 100-B-8 \\
\hline 5623 & 14474 & UNKNOWN & 565300.636 & 144833.131 & HEISPROD & ESTIMATED & ONSITE & & 100-B-8 \\
\hline 5624 & 14475 & UNKNOWN & 565300.563 & 144825.347 & HEISPROD & ESTIMATED & ONSITE & & 100-B-8 \\
\hline \begin{tabular}{|l|}
5625 \\
\end{tabular} & 14476 & UNKNOWN & 565307.341 & 145092.251 & HEISPROD & ESTIMATED & ONSITE & & 100-B-8 \\
\hline
\end{tabular}


A

$\mathrm{B}$

\begin{tabular}{l|l}
$\mathrm{C}$ & $\mathrm{D}$ \\
\hline
\end{tabular}

$\mathrm{E}$ \begin{tabular}{l|l|l|l|}
\hline EW_COORD & NS_COORD & OWNER_ID & COORD_SOURCE
\end{tabular} 14477 UNKNOWN

14478 UNKNOWN

565311.543145091 .669 HEISPROD ESTIMATED

14479 UNKNOWN

14480 UNKNOWN

\begin{tabular}{l|l}
565322.931 & 145089.239 \\
$5 E I S P R O D$ & ESTIMATED
\end{tabular}

\begin{tabular}{|l|l|l}
565308.107 & 145080.686 HEISPROD & ESTIMATED
\end{tabular}

\begin{tabular}{|l|l|l}
565309.043 & 145072.159 HEISPROD & ESTIMATED
\end{tabular}

14481 UNKNOWN

14482 UNKNOWN

14483 UNKNOWN

565325.18145071 .461 HEISPROD ESTIMATED

\begin{tabular}{l|l}
565309.186 & 145043.89 HEISPROD \\
\hline
\end{tabular}

$565309.344 \quad 145034.266$ HEISPROD ESTIMATED

565323.679145018 .088 HEISPROD ESTIMATED

14484 UNKNOWN

14485 UNKNOWN

14486 UNKNOWN

14487 UNKNOWN

\begin{tabular}{|l|l|l}
565313.816 & 145009.141 HEISPROD & ESTIMATED
\end{tabular}

565324.285145004 .955 HEISPROD ESTIMATED

565314.5 144998.08 HEISPROD ESTIMATED

\begin{tabular}{|l|r|r|r|}
\hline 14488 & 565314.462 & 144985.958 \\
HEISPROD & ESTIMATED
\end{tabular}

14489 UNKNOWN

14490 UNKNOWN

$\begin{array}{lll}565323.391 & 144986.958 \text { HEISPROD } & \text { ESTIMATED }\end{array}$

$565322.567 \quad 144954.121$ HEISPROD ESTIMATED

565326.94144967 .268 HEISPROD ESTIMATED

14491 UNKNOWN

14492 UNKNOWN

14493 UNKNOWN

14494 UNKNOWN

565336.938 144981.161 HEISPROD ESTIMATED

$\begin{array}{lll}565343.086 & 144963.287 \text { HEISPROD } & \text { ESTIMATED }\end{array}$

$565324.408 \quad 144942883$ HEISPROD ESTIMATED

14495 UNKNOWN

14496 UNKNOWN

14497 UNKNOWN

565332.318144901 .695 HEISPROD ESTIMATED

\begin{tabular}{|l|l|l|}
565332.977 & 144890.602 \\
HEISPROD & ESTIMATED
\end{tabular}

565327.55144882 .456 HEISPROD ESTIMATED

14498 UNKNOWN

$\begin{array}{lll}565329.081 & 144869.855 \\ & \text { HEISPROD } & \text { ESTIMATED }\end{array}$

14499 UNKNOWN

\begin{tabular}{|l|l|l|l}
565329.081 & 144869.855 & HEISPROD & ESTIMATED \\
\hline 565333.868 & 144870.283 HEISPROD & ESTIMATED
\end{tabular}

14500

HEISPROD

HEISPROD

\begin{tabular}{|l|l|l|}
\hline 14501 & HEISPROD \\
\hline 14502 & & HEISPROD \\
\hline 14503 & HEISPROD
\end{tabular}

14504

HEISPROD

HEISPROD

14505

14506

14507

HEISPROD

HEISPROD

HEISPROD

14508

HEISPROD

14509

14511 UNKNOWN

14512 UNKNOWN HEISPROD

14513 UNKNOWN

14514 UNKNOWN

571524

571524

149801 HEISPROD ESTIMATED

149801 HEISPROD ESTIMATED

$571524 \quad 149801$ HEISPROD ESTIMATED

149801 HEISPROD ESTIMATED

G

$\mathrm{H}$

I

DIST_CLASS $\quad$ RAD_LEVEL

ONSITE

SAMP_SITE_NAME

ONSITE

ONSITE

\begin{tabular}{l|l}
$100-B-8$ \\
$100-B-8$
\end{tabular}

ONSITE $100-B-8$

\begin{tabular}{l|l}
\hline ONSITE & $100-\mathrm{B}-8$ \\
\hline
\end{tabular}

ONSITE $100-B-8$

ONSITE $100-B-8$

\begin{tabular}{l|l} 
ONSITE & $100-\mathrm{B}-8$ \\
\hline
\end{tabular}

ONSITE $100-B-8$

ONSITE $100-B-8$

\begin{tabular}{l|l} 
ONSITE & $100-B-8$
\end{tabular}

\begin{tabular}{|l|l|}
\hline ONSITE & $100-B-8$ \\
\hline
\end{tabular}

ONSITE $100-B-8$

ONSITE $100-B-8$

\begin{tabular}{l|l}
\hline ONSITE & $100-\mathrm{B}-8$ \\
\hline
\end{tabular}

\begin{tabular}{l|l} 
ONSITE & $100-B-8$
\end{tabular}

ONSITE $100-B-8$

\begin{tabular}{l|l} 
ONSITE & $100-B-8$
\end{tabular}

\begin{tabular}{|l|l|}
\hline ONSITE & $100-B-8$ \\
\hline
\end{tabular}

ONSITE $100-B-8$

ONSITE $100-\mathrm{B}-8$

\begin{tabular}{|l|l|}
\hline ONSITE & $100-\mathrm{B}-8$ \\
\hline
\end{tabular}

100-B-8

1607-F2

1607-F2

$1607-\mathrm{F} 2$

1607-F2

1607-F2

1607-F2

1607-F6

1607-F6

1607-F2

1607-F2

ONSITE

$116-\mathrm{N}-1$

ONSITE

ONSITE

ONSITE

$116-\mathrm{N}-1$

$116-\mathrm{N}-1$

\begin{tabular}{rrr}
571524 & $149801 \mathrm{HEISPROD}$ & ESTIMATED \\
\hline & Page 149
\end{tabular}

$116-\mathrm{N}-1$

(n)

(20)

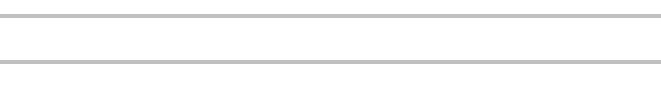

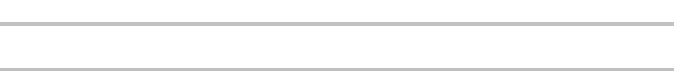

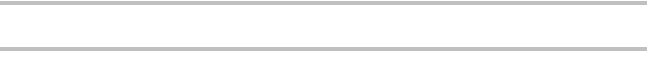

(2)

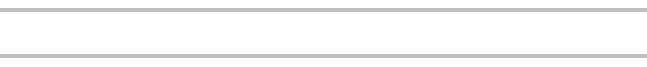

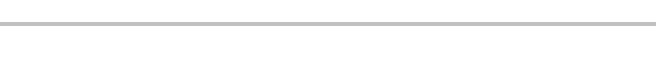

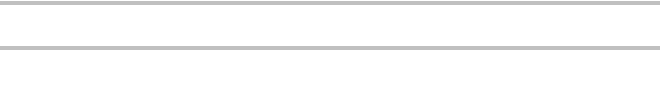

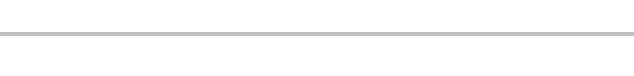

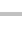

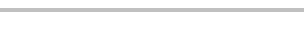

$+2$

rar

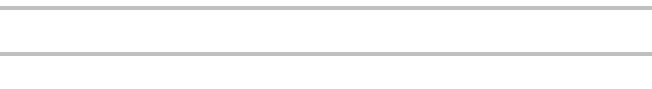

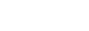


A

B

C 1 D

\begin{tabular}{|l|l|l|}
$\mathrm{D}$ & $\mathrm{E}$ & $\mathrm{F}$ \\
\hline
\end{tabular} F 14515 UNKNOWN

14516 UNKNOWN 571690

149866 HEISPROD ESTIMATED

$\begin{array}{ll}571712 & 149854 \text { HEISPROD } \\ 571702 & \text { ESTIMATED }\end{array}$ 14517 UNKNOWN

14518 UNKNOWN 149885 HEISPROD ESTIMATED

\begin{tabular}{ll}
571727 & 149871 HEISPROD \\
\hline
\end{tabular}

\begin{tabular}{|l|l|l|l}
14519 UNKNOWN & 571688 & 149830 HEISPROD & ESTIMATED
\end{tabular}

14520 UNKNOWN

14521 UNKNOWN

571702149812 HEISPROD ESTIMATED

14522 UNKNOWN

149809 HEISPROD ESTIMATED

571699149792 HEISPROD ESTIMATED

14523 UNKNOWN

14524 UNKNOWN

14525 UNKNOWN

\begin{tabular}{llll}
571699 & 149792 HEISPROD & ESTIMATED \\
\hline 571539 & 149739 HEISPROD & ESTIMATED
\end{tabular}

571535149737 HEISPROD ESTIMATED

573530149738 HEISPROD ESTIMATED

14526 UNKNOWN

14527 NAD83(91)

571525149745 HEISPROD ESTIMATED

14528 UNKNOWN

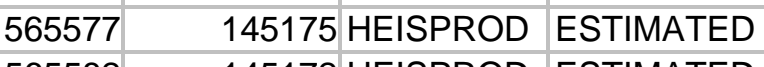

565582145172 HEISPROD ESTIMATED

14529 NAD83(91)

145178 HEISPROD ESTIMATED

\begin{tabular}{llll}
565603 & 145178 HEISPROD & ESTIMATED \\
\hline 565670 & 145204 HEISPROD & ESTIMATED
\end{tabular}

565635145211 HEISPROD ESTIMATED

14531 UNKNOWN

14532 UNKNOWN

145225 HEISPROD ESTIMATED

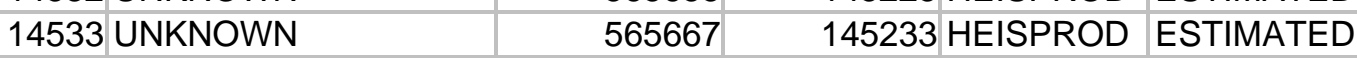

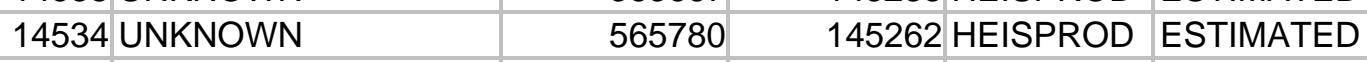

14535 UNKNOWN

14536 UNKNOWN

14537 UNKNOWN

14538 UNKNOWN

14539 UNKNOWN

145262 HEISPROD ESTIMATED

$565717 \quad 145345$ HEISPROD ESTIMATED

$565724 \quad 145472$ HEISPROD ESTIMATED

$565300.857 \quad 144795.287$ HEISPROD ESTIMATED

$565300.648 \quad 144787.925$ HEISPROD ESTIMATED

$565300.607 \quad 144780.354$ HEISPROD ESTIMATED

565313.908 144883.285 HEISPROD ESTIMATED

565311.804144856 .207 HEISPROD ESTIMATED

565313.165144811 .047 HEISPROD ESTIMATED

565319.569 144813.201 HEISPROD ESTIMATED

$565322.224 \quad 144782.685$ HEISPROD ESTIMATED

565330.614144853 .375 HEISPROD ESTIMATED

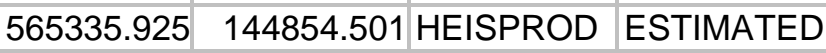

$565331.596 \quad 144842.491$ HEISPROD ESTIMATED

565339.484144820 .499 HEISPROD ESTIMATED

565342.044144797 .566 HEISPROD ESTIMATED

$\begin{array}{llll}565343.409 & 144785.068 \text { HEISPROD } & \text { ESTIMATED }\end{array}$

G

$\mathrm{H}$

I

\begin{tabular}{|l|r|}
\hline \multicolumn{1}{|c|}{ DIST_CLASS } & RAD_LEVEL \\
\hline ONSITE & \\
\hline ONSITE & \\
\hline ONSITE & \\
\hline ONSITE
\end{tabular}

ONSITE

ONSITE

ONSITE

ONSITE

ONSITE

ONSITE

ONSITE

ONSITE

ONSITE

ONSITE

ONSITE

ONSITE

ONSITE

ONSITE

ONSITE

ONSITE

ONSITE

ONSITE

ONSITE

ONSITE

ONSITE

ONSITE

ONSITE

ONSITE

ONSITE

ONSITE

ONSITE

ONSITE

ONSITE

ONSITE

ONSITE

OFFSITE

ONSITE

14549 NAD83(91)

ONSITE

14551 UNKNOWN

14552 UNKNOWN

$\begin{array}{lll}565483.598 & 145199.477 \mathrm{HEISPROD} & \text { ESTIMATED }\end{array}$

ONSITE

ONSITE

$116-\mathrm{N}-1$

I

116-N-1

$116-\mathrm{N}-1$

116-N-1

$116-\mathrm{N}-1$

116-N-1

$116-\mathrm{N}-1$

$116-\mathrm{N}-1$

116-N-1

$116-\mathrm{N}-1$

$116-\mathrm{N}-1$

$116-\mathrm{N}-1$

100-C-6

100-C-6

100-C-6

100-C-6

100-C-6

100-C-6

100-C-6

100-C-6

100-C-6

132-C-2

132-C-2

100-B-8

100-B-8

100-B-8

100-B-8

100-B-8

100-B-8

100-B-8

100-B-8

100-B-8

100-B-8

100-B-8

100-B-8

100-B-8

Page 150

100-C-6 


\begin{tabular}{|c|c|c|c|c|c|c|c|c|c|}
\hline & $A$ & $\mathrm{~B}$ & $\mathrm{C}$ & $\mathrm{D}$ & $E$ & $\mathrm{~F}$ & $\mathrm{G}$ & $\mathrm{H}$ & $\mathrm{I}$ \\
\hline 1 & SAMP_SITE_ID & HORIZ_COORD_TYPE & EW_COORD & NS_COORD & OWNER_ID & COORD_SOURCE & DIST_CLASS & RAD_LEVEL & SAMP_SITE_NAME \\
\hline 5702 & 14553 & UNKNOWN & 565483.264 & 145193.264 & HEISPROD & ESTIMATED & ONSITE & & 100-B-8 \\
\hline 5703 & 14554 & UNKNOWN & 565483.553 & 145234.268 & HEISPROD & ESTIMATED & ONSITE & & $100-C-6$ \\
\hline 5704 & 14555 & UNKNOWN & 565484.786 & 145249.309 & HEISPROD & ESTIMATED & ONSITE & & $100-C-6$ \\
\hline 5705 & 14556 & UNKNOWN & 565476.272 & 145245.352 & HEISPROD & ESTIMATED & ONSITE & & $100-C-6$ \\
\hline 5706 & 14557 & UNKNOWN & 565470.363 & 145249.239 & HEISPROD & ESTIMATED & ONSITE & & $100-C-6$ \\
\hline 5707 & 14558 & UNKNOWN & 565494.744 & 145256.425 & HEISPROD & ESTIMATED & ONSITE & & $100-C-6$ \\
\hline 5708 & 14559 & UNKNOWN & 565501.409 & 145259.216 & HEISPROD & ESTIMATED & ONSITE & & $100-C-6$ \\
\hline 5709 & 14560 & UNKNOWN & 565493.471 & 145222.554 & HEISPROD & ESTIMATED & ONSITE & & $100-C-6$ \\
\hline 5710 & 14561 & UNKNOWN & 565498.577 & 145205.995 & HEISPROD & ESTIMATED & ONSITE & & $100-C-6$ \\
\hline 5711 & 14562 & UNKNOWN & 565697.324 & 145471.622 & HEISPROD & ESTIMATED & ONSITE & & $100-C-6$ \\
\hline 5712 & 14563 & UNKNOWN & 565699.363 & 145459.052 & HEISPROD & ESTIMATED & ONSITE & & $100-C-6$ \\
\hline 5713 & 14564 & UNKNOWN & 565700.826 & 145440.271 & HEISPROD & ESTIMATED & ONSITE & & $100-C-6$ \\
\hline 5714 & 14565 & UNKNOWN & 565706.657 & 145446.589 & HEISPROD & ESTIMATED & ONSITE & & $100-C-6$ \\
\hline 5715 & 14566 & UNKNOWN & 565708.119 & 145430.378 & HEISPROD & ESTIMATED & ONSITE & & $100-C-6$ \\
\hline 5716 & 14567 & UNKNOWN & 565708.066 & 145412.634 & HEISPROD & ESTIMATED & ONSITE & & $100-C-6$ \\
\hline \begin{tabular}{|l|}
5717 \\
\end{tabular} & 14568 & UNKNOWN & 565703.667 & 145399.74 & HEISPROD & ESTIMATED & ONSITE & & $100-C-6$ \\
\hline 5718 & 14569 & UNKNOWN & 565712.736 & 145454.739 & HEISPROD & ESTIMATED & ONSITE & & $100-C-6$ \\
\hline 5719 & 14570 & UNKNOWN & 565736.123 & 145468.62 & HEISPROD & ESTIMATED & ONSITE & & $100-C-6$ \\
\hline 5720 & 14571 & UNKNOWN & 565730.526 & 145464.544 & HEISPROD & ESTIMATED & ONSITE & & $100-C-6$ \\
\hline \begin{tabular}{|l|}
5721 \\
\end{tabular} & 14572 & UNKNOWN & 565712.554 & 145437.337 & HEISPROD & ESTIMATED & ONSITE & & $100-C-6$ \\
\hline 5722 & 14573 & UNKNOWN & 565715.164 & 145422.349 & HEISPROD & ESTIMATED & ONSITE & & $100-C-6$ \\
\hline 5723 & 14574 & UNKNOWN & 565702.177 & 145377.73 & HEISPROD & ESTIMATED & ONSITE & & $100-C-6$ \\
\hline 5724 & 14575 & UNKNOWN & 565707.95 & 145380.828 & HEISPROD & ESTIMATED & ONSITE & & $100-C-6$ \\
\hline 5725 & 14576 & UNKNOWN & 565697.646 & 145353.692 & HEISPROD & ESTIMATED & ONSITE & & $100-C-6$ \\
\hline 5726 & 14577 & UNKNOWN & 565706.33 & 145338.487 & HEISPROD & ESTIMATED & ONSITE & & $100-C-6$ \\
\hline 5727 & 14578 & UNKNOWN & 565698.35 & 145340.592 & HEISPROD & ESTIMATED & ONSITE & & $100-C-6$ \\
\hline 5728 & 14579 & UNKNOWN & 565702.165 & 145316.197 & HEISPROD & ESTIMATED & ONSITE & & $100-C-6$ \\
\hline 5729 & 14580 & UNKNOWN & 565700.715 & 145295.039 & HEISPROD & ESTIMATED & ONSITE & & $100-C-6$ \\
\hline 5730 & 14581 & UNKNOWN & 565700.034 & 145283.711 & HEISPROD & ESTIMATED & ONSITE & & $100-C-6$ \\
\hline 5731 & 14582 & UNKNOWN & 565673.983 & 145264.485 & HEISPROD & ESTIMATED & ONSITE & & $100-C-6$ \\
\hline 5732 & 14583 & NAD83(91) & 565640.106 & 145205.079 & HEISPROD & ESTIMATED & ONSITE & & $100-C-6$ \\
\hline 5733 & 14584 & UNKNOWN & 565649.494 & 145207.132 & HEISPROD & ESTIMATED & ONSITE & & $100-C-6$ \\
\hline 5734 & 14585 & UNKNOWN & 565659.095 & 145210.782 & HEISPROD & ESTIMATED & ONSITE & & $100-C-6$ \\
\hline 5735 & 14586 & UNKNOWN & 565667.194 & 145214.712 & HEISPROD & ESTIMATED & ONSITE & & $100-C-6$ \\
\hline 5736 & 14587 & NAD83(91) & 565664.884 & 145222.217 & HEISPROD & ESTIMATED & ONSITE & & $100-C-6$ \\
\hline \begin{tabular}{|l|}
5737 \\
\end{tabular} & 14588 & UNKNOWN & 565674.013 & 145218.381 & HEISPROD & ESTIMATED & ONSITE & & $100-C-6$ \\
\hline 5738 & 14589 & UNKNOWN & 565680.239 & 145222.209 & HEISPROD & ESTIMATED & ONSITE & & $100-C-6$ \\
\hline 5739 & 14590 & UNKNOWN & 565676.22 & 145229.445 & HEISPROD & ESTIMATED & ONSITE & & 100-C-6 \\
\hline
\end{tabular}


A

$\mathrm{B}$

$\mathrm{C}$

D

$\mathrm{E}$

\begin{tabular}{|l|c|}
\hline & F \\
\hline
\end{tabular}
14591 UNKNOWN 14592 UNKNOWN 14593 NAD83(91) $\begin{array}{ll}565686.424 & 145225.255 \text { HEISPROD }\end{array}$ \begin{tabular}{|l|l|l|l|}
\hline 565686.424 & 145225.255 & HEISPROD & ESTIMATED \\
\hline 565686.567 & 145236.247 HEISPROD & ESTIMATED
\end{tabular} $\begin{array}{lll}565701.076 & 145232.077 \text { HEISPROD } & \text { ESTIMATED }\end{array}$ 14594 UNKNOWN 14595 UNKNOWN 14596 UNKNOWN 14597 UNKNOWN $565712.172 \quad 145239.612$ HEISPROD ESTIMATED $\begin{array}{ll}565712.766 & 145253.237 \text { HEISPROD } \\ \text { ESTIMATED }\end{array}$ \begin{tabular}{|l|l|l}
565717.016 & 145242.056 HEISPROD & ESTIMATED
\end{tabular} 565726.187 145243.94 HEISPROD ESTIMATED 565739.259145247 .589 HEISPROD ESTIMATED \begin{tabular}{l|l|l}
565745.525 & 145255.412 \\
HEISPROD & ESTIMATED
\end{tabular} $\begin{array}{lll}565721.044 & 145302.184 \mathrm{HEISPROD} & \text { ESTIMATED }\end{array}$ $\begin{array}{lll}565721.126 & 145286.328 \text { HEISPROD } & \text { ESTIMATED }\end{array}$ $\begin{array}{lll}565722.972 & 145274.151 \text { HEISPROD } & \text { ESTIMATED }\end{array}$ \begin{tabular}{|l|l|l}
565743.215 & 145268.448 HEISPROD & ESTIMATED
\end{tabular} $565762.742 \quad 145269.86$ HEISPROD ESTIMATED $565779.238 \quad 145274.751$ HEISPROD ESTIMATED \begin{tabular}{|c|c|c|c|}
565785.868 & 145253.628 HEISPROD & ESTIMATED
\end{tabular} 565794.624 145249.783 HEISPROD ESTIMATED $565793.498 \quad 145236.548$ HEISPROD ESTIMATED $\begin{array}{lll}565787.649 & 145236.368 \text { HEISPROD } & \text { ESTIMATED }\end{array}$ \begin{tabular}{rl}
565771.26 & 145234.511 HEISPROD \\
\hline
\end{tabular} 565754.028145230 .747 HEISPROD ESTIMATED 565744.447145233 .296 HEISPROD ESTIMATED $565745.356 \quad 145226.577$ HEISPROD ESTIMATED 565719.711 145213.78 HEISPROD ESTIMATED 565705.71 145214.32 HEISPROD ESTIMATED $\begin{array}{lll}565700.397 & 145202.947 \text { HEISPROD } & \text { ESTIMATED }\end{array}$ 565677.712145199 .959 HEISPROD ESTIMATED 565674.729145192 .403 HEISPROD ESTIMATED 565658.795145190 .984 HEISPROD ESTIMATED 565656.923145183 .477 HEISPROD ESTIMATED $565637.53 \quad 145173.554$ HEISPROD ESTIMATED

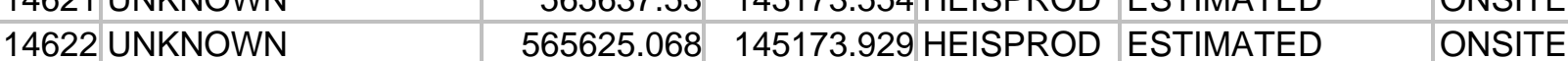

\section{G} $\mathrm{H}$ \begin{tabular}{l|l} 
CLASS & RAD_LEVEL \\
\hline
\end{tabular} ONSITE $100-\mathrm{C}-6$ $100-\mathrm{C}-6$ \begin{tabular}{l|l} 
ONSITE & $100-\mathrm{C}-6$ \\
\hline
\end{tabular} ONSITE $100-\mathrm{C}-6$ \begin{tabular}{l|l}
\hline ONSITE & $100-\mathrm{C}-6$ \\
\hline
\end{tabular} ONSITE $100-\mathrm{C}-6$ \begin{tabular}{l|l} 
ONSITE & $100-\mathrm{C}-6$ \\
\hline
\end{tabular} \begin{tabular}{l|l|l|}
\hline ONSITE & $100-\mathrm{C}-6$ \\
\hline
\end{tabular} ONSITE $100-\mathrm{C}-6$ ONSITE $100-\mathrm{C}-6$ \begin{tabular}{l|l} 
ONSITE & $100-C-6$
\end{tabular} \begin{tabular}{l|l} 
ONSITE & $100-\mathrm{C}-6$ \\
\hline
\end{tabular} ONSITE $100-\mathrm{C}-6$ ONSITE \begin{tabular}{l|l} 
ONSITE & $100-\mathrm{C}-6$ \\
\hline
\end{tabular} ONSITE $100-\mathrm{C}-6$ ONSITE $100-\mathrm{C}-6$ ONSITE $100-\mathrm{C}-6$ \begin{tabular}{l|l|l|}
\hline ONSITE & $100-\mathrm{C}-6$ \\
\hline
\end{tabular} ONSITE $100-\mathrm{C}-6$ ONSITE \begin{tabular}{l|l} 
ONSITE & $100-\mathrm{C}-6$
\end{tabular} ONSITE $100-\mathrm{C}-6$ ONSITE $100-\mathrm{C}-6$ \begin{tabular}{l|l} 
ONSITE & $100-\mathrm{C}-6$ \\
\hline
\end{tabular} \begin{tabular}{|l|l|}
\hline ONSITE & $100-\mathrm{C}-6$ \\
\hline ONSITE & $100-\mathrm{C}-6$ \\
\hline
\end{tabular} ONSITE $100-\mathrm{C}-6$ ONSITE $100-\mathrm{C}-6$ \begin{tabular}{l|l|l} 
ONSITE & $100-\mathrm{C}-6$ \\
\hline
\end{tabular} ONSITE $100-\mathrm{C}-6$ \begin{tabular}{|l|r|r|r|}
\hline 14622 & UNKNOWN & 565625.068 & 145173.929 \\
\hline
\end{tabular} \begin{tabular}{|l|l|l|}
\hline 14623 & HEISPROD \\
\hline 14624 & HEISPROD \\
\hline 14625 & HEISPROD
\end{tabular} 14625 HEISPROD \begin{tabular}{|l|l|}
\hline 14626 & HEISPROD
\end{tabular} 14627 14628

\begin{tabular}{|l|l|l|}
\hline & & $126-\mathrm{F}-1$ \\
\hline & & $126-\mathrm{F}-1$ \\
\hline & & $126-\mathrm{F}-1$ \\
\hline & & $126-\mathrm{F}-1$ \\
\hline & & $126-\mathrm{F}-1$ \\
\hline
\end{tabular}




\begin{tabular}{|c|c|c|c|c|c|c|c|c|c|}
\hline & $A$ & $\mathrm{~B}$ & $\mathrm{C}$ & $\mathrm{D}$ & $E$ & $\mathrm{~F}$ & G & $\mathrm{H}$ & $\mathrm{I}$ \\
\hline 1 & SAMP_SITE_ID & HORIZ_COORD_TYPE & EW_COORD & NS_COORD & OWNER_ID & COORD_SOURCE & DIST_CLASS & RAD_LEVEL & SAMP_SITE_NAME \\
\hline 5778 & 14629 & & & & HEISPROD & & & & 126-F-1 \\
\hline 5779 & 14630 & & & & HEISPROD & & & & 126-F-1 \\
\hline 5780 & 14631 & & & & HEISPROD & & & & 126-F-1 \\
\hline \begin{tabular}{|l|}
5781 \\
\end{tabular} & 14632 & & & & HEISPROD & & & & 126-F-1 \\
\hline 5782 & 14633 & & & & HEISPROD & & & & 126-F-1 \\
\hline 5783 & 14634 & & & & HEISPROD & & & & 126-F-1 \\
\hline 5784 & 14635 & & & & HEISPROD & & & & 126-F-1 \\
\hline 5785 & 14636 & & & & HEISPROD & & & & 126-F-1 \\
\hline 5786 & 14637 & & & & HEISPROD & & & & 126-F-1 \\
\hline \begin{tabular}{|l|}
5787 \\
\end{tabular} & 14638 & & & & HEISPROD & & & & 100-B-8 \\
\hline \begin{tabular}{|l|}
5788 \\
\end{tabular} & 14639 & & & & HEISPROD & & & & 100-B-8 \\
\hline 5789 & 14640 & & & & HEISPROD & & & & 100-B-8 \\
\hline 5790 & 14641 & & & & HEISPROD & & & & 100-B-8 \\
\hline 5791 & 14642 & & & & HEISPROD & & & & 100-B-8 \\
\hline 5792 & 14643 & & & & HEISPROD & & & & 100-B-8 \\
\hline 5793 & 14644 & & & & HEISPROD & & & & 100-B-8 \\
\hline 5794 & 14645 & & & & HEISPROD & & & & 100-B-8 \\
\hline 5795 & 14646 & UNKNOWN & 565445 & 144972 & 2 HEISPROD & ESTIMATED & ONSITE & & $100-C-6$ \\
\hline 5796 & 14647 & NAD83(91) & 565445 & 144972 & 2 HEISPROD & ESTIMATED & ONSITE & & $100-C-6$ \\
\hline \begin{tabular}{|l|}
5797 \\
\end{tabular} & 14648 & UNKNOWN & 565449 & 144972 & 2 HEISPROD & ESTIMATED & ONSITE & & $100-C-6$ \\
\hline \begin{tabular}{|l|}
5798 \\
\end{tabular} & 14649 & UNKNOWN & 565451 & 144977 & 7 HEISPROD & ESTIMATED & ONSITE & & $100-C-6$ \\
\hline 5799 & 14650 & UNKNOWN & 565443 & 144977 & 7 HEISPROD & ESTIMATED & ONSITE & & $100-C-6$ \\
\hline 5800 & 14651 & UNKNOWN & 565437 & 144976 & HEISPROD & ESTIMATED & ONSITE & & $100-C-6$ \\
\hline 5801 & 14652 & NAD83(91) & 565423 & 145001 & HEISPROD & ESTIMATED & ONSITE & & 100-B-8 \\
\hline 5802 & 14653 & UNKNOWN & 565382 & 144974 & 4 HEISPROD & ESTIMATED & ONSITE & & 100-B-8 \\
\hline 5803 & 14654 & UNKNOWN & 565344 & 145017 & 7 HEISPROD & ESTIMATED & OFFSITE & & 100-B-8 \\
\hline 5804 & 14655 & UNKNOWN & 565323 & 145043 & 3 HEISPROD & ESTIMATED & ONSITE & & 100-B-8 \\
\hline 5805 & 14656 & UNKNOWN & 565449 & 144972 & 2 HEISPROD & ESTIMATED & ONSITE & & $100-C-6$ \\
\hline 5806 & 14657 & UNKNOWN & 565604 & 145177 & 7 HEISPROD & ESTIMATED & ONSITE & & $100-C-6$ \\
\hline 5807 & 14658 & NAD83(91) & 565578 & 145170 & HEISPROD & ESTIMATED & ONSITE & & $100-C-6$ \\
\hline 5808 & 14659 & UNKNOWN & 565577 & 145173 & 3 HEISPROD & ESTIMATED & ONSITE & & $100-C-6$ \\
\hline 5809 & 14660 & UNKNOWN & 571735 & 149997 & 7 HEISPROD & ESTIMATED & ONSITE & & $116-\mathrm{N}-1$ \\
\hline 5810 & 14661 & NAD83(91) & 571735 & 149997 & 7 HEISPROD & ESTIMATED & ONSITE & & $116-\mathrm{N}-1$ \\
\hline 5811 & 14662 & UNKNOWN & 571735 & 149997 & 7 HEISPROD & ESTIMATED & ONSITE & & $116-\mathrm{N}-1$ \\
\hline 5812 & 14663 & UNKNOWN & 571735 & 149997 & 7 HEISPROD & ESTIMATED & ONSITE & & $116-\mathrm{N}-1$ \\
\hline 5813 & 14664 & UNKNOWN & 571705 & 149980 & HEISPROD & ESTIMATED & ONSITE & & $116-\mathrm{N}-1$ \\
\hline 5814 & 14665 & NAD83(91) & 571705 & 149980 & HEISPROD & ESTIMATED & ONSITE & & $116-\mathrm{N}-1$ \\
\hline 5815 & 14666 & UNKNOWN & 571705 & 149980 & HEISPROD & ESTIMATED & ONSITE & & $116-\mathrm{N}-1$ \\
\hline
\end{tabular}


A

$\mathrm{B}$

\begin{tabular}{l|l}
$\mathrm{C}$ & $\mathrm{D}$ \\
\hline
\end{tabular}

\begin{tabular}{l|l|l}
$D$ & $E$ & $F$
\end{tabular} F 14667 UNKNOWN 14668 UNKNOWN

\begin{tabular}{l|l} 
& $\mathrm{C}$ \\
\hline
\end{tabular}

\section{6} 14669 UNKNOWN 14670 NAD83(91) 14671 UNKNOWN 14672 UNKNOWN 14673 NAD83(91) 14674 NAD83(91) 14675 NAD83(91) 14676 UNKNOWN 14677 UNKNOWN 14678 NAD83(91) 14679 UNKNOWN 14680 NAD83(91) 14681 NAD83(91) 14682 UNKNOWN 14683 UNKNOWN 14684 UNKNOWN $571675 \quad 149939$ HEISPROD ESTIMATED $571675 \quad 149939$ HEISPROD $\quad$ ESTIMATED 571675149939 HEISPROD ESTIMATED $571675 \quad 149939$ HEISPROD ESTIMATED 571696149955 HEISPROD ESTIMATED $571696 \quad 149955$ HEISPROD ESTIMATED 571696149955 HEISPROD ESTIMATED 571696149955 HEISPROD ESTIMATED

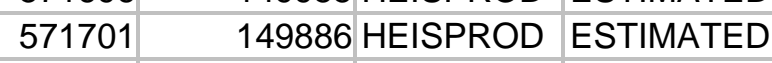
571691149884 HEISPROD ESTIMATED $571690 \quad 149863$ HEISPROD ESTIMATED 571683149847 HEISPROD ESTIMATED 571718149860 HEISPROD $\quad$ ESTIMATED 571737149848 HEISPROD $\quad$ ESTIMATED $571660 \quad 149814$ HEISPROD ESTIMATED $571626 \quad 149800$ HEISPROD ESTIMATED 571454149747 HEISPROD ESTIMATED $571432 \quad 149708$ HEISPROD ESTIMATED

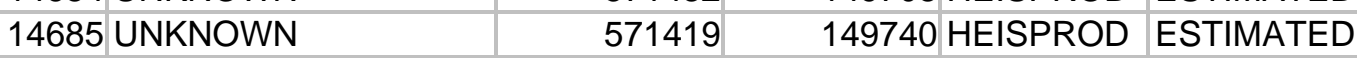

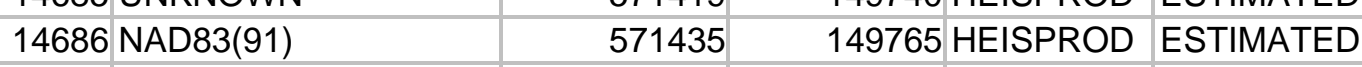
14687 UNKNOWN $571426 \quad 149753$ HEISPROD ESTIMATED \begin{tabular}{|l|r|r|r|}
\hline 14688 UNKNOWN & 571425 & 149754 HEISPROD & ESTIMATED \\
\hline 14689 UNKNOWN & 571424 & 149755 HEISPROD & ESTIMATED \\
\hline
\end{tabular} 14690 NAD83(91) 14691 NAD83(91) 571308.48 49226.13 HEISPROD GPS 14692 NAD83(91) 14693 NAD83(91) 14694

14695 UNKNOWN 14696 UNKNOWN 14697 UNKNOWN 14698 UNKNOWN 14699 UNKNOWN 14700 UNKNOWN 14701 UNKNOWN 14702 UNKNOWN 14703 UNKNOWN 14704 UNKNOWN

\begin{tabular}{|l|l|l}
571244 & 149228 HEISPROD & GPS
\end{tabular}

\begin{tabular}{|l|r|}
571146.5 & 149336.5 \\
HEISPROD & GPS
\end{tabular} HEISPROD

565528.38145246 .02 HEISPROD ESTIMATED 565534.11145250 .15 HEISPROD ESTIMATED 565550.28 145251.3 HEISPROD ESTIMATED $565563.61 \quad 145246.66 \mathrm{HEISPROD}$ ESTIMATED

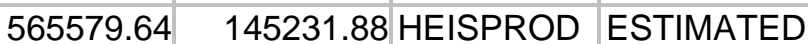
$\begin{array}{lll}565564.93 & 145256.51 \text { HEISPROD } & \text { ESTIMATED }\end{array}$ 565569.74 145251.16 HEISPROD ESTIMATED $565588.92 \quad 145231.25$ HEISPROD ESTIMATED 565565.63145267 .66 HEISPROD ESTIMATED $\begin{array}{lll}565585.72 & 145245.72 \\ \end{array}$

G $\mathrm{H}$ I DIST_CLASS $\quad$ RAD_LEVEL ONSITE $116-\mathrm{N}-1$ \begin{tabular}{l|l} 
ONSITE & $116-\mathrm{N}-1$
\end{tabular} ONSITE $116-\mathrm{N}-1$

\begin{tabular}{l|l} 
ONSITE & $116-\mathrm{N}-1$
\end{tabular}

\begin{tabular}{|l|l|}
\hline ONSITE & $116-\mathrm{N}-1$ \\
\hline
\end{tabular} ONSITE ONSITE ONSITE ONSITE ONSITE ONSITE ONSITE OFFSITE ONSITE OFFSITE ONSITE

ONSITE

ONSITE

ONSITE ONSITE ONSITE ONSITE ONSITE

ONSITE

ONSITE ONSITE

ONSITE ONSITE ONSITE

ONSITE

ONSITE

ONSITE ONSITE

ONSITE

ONSITE ONSITE 


\begin{tabular}{|c|c|c|c|c|c|c|c|c|c|}
\hline & $\bar{A}$ & $\mathrm{~B}$ & $\mathrm{C}$ & $\mathrm{D}$ & $E$ & $\mathrm{~F}$ & G & $\mathrm{H}$ & $\mathrm{I}$ \\
\hline 1 & SAMP_SITE_ID & HORIZ_COORD_TYPE & EW_COORD & NS_COORD & OWNER_ID & COORD_SOURCE & DIST_CLASS & RAD_LEVEL & SAMP_SITE_NAME \\
\hline 5854 & 14705 & UNKNOWN & 565595.38 & 145234.66 & HEISPROD & ESTIMATED & ONSITE & & $100-C-6$ \\
\hline 5855 & 14706 & UNKNOWN & 565572.15 & 145271.92 & HEISPROD & ESTIMATED & ONSITE & & $100-C-6$ \\
\hline 5856 & 14707 & UNKNOWN & 565577.42 & 145266.08 & HEISPROD & ESTIMATED & ONSITE & & $100-C-6$ \\
\hline 5857 & 14708 & UNKNOWN & 565582.65 & 145260.28 & HEISPROD & ESTIMATED & ONSITE & & $100-C-6$ \\
\hline 5858 & 14709 & UNKNOWN & 565577.07 & 145277.58 & HEISPROD & ESTIMATED & ONSITE & & $100-C-6$ \\
\hline 5859 & 14710 & UNKNOWN & 565592.6 & 145260.36 & HEISPROD & ESTIMATED & ONSITE & & $100-C-6$ \\
\hline 5860 & 14711 & UNKNOWN & 565588.15 & 145276.45 & HEISPROD & ESTIMATED & ONSITE & & $100-C-6$ \\
\hline 5861 & 14712 & UNKNOWN & 565604.04 & 145258.46 & HEISPROD & ESTIMATED & ONSITE & & $100-C-6$ \\
\hline 5862 & 14713 & UNKNOWN & 565609.43 & 145252.37 & HEISPROD & ESTIMATED & ONSITE & & $100-C-6$ \\
\hline 5863 & 14714 & UNKNOWN & 565588.74 & 145286.7 & HEISPROD & ESTIMATED & ONSITE & & $100-C-6$ \\
\hline 5864 & 14715 & UNKNOWN & 565610.34 & 145262.17 & HEISPROD & ESTIMATED & ONSITE & & $100-C-6$ \\
\hline 5865 & 14716 & UNKNOWN & 565615.68 & 145256.1 & HEISPROD & ESTIMATED & ONSITE & & $100-C-6$ \\
\hline 5866 & 14717 & UNKNOWN & 565599 & 145315.33 & HEISPROD & ESTIMATED & ONSITE & & $100-C-6$ \\
\hline 5867 & 14718 & UNKNOWN & 565595.64 & 145310.02 & HEISPROD & ESTIMATED & ONSITE & & $100-C-6$ \\
\hline 5868 & 14719 & UNKNOWN & 565588.23 & 145297.39 & HEISPROD & ESTIMATED & ONSITE & & $100-C-6$ \\
\hline 5869 & 14720 & UNKNOWN & 565607.19 & 145312.53 & HEISPROD & ESTIMATED & ONSITE & & $100-C-6$ \\
\hline 5870 & 14721 & UNKNOWN & 565614.02 & 145310.9 & HEISPROD & ESTIMATED & ONSITE & & $100-C-6$ \\
\hline 5871 & 14722 & UNKNOWN & 565603.4 & 145295.5 & HEISPROD & ESTIMATED & ONSITE & & $100-C-6$ \\
\hline 5872 & 14723 & UNKNOWN & 565612.84 & 145288.84 & HEISPROD & ESTIMATED & ONSITE & & $100-C-6$ \\
\hline 5873 & 14724 & UNKNOWN & 565607.08 & 145279.44 & HEISPROD & ESTIMATED & ONSITE & & $100-C-6$ \\
\hline 5874 & 14725 & UNKNOWN & 565616.91 & 145285.7 & HEISPROD & ESTIMATED & ONSITE & & $100-C-6$ \\
\hline 5875 & 14726 & UNKNOWN & 565634.91 & 145303.66 & HEISPROD & ESTIMATED & ONSITE & & $100-C-6$ \\
\hline 5876 & 14727 & UNKNOWN & 565638.57 & 145302.07 & HEISPROD & ESTIMATED & ONSITE & & $100-C-6$ \\
\hline 5877 & 14728 & UNKNOWN & 565639.03 & 145297.12 & HEISPROD & ESTIMATED & ONSITE & & $100-C-6$ \\
\hline 5878 & 14729 & UNKNOWN & 565632.78 & 145284.29 & HEISPROD & ESTIMATED & ONSITE & & $100-C-6$ \\
\hline 5879 & 14730 & UNKNOWN & 565638.88 & 145283.79 & HEISPROD & ESTIMATED & ONSITE & & $100-C-6$ \\
\hline 5880 & 14731 & UNKNOWN & 565628.74 & 145267.82 & HEISPROD & ESTIMATED & ONSITE & & $100-C-6$ \\
\hline 5881 & 14732 & UNKNOWN & 565642.26 & 145266.82 & HEISPROD & ESTIMATED & ONSITE & & $100-C-6$ \\
\hline 5882 & 14733 & UNKNOWN & 565637.12 & 145311.39 & HEISPROD & ESTIMATED & ONSITE & & $100-C-6$ \\
\hline 5883 & 14734 & UNKNOWN & 565610.57 & 145334.86 & HEISPROD & ESTIMATED & ONSITE & & $100-C-6$ \\
\hline 5884 & 14735 & UNKNOWN & 565605.97 & 145335.2 & HEISPROD & ESTIMATED & ONSITE & & $100-C-6$ \\
\hline 5885 & 14736 & UNKNOWN & 565588.21 & 145366.92 & HEISPROD & ESTIMATED & ONSITE & & $100-C-6$ \\
\hline 5886 & 14737 & UNKNOWN & 565599.38 & 145376.15 & HEISPROD & ESTIMATED & ONSITE & & $100-C-6$ \\
\hline 5887 & 14738 & UNKNOWN & 565599.97 & 145369.63 & HEISPROD & ESTIMATED & ONSITE & & $100-C-6$ \\
\hline 5888 & 14739 & UNKNOWN & 565613.11 & 145382 & HEISPROD & ESTIMATED & ONSITE & & $100-C-6$ \\
\hline 5889 & 14740 & UNKNOWN & 565609.24 & 145377.04 & HEISPROD & ESTIMATED & ONSITE & & $100-C-6$ \\
\hline 5890 & 14741 & UNKNOWN & 565638.62 & 145383.44 & HEISPROD & ESTIMATED & ONSITE & & $100-C-6$ \\
\hline 5891 & 14742 & UNKNOWN & 565629.77 & 145375.22 & HEISPROD & ESTIMATED & ONSITE & & $100-C-6$ \\
\hline
\end{tabular}




\begin{tabular}{|c|c|c|c|c|c|c|c|c|c|}
\hline & A & $\mathrm{B}$ & C & $\mathrm{D}$ & $E$ & $\mathrm{~F}$ & $\mathrm{G}$ & $\mathrm{H}$ & $\mathrm{I}$ \\
\hline 1 & SAMP_SITE_ID & HORIZ_COORD_TYPE & EW_COORD & NS_COORD & OWNER_ID & COORD_SOURCE & DIST_CLASS & RAD_LEVEL & SAMP_SITE_NAME \\
\hline 5930 & 14781 & & & & HEISPROD & & & & 100-B-8 \\
\hline 5931 & 14782 & & & & HEISPROD & & & & 100-B-8 \\
\hline 5932 & 14783 & & & & HEISPROD & & & & 100-B-8 \\
\hline 5933 & 14784 & & & & HEISPROD & & & & 100-B-8 \\
\hline 5934 & 14785 & & & & HEISPROD & & & & 100-B-8 \\
\hline 5935 & 14786 & & & & HEISPROD & & & & 100-B-8 \\
\hline 5936 & 14787 & & & & HEISPROD & & & & $100-C-6$ \\
\hline \begin{tabular}{|l|}
5937 \\
\end{tabular} & 14796 & & & & CENTPLAT & & & & 100-HR-3 PUMP \& TREAT \\
\hline 5938 & 14797 & & & & CENTPLAT & & & & 100-HR-3 PUMP \& TREAT \\
\hline 5939 & 14798 & & & & CENTPLAT & & & & 100-HR-3 PUMP \& TREAT \\
\hline 5940 & 14799 & & & & CENTPLAT & & & & 100-HR-3 PUMP \& TREAT \\
\hline 5941 & 14816 & & & & CENTPLAT & & & & 100-KR-4 PUMP \& TREAT \\
\hline 5942 & 14817 & & & & CENTPLAT & & & & 100-KR-4 PUMP \& TREAT \\
\hline 5943 & 14818 & & & & CENTPLAT & & & & 100-KR-4 PUMP \& TREAT \\
\hline 5944 & 14819 & & & & CENTPLAT & & & & 100-KR-4 PUMP \& TREAT \\
\hline 5945 & 14820 & & & & CENTPLAT & & & & 100-KR-4 PUMP \& TREAT \\
\hline 5946 & 14821 & & & & CENTPLAT & & & & 100-KR-4 PUMP \& TREAT \\
\hline \begin{tabular}{|l|}
5947 \\
\end{tabular} & 14822 & & & & CENTPLAT & & & & 100-HR-3 PUMP \& TREAT \\
\hline 5948 & 14823 & & & & CENTPLAT & & & & 100-HR-3 PUMP \& TREAT \\
\hline 5949 & 14824 & & & & CENTPLAT & & & & 100-HR-3 PUMP \& TREAT \\
\hline 5950 & 14825 & & & & CENTPLAT & & & & 100-HR-3 PUMP \& TREAT \\
\hline 5951 & 14836 & & & & CENTPLAT & & & & 100-HR-3 PUMP \& TREAT \\
\hline 5952 & 14837 & & & & CENTPLAT & & & & 100-HR-3 PUMP \& TREAT \\
\hline \begin{tabular}{|l|}
5953 \\
\end{tabular} & 14838 & & & & CENTPLAT & & & & 100-HR-3 PUMP \& TREAT \\
\hline 5954 & 14839 & & & & CENTPLAT & & & & 100-HR-3 PUMP \& TREAT \\
\hline 5955 & 14856 & & & & CENTPLAT & & & & 200-ZP-1 PUMP \& TREAT \\
\hline
\end{tabular}




\begin{tabular}{|r|l|l|l|}
\hline & \multicolumn{1}{|c|}{ J } & \multicolumn{1}{c|}{ K } & L \\
\hline 1 & SAMP_SITE_TYPE & SCHED_LABEL_CODE & TRANSFORM_CODE \\
\hline 2 & NAMED_POINT & & \\
\hline 3 & NAMED_POINT & & \\
\hline 4 & NAMED_POINT & C & \\
\hline 5 & NAMED_POINT & & \\
\hline 6 & NAMED_POINT & & \\
\hline 7 & NAMED_POINT & & \\
\hline 8 & NAMED_POINT & & \\
\hline 9 & NAMED_POINT & & \\
\hline 10 & NAMED_POINT & & \\
\hline 11 & NAMED_POINT & & \\
\hline 12 & NAMED_POINT & D & \\
\hline 13 & NAMED_POINT & & \\
\hline 14 & NAMED_POINT & & \\
\hline 15 & NAMED_POINT & & \\
\hline 16 & NAMED_POINT & G & \\
\hline 17 & NAMED_POINT & & \\
\hline 18 & NAMED_POINT & & \\
\hline 19 & NAMED_POINT & & \\
\hline 20 & NAMED_POINT & & \\
\hline 21 & NAMED_POINT & & \\
\hline 22 & NAMED_POINT & & \\
\hline 23 & NAMED_POINT & & \\
\hline 24 & NAMED_POINT & D & \\
\hline 25 & NAMED_POINT & & \\
\hline 26 & NAMED_POINT & D & \\
\hline 27 & NAMED_POINT & D & \\
\hline 28 & NAMED_POINT & D & \\
\hline 29 & NAMED_POINT & & \\
\hline 30 & NAMED_POINT & A & \\
\hline 31 & NAMED_POINT & & \\
\hline 32 & NAMED_POINT & A & \\
\hline 33 & NAMED_POINT & & \\
\hline 34 & NAMED_POINT & & \\
\hline 35 & NAMED_POINT & & \\
\hline 36 & NAMED_POINT & & \\
\hline 37 & NAMED_POINT & & \\
\hline 38 & NAMED_POINT & & \\
\hline 39 & NAMED_POINT & A & \\
\hline & & & \\
\hline
\end{tabular}




\begin{tabular}{|c|l|l|l|}
\hline & \multicolumn{1}{|c|}{ J } & \multicolumn{1}{c|}{ K } & L \\
\hline 1 & SAMP_SITE_TYPE & SCHED_LABEL_CODE & TRANSFORM_CODE \\
\hline 40 & NAMED_POINT & A & \\
\hline 41 & NAMED_POINT & & \\
\hline 42 & NAMED_POINT & & \\
\hline 43 & NAMED_POINT & A & \\
\hline 44 & NAMED_POINT & & \\
\hline 45 & NAMED_POINT & G & \\
\hline 46 & NAMED_POINT & & \\
\hline 47 & NAMED_POINT & & \\
\hline 48 & NAMED_POINT & & \\
\hline 49 & NAMED_POINT & & \\
\hline 50 & NAMED_POINT & A & \\
\hline 51 & NAMED_POINT & A & \\
\hline 52 & NAMED_POINT & A & \\
\hline 53 & NAMED_POINT & A & \\
\hline 54 & NAMED_POINT & & \\
\hline 55 & NAMED_POINT & & \\
\hline 56 & NAMED_POINT & A & \\
\hline 57 & NAMED_POINT & & \\
\hline 58 & NAMED_POINT & A & \\
\hline 59 & NAMED_POINT & A & \\
\hline 60 & NAMED_POINT & A & \\
\hline 61 & NAMED_POINT & & \\
\hline 62 & NAMED_POINT & A & \\
\hline 63 & NAMED_POINT & & \\
\hline 64 & NAMED_POINT & & \\
\hline 65 & NAMED_POINT & & \\
\hline 66 & NAMED_POINT & D & \\
\hline 67 & NAMED_POINT & & \\
\hline 68 & NAMED_POINT & D & \\
\hline 69 & NAMED_POINT & & \\
\hline 70 & NAMED_POINT & & \\
\hline 71 & NAMED_POINT & D & \\
\hline 72 & NAMED_POINT & D & \\
\hline 73 & NAMED_POINT & & \\
\hline 74 & NAMED_POINT & & \\
\hline 75 & NAMED_POINT & A & \\
\hline 76 & NAMED_POINT & & \\
\hline 77 & NAMED_POINT & & \\
\hline & & & \\
\hline
\end{tabular}




\begin{tabular}{|r|l|l|l|}
\hline & \multicolumn{1}{|c|}{ J } & \multicolumn{1}{c|}{ K } & L \\
\hline 1 & SAMP_SITE_TYPE & SCHED_LABEL_CODE & TRANSFORM_CODE \\
\hline 78 & NAMED_POINT & D & \\
\hline 79 & NAMED_POINT & A & \\
\hline 80 & NAMED_POINT & A & \\
\hline 81 & NAMED_POINT & A & \\
\hline 82 & NAMED_POINT & A & \\
\hline 83 & NAMED_POINT & & \\
\hline 84 & NAMED_POINT & & \\
\hline 85 & NAMED_POINT & & \\
\hline 86 & NAMED_POINT & A & \\
\hline 87 & NAMED_POINT & & \\
\hline 88 & NAMED_POINT & A & \\
\hline 89 & NAMED_POINT & & \\
\hline 90 & NAMED_POINT & A & \\
\hline 91 & NAMED_POINT & & \\
\hline 92 & NAMED_POINT & & \\
\hline 93 & NAMED_POINT & A & \\
\hline 94 & NAMED_POINT & & \\
\hline 95 & NAMED_POINT & A & \\
\hline 96 & NAMED_POINT & & \\
\hline 97 & NAMED_POINT & C & \\
\hline 98 & NAMED_POINT & & \\
\hline 99 & NAMED_POINT & A & \\
\hline 100 & NAMED_POINT & & \\
\hline 101 & NAMED_POINT & & \\
\hline 102 & NAMED_POINT & A & \\
\hline 103 & NAMED_POINT & & \\
\hline 104 & NAMED_POINT & & \\
\hline 105 & NAMED_POINT & & \\
\hline 106 & NAMED_POINT & A & \\
\hline 107 & NAMED_POINT & & \\
\hline 108 & NAMED_POINT & A & \\
\hline 109 & NAMED_POINT & A & \\
\hline 110 & NAMED_POINT & & \\
\hline 111 & NAMED_POINT & & \\
\hline 112 & NAMED_POINT & A & \\
\hline 113 & NAMED_POINT & & \\
\hline 114 & NAMED_POINT & A & \\
\hline 115 & NAMED_POINT & A & \\
\hline & & & \\
\hline
\end{tabular}




\begin{tabular}{|c|c|c|c|}
\hline & $\mathrm{J}$ & $\mathrm{K}$ & $\mathrm{L}$ \\
\hline 1 & SAMP_SITE_TYPE & SCHED_LABEL_CODE & TRANSFORM_CODE \\
\hline 116 & NAMED_POINT & & \\
\hline 117 & NAMED_POINT & & \\
\hline 118 & NAMED_POINT & & \\
\hline 119 & NAMED_POINT & A & \\
\hline 120 & NAMED_POINT & A & \\
\hline 121 & NAMED_POINT & A & \\
\hline 122 & NAMED_POINT & A & \\
\hline 123 & NAMED_POINT & A & \\
\hline 124 & NAMED_POINT & & \\
\hline 125 & NAMED_POINT & & \\
\hline 126 & NAMED_POINT & & \\
\hline 127 & NAMED_POINT & & \\
\hline 128 & NAMED_POINT & A & \\
\hline 129 & NAMED_POINT & & \\
\hline 130 & NAMED_POINT & & \\
\hline 131 & NAMED_POINT & & \\
\hline 132 & NAMED_POINT & A & \\
\hline 133 & NAMED_POINT & A & \\
\hline 134 & NAMED_POINT & A & \\
\hline 135 & NAMED_POINT & A & \\
\hline 136 & NAMED_POINT & A & \\
\hline 137 & NAMED_POINT & & \\
\hline 138 & NAMED_POINT & C & \\
\hline 139 & NAMED_POINT & C & \\
\hline 140 & NAMED_POINT & & \\
\hline 141 & NAMED_POINT & C & \\
\hline 142 & NAMED_POINT & C & \\
\hline 143 & NAMED_POINT & & \\
\hline 144 & NAMED_POINT & C & \\
\hline 145 & NAMED_POINT & C & \\
\hline 146 & NAMED_POINT & C & \\
\hline 147 & NAMED_POINT & C & \\
\hline 148 & NAMED_POINT & & \\
\hline 149 & NAMED_POINT & & \\
\hline 150 & NAMED_POINT & C & \\
\hline 151 & NAMED_POINT & & \\
\hline 152 & NAMED_POINT & C & \\
\hline 153 & NAMED_POINT & & \\
\hline
\end{tabular}




\begin{tabular}{|c|l|l|l|}
\hline & \multicolumn{1}{|c|}{ J } & \multicolumn{1}{c|}{ K } & L \\
\hline 1 & SAMP_SITE_TYPE & SCHED_LABEL_CODE & TRANSFORM_CODE \\
\hline 154 & NAMED_POINT & C & \\
\hline 155 & NAMED_POINT & & \\
\hline 156 & NAMED_POINT & E & \\
\hline 157 & NAMED_POINT & & \\
\hline 158 & NAMED_POINT & & \\
\hline 159 & NAMED_POINT & & \\
\hline 160 & NAMED_POINT & & \\
\hline 161 & NAMED_POINT & & \\
\hline 162 & NAMED_POINT & & \\
\hline 163 & NAMED_POINT & D & \\
\hline 164 & NAMED_POINT & A & \\
\hline 165 & NAMED_POINT & & \\
\hline 166 & NAMED_POINT & & \\
\hline 167 & NAMED_POINT & & \\
\hline 168 & NAMED_POINT & & \\
\hline 169 & NAMED_POINT & & \\
\hline 170 & NAMED_POINT & & \\
\hline 171 & NAMED_POINT & & \\
\hline 172 & NAMED_POINT & & \\
\hline 173 & NAMED_POINT & & \\
\hline 174 & NAMED_POINT & & \\
\hline 175 & NAMED_POINT & & \\
\hline 176 & NAMED_POINT & & \\
\hline 177 & NAMED_POINT & & \\
\hline 178 & NAMED_POINT & A & \\
\hline 179 & NAMED_POINT & A & \\
\hline 180 & NAMED_POINT & A & \\
\hline 181 & NAMED_POINT & & \\
\hline 182 & NAMED_POINT & & \\
\hline 183 & NAMED_POINT & & \\
\hline 184 & NAMED_POINT & & \\
\hline 185 & NAMED_POINT & & \\
\hline 186 & NAMED_POINT & & \\
\hline 187 & NAMED_POINT & & \\
\hline 188 & NAMED_POINT & & \\
\hline 189 & NAMED_POINT & & \\
\hline 190 & NAMED_POINT & A & \\
\hline 191 & NAMED_POINT & & \\
\hline & & & \\
\hline
\end{tabular}




\begin{tabular}{|c|l|l|l|}
\hline & \multicolumn{1}{|c|}{ J } & \multicolumn{1}{c|}{ K } & L \\
\hline 1 & SAMP_SITE_TYPE & SCHED_LABEL_CODE & TRANSFORM_CODE \\
\hline 192 & NAMED_POINT & A & \\
\hline 193 & NAMED_POINT & & \\
\hline 194 & NAMED_POINT & & \\
\hline 195 & NAMED_POINT & A & \\
\hline 196 & NAMED_POINT & & \\
\hline 197 & NAMED_POINT & A & \\
\hline 198 & NAMED_POINT & A & \\
\hline 199 & NAMED_POINT & A & \\
\hline 200 & NAMED_POINT & & \\
\hline 201 & NAMED_POINT & & \\
\hline 202 & NAMED_POINT & & \\
\hline 203 & NAMED_POINT & & \\
\hline 204 & NAMED_POINT & & \\
\hline 205 & NAMED_POINT & & \\
\hline 206 & NAMED_POINT & & \\
\hline 207 & NAMED_POINT & & \\
\hline 208 & NAMED_POINT & C & \\
\hline 209 & NAMED_POINT & C & \\
\hline 210 & NAMED_POINT & C & \\
\hline 211 & NAMED_POINT & C & \\
\hline 212 & NAMED_POINT & & \\
\hline 213 & NAMED_POINT & D & \\
\hline 214 & NAMED_POINT & C & \\
\hline 215 & NAMED_POINT & C & \\
\hline 216 & NAMED_POINT & & \\
\hline 217 & NAMED_POINT & B & \\
\hline 218 & NAMED_POINT & & \\
\hline 219 & NAMED_POINT & C & \\
\hline 220 & NAMED_POINT & & \\
\hline 221 & NAMED_POINT & & \\
\hline 222 & NAMED_POINT & & \\
\hline 223 & NAMED_POINT & & \\
\hline 224 & NAMED_POINT & & \\
\hline 225 & NAMED_POINT & C & \\
\hline 226 & NAMED_POINT & & \\
\hline 227 & NAMED_POINT & & \\
\hline 228 & NAMED_POINT & & \\
\hline 229 & NAMED_POINT & & \\
\hline & & & \\
\hline
\end{tabular}




\begin{tabular}{|c|l|l|l|}
\hline & \multicolumn{1}{|c|}{ J } & \multicolumn{1}{c|}{ K } & L \\
\hline 1 & SAMP_SITE_TYPE & SCHED_LABEL_CODE & TRANSFORM_CODE \\
\hline 230 & NAMED_POINT & & \\
\hline 231 & NAMED_POINT & & \\
\hline 232 & NAMED_POINT & & \\
\hline 233 & NAMED_POINT & & \\
\hline 234 & NAMED_POINT & & \\
\hline 235 & NAMED_POINT & & \\
\hline 236 & NAMED_POINT & & \\
\hline 237 & NAMED_POINT & & \\
\hline 238 & NAMED_POINT & & \\
\hline 239 & NAMED_POINT & & \\
\hline 240 & NAMED_POINT & & \\
\hline 241 & NAMED_POINT & & \\
\hline 242 & NAMED_POINT & & \\
\hline 243 & NAMED_POINT & & \\
\hline 244 & NAMED_POINT & & \\
\hline 245 & NAMED_POINT & & \\
\hline 246 & NAMED_POINT & & \\
\hline 247 & NAMED_POINT & & \\
\hline 248 & NAMED_POINT & & \\
\hline 249 & NAMED_POINT & & \\
\hline 250 & NAMED_POINT & D & \\
\hline 251 & NAMED_POINT & & \\
\hline 252 & NAMED_POINT & \\
\hline 253 & NAMED_POINT & & \\
\hline 254 & NAMED_POINT & A & \\
\hline 255 & NAMED_POINT & A & \\
\hline 256 & NAMED_POINT & & \\
\hline 257 & NAMED_POINT & & \\
\hline 258 & NAMED_POINT & \\
\hline 259 & NAMED_POINT & & \\
\hline 260 & NAMED_POINT & A & \\
\hline 261 & NAMED_POINT & A & \\
\hline 262 & NAMED_POINT & A & \\
\hline 263 & NAMED_POINT & & \\
\hline 264 & NAMED_POINT & A & \\
\hline 265 & NAMED_POINT & A & \\
\hline 266 & NAMED_POINT & A & \\
\hline 267 & NAMED_POINT & A & \\
\hline & & & \\
\hline
\end{tabular}




\begin{tabular}{|c|c|c|c|}
\hline & $\mathrm{J}$ & $\mathrm{K}$ & $\mathrm{L}$ \\
\hline 1 & SAMP_SITE_TYPE & SCHED_LABEL_CODE & TRANSFORM_CODE \\
\hline 268 & NAMED_POINT & A & \\
\hline 269 & NAMED_POINT & A & \\
\hline 270 & NAMED_POINT & A & \\
\hline 271 & NAMED_POINT & E & \\
\hline 272 & NAMED_POINT & C & \\
\hline 273 & NAMED_POINT & C & \\
\hline 274 & NAMED_POINT & C & \\
\hline 275 & NAMED_POINT & C & \\
\hline 276 & NAMED_POINT & C & \\
\hline 277 & NAMED_POINT & C & \\
\hline 278 & NAMED_POINT & C & \\
\hline 279 & NAMED_POINT & C & \\
\hline 280 & NAMED_POINT & C & \\
\hline 281 & NAMED_POINT & C & \\
\hline 282 & NAMED_POINT & C & \\
\hline 283 & NAMED_POINT & C & \\
\hline 284 & NAMED_POINT & C & \\
\hline 285 & NAMED_POINT & B & \\
\hline 286 & NAMED_POINT & A & \\
\hline 287 & NAMED_POINT & A & \\
\hline 288 & NAMED_POINT & A & \\
\hline 289 & NAMED_POINT & A & \\
\hline 290 & NAMED_POINT & A & \\
\hline 291 & NAMED_POINT & $\mathrm{F}$ & \\
\hline 292 & NAMED_POINT & D & \\
\hline 293 & NAMED_POINT & D & \\
\hline 294 & NAMED_POINT & D & \\
\hline 295 & NAMED_POINT & D & \\
\hline 296 & NAMED_POINT & & \\
\hline 297 & NAMED_POINT & & \\
\hline 298 & NAMED_POINT & & \\
\hline 299 & NAMED_POINT & & \\
\hline 300 & NAMED_POINT & & \\
\hline 301 & NAMED_POINT & & \\
\hline 302 & NAMED_POINT & & \\
\hline 303 & NAMED_POINT & & \\
\hline 304 & NAMED_POINT & & \\
\hline 305 & NAMED_POINT & & \\
\hline
\end{tabular}




\begin{tabular}{|c|l|l|l|}
\hline & \multicolumn{1}{|c|}{ J } & \multicolumn{1}{c|}{ K } & L \\
\hline 1 & SAMP_SITE_TYPE & SCHED_LABEL_CODE & TRANSFORM_CODE \\
\hline 306 & NAMED_POINT & & \\
\hline 307 & NAMED_POINT & & \\
\hline 308 & NAMED_POINT & & \\
\hline 309 & NAMED_POINT & & \\
\hline 310 & NAMED_POINT & & \\
\hline 311 & NAMED_POINT & & \\
\hline 312 & NAMED_POINT & & \\
\hline 313 & NAMED_POINT & & \\
\hline 314 & NAMED_POINT & & \\
\hline 315 & NAMED_POINT & G & \\
\hline 316 & NAMED_POINT & & \\
\hline 317 & NAMED_POINT & & \\
\hline 318 & NAMED_POINT & & \\
\hline 319 & NAMED_POINT & & \\
\hline 320 & NAMED_POINT & & \\
\hline 321 & NAMED_POINT & G & \\
\hline 322 & NAMED_POINT & & \\
\hline 323 & NAMED_POINT & & \\
\hline 324 & NAMED_POINT & & \\
\hline 325 & NAMED_POINT & & \\
\hline 326 & NAMED_POINT & A & \\
\hline 327 & NAMED_POINT & & \\
\hline 328 & NAMED_POINT & & \\
\hline 329 & NAMED_POINT & A & \\
\hline 330 & NAMED_POINT & & \\
\hline 331 & NAMED_POINT & & \\
\hline 332 & NAMED_POINT & A & \\
\hline 333 & NAMED_POINT & & \\
\hline 334 & NAMED_POINT & \\
\hline 335 & NAMED_POINT & & \\
\hline 336 & NAMED_POINT & & \\
\hline 337 & NAMED_POINT & & \\
\hline 338 & NAMED_POINT & A & \\
\hline 339 & NAMED_POINT & A & \\
\hline 340 & NAMED_POINT & & \\
\hline 341 & NAMED_POINT & & \\
\hline 342 & NAMED_POINT & & \\
\hline 343 & NAMED_POINT & A & \\
\hline & & & \\
\hline
\end{tabular}




\begin{tabular}{|c|l|l|l|}
\hline & \multicolumn{1}{|c|}{ J } & \multicolumn{1}{c|}{ K } & L \\
\hline 1 & SAMP_SITE_TYPE & SCHED_LABEL_CODE & TRANSFORM_CODE \\
\hline 344 & NAMED_POINT & & \\
\hline 345 & NAMED_POINT & A & \\
\hline 346 & NAMED_POINT & D & \\
\hline 347 & NAMED_POINT & D & \\
\hline 348 & NAMED_POINT & D & \\
\hline 349 & NAMED_POINT & & \\
\hline 350 & NAMED_POINT & & \\
\hline 351 & NAMED_POINT & & \\
\hline 352 & NAMED_POINT & D & \\
\hline 353 & NAMED_POINT & & \\
\hline 354 & NAMED_POINT & & \\
\hline 355 & NAMED_POINT & & \\
\hline 356 & NAMED_POINT & & \\
\hline 357 & NAMED_POINT & & \\
\hline 358 & NAMED_POINT & & \\
\hline 359 & NAMED_POINT & & \\
\hline 360 & NAMED_POINT & D & \\
\hline 361 & NAMED_POINT & D & \\
\hline 362 & NAMED_POINT & D & \\
\hline 363 & NAMED_POINT & & \\
\hline 364 & NAMED_POINT & D & \\
\hline 365 & NAMED_POINT & & \\
\hline 366 & NAMED_POINT & & \\
\hline 367 & NAMED_POINT & A & \\
\hline 368 & NAMED_POINT & & \\
\hline 369 & NAMED_POINT & A & \\
\hline 370 & NAMED_POINT & A & \\
\hline 371 & NAMED_POINT & & \\
\hline 372 & NAMED_POINT & A & \\
\hline 373 & NAMED_POINT & & \\
\hline 374 & NAMED_POINT & & \\
\hline 375 & NAMED_POINT & & \\
\hline 376 & NAMED_POINT & A & \\
\hline 377 & NAMED_POINT & & \\
\hline 378 & NAMED_POINT & & \\
\hline 379 & NAMED_POINT & A & \\
\hline 380 & NAMED_POINT & A & \\
\hline 381 & NAMED_POINT & A & \\
\hline & & & \\
\hline
\end{tabular}




\begin{tabular}{|c|l|l|l|}
\hline & \multicolumn{1}{|c|}{ J } & \multicolumn{1}{c|}{ K } & L \\
\hline 1 & SAMP_SITE_TYPE & SCHED_LABEL_CODE & TRANSFORM_CODE \\
\hline 382 & NAMED_POINT & & \\
\hline 383 & NAMED_POINT & A & \\
\hline 384 & NAMED_POINT & & \\
\hline 385 & NAMED_POINT & C & \\
\hline 386 & NAMED_POINT & C & \\
\hline 387 & NAMED_POINT & & \\
\hline 388 & NAMED_POINT & & \\
\hline 389 & NAMED_POINT & C & \\
\hline 390 & NAMED_POINT & & \\
\hline 391 & NAMED_POINT & & \\
\hline 392 & NAMED_POINT & A & \\
\hline 393 & NAMED_POINT & & \\
\hline 394 & NAMED_POINT & & \\
\hline 395 & NAMED_POINT & & \\
\hline 396 & NAMED_POINT & A & \\
\hline 397 & NAMED_POINT & A & \\
\hline 398 & NAMED_POINT & & \\
\hline 399 & NAMED_POINT & A & \\
\hline 400 & NAMED_POINT & & \\
\hline 401 & NAMED_POINT & & \\
\hline 402 & NAMED_POINT & & \\
\hline 403 & NAMED_POINT & & \\
\hline 404 & NAMED_POINT & & \\
\hline 405 & NAMED_POINT & & \\
\hline 406 & NAMED_POINT & A & \\
\hline 407 & NAMED_POINT & & \\
\hline 408 & NAMED_POINT & & \\
\hline 409 & NAMED_POINT & & \\
\hline 410 & NAMED_POINT & C & \\
\hline 411 & NAMED_POINT & A & \\
\hline 412 & NAMED_POINT & A & \\
\hline 413 & NAMED_POINT & D & \\
\hline 414 & NAMED_POINT & D & \\
\hline 415 & NAMED_POINT & & \\
\hline 416 & NAMED_POINT & & \\
\hline 417 & NAMED_POINT & & \\
\hline 418 & NAMED_POINT & & \\
\hline 419 & NAMED_POINT & & \\
\hline & & & \\
\hline
\end{tabular}




\begin{tabular}{|c|l|l|l|}
\hline & \multicolumn{1}{|c|}{ J } & \multicolumn{1}{c|}{ K } & L \\
\hline 1 & SAMP_SITE_TYPE & SCHED_LABEL_CODE & TRANSFORM_CODE \\
\hline 420 & NAMED_POINT & & \\
\hline 421 & NAMED_POINT & & \\
\hline 422 & NAMED_POINT & & \\
\hline 423 & NAMED_POINT & D & \\
\hline 424 & NAMED_POINT & & \\
\hline 425 & NAMED_POINT & & \\
\hline 426 & NAMED_POINT & & \\
\hline 427 & NAMED_POINT & & \\
\hline 428 & NAMED_POINT & & \\
\hline 429 & NAMED_POINT & & \\
\hline 430 & NAMED_POINT & A & \\
\hline 431 & NAMED_POINT & D & \\
\hline 432 & NAMED_POINT & & \\
\hline 433 & NAMED_POINT & & \\
\hline 434 & NAMED_POINT & & \\
\hline 435 & NAMED_POINT & & \\
\hline 436 & NAMED_POINT & & \\
\hline 437 & NAMED_POINT & & \\
\hline 438 & NAMED_POINT & & \\
\hline 439 & NAMED_POINT & & \\
\hline 440 & NAMED_POINT & & \\
\hline 441 & NAMED_POINT & & \\
\hline 442 & NAMED_POINT & & \\
\hline 443 & NAMED_POINT & A & \\
\hline 444 & NAMED_POINT & A & \\
\hline 445 & NAMED_POINT & & \\
\hline 446 & NAMED_POINT & & \\
\hline 447 & NAMED_POINT & & \\
\hline 448 & NAMED_POINT & A & \\
\hline 449 & NAMED_POINT & & \\
\hline 450 & NAMED_POINT & & \\
\hline 451 & NAMED_POINT & D & \\
\hline 452 & NAMED_POINT & A & \\
\hline 453 & NAMED_POINT & & \\
\hline 454 & NAMED_POINT & A & \\
\hline 455 & NAMED_POINT & A & \\
\hline 456 & NAMED_POINT & & \\
\hline 457 & NAMED_POINT & & \\
\hline & & & \\
\hline
\end{tabular}




\begin{tabular}{|c|l|l|l|}
\hline & \multicolumn{1}{|c|}{ J } & \multicolumn{1}{c|}{ K } & L \\
\hline 1 & SAMP_SITE_TYPE & SCHED_LABEL_CODE & TRANSFORM_CODE \\
\hline 458 & NAMED_POINT & & \\
\hline 459 & NAMED_POINT & & \\
\hline 460 & NAMED_POINT & & \\
\hline 461 & NAMED_POINT & & \\
\hline 462 & NAMED_POINT & A & \\
\hline 463 & NAMED_POINT & & \\
\hline 464 & NAMED_POINT & A & \\
\hline 465 & NAMED_POINT & & \\
\hline 466 & NAMED_POINT & A & \\
\hline 467 & NAMED_POINT & A & \\
\hline 468 & NAMED_POINT & A & \\
\hline 469 & NAMED_POINT & & \\
\hline 470 & NAMED_POINT & & \\
\hline 471 & NAMED_POINT & A & \\
\hline 472 & NAMED_POINT & & \\
\hline 473 & NAMED_POINT & C & \\
\hline 474 & NAMED_POINT & C & \\
\hline 475 & NAMED_POINT & & \\
\hline 476 & NAMED_POINT & C & \\
\hline 477 & NAMED_POINT & C & \\
\hline 478 & NAMED_POINT & C & \\
\hline 479 & NAMED_POINT & & \\
\hline 480 & NAMED_POINT & & \\
\hline 481 & NAMED_POINT & & \\
\hline 482 & NAMED_POINT & & \\
\hline 483 & NAMED_POINT & A & \\
\hline 484 & NAMED_POINT & & \\
\hline 485 & NAMED_POINT & & \\
\hline 486 & NAMED_POINT & & \\
\hline 487 & NAMED_POINT & A & \\
\hline 488 & NAMED_POINT & & \\
\hline 489 & NAMED_POINT & & \\
\hline 490 & NAMED_POINT & & \\
\hline 491 & NAMED_POINT & & \\
\hline 492 & NAMED_POINT & A & \\
\hline 493 & NAMED_POINT & & \\
\hline 494 & NAMED_POINT & A & \\
\hline 495 & NAMED_POINT & C & \\
\hline & & & \\
\hline
\end{tabular}




\begin{tabular}{|c|c|c|c|}
\hline & $\mathrm{J}$ & $\mathrm{K}$ & $\mathrm{L}$ \\
\hline 1 & SAMP_SITE_TYPE & SCHED_LABEL_CODE & TRANSFORM_CODE \\
\hline 496 & NAMED_POINT & & \\
\hline 497 & NAMED_POINT & & \\
\hline 498 & NAMED_POINT & C & \\
\hline 499 & NAMED_POINT & & \\
\hline 500 & NAMED_POINT & & \\
\hline 501 & NAMED_POINT & & \\
\hline 502 & NAMED_POINT & & \\
\hline 503 & NAMED_POINT & A & \\
\hline 504 & NAMED_POINT & & \\
\hline 505 & NAMED_POINT & C & \\
\hline 506 & NAMED_POINT & & \\
\hline 507 & NAMED_POINT & & \\
\hline 508 & NAMED_POINT & & \\
\hline 509 & NAMED_POINT & & \\
\hline 510 & NAMED_POINT & & \\
\hline 511 & NAMED_POINT & & \\
\hline 512 & NAMED_POINT & A & \\
\hline 513 & NAMED_POINT & A & \\
\hline 514 & NAMED_POINT & A & \\
\hline 515 & NAMED_POINT & A & \\
\hline 516 & NAMED_POINT & C & \\
\hline 517 & NAMED_POINT & C & \\
\hline 518 & NAMED_POINT & C & \\
\hline 519 & NAMED_POINT & C & \\
\hline 520 & NAMED_POINT & B & \\
\hline 521 & NAMED_POINT & A & \\
\hline 522 & NAMED_POINT & D & \\
\hline 523 & NAMED_POINT & D & \\
\hline 524 & NAMED_POINT & & \\
\hline 525 & NAMED_POINT & & \\
\hline 526 & NAMED_POINT & & \\
\hline 527 & NAMED_POINT & & \\
\hline 528 & NAMED_POINT & & \\
\hline 529 & NAMED_POINT & & \\
\hline 530 & NAMED_POINT & & \\
\hline 531 & NAMED_POINT & & \\
\hline 532 & NAMED_POINT & & \\
\hline 533 & NAMED_POINT & D & \\
\hline
\end{tabular}




\begin{tabular}{|c|c|c|c|}
\hline & $\mathrm{J}$ & $\mathrm{K}$ & $\mathrm{L}$ \\
\hline 1 & SAMP_SITE_TYPE & SCHED_LABEL_CODE & TRANSFORM_CODE \\
\hline 534 & NAMED_POINT & & \\
\hline 535 & NAMED_POINT & & \\
\hline 536 & NAMED_POINT & & \\
\hline 537 & NAMED_POINT & & \\
\hline 538 & NAMED_POINT & A & \\
\hline 539 & NAMED_POINT & & \\
\hline 540 & NAMED_POINT & & \\
\hline 541 & NAMED_POINT & & \\
\hline 542 & NAMED_POINT & & \\
\hline 543 & NAMED_POINT & & \\
\hline 544 & NAMED_POINT & & \\
\hline 545 & NAMED_POINT & A & \\
\hline 546 & NAMED_POINT & A & \\
\hline 547 & NAMED_POINT & A & \\
\hline 548 & NAMED_POINT & C & \\
\hline 549 & NAMED_POINT & D & \\
\hline 550 & NAMED_POINT & & \\
\hline 551 & NAMED_POINT & & \\
\hline 552 & NAMED_POINT & & \\
\hline 553 & NAMED_POINT & & \\
\hline 554 & NAMED_POINT & & \\
\hline 555 & NAMED_POINT & & \\
\hline 556 & NAMED_POINT & & \\
\hline 557 & NAMED_POINT & & \\
\hline 558 & NAMED_POINT & & \\
\hline 559 & NAMED_POINT & & \\
\hline 560 & NAMED_POINT & & \\
\hline 561 & NAMED_POINT & & \\
\hline 562 & NAMED_POINT & & \\
\hline 563 & NAMED_POINT & & \\
\hline 564 & NAMED_POINT & & \\
\hline 565 & NAMED_POINT & & \\
\hline 566 & NAMED_POINT & & \\
\hline 567 & NAMED_POINT & & \\
\hline 568 & NAMED_POINT & & \\
\hline 569 & NAMED_POINT & & \\
\hline 570 & NAMED_POINT & & \\
\hline 571 & NAMED_POINT & & \\
\hline
\end{tabular}




\begin{tabular}{|c|l|l|l|}
\hline & \multicolumn{1}{|c|}{ J } & K & L \\
\hline 1 & SAMP_SITE_TYPE & SCHED_LABEL_CODE & TRANSFORM_CODE \\
\hline 572 & NAMED_POINT & & \\
\hline 573 & NAMED_POINT & & \\
\hline 574 & NAMED_POINT & & \\
\hline 575 & NAMED_POINT & & \\
\hline 576 & NAMED_POINT & & \\
\hline 577 & NAMED_POINT & & \\
\hline 578 & NAMED_POINT & & \\
\hline 579 & NAMED_POINT & & \\
\hline 580 & NAMED_POINT & & \\
\hline 581 & NAMED_POINT & & \\
\hline 582 & NAMED_POINT & & \\
\hline 583 & NAMED_POINT & & \\
\hline 584 & NAMED_POINT & & \\
\hline 585 & NAMED_POINT & & \\
\hline 586 & NAMED_POINT & & \\
\hline 587 & NAMED_POINT & & \\
\hline 588 & NAMED_POINT & & \\
\hline 589 & NAMED_POINT & & \\
\hline 590 & NAMED_POINT & & \\
\hline 591 & NAMED_POINT & & \\
\hline 592 & NAMED_POINT & & \\
\hline 593 & NAMED_POINT & & \\
\hline 594 & NAMED_POINT & & \\
\hline 595 & NAMED_POINT & & \\
\hline 596 & NAMED_POINT & & \\
\hline 597 & NAMED_POINT & & \\
\hline 598 & NAMED_POINT & & \\
\hline 599 & NAMED_POINT & & \\
\hline 600 & NAMED_POINT & & \\
\hline 601 & NAMED_POINT & & \\
\hline 602 & NAMED_POINT & & \\
\hline 603 & NAMED_POINT & & \\
\hline 604 & NAMED_POINT & & \\
\hline 605 & NAMED_POINT & & \\
\hline 606 & NAMED_POINT & & \\
\hline 607 & NAMED_POINT & & \\
\hline 608 & NAMED_POINT & & \\
\hline 609 & NAMED_POINT & & \\
\hline & & & \\
\hline
\end{tabular}




\begin{tabular}{|c|l|l|l|}
\hline & \multicolumn{1}{|c|}{ J } & K & L \\
\hline 1 & SAMP_SITE_TYPE & SCHED_LABEL_CODE & TRANSFORM_CODE \\
\hline 610 & NAMED_POINT & & \\
\hline 611 & NAMED_POINT & & \\
\hline 612 & NAMED_POINT & & \\
\hline 613 & NAMED_POINT & & \\
\hline 614 & NAMED_POINT & & \\
\hline 615 & NAMED_POINT & & \\
\hline 616 & AREA & & \\
\hline 617 & NAMED_POINT & & \\
\hline 618 & NAMED_POINT & & \\
\hline 619 & NAMED_POINT & & \\
\hline 620 & NAMED_POINT & & \\
\hline 621 & NAMED_POINT & & \\
\hline 622 & NAMED_POINT & & \\
\hline 623 & NAMED_POINT & & \\
\hline 624 & AREA & & \\
\hline 625 & AREA & & \\
\hline 626 & AREA & & \\
\hline 627 & AREA & & \\
\hline 628 & NAMED_POINT & & \\
\hline 629 & NAMED_POINT & & \\
\hline 630 & NAMED_POINT & & \\
\hline 631 & NAMED_POINT & & \\
\hline 632 & NAMED_POINT & & \\
\hline 633 & NAMED_POINT & & \\
\hline 634 & NAMED_POINT & & \\
\hline 635 & NAMED_POINT & & \\
\hline 636 & NAMED_POINT & & \\
\hline 637 & NAMED_POINT & & \\
\hline 638 & NAMED_POINT & & \\
\hline 639 & NAMED_POINT & & \\
\hline 640 & NAMED_POINT & & \\
\hline 641 & NAMED_POINT & & \\
\hline 642 & NAMED_POINT & & \\
\hline 643 & NAMED_POINT & & \\
\hline 644 & NAMED_POINT & & \\
\hline 645 & NAMED_POINT & & \\
\hline 646 & NAMED_POINT & & \\
\hline 647 & NAMED_POINT & & \\
\hline & & & \\
\hline
\end{tabular}




\begin{tabular}{|c|l|l|l|}
\hline & \multicolumn{1}{|c|}{ J } & \multicolumn{1}{|c|}{ K } & L \\
\hline 1 & SAMP_SITE_TYPE & SCHED_LABEL_CODE & TRANSFORM_CODE \\
\hline 648 & NAMED_POINT & & \\
\hline 649 & NAMED_POINT & & \\
\hline 650 & NAMED_POINT & & \\
\hline 651 & NAMED_POINT & & \\
\hline 652 & NAMED_POINT & & \\
\hline 653 & NAMED_POINT & & \\
\hline 654 & NAMED_POINT & & \\
\hline 655 & NAMED_POINT & & \\
\hline 656 & NAMED_POINT & & \\
\hline 657 & NAMED_POINT & & \\
\hline 658 & NAMED_POINT & & \\
\hline 659 & NAMED_POINT & & \\
\hline 660 & NAMED_POINT & & \\
\hline 661 & NAMED_POINT & & \\
\hline 662 & NAMED_POINT & & \\
\hline 663 & NAMED_POINT & & \\
\hline 664 & NAMED_POINT & & \\
\hline 665 & NAMED_POINT & & \\
\hline 666 & NAMED_POINT & & \\
\hline 667 & NAMED_POINT & & \\
\hline 668 & NAMED_POINT & & \\
\hline 669 & NAMED_POINT & & \\
\hline 670 & NAMED_POINT & & \\
\hline 671 & NAMED_POINT & & \\
\hline 672 & NAMED_POINT & & \\
\hline 673 & NAMED_POINT & & \\
\hline 674 & NAMED_POINT & & \\
\hline 675 & NAMED_POINT & & \\
\hline 676 & NAMED_POINT & & \\
\hline 677 & NAMED_POINT & & \\
\hline 678 & NAMED_POINT & & \\
\hline 679 & NAMED_POINT & & \\
\hline 680 & NAMED_POINT & & \\
\hline 681 & NAMED_POINT & & \\
\hline 682 & NAMED_POINT & & \\
\hline 683 & NAMED_POINT & & \\
\hline 684 & NAMED_POINT & & \\
\hline 685 & AREA & & \\
\hline & & & \\
\hline
\end{tabular}




\begin{tabular}{|c|l|l|l|}
\hline & \multicolumn{1}{|c|}{ J } & \multicolumn{1}{c|}{ K } & L \\
\hline 1 & SAMP_SITE_TYPE & SCHED_LABEL_CODE & TRANSFORM_CODE \\
\hline 686 & AREA & & \\
\hline 687 & AREA & & \\
\hline 688 & NAMED_POINT & & \\
\hline 689 & NAMED_POINT & & \\
\hline 690 & NAMED_POINT & & \\
\hline 691 & NAMED_POINT & & \\
\hline 692 & NAMED_POINT & & \\
\hline 693 & NAMED_POINT & & \\
\hline 694 & NAMED_POINT & & \\
\hline 695 & NAMED_POINT & & \\
\hline 696 & NAMED_POINT & & \\
\hline 697 & NAMED_POINT & & \\
\hline 698 & NAMED_POINT & & \\
\hline 699 & NAMED_POINT & & \\
\hline 700 & NAMED_POINT & & \\
\hline 701 & NAMED_POINT & & \\
\hline 702 & NAMED_POINT & & \\
\hline 703 & NAMED_POINT & & \\
\hline 704 & NAMED_POINT & & \\
\hline 705 & NAMED_POINT & & \\
\hline 706 & NAMED_POINT & & \\
\hline 707 & NAMED_POINT & & \\
\hline 708 & NAMED_POINT & & \\
\hline 709 & NAMED_POINT & & \\
\hline 710 & NAMED_POINT & & \\
\hline 711 & NAMED_POINT & & \\
\hline 712 & NAMED_POINT & & \\
\hline 713 & NAMED_POINT & & \\
\hline 714 & NAMED_POINT & & \\
\hline 715 & NAMED_POINT & & \\
\hline 716 & NAMED_POINT & & \\
\hline 717 & NAMED_POINT & & \\
\hline 718 & AREA & & \\
\hline 719 & AREA & & \\
\hline 720 & AREA & & \\
\hline 721 & AREA & & \\
\hline 722 & AREA & & \\
\hline 723 & AREA & & \\
\hline & & & \\
\hline
\end{tabular}




\begin{tabular}{|c|c|c|c|}
\hline & $\mathrm{J}$ & $\mathrm{K}$ & $\mathrm{L}$ \\
\hline 1 & SAMP_SITE_TYPE & SCHED_LABEL_CODE & TRANSFORM_CODE \\
\hline 724 & AREA & G & \\
\hline 725 & AREA & G & \\
\hline 726 & NAMED_POINT & G & \\
\hline 727 & NAMED_POINT & G & \\
\hline 728 & NAMED_POINT & G & \\
\hline 729 & AREA & & \\
\hline 730 & AREA & & \\
\hline 731 & AREA & & \\
\hline 732 & AREA & & \\
\hline 733 & AREA & & \\
\hline 734 & AREA & & \\
\hline 735 & AREA & & \\
\hline 736 & AREA & & \\
\hline 737 & AREA & & \\
\hline 738 & AREA & & \\
\hline 739 & AREA & & \\
\hline 740 & AREA & & \\
\hline 741 & AREA & & \\
\hline 742 & AREA & & \\
\hline 743 & AREA & & \\
\hline 744 & AREA & & \\
\hline 745 & AREA & & \\
\hline 746 & AREA & & \\
\hline 747 & NAMED_POINT & C & \\
\hline 748 & NAMED_POINT & C & \\
\hline 749 & NAMED_POINT & C & \\
\hline 750 & NAMED_POINT & C & \\
\hline 751 & NAMED_POINT & C & \\
\hline 752 & NAMED_POINT & C & \\
\hline 753 & NAMED_POINT & C & \\
\hline 754 & NAMED_POINT & C & \\
\hline 755 & NAMED_POINT & C & \\
\hline 756 & NAMED_POINT & C & \\
\hline 757 & NAMED_POINT & C & \\
\hline 758 & NAMED_POINT & C & \\
\hline 759 & NAMED_POINT & C & \\
\hline 760 & NAMED_POINT & C & \\
\hline 761 & NAMED_POINT & C & \\
\hline
\end{tabular}




\begin{tabular}{|c|l|l|l|}
\hline & \multicolumn{1}{|c|}{ J } & \multicolumn{1}{|c|}{ K } & L \\
\hline 1 & SAMP_SITE_TYPE & SCHED_LABEL_CODE & TRANSFORM_CODE \\
\hline 762 & NAMED_POINT & C & \\
\hline 763 & NAMED_POINT & C & \\
\hline 764 & NAMED_POINT & C & \\
\hline 765 & NAMED_POINT & C & \\
\hline 766 & NAMED_POINT & C & \\
\hline 767 & NAMED_POINT & C & \\
\hline 768 & NAMED_POINT & C & \\
\hline 769 & NAMED_POINT & C & \\
\hline 770 & NAMED_POINT & C & \\
\hline 771 & NAMED_POINT & C & \\
\hline 772 & NAMED_POINT & C & \\
\hline 773 & NAMED_POINT & C & \\
\hline 774 & NAMED_POINT & C & \\
\hline 775 & NAMED_POINT & A & \\
\hline 776 & NAMED_POINT & & \\
\hline 777 & NAMED_POINT & & \\
\hline 778 & NAMED_POINT & & \\
\hline 779 & NAMED_POINT & & \\
\hline 780 & AREA & G & \\
\hline 781 & NAMED_POINT & G & \\
\hline 782 & NAMED_POINT & G & \\
\hline 783 & NAMED_POINT & A & \\
\hline 784 & AREA & & \\
\hline 785 & NAMED_POINT & & \\
\hline 786 & NAMED_POINT & & \\
\hline 787 & NAMED_POINT & & \\
\hline 788 & NAMED_POINT & & \\
\hline 789 & NAMED_POINT & & \\
\hline 790 & NAMED_POINT & & \\
\hline 791 & NAMED_POINT & & \\
\hline 792 & NAMED_POINT & & \\
\hline 793 & NAMED_POINT & & \\
\hline 794 & NAMED_POINT & & \\
\hline 795 & NAMED_POINT & & \\
\hline 796 & NAMED_POINT & & \\
\hline 797 & NAMED_POINT & & \\
\hline 798 & NAMED_POINT & & \\
\hline 799 & NAMED_POINT & & \\
\hline & & & \\
\hline
\end{tabular}




\begin{tabular}{|c|l|l|l|}
\hline & \multicolumn{1}{|c|}{ J } & K & L \\
\hline 1 & SAMP_SITE_TYPE & SCHED_LABEL_CODE & TRANSFORM_CODE \\
\hline 800 & NAMED_POINT & & \\
\hline 801 & NAMED_POINT & & \\
\hline 802 & NAMED_POINT & & \\
\hline 803 & NAMED_POINT & & \\
\hline 804 & NAMED_POINT & & \\
\hline 805 & NAMED_POINT & & \\
\hline 806 & NAMED_POINT & & \\
\hline 807 & NAMED_POINT & & \\
\hline 808 & NAMED_POINT & & \\
\hline 809 & NAMED_POINT & & \\
\hline 810 & NAMED_POINT & & \\
\hline 811 & NAMED_POINT & & \\
\hline 812 & NAMED_POINT & & \\
\hline 813 & NAMED_POINT & & \\
\hline 814 & NAMED_POINT & & \\
\hline 815 & NAMED_POINT & & \\
\hline 816 & NAMED_POINT & & \\
\hline 817 & NAMED_POINT & & \\
\hline 818 & AREA & & \\
\hline 819 & NAMED_POINT & & \\
\hline 820 & NAMED_POINT & & \\
\hline 821 & NAMED_POINT & & \\
\hline 822 & NAMED_POINT & & \\
\hline 823 & NAMED_POINT & & \\
\hline 824 & NAMED_POINT & & \\
\hline 825 & NAMED_POINT & & \\
\hline 826 & NAMED_POINT & & \\
\hline 827 & NAMED_POINT & & \\
\hline 828 & NAMED_POINT & & \\
\hline 829 & NAMED_POINT & & \\
\hline 830 & NAMED_POINT & & \\
\hline 831 & NAMED_POINT & & \\
\hline 832 & NAMED_POINT & & \\
\hline 833 & NAMED_POINT & & \\
\hline 834 & NAMED_POINT & & \\
\hline 835 & NAMED_POINT & & \\
\hline 836 & NAMED_POINT & & \\
\hline 837 & NAMED_POINT & & \\
\hline & & & \\
\hline
\end{tabular}




\begin{tabular}{|c|l|l|l|}
\hline & \multicolumn{1}{|c|}{ J } & K & L \\
\hline 1 & SAMP_SITE_TYPE & SCHED_LABEL_CODE & TRANSFORM_CODE \\
\hline 838 & NAMED_POINT & & \\
\hline 839 & NAMED_POINT & & \\
\hline 840 & NAMED_POINT & & \\
\hline 841 & NAMED_POINT & & \\
\hline 842 & NAMED_POINT & & \\
\hline 843 & NAMED_POINT & & \\
\hline 844 & NAMED_POINT & & \\
\hline 845 & NAMED_POINT & & \\
\hline 846 & NAMED_POINT & & \\
\hline 847 & NAMED_POINT & & \\
\hline 848 & NAMED_POINT & & \\
\hline 849 & NAMED_POINT & & \\
\hline 850 & NAMED_POINT & & \\
\hline 851 & NAMED_POINT & & \\
\hline 852 & NAMED_POINT & & \\
\hline 853 & NAMED_POINT & & \\
\hline 854 & NAMED_POINT & & \\
\hline 855 & NAMED_POINT & G & \\
\hline 856 & NAMED_POINT & G & \\
\hline 857 & NAMED_POINT & & \\
\hline 858 & NAMED_POINT & & \\
\hline 859 & NAMED_POINT & & \\
\hline 860 & NAMED_POINT & & \\
\hline 861 & NAMED_POINT & & \\
\hline 862 & NAMED_POINT & & \\
\hline 863 & NAMED_POINT & & \\
\hline 864 & NAMED_POINT & & \\
\hline 865 & NAMED_POINT & & \\
\hline 866 & NAMED_POINT & & \\
\hline 867 & NAMED_POINT & & \\
\hline 868 & NAMED_POINT & & \\
\hline 869 & NAMED_POINT & & \\
\hline 870 & NAMED_POINT & & \\
\hline 871 & NAMED_POINT & & \\
\hline 872 & AREA & & \\
\hline 873 & NAMED_POINT & & \\
\hline 874 & NAMED_POINT & & \\
\hline 875 & NAMED_POINT & & \\
\hline & & & \\
\hline
\end{tabular}




\begin{tabular}{|c|l|l|l|}
\hline & \multicolumn{1}{|c|}{ J } & K & L \\
\hline 1 & SAMP_SITE_TYPE & SCHED_LABEL_CODE & TRANSFORM_CODE \\
\hline 876 & NAMED_POINT & & \\
\hline 877 & NAMED_POINT & & \\
\hline 878 & NAMED_POINT & & \\
\hline 879 & NAMED_POINT & & \\
\hline 880 & NAMED_POINT & & \\
\hline 881 & NAMED_POINT & & \\
\hline 882 & NAMED_POINT & & \\
\hline 883 & NAMED_POINT & & \\
\hline 884 & NAMED_POINT & & \\
\hline 885 & NAMED_POINT & & \\
\hline 886 & NAMED_POINT & & \\
\hline 887 & NAMED_POINT & & \\
\hline 888 & NAMED_POINT & & \\
\hline 889 & NAMED_POINT & & \\
\hline 890 & NAMED_POINT & & \\
\hline 891 & NAMED_POINT & & \\
\hline 892 & NAMED_POINT & & \\
\hline 893 & NAMED_POINT & & \\
\hline 894 & NAMED_POINT & & \\
\hline 895 & NAMED_POINT & & \\
\hline 896 & NAMED_POINT & & \\
\hline 897 & NAMED_POINT & & \\
\hline 898 & NAMED_POINT & & \\
\hline 899 & NAMED_POINT & & \\
\hline 900 & NAMED_POINT & & \\
\hline 901 & NAMED_POINT & & \\
\hline 902 & NAMED_POINT & & \\
\hline 903 & NAMED_POINT & & \\
\hline 904 & NAMED_POINT & & \\
\hline 905 & NAMED_POINT & & \\
\hline 906 & NAMED_POINT & & \\
\hline 907 & NAMED_POINT & & \\
\hline 908 & NAMED_POINT & & \\
\hline 909 & NAMED_POINT & & \\
\hline 910 & NAMED_POINT & & \\
\hline 911 & NAMED_POINT & & \\
\hline 912 & NAMED_POINT & & \\
\hline 913 & NAMED_POINT & & \\
\hline & & & \\
\hline
\end{tabular}




\begin{tabular}{|c|l|l|l|}
\hline & \multicolumn{1}{|c|}{ J } & K & L \\
\hline 1 & SAMP_SITE_TYPE & SCHED_LABEL_CODE & TRANSFORM_CODE \\
\hline 914 & NAMED_POINT & & \\
\hline 915 & NAMED_POINT & & \\
\hline 916 & NAMED_POINT & & \\
\hline 917 & NAMED_POINT & & \\
\hline 918 & NAMED_POINT & & \\
\hline 919 & NAMED_POINT & & \\
\hline 920 & NAMED_POINT & & \\
\hline 921 & NAMED_POINT & & \\
\hline 922 & NAMED_POINT & & \\
\hline 923 & NAMED_POINT & & \\
\hline 924 & NAMED_POINT & & \\
\hline 925 & NAMED_POINT & & \\
\hline 926 & NAMED_POINT & & \\
\hline 927 & AREA & & \\
\hline 928 & NAMED_POINT & & \\
\hline 929 & NAMED_POINT & & \\
\hline 930 & NAMED_POINT & & \\
\hline 931 & NAMED_POINT & & \\
\hline 932 & NAMED_POINT & & \\
\hline 933 & NAMED_POINT & & \\
\hline 934 & NAMED_POINT & & \\
\hline 935 & NAMED_POINT & & \\
\hline 936 & NAMED_POINT & & \\
\hline 937 & NAMED_POINT & & \\
\hline 938 & NAMED_POINT & & \\
\hline 939 & NAMED_POINT & & \\
\hline 940 & NAMED_POINT & & \\
\hline 941 & NAMED_POINT & & \\
\hline 942 & NAMED_POINT & & \\
\hline 943 & NAMED_POINT & & \\
\hline 944 & NAMED_POINT & & \\
\hline 945 & NAMED_POINT & & \\
\hline 946 & NAMED_POINT & & \\
\hline 947 & NAMED_POINT & & \\
\hline 948 & NAMED_POINT & & \\
\hline 949 & NAMED_POINT & & \\
\hline 950 & NAMED_POINT & & \\
\hline 951 & NAMED_POINT & & \\
\hline & & & \\
\hline
\end{tabular}




\begin{tabular}{|c|l|l|l|}
\hline & \multicolumn{1}{|c|}{ J } & K & L \\
\hline 1 & SAMP_SITE_TYPE & SCHED_LABEL_CODE & TRANSFORM_CODE \\
\hline 952 & NAMED_POINT & & \\
\hline 953 & NAMED_POINT & & \\
\hline 954 & NAMED_POINT & & \\
\hline 955 & NAMED_POINT & & \\
\hline 956 & NAMED_POINT & & \\
\hline 957 & NAMED_POINT & & \\
\hline 958 & NAMED_POINT & & \\
\hline 959 & NAMED_POINT & & \\
\hline 960 & NAMED_POINT & & \\
\hline 961 & NAMED_POINT & & \\
\hline 962 & NAMED_POINT & & \\
\hline 963 & NAMED_POINT & & \\
\hline 964 & NAMED_POINT & & \\
\hline 965 & NAMED_POINT & & \\
\hline 966 & NAMED_POINT & & \\
\hline 967 & NAMED_POINT & & \\
\hline 968 & NAMED_POINT & & \\
\hline 969 & NAMED_POINT & & \\
\hline 970 & NAMED_POINT & & \\
\hline 971 & NAMED_POINT & & \\
\hline 972 & NAMED_POINT & & \\
\hline 973 & NAMED_POINT & & \\
\hline 974 & NAMED_POINT & & \\
\hline 975 & NAMED_POINT & & \\
\hline 976 & NAMED_POINT & & \\
\hline 977 & NAMED_POINT & & \\
\hline 978 & NAMED_POINT & & \\
\hline 979 & NAMED_POINT & & \\
\hline 980 & NAMED_POINT & & \\
\hline 981 & NAMED_POINT & & \\
\hline 982 & NAMED_POINT & & \\
\hline 983 & NAMED_POINT & & \\
\hline 984 & NAMED_POINT & & \\
\hline 985 & NAMED_POINT & & \\
\hline 986 & NAMED_POINT & & \\
\hline 987 & NAMED_POINT & & \\
\hline 988 & NAMED_POINT & & \\
\hline 989 & NAMED_POINT & & \\
\hline & & & \\
\hline
\end{tabular}




\begin{tabular}{|c|l|l|l|}
\hline & \multicolumn{1}{|c|}{ J } & \multicolumn{1}{|c|}{ K } & \\
\hline 1 & SAMP_SITE_TYPE & SCHED_LABEL_CODE & TRANSFORM_CODE \\
\hline 990 & NAMED_POINT & & \\
\hline 991 & NAMED_POINT & & \\
\hline 992 & NAMED_POINT & & \\
\hline 993 & NAMED_POINT & & \\
\hline 994 & NAMED_POINT & & \\
\hline 995 & NAMED_POINT & & \\
\hline 996 & NAMED_POINT & & \\
\hline 997 & NAMED_POINT & & \\
\hline 998 & NAMED_POINT & & \\
\hline 999 & NAMED_POINT & & \\
\hline 1000 & NAMED_POINT & & \\
\hline 1001 & NAMED_POINT & & \\
\hline 1002 & NAMED_POINT & & \\
\hline 1003 & AREA & & \\
\hline 1004 & NAMED_POINT & & \\
\hline 1005 & NAMED_POINT & & \\
\hline 1006 & NAMED_POINT & & \\
\hline 1007 & NAMED_POINT & & \\
\hline 1008 & NAMED_POINT & & \\
\hline 1009 & NAMED_POINT & & \\
\hline 1010 & NAMED_POINT & & \\
\hline 1011 & NAMED_POINT & & \\
\hline 1012 & NAMED_POINT & & \\
\hline 1013 & NAMED_POINT & & \\
\hline 1014 & NAMED_POINT & & \\
\hline 1015 & NAMED_POINT & & \\
\hline 1016 & NAMED_POINT & & \\
\hline 1017 & NAMED_POINT & & \\
\hline 1018 & NAMED_POINT & & \\
\hline 1019 & NAMED_POINT & & \\
\hline 1020 & NAMED_POINT & & \\
\hline 1021 & NAMED_POINT & & \\
\hline 1022 & NAMED_POINT & & \\
\hline 1023 & NAMED_POINT & & \\
\hline 1024 & NAMED_POINT & & \\
\hline 1025 & NAMED_POINT & & \\
\hline 1026 & NAMED_POINT & & \\
\hline 1027 & NAMED_POINT & & \\
\hline & & & \\
\hline
\end{tabular}




\begin{tabular}{|c|l|l|l|}
\hline & \multicolumn{1}{|c|}{$J$} & \multicolumn{1}{c|}{ K } & L \\
\hline 1 & SAMP_SITE_TYPE & SCHED_LABEL_CODE & TRANSFORM_CODE \\
\hline 1028 & NAMED_POINT & & \\
\hline 1029 & NAMED_POINT & & \\
\hline 1030 & NAMED_POINT & & \\
\hline 1031 & NAMED_POINT & & \\
\hline 1032 & NAMED_POINT & & \\
\hline 1033 & NAMED_POINT & & \\
\hline 1034 & NAMED_POINT & & \\
\hline 1035 & NAMED_POINT & & \\
\hline 1036 & NAMED_POINT & & \\
\hline 1037 & NAMED_POINT & & \\
\hline 1038 & NAMED_POINT & & \\
\hline 1039 & NAMED_POINT & & \\
\hline 1040 & NAMED_POINT & & \\
\hline 1041 & NAMED_POINT & & \\
\hline 1042 & NAMED_POINT & & \\
\hline 1043 & NAMED_POINT & & \\
\hline 1044 & NAMED_POINT & & \\
\hline 1045 & NAMED_POINT & & \\
\hline 1046 & NAMED_POINT & & \\
\hline 1047 & NAMED_POINT & & \\
\hline 1048 & NAMED_POINT & & \\
\hline 1049 & NAMED_POINT & & \\
\hline 1050 & NAMED_POINT & & \\
\hline 1051 & NAMED_POINT & & \\
\hline 1052 & NAMED_POINT & & \\
\hline 1053 & NAMED_POINT & & \\
\hline 1054 & NAMED_POINT & & \\
\hline 1055 & NAMED_POINT & & \\
\hline 1056 & NAMED_POINT & & \\
\hline 1057 & NAMED_POINT & & \\
\hline 1058 & NAMED_POINT & & \\
\hline 1059 & NAMED_POINT & & \\
\hline 1060 & NAMED_POINT & & \\
\hline 1061 & NAMED_POINT & & \\
\hline 1062 & NAMED_POINT & & \\
\hline 1063 & NAMED_POINT & & \\
\hline 1064 & NAMED_POINT & & \\
\hline 1065 & NAMED_POINT & & \\
\hline
\end{tabular}




\begin{tabular}{|c|l|l|l|}
\hline & \multicolumn{1}{|c|}{$J$} & \multicolumn{1}{c|}{ K } & L \\
\hline 1 & SAMP_SITE_TYPE & SCHED_LABEL_CODE & TRANSFORM_CODE \\
\hline 1066 & NAMED_POINT & & \\
\hline 1067 & NAMED_POINT & & \\
\hline 1068 & NAMED_POINT & & \\
\hline 1069 & NAMED_POINT & & \\
\hline 1070 & NAMED_POINT & & \\
\hline 1071 & NAMED_POINT & & \\
\hline 1072 & NAMED_POINT & & \\
\hline 1073 & NAMED_POINT & & \\
\hline 1074 & NAMED_POINT & & \\
\hline 1075 & NAMED_POINT & & \\
\hline 1076 & NAMED_POINT & & \\
\hline 1077 & NAMED_POINT & & \\
\hline 1078 & NAMED_POINT & & \\
\hline 1079 & NAMED_POINT & & \\
\hline 1080 & NAMED_POINT & & \\
\hline 1081 & NAMED_POINT & & \\
\hline 1082 & NAMED_POINT & & \\
\hline 1083 & NAMED_POINT & & \\
\hline 1084 & NAMED_POINT & & \\
\hline 1085 & NAMED_POINT & & \\
\hline 1086 & NAMED_POINT & & \\
\hline 1087 & NAMED_POINT & & \\
\hline 1088 & NAMED_POINT & & \\
\hline 1089 & NAMED_POINT & & \\
\hline 1090 & NAMED_POINT & & \\
\hline 1091 & NAMED_POINT & & \\
\hline 1092 & NAMED_POINT & & \\
\hline 1093 & NAMED_POINT & & \\
\hline 1094 & NAMED_POINT & & \\
\hline 1095 & NAMED_POINT & & \\
\hline 1096 & NAMED_POINT & & \\
\hline 1097 & NAMED_POINT & & \\
\hline 1098 & NAMED_POINT & & \\
\hline 1099 & NAMED_POINT & & \\
\hline 1100 & NAMED_POINT & & \\
\hline 1101 & NAMED_POINT & & \\
\hline 1102 & NAMED_POINT & & \\
\hline 1103 & NAMED_POINT & & \\
\hline
\end{tabular}




\begin{tabular}{|c|l|l|l|}
\hline & \multicolumn{1}{|c|}{$J$} & \multicolumn{1}{c|}{ K } & L \\
\hline 1 & SAMP_SITE_TYPE & SCHED_LABEL_CODE & TRANSFORM_CODE \\
\hline 1104 & NAMED_POINT & & \\
\hline 1105 & NAMED_POINT & & \\
\hline 1106 & NAMED_POINT & & \\
\hline 1107 & NAMED_POINT & & \\
\hline 1108 & NAMED_POINT & & \\
\hline 1109 & NAMED_POINT & & \\
\hline 1110 & NAMED_POINT & & \\
\hline 1111 & NAMED_POINT & & \\
\hline 1112 & NAMED_POINT & & \\
\hline 1113 & NAMED_POINT & & \\
\hline 1114 & NAMED_POINT & & \\
\hline 1115 & NAMED_POINT & & \\
\hline 1116 & NAMED_POINT & & \\
\hline 1117 & NAMED_POINT & & \\
\hline 1118 & NAMED_POINT & & \\
\hline 1119 & NAMED_POINT & & \\
\hline 1120 & NAMED_POINT & & \\
\hline 1121 & NAMED_POINT & & \\
\hline 1122 & NAMED_POINT & & \\
\hline 1123 & NAMED_POINT & & \\
\hline 1124 & NAMED_POINT & & \\
\hline 1125 & NAMED_POINT & & \\
\hline 1126 & NAMED_POINT & & \\
\hline 1127 & NAMED_POINT & & \\
\hline 1128 & NAMED_POINT & & \\
\hline 1129 & NAMED_POINT & & \\
\hline 1130 & NAMED_POINT & & \\
\hline 1131 & NAMED_POINT & & \\
\hline 1132 & NAMED_POINT & & \\
\hline 1133 & NAMED_POINT & & \\
\hline 1134 & NAMED_POINT & & \\
\hline 1135 & NAMED_POINT & & \\
\hline 1136 & NAMED_POINT & & \\
\hline 1137 & NAMED_POINT & & \\
\hline 1138 & NAMED_POINT & & \\
\hline 1139 & NAMED_POINT & & \\
\hline 1140 & NAMED_POINT & & \\
\hline 1141 & NAMED_POINT & & \\
\hline
\end{tabular}




\begin{tabular}{|c|l|l|l|}
\hline & \multicolumn{1}{|c|}{$J$} & \multicolumn{1}{c|}{ K } & L \\
\hline 1 & SAMP_SITE_TYPE & SCHED_LABEL_CODE & TRANSFORM_CODE \\
\hline 1142 & NAMED_POINT & & \\
\hline 1143 & NAMED_POINT & & \\
\hline 1144 & NAMED_POINT & & \\
\hline 1145 & NAMED_POINT & & \\
\hline 1146 & NAMED_POINT & & \\
\hline 1147 & NAMED_POINT & & \\
\hline 1148 & NAMED_POINT & & \\
\hline 1149 & NAMED_POINT & & \\
\hline 1150 & NAMED_POINT & & \\
\hline 1151 & NAMED_POINT & & \\
\hline 1152 & NAMED_POINT & & \\
\hline 1153 & NAMED_POINT & & \\
\hline 1154 & NAMED_POINT & & \\
\hline 1155 & NAMED_POINT & & \\
\hline 1156 & NAMED_POINT & & \\
\hline 1157 & NAMED_POINT & & \\
\hline 1158 & NAMED_POINT & & \\
\hline 1159 & NAMED_POINT & & \\
\hline 1160 & NAMED_POINT & & \\
\hline 1161 & NAMED_POINT & & \\
\hline 1162 & NAMED_POINT & & \\
\hline 1163 & NAMED_POINT & & \\
\hline 1164 & NAMED_POINT & & \\
\hline 1165 & NAMED_POINT & & \\
\hline 1166 & NAMED_POINT & & \\
\hline 1167 & NAMED_POINT & & \\
\hline 1168 & NAMED_POINT & & \\
\hline 1169 & NAMED_POINT & & \\
\hline 1170 & NAMED_POINT & & \\
\hline 1171 & NAMED_POINT & & \\
\hline 1172 & NAMED_POINT & & \\
\hline 1173 & NAMED_POINT & & \\
\hline 1174 & NAMED_POINT & & \\
\hline 1175 & NAMED_POINT & & \\
\hline 1176 & NAMED_POINT & & \\
\hline 1177 & NAMED_POINT & & \\
\hline 1178 & NAMED_POINT & & \\
\hline 1179 & NAMED_POINT & & \\
\hline
\end{tabular}




\begin{tabular}{|c|l|l|l|}
\hline & \multicolumn{1}{|c|}{$J$} & \multicolumn{1}{c|}{ K } & L \\
\hline 1 & SAMP_SITE_TYPE & SCHED_LABEL_CODE & TRANSFORM_CODE \\
\hline 1180 & NAMED_POINT & & \\
\hline 1181 & NAMED_POINT & & \\
\hline 1182 & NAMED_POINT & & \\
\hline 1183 & NAMED_POINT & & \\
\hline 1184 & NAMED_POINT & & \\
\hline 1185 & NAMED_POINT & & \\
\hline 1186 & NAMED_POINT & & \\
\hline 1187 & NAMED_POINT & & \\
\hline 1188 & NAMED_POINT & & \\
\hline 1189 & NAMED_POINT & & \\
\hline 1190 & NAMED_POINT & & \\
\hline 1191 & NAMED_POINT & & \\
\hline 1192 & NAMED_POINT & & \\
\hline 1193 & NAMED_POINT & & \\
\hline 1194 & NAMED_POINT & & \\
\hline 1195 & NAMED_POINT & & \\
\hline 1196 & NAMED_POINT & & \\
\hline 1197 & NAMED_POINT & & \\
\hline 1198 & NAMED_POINT & & \\
\hline 1199 & NAMED_POINT & & \\
\hline 1200 & NAMED_POINT & & \\
\hline 1201 & NAMED_POINT & & \\
\hline 1202 & NAMED_POINT & & \\
\hline 1203 & NAMED_POINT & & \\
\hline 1204 & NAMED_POINT & & \\
\hline 1205 & NAMED_POINT & & \\
\hline 1206 & NAMED_POINT & & \\
\hline 1207 & NAMED_POINT & & \\
\hline 1208 & NAMED_POINT & & \\
\hline 1209 & NAMED_POINT & & \\
\hline 1210 & NAMED_POINT & & \\
\hline 1211 & NAMED_POINT & & \\
\hline 1212 & NAMED_POINT & & \\
\hline 1213 & NAMED_POINT & & \\
\hline 1214 & NAMED_POINT & & \\
\hline 1215 & NAMED_POINT & & \\
\hline 1216 & NAMED_POINT & & \\
\hline 1217 & NAMED_POINT & & \\
\hline
\end{tabular}




\begin{tabular}{|c|l|l|l|}
\hline & \multicolumn{1}{|c|}{$J$} & \multicolumn{1}{c|}{ K } & L \\
\hline 1 & SAMP_SITE_TYPE & SCHED_LABEL_CODE & TRANSFORM_CODE \\
\hline 1218 & NAMED_POINT & & \\
\hline 1219 & NAMED_POINT & & \\
\hline 1220 & NAMED_POINT & & \\
\hline 1221 & NAMED_POINT & & \\
\hline 1222 & NAMED_POINT & & \\
\hline 1223 & NAMED_POINT & & \\
\hline 1224 & NAMED_POINT & & \\
\hline 1225 & NAMED_POINT & & \\
\hline 1226 & NAMED_POINT & & \\
\hline 1227 & NAMED_POINT & & \\
\hline 1228 & NAMED_POINT & & \\
\hline 1229 & NAMED_POINT & & \\
\hline 1230 & NAMED_POINT & & \\
\hline 1231 & NAMED_POINT & & \\
\hline 1232 & NAMED_POINT & & \\
\hline 1233 & NAMED_POINT & & \\
\hline 1234 & NAMED_POINT & & \\
\hline 1235 & NAMED_POINT & & \\
\hline 1236 & NAMED_POINT & & \\
\hline 1237 & NAMED_POINT & & \\
\hline 1238 & NAMED_POINT & & \\
\hline 1239 & NAMED_POINT & & \\
\hline 1240 & NAMED_POINT & & \\
\hline 1241 & NAMED_POINT & & \\
\hline 1242 & NAMED_POINT & & \\
\hline 1243 & NAMED_POINT & & \\
\hline 1244 & NAMED_POINT & & \\
\hline 1245 & NAMED_POINT & & \\
\hline 1246 & NAMED_POINT & & \\
\hline 1247 & NAMED_POINT & & \\
\hline 1248 & NAMED_POINT & & \\
\hline 1249 & AREA & & \\
\hline 1250 & AREA & & \\
\hline 1251 & AREA & & \\
\hline 1252 & AREA & \\
\hline 1253 & AREA & & \\
\hline 1254 & NAMED_POINT & & \\
\hline 1255 & NAMED_POINT & & \\
\hline
\end{tabular}




\begin{tabular}{|c|l|l|l|}
\hline & \multicolumn{1}{|c|}{$J$} & \multicolumn{1}{c|}{ K } & L \\
\hline 1 & SAMP_SITE_TYPE & SCHED_LABEL_CODE & TRANSFORM_CODE \\
\hline 1256 & NAMED_POINT & & \\
\hline 1257 & NAMED_POINT & & \\
\hline 1258 & NAMED_POINT & & \\
\hline 1259 & NAMED_POINT & & \\
\hline 1260 & NAMED_POINT & & \\
\hline 1261 & NAMED_POINT & & \\
\hline 1262 & NAMED_POINT & & \\
\hline 1263 & NAMED_POINT & & \\
\hline 1264 & NAMED_POINT & & \\
\hline 1265 & NAMED_POINT & & \\
\hline 1266 & NAMED_POINT & & \\
\hline 1267 & NAMED_POINT & & \\
\hline 1268 & NAMED_POINT & & \\
\hline 1269 & NAMED_POINT & & \\
\hline 1270 & NAMED_POINT & & \\
\hline 1271 & NAMED_POINT & & \\
\hline 1272 & NAMED_POINT & & \\
\hline 1273 & NAMED_POINT & & \\
\hline 1274 & NAMED_POINT & & \\
\hline 1275 & NAMED_POINT & & \\
\hline 1276 & NAMED_POINT & & \\
\hline 1277 & NAMED_POINT & & \\
\hline 1278 & NAMED_POINT & & \\
\hline 1279 & NAMED_POINT & & \\
\hline 1280 & NAMED_POINT & & \\
\hline 1281 & NAMED_POINT & & \\
\hline 1282 & NAMED_POINT & & \\
\hline 1283 & NAMED_POINT & & \\
\hline 1284 & NAMED_POINT & & \\
\hline 1285 & NAMED_POINT & & \\
\hline 1286 & NAMED_POINT & & \\
\hline 1287 & NAMED_POINT & & \\
\hline 1288 & NAMED_POINT & & \\
\hline 1289 & NAMED_POINT & & \\
\hline 1290 & NAMED_POINT & & \\
\hline 1291 & NAMED_POINT & & \\
\hline 1292 & NAMED_POINT & & \\
\hline 1293 & NAMED_POINT & & \\
\hline
\end{tabular}




\begin{tabular}{|c|l|l|l|}
\hline & \multicolumn{1}{|c|}{$J$} & \multicolumn{1}{c|}{ K } & L \\
\hline 1 & SAMP_SITE_TYPE & SCHED_LABEL_CODE & TRANSFORM_CODE \\
\hline 1294 & NAMED_POINT & & \\
\hline 1295 & NAMED_POINT & & \\
\hline 1296 & NAMED_POINT & & \\
\hline 1297 & NAMED_POINT & & \\
\hline 1298 & NAMED_POINT & & \\
\hline 1299 & NAMED_POINT & & \\
\hline 1300 & NAMED_POINT & & \\
\hline 1301 & NAMED_POINT & & \\
\hline 1302 & NAMED_POINT & & \\
\hline 1303 & NAMED_POINT & & \\
\hline 1304 & NAMED_POINT & & \\
\hline 1305 & NAMED_POINT & & \\
\hline 1306 & NAMED_POINT & & \\
\hline 1307 & NAMED_POINT & & \\
\hline 1308 & NAMED_POINT & & \\
\hline 1309 & NAMED_POINT & & \\
\hline 1310 & NAMED_POINT & & \\
\hline 1311 & NAMED_POINT & & \\
\hline 1312 & NAMED_POINT & & \\
\hline 1313 & NAMED_POINT & & \\
\hline 1314 & NAMED_POINT & & \\
\hline 1315 & NAMED_POINT & & \\
\hline 1316 & NAMED_POINT & & \\
\hline 1317 & NAMED_POINT & & \\
\hline 1318 & NAMED_POINT & & \\
\hline 1319 & NAMED_POINT & & \\
\hline 1320 & NAMED_POINT & & \\
\hline 1321 & NAMED_POINT & & \\
\hline 1322 & NAMED_POINT & & \\
\hline 1323 & NAMED_POINT & & \\
\hline 1324 & NAMED_POINT & & \\
\hline 1325 & NAMED_POINT & & \\
\hline 1326 & NAMED_POINT & & \\
\hline 1327 & NAMED_POINT & & \\
\hline 1328 & NAMED_POINT & & \\
\hline 1329 & NAMED_POINT & & \\
\hline 1330 & NAMED_POINT & & \\
\hline 1331 & NAMED_POINT & & \\
\hline
\end{tabular}




\begin{tabular}{|c|l|l|l|}
\hline & \multicolumn{1}{|c|}{$J$} & \multicolumn{1}{c|}{ K } & L \\
\hline 1 & SAMP_SITE_TYPE & SCHED_LABEL_CODE & TRANSFORM_CODE \\
\hline 1332 & NAMED_POINT & & \\
\hline 1333 & AREA & & \\
\hline 1334 & NAMED_POINT & & \\
\hline 1335 & NAMED_POINT & & \\
\hline 1336 & NAMED_POINT & & \\
\hline 1337 & NAMED_POINT & & \\
\hline 1338 & NAMED_POINT & & \\
\hline 1339 & NAMED_POINT & & \\
\hline 1340 & NAMED_POINT & & \\
\hline 1341 & NAMED_POINT & & \\
\hline 1342 & NAMED_POINT & & \\
\hline 1343 & NAMED_POINT & & \\
\hline 1344 & NAMED_POINT & & \\
\hline 1345 & NAMED_POINT & & \\
\hline 1346 & NAMED_POINT & & \\
\hline 1347 & NAMED_POINT & & \\
\hline 1348 & NAMED_POINT & & \\
\hline 1349 & NAMED_POINT & & \\
\hline 1350 & NAMED_POINT & & \\
\hline 1351 & NAMED_POINT & & \\
\hline 1352 & NAMED_POINT & & \\
\hline 1353 & NAMED_POINT & & \\
\hline 1354 & NAMED_POINT & & \\
\hline 1355 & NAMED_POINT & & \\
\hline 1356 & NAMED_POINT & & \\
\hline 1357 & NAMED_POINT & & \\
\hline 1358 & NAMED_POINT & & \\
\hline 1359 & NAMED_POINT & & \\
\hline 1360 & NAMED_POINT & & \\
\hline 1361 & NAMED_POINT & & \\
\hline 1362 & NAMED_POINT & & \\
\hline 1363 & NAMED_POINT & & \\
\hline 1364 & NAMED_POINT & & \\
\hline 1365 & NAMED_POINT & & \\
\hline 1366 & NAMED_POINT & & \\
\hline 1367 & NAMED_POINT & & \\
\hline 1368 & NAMED_POINT & & \\
\hline 1369 & NAMED_POINT & & \\
\hline
\end{tabular}




\begin{tabular}{|c|l|l|l|}
\hline & \multicolumn{1}{|c|}{$J$} & \multicolumn{1}{c|}{ K } & L \\
\hline 1 & SAMP_SITE_TYPE & SCHED_LABEL_CODE & TRANSFORM_CODE \\
\hline 1370 & NAMED_POINT & & \\
\hline 1371 & NAMED_POINT & & \\
\hline 1372 & NAMED_POINT & & \\
\hline 1373 & NAMED_POINT & & \\
\hline 1374 & NAMED_POINT & & \\
\hline 1375 & NAMED_POINT & & \\
\hline 1376 & NAMED_POINT & & \\
\hline 1377 & NAMED_POINT & & \\
\hline 1378 & NAMED_POINT & & \\
\hline 1379 & NAMED_POINT & & \\
\hline 1380 & NAMED_POINT & & \\
\hline 1381 & NAMED_POINT & & \\
\hline 1382 & NAMED_POINT & & \\
\hline 1383 & NAMED_POINT & & \\
\hline 1384 & NAMED_POINT & & \\
\hline 1385 & NAMED_POINT & & \\
\hline 1386 & NAMED_POINT & & \\
\hline 1387 & NAMED_POINT & & \\
\hline 1388 & NAMED_POINT & & \\
\hline 1389 & NAMED_POINT & & \\
\hline 1390 & NAMED_POINT & & \\
\hline 1391 & NAMED_POINT & & \\
\hline 1392 & NAMED_POINT & & \\
\hline 1393 & NAMED_POINT & & \\
\hline 1394 & NAMED_POINT & & \\
\hline 1395 & NAMED_POINT & & \\
\hline 1396 & NAMED_POINT & & \\
\hline 1397 & NAMED_POINT & & \\
\hline 1398 & NAMED_POINT & & \\
\hline 1399 & NAMED_POINT & & \\
\hline 1400 & NAMED_POINT & & \\
\hline 1401 & NAMED_POINT & & \\
\hline 1402 & NAMED_POINT & & \\
\hline 1403 & NAMED_POINT & & \\
\hline 1404 & NAMED_POINT & & \\
\hline 1405 & NAMED_POINT & & \\
\hline 1406 & NAMED_POINT & & \\
\hline 1407 & NAMED_POINT & & \\
\hline
\end{tabular}




\begin{tabular}{|c|l|l|l|}
\hline & \multicolumn{1}{|c|}{$J$} & \multicolumn{1}{c|}{ K } & L \\
\hline 1 & SAMP_SITE_TYPE & SCHED_LABEL_CODE & TRANSFORM_CODE \\
\hline 1408 & NAMED_POINT & & \\
\hline 1409 & NAMED_POINT & & \\
\hline 1410 & NAMED_POINT & & \\
\hline 1411 & NAMED_POINT & & \\
\hline 1412 & NAMED_POINT & & \\
\hline 1413 & NAMED_POINT & & \\
\hline 1414 & NAMED_POINT & & \\
\hline 1415 & NAMED_POINT & & \\
\hline 1416 & NAMED_POINT & & \\
\hline 1417 & NAMED_POINT & & \\
\hline 1418 & NAMED_POINT & & \\
\hline 1419 & NAMED_POINT & & \\
\hline 1420 & NAMED_POINT & & \\
\hline 1421 & NAMED_POINT & & \\
\hline 1422 & NAMED_POINT & & \\
\hline 1423 & NAMED_POINT & & \\
\hline 1424 & NAMED_POINT & & \\
\hline 1425 & NAMED_POINT & & \\
\hline 1426 & NAMED_POINT & & \\
\hline 1427 & NAMED_POINT & & \\
\hline 1428 & NAMED_POINT & & \\
\hline 1429 & NAMED_POINT & & \\
\hline 1430 & NAMED_POINT & & \\
\hline 1431 & NAMED_POINT & & \\
\hline 1432 & NAMED_POINT & & \\
\hline 1433 & NAMED_POINT & & \\
\hline 1434 & NAMED_POINT & & \\
\hline 1435 & NAMED_POINT & & \\
\hline 1436 & NAMED_POINT & & \\
\hline 1437 & NAMED_POINT & & \\
\hline 1438 & NAMED_POINT & & \\
\hline 1439 & NAMED_POINT & & \\
\hline 1440 & NAMED_POINT & & \\
\hline 1441 & NAMED_POINT & & \\
\hline 1442 & NAMED_POINT & & \\
\hline 1443 & NAMED_POINT & & \\
\hline 1444 & NAMED_POINT & & \\
\hline 1445 & NAMED_POINT & & \\
\hline
\end{tabular}




\begin{tabular}{|c|l|l|l|}
\hline & \multicolumn{1}{|c|}{$J$} & \multicolumn{1}{c|}{ K } & L \\
\hline 1 & SAMP_SITE_TYPE & SCHED_LABEL_CODE & TRANSFORM_CODE \\
\hline 1446 & NAMED_POINT & & \\
\hline 1447 & NAMED_POINT & & \\
\hline 1448 & NAMED_POINT & & \\
\hline 1449 & NAMED_POINT & & \\
\hline 1450 & NAMED_POINT & & \\
\hline 1451 & NAMED_POINT & & \\
\hline 1452 & NAMED_POINT & & \\
\hline 1453 & NAMED_POINT & & \\
\hline 1454 & NAMED_POINT & & \\
\hline 1455 & NAMED_POINT & & \\
\hline 1456 & NAMED_POINT & & \\
\hline 1457 & NAMED_POINT & & \\
\hline 1458 & NAMED_POINT & & \\
\hline 1459 & NAMED_POINT & & \\
\hline 1460 & NAMED_POINT & & \\
\hline 1461 & NAMED_POINT & & \\
\hline 1462 & NAMED_POINT & & \\
\hline 1463 & NAMED_POINT & & \\
\hline 1464 & NAMED_POINT & & \\
\hline 1465 & NAMED_POINT & & \\
\hline 1466 & NAMED_POINT & & \\
\hline 1467 & NAMED_POINT & & \\
\hline 1468 & NAMED_POINT & & \\
\hline 1469 & NAMED_POINT & & \\
\hline 1470 & NAMED_POINT & & \\
\hline 1471 & NAMED_POINT & & \\
\hline 1472 & NAMED_POINT & & \\
\hline 1473 & NAMED_POINT & & \\
\hline 1474 & NAMED_POINT & & \\
\hline 1475 & NAMED_POINT & & \\
\hline 1476 & NAMED_POINT & & \\
\hline 1477 & NAMED_POINT & & \\
\hline 1478 & NAMED_POINT & & \\
\hline 1479 & NAMED_POINT & & \\
\hline 1480 & NAMED_POINT & & \\
\hline 1481 & NAMED_POINT & & \\
\hline 1482 & NAMED_POINT & & \\
\hline 1483 & NAMED_POINT & & \\
\hline
\end{tabular}




\begin{tabular}{|c|l|l|l|}
\hline & \multicolumn{1}{|c|}{$J$} & \multicolumn{1}{c|}{ K } & L \\
\hline 1 & SAMP_SITE_TYPE & SCHED_LABEL_CODE & TRANSFORM_CODE \\
\hline 1484 & NAMED_POINT & & \\
\hline 1485 & NAMED_POINT & & \\
\hline 1486 & NAMED_POINT & & \\
\hline 1487 & NAMED_POINT & & \\
\hline 1488 & NAMED_POINT & & \\
\hline 1489 & NAMED_POINT & & \\
\hline 1490 & NAMED_POINT & & \\
\hline 1491 & NAMED_POINT & & \\
\hline 1492 & NAMED_POINT & & \\
\hline 1493 & NAMED_POINT & & \\
\hline 1494 & NAMED_POINT & & \\
\hline 1495 & NAMED_POINT & & \\
\hline 1496 & NAMED_POINT & & \\
\hline 1497 & NAMED_POINT & & \\
\hline 1498 & NAMED_POINT & & \\
\hline 1499 & NAMED_POINT & & \\
\hline 1500 & NAMED_POINT & & \\
\hline 1501 & NAMED_POINT & & \\
\hline 1502 & NAMED_POINT & & \\
\hline 1503 & NAMED_POINT & & \\
\hline 1504 & NAMED_POINT & & \\
\hline 1505 & NAMED_POINT & & \\
\hline 1506 & NAMED_POINT & & \\
\hline 1507 & NAMED_POINT & & \\
\hline 1508 & NAMED_POINT & & \\
\hline 1509 & NAMED_POINT & & \\
\hline 1510 & NAMED_POINT & & \\
\hline 1511 & NAMED_POINT & & \\
\hline 1512 & NAMED_POINT & & \\
\hline 1513 & NAMED_POINT & & \\
\hline 1514 & NAMED_POINT & & \\
\hline 1515 & NAMED_POINT & & \\
\hline 1516 & NAMED_POINT & & \\
\hline 1517 & NAMED_POINT & & \\
\hline 1518 & NAMED_POINT & & \\
\hline 1519 & NAMED_POINT & & \\
\hline 1520 & NAMED_POINT & & \\
\hline 1521 & NAMED_POINT & & \\
\hline
\end{tabular}




\begin{tabular}{|c|l|l|l|}
\hline & \multicolumn{1}{|c|}{ J } & \multicolumn{1}{c|}{ K } & L \\
\hline 1 & SAMP_SITE_TYPE & SCHED_LABEL_CODE & TRANSFORM_CODE \\
\hline 1522 & NAMED_POINT & & \\
\hline 1523 & NAMED_POINT & & \\
\hline 1524 & NAMED_POINT & & \\
\hline 1525 & NAMED_POINT & & \\
\hline 1526 & NAMED_POINT & & \\
\hline 1527 & NAMED_POINT & & \\
\hline 1528 & NAMED_POINT & & \\
\hline 1529 & NAMED_POINT & & \\
\hline 1530 & NAMED_POINT & & \\
\hline 1531 & NAMED_POINT & & \\
\hline 1532 & NAMED_POINT & & \\
\hline 1533 & NAMED_POINT & & \\
\hline 1534 & NAMED_POINT & & \\
\hline 1535 & NAMED_POINT & & \\
\hline 1536 & NAMED_POINT & & \\
\hline 1537 & NAMED_POINT & & \\
\hline 1538 & NAMED_POINT & & \\
\hline 1539 & NAMED_POINT & & \\
\hline 1540 & NAMED_POINT & & \\
\hline 1541 & NAMED_POINT & & \\
\hline 1542 & NAMED_POINT & & \\
\hline 1543 & NAMED_POINT & & \\
\hline 1544 & NAMED_POINT & & \\
\hline 1545 & NAMED_POINT & & \\
\hline 1546 & NAMED_POINT & & \\
\hline 1547 & NAMED_POINT & & \\
\hline 1548 & NAMED_POINT & & \\
\hline 1549 & NAMED_POINT & & \\
\hline 1550 & NAMED_POINT & & \\
\hline 1551 & NAMED_POINT & & \\
\hline 1552 & NAMED_POINT & & \\
\hline 1553 & NAMED_POINT & & \\
\hline 1554 & NAMED_POINT & & \\
\hline 1555 & NAMED_POINT & & \\
\hline 1556 & NAMED_POINT & & \\
\hline 1557 & NAMED_POINT & & \\
\hline 1558 & NAMED_POINT & & \\
\hline 1559 & NAMED_POINT & & \\
\hline & & & \\
\hline
\end{tabular}




\begin{tabular}{|c|l|l|l|}
\hline & \multicolumn{1}{|c|}{$J$} & \multicolumn{1}{c|}{ K } & L \\
\hline 1 & SAMP_SITE_TYPE & SCHED_LABEL_CODE & TRANSFORM_CODE \\
\hline 1560 & NAMED_POINT & & \\
\hline 1561 & NAMED_POINT & & \\
\hline 1562 & NAMED_POINT & & \\
\hline 1563 & NAMED_POINT & & \\
\hline 1564 & NAMED_POINT & & \\
\hline 1565 & NAMED_POINT & & \\
\hline 1566 & NAMED_POINT & & \\
\hline 1567 & NAMED_POINT & & \\
\hline 1568 & NAMED_POINT & & \\
\hline 1569 & NAMED_POINT & & \\
\hline 1570 & NAMED_POINT & & \\
\hline 1571 & NAMED_POINT & & \\
\hline 1572 & NAMED_POINT & & \\
\hline 1573 & NAMED_POINT & & \\
\hline 1574 & NAMED_POINT & & \\
\hline 1575 & NAMED_POINT & & \\
\hline 1576 & NAMED_POINT & & \\
\hline 1577 & NAMED_POINT & & \\
\hline 1578 & NAMED_POINT & & \\
\hline 1579 & NAMED_POINT & & \\
\hline 1580 & NAMED_POINT & & \\
\hline 1581 & NAMED_POINT & & \\
\hline 1582 & NAMED_POINT & & \\
\hline 1583 & NAMED_POINT & & \\
\hline 1584 & NAMED_POINT & & \\
\hline 1585 & NAMED_POINT & & \\
\hline 1586 & NAMED_POINT & & \\
\hline 1587 & NAMED_POINT & & \\
\hline 1588 & NAMED_POINT & & \\
\hline 1589 & NAMED_POINT & & \\
\hline 1590 & NAMED_POINT & & \\
\hline 1591 & NAMED_POINT & & \\
\hline 1592 & NAMED_POINT & & \\
\hline 1593 & NAMED_POINT & & \\
\hline 1594 & NAMED_POINT & & \\
\hline 1595 & NAMED_POINT & & \\
\hline 1596 & NAMED_POINT & & \\
\hline 1597 & NAMED_POINT & & \\
\hline
\end{tabular}




\begin{tabular}{|c|l|l|l|}
\hline & \multicolumn{1}{|c|}{ J } & \multicolumn{1}{c|}{ K } & L \\
\hline 1 & SAMP_SITE_TYPE & SCHED_LABEL_CODE & TRANSFORM_CODE \\
\hline 1598 & NAMED_POINT & & \\
\hline 1599 & NAMED_POINT & & \\
\hline 1600 & NAMED_POINT & & \\
\hline 1601 & NAMED_POINT & & \\
\hline 1602 & NAMED_POINT & & \\
\hline 1603 & NAMED_POINT & & \\
\hline 1604 & NAMED_POINT & & \\
\hline 1605 & NAMED_POINT & & \\
\hline 1606 & NAMED_POINT & & \\
\hline 1607 & NAMED_POINT & & \\
\hline 1608 & NAMED_POINT & & \\
\hline 1609 & NAMED_POINT & & \\
\hline 1610 & NAMED_POINT & & \\
\hline 1611 & NAMED_POINT & & \\
\hline 1612 & NAMED_POINT & & \\
\hline 1613 & NAMED_POINT & & \\
\hline 1614 & NAMED_POINT & & \\
\hline 1615 & NAMED_POINT & & \\
\hline 1616 & NAMED_POINT & & \\
\hline 1617 & NAMED_POINT & & \\
\hline 1618 & NAMED_POINT & & \\
\hline 1619 & NAMED_POINT & & \\
\hline 1620 & NAMED_POINT & & \\
\hline 1621 & NAMED_POINT & & \\
\hline 1622 & NAMED_POINT & & \\
\hline 1623 & NAMED_POINT & & \\
\hline 1624 & NAMED_POINT & & \\
\hline 1625 & NAMED_POINT & & \\
\hline 1626 & NAMED_POINT & & \\
\hline 1627 & NAMED_POINT & & \\
\hline 1628 & NAMED_POINT & & \\
\hline 1629 & NAMED_POINT & & \\
\hline 1630 & NAMED_POINT & & \\
\hline 1631 & NAMED_POINT & & \\
\hline 1632 & NAMED_POINT & & \\
\hline 1633 & NAMED_POINT & & \\
\hline 1634 & NAMED_POINT & & \\
\hline 1635 & NAMED_POINT & & \\
\hline & & & \\
\hline
\end{tabular}




\begin{tabular}{|c|l|l|l|}
\hline & \multicolumn{1}{|c|}{$J$} & \multicolumn{1}{c|}{ K } & L \\
\hline 1 & SAMP_SITE_TYPE & SCHED_LABEL_CODE & TRANSFORM_CODE \\
\hline 1636 & NAMED_POINT & & \\
\hline 1637 & NAMED_POINT & & \\
\hline 1638 & NAMED_POINT & & \\
\hline 1639 & NAMED_POINT & & \\
\hline 1640 & NAMED_POINT & & \\
\hline 1641 & NAMED_POINT & & \\
\hline 1642 & NAMED_POINT & & \\
\hline 1643 & NAMED_POINT & & \\
\hline 1644 & NAMED_POINT & & \\
\hline 1645 & NAMED_POINT & & \\
\hline 1646 & NAMED_POINT & & \\
\hline 1647 & NAMED_POINT & & \\
\hline 1648 & NAMED_POINT & & \\
\hline 1649 & NAMED_POINT & & \\
\hline 1650 & NAMED_POINT & & \\
\hline 1651 & NAMED_POINT & & \\
\hline 1652 & NAMED_POINT & & \\
\hline 1653 & NAMED_POINT & & \\
\hline 1654 & NAMED_POINT & & \\
\hline 1655 & NAMED_POINT & & \\
\hline 1656 & NAMED_POINT & & \\
\hline 1657 & NAMED_POINT & & \\
\hline 1658 & NAMED_POINT & & \\
\hline 1659 & NAMED_POINT & & \\
\hline 1660 & NAMED_POINT & & \\
\hline 1661 & NAMED_POINT & & \\
\hline 1662 & NAMED_POINT & & \\
\hline 1663 & NAMED_POINT & & \\
\hline 1664 & NAMED_POINT & & \\
\hline 1665 & NAMED_POINT & & \\
\hline 1666 & NAMED_POINT & & \\
\hline 1667 & NAMED_POINT & & \\
\hline 1668 & NAMED_POINT & & \\
\hline 1669 & NAMED_POINT & & \\
\hline 1670 & NAMED_POINT & & \\
\hline 1671 & NAMED_POINT & & \\
\hline 1672 & NAMED_POINT & & \\
\hline 1673 & NAMED_POINT & & \\
\hline
\end{tabular}




\begin{tabular}{|c|l|l|l|}
\hline & \multicolumn{1}{|c|}{$J$} & \multicolumn{1}{c|}{ K } & L \\
\hline 1 & SAMP_SITE_TYPE & SCHED_LABEL_CODE & TRANSFORM_CODE \\
\hline 1674 & NAMED_POINT & & \\
\hline 1675 & NAMED_POINT & & \\
\hline 1676 & NAMED_POINT & & \\
\hline 1677 & NAMED_POINT & & \\
\hline 1678 & NAMED_POINT & & \\
\hline 1679 & NAMED_POINT & & \\
\hline 1680 & NAMED_POINT & & \\
\hline 1681 & NAMED_POINT & & \\
\hline 1682 & NAMED_POINT & & \\
\hline 1683 & NAMED_POINT & & \\
\hline 1684 & NAMED_POINT & & \\
\hline 1685 & NAMED_POINT & & \\
\hline 1686 & NAMED_POINT & & \\
\hline 1687 & NAMED_POINT & & \\
\hline 1688 & NAMED_POINT & & \\
\hline 1689 & NAMED_POINT & & \\
\hline 1690 & NAMED_POINT & & \\
\hline 1691 & NAMED_POINT & & \\
\hline 1692 & NAMED_POINT & & \\
\hline 1693 & NAMED_POINT & & \\
\hline 1694 & NAMED_POINT & & \\
\hline 1695 & NAMED_POINT & & \\
\hline 1696 & NAMED_POINT & & \\
\hline 1697 & NAMED_POINT & & \\
\hline 1698 & NAMED_POINT & & \\
\hline 1699 & NAMED_POINT & & \\
\hline 1700 & NAMED_POINT & & \\
\hline 1701 & NAMED_POINT & & \\
\hline 1702 & NAMED_POINT & & \\
\hline 1703 & NAMED_POINT & & \\
\hline 1704 & NAMED_POINT & & \\
\hline 1705 & NAMED_POINT & & \\
\hline 1706 & NAMED_POINT & & \\
\hline 1707 & NAMED_POINT & & \\
\hline 1708 & NAMED_POINT & & \\
\hline 1709 & NAMED_POINT & & \\
\hline 1710 & NAMED_POINT & & \\
\hline 1711 & NAMED_POINT & & \\
\hline
\end{tabular}




\begin{tabular}{|c|l|l|l|}
\hline & \multicolumn{1}{|c|}{$J$} & \multicolumn{1}{c|}{ K } & L \\
\hline 1 & SAMP_SITE_TYPE & SCHED_LABEL_CODE & TRANSFORM_CODE \\
\hline 1712 & NAMED_POINT & & \\
\hline 1713 & NAMED_POINT & & \\
\hline 1714 & NAMED_POINT & & \\
\hline 1715 & NAMED_POINT & & \\
\hline 1716 & NAMED_POINT & & \\
\hline 1717 & NAMED_POINT & & \\
\hline 1718 & NAMED_POINT & & \\
\hline 1719 & NAMED_POINT & & \\
\hline 1720 & NAMED_POINT & & \\
\hline 1721 & NAMED_POINT & & \\
\hline 1722 & NAMED_POINT & & \\
\hline 1723 & NAMED_POINT & & \\
\hline 1724 & NAMED_POINT & & \\
\hline 1725 & NAMED_POINT & & \\
\hline 1726 & NAMED_POINT & & \\
\hline 1727 & NAMED_POINT & & \\
\hline 1728 & NAMED_POINT & & \\
\hline 1729 & NAMED_POINT & & \\
\hline 1730 & NAMED_POINT & & \\
\hline 1731 & NAMED_POINT & & \\
\hline 1732 & NAMED_POINT & & \\
\hline 1733 & NAMED_POINT & & \\
\hline 1734 & NAMED_POINT & & \\
\hline 1735 & NAMED_POINT & & \\
\hline 1736 & NAMED_POINT & & \\
\hline 1737 & NAMED_POINT & & \\
\hline 1738 & NAMED_POINT & & \\
\hline 1739 & NAMED_POINT & & \\
\hline 1740 & NAMED_POINT & & \\
\hline 1741 & NAMED_POINT & & \\
\hline 1742 & NAMED_POINT & & \\
\hline 1743 & NAMED_POINT & & \\
\hline 1744 & NAMED_POINT & & \\
\hline 1745 & NAMED_POINT & & \\
\hline 1746 & NAMED_POINT & & \\
\hline 1747 & NAMED_POINT & & \\
\hline 1748 & NAMED_POINT & & \\
\hline 1749 & NAMED_POINT & & \\
\hline
\end{tabular}




\begin{tabular}{|c|l|l|l|}
\hline & \multicolumn{1}{|c|}{ J } & \multicolumn{1}{c|}{ K } & L \\
\hline 1 & SAMP_SITE_TYPE & SCHED_LABEL_CODE & TRANSFORM_CODE \\
\hline 1750 & NAMED_POINT & & \\
\hline 1751 & NAMED_POINT & & \\
\hline 1752 & NAMED_POINT & & \\
\hline 1753 & NAMED_POINT & G & \\
\hline 1754 & NAMED_POINT & & \\
\hline 1755 & NAMED_POINT & & \\
\hline 1756 & NAMED_POINT & & \\
\hline 1757 & NAMED_POINT & & \\
\hline 1758 & NAMED_POINT & & \\
\hline 1759 & NAMED_POINT & & \\
\hline 1760 & NAMED_POINT & & \\
\hline 1761 & NAMED_POINT & & \\
\hline 1762 & NAMED_POINT & & \\
\hline 1763 & NAMED_POINT & & \\
\hline 1764 & NAMED_POINT & & \\
\hline 1765 & NAMED_POINT & & \\
\hline 1766 & NAMED_POINT & & \\
\hline 1767 & NAMED_POINT & & \\
\hline 1768 & NAMED_POINT & & \\
\hline 1769 & NAMED_POINT & & \\
\hline 1770 & NAMED_POINT & & \\
\hline 1771 & NAMED_POINT & & \\
\hline 1772 & NAMED_POINT & & \\
\hline 1773 & NAMED_POINT & & \\
\hline 1774 & NAMED_POINT & & \\
\hline 1775 & NAMED_POINT & & \\
\hline 1776 & NAMED_POINT & & \\
\hline 1777 & NAMED_POINT & & \\
\hline 1778 & NAMED_POINT & & \\
\hline 1779 & NAMED_POINT & & \\
\hline 1780 & & & \\
\hline 1781 & NAMED_POINT & & \\
\hline 1782 & NAMED_POINT & & \\
\hline 1783 & NAMED_POINT & & \\
\hline 1784 & NAMED_POINT & & \\
\hline 1785 & NAMED_POINT & & \\
\hline 1786 & NAMED_POINT & & \\
\hline 1787 & NAMED_POINT & & \\
\hline
\end{tabular}




\begin{tabular}{|c|l|l|l|}
\hline & \multicolumn{1}{|c|}{$J$} & \multicolumn{1}{c|}{ K } & L \\
\hline 1 & SAMP_SITE_TYPE & SCHED_LABEL_CODE & TRANSFORM_CODE \\
\hline 1788 & NAMED_POINT & & \\
\hline 1789 & NAMED_POINT & & \\
\hline 1790 & NAMED_POINT & & \\
\hline 1791 & NAMED_POINT & & \\
\hline 1792 & NAMED_POINT & & \\
\hline 1793 & NAMED_POINT & & \\
\hline 1794 & NAMED_POINT & & \\
\hline 1795 & NAMED_POINT & & \\
\hline 1796 & NAMED_POINT & & \\
\hline 1797 & NAMED_POINT & & \\
\hline 1798 & NAMED_POINT & & \\
\hline 1799 & NAMED_POINT & & \\
\hline 1800 & NAMED_POINT & & \\
\hline 1801 & NAMED_POINT & & \\
\hline 1802 & NAMED_POINT & & \\
\hline 1803 & AREA & & \\
\hline 1804 & NAMED_POINT & G & \\
\hline 1805 & NAMED_POINT & & \\
\hline 1806 & NAMED_POINT & & \\
\hline 1807 & NAMED_POINT & & \\
\hline 1808 & NAMED_POINT & & \\
\hline 1809 & NAMED_POINT & & \\
\hline 1810 & NAMED_POINT & & \\
\hline 1811 & NAMED_POINT & & \\
\hline 1812 & NAMED_POINT & & \\
\hline 1813 & NAMED_POINT & & \\
\hline 1814 & NAMED_POINT & & \\
\hline 1815 & NAMED_POINT & & \\
\hline 1816 & NAMED_POINT & & \\
\hline 1817 & NAMED_POINT & & \\
\hline 1818 & NAMED_POINT & & \\
\hline 1819 & NAMED_POINT & & \\
\hline 1820 & NAMED_POINT & & \\
\hline 1821 & NAMED_POINT & & \\
\hline 1822 & NAMED_POINT & & \\
\hline 1823 & NAMED_POINT & & \\
\hline 1824 & NAMED_POINT & & \\
\hline 1825 & NAMED_POINT & & \\
\hline
\end{tabular}




\begin{tabular}{|c|l|l|l|}
\hline & \multicolumn{1}{|c|}{ J } & \multicolumn{1}{c|}{ K } & L \\
\hline 1 & SAMP_SITE_TYPE & SCHED_LABEL_CODE & TRANSFORM_CODE \\
\hline 1826 & NAMED_POINT & & \\
\hline 1827 & NAMED_POINT & & \\
\hline 1828 & NAMED_POINT & & \\
\hline 1829 & NAMED_POINT & & \\
\hline 1830 & NAMED_POINT & & \\
\hline 1831 & NAMED_POINT & & \\
\hline 1832 & NAMED_POINT & & \\
\hline 1833 & NAMED_POINT & & \\
\hline 1834 & NAMED_POINT & & \\
\hline 1835 & NAMED_POINT & & \\
\hline 1836 & NAMED_POINT & & \\
\hline 1837 & NAMED_POINT & & \\
\hline 1838 & NAMED_POINT & & \\
\hline 1839 & NAMED_POINT & & \\
\hline 1840 & NAMED_POINT & & \\
\hline 1841 & NAMED_POINT & & \\
\hline 1842 & NAMED_POINT & & \\
\hline 1843 & NAMED_POINT & & \\
\hline 1844 & NAMED_POINT & & \\
\hline 1845 & NAMED_POINT & & \\
\hline 1846 & NAMED_POINT & & \\
\hline 1847 & NAMED_POINT & & \\
\hline 1848 & NAMED_POINT & & \\
\hline 1849 & NAMED_POINT & & \\
\hline 1850 & NAMED_POINT & & \\
\hline 1851 & NAMED_POINT & & \\
\hline 1852 & NAMED_POINT & & \\
\hline 1853 & NAMED_POINT & & \\
\hline 1854 & NAMED_POINT & & \\
\hline 1855 & NAMED_POINT & & \\
\hline 1856 & NAMED_POINT & & \\
\hline 1857 & NAMED_POINT & & \\
\hline 1858 & NAMED_POINT & & \\
\hline 1859 & NAMED_POINT & & \\
\hline 1860 & NAMED_POINT & & \\
\hline 1861 & NAMED_POINT & & \\
\hline 1862 & NAMED_POINT & & \\
\hline 1863 & NAMED_POINT & & \\
\hline & & & \\
\hline
\end{tabular}




\begin{tabular}{|c|l|l|l|}
\hline & \multicolumn{1}{|c|}{ J } & \multicolumn{1}{|c|}{ K } & L \\
\hline 1 & SAMP_SITE_TYPE & SCHED_LABEL_CODE & TRANSFORM_CODE \\
\hline 1864 & NAMED_POINT & & \\
\hline 1865 & NAMED_POINT & & \\
\hline 1866 & NAMED_POINT & & \\
\hline 1867 & NAMED_POINT & & \\
\hline 1868 & NAMED_POINT & & \\
\hline 1869 & NAMED_POINT & & \\
\hline 1870 & NAMED_POINT & & \\
\hline 1871 & NAMED_POINT & & \\
\hline 1872 & NAMED_POINT & & \\
\hline 1873 & NAMED_POINT & & \\
\hline 1874 & NAMED_POINT & & \\
\hline 1875 & NAMED_POINT & & \\
\hline 1876 & NAMED_POINT & & \\
\hline 1877 & NAMED_POINT & & \\
\hline 1878 & NAMED_POINT & & \\
\hline 1879 & NAMED_POINT & & \\
\hline 1880 & NAMED_POINT & & \\
\hline 1881 & NAMED_POINT & & \\
\hline 1882 & NAMED_POINT & & \\
\hline 1883 & NAMED_POINT & & \\
\hline 1884 & NAMED_POINT & G & \\
\hline 1885 & NAMED_POINT & G & \\
\hline 1886 & NAMED_POINT & G & \\
\hline 1887 & NAMED_POINT & G & \\
\hline 1888 & NAMED_POINT & G & \\
\hline 1889 & NAMED_POINT & G & \\
\hline 1890 & NAMED_POINT & G & \\
\hline 1891 & NAMED_POINT & G & \\
\hline 1892 & NAMED_POINT & G & \\
\hline 1893 & NAMED_POINT & G & \\
\hline 1894 & NAMED_POINT & G & \\
\hline 1895 & NAMED_POINT & G & \\
\hline 1896 & NAMED_POINT & G & \\
\hline 1897 & NAMED_POINT & G & \\
\hline 1898 & NAMED_POINT & G & \\
\hline 1899 & NAMED_POINT & G & \\
\hline 1900 & NAMED_POINT & G & \\
\hline 1901 & NAMED_POINT & G & \\
\hline & & & \\
\hline
\end{tabular}




\begin{tabular}{|c|c|c|c|}
\hline & $\mathrm{J}$ & $\mathrm{K}$ & $\mathrm{L}$ \\
\hline 1 & SAMP_SITE_TYPE & SCHED_LABEL_CODE & TRANSFORM_CODE \\
\hline 1902 & NAMED_POINT & G & \\
\hline 1903 & NAMED_POINT & G & \\
\hline 1904 & NAMED_POINT & G & \\
\hline 1905 & NAMED_POINT & G & \\
\hline 1906 & NAMED_POINT & G & \\
\hline 1907 & AREA & & \\
\hline 1908 & AREA & & \\
\hline 1909 & AREA & & \\
\hline 1910 & AREA & & \\
\hline 1911 & AREA & & \\
\hline 1912 & AREA & & \\
\hline 1913 & AREA & & \\
\hline 1914 & AREA & & \\
\hline 1915 & AREA & & \\
\hline 1916 & AREA & & \\
\hline 1917 & AREA & & \\
\hline 1918 & AREA & & \\
\hline 1919 & AREA & & \\
\hline 1920 & AREA & & \\
\hline 1921 & AREA & & \\
\hline 1922 & AREA & & \\
\hline 1923 & AREA & & \\
\hline 1924 & AREA & & \\
\hline 1925 & AREA & & \\
\hline 1926 & AREA & & \\
\hline 1927 & AREA & & \\
\hline 1928 & AREA & & \\
\hline 1929 & AREA & & \\
\hline 1930 & AREA & & \\
\hline 1931 & AREA & & \\
\hline 1932 & AREA & & \\
\hline 1933 & AREA & & \\
\hline 1934 & AREA & & \\
\hline 1935 & AREA & & \\
\hline 1936 & AREA & & \\
\hline 1937 & AREA & & \\
\hline 1938 & AREA & & \\
\hline 1939 & AREA & & \\
\hline
\end{tabular}




\begin{tabular}{|c|c|c|c|}
\hline & $\mathrm{J}$ & $\mathrm{K}$ & $\mathrm{L}$ \\
\hline 1 & SAMP_SITE_TYPE & SCHED_LABEL_CODE & TRANSFORM_CODE \\
\hline 1940 & AREA & & \\
\hline 1941 & AREA & & \\
\hline 1942 & AREA & & \\
\hline 1943 & AREA & & \\
\hline 1944 & AREA & & \\
\hline 1945 & AREA & & \\
\hline 1946 & AREA & & \\
\hline 1947 & AREA & & \\
\hline 1948 & AREA & & \\
\hline 1949 & AREA & & \\
\hline 1950 & AREA & & \\
\hline 1951 & AREA & & \\
\hline 1952 & AREA & & \\
\hline 1953 & AREA & & \\
\hline 1954 & AREA & & \\
\hline 1955 & AREA & & \\
\hline 1956 & AREA & & \\
\hline 1957 & AREA & & \\
\hline 1958 & AREA & & \\
\hline 1959 & AREA & & \\
\hline 1960 & AREA & & \\
\hline 1961 & AREA & & \\
\hline 1962 & AREA & & \\
\hline 1963 & AREA & & \\
\hline 1964 & AREA & & \\
\hline 1965 & AREA & & \\
\hline 1966 & AREA & & \\
\hline 1967 & AREA & & \\
\hline 1968 & AREA & & \\
\hline 1969 & AREA & & \\
\hline 1970 & AREA & & \\
\hline 1971 & AREA & & \\
\hline 1972 & AREA & & \\
\hline 1973 & AREA & & \\
\hline 1974 & AREA & & \\
\hline 1975 & AREA & & \\
\hline 1976 & AREA & & \\
\hline 1977 & AREA & & \\
\hline
\end{tabular}




\begin{tabular}{|c|c|c|c|}
\hline & $\mathrm{J}$ & $\mathrm{K}$ & $\mathrm{L}$ \\
\hline 1 & SAMP_SITE_TYPE & SCHED_LABEL_CODE & TRANSFORM_CODE \\
\hline \begin{tabular}{|l|}
1978 \\
\end{tabular} & AREA & & \\
\hline 1979 & AREA & & \\
\hline 1980 & AREA & & \\
\hline 1981 & AREA & & \\
\hline 1982 & AREA & & \\
\hline 1983 & AREA & & \\
\hline 1984 & AREA & & \\
\hline 1985 & AREA & & \\
\hline 1986 & AREA & & \\
\hline 1987 & AREA & & \\
\hline 1988 & AREA & & \\
\hline 1989 & AREA & & \\
\hline 1990 & AREA & & \\
\hline 1991 & AREA & & \\
\hline 1992 & AREA & & \\
\hline 1993 & AREA & & \\
\hline 1994 & AREA & & \\
\hline 1995 & AREA & & \\
\hline 1996 & AREA & & \\
\hline 1997 & AREA & & \\
\hline 1998 & AREA & & \\
\hline 1999 & AREA & & \\
\hline 2000 & AREA & & \\
\hline 2001 & AREA & & \\
\hline 2002 & AREA & & \\
\hline 2003 & AREA & & \\
\hline 2004 & AREA & & \\
\hline 2005 & AREA & & \\
\hline 2006 & AREA & & \\
\hline 2007 & AREA & & \\
\hline 2008 & AREA & & \\
\hline 2009 & AREA & & \\
\hline 2010 & AREA & & \\
\hline 2011 & AREA & & \\
\hline 2012 & AREA & & \\
\hline 2013 & AREA & & \\
\hline 2014 & AREA & & \\
\hline 2015 & AREA & & \\
\hline
\end{tabular}




\begin{tabular}{|c|l|l|l|}
\hline & \multicolumn{1}{|c|}{ J } & \multicolumn{1}{|c|}{ K } & L \\
\hline 1 & SAMP_SITE_TYPE & SCHED_LABEL_CODE & TRANSFORM_CODE \\
\hline 2016 & AREA & & \\
\hline 2017 & AREA & & \\
\hline 2018 & AREA & & \\
\hline 2019 & AREA & & \\
\hline 2020 & NAMED_POINT & & \\
\hline 2021 & NAMED_POINT & & \\
\hline 2022 & NAMED_POINT & & \\
\hline 2023 & NAMED_POINT & & \\
\hline 2024 & NAMED_POINT & & \\
\hline 2025 & NAMED_POINT & & \\
\hline 2026 & & & \\
\hline 2027 & & & \\
\hline 2028 & NAMED_POINT & & \\
\hline 2029 & & & \\
\hline 2030 & & & \\
\hline 2031 & & & \\
\hline 2032 & & & \\
\hline 2033 & & & \\
\hline 2034 & NAMED_POINT & & \\
\hline 2035 & NAMED_POINT & & \\
\hline 2036 & NAMED_POINT & & \\
\hline 2037 & NAMED_POINT & & \\
\hline 2038 & NAMED_POINT & & \\
\hline 2039 & NAMED_POINT & & \\
\hline 2040 & & & \\
\hline 2041 & & & \\
\hline 2042 & & & \\
\hline 2043 & & & \\
\hline 2044 & & & \\
\hline 2045 & & & \\
\hline 2046 & & & \\
\hline 2047 & NAMED_POINT & & \\
\hline 2048 & & & \\
\hline 2049 & & & \\
\hline 2050 & & & \\
\hline 2051 & & & \\
\hline 2052 & & & \\
\hline 2053 & & & \\
\hline
\end{tabular}




$$
\Gamma
$$




\begin{tabular}{|c|l|l|l|}
\hline & \multicolumn{1}{|c|}{ J } & \multicolumn{1}{c|}{ K } & L \\
\hline 1 & SAMP_SITE_TYPE & SCHED_LABEL_CODE & TRANSFORM_CODE \\
\hline 2092 & & & \\
\hline 2093 & & & \\
\hline 2094 & & & \\
\hline 2095 & NAMED_POINT & A & \\
\hline 2096 & NAMED_POINT & A & \\
\hline 2097 & NAMED_POINT & A & \\
\hline 2098 & NAMED_POINT & A & \\
\hline 2099 & NAMED_POINT & A & \\
\hline 2100 & NAMED_POINT & A & \\
\hline 2101 & NAMED_POINT & A & \\
\hline 2102 & NAMED_POINT & G & \\
\hline 2103 & NAMED_POINT & A & \\
\hline 2104 & NAMED_POINT & G & \\
\hline 2105 & AREA & & \\
\hline 2106 & NAMED_POINT & A & \\
\hline 2107 & NAMED_POINT & A & \\
\hline 2108 & NAMED_POINT & A & \\
\hline 2109 & NAMED_POINT & A & \\
\hline 2110 & NAMED_POINT & A & \\
\hline 2111 & & & \\
\hline 2112 & & & \\
\hline 2113 & & & \\
\hline 2114 & NAMED_POINT & C & \\
\hline 2115 & NAMED_POINT & A & \\
\hline 2116 & NAMED_POINT & G & \\
\hline 2117 & NAMED_POINT & D & \\
\hline 2118 & & & \\
\hline 2119 & NAMED_POINT & G & \\
\hline 2120 & NAMED_POINT & A & \\
\hline 2121 & & & \\
\hline 2122 & & & \\
\hline 2123 & NAMED_POINT & & \\
\hline 2124 & NAMED_POINT & & \\
\hline 2125 & NAMED_POINT & & \\
\hline 2126 & NAMED_POINT & & \\
\hline 2127 & NAMED_POINT & & \\
\hline 2128 & NAMED_POINT & & \\
\hline 2129 & NAMED_POINT & & \\
\hline & & & \\
\hline
\end{tabular}




\begin{tabular}{|c|c|c|c|}
\hline & $\mathrm{J}$ & $\mathrm{K}$ & $\underline{L}$ \\
\hline 1 & SAMP_SITE_TYPE & SCHED_LABEL_CODE & TRANSFORM_CODE \\
\hline 2130 & NAMED_POINT & & \\
\hline 2131 & NAMED_POINT & & \\
\hline 2132 & NAMED_POINT & & \\
\hline 2133 & NAMED_POINT & & \\
\hline 2134 & NAMED_POINT & & \\
\hline 2135 & NAMED_POINT & & \\
\hline 2136 & NAMED_POINT & & \\
\hline 2137 & NAMED_POINT & & \\
\hline 2138 & NAMED_POINT & & \\
\hline 2139 & NAMED_POINT & & \\
\hline 2140 & NAMED_POINT & & \\
\hline 2141 & NAMED_POINT & & \\
\hline 2142 & NAMED_POINT & & \\
\hline 2143 & NAMED_POINT & & \\
\hline 2144 & NAMED_POINT & & \\
\hline 2145 & NAMED_POINT & & \\
\hline 2146 & NAMED_POINT & & \\
\hline 2147 & NAMED_POINT & & \\
\hline 2148 & NAMED_POINT & & \\
\hline 2149 & NAMED_POINT & & \\
\hline 2150 & NAMED_POINT & & \\
\hline 2151 & NAMED_POINT & & \\
\hline 2152 & NAMED_POINT & & \\
\hline 2153 & NAMED_POINT & & \\
\hline 2154 & NAMED_POINT & & \\
\hline 2155 & NAMED_POINT & & \\
\hline 2156 & NAMED_POINT & B & \\
\hline 2157 & NAMED_POINT & B & \\
\hline 2158 & NAMED_POINT & A & \\
\hline 2159 & NAMED_POINT & $B$ & \\
\hline 2160 & & & \\
\hline 2161 & & & \\
\hline 2162 & NAMED_POINT & G & \\
\hline 2163 & NAMED_POINT & G & \\
\hline 2164 & NAMED_POINT & G & \\
\hline 2165 & NAMED_POINT & G & \\
\hline 2166 & NAMED_POINT & G & \\
\hline 2167 & NAMED_POINT & G & \\
\hline
\end{tabular}




\begin{tabular}{|c|c|c|c|}
\hline & $\mathrm{J}$ & $\mathrm{K}$ & $\mathrm{L}$ \\
\hline 1 & SAMP_SITE_TYPE & SCHED_LABEL_CODE & TRANSFORM_CODE \\
\hline 2168 & NAMED_POINT & G & \\
\hline 2169 & NAMED_POINT & C & \\
\hline 2170 & NAMED_POINT & C & \\
\hline 2171 & NAMED_POINT & C & \\
\hline 2172 & NAMED_POINT & C & \\
\hline 2173 & NAMED_POINT & D & \\
\hline 2174 & NAMED_POINT & C & \\
\hline 2175 & NAMED_POINT & C & \\
\hline 2176 & NAMED_POINT & C & \\
\hline 2177 & NAMED_POINT & C & \\
\hline 2178 & NAMED_POINT & C & \\
\hline 2179 & NAMED_POINT & C & \\
\hline 2180 & NAMED_POINT & C & \\
\hline 2181 & NAMED_POINT & C & \\
\hline 2182 & AREA & A & \\
\hline 2183 & AREA & A & \\
\hline 2184 & NAMED_POINT & A & \\
\hline 2185 & NAMED_POINT & A & \\
\hline 2186 & NAMED_POINT & A & \\
\hline 2187 & NAMED_POINT & A & \\
\hline 2188 & NAMED_POINT & $\mathrm{F}$ & \\
\hline 2189 & NAMED_POINT & $\mathrm{F}$ & \\
\hline 2190 & NAMED_POINT & $\mathrm{F}$ & \\
\hline 2191 & NAMED_POINT & $\mathrm{F}$ & \\
\hline 2192 & NAMED_POINT & $E$ & \\
\hline 2193 & NAMED_POINT & E & \\
\hline 2194 & NAMED_POINT & E & \\
\hline 2195 & NAMED_POINT & $\mathrm{E}$ & \\
\hline 2196 & NAMED_POINT & D & \\
\hline 2197 & AREA & A & \\
\hline \multicolumn{4}{|l|}{2198} \\
\hline \multicolumn{4}{|l|}{2199} \\
\hline \multicolumn{4}{|l|}{2200} \\
\hline \multicolumn{4}{|l|}{2201} \\
\hline \multicolumn{4}{|l|}{2202} \\
\hline \multicolumn{4}{|c|}{\begin{tabular}{|l|}
2203 \\
\end{tabular}} \\
\hline \multicolumn{4}{|c|}{2204} \\
\hline 2205 & & & \\
\hline
\end{tabular}




$$
\Gamma
$$




$$
\Gamma
$$




\begin{tabular}{|c|c|c|c|}
\hline & $\mathrm{J}$ & $\mathrm{K}$ & $\mathrm{L}$ \\
\hline 1 & SAMP_SITE_TYPE & SCHED_LABEL_CODE & TRANSFORM_CODE \\
\hline \multicolumn{4}{|c|}{\begin{tabular}{|l|l|}
2282 & $=$ \\
\end{tabular}} \\
\hline \multicolumn{4}{|c|}{2283} \\
\hline \multicolumn{4}{|c|}{2284} \\
\hline \multicolumn{4}{|c|}{2285} \\
\hline \multicolumn{4}{|c|}{2286} \\
\hline \multicolumn{4}{|c|}{2287} \\
\hline \multicolumn{4}{|c|}{2288} \\
\hline \multicolumn{4}{|c|}{2289} \\
\hline 2290 & NAMED_POINT & A & \\
\hline 2291 & NAMED_POINT & A & \\
\hline 2292 & NAMED_POINT & A & \\
\hline 2293 & & B & \\
\hline \multicolumn{4}{|c|}{2294} \\
\hline \multicolumn{4}{|c|}{2295} \\
\hline \multicolumn{4}{|c|}{2296} \\
\hline \multicolumn{4}{|c|}{\begin{tabular}{|l|l|}
2297 & NAMED_POINT \\
\end{tabular}} \\
\hline 2298 & NAMED_POINT & D & \\
\hline 2299 & NAMED_POINT & D & \\
\hline 2300 & NAMED_POINT & D & \\
\hline 2301 & NAMED_POINT & A & \\
\hline 2302 & NAMED_POINT & A & \\
\hline 2303 & NAMED_POINT & A & \\
\hline 2304 & NAMED_POINT & A & \\
\hline 2305 & NAMED_POINT & A & \\
\hline 2306 & NAMED_POINT & C & \\
\hline 2307 & NAMED_POINT & C & \\
\hline 2308 & NAMED_POINT & C & \\
\hline 2309 & NAMED_POINT & A & \\
\hline 2310 & NAMED_POINT & A & \\
\hline 2311 & NAMED_POINT & A & \\
\hline 2312 & NAMED_POINT & A & \\
\hline 2313 & NAMED_POINT & A & \\
\hline 2314 & NAMED_POINT & A & \\
\hline 2315 & NAMED_POINT & A & \\
\hline 2316 & NAMED_POINT & A & \\
\hline 2317 & NAMED_POINT & A & \\
\hline 2318 & NAMED_POINT & A & \\
\hline 2319 & NAMED_POINT & A & \\
\hline
\end{tabular}




\begin{tabular}{|c|l|l|l|}
\hline & \multicolumn{1}{|c|}{$J$} & \multicolumn{1}{|c|}{ K } & \\
\hline 1 & SAMP_SITE_TYPE & SCHED_LABEL_CODE & TRANSFORM_CODE \\
\hline 2320 & NAMED_POINT & A & L \\
\hline 2321 & AREA & A & \\
\hline 2322 & NAMED_POINT & D & \\
\hline 2323 & NAMED_POINT & C & \\
\hline 2324 & NAMED_POINT & A & \\
\hline 2325 & NAMED_POINT & A & \\
\hline 2326 & NAMED_POINT & A & \\
\hline 2327 & NAMED_POINT & & \\
\hline 2328 & NAMED_POINT & & \\
\hline 2329 & NAMED_POINT & B & \\
\hline 2330 & NAMED_POINT & & \\
\hline 2331 & NAMED_POINT & & \\
\hline 2332 & NAMED_POINT & & \\
\hline 2333 & NAMED_POINT & & NONE \\
\hline 2334 & NAMED_POINT & & NONE \\
\hline 2335 & NAMED_POINT & & NONE \\
\hline 2336 & NAMED_POINT & & NONE \\
\hline 2337 & NAMED_POINT & & NONE \\
\hline 2338 & NAMED_POINT & & NONE \\
\hline 2339 & NAMED_POINT & & NONE \\
\hline 2340 & NAMED_POINT & & NONE \\
\hline 2341 & AREA & NONE & NONE \\
\hline 2342 & AREA & & NONE \\
\hline 2343 & NAMED_POINT & & NONE \\
\hline 2344 & AREA & & NONE \\
\hline 2345 & NAMED_POINT & & NONE \\
\hline 2346 & NAMED_POINT & & \\
\hline 2347 & NAMED_POINT & & \\
\hline 2348 & AREA & & \\
\hline 2349 & NAMED_POINT & & \\
\hline 2350 & NAMED_POINT & & \\
\hline 2351 & AREA & & \\
\hline 2352 & NAMED_POINT & & \\
\hline 2353 & NAMED_POINT & & \\
\hline 2354 & AREA & & \\
\hline 2355 & AREA & & \\
\hline 2356 & NAMED_POINT & & \\
\hline 2357 & NAMED_POINT & & \\
\hline & & & \\
\hline
\end{tabular}




\begin{tabular}{|c|l|l|l|}
\hline & \multicolumn{1}{|c|}{$J$} & \multicolumn{1}{|c|}{ K } & \\
\hline 1 & SAMP_SITE_TYPE & SCHED_LABEL_CODE & TRANSFORM_CODE \\
\hline 2358 & NAMED_POINT & NONE \\
\hline 2359 & NAMED_POINT & NONE \\
\hline 2360 & NAMED_POINT & NONE \\
\hline 2361 & NAMED_POINT & NONE \\
\hline 2362 & NAMED_POINT & NONE \\
\hline 2363 & NAMED_POINT & NONE \\
\hline 2364 & NAMED_POINT & NONE \\
\hline 2365 & NAMED_POINT & NONE \\
\hline 2366 & NAMED_POINT & NONE \\
\hline 2367 & NAMED_POINT & NONE \\
\hline 2368 & NAMED_POINT & NONE \\
\hline 2369 & NAMED_POINT & NONE \\
\hline 2370 & NAMED_POINT & NONE \\
\hline 2371 & NAMED_POINT & NONE \\
\hline 2372 & NAMED_POINT & NONE \\
\hline 2373 & NAMED_POINT & NONE \\
\hline 2374 & NAMED_POINT & NONE \\
\hline 2375 & NAMED_POINT & NONE \\
\hline 2376 & NAMED_POINT & NONE \\
\hline 2377 & NAMED_POINT & NONE \\
\hline 2378 & NAMED_POINT & NONE \\
\hline 2379 & NAMED_POINT & NONE \\
\hline 2380 & AREA & NONE \\
\hline 2381 & AREA & NONE \\
\hline 2382 & AREA & NONE \\
\hline 2383 & AREA & NONE \\
\hline 2384 & AREA & NONE \\
\hline 2385 & AREA & NONE \\
\hline 2386 & AREA & NONE \\
\hline 2387 & AREA & NONE \\
\hline 2388 & AREA & NONE \\
\hline 2389 & AREA & NONE \\
\hline 2390 & AREA & NONE \\
\hline 2391 & AREA & NONE \\
\hline 2392 & AREA & NONE \\
\hline 2393 & AREA & NONE \\
\hline 2394 & AREA & NONE \\
\hline 2395 & AREA & NONE \\
\hline & & \\
\hline & & \\
\hline
\end{tabular}




\begin{tabular}{|c|l|l|l|}
\hline & \multicolumn{1}{|c|}{$J$} & \multicolumn{1}{|c|}{ K } & \multicolumn{1}{|c|}{ L } \\
\hline 1 & SAMP_SITE_TYPE & SCHED_LABEL_CODE & TRANSFORM_CODE \\
\hline 2396 & AREA & NONE \\
\hline 2397 & AREA & NONE \\
\hline 2398 & AREA & NONE \\
\hline 2399 & AREA & NONE \\
\hline 2400 & NAMED_POINT & NONE \\
\hline 2401 & NAMED_POINT & NONE \\
\hline 2402 & NAMED_POINT & NONE \\
\hline 2403 & AREA & NONE \\
\hline 2404 & NAMED_POINT & NONE \\
\hline 2405 & NAMED_POINT & NONE \\
\hline 2406 & NAMED_POINT & NONE \\
\hline 2407 & NAMED_POINT & NONE \\
\hline 2408 & NAMED_POINT & NONE \\
\hline 2409 & NAMED_POINT & NONE \\
\hline 2410 & NAMED_POINT & NONE \\
\hline 2411 & NAMED_POINT & NONE \\
\hline 2412 & NAMED_POINT & NONE \\
\hline 2413 & NAMED_POINT & NONE \\
\hline 2414 & NAMED_POINT & NONE \\
\hline 2415 & NAMED_POINT & NONE \\
\hline 2416 & NAMED_POINT & NONE \\
\hline 2417 & NAMED_POINT & NONE \\
\hline 2418 & AREA & NONE \\
\hline 2419 & AREA & NONE \\
\hline 2420 & AREA & NONE \\
\hline 2421 & AREA & NONE \\
\hline 2422 & AREA & NONE \\
\hline 2423 & AREA & NONE \\
\hline 2424 & AREA & NONE \\
\hline 2425 & AREA & NONE \\
\hline 2426 & AREA & NONE \\
\hline 2427 & AREA & NONE \\
\hline 2428 & AREA & NONE \\
\hline 2429 & AREA & NONE \\
\hline 2430 & AREA & NONE \\
\hline 2431 & NAMED_POINT & NONE \\
\hline 2432 & AREA & NONE \\
\hline 2433 & AREA & NONE \\
\hline & & \\
\hline & & \\
\hline
\end{tabular}




\begin{tabular}{|c|l|l|l|}
\hline & \multicolumn{1}{|c|}{$J$} & \multicolumn{1}{|c|}{ K } & \multicolumn{1}{|c|}{ L } \\
\hline 1 & SAMP_SITE_TYPE & SCHED_LABEL_CODE & TRANSFORM_CODE \\
\hline 2434 & AREA & NONE \\
\hline 2435 & AREA & NONE \\
\hline 2436 & NAMED_POINT & NONE \\
\hline 2437 & NAMED_POINT & NONE \\
\hline 2438 & NAMED_POINT & NONE \\
\hline 2439 & NAMED_POINT & NONE \\
\hline 2440 & NAMED_POINT & NONE \\
\hline 2441 & NAMED_POINT & NONE \\
\hline 2442 & NAMED_POINT & NONE \\
\hline 2443 & NAMED_POINT & NONE \\
\hline 2444 & NAMED_POINT & NONE \\
\hline 2445 & NAMED_POINT & NONE \\
\hline 2446 & NAMED_POINT & NONE \\
\hline 2447 & NAMED_POINT & NONE \\
\hline 2448 & NAMED_POINT & NONE \\
\hline 2449 & NAMED_POINT & NONE \\
\hline 2450 & NAMED_POINT & NONE \\
\hline 2451 & NAMED_POINT & NONE \\
\hline 2452 & NAMED_POINT & NONE \\
\hline 2453 & NAMED_POINT & NONE \\
\hline 2454 & NAMED_POINT & NONE \\
\hline 2455 & NAMED_POINT & NONE \\
\hline 2456 & NAMED_POINT & NONE \\
\hline 2457 & NAMED_POINT & NONE \\
\hline 2458 & NAMED_POINT & NONE \\
\hline 2459 & NAMED_POINT & NONE \\
\hline 2460 & AREA & NONE \\
\hline 2461 & NAMED_POINT & NONE \\
\hline 2462 & AREA & NONE \\
\hline 2463 & AREA & NONE \\
\hline 2464 & AREA & NONE \\
\hline 2465 & AREA & NONE \\
\hline 2466 & NAMED_POINT & NONE \\
\hline 2467 & NAMED_POINT & NONE \\
\hline 2468 & NAMED_POINT & NONE \\
\hline 2469 & NAMED_POINT & NONE \\
\hline 2470 & NAMED_POINT & NONE \\
\hline 2471 & NAMED_POINT & NONE \\
\hline & & \\
\hline & & \\
\hline
\end{tabular}




\begin{tabular}{|c|l|l|l|}
\hline & \multicolumn{1}{|c|}{ J } & \multicolumn{1}{|c|}{ K } & \\
\hline 1 & SAMP_SITE_TYPE & SCHED_LABEL_CODE & TRANSFORM_CODE \\
\hline 2472 & NAMED_POINT & NONE \\
\hline 2473 & NAMED_POINT & NONE \\
\hline 2474 & NAMED_POINT & NONE \\
\hline 2475 & NAMED_POINT & NONE \\
\hline 2476 & NAMED_POINT & NONE \\
\hline 2477 & NAMED_POINT & NONE \\
\hline 2478 & NAMED_POINT & NONE \\
\hline 2479 & AREA & NONE \\
\hline 2480 & NAMED_POINT & NONE \\
\hline 2481 & NAMED_POINT & NONE \\
\hline 2482 & NAMED_POINT & NONE \\
\hline 2483 & NAMED_POINT & NONE \\
\hline 2484 & NAMED_POINT & NONE \\
\hline 2485 & NAMED_POINT & NONE \\
\hline 2486 & NAMED_POINT & NONE \\
\hline 2487 & NAMED_POINT & NONE \\
\hline 2488 & NAMED_POINT & NONE \\
\hline 2489 & NAMED_POINT & NONE \\
\hline 2490 & NAMED_POINT & NONE \\
\hline 2491 & NAMED_POINT & NONE \\
\hline 2492 & NAMED_POINT & NONE \\
\hline 2493 & NAMED_POINT & NONE \\
\hline 2494 & NAMED_POINT & NONE \\
\hline 2495 & NAMED_POINT & NONE \\
\hline 2496 & NAMED_POINT & NONE \\
\hline 2497 & NAMED_POINT & NONE \\
\hline 2498 & NAMED_POINT & NONE \\
\hline 2499 & NAMED_POINT & NONE \\
\hline 2500 & NAMED_POINT & NONE \\
\hline 2501 & NAMED_POINT & NONE \\
\hline 2502 & NAMED_POINT & NONE \\
\hline 2503 & NAMED_POINT & NONE \\
\hline 2504 & NAMED_POINT & NONE \\
\hline 2505 & NAMED_POINT & NONE \\
\hline 2506 & NAMED_POINT & NONE \\
\hline 2507 & NAMED_POINT & NONE \\
\hline 2508 & NAMED_POINT & NONE \\
\hline 2509 & NAMED_POINT & \\
\hline & & & \\
\hline
\end{tabular}




\begin{tabular}{|c|l|l|l|}
\hline & \multicolumn{1}{|c|}{ J } & \multicolumn{1}{|c|}{ K } & \\
\hline 1 & SAMP_SITE_TYPE & SCHED_LABEL_CODE & TRANSFORM_CODE \\
\hline 2510 & NAMED_POINT & NONE \\
\hline 2511 & NAMED_POINT & NONE \\
\hline 2512 & NAMED_POINT & NONE \\
\hline 2513 & NAMED_POINT & NONE \\
\hline 2514 & NAMED_POINT & NONE \\
\hline 2515 & NAMED_POINT & NONE \\
\hline 2516 & NAMED_POINT & NONE \\
\hline 2517 & NAMED_POINT & NONE \\
\hline 2518 & NAMED_POINT & NONE \\
\hline 2519 & NAMED_POINT & NONE \\
\hline 2520 & NAMED_POINT & NONE \\
\hline 2521 & NAMED_POINT & NONE \\
\hline 2522 & NAMED_POINT & NONE \\
\hline 2523 & NAMED_POINT & NONE \\
\hline 2524 & NAMED_POINT & NONE \\
\hline 2525 & NAMED_POINT & NONE \\
\hline 2526 & NAMED_POINT & NONE \\
\hline 2527 & NAMED_POINT & NONE \\
\hline 2528 & NAMED_POINT & NONE \\
\hline 2529 & NAMED_POINT & NONE \\
\hline 2530 & NAMED_POINT & NONE \\
\hline 2531 & NAMED_POINT & NONE \\
\hline 2532 & NAMED_POINT & NONE \\
\hline 2533 & NAMED_POINT & NONE \\
\hline 2534 & NAMED_POINT & NONE \\
\hline 2535 & NAMED_POINT & NONE \\
\hline 2536 & NAMED_POINT & NONE \\
\hline 2537 & NAMED_POINT & NONE \\
\hline 2538 & NAMED_POINT & NONE \\
\hline 2539 & NAMED_POINT & NONE \\
\hline 2540 & NAMED_POINT & NONE \\
\hline 2541 & NAMED_POINT & NONE \\
\hline 2542 & NAMED_POINT & NONE \\
\hline 2543 & NAMED_POINT & NONE \\
\hline 2544 & NAMED_POINT & NONE \\
\hline 2545 & NAMED_POINT & NONE \\
\hline 2546 & NAMED_POINT & & \\
\hline 2547 & NAMED_POINT & & \\
\hline & & & \\
\hline
\end{tabular}




\begin{tabular}{|c|l|l|l|}
\hline & \multicolumn{1}{|c|}{$J$} & \multicolumn{1}{|c|}{ K } & \\
\hline 1 & SAMP_SITE_TYPE & SCHED_LABEL_CODE & TRANSFORM_CODE \\
\hline 2548 & NAMED_POINT & NONE \\
\hline 2549 & NAMED_POINT & NONE \\
\hline 2550 & NAMED_POINT & NONE \\
\hline 2551 & NAMED_POINT & NONE \\
\hline 2552 & NAMED_POINT & NONE \\
\hline 2553 & NAMED_POINT & NONE \\
\hline 2554 & AREA & NONE \\
\hline 2555 & NAMED_POINT & NONE \\
\hline 2556 & NAMED_POINT & NONE \\
\hline 2557 & NAMED_POINT & NONE \\
\hline 2558 & NAMED_POINT & NONE \\
\hline 2559 & NAMED_POINT & NONE \\
\hline 2560 & NAMED_POINT & NONE \\
\hline 2561 & NAMED_POINT & NONE \\
\hline 2562 & NAMED_POINT & NONE \\
\hline 2563 & NAMED_POINT & NONE \\
\hline 2564 & NAMED_POINT & NONE \\
\hline 2565 & NAMED_POINT & NONE \\
\hline 2566 & NAMED_POINT & NONE \\
\hline 2567 & NAMED_POINT & NONE \\
\hline 2568 & NAMED_POINT & NONE \\
\hline 2569 & NAMED_POINT & NONE \\
\hline 2570 & NAMED_POINT & NONE \\
\hline 2571 & NAMED_POINT & NONE \\
\hline 2572 & NAMED_POINT & NONE \\
\hline 2573 & NAMED_POINT & NONE \\
\hline 2574 & NAMED_POINT & NONE \\
\hline 2575 & NAMED_POINT & NONE \\
\hline 2576 & AREA & NONE \\
\hline 2577 & AREA & NONE \\
\hline 2578 & AREA & NONE \\
\hline 2579 & AREA & NONE \\
\hline 2580 & AREA & NONE \\
\hline 2581 & AREA & NONE \\
\hline 2582 & AREA & NONE \\
\hline 2583 & AREA & NONE \\
\hline 2584 & AREA & NONE \\
\hline 2585 & NAMED_POINT & NONE \\
\hline & & \\
\hline
\end{tabular}




\begin{tabular}{|c|l|l|l|}
\hline & \multicolumn{1}{|c|}{ J } & \multicolumn{1}{|c|}{ K } & \\
\hline 1 & SAMP_SITE_TYPE & SCHED_LABEL_CODE & TRANSFORM_CODE \\
\hline 2586 & NAMED_POINT & NONE \\
\hline 2587 & NAMED_POINT & NONE \\
\hline 2588 & NAMED_POINT & NONE \\
\hline 2589 & NAMED_POINT & NONE \\
\hline 2590 & NAMED_POINT & NONE \\
\hline 2591 & NAMED_POINT & NONE \\
\hline 2592 & NAMED_POINT & NONE \\
\hline 2593 & NAMED_POINT & NONE \\
\hline 2594 & NAMED_POINT & NONE \\
\hline 2595 & NAMED_POINT & NONE \\
\hline 2596 & NAMED_POINT & NONE \\
\hline 2597 & NAMED_POINT & NONE \\
\hline 2598 & NAMED_POINT & NONE \\
\hline 2599 & NAMED_POINT & NONE \\
\hline 2600 & NAMED_POINT & NONE \\
\hline 2601 & NAMED_POINT & NONE \\
\hline 2602 & NAMED_POINT & NONE \\
\hline 2603 & NAMED_POINT & NONE \\
\hline 2604 & NAMED_POINT & NONE \\
\hline 2605 & NAMED_POINT & NONE \\
\hline 2606 & NAMED_POINT & NONE \\
\hline 2607 & NAMED_POINT & NONE \\
\hline 2608 & NAMED_POINT & NONE \\
\hline 2609 & NAMED_POINT & NONE \\
\hline 2610 & NAMED_POINT & NONE \\
\hline 2611 & NAMED_POINT & NONE \\
\hline 2612 & NAMED_POINT & NONE \\
\hline 2613 & NAMED_POINT & NONE \\
\hline 2614 & NAMED_POINT & NONE \\
\hline 2615 & NAMED_POINT & NONE \\
\hline 2616 & NAMED_POINT & NONE \\
\hline 2617 & NAMED_POINT & NONE \\
\hline 2618 & NAMED_POINT & NONE \\
\hline 2619 & NAMED_POINT & NONE \\
\hline 2620 & NAMED_POINT & NONE \\
\hline 2621 & NAMED_POINT & NONE \\
\hline 2622 & NAMED_POINT & \\
\hline 2623 & NAMED_POINT & \\
\hline & & \\
\hline
\end{tabular}




\begin{tabular}{|c|l|l|l|}
\hline & \multicolumn{1}{|c|}{$J$} & \multicolumn{1}{|c|}{ K } & \\
\hline 1 & SAMP_SITE_TYPE & SCHED_LABEL_CODE & TRANSFORM_CODE \\
\hline 2624 & NAMED_POINT & NONE \\
\hline 2625 & NAMED_POINT & NONE \\
\hline 2626 & NAMED_POINT & NONE \\
\hline 2627 & NAMED_POINT & NONE \\
\hline 2628 & NAMED_POINT & NONE \\
\hline 2629 & NAMED_POINT & NONE \\
\hline 2630 & NAMED_POINT & NONE \\
\hline 2631 & NAMED_POINT & NONE \\
\hline 2632 & NAMED_POINT & NONE \\
\hline 2633 & NAMED_POINT & NONE \\
\hline 2634 & NAMED_POINT & NONE \\
\hline 2635 & NAMED_POINT & NONE \\
\hline 2636 & NAMED_POINT & NONE \\
\hline 2637 & NAMED_POINT & NONE \\
\hline 2638 & NAMED_POINT & NONE \\
\hline 2639 & NAMED_POINT & NONE \\
\hline 2640 & NAMED_POINT & NONE \\
\hline 2641 & NAMED_POINT & NONE \\
\hline 2642 & NAMED_POINT & NONE \\
\hline 2643 & AREA & NONE \\
\hline 2644 & & NONE \\
\hline 2645 & & NONE \\
\hline 2646 & NAMED_POINT & NONE \\
\hline 2647 & NAMED_POINT & NONE \\
\hline 2648 & NAMED_POINT & NONE \\
\hline 2649 & AREA & NONE \\
\hline 2650 & AREA & NONE \\
\hline 2651 & AREA & NONE \\
\hline 2652 & AREA & NONE \\
\hline 2653 & AREA & NONE \\
\hline 2654 & AREA & NONE \\
\hline 2655 & AREA & NONE \\
\hline 2656 & AREA & NONE \\
\hline 2657 & AREA & NONE \\
\hline 2658 & AREA & NONE \\
\hline 2659 & AREA & NONE \\
\hline 2660 & AREA & NONE \\
\hline 2661 & AREA & NONE \\
\hline & & \\
\hline & & \\
\hline
\end{tabular}




\begin{tabular}{|c|l|l|l|}
\hline & \multicolumn{1}{|c|}{$J$} & \multicolumn{1}{|c|}{ K } & \\
\hline 1 & SAMP_SITE_TYPE & SCHED_LABEL_CODE & TRANSFORM_CODE \\
\hline 2662 & AREA & NONE \\
\hline 2663 & AREA & NONE \\
\hline 2664 & AREA & NONE \\
\hline 2665 & AREA & NONE \\
\hline 2666 & AREA & NONE \\
\hline 2667 & AREA & NONE \\
\hline 2668 & AREA & NONE \\
\hline 2669 & AREA & NONE \\
\hline 2670 & AREA & NONE \\
\hline 2671 & AREA & NONE \\
\hline 2672 & AREA & NONE \\
\hline 2673 & AREA & NONE \\
\hline 2674 & AREA & NONE \\
\hline 2675 & AREA & NONE \\
\hline 2676 & AREA & NONE \\
\hline 2677 & AREA & NONE \\
\hline 2678 & AREA & NONE \\
\hline 2679 & AREA & NONE \\
\hline 2680 & AREA & NONE \\
\hline 2681 & AREA & NONE \\
\hline 2682 & AREA & NONE \\
\hline 2683 & & NONE \\
\hline 2684 & & NONE \\
\hline 2685 & NAMED_POINT & NONE \\
\hline 2686 & NAMED_POINT & NONE \\
\hline 2687 & NAMED_POINT & NONE \\
\hline 2688 & NAMED_POINT & NONE \\
\hline 2689 & NAMED_POINT & NONE \\
\hline 2690 & NAMED_POINT & NONE \\
\hline 2691 & NAMED_POINT & NONE \\
\hline 2692 & NAMED_POINT & NONE \\
\hline 2693 & NAMED_POINT & NONE \\
\hline 2694 & NAMED_POINT & NONE \\
\hline 2695 & NAMED_POINT & NONE \\
\hline 2696 & NAMED_POINT & NONE \\
\hline 2697 & NAMED_POINT & NONE \\
\hline 2698 & NAMED_POINT & NONE \\
\hline 2699 & NAMED_POINT & NONE \\
\hline & & \\
\hline & & & \\
\hline & & & \\
\hline & & & \\
\hline & & & \\
\hline
\end{tabular}




\begin{tabular}{|c|l|l|l|}
\hline & \multicolumn{1}{|c|}{ J } & \multicolumn{1}{|c|}{ K } & \\
\hline 1 & SAMP_SITE_TYPE & SCHED_LABEL_CODE & TRANSFORM_CODE \\
\hline 2700 & NAMED_POINT & NONE \\
\hline 2701 & NAMED_POINT & NONE \\
\hline 2702 & NAMED_POINT & NONE \\
\hline 2703 & NAMED_POINT & NONE \\
\hline 2704 & NAMED_POINT & NONE \\
\hline 2705 & NAMED_POINT & NONE \\
\hline 2706 & NAMED_POINT & NONE \\
\hline 2707 & NAMED_POINT & NONE \\
\hline 2708 & AREA & NONE \\
\hline 2709 & AREA & NONE \\
\hline 2710 & AREA & NONE \\
\hline 2711 & NAMED_POINT & NONE \\
\hline 2712 & NAMED_POINT & NONE \\
\hline 2713 & NAMED_POINT & NONE \\
\hline 2714 & NAMED_POINT & NONE \\
\hline 2715 & NAMED_POINT & NONE \\
\hline 2716 & NAMED_POINT & NONE \\
\hline 2717 & NAMED_POINT & NONE \\
\hline 2718 & NAMED_POINT & NONE \\
\hline 2719 & NAMED_POINT & NONE \\
\hline 2720 & NAMED_POINT & NONE \\
\hline 2721 & NAMED_POINT & NONE \\
\hline 2722 & NAMED_POINT & NONE \\
\hline 2723 & NAMED_POINT & NONE \\
\hline 2724 & NAMED_POINT & NONE \\
\hline 2725 & NAMED_POINT & NONE \\
\hline 2726 & NAMED_POINT & NONE \\
\hline 2727 & NAMED_POINT & NONE \\
\hline 2728 & NAMED_POINT & NONE \\
\hline 2729 & NAMED_POINT & NONE \\
\hline 2730 & NAMED_POINT & NONE \\
\hline 2731 & NAMED_POINT & NONE \\
\hline 2732 & NAMED_POINT & NONE \\
\hline 2733 & NAMED_POINT & NONE \\
\hline 2734 & NAMED_POINT & NONE \\
\hline 2735 & NAMED_POINT & NONE \\
\hline 2736 & NAMED_POINT & NONE \\
\hline 2737 & NAMED_POINT & \\
\hline & & & \\
\hline
\end{tabular}




\begin{tabular}{|c|l|l|l|}
\hline & \multicolumn{1}{|c|}{ J } & \multicolumn{1}{|c|}{ K } & \\
\hline 1 & SAMP_SITE_TYPE & SCHED_LABEL_CODE & TRANSFORM_CODE \\
\hline 2738 & NAMED_POINT & NONE \\
\hline 2739 & NAMED_POINT & NONE \\
\hline 2740 & NAMED_POINT & NONE \\
\hline 2741 & & NONE \\
\hline 2742 & NAMED_POINT & NONE \\
\hline 2743 & NAMED_POINT & NONE \\
\hline 2744 & NAMED_POINT & NONE \\
\hline 2745 & NAMED_POINT & NONE \\
\hline 2746 & NAMED_POINT & NONE \\
\hline 2747 & NAMED_POINT & NONE \\
\hline 2748 & NAMED_POINT & NONE \\
\hline 2749 & NAMED_POINT & NONE \\
\hline 2750 & NAMED_POINT & NONE \\
\hline 2751 & NAMED_POINT & NONE \\
\hline 2752 & NAMED_POINT & NONE \\
\hline 2753 & NAMED_POINT & NONE \\
\hline 2754 & NAMED_POINT & NONE \\
\hline 2755 & NAMED_POINT & NONE \\
\hline 2756 & NAMED_POINT & NONE \\
\hline 2757 & NAMED_POINT & NONE \\
\hline 2758 & NAMED_POINT & NONE \\
\hline 2759 & NAMED_POINT & NONE \\
\hline 2760 & NAMED_POINT & NONE \\
\hline 2761 & NAMED_POINT & NONE \\
\hline 2762 & NAMED_POINT & NONE \\
\hline 2763 & NAMED_POINT & NONE \\
\hline 2764 & NAMED_POINT & NONE \\
\hline 2765 & NAMED_POINT & NONE \\
\hline 2766 & NAMED_POINT & NONE \\
\hline 2767 & NAMED_POINT & NONE \\
\hline 2768 & NAMED_POINT & NONE \\
\hline 2769 & NAMED_POINT & NONE \\
\hline 2770 & NAMED_POINT & NONE \\
\hline 2771 & NAMED_POINT & NONE \\
\hline 2772 & NAMED_POINT & NONE \\
\hline 2773 & NAMED_POINT & & \\
\hline 2774 & NAMED_POINT & NANE \\
\hline 2775 & NAMED_POINT & & \\
\hline & & & \\
\hline
\end{tabular}




\begin{tabular}{|c|l|l|l|}
\hline & \multicolumn{1}{|c|}{ J } & \multicolumn{1}{|c|}{ K } & \\
\hline 1 & SAMP_SITE_TYPE & SCHED_LABEL_CODE & TRANSFORM_CODE \\
\hline 2776 & NAMED_POINT & NONE \\
\hline 2777 & NAMED_POINT & NONE \\
\hline 2778 & NAMED_POINT & NONE \\
\hline 2779 & NAMED_POINT & NONE \\
\hline 2780 & NAMED_POINT & NONE \\
\hline 2781 & NAMED_POINT & NONE \\
\hline 2782 & NAMED_POINT & NONE \\
\hline 2783 & NAMED_POINT & NONE \\
\hline 2784 & NAMED_POINT & NONE \\
\hline 2785 & NAMED_POINT & NONE \\
\hline 2786 & NAMED_POINT & NONE \\
\hline 2787 & NAMED_POINT & NONE \\
\hline 2788 & NAMED_POINT & NONE \\
\hline 2789 & NAMED_POINT & NONE \\
\hline 2790 & NAMED_POINT & NONE \\
\hline 2791 & NAMED_POINT & NONE \\
\hline 2792 & NAMED_POINT & NONE \\
\hline 2793 & NAMED_POINT & NONE \\
\hline 2794 & NAMED_POINT & NONE \\
\hline 2795 & NAMED_POINT & NONE \\
\hline 2796 & NAMED_POINT & NONE \\
\hline 2797 & NAMED_POINT & NONE \\
\hline 2798 & NAMED_POINT & NONE \\
\hline 2799 & NAMED_POINT & NONE \\
\hline 2800 & NAMED_POINT & NONE \\
\hline 2801 & NAMED_POINT & NONE \\
\hline 2802 & NAMED_POINT & NONE \\
\hline 2803 & NAMED_POINT & NONE \\
\hline 2804 & NAMED_POINT & NONE \\
\hline 2805 & NAMED_POINT & NONE \\
\hline 2806 & NAMED_POINT & NONE \\
\hline 2807 & NAMED_POINT & NONE \\
\hline 2808 & NAMED_POINT & NONE \\
\hline 2809 & NAMED_POINT & NONE \\
\hline 2810 & NAMED_POINT & NONE \\
\hline 2811 & NAMED_POINT & NONE \\
\hline 2812 & NAMED_POINT & NONE \\
\hline 2813 & NAMED_POINT & \\
\hline & & & \\
\hline
\end{tabular}




\begin{tabular}{|c|l|l|l|}
\hline & \multicolumn{1}{|c|}{ J } & \multicolumn{1}{|c|}{ K } & \\
\hline 1 & SAMP_SITE_TYPE & SCHED_LABEL_CODE & TRANSFORM_CODE \\
\hline 2814 & NAMED_POINT & NONE \\
\hline 2815 & NAMED_POINT & NONE \\
\hline 2816 & NAMED_POINT & NONE \\
\hline 2817 & NAMED_POINT & NONE \\
\hline 2818 & NAMED_POINT & NONE \\
\hline 2819 & NAMED_POINT & NONE \\
\hline 2820 & NAMED_POINT & NONE \\
\hline 2821 & NAMED_POINT & NONE \\
\hline 2822 & NAMED_POINT & NONE \\
\hline 2823 & NAMED_POINT & NONE \\
\hline 2824 & NAMED_POINT & NONE \\
\hline 2825 & NAMED_POINT & NONE \\
\hline 2826 & NAMED_POINT & NONE \\
\hline 2827 & NAMED_POINT & NONE \\
\hline 2828 & NAMED_POINT & NONE \\
\hline 2829 & NAMED_POINT & NONE \\
\hline 2830 & NAMED_POINT & NONE \\
\hline 2831 & NAMED_POINT & NONE \\
\hline 2832 & NAMED_POINT & NONE \\
\hline 2833 & NAMED_POINT & NONE \\
\hline 2834 & NAMED_POINT & NONE \\
\hline 2835 & NAMED_POINT & NONE \\
\hline 2836 & NAMED_POINT & NONE \\
\hline 2837 & NAMED_POINT & NONE \\
\hline 2838 & NAMED_POINT & NONE \\
\hline 2839 & NAMED_POINT & NONE \\
\hline 2840 & AREA & NONE \\
\hline 2841 & AREA & NONE \\
\hline 2842 & NAMED_POINT & NONE \\
\hline 2843 & NAMED_POINT & NONE \\
\hline 2844 & NAMED_POINT & NONE \\
\hline 2845 & NAMED_POINT & NONE \\
\hline 2846 & NAMED_POINT & NONE \\
\hline 2847 & NAMED_POINT & NONE \\
\hline 2848 & NAMED_POINT & NONE \\
\hline 2849 & NAMED_POINT & NONE \\
\hline 2850 & & NONE \\
\hline 2851 & NAMED_POINT & \\
\hline & & & \\
\hline
\end{tabular}




\begin{tabular}{|c|c|c|c|}
\hline & $\mathrm{J}$ & $\mathrm{K}$ & $\mathrm{L}$ \\
\hline 1 & SAMP_SITE_TYPE & SCHED_LABEL_CODE & TRANSFORM_CODE \\
\hline 2852 & NAMED_POINT & & NONE \\
\hline 2853 & AREA & & NONE \\
\hline 2854 & NAMED_POINT & & NONE \\
\hline 2855 & NAMED_POINT & & NONE \\
\hline 2856 & NAMED_POINT & & NONE \\
\hline 2857 & NAMED_POINT & & NONE \\
\hline 2858 & NAMED_POINT & & NONE \\
\hline 2859 & NAMED_POINT & & NONE \\
\hline 2860 & AREA & & NONE \\
\hline 2861 & AREA & & NONE \\
\hline 2862 & AREA & & NONE \\
\hline 2863 & AREA & & NONE \\
\hline 2864 & AREA & & NONE \\
\hline 2865 & & & NONE \\
\hline 2866 & & & NONE \\
\hline 2867 & NAMED_POINT & & NONE \\
\hline 2868 & NAMED_POINT & & NONE \\
\hline 2869 & NAMED_POINT & & NONE \\
\hline 2870 & NAMED_POINT & & NONE \\
\hline 2871 & NAMED_POINT & & NONE \\
\hline 2872 & NAMED_POINT & & NONE \\
\hline 2873 & NAMED_POINT & & \\
\hline 2874 & NAMED_POINT & & \\
\hline 2875 & NAMED_POINT & & \\
\hline 2876 & NAMED_POINT & & \\
\hline 2877 & & & NONE \\
\hline 2878 & & & NONE \\
\hline 2879 & & & NONE \\
\hline 2880 & & & NONE \\
\hline 2881 & & & NONE \\
\hline 2882 & NAMED_POINT & C & \\
\hline 2883 & NAMED_POINT & C & \\
\hline 2884 & NAMED_POINT & & NONE \\
\hline 2885 & NAMED_POINT & & NONE \\
\hline 2886 & NAMED_POINT & G & \\
\hline 2887 & NAMED_POINT & & NONE \\
\hline 2888 & NAMED_POINT & G & \\
\hline 2889 & NAMED_POINT & G & \\
\hline
\end{tabular}




\begin{tabular}{|c|l|l|l|}
\hline & \multicolumn{1}{|c|}{ J } & \multicolumn{1}{|c|}{ K } & \multicolumn{1}{c|}{ L } \\
\hline 1 & SAMP_SITE_TYPE & SCHED_LABEL_CODE & TRANSFORM_CODE \\
\hline 2890 & NAMED_POINT & D & \\
\hline 2891 & NAMED_POINT & D & \\
\hline 2892 & NAMED_POINT & D & \\
\hline 2893 & NAMED_POINT & D & \\
\hline 2894 & NAMED_POINT & D & \\
\hline 2895 & NAMED_POINT & D & \\
\hline 2896 & NAMED_POINT & D & \\
\hline 2897 & NAMED_POINT & D & \\
\hline 2898 & NAMED_POINT & D & \\
\hline 2899 & NAMED_POINT & D & \\
\hline 2900 & NAMED_POINT & D & \\
\hline 2901 & NAMED_POINT & D & \\
\hline 2902 & NAMED_POINT & D & \\
\hline 2903 & NAMED_POINT & D & \\
\hline 2904 & NAMED_POINT & D & \\
\hline 2905 & NAMED_POINT & D & NONE \\
\hline 2906 & NAMED_POINT & D & \\
\hline 2907 & NAMED_POINT & D & \\
\hline 2908 & NAMED_POINT & D & NONE \\
\hline 2909 & NAMED_POINT & D & NONE \\
\hline 2910 & NAMED_POINT & D & \\
\hline 2911 & NAMED_POINT & D & NONE \\
\hline 2912 & NAMED_POINT & D & \\
\hline 2913 & NAMED_POINT & D & \\
\hline 2914 & NAMED_POINT & D & \\
\hline 2915 & NAMED_POINT & A & \\
\hline 2916 & NAMED_POINT & A & \\
\hline 2917 & NAMED_POINT & A & \\
\hline 2918 & NAMED_POINT & & \\
\hline 2919 & NAMED_POINT & & \\
\hline 2920 & AREA & & \\
\hline 2921 & AREA & & \\
\hline 2922 & NAMED_POINT & & \\
\hline 2923 & NAMED_POINT & & \\
\hline 2924 & NAMED_POINT & & \\
\hline 2925 & AREA & & \\
\hline 2926 & AREA & NONE \\
\hline 2927 & AREA & & \\
\hline & & & \\
\hline
\end{tabular}




\begin{tabular}{|c|c|c|c|}
\hline & $\mathrm{J}$ & $\mathrm{K}$ & $\mathrm{L}$ \\
\hline 1 & SAMP_SITE_TYPE & SCHED_LABEL_CODE & TRANSFORM_CODE \\
\hline 2928 & AREA & & NONE \\
\hline 2929 & AREA & & NONE \\
\hline 2930 & AREA & & NONE \\
\hline 2931 & AREA & & NONE \\
\hline 2932 & AREA & & NONE \\
\hline 2933 & AREA & & NONE \\
\hline 2934 & AREA & & NONE \\
\hline 2935 & AREA & & NONE \\
\hline 2936 & AREA & & NONE \\
\hline 2937 & AREA & & NONE \\
\hline 2938 & AREA & & NONE \\
\hline 2939 & NAMED_POINT & & NONE \\
\hline 2940 & NAMED_POINT & & NONE \\
\hline 2941 & NAMED_POINT & D & \\
\hline 2942 & NAMED_POINT & D & \\
\hline 2943 & NAMED_POINT & D & \\
\hline 2944 & NAMED_POINT & D & \\
\hline 2945 & NAMED_POINT & D & \\
\hline 2946 & NAMED_POINT & D & \\
\hline 2947 & NAMED_POINT & D & \\
\hline 2948 & NAMED_POINT & A & \\
\hline 2949 & NAMED_POINT & & NONE \\
\hline 2950 & NAMED_POINT & & NONE \\
\hline 2951 & NAMED_POINT & & NONE \\
\hline 2952 & NAMED_POINT & & NONE \\
\hline 2953 & NAMED_POINT & & NONE \\
\hline 2954 & NAMED_POINT & & NONE \\
\hline 2955 & NAMED_POINT & & NONE \\
\hline 2956 & NAMED_POINT & & NONE \\
\hline 2957 & NAMED_POINT & & NONE \\
\hline 2958 & NAMED_POINT & & NONE \\
\hline 2959 & NAMED_POINT & & NONE \\
\hline 2960 & NAMED_POINT & & NONE \\
\hline 2961 & NAMED_POINT & & NONE \\
\hline 2962 & NAMED_POINT & & NONE \\
\hline 2963 & NAMED_POINT & & NONE \\
\hline 2964 & NAMED_POINT & & NONE \\
\hline 2965 & NAMED_POINT & & NONE \\
\hline
\end{tabular}




\begin{tabular}{|c|l|l|l|}
\hline & \multicolumn{1}{|c|}{ J } & \multicolumn{1}{|c|}{ K } & \\
\hline 1 & SAMP_SITE_TYPE & SCHED_LABEL_CODE & TRANSFORM_CODE \\
\hline 2966 & NAMED_POINT & NONE \\
\hline 2967 & NAMED_POINT & NONE \\
\hline 2968 & NAMED_POINT & NONE \\
\hline 2969 & NAMED_POINT & NONE \\
\hline 2970 & NAMED_POINT & NONE \\
\hline 2971 & NAMED_POINT & NONE \\
\hline 2972 & NAMED_POINT & NONE \\
\hline 2973 & NAMED_POINT & NONE \\
\hline 2974 & NAMED_POINT & NONE \\
\hline 2975 & NAMED_POINT & NONE \\
\hline 2976 & NAMED_POINT & NONE \\
\hline 2977 & NAMED_POINT & NONE \\
\hline 2978 & NAMED_POINT & NONE \\
\hline 2979 & NAMED_POINT & NONE \\
\hline 2980 & NAMED_POINT & NONE \\
\hline 2981 & NAMED_POINT & NONE \\
\hline 2982 & NAMED_POINT & NONE \\
\hline 2983 & NAMED_POINT & NONE \\
\hline 2984 & NAMED_POINT & NONE \\
\hline 2985 & NAMED_POINT & NONE \\
\hline 2986 & NAMED_POINT & NONE \\
\hline 2987 & NAMED_POINT & NONE \\
\hline 2988 & NAMED_POINT & NONE \\
\hline 2989 & NAMED_POINT & NONE \\
\hline 2990 & NAMED_POINT & NONE \\
\hline 2991 & NAMED_POINT & NONE \\
\hline 2992 & NAMED_POINT & NONE \\
\hline 2993 & NAMED_POINT & NONE \\
\hline 2994 & NAMED_POINT & NONE \\
\hline 2995 & NAMED_POINT & NONE \\
\hline 2996 & NAMED_POINT & NONE \\
\hline 2997 & NAMED_POINT & NONE \\
\hline 2998 & NAMED_POINT & NONE \\
\hline 2999 & NAMED_POINT & NONE \\
\hline 3000 & NAMED_POINT & NONE \\
\hline 3001 & NAMED_POINT & NONE \\
\hline 3002 & NAMED_POINT & NONE \\
\hline 3003 & NAMED_POINT & \\
\hline & & & \\
\hline
\end{tabular}




\begin{tabular}{|c|l|l|l|}
\hline & \multicolumn{1}{|c|}{ J } & \multicolumn{1}{|c|}{ K } & \\
\hline 1 & SAMP_SITE_TYPE & SCHED_LABEL_CODE & TRANSFORM_CODE \\
\hline 3004 & NAMED_POINT & NONE \\
\hline 3005 & NAMED_POINT & NONE \\
\hline 3006 & NAMED_POINT & NONE \\
\hline 3007 & NAMED_POINT & NONE \\
\hline 3008 & NAMED_POINT & NONE \\
\hline 3009 & NAMED_POINT & NONE \\
\hline 3010 & NAMED_POINT & NONE \\
\hline 3011 & NAMED_POINT & NONE \\
\hline 3012 & NAMED_POINT & NONE \\
\hline 3013 & AREA & NONE \\
\hline 3014 & AREA & NONE \\
\hline 3015 & NAMED_POINT & NONE \\
\hline 3016 & NAMED_POINT & NONE \\
\hline 3017 & NAMED_POINT & NONE \\
\hline 3018 & NAMED_POINT & NONE \\
\hline 3019 & NAMED_POINT & NONE \\
\hline 3020 & NAMED_POINT & NONE \\
\hline 3021 & NAMED_POINT & NONE \\
\hline 3022 & NAMED_POINT & NONE \\
\hline 3023 & NAMED_POINT & NONE \\
\hline 3024 & NAMED_POINT & NONE \\
\hline 3025 & NAMED_POINT & NONE \\
\hline 3026 & NAMED_POINT & NONE \\
\hline 3027 & NAMED_POINT & NONE \\
\hline 3028 & NAMED_POINT & NONE \\
\hline 3029 & NAMED_POINT & NONE \\
\hline 3030 & NAMED_POINT & NONE \\
\hline 3031 & NAMED_POINT & NONE \\
\hline 3032 & NAMED_POINT & NONE \\
\hline 3033 & NAMED_POINT & NONE \\
\hline 3034 & NAMED_POINT & NONE \\
\hline 3035 & NAMED_POINT & NONE \\
\hline 3036 & NAMED_POINT & NONE \\
\hline 3037 & NAMED_POINT & NONE \\
\hline 3038 & NAMED_POINT & NONE \\
\hline 3039 & NAMED_POINT & NONE \\
\hline 3040 & NAMED_POINT & \\
\hline 3041 & NAMED_POINT & \\
\hline & & \\
\hline
\end{tabular}




\begin{tabular}{|c|l|l|l|}
\hline & \multicolumn{1}{|c|}{ J } & \multicolumn{1}{|c|}{ K } & \\
\hline 1 & SAMP_SITE_TYPE & SCHED_LABEL_CODE & TRANSFORM_CODE \\
\hline 3042 & NAMED_POINT & NONE \\
\hline 3043 & NAMED_POINT & NONE \\
\hline 3044 & NAMED_POINT & NONE \\
\hline 3045 & NAMED_POINT & NONE \\
\hline 3046 & NAMED_POINT & NONE \\
\hline 3047 & NAMED_POINT & NONE \\
\hline 3048 & NAMED_POINT & NONE \\
\hline 3049 & NAMED_POINT & NONE \\
\hline 3050 & NAMED_POINT & NONE \\
\hline 3051 & NAMED_POINT & NONE \\
\hline 3052 & NAMED_POINT & NONE \\
\hline 3053 & NAMED_POINT & NONE \\
\hline 3054 & NAMED_POINT & NONE \\
\hline 3055 & NAMED_POINT & NONE \\
\hline 3056 & NAMED_POINT & NONE \\
\hline 3057 & NAMED_POINT & NONE \\
\hline 3058 & NAMED_POINT & NONE \\
\hline 3059 & NAMED_POINT & NONE \\
\hline 3060 & NAMED_POINT & NONE \\
\hline 3061 & NAMED_POINT & NONE \\
\hline 3062 & NAMED_POINT & NONE \\
\hline 3063 & NAMED_POINT & NONE \\
\hline 3064 & NAMED_POINT & NONE \\
\hline 3065 & NAMED_POINT & NONE \\
\hline 3066 & NAMED_POINT & NONE \\
\hline 3067 & NAMED_POINT & NONE \\
\hline 3068 & NAMED_POINT & NONE \\
\hline 3069 & NAMED_POINT & NONE \\
\hline 3070 & NAMED_POINT & NONE \\
\hline 3071 & NAMED_POINT & NONE \\
\hline 3072 & NAMED_POINT & NONE \\
\hline 3073 & NAMED_POINT & NONE \\
\hline 3074 & NAMED_POINT & NONE \\
\hline 3075 & NAMED_POINT & NONE \\
\hline 3076 & NAMED_POINT & NONE \\
\hline 3077 & NAMED_POINT & NONE \\
\hline 3078 & NAMED_POINT & & \\
\hline 3079 & NAMED_POINT & & \\
\hline & & & \\
\hline
\end{tabular}




\begin{tabular}{|c|l|l|l|}
\hline & \multicolumn{1}{|c|}{ J } & \multicolumn{1}{|c|}{ K } & \\
\hline 1 & SAMP_SITE_TYPE & SCHED_LABEL_CODE & TRANSFORM_CODE \\
\hline 3080 & NAMED_POINT & NONE \\
\hline 3081 & NAMED_POINT & NONE \\
\hline 3082 & NAMED_POINT & NONE \\
\hline 3083 & NAMED_POINT & NONE \\
\hline 3084 & NAMED_POINT & NONE \\
\hline 3085 & NAMED_POINT & NONE \\
\hline 3086 & NAMED_POINT & NONE \\
\hline 3087 & NAMED_POINT & NONE \\
\hline 3088 & NAMED_POINT & NONE \\
\hline 3089 & NAMED_POINT & NONE \\
\hline 3090 & NAMED_POINT & NONE \\
\hline 3091 & NAMED_POINT & NONE \\
\hline 3092 & NAMED_POINT & NONE \\
\hline 3093 & NAMED_POINT & NONE \\
\hline 3094 & NAMED_POINT & NONE \\
\hline 3095 & NAMED_POINT & NONE \\
\hline 3096 & NAMED_POINT & NONE \\
\hline 3097 & NAMED_POINT & NONE \\
\hline 3098 & NAMED_POINT & NONE \\
\hline 3099 & NAMED_POINT & NONE \\
\hline 3100 & NAMED_POINT & NONE \\
\hline 3101 & NAMED_POINT & NONE \\
\hline 3102 & NAMED_POINT & NONE \\
\hline 3103 & NAMED_POINT & NONE \\
\hline 3104 & NAMED_POINT & NONE \\
\hline 3105 & NAMED_POINT & NONE \\
\hline 3106 & NAMED_POINT & NONE \\
\hline 3107 & NAMED_POINT & NONE \\
\hline 3108 & NAMED_POINT & NONE \\
\hline 3109 & NAMED_POINT & NONE \\
\hline 3110 & NAMED_POINT & NONE \\
\hline 3111 & NAMED_POINT & NONE \\
\hline 3112 & NAMED_POINT & NONE \\
\hline 3113 & NAMED_POINT & NONE \\
\hline 3114 & NAMED_POINT & NONE \\
\hline 3115 & NAMED_POINT & NONE \\
\hline 3116 & NAMED_POINT & & \\
\hline 3117 & NAMED_POINT & & \\
\hline & & & \\
\hline
\end{tabular}




\begin{tabular}{|c|l|l|l|}
\hline & \multicolumn{1}{|c|}{ J } & \multicolumn{1}{|c|}{ K } & \multicolumn{1}{c|}{ L } \\
\hline 1 & SAMP_SITE_TYPE & SCHED_LABEL_CODE & TRANSFORM_CODE \\
\hline 3118 & NAMED_POINT & & NONE \\
\hline 3119 & NAMED_POINT & D & \\
\hline 3120 & NAMED_POINT & D & \\
\hline 3121 & NAMED_POINT & D & \\
\hline 3122 & NAMED_POINT & D & \\
\hline 3123 & NAMED_POINT & D & \\
\hline 3124 & NAMED_POINT & D & \\
\hline 3125 & NAMED_POINT & D & \\
\hline 3126 & NAMED_POINT & D & \\
\hline 3127 & NAMED_POINT & D & \\
\hline 3128 & NAMED_POINT & D & \\
\hline 3129 & NAMED_POINT & & \\
\hline 3130 & NAMED_POINT & & \\
\hline 3131 & NAMED_POINT & & \\
\hline 3132 & NAMED_POINT & & NONE \\
\hline 3133 & NAMED_POINT & A & \\
\hline 3134 & NAMED_POINT & A & \\
\hline 3135 & NAMED_POINT & A & NONE \\
\hline 3136 & NAMED_POINT & A & NONE \\
\hline 3137 & NAMED_POINT & A & NONE \\
\hline 3138 & NAMED_POINT & A & \\
\hline 3139 & NAMED_POINT & A & NONE \\
\hline 3140 & NAMED_POINT & A & \\
\hline 3141 & NAMED_POINT & A & \\
\hline 3142 & NAMED_POINT & D & \\
\hline 3143 & NAMED_POINT & & NONE \\
\hline 3144 & NAMED_POINT & & \\
\hline 3145 & NAMED_POINT & & \\
\hline 3146 & NAMED_POINT & & \\
\hline 3147 & NAMED_POINT & & \\
\hline 3148 & NAMED_POINT & & \\
\hline 3149 & NAMED_POINT & & \\
\hline 3150 & NAMED_POINT & & \\
\hline 3151 & NAMED_POINT & & \\
\hline 3152 & NAMED_POINT & & \\
\hline 3153 & NAMED_POINT & & \\
\hline 3154 & NAMED_POINT & & \\
\hline 3155 & NAMED_POINT & & \\
\hline & & & \\
\hline
\end{tabular}




\begin{tabular}{|c|c|c|c|}
\hline & $\mathrm{J}$ & $\mathrm{K}$ & $\mathrm{L}$ \\
\hline 1 & SAMP_SITE_TYPE & SCHED_LABEL_CODE & TRANSFORM_CODE \\
\hline 3156 & NAMED_POINT & & NONE \\
\hline 3157 & NAMED_POINT & & NONE \\
\hline 3158 & & & NONE \\
\hline 3159 & NAMED_POINT & & NONE \\
\hline 3160 & NAMED_POINT & & NONE \\
\hline 3161 & NAMED_POINT & & NONE \\
\hline 3162 & NAMED_POINT & & NONE \\
\hline 3163 & NAMED_POINT & & NONE \\
\hline 3164 & NAMED_POINT & & NONE \\
\hline 3165 & NAMED_POINT & & NONE \\
\hline 3166 & NAMED_POINT & & NONE \\
\hline 3167 & NAMED_POINT & & NONE \\
\hline 3168 & NAMED_POINT & & NONE \\
\hline 3169 & & & NONE \\
\hline 3170 & NAMED_POINT & & NONE \\
\hline 3171 & NAMED_POINT & & NONE \\
\hline 3172 & NAMED_POINT & & NONE \\
\hline 3173 & NAMED_POINT & & NONE \\
\hline 3174 & NAMED_POINT & & NONE \\
\hline 3175 & NAMED_POINT & & NONE \\
\hline 3176 & NAMED_POINT & & NONE \\
\hline 3177 & NAMED_POINT & & NONE \\
\hline 3178 & NAMED_POINT & & NONE \\
\hline 3179 & NAMED_POINT & & NONE \\
\hline 3180 & NAMED_POINT & & NONE \\
\hline 3181 & NAMED_POINT & & NONE \\
\hline 3182 & NAMED_POINT & & NONE \\
\hline 3183 & NAMED_POINT & & NONE \\
\hline 3184 & NAMED_POINT & & NONE \\
\hline 3185 & NAMED_POINT & & NONE \\
\hline 3186 & NAMED_POINT & & NONE \\
\hline 3187 & NAMED_POINT & & NONE \\
\hline 3188 & & & NONE \\
\hline 3189 & & & NONE \\
\hline 3190 & & & NONE \\
\hline 3191 & & & NONE \\
\hline 3192 & & & NONE \\
\hline 3193 & & & NONE \\
\hline
\end{tabular}




\begin{tabular}{|c|l|l|l|}
\hline & \multicolumn{1}{|c|}{$J$} & \multicolumn{1}{|c|}{$\mathrm{K}$} & \\
\hline 1 & SAMP_SITE_TYPE & SCHED_LABEL_CODE & TRANSFORM_CODE \\
\hline 3194 & & NONE \\
\hline 3195 & & NONE \\
\hline 3196 & & NONE \\
\hline 3197 & & NONE \\
\hline 3198 & & NONE \\
\hline 3199 & & NONE \\
\hline 3200 & AREA & NREA & NONE \\
\hline 3201 & AREA & NONE \\
\hline 3202 & AREA & AREA & NONE \\
\hline 3203 & AREA & NONE \\
\hline 3204 & ARRAA & NONE \\
\hline 3205 & AREA & NONE \\
\hline 3206 & AREA & AREA & NONE \\
\hline 3207 & AREA & NONE \\
\hline 3208 & NAMED_POINT & NONE \\
\hline 3209 & NAMED_POINT & NONE \\
\hline 3210 & NAMED_POINT & NONE \\
\hline 3211 & NAMED_POINT & NONE \\
\hline 3212 & NAMED_POINT & NREA & NONE \\
\hline 3213 & AREA & NONE \\
\hline 3214 & AREA & NONE \\
\hline 3215 & AREA & AREA & NONE \\
\hline 3216 & AREA & NONE \\
\hline 3217 & AREA & AREA & NONE \\
\hline 3218 & AREA & NONE \\
\hline 3219 & AREA & NONE \\
\hline 3220 & AREA & NONE \\
\hline 3221 & AREA & NONE \\
\hline 3223 & AREA & AREA & NONE \\
\hline 3224 & AREA & NONE \\
\hline 3225 & AREA & NONE \\
\hline 3226 & AREA & NONE \\
\hline 3228 & AREA & NONE \\
\hline 3229 & AREA & NONE \\
\hline 3231 & AREA & \\
\hline
\end{tabular}




\begin{tabular}{|c|c|c|c|}
\hline & $\mathrm{J}$ & $\mathrm{K}$ & $\mathrm{L}$ \\
\hline 1 & SAMP_SITE_TYPE & SCHED_LABEL_CODE & TRANSFORM_CODE \\
\hline 3232 & AREA & & NONE \\
\hline 3233 & AREA & & NONE \\
\hline 3234 & AREA & & NONE \\
\hline 3235 & AREA & & NONE \\
\hline 3236 & AREA & & NONE \\
\hline 3237 & AREA & & NONE \\
\hline 3238 & AREA & & NONE \\
\hline 3239 & AREA & & NONE \\
\hline 3240 & AREA & & NONE \\
\hline 3241 & AREA & & NONE \\
\hline 3242 & AREA & & NONE \\
\hline 3243 & AREA & & NONE \\
\hline 3244 & AREA & & NONE \\
\hline 3245 & AREA & & NONE \\
\hline 3246 & AREA & & NONE \\
\hline 3247 & AREA & & NONE \\
\hline 3248 & AREA & & NONE \\
\hline 3249 & AREA & & NONE \\
\hline 3250 & AREA & & NONE \\
\hline 3251 & & & NONE \\
\hline 3252 & & & NONE \\
\hline 3253 & & & NONE \\
\hline 3254 & & & NONE \\
\hline 3255 & & & NONE \\
\hline 3256 & & & NONE \\
\hline 3257 & & & NONE \\
\hline 3258 & & & NONE \\
\hline 3259 & & & NONE \\
\hline 3260 & & & NONE \\
\hline 3261 & & & NONE \\
\hline 3262 & & & NONE \\
\hline 3263 & & & NONE \\
\hline 3264 & & & NONE \\
\hline 3265 & AREA & & NONE \\
\hline 3266 & NAMED_POINT & & NONE \\
\hline 3267 & AREA & & NONE \\
\hline 3268 & AREA & & NONE \\
\hline 3269 & AREA & & NONE \\
\hline
\end{tabular}




\begin{tabular}{|c|l|l|l|}
\hline & \multicolumn{1}{|c|}{$J$} & \multicolumn{1}{|c|}{ K } & \multicolumn{1}{|c|}{ L } \\
\hline 1 & SAMP_SITE_TYPE & SCHED_LABEL_CODE & TRANSFORM_CODE \\
\hline 3270 & AREA & NONE \\
\hline 3271 & AREA & NONE \\
\hline 3272 & NAMED_POINT & NONE \\
\hline 3273 & NAMED_POINT & NONE \\
\hline 3274 & NAMED_POINT & NONE \\
\hline 3275 & NAMED_POINT & NONE \\
\hline 3276 & NAMED_POINT & NONE \\
\hline 3277 & AREA & NONE \\
\hline 3278 & AREA & NONE \\
\hline 3279 & NAMED_POINT & NONE \\
\hline 3280 & NAMED_POINT & NONE \\
\hline 3281 & NAMED_POINT & NONE \\
\hline 3282 & NAMED_POINT & NONE \\
\hline 3283 & NAMED_POINT & NONE \\
\hline 3284 & NAMED_POINT & NONE \\
\hline 3285 & NAMED_POINT & NONE \\
\hline 3286 & NAMED_POINT & NONE \\
\hline 3287 & NAMED_POINT & NONE \\
\hline 3288 & & NONE \\
\hline 3289 & AREA & NONE \\
\hline 3290 & AREA & NONE \\
\hline 3291 & AREA & NONE \\
\hline 3292 & NAMED_POINT & NONE \\
\hline 3293 & NAMED_POINT & NONE \\
\hline 3294 & NAMED_POINT & NONE \\
\hline 3295 & NAMED_POINT & NONE \\
\hline 3296 & NAMED_POINT & NONE \\
\hline 3297 & NAMED_POINT & NONE \\
\hline 3298 & NAMED_POINT & NONE \\
\hline 3299 & NAMED_POINT & NONE \\
\hline 3300 & NAMED_POINT & NONE \\
\hline 3301 & NAMED_POINT & NONE \\
\hline 3302 & NAMED_POINT & NONE \\
\hline 3303 & NAMED_POINT & NONE \\
\hline 3304 & NAMED_POINT & NONE \\
\hline 3305 & NAMED_POINT & NONE \\
\hline 3306 & NAMED_POINT & NONE \\
\hline 3307 & NAMED_POINT & NONE \\
\hline & & \\
\hline & & \\
\hline
\end{tabular}




\begin{tabular}{|c|l|l|l|}
\hline & \multicolumn{1}{|c|}{$J$} & \multicolumn{1}{|c|}{$\mathrm{K}$} & \\
\hline 1 & SAMP_SITE_TYPE & SCHED_LABEL_CODE & TRANSFORM_CODE \\
\hline 3308 & NAMED_POINT & NONE \\
\hline 3309 & NAMED_POINT & NONE \\
\hline 3310 & NAMED_POINT & NONE \\
\hline 3311 & NAMED_POINT & NONE \\
\hline 3312 & NAMED_POINT & NONE \\
\hline 3313 & NAMED_POINT & NONE \\
\hline 3314 & NAMED_POINT & NONE \\
\hline 3315 & NAMED_POINT & NONE \\
\hline 3316 & AREA & NONE \\
\hline 3317 & AREA & NONE \\
\hline 3318 & AREA & NONE \\
\hline 3319 & AREA & NONE \\
\hline 3320 & AREA & NONE \\
\hline 3321 & AREA & NONE \\
\hline 3322 & AREA & NONE \\
\hline 3323 & AREA & NONE \\
\hline 3324 & AREA & NONE \\
\hline 3325 & AREA & NONE \\
\hline 3326 & AREA & NONE \\
\hline 3327 & AREA & NONE \\
\hline 3328 & AREA & NONE \\
\hline 3329 & AREA & NONE \\
\hline 3330 & AREA & NONE \\
\hline 3331 & AREA & NONE \\
\hline 3332 & AREA & NONE \\
\hline 3333 & AREA & NONE \\
\hline 3334 & AREA & NONE \\
\hline 3335 & AREA & NONE \\
\hline 3336 & AREA & NONE \\
\hline 3337 & AREA & NONE \\
\hline 3338 & AREA & NONE \\
\hline 3339 & AREA & NONE \\
\hline 3340 & AREA & NONE \\
\hline 3341 & AREA & NONE \\
\hline 3342 & AREA & NONE \\
\hline 3343 & AREA & & NONE \\
\hline 3344 & AREA & & \\
\hline 3345 & AREA & & \\
\hline & & & \\
\hline
\end{tabular}




\begin{tabular}{|c|c|c|c|}
\hline & $\mathrm{J}$ & $\mathrm{K}$ & $\mathrm{L}$ \\
\hline 1 & SAMP_SITE_TYPE & SCHED_LABEL_CODE & TRANSFORM_CODE \\
\hline 3346 & AREA & & NONE \\
\hline 3347 & AREA & & NONE \\
\hline 3348 & AREA & & NONE \\
\hline 3349 & AREA & & NONE \\
\hline 3350 & AREA & & NONE \\
\hline 3351 & AREA & & NONE \\
\hline 3352 & AREA & & NONE \\
\hline 3353 & AREA & & \\
\hline 3354 & AREA & & NONE \\
\hline 3355 & AREA & & NONE \\
\hline 3356 & AREA & & NONE \\
\hline 3357 & AREA & & NONE \\
\hline 3358 & AREA & & NONE \\
\hline 3359 & NAMED_POINT & & NONE \\
\hline 3360 & NAMED_POINT & & NONE \\
\hline 3361 & AREA & & NONE \\
\hline 3362 & AREA & & NONE \\
\hline 3363 & AREA & & NONE \\
\hline 3364 & AREA & & NONE \\
\hline 3365 & AREA & & NONE \\
\hline 3366 & AREA & & \\
\hline 3367 & AREA & & NONE \\
\hline 3368 & AREA & & NONE \\
\hline 3369 & AREA & & NONE \\
\hline 3370 & AREA & & NONE \\
\hline 3371 & AREA & & NONE \\
\hline 3372 & AREA & & NONE \\
\hline 3373 & AREA & & NONE \\
\hline 3374 & AREA & & NONE \\
\hline 3375 & AREA & & NONE \\
\hline 3376 & AREA & & NONE \\
\hline 3377 & AREA & & NONE \\
\hline 3378 & AREA & & NONE \\
\hline 3379 & AREA & & NONE \\
\hline 3380 & AREA & & NONE \\
\hline 3381 & AREA & & NONE \\
\hline 3382 & AREA & & NONE \\
\hline 3383 & AREA & & NONE \\
\hline
\end{tabular}




\begin{tabular}{|c|l|l|l|}
\hline & \multicolumn{1}{|c|}{$J$} & \multicolumn{1}{|c|}{ K } & \multicolumn{1}{|c|}{ L } \\
\hline 1 & SAMP_SITE_TYPE & SCHED_LABEL_CODE & TRANSFORM_CODE \\
\hline 3384 & AREA & NONE \\
\hline 3385 & NAMED_POINT & NONE \\
\hline 3386 & NAMED_POINT & NONE \\
\hline 3387 & NAMED_POINT & NONE \\
\hline 3388 & NAMED_POINT & NONE \\
\hline 3389 & AREA & NONE \\
\hline 3390 & AREA & NONE \\
\hline 3391 & AREA & NONE \\
\hline 3392 & AREA & NONE \\
\hline 3393 & AREA & NONE \\
\hline 3394 & AREA & NONE \\
\hline 3395 & NAMED_POINT & NONE \\
\hline 3396 & NAMED_POINT & NONE \\
\hline 3397 & NAMED_POINT & NONE \\
\hline 3398 & NAMED_POINT & NONE \\
\hline 3399 & NAMED_POINT & NONE \\
\hline 3400 & NAMED_POINT & NONE \\
\hline 3401 & NAMED_POINT & NONE \\
\hline 3402 & NAMED_POINT & NONE \\
\hline 3403 & NAMED_POINT & NONE \\
\hline 3404 & NAMED_POINT & NONE \\
\hline 3405 & NAMED_POINT & NONE \\
\hline 3406 & NAMED_POINT & NONE \\
\hline 3407 & NAMED_POINT & NONE \\
\hline 3408 & NAMED_POINT & NONE \\
\hline 3409 & NAMED_POINT & NONE \\
\hline 3410 & NAMED_POINT & NONE \\
\hline 3411 & NAMED_POINT & NONE \\
\hline 3412 & NAMED_POINT & NONE \\
\hline 3413 & NAMED_POINT & NONE \\
\hline 3414 & NAMED_POINT & NONE \\
\hline 3415 & AREA & NONE \\
\hline 3416 & AREA & NONE \\
\hline 3417 & AREA & NONE \\
\hline 3418 & AREA & NONE \\
\hline 3419 & AREA & NONE \\
\hline 3420 & AREA & NONE \\
\hline 3421 & AREA & NONE \\
\hline & & \\
\hline
\end{tabular}




\begin{tabular}{|c|l|l|l|}
\hline & \multicolumn{1}{|c|}{$J$} & \multicolumn{1}{|c|}{ K } & \\
\hline 1 & SAMP_SITE_TYPE & SCHED_LABEL_CODE & TRANSFORM_CODE \\
\hline 3422 & NAMED_POINT & NONE \\
\hline 3423 & NAMED_POINT & NONE \\
\hline 3424 & AREA & NONE \\
\hline 3425 & AREA & NONE \\
\hline 3426 & AREA & NONE \\
\hline 3427 & NAMED_POINT & NONE \\
\hline 3428 & AREA & NONE \\
\hline 3429 & NAMED_POINT & NONE \\
\hline 3430 & NAMED_POINT & NONE \\
\hline 3431 & NAMED_POINT & NONE \\
\hline 3432 & AREA & NONE \\
\hline 3433 & NAMED_POINT & NONE \\
\hline 3434 & NAMED_POINT & NONE \\
\hline 3435 & NAMED_POINT & NONE \\
\hline 3436 & NAMED_POINT & NONE \\
\hline 3437 & NAMED_POINT & NONE \\
\hline 3438 & NAMED_POINT & NONE \\
\hline 3439 & NAMED_POINT & NONE \\
\hline 3440 & NAMED_POINT & NONE \\
\hline 3441 & NAMED_POINT & NONE \\
\hline 3442 & AREA & NONE \\
\hline 3443 & AREA & NONE \\
\hline 3444 & AREA & NONE \\
\hline 3445 & AREA & NONE \\
\hline 3446 & AREA & NONE \\
\hline 3447 & AREA & NONE \\
\hline 3448 & AREA & NONE \\
\hline 3449 & AREA & NONE \\
\hline 3450 & AREA & NONE \\
\hline 3451 & AREA & NONE \\
\hline 3452 & AREA & NONE \\
\hline 3453 & AREA & NONE \\
\hline 3454 & AREA & NONE \\
\hline 3455 & AREA & NONE \\
\hline 3456 & AREA & NONE \\
\hline 3457 & AREA & NONE \\
\hline 3458 & AREA & NONE \\
\hline 3459 & AREA & \\
\hline & & & \\
\hline & & \\
\hline
\end{tabular}




\begin{tabular}{|c|l|l|l|}
\hline & \multicolumn{1}{|c|}{$J$} & \multicolumn{1}{|c|}{ K } & \\
\hline 1 & SAMP_SITE_TYPE & SCHED_LABEL_CODE & TRANSFORM_CODE \\
\hline 3460 & AREA & NONE \\
\hline 3461 & AREA & NONE \\
\hline 3462 & AREA & NONE \\
\hline 3463 & AREA & NONE \\
\hline 3464 & AREA & NONE \\
\hline 3465 & AREA & NONE \\
\hline 3466 & AREA & NONE \\
\hline 3467 & AREA & NONE \\
\hline 3468 & AREA & NONE \\
\hline 3469 & AREA & NONE \\
\hline 3470 & AREA & NONE \\
\hline 3471 & AREA & NONE \\
\hline 3472 & AREA & NONE \\
\hline 3473 & AREA & NONE \\
\hline 3474 & AREA & NONE \\
\hline 3475 & NAMED_POINT & NONE \\
\hline 3476 & NAMED_POINT & NONE \\
\hline 3477 & NAMED_POINT & NONE \\
\hline 3478 & NAMED_POINT & NONE \\
\hline 3479 & NAMED_POINT & NONE \\
\hline 3480 & NAMED_POINT & NONE \\
\hline 3481 & NAMED_POINT & NONE \\
\hline 3482 & NAMED_POINT & NONE \\
\hline 3483 & NAMED_POINT & NONE \\
\hline 3484 & NAMED_POINT & NONE \\
\hline 3485 & NAMED_POINT & NONE \\
\hline 3486 & NAMED_POINT & NONE \\
\hline 3487 & NAMED_POINT & NONE \\
\hline 3488 & NAMED_POINT & NONE \\
\hline 3489 & NAMED_POINT & NONE \\
\hline 3490 & NAMED_POINT & NONE \\
\hline 3491 & NAMED_POINT & NONE \\
\hline 3492 & NAMED_POINT & NONE \\
\hline 3493 & NAMED_POINT & NONE \\
\hline 3494 & NAMED_POINT & NONE \\
\hline 3495 & NAMED_POINT & NONE \\
\hline 3496 & NAMED_POINT & NONE \\
\hline 3497 & NAMED_POINT & \\
\hline & & & \\
\hline
\end{tabular}




\begin{tabular}{|c|l|l|l|}
\hline & \multicolumn{1}{|c|}{ J } & \multicolumn{1}{|c|}{ K } & \\
\hline 1 & SAMP_SITE_TYPE & SCHED_LABEL_CODE & TRANSFORM_CODE \\
\hline 3498 & NAMED_POINT & NONE \\
\hline 3499 & NAMED_POINT & NONE \\
\hline 3500 & NAMED_POINT & NONE \\
\hline 3501 & NAMED_POINT & NONE \\
\hline 3502 & NAMED_POINT & NONE \\
\hline 3503 & NAMED_POINT & NONE \\
\hline 3504 & NAMED_POINT & NONE \\
\hline 3505 & NAMED_POINT & NONE \\
\hline 3506 & NAMED_POINT & NONE \\
\hline 3507 & NAMED_POINT & NONE \\
\hline 3508 & NAMED_POINT & NONE \\
\hline 3509 & NAMED_POINT & NONE \\
\hline 3510 & NAMED_POINT & NONE \\
\hline 3511 & NAMED_POINT & NONE \\
\hline 3512 & NAMED_POINT & NONE \\
\hline 3513 & NAMED_POINT & NONE \\
\hline 3514 & NAMED_POINT & NONE \\
\hline 3515 & NAMED_POINT & NONE \\
\hline 3516 & NAMED_POINT & NONE \\
\hline 3517 & NAMED_POINT & NONE \\
\hline 3518 & NAMED_POINT & NONE \\
\hline 3519 & NAMED_POINT & NONE \\
\hline 3520 & NAMED_POINT & NONE \\
\hline 3521 & NAMED_POINT & NONE \\
\hline 3522 & NAMED_POINT & NONE \\
\hline 3523 & NAMED_POINT & NONE \\
\hline 3524 & NAMED_POINT & NONE \\
\hline 3525 & NAMED_POINT & NONE \\
\hline 3526 & NAMED_POINT & NONE \\
\hline 3527 & NAMED_POINT & NONE \\
\hline 3528 & NAMED_POINT & NONE \\
\hline 3529 & NAMED_POINT & NONE \\
\hline 3530 & NAMED_POINT & NONE \\
\hline 3531 & NAMED_POINT & NONE \\
\hline 3532 & NAMED_POINT & NONE \\
\hline 3533 & NAMED_POINT & NONE \\
\hline 3534 & NAMED_POINT & & \\
\hline 3535 & NAMED_POINT & & \\
\hline & & & \\
\hline
\end{tabular}




\begin{tabular}{|c|l|l|l|}
\hline & \multicolumn{1}{|c|}{ J } & \multicolumn{1}{|c|}{ K } & \\
\hline 1 & SAMP_SITE_TYPE & SCHED_LABEL_CODE & TRANSFORM_CODE \\
\hline 3536 & NAMED_POINT & NONE \\
\hline 3537 & NAMED_POINT & NONE \\
\hline 3538 & NAMED_POINT & NONE \\
\hline 3539 & NAMED_POINT & NONE \\
\hline 3540 & NAMED_POINT & NONE \\
\hline 3541 & NAMED_POINT & NONE \\
\hline 3542 & NAMED_POINT & NONE \\
\hline 3543 & NAMED_POINT & NONE \\
\hline 3544 & NAMED_POINT & NONE \\
\hline 3545 & NAMED_POINT & NONE \\
\hline 3546 & NAMED_POINT & NONE \\
\hline 3547 & NAMED_POINT & NONE \\
\hline 3548 & NAMED_POINT & NONE \\
\hline 3549 & NAMED_POINT & NONE \\
\hline 3550 & NAMED_POINT & NONE \\
\hline 3551 & NAMED_POINT & NONE \\
\hline 3552 & NAMED_POINT & NONE \\
\hline 3553 & NAMED_POINT & NONE \\
\hline 3554 & NAMED_POINT & NONE \\
\hline 3555 & NAMED_POINT & NONE \\
\hline 3556 & NAMED_POINT & NONE \\
\hline 3557 & NAMED_POINT & NONE \\
\hline 3558 & NAMED_POINT & NONE \\
\hline 3559 & NAMED_POINT & NONE \\
\hline 3560 & NAMED_POINT & NONE \\
\hline 3561 & NAMED_POINT & NONE \\
\hline 3562 & NAMED_POINT & NONE \\
\hline 3563 & NAMED_POINT & NONE \\
\hline 3564 & NAMED_POINT & NONE \\
\hline 3565 & NAMED_POINT & NONE \\
\hline 3566 & NAMED_POINT & NONE \\
\hline 3567 & NAMED_POINT & NONE \\
\hline 3568 & NAMED_POINT & NONE \\
\hline 3569 & NAMED_POINT & & \\
\hline 3570 & NAMED_POINT & NONE \\
\hline 3571 & NAMED_POINT & NAME_POINT & \\
\hline 3572 & NAMED_POINT & NAMED_POINT & \\
\hline 3573 & & \\
\hline & & & \\
\hline
\end{tabular}




\begin{tabular}{|c|l|l|l|}
\hline & \multicolumn{1}{|c|}{ J } & \multicolumn{1}{|c|}{ K } & \\
\hline 1 & SAMP_SITE_TYPE & SCHED_LABEL_CODE & TRANSFORM_CODE \\
\hline 3574 & NAMED_POINT & NONE \\
\hline 3575 & NAMED_POINT & NONE \\
\hline 3576 & NAMED_POINT & NONE \\
\hline 3577 & NAMED_POINT & NONE \\
\hline 3578 & NAMED_POINT & NONE \\
\hline 3579 & NAMED_POINT & NONE \\
\hline 3580 & NAMED_POINT & NONE \\
\hline 3581 & NAMED_POINT & NONE \\
\hline 3582 & NAMED_POINT & NONE \\
\hline 3583 & NAMED_POINT & NONE \\
\hline 3584 & NAMED_POINT & NONE \\
\hline 3585 & NAMED_POINT & NONE \\
\hline 3586 & NAMED_POINT & NONE \\
\hline 3587 & NAMED_POINT & NONE \\
\hline 3588 & NAMED_POINT & NONE \\
\hline 3589 & NAMED_POINT & NONE \\
\hline 3590 & NAMED_POINT & NONE \\
\hline 3591 & NAMED_POINT & NONE \\
\hline 3592 & NAMED_POINT & NONE \\
\hline 3593 & NAMED_POINT & NONE \\
\hline 3594 & NAMED_POINT & NONE \\
\hline 3595 & NAMED_POINT & NONE \\
\hline 3596 & NAMED_POINT & NONE \\
\hline 3597 & NAMED_POINT & NONE \\
\hline 3598 & NAMED_POINT & NONE \\
\hline 3599 & NAMED_POINT & NONE \\
\hline 3600 & NAMED_POINT & NONE \\
\hline 3601 & NAMED_POINT & NONE \\
\hline 3602 & NAMED_POINT & NONE \\
\hline 3603 & NAMED_POINT & NONE \\
\hline 3604 & NAMED_POINT & NONE \\
\hline 3605 & NAMED_POINT & NONE \\
\hline 3606 & NAMED_POINT & NONE \\
\hline 3607 & NAMED_POINT & NONE \\
\hline 3608 & NAMED_POINT & NONE \\
\hline 3609 & NAMED_POINT & NONE \\
\hline 3610 & NAMED_POINT & \\
\hline 3611 & NAMED_POINT & \\
\hline & & \\
\hline
\end{tabular}




\begin{tabular}{|c|l|l|l|}
\hline & \multicolumn{1}{|c|}{ J } & \multicolumn{1}{|c|}{ K } & \\
\hline 1 & SAMP_SITE_TYPE & SCHED_LABEL_CODE & TRANSFORM_CODE \\
\hline 3612 & NAMED_POINT & NONE \\
\hline 3613 & NAMED_POINT & NONE \\
\hline 3614 & NAMED_POINT & NONE \\
\hline 3615 & NAMED_POINT & NONE \\
\hline 3616 & NAMED_POINT & NONE \\
\hline 3617 & NAMED_POINT & NONE \\
\hline 3618 & NAMED_POINT & NONE \\
\hline 3619 & NAMED_POINT & NONE \\
\hline 3620 & NAMED_POINT & NONE \\
\hline 3621 & NAMED_POINT & NONE \\
\hline 3622 & NAMED_POINT & NONE \\
\hline 3623 & NAMED_POINT & NONE \\
\hline 3624 & NAMED_POINT & NONE \\
\hline 3625 & NAMED_POINT & NONE \\
\hline 3626 & NAMED_POINT & NONE \\
\hline 3627 & NAMED_POINT & NONE \\
\hline 3628 & NAMED_POINT & NONE \\
\hline 3629 & NAMED_POINT & NONE \\
\hline 3630 & NAMED_POINT & NONE \\
\hline 3631 & NAMED_POINT & NONE \\
\hline 3632 & NAMED_POINT & NONE \\
\hline 3633 & NAMED_POINT & NONE \\
\hline 3634 & NAMED_POINT & NONE \\
\hline 3635 & NAMED_POINT & NONE \\
\hline 3636 & NAMED_POINT & NONE \\
\hline 3637 & NAMED_POINT & NONE \\
\hline 3638 & NAMED_POINT & NONE \\
\hline 3639 & NAMED_POINT & NONE \\
\hline 3640 & NAMED_POINT & NONE \\
\hline 3641 & NAMED_POINT & NONE \\
\hline 3642 & NAMED_POINT & NONE \\
\hline 3643 & NAMED_POINT & NONE \\
\hline 3644 & NAMED_POINT & NONE \\
\hline 3645 & NAMED_POINT & NONE \\
\hline 3646 & NAMED_POINT & NONE \\
\hline 3647 & NAMED_POINT & NONE \\
\hline 3648 & NAMED_POINT & \\
\hline 3649 & NAMED_POINT & \\
\hline & & \\
\hline
\end{tabular}




\begin{tabular}{|c|l|l|l|}
\hline & \multicolumn{1}{|c|}{ J } & \multicolumn{1}{|c|}{ K } & \\
\hline 1 & SAMP_SITE_TYPE & SCHED_LABEL_CODE & TRANSFORM_CODE \\
\hline 3650 & NAMED_POINT & NONE \\
\hline 3651 & NAMED_POINT & NONE \\
\hline 3652 & NAMED_POINT & NONE \\
\hline 3653 & NAMED_POINT & NONE \\
\hline 3654 & NAMED_POINT & NONE \\
\hline 3655 & NAMED_POINT & NONE \\
\hline 3656 & NAMED_POINT & NONE \\
\hline 3657 & NAMED_POINT & NONE \\
\hline 3658 & NAMED_POINT & NONE \\
\hline 3659 & NAMED_POINT & NONE \\
\hline 3660 & NAMED_POINT & NONE \\
\hline 3661 & NAMED_POINT & NONE \\
\hline 3662 & NAMED_POINT & NONE \\
\hline 3663 & NAMED_POINT & NONE \\
\hline 3664 & NAMED_POINT & NONE \\
\hline 3665 & NAMED_POINT & NONE \\
\hline 3666 & NAMED_POINT & NONE \\
\hline 3667 & NAMED_POINT & NONE \\
\hline 3668 & NAMED_POINT & NONE \\
\hline 3669 & NAMED_POINT & NONE \\
\hline 3670 & NAMED_POINT & NONE \\
\hline 3671 & NAMED_POINT & NONE \\
\hline 3672 & NAMED_POINT & NONE \\
\hline 3673 & NAMED_POINT & NONE \\
\hline 3674 & NAMED_POINT & NONE \\
\hline 3675 & NAMED_POINT & NONE \\
\hline 3676 & NAMED_POINT & NONE \\
\hline 3677 & NAMED_POINT & NONE \\
\hline 3678 & NAMED_POINT & NONE \\
\hline 3679 & NAMED_POINT & NONE \\
\hline 3680 & NAMED_POINT & NONE \\
\hline 3681 & NAMED_POINT & NONE \\
\hline 3682 & NAMED_POINT & NONE \\
\hline 3683 & NAMED_POINT & NONE \\
\hline 3684 & NAMED_POINT & NONE \\
\hline 3685 & NAMED_POINT & NONE \\
\hline 3686 & NAMED_POINT & \\
\hline 3687 & NAMED_POINT & \\
\hline & & \\
\hline
\end{tabular}




\begin{tabular}{|c|l|l|l|}
\hline & \multicolumn{1}{|c|}{ J } & \multicolumn{1}{|c|}{ K } & \\
\hline 1 & SAMP_SITE_TYPE & SCHED_LABEL_CODE & TRANSFORM_CODE \\
\hline 3688 & NAMED_POINT & NONE \\
\hline 3689 & NAMED_POINT & NONE \\
\hline 3690 & NAMED_POINT & NONE \\
\hline 3691 & NAMED_POINT & NONE \\
\hline 3692 & NAMED_POINT & NONE \\
\hline 3693 & NAMED_POINT & NONE \\
\hline 3694 & NAMED_POINT & NONE \\
\hline 3695 & NAMED_POINT & NONE \\
\hline 3696 & NAMED_POINT & NONE \\
\hline 3697 & NAMED_POINT & NONE \\
\hline 3698 & NAMED_POINT & NONE \\
\hline 3699 & NAMED_POINT & NONE \\
\hline 3700 & NAMED_POINT & NONE \\
\hline 3701 & NAMED_POINT & NONE \\
\hline 3702 & NAMED_POINT & NONE \\
\hline 3703 & NAMED_POINT & NONE \\
\hline 3704 & NAMED_POINT & NONE \\
\hline 3705 & NAMED_POINT & NONE \\
\hline 3706 & NAMED_POINT & NONE \\
\hline 3707 & NAMED_POINT & NONE \\
\hline 3708 & NAMED_POINT & NONE \\
\hline 3709 & NAMED_POINT & NONE \\
\hline 3710 & NAMED_POINT & NONE \\
\hline 3711 & NAMED_POINT & NONE \\
\hline 3712 & NAMED_POINT & NONE \\
\hline 3713 & NAMED_POINT & NONE \\
\hline 3714 & NAMED_POINT & NONE \\
\hline 3715 & NAMED_POINT & NONE \\
\hline 3716 & NAMED_POINT & NONE \\
\hline 3717 & NAMED_POINT & NONE \\
\hline 3718 & NAMED_POINT & NONE \\
\hline 3719 & NAMED_POINT & NONE \\
\hline 3720 & NAMED_POINT & NONE \\
\hline 3721 & NAMED_POINT & NONE \\
\hline 3722 & NAMED_POINT & NONE \\
\hline 3723 & NAMED_POINT & NONE \\
\hline 3724 & NAMED_POINT & & \\
\hline 3725 & NAMED_POINT & & \\
\hline & & & \\
\hline
\end{tabular}




\begin{tabular}{|c|l|l|l|}
\hline & \multicolumn{1}{|c|}{ J } & \multicolumn{1}{|c|}{ K } & \\
\hline 1 & SAMP_SITE_TYPE & SCHED_LABEL_CODE & TRANSFORM_CODE \\
\hline 3726 & NAMED_POINT & NONE \\
\hline 3727 & NAMED_POINT & NONE \\
\hline 3728 & NAMED_POINT & NONE \\
\hline 3729 & NAMED_POINT & NONE \\
\hline 3730 & NAMED_POINT & NONE \\
\hline 3731 & NAMED_POINT & NONE \\
\hline 3732 & NAMED_POINT & NONE \\
\hline 3733 & NAMED_POINT & NONE \\
\hline 3734 & NAMED_POINT & NONE \\
\hline 3735 & AREA & NONE \\
\hline 3736 & AREA & NONE \\
\hline 3737 & NAMED_POINT & NONE \\
\hline 3738 & NAMED_POINT & NONE \\
\hline 3739 & NAMED_POINT & NONE \\
\hline 3740 & NAMED_POINT & NONE \\
\hline 3741 & NAMED_POINT & NONE \\
\hline 3742 & NAMED_POINT & NONE \\
\hline 3743 & NAMED_POINT & NONE \\
\hline 3744 & NAMED_POINT & NONE \\
\hline 3745 & NAMED_POINT & NONE \\
\hline 3746 & NAMED_POINT & NONE \\
\hline 3747 & NAMED_POINT & NONE \\
\hline 3748 & NAMED_POINT & NONE \\
\hline 3749 & NAMED_POINT & NONE \\
\hline 3750 & NAMED_POINT & NONE \\
\hline 3751 & NAMED_POINT & NONE \\
\hline 3752 & NAMED_POINT & NONE \\
\hline 3753 & NAMED_POINT & NONE \\
\hline 3754 & NAMED_POINT & NONE \\
\hline 3755 & NAMED_POINT & NONE \\
\hline 3756 & NAMED_POINT & NONE \\
\hline 3757 & NAMED_POINT & NONE \\
\hline 3758 & NAMED_POINT & NONE \\
\hline 3759 & NAMED_POINT & NONE \\
\hline 3760 & NAMED_POINT & NONE \\
\hline 3761 & NAMED_POINT & NONE \\
\hline 3762 & NAMED_POINT & \\
\hline 3763 & NAMED_POINT & \\
\hline & & \\
\hline
\end{tabular}




\begin{tabular}{|c|l|l|l|}
\hline & \multicolumn{1}{|c|}{$J$} & \multicolumn{1}{|c|}{ K } & \multicolumn{1}{|c|}{ L } \\
\hline 1 & SAMP_SITE_TYPE & SCHED_LABEL_CODE & TRANSFORM_CODE \\
\hline 3764 & AREA & NONE \\
\hline 3765 & AREA & NONE \\
\hline 3766 & AREA & NONE \\
\hline 3767 & AREA & NONE \\
\hline 3768 & AREA & NONE \\
\hline 3769 & AREA & NONE \\
\hline 3770 & AREA & NONE \\
\hline 3771 & NAMED_POINT & NONE \\
\hline 3772 & NAMED_POINT & NONE \\
\hline 3773 & NAMED_POINT & NONE \\
\hline 3774 & NAMED_POINT & \\
\hline 3775 & NAMED_POINT & NONE \\
\hline 3776 & NAMED_POINT & NONE \\
\hline 3777 & NAMED_POINT & NONE \\
\hline 3778 & NAMED_POINT & NONE \\
\hline 3779 & NAMED_POINT & NONE \\
\hline 3780 & NAMED_POINT & NONE \\
\hline 3781 & AREA & NONE \\
\hline 3782 & AREA & NONE \\
\hline 3783 & NAMED_POINT & NONE \\
\hline 3784 & NAMED_POINT & NONE \\
\hline 3785 & NAMED_POINT & NONE \\
\hline 3786 & NAMED_POINT & NONE \\
\hline 3787 & NAMED_POINT & NONE \\
\hline 3788 & NAMED_POINT & NONE \\
\hline 3789 & NAMED_POINT & NONE \\
\hline 3790 & NAMED_POINT & NONE \\
\hline 3791 & NAMED_POINT & NONE \\
\hline 3792 & NAMED_POINT & NONE \\
\hline 3793 & NAMED_POINT & NONE \\
\hline 3794 & NAMED_POINT & NONE \\
\hline 3795 & NAMED_POINT & NONE \\
\hline 3796 & NAMED_POINT & NONE \\
\hline 3797 & NAMED_POINT & NONE \\
\hline 3798 & NAMED_POINT & NONE \\
\hline 3799 & NAMED_POINT & NONE \\
\hline 3800 & NAMED_POINT & NONE \\
\hline 3801 & NAMED_POINT & NONE \\
\hline & & \\
\hline
\end{tabular}




\begin{tabular}{|c|l|l|l|}
\hline & \multicolumn{1}{|c|}{ J } & \multicolumn{1}{|c|}{ K } & \\
\hline 1 & SAMP_SITE_TYPE & SCHED_LABEL_CODE & TRANSFORM_CODE \\
\hline 3802 & NAMED_POINT & NONE \\
\hline 3803 & NAMED_POINT & NONE \\
\hline 3804 & NAMED_POINT & NONE \\
\hline 3805 & NAMED_POINT & NONE \\
\hline 3806 & NAMED_POINT & NONE \\
\hline 3807 & NAMED_POINT & NONE \\
\hline 3808 & NAMED_POINT & NONE \\
\hline 3809 & NAMED_POINT & NONE \\
\hline 3810 & NAMED_POINT & NONE \\
\hline 3811 & NAMED_POINT & NONE \\
\hline 3812 & NAMED_POINT & NONE \\
\hline 3813 & NAMED_POINT & NONE \\
\hline 3814 & NAMED_POINT & NONE \\
\hline 3815 & NAMED_POINT & NONE \\
\hline 3816 & NAMED_POINT & NONE \\
\hline 3817 & NAMED_POINT & NONE \\
\hline 3818 & NAMED_POINT & NONE \\
\hline 3819 & NAMED_POINT & NONE \\
\hline 3820 & NAMED_POINT & NONE \\
\hline 3821 & NAMED_POINT & NONE \\
\hline 3822 & NAMED_POINT & NONE \\
\hline 3823 & NAMED_POINT & NONE \\
\hline 3824 & NAMED_POINT & NONE \\
\hline 3825 & NAMED_POINT & NONE \\
\hline 3826 & NAMED_POINT & NONE \\
\hline 3827 & NAMED_POINT & NONE \\
\hline 3828 & NAMED_POINT & NONE \\
\hline 3829 & NAMED_POINT & NONE \\
\hline 3830 & NAMED_POINT & NONE \\
\hline 3831 & NAMED_POINT & NONE \\
\hline 3832 & NAMED_POINT & NONE \\
\hline 3833 & NAMED_POINT & NONE \\
\hline 3834 & NAMED_POINT & NONE \\
\hline 3835 & NAMED_POINT & NONE \\
\hline 3836 & NAMED_POINT & NONE \\
\hline 3837 & NAMED_POINT & NONE \\
\hline 3838 & AREA & \\
\hline 3839 & AREA & & \\
\hline & & & \\
\hline
\end{tabular}




\begin{tabular}{|c|l|l|l|}
\hline & \multicolumn{1}{|c|}{$J$} & \multicolumn{1}{|c|}{ K } & \\
\hline 1 & SAMP_SITE_TYPE & SCHED_LABEL_CODE & TRANSFORM_CODE \\
\hline 3840 & AREA & NONE \\
\hline 3841 & AREA & NONE \\
\hline 3842 & AREA & NONE \\
\hline 3843 & AREA & NONE \\
\hline 3844 & AREA & NONE \\
\hline 3845 & AREA & NONE \\
\hline 3846 & AREA & NONE \\
\hline 3847 & AREA & NONE \\
\hline 3848 & AREA & NONE \\
\hline 3849 & AREA & NONE \\
\hline 3850 & AREA & NONE \\
\hline 3851 & AREA & NONE \\
\hline 3852 & NAMED_POINT & NONE \\
\hline 3853 & NAMED_POINT & NONE \\
\hline 3854 & NAMED_POINT & NONE \\
\hline 3855 & NAMED_POINT & NONE \\
\hline 3856 & NAMED_POINT & NONE \\
\hline 3857 & NAMED_POINT & NONE \\
\hline 3858 & NAMED_POINT & NONE \\
\hline 3859 & NAMED_POINT & NONE \\
\hline 3860 & NAMED_POINT & NONE \\
\hline 3861 & NAMED_POINT & NONE \\
\hline 3862 & NAMED_POINT & NONE \\
\hline 3863 & NAMED_POINT & NONE \\
\hline 3864 & NAMED_POINT & NONE \\
\hline 3865 & NAMED_POINT & NONE \\
\hline 3866 & NAMED_POINT & NONE \\
\hline 3867 & NAMED_POINT & NONE \\
\hline 3868 & NAMED_POINT & NONE \\
\hline 3869 & NAMED_POINT & NONE \\
\hline 3870 & NAMED_POINT & NONE \\
\hline 3871 & NAMED_POINT & NONE \\
\hline 3872 & NAMED_POINT & NONE \\
\hline 3873 & NAMED_POINT & NONE \\
\hline 3874 & NAMED_POINT & NONE \\
\hline 3875 & NAMED_POINT & NONE \\
\hline 3876 & NAMED_POINT & NONE \\
\hline 3877 & NAMED_POINT & \\
\hline & & & \\
\hline
\end{tabular}




\begin{tabular}{|c|l|l|l|}
\hline & \multicolumn{1}{|c|}{ J } & \multicolumn{1}{|c|}{ K } & \\
\hline 1 & SAMP_SITE_TYPE & SCHED_LABEL_CODE & TRANSFORM_CODE \\
\hline 3878 & NAMED_POINT & NONE \\
\hline 3879 & NAMED_POINT & NONE \\
\hline 3880 & NAMED_POINT & NONE \\
\hline 3881 & NAMED_POINT & NONE \\
\hline 3882 & NAMED_POINT & NONE \\
\hline 3883 & NAMED_POINT & NONE \\
\hline 3884 & NAMED_POINT & NONE \\
\hline 3885 & NAMED_POINT & NONE \\
\hline 3886 & NAMED_POINT & NONE \\
\hline 3887 & NAMED_POINT & NONE \\
\hline 3888 & NAMED_POINT & NONE \\
\hline 3889 & NAMED_POINT & NONE \\
\hline 3890 & NAMED_POINT & NONE \\
\hline 3891 & NAMED_POINT & NONE \\
\hline 3892 & NAMED_POINT & NONE \\
\hline 3893 & AREA & NONE \\
\hline 3894 & AREA & NONE \\
\hline 3895 & AREA & NONE \\
\hline 3896 & NAMED_POINT & NONE \\
\hline 3897 & NAMED_POINT & NONE \\
\hline 3898 & NAMED_POINT & NONE \\
\hline 3899 & NAMED_POINT & NONE \\
\hline 3900 & NAMED_POINT & NONE \\
\hline 3901 & NAMED_POINT & NONE \\
\hline 3902 & NAMED_POINT & NONE \\
\hline 3903 & NAMED_POINT & NONE \\
\hline 3904 & NAMED_POINT & NONE \\
\hline 3905 & NAMED_POINT & NONE \\
\hline 3906 & NAMED_POINT & NONE \\
\hline 3907 & NAMED_POINT & NONE \\
\hline 3908 & NAMED_POINT & NONE \\
\hline 3909 & NAMED_POINT & NONE \\
\hline 3910 & NAMED_POINT & NONE \\
\hline 3911 & NAMED_POINT & NONE \\
\hline 3912 & NAMED_POINT & NONE \\
\hline 3913 & NAMED_POINT & NONE \\
\hline 3914 & NAMED_POINT & NONE \\
\hline 3915 & NAMED_POINT & \\
\hline & & & \\
\hline
\end{tabular}




\begin{tabular}{|c|l|l|l|}
\hline & \multicolumn{1}{|c|}{ J } & \multicolumn{1}{|c|}{ K } & \\
\hline 1 & SAMP_SITE_TYPE & SCHED_LABEL_CODE & TRANSFORM_CODE \\
\hline 3916 & NAMED_POINT & NONE \\
\hline 3917 & NAMED_POINT & NONE \\
\hline 3918 & NAMED_POINT & NONE \\
\hline 3919 & NAMED_POINT & NONE \\
\hline 3920 & NAMED_POINT & NONE \\
\hline 3921 & NAMED_POINT & NONE \\
\hline 3922 & NAMED_POINT & NONE \\
\hline 3923 & NAMED_POINT & NONE \\
\hline 3924 & NAMED_POINT & NONE \\
\hline 3925 & NAMED_POINT & NONE \\
\hline 3926 & NAMED_POINT & NONE \\
\hline 3927 & NAMED_POINT & NONE \\
\hline 3928 & NAMED_POINT & NONE \\
\hline 3929 & NAMED_POINT & NONE \\
\hline 3930 & NAMED_POINT & NONE \\
\hline 3931 & NAMED_POINT & NONE \\
\hline 3932 & NAMED_POINT & NONE \\
\hline 3933 & AREA & NONE \\
\hline 3934 & AREA & NONE \\
\hline 3935 & AREA & NONE \\
\hline 3936 & NAMED_POINT & NONE \\
\hline 3937 & NAMED_POINT & NONE \\
\hline 3938 & NAMED_POINT & NONE \\
\hline 3939 & NAMED_POINT & NONE \\
\hline 3940 & NAMED_POINT & NONE \\
\hline 3941 & NAMED_POINT & NONE \\
\hline 3942 & NAMED_POINT & NONE \\
\hline 3943 & NAMED_POINT & NONE \\
\hline 3944 & NAMED_POINT & NONE \\
\hline 3945 & NAMED_POINT & NONE \\
\hline 3946 & NAMED_POINT & NONE \\
\hline 3947 & NAMED_POINT & NONE \\
\hline 3948 & NAMED_POINT & NONE \\
\hline 3949 & NAMED_POINT & NONE \\
\hline 3950 & AREA & NONE \\
\hline 3951 & NAMED_POINT & NONE \\
\hline 3952 & NAMED_POINT & NONE \\
\hline 3953 & NAMED_POINT & \\
\hline & & & \\
\hline
\end{tabular}




\begin{tabular}{|c|l|l|l|}
\hline & \multicolumn{1}{|c|}{ J } & \multicolumn{1}{|c|}{ K } & \\
\hline 1 & SAMP_SITE_TYPE & SCHED_LABEL_CODE & TRANSFORM_CODE \\
\hline 3954 & NAMED_POINT & NONE \\
\hline 3955 & NAMED_POINT & NONE \\
\hline 3956 & NAMED_POINT & NONE \\
\hline 3957 & NAMED_POINT & NONE \\
\hline 3958 & NAMED_POINT & NONE \\
\hline 3959 & NAMED_POINT & NONE \\
\hline 3960 & NAMED_POINT & NONE \\
\hline 3961 & NAMED_POINT & NONE \\
\hline 3962 & NAMED_POINT & NONE \\
\hline 3963 & NAMED_POINT & NONE \\
\hline 3964 & NAMED_POINT & NONE \\
\hline 3965 & NAMED_POINT & NONE \\
\hline 3966 & AREA & NONE \\
\hline 3967 & AREA & NONE \\
\hline 3968 & NAMED_POINT & NONE \\
\hline 3969 & NAMED_POINT & NONE \\
\hline 3970 & AREA & NONE \\
\hline 3971 & AREA & NONE \\
\hline 3972 & NAMED_POINT & NONE \\
\hline 3973 & NAMED_POINT & NONE \\
\hline 3974 & NAMED_POINT & NONE \\
\hline 3975 & NAMED_POINT & NONE \\
\hline 3976 & NAMED_POINT & NONE \\
\hline 3977 & NAMED_POINT & NONE \\
\hline 3978 & NAMED_POINT & NONE \\
\hline 3979 & NAMED_POINT & NONE \\
\hline 3980 & NAMED_POINT & NONE \\
\hline 3981 & NAMED_POINT & NONE \\
\hline 3982 & NAMED_POINT & NONE \\
\hline 3983 & NAMED_POINT & NONE \\
\hline 3984 & NAMED_POINT & NONE \\
\hline 3985 & AREA & NONE \\
\hline 3986 & AREA & NONE \\
\hline 3987 & AREA & NONE \\
\hline 3988 & NAMED_POINT & NONE \\
\hline 3989 & NAMED_POINT & NONE \\
\hline 3990 & NAMED_POINT & NONE \\
\hline 3991 & NAMED_POINT & \\
\hline & & & \\
\hline
\end{tabular}




\begin{tabular}{|c|l|l|l|}
\hline & \multicolumn{1}{|c|}{$J$} & \multicolumn{1}{|c|}{ K } & \\
\hline 1 & SAMP_SITE_TYPE & SCHED_LABEL_CODE & TRANSFORM_CODE \\
\hline 3992 & NAMED_POINT & NONE \\
\hline 3993 & NAMED_POINT & NONE \\
\hline 3994 & NAMED_POINT & NONE \\
\hline 3995 & NAMED_POINT & NONE \\
\hline 3996 & NAMED_POINT & NONE \\
\hline 3997 & NAMED_POINT & NONE \\
\hline 3998 & NAMED_POINT & NONE \\
\hline 3999 & NAMED_POINT & NONE \\
\hline 4000 & NAMED_POINT & NONE \\
\hline 4001 & NAMED_POINT & NONE \\
\hline 4002 & NAMED_POINT & NONE \\
\hline 4003 & NAMED_POINT & NONE \\
\hline 4004 & AREA & NONE \\
\hline 4005 & AREA & NONE \\
\hline 4006 & AREA & NONE \\
\hline 4007 & AREA & NONE \\
\hline 4008 & AREA & NONE \\
\hline 4009 & AREA & NONE \\
\hline 4010 & AREA & NONE \\
\hline 4011 & AREA & NONE \\
\hline 4012 & AREA & NONE \\
\hline 4013 & AREA & NONE \\
\hline 4014 & AREA & NONE \\
\hline 4015 & NAMED_POINT & NONE \\
\hline 4016 & NAMED_POINT & NONE \\
\hline 4017 & NAMED_POINT & NONE \\
\hline 4018 & NAMED_POINT & NONE \\
\hline 4019 & NAMED_POINT & NONE \\
\hline 4020 & NAMED_POINT & NONE \\
\hline 4021 & NAMED_POINT & NONE \\
\hline 4022 & NAMED_POINT & NONE \\
\hline 4023 & NAMED_POINT & NONE \\
\hline 4024 & NAMED_POINT & NONE \\
\hline 4025 & NAMED_POINT & NONE \\
\hline 4026 & NAMED_POINT & NONE \\
\hline 4027 & NAMED_POINT & NONE \\
\hline 4028 & NAMED_POINT & NONE \\
\hline 4029 & NAMED_POINT & \\
\hline & & & \\
\hline
\end{tabular}




\begin{tabular}{|c|l|l|l|}
\hline & \multicolumn{1}{|c|}{ J } & \multicolumn{1}{|c|}{ K } & \\
\hline 1 & SAMP_SITE_TYPE & SCHED_LABEL_CODE & TRANSFORM_CODE \\
\hline 4030 & NAMED_POINT & NONE \\
\hline 4031 & AREA & NONE \\
\hline 4032 & AREA & NONE \\
\hline 4033 & AREA & NONE \\
\hline 4034 & AREA & NONE \\
\hline 4035 & AREA & NONE \\
\hline 4036 & AREA & NONE \\
\hline 4037 & AREA & NONE \\
\hline 4038 & AREA & NONE \\
\hline 4039 & AREA & NONE \\
\hline 4040 & AREA & NONE \\
\hline 4041 & NAMED_POINT & NONE \\
\hline 4042 & NAMED_POINT & NONE \\
\hline 4043 & NAMED_POINT & NONE \\
\hline 4044 & NAMED_POINT & NONE \\
\hline 4045 & NAMED_POINT & NONE \\
\hline 4046 & NAMED_POINT & NONE \\
\hline 4047 & NAMED_POINT & NONE \\
\hline 4048 & NAMED_POINT & NONE \\
\hline 4049 & NAMED_POINT & NONE \\
\hline 4050 & NAMED_POINT & NONE \\
\hline 4051 & NAMED_POINT & NONE \\
\hline 4052 & NAMED_POINT & NONE \\
\hline 4053 & NAMED_POINT & NONE \\
\hline 4054 & NAMED_POINT & NONE \\
\hline 4055 & NAMED_POINT & NONE \\
\hline 4056 & NAMED_POINT & NONE \\
\hline 4057 & NAMED_POINT & NONE \\
\hline 4058 & NAMED_POINT & NONE \\
\hline 4059 & NAMED_POINT & NONE \\
\hline 4060 & NAMED_POINT & NONE \\
\hline 4061 & NAMED_POINT & NONE \\
\hline 4062 & NAMED_POINT & \\
\hline 4063 & NAMED_POINT & NONE \\
\hline 4064 & AREA & NONE \\
\hline 4065 & NAMED_POINT & & \\
\hline 4066 & NAMED_POINT & NAMED_POINT & \\
\hline 4067 & NAMEDE & \\
\hline & & & \\
\hline
\end{tabular}




\begin{tabular}{|c|l|l|l|}
\hline & \multicolumn{1}{|c|}{ J } & \multicolumn{1}{|c|}{ K } & \\
\hline 1 & SAMP_SITE_TYPE & SCHED_LABEL_CODE & TRANSFORM_CODE \\
\hline 4068 & NAMED_POINT & NONE \\
\hline 4069 & NAMED_POINT & NONE \\
\hline 4070 & NAMED_POINT & NONE \\
\hline 4071 & NAMED_POINT & NONE \\
\hline 4072 & NAMED_POINT & NONE \\
\hline 4073 & NAMED_POINT & NONE \\
\hline 4074 & NAMED_POINT & NONE \\
\hline 4075 & NAMED_POINT & NONE \\
\hline 4076 & NAMED_POINT & NONE \\
\hline 4077 & NAMED_POINT & NONE \\
\hline 4078 & NAMED_POINT & NONE \\
\hline 4079 & NAMED_POINT & NONE \\
\hline 4080 & NAMED_POINT & NONE \\
\hline 4081 & NAMED_POINT & NONE \\
\hline 4082 & NAMED_POINT & NONE \\
\hline 4083 & NAMED_POINT & NONE \\
\hline 4084 & NAMED_POINT & NONE \\
\hline 4085 & NAMED_POINT & NONE \\
\hline 4086 & NAMED_POINT & NONE \\
\hline 4087 & NAMED_POINT & NONE \\
\hline 4088 & NAMED_POINT & NONE \\
\hline 4089 & NAMED_POINT & NONE \\
\hline 4090 & NAMED_POINT & NONE \\
\hline 4091 & NAMED_POINT & NONE \\
\hline 4092 & NAMED_POINT & NONE \\
\hline 4093 & NAMED_POINT & NONE \\
\hline 4094 & NAMED_POINT & NONE \\
\hline 4095 & NAMED_POINT & NONE \\
\hline 4096 & NAMED_POINT & NONE \\
\hline 4097 & NAMED_POINT & NONE \\
\hline 4098 & NAMED_POINT & NONE \\
\hline 4099 & NAMED_POINT & NONE \\
\hline 4100 & NAMED_POINT & NONE \\
\hline 4101 & NAMED_POINT & NONE \\
\hline 4102 & NAMED_POINT & NONE \\
\hline 4103 & NAMED_POINT & NONE \\
\hline 4104 & NAMED_POINT & \\
\hline 4105 & NAMED_POINT & \\
\hline & & \\
\hline
\end{tabular}




\begin{tabular}{|c|l|l|l|}
\hline & \multicolumn{1}{|c|}{ J } & \multicolumn{1}{|c|}{ K } & \\
\hline 1 & SAMP_SITE_TYPE & SCHED_LABEL_CODE & TRANSFORM_CODE \\
\hline 4106 & NAMED_POINT & NONE \\
\hline 4107 & NAMED_POINT & NONE \\
\hline 4108 & NAMED_POINT & NONE \\
\hline 4109 & NAMED_POINT & NONE \\
\hline 4110 & & NONE \\
\hline 4111 & NAMED_POINT & NONE \\
\hline 4112 & NAMED_POINT & NONE \\
\hline 4113 & NAMED_POINT & NONE \\
\hline 4114 & NAMED_POINT & NONE \\
\hline 4115 & NAMED_POINT & NONE \\
\hline 4116 & NAMED_POINT & NONE \\
\hline 4117 & NAMED_POINT & NONE \\
\hline 4118 & NAMED_POINT & NONE \\
\hline 4119 & NAMED_POINT & NONE \\
\hline 4120 & NAMED_POINT & NONE \\
\hline 4121 & NAMED_POINT & NONE \\
\hline 4122 & NAMED_POINT & NONE \\
\hline 4123 & NAMED_POINT & NONE \\
\hline 4124 & NAMED_POINT & NONE \\
\hline 4125 & NAMED_POINT & NONE \\
\hline 4126 & NAMED_POINT & NONE \\
\hline 4127 & NAMED_POINT & NONE \\
\hline 4128 & NAMED_POINT & NONE \\
\hline 4129 & NAMED_POINT & NONE \\
\hline 4130 & NAMED_POINT & NONE \\
\hline 4131 & NAMED_POINT & NONE \\
\hline 4132 & NAMED_POINT & NONE \\
\hline 4133 & NAMED_POINT & NONE \\
\hline 4134 & NAMED_POINT & NONE \\
\hline 4135 & NAMED_POINT & NONE \\
\hline 4136 & NAMED_POINT & NONE \\
\hline 4137 & NAMED_POINT & NONE \\
\hline 4138 & NAMED_POINT & NONE \\
\hline 4139 & NAMED_POINT & NONE \\
\hline 4140 & NAMED_POINT & NONE \\
\hline 4141 & NAMED_POINT & NONE \\
\hline 4142 & NAMED_POINT & \\
\hline 4143 & NAMED_POINT & \\
\hline & & \\
\hline
\end{tabular}




\begin{tabular}{|c|l|l|l|}
\hline & \multicolumn{1}{|c|}{$J$} & \multicolumn{1}{|c|}{ K } & \multicolumn{1}{|c|}{ L } \\
\hline 1 & SAMP_SITE_TYPE & SCHED_LABEL_CODE & TRANSFORM_CODE \\
\hline 4144 & NAMED_POINT & NONE \\
\hline 4145 & NAMED_POINT & NONE \\
\hline 4146 & NAMED_POINT & NONE \\
\hline 4147 & NAMED_POINT & NONE \\
\hline 4148 & NAMED_POINT & NONE \\
\hline 4149 & NAMED_POINT & NONE \\
\hline 4150 & NAMED_POINT & NONE \\
\hline 4151 & NAMED_POINT & NONE \\
\hline 4152 & NAMED_POINT & NONE \\
\hline 4153 & NAMED_POINT & NONE \\
\hline 4154 & NAMED_POINT & NONE \\
\hline 4155 & NAMED_POINT & NONE \\
\hline 4156 & NAMED_POINT & NONE \\
\hline 4157 & NAMED_POINT & NONE \\
\hline 4158 & NAMED_POINT & NONE \\
\hline 4159 & NAMED_POINT & NONE \\
\hline 4160 & NAMED_POINT & NONE \\
\hline 4161 & NAMED_POINT & NONE \\
\hline 4162 & NAMED_POINT & NONE \\
\hline 4163 & NAMED_POINT & NONE \\
\hline 4164 & NAMED_POINT & NONE \\
\hline 4165 & NAMED_POINT & NONE \\
\hline 4166 & NAMED_POINT & NONE \\
\hline 4167 & NAMED_POINT & NONE \\
\hline 4168 & NAMED_POINT & NONE \\
\hline 4169 & NAMED_POINT & NONE \\
\hline 4170 & NAMED_POINT & NONE \\
\hline 4171 & NAMED_POINT & NONE \\
\hline 4172 & NAMED_POINT & NONE \\
\hline 4173 & NAMED_POINT & NONE \\
\hline 4174 & NAMED_POINT & NONE \\
\hline 4175 & NAMED_POINT & NONE \\
\hline 4176 & NAMED_POINT & NONE \\
\hline 4177 & NAMED_POINT & NONE \\
\hline 4178 & NAMED_POINT & NONE \\
\hline 4179 & NAMED_POINT & NONE \\
\hline 4180 & NAMED_POINT & NONE \\
\hline 4181 & NAMED_POINT & NONE \\
\hline & & \\
\hline
\end{tabular}




\begin{tabular}{|c|l|l|l|}
\hline & \multicolumn{1}{|c|}{$J$} & \multicolumn{1}{|c|}{ K } & \\
\hline 1 & SAMP_SITE_TYPE & SCHED_LABEL_CODE & TRANSFORM_CODE \\
\hline 4182 & NAMED_POINT & NONE \\
\hline 4183 & NAMED_POINT & NONE \\
\hline 4184 & NAMED_POINT & NONE \\
\hline 4185 & NAMED_POINT & NONE \\
\hline 4186 & NAMED_POINT & NONE \\
\hline 4187 & NAMED_POINT & NONE \\
\hline 4188 & NAMED_POINT & NONE \\
\hline 4189 & NAMED_POINT & NONE \\
\hline 4190 & NAMED_POINT & NONE \\
\hline 4191 & NAMED_POINT & NONE \\
\hline 4192 & NAMED_POINT & NONE \\
\hline 4193 & NAMED_POINT & NONE \\
\hline 4194 & NAMED_POINT & NONE \\
\hline 4195 & NAMED_POINT & NONE \\
\hline 4196 & NAMED_POINT & NONE \\
\hline 4197 & NAMED_POINT & NONE \\
\hline 4198 & NAMED_POINT & NONE \\
\hline 4199 & NAMED_POINT & NONE \\
\hline 4200 & AREA & NONE \\
\hline 4201 & AREA & NONE \\
\hline 4202 & AREA & NONE \\
\hline 4203 & AREA & NONE \\
\hline 4204 & AREA & NONE \\
\hline 4205 & AREA & NONE \\
\hline 4206 & AREA & NONE \\
\hline 4207 & AREA & NONE \\
\hline 4208 & AREA & NONE \\
\hline 4209 & AREA & NONE \\
\hline 4210 & NAMED_POINT & NONE \\
\hline 4211 & NAMED_POINT & NONE \\
\hline 4212 & NAMED_POINT & NONE \\
\hline 4213 & NAMED_POINT & NONE \\
\hline 4214 & NAMED_POINT & NONE \\
\hline 4215 & NAMED_POINT & NONE \\
\hline 4216 & NAMED_POINT & NONE \\
\hline 4217 & AREA & NONE \\
\hline 4218 & AREA & NONE \\
\hline 4219 & AREA & \\
\hline & & & \\
\hline & & & \\
\hline & & & \\
\hline & & & \\
\hline
\end{tabular}




\begin{tabular}{|c|l|l|l|}
\hline & \multicolumn{1}{|c|}{$J$} & \multicolumn{1}{|c|}{ K } & \multicolumn{1}{|c|}{ L } \\
\hline 1 & SAMP_SITE_TYPE & SCHED_LABEL_CODE & TRANSFORM_CODE \\
\hline 4220 & AREA & & NONE \\
\hline 4221 & AREA & & NONE \\
\hline 4222 & AREA & & NONE \\
\hline 4223 & AREA & NONE \\
\hline 4224 & AREA & NONE \\
\hline 4225 & AREA & NONE \\
\hline 4226 & AREA & & NONE \\
\hline 4227 & AREA & NONE \\
\hline 4228 & AREA & NONE \\
\hline 4229 & NAMED_POINT & A & \\
\hline 4230 & NAMED_POINT & A & \\
\hline 4231 & NAMED_POINT & A & \\
\hline 4232 & NAMED_POINT & A & \\
\hline 4233 & NAMED_POINT & A & \\
\hline 4234 & NAMED_POINT & A & \\
\hline 4235 & NAMED_POINT & A & \\
\hline 4236 & NAMED_POINT & A & NONE \\
\hline 4237 & NAMED_POINT & A & NONE \\
\hline 4238 & NAMED_POINT & A & NONE \\
\hline 4239 & NAMED_POINT & & NONE \\
\hline 4240 & NAMED_POINT & & NONE \\
\hline 4241 & & & NONE \\
\hline 4242 & & & NONE \\
\hline 4243 & & & \\
\hline 4244 & & & \\
\hline 4245 & & & \\
\hline 4246 & & & \\
\hline 4247 & & & \\
\hline 4248 & AREA & & \\
\hline 4249 & AREA & & \\
\hline 4250 & AREA & & \\
\hline 4251 & AREA & & \\
\hline 4252 & AREA & & \\
\hline 4253 & AREA & & \\
\hline 4254 & AREA & & \\
\hline 4255 & AREA & & \\
\hline 4256 & AREA & & \\
\hline 4257 & AREA & & \\
\hline & & & \\
\hline
\end{tabular}




\begin{tabular}{|c|l|l|l|}
\hline & \multicolumn{1}{|c|}{$\mathrm{J}$} & \multicolumn{1}{|c|}{$\mathrm{K}$} & \\
\hline 1 & SAMP_SITE_TYPE & SCHED_LABEL_CODE & TRANSFORM_CODE \\
\hline 4258 & AREA & NONE \\
\hline 4259 & AREA & NONE \\
\hline 4260 & AREA & NONE \\
\hline 4261 & AREA & NONE \\
\hline 4262 & AREA & NONE \\
\hline 4263 & AREA & NONE \\
\hline 4264 & AREA & NONE \\
\hline 4265 & AREA & NONE \\
\hline 4266 & AREA & NONE \\
\hline 4267 & AREA & NONE \\
\hline 4268 & AREA & NONE \\
\hline 4269 & AREA & NONE \\
\hline 4270 & AREA & NONE \\
\hline 4271 & AREA & NONE \\
\hline 4272 & AREA & NONE \\
\hline 4273 & AREA & NONE \\
\hline 4274 & AREA & NONE \\
\hline 4275 & AREA & NONE \\
\hline 4276 & AREA & NONE \\
\hline 4277 & AREA & NONE \\
\hline 4278 & AREA & NONE \\
\hline 4279 & AREA & NONE \\
\hline 4280 & AREA & NONE \\
\hline 4281 & AREA & NONE \\
\hline 4282 & AREA & NONE \\
\hline 4283 & AREA & NONE \\
\hline 4284 & AREA & NONE \\
\hline 4285 & AREA & NONE \\
\hline 4286 & AREA & NONE \\
\hline 4287 & AREA & NONE \\
\hline 4288 & AREA & NONE \\
\hline 4289 & AREA & NONE \\
\hline 4290 & AREA & NONE \\
\hline 4291 & AREA & NONE \\
\hline 4292 & AREA & NONE \\
\hline 4293 & AREA & & NONE \\
\hline 4294 & AREA & & \\
\hline 4295 & AREA & & \\
\hline & & & \\
\hline
\end{tabular}




\begin{tabular}{|c|l|l|l|}
\hline & \multicolumn{1}{|c|}{$\mathrm{J}$} & \multicolumn{1}{|c|}{$\mathrm{K}$} & \\
\hline 1 & SAMP_SITE_TYPE & SCHED_LABEL_CODE & TRANSFORM_CODE \\
\hline 4296 & AREA & NONE \\
\hline 4297 & AREA & NONE \\
\hline 4298 & AREA & NONE \\
\hline 4299 & AREA & NONE \\
\hline 4300 & AREA & NONE \\
\hline 4301 & AREA & NONE \\
\hline 4302 & AREA & NONE \\
\hline 4303 & AREA & NONE \\
\hline 4304 & AREA & NONE \\
\hline 4305 & AREA & NONE \\
\hline 4306 & AREA & NONE \\
\hline 4307 & AREA & NONE \\
\hline 4308 & AREA & NONE \\
\hline 4309 & AREA & NONE \\
\hline 4310 & AREA & NONE \\
\hline 4311 & AREA & NONE \\
\hline 4312 & AREA & NONE \\
\hline 4313 & AREA & NONE \\
\hline 4314 & AREA & NONE \\
\hline 4315 & AREA & NONE \\
\hline 4316 & AREA & NONE \\
\hline 4317 & AREA & NONE \\
\hline 4318 & AREA & NONE \\
\hline 4319 & AREA & NONE \\
\hline 4320 & AREA & NONE \\
\hline 4321 & AREA & NONE \\
\hline 4322 & AREA & NONE \\
\hline 4323 & AREA & NONE \\
\hline 4324 & AREA & NONE \\
\hline 4325 & AREA & NONE \\
\hline 4326 & AREA & NONE \\
\hline 4327 & AREA & & NONE \\
\hline 4328 & AREA & NONE \\
\hline 4329 & AREA & NONE \\
\hline 4330 & AREA & NONE \\
\hline 4331 & AREA & & \\
\hline 4332 & AREA & & NONE \\
\hline 4333 & AREA & & \\
\hline & & & \\
\hline
\end{tabular}




\begin{tabular}{|c|l|l|l|}
\hline & \multicolumn{1}{|c|}{$\mathrm{J}$} & \multicolumn{1}{|c|}{$\mathrm{K}$} & \\
\hline 1 & SAMP_SITE_TYPE & SCHED_LABEL_CODE & TRANSFORM_CODE \\
\hline 4334 & AREA & NONE \\
\hline 4335 & AREA & NONE \\
\hline 4336 & AREA & NONE \\
\hline 4337 & AREA & NONE \\
\hline 4338 & AREA & NONE \\
\hline 4339 & AREA & NONE \\
\hline 4340 & AREA & NONE \\
\hline 4341 & AREA & NONE \\
\hline 4342 & AREA & NONE \\
\hline 4343 & AREA & NONE \\
\hline 4344 & AREA & NONE \\
\hline 4345 & AREA & NONE \\
\hline 4346 & AREA & NONE \\
\hline 4347 & AREA & NONE \\
\hline 4348 & AREA & NONE \\
\hline 4349 & AREA & NONE \\
\hline 4350 & AREA & NONE \\
\hline 4351 & AREA & NONE \\
\hline 4352 & AREA & NONE \\
\hline 4353 & AREA & NONE \\
\hline 4354 & AREA & NONE \\
\hline 4355 & AREA & NONE \\
\hline 4356 & AREA & NONE \\
\hline 4357 & AREA & NONE \\
\hline 4358 & AREA & NONE \\
\hline 4359 & AREA & NONE \\
\hline 4360 & AREA & NONE \\
\hline 4361 & AREA & NONE \\
\hline 4362 & AREA & NONE \\
\hline 4363 & AREA & NONE \\
\hline 4364 & AREA & NONE \\
\hline 4365 & AREA & NONE \\
\hline 4366 & AREA & NONE \\
\hline 4367 & AREA & NONE \\
\hline 4368 & AREA & NONE \\
\hline 4369 & AREA & & NONE \\
\hline 4370 & AREA & \\
\hline 4371 & AREA & & \\
\hline & & & \\
\hline
\end{tabular}




\begin{tabular}{|c|c|c|c|}
\hline & $\mathrm{J}$ & $\mathrm{K}$ & $\mathrm{L}$ \\
\hline 1 & SAMP_SITE_TYPE & SCHED_LABEL_CODE & TRANSFORM_CODE \\
\hline 4372 & AREA & & NONE \\
\hline 4373 & AREA & & NONE \\
\hline 4374 & AREA & & NONE \\
\hline 4375 & AREA & & NONE \\
\hline 4376 & AREA & & NONE \\
\hline 4377 & AREA & & NONE \\
\hline 4378 & AREA & & NONE \\
\hline 4379 & AREA & & NONE \\
\hline 4380 & AREA & & NONE \\
\hline 4381 & AREA & & NONE \\
\hline 4382 & AREA & & NONE \\
\hline 4383 & AREA & & NONE \\
\hline 4384 & AREA & & NONE \\
\hline 4385 & AREA & & NONE \\
\hline 4386 & AREA & & NONE \\
\hline 4387 & AREA & & NONE \\
\hline 4388 & AREA & & NONE \\
\hline 4389 & AREA & & NONE \\
\hline 4390 & AREA & & NONE \\
\hline 4391 & AREA & & NONE \\
\hline 4392 & AREA & & NONE \\
\hline 4393 & AREA & & NONE \\
\hline 4394 & AREA & & NONE \\
\hline 4395 & AREA & & NONE \\
\hline 4396 & AREA & & NONE \\
\hline 4397 & AREA & & NONE \\
\hline 4398 & AREA & & NONE \\
\hline 4399 & AREA & & NONE \\
\hline 4400 & AREA & & NONE \\
\hline 4401 & AREA & & NONE \\
\hline 4402 & AREA & & NONE \\
\hline 4403 & AREA & & NONE \\
\hline 4404 & AREA & & NONE \\
\hline 4405 & AREA & & NONE \\
\hline 4406 & AREA & & NONE \\
\hline 4407 & AREA & & NONE \\
\hline 4408 & AREA & & NONE \\
\hline 4409 & AREA & & NONE \\
\hline
\end{tabular}




\begin{tabular}{|c|l|l|l|}
\hline & \multicolumn{1}{|c|}{$\mathrm{J}$} & \multicolumn{1}{|c|}{$\mathrm{K}$} & \\
\hline 1 & SAMP_SITE_TYPE & SCHED_LABEL_CODE & TRANSFORM_CODE \\
\hline 4410 & AREA & NONE \\
\hline 4411 & AREA & NONE \\
\hline 4412 & AREA & NONE \\
\hline 4413 & AREA & NONE \\
\hline 4414 & AREA & NONE \\
\hline 4415 & AREA & NONE \\
\hline 4416 & AREA & NONE \\
\hline 4417 & AREA & NONE \\
\hline 4418 & AREA & NONE \\
\hline 4419 & AREA & NONE \\
\hline 4420 & AREA & NONE \\
\hline 4421 & AREA & NONE \\
\hline 4422 & AREA & NONE \\
\hline 4423 & AREA & NONE \\
\hline 4424 & AREA & NONE \\
\hline 4425 & AREA & NONE \\
\hline 4426 & AREA & NONE \\
\hline 4427 & AREA & NONE \\
\hline 4428 & AREA & NONE \\
\hline 4429 & AREA & NONE \\
\hline 4430 & AREA & NONE \\
\hline 4431 & AREA & NONE \\
\hline 4432 & AREA & NONE \\
\hline 4433 & AREA & NONE \\
\hline 4434 & AREA & NONE \\
\hline 4435 & AREA & NONE \\
\hline 4436 & AREA & NONE \\
\hline 4437 & AREA & NONE \\
\hline 4438 & AREA & NONE \\
\hline 4439 & AREA & NONE \\
\hline 4440 & AREA & NONE \\
\hline 4441 & AREA & & NONE \\
\hline 4442 & AREA & NONE \\
\hline 4443 & AREA & NONE \\
\hline 4444 & AREA & NONE \\
\hline 4445 & AREA & & \\
\hline 4446 & AREA & & NONE \\
\hline 4447 & AREA & & \\
\hline & & & \\
\hline
\end{tabular}




\begin{tabular}{|c|l|l|l|}
\hline & \multicolumn{1}{|c|}{$\mathrm{J}$} & \multicolumn{1}{|c|}{$\mathrm{K}$} & \\
\hline 1 & SAMP_SITE_TYPE & SCHED_LABEL_CODE & TRANSFORM_CODE \\
\hline 4448 & AREA & NONE \\
\hline 4449 & AREA & NONE \\
\hline 4450 & AREA & NONE \\
\hline 4451 & AREA & NONE \\
\hline 4452 & AREA & NONE \\
\hline 4453 & AREA & NONE \\
\hline 4454 & AREA & NONE \\
\hline 4455 & AREA & NONE \\
\hline 4456 & AREA & NONE \\
\hline 4457 & AREA & NONE \\
\hline 4458 & AREA & NONE \\
\hline 4459 & AREA & NONE \\
\hline 4460 & AREA & NONE \\
\hline 4461 & AREA & NONE \\
\hline 4462 & AREA & NONE \\
\hline 4463 & AREA & NONE \\
\hline 4464 & AREA & NONE \\
\hline 4465 & AREA & NONE \\
\hline 4466 & AREA & NONE \\
\hline 4467 & AREA & NONE \\
\hline 4468 & AREA & NONE \\
\hline 4469 & AREA & NONE \\
\hline 4470 & AREA & NONE \\
\hline 4471 & AREA & NONE \\
\hline 4472 & AREA & NONE \\
\hline 4473 & AREA & NONE \\
\hline 4474 & AREA & NONE \\
\hline 4475 & AREA & NONE \\
\hline 4476 & AREA & NONE \\
\hline 4477 & & NONE \\
\hline 4478 & AREA & NONE \\
\hline 4479 & AREA & & NONE \\
\hline 4480 & AREA & NONE \\
\hline 4481 & AREA & NONE \\
\hline 4482 & AREA & NONE \\
\hline 4483 & AREA & & \\
\hline 4484 & AREA & & NONE \\
\hline 4485 & AREA & & \\
\hline & & & \\
\hline
\end{tabular}




\begin{tabular}{|c|l|l|l|}
\hline & \multicolumn{1}{|c|}{$\mathrm{J}$} & \multicolumn{1}{|c|}{$\mathrm{K}$} & \\
\hline 1 & SAMP_SITE_TYPE & SCHED_LABEL_CODE & TRANSFORM_CODE \\
\hline 4486 & AREA & NONE \\
\hline 4487 & AREA & NONE \\
\hline 4488 & AREA & NONE \\
\hline 4489 & AREA & NONE \\
\hline 4490 & AREA & NONE \\
\hline 4491 & AREA & NONE \\
\hline 4492 & AREA & NONE \\
\hline 4493 & AREA & NONE \\
\hline 4494 & AREA & NONE \\
\hline 4495 & AREA & NONE \\
\hline 4496 & AREA & NONE \\
\hline 4497 & AREA & NONE \\
\hline 4498 & AREA & NONE \\
\hline 4499 & AREA & NONE \\
\hline 4500 & AREA & NONE \\
\hline 4501 & AREA & NONE \\
\hline 4502 & AREA & NONE \\
\hline 4503 & AREA & NONE \\
\hline 4504 & AREA & NONE \\
\hline 4505 & AREA & NONE \\
\hline 4506 & AREA & NONE \\
\hline 4507 & AREA & NONE \\
\hline 4508 & AREA & NONE \\
\hline 4509 & AREA & NONE \\
\hline 4510 & AREA & NONE \\
\hline 4511 & AREA & NONE \\
\hline 4512 & AREA & NONE \\
\hline 4513 & AREA & NONE \\
\hline 4514 & AREA & NONE \\
\hline 4515 & AREA & NONE \\
\hline 4516 & AREA & NONE \\
\hline 4517 & AREA & & NONE \\
\hline 4518 & AREA & NONE \\
\hline 4519 & AREA & NONE \\
\hline 4520 & AREA & NONE \\
\hline 4521 & AREA & & \\
\hline 4522 & AREA & & NONE \\
\hline 4523 & AREA & & \\
\hline & & & \\
\hline
\end{tabular}




\begin{tabular}{|c|l|l|l|}
\hline & \multicolumn{1}{|c|}{$\mathrm{J}$} & \multicolumn{1}{|c|}{$\mathrm{K}$} & \\
\hline 1 & SAMP_SITE_TYPE & SCHED_LABEL_CODE & TRANSFORM_CODE \\
\hline 4524 & AREA & NONE \\
\hline 4525 & AREA & NONE \\
\hline 4526 & AREA & NONE \\
\hline 4527 & AREA & NONE \\
\hline 4528 & AREA & NONE \\
\hline 4529 & AREA & NONE \\
\hline 4530 & AREA & NONE \\
\hline 4531 & AREA & NONE \\
\hline 4532 & & NONE \\
\hline 4533 & AREA & NONE \\
\hline 4534 & AREA & NONE \\
\hline 4535 & AREA & NONE \\
\hline 4536 & AREA & NONE \\
\hline 4537 & AREA & NONE \\
\hline 4538 & AREA & NONE \\
\hline 4539 & AREA & NONE \\
\hline 4540 & AREA & NONE \\
\hline 4541 & AREA & NONE \\
\hline 4542 & AREA & NONE \\
\hline 4543 & AREA & NONE \\
\hline 4544 & AREA & NONE \\
\hline 4545 & AREA & NONE \\
\hline 4546 & AREA & NONE \\
\hline 4547 & AREA & NONE \\
\hline 4548 & AREA & NONE \\
\hline 4549 & AREA & NONE \\
\hline 4550 & AREA & NONE \\
\hline 4551 & AREA & NONE \\
\hline 4552 & AREA & NONE \\
\hline 4553 & AREA & NONE \\
\hline 4554 & AREA & NONE \\
\hline 4555 & AREA & & NONE \\
\hline 4556 & AREA & NONE \\
\hline 4557 & AREA & NONE \\
\hline 4558 & AREA & & \\
\hline 4559 & AREA & & NONE \\
\hline 4560 & AREA & & NONE \\
\hline 4561 & AREA & & \\
\hline & & & \\
\hline
\end{tabular}




\begin{tabular}{|c|l|l|l|}
\hline & \multicolumn{1}{|c|}{$\mathrm{J}$} & \multicolumn{1}{|c|}{$\mathrm{K}$} & \\
\hline 1 & SAMP_SITE_TYPE & SCHED_LABEL_CODE & TRANSFORM_CODE \\
\hline 4562 & AREA & NONE \\
\hline 4563 & AREA & NONE \\
\hline 4564 & AREA & NONE \\
\hline 4565 & AREA & NONE \\
\hline 4566 & AREA & NONE \\
\hline 4567 & AREA & NONE \\
\hline 4568 & AREA & NONE \\
\hline 4569 & AREA & NONE \\
\hline 4570 & AREA & NONE \\
\hline 4571 & AREA & NONE \\
\hline 4572 & AREA & NONE \\
\hline 4573 & AREA & NONE \\
\hline 4574 & AREA & NONE \\
\hline 4575 & AREA & NONE \\
\hline 4576 & AREA & NONE \\
\hline 4577 & AREA & NONE \\
\hline 4578 & AREA & NONE \\
\hline 4579 & AREA & NONE \\
\hline 4580 & AREA & NONE \\
\hline 4581 & AREA & NONE \\
\hline 4582 & AREA & NONE \\
\hline 4583 & AREA & NONE \\
\hline 4584 & AREA & NONE \\
\hline 4585 & AREA & NONE \\
\hline 4586 & AREA & NONE \\
\hline 4587 & AREA & NONE \\
\hline 4588 & AREA & NONE \\
\hline 4589 & AREA & NONE \\
\hline 4590 & AREA & NONE \\
\hline 4591 & AREA & NONE \\
\hline 4592 & AREA & NONE \\
\hline 4593 & AREA & & NONE \\
\hline 4594 & AREA & NONE \\
\hline 4595 & AREA & NONE \\
\hline 4596 & AREA & NONE \\
\hline 4597 & AREA & & \\
\hline 4598 & AREA & & NONE \\
\hline 4599 & AREA & & \\
\hline & & & \\
\hline
\end{tabular}




\begin{tabular}{|c|c|c|c|}
\hline & $\mathrm{J}$ & $\mathrm{K}$ & $\mathrm{L}$ \\
\hline 1 & SAMP_SITE_TYPE & SCHED_LABEL_CODE & TRANSFORM_CODE \\
\hline 4600 & AREA & & NONE \\
\hline 4601 & AREA & & NONE \\
\hline 4602 & AREA & & NONE \\
\hline 4603 & AREA & & NONE \\
\hline 4604 & AREA & & NONE \\
\hline 4605 & AREA & & NONE \\
\hline 4606 & AREA & & NONE \\
\hline 4607 & AREA & & NONE \\
\hline 4608 & AREA & & NONE \\
\hline 4609 & AREA & & NONE \\
\hline 4610 & AREA & & NONE \\
\hline 4611 & AREA & & NONE \\
\hline 4612 & AREA & & NONE \\
\hline 4613 & AREA & & NONE \\
\hline 4614 & AREA & & NONE \\
\hline 4615 & AREA & & NONE \\
\hline 4616 & AREA & & NONE \\
\hline 4617 & AREA & & NONE \\
\hline 4618 & AREA & & NONE \\
\hline 4619 & AREA & & NONE \\
\hline 4620 & AREA & & NONE \\
\hline 4621 & AREA & & NONE \\
\hline 4622 & AREA & & NONE \\
\hline 4623 & AREA & & NONE \\
\hline 4624 & AREA & & NONE \\
\hline 4625 & AREA & & NONE \\
\hline 4626 & AREA & & NONE \\
\hline 4627 & AREA & & NONE \\
\hline 4628 & AREA & & NONE \\
\hline 4629 & AREA & & NONE \\
\hline 4630 & AREA & & NONE \\
\hline 4631 & AREA & & NONE \\
\hline 4632 & AREA & & NONE \\
\hline 4633 & AREA & & NONE \\
\hline 4634 & AREA & & NONE \\
\hline 4635 & AREA & & NONE \\
\hline 4636 & AREA & & NONE \\
\hline 4637 & AREA & & NONE \\
\hline
\end{tabular}




\begin{tabular}{|c|l|l|l|}
\hline & \multicolumn{1}{|c|}{$\mathrm{J}$} & \multicolumn{1}{|c|}{$\mathrm{K}$} & \\
\hline 1 & SAMP_SITE_TYPE & SCHED_LABEL_CODE & TRANSFORM_CODE \\
\hline 4638 & AREA & NONE \\
\hline 4639 & AREA & NONE \\
\hline 4640 & AREA & NONE \\
\hline 4641 & AREA & NONE \\
\hline 4642 & AREA & NONE \\
\hline 4643 & AREA & NONE \\
\hline 4644 & AREA & NONE \\
\hline 4645 & AREA & NONE \\
\hline 4646 & AREA & NONE \\
\hline 4647 & AREA & NONE \\
\hline 4648 & AREA & NONE \\
\hline 4649 & AREA & NONE \\
\hline 4650 & AREA & NONE \\
\hline 4651 & AREA & NONE \\
\hline 4652 & AREA & NONE \\
\hline 4653 & AREA & NONE \\
\hline 4654 & AREA & NONE \\
\hline 4655 & AREA & NONE \\
\hline 4656 & AREA & NONE \\
\hline 4657 & AREA & NONE \\
\hline 4658 & AREA & NONE \\
\hline 4659 & AREA & NONE \\
\hline 4660 & AREA & NONE \\
\hline 4661 & AREA & NONE \\
\hline 4662 & AREA & NONE \\
\hline 4663 & AREA & NONE \\
\hline 4664 & AREA & NONE \\
\hline 4665 & AREA & NONE \\
\hline 4666 & AREA & NONE \\
\hline 4667 & AREA & NONE \\
\hline 4668 & AREA & NONE \\
\hline 4669 & AREA & & NONE \\
\hline 4670 & AREA & NONE \\
\hline 4671 & AREA & & NONE \\
\hline 4672 & AREA & & NONE \\
\hline 4673 & AREA & & \\
\hline 4674 & AREA & & \\
\hline 4675 & AREA & & \\
\hline & & & \\
\hline
\end{tabular}




\begin{tabular}{|c|l|l|l|}
\hline & \multicolumn{1}{|c|}{$\mathrm{J}$} & \multicolumn{1}{|c|}{$\mathrm{K}$} & \\
\hline 1 & SAMP_SITE_TYPE & SCHED_LABEL_CODE & TRANSFORM_CODE \\
\hline 4676 & AREA & NONE \\
\hline 4677 & AREA & NONE \\
\hline 4678 & AREA & NONE \\
\hline 4679 & AREA & NONE \\
\hline 4680 & AREA & NONE \\
\hline 4681 & AREA & NONE \\
\hline 4682 & AREA & NONE \\
\hline 4683 & AREA & NONE \\
\hline 4684 & AREA & NONE \\
\hline 4685 & AREA & NONE \\
\hline 4686 & AREA & NONE \\
\hline 4687 & AREA & NONE \\
\hline 4688 & AREA & NONE \\
\hline 4689 & AREA & NONE \\
\hline 4690 & AREA & NONE \\
\hline 4691 & AREA & NONE \\
\hline 4692 & AREA & NONE \\
\hline 4693 & AREA & NONE \\
\hline 4694 & AREA & NONE \\
\hline 4695 & AREA & NONE \\
\hline 4696 & AREA & NONE \\
\hline 4697 & AREA & NONE \\
\hline 4698 & AREA & NONE \\
\hline 4699 & AREA & NONE \\
\hline 4700 & AREA & NONE \\
\hline 4701 & AREA & NONE \\
\hline 4702 & AREA & NONE \\
\hline 4703 & AREA & NONE \\
\hline 4704 & AREA & NONE \\
\hline 4705 & AREA & NONE \\
\hline 4706 & AREA & NONE \\
\hline 4707 & AREA & NONE \\
\hline 4708 & AREA & NONE \\
\hline 4709 & AREA & NONE \\
\hline 4710 & AREA & NONE \\
\hline 4711 & AREA & & \\
\hline 4712 & AREA & & NONE \\
\hline 4713 & AREA & & \\
\hline & & & \\
\hline
\end{tabular}




$$
\prod
$$




\begin{tabular}{|c|l|l|l|}
\hline & \multicolumn{1}{|c|}{$\mathrm{J}$} & \multicolumn{1}{|c|}{$\mathrm{K}$} & \\
\hline 1 & SAMP_SITE_TYPE & SCHED_LABEL_CODE & TRANSFORM_CODE \\
\hline 4752 & AREA & NONE \\
\hline 4753 & AREA & NONE \\
\hline 4754 & AREA & NONE \\
\hline 4755 & AREA & NONE \\
\hline 4756 & AREA & NONE \\
\hline 4757 & AREA & NONE \\
\hline 4758 & AREA & NONE \\
\hline 4759 & AREA & NONE \\
\hline 4760 & AREA & NONE \\
\hline 4761 & AREA & NONE \\
\hline 4762 & AREA & NONE \\
\hline 4763 & AREA & NONE \\
\hline 4764 & AREA & NONE \\
\hline 4765 & AREA & NONE \\
\hline 4766 & AREA & NONE \\
\hline 4767 & AREA & NONE \\
\hline 4768 & AREA & NONE \\
\hline 4769 & AREA & NONE \\
\hline 4770 & AREA & NONE \\
\hline 4771 & AREA & NONE \\
\hline 4772 & AREA & NONE \\
\hline 4773 & AREA & NONE \\
\hline 4774 & AREA & NONE \\
\hline 4775 & AREA & NONE \\
\hline 4776 & AREA & NONE \\
\hline 4777 & AREA & NONE \\
\hline 4778 & AREA & NONE \\
\hline 4779 & AREA & NONE \\
\hline 4780 & AREA & NONE \\
\hline 4781 & AREA & NONE \\
\hline 4782 & AREA & NONE \\
\hline 4783 & AREA & NONE \\
\hline 4784 & AREA & NONE \\
\hline 4785 & AREA & NONE \\
\hline 4786 & AREA & NONE \\
\hline 4787 & AREA & & \\
\hline 4788 & AREA & & \\
\hline 4789 & AREA & & \\
\hline & & & \\
\hline
\end{tabular}




\begin{tabular}{|c|l|l|l|}
\hline & \multicolumn{1}{|c|}{$\mathrm{J}$} & \multicolumn{1}{|c|}{$\mathrm{K}$} & \\
\hline 1 & SAMP_SITE_TYPE & SCHED_LABEL_CODE & TRANSFORM_CODE \\
\hline 4790 & AREA & NONE \\
\hline 4791 & AREA & NONE \\
\hline 4792 & AREA & NONE \\
\hline 4793 & AREA & NONE \\
\hline 4794 & AREA & NONE \\
\hline 4795 & AREA & NONE \\
\hline 4796 & AREA & NONE \\
\hline 4797 & AREA & NONE \\
\hline 4798 & AREA & NONE \\
\hline 4799 & AREA & NONE \\
\hline 4800 & AREA & NONE \\
\hline 4801 & AREA & NONE \\
\hline 4802 & AREA & NONE \\
\hline 4803 & AREA & NONE \\
\hline 4804 & AREA & NONE \\
\hline 4805 & AREA & NONE \\
\hline 4806 & AREA & NONE \\
\hline 4807 & AREA & NONE \\
\hline 4808 & AREA & NONE \\
\hline 4809 & AREA & NONE \\
\hline 4810 & AREA & NONE \\
\hline 4811 & AREA & NONE \\
\hline 4812 & AREA & NONE \\
\hline 4813 & AREA & NONE \\
\hline 4814 & AREA & NONE \\
\hline 4815 & AREA & NONE \\
\hline 4816 & AREA & NONE \\
\hline 4817 & AREA & NONE \\
\hline 4818 & AREA & NONE \\
\hline 4819 & AREA & NONE \\
\hline 4820 & AREA & NONE \\
\hline 4821 & AREA & & NONE \\
\hline 4822 & AREA & NONE \\
\hline 4823 & AREA & & NONE \\
\hline 4824 & AREA & & \\
\hline 4825 & AREA & & NONE \\
\hline 4826 & AREA & & \\
\hline 4827 & AREA & & \\
\hline & & & \\
\hline
\end{tabular}




\begin{tabular}{|c|l|l|l|}
\hline & \multicolumn{1}{|c|}{$\mathrm{J}$} & \multicolumn{1}{|c|}{$\mathrm{K}$} & \\
\hline 1 & SAMP_SITE_TYPE & SCHED_LABEL_CODE & TRANSFORM_CODE \\
\hline 4828 & AREA & NONE \\
\hline 4829 & AREA & NONE \\
\hline 4830 & AREA & NONE \\
\hline 4831 & AREA & NONE \\
\hline 4832 & AREA & NONE \\
\hline 4833 & AREA & NONE \\
\hline 4834 & AREA & NONE \\
\hline 4835 & AREA & NONE \\
\hline 4836 & AREA & NONE \\
\hline 4837 & AREA & NONE \\
\hline 4838 & AREA & NONE \\
\hline 4839 & AREA & NONE \\
\hline 4840 & AREA & NONE \\
\hline 4841 & AREA & NONE \\
\hline 4842 & AREA & NONE \\
\hline 4843 & AREA & NONE \\
\hline 4844 & AREA & NONE \\
\hline 4845 & AREA & NONE \\
\hline 4846 & AREA & NONE \\
\hline 4847 & AREA & NONE \\
\hline 4848 & AREA & NONE \\
\hline 4849 & AREA & NONE \\
\hline 4850 & AREA & NONE \\
\hline 4851 & AREA & NONE \\
\hline 4852 & AREA & NONE \\
\hline 4853 & AREA & NONE \\
\hline 4854 & AREA & NONE \\
\hline 4855 & AREA & NONE \\
\hline 4856 & AREA & NONE \\
\hline 4857 & AREA & NONE \\
\hline 4858 & AREA & NONE \\
\hline 4859 & AREA & & NONE \\
\hline 4860 & AREA & NONE \\
\hline 4861 & AREA & NONE \\
\hline 4862 & AREA & NONE \\
\hline 4863 & AREA & & \\
\hline 4864 & AREA & & NONE \\
\hline 4865 & AREA & & \\
\hline & & & \\
\hline
\end{tabular}




\begin{tabular}{|c|l|l|l|}
\hline & \multicolumn{1}{|c|}{$\mathrm{J}$} & \multicolumn{1}{|c|}{$\mathrm{K}$} & \\
\hline 1 & SAMP_SITE_TYPE & SCHED_LABEL_CODE & TRANSFORM_CODE \\
\hline 4866 & AREA & NONE \\
\hline 4867 & AREA & NONE \\
\hline 4868 & AREA & NONE \\
\hline 4869 & AREA & NONE \\
\hline 4870 & AREA & NONE \\
\hline 4871 & AREA & NONE \\
\hline 4872 & AREA & NONE \\
\hline 4873 & AREA & NONE \\
\hline 4874 & AREA & NONE \\
\hline 4875 & AREA & NONE \\
\hline 4876 & AREA & NONE \\
\hline 4877 & AREA & NONE \\
\hline 4878 & AREA & NONE \\
\hline 4879 & AREA & NONE \\
\hline 4880 & AREA & NONE \\
\hline 4881 & AREA & NONE \\
\hline 4882 & AREA & NONE \\
\hline 4883 & AREA & NONE \\
\hline 4884 & AREA & NONE \\
\hline 4885 & AREA & NONE \\
\hline 4886 & AREA & NONE \\
\hline 4887 & AREA & NONE \\
\hline 4888 & AREA & NONE \\
\hline 4889 & AREA & NONE \\
\hline 4890 & AREA & NONE \\
\hline 4891 & AREA & NONE \\
\hline 4892 & AREA & NONE \\
\hline 4893 & AREA & NONE \\
\hline 4894 & AREA & NONE \\
\hline 4895 & AREA & NONE \\
\hline 4896 & AREA & NONE \\
\hline 4897 & AREA & NONE \\
\hline 4898 & AREA & NONE \\
\hline 4899 & AREA & NONE \\
\hline 4900 & AREA & NONE \\
\hline 4901 & AREA & & \\
\hline 4902 & AREA & & NONE \\
\hline 4903 & AREA & & \\
\hline & & & \\
\hline
\end{tabular}




\begin{tabular}{|c|l|l|l|}
\hline & \multicolumn{1}{|c|}{$\mathrm{J}$} & \multicolumn{1}{|c|}{$\mathrm{K}$} & \\
\hline 1 & SAMP_SITE_TYPE & SCHED_LABEL_CODE & TRANSFORM_CODE \\
\hline 4904 & AREA & NONE \\
\hline 4905 & AREA & NONE \\
\hline 4906 & AREA & NONE \\
\hline 4907 & AREA & NONE \\
\hline 4908 & AREA & NONE \\
\hline 4909 & AREA & NONE \\
\hline 4910 & AREA & NONE \\
\hline 4911 & AREA & NONE \\
\hline 4912 & AREA & NONE \\
\hline 4913 & AREA & NONE \\
\hline 4914 & AREA & NONE \\
\hline 4915 & AREA & NONE \\
\hline 4916 & AREA & NONE \\
\hline 4917 & AREA & NONE \\
\hline 4918 & AREA & NONE \\
\hline 4919 & AREA & NONE \\
\hline 4920 & AREA & NONE \\
\hline 4921 & AREA & NONE \\
\hline 4922 & AREA & NONE \\
\hline 4923 & AREA & NONE \\
\hline 4924 & AREA & NONE \\
\hline 4925 & AREA & NONE \\
\hline 4926 & AREA & NONE \\
\hline 4927 & AREA & NONE \\
\hline 4928 & AREA & NONE \\
\hline 4929 & AREA & NONE \\
\hline 4930 & AREA & NONE \\
\hline 4931 & AREA & NONE \\
\hline 4932 & AREA & NONE \\
\hline 4933 & AREA & NONE \\
\hline 4934 & AREA & NONE \\
\hline 4935 & AREA & NONE \\
\hline 4936 & AREA & NONE \\
\hline 4937 & AREA & NONE \\
\hline 4938 & AREA & NONE \\
\hline 4939 & AREA & & \\
\hline 4940 & AREA & & NONE \\
\hline 4941 & AREA & & \\
\hline & & & \\
\hline
\end{tabular}




\begin{tabular}{|c|l|l|l|}
\hline & \multicolumn{1}{|c|}{$\mathrm{J}$} & \multicolumn{1}{|c|}{$\mathrm{K}$} & \\
\hline 1 & SAMP_SITE_TYPE & SCHED_LABEL_CODE & TRANSFORM_CODE \\
\hline 4942 & AREA & NONE \\
\hline 4943 & AREA & NONE \\
\hline 4944 & AREA & NONE \\
\hline 4945 & AREA & NONE \\
\hline 4946 & AREA & NONE \\
\hline 4947 & AREA & NONE \\
\hline 4948 & AREA & NONE \\
\hline 4949 & AREA & NONE \\
\hline 4950 & AREA & NONE \\
\hline 4951 & AREA & NONE \\
\hline 4952 & AREA & NONE \\
\hline 4953 & AREA & NONE \\
\hline 4954 & AREA & NONE \\
\hline 4955 & AREA & NONE \\
\hline 4956 & AREA & NONE \\
\hline 4957 & AREA & NONE \\
\hline 4958 & AREA & NONE \\
\hline 4959 & AREA & NONE \\
\hline 4960 & AREA & NONE \\
\hline 4961 & AREA & NONE \\
\hline 4962 & AREA & NONE \\
\hline 4963 & AREA & NONE \\
\hline 4964 & AREA & NONE \\
\hline 4965 & AREA & NONE \\
\hline 4966 & AREA & NONE \\
\hline 4967 & AREA & NONE \\
\hline 4968 & AREA & NONE \\
\hline 4969 & AREA & NONE \\
\hline 4970 & AREA & NONE \\
\hline 4971 & AREA & NONE \\
\hline 4972 & AREA & NONE \\
\hline 4973 & AREA & & NONE \\
\hline 4974 & AREA & NONE \\
\hline 4975 & AREA & NONE \\
\hline 4976 & AREA & NONE \\
\hline 4977 & AREA & & \\
\hline 4978 & AREA & & \\
\hline 4979 & AREA & & \\
\hline & & & \\
\hline
\end{tabular}




\begin{tabular}{|c|l|l|l|}
\hline & \multicolumn{1}{|c|}{$\mathrm{J}$} & \multicolumn{1}{|c|}{$\mathrm{K}$} & \\
\hline 1 & SAMP_SITE_TYPE & SCHED_LABEL_CODE & TRANSFORM_CODE \\
\hline 4980 & AREA & NONE \\
\hline 4981 & AREA & NONE \\
\hline 4982 & AREA & NONE \\
\hline 4983 & AREA & NONE \\
\hline 4984 & AREA & NONE \\
\hline 4985 & AREA & NONE \\
\hline 4986 & AREA & NONE \\
\hline 4987 & AREA & NONE \\
\hline 4988 & AREA & NONE \\
\hline 4989 & AREA & NONE \\
\hline 4990 & AREA & NONE \\
\hline 4991 & AREA & NONE \\
\hline 4992 & AREA & NONE \\
\hline 4993 & AREA & NONE \\
\hline 4994 & AREA & NONE \\
\hline 4995 & AREA & NONE \\
\hline 4996 & AREA & NONE \\
\hline 4997 & AREA & NONE \\
\hline 4998 & AREA & NONE \\
\hline 4999 & AREA & NONE \\
\hline 5000 & AREA & NONE \\
\hline 5001 & AREA & NONE \\
\hline 5002 & AREA & NONE \\
\hline 5003 & AREA & NONE \\
\hline 5004 & AREA & NONE \\
\hline 5005 & AREA & NONE \\
\hline 5006 & AREA & NONE \\
\hline 5007 & AREA & NONE \\
\hline 5008 & AREA & NONE \\
\hline 5009 & AREA & & NONE \\
\hline 5010 & AREA & NONE \\
\hline 5011 & AREA & & NONE \\
\hline 5012 & AREA & & \\
\hline 5013 & AREA & & NONE \\
\hline 5014 & AREA & & NONE \\
\hline 5015 & AREA & & \\
\hline 5016 & AREA & & \\
\hline 5017 & AREA & & \\
\hline & & & \\
\hline
\end{tabular}




\begin{tabular}{|c|l|l|l|}
\hline & \multicolumn{1}{|c|}{$\mathrm{J}$} & \multicolumn{1}{|c|}{$\mathrm{K}$} & \\
\hline 1 & SAMP_SITE_TYPE & SCHED_LABEL_CODE & TRANSFORM_CODE \\
\hline 5018 & AREA & NONE \\
\hline 5019 & AREA & NONE \\
\hline 5020 & AREA & NONE \\
\hline 5021 & AREA & NONE \\
\hline 5022 & AREA & NONE \\
\hline 5023 & AREA & NONE \\
\hline 5024 & AREA & NONE \\
\hline 5025 & AREA & NONE \\
\hline 5026 & AREA & NONE \\
\hline 5027 & AREA & NONE \\
\hline 5028 & AREA & NONE \\
\hline 5029 & AREA & NONE \\
\hline 5030 & AREA & NONE \\
\hline 5031 & AREA & & NONE \\
\hline 5032 & AREA & NONE \\
\hline 5033 & AREA & NONE \\
\hline 5034 & AREA & NONE \\
\hline 5035 & AREA & NONE \\
\hline 5036 & AREA & NONE \\
\hline 5037 & AREA & & NONE \\
\hline 5038 & AREA & NONE \\
\hline 5039 & AREA & NONE \\
\hline 5040 & AREA & NONE \\
\hline 5041 & AREA & NONE \\
\hline 5042 & AREA & NONE \\
\hline 5043 & AREA & NONE \\
\hline 5044 & AREA & NONE \\
\hline 5045 & AREA & NONE \\
\hline 5046 & AREA & NONE \\
\hline 5047 & AREA & & NONE \\
\hline 5048 & AREA & NONE \\
\hline 5049 & AREA & & NONE \\
\hline 5050 & AREA & & NONE \\
\hline 5051 & AREA & & NONE \\
\hline 5052 & AREA & & NONE \\
\hline 5053 & AREA & & \\
\hline 5054 & AREA & & \\
\hline 5055 & AREA & & \\
\hline & & & \\
\hline
\end{tabular}




\begin{tabular}{|c|c|c|c|}
\hline & $\mathrm{J}$ & $\mathrm{K}$ & $\mathrm{L}$ \\
\hline 1 & SAMP_SITE_TYPE & SCHED_LABEL_CODE & TRANSFORM_CODE \\
\hline 5056 & AREA & & NONE \\
\hline 5057 & AREA & & NONE \\
\hline 5058 & AREA & & NONE \\
\hline 5059 & AREA & & NONE \\
\hline 5060 & AREA & & NONE \\
\hline 5061 & AREA & & NONE \\
\hline 5062 & AREA & & NONE \\
\hline 5063 & AREA & & NONE \\
\hline 5064 & AREA & & NONE \\
\hline 5065 & AREA & & NONE \\
\hline 5066 & AREA & & NONE \\
\hline 5067 & AREA & & NONE \\
\hline 5068 & AREA & & NONE \\
\hline 5069 & AREA & & NONE \\
\hline 5070 & AREA & & NONE \\
\hline 5071 & AREA & & NONE \\
\hline 5072 & AREA & & NONE \\
\hline 5073 & AREA & & NONE \\
\hline 5074 & AREA & & NONE \\
\hline 5075 & AREA & & NONE \\
\hline 5076 & AREA & & NONE \\
\hline 5077 & AREA & & NONE \\
\hline 5078 & AREA & & NONE \\
\hline 5079 & AREA & & NONE \\
\hline 5080 & AREA & & NONE \\
\hline 5081 & AREA & & NONE \\
\hline 5082 & AREA & & NONE \\
\hline 5083 & AREA & & NONE \\
\hline 5084 & AREA & & NONE \\
\hline 5085 & AREA & & NONE \\
\hline 5086 & AREA & & NONE \\
\hline 5087 & AREA & & NONE \\
\hline 5088 & AREA & & NONE \\
\hline 5089 & AREA & & NONE \\
\hline 5090 & AREA & & NONE \\
\hline 5091 & AREA & & NONE \\
\hline 5092 & AREA & & NONE \\
\hline 5093 & AREA & & NONE \\
\hline
\end{tabular}




\begin{tabular}{|c|l|l|l|}
\hline & \multicolumn{1}{|c|}{$\mathrm{J}$} & \multicolumn{1}{|c|}{$\mathrm{K}$} & \\
\hline 1 & SAMP_SITE_TYPE & SCHED_LABEL_CODE & TRANSFORM_CODE \\
\hline 5094 & AREA & & NONE \\
\hline 5095 & AREA & & NONE \\
\hline 5096 & AREA & & NONE \\
\hline 5097 & AREA & & NONE \\
\hline 5098 & AREA & & NONE \\
\hline 5099 & AREA & & NONE \\
\hline 5100 & AREA & & NONE \\
\hline 5101 & AREA & & NONE \\
\hline 5102 & AREA & & NONE \\
\hline 5103 & AREA & & NONE \\
\hline 5104 & AREA & & NONE \\
\hline 5105 & AREA & & NONE \\
\hline 5106 & AREA & & NONE \\
\hline 5107 & AREA & & NONE \\
\hline 5108 & AREA & & NONE \\
\hline 5109 & AREA & & NONE \\
\hline 5110 & AREA & & NONE \\
\hline 5111 & AREA & & NONE \\
\hline 5112 & AREA & & NONE \\
\hline 5113 & AREA & & NONE \\
\hline 5114 & AREA & & NONE \\
\hline 5115 & AREA & & NONE \\
\hline 5116 & AREA & & NONE \\
\hline 5117 & AREA & & NONE \\
\hline 5118 & AREA & & NONE \\
\hline 5119 & NAMED_POINT & $\mathrm{C}$ & NONE \\
\hline 5120 & AREA & & NONE \\
\hline 5121 & AREA & & NONE \\
\hline 5122 & AREA & & NONE \\
\hline 5123 & AREA & & NONE \\
\hline 5124 & AREA & & NONE \\
\hline 5125 & AREA & & NONE \\
\hline 5126 & AREA & & NONE \\
\hline 5127 & AREA & & \\
\hline 5128 & AREA & & \\
\hline 5129 & AREA & & \\
\hline 5130 & AREA & & \\
\hline 5131 & AREA & & \\
\hline & & & \\
\hline
\end{tabular}




\begin{tabular}{|c|l|l|l|}
\hline & \multicolumn{1}{|c|}{$\mathrm{J}$} & \multicolumn{1}{|c|}{$\mathrm{K}$} & \\
\hline 1 & SAMP_SITE_TYPE & SCHED_LABEL_CODE & TRANSFORM_CODE \\
\hline 5132 & AREA & $\mathrm{G}$ & \\
\hline 5133 & AREA & $\mathrm{G}$ & \\
\hline 5134 & AREA & & NONE \\
\hline 5135 & AREA & & NONE \\
\hline 5136 & AREA & NONE \\
\hline 5137 & AREA & & NONE \\
\hline 5138 & AREA & & NONE \\
\hline 5139 & AREA & & NONE \\
\hline 5140 & AREA & & NONE \\
\hline 5141 & AREA & & NONE \\
\hline 5142 & AREA & & NONE \\
\hline 5143 & AREA & & NONE \\
\hline 5144 & AREA & & NONE \\
\hline 5145 & AREA & & NONE \\
\hline 5146 & AREA & & NONE \\
\hline 5147 & AREA & & NONE \\
\hline 5148 & AREA & & NONE \\
\hline 5149 & AREA & & NONE \\
\hline 5150 & AREA & & NONE \\
\hline 5151 & AREA & & NONE \\
\hline 5152 & AREA & & NONE \\
\hline 5153 & AREA & & NONE \\
\hline 5154 & AREA & & NONE \\
\hline 5155 & AREA & & NONE \\
\hline 5156 & AREA & & NONE \\
\hline 5157 & AREA & & NONE \\
\hline 5158 & AREA & & NONE \\
\hline 5159 & AREA & & NONE \\
\hline 5160 & AREA & & NONE \\
\hline 5161 & AREA & & NONE \\
\hline 5162 & AREA & & NONE \\
\hline 5163 & AREA & & NONE \\
\hline 5164 & AREA & & NONE \\
\hline 5165 & AREA & & \\
\hline 5166 & AREA & & \\
\hline 5167 & AREA & & \\
\hline 5168 & AREA & & \\
\hline 5169 & AREA & & \\
\hline & & & \\
\hline
\end{tabular}




\begin{tabular}{|c|c|c|c|}
\hline & $\mathrm{J}$ & $\mathrm{K}$ & $\mathrm{L}$ \\
\hline 1 & SAMP_SITE_TYPE & SCHED_LABEL_CODE & TRANSFORM_CODE \\
\hline 5170 & AREA & & NONE \\
\hline 5171 & AREA & & NONE \\
\hline 5172 & AREA & & NONE \\
\hline 5173 & AREA & & NONE \\
\hline 5174 & AREA & & NONE \\
\hline 5175 & AREA & & NONE \\
\hline 5176 & AREA & & NONE \\
\hline 5177 & AREA & & NONE \\
\hline 5178 & AREA & & NONE \\
\hline 5179 & AREA & & NONE \\
\hline 5180 & AREA & & NONE \\
\hline 5181 & AREA & & NONE \\
\hline 5182 & AREA & & NONE \\
\hline 5183 & AREA & & NONE \\
\hline 5184 & NAMED_POINT & C & \\
\hline 5185 & AREA & & NONE \\
\hline 5186 & AREA & & NONE \\
\hline 5187 & AREA & & NONE \\
\hline 5188 & AREA & & NONE \\
\hline 5189 & AREA & & NONE \\
\hline 5190 & AREA & & NONE \\
\hline 5191 & AREA & & NONE \\
\hline 5192 & AREA & & NONE \\
\hline 5193 & AREA & & NONE \\
\hline 5194 & AREA & & NONE \\
\hline 5195 & AREA & & NONE \\
\hline 5196 & AREA & & NONE \\
\hline 5197 & AREA & & NONE \\
\hline 5198 & AREA & & NONE \\
\hline 5199 & AREA & & NONE \\
\hline 5200 & AREA & & NONE \\
\hline 5201 & AREA & & NONE \\
\hline 5202 & AREA & & NONE \\
\hline 5203 & AREA & & NONE \\
\hline 5204 & AREA & & NONE \\
\hline 5205 & AREA & & NONE \\
\hline 5206 & AREA & & NONE \\
\hline 5207 & AREA & & NONE \\
\hline
\end{tabular}




\begin{tabular}{|c|l|l|l|}
\hline & \multicolumn{1}{|c|}{$\mathrm{J}$} & \multicolumn{1}{|c|}{$\mathrm{K}$} & \\
\hline 1 & SAMP_SITE_TYPE & SCHED_LABEL_CODE & TRANSFORM_CODE \\
\hline 5208 & AREA & NONE \\
\hline 5209 & AREA & NONE \\
\hline 5210 & AREA & NONE \\
\hline 5211 & AREA & NONE \\
\hline 5212 & AREA & NONE \\
\hline 5213 & AREA & NONE \\
\hline 5214 & AREA & NONE \\
\hline 5215 & AREA & NONE \\
\hline 5216 & AREA & NONE \\
\hline 5217 & AREA & NONE \\
\hline 5218 & AREA & NONE \\
\hline 5219 & AREA & NONE \\
\hline 5220 & AREA & NONE \\
\hline 5221 & AREA & NONE \\
\hline 5222 & AREA & NONE \\
\hline 5223 & AREA & NONE \\
\hline 5224 & AREA & NONE \\
\hline 5225 & AREA & NONE \\
\hline 5226 & AREA & NONE \\
\hline 5227 & NAMED_POINT & NONE \\
\hline 5228 & AREA & & NONE \\
\hline 5229 & AREA & NONE \\
\hline 5230 & AREA & NONE \\
\hline 5231 & AREA & NONE \\
\hline 5232 & AREA & NONE \\
\hline 5233 & AREA & NONE \\
\hline 5234 & AREA & NONE \\
\hline 5235 & AREA & & NONE \\
\hline 5236 & AREA & NONE \\
\hline 5237 & AREA & & NONE \\
\hline 5238 & AREA & NONE \\
\hline 5239 & AREA & & NONE \\
\hline 5240 & AREA & & \\
\hline 5241 & AREA & & NONE \\
\hline 5242 & AREA & & NONE \\
\hline 5243 & AREA & & \\
\hline 5244 & AREA & & \\
\hline 5245 & AREA & & \\
\hline & & & \\
\hline
\end{tabular}




\begin{tabular}{|c|l|l|l|}
\hline & \multicolumn{1}{|c|}{$\mathrm{J}$} & \multicolumn{1}{|c|}{$\mathrm{K}$} & \\
\hline 1 & SAMP_SITE_TYPE & SCHED_LABEL_CODE & TRANSFORM_CODE \\
\hline 5246 & AREA & NONE \\
\hline 5247 & AREA & NONE \\
\hline 5248 & AREA & NONE \\
\hline 5249 & AREA & NONE \\
\hline 5250 & AREA & NONE \\
\hline 5251 & AREA & NONE \\
\hline 5252 & AREA & NONE \\
\hline 5253 & AREA & NONE \\
\hline 5254 & AREA & NONE \\
\hline 5255 & AREA & NONE \\
\hline 5256 & AREA & NONE \\
\hline 5257 & AREA & NONE \\
\hline 5258 & AREA & NONE \\
\hline 5259 & AREA & NONE \\
\hline 5260 & AREA & NONE \\
\hline 5261 & AREA & NONE \\
\hline 5262 & AREA & NONE \\
\hline 5263 & AREA & NONE \\
\hline 5264 & AREA & NONE \\
\hline 5265 & AREA & NONE \\
\hline 5266 & AREA & NONE \\
\hline 5267 & AREA & NONE \\
\hline 5268 & AREA & NONE \\
\hline 5269 & AREA & NONE \\
\hline 5270 & AREA & NONE \\
\hline 5271 & AREA & NONE \\
\hline 5272 & AREA & NONE \\
\hline 5273 & AREA & & NONE \\
\hline 5274 & AREA & NONE \\
\hline 5275 & AREA & & NONE \\
\hline 5276 & NAMED_POINT & NONE \\
\hline 5277 & AREA & & \\
\hline 5278 & & & NONE \\
\hline 5279 & & & NONE \\
\hline 5280 & & & NONE \\
\hline 5281 & & & \\
\hline 5282 & & & \\
\hline 5283 & & & \\
\hline & & & \\
\hline
\end{tabular}




\begin{tabular}{|c|l|l|l|}
\hline & \multicolumn{1}{|c|}{ J } & \multicolumn{1}{|c|}{ K } & \\
\hline 1 & SAMP_SITE_TYPE & SCHED_LABEL_CODE & TRANSFORM_CODE \\
\hline 5284 & & NONE \\
\hline 5285 & NAMED_POINT & NONE \\
\hline 5286 & NAMED_POINT & NONE \\
\hline 5287 & NAMED_POINT & NONE \\
\hline 5288 & NAMED_POINT & NONE \\
\hline 5289 & NAMED_POINT & NONE \\
\hline 5290 & NAMED_POINT & NONE \\
\hline 5291 & NAMED_POINT & NONE \\
\hline 5292 & NAMED_POINT & NONE \\
\hline 5293 & NAMED_POINT & NONE \\
\hline 5294 & NAMED_POINT & NONE \\
\hline 5295 & NAMED_POINT & NONE \\
\hline 5296 & NAMED_POINT & NONE \\
\hline 5297 & NAMED_POINT & NONE \\
\hline 5298 & NAMED_POINT & NONE \\
\hline 5299 & NAMED_POINT & NONE \\
\hline 5300 & NAMED_POINT & NONE \\
\hline 5301 & NAMED_POINT & NONE \\
\hline 5302 & NAMED_POINT & NONE \\
\hline 5303 & NAMED_POINT & NONE \\
\hline 5304 & NAMED_POINT & NONE \\
\hline 5305 & NAMED_POINT & NONE \\
\hline 5306 & NAMED_POINT & NONE \\
\hline 5307 & NAMED_POINT & NONE \\
\hline 5308 & NAMED_POINT & NONE \\
\hline 5309 & AREA & NONE \\
\hline 5310 & AREA & NONE \\
\hline 5311 & AREA & NONE \\
\hline 5312 & AREA & NONE \\
\hline 5313 & AREA & NONE \\
\hline 5314 & & NONE \\
\hline 5315 & & NONE \\
\hline 5316 & NAMED_POINT & NONE \\
\hline 5317 & NAMED_POINT & NONE \\
\hline 5318 & NAMED_POINT & NONE \\
\hline 5319 & NAMED_POINT & NONE \\
\hline 5320 & NAMED_POINT & & \\
\hline 5321 & NAMED_POINT & & \\
\hline & & & \\
\hline
\end{tabular}




\begin{tabular}{|c|l|l|l|}
\hline & \multicolumn{1}{|c|}{ J } & \multicolumn{1}{|c|}{ K } & \\
\hline 1 & SAMP_SITE_TYPE & SCHED_LABEL_CODE & TRANSFORM_CODE \\
\hline 5322 & NAMED_POINT & NONE \\
\hline 5323 & NAMED_POINT & NONE \\
\hline 5324 & NAMED_POINT & NONE \\
\hline 5325 & NAMED_POINT & NONE \\
\hline 5326 & NAMED_POINT & NONE \\
\hline 5327 & NAMED_POINT & NONE \\
\hline 5328 & NAMED_POINT & NONE \\
\hline 5329 & NAMED_POINT & NONE \\
\hline 5330 & NAMED_POINT & NONE \\
\hline 5331 & NAMED_POINT & NONE \\
\hline 5332 & NAMED_POINT & NONE \\
\hline 5333 & NAMED_POINT & NONE \\
\hline 5334 & NAMED_POINT & NONE \\
\hline 5335 & NAMED_POINT & NONE \\
\hline 5336 & NAMED_POINT & NONE \\
\hline 5337 & NAMED_POINT & NONE \\
\hline 5338 & NAMED_POINT & NONE \\
\hline 5339 & NAMED_POINT & NONE \\
\hline 5340 & NAMED_POINT & NONE \\
\hline 5341 & NAMED_POINT & NONE \\
\hline 5342 & NAMED_POINT & NONE \\
\hline 5343 & NAMED_POINT & NONE \\
\hline 5344 & NAMED_POINT & NONE \\
\hline 5345 & NAMED_POINT & NONE \\
\hline 5346 & NAMED_POINT & NONE \\
\hline 5347 & NAMED_POINT & NONE \\
\hline 5348 & NAMED_POINT & NONE \\
\hline 5349 & NAMED_POINT & NONE \\
\hline 5350 & NAMED_POINT & NONE \\
\hline 5351 & NAMED_POINT & NONE \\
\hline 5352 & NAMED_POINT & NONE \\
\hline 5353 & NAMED_POINT & NONE \\
\hline 5354 & NAMED_POINT & NONE \\
\hline 5355 & NAMED_POINT & NONE \\
\hline 5356 & NAMED_POINT & NONE \\
\hline 5357 & NAMED_POINT & & \\
\hline 5358 & NAMED_POINT & NONE \\
\hline 5359 & NAMED_POINT & & \\
\hline & & & \\
\hline
\end{tabular}




\begin{tabular}{|c|l|l|l|}
\hline & \multicolumn{1}{|c|}{ J } & \multicolumn{1}{|c|}{ K } & \\
\hline 1 & SAMP_SITE_TYPE & SCHED_LABEL_CODE & TRANSFORM_CODE \\
\hline 5360 & NAMED_POINT & NONE \\
\hline 5361 & NAMED_POINT & NONE \\
\hline 5362 & NAMED_POINT & NONE \\
\hline 5363 & NAMED_POINT & NONE \\
\hline 5364 & NAMED_POINT & NONE \\
\hline 5365 & NAMED_POINT & NONE \\
\hline 5366 & NAMED_POINT & NONE \\
\hline 5367 & NAMED_POINT & NONE \\
\hline 5368 & NAMED_POINT & NONE \\
\hline 5369 & NAMED_POINT & NONE \\
\hline 5370 & NAMED_POINT & NONE \\
\hline 5371 & NAMED_POINT & NONE \\
\hline 5372 & NAMED_POINT & NONE \\
\hline 5373 & NAMED_POINT & NONE \\
\hline 5374 & NAMED_POINT & NONE \\
\hline 5375 & NAMED_POINT & NONE \\
\hline 5376 & NAMED_POINT & NONE \\
\hline 5377 & NAMED_POINT & NONE \\
\hline 5378 & NAMED_POINT & NONE \\
\hline 5379 & NAMED_POINT & NONE \\
\hline 5380 & NAMED_POINT & NONE \\
\hline 5381 & NAMED_POINT & NONE \\
\hline 5382 & NAMED_POINT & NONE \\
\hline 5383 & NAMED_POINT & NONE \\
\hline 5384 & NAMED_POINT & NONE \\
\hline 5385 & NAMED_POINT & NONE \\
\hline 5386 & NAMED_POINT & NONE \\
\hline 5387 & NAMED_POINT & NONE \\
\hline 5388 & NAMED_POINT & NONE \\
\hline 5389 & NAMED_POINT & NONE \\
\hline 5390 & NAMED_POINT & NONE \\
\hline 5391 & NAMED_POINT & NONE \\
\hline 5392 & NAMED_POINT & NONE \\
\hline 5393 & NAMED_POINT & NONE \\
\hline 5394 & NAMED_POINT & NONE \\
\hline 5395 & NAMED_POINT & NONE \\
\hline 5396 & NAMED_POINT & & \\
\hline 5397 & NAMED_POINT & & \\
\hline & & & \\
\hline
\end{tabular}




\begin{tabular}{|c|l|l|l|}
\hline & \multicolumn{1}{|c|}{$J$} & \multicolumn{1}{|c|}{ K } & \\
\hline 1 & SAMP_SITE_TYPE & SCHED_LABEL_CODE & TRANSFORM_CODE \\
\hline 5398 & NAMED_POINT & NONE \\
\hline 5399 & NAMED_POINT & NONE \\
\hline 5400 & NAMED_POINT & NONE \\
\hline 5401 & NAMED_POINT & NONE \\
\hline 5402 & NAMED_POINT & NONE \\
\hline 5403 & NAMED_POINT & NONE \\
\hline 5404 & NAMED_POINT & NONE \\
\hline 5405 & NAMED_POINT & NONE \\
\hline 5406 & NAMED_POINT & NONE \\
\hline 5407 & NAMED_POINT & NONE \\
\hline 5408 & AREA & NONE \\
\hline 5409 & AREA & NONE \\
\hline 5410 & NAMED_POINT & NONE \\
\hline 5411 & AREA & NONE \\
\hline 5412 & AREA & NONE \\
\hline 5413 & AREA & NONE \\
\hline 5414 & AREA & NONE \\
\hline 5415 & AREA & NONE \\
\hline 5416 & AREA & NONE \\
\hline 5417 & AREA & NONE \\
\hline 5418 & AREA & NONE \\
\hline 5419 & AREA & NONE \\
\hline 5420 & AREA & NONE \\
\hline 5421 & AREA & NONE \\
\hline 5422 & AREA & NONE \\
\hline 5423 & AREA & NONE \\
\hline 5424 & AREA & NONE \\
\hline 5425 & AREA & NONE \\
\hline 5426 & AREA & NONE \\
\hline 5427 & AREA & NONE \\
\hline 5428 & AREA & NONE \\
\hline 5429 & & NONE \\
\hline 5430 & & NONE \\
\hline 5431 & AREA & NONE \\
\hline 5432 & AREA & NONE \\
\hline 5433 & AREA & & \\
\hline 5434 & AREA & & NONE \\
\hline 5435 & AREA & & \\
\hline & & & \\
\hline
\end{tabular}




\begin{tabular}{|c|l|l|l|}
\hline & \multicolumn{1}{|c|}{$J$} & \multicolumn{1}{|c|}{ K } & \\
\hline 1 & SAMP_SITE_TYPE & SCHED_LABEL_CODE & TRANSFORM_CODE \\
\hline 5436 & AREA & NONE \\
\hline 5437 & AREA & NONE \\
\hline 5438 & AREA & NONE \\
\hline 5439 & AREA & NONE \\
\hline 5440 & AREA & NONE \\
\hline 5441 & AREA & NONE \\
\hline 5442 & AREA & NONE \\
\hline 5443 & AREA & NONE \\
\hline 5444 & AREA & NONE \\
\hline 5445 & AREA & NONE \\
\hline 5446 & AREA & NONE \\
\hline 5447 & AREA & NONE \\
\hline 5448 & AREA & NONE \\
\hline 5449 & & NONE \\
\hline 5450 & & NONE \\
\hline 5451 & NAMED_POINT & NONE \\
\hline 5452 & NAMED_POINT & NONE \\
\hline 5453 & NAMED_POINT & NONE \\
\hline 5454 & NAMED_POINT & NONE \\
\hline 5455 & NAMED_POINT & NONE \\
\hline 5456 & NAMED_POINT & NONE \\
\hline 5457 & NAMED_POINT & NONE \\
\hline 5458 & NAMED_POINT & NONE \\
\hline 5459 & NAMED_POINT & NONE \\
\hline 5460 & NAMED_POINT & NONE \\
\hline 5461 & NAMED_POINT & NONE \\
\hline 5462 & NAMED_POINT & NONE \\
\hline 5463 & NAMED_POINT & NONE \\
\hline 5464 & NAMED_POINT & NONE \\
\hline 5465 & NAMED_POINT & NONE \\
\hline 5466 & NAMED_POINT & NONE \\
\hline 5467 & NAMED_POINT & NONE \\
\hline 5468 & NAMED_POINT & NONE \\
\hline 5469 & NAMED_POINT & NONE \\
\hline 5470 & NAMED_POINT & NONE \\
\hline 5471 & NAMED_POINT & NONE \\
\hline 5472 & NAMED_POINT & \\
\hline 5473 & NAMED_POINT & & \\
\hline & & & \\
\hline
\end{tabular}




\begin{tabular}{|c|l|l|l|}
\hline & \multicolumn{1}{|c|}{ J } & \multicolumn{1}{|c|}{ K } & \\
\hline 1 & SAMP_SITE_TYPE & SCHED_LABEL_CODE & TRANSFORM_CODE \\
\hline 5474 & NAMED_POINT & NONE \\
\hline 5475 & NAMED_POINT & NONE \\
\hline 5476 & NAMED_POINT & NONE \\
\hline 5477 & NAMED_POINT & NONE \\
\hline 5478 & NAMED_POINT & NONE \\
\hline 5479 & NAMED_POINT & NONE \\
\hline 5480 & NAMED_POINT & NONE \\
\hline 5481 & NAMED_POINT & NONE \\
\hline 5482 & NAMED_POINT & NONE \\
\hline 5483 & NAMED_POINT & NONE \\
\hline 5484 & NAMED_POINT & NONE \\
\hline 5485 & NAMED_POINT & NONE \\
\hline 5486 & NAMED_POINT & NONE \\
\hline 5487 & NAMED_POINT & NONE \\
\hline 5488 & NAMED_POINT & NONE \\
\hline 5489 & NAMED_POINT & NONE \\
\hline 5490 & NAMED_POINT & NONE \\
\hline 5491 & NAMED_POINT & NONE \\
\hline 5492 & NAMED_POINT & NONE \\
\hline 5493 & NAMED_POINT & NONE \\
\hline 5494 & NAMED_POINT & NONE \\
\hline 5495 & NAMED_POINT & NONE \\
\hline 5496 & NAMED_POINT & NONE \\
\hline 5497 & NAMED_POINT & NONE \\
\hline 5498 & NAMED_POINT & NONE \\
\hline 5499 & NAMED_POINT & NONE \\
\hline 5500 & NAMED_POINT & NONE \\
\hline 5501 & NAMED_POINT & NONE \\
\hline 5502 & NAMED_POINT & NONE \\
\hline 5503 & NAMED_POINT & NONE \\
\hline 5504 & NAMED_POINT & NONE \\
\hline 5505 & NAMED_POINT & NONE \\
\hline 5506 & NAMED_POINT & NONE \\
\hline 5507 & NAMED_POINT & NONE \\
\hline 5508 & NAMED_POINT & NONE \\
\hline 5509 & NAMED_POINT & & \\
\hline 5510 & NAMED_POINT & NONE \\
\hline 5511 & NAMED_POINT & & \\
\hline & & & \\
\hline
\end{tabular}




\begin{tabular}{|c|l|l|l|}
\hline & \multicolumn{1}{|c|}{ J } & \multicolumn{1}{|c|}{ K } & \\
\hline 1 & SAMP_SITE_TYPE & SCHED_LABEL_CODE & TRANSFORM_CODE \\
\hline 5512 & NAMED_POINT & NONE \\
\hline 5513 & NAMED_POINT & NONE \\
\hline 5514 & NAMED_POINT & NONE \\
\hline 5515 & NAMED_POINT & NONE \\
\hline 5516 & NAMED_POINT & NONE \\
\hline 5517 & NAMED_POINT & NONE \\
\hline 5518 & NAMED_POINT & NONE \\
\hline 5519 & NAMED_POINT & NONE \\
\hline 5520 & NAMED_POINT & NONE \\
\hline 5521 & NAMED_POINT & NONE \\
\hline 5522 & NAMED_POINT & NONE \\
\hline 5523 & NAMED_POINT & NONE \\
\hline 5524 & NAMED_POINT & NONE \\
\hline 5525 & NAMED_POINT & NONE \\
\hline 5526 & NAMED_POINT & NONE \\
\hline 5527 & NAMED_POINT & NONE \\
\hline 5528 & NAMED_POINT & NONE \\
\hline 5529 & NAMED_POINT & NONE \\
\hline 5530 & NAMED_POINT & NONE \\
\hline 5531 & NAMED_POINT & NONE \\
\hline 5532 & NAMED_POINT & NONE \\
\hline 5533 & NAMED_POINT & NONE \\
\hline 5534 & NAMED_POINT & NONE \\
\hline 5535 & NAMED_POINT & NONE \\
\hline 5536 & NAMED_POINT & NONE \\
\hline 5537 & NAMED_POINT & NONE \\
\hline 5538 & NAMED_POINT & NONE \\
\hline 5539 & NAMED_POINT & NONE \\
\hline 5540 & NAMED_POINT & NONE \\
\hline 5541 & NAMED_POINT & NONE \\
\hline 5542 & NAMED_POINT & NONE \\
\hline 5543 & NAMED_POINT & NONE \\
\hline 5544 & NAMED_POINT & NONE \\
\hline 5545 & NAMED_POINT & NONE \\
\hline 5546 & NAMED_POINT & NONE \\
\hline 5547 & NAMED_POINT & & \\
\hline 5548 & NAMED_POINT & NONE \\
\hline 5549 & NAMED_POINT & & \\
\hline & & & \\
\hline
\end{tabular}




\begin{tabular}{|c|l|l|l|}
\hline & \multicolumn{1}{|c|}{ J } & \multicolumn{1}{|c|}{ K } & \\
\hline 1 & SAMP_SITE_TYPE & SCHED_LABEL_CODE & TRANSFORM_CODE \\
\hline 5550 & NAMED_POINT & NONE \\
\hline 5551 & NAMED_POINT & NONE \\
\hline 5552 & NAMED_POINT & NONE \\
\hline 5553 & NAMED_POINT & NONE \\
\hline 5554 & NAMED_POINT & NONE \\
\hline 5555 & NAMED_POINT & NONE \\
\hline 5556 & NAMED_POINT & NONE \\
\hline 5557 & NAMED_POINT & NONE \\
\hline 5558 & NAMED_POINT & NONE \\
\hline 5559 & NAMED_POINT & NONE \\
\hline 5560 & NAMED_POINT & NONE \\
\hline 5561 & NAMED_POINT & NONE \\
\hline 5562 & NAMED_POINT & NONE \\
\hline 5563 & NAMED_POINT & NONE \\
\hline 5564 & NAMED_POINT & NONE \\
\hline 5565 & NAMED_POINT & NONE \\
\hline 5566 & NAMED_POINT & NONE \\
\hline 5567 & NAMED_POINT & NONE \\
\hline 5568 & NAMED_POINT & NONE \\
\hline 5569 & NAMED_POINT & NONE \\
\hline 5570 & NAMED_POINT & NONE \\
\hline 5571 & NAMED_POINT & NONE \\
\hline 5572 & NAMED_POINT & NONE \\
\hline 5573 & NAMED_POINT & NONE \\
\hline 5574 & NAMED_POINT & NONE \\
\hline 5575 & NAMED_POINT & NONE \\
\hline 5576 & NAMED_POINT & NONE \\
\hline 5577 & NAMED_POINT & NONE \\
\hline 5578 & NAMED_POINT & NONE \\
\hline 5579 & NAMED_POINT & NONE \\
\hline 5580 & NAMED_POINT & NONE \\
\hline 5581 & NAMED_POINT & NONE \\
\hline 5582 & NAMED_POINT & NONE \\
\hline 5583 & NAMED_POINT & NONE \\
\hline 5584 & NAMED_POINT & NONE \\
\hline 5585 & NAMED_POINT & & \\
\hline 5586 & NAMED_POINT & NONE \\
\hline 5587 & NAMED_POINT & & \\
\hline & & & \\
\hline
\end{tabular}




\begin{tabular}{|c|l|l|l|}
\hline & \multicolumn{1}{|c|}{ J } & \multicolumn{1}{|c|}{ K } & \\
\hline 1 & SAMP_SITE_TYPE & SCHED_LABEL_CODE & TRANSFORM_CODE \\
\hline 5588 & NAMED_POINT & NONE \\
\hline 5589 & NAMED_POINT & NONE \\
\hline 5590 & NAMED_POINT & NONE \\
\hline 5591 & NAMED_POINT & NONE \\
\hline 5592 & NAMED_POINT & NONE \\
\hline 5593 & NAMED_POINT & NONE \\
\hline 5594 & NAMED_POINT & NONE \\
\hline 5595 & NAMED_POINT & NONE \\
\hline 5596 & NAMED_POINT & NONE \\
\hline 5597 & NAMED_POINT & NONE \\
\hline 5598 & NAMED_POINT & NONE \\
\hline 5599 & NAMED_POINT & NONE \\
\hline 5600 & NAMED_POINT & NONE \\
\hline 5601 & NAMED_POINT & NONE \\
\hline 5602 & NAMED_POINT & NONE \\
\hline 5603 & NAMED_POINT & NONE \\
\hline 5604 & NAMED_POINT & NONE \\
\hline 5605 & NAMED_POINT & NONE \\
\hline 5606 & NAMED_POINT & NONE \\
\hline 5607 & NAMED_POINT & NONE \\
\hline 5608 & NAMED_POINT & NONE \\
\hline 5609 & NAMED_POINT & NONE \\
\hline 5610 & NAMED_POINT & NONE \\
\hline 5611 & NAMED_POINT & NONE \\
\hline 5612 & NAMED_POINT & NONE \\
\hline 5613 & NAMED_POINT & NONE \\
\hline 5614 & NAMED_POINT & NONE \\
\hline 5615 & NAMED_POINT & NONE \\
\hline 5616 & NAMED_POINT & NONE \\
\hline 5617 & NAMED_POINT & NONE \\
\hline 5618 & NAMED_POINT & NONE \\
\hline 5619 & NAMED_POINT & NONE \\
\hline 5620 & NAMED_POINT & NONE \\
\hline 5621 & NAMED_POINT & NONE \\
\hline 5622 & NAMED_POINT & NONE \\
\hline 5623 & NAMED_POINT & \\
\hline 5624 & NAMED_POINT & NONE \\
\hline 5625 & NAMED_POINT & \\
\hline & & \\
\hline
\end{tabular}




\begin{tabular}{|c|l|l|l|}
\hline & \multicolumn{1}{|c|}{$J$} & \multicolumn{1}{|c|}{ K } & \\
\hline 1 & SAMP_SITE_TYPE & SCHED_LABEL_CODE & TRANSFORM_CODE \\
\hline 5626 & NAMED_POINT & NONE \\
\hline 5627 & NAMED_POINT & NONE \\
\hline 5628 & NAMED_POINT & NONE \\
\hline 5629 & NAMED_POINT & NONE \\
\hline 5630 & NAMED_POINT & NONE \\
\hline 5631 & NAMED_POINT & NONE \\
\hline 5632 & NAMED_POINT & NONE \\
\hline 5633 & NAMED_POINT & NONE \\
\hline 5634 & NAMED_POINT & NONE \\
\hline 5635 & NAMED_POINT & NONE \\
\hline 5636 & NAMED_POINT & NONE \\
\hline 5637 & NAMED_POINT & NONE \\
\hline 5638 & NAMED_POINT & NONE \\
\hline 5639 & NAMED_POINT & NONE \\
\hline 5640 & NAMED_POINT & NONE \\
\hline 5641 & NAMED_POINT & NONE \\
\hline 5642 & NAMED_POINT & NONE \\
\hline 5643 & NAMED_POINT & NONE \\
\hline 5644 & NAMED_POINT & NONE \\
\hline 5645 & NAMED_POINT & NONE \\
\hline 5646 & NAMED_POINT & NONE \\
\hline 5647 & NAMED_POINT & NONE \\
\hline 5648 & NAMED_POINT & NONE \\
\hline 5649 & AREA & NONE \\
\hline 5650 & AREA & NONE \\
\hline 5651 & AREA & NONE \\
\hline 5652 & AREA & NONE \\
\hline 5653 & AREA & NONE \\
\hline 5654 & AREA & NONE \\
\hline 5655 & AREA & NONE \\
\hline 5656 & AREA & NONE \\
\hline 5657 & AREA & NONE \\
\hline 5658 & AREA & NONE \\
\hline 5659 & AREA & NONE \\
\hline 5660 & NAMED_POINT & NONE \\
\hline 5661 & NAMED_POINT & NONE \\
\hline 5662 & NAMED_POINT & & \\
\hline 5663 & NAMED_POINT & & \\
\hline & & & \\
\hline
\end{tabular}




\begin{tabular}{|c|l|l|l|}
\hline & \multicolumn{1}{|c|}{ J } & \multicolumn{1}{|c|}{ K } & \\
\hline 1 & SAMP_SITE_TYPE & SCHED_LABEL_CODE & TRANSFORM_CODE \\
\hline 5664 & NAMED_POINT & NONE \\
\hline 5665 & NAMED_POINT & NONE \\
\hline 5666 & NAMED_POINT & NONE \\
\hline 5667 & NAMED_POINT & NONE \\
\hline 5668 & NAMED_POINT & NONE \\
\hline 5669 & NAMED_POINT & NONE \\
\hline 5670 & NAMED_POINT & NONE \\
\hline 5671 & NAMED_POINT & NONE \\
\hline 5672 & NAMED_POINT & NONE \\
\hline 5673 & NAMED_POINT & NONE \\
\hline 5674 & NAMED_POINT & NONE \\
\hline 5675 & NAMED_POINT & NONE \\
\hline 5676 & NAMED_POINT & NONE \\
\hline 5677 & NAMED_POINT & NONE \\
\hline 5678 & NAMED_POINT & NONE \\
\hline 5679 & NAMED_POINT & NONE \\
\hline 5680 & NAMED_POINT & NONE \\
\hline 5681 & NAMED_POINT & NONE \\
\hline 5682 & NAMED_POINT & NONE \\
\hline 5683 & NAMED_POINT & NONE \\
\hline 5684 & NAMED_POINT & NONE \\
\hline 5685 & NAMED_POINT & NONE \\
\hline 5686 & NAMED_POINT & NONE \\
\hline 5687 & NAMED_POINT & NONE \\
\hline 5688 & NAMED_POINT & NONE \\
\hline 5689 & NAMED_POINT & NONE \\
\hline 5690 & NAMED_POINT & NONE \\
\hline 5691 & NAMED_POINT & NONE \\
\hline 5692 & NAMED_POINT & NONE \\
\hline 5693 & NAMED_POINT & NONE \\
\hline 5694 & NAMED_POINT & NONE \\
\hline 5695 & NAMED_POINT & NONE \\
\hline 5696 & NAMED_POINT & NONE \\
\hline 5697 & NAMED_POINT & NONE \\
\hline 5698 & NAMED_POINT & NONE \\
\hline 5699 & NAMED_POINT & & \\
\hline 5700 & NAMED_POINT & NONE \\
\hline 5701 & NAMED_POINT & & \\
\hline & & & \\
\hline
\end{tabular}




\begin{tabular}{|c|l|l|l|}
\hline & \multicolumn{1}{|c|}{ J } & \multicolumn{1}{|c|}{ K } & \\
\hline 1 & SAMP_SITE_TYPE & SCHED_LABEL_CODE & TRANSFORM_CODE \\
\hline 5702 & NAMED_POINT & NONE \\
\hline 5703 & NAMED_POINT & NONE \\
\hline 5704 & NAMED_POINT & NONE \\
\hline 5705 & NAMED_POINT & NONE \\
\hline 5706 & NAMED_POINT & NONE \\
\hline 5707 & NAMED_POINT & NONE \\
\hline 5708 & NAMED_POINT & NONE \\
\hline 5709 & NAMED_POINT & NONE \\
\hline 5710 & NAMED_POINT & NONE \\
\hline 5711 & NAMED_POINT & NONE \\
\hline 5712 & NAMED_POINT & NONE \\
\hline 5713 & NAMED_POINT & NONE \\
\hline 5714 & NAMED_POINT & NONE \\
\hline 5715 & NAMED_POINT & NONE \\
\hline 5716 & NAMED_POINT & NONE \\
\hline 5717 & NAMED_POINT & NONE \\
\hline 5718 & NAMED_POINT & NONE \\
\hline 5719 & NAMED_POINT & NONE \\
\hline 5720 & NAMED_POINT & NONE \\
\hline 5721 & NAMED_POINT & NONE \\
\hline 5722 & NAMED_POINT & NONE \\
\hline 5723 & NAMED_POINT & NONE \\
\hline 5724 & NAMED_POINT & NONE \\
\hline 5725 & NAMED_POINT & NONE \\
\hline 5726 & NAMED_POINT & NONE \\
\hline 5727 & NAMED_POINT & NONE \\
\hline 5728 & NAMED_POINT & NONE \\
\hline 5729 & NAMED_POINT & NONE \\
\hline 5730 & NAMED_POINT & NONE \\
\hline 5731 & NAMED_POINT & NONE \\
\hline 5732 & NAMED_POINT & NONE \\
\hline 5733 & NAMED_POINT & NONE \\
\hline 5734 & NAMED_POINT & NONE \\
\hline 5735 & NAMED_POINT & NONE \\
\hline 5736 & NAMED_POINT & NONE \\
\hline 5737 & NAMED_POINT & & \\
\hline 5738 & NAMED_POINT & NONE \\
\hline 5739 & NAMED_POINT & & \\
\hline & & & \\
\hline
\end{tabular}




\begin{tabular}{|c|l|l|l|}
\hline & \multicolumn{1}{|c|}{ J } & \multicolumn{1}{|c|}{ K } & \\
\hline 1 & SAMP_SITE_TYPE & SCHED_LABEL_CODE & TRANSFORM_CODE \\
\hline 5740 & NAMED_POINT & NONE \\
\hline 5741 & NAMED_POINT & NONE \\
\hline 5742 & NAMED_POINT & NONE \\
\hline 5743 & NAMED_POINT & NONE \\
\hline 5744 & NAMED_POINT & NONE \\
\hline 5745 & NAMED_POINT & NONE \\
\hline 5746 & NAMED_POINT & NONE \\
\hline 5747 & NAMED_POINT & NONE \\
\hline 5748 & NAMED_POINT & NONE \\
\hline 5749 & NAMED_POINT & NONE \\
\hline 5750 & NAMED_POINT & NONE \\
\hline 5751 & NAMED_POINT & NONE \\
\hline 5752 & NAMED_POINT & NONE \\
\hline 5753 & NAMED_POINT & NONE \\
\hline 5754 & NAMED_POINT & NONE \\
\hline 5755 & NAMED_POINT & NONE \\
\hline 5756 & NAMED_POINT & NONE \\
\hline 5757 & NAMED_POINT & NONE \\
\hline 5758 & NAMED_POINT & NONE \\
\hline 5759 & NAMED_POINT & NONE \\
\hline 5760 & NAMED_POINT & NONE \\
\hline 5761 & NAMED_POINT & NONE \\
\hline 5762 & NAMED_POINT & NONE \\
\hline 5763 & NAMED_POINT & NONE \\
\hline 5764 & NAMED_POINT & NONE \\
\hline 5765 & NAMED_POINT & NONE \\
\hline 5766 & NAMED_POINT & NONE \\
\hline 5767 & NAMED_POINT & NONE \\
\hline 5768 & NAMED_POINT & NONE \\
\hline 5769 & NAMED_POINT & NONE \\
\hline 5770 & NAMED_POINT & NONE \\
\hline 5771 & NAMED_POINT & NONE \\
\hline 5772 & AREA & NONE \\
\hline 5773 & AREA & NONE \\
\hline 5774 & AREA & NONE \\
\hline 5775 & AREA & NONE \\
\hline 5776 & AREA & \\
\hline 5777 & AREA & & \\
\hline & & & \\
\hline
\end{tabular}




\begin{tabular}{|c|l|l|l|}
\hline & \multicolumn{1}{|c|}{$J$} & \multicolumn{1}{|c|}{ K } & \\
\hline 1 & SAMP_SITE_TYPE & SCHED_LABEL_CODE & TRANSFORM_CODE \\
\hline 5778 & AREA & NONE \\
\hline 5779 & AREA & NONE \\
\hline 5780 & AREA & NONE \\
\hline 5781 & AREA & NONE \\
\hline 5782 & AREA & NONE \\
\hline 5783 & AREA & NONE \\
\hline 5784 & AREA & NONE \\
\hline 5785 & AREA & NONE \\
\hline 5786 & AREA & NONE \\
\hline 5787 & AREA & NONE \\
\hline 5788 & AREA & NONE \\
\hline 5789 & AREA & NONE \\
\hline 5790 & AREA & NONE \\
\hline 5791 & AREA & NONE \\
\hline 5792 & AREA & NONE \\
\hline 5793 & AREA & NONE \\
\hline 5794 & AREA & NONE \\
\hline 5795 & NAMED_POINT & NONE \\
\hline 5796 & NAMED_POINT & NONE \\
\hline 5797 & NAMED_POINT & NONE \\
\hline 5798 & NAMED_POINT & NONE \\
\hline 5799 & NAMED_POINT & NONE \\
\hline 5800 & NAMED_POINT & NONE \\
\hline 5801 & NAMED_POINT & NONE \\
\hline 5802 & NAMED_POINT & NONE \\
\hline 5803 & NAMED_POINT & NONE \\
\hline 5804 & NAMED_POINT & NONE \\
\hline 5805 & NAMED_POINT & NONE \\
\hline 5806 & NAMED_POINT & NONE \\
\hline 5807 & NAMED_POINT & NONE \\
\hline 5808 & NAMED_POINT & NONE \\
\hline 5809 & NAMED_POINT & NONE \\
\hline 5810 & NAMED_POINT & NONE \\
\hline 5811 & NAMED_POINT & NONE \\
\hline 5812 & NAMED_POINT & NONE \\
\hline 5813 & NAMED_POINT & NONE \\
\hline 5814 & NAMED_POINT & & \\
\hline 5815 & NAMED_POINT & & \\
\hline & & & \\
\hline
\end{tabular}




\begin{tabular}{|c|l|l|l|}
\hline & \multicolumn{1}{|c|}{ J } & \multicolumn{1}{|c|}{ K } & \\
\hline 1 & SAMP_SITE_TYPE & SCHED_LABEL_CODE & TRANSFORM_CODE \\
\hline 5816 & NAMED_POINT & NONE \\
\hline 5817 & NAMED_POINT & NONE \\
\hline 5818 & NAMED_POINT & NONE \\
\hline 5819 & NAMED_POINT & NONE \\
\hline 5820 & NAMED_POINT & NONE \\
\hline 5821 & NAMED_POINT & NONE \\
\hline 5822 & NAMED_POINT & NONE \\
\hline 5823 & NAMED_POINT & NONE \\
\hline 5824 & NAMED_POINT & NONE \\
\hline 5825 & NAMED_POINT & NONE \\
\hline 5826 & NAMED_POINT & NONE \\
\hline 5827 & NAMED_POINT & NONE \\
\hline 5828 & NAMED_POINT & NONE \\
\hline 5829 & NAMED_POINT & NONE \\
\hline 5830 & NAMED_POINT & NONE \\
\hline 5831 & NAMED_POINT & NONE \\
\hline 5832 & NAMED_POINT & NONE \\
\hline 5833 & NAMED_POINT & NONE \\
\hline 5834 & NAMED_POINT & NONE \\
\hline 5835 & NAMED_POINT & NONE \\
\hline 5836 & NAMED_POINT & NONE \\
\hline 5837 & NAMED_POINT & NONE \\
\hline 5838 & NAMED_POINT & NONE \\
\hline 5839 & NAMED_POINT & NONE \\
\hline 5840 & NAMED_POINT & NONE \\
\hline 5841 & NAMED_POINT & NONE \\
\hline 5842 & NAMED_POINT & NONE \\
\hline 5843 & NAMED_POINT & NONE \\
\hline 5844 & NAMED_POINT & NONE \\
\hline 5845 & NAMED_POINT & NONE \\
\hline 5846 & NAMED_POINT & NONE \\
\hline 5847 & NAMED_POINT & NONE \\
\hline 5848 & NAMED_POINT & NONE \\
\hline 5849 & NAMED_POINT & NONE \\
\hline 5850 & NAMED_POINT & NONE \\
\hline 5851 & NAMED_POINT & & \\
\hline 5852 & NAMED_POINT & NONE \\
\hline 5853 & NAMED_POINT & & \\
\hline & & & \\
\hline
\end{tabular}




\begin{tabular}{|c|l|l|l|}
\hline & \multicolumn{1}{|c|}{ J } & \multicolumn{1}{|c|}{ K } & \\
\hline 1 & SAMP_SITE_TYPE & SCHED_LABEL_CODE & TRANSFORM_CODE \\
\hline 5854 & NAMED_POINT & NONE \\
\hline 5855 & NAMED_POINT & NONE \\
\hline 5856 & NAMED_POINT & NONE \\
\hline 5857 & NAMED_POINT & NONE \\
\hline 5858 & NAMED_POINT & NONE \\
\hline 5859 & NAMED_POINT & NONE \\
\hline 5860 & NAMED_POINT & NONE \\
\hline 5861 & NAMED_POINT & NONE \\
\hline 5862 & NAMED_POINT & NONE \\
\hline 5863 & NAMED_POINT & NONE \\
\hline 5864 & NAMED_POINT & NONE \\
\hline 5865 & NAMED_POINT & NONE \\
\hline 5866 & NAMED_POINT & NONE \\
\hline 5867 & NAMED_POINT & NONE \\
\hline 5868 & NAMED_POINT & NONE \\
\hline 5869 & NAMED_POINT & NONE \\
\hline 5870 & NAMED_POINT & NONE \\
\hline 5871 & NAMED_POINT & NONE \\
\hline 5872 & NAMED_POINT & NONE \\
\hline 5873 & NAMED_POINT & NONE \\
\hline 5874 & NAMED_POINT & NONE \\
\hline 5875 & NAMED_POINT & NONE \\
\hline 5876 & NAMED_POINT & NONE \\
\hline 5877 & NAMED_POINT & NONE \\
\hline 5878 & NAMED_POINT & NONE \\
\hline 5879 & NAMED_POINT & NONE \\
\hline 5880 & NAMED_POINT & NONE \\
\hline 5881 & NAMED_POINT & NONE \\
\hline 5882 & NAMED_POINT & NONE \\
\hline 5883 & NAMED_POINT & NONE \\
\hline 5884 & NAMED_POINT & NONE \\
\hline 5885 & NAMED_POINT & NONE \\
\hline 5886 & NAMED_POINT & NONE \\
\hline 5887 & NAMED_POINT & NONE \\
\hline 5888 & NAMED_POINT & NONE \\
\hline 5889 & NAMED_POINT & NONE \\
\hline 5890 & NAMED_POINT & & \\
\hline 5891 & NAMED_POINT & & \\
\hline & & & \\
\hline
\end{tabular}




\begin{tabular}{|c|l|l|l|}
\hline & \multicolumn{1}{|c|}{$J$} & \multicolumn{1}{|c|}{ K } & \\
\hline 1 & SAMP_SITE_TYPE & SCHED_LABEL_CODE & TRANSFORM_CODE \\
\hline 5892 & NAMED_POINT & NONE \\
\hline 5893 & NAMED_POINT & NONE \\
\hline 5894 & NAMED_POINT & NONE \\
\hline 5895 & NAMED_POINT & NONE \\
\hline 5896 & NAMED_POINT & NONE \\
\hline 5897 & NAMED_POINT & NONE \\
\hline 5898 & NAMED_POINT & NONE \\
\hline 5899 & NAMED_POINT & NONE \\
\hline 5900 & NAMED_POINT & NONE \\
\hline 5901 & NAMED_POINT & NONE \\
\hline 5902 & NAMED_POINT & NONE \\
\hline 5903 & NAMED_POINT & NONE \\
\hline 5904 & NAMED_POINT & NONE \\
\hline 5905 & NAMED_POINT & NONE \\
\hline 5906 & NAMED_POINT & NONE \\
\hline 5907 & NAMED_POINT & NONE \\
\hline 5908 & NAMED_POINT & NONE \\
\hline 5909 & NAMED_POINT & NONE \\
\hline 5910 & NAMED_POINT & NONE \\
\hline 5911 & NAMED_POINT & NONE \\
\hline 5912 & NAMED_POINT & NONE \\
\hline 5913 & NAMED_POINT & NONE \\
\hline 5914 & NAMED_POINT & NONE \\
\hline 5915 & & NONE \\
\hline 5916 & AREA & NONE \\
\hline 5917 & AREA & NONE \\
\hline 5918 & & NONE \\
\hline 5919 & AREA & NONE \\
\hline 5920 & AREA & NONE \\
\hline 5921 & AREA & NONE \\
\hline 5922 & AREA & NONE \\
\hline 5923 & AREA & NONE \\
\hline 5924 & AREA & NONE \\
\hline 5925 & AREA & NONE \\
\hline 5926 & AREA & NONE \\
\hline 5927 & AREA & NONE \\
\hline 5928 & AREA & & \\
\hline 5929 & & & \\
\hline & & & \\
\hline
\end{tabular}




\begin{tabular}{|c|l|l|l|}
\hline & \multicolumn{1}{|c|}{$J$} & \multicolumn{1}{|c|}{$\mathrm{K}$} & \multicolumn{1}{c|}{$\mathrm{L}$} \\
\hline 1 & SAMP_SITE_TYPE & SCHED_LABEL_CODE & TRANSFORM_CODE \\
\hline 5930 & AREA & NONE \\
\hline 5931 & AREA & NONE \\
\hline 5932 & AREA & NONE \\
\hline 5933 & AREA & NONE \\
\hline 5934 & AREA & NONE \\
\hline 5935 & AREA & NONE \\
\hline 5936 & AREA & NONE \\
\hline 5937 & AREA & NONE \\
\hline 5938 & AREA & NONE \\
\hline 5939 & AREA & NONE \\
\hline 5940 & AREA & NONE \\
\hline 5941 & AREA & NONE \\
\hline 5942 & AREA & NONE \\
\hline 5943 & AREA & NONEA & NONE \\
\hline 5944 & AREA & NREA & NONE \\
\hline 5945 & AREA & NONE \\
\hline 5946 & AREA & NONE \\
\hline 5947 & AREA & NREA & NONE \\
\hline 5948 & AREA & NONE \\
\hline 5949 & AREA & NREA & NONE \\
\hline 5950 & AREA & NONE \\
\hline 5951 & AREA & NONE \\
\hline 5952 & AREA & NONE \\
\hline 5953 & AREA & NRA & NONE \\
\hline 5954 & AREA & \\
\hline 5955 & AREA & & \\
\hline & & & \\
\hline
\end{tabular}




\begin{tabular}{|c|c|c|}
\hline & & \\
\hline 1 & 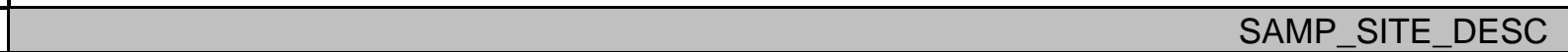 & SITE_ELEVATION \\
\hline 2 & 4817 & \\
\hline 3 & A010 & \\
\hline 4 & A012 & \\
\hline 5 & A030 & \\
\hline 6 & A040 & \\
\hline 7 & A045 & \\
\hline 8 & A050 & \\
\hline 9 & AG01 & \\
\hline 10 & ANO2 & \\
\hline 11 & B020 & \\
\hline 12 & B025 & 94.96 \\
\hline 13 & B026 & \\
\hline 14 & B030 & \\
\hline 15 & B041 & \\
\hline 16 & B050 & \\
\hline 17 & B060 & \\
\hline 18 & B070 & \\
\hline 19 & B085 & \\
\hline 22 & B110 & \\
\hline 23 & B111 & \\
\hline 24 & B120 & 101.51 \\
\hline 25 & B130 & 98.95 \\
\hline 26 & B145 & 114 \\
\hline 27 & B150 & \\
\hline 28 & B160 & \\
\hline 29 & C030 & \\
\hline 30 & C035 & \\
\hline 31 & $\mathrm{CO} 40$ & \\
\hline 32 & C060 & 317.83 \\
\hline 33 & C070 & \\
\hline 34 & C071 & \\
\hline 35 & C073 & \\
\hline 36 & C074 & \\
\hline 37 & C075 & \\
\hline 38 & C090 & \\
\hline 39 & C100 & \\
\hline
\end{tabular}




\begin{tabular}{|c|c|c|}
\hline & 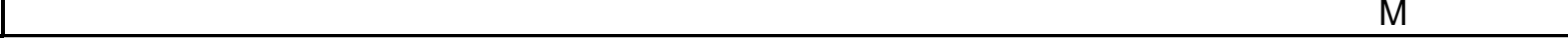 & \\
\hline 1 & $\begin{array}{ll} & \text { SAMP_SITE_DESC } \\
\end{array}$ & SITE_ELEVATION \\
\hline 40 & C110 & \\
\hline 41 & C130 & \\
\hline 42 & C140 & \\
\hline 43 & C150 & 174 \\
\hline 44 & C160 & \\
\hline 45 & D000 & \\
\hline 46 & D010 & \\
\hline 47 & D020 & \\
\hline 48 & D050 & \\
\hline 49 & D051 & \\
\hline 50 & D060 & \\
\hline 51 & D085 & \\
\hline 52 & D086 & \\
\hline 53 & D088 & \\
\hline 54 & D095 & \\
\hline 55 & D110 & \\
\hline 56 & D120 & \\
\hline 57 & $\mathrm{D} 140$ & \\
\hline 60 & D175 & \\
\hline 61 & D180 & \\
\hline 62 & D182 & \\
\hline 63 & D300 & \\
\hline 64 & D301 & \\
\hline 65 & D302 & \\
\hline 66 & E010 & \\
\hline 67 & E030 & \\
\hline 68 & E035 & 191.31 \\
\hline 69 & E040 & \\
\hline 70 & E060 & \\
\hline 71 & E070 & 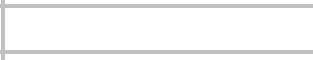 \\
\hline 72 & E075 & 159.02 \\
\hline 73 & E090 & \\
\hline 74 & E100 & \\
\hline 75 & E110 & \\
\hline 76 & E111 & \\
\hline 77 & E180 & \\
\hline
\end{tabular}




\begin{tabular}{|c|c|c|}
\hline & & \\
\hline 1 & SAMP_SITE_DESC & SITE_ELEVATION \\
\hline 78 & EG01 & \\
\hline 79 & EP02 & \\
\hline 80 & F010 & \\
\hline 81 & F040 & 155.5 \\
\hline 82 & F060 & \\
\hline 83 & F076 & \\
\hline 84 & F077 & \\
\hline 85 & F100 & \\
\hline 86 & F110 & \\
\hline 87 & F130 & \\
\hline 88 & F140 & 136 \\
\hline 89 & F145 & \\
\hline 90 & F150 & \\
\hline 91 & G020 & \\
\hline 92 & G030 & \\
\hline 93 & G050 & \\
\hline 94 & G051 & \\
\hline 95 & G060 & \\
\hline 98 & G090 & \\
\hline 99 & GN01 & \\
\hline 100 & $\mathrm{HO} 20$ & \\
\hline 101 & $\mathrm{H} 029$ & \\
\hline 102 & $\mathrm{H} 030$ & \\
\hline 103 & $\mathrm{H} 040$ & \\
\hline 104 & $\mathrm{H} 050$ & \\
\hline 105 & $\mathrm{H} 051$ & \\
\hline 106 & $\mathrm{H} 070$ & \\
\hline 107 & $\mathrm{H} 080$ & \\
\hline 108 & $\mathrm{H} 090$ & \\
\hline 109 & H095 & \\
\hline 110 & $\mathrm{H} 110$ & \\
\hline 111 & $\mathrm{H} 130$ & 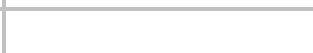 \\
\hline 112 & $\mathrm{H} 140$ & 139.7 \\
\hline 113 & $\mathrm{H} 170$ & \\
\hline 114 & $\mathrm{H} 175$ & \\
\hline 115 & 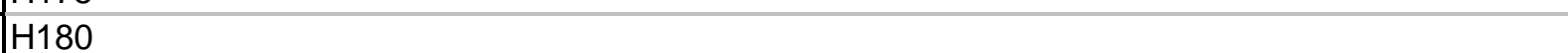 & \\
\hline
\end{tabular}




\begin{tabular}{|c|c|c|}
\hline & 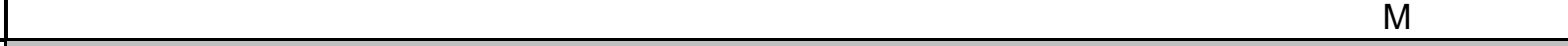 & $\mathrm{N}$ \\
\hline 1 & SAMP_SITE_DESC & SITE_ELEVATION \\
\hline 116 & $\mathrm{H} 200$ & \\
\hline 117 & $\mathrm{H} 22 \mathrm{O}$ & \\
\hline 118 & $\mathrm{H} 250$ & \\
\hline 119 & $\mathrm{H} 251$ & \\
\hline 120 & H252 & \\
\hline 121 & $\mathrm{H} 270$ & 262.15 \\
\hline 122 & $\mathrm{H} 275$ & 200.36 \\
\hline 123 & K010 & 190 \\
\hline 124 & K015 & \\
\hline 125 & K030 & \\
\hline 126 & K040 & \\
\hline 127 & K050 & \\
\hline 128 & K070 & \\
\hline 129 & K080 & \\
\hline 130 & K090 & \\
\hline 131 & K091 & \\
\hline 132 & K110 & \\
\hline 133 & K120 & \\
\hline 134 & K130 & 179.808 \\
\hline 135 & K170 & \\
\hline 136 & K180 & \\
\hline 137 & KA01 & \\
\hline 138 & N020 & \\
\hline 139 & N033 & \\
\hline 140 & N035 & \\
\hline 141 & N045 & \\
\hline 142 & N055 & \\
\hline 143 & N060 & \\
\hline 144 & N092 & \\
\hline 145 & N100 & \\
\hline 146 & N110 & \\
\hline 147 & N115 & \\
\hline 148 & $\mathrm{~N} 120$ & \\
\hline 149 & N130 & \\
\hline 150 & N135 & \\
\hline 151 & N140 & \\
\hline 152 & N141 & \\
\hline 153 & N150 & \\
\hline
\end{tabular}




\begin{tabular}{|c|c|c|}
\hline 1 & $\begin{array}{ll} & \text { SAMP SITE DESC }\end{array}$ & SITE ELEVATION \\
\hline 154 & N151 & 124 \\
\hline 155 & N160 & \\
\hline 156 & N170 & \\
\hline 157 & N190 & \\
\hline 158 & P010 & \\
\hline 159 & P016 & 213.97 \\
\hline 160 & P018 & 211.41 \\
\hline 161 & P019 & 202.14 \\
\hline 162 & P020 & \\
\hline 163 & P030 & 202.1 \\
\hline 164 & P060 & \\
\hline 165 & P061 & \\
\hline 166 & $\mathrm{P} 070$ & \\
\hline 167 & QA01 & \\
\hline 168 & QA02 & \\
\hline 169 & QA03 & \\
\hline 170 & QA05 & \\
\hline 171 & QA07 & \\
\hline \begin{tabular}{|l|}
172 \\
\end{tabular} & QA09 & \\
\hline 173 & QA10 & \\
\hline 174 & QA13 & \\
\hline \begin{tabular}{|l|}
175 \\
\end{tabular} & QA14 & \\
\hline 176 & QA16 & \\
\hline \begin{tabular}{|l|}
177 \\
\end{tabular} & QA19 & \\
\hline \begin{tabular}{|l|}
178 \\
\end{tabular} & QA20 & \\
\hline \begin{tabular}{|l|}
179 \\
\end{tabular} & QA21 & \\
\hline 180 & QA23 & \\
\hline 181 & QA24 & \\
\hline \begin{tabular}{|l|}
182 \\
\end{tabular} & QA25 & \\
\hline \begin{tabular}{|l|}
183 \\
\end{tabular} & QA27 & \\
\hline 184 & QA28 & \\
\hline 185 & QA30 & \\
\hline 186 & QA31 & \\
\hline 187 & QA32 & \\
\hline \begin{tabular}{|l|}
188 \\
\end{tabular} & QA34 & \\
\hline \begin{tabular}{|l|}
189 \\
\end{tabular} & QA38 & \\
\hline 190 & QA39 & \\
\hline 191 & QA41 & \\
\hline
\end{tabular}




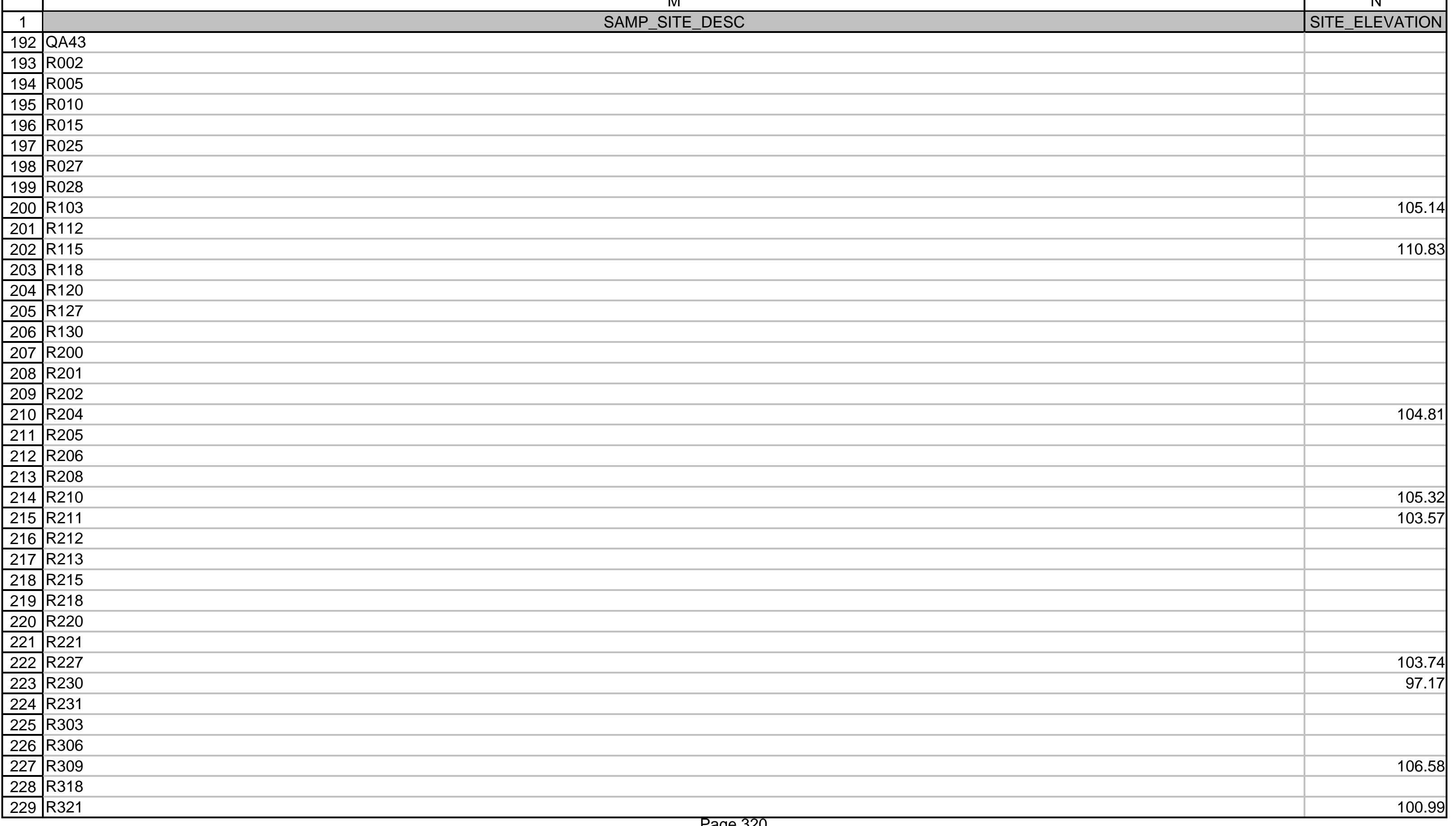




\begin{tabular}{|c|c|c|}
\hline & & \\
\hline 1 & SAMP_SITE_DESC & SITE_ELEVATION \\
\hline 230 & R324 & \\
\hline 231 & R330 & \\
\hline 232 & R336 & 98.76 \\
\hline 233 & R403 & \\
\hline 234 & R406 & \\
\hline 235 & R409 & 99.7 \\
\hline 236 & R412 & 110 \\
\hline 237 & R415 & 95.92 \\
\hline 238 & R418 & \\
\hline 239 & R424 & 97.48 \\
\hline 240 & R427 & \\
\hline 241 & R500 & \\
\hline 242 & R506 & \\
\hline 243 & R509 & 92.35 \\
\hline 244 & R512 & 88.78 \\
\hline 245 & R515 & \\
\hline 246 & R521 & \\
\hline 247 & R522 & \\
\hline 248 & R600 & \\
\hline 249 & R603 & \\
\hline 250 & R609 & \\
\hline 251 & R614 & 103.14 \\
\hline 252 & R615 & 89.05 \\
\hline 253 & R618 & \\
\hline 254 & R701 & \\
\hline 255 & R702 & \\
\hline 256 & R703 & \\
\hline 257 & R712 & \\
\hline 258 & R715 & \\
\hline 259 & R718 & \\
\hline 260 & R801 & \\
\hline 261 & R803 & \\
\hline 262 & R804 & \\
\hline 263 & R821 & \\
\hline 264 & R832 & \\
\hline 265 & R833 & \\
\hline 266 & R835 & \\
\hline 267 & R836 & \\
\hline
\end{tabular}




\begin{tabular}{|c|c|c|}
\hline & & \begin{tabular}{|c}
$\mathrm{N}$ \\
\end{tabular} \\
\hline 1 & $\begin{array}{ll} & \text { SAMP_SITE_DESC } \\
\end{array}$ & SITE_ELEVATION \\
\hline 268 & R837 & \\
\hline 269 & R838 & \\
\hline 270 & R840 & \\
\hline 271 & R851 & \\
\hline 272 & R852 & \\
\hline 273 & R853 & \\
\hline 274 & R855 & \\
\hline 275 & R856 & \\
\hline 276 & R857 & \\
\hline 277 & R859 & \\
\hline 278 & R860 & \\
\hline 279 & R864 & \\
\hline 280 & R866 & \\
\hline 281 & R867 & \\
\hline 282 & R870 & \\
\hline 283 & R871 & \\
\hline 284 & R873 & \\
\hline 285 & R878 & \\
\hline 288 & R882 & \\
\hline 289 & R885 & \\
\hline 290 & R886 & \\
\hline 291 & R889 & \\
\hline 292 & R892 & \\
\hline 293 & R894 & \\
\hline 294 & R895 & \\
\hline 295 & R898 & \\
\hline 296 & R900 & \\
\hline 297 & R902 & \\
\hline 298 & R903 & \\
\hline 299 & R904 & \\
\hline 300 & R906 & \\
\hline 301 & R907 & \\
\hline 302 & R908 & \\
\hline 303 & R910 & \\
\hline 304 & R911 & \\
\hline 305 & R915 & \\
\hline
\end{tabular}




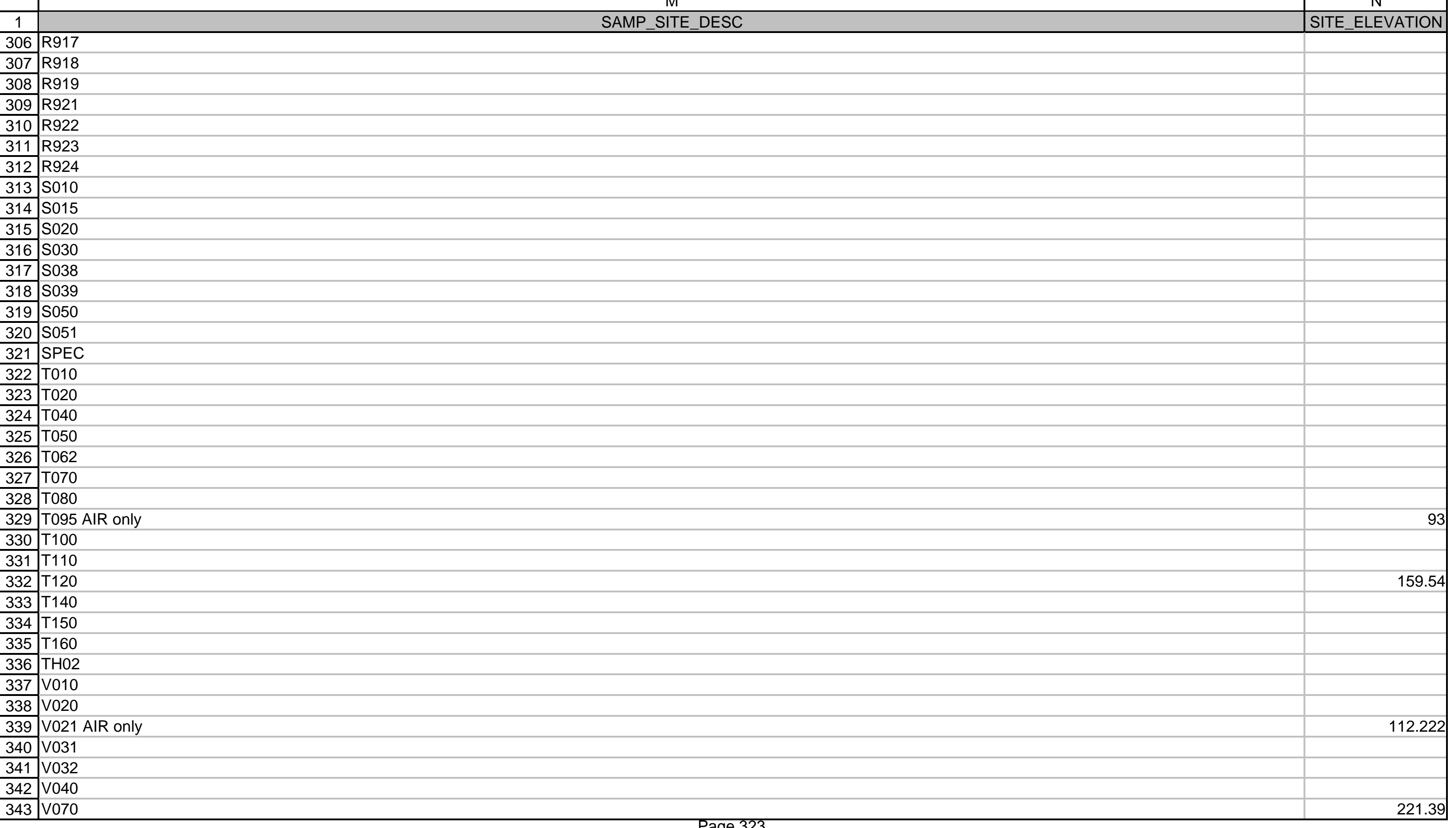




\begin{tabular}{|c|c|c|}
\hline & $\mathrm{M}$ & $\mathrm{N}$ \\
\hline 1 & SAMP_SITE_DESC & SITE_ELEVATION \\
\hline 344 & V071 & \\
\hline 345 & V072 & \\
\hline 346 & W015 & 205.94 \\
\hline 347 & W020 & \\
\hline 348 & W024 & \\
\hline 349 & w030 & \\
\hline 350 & W040 & \\
\hline 351 & W060 & \\
\hline 352 & W090 & \\
\hline 353 & W100 & \\
\hline 354 & W109 & \\
\hline 355 & W130 & \\
\hline 356 & W140 & \\
\hline 357 & W170 & \\
\hline 358 & W180 & \\
\hline 359 & W190 & \\
\hline 360 & W200 & \\
\hline 361 & W210 & 174.23 \\
\hline 364 & B105 & 92.54 \\
\hline \begin{tabular}{|l|}
365 \\
\end{tabular} & B121 & 105.31 \\
\hline 366 & B146 & \\
\hline 367 & $\mathrm{C} 010$ & \\
\hline 368 & D030 & \\
\hline 369 & D070 & \\
\hline 370 & D087 & \\
\hline 371 & D100 & \\
\hline 372 & D160 & \\
\hline 373 & E140 & \\
\hline \begin{tabular}{|l|}
374 \\
\end{tabular} & E170 & \\
\hline 375 & E200 & 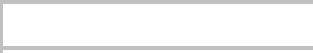 \\
\hline 376 & F050 & 145.4 \\
\hline \begin{tabular}{|l|}
377 \\
\end{tabular} & F075 & \\
\hline 378 & F090 & \\
\hline 379 & F115 & \\
\hline 380 & H081 & 247.24 \\
\hline 381 & $\mathrm{H} 120$ & \\
\hline
\end{tabular}




\begin{tabular}{|c|c|c|}
\hline 1 & $\begin{array}{ll} & \text { SAMP_SITE_DESC }\end{array}$ & SITE_ELEVATION \\
\hline 382 & 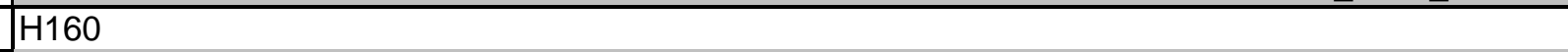 & \\
\hline 383 & $\mathrm{H} 190$ & \\
\hline 384 & $\mathrm{H} 230$ & \\
\hline 385 & N010 & \\
\hline 386 & N034 & \\
\hline 387 & N050 & \\
\hline 388 & N070 & \\
\hline 389 & N095 & \\
\hline 390 & P050 & \\
\hline 391 & PF05 & \\
\hline 392 & QA04 & \\
\hline 393 & QA08 & \\
\hline 394 & QA11 & \\
\hline 395 & QA15 & \\
\hline 396 & QA42 & \\
\hline 397 & R004 & \\
\hline 398 & R020 & \\
\hline 399 & R100 & \\
\hline 400 & R109 & 105.56 \\
\hline 402 & R300 & \\
\hline 403 & R312 & \\
\hline 404 & R327 & \\
\hline 405 & R400 & 96.07 \\
\hline 406 & R700 & \\
\hline 407 & R709 AIR only & 88.03 \\
\hline 408 & R721 & \\
\hline 409 & R820 & \\
\hline 410 & R872 & \\
\hline 411 & R879 & \\
\hline 412 & R883 & \\
\hline 413 & $R 890$ & \\
\hline 414 & R897 & \\
\hline 415 & R926 & \\
\hline 416 & s031 & \\
\hline 417 & s040 & 101.98 \\
\hline 418 & S060 & \\
\hline 419 & T015 & \\
\hline
\end{tabular}




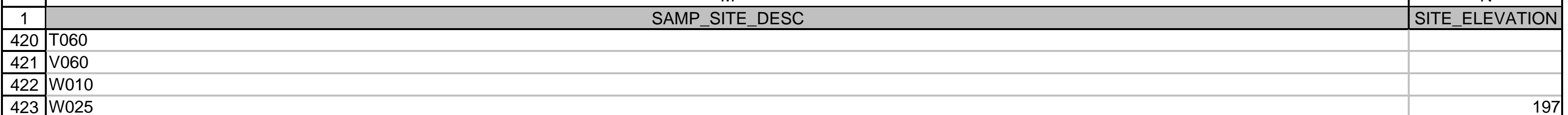

423 W025

425 W080

426 W110

427 W160

\begin{tabular}{|l|l|}
4228 & 4482 \\
\hline 429 &
\end{tabular}

429 A020

430 A060

431 B015

432 B040

433 B071

434 B100

\begin{tabular}{|l|l|}
\hline 435 & B140 \\
\hline 436 & C020
\end{tabular}

436 C020

437 C050

\begin{tabular}{|l|l|}
\hline 438 & $\mathrm{C} 072$ \\
\hline
\end{tabular}

439 C080

440 C120

441 C151

\begin{tabular}{|l|l|}
\hline 442 & D040 \\
\hline 443 & $D 080$
\end{tabular}

\begin{tabular}{|l|l|}
\hline 443 & $D 080$ \\
\hline 444 & $D 090$ \\
\hline
\end{tabular}

444 D090

\begin{tabular}{|l|l|}
445 & D130
\end{tabular}

\begin{tabular}{|l|l|}
4446 & D161 \\
\hline
\end{tabular}

447 D170

448 D185

449 E020

450 E050

451 E095

\begin{tabular}{|l|l|}
452 & E120
\end{tabular}

\begin{tabular}{l|l|l}
453 & E190 \\
\hline 454 & F030
\end{tabular}

454 F030

455 F070

\begin{tabular}{|l|l|}
456 & $F 080$
\end{tabular}

\begin{tabular}{ll|l}
457 & F135
\end{tabular} 


\begin{tabular}{|c|c|c|}
\hline & & \\
\hline 1 & 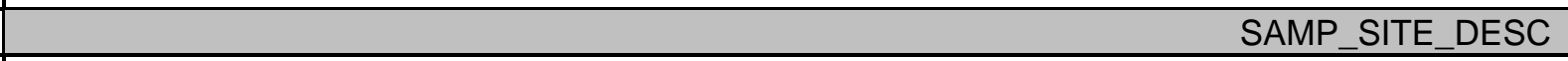 & SITE_ELEVATION \\
\hline 458 & G010 & \\
\hline \begin{tabular}{|l|l|} 
\\
\end{tabular} & G040 & \\
\hline 460 & G070 & \\
\hline 461 & $\mathrm{H} 010$ & \\
\hline 462 & $\mathrm{H} 035$ & \\
\hline 463 & $\mathrm{H} 061$ & \\
\hline 464 & $\mathrm{H} 100$ & \\
\hline 465 & $\mathrm{H} 150$ & \\
\hline 466 & $\mathrm{H} 210$ & 112.88 \\
\hline 467 & $\mathrm{H} 240$ & \\
\hline 468 & $\mathrm{H} 260$ & \\
\hline \begin{tabular}{|l|}
469 \\
\end{tabular} & K020 & \\
\hline 470 & K060 & \\
\hline 471 & K100 & \\
\hline 472 & K140 & \\
\hline 473 & N030 & \\
\hline 474 & N040 & \\
\hline 475 & N080 & \\
\hline \begin{tabular}{|l|}
476 \\
\end{tabular} & N102 & \\
\hline 477 & N111 & \\
\hline 478 & N131 & \\
\hline \begin{tabular}{|l|}
479 \\
\end{tabular} & N145 & \\
\hline \begin{tabular}{|l|}
480 \\
\end{tabular} & N180 & \\
\hline 481 & P017 & 203.72 \\
\hline 482 & P040 & \\
\hline 483 & P080 & \\
\hline \begin{tabular}{|l|}
484 \\
\end{tabular} & QA06 & \\
\hline 485 & QA12 & \\
\hline 486 & QA18 & \\
\hline 487 & QA22 & \\
\hline 488 & QA26 & \\
\hline 489 & QA29 & \\
\hline 490 & QA33 & \\
\hline 491 & QA36 & \\
\hline \begin{tabular}{|l|}
492 \\
\end{tabular} & QA40 & \\
\hline 493 & R003 & \\
\hline 494 & R026 & \\
\hline 495 & R106 & \\
\hline
\end{tabular}




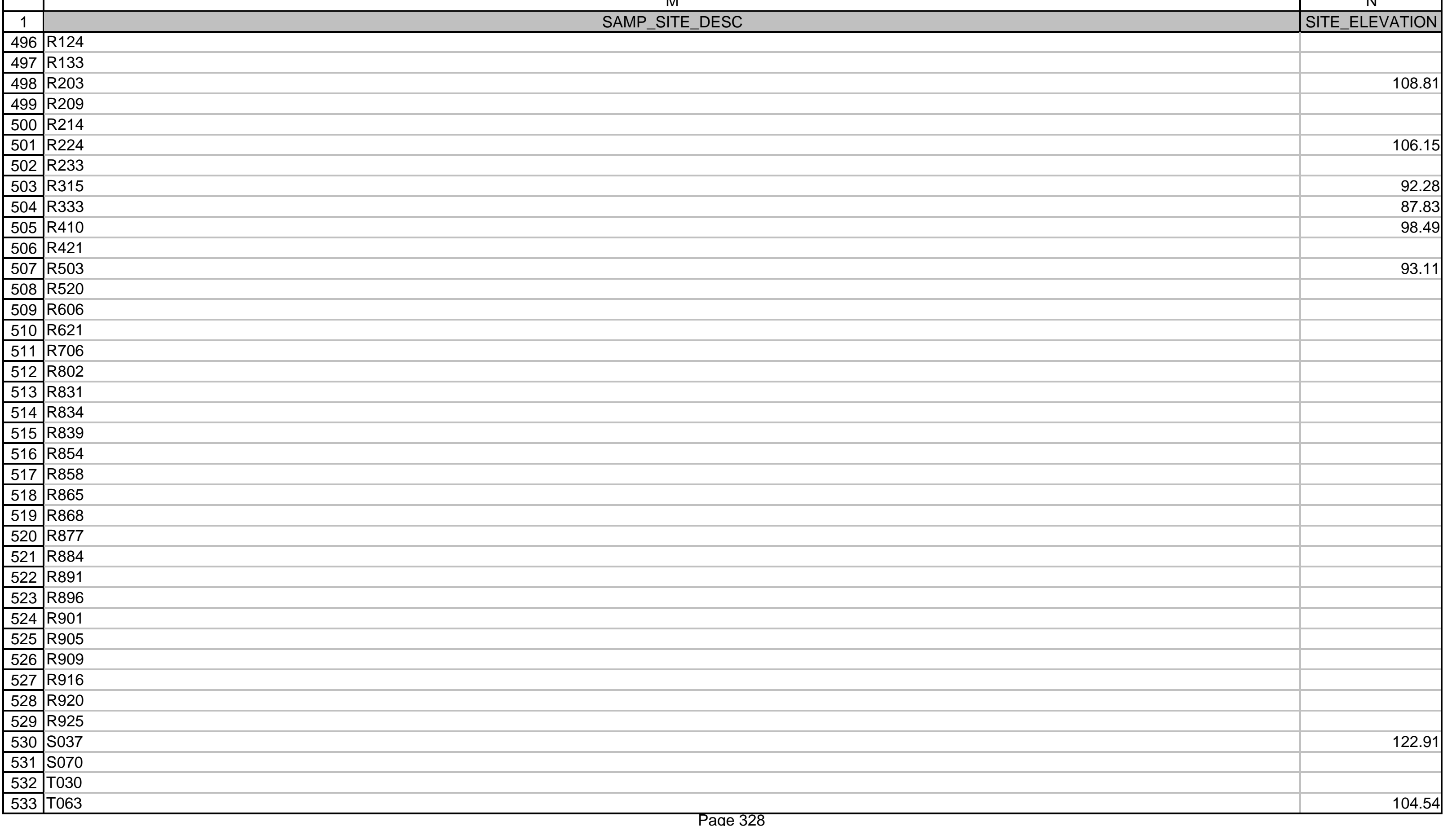




\begin{tabular}{|c|c|c|}
\hline 1 & $\begin{array}{ll} & \text { SAMP SITE DESC }\end{array}$ & SITE ELEVATION \\
\hline 534 & 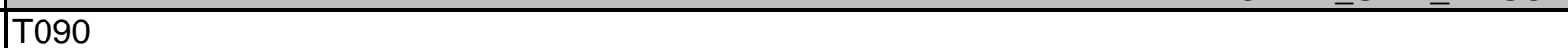 & \\
\hline 535 & T130 & \\
\hline 536 & TH01 & \\
\hline 537 & V030 & \\
\hline 538 & J050 & 189.39 \\
\hline 539 & W005 & \\
\hline 540 & W026 & \\
\hline 541 & W070 & \\
\hline 542 & W120 & \\
\hline 543 & W150 & \\
\hline 544 & D150 & \\
\hline 545 & F020 & 157.22 \\
\hline 546 & $\mathrm{H} 060$ & \\
\hline 547 & K160 & 135.58 \\
\hline 548 & R869 & \\
\hline 549 & R893 & \\
\hline 550 & Macrophyte & \\
\hline 551 & Macrophyte & \\
\hline 552 & Macrophyte & \\
\hline 553 & Macrophyte & \\
\hline 554 & Macrophyte & \\
\hline 555 & & \\
\hline 556 & & \\
\hline 557 & & \\
\hline 558 & & \\
\hline 559 & & \\
\hline 560 & & \\
\hline 561 & & \\
\hline 562 & & \\
\hline 563 & & \\
\hline 564 & & \\
\hline 565 & Below F Area & \\
\hline 566 & Below H Area & \\
\hline 567 & Above D area & \\
\hline \begin{tabular}{|l|}
568 \\
\end{tabular} & Below K Area & \\
\hline 569 & Below BC Area & \\
\hline 570 & Above BC Area & \\
\hline 571 & North Side Vernita & \\
\hline
\end{tabular}


572100 F Area/100-FR-2/126-F-1

SAMP SITE DESC

573100 F Area/100-FR-2/126-F-1

574100 F Area/100-FR-1/1607-F2

575100 R Area/100-FR-1/116-F-1

\begin{tabular}{|l|l}
576 & $100 \mathrm{~F}$ Area/100-FR-2/118-F-2
\end{tabular}

\begin{tabular}{|l|l|}
577 & 100 F Area/100-FR-2/118-F-2 \\
\hline 578 & 100 H Area/100-HR-2/118-H-3 \\
\hline
\end{tabular}

$578100 \mathrm{H}$ Area/100-HR-2/118-H-3

$579100 \mathrm{H}$ Area/100-HR-2/118-H-3

\begin{tabular}{|l|l|}
5580 & $100 \mathrm{H}$ Area/North of $\mathrm{H}-7$
\end{tabular}

\begin{tabular}{|l|l}
581 & $100 \mathrm{H}$ Area/100-HR-2/118-H-2
\end{tabular}

$582100 \mathrm{D}$ Area East of $116-\mathrm{D}-7$

\begin{tabular}{|l|l|}
583 & 100 D Area East of 116-D-7 \\
\hline 584 & 100 D Area
\end{tabular}

\begin{tabular}{|l|l}
584 & 100 D Area \\
\hline 585 & Belo
\end{tabular}

585 Below D Area (Coyote Rapids)

586 North Slope, H-83L, Army bunk near Vernita Bridge

\begin{tabular}{|l|l}
587 & North Slope, HO-4, Locke Island \\
\hline 588 & Norh
\end{tabular}

588 North Slope, H-06-C, Corfu

589 H Area, road 2 between $2 \& 3$

590100 F Area

\begin{tabular}{|l|l}
591 & 100 BC Area \\
\hline 592 & 100 BC Area \\
\hline
\end{tabular}

592100 BC Area

593 100 K Area

$594100 \mathrm{~N}$ Area

5951 mile SE of $\mathrm{H}$ Area - tree

596 White Bluffs Ferry Landing

597 North Slope, H-90

598 N-Spring

599 Control Sample, above Vernita +- 50 Meters for estimated coordinates

600 Below B/C Area

601 Below K Area

602 Above K Area, Allard pump station

$603100 \mathrm{~N}$ Area

604 


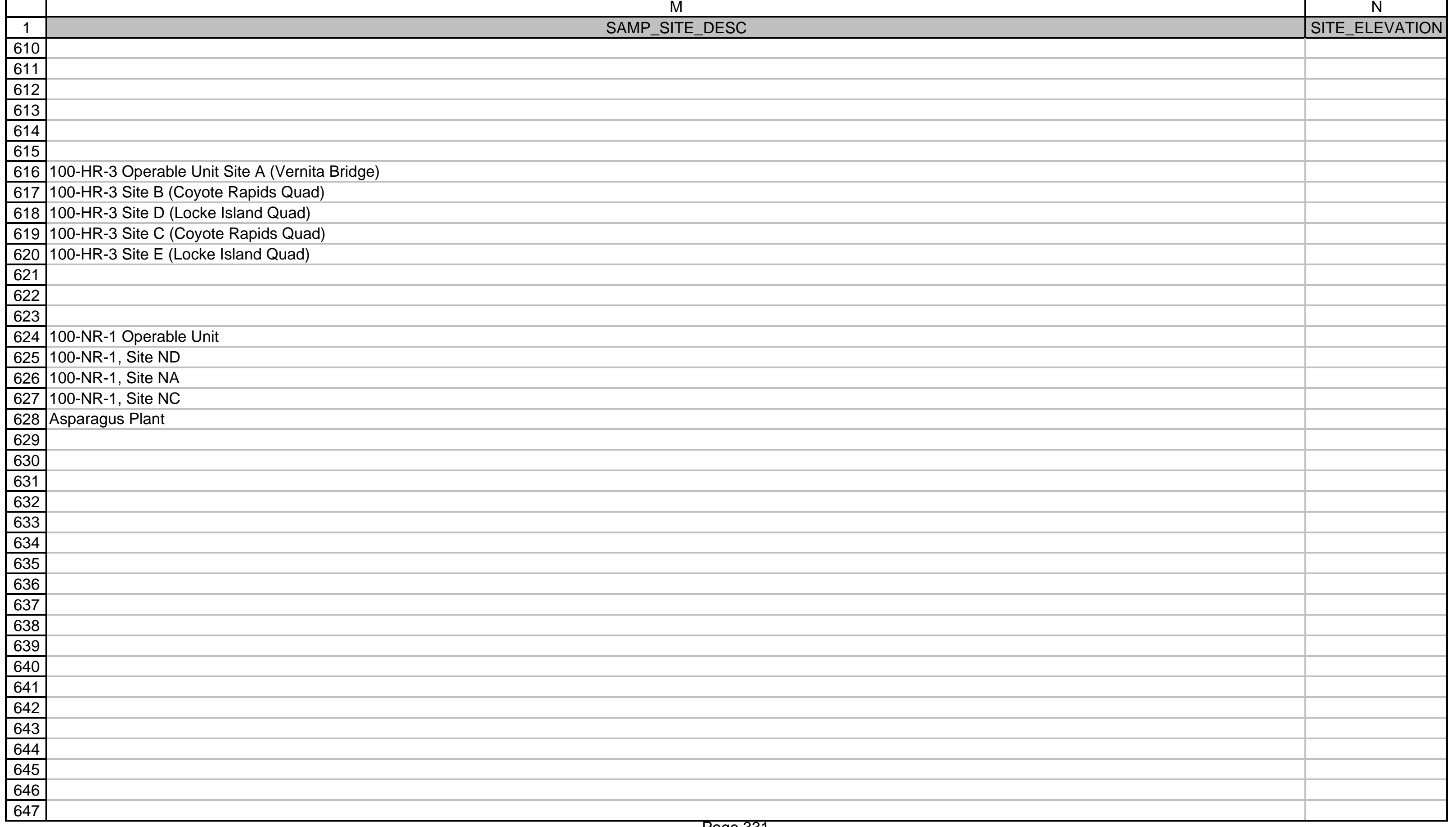




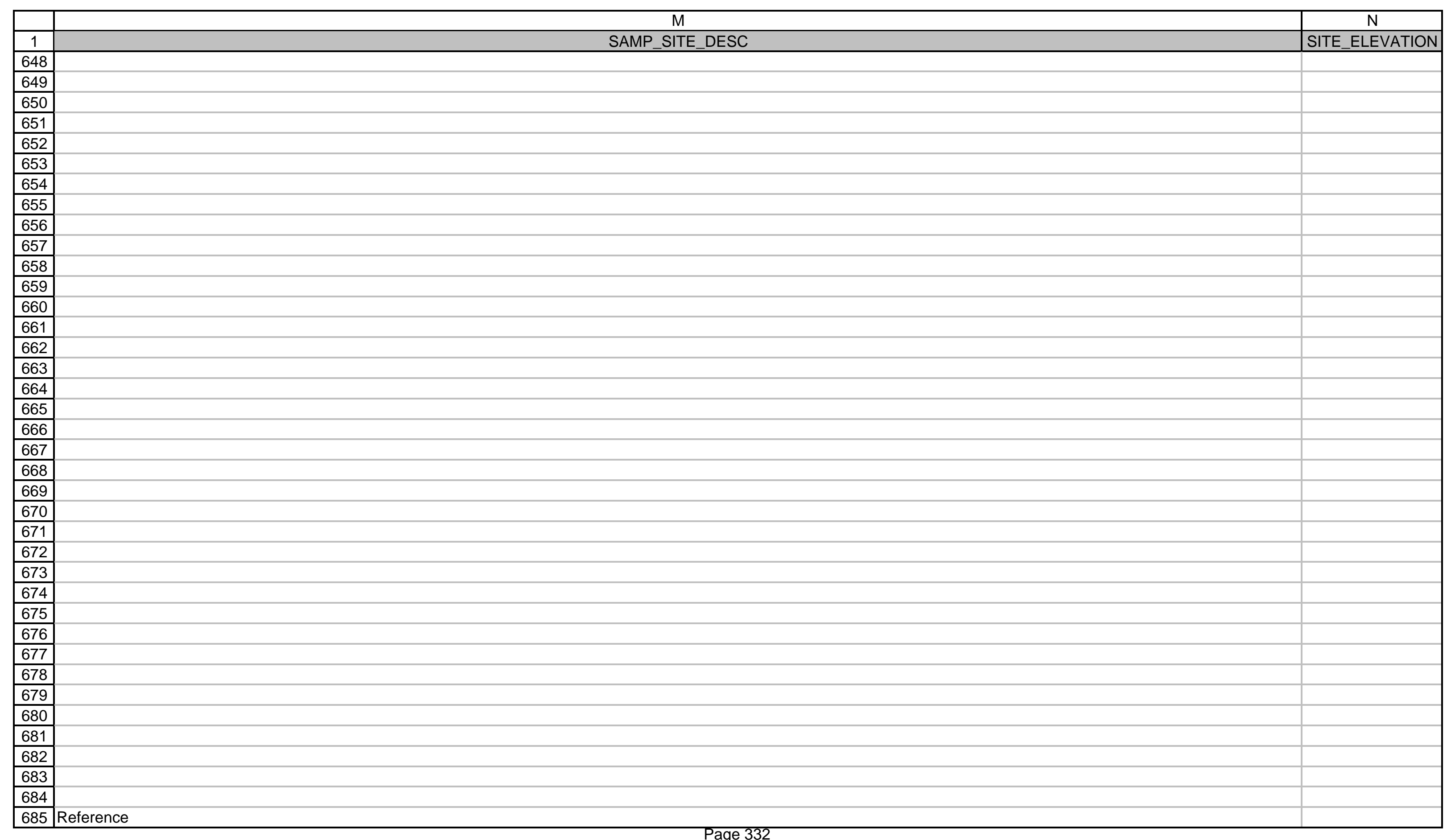




\begin{tabular}{|c|c|c|}
\hline & 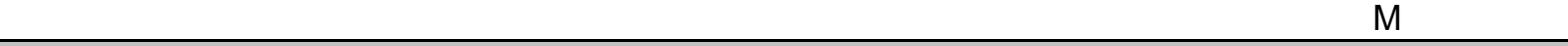 & $\mathrm{N}$ \\
\hline 1 & 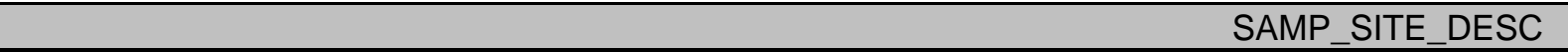 & SITE_ELEVATION \\
\hline 686 & TRAP SITE \#1 & \\
\hline 687 & TRAP SITE \#2 & \\
\hline 688 & Plant Material & \\
\hline 690 & Plant Material & \\
\hline 691 & Plant Material & \\
\hline 692 & Plant Material & \\
\hline 693 & Plant Material & \\
\hline 695 & Plant Material & \\
\hline 696 & Plant Material & \\
\hline 697 & plant matrix & \\
\hline 698 & plant matrix & \\
\hline 699 & plant matrix & \\
\hline 700 & plant matrix & \\
\hline 701 & plant matrix & \\
\hline 702 & plant matrix & \\
\hline 703 & plant matrix & \\
\hline 705 & plant matrix & \\
\hline 706 & plant matrix & \\
\hline 707 & plant matrix & \\
\hline 708 & plant matrix & \\
\hline 709 & plant matrix & \\
\hline 710 & plant matrix & \\
\hline 711 & plant matrix & \\
\hline 712 & plant matrix & \\
\hline 713 & plant matrix & \\
\hline 714 & plant matrix & \\
\hline 715 & plant matrix & \\
\hline 716 & plant matrix & \\
\hline 717 & plant matrix & \\
\hline 718 & & \\
\hline 719 & & \\
\hline 720 & & \\
\hline 721 & & \\
\hline 722 & & \\
\hline 723 & & \\
\hline
\end{tabular}




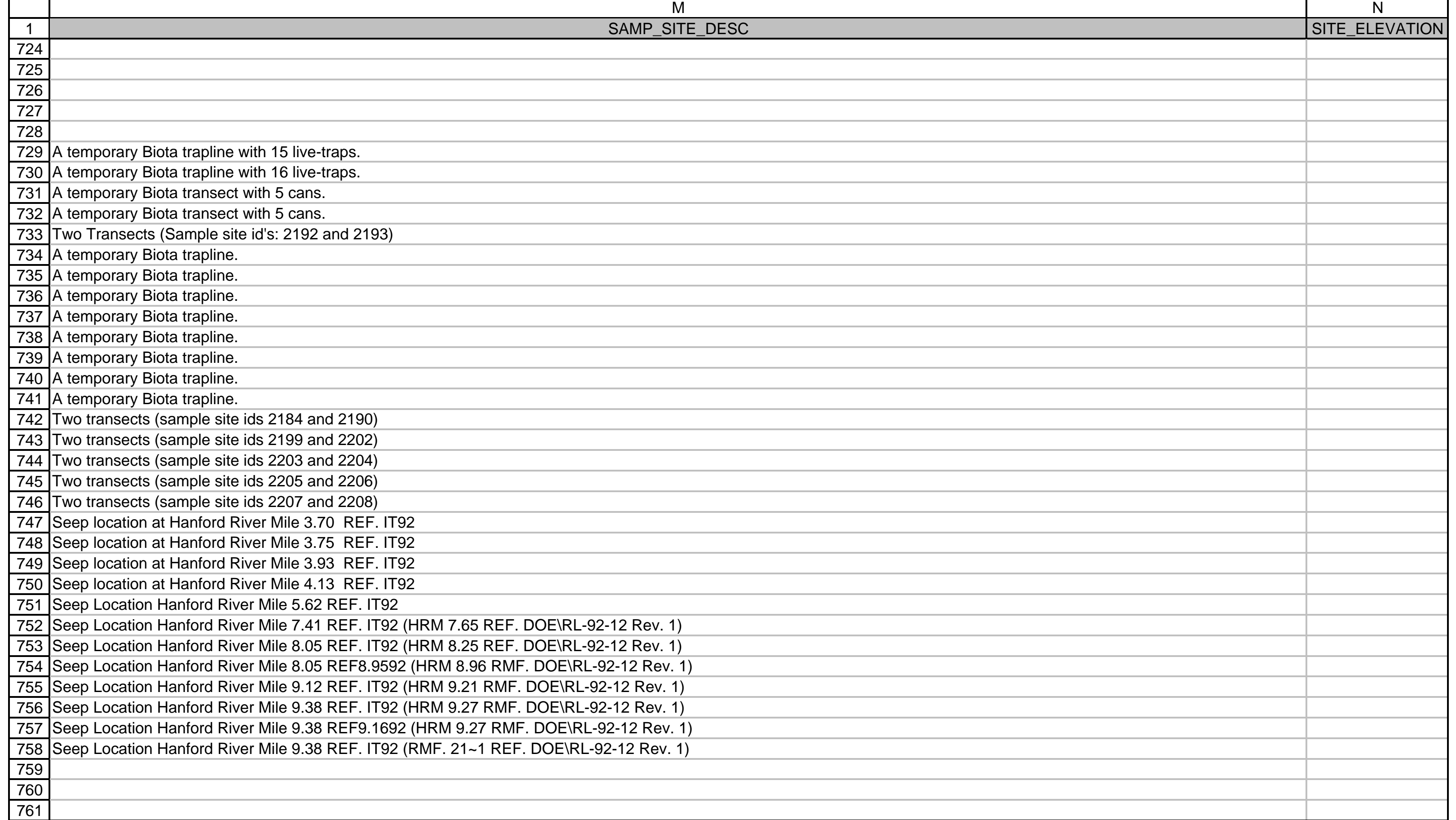




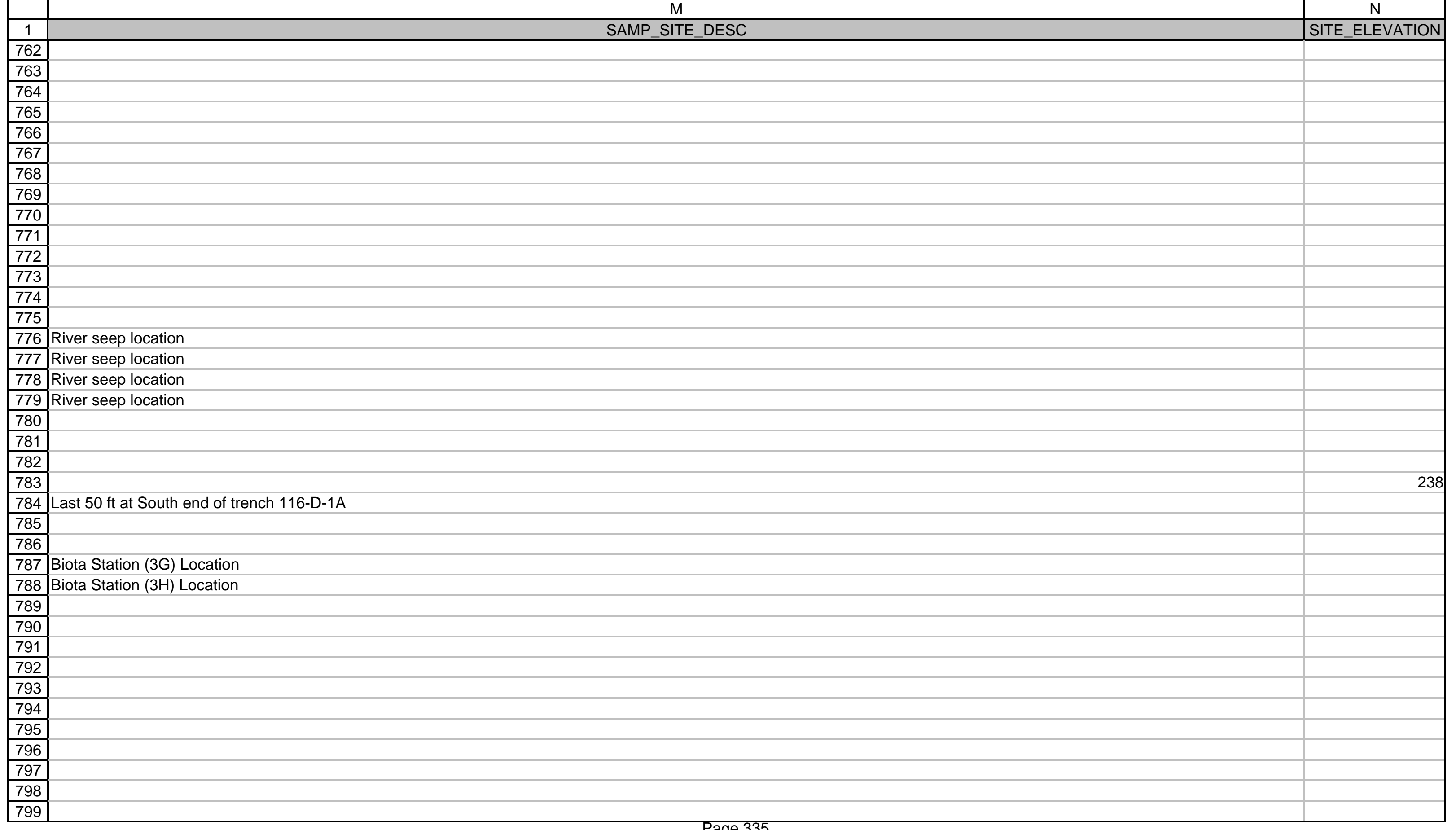




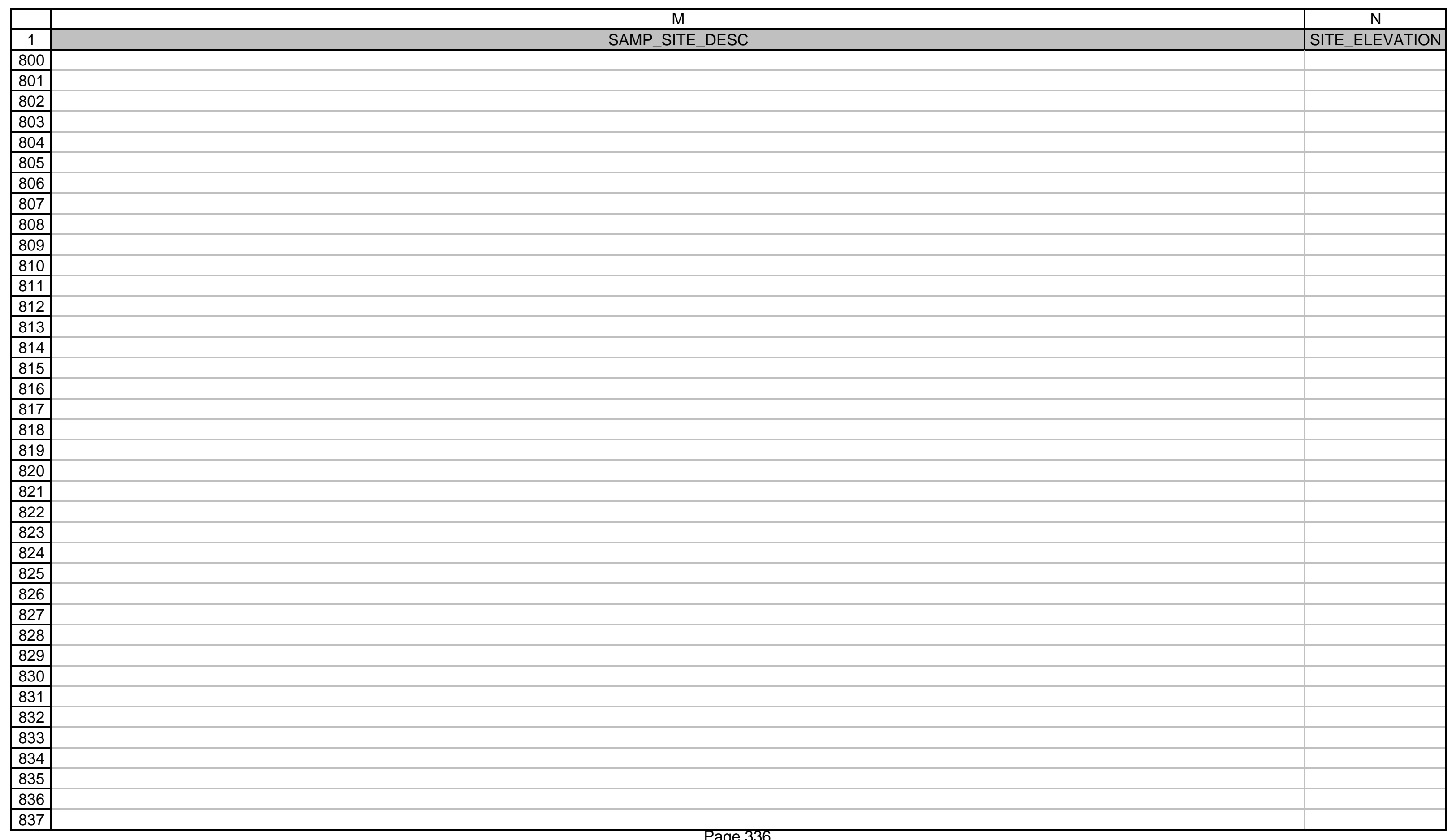




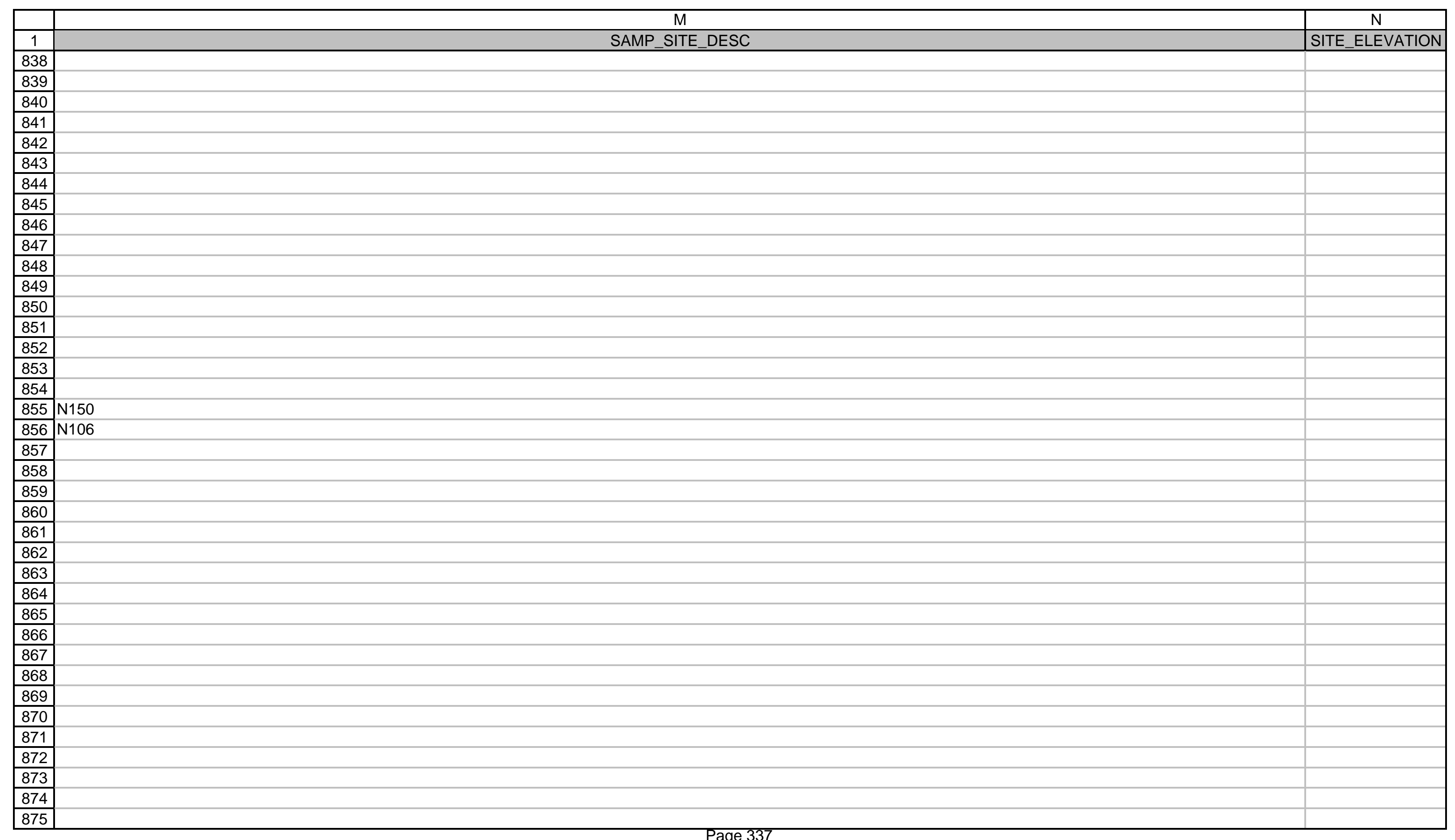




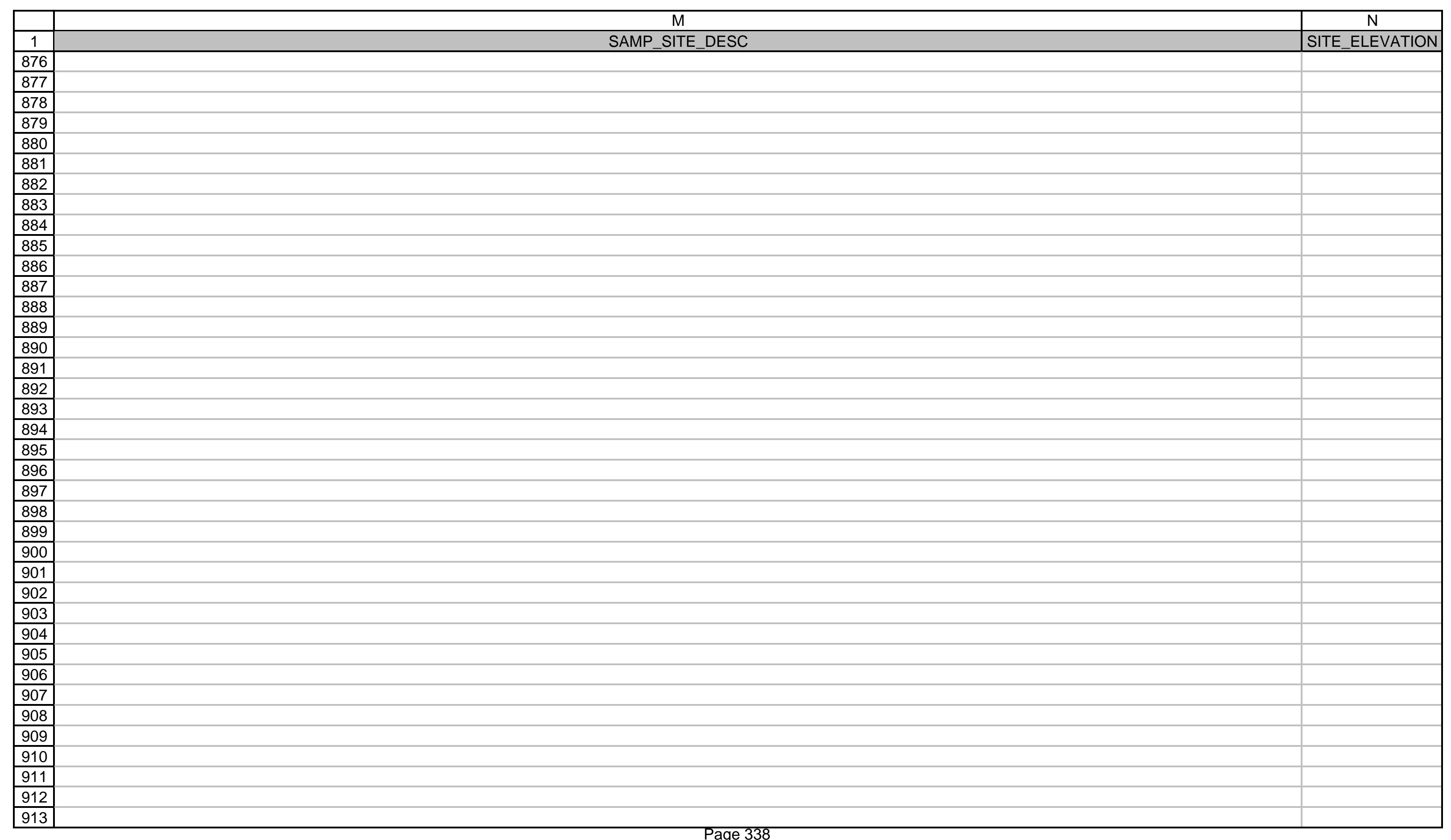




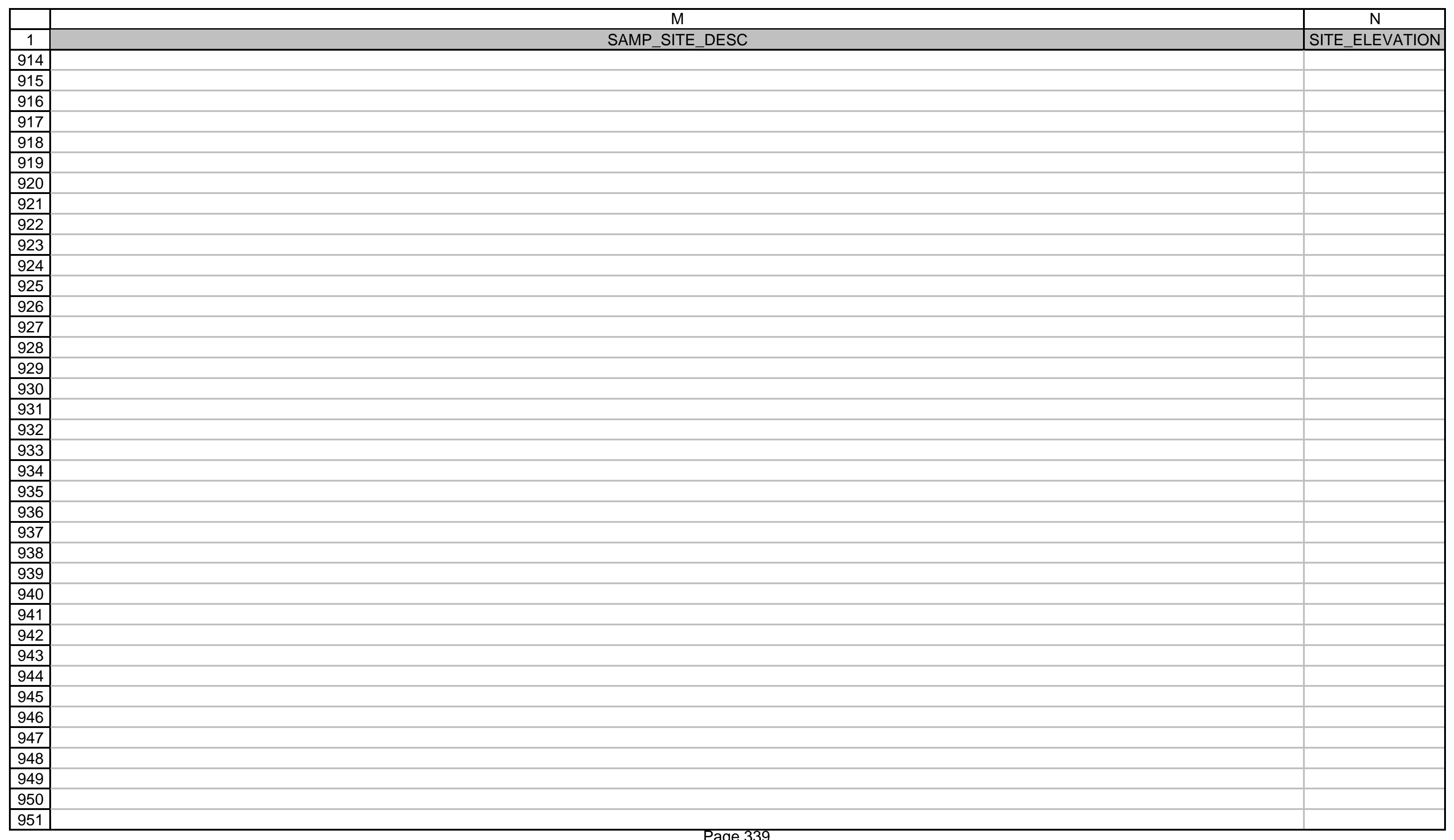




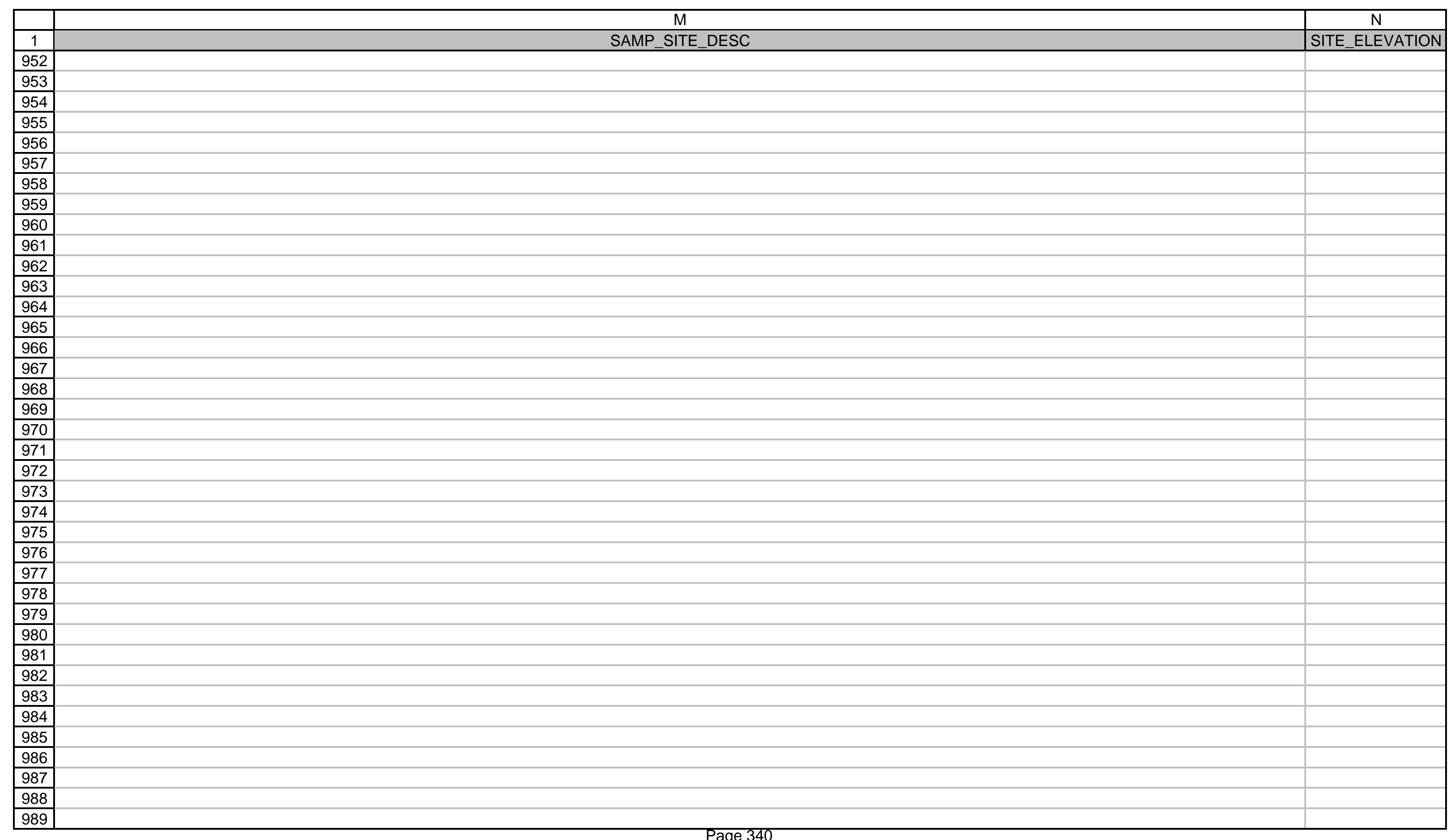




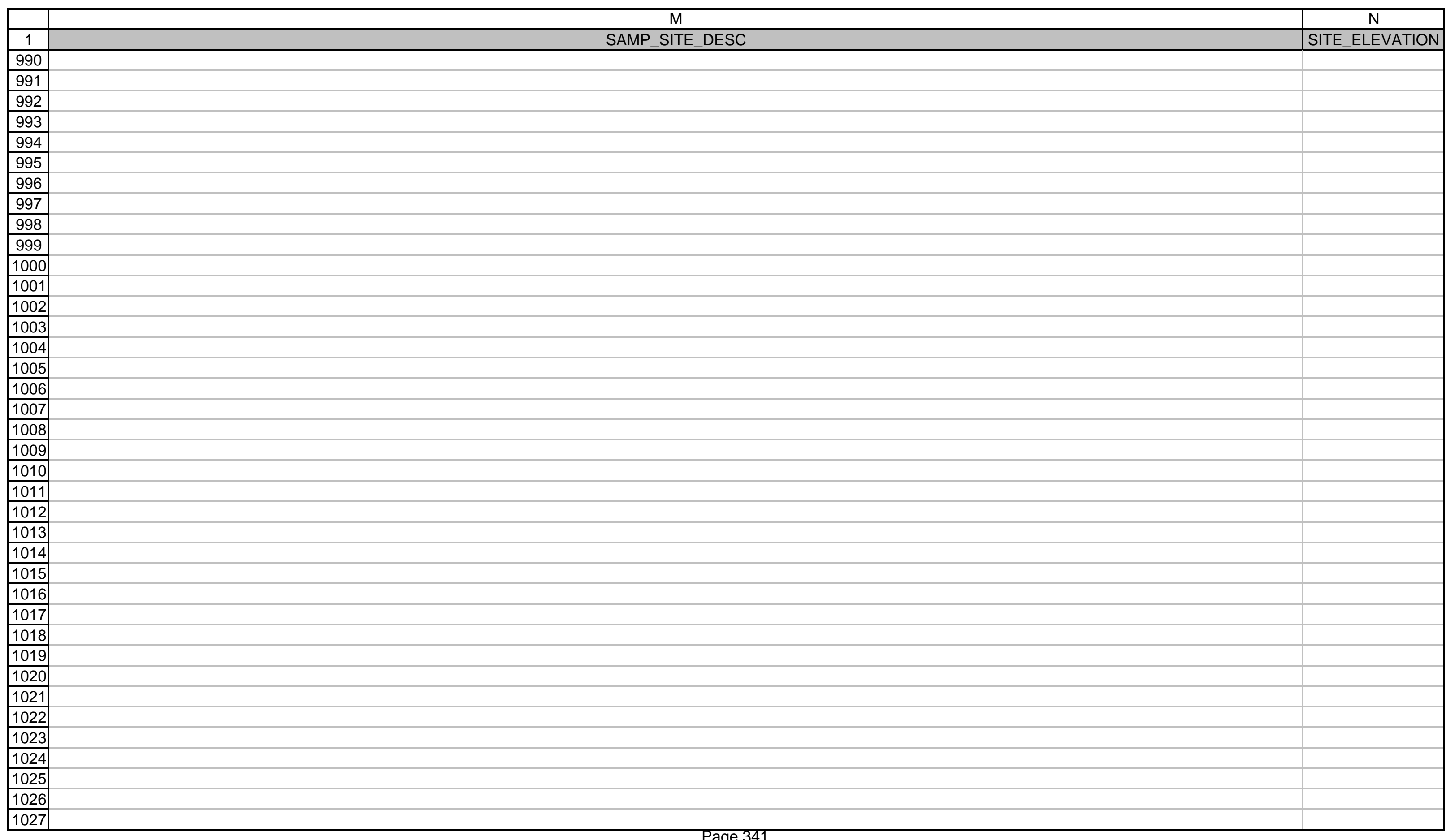




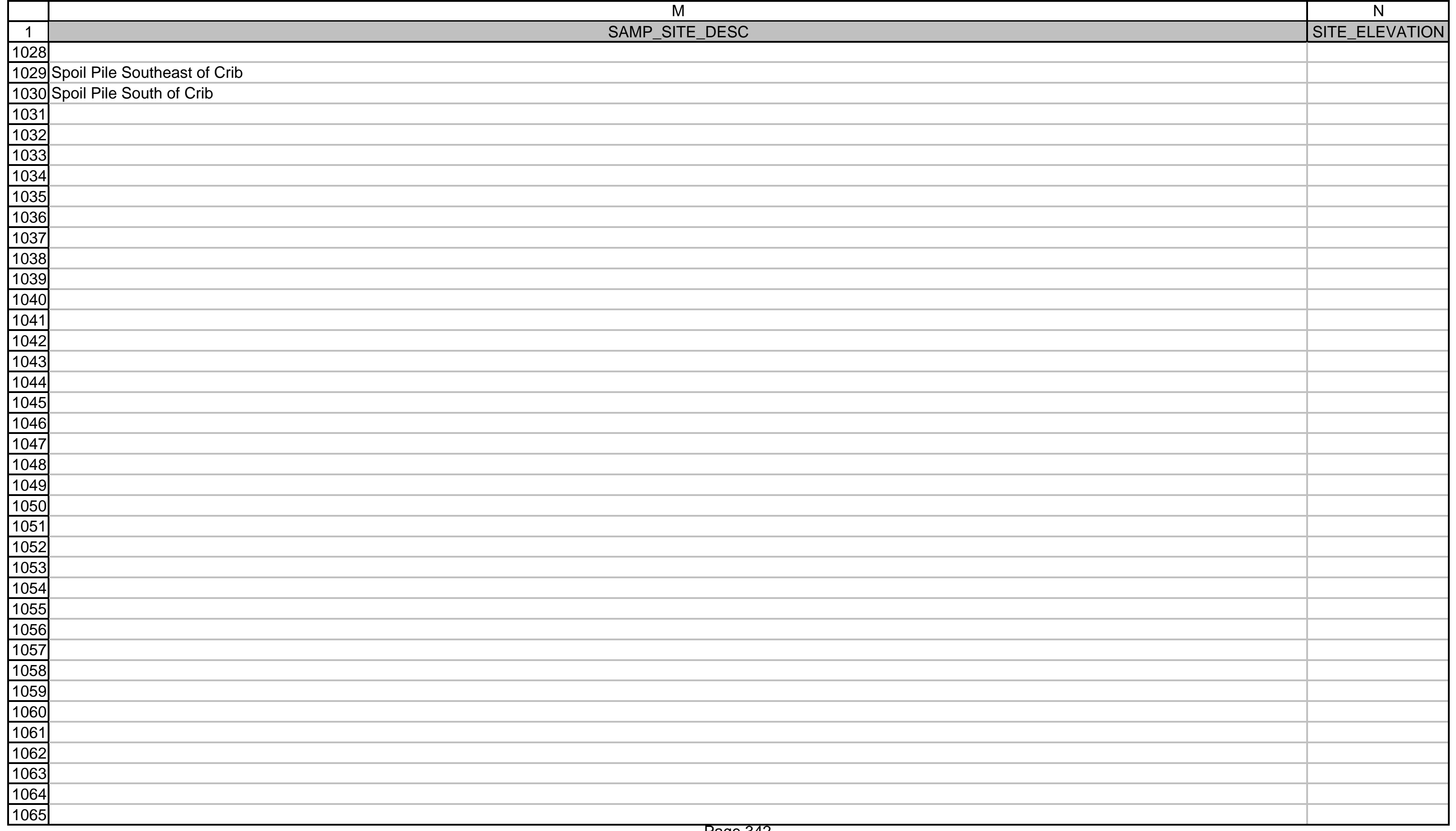




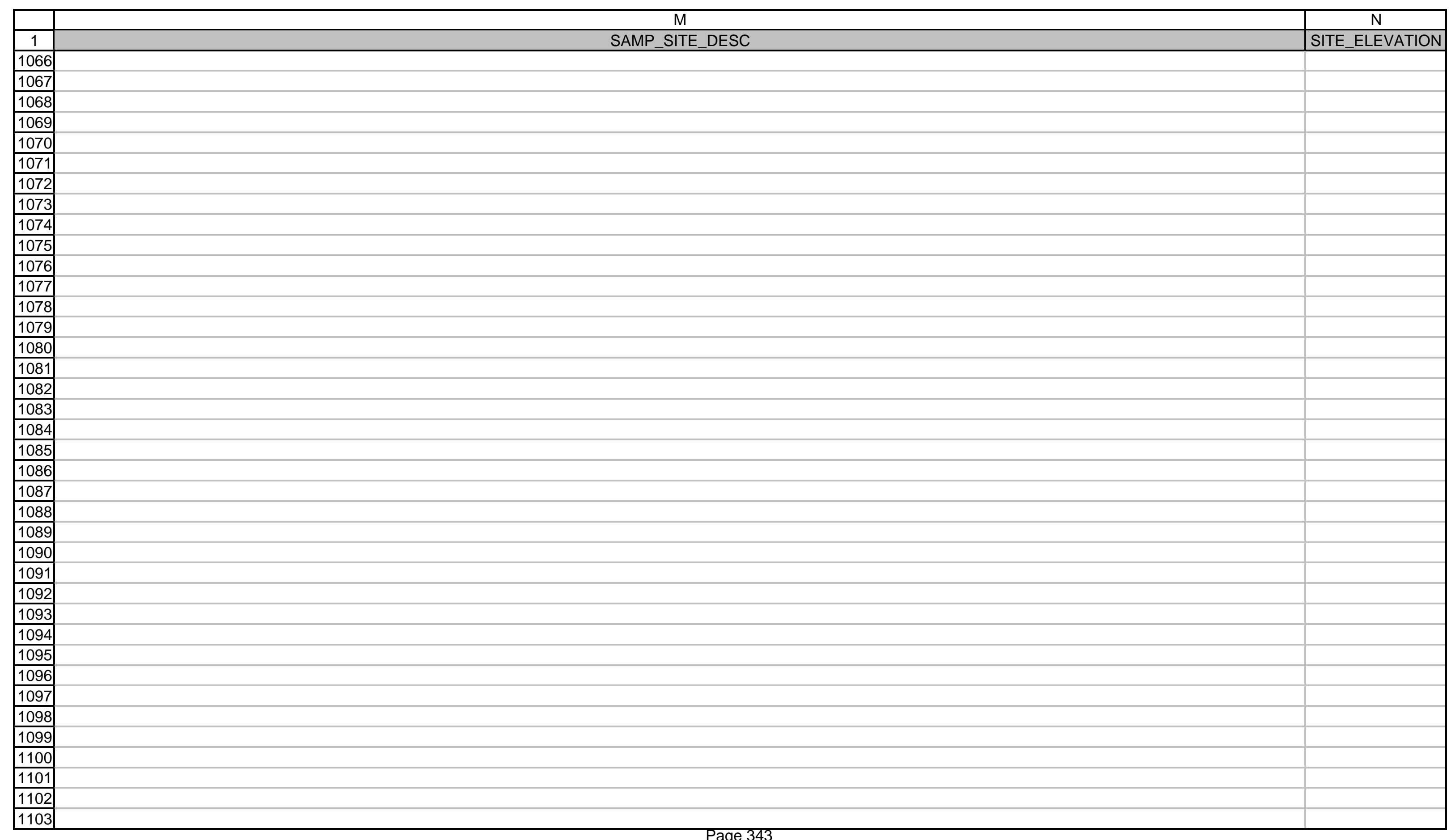




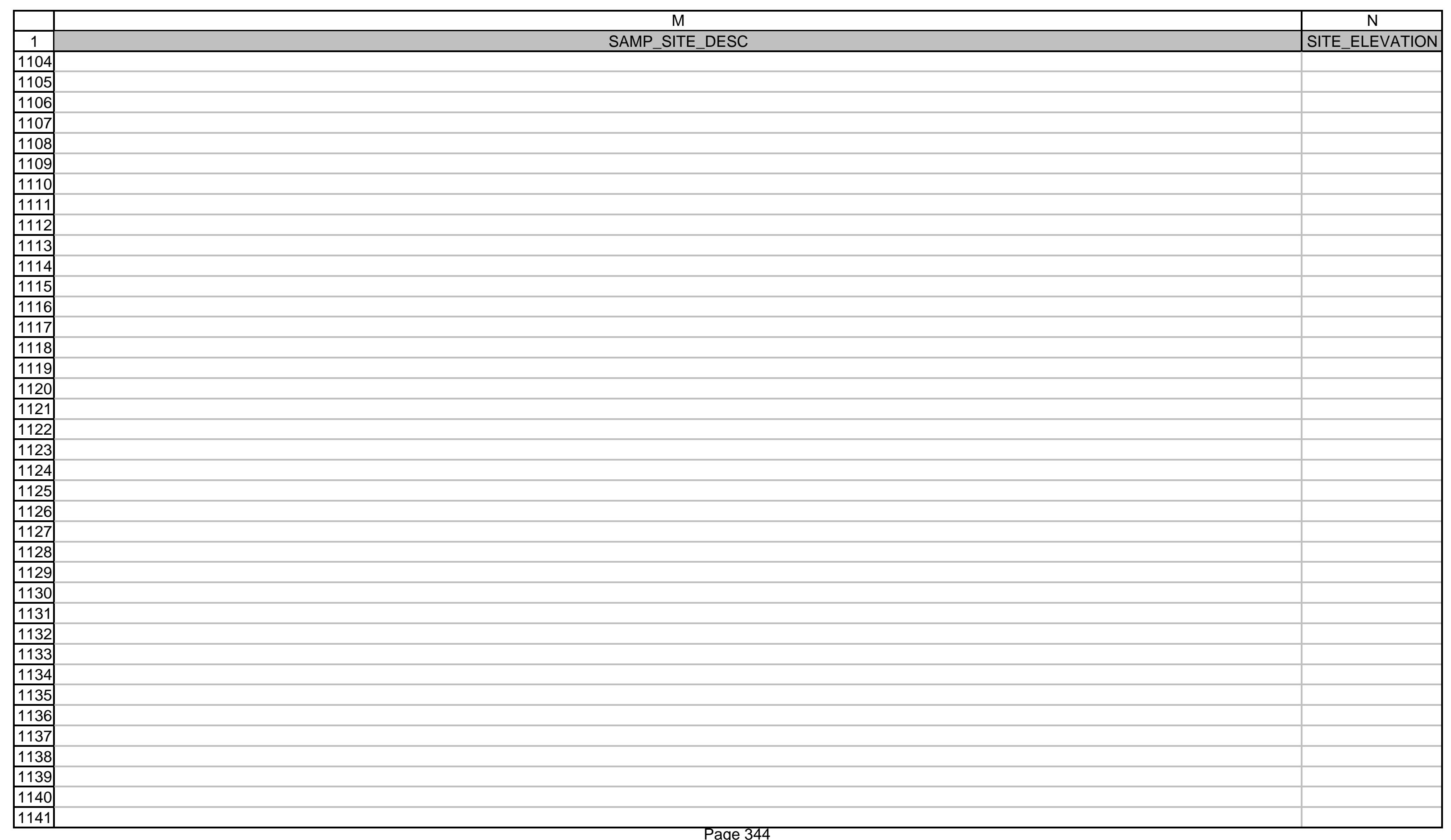




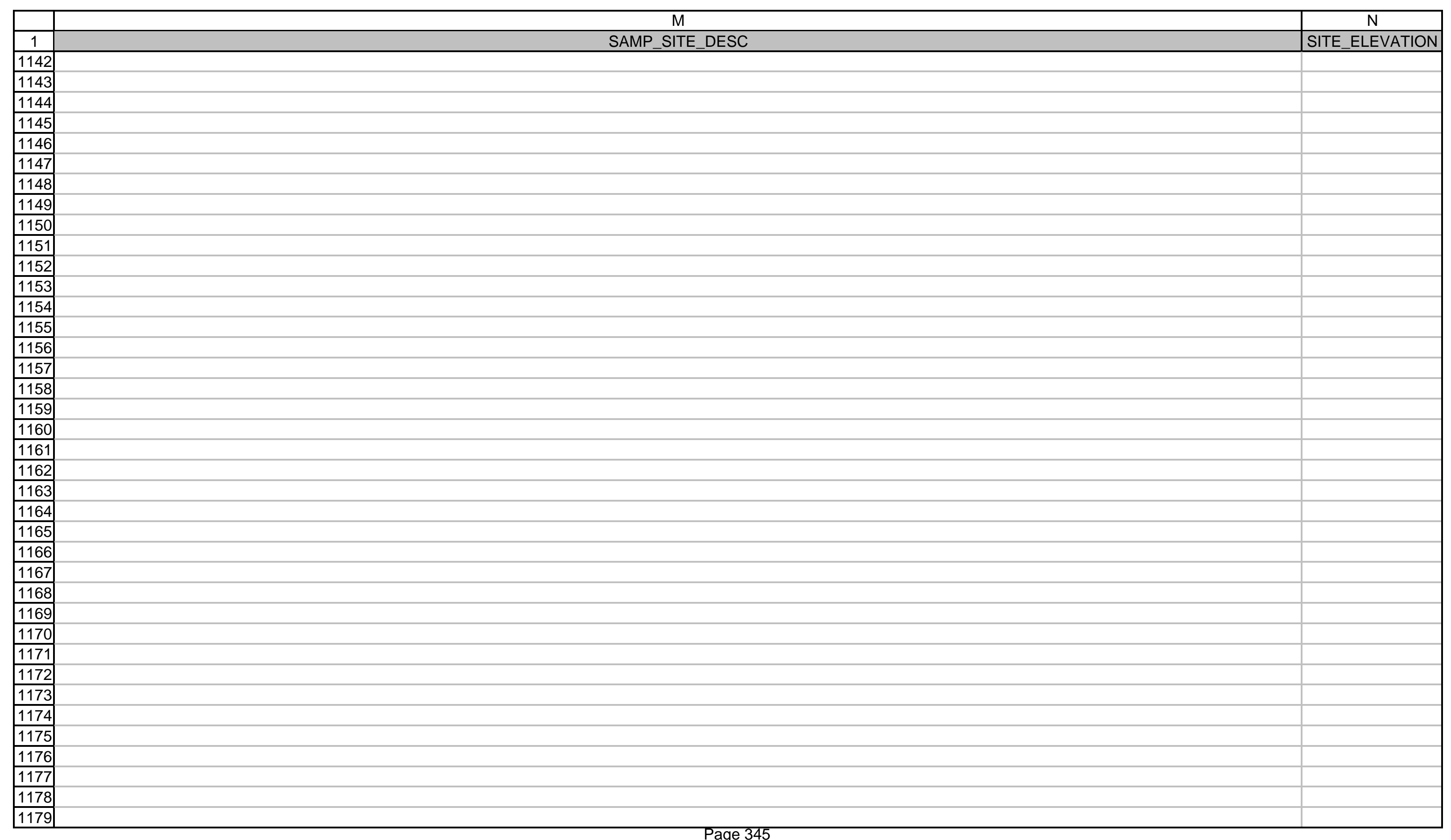




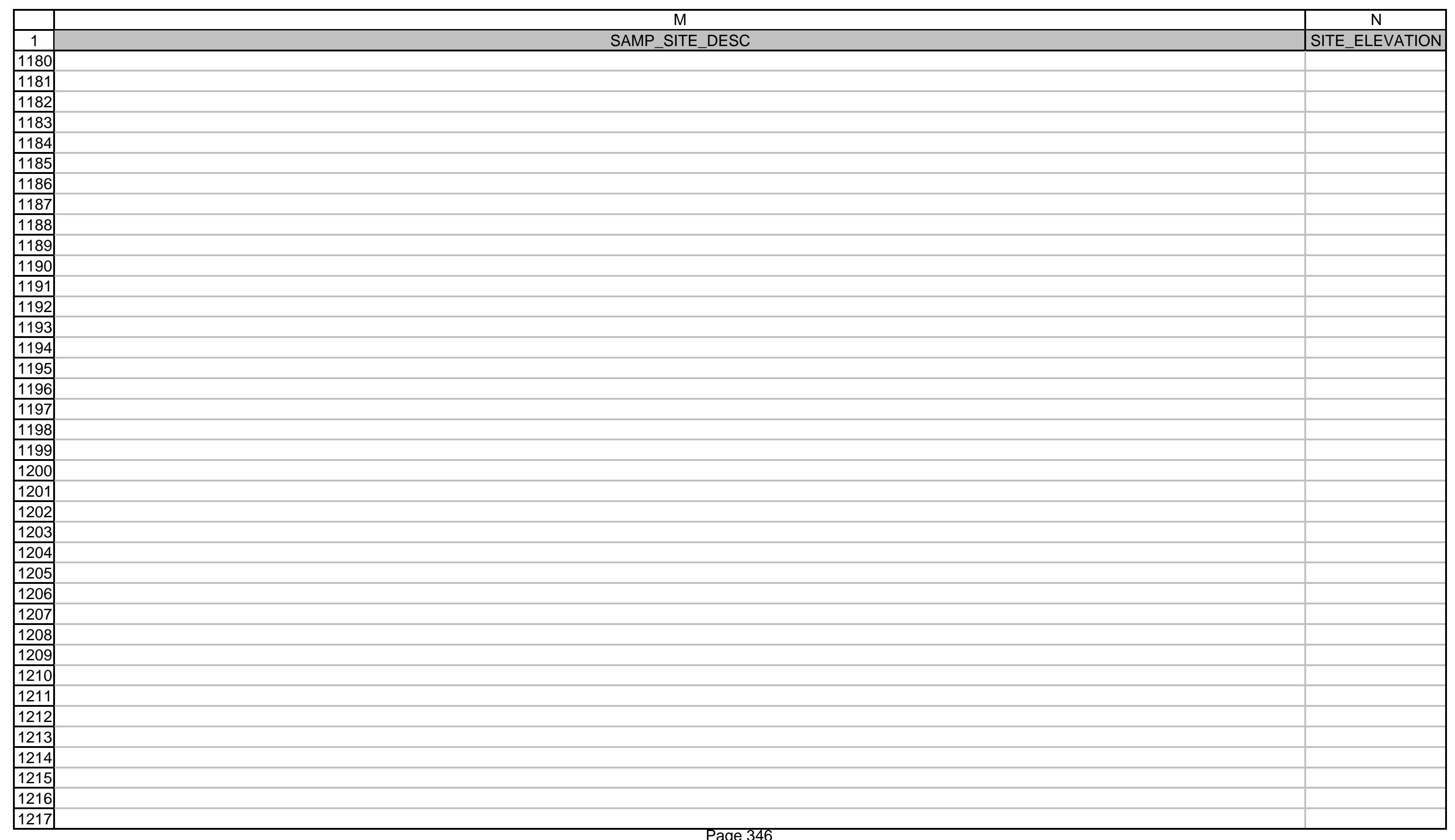




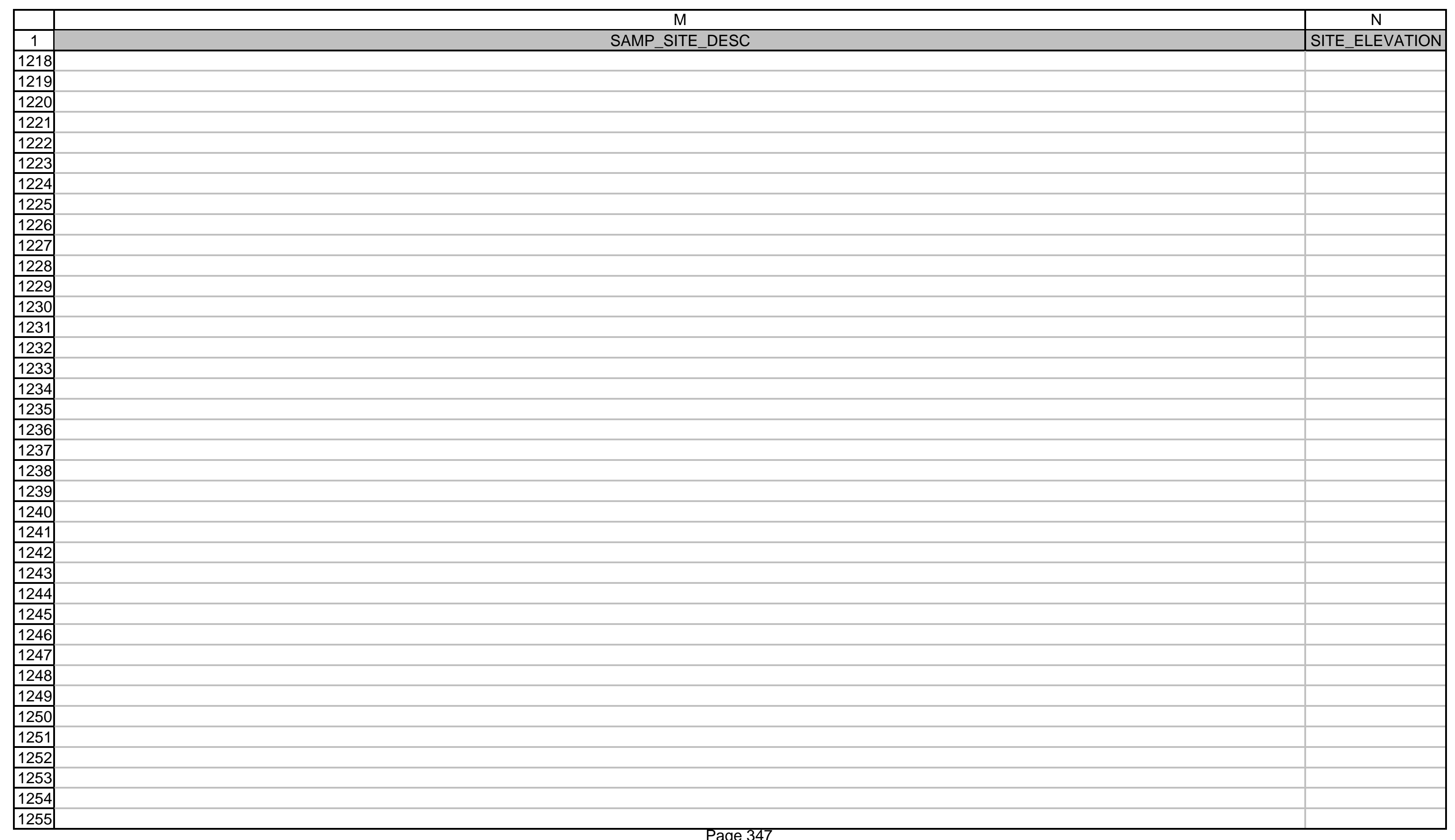




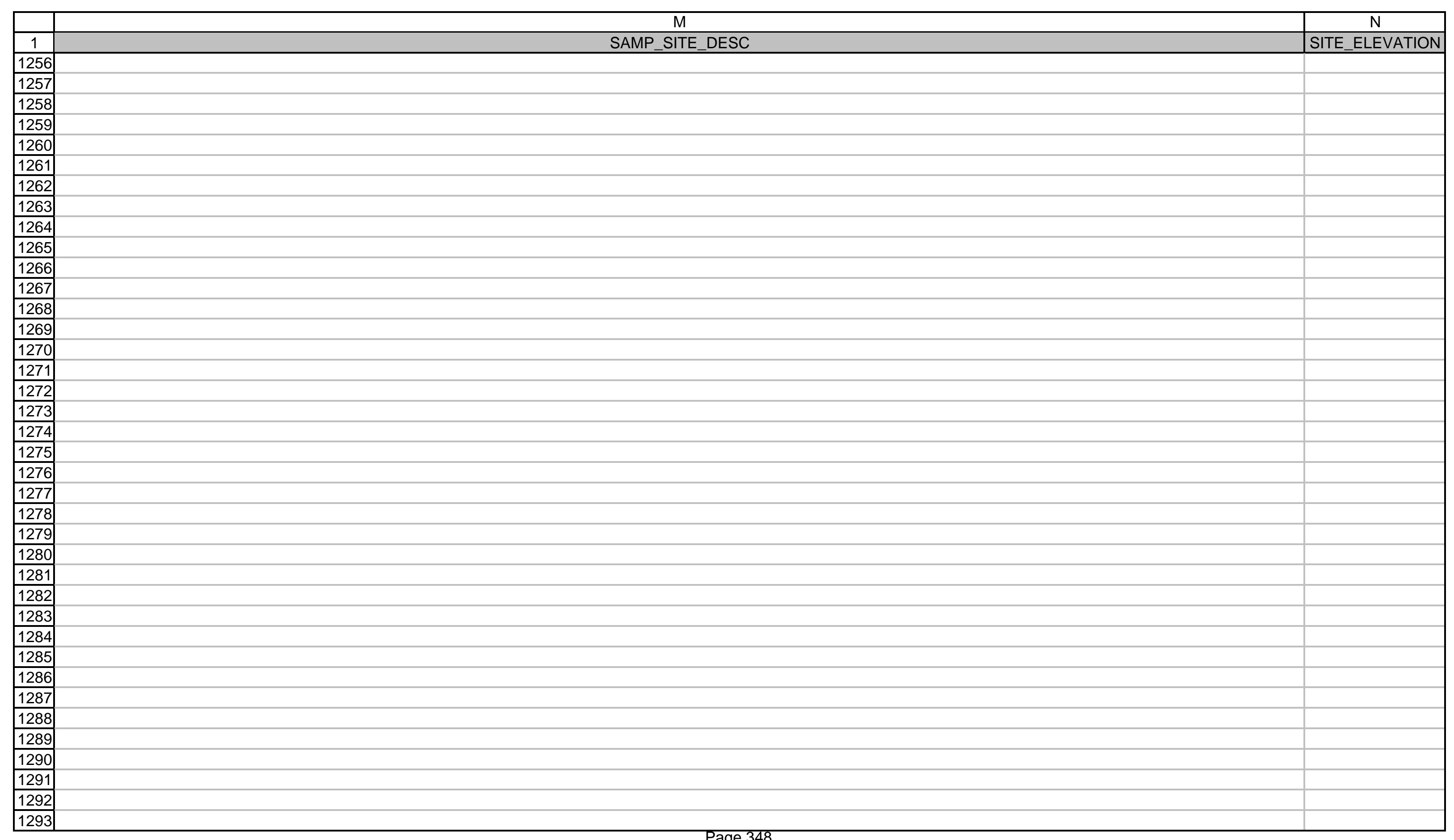




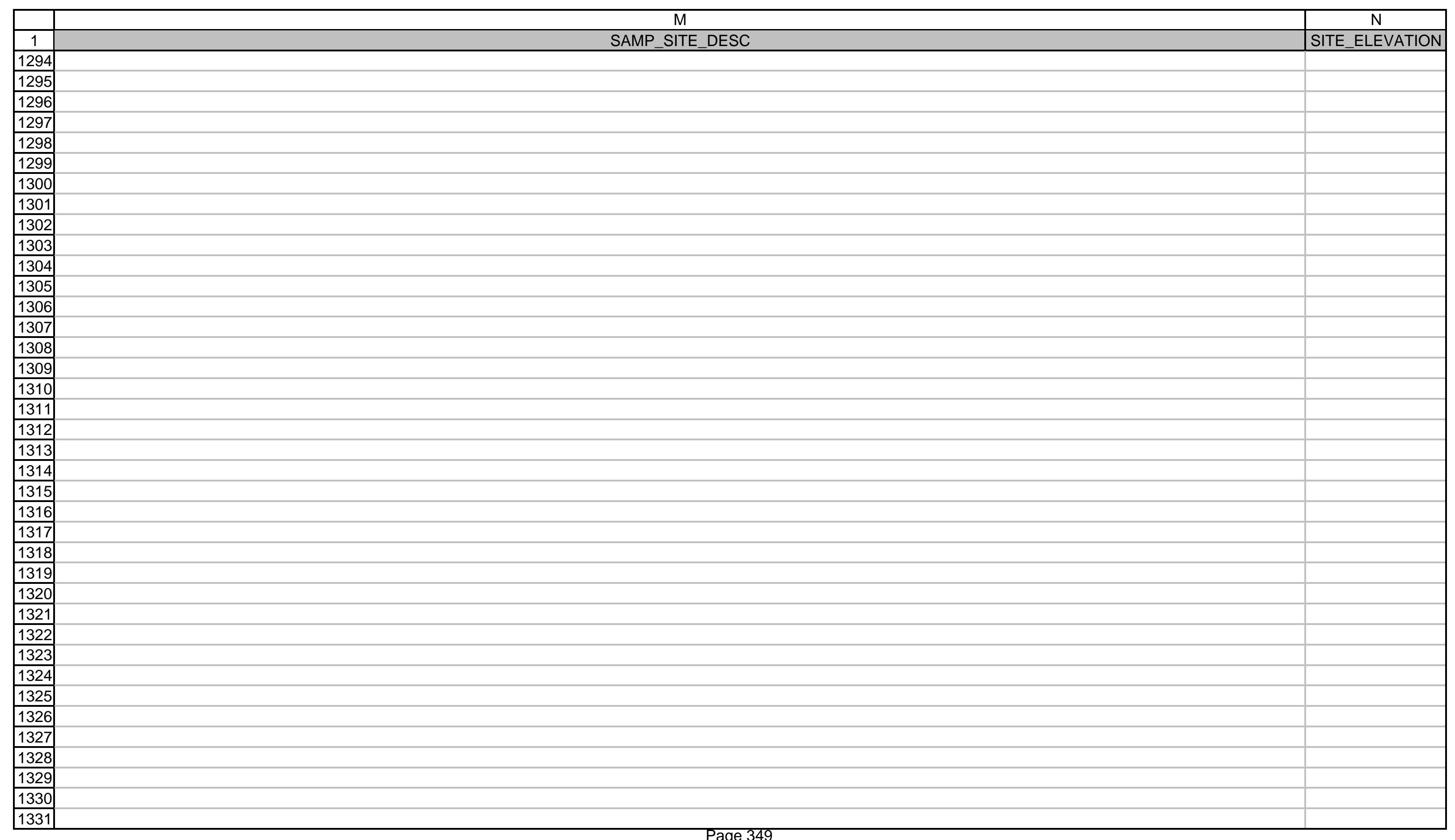




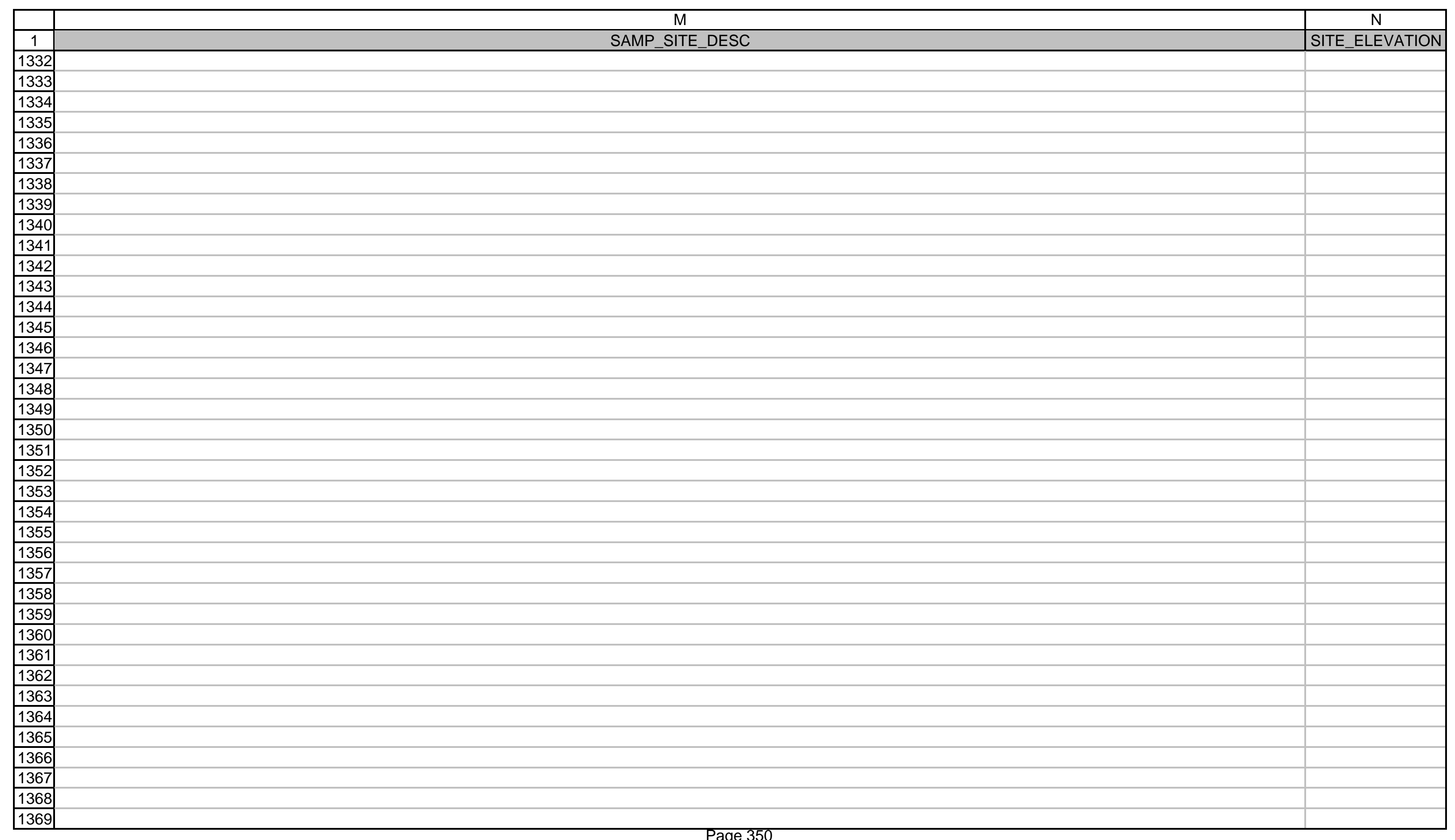




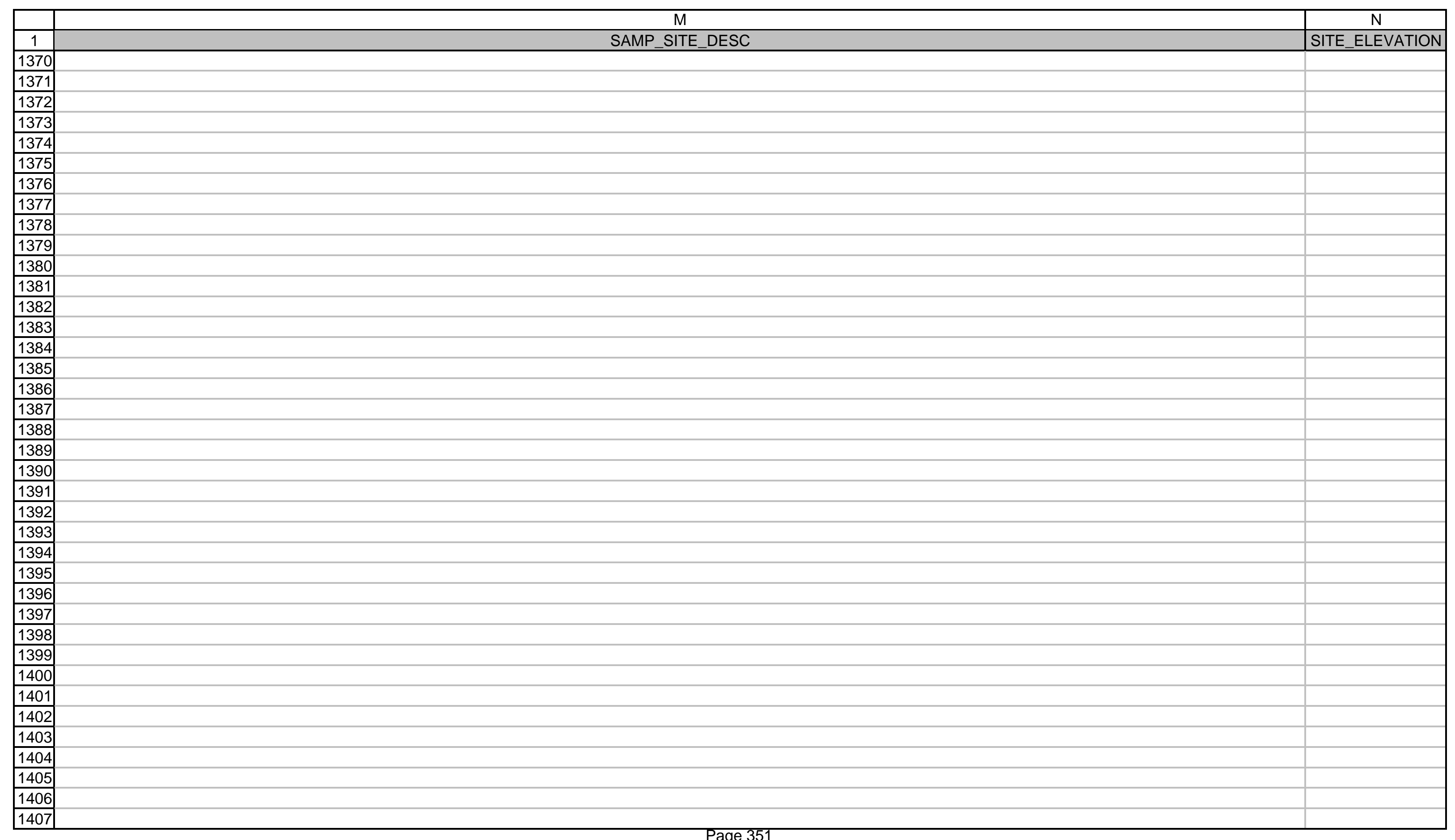




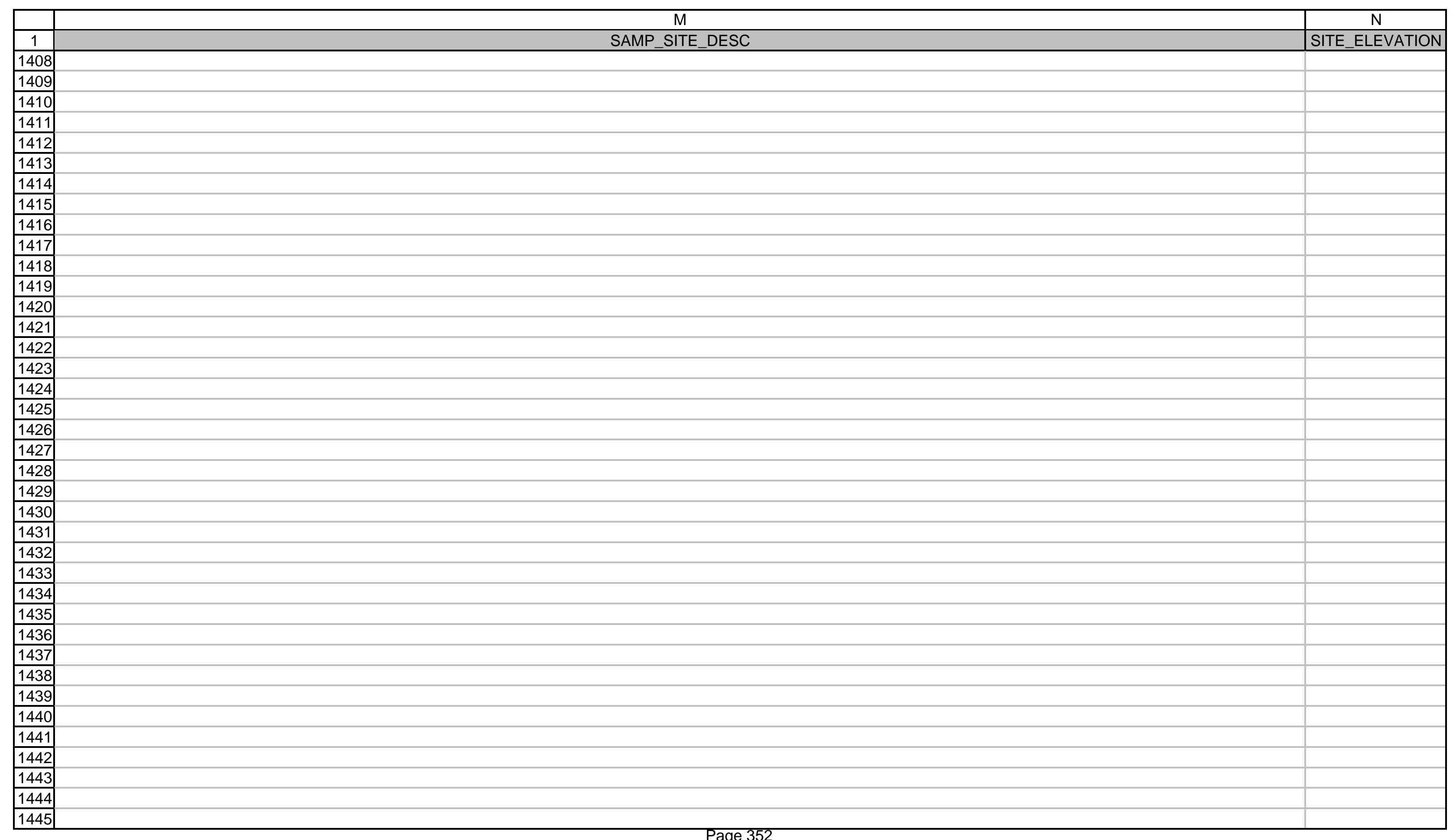




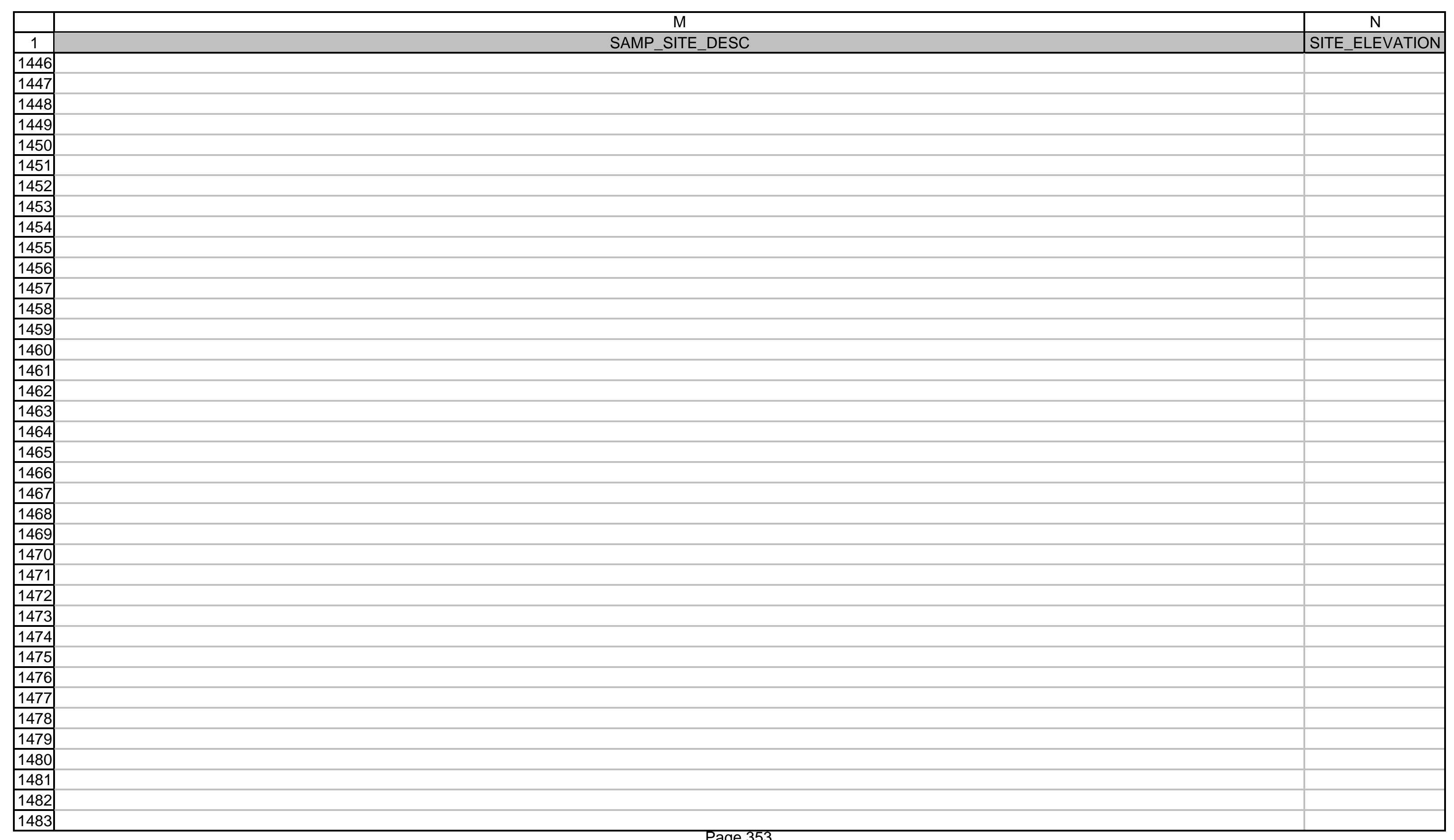




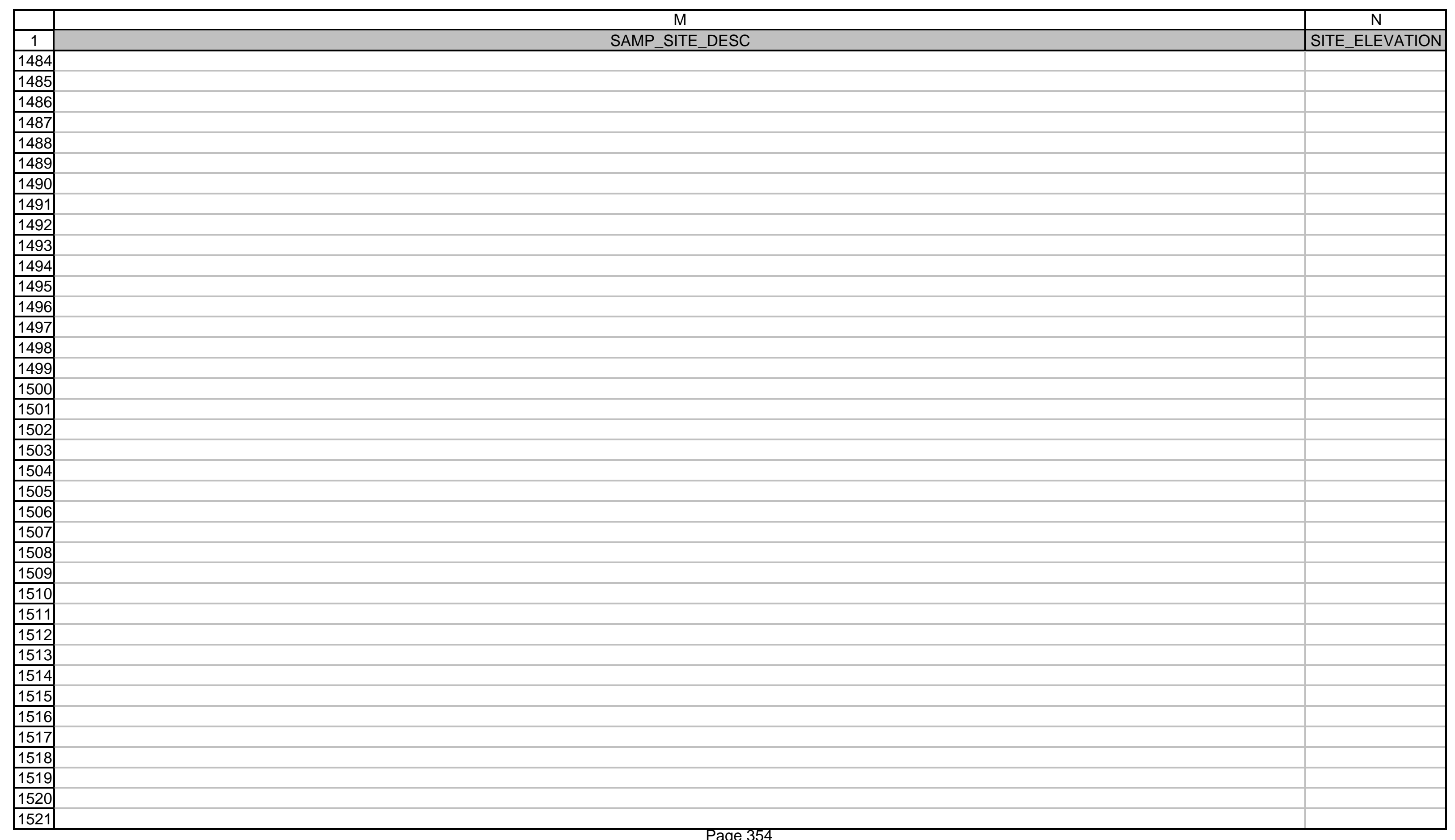




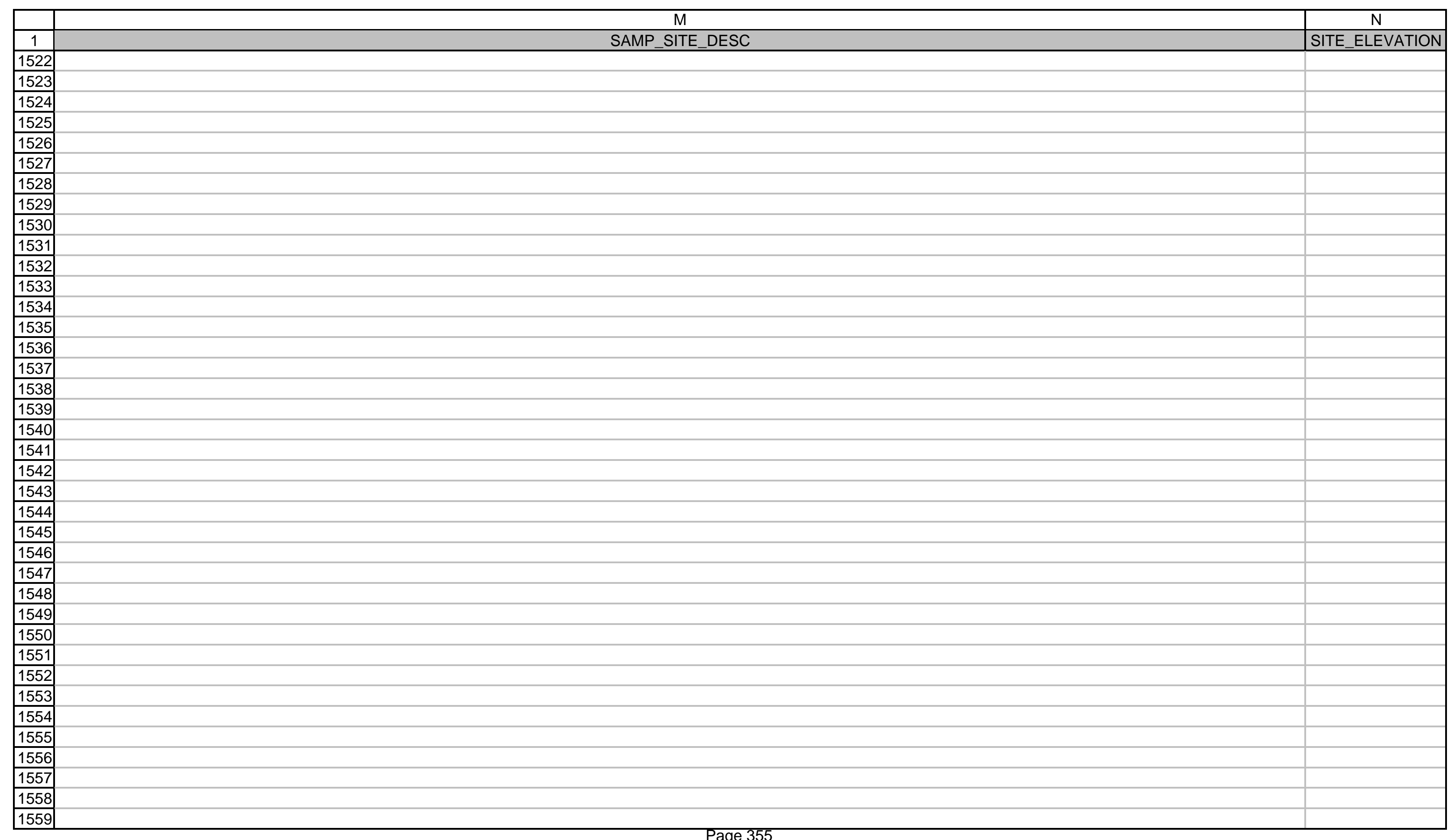




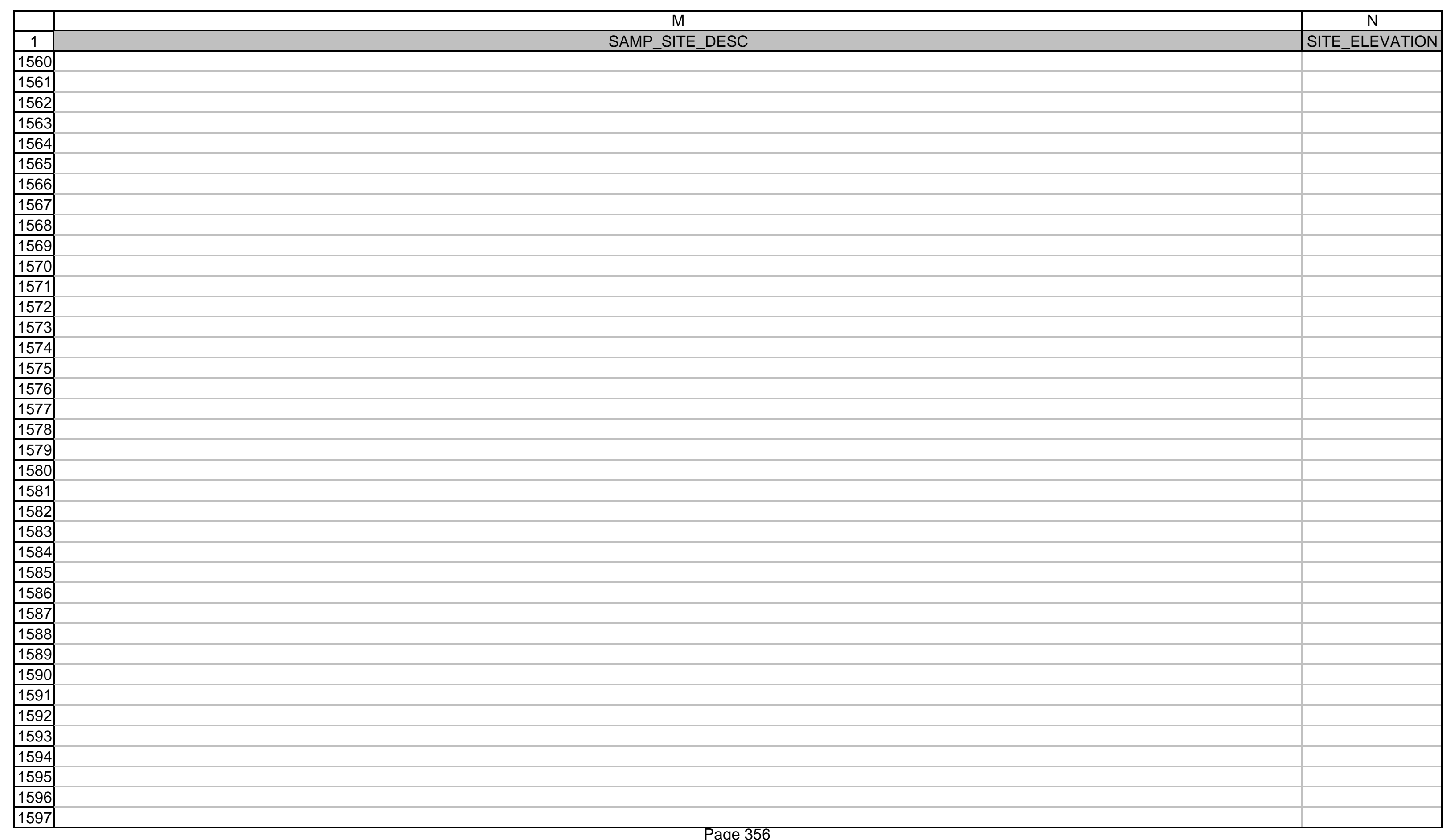




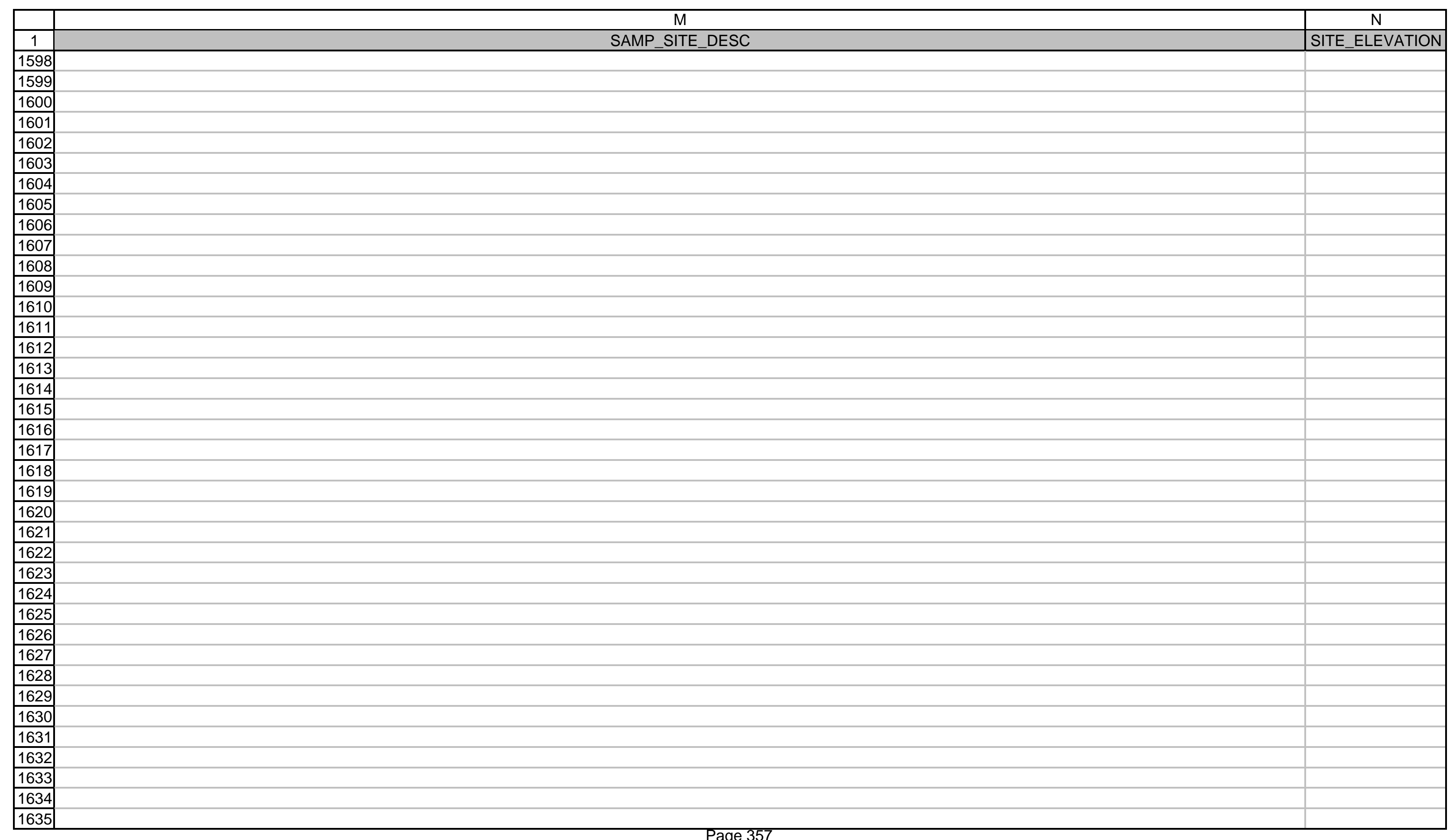




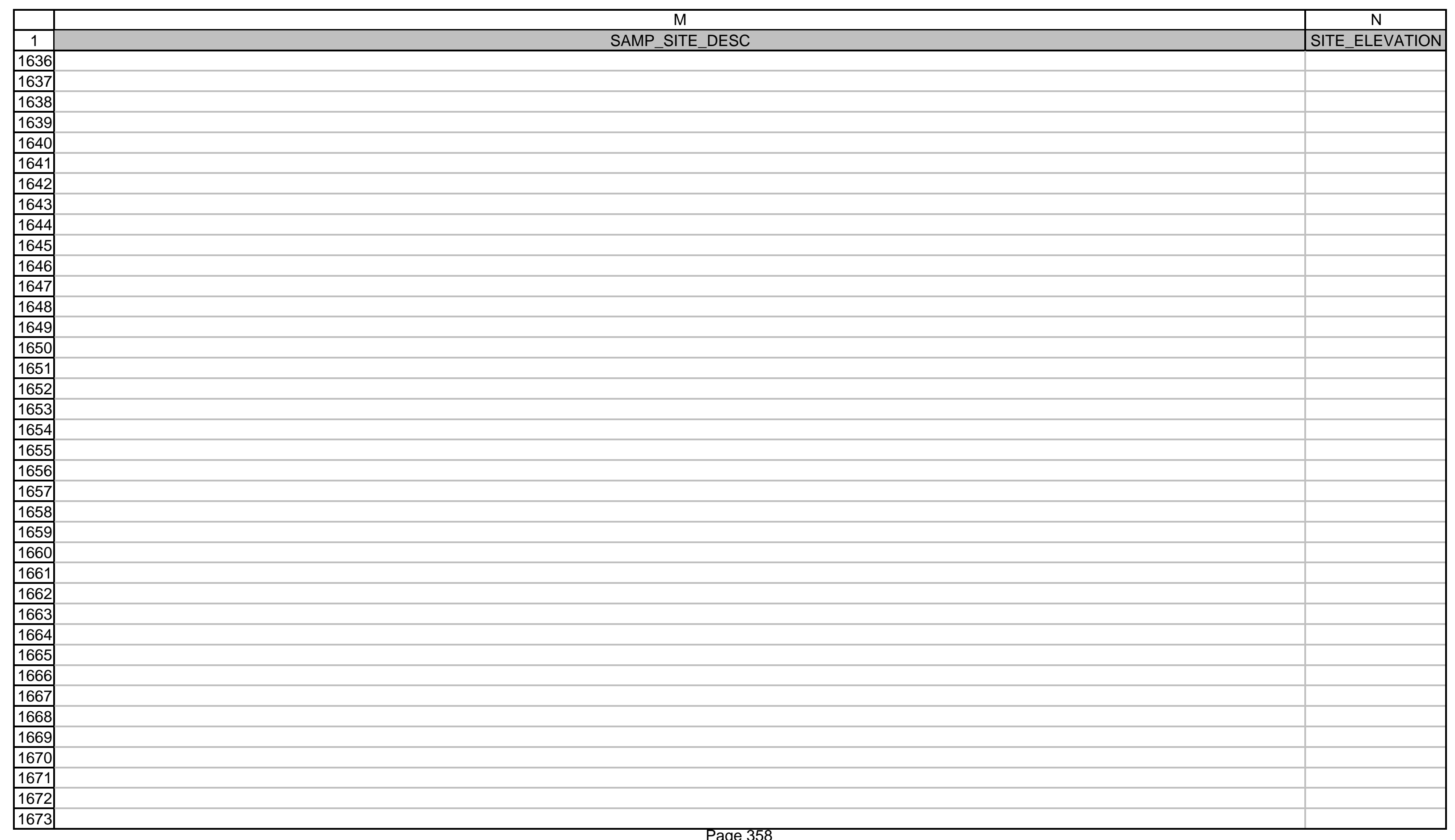




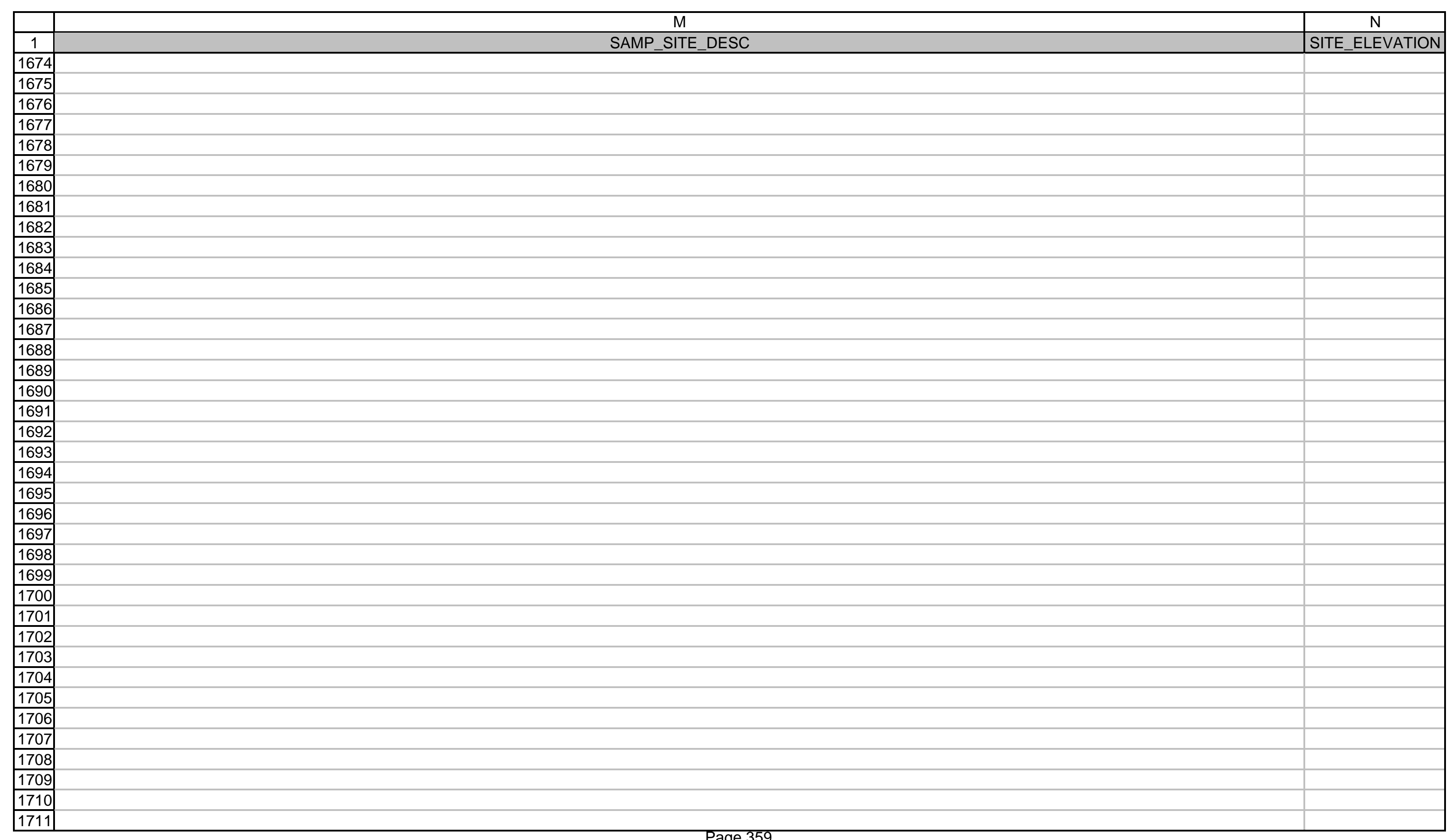




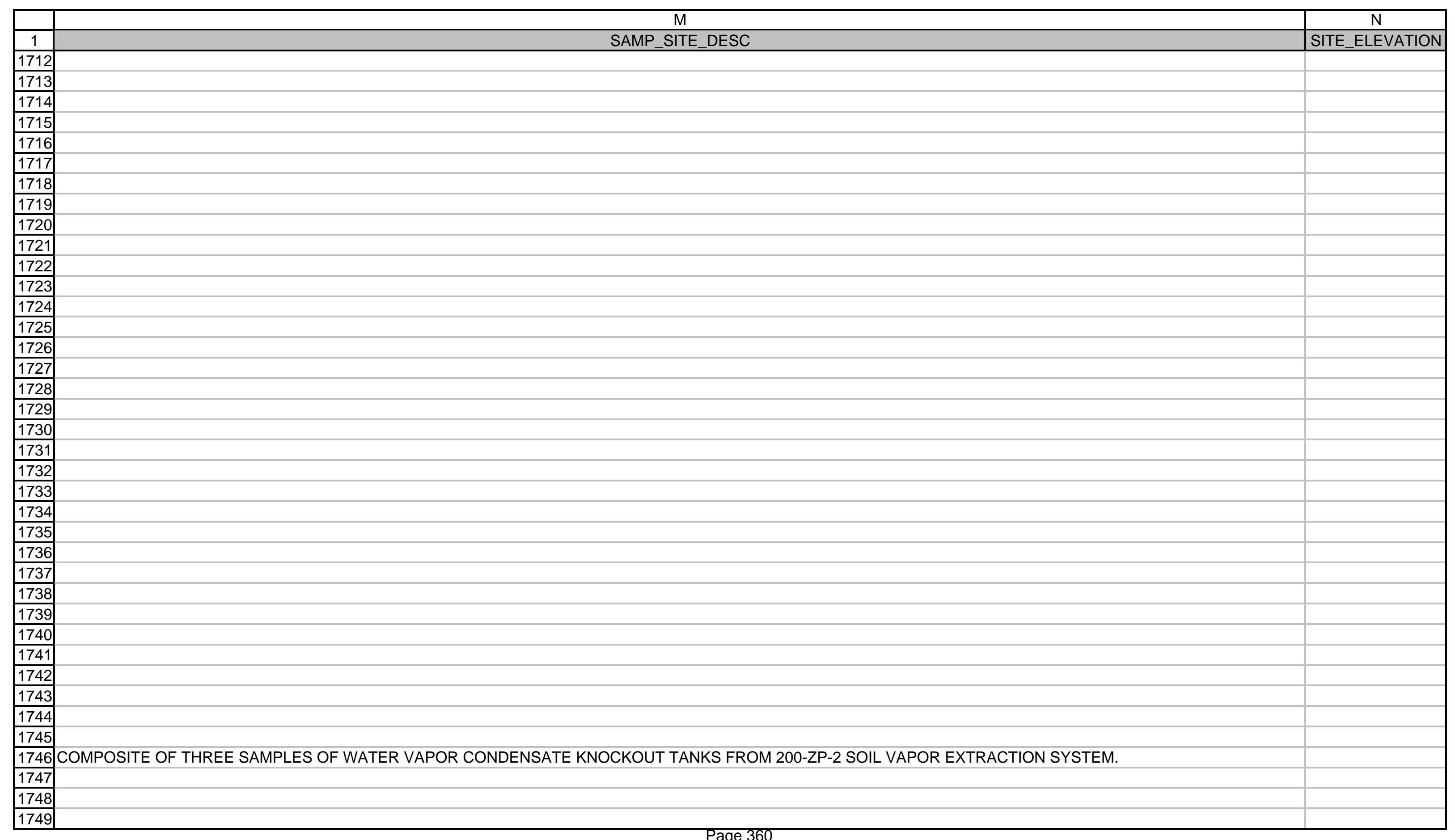




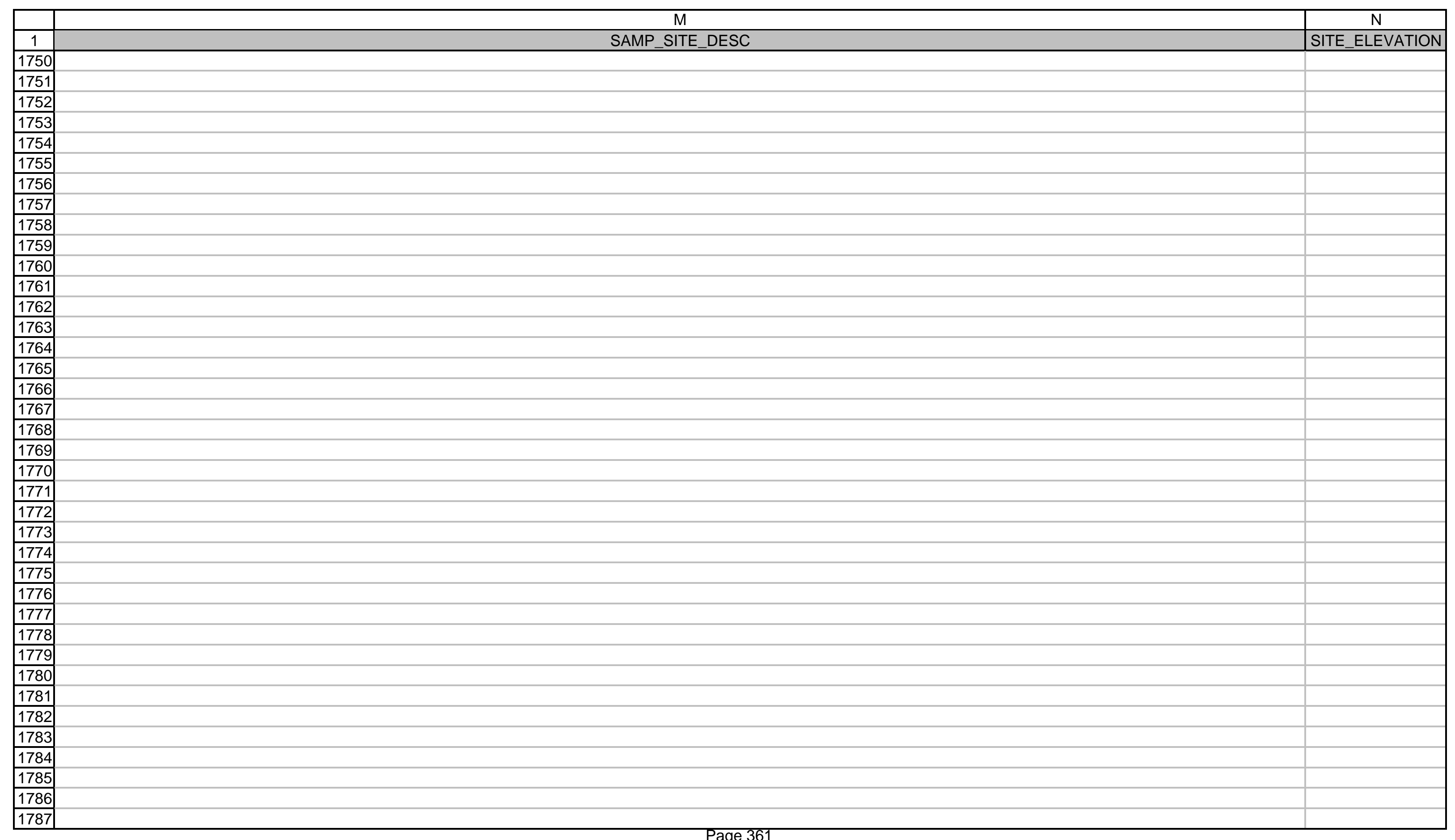




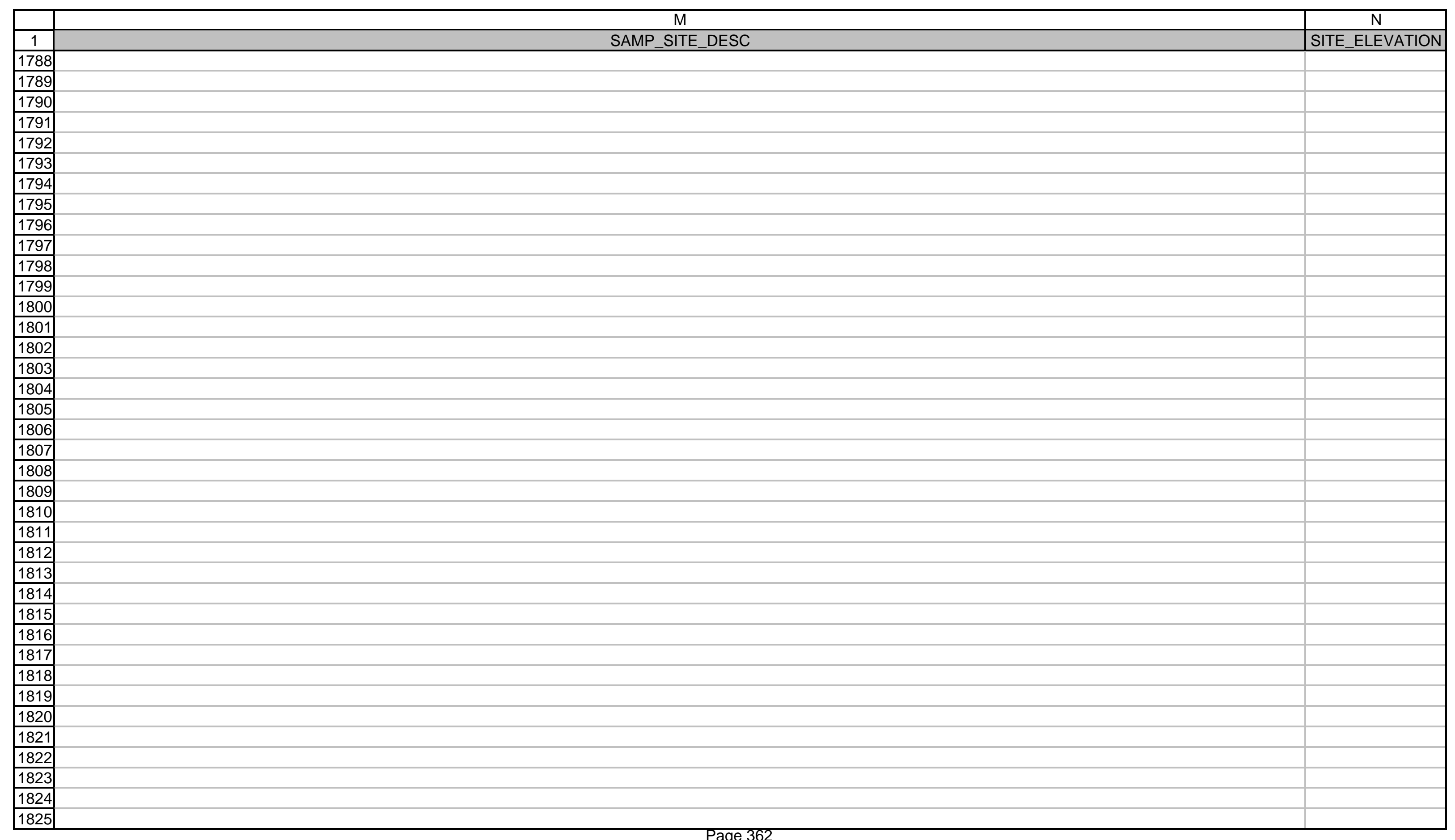




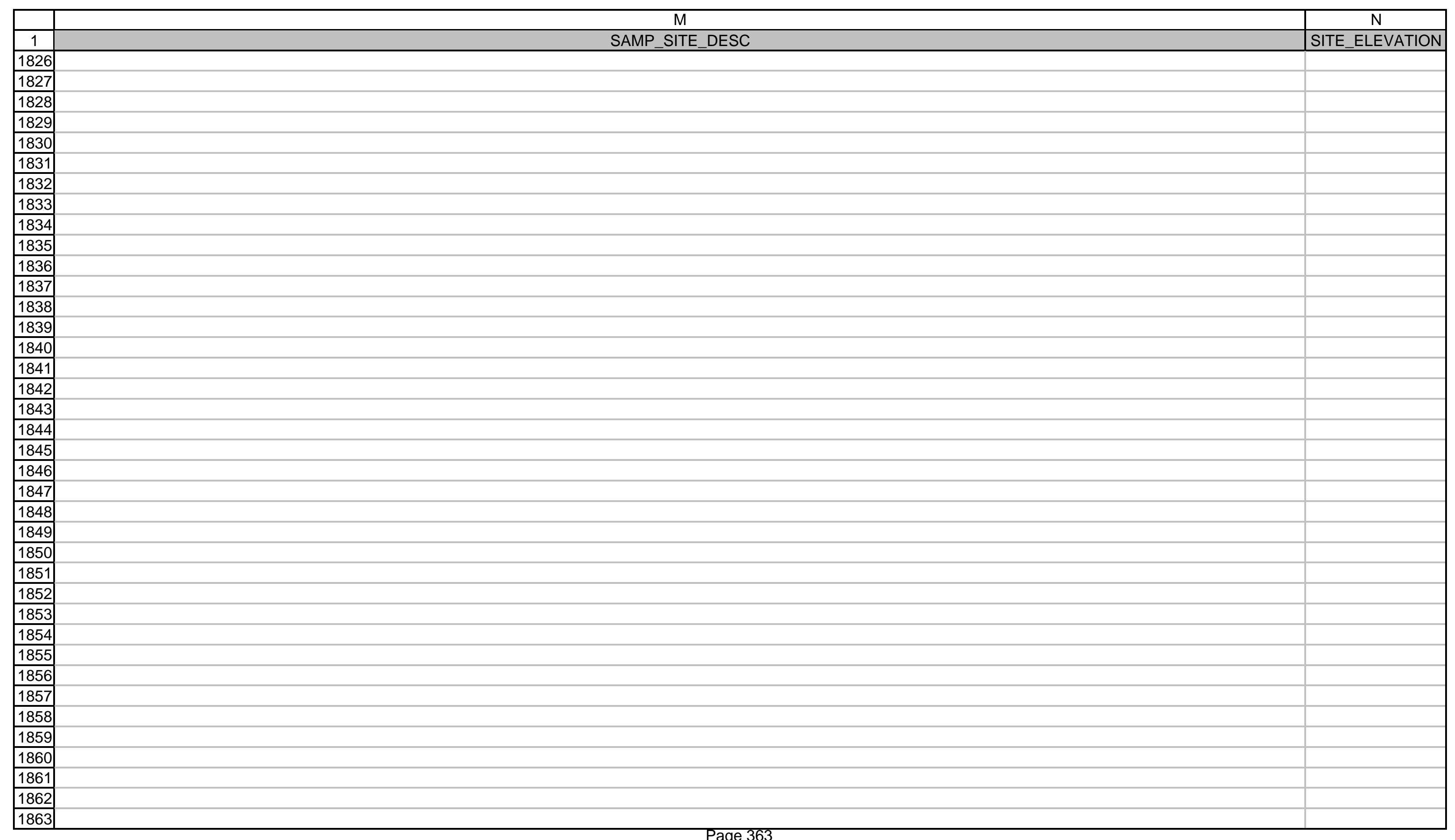




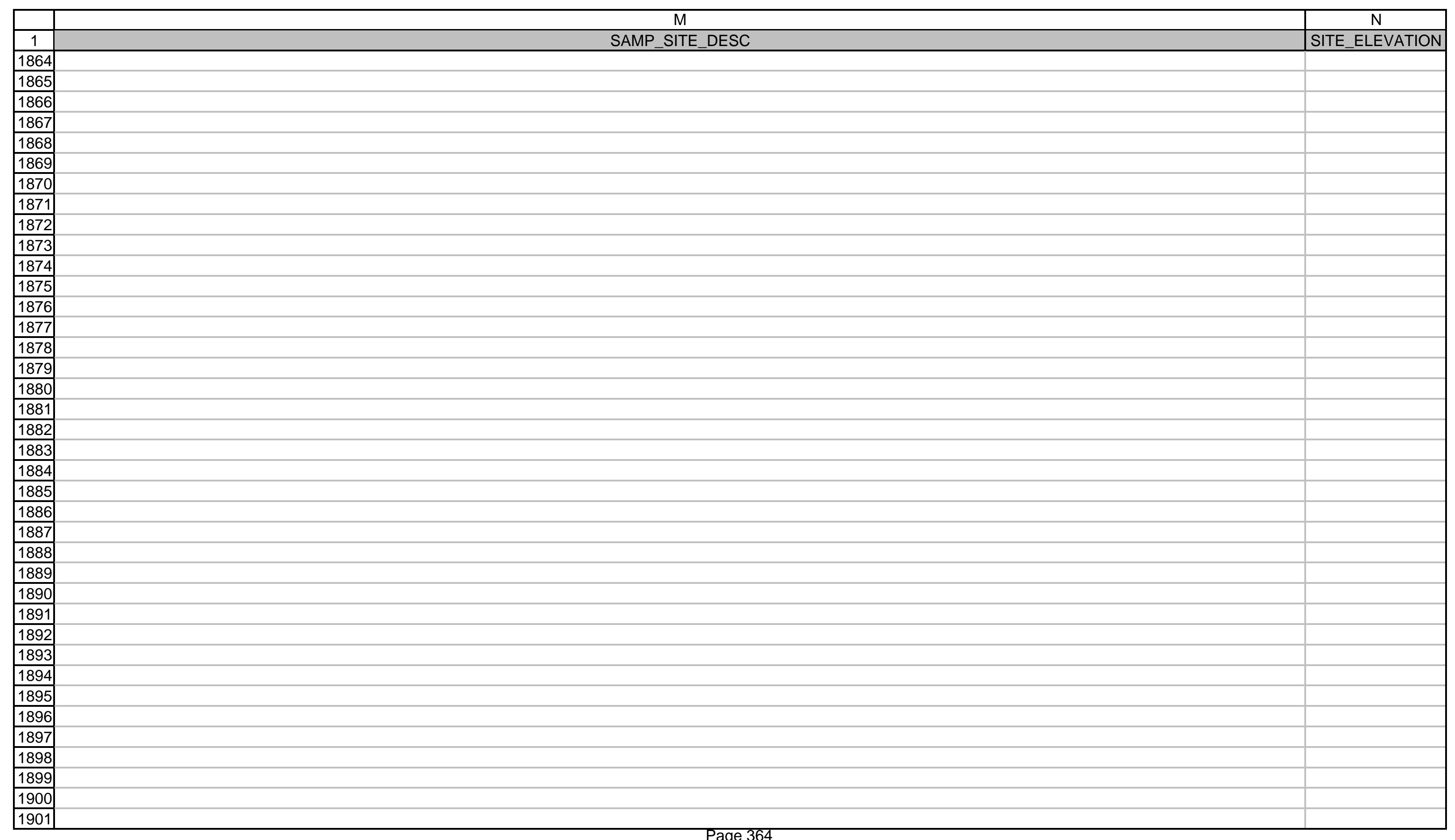




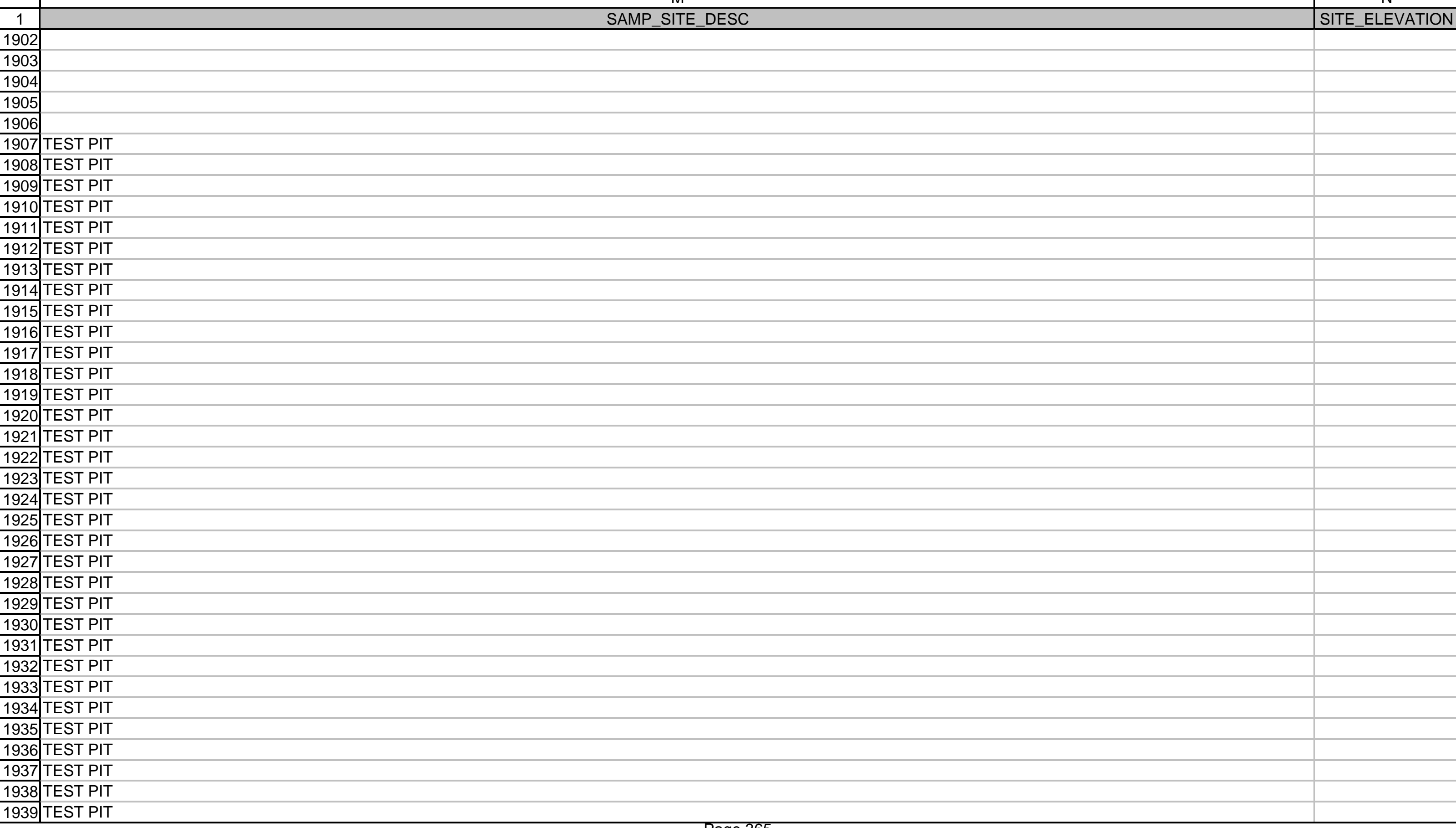




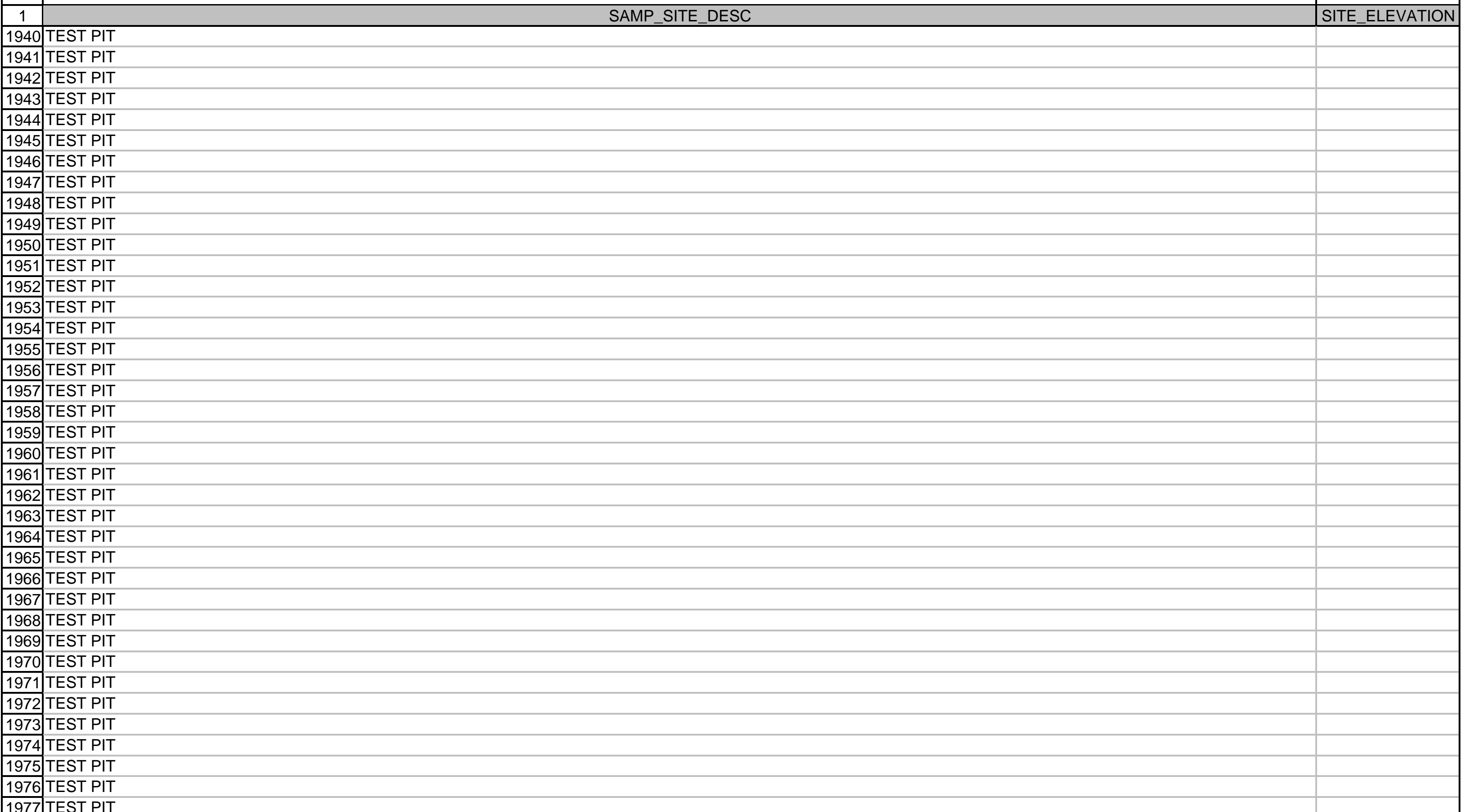

\begin{tabular}{|l|l|}
1977 & TEST PIT \\
\hline
\end{tabular} 
1979 TEST PIT

1980 TEST PIT

1981 TEST PIT

1982 TEST PIT

1983 TEST PIT

1984 TEST PIT

1985 TEST PIT

\begin{tabular}{|l|l|}
1986 & TEST PIT \\
\hline
\end{tabular}

1987 TEST PIT

1988 TEST PIT

1989 TEST PIT

1990 TEST PIT

1991 TEST PIT

1992 TEST PIT

\begin{tabular}{l|l|l}
1993 & TEST PIT \\
\hline
\end{tabular}

1994 TEST PIT

1995 TEST PIT

1996 TEST PIT

1997 TEST PIT

1998 TEST PIT

1999 TEST PIT

2000 TEST PIT

2001 TEST PIT

2002 TEST PIT

2003 TEST PIT

\begin{tabular}{|l|l|}
\hline 2004 & TEST PIT \\
\hline 2005 & TEST PIT
\end{tabular}

2005 TEST PIT

2006 TEST PIT

2007 TEST PIT

2008 TEST PIT

2009 TEST PIT

2010 TEST PIT

\begin{tabular}{|l|l|}
\hline 2011 & TEST PIT \\
\hline 2012 &
\end{tabular}

2012 TEST PIT

2013 TEST PIT

2014 TEST PIT

2015 TEST PIT 


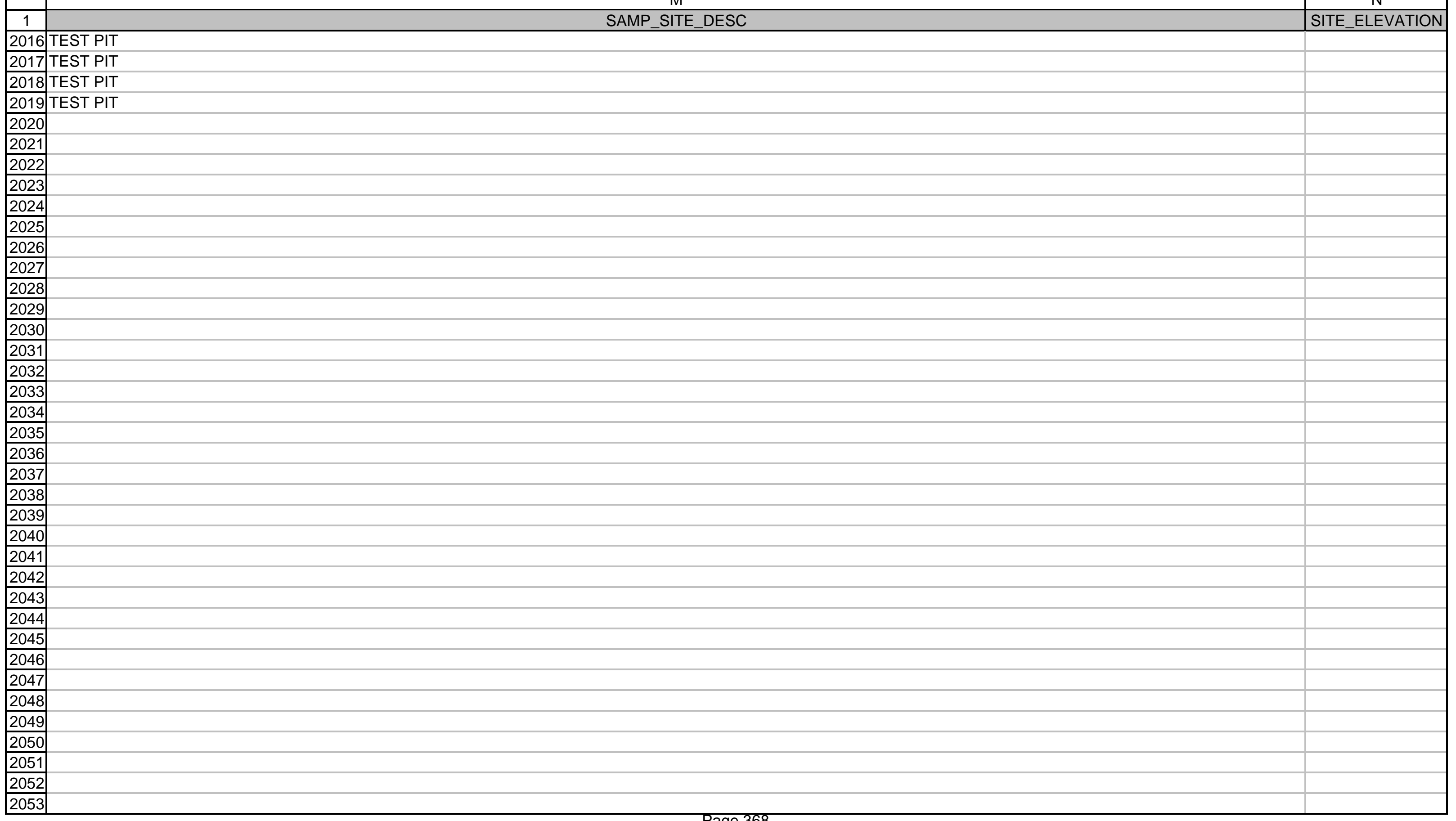




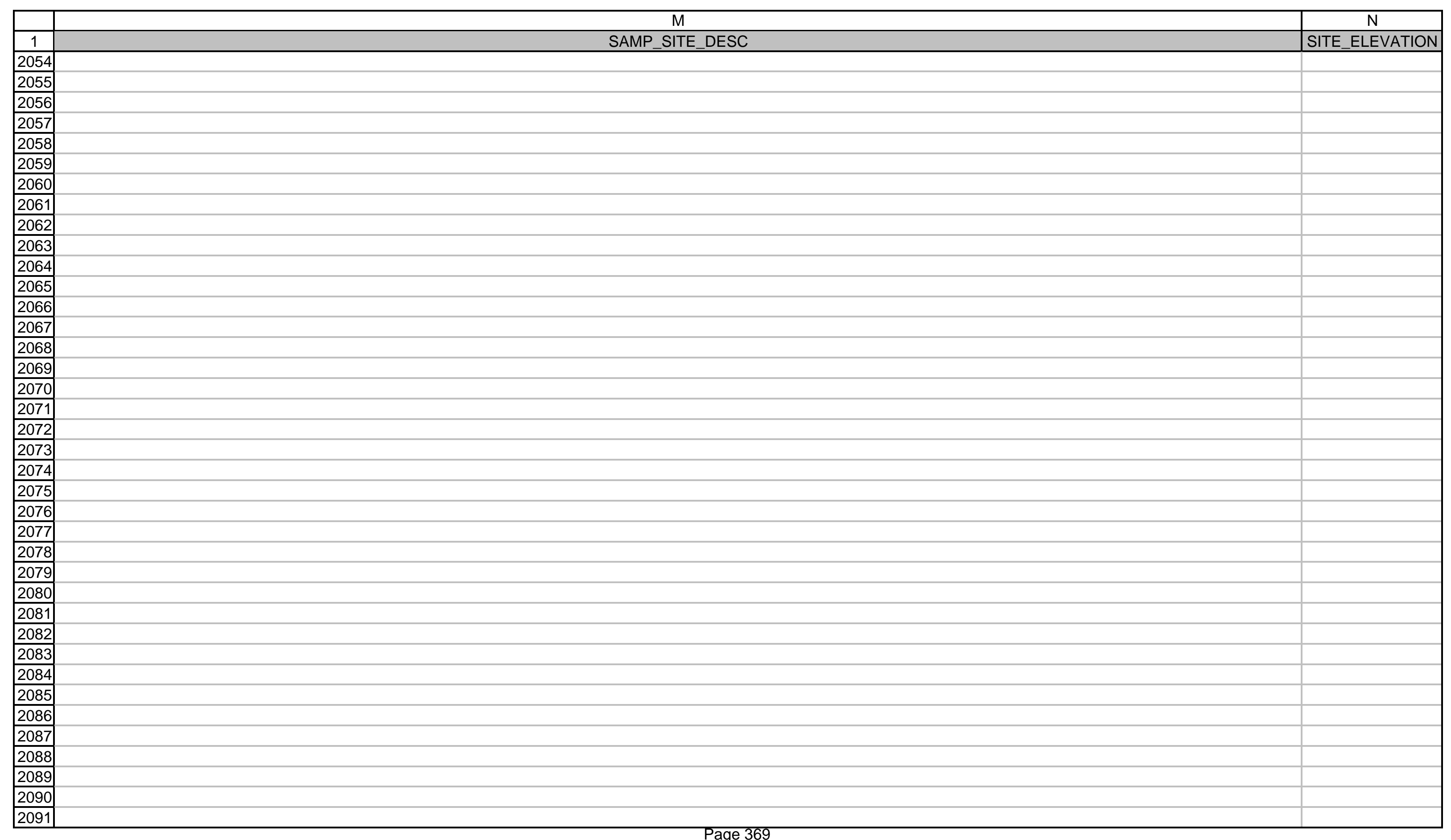




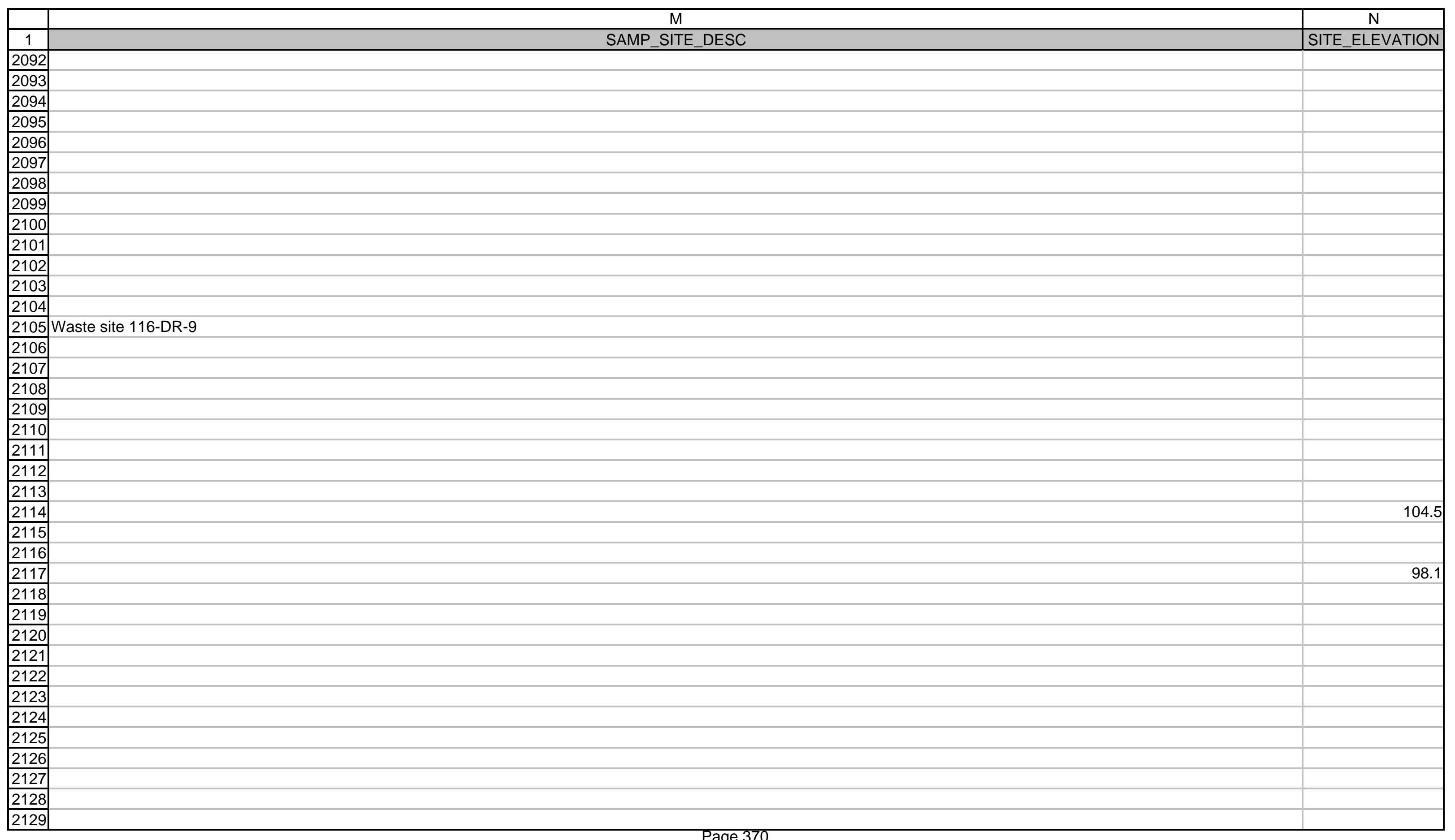




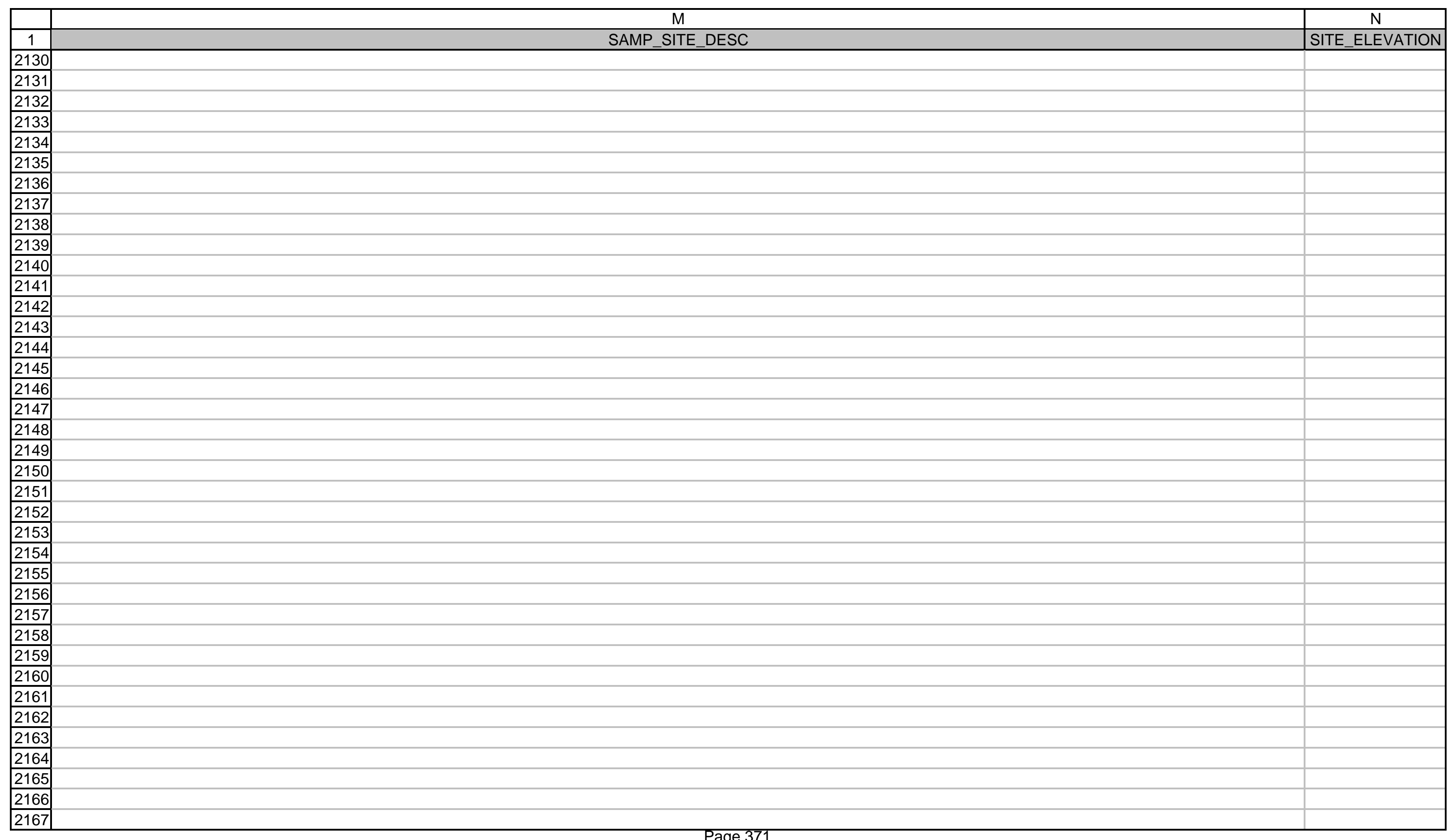




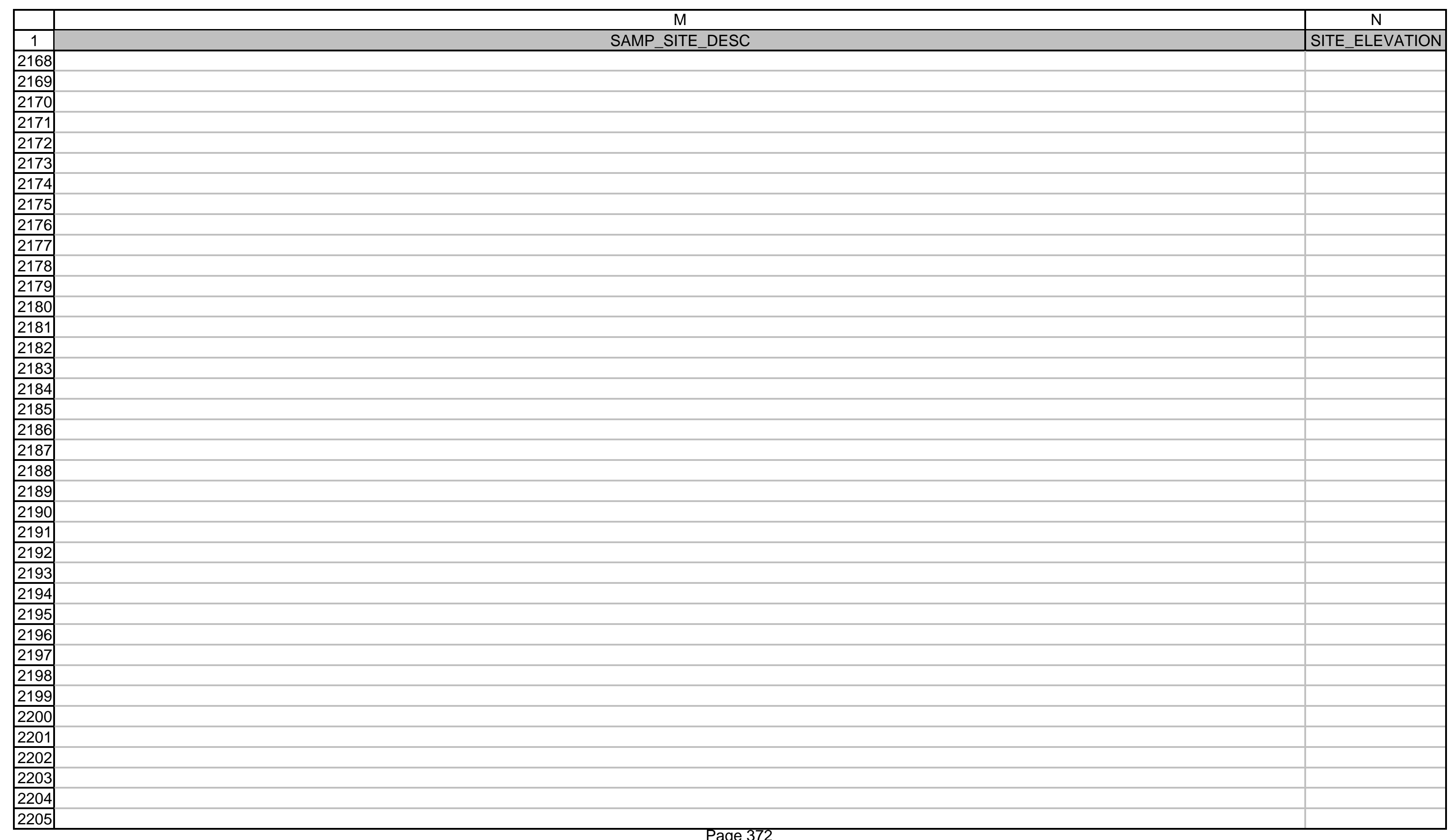




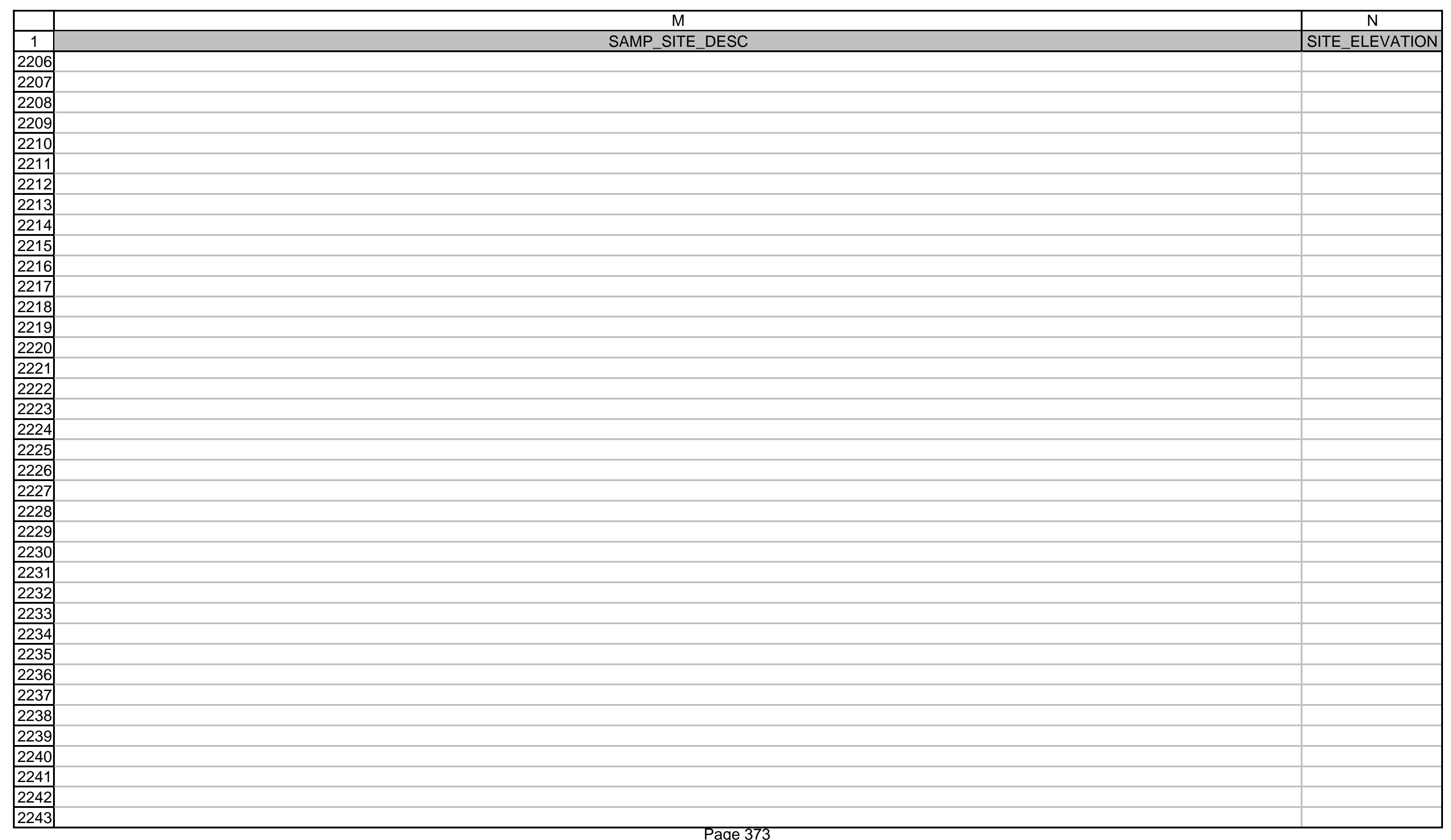




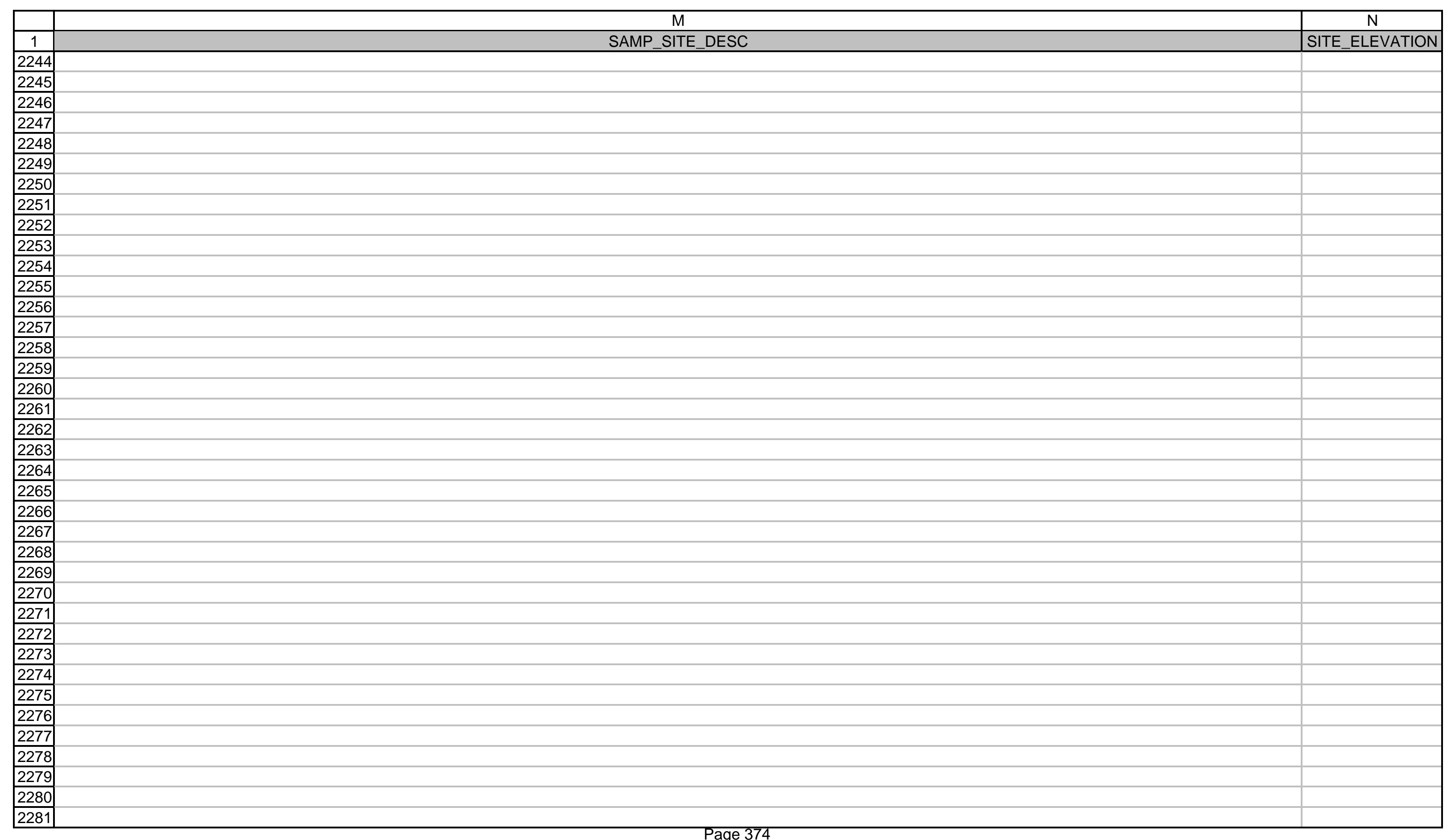




\begin{tabular}{|c|c|c|}
\hline & +2 & $\mathrm{~N}$ \\
\hline 1 & $\begin{array}{r}\text { SAMP_SITE_DESC } \\
\end{array}$ & SITE_ELEVATION \\
\hline \multicolumn{3}{|c|}{ 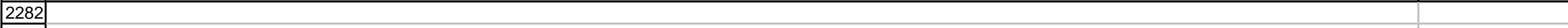 } \\
\hline \multicolumn{3}{|l|}{\begin{tabular}{|l|}
2283 \\
\end{tabular}} \\
\hline \multicolumn{3}{|l|}{\begin{tabular}{|l|}
2284 \\
\end{tabular}} \\
\hline \multicolumn{3}{|l|}{\begin{tabular}{|l|}
2285 \\
\end{tabular}} \\
\hline \multicolumn{3}{|l|}{\begin{tabular}{|l|}
2286 \\
\end{tabular}} \\
\hline \multicolumn{3}{|l|}{2287} \\
\hline \multicolumn{3}{|c|}{2288 Eastern half of $\mathrm{H}-06-\mathrm{LE}$ grid } \\
\hline \multicolumn{3}{|c|}{2289 Western half of $\mathrm{H}-06-\mathrm{LE}$ grid } \\
\hline 2291 & & 84.9 \\
\hline 2292 & & 86.2 \\
\hline \multicolumn{3}{|c|}{\begin{tabular}{|c|c|}
2293 & Holder for QC samples. \\
2294 &
\end{tabular}} \\
\hline \multicolumn{3}{|c|}{\begin{tabular}{|l|}
2294 \\
\end{tabular}} \\
\hline \multicolumn{3}{|l|}{2295} \\
\hline \multicolumn{3}{|l|}{\begin{tabular}{|l|}
2296 \\
\end{tabular}} \\
\hline \multicolumn{3}{|c|}{\begin{tabular}{|l|l|}
2297 & TLD only \\
\end{tabular}} \\
\hline \begin{tabular}{|l|}
2298 \\
\end{tabular} & & 180.54 \\
\hline 2299 & & 176.8 \\
\hline \multicolumn{3}{|l|}{2301} \\
\hline \multicolumn{3}{|l|}{2302} \\
\hline \multicolumn{3}{|l|}{2303} \\
\hline \multicolumn{3}{|l|}{2304} \\
\hline \multicolumn{3}{|l|}{2305} \\
\hline \multicolumn{3}{|l|}{\begin{tabular}{|l|}
2306 \\
\end{tabular}} \\
\hline \multicolumn{3}{|l|}{\begin{tabular}{|l|}
2307 \\
\end{tabular}} \\
\hline \multicolumn{3}{|l|}{\begin{tabular}{|l|}
2308 \\
\end{tabular}} \\
\hline \multicolumn{3}{|l|}{\begin{tabular}{|l|}
2309 \\
\end{tabular}} \\
\hline \multicolumn{3}{|l|}{\begin{tabular}{|l|}
2310 \\
\end{tabular}} \\
\hline \multicolumn{3}{|l|}{\begin{tabular}{|l|}
2311 \\
\end{tabular}} \\
\hline \multicolumn{3}{|l|}{\begin{tabular}{|l|}
2312 \\
\end{tabular}} \\
\hline \multicolumn{3}{|l|}{2313} \\
\hline \multicolumn{3}{|l|}{2314} \\
\hline \multicolumn{3}{|l|}{2315} \\
\hline \begin{tabular}{|l|}
2316 \\
\end{tabular} & & \\
\hline 2317 & & \\
\hline 2318 & & \\
\hline
\end{tabular}




\begin{tabular}{|c|c|c|}
\hline 1 & $\begin{array}{ll} & \text { SAMP_SITE_DESC } \\
\end{array}$ & SITE_ELEVATION \\
\hline \multicolumn{3}{|c|}{ 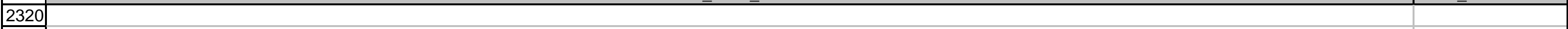 } \\
\hline \multicolumn{3}{|l|}{2321} \\
\hline \multicolumn{3}{|l|}{ 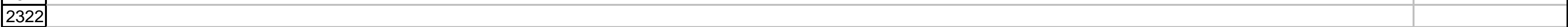 } \\
\hline \multicolumn{3}{|l|}{2323} \\
\hline \multicolumn{3}{|l|}{2324} \\
\hline \multicolumn{3}{|l|}{2325} \\
\hline \multicolumn{3}{|l|}{2326} \\
\hline 2327 & TLD only & 100.09 \\
\hline 2328 & TLD only & 98.4 \\
\hline \multicolumn{3}{|l|}{2329} \\
\hline 2330 & & 125.664 \\
\hline 2331 & & 123.352 \\
\hline 2332 & & 122.832 \\
\hline 2333 & & 119.7 \\
\hline 2334 & & 115.7 \\
\hline \multicolumn{3}{|c|}{\begin{tabular}{|l|l|l|l|l}
2335 & Node: S-A2-14 \\
\end{tabular}} \\
\hline 2336 & Node: S-A2-15 & \\
\hline 2337 & Node: S-A3-1 & \\
\hline 2338 & Node: S-A3-2 & \\
\hline \multicolumn{3}{|c|}{2340 Node: S-A3-5 } \\
\hline \multicolumn{3}{|c|}{2341 COMPOSITE SAMPLE FROM NODES:E7-1, E7-3, E7-4, E7-5, E7-8, E7-11. } \\
\hline \multicolumn{3}{|c|}{2342 COMPOSITE SAMPLE FROM NODES:D3-1, D3-2, D3-4, D3-5, D3-9, D3-11. } \\
\hline \multicolumn{3}{|c|}{2343 Node: S-A3-9 } \\
\hline \multicolumn{3}{|c|}{2344 COMPOSITE SAMPLE FROM NODES:D4-3, D4-4, D4-7, D4-9, D4-12, D4-13. } \\
\hline \multicolumn{3}{|c|}{\begin{tabular}{|l|l|}
2345 & Node: S-A3-11 \\
\end{tabular}} \\
\hline \multicolumn{3}{|c|}{2346 Node: S-A4-3 } \\
\hline \multicolumn{3}{|c|}{2347 Node: S-A4-4 } \\
\hline \multicolumn{3}{|c|}{2348 COMPOSITE SAMPLE FROM NODES:D5-1, D5-2, D5-5, D5-7, D5-13, D5-15. } \\
\hline \multicolumn{3}{|c|}{\begin{tabular}{l|l}
2349 & Node: S-A4-7
\end{tabular}} \\
\hline \multicolumn{3}{|c|}{ 2350 Node: S-A4-9 } \\
\hline \multicolumn{3}{|c|}{2351 COMPOSITE SAMPLE FROM NODES:D6-1, D6-10, D6-12, D6-13, D6-13A, D6-15, D6-16. } \\
\hline \multicolumn{3}{|c|}{2352 Node: S-A4-12 } \\
\hline \multicolumn{3}{|c|}{2353 Node: S-A4-13 } \\
\hline \multicolumn{3}{|c|}{\begin{tabular}{|l|l|l|}
2354 & CCOMPOSITE SAMPLE FROM NODES:E8-2, E8-3, E8-4, E8-8, E8-12, E8-13. \\
\end{tabular}} \\
\hline \multirow{2}{*}{\begin{tabular}{|l|}
2355 \\
2356 \\
\end{tabular}} & COMPOSITE SAMPLE FROM NODES:E9-3, E9-4, E9-5, E9-10, E9-14, E9-16. & \\
\hline & Node: S-B5-1 & \\
\hline \begin{tabular}{|l|}
2356 \\
2357 \\
\end{tabular} & Node: S-B5-2 & \\
\hline
\end{tabular}

2335 Node: S-A2-14

2336 Node: S-A2-15

2339 Node: S-A3-4

Node: S-A3- 5

2342 COMPOSITE SAMPLE FROM NODES:D3-1, D3-2, D3-4, D3-5, D3-9, D3-11.

Node: S-A3-9

COMPOSITE SAMPLE FROM NODES:D5-1, D5-2, D5-5, D5-7, D5-13, D5-15.

2349 Node: S-A4-7

2352 Node: S-A4-12

2357 Node: S-B5-2 


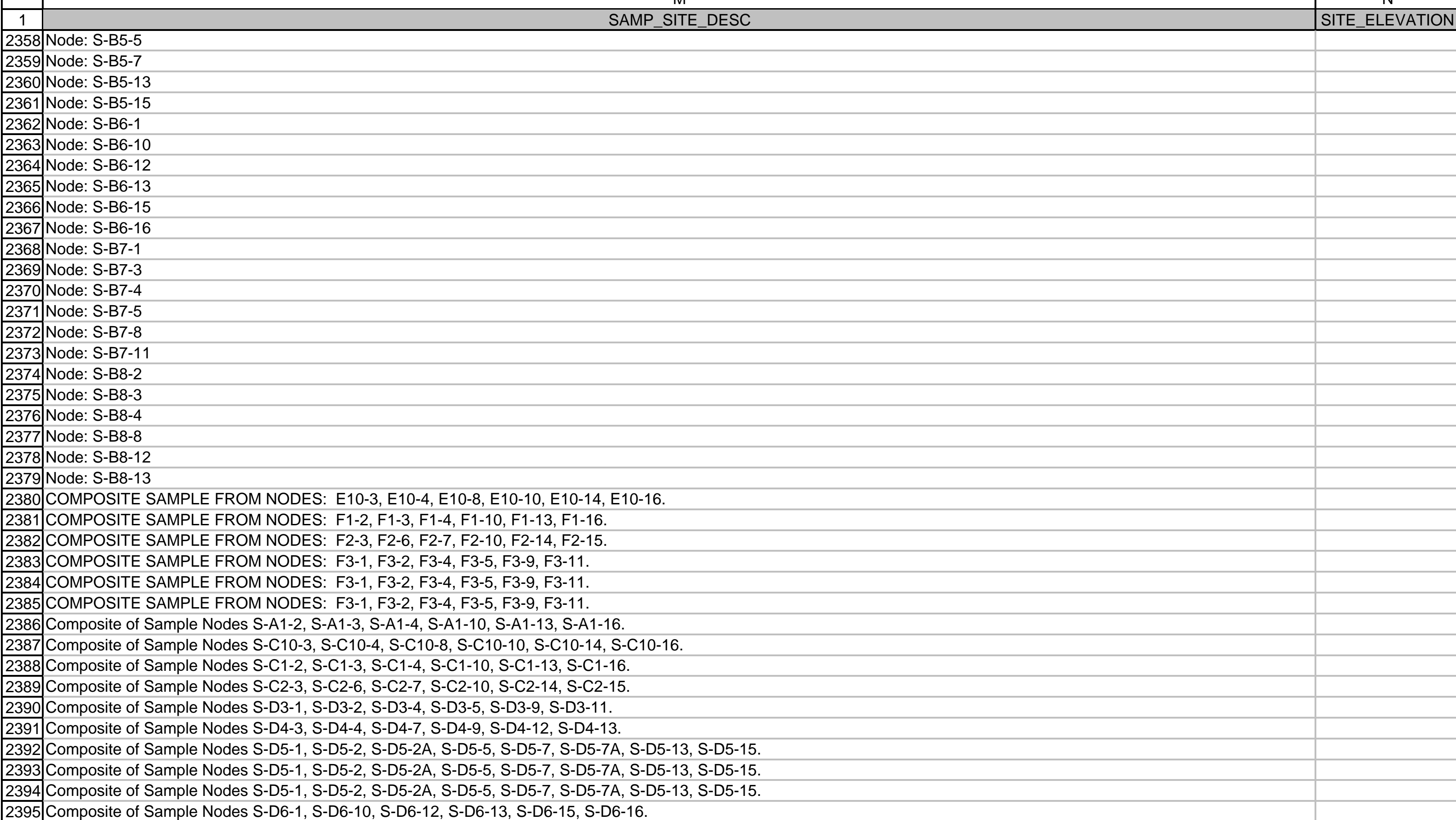

\begin{tabular}{l|l}
2395 & Composite of Sample Nodes S-D6-1, S-D6-10, S-D6-12, S-D6-13, S-D6-15, S-D6-16.
\end{tabular} 
2396 Composite of Sample Nodes S-E7-1, S-E7-3, S-E7-4, S-E7-5, S-E7-8, S-E7-11.

2397 Composite of Sample Nodes S-E8-2, S-E8-3, S-E8-4, S-E8-8, S-E8-12, S-E8-13.

2398 Composite of Sample Nodes S-E9-3, S-E9-4, S-E9-5, S-E9-10, S-E9-14, S-E9-16.

2399 Composite of Sample Nodes S-E10-3, S-E10-4, S-E10-8, S-E10-10, S-E10-14, S-E10-16.

2400 100-F-19 Shallow Zone

\begin{tabular}{|l|l|l}
2401 & $100-F-19$ & Shallow Zone
\end{tabular}

2402 100-F-19 Shallow Zone

2403 Composite from sample

\begin{tabular}{|l|l}
2404 & $100-F-19$ \\
2 Shallow Zone
\end{tabular}

2405 100-F-19 Shallow Zone

2406 100-F-19 Shallow Zone

2407 100-F-19 Shallow Zone

2408 100-F-19 Shallow Zone

2409 100-F-19 Shallow Zone

\begin{tabular}{l|l}
2410 & $100-F-19$ Shallow Zone
\end{tabular}

\begin{tabular}{|l|l|}
2411 & $100-F-19$ \\
\hline 2412 & Shallow Zone
\end{tabular}

2412 100-F-19 Shallow Zone

2413 100-F-19 Shallow Zone

\begin{tabular}{|l|l|l|l}
2414 & $100-F-19$ & Shallow Zone
\end{tabular}

2415 100-F-19 Shallow Zone

2416 100-F-19 Shallow Zone

2417 100-F-19 Shallow Zone

2418 Composite from sample nodes D-A2-3, D-A2-6, D-A2-7, D-A2-15

2419 Composite from sample nodes D-A3-1, D-A3-2, D-A3-4, D-A3-11

2420 Composite from sample nodes D-B5-1, D-B5-5, D-B5-7, D-B5-15

2421 Composite from sample nodes D-B4-3, D-B4-4, D-B4-7, D-B4-12

2422 Composite from sample nodes D-B6-1, D-B6-10, D-B6-13, D-B6-15

2423 Composite from sample nodes D-C7-3, D-C7-4, D-C7-5, D-C7-11

2424 Composite from sample nodes D-C8-3, D-C8-4, D-C8-8, D-C8-13

\begin{tabular}{|l|l}
2425 & Composite from sample nodes D-C9-3, D-C9-4, D-C9-10, D-C9-16
\end{tabular}

2426 Composite from sample nodes D-D10-4, D-D10-10, D-D10-14, D-D10-16

2427 Composite from sample nodes D-D1-3, D-D1-4, D-D1-10, D-D1-16

2428 Composite from sample nodes D-D2-3, D-D2-6, D-D2-7, D-D2-15

2429 Composite from sample nodes D-E3-1, D-E3-2, D-E3-4, D-E3-11

2430 Composite from sample nodes D-E4-3, D-E4-4, D-E4-7, D-E4-12

2431 100-F-19 Shallow Zone

2432 Composite from sample nodes D-E5-1, D-E5-5, D-E5-7, D-E5-15

\begin{tabular}{|l|l}
2433 & Composite from sample nodes D-B6-1, D-B6-10, D-B6-13, D-B6-15
\end{tabular} 
2434 Composite from sample nodes D-B6-1, D-B6-10, D-B6-13, D-B6-15

2435 Composite from sample nodes D-B6-1, D-B6-10, D-B6-13, D-B6-15

2436 100-F-19 Shallow Zone

2437 Node: S-B8-4

\begin{tabular}{l|l}
2438 & $100-F-19$ Shallow Zone
\end{tabular}

2439 Node: S-B8-8

2440 100-F-19 Shallow Zone

2441 100-F-19 Shallow Zone

2442 Node: S-B8-12

2443 100-F-19 Shallow Zone

2444 100-F-19 Shallow Zone

2445 Node: S-B8-13

2446 Node: S-D3-1

2447 Node: S-D3-2

2448 Node: S-D3-4

2449 Node: S-D3-5

2450 Node: S-D3-9

2451 Node: S-D3-11

2452 Node: S-D4-3

2453 Node: S-D4-4

2454 Node: S-D4-7

2455 Node: S-D4-9

2456 Node: S-D4-12

2457 Shallow Zone

2458 Node: S-D5-1

2459 Shallow Zone

2460 Composite from sample nodes S-D5-2 AND S-D5-2A

2461 Node: S-D4-13

2462 Ash Pit

2463 Ash Pit

2464 Ash Pit

2465 Ash Pit

2466 Node: S-E8-12

2467 Node: S-E8-13

2468 Node: S-E9-3

2469 Node: S-E9-4

2470 Node: S-E9-5

2471 Pipeline \#16 
2473 Node: S-E9-10

2474 116-N-3 Pipeline Valves

2475 116-N-3 Pipeline Valves

2476 Node: S-E9-14

2477 Node: S-E9-16

2478 Node: S-D5-5

2479 Composite of Nodes: S-D5-7 and S-D5-7A

2480 Node: S-D5-13

2481 Node: S-D5-15

2482 Phase I Overburden Piles

2483 Phase I Overburden Piles

2484 Phase I Overburden Piles

2485 Phase I Overburden Piles

2486

\begin{tabular}{|l|l|}
\hline 2487 & Phase I Overburden Piles \\
\hline 2488 & Phase I Overburden Piles
\end{tabular}

2488 Phase I Overburden Piles

2489 Phase I Overburden Piles

2490 Phase I Overburden Piles

\begin{tabular}{|l|l|}
\hline 2491 & Phase I Overburden Piles \\
\hline 2492 & Phase I Oveburden Ples
\end{tabular}

2492 Phase I Overburden Piles

2493 Phase I Overburden Piles

\begin{tabular}{|l|l|}
\hline 2494 & Phase I Overburden Piles \\
\hline 2495 & Phase I Overburden Piles
\end{tabular}

2495 Phase I Overburden Piles

2496 Phase I Overburden Piles

2497 Phase I Overburden Piles

\begin{tabular}{|l|l}
\hline 2498 & Phase I Overburden Piles \\
\hline 2499 & Phase I Overburden Piles
\end{tabular}

2499 Phase I Overburden Piles

2500 Phase I Overburden Piles

\begin{tabular}{|l|l}
\hline 2501 & Phase I Overburden Piles
\end{tabular}

2502 Phase I Overburden Piles

2503 Phase I Overburden Piles

2504 Phase I Overburden Piles

2505 Phase I Overburden Piles

2506 Phase I Overburden Piles

2507 Phase I Overburden Piles

2508 Phase I Overburden Piles

\begin{tabular}{|l|l|}
\hline 2509 & Phase I Overburden Piles \\
\hline
\end{tabular}

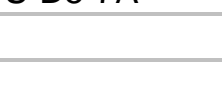

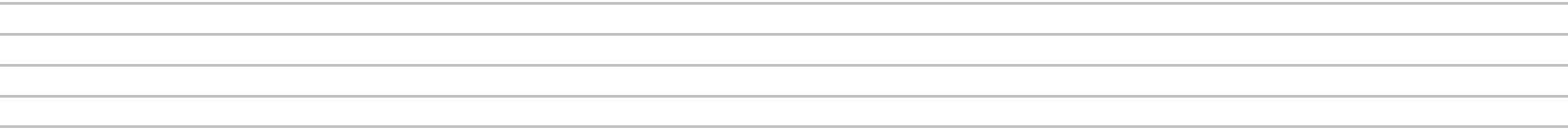

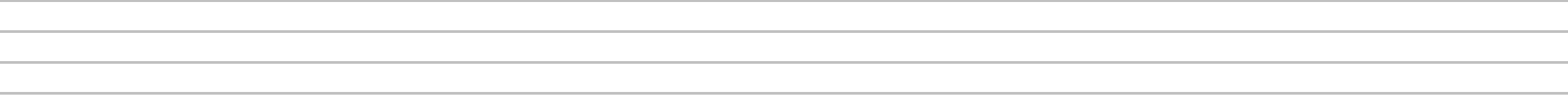

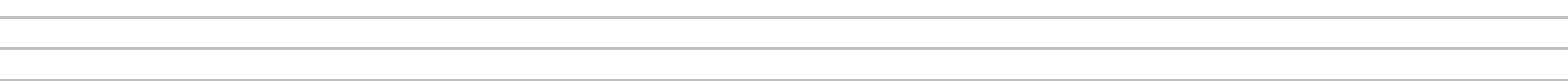

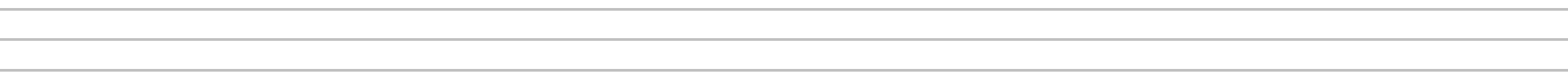

(20)

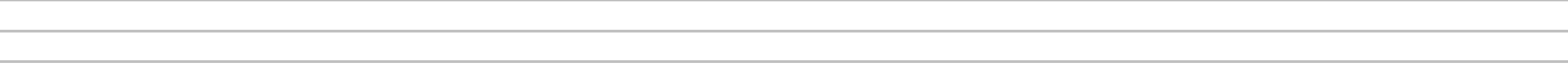

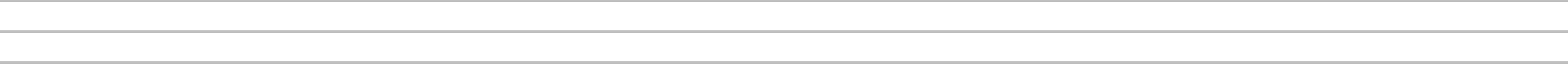

(20)

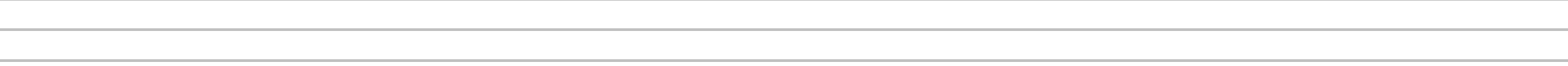

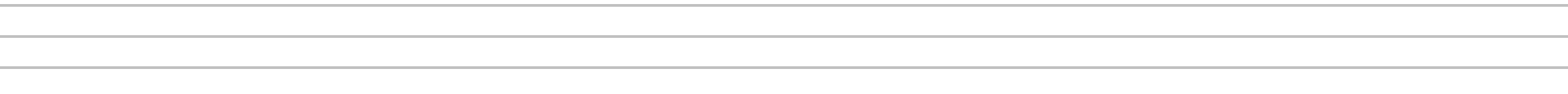

Page 380 
2511 Phase I Overburden Piles 2512 Phase I Overburden Piles

2513 Phase I Overburden Piles

2514 Phase I Overburden Piles

2515 Phase I Overburden Piles

2516 Phase I Overburden Piles

2517 Phase I Overburden Piles

2518 Phase I Overburden Piles

2519 Phase I Overburden Piles

2520 Phase I Overburden Piles

2521 Phase I Overburden Piles

2522 Phase I Overburden Piles

2523 Phase I Overburden Piles

2524 Phase I Overburden Piles

2525 Phase I Overburden Piles

2526 Phase I Overburden Piles

2527 Phase I Overburden Piles

2528 Phase I Overburden Piles

2529 Phase I Overburden Piles

2530 Phase I Overburden Piles

2531 Node: S-A3-9

2532 Node: S-A3-11

2533 Node: S-B5-1

2534 Node: S-B5-2

2535 Node: S-B5-5

2536 Node: S-B5-7

2537 Node: S-B5-13

2538 Node: S-B5-15

2539 Node: S-B6-1

2540 Node: S-B6-10

2541 Node: S-B6-12

2542 Node: S-B6-13

2543 Node: S-B6-15

2544 Node: S-B6-16

2545 Node: S-B7-1

2546 Node: S-B7-3

2547 Node: S-B7-4 
2576 Composite from sample nodes D-A1-3, D-A1-4, D-A1-10, D-A1-16

2577 Composite from sample nodes D-A2-3, D-A2-6, D-A2-7, D-A2-15

2578 Composite from sample nodes D-A3-1, D-A3-2, D-A3-4, D-A3-11

2579 Composite from sample nodes D-B4-3, D-B4-4, D-B4-7, D-B4-12

2580 Composite from sample nodes D-B5-1, D-B5-5, D-B5-7, D-B5-15

2581 Composite from sample nodes D-B6-1, D-B6-10, D-B6-13, D-B6-15

2582 Composite from sample nodes D-A2-3, D-A2-6, D-A2-7, D-A2-15

2583 Composite from sample nodes D-A2-3, D-A2-6, D-A2-7, D-A2-15

2584 Composite from sample nodes D-A2-3, D-A2-6, D-A2-7, D-A2-15

2585 Node: A-A1-2 
\begin{tabular}{|l|l}
2608 & From envelope of outfall sturcture \\
\hline 2609 & Composito
\end{tabular}

2609 Composite sample at overburden of pipeline \#4.

2610 Composite sample of soils removed from the excavation envelope of pipeline \#4.

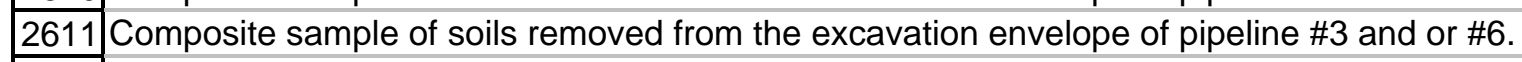

2612 sample from excavatio associated with outfall 132-B-6

2613 Sample from excavation associated with contamination on stockpile 11.

2614 From 116-B-7 outfall structure

2615 Sample from excavation associated with outfall of 132-B-6

2616 Sample from excavation associated with outfall of 132-B-6

2617 Sample from excavation associated with outfall of 132-B-6

2618 Sample from excavation associated with outfall and pipeline \#4.

2619 Sample from excavation associated with pipeline \#3.

2620 Composite sample of soils removed from west side wall of the excavation of pipelines \#3 and \#4.

2621 Composite sample of soils removed from west side wall of the excavation of pipelines \#3 and \#4.

2622 Composite sample of soils removed from west side wall of the excavation of pipelines \#3 and \#4.

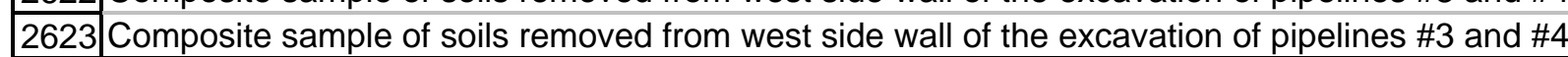


2624 Composite sample of soils removed from west side wall of the excavation of pipelines \#3 and \#4.

2625 Composite sample of soils removed from west side wall of the excavation of pipelines \#3 and \#4.

2626 Composite sample of soils removed from west side wall of the excavation of pipelines \#3 and \#4.

2627 Composite sample of soils removed from west side wall of the excavation of pipelines \#3 and \#4.

2628 Composite sample of soils removed from west side wall of the excavation of pipelines \#3 and \#4.

2629 Composite sample of soils removed from west side wall of the excavation of pipelines \#3 and \#4.

2630 Composite sample of soils removed from west side wall of the excavation of pipelines \#3 and \#4.

2631 Sample of soils removed from west side wall of the excavation of pipelines \#4.

2632 116-N-3 By-Pass Trench (at Original Main Roadway)

2633 116-N-3 Pipeline Trench (Near Camera Man-hole \#2)

2634 100-C-6

$2635100-\mathrm{C}-6$

2636

2637 Potholes

2638 Potholes

2639 Potholes

2640 Potholes

2641 Potholes

2642

\begin{tabular}{|l|l|l|}
\hline 2643 & Composite from nodes: S-B5-1, S-B5-2, S-B5-5, S-B5-7, S-B5-13, S-B5-15 \\
\hline 2644 & Decon Pad Sump
\end{tabular}

2644 Decon Pad Sump

2645 Decon Pad Sump

2646 116-N-3 Trench Shallow Zone

2647 116-n-3 Trench Shallow Zone

2648 116-N-3 Decon-Pad Sump

2649 AD-3 Test pit for the 216-A-29 Ditch

2650 AD-3 Test pit for the 216-A-29 Ditch

2651 AD-3 Test pit for the 216-A-29 Ditch

2652 AD-3 Test pit for the 216-A-29 Ditch

2653 AD-1 Test pit for the 216-A-29 Ditch

2654 AD-1 Test pit for the 216-A-29 Ditch

2655 AD-1 Test pit for the 216-A-29 Ditch

2656 AD-1 Test pit for the 216-A-29 Ditch

2657 AD-2 Test pit for the 216-A-29 Ditch

2658 AD-2 Test pit for the 216-A-29 Ditch

2659 AD-2 Test pit for the 216-A-29 Ditch

\begin{tabular}{|l|l}
2660 & AD-2 Test pit for the 216-A-29 Ditch \\
\hline 2661 & AD-2 Test pit for the $216-A-29$ Ditch \\
\hline
\end{tabular}

\begin{tabular}{|l|l|l}
2661 & AD-2 Test pit for the 216-A-29 Ditch
\end{tabular}

(n) 
2663 BT-2 Test Pit from the 216-B-63 Ditch

2664 BT-2 Test Pit from the 216-B-63 Ditch

2665 AD-3 Test pit for the 216-A-29 Ditch

2666 AD-1 Test pit for the 216-A-29 Ditch

2667 AD-2 Test pit for the 216-A-29 Ditch

2668 BT-2 Test Pit from the 216-B-63 Ditch

2669 AD-3 Test pit for the 216-A-29 Ditch

2670 AD-1 Test pit for the 216-A-29 Ditch

2671 AD-2 Test pit for the 216-A-29 Ditch

2672 BT-2 Test Pit from the 216-B-63 Ditch

2673 126-F-1

2674 126-F-1

2675 126-F-1

2676 126-F-1

2677 126-F-1

2678 126-F-1

2679 126-F-1

2680 126-F-1

2681 126-F-1

2682 126-F-1

2683

2685 Potholes around the perimeter of 116-N-1 Crib \& Trench/N1PH-13-L1

2686 Potholes around the perimeter of 116-N-1 Crib \& Trench/N1PH-13-L2

2687 Potholes around the perimeter of 116-N-1 Crib \& Trench/N1PH-13-L3

2688 Potholes around the perimeter of 116-N-1 Crib \& Trench/N1PH-13-L4

2689 Potholes around the perimeter of 116-N-1 Crib \& Trench/N1PH-13-L5

2690 Potholes around the perimeter of 116-N-1 Crib \& Trench/N1PH-13-L5

2691 Potholes around the perimeter of 116-N-1 Crib \& Trench/N1PH-12-L5

2692 Potholes around the perimeter of 116-N-1 Crib \& Trench/N1PH-12-L1

2693 Potholes around the perimeter of 116-N-1 Crib \& Trench/N1PH-14-L5

2694 Potholes around the perimeter of 116-N-1 Crib \& Trench/N1PH-12-L2

2695 Potholes around the perimeter of 116-N-1 Crib \& Trench/N1PH-12-L3

2696 Potholes around the perimeter of 116-N-1 Crib \& Trench/N1PH-12-L4

2697 Potholes around the perimeter of 116-N-1 Crib \& Trench/N1PH-12-L5

2698 Potholes around the perimeter of 116-N-1 Crib \& Trench/N1PH-15-L1

\begin{tabular}{|l|l|l|}
2699 & Potholes around the perimeter of 116-N-1 Crib \& Trench/N1PH-15-L2
\end{tabular} 
2701 Potholes around the perimeter of 116-N-1 Crib \& Trench/N1PH-15-L4 2702 Potholes around the perimeter of 116-N-1 Crib \& Trench/N1PH-15-L5 2703 Potholes around the perimeter of 116-N-1 Crib \& Trench/N1PH-14-L1

2704 Potholes around the perimeter of 116-N-1 Crib \& Trench/N1PH-14-L2

2705 Potholes around the perimeter of 116-N-1 Crib \& Trench/N1PH-14-L3

2706 Potholes around the perimeter of 116-N-1 Crib \& Trench/N1PH-14-L4

2707 Potholes around the perimeter of 116-N-1 Crib \& Trench/N1PH-14-L5

\begin{tabular}{|l|l|}
\hline 2708 & Overburden stockpile \#11 \\
\hline 2709 & Overburden stockpile \#11
\end{tabular}

2709 Overburden stockpile \#11

2710 Overburden stockpile \#11

2711 Hot spot in pipelines 3 \& 4

2712 Potholes around the perimeter of 116-N-1 Crib \& Trench/N1PH-01-L1

2713 Potholes around the perimeter of 116-N-1 Crib \& Trench/N1PH-01-L2

2714 Potholes around the perimeter of 116-N-1 Crib \& Trench/N1PH-01-L3

2715 Potholes around the perimeter of 116-N-1 Crib \& Trench/N1PH-01-L4

2716 Potholes around the perimeter of 116-N-1 Crib \& Trench/N1PH-01-L5

2717 Potholes around the perimeter of 116-N-1 Crib \& Trench/N1PH-01-L5

2718 Potholes around the perimeter of 116-N-1 Crib \& Trench/N1PH-02-L1

2719 Potholes around the perimeter of $116-\mathrm{N}-1$ Crib \& Trench/N1PH-02-L2

2720 Potholes around the perimeter of 116-N-1 Crib \& Trench/N1PH-02-L3

2721 Potholes around the perimeter of 116-N-1 Crib \& Trench/N1PH-02-L4

\begin{tabular}{|l|l}
2722 & Potholes around the perimeter of 116-N-1 Crib \& Trench/N1PH-02-L5
\end{tabular}

2723 Potholes around the perimeter of 116-N-1 Crib \& Trench/N1PH-03-L1

2724 116-N-3 By-Pass Trench (at Original Main Roadway)

$2725132-\mathrm{B}-6$

$2726132-\mathrm{C}-2$

2727 132-C-2

2728 Hot spot in pipelines $3 \& 4$

2729 Potholes around the perimeter of 116-N-1 Crib \& Trench/N1PH-02-L5

2730 Potholes around the perimeter of 116-N-1 Crib \& Trench/N1PH-03-L2

2731 Potholes around the perimeter of 116-N-1 Crib \& Trench/N1PH-03-L3

2732 Potholes around the perimeter of 116-N-1 Crib \& Trench/N1PH-03-L4

2733 Potholes around the perimeter of 116-N-1 Crib \& Trench/N1PH-03-L5

2734 Potholes around the perimeter of 116-N-1 Crib \& Trench/N1PH-03-L5

2735 Potholes around the perimeter of 116-N-1 Crib \& Trench/N1PH-04-L1

2736 Potholes around the perimeter of 116-N-1 Crib \& Trench/N1PH-04-L2

2737 Potholes around the perimeter of 116-N-1 Crib \& Trench/N1PH-04-L3

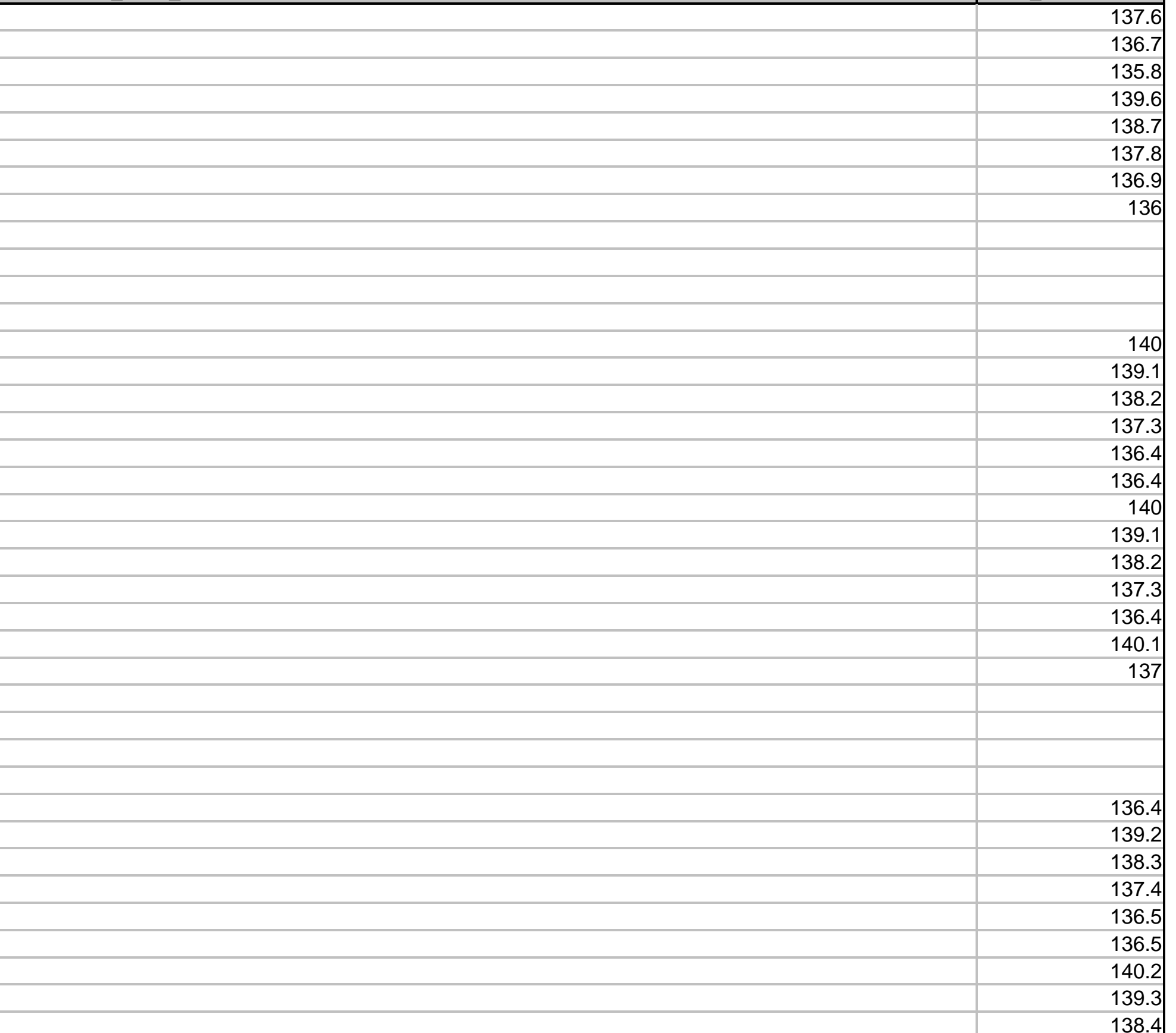


2741 Transition Zone-01

2742 Node: SC10-3

2743 116-N-3 Decon-Pad Sump

2744 NODE: SC10-4

2745 NODE: SC10-8

2746 NODE: SC10-10

2747 NODE: SC10-14

2748 NODE: SC10-16

2749 NODE: SC1-2

2750 NODE: SC1-3

2751 NODE: SC1-4

2752 NODE: SC1-10

2753 NODE: SC1-13

2754 NODE: SC1-16

2755 NODE: SC2-63

2756 NODE: SC2-6

2757 NODE: SC2-7

2758 NODE: SC2-10

2759 NODE: SC2-14

2760 NODE: SC2-15

2761 NODE: SD3-1

2762 NODE: SD3-2

2763 NODE: SD3-4

2764 NODE: SD3-5

2765 NODE: SD3-9

2766 NODE: SD3-11

2767 NODE: SD4-3

2768 NODE: SD4-4

2769 NODE: SD4-7

2770 NODE: SD4-9

2771 NODE: SD4-12

2772 NODE: SD4-13

2773 NODE: SD5-1

2774 NODE: SD5-2

\begin{tabular}{|l|l|}
2775 & NODE: SD5-5
\end{tabular} 


\begin{tabular}{|c|c|c|}
\hline 1 & $\begin{array}{ll} & \text { SAMP_SITE_DESC } \\
\end{array}$ & SITE_ELEVATION \\
\hline 2776 & NODE: SD5-7 & 127.433 \\
\hline 2777 & NODE: SD5-13 & 131.295 \\
\hline 2778 & NODE: SD5-15 & 129.894 \\
\hline 2779 & NODE: SD6-1 & 129.004 \\
\hline 2780 & NODE: SD6-10 & 131.327 \\
\hline 2781 & NODE: SD6-12 & 132.741 \\
\hline 2782 & NODE: SD6-13 & 131.886 \\
\hline 2783 & NODE: SD6-15 & 132.365 \\
\hline 2784 & NODE: SD6-16 & 133.34 \\
\hline 2786 & NODE: SB6-13 & 128.72 \\
\hline 2787 & NODE: SB6-15 & 129 \\
\hline 2788 & NODE: SB6-16 & 129.123 \\
\hline 2789 & NODE: SB7-1 & 130.267 \\
\hline 2790 & NODE: SB7-3 & 126.651 \\
\hline 2791 & NODE: SB7-4 & 127.096 \\
\hline 2792 & NODE: SB7-5 & 127.487 \\
\hline 2793 & NODE: SB7-8 & 128.615 \\
\hline 2794 & NODE: SB7-11 & 126.391 \\
\hline 2796 & NODE: SB8-3 & 129.995 \\
\hline 2797 & NODE: SB8-4 & 130.169 \\
\hline 2798 & NODE: SB8-8 & 129.479 \\
\hline 2799 & NODE: SB8-12 & 129.167 \\
\hline 2800 & NODE: SB8-13 & 129.282 \\
\hline 2801 & NODE: SC9-3 & 126.695 \\
\hline 2802 & NODE: SC9-4 & 127.791 \\
\hline 2803 & NODE: SC9-5 & 125.176 \\
\hline 2804 & NODE: SC9-10 & 125.57 \\
\hline 2805 & NODE: SC9-14 & 125.532 \\
\hline 2806 & NODE: SC9-16 & 127.524 \\
\hline 2807 & NODE: S-A1-2 & 130.335 \\
\hline 2808 & NODE: SB7-1 & 130.267 \\
\hline 2809 & NODE: S-A1-3 & 129.726 \\
\hline 2810 & NODE: S-A1-4 & 128.676 \\
\hline 2811 & NODE: S-A1-10 & 130.293 \\
\hline 2812 & NODE: S-A1-13 & 130.557 \\
\hline 2813 & NODE: S-A1-16 & 127.688 \\
\hline
\end{tabular}


2842 Discovery areas DA-100BC-001 a.k.a. "Al"

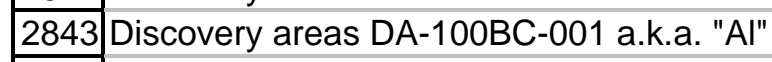

\begin{tabular}{|l|l}
2844 & Discovery areas DA-100BC-002 a.k.a. "George"
\end{tabular}

2845 From off of pipeline 20 and 42

\begin{tabular}{|l|l|}
2846 & From off of pipeline 20 and 42
\end{tabular}

2847 From plume 100BC-4-1/from northern extreme of pipeline 4

2848 From plume 100BC-4-10/just north of the expansion box excavation

2849 Potholes around the perimeter of 116-N-1 Crib \& Trench/N1PH-15-L5

\begin{tabular}{|l|l|l}
2850 & $116-N-3$ By-pass \\
\hline 2851 & $116-N-1$ Trenching
\end{tabular} 


\begin{tabular}{|c|c|c|}
\hline 1 & $\begin{array}{l}\text { SAMP_SITE_DESC } \\
\end{array}$ & SITE_ELEVATION \\
\hline 2852 & 116-N-1 Trenching Soil Samples & 140 \\
\hline 2853 & Test Pit & \\
\hline 2854 & Northern end of 116-N-1 Trench Potholes & 137.7 \\
\hline 2855 & Northern End of 116-N-1 Trench Potholes & 138.5 \\
\hline 2856 & Northern End of 116-N-1 Trench Potholes & 137 \\
\hline 2857 & Middle section of Trench Pothole 18 & 138.5 \\
\hline \begin{tabular}{|l|l|}
2858 \\
\end{tabular} & Middle section of trench Pothole 18 & 137.6 \\
\hline 2859 & Middle section of trench Pothole 18 & 137 \\
\hline 2860 & Test Pit & \\
\hline 2861 & Test Pit & \\
\hline 2862 & Test Pit & \\
\hline 2863 & Test Pit & \\
\hline 2864 & Test Pit & \\
\hline 2865 & & \\
\hline 2866 & 116-N-3 By-pass & \\
\hline 2867 & 116-N-1 Trenching Soil Samples & \\
\hline 2868 & 116-N-1 Trenching Soil Samples & \\
\hline 2869 & 116-N-1 Trenching Soil Samples & \\
\hline 2870 & 116-N-1 Trenching Soil Samples & \\
\hline 2871 & 116-N-1 Trenching Soil Samples & \\
\hline 2872 & 116-N-1 Trenching Soil Samples & \\
\hline 2873 & & 119.3 \\
\hline 2874 & & 118.314 \\
\hline 2875 & & 119.766 \\
\hline 2876 & & 121.145 \\
\hline 2877 & & \\
\hline 2878 & & \\
\hline 2879 & & \\
\hline 2880 & & \\
\hline 2881 & & \\
\hline 2882 & & \\
\hline 2883 & 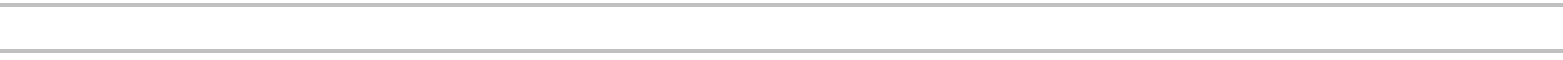 & \\
\hline 2884 & Stockpile 11, Lift 3 & \\
\hline 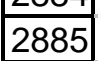 & North end of pipeline 4 & \\
\hline 2886 & & \\
\hline 2887 & 116-N-3 Trench Plume Sidewall (Plume \#3) & \\
\hline 2888 & 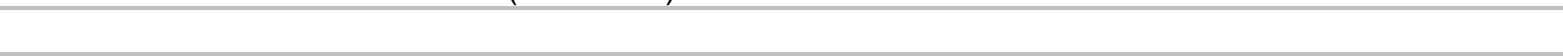 & \\
\hline
\end{tabular}

2860 Test Pit

Test Pit

2867 116-N-1 Trenching Soil Samples

2868 116-N-1 Trenching Soil Samples

-1 Trenching Soil Samples

2870 116-N-1 Trenching Soil Samples

(ching Soil Samples 


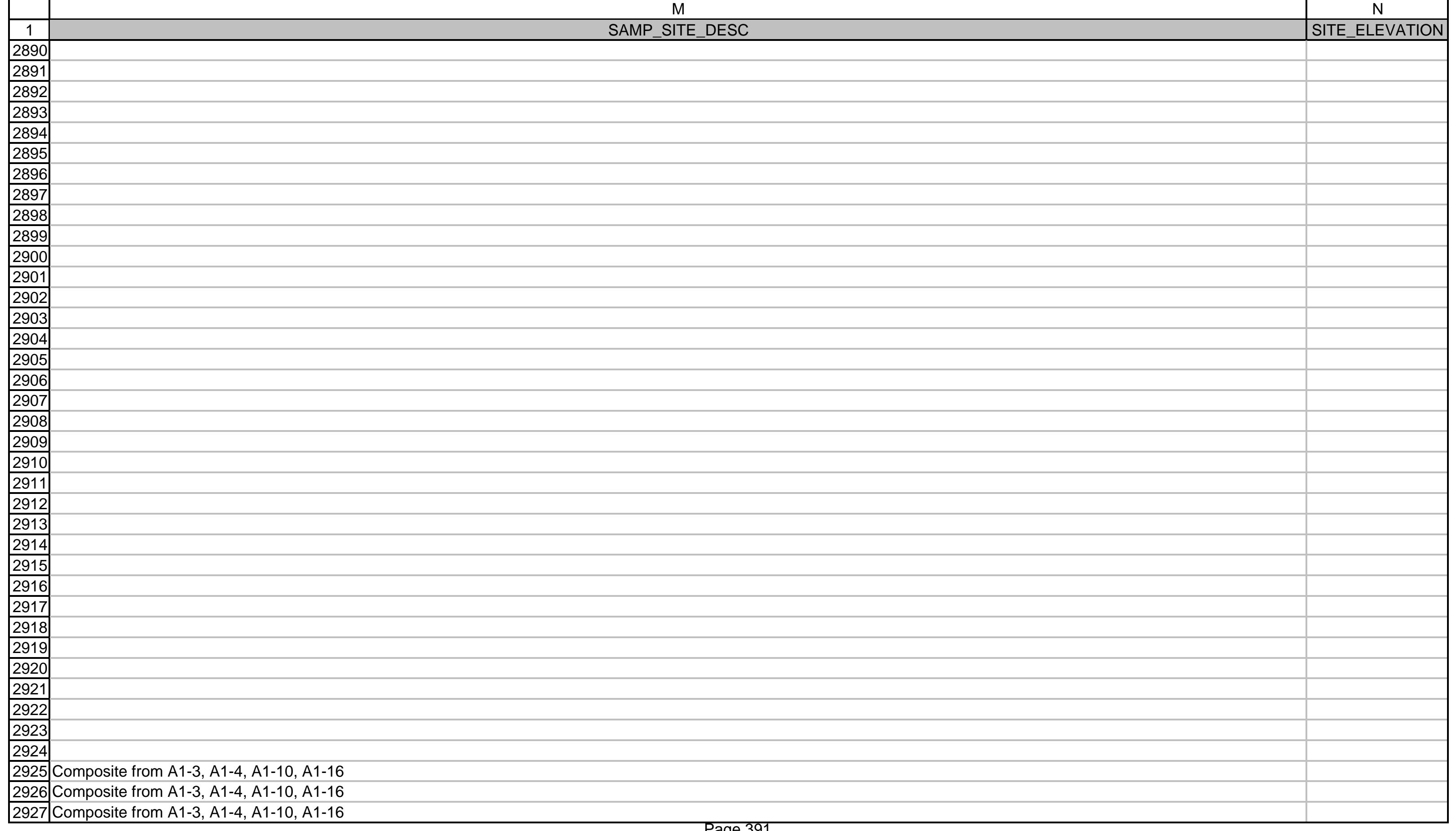


2928 Composite from A2-3, A2-6, A2-7, A2-15

2929 Composite from A3-1, A3-2, A3-4, A3-11

2930 Composite from A4-3, A4-4, A4-7, A4-12

2931 Composite from B5-1, B5-5, B5-7, B5-15

\begin{tabular}{|l|l|}
2932 & Composite from B6-1, B6-10, B6-13, B6-15 \\
\hline
\end{tabular}

\begin{tabular}{|l|l|}
2933 & Composite from B7-3, B7-4, B7-5, B7-11 \\
\hline
\end{tabular}

2934 Composite from B8-3, B8-4, B8-8, B8-13

2935 Composite from C9-3, C9-4, C9-10, C9-16

2936 Composite from C10-4, C10-10, C10-14, C10-16

2937 Composite from C1-3, C1-4, C1-10, C1-16

2938 Composite from C2-3, C2-6, C2-7, C2-15

2952 Phase I Overburden Piles/Lewis Canal North Lateral and partial South Lateral

2953 Phase I Overburden Piles/Lewis Canal North Lateral and partial South Lateral

2954 Phase I Overburden Piles/Lewis Canal North Lateral and partial South Lateral

2955 Phase I Overburden Piles/Lewis Canal North Lateral and partial South Latera

2956 Phase I Overburden Piles/Lewis Canal North Lateral and partial South Latera

2957 Phase I Overburden Piles/Lewis Canal North Lateral and partial South Lateral

2958 Phase I Overburden Piles/Lewis Canal North Lateral and partial South Latera

2959 Phase I Overburden Piles/Lewis Canal North Lateral and partial South Latera

2960 Phase I Overburden Piles/Lewis Canal North Lateral and partial South Lateral

2961 Phase I Overburden Piles/Lewis Canal North Lateral and partial South Lateral

2962 Phase I Overburden Piles/Lewis Canal North Lateral and partial South Latera

2963 Phase I Overburden Piles/Lewis Canal North Lateral and partial South Lateral

2964 Phase I Overburden Piles/Lewis Canal North Lateral and partial South Lateral

\begin{tabular}{|l|l|l|}
2965 & Phase I Overburden Piles/Lewis Canal North Lateral and partial South Lateral
\end{tabular} 
(n) 
3042 Phase I Overburden Piles/Lewis Canal North Lateral and partial South Lateral

3043 Phase I Overburden Piles/Lewis Canal North Lateral and partial South Latera 3044 Phase I Overburden Piles/Lewis Canal North Lateral and partial South Lateral

3045 FROM DEEP ZONE

\begin{tabular}{|l|l|l|}
\hline 3046 & FROM DEEP ZONE \\
\hline 3047 & FROM SHALLOW ZONE & \\
\hline
\end{tabular}

\begin{tabular}{|l|l|l|}
\hline 3047 & FROM SHALLOW ZONE \\
\hline 3048 & FROM DEEP ZONE
\end{tabular}

3048 FROM DEEP ZONE

3049 FROM SHALLOW ZONE

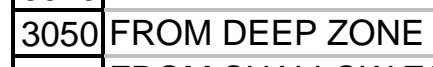

3051 FROM SHALLOW ZONE

3052 FROM SHALLOW ZONE

3053 FROM SHALLOW ZONE

3054 FROM SHALLOW ZONE

3055 FROM DEEP ZONE

3056 FROM SHALLOW ZONE

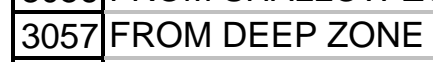

3058 FROM SHALLOW ZONE

3059 FROM SHALLOW ZONE

\begin{tabular}{|l|l}
3060 & FROM DEEP ZONE \\
\hline 3061 & FROM SHALLOW ZON
\end{tabular}

\begin{tabular}{l|l}
3061 & FROM SHALLOW ZONE \\
\hline 3062 & ROM SHALLOW ZONE
\end{tabular}

3062 FROM SHALLOW ZONE

\begin{tabular}{|l|l|l|l}
3063 & FROM DEEP ZONE \\
\hline 3064 & FROM SHALLOW ZON
\end{tabular}

3064 FROM SHALLOW ZONE

3065 FROM DEEP ZONE

3066 FROM SHALLOW ZONE

3067 FROM DEEP ZONE

3068 FROM SHALLOW ZONE

3069 FROM SHALLOW ZONE

3070 FROM DEEP ZONE

3071 FROM SHALLOW ZONE

3072 FROM DEEP ZONE

3073 FROM SHALLOW ZONE

3074 FROM SHALLOW ZONE

3075 FROM SHALLOW ZONE

3076 FROM SHALLOW ZONE

3077 FROM SHALLOW ZONE

\begin{tabular}{|l|l}
3078 & FROM SHALLOW ZONE \\
\hline 307 & FROM DEEP ZONEE \\
\hline
\end{tabular}

\begin{tabular}{|l|l|}
\hline 3079 & FROM DEEP ZONE \\
\hline
\end{tabular} 


\begin{tabular}{|c|c|c|}
\hline 1 & SAMP_SITE_DESC & SITE_ELEVATION \\
\hline 3080 & FROM DEEP ZONE & \\
\hline 3081 & FROM DEEP ZONE & \\
\hline 3082 & FROM DEEP ZONE & \\
\hline 3083 & FROM DEEP ZONE & \\
\hline 3084 & FROM DEEP ZONE & \\
\hline 3085 & FROM DEEP ZONE & \\
\hline 3086 & FROM DEEP ZONE & \\
\hline 3087 & FROM DEEP ZONE & \\
\hline 3088 & FROM DEEP ZONE & \\
\hline 3089 & Node: S-B7-8 & \\
\hline 3090 & Node: S-B7-11 & \\
\hline 3091 & Node: S-B8-2 & \\
\hline 3092 & Node: S-B8-3 & \\
\hline 3093 & Composite sample at 116-B-7 outfall & \\
\hline 3094 & Suspected asbestos containing material at $116-B-7$ outfall & \\
\hline 3095 & Suspected asbestos containing material at $116-\mathrm{B}-7$ outfall & \\
\hline 3096 & From "Hot Spot" in bottom of pipeline 1 \& 2 CA & \\
\hline 3097 & from side of pipe \#12 within layback of 132-B-6 outfall & \\
\hline 3100 & & \\
\hline 3101 & & \\
\hline 3102 & & \\
\hline 3103 & & \\
\hline 3104 & & \\
\hline 3105 & & \\
\hline 3106 & & \\
\hline 3107 & & \\
\hline 3108 & & \\
\hline 3109 & & \\
\hline 3110 & & \\
\hline 3111 & & \\
\hline 3112 & & \\
\hline 3113 & & \\
\hline 3114 & & \\
\hline 3115 & & \\
\hline 3116 & & \\
\hline 3117 & & \\
\hline
\end{tabular}




\begin{tabular}{|c|c|c|}
\hline & $\mathrm{M}$ & \\
\hline 1 & SAMP_SITE_DESC & SITE_ELEVATION \\
\hline \multicolumn{3}{|l|}{3118} \\
\hline \multicolumn{3}{|l|}{3119} \\
\hline \multicolumn{3}{|l|}{3120} \\
\hline \multicolumn{3}{|l|}{3121} \\
\hline \multicolumn{3}{|l|}{3122} \\
\hline \multicolumn{3}{|l|}{3123} \\
\hline \multicolumn{3}{|l|}{3124} \\
\hline \multicolumn{3}{|l|}{3125} \\
\hline \multicolumn{3}{|l|}{3126} \\
\hline \multicolumn{3}{|l|}{3127} \\
\hline \multicolumn{3}{|l|}{3128} \\
\hline \multicolumn{3}{|c|}{ 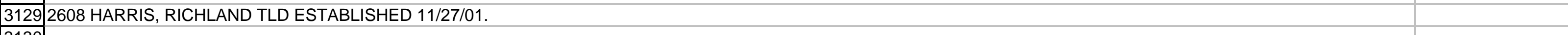 } \\
\hline \multicolumn{3}{|l|}{3130} \\
\hline \multicolumn{3}{|l|}{3131} \\
\hline \multicolumn{3}{|l|}{3132} \\
\hline \multicolumn{3}{|l|}{3133} \\
\hline \multicolumn{3}{|l|}{3134} \\
\hline \multicolumn{3}{|l|}{3135} \\
\hline \multicolumn{3}{|l|}{3138} \\
\hline \multicolumn{3}{|l|}{3139} \\
\hline \multicolumn{3}{|l|}{3140} \\
\hline \multicolumn{3}{|l|}{3141} \\
\hline \multicolumn{3}{|l|}{3142} \\
\hline \multicolumn{3}{|c|}{\begin{tabular}{|l|l|}
3143 & Node: $S-A 1-2$ \\
\end{tabular}} \\
\hline 3144 & Node: S-A1-3 & \\
\hline 3145 & Node: S-A1-4 & \\
\hline 3146 & Node: S-A1-10 & \\
\hline 3147 & Node: S-A1-13 & \\
\hline 3148 & Node: S-A1-16 & \\
\hline 3149 & Node: S-A2-3 & \\
\hline 3150 & Node: S-A2-6 & \\
\hline 3151 & Node: S-A2-7 & \\
\hline 3152 & Node: S-A2-10 & \\
\hline 3153 & 116-N-1 Trenching Soil Samples & \\
\hline 3154 & 116-N-3 Pipeline Trench (segment Z1). & 136.8 \\
\hline 3155 & 116-N-3 Pipeline Trench (segment Z1). & 138 \\
\hline
\end{tabular}


3156 116-N-3 Pipeline Trench (segment Z1)

SAMP SITE DESC

3158

3159 Sample Point: SP-01

3160 Sample Point: SP-02

3161 Sample Point: SP-03

3162 Sample Point: SP-04

3163 Sample Point: SP-05

3164 Sample Point: SP-06

3165 Sample Point: SP-07

3166 Sample Point: SP-08

3167 Sample Point: SP-09

3168 Sample Point: SP-02

3169

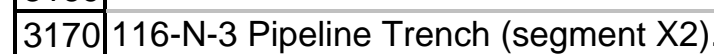

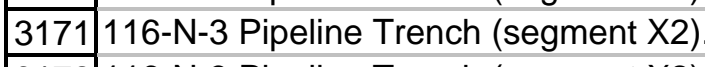

3172 116-N-3 Pipeline Trench (segment X2).

3173 116-N-3 Pipeline Trench (segment X2).

3174 Sample Point \#6

3175 Sample Point \#7

3176 Sample Point \#8

3177 Sample Point \#9

\begin{tabular}{|l|l|}
\hline 3178 & Sample Point \#10 \\
\hline 3179 & Sample Point \#11
\end{tabular}

3179 Sample Point \#11

3180 Sample Point \#1

3181 Sample Point \#2 at Plume \#12

3182 Sample Point \#3 at Plume \#12

3183 Sample Point \#4 at Plume \#12

3184 Sample Point \#5

3185 116-N-3 Pipeline Trench (segment X2)

3186 116-N-3 Pipeline Trench (segment X2).

3187 116-N-3 Pipeline Trench (segment X2).

3188 116-N-3 Crib Sidewalls

3189 116-N-3 Crib Sidewalls

3190 116-N-3 Crib Sidewalls

3191 116-N-3 Crib Sidewalls

3192 116-N-3 Crib Sidewalls

$\begin{array}{ll}3193 & 116-N-3 \text { Crib Sidewalls }\end{array}$ 
3203 Composite of 20 increments from 5 sections ( 4 on bottom of excavation and 1 from East slope)

3204 Composite of 20 increments from 5 sections (4 on bottom of excavation and 1 from East slope)

3205 Composite of 20 increments from 5 sections ( 4 on bottom of excavation and 1 from East slope)

3206 Composite of 20 increments from 5 sections (4 on bottom of excavation and 1 from East slope)

3207 Composite of 20 increments from 5 sections ( 4 on bottom of excavation and 1 from East slope)

\begin{tabular}{|l|l|l}
3208 & 200 yards east of the F-Area Queue \\
\hline 3209 & Outfall
\end{tabular}

3209 Outfall ACM

3210 Outfall ACM

3211 Outfall ACM

3212 Outfall ACM

3213 Shallow Zone Composite of nodes A1-3, A1-4, A1-10 and A1-16

3214 Shallow Zone Composite of nodes A2-3, A2-6, A2-7 and A2-15

3215 Shallow Zone Composite of nodes A3-1, A3-2, A3-4 and A3-11

\begin{tabular}{l|l}
3216 & Shallow Zone Composite of nodes A4-3, A4-4, A4-7 and A4-12
\end{tabular}

3217 Shallow Zone Composite of nodes B5-1, B5-5, B5-7 and B5-15

3218 Shallow Zone Composite of nodes B6-1, B6-10, B6-13 and B6-15

3219 Shallow Zone Composite of nodes B7-3, B7-4, B7-5 and B7-11

3220 Shallow Zone Composite of nodes B8-3, B8-4, B8-8 and B8-13

3221 Shallow Zone Composite of nodes C9-3, C9-4, C9-10 and C9-16

3222 Shallow Zone Composite of nodes C10-4, C10-10, C10-14, and C10-16

\begin{tabular}{|l|l}
3223 & Shallow Zone Composite of nodes C1-3, C1-4, C1-10, C1-16
\end{tabular}

3224 Shallow Zone Composite of nodes C2-3, C2-6, C2-7, C2-15

3225 Shallow Zone Composite of nodes D3-1, D3-2, D3-4, D3-11

3226 Shallow Zone Composite of nodes D4-3, D4-4, D4-7, D4-12

3227 Shallow Zone Composite of nodes D5-1, D5-5, D5-7, D5-15

3228 Shallow Zone Composite of nodes D6-1, D6-10, D6-13, D6-15

3229 Shallow Zone Composite of nodes A3-1, A3-2, A3-4 and A3-11

3230 Shallow Zone Composite of nodes D3-1, D3-2, D3-4, D3-11

\begin{tabular}{|l|l|}
3231 & Deep Zone Composite of nodes A1-3, A1-4, A1-10 and A1-16
\end{tabular} 
3232 Deep Zone Composite of nodes A2-3, A2-6, A2-7, A2-15

SAMP SITE DESC

3233 Deep Zone Composite of nodes A3-1, A3-2, A3-4, A3-11

3234 Deep Zone Composite of nodes A1-3, A1-4, A1-10 and A1-16

3235 Deep Zone Composite of nodes A1-3, A1-4, A1-10 and A1-16

\begin{tabular}{|l|l|l|}
\hline 3236 & Test Pit \\
\hline 3237 & Test Pit
\end{tabular}

3237 Test Pit

3238 Node A2-3

3239 Node A2-6

\begin{tabular}{l|l}
3240 & Node A2-7 \\
\hline 3241 & Node A2-15 \\
\hline
\end{tabular}

3241 Node A2-15

3242 Node B7-3

3243 Node B7-5

3244 Node B7-4

3245 Node B7-11

3246 Test Pit

\begin{tabular}{|l|l|l}
3247 & Test Pit \\
\hline
\end{tabular}

3248 Test Pit

3249 Test Pit

3250 Test Pit

3251 116-N-3 Crib Sidewalls

3252 116-N-3 Crib Sidewalls

3253 116-N-3 Crib Sidewalls

\begin{tabular}{|l|l}
3254 & $116-\mathrm{N}-3$ Crib Sidewalls \\
\hline 3255 & $116-\mathrm{N}-3$ Crib Sidewalls \\
\hline
\end{tabular}

3255 116-N-3 Crib Sidewalls

3256 116-N-3 Crib Sidewalls

3257 116-N-3 Crib Sidewalls

3258 116-N-3 Crib Sidewalls

3259 116-N-3 Crib Sidewalls

3260 116-N-3 Crib Sidewalls

\begin{tabular}{|l|l}
3261 & $116-N-3$ Crib Sidewalls \\
\hline 326 & $116-N-3$ Crib Sidd
\end{tabular}

3262 116-N-3 Crib Sidewalls

3263 116-N-3 Crib Sidewalls

3264 116-N-3 Crib Sidewalls

\begin{tabular}{|l|l}
\hline 3265 & $100-N R-1$ Vegetation \\
\hline 3266 & $116-N-3$ Cride
\end{tabular}

3266 116-N-3 Crib Sidewalls Sample

3267 116-NR-1 Vegetation 


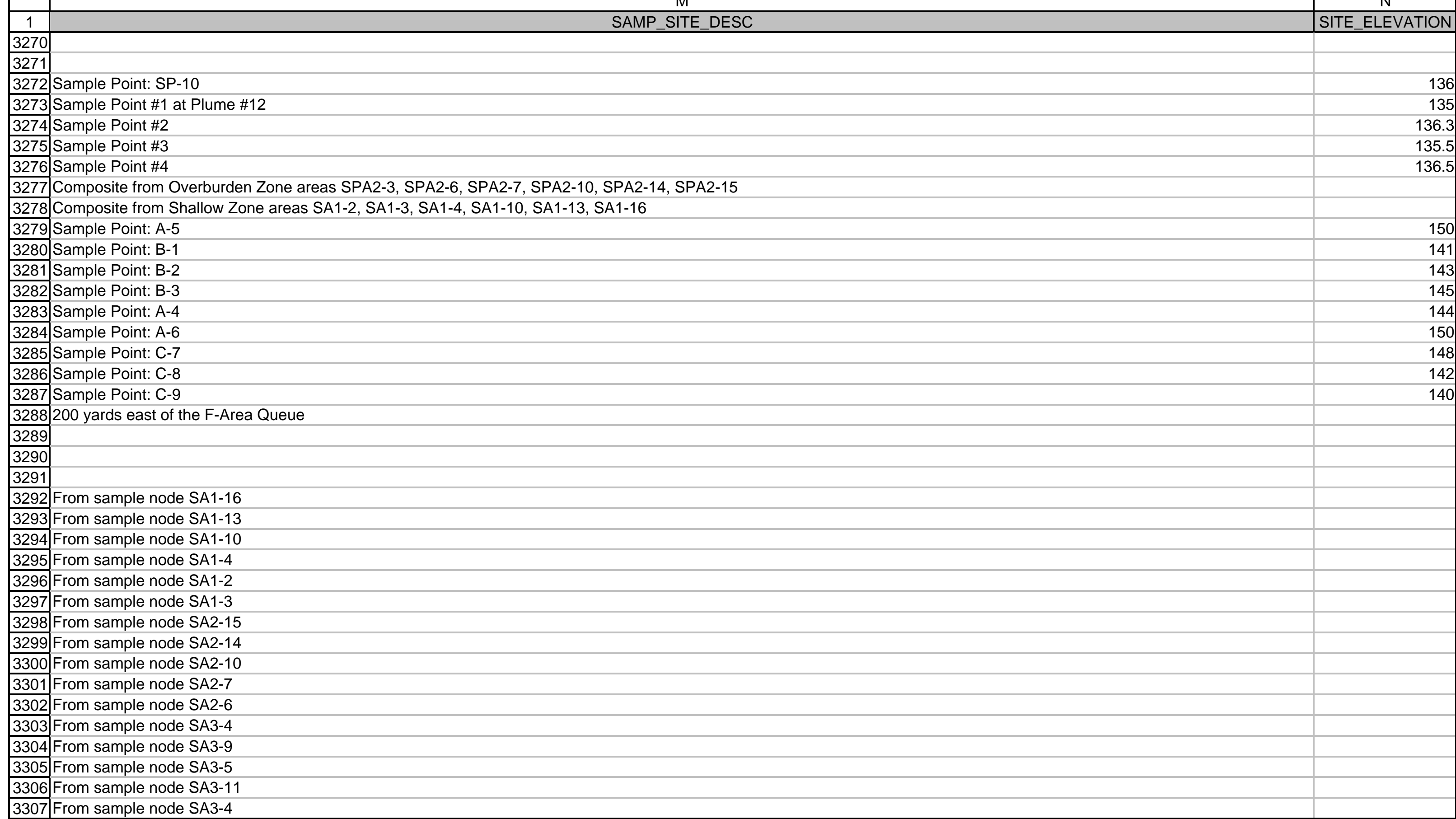

\begin{tabular}{|l|l|}
3306 & From sample node SA3-11 \\
\hline 3307 & From sample node SA3-4 \\
\hline
\end{tabular} 
3309 From sample node SA3-1

3310 From sample node SA4-13

3311 From sample node SA4-12

3312 From sample node SA4-9

3313 From sample node SA4-7

3314 From sample node SA4-4

3315 From sample node SA4-3

\begin{tabular}{|l|l|l}
3316 & Composite from Overburden Zone areas SPA2-3, SPA2-6, SPA2-7, SPA2-10, SPA2-14, SPA2-15
\end{tabular}

3317 Composite from Overburden Zone areas SPA2-3, SPA2-6, SPA2-7, SPA2-10, SPA2-14, SPA2-15

3318 Composite from Overburden Zone areas SPA1-2, SPA1-3, SPA1-4, SPA1-10, SPA1-13, SPA1-16

3319 Composite from Overburden Zone areas SPA3-1, SPA3-2, SPA3-4, SPA3-5, SPA3-9, SPA3-11

3320 Composite from Overburden Zone areas SPA4-3, SPA4-4, SPA4-7, SPA4-9, SPA4-12, SPA4-13

3321 Composite from Shallow Zone areas SA1-2, SA1-3, SA1-4, SA1-10, SA1-13, SA1-16

3322 Composite from Shallow Zone areas SA1-2, SA1-3, SA1-4, SA1-10, SA1-13, SA1-16

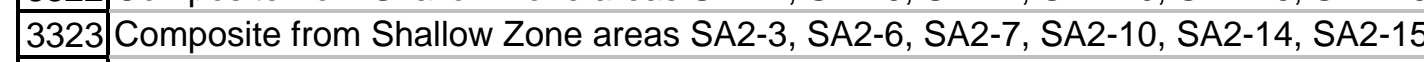

3324 Composite from Shallow Zone areas SA3-1, SA3-2, SA3-4, SA3-5, SA3-9, SA3-11

3325 Composite from Shallow Zone areas SA4-3, SA4-4, SA4-7, SA4-9, SA4-12, SA4-13

\begin{tabular}{|l|l|l|}
\hline 3326 & From an exploratory trench at the SE end of 116-F-9 main trench
\end{tabular} 
3347 Pothole sampling/Test Pit \#4

3348 Pothole sampling/Test Pit \#5

3349 Pothole sampling/Test Pit \#6

3350 Pothole sampling/Test Pit \#7

3351 Pothole sampling/Test Pit \#8

3352 Pothole sampling/Test Pit \#8

3353 From composite sample from nodes SA1-2, SA1-3, SA1-4, SA1-10, SA1-13, SA1-16

3354 From composite sample from nodes SA2-3, SA2-6, SA2-7, SA2-10, SA2-14, SA2-15

3355 From composite sample from nodes SA3-1, SA3-2, SA3-4, SA3-5, SA3-9, SA3-11

3356 From composite sample from nodes SA4-3, SA4-4, SA4-7, SA4-9, SA4-12, SA4-13

3357 From composite sample from nodes SA1-2, SA1-3, SA1-4, SA1-10, SA1-13, SA1-16

3358 From composite sample from nodes SA1-2, SA1-3, SA1-4, SA1-10, SA1-13, SA1-16

3359 Sample Point \#1 at Plume \#15

3360 Sample Point \#2 at Plume \#15

3367 Lewis Canal Pipe Trench crossed by 10 -inch water protection line

3368 Lewis Canal Pipe Trench crossed by 10 -inch water protection line

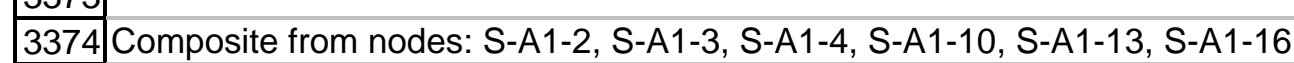

3375 Composite from nodes: S-A2-3, S-A2-6, S-A2-7, S-A2-10, S-A2-14, S-A2-15

3376 Composite from nodes: S-A3-1, S-A3-2, S-A3-4, S-A3-5, S-A3-9, S-A3-11

3377 Composite from nodes: S-A4-3, S-A4-4, S-A4-7, S-A4-9, S-A4-12, S-A4-13

3378 Composite from nodes: S-B5-1, S-B5-2, S-B5-5, S-B5-7, S-B5-13, S-B5-15

3379 Composite from nodes: S-B5-1, S-B5-2, S-B5-5, S-B5-7, S-B5-13, S-B5-15

3380 Composite from nodes: S-B6-1, S-B6-10, S-B6-12, S-B6-13, S-B6-15, S-B6-16

3381 Composite from nodes: S-B7-1, S-B7-3, S-B7-4, S-B7-5, S-B7-8, S-B7-11

3382 Composite from nodes: S-B8-2, S-B8-3, S-B8-4, S-B8-8, S-B8-12, S-B8-13

\begin{tabular}{|l|l|}
\hline 3383 & Pothole sampling/Test Pit \#7 \\
\hline
\end{tabular} 
3385 From Node: B5-1

3386 From Node: B5-6

3387 From Node: B5-7

3388 From Node: B5-15

3389 From Nodes: B5-1, B5-6, B5-7, B5-15

3390 From Nodes: B5-1, B5-6, B5-7, B5-15

3391 From Nodes: B5-1, B5-6, B5-7, B5-15

3392.

3393

3394

3395 Sample Point \#1 at Plume \#10

3396 Sample Point \#2 at Plume \#10

3397 Sample Point \#3 at Plume \#10

3398 Sample Point \#1 at Plume \#11

3399 Sample Point \#2 at Plume \#11

3400 Sample Point \#3 at Plume \#11

3401 Sample Point \#1 at Crossover at Haul Road

3402 Sample Point \#2 at Crossover at Haul Road

3403 Sample Point \#3 at Plume \#15

3404 Sample Point \#4 at Plume \#15

3405 Sample Point \#5 at Plume \#15

3406

\begin{tabular}{|l|}
\hline 3407 \\
\hline 3408 \\
\hline
\end{tabular}

3408

\begin{tabular}{|l|}
\hline 3408 \\
\hline 3410 \\
\hline
\end{tabular}

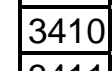

3411

\begin{tabular}{|l|l|}
\hline 3412 \\
\hline 3413 \\
\hline
\end{tabular}

3413

3414

3415 COMPOSITE OF SOILS FROM NORTHING 144914 TO 144950 AND EASTING 565319

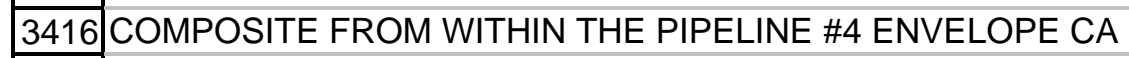

3417

3418 Composite of overburden stockpiles - located south of 116-B-1 and on the northern edge o project overburden stockpile \#5

3419 Composite of overburden stockpiles - located south of 116-B-1 and on the northern edge o project overburden stockpile \#5

3420 Composite of overburden stockpiles - located south of 116-B-1 and on the northern edge o project overburden stockpile \#5

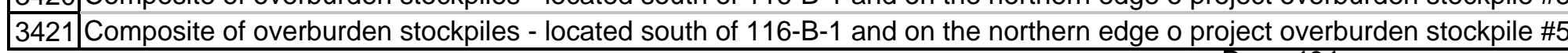

Page 404 


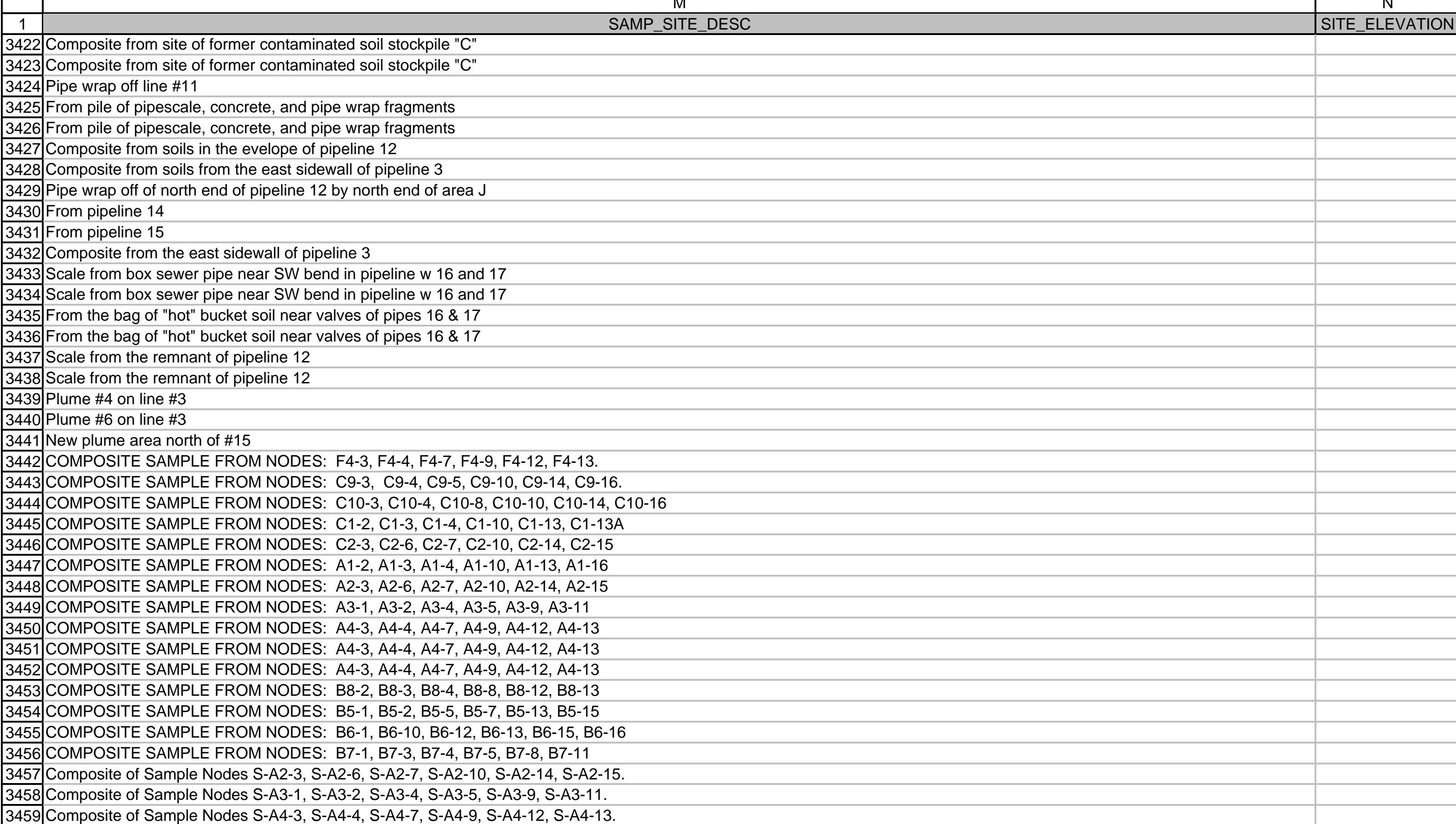

\begin{tabular}{l|l}
3459 & Composite of Sample Nodes S-A4-3, S-A4-4, S-A4-7, S-A4-9, S-A4-12, S-A4-13. \\
\hline
\end{tabular} 
3460 Composite of Sample Nodes S-B5-1, S-B5-2, S-B5-5, S-B5-7, S-B5-13, S-B5-15.

3461 Composite of Sample Nodes S-B6-1, S-B6-10, S-B6-12, S-B6-13, S-B6-15, S-B6-16.

3462 Composite of Sample Nodes S-B7-1, S-B7-3, S-B7-4, S-B7-5, S-B7-8, S-B7-11.

3463 Composite of Sample Nodes S-B8-2, S-B8-3, S-B8-4, S-B8-8, S-B8-12, S-B8-13.

3464 Composite of Sample Nodes S-C9-3, S-C9-4, S-C9-5, S-C9-10, S-C-9-14, S-C9-16.

3465

3466

3467

3468

3469

3470

3471

3472

3473

3474

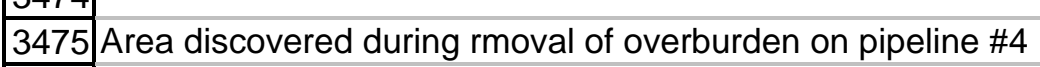

3476 Area discovered during rmoval of overburden on pipeline \#4

3477 From the bottom of the excavation.

3478 Site B

\begin{tabular}{l|l|l|l}
\hline 3479 & Site B \\
\hline 3480 & Site B
\end{tabular}

3480 Site B

3481 Site B

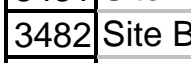

3483 Site B

3484 Site B

\begin{tabular}{ll|l}
3485 & Site $B$ \\
\hline 3486 & Site B
\end{tabular}

\begin{tabular}{|l|l|l|l|}
\hline 3485 & Site $B$ \\
\hline 3486 & Site $B$ \\
\hline 3487 & Site $B$
\end{tabular}

3487 Site B

3488 Site B

\begin{tabular}{|l|l|l|}
3488 & Site B \\
\hline 3489 & Site B \\
\hline 3490 & Site B
\end{tabular}

3490 Site B

3491 Site B

\begin{tabular}{l|l}
3492 & Site B \\
\hline 3493 &
\end{tabular}

\begin{tabular}{|l|l|}
\hline 3492 & Site B \\
\hline 3493 & Site B \\
\hline 3494 & Site B
\end{tabular}

\begin{tabular}{ll|l}
3494 & Site B \\
\hline
\end{tabular}

3495 Site B

\begin{tabular}{|l|l|l|}
\hline 3496 & Site B \\
\hline 3497 & Sit B \\
\hline
\end{tabular}

\begin{tabular}{|l|l|}
\hline 3497 & Site B \\
\hline
\end{tabular} 


\begin{tabular}{|c|c|c|}
\hline 1 & $\frac{\mathrm{Ml}}{\mathrm{CAMP}} \mathrm{CITC} \mathrm{DECC}$ & $\frac{\mathrm{N}}{\text { SITF FIFVATION }}$ \\
\hline \begin{tabular}{|c|c|} 
\\
3498
\end{tabular} & Site B & 900.2 \\
\hline 3499 & Site B & 896.9 \\
\hline 3500 & Site B & 896.9 \\
\hline 3501 & Site B & 888.3 \\
\hline 3502 & Site B & 888.3 \\
\hline 3503 & Site B & 888.3 \\
\hline 3504 & Site B & 888.6 \\
\hline 3505 & Site B & 888.6 \\
\hline 3506 & Site B & 874.4 \\
\hline 3507 & Site B & 874.4 \\
\hline 3508 & Site B & 867.2 \\
\hline 3509 & Site B & 867.2 \\
\hline 3510 & Site B & 864.7 \\
\hline 3511 & Site B & 864.7 \\
\hline 3512 & Site B & 864.7 \\
\hline 3513 & Site B & 897.3 \\
\hline \begin{tabular}{|l|}
3514 \\
\end{tabular} & Site B & 897.3 \\
\hline \begin{tabular}{|l|}
3515 \\
\end{tabular} & Site B & 854.2 \\
\hline 3518 & Site B & $840 . c$ \\
\hline 3519 & Site B & 840.9 \\
\hline 3520 & Site B & 840.9 \\
\hline 3521 & Site B & 833.5 \\
\hline \begin{tabular}{|l}
3522 \\
\end{tabular} & Site B & 833.5 \\
\hline 3523 & Site B & 833.5 \\
\hline 3524 & Site B & 833.5 \\
\hline \begin{tabular}{|l|}
3525 \\
\end{tabular} & Site B & 1020.6 \\
\hline 3526 & Site B & 993 \\
\hline \begin{tabular}{|l|}
3527 \\
\end{tabular} & Site B & 997.6 \\
\hline 3528 & Site B & 963.1 \\
\hline 3529 & Site B & 963.1 \\
\hline 3530 & Site B & 963.1 \\
\hline 3531 & Site B & 963.1 \\
\hline 3532 & Site B & 943.7 \\
\hline 3533 & Site B & 943.7 \\
\hline \begin{tabular}{|l|}
3534 \\
\end{tabular} & Site B & 924.7 \\
\hline 3535 & Site B & 929.2 \\
\hline
\end{tabular}




\begin{tabular}{|c|c|c|}
\hline & $\mathrm{M}$ & $\mathrm{N}$ \\
\hline 1 & SAMP_SITE_DESC & SITE_ELEVATION \\
\hline 3536 & Site B & 929.2 \\
\hline 3537 & Site B & 916.6 \\
\hline 3538 & Site B & 916.6 \\
\hline 3539 & Site B & 912.3 \\
\hline 3540 & Site B & 912.3 \\
\hline 3541 & Site B & 901.3 \\
\hline 3542 & Site B & 901.3 \\
\hline 3543 & Site B & 880.4 \\
\hline 3544 & Site B & 880.4 \\
\hline 3545 & Site B & 865.8 \\
\hline 3546 & Site B & 847.8 \\
\hline 3547 & Site B & 840.7 \\
\hline 3548 & Site B & 828.8 \\
\hline 3549 & Site B & 843 \\
\hline 3550 & Site B & 843 \\
\hline 3551 & Site B & 823.8 \\
\hline 3552 & Site B & 823.8 \\
\hline 3553 & Site B & 812.6 \\
\hline 3556 & From Shallow Zone Node SG5-2 & \\
\hline 3557 & From Shallow Zone Node SG5-5 & \\
\hline 3558 & From Shallow Zone Node SG5-7 & \\
\hline 3559 & From Shallow Zone Node SG5-13 & \\
\hline 3560 & From Shallow Zone Node SG5-15 & \\
\hline 3561 & From Shallow Zone Node SG6-1 & \\
\hline 3562 & From Shallow Zone Node SG6-10 & \\
\hline 3563 & From Shallow Zone Node SG6-12 & \\
\hline 3564 & From Shallow Zone Node SG6-13 & \\
\hline 3565 & From Shallow Zone Node SG6-15 & \\
\hline 3566 & From Shallow Zone Node SG6-16 & \\
\hline 3567 & From Shallow Zone Node SG7-1 & \\
\hline 3568 & From Shallow Zone Node SG7-3 & \\
\hline 3569 & From Shallow Zone Node SG7-4 & \\
\hline 3570 & From Shallow Zone Node SG7-5 & \\
\hline 3571 & From Shallow Zone Node SG7-8 & \\
\hline 3572 & From Shallow Zone Node SG7-11 & \\
\hline 3573 & From Shallow Zone Node SG8-2 & \\
\hline
\end{tabular}


3575 From Shallow Zone Node SG8-4

3576 From Shallow Zone Node SG8-8

3577 From Shallow Zone Node SG8-12

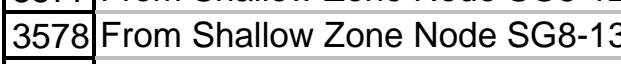

3579 From Shallow Zone Node SI3-1

3580 From Shallow Zone Node SI3-2

3581 From Shallow Zone Node SI3-4

\begin{tabular}{|l|l}
3582 & From Shallow Zone Node SI3-5 \\
\hline 3583 &
\end{tabular}

3583 From Shallow Zone Node SI3-9

3584 From Shallow Zone Node SI3-11

3585 From Shallow Zone Node SI4-3

\begin{tabular}{|l|l}
3586 & From Shallow Zone Node SI4-4
\end{tabular}

3587 From Shallow Zone Node SI4-7

3588 From Shallow Zone Node SI4-9

\begin{tabular}{|l|l}
3589 & From Shallow Zone Node SI4-12
\end{tabular}

3590 From Shallow Zone Node SI4-13

3591 From Shallow Zone Node SI5-1

3592 From Shallow Zone Node SI5-2

\begin{tabular}{|l|l}
3593 & From Shallow Zone Node SI5-5 \\
\hline
\end{tabular}

3594 From Shallow Zone Node SI5-7

3595 From Shallow Zone Node SI5-13

\begin{tabular}{|l|l}
3596 & From Shallow Zone Node SI5-15 \\
\hline 3597 & From Shallow Zone Node SI6-1
\end{tabular}

3597 From Shallow Zone Node SI6-1

3598 From Shallow Zone Node SI6-10

3599 From Shallow Zone Node SI6-12

3600 From Shallow Zone Node SI6-13

3601 From Shallow Zone Node SI6-15

3602 From Shallow Zone Node SI6-16

\begin{tabular}{|l|l|}
\hline 3603 & Shallow Zone sample point: VSZ-01 \\
\hline 3604 & Shallow Zone sampla pont: VSZ-02
\end{tabular}

3604 Shallow Zone sample point: VSZ-02

3605 Shallow Zone sample point: VSZ-03

3606 Shallow Zone sample point: VSZ-03

3607 Shallow Zone sample point: VSZ-05

3608 Shallow Zone sample point: VSZ-07

3609 Shallow Zone sample point: VSZ-08

Shallow Zone sample point: VSZ-09

\begin{tabular}{|l|l|}
\hline 3611 & Shallow Zone sample point: VSZ-17 \\
\hline
\end{tabular} 
3613 Shallow Zone sample point: VSZ-20

3614 Shallow Zone sample point: VSZ-21

3615 Shallow Zone sample point: VSZ-22

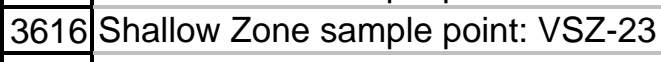

\begin{tabular}{|l|l}
\hline 3617 & Shallow Zone sample point: VSZ-24
\end{tabular}

3618 Shallow Zone sample point: VSZ-25

3619 Shallow Zone sample point: VSZ-26

3620 Shallow Zone sample point: VSZ-29

3621 Shallow Zone sample point: VSZ-10

3622 Shallow Zone sample point: VSZ-12

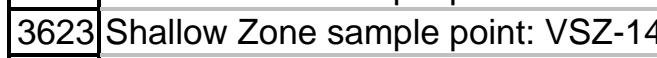

\begin{tabular}{l|l}
3624 & Shallow Zone sample point: VSZ-15
\end{tabular}

3625 Shallow Zone sample point: VSZ-16

3626 Shallow Zone sample point: VSZ-28

\begin{tabular}{|l|l|}
3627 & Pipeline Sample Point N3P-Z07 \\
\hline 3628 & Pipeline Sample Point N3P-Z08
\end{tabular}

3628 Pipeline Sample Point N3P-Z08

3629 Shallow Zone sample point: VSZ-06

S630 Shallow Zone sample point: VSZ-11

3631 Shallow Zone sample point: VSZ-13

3632 Shallow Zone sample point: VSZ-19

3633 Shallow Zone sample point: VSZ-27

3634 Shallow Zone sample point: VSZ-30

3635 Pipeline Sample Point N3P-Z09

3636 Pipeline Sample Point N3P-Z10

3637 Overburden Stockpile Sample Point: OVB -01

3638 Overburden Stockpile Sample Point: OVB -02

3639 Overburden Stockpile Sample Point: OVB -02

3640 Overburden Stockpile Sample Point: OVB -02

3641 Overburden Stockpile Sample Point: OVB -03

3642 Overburden Stockpile Sample Point: OVB -04

3643 Overburden Stockpile Sample Point: OVB -05

3644 Overburden Stockpile Sample Point: OVB -06

3645 Overburden Stockpile Sample Point: OVB -07

3646 Overburden Stockpile Sample Point: OVB -08

3647 Overburden Stockpile Sample Point: OVB -09

3648 Overburden Stockpile Sample Point: OVB -10

3649 Overburden Stockpile Sample Point: OVB -02

(n) 
3651 From Shallow Zone Node SA2-6

3652 From Shallow Zone Node SA2-7

3653 From Shallow Zone Node SA2-10

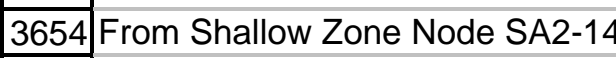

3655 From Shallow Zone Node SA2-15

3656 From Shallow Zone Node SA3-1

3657 From Shallow Zone Node SA3-2

\begin{tabular}{|l|l}
3658 & From Shallow Zone Node SA3-4 \\
\hline
\end{tabular}

3659 From Shallow Zone Node SA3-5

3660 From Shallow Zone Node SA3-9

3661 From Shallow Zone Node SA3-11

\begin{tabular}{l|l}
3662 & From Shallow Zone Node SA4-3
\end{tabular}

3663 From Shallow Zone Node SA4-4

3664 From Shallow Zone Node SA4-7

\begin{tabular}{|l|l|}
3665 & From Shallow Zone Node SA4-9 \\
\hline 3666 & Fom Shallow Zone Node SA4-
\end{tabular}

3666 From Shallow Zone Node SA4-12

3667 From Shallow Zone Node SA4-13

3668 From Shallow Zone Node SB6-15

3669 From Shallow Zone Node SB6-16

3670 From Shallow Zone Node SB7-8

3671 From Shallow Zone Node SB8-8

\begin{tabular}{|l|l}
3672 & From Shallow Zone Node SC9-14 \\
\hline 3673 & From Shallow Zone Node SC9-16
\end{tabular}

3673 From Shallow Zone Node SC9-16

3674 From Shallow Zone Node SC10-14

3675 From Shallow Zone Node SC10-16

3676 From Shallow Zone Node SC1-13

3677 From Shallow Zone Node SC1-16

3678 From Shallow Zone Node SC2-14

3679 From Shallow Zone Node SC2-15

3680 From Shallow Zone Node SD4-13

3681 From Shallow Zone Node SD5-13

3682 From Shallow Zone Node SD5-15

3683 From Shallow Zone Node SD6-13

3684 From Shallow Zone Node SD6-15

3685 From Shallow Zone Node SD6-16

3686 From Shallow Zone Node SE9-14

3687 From Shallow Zone Node SE9-16 
3689 From Shallow Zone Node SH9-4

3690 From Shallow Zone Node SH9-10

3691 From Shallow Zone Node SH9-14

\begin{tabular}{|l|l}
3692 & From Shallow Zone Node SH9-16 \\
\hline 3693 & Srom Shallow Zone Node SH10-3 \\
\hline
\end{tabular}

3693 From Shallow Zone Node SH10-3

3694 From Shallow Zone Node SH10-4

3695 From Shallow Zone Node SH10-8

\begin{tabular}{|l|l}
3696 & From Shallow Zone Node SH10-10
\end{tabular}

3697 From Shallow Zone Node SH10-14

3698 From Shallow Zone Node SH10-16

3699 From Shallow Zone Node SH1-2

\begin{tabular}{l|l}
3700 & From Shallow Zone Node SH1-3
\end{tabular}

3701 From Shallow Zone Node SH1-4

3702 From Shallow Zone Node SH1-10

\begin{tabular}{|l|l}
3703 & From Shallow Zone Node SH1-13 \\
\hline 3704 & From Shallow Zone Node SH1-16
\end{tabular}

3704 From Shallow Zone Node SH1-16

3705 From Shallow Zone Node SH2-3

3706 From Shallow Zone Node SH2-6

3707 From Shallow Zone Node SH2-7

3708 From Shallow Zone Node SH2-10

3709 From Shallow Zone Node SH2-14

\begin{tabular}{l|l}
3710 & From Shallow Zone Node SH2-15
\end{tabular}

3711 From Overburden Zone Node OA1-2

3712 From Overburden Zone Node OA1-3

3713 From Overburden Zone Node OA1-4

3714 From Overburden Zone Node OA1-10

3715 From Overburden Zone Node OA1-13

3716 From Overburden Zone Node OA1-16

\begin{tabular}{|l|l}
3717 & From Overburden Zone Node OA2-3 \\
\hline
\end{tabular}

3718 From Overburden Zone Node OA2-6

3719 From Overburden Zone Node OA2-7

3720 From Overburden Zone Node OA2-10

\begin{tabular}{l|l}
3721 & From Overburden Zone Node OA2-14 \\
\hline 3722 & From Over
\end{tabular}

3722 From Overburden Zone Node OA2-15

3723 From Overburden Zone Node OA3-1

3724 From Overburden Zone Node OA3-2

\begin{tabular}{|l|l|}
\hline 3725 & From Overburden Zone Node OA3-4 \\
\hline
\end{tabular} 
3727 From Overburden Zone Node OA3-9

3728 From Overburden Zone Node OA3-11

3729 From Overburden Zone Node OA4-3

3730 From Overburden Zone Node OA4-4

3731 From Overburden Zone Node OA4-7

3732 From Overburden Zone Node OA4-9

3733 From Overburden Zone Node OA4-12

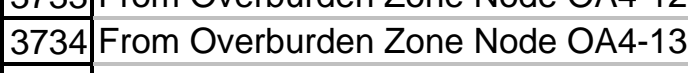

3735 Composite of Sample Nodes A3-1, A3-2, A3-4, and A3-11.

3736 Composite of Sample Nodes B5-1, B5-5, B5-7, B5-15.

3737 From Overburden Zone Node OB5-1

3738 From Overburden Zone Node OB5-2

3739 From Overburden Zone Node OB5-5

3740 From Overburden Zone Node OB5-7

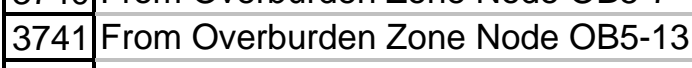

3742 From Overburden Zone Node OB5-15

3743 From Overburden Zone Node OB6-1

3744 From Overburden Zone Node OB6-10

3745 From Overburden Zone Node OB6-12

3746 From Overburden Zone Node OB6-13

3747 From Overburden Zone Node OB6-15

\begin{tabular}{|l|l|l|}
\hline 3748 & From Overburden Zone Node OB6-16 \\
\hline 3749 & From Overden
\end{tabular}

3749 From Overburden Zone Node OB7-1

3750 From Overburden Zone Node OB7-3

3751 From Overburden Zone Node OB7-4

\begin{tabular}{|l|l}
3752 & From Overburden Zone Node OB7-5
\end{tabular}

3753 From Overburden Zone Node OB7-8

3754 From Overburden Zone Node OB7-11

\begin{tabular}{l|l}
3755 & From Overburden Zone Node OB8-2
\end{tabular}

3756 From Overburden Zone Node OB8-3

3757 From Overburden Zone Node OB8-4

3758 From Overburden Zone Node OB8-8

3759 From Overburden Zone Node OB8-12

3760 From Overburden Zone Node OB8-13

3761 116-N-1 Trenching Soil Samples from Sample Point N1-DRPH-021a

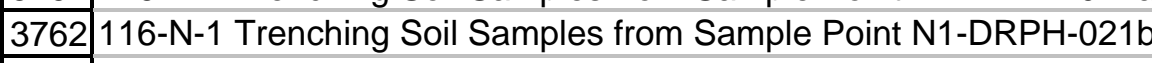

\begin{tabular}{|l|l|l}
3763 & $116-N-1$ Trenching Soil Samples from Sample Point N1-DRPH-021c \\
\hline
\end{tabular} 
3765 Scale sample from pipeline

3766 Composite of soils from the "orphan stockpile" from previous remediation work at $100 \mathrm{BC}$

3767 Composite of soils from the "orphan stockpile" from previous remediation work at $100 \mathrm{BC}$

3768 Composite of soils from the "orphan stockpile" from previous remediation work at $100 \mathrm{BC}$

3769 Composite of soils from the "orphan stockpile" from previous remediation work at $100 \mathrm{BC}$

3770 Composite of soils from the "orphan stockpile" from previous remediation work at $100 \mathrm{BC}$

3771 From south of line 11

\begin{tabular}{|l|l|l|}
3772 & From SW of W 116-C-5 BASIN
\end{tabular}

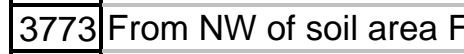

3774 From West of pipe 22

3775 From between pipes $22 \& 23$

\begin{tabular}{l|l}
3776 & From south of soil area $\mathrm{K}$ \\
\hline 3777 & From east
\end{tabular}

3777 From east of pipe \#26

3778 From east of "floor"

\begin{tabular}{|l|l|l}
3779 & From south of "floor" east of pipe 26
\end{tabular}

3780 From between pipes 25 \& 26

3781 Burial Ground - Test Pit

3782 Burial Ground - Test Pit

\begin{tabular}{|l|l}
3783 & From Sample Node: S-A1-2
\end{tabular}

3784 From Sample Node: S-A1-3

3785 From Sample Node: S-A1-4

3786 From Sample Node: S-A1-10

3787 From Sample Node: S-A1-13

3788 From Sample Node: S-A1-16

3789 From Sample Node: S-A2-3

3790 From Sample Node: S-A2-6

3791 From Sample Node: S-A2-7

3792 From Sample Node: S-A2-10

\begin{tabular}{|l|l}
3793 & From Sample Node: S-A2-14 \\
\hline 3794 & From Sample
\end{tabular}

3794 From Sample Node: S-A2-15

3795 From Sample Node: S-A3-1

3796 From Sample Node: S-A3-2

3797 From Sample Node: S-A3-4

3798 From Sample Node: S-A3-5

3799 From Sample Node: S-A3-9

3800 From Sample Node: S-A3-11

3801 From Sample Node: S-A4-3 
3807 116-N-1 Trench Pothole (Zone 1, east of "dig road" Sample Point: N1-DRPH-022a

3808 116-N-1 Trench Pothole (Zone 1, east of "dig road" Sample Point: N1-DRPH-022b

3809 116-N-1 Trench Pothole (Zone 1, east of "dig road" Sample Point: N1-DRPH-022c

3810 116-N-1 Trench Pothole (Zone 1, east of "dig road" Sample Point: N1-DRPH-022d

3811 From Shallow Zone Node SF4-13

3812 From Overburden Zone Node OC9-3

\begin{tabular}{|l|l}
3813 & From Overburden Zone Node OC9-4 \\
\hline 3814 & From Overburden Zone Node OC9-5
\end{tabular}

3814 From Overburden Zone Node OC9-5

3815 From Overburden Zone Node OC9-10

3816 From Overburden Zone Node OC9-14

3817 From Overburden Zone Node OC9-16

3818 From Overburden Zone Node OC10-3

3819 From Overburden Zone Node OC10-4

3820 From Overburden Zone Node OC10-8

\begin{tabular}{l|l}
3821 & From Overburden Zone Node OC10-10
\end{tabular}

3822 From Overburden Zone Node OC10-14

3823 From Overburden Zone Node OC10-16

3824 From Overburden Zone Node OC1-2

3825 From Overburden Zone Node OC1-3

3826 From Overburden Zone Node OC1-4

3827 From Overburden Zone Node OC1-10

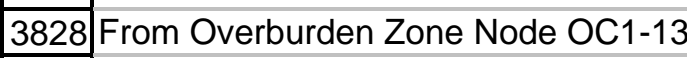

3829 From Overburden Zone Node OC1-16

3830 From Overburden Zone Node OC2-3

3831 From Overburden Zone Node OC2-6

3832 From Overburden Zone Node OC2-7

3833 From Overburden Zone Node OC2-10

3834 From Overburden Zone Node OC2-14

\begin{tabular}{l|l}
3835 & From Overburden Zone Node OC2-15 \\
\hline 383 & From
\end{tabular}

3836 From Shallow Zone Node SB5-7

3837 From Shallow Zone Node SB5-15

3838 Composite of sample nodes: D-A1-3, D-A1-4, D-A1-10, and D-A1-16

3839 Composite of sample nodes: D-A1-3, D-A1-4, D-A1-10, and D-A1-16 
3840 Composite of sample nodes: D-A1-3, D-A1-4, D-A1-10, and D-A1-16

SAMP SITE_DESC

3841 Composite of sample nodes: D-A2-3, D-A2-6, D-A2-7, and D-A2-15

3842 Composite of sample nodes: D-A3-1, D-A3-2, D-A3-4, and D-A3-11

3843 Composite of sample nodes: D-B4-3, D-B4-4, D-B4-7, and D-B4-12

3844 Composite of sample nodes: D-B5-1, D-B5-5, D-B5-7, and D-B5-15

3845 Composite of sample nodes: D-B6-1, D-B6-10, D-B6-13, and D-B6-15

3846 Composite of sample nodes: D-C7-3, D-C7-4, D-C7-5, and D-C7-11

3847 Composite of sample nodes: D-C8-3, D-C8-4, D-C8-8, and D-C8-13

3848 Composite of sample nodes: D-C9-3, D-C9-4, D-C9-10, and D-C9-16

3849 Composite of sample nodes: D-D10-4, D-D10-10, D-D10-14, and D-D10-16

3850 Composite of sample nodes: D-D1-3, D-D1-4, D-D1-10, and D-D1-16

3851 Composite of sample nodes: D-D2-3, D-D2-6, D-D2-7, and D-D2-15

3852 116-N-3 Crib floor - sample point N3C-DZ01

3853 116-N-3 Crib floor - sample point N3C-DZ02

3854 116-N-3 Crib floor - sample point N3C-DZ03

3855 116-N-3 Crib floor - sample point N3C-DZ04

3856 116-N-3 Crib floor - sample point N3C-DZ05

3857 116-N-3 Crib floor - sample point N3C-DZ06

3858 116-N-3 Crib floor - sample point N3C-DZ07

3859 116-N-3 Crib floor - sample point N3C-DZ08

3860 116-N-3 Crib floor - sample point N3C-DZ09

3861 116-N-3 Crib floor - sample point N3C-DZ10

\begin{tabular}{|l|l|}
3862 & From sample node: S-B5-1 \\
\hline 3863 & From sample node: S-B5-2
\end{tabular}

3863 From sample node: S-B5-2

3864 From sample node: S-B5-5

3865 From sample node: S-B5-7

3866 From sample node: S-B5-13

3867 From sample node: S-B5-15

3868 From sample node: S-B6-1

3869 From sample node: S-B6-10

3870 From sample node: S-B6-12

3871 From sample node: S-B6-13

3872 From sample node: S-B6-15

\begin{tabular}{l|l}
3873 & From sample node: S-B6-16
\end{tabular}

3874 From sample node: S-B7-1

3875 From sample node: S-B7-3

3876 From sample node: S-B7-4

3877 From sample node: S-B7-5

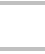

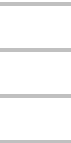

(20)

-


3878 From sample node: S-B7-8

\begin{tabular}{|l|l}
3879 & From sample node: S-B7-11 \\
\hline 3880 & From sample note: S-B8-2
\end{tabular}

3880 From sample node: S-B8-2

3881 From sample node: S-B8-3

3882 From sample node: S-B8-4

\begin{tabular}{|l|l}
3883 & From sample node: S-B8-8
\end{tabular}

3884 From sample node: S-B8-12

3885 From sample node: S-B8-13

\begin{tabular}{|l|l|}
\hline 3886 & 72 " sewer pipe \\
\hline 3887 & 72 " sewer pipe \\
\hline
\end{tabular}

3887 72 " sewer pipe

3888 Plume \#15 on line \#7

3889 Plume \#15 on line \#7

3890 116-N-3 Decon Pad - Sump

3891 FROM SE CORNER OF SOUTHERN MOST EXCAVATION

3892 FROM NW CORNER OF NORTHERN MOST EXCAVATION

\begin{tabular}{|l|l}
3893 & Deep Zone Composite at the A3 deep zone
\end{tabular}

3894 Deep Zone Composite at the A3 deep zone

3895 Deep Zone Composite at the A3 deep zone

3896 Sample Point \#1, Surface

3897 Sample Point \#1, Deep

3898 Sample Point \#2, Surface

3899 Sample Point \#2, Deep

3900 Sample Point \#3, Surface

3901 Sample Point \#3, Deep

3902 Sample Point \#4, Surface

3903 Sample Point \#4, Deep

3904 Sample Point \#1, Surface

3905 Sample Point \#1, Deep

3906 Contaminated soil area "G"

\begin{tabular}{|l|l}
3907 & Contaminated soil area "G" \\
\hline 3908 &
\end{tabular}

3908

3909

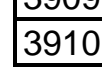

3911

3912

3913 Sample Location: Area H-1

3914 Sample Location: Area H-2

3915 Sample Location: Area H-3 
3917 Sample Location: Area I-2 3918 Sample Location: Area I-3

3919 Sample Location: Pipe C-1

3920 Sample Location: Pipe C-2

3921 Sample Location: Pipe C-3

3922 Sample Location: Pipe C-4

3923 Sample Location: Pipe C-5

\begin{tabular}{|l|l|}
3924 & Pipeline 3 \\
\hline
\end{tabular}

3925 Sample Location: I-1

3926 Sample Location: I-2

3927 Sample Location: G-1

3928 Sample Location: G-2

3929 Sample Location: $\mathrm{H}-1$

3930 Sample Location: $\mathrm{H}-2$

3931 Sample Location: G-1

3932 Sample Location: G-2

3933 Sample from Soil Area "G"

3934 Sample from Soil Area "G"

3935 Sample from Soil Area "G"

3936 Sample from Soil Area G-1

3937 Sample from Soil Area G-2

\begin{tabular}{l|l}
3938 & Sample from Soil Area G-3 \\
\hline 3939 & Sample from Soll Area G-4
\end{tabular}

3939 Sample from Soil Area G-4

3940 Sample from Soil Area G-5

3941 Sample from Soil Area $\mathrm{H}-1$

3942 Sample from Soil Area $\mathrm{H}-2$

3943 Sample from Soil Area $\mathrm{H}-3$

3944 Sample from Soil Area $\mathrm{H}-4$

3945 Sample from Soil Area $\mathrm{H}-5$

3946 Sample from near soil area "G"

3947 Sample from near soil area "G"

3948 Sample from near soil area "G"

3949 Sample from near soil area "G"

3950 From sample point SP12, \#1 inside area "G"

3951 From Pipes 3 \& 4 just north of the railroad tracks in area "G"

3952 Sample from North of sample area "G"

3953 Sample from North of sample area "G" hot spot 
3960 Sample from north of soil area "G"

3961 Sample from north of soil area "G"

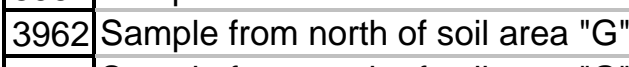

3963 Sample from north of soil area "G"

3964 Sample from north of soil area "G"

3965 Sample from north of soil area "G"

3966 From overburden stockpile \#11

3967 From overburden stockpile \#11

\begin{tabular}{|l|l}
3968 & From overburden stockpile \#7
\end{tabular}

3969 From pipe envelope of line \#3

3970 From pipe envelope of line \#3

3971 From pipe envelope of line \#3

3972 116-N-3 Pipeline Trench - western most segment adjacent to 1316-NB - Sample Point N3P-Z01

3973 116-N-3 Pipeline Trench - western most segment adjacent to 1316-NB - Sample Point N3P-Z02

3974 116-N-3 Pipeline Trench - western most segment adjacent to 1316-NB - Sample Point N3P-Z03

3975 116-N-3 Pipeline Trench - western most segment adjacent to 1316-NB - Sample Point N3P-Z04

3976 116-N-3 Pipeline Trench - western most segment adjacent to 1316-NB - Sample Point N3P-Z05

3977 116-N-3 Pipeline Trench - western most segment adjacent to 1316-NB - Sample Point N3P-Z06

3978 116-N-3 Crib - Plume \#14 sidewall (sample point Plume 14-01)

3979 116-N-3 Crib - Plume \#14 sidewall (sample point Plume 14-02)

3980 116-N-3 Crib - Plume \#14 sidewall (sample point Plume 14-03)

3981 116-N-3 Decon Pad - Sump (Round 4)

3982 116-N-3 Decon Pad - Sump (Round 4)

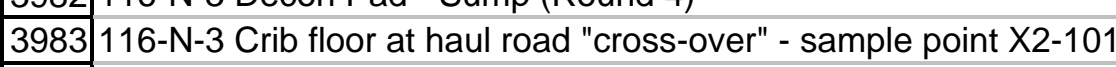

3984 116-N-3 Crib floor at haul road "cross-over" - sample point X2-102

3985 From the tile field - shallow zone in red stained soil

3986 From the tile field - shallow zone in red stained soil

3987 From the tile field - shallow zone in red stained soi

3988 116-N-3 Crib Shallow Zone - sample point SZ-01

3989 116-N-3 Crib Shallow Zone - sample point SZ-01

3990 116-N-3 Crib Shallow Zone - sample point SZ-01

\begin{tabular}{|l|l|}
\hline 3991 & $116-N-3$ Crib Shallow Zone - sample point SZ-01
\end{tabular} 
3992 116-N-3 Crib Shallow Zone - sample point SZ-01

SAMP SITE DESC

3993 116-N-3 Crib Shallow Zone - sample point SZ-02

3994 116-N-3 Crib Shallow Zone - sample point SZ-03

3995 116-N-3 Crib Shallow Zone - sample point SZ-04

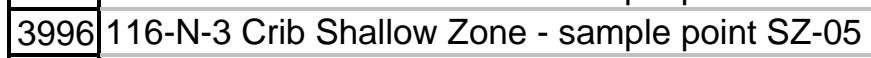

3997 116-N-3 Crib Shallow Zone - sample point SZ-06

3998 116-N-3 Crib Shallow Zone - sample point SZ-06

3999 116-N-3 Crib Shallow Zone - sample point SZ-07

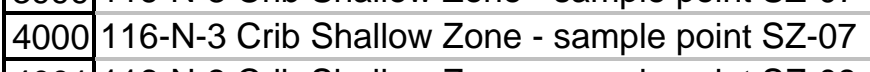

4001 116-N-3 Crib Shallow Zone - sample point SZ-08

4002 116-N-3 Crib Shallow Zone - sample point SZ-09

4003 116-N-3 Crib Shallow Zone - sample point SZ-10

4004

4005

4005

4006

4008

4009 Sample from load out area on pipeline 16/17

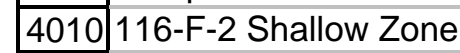

\begin{tabular}{|l|l}
4011 & $116-F-2$ \\
4012 & Shallow Zone
\end{tabular}

4012 116-F-2 Shallow Zone

\begin{tabular}{|l|l}
\hline 4013 & $116-F-2$ \\
\hline 4014 & Shallow Zone
\end{tabular}

\begin{tabular}{|l|l|}
\hline 4014 & $116-F-2$ \\
\hline 4015 & $116-N-3$ Crlow Zone
\end{tabular}

4015 116-N-3 Crib Deep Zone - sample point DZ-01

4016 116-N-3 Crib Deep Zone - sample point DZ-02

4017 116-N-3 Crib Deep Zone - sample point DZ-02

\begin{tabular}{|l|l|}
\hline 4018 & $116-N-3$ Crib Deep Zone - sample point DZ-02 \\
\hline 4019 & $116-N-3$ Crib Deep Zone - sample poin DZ-01
\end{tabular}

4019 116-N-3 Crib Deep Zone - sample point DZ-0

4020 116-N-3 Crib Deep Zone - sample point DZ-03

4021 116-N-3 Crib Deep Zone - sample point DZ-04

4022 116-N-3 Crib Deep Zone - sample point DZ-05

4023 116-N-3 Crib Deep Zone - sample point DZ-06

4024 116-N-3 Crib Deep Zone - sample point DZ-07

\begin{tabular}{|l|l}
4025 & $116-N-3$ Crib Deep Zone - sample point DZ-08 \\
\hline 4026 & $116-N-3$ Crib Deep Zone - sample point DZ-09
\end{tabular}

4026 116-N-3 Crib Deep Zone - sample point DZ-09

4027 116-N-3 Crib Deep Zone - sample point DZ-10

4028 116-N-3 Crib Deep Zone - sample point DZ-01

\begin{tabular}{|l|l}
\hline 4029 & $116-N-3$ Crib Deep Zone - sample point DZ-03 \\
\hline
\end{tabular} 
4031 Composite from Sample Nodes: S-A1-2, S-A1-3, S-A1-4, S-A1-10, S-A1-13, S-A1-16

4032 Composite from Sample Nodes: S-A1-2, S-A1-3, S-A1-4, S-A1-10, S-A1-13, S-A1-16

4033 Composite from Sample Nodes: S-A1-2, S-A1-3, S-A1-4, S-A1-10, S-A1-13, S-A1-16

4034 Composite from Sample Nodes: S-A2-3, S-A2-6, S-A2-7, S-A2-10, S-A2-14, S-A2-15

4035 Composite from Sample Nodes: S-A3-1, S-A3-2, S-A3-4, S-A3-5, S-A3-9, S-A3-11

4036 Composite from Sample Nodes: S-A4-3, S-A4-4, S-A4-7, S-A4-9, S-A4-12, S-A4-15

4037 Composite from Sample Nodes: S-B5-1, S-B5-2, S-B5-5, S-B5-7, S-B5-13, S-B5-15

4038 Composite from Sample Nodes: S-B6-1, S-B6-10, S-B6-12, S-B6-13, S-B6-15, S-B6-16

4039 Composite from Sample Nodes: S-B7-1, S-B7-3, S-B7-4, S-B7-5, S-B7-8, S-B7-11

4040 Composite from Sample Nodes: S-B8-2, S-B8-3, S-B8-4, S-B8-8, S-B8-12, S-B8-13

4041 From off of floor of plume 15 on pipeline 4

4042 From off of floor of plume 15 on pipeline 4

4043 From off of floor of plume 15 on pipeline 4

4044 From off of floor of plume 15 on pipeline 4

\begin{tabular}{|l|l|}
4045 & From off of floor of plume 15 on pipeline 4
\end{tabular}

4046 From off of floor of plume 15 on pipeline 4

4047 From off of floor of plume 15 on pipeline 4

4048 From off of floor of plume 15 on pipeline 4

4049 From off of floor of plume 15 on pipeline 4

4050 From off of floor of plume 15 on pipeline 4

4051 From off of floor of plume 15 on pipeline 4

\begin{tabular}{|l|l|l}
4051 & From off of floor of plume 15 on pipeline 4
\end{tabular}

4053 116-N-3 Size Reduction Area - Sample Point: SRA-11

4054 116-N-3 Size Reduction Area - Sample Point: SRA-12

4055 116-N-3 Size Reduction Area - Sample Point: SRA-13

4056 116-N-3 Size Reduction Area - Sample Point: SRA-14

4057 116-N-3 Size Reduction Area - Sample Point: SRA-15

4058 116-N-3 Size Reduction Area - Sample Point: SRA-16

4059 116-N-3 Size Reduction Area - Sample Point: SRA-17

4060 116-N-3 Size Reduction Area - Sample Point: SRA-18

4061 Burial Ground - Test Pit

4062 Burial Ground - Test Pit (B1-TP1)

4063 Burial Ground - Test Pit

4064 Burial Ground - Test Pit

4065 Burial Ground - Test Pit (B1-TP1)

\begin{tabular}{|l|l}
4066 & Burial Ground - Test Pit (B1-TP2) \\
\hline 4067 & Buil Ground - Test Pit (B1-TP3)
\end{tabular}

\begin{tabular}{|l|l|}
\hline 4067 & Burial Ground - Test Pit (B1-TP3) \\
\hline
\end{tabular} 
4070 116-N-3 Size Reduction Area - Sample Point: SRA-3-01

4071 116-N-3 Size Reduction Area - Sample Point: SRA-3-02

4072 116-N-3 Size Reduction Area - Sample Point: SRA-3-03

4073 116-N-3 Size Reduction Area - Sample Point: SRA-3-04

4074 116-N-3 Size Reduction Area - Sample Point: SRA-3-05

4075 116-N-3 Size Reduction Area - Sample Point: SRA-3-06

\begin{tabular}{|l|l}
4076 & "Load-Out" Pile, at east end of zone 5 \\
\hline 4077 & Load-Out" Plle, at east end of zone 5
\end{tabular}

4077 "Load-Out" Pile, at east end of zone 5

4078 116-F-2 Shallow Zone Node: S-A1-2

\begin{tabular}{|l|l|l|}
4079 & $116-F-2$ & Shallow Zone Node: S-A1-3
\end{tabular}

\begin{tabular}{|l|l}
4080 & $116-F-2$ \\
408 & Shallow Zone Node: S-A1-4
\end{tabular}

4081 116-F-2 Shallow Zone Node: S-A1-4

4082 "Load-Out" Pile, at east end of zone 5

4083 "Load-Out" Pile, at east end of zone 5

4084 "Load-Out" Pile, at east end of zone 5

4085 "Load-Out" Pile, at east end of zone 5

4086 "Load-Out" Pile, at east end of zone 5

4087 $116-\mathrm{N}-3$ Size Reduction Area - Sample Point: SRA-3-04 (After area was leveled)

4088 116-N-3 Size Reduction Area - Sample Point: SRA-3-05 (After area was leveled)

4089 116-F-2 Shallow Zone Node: S-A1-13

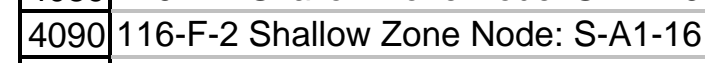

\begin{tabular}{|l|l}
4091 & $116-F-2$ \\
\hline
\end{tabular}

4092 116-F-2 Shallow Zone Node: S-A2-6

4093 116-F-2 Shallow Zone Node: S-A2-7

4094 116-F-2 Shallow Zone Node: S-A2-10

4095 116-F-2 Shallow Zone Node: S-A2-14

4096 116-F-2 Shallow Zone Node: S-A2-15

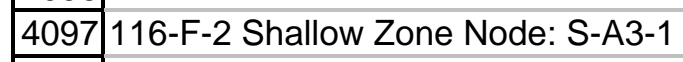

\begin{tabular}{|l|l|}
4098 & $116-F-2$ \\
\hline
\end{tabular}

4099 116-F-2 Shallow Zone Node: S-A3-4

\begin{tabular}{l|l}
4100 & $116-F-2$ \\
\end{tabular}

\begin{tabular}{|l|l|l|}
\hline 4101 & $116-F-2$ & Shallow Zone Node: S-A3-9
\end{tabular}

4102 116-F-2 Shallow Zone Node: S-A3-11

4103 116-F-2 Shallow Zone Node: S-A4-3

\begin{tabular}{|l|l}
4104 & $116-F-2$ \\
\hline 4105 & Shallow Zone Node: S-A4-4
\end{tabular}

\begin{tabular}{|l|l|l}
\hline 4105 & $116-F-2$ & Shallow Zone Node: S-A4-7 \\
\hline
\end{tabular}

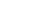

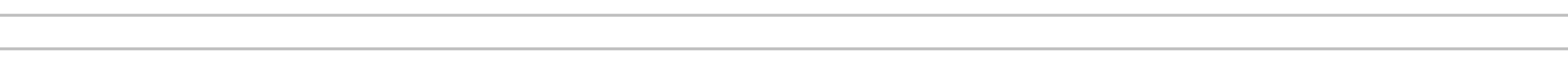

20

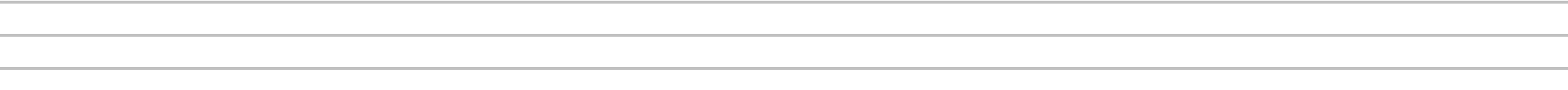

(14)

(1)

(2)

1


4106 116-F-2 Shallow Zone Node: S-A4-9

SAMP SITE DESC

4107 116-F-2 Shallow Zone Node: S-A4-12

4108 116-F-2 Shallow Zone Node: S-A4-13

4109 116-N-3 Decon Pad - Sump

4110 116-N-3 Decon Pad

4111 Pipeline coating from line 26

4112 Pipeline coating from line 26

4113 From off of floor of plume 15 on pipeline 4

\begin{tabular}{|l|l|l|}
4114 & From off of floor of plume 15 on pipeline 4
\end{tabular}

4115 From off of floor of plume 15 on pipeline 4

4116 From off of floor of plume 15 on pipeline 4

4117 From off of floor of plume 15 on pipeline 4

\begin{tabular}{|l|l}
4118 & From off of floor of plume 15 on pipeline 4
\end{tabular}

4119 From off of floor of plume 15 on pipeline 4

\begin{tabular}{|l|l}
4120 & From off of floor of plume 15 on pipeline 4
\end{tabular}

\begin{tabular}{|l|l}
4121 & From off of floor of plume 15 on pipeline 4
\end{tabular}

4122 From off of floor of plume 15 on pipeline 4

4123 From off of floor of plume 15 on pipeline 4

4124 116-F-2 Shallow Zone Node: S-B5-1

4125 116-F-2 Shallow Zone Node: S-B5-2

4126 116-F-2 Shallow Zone Node: S-B5-5

4127 116-F-2 Shallow Zone Node: S-B5-7

\begin{tabular}{|l|l|}
4128 & $116-F-2$ \\
Shallow Zone Node: S-B5-13
\end{tabular}

4129 116-F-2 Shallow Zone Node: S-B5-15

4130 116-F-2 Shallow Zone Node: S-B6-1

4131 116-F-2 Shallow Zone Node: S-B6-10

4132 116-F-2 Shallow Zone Node: S-B6-12

4133 116-F-2 Shallow Zone Node: S-B6-13

4134 116-F-2 Shallow Zone Node: S-B6-15

\begin{tabular}{|l|l}
4135 & $116-F-2$ \\
Shallow Zone Node: S-B6-16
\end{tabular}

4136 116-F-2 Shallow Zone Node: S-B7-1

4137 116-F-2 Shallow Zone Node: S-B7-3

4138 116-F-2 Shallow Zone Node: S-B7-4

\begin{tabular}{|l|l}
4139 & $116-F-2$ \\
\hline 4140 & Shallow Zone Node: S-B7-5
\end{tabular}

4140 116-F-2 Shallow Zone Node: S-B7-8

4141 116-F-2 Shallow Zone Node: S-B7-11

\begin{tabular}{|l|l|}
4142 & $116-F-2$ \\
4
\end{tabular} \begin{tabular}{|l|l|l}
\hline 4143 & $116-F-2$ & Shallow Zone Node: S-B8-3
\end{tabular}

$(20)$

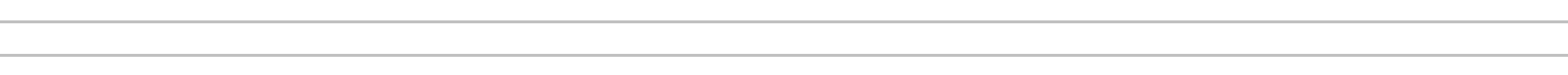

(20)

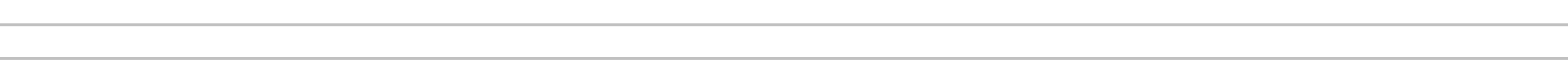

(20)

(20) 
4145 116-F-2 Shallow Zone Node: S-B8-8

4146 116-F-2 Shallow Zone Node: S-B8-12

4147 116-F-2 Shallow Zone Node: S-B8-13

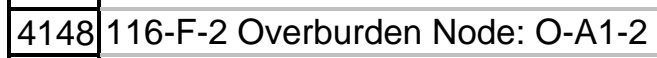

\begin{tabular}{|l|l|}
4149 & $116-F-2$ \\
\hline
\end{tabular}

\begin{tabular}{l|l}
4150 & $116-F-2$ \\
\end{tabular}

4151 116-F-2 Overburden Node: O-A1-10

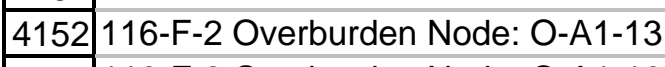

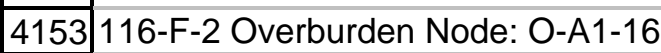

4154 116-F-2 Overburden Node: O-A2-3

4155 116-F-2 Overburden Node: O-A2-6

4156 116-F-2 Overburden Node: O-A2-7

4157 116-F-2 Overburden Node: O-A2-10

4158 116-F-2 Overburden Node: O-A2-14

\begin{tabular}{|l|l|l|}
\hline 4159 & $116-F-2$ & Overburden Node: O-A2-15 \\
\hline 4160 & $116-F-2$ & Overburden Node: O-A3-1
\end{tabular}

4160 116-F-2 Overburden Node: O-A3-1

4161 116-F-2 Overburden Node: O-A3-2

4162 116-F-2 Overburden Node: O-A3-4

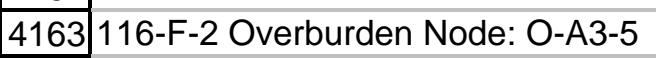

4164 116-F-2 Overburden Node: O-A3-9

4165 116-F-2 Overburden Node: O-A3-11

\begin{tabular}{|l|l}
\hline 4166 & $116-F-2$ \\
\hline 4167 & Overburden Node: O-A4-3
\end{tabular}

4167 116-F-2 Overburden Node: O-A4-4

4168 116-F-2 Overburden Node: O-A4-7

4169 116-F-2 Overburden Node: O-A4-9

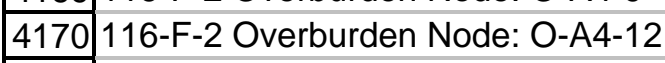

4171 116-F-2 Overburden Node: O-A4-13

4172 116-F-2 Overburden Node: O-B5-1

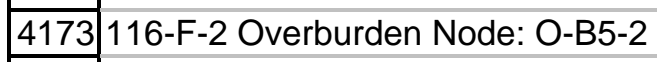

4174 116-F-2 Overburden Node: O-B5-5

4175 116-F-2 Overburden Node: O-B5-7

4176 116-F-2 Overburden Node: O-B5-13

\begin{tabular}{|l|l|l|}
\hline 4177 & $116-F-2$ & Overburden Node: O-B5-15 \\
\hline 4178 & $116-F-2$ Overburden Node: O-B6-1
\end{tabular}

4178 116-F-2 Overburden Node: O-B6-1

4179 116-F-2 Overburden Node: O-B6-10

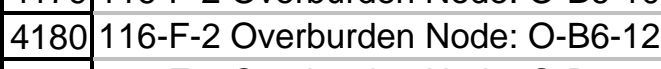

\begin{tabular}{|l|l|l|}
4181 & $116-F-2$ & Overburden Node: O-B6-13
\end{tabular} 
4183 116-F-2 Overburden Node: O-B6-16

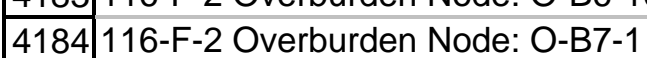

\begin{tabular}{l|l}
4185 & $116-F-2$ \\
\end{tabular}

4186 116-F-2 Overburden Node: O-B7-4

4187 116-F-2 Overburden Node: O-B7-5

4188 116-F-2 Overburden Node: O-B7-8

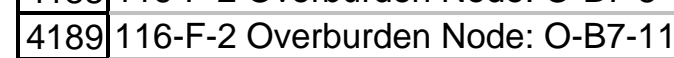

4190 116-F-2 Overburden Node: O-B8-2

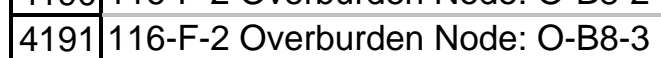

4192 116-F-2 Overburden Node: O-B8-4

4193 116-F-2 Overburden Node: O-B8-8

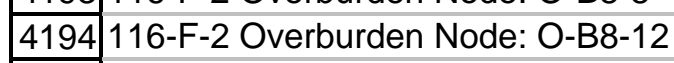

4195 116-F-2 Overburden Node: O-B8-13

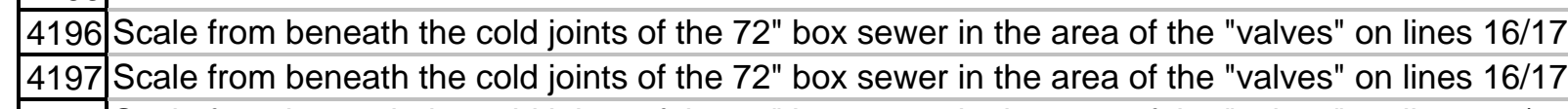

4198 Scale from beneath the cold joints of the 72" box sewer in the area of the "valves" on lines 16/17.

4199 From the black tarry viscous material observed seeping from the ground in the vicinity of where pipelines 18 \& 21 cross under the 72 " twin box sewer.

4200 116-F-2 Overburden Composite of OA1-3, OA1-4, OA1-10 and 0A1-16

4201 116-F-2 Overburden Composite of OA1-3, OA1-4, OA1-10 and OA1-16

4202 116-F-2 Overburden Composite of OA1-3, OA1-4, OA1-10 and 0A1-16

4203 116-F-2 Overburden Composite of OB2-3, OB2-6, OB2-7 and OB2-15

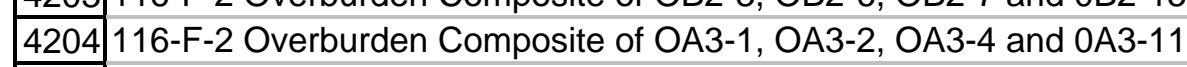

4205 116-F-2 Overburden Composite of OA4-3, OA4-4, OA4-7 and 0A4-12

4206 116-F-2 Overburden Composite of OB5-1, OB5-5, OB5-7 and 0B5-15

4207 116-F-2 Overburden Composite of OB6-1, OB6-10, OB6-13 and 0B6-15

4208 116-F-2 Overburden Composite of OB7-3, OB7-4, OB7-5 and 0B7-11

4209 116-F-2 Overburden Composite of OB8-3, OB8-4, OB8-8 and 0B8-13

4210 From the black tarry viscous material observed seeping from the ground in the vicinity of where pipelines 18 \& 21 cross under the 72 " twin box sewer.

4211 From the black tarry viscous material observed seeping from the ground in the vicinity of where pipelines 18 \& 21 cross under the 72 " twin box sewer.

4212 Sample Point: N1-DRPH-040-1

4213 Sample Point N1-DRPH-040-2

\begin{tabular}{|l|l|}
4213 & Sample Point N1-DRPH-023-1 \\
\hline 4215 & Samplng Point N1-DRPH-026-1 \\
\hline
\end{tabular}

4215 Sampling Point N1-DRPH-026-1

4216 Sample Point N1-DRPH-028-1

4217 Composite soil sample collected from the excavation floor as soil is loaded out

\begin{tabular}{|l|l}
\hline 4218 & Pipeline 11 Hotspot Sampling \\
\hline
\end{tabular}

4219 Composite from Sample Nodes SA1-3, SA1-4, SA1-10, SA1-16 
4220 Composite from Sample Nodes S-A1-3, S-A1-4, S-A1-10, S-A1-16

SAMP SITE DESC

4221 Composite from Sample Nodes S-A1-3, S-A1-4, S-A1-10, A-1-16

4222 Composite from Sample Nodes SA2-3, SA2-6, SA2-7, SA2-15

4223 Composite from Sample Nodes SA3-1, SA3-2, SA3-4, SA3-11

4224 Composite from Sample Nodes: SA4-3, SA4-4, SA4-7, SA4-12

4225 Composite from Sample Nodes: SB5-1, SB5-5, SB5-7, SB5-15

4226 Composite from Sampling Nodes: SB6-1, SB6-10, SB6-13, SB6-15

4227 Composite from Sampling Nodes: SB7-3, SB7-4, SB7-5, SB7-11

4228 Composite of Sampling Nodes: SB8-3, SB8-4, SB8-8, SB8-13

4229

4230

4231

4232

4233

\begin{tabular}{|l}
4233 \\
\hline 4235 \\
\hline
\end{tabular}

4235

4236

4237

4238

\begin{tabular}{|l|l|}
4239 & $116-N-3$ DECON PAD SUMP \\
\hline 4240 & $116-N-3$ DECON PAD SUMP \\
\hline
\end{tabular}

\begin{tabular}{|l|l|}
\hline 4240 & $116-N-3$ DECON PAD SUMP \\
\hline
\end{tabular}

4241

4242

4243

4244

\begin{tabular}{|l|l|}
\hline 4245 \\
\hline 4246 \\
\hline
\end{tabular}

4246

4247

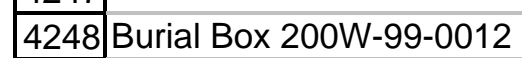

\begin{tabular}{|l|l|}
4249 & Burial Box 200W-99-0012 \\
\hline 4250 & Burial Box 200W-98-0010
\end{tabular}

4250 Burial Box 200W-98-0010

4251 Burial Box 200W-98-0010

4252 Waste Container with no identifier

4253 Waste Container with no identifier

4254 Waste Container with no identifier

4255 Burial Box 200W-98-0010

\begin{tabular}{|l|l|}
\hline 4256 & Bag with no identifier \\
\hline 4257 & Bag with no identier \\
\hline
\end{tabular}

\begin{tabular}{|l||l|}
\hline 4257 & Bag with no identifier \\
\hline
\end{tabular} 
4260 Composite of sample material from drum 202S-95-0025 and the unidentified drum

4261 From Core 36-E and 36-F/sample designated as PC 36-1

4262 From Core 36-C and 36-D/sample designated as PC 36-2

4263 From Core 36-E and 36-F/sample designated as PC 36-1

4264 From Core 36-E and 36-F/sample designated as PC 36-1

4265 From Core $36-\mathrm{C}$ and 36-D/sample designated as PC 36-2

4266 From Core 36-C and 36-D/sample designated as PC 36-2

4267 From Core 6-J, 6-K and 6-M/sample designated as PC 36-2

4268 From Core 6-J, 6-K and 6-M/sample designated as PC 36-2

4269 From Core 6-J, 6-K and 6-M/sample designated as PC 36-2

4270 From Core 6-A and 6-B/sample designated as PC 6-2

4271 From Core 6-A and 6-B/sample designated as PC 6-2

4272 From Core 6-A and 6-B/sample designated as PC 6-2

\begin{tabular}{|l|l}
4273 & From Core 26-D and 26-J/sample designated as PC 26-1
\end{tabular}

4274 From Core 26-D and 26-J/sample designated as PC 26-1

4275 From Core 26-A and 26-B/sample designated as PC 26-1

4276 From Core 26-A and 26-B/sample designated as PC 26-1

4277 From Core 26-C, 26-G, and 26-H/sample designated as PC 26-2

4278 From Core 26-C, 26-G, and 26-H/sample designated as PC 26-2

4279 From Core 26-C, 26-G, and 26-H/sample designated as PC 26-2

4280 From Core 5-C, and 5-D/sample designated as PC 5-1

4281 From Core 5-C, and 5-D/sample designated as PC 5-1

4282 From Core 5-C, and 5-D/sample designated as PC 5-1

4283 From Core 5-A, and 5-E/sample designated as PC 5-2

4284 From Core 5-A, and 5-E/sample designated as PC 5-2

4285 From Core 26-A and 26-B/sample designated as PC 26-1

\begin{tabular}{|l|l|l|}
\hline 4286 & $24-i n c h$ drain pipe \\
\hline 4287 & $24-$ inch drain pipe - fr
\end{tabular}

\begin{tabular}{|l|l}
4287 & 24 -inch drain pipe - from track on robot used to retrieve samples
\end{tabular}

\begin{tabular}{|l|l|}
\hline 4288 & $24-$ inch drain pipe \\
\hline
\end{tabular}

4289 24-inch drain pipe - from track on robot used to retrieve samples

\begin{tabular}{|l|l|}
\hline 4290 & $24-$ inch drain pipe \\
\hline 4291 & $24-$ inch drain pipe
\end{tabular}

4291 24-inch drain pipe

\begin{tabular}{|l|l}
4292 & $24-i n c h$ drain pipe \\
\hline
\end{tabular}

4293 24-inch drain pipe

\begin{tabular}{|l|l|l|}
\hline 4294 & $24-$ inch drain pipe \\
\hline 4295 & $24-$ inch drain pipe \\
\hline
\end{tabular}

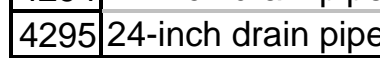


4297 24-inch drain pipe

4298 24-inch drain pipe

4299 Site \#8 on south side of facility

\begin{tabular}{|l|l|l|}
\hline 4300 & Site $\# 3$ at the pumphouse \\
\hline 4301 & From
\end{tabular}

\begin{tabular}{|l|l}
4301 & From the NW corner of the 308 building
\end{tabular}

4302 CAB shack in the the NW corner of the 291-U fence enclosrue

4303 CAB shack in the the NW corner of the 291-U fence enclosrue

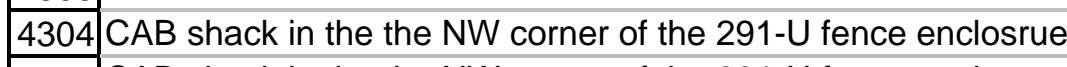

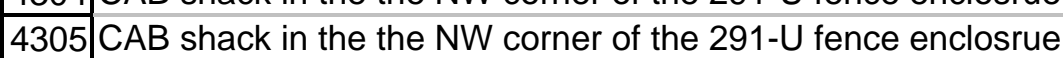

4306 Composite from each end of overhead pipe near 293-S and 292-S, and from 8" overhead pipe just N of large smoke stack that is N of 291-U

4307 Composite from each end of overhead pipe near 293-S and 292-S, and from 8" overhead pipe just $\mathrm{N}$ of large smoke stack that is $\mathrm{N}$ of $291-\mathrm{U}$

4308 From pipe 18" off ground on E side of building

4309 From pipe 18" off ground on E side of building

4310 From pipe 18" off ground on E side of building

4311 From pipes on NE corner of building

4312 From pipes on NE corner of building

4313 From east side of building

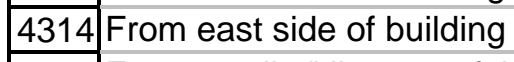

4315 From small 3" line out of the back of the builing on the west side

4316 From small 3" line out of the back of the builing on the west side

4317 from 8" pipe

4318 from 8" pipe

4319 from East side of building

4320 from East side of building

4321 From 8" elbow, two different 4" lines

4322 From 8" elbow, two different 4 " lines

4323 From inside the building, 2nd story - from door by fire extinguisher station \#4

4324 From inside the building, 2nd story - from door by fire extinguisher station \#4

4325 From Cell 30

4326 From Cell 24

4327 From Cell 24

\begin{tabular}{|l|l|}
4328 & From Cell 24 \\
\hline 4329 & From
\end{tabular}

4329 From tank 5-6 in cell 10

4330 From tank 5-6 in cell 10

4331 From tank 5-6 in cell 10

4332 From tank 5-6 in cell 10

\begin{tabular}{|l|l}
\hline 4333 & From tank 5-6 in cell 10
\end{tabular} 
4335 CRIB WITHIN THE 200-TW-1 OU 4336 CRIB WITHIN THE 200-TW-1 OU 4337 CRIB WITHIN THE 200-TW-1 OU \begin{tabular}{|l|l|}
\hline 4338 & CRIB WITHIN THE 200-TW-1 OU \\
\hline 4339 & CRIB WITHIN THE 200-TW-1 OU
\end{tabular} 4339 CRIB WITHIN THE 200-TW-1 OU 4340 CRIB WITHIN THE 200-TW-1 OU 4341 CRIB WITHIN THE 200-TW-2 OU \begin{tabular}{|l|l|l|}
\hline 4342 & CRIB WITHIN THE 200-TW-2 OU \\
\hline 4343 & CRIB WITHIN THE 200-TW-2 OU
\end{tabular}

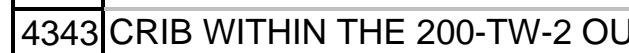
4344 CRIB WITHIN THE 200-TW-2 OU \begin{tabular}{|l|l}
43445 & CRIB WITHIN THE 200-TW-2 OU
\end{tabular} \begin{tabular}{|l|l|}
\hline 4346 & CRIB WITHIN THE 200-TW-1 OU \\
\hline
\end{tabular} 4347 CRIB WITHIN THE 200-TW-2 OU \begin{tabular}{|l|l}
4348 & CRIB WITHIN THE 200-TW-2 OU \\
\hline 4349 & CRIB WITHIN THE 200-TW-1 OU
\end{tabular} \begin{tabular}{|l|l|}
4348 & CRIB WITHIN THE 200-TW-2 OU \\
\hline 4349 & CRIB WITHIN THE 200-TW-1 OU
\end{tabular} 4350 CRIB WITHIN THE 200-TW-1 OU 4351 CRIB WITHIN THE 200-TW-1 OU 4352 CRIB WITHIN THE 200-TW-1 OU

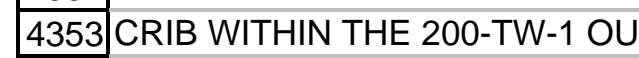
4354 CRIB WITHIN THE 200-TW-1 OU 4355 CRIB WITHIN THE 200-TW-1 OU \begin{tabular}{|l|l|}
\hline 4356 & CRIB WITHIN THE 200-TW-1 OU \\
\hline 4357 & CRIB WITHIN THE 200-TW-1 OU
\end{tabular} 4357 CRIB WITHIN THE 200-TW-1 OU 4358 CRIB WITHIN THE 200-TW-1 OU 4359 CRIB WITHIN THE 200-TW-1 OU \begin{tabular}{|l|l|}
4360 & CRIB WITHIN THE 200-TW-1 OU \\
\hline 4361 & CRIB WITHIN THE 200-TW-2 OU
\end{tabular} 4361 CRIB WITHIN THE 200-TW-2 OU

4362 CRIB WITHIN THE 200-TW-2 OU

\begin{tabular}{|l|l|}
\hline 4363 & CRIB WITHIN THE 200-TW-2 OU \\
\hline 4364 & CRIB WITHN THE 200-TW-2 OU
\end{tabular}

4364 CRIB WITHIN THE 200-TW-2 OU

4365 CRIB WITHIN THE 200-TW-2 OU

4366 CRIB WITHIN THE 200-TW-2 OU

\begin{tabular}{|l|l|}
\hline 4367 & CRIB WITHIN THE 200-TW-2 OU \\
\hline 4368 & CRIB WITHN THE 200-TW-2 OU
\end{tabular}

4368 CRIB WITHIN THE 200-TW-2 OU

4369 CRIB WITHIN THE 200-TW-2 OU 
4373 CRIB WITHIN THE 200-TW-2 OU 4374 CRIB WITHIN THE 200-TW-2 OU 4375 CRIB WITHIN THE 200-TW-2 OU 4376 CRIB WITHIN THE 200-TW-2 OU 4377 CRIB WITHIN THE 200-TW-2 OU 4378 CRIB WITHIN THE 200-TW-2 OU 4379 CRIB WITHIN THE 200-TW-2 OU $\begin{array}{lll}4379 & \text { CRIB WIB WITHIN THE 200-TW-2 OU }\end{array}$ 4381 CRIB WITHIN THE 200-TW-2 OU 4382 CRIB WITHIN THE 200-TW-2 OU \begin{tabular}{|l|l|}
\hline 4383 & CRIB WITHIN THE 200-TW-2 OU \\
\hline
\end{tabular} 4384 CRIB WITHIN THE 200-TW-2 OU 4385 CRIB WITHIN THE 200-TW-2 OU 4386 CRIB WITHIN THE 200-TW-2 OU \begin{tabular}{l|l}
4386 & CRIB WITHIN THE 200-TW-2 OU \\
\hline 4387 & CRIB WITHIN THE 200-TW-2 OU
\end{tabular} 4388 CRIB WITHIN THE 200-TW-2 OU 4389 CRIB WITHIN THE 200-TW-2 OU

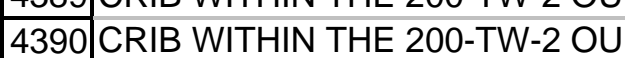
4391 CRIB WITHIN THE 200-TW-1 OU 4392 CRIB WITHIN THE 200-TW-2 OU

4393 CRIB WITHIN THE 200-TW-2 OU 4394 CRIB WITHIN THE 200-TW-2 OU 4395 CRIB WITHIN THE 200-TW-2 OU 4396 CRIB WITHIN THE 200-TW-2 OU 4397 CRIB WITHIN THE 200-TW-2 OU

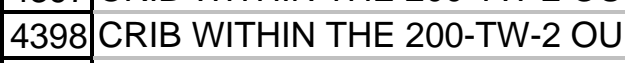
4399 CRIB WITHIN THE 200-TW-2 OU

4400 CRIB WITHIN THE 200-TW-2 OU 4401 CRIB WITHIN THE 200-TW-2 OU 4402 CRIB WITHIN THE 200-TW-2 OU 4403 CRIB WITHIN THE 200-TW-2 OU 4404 CRIB WITHIN THE 200-TW-2 OU \begin{tabular}{|l|l}
44405 & CRIB WITHIN THE 200-TW-2 OU \\
\hline 4406 & CRRB WITHN THE 200-TW-2 OU
\end{tabular} 4406 CRIB WITHIN THE 200-TW-2 OU 4407 CRIB WITHIN THE 200-TW-1 OU 4408 CRIB WITHIN THE 200-TW-1 OU \begin{tabular}{|l|l|l|}
\hline 4409 & CRIB WITHIN THE 200-TW-1 OU \\
\hline
\end{tabular} 
4431 CRIB WITHIN THE 200-TW-2 OU

\begin{tabular}{|l|l|}
\hline 4432 & CRIB WITHIN THE 200-TW-2 OU \\
\hline 4433 & CRIB WITHN THE 200-TW-2 OU
\end{tabular}

\begin{tabular}{|l|l}
\hline 4433 & CRIB WITHIN THE 200-TW-2 OU \\
\hline
\end{tabular}

4434 CRIB WITHIN THE 200-TW-1 OU

4435 CRIB WITHIN THE 200-TW-2 OU

\begin{tabular}{|l|l|l|}
\hline 4436 & CRIB WITHIN THE 200-TW-2 OU \\
\hline 4437 & CRIB WITHN THE 200-TW-2 OU \\
\hline
\end{tabular}

4437 CRIB WITHIN THE 200-TW-2 OU

4438 CRIB WITHIN THE 200-TW-2 OU

\begin{tabular}{|l|l|}
\hline 4439 & CRIB WITHIN THE 200-TW-2 OU \\
\hline 444 & CRIB WITHN THE 200-TW-2 OU
\end{tabular}

4440 CRIB WITHIN THE 200-TW-2 OU

4441 CRIB WITHIN THE 200-TW-2 OU

4442 CRIB WITHIN THE 200-TW-2 OU

\begin{tabular}{|l|l|l|}
\hline 4443 & CRIB WITHIN THE 200-TW-2 OU \\
\hline 4444 & CRB WITHIN THE 200-TW-2 OU
\end{tabular}

4444 CRIB WITHIN THE 200-TW-2 OU

4445 CRIB WITHIN THE 200-TW-2 OU

4446 CRIB WITHIN THE 200-TW-2 OU 
4449 CRIB WITHIN THE 200-TW-2 OU

4450 CRIB WITHIN THE 200-TW-2 OU

4451 CRIB WITHIN THE 200-TW-2 OU

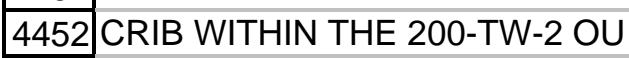

4453 CRIB WITHIN THE 200-TW-2 OU

4454 CRIB WITHIN THE 200-TW-2 OU

4455 CRIB WITHIN THE 200-TW-2 OU

\begin{tabular}{|l|l|}
\hline 4456 & CRIB WITHIN THE 200-TW-2 OU \\
\hline 4455 & CRIB WITHIN THE 200-TW-2 OU \\
\hline
\end{tabular}

\begin{tabular}{|l|l}
4457 & CRIB WITHIN THE 200-TW-2 OU \\
\hline 4458 & CRIB WITHIN THE 200-TW-2 OU
\end{tabular}

4458 CRIB WITHIN THE 200-TW-2 OU

4459 CRIB WITHIN THE 200-TW-2 OU

4460 CRIB WITHIN THE 200-TW-2 OU

4461 CRIB WITHIN THE 200-TW-2 OU

4462 CRIB WITHIN THE 200-TW-2 OU

\begin{tabular}{|l|l|}
\hline 4463 & CRIB WITHIN THE 200-TW-2 OU \\
\hline 4464 & CRIB WITHIN THE 200-TW-2 OU
\end{tabular}

4464 CRIB WITHIN THE 200-TW-2 OU

4465 CRIB WITHIN THE 200-TW-2 OU

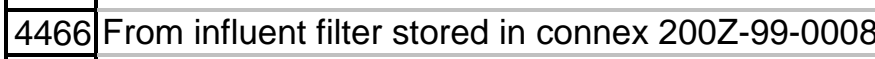

4467 From carbon stored in vessel marked AE-1090 - 200Z-00-0027 VO2A

4468 From influent filter stored in connex 200Z-99-0008

4469 From carbon stored in vessel marked AE-1090 - 200Z-00-0027 VO2A

4470 From cannister ae 1075

4471 From a cardboard box stored in an EPA trailer

4472 From a cardboard box stored in an EPA trailer

4473 Composite from 5 blue poly 55 gallon drums that are all lacking unique identifeirs

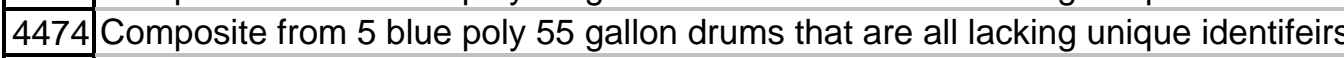

4475 Composite from 5 blue poly 55 gallon drums that are all lacking unique identifeirs

4476 Stored in the EPA trailer in the fenced in area of Pit 6.

4477 From an uncharacterized area of $271-\mathrm{U}$

4478 From the Purex and Redox craneways

4479 From the Purex and Redox craneways

4480 From the Purex and Redox craneways

4481 From the Purex and Redox craneways

4482 From the Purex and Redox craneways

4483 From the Purex and Redox craneways

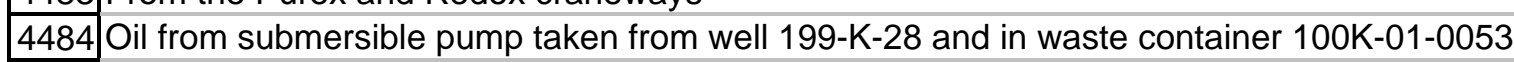

4485 Oil from submersible pump from well 299-10-E-12 and stored in the connex box loacated on the North side of the 308 Building 
4487 Casing from well 699-42-10

\begin{tabular}{|l|l|l|}
\hline 4488 & Casing from well 699-16-E4A \\
\hline
\end{tabular}

4489 Casing from well 699-7-E1A

\begin{tabular}{|l|l|}
\hline 4490 & Casing from well 699-7-E2 \\
\hline 4491 & Casing from well 699-11-E8A
\end{tabular}

44491 Casing from well 699-11-E8A

4492 Casing from well 699-14-3

4493 Casing from well 699-15-3

\begin{tabular}{|l|l|l|}
\hline 4494 & Casing from well 699-37-4 \\
\hline 4495 & Casing from well 699-38-E0 \\
\hline
\end{tabular}

4495 Casing from well 699-38-E0

4496 Casing from well HWDS53 (Well ID C3353)

\begin{tabular}{|l|l|l|}
\hline 4497 & Casing from well 699-39-1A \\
\hline 4498 & Casing from well HWD51 (Wel
\end{tabular}

\begin{tabular}{|l|l}
4498 & Casing from well HWDS51 (Well ID C3352) \\
\hline
\end{tabular}

4499 Casing from well 699-46-3

4500 Casing from well 699-40-12 G

\begin{tabular}{|l|l}
\hline 4501 & Casing from well HWDS52 Well ID C3351) \\
\hline 4502 & Casing from
\end{tabular}

\begin{tabular}{|l|l|l|}
\hline 4502 & Casing from well 699-15-4 \\
\hline 4503 & Casing from
\end{tabular}

4503 Casing from well 699-16-E4A

4504 Casing from well 699-36-1

\begin{tabular}{|l|l}
4505 & Casing from well 699-41-5 \\
\hline 4506 & Casing from
\end{tabular}

4506 Casing from well 699-40-6

4507 Casing from well 699-42-10

\begin{tabular}{|l|l|l|}
\hline 4508 & Casing from well 699-42-10 \\
\hline 4509 & Casing from well 699-16-E4 \\
\hline
\end{tabular}

4509 Casing from well 699-16-E4A

4510 Casing from well 699-7-E1A

\begin{tabular}{|l|l|}
\hline 4511 & Casing from well 699-7-E2
\end{tabular}

4512 Casing from well 699-11-E8A

4513 Casing from well 699-14-3

4514 Casing from well 699-37-4

4515 Casing from well 699-37-4

\begin{tabular}{|l|l}
4516 & Casing from well 699-37-4
\end{tabular}

4517 Casing from well 699-37-4

4518 Casing from well 699-15-3

\begin{tabular}{|l|l|l|}
4519 & Casing from well 699-37-4 \\
\hline 4520 & Casing from well 699-38-E0
\end{tabular}

4520 Casing from well 699-38-E0

4521 Casing from well HWDS53 (Well ID C3353)

\begin{tabular}{|l|l|}
\hline 4522 & Casing from well 699-39-1A \\
\hline 4523 & Casing from well HWDS51 \\
\hline
\end{tabular}

\begin{tabular}{|l|l|l|}
\hline 4523 & Casing from well HWDS51 (Well ID C3352) \\
\hline
\end{tabular} 
4525 Casing from well 699-40-12 G

4526 Casing from well HWDS52 Well ID C3351)

\begin{tabular}{|l|l}
\hline 4527 & Casing from well 699-15-4 \\
\hline
\end{tabular}

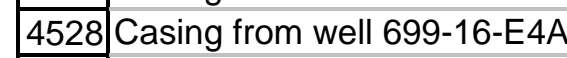

\begin{tabular}{|l|l}
4529 & Casing from well 699-36-1
\end{tabular}

4530 Casing from well 699-41-5

4531 Casing from well 699-40-6

\begin{tabular}{|l|l|l}
4532 & From a beaker locted in the space behind HVAC duct
\end{tabular}

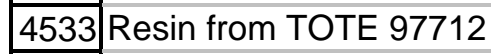

4534 Resin from TOTE 97742

4535 Resin from TOTE 99718

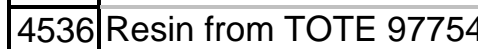

4537 Resin from TOTE 97729

\begin{tabular}{|l|l}
4538 & Resin from TOTE 97715 \\
\hline
\end{tabular}

\begin{tabular}{|l|l}
4539 & Resin from TOTE 97728 \\
\hline 4540 & Resin from TOTE 97720 \\
\hline
\end{tabular}

4540 Resin from TOTE 97720

4541 Resin from TOTE 97739

4542 Resin from TOTE 97726

\begin{tabular}{|l|l}
\hline 4543 & Resin from TOTE 97734 \\
\hline 4544 & Resin fom TOTE 97755 \\
\hline
\end{tabular}

4544 Resin from TOTE 97755

\begin{tabular}{|l|l}
4545 & Resin from TOTE 97719
\end{tabular}

\begin{tabular}{|l|l}
45546 & Resin from TOTE 97705 \\
\hline 4547 & Resin from TOTE 97746
\end{tabular}

\begin{tabular}{|l|l}
4547 & Resin from TOTE 97746 \\
\hline 4548
\end{tabular}

4548 Resin from TOTE 97744

4549 Resin from TOTE 97753

\begin{tabular}{|l|l}
\hline 4550 & Resin from TOTE 97747 \\
\hline
\end{tabular}

4551 Resin from TOTE 97731

4552 Resin from TOTE 97738

\begin{tabular}{|l|l}
4553 & Resin from TOTE 97707 marked K100-01-0055 \\
\hline
\end{tabular}

4554 Resin from TOTE 97710 marked K100-01-0057

4555 Resin from TOTE 97713 marked K100-01-0061

4556 Resin from TOTE 97724 marked K100-01-0058

\begin{tabular}{|l|l}
45557 & Resin from TOTE 97736 marked K100-01-0062 \\
\hline
\end{tabular}

4558 Resin from TOTE 97745 marked K100-01-0054

4559 Resin from TOTE 97751 marked K100-01-0059

4560 Resin from TOTE 97752 marked K100-01-0060

\begin{tabular}{|l|l}
45661 & Resin from TOTE 97706 marked K100-01-0066 \\
\hline
\end{tabular} 
4562 Resin from TOTE 97712 marked K100-01-0072

SAMP SITE DESC

4563 Resin from TOTE 97730 marked K100-01-0068 4564 Resin from TOTE 97741 marked K100-01-0065 4565 Resin from TOTE 97742 marked K100-01-0069 4566 Resin from TOTE 97754 marked K100-01-0071 4567 Resin from TOTE 97729 marked K100-01-0103 4568 Resin from TOTE 97726 marked K100-01-0104 4569 Resin from TOTE 97716 marked K100-01-0107 \begin{tabular}{|l|l}
4570 & Resin from TOTE 97717 marked K100-01-0106 \\
\hline
\end{tabular} 4571 Resin from TOTE 97721 marked K100-02-0002 4572 Resin from TOTE 97715 marked K100-02-0001 4573 Resin from TOTE 97710 marked K100-02-0013 4574 Resin from TOTE 97720 marked K100-02-0006 4575 Resin from TOTE 97724 marked K100-02-0012 4576 Resin from TOTE 97728 marked K100-02-0005 \begin{tabular}{|l|l}
4577 & Resin from TOTE 97747 marked K100-02-0010 \\
\hline
\end{tabular} 4578 Resin from TOTE 97753 marked K100-02-0011 4579 Resin from TOTE 97730 marked K100-02-0016

4580 Resin from TOTE 97742 marked K100-02-0015

4581 Resin from TOTE 97751 marked K100-02-0018

4582 Resin from TOTE 97752 marked K100-02-0017

4583 Resin from TOTE 97734

\begin{tabular}{|l|l}
4588 & Resin from TOTE 97746 \\
\hline 4585 & Resin from TOTE 97731
\end{tabular}

4585 Resin from TOTE 97731

4586 Resin from TOTE 97713

4587 Resin from TOTE 97723

\begin{tabular}{|l|l}
4588 & Resin from TOTE 97730
\end{tabular}

4589 Resin from BURIAL BOX 100H-01-0059

4590 Resin from BURIAL BOX 100H-01-0059

\begin{tabular}{|l|l}
4591 & Resin from TOTE 97749
\end{tabular}

4592 Resin from TOTE 97743

4593 Resin from TOTE 97717

4594 Resin from TOTE 97740

\begin{tabular}{|l|l|l}
\hline 4595 & Resin from TOTE 97725 \\
\hline 4596 & Resi from
\end{tabular}

4596 Resin from TOTE 97732

\begin{tabular}{|l|l|}
\hline 4597 & Resin from BURIAL BOX 100H-01-0097 \\
\hline 4598 & Resin from BURIAL BOX 100H-01-0097 \\
\hline 4599 & Resin from BURIAL BOX 100H-01-0100 \\
\hline
\end{tabular}

4599 Resin from BURIAL BOX $100 \mathrm{H}-01-0100$ 
4601 Resin from BURIAL BOX 100H-02-003

4602 Resin from TOTE 97708

4603 Resin from BURIAL BOX $100 \mathrm{H}-98-0157$

4604 Resin from BURIAL BOX $100 \mathrm{H}-98-0151$

\begin{tabular}{|l|l}
4605 & Resin from BURIAL BOX $100 \mathrm{H}-98-0153$ \\
\hline 4606 & Resin from
\end{tabular}

4606 Resin from BURIAL BOX $100 \mathrm{H}-98-0153$

4607 Resin from BURIAL BOX 100H-98-0178

\begin{tabular}{|l|l|l|}
4608 & Resin from BURIAL BOX $100 \mathrm{H}-98-0178$ \\
\hline 4609 & Resin from BURIAL BOX $100 \mathrm{H}-98-0139$ \\
\hline
\end{tabular}

4609 Resin from BURIAL BOX 100H-98-0139

4610 Resin from BURIAL BOX 100H-98-0139

4611 Resin from BURIAL BOX 100H-01-0129

\begin{tabular}{|l|l}
4612 & Resin from BURIAL BOX 100H-01-0129 \\
\hline 4613 & Resin from BURIAL BOX 100H -01-0116
\end{tabular}

4613 Resin from BURIAL BOX 100H-01-0116

4614 Resin from BURIAL BOX 100H-01-0116

\begin{tabular}{|l|l|l}
4615 & Resin from BURIAL BOX 100H-01-0104 \\
\hline 4615 & Resin from BURIAL BOX 100H -01-0104
\end{tabular}

4616 Resin from BURIAL BOX 100H-01-0104

4617 Resin from BURIAL BOX 100H-01-0115

4618 Resin from BURIAL BOX 100H-01-0115

\begin{tabular}{|l|l}
4619 & Resin from BURIAL BOX $100 \mathrm{H}-01-0113$ \\
\hline
\end{tabular}

4620 Resin from BURIAL BOX 100H-01-0113

4621 Resin from BURIAL BOX 100H-01-0133

\begin{tabular}{|l|l|l}
4622 & Resin from BURIAL BOX $100 \mathrm{H}-01-0134$ \\
\hline
\end{tabular}

4623 Resin from BURIAL BOX $100 \mathrm{H}-01-0132$

4624 Resin from BURIAL BOX 100H-01-0137

4625 Resin from BURIAL BOX 100H-01-0136

\begin{tabular}{|l|l}
4626 & Resin from BURIAL BOX 100H-01-0135 \\
\hline 4627 & Resin from BURIAL BOX $100 \mathrm{H}-01-0142$
\end{tabular}

4627 Resin from BURIAL BOX 100H-01-0142

4628 Resin from BURIAL BOX 100H-01-0142

4629 Resin from BURIAL BOX 100H-01-0167

4630 Resin from BURIAL BOX 100H-01-0167

4631 Resin from BURIAL BOX 100H-01-0093

4632 Resin from BURIAL BOX 100H-01-0094

\begin{tabular}{l|l}
4633 & Resin from BURIAL BOX $100 \mathrm{H}-02-0002$ \\
\hline 4634 & Resin from BURIAL BOX 100H-02-0001
\end{tabular}

4634 Resin from BURIAL BOX 100H-02-0001

4635 Resin from TOTE 97708

4636 Resin from BURIAL BOX 100H-01-0165

\begin{tabular}{|l|l|}
44637 & Resin from BURIAL BOX $100 \mathrm{H}-02-0028$ \\
\hline
\end{tabular}

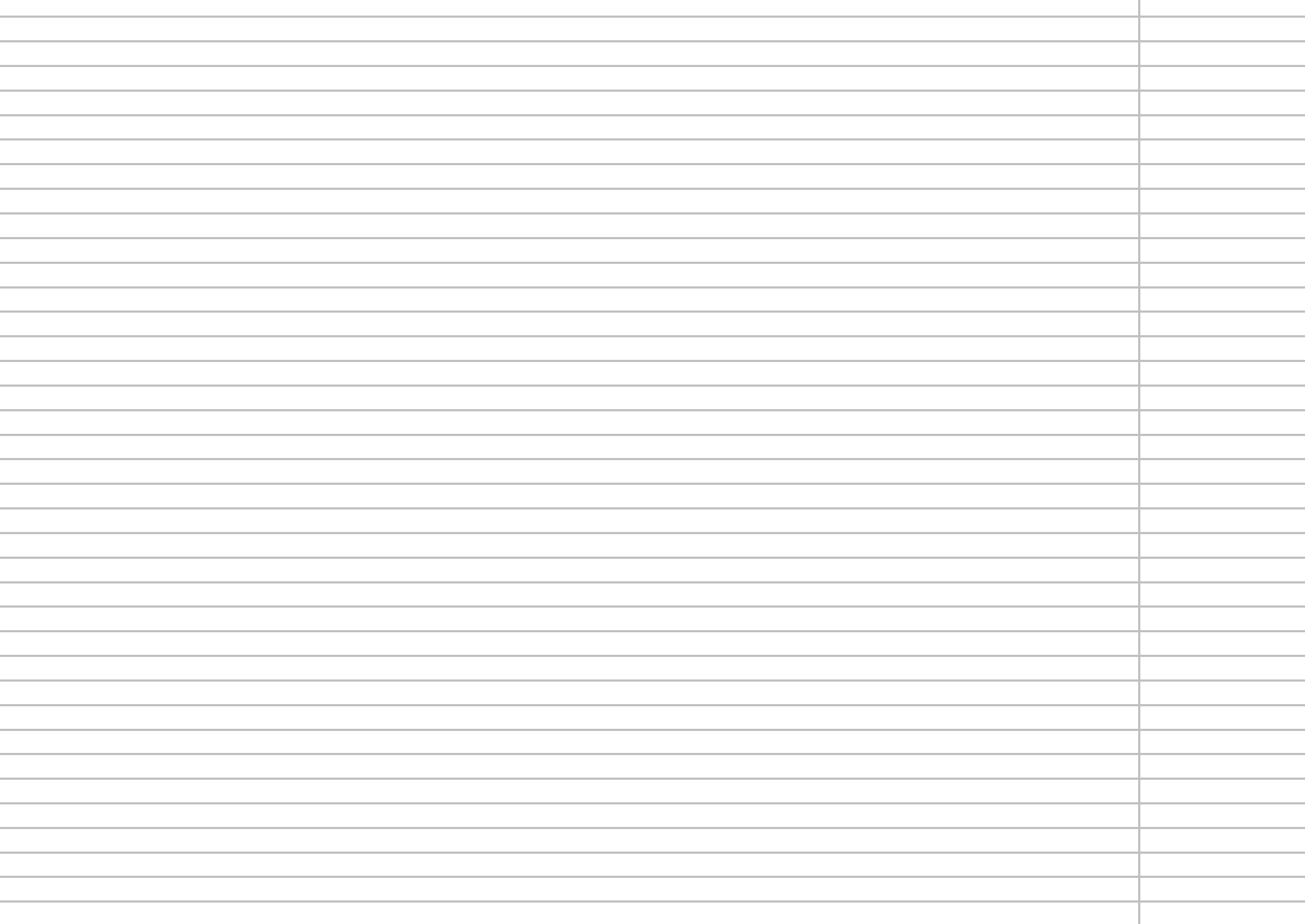


4639 Resin from BURIAL BOX 100H-02-0096

4640 Resin from BURIAL BOX 100H-01-0059

4641 Resin from BURIAL BOX $100 \mathrm{H}-02-0072$

4642 Resin from BURIAL BOX 100H-02-0072

\begin{tabular}{|l|l|l}
\hline 4643 & Resin from BURIAL BOX $100 \mathrm{H}-02-0100$ \\
\hline 4644 & Resin from BURIAL BOX $100 \mathrm{H}-02-0094$
\end{tabular}

4644 Resin from BURIAL BOX $100 \mathrm{H}-02-0094$

4645 Resin from BURIAL BOX $100 \mathrm{H}-02-0095$

\begin{tabular}{|l|l|l}
4646 & Resin from BURIAL BOX $100 \mathrm{H}-02-0101$ \\
\hline 4647 & Resin from
\end{tabular}

4647 Resin from BURIAL BOX 100H-02-0108

4648 Resin from BURIAL BOX 100H-02-0108

4649 Resin from TOTE 97708

4650 Resin from TOTE 97745

4651

4652

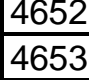

46554

4655

46556

4657 Smear of drum 600A-01-0193

4658 Smear of drum 600A-01-0192

4659 Smear of drum 600A-01-0178

\begin{tabular}{|l|l|l|}
46560 & Smear of drum 600A-01-0184 \\
\hline
\end{tabular}

4661 Smear of drum 600A-01-0171

4662 FROM DRUM 200W-01-0025

4663 FROM DRUM 200W-00-0008

4664 FROM DRUM 600A-01-0130

4665 FROM DRUM 200W-01-0115

4666 CRIB WITHIN THE 200-TW-2 OU

\begin{tabular}{|l|}
4667 \\
\hline 4668 \\
\hline
\end{tabular}

4668

\begin{tabular}{|l|}
\hline 4668 \\
\hline 4669 \\
\hline 4670 \\
\hline
\end{tabular}

4670 


\begin{tabular}{|c|c|c|}
\hline & 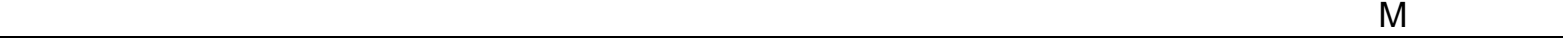 & \\
\hline \multirow{2}{*}{$\frac{1}{4676}$} & $\begin{array}{ll} & \text { SAMP_SITE_DESC } \\
\end{array}$ & SITE_ELEVATION \\
\hline & & \\
\hline \multicolumn{3}{|c|}{4677} \\
\hline \multicolumn{3}{|c|}{\begin{tabular}{|l|l|l}
4678 \\
\end{tabular}} \\
\hline \multicolumn{3}{|c|}{4679} \\
\hline \multicolumn{3}{|c|}{4680} \\
\hline \multicolumn{3}{|l|}{4681} \\
\hline \multicolumn{3}{|l|}{4682} \\
\hline \multicolumn{3}{|l|}{4683} \\
\hline \multicolumn{3}{|l|}{4684} \\
\hline \multicolumn{3}{|l|}{4685} \\
\hline \multicolumn{3}{|c|}{4686} \\
\hline \multicolumn{3}{|c|}{4687} \\
\hline \multicolumn{3}{|l|}{4688} \\
\hline \multicolumn{3}{|c|}{4689} \\
\hline \multicolumn{3}{|l|}{4690} \\
\hline \multicolumn{3}{|l|}{4691} \\
\hline \multicolumn{3}{|l|}{4692} \\
\hline \multicolumn{3}{|l|}{4693} \\
\hline \multicolumn{3}{|l|}{4694} \\
\hline \multicolumn{3}{|l|}{4695} \\
\hline \multicolumn{3}{|l|}{4696} \\
\hline \multicolumn{3}{|l|}{4697} \\
\hline \multicolumn{3}{|l|}{4698} \\
\hline \multicolumn{3}{|l|}{4699} \\
\hline \multicolumn{3}{|l|}{4700} \\
\hline \multicolumn{3}{|l|}{4701} \\
\hline \multicolumn{3}{|l|}{4702} \\
\hline 4703 & From the Influent Valve & \\
\hline 4704 & From the Effluent valve & \\
\hline 4705 & From the Influent Valve & \\
\hline 4706 & From the Effluent valve & \\
\hline 4707 & From the Influent Valve & \\
\hline 4708 & From the Effluent valve & \\
\hline 4709 & From the Influent Valve & \\
\hline 4710 & From the Effluent valve & \\
\hline 4711 & From the Influent Valve & \\
\hline 4712 & From the Effluent valve & \\
\hline 4713 & From the Influent Valve & \\
\hline
\end{tabular}


\begin{tabular}{|l|l|}
4715 & From the Influent Valve
\end{tabular}

\begin{tabular}{|l|l}
4716 & From the Effluent valve
\end{tabular}

4717 From Ion Exchange Column H-IX-A2

4718 From Ion Exchange Column H-IX-C3

4719 From $\mathrm{H}$-Influent valve

4720 From D-Influent valve

4721 From HR-3 Combined Effluent

\begin{tabular}{|l|l|l}
4722 & From Ion Exchange Column H-IX-D1 \\
\hline 4723 & From Ion Exchange Column H-IX-A2 \\
\hline
\end{tabular}

4723 From Ion Exchange Column H-IX-A2

4724 From Ion Exchange Column H-IX-C3

4725 From $\mathrm{H}$-Influent valve

\begin{tabular}{|l|l}
4726 & From D-Influent valve \\
\hline 4727 & From
\end{tabular}

4727 From HR-3 Combined Effluent

4728 From Ion Exchange Column H-IX-D2

\begin{tabular}{|l|l}
4729 & From K-Influent valve \\
\hline 4730 & From K-Efluent valve
\end{tabular}

4730 From K-Effluent valve

4731 From Ion Exchange Column K-IX-A4

\begin{tabular}{|l|l}
4732 & From Ion Exchange Column K-IX-B3 \\
\hline 4733 & From Ion Exchange Column K-IX-C3
\end{tabular}

4733 From Ion Exchange Column K-IX-C3

4734 From K-Influent valve

4735 From K-Effluent valve

\begin{tabular}{|l|l}
\hline 4736 & From Ion Exchange Column K-IX-A4 \\
\hline 4737 & From Ion Exchange Column K-IX-B3
\end{tabular}

4737 From Ion Exchange Column K-IX-B3

4738 From Ion Exchange Column K-IX-C3

4739 From the Influent Valve

\begin{tabular}{|l|l|}
\hline 4740 & From the Effluent valve
\end{tabular}

4741 From $\mathrm{H}$-Influent valve

4742 From $\mathrm{H}$-Influent valve

\begin{tabular}{|l|l}
4743 & From $\mathrm{H}$-Influent valve \\
\hline 4744 & From H-Iflent valve
\end{tabular}

4744 From $\mathrm{H}$-Influent valve

4745 From D-Influent valve

4746 From D-Influent valve

\begin{tabular}{|l|l}
4747 & From D-Influent valve
\end{tabular}

4748 From D-Influent valve

4749 From D-Influent valve

\begin{tabular}{|l|l}
4750 & From D-Influent valve \\
\hline 4751 & From HR-3 Combined Ef \\
\hline
\end{tabular}

\begin{tabular}{|l|l|}
44751 & From HR-3 Combined Effluent \\
\hline
\end{tabular} 
\begin{tabular}{|l|l|}
4753 & From HR-3 Combined Effluent \\
\hline
\end{tabular}

4754 From HR-3 Combined Effluent

4755 From H-Influent valve

\begin{tabular}{|l|l|}
\hline 4756 & From HR-3 Combined Effluent \\
\hline 4757 & From H-Infuent vave
\end{tabular}

\begin{tabular}{|l|l|}
44757 & From $\mathrm{H}-$ Influent valve \\
\hline 4758 & From HR-3 Combined
\end{tabular}

4758 From HR-3 Combined Effluent

4759 From HR-3 Combined Effluent

\begin{tabular}{|l|l|}
\hline 4760 & From HR-3 Combined Effluent \\
\hline 4761 & From Drum
\end{tabular}

4761 From Drum 200Z-01-0018

4762 From exterior of a heat exchanger building located between the PUREX R-cell and the gas bottle rack

\begin{tabular}{|l|l}
4763 & From exterior of a heat exchanger building located between the PUREX R-cell and the gas bottle rack
\end{tabular}

4764 From exterior of a heat exchanger building located between the PUREX R-cell and the gas bottle rack

4765 Box 233S-01-0039

4766 Box 233S-01-0039

\begin{tabular}{|l|l|l|}
\hline 4767 & Box 233S-01-0039 \\
\hline 4768 & Box 2335-01-0039 \\
\hline
\end{tabular}

4768 Box 233S-01-0039

4769 Box 233S-01-0039

4770 Box 233S-01-0039

4771 From Waste Container 233-S-01-0028

4772 From Waste Container 233-S-01-0029

4773 From Waste Container 233S-01-0030

\begin{tabular}{|l|l|l|}
\hline 4774 & From Waste Container 233S-01-0031
\end{tabular}

4775 From Waste Container 233S-01-0002

4776 From Waste Container 233S-01-0003

4777 From Waste Container 233S-01-0005

4778 From Waste Container 233S-01-0006

4779 From Waste Container 233S-01-0008

4780 From Waste Container 233S-01-0010

4781 From Waste Container 233S-01-0011

4782 From Waste Container 233S-01-0014

4783 From Waste Container 233S-01-0021

4784 From Waste Container 233S-01-0023

\begin{tabular}{|l|l|l|}
\hline 4785 & From Waste Container 233S-01-0023 \\
\hline 4786 & From Waste Contaner 233S-01-0024
\end{tabular}

4786 From Waste Container 233S-01-0024

4787 From Waste Container 233S-01-0025

4788 From Waste Container 233S-01-0026

\begin{tabular}{|l|l}
\hline 4789 & From Waste Container 233S-01-0027
\end{tabular} 
4791 From Waste Container 233S-01-0044

4792 From Waste Container 233S-01-0045

4793 From Waste Container 233S-01-0047

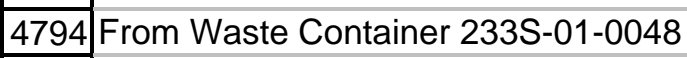

4 From Waste Container 2335-01-0049

4796 From Waste Container 233S-01-0085

4797 From Waste Container 233S-01-0093

\begin{tabular}{|l|l|}
\hline 4798 & From Waste Container 233S-01-0094 \\
\hline 4799 & From Waste Contaner 233S-01-0095
\end{tabular}

4799 From Waste Container 233S-01-0095

4800 From Waste Container 233S-01-0096

4801 From Waste Container 233S-01-0097

\begin{tabular}{|l|l}
\hline 4802 & From Waste Container 233S-01-0098
\end{tabular}

4803 From Waste Container 233S-01-0099

4804 From Waste Container 233S-01-0130

\begin{tabular}{|l|l}
4805 & From Waste Container 233S-01-0130
\end{tabular}

4806 Miscellaneous objects surveyed using the In Situ Object Counting System

4807 Miscellaneous objects surveyed using the In Situ Object Counting System

4808 Miscellaneous objects surveyed using the In Situ Object Counting System

4809 Miscellaneous objects surveyed using the In Situ Object Counting System

4810 From Waste Container 233S-01-0043

4811 From Waste Container 233S-01-0043

\begin{tabular}{|l|l}
4812 & From Waste Container 233S-01-0100 \\
\hline 4813 & From Waste Container 2335-01-0101
\end{tabular}

4813 From Waste Container 233S-01-0101

4814 From Waste Container 233S-01-0102

4815 From Waste Container 233S-01-0103

\begin{tabular}{|l|l}
4816 & From Waste Container 233S-01-0104
\end{tabular}

4817 From Waste Container 233S-01-0105

4818 From Waste Container 233S-01-0106

\begin{tabular}{|l|l}
4819 & From Waste Container 233S-01-0108
\end{tabular}

4820 From Waste Container 233S-01-0109

4821 From Waste Container 233S-01-0110

\begin{tabular}{|l|l|}
4822 & From Waste Container 233S-01-0119 \\
\hline
\end{tabular}

\begin{tabular}{|l|l}
\hline 4823 & From Waste Container 233S-01-0121 \\
\hline 4824 & From Waste Container $2335-01-0122$ \\
\hline
\end{tabular}

4824 From Waste Container 233S-01-0122

4825 From Waste Container 2233S-01-0123

\begin{tabular}{|l|l}
\hline 4826 & From Waste Container 233S-01-0051 \\
\hline 4827 & From Waste Container $2335-01-0052$ \\
\hline
\end{tabular}

4 4827 From Waste Container 233S-01-0052 
4829 From Waste Container 233S-01-0001

4830 From Waste Container 233S-01-0001

4831 Miscellaneous objects surveyed using the In Situ Object Counting System

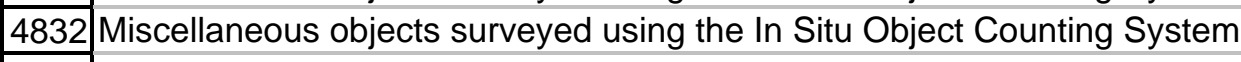

4833 Miscellaneous objects surveyed using the In Situ Object Counting System

4834 Miscellaneous objects surveyed using the In Situ Object Counting System

4835 Miscellaneous objects surveyed using the In Situ Object Counting System

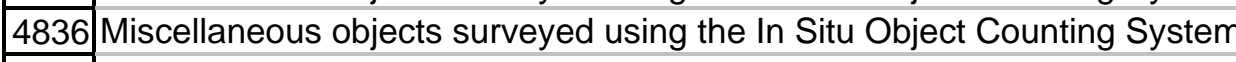

4837 Miscellaneous objects surveyed using the In Situ Object Counting System

4838 Miscellaneous objects surveyed using the In Situ Object Counting System

4839 Miscellaneous objects surveyed using the In Situ Object Counting System

4840 Miscellaneous objects surveyed using the In Situ Object Counting System

4841 Miscellaneous objects surveyed using the In Situ Object Counting System

4842 Miscellaneous objects surveyed using the In Situ Object Counting System

\begin{tabular}{|l|l}
4843 & Miscellaneous objects surveyed using the In Situ Object Counting System
\end{tabular}

4844 Miscellaneous objects surveyed using the In Situ Object Counting System

4845 Miscellaneous objects surveyed using the In Situ Object Counting System

4846 Miscellaneous objects surveyed using the In Situ Object Counting System

4847 Miscellaneous objects surveyed using the In Situ Object Counting System

4848 Miscellaneous objects surveyed using the In Situ Object Counting System

4849 Miscellaneous objects surveyed using the In Situ Object Counting System

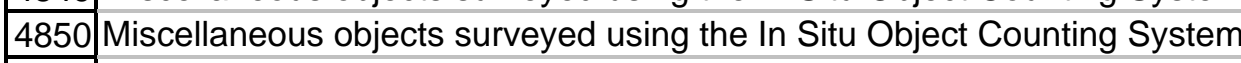

4851 Miscellaneous objects surveyed using the In Situ Object Counting System

4852 Miscellaneous objects surveyed using the In Situ Object Counting System

4853 Miscellaneous objects surveyed using the In Situ Object Counting System

4854 Miscellaneous objects surveyed using the In Situ Object Counting System

4855 Miscellaneous objects surveyed using the In Situ Object Counting System

4856 Miscellaneous objects surveyed using the In Situ Object Counting System

4857 Miscellaneous objects surveyed using the In Situ Object Counting System

4858 Miscellaneous objects surveyed using the In Situ Object Counting System

4859 Miscellaneous objects surveyed using the In Situ Object Counting System

4860 Miscellaneous objects surveyed using the In Situ Object Counting System

4861 Miscellaneous objects surveyed using the In Situ Object Counting System

4862 Miscellaneous objects surveyed using the In Situ Object Counting System

4863 Miscellaneous objects surveyed using the In Situ Object Counting System

4864 Miscellaneous objects surveyed using the In Situ Object Counting System

4865 Miscellaneous objects surveyed using the In Situ Object Counting System

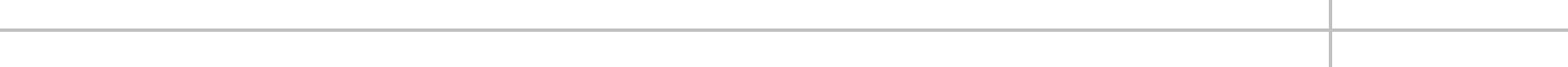

(20)

(20)

(20)

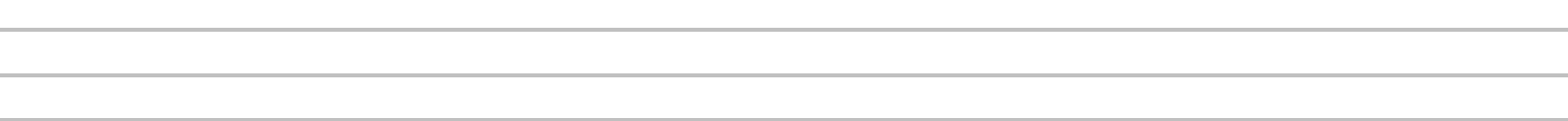

(10)

(2)

(n)

(20)

(2)

(20)

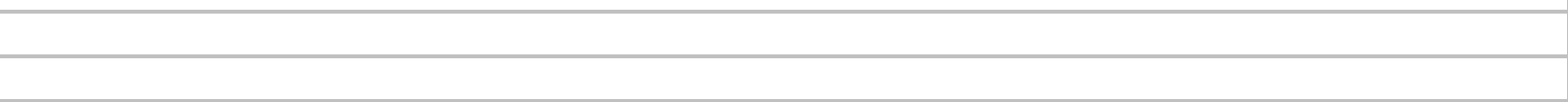

(2)

\begin{tabular}{|l|l|}
\hline & \\
\hline & \\
\hline & \\
\hline
\end{tabular}


4866 Miscellaneous objects surveyed using the In Situ Object Counting System

4867 Miscellaneous objects surveyed using the In Situ Object Counting System 4868 Miscellaneous objects surveyed using the In Situ Object Counting System 4869 Miscellaneous objects surveyed using the In Situ Object Counting System 4870 Miscellaneous objects surveyed using the In Situ Object Counting System 4871 Miscellaneous objects surveyed using the In Situ Object Counting System 4872 Miscellaneous objects surveyed using the In Situ Object Counting System 4873 Miscellaneous objects surveyed using the In Situ Object Counting System 4874 Miscellaneous objects surveyed using the In Situ Object Counting System 4875 Miscellaneous objects surveyed using the In Situ Object Counting System 4876 Miscellaneous objects surveyed using the In Situ Object Counting System 4877 Miscellaneous objects surveyed using the In Situ Object Counting System 4878 Miscellaneous objects surveyed using the In Situ Object Counting System 4879 Miscellaneous objects surveyed using the In Situ Object Counting System 4880 Miscellaneous objects surveyed using the In Situ Object Counting System

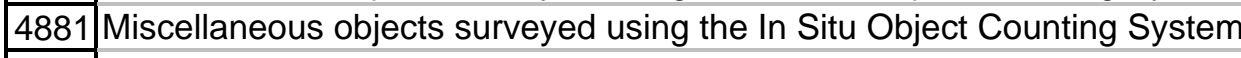
4882 Miscellaneous objects surveyed using the In Situ Object Counting System 4883 Miscellaneous objects surveyed using the In Situ Object Counting System 4884 Miscellaneous objects surveyed using the In Situ Object Counting System 4885 Miscellaneous objects surveyed using the In Situ Object Counting System 4886 Miscellaneous objects surveyed using the In Situ Object Counting System 4887 Miscellaneous objects surveyed using the In Situ Object Counting System 4888 Miscellaneous objects surveyed using the In Situ Object Counting System 4889 Miscellaneous objects surveyed using the In Situ Object Counting System 4890 Miscellaneous objects surveyed using the In Situ Object Counting System 4891 Miscellaneous objects surveyed using the In Situ Object Counting System 4892 Miscellaneous objects surveyed using the In Situ Object Counting System 4893 Miscellaneous objects surveyed using the In Situ Object Counting System 4894 Miscellaneous objects surveyed using the In Situ Object Counting System 4895 Miscellaneous objects surveyed using the In Situ Object Counting System 4896 Miscellaneous objects surveyed using the In Situ Object Counting System 4897 Miscellaneous objects surveyed using the In Situ Object Counting System

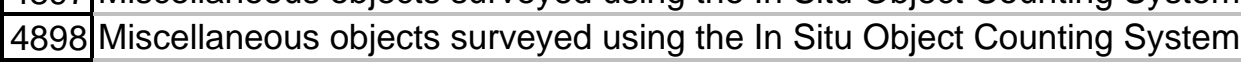
4899 Miscellaneous objects surveyed using the In Situ Object Counting System 4900 Miscellaneous objects surveyed using the In Situ Object Counting System 4901 Miscellaneous objects surveyed using the In Situ Object Counting System 4902 Miscellaneous objects surveyed using the In Situ Object Counting System 4903 Miscellaneous objects surveyed using the In Situ Object Counting System

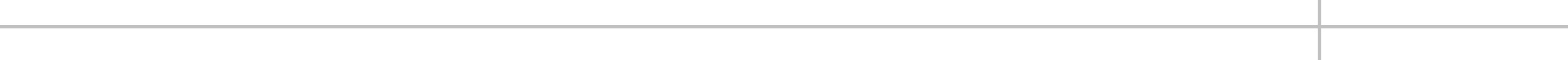

(20)

(20)

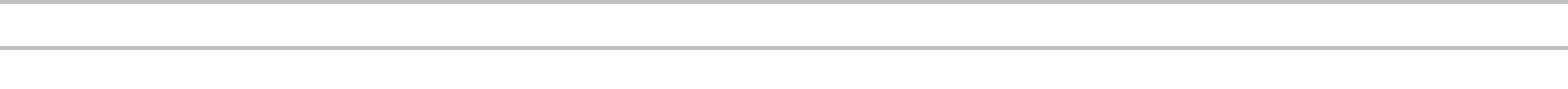

(20)

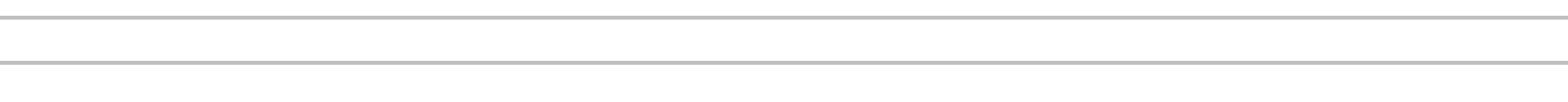

(20)

(20)

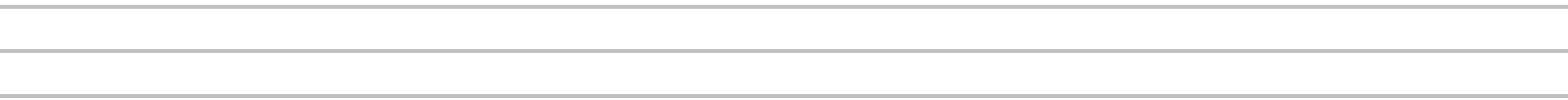

(2)

(2)

(2)

(20)

(n)

(20)

(20)

(2)


4904 Miscellaneous objects surveyed using the In Situ Object Counting System

4905 Miscellaneous objects surveyed using the In Situ Object Counting System 4906 Miscellaneous objects surveyed using the In Situ Object Counting System 4907 Miscellaneous objects surveyed using the In Situ Object Counting System

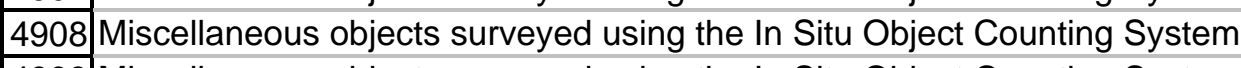
4909 Miscellaneous objects surveyed using the In Situ Object Counting System 4910 Miscellaneous objects surveyed using the In Situ Object Counting System 4911 Miscellaneous objects surveyed using the In Situ Object Counting System 4912 Miscellaneous objects surveyed using the In Situ Object Counting System 4913 Miscellaneous objects surveyed using the In Situ Object Counting System 4914 Miscellaneous objects surveyed using the In Situ Object Counting System 4915 Miscellaneous objects surveyed using the In Situ Object Counting System 44916 Miscellaneous objects surveyed using the In Situ Object Counting System 4917 Miscellaneous objects surveyed using the In Situ Object Counting System 4918 Miscellaneous objects surveyed using the In Situ Object Counting System 4919 Miscellaneous objects surveyed using the In Situ Object Counting System 4920 Miscellaneous objects surveyed using the In Situ Object Counting System 4921 Miscellaneous objects surveyed using the In Situ Object Counting System 4922 Miscellaneous objects surveyed using the In Situ Object Counting System 4923 Miscellaneous objects surveyed using the In Situ Object Counting System 4924 From Waste Container 233S-01-007

4925 From Waste Container 233S-01-0004

4926 From Waste Container 233S-01-0118

4927 From Waste Container 233S-01-0117

4928 From Waste Container 233S-01-0107

4929 From Waste Container 233S-01-0111

4930 From Waste Container 233S-01-0111

4931 From Waste Container 233S-01-0092

4932 From Waste Container 233S-01-0115

\begin{tabular}{|l|l}
4933 & From Waste Container 233S-01-0086
\end{tabular}

4934 From Waste Container 233S-01-0134

4935 From Waste Container 233S-01-0120

4936 From Waste Container 233S-01-0053

\begin{tabular}{|l|l|}
44937 & From Waste Container 233S-01-0054 \\
\hline
\end{tabular}

4938 From Waste Container 233S-01-0022

4939 From Waste Container 233S-01-0087

44940 From Waste Container 233S-01-0112

\begin{tabular}{|l|l}
\hline 4941 & From Waste Container 233S-01-0115 \\
\hline
\end{tabular}

SAMP SITE DESC

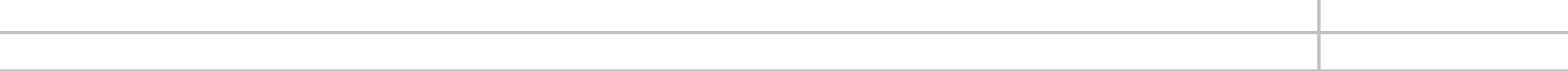

(20)

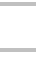

(20)

(20)

(20)

(20)

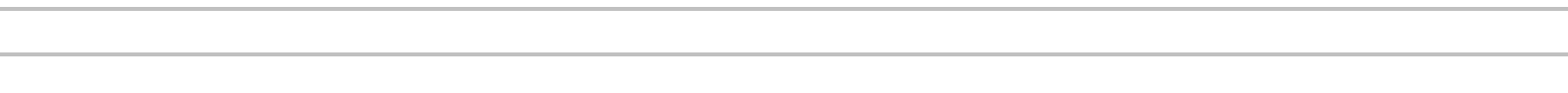

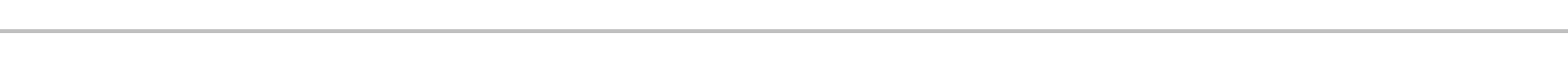

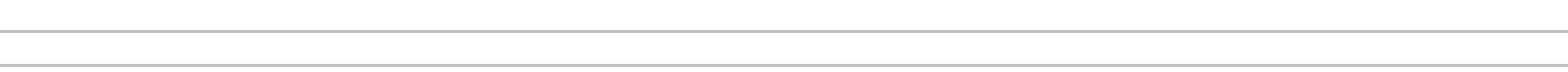

(20)

(20)

(20)

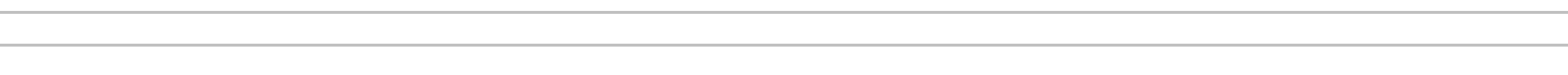

(20)

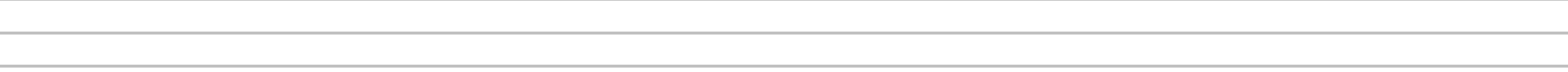

Page 444 
4943 From Waste Container 233S-01-0125

4944 From Waste Container 233S-01-0127

4945 From Waste Container 233S-01-0128

4946 From Waste Container 233S-01-0129

4 From Waste Container233S-01-0129

4948 From Waste Container 233S-01-0131

4949 From Waste Container 233S-01-0132

4950 From Waste Container 233S-01-0133

4951 Miscellaneous objects surveyed using the In Situ Object Counting System

4952 Miscellaneous objects surveyed using the In Situ Object Counting System

4953 Miscellaneous objects surveyed using the In Situ Object Counting System

4954 Miscellaneous objects surveyed using the In Situ Object Counting System

4955 Miscellaneous objects surveyed using the In Situ Object Counting System

4956 Miscellaneous objects surveyed using the In Situ Object Counting System

4957 Miscellaneous objects surveyed using the In Situ Object Counting System

4958 Miscellaneous objects surveyed using the In Situ Object Counting System

4959 Miscellaneous objects surveyed using the In Situ Object Counting System

4960 Miscellaneous objects surveyed using the In Situ Object Counting System

4961 Miscellaneous objects surveyed using the In Situ Object Counting System

4962 Miscellaneous objects surveyed using the In Situ Object Counting System

4963 Miscellaneous objects surveyed using the In Situ Object Counting System

\begin{tabular}{|l|l}
4964 & Miscellaneous objects surveyed using the In Situ Object Counting System
\end{tabular}

4965 Miscellaneous objects surveyed using the In Situ Object Counting System

4966 Miscellaneous objects surveyed using the In Situ Object Counting System

4967 Miscellaneous objects surveyed using the In Situ Object Counting System

4968 Miscellaneous objects surveyed using the In Situ Object Counting System

4969 Miscellaneous objects surveyed using the In Situ Object Counting System

4970 Miscellaneous objects surveyed using the In Situ Object Counting System

4971 Miscellaneous objects surveyed using the In Situ Object Counting System

4972 Miscellaneous objects surveyed using the In Situ Object Counting System

4973 Miscellaneous objects surveyed using the In Situ Object Counting System

4974 Miscellaneous objects surveyed using the In Situ Object Counting System

4975 Miscellaneous objects surveyed using the In Situ Object Counting System

4976 Miscellaneous objects surveyed using the In Situ Object Counting System

4977 From Waste Container 233S-00-0026

4978 From Waste Container 233S-01-0009

\begin{tabular}{|l|l|}
44979 & From Waste Container 233S-01-0020 \\
\hline
\end{tabular}

\begin{tabular}{|l|l|l|}
\hline & & \\
\hline & & \\
\hline & & \\
\hline & & \\
\hline & & \\
\hline & & \\
\hline & & \\
\hline & & \\
\hline
\end{tabular}

Page 445 
4981 From Waste Container 233S-01-0089

4982 From Waste Container 233S-01-0017

4983 From Waste Container 233S-01-0113

4984 From Waste Container 233S-01-0113

\begin{tabular}{|l|l}
4985 & Miscellaneous objects surveyed using the In Situ Object Counting System
\end{tabular}

4986 Miscellaneous objects surveyed using the In Situ Object Counting System

4987 Miscellaneous objects surveyed using the In Situ Object Counting System

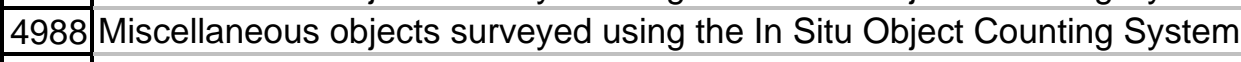

4989 Miscellaneous objects surveyed using the In Situ Object Counting System

4990 Miscellaneous objects surveyed using the In Situ Object Counting System

4991 From Waste Container 233S-01-0016

4992 From Waste Container 233S-01-0091

4993 From Waste Container 233S-01-0094

4994 From Waste Container 233S-01-0088

4995 From Waste Container 233S-01-0114

4996 From Waste Container 233S-01-0055SWB

4997 Miscellaneous objects surveyed using the In Situ Object Counting System

4998 Miscellaneous objects surveyed using the In Situ Object Counting System

4999 Miscellaneous objects surveyed using the In Situ Object Counting System

5000 Miscellaneous objects surveyed using the In Situ Object Counting System

5001 From Waste Container 233S-02-0032

5002 Miscellaneous objects surveyed using the In Situ Object Counting System

5003 Miscellaneous objects surveyed using the In Situ Object Counting System

5004 Miscellaneous objects surveyed using the In Situ Object Counting System

5005 Miscellaneous objects surveyed using the In Situ Object Counting System

5006 221-U Vent Tunnel

5007 221-U Vent Tunnel

5008 221-U Vent Tunnel

\begin{tabular}{|l|l|l}
5009 & $221-U$ Vent Tunnel \\
\hline 5010 &
\end{tabular}

5010 From K-Influent valve/

5011 From H-Influent valve

5012

\begin{tabular}{|l|l}
5013 & EVAPORATION POND \\
\hline 5014 & EVAPORATION POND \\
\hline
\end{tabular}

5014 EVAPORATION POND

5015 EVAPORATION POND

5016 From DRUM 200W-00-0011

5017 From DRUM 200W-00-0011 
5018 Composite from Drums 200W-00-0011, 200W-00-0009, and 200W-00-0010

SAMP SITE DESC

5019 Composite from Drums 200W-00-0011, 200W-00-0009, and 200W-00-0010

5020 B Plant Downwind Exhaust

5021 B Plant Downstream Exhauster

5022 221-B Downwind Exhauster

\begin{tabular}{|l|l|l}
5023 & B Plant \\
\hline 5024 & Resin from \\
\hline
\end{tabular}

5024 Resin from K100-02-0042

5025 Resin from TOTE K100-02-0041

\begin{tabular}{|l|l}
5026 & Resin from TOTE K100-02-0036 \\
\hline 5027 & Resin from TOTE K100-02-0035
\end{tabular}

5027 Resin from TOTE K100-02-0035

5028 Resin from TOTE K100-02-0039

\begin{tabular}{l|l}
5029 & Resin from TOTE K100-02-0040
\end{tabular}

\begin{tabular}{l|l}
5030 & Resin from TOTE K100-02-0045
\end{tabular}

5031 Resin from TOTE K100-02-0046

5032 Resin from TOTE K100-02-0043

\begin{tabular}{|l|l|}
5033 & Resin from TOTE K100-02-0044 \\
\hline 5034 & Resin fom TOTE KR4-02-005 (9774
\end{tabular}

5034 Resin from TOTE KR4-02-005 (97743)

5035 Resin from TOTE KR4-02-006 (97747)

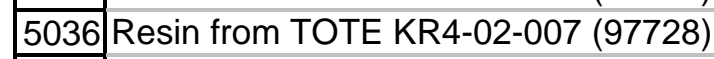

5037 Resin from TOTE KR4-02-008 (97721)

5038 Resin from TOTE KR4-02-011 (97738)

5039 Resin from TOTE KR4-02-010 (97711)

\begin{tabular}{l|l}
5040 & Resin from BURIAL BOX 100H-02-0111
\end{tabular}

5041 Resin from BURIAL BOX 100H-02-0111

5042 Resin from BURIAL BOX HR3-02-001

\begin{tabular}{|l|l}
5043 & Resin from BURIAL BOX HR3-02-001
\end{tabular}

\begin{tabular}{|l|l}
5044 & Resin from TOTE $100 \mathrm{H}-02-0114$ \\
\hline 5045 & Resin from
\end{tabular}

5045 Resin from TOTE $100 \mathrm{H}-02-0113$

5046 Resin from BURIAL BOX HR3-02-006

\begin{tabular}{|l|l}
5047 & Resin from BURIAL BOX HR3-02-005 \\
\hline 5048 & Resin from
\end{tabular}

\begin{tabular}{|l|l}
5048 & Resin from TOTE HR3-02-008 (97729)
\end{tabular}

5049 Resin from TOTE HR3-02-009 (97724)

5050 Resin from TOTE HR3-02-004 (97740)

5051 Borehole CW-5

5052 From location T1-01 (Trench 1, first sample collected)

5053 From location T1-02 (Trench 1, second sample collected)

5054 From location T1-03 (Trench 1, third sample collected)

\begin{tabular}{|l|l}
5055 & From location T1-04 (Trench 1, fourth sample collected)
\end{tabular} 
5057 From location T4-02 (Trench 4, second sample collected)

5058 From location T4-03 (Trench 4, third sample collected)

5059 From location T4-04 (Trench 4, fourth sample collected)

5060 From location T4-05 (Trench 4, fifth sample collected)

5061 From location T4-06 (Trench 4, sixth sample collected)

5062 From location T4-07 (Trench 4, seventh sample collected)

5063 From location T4-04 (Trench 4, fourth sample collected)

5064 From location T4-08 (Trench 4, eighth sample collected)

5065 From location T4-09 (Trench 4, ninth sample collected)

5066 From location T4-10 (Trench 4, tenth sample collected)

5067 From location T4-11 (Trench 4, eleventh sample collected)

5068 From location T4-12 (Trench 4, twelvth sample collected)

5069 From location T4-13 (Trench 4, thirteenth sample collected)

5070 From location T4-14 (Trench 4, fourteenth sample collected)

5071 From location T4-15 (Trench 4, fifteenth sample collected)

5072 From location T4-16 (Trench 4, sixteenth sample collected)

5073 From location T4-14 (Trench 4, fourteenth sample collected)

5074 From location T7-01 (Trench 7, first sample collected)

5075 From location T7-02 (Trench 7, second sample collected)

5076 From location T7-03 (Trench 7, third sample collected)

5077 From location T7-04 (Trench 7, fourth sample collected)

5078 From location T7-05 (Trench 7, fifth sample collected)

5079 From location T4-05A (Trench 7, from riser between T4-05 and T4-06 - located 20 feet west of T4-05)

5080 From location T4-04A (Trench 7, from riser between T4-05 and T4-06 - located 27 feet east of T4-05)

5081 Borehole CW-5

5082 Borehole CW-5

5083 Borehole CW-5

5084 Borehole CW-5

\begin{tabular}{|l|l|l}
5085 & Borehole CW-5 \\
\hline 5086 & Borehole $\mathrm{CW}-5$
\end{tabular}

5086 Borehole CW-5

5087 Borehole CW-5

\begin{tabular}{|l|l|}
5088 & Borehole CW-5 \\
\hline
\end{tabular}

5089 Borehole CW-5

5090 Borehole CW- 5

5091 Borehole CW- 5

5092 Borehole CW-5 
5095 From DRUM 200W-01-0126

5096 From DRUM 200W-01-0125

5097 From K-Influent valve

\begin{tabular}{|l|l|}
\hline 5098 & From the Effluent valve \\
\hline 5099 & From
\end{tabular}

5099 From Ion Exchange Column K-IX-A4

5100 From Ion Exchange Column K-IX-B3

5101 From Ion Exchange Column K-IX-C3

\begin{tabular}{|l|l}
5102 & From Ion Exchange Column H-IX-A2 \\
\hline 5103 & From Ion Exchange Column H-IX-C3
\end{tabular}

5103 From Ion Exchange Column H-IX-C3

5104 From $\mathrm{H}$-Influent valve

5105 From D-Influent valve

5106 From HR-3 Combined Effluent

5107 From HR-3 Combined Effluent

5108

5109

5110 Virgin GAC from cylinder with no number

5111 From cylinder ZP-1-02-004

\begin{tabular}{|l|l|}
5112 & Filter paper from the influent location \\
\hline 5113 & Firm
\end{tabular}

\begin{tabular}{|l|l|}
5113 & From cylinder ZP-1-02-004 \\
\hline 5114 & Fin
\end{tabular}

5114 Filter paper from the influent location

5115 FROM TANK T2-01

\begin{tabular}{|l|l}
5116 & FROM EFFLUENT FILTER B2-01
\end{tabular}

\begin{tabular}{|l|l}
5117 & FROM POST VALVE V1-02 \\
\hline 5118 & FROM POST TANK T1-01
\end{tabular}

\begin{tabular}{|l|l}
5118 & FROM POST TANK T1-01
\end{tabular}

5119

5120 From the K-Influent valve

5121 From Ion Exchange Column K-IX-A4

5122 From K-Effluent valve

\begin{tabular}{|l|l|}
5123 & From Ion Exchange Column H-IX-A2 \\
\hline 5124 & From Ion
\end{tabular}

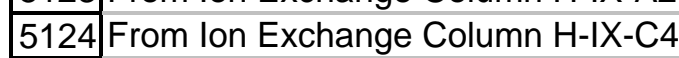

5125 From $\mathrm{H}$-Influent valve

5126 From D-Influent valve

5127 From HR-3 Combined Effluent

5128 From Ion Exchange Column H-IX-D2

5129

5130

5131 


\begin{tabular}{|c|c|c|}
\hline 1 & $\begin{array}{ll} & \text { SAMP_SITE_DESC } \\
\end{array}$ & SITE_ELEVATION \\
\hline \multicolumn{3}{|l|}{5132} \\
\hline \multicolumn{3}{|l|}{5133} \\
\hline 5134 & FROM SWL-01A & \\
\hline 5135 & FROM SWL-01B & \\
\hline 5136 & FROM SWL-02A & \\
\hline 5137 & FROM SWL-02B & \\
\hline 5138 & FROM SWL-03A & \\
\hline 5139 & FROM SWL-03B & \\
\hline 5140 & FROM SWL-04A & \\
\hline 5141 & FROM SWL-04B & \\
\hline 5142 & FROM SWL-04X & \\
\hline 5143 & FROM SWL-DW2 & \\
\hline 5144 & FROM SWL-DE1 & \\
\hline 5145 & FROM SWL-05B & \\
\hline 5146 & FROM SWL-06A & \\
\hline 5147 & FROM SWL-06B & \\
\hline 5148 & FROM SWL-07A & \\
\hline 5149 & FROM SWL-07B & \\
\hline 5150 & FROM SWL-07B & \\
\hline 5152 & FROM SWL-08A & \\
\hline 5153 & FROM SWL-08B & \\
\hline 5154 & ZERO AIR & \\
\hline 5155 & CAL CHECK & \\
\hline 5156 & LCS & \\
\hline 5157 & CAL STANDARD & \\
\hline 5158 & FROM SWL-05A & \\
\hline 5159 & FROM POST TANK T-02-01 & \\
\hline 5160 & FROM POST VALVE V-01-01 & \\
\hline 5161 & FROM EFFLUENT FILTER B2-01 & \\
\hline 5162 & FROM POST TANK T-01-01 & \\
\hline 5163 & FROM POST TANK T-02-01 & \\
\hline 5164 & FROM POST VALVE V-01-01 & \\
\hline 5165 & FROM EFFLUENT FILTER B2-01 & \\
\hline 5166 & FROM POST TANK T-01-01 & \\
\hline 5167 & POST CHILLER AIR & \\
\hline 5168 & STACK EFFLUENT & \\
\hline 5169 & POST CHILLER AIR & \\
\hline
\end{tabular}

\begin{tabular}{|l|l|}
5168 & STACK EFFU \\
\hline 5169 & POST CHILLER AIR \\
\hline
\end{tabular} 
5171 From Ion Exchange Column K-IX-B4

5172 From Ion Exchange Column K-IX-C4

5173 From K-Influent valve

5174 From Ion Exchange Column K-IX-A1

5175 From Ion Exchange Column K-IX-B4

5176 From Ion Exchange Column K-IX-C1

5177 From K-Effluent valve

5178 From Ion Exchange Column H-IX-A3

5179 From Ion Exchange Column H-IX-C4

5180 From $\mathrm{H}$-Influent valve

5181 From D-Influent valve

\begin{tabular}{|l|l|}
5182 & From HR-3 Combined Effluent \\
\hline 5183 & From
\end{tabular}

5183 From Ion Exchange Column H-IX-D2

5184

\begin{tabular}{|l|l|}
5185 & FROM POST TANK T-02-01 \\
\hline 5186 & FROM
\end{tabular}

5186 FROM: INFLUENT

5187 FROM: EFFLUENT

\begin{tabular}{|l|l}
5188 & From the Effluent valve \\
\hline 5189 & From the
\end{tabular}

\begin{tabular}{|l|l}
5189 & From the Influent Valve \\
\hline 5190 & From HR-3 Combned
\end{tabular}

5190 From HR-3 Combined Effluent

5191 From $\mathrm{H}$-Influent valve

\begin{tabular}{|l|l}
5192 & From Ion Exchange Column H-IX-A3 \\
\hline 5193 & From Ion Exchange Column H-IX-C4
\end{tabular}

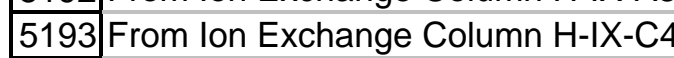

5194 From $\mathrm{H}$-Influent valve

5195 From D-Influent valve

5196 From HR-3 Combined Effluent

5197 From Ion Exchange Column H-IX-D3

5198 From K-Influent valve/

5199 From Ion Exchange Column K-IX-A2

5200 From Ion Exchange Column K-IX-B4

5201 From Ion Exchange Column K-IX-C1

5202 From K-Effluent valve

\begin{tabular}{|l|l|}
5203 & Resin from TOTE marked KR4-02-003 \\
\hline 5204 & Resin fom TOTE makk KR4-02-015
\end{tabular}

5204 Resin from TOTE marked KR4-02-015

5205 Resin from TOTE marked KR4-02-002

\begin{tabular}{|l|l}
5206 & Resin from TOTE marked KR4-02-014 \\
\hline 5207 & Resin from
\end{tabular}

\begin{tabular}{|l|l|}
5207 & Resin from TOTE marked KR4-02-013 \\
\hline
\end{tabular} 
5208 Resin from TOTE marked KR4-02-012

SAMP SITE DESC

5209 Resin from TOTE marked KR4-02-029

5210 Resin from TOTE marked KR4-02-028

5211 Resin from TOTE marked KR4-02-025

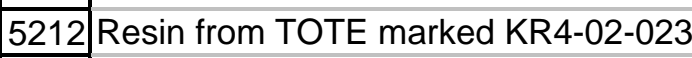

\begin{tabular}{|l|l}
5213 & Resin from TOTE marked KR4-02-026 \\
\hline 5214 & Resin from TOTE marked KR4-02-022
\end{tabular}

5214 Resin from TOTE marked KR4-02-022

5215 Resin from TOTE marked KR4-02-024

\begin{tabular}{|l|l}
5216 & Resin from TOTE marked KR4-02-021
\end{tabular}

5217 Resin from TOTE marked HR3-02-011

5218 Resin from TOTE marked HR3-02-012

5219 FROM POST TANK T-02-01

\begin{tabular}{|l|l}
5220 & FROM POST VALVE V-01-01
\end{tabular}

5221 FROM EFFLUENT FILTER B2-01

5222 FROM POST TANK T-01-01

\begin{tabular}{|l|l|}
5223 & Resin from TOTE marked HR3-02-010 \\
\hline 5224 & Resin from
\end{tabular}

5224 Resin from TOTE marked HR3-02-010

5225 Resin from TOTE marked HR3-02-014

5226 Resin from TOTE marked HR3-02-013

\begin{tabular}{|l|l}
5227 & FROM INJECTION WELL 299-W18-36
\end{tabular}

5228 FROM POST TANK T-02-01

5229 FROM POST VALVE V-01-01

\begin{tabular}{|l|l}
5230 & FROM EFFLUENT FILTER B2-01
\end{tabular}

5231 FROM POST TANK T-01-01

5232 FROM POST TANK T-02-01

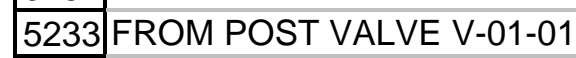

\begin{tabular}{|l|l|}
5234 & FROM EFFLUENT FILTER B2-01 \\
\hline 5235 & FROM POST TANK T-01-01
\end{tabular}

5235 FROM POST TANK T-01-01

5236 FROM POST TANK T-02-01

\begin{tabular}{|l|l|}
5237 & FROM POST VALVE V-01-01 \\
\hline 5238
\end{tabular}

5238 FROM EFFLUENT FILTER B2-01

5239 FROM POST TANK T-01-01

5240 EVAPORATION POND

\begin{tabular}{|l|l}
5241 & EVAPORATION POND \\
\hline 5242 &
\end{tabular}

5242 From the K-Influent valve

5243 From Ion Exchange Column K-IX-A1

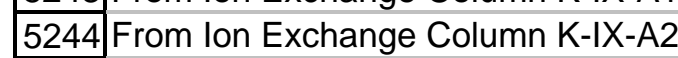

5245 From Ion Exchange Column K-IX-A3 
5247 From Ion Exchange Column K-IX-C2

5248 From Ion Exchange Column K-IX-B1

5249 From Ion Exchange Column H-IX-A1

5250 From Ion Exchange Column $\mathrm{H}-\mathrm{IX}-\mathrm{A} 4$

5251 From Ion Exchange Column H-IX-C1

5252 From Ion Exchange Column H-IX-C2

5253 From Ion Exchange Column H-IX-D4

5254 From Ion Exchange Column H-IX-D3

5255 From Influent D-Transfer/Valve $\mathrm{HI}-03$

5256 From Influent $\mathrm{H}$-Transfer/Valve HP-14

5257 From Effluent

5258 POST CHILLER AIR

5259 STACK EFFLUENT

5260 FROM POST TANK T-02-01

5261 FROM POST VALVE V-01-01

5262 FROM EFFLUENT FILTER B2-01

5263 FROM POST TANK T-01-01

5264 From the Post Chiller Air

5265 Resin from TOTE 97719

5266 Resin from TOTE 97707

5267 Resin from TOTE 97750

\begin{tabular}{|l|l}
5268 & Resin from TOTE 97744 \\
\hline 5269 & Resin from
\end{tabular}

5269 Resin from BURIAL BOX marked HR3-02-021

5270 Resin from TOTE 97706

5271 Resin from TOTE 97733

5272 Resin from TOTE 97741

5273 Resin from TOTE 97746

5274 Resin from TOTE 97708

5275 Resin from TOTE 97739

5276

5277 BT-1 Test Pit from the 216-B-63 Ditch

5278 TEST PIT SP-1

5279 TEST PIT SP-2

5280 TEST PIT SP-3

5281 TEST PIT SD-1

5282 TEST PIT SD-3

5283 TEST PIT SP-4 
5285 126-F-1 Shallow Zone - Sample Node SA1-3

5286 126-F-1 Shallow Zone - Sampling Node SA1-3

5287 126-F-1 Shallow Zone - Sampling Node SA1-4

\begin{tabular}{|l|l|}
\hline 5288 & $126-F-1$ Shallow Zone - Sampling Node SA1-10 \\
\hline 528 & $126-F-1$ Shallow Zone - Samplng Node SA1-13
\end{tabular}

5289 126-F-1 Shallow Zone - Sampling Node SA1-13

5290 126-F-1 Shallow Zone - Sampling Node SA1-16

5291 126-F-1 Shallow Zone - Sampling Node SB5-1

\begin{tabular}{|l|l|}
5292 & $126-F-1$ Shallow Zone - Sampling Node SB5-2 \\
\hline 529 & $126-F-1$ Shallow Zone - Sampling Node SB5-5
\end{tabular}

\begin{tabular}{|l|l}
5293 & $126-F-1$ Shallow Zone - Sampling Node SB5-5
\end{tabular}

\begin{tabular}{l|l}
5294 & $126-F-1$ \\
\end{tabular}

\begin{tabular}{|l|l}
5295 & $126-F-1$ Shallow Zone - Sampling Node SB6-1
\end{tabular}

\begin{tabular}{|l|l|}
5296 & $126-F-1$ \\
\hline
\end{tabular}

5297 126-F-1 Shallow Zone - Sampling Node SB6-12

5298 $126-\mathrm{F}-1$ Shallow Zone - Sampling Node SB6-13

\begin{tabular}{|l|l}
5299 & $126-F-1$ Shallow Zone - Sampling Node SB7-1 \\
\hline 5300 & $126-F-1$
\end{tabular}

\begin{tabular}{|l|l}
5300 & $126-F-1$ Shallow Zone - Sampling Node SB7-3
\end{tabular}

5301 126-F-1 Shallow Zone - Sampling Node SB7-4

\begin{tabular}{|l|l|}
5302 & $126-F-1$ Shallow Zone - Sampling Node SB7-5 \\
\hline 5303 & $126-$ F-1 Shallow Zone - Sampling Node SB7-11
\end{tabular}

\begin{tabular}{|l|l|}
5303 & $126-F-1$ Shallow Zone - Sampling Node SB7-11 \\
\hline 5304 & 26-F-1 Shallow Zone - Sampling Node SB8-2 \\
\hline
\end{tabular}

5304 126-F-1 Shallow Zone - Sampling Node SB8-2

\begin{tabular}{l|l}
5305 & $126-F-1$ Shallow Zone - Sampling Node SB8-3
\end{tabular}

\begin{tabular}{|l|l|}
5306 & $126-F-1$ Shallow Zone - Sampling Node SB8-4 \\
\hline 5307 & $126-$ F-1 Shallow Zone - Sampling Node SB8-12 \\
\hline
\end{tabular}

\begin{tabular}{|l|l|}
5307 & $126-F-1$ Shallow Zone - Sampling Node SB8-12 \\
\hline 5308 & $126-F-1$ Shallow Zone - Sampling Node SB8-13 \\
\hline
\end{tabular}

5308 126-F-1 Shallow Zone - Sampling Node SB8-13

\begin{tabular}{|l|l|l|}
\hline 5309 & $116-F-2$ & Deep Zone - Composite of Sampling Nodes DA2-3, DA2-6, DA2-7 \& DA2-15 \\
\hline 5310 & $116-F-2$ Deep Zone - Composite of Sampling Nodes DA3-1, DA3-2, DA3-4 \& DA3-11 \\
\hline 5311 & $116-F$
\end{tabular}

5311 116-F-2 Deep Zone - Composite of Sampling Nodes DB4-3, DB4-4, DB4-7 \& DB4-12

5312 116-F-2 Deep Zone - Composite of Sampling Nodes DB5-1, DB5-5, DB5-7, \& DB5-15

5313 116-F-2 Deep Zone - Composite of Sampling Nodes DB6-1, DB6-13, DB6-10 \& DB6-15

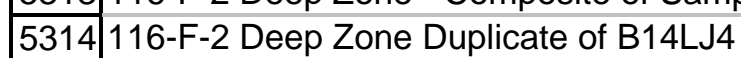

5315 116-F-2 Deep Zone - Equipment Blank

\begin{tabular}{|l|l|}
\hline 5316 & $126-F-1$ Shallow Zone - Sampling Node SC9-3
\end{tabular}

\begin{tabular}{|l|l|}
5317 & $126-F-1$ Shallow Zone - Sampling Node SC9-4 \\
\hline 5318 & $126--1$ \\
\hline
\end{tabular}

5318 126-F-1 Shallow Zone - Sampling Node SC9-5

\begin{tabular}{|l|l}
5319 & $126-F-1$ \\
5
\end{tabular}

\begin{tabular}{|l|l}
5320 & $126-F-1$ \\
\hline 53 & Shallow Zone - Sampling Node SC10-3
\end{tabular}

\begin{tabular}{|l}
5321 \\
126-F-1 Shallow Zone - Sampling Node SC10-4
\end{tabular} 
5322 126-F-1 Shallow Zone - Sampling Node SC10-8

SAMP SITE DESC

5323 126-F-1 Shallow Zone - Sampling Node SC10-10

5324 126-F-1 Shallow Zone - Sampling Node SC1-2

5325 126-F-1 Shallow Zone - Sampling Node SC1-3

\begin{tabular}{|l|l|}
5326 & $126-F-1$ Shallow Zone - Sampling Node SC1-4 \\
\hline 5327 & $126-F-1$ Shalow Zone - Sampling Node SC1-10
\end{tabular}

\begin{tabular}{|l|l}
5327 & $126-F-1$ Shallow Zone - Sampling Node SC1-10
\end{tabular}

5328 126-F-1 Shallow Zone - Sampling Node SC2-3

5329 126-F-1 Shallow Zone - Sampling Node SC2-6

5330 126-F-1 Shallow Zone - Sampling Node SC2-7

5331 126-F-1 Shallow Zone - Sampling Node SC2-10

5332 126-F-1 Shallow Zone - Sampling Node SD3-1

\begin{tabular}{|l|l}
5333 & $126-F-1$ Shallow Zone - Sampling Node SD3-2
\end{tabular}

\begin{tabular}{|l|l}
5334 & $126-F-1$ Shallow Zone - Sampling Node SD3-4
\end{tabular}

5335 126-F-1 Shallow Zone - Sampling Node SD3-5

\begin{tabular}{|l|l}
5336 & $126-F-1$ Shallow Zone - Sampling Node SD3-9
\end{tabular}

\begin{tabular}{|l|l|}
5337 & $126-F-1$ Shallow Zone - Sampling Node SD3-9 \\
\hline 5338 & 126-F- Shallow Zone - Sampling Node SD4-3
\end{tabular}

5338 126-F-1 Shallow Zone - Sampling Node SD4-3

5339 126-F-1 Shallow Zone - Sampling Node SD4-4

\begin{tabular}{|l|l}
5340 & $126-F-1$ Shallow Zone - Sampling Node SD4-7
\end{tabular}

\begin{tabular}{|l|l}
5341 & $126-F-1$ \\
5
\end{tabular}

5342 126-F-1 Shallow Zone - Sampling Node SD4-9

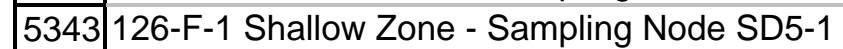

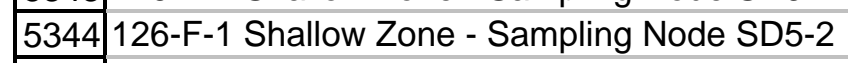

5345 126-F-1 Shallow Zone - Sampling Node SD5-5

5346 126-F-1 Shallow Zone - Sampling Node SD5-7

5347 126-F-1 Shallow Zone - Sampling Node SD6-1

\begin{tabular}{|l|l|}
\hline 5348 & $126-F-1$ Shallow Zone - Sampling Node SD6-10 \\
\hline 5534 & $126-F-1$ Shallow Zone - Sampling Node SD6-12
\end{tabular}

5349 126-F-1 Shallow Zone - Sampling Node SD6-12

5350 126-F-1 Shallow Zone - Sampling Node SE7-1

\begin{tabular}{|l|l}
5351 & $126-F-1$ Shallow Zone - Sampling Node SE7-3 \\
\hline 5352 & $126-$ -
\end{tabular}

5352 126-F-1 Shallow Zone - Sampling Node SE7-4

5353 126-F-1 Shallow Zone - Sampling Node SE7-5

\begin{tabular}{|l|l}
5354 & $126-F-1$ Shallow Zone - Sampling Node SE7-8
\end{tabular}

\begin{tabular}{|l|l|}
5355 & $126-F-1$ Shallow Zone \\
\hline 5356 & $126-F-1$ Shallow Zone \\
\hline
\end{tabular}

5356 126-F-1 Shallow Zone - Sampling Node SE8-2

5357 126-F-1 Shallow Zone - Sampling Node SE8-3

\begin{tabular}{|l|l|}
5358 & $126-F-1$ Shallow Zone - Sampling Node SE8-4
\end{tabular}

\begin{tabular}{|l|l|l|}
\hline 5359 & $126-\mathrm{F}-1$ \\
\hline
\end{tabular} 
5361 126-F-1 Shallow Zone - Sampling Node SE8-13

5362 126-F-1 Shallow Zone - Sampling Node SE9-3

5363 126-F-1 Shallow Zone - Sampling Node SE9-4

5364 126-F-1 Shallow Zone - Sampling Node SE9-5

\begin{tabular}{|l|l|l}
5365 & $126-F-1$ Shallow Zone - Sampling Node SE9-10
\end{tabular}

5366 126-F-1 Shallow Zone - Sampling Node SE10-3

5367 126-F-1 Shallow Zone - Sampling Node SE10-4

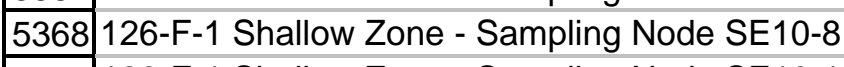

5369 126-F-1 Shallow Zone - Sampling Node SE10-10

5370 126-F-1 Shallow Zone - Sampling Node SE10-14

5371 126 -F-1 Shallow Zone - Sampling Node SE10-16

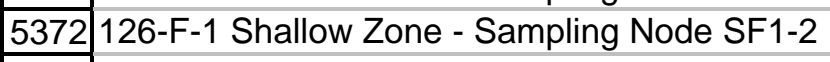

5373 126-F-1 Shallow Zone - Sampling Node SF1-3

\begin{tabular}{l|l}
5374 & $126-F-1$ Shallow Zone - Sampling Node SF1-4
\end{tabular}

5375 126-F-1 Shallow Zone - Sampling Node SF1-10

5376 126-F-1 Shallow Zone - Sampling Node SF1-13

5377 126-F-1 Shallow Zone - Sampling Node SF1-16

\begin{tabular}{|l|l}
5378 & $126-F-1$ Shallow Zone - Sampling Node SF2-3
\end{tabular}

\begin{tabular}{l|l}
5379 & $126-F-1$ Shallow Zone - Sampling Node SF2-6
\end{tabular}

5380 126-F-1 Shallow Zone - Sampling Node SF2-7

5381 126-F-1 Shallow Zone - Sampling Node SF2-10

\begin{tabular}{|l|l}
5382 & $126-F-1$ Shallow Zone - Sampling Node SF2-14 \\
\hline 5383 & $26-F-1$
\end{tabular}

\begin{tabular}{|l|l}
5383 & $126-F-1$ Shallow Zone - Sampling Node SF2-15 \\
\hline 5384 & 126-F- Shallow Zone - Sampling Node SF3-1
\end{tabular}

5384 126-F-1 Shallow Zone - Sampling Node SF3-1

5385 126-F-1 Shallow Zone - Sampling Node SF3-2

\begin{tabular}{|l|l}
5386 & $126-F-1$ Shallow Zone - Sampling Node SF3-4 \\
\hline
\end{tabular}

5387 126-F-1 Shallow Zone - Sampling Node SF3-5

\begin{tabular}{|l|l}
5388 & $126-F-1$ \\
5
\end{tabular}

\begin{tabular}{|l|l|l}
5389 & $126-\mathrm{F}-1$ \\
\hline 5390 & $126-\mathrm{F}-1 \mathrm{Sh}$
\end{tabular}

5390 126-F-1 Shallow Zone - Sampling Node SF4-3

5391 126-F-1 Shallow Zone - Sampling Node SF4-4

5392 $126-F-1$ Shallow Zone - Sampling Node SF4-7

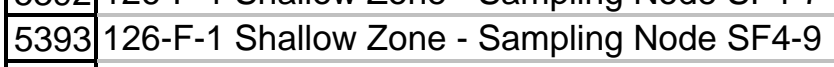

5394 126-F-1 Shallow Zone - Sampling Node SF4-12

5395 126-F-1 Shallow Zone - Sampling Node SH9-5

\begin{tabular}{|l|l|}
5396 & $116-N-1$ TRENCH SIDEWALL N1P4/5-01 \\
\hline 539 & $116-N-1$ TENCH SIDEWALL - N1P4/5-02 \\
\hline
\end{tabular}

\begin{tabular}{|l|l|}
5397 & $116-N-1$ TRENCH SIDEWALL - N1P4/5-02 \\
\hline
\end{tabular} 
5411 126-F-1 Shallow Zone Verification Sampling - Composition of Sampling Nodes SA1-3, SA1-4, SA1-10 \& SA1-16

5412 126-F-1 Shallow Zone Verification Sampling - Composition of Sampling Nodes SA2-3, SA2-6, SA2-7, SA2-15

5413 126-F-1 Shallow Zone Verification Sampling - Composite of Sampling Nodes SA3-1, SA3-2, SA\#-4, SA3-11

\begin{tabular}{|l|l}
5414 & $126-F-1$ \\
\hline 5415 & Shallow Zone Verification Sampling - Composite of Sampling Nodes SA4-3, SA4-4, SA4-7, SA4-12
\end{tabular}

5415 126-F-1 Shallow Zone Verification Sampling - Composite of Sampling Nodes SB5-1, SB5-5, SB5-7, SB5-15

5416 126-F-1 Shallow Zone Verification Sampling - Composite of Sampling Nodes SB6-1, SB6-10, SB6-13, SB6-15

5417 126-F-1 Shallow Zone Verification Sampling - Composite of Sampling Nodes SB7-3, SB7-4, SB7-5, SB7-11

5418 126-F-1 Shallow Zone Verification Sampling - Composite of Sampling Nodes SB8-3, SB8-4, SB8-8, SB8-13

5419 126-F-1 Shallow Zone Verification Sampling - Composite of Sampling Nodes SC9-3, SC9-4, SC9-10, SC9-16

5420 126-f-1 Shallow Zone Verification Sampling - Composite of Sampling Nodes SC10-4, SC10-10, SC10-14, SC10-16

5421 126-F-1 Shallow Zone Verification Sampling - Composite of Sampling Nodes SC1-3, SC1-4, SC1-10, SC1-16

5422 126-F-1 Shallow Zone Verification Sampling - Composite of Sampling Nodes SC2-3, SC2-6, SC2-7, SC2-15

5423 126-F-1 Shallow Zone Verification Sampling - Composite of Sampling Nodes SD3-1, SD3-2, SD3-4, SD3-11

\begin{tabular}{|l|l}
5424 & $126-F-1$ Shallow Zone Verification Sampling - Composite of Sampling Nodes SD4-3, SD4-4, SD4-7. SD4-12
\end{tabular}

5425 126-F-1 Shallow Zone Verification Sampling - Composite of Sampling Nodes SD5-1, SD5-5, SD5-7, SD5-15

5426 126-F-1 Shallow Zone Verification Sampling - Composite of Sampling Nodes SD6-1, SD6-10, SD6-13, SD6-15

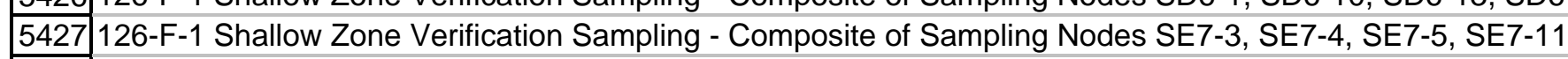

5428 126-F-1 Shallow Zone Verification Sampling - Composite of Sampling Nodes SE8-3, SE8-4, SE8-8, SE8-13

5429 126-F-1 Shallow Zone Verification Sampling - Duplicate of J000C2

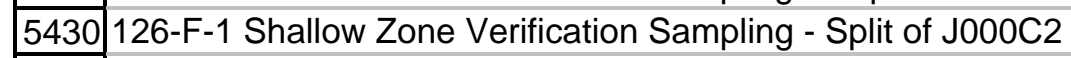

5431 126-F-1 Shallow Zone Verification Sampling - Composite of Sampling Nodes SE9-3, SE9-4, SE9-10, SE9-16

5432 126-F-1 Shallow Zone Verification Sampling - Composite of Sampling Nodes SE10-4, SE10-10, SE10-14, SE10-16

5433 126-F-1 Shallow Zone Verification Sampling - Composite of Sampling Nodes SF1-3, SF1-4, SF1-10, SF1-16

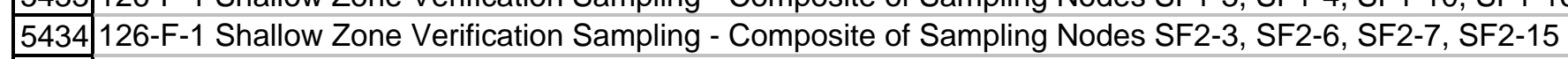

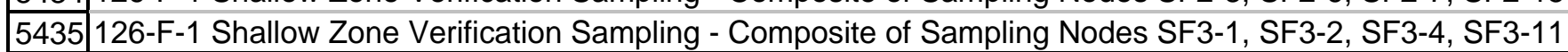


5436 126-F-1 Shallow Zone Verification Sampling - Composite of Sampling Nodes SF4-3, SF4-4, SF4-7, SF4-12

5437 126-F-1 Shallow Zone Verification Sampling - Composite of Sampling Nodes SG5-1, SG5-5, SG5-7, SG5-11

5438 126-F-1 Shallow Zone Verification Sampling - Composite of Sampling Nodes SG6-1, SG6-10, SG6-13, SG6-15

5439 126-f-1 Shallow Zone Verification Sampling - Composite of Sampling Nodes SG7-3, SG7-4, SG7-5, SG7-11

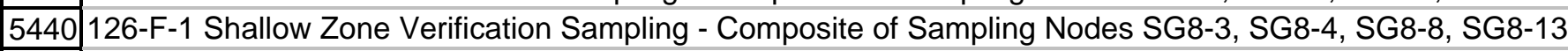

5441 126-F-1 Shallow Zone Verification Sampling - Composite of Sampling Nodes SH9-3, SH9-4, SH9-10, SH9-16

5442 126-F-1 Shallow Zone Verification Sampling - Composite of Sampling Nodes SH10-4, SH10-10, SH10-14, SH10-16

5443 126-F-1 Shallow Zone Verification Sampling - Composite of sampling nodes SH1-3, SH-4, SH1-10, SH1-16

5444 126-F-1 Shallow Zone Verification Sampling - Composite of Sampling Nodes SH2-3, SH2-6, SH2-7, SH2-15

5445 126-F-1 Shallow Zone Verification Sampling - Composite of Sampling Nodes SI3-1, SI3-2, SI3-4, SI3-11

5446 126-F-1 Shallow Zone Verification Sampling - Composite of Sampling Nodes SI4-3, SI4-4, SI4-7, SI4-12

5447 126-F-1 Shallow Zone Verification Sampling - Composite of Sampling Nodes SI5-2, SI5-5, SI5-7, SI5-15

5448 126-F-1 Shallow Zone Verification Sampling - Composite of Sampling Nodes SI6-1, SI6-10, SI6-13, SI6-15

5449 126-F-1 Shallow Zone - Duplicate of J000F1

5450 126-F-1 Shallow Zone - Split of J000F1

5451 100-B-8 Sample Node O-A1-2

5452 100-B-8 Sample Node O-A1-3

5453 100-B-8 Sample Node O-A1-4

5454 100-B-8 Sample Node O-A1-10

\begin{tabular}{|l}
5455 \\
$100-B-8$ Sample Node O-A1-13
\end{tabular}

5456 100-B-8 Sample Node O-A1-16

5457 100-B-8 Sample Node O-A2-3

5458 100-B-8 Sampling Node O-A2-6

5459 100-B-8 Sampling Node O-A2-7

5460 100-B-8 Sampling Node O-A2-10

\begin{tabular}{|l|l}
5461 & $100-B-8$ Sample Node O-A2-14
\end{tabular}

\begin{tabular}{|l|l}
5462 & $100-B-8$ Sample Node O-A2-15 \\
\hline 5463 & No0-B-8 Sampe Node O-A3-1
\end{tabular}

5463 100-B-8 Sample Node O-A3-1

5464 100-B-8 Sample Node O-A3-2

\begin{tabular}{|l|l|}
\hline 5465 & $100-B-8$ Sampling Node O-A3-2 \\
\hline 5466 & $100-B-8$ Sampling
\end{tabular}

5466 100-B-8 Sampling Node O-A3-5

5467 100-B-8 Sampling Node O-A3-9

5468 $100-B-8$ Sampling Node O-A3-11

\begin{tabular}{|l|l|l|}
\hline 5469 & $100-B-8$ Sampling Node O-A4-3 \\
\hline 5440 & No-B-8 Sampling
\end{tabular}

5470 100-B-8 Sample Node O-A4-4

5471 100-B-8 Sample Node O-A4-7

\begin{tabular}{|l|l}
5472 & $100-B-8$ Sample Node O-A4-9
\end{tabular}

\begin{tabular}{|l|l|}
\hline 5473 & $100-B-8$ Sample Node O-A4-9 \\
\hline
\end{tabular} 
5476 100-B-8 Sampling Node O-B5-1a

5477 100-B-8 Sample Node O-B5-2

\begin{tabular}{|l|l}
5478 & $100-B-8$ Sample Node O-B5-5
\end{tabular}

5479 100-B-8 Sampling Node O-B5-7

5480 100-B-8 Sampling Node O-B5-13

5481 100-B-8 Sample Node O-B5-15

\begin{tabular}{|l|l|}
\hline 5482 & $100-B-8$ Sample Node O-B6-1 \\
\hline 5483 & $100-B-8$ Samplng Node O-B6-10
\end{tabular}

5483 100-B-8 Sampling Node O-B6-10

5484 100-B-8 Sampling Node O-B6-12

5485 100-B-8 Sample Node O-B6-13

5486 100-B-8 Sample Node O-B6-15

5487 100-B-8 Sampling Node O-B6-16

5488 100-B-8 Sample Node O-B7-1

\begin{tabular}{|l|l|}
5489 & $100-B-8$ Sample Node O-B7-1 \\
\hline 5490 & $100-B-8$ Sampling Node O-B7-3
\end{tabular}

5490 100-B-8 Sampling Node O-B7-3

5491 100-B-8 Sample Node O-B7-4

5492 100-B-8 Sample Node O-B7-5

5493 100-b-8 Sample Node O-B7-8

5494 100-b-8 Sample Node O-B7-11

5495 100-b-8 Sample Node O-B8-2

\begin{tabular}{|l|l|}
\hline 5496 & $100-B-8$ Sample Node O-B8-3 \\
\hline 5497 & $100-b-8$ Sample Node O-B8-4
\end{tabular}

5497 100-b-8 Sample Node O-B8-4

5498 100-B-8 Sample Node O-B8-8

5499 100-B-8 Sample Node O-B8-8

5500 100-B-8 Sampling Node O-B8-13

5501 Soil near a collection of red rocks by pipelines 22,23 and 25

5502 Soil near a collection of red rocks by pipelines $22,23 \& 25$

\begin{tabular}{|l|l|}
\hline 5503 & Pipes 11, 22 \& 23 \\
\hline 5504 & Pipes 11, 22 \& 23
\end{tabular}

5504 Pipes 11, 22 \& 23

5505 1607-F2 Shallow Zone Sample Node S-A1-2

5506 1607-F2 Shallow Zone Sampling Node S-A1-3

5507 1607-F2 Shallow Zone Sampling Node S-A1-4

5508 1607-F2 Shallow Zone Sampling Node S-A1-10

5509 1607-F2 Shallow Zone Sampling Node S-A1-13

\begin{tabular}{|l|l}
5510 & $1607-F 2$ Shallow Zone Sampling Node S-A1-16
\end{tabular}

\begin{tabular}{|l|l}
5511 & $1607-F 2$ \\
\hline
\end{tabular} 
5512 1607-F2 Shallow Zone Sampling Node S-A2-6

SAMP SITE DESC

5513 1607-F2 Shallow Zone Sampling Node S-A2-7

5514 1607-F2 Shallow Zone Sampling Node S-A2-10

5515 1607-F2 Shallow Zone Sampling Node S-A2-14

5516 1607-F2 Shallow Zone Sampling Node S-A2-15

5517 1607-F2 Shallow Zone Sampling Node S-A3-1

5518 1607-F2 Shallow Zone Sampling Node S-A3-2

5519 1607-F2 Shallow Zone Sampling Node S-A3-4

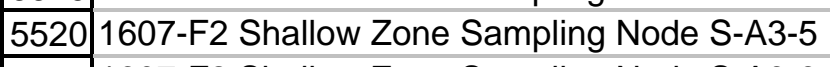

5521 1607-F2 Shallow Zone Sampling Node S-A3-9

5522 1607-F2 Shallow Zone Sampling Node S-A3-11

\begin{tabular}{|l|l}
5523 & $1607-F 2$ Shallow Zone Sampling Node S-A4-3
\end{tabular}

\begin{tabular}{|l|l}
5524 & $1607-F 2$ Shallow Zone Sampling Node S-A4-4
\end{tabular}

5525 1607-F2 Shallow Zone Sampling Node S-A4-7

5526 1607-F2 Shallow Zone Sampling Node S-A4-9

\begin{tabular}{|l|l}
5527 & $1607-F 2$ Shallow Zone Sampling Node S-A4-12
\end{tabular}

\begin{tabular}{|l|l|}
5528 & $1607-F 2$ \\
5 & Shallow Zone Sampling Node S-A4-13
\end{tabular}

5529 1607-F2 Shallow Zone Sampling Node S-B5-1

5530 1607-F2 Shallow Zone Sampling Node S-B5-2

\begin{tabular}{|l|l}
5531 & $1607-F 2$ \\
553 & Shallow Zone Sampling Node S-B5-5
\end{tabular}

5532 1607-F2 Shallow Zone Sampling Node S-B5-7

\begin{tabular}{|l|l}
5533 & $1607-F 2$ \\
\hline
\end{tabular}

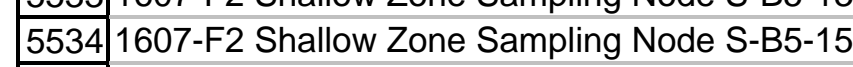

\begin{tabular}{|l|l}
5535 & $1607-F 2$ \\
5 & Shallow Zone Sampling Node S-B6-1
\end{tabular}

5536 1607-F2 Shallow Zone Sampling Node S-B6-10

5537 1607-F2 Shallow Zone Sampling Node S-B6-12

5538 1607-F2 Shallow Zone Sampling Node S-B6-13

5539 1607-F2 Shallow Zone Sampling Node S-B6-15

5540 1607-F2 Shallow Zone Sampling Node S-B6-16

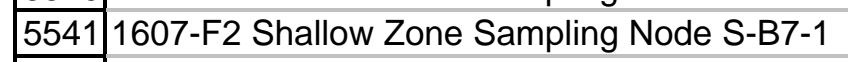

\begin{tabular}{|l|l|}
5542 & $1607-F 2$ Shallow Zone Sampling Node S-B7-3
\end{tabular}

5543 1607-F2 Shallow Zone Sampling Node S-B7-4

\begin{tabular}{|l|l|l|}
5544 & $1607-F 2$ \\
\hline
\end{tabular}

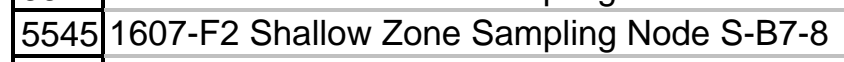

5546 1607-F2 Shallow Zone Sampling Node S-B7-11

5547 1607-F2 Shallow Zone Sampling Node S-B8-2

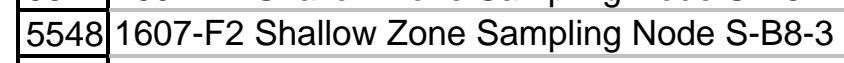

\begin{tabular}{|l|l|}
5549 & $1607-F 2$ Shallow Zone Sampling Node S-B8-4
\end{tabular} 
5553 100-B-8 Sampling Node S-A1-2

\begin{tabular}{|l|l|}
5554 & $100-B-8$ Sampling Node S-A1-3 \\
\hline 5555 & $100-B-8$ Sampling Node S-A1-4
\end{tabular}

\begin{tabular}{|l|l}
5555 & $100-B-8$ Sampling Node S-A1-4
\end{tabular}

5556 100-B-8 Sampling Node S-A1-10

5557 100-C-6 Sampling Node S-A!-13

\begin{tabular}{|l|l}
5558 & $100-C-6$ Sampling Node S-A!-16 \\
\hline 5559 & $100-C-6$ Sampling Node S-A2-3
\end{tabular}

\begin{tabular}{l|l}
5559 & $100-C-6$ Sampling Node S-A2-3
\end{tabular}

5560 100-C-6 Sampling Node S-A2-6

5561 $100-C-6$ Sampling Node S-A2-7

\begin{tabular}{l|l}
5562 & $100-C-6$ Sampling Node S-A2-10
\end{tabular}

5563 100-C-6 Sampling Node S-A2-14

5564 100-C-6 Sampling Node S-A1-16

\begin{tabular}{|l|l}
5565 & $100-C-6$ Sampling Node S-A3-1 \\
\hline 5566 & $100-C-6$ Sampling Node S-A3-2
\end{tabular}

5566 100-C-6 Sampling Node S-A3-2

5567 100-C-6 Sampling Node S-A3-4

5568 100-C-6 Sampling Node S-A\#-5

5569 100-C-6 Sampling Node S-A3-9

5570 100-C-6 Sampling Node S-A3-11

5571 100-C-6 Sampling Node S-A4-3

\begin{tabular}{|l|l|}
5572 & $100-C-6$ Sampling Node S-A4-4 \\
\hline 5573 & $100-C-6$ Sampling Node S-A4-7
\end{tabular}

5573 100-C-6 Sampling Node S-A4-7

5574 100-C-6 Sampling Node S-A4-9

5575 100-C-6 Sampling Node S-A4-12

5576 100-C-6 Sampling Node S-A4-13

5577 100-C-6 Sampling Node S-B5-1

5578 100-C-6 Sampling Node S-B5-2

\begin{tabular}{|l|l}
5579 & $100-C-6$ Sampling Node S-B5-5 \\
\hline 5580 & $100-C-6$ Sampling Node S-B5-7
\end{tabular}

5580 100-C-6 Sampling Node S-B5-7

5581 100-C-6 Sampling Node S-B5-13

5582 100-C-6 Sampling Node S-B5-15

\begin{tabular}{|l|l}
5583 & $100-C-6$ Sampling Node S-B6-1 \\
\hline 5584 & $100-B-8$ Sampling Node
\end{tabular}

5584 100-B-8 Sampling Node S-B6-10

5585 100-B-8 Sampling Node S-B6-12

5586 100-B-8 Sampling Node S-B6-13

\begin{tabular}{|l|l}
5587 & $100-B-8$ Sampling Node S-B6-15 \\
\hline
\end{tabular} 


\begin{tabular}{|c|c|c|}
\hline 1 & SAMP_SITE_DESC & SITE_ELEVATION \\
\hline 5588 & 100-B-8 Sampling Node S-B6-16 & 133.257 \\
\hline 5589 & 100-B-8 Sampling Node S-B7-1 & 131.486 \\
\hline 5590 & 100-B-8 Sampling Node S-B7-3 & 130.273 \\
\hline$\overline{5591}$ & 100-B-8 Sampling Node S-B7-4 & 132.671 \\
\hline$\overline{5592}$ & 100-B-8 Sampling Node S-B7-5 & 129.884 \\
\hline 5593 & 100-B-8 Sampling Node S-B7-8 & 133.299 \\
\hline 5594 & 100-B-8 Sampling Node S-B7-11 & 130.705 \\
\hline 5595 & 100-B-8 Sampling Node S-B8-2 & 132.971 \\
\hline 5596 & 100-B-8 Sampling Node S-B8-3 & 132.871 \\
\hline 5598 & 100-B-8 Sampling Node S-B8-8 & 131.494 \\
\hline 5599 & 100-B-8 Sampling Node S-B8-12 & 131.834 \\
\hline 5600 & 100-B-8 Sampling Node S-B8-13 & 132.447 \\
\hline 5601 & 100-B-8 Sampling Node S-C9-3 & 132.048 \\
\hline 5602 & 100-B-8 & 133.912 \\
\hline 5603 & 100-B-8 Sampling Node S-C9-5 & 134.334 \\
\hline$\overline{5604}$ & 100-B-8 Sampling Node S-C9-10 & 132622 \\
\hline 5605 & 100-B-8 Sampling Node S-C9-14 & 133.949 \\
\hline 5606 & 100-B-8 Sampling Node S-C9-16 & 134.171 \\
\hline 5608 & 100-B-8 Sampling Node S-C10-4 & 132.102 \\
\hline 5609 & 100-B-8 Sampling Node S-C10-8 & 132.696 \\
\hline 5610 & 100-B-8 Sampling Node S-C10--10 & 132.899 \\
\hline 5611 & 100-B-8 Sampling Node S-C10-14 & 134.07 \\
\hline 5612 & 100-B-8 Sampling Node S-C10-6 & 134.107 \\
\hline 5613 & 100-B-8 Sampling Node S-C1-2 & 136.304 \\
\hline 5614 & 100-B-8 Sampling Node S-C1-3 & 136.162 \\
\hline$\overline{5615}$ & 100-B-8 Sampling Node S-C1-4 & 136.13 \\
\hline$\overline{5616}$ & 100-B-8 Sampling Node S-C1-10 & 138.187 \\
\hline$\overline{5617}$ & 100-B-8 Sampling Node S-C1-13 & 136.78 \\
\hline 5618 & 100-B-8 Sampling Node S-C1-16 & 137.114 \\
\hline 5619 & 100-B-8 Sampling Node S-C2-3 & 137.242 \\
\hline 5620 & 100-B-8 Sampling Node S-C2-6 & 137.118 \\
\hline 5621 & 100-B-8 Sampling Node S-C2-7 & 138.288 \\
\hline 5622 & 100-B-8 Sampling Node S-C2-10 & 138.544 \\
\hline 5623 & 100-B-8 Sampling Node S-C2-14 & 138.724 \\
\hline 5624 & 100-B-8 Sampling Node S-C2-15 & 138.907 \\
\hline 5625 & 100-B-8 Sampling Node S-D3-1 & 132.275 \\
\hline
\end{tabular}


5633 100-B-8 Sampling Node S-D4-7

\begin{tabular}{|l|l|}
\hline 5634 & $100-B-8$ Sampling Node S-D4-9 \\
\hline 5635 & No0-B-8 Sampling Node S-D4-12
\end{tabular}

5635 100-B-8 Sampling Node S-D4-12

5636 100-B-8 Sampling Node S-D4-13

5637 100-B-8 Sampling Node S-D5-1

5638 100-B-8 Sampling Node S-D5-1

5639 100-B-8 Sampling Node S-D5-5

\begin{tabular}{|l|l}
5640 & $100-B-8$ Sampling Node S-D5-7
\end{tabular}

\begin{tabular}{|l|l|}
5641 & $100-B-8$ Sampling Node S-D5-13 \\
\hline 5642 & 100-B-8 Sampling Node S-D5-15
\end{tabular}

5642 100-B-8 Sampling Node S-D5-15

5643 100-B-8 Sampling Node S-D6-1

5644 100-B-8 Sampling Node S-D6-10

5645 100-B-8 Sampling Node S-D6-12

5646 100-B-8 Sampling Node S-D6-13

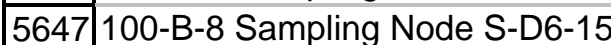

\begin{tabular}{|l|l|}
5648 & $100-B-8$ Sampling Node S-D6-16 \\
\hline 5649 & $100-B-8$ Dedis
\end{tabular}

5649 100-B-8 Debris material suspected of containing asbestos from the side of an excavation north of pipeline 11 . The material is most likely from off of phamtom pipeline 002.

5650 1607-F2 Shallow Zone Verification Composite Sample from Sample Nodes SA1-3, SA1-4, SA1-10, SA1-16

5651 1607-F2 Shallow Zone Verification Composite Sample from Sample Nodes SA2-3, SA2-6, SA2-7, SA2-15

5652 1607-F2 Shallow Zone Verification Composite Sample from Sample Nodes SA3-1, SA3-2, SA3-4, SA3-11

5653 1607-F2 Shallow Zone Verification Composite Sample from Sample Nodes SA4-3, SA4-4, SA4-7, SA4-12

5654 1607-F2 Shallow Zone Verification Composite Sample from Sample Nodes SB5-1, SB5-5, SB5-7, SB5-15

5655 1607-F2 Shallow Zone Verification Composite Sample from Sample Nodes SB6-1, SB6-10, SB6-13, SB6-15

5656 1607-F2 Shallow Zone Verification Composite Sample from Sample Nodes SB8-3, SB8-4, SB8-8, SB8-13

5657 1607-F2 Shallow Zone Verification Equipment Blank Associated with Sample J002X0

5658 1607-F2 Shallow Zone Verification Duplicate Associated with Sample J002X0

5659 1607-F2 Shallow Zone Verification Split Associated with Sample J002X0

5660 116-N-1 Trench, Plume 4 Sidewall, Sampling Point N1P4/5-02A

5661 116-N-1 Trench, Plume 4 Sidewall, Sampling Point N1P4/5-02A

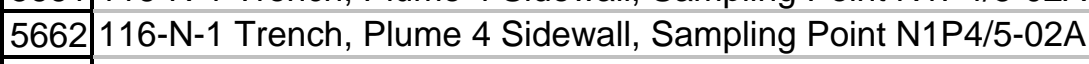


5664 116-N-1 Trench Overburden, Stockpile A, Sampling Point N1-OVB-A0-1

SAMP SITE DESC

5665 116-N-1 Trench Overburden, Stockpile A, Sampling Point N1-OVB-A

5666 116-N-1 Trench Overburden, Stockpile A, Sample Point N1-OVB-A1A-1

5667 116-N-1 Trench Overburden, Stockpile A, Sampling Point N1-OVB-A1A-2

5668 116-N-1 Trench Overburden, Stockpile A, Sampling Point N1-OVB-B0-1

5669 116-N-1 Trench Overburden, Stockpile A, Sampling Point N1-OVB-B0-2

5670 116-N-1 Trench Overburden, Stockpile A, Sampling Point N1-OVB-B0-3

5671 116-N-1 Trench Overburden, Stock A, Sampling Point N1-OVB-B0-4

\begin{tabular}{|l|l|}
5672 & $116-N-1$ Trench, Pume 3 Sidewall, Sampling Point N1P3-01 \\
\hline 5673 & $116-N-1$ Trench, Plame 3 Sidewal, Samplng Pon N1P3-02
\end{tabular}

5673 116-N-1 Trench, Plume 3 Sidewall, Sampling Point N1P3-02

5674 116-N-1 Trench, Plume 3 Sidewall, Sampling Point N1P3-03

5675 116-N-1 Trench, Plume 3 Sidewall, Sampling Point N1P3-04

5676 100-C-6 Floor Hot Spot Grab Soil Sample

5677 100-C-6 Floor Hot Spot Grab Sample

5678 100-C-6 Floor Hot Spot Grab Sample

\begin{tabular}{|l|l}
5679 & $100-C-6$ Floor Hot Spot Grab Sample
\end{tabular}

5680 100-C-6 Floor Hot Spot Grab Sample

5681 100-C-6 Floor Hot Spot Grab Sample

5682 100-C-6 Floor Hot Spot Grab Sample

\begin{tabular}{|l|l}
5683 & $100-C-6$ Floor Hot Spot Grab Sample
\end{tabular}

5684 100-C-6 Floor Hot Spot Grab Sample

5685 132-C-2 Outfall Floor Hot Spot Grab Sample

\begin{tabular}{|l|l}
5686 & $132-C-2$ \\
\hline 5687 & Outfall Floor Hot Spot Grab Sample
\end{tabular}

5687 100-B-8 Sampling Node S-E7-3

5688 100-B-8 Sampling Node S-E7-4

5689 100-B-8 Sampling Node S-E7-5

\begin{tabular}{|l|l}
5690 & $100-B-8$ Sampling Node S-E7-8
\end{tabular}

5691 100-B-8

5692 100-B-8 Sampling Node S-E8-3

\begin{tabular}{|l|l}
5693 & $100-B-8$ Sampling Node S-E8-4 \\
\hline 5694 & No0-B-8 Sampling Node S-E8-12
\end{tabular}

5694 100-B-8 Sampling Node S-E8-12

5695 100-B-8 Sampling Node S-E9-3

5696 100-B-8 Sampling Node S-E9-4

\begin{tabular}{|l|l|}
\hline 5697 & $100-B-8$ Sampling Node S-E9-5 \\
\hline 5698 & $100-B-8$ Sampling Node S-E9-10 \\
\hline
\end{tabular}

5698 100-B-8 Sampling Node S-E9-10

5699 100-B-8 Sampling Node S-E9-14

5700 100-B-8 Sampling Node S-E9-16

5701 100-C-6 Sampling Node S-E10-4 


\begin{tabular}{|c|c|c|}
\hline 1 & SAMP_SITE_DESC & SITE_ELEVATION \\
\hline 5702 & 100-B-8 Sampling Node S-E10-3 & 132217 \\
\hline 5703 & 100-C-6 Sampling Node S-E10-8 & 132.363 \\
\hline 5704 & 100-C-6 Sampling Node S-E10-10 & 131.337 \\
\hline$\overline{5705}$ & 100-C-6 Sampling Node S-E10-14 & 134.426 \\
\hline$\overline{5706}$ & $100-C-6$ & 134.334 \\
\hline 5707 & 100-C-6 Sampling Node S-F1-2 & 132.878 \\
\hline 5708 & 100-C-6 Sampling Node S-F1-3 & 134.044 \\
\hline 5709 & 100-C-6 Sampling Node S-F1-10 & 132.907 \\
\hline 5710 & 100-C-6 Sampling Node S-F1-13 & 134.035 \\
\hline 5712 & 100-C-6 Sampling Node S-F2-3 & 132.418 \\
\hline 5713 & 100-C-6 Sampling Node S-F2-6 & 132.986 \\
\hline$\overline{5714}$ & 100-C-6 Sampling Node S-F2-7 & 132.231 \\
\hline 5715 & 100-C-6 Sampling Node S-F2-10 & 131.528 \\
\hline$\overline{5716}$ & 100-C-6 Sampling Node S-F2-16 & 132.32 \\
\hline$\overline{5717}$ & 100-C-6 Sampling Node S-F2-15 & 132.999 \\
\hline$\overline{5718}$ & 100-C-6 Sampling Node S-F3-1 & 129.943 \\
\hline 5719 & 100-C-6 Sampling Node S-F3-4 & 130.684 \\
\hline 5720 & 100-C-6 Sampling Node S-F3-5 & 129.733 \\
\hline 5722 & 100-C-6 Sampling Node S-F3-11 & 129.77 \\
\hline 5723 & 100-C-6 Sampling Node S-F4-3 & 133997 \\
\hline 5724 & 100-C-6 Sampling Node S-F4-4 & 132.044 \\
\hline 5725 & 100-C-6 Sampling Node S-F4-9 & 134747 \\
\hline$\overline{5726}$ & 100-C-6 Sampling Node S-F4-12 & 132.814 \\
\hline 5727 & 100-C-6 Sampling Node S-F4-13 & 135.252 \\
\hline 5728 & 100-C-6 Sampling Node S-G5-1 & 134.006 \\
\hline 5729 & 100-C-6 Sampling Node S-G5-5 & 134.743 \\
\hline 5730 & 100-C-6 Sampling Node S-G5-7 & 134.256 \\
\hline$\overline{5731}$ & 100-C-6 Sampling Node S-G5-15 & 135.475 \\
\hline 5732 & 100-C-6 Sampling Node S-C6-10 & 132.191 \\
\hline 5733 & 100-C-6 Sampling Node S-G6-12 & 132.396 \\
\hline$\overline{5734}$ & 100-C-6 Sampling Node S-G6-13 & 132.648 \\
\hline 5735 & 100-C-6 Sampling Node S-G6-15 & 132.824 \\
\hline$\overline{5736}$ & 100-C-6 Sampling Node S-G6-16 & 132.65 \\
\hline 5737 & 100-C-6 Sampling Node S-G7-1 & 133.016 \\
\hline 5738 & 100-C-6 Sampling Node S-G7-3 & 133305 \\
\hline 5739 & 100-C-6 Sampling Node S-G7-4 & 132.713 \\
\hline
\end{tabular}




\begin{tabular}{|c|c|c|}
\hline 1 & \multirow{2}{*}{$\begin{array}{|lc|} & \text { SAMP_SITE_DESC } \\
\text { 100-C-6 Sampling Node S-G7-5 } & \end{array}$} & SITE_ELEVATION \\
\hline \multirow{2}{*}{5740} & & 133.266 \\
\hline & 100-C-6 Sampling Node S-G7-8 & 132.964 \\
\hline 5742 & 100-C-6 Sampling Node S-G7-11 & 132.59 \\
\hline 5743 & 100-C-6 Sampling Node S-G8-2 & 133.15 \\
\hline 5744 & 100-C-6 Sampling Node S-G8-3 & 133.926 \\
\hline 5745 & 100-C-6 Sampling Node S-G8-4 & 134.949 \\
\hline 5746 & 100-C-6 Sampling Node S-G8-8 & 133.621 \\
\hline 5747 & 100-C-6 Sampling Node S-G8-12 & 132.341 \\
\hline 5748 & 100-C-6 Sampling Node S-G8-13 & 132.552 \\
\hline 5750 & 100-C-6 Sampling Node S-H9-4 & 132.681 \\
\hline 5751 & 100-C-6 Sampling Node S-H9-5 & 133.71 \\
\hline 5752 & 100-C-6 Sampling Node S-H9-10 & 133.12 \\
\hline 5753 & 100-C-6 Sampling Node S-H9-14 & 133.005 \\
\hline 5754 & 100-C-6 Sampling Node S-H9-16 & 132.96 \\
\hline 5755 & 100-C-6 Sampling Node S-H10-3 & 132.642 \\
\hline 5756 & 100-C-6 Sampling Node S-H10-4 & 134.094 \\
\hline 5757 & 100-C-6 Sampling Node S-H10-8 & 133.762 \\
\hline 5758 & 100-C-6 Sampling Node S-H10-10 & 133.668 \\
\hline 5760 & 100-C-6 Sampling Node S-H1-2 & 133.907 \\
\hline 5761 & 100-C-6 Sampling Node S-H1-3 & 132.888 \\
\hline 5762 & 100-C-6 Sampling Node S-H1-4 & 134.448 \\
\hline 5763 & 100-C-6 Sampling Node S-H1-10 & 134.48 \\
\hline 5764 & 100-C-6 Sampling Node S-H1-13 & 132.614 \\
\hline 5765 & 100-C-6 Sampling Node S-H1-16 & 136.184 \\
\hline 5766 & 100-C-6 Sampling Node S-H2-3 & 133.214 \\
\hline 5767 & 100-C-6 Sampling Node S-H2-6 & 136.157 \\
\hline 5768 & 100-C-6 Sampling Node S-H2-7 & 133.286 \\
\hline 5769 & 100-C-6 Sampling Node S-H2-10 & 136.445 \\
\hline 5770 & 100-C-6 Sampling Site S-H2-14 & 136.577 \\
\hline 5771 & 100-C-6 Sampling Node S-H2-15 & 133.334 \\
\hline 5772 & 126-F-1 Overburden Zone Verification Composite Sampling - Nodes OA1-3, OA1-4, OA1-10, OA1-16 & \\
\hline 5773 & 126-F-1 Overburden Zone Verification Composite Sampling - Nodes OA2-3, OA2-6, OA2-7, OA2-15 & \\
\hline 5774 & 126-F-1 Overburden Zone Verification Composite Sampling - Nodes OA3-1, OA3-2, OA3-4, OA3-11 & \\
\hline 5775 & 126-F-1 Overburden Zone Verification Composite Sampling - Nodes OA4-3, OA4-4, OA4-7, OA4-15 & \\
\hline 5776 & 126-F-1 Overburden Zone Verification Composite Sampling - Nodes OB5-1, OB5-5, OB5-7, OB5-15 & \\
\hline 5777 & 126-F-1 Overburden Zone Verification Composite Sampling - Nodes OB6 & \\
\hline
\end{tabular}

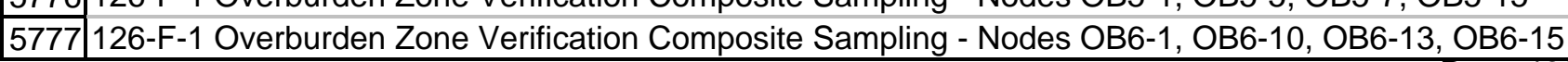


5779 126-F-1 Overburden Zone Verification Composite Sampling - Nodes OB8-3, OB8-4, OB8-8, OB8-13

5780 126-F-1 Overburden Verification Composite Sampling - Nodes OC9-3, OC9-4, OC9-10, OC9-16

5781 126-F-1 Overburden Zone Verification Composite Sampling - Nodes OC10-4, OC10-10, OC10-14, OC10-16

5782 126-F-1 Overburden Zone Verification Composite Sampling - Nodes OC1-3, OC1-4, OC1-10, OC1-16

5783 126-F-1 Overburden Zone Verification Composite Sampling - Nodes OC2-3, OC2-6, OC2-7, OC2-15

5784 126-F-1 Overburden Zone Verification Compositie Sampling - Duplicate of J00097

5785 126-F-1 Overburden Zone Verification Composite Sampling - Equipment Blank for J00097

5786 126-F-1 Overburden Verification Composite Sampling - Split of J00097

5787 100-B-8 Verification Composite Sampling

5788 100-B-8 Verification Composite Sampling

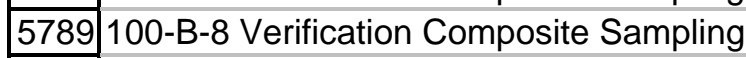

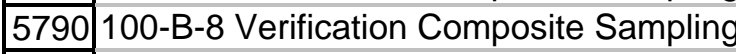

5791 100-B-8 Verification Composite Sampling

5792 100-B-8 Verification Composite Sampling

\begin{tabular}{|l|l}
5793 & $100-B-8$ Verification Composite Sampling \\
\hline 5794 & $00-B-8$ Veification Composite Sampling \\
\hline
\end{tabular}

5794 100-B-8 Verification Composite Sampling

5795 100-C-6 Floor Hot Spots

\begin{tabular}{|l|l}
5796 & $100-\mathrm{C}-6$ Floor Hot Spots
\end{tabular}

5797 100-C-6 Floor Hot Spots

5798 100-C-6 Floor Hot Spots

5799 100-C-6 Floor Hot Spots

\begin{tabular}{|l|l|}
5800 & $100-C-6$ Floor Hot Spots \\
\hline 5801 & $100-B-8$ Floor Hot Spots
\end{tabular}

5801 100-B-8 Floor Hot Spots

5802 100-B-8 Floor Hot Spots - Line 11 Elbow

\begin{tabular}{|l|l}
5803 & $100-B-8$ Floor Hot Spots - Line 11 Expansion Box
\end{tabular}

5804 100-B-8 Floor Hot Spots - Distal end of Line 11 Silty soil

5805 100-C-9 Potholing activity east of box sewer

5806 Deep plumes on pipelines 17,18 , and 19

\begin{tabular}{|l|l|l}
5807 & $100-C-6$ Deep plumes on pipelines 17,18 \& 19
\end{tabular}

5808 100-C-6 Deep plumes on pipelines 17, 18 \& 19

5809 116-N-1 potholes on east side of trench adjacent to zone 1 - pothole \#51 - sample point N1-PH-051

5810 116-N-1 potholes on east side of trench adjacent to zone 1 - pothole \#51 - sample point N1-PH-051

5811 116- $\mathrm{N}-1$ potholes on east side of trench adjacent to zone 1 - pothole \#51 - sample point N1-PH-051

5812 116-N-1 potholes on east side of trench adjacent to zone 1 - pothole \#51 - sample point N1-PH-051

5813 Potholes adjacent to 116-N-1 Trench (\#52,53,\&54) - Sample Point N1-PH-052

5814 Potholes adjacent to 116-N-1 Trench (\#52,53,\&54) - Sample Point N1-PH-052

5815 Potholes adjacent to 116-N-1 Trench (\#52,53,\&54) - Sample Point N1-PH-052 
\begin{tabular}{|l|l|}
\hline 5816 & Potholes adjacent to $116-N-1$ Trench $(\# 52,53, \& 54)$ - Sample Point N1-PH-054 \\
\hline 5817 & Potholes adjacent to 116-N-1 Trench $(\# 52,53, \& 54)$ - Sample Point N1-PH-054
\end{tabular}

SAMP SITE

5818 Potholes adjacent to $116-\mathrm{N}-1$ Trench $(\# 52,53, \& 54)$ - Sample Point N1-PH-054

5819 Potholes adjacent to 116-N-1 Trench (\#52,53,\&54) - Sample Point N1-PH-054

5820 Potholes adjacent to 116-N-1 Trench (\#55,56,\&57) - Sample Point N1-PH-055

5821 Potholes adjacent to $116-\mathrm{N}-1$ Trench $(\# 55,56, \& 57)$ - Sample Point N1-PH-055

5822 Potholes adjacent to 116-N-1 Trench (\#55,56,\&57) - Sample Point N1-PH-055

5823 Potholes adjacent to $116-\mathrm{N}-1$ Trench $(\# 55,56, \& 57)$ - Sample Point N1-PH-055

5824 116-N-1 Trench Overburden Stockpile A, Lift 1 (A1b) and Stockpile D, Lift 1 - Sample Point N1-OVB-A1b-01

5825 116-N-1 Trench Overburden, Stockpile A, Lift 1 (A1b) and Stockpile D, Lift 1 - Sample Point N1-OVB-A1b-02

5826 116-N-1 Trench Overburden, Stockpile A, Lift 1 (A1b) and Stockpile D, Lift 1 - Sample Point N1-OVB-A1b-03

5827 116-N-1 Trench Overburden, Stockpile A, Lift 1 (A1b) and Stockpile D, Lift 1 - Sample Point N1-OVB-A1b-04

5828 116-N-1 Trench Overburden, Stockpile A, Lift 1 (A1b) and Stockpile D, Lift 1 - Sample Point N1-OVB-A1b-05

5829 116-N-1 Trench Overburden, Stockpile A, Lift 1 (A1b) and Stockpile D, Lift 1 - Sample Point N1-OVB-A1b-06

5830 116-N-1 Trench Overburden, Stockpile A, Lift 1 (A1b) and Stockpile D, Lift 1 - Sample Point N1-OVB-D1-01

5831 $116-N-1$ Trench Overburden, Stockpile A, Lift 1 (A1b) and Stockpile D, Lift 1 - Sample Point N1-OVB-D1-02

5832 West side of $116-\mathrm{N}-1$ crib in the vacinity of potholes \#1 and \#2

5833 West side of $116-\mathrm{N}-1$ crib in the vacinity of potholes \#1 and \#2

5834 West side of $116-\mathrm{N}-1$ crib in the vacinity of potholes \#1 and \#2

5835 West side of 116-N-1 crib in the vacinity of potholes \#1 and \#2

5836 Insulation material samples from covering on the PVC pipeline near UPR-100-N-31

5837 Insulation material samples from covering on the PVC pipeline near UPR-100-N-31

5838 Insulation material samples from covering on the PVC pipeline near UPR-100-N-31

5839 120-N-2,1324-N Surface Impoundment

5840 120-N-1,1324-NA Percolation Pond

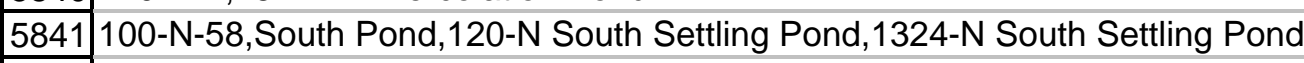

5842 100-N-58,South Pond,120-N South Settling Pond,1324-N South Settling Pond

5843 120-N-2,1324-N Surface Impoundment

5844 100-C-6 Stockpiles 1, 2 \& 5 Sampling Node O-C9-3

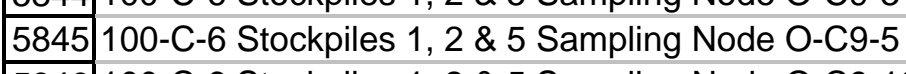

5846 100-C-6 Stockpiles 1, 2 \& 5 Sampling Node O-C9-10

5847 100-C-6 Stockpiles 1, 2 \& 5 Sampling Node O-C9-14

5848 $100-$ - -6 Stockpiles 1, 2 \& 5 Sampling Node O-C9-16

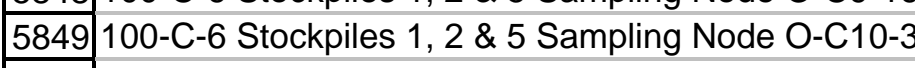

5850 100-C-6 Stockpiles 1, 2 \& 5 Sampling Node O-C10-4

5851 100-C-6 Stockpiles 1, 2 \& 5 Sampling Node O-C10-8

5852 100-C-6 Stockpiles 1, 2 \& 5 Sampling Node O-C10-10

\begin{tabular}{|l|l|}
5853 & $100-C-6$ Stockpiles 1, 2 \& 5 Sampling Node O-C10-14 \\
\hline
\end{tabular}

\begin{tabular}{|r|r|}
\hline & 138.256 \\
\hline & 138.857 \\
139.59 \\
138.437 \\
\hline 136.836 \\
139.781 \\
\hline 139.191 \\
\hline \\
137.308 \\
139.895 \\
138.457 \\
\hline
\end{tabular}

Page 468

\begin{tabular}{|r|}
\hline SITE_ELEVATION \\
\hline 136.4 \\
\hline 136 \\
\hline 149939 \\
\hline 135.1 \\
\hline 136.4 \\
\hline 136 \\
\hline 135.4 \\
\hline 135.1 \\
\hline 1425 \\
\hline 1425 \\
\hline 142.5 \\
\hline 142.5 \\
\hline 142.5 \\
\hline 142.5 \\
\hline 142.5 \\
\hline 142.5 \\
\hline 139.3 \\
\hline 139.3 \\
\hline 139.5 \\
\hline 139.5 \\
\hline 139.8 \\
\hline 139.8 \\
\hline 139.8 \\
\hline 140.3 \\
\hline 137.9 \\
\hline 138.8 \\
\hline 138.1 \\
\hline 138.256 \\
\hline 138.857 \\
\hline 139.59 \\
\hline 138.437 \\
\hline 136.836 \\
\hline 139.781 \\
\hline 139.191 \\
\hline 137.308 \\
\hline 139.895 \\
\hline 138.457 \\
\hline
\end{tabular}




\begin{tabular}{|c|c|c|}
\hline \multirow[b]{2}{*}{1} & \multirow{2}{*}{ SAMP_SITE_DESC } & \multirow{2}{*}{ SITE_ELEVATION } \\
\hline & & \\
\hline 5854 & 100-C-6 Stockpiles 1, 2 \& 5 Sampling Node O-C10-16 & 137.972 \\
\hline 5855 & 100-C-6 Stockpiles 1, 2 \& 5 Sampling Node O-C1-2 & 140.084 \\
\hline 5856 & 100-C-6 Stockpiles 1, 2 \& 5 Sampling Node O-C1-3 & 139.929 \\
\hline 5857 & 100-C-6 Stockpiles 1, 2 \& 5 Sampling Node O-C1-4 & 138.722 \\
\hline 5858 & 100-C-6 Stockpiles 1, 2 \& 5 Sampling Node O-C1-10 & 140.208 \\
\hline 5859 & 100-C-6 Stockpiles 1, 2 \& 5 Sampling Node O-C1-13 & 139.006 \\
\hline 5860 & 100-C-6 Stockpiles 1, 2 \& 5 Sampling Node O-C2-3 & 138.889 \\
\hline 5861 & 100-C-6 Stockpiles 1, 2 \& 5 Sampling Node O-C2-6 & 139.157 \\
\hline 5862 & 100-C-6 Stockpiles 1, 2 \& 5 Sampling Node O-C2-7 & 139.155 \\
\hline 5863 & 100-C-6 Stockpiles 1, 2 \& 5 Sampling Node O-C2-10 & 139.462 \\
\hline 5864 & 100-C-6 Stockpiles 1, 2 \& 5 Sampling Node O-C2-14 & 139.184 \\
\hline 5865 & 100-C-6 Stockpiles 1, 2 \& 5 Sampling Node O-C2-15 & 139.171 \\
\hline 5866 & 100-C-6 Stockpiles 1, 2 \& 5 Sampling Node O-D3-1 & 139.599 \\
\hline 5867 & 100-C-6 Stockpiles 1, 2 \& 5 Sampling Node O-D3-2 & 139.929 \\
\hline 5868 & 100-C-6 Stockpiles 1, 2 \& 5 Sampling Node O-D3-4 & 140.437 \\
\hline 5869 & 100-C-6 Stockpiles 1, 2 \& 5 Sampling Node O-D3-5 & 138.64 \\
\hline 5870 & 100-C-6 Stockpiles 1, 2 \& 5 Sampling Node O-D3-9 & 137.081 \\
\hline 5871 & 100-C-6 Stockpiles 1, 2 \& 5 Sampling Node O-D3-11 & 138.191 \\
\hline 5874 & 100-C-6 Stockpiles 1, 2 \& 5 Sampling Node O-D4-7 & 138.224 \\
\hline 5875 & 100-C-6 Stockpiles 1, 2 \& 5 Sampling Node O-D4-9 & 136.619 \\
\hline 5876 & 100-C-6 Stockpiles 1, 2 \& 5 Sampling Node O-D4-13 & 136.546 \\
\hline 5877 & 100-C-6 Stockpiles 1, 2 \& 5 Sampling Node O-D5-1 & 136.388 \\
\hline 5878 & 100-C-6 Stockpiles 1, 2 \& 5 Sampling Node O-D5-2 & 137.691 \\
\hline 5879 & 100-C-6 Stockpiles 1, 2 \& 5 Sampling Node O-D5-5 & 137.265 \\
\hline 5880 & 100-C-6 Stockpiles 1, 2 \& 5 Sampling Node O-D5-7 & 138.578 \\
\hline 5881 & 100-C-6 Stockpiles 1, 2 \& 5 Sampling Node O-D5-15 & 138.113 \\
\hline 5882 & 100-C-6 Stockpiles 1, 2 \& 5 Sampling Node O-D6-1 & 136.134 \\
\hline 5883 & 100-C-6 Stockpiles 1, 2 \& 5 Sampling Node O-D6-10 & 137.594 \\
\hline 5884 & 100-C-6 Stockpiles 1, 2 \& 5 Sampling Node O-D6-12 & 137.616 \\
\hline 5885 & 100-C-6 Stockpiles 1, 2 \& 5 Sampling Node O-D6-13 & 135.422 \\
\hline 5886 & 100-C-6 Stockpiles 1, 2 \& 5 Sampling Node O-D6-15 & 135.868 \\
\hline 5887 & 100-C-6 Stockpiles 1, 2 \& 5 Sampling Node O-D6-16 & 135759 \\
\hline 5888 & 100-C-6 Stockpiles 1, 2 \& 5 Sampling Node O-E7-1 & 135.875 \\
\hline 5889 & 100-C-6 Stockpiles 1, 2 \& 5 Sampling Node O-E7-5 & 135.954 \\
\hline 5890 & 100-C-6 Stockpiles 1, 2 \& 5 Sampling Node O-E7-8 & 136.744 \\
\hline 589 & 100-C-6 Stockpiles 1, 2 \& 5 Sampling Node O-E7-11 & 136.714 \\
\hline
\end{tabular}


5895 100-C-6 Stockpiles 1, 2 \& 5 Sampling Node O-E8-8

5896 100-C-6 Stockpiles 1, 2 \& 5 Sampling Node O-E8-12

5898 100-C-6 Stockpiles 1, 2 \& 5 Sampling Node O-E9-10

5899 100-C-

5900 100-C-6 Stockpiles 1, 2 \& 5 Sampling Node O-E10-4

5901 100-C-6 Stockpiles 1, 2 \& 5 Sampling Node O-E10-8

5902 100-C-6 Stockpiles 1, 2 \& 5 Sampling Node O-E10-10

5903 100-C-6 Stockpiles 1, 2 \& 5 Sampling Node O-F1-2

5904 100-C-6 Stockpiles 1, 2 \& 5 Sampling Node O-F1-3

5905 100-C-6 Stockpiles 1, 2 \& 5 Sampling Node O-F1-16

5906 100-C-6 Stockpiles 1, 2 \& 5 Sampling Node O-F2-10

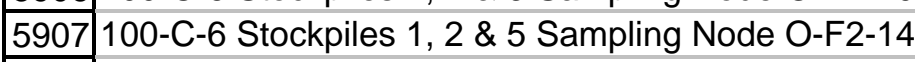

|5908 $100-$ - -6 Stockpiles 1, 2 \& 5 Sampling Node O-F2-15

5909 100-C-6 Stockpiles 1, 2 \& 5 Sampling Node O-F3-2

5910 100-C-6 Stockpiles 1, 2 \& 5 Sampling Node O-F3-9

5911 100-C-6 Stockpiles 1, 2 \& 5 Sampling Node O-F3-11

5912 100-C-6 Stockpiles 1, 2 \& 5 Sampling Node O-F4-7

5913 100-C-6 Stockpiles 1, 2 \& 5 Sampling Node O-F4-9

\begin{tabular}{|l|l|l}
5914 & $116-F-11$ Verification Sampling
\end{tabular}

5915 100-B-8 Verification Sampling

5916 100-B-8 Verification Sampling Shallow Zone Sampling Area A1 - Sampling Nodes 3, 4, 10 \& 16

5917 100-B-8 Verification Sampling Shallow Zone Sampling Area A2 - Sampling Nodes 3, 6, 7, \& 15

5918 100-B-8 Verification Sampling Shallow Zone Sampling Area A3 - Sampling Area 1, 2, 4, 11

5919 100-B-8 Verification Sampling Shallow Zone Sampling Area A4 - Sampling Nodes 3, 4, 7 \& 12

5920 100-B-8 Verification Sampling Shallow Zone Sampling Area B5 - Sampling Nodes 1, 5, 7, \& 15

5921 100-B-8 Verification Sampling Shallow Zone Sampling Area B6 - Sampling Nodes 1, 10, 13 \& 15

5922 100-B-8 Verification Sampling Shallow Zone Sampling Area B7 - Sampling Nodes 3, 4, 5, \& 11

5923 100-B-8 Verification Sampling Shallow Zone Sampling Area B8 - Sampling Nodes 3, 4, 8 \& 13

5924 100-B-8 Verification Sampling Shallow Zone Sampling Area C9 - Sampling Nodes 3, 4, 10 \& 16

5925 100-B-8 Verification Sampling Shallow Zone Sampling Area C10 - Sampling Nodes 4, 10, 14 \& 16

5926 100-B-8 Verification Sampling Shallow Zone Sampling Area C1 - Sampling Nodes 3, 4, 10 \& 16

5927 100-B-8 Verification Sampling Shallow Zone Sampling Area A1 - Sampling Nodes 3, 6, 7, \& 15

5928 100-B-8 Verification Sampling Shallow Zone Sampling Area D3 - Sampling Nodes 1, 2, 4, \& 11

5929 100-B-8 Verification Sampling - Shallow Zone - Sampling Area D4 - Sampling Nodes 3, 4, 7 \& 12 
5930 100-B-8 Verification Sampling - Shallow Zone - Sampling Area D5 - Sampling Nodes 1, 5, 7, 15

5931 100-B-8 Verification Sampling - Shallow Zone - Sampling Area A1 - Sampling Nodes 3, 4, 10 \& 16

5932 100-B-8 Verification Sampling - Shallow Zone - Sampling Area A2 - Sampling Nodes 3, 6, 7, 15

5933 100-B-8 Verification Sampling - Shallow Zone - Sampling Area D4 - Sampling Nodes 3, 6, 7 \& 15

5934 100-B-8 Verification Sampling - Shallow Zone - Sampling Area D4 - Sampling Nodes 3, 6, 7, \& 15

5935 100-B-8 Verification Sampling - Shallow Zone - Sampling Area D4 - Sampling Nodes 1, 2, 4, 11

5936 100-C-6

5937 From Ion Exchange Column H-IX-B1

5938 From Ion Exchange Column H-IX-B2

5939 From lon Exchange Column H-IX-B3

5940 From Ion Exchange Column H-IX-B4

5941 Resin from TOTE 97722

5942 Resin from TOTE 97725

5943 Resin from TOTE 97723

5944 Resin from TOTE 97732

\begin{tabular}{l|l}
5945 & Resin from TOTE 97730
\end{tabular}

5946 Resin from TOTE 97755

5947 Resin from TOTE 97716

5948 Resin from TOTE 97748

5949 Resin from TOTE 97745

5950 Resin from TOTE 97754

5951 Resin from TOTE 97727

5952 Resin from BURIAL BOX marked HR3-02-026

5953 Resin from TOTE 97717

5954 Resin from TOTE 97712

\begin{tabular}{|l|l|}
\hline 5955 & FROM TSL04 VALVE \\
\hline
\end{tabular} 


\begin{tabular}{|c|c|c|c|c|}
\hline & $\mathrm{O}$ & $P$ & $\bar{Q}$ & $\mathrm{R}$ \\
\hline 1 & SITE_CODE & ELEVATION_UNITS & VERT_COORD_TYPE & DATE_LAST_MODIFIED \\
\hline 2 & & & & \\
\hline 3 & & & & \\
\hline 4 & & & & \\
\hline 5 & & & & \\
\hline 6 & & & & \\
\hline 7 & & & & \\
\hline 8 & & & & \\
\hline 9 & & & & \\
\hline 10 & & & & \\
\hline 11 & & & & \\
\hline 12 & & $\mathrm{~m}$ & & \\
\hline 13 & & & & \\
\hline 14 & & & & \\
\hline 15 & & & & \\
\hline 16 & & & & \\
\hline 17 & & & & \\
\hline 18 & & & & \\
\hline 19 & & & & \\
\hline 20 & & & & \\
\hline 21 & & $\mathrm{~m}$ & & \\
\hline 22 & & & & \\
\hline 23 & & & & \\
\hline 24 & & $\mathrm{~m}$ & & \\
\hline 25 & & $\mathrm{~m}$ & & \\
\hline 26 & & $\mathrm{~m}$ & & \\
\hline 27 & & & & \\
\hline 28 & & & & \\
\hline 29 & & & & \\
\hline 30 & & & & \\
\hline 31 & & & & \\
\hline 32 & & $\mathrm{~m}$ & & \\
\hline 33 & & & & \\
\hline 34 & & & & \\
\hline 35 & & & & \\
\hline 36 & & & & \\
\hline 37 & & & & \\
\hline 38 & & & & \\
\hline 39 & & & & \\
\hline
\end{tabular}




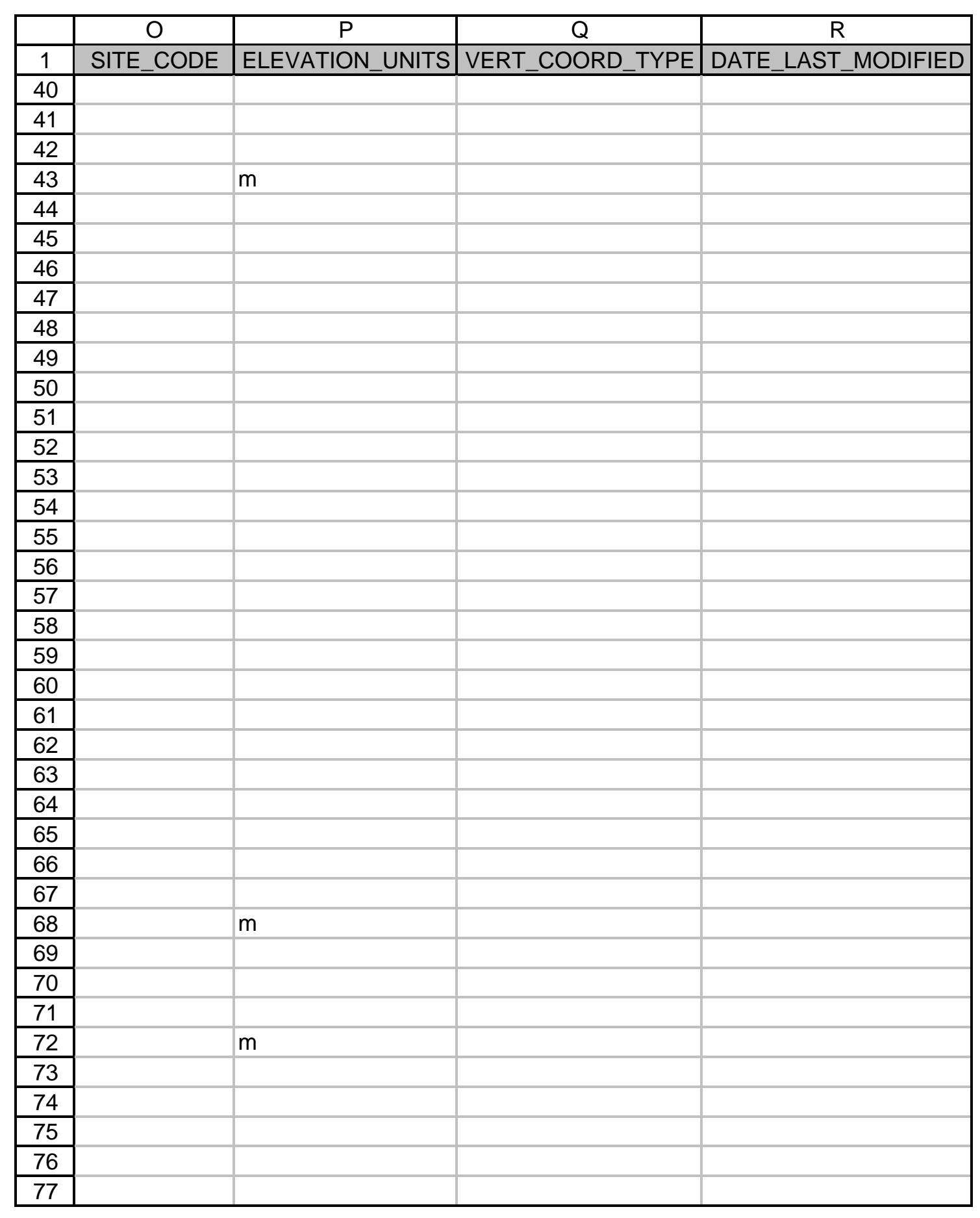




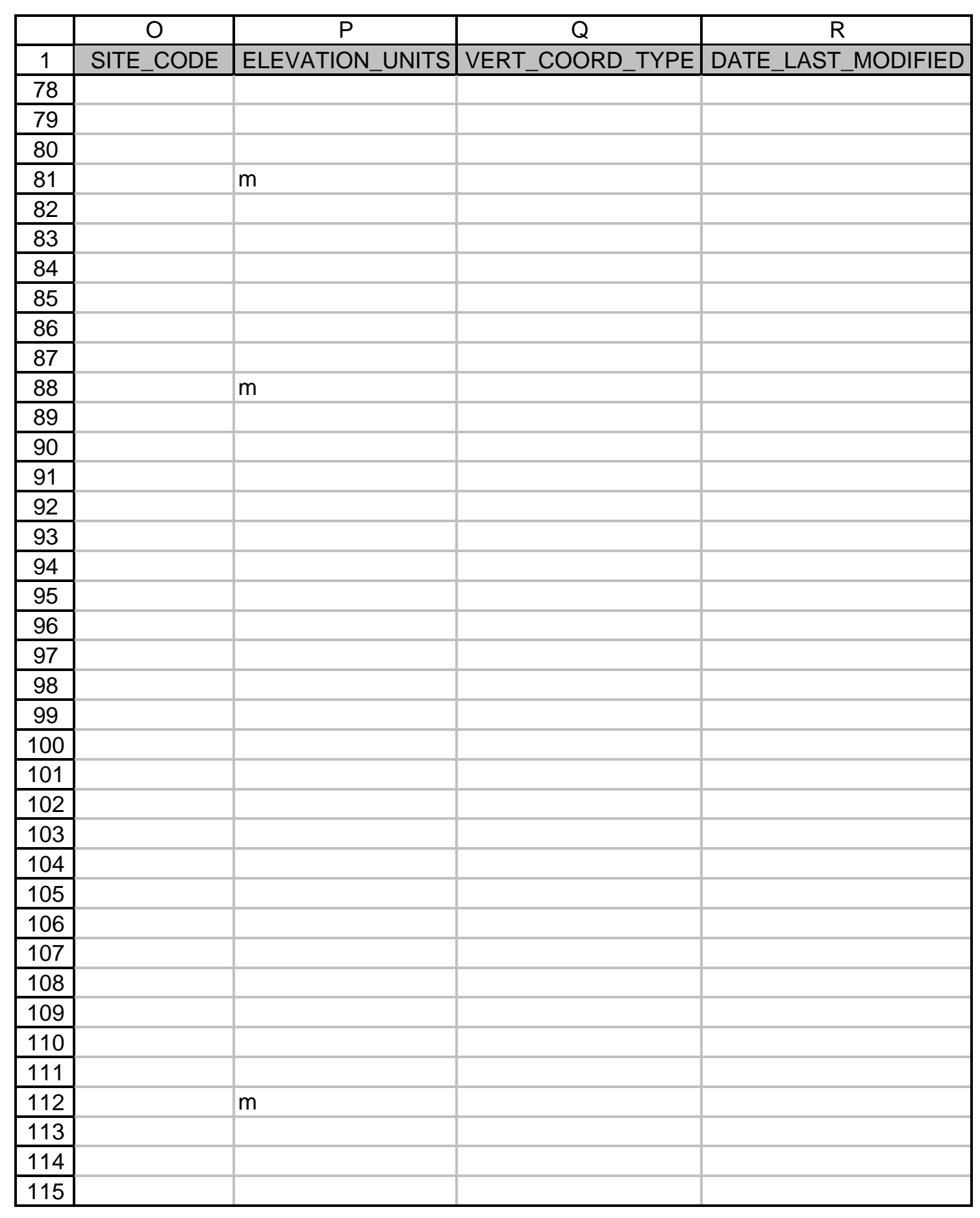




\begin{tabular}{|c|c|c|c|c|}
\hline & O & P & Q & R \\
\hline 1 & SITE_CODE & ELEVATION_UNITS & VERT_COORD_TYPE & DATE_LAST_MODIFIED \\
\hline 116 & & & & \\
\hline 117 & & & & \\
\hline 118 & & & & \\
\hline 119 & & $m$ & & \\
\hline 120 & & $m$ & & \\
\hline 121 & & & & \\
\hline 122 & & & & \\
\hline 123 & & & & \\
\hline 124 & & & & \\
\hline 125 & & & & \\
\hline 126 & & & & \\
\hline 127 & & & & \\
\hline 128 & & & & \\
\hline 129 & & & & \\
\hline 130 & & & & \\
\hline 131 & & & & \\
\hline 132 & & & & \\
\hline 133 & & & & \\
\hline 134 & & & & \\
\hline 135 & & & & \\
\hline 136 & & & & \\
\hline 137 & & & & \\
\hline 138 & & & & \\
\hline 139 & & & & \\
\hline 140 & & & & \\
\hline 141 & & & & \\
\hline 142 & & & & \\
\hline 143 & & & & \\
\hline 144 & & & & \\
\hline 145 & & & & \\
\hline 146 & & & & \\
\hline 148 & & & & \\
\hline 149 & & & & \\
\hline 150 & & & & \\
\hline 152 & & & & \\
\hline 153 & & & & \\
\hline
\end{tabular}




\begin{tabular}{|c|c|c|c|c|}
\hline & O & P & Q & R \\
\hline 1 & SITE_CODE & ELEVATION_UNITS & VERT_COORD_TYPE & DATE_LAST_MODIFIED \\
\hline 154 & & m & & \\
\hline 155 & & & & \\
\hline 156 & & & & \\
\hline 157 & & $m$ & & \\
\hline 158 & & $m$ & & \\
\hline 159 & & $m$ & & \\
\hline 160 & & & & \\
\hline 161 & & & & \\
\hline 162 & & & & \\
\hline 163 & & & & \\
\hline 164 & & & & \\
\hline 165 & & & & \\
\hline 166 & & & & \\
\hline 167 & & & & \\
\hline 168 & & & & \\
\hline 169 & & & & \\
\hline 170 & & & & \\
\hline 171 & & & & \\
\hline 172 & & & & \\
\hline 173 & & & & \\
\hline 174 & & & & \\
\hline 175 & & & & \\
\hline 176 & & & & \\
\hline 177 & & & & \\
\hline 178 & & & & \\
\hline 179 & & & & \\
\hline 180 & & & & \\
\hline 181 & & & & \\
\hline 182 & & & & \\
\hline 183 & & & & \\
\hline 184 & & & & \\
\hline 185 & & & & \\
\hline 186 & & & & \\
\hline 187 & & & & \\
\hline 188 & & & & \\
\hline 189 & & & & \\
\hline 190 & & & & \\
\hline
\end{tabular}




\begin{tabular}{|c|c|c|c|c|}
\hline & O & P & Q & R \\
\hline 1 & SITE_CODE & ELEVATION_UNITS & VERT_COORD_TYPE & DATE_LAST_MODIFIED \\
\hline 192 & & & & \\
\hline 193 & & & & \\
\hline 194 & & & & \\
\hline 195 & & & & \\
\hline 196 & & & & \\
\hline 197 & & & & \\
\hline 198 & & & & \\
\hline 199 & & & & \\
\hline 200 & & & & \\
\hline 201 & & & & \\
\hline 202 & & & & \\
\hline 203 & & & & \\
\hline 204 & & & & \\
\hline 205 & & & & \\
\hline 206 & & & & \\
\hline 207 & & & & \\
\hline 208 & & & & \\
\hline 209 & & & & \\
\hline 210 & & & & \\
\hline 211 & & & & \\
\hline 212 & & & & \\
\hline 213 & & & & \\
\hline 214 & & & & \\
\hline 215 & & & & \\
\hline 216 & & & & \\
\hline 217 & & & & \\
\hline 218 & & & & \\
\hline 219 & & & & \\
\hline 220 & & & & \\
\hline 221 & & & & \\
\hline 222 & & & & \\
\hline 223 & & & & \\
\hline 225 & & & & \\
\hline 226 & & & & \\
\hline 227 & & & & \\
\hline 29 & & & & \\
\hline
\end{tabular}




\begin{tabular}{|c|c|c|c|c|}
\hline & O & P & Q & R \\
\hline 1 & SITE_CODE & ELEVATION_UNITS & VERT_COORD_TYPE & DATE_LAST_MODIFIED \\
\hline 230 & & & & \\
\hline 231 & & $m$ & & \\
\hline 232 & & & & \\
\hline 233 & & $m$ & & \\
\hline 234 & & $m$ & & \\
\hline 235 & & $m$ & & \\
\hline 236 & & & & \\
\hline 237 & & & & \\
\hline 238 & & & & \\
\hline 239 & & & & \\
\hline 240 & & & & \\
\hline 241 & & & & \\
\hline 242 & & & & \\
\hline 243 & & & & \\
\hline 244 & & & & \\
\hline 245 & & & & \\
\hline 246 & & & & \\
\hline 247 & & & & \\
\hline 248 & & & & \\
\hline 249 & & & & \\
\hline 250 & & & & \\
\hline 251 & & & & \\
\hline 252 & & & & \\
\hline 253 & & & & \\
\hline 254 & & & & \\
\hline 255 & & & & \\
\hline 256 & & & & \\
\hline 257 & & & & \\
\hline 259 & & & & \\
\hline 260 & & & & \\
\hline 261 & & & & \\
\hline 262 & & & & \\
\hline 263 & & & & \\
\hline 264 & & & & \\
\hline 265 & & & & \\
\hline 266 & & & & \\
\hline 267 & & & & \\
\hline
\end{tabular}




\begin{tabular}{|c|c|c|c|c|}
\hline & O & P & Q & R \\
\hline 1 & SITE_CODE & ELEVATION_UNITS & VERT_COORD_TYPE & DATE_LAST_MODIFIED \\
\hline 268 & & & & \\
\hline 269 & & & & \\
\hline 270 & & & & \\
\hline 271 & & & & \\
\hline 272 & & & & \\
\hline 273 & & & & \\
\hline 274 & & & & \\
\hline 275 & & & \\
\hline 276 & & & & \\
\hline 277 & & & & \\
\hline 278 & & & & \\
\hline 279 & & & & \\
\hline 280 & & & & \\
\hline 281 & & & & \\
\hline 282 & & & & \\
\hline 283 & & & & \\
\hline 284 & & & & \\
\hline 285 & & & & \\
\hline 286 & & & & \\
\hline 287 & & & & \\
\hline 288 & & & & \\
\hline 289 & & & & \\
\hline 290 & & & & \\
\hline 291 & & & & \\
\hline 292 & & & & \\
\hline 293 & & & & \\
\hline 294 & & & & \\
\hline 295 & & & & \\
\hline 296 & & & & \\
\hline 297 & & & & \\
\hline 298 & & & & \\
\hline 299 & & & & \\
\hline 300 & & & & \\
\hline 301 & & & & \\
\hline 302 & & & & \\
\hline 303 & & & & \\
\hline 305 & & & & \\
\hline
\end{tabular}




\begin{tabular}{|c|c|c|c|c|}
\hline & O & P & Q & R \\
\hline 1 & SITE_CODE & ELEVATION_UNITS & VERT_COORD_TYPE & DATE_LAST_MODIFIED \\
\hline 306 & & & & \\
\hline 307 & & & & \\
\hline 308 & & & & \\
\hline 309 & & & & \\
\hline 310 & & & & \\
\hline 311 & & & & \\
\hline 312 & & & & \\
\hline 313 & & & & \\
\hline 314 & & & & \\
\hline 315 & & & & \\
\hline 316 & & & & \\
\hline 317 & & & & \\
\hline 318 & & & & \\
\hline 319 & & & & \\
\hline 320 & & & & \\
\hline 321 & & & & \\
\hline 322 & & & & \\
\hline 323 & & & & \\
\hline 324 & & & & \\
\hline 325 & & & & \\
\hline 326 & & & & \\
\hline 327 & & & & \\
\hline 328 & & & & \\
\hline 329 & & & & \\
\hline 330 & & & & \\
\hline 331 & & & & \\
\hline 332 & & & & \\
\hline 333 & & & & \\
\hline 334 & & & & \\
\hline 335 & & & & \\
\hline 336 & & & & \\
\hline 338 & & & & \\
\hline 339 & & & & \\
\hline 340 & & & & \\
\hline 342 & & & & \\
\hline
\end{tabular}




\begin{tabular}{|c|c|c|c|c|}
\hline & O & P & Q & R \\
\hline 1 & SITE_CODE & ELEVATION_UNITS & VERT_COORD_TYPE & DATE_LAST_MODIFIED \\
\hline 344 & & & & \\
\hline 345 & & $m$ & & \\
\hline 346 & & & & \\
\hline 347 & & & & \\
\hline 348 & & & & \\
\hline 349 & & & & \\
\hline 350 & & & & \\
\hline 351 & & & & \\
\hline 352 & & & & \\
\hline 353 & & & & \\
\hline 354 & & & & \\
\hline 355 & & & & \\
\hline 356 & & & & \\
\hline 357 & & & & \\
\hline 358 & & & & \\
\hline 359 & & & & \\
\hline 360 & & & & \\
\hline 361 & & & & \\
\hline 362 & & & & \\
\hline 363 & & & & \\
\hline 364 & & & & \\
\hline 365 & & & & \\
\hline 366 & & & & \\
\hline 367 & & & & \\
\hline 368 & & & & \\
\hline 369 & & & & \\
\hline 370 & & & & \\
\hline 371 & & & & \\
\hline 372 & & & & \\
\hline 373 & & & & \\
\hline 375 & & & & \\
\hline 376 & & & & \\
\hline 377 & & & & \\
\hline 378 & & & & \\
\hline 380 & & & & \\
\hline
\end{tabular}




\begin{tabular}{|c|c|c|c|c|}
\hline & O & P & & Q \\
\hline 1 & SITE_CODE & ELEVATION_UNITS & VERT_COORD_TYPE & DATE_LAST_MODIFIED \\
\hline 382 & & & & \\
\hline 383 & & & & \\
\hline 384 & & & & \\
\hline 385 & & & & \\
\hline 386 & & & & \\
\hline 387 & & & & \\
\hline 388 & & & & \\
\hline 389 & & & & \\
\hline 390 & & & & \\
\hline 391 & & & & \\
\hline 392 & & & & \\
\hline 393 & & & & \\
\hline 394 & & & & \\
\hline 395 & & & & \\
\hline 396 & & & & \\
\hline 397 & & & & \\
\hline 398 & & & & \\
\hline 399 & & & & \\
\hline 400 & & & & \\
\hline 401 & & & & \\
\hline 402 & & & & \\
\hline 403 & & & & \\
\hline 404 & & & & \\
\hline 405 & & & & \\
\hline 406 & & & & \\
\hline 407 & & & & \\
\hline 408 & & & & \\
\hline 409 & & & & \\
\hline 410 & & & & \\
\hline 411 & & & & \\
\hline 412 & & & & \\
\hline 413 & & & & \\
\hline 414 & & & & \\
\hline 415 & & & & \\
\hline 416 & & & & \\
\hline 417 & & & & \\
\hline 418 & & & & \\
\hline 419 & & & & \\
\hline
\end{tabular}




\begin{tabular}{|c|c|c|c|c|}
\hline & O & \multicolumn{1}{|c|}{ P } & Q & R \\
\hline 1 & SITE_CODE & ELEVATION_UNITS & VERT_COORD_TYPE & DATE_LAST_MODIFIED \\
\hline 420 & & & & \\
\hline 421 & & & & \\
\hline 422 & & & & \\
\hline 423 & & & & \\
\hline 424 & & & & \\
\hline 425 & & & & \\
\hline 426 & & & & \\
\hline 427 & & & & \\
\hline 428 & & & & \\
\hline 429 & & & & \\
\hline 430 & & & & \\
\hline 431 & & & & \\
\hline 432 & & & & \\
\hline 433 & & & & \\
\hline 434 & & & & \\
\hline 435 & & & & \\
\hline 436 & & & & \\
\hline 437 & & & & \\
\hline 438 & & & & \\
\hline 439 & & & & \\
\hline 440 & & & & \\
\hline 441 & & & & \\
\hline 442 & & & & \\
\hline 443 & & & & \\
\hline 444 & & & & \\
\hline 445 & & & & \\
\hline 446 & & & & \\
\hline 447 & & & & \\
\hline 448 & & & & \\
\hline 449 & & & & \\
\hline 450 & & & & \\
\hline 451 & & & & \\
\hline 452 & & & & \\
\hline 453 & & & & \\
\hline 454 & & & & \\
\hline 455 & & & & \\
\hline 456 & & & & \\
\hline
\end{tabular}




\begin{tabular}{|c|c|c|c|c|}
\hline & O & \multicolumn{1}{|c|}{ P } & Q & R \\
\hline 1 & SITE_CODE & ELEVATION_UNITS & VERT_COORD_TYPE & DATE_LAST_MODIFIED \\
\hline 458 & & & & \\
\hline 459 & & & & \\
\hline 460 & & & & \\
\hline 461 & & & & \\
\hline 462 & & & & \\
\hline 463 & & & & \\
\hline 464 & & & & \\
\hline 465 & & & & \\
\hline 466 & & & & \\
\hline 467 & & & & \\
\hline 468 & & & & \\
\hline 469 & & & & \\
\hline 470 & & & & \\
\hline 471 & & & & \\
\hline 472 & & & & \\
\hline 473 & & & & \\
\hline 474 & & & & \\
\hline 475 & & & & \\
\hline 476 & & & & \\
\hline 477 & & & & \\
\hline 478 & & & & \\
\hline 479 & & & & \\
\hline 480 & & & & \\
\hline 481 & & & & \\
\hline 482 & & & & \\
\hline 483 & & & & \\
\hline 484 & & & & \\
\hline 485 & & & & \\
\hline 486 & & & & \\
\hline 487 & & & & \\
\hline 488 & & & & \\
\hline 489 & & & & \\
\hline 490 & & & & \\
\hline 491 & & & & \\
\hline 492 & & & & \\
\hline 493 & & & & \\
\hline 494 & & & & \\
\hline 495 & & & & \\
\hline
\end{tabular}




\begin{tabular}{|c|c|c|c|c|}
\hline & O & P & Q & R \\
\hline 1 & SITE_CODE & ELEVATION_UNITS & VERT_COORD_TYPE & DATE_LAST_MODIFIED \\
\hline 496 & & & & \\
\hline 497 & & $m$ & & \\
\hline 498 & & & & \\
\hline 499 & & $m$ & & \\
\hline 500 & & & & \\
\hline 501 & & $m$ & & \\
\hline 502 & & $m$ & & \\
\hline 503 & & & & \\
\hline 504 & & & & \\
\hline 505 & & & & \\
\hline 506 & & & & \\
\hline 507 & & & & \\
\hline 508 & & & & \\
\hline 509 & & & & \\
\hline 510 & & & & \\
\hline 511 & & & & \\
\hline 512 & & & & \\
\hline 513 & & & & \\
\hline 514 & & & & \\
\hline 515 & & & & \\
\hline 516 & & & & \\
\hline 517 & & & & \\
\hline 518 & & & & \\
\hline 519 & & & & \\
\hline 520 & & & & \\
\hline 521 & & & & \\
\hline 522 & & & & \\
\hline 523 & & & & \\
\hline 524 & & & & \\
\hline 525 & & & & \\
\hline 526 & & & & \\
\hline 527 & & & & \\
\hline 528 & & & & \\
\hline 530 & & & & \\
\hline 531 & & & & \\
\hline 533 & & & & \\
\hline
\end{tabular}




\begin{tabular}{|c|c|c|c|c|}
\hline & O & P & Q & R \\
\hline 1 & SITE_CODE & ELEVATION_UNITS & VERT_COORD_TYPE & DATE_LAST_MODIFIED \\
\hline 534 & & & & \\
\hline 535 & & & & \\
\hline 536 & & $m$ & & \\
\hline 537 & & & & \\
\hline 538 & & & & \\
\hline 539 & & & & \\
\hline 540 & & & & \\
\hline 541 & & & & \\
\hline 542 & & & & \\
\hline 543 & & & & \\
\hline 544 & & & & \\
\hline 545 & & & & \\
\hline 546 & & & & \\
\hline 547 & & & & \\
\hline 548 & & & & \\
\hline 549 & & & & \\
\hline 550 & & & & \\
\hline 551 & & & & \\
\hline 552 & & & & \\
\hline 553 & & & & \\
\hline 554 & & & & \\
\hline 555 & & & & \\
\hline 556 & & & & \\
\hline 557 & & & & \\
\hline 558 & & & & \\
\hline 559 & & & & \\
\hline 560 & & & & \\
\hline 561 & & & & \\
\hline 562 & & & & \\
\hline 563 & & & & \\
\hline 564 & & & & \\
\hline 565 & & & & \\
\hline 567 & & & & \\
\hline 568 & & & & \\
\hline 569 & & & & \\
\hline 571 & & & & \\
\hline
\end{tabular}




\begin{tabular}{|c|c|c|c|c|}
\hline & $\mathrm{O}$ & $P$ & $\mathrm{Q}$ & $R$ \\
\hline 1 & SITE_CODE & ELEVATION_UNITS & VERT_COORD_TYPE & DATE_LAST_MODIFIED \\
\hline \multicolumn{5}{|c|}{ 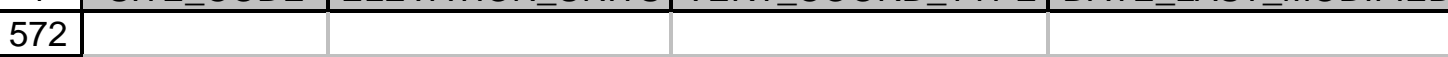 } \\
\hline \multicolumn{5}{|l|}{573} \\
\hline \multicolumn{5}{|l|}{574} \\
\hline \multicolumn{5}{|l|}{575} \\
\hline \multicolumn{5}{|l|}{576} \\
\hline \multicolumn{5}{|l|}{577} \\
\hline \multicolumn{5}{|l|}{578} \\
\hline \multicolumn{5}{|l|}{579} \\
\hline \multicolumn{5}{|l|}{580} \\
\hline \multicolumn{5}{|l|}{581} \\
\hline \multicolumn{5}{|l|}{582} \\
\hline \multicolumn{5}{|l|}{583} \\
\hline \multicolumn{5}{|l|}{584} \\
\hline \multicolumn{5}{|l|}{585} \\
\hline \multicolumn{5}{|l|}{586} \\
\hline \multicolumn{5}{|l|}{587} \\
\hline \multicolumn{5}{|l|}{588} \\
\hline \multicolumn{5}{|l|}{589} \\
\hline \multicolumn{5}{|l|}{590} \\
\hline \multicolumn{5}{|l|}{591} \\
\hline \multicolumn{5}{|l|}{592} \\
\hline \multicolumn{5}{|l|}{593} \\
\hline \multicolumn{5}{|l|}{594} \\
\hline \multicolumn{5}{|c|}{595} \\
\hline \multicolumn{5}{|l|}{596} \\
\hline \multicolumn{5}{|l|}{597} \\
\hline \multicolumn{5}{|c|}{598} \\
\hline 599 & & & & \\
\hline 600 & & & & \\
\hline 601 & & & & \\
\hline 602 & & & & \\
\hline 603 & & & & \\
\hline 604 & & & & \\
\hline 605 & & & & \\
\hline 606 & & & & \\
\hline 607 & & & & \\
\hline 608 & & & & \\
\hline 609 & & & & \\
\hline
\end{tabular}




\begin{tabular}{|c|c|c|c|c|}
\hline & $\mathrm{O}$ & $\mathrm{P}$ & $\mathrm{Q}$ & $\mathrm{R}$ \\
\hline 1 & SITE_CODE & ELEVATION_UNITS & VERT_COORD_TYPE & DATE_LAST_MODIFIED \\
\hline 610 & & & & \\
\hline 611 & & & & \\
\hline 612 & & & & \\
\hline 613 & & & & \\
\hline 614 & & & & \\
\hline 615 & & & & \\
\hline 616 & & & & \\
\hline 617 & & & & \\
\hline 618 & & & & \\
\hline 619 & & & & \\
\hline 620 & & & & \\
\hline 621 & & & & \\
\hline 622 & & & & \\
\hline 623 & & & & \\
\hline 624 & & & & \\
\hline 625 & & & & \\
\hline 626 & & & & \\
\hline 627 & & & & \\
\hline 628 & & & & \\
\hline 629 & & & & \\
\hline 630 & & & & \\
\hline 631 & & & & \\
\hline 632 & & & & \\
\hline 633 & & & & \\
\hline 634 & & & & \\
\hline 635 & & & & \\
\hline 636 & & & & \\
\hline 637 & & & & \\
\hline 638 & & & & \\
\hline 639 & & & & \\
\hline 640 & & & & \\
\hline 641 & & & & \\
\hline 642 & & & & \\
\hline 643 & & & & \\
\hline 644 & & & & \\
\hline 645 & & & & \\
\hline 646 & & & & \\
\hline 647 & & & & \\
\hline
\end{tabular}




\begin{tabular}{|c|c|c|c|c|}
\hline & $\mathrm{O}$ & $\mathrm{P}$ & $\mathrm{Q}$ & $\mathrm{R}$ \\
\hline 1 & SITE_CODE & ELEVATION_UNITS & VERT_COORD_TYPE & DATE_LAST_MODIFIED \\
\hline \multicolumn{5}{|l|}{648} \\
\hline \multicolumn{5}{|l|}{649} \\
\hline \multicolumn{5}{|l|}{650} \\
\hline \multicolumn{5}{|l|}{651} \\
\hline \multicolumn{5}{|l|}{652} \\
\hline \multicolumn{5}{|l|}{653} \\
\hline \multicolumn{5}{|l|}{654} \\
\hline \multicolumn{5}{|l|}{655} \\
\hline \multicolumn{5}{|l|}{656} \\
\hline \multicolumn{5}{|l|}{657} \\
\hline \multicolumn{5}{|l|}{658} \\
\hline \multicolumn{5}{|l|}{659} \\
\hline \multicolumn{5}{|l|}{660} \\
\hline \multicolumn{5}{|l|}{661} \\
\hline \multicolumn{5}{|l|}{662} \\
\hline \multicolumn{5}{|l|}{663} \\
\hline \multicolumn{5}{|l|}{664} \\
\hline \multicolumn{5}{|l|}{665} \\
\hline \multicolumn{5}{|l|}{666} \\
\hline \multicolumn{5}{|l|}{667} \\
\hline \multicolumn{5}{|l|}{668} \\
\hline \multicolumn{5}{|l|}{669} \\
\hline \multicolumn{5}{|l|}{670} \\
\hline \multicolumn{5}{|l|}{671} \\
\hline \multicolumn{5}{|l|}{672} \\
\hline \multicolumn{5}{|l|}{673} \\
\hline \multicolumn{5}{|l|}{674} \\
\hline 675 & & & & \\
\hline 676 & & & & \\
\hline 677 & & & & \\
\hline 678 & & & & \\
\hline 679 & & & & \\
\hline 680 & & & & \\
\hline 681 & & & & \\
\hline 682 & & & & \\
\hline 683 & & & & \\
\hline 684 & & & & \\
\hline 685 & & & & \\
\hline
\end{tabular}




\begin{tabular}{|c|c|c|c|c|}
\hline & $\mathrm{O}$ & $\mathrm{P}$ & $\mathrm{Q}$ & $\mathrm{R}$ \\
\hline 1 & SITE_CODE & ELEVATION_UNITS & VERT_COORD_TYPE & DATE_LAST_MODIFIED \\
\hline \multicolumn{5}{|l|}{686} \\
\hline \multicolumn{5}{|l|}{687} \\
\hline \multicolumn{5}{|l|}{688} \\
\hline \multicolumn{5}{|l|}{689} \\
\hline \multicolumn{5}{|l|}{690} \\
\hline \multicolumn{5}{|l|}{691} \\
\hline \multicolumn{5}{|l|}{692} \\
\hline \multicolumn{5}{|l|}{693} \\
\hline \multicolumn{5}{|l|}{694} \\
\hline \multicolumn{5}{|l|}{695} \\
\hline \multicolumn{5}{|l|}{696} \\
\hline \multicolumn{5}{|l|}{697} \\
\hline \multicolumn{5}{|l|}{698} \\
\hline \multicolumn{5}{|l|}{699} \\
\hline \multicolumn{5}{|l|}{700} \\
\hline \multicolumn{5}{|l|}{701} \\
\hline \multicolumn{5}{|l|}{702} \\
\hline \multicolumn{5}{|l|}{703} \\
\hline \multicolumn{5}{|l|}{704} \\
\hline \multicolumn{5}{|l|}{705} \\
\hline \multicolumn{5}{|l|}{706} \\
\hline \multicolumn{5}{|l|}{707} \\
\hline \multicolumn{5}{|l|}{708} \\
\hline \multicolumn{5}{|l|}{709} \\
\hline \multicolumn{5}{|l|}{710} \\
\hline \multicolumn{5}{|l|}{711} \\
\hline \multicolumn{5}{|l|}{712} \\
\hline 713 & & & & \\
\hline 714 & & & & \\
\hline 715 & & & & \\
\hline 716 & & & & \\
\hline 717 & & & & \\
\hline 718 & & & & \\
\hline 719 & & & & \\
\hline 720 & & & & \\
\hline 721 & & & & \\
\hline 722 & & & & \\
\hline 723 & & & & \\
\hline
\end{tabular}




\begin{tabular}{|c|c|c|c|c|}
\hline & O & P & & Q \\
\hline 1 & SITE_CODE & ELEVATION_UNITS & VERT_COORD_TYPE & DATE_LAST_MODIFIED \\
\hline 724 & & & & \\
\hline 725 & & & & \\
\hline 726 & & & & \\
\hline 727 & & & & \\
\hline 728 & & & & \\
\hline 729 & & & & \\
\hline 730 & & & & \\
\hline 731 & & & & \\
\hline 732 & & & & \\
\hline 733 & & & & \\
\hline 734 & & & & \\
\hline 735 & & & & \\
\hline 736 & & & & \\
\hline 737 & & & & \\
\hline 738 & & & & \\
\hline 739 & & & & \\
\hline 740 & & & & \\
\hline 741 & & & & \\
\hline 742 & & & & \\
\hline 743 & & & & \\
\hline 744 & & & & \\
\hline 745 & & & & \\
\hline 746 & & & & \\
\hline 747 & & & & \\
\hline 748 & & & & \\
\hline 749 & & & & \\
\hline 750 & & & & \\
\hline 751 & & & & \\
\hline 752 & & & & \\
\hline 753 & & & & \\
\hline 754 & & & & \\
\hline 755 & & & & \\
\hline 756 & & & & \\
\hline 757 & & & & \\
\hline 758 & & & & \\
\hline 759 & & & & \\
\hline 760 & & & & \\
\hline 761 & & & & \\
\hline
\end{tabular}




\begin{tabular}{|c|c|c|c|c|}
\hline & O & P & Q & R \\
\hline 1 & SITE_CODE & ELEVATION_UNITS & VERT_COORD_TYPE & DATE_LAST_MODIFIED \\
\hline 762 & & & & \\
\hline 763 & & & & \\
\hline 764 & & & & \\
\hline 765 & & & & \\
\hline 766 & & & & \\
\hline 767 & & & & \\
\hline 768 & & & & \\
\hline 769 & & & & \\
\hline 770 & & & & \\
\hline 771 & & & & \\
\hline 772 & & & & \\
\hline 773 & & & & \\
\hline 774 & & & & \\
\hline 775 & & & & \\
\hline 776 & & & & \\
\hline 777 & & & & \\
\hline 778 & & & & \\
\hline 779 & & & & \\
\hline 780 & & & & \\
\hline 781 & & & & \\
\hline 782 & & & & \\
\hline 783 & & & & \\
\hline 784 & & & & \\
\hline 785 & & & & \\
\hline 786 & & & & \\
\hline 787 & & & & \\
\hline 788 & & & & \\
\hline 789 & & & & \\
\hline 790 & & & & \\
\hline 791 & & & & \\
\hline 792 & & & & \\
\hline 793 & & & & \\
\hline 794 & & & & \\
\hline 795 & & & & \\
\hline 796 & & & & \\
\hline 797 & & & & \\
\hline 799 & & & & \\
\hline
\end{tabular}




\begin{tabular}{|c|c|c|c|c|}
\hline & O & P & & Q \\
\hline 1 & SITE_CODE & ELEVATION_UNITS & VERT_COORD_TYPE & DATE_LAST_MODIFIED \\
\hline 800 & & & & \\
\hline 801 & & & & \\
\hline 802 & & & & \\
\hline 803 & & & & \\
\hline 804 & & & & \\
\hline 805 & & & & \\
\hline 806 & & & & \\
\hline 807 & & & & \\
\hline 808 & & & & \\
\hline 809 & & & & \\
\hline 810 & & & & \\
\hline 811 & & & & \\
\hline 812 & & & & \\
\hline 813 & & & & \\
\hline 814 & & & & \\
\hline 815 & & & & \\
\hline 816 & & & & \\
\hline 817 & & & & \\
\hline 818 & & & & \\
\hline 819 & & & & \\
\hline 820 & & & & \\
\hline 821 & & & & \\
\hline 822 & & & & \\
\hline 823 & & & & \\
\hline 824 & & & & \\
\hline 825 & & & & \\
\hline 826 & & & & \\
\hline 827 & & & & \\
\hline 828 & & & & \\
\hline 829 & & & & \\
\hline 830 & & & & \\
\hline 831 & & & & \\
\hline 832 & & & & \\
\hline 833 & & & & \\
\hline 834 & & & & \\
\hline 835 & & & & \\
\hline 836 & & & & \\
\hline 837 & & & & \\
\hline
\end{tabular}




\begin{tabular}{|c|c|c|c|c|}
\hline & $\mathrm{O}$ & $\mathrm{P}$ & $\mathrm{Q}$ & $\mathrm{R}$ \\
\hline 1 & SITE_CODE & ELEVATION_UNITS & VERT_COORD_TYPE & DATE_LAST_MODIFIED \\
\hline \multicolumn{5}{|c|}{\begin{tabular}{l|l|l|l|l|}
838 & & & -2 \\
\end{tabular}} \\
\hline \multicolumn{5}{|l|}{839} \\
\hline \multicolumn{5}{|l|}{840} \\
\hline \multicolumn{5}{|l|}{841} \\
\hline \multicolumn{5}{|l|}{842} \\
\hline \multicolumn{5}{|l|}{843} \\
\hline \multicolumn{5}{|l|}{844} \\
\hline \multicolumn{5}{|l|}{845} \\
\hline \multicolumn{5}{|l|}{846} \\
\hline \multicolumn{5}{|l|}{847} \\
\hline \multicolumn{5}{|l|}{848} \\
\hline \multicolumn{5}{|l|}{849} \\
\hline \multicolumn{5}{|l|}{850} \\
\hline \multicolumn{5}{|l|}{851} \\
\hline \multicolumn{5}{|l|}{852} \\
\hline \multicolumn{5}{|l|}{853} \\
\hline 854 & & & & 26-Jul-02 \\
\hline \multicolumn{5}{|l|}{855} \\
\hline \multicolumn{5}{|l|}{856} \\
\hline \multicolumn{5}{|l|}{857} \\
\hline \multicolumn{5}{|l|}{858} \\
\hline \multicolumn{5}{|l|}{859} \\
\hline 860 & & & & 26-Jul-02 \\
\hline \multirow{2}{*}{\multicolumn{5}{|c|}{$\frac{861}{862}$}} \\
\hline & & & & \\
\hline 863 & & & & 26-Jul-02 \\
\hline 864 & & & & 26-Jul-02 \\
\hline 865 & & & & 26-Jul-02 \\
\hline 866 & & & & 26-Jul-02 \\
\hline 867 & & & & 26-Jul-02 \\
\hline 868 & & & & 26-Jul-02 \\
\hline 869 & & & & 26-Jul-02 \\
\hline 870 & & & & 26-Jul-02 \\
\hline 871 & & & & 26-Jul-02 \\
\hline 872 & & & & \\
\hline 873 & & & & \\
\hline 874 & & & & \\
\hline 875 & & & & \\
\hline
\end{tabular}




\begin{tabular}{|c|c|c|c|c|}
\hline & O & \multicolumn{1}{|c|}{ P } & Q & \\
\hline 1 & SITE_CODE & ELEVATION_UNITS & VERT_COORD_TYPE & DATE_LAST_MODIFIED \\
\hline 876 & & & & \\
\hline 877 & & & & \\
\hline 878 & & & & \\
\hline 879 & & & & \\
\hline 880 & & & & \\
\hline 881 & & & & \\
\hline 882 & & & & \\
\hline 883 & & & & \\
\hline 884 & & & & \\
\hline 885 & & & & \\
\hline 886 & & & & \\
\hline 887 & & & & \\
\hline 888 & & & & \\
\hline 889 & & & & \\
\hline 890 & & & & \\
\hline 891 & & & & \\
\hline 892 & & & & \\
\hline 893 & & & & \\
\hline 894 & & & & \\
\hline 895 & & & & \\
\hline 896 & & & & \\
\hline 897 & & & & \\
\hline 898 & & & & \\
\hline 899 & & & & \\
\hline 900 & & & & \\
\hline 901 & & & & \\
\hline 902 & & & & \\
\hline 903 & & & & \\
\hline 904 & & & & \\
\hline 905 & & & & \\
\hline 906 & & & & \\
\hline 907 & & & & \\
\hline 908 & & & & \\
\hline 909 & & & & \\
\hline 910 & & & & \\
\hline 911 & & & & \\
\hline 913 & & & & \\
\hline
\end{tabular}




\begin{tabular}{|c|c|c|c|c|}
\hline & O & P & & Q \\
\hline 1 & SITE_CODE & ELEVATION_UNITS & VERT_COORD_TYPE & DATE_LAST_MODIFIED \\
\hline 914 & & & & \\
\hline 915 & & & & \\
\hline 916 & & & & \\
\hline 917 & & & & \\
\hline 918 & & & & \\
\hline 919 & & & & \\
\hline 920 & & & & \\
\hline 921 & & & & \\
\hline 922 & & & & \\
\hline 923 & & & & \\
\hline 924 & & & & \\
\hline 925 & & & & \\
\hline 926 & & & & \\
\hline 927 & & & & \\
\hline 928 & & & & \\
\hline 929 & & & & \\
\hline 930 & & & & \\
\hline 931 & & & & \\
\hline 932 & & & & \\
\hline 933 & & & & \\
\hline 934 & & & & \\
\hline 935 & & & & \\
\hline 936 & & & & \\
\hline 937 & & & & \\
\hline 938 & & & & \\
\hline 939 & & & & \\
\hline 940 & & & & \\
\hline 941 & & & & \\
\hline 942 & & & & \\
\hline 943 & & & & \\
\hline 944 & & & & \\
\hline 945 & & & & \\
\hline 946 & & & & \\
\hline 947 & & & & \\
\hline 948 & & & & \\
\hline 949 & & & & \\
\hline 950 & & & & \\
\hline 951 & & & & \\
\hline
\end{tabular}




\begin{tabular}{|c|c|c|c|c|}
\hline & 0 & $P$ & $\mathrm{Q}$ & $R$ \\
\hline 1 & SITE_CODE & ELEVATION_UNITS & VERT_COORD_TYPE & DATE_LAST_MODIFIED \\
\hline \multicolumn{5}{|c|}{ 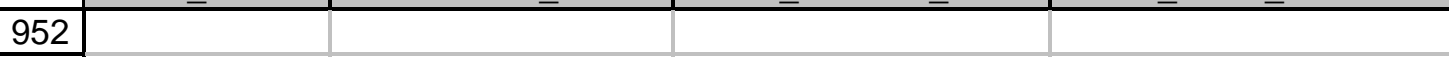 } \\
\hline \multicolumn{5}{|c|}{953} \\
\hline \multicolumn{5}{|l|}{954} \\
\hline \multicolumn{5}{|l|}{955} \\
\hline \multicolumn{5}{|c|}{956} \\
\hline \multicolumn{5}{|l|}{957} \\
\hline \multicolumn{5}{|l|}{958} \\
\hline \multicolumn{5}{|l|}{959} \\
\hline \multicolumn{5}{|l|}{960} \\
\hline \multicolumn{5}{|c|}{961} \\
\hline \multicolumn{5}{|c|}{962} \\
\hline \multicolumn{5}{|c|}{963} \\
\hline \multicolumn{5}{|c|}{964} \\
\hline \multicolumn{5}{|c|}{965} \\
\hline \multicolumn{5}{|c|}{966} \\
\hline \multicolumn{5}{|c|}{967} \\
\hline \multicolumn{5}{|c|}{968} \\
\hline \multicolumn{5}{|l|}{969} \\
\hline \multicolumn{5}{|c|}{$\overline{970}$} \\
\hline \multicolumn{5}{|l|}{971} \\
\hline \multicolumn{5}{|l|}{972} \\
\hline \multicolumn{5}{|l|}{973} \\
\hline \multicolumn{5}{|c|}{974} \\
\hline \multicolumn{5}{|c|}{975} \\
\hline \multicolumn{5}{|c|}{976} \\
\hline \multicolumn{5}{|c|}{977} \\
\hline \multicolumn{5}{|c|}{978} \\
\hline 979 & & & & \\
\hline 980 & & & & \\
\hline 981 & & & & \\
\hline 982 & & & & \\
\hline 983 & & & & \\
\hline 984 & & & & \\
\hline 985 & & & & \\
\hline 986 & & & & \\
\hline 987 & & & & \\
\hline 988 & & & & \\
\hline 989 & & & & \\
\hline
\end{tabular}




\begin{tabular}{|c|c|c|c|c|}
\hline & $\mathrm{O}$ & $\mathrm{P}$ & Q & $\mathrm{R}$ \\
\hline 1 & SITE_CODE & ELEVATION_UNITS & VERT_COORD_TYPE & DATE_LAST_MODIFIED \\
\hline \multicolumn{5}{|c|}{\begin{tabular}{|l|l|l|l|}
990 & &
\end{tabular}} \\
\hline \multicolumn{5}{|l|}{991} \\
\hline \multicolumn{5}{|l|}{992} \\
\hline \multicolumn{5}{|l|}{993} \\
\hline 994 & $1607-\mathrm{H} 4$ & & & 04-Jun-02 \\
\hline \multicolumn{5}{|l|}{995} \\
\hline \multicolumn{5}{|l|}{996} \\
\hline \multicolumn{5}{|l|}{997} \\
\hline \multicolumn{5}{|l|}{998} \\
\hline \multicolumn{5}{|l|}{999} \\
\hline \multicolumn{5}{|l|}{1000} \\
\hline \multicolumn{5}{|l|}{1001} \\
\hline \multicolumn{5}{|l|}{1002} \\
\hline \multicolumn{5}{|l|}{1003} \\
\hline \multicolumn{5}{|l|}{1004} \\
\hline \multicolumn{5}{|l|}{1005} \\
\hline \multicolumn{5}{|l|}{1006} \\
\hline \multicolumn{5}{|l|}{1007} \\
\hline \multicolumn{5}{|l|}{1008} \\
\hline \multicolumn{5}{|l|}{1009} \\
\hline \multicolumn{5}{|l|}{1010} \\
\hline \multicolumn{5}{|l|}{1011} \\
\hline \multicolumn{5}{|l|}{1012} \\
\hline \multicolumn{5}{|l|}{1013} \\
\hline \multicolumn{5}{|l|}{1014} \\
\hline \multicolumn{5}{|l|}{1015} \\
\hline \multicolumn{5}{|l|}{\begin{tabular}{|l|}
1016 \\
\end{tabular}} \\
\hline \multicolumn{5}{|l|}{1017} \\
\hline 1018 & & & & \\
\hline 1019 & & & & \\
\hline 1020 & & & & \\
\hline 1021 & & & & \\
\hline 1022 & & & & \\
\hline 1023 & & & & \\
\hline 1024 & & & & \\
\hline 1025 & & & & \\
\hline 1026 & & & & \\
\hline 1027 & & & & \\
\hline
\end{tabular}




\begin{tabular}{|c|c|c|c|c|}
\hline & $\mathrm{O}$ & $P$ & $\mathrm{Q}$ & $\mathrm{R}$ \\
\hline 1 & SITE_CODE & ELEVATION_UNITS & VERT_COORD_TYPE & DATE_LAST_MODIFIED \\
\hline 1028 & & & & \\
\hline 1029 & & & & \\
\hline 1030 & & & & \\
\hline 1031 & & & & \\
\hline 1032 & & & & \\
\hline 1033 & & & & \\
\hline 1034 & & & & \\
\hline 1035 & & & & \\
\hline 1036 & & & & \\
\hline 1037 & & & & \\
\hline 1038 & & & & \\
\hline 1039 & & & & \\
\hline 1040 & & & & \\
\hline 1041 & & & & \\
\hline 1042 & & & & \\
\hline 1043 & & & & \\
\hline 1044 & & & & \\
\hline 1045 & & & & \\
\hline 1046 & & & & \\
\hline 1047 & & & & \\
\hline 1048 & & & & \\
\hline 1049 & & & & \\
\hline 1050 & & & & \\
\hline 1051 & & & & \\
\hline 1052 & & & & \\
\hline 1053 & & & & \\
\hline 1054 & & & & \\
\hline 1055 & & & & \\
\hline 1056 & & & & \\
\hline 1057 & & & & \\
\hline 1058 & & & & \\
\hline 1059 & & & & \\
\hline 1060 & & & & \\
\hline 1061 & & & & \\
\hline 1062 & & & & \\
\hline 1063 & & & & \\
\hline 1064 & & & & \\
\hline 1065 & & & & \\
\hline
\end{tabular}




\begin{tabular}{|c|c|c|c|c|}
\hline & $\mathrm{O}$ & $P$ & $\mathrm{Q}$ & R \\
\hline 1 & SITE_CODE & ELEVATION_UNITS & VERT_COORD_TYPE & DATE_LAST_MODIFIED \\
\hline 1066 & & & & \\
\hline \begin{tabular}{|c|}
1067 \\
\end{tabular} & & & & \\
\hline 1068 & & & & \\
\hline 1069 & & & & \\
\hline 1070 & & & & \\
\hline 1071 & & & & \\
\hline 1072 & & & & \\
\hline 1073 & & & & \\
\hline 1074 & & & & \\
\hline 1075 & & & & \\
\hline 1076 & & & & \\
\hline 1077 & & & & \\
\hline 1078 & & & & \\
\hline \begin{tabular}{|l|l}
1079 \\
\end{tabular} & & & & \\
\hline 1080 & & & & \\
\hline 1081 & & & & \\
\hline 1082 & & & & \\
\hline 1083 & & & & \\
\hline 1084 & & & & \\
\hline 1085 & & & & \\
\hline 1086 & & & & \\
\hline 1087 & & & & \\
\hline 1088 & & & & \\
\hline 1089 & & & & \\
\hline 1090 & & & & \\
\hline 1091 & & & & \\
\hline 1092 & & & & \\
\hline 1093 & & & & \\
\hline 1094 & & & & \\
\hline 1095 & & & & \\
\hline 1096 & & & & \\
\hline 1097 & & & & \\
\hline 1098 & & & & \\
\hline 1099 & & & & \\
\hline 1100 & & & & \\
\hline 1101 & & & & \\
\hline 1102 & & & & \\
\hline 1103 & & & & \\
\hline
\end{tabular}




\begin{tabular}{|c|c|c|c|c|}
\hline & $\mathrm{O}$ & $P$ & $\mathrm{Q}$ & R \\
\hline 1 & SITE_CODE & ELEVATION_UNITS & VERT_COORD_TYPE & DATE_LAST_MODIFIED \\
\hline 1104 & & & & \\
\hline 1105 & & & & \\
\hline 1106 & & & & \\
\hline 1107 & & & & \\
\hline 1108 & & & & \\
\hline 1109 & & & & \\
\hline 1110 & & & & \\
\hline 1111 & & & & \\
\hline 1112 & & & & \\
\hline 1113 & & & & \\
\hline 1114 & & & & \\
\hline 1115 & & & & \\
\hline 1116 & & & & \\
\hline 1117 & & & & \\
\hline 1118 & & & & \\
\hline 1119 & & & & \\
\hline 1120 & & & & \\
\hline 1121 & & & & \\
\hline 1122 & & & & \\
\hline 1123 & & & & \\
\hline 1124 & & & & \\
\hline 1125 & & & & \\
\hline 1126 & & & & \\
\hline 1127 & & & & \\
\hline 1128 & & & & \\
\hline 1129 & & & & \\
\hline 1130 & & & & \\
\hline 1131 & & & & \\
\hline 1132 & & & & \\
\hline 1133 & & & & \\
\hline 1134 & & & & \\
\hline 1135 & & & & \\
\hline 1136 & & & & \\
\hline 1137 & & & & \\
\hline 1138 & & & & \\
\hline 1139 & & & & \\
\hline 1140 & & & & \\
\hline 1141 & & & & \\
\hline
\end{tabular}




\begin{tabular}{|c|c|c|c|c|}
\hline & $\mathrm{O}$ & $P$ & $\mathrm{Q}$ & R \\
\hline 1 & SITE_CODE & ELEVATION_UNITS & VERT_COORD_TYPE & DATE_LAST_MODIFIED \\
\hline 1142 & & & & \\
\hline 1143 & & & & \\
\hline 1144 & & & & \\
\hline 1145 & & & & \\
\hline 1146 & & & & \\
\hline 1147 & & & & \\
\hline 1148 & & & & \\
\hline 1149 & & & & \\
\hline 1150 & & & & \\
\hline 1151 & & & & \\
\hline 1152 & & & & \\
\hline 1153 & & & & \\
\hline 1154 & & & & \\
\hline 1155 & & & & \\
\hline 1156 & & & & \\
\hline 1157 & & & & \\
\hline 1158 & & & & \\
\hline 1159 & & & & \\
\hline 1160 & & & & \\
\hline 1161 & & & & \\
\hline 1162 & & & & \\
\hline 1163 & & & & \\
\hline 1164 & & & & \\
\hline 1165 & & & & \\
\hline 1166 & & & & \\
\hline 1167 & & & & \\
\hline 1168 & & & & \\
\hline 1169 & & & & \\
\hline 1170 & & & & \\
\hline 1171 & & & & \\
\hline 1172 & & & & \\
\hline 1173 & & & & \\
\hline 1174 & & & & \\
\hline 1175 & & & & \\
\hline 1176 & & & & \\
\hline 1177 & & & & \\
\hline 1178 & & & & \\
\hline 1179 & & & & \\
\hline
\end{tabular}




\begin{tabular}{|c|c|c|c|c|}
\hline & $\mathrm{O}$ & $P$ & $\mathrm{Q}$ & R \\
\hline 1 & SITE_CODE & ELEVATION_UNITS & VERT_COORD_TYPE & DATE_LAST_MODIFIED \\
\hline 1180 & & & & \\
\hline 1181 & & & & \\
\hline 1182 & & & & \\
\hline 1183 & & & & \\
\hline 1184 & & & & \\
\hline 1185 & & & & \\
\hline 1186 & & & & \\
\hline 1187 & & & & \\
\hline 1188 & & & & \\
\hline 1189 & & & & \\
\hline 1190 & & & & \\
\hline 1191 & & & & \\
\hline 1192 & & & & \\
\hline 1193 & & & & \\
\hline 1194 & & & & \\
\hline 1195 & & & & \\
\hline 1196 & & & & \\
\hline 1197 & & & & \\
\hline 1198 & & & & \\
\hline 1199 & & & & \\
\hline 1200 & & & & \\
\hline 1201 & & & & \\
\hline 1202 & & & & \\
\hline 1203 & & & & \\
\hline 1204 & & & & \\
\hline 1205 & & & & \\
\hline 1206 & & & & \\
\hline 1207 & & & & \\
\hline 1208 & & & & \\
\hline 1209 & & & & \\
\hline 1210 & & & & \\
\hline 1211 & & & & \\
\hline 1212 & & & & \\
\hline 1213 & & & & \\
\hline 1214 & & & & \\
\hline 1215 & & & & \\
\hline 1216 & & & & \\
\hline 1217 & & & & \\
\hline
\end{tabular}




\begin{tabular}{|c|c|c|c|c|}
\hline & O & P & \multicolumn{1}{c|}{ Q } & \\
\hline 1 & SITE_CODE & ELEVATION_UNITS & VERT_COORD_TYPE & DATE_LAST_MODIFIED \\
\hline 1218 & & & & \\
\hline 1219 & & & & \\
\hline 1220 & & & & \\
\hline 1221 & & & & \\
\hline 1222 & & & & \\
\hline 1223 & & & & \\
\hline 1224 & & & & \\
\hline 1225 & & & & \\
\hline 1226 & & & & \\
\hline 1227 & & & & \\
\hline 1228 & & & & \\
\hline 1229 & & & & \\
\hline 1230 & & & & \\
\hline 1231 & & & & \\
\hline 1232 & & & & \\
\hline 1233 & & & & \\
\hline 1234 & & & & \\
\hline 1235 & & & & \\
\hline 1236 & & & & \\
\hline 1237 & & & & \\
\hline 1238 & & & & \\
\hline 1239 & & & & \\
\hline 1240 & & & & \\
\hline 1241 & & & & \\
\hline 1242 & & & & \\
\hline 1243 & & & & \\
\hline 1244 & & & & \\
\hline 1245 & & & & \\
\hline 1246 & & & & \\
\hline 1247 & & & & \\
\hline 1248 & & & & \\
\hline 1249 & & & & \\
\hline 1250 & & & & \\
\hline 1251 & & & & \\
\hline 1252 & & & & \\
\hline 1253 & & & & \\
\hline 1255 & & & & \\
\hline
\end{tabular}




\begin{tabular}{|c|c|c|c|c|}
\hline & $\mathrm{O}$ & $P$ & $\mathrm{Q}$ & $\mathrm{R}$ \\
\hline 1 & SITE_CODE & ELEVATION_UNITS & VERT_COORD_TYPE & DATE_LAST_MODIFIED \\
\hline 1256 & & & & \\
\hline \begin{tabular}{|l|}
1257 \\
\end{tabular} & & & & \\
\hline 1258 & & & & \\
\hline 1259 & & & & \\
\hline 1260 & & & & \\
\hline 1261 & & & & \\
\hline 1262 & & & & \\
\hline 1263 & & & & \\
\hline 1264 & & & & \\
\hline 1265 & & & & \\
\hline 1266 & & & & \\
\hline 1267 & & & & 26-Jul-02 \\
\hline 1268 & & & & \\
\hline 1269 & & & & \\
\hline 1270 & & & & \\
\hline 1271 & & & & \\
\hline 1272 & & & & \\
\hline 1273 & & & & \\
\hline 1274 & & & & \\
\hline 1275 & & & & \\
\hline 1276 & & & & \\
\hline 1277 & & & & \\
\hline 1278 & & & & \\
\hline 1279 & & & & \\
\hline 1280 & & & & \\
\hline 1281 & & & & \\
\hline 1282 & & & & \\
\hline 1283 & & & & \\
\hline 1284 & & & & \\
\hline 1285 & & & & \\
\hline 1286 & & & & \\
\hline 1287 & & & & \\
\hline 1288 & & & & \\
\hline 1289 & & & & \\
\hline 1290 & & & & \\
\hline 1291 & & & & \\
\hline 1292 & & & & \\
\hline 1293 & & & & \\
\hline
\end{tabular}




\begin{tabular}{|c|c|c|c|c|}
\hline & $\mathrm{O}$ & $P$ & $\mathrm{Q}$ & $\mathrm{R}$ \\
\hline 1 & SITE_CODE & ELEVATION_UNITS & VERT_COORD_TYPE & DATE_LAST_MODIFIED \\
\hline 1294 & & & & \\
\hline 1295 & & & & \\
\hline 1296 & & & & \\
\hline 1297 & & & & \\
\hline 1298 & & & & \\
\hline 1299 & & & & \\
\hline 1300 & & & & \\
\hline 1301 & & & & \\
\hline 1302 & & & & \\
\hline 1303 & & & & \\
\hline 1304 & & & & \\
\hline 1305 & & & & \\
\hline 1306 & & & & \\
\hline 1307 & & & & \\
\hline 1308 & & & & \\
\hline 1309 & & & & \\
\hline 1310 & & & & \\
\hline 1311 & & & & \\
\hline 1312 & & & & \\
\hline 1313 & & & & \\
\hline 1314 & & & & \\
\hline 1315 & & & & \\
\hline 1316 & & & & \\
\hline 1317 & & & & \\
\hline 1318 & & & & \\
\hline 1319 & & & & \\
\hline 1320 & & & & \\
\hline 1321 & & & & \\
\hline 1322 & & & & 26-Jul-02 \\
\hline 1323 & & & & 26-Jul-02 \\
\hline 1324 & & & & 26-Jul-02 \\
\hline 1325 & & & & \\
\hline 1326 & & & & \\
\hline 1327 & & & & \\
\hline 1328 & & & & \\
\hline 1329 & & & & \\
\hline 1330 & & & & \\
\hline 1331 & & & & \\
\hline
\end{tabular}




\begin{tabular}{|c|c|c|c|c|}
\hline & $\mathrm{O}$ & $P$ & $\mathrm{Q}$ & $R$ \\
\hline 1 & SITE CODE & ELEVATION UNITS & VERT COORD TYPE & DATE LAST MODIFIED \\
\hline 1332 & & & & 26-Jul-02 \\
\hline 1333 & & & & 26-Jul-02 \\
\hline 1334 & & & & \\
\hline 1335 & & & & \\
\hline 1336 & & & & \\
\hline 1337 & & & & \\
\hline 1338 & & & & \\
\hline 1339 & & & & \\
\hline 1340 & & & & \\
\hline 1341 & & & & \\
\hline 1342 & & & & \\
\hline 1343 & & & & 26-Jul-02 \\
\hline 1344 & & & & 26-Jul-02 \\
\hline 1345 & & & & 26-Jul-02 \\
\hline 1346 & & & & 26-Jul-02 \\
\hline 1347 & & & & 26-Jul-02 \\
\hline 1348 & & & & 26-Jul-02 \\
\hline 1349 & & & & 26-Jul-02 \\
\hline 1350 & & & & 26-Jul-02 \\
\hline 1351 & & & & 26-Jul-02 \\
\hline 1352 & & & & 26-Jul-02 \\
\hline 1353 & & & & 26-Jul-02 \\
\hline 1354 & & & & 26-Jul-02 \\
\hline 1355 & & & & 26-Jul-02 \\
\hline 1356 & & & & 26-Jul-02 \\
\hline 1357 & & & & 26-Jul-02 \\
\hline 1358 & & & & 26-Jul-02 \\
\hline 1359 & & & & 26-Jul-02 \\
\hline 1360 & & & & 26-Jul-02 \\
\hline 1361 & & & & 26-Jul-02 \\
\hline 1362 & & & & 26-Jul-02 \\
\hline 1363 & & & & 26-Jul-02 \\
\hline 1364 & & & & 26-Jul-02 \\
\hline 1365 & & & & 26-Jul-02 \\
\hline 1366 & & & & 26-Jul-02 \\
\hline 1367 & & & & 26-Jul-02 \\
\hline 1368 & & & & 26-Jul-02 \\
\hline 1369 & & & & 26-Jul-02 \\
\hline
\end{tabular}




\begin{tabular}{|c|c|c|c|c|}
\hline & $\mathrm{O}$ & $P$ & $\bar{Q}$ & $\bar{R}$ \\
\hline 1 & SITE_CODE & ELEVATION_UNITS & VERT_COORD_TYPE & DATE_LAST_MODIFIED \\
\hline 1370 & & & & 26-Jul-02 \\
\hline 1371 & & & & 26-Jul-02 \\
\hline 1372 & & & & 26-Jul-02 \\
\hline 1373 & & & & 26-Jul-02 \\
\hline 1374 & & & & 26-Jul-02 \\
\hline 1375 & & & & 26-Jul-02 \\
\hline 1376 & & & & 26-Jul-02 \\
\hline 1377 & & & & 26-Jul-02 \\
\hline 1378 & & & & 26-Jul-02 \\
\hline 1379 & & & & 26-Jul-02 \\
\hline 1380 & & & & $26-J u l-02$ \\
\hline 1381 & & & & 26-Jul-02 \\
\hline 1382 & & & & 26-Jul-02 \\
\hline 1383 & & & & 26-Jul-02 \\
\hline 1384 & & & & 26-Jul-02 \\
\hline 1385 & & & & 26-Jul-02 \\
\hline 1386 & & & & 26-Jul-02 \\
\hline 1387 & & & & 26-Jul-02 \\
\hline 1388 & & & & 26-Jul-02 \\
\hline 1389 & & & & 26-Jul-02 \\
\hline 1390 & & & & 26-Jul-02 \\
\hline 1391 & & & & 26-Jul-02 \\
\hline 1392 & & & & 26-Jul-02 \\
\hline 1393 & & & & 26-Jul-02 \\
\hline 1394 & & & & 26-Jul-02 \\
\hline 1395 & & & & 26-Jul-02 \\
\hline 1396 & & & & 26-Jul-02 \\
\hline 1397 & & & & 26-Jul-02 \\
\hline 1398 & & & & 26-Jul-02 \\
\hline 1399 & & & & 26-Jul-02 \\
\hline 1400 & & & & 26-Jul-02 \\
\hline 1401 & & & & 26-Jul-02 \\
\hline 1402 & & & & 26-Jul-02 \\
\hline 1403 & & & & 26-Jul-02 \\
\hline 1404 & & & & 26-Jul-02 \\
\hline 1405 & & & & $26-J u l-02$ \\
\hline 1406 & & & & 26-Jul-02 \\
\hline 1407 & & & & 26-Jul-02 \\
\hline
\end{tabular}




\begin{tabular}{|c|c|c|c|c|}
\hline & $\mathrm{O}$ & $P$ & $\bar{Q}$ & $\bar{R}$ \\
\hline 1 & SITE_CODE & ELEVATION_UNITS & VERT_COORD_TYPE & DATE_LAST_MODIFIED \\
\hline 1408 & & & & 26-Jul-02 \\
\hline 1409 & & & & 26-Jul-02 \\
\hline 1410 & & & & 26-Jul-02 \\
\hline 1411 & & & & 26-Jul-02 \\
\hline 1412 & & & & 26-Jul-02 \\
\hline 1413 & & & & 26-Jul-02 \\
\hline 1414 & & & & 26-Jul-02 \\
\hline 1415 & & & & $26-\mathrm{Jul}-02$ \\
\hline 1416 & & & & 26-Jul-02 \\
\hline 1417 & & & & 26-Jul-02 \\
\hline 1418 & & & & 26-Jul-02 \\
\hline 1419 & & & & 26-Jul-02 \\
\hline 1420 & & & & 26-Jul-02 \\
\hline 1421 & & & & 26-Jul-02 \\
\hline 1422 & & & & 26-Jul-02 \\
\hline 1423 & & & & 26-Jul-02 \\
\hline 1424 & & & & 26-Jul-02 \\
\hline 1425 & & & & 26-Jul-02 \\
\hline 1426 & & & & 26-Jul-02 \\
\hline 1427 & & & & 26-Jul-02 \\
\hline 1428 & & & & 26-Jul-02 \\
\hline 1429 & & & & 26-Jul-02 \\
\hline 1430 & & & & 26-Jul-02 \\
\hline 1431 & & & & 26-Jul-02 \\
\hline 1432 & & & & 26-Jul-02 \\
\hline 1433 & & & & 26-Jul-02 \\
\hline 1434 & & & & 26-Jul-02 \\
\hline 1435 & & & & 26-Jul-02 \\
\hline 1436 & & & & 26-Jul-02 \\
\hline 1437 & & & & 26-Jul-02 \\
\hline 1438 & & & & 26-Jul-02 \\
\hline 1439 & & & & 26-Jul-02 \\
\hline 1440 & & & & 26-Jul-02 \\
\hline \multicolumn{5}{|l|}{1441} \\
\hline \multicolumn{5}{|l|}{1442} \\
\hline \multicolumn{5}{|l|}{1443} \\
\hline \multicolumn{5}{|l|}{1444} \\
\hline 1445 & & & & \\
\hline
\end{tabular}




\begin{tabular}{|c|c|c|c|c|}
\hline & $\mathrm{O}$ & $P$ & $\mathrm{Q}$ & R \\
\hline 1 & SITE_CODE & ELEVATION_UNITS & VERT_COORD_TYPE & DATE_LAST_MODIFIED \\
\hline 1446 & & & & \\
\hline 1447 & & & & \\
\hline 1448 & & & & \\
\hline 1449 & & & & \\
\hline 1450 & & & & \\
\hline 1451 & & & & \\
\hline 1452 & & & & \\
\hline 1453 & & & & \\
\hline 1454 & & & & \\
\hline 1455 & & & & \\
\hline 1456 & & & & \\
\hline 1457 & & & & \\
\hline 1458 & & & & \\
\hline 1459 & & & & \\
\hline 1460 & & & & \\
\hline 1461 & & & & \\
\hline 1462 & & & & \\
\hline 1463 & & & & \\
\hline 1464 & & & & \\
\hline 1465 & & & & \\
\hline 1466 & & & & \\
\hline 1467 & & & & \\
\hline 1468 & & & & \\
\hline 1469 & & & & \\
\hline 1470 & & & & \\
\hline 1471 & & & & \\
\hline 1472 & & & & \\
\hline 1473 & & & & \\
\hline 1474 & & & & \\
\hline 1475 & & & & \\
\hline 1476 & & & & \\
\hline 1477 & & & & \\
\hline 1478 & & & & \\
\hline 1479 & & & & \\
\hline 1480 & & & & \\
\hline 1481 & & & & \\
\hline 1482 & & & & \\
\hline 1483 & & & & \\
\hline
\end{tabular}




\begin{tabular}{|c|c|c|c|c|}
\hline & $\mathrm{O}$ & $P$ & $\mathrm{Q}$ & $\mathrm{R}$ \\
\hline 1 & SITE_CODE & ELEVATION_UNITS & VERT_COORD_TYPE & DATE_LAST_MODIFIED \\
\hline 1484 & & & & \\
\hline 1485 & & & & \\
\hline 1486 & & & & \\
\hline 1487 & & & & \\
\hline 1488 & & & & \\
\hline 1489 & & & & \\
\hline 1490 & & & & \\
\hline 1491 & & & & \\
\hline 1492 & & & & \\
\hline 1493 & & & & \\
\hline 1494 & & & & \\
\hline 1495 & & & & \\
\hline 1496 & & & & \\
\hline 1497 & & & & \\
\hline 1498 & & & & \\
\hline 1499 & & & & \\
\hline 1500 & & & & \\
\hline 1501 & & & & \\
\hline 1502 & & & & \\
\hline 1503 & & & & \\
\hline 1504 & & & & \\
\hline 1505 & & & & \\
\hline 1506 & & & & \\
\hline 1507 & & & & \\
\hline 1508 & & & & \\
\hline 1509 & & & & \\
\hline 1510 & & & & \\
\hline 1511 & & & & \\
\hline 1512 & & & & \\
\hline 1513 & & & & \\
\hline 1514 & & & & \\
\hline 1515 & & & & \\
\hline 1516 & & & & \\
\hline 1517 & & & & \\
\hline 1518 & & & & \\
\hline 1519 & & & & \\
\hline 1520 & & & & \\
\hline 1521 & & & & \\
\hline
\end{tabular}




\begin{tabular}{|c|c|c|c|c|}
\hline & \multicolumn{1}{|c|}{ O } & P & Q & R \\
\hline 1 & SITE_CODE & ELEVATION_UNITS & VERT_COORD_TYPE & DATE_LAST_MODIFIED \\
\hline 1522 & & & & \\
\hline 1523 & & & & \\
\hline 1524 & & & & \\
\hline 1525 & & & & \\
\hline 1526 & & & & \\
\hline 1527 & & & & \\
\hline 1528 & & & & \\
\hline 1529 & & & & \\
\hline 1530 & & & \\
\hline 1531 & & & & \\
\hline 1532 & & & & \\
\hline 1533 & & & & \\
\hline 1534 & & & & \\
\hline 1535 & & & & \\
\hline 1536 & & & & \\
\hline 1537 & & & & \\
\hline 1538 & & & & \\
\hline 1539 & & & & \\
\hline 1540 & & & & \\
\hline 1541 & & & & \\
\hline 1542 & & & & \\
\hline 1543 & & & & \\
\hline 1544 & & & & \\
\hline 1545 & & & & \\
\hline 1546 & & & & \\
\hline 1547 & & & & \\
\hline 1548 & & & & \\
\hline 1549 & $100-H-24$ & & & \\
\hline 1550 & $100-H-24$ & & & \\
\hline 1551 & $100-H-24$ & & & \\
\hline 1552 & $100-H-24$ & & & \\
\hline 1553 & $100-H-24$ & & & \\
\hline 1554 & $118-H-6$ & & & \\
\hline 1555 & & & & \\
\hline 1556 & & & & \\
\hline 1557 & & & & \\
\hline 1558 & & & & \\
\hline 1559 & & & & \\
\hline
\end{tabular}




\begin{tabular}{|c|c|c|c|c|}
\hline & $\mathrm{O}$ & $\mathrm{P}$ & $\mathrm{Q}$ & $\mathrm{R}$ \\
\hline 1 & SITE_CODE & ELEVATION_UNITS & VERT_COORD_TYPE & DATE_LAST_MODIFIED \\
\hline \multicolumn{5}{|c|}{1560} \\
\hline \multicolumn{5}{|l|}{1561} \\
\hline \multicolumn{5}{|l|}{1562} \\
\hline \multicolumn{5}{|l|}{1563} \\
\hline \multicolumn{5}{|c|}{1564} \\
\hline \multicolumn{5}{|l|}{1565} \\
\hline \multicolumn{5}{|l|}{1566} \\
\hline \multicolumn{5}{|c|}{\begin{tabular}{|l|}
1567 \\
\end{tabular}} \\
\hline \multicolumn{5}{|l|}{1568} \\
\hline \multicolumn{5}{|c|}{1569} \\
\hline \multicolumn{5}{|l|}{1570} \\
\hline \multicolumn{5}{|l|}{1571} \\
\hline \multicolumn{5}{|c|}{1572} \\
\hline \multicolumn{5}{|l|}{1573} \\
\hline \multicolumn{5}{|l|}{\begin{tabular}{|l|}
1574 \\
\end{tabular}} \\
\hline \multicolumn{5}{|l|}{1575} \\
\hline \multicolumn{5}{|l|}{1576} \\
\hline \multicolumn{5}{|l|}{1577} \\
\hline \multicolumn{5}{|l|}{1578} \\
\hline \multicolumn{5}{|l|}{1579} \\
\hline \multicolumn{5}{|c|}{1580} \\
\hline \multicolumn{5}{|l|}{1581} \\
\hline \multicolumn{5}{|l|}{1582} \\
\hline \multicolumn{5}{|c|}{1583} \\
\hline \multicolumn{5}{|l|}{1584} \\
\hline \multicolumn{5}{|l|}{1585} \\
\hline \multicolumn{5}{|c|}{1586} \\
\hline 1587 & & & & \\
\hline 1588 & & & & \\
\hline 1589 & & & & \\
\hline 1590 & & & & \\
\hline \begin{tabular}{|l|}
1591 \\
\end{tabular} & & & & \\
\hline 1592 & & & & \\
\hline 1593 & & & & \\
\hline 1594 & & & & \\
\hline 1595 & & & & \\
\hline 1596 & & & & \\
\hline 1597 & & & & \\
\hline
\end{tabular}




\begin{tabular}{|c|c|c|c|c|}
\hline & $\mathrm{O}$ & $P$ & $\mathrm{Q}$ & $\mathrm{R}$ \\
\hline 1 & SITE_CODE & ELEVATION_UNITS & VERT_COORD_TYPE & \begin{tabular}{|l} 
DATE_LAST_MODIFIED \\
\end{tabular} \\
\hline 1598 & & & & \\
\hline 1599 & & & & \\
\hline 1600 & & & & \\
\hline 1601 & & & & \\
\hline 1602 & & & & \\
\hline 1603 & & & & \\
\hline 1604 & & & & \\
\hline 1605 & & & & \\
\hline 1606 & & & & \\
\hline 1607 & & & & \\
\hline 1608 & & & & \\
\hline 1609 & & & & \\
\hline 1610 & & & & \\
\hline 1611 & & & & \\
\hline 1612 & & & & \\
\hline 1613 & & & & \\
\hline 1614 & & & & \\
\hline 1615 & & & & \\
\hline 1616 & & & & \\
\hline 1617 & & & & \\
\hline 1618 & & & & \\
\hline 1619 & & & & \\
\hline 1620 & & & & \\
\hline 1621 & & & & \\
\hline 1622 & & & & \\
\hline 1623 & & & & \\
\hline 1624 & & & & \\
\hline 1625 & & & & \\
\hline 1626 & & & & \\
\hline 1627 & & & & \\
\hline 1628 & & & & \\
\hline 1629 & & & & \\
\hline 1630 & & & & \\
\hline 1631 & & & & \\
\hline 1632 & & & & \\
\hline 1633 & & & & \\
\hline 1634 & & & & \\
\hline 1635 & & & & \\
\hline
\end{tabular}




\begin{tabular}{|c|c|c|c|c|}
\hline & $\mathrm{O}$ & $P$ & $\mathrm{Q}$ & R \\
\hline 1 & SITE_CODE & ELEVATION_UNITS & VERT_COORD_TYPE & DATE_LAST_MODIFIED \\
\hline 1636 & & & & \\
\hline \begin{tabular}{|l|}
1637 \\
\end{tabular} & & & & \\
\hline \begin{tabular}{|l|}
1638 \\
\end{tabular} & & & & \\
\hline 1639 & & & & \\
\hline 1640 & & & & \\
\hline 1641 & & & & \\
\hline 1642 & & & & \\
\hline 1643 & & & & \\
\hline 1644 & & & & \\
\hline 1645 & & & & \\
\hline 1646 & & & & \\
\hline 1647 & & & & \\
\hline 1648 & & & & \\
\hline 1649 & & & & \\
\hline 1650 & & & & \\
\hline 1651 & & & & \\
\hline 1652 & & & & \\
\hline 1653 & & & & \\
\hline 1654 & & & & \\
\hline 1655 & & & & \\
\hline 1656 & & & & \\
\hline 1657 & & & & \\
\hline 1658 & & & & \\
\hline 1659 & & & & \\
\hline 1660 & & & & \\
\hline 1661 & & & & \\
\hline 1662 & & & & \\
\hline 1663 & & & & \\
\hline 1664 & & & & \\
\hline 1665 & & & & \\
\hline 1666 & & & & \\
\hline 1667 & & & & \\
\hline 1668 & & & & \\
\hline 1669 & & & & \\
\hline 1670 & & & & \\
\hline 1671 & & & & \\
\hline 1672 & & & & \\
\hline 1673 & & & & \\
\hline
\end{tabular}




\begin{tabular}{|c|c|c|c|c|}
\hline & $\mathrm{O}$ & $P$ & $\mathrm{Q}$ & R \\
\hline 1 & SITE_CODE & ELEVATION_UNITS & VERT_COORD_TYPE & DATE_LAST_MODIFIED \\
\hline 1674 & & & & \\
\hline 1675 & & & & \\
\hline 1676 & & & & \\
\hline 1677 & & & & \\
\hline 1678 & & & & \\
\hline 1679 & & & & \\
\hline 1680 & & & & \\
\hline 1681 & & & & \\
\hline 1682 & & & & \\
\hline 1683 & & & & \\
\hline 1684 & & & & \\
\hline 1685 & & & & \\
\hline 1686 & & & & \\
\hline 1687 & & & & \\
\hline 1688 & & & & \\
\hline 1689 & & & & \\
\hline 1690 & & & & \\
\hline 1691 & & & & \\
\hline 1692 & & & & \\
\hline 1693 & & & & \\
\hline 1694 & & & & \\
\hline 1695 & & & & \\
\hline 1696 & & & & \\
\hline 1697 & & & & \\
\hline 1698 & & & & 26-Jul-02 \\
\hline 1699 & & & & \\
\hline 1700 & & & & \\
\hline 1701 & & & & \\
\hline 1702 & & & & 26-Jul-02 \\
\hline 1703 & & & & \\
\hline 1704 & & & & \\
\hline 1705 & & & & \\
\hline 1706 & & & & \\
\hline 1707 & & & & \\
\hline 1708 & & & & \\
\hline 1709 & & & & \\
\hline 1710 & & & & \\
\hline 1711 & & & & \\
\hline
\end{tabular}




\begin{tabular}{|c|c|c|c|c|}
\hline & $\mathrm{O}$ & $P$ & $\mathrm{Q}$ & $\mathrm{R}$ \\
\hline 1 & SITE_CODE & ELEVATION_UNITS & VERT_COORD_TYPE & DATE_LAST_MODIFIED \\
\hline 1712 & & & & \\
\hline 1713 & & & & \\
\hline 1714 & & & & 26-Jul-02 \\
\hline 1715 & & & & \\
\hline 1716 & & & & 26-Jul-02 \\
\hline 1717 & & & & 26-Jul-02 \\
\hline 1718 & & & & \\
\hline 1719 & & & & \\
\hline 1720 & & & & \\
\hline 1721 & & & & \\
\hline 1722 & & & & 26-Jul-02 \\
\hline 1723 & & & & 26-Jul-02 \\
\hline 1724 & & & & 26-Jul-02 \\
\hline 1725 & & & & 26-Jul-02 \\
\hline 1726 & & & & \\
\hline 1727 & & & & \\
\hline 1728 & & & & \\
\hline 1729 & & & & \\
\hline 1730 & & & & \\
\hline 1731 & & & & 26-Jul-02 \\
\hline 1732 & & & & 26-Jul-02 \\
\hline 1733 & & & & 26-Jul-02 \\
\hline 1734 & & & & \\
\hline 1735 & & & & \\
\hline 1736 & & & & \\
\hline 1737 & & & & \\
\hline 1738 & & & & \\
\hline 1739 & & & & 26-Jul-02 \\
\hline 1740 & & & & \\
\hline 1741 & & & & \\
\hline 1742 & & & & \\
\hline 1743 & & & & \\
\hline 1744 & & & & \\
\hline 1745 & & & & \\
\hline 1746 & & & & 26-Jul-02 \\
\hline 1747 & & & & \\
\hline 1748 & & & & \\
\hline 1749 & & & & \\
\hline
\end{tabular}




\begin{tabular}{|c|c|c|c|c|}
\hline & $\mathrm{O}$ & $\mathrm{P}$ & $\mathrm{Q}$ & $\mathrm{R}$ \\
\hline 1 & SITE_CODE & ELEVATION_UNITS & VERT_COORD_TYPE & DATE_LAST_MODIFIED \\
\hline \multicolumn{5}{|c|}{1750} \\
\hline \multicolumn{5}{|l|}{1751} \\
\hline \multicolumn{5}{|l|}{1752} \\
\hline \multicolumn{5}{|l|}{1753} \\
\hline \multicolumn{5}{|c|}{1754} \\
\hline \multicolumn{5}{|l|}{1755} \\
\hline \multicolumn{5}{|l|}{1756} \\
\hline \multicolumn{5}{|c|}{\begin{tabular}{|l|}
1757 \\
\end{tabular}} \\
\hline \multicolumn{5}{|l|}{1758} \\
\hline \multicolumn{5}{|c|}{1759} \\
\hline \multicolumn{5}{|l|}{1760} \\
\hline \multicolumn{5}{|l|}{1761} \\
\hline \multicolumn{5}{|c|}{1762} \\
\hline \multicolumn{5}{|l|}{1763} \\
\hline \multicolumn{5}{|l|}{1764} \\
\hline \multicolumn{5}{|l|}{1765} \\
\hline \multicolumn{5}{|l|}{1766} \\
\hline \multicolumn{5}{|l|}{1767} \\
\hline \multicolumn{5}{|l|}{1768} \\
\hline \multicolumn{5}{|l|}{\begin{tabular}{|l|}
1769 \\
\end{tabular}} \\
\hline \multicolumn{5}{|c|}{\begin{tabular}{|l|}
1770 \\
\end{tabular}} \\
\hline \multicolumn{5}{|l|}{1771} \\
\hline \multicolumn{5}{|l|}{1772} \\
\hline \multicolumn{5}{|c|}{1773} \\
\hline \multicolumn{5}{|l|}{1774} \\
\hline \multicolumn{5}{|l|}{1775} \\
\hline \multicolumn{5}{|c|}{1776} \\
\hline 1777 & & & & \\
\hline 1778 & & & & \\
\hline 1779 & & & & \\
\hline 1780 & & & & \\
\hline 1781 & & & & \\
\hline 1782 & & & & \\
\hline 1783 & & & & \\
\hline 1784 & & & & \\
\hline 1785 & & & & \\
\hline 1786 & & & & \\
\hline 1787 & & & & \\
\hline
\end{tabular}




\begin{tabular}{|c|c|c|c|c|}
\hline & $\mathrm{O}$ & $P$ & $\mathrm{Q}$ & R \\
\hline 1 & SITE_CODE & ELEVATION_UNITS & VERT_COORD_TYPE & DATE_LAST_MODIFIED \\
\hline 1788 & & & & \\
\hline 1789 & & & & \\
\hline 1790 & & & & \\
\hline 1791 & & & & \\
\hline 1792 & & & & \\
\hline 1793 & & & & \\
\hline 1794 & & & & \\
\hline 1795 & & & & \\
\hline 1796 & & & & \\
\hline 1797 & & & & \\
\hline 1798 & & & & \\
\hline 1799 & & & & \\
\hline 1800 & & & & \\
\hline \begin{tabular}{|l|}
1801 \\
\end{tabular} & & & & \\
\hline 1802 & & & & \\
\hline 1803 & & & & \\
\hline 1804 & & & & \\
\hline 1805 & & & & \\
\hline 1806 & & & & 26-Jul-02 \\
\hline 1807 & & & & \\
\hline 1808 & & & & 26-Jul-02 \\
\hline 1809 & & & & \\
\hline 1810 & & & & \\
\hline 1811 & & & & \\
\hline 1812 & & & & \\
\hline 1813 & & & & \\
\hline 1814 & & & & \\
\hline 1815 & & & & \\
\hline 1816 & & & & \\
\hline 1817 & & & & \\
\hline 1818 & & & & \\
\hline 1819 & & & & \\
\hline 1820 & & & & \\
\hline 1821 & & & & \\
\hline 1822 & & & & \\
\hline 1823 & & & & \\
\hline 1824 & & & & \\
\hline 1825 & & & & \\
\hline
\end{tabular}




\begin{tabular}{|c|c|c|c|c|}
\hline & $\mathrm{O}$ & $P$ & $\mathrm{Q}$ & R \\
\hline 1 & SITE_CODE & ELEVATION_UNITS & VERT_COORD_TYPE & DATE_LAST_MODIFIED \\
\hline 1826 & & & & \\
\hline 1827 & & & & \\
\hline 1828 & & & & \\
\hline 1829 & & & & \\
\hline 1830 & & & & \\
\hline 1831 & & & & \\
\hline 1832 & & & & \\
\hline 1833 & & & & \\
\hline 1834 & & & & \\
\hline 1835 & & & & \\
\hline 1836 & & & & \\
\hline 1837 & & & & \\
\hline 1838 & & & & \\
\hline \begin{tabular}{|l|}
1839 \\
\end{tabular} & & & & \\
\hline 1840 & & & & \\
\hline 1841 & & & & \\
\hline 1842 & & & & \\
\hline 1843 & & & & \\
\hline 1844 & & & & \\
\hline 1845 & & & & \\
\hline 1846 & & & & \\
\hline 1847 & & & & \\
\hline 1848 & & & & \\
\hline 1849 & & & & \\
\hline 1850 & & & & \\
\hline 1851 & & & & \\
\hline 1852 & & & & \\
\hline 1853 & & & & \\
\hline 1854 & & & & \\
\hline 1855 & & & & \\
\hline 1856 & & & & \\
\hline 1857 & & & & \\
\hline 1858 & & & & \\
\hline 1859 & & & & \\
\hline 1860 & & & & \\
\hline 1861 & & & & \\
\hline 1862 & & & & \\
\hline 1863 & & & & \\
\hline
\end{tabular}




\begin{tabular}{|c|c|c|c|c|}
\hline & $\mathrm{O}$ & $P$ & $\mathrm{Q}$ & $\mathrm{R}$ \\
\hline 1 & SITE_CODE & ELEVATION_UNITS & \begin{tabular}{|l} 
VERT_COORD_TYPE \\
\end{tabular} & \begin{tabular}{|l|} 
DATE_LAST_MODIFIED \\
\end{tabular} \\
\hline \multicolumn{5}{|c|}{\begin{tabular}{|l|l|l|l|l|}
1864 & & -1
\end{tabular}} \\
\hline 1865 & & & & 26-Jul-02 \\
\hline 1866 & & & & 26-Jul-02 \\
\hline 1867 & & & & 26-Jul-02 \\
\hline 1868 & & & & 26-Jul-02 \\
\hline 1869 & & & & 26-Jul-02 \\
\hline 1870 & & & & 26-Jul-02 \\
\hline 1871 & & & & 26-Jul-02 \\
\hline 1872 & & & & 26-Jul-02 \\
\hline 1873 & & & & 26-Jul-02 \\
\hline \multicolumn{5}{|l|}{1874} \\
\hline \multicolumn{5}{|c|}{1875} \\
\hline \multicolumn{5}{|l|}{1876} \\
\hline \multicolumn{5}{|l|}{1877} \\
\hline \multicolumn{5}{|l|}{1878} \\
\hline \multicolumn{5}{|l|}{1879} \\
\hline \multicolumn{5}{|l|}{1880} \\
\hline \multicolumn{5}{|l|}{1881} \\
\hline \multicolumn{5}{|l|}{1882} \\
\hline \multicolumn{5}{|l|}{1883} \\
\hline \multicolumn{5}{|c|}{1884} \\
\hline \multicolumn{5}{|l|}{1885} \\
\hline \multicolumn{5}{|l|}{1886} \\
\hline \multicolumn{5}{|l|}{1887} \\
\hline \multicolumn{5}{|l|}{1888} \\
\hline \multicolumn{5}{|l|}{1889} \\
\hline \multicolumn{5}{|l|}{1890} \\
\hline \multicolumn{5}{|l|}{1891} \\
\hline \multicolumn{5}{|l|}{1892} \\
\hline \multicolumn{5}{|l|}{1893} \\
\hline \multicolumn{5}{|l|}{1894} \\
\hline \multicolumn{5}{|l|}{1895} \\
\hline \multicolumn{5}{|l|}{1896} \\
\hline \multicolumn{5}{|l|}{1897} \\
\hline \multicolumn{5}{|l|}{1898} \\
\hline \multicolumn{5}{|l|}{1899} \\
\hline 1900 & & & & \\
\hline 1901 & & & & \\
\hline
\end{tabular}




\begin{tabular}{|c|c|c|c|c|}
\hline & $\mathrm{O}$ & $\mathrm{P}$ & $\mathrm{Q}$ & $\mathrm{R}$ \\
\hline 1 & SITE_CODE & ELEVATION_UNITS & VERT_COORD_TYPE & DATE_LAST_MODIFIED \\
\hline \multicolumn{5}{|c|}{1902} \\
\hline \multicolumn{5}{|l|}{1903} \\
\hline \multicolumn{5}{|l|}{1904} \\
\hline \multicolumn{5}{|l|}{1905} \\
\hline \multicolumn{5}{|c|}{1906} \\
\hline \multicolumn{5}{|l|}{1907} \\
\hline \multicolumn{5}{|c|}{\begin{tabular}{|l|}
1908 \\
\end{tabular}} \\
\hline \multicolumn{5}{|c|}{\begin{tabular}{|l|}
1909 \\
\end{tabular}} \\
\hline \multicolumn{5}{|l|}{1910} \\
\hline \multicolumn{5}{|c|}{1911} \\
\hline \multicolumn{5}{|l|}{1912} \\
\hline \multicolumn{5}{|l|}{1913} \\
\hline \multicolumn{5}{|c|}{1914} \\
\hline \multicolumn{5}{|l|}{1915} \\
\hline \multicolumn{5}{|l|}{\begin{tabular}{|l|l|}
1916 \\
\end{tabular}} \\
\hline \multicolumn{5}{|c|}{1917} \\
\hline \multicolumn{5}{|l|}{1918} \\
\hline \multicolumn{5}{|l|}{1919} \\
\hline \multicolumn{5}{|l|}{1920} \\
\hline \multicolumn{5}{|l|}{1921} \\
\hline \multicolumn{5}{|c|}{\begin{tabular}{|l|}
1922 \\
\end{tabular}} \\
\hline \multicolumn{5}{|l|}{1923} \\
\hline \multicolumn{5}{|l|}{1924} \\
\hline \multicolumn{5}{|c|}{1925} \\
\hline \multicolumn{5}{|l|}{1926} \\
\hline \multicolumn{5}{|l|}{1927} \\
\hline \multicolumn{5}{|c|}{1928} \\
\hline 1929 & & & & \\
\hline 1930 & & & & \\
\hline 1931 & & & & \\
\hline 1932 & & & & \\
\hline 1933 & & & & \\
\hline 1934 & & & & \\
\hline 1935 & & & & \\
\hline 1936 & & & & \\
\hline 1937 & & & & \\
\hline 1938 & & & & \\
\hline 1939 & & & & \\
\hline
\end{tabular}




\begin{tabular}{|c|c|c|c|c|}
\hline & $\mathrm{O}$ & $P$ & $\mathrm{Q}$ & $\mathrm{R}$ \\
\hline 1 & SITE_CODE & ELEVATION_UNITS & VERT_COORD_TYPE & DATE_LAST_MODIFIED \\
\hline 1940 & & & & \\
\hline 1941 & & & & \\
\hline 1942 & & & & \\
\hline \begin{tabular}{|l|}
1943 \\
\end{tabular} & & & & \\
\hline 1944 & & & & \\
\hline 1945 & & & & \\
\hline 1946 & & & & \\
\hline 1947 & & & & \\
\hline 1948 & & & & \\
\hline 1949 & & & & \\
\hline 1950 & & & & \\
\hline 1951 & & & & \\
\hline 1952 & & & & \\
\hline 1953 & & & & \\
\hline 1954 & & & & \\
\hline 1955 & & & & \\
\hline 1956 & & & & \\
\hline 1957 & & & & \\
\hline 1958 & & & & \\
\hline 1959 & & & & \\
\hline 1960 & $316-2$ & & & 09-Jul-02 \\
\hline 1961 & $316-2$ & & & 09-Jul-02 \\
\hline 1962 & $316-2$ & & & 09-Jul-02 \\
\hline 1963 & & & & \\
\hline 1964 & & & & \\
\hline 1965 & & & & \\
\hline 1966 & & & & \\
\hline 1967 & $316-1$ & & & 09-Jul-02 \\
\hline 1968 & & & & \\
\hline 1969 & & & & \\
\hline 1970 & & & & \\
\hline 1971 & & & & \\
\hline 1972 & & & & \\
\hline 1973 & & & & \\
\hline 1974 & $316-5$ & & & 09-Jul-02 \\
\hline 1975 & $316-5$ & & & 09-Jul-02 \\
\hline 1976 & $316-5$ & & & 09-Jul-02 \\
\hline 1977 & $316-5$ & & & 09-Jul-02 \\
\hline
\end{tabular}




\begin{tabular}{|c|c|c|c|c|}
\hline & $\mathrm{O}$ & $P$ & $\mathrm{Q}$ & $\mathrm{R}$ \\
\hline 1 & SITE_CODE & ELEVATION_UNITS & VERT_COORD_TYPE & DATE_LAST_MODIFIED \\
\hline 1978 & $316-5$ & & & 09-Jul-02 \\
\hline 1979 & $316-5$ & & & 09-Jul-02 \\
\hline 1980 & $316-5$ & & & 09-Jul-02 \\
\hline 1981 & $316-5$ & & & 09-Jul-02 \\
\hline 1982 & $316-5$ & & & 09-Jul-02 \\
\hline 1983 & $316-5$ & & & 09-Jul-02 \\
\hline 1984 & $316-1$ & & & 09-Jul-02 \\
\hline 1985 & $316-1$ & & & 09-Jul-02 \\
\hline 1986 & & & & \\
\hline 1987 & & & & \\
\hline 1988 & & & & \\
\hline 1989 & & & & \\
\hline 1990 & & & & \\
\hline 1991 & & & & \\
\hline 1992 & & & & \\
\hline 1993 & & & & \\
\hline 1994 & & & & \\
\hline 1995 & & & & \\
\hline 1996 & & & & \\
\hline 1997 & & & & \\
\hline 1998 & & & & \\
\hline 1999 & & & & \\
\hline 2000 & & & & \\
\hline 2001 & & & & \\
\hline 2002 & & & & \\
\hline 2003 & & & & \\
\hline 2004 & & & & \\
\hline 2005 & & & & \\
\hline 2006 & & & & \\
\hline 2007 & & & & \\
\hline 2008 & & & & \\
\hline 2009 & & & & \\
\hline 2010 & & & & \\
\hline 2011 & & & & \\
\hline 2012 & & & & \\
\hline 2013 & & & & \\
\hline 2014 & & & & \\
\hline 2015 & & & & \\
\hline
\end{tabular}




\begin{tabular}{|c|c|c|c|c|}
\hline & $\mathrm{O}$ & $P$ & $\mathrm{Q}$ & $\mathrm{R}$ \\
\hline 1 & SITE_CODE & ELEVATION_UNITS & VERT_COORD_TYPE & DATE_LAST_MODIFIED \\
\hline 2016 & & & & \\
\hline 2017 & & & & \\
\hline 2018 & & & & \\
\hline 2019 & & & & \\
\hline 2020 & & & & \\
\hline 2021 & & & & \\
\hline 2022 & & & & \\
\hline 2023 & & & & \\
\hline 2024 & & & & \\
\hline 2025 & & & & \\
\hline 2026 & & & & \\
\hline 2027 & & & & \\
\hline 2028 & & & & \\
\hline 2029 & 216-B-2-2 & & & 14-Aug-02 \\
\hline 2030 & & & & 26-Jul-02 \\
\hline 2031 & & & & 29-Jan-03 \\
\hline 2032 & & & & 26-Jul-02 \\
\hline 2033 & & & & \\
\hline 2034 & & & & \\
\hline 2035 & & & & \\
\hline 2036 & & & & \\
\hline 2037 & & & & \\
\hline 2038 & & & & \\
\hline 2039 & & & & \\
\hline 2040 & & & & 14-Aug-02 \\
\hline 2041 & & & & \\
\hline 2042 & & & & \\
\hline 2043 & & & & \\
\hline 2044 & & & & \\
\hline 2045 & & & & \\
\hline 2046 & & & & \\
\hline 2047 & & & & \\
\hline 2048 & & & & \\
\hline 2049 & & & & \\
\hline 2050 & & & & \\
\hline 2051 & & & & \\
\hline 2052 & & & & \\
\hline 2053 & & & & \\
\hline
\end{tabular}




\begin{tabular}{|c|c|c|c|c|}
\hline & $\mathrm{O}$ & $P$ & $\mathrm{Q}$ & R \\
\hline 1 & SITE_CODE & ELEVATION_UNITS & VERT_COORD_TYPE & DATE_LAST_MODIFIED \\
\hline 2054 & & & & \\
\hline 2055 & & & & \\
\hline 2056 & & & & \\
\hline 2057 & & & & \\
\hline 2058 & & & & \\
\hline 2059 & & & & \\
\hline 2060 & & & & \\
\hline 2061 & & & & \\
\hline 2062 & & & & \\
\hline 2063 & & & & \\
\hline 2064 & & & & \\
\hline 2065 & & & & \\
\hline 2066 & & & & \\
\hline 2067 & & & & \\
\hline 2068 & & & & \\
\hline 2069 & & & & \\
\hline 2070 & & & & \\
\hline 2071 & & & & \\
\hline 2072 & & & & \\
\hline 2073 & & & & \\
\hline 2074 & & & & \\
\hline 2075 & & & & \\
\hline 2076 & & & & \\
\hline 2077 & & & & \\
\hline 2078 & & & & \\
\hline 2079 & & & & \\
\hline 2080 & & & & \\
\hline 2081 & & & & \\
\hline 2082 & & & & \\
\hline 2083 & & & & \\
\hline 2084 & & & & \\
\hline 2085 & & & & \\
\hline 2086 & & & & \\
\hline 2087 & & & & \\
\hline 2088 & & & & \\
\hline 2089 & & & & \\
\hline 2090 & & & & \\
\hline 2091 & & & & \\
\hline
\end{tabular}




\begin{tabular}{|c|c|c|c|c|}
\hline & O & P & Q & R \\
\hline 1 & SITE_CODE & ELEVATION_UNITS & VERT_COORD_TYPE & DATE_LAST_MODIFIED \\
\hline 2092 & & & & \\
\hline 2093 & & & & \\
\hline 2094 & & & & \\
\hline 2095 & & & & \\
\hline 2096 & & & & \\
\hline 2097 & & & & \\
\hline 2098 & & & & \\
\hline 2099 & & & & \\
\hline 2100 & & & & \\
\hline 2101 & & & & \\
\hline 2102 & & & & \\
\hline 2103 & & & & \\
\hline 2104 & & & & \\
\hline 2105 & & & & \\
\hline 2106 & & & & \\
\hline 2107 & & & & \\
\hline 2108 & & & & \\
\hline 2109 & & & & \\
\hline 2110 & & & & \\
\hline 2111 & & & \\
\hline 2112 & & & & \\
\hline 2113 & & & & \\
\hline 2114 & & & & \\
\hline 2115 & & & & \\
\hline 2116 & & & & \\
\hline 2117 & & & & \\
\hline 2118 & & & & \\
\hline 2119 & & & & \\
\hline 2120 & & & & \\
\hline 2121 & & & & \\
\hline 2122 & & & & \\
\hline 2123 & & & & \\
\hline 2124 & & & & \\
\hline 2125 & & & & \\
\hline 2126 & & & & \\
\hline 2127 & & & & \\
\hline 2128 & & & & \\
\hline 2129 & & & & \\
\hline
\end{tabular}




\begin{tabular}{|c|c|c|c|c|}
\hline & $\mathrm{O}$ & $\mathrm{P}$ & $\mathrm{Q}$ & $\mathrm{R}$ \\
\hline 1 & SITE_CODE & ELEVATION_UNITS & VERT_COORD_TYPE & DATE_LAST_MODIFIED \\
\hline \multicolumn{5}{|c|}{2130} \\
\hline \multicolumn{5}{|c|}{2131} \\
\hline \multicolumn{5}{|c|}{2132} \\
\hline \multicolumn{5}{|l|}{2133} \\
\hline \multicolumn{5}{|c|}{2134} \\
\hline \multicolumn{5}{|l|}{2135} \\
\hline \multicolumn{5}{|l|}{2136} \\
\hline \multicolumn{5}{|c|}{\begin{tabular}{|l|}
2137 \\
\end{tabular}} \\
\hline \multicolumn{5}{|l|}{2138} \\
\hline \multicolumn{5}{|c|}{2139} \\
\hline \multicolumn{5}{|l|}{2140} \\
\hline \multicolumn{5}{|l|}{2141} \\
\hline \multicolumn{5}{|c|}{2142} \\
\hline \multicolumn{5}{|l|}{2143} \\
\hline \multicolumn{5}{|c|}{\begin{tabular}{|l|}
2144 \\
\end{tabular}} \\
\hline \multicolumn{5}{|l|}{2145} \\
\hline \multicolumn{5}{|l|}{2146} \\
\hline \multicolumn{5}{|l|}{2147} \\
\hline \multicolumn{5}{|l|}{2148} \\
\hline \multicolumn{5}{|l|}{2149} \\
\hline \multicolumn{5}{|c|}{\begin{tabular}{|l|}
2150 \\
\end{tabular}} \\
\hline \multicolumn{5}{|l|}{2151} \\
\hline \multicolumn{5}{|l|}{2152} \\
\hline \multicolumn{5}{|c|}{2153} \\
\hline \multicolumn{5}{|l|}{2154} \\
\hline \multicolumn{5}{|l|}{\begin{tabular}{|l|}
2155 \\
\end{tabular}} \\
\hline \multicolumn{5}{|c|}{\begin{tabular}{|l|}
2156 \\
\end{tabular}} \\
\hline 2157 & & & & \\
\hline 2158 & & & & \\
\hline 2159 & & & & \\
\hline 2160 & & & & \\
\hline 2161 & & & & \\
\hline 2162 & & & & \\
\hline 2163 & & & & \\
\hline 2164 & & & & \\
\hline 2165 & & & & \\
\hline 2166 & & & & \\
\hline 2167 & & & & \\
\hline
\end{tabular}




\begin{tabular}{|c|c|c|c|c|}
\hline & $\mathrm{O}$ & $P$ & $\mathrm{Q}$ & $\mathrm{R}$ \\
\hline 1 & SITE_CODE & ELEVATION_UNITS & VERT_COORD_TYPE & DATE_LAST_MODIFIED \\
\hline 2168 & & & & \\
\hline 2169 & & & & \\
\hline 2170 & & & & \\
\hline 2171 & & & & \\
\hline 2172 & & & & \\
\hline 2173 & & & & \\
\hline 2174 & & & & \\
\hline 2175 & & & & \\
\hline 2176 & & & & \\
\hline 2177 & & & & \\
\hline 2178 & & & & 13-Nov-02 \\
\hline 2179 & & & & 13-Nov-02 \\
\hline 2180 & & & & \\
\hline 2181 & & & & 13-Nov-02 \\
\hline 2182 & & & & \\
\hline 2183 & & & & \\
\hline 2184 & & & & \\
\hline 2185 & & & & \\
\hline 2186 & & & & \\
\hline 2187 & & & & \\
\hline 2188 & & & & \\
\hline 2189 & & & & \\
\hline 2190 & & & & \\
\hline 2191 & & & & \\
\hline 2192 & & & & \\
\hline 2193 & & & & \\
\hline 2194 & & & & \\
\hline 2195 & & & & \\
\hline 2196 & & & & \\
\hline 2197 & & & & \\
\hline 2198 & & & & \\
\hline 2199 & & & & \\
\hline 2200 & & & & \\
\hline 2201 & & & & \\
\hline 2202 & & & & \\
\hline 2203 & & & & \\
\hline 2204 & & & & \\
\hline 2205 & & & & \\
\hline
\end{tabular}




\begin{tabular}{|c|c|c|c|c|}
\hline & $\mathrm{O}$ & $P$ & $\mathrm{Q}$ & $\mathrm{R}$ \\
\hline 1 & SITE_CODE & ELEVATION_UNITS & VERT_COORD_TYPE & DATE_LAST_MODIFIED \\
\hline 2206 & & & & \\
\hline 2207 & & & & \\
\hline 2208 & & & & \\
\hline 2209 & & & & \\
\hline 2210 & & & & \\
\hline 2211 & & & & \\
\hline \begin{tabular}{|l|}
2212 \\
\end{tabular} & & & & \\
\hline 2213 & & & & \\
\hline 2214 & & & & \\
\hline 2215 & & & & \\
\hline 2216 & & & & \\
\hline 2217 & & & & \\
\hline \begin{tabular}{|l|}
2218 \\
\end{tabular} & & & & \\
\hline 2219 & & & & \\
\hline 2220 & & & & \\
\hline 2221 & & & & \\
\hline 2222 & & & & \\
\hline 2223 & & & & \\
\hline 2224 & & & & \\
\hline 2225 & & & & \\
\hline 2226 & & & & \\
\hline 2227 & & & & \\
\hline \begin{tabular}{|l|}
2228 \\
\end{tabular} & & & & \\
\hline \begin{tabular}{|l|}
2229 \\
\end{tabular} & & & & \\
\hline 2230 & & & & \\
\hline 22 & & & & \\
\hline 2232 & & & & \\
\hline 2233 & & & & \\
\hline 2234 & & & & \\
\hline 2235 & & & & \\
\hline 2236 & & & & \\
\hline 2237 & & & & \\
\hline \begin{tabular}{|l|}
2238 \\
\end{tabular} & & & & \\
\hline 2239 & & & & \\
\hline 2240 & & & & \\
\hline 2241 & & & & \\
\hline 2242 & & & & \\
\hline 2243 & & & & \\
\hline
\end{tabular}




\begin{tabular}{|c|c|c|c|c|}
\hline & $\mathrm{O}$ & $\mathrm{P}$ & $\mathrm{Q}$ & $\mathrm{R}$ \\
\hline 1 & SITE_CODE & ELEVATION_UNITS & VERT_COORD_TYPE & DATE_LAST_MODIFIED \\
\hline 2244 & & & & \\
\hline 2245 & & & & \\
\hline 2246 & & & & \\
\hline 2247 & & & & \\
\hline 2248 & & & & \\
\hline 2249 & & & & \\
\hline 2250 & & & & \\
\hline 2251 & & & & \\
\hline 2252 & & & & \\
\hline 2253 & & & & \\
\hline 2254 & & & & \\
\hline 2255 & & & & \\
\hline 2256 & & & & \\
\hline 2257 & & & & \\
\hline 2258 & & & & \\
\hline 2259 & & & & \\
\hline 2260 & & & & \\
\hline 2261 & & & & \\
\hline 2262 & & & & \\
\hline 2263 & & & & \\
\hline 2264 & & & & \\
\hline 2265 & & & & \\
\hline 2266 & & & & \\
\hline 2267 & & & & \\
\hline 2268 & & & & \\
\hline 2269 & & & & \\
\hline 2270 & & & & \\
\hline 2271 & & & & \\
\hline 2272 & & & & \\
\hline 2273 & & & & \\
\hline 2274 & & & & \\
\hline 2275 & & & & \\
\hline 2276 & & & & \\
\hline 2277 & & & & \\
\hline 2278 & & & & \\
\hline 2279 & & & & \\
\hline 2280 & & & & \\
\hline 2281 & & & & \\
\hline
\end{tabular}




\begin{tabular}{|c|c|c|c|c|}
\hline & $\mathrm{O}$ & $\mathrm{P}$ & $\mathrm{Q}$ & $\mathrm{R}$ \\
\hline 1 & SITE_CODE & ELEVATION_UNITS & VERT_COORD_TYPE & DATE_LAST_MODIFIED \\
\hline \multicolumn{5}{|l|}{2282} \\
\hline \multicolumn{5}{|l|}{2283} \\
\hline \multicolumn{5}{|l|}{2284} \\
\hline \multicolumn{5}{|l|}{\begin{tabular}{|l|}
2285 \\
\end{tabular}} \\
\hline \multicolumn{5}{|l|}{2286} \\
\hline \multicolumn{5}{|l|}{2287} \\
\hline \multicolumn{5}{|l|}{2288} \\
\hline \multicolumn{5}{|l|}{2289} \\
\hline 2290 & & $\mathrm{~m}$ & & \\
\hline 2291 & & m & & \\
\hline 2292 & & $\mathrm{~m}$ & & \\
\hline \multicolumn{5}{|l|}{2293} \\
\hline \multicolumn{5}{|l|}{2294} \\
\hline \multicolumn{5}{|l|}{\begin{tabular}{|l|}
2295 \\
\end{tabular}} \\
\hline \multicolumn{5}{|l|}{2296} \\
\hline \multicolumn{5}{|l|}{\begin{tabular}{|l|}
2297 \\
\end{tabular}} \\
\hline 2298 & & $\mathrm{~m}$ & & \\
\hline 2299 & & $\mathrm{~m}$ & & \\
\hline 2300 & & $\mathrm{~m}$ & & \\
\hline \multicolumn{5}{|l|}{2301} \\
\hline \multicolumn{5}{|l|}{\begin{tabular}{|l|}
2302 \\
\end{tabular}} \\
\hline \multicolumn{5}{|l|}{\begin{tabular}{|l|}
2303 \\
\end{tabular}} \\
\hline \multicolumn{5}{|l|}{2304} \\
\hline \multicolumn{5}{|l|}{2305} \\
\hline \multicolumn{5}{|l|}{2306} \\
\hline \multicolumn{5}{|l|}{\begin{tabular}{|l|}
2307 \\
\end{tabular}} \\
\hline \multicolumn{5}{|l|}{\begin{tabular}{|l|}
2308 \\
\end{tabular}} \\
\hline \multicolumn{5}{|l|}{\begin{tabular}{|l|}
2309 \\
\end{tabular}} \\
\hline \multicolumn{5}{|l|}{2310} \\
\hline \multicolumn{5}{|l|}{2311} \\
\hline \multicolumn{5}{|l|}{2312} \\
\hline \multicolumn{5}{|l|}{2313} \\
\hline \multicolumn{5}{|l|}{\begin{tabular}{|l|}
2314 \\
\end{tabular}} \\
\hline 2315 & & & & \\
\hline 2316 & & & & \\
\hline 2317 & & & & \\
\hline 2318 & & & & \\
\hline 2319 & & & & \\
\hline
\end{tabular}




\begin{tabular}{|c|c|c|c|c|}
\hline & $\mathrm{O}$ & $P$ & $\mathrm{Q}$ & $\mathrm{R}$ \\
\hline 1 & SITE_CODE & ELEVATION_UNITS & VERT_COORD_TYPE & DATE_LAST_MODIFIED \\
\hline \multicolumn{5}{|l|}{2320} \\
\hline \multicolumn{5}{|l|}{2321} \\
\hline \multicolumn{5}{|c|}{\begin{tabular}{|l|}
2322 \\
\end{tabular}} \\
\hline 2323 & & & & 13-Nov-02 \\
\hline \multicolumn{5}{|l|}{2324} \\
\hline \multicolumn{5}{|l|}{2325} \\
\hline \multicolumn{5}{|l|}{2326} \\
\hline 2327 & & $\mathrm{~m}$ & & \\
\hline 2328 & & $\mathrm{~m}$ & & \\
\hline \multicolumn{5}{|c|}{2329} \\
\hline 2330 & & $\mathrm{~m}$ & & \\
\hline 2331 & & $\mathrm{~m}$ & & \\
\hline 2332 & & $\mathrm{~m}$ & & \\
\hline 2333 & & $\mathrm{~m}$ & & \\
\hline 2334 & & $\mathrm{~m}$ & & \\
\hline 2335 & $116-F-14$ & & UNKNOWN & \\
\hline 2336 & $116-F-14$ & & UNKNOWN & \\
\hline 2337 & $116-F-14$ & & UNKNOWN & \\
\hline 2338 & $116-F-14$ & & UNKNOWN & \\
\hline 2339 & $116-F-14$ & & UNKNOWN & \\
\hline 2340 & $116-F-14$ & & UNKNOWN & \\
\hline \multicolumn{5}{|c|}{\begin{tabular}{|l|l|}
2341 & $100-F-19$ \\
\end{tabular}} \\
\hline \multicolumn{5}{|c|}{\begin{tabular}{|l|l|}
2342 & $100-F-19$ \\
\end{tabular}} \\
\hline 2343 & $116-F-14$ & & UNKNOWN & \\
\hline \multicolumn{5}{|c|}{\begin{tabular}{|l|l|}
2344 & $100-F-19$ \\
\end{tabular}} \\
\hline 2345 & $116-F-14$ & & UNKNOWN & \\
\hline 2346 & $116-F-14$ & & UNKNOWN & \\
\hline 2347 & $116-F-14$ & & UNKNOWN & \\
\hline \multicolumn{5}{|c|}{\begin{tabular}{|l|l|}
2348 & $100-F-19$
\end{tabular}} \\
\hline 2349 & $116-F-14$ & & UNKNOWN & \\
\hline 2350 & $116-F-14$ & & UNKNOWN & \\
\hline \multicolumn{5}{|c|}{\begin{tabular}{|l|l|}
2351 & $100-F-19$ \\
\end{tabular}} \\
\hline 2352 & 116-F-14 & & UNKNOWN & \\
\hline 2353 & 116-F-14 & & UNKNOWN & \\
\hline \multicolumn{5}{|c|}{\begin{tabular}{|l|l|}
2354 & $100-F-19$ \\
\end{tabular}} \\
\hline \multicolumn{5}{|c|}{\begin{tabular}{|l|l|}
2355 & $100-F-19$ \\
\end{tabular}} \\
\hline 2356 & $116-F-14$ & & UNKNOWN & \\
\hline 2357 & 116-F-14 & & UNKNOWN & \\
\hline
\end{tabular}




\begin{tabular}{|c|c|c|c|c|}
\hline & $\mathrm{O}$ & $\mathrm{P}$ & $\bar{Q}$ & $\bar{R}$ \\
\hline 1 & SITE_CODE & ELEVATION_UNITS & VERT_COORD_TYPE & DATE_LAST_MODIFIED \\
\hline 2358 & $116-F-14$ & & UNKNOWN & \\
\hline 2359 & $116-F-14$ & & UNKNOWN & \\
\hline 2360 & 116-F-14 & & UNKNOWN & \\
\hline 2361 & 116-F-14 & & UNKNOWN & \\
\hline 2362 & $116-F-14$ & & UNKNOWN & \\
\hline 2363 & 116-F-14 & & UNKNOWN & \\
\hline 2364 & 116-F-14 & & UNKNOWN & \\
\hline 2365 & 116-F-14 & & UNKNOWN & \\
\hline 2366 & 116-F-14 & & UNKNOWN & \\
\hline 2367 & 116-F-14 & & UNKNOWN & \\
\hline 2368 & $116-F-14$ & & UNKNOWN & \\
\hline 2369 & $116-F-14$ & & UNKNOWN & \\
\hline 2370 & 116-F-14 & & UNKNOWN & \\
\hline 2371 & 116-F-14 & & UNKNOWN & \\
\hline 2372 & 116-F-14 & & UNKNOWN & \\
\hline 2373 & 116-F-14 & & UNKNOWN & \\
\hline 2374 & 116-F-14 & & UNKNOWN & \\
\hline 2375 & 116-F-14 & & UNKNOWN & \\
\hline 2376 & 116-F-14 & & UNKNOWN & \\
\hline 2377 & 116-F-14 & & UNKNOWN & \\
\hline 2378 & 116-F-14 & & UNKNOWN & \\
\hline 2379 & $116-F-14$ & & UNKNOWN & \\
\hline 2380 & $100-F-19$ & & & \\
\hline 2381 & $100-F-19$ & & & \\
\hline 2382 & 100-F-19 & & & \\
\hline 2383 & 100-F-19 & & & \\
\hline 2384 & 100-F-19 & & & \\
\hline 2385 & 100-F-19 & & & \\
\hline 2386 & 100-F-19 & & & \\
\hline 2387 & 100-F-19 & & & \\
\hline 2388 & 100-F-19 & & & \\
\hline 2389 & 100-F-19 & & & \\
\hline 2390 & 100-F-19 & & & \\
\hline 2391 & 100-F-19 & & & \\
\hline 2392 & $100-F-19$ & & & \\
\hline 2393 & $100-F-19$ & & & \\
\hline 2394 & 100-F-19 & & & \\
\hline 2395 & 100-F-19 & & & \\
\hline
\end{tabular}




\begin{tabular}{|c|c|c|c|c|}
\hline & $\mathrm{O}$ & $P$ & $\mathrm{Q}$ & $R$ \\
\hline 1 & SITE CODE & ELEVATION UNITS & VERT COORD TYPE & DATE LAST MODIFIED \\
\hline 2396 & $100-F-19$ & & & \\
\hline 2397 & 100-F-19 & & & \\
\hline 2398 & $100-F-19$ & & & \\
\hline 2399 & $100-F-19$ & & & \\
\hline 2400 & $100-F-19$ & & UNKNOWN & \\
\hline 2401 & 100-F-19 & & UNKNOWN & \\
\hline 2402 & 100-F-19 & & UNKNOWN & \\
\hline 2403 & $116-F-14$ & & & \\
\hline 2404 & 100-F-19 & & UNKNOWN & \\
\hline 2405 & $100-F-19$ & & UNKNOWN & \\
\hline 2406 & 100-F-19 & & UNKNOWN & \\
\hline 2407 & $100-F-19$ & & UNKNOWN & \\
\hline 2408 & 100-F-19 & & UNKNOWN & \\
\hline 2409 & $100-F-19$ & & UNKNOWN & \\
\hline 2410 & $100-F-19$ & & UNKNOWN & \\
\hline 2411 & $100-F-19$ & & UNKNOWN & \\
\hline 2412 & 100-F-19 & & UNKNOWN & \\
\hline 2413 & $100-F-19$ & & UNKNOWN & \\
\hline 2414 & $100-F-19$ & & UNKNOWN & \\
\hline 2415 & 100-F-19 & & UNKNOWN & \\
\hline 2416 & $100-F-19$ & & UNKNOWN & \\
\hline 2417 & $100-F-19$ & & UNKNOWN & \\
\hline 2418 & $116-F-14$ & & & \\
\hline 2419 & 116-F-14 & & & \\
\hline 2420 & $116-F-14$ & & & \\
\hline 2421 & 116-F-14 & & & \\
\hline 2422 & $116-F-14$ & & & \\
\hline 2423 & $116-F-14$ & & & \\
\hline 2424 & $116-F-14$ & & & \\
\hline 2425 & $116-F-14$ & & & \\
\hline 2426 & 116-F-14 & & & \\
\hline 2427 & 116-F-14 & & & \\
\hline 2428 & 116-F-14 & & & \\
\hline 2429 & $116-F-14$ & & & \\
\hline 2430 & $116-F-14$ & & & \\
\hline 2431 & $100-F-19$ & & UNKNOWN & \\
\hline 2432 & $116-F-14$ & & & \\
\hline 2433 & 116-F-14 & & & \\
\hline
\end{tabular}




\begin{tabular}{|c|c|c|c|c|}
\hline & $\mathrm{O}$ & $P$ & $\mathrm{Q}$ & $\mathrm{R}$ \\
\hline 1 & SITE_CODE & ELEVATION_UNITS & VERT_COORD_TYPE & DATE_LAST_MODIFIED \\
\hline 2434 & $116-F-14$ & & & \\
\hline 2435 & 116-F-14 & & & \\
\hline 2436 & $100-F-19$ & & UNKNOWN & \\
\hline 2437 & $100-F-19$ & & UNKNOWN & \\
\hline 2438 & $100-F-19$ & & UNKNOWN & \\
\hline 2439 & 100-F-19 & & UNKNOWN & \\
\hline 2440 & $100-F-19$ & & UNKNOWN & \\
\hline 2441 & 100-F-19 & & UNKNOWN & \\
\hline 2442 & 100-F-19 & & UNKNOWN & \\
\hline 2443 & $100-F-19$ & & UNKNOWN & \\
\hline 2444 & $100-F-19$ & & UNKNOWN & \\
\hline 2445 & $100-F-19$ & & UNKNOWN & \\
\hline 2446 & 100-F-19 & & UNKNOWN & \\
\hline 2447 & $100-F-19$ & & UNKNOWN & \\
\hline 2448 & 100-F-19 & & UNKNOWN & \\
\hline 2449 & 100-F-19 & & UNKNOWN & \\
\hline 2450 & $100-F-19$ & & UNKNOWN & \\
\hline 2451 & $100-F-19$ & & UNKNOWN & \\
\hline 2452 & $100-F-19$ & & UNKNOWN & \\
\hline 2453 & 100-F-19 & & UNKNOWN & \\
\hline 2454 & 100-F-19 & & UNKNOWN & \\
\hline 2455 & $100-F-19$ & & UNKNOWN & \\
\hline 2456 & $100-F-19$ & & UNKNOWN & \\
\hline 2457 & 100-F-19 & & UNKNOWN & \\
\hline 2458 & $100-F-19$ & & UNKNOWN & \\
\hline 2459 & $100-F-19$ & & UNKNOWN & \\
\hline 2460 & & & & \\
\hline 2461 & $100-F-19$ & & UNKNOWN & \\
\hline 2462 & & & & \\
\hline 2463 & & & & \\
\hline 2464 & & & & \\
\hline 2465 & & & & \\
\hline 2466 & $100-F-19$ & & UNKNOWN & \\
\hline 2467 & $100-F-19$ & & UNKNOWN & \\
\hline 2468 & $100-F-19$ & & UNKNOWN & \\
\hline 2469 & $100-F-19$ & & UNKNOWN & \\
\hline 2470 & 100-F-19 & & UNKNOWN & \\
\hline 2471 & $100-C-6$ & & UNKNOWN & \\
\hline
\end{tabular}




\begin{tabular}{|c|c|c|c|c|}
\hline & $\mathrm{O}$ & $P$ & $\mathrm{Q}$ & $R$ \\
\hline 1 & SITE CODE & ELEVATION UNITS & VERT COORD TYPE & DATE LAST MODIFIED \\
\hline 2472 & $100-C-6$ & & UNKNOWN & \\
\hline 2473 & $100-F-19$ & & UNKNOWN & \\
\hline 2474 & $116-\mathrm{N}-3$ & $\mathrm{~m}$ & NAVD88 & \\
\hline 2475 & $116-\mathrm{N}-3$ & $\mathrm{~m}$ & NAVD88 & \\
\hline 2476 & $100-F-19$ & & UNKNOWN & \\
\hline 2477 & $100-F-19$ & & UNKNOWN & \\
\hline 2478 & $100-F-19$ & & UNKNOWN & \\
\hline \multicolumn{5}{|l|}{2479} \\
\hline 2480 & 100-F-19 & & UNKNOWN & \\
\hline 2481 & 100-F-19 & & UNKNOWN & \\
\hline 2482 & 100-F-19 & & UNKNOWN & \\
\hline 2483 & $100-F-19$ & & UNKNOWN & \\
\hline 2484 & 100-F-19 & & UNKNOWN & \\
\hline 2485 & 100-F-19 & & UNKNOWN & \\
\hline \multicolumn{5}{|c|}{\begin{tabular}{|l|}
2486 \\
\end{tabular}} \\
\hline 2487 & 100-F-19 & & UNKNOWN & \\
\hline 2488 & 100-F-19 & & UNKNOWN & \\
\hline 2489 & $100-F-19$ & & UNKNOWN & \\
\hline 2490 & $100-F-19$ & & UNKNOWN & \\
\hline 2491 & 100-F-19 & & UNKNOWN & \\
\hline 2492 & $100-F-19$ & & UNKNOWN & \\
\hline 2493 & 100-F-19 & & UNKNOWN & \\
\hline 2494 & 100-F-19 & & UNKNOWN & \\
\hline 2495 & $100-F-19$ & & UNKNOWN & \\
\hline 2496 & 100-F-19 & & UNKNOWN & \\
\hline 2497 & $100-F-19$ & & UNKNOWN & \\
\hline 2498 & $100-F-19$ & & UNKNOWN & \\
\hline 2499 & 100-F-19 & & UNKNOWN & \\
\hline 2500 & 100-F-19 & & UNKNOWN & \\
\hline 2501 & 100-F-19 & & UNKNOWN & \\
\hline 2502 & 100-F-19 & & UNKNOWN & \\
\hline 2503 & 100-F-19 & & UNKNOWN & \\
\hline 2504 & $100-F-19$ & & UNKNOWN & \\
\hline 2505 & 100-F-19 & & UNKNOWN & \\
\hline 2506 & 100-F-19 & & UNKNOWN & \\
\hline 2507 & 100-F-19 & & UNKNOWN & \\
\hline 2508 & $100-F-19$ & & UNKNOWN & \\
\hline 2509 & 100-F-19 & & UNKNOWN & \\
\hline
\end{tabular}




\begin{tabular}{|c|c|c|c|c|}
\hline & $\mathrm{O}$ & $P$ & $\mathrm{Q}$ & $R$ \\
\hline 1 & SITE CODE & ELEVATION UNITS & VERT COORD TYPE & DATE LAST MODIFIED \\
\hline 2510 & $100-F-19$ & & UNKNOWN & \\
\hline 2511 & $100-F-19$ & & UNKNOWN & \\
\hline 2512 & $100-F-19$ & & UNKNOWN & \\
\hline 2513 & 100-F-19 & & UNKNOWN & \\
\hline 2514 & $100-F-19$ & & UNKNOWN & \\
\hline 2515 & $100-F-19$ & & UNKNOWN & \\
\hline 2516 & $100-F-19$ & & UNKNOWN & \\
\hline 2517 & $100-F-19$ & & UNKNOWN & \\
\hline 2518 & $100-F-19$ & & UNKNOWN & \\
\hline 2519 & 100-F-19 & & UNKNOWN & \\
\hline 2520 & $100-F-19$ & & UNKNOWN & \\
\hline 2521 & $100-F-19$ & & UNKNOWN & \\
\hline 2522 & $100-F-19$ & & UNKNOWN & \\
\hline 2523 & $100-F-19$ & & UNKNOWN & \\
\hline 2524 & $100-F-19$ & & UNKNOWN & \\
\hline 2525 & $100-F-19$ & & UNKNOWN & \\
\hline 2526 & $100-F-19$ & & UNKNOWN & \\
\hline 2527 & $100-F-19$ & & UNKNOWN & \\
\hline 2528 & $100-F-19$ & & UNKNOWN & \\
\hline 2529 & $100-F-19$ & & UNKNOWN & \\
\hline 2530 & $100-F-19$ & & UNKNOWN & \\
\hline 2531 & $100-F-19$ & & UNKNOWN & \\
\hline 2532 & $100-F-19$ & & UNKNOWN & \\
\hline 2533 & $100-F-19$ & & UNKNOWN & \\
\hline 2534 & $100-F-19$ & & UNKNOWN & \\
\hline 2535 & $100-F-19$ & & UNKNOWN & \\
\hline 2536 & $100-F-19$ & & UNKNOWN & \\
\hline 2537 & $100-F-19$ & & UNKNOWN & \\
\hline 2538 & $100-F-19$ & & UNKNOWN & \\
\hline 2539 & $100-F-19$ & & UNKNOWN & \\
\hline 2540 & $100-F-19$ & & UNKNOWN & \\
\hline 2541 & $100-F-19$ & & UNKNOWN & \\
\hline 2542 & $100-F-19$ & & UNKNOWN & \\
\hline 2543 & $100-F-19$ & & UNKNOWN & \\
\hline 2544 & $100-F-19$ & & UNKNOWN & \\
\hline 2545 & $100-F-19$ & & UNKNOWN & \\
\hline 2546 & $100-F-19$ & & UNKNOWN & \\
\hline 2547 & 100-F-19 & & UNKNOWN & \\
\hline
\end{tabular}




\begin{tabular}{|c|c|c|c|c|}
\hline & $\mathrm{O}$ & $P$ & $\mathrm{Q}$ & $R$ \\
\hline 1 & SITE CODE & ELEVATION UNITS & VERT COORD TYPE & DATE LAST MODIFIED \\
\hline 2548 & $100-F-19$ & & UNKNOWN & \\
\hline 2549 & $100-F-19$ & & UNKNOWN & \\
\hline 2550 & 100-F-19 & & UNKNOWN & \\
\hline 2551 & 132-B-6 & & UNKNOWN & \\
\hline 2552 & 132-B-6 & & UNKNOWN & \\
\hline 2553 & 132-B-6 & & UNKNOWN & \\
\hline 2554 & 132-B-6 & & & \\
\hline 2555 & 100-F-19 & & UNKNOWN & \\
\hline 2556 & 100-F-19 & & UNKNOWN & \\
\hline 2557 & 100-F-19 & & UNKNOWN & \\
\hline 2558 & 100-F-19 & & UNKNOWN & \\
\hline 2559 & $100-F-19$ & & UNKNOWN & \\
\hline 2560 & 100-F-19 & & UNKNOWN & \\
\hline 2561 & 100-F-19 & & UNKNOWN & \\
\hline 2562 & $100-F-19$ & & UNKNOWN & \\
\hline 2563 & 100-F-19 & & UNKNOWN & \\
\hline 2564 & 100-F-19 & & UNKNOWN & \\
\hline 2565 & $100-F-19$ & & UNKNOWN & \\
\hline 2566 & $100-F-19$ & & UNKNOWN & \\
\hline 2567 & 100-F-19 & & UNKNOWN & \\
\hline 2568 & $100-F-19$ & & UNKNOWN & \\
\hline 2569 & $100-F-19$ & & UNKNOWN & \\
\hline 2570 & 100-F-19 & & UNKNOWN & \\
\hline 2571 & $100-F-19$ & & UNKNOWN & \\
\hline 2572 & $100-F-19$ & & UNKNOWN & \\
\hline 2573 & $100-F-19$ & & UNKNOWN & \\
\hline 2574 & $100-F-19$ & & UNKNOWN & \\
\hline 2575 & 100-F-19 & & UNKNOWN & \\
\hline 2576 & 100-F-19 & & & \\
\hline 2577 & 100-F-19 & & & \\
\hline 2578 & 100-F-19 & & & \\
\hline 2579 & 100-F-19 & & & \\
\hline 2580 & $100-F-19$ & & & \\
\hline 2581 & 100-F-19 & & & \\
\hline 2582 & 100-F-19 & & & \\
\hline 2583 & 100-F-19 & & & \\
\hline 2584 & $100-F-19$ & & & \\
\hline 2585 & 100-F-19 & & UNKNOWN & \\
\hline
\end{tabular}




\begin{tabular}{|c|c|c|c|c|}
\hline & $\mathrm{O}$ & $\mathrm{P}$ & $\bar{Q}$ & $\bar{R}$ \\
\hline 1 & SITE_CODE & ELEVATION_UNITS & VERT_COORD_TYPE & DATE_LAST_MODIFIED \\
\hline 2586 & 100-F-19 & & UNKNOWN & \\
\hline 2587 & $100-F-19$ & & UNKNOWN & \\
\hline 2588 & 100-F-19 & & UNKNOWN & \\
\hline 2589 & 100-F-19 & & UNKNOWN & \\
\hline 2590 & $100-F-19$ & & UNKNOWN & \\
\hline 2591 & 100-F-19 & & UNKNOWN & \\
\hline 2592 & 100-F-19 & & UNKNOWN & \\
\hline 2593 & 100-F-19 & & UNKNOWN & \\
\hline 2594 & 100-F-19 & & UNKNOWN & \\
\hline 2595 & 100-F-19 & & UNKNOWN & \\
\hline 2596 & $100-F-19$ & & UNKNOWN & \\
\hline 2597 & $100-F-19$ & & UNKNOWN & \\
\hline 2598 & 100-F-19 & & UNKNOWN & \\
\hline 2599 & 100-F-19 & & UNKNOWN & \\
\hline 2600 & 100-F-19 & & UNKNOWN & \\
\hline 2601 & 100-F-19 & & UNKNOWN & \\
\hline 2602 & 100-F-19 & & UNKNOWN & \\
\hline 2603 & 100-F-19 & & UNKNOWN & \\
\hline 2604 & 100-F-19 & & UNKNOWN & \\
\hline 2605 & 100-F-19 & & UNKNOWN & \\
\hline 2606 & 100-F-19 & & UNKNOWN & \\
\hline 2607 & 100-B-8 & & UNKNOWN & \\
\hline 2608 & 116-B-7 & & & \\
\hline 2609 & 100-B-8 & & & \\
\hline 2610 & 100-B-8 & & & \\
\hline 2611 & 100-B-8 & & & \\
\hline 2612 & 132-B-6 & & & \\
\hline 2613 & 100-B-8 & & & \\
\hline 2614 & 116-B-7 & & & \\
\hline 2615 & 132-B-6 & & & \\
\hline 2616 & 132-B-6 & & & \\
\hline 2617 & 132-B-6 & & & \\
\hline 2618 & 100-B-8 & & & \\
\hline 2619 & 100-B-8 & & & \\
\hline 2620 & 100-B-8 & & & \\
\hline 2621 & 100-B-8 & & & \\
\hline 2622 & 100-B-8 & & & \\
\hline 2623 & 100-B-8 & & & \\
\hline
\end{tabular}




\begin{tabular}{|c|c|c|c|c|}
\hline & $\mathrm{O}$ & $P$ & $\mathrm{Q}$ & $\mathrm{R}$ \\
\hline 1 & SITE CODE & ELEVATION UNITS & VERT COORD TYPE & DATE LAST MODIFIED \\
\hline 2624 & 100-B-8 & & & \\
\hline 2625 & 100-B-8 & & & \\
\hline 2626 & 100-B-8 & & & \\
\hline 2627 & 100-B-8 & & & \\
\hline 2628 & 100-B-8 & & & \\
\hline 2629 & 100-B-8 & & & \\
\hline 2630 & 100-B-8 & & & \\
\hline 2631 & 100-B-8 & & & \\
\hline 2632 & $116-\mathrm{N}-3$ & $\mathrm{~m}$ & NAVD88 & \\
\hline 2633 & $116-\mathrm{N}-3$ & $\mathrm{~m}$ & NAVD88 & \\
\hline 2634 & $100-C-6$ & & UNKNOWN & \\
\hline 2635 & $100-C-6$ & & UNKNOWN & \\
\hline 2636 & & & & \\
\hline 2637 & $116-\mathrm{N}-1$ & & & \\
\hline 2638 & $116-\mathrm{N}-1$ & & & \\
\hline 2639 & $116-\mathrm{N}-1$ & & & \\
\hline 2640 & $116-\mathrm{N}-1$ & & & \\
\hline 2641 & $116-\mathrm{N}-1$ & & & \\
\hline 2642 & $116-\mathrm{N}-1$ & & & \\
\hline 2643 & 116-F-14 & & & \\
\hline 2644 & $116-\mathrm{N}-3$ & & & \\
\hline 2645 & $116-\mathrm{N}-3$ & & & \\
\hline 2646 & $116-N-3$ & & & \\
\hline 2647 & $116-\mathrm{N}-3$ & & & \\
\hline 2648 & $116-\mathrm{N}-3$ & $\mathrm{~m}$ & NAVD88 & \\
\hline 2649 & $216-A-29$ & & & 26-Jul-02 \\
\hline 2650 & $216-A-29$ & & & 26-Jul-02 \\
\hline 2651 & 216-A-29 & & & 26-Jul-02 \\
\hline 2652 & $216-A-29$ & & & 26-Jul-02 \\
\hline 2653 & 216-A-29 & & & 26-Jul-02 \\
\hline 2654 & $216-A-29$ & & & 26-Jul-02 \\
\hline 2655 & $216-A-29$ & & & 26-Jul-02 \\
\hline 2656 & $216-A-29$ & & & 26-Jul-02 \\
\hline 2657 & $216-A-29$ & & & 26-Jul-02 \\
\hline 2658 & 216-A-29 & & & 26-Jul-02 \\
\hline 2659 & 216-A-29 & & & 26-Jul-02 \\
\hline 2660 & $216-A-29$ & & & 26-Jul-02 \\
\hline 2661 & 216-A-29 & & & 26-Jul-02 \\
\hline
\end{tabular}




\begin{tabular}{|c|c|c|c|c|}
\hline & $\mathrm{O}$ & $P$ & $\bar{Q}$ & $\bar{R}$ \\
\hline 1 & SITE_CODE & ELEVATION_UNITS & VERT_COORD_TYPE & DATE_LAST_MODIFIED \\
\hline 2662 & 216-A-29 & & & 26-Jul-02 \\
\hline 2663 & 216-B-63 & & & 26-Jul-02 \\
\hline 2664 & 216-B-63 & & & 26-Jul-02 \\
\hline 2665 & 216-A-29 & & & 26-Jul-02 \\
\hline 2666 & $216-A-29$ & & & 26-Jul-02 \\
\hline 2667 & $216-A-29$ & & & 26-Jul-02 \\
\hline 2668 & 216-B-63 & & & 26-Jul-02 \\
\hline 2669 & 216-A-29 & & & 26-Jul-02 \\
\hline 2670 & 216-A-29 & & & 26-Jul-02 \\
\hline 2671 & 216-A-29 & & & 26-Jul-02 \\
\hline 2672 & 216-B-63 & & & 26-Jul-02 \\
\hline 2673 & 126-F-1 & & & \\
\hline 2674 & 126-F-1 & & & \\
\hline 2675 & $126-\mathrm{F}-1$ & & & \\
\hline 2676 & $126-F-1$ & & & \\
\hline 2677 & $126-\mathrm{F}-1$ & & & \\
\hline 2678 & 126-F-1 & & & \\
\hline 2679 & 126-F-1 & & & \\
\hline 2680 & $126-\mathrm{F}-1$ & & & \\
\hline 2681 & 126-F-1 & & & \\
\hline 2682 & 126-F-1 & & & \\
\hline 2683 & $116-\mathrm{F}-9$ & & & \\
\hline 2684 & 100-F-19 & & & \\
\hline 2685 & $116-\mathrm{N}-1$ & $\mathrm{~m}$ & NAVD88 & \\
\hline 2686 & $116-\mathrm{N}-1$ & $\mathrm{~m}$ & NAVD88 & \\
\hline 2687 & $116-\mathrm{N}-1$ & $\mathrm{~m}$ & NAVD88 & \\
\hline 2688 & $116-\mathrm{N}-1$ & $\mathrm{~m}$ & NAVD88 & \\
\hline 2689 & $116-\mathrm{N}-1$ & $\mathrm{~m}$ & NAVD88 & \\
\hline 2690 & $116-\mathrm{N}-1$ & $\mathrm{~m}$ & NAVD88 & \\
\hline 2691 & $116-\mathrm{N}-1$ & $\mathrm{~m}$ & NAVD88 & \\
\hline 2692 & $116-\mathrm{N}-1$ & $\mathrm{~m}$ & NAVD88 & \\
\hline 2693 & $116-\mathrm{N}-1$ & $\mathrm{~m}$ & NAVD88 & \\
\hline 2694 & $116-\mathrm{N}-1$ & $\mathrm{~m}$ & NAVD88 & \\
\hline 2695 & $116-\mathrm{N}-1$ & $\mathrm{~m}$ & NAVD88 & \\
\hline 2696 & $116-\mathrm{N}-1$ & $\mathrm{~m}$ & NAVD88 & \\
\hline 2697 & $116-\mathrm{N}-1$ & $\mathrm{~m}$ & NAVD88 & \\
\hline 2698 & $116-\mathrm{N}-1$ & $\mathrm{~m}$ & NAVD88 & \\
\hline 2699 & $116-\mathrm{N}-1$ & $\mathrm{~m}$ & NAVD88 & \\
\hline
\end{tabular}




\begin{tabular}{|c|l|l|l|l|}
\hline & \multicolumn{1}{|c|}{ O } & \multicolumn{1}{|c|}{$\mathrm{P}$} & \multicolumn{1}{|c|}{$\mathrm{Q}$} & \\
\hline 1 & SITE_CODE & ELEVATION_UNITS & VERT_COORD_TYPE & DATE_LAST_MODIFIED \\
\hline 2700 & $116-\mathrm{N}-1$ & $\mathrm{~m}$ & NAVD88 & \\
\hline 2701 & $116-\mathrm{N}-1$ & $\mathrm{~m}$ & NAVD88 & \\
\hline 2702 & $116-\mathrm{N}-1$ & $\mathrm{~m}$ & NAVD88 & \\
\hline 2703 & $116-\mathrm{N}-1$ & $\mathrm{~m}$ & NAVD88 & \\
\hline 2704 & $116-\mathrm{N}-1$ & $\mathrm{~m}$ & NAVD88 & \\
\hline 2705 & $116-\mathrm{N}-1$ & $\mathrm{~m}$ & NAVD88 & \\
\hline 2706 & $116-\mathrm{N}-1$ & $\mathrm{~m}$ & NAVD88 & \\
\hline 2707 & $116-\mathrm{N}-1$ & $\mathrm{~m}$ & & \\
\hline 2708 & & & & \\
\hline 2709 & & & & \\
\hline 2710 & & & NAVD88 & \\
\hline 2711 & $100-\mathrm{B}-8$ & & NAVD88 & \\
\hline 2712 & $116-\mathrm{N}-1$ & $\mathrm{~m}$ & NAVD88 & \\
\hline 2713 & $116-\mathrm{N}-1$ & $\mathrm{~m}$ & NAVD88 & \\
\hline 2714 & $116-\mathrm{N}-1$ & $\mathrm{~m}$ & NAVD88 & \\
\hline 2715 & $116-\mathrm{N}-1$ & $\mathrm{~m}$ & NAVD88 & \\
\hline 2716 & $116-\mathrm{N}-1$ & $\mathrm{~m}$ & NAVD88 & \\
\hline 2717 & $116-\mathrm{N}-1$ & $\mathrm{~m}$ & NAVD88 & \\
\hline 2718 & $116-\mathrm{N}-1$ & $\mathrm{~m}$ & NAVD88 & \\
\hline 2719 & $116-\mathrm{N}-1$ & $\mathrm{~m}$ & NAVD88 & \\
\hline 2720 & $116-\mathrm{N}-1$ & $\mathrm{~m}$ & NAVD88 & \\
\hline 2721 & $116-\mathrm{N}-1$ & $\mathrm{~m}$ & NAVD88 & \\
\hline 2722 & $116-\mathrm{N}-1$ & $\mathrm{~m}$ & NAVD88 & \\
\hline 2723 & $116-\mathrm{N}-1$ & $\mathrm{~m}$ & UNKNOWN & \\
\hline 2724 & $116-\mathrm{N}-3$ & $\mathrm{~m}$ & UNKNOWN & \\
\hline 2725 & $100-\mathrm{B}-8$ & & UNKNOWN & \\
\hline 2726 & $100-\mathrm{B}-8$ & & UNKNOWN & \\
\hline 2727 & $100-\mathrm{B}-8$ & $\mathrm{~m}$ & NAVD88 & \\
\hline 2728 & $100-\mathrm{B}-8$ & & NAVD88 & \\
\hline 2729 & $116-\mathrm{N}-1$ & $\mathrm{~m}$ & NAVD88 & \\
\hline 2730 & $116-\mathrm{N}-1$ & $\mathrm{~m}$ & NAVD88 & \\
\hline 2731 & $116-\mathrm{N}-1$ & $\mathrm{~m}$ & & \\
\hline 2732 & $116-\mathrm{N}-1$ & $\mathrm{~m}$ & & \\
\hline 2733 & $116-\mathrm{N}-1$ & $\mathrm{~m}$ & & \\
\hline 2734 & $116-\mathrm{N}-1$ & $\mathrm{~m}$ & & \\
\hline 2735 & $116-\mathrm{N}-1$ & $\mathrm{~m}$ & & \\
\hline 2736 & $116-\mathrm{N}-1$ & $\mathrm{~m}-1$ & & \\
\hline 2737 & $116-\mathrm{N}-1$ & $\mathrm{~m}$ & & \\
\hline & & & & \\
\hline
\end{tabular}




\begin{tabular}{|c|c|c|c|c|}
\hline & $\mathrm{O}$ & $P$ & $\mathrm{Q}$ & $\mathrm{R}$ \\
\hline 1 & SITE_CODE & ELEVATION_UNITS & VERT_COORD_TYPE & DATE_LAST_MODIFIED \\
\hline 2738 & $116-\mathrm{N}-1$ & $\mathrm{~m}$ & NAVD88 & \\
\hline 2739 & $116-\mathrm{N}-1$ & $\mathrm{~m}$ & NAVD88 & \\
\hline 2740 & $116-\mathrm{N}-1$ & $\mathrm{~m}$ & NAVD88 & \\
\hline 2741 & $116-\mathrm{N}-3$ & & & \\
\hline 2742 & 132-B-6 & $\mathrm{m}$ & NAVD88 & \\
\hline 2743 & $116-\mathrm{N}-3$ & $\mathrm{~m}$ & NAVD88 & \\
\hline 2744 & 132-B-6 & $\mathrm{m}$ & NAVD88 & \\
\hline 2745 & 132-B-6 & $\mathrm{m}$ & NAVD88 & \\
\hline 2746 & 132-B-6 & $\mathrm{m}$ & NAVD88 & \\
\hline 2747 & 132-B-6 & $\mathrm{m}$ & NAVD88 & \\
\hline 2748 & 132-B-6 & $\mathrm{m}$ & NAVD88 & \\
\hline 2749 & 132-B-6 & $\mathrm{m}$ & NAVD88 & \\
\hline 2750 & 132-B-6 & $\mathrm{m}$ & NAVD88 & \\
\hline 2751 & 132-B-6 & $\mathrm{m}$ & NAVD88 & \\
\hline 2752 & 132-B-6 & $\mathrm{m}$ & NAVD88 & \\
\hline 2753 & 132-B-6 & $\mathrm{m}$ & NAVD88 & \\
\hline 2754 & 132-B-6 & $\mathrm{m}$ & NAVD88 & \\
\hline 2755 & 132-B-6 & $\mathrm{m}$ & NAVD88 & \\
\hline 2756 & 132-B-6 & $\mathrm{m}$ & NAVD88 & \\
\hline 2757 & 132-B-6 & $\mathrm{m}$ & NAVD88 & \\
\hline 2758 & 132-B-6 & $\mathrm{m}$ & NAVD88 & \\
\hline 2759 & 132-B-6 & $\mathrm{m}$ & NAVD88 & \\
\hline 2760 & 132-B-6 & $\mathrm{m}$ & NAVD88 & \\
\hline 2761 & 132-B-6 & $\mathrm{m}$ & NAVD88 & \\
\hline 2762 & 132-B-6 & $\mathrm{m}$ & NAVD88 & \\
\hline 2763 & 132-B-6 & $\mathrm{m}$ & NAVD88 & \\
\hline 2764 & 132-B-6 & $\mathrm{m}$ & NAVD88 & \\
\hline 2765 & 132-B-6 & $\mathrm{m}$ & NAVD88 & \\
\hline 2766 & 132-B-6 & $\mathrm{m}$ & NAVD88 & \\
\hline 2767 & 132-C-2 & $\mathrm{m}$ & NAVD88 & \\
\hline 2768 & 132-C-2 & $\mathrm{m}$ & NAVD88 & \\
\hline 2769 & $132-C-2$ & $\mathrm{~m}$ & NAVD88 & \\
\hline 2770 & 132-C-2 & $\mathrm{m}$ & NAVD88 & \\
\hline 2771 & $132-C-2$ & $\mathrm{~m}$ & NAVD88 & \\
\hline 2772 & 132-C-2 & $\mathrm{m}$ & NAVD88 & \\
\hline 2773 & 132-C-2 & $\mathrm{m}$ & NAVD88 & \\
\hline 2774 & 132-C-2 & $\mathrm{m}$ & NAVD88 & \\
\hline 2775 & $132-C-2$ & $\mathrm{~m}$ & NAVD88 & \\
\hline
\end{tabular}




\begin{tabular}{|c|c|c|c|c|}
\hline & $\mathrm{O}$ & $P$ & $\mathrm{Q}$ & $R$ \\
\hline 1 & SITE CODE & ELEVATION UNITS & VERT COORD TYPE & DATE LAST MODIFIED \\
\hline 2776 & $132-C-2$ & $\mathrm{~m}$ & NAVD88 & \\
\hline 2777 & $132-C-2$ & $\mathrm{~m}$ & NAVD88 & \\
\hline 2778 & $132-C-2$ & $\mathrm{~m}$ & NAVD88 & \\
\hline 2779 & $132-C-2$ & $\mathrm{~m}$ & NAVD88 & \\
\hline 2780 & $132-C-2$ & $\mathrm{~m}$ & NAVD88 & \\
\hline 2781 & $132-C-2$ & $\mathrm{~m}$ & NAVD88 & \\
\hline 2782 & $132-C-2$ & $\mathrm{~m}$ & NAVD88 & \\
\hline 2783 & $132-C-2$ & $\mathrm{~m}$ & NAVD88 & \\
\hline 2784 & $132-C-2$ & $\mathrm{~m}$ & NAVD88 & \\
\hline 2785 & 116-B-7 & $\mathrm{m}$ & NAVD88 & \\
\hline 2786 & 116-B-7 & $\mathrm{m}$ & NAVD88 & \\
\hline 2787 & 116-B-7 & $\mathrm{m}$ & NAVD88 & \\
\hline 2788 & 116-B-7 & $\mathrm{m}$ & NAVD88 & \\
\hline 2789 & 116-B-7 & $\mathrm{m}$ & NAVD88 & \\
\hline 2790 & 132-B-6 & $\mathrm{m}$ & NAVD88 & \\
\hline 2791 & 132-B-6 & $\mathrm{m}$ & NAVD88 & \\
\hline 2792 & 132-B-6 & $\mathrm{m}$ & NAVD88 & \\
\hline 2793 & 132-B-6 & $\mathrm{m}$ & NAVD88 & \\
\hline 2794 & 132-B-6 & $\mathrm{m}$ & NAVD88 & \\
\hline 2795 & 132-B-6 & $\mathrm{m}$ & NAVD88 & \\
\hline 2796 & 132-B-6 & $\mathrm{m}$ & NAVD88 & \\
\hline 2797 & 132-B-6 & $\mathrm{m}$ & NAVD88 & \\
\hline 2798 & 132-B-6 & $\mathrm{m}$ & NAVD88 & \\
\hline 2799 & 132-B-6 & $\mathrm{m}$ & NAVD88 & \\
\hline 2800 & 132-B-6 & $\mathrm{m}$ & NAVD88 & \\
\hline 2801 & 132-B-6 & $\mathrm{m}$ & NAVD88 & \\
\hline 2802 & 132-B-6 & $\mathrm{m}$ & NAVD88 & \\
\hline 2803 & 132-B-6 & $\mathrm{m}$ & NAVD88 & \\
\hline 2804 & 132-B-6 & $\mathrm{m}$ & NAVD88 & \\
\hline 2805 & 132-B-6 & $\mathrm{m}$ & NAVD88 & \\
\hline 2806 & 132-B-6 & $\mathrm{m}$ & NAVD88 & \\
\hline 2807 & 116-B-7 & $\mathrm{m}$ & NAVD88 & \\
\hline 2808 & 116-B-7 & $\mathrm{m}$ & NAVD88 & \\
\hline 2809 & 116-B-7 & $\mathrm{m}$ & NAVD88 & \\
\hline 2810 & 116-B-7 & $\mathrm{m}$ & NAVD88 & \\
\hline 2811 & 116-B-7 & $\mathrm{m}$ & NAVD88 & \\
\hline 2812 & 116-B-7 & $\mathrm{m}$ & NAVD88 & \\
\hline 2813 & 116-B-7 & $\mathrm{m}$ & NAVD88 & \\
\hline
\end{tabular}




\begin{tabular}{|c|c|c|c|c|}
\hline & $\mathrm{O}$ & $P$ & $\mathrm{Q}$ & $R$ \\
\hline 1 & SITE CODE & ELEVATION UNITS & VERT COORD TYPE & DATE LAST MODIFIED \\
\hline 2814 & 116-B-7 & $\mathrm{m}$ & NAVD88 & \\
\hline 2815 & 116-B-7 & $\mathrm{m}$ & NAVD88 & \\
\hline 2816 & 116-B-7 & $\mathrm{m}$ & NAVD88 & \\
\hline 2817 & 116-B-7 & $\mathrm{m}$ & NAVD88 & \\
\hline 2818 & 116-B-7 & $\mathrm{m}$ & NAVD88 & \\
\hline 2819 & 116-B-7 & $\mathrm{m}$ & NAVD88 & \\
\hline 2820 & 116-B-7 & $\mathrm{m}$ & NAVD88 & \\
\hline 2821 & 116-B-7 & $\mathrm{m}$ & NAVD88 & \\
\hline 2822 & 116-B-7 & $\mathrm{m}$ & NAVD88 & \\
\hline 2823 & 116-B-7 & $\mathrm{m}$ & NAVD88 & \\
\hline 2824 & 116-B-7 & $\mathrm{m}$ & NAVD88 & \\
\hline 2825 & 116-B-7 & $\mathrm{m}$ & NAVD88 & \\
\hline 2826 & 116-B-7 & $\mathrm{m}$ & NAVD88 & \\
\hline 2827 & 116-B-7 & $\mathrm{m}$ & NAVD88 & \\
\hline 2828 & 116-B-7 & $\mathrm{m}$ & NAVD88 & \\
\hline 2829 & 116-B-7 & $\mathrm{m}$ & NAVD88 & \\
\hline 2830 & 116-B-7 & $\mathrm{m}$ & NAVD88 & \\
\hline 2831 & 116-B-7 & $\mathrm{m}$ & NAVD88 & \\
\hline 2832 & 116-B-7 & $\mathrm{m}$ & NAVD88 & \\
\hline 2833 & 116-B-7 & $\mathrm{m}$ & NAVD88 & \\
\hline 2834 & 116-B-7 & $\mathrm{m}$ & NAVD88 & \\
\hline 2835 & 116-B-7 & $\mathrm{m}$ & NAVD88 & \\
\hline 2836 & 116-B-7 & $\mathrm{m}$ & NAVD88 & \\
\hline 2837 & 116-B-7 & $\mathrm{m}$ & NAVD88 & \\
\hline 2838 & 116-B-7 & $\mathrm{m}$ & NAVD88 & \\
\hline 2839 & 116-B-7 & $\mathrm{m}$ & NAVD88 & \\
\hline 2840 & $116-F-14$ & & & \\
\hline 2841 & 116-F-14 & & & \\
\hline 2842 & $100-C-6$ & & UNKNOWN & \\
\hline 2843 & 100-C-6 & & UNKNOWN & \\
\hline 2844 & $100-C-6$ & & UNKNOWN & \\
\hline 2845 & 100-C-6 & & UNKNOWN & \\
\hline 2846 & $100-C-6$ & & UNKNOWN & \\
\hline 2847 & 100-B-8 & & UNKNOWN & \\
\hline 2848 & 100-B-8 & & UNKNOWN & \\
\hline 2849 & $116-\mathrm{N}-1$ & $\mathrm{~m}$ & NAVD88 & \\
\hline 2850 & $116-\mathrm{N}-3$ & & NAVD88 & \\
\hline 2851 & $116-\mathrm{N}-1$ & $\mathrm{~m}$ & NAVD88 & \\
\hline
\end{tabular}




\begin{tabular}{|c|l|l|l|l|}
\hline & \multicolumn{1}{|c|}{ O } & \multicolumn{1}{|c|}{$\mathrm{P}$} & \multicolumn{1}{c|}{ Q } & R \\
\hline 1 & SITE_CODE & ELEVATION_UNITS & VERT_COORD_TYPE & DATE_LAST_MODIFIED \\
\hline 2852 & $116-\mathrm{N}-1$ & $\mathrm{~m}$ & NAVD88 & \\
\hline 2853 & $116-\mathrm{F}-14$ & & NAVD88 & \\
\hline 2854 & $116-\mathrm{N}-1$ & $\mathrm{~m}$ & NAVD88 & \\
\hline 2855 & $116-\mathrm{N}-1$ & $\mathrm{~m}$ & NAVD88 & \\
\hline 2856 & $116-\mathrm{N}-1$ & $\mathrm{~m}$ & NAVD88 & \\
\hline 2857 & $116-\mathrm{N}-1$ & $\mathrm{~m}$ & UNKNOWN & \\
\hline 2858 & $116-\mathrm{N}-1$ & $\mathrm{~m}$ & UNKNOWN & \\
\hline 2859 & $116-\mathrm{N}-1$ & $\mathrm{~m}$ & & \\
\hline 2860 & $116-\mathrm{F}-14$ & & & \\
\hline 2861 & $116-\mathrm{F}-14$ & & & \\
\hline 2862 & $116-\mathrm{F}-14$ & & & \\
\hline 2863 & $116-\mathrm{F}-14$ & & & \\
\hline 2864 & $116-\mathrm{F}-14$ & & NAVD88 & \\
\hline 2865 & $116-\mathrm{N}-3$ & & NAVD88 & \\
\hline 2866 & $116-\mathrm{N}-3$ & & NAVD88 & \\
\hline 2867 & $116-\mathrm{N}-1$ & & NAVD88 & \\
\hline 2868 & $116-\mathrm{N}-1$ & & NAVD88 & \\
\hline 2869 & $116-\mathrm{N}-1$ & & NAVD88 & \\
\hline 2870 & $116-\mathrm{N}-1$ & & NAVD88 & \\
\hline 2871 & $116-\mathrm{N}-1$ & & & \\
\hline 2872 & $116-\mathrm{N}-1$ & & & \\
\hline 2873 & & $\mathrm{~m}$ & & \\
\hline 2874 & & $\mathrm{~m}$ & & \\
\hline 2875 & & $\mathrm{~m}$ & & \\
\hline 2876 & & & & \\
\hline 2877 & $100-\mathrm{B}-1$ & & & \\
\hline 2878 & $100-\mathrm{D}-49: 1$ & & & \\
\hline 2879 & $100-\mathrm{D}-55$ & & & \\
\hline 2880 & $100-\mathrm{D}-57$ & & & \\
\hline 2881 & $100-\mathrm{D}-48: 1$ & & & \\
\hline 2882 & & & & \\
\hline 2883 & & & & \\
\hline 2884 & $100-\mathrm{B}-8$ & & & \\
\hline 2885 & $100-\mathrm{B}-8$ & & & \\
\hline 2886 & & & & \\
\hline 2887 & $116-\mathrm{N}-3$ & & & \\
\hline 2888 & & & & \\
\hline 2889 & & & & \\
\hline & & & & \\
\hline
\end{tabular}




\begin{tabular}{|c|c|c|c|c|}
\hline & \multicolumn{1}{|c|}{ O } & P & \multicolumn{1}{c|}{ Q } & R \\
\hline 1 & SITE_CODE & ELEVATION_UNITS & VERT_COORD_TYPE & DATE_LAST_MODIFIED \\
\hline 2890 & & & & \\
\hline 2891 & & & & \\
\hline 2892 & & & & \\
\hline 2893 & & & & \\
\hline 2894 & & & & \\
\hline 2895 & & & & \\
\hline 2896 & & & & \\
\hline 2897 & & & & \\
\hline 2898 & & & & \\
\hline 2899 & & & & \\
\hline 2900 & & & & \\
\hline 2901 & & & & \\
\hline 2902 & & & & \\
\hline 2903 & & & & \\
\hline 2904 & & & & \\
\hline 2905 & & & & \\
\hline 2906 & & & & \\
\hline 2907 & & & & \\
\hline 2908 & & & & \\
\hline 2909 & & & & \\
\hline 2910 & & & & \\
\hline 2911 & & & & \\
\hline 2912 & & & & \\
\hline 2913 & & & & \\
\hline 2914 & & & & \\
\hline 2915 & & & & \\
\hline 2916 & & & & \\
\hline 2917 & & & & \\
\hline 2918 & $132-B-6$ & & & \\
\hline 2919 & $132-B-6$ & & & \\
\hline 2920 & $132-C-2$ & & & \\
\hline 2921 & $132-C-2$ & & & \\
\hline 2922 & $132-B-6$ & & & \\
\hline 2923 & $132-B-6$ & & & \\
\hline 2924 & $132-B-6$ & & & \\
\hline 2925 & UPR-100-F-2 & & & \\
\hline 2926 & UPR-100-F-2 & & & \\
\hline 2927 & UPR-100-F-2 & & & \\
\hline
\end{tabular}




\begin{tabular}{|c|c|c|c|c|}
\hline & $\mathrm{O}$ & $P$ & $\bar{Q}$ & $\bar{R}$ \\
\hline 1 & SITE CODE & ELEVATION UNITS & VERT COORD TYPE & DATE LAST MODIFIED \\
\hline 2928 & UPR-100-F-2 & & & \\
\hline 2929 & UPR-100-F-2 & & & \\
\hline 2930 & UPR-100-F-2 & & & \\
\hline 2931 & UPR-100-F-2 & & & \\
\hline 2932 & UPR-100-F-2 & & & \\
\hline 2933 & UPR-100-F-2 & & & \\
\hline 2934 & UPR-100-F-2 & & & \\
\hline 2935 & UPR-100-F-2 & & & \\
\hline 2936 & UPR-100-F-2 & & & \\
\hline 2937 & UPR-100-F-2 & & & \\
\hline 2938 & UPR-100-F-2 & & & \\
\hline 2939 & $116-F-14$ & & & \\
\hline 2940 & 116-F-14 & & & \\
\hline 2941 & & & & \\
\hline 2942 & & & & \\
\hline 2943 & & & & \\
\hline 2944 & & & & \\
\hline 2945 & & & & \\
\hline 2946 & & & & \\
\hline 2947 & & & & \\
\hline 2948 & & & & \\
\hline 2949 & 100-F-19 & & UNKNOWN & \\
\hline 2950 & 100-F-19 & & UNKNOWN & \\
\hline 2951 & 100-F-19 & & UNKNOWN & \\
\hline 2952 & 100-F-19 & & UNKNOWN & \\
\hline 2953 & $100-F-19$ & & UNKNOWN & \\
\hline 2954 & $100-F-19$ & & UNKNOWN & \\
\hline 2955 & 100-F-19 & & UNKNOWN & \\
\hline 2956 & 100-F-19 & & UNKNOWN & \\
\hline 2957 & $100-F-19$ & & UNKNOWN & \\
\hline 2958 & $100-F-19$ & & UNKNOWN & \\
\hline 2959 & 100-F-19 & & UNKNOWN & \\
\hline 2960 & $100-F-19$ & & UNKNOWN & \\
\hline 2961 & 100-F-19 & & UNKNOWN & \\
\hline 2962 & 100-F-19 & & UNKNOWN & \\
\hline 2963 & $100-F-19$ & & UNKNOWN & \\
\hline 2964 & 100-F-19 & & UNKNOWN & \\
\hline 2965 & $100-F-19$ & & UNKNOWN & \\
\hline
\end{tabular}




\begin{tabular}{|c|l|l|l|l|}
\hline & \multicolumn{1}{|c|}{ O } & \multicolumn{1}{|c|}{ P } & & R \\
\hline 1 & SITE_CODE & ELEVATION_UNITS & VERT_COORD_TYPE & DATE_LAST_MODIFIED \\
\hline 2966 & $100-F-19$ & UNKNOWN & \\
\hline 2967 & $100-F-19$ & UNKNOWN & \\
\hline 2968 & $100-F-19$ & UNKNOWN & \\
\hline 2969 & $100-F-19$ & UNKNOWN & \\
\hline 2970 & $100-F-19$ & UNKNOWN & \\
\hline 2971 & $100-F-19$ & UNKNOWN & \\
\hline 2972 & $100-F-19$ & UNKNOWN & \\
\hline 2973 & $100-F-19$ & UNKNOWN & \\
\hline 2974 & $100-F-19$ & UNKNOWN & \\
\hline 2975 & $100-F-19$ & UNKNOWN & \\
\hline 2976 & $100-F-19$ & UNKNOWN & \\
\hline 2977 & $100-F-19$ & UNKNOWN & \\
\hline 2978 & $100-F-19$ & UNKNOWN & \\
\hline 2979 & $100-F-19$ & UNKNOWN & \\
\hline 2980 & $100-F-19$ & UNKNOWN & \\
\hline 2981 & $100-F-19$ & UNKNOWN & \\
\hline 2982 & $100-F-19$ & UNKNOWN & \\
\hline 2983 & $100-F-19$ & UNKNOWN & \\
\hline 2984 & $100-F-19$ & UNKNOWN & \\
\hline 2985 & $100-F-19$ & UNKNOWN & \\
\hline 2986 & $100-F-19$ & UNKNOWN & \\
\hline 2987 & $100-F-19$ & UNKNOWN & \\
\hline 2988 & $100-F-19$ & UNKNOWN & \\
\hline 2989 & $100-F-19$ & UNKNOWN & \\
\hline 2990 & $100-F-19$ & UNKNOWN & \\
\hline 2991 & $100-F-19$ & UNKNOWN & \\
\hline 2992 & $100-F-19$ & UNKNOWN & \\
\hline 2993 & $100-F-19$ & UNKNOWN & \\
\hline 2994 & $100-F-19$ & UNKNOWN & \\
\hline 2995 & $100-F-19$ & UNKNOWN & \\
\hline 2996 & $100-F-19$ & UNKNOWN & \\
\hline 2997 & $100-F-19$ & UNKNOWN & \\
\hline 2998 & $100-F-19$ & UNKNOWN & \\
\hline 2999 & $100-F-19$ & UNKNOWN & \\
\hline 3000 & $100-F-19$ & UNKNOWN & \\
\hline 3001 & $100-F-19$ & $100-F-19$ & UNKNOWN & \\
\hline 3002 & $100-F-19$ & & & \\
\hline 3003 & & & \\
\hline
\end{tabular}




\begin{tabular}{|c|c|c|c|c|}
\hline & $\mathrm{O}$ & $P$ & $\mathrm{Q}$ & $\mathrm{R}$ \\
\hline 1 & SITE_CODE & ELEVATION_UNITS & VERT_COORD_TYPE & DATE_LAST_MODIFIED \\
\hline 3004 & $100-F-19$ & & UNKNOWN & \\
\hline 3005 & 100-F-19 & & UNKNOWN & \\
\hline 3006 & $100-F-19$ & & UNKNOWN & \\
\hline 3007 & 100-F-19 & & UNKNOWN & \\
\hline 3008 & $100-F-19$ & & UNKNOWN & \\
\hline 3009 & 100-F-19 & & UNKNOWN & \\
\hline 3010 & $100-F-19$ & & UNKNOWN & \\
\hline 3011 & $100-F-19$ & & UNKNOWN & \\
\hline 3012 & $100-F-19$ & & UNKNOWN & \\
\hline 3013 & $100-F-19$ & & & \\
\hline 3014 & $100-F-19$ & & & \\
\hline 3015 & $100-F-19$ & & UNKNOWN & \\
\hline 3016 & 100-F-19 & & UNKNOWN & \\
\hline 3017 & $100-F-19$ & & UNKNOWN & \\
\hline 3018 & 100-F-19 & & UNKNOWN & \\
\hline 3019 & $100-F-19$ & & UNKNOWN & \\
\hline 3020 & $100-F-19$ & & UNKNOWN & \\
\hline 3021 & $100-F-19$ & & UNKNOWN & \\
\hline 3022 & $100-F-19$ & & UNKNOWN & \\
\hline 3023 & 100-F-19 & & UNKNOWN & \\
\hline 3024 & 100-F-19 & & UNKNOWN & \\
\hline 3025 & $100-F-19$ & & UNKNOWN & \\
\hline 3026 & $100-F-19$ & & UNKNOWN & \\
\hline 3027 & $100-F-19$ & & UNKNOWN & \\
\hline 3028 & $100-F-19$ & & UNKNOWN & \\
\hline 3029 & $100-F-19$ & & UNKNOWN & \\
\hline 3030 & 100-F-19 & & UNKNOWN & \\
\hline 3031 & $100-F-19$ & & UNKNOWN & \\
\hline 3032 & $100-F-19$ & & UNKNOWN & \\
\hline 3033 & $100-F-19$ & & UNKNOWN & \\
\hline 3034 & 100-F-19 & & UNKNOWN & \\
\hline 3035 & $100-F-19$ & & UNKNOWN & \\
\hline 3036 & $100-F-19$ & & UNKNOWN & \\
\hline 3037 & $100-F-19$ & & UNKNOWN & \\
\hline 3038 & $100-F-19$ & & UNKNOWN & \\
\hline 3039 & $100-F-19$ & & UNKNOWN & \\
\hline 3040 & 100-F-19 & & UNKNOWN & \\
\hline 3041 & 100-F-19 & & UNKNOWN & \\
\hline
\end{tabular}




\begin{tabular}{|c|l|l|l|l|}
\hline & \multicolumn{1}{|c|}{ O } & \multicolumn{1}{|c|}{ P } & & R \\
\hline 1 & SITE_CODE & ELEVATION_UNITS & VERT_COORD_TYPE & DATE_LAST_MODIFIED \\
\hline 3042 & $100-\mathrm{F}-19$ & UNKNOWN & \\
\hline 3043 & $100-\mathrm{F}-19$ & UNKNOWN & \\
\hline 3044 & $100-\mathrm{F}-19$ & UNKNOWN & \\
\hline 3045 & $116-\mathrm{N}-3$ & UNKNOWN & \\
\hline 3046 & $116-\mathrm{N}-3$ & UNKNOWN & \\
\hline 3047 & $116-\mathrm{N}-3$ & UNKNOWN & \\
\hline 3048 & $116-\mathrm{N}-3$ & UNKNOWN & \\
\hline 3049 & $116-\mathrm{N}-3$ & UNKNOWN & \\
\hline 3050 & $116-\mathrm{N}-3$ & UNKNOWN & \\
\hline 3051 & $116-\mathrm{N}-3$ & UNKNOWN & \\
\hline 3052 & $116-\mathrm{N}-3$ & UNKNOWN & \\
\hline 3053 & $116-\mathrm{N}-3$ & UNKNOWN & \\
\hline 3054 & $116-\mathrm{N}-3$ & UNKNOWN & \\
\hline 3055 & $116-\mathrm{N}-3$ & UNKNOWN & \\
\hline 3056 & $116-\mathrm{N}-3$ & UNKNOWN & \\
\hline 3057 & $116-\mathrm{N}-3$ & UNKNOWN & \\
\hline 3058 & $116-\mathrm{N}-3$ & UNKNOWN & \\
\hline 3059 & $116-\mathrm{N}-3$ & UNKNOWN & \\
\hline 3060 & $116-\mathrm{N}-3$ & UNKNOWN & \\
\hline 3061 & $116-\mathrm{N}-3$ & UNKNOWN & \\
\hline 3062 & $116-\mathrm{N}-3$ & UNKNOWN & \\
\hline 3063 & $116-\mathrm{N}-3$ & UNKNOWN & \\
\hline 3064 & $116-\mathrm{N}-3$ & UNKNOWN & \\
\hline 3065 & $116-\mathrm{N}-3$ & UNKNOWN & \\
\hline 3066 & $116-\mathrm{N}-3$ & UNKNOWN & \\
\hline 3067 & $116-\mathrm{N}-3$ & UNKNOWN & \\
\hline 3068 & $116-\mathrm{N}-3$ & UNKNOWN & \\
\hline 3069 & $116-\mathrm{N}-3$ & UNKNOWN & \\
\hline 3070 & $116-\mathrm{N}-3$ & UNKNOWN & \\
\hline 3071 & $116-\mathrm{N}-3$ & UNKNOWN & \\
\hline 3072 & $116-\mathrm{N}-3$ & UNKNOWN & \\
\hline 3073 & $116-\mathrm{N}-3$ & UNKNOWN & \\
\hline 3074 & $116-\mathrm{N}-3$ & UNKNOWN & \\
\hline 3075 & $116-\mathrm{N}-3$ & UNKNOWN & \\
\hline 3076 & $116-\mathrm{N}-3$ & $116-\mathrm{N}-3$ & UNKNOWN & \\
\hline 3077 & $116-\mathrm{N}-3$ & & \\
\hline 3078 & & & \\
\hline 3079 & $116-\mathrm{N}-3$ & & & \\
\hline & & & & \\
\hline
\end{tabular}




\begin{tabular}{|c|l|l|l|l|}
\hline & \multicolumn{1}{|c|}{ O } & \multicolumn{1}{|c|}{ P } & \multicolumn{1}{|c|}{ R } & \\
\hline 1 & SITE_CODE & ELEVATION_UNITS & VERT_COORD_TYPE & DATE_LAST_MODIFIED \\
\hline 3080 & $116-N-3$ & UNKNOWN & \\
\hline 3081 & $116-\mathrm{N}-3$ & & UNKNOWN & \\
\hline 3082 & $116-\mathrm{N}-3$ & UNKNOWN & \\
\hline 3083 & $116-\mathrm{N}-3$ & UNKNOWN & \\
\hline 3084 & $116-\mathrm{N}-3$ & UNKNOWN & \\
\hline 3085 & $116-\mathrm{N}-3$ & & UNKNOWN & \\
\hline 3086 & $116-\mathrm{N}-3$ & UNKNOWN & \\
\hline 3087 & $116-\mathrm{N}-3$ & UNKNOWN & \\
\hline 3088 & $116-\mathrm{N}-3$ & & UNKNOWN & \\
\hline 3089 & $100-\mathrm{F}-19$ & & UNKNOWN & \\
\hline 3090 & $100-\mathrm{F}-19$ & & UNKNOWN & \\
\hline 3091 & $100-\mathrm{F}-19$ & & UNKNOWN & \\
\hline 3092 & $100-\mathrm{F}-19$ & & & \\
\hline 3093 & $100-\mathrm{B}-8$ & & & \\
\hline 3094 & $100-\mathrm{B}-8$ & & & \\
\hline 3095 & $100-\mathrm{B}-8$ & & & \\
\hline 3096 & $116-\mathrm{B}-7$ & & & \\
\hline 3097 & $132-\mathrm{B}-6$ & & & \\
\hline 3098 & $100-\mathrm{B}-8$ & & & \\
\hline 3099 & $100-\mathrm{B}-14$ & & & \\
\hline 3100 & $100-\mathrm{B}-8$ & & & \\
\hline 3101 & $100-\mathrm{B}-8$ & & & \\
\hline 3102 & $100-\mathrm{B}-8$ & & & \\
\hline 3103 & $100-\mathrm{B}-8$ & & & \\
\hline 3104 & $132-\mathrm{B}-6$ & & & \\
\hline 3105 & $132-\mathrm{B}-6$ & & & \\
\hline 3106 & $132-\mathrm{B}-6$ & & & \\
\hline 3107 & $132-\mathrm{B}-6$ & & & \\
\hline 3108 & $132-\mathrm{B}-6$ & & & \\
\hline 3109 & $100-\mathrm{B}-8$ & & & \\
\hline 3110 & $100-\mathrm{B}-8$ & & & \\
\hline 3111 & $100-\mathrm{B}-8$ & & & \\
\hline 3112 & $100-\mathrm{B}-8$ & & & \\
\hline 3113 & $100-\mathrm{B}-8$ & & & \\
\hline 3114 & $100-\mathrm{B}-8$ & & & \\
\hline 3115 & $100-\mathrm{B}-8$ & & & \\
\hline 3116 & $100-\mathrm{B}-8$ & & & \\
\hline 3117 & $100-\mathrm{B}-8$ & & & \\
\hline
\end{tabular}




\begin{tabular}{|c|c|c|c|c|}
\hline & $\mathrm{O}$ & $P$ & $\mathrm{Q}$ & $\mathrm{R}$ \\
\hline 1 & SITE_CODE & ELEVATION_UNITS & |VERT_COORD_TYPE & DATE_LAST_MODIFIED \\
\hline 3118 & 100-B-8 & & & \\
\hline 3119 & & & & \\
\hline 3120 & & & & \\
\hline 3121 & & & & \\
\hline 3122 & & & & \\
\hline 3123 & & & & \\
\hline 3124 & & & & \\
\hline 3125 & & & & \\
\hline 3126 & & & & \\
\hline 3127 & & & & \\
\hline 3128 & & & & \\
\hline 3129 & & & & \\
\hline 3130 & & & & \\
\hline 3131 & & & & \\
\hline 3132 & & & & \\
\hline 3133 & & & & \\
\hline 3134 & & & & \\
\hline 3135 & & & & \\
\hline 3136 & & & & \\
\hline 3137 & & & & \\
\hline 3138 & & & & \\
\hline 3139 & & & & \\
\hline 3140 & & & & \\
\hline 3141 & & & & \\
\hline 3142 & & & & \\
\hline 3143 & $116-F-14$ & & UNKNOWN & \\
\hline 3144 & $116-F-14$ & & UNKNOWN & \\
\hline 3145 & $116-F-14$ & & UNKNOWN & \\
\hline 3146 & $116-F-14$ & & UNKNOWN & \\
\hline 3147 & $116-F-14$ & & UNKNOWN & \\
\hline 3148 & $116-F-14$ & & UNKNOWN & \\
\hline 3149 & $116-F-14$ & & UNKNOWN & \\
\hline 3150 & $116-F-14$ & & UNKNOWN & \\
\hline 3151 & $116-F-14$ & & UNKNOWN & \\
\hline 3152 & $116-F-14$ & & UNKNOWN & \\
\hline 3153 & $116-\mathrm{N}-1$ & & NAVD88 & \\
\hline 3154 & $116-\mathrm{N}-3$ & $\mathrm{~m}$ & NAVD88 & \\
\hline 3155 & $116-N-3$ & $\mathrm{~m}$ & NAVD88 & \\
\hline
\end{tabular}




\begin{tabular}{|c|c|c|c|c|}
\hline & $\mathrm{O}$ & $P$ & $\mathrm{Q}$ & $\mathrm{R}$ \\
\hline 1 & SITE_CODE & ELEVATION_UNITS & VERT_COORD_TYPE & DATE_LAST_MODIFIED \\
\hline 3156 & $116-\mathrm{N}-3$ & $\mathrm{~m}$ & NAVD88 & \\
\hline 3157 & $116-\mathrm{N}-3$ & $\mathrm{~m}$ & NAVD88 & \\
\hline 3158 & $116-\mathrm{N}-3$ & & & \\
\hline 3159 & $116-\mathrm{N}-3$ & & UNKNOWN & \\
\hline 3160 & $116-\mathrm{N}-3$ & & UNKNOWN & \\
\hline 3161 & $116-\mathrm{N}-3$ & & UNKNOWN & \\
\hline 3162 & $116-\mathrm{N}-3$ & & UNKNOWN & \\
\hline 3163 & $116-\mathrm{N}-3$ & & UNKNOWN & \\
\hline 3164 & $116-\mathrm{N}-3$ & & UNKNOWN & \\
\hline 3165 & $116-\mathrm{N}-3$ & & UNKNOWN & \\
\hline 3166 & $116-N-3$ & & UNKNOWN & \\
\hline 3167 & $116-\mathrm{N}-3$ & & UNKNOWN & \\
\hline 3168 & $116-\mathrm{N}-3$ & & UNKNOWN & \\
\hline 3169 & $116-\mathrm{N}-3$ & & & \\
\hline 3170 & $116-\mathrm{N}-3$ & $\mathrm{~m}$ & NAVD88 & \\
\hline 3171 & $116-\mathrm{N}-3$ & $\mathrm{~m}$ & NAVD88 & \\
\hline 3172 & $116-N-3$ & $\mathrm{~m}$ & NAVD88 & \\
\hline 3173 & $116-\mathrm{N}-3$ & $\mathrm{~m}$ & NAVD88 & \\
\hline 3174 & $116-\mathrm{N}-3$ & $\mathrm{~m}$ & UNKNOWN & \\
\hline 3175 & $116-\mathrm{N}-3$ & $\mathrm{~m}$ & UNKNOWN & \\
\hline 3176 & $116-\mathrm{N}-3$ & $\mathrm{~m}$ & UNKNOWN & \\
\hline 3177 & $116-\mathrm{N}-3$ & $\mathrm{~m}$ & UNKNOWN & \\
\hline 3178 & $116-N-3$ & $\mathrm{~m}$ & UNKNOWN & \\
\hline 3179 & $116-N-3$ & $\mathrm{~m}$ & UNKNOWN & \\
\hline 3180 & $116-\mathrm{N}-3$ & $\mathrm{~m}$ & UNKNOWN & \\
\hline 3181 & $116-\mathrm{N}-3$ & $\mathrm{~m}$ & UNKNOWN & \\
\hline 3182 & $116-\mathrm{N}-3$ & $\mathrm{~m}$ & UNKNOWN & \\
\hline 3183 & $116-\mathrm{N}-3$ & $\mathrm{~m}$ & UNKNOWN & \\
\hline 3184 & $116-N-3$ & $\mathrm{~m}$ & UNKNOWN & \\
\hline 3185 & $116-N-3$ & $\mathrm{~m}$ & NAVD88 & \\
\hline 3186 & $116-N-3$ & $\mathrm{~m}$ & NAVD88 & \\
\hline 3187 & $116-\mathrm{N}-3$ & $\mathrm{~m}$ & NAVD88 & \\
\hline 3188 & $116-\mathrm{N}-3$ & $\mathrm{~m}$ & NAVD88 & \\
\hline 3189 & $116-\mathrm{N}-3$ & $\mathrm{~m}$ & NAVD88 & \\
\hline 3190 & $116-\mathrm{N}-3$ & $\mathrm{~m}$ & NAVD88 & \\
\hline 3191 & $116-N-3$ & $\mathrm{~m}$ & NAVD88 & \\
\hline 3192 & $116-N-3$ & $\mathrm{~m}$ & NAVD88 & \\
\hline 3193 & $116-N-3$ & $\mathrm{~m}$ & NAVD88 & \\
\hline
\end{tabular}




\begin{tabular}{|c|l|l|l|l|}
\hline & \multicolumn{1}{|c|}{ O } & \multicolumn{1}{|c|}{$\mathrm{P}$} & \multicolumn{1}{c|}{ Q } & \\
\hline 1 & SITE_CODE & ELEVATION_UNITS & VERT_COORD_TYPE & DATE_LAST_MODIFIED \\
\hline 3194 & $116-\mathrm{N}-3$ & $\mathrm{~m}$ & NAVD88 & \\
\hline 3195 & $116-\mathrm{N}-3$ & $\mathrm{~m}$ & NAVD88 & \\
\hline 3196 & $116-\mathrm{N}-3$ & $\mathrm{~m}$ & NAVD88 & \\
\hline 3197 & $116-\mathrm{N}-3$ & $\mathrm{~m}$ & NAVD88 & \\
\hline 3198 & $116-\mathrm{N}-3$ & $\mathrm{~m}$ & NAVD88 & \\
\hline 3199 & $116-\mathrm{N}-3$ & $\mathrm{~m}$ & & \\
\hline 3200 & $126-\mathrm{F}-1$ & & & \\
\hline 3201 & $100-\mathrm{F}-2$ & & & \\
\hline 3202 & $116-\mathrm{F}-2$ & & & \\
\hline 3203 & $126-\mathrm{F}-1$ & & & \\
\hline 3204 & $126-\mathrm{F}-1$ & & & \\
\hline 3205 & $126-\mathrm{F}-1$ & & & \\
\hline 3206 & $126-\mathrm{F}-1$ & & & \\
\hline 3207 & $126-\mathrm{F}-1$ & & & \\
\hline 3208 & & & & \\
\hline 3209 & $132-\mathrm{B}-6$ & & & \\
\hline 3210 & $132-\mathrm{B}-6$ & & & \\
\hline 3211 & $132-\mathrm{B}-6$ & & & \\
\hline 3212 & $132-\mathrm{B}-6$ & & & \\
\hline 3213 & $116-\mathrm{B}-7$ & & & \\
\hline 3214 & $116-\mathrm{B}-7$ & & & \\
\hline 3215 & $116-\mathrm{B}-7$ & & & \\
\hline 3216 & $116-\mathrm{B}-7$ & & & \\
\hline 3217 & $116-\mathrm{B}-7$ & & & \\
\hline 3218 & $116-\mathrm{B}-7$ & & & \\
\hline 3219 & $132-\mathrm{B}-6$ & & & \\
\hline 3220 & $132-\mathrm{B}-6$ & & & \\
\hline 3221 & $132-\mathrm{B}-6$ & & & \\
\hline 3222 & $132-\mathrm{B}-6$ & & & \\
\hline 3223 & $132-\mathrm{B}-6$ & & & \\
\hline 3224 & $132-\mathrm{B}-6$ & & & \\
\hline 3225 & $132-\mathrm{B}-6$ & & & \\
\hline 3226 & $132-\mathrm{C}-2$ & & & \\
\hline 3227 & $132-\mathrm{C}-2$ & & & \\
\hline 3228 & $132-\mathrm{C}-2$ & & & \\
\hline 3229 & $116-\mathrm{B}-7$ & & & \\
\hline 3230 & $132-\mathrm{B}-6$ & & & \\
\hline 3231 & $116-\mathrm{B}-7$ & & & \\
\hline
\end{tabular}




\begin{tabular}{|c|c|c|c|c|}
\hline & $\mathrm{O}$ & $P$ & $\mathrm{Q}$ & $\mathrm{R}$ \\
\hline 1 & SITE_CODE & ELEVATION_UNITS & VERT_COORD_TYPE & DATE_LAST_MODIFIED \\
\hline 3232 & $132-B-6$ & & & \\
\hline 3233 & $132-C-2$ & & & \\
\hline 3234 & $116-\mathrm{B}-7$ & & & \\
\hline 3235 & $116-B-7$ & & & \\
\hline 3236 & $116-F-14$ & & & \\
\hline 3237 & $116-F-14$ & & & \\
\hline 3238 & $116-F-14$ & & & \\
\hline 3239 & $116-F-14$ & & & \\
\hline 3240 & $116-F-14$ & & & \\
\hline 3241 & $116-F-14$ & & & \\
\hline 3242 & $116-F-14$ & & & \\
\hline 3243 & $116-F-14$ & & & \\
\hline 3244 & $116-F-14$ & & & \\
\hline 3245 & $116-F-14$ & & & \\
\hline 3246 & $116-F-14$ & & & \\
\hline 3247 & $116-F-14$ & & & \\
\hline 3248 & $116-F-14$ & & & \\
\hline 3249 & $116-F-14$ & & & \\
\hline 3250 & $116-F-14$ & & & \\
\hline 3251 & $116-N-3$ & $\mathrm{~m}$ & NAVD88 & \\
\hline 3252 & $116-\mathrm{N}-3$ & $\mathrm{~m}$ & NAVD88 & \\
\hline 3253 & $116-\mathrm{N}-3$ & $\mathrm{~m}$ & NAVD88 & \\
\hline 3254 & $116-\mathrm{N}-3$ & $\mathrm{~m}$ & NAVD88 & \\
\hline 3255 & $116-\mathrm{N}-3$ & $\mathrm{~m}$ & NAVD88 & \\
\hline 3256 & $116-\mathrm{N}-3$ & $\mathrm{~m}$ & NAVD88 & \\
\hline 3257 & $116-\mathrm{N}-3$ & $\mathrm{~m}$ & NAVD88 & \\
\hline 3258 & $116-\mathrm{N}-3$ & $\mathrm{~m}$ & NAVD88 & \\
\hline 3259 & $116-\mathrm{N}-3$ & $\mathrm{~m}$ & NAVD88 & \\
\hline 3260 & $116-\mathrm{N}-3$ & $\mathrm{~m}$ & NAVD88 & \\
\hline 3261 & $116-\mathrm{N}-3$ & $\mathrm{~m}$ & NAVD88 & \\
\hline 3262 & $116-\mathrm{N}-3$ & $\mathrm{~m}$ & NAVD88 & \\
\hline 3263 & $116-\mathrm{N}-3$ & $\mathrm{~m}$ & NAVD88 & \\
\hline 3264 & $116-\mathrm{N}-3$ & $\mathrm{~m}$ & NAVD88 & \\
\hline 3265 & & & & \\
\hline 3266 & $116-\mathrm{N}-3$ & $\mathrm{~m}$ & NAVD88 & \\
\hline 3267 & & & & \\
\hline 3268 & $116-F-9$ & & & \\
\hline 3269 & $116-F-9$ & & & \\
\hline
\end{tabular}




\begin{tabular}{|c|c|c|c|c|}
\hline & $\mathrm{O}$ & $P$ & $\mathrm{Q}$ & $\mathrm{R}$ \\
\hline 1 & SITE_CODE & ELEVATION_UNITS & VERT_COORD_TYPE & DATE_LAST_MODIFIED \\
\hline 3270 & $116-\mathrm{F}-9$ & & & \\
\hline 3271 & $116-\mathrm{F}-9$ & & & \\
\hline 3272 & $116-\mathrm{N}-3$ & $\mathrm{~m}$ & UNKNOWN & \\
\hline 3273 & $116-\mathrm{N}-3$ & $\mathrm{~m}$ & UNKNOWN & \\
\hline 3274 & $116-\mathrm{N}-3$ & $\mathrm{~m}$ & UNKNOWN & \\
\hline 3275 & $116-\mathrm{N}-3$ & $\mathrm{~m}$ & UNKNOWN & \\
\hline 3276 & $116-\mathrm{N}-3$ & $\mathrm{~m}$ & UNKNOWN & \\
\hline 3277 & $100-F-15$ & & & \\
\hline 3278 & $100-F-15$ & & & \\
\hline 3279 & $116-\mathrm{N}-3$ & $\mathrm{~m}$ & UNKNOWN & \\
\hline 3280 & $116-N-3$ & $\mathrm{~m}$ & UNKNOWN & \\
\hline 3281 & $116-\mathrm{N}-3$ & $\mathrm{~m}$ & UNKNOWN & \\
\hline 3282 & $116-\mathrm{N}-3$ & $\mathrm{~m}$ & UNKNOWN & \\
\hline 3283 & $116-\mathrm{N}-3$ & $\mathrm{~m}$ & UNKNOWN & \\
\hline 3284 & $116-\mathrm{N}-3$ & $\mathrm{~m}$ & UNKNOWN & \\
\hline 3285 & $116-\mathrm{N}-3$ & $\mathrm{~m}$ & UNKNOWN & \\
\hline 3286 & $116-N-3$ & $\mathrm{~m}$ & UNKNOWN & \\
\hline 3287 & $116-\mathrm{N}-3$ & $\mathrm{~m}$ & UNKNOWN & \\
\hline 3288 & & & & \\
\hline 3289 & $100-F-11$ & & & \\
\hline 3290 & $100-F-16$ & & & \\
\hline 3291 & $100-F-4$ & & & \\
\hline 3292 & $100-F-2$ & & UNKNOWN & \\
\hline 3293 & $100-F-2$ & & UNKNOWN & \\
\hline 3294 & $100-F-2$ & & UNKNOWN & \\
\hline 3295 & $100-\mathrm{F}-2$ & & UNKNOWN & \\
\hline 3296 & $100-F-2$ & & UNKNOWN & \\
\hline 3297 & $100-F-2$ & & UNKNOWN & \\
\hline 3298 & $100-F-2$ & & UNKNOWN & \\
\hline 3299 & $100-F-2$ & & UNKNOWN & \\
\hline 3300 & 100-F-2 & & UNKNOWN & \\
\hline 3301 & $100-\mathrm{F}-2$ & & UNKNOWN & \\
\hline 3302 & $100-\mathrm{F}-2$ & & UNKNOWN & \\
\hline 3303 & $100-F-2$ & & UNKNOWN & \\
\hline 3304 & $100-F-2$ & & UNKNOWN & \\
\hline 3305 & $100-F-2$ & & UNKNOWN & \\
\hline 3306 & 100-F-2 & & UNKNOWN & \\
\hline 3307 & $100-F-2$ & & UNKNOWN & \\
\hline
\end{tabular}




\begin{tabular}{|c|l|l|l|l|}
\hline & \multicolumn{1}{|c|}{ O } & \multicolumn{1}{|c|}{ P } & \multicolumn{1}{|c|}{ R } \\
\hline 1 & SITE_CODE & ELEVATION_UNITS & VERT_COORD_TYPE & DATE_LAST_MODIFIED \\
\hline 3308 & $100-F-2$ & UNKNOWN & \\
\hline 3309 & $100-F-2$ & & UNKNOWN & \\
\hline 3310 & $100-F-2$ & UNKNOWN & \\
\hline 3311 & $100-F-2$ & UNKNOWN & \\
\hline 3312 & $100-F-2$ & UNKNOWN & \\
\hline 3313 & $100-F-2$ & & UNKNOWN & \\
\hline 3314 & $100-F-2$ & & UNKNOWN & \\
\hline 3315 & $100-F-2$ & & & \\
\hline 3316 & $100-F-15$ & & & \\
\hline 3317 & $100-F-15$ & & & \\
\hline 3318 & $100-F-15$ & & & \\
\hline 3319 & $100-F-15$ & & & \\
\hline 3320 & $100-F-15$ & & & \\
\hline 3321 & $100-F-15$ & & & \\
\hline 3322 & $100-F-15$ & & & \\
\hline 3323 & $100-F-15$ & & & \\
\hline 3324 & $100-F-15$ & & & \\
\hline 3325 & $100-F-15$ & & & \\
\hline 3326 & $116-F-9$ & & & \\
\hline 3327 & $100-F-2$ & & & \\
\hline 3328 & $100-F-2$ & & & \\
\hline 3329 & $100-F-2$ & & & \\
\hline 3330 & $100-F-2$ & & & \\
\hline 3331 & $100-F-2$ & & & \\
\hline 3332 & $100-F-2$ & & & \\
\hline 3333 & $100-F-2$ & & & \\
\hline 3334 & $100-F-2$ & & & \\
\hline 3335 & $100-F-2$ & & & \\
\hline 3336 & $100-F-2$ & & & \\
\hline 3337 & $116-F-9$ & & & \\
\hline 3338 & $116-F-9$ & & & \\
\hline 3339 & $116-F-9$ & & & \\
\hline 3340 & $116-F-9$ & & & \\
\hline 3341 & $116-F-9$ & & & \\
\hline 3342 & $116-F-14$ & & & \\
\hline 3343 & $116-F-14$ & & & \\
\hline 3344 & $100-F-2$ & & & \\
\hline 3345 & $100-F-2$ & & & \\
\hline
\end{tabular}




\begin{tabular}{|c|c|c|c|c|}
\hline & $\mathrm{O}$ & $\mathrm{P}$ & Q & $\mathrm{R}$ \\
\hline 1 & SITE_CODE & ELEVATION_UNITS & VERT_COORD_TYPE & DATE_LAST_MODIFIED \\
\hline 3346 & $116-F-1$ & & & \\
\hline 3347 & 116-F-1 & & & \\
\hline 3348 & 116-F-1 & & & \\
\hline 3349 & 116-F-1 & & & \\
\hline 3350 & $116-F-1$ & & & \\
\hline 3351 & 116-F-1 & & & \\
\hline 3352 & 116-F-1 & & & \\
\hline 3353 & $100-F-2$ & & & \\
\hline 3354 & $100-F-2$ & & & \\
\hline 3355 & $100-F-2$ & & & \\
\hline 3356 & $100-F-2$ & & & \\
\hline 3357 & $100-F-2$ & & & \\
\hline 3358 & $100-F-2$ & & & \\
\hline 3359 & $116-\mathrm{N}-3$ & $\mathrm{~m}$ & UNKNOWN & \\
\hline 3360 & $116-\mathrm{N}-3$ & $\mathrm{~m}$ & UNKNOWN & \\
\hline 3361 & $116-F-9$ & & & \\
\hline 3362 & $116-F-9$ & & & \\
\hline 3363 & $116-F-9$ & & & \\
\hline 3364 & $116-F-9$ & & & \\
\hline 3365 & 116-F-9 & & & \\
\hline 3366 & 100-F-19 & & & \\
\hline 3367 & 100-F-19 & & & \\
\hline 3368 & 100-F-19 & & & \\
\hline 3369 & 116-F-14 & & & \\
\hline 3370 & $116-F-2$ & & & \\
\hline 3371 & $116-\mathrm{F}-2$ & & & \\
\hline 3372 & $116-F-2$ & & & \\
\hline 3373 & 116-F-2 & & & \\
\hline 3374 & 116-F-14 & & & \\
\hline 3375 & $116-F-14$ & & & \\
\hline 3376 & 116-F-14 & & & \\
\hline 3377 & 116-F-14 & & & \\
\hline 3378 & 116-F-14 & & & \\
\hline 3379 & 116-F-14 & & & \\
\hline 3380 & 116-F-14 & & & \\
\hline 3381 & 116-F-14 & & & \\
\hline 3382 & 116-F-14 & & & \\
\hline 3383 & $116-\mathrm{F}-1$ & & & \\
\hline
\end{tabular}




\begin{tabular}{|c|c|c|c|c|}
\hline & $\mathrm{O}$ & $P$ & $\mathrm{Q}$ & $\mathrm{R}$ \\
\hline 1 & SITE CODE & ELEVATION UNITS & VERT COORD TYPE & DATE LAST MODIFIED \\
\hline 3384 & $116-\mathrm{F}-1$ & & & \\
\hline 3385 & $116-F-14$ & & & \\
\hline 3386 & $116-F-14$ & & & \\
\hline 3387 & $116-F-14$ & & & \\
\hline 3388 & $116-F-14$ & & & \\
\hline 3389 & 116-F-14 & & & \\
\hline 3390 & $116-F-14$ & & & \\
\hline 3391 & $116-F-14$ & & & \\
\hline 3392 & $116-\mathrm{F}-9$ & & & \\
\hline 3393 & $100-F-19$ & & & \\
\hline 3394 & 100-F-19 & & & \\
\hline 3395 & $116-\mathrm{N}-3$ & $\mathrm{~m}$ & UNKNOWN & \\
\hline 3396 & $116-\mathrm{N}-3$ & $\mathrm{~m}$ & UNKNOWN & \\
\hline 3397 & $116-\mathrm{N}-3$ & $\mathrm{~m}$ & UNKNOWN & \\
\hline 3398 & $116-\mathrm{N}-3$ & $\mathrm{~m}$ & UNKNOWN & \\
\hline 3399 & $116-\mathrm{N}-3$ & $\mathrm{~m}$ & UNKNOWN & \\
\hline 3400 & $116-\mathrm{N}-3$ & $\mathrm{~m}$ & UNKNOWN & \\
\hline 3401 & $116-\mathrm{N}-3$ & $\mathrm{~m}$ & UNKNOWN & \\
\hline 3402 & $116-\mathrm{N}-3$ & $\mathrm{~m}$ & UNKNOWN & \\
\hline 3403 & $116-\mathrm{N}-3$ & $\mathrm{~m}$ & UNKNOWN & \\
\hline 3404 & $116-\mathrm{N}-3$ & $\mathrm{~m}$ & UNKNOWN & \\
\hline 3405 & $116-\mathrm{N}-3$ & $\mathrm{~m}$ & UNKNOWN & \\
\hline 3406 & 100-B-8 & & UNKNOWN & \\
\hline 3407 & 100-B-8 & & UNKNOWN & \\
\hline 3408 & 100-B-8 & & UNKNOWN & \\
\hline 3409 & 100-B-8 & & UNKNOWN & \\
\hline 3410 & 100-B-8 & & UNKNOWN & \\
\hline 3411 & 100-B-8 & & UNKNOWN & \\
\hline 3412 & 100-B-8 & & UNKNOWN & \\
\hline 3413 & 100-B-8 & & UNKNOWN & \\
\hline 3414 & 100-B-8 & & UNKNOWN & \\
\hline 3415 & 100-B-8 & & & \\
\hline 3416 & 100-B-8 & & & \\
\hline 3417 & 132-B-6 & & & \\
\hline 3418 & 100-B-8 & & & \\
\hline 3419 & 100-B-8 & & & \\
\hline 3420 & 100-B-8 & & & \\
\hline 3421 & 100-B-8 & & & \\
\hline
\end{tabular}




\begin{tabular}{|c|c|c|c|c|}
\hline & $\mathrm{O}$ & $P$ & $\mathrm{Q}$ & $\mathrm{R}$ \\
\hline 1 & SITE CODE & ELEVATION UNITS & VERT COORD TYPE & DATE LAST MODIFIED \\
\hline 3422 & $100-C-6$ & & UNKNOWN & \\
\hline 3423 & $100-C-6$ & & UNKNOWN & \\
\hline 3424 & 100-B-8 & & & \\
\hline 3425 & 100-B-8 & & & \\
\hline 3426 & 100-B-8 & & & \\
\hline 3427 & $100-C-6$ & & UNKNOWN & \\
\hline 3428 & 100-B-8 & & & \\
\hline 3429 & $100-C-6$ & & UNKNOWN & \\
\hline 3430 & $100-C-6$ & & UNKNOWN & \\
\hline 3431 & $100-C-6$ & & UNKNOWN & \\
\hline 3432 & $100-C-6$ & & & \\
\hline 3433 & $100-C-9$ & & UNKNOWN & 16-May-02 \\
\hline 3434 & $100-C-9$ & & UNKNOWN & 16-May-02 \\
\hline 3435 & $100-C-6$ & & UNKNOWN & \\
\hline 3436 & $100-C-6$ & & UNKNOWN & \\
\hline 3437 & $100-C-6$ & & UNKNOWN & \\
\hline 3438 & $100-C-6$ & & UNKNOWN & \\
\hline 3439 & 100-B-8 & & UNKNOWN & \\
\hline 3440 & 100-B-8 & & UNKNOWN & \\
\hline 3441 & 100-B-8 & & UNKNOWN & \\
\hline 3442 & $100-F-19$ & & & \\
\hline 3443 & $100-F-19$ & & & \\
\hline 3444 & $100-F-19$ & & & \\
\hline 3445 & 100-F-19 & & & \\
\hline 3446 & $100-F-19$ & & & \\
\hline 3447 & 100-F-19 & & & \\
\hline 3448 & $100-F-19$ & & & \\
\hline 3449 & $100-F-19$ & & & \\
\hline 3450 & $100-F-19$ & & & \\
\hline 3451 & 100-F-19 & & & \\
\hline 3452 & 100-F-19 & & & \\
\hline 3453 & 100-F-19 & & & \\
\hline 3454 & $100-F-19$ & & & \\
\hline 3455 & $100-F-19$ & & & \\
\hline 3456 & $100-F-19$ & & & \\
\hline 3457 & $100-F-19$ & & & \\
\hline 3458 & $100-F-19$ & & & \\
\hline 3459 & $100-F-19$ & & & \\
\hline
\end{tabular}




\begin{tabular}{|c|c|c|c|c|}
\hline & $\mathrm{O}$ & $P$ & $\bar{Q}$ & $\overline{\mathrm{R}}$ \\
\hline 1 & SITE_CODE & ELEVATION_UNITS & VERT_COORD_TYPE & DATE_LAST_MODIFIED \\
\hline 3460 & $100-F-19$ & & & \\
\hline 3461 & 100-F-19 & & & \\
\hline 3462 & $100-F-19$ & & & \\
\hline 3463 & 100-F-19 & & & \\
\hline 3464 & $100-F-19$ & & & \\
\hline 3465 & 100-F-19 & & & \\
\hline 3466 & $100-F-19$ & & & \\
\hline 3467 & 100-F-19 & & & \\
\hline 3468 & 100-F-19 & & & \\
\hline 3469 & 100-F-19 & & & \\
\hline 3470 & 100-F-19 & & & \\
\hline 3471 & $100-F-19$ & & & \\
\hline 3472 & 100-F-19 & & & \\
\hline 3473 & $116-F-9$ & & & \\
\hline 3474 & $100-F-2$ & & & \\
\hline 3475 & 100-B-8 & & UNKNOWN & \\
\hline 3476 & 100-B-8 & & UNKNOWN & \\
\hline 3477 & 100-F-19 & $\mathrm{m}$ & UNKNOWN & \\
\hline 3478 & & $\mathrm{ft}$ & NGVD29 & \\
\hline 3479 & & $\mathrm{ft}$ & NGVD29 & \\
\hline 3480 & & $\mathrm{ft}$ & NGVD29 & \\
\hline 3481 & & $\mathrm{ft}$ & NGVD29 & \\
\hline 3482 & & $\mathrm{ft}$ & NGVD29 & \\
\hline 3483 & & $\mathrm{ft}$ & NGVD29 & \\
\hline 3484 & & $\mathrm{ft}$ & NGVD29 & \\
\hline 3485 & & $\mathrm{ft}$ & NGVD29 & \\
\hline 3486 & & $\mathrm{ft}$ & NGVD29 & \\
\hline 3487 & & $\mathrm{ft}$ & NGVD29 & \\
\hline 3488 & & $\mathrm{ft}$ & NGVD29 & \\
\hline 3489 & & $\mathrm{ft}$ & NGVD29 & \\
\hline 3490 & & $\mathrm{ft}$ & NGVD29 & \\
\hline 3491 & & $\mathrm{ft}$ & NGVD29 & \\
\hline 3492 & & $\mathrm{ft}$ & NGVD29 & \\
\hline 3493 & & $\mathrm{ft}$ & NGVD29 & \\
\hline 3494 & & $\mathrm{ft}$ & NGVD29 & \\
\hline 3495 & & $\mathrm{ft}$ & NGVD29 & \\
\hline 3496 & & $\mathrm{ft}$ & NGVD29 & \\
\hline 3497 & & $\mathrm{ft}$ & NGVD29 & \\
\hline
\end{tabular}




\begin{tabular}{|c|c|c|c|c|}
\hline & $\mathrm{O}$ & $P$ & $\mathrm{Q}$ & $\mathrm{R}$ \\
\hline 1 & SITE CODE & ELEVATION UNITS & VERT COORD TYPE & DATE LAST MODIFIED \\
\hline 3498 & & $\mathrm{ft}$ & NGVD29 & \\
\hline 3499 & & $\mathrm{ft}$ & NGVD29 & \\
\hline 3500 & & $\mathrm{ft}$ & NGVD29 & \\
\hline 3501 & & $\mathrm{ft}$ & NGVD29 & \\
\hline 3502 & & $\mathrm{ft}$ & NGVD29 & \\
\hline 3503 & & $\mathrm{ft}$ & NGVD29 & \\
\hline 3504 & & $\mathrm{ft}$ & NGVD29 & \\
\hline 3505 & & $\mathrm{ft}$ & NGVD29 & \\
\hline 3506 & & $\mathrm{ft}$ & NGVD29 & \\
\hline 3507 & & $\mathrm{ft}$ & NGVD29 & \\
\hline 3508 & & $\mathrm{ft}$ & NGVD29 & \\
\hline 3509 & & $\mathrm{ft}$ & NGVD29 & \\
\hline 3510 & & $\mathrm{ft}$ & NGVD29 & \\
\hline 3511 & & $\mathrm{ft}$ & NGVD29 & \\
\hline 3512 & & $\mathrm{ft}$ & NGVD29 & \\
\hline 3513 & & $\mathrm{ft}$ & NGVD29 & \\
\hline 3514 & & $\mathrm{ft}$ & NGVD29 & \\
\hline 3515 & & $\mathrm{ft}$ & NGVD29 & \\
\hline 3516 & & $\mathrm{ft}$ & NGVD29 & \\
\hline 3517 & & $\mathrm{ft}$ & NGVD29 & \\
\hline 3518 & & $\mathrm{ft}$ & NGVD29 & \\
\hline 3519 & & $\mathrm{ft}$ & NGVD29 & \\
\hline 3520 & & $\mathrm{ft}$ & NGVD29 & \\
\hline 3521 & & $\mathrm{ft}$ & NGVD29 & \\
\hline 3522 & & $\mathrm{ft}$ & NGVD29 & \\
\hline 3523 & & $\mathrm{ft}$ & NGVD29 & \\
\hline 3524 & & $\mathrm{ft}$ & NGVD29 & \\
\hline 3525 & & $\mathrm{ft}$ & NGVD29 & \\
\hline 3526 & & $\mathrm{ft}$ & NGVD29 & \\
\hline 3527 & & $\mathrm{ft}$ & NGVD29 & \\
\hline 3528 & & $\mathrm{ft}$ & NGVD29 & \\
\hline 3529 & & $\mathrm{ft}$ & NGVD29 & \\
\hline 3530 & & $\mathrm{ft}$ & NGVD29 & \\
\hline 3531 & & $\mathrm{ft}$ & NGVD29 & \\
\hline 3532 & & $\mathrm{ft}$ & NGVD29 & \\
\hline 3533 & & $\mathrm{ft}$ & NGVD29 & \\
\hline 3534 & & $\mathrm{ft}$ & NGVD29 & \\
\hline 3535 & & $\mathrm{ft}$ & NGVD29 & \\
\hline
\end{tabular}




\begin{tabular}{|c|c|c|c|c|}
\hline & $\mathrm{O}$ & $\mathrm{P}$ & $\mathrm{Q}$ & $\mathrm{R}$ \\
\hline 1 & SITE_CODE & ELEVATION_UNITS & VERT_COORD_TYPE & DATE_LAST_MODIFIED \\
\hline 3536 & & $\mathrm{ft}$ & NGVD29 & \\
\hline 3537 & & $\mathrm{ft}$ & NGVD29 & \\
\hline 3538 & & $\mathrm{ft}$ & NGVD29 & \\
\hline 3539 & & $\mathrm{ft}$ & NGVD29 & \\
\hline 3540 & & $\mathrm{ft}$ & NGVD29 & \\
\hline 3541 & & $\mathrm{ft}$ & NGVD29 & \\
\hline 3542 & & $\mathrm{ft}$ & NGVD29 & \\
\hline 3543 & & $\mathrm{ft}$ & NGVD29 & \\
\hline 3544 & & $\mathrm{ft}$ & NGVD29 & \\
\hline 3545 & & $\mathrm{ft}$ & NGVD29 & \\
\hline 3546 & & $\mathrm{ft}$ & NGVD29 & \\
\hline 3547 & & $\mathrm{ft}$ & NGVD29 & \\
\hline 3548 & & $\mathrm{ft}$ & NGVD29 & \\
\hline 3549 & & $\mathrm{ft}$ & NGVD29 & \\
\hline 3550 & & $\mathrm{ft}$ & NGVD29 & \\
\hline 3551 & & $\mathrm{ft}$ & NGVD29 & \\
\hline 3552 & & $\mathrm{ft}$ & NGVD29 & \\
\hline 3553 & & $\mathrm{ft}$ & NGVD29 & \\
\hline 3554 & & $\mathrm{ft}$ & NGVD29 & \\
\hline 3555 & 126-F-1 & & UNKNOWN & \\
\hline 3556 & 126-F-1 & & UNKNOWN & \\
\hline 3557 & 126-F-1 & & UNKNOWN & \\
\hline 3558 & 126-F-1 & & UNKNOWN & \\
\hline 3559 & 126-F-1 & & UNKNOWN & \\
\hline 3560 & $126-\mathrm{F}-1$ & & UNKNOWN & \\
\hline 3561 & 126-F-1 & & UNKNOWN & \\
\hline 3562 & 126-F-1 & & UNKNOWN & \\
\hline 3563 & 126-F-1 & & UNKNOWN & \\
\hline 3564 & 126-F-1 & & UNKNOWN & \\
\hline 3565 & 126-F-1 & & UNKNOWN & \\
\hline 3566 & 126-F-1 & & UNKNOWN & \\
\hline 3567 & 126-F-1 & & UNKNOWN & \\
\hline 3568 & 126-F-1 & & UNKNOWN & \\
\hline 3569 & 126-F-1 & & UNKNOWN & \\
\hline 3570 & 126-F-1 & & UNKNOWN & \\
\hline 3571 & $126-F-1$ & & UNKNOWN & \\
\hline 3572 & $126-F-1$ & & UNKNOWN & \\
\hline 3573 & $126-\mathrm{F}-1$ & & UNKNOWN & \\
\hline
\end{tabular}




\begin{tabular}{|c|c|c|c|c|}
\hline & 0 & $\mathrm{P}$ & $\mathrm{Q}$ & $\mathrm{R}$ \\
\hline 1 & SITE_CODE & ELEVATION_UNITS & VERT_COORD_TYPE & DATE_LAST_MODIFIED \\
\hline 3574 & $126-\mathrm{F}-1$ & & UNKNOWN & \\
\hline 3575 & $126-\mathrm{F}-1$ & & UNKNOWN & \\
\hline 3576 & $126-\mathrm{F}-1$ & & UNKNOWN & \\
\hline 3577 & $126-\mathrm{F}-1$ & & UNKNOWN & \\
\hline 3578 & 126-F-1 & & UNKNOWN & \\
\hline 3579 & 126-F-1 & & UNKNOWN & \\
\hline 3580 & $126-\mathrm{F}-1$ & & UNKNOWN & \\
\hline 3581 & 126-F-1 & & UNKNOWN & \\
\hline 3582 & 126-F-1 & & UNKNOWN & \\
\hline 3583 & $126-F-1$ & & UNKNOWN & \\
\hline 3584 & 126-F-1 & & UNKNOWN & \\
\hline 3585 & $126-F-1$ & & UNKNOWN & \\
\hline 3586 & $126-F-1$ & & UNKNOWN & \\
\hline 3587 & 126-F-1 & & UNKNOWN & \\
\hline 3588 & 126-F-1 & & UNKNOWN & \\
\hline 3589 & 126-F-1 & & UNKNOWN & \\
\hline 3590 & $126-F-1$ & & UNKNOWN & \\
\hline 3591 & 126-F-1 & & UNKNOWN & \\
\hline 3592 & 126-F-1 & & UNKNOWN & \\
\hline 3593 & 126-F-1 & & UNKNOWN & \\
\hline 3594 & 126-F-1 & & UNKNOWN & \\
\hline 3595 & 126-F-1 & & UNKNOWN & \\
\hline 3596 & 126-F-1 & & UNKNOWN & \\
\hline 3597 & $126-\mathrm{F}-1$ & & UNKNOWN & \\
\hline 3598 & 126-F-1 & & UNKNOWN & \\
\hline 3599 & 126-F-1 & & UNKNOWN & \\
\hline 3600 & 126-F-1 & & UNKNOWN & \\
\hline 3601 & 126-F-1 & & UNKNOWN & \\
\hline 3602 & $126-F-1$ & & UNKNOWN & \\
\hline 3603 & $116-\mathrm{N}-3$ & & UNKNOWN & \\
\hline 3604 & $116-\mathrm{N}-3$ & & UNKNOWN & \\
\hline 3605 & $116-\mathrm{N}-3$ & & UNKNOWN & \\
\hline 3606 & $116-\mathrm{N}-3$ & & UNKNOWN & \\
\hline 3607 & $116-\mathrm{N}-3$ & & UNKNOWN & \\
\hline 3608 & $116-\mathrm{N}-3$ & & UNKNOWN & \\
\hline 3609 & $116-\mathrm{N}-3$ & & UNKNOWN & \\
\hline 3610 & $116-\mathrm{N}-3$ & & UNKNOWN & \\
\hline 3611 & $116-\mathrm{N}-3$ & & UNKNOWN & \\
\hline
\end{tabular}




\begin{tabular}{|c|c|c|c|c|}
\hline & $\mathrm{O}$ & $P$ & $\mathrm{Q}$ & $R$ \\
\hline 1 & SITE CODE & ELEVATION UNITS & VERT COORD TYPE & DATE LAST MODIFIED \\
\hline 3612 & $116-\mathrm{N}-3$ & & UNKNOWN & \\
\hline 3613 & $116-\mathrm{N}-3$ & & UNKNOWN & \\
\hline 3614 & $116-\mathrm{N}-3$ & & UNKNOWN & \\
\hline 3615 & $116-\mathrm{N}-3$ & & UNKNOWN & \\
\hline 3616 & $116-\mathrm{N}-3$ & & UNKNOWN & \\
\hline 3617 & $116-\mathrm{N}-3$ & & UNKNOWN & \\
\hline 3618 & $116-\mathrm{N}-3$ & & UNKNOWN & \\
\hline 3619 & $116-\mathrm{N}-3$ & & UNKNOWN & \\
\hline 3620 & $116-\mathrm{N}-3$ & & UNKNOWN & \\
\hline 3621 & $116-\mathrm{N}-3$ & & UNKNOWN & \\
\hline 3622 & $116-\mathrm{N}-3$ & & UNKNOWN & \\
\hline 3623 & $116-\mathrm{N}-3$ & & UNKNOWN & \\
\hline 3624 & $116-\mathrm{N}-3$ & & UNKNOWN & \\
\hline 3625 & $116-\mathrm{N}-3$ & & UNKNOWN & \\
\hline 3626 & $116-\mathrm{N}-3$ & & UNKNOWN & \\
\hline 3627 & $116-\mathrm{N}-3$ & $\mathrm{~m}$ & UNKNOWN & \\
\hline 3628 & $116-\mathrm{N}-3$ & $\mathrm{~m}$ & UNKNOWN & \\
\hline 3629 & $116-\mathrm{N}-3$ & & UNKNOWN & \\
\hline 3630 & $116-N-3$ & & UNKNOWN & \\
\hline 3631 & $116-\mathrm{N}-3$ & & UNKNOWN & \\
\hline 3632 & $116-\mathrm{N}-3$ & & UNKNOWN & \\
\hline 3633 & $116-\mathrm{N}-3$ & & UNKNOWN & \\
\hline 3634 & $116-\mathrm{N}-3$ & & UNKNOWN & \\
\hline 3635 & $116-\mathrm{N}-3$ & $\mathrm{~m}$ & UNKNOWN & \\
\hline 3636 & $116-\mathrm{N}-3$ & $\mathrm{~m}$ & UNKNOWN & \\
\hline 3637 & $116-\mathrm{N}-3$ & & UNKNOWN & \\
\hline 3638 & $116-\mathrm{N}-3$ & & UNKNOWN & \\
\hline 3639 & $116-\mathrm{N}-3$ & & UNKNOWN & \\
\hline 3640 & $116-\mathrm{N}-3$ & & UNKNOWN & \\
\hline 3641 & $116-\mathrm{N}-3$ & & UNKNOWN & \\
\hline 3642 & $116-\mathrm{N}-3$ & & UNKNOWN & \\
\hline 3643 & $116-\mathrm{N}-3$ & & UNKNOWN & \\
\hline 3644 & $116-\mathrm{N}-3$ & & UNKNOWN & \\
\hline 3645 & $116-\mathrm{N}-3$ & & UNKNOWN & \\
\hline 3646 & $116-\mathrm{N}-3$ & & UNKNOWN & \\
\hline 3647 & $116-\mathrm{N}-3$ & & UNKNOWN & \\
\hline 3648 & $116-\mathrm{N}-3$ & & UNKNOWN & \\
\hline 3649 & $116-\mathrm{N}-3$ & & UNKNOWN & \\
\hline
\end{tabular}




\begin{tabular}{|c|l|l|l|l|}
\hline & \multicolumn{1}{|c|}{ O } & \multicolumn{1}{|c|}{ P } & & R \\
\hline 1 & SITE_CODE & ELEVATION_UNITS & VERT_COORD_TYPE & DATE_LAST_MODIFIED \\
\hline 3650 & $126-F-1$ & UNKNOWN & \\
\hline 3651 & $126-F-1$ & UNKNOWN & \\
\hline 3652 & $126-F-1$ & UNKNOWN & \\
\hline 3653 & $126-F-1$ & UNKNOWN & \\
\hline 3654 & $126-F-1$ & UNKNOWN & \\
\hline 3655 & $126-F-1$ & UNKNOWN & \\
\hline 3656 & $126-F-1$ & UNKNOWN & \\
\hline 3657 & $126-F-1$ & UNKNOWN & \\
\hline 3658 & $126-F-1$ & UNKNOWN & \\
\hline 3659 & $126-F-1$ & UNKNOWN & \\
\hline 3660 & $126-F-1$ & UNKNOWN & \\
\hline 3661 & $126-F-1$ & UNKNOWN & \\
\hline 3662 & $126-F-1$ & UNKNOWN & \\
\hline 3663 & $126-F-1$ & UNKNOWN & \\
\hline 3664 & $126-F-1$ & UNKNOWN & \\
\hline 3665 & $126-F-1$ & UNKNOWN & \\
\hline 3666 & $126-F-1$ & UNKNOWN & \\
\hline 3667 & $126-F-1$ & UNKNOWN & \\
\hline 3668 & $126-F-1$ & UNKNOWN & \\
\hline 3669 & $126-F-1$ & UNKNOWN & \\
\hline 3670 & $126-F-1$ & UNKNOWN & \\
\hline 3671 & $126-F-1$ & UNKNOWN & \\
\hline 3672 & $126-F-1$ & UNKNOWN & \\
\hline 3673 & $126-F-1$ & UNKNOWN & \\
\hline 3674 & $126-F-1$ & UNKNOWN & \\
\hline 3675 & $126-F-1$ & UNKNOWN & \\
\hline 3676 & $126-F-1$ & UNKNOWN & \\
\hline 3677 & $126-F-1$ & UNKNOWN & \\
\hline 3678 & $126-F-1$ & UNKNOWN & \\
\hline 3679 & $126-F-1$ & UNKNOWN & \\
\hline 3680 & $126-F-1$ & UNKNOWN & \\
\hline 3681 & $126-F-1$ & UNKNOWN & \\
\hline 3682 & $126-F-1$ & UNKNOWN & \\
\hline 3683 & $126-F-1$ & UNKNOWN & \\
\hline 3684 & $126-F-1$ & $126-F-1$ & UNKNOWN & \\
\hline 3685 & $126-F-1$ & & & \\
\hline 3686 & & & \\
\hline 3687 & $126-F-1$ & & & \\
\hline
\end{tabular}




\begin{tabular}{|c|l|l|l|l|}
\hline & \multicolumn{1}{|c|}{ O } & \multicolumn{1}{|c|}{ P } & & R \\
\hline 1 & SITE_CODE & ELEVATION_UNITS & VERT_COORD_TYPE & DATE_LAST_MODIFIED \\
\hline 3688 & $126-F-1$ & UNKNOWN & \\
\hline 3689 & $126-F-1$ & UNKNOWN & \\
\hline 3690 & $126-F-1$ & UNKNOWN & \\
\hline 3691 & $126-F-1$ & UNKNOWN & \\
\hline 3692 & $126-F-1$ & UNKNOWN & \\
\hline 3693 & $126-F-1$ & UNKNOWN & \\
\hline 3694 & $126-F-1$ & UNKNOWN & \\
\hline 3695 & $126-F-1$ & UNKNOWN & \\
\hline 3696 & $126-F-1$ & UNKNOWN & \\
\hline 3697 & $126-F-1$ & UNKNOWN & \\
\hline 3698 & $126-F-1$ & UNKNOWN & \\
\hline 3699 & $126-F-1$ & UNKNOWN & \\
\hline 3700 & $126-F-1$ & UNKNOWN & \\
\hline 3701 & $126-F-1$ & UNKNOWN & \\
\hline 3702 & $126-F-1$ & UNKNOWN & \\
\hline 3703 & $126-F-1$ & UNKNOWN & \\
\hline 3704 & $126-F-1$ & UNKNOWN & \\
\hline 3705 & $126-F-1$ & UNKNOWN & \\
\hline 3706 & $126-F-1$ & UNKNOWN & \\
\hline 3707 & $126-F-1$ & UNKNOWN & \\
\hline 3708 & $126-F-1$ & UNKNOWN & \\
\hline 3709 & $126-F-1$ & UNKNOWN & \\
\hline 3710 & $126-F-1$ & UNKNOWN & \\
\hline 3711 & $126-F-1$ & UNKNOWN & \\
\hline 3712 & $126-F-1$ & UNKNOWN & \\
\hline 3713 & $126-F-1$ & UNKNOWN & \\
\hline 3714 & $126-F-1$ & UNKNOWN & \\
\hline 3715 & $126-F-1$ & UNKNOWN & \\
\hline 3716 & $126-F-1$ & UNKNOWN & \\
\hline 3717 & $126-F-1$ & UNKNOWN & \\
\hline 3718 & $126-F-1$ & UNKNOWN & \\
\hline 3719 & $126-F-1$ & UNKNOWN & \\
\hline 3720 & $126-F-1$ & UNKNOWN & \\
\hline 3721 & $126-F-1$ & UNKNOWN & \\
\hline 3722 & $126-F-1$ & $126-F-1$ & UNKNOWN & \\
\hline 3723 & $126-F-1$ & & & \\
\hline 3724 & $126-F-1$ & & & \\
\hline 3725 & & & \\
\hline
\end{tabular}




\begin{tabular}{|c|c|c|c|c|}
\hline & $\mathrm{O}$ & $P$ & $\mathrm{Q}$ & $\mathrm{R}$ \\
\hline 1 & SITE_CODE & ELEVATION_UNITS & VERT_COORD_TYPE & DATE_LAST_MODIFIED \\
\hline 3726 & $126-\mathrm{F}-1$ & & UNKNOWN & \\
\hline 3727 & 126-F-1 & & UNKNOWN & \\
\hline 3728 & $126-\mathrm{F}-1$ & & UNKNOWN & \\
\hline 3729 & 126-F-1 & & UNKNOWN & \\
\hline 3730 & $126-\mathrm{F}-1$ & & UNKNOWN & \\
\hline 3731 & 126-F-1 & & UNKNOWN & \\
\hline 3732 & $126-\mathrm{F}-1$ & & UNKNOWN & \\
\hline 3733 & $126-\mathrm{F}-1$ & & UNKNOWN & \\
\hline 3734 & $126-\mathrm{F}-1$ & & UNKNOWN & \\
\hline 3735 & $100-F-19$ & & & \\
\hline 3736 & $100-F-19$ & & & \\
\hline 3737 & $126-\mathrm{F}-1$ & & UNKNOWN & \\
\hline 3738 & $126-\mathrm{F}-1$ & & UNKNOWN & \\
\hline 3739 & $126-\mathrm{F}-1$ & & UNKNOWN & \\
\hline 3740 & 126-F-1 & & UNKNOWN & \\
\hline 3741 & $126-\mathrm{F}-1$ & & UNKNOWN & \\
\hline 3742 & $126-\mathrm{F}-1$ & & UNKNOWN & \\
\hline 3743 & $126-\mathrm{F}-1$ & & UNKNOWN & \\
\hline 3744 & $126-\mathrm{F}-1$ & & UNKNOWN & \\
\hline 3745 & $126-\mathrm{F}-1$ & & UNKNOWN & \\
\hline 3746 & $126-\mathrm{F}-1$ & & UNKNOWN & \\
\hline 3747 & $126-\mathrm{F}-1$ & & UNKNOWN & \\
\hline 3748 & $126-\mathrm{F}-1$ & & UNKNOWN & \\
\hline 3749 & $126-\mathrm{F}-1$ & & UNKNOWN & \\
\hline 3750 & $126-\mathrm{F}-1$ & & UNKNOWN & \\
\hline 3751 & $126-\mathrm{F}-1$ & & UNKNOWN & \\
\hline 3752 & $126-\mathrm{F}-1$ & & UNKNOWN & \\
\hline 3753 & $126-\mathrm{F}-1$ & & UNKNOWN & \\
\hline 3754 & $126-\mathrm{F}-1$ & & UNKNOWN & \\
\hline 3755 & $126-\mathrm{F}-1$ & & UNKNOWN & \\
\hline 3756 & 126-F-1 & & UNKNOWN & \\
\hline 3757 & 126-F-1 & & UNKNOWN & \\
\hline 3758 & $126-\mathrm{F}-1$ & & UNKNOWN & \\
\hline 3759 & $126-\mathrm{F}-1$ & & UNKNOWN & \\
\hline 3760 & $126-\mathrm{F}-1$ & & UNKNOWN & \\
\hline 3761 & $116-\mathrm{N}-1$ & $\mathrm{ft}$ & NAVD88 & \\
\hline 3762 & $116-\mathrm{N}-1$ & $\mathrm{ft}$ & NAVD88 & \\
\hline 3763 & $116-\mathrm{N}-1$ & $\mathrm{ft}$ & NAVD88 & \\
\hline
\end{tabular}




\begin{tabular}{|c|c|c|c|c|}
\hline & $\mathrm{O}$ & $P$ & $\mathrm{Q}$ & $\mathrm{R}$ \\
\hline 1 & SITE_CODE & ELEVATION_UNITS & VERT_COORD_TYPE & DATE_LAST_MODIFIED \\
\hline 3764 & $100-C-6$ & & & \\
\hline 3765 & $100-C-6$ & & & \\
\hline 3766 & 116-B-1 & & & \\
\hline 3767 & 116-B-1 & & & \\
\hline 3768 & 116-B-1 & & & \\
\hline 3769 & 116-B-1 & & & \\
\hline 3770 & 116-B-1 & & & \\
\hline 3771 & $100-C-6$ & & UNKNOWN & \\
\hline 3772 & $100-C-6$ & & UNKNOWN & \\
\hline 3773 & $100-C-6$ & & UNKNOWN & \\
\hline 3774 & $100-C-6$ & & UNKNOWN & \\
\hline 3775 & $100-C-6$ & & UNKNOWN & \\
\hline 3776 & $100-C-6$ & & UNKNOWN & \\
\hline 3777 & $100-C-6$ & & UNKNOWN & \\
\hline 3778 & $100-C-6$ & & UNKNOWN & \\
\hline 3779 & $100-C-6$ & & UNKNOWN & \\
\hline 3780 & $100-C-6$ & & UNKNOWN & \\
\hline 3781 & 118-B-3 & & & \\
\hline 3782 & 118-B-3 & & & \\
\hline 3783 & $116-\mathrm{F}-9$ & & UNKNOWN & \\
\hline 3784 & $116-\mathrm{F}-9$ & & UNKNOWN & \\
\hline 3785 & $116-F-9$ & & UNKNOWN & \\
\hline 3786 & $116-F-9$ & & UNKNOWN & \\
\hline 3787 & $116-\mathrm{F}-9$ & & UNKNOWN & \\
\hline 3788 & $116-\mathrm{F}-9$ & & UNKNOWN & \\
\hline 3789 & $116-\mathrm{F}-9$ & & UNKNOWN & \\
\hline 3790 & $116-F-9$ & & UNKNOWN & \\
\hline 3791 & $116-F-9$ & & UNKNOWN & \\
\hline 3792 & $116-F-9$ & & UNKNOWN & \\
\hline 3793 & $116-\mathrm{F}-9$ & & UNKNOWN & \\
\hline 3794 & 116-F-9 & & UNKNOWN & \\
\hline 3795 & $116-\mathrm{F}-9$ & & UNKNOWN & \\
\hline 3796 & 116-F-9 & & UNKNOWN & \\
\hline 3797 & $116-F-9$ & & UNKNOWN & \\
\hline 3798 & $116-F-9$ & & UNKNOWN & \\
\hline 3799 & $116-\mathrm{F}-9$ & & UNKNOWN & \\
\hline 3800 & $116-F-9$ & & UNKNOWN & \\
\hline 3801 & $116-\mathrm{F}-9$ & & UNKNOWN & \\
\hline
\end{tabular}




\begin{tabular}{|c|c|c|c|c|}
\hline & $\mathrm{O}$ & $P$ & $\bar{Q}$ & $\bar{R}$ \\
\hline 1 & SITE_CODE & ELEVATION_UNITS & VERT_COORD_TYPE & DATE_LAST_MODIFIED \\
\hline 3802 & $116-\mathrm{F}-9$ & & UNKNOWN & \\
\hline 3803 & 116-F-9 & & UNKNOWN & \\
\hline 3804 & $116-\mathrm{F}-9$ & & UNKNOWN & \\
\hline 3805 & $116-\mathrm{F}-9$ & & UNKNOWN & \\
\hline 3806 & $116-\mathrm{F}-9$ & & UNKNOWN & \\
\hline 3807 & $116-\mathrm{N}-1$ & $\mathrm{ft}$ & NAVD88 & \\
\hline 3808 & $116-\mathrm{N}-1$ & $\mathrm{ft}$ & NAVD88 & \\
\hline 3809 & $116-\mathrm{N}-1$ & $\mathrm{ft}$ & NAVD88 & \\
\hline 3810 & $116-\mathrm{N}-1$ & $\mathrm{ft}$ & NAVD88 & \\
\hline 3811 & 126-F-1 & & UNKNOWN & \\
\hline 3812 & 126-F-1 & & UNKNOWN & \\
\hline 3813 & 126-F-1 & & UNKNOWN & \\
\hline 3814 & $126-F-1$ & & UNKNOWN & \\
\hline 3815 & $126-F-1$ & & UNKNOWN & \\
\hline 3816 & 126-F-1 & & UNKNOWN & \\
\hline 3817 & 126-F-1 & & UNKNOWN & \\
\hline 3818 & $126-\mathrm{F}-1$ & & UNKNOWN & \\
\hline 3819 & 126-F-1 & & UNKNOWN & \\
\hline 3820 & 126-F-1 & & UNKNOWN & \\
\hline 3821 & 126-F-1 & & UNKNOWN & \\
\hline 3822 & 126-F-1 & & UNKNOWN & \\
\hline 3823 & 126-F-1 & & UNKNOWN & \\
\hline 3824 & $126-\mathrm{F}-1$ & & UNKNOWN & \\
\hline 3825 & 126-F-1 & & UNKNOWN & \\
\hline 3826 & $126-\mathrm{F}-1$ & & UNKNOWN & \\
\hline 3827 & 126-F-1 & & UNKNOWN & \\
\hline 3828 & 126-F-1 & & UNKNOWN & \\
\hline 3829 & 126-F-1 & & UNKNOWN & \\
\hline 3830 & 126-F-1 & & UNKNOWN & \\
\hline 3831 & $126-F-1$ & & UNKNOWN & \\
\hline 3832 & 126-F-1 & & UNKNOWN & \\
\hline 3833 & 126-F-1 & & UNKNOWN & \\
\hline 3834 & 126-F-1 & & UNKNOWN & \\
\hline 3835 & 126-F-1 & & UNKNOWN & \\
\hline 3836 & $126-\mathrm{F}-1$ & & UNKNOWN & \\
\hline 3837 & 126-F-1 & & UNKNOWN & \\
\hline 3838 & $116-F-9$ & & & \\
\hline 3839 & 116-F-9 & & & \\
\hline
\end{tabular}




\begin{tabular}{|c|l|l|l|l|}
\hline & \multicolumn{1}{|c|}{ O } & \multicolumn{1}{|c|}{$\mathrm{P}$} & \multicolumn{1}{|c|}{$\mathrm{Q}$} & \\
\hline 1 & SITE_CODE & ELEVATION_UNITS & VERT_COORD_TYPE & DATE_LAST_MODIFIED \\
\hline 3840 & $116-\mathrm{F}-9$ & & & \\
\hline 3841 & $116-\mathrm{F}-9$ & & & \\
\hline 3842 & $116-\mathrm{F}-9$ & & & \\
\hline 3843 & $116-\mathrm{F}-9$ & & & \\
\hline 3844 & $116-\mathrm{F}-9$ & & & \\
\hline 3845 & $116-\mathrm{F}-9$ & & & \\
\hline 3846 & $116-\mathrm{F}-9$ & & & \\
\hline 3847 & $116-\mathrm{F}-9$ & & & \\
\hline 3848 & $116-\mathrm{F}-9$ & & & \\
\hline 3849 & $116-\mathrm{F}-9$ & & & \\
\hline 3850 & $116-\mathrm{F}-9$ & & NAVD88 & \\
\hline 3851 & $116-\mathrm{F}-9$ & & NAVD88 & \\
\hline 3852 & $116-\mathrm{N}-3$ & $\mathrm{~m}$ & NAVD88 & \\
\hline 3853 & $116-\mathrm{N}-3$ & $\mathrm{~m}$ & NAVD88 & \\
\hline 3854 & $116-\mathrm{N}-3$ & $\mathrm{~m}$ & NAVD88 & \\
\hline 3855 & $116-\mathrm{N}-3$ & $\mathrm{~m}$ & NAVD88 & \\
\hline 3856 & $116-\mathrm{N}-3$ & $\mathrm{~m}$ & NAVD88 & \\
\hline 3857 & $116-\mathrm{N}-3$ & $\mathrm{~m}$ & NAVD88 & \\
\hline 3858 & $116-\mathrm{N}-3$ & $\mathrm{~m}$ & NAVD88 & \\
\hline 3859 & $116-\mathrm{N}-3$ & $\mathrm{~m}$ & NAVD88 & \\
\hline 3860 & $116-\mathrm{N}-3$ & $\mathrm{~m}$ & UNKNOWN & \\
\hline 3861 & $116-\mathrm{N}-3$ & $\mathrm{~m}$ & UNKNOWN & \\
\hline 3862 & $116-\mathrm{F}-9$ & & UNKNOWN & \\
\hline 3863 & $116-\mathrm{F}-9$ & & UNKNOWN & \\
\hline 3864 & $116-\mathrm{F}-9$ & & UNKNOWN & \\
\hline 3865 & $116-\mathrm{F}-9$ & & UNKNOWN & \\
\hline 3866 & $116-\mathrm{F}-9$ & & UNKNOWN & \\
\hline 3867 & $116-\mathrm{F}-9$ & & UNKNOWN & \\
\hline 3868 & $116-\mathrm{F}-9$ & & UNKNOWN & \\
\hline 3869 & $116-\mathrm{F}-9$ & & UNKNOWN & \\
\hline 3870 & $116-\mathrm{F}-9$ & & & \\
\hline 3871 & $116-\mathrm{F}-9$ & & & \\
\hline 3872 & $116-\mathrm{F}-9$ & & & \\
\hline 3873 & $116-\mathrm{F}-9$ & & & \\
\hline 3874 & $116-\mathrm{F}-9$ & & & \\
\hline 3875 & $116-\mathrm{F}-9$ & & & \\
\hline 3876 & $116-\mathrm{F}-9$ & & & \\
\hline 3877 & $116-\mathrm{F}-9$ & & & \\
\hline & & & & \\
\hline
\end{tabular}




\begin{tabular}{|c|c|c|c|c|}
\hline & $\mathrm{O}$ & $\mathrm{P}$ & $\bar{Q}$ & $\bar{R}$ \\
\hline 1 & SITE_CODE & ELEVATION_UNITS & VERT_COORD_TYPE & DATE_LAST_MODIFIED \\
\hline 3878 & $116-\mathrm{F}-9$ & & UNKNOWN & \\
\hline 3879 & $116-\mathrm{F}-9$ & & UNKNOWN & \\
\hline 3880 & $116-F-9$ & & UNKNOWN & \\
\hline 3881 & $116-\mathrm{F}-9$ & & UNKNOWN & \\
\hline 3882 & $116-\mathrm{F}-9$ & & UNKNOWN & \\
\hline 3883 & 116-F-9 & & UNKNOWN & \\
\hline 3884 & 116-F-9 & & UNKNOWN & \\
\hline 3885 & 116-F-9 & & UNKNOWN & \\
\hline 3886 & 100-B-14 & & UNKNOWN & \\
\hline 3887 & 100-B-14 & & UNKNOWN & \\
\hline 3888 & 100-B-8 & & UNKNOWN & \\
\hline 3889 & 100-B-8 & & UNKNOWN & \\
\hline 3890 & $116-\mathrm{N}-3$ & $\mathrm{~m}$ & NAVD88 & \\
\hline 3891 & 132-B-6 & & UNKNOWN & \\
\hline 3892 & 132-B-6 & & UNKNOWN & \\
\hline 3893 & $132-C-2$ & & & \\
\hline 3894 & $132-C-2$ & & & \\
\hline 3895 & $132-C-2$ & & & \\
\hline 3896 & 100-B-8 & & UNKNOWN & \\
\hline 3897 & 100-B-8 & & UNKNOWN & \\
\hline 3898 & 100-B-8 & & UNKNOWN & \\
\hline 3899 & 100-B-8 & & UNKNOWN & \\
\hline 3900 & $100-C-6$ & & UNKNOWN & \\
\hline 3901 & $100-C-6$ & & UNKNOWN & \\
\hline 3902 & $100-C-6$ & & UNKNOWN & \\
\hline 3903 & $100-C-6$ & & UNKNOWN & \\
\hline 3904 & $100-C-6$ & & UNKNOWN & \\
\hline 3905 & $100-C-6$ & & UNKNOWN & \\
\hline 3906 & $100-C-6$ & & UNKNOWN & \\
\hline 3907 & $100-C-6$ & & UNKNOWN & \\
\hline 3908 & 100-B-8 & & UNKNOWN & \\
\hline 3909 & 100-B-8 & & UNKNOWN & \\
\hline 3910 & 100-B-8 & & UNKNOWN & \\
\hline 3911 & 100-B-8 & & UNKNOWN & \\
\hline 3912 & 100-B-8 & & UNKNOWN & \\
\hline 3913 & $100-C-6$ & & UNKNOWN & \\
\hline 3914 & $100-C-6$ & & UNKNOWN & \\
\hline 3915 & $100-C-6$ & & UNKNOWN & \\
\hline
\end{tabular}




\begin{tabular}{|c|c|c|c|c|}
\hline & $\mathrm{O}$ & $\mathrm{P}$ & $\bar{Q}$ & $\bar{R}$ \\
\hline 1 & SITE_CODE & ELEVATION_UNITS & VERT_COORD_TYPE & DATE_LAST_MODIFIED \\
\hline 3916 & $100-C-6$ & & UNKNOWN & \\
\hline 3917 & $100-C-6$ & & UNKNOWN & \\
\hline 3918 & $100-C-6$ & & UNKNOWN & \\
\hline 3919 & $100-C-6$ & & UNKNOWN & \\
\hline 3920 & $100-C-6$ & & UNKNOWN & \\
\hline 3921 & $100-C-6$ & & UNKNOWN & \\
\hline 3922 & $100-C-6$ & & UNKNOWN & \\
\hline 3923 & $100-C-6$ & & UNKNOWN & \\
\hline 3924 & 100-B-8 & & UNKNOWN & \\
\hline 3925 & $100-C-6$ & & UNKNOWN & \\
\hline 3926 & $100-C-6$ & & UNKNOWN & \\
\hline 3927 & $100-C-6$ & & UNKNOWN & \\
\hline 3928 & $100-C-6$ & & UNKNOWN & \\
\hline 3929 & $100-C-6$ & & UNKNOWN & \\
\hline 3930 & $100-C-6$ & & UNKNOWN & \\
\hline 3931 & $100-C-6$ & & UNKNOWN & \\
\hline 3932 & $100-C-6$ & & UNKNOWN & \\
\hline 3933 & $100-C-6$ & & & \\
\hline 3934 & $100-C-6$ & & & \\
\hline 3935 & $100-C-6$ & & & \\
\hline 3936 & $100-C-6$ & & UNKNOWN & \\
\hline 3937 & $100-C-6$ & & UNKNOWN & \\
\hline 3938 & $100-C-6$ & & UNKNOWN & \\
\hline 3939 & $100-C-6$ & & UNKNOWN & \\
\hline 3940 & $100-C-6$ & & UNKNOWN & \\
\hline 3941 & $100-C-6$ & & UNKNOWN & \\
\hline 3942 & $100-C-6$ & & UNKNOWN & \\
\hline 3943 & $100-C-6$ & & UNKNOWN & \\
\hline 3944 & $100-C-6$ & & UNKNOWN & \\
\hline 3945 & $100-C-6$ & & UNKNOWN & \\
\hline 3946 & 100-C-6 & & UNKNOWN & \\
\hline 3947 & $100-C-6$ & & UNKNOWN & \\
\hline 3948 & $100-C-6$ & & UNKNOWN & \\
\hline 3949 & $100-C-6$ & & UNKNOWN & \\
\hline 3950 & 100-B-8 & & & \\
\hline 3951 & 100-B-8 & & UNKNOWN & \\
\hline 3952 & $100-C-6$ & & UNKNOWN & \\
\hline 3953 & $100-C-6$ & & UNKNOWN & \\
\hline
\end{tabular}




\begin{tabular}{|c|c|c|c|c|}
\hline & $\mathrm{O}$ & $P$ & $\mathrm{Q}$ & $R$ \\
\hline 1 & SITE CODE & ELEVATION UNITS & VERT COORD TYPE & DATE LAST MODIFIED \\
\hline 3954 & $100-C-6$ & & UNKNOWN & \\
\hline 3955 & $100-C-6$ & & UNKNOWN & \\
\hline 3956 & 100-B-8 & & UNKNOWN & \\
\hline 3957 & 100-B-8 & & UNKNOWN & \\
\hline 3958 & $100-C-6$ & & UNKNOWN & \\
\hline 3959 & $100-C-6$ & & UNKNOWN & \\
\hline 3960 & $100-C-6$ & & UNKNOWN & \\
\hline 3961 & $100-C-6$ & & UNKNOWN & \\
\hline 3962 & $100-C-6$ & & UNKNOWN & \\
\hline 3963 & $100-C-6$ & & UNKNOWN & \\
\hline 3964 & 100-C-6 & & UNKNOWN & \\
\hline 3965 & $100-C-6$ & & UNKNOWN & \\
\hline 3966 & 100-B-8 & & & \\
\hline 3967 & 100-B-8 & & & \\
\hline 3968 & 100-B-8 & & UNKNOWN & \\
\hline 3969 & 100-B-8 & & UNKNOWN & \\
\hline 3970 & 100-B-8 & & & \\
\hline 3971 & 100-B-8 & & & \\
\hline 3972 & $116-N-3$ & $\mathrm{~m}$ & NAVD88 & \\
\hline 3973 & $116-\mathrm{N}-3$ & $\mathrm{~m}$ & NAVD88 & \\
\hline 3974 & $116-\mathrm{N}-3$ & $\mathrm{~m}$ & NAVD88 & \\
\hline 3975 & $116-\mathrm{N}-3$ & $\mathrm{~m}$ & NAVD88 & \\
\hline 3976 & $116-\mathrm{N}-3$ & $\mathrm{~m}$ & NAVD88 & \\
\hline 3977 & $116-\mathrm{N}-3$ & $\mathrm{~m}$ & NAVD88 & \\
\hline 3978 & $116-\mathrm{N}-3$ & $\mathrm{~m}$ & NAVD88 & \\
\hline 3979 & $116-\mathrm{N}-3$ & $\mathrm{~m}$ & NAVD88 & \\
\hline 3980 & $116-\mathrm{N}-3$ & $\mathrm{~m}$ & NAVD88 & \\
\hline 3981 & $116-\mathrm{N}-3$ & $\mathrm{~m}$ & NAVD88 & \\
\hline 3982 & $116-\mathrm{N}-3$ & $\mathrm{~m}$ & NAVD88 & \\
\hline 3983 & $116-\mathrm{N}-3$ & $\mathrm{~m}$ & NAVD88 & \\
\hline 3984 & $116-\mathrm{N}-3$ & $\mathrm{~m}$ & NAVD88 & \\
\hline 3985 & 1607-F2 & & & \\
\hline 3986 & 1607-F2 & & & \\
\hline 3987 & 1607-F2 & & & \\
\hline 3988 & $116-\mathrm{N}-3$ & & UNKNOWN & \\
\hline 3989 & $116-\mathrm{N}-3$ & & UNKNOWN & \\
\hline 3990 & $116-\mathrm{N}-3$ & & UNKNOWN & \\
\hline 3991 & $116-\mathrm{N}-3$ & & UNKNOWN & \\
\hline
\end{tabular}




\begin{tabular}{|c|c|c|c|c|}
\hline & $\mathrm{O}$ & $P$ & $\bar{Q}$ & $\bar{R}$ \\
\hline 1 & SITE_CODE & ELEVATION_UNITS & VERT_COORD_TYPE & DATE_LAST_MODIFIED \\
\hline 3992 & $116-\mathrm{N}-3$ & & UNKNOWN & \\
\hline 3993 & $116-\mathrm{N}-3$ & & UNKNOWN & \\
\hline 3994 & $116-\mathrm{N}-3$ & & UNKNOWN & \\
\hline 3995 & $116-\mathrm{N}-3$ & & UNKNOWN & \\
\hline 3996 & $116-\mathrm{N}-3$ & & UNKNOWN & \\
\hline 3997 & $116-\mathrm{N}-3$ & & UNKNOWN & \\
\hline 3998 & $116-\mathrm{N}-3$ & & UNKNOWN & \\
\hline 3999 & $116-\mathrm{N}-3$ & & UNKNOWN & \\
\hline 4000 & $116-\mathrm{N}-3$ & & UNKNOWN & \\
\hline 4001 & $116-\mathrm{N}-3$ & & UNKNOWN & \\
\hline 4002 & $116-\mathrm{N}-3$ & & UNKNOWN & \\
\hline 4003 & $116-\mathrm{N}-3$ & & UNKNOWN & \\
\hline 4004 & $126-F-1$ & & & \\
\hline 4005 & $126-\mathrm{F}-1$ & & & \\
\hline 4006 & 126-F-1 & & & \\
\hline 4007 & 126-F-1 & & & \\
\hline 4008 & 126-F-1 & & & \\
\hline 4009 & $100-C-6$ & & & \\
\hline 4010 & $116-F-2$ & & & \\
\hline 4011 & $116-F-2$ & & & \\
\hline 4012 & 116-F-2 & & & \\
\hline 4013 & 116-F-2 & & & \\
\hline 4014 & $116-F-2$ & & & \\
\hline 4015 & $116-\mathrm{N}-3$ & $\mathrm{~m}$ & NAVD88 & \\
\hline 4016 & $116-N-3$ & $\mathrm{~m}$ & NAVD88 & \\
\hline 4017 & $116-\mathrm{N}-3$ & $\mathrm{~m}$ & NAVD88 & \\
\hline 4018 & $116-\mathrm{N}-3$ & $\mathrm{~m}$ & NAVD88 & \\
\hline 4019 & $116-\mathrm{N}-3$ & $\mathrm{~m}$ & NAVD88 & \\
\hline 4020 & $116-\mathrm{N}-3$ & $\mathrm{~m}$ & NAVD88 & \\
\hline 4021 & $116-\mathrm{N}-3$ & $\mathrm{~m}$ & NAVD88 & \\
\hline 4022 & $116-\mathrm{N}-3$ & $\mathrm{~m}$ & NAVD88 & \\
\hline 4023 & $116-\mathrm{N}-3$ & $\mathrm{~m}$ & NAVD88 & \\
\hline 4024 & $116-\mathrm{N}-3$ & $\mathrm{~m}$ & NAVD88 & \\
\hline 4025 & $116-\mathrm{N}-3$ & $\mathrm{~m}$ & NAVD88 & \\
\hline 4026 & $116-\mathrm{N}-3$ & $\mathrm{~m}$ & NAVD88 & \\
\hline 4027 & $116-\mathrm{N}-3$ & $\mathrm{~m}$ & NAVD88 & \\
\hline 4028 & $116-\mathrm{N}-3$ & $\mathrm{~m}$ & NAVD88 & \\
\hline 4029 & $116-\mathrm{N}-3$ & $\mathrm{~m}$ & NAVD88 & \\
\hline
\end{tabular}




\begin{tabular}{|c|c|c|c|c|}
\hline & $\mathrm{O}$ & $P$ & $\mathrm{Q}$ & $R$ \\
\hline 1 & SITE CODE & ELEVATION UNITS & VERT COORD TYPE & DATE LAST MODIFIED \\
\hline 4030 & $116-\mathrm{N}-3$ & $\mathrm{~m}$ & NAVD88 & \\
\hline 4031 & $116-\mathrm{F}-9$ & & & \\
\hline 4032 & $116-\mathrm{F}-9$ & & & \\
\hline 4033 & 116-F-9 & & & \\
\hline 4034 & $116-F-9$ & & & \\
\hline 4035 & $116-F-9$ & & & \\
\hline 4036 & $116-F-9$ & & & \\
\hline 4037 & 116-F-9 & & & \\
\hline 4038 & $116-\mathrm{F}-9$ & & & \\
\hline 4039 & 116-F-9 & & & \\
\hline 4040 & 116-F-9 & & & \\
\hline 4041 & 100-B-8 & & UNKNOWN & \\
\hline 4042 & 100-B-8 & & UNKNOWN & \\
\hline 4043 & 100-B-8 & & UNKNOWN & \\
\hline 4044 & 100-B-8 & & UNKNOWN & \\
\hline 4045 & 100-B-8 & & UNKNOWN & \\
\hline 4046 & 100-B-8 & & UNKNOWN & \\
\hline 4047 & 100-B-8 & & UNKNOWN & \\
\hline 4048 & 100-B-8 & & UNKNOWN & \\
\hline 4049 & 100-B-8 & & UNKNOWN & \\
\hline 4050 & 100-B-8 & & UNKNOWN & \\
\hline 4051 & 100-B-8 & & UNKNOWN & \\
\hline 4052 & 100-B-8 & & UNKNOWN & \\
\hline 4053 & $116-\mathrm{N}-3$ & $\mathrm{~m}$ & NAVD88 & \\
\hline 4054 & $116-\mathrm{N}-3$ & $\mathrm{~m}$ & NAVD88 & \\
\hline 4055 & $116-\mathrm{N}-3$ & $\mathrm{~m}$ & NAVD88 & \\
\hline 4056 & $116-\mathrm{N}-3$ & $\mathrm{~m}$ & NAVD88 & \\
\hline 4057 & $116-\mathrm{N}-3$ & $\mathrm{~m}$ & NAVD88 & \\
\hline 4058 & $116-\mathrm{N}-3$ & $\mathrm{~m}$ & NAVD88 & \\
\hline 4059 & $116-\mathrm{N}-3$ & $\mathrm{~m}$ & NAVD88 & \\
\hline 4060 & $116-\mathrm{N}-3$ & $\mathrm{~m}$ & NAVD88 & \\
\hline 4061 & 118-B-1 & & UNKNOWN & \\
\hline 4062 & 118-B-1 & & UNKNOWN & \\
\hline 4063 & 118-B-1 & & UNKNOWN & \\
\hline 4064 & 118-B-3 & & & \\
\hline 4065 & 118-B-1 & & UNKNOWN & \\
\hline 4066 & 118-B-1 & & UNKNOWN & \\
\hline 4067 & 118-B-1 & & UNKNOWN & \\
\hline
\end{tabular}




\begin{tabular}{|c|c|c|c|c|}
\hline & $\mathrm{O}$ & $P$ & $\bar{Q}$ & $\bar{R}$ \\
\hline 1 & SITE_CODE & ELEVATION_UNITS & VERT_COORD_TYPE & DATE_LAST_MODIFIED \\
\hline 4068 & 132-B-6 & & UNKNOWN & 08-May-02 \\
\hline 4069 & $100-C-6$ & & UNKNOWN & 08-May-02 \\
\hline 4070 & $116-\mathrm{N}-3$ & $\mathrm{~m}$ & NAVD88 & 20-May-02 \\
\hline 4071 & $116-\mathrm{N}-3$ & $\mathrm{~m}$ & NAVD88 & 20-May-02 \\
\hline 4072 & $116-\mathrm{N}-3$ & $\mathrm{~m}$ & NAVD88 & 20-May-02 \\
\hline 4073 & $116-\mathrm{N}-3$ & $\mathrm{~m}$ & NAVD88 & 20-May-02 \\
\hline 4074 & $116-\mathrm{N}-3$ & $\mathrm{~m}$ & NAVD88 & 21-May-02 \\
\hline 4075 & $116-\mathrm{N}-3$ & $\mathrm{~m}$ & NAVD88 & 21-May-02 \\
\hline 4076 & $116-\mathrm{N}-1$ & $\mathrm{~m}$ & NAVD88 & 21-May-02 \\
\hline 4077 & $116-\mathrm{N}-1$ & $\mathrm{~m}$ & NAVD88 & 21-May-02 \\
\hline 4078 & 116-F-2 & & & 21-May-02 \\
\hline 4079 & $116-F-2$ & & & 21-May-02 \\
\hline 4080 & $116-F-2$ & & & 21-May-02 \\
\hline 4081 & $116-F-2$ & & & 21-May-02 \\
\hline 4082 & $116-\mathrm{N}-1$ & $\mathrm{~m}$ & NAVD88 & 21-May-02 \\
\hline 4083 & $116-\mathrm{N}-1$ & $\mathrm{~m}$ & NAVD88 & 21-May-02 \\
\hline 4084 & $116-\mathrm{N}-1$ & $\mathrm{~m}$ & NAVD88 & 21-May-02 \\
\hline 4085 & $116-\mathrm{N}-1$ & $\mathrm{~m}$ & NAVD88 & 21-May-02 \\
\hline 4086 & $116-\mathrm{N}-1$ & $\mathrm{~m}$ & NAVD88 & 21-May-02 \\
\hline 4087 & $116-\mathrm{N}-3$ & $\mathrm{~m}$ & NAVD88 & 21-May-02 \\
\hline 4088 & $116-\mathrm{N}-3$ & $\mathrm{~m}$ & NAVD88 & 21-May-02 \\
\hline 4089 & $116-F-2$ & & & 21-May-02 \\
\hline 4090 & 116-F-2 & & & 21-May-02 \\
\hline 4091 & $116-F-2$ & & & 21-May-02 \\
\hline 4092 & $116-F-2$ & & & 21-May-02 \\
\hline 4093 & 116-F-2 & & & 21-May-02 \\
\hline 4094 & $116-F-2$ & & & 21-May-02 \\
\hline 4095 & $116-F-2$ & & & 21-May-02 \\
\hline 4096 & $116-F-2$ & & & 21-May-02 \\
\hline 4097 & 116-F-2 & & & 21-May-02 \\
\hline 4098 & 116-F-2 & & & 21-May-02 \\
\hline 4099 & 116-F-2 & & & 21-May-02 \\
\hline 4100 & 116-F-2 & & & 21-May-02 \\
\hline 4101 & $116-F-2$ & & & 21-May-02 \\
\hline 4102 & $116-F-2$ & & & 21-May-02 \\
\hline 4103 & $116-F-2$ & & & 21-May-02 \\
\hline 4104 & $116-F-2$ & & & 21-May-02 \\
\hline 4105 & $116-\mathrm{F}-2$ & & & 21-May-02 \\
\hline
\end{tabular}




\begin{tabular}{|c|c|c|c|c|}
\hline & $\mathrm{O}$ & $P$ & $\bar{Q}$ & $\bar{R}$ \\
\hline 1 & SITE_CODE & ELEVATION_UNITS & VERT_COORD_TYPE & DATE_LAST_MODIFIED \\
\hline 4106 & $116-\mathrm{F}-2$ & & & 21-May-02 \\
\hline 4107 & $116-F-2$ & & & 21-May-02 \\
\hline 4108 & $116-F-2$ & & & 21-May-02 \\
\hline 4109 & $116-\mathrm{N}-3$ & $\mathrm{~m}$ & NAVD88 & 21-May-02 \\
\hline 4110 & $116-\mathrm{N}-3$ & $\mathrm{~m}$ & NAVD88 & 21-May-02 \\
\hline 4111 & $100-C-6$ & & UNKNOWN & 21-May-02 \\
\hline 4112 & $100-C-6$ & & UNKNOWN & 21-May-02 \\
\hline 4113 & 100-B-8 & & UNKNOWN & 21-May-02 \\
\hline 4114 & 100-B-8 & & UNKNOWN & 21-May-02 \\
\hline 4115 & 100-B-8 & & UNKNOWN & 21-May-02 \\
\hline 4116 & 100-B-8 & & UNKNOWN & 21-May-02 \\
\hline 4117 & 100-B-8 & & UNKNOWN & 21-May-02 \\
\hline 4118 & 100-B-8 & & UNKNOWN & 21-May-02 \\
\hline 4119 & 100-B-8 & & UNKNOWN & 21-May-02 \\
\hline 4120 & 100-B-8 & & UNKNOWN & 21-May-02 \\
\hline 4121 & 100-B-8 & & UNKNOWN & 21-May-02 \\
\hline 4122 & 100-B-8 & & UNKNOWN & 21-May-02 \\
\hline 4123 & 100-B-8 & & UNKNOWN & 21-May-02 \\
\hline 4124 & $116-F-2$ & & & 21-May-02 \\
\hline 4125 & 116-F-2 & & & 21-May-02 \\
\hline 4126 & $116-F-2$ & & & 21-May-02 \\
\hline 4127 & $116-F-2$ & & & 21-May-02 \\
\hline 4128 & 116-F-2 & & & 21-May-02 \\
\hline 4129 & $116-F-2$ & & & 21-May-02 \\
\hline 4130 & $116-F-2$ & & & 21-May-02 \\
\hline 4131 & 116-F-2 & & & 21-May-02 \\
\hline 4132 & $116-F-2$ & & & 21-May-02 \\
\hline 4133 & $116-F-2$ & & & 21-May-02 \\
\hline 4134 & $116-\mathrm{F}-2$ & & & 21-May-02 \\
\hline 4135 & 116-F-2 & & & 21-May-02 \\
\hline 4136 & 116-F-2 & & & 21-May-02 \\
\hline 4137 & 116-F-2 & & & 21-May-02 \\
\hline 4138 & 116-F-2 & & & 21-May-02 \\
\hline 4139 & $116-F-2$ & & & 21-May-02 \\
\hline 4140 & $116-F-2$ & & & 21-May-02 \\
\hline 4141 & $116-F-2$ & & & 21-May-02 \\
\hline 4142 & $116-F-2$ & & & 21-May-02 \\
\hline 4143 & 116-F-2 & & & 21-May-02 \\
\hline
\end{tabular}




\begin{tabular}{|c|c|c|c|c|}
\hline & $\mathrm{O}$ & $P$ & $\mathrm{Q}$ & $\mathrm{R}$ \\
\hline 1 & SITE_CODE & ELEVATION_UNITS & VERT_COORD_TYPE & DATE_LAST_MODIFIED \\
\hline 4144 & $116-\mathrm{F}-2$ & & & 21-May-02 \\
\hline 4145 & $116-\mathrm{F}-2$ & & & 21-May-02 \\
\hline 4146 & $116-\mathrm{F}-2$ & & & 21-May-02 \\
\hline 4147 & $116-\mathrm{F}-2$ & & & 21-May-02 \\
\hline 4148 & $116-\mathrm{F}-2$ & & & 21-May-02 \\
\hline 4149 & $116-\mathrm{F}-2$ & & & 21-May-02 \\
\hline 4150 & $116-\mathrm{F}-2$ & & & 21-May-02 \\
\hline 4151 & $116-\mathrm{F}-2$ & & & 21-May-02 \\
\hline 4152 & $116-\mathrm{F}-2$ & & & 21-May-02 \\
\hline 4153 & 116-F-2 & & & 21-May-02 \\
\hline 4154 & 116-F-2 & & & 21-May-02 \\
\hline 4155 & 116-F-2 & & & 21-May-02 \\
\hline 4156 & 116-F-2 & & & 21-May-02 \\
\hline 4157 & $116-\mathrm{F}-2$ & & & 21-May-02 \\
\hline 4158 & $116-F-2$ & & & 21-May-02 \\
\hline 4159 & $116-F-2$ & & & 21-May-02 \\
\hline 4160 & 116-F-2 & & & 21-May-02 \\
\hline 4161 & $116-\mathrm{F}-2$ & & & 21-May-02 \\
\hline 4162 & 116-F-2 & & & 21-May-02 \\
\hline 4163 & $116-\mathrm{F}-2$ & & & 21-May-02 \\
\hline 4164 & $116-\mathrm{F}-2$ & & & 21-May-02 \\
\hline 4165 & $116-F-2$ & & & 21-May-02 \\
\hline 4166 & $116-\mathrm{F}-2$ & & & 21-May-02 \\
\hline 4167 & 116-F-2 & & & 21-May-02 \\
\hline 4168 & 116-F-2 & & & 21-May-02 \\
\hline 4169 & $116-\mathrm{F}-2$ & & & 21-May-02 \\
\hline 4170 & 116-F-2 & & & 21-May-02 \\
\hline 4171 & $116-\mathrm{F}-2$ & & & 21-May-02 \\
\hline 4172 & $116-\mathrm{F}-2$ & & & 21-May-02 \\
\hline 4173 & $116-F-2$ & & & 21-May-02 \\
\hline 4174 & $116-\mathrm{F}-2$ & & & 21-May-02 \\
\hline 4175 & $116-\mathrm{F}-2$ & & & 21-May-02 \\
\hline 4176 & $116-\mathrm{F}-2$ & & & 21-May-02 \\
\hline 4177 & $116-F-2$ & & & 21-May-02 \\
\hline 4178 & $116-\mathrm{F}-2$ & & & 21-May-02 \\
\hline 4179 & $116-F-2$ & & & 21-May-02 \\
\hline 4180 & 116-F-2 & & & 21-May-02 \\
\hline 4181 & $116-\mathrm{F}-2$ & & & 21-May-02 \\
\hline
\end{tabular}




\begin{tabular}{|c|c|c|c|c|}
\hline & $\mathrm{O}$ & $P$ & $\mathrm{Q}$ & $R$ \\
\hline 1 & SITE CODE & ELEVATION UNITS & VERT COORD TYPE & DATE LAST MODIFIED \\
\hline 4182 & $116-\mathrm{F}-2$ & & & 21-May-02 \\
\hline 4183 & $116-\mathrm{F}-2$ & & & 21-May-02 \\
\hline 4184 & $116-\mathrm{F}-2$ & & & 21-May-02 \\
\hline 4185 & 116-F-2 & & & 21-May-02 \\
\hline 4186 & $116-F-2$ & & & 21-May-02 \\
\hline 4187 & $116-F-2$ & & & 21-May-02 \\
\hline 4188 & $116-\mathrm{F}-2$ & & & 21-May-02 \\
\hline 4189 & $116-F-2$ & & & 21-May-02 \\
\hline 4190 & 116-F-2 & & & 21-May-02 \\
\hline 4191 & $116-\mathrm{F}-2$ & & & 21-May-02 \\
\hline 4192 & $116-F-2$ & & & 21-May-02 \\
\hline 4193 & 116-F-2 & & & 21-May-02 \\
\hline 4194 & $116-F-2$ & & & 21-May-02 \\
\hline 4195 & $116-F-2$ & & & 22-May-02 \\
\hline 4196 & $100-C-9$ & & UNKNOWN & 31-May-02 \\
\hline 4197 & $100-C-9$ & & UNKNOWN & 31-May-02 \\
\hline 4198 & $100-C-9$ & & UNKNOWN & 31-May-02 \\
\hline 4199 & $100-C-9$ & & UNKNOWN & 31-May-02 \\
\hline 4200 & $116-F-2$ & & & 11-Jun-02 \\
\hline 4201 & $116-F-2$ & & & 10-Jun-02 \\
\hline 4202 & 116-F-2 & & & 10-Jun-02 \\
\hline 4203 & $116-F-2$ & & & 10-Jun-02 \\
\hline 4204 & 116-F-2 & & & 10-Jun-02 \\
\hline 4205 & $116-F-2$ & & & 10-Jun-02 \\
\hline 4206 & $116-F-2$ & & & 10-Jun-02 \\
\hline 4207 & 116-F-2 & & & 10-Jun-02 \\
\hline 4208 & $116-\mathrm{F}-2$ & & & 10-Jun-02 \\
\hline 4209 & $116-F-2$ & & & 10-Jun-02 \\
\hline 4210 & $100-C-9$ & & UNKNOWN & 10-Jun-02 \\
\hline 4211 & 100-C-9 & & UNKNOWN & 10-Jun-02 \\
\hline 4212 & $116-\mathrm{N}-1$ & $\mathrm{~m}$ & UNKNOWN & 20-Jun-02 \\
\hline 4213 & $116-\mathrm{N}-1$ & $\mathrm{~m}$ & UNKNOWN & 20-Jun-02 \\
\hline 4214 & $116-\mathrm{N}-1$ & $\mathrm{~m}$ & UNKNOWN & 20-Jun-02 \\
\hline 4215 & $116-\mathrm{N}-1$ & $\mathrm{~m}$ & UNKNOWN & 20-Jun-02 \\
\hline 4216 & $116-\mathrm{N}-1$ & $\mathrm{~m}$ & UNKNOWN & 20-Jun-02 \\
\hline 4217 & 100-B-8 & & UNKNOWN & 21-Jun-02 \\
\hline 4218 & 100-B-8 & & UNKNOWN & 21-Jun-02 \\
\hline 4219 & $116-F-2$ & & & 24-Jun-02 \\
\hline
\end{tabular}




\begin{tabular}{|c|c|c|c|c|}
\hline & $\mathrm{O}$ & $\mathrm{P}$ & $\mathrm{Q}$ & $\mathrm{R}$ \\
\hline 1 & SITE_CODE & ELEVATION_UNITS & VERT_COORD_TYPE & DATE_LAST_MODIFIED \\
\hline 4220 & $116-\mathrm{F}-2$ & & & 24-Jun-02 \\
\hline 4221 & $116-F-2$ & & & 24-Jun-02 \\
\hline 4222 & $116-\mathrm{F}-2$ & & & 24-Jun-02 \\
\hline 4223 & 116-F-2 & & & 24-Jun-02 \\
\hline 4224 & $116-\mathrm{F}-2$ & & & 24-Jun-02 \\
\hline 4225 & $116-\mathrm{F}-2$ & & & 24-Jun-02 \\
\hline 4226 & 116-F-2 & & & 24-Jun-02 \\
\hline 4227 & $116-\mathrm{F}-2$ & & & 24-Jun-02 \\
\hline 4228 & 116-F-2 & & & 24-Jun-02 \\
\hline 4229 & & & & 26-Jun-02 \\
\hline 4230 & & & & 26-Jun-02 \\
\hline 4231 & & & & 26-Jun-02 \\
\hline 4232 & & & & 26-Jun-02 \\
\hline 4233 & & & & 26-Jun-02 \\
\hline 4234 & & & & 26-Jun-02 \\
\hline 4235 & & & & 26-Jun-02 \\
\hline 4236 & & & & 26-Jun-02 \\
\hline 4237 & & & & 26-Jun-02 \\
\hline 4238 & & & & 26-Jun-02 \\
\hline 4239 & $116-\mathrm{N}-3$ & $\mathrm{~mm}$ & NAVD88 & 27-Jun-02 \\
\hline 4240 & $116-\mathrm{N}-3$ & $\mathrm{~mm}$ & NAVD88 & 27-Jun-02 \\
\hline 4241 & $116-F-2$ & & & 27-Jun-02 \\
\hline 4242 & $276-S$ & & & 29-Jul-02 \\
\hline 4243 & $276-S$ & & & 29-Jul-02 \\
\hline 4244 & $276-S$ & & & 29-Jul-02 \\
\hline 4245 & 293-S & & & 14-Aug-02 \\
\hline 4246 & $271-U$ & & & 30-Jul-02 \\
\hline 4247 & 233-SA & & & 14-Aug-02 \\
\hline 4248 & & & & 07-Aug-02 \\
\hline 4249 & & & & 07-Aug-02 \\
\hline 4250 & & & & 07-Aug-02 \\
\hline 4251 & & & & 07-Aug-02 \\
\hline 4252 & & & & 07-Aug-02 \\
\hline 4253 & & & & 07-Aug-02 \\
\hline 4254 & & & & 07-Aug-02 \\
\hline 4255 & & & & 07-Aug-02 \\
\hline 4256 & & & & 07-Aug-02 \\
\hline 4257 & & & & 07-Aug-02 \\
\hline
\end{tabular}




\begin{tabular}{|c|l|l|r|r|}
\hline & \multicolumn{1}{|c|}{ O } & P & Q & R \\
\hline 1 & SITE_CODE & ELEVATION_UNITS & VERT_COORD_TYPE & DATE_LAST_MODIFIED \\
\hline 4258 & & & $07-A u g-02$ \\
\hline 4259 & & & $07-A u g-02$ \\
\hline 4260 & & & $07-A u g-02$ \\
\hline 4261 & $221-U$ & & $14-A u g-02$ \\
\hline 4262 & $221-U$ & & $14-A u g-02$ \\
\hline 4263 & $221-U$ & & $14-A u g-02$ \\
\hline 4264 & $221-U$ & & $14-A u g-02$ \\
\hline 4265 & $221-U$ & & $14-A u g-02$ \\
\hline 4266 & $221-U$ & & $14-A u g-02$ \\
\hline 4267 & $221-U$ & & $14-A u g-02$ \\
\hline 4268 & $221-U$ & & $14-A u g-02$ \\
\hline 4269 & $221-U$ & & $14-A u g-02$ \\
\hline 4270 & $221-U$ & & $14-A u g-02$ \\
\hline 4271 & $221-U$ & & $14-A u g-02$ \\
\hline 4272 & $221-U$ & & $14-A u g-02$ \\
\hline 4273 & $221-U$ & & $14-A u g-02$ \\
\hline 4274 & $221-U$ & & $14-A u g-02$ \\
\hline 4275 & $221-U$ & & $14-A u g-02$ \\
\hline 4276 & $221-U$ & & $14-A u g-02$ \\
\hline 4277 & $221-U$ & & $14-A u g-02$ \\
\hline 4278 & $221-U$ & & $14-A u g-02$ \\
\hline 4279 & $221-U$ & & $14-A u g-02$ \\
\hline 4280 & $221-U$ & & $14-A u g-02$ \\
\hline 4281 & $221-U$ & & $14-A u g-02$ \\
\hline 4282 & $221-U$ & & $14-A u g-02$ \\
\hline 4283 & $221-U$ & & $14-A u g-02$ \\
\hline 4284 & $221-U$ & & $14-A u g-02$ \\
\hline 4285 & $221-U$ & & $14-A u g-02$ \\
\hline 4286 & $221-U$ & & $14-A u g-02$ \\
\hline 4287 & $221-U$ & & $14-A u g-02$ \\
\hline 4288 & $221-U$ & & $14-$ Aug-02 \\
\hline 4289 & $221-U$ & & $14-A u g-02$ \\
\hline 4290 & $221-U$ & & $14-A u g-02$ \\
\hline 4291 & $221-U$ & & $14-A u g-02$ \\
\hline 4292 & $221-U$ & & $14-A u g-02$ \\
\hline 4293 & $221-U$ & & $14-A u g-02$ \\
\hline 4294 & $221-U$ & & $14-A u g-02$ \\
\hline 4295 & $221-U$ & & \\
\hline & & & & \\
\hline
\end{tabular}




\begin{tabular}{|c|c|c|c|c|}
\hline & $\mathrm{O}$ & $\mathrm{P}$ & $\bar{Q}$ & $\bar{R}$ \\
\hline 1 & SITE_CODE & ELEVATION_UNITS & VERT_COORD_TYPE & DATE_LAST_MODIFIED \\
\hline 4296 & $221-U$ & & & 14-Aug-02 \\
\hline 4297 & $221-U$ & & & 14-Aug-02 \\
\hline 4298 & $221-U$ & & & 14-Aug-02 \\
\hline 4299 & $224-B$ & & & 14-Aug-02 \\
\hline 4300 & & & & 14-Aug-02 \\
\hline 4301 & & & & 14-Aug-02 \\
\hline 4302 & $291-U$ & & & 09-Aug-02 \\
\hline 4303 & $291-U$ & & & 09-Aug-02 \\
\hline 4304 & $291-U$ & & & 09-Aug-02 \\
\hline 4305 & $291-U$ & & & 09-Aug-02 \\
\hline 4306 & & & & 14-Aug-02 \\
\hline 4307 & & & & 14-Aug-02 \\
\hline 4308 & $2718-S$ & & & 09-Aug-02 \\
\hline 4309 & $2718-S$ & & & 09-Aug-02 \\
\hline 4310 & $2718-S$ & & & 09-Aug-02 \\
\hline 4311 & $202-S$ & & & 09-Aug-02 \\
\hline 4312 & $202-S$ & & & 09-Aug-02 \\
\hline 4313 & $276-S$ & & & 09-Aug-02 \\
\hline 4314 & $276-S$ & & & 09-Aug-02 \\
\hline 4315 & & & & 09-Aug-02 \\
\hline 4316 & & & & 09-Aug-02 \\
\hline 4317 & & & & 09-Aug-02 \\
\hline 4318 & & & & 09-Aug-02 \\
\hline 4319 & & & & 09-Aug-02 \\
\hline 4320 & & & & 09-Aug-02 \\
\hline 4321 & $215-C$ & & & 09-Aug-02 \\
\hline 4322 & $215-C$ & & & 09-Aug-02 \\
\hline 4323 & $224-B$ & & & 09-Aug-02 \\
\hline 4324 & $224-B$ & & & 09-Aug-02 \\
\hline 4325 & $221-U$ & & & 14-Aug-02 \\
\hline 4326 & $221-U$ & & & 14-Aug-02 \\
\hline 4327 & $221-U$ & & & 14-Aug-02 \\
\hline 4328 & $221-U$ & & & 14-Aug-02 \\
\hline 4329 & $221-U$ & & & 14-Aug-02 \\
\hline 4330 & $221-U$ & & & 14-Aug-02 \\
\hline 4331 & $221-U$ & & & 14-Aug-02 \\
\hline 4332 & $221-U$ & & & 14-Aug-02 \\
\hline 4333 & $221-U$ & & & 14-Aug-02 \\
\hline
\end{tabular}




\begin{tabular}{|c|c|c|c|c|}
\hline & $\mathrm{O}$ & $P$ & $\mathrm{Q}$ & $\mathrm{R}$ \\
\hline 1 & SITE_CODE & ELEVATION_UNITS & VERT_COORD_TYPE & DATE_LAST_MODIFIED \\
\hline 4334 & $221-U$ & & & 14-Aug-02 \\
\hline 4335 & 216-T-26 & & & 14-Aug-02 \\
\hline 4336 & 216-T-26 & & & 14-Aug-02 \\
\hline 4337 & 216-T-26 & & & 14-Aug-02 \\
\hline 4338 & 216-T-26 & & & 14-Aug-02 \\
\hline 4339 & 216-T-26 & & & 14-Aug-02 \\
\hline 4340 & 216-T-26 & & & 14-Aug-02 \\
\hline 4341 & 216-B-7A\&B & & & 14-Aug-02 \\
\hline 4342 & 216-B-7A\&B & & & 14-Aug-02 \\
\hline 4343 & 216-B-7A\&B & & & 14-Aug-02 \\
\hline 4344 & 216-B-38 & & & 12-Aug-02 \\
\hline 4345 & 216-B-38 & & & 12-Aug-02 \\
\hline 4346 & 216-T-26 & & & 14-Aug-02 \\
\hline 4347 & 216-B-7A\&B & & & 14-Aug-02 \\
\hline 4348 & 216-B-38 & & & 12-Aug-02 \\
\hline 4349 & 216-T-26 & & & 14-Aug-02 \\
\hline 4350 & 216-T-26 & & & 14-Aug-02 \\
\hline 4351 & 216-T-26 & & & 14-Aug-02 \\
\hline 4352 & 216-T-26 & & & 14-Aug-02 \\
\hline 4353 & 216-T-26 & & & 14-Aug-02 \\
\hline 4354 & 216-T-26 & & & 14-Aug-02 \\
\hline 4355 & 216-T-26 & & & 14-Aug-02 \\
\hline 4356 & 216-T-26 & & & 14-Aug-02 \\
\hline 4357 & 216-T-26 & & & 14-Aug-02 \\
\hline 4358 & 216-T-26 & & & 14-Aug-02 \\
\hline 4359 & 216-T-26 & & & 14-Aug-02 \\
\hline 4360 & 216-T-26 & & & 14-Aug-02 \\
\hline 4361 & 216-B-38 & & & 12-Aug-02 \\
\hline 4362 & 216-B-38 & & & 12-Aug-02 \\
\hline 4363 & 216-B-38 & & & 12-Aug-02 \\
\hline 4364 & 216-B-38 & & & 12-Aug-02 \\
\hline 4365 & 216-B-38 & & & 12-Aug-02 \\
\hline 4366 & 216-B-38 & & & 12-Aug-02 \\
\hline 4367 & 216-B-38 & & & 12-Aug-02 \\
\hline 4368 & 216-B-38 & & & 12-Aug-02 \\
\hline 4369 & 216-B-38 & & & 12-Aug-02 \\
\hline 4370 & 216-B-38 & & & 12-Aug-02 \\
\hline 4371 & 216-B-38 & & & 12-Aug-02 \\
\hline
\end{tabular}




\begin{tabular}{|c|c|c|c|c|}
\hline & $\mathrm{O}$ & $P$ & $\mathrm{Q}$ & $R$ \\
\hline 1 & SITE_CODE & ELEVATION_UNITS & VERT_COORD_TYPE & DATE_LAST_MODIFIED \\
\hline 4372 & $216-B-38$ & & & 12-Aug-02 \\
\hline 4373 & 216-B-7A\&B & & & 14-Aug-02 \\
\hline 4374 & 216-B-7A\&B & & & 14-Aug-02 \\
\hline 4375 & 216-B-7A\&B & & & 14-Aug-02 \\
\hline 4376 & 216-B-7A\&B & & & 14-Aug-02 \\
\hline 4377 & 216-B-7A\&B & & & 14-Aug-02 \\
\hline 4378 & 216-B-7A\&B & & & 14-Aug-02 \\
\hline 4379 & 216-B-7A\&B & & & 14-Aug-02 \\
\hline 4380 & 216-B-7A\&B & & & 14-Aug-02 \\
\hline 4381 & 216-B-7A\&B & & & 14-Aug-02 \\
\hline 4382 & 216-B-7A\&B & & & 14-Aug-02 \\
\hline 4383 & 216-B-7A\&B & & & 14-Aug-02 \\
\hline 4384 & 216-B-7A\&B & & & 14-Aug-02 \\
\hline 4385 & 216-B-7A\&B & & & 14-Aug-02 \\
\hline 4386 & 216-B-7A\&B & & & 14-Aug-02 \\
\hline 4387 & 216-B-7A\&B & & & 14-Aug-02 \\
\hline 4388 & 216-B-7A\&B & & & 14-Aug-02 \\
\hline 4389 & 216-B-7A\&B & & & 14-Aug-02 \\
\hline 4390 & 216-B-7A\&B & & & 14-Aug-02 \\
\hline 4391 & 216-T-26 & & & 14-Aug-02 \\
\hline 4392 & 216-B-38 & & & 12-Aug-02 \\
\hline 4393 & 216-B-38 & & & 12-Aug-02 \\
\hline 4394 & 216-B-38 & & & 12-Aug-02 \\
\hline 4395 & 216-B-7A\&B & & & 14-Aug-02 \\
\hline 4396 & 216-B-38 & & & 12-Aug-02 \\
\hline 4397 & 216-B-38 & & & 12-Aug-02 \\
\hline 4398 & 216-B-38 & & & 12-Aug-02 \\
\hline 4399 & 216-B-7A\&B & & & 14-Aug-02 \\
\hline 4400 & 216-B-7A\&B & & & 14-Aug-02 \\
\hline 4401 & 216-B-7A\&B & & & 14-Aug-02 \\
\hline 4402 & 216-B-7A\&B & & & 14-Aug-02 \\
\hline 4403 & 216-B-7A\&B & & & 14-Aug-02 \\
\hline 4404 & 216-B-7A\&B & & & 14-Aug-02 \\
\hline 4405 & 216-B-7A\&B & & & 14-Aug-02 \\
\hline 4406 & 216-B-7A\&B & & & 14-Aug-02 \\
\hline 4407 & 216-T-26 & & & 14-Aug-02 \\
\hline 4408 & 216-T-26 & & & 14-Aug-02 \\
\hline 4409 & 216-T-26 & & & 14-Aug-02 \\
\hline
\end{tabular}




\begin{tabular}{|c|c|c|c|c|}
\hline & $\mathrm{O}$ & $P$ & $\bar{Q}$ & $\bar{R}$ \\
\hline 1 & SITE_CODE & ELEVATION_UNITS & VERT_COORD_TYPE & DATE_LAST_MODIFIED \\
\hline 4410 & 216-T-26 & & & 14-Aug-02 \\
\hline 4411 & 216-T-26 & & & 14-Aug-02 \\
\hline 4412 & 216-T-26 & & & 14-Aug-02 \\
\hline 4413 & 216-T-26 & & & 14-Aug-02 \\
\hline 4414 & 216-T-26 & & & 14-Aug-02 \\
\hline 4415 & 216-T-26 & & & 14-Aug-02 \\
\hline 4416 & 216-T-26 & & & 14-Aug-02 \\
\hline 4417 & 216-T-26 & & & 14-Aug-02 \\
\hline 4418 & 216-B-7A\&B & & & 14-Aug-02 \\
\hline 4419 & 216-B-38 & & & 12-Aug-02 \\
\hline 4420 & 216-T-26 & & & 14-Aug-02 \\
\hline 4421 & 216-B-38 & & & 12-Aug-02 \\
\hline 4422 & 216-B-38 & & & 12-Aug-02 \\
\hline 4423 & 216-B-38 & & & 12-Aug-02 \\
\hline 4424 & 216-B-38 & & & 12-Aug-02 \\
\hline 4425 & 216-B-38 & & & 12-Aug-02 \\
\hline 4426 & 216-B-38 & & & 12-Aug-02 \\
\hline 4427 & 216-B-38 & & & 12-Aug-02 \\
\hline 4428 & 216-B-38 & & & 12-Aug-02 \\
\hline 4429 & 216-B-38 & & & 12-Aug-02 \\
\hline 4430 & 216-B-38 & & & 12-Aug-02 \\
\hline 4431 & 216-B-38 & & & 12-Aug-02 \\
\hline 4432 & 216-B-7A\&B & & & 14-Aug-02 \\
\hline 4433 & 216-B-7A\&B & & & 14-Aug-02 \\
\hline 4434 & 216-T-26 & & & 14-Aug-02 \\
\hline 4435 & 216-B-38 & & & 12-Aug-02 \\
\hline 4436 & 216-B-38 & & & 12-Aug-02 \\
\hline 4437 & 216-B-38 & & & 12-Aug-02 \\
\hline 4438 & 216-B-7A\&B & & & 14-Aug-02 \\
\hline 4439 & 216-B-7A\&B & & & 14-Aug-02 \\
\hline 4440 & 216-B-7A\&B & & & 14-Aug-02 \\
\hline 4441 & 216-B-7A\&B & & & 14-Aug-02 \\
\hline 4442 & 216-B-7A\&B & & & 14-Aug-02 \\
\hline 4443 & 216-B-7A\&B & & & 14-Aug-02 \\
\hline 4444 & 216-B-7A\&B & & & 14-Aug-02 \\
\hline 4445 & 216-B-7A\&B & & & 14-Aug-02 \\
\hline 4446 & 216-B-7A\&B & & & 14-Aug-02 \\
\hline 4447 & 216-B-7A\&B & & & 14-Aug-02 \\
\hline
\end{tabular}




\begin{tabular}{|c|c|c|r|r|}
\hline & \multicolumn{1}{|c|}{ O } & P & Q & R \\
\hline 1 & SITE_CODE & ELEVATION_UNITS & VERT_COORD_TYPE & DATE_LAST_MODIFIED \\
\hline 4448 & $216-B-7 A \& B$ & & $14-A u g-02$ \\
\hline 4449 & $216-B-7 A \& B$ & & $14-A u g-02$ \\
\hline 4450 & $216-B-7 A \& B$ & & $14-A u g-02$ \\
\hline 4451 & $216-B-7 A \& B$ & & $14-A u g-02$ \\
\hline 4452 & $216-B-7 A \& B$ & & $14-A u g-02$ \\
\hline 4453 & $216-B-7 A \& B$ & & $14-A u g-02$ \\
\hline 4454 & $216-B-7 A \& B$ & & $14-A u g-02$ \\
\hline 4455 & $216-B-7 A \& B$ & & $14-A u g-02$ \\
\hline 4456 & $216-B-7 A \& B$ & & $14-A u g-02$ \\
\hline 4457 & $216-B-7 A \& B$ & & $14-A u g-02$ \\
\hline 4458 & $216-B-7 A \& B$ & & $14-A u g-02$ \\
\hline 4459 & $216-B-7 A \& B$ & & $14-A u g-02$ \\
\hline 4460 & $216-B-7 A \& B$ & & $14-A u g-02$ \\
\hline 4461 & $216-B-7 A \& B$ & & $14-A u g-02$ \\
\hline 4462 & $216-B-7 A \& B$ & & $14-A u g-02$ \\
\hline 4463 & $216-B-7 A \& B$ & & $14-A u g-02$ \\
\hline 4464 & $216-B-7 A \& B$ & & $14-A u g-02$ \\
\hline 4465 & $216-B-7 A \& B$ & & $14-A u g-02$ \\
\hline 4466 & & & $12-A u g-02$ \\
\hline 4467 & & & $12-A u g-02$ \\
\hline 4468 & & & $12-A u g-02$ \\
\hline 4469 & & & $12-A u g-02$ \\
\hline 4470 & & & $12-A u g-02$ \\
\hline 4471 & & & $12-A u g-02$ \\
\hline 4472 & & & $12-A u g-02$ \\
\hline 4473 & & & $12-A u g-02$ \\
\hline 4474 & & & $12-A u g-02$ \\
\hline 4475 & & & $12-A u g-02$ \\
\hline 4476 & & & $12-A u g-02$ \\
\hline 4477 & $271-U$ & & $13-A u g-02$ \\
\hline 4478 & $271-U$ & & $13-A u g-02$ \\
\hline 4479 & $271-U$ & & $13-A u g-02$ \\
\hline 4480 & $271-U$ & & $13-A u g-02$ \\
\hline 4481 & $271-U$ & & $13-A u g-02$ \\
\hline 4482 & $271-U$ & & $13-A u g-02$ \\
\hline 4483 & $271-U$ & & $13-A u g-02$ \\
\hline 4484 & & & $14-A u g-02$ \\
\hline 4485 & & & $14-A u g-02$ \\
\hline & & & \\
\hline
\end{tabular}




\begin{tabular}{|c|c|c|c|c|}
\hline & $\mathrm{O}$ & $\mathrm{P}$ & $\bar{Q}$ & $\mathrm{R}$ \\
\hline 1 & SITE_CODE & ELEVATION_UNITS & VERT_COORD_TYPE & DATE_LAST_MODIFIED \\
\hline 4486 & & & & 13-Aug-02 \\
\hline 4487 & & & & 13-Aug-02 \\
\hline 4488 & & & & 13-Aug-02 \\
\hline 4489 & & & & 13-Aug-02 \\
\hline 4490 & & & & 13-Aug-02 \\
\hline 4491 & & & & 13-Aug-02 \\
\hline 4492 & & & & 13-Aug-02 \\
\hline 4493 & & & & 13-Aug-02 \\
\hline 4494 & & & & 13-Aug-02 \\
\hline 4495 & & & & 13-Aug-02 \\
\hline 4496 & & & & 13-Aug-02 \\
\hline 4497 & & & & 13-Aug-02 \\
\hline 4498 & & & & 13-Aug-02 \\
\hline 4499 & & & & 13-Aug-02 \\
\hline 4500 & & & & 13-Aug-02 \\
\hline 4501 & & & & 13-Aug-02 \\
\hline 4502 & & & & 13-Aug-02 \\
\hline 4503 & & & & 13-Aug-02 \\
\hline 4504 & & & & 13-Aug-02 \\
\hline 4505 & & & & 13-Aug-02 \\
\hline 4506 & & & & 13-Aug-02 \\
\hline 4507 & & & & 13-Aug-02 \\
\hline 4508 & & & & 13-Aug-02 \\
\hline 4509 & & & & 13-Aug-02 \\
\hline 4510 & & & & 13-Aug-02 \\
\hline 4511 & & & & 13-Aug-02 \\
\hline 4512 & & & & 13-Aug-02 \\
\hline 4513 & & & & 13-Aug-02 \\
\hline 4514 & & & & 13-Aug-02 \\
\hline 4515 & & & & 13-Aug-02 \\
\hline 4516 & & & & 13-Aug-02 \\
\hline 4517 & & & & 13-Aug-02 \\
\hline 4518 & & & & 13-Aug-02 \\
\hline 4519 & & & & 13-Aug-02 \\
\hline 4520 & & & & 13-Aug-02 \\
\hline 4521 & & & & 13-Aug-02 \\
\hline 4522 & & & & 13-Aug-02 \\
\hline 4523 & & & & 13-Aug-02 \\
\hline
\end{tabular}




\begin{tabular}{|c|c|c|c|c|}
\hline & $\mathrm{O}$ & $P$ & $\mathrm{Q}$ & $R$ \\
\hline 1 & SITE CODE & ELEVATION UNITS & VERT COORD TYPE & DATE LAST MODIFIED \\
\hline 4524 & & & & 13-Aug-02 \\
\hline 4525 & & & & 13-Aug-02 \\
\hline 4526 & & & & 13-Aug-02 \\
\hline 4527 & & & & 13-Aug-02 \\
\hline 4528 & & & & 13-Aug-02 \\
\hline 4529 & & & & 13-Aug-02 \\
\hline 4530 & & & & 13-Aug-02 \\
\hline 4531 & & & & 13-Aug-02 \\
\hline 4532 & $271-U$ & & & 13-Aug-02 \\
\hline 4533 & & & & 14-Aug-02 \\
\hline 4534 & & & & 14-Aug-02 \\
\hline 4535 & & & & 14-Aug-02 \\
\hline 4536 & & & & 14-Aug-02 \\
\hline 4537 & & & & 14-Aug-02 \\
\hline 4538 & & & & 14-Aug-02 \\
\hline 4539 & & & & 14-Aug-02 \\
\hline 4540 & & & & 14-Aug-02 \\
\hline 4541 & & & & 14-Aug-02 \\
\hline 4542 & & & & 14-Aug-02 \\
\hline 4543 & & & & 14-Aug-02 \\
\hline 4544 & & & & 14-Aug-02 \\
\hline 4545 & & & & 14-Aug-02 \\
\hline 4546 & & & & 14-Aug-02 \\
\hline 4547 & & & & 14-Aug-02 \\
\hline 4548 & & & & 14-Aug-02 \\
\hline 4549 & & & & 14-Aug-02 \\
\hline 4550 & & & & 14-Aug-02 \\
\hline 4551 & & & & 14-Aug-02 \\
\hline 4552 & & & & 14-Aug-02 \\
\hline 4553 & & & & 14-Aug-02 \\
\hline 4554 & & & & 14-Aug-02 \\
\hline 4555 & & & & 14-Aug-02 \\
\hline 4556 & & & & 14-Aug-02 \\
\hline 4557 & & & & 14-Aug-02 \\
\hline 4558 & & & & 14-Aug-02 \\
\hline 4559 & & & & 14-Aug-02 \\
\hline 4560 & & & & 14-Aug-02 \\
\hline 4561 & & & & 14-Aug-02 \\
\hline
\end{tabular}




\begin{tabular}{|c|c|c|c|c|}
\hline & $\mathrm{O}$ & $\mathrm{P}$ & $\bar{Q}$ & $\mathrm{R}$ \\
\hline 1 & SITE_CODE & ELEVATION_UNITS & VERT_COORD_TYPE & DATE_LAST_MODIFIED \\
\hline 4562 & & & & 14-Aug-02 \\
\hline 4563 & & & & 14-Aug-02 \\
\hline 4564 & & & & 14-Aug-02 \\
\hline 4565 & & & & 14-Aug-02 \\
\hline 4566 & & & & 14-Aug-02 \\
\hline 4567 & & & & 14-Aug-02 \\
\hline 4568 & & & & 14-Aug-02 \\
\hline 4569 & & & & 14-Aug-02 \\
\hline 4570 & & & & 14-Aug-02 \\
\hline 4571 & & & & 14-Aug-02 \\
\hline 4572 & & & & 14-Aug-02 \\
\hline 4573 & & & & 14-Aug-02 \\
\hline 4574 & & & & 14-Aug-02 \\
\hline 4575 & & & & 14-Aug-02 \\
\hline 4576 & & & & 14-Aug-02 \\
\hline 4577 & & & & 14-Aug-02 \\
\hline 4578 & & & & 14-Aug-02 \\
\hline 4579 & & & & 14-Aug-02 \\
\hline 4580 & & & & 14-Aug-02 \\
\hline 4581 & & & & 14-Aug-02 \\
\hline 4582 & & & & 14-Aug-02 \\
\hline 4583 & & & & 14-Aug-02 \\
\hline 4584 & & & & 14-Aug-02 \\
\hline 4585 & & & & 14-Aug-02 \\
\hline 4586 & & & & 14-Aug-02 \\
\hline 4587 & & & & 14-Aug-02 \\
\hline 4588 & & & & 14-Aug-02 \\
\hline 4589 & & & & 14-Aug-02 \\
\hline 4590 & & & & 14-Aug-02 \\
\hline 4591 & & & & 14-Aug-02 \\
\hline 4592 & & & & 14-Aug-02 \\
\hline 4593 & & & & 14-Aug-02 \\
\hline 4594 & & & & 14-Aug-02 \\
\hline 4595 & & & & 14-Aug-02 \\
\hline 4596 & & & & 14-Aug-02 \\
\hline 4597 & & & & 14-Aug-02 \\
\hline 4598 & & & & 14-Aug-02 \\
\hline 4599 & & & & 14-Aug-02 \\
\hline
\end{tabular}




\begin{tabular}{|c|c|c|c|c|}
\hline & $\mathrm{O}$ & $\mathrm{P}$ & $\bar{Q}$ & $\mathrm{R}$ \\
\hline 1 & SITE_CODE & ELEVATION_UNITS & VERT_COORD_TYPE & DATE_LAST_MODIFIED \\
\hline 4600 & & & & 14-Aug-02 \\
\hline 4601 & & & & 14-Aug-02 \\
\hline 4602 & & & & 14-Aug-02 \\
\hline 4603 & & & & 14-Aug-02 \\
\hline 4604 & & & & 14-Aug-02 \\
\hline 4605 & & & & 14-Aug-02 \\
\hline 4606 & & & & 14-Aug-02 \\
\hline 4607 & & & & 14-Aug-02 \\
\hline 4608 & & & & 14-Aug-02 \\
\hline 4609 & & & & 14-Aug-02 \\
\hline 4610 & & & & 14-Aug-02 \\
\hline 4611 & & & & 14-Aug-02 \\
\hline 4612 & & & & 14-Aug-02 \\
\hline 4613 & & & & 14-Aug-02 \\
\hline 4614 & & & & 14-Aug-02 \\
\hline 4615 & & & & 14-Aug-02 \\
\hline 4616 & & & & 14-Aug-02 \\
\hline 4617 & & & & 14-Aug-02 \\
\hline 4618 & & & & 14-Aug-02 \\
\hline 4619 & & & & 14-Aug-02 \\
\hline 4620 & & & & 14-Aug-02 \\
\hline 4621 & & & & 14-Aug-02 \\
\hline 4622 & & & & 14-Aug-02 \\
\hline 4623 & & & & 14-Aug-02 \\
\hline 4624 & & & & 14-Aug-02 \\
\hline 4625 & & & & 14-Aug-02 \\
\hline 4626 & & & & 14-Aug-02 \\
\hline 4627 & & & & 14-Aug-02 \\
\hline 4628 & & & & 14-Aug-02 \\
\hline 4629 & & & & 14-Aug-02 \\
\hline 4630 & & & & 14-Aug-02 \\
\hline 4631 & & & & 14-Aug-02 \\
\hline 4632 & & & & 14-Aug-02 \\
\hline 4633 & & & & 14-Aug-02 \\
\hline 4634 & & & & 14-Aug-02 \\
\hline 4635 & & & & 14-Aug-02 \\
\hline 4636 & & & & 14-Aug-02 \\
\hline 4637 & & & & 14-Aug-02 \\
\hline
\end{tabular}




\begin{tabular}{|c|c|c|c|c|}
\hline & $\mathrm{O}$ & $\mathrm{P}$ & $\mathrm{Q}$ & $\mathrm{R}$ \\
\hline 1 & SITE_CODE & ELEVATION_UNITS & VERT_COORD_TYPE & DATE_LAST_MODIFIED \\
\hline 4638 & & & & 14-Aug-02 \\
\hline 4639 & & & & 14-Aug-02 \\
\hline 4640 & & & & 14-Aug-02 \\
\hline 4641 & & & & 14-Aug-02 \\
\hline 4642 & & & & 14-Aug-02 \\
\hline 4643 & & & & 14-Aug-02 \\
\hline 4644 & & & & 14-Aug-02 \\
\hline 4645 & & & & 14-Aug-02 \\
\hline 4646 & & & & 14-Aug-02 \\
\hline 4647 & & & & 14-Aug-02 \\
\hline 4648 & & & & 14-Aug-02 \\
\hline 4649 & & & & 14-Aug-02 \\
\hline 4650 & & & & 14-Aug-02 \\
\hline 4651 & $202-S$ & & & 14-Aug-02 \\
\hline 4652 & $202-S$ & & & 14-Aug-02 \\
\hline 4653 & $202-S$ & & & 14-Aug-02 \\
\hline 4654 & $202-S$ & & & 14-Aug-02 \\
\hline 4655 & $202-S$ & & & 14-Aug-02 \\
\hline 4656 & $202-S$ & & & 14-Aug-02 \\
\hline 4657 & $618-11$ & & & 14-Aug-02 \\
\hline 4658 & $618-11$ & & & 14-Aug-02 \\
\hline 4659 & $618-11$ & & & 14-Aug-02 \\
\hline 4660 & $618-11$ & & & 14-Aug-02 \\
\hline 4661 & $618-11$ & & & 14-Aug-02 \\
\hline 4662 & & & & 15-Aug-02 \\
\hline 4663 & & & & 15-Aug-02 \\
\hline 4664 & & & & 15-Aug-02 \\
\hline 4665 & & & & 15-Aug-02 \\
\hline 4666 & 216-B-7A\&B & & & 19-Aug-02 \\
\hline 4667 & & & & 19-Aug-02 \\
\hline 4668 & & & & 19-Aug-02 \\
\hline 4669 & & & & 19-Aug-02 \\
\hline 4670 & & & & 19-Aug-02 \\
\hline 4671 & & & & 19-Aug-02 \\
\hline 4672 & & & & 19-Aug-02 \\
\hline 4673 & & & & 19-Aug-02 \\
\hline 4674 & & & & 19-Aug-02 \\
\hline 4675 & & & & 19-Aug-02 \\
\hline
\end{tabular}




\begin{tabular}{|c|c|c|c|c|}
\hline & $\mathrm{O}$ & $P$ & $\mathrm{Q}$ & $R$ \\
\hline 1 & SITE CODE & ELEVATION UNITS & VERT COORD TYPE & DATE LAST MODIFIED \\
\hline 4676 & & & & 19-Aug-02 \\
\hline 4677 & & & & 19-Aug-02 \\
\hline 4678 & & & & 19-Aug-02 \\
\hline 4679 & & & & 19-Aug-02 \\
\hline 4680 & & & & 19-Aug-02 \\
\hline 4681 & & & & 19-Aug-02 \\
\hline 4682 & & & & 19-Aug-02 \\
\hline 4683 & & & & 19-Aug-02 \\
\hline 4684 & & & & 19-Aug-02 \\
\hline 4685 & & & & 19-Aug-02 \\
\hline 4686 & & & & 19-Aug-02 \\
\hline 4687 & & & & 19-Aug-02 \\
\hline 4688 & & & & 19-Aug-02 \\
\hline 4689 & & & & 19-Aug-02 \\
\hline 4690 & & & & 19-Aug-02 \\
\hline 4691 & & & & 19-Aug-02 \\
\hline 4692 & & & & 19-Aug-02 \\
\hline 4693 & & & & 19-Aug-02 \\
\hline 4694 & & & & 19-Aug-02 \\
\hline 4695 & & & & 19-Aug-02 \\
\hline 4696 & & & & 19-Aug-02 \\
\hline 4697 & & & & 19-Aug-02 \\
\hline 4698 & & & & 19-Aug-02 \\
\hline 4699 & & & & 19-Aug-02 \\
\hline 4700 & & & & 19-Aug-02 \\
\hline 4701 & & & & 19-Aug-02 \\
\hline 4702 & & & & 19-Aug-02 \\
\hline 4703 & & & & 19-Aug-02 \\
\hline 4704 & & & & 19-Aug-02 \\
\hline 4705 & & & & 19-Aug-02 \\
\hline 4706 & & & & 19-Aug-02 \\
\hline 4707 & & & & 19-Aug-02 \\
\hline 4708 & & & & 19-Aug-02 \\
\hline 4709 & & & & 19-Aug-02 \\
\hline 4710 & & & & 19-Aug-02 \\
\hline 4711 & & & & 19-Aug-02 \\
\hline 4712 & & & & 19-Aug-02 \\
\hline 4713 & & & & 19-Aug-02 \\
\hline
\end{tabular}




\begin{tabular}{|c|c|c|c|c|}
\hline & $\mathrm{O}$ & $\mathrm{P}$ & $\bar{Q}$ & $\mathrm{R}$ \\
\hline 1 & SITE_CODE & ELEVATION_UNITS & VERT_COORD_TYPE & DATE_LAST_MODIFIED \\
\hline 4714 & & & & 19-Aug-02 \\
\hline 4715 & & & & 19-Aug-02 \\
\hline 4716 & & & & 19-Aug-02 \\
\hline 4717 & & & & 21-Aug-02 \\
\hline 4718 & & & & 21-Aug-02 \\
\hline 4719 & & & & 21-Aug-02 \\
\hline 4720 & & & & 21-Aug-02 \\
\hline 4721 & & & & 21-Aug-02 \\
\hline 4722 & & & & 21-Aug-02 \\
\hline 4723 & & & & 21-Aug-02 \\
\hline 4724 & & & & 21-Aug-02 \\
\hline 4725 & & & & 21-Aug-02 \\
\hline 4726 & & & & 21-Aug-02 \\
\hline 4727 & & & & 21-Aug-02 \\
\hline 4728 & & & & 21-Aug-02 \\
\hline 4729 & & & & 21-Aug-02 \\
\hline 4730 & & & & 21-Aug-02 \\
\hline 4731 & & & & 21-Aug-02 \\
\hline 4732 & & & & 21-Aug-02 \\
\hline 4733 & & & & 21-Aug-02 \\
\hline 4734 & & & & 21-Aug-02 \\
\hline 4735 & & & & 21-Aug-02 \\
\hline 4736 & & & & 21-Aug-02 \\
\hline 4737 & & & & 21-Aug-02 \\
\hline 4738 & & & & 21-Aug-02 \\
\hline 4739 & & & & 22-Aug-02 \\
\hline 4740 & & & & 22-Aug-02 \\
\hline 4741 & & & & 22-Aug-02 \\
\hline 4742 & & & & 22-Aug-02 \\
\hline 4743 & & & & 22-Aug-02 \\
\hline 4744 & & & & 22-Aug-02 \\
\hline 4745 & & & & 22-Aug-02 \\
\hline 4746 & & & & 22-Aug-02 \\
\hline 4747 & & & & 22-Aug-02 \\
\hline 4748 & & & & 22-Aug-02 \\
\hline 4749 & & & & 22-Aug-02 \\
\hline 4750 & & & & 22-Aug-02 \\
\hline 4751 & & & & 22-Aug-02 \\
\hline
\end{tabular}




\begin{tabular}{|c|c|c|c|c|}
\hline & $\mathrm{O}$ & $P$ & $\mathrm{Q}$ & $R$ \\
\hline 1 & SITE CODE & ELEVATION UNITS & VERT COORD TYPE & DATE LAST MODIFIED \\
\hline 4752 & & & & 22-Aug-02 \\
\hline 4753 & & & & 22-Aug-02 \\
\hline 4754 & & & & 22-Aug-02 \\
\hline 4755 & & & & 22-Aug-02 \\
\hline 4756 & & & & 22-Aug-02 \\
\hline 4757 & & & & 22-Aug-02 \\
\hline 4758 & & & & 22-Aug-02 \\
\hline 4759 & & & & 22-Aug-02 \\
\hline 4760 & & & & 22-Aug-02 \\
\hline 4761 & & & & 22-Aug-02 \\
\hline 4762 & & & & 22-Aug-02 \\
\hline 4763 & & & & 22-Aug-02 \\
\hline 4764 & & & & 22-Aug-02 \\
\hline 4765 & 233-S & & & 22-Aug-02 \\
\hline 4766 & $233-S$ & & & 22-Aug-02 \\
\hline 4767 & $233-S$ & & & 22-Aug-02 \\
\hline 4768 & $233-S$ & & & 22-Aug-02 \\
\hline 4769 & $233-S$ & & & 22-Aug-02 \\
\hline 4770 & $233-5$ & & & 22-Aug-02 \\
\hline 4771 & $233-S$ & & & 22-Aug-02 \\
\hline 4772 & $233-S$ & & & 22-Aug-02 \\
\hline 4773 & $233-S$ & & & 22-Aug-02 \\
\hline 4774 & $233-S$ & & & 22-Aug-02 \\
\hline 4775 & $233-S$ & & & 22-Aug-02 \\
\hline 4776 & $233-S$ & & & 22-Aug-02 \\
\hline 4777 & $233-S$ & & & 22-Aug-02 \\
\hline 4778 & 233-S & & & 22-Aug-02 \\
\hline 4779 & 233-S & & & 22-Aug-02 \\
\hline 4780 & $233-S$ & & & 22-Aug-02 \\
\hline 4781 & $233-S$ & & & 22-Aug-02 \\
\hline 4782 & $233-S$ & & & 22-Aug-02 \\
\hline 4783 & 233-S & & & 22-Aug-02 \\
\hline 4784 & $233-S$ & & & 22-Aug-02 \\
\hline 4785 & $233-S$ & & & 22-Aug-02 \\
\hline 4786 & 233-S & & & 22-Aug-02 \\
\hline 4787 & $233-S$ & & & 22-Aug-02 \\
\hline 4788 & $233-S$ & & & 22-Aug-02 \\
\hline 4789 & $233-5$ & & & 22-Aug-02 \\
\hline
\end{tabular}




\begin{tabular}{|c|c|c|c|c|}
\hline & $\mathrm{O}$ & $\mathrm{P}$ & $\bar{Q}$ & $\bar{R}$ \\
\hline 1 & SITE_CODE & ELEVATION_UNITS & VERT_COORD_TYPE & DATE_LAST_MODIFIED \\
\hline 4790 & $233-S$ & & & 22-Aug-02 \\
\hline 4791 & 233-S & & & 22-Aug-02 \\
\hline 4792 & $233-S$ & & & 22-Aug-02 \\
\hline 4793 & 233-S & & & 22-Aug-02 \\
\hline 4794 & $233-S$ & & & 22-Aug-02 \\
\hline 4795 & $233-S$ & & & 22-Aug-02 \\
\hline 4796 & $233-S$ & & & 22-Aug-02 \\
\hline 4797 & $233-S$ & & & 22-Aug-02 \\
\hline 4798 & $233-S$ & & & 22-Aug-02 \\
\hline 4799 & $233-S$ & & & 22-Aug-02 \\
\hline 4800 & 233-S & & & 22-Aug-02 \\
\hline 4801 & 233-S & & & 22-Aug-02 \\
\hline 4802 & $233-S$ & & & 22-Aug-02 \\
\hline 4803 & $233-S$ & & & 22-Aug-02 \\
\hline 4804 & $233-S$ & & & 22-Aug-02 \\
\hline 4805 & $233-S$ & & & 22-Aug-02 \\
\hline 4806 & $233-S$ & & & 22-Aug-02 \\
\hline 4807 & $233-S$ & & & 22-Aug-02 \\
\hline 4808 & $233-S$ & & & 22-Aug-02 \\
\hline 4809 & $233-S$ & & & 22-Aug-02 \\
\hline 4810 & $233-S$ & & & 22-Aug-02 \\
\hline 4811 & $233-S$ & & & 22-Aug-02 \\
\hline 4812 & $233-5$ & & & 22-Aug-02 \\
\hline 4813 & $233-S$ & & & 22-Aug-02 \\
\hline 4814 & $233-S$ & & & 22-Aug-02 \\
\hline 4815 & $233-S$ & & & 22-Aug-02 \\
\hline 4816 & $233-S$ & & & 22-Aug-02 \\
\hline 4817 & $233-S$ & & & 22-Aug-02 \\
\hline 4818 & $233-S$ & & & 22-Aug-02 \\
\hline 4819 & $233-S$ & & & 22-Aug-02 \\
\hline 4820 & $233-S$ & & & 22-Aug-02 \\
\hline 4821 & $233-S$ & & & 22-Aug-02 \\
\hline 4822 & $233-S$ & & & 22-Aug-02 \\
\hline 4823 & $233-S$ & & & 22-Aug-02 \\
\hline 4824 & $233-S$ & & & 22-Aug-02 \\
\hline 4825 & $233-S$ & & & 22-Aug-02 \\
\hline 4826 & $233-S$ & & & 22-Aug-02 \\
\hline 4827 & 233-S & & & 22-Aug-02 \\
\hline
\end{tabular}




\begin{tabular}{|c|c|c|c|c|}
\hline & $\mathrm{O}$ & $P$ & $\mathrm{Q}$ & $R$ \\
\hline 1 & SITE CODE & ELEVATION UNITS & VERT COORD TYPE & DATE LAST MODIFIED \\
\hline 4828 & $233-\mathrm{S}$ & & & 22-Aug-02 \\
\hline 4829 & 233-S & & & 22-Aug-02 \\
\hline 4830 & $233-S$ & & & 22-Aug-02 \\
\hline 4831 & $233-S$ & & & 22-Aug-02 \\
\hline 4832 & $233-S$ & & & 22-Aug-02 \\
\hline 4833 & $233-S$ & & & 22-Aug-02 \\
\hline 4834 & 233-S & & & 22-Aug-02 \\
\hline 4835 & $233-S$ & & & 22-Aug-02 \\
\hline 4836 & $233-S$ & & & 22-Aug-02 \\
\hline 4837 & 233-S & & & 22-Aug-02 \\
\hline 4838 & $233-S$ & & & 22-Aug-02 \\
\hline 4839 & 233-S & & & 22-Aug-02 \\
\hline 4840 & $233-S$ & & & 22-Aug-02 \\
\hline 4841 & 233-S & & & 22-Aug-02 \\
\hline 4842 & $233-S$ & & & 22-Aug-02 \\
\hline 4843 & $233-S$ & & & 22-Aug-02 \\
\hline 4844 & $233-S$ & & & 22-Aug-02 \\
\hline 4845 & $233-S$ & & & 22-Aug-02 \\
\hline 4846 & $233-5$ & & & 22-Aug-02 \\
\hline 4847 & $233-S$ & & & 22-Aug-02 \\
\hline 4848 & $233-S$ & & & 22-Aug-02 \\
\hline 4849 & 233-S & & & 22-Aug-02 \\
\hline 4850 & $233-S$ & & & 22-Aug-02 \\
\hline 4851 & $233-S$ & & & 22-Aug-02 \\
\hline 4852 & 233-S & & & 22-Aug-02 \\
\hline 4853 & $233-S$ & & & 22-Aug-02 \\
\hline 4854 & 233-S & & & 22-Aug-02 \\
\hline 4855 & 233-S & & & 22-Aug-02 \\
\hline 4856 & $233-S$ & & & 22-Aug-02 \\
\hline 4857 & $233-S$ & & & 22-Aug-02 \\
\hline 4858 & $233-S$ & & & 22-Aug-02 \\
\hline 4859 & 233-S & & & 22-Aug-02 \\
\hline 4860 & $233-S$ & & & 22-Aug-02 \\
\hline 4861 & $233-S$ & & & 22-Aug-02 \\
\hline 4862 & $233-S$ & & & 22-Aug-02 \\
\hline 4863 & $233-S$ & & & 22-Aug-02 \\
\hline 4864 & 233-S & & & 22-Aug-02 \\
\hline 4865 & 233-S & & & 22-Aug-02 \\
\hline
\end{tabular}




\begin{tabular}{|c|c|c|c|c|}
\hline & $\mathrm{O}$ & $P$ & $\mathrm{Q}$ & $R$ \\
\hline 1 & SITE CODE & ELEVATION UNITS & VERT COORD TYPE & DATE LAST MODIFIED \\
\hline 4866 & $233-S$ & & & 22-Aug-02 \\
\hline 4867 & 233-S & & & 22-Aug-02 \\
\hline 4868 & $233-S$ & & & 22-Aug-02 \\
\hline 4869 & $233-S$ & & & 22-Aug-02 \\
\hline 4870 & $233-S$ & & & 22-Aug-02 \\
\hline 4871 & $233-S$ & & & 22-Aug-02 \\
\hline 4872 & 233-S & & & 22-Aug-02 \\
\hline 4873 & $233-S$ & & & 22-Aug-02 \\
\hline 4874 & $233-S$ & & & 22-Aug-02 \\
\hline 4875 & 233-S & & & 22-Aug-02 \\
\hline 4876 & $233-S$ & & & 22-Aug-02 \\
\hline 4877 & 233-S & & & 22-Aug-02 \\
\hline 4878 & $233-S$ & & & 22-Aug-02 \\
\hline 4879 & 233-S & & & 22-Aug-02 \\
\hline 4880 & $233-S$ & & & 22-Aug-02 \\
\hline 4881 & $233-S$ & & & 22-Aug-02 \\
\hline 4882 & $233-S$ & & & 22-Aug-02 \\
\hline 4883 & $233-S$ & & & 22-Aug-02 \\
\hline 4884 & $233-5$ & & & 22-Aug-02 \\
\hline 4885 & $233-S$ & & & 22-Aug-02 \\
\hline 4886 & $233-S$ & & & 22-Aug-02 \\
\hline 4887 & 233-S & & & 22-Aug-02 \\
\hline 4888 & $233-S$ & & & 22-Aug-02 \\
\hline 4889 & $233-S$ & & & 22-Aug-02 \\
\hline 4890 & $233-S$ & & & 22-Aug-02 \\
\hline 4891 & $233-S$ & & & 22-Aug-02 \\
\hline 4892 & 233-S & & & 22-Aug-02 \\
\hline 4893 & 233-S & & & 22-Aug-02 \\
\hline 4894 & $233-S$ & & & 22-Aug-02 \\
\hline 4895 & $233-S$ & & & 22-Aug-02 \\
\hline 4896 & $233-S$ & & & 22-Aug-02 \\
\hline 4897 & 233-S & & & 22-Aug-02 \\
\hline 4898 & $233-S$ & & & 22-Aug-02 \\
\hline 4899 & $233-S$ & & & 22-Aug-02 \\
\hline 4900 & $233-S$ & & & 22-Aug-02 \\
\hline 4901 & $233-S$ & & & 22-Aug-02 \\
\hline 4902 & 233-S & & & 22-Aug-02 \\
\hline 4903 & 233-S & & & 22-Aug-02 \\
\hline
\end{tabular}




\begin{tabular}{|c|c|c|c|c|}
\hline & $\mathrm{O}$ & $\mathrm{P}$ & $\bar{Q}$ & $\bar{R}$ \\
\hline 1 & SITE_CODE & ELEVATION_UNITS & VERT_COORD_TYPE & DATE_LAST_MODIFIED \\
\hline 4904 & 233-S & & & 22-Aug-02 \\
\hline 4905 & 233-S & & & 22-Aug-02 \\
\hline 4906 & $233-S$ & & & 22-Aug-02 \\
\hline 4907 & 233-S & & & 22-Aug-02 \\
\hline 4908 & $233-S$ & & & 22-Aug-02 \\
\hline 4909 & $233-S$ & & & 22-Aug-02 \\
\hline 4910 & $233-S$ & & & 22-Aug-02 \\
\hline 4911 & $233-S$ & & & 22-Aug-02 \\
\hline 4912 & $233-S$ & & & 22-Aug-02 \\
\hline 4913 & $233-S$ & & & 22-Aug-02 \\
\hline 4914 & 233-S & & & 22-Aug-02 \\
\hline 4915 & 233-S & & & 22-Aug-02 \\
\hline 4916 & $233-S$ & & & 22-Aug-02 \\
\hline 4917 & $233-S$ & & & 22-Aug-02 \\
\hline 4918 & $233-S$ & & & 22-Aug-02 \\
\hline 4919 & $233-S$ & & & 22-Aug-02 \\
\hline 4920 & $233-S$ & & & 22-Aug-02 \\
\hline 4921 & $233-S$ & & & 22-Aug-02 \\
\hline 4922 & $233-S$ & & & 22-Aug-02 \\
\hline 4923 & $233-S$ & & & 22-Aug-02 \\
\hline 4924 & $233-S$ & & & 22-Aug-02 \\
\hline 4925 & $233-S$ & & & 22-Aug-02 \\
\hline 4926 & $233-5$ & & & 22-Aug-02 \\
\hline 4927 & 233-S & & & 22-Aug-02 \\
\hline 4928 & $233-S$ & & & 22-Aug-02 \\
\hline 4929 & $233-S$ & & & 22-Aug-02 \\
\hline 4930 & $233-S$ & & & 22-Aug-02 \\
\hline 4931 & $233-S$ & & & 22-Aug-02 \\
\hline 4932 & $233-S$ & & & 22-Aug-02 \\
\hline 4933 & $233-S$ & & & 22-Aug-02 \\
\hline 4934 & $233-S$ & & & 22-Aug-02 \\
\hline 4935 & $233-S$ & & & 22-Aug-02 \\
\hline 4936 & $233-S$ & & & 22-Aug-02 \\
\hline 4937 & $233-S$ & & & 22-Aug-02 \\
\hline 4938 & $233-S$ & & & 22-Aug-02 \\
\hline 4939 & $233-S$ & & & 22-Aug-02 \\
\hline 4940 & $233-S$ & & & 22-Aug-02 \\
\hline 4941 & 233-S & & & 22-Aug-02 \\
\hline
\end{tabular}




\begin{tabular}{|c|c|c|c|c|}
\hline & $\mathrm{O}$ & $\mathrm{P}$ & $\bar{Q}$ & $\bar{R}$ \\
\hline 1 & SITE_CODE & ELEVATION_UNITS & VERT_COORD_TYPE & DATE_LAST_MODIFIED \\
\hline 4942 & 233-S & & & 22-Aug-02 \\
\hline 4943 & 233-S & & & 22-Aug-02 \\
\hline 4944 & $233-S$ & & & 22-Aug-02 \\
\hline 4945 & 233-S & & & 22-Aug-02 \\
\hline 4946 & $233-S$ & & & 22-Aug-02 \\
\hline 4947 & $233-S$ & & & 22-Aug-02 \\
\hline 4948 & $233-S$ & & & 22-Aug-02 \\
\hline 4949 & $233-S$ & & & 22-Aug-02 \\
\hline 4950 & $233-S$ & & & 22-Aug-02 \\
\hline 4951 & $233-S$ & & & 22-Aug-02 \\
\hline 4952 & 233-S & & & 22-Aug-02 \\
\hline 4953 & 233-S & & & 22-Aug-02 \\
\hline 4954 & $233-S$ & & & 22-Aug-02 \\
\hline 4955 & $233-S$ & & & 22-Aug-02 \\
\hline 4956 & $233-S$ & & & 22-Aug-02 \\
\hline 4957 & $233-S$ & & & 22-Aug-02 \\
\hline 4958 & $233-S$ & & & 22-Aug-02 \\
\hline 4959 & $233-S$ & & & 22-Aug-02 \\
\hline 4960 & $233-S$ & & & 22-Aug-02 \\
\hline 4961 & $233-S$ & & & 22-Aug-02 \\
\hline 4962 & $233-S$ & & & 22-Aug-02 \\
\hline 4963 & $233-S$ & & & 22-Aug-02 \\
\hline 4964 & $233-5$ & & & 22-Aug-02 \\
\hline 4965 & $233-S$ & & & 22-Aug-02 \\
\hline 4966 & $233-S$ & & & 22-Aug-02 \\
\hline 4967 & $233-S$ & & & 22-Aug-02 \\
\hline 4968 & $233-S$ & & & 22-Aug-02 \\
\hline 4969 & $233-S$ & & & 22-Aug-02 \\
\hline 4970 & $233-S$ & & & 22-Aug-02 \\
\hline 4971 & $233-S$ & & & 22-Aug-02 \\
\hline 4972 & $233-S$ & & & 22-Aug-02 \\
\hline 4973 & $233-S$ & & & 22-Aug-02 \\
\hline 4974 & $233-S$ & & & 22-Aug-02 \\
\hline 4975 & $233-S$ & & & 22-Aug-02 \\
\hline 4976 & $233-S$ & & & 22-Aug-02 \\
\hline 4977 & 233-S & & & 22-Aug-02 \\
\hline 4978 & $233-S$ & & & 22-Aug-02 \\
\hline 4979 & 233-S & & & 22-Aug-02 \\
\hline
\end{tabular}




\begin{tabular}{|c|c|c|c|c|}
\hline & $\mathrm{O}$ & $P$ & $\bar{Q}$ & $\bar{R}$ \\
\hline 1 & SITE_CODE & ELEVATION_UNITS & VERT_COORD_TYPE & DATE_LAST_MODIFIED \\
\hline 4980 & 233-S & & & 22-Aug-02 \\
\hline 4981 & 233-S & & & 22-Aug-02 \\
\hline 4982 & $233-S$ & & & 22-Aug-02 \\
\hline 4983 & 233-S & & & 22-Aug-02 \\
\hline 4984 & $233-S$ & & & 22-Aug-02 \\
\hline 4985 & $233-S$ & & & 22-Aug-02 \\
\hline 4986 & $233-S$ & & & 22-Aug-02 \\
\hline 4987 & $233-S$ & & & 22-Aug-02 \\
\hline 4988 & $233-S$ & & & 22-Aug-02 \\
\hline 4989 & $233-S$ & & & 22-Aug-02 \\
\hline 4990 & 233-S & & & 22-Aug-02 \\
\hline 4991 & 233-S & & & 22-Aug-02 \\
\hline 4992 & $233-S$ & & & 22-Aug-02 \\
\hline 4993 & $233-S$ & & & 22-Aug-02 \\
\hline 4994 & $233-S$ & & & 22-Aug-02 \\
\hline 4995 & $233-S$ & & & 22-Aug-02 \\
\hline 4996 & $233-S$ & & & 22-Aug-02 \\
\hline 4997 & $233-S$ & & & 22-Aug-02 \\
\hline 4998 & $233-5$ & & & 22-Aug-02 \\
\hline 4999 & $233-S$ & & & 22-Aug-02 \\
\hline 5000 & $233-S$ & & & 22-Aug-02 \\
\hline 5001 & $233-S$ & & & 22-Aug-02 \\
\hline 5002 & $233-5$ & & & 22-Aug-02 \\
\hline 5003 & 233-S & & & 22-Aug-02 \\
\hline 5004 & $233-S$ & & & 22-Aug-02 \\
\hline 5005 & $233-S$ & & & 22-Aug-02 \\
\hline 5006 & $221-U$ & & & 23-Aug-02 \\
\hline 5007 & $221-U$ & & & 23-Aug-02 \\
\hline 5008 & $221-U$ & & & 23-Aug-02 \\
\hline 5009 & $221-U$ & & & 23-Aug-02 \\
\hline 5010 & & & & 23-Aug-02 \\
\hline 5011 & & & & 23-Aug-02 \\
\hline 5012 & $221-U$ & & & 23-Aug-02 \\
\hline 5013 & & & & 23-Aug-02 \\
\hline 5014 & & & & 23-Aug-02 \\
\hline 5015 & & & & 23-Aug-02 \\
\hline 5016 & & & & 23-Aug-02 \\
\hline 5017 & & & & 23-Aug-02 \\
\hline
\end{tabular}




\begin{tabular}{|c|c|c|c|c|}
\hline & $\mathrm{O}$ & $P$ & $\mathrm{Q}$ & $R$ \\
\hline 1 & SITE CODE & ELEVATION UNITS & VERT COORD TYPE & DATE LAST MODIFIED \\
\hline 5018 & & & & 23-Aug-02 \\
\hline 5019 & & & & 23-Aug-02 \\
\hline 5020 & & & & 23-Aug-02 \\
\hline 5021 & & & & 23-Aug-02 \\
\hline 5022 & & & & 23-Aug-02 \\
\hline 5023 & & & & 23-Aug-02 \\
\hline 5024 & & & & 23-Aug-02 \\
\hline 5025 & & & & 23-Aug-02 \\
\hline 5026 & & & & 23-Aug-02 \\
\hline 5027 & & & & 23-Aug-02 \\
\hline 5028 & & & & 23-Aug-02 \\
\hline 5029 & & & & 23-Aug-02 \\
\hline 5030 & & & & 23-Aug-02 \\
\hline 5031 & & & & 23-Aug-02 \\
\hline 5032 & & & & 23-Aug-02 \\
\hline 5033 & & & & 23-Aug-02 \\
\hline 5034 & & & & 18-Nov-02 \\
\hline 5035 & & & & 18-Nov-02 \\
\hline 5036 & & & & 18-Nov-02 \\
\hline 5037 & & & & 18-Nov-02 \\
\hline 5038 & & & & 18-Nov-02 \\
\hline 5039 & & & & 18-Nov-02 \\
\hline 5040 & & & & 26-Aug-02 \\
\hline 5041 & & & & 26-Aug-02 \\
\hline 5042 & & & & 26-Aug-02 \\
\hline 5043 & & & & 26-Aug-02 \\
\hline 5044 & & & & 26-Aug-02 \\
\hline 5045 & & & & 26-Aug-02 \\
\hline 5046 & & & & 26-Aug-02 \\
\hline 5047 & & & & 18-Nov-02 \\
\hline 5048 & & & & 18-Nov-02 \\
\hline 5049 & & & & 18-Nov-02 \\
\hline 5050 & & & & 18-Nov-02 \\
\hline 5051 & 216-Z-11 & & & 26-Aug-02 \\
\hline 5052 & & & & 26-Aug-02 \\
\hline 5053 & & & & 26-Aug-02 \\
\hline 5054 & & & & 26-Aug-02 \\
\hline 5055 & & & & 26-Aug-02 \\
\hline
\end{tabular}




\begin{tabular}{|c|c|c|c|c|}
\hline & $\mathrm{O}$ & $P$ & $\mathrm{Q}$ & $R$ \\
\hline 1 & SITE CODE & ELEVATION UNITS & VERT COORD TYPE & DATE LAST MODIFIED \\
\hline 5056 & & & & 26-Aug-02 \\
\hline 5057 & & & & 26-Aug-02 \\
\hline 5058 & & & & 26-Aug-02 \\
\hline 5059 & & & & 26-Aug-02 \\
\hline 5060 & & & & 26-Aug-02 \\
\hline 5061 & & & & 26-Aug-02 \\
\hline 5062 & & & & 26-Aug-02 \\
\hline 5063 & & & & 26-Aug-02 \\
\hline 5064 & & & & 26-Aug-02 \\
\hline 5065 & & & & 26-Aug-02 \\
\hline 5066 & & & & 26-Aug-02 \\
\hline 5067 & & & & 26-Aug-02 \\
\hline 5068 & & & & 26-Aug-02 \\
\hline 5069 & & & & 26-Aug-02 \\
\hline 5070 & & & & 26-Aug-02 \\
\hline 5071 & & & & 26-Aug-02 \\
\hline 5072 & & & & 26-Aug-02 \\
\hline 5073 & & & & 26-Aug-02 \\
\hline 5074 & & & & 26-Aug-02 \\
\hline 5075 & & & & 26-Aug-02 \\
\hline 5076 & & & & 26-Aug-02 \\
\hline 5077 & & & & 26-Aug-02 \\
\hline 5078 & & & & 26-Aug-02 \\
\hline 5079 & & & & 26-Aug-02 \\
\hline 5080 & & & & 26-Aug-02 \\
\hline 5081 & 216-Z-11 & & & 26-Aug-02 \\
\hline 5082 & $216-Z-11$ & & & 26-Aug-02 \\
\hline 5083 & $216-Z-11$ & & & 26-Aug-02 \\
\hline 5084 & 216-Z-11 & & & 26-Aug-02 \\
\hline 5085 & $216-Z-11$ & & & 26-Aug-02 \\
\hline 5086 & $216-Z-11$ & & & 26-Aug-02 \\
\hline 5087 & $216-Z-11$ & & & 26-Aug-02 \\
\hline 5088 & $216-Z-11$ & & & 26-Aug-02 \\
\hline 5089 & $216-Z-11$ & & & 26-Aug-02 \\
\hline 5090 & $216-Z-11$ & & & 26-Aug-02 \\
\hline 5091 & 216-Z-11 & & & 26-Aug-02 \\
\hline 5092 & $216-Z-11$ & & & 26-Aug-02 \\
\hline 5093 & & & & 26-Aug-02 \\
\hline
\end{tabular}




\begin{tabular}{|c|c|c|c|c|}
\hline & $\mathrm{O}$ & $P$ & $\mathrm{Q}$ & $R$ \\
\hline 1 & SITE CODE & ELEVATION UNITS & VERT COORD TYPE & DATE LAST MODIFIED \\
\hline 5094 & & & & $26-$ Aug-02 \\
\hline 5095 & 216-T-26 & & & 26-Aug-02 \\
\hline 5096 & 216-T-26 & & & 26-Aug-02 \\
\hline 5097 & & & & 03-Sep-02 \\
\hline 5098 & & & & 03-Sep-02 \\
\hline 5099 & & & & 03-Sep-02 \\
\hline 5100 & & & & 03-Sep-02 \\
\hline 5101 & & & & 03-Sep-02 \\
\hline 5102 & & & & 03-Sep-02 \\
\hline 5103 & & & & 03-Sep-02 \\
\hline 5104 & & & & 03-Sep-02 \\
\hline 5105 & & & & 03-Sep-02 \\
\hline 5106 & & & & 03-Sep-02 \\
\hline 5107 & & & & 03-Sep-02 \\
\hline 5108 & & & & 03-Sep-02 \\
\hline 5109 & & & & 03-Sep-02 \\
\hline 5110 & & & & 04-Sep-02 \\
\hline 5111 & & & & 04-Sep-02 \\
\hline 5112 & & & & 04-Sep-02 \\
\hline 5113 & & & & 04-Sep-02 \\
\hline 5114 & & & & 04-Sep-02 \\
\hline 5115 & & & & 11-Sep-02 \\
\hline 5116 & & & & 11-Sep-02 \\
\hline 5117 & & & & 08-Jan-03 \\
\hline 5118 & & & & 08-Jan-03 \\
\hline 5119 & & & & 11-Sep-02 \\
\hline 5120 & & & & 16-Sep-02 \\
\hline 5121 & & & & 16-Sep-02 \\
\hline 5122 & & & & 16-Sep-02 \\
\hline 5123 & & & & 16-Sep-02 \\
\hline 5124 & & & & 16-Sep-02 \\
\hline 5125 & & & & 16-Sep-02 \\
\hline 5126 & & & & 16-Sep-02 \\
\hline 5127 & & & & 16-Sep-02 \\
\hline 5128 & & & & 16-Sep-02 \\
\hline 5129 & & & & 16-Sep-02 \\
\hline 5130 & & & & 16-Sep-02 \\
\hline 5131 & & & & 16-Sep-02 \\
\hline
\end{tabular}




\begin{tabular}{|c|c|c|c|c|}
\hline & $\mathrm{O}$ & $P$ & $\bar{Q}$ & $\bar{R}$ \\
\hline 1 & SITE_CODE & ELEVATION_UNITS & VERT_COORD_TYPE & DATE_LAST_MODIFIED \\
\hline 5132 & & & & 16-Sep-02 \\
\hline 5133 & & & & 16-Sep-02 \\
\hline 5134 & & & & 19-Sep-02 \\
\hline 5135 & & & & 19-Sep-02 \\
\hline 5136 & & & & 19-Sep-02 \\
\hline 5137 & & & & 19-Sep-02 \\
\hline 5138 & & & & 19-Sep-02 \\
\hline 5139 & & & & 19-Sep-02 \\
\hline 5140 & & & & 19-Sep-02 \\
\hline 5141 & & & & 19-Sep-02 \\
\hline 5142 & & & & 19-Sep-02 \\
\hline 5143 & & & & 19-Sep-02 \\
\hline 5144 & & & & 19-Sep-02 \\
\hline 5145 & & & & 19-Sep-02 \\
\hline 5146 & & & & 19-Sep-02 \\
\hline 5147 & & & & 19-Sep-02 \\
\hline 5148 & & & & 19-Sep-02 \\
\hline 5149 & & & & 19-Sep-02 \\
\hline 5150 & & & & 19-Sep-02 \\
\hline 5151 & & & & 19-Sep-02 \\
\hline 5152 & & & & 19-Sep-02 \\
\hline 5153 & & & & 19-Sep-02 \\
\hline 5154 & & & & 19-Sep-02 \\
\hline 5155 & & & & 19-Sep-02 \\
\hline 5156 & & & & 19-Sep-02 \\
\hline 5157 & & & & 19-Sep-02 \\
\hline 5158 & & & & 19-Sep-02 \\
\hline 5159 & & & & 19-Sep-02 \\
\hline 5160 & & & & 19-Sep-02 \\
\hline 5161 & & & & 19-Sep-02 \\
\hline 5162 & & & & 19-Sep-02 \\
\hline 5163 & & & & 19-Sep-02 \\
\hline 5164 & & & & 19-Sep-02 \\
\hline 5165 & & & & 19-Sep-02 \\
\hline 5166 & & & & 19-Sep-02 \\
\hline 5167 & & & & 19-Sep-02 \\
\hline 5168 & & & & 19-Sep-02 \\
\hline 5169 & & & & 19-Sep-02 \\
\hline
\end{tabular}




\begin{tabular}{|c|c|c|c|c|}
\hline & $\mathrm{O}$ & $P$ & $\bar{Q}$ & $\mathrm{R}$ \\
\hline 1 & SITE_CODE & ELEVATION_UNITS & VERT_COORD_TYPE & DATE_LAST_MODIFIED \\
\hline 5170 & & & & 19-Sep-02 \\
\hline 5171 & & & & 19-Sep-02 \\
\hline 5172 & & & & 19-Sep-02 \\
\hline 5173 & & & & 19-Sep-02 \\
\hline 5174 & & & & 19-Sep-02 \\
\hline 5175 & & & & 19-Sep-02 \\
\hline 5176 & & & & 19-Sep-02 \\
\hline 5177 & & & & 19-Sep-02 \\
\hline 5178 & & & & 19-Sep-02 \\
\hline 5179 & & & & 19-Sep-02 \\
\hline 5180 & & & & 19-Sep-02 \\
\hline 5181 & & & & 19-Sep-02 \\
\hline 5182 & & & & 19-Sep-02 \\
\hline 5183 & & & & 19-Sep-02 \\
\hline 5184 & & & & 23-Sep-02 \\
\hline 5185 & & & & 24-Sep-02 \\
\hline 5186 & & & & 24-Sep-02 \\
\hline 5187 & & & & 24-Sep-02 \\
\hline 5188 & & & & 26-Sep-02 \\
\hline 5189 & & & & 26-Sep-02 \\
\hline 5190 & & & & 26-Sep-02 \\
\hline 5191 & & & & 26-Sep-02 \\
\hline 5192 & & & & 26-Sep-02 \\
\hline 5193 & & & & 26-Sep-02 \\
\hline 5194 & & & & 26-Sep-02 \\
\hline 5195 & & & & 26-Sep-02 \\
\hline 5196 & & & & 26-Sep-02 \\
\hline 5197 & & & & 26-Sep-02 \\
\hline 5198 & & & & 26-Sep-02 \\
\hline 5199 & & & & 26-Sep-02 \\
\hline 5200 & & & & 26-Sep-02 \\
\hline 5201 & & & & 26-Sep-02 \\
\hline 5202 & & & & 26-Sep-02 \\
\hline 5203 & & & & 01-Oct-02 \\
\hline 5204 & & & & 01-Oct-02 \\
\hline 5205 & & & & 01-Oct-02 \\
\hline 5206 & & & & $01-$ Oct-02 \\
\hline 5207 & & & & 01-Oct-02 \\
\hline
\end{tabular}




\begin{tabular}{|c|c|c|c|c|}
\hline & $\mathrm{O}$ & $P$ & $\mathrm{Q}$ & $R$ \\
\hline 1 & SITE CODE & ELEVATION UNITS & VERT COORD TYPE & DATE LAST MODIFIED \\
\hline 5208 & & & & 01-Oct-02 \\
\hline 5209 & & & & 01-Oct-02 \\
\hline 5210 & & & & 01-Oct-02 \\
\hline 5211 & & & & 01-Oct-02 \\
\hline 5212 & & & & 01-Oct-02 \\
\hline 5213 & & & & 01-Oct-02 \\
\hline 5214 & & & & 01-Oct-02 \\
\hline 5215 & & & & 01-Oct-02 \\
\hline 5216 & & & & 01-Oct-02 \\
\hline 5217 & & & & 01-Oct-02 \\
\hline 5218 & & & & 01-Oct-02 \\
\hline 5219 & & & & 04-Oct-02 \\
\hline 5220 & & & & 04-Oct-02 \\
\hline 5221 & & & & 04-Oct-02 \\
\hline 5222 & & & & 04-Oct-02 \\
\hline 5223 & & & & 07-Oct-02 \\
\hline 5224 & & & & 07-Oct-02 \\
\hline 5225 & & & & 07-Oct-02 \\
\hline 5226 & & & & 07-Oct-02 \\
\hline 5227 & & & & 07-Oct-02 \\
\hline 5228 & & & & 07-Oct-02 \\
\hline 5229 & & & & $07-$ Oct-02 \\
\hline 5230 & & & & 07-Oct-02 \\
\hline 5231 & & & & 07-Oct-02 \\
\hline 5232 & & & & 07-Oct-02 \\
\hline 5233 & & & & 07-Oct-02 \\
\hline 5234 & & & & 07-Oct-02 \\
\hline 5235 & & & & 07-Oct-02 \\
\hline 5236 & & & & 07-Oct-02 \\
\hline 5237 & & & & 07-Oct-02 \\
\hline 5238 & & & & 07-Oct-02 \\
\hline 5239 & & & & 07-Oct-02 \\
\hline 5240 & & & & 08-Oct-02 \\
\hline 5241 & & & & 08-Oct-02 \\
\hline 5242 & & & & 09-Oct-02 \\
\hline 5243 & & & & 09-Oct-02 \\
\hline 5244 & & & & 09-Oct-02 \\
\hline 5245 & & & & 09-Oct-02 \\
\hline
\end{tabular}




\begin{tabular}{|c|c|c|c|c|}
\hline & $\mathrm{O}$ & $P$ & $\mathrm{Q}$ & $R$ \\
\hline 1 & SITE CODE & ELEVATION UNITS & VERT COORD TYPE & DATE LAST MODIFIED \\
\hline 5246 & & & & 09-Oct-02 \\
\hline 5247 & & & & 09-Oct-02 \\
\hline 5248 & & & & 09-Oct-02 \\
\hline 5249 & & & & 09-Oct-02 \\
\hline 5250 & & & & 09-Oct-02 \\
\hline 5251 & & & & 09-Oct-02 \\
\hline 5252 & & & & 09-Oct-02 \\
\hline 5253 & & & & 09-Oct-02 \\
\hline 5254 & & & & 09-Oct-02 \\
\hline 5255 & & & & 14-Oct-02 \\
\hline 5256 & & & & 14-Oct-02 \\
\hline 5257 & & & & 14-Oct-02 \\
\hline 5258 & & & & 21-Oct-02 \\
\hline 5259 & & & & 21-Oct-02 \\
\hline 5260 & & & & 21-Oct-02 \\
\hline 5261 & & & & 21-Oct-02 \\
\hline 5262 & & & & 21-Oct-02 \\
\hline 5263 & & & & 21-Oct-02 \\
\hline 5264 & & & & 07-Nov-02 \\
\hline 5265 & & & & 12-Nov-02 \\
\hline 5266 & & & & 12-Nov-02 \\
\hline 5267 & & & & 12-Nov-02 \\
\hline 5268 & & & & 12-Nov-02 \\
\hline 5269 & & & & 12-Nov-02 \\
\hline 5270 & & & & 12-Nov-02 \\
\hline 5271 & & & & 12-Nov-02 \\
\hline 5272 & & & & 12-Nov-02 \\
\hline 5273 & & & & 12-Nov-02 \\
\hline 5274 & & & & 12-Nov-02 \\
\hline 5275 & & & & 12-Nov-02 \\
\hline 5276 & & & & 12-Nov-02 \\
\hline 5277 & 216-B-63 & & & 18-Nov-02 \\
\hline 5278 & 216-S-10P & & & 25-Nov-02 \\
\hline 5279 & 216-S-10P & & & 25-Nov-02 \\
\hline 5280 & $216-S-10 P$ & & & $25-N o v-02$ \\
\hline 5281 & 216-S-10P & & & 02-Dec-02 \\
\hline 5282 & 216-S-10P & & & 02-Dec-02 \\
\hline 5283 & $216-S-10 P$ & & & 02-Dec-02 \\
\hline
\end{tabular}




\begin{tabular}{|c|c|c|c|c|}
\hline & $\mathrm{O}$ & $P$ & $\mathrm{Q}$ & $R$ \\
\hline 1 & SITE CODE & ELEVATION UNITS & VERT COORD TYPE & DATE LAST MODIFIED \\
\hline 5284 & $216-S-10 P$ & & & 02-Dec-02 \\
\hline 5285 & $126-\mathrm{F}-1$ & & UNKNOWN & 05-Dec-02 \\
\hline 5286 & $126-\mathrm{F}-1$ & & UNKNOWN & 05-Dec-02 \\
\hline 5287 & 126-F-1 & & UNKNOWN & 05-Dec-02 \\
\hline 5288 & $126-\mathrm{F}-1$ & & UNKNOWN & 05-Dec-02 \\
\hline 5289 & $126-\mathrm{F}-1$ & & UNKNOWN & 05-Dec-02 \\
\hline 5290 & $126-\mathrm{F}-1$ & & UNKNOWN & 05-Dec-02 \\
\hline 5291 & 126-F-1 & & NGVD29 & 05-Dec-02 \\
\hline 5292 & $126-\mathrm{F}-1$ & & UNKNOWN & 05-Dec-02 \\
\hline 5293 & $126-\mathrm{F}-1$ & & UNKNOWN & 05-Dec-02 \\
\hline 5294 & $126-\mathrm{F}-1$ & & UNKNOWN & 05-Dec-02 \\
\hline 5295 & $126-\mathrm{F}-1$ & & UNKNOWN & 05-Dec-02 \\
\hline 5296 & 126-F-1 & & UNKNOWN & 05-Dec-02 \\
\hline 5297 & $126-\mathrm{F}-1$ & & UNKNOWN & 05-Dec-02 \\
\hline 5298 & $126-\mathrm{F}-1$ & & UNKNOWN & 05-Dec-02 \\
\hline 5299 & 126-F-1 & & UNKNOWN & 05-Dec-02 \\
\hline 5300 & 126-F-1 & & UNKNOWN & 05-Dec-02 \\
\hline 5301 & $126-\mathrm{F}-1$ & & UNKNOWN & 05-Dec-02 \\
\hline 5302 & $126-\mathrm{F}-1$ & & UNKNOWN & 05-Dec-02 \\
\hline 5303 & 126-F-1 & & UNKNOWN & 05-Dec-02 \\
\hline 5304 & $126-\mathrm{F}-1$ & & UNKNOWN & 05-Dec-02 \\
\hline 5305 & $126-\mathrm{F}-1$ & & UNKNOWN & 05-Dec-02 \\
\hline 5306 & 126-F-1 & & UNKNOWN & 05-Dec-02 \\
\hline 5307 & $126-\mathrm{F}-1$ & & UNKNOWN & 05-Dec-02 \\
\hline 5308 & 126-F-1 & & UNKNOWN & 05-Dec-02 \\
\hline 5309 & 116-F-2 & & & 05-Dec-02 \\
\hline 5310 & $116-\mathrm{F}-2$ & & & 05-Dec-02 \\
\hline 5311 & $116-\mathrm{F}-2$ & & & 05-Dec-02 \\
\hline 5312 & $116-F-2$ & & & 05-Dec-02 \\
\hline 5313 & $116-F-2$ & & & 05-Dec-02 \\
\hline 5314 & 116-F-2 & & & 05-Dec-02 \\
\hline 5315 & $116-\mathrm{F}-2$ & & & 05-Dec-02 \\
\hline 5316 & 126-F-1 & & UNKNOWN & 05-Dec-02 \\
\hline 5317 & $126-\mathrm{F}-1$ & & UNKNOWN & 05-Dec-02 \\
\hline 5318 & 126-F-1 & & UNKNOWN & 05-Dec-02 \\
\hline 5319 & 126-F-1 & & UNKNOWN & 05-Dec-02 \\
\hline 5320 & $126-\mathrm{F}-1$ & & UNKNOWN & 05-Dec-02 \\
\hline 5321 & $126-\mathrm{F}-1$ & & UNKNOWN & 05-Dec-02 \\
\hline
\end{tabular}




\begin{tabular}{|r|l|l|l|r|}
\hline & \multicolumn{1}{|c|}{ O } & \multicolumn{1}{|c|}{ Q } & \multicolumn{1}{|c|}{ R } \\
\hline 1 & SITE_CODE & ELEVATION_UNITS & VERT_COORD_TYPE & DATE_LAST_MODIFIED \\
\hline 5322 & $126-F-1$ & UNKNOWN & $05-$ Dec-02 \\
\hline 5323 & $126-F-1$ & UNKNOWN & $05-$ Dec-02 \\
\hline 5324 & $126-F-1$ & UNKNOWN & $05-$ Dec-02 \\
\hline 5325 & $126-F-1$ & UNKNOWN & $05-$ Dec-02 \\
\hline 5326 & $126-F-1$ & UNKNOWN & $05-$ Dec-02 \\
\hline 5327 & $126-F-1$ & UNKNOWN & $05-$ Dec-02 \\
\hline 5328 & $126-F-1$ & UNKNOWN & $05-$ Dec-02 \\
\hline 5329 & $126-F-1$ & UNKNOWN & $05-$ Dec-02 \\
\hline 5330 & $126-F-1$ & UNKNOWN & $05-$ Dec-02 \\
\hline 5331 & $126-F-1$ & UNKNOWN & $05-$ Dec-02 \\
\hline 5332 & $126-F-1$ & UNKNOWN & $05-$ Dec-02 \\
\hline 5333 & $126-F-1$ & UNKNOWN & $05-$ Dec-02 \\
\hline 5334 & $126-F-1$ & UNKNOWN & $05-$ Dec-02 \\
\hline 5335 & $126-F-1$ & UNKNOWN & $05-$ Dec-02 \\
\hline 5336 & $126-F-1$ & UNKNOWN & $05-$ Dec-02 \\
\hline 5337 & $126-F-1$ & UNKNOWN & $05-$ Dec-02 \\
\hline 5338 & $126-F-1$ & UNKNOWN & $05-$ Dec-02 \\
\hline 5339 & $126-F-1$ & UNKNOWN & $05-$ Dec-02 \\
\hline 5340 & $126-F-1$ & UNKNOWN & $05-$ Dec-02 \\
\hline 5341 & $126-F-1$ & UNKNOWN & $05-$ Dec-02 \\
\hline 5342 & $126-F-1$ & UNKNOWN & $05-$ Dec-02 \\
\hline 5343 & $126-F-1$ & UNKNOWN & $05-$ Dec-02 \\
\hline 5344 & $126-F-1$ & UNKNOWN & $05-$ Dec-02 \\
\hline 5345 & $126-F-1$ & UNKNOWN & $05-$ Dec-02 \\
\hline 5346 & $126-F-1$ & UNKNOWN & $05-$ Dec-02 \\
\hline 5347 & $126-F-1$ & UNKNOWN & $05-$ Dec-02 \\
\hline 5348 & $126-F-1$ & UNKNOWN & $05-$ Dec-02 \\
\hline 5349 & $126-F-1$ & UNKNOWN & $05-$ Dec-02 \\
\hline 5350 & $126-F-1$ & UNKNOWN & $05-$ Dec-02 \\
\hline 5351 & $126-F-1$ & UNKNOWN & $05-$ Dec-02 \\
\hline 5352 & $126-F-1$ & UNKNOWN & $05-$ Dec-02 \\
\hline 5353 & $126-F-1$ & UNKNOWN & $05-$ Dec-02 \\
\hline 5354 & $126-F-1$ & UNKNOWN & $05-$ Dec-02 \\
\hline 5355 & $126-F-1$ & UNKNOWN & $05-$ Dec-02 \\
\hline 5356 & $126-F-1$ & UNKNOWN & $05-$ Dec-02 \\
\hline 5357 & $126-F-1$ & $126-F-1$ & UNKNOWN & 05 Dec-02 \\
\hline 5358 & & &
\end{tabular}




\begin{tabular}{|c|c|c|c|c|}
\hline & $\mathrm{O}$ & $P$ & $\bar{Q}$ & $\bar{R}$ \\
\hline 1 & SITE_CODE & ELEVATION_UNITS & VERT_COORD_TYPE & DATE_LAST_MODIFIED \\
\hline 5360 & $126-F-1$ & & UNKNOWN & 05-Dec-02 \\
\hline 5361 & $126-F-1$ & & UNKNOWN & 05-Dec-02 \\
\hline 5362 & $126-\mathrm{F}-1$ & & UNKNOWN & 05-Dec-02 \\
\hline 5363 & $126-F-1$ & & UNKNOWN & 05-Dec-02 \\
\hline 5364 & $126-\mathrm{F}-1$ & & UNKNOWN & 05-Dec-02 \\
\hline 5365 & $126-\mathrm{F}-1$ & & UNKNOWN & 05-Dec-02 \\
\hline 5366 & 126-F-1 & & UNKNOWN & 05-Dec-02 \\
\hline 5367 & $126-\mathrm{F}-1$ & & UNKNOWN & 05-Dec-02 \\
\hline 5368 & 126-F-1 & & UNKNOWN & 05-Dec-02 \\
\hline 5369 & 126-F-1 & & UNKNOWN & 05-Dec-02 \\
\hline 5370 & $126-\mathrm{F}-1$ & & UNKNOWN & 05-Dec-02 \\
\hline 5371 & $126-F-1$ & & UNKNOWN & 05-Dec-02 \\
\hline 5372 & 126-F-1 & & UNKNOWN & 05-Dec-02 \\
\hline 5373 & $126-F-1$ & & UNKNOWN & 05-Dec-02 \\
\hline 5374 & $126-F-1$ & & UNKNOWN & 05-Dec-02 \\
\hline 5375 & 126-F-1 & & UNKNOWN & 05-Dec-02 \\
\hline 5376 & 126-F-1 & & UNKNOWN & 05-Dec-02 \\
\hline 5377 & $126-\mathrm{F}-1$ & & UNKNOWN & 05-Dec-02 \\
\hline 5378 & 126-F-1 & & UNKNOWN & 05-Dec-02 \\
\hline 5379 & 126-F-1 & & UNKNOWN & 05-Dec-02 \\
\hline 5380 & $126-F-1$ & & UNKNOWN & 05-Dec-02 \\
\hline 5381 & $126-F-1$ & & UNKNOWN & 05-Dec-02 \\
\hline 5382 & 126-F-1 & & UNKNOWN & 05-Dec-02 \\
\hline 5383 & 126-F-1 & & UNKNOWN & 05-Dec-02 \\
\hline 5384 & $126-\mathrm{F}-1$ & & UNKNOWN & 05-Dec-02 \\
\hline 5385 & $126-\mathrm{F}-1$ & & UNKNOWN & 05-Dec-02 \\
\hline 5386 & 126-F-1 & & UNKNOWN & 05-Dec-02 \\
\hline 5387 & 126-F-1 & & UNKNOWN & 05-Dec-02 \\
\hline 5388 & 126-F-1 & & UNKNOWN & 05-Dec-02 \\
\hline 5389 & 126-F-1 & & UNKNOWN & 05-Dec-02 \\
\hline 5390 & $126-\mathrm{F}-1$ & & UNKNOWN & 05-Dec-02 \\
\hline 5391 & 126-F-1 & & UNKNOWN & 05-Dec-02 \\
\hline 5392 & $126-\mathrm{F}-1$ & & UNKNOWN & 05-Dec-02 \\
\hline 5393 & 126-F-1 & & UNKNOWN & 05-Dec-02 \\
\hline 5394 & 126-F-1 & & UNKNOWN & 05-Dec-02 \\
\hline 5395 & $126-\mathrm{F}-1$ & & UNKNOWN & 05-Dec-02 \\
\hline 5396 & $116-\mathrm{N}-1$ & & UNKNOWN & 05-Dec-02 \\
\hline 5397 & $116-\mathrm{N}-1$ & & UNKNOWN & 05-Dec-02 \\
\hline
\end{tabular}




\begin{tabular}{|c|c|c|c|c|}
\hline & $\mathrm{O}$ & $P$ & $\mathrm{Q}$ & $\bar{R}$ \\
\hline 1 & SITE_CODE & ELEVATION_UNITS & VERT_COORD_TYPE & DATE_LAST_MODIFIED \\
\hline 5398 & $116-\mathrm{N}-1$ & & UNKNOWN & 05-Dec-02 \\
\hline 5399 & $116-\mathrm{N}-1$ & & UNKNOWN & 05-Dec-02 \\
\hline 5400 & $116-\mathrm{N}-1$ & & UNKNOWN & 05-Dec-02 \\
\hline 5401 & $116-\mathrm{N}-1$ & & UNKNOWN & 05-Dec-02 \\
\hline 5402 & $116-\mathrm{N}-1$ & $\mathrm{~mm}$ & UNKNOWN & 05-Dec-02 \\
\hline 5403 & $116-\mathrm{N}-1$ & $\mathrm{~mm}$ & UNKNOWN & 05-Dec-02 \\
\hline 5404 & $116-\mathrm{N}-1$ & $\mathrm{~mm}$ & UNKNOWN & 05-Dec-02 \\
\hline 5405 & $116-\mathrm{N}-1$ & $\mathrm{~mm}$ & UNKNOWN & 05-Dec-02 \\
\hline 5406 & $116-\mathrm{N}-1$ & $\mathrm{~mm}$ & UNKNOWN & 05-Dec-02 \\
\hline 5407 & $116-\mathrm{N}-1$ & $\mathrm{~mm}$ & UNKNOWN & 05-Dec-02 \\
\hline 5408 & 116-F-1 & & UNKNOWN & 05-Dec-02 \\
\hline 5409 & 116-F-1 & & UNKNOWN & 05-Dec-02 \\
\hline 5410 & $116-\mathrm{N}-1$ & $\mathrm{~mm}$ & UNKNOWN & 05-Dec-02 \\
\hline 5411 & $126-F-1$ & & & 05-Dec-02 \\
\hline 5412 & 126-F-1 & & & 05-Dec-02 \\
\hline 5413 & 126-F-1 & & & 05-Dec-02 \\
\hline 5414 & $126-\mathrm{F}-1$ & & & 05-Dec-02 \\
\hline 5415 & 126-F-1 & & & 05-Dec-02 \\
\hline 5416 & 126-F-1 & & & 05-Dec-02 \\
\hline 5417 & 126-F-1 & & & 05-Dec-02 \\
\hline 5418 & 126-F-1 & & & 05-Dec-02 \\
\hline 5419 & $126-F-1$ & & & 05-Dec-02 \\
\hline 5420 & $126-F-1$ & & & 05-Dec-02 \\
\hline 5421 & 126-F-1 & & & 05-Dec-02 \\
\hline 5422 & $126-F-1$ & & & 05-Dec-02 \\
\hline 5423 & $126-F-1$ & & & 05-Dec-02 \\
\hline 5424 & 126-F-1 & & & 05-Dec-02 \\
\hline 5425 & 126-F-1 & & & 05-Dec-02 \\
\hline 5426 & 126-F-1 & & & 05-Dec-02 \\
\hline 5427 & 126-F-1 & & & 05-Dec-02 \\
\hline 5428 & 126-F-1 & & & 05-Dec-02 \\
\hline 5429 & $126-F-1$ & & & 05-Dec-02 \\
\hline 5430 & 126-F-1 & & & 05-Dec-02 \\
\hline 5431 & 126-F-1 & & & 05-Dec-02 \\
\hline 5432 & $126-F-1$ & & & 05-Dec-02 \\
\hline 5433 & $126-F-1$ & & & 05-Dec-02 \\
\hline 5434 & $126-F-1$ & & & 05-Dec-02 \\
\hline 5435 & $126-F-1$ & & & 05-Dec-02 \\
\hline
\end{tabular}




\begin{tabular}{|c|c|c|c|c|}
\hline & $\mathrm{O}$ & $P$ & $\mathrm{Q}$ & $\bar{R}$ \\
\hline 1 & SITE_CODE & ELEVATION_UNITS & VERT_COORD_TYPE & DATE_LAST_MODIFIED \\
\hline 5436 & $126-\mathrm{F}-1$ & & & 05-Dec-02 \\
\hline 5437 & $126-\mathrm{F}-1$ & & & 05-Dec-02 \\
\hline 5438 & $126-\mathrm{F}-1$ & & & 05-Dec-02 \\
\hline 5439 & $126-\mathrm{F}-1$ & & & 05-Dec-02 \\
\hline 5440 & 126-F-1 & & & 05-Dec-02 \\
\hline 5441 & 126-F-1 & & & 05-Dec-02 \\
\hline 5442 & 126-F-1 & & & 05-Dec-02 \\
\hline 5443 & 126-F-1 & & & 05-Dec-02 \\
\hline 5444 & $126-\mathrm{F}-1$ & & & 05-Dec-02 \\
\hline 5445 & $126-F-1$ & & & 05-Dec-02 \\
\hline 5446 & $126-F-1$ & & & 05-Dec-02 \\
\hline 5447 & $126-F-1$ & & & 05-Dec-02 \\
\hline 5448 & $126-F-1$ & & & 05-Dec-02 \\
\hline 5449 & 126-F-1 & & & 05-Dec-02 \\
\hline 5450 & 126-F-1 & & & 05-Dec-02 \\
\hline 5451 & 100-B-8 & $\mathrm{mm}$ & UNKNOWN & 05-Dec-02 \\
\hline 5452 & 100-B-8 & $\mathrm{mm}$ & UNKNOWN & 05-Dec-02 \\
\hline 5453 & 100-B-8 & $\mathrm{mm}$ & UNKNOWN & 05-Dec-02 \\
\hline 5454 & 100-B-8 & $\mathrm{mm}$ & UNKNOWN & 05-Dec-02 \\
\hline 5455 & 100-B-8 & $\mathrm{mm}$ & UNKNOWN & 05-Dec-02 \\
\hline 5456 & 100-B-8 & $\mathrm{mm}$ & UNKNOWN & 05-Dec-02 \\
\hline 5457 & 100-B-8 & $\mathrm{mm}$ & UNKNOWN & 05-Dec-02 \\
\hline 5458 & 100-B-8 & $\mathrm{mm}$ & UNKNOWN & 05-Dec-02 \\
\hline 5459 & 100-B-8 & $\mathrm{mm}$ & UNKNOWN & 05-Dec-02 \\
\hline 5460 & 100-B-8 & $\mathrm{mm}$ & UNKNOWN & 05-Dec-02 \\
\hline 5461 & 100-B-8 & $\mathrm{mm}$ & UNKNOWN & 05-Dec-02 \\
\hline 5462 & 100-B-8 & $\mathrm{mm}$ & UNKNOWN & 05-Dec-02 \\
\hline 5463 & 100-B-8 & $\mathrm{mm}$ & UNKNOWN & 05-Dec-02 \\
\hline 5464 & 100-B-8 & $\mathrm{mm}$ & UNKNOWN & 05-Dec-02 \\
\hline 5465 & 100-B-8 & $\mathrm{mm}$ & UNKNOWN & 05-Dec-02 \\
\hline 5466 & 100-B-8 & $\mathrm{mm}$ & UNKNOWN & 05-Dec-02 \\
\hline 5467 & 100-B-8 & $\mathrm{mm}$ & UNKNOWN & 05-Dec-02 \\
\hline 5468 & 100-B-8 & $\mathrm{mm}$ & UNKNOWN & 05-Dec-02 \\
\hline 5469 & 100-B-8 & $\mathrm{mm}$ & UNKNOWN & 05-Dec-02 \\
\hline 5470 & 100-B-8 & $\mathrm{mm}$ & UNKNOWN & 05-Dec-02 \\
\hline 5471 & 100-B-8 & $\mathrm{mm}$ & UNKNOWN & 05-Dec-02 \\
\hline 5472 & 100-B-8 & $\mathrm{mm}$ & UNKNOWN & 05-Dec-02 \\
\hline 5473 & 100-B-8 & $\mathrm{mm}$ & UNKNOWN & 05-Dec-02 \\
\hline
\end{tabular}




\begin{tabular}{|c|c|c|c|c|}
\hline & $\mathrm{O}$ & $P$ & $\bar{Q}$ & $\bar{R}$ \\
\hline 1 & SITE_CODE & ELEVATION_UNITS & VERT_COORD_TYPE & DATE_LAST_MODIFIED \\
\hline 5474 & 100-B-8 & $\mathrm{mm}$ & UNKNOWN & 05-Dec-02 \\
\hline 5475 & 100-B-8 & $\mathrm{mm}$ & NGVD29 & 05-Dec-02 \\
\hline 5476 & 100-B-8 & $\mathrm{mm}$ & UNKNOWN & 05-Dec-02 \\
\hline 5477 & 100-B-8 & $\mathrm{mm}$ & UNKNOWN & 05-Dec-02 \\
\hline 5478 & 100-B-8 & $\mathrm{mm}$ & UNKNOWN & 05-Dec-02 \\
\hline 5479 & 100-B-8 & $\mathrm{mm}$ & UNKNOWN & 05-Dec-02 \\
\hline 5480 & 100-B-8 & $\mathrm{mm}$ & UNKNOWN & 05-Dec-02 \\
\hline 5481 & 100-B-8 & $\mathrm{mm}$ & UNKNOWN & 05-Dec-02 \\
\hline 5482 & 100-B-8 & $\mathrm{mm}$ & UNKNOWN & 05-Dec-02 \\
\hline 5483 & 100-B-8 & $\mathrm{mm}$ & UNKNOWN & 05-Dec-02 \\
\hline 5484 & 100-B-8 & $\mathrm{mm}$ & UNKNOWN & 05-Dec-02 \\
\hline 5485 & 100-B-8 & $\mathrm{mm}$ & UNKNOWN & 05-Dec-02 \\
\hline 5486 & 100-B-8 & $\mathrm{mm}$ & UNKNOWN & 05-Dec-02 \\
\hline 5487 & 100-B-8 & $\mathrm{mm}$ & UNKNOWN & 05-Dec-02 \\
\hline 5488 & 100-B-8 & $\mathrm{mm}$ & UNKNOWN & 05-Dec-02 \\
\hline 5489 & 100-B-8 & $\mathrm{mm}$ & UNKNOWN & 05-Dec-02 \\
\hline 5490 & 100-B-8 & $\mathrm{mm}$ & UNKNOWN & 05-Dec-02 \\
\hline 5491 & 100-B-8 & $\mathrm{mm}$ & UNKNOWN & 05-Dec-02 \\
\hline 5492 & 100-B-8 & $\mathrm{mm}$ & UNKNOWN & 05-Dec-02 \\
\hline 5493 & 100-B-8 & $\mathrm{mm}$ & UNKNOWN & 05-Dec-02 \\
\hline 5494 & 100-B-8 & $\mathrm{mm}$ & UNKNOWN & 05-Dec-02 \\
\hline 5495 & 100-B-8 & $\mathrm{mm}$ & UNKNOWN & 05-Dec-02 \\
\hline 5496 & 100-B-8 & $\mathrm{mm}$ & UNKNOWN & 05-Dec-02 \\
\hline 5497 & 100-B-8 & $\mathrm{mm}$ & UNKNOWN & 05-Dec-02 \\
\hline 5498 & 100-B-8 & $\mathrm{mm}$ & UNKNOWN & 05-Dec-02 \\
\hline 5499 & 100-B-8 & $\mathrm{mm}$ & UNKNOWN & 05-Dec-02 \\
\hline 5500 & 100-B-8 & $\mathrm{mm}$ & UNKNOWN & 05-Dec-02 \\
\hline 5501 & $100-C-6$ & & UNKNOWN & 05-Dec-02 \\
\hline 5502 & $100-C-6$ & & UNKNOWN & 05-Dec-02 \\
\hline 5503 & 100-B-8 & & UNKNOWN & 05-Dec-02 \\
\hline 5504 & 100-B-8 & & UNKNOWN & 05-Dec-02 \\
\hline 5505 & 1607-F2 & & UNKNOWN & 05-Dec-02 \\
\hline 5506 & 1607-F2 & & UNKNOWN & 05-Dec-02 \\
\hline 5507 & 1607-F2 & & UNKNOWN & 05-Dec-02 \\
\hline 5508 & 1607-F2 & & UNKNOWN & 05-Dec-02 \\
\hline 5509 & 1607-F2 & & UNKNOWN & 05-Dec-02 \\
\hline 5510 & 1607-F2 & & UNKNOWN & 05-Dec-02 \\
\hline 5511 & 1607-F2 & & UNKNOWN & 05-Dec-02 \\
\hline
\end{tabular}




\begin{tabular}{|c|c|c|c|c|}
\hline & $\mathrm{O}$ & $P$ & $\bar{Q}$ & $\bar{R}$ \\
\hline 1 & SITE_CODE & ELEVATION_UNITS & VERT_COORD_TYPE & DATE_LAST_MODIFIED \\
\hline 5512 & 1607-F2 & & UNKNOWN & 05-Dec-02 \\
\hline 5513 & 1607-F2 & & UNKNOWN & 05-Dec-02 \\
\hline 5514 & 1607-F2 & & UNKNOWN & 05-Dec-02 \\
\hline 5515 & 1607-F2 & & UNKNOWN & 05-Dec-02 \\
\hline 5516 & 1607-F2 & & UNKNOWN & 05-Dec-02 \\
\hline 5517 & $1607-F 2$ & & UNKNOWN & 05-Dec-02 \\
\hline 5518 & 1607-F2 & & UNKNOWN & 05-Dec-02 \\
\hline 5519 & 1607-F2 & & UNKNOWN & 05-Dec-02 \\
\hline 5520 & 1607-F2 & & UNKNOWN & 05-Dec-02 \\
\hline 5521 & 1607-F2 & & UNKNOWN & 05-Dec-02 \\
\hline 5522 & 1607-F2 & & UNKNOWN & 05-Dec-02 \\
\hline 5523 & 1607-F2 & & UNKNOWN & 05-Dec-02 \\
\hline 5524 & 1607-F2 & & UNKNOWN & 05-Dec-02 \\
\hline 5525 & 1607-F2 & & UNKNOWN & 05-Dec-02 \\
\hline 5526 & 1607-F2 & & UNKNOWN & 05-Dec-02 \\
\hline 5527 & 1607-F2 & & UNKNOWN & 05-Dec-02 \\
\hline 5528 & 1607-F2 & & UNKNOWN & 05-Dec-02 \\
\hline 5529 & 1607-F2 & & UNKNOWN & 05-Dec-02 \\
\hline 5530 & 1607-F2 & & UNKNOWN & 05-Dec-02 \\
\hline 5531 & 1607-F2 & & UNKNOWN & 05-Dec-02 \\
\hline 5532 & 1607-F2 & & UNKNOWN & 05-Dec-02 \\
\hline 5533 & 1607-F2 & & UNKNOWN & 05-Dec-02 \\
\hline 5534 & 1607-F2 & & UNKNOWN & 05-Dec-02 \\
\hline 5535 & 1607-F2 & & UNKNOWN & 05-Dec-02 \\
\hline 5536 & 1607-F2 & & UNKNOWN & 05-Dec-02 \\
\hline 5537 & 1607-F2 & & UNKNOWN & 05-Dec-02 \\
\hline 5538 & 1607-F2 & & UNKNOWN & 05-Dec-02 \\
\hline 5539 & 1607-F2 & & UNKNOWN & 05-Dec-02 \\
\hline 5540 & 1607-F2 & & UNKNOWN & 05-Dec-02 \\
\hline 5541 & 1607-F2 & & UNKNOWN & 05-Dec-02 \\
\hline 5542 & $1607-F 2$ & & UNKNOWN & 05-Dec-02 \\
\hline 5543 & 1607-F2 & & UNKNOWN & 05-Dec-02 \\
\hline 5544 & 1607-F2 & & UNKNOWN & 05-Dec-02 \\
\hline 5545 & 1607-F2 & & UNKNOWN & 05-Dec-02 \\
\hline 5546 & 1607-F2 & & UNKNOWN & 05-Dec-02 \\
\hline 5547 & 1607-F2 & & UNKNOWN & 05-Dec-02 \\
\hline 5548 & 1607-F2 & & UNKNOWN & 05-Dec-02 \\
\hline 5549 & $1607-F 2$ & & UNKNOWN & 05-Dec-02 \\
\hline
\end{tabular}




\begin{tabular}{|c|c|c|c|c|}
\hline & $\mathrm{O}$ & $P$ & $\mathrm{Q}$ & $R$ \\
\hline 1 & SITE CODE & ELEVATION UNITS & VERT COORD TYPE & DATE LAST MODIFIED \\
\hline 5550 & $1607-\bar{F} 2$ & & UNKNOWN & 05-Dec-02 \\
\hline 5551 & 1607-F2 & & UNKNOWN & 05-Dec-02 \\
\hline 5552 & 1607-F2 & & UNKNOWN & 05-Dec-02 \\
\hline 5553 & 100-B-8 & $\mathrm{mm}$ & UNKNOWN & 05-Dec-02 \\
\hline 5554 & 100-B-8 & $\mathrm{mm}$ & UNKNOWN & 05-Dec-02 \\
\hline 5555 & 100-B-8 & $\mathrm{mm}$ & UNKNOWN & 05-Dec-02 \\
\hline 5556 & 100-B-8 & $\mathrm{mm}$ & UNKNOWN & 05-Dec-02 \\
\hline 5557 & 100-C-6 & $\mathrm{mm}$ & UNKNOWN & 05-Dec-02 \\
\hline 5558 & $100-C-6$ & $\mathrm{~mm}$ & UNKNOWN & 05-Dec-02 \\
\hline 5559 & $100-C-6$ & $\mathrm{~mm}$ & UNKNOWN & 05-Dec-02 \\
\hline 5560 & 100-C-6 & $\mathrm{mm}$ & UNKNOWN & 05-Dec-02 \\
\hline 5561 & $100-C-6$ & $\mathrm{~mm}$ & UNKNOWN & 05-Dec-02 \\
\hline 5562 & $100-C-6$ & $\mathrm{~mm}$ & UNKNOWN & 05-Dec-02 \\
\hline 5563 & $100-C-6$ & $\mathrm{~mm}$ & UNKNOWN & 05-Dec-02 \\
\hline 5564 & $100-C-6$ & $\mathrm{~mm}$ & UNKNOWN & 05-Dec-02 \\
\hline 5565 & $100-C-6$ & $\mathrm{~mm}$ & UNKNOWN & 05-Dec-02 \\
\hline 5566 & 100-C-6 & $\mathrm{mm}$ & UNKNOWN & 05-Dec-02 \\
\hline 5567 & $100-C-6$ & $\mathrm{~mm}$ & UNKNOWN & 05-Dec-02 \\
\hline 5568 & $100-C-6$ & $\mathrm{~mm}$ & UNKNOWN & 05-Dec-02 \\
\hline 5569 & 100-C-6 & $\mathrm{mm}$ & UNKNOWN & 05-Dec-02 \\
\hline 5570 & $100-C-6$ & $\mathrm{~mm}$ & UNKNOWN & 05-Dec-02 \\
\hline 5571 & $100-C-6$ & $\mathrm{~mm}$ & UNKNOWN & 05-Dec-02 \\
\hline 5572 & 100-C-6 & $\mathrm{mm}$ & UNKNOWN & 05-Dec-02 \\
\hline 5573 & $100-C-6$ & $\mathrm{~mm}$ & UNKNOWN & 05-Dec-02 \\
\hline 5574 & $100-C-6$ & $\mathrm{~mm}$ & UNKNOWN & 05-Dec-02 \\
\hline 5575 & $100-C-6$ & $\mathrm{~mm}$ & UNKNOWN & 05-Dec-02 \\
\hline 5576 & $100-C-6$ & $\mathrm{~mm}$ & UNKNOWN & 05-Dec-02 \\
\hline 5577 & $100-C-6$ & $\mathrm{~mm}$ & UNKNOWN & 05-Dec-02 \\
\hline 5578 & $100-C-6$ & $\mathrm{~mm}$ & UNKNOWN & 05-Dec-02 \\
\hline 5579 & 100-C-6 & $\mathrm{mm}$ & UNKNOWN & 05-Dec-02 \\
\hline 5580 & 100-C-6 & $\mathrm{mm}$ & UNKNOWN & 05-Dec-02 \\
\hline 5581 & $100-C-6$ & $\mathrm{~mm}$ & UNKNOWN & 05-Dec-02 \\
\hline 5582 & 100-C-6 & $\mathrm{mm}$ & UNKNOWN & 05-Dec-02 \\
\hline 5583 & $100-C-6$ & $\mathrm{~mm}$ & UNKNOWN & 05-Dec-02 \\
\hline 5584 & 100-B-8 & $\mathrm{mm}$ & UNKNOWN & 05-Dec-02 \\
\hline 5585 & 100-B-8 & $\mathrm{mm}$ & UNKNOWN & 05-Dec-02 \\
\hline 5586 & 100-B-8 & $\mathrm{mm}$ & UNKNOWN & 05-Dec-02 \\
\hline 5587 & 100-B-8 & $\mathrm{mm}$ & UNKNOWN & 05-Dec-02 \\
\hline
\end{tabular}




\begin{tabular}{|c|c|c|c|c|}
\hline & $\mathrm{O}$ & $P$ & $\mathrm{Q}$ & $R$ \\
\hline 1 & SITE CODE & ELEVATION UNITS & VERT COORD TYPE & DATE LAST MODIFIED \\
\hline 5588 & $100-B-8$ & $\mathrm{~mm}$ & UNKNOWN & 05-Dec-02 \\
\hline 5589 & 100-B-8 & $\mathrm{mm}$ & UNKNOWN & 05-Dec-02 \\
\hline 5590 & 100-B-8 & $\mathrm{mm}$ & UNKNOWN & 05-Dec-02 \\
\hline 5591 & 100-B-8 & $\mathrm{mm}$ & UNKNOWN & 05-Dec-02 \\
\hline 5592 & 100-B-8 & $\mathrm{mm}$ & UNKNOWN & 05-Dec-02 \\
\hline 5593 & 100-B-8 & $\mathrm{mm}$ & UNKNOWN & 05-Dec-02 \\
\hline 5594 & 100-B-8 & $\mathrm{mm}$ & UNKNOWN & 05-Dec-02 \\
\hline 5595 & 100-B-8 & $\mathrm{mm}$ & UNKNOWN & 05-Dec-02 \\
\hline 5596 & 100-B-8 & $\mathrm{mm}$ & UNKNOWN & 05-Dec-02 \\
\hline 5597 & 100-B-8 & $\mathrm{mm}$ & UNKNOWN & 05-Dec-02 \\
\hline 5598 & 100-B-8 & $\mathrm{mm}$ & UNKNOWN & 05-Dec-02 \\
\hline 5599 & 100-B-8 & $\mathrm{mm}$ & UNKNOWN & 05-Dec-02 \\
\hline 5600 & 100-B-8 & $\mathrm{mm}$ & UNKNOWN & 05-Dec-02 \\
\hline 5601 & 100-B-8 & $\mathrm{mm}$ & UNKNOWN & 05-Dec-02 \\
\hline 5602 & 100-B-8 & $\mathrm{mm}$ & UNKNOWN & 05-Dec-02 \\
\hline 5603 & 100-B-8 & $\mathrm{mm}$ & UNKNOWN & 05-Dec-02 \\
\hline 5604 & 100-B-8 & $\mathrm{mm}$ & UNKNOWN & 05-Dec-02 \\
\hline 5605 & 100-B-8 & $\mathrm{mm}$ & UNKNOWN & 05-Dec-02 \\
\hline 5606 & 100-B-8 & $\mathrm{mm}$ & UNKNOWN & 05-Dec-02 \\
\hline 5607 & 100-B-8 & $\mathrm{mm}$ & UNKNOWN & 05-Dec-02 \\
\hline 5608 & 100-B-8 & $\mathrm{mm}$ & UNKNOWN & 05-Dec-02 \\
\hline 5609 & 100-B-8 & $\mathrm{mm}$ & UNKNOWN & 05-Dec-02 \\
\hline 5610 & 100-B-8 & $\mathrm{mm}$ & UNKNOWN & 05-Dec-02 \\
\hline 5611 & 100-B-8 & $\mathrm{mm}$ & UNKNOWN & 05-Dec-02 \\
\hline 5612 & 100-B-8 & $\mathrm{mm}$ & UNKNOWN & 05-Dec-02 \\
\hline 5613 & 100-B-8 & $\mathrm{mm}$ & UNKNOWN & 05-Dec-02 \\
\hline 5614 & 100-B-8 & $\mathrm{mm}$ & UNKNOWN & 05-Dec-02 \\
\hline 5615 & 100-B-8 & $\mathrm{mm}$ & UNKNOWN & 05-Dec-02 \\
\hline 5616 & 100-B-8 & $\mathrm{mm}$ & UNKNOWN & 05-Dec-02 \\
\hline 5617 & 100-B-8 & $\mathrm{mm}$ & UNKNOWN & 05-Dec-02 \\
\hline 5618 & 100-B-8 & $\mathrm{mm}$ & UNKNOWN & 05-Dec-02 \\
\hline 5619 & 100-B-8 & $\mathrm{mm}$ & UNKNOWN & 05-Dec-02 \\
\hline 5620 & 100-B-8 & $\mathrm{mm}$ & UNKNOWN & 05-Dec-02 \\
\hline 5621 & 100-B-8 & $\mathrm{mm}$ & UNKNOWN & 05-Dec-02 \\
\hline 5622 & 100-B-8 & $\mathrm{mm}$ & UNKNOWN & 05-Dec-02 \\
\hline 5623 & 100-B-8 & $\mathrm{mm}$ & UNKNOWN & 05-Dec-02 \\
\hline 5624 & 100-B-8 & $\mathrm{mm}$ & UNKNOWN & 05-Dec-02 \\
\hline 5625 & 100-B-8 & $\mathrm{mm}$ & UNKNOWN & 05-Dec-02 \\
\hline
\end{tabular}




\begin{tabular}{|c|c|c|c|c|}
\hline & $\mathrm{O}$ & $P$ & $\mathrm{Q}$ & $\bar{R}$ \\
\hline 1 & SITE_CODE & ELEVATION_UNITS & VERT_COORD_TYPE & DATE_LAST_MODIFIED \\
\hline 5626 & 100-B-8 & $\mathrm{mm}$ & UNKNOWN & 05-Dec-02 \\
\hline 5627 & 100-B-8 & $\mathrm{mm}$ & UNKNOWN & 05-Dec-02 \\
\hline 5628 & 100-B-8 & $\mathrm{mm}$ & UNKNOWN & 05-Dec-02 \\
\hline 5629 & 100-B-8 & $\mathrm{mm}$ & UNKNOWN & 05-Dec-02 \\
\hline 5630 & 100-B-8 & $\mathrm{mm}$ & UNKNOWN & 05-Dec-02 \\
\hline 5631 & 100-B-8 & $\mathrm{mm}$ & UNKNOWN & 05-Dec-02 \\
\hline 5632 & 100-B-8 & $\mathrm{mm}$ & UNKNOWN & 05-Dec-02 \\
\hline 5633 & 100-B-8 & $\mathrm{mm}$ & UNKNOWN & 05-Dec-02 \\
\hline 5634 & 100-B-8 & $\mathrm{mm}$ & UNKNOWN & 05-Dec-02 \\
\hline 5635 & 100-B-8 & $\mathrm{mm}$ & UNKNOWN & 05-Dec-02 \\
\hline 5636 & 100-B-8 & $\mathrm{mm}$ & UNKNOWN & 05-Dec-02 \\
\hline 5637 & 100-B-8 & $\mathrm{mm}$ & UNKNOWN & 05-Dec-02 \\
\hline 5638 & 100-B-8 & $\mathrm{mm}$ & UNKNOWN & 05-Dec-02 \\
\hline 5639 & 100-B-8 & $\mathrm{mm}$ & UNKNOWN & 05-Dec-02 \\
\hline 5640 & 100-B-8 & $\mathrm{mm}$ & UNKNOWN & 05-Dec-02 \\
\hline 5641 & 100-B-8 & $\mathrm{mm}$ & UNKNOWN & 05-Dec-02 \\
\hline 5642 & 100-B-8 & $\mathrm{mm}$ & UNKNOWN & 05-Dec-02 \\
\hline 5643 & 100-B-8 & $\mathrm{mm}$ & UNKNOWN & 05-Dec-02 \\
\hline 5644 & 100-B-8 & $\mathrm{mm}$ & UNKNOWN & 05-Dec-02 \\
\hline 5645 & 100-B-8 & $\mathrm{mm}$ & UNKNOWN & 05-Dec-02 \\
\hline 5646 & 100-B-8 & $\mathrm{mm}$ & UNKNOWN & 05-Dec-02 \\
\hline 5647 & 100-B-8 & $\mathrm{mm}$ & UNKNOWN & 05-Dec-02 \\
\hline 5648 & 100-B-8 & $\mathrm{mm}$ & UNKNOWN & 05-Dec-02 \\
\hline 5649 & 100-B-8 & & & 05-Dec-02 \\
\hline 5650 & 1607-F2 & & & 05-Dec-02 \\
\hline 5651 & 1607-F2 & & & 05-Dec-02 \\
\hline 5652 & 1607-F2 & & & 05-Dec-02 \\
\hline 5653 & 1607-F2 & & & 05-Dec-02 \\
\hline 5654 & 1607-F2 & & & 05-Dec-02 \\
\hline 5655 & 1607-F2 & & & 05-Dec-02 \\
\hline 5656 & 1607-F6 & & & 05-Dec-02 \\
\hline 5657 & 1607-F6 & & & 05-Dec-02 \\
\hline 5658 & 1607-F2 & & & 05-Dec-02 \\
\hline 5659 & 1607-F2 & & & 05-Dec-02 \\
\hline 5660 & $116-\mathrm{N}-1$ & $\mathrm{~mm}$ & UNKNOWN & 05-Dec-02 \\
\hline 5661 & $116-\mathrm{N}-1$ & $\mathrm{~mm}$ & UNKNOWN & 05-Dec-02 \\
\hline 5662 & $116-\mathrm{N}-1$ & $\mathrm{~mm}$ & UNKNOWN & 05-Dec-02 \\
\hline 5663 & $116-\mathrm{N}-1$ & $\mathrm{~mm}$ & UNKNOWN & 05-Dec-02 \\
\hline
\end{tabular}




\begin{tabular}{|c|c|c|c|c|}
\hline & $\mathrm{O}$ & $P$ & $\mathrm{Q}$ & $R$ \\
\hline 1 & SITE CODE & ELEVATION UNITS & VERT COORD TYPE & DATE LAST MODIFIED \\
\hline 5664 & $116-\mathrm{N}-1$ & $\mathrm{~mm}$ & UNKNOWN & 05-Dec-02 \\
\hline 5665 & $116-\mathrm{N}-1$ & $\mathrm{~mm}$ & UNKNOWN & 05-Dec-02 \\
\hline 5666 & $116-\mathrm{N}-1$ & $\mathrm{~mm}$ & UNKNOWN & 05-Dec-02 \\
\hline 5667 & $116-\mathrm{N}-1$ & $\mathrm{~mm}$ & UNKNOWN & 05-Dec-02 \\
\hline 5668 & $116-\mathrm{N}-1$ & $\mathrm{~mm}$ & UNKNOWN & 05-Dec-02 \\
\hline 5669 & $116-\mathrm{N}-1$ & $\mathrm{~mm}$ & UNKNOWN & 05-Dec-02 \\
\hline 5670 & $116-\mathrm{N}-1$ & $\mathrm{~mm}$ & UNKNOWN & 05-Dec-02 \\
\hline 5671 & $116-\mathrm{N}-1$ & $\mathrm{~mm}$ & UNKNOWN & 05-Dec-02 \\
\hline 5672 & $116-\mathrm{N}-1$ & $\mathrm{~mm}$ & UNKNOWN & 05-Dec-02 \\
\hline 5673 & $116-\mathrm{N}-1$ & $\mathrm{~mm}$ & UNKNOWN & 05-Dec-02 \\
\hline 5674 & $116-\mathrm{N}-1$ & $\mathrm{~mm}$ & UNKNOWN & 05-Dec-02 \\
\hline 5675 & $116-\mathrm{N}-1$ & $\mathrm{~mm}$ & UNKNOWN & 05-Dec-02 \\
\hline 5676 & $100-C-6$ & & UNKNOWN & 05-Dec-02 \\
\hline 5677 & $100-C-6$ & & UNKNOWN & 05-Dec-02 \\
\hline 5678 & $100-C-6$ & & UNKNOWN & 05-Dec-02 \\
\hline 5679 & $100-C-6$ & & UNKNOWN & 05-Dec-02 \\
\hline 5680 & 100-C-6 & & UNKNOWN & 05-Dec-02 \\
\hline 5681 & 100-C-6 & & UNKNOWN & 05-Dec-02 \\
\hline 5682 & $100-C-6$ & & UNKNOWN & 05-Dec-02 \\
\hline 5683 & 100-C-6 & & UNKNOWN & 05-Dec-02 \\
\hline 5684 & $100-C-6$ & & UNKNOWN & 05-Dec-02 \\
\hline 5685 & $132-C-2$ & & UNKNOWN & 05-Dec-02 \\
\hline 5686 & $132-C-2$ & & UNKNOWN & 05-Dec-02 \\
\hline 5687 & 100-B-8 & $\mathrm{mm}$ & UNKNOWN & 05-Dec-02 \\
\hline 5688 & 100-B-8 & $\mathrm{mm}$ & UNKNOWN & 05-Dec-02 \\
\hline 5689 & 100-B-8 & $\mathrm{mm}$ & UNKNOWN & 05-Dec-02 \\
\hline 5690 & 100-B-8 & $\mathrm{mm}$ & UNKNOWN & 05-Dec-02 \\
\hline 5691 & 100-B-8 & $\mathrm{mm}$ & UNKNOWN & 05-Dec-02 \\
\hline 5692 & 100-B-8 & $\mathrm{mm}$ & UNKNOWN & 05-Dec-02 \\
\hline 5693 & 100-B-8 & $\mathrm{mm}$ & UNKNOWN & 05-Dec-02 \\
\hline 5694 & 100-B-8 & $\mathrm{mm}$ & UNKNOWN & 05-Dec-02 \\
\hline 5695 & 100-B-8 & $\mathrm{mm}$ & UNKNOWN & 05-Dec-02 \\
\hline 5696 & 100-B-8 & $\mathrm{mm}$ & UNKNOWN & 05-Dec-02 \\
\hline 5697 & 100-B-8 & $\mathrm{mm}$ & UNKNOWN & 05-Dec-02 \\
\hline 5698 & 100-B-8 & $\mathrm{mm}$ & UNKNOWN & 05-Dec-02 \\
\hline 5699 & 100-B-8 & $\mathrm{mm}$ & UNKNOWN & 05-Dec-02 \\
\hline 5700 & 100-B-8 & $\mathrm{mm}$ & UNKNOWN & 05-Dec-02 \\
\hline 5701 & $100-C-6$ & $\mathrm{~mm}$ & UNKNOWN & 05-Dec-02 \\
\hline
\end{tabular}




\begin{tabular}{|c|c|c|c|c|}
\hline & $\mathrm{O}$ & $P$ & $\mathrm{Q}$ & $\bar{R}$ \\
\hline 1 & SITE_CODE & ELEVATION_UNITS & VERT_COORD_TYPE & DATE_LAST_MODIFIED \\
\hline 5702 & 100-B-8 & $\mathrm{mm}$ & UNKNOWN & 05-Dec-02 \\
\hline 5703 & $100-C-6$ & $\mathrm{~mm}$ & UNKNOWN & 05-Dec-02 \\
\hline 5704 & $100-C-6$ & $\mathrm{~mm}$ & UNKNOWN & 05-Dec-02 \\
\hline 5705 & $100-C-6$ & $\mathrm{~mm}$ & UNKNOWN & 05-Dec-02 \\
\hline 5706 & $100-C-6$ & $\mathrm{~mm}$ & UNKNOWN & 05-Dec-02 \\
\hline 5707 & $100-C-6$ & $\mathrm{~mm}$ & UNKNOWN & 05-Dec-02 \\
\hline 5708 & $100-C-6$ & $\mathrm{~mm}$ & UNKNOWN & 05-Dec-02 \\
\hline 5709 & $100-C-6$ & $\mathrm{~mm}$ & UNKNOWN & 05-Dec-02 \\
\hline 5710 & $100-C-6$ & $\mathrm{~mm}$ & UNKNOWN & 05-Dec-02 \\
\hline 5711 & $100-C-6$ & $\mathrm{~mm}$ & UNKNOWN & 05-Dec-02 \\
\hline 5712 & $100-C-6$ & $\mathrm{~mm}$ & UNKNOWN & 05-Dec-02 \\
\hline 5713 & $100-C-6$ & $\mathrm{~mm}$ & UNKNOWN & 05-Dec-02 \\
\hline 5714 & $100-C-6$ & $\mathrm{~mm}$ & UNKNOWN & 05-Dec-02 \\
\hline 5715 & $100-C-6$ & $\mathrm{~mm}$ & UNKNOWN & 05-Dec-02 \\
\hline 5716 & $100-C-6$ & $\mathrm{~mm}$ & UNKNOWN & 05-Dec-02 \\
\hline 5717 & $100-C-6$ & $\mathrm{~mm}$ & UNKNOWN & 05-Dec-02 \\
\hline 5718 & $100-C-6$ & $\mathrm{~mm}$ & UNKNOWN & 05-Dec-02 \\
\hline 5719 & $100-C-6$ & $\mathrm{~mm}$ & UNKNOWN & 05-Dec-02 \\
\hline 5720 & 100-C-6 & $\mathrm{mm}$ & UNKNOWN & 05-Dec-02 \\
\hline 5721 & $100-C-6$ & $\mathrm{~mm}$ & UNKNOWN & 05-Dec-02 \\
\hline 5722 & $100-C-6$ & $\mathrm{~mm}$ & UNKNOWN & 05-Dec-02 \\
\hline 5723 & $100-C-6$ & $\mathrm{~mm}$ & UNKNOWN & 05-Dec-02 \\
\hline 5724 & $100-C-6$ & $\mathrm{~mm}$ & UNKNOWN & 05-Dec-02 \\
\hline 5725 & $100-C-6$ & $\mathrm{~mm}$ & UNKNOWN & 05-Dec-02 \\
\hline 5726 & $100-C-6$ & $\mathrm{~mm}$ & UNKNOWN & 05-Dec-02 \\
\hline 5727 & $100-C-6$ & $\mathrm{~mm}$ & UNKNOWN & 05-Dec-02 \\
\hline 5728 & $100-C-6$ & $\mathrm{~mm}$ & UNKNOWN & 05-Dec-02 \\
\hline 5729 & $100-C-6$ & $\mathrm{~mm}$ & UNKNOWN & 05-Dec-02 \\
\hline 5730 & $100-C-6$ & $\mathrm{~mm}$ & UNKNOWN & 05-Dec-02 \\
\hline 5731 & $100-C-6$ & $\mathrm{~mm}$ & UNKNOWN & 05-Dec-02 \\
\hline 5732 & $100-C-6$ & $\mathrm{~mm}$ & UNKNOWN & 05-Dec-02 \\
\hline 5733 & $100-C-6$ & $\mathrm{~mm}$ & UNKNOWN & 05-Dec-02 \\
\hline 5734 & $100-C-6$ & $\mathrm{~mm}$ & UNKNOWN & 05-Dec-02 \\
\hline 5735 & $100-C-6$ & $\mathrm{~mm}$ & UNKNOWN & 05-Dec-02 \\
\hline 5736 & $100-C-6$ & $\mathrm{~mm}$ & UNKNOWN & 05-Dec-02 \\
\hline 5737 & $100-C-6$ & $\mathrm{~mm}$ & UNKNOWN & 05-Dec-02 \\
\hline 5738 & $100-C-6$ & $\mathrm{~mm}$ & UNKNOWN & 05-Dec-02 \\
\hline 5739 & $100-C-6$ & $\mathrm{~mm}$ & UNKNOWN & 05-Dec-02 \\
\hline
\end{tabular}




\begin{tabular}{|c|c|c|c|c|}
\hline & $\mathrm{O}$ & $P$ & $\mathrm{Q}$ & $R$ \\
\hline 1 & SITE CODE & ELEVATION UNITS & VERT COORD TYPE & DATE LAST MODIFIED \\
\hline 5740 & $100-C-6$ & $\mathrm{~mm}$ & UNKNOWN & 05-Dec-02 \\
\hline 5741 & $100-C-6$ & $\mathrm{~mm}$ & UNKNOWN & 05-Dec-02 \\
\hline 5742 & $100-C-6$ & $\mathrm{~mm}$ & UNKNOWN & 05-Dec-02 \\
\hline 5743 & 100-C-6 & $\mathrm{mm}$ & UNKNOWN & 05-Dec-02 \\
\hline 5744 & $100-C-6$ & $\mathrm{~mm}$ & UNKNOWN & 05-Dec-02 \\
\hline 5745 & $100-C-6$ & $\mathrm{~mm}$ & UNKNOWN & 05-Dec-02 \\
\hline 5746 & $100-C-6$ & $\mathrm{~mm}$ & UNKNOWN & 05-Dec-02 \\
\hline 5747 & 100-C-6 & $\mathrm{mm}$ & UNKNOWN & 05-Dec-02 \\
\hline 5748 & $100-C-6$ & $\mathrm{~mm}$ & UNKNOWN & 05-Dec-02 \\
\hline 5749 & $100-C-6$ & $\mathrm{~mm}$ & UNKNOWN & 05-Dec-02 \\
\hline 5750 & $100-C-6$ & $\mathrm{~mm}$ & UNKNOWN & 05-Dec-02 \\
\hline 5751 & $100-C-6$ & $\mathrm{~mm}$ & UNKNOWN & 05-Dec-02 \\
\hline 5752 & $100-C-6$ & $\mathrm{~mm}$ & UNKNOWN & 05-Dec-02 \\
\hline 5753 & $100-C-6$ & $\mathrm{~mm}$ & UNKNOWN & 05-Dec-02 \\
\hline 5754 & $100-C-6$ & $\mathrm{~mm}$ & UNKNOWN & 05-Dec-02 \\
\hline 5755 & $100-C-6$ & $\mathrm{~mm}$ & UNKNOWN & 05-Dec-02 \\
\hline 5756 & 100-C-6 & $\mathrm{mm}$ & UNKNOWN & 05-Dec-02 \\
\hline 5757 & 100-C-6 & $\mathrm{mm}$ & UNKNOWN & 05-Dec-02 \\
\hline 5758 & $100-C-6$ & $\mathrm{~mm}$ & UNKNOWN & 05-Dec-02 \\
\hline 5759 & 100-C-6 & $\mathrm{mm}$ & UNKNOWN & 05-Dec-02 \\
\hline 5760 & $100-C-6$ & $\mathrm{~mm}$ & UNKNOWN & 05-Dec-02 \\
\hline 5761 & $100-C-6$ & $\mathrm{~mm}$ & UNKNOWN & 05-Dec-02 \\
\hline 5762 & 100-C-6 & $\mathrm{mm}$ & UNKNOWN & 05-Dec-02 \\
\hline 5763 & $100-C-6$ & $\mathrm{~mm}$ & UNKNOWN & 05-Dec-02 \\
\hline 5764 & $100-C-6$ & $\mathrm{~mm}$ & UNKNOWN & 05-Dec-02 \\
\hline 5765 & $100-C-6$ & $\mathrm{~mm}$ & UNKNOWN & 05-Dec-02 \\
\hline 5766 & $100-C-6$ & $\mathrm{~mm}$ & UNKNOWN & 05-Dec-02 \\
\hline 5767 & $100-C-6$ & $\mathrm{~mm}$ & UNKNOWN & 05-Dec-02 \\
\hline 5768 & $100-C-6$ & $\mathrm{~mm}$ & UNKNOWN & 05-Dec-02 \\
\hline 5769 & $100-C-6$ & $\mathrm{~mm}$ & UNKNOWN & 05-Dec-02 \\
\hline 5770 & 100-C-6 & $\mathrm{mm}$ & UNKNOWN & 05-Dec-02 \\
\hline 5771 & $100-C-6$ & $\mathrm{~mm}$ & UNKNOWN & 05-Dec-02 \\
\hline 5772 & 126-F-1 & & & 05-Dec-02 \\
\hline 5773 & $126-\mathrm{F}-1$ & & & 05-Dec-02 \\
\hline 5774 & 126-F-1 & & & 05-Dec-02 \\
\hline 5775 & 126-F-1 & & & 05-Dec-02 \\
\hline 5776 & 126-F-1 & & & 05-Dec-02 \\
\hline 5777 & 126-F-1 & & & 05-Dec-02 \\
\hline
\end{tabular}




\begin{tabular}{|c|c|c|c|c|}
\hline & $\mathrm{O}$ & $P$ & $\mathrm{Q}$ & $\bar{R}$ \\
\hline 1 & SITE_CODE & ELEVATION_UNITS & VERT_COORD_TYPE & DATE_LAST_MODIFIED \\
\hline 5778 & $126-\mathrm{F}-1$ & & & 05-Dec-02 \\
\hline 5779 & $126-F-1$ & & & 05-Dec-02 \\
\hline 5780 & 126-F-1 & & & 05-Dec-02 \\
\hline 5781 & 126-F-1 & & & 05-Dec-02 \\
\hline 5782 & 126-F-1 & & & 05-Dec-02 \\
\hline 5783 & 126-F-1 & & & 05-Dec-02 \\
\hline 5784 & 126-F-1 & & & 05-Dec-02 \\
\hline 5785 & 126-F-1 & & & 05-Dec-02 \\
\hline 5786 & 126-F-1 & & & 05-Dec-02 \\
\hline 5787 & 100-B-8 & & & 05-Dec-02 \\
\hline 5788 & 100-B-8 & & & 05-Dec-02 \\
\hline 5789 & 100-B-8 & & & 05-Dec-02 \\
\hline 5790 & 100-B-8 & & & 05-Dec-02 \\
\hline 5791 & 100-B-8 & & & 05-Dec-02 \\
\hline 5792 & 100-B-8 & & & 05-Dec-02 \\
\hline 5793 & 100-B-8 & & & 05-Dec-02 \\
\hline 5794 & 100-B-8 & & & 05-Dec-02 \\
\hline 5795 & $100-C-6$ & & UNKNOWN & 05-Dec-02 \\
\hline 5796 & 100-C-6 & & UNKNOWN & 05-Dec-02 \\
\hline 5797 & $100-C-6$ & & UNKNOWN & 05-Dec-02 \\
\hline 5798 & $100-C-6$ & & UNKNOWN & 05-Dec-02 \\
\hline 5799 & $100-C-6$ & & UNKNOWN & 05-Dec-02 \\
\hline 5800 & $100-C-6$ & & UNKNOWN & 05-Dec-02 \\
\hline 5801 & 100-B-8 & & UNKNOWN & 05-Dec-02 \\
\hline 5802 & 100-B-8 & & UNKNOWN & 05-Dec-02 \\
\hline 5803 & 100-B-8 & & UNKNOWN & 05-Dec-02 \\
\hline 5804 & 100-B-8 & & UNKNOWN & 05-Dec-02 \\
\hline 5805 & $100-C-6$ & & UNKNOWN & 05-Dec-02 \\
\hline 5806 & $100-C-6$ & & UNKNOWN & 05-Dec-02 \\
\hline 5807 & $100-C-6$ & & UNKNOWN & 05-Dec-02 \\
\hline 5808 & $100-C-6$ & & UNKNOWN & 05-Dec-02 \\
\hline 5809 & $116-\mathrm{N}-1$ & $\mathrm{~mm}$ & UNKNOWN & 05-Dec-02 \\
\hline 5810 & $116-\mathrm{N}-1$ & $\mathrm{~mm}$ & UNKNOWN & 05-Dec-02 \\
\hline 5811 & $116-\mathrm{N}-1$ & $\mathrm{~mm}$ & UNKNOWN & 05-Dec-02 \\
\hline 5812 & $116-\mathrm{N}-1$ & $\mathrm{~mm}$ & UNKNOWN & 05-Dec-02 \\
\hline 5813 & $116-\mathrm{N}-1$ & $\mathrm{~mm}$ & UNKNOWN & 05-Dec-02 \\
\hline 5814 & $116-\mathrm{N}-1$ & $\mathrm{~mm}$ & UNKNOWN & 05-Dec-02 \\
\hline 5815 & $116-\mathrm{N}-1$ & $\mathrm{~mm}$ & UNKNOWN & 05-Dec-02 \\
\hline
\end{tabular}




\begin{tabular}{|c|c|c|c|c|}
\hline & $\mathrm{O}$ & $P$ & $\mathrm{Q}$ & $R$ \\
\hline 1 & SITE CODE & ELEVATION UNITS & VERT COORD TYPE & DATE LAST MODIFIED \\
\hline 5816 & $116-\mathrm{N}-1$ & $\mathrm{~mm}$ & UNKNOWN & 05-Dec-02 \\
\hline 5817 & $116-\mathrm{N}-1$ & $\mathrm{~mm}$ & UNKNOWN & 05-Dec-02 \\
\hline 5818 & $116-\mathrm{N}-1$ & $\mathrm{~mm}$ & UNKNOWN & 05-Dec-02 \\
\hline 5819 & $116-\mathrm{N}-1$ & $\mathrm{~mm}$ & UNKNOWN & 05-Dec-02 \\
\hline 5820 & $116-\mathrm{N}-1$ & $\mathrm{~mm}$ & UNKNOWN & 05-Dec-02 \\
\hline 5821 & $116-\mathrm{N}-1$ & $\mathrm{~mm}$ & UNKNOWN & 05-Dec-02 \\
\hline 5822 & $116-\mathrm{N}-1$ & $\mathrm{~mm}$ & UNKNOWN & 05-Dec-02 \\
\hline 5823 & $116-\mathrm{N}-1$ & $\mathrm{~mm}$ & UNKNOWN & 05-Dec-02 \\
\hline 5824 & $116-\mathrm{N}-1$ & $\mathrm{~mm}$ & UNKNOWN & 05-Dec-02 \\
\hline 5825 & $116-\mathrm{N}-1$ & $\mathrm{~mm}$ & UNKNOWN & 05-Dec-02 \\
\hline 5826 & $116-\mathrm{N}-1$ & $\mathrm{~mm}$ & UNKNOWN & 05-Dec-02 \\
\hline 5827 & $116-\mathrm{N}-1$ & $\mathrm{~mm}$ & UNKNOWN & 05-Dec-02 \\
\hline 5828 & $116-\mathrm{N}-1$ & $\mathrm{~mm}$ & UNKNOWN & 05-Dec-02 \\
\hline 5829 & $116-\mathrm{N}-1$ & $\mathrm{~mm}$ & UNKNOWN & 05-Dec-02 \\
\hline 5830 & $116-\mathrm{N}-1$ & $\mathrm{~mm}$ & UNKNOWN & 05-Dec-02 \\
\hline 5831 & $116-\mathrm{N}-1$ & $\mathrm{~mm}$ & UNKNOWN & 05-Dec-02 \\
\hline 5832 & $116-\mathrm{N}-1$ & $\mathrm{~mm}$ & UNKNOWN & 05-Dec-02 \\
\hline 5833 & $116-\mathrm{N}-1$ & $\mathrm{~mm}$ & UNKNOWN & 05-Dec-02 \\
\hline 5834 & $116-\mathrm{N}-1$ & $\mathrm{~mm}$ & UNKNOWN & 05-Dec-02 \\
\hline 5835 & $116-\mathrm{N}-1$ & $\mathrm{~mm}$ & UNKNOWN & 05-Dec-02 \\
\hline 5836 & UPR-100-N-31 & $\mathrm{mm}$ & UNKNOWN & 05-Dec-02 \\
\hline 5837 & UPR-100-N-31 & $\mathrm{mm}$ & UNKNOWN & 05-Dec-02 \\
\hline 5838 & UPR-100-N-31 & $\mathrm{mm}$ & UNKNOWN & 05-Dec-02 \\
\hline 5839 & $120-\mathrm{N}-2$ & $\mathrm{~m}$ & NAVD88 & 05-Dec-02 \\
\hline 5840 & $120-\mathrm{N}-1$ & $\mathrm{~m}$ & NAVD88 & 05-Dec-02 \\
\hline 5841 & $100-N-58$ & $\mathrm{~m}$ & NAVD88 & 05-Dec-02 \\
\hline 5842 & $100-N-58$ & $\mathrm{~m}$ & NAVD88 & 05-Dec-02 \\
\hline 5843 & $120-\mathrm{N}-2$ & & & 05-Dec-02 \\
\hline 5844 & $100-C-6$ & $\mathrm{~mm}$ & UNKNOWN & 05-Dec-02 \\
\hline 5845 & $100-C-6$ & $\mathrm{~mm}$ & UNKNOWN & 05-Dec-02 \\
\hline 5846 & $100-C-6$ & $\mathrm{~mm}$ & UNKNOWN & 05-Dec-02 \\
\hline 5847 & $100-C-6$ & $\mathrm{~mm}$ & UNKNOWN & 05-Dec-02 \\
\hline 5848 & $100-C-6$ & $\mathrm{~mm}$ & UNKNOWN & 05-Dec-02 \\
\hline 5849 & $100-C-6$ & $\mathrm{~mm}$ & UNKNOWN & 05-Dec-02 \\
\hline 5850 & $100-C-6$ & $\mathrm{~mm}$ & UNKNOWN & 05-Dec-02 \\
\hline 5851 & $100-C-6$ & $\mathrm{~mm}$ & UNKNOWN & 05-Dec-02 \\
\hline 5852 & $100-C-6$ & $\mathrm{~mm}$ & UNKNOWN & 05-Dec-02 \\
\hline 5853 & $100-C-6$ & $\mathrm{~mm}$ & UNKNOWN & 05-Dec-02 \\
\hline
\end{tabular}




\begin{tabular}{|c|c|c|c|c|}
\hline & $\mathrm{O}$ & $P$ & $\mathrm{Q}$ & $R$ \\
\hline 1 & SITE CODE & ELEVATION UNITS & VERT COORD TYPE & DATE LAST MODIFIED \\
\hline 5854 & $100-C-6$ & $\mathrm{~mm}$ & UNKNOWN & 05-Dec-02 \\
\hline 5855 & $100-C-6$ & $\mathrm{~mm}$ & UNKNOWN & 05-Dec-02 \\
\hline 5856 & $100-C-6$ & $\mathrm{~mm}$ & UNKNOWN & 05-Dec-02 \\
\hline 5857 & 100-C-6 & $\mathrm{mm}$ & UNKNOWN & 05-Dec-02 \\
\hline 5858 & $100-C-6$ & $\mathrm{~mm}$ & UNKNOWN & 05-Dec-02 \\
\hline 5859 & $100-C-6$ & $\mathrm{~mm}$ & UNKNOWN & 05-Dec-02 \\
\hline 5860 & $100-C-6$ & $\mathrm{~mm}$ & UNKNOWN & 05-Dec-02 \\
\hline 5861 & 100-C-6 & $\mathrm{mm}$ & UNKNOWN & 05-Dec-02 \\
\hline 5862 & $100-C-6$ & $\mathrm{~mm}$ & UNKNOWN & 05-Dec-02 \\
\hline 5863 & $100-C-6$ & $\mathrm{~mm}$ & UNKNOWN & 05-Dec-02 \\
\hline 5864 & $100-C-6$ & $\mathrm{~mm}$ & UNKNOWN & 05-Dec-02 \\
\hline 5865 & $100-C-6$ & $\mathrm{~mm}$ & UNKNOWN & 05-Dec-02 \\
\hline 5866 & $100-C-6$ & $\mathrm{~mm}$ & UNKNOWN & 05-Dec-02 \\
\hline 5867 & $100-C-6$ & $\mathrm{~mm}$ & UNKNOWN & 05-Dec-02 \\
\hline 5868 & $100-C-6$ & $\mathrm{~mm}$ & UNKNOWN & 05-Dec-02 \\
\hline 5869 & $100-C-6$ & $\mathrm{~mm}$ & UNKNOWN & 05-Dec-02 \\
\hline 5870 & 100-C-6 & $\mathrm{mm}$ & UNKNOWN & 05-Dec-02 \\
\hline 5871 & 100-C-6 & $\mathrm{mm}$ & UNKNOWN & 05-Dec-02 \\
\hline 5872 & $100-C-6$ & $\mathrm{~mm}$ & UNKNOWN & 05-Dec-02 \\
\hline 5873 & 100-C-6 & $\mathrm{mm}$ & UNKNOWN & 05-Dec-02 \\
\hline 5874 & $100-C-6$ & $\mathrm{~mm}$ & UNKNOWN & 05-Dec-02 \\
\hline 5875 & $100-C-6$ & $\mathrm{~mm}$ & UNKNOWN & 05-Dec-02 \\
\hline 5876 & 100-C-6 & $\mathrm{mm}$ & UNKNOWN & 05-Dec-02 \\
\hline 5877 & $100-C-6$ & $\mathrm{~mm}$ & UNKNOWN & 05-Dec-02 \\
\hline 5878 & $100-C-6$ & $\mathrm{~mm}$ & UNKNOWN & 05-Dec-02 \\
\hline 5879 & $100-C-6$ & $\mathrm{~mm}$ & UNKNOWN & 05-Dec-02 \\
\hline 5880 & $100-C-6$ & $\mathrm{~mm}$ & UNKNOWN & 05-Dec-02 \\
\hline 5881 & $100-C-6$ & $\mathrm{~mm}$ & UNKNOWN & 05-Dec-02 \\
\hline 5882 & $100-C-6$ & $\mathrm{~mm}$ & UNKNOWN & 05-Dec-02 \\
\hline 5883 & $100-C-6$ & $\mathrm{~mm}$ & UNKNOWN & 05-Dec-02 \\
\hline 5884 & 100-C-6 & $\mathrm{mm}$ & UNKNOWN & 05-Dec-02 \\
\hline 5885 & $100-C-6$ & $\mathrm{~mm}$ & UNKNOWN & 05-Dec-02 \\
\hline 5886 & 100-C-6 & $\mathrm{mm}$ & UNKNOWN & 05-Dec-02 \\
\hline 5887 & $100-C-6$ & $\mathrm{~mm}$ & UNKNOWN & 05-Dec-02 \\
\hline 5888 & $100-C-6$ & $\mathrm{~mm}$ & UNKNOWN & 05-Dec-02 \\
\hline 5889 & $100-C-6$ & $\mathrm{~mm}$ & UNKNOWN & 05-Dec-02 \\
\hline 5890 & $100-C-6$ & $\mathrm{~mm}$ & UNKNOWN & 05-Dec-02 \\
\hline 5891 & $100-C-6$ & $\mathrm{~mm}$ & UNKNOWN & 05-Dec-02 \\
\hline
\end{tabular}




\begin{tabular}{|c|c|c|c|c|}
\hline & $\mathrm{O}$ & $P$ & $\mathrm{Q}$ & $R$ \\
\hline 1 & SITE CODE & ELEVATION UNITS & VERT COORD TYPE & DATE LAST MODIFIED \\
\hline 5892 & $100-C-6$ & $\mathrm{~mm}$ & UNKNOWN & 05-Dec-02 \\
\hline 5893 & $100-C-6$ & $\mathrm{~mm}$ & UNKNOWN & 05-Dec-02 \\
\hline 5894 & $100-C-6$ & $\mathrm{~mm}$ & UNKNOWN & 05-Dec-02 \\
\hline 5895 & 100-C-6 & $\mathrm{mm}$ & UNKNOWN & 05-Dec-02 \\
\hline 5896 & $100-C-6$ & $\mathrm{~mm}$ & UNKNOWN & 05-Dec-02 \\
\hline 5897 & $100-C-6$ & $\mathrm{~mm}$ & UNKNOWN & 05-Dec-02 \\
\hline 5898 & $100-C-6$ & $\mathrm{~mm}$ & UNKNOWN & 05-Dec-02 \\
\hline 5899 & 100-C-6 & $\mathrm{mm}$ & UNKNOWN & 05-Dec-02 \\
\hline 5900 & $100-C-6$ & $\mathrm{~mm}$ & UNKNOWN & 05-Dec-02 \\
\hline 5901 & $100-C-6$ & $\mathrm{~mm}$ & UNKNOWN & 05-Dec-02 \\
\hline 5902 & 100-C-6 & $\mathrm{mm}$ & UNKNOWN & 05-Dec-02 \\
\hline 5903 & $100-C-6$ & $\mathrm{~mm}$ & UNKNOWN & 05-Dec-02 \\
\hline 5904 & $100-C-6$ & $\mathrm{~mm}$ & UNKNOWN & 05-Dec-02 \\
\hline 5905 & $100-C-6$ & $\mathrm{~mm}$ & UNKNOWN & 05-Dec-02 \\
\hline 5906 & $100-C-6$ & $\mathrm{~mm}$ & UNKNOWN & 05-Dec-02 \\
\hline 5907 & $100-C-6$ & $\mathrm{~mm}$ & UNKNOWN & 05-Dec-02 \\
\hline 5908 & 100-C-6 & $\mathrm{mm}$ & UNKNOWN & 05-Dec-02 \\
\hline 5909 & 100-C-6 & $\mathrm{mm}$ & UNKNOWN & 05-Dec-02 \\
\hline 5910 & $100-C-6$ & $\mathrm{~mm}$ & UNKNOWN & 05-Dec-02 \\
\hline 5911 & 100-C-6 & $\mathrm{mm}$ & UNKNOWN & 05-Dec-02 \\
\hline 5912 & $100-C-6$ & $\mathrm{~mm}$ & UNKNOWN & 05-Dec-02 \\
\hline 5913 & $100-C-6$ & $\mathrm{~mm}$ & UNKNOWN & 05-Dec-02 \\
\hline 5914 & 116-F-11 & & UNKNOWN & 05-Dec-02 \\
\hline 5915 & 100-B-8 & & & 05-Dec-02 \\
\hline 5916 & 100-B-8 & & & 05-Dec-02 \\
\hline 5917 & 100-B-8 & & & 05-Dec-02 \\
\hline 5918 & 100-B-8 & & & 05-Dec-02 \\
\hline 5919 & 100-B-8 & & & 05-Dec-02 \\
\hline 5920 & 100-B-8 & & & 05-Dec-02 \\
\hline 5921 & 100-B-8 & & & 05-Dec-02 \\
\hline 5922 & 100-B-8 & & & 05-Dec-02 \\
\hline 5923 & 100-B-8 & & & 05-Dec-02 \\
\hline 5924 & 100-B-8 & & & 05-Dec-02 \\
\hline 5925 & 100-B-8 & & & 05-Dec-02 \\
\hline 5926 & 100-B-8 & & & 05-Dec-02 \\
\hline 5927 & 100-B-8 & & & 05-Dec-02 \\
\hline 5928 & 100-B-8 & & & 05-Dec-02 \\
\hline 5929 & 100-B-8 & & & 05-Dec-02 \\
\hline
\end{tabular}




\begin{tabular}{|c|c|c|c|c|}
\hline & $\mathrm{O}$ & $P$ & $\mathrm{Q}$ & $\mathrm{R}$ \\
\hline 1 & SITE_CODE & ELEVATION_UNITS & VERT_COORD_TYPE & DATE_LAST_MODIFIED \\
\hline 5930 & 100-B-8 & & & 05-Dec-02 \\
\hline 5931 & 100-B-8 & & & 05-Dec-02 \\
\hline 5932 & 100-B-8 & & & 05-Dec-02 \\
\hline 5933 & 100-B-8 & & & 05-Dec-02 \\
\hline 5934 & 100-B-8 & & & 05-Dec-02 \\
\hline 5935 & 100-B-8 & & & 05-Dec-02 \\
\hline 5936 & $100-C-6$ & & & 05-Dec-02 \\
\hline 5937 & & & & 23-Dec-02 \\
\hline 5938 & & & & 23-Dec-02 \\
\hline 5939 & & & & 23-Dec-02 \\
\hline 5940 & & & & 23-Dec-02 \\
\hline 5941 & & & & 27-Dec-02 \\
\hline 5942 & & & & 27-Dec-02 \\
\hline 5943 & & & & 27-Dec-02 \\
\hline 5944 & & & & 27-Dec-02 \\
\hline 5945 & & & & 27-Dec-02 \\
\hline 5946 & & & & 27-Dec-02 \\
\hline 5947 & & & & 27-Dec-02 \\
\hline 5948 & & & & 27-Dec-02 \\
\hline 5949 & & & & 27-Dec-02 \\
\hline 5950 & & & & 27-Dec-02 \\
\hline 5951 & & & & 07-Jan-03 \\
\hline 5952 & & & & 07-Jan-03 \\
\hline 5953 & & & & 07-Jan-03 \\
\hline 5954 & & & & 07-Jan-03 \\
\hline 5955 & & & & 20-Jan-03 \\
\hline
\end{tabular}

